Prepared for the U.S. Department of Energy under Contract DE-AC05-76RL01830

\title{
PEP Run Report for Integrated Test A; Caustic Leaching in UFP-VSL-T01A, Oxidative Leaching in UFP-VSL-T02A
}

$\begin{array}{lll}\text { CE Guzman-Leong } & \text { JGH Geeting } & \text { DM Smith } \\ \text { OP Bredt } & \text { EC Golovich } & \text { PLJ Valdez } \\ \text { CA Burns } & \text { GB Josephson } & \text { ST Yokuda } \\ \text { RC Daniel } & \text { DE Kurath } & \text { JK Young } \\ \text { YF Su } & \text { GJ Sevigny } & \end{array}$

December 2009

Pacific Northwest NATIONAL LABORATORY

Proudly Operated by Battelle Since 1965 


\section{PEP Run Report for Integrated Test A; Caustic Leaching in UFP-VSL-T01A, Oxidative Leaching in UFP-VSL-T02A}

$\begin{array}{llll}\text { CE Guzman-Leong } & \text { YF Su } & \text { DE Kurath } & \text { ST Yokuda } \\ \text { OP Bredt } & \text { JGH Geeting } & \text { GJ Sevigny } & \text { JK Young } \\ \text { CA Burns } & \text { EC Golovich } & \text { DM Smith } & \\ \text { RC Daniel } & \text { GB Josephson } & \text { PLJ Valdez } & \end{array}$

December 2009

Test Specification: 24590-PTF-TSP-RT-07-001 Rev 2

Work Authorization: WA\# 2007-024

Test Plan: TP-RPP-WTP-506, Rev. 0.4; TP-WTP-PEP-044, Rev 0.2

Test Exceptions: $\quad$ 24590-PTF-TEF-RT-08-00002

24590-WTP-TEF-RT-09-00003

24590-PTF-TEF-RT-09-00001

24590-WTP-TEF-RT-09-00002 Rev 0

24590-WTP-TEF-RT-09-00001 Rev 1

R\&T focus area: Pretreatment

Test Scoping Statement: N/A

Prepared for

the U.S. Department of Energy

Under Contract DE-AC05-76RL01830

Pacific Northwest National Laboratory

Richland, Washington 99352 
WTP-RPT-191, Rev 0

\section{Completeness of Testing}

This report describes the results of work and testing specified by Test Specification 24590-PTF-TSP-RT-07-001 Rev 2 "Pretreatment Engineering Platform (PEP) Testing (Phase 1)" and Test Plans TP-RPP-WTP-506 Rev 0.4 "Pretreatment Engineering Platform (PEP) Testing (Phase 1) and TP-WTP-PEP-044 Rev 0.2" Test Plan for the PEP Parallel Laboratory Testing." The work and any associated testing followed the quality assurance requirements outlined in the Test Specification/Plan. The descriptions provided in this test report are an accurate account of both the conduct of the work and the data collected. Test plan results are reported. Also reported are any unusual or anomalous occurrences that are different from expected results. The test results and this report have been reviewed and verified.

\section{Approved:}

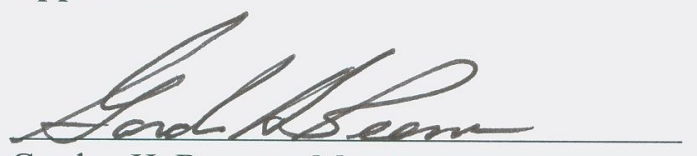

Gordon H. Beeman, Manager WTP R\&T Support Project

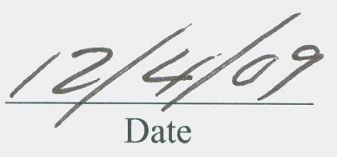

Date 


\section{Contents}

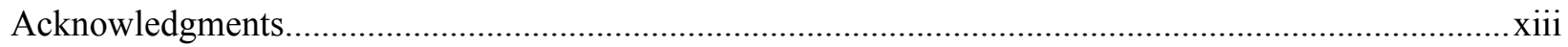

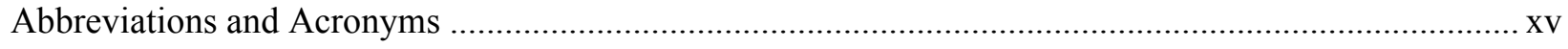

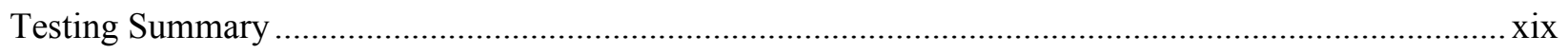

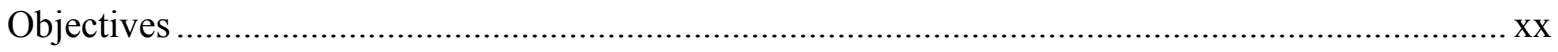

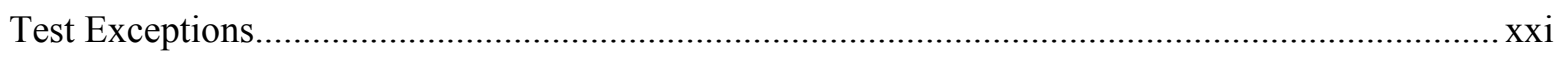

Results and Performance Against Success Criteria ................................................................. Xxv

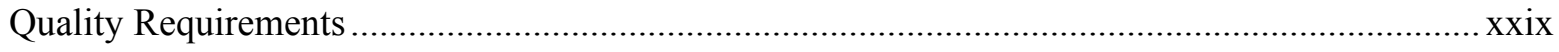

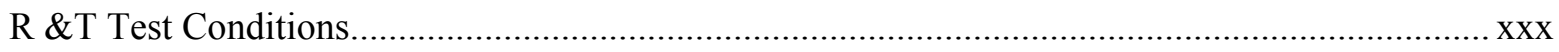

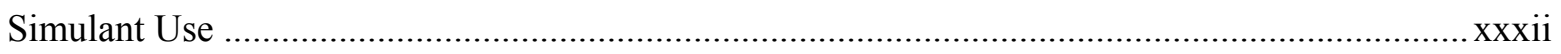

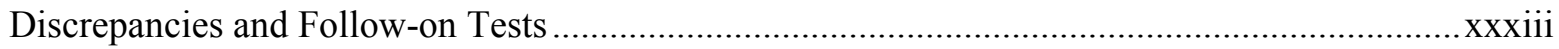

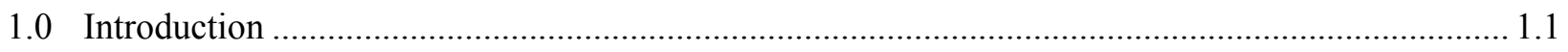

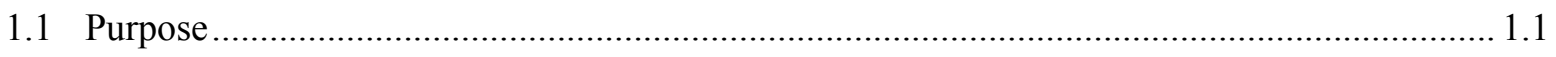

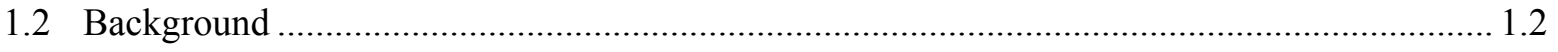

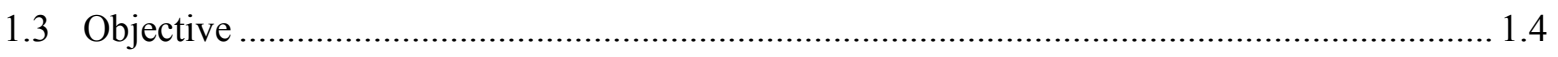

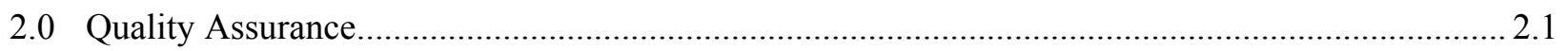

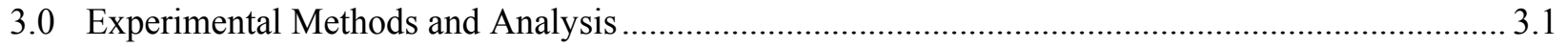

3.1 Pretreatment Engineering Platform Description ................................................................ 3.1

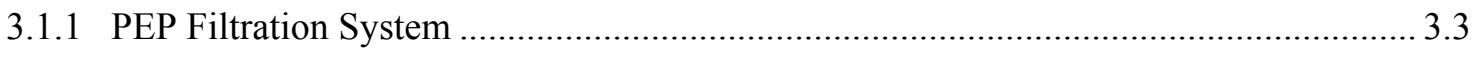

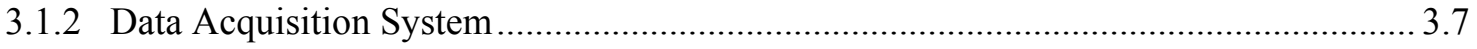

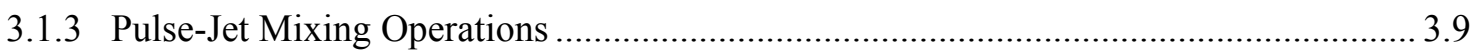

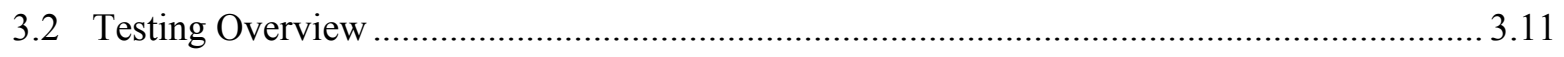

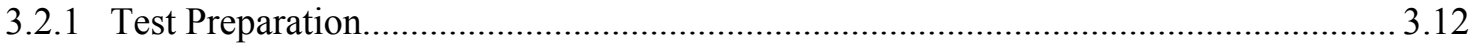

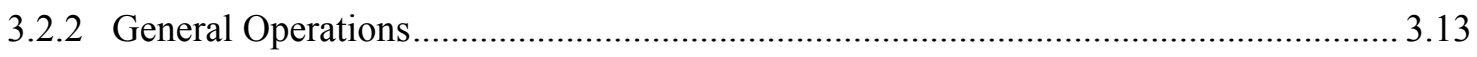

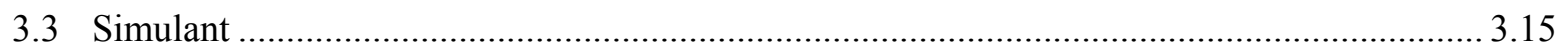

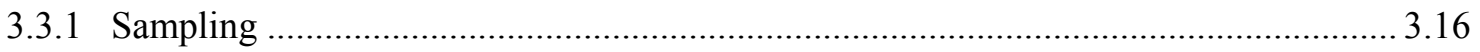

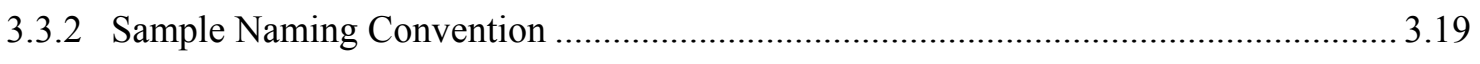

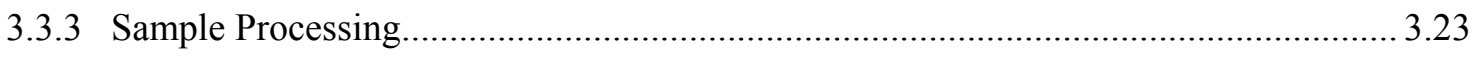

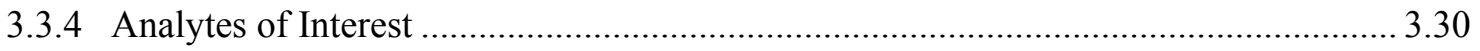

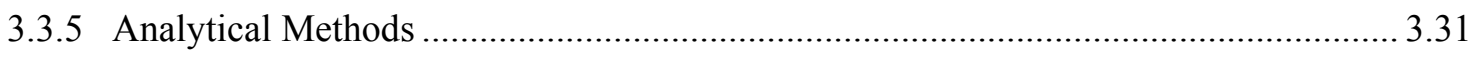

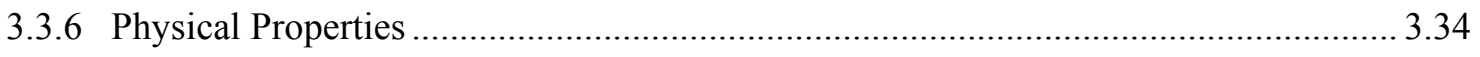

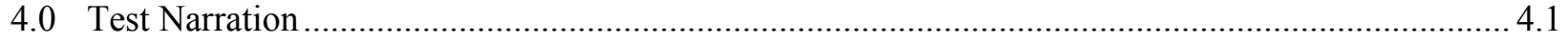

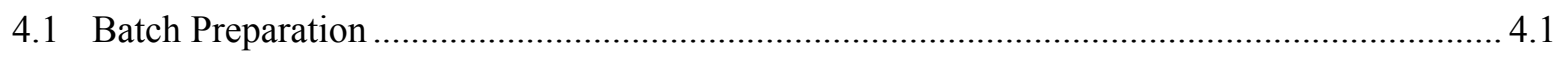

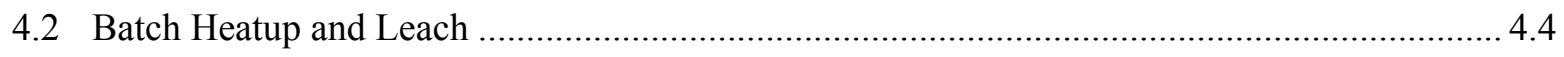

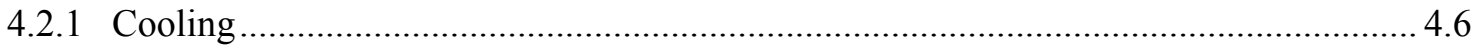

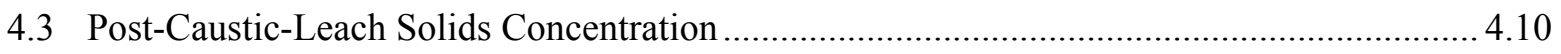

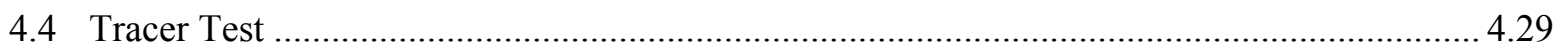




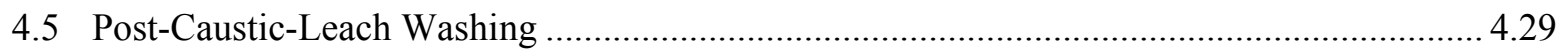

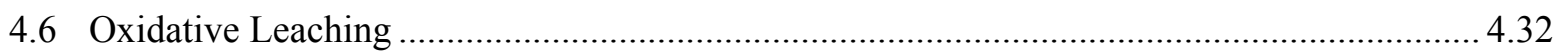

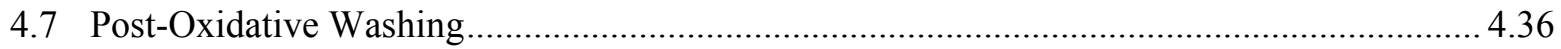

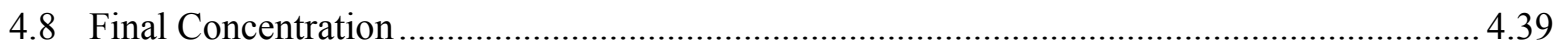

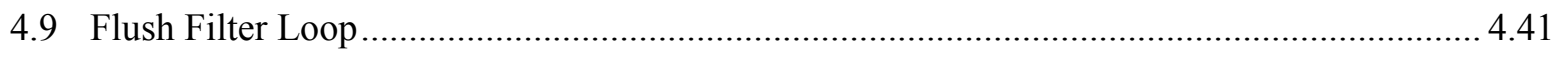

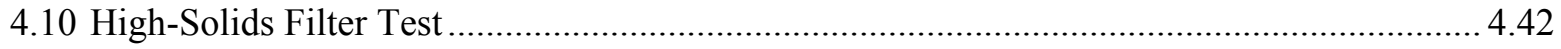

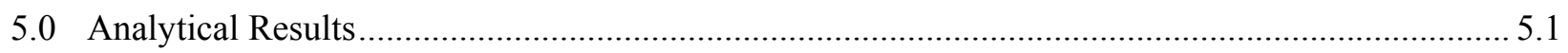

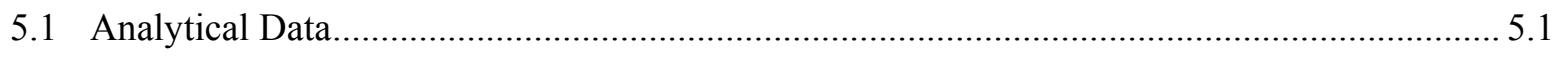

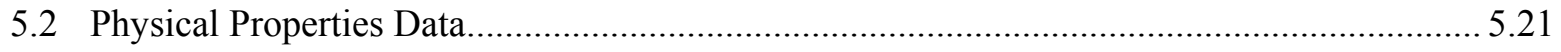

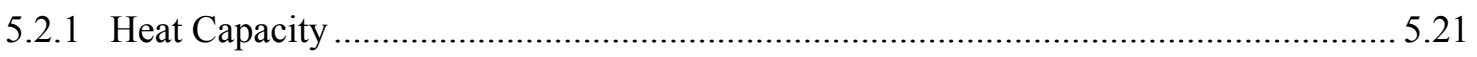

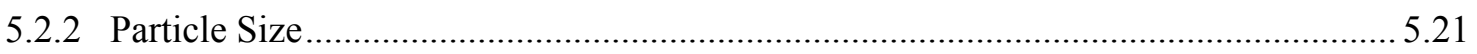

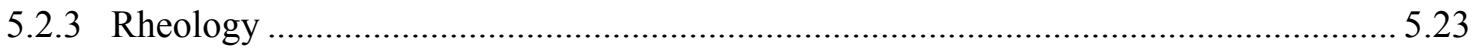

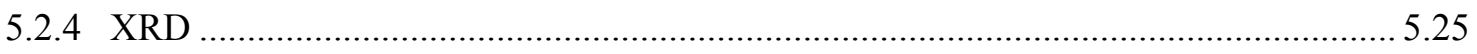

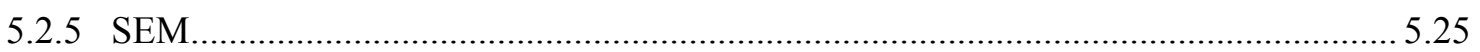

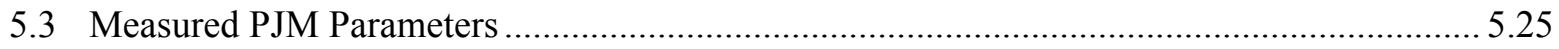

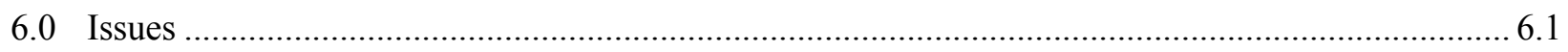

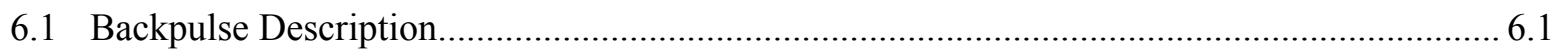

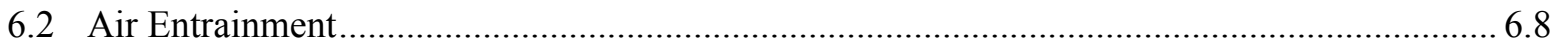

6.2.1 Summary of Slurry Degassing and Filter-Loop Pump Exercises.............................. 6.13

6.2.2 Air Entrainment Operational Guidance and Recommendations ................................ 6.25

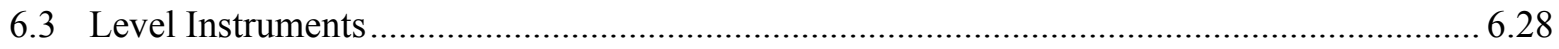

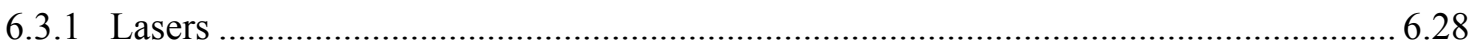

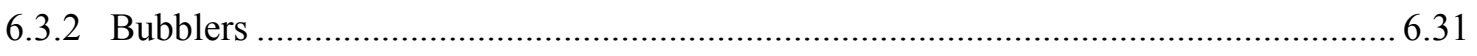

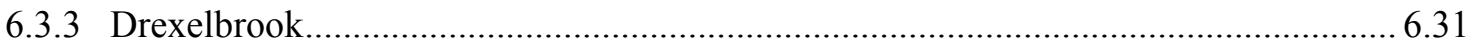

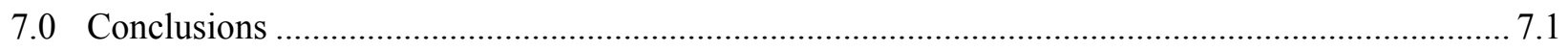

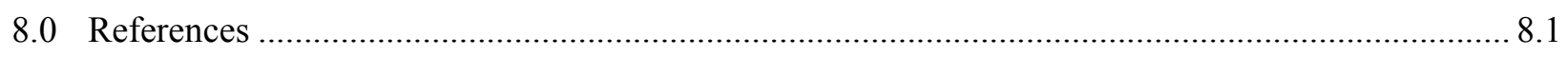

Appendix A: Process Instrumentation Summary …..................................................................... A.1

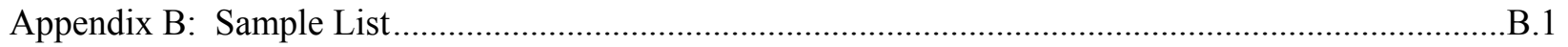

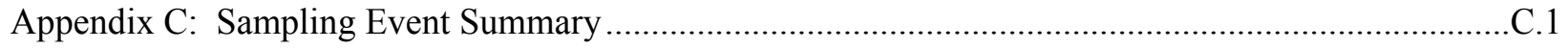

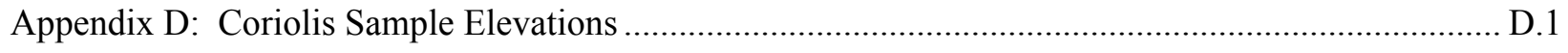

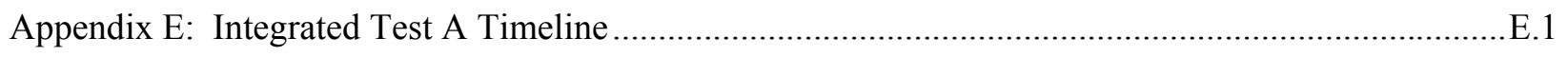

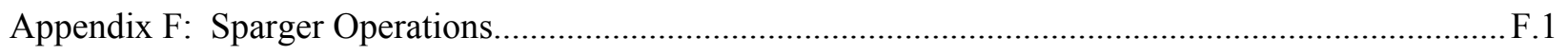

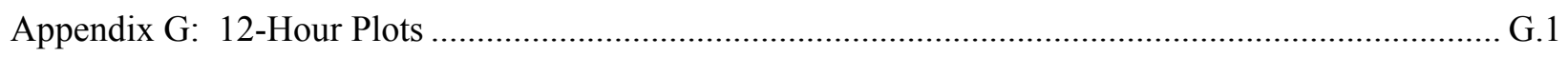

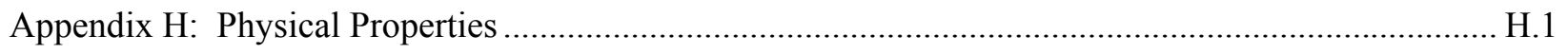

Appendix I: Operational Control Systems for PEP.........................................................................

Appendix J: Operational Process Sheet, Integrated Test A Parameters .....................................................1 


\section{Figures}

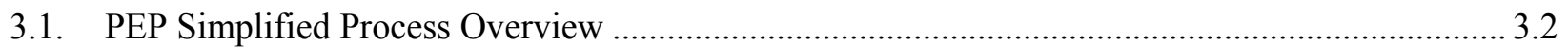

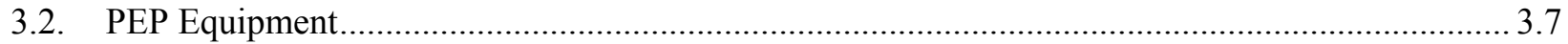

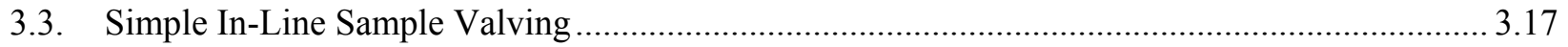

3.4. In-Tank Sampling, Showing the Three Radial Positions at Three Heights and Sampling

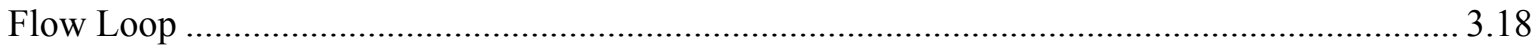

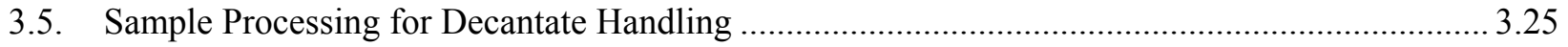

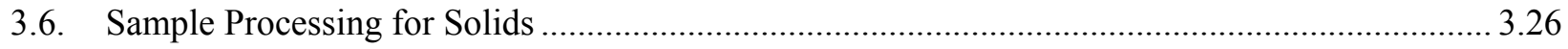

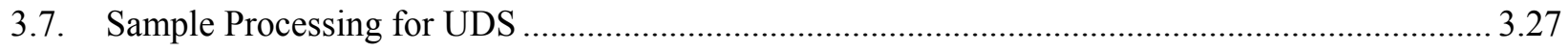

4.1. Integrated Test A Schedule of Completion .............................................................................. 4.2

4.2. UFP-VSL-T01A Caustic Leach Batch 1 Heating and Partial Cooling......................................... 4.7

4.3. UFP-VSL-T01B Caustic Leach Batch 2 Heating and Partial Cooling........................................... 4.8

4.4. UFP-VSL-T01A Caustic Leach Batch 3 Heating and Partial Cooling......................................... 4.8

4.5. UFP-VSL-T01B Caustic Leach Batch 4 Heating and Partial Cooling .......................................... 4.9

4.6. UFP-VSL-T01A Caustic Leach Batch 5 Heating and Partial Cooling ......................................... 4.9

4.7. UFP-VSL-T01B Caustic Leach Batch 6 Heating and Partial Cooling ....................................... 4.10

4.8. Axial Velocity Based on Flow Measurements at the Suction to Pump T42A and the Discharge to Pump T43A for Post-Caustic-Leach Concentration, Batch \#1; Valve SV-0609 Setting (for information only) Scale 0 to 2 Correlates to $0 \%$ and $100 \%$ Open, Respectively ....... 4.12

4.9. TMP for Post-Caustic-Leach Concentration, Batch \#1 .............................................................. 4.12

4.10. Tank T02A (TTK-0619) Temperature for Post-Caustic-Leach Concentration, Batch \#1 ............. 4.13

4.11. Permeate Production Rate for Post-Caustic-Leach Concentration, Batch \#1 ….......................... 4.13

4.12. Axial Velocity Based on Flow Measurements at the Suction to Pump T42A and the Discharge to Pump T43A for Post-Caustic-Leach Concentration, Batch \#2; Valve SV-0609 Setting (for information only) Scale 0 to 2 Correlates to $0 \%$ and $100 \%$ Open, Respectively....... 4.14

4.13. TMP for Post-Caustic-Leach Concentration, Batch \#2 …...................................................... 4.14

4.14. Tank T02A (TTK-0619) Temperature for Post-Caustic-Leach Concentration, Batch \#2 ............ 4.15

4.15. Permeate Production Rate for Post-Caustic-Leach Concentration, Batch \#2 .............................. 4.15

4.16. Axial Velocity Based on Flow Measurements At the Suction to Pump T42A and the Discharge to Pump T43A for Post-Caustic-Leach Concentration, Batch \#3; Valve SV-0609 Setting (for information only) Scale 0 to 2 Correlates to $0 \%$ and $100 \%$ Open, Respectively ....... 4.16

4.17. TMP for Post-Caustic-Leach Concentration, Batch \#3 ........................................................... 4.16

4.18. Tank T02A (TTK-0619) Temperature for Post-Caustic-Leach Concentration, Batch \#3 ............ 4.17

4.19. Permeate Production Rate for Post-Caustic-Leach Concentration, Batch \#3 …........................... 4.17 


\section{Figures}

4.20. Axial Velocity Based on Flow Measurements at the Suction to Pump T42A and the Discharge to Pump T43A for Post-Caustic-Leach Concentration, Batch \#4; Valve SV-0609 Setting (for information only) Scale 0 to 2 Correlates to $\% \%$ and $100 \%$ Open, Respectively....... 4.18

4.21. TMP for Post-Caustic-Leach Concentration, Batch \#4 ........................................................... 4.19

4.22. Tank T02A (TTK-0619) Temperature for Post-Caustic-Leach Concentration, Batch \#4 ............ 4.19

4.23. Permeate Production Rate for Post-Caustic-Leach Concentration, Batch \#4 .............................. 4.20

4.24. Axial Velocity Based on Flow Measurements at the Suction to Pump T42A and the Discharge to Pump T43A for Post-Caustic-Leach Concentration, Batch \#5; Valve SV-0609 Setting (for information only) Scale 0 to 2 Correlates to $0 \%$ and $100 \%$ Open, Respectively ....... 4.21

4.25. TMP for Post-Caustic-Leach Concentration, Batch \#5 .

4.26. Tank T02A (TTK-0619) Temperature for Post-Caustic-Leach Concentration, Batch \#5 ............ 4.22

4.27. Permeate Production Rate for Post-Caustic-Leach Concentration, Batch \#5. 4.22

4.28. Axial Velocity Based on Flow Measurements at the Suction to Pump T42A and the Discharge to Pump T43A for Post-Caustic-Leach Concentration, Batch \#6; Valve SV-0609 Setting (for information only) Scale 0 to 2 Correlates to $0 \%$ and $100 \%$ Open, Respectively ....... 4.23

4.29. TMP for Post-Caustic-Leach Concentration, Batch \#6 4.23

4.30. Tank T02A (TTK-0619) Temperature for Post-Caustic-Leach Concentration, Batch \#6.

4.31. Permeate Production Rate for Post-Caustic-Leach Concentration, Batch \#6. 4.24

4.32. Axial Velocity Based on Flow Measurements at the Suction to Pump T42A and the Discharge to Pump T43A for Post-Caustic-Leach Concentration, All Batches; Valve SV-0609 Setting (for information only) Scale 0 to 2 Correlates to $0 \%$ and $100 \%$ Open, Respectively....... 4.25

4.33. TMP for Post-Caustic-Leach Concentration, All Batches.......................................................... 4.25

4.34. Tank T02A (TTK-0619) Temperature for Post-Caustic-Leach Concentration, All Batches ........ 4.26

4.35. Permeate Production Rate for Post-Caustic-Leach Concentration, All Batches ......................... 4.26

4.36. Permeate Production Rate During a Select Time Period in Integrated Test A............................ 4.27

4.37. Filter TMP During a Select Time Period in Integrated Test A................................................ 4.28

4.38. Slurry Flow rate and Valve SV-0609 Setting During a Select Time Period in Integrated Test A; Valve SV-0609 Setting (for information only) Scale 0 to 2 Correlates to $0 \%$ and 100\% Open, Respectively

4.39. Axial Velocity Based on Flow Measurements at the Suction to Pump T42A and the Discharge to Pump T43A for Post-Caustic-Leach Slurry Wash; Valve SV-0609 Setting (for information only) Scale 0 to 2 Correlates to $0 \%$ and $100 \%$ Open, Respectively

4.40. TMP for Post-Caustic-Leach Slurry Wash ............................................................................ 4.31

4.41. Tank T02A (TTK-0619) Temperature for Post-Caustic-Leach Slurry Wash.............................. 4.31

4.42. Permeate Production Rate for Post-Caustic-Leach Slurry Wash ................................................ 4.32 


\section{Figures}

4.43. Axial Velocity Based on Flow Measurements at the Suction to Pump T42A and the Discharge to Pump T43A for the Reconcentration and Wash Operations Following Chromium Slurry Addition; Valve SV-0609 Setting (for information only) Scale 0 to 2

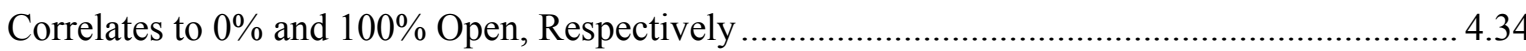

4.44. TMP for the Reconcentration and Wash Operations Following Chromium Slurry Addition ....... 4.35

4.45. Tank T02A (TTK-0619) Temperature for the Reconcentration and Wash Operations Following Chromium Slurry Addition

4.46. Permeate Production Rate for the Reconcentration and Wash Operations Following Chromium Slurry Addition.

4.47. Axial Velocity Based on Flow Measurements at the Suction to Pump T42A and the Discharge to Pump T43A for Post-Oxidative-Leach Slurry Wash; Valve SV-0609 Setting (for information only) Scale 0 to 2 Correlates to $0 \%$ and $100 \%$ Open, Respectively

4.48. TMP for Post-Oxidative-Leach Slurry Wash .............................................................................. 4.37

4.49. Tank T02A (TTK-0619) Temperature for Post-Oxidative-Leach Slurry Wash.......................... 4.38

4.50. Permeate Production Rate for Post-Oxidative-Leach Slurry Wash............................................. 4.38

4.51. Axial Velocity Based on Flow Measurements at the Suction to Pump T42A and the Discharge to Pump T43A for Post-Oxidative-Leach Slurry Concentration; Valve SV-0609 Setting (for information only) Scale 0 to 2 Correlates to $0 \%$ and $100 \%$ Open, Respectively ....... 4.39

4.52. TMP for Post-Oxidative-Leach Slurry Concentration.............................................................. 4.40

4.53. Tank T02A (TTK-0619) Temperature for Post-Oxidative-Leach Slurry Concentration .............. 4.40

4.54. Permeate Production Rate for Post-Oxidative-Leach Slurry Concentration ............................... 4.41

4.55. Axial Velocity Based on Flow Measurements at the Suction to Pump T42A and the Discharge to Pump T43A for the High-Solids Scaling Test Conducted on 3/21/2009; Valve SV-0609 Setting (for information only) Scale 0 to 2 Correlates to $\% \%$ and 100\% Open,

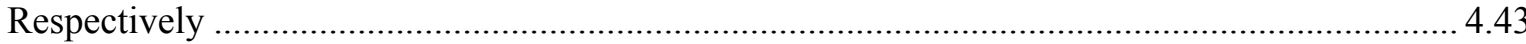

4.56. TMP for the High-Solids Scaling Test Conducted on 3/21/2009 ….........................................4.44

4.57. Tank T02A (TTK-0619) Temperature for the High-Solids Scaling Test Conducted on $3 / 21 / 2009$. 4.44

4.58. Permeate Production Rate for the High-Solids Scaling Test Conducted on 3/21/2009 ............... 4.45

5.1. Tank T01A PJM Peak Average Velocity After Batch 1 Caustic Addition ................................. 5.27

5.2. Tank T01A PJM Peak Average Velocity at the start of Batch 1 Caustic Leaching .................... 5.27

5.3. Tank T01A PJM Peak Average Velocity During Batch 1 Caustic Leaching ............................. 5.28

5.4. Tank T02A Peak Average Velocity After Initial Transfer from UFP-VSL-T01A...................... 5.28

5.5. Tank T01B PJM Peak Average Velocity After Batch 2 Caustic Addition.................................. 5.29

5.6. Tank T01B PJM Peak Average Velocity at the start of Batch 2 Caustic Leach.......................... 5.29

5.7. Tank T01B PJM Peak Average Velocity During Batch 2 Caustic Leach .................................. 5.30

5.8. Tank T01A PJM Peak Average Velocity After Batch 3 Caustic Addition .................................. 5.30 


\section{Figures}

5.9. Tank T01A PJM Peak Average Velocity at the Start of Batch 3 Caustic Leach..........................5.31

5.10. Tank T01A PJM Peak Average Velocity During Batch 3 Caustic Leach ................................... 5.31

5.11. Tank T01B Peak Average Velocity Towards the End of Batch 4 Initial Heatup ........................ 5.32

5.12. Tank T01B PJM Peak Average Velocity During Batch 4 Caustic Leach .................................. 5.32

5.13. Tank T01A PJM Peak Average Velocity after Batch 5 Caustic Addition................................... 5.33

5.14. Tank T01A PJM Peak Average Velocity During Batch 5 Caustic Leaching .............................. 5.33

5.15. Tank T01B PJM Peak Average Velocity After Batch 6 Caustic Addition................................... 5.34

5.16. Tank T01B PJM Peak Average Velocity During Batch 6 Caustic Leaching ............................. 5.34

5.17. Tank T02A PJM Peak Average Velocity Before the Tracer Test .............................................5.35

5.18. Tank T02A PJM Peak Average Velocity Before the Tracer Test ............................................. 5.35

5.19. Nozzle Velocity Distributions Used to Evaluate the Loss Coefficients for Tank T01A Tank at the Peak Average Velocity of $4.9(\mathrm{~m} / \mathrm{s}): \mathrm{k} f=0.25$ is the Loss Coefficient of the Forward Flow, and k_b = 1.2 is the Loss Coefficient of the Backward Flow ............................. 5.36

5.20. Nozzle Velocity Distributions Used to Evaluate the Loss Coefficients for Tank T01B Tank at the Peak Average Velocity of $4.9(\mathrm{~m} / \mathrm{s}): \mathrm{k} f=0.38$ is the Loss Coefficient of the Forward Flow, and k_b $=1.3$ is the Loss Coefficient of the Backward Flow.....

5.21. Nozzle Velocity Distributions Used to Evaluate the Loss Coefficients for Tank T02A Tank at the Peak Average Velocity of $7.1(\mathrm{~m} / \mathrm{s}): \mathrm{k}_{-} \mathrm{f}=0.34$ is the Loss Coefficient of the Forward Flow, and k_b $=0.1$ is the Loss Coefficient of the Backward Flow.....

5.22. Nozzle Velocity Distributions Used to Evaluate the Loss Coefficients for Tank T02A Tank at the Peak Average Velocity of $11.5(\mathrm{~m} / \mathrm{s}): \mathrm{k}_{-} \mathrm{f}=0.1$ is the Loss Coefficient of the Forward Flow, and k_b $=0.1$ is the Loss Coefficient of the Backward Flow ............................ 5.38

6.1. Filter T01A Backpulse (1) Using Deadband and Overpressures of 5 and 20 psi, 2/6/2009........... 6.2

6.2. Two Consecutive Backpulses (3) on Filter T01A Backpulses Using Deadband and Overpressures of 5 and 20 psi, 2/6/2009

6.3. Two Consecutive Backpulses (4) on Filter T01A Using Deadband and Overpressures of 5 and 40 psi, $2 / 5 / 2009$

6.4. Filter T01A Backpulse (5a) Using Deadband and Overpressures of 20.7 and 60 psi, $2 / 6 / 2009$

6.5. Filter T01A Backpulse (5b) Using Deadband and Overpressures of 20.7 and 60 psi, 2/7/2009.... 6.5

6.6. Five Consecutive Backpulses (5c) on Filter T01A Using Deadband and Overpressures of 20.7 and 60 psi, 2/7/2009 ...

6.7. Filter T01A Backpulse (5d) Using Deadband and Overpressures of 20.7 and 60 psi, 2/7/2009.... 6.7

6.8. Filter T01A Backpulse (7) Using Deadband and Overpressures of 32.5 and 75 psi, 2/7/2009...... 6.8

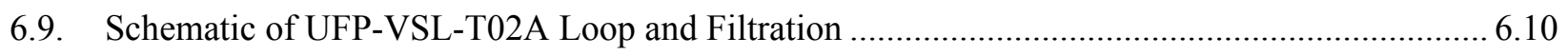

6.10. UFP-VSL-T02A Gas Accumulation and Gas Release Operations Using PJM and Lower Spargers, in Reference to Table 6.2 and Table 6.3 


\section{Figures}

6.11. UFP-VSL-T02A Gas Release Operations, in Reference to Table 6.4, Steps 1 Through 5

6.12. UFP-VSL-T02A Gas Release Operations, in Reference to Table 6.4, Steps 6 Through 9

6.13. UFP-VSL-T02A Gas Release Operations, in Reference to Table 6.6

6.14. Operations for Restoring Target Filter-Loop Flow Rate, in Reference to Table 6.7, Step 1

6.15. Operations for Restoring Target Filter-Loop Flow Rate, in Reference to Table 6.7, Step 2

6.16. Operations for Restoring Target Filter-Loop Flow Rate, in Reference to Table 6.7, Steps A Through F

6.17. Operations for Restoring Target Filter-Loop Flow Rate, in Reference to Table 6.8, Steps 2 and 3

6.18. Operations for Restoring Target Filter-Loop Flow Rate, in Reference to Table 6.8, Steps 4 Through 7

6.19. UFP-VSL-T01B Laser Performance Improved with Sample Port Open

6.20. UFP-VSL-T02A Laser Performance Improved After Opening Chemical Addition Vent Flange. 


\section{Tables}

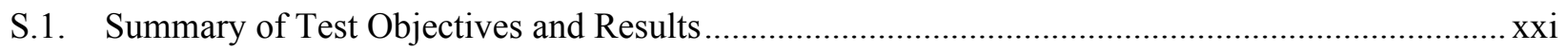

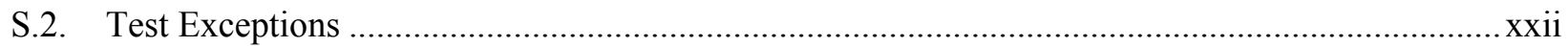

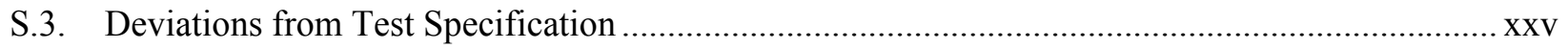

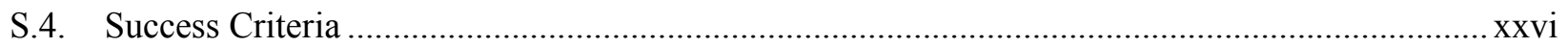

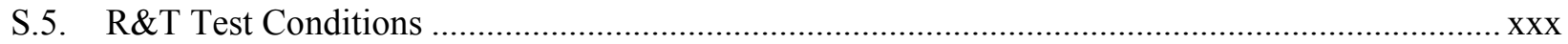

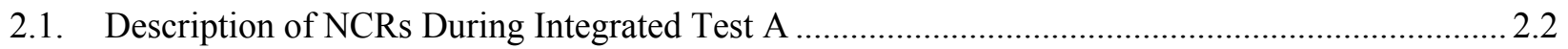

3.1. Specifications of the Five PEP Cross-Flow Filtration Bundles ......................................................... 3.5

3.2. Permeate Metering and Pulse Pot Configurations for PEP ........................................................... 3.6

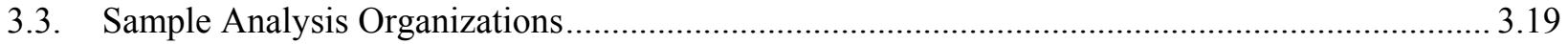

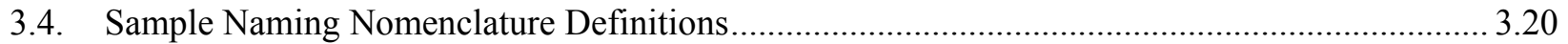

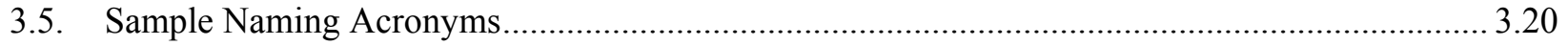

3.6. Estimated Quantitative Limits for Solids and Supernatants in Sample Loop Testing, PEP

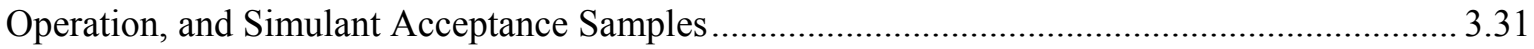

3.7. Vane Immersion Depth and Container Geometry Constraints for Shear-Strength Tests Using

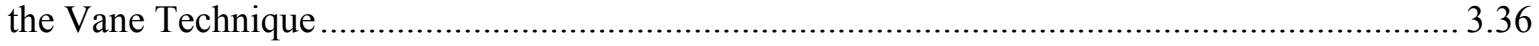

3.8. Vane and Cup and Rotor Measuring System Dimensions........................................................ 3.39

3.9. Properties Applied to Group 8 Test Materials ............................................................................ 3.4

4.1. Integrated Test A UFP Batch Transfer Times ............................................................................ 4.3

4.2. Transfer Volumes Integrated Test A Batches.......................................................................... 4.4

4.3. Start and Stop Times to Heat, and Caustic Leach Integrated Test A Batches .............................. 4.5

4.4. Start/Stop Times to Partially Cool Integrated Test A Batches ...................................................... 4.7

4.5. Batch 1 Chromium Slurry Composition for Integrated Test A ….............................................. 4.33

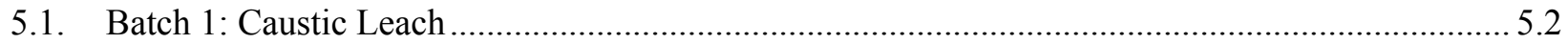

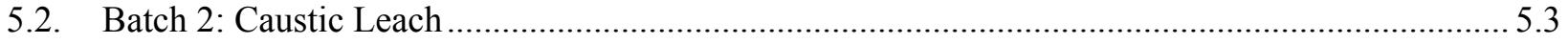

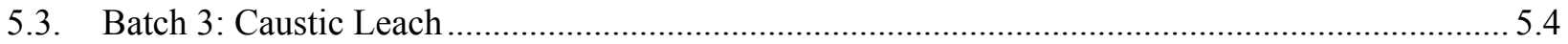

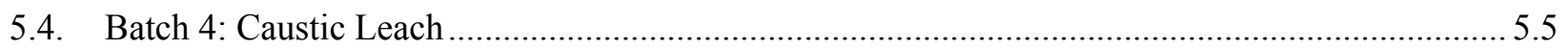

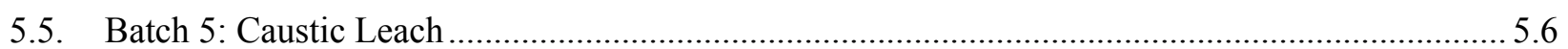

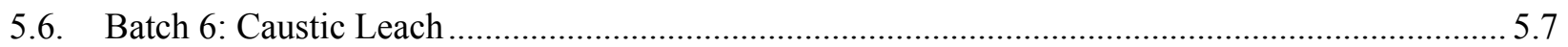

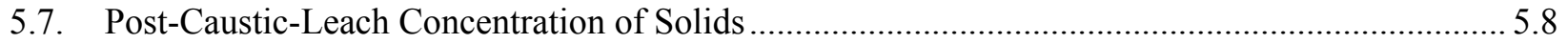

5.8. Evaluation By-Pass of Filter-Loop Jet within Tank T02A on 02/14/09 ................................... 5.8

5.9. Evaluation By-Pass of Filter-Loop Jet within Tank T02A on 3/21/09 …................................... 5.9

5.10. Post-Caustic-Leach Wash....................................................................................................... 5.10

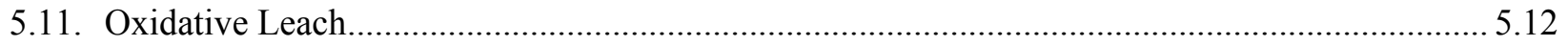

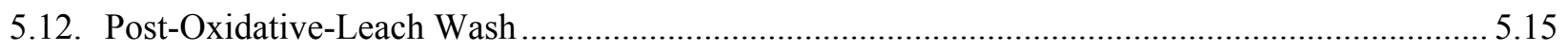




\section{Tables}

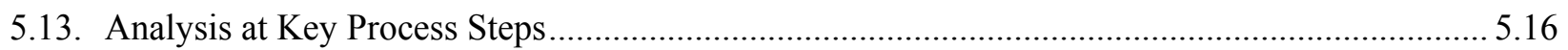

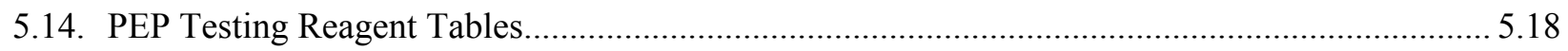

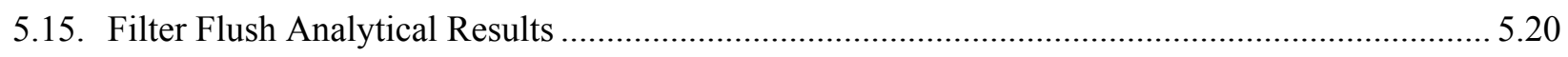

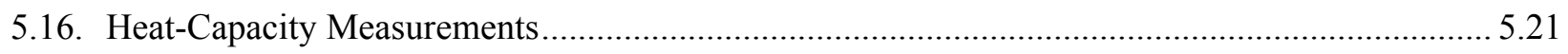

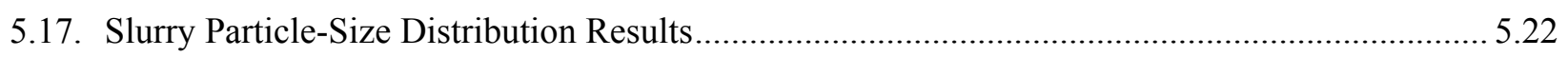

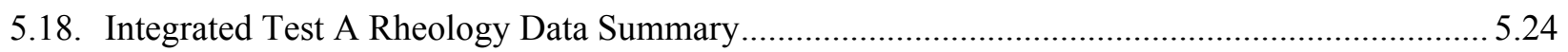

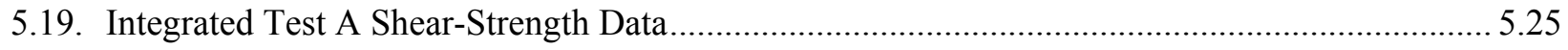

5.20. PJM Parameters for Integrated Test A (TI-WTP-PEP-065) .................................................... 5.26

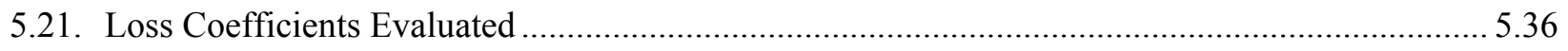

6.1. Initial Backpulses Performed During Post-Caustic-Leach Concentration ................................... 6.1

6.2. Gas Accumulation and Degassing Instructions per Guidance Section 1 1................................. 6.15

6.3. Gas Accumulation and Degassing Instructions per Guidance Section 2 ................................... 6.15

6.4. Gas Accumulation and Degassing Instructions per Guidance ................................................... 6.16

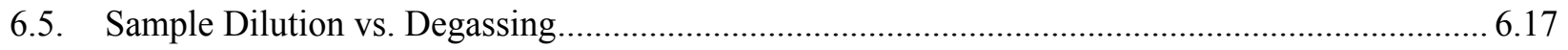

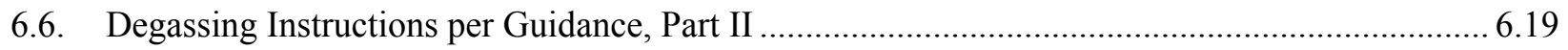

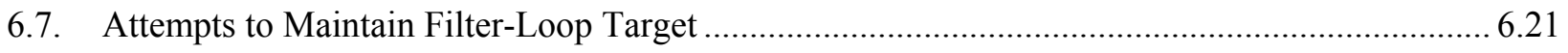

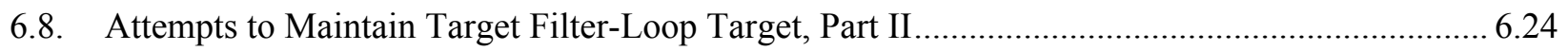





\section{Acknowledgments}

In addition to the authors, this report was supported by many dedicated staff involved in laboratory experiments, sampling, data acquisition, and technical reviews.

$\begin{array}{lll}\text { RL Aaberg } & \text { BM Dunn } & \text { RP Pires } \\ \text { JM Alzheimer } & \text { A Edmondson } & \text { KD Quigley } \\ \text { EJ Antonio } & \text { RP Elmore } & \text { BJ Riley } \\ \text { EBK Baer } & \text { MS Fountain } & \text { CP Rodriguez } \\ \text { R Bang } & \text { KC Groves } & \text { JH Sachs } \\ \text { M Barnett } & \text { AD Guzman } & \text { RC Shannon } \\ \text { NN Bauman } & \text { SD Halstead } & \text { SV Shoemaker } \\ \text { JD Bohlke } & \text { GL Harvey } & \text { DS Sklarew } \\ \text { JR Bontha } & \text { RE Hohimer } & \text { CT Smith } \\ \text { CF Brown } & \text { DE Hurley } & \text { SA Smith } \\ \text { WC Buchmiller } & \text { SD Lehrman } & \text { JJ Soelberg } \\ \text { KJ Cantrell } & \text { ML Kimura } & \text { BA Stiver } \\ \text { CC Chapman } & \text { SN Kundu } & \text { JC Tucker } \\ \text { DA Clark } & \text { JB Lang } & \text { D Vonghalath } \\ \text { E Cordova } & \text { JS Loveland } & \text { BE Wells } \\ \text { BP Crume } & \text { JR Markillie } & \text { AD Woodstock } \\ \text { JM Cuta } & \text { ES Mast } & \text { SA Wright } \\ \text { RL Daubert } & \text { DP Mendoza } & \text { L Zhong } \\ \text { CS Dreyer } & \text { KD Miller } & \text { MR Zumhoff } \\ \text { PE Dresel } & \text { KE Parker } & \end{array}$

Individuals on the operations and sampling crew are especially recognized for supporting the Pretreatment Engineering Platform Project by working unpredictable, long, and off-hour shifts for the ten months of PEP operations. The tests and reports could not have gotten this far without their extraordinary effort.

The authors would also like to thank Wayne Cosby for valuable editorial support, Dave MacPherson and Kirsten Meier for Quality Assurance support throughout the project, Chrissy Charron, Andrea Boehler, and Mona Champion for their administrative support through the months of PEP testing, and Wesley Lawrence, James Huckaby, Steve Barnes, Donald Bachand, Robert Burke, and Gordon Beeman for their leadership and technical guidance during PEP operations. We are also grateful to Brady Hanson and Michael Minette for the management and coordination of data analysis activities. 



\section{Abbreviations and Acronyms}

\begin{tabular}{|c|c|}
\hline AFA & anti-foam agent \\
\hline APEL & Applied Processing Engineering Laboratory \\
\hline ASME & American Society of Mechanical Engineers \\
\hline BNI & Bechtel National Inc. \\
\hline $\mathrm{BS}$ & Blank Spike \\
\hline $\mathrm{CD}$ & Coriolis densitometer \\
\hline CFR & Code of Federal Regulations \\
\hline CUF & Cells Unit Filter \\
\hline DAS & Data Acquisition System \\
\hline DOE & U.S. Department of Energy \\
\hline EFRT & External Flowsheet Review Team \\
\hline $\mathrm{ft}$ & feet \\
\hline G & gravity \\
\hline GPC & gel permeation chromatography \\
\hline GPM & gallons per minute \\
\hline GPS & global positioning system \\
\hline GUI & graphical user interface \\
\hline HASQARD & Hanford Analytical Services Quality Assurance Requirements Documents \\
\hline HDI & “How Do I...?” \\
\hline HMI & human machine interface \\
\hline IC & ion chromatography \\
\hline ICDD & International Centre for Diffraction Data \\
\hline ICP & inductively coupled plasma \\
\hline ICP-AES & inductively coupled plasma-atomic emissions spectroscopy \\
\hline ICP-MS & inductively coupled plasma-mass spectrometry \\
\hline ICSD & Inorganic Crystal Structure Database \\
\hline IW & inhibited water \\
\hline JTG & Joint Test Group \\
\hline LCS & laboratory control sample \\
\hline LRB & laboratory record book \\
\hline LSB & Laboratory Support Building \\
\hline $\mathrm{m}$ & meter \\
\hline M\&TE & measuring and test equipment \\
\hline
\end{tabular}




\begin{tabular}{|c|c|}
\hline MS & matrix spike \\
\hline $\mathrm{NaOH}$ & sodium hydroxide \\
\hline NCR & nonconformance report \\
\hline NQA & nuclear quality assurance \\
\hline $\mathrm{OH}$ & free hydroxide \\
\hline P\&ID & piping and flow diagram \\
\hline PB & preparation blank \\
\hline PDL-W & Process Development Laboratory-West \\
\hline PDMS & polydimethylsiloxane \\
\hline PEP & Pretreatment Engineering Platform \\
\hline PJM & pulse-jet mixer \\
\hline PLM & polarized light microscopy \\
\hline PNNL & Pacific Northwest National Laboratory \\
\hline PNWD & Battelle_Pacific Northwest Division \\
\hline PPG & polypropylene glycol \\
\hline PSD & particle-size distribution \\
\hline psid & pounds per square inch differential \\
\hline psig & pounds per square inch gauge \\
\hline PTF & Pretreatment Facility \\
\hline QA & quality assurance \\
\hline QC & quality control \\
\hline QAM & Quality Assurance Manual \\
\hline QARD & Quality Assurance Requirements and Descriptions \\
\hline RPD & relative percent difference \\
\hline RPP-WTP & $\begin{array}{l}\text { River Protection Project-Hanford Tank Waste Treatment and Immobilization Plant } \\
\text { Support Program }\end{array}$ \\
\hline RTD & resistance temperature detector \\
\hline R\&T & research and technology \\
\hline SEM & scanning electron microscopy \\
\hline SQL & structured query language \\
\hline SwRI & Southwest Research Institute \\
\hline TDS & total dissolved solids \\
\hline TEM & transmission electron microscopy \\
\hline THF & tetrahydrofuran \\
\hline TIC/TOC & total inorganic carbon and total organic carbon \\
\hline TMP & transmembrane pressure \\
\hline
\end{tabular}


UDS undissolved solids

UFP ultrafiltration process

$\mathrm{Wt} \% \quad$ weight percent

WTP Waste Treatment and Immobilization Plant

XRD X-ray diffraction 



\section{Testing Summary}

Pacific Northwest National Laboratory (PNNL) was tasked by Bechtel National Inc. (BNI) on the River Protection Project-Hanford Tank Waste Treatment and Immobilization Plant (RPP-WTP) project to perform research and development activities to resolve technical issues identified for the Pretreatment Facility (PTF). The Pretreatment Engineering Platform (PEP) was designed, constructed, and operated as part of a plan to respond to issue M12, "Undemonstrated Leaching Processes." The PEP, located in the Process Development Laboratory-West (PDL-W) in Richland, Washington, is a ${ }^{1} / 4.5$-scale test platform ${ }^{(a)}$ designed to simulate the WTP pretreatment caustic leaching, oxidative leaching, ultrafiltration solids concentration, and slurry washing processes. The PEP replicates the WTP leaching processes using prototypic equipment and control strategies. The PEP also includes nonprototypic ancillary equipment to support the core processing.

The PEP testing program was conducted with a waste simulant that was developed in response to Task 5 from the M12 External Flowsheet Review Team (EFRT) issue response plan. ${ }^{\text {(b) }}$ The testing included the following tests with simulated Hanford tank waste:

- Shakedown/Functional testing: tested process operations (e.g., slurry transfers, steam heating of the vessels and the accumulation of condensate, filter backpulsing and flushing), process controls (e.g., transmembrane pressure [TMP] and axial flow velocity in the filter loop), and certain test functions (e.g., in-line slurry sampling accuracy and precision).

- Integrated Test A: demonstrated integrated processing when caustic leaching $\left(98^{\circ} \mathrm{C}\right)$ is performed in UFP-VSL-00001A/B with the Cr simulant component added after the post-caustic-leach washing step.

- Integrated Test B: demonstrated integrated processing when the caustic leaching $\left(98^{\circ} \mathrm{C}\right)$ is performed in UFP-VSL-00002A with the Cr simulant component added after the post-caustic-leach washing step.

- Integrated Test D: demonstrated integrated processing when the caustic leaching is performed at a lower temperature $\left(85^{\circ} \mathrm{C}\right)$ in UFP-VSL-00002A and with the Cr simulant component added to the initial batch of simulant.

Integrated Test $\mathrm{C}$ was deleted from the scope of the testing as per ICN-TP-RPP-WTP-506_R0.2. ${ }^{(\mathrm{c})}$

(a) The scale of $1 / 4.5$ was chosen because this scale enables the ultrafiltration loop to be configured to meet two important criteria: 1) using one filter bundle, the ratio of solids in the feed tank to filter surface area will be the same as in the plant, and 2) using five filter bundles, the type and extent of mixing in the feed vessel will be approximately prototypic during the solids washing processes.

(b) SM Barnes, and R Voke. 2006. Issue Response Plan for Implementation of External Flowsheet Review Team (EFRT) Recommendations - M12: Undemonstrated Leaching Process. 24590-WTP-PL-ENG-06-0024 Rev. 0, Bechtel National, Inc., Richland, Washington.

(c) GB Josephson, OP Bredt, JK Young, and DE Kurath. 2009. Test Plan for Pretreatment Engineering Platform (PEP) Testing (Phase I). TP-RPP-WTP-506, Rev 0.2, Pacific Northwest National Laboratory, Richland, Washington. 
Two process flowsheets are currently being evaluated for the ultrafiltration process (UFP) and leaching operations. The baseline flowsheet has caustic leaching conducted in the UFP-1 ultrafiltration feed preparation vessels (i.e., vessels UFP-VSL-T01A and B in the PEP; vessels UFP-VSL-00001A and $\mathrm{B}$ [UFP-1] in the WTP PTF). The alternative scenario has caustic leaching performed in the UFP-2 ultrafiltration feed vessels (i.e., vessel UFP-VSL-T02A ${ }^{(a)}$ in the PEP and vessels UFP-VSL-00002A and B [UFP-2] in the WTP PTF).

With both flowsheets, 19-M sodium hydroxide solution ( $\mathrm{NaOH}$, caustic) is added to the waste slurry to leach solid aluminum compounds (e.g., gibbsite, boehmite). Caustic addition is followed by a heating step that uses direct injection of steam to accelerate the leaching process. Following the caustic leach, the vessel contents are cooled using vessel cooling jackets and/or external heat exchangers. The main difference between the two scenarios is that for leaching in UFP-1, the 19-M NaOH is added to unconcentrated waste slurry (3 to 8-wt $\%$ solids), while for leaching in UFP-2, the slurry is concentrated to nominally 20 -wt $\%$ solids using cross-flow ultrafiltration before adding caustic. After cooling, the leached slurry is concentrated and washed with an aqueous solution of $0.01-\mathrm{M} \mathrm{NaOH}$ (referred to as inhibited water) to remove soluble salts. If the resulting waste solids remain high in chromium, sodium permanganate reagent is added, and the slurry is circulated to oxidize and dissolve the chromium solids. Following the oxidative leaching of chromium-containing solids, the slurry is washed to remove the dissolved chromium and concentrated.

The work described in this report includes a narrative of the conduct of Integrated Test A and the data obtained during the test. This report summarizes test operations and difficulties encountered during Integrated Test A. For test summary operations for the other tests, refer to WTP-RPT-190 for Shakedown/Functional Testing, WTP-RPT-192 for Integrated Test B, and WTP-RPT-193 for Integrated Test D.

In addition, reports have been written that specifically address the following:

1. Filtration Scale-Up (WTP-RPT-185)

2. Caustic Leaching Scale-Up (WTP-RPT-186)

3. Solids Washing (WTP-RPT-187)

4. Oxidative Leaching Scale-Up (WTP-RPT-188).

This report only includes very limited data analysis. Supporting data files have been supplied on transportable hard drives because of the large amount of data.

\section{Objectives}

Table S.1 summarizes the objectives for the entire PEP testing program along with a discussion of contributions made by the results of Integrated Test A to meeting these objectives.

(a) In this report, the UFP vessels are generally denoted as Tank T01A/B and Tank T02A. In some cases alternative designations are used to maintain continuity with previous documentation (e.g., test specification/plan). 
Table S.1. Summary of Test Objectives and Results

\begin{tabular}{|c|c|c|}
\hline Test Objective & \begin{tabular}{|l|} 
Objective \\
Met?
\end{tabular} & Discussion \\
\hline $\begin{array}{l}\text { Caustic leach process: Compare } \\
\text { engineering- and laboratory-scale } \\
\text { results to determine impact of } \\
\text { scale-up. }\end{array}$ & Yes & $\begin{array}{l}\text { Integrated Test A data were analyzed and compared with } \\
\text { laboratory-scale data with results presented in reports WTP-RPT-186 } \\
\text { and WTP-RPT-197. }\end{array}$ \\
\hline $\begin{array}{l}\text { Oxidative leach process: Compare } \\
\text { engineering- and laboratory-scale } \\
\text { results to determine impact of } \\
\text { scale-up. }\end{array}$ & Yes & $\begin{array}{l}\text { Integrated Test A data were analyzed and compared with } \\
\text { laboratory-scale data with results presented in reports WTP-RPT-188 } \\
\text { and WTP-RPT-197. }\end{array}$ \\
\hline $\begin{array}{l}\text { Cross-flow ultrafiltration: Monitor } \\
\text { cross-flow filter performance at } \\
\text { engineering- and laboratory-scale } \\
\text { to determine scale-up. }\end{array}$ & Yes & $\begin{array}{l}\text { Integrated Test A data were analyzed and compared with } \\
\text { laboratory-scale data with results presented in reports WTP-RPT-185 } \\
\text { and WTP-RPT-197. }\end{array}$ \\
\hline $\begin{array}{l}\text { Slurry wash process: Determine } \\
\text { the post-caustic and } \\
\text { oxidative-leaching slurry wash } \\
\text { efficiencies. }\end{array}$ & Yes & $\begin{array}{l}\text { Integrated Test A data were analyzed with results presented in reports } \\
\text { WTP-RPT-187 and WTP-RPT-197. }\end{array}$ \\
\hline $\begin{array}{l}\text { Process integration: Evaluate the } \\
\text { chemical addition, filter operation } \\
\text { cycle performance, and pressure } \\
\text { pot operations. Also perform mass } \\
\text { balances for aluminum, chromium, } \\
\text { manganese, sodium, hydroxide, } \\
\text { oxalate, phosphate, sulfate, and } \\
\text { water and monitor permeates for } \\
\text { post-filtration precipitation. }\end{array}$ & Yes & $\begin{array}{l}\text { Supporting data from Integrated Test A are presented, and results to } \\
\text { meet this objective are discussed in WTP-RPT- } 197 .\end{array}$ \\
\hline $\begin{array}{l}\text { Monitor the performance of the } \\
\text { recirculation system pumps, filters, } \\
\text { and heat exchanger to support } \\
\text { engineering fabrication decisions } \\
\text { for these components. }\end{array}$ & Yes & $\begin{array}{l}\text { The data required to meet this objective were provided on compact } \\
\text { discs transmitted in the following reference: Letter from GH Beeman } \\
\text { to H Hazen, "Subcontract No. 24590-QL-HC9-WA49-00001, Project } \\
\text { No. } 53569 \text { (WA-024) Engineering Ties Data Transmittal: The } \\
\text { Electronic File Enclosed With This Letter Has Been Reviewed for } \\
\text { Technical Accuracy Per the QA Program, } \\
\text { WTP/RPP-MOA-PNNL-00392, dated 4/10/09. }\end{array}$ \\
\hline
\end{tabular}

\section{Test Exceptions}

A summary description of the Test Exceptions applied to these tests is shown in Table S.2. 
Table S.2. Test Exceptions

\begin{tabular}{|c|c|}
\hline Test Exceptions & Description of Test Exceptions \\
\hline $\begin{array}{l}\text { 1) } 24590 \text {-PTF-TEF-RT-08- } \\
\text { 00002 incorporated into ICN-1 } \\
\text { to Test Plan TP-RPP-WTP-506. }\end{array}$ & $\begin{array}{l}\text { This test exception: } \\
\text { 1. Added a stage during the filter conditioning section of the } \\
\text { Shakedown/Functional Test where the simulant slurry is concentrated from } \\
\text { approximately } 5 \text {-wt\% solids to } 20 \text {-wt\% solids in one operation. This is in } \\
\text { addition to the previously specified low-solids filter and high-solids filter } \\
\text { testing. } \\
\text { 2. Documented the Joint Test Group (JTG) decision regarding the number of } \\
\text { replicate samples to be collected at various processing times. } \\
\text { 3. Revised the terminology specifying the Coriolis densitometer (CD) sample } \\
\text { locations that were changed to be consistent with PEP operating procedures. } \\
\text { Renamed the "center" array to "inner." } \\
\text { The sampling specified in the low-solids filtration test over-specifies the } \\
\text { sample collection timing required. The technical requirement is to get } 30 \\
\text { unique samples. The sampling schedule specified is not required to achieve } \\
\text { this test objective. }\end{array}$ \\
\hline $\begin{array}{l}\text { 2) 24590-PTF-TEF-RT-09- } \\
\text { 00001 incorporated into ICN-2 } \\
\text { and ICN-3 to Test Plan } \\
\text { TP-RPP-WTP-506. }\end{array}$ & $\begin{array}{l}\text { 1. In several steps, the sampling location was changed from the filer-loop } \\
\text { in-line location to a middle-low CD sample loop location in the } \\
\text { UFP-VSL-T02A vessel. This change impacted sampling in the } \\
\text { Shakedown/Functional and all of the Integrated tests (ref CCN 187749). } \\
\text { 2. Added a step to the Shakedown/Functional Test (step A.1.31) to add sodium } \\
\text { permanganate to UFP-VSL-T02A to assess possible foaming issue (ref CCN } \\
\text { 187749). } \\
\text { 3. Changed location of second sample for parallel laboratory-scale Cells Unit } \\
\text { Filter (CUF) testing from the in-line filter loop to the middle-low CD port in } \\
\text { the UFP-VSL-T02A (Step A.1.10; Shakedown/Functional Test) (ref CCN } \\
\text { 187749). } \\
\text { 4. Collected samples for parallel laboratory leaching test before and after caustic } \\
\text { addition in UFP-VSL-T01A (A.1.20; Shakedown/Functional Test) and } \\
\text { UFP-VSL-T02A (Step A.1.15; Shakedown/Functional Test), and in the } \\
\text { Integrated Test steps (B.1.2; Integrated Test A; B.2.6; Integrated Tests B/D) } \\
\text { (ref CCN 192734). } \\
\text { 5. Deleted reconfiguration of the filter loop to bypass UFP-VSL-T02A and } \\
\text { circulate flush water with UFP-PMP-T42A and/or UFP-PMP-T43A to allow } \\
\text { a representative in-line sample to be collected. This step (Step A.1.17; } \\
\text { Shakedown/Functional Test) could not be done under the operating } \\
\text { restrictions in place on the operation of the filter loop (ref CCN 192734.) } \\
\text { 6liminated Step A.1.25 (filter-loop bypass test with tracer) from the } \\
\text { Shakedown/Functional Test. This test was conducted after Integrated Test B } \\
\text { was completed (ref CCN 187753). } \\
\text { 7. Modified Step A.1.29 (Shakedown/Functional Test) to eliminate the removal } \\
\text { of solids from UFP-VSL-T02A before the high-solids filter test. This step } \\
\text { was not needed as the amount of solids is less than anticipated (ref CCN } \\
\text { 187752). } \\
\text { 8. Modified Step A.1.30 (Shakedown/Functional Test) to include five filter } \\
\text { backpulses before starting the high-solids filter test (ref CCN 187752). } \\
\text { 9. Modify Step B.1.8 (Integrated Test A) to allow 80\% of caustic to be added } \\
\text { during in-line simulant transfers to UFP-VSL-T01B and 20\% to be added } \\
\text { directly to UFP-VSL-T01B (ref CCN 187748). }\end{array}$ \\
\hline
\end{tabular}


Table S.2. Test Exceptions

\begin{tabular}{|c|c|}
\hline Test Exceptions & Description of Test Exceptions \\
\hline & 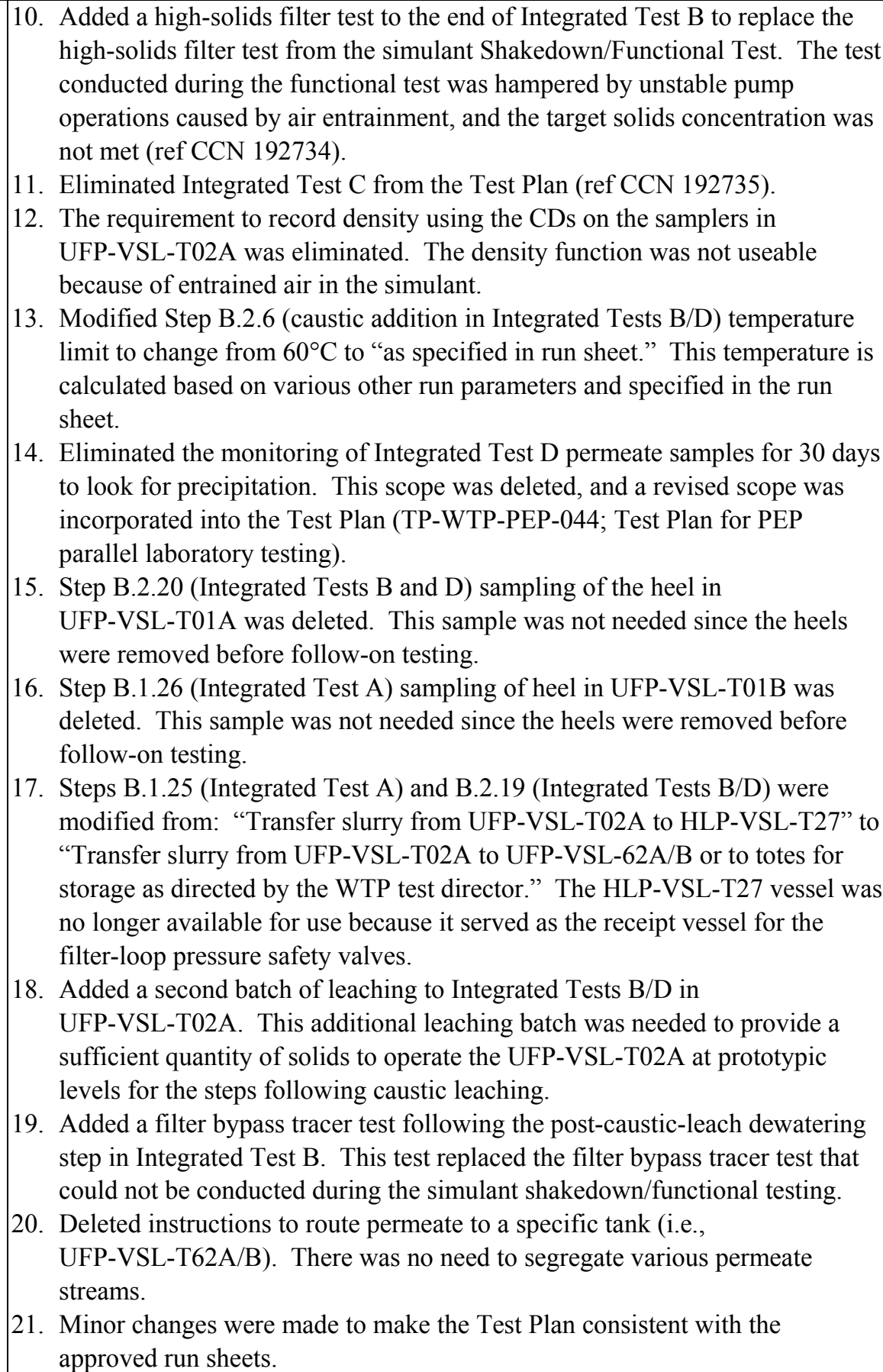 \\
\hline $\begin{array}{l}\text { 3) } 24590 \text {-WTP-TEF-RT-09- } \\
\text { 00003 incorporated into ICN-1 } \\
\text { to Test Plan TP-WTP-PEP-044. }\end{array}$ & $\begin{array}{l}\text { This Test Exception specified activities to be performed with permeate samples } \\
\text { obtained from Integrated Test D. The Integrated Test D permeate samples were } \\
\text { originally stored in a temperature-controlled environment and then moved to a } \\
\text { location with a reduced temperature where precipitation was likely to occur. The } \\
\text { Test Exception requested that the approximate size distribution of the solids be } \\
\text { measured in several (three or four) selected PEP samples from Integrated Test D } \\
\text { using polarized light microscopy (PLM). Size-calibrated photographs should be }\end{array}$ \\
\hline
\end{tabular}


Table S.2. Test Exceptions

\begin{tabular}{|c|c|}
\hline Test Exceptions & Description of Test Exceptions \\
\hline & $\begin{array}{l}\text { provided along with the analysis. If possible, record the mineral identification of } \\
\text { the solids phase(s) along with the particle-size distribution. Samples will be } \\
\text { selected by WTP personnel in consultation with the subcontractor and will be } \\
\text { based in part on the observation of which samples contain the most solids or } \\
\text { appear to contain different types of solids. Repeat the size distribution analysis } \\
\text { approximately } 1 \text { week after the initial measurements to determine whether there } \\
\text { was a significant change in crystal size, habit, or composition. } \\
\text { Perform each size distribution analysis by measuring the diameter (or length and } \\
\text { width for elongated crystals) of approximately } 100 \text { individual particles in each } \\
\text { sample. The size may be measured either on the microscope slide, using a } \\
\text { calibrated ocular scale, or on the size-calibrated photographs. The program } \\
\text { recognizes the limitations of the statistical significance of a size distribution } \\
\text { measurement based on such a small population. This Test Exception did not } \\
\text { affect any of the existing test plan objectives. }\end{array}$ \\
\hline $\begin{array}{l}\text { 4) } 24590 \text {-WTP-TEF-RT-09- } \\
\text { 00002 Rev 0, incorporated into } \\
\text { ICN-4 to Test Plan } \\
\text { TP-RPP-WTP-506. }\end{array}$ & $\begin{array}{l}\text { This test exception: } \\
\text { 1. Requests a report summarizing the lessons learned during scale-up, } \\
\text { manufacture, and transport of the PEP simulant. } \\
\text { 2. Specifies the sampling and analysis scope to be performed to complete the } \\
\text { prototypic nitric acid PEP filter cleaning process. } \\
\text { 3. Deletes the Engineering Ties report scope. } \\
\text { 4. Specifies additional experimental and analytical work required to estimate the } \\
\text { amount of excess caustic in caustic-leachate samples and post-caustic-leach } \\
\text { wash solutions containing } \approx 3.5-\mathrm{M} \mathrm{Na.}\end{array}$ \\
\hline $\begin{array}{l}\text { 5) } 24590 \text {-WTP-TEF-RT-09- } \\
\text { 00001 Rev } 1 \text { incorporated into } \\
\text { ICN-2 to Test Plan } \\
\text { TP-WTP-PEP-044. }\end{array}$ & $\begin{array}{l}\text { This Test Exception specifies additional work to be conducted with caustic-leach } \\
\text { solutions and post-caustic-leach washing permeate samples obtained from PEP } \\
\text { Integrated Tests A, B, and D. It contains the following tasks: } \\
\text { 1. Determine precipitate mineralogy, precipitate phase compositions, and } \\
\text { solution saturation composition. } \\
\text { 2. Determine rate of approach to saturation concentrations. } \\
\text { 3. Identify and characterize precipitates formed in post-caustic-leach filtrate. } \\
\text { 4. Determine the dilution required to redissolve the precipitate. } \\
\text { 5. Determine super-saturation in post-caustic-leach filtrates from Integrated } \\
\text { Test B in the PEP. } \\
\text { 6. Determine the effects of blending during the post-caustic-leach dewatering } \\
\text { and wash cycle. }\end{array}$ \\
\hline
\end{tabular}

As documented in the PEP Test Plan, the deviations from the Test Specification are provided in Table S.3. 
Table S.3. Deviations from Test Specification

\begin{tabular}{|c|c|}
\hline Test Specification Reference & Exception Taken \\
\hline $\begin{array}{l}\text { Section } 6.4 .4 \text { "Analytical measurements will be } \\
\text { made in conformance to the Guidelines for } \\
\text { Performing Chemical Physical, and Rheological } \\
\text { Properties Measurements (a) as applicable." }\end{array}$ & $\begin{array}{l}\text { Three method exceptions are required under this Test Plan: } \\
\text { 1. Caustic leach and oxidative leach samples taken during this } \\
\text { testing must be separated more quickly than the standard } \\
\text { method using syringes. This testing will use a modified } \\
\text { method with a shorter centrifuge time and will apply higher } \\
g \text { forces (e.g., } 4000 \mathrm{~g} \text { vs. } 1000 \mathrm{~g} \text { ). } \\
\text { Impact on results: If the standard method were used, the } \\
\text { longer time could very well lead to greater dissolution and } \\
\text { inaccurate results. Laboratory testing will be conducted } \\
\text { with simulants to confirm that this method of sample } \\
\text { handling is adequate. } \\
\text { Densities of samples smaller than } 10 \text {-mL can only be } \\
\text { established within two significant figures of accuracy. } \\
\text { Density measurements for this Test Plan require greater } \\
\text { accuracy. Therefore, a more accurate method employing a } \\
\text { pycnometer will be used. } \\
\text { Impact on results: The change to a pycnometer will } \\
\text { generate more precise results than the standard method. } \\
\text { The main impact is expected to be on analysis time. The } \\
\text { pycnometer method will be slower. } \\
\text { The process for determining the wt\% undissolved solids } \\
\text { (UDS) content of the slurries will in some cases be } \\
\text { determined with the use of a moisture analyzer. In } \\
\text { addition, the method of drying samples will be modified to } \\
\text { allow glass fiber filters to be used in drying the samples. } \\
\text { Impact on results: Both modifications are intended to } \\
\text { decrease the time required to obtain results. }\end{array}$ \\
\hline
\end{tabular}

\section{Results and Performance Against Success Criteria}

The PEP system tests were designed to generate the data necessary to:

- Provide engineering-scale system performance data. This information is used to support the projections of the WTP computer process models for the waste-processing campaign.

- Confirm the operability and functionality of UFP system components.

The WTP Research and Technology (R\&T) success criteria for achieving these objectives are discussed in Table S.4. The success criteria for the entire PEP testing program are provided with discussion limited to the success criteria covered by the scope of this report. The success criteria not addressed in this report are shaded in gray.

(a) GL Smith and K Prindiville. 2002. Guidelines for Performing Chemical, Physical, and Rheological Properties Measurements. 24590-WTP-GPG-RTD-001 Rev. 0, Bechtel National, Inc., Richland Washington. 
Table S.4. Success Criteria

\begin{tabular}{|c|c|}
\hline Success Criteria & How Testing Did or Did Not Meet Success Criteria \\
\hline \multicolumn{2}{|l|}{ UFP System Process Performance } \\
\hline $\begin{array}{l}\text { Measure the aluminum leaching } \\
\text { performance of the PEP and laboratory } \\
\text { systems as a function of time under WTP } \\
\text { UFP- } 1 \text { and UFP- } 2 \text { projected leaching } \\
\text { conditions at bounding high and low } \\
\text { process temperatures (nominally } 100^{\circ} \mathrm{C} \\
\text { and } 80^{\circ} \mathrm{C} \text { ). }\end{array}$ & $\begin{array}{l}\text { Aluminum leaching at } 98^{\circ} \mathrm{C} \text { was measured as a function of time under } \\
\text { WTP UFP-1 projected leaching conditions during Integrated Test A. } \\
\text { Results are presented in this report and in reports WTP-RPT-186 and } \\
\text { WTP-RPT-197. }\end{array}$ \\
\hline $\begin{array}{l}\text { Compare aluminum leach performance in } \\
\text { UFP-1 where all of the } \mathrm{NaOH} \text { is added } \\
\text { in-line to the case where a fraction of the } \\
\text { total } \mathrm{NaOH} \text { is added directly to the tank. }\end{array}$ & $\begin{array}{l}\text { Results to meet this success criterion are discussed in report } \\
\text { WTP-RPT-197. }\end{array}$ \\
\hline $\begin{array}{l}\text { Measure chromium leaching performance } \\
\text { in the PEP and laboratory systems as a } \\
\text { function of time at the WTP projected } \\
\text { conditions in UFP-2 for both the UFP-1 } \\
\text { and UFP-2 aluminum leaching flowsheets. }\end{array}$ & $\begin{array}{l}\text { Chromium leaching was measured as a function of time under WTP } \\
\text { projected leaching conditions during Integrated Test A. Results to } \\
\text { meet this success criterion are discussed in reports WTP-RPT-188 and } \\
\text { WTP-RPT-197. }\end{array}$ \\
\hline $\begin{array}{l}\text { Evaluate the process control strategy for } \\
\text { specification of required reagent additions, } \\
\text { including } \mathrm{NaOH}, \mathrm{NaMnO}_{4} \text {, and wash } \\
\text { solutions provided in the PEP Phase } 1 \\
\text { Testing Process Description. }\end{array}$ & $\begin{array}{l}\text { The process control strategy for specifying the amount of reagent was } \\
\text { to analyze a sample of the simulant feed for aluminum and chromium } \\
\text { content and specify the amount of } \mathrm{NaOH} \text { and } \mathrm{NaMnO}_{4} \text { based on the } \\
\text { result. The amount of reagent added was correct within the analytical } \\
\text { error of the analysis method. A comparison of targeted and delivered } \\
\text { reagent additions is provided in report WTP-RPT-188 for Integrated } \\
\text { Tests A and B. Additional discussion and results for Integrated } \\
\text { Test D are provided in report WTP-RPT-197. }\end{array}$ \\
\hline $\begin{array}{l}\text { Measure the filter system performance at } \\
\text { the nominal flow velocity and TMPs for } \\
\text { the solids concentration and washing } \\
\text { stages for the UFP-1 and UFP-2 aluminum } \\
\text { leaching flowsheets. }\end{array}$ & $\begin{array}{l}\text { Filter performance was measured at a nominal axial flow velocity of } \\
15 \mathrm{ft} / \mathrm{sec} \text { and a TMP of } 40 \text { psid during solids concentration and } \\
\text { washing during Integrated Test A. Actual permeate flux and filtration } \\
\text { conditions are provided in this report. A summary of filter system } \\
\text { performance is provided in WTP-RPT- } 197 \text {. }\end{array}$ \\
\hline $\begin{array}{l}\text { Evaluate the control strategy for make-up } \\
\text { additions from UFP-VSL-00001A/B to } \\
\text { UFP-VSL-00002A/B during initial } \\
\text { dewatering process. }\end{array}$ & $\begin{array}{l}\text { The control strategy for make-up additions between } \\
\text { UFP-VSL-T01A/B and UFP-VSL-T02A was demonstrated during } \\
\text { post-caustic-leach concentration in Integrated Test A. The control } \\
\text { strategy was determined to be adequate, although maintaining a high } \\
\text { operating level in UFP-VSL-T02A until the end of processing should } \\
\text { reduce the possibility of entraining air into the recirculation pumps. } \\
\text { Results to meet this success criterion are discussed in report } \\
\text { WTP-RPT-197. }\end{array}$ \\
\hline $\begin{array}{l}\text { Measure the wash water volumes required } \\
\text { to remove or reduce the free hydroxide } \\
\text { following the aluminum leaching stage and } \\
\text { dissolved chromium after the oxidative } \\
\text { leaching process to the specified } \\
\text { concentrations. }\end{array}$ & $\begin{array}{l}\text { The volume of wash water required to reduce free hydroxide } \\
\text { following the aluminum leach was evaluated for Integrated Test A, } \\
\text { and results to meet this success criterion are discussed in reports } \\
\text { WTP-RPT-187 and WTP-RPT-197. }\end{array}$ \\
\hline
\end{tabular}


Table S.4. Success Criteria

\begin{tabular}{|c|c|}
\hline Success Criteria & How Testing Did or Did Not Meet Success Criteria \\
\hline $\begin{array}{l}\text { Perform mass balances for selected } \\
\text { constituents, including aluminum, } \\
\text { chromium, manganese, sodium, hydroxide, } \\
\text { oxalate, phosphate, sulfate, and water to } \\
\text { evaluate leaching and washing process } \\
\text { performance. }\end{array}$ & $\begin{array}{l}\text { Results necessary for performing mass balances for selected } \\
\text { constituents for Integrated Test A are presented in this report. Results } \\
\text { to meet this success criterion are discussed for Cr in the oxidative } \\
\text { leaching process for Integrated Tests A and B in report } \\
\text { WTP-RPT-188 and are fully discussed for all constituents in report } \\
\text { WTP-RPT-197. }\end{array}$ \\
\hline $\begin{array}{l}\text { Measure solids distribution under scaled } \\
\text { mixing conditions before and after caustic } \\
\text { leaching evolutions. }\end{array}$ & $\begin{array}{l}\text { Solids distribution before and after caustic leaching is presented in } \\
\text { reports WTP-RPT-190 and WTP-RPT-197. }\end{array}$ \\
\hline $\begin{array}{l}\text { Measure the rheology of the slurry } \\
\text { simulant and shear strength of the settled } \\
\text { solids before and after each leaching and } \\
\text { washing unit operation and following final } \\
\text { concentration. }\end{array}$ & $\begin{array}{l}\text { The rheology of the slurry simulant was measured for Integrated } \\
\text { Test A and is provided in this report. }\end{array}$ \\
\hline $\begin{array}{l}\text { Estimate the quantity of excess hydroxide } \\
\text { added in the process that may not be } \\
\text { needed to keep aluminate in solution } \\
\text { following filtration. }\end{array}$ & $\begin{array}{l}\text { Results to meet this success criterion are discussed in report } \\
\text { WTP-RPT-197. }\end{array}$ \\
\hline $\begin{array}{l}\text { Collect and retain permeate samples for } \\
\text { extended precipitation studies (including } \\
\text { permeate/simulated supernatant blended } \\
\text { cases) from each concentration cycle. }\end{array}$ & $\begin{array}{l}\text { Permeate samples were collected during Integrated Test A for } \\
\text { precipitation studies. The results of the precipitation studies are } \\
\text { discussed in reports WTP-RPT-197, WTP-RPT-200, and } \\
\text { WTP-RPT-205. }\end{array}$ \\
\hline \multicolumn{2}{|l|}{ UFP System Operability and Functionality } \\
\hline $\begin{array}{l}\text { Verify that the dual, in-series pump } \\
\text { configuration is controllable and maintains } \\
\text { the required slurry velocity and pressures } \\
\text { for ultrafilter operation. }\end{array}$ & $\begin{array}{l}\text { The data required to meet this success criterion were provided on } \\
\text { compact disks transmitted in the following reference: Letter from } \\
\text { GH Beeman to H Hazen, "Subcontract No. } \\
\text { 24590-QL-HC9-WA49-00001, Project No. } 53569 \text { (WA-024) } \\
\text { Engineering Ties Data Transmittal: The Electronic File Enclosed } \\
\text { With This Letter Has Been Reviewed For Technical Accuracy Per the } \\
\text { QA Program," WTP/RPP-MOA-PNNL-00392, dated 4/10/09. }\end{array}$ \\
\hline $\begin{array}{l}\text { Measure the operating characteristics for } \\
\text { the cooling heat exchanger for the } \\
\text { UFP-VSL-00002 filter recirculation loop } \\
\text { (temperature changes as a function of flow } \\
\text { to determine how to achieve the desired } \\
\text { performance in the PTF analog). }\end{array}$ & $\begin{array}{l}\text { The data required to meet this success criterion were provided on } \\
\text { compact disks transmitted in the following reference: Letter from } \\
\text { GH Beeman to H Hazen, "Subcontract No. } \\
\text { 24590-QL-HC9-WA49-00001, Project No. } 53569 \text { (WA-024) } \\
\text { Engineering Ties Data Transmittal: The Electronic File Enclosed } \\
\text { With This Letter Has Been Reviewed For Technical Accuracy Per the } \\
\text { QA Program," WTP/RPP-MOA-PNNL-00392, dated 4/10/09. }\end{array}$ \\
\hline
\end{tabular}


Table S.4. Success Criteria

\begin{tabular}{|c|c|}
\hline Success Criteria & How Testing Did or Did Not Meet Success Criteria \\
\hline $\begin{array}{l}\text { Confirm whether the WTP process control } \\
\text { strategies for ultrafilter system filling, } \\
\text { operating, backpulsing, draining, flushing, } \\
\text { and cleaning are adequate for stable } \\
\text { operation. Provide to WTP data to } \\
\text { determine whether backpulsing is a } \\
\text { required and effective means of restoring } \\
\text { the filter permeate rates to make certain } \\
\text { that production throughput is maintained } \\
\text { and determine whether operation of the } \\
\text { backpulse system induces any process or } \\
\text { equipment operations issues. }\end{array}$ & $\begin{array}{l}\text { Results to meet this success criterion are discussed in report } \\
\text { WTP-RPT-197. }\end{array}$ \\
\hline $\begin{array}{l}\text { Use only the process information and data } \\
\text { available to the WTP PTF operating staff } \\
\text { during WTP operations (e.g., caustic and } \\
\text { permanganate addition volumes, permeate } \\
\text { mass balances for solids concentration) to } \\
\text { operate the PEP. }\end{array}$ & $\begin{array}{l}\text { This success criterion was met by developing a run sheet of all the } \\
\text { operating parameters (e.g., transfer volumes, reagent addition } \\
\text { volumes, control levels) based on prototypic characterization data } \\
\text { before the start of Integrated Test A. Changes to the run sheet made } \\
\text { during the test itself were based only on data that would be available } \\
\text { to the plant, and were not, for example, based on information from } \\
\text { nonprototypic samples. This success criterion is fully discussed in } \\
\text { WTP-RPT-197. }\end{array}$ \\
\hline $\begin{array}{l}\text { Confirm whether the elevated temperature } \\
\text { pulse-jet mixer (PJM) operating strategy is } \\
\text { adequate for stable PEP and WTP } \\
\text { operation. }\end{array}$ & $\begin{array}{l}\text { Results to meet this success criterion are discussed in report } \\
\text { WTP-RPT-197. }\end{array}$ \\
\hline $\begin{array}{l}\text { Measure the heat-up rate and } \\
\text { controllability of the PEP UFP-VSL- } 00001 \\
\text { and UFP-VSL-00002 vessels and the } \\
\text { cooling performance for UFP vessels. }\end{array}$ & $\begin{array}{l}\text { The heat up, thermal control at } 98^{\circ} \mathrm{C} \text {, and cool-down performance of } \\
\text { UFP-VSL-T01A/B was measured during Integrated Test A. Thermal } \\
\text { profiles are provided in this report. This information for the other } \\
\text { tests is discussed in the other run reports and summarized in } \\
\text { WTP-RPT-197. }\end{array}$ \\
\hline $\begin{array}{l}\text { Measure the performance of the in-line } \\
\text { addition of process chemicals into the } \\
\text { simulated wastes and determine the extent } \\
\text { of blending in the process vessels. }\end{array}$ & $\begin{array}{l}\text { The blending of process chemicals added in-line is presented in } \\
\text { reports WTP-RPT-190 and WTP-RPT- } 197 .\end{array}$ \\
\hline $\begin{array}{l}\text { Monitor ultrafilter performance (to include } \\
\text { visual inspection of the filter tubes, tube } \\
\text { sheets, and heads from an ultrafilter for } \\
\text { any evidence of flow mal-distribution } \\
\text { and/or solids buildup at least once during } \\
\text { Phase 1). }\end{array}$ & $\begin{array}{l}\text { Ultrafilter visual inspection results are presented in reports } \\
\text { WTP-RPT-193 and WTP-RPT-197. }\end{array}$ \\
\hline $\begin{array}{l}\text { Measure, record, and control ultrafiltration } \\
\text { temperature, TMP, and slurry flow during } \\
\text { filter-loop operations. }\end{array}$ & $\begin{array}{l}\text { Slurry flow rate, temperature, and TMP were recorded and controlled } \\
\text { during Integrated Test A. Results are provided in this report. }\end{array}$ \\
\hline $\begin{array}{l}\text { Record any solids accumulations observed } \\
\text { during any operating stage or maintenance } \\
\text { evolution. }\end{array}$ & $\begin{array}{l}\text { Results to meet this success criterion are discussed in report } \\
\text { WTP-RPT-197. }\end{array}$ \\
\hline
\end{tabular}


Table S.4. Success Criteria

\begin{tabular}{|l|l||}
\hline Success Criteria & How Testing Did or Did Not Meet Success Criteria \\
\hline $\begin{array}{l}\text { Monitor the permeate production rate of } \\
\text { each ultrafilter assembly in operation. }\end{array}$ & $\begin{array}{l}\text { The permeate production rate of each ultrafilter was recorded during } \\
\text { Integrated Test A. Results are provided in this report. The permeate } \\
\text { production rates for each test are presented in the relevant run report. }\end{array}$ \\
\hline $\begin{array}{l}\text { Record operating time of each ultrafilter } \\
\text { assembly. }\end{array}$ & $\begin{array}{l}\text { The operating time of each ultrafilter assembly was recorded during } \\
\text { Integrated Test A. Results are provided in this report. The operating } \\
\text { time of each filter assembly for each test is discussed in the relevant } \\
\text { run report and summarized in report WTP-RPT-197. }\end{array}$ \\
\hline $\begin{array}{l}\text { Record each ultrafilter assembly cleaning } \\
\text { event (backpulse, flush, chemical cleaning, } \\
\text { etc.). }\end{array}$ & $\begin{array}{l}\text { Results to meet this success criterion are discussed in reports } \\
\text { WTP-RPT-190 and WTP-RPT-193 and are summarized in } \\
\text { WTP-RPT-197. }\end{array}$ \\
\hline $\begin{array}{l}\text { Evaluate the pulse pot operation and } \\
\text { backpulse operation strategies contained in } \\
\text { Pretreatment Engineering Platform (PEP) } \\
\text { Phase 1 Testing Process Description. }\end{array}$ & $\begin{array}{l}\text { Backpulsing was conducted to increase the declining permeate rate } \\
\text { through the course of post-caustic-leach concentration. Backpulse } \\
\text { operations are evaluated in report WTP-RPT-197. }\end{array}$ \\
\hline $\begin{array}{l}\text { Evaluate permeate and permeate blends for } \\
\text { precipitation of solids, particularly } \\
\text { aluminum and oxalate solids. }\end{array}$ & $\begin{array}{l}\text { Permeate samples were collected during Integrated Test A for } \\
\text { precipitation studies. Results to meet this success criterion are } \\
\text { discussed in reports WTP-RPT-197, WTP-RPT-200, and } \\
\text { WTP-RPT-205. }\end{array}$ \\
\hline
\end{tabular}

\section{Quality Requirements}

The PNNL Quality Assurance (QA) Program is based upon the requirements as defined in the U.S. Department of Energy (DOE) Order 414.1C, Quality Assurance and 10 CFR 830, Energy/Nuclear Safety Management, Subpart A-Quality Assurance Requirements (a.k.a. the Quality Rule). PNNL has chosen to implement the following consensus standards in a graded approach:

- ASME NQA-1-2000: Quality Assurance Requirements for Nuclear Facility Applications, Part 1, Requirements for Quality Assurance Programs for Nuclear Facilities

- ASME NQA-1-2000, Part II, Subpart 2.7: Quality Assurance Requirements for Computer Software for Nuclear Facility Applications

- ASME NQA-1-2000, Part IV, Subpart 4.2: Graded Approach Application of Quality Assurance Requirements for Research and Development.

The procedures necessary to implement the requirements are documented in PNNL's "How Do I...?" (HDI). ${ }^{(a)}$

The RPP-WTP quality requirements are implemented by performing work in accordance with the River Protection Project-Hanford Tank Waste Treatment and Immobilization Plant Support Program (RPP-WTP) Quality Assurance Plan (RPP-WTP-QA-001, QAP). Work was performed to the quality requirements of NQA-1-1989 Part I, Basic and Supplementary Requirements, NQA-2a-1990, Part 2.7, and DOE/RW-0333P, Rev 13, Quality Assurance Requirements and Descriptions (QARD), as applicable. These quality requirements are implemented through the River Protection Project-Waste Treatment

(a) The system for managing the delivery of laboratory-level policies, requirements, and procedures. 
Plant Support Program (RPP-WTP) Quality Assurance Manual (RPP-WTP-QA-003, QAM). The requirements of DOE/RW-0333P Rev 13, Quality Assurance Requirements and Descriptions (QARD) and 10 CFR 830 Subpart A were not required for this work.

The RPP-WTP addresses internal verification and validation activities by conducting an independent technical review of the final data report in accordance with RPP-WTP's procedure QA-RPP-WTP-604. This review procedure is part of PNNL's RPP-WTP Quality Assurance Manual (RPP-WTP-QA-003). Following this procedure, a technical review would verify that the reported results are traceable, that inferences and conclusions are soundly based, and the reported work satisfies the objectives.

\section{R\&T Test Conditions}

The R\&T test conditions as defined in the Test Specification are summarized in Table S.5. The R\&T test conditions for the entire PEP testing program are provided with discussion limited to the R\&T test conditions covered by the scope of this report. R\&T test conditions not addressed in this report are shaded in gray.

Table S.5. R\&T Test Conditions

\begin{tabular}{|c|c|}
\hline List R\&T Test Conditions & Were Test Conditions Followed? \\
\hline \multicolumn{2}{|l|}{ General Requirements } \\
\hline $\begin{array}{l}\text { Perform mass balances for selected constituents, } \\
\text { including aluminum, chromium, manganese, sodium, } \\
\text { hydroxide, oxalate, phosphate, sulfate, and water, to } \\
\text { evaluate leaching and washing process performance. }\end{array}$ & $\begin{array}{l}\text { Necessary samples were taken to conduct a mass } \\
\text { balance for Integrated Test A. Mass balance results are } \\
\text { provided in report WTP-RPT-197. }\end{array}$ \\
\hline $\begin{array}{l}\text { Evaluate ultrafilter performance (to include visual } \\
\text { inspection of the filter tubes, tube sheets, and heads from } \\
\text { an ultrafilter for any evidence of flow mal-distribution } \\
\text { and/or solids buildup or evidence of potential failure). }\end{array}$ & $\begin{array}{l}\text { This R\&T test condition is discussed in reports } \\
\text { WTP-RPT-193 and WTP-RPT- } 197 .\end{array}$ \\
\hline $\begin{array}{l}\text { Assess the blending achieved during in-line additions of } \\
\text { leaching and washing solutions. }\end{array}$ & $\begin{array}{l}\text { In-line addition of wash water during Integrated } \\
\text { Tests A and B is discussed in reports WTP-RPT-187 } \\
\text { and WTP-RPT-197. }\end{array}$ \\
\hline $\begin{array}{l}\text { Record any solids accumulations observed during any } \\
\text { operating stage or maintenance evolution (e.g., } \\
\text { photography, particle-size distribution). }\end{array}$ & $\begin{array}{l}\text { This R\&T test condition is discussed in report } \\
\text { WTP-RPT-197. }\end{array}$ \\
\hline \multicolumn{2}{|l|}{ Leaching Operations } \\
\hline $\begin{array}{l}\text { Maintain caustic leaching temperature at the required } \\
\text { setpoint and record steam usage to remain in the } \\
\text { temperature range. }\end{array}$ & $\begin{array}{l}\text { The temperature during caustic leaching was } \\
\text { maintained at setpoint with steam usage measured } \\
\text { during Integrated Test A with the exceptions provided } \\
\text { in this report. Additional discussion of this condition is } \\
\text { provided in reports WTP-RPT-186 and WTP-RPT-197. }\end{array}$ \\
\hline $\begin{array}{l}\text { Maintain oxidative leaching temperature at the required } \\
\text { setpoint. }\end{array}$ & $\begin{array}{l}\text { The temperature during oxidative leaching was } \\
\text { maintained at setpoint during Integrated Test A. } \\
\text { Additional discussion of this condition is provided in } \\
\text { reports WTP-RPT-188 and WTP-RPT-197. }\end{array}$ \\
\hline $\begin{array}{l}\text { Obtain periodic samples during the leaching operations to } \\
\text { monitor the amount of aluminum or chromium that has } \\
\text { dissolved and concentrations of the reactants and } \\
\text { products in the liquid fraction in the vessel. }\end{array}$ & $\begin{array}{l}\text { This R\&T condition was met for the caustic-leaching } \\
\text { tests discussed in this report. Additional discussion of } \\
\text { this R\&T condition is provided in reports } \\
\text { WTP-RPT-186, WTP-RPT-188, and WTP-RPT-197. }\end{array}$ \\
\hline
\end{tabular}


Table S.5. R\&T Test Conditions

\begin{tabular}{|c|c|}
\hline List R\&T Test Conditions & Were Test Conditions Followed? \\
\hline $\begin{array}{l}\text { Provide data to demonstrate the WTP process control } \\
\text { strategy for the caustic and permanganate addition. }\end{array}$ & $\begin{array}{l}\text { This R\&T test condition is discussed in report } \\
\text { WTP-RPT- } 197 .\end{array}$ \\
\hline $\begin{array}{l}\text { Measure the rheology of the slurry simulant and the shear } \\
\text { strength of the settled solids before and following each } \\
\text { leaching unit operation. }\end{array}$ & $\begin{array}{l}\text { Slurry rheology was measured with samples taken } \\
\text { before and after each leaching unit operation. Results } \\
\text { are provided in this report. Rheology results for the } \\
\text { other tests are included in the relevant run reports. }\end{array}$ \\
\hline \multicolumn{2}{|l|}{ Concentration Operations } \\
\hline $\begin{array}{l}\text { Monitor the permeate production rate of each ultrafilter } \\
\text { assembly in operation. }\end{array}$ & $\begin{array}{l}\text { Permeate production for each ultrafilter assembly was } \\
\text { monitored during testing, and results are provided in } \\
\text { this report. Permeate production rates for the other } \\
\text { tests are included in the relevant run reports. }\end{array}$ \\
\hline Record operating time of each ultrafilter assembly. & $\begin{array}{l}\text { The operating time of each ultrafilter was recorded } \\
\text { during Integrated Test A, with results discussed in } \\
\text { report WTP-RPT-197. }\end{array}$ \\
\hline $\begin{array}{l}\text { Record each ultrafilter assembly "cleaning" event } \\
\text { (backpulse, flush, chemical cleaning, etc.). }\end{array}$ & $\begin{array}{l}\text { Ultrafilter cleaning events were not part of Integrated } \\
\text { Test A efforts; therefore, they are not included in this } \\
\text { report. However, backpulse events are summarized in } \\
\text { this report. A summary of the filter cleaning events is } \\
\text { provided in report WTP-RPT-197, and details of the } \\
\text { final prototypic filter cleaning are presented in report } \\
\text { WTP-RPT-193. Additional filter cleaning events are } \\
\text { also discussed in reports WTP-RPT-190 and } \\
\text { WTP-RPT-192. }\end{array}$ \\
\hline $\begin{array}{l}\text { Confirm pulse pot operation and backpulse operation } \\
\text { strategies. }\end{array}$ & $\begin{array}{l}\text { This R\&T test condition is discussed in report } \\
\text { WTP-RPT- } 197 .\end{array}$ \\
\hline $\begin{array}{l}\text { Control ultrafiltration temperature, TMP, and slurry flow } \\
\text { as specified in test-specific run sheets. }\end{array}$ & $\begin{array}{l}\text { Ultrafilter temperature, TMP, and slurry flow rate were } \\
\text { controlled. Deviations from values specified in the } \\
\text { Integrated Test A run sheet are noted in this report. } \\
\text { R\&T conditions for the other tests are included in the } \\
\text { relevant run reports. }\end{array}$ \\
\hline $\begin{array}{l}\text { Collect and retain permeate samples for extended } \\
\text { precipitation studies (including permeate/simulated } \\
\text { supernatant blended cases) from each concentration } \\
\text { cycle. }\end{array}$ & $\begin{array}{l}\text { Samples were collected and retained for extended } \\
\text { precipitation studies. The results of the precipitation } \\
\text { studies are discussed in reports WTP-RPT-197, } \\
\text { WTP-RPT-200, and WTP-RPT-205. }\end{array}$ \\
\hline $\begin{array}{l}\text { Demonstrate WTP ultrafiltration system control scheme } \\
\text { in normal operating modes (e.g., fill and startup, } \\
\text { operation, backpulsing, flush and drain, cleaning and } \\
\text { return to service). }\end{array}$ & $\begin{array}{l}\text { This R\&T test condition is discussed in report } \\
\text { WTP-RPT-197. }\end{array}$ \\
\hline \multicolumn{2}{|l|}{ Washing Operations } \\
\hline $\begin{array}{l}\text { Wash slurries using a washing protocol to be specified in } \\
\text { test specific run sheets. }\end{array}$ & $\begin{array}{l}\text { Slurries were washed as specified in the Integrated } \\
\text { Test A run sheet. Washing results are discussed in } \\
\text { reports WTP-RPT-187 and WTP-RPT-197. }\end{array}$ \\
\hline $\begin{array}{l}\text { Sample permeate immediately before each wash solution } \\
\text { addition to monitor washing performance/efficiency. }\end{array}$ & $\begin{array}{l}\text { Permeate was sampled and monitored during washing } \\
\text { with results provided in reports WTP-RPT-187 and } \\
\text { WTP-RPT-197. }\end{array}$ \\
\hline
\end{tabular}


Table S.5. R\&T Test Conditions

\begin{tabular}{|l|l|}
\hline List R\&T Test Conditions & Were Test Conditions Followed? \\
\hline Measure rheology of the washed solids. & $\begin{array}{l}\text { The rheology of washed solids was measured with } \\
\text { results provided in this report. Rheology results for the } \\
\text { other tests are included in the relevant run reports with } \\
\text { selected results also discussed in reports WTP-RPT-187 } \\
\text { and WTP-RPT-197. }\end{array}$ \\
\hline
\end{tabular}

\section{Simulant Use}

PEP process testing was performed with a nonradioactive aqueous slurry of simulant waste chemicals and solids. The simulant composition and make-up recipe were provided by WTP as documented in Simulant Recommendation for Phase 1 Testing in the Pretreatment Engineering Platform. ${ }^{\text {(a) }}$ Aqueous chemical concentrations were within the ranges expected for waste feeds to the PTF. The hydroxide concentration was marginally one standard deviation lower than the average concentration expected in the feeds to the plant. The oxalate and phosphate components were at the lower end of the expected ranges, but the oxalate component was at the solubility limit, and the phosphate component was at or near the solubility limit. The solids components and blend were selected to obtain targeted solids mass loss (aluminum and chromium leaching and oxalate washing) and treatment time. The simulant was not selected to represent any particular Hanford tank waste type.

The simulant was blended from the components listed below. The basis for selecting the individual components and the comparison to actual waste behavior is provided where applicable in the indicated references:

- Boehmite (for Al) (Russell et al. 2009a)

- Gibbsite (for Al) (Russell et al. 2009b)

- Chromium oxyhydroxide (CrOOH) slurry (Rapko et al. 2007)

- Sodium oxalate

- Filtration simulant (Russell et al. 2009c)

- Supernate.

A separate chromium solids slurry simulant was prepared and added to the PEP process after post-caustic-leach washing (a nonprototypic addition) during the Shakedown/Functional Tests and Integrated Tests A and B. This approach was taken because laboratory-scale tests had shown that the high-temperature caustic leaching step dissolved significant amounts of the $\mathrm{CrOOH}$ solids (Russell et al. 2009a). In Integrated Test D, the chromium solids component of the simulant was added during the simulant make-up process to demonstrate the PTF permanganate addition strategy.

(a) P Sundar. 2008. Simulant Recommendation for Phase 1 Testing in the Pretreatment Engineering Platform. 24590-PTF-RPT-RT-08-006, Rev 0, Bechtel National, Inc., Richland, WA. 
Simulant was procured from NOAH Technologies Corporation (San Antonio, TX). Samples of each simulant batch were characterized to make certain that chemical and physical properties requirements were met. Batches of the simulant were procured as follows:

- A 15-gallon trial batch of the blended simulant for laboratory testing to demonstrate the efficacy of the simulant fabrication procedure.

- A 250-gallon scale-up batch of the blended simulant to demonstrate scale-up of the simulant fabrication procedure to an intermediate scale.

- Batches 0, 1, and 2, each nominally 3500 gallons, of blended simulant for the Shakedown/Functional Tests and Integrated Tests A and B. These batches did not contain the CrOOH component.

- The CrOOH solids slurry for the Shakedown/Functional Test and Integrated Tests A and B was obtained in two separate batches, containing nominally 18 and $36 \mathrm{~kg}$ of $\mathrm{Cr}$ as $\mathrm{CrOOH}$.

\section{Discrepancies and Follow-on Tests}

No discrepancies or follow-on tests were identified. 



\subsection{Introduction}

This report summarizes test activities for Integrated Test A. Integrated Test A is the first of three tests aimed to address concerns raised by the External Flowsheet Review Team (EFRT). In October 2005, the EFRT reviewed the design of the Hanford Tank Waste Treatment and Immobilization Plant (WTP) and identified several issues that could impact operation of the plant if not addressed. In response to the EFRT issues, an engineering-scale integrated platform — named the Pretreatment Engineering Platform (PEP) — was built to demonstrate the WTP system design and treatment process flowsheet.

The PEP is a $1 / 4.5$ linear scale test platform located in the Process Development Engineering Laboratory-West (PDL-W) at Pacific Northwest National Laboratory (PNNL). Area dimensions are $1 / 4.5^{2}$ scale, and volume dimensions are $1 / 4.5^{3}$ scale. The PEP is designed to simulate the WTP pretreatment caustic leaching, oxidative leaching, ultrafiltration solids concentration, and slurry washing processes. In addition, PEP operations collect data needed for design input and to improve prediction of full-scale plant performance and demonstrate component operation and operating characteristics. PEP equipment for conducting these core processes has been designed to be prototypic of the plant; however, it also includes nonprototypic ancillary equipment.

This is one in a series of reports that summarize Phase 1 testing results from PEP. Separate run reports have been prepared for each Phase 1 test (i.e., Shakedown/Functional Testing [Josephson et al. 2009], Integrated Test B [Geeting et al. 2009], and Integrated Test D [Sevigny et al. 2009]). In addition, reports have been written that specifically address the following:

1. Filtration Scale-Up (Daniel et al. 2009)

2. Caustic Leaching Scale-Up (Mahoney et al. 2009)

3. Solids Washing (Baldwin et al. 2009)

4. Oxidative Leaching Scale-Up (Rapko et al. 2009).

Integrated Test A was conducted per TI-WTP-PEP-065, written in accordance with Test Plan TP-RPP-WTP-506, ${ }^{\text {(a) }}$ which was written in response to Test Specification 24590-PTF-TSP-RT-07-001, Rev 2. ${ }^{\left({ }^{\prime}\right)}$ Integrated Test $A$ demonstrated the integrated processes of caustic leaching in UFP-VSL-T01A/B at $98^{\circ} \mathrm{C}$ and oxidative leaching in UFP-VSL-T02A.

\subsection{Purpose}

The purpose of this testing program is to provide WTP with data to "...confirm the ultrafiltration process (UFP) system design and sludge treatment process flowsheet."(c) The system design and

(a) G Josephson, O Bredt, J Young, and D Kurath. 2008. Test Plan: Pretreatment Engineering Platform (PEP) Testing (Phase I). TP-RPP-WTP-506, Rev 0, Pacific Northwest National Laboratory, Richland, WA, 99352.

(b) JL Huckaby and JR Markillie. 2008. Pretreatment Engineering Platform (PEP) Testing (Phase I). 24590-PTF-TSP-RT-07-001, Rev 2, Bechtel National, Inc., Richland, Washington.

(c) SM Barnes and R Voke. 2006. Issue Response Plan for Implementation of External Flowsheet Review Team (EFRT) Recommendations-M12, Undemonstrated Leaching Processes. 24590-WTP-PL-ENG-06-0024 Rev. 0, p. 24; Bechtel National, Inc., Richland, Washington. 
treatment process flowsheet will be confirmed by evaluating the following operations on an engineering-scale integrated platform called the PEP:

- Solids concentration

- Aluminum leaching

- Washing

- Chromium leaching.

Tests were used to collect data needed for design input and to improve prediction of full-scale plant performance:

- Demonstration of component operation and determination of operating characteristics

- Integrated simulations of leaching scenarios.

The PEP data will be used by WTP along with laboratory-scale data and appropriate application of scaling to improve the WTP plant models used to predict plant operating performance.

\subsection{Background}

In October 2005, an EFRT was assembled to challenge and provide a critical review of the WTP design. The review identified a number of issues and potential issues that if not addressed could impact the operation of the plant. ${ }^{\text {(a) }}$ One of the issues that must be addressed in the Pretreatment Facility (PTF) is "Undemonstrated Leaching Processes." The EFRT reported that neither the caustic leaching nor the oxidative leaching process has been demonstrated at greater than bench scale. Without a scale-up study, the EFRT indicated that the ability to predict the effectiveness of these processes is limited.

The WTP project under BNI and the U.S. Department of Energy (DOE) developed an issue response plan for implementation of EFRT recommendations: M12, Undemonstrated Leaching Processes to resolve the "Undemonstrated Leaching Processes" issue. ${ }^{(b)}$ The plan addresses related topics that are not specifically in response to EFRT concerns. These include caustic addition and leaching concerns that were better understood after the EFRT report was issued, information to support revision of the contract design basis for the PTF, including system capacities, and earlier initiatives on enhancing plant throughput capacity. The solution for closing this issue includes conducting engineering-scale testing of all leaching (caustic and oxidative), washing, and filtration processes. ${ }^{\text {(a) }}$

The flowsheet and equipment design demonstration will be performed in the following two phases:

Phase $1-$ Confirm the UFP system design and sludge treatment process flowsheet. ${ }^{(a)}$

(a) JP Henschel. March 17, 2006. Contract No. DE-AC27-01RV14136 - Report of External Flowsheet Review Team for the Hanford Tank Waste Treatment and Immobilization Plant: "Comprehensive Review of the Waste Treatment Plant Flowsheet and Throughput.” Letter to RJ Schepens. CCN 132846, Bechtel National, Inc., Richland, Washington.

(b) S Barnes and R Voke. 2006. Issue Response Plan for Implementation of External Flowsheet Review Team (EFRT) Recommendations-M12, Undemonstrated Leaching Processes. 24590-WTP-PL-ENG-06-0024, Rev 0, p. 5; Bechtel National, Inc., Richland, Washington. 
Phase 2-Confirm the UFP system performance over a range of anticipated plant process conditions including the principal types of WTP feeds.

The data provided by the Phase 1 engineering-scale testing will be used to confirm the performance of the selected process flowsheet design and equipment based on post-Phase 1 test modeling.

On October 23 and 24, 2006, a WTP Project ultrafiltration leaching process technical review meeting was held to review the Issue Response Plan for M12. ${ }^{\text {(a) }}$ Participants included members of DOE, EFRT, the WTP project, and PNNL. At the meeting, PNNL made recommendations on the methodology and scale for the leaching processes. ${ }^{\text {(a) }}$

Members of the EFRT also presented their assessment (Integrated Test Platform, M12-Test Review) with specific recommendations. ${ }^{\text {(a) }}$ The general system requirements recommended by EFRT and PNNL staff members included the following:

- Principal processing equipment elements of the UFP system need to be included in the demonstration (including UFP-VSL-00001A/B, UFP-VSL-00002, the ultrafiltration loop recirculation pumps, and the ultrafilters with supporting equipment).

- The demonstration system needs to be flexible to accommodate testing of the baseline processes and process options for a range of operating conditions and processing times.

- Geometric similarity (prototypic) is needed in the key pieces of processing equipment (including UFP-VSL-00001A/B and UFP-VSL-00002).

- Ultrafilter elements must be a prototypic length and diameter to obtain expected filter performance data. The test equipment should be scaled down by using fewer filter elements in each assembly.

The following system parameters were developed and approved by the EFRT consultants:

- The engineering-scale test facility should be a factor of 4.5 smaller than the full-scale PTF.

- Each ultrafilter assembly should contain 12 full-scale elements.

- Five ultrafilter assemblies should be provided (based on M-13 recommendations).

Additional bases for the engineering-scale test system factor of 4.5 are provided in Scaling Relationships for the Pretreatment Engineering Platform (Kuhn et al. 2008). Some of the scaling factors are listed below.

- Selected processes within the PEP will be operated at both scale-time (4.5 times shorter than plant time) and plant time in separate test runs to address scaling issues.

- Prototypic tanks will be dimensionally scaled with a radius $1 / 4.5$ times their full-scale radius and operating height $1 / 4.5$ that of full-scale. The tank volume will be $1 /(4.5)^{3}$ or $\sim^{1} / 90^{\text {th }}$ of the plant.

- The filtration design is scaled by filter area. The total filtration area is $1 /(4.5)^{2}$ or $\sim 1 / 20^{\text {th }}$ that of the plant.

(a) S Barnes and R Voke. 2006. Issue Response Plan for Implementation of External Flowsheet Review Team (EFRT) Recommendations-M12, Undemonstrated Leaching Processes. 24590-WTP-PL-ENG-06-0024 Rev 0, Bechtel National, Inc., Richland, Washington. 
- Fluid velocities in pipes are kept approximately the same (using commercially available pipe diameters) to mimic solids settling that may occur in plant piping.

- Chemical reaction times are not scaled.

- The pulse-jet mixer (PJM) nozzle velocity in the PEP will be scaled to provide the same mixing power per volume of tank contents. The plant targets a nozzle discharge velocity of $8 \mathrm{~m} / \mathrm{s}$ in UFP-VSL-00001A/B and $12 \mathrm{~m} / \mathrm{s}$ in UFP-VSL-00002A/B. For non-Newtonian fluids, the nozzle velocity will be the same in the PEP and WTP. For Newtonian fluids, the velocity is scaled by $1 /(4.5)^{0.333}$. The total PJM cycle time will be scaled to maintain the fraction of the cycle in active drive mode. It is recognized that there is not a sharp distinction from Newtonian to non-Newtonian behavior. For the purpose of Phase 1 testing, we will scale the PJM velocities to maintain equal power per volume for the initial concentration and caustic leach. After the slurry is concentrated following the caustic leach, the PJMs will be adjusted to have the same PJM nozzle velocities as the plant (non-Newtonian scaling) and kept in that regime for the remainder of the process (post-caustic-leach wash, slurry wash, oxidative leaching, final slurry washing, and final concentration).

Section 6.4 of Pretreatment Engineering Platform (PEP) Testing (Phase 1) Test Specification (24590-PTF-TSP-RT-07-001 Rev 2) identifies the requirements for engineering-scale tests. ${ }^{(a)}$ The PEP data will be used by WTP along with laboratory-scale data and appropriate application of scaling to improve the WTP plant models used to predict plant operating performance.

\subsection{Objective}

The objective of this report is to present the results of Integrated Test A with only very limited data analysis. This includes a test narrative, sample analyses, summaries of PEP monitoring data, evaluations or summaries of problems encountered, and deviations from the test protocols. Specific test objectives and summary results are provided in the summary section of this report.

(a) JL Huckaby and JR Markillie. 2008. Pretreatment Engineering Platform (PEP) Testing (Phase I). 24590-PTF-TSP-RT-07-001, Rev 2, Bechtel National, Inc., Richland, Washington. 


\subsection{Quality Assurance}

The PNNL QA Program is based upon the requirements as defined in the U.S. Department of Energy (DOE) Order 414.1C, Quality Assurance and 10 CFR 830, Energy/Nuclear Safety Management, Subpart A-Quality Assurance Requirements (a.k.a. the Quality Rule). PNNL has chosen to implement the following consensus standards in a graded approach:

- ASME NQA-1-2000: Quality Assurance Requirements for Nuclear Facility Applications, Part 1, Requirements for Quality Assurance Programs for Nuclear Facilities

- ASME NQA-1-2000, Part II, Subpart 2.7: Quality Assurance Requirements for Computer Software for Nuclear Facility Applications

- ASME NQA-1-2000, Part IV, Subpart 4.2: Graded Approach Application of Quality Assurance Requirements for Research and Development.

The procedures necessary to implement the requirements are documented in PNNL's "How Do I...?" (HDI). ${ }^{\text {(a) }}$

The RPP-WTP quality requirements are implemented by performing work in accordance with the River Protection Project-Waste Treatment Plant Support Program (RPP-WTP) Quality Assurance Plan (RPP-WTP-QA-001, QAP). Work was performed to the quality requirements of NQA-1-1989 Part I, Basic and Supplementary Requirements, NQA-2a-1990, Part 2.7, and DOE/RW-0333P, Rev 13, Quality Assurance Requirements and Descriptions (QARD), as applicable. These quality requirements are implemented through the River Protection Project-Waste Treatment Plant Support Program (RPP-WTP) Quality Assurance Manual (RPP-WTP-QA-003, QAM). The requirements of DOE/RW-0333P Rev 13, Quality Assurance Requirements and Descriptions (QARD), and 10 CFR 830 Subpart A were not required for this work.

The RPP-WTP addresses internal verification and validation activities by conducting an independent technical review of the final data report in accordance with RPP-WTP's procedure QA-RPP-WTP-604. This review procedure is part of PNNL's RPP-WTP Quality Assurance Manual (RPP-WTP-QA-003). Following this procedure, a technical review would verify that the reported results are traceable, that inferences and conclusions are soundly based, and the reported work satisfies the objectives.

Table 2.1 lists nonconformance reports (NCRs) active during Integrated Test A.

(a) The system for managing the delivery of laboratory-level policies, requirements, and procedures. 
Table 2.1. Description of NCRs During Integrated Test A

\begin{tabular}{|c|c|}
\hline $\begin{array}{l}\text { Nonconformance } \\
\text { Report Number }\end{array}$ & Description \\
\hline \multirow[t]{4}{*}{ NCR 38767.1} & Measurement: UFP-VSL-T02A spargers air flow rate. \\
\hline & $\begin{array}{l}\text { Issue: Micro-Motion identifies flow rates below which the uncertainty is greater } \\
\text { than } 0.5 \% \text {. In the case of the PEP, air flow rates below } 0.090 \mathrm{~kg} / \mathrm{min} \text { have } \\
\text { uncertainties greater than } 0.5 \% \text {. For the lowest flow rate reported }(0.012 \mathrm{~kg} / \mathrm{min} \\
\text { on FT-1977), the estimated uncertainty is } \sim 4 \% \text {. }\end{array}$ \\
\hline & $\begin{array}{l}\text { Affected instruments follow: FT-1973 (UFP-VSL-T01B steam ring purge), } \\
\text { FT-1977 (UFP-VSL-T02A bottom sparger), FT-1981 (UFP-VSL-T01A steam } \\
\text { ring purge), FT-1901 (UFP-VSL-T02A upper sparger), and FT-1995 } \\
\text { (UFP-VSL-T02A steam ring purge). }\end{array}$ \\
\hline & $\begin{array}{l}\text { Consequence: There is greater uncertainty associated with data for air flow rates } \\
<0.090 \mathrm{~kg} / \mathrm{min} \text {. }\end{array}$ \\
\hline \multirow[t]{2}{*}{ NCR 43398.1} & Measurement: UFP-VSL-T01B temperature. \\
\hline & $\begin{array}{l}\text { Issue and Consequence: TTK- } 0427 \text { failed at installation. All data from } \\
\text { TTK- } 0427 \text { are invalid and unusable. However, there are } 19 \text { other temperature } \\
\text { measurements available within vessel UFP-VSL-T01B, with TTK- } 0425 \text { as the } \\
\text { prototypic measurement. }\end{array}$ \\
\hline \multirow[t]{5}{*}{ NCR 42402.1} & Measurement: Slurry temperature in the filter loop. \\
\hline & $\begin{array}{l}\text { Issues and Consequences: RTD thermowells in the filter loop do not extend into } \\
\text { the process stream: TT-0513: UFP-HX-T02A; (cooling) slurry outlet } \\
\text { temperature. No data are to be used for quality-affecting work. Data may be } \\
\text { used for qualitative purposes only. }\end{array}$ \\
\hline & $\begin{array}{l}\text { TT-0515: UFP-HX-T03A; (heating) slurry outlet temperature. No data are to be } \\
\text { used for quality-affecting work. Data may be used for qualitative purposes only. }\end{array}$ \\
\hline & $\begin{array}{l}\text { TT-0537: Filter UFP-FILT-T05A; outlet temperature (UFP-HX-T02A inlet). No } \\
\text { data are to be used for quality-affecting work. Data may be used for qualitative } \\
\text { purposes only. }\end{array}$ \\
\hline & $\begin{array}{l}\text { TT-0791: Filter UFP-FILT-T01A inlet temperature. Data from this should not } \\
\text { be used at all. Tank temperature data will be used for quality-affecting filter } \\
\text { processing data. }\end{array}$ \\
\hline \multirow[t]{2}{*}{ NCR 42317.1} & Measurement: Filter-loop flow rate downstream from UFP-PMP-T43A. \\
\hline & $\begin{array}{l}\text { Issue and Consequence: FE- } 0635 \text { is reporting suspect flow values. Data from } \\
\text { FE-0635 are unusable from } 2 / 14 / 2009 \text { through } 2 / 27 / 2009 \text {. }\end{array}$ \\
\hline \multirow[t]{3}{*}{ NCR 41589.1} & Measurement: UFP-VSL-T01B Coriolis sample station on-line density. \\
\hline & Issue: DT-2101 failed. \\
\hline & Consequence: All readings after 12/23/2009 are not valid. \\
\hline
\end{tabular}




\subsection{Experimental Methods and Analysis}

The sections below describe PEP operations, testing overview, and the simulant and sample processing used for Integrated Test A.

\subsection{Pretreatment Engineering Platform Description}

The PEP test system is designed to perform engineering-scale demonstrations of most WTP pretreatment processes. These include (but are not limited to) vessel-to-vessel transfers, waste pumping, cross-flow filtration, filter cleaning, waste solids washing, chemical reagent addition and mixing, waste slurry heating and cooling, and waste chemical leaching. Refer to Sections 3.1.2 and 3.1.3 for a description of filter pulse pot and PJM operations, respectively.

The PEP is composed of prototypic and nonprototypic equipment as well as auxiliary bulk chemical and utility systems. Slurry is received at feed receipt vessels FRP-VSL-T01, FEP-VSL-T01, and HLP-VSL-T22. For Integrated Test A, only simulant in HLP-VSL-T22 was used. Simulant is then transferred to the upfront ultrafeed vessels UFP-VSL-T01A and B. For Integrated Test A, caustic leaching was done in the upfront vessels, and then it was transferred to UFP-VSL-T02A for post-caustic-leach concentration using the recirculation loop filter(s). The filters purchased for the PEP were obtained from the Mott Corporation (Farmington, CT) using the same specifications for the filters being purchased for the WTP-PTF. The filters are constructed of porous sintered 316 stainless steel with an effective filtration rating of $0.1 \mu \mathrm{m}$. The PEP test system employs a combination of 8-ft-long and 10 -ft-long filter elements (which were formed by welding either four or five 2-ft filter elements together). As such, the PEP elements have the same radial dimensions and filtration ratings as the 2-ft elements, but have a longer filtration length of either 96 in. or 120 in.

Cooling temperature control is done by cooling heat exchangers UFP-HX-T05A and B for UFP-VSL-T01A and B, respectively, and UFP-HX-T02A or the cooling jacket for UFP-VSL-T02A. For high-temperature processing, direct steam injection and heat exchangers UFP-HX-T04A and B are used for UFP-VSL-T01A and B, respectively, while direct steam injection or UFP-HX-T03A is used for UFP-VSL-T02A. Simulant can also be concentrated before it is caustic leached in UFP-VSL-T02A (Integrated Tests B and D). During concentration, permeate is sent to permeate vessels UFP-VSL-T62A and/or B. Once simulant is in UFP-VSL-T02A, further processing, such as oxidative leaching and washing (before and after oxidative leaching), followed by a final concentration, occurs. After processing is complete, the high-solids material is sent to waste storage vessel HLP-VSL-T27. For Integrated Test A, however, the high-solids material was not sent to HLP-VSL-T27. Refer to Figure 3.1 for an overview of the PEP processes and components. 


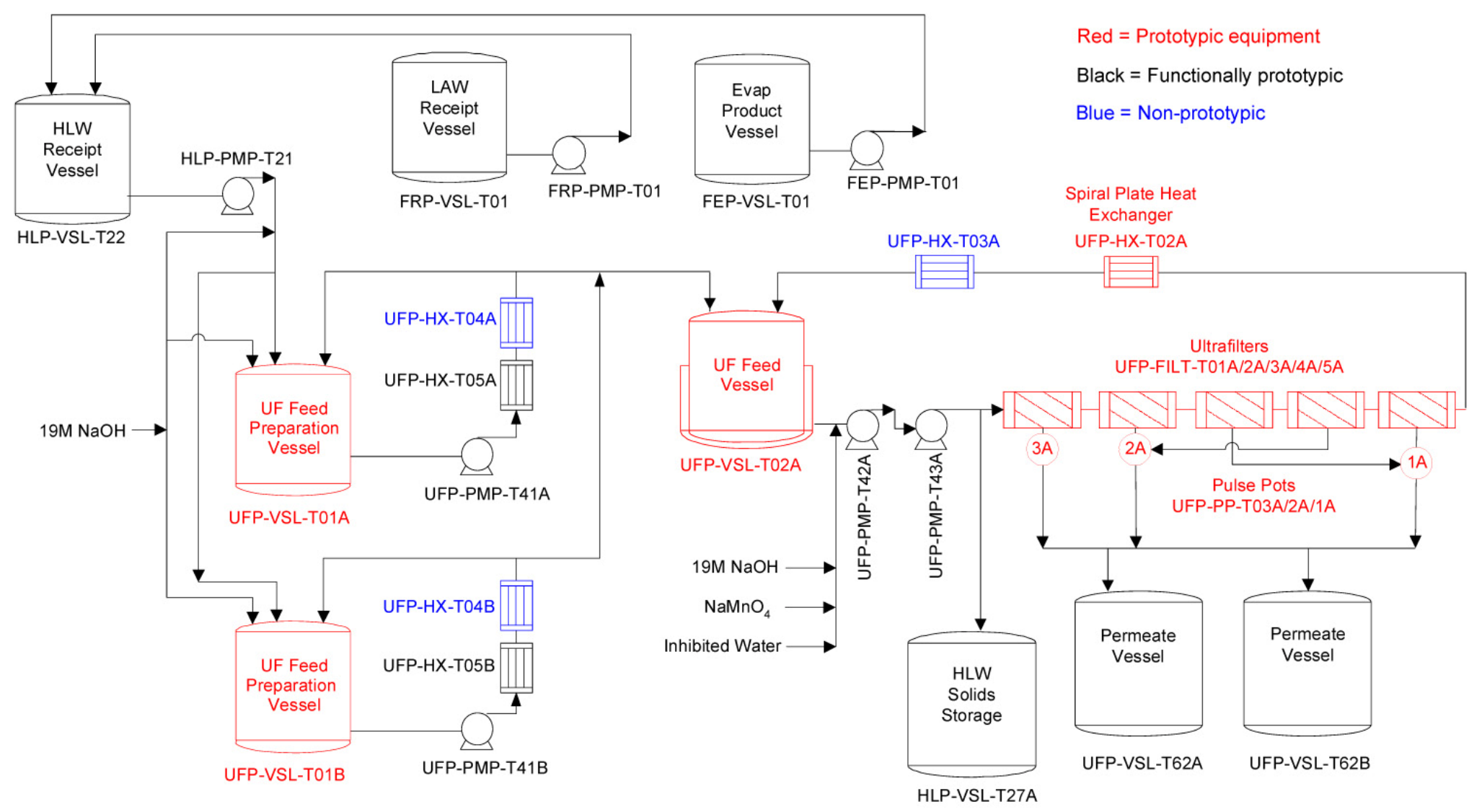

Figure 3.1. PEP Simplified Process Overview 
Equipment that was considered critical to evaluate the integrated system performance was scaled to be prototypic in the PEP. Vessels were scaled to be geometrically similar with a $1 / 4.5$ scale; the working height and diameter were scaled $1 / 4.5$. Pipe sizes are scaled to have approximately $1 / 4.5$ the diameter, but the fluid velocity was approximately the same as the full-scale plant because solids settling does not scale. The pipe diameters in the scaled system were sometimes further reduced if the Reynolds number for fluid transfers in the scaled system was projected to drop below 2100. The PEP also contains equipment to provide feed, accept processed products, and provide services to support operations. The additional equipment was not designed to be prototypic of corresponding tanks, pumps, and services in the full-scale WTP. Refer to the Test Plan for the complete listing of prototypic equipment. ${ }^{(a)}$

Two types of instrumentation were used on the PEP, instruments for monitoring and controlling the process and instruments for collecting quality-affecting data (Nuclear Quality Assurance [NQA]-1 instruments). The instrumentation and control for the test system were functionally prototypic to those specified for the plant with the exception that the PJMs and pulse pots had additional data capabilities to meet the PEP functional requirements. ${ }^{(b)}$ Refer to Appendix A for a summary of pertinent process instruments.

Process control strategies and control ranges were as specified in 24590-WTP-RPT-PET-07-002, Rev 1, Pretreatment Engineering Platform (PEP) Phase I Testing Process Description. ${ }^{(\mathrm{c})}$ The temperature bands for caustic leaching and oxidative leaching are given in the Test Specification, Pretreatment Engineering Platform (PEP) Testing (Phase I draft), 24590-PTF-TSP-RT-07-001 Rev 2.

For detailed information on the PEP components and operations, refer to Pretreatment Engineering Platform (PEP) Phase I Testing Process Description, 24590-WTP-RPT-PET-07-002, Rev $1{ }^{(\mathrm{c})}$ and Functional Requirements for the Pretreatment Engineering Platform (PEP), 24590-PTF-3YD-UFP-00002, $\operatorname{Rev} 1 .^{(b)}$

\subsubsection{PEP Filtration System}

The PEP filtration system is composed of an ultrafiltration feed tank (UFP-VSL-T02A; henceforth also referred to as Tank T02A), a slurry circulation and filtration loop, a permeate metering and collection system, and a filter backpulse and cleaning system. The PEP filtration system is instrumented to measure the feed flow rate, temperature at four locations, and axial and transmembrane pressure (TMP) drop across each filter bundle. In addition, the system is configurable such that filter bundles 1 through 5 may be connected in series to the slurry circulation loop or bypassed such that flow is directed through filter bundle 1 or through filter bundles 2 through 5. A summary of process instrumentation is provided in Appendix A. In the following paragraphs, key process equipment for slurry filtration operations is identified and discussed. Interested readers are referred to the documents listed in Section 3.1 if more information is needed.

(a) G Josephson, O Bredt, J Young, and D Kurath. 2008. Test Plan: Pretreatment Engineering Platform (PEP) Testing (Phase I). TP-RPP-WTP-506, Rev. 0, Pacific Northwest National Laboratory, Richland, WA, 99352.

(b) B Stiver. 2007. Functional Requirements for Pretreatment Engineering Platform (PEP), 24590-PTF-3YD-UFP-00002 Rev. 1, Bechtel National Incorporated, Richland, Washington.

(c) SD Lehrman SD. 2008. Pretreatment Engineering Platform (PEP) Phase I Testing Process Description. 24590-WTP-RPT-PET-07-002, Rev. 1, Bechtel National Incorporated, Richland, Washington. 


\subsubsection{Ultrafiltration Feed Tank}

Tank T02A serves as a primary supply and mixing reservoir for slurry being circulated through the filtration loop. The contents of this tank are mixed with an array of six PJMs. Ancillary systems for Tank T02A include air spargers to limit flammable gas hold-up in the actual waste treatment system, bubblers to measure slurry density and level, laser-level sensors, and an array of resistance temperature detectors (RTDs) to measure the tank temperature profile. (Specific locations of the RTD arrays for tanks UFP-VSL-T02A and UFP-VSL-T01A are available in laboratory record book [LRB] 59944, pages 12-17.) Tank T02A is equipped with a water jacket supplied with chilled water to cool the contained slurry.

\subsubsection{Slurry Filtration Circulation Loop}

The filtration loop contains process equipment that is key to slurry dewatering and washing operations. It is composed of two slurry pumps, a series of five filter bundles, and two heat exchangers.

Two centrifugal slurry pumps, UFP-PMP-T42A and UFP-PMP-T43A (hereafter also referred to as Pumps T42A and T43A, respectively), are operated in series to provide the required slurry flow rate and pressure for the cross-flow filter bundles. The suction to Pump T42A is fed from Tank T02A. In addition, the feed to Pump T42A is connected to process inhibited water (IW) ${ }^{(\mathrm{a})}$ supplies used for slurry washing and dilution operations. The discharge from Pump T42A feeds Pump T43A. Slurry discharge from Pump T43A can be fed through, or bypassed around, the cross-flow filter banks. Pumps T42A and T43A provide a combined filtration-loop flow rate and pressure of up to $150 \mathrm{gpm}$ and $250 \mathrm{psig}$.

The cross-flow filter system is the core of slurry liquid-solid separations. It is composed of five filter bundles connected in series. These filter bundles are designated as UFP-FILT-T01A to -T05A (hereafter also referred to as Filters T01A through T05A). The filter loop is equipped with slurry bypass valves to allow slurry to flow through Filter Bundle 1 and/or Filters T02A through T05A.

The filters purchased for the PEP were obtained from the Mott Corporation (Farmington, CT) using the same specifications for the filters being purchased for the PTF. The filters are constructed of porous sintered 316 stainless steel with an effective filtration rating of $0.1 \mu \mathrm{m}$. The PEP test system employs a combination of 8-ft-long and 10-ft-long filter elements (which were formed by welding either four or five 2 -ft filter elements together). As such, the PEP elements have the same radial dimensions and filtration ratings as the 2-ft elements, but have a longer filtration length of either 96 in. or 120 in. A summary of the geometries of the five filter bundles is provided in Table 3.1.

The tubeside slurry flow rate and pressure are monitored by a series of flow meters and pressure transducers. Slurry flow to Pump T42A is measured by magnetic flow meter FT-0623. Slurry discharge flow from Pump T43A is measured by a second magnetic flow meter (FT-0635). Circulation loop pressure is monitored by a series of pressure transducers located at the entrance to each slurry pump, filter bundle, and heat exchanger.

The temperature in both Tank T02A and the slurry filtration loop is monitored with a combination of two in-line heat exchangers. The first heat exchanger, UFP-HX-T02A, is a spiral plate heat exchanger

(a) Inhibited water typically refers to a $0.01-\mathrm{M}$ solution of $\mathrm{NaOH}$. 
that uses chilled water to cool the circulating slurry. Heat exchanger UFP-HX-T02A controls the temperature of Tank T02A and the filtration loop. The second heat exchanger, UFP-HX-T03A, is a steam exchanger intended to heat the circulating slurry if needed. Both heat exchangers are equipped with a bypass loop so that they can be isolated from slurry flow. RTDs installed in thermowells monitor and control the performance of the heat exchanger. ${ }^{\text {(a) }}$ For the current testing, UFP-HX-T03A was not used and was bypassed. The final process element in the slurry circulation loop is a pressure control valve (SV-0609) that can be adjusted in combination with the slurry pumps to provide adequate backpressure for permeate production. After passing through SV-0609, the dewatered circulating slurry is recycled back into Tank T02A.

Table 3.1. Specifications of the Five PEP Cross-Flow Filtration Bundles

\begin{tabular}{cccccc}
\hline Filter \# & Filter ID & $\begin{array}{c}\text { Number of } \\
\text { Elements in } \\
\text { Bundle }\end{array}$ & $\begin{array}{c}\text { Element } \\
\text { Inside } \\
\text { Diameter } \\
\text { (inches) }\end{array}$ & $\begin{array}{c}\text { Element } \\
\text { Length }(\mathrm{ft})\end{array}$ & $\begin{array}{c}\text { Bundle } \\
\text { Surface Area } \\
\left(\mathrm{ft}^{2}\right)\end{array}$ \\
\hline 1 & UFP-FILT-T01A & 12 & 0.5 & 10 & 15.7 \\
2 & UFP-FILT-T02A & 12 & 0.5 & 10 & 15.7 \\
3 & UFP-FILT-T03A & 12 & 0.5 & 10 & 15.7 \\
4 & UFP-FILT-T04A & 12 & 0.5 & 8 & 12.6 \\
5 & UFP-FILT-T05A & 12 & 0.5 & 8 & 12.6 \\
\hline Total & -- & -- & -- & -- & 72.3 \\
\hline
\end{tabular}

\subsubsection{Permeate Metering and Collection Systems/Filtration Backpulse Systems}

The permeate metering and collection systems consist of Coriolis mass flow meters for monitoring permeate production rates and permeate collection tanks, and three pulse pots are connected to high-pressure air supplies for backpulsing the filter bundles.

Permeate (shell-side) mass production rates from Filters T01A through T05A are monitored by Coriolis flow meters. Permeate flow from each of the filter bundles is directed to three pulse pots (designated as UFP-PP-T01A to UFP-PP-T03A). Pulse pot UFP-PP-T03A serves filter bundle 1, pulse pot UFP-PP-T02A serves filter bundles 2 and 4, and pulse pot UFP-PP-T01A serves filter bundles 3 and 5. The pulse pots are filled with a sufficient volume of collected permeate to backpulse the filter bundles. Overflow from the pulse pots may be directed to 1) permeate or process slurry collection tanks (UFP-VSL-T62A and -T62B) during slurry dewatering operations, or 2) a return line to Tank T02A during continuous recycle filtration operations. Table 3.2 summarizes the permeate metering and pulse pot systems.

(a) The RTD measuring the slurry outlet temperature (TT-0513) did not extend into the process stream, and data were to be used for qualitative purposes only. See discussion in Section 2 for a description of this nonconformance (NCR 42402.1). 
Table 3.2. Permeate Metering and Pulse Pot Configurations for PEP

\begin{tabular}{lll}
\hline Filter Bundle No./ID & $\begin{array}{l}\text { Permeate } \\
\text { Coriolis Meter }\end{array}$ & $\begin{array}{l}\text { Associated Pulse } \\
\text { Pot }\end{array}$ \\
\hline 1- UFP-FILT-T01A & FT-0720 & UFP-PP-T03A \\
2- UFP-FILT-T02A & FT-0755 & UFP-PP-T02A \\
3 - UFP-FILT-T03A & FT-0765 & UFP-PP-T01A \\
4- UFP-FILT-T04A & FT-0775 & UFP-PP-T02A \\
5 - UFP-FILT-T05A & FT-0785 & UFP-PP-T01A \\
\hline
\end{tabular}

During backpulsing, one of the pulse pots is isolated and charged with high-pressure air until the pulse pot pressure exceeds the filter bundle inlet pressure to be backpulsed by a given amount (typically 40 psid). After the target pulse pot pressure is reached, the valve isolating the pulse pot from the filter is opened, and the permeate collected in the pulse pot flows back through the filter element. The backflow of permeate forces any particles loose that are weakly entrained in the filter pores or that have caked on the filter surface. A description of the backpulsing process, as it was implemented in the PEP, is provided in the next section.

\subsubsection{Backpulse Operations}

The permeate metering and collection systems consist of Coriolis mass flow meters for monitoring permeate.

Backpulsing was employed to restore filter fluxes during solids concentration in the UFP-VSL-T02A recirculation loop. The backpulsing function of the filter loop can be operated only when actively filtering UFP-VSL-T02A contents. There are three variables that can be set by the operator: Level Drain Set Point, Backpulse Pressure Set Point, and Pressure Deadband for Completion.

The Level Drain Set Point is the height of the fluid in the pulse pot used for the backpulse. The Backpulse Pressure Set Point is the amount above the filter inlet pressure that the pulse pot should be charged to (i.e., if the inlet pressure is 100 psig and the Backpulse Pressure Set Point is set to 40 psig, the control system will charge the pulse pot to $140 \mathrm{psig}$ ).

The Pressure Deadband for Completion is the amount above the filter inlet pressure that will cause the backpulse to be marked as finished (i.e., if the inlet pressure is 100 psig and the Deadband is set to $10 \mathrm{psig}$, the backpulse will complete when the pulse pot pressure indicator gets down to $110 \mathrm{psig}$ ).

During filtering, the operator presses the "Start Backpulse" button in the human-machine interface (HMI), which initiates the backpulse cycle. The first step is to close all valves entering and leaving the pulse pot. Next, the high-pressure air line is opened, and the pulse pot is pressurized to $50 \mathrm{psig}$. The high-pressure air valve is closed, and the drain valve to UFP-VSL-T62A/B is opened. The pulse pot fluid level falls until reaching the Level Drain Set Point when the drain valve is closed. The high-pressure air valve is opened again and pressurizes the pulse pot to the sum of the filter inlet pressure plus the Backpulse Pressure Set Point (100 psig +40 psig $=140$ psig in the above example). The air valve is closed, and the backpulse cycle pauses for 15 seconds. The fast-acting valve then opens, and the pressure in the pulse pot pushes fluid back through the filter until the pressure in the pulse pot is equal to the filter inlet pressure plus the Deadband ( $100 \mathrm{psig}+10 \mathrm{psig}=110 \mathrm{psig}$ in the above example). The final step is 
to return to filtering conditions. The fast-acting valve closes, and the filter outlet valve and pulse pot outlet valve to UFP-VSL-T62A/B (not the drain valve) are opened.

\subsubsection{Data Acquisition System}

The Data Acquisition System (DAS) for PEP is composed of four data acquisition servers and one graphical user interface (GUI) personal computer. They are networked together, along with the rest of the PEP equipment as depicted in Figure 3.2.

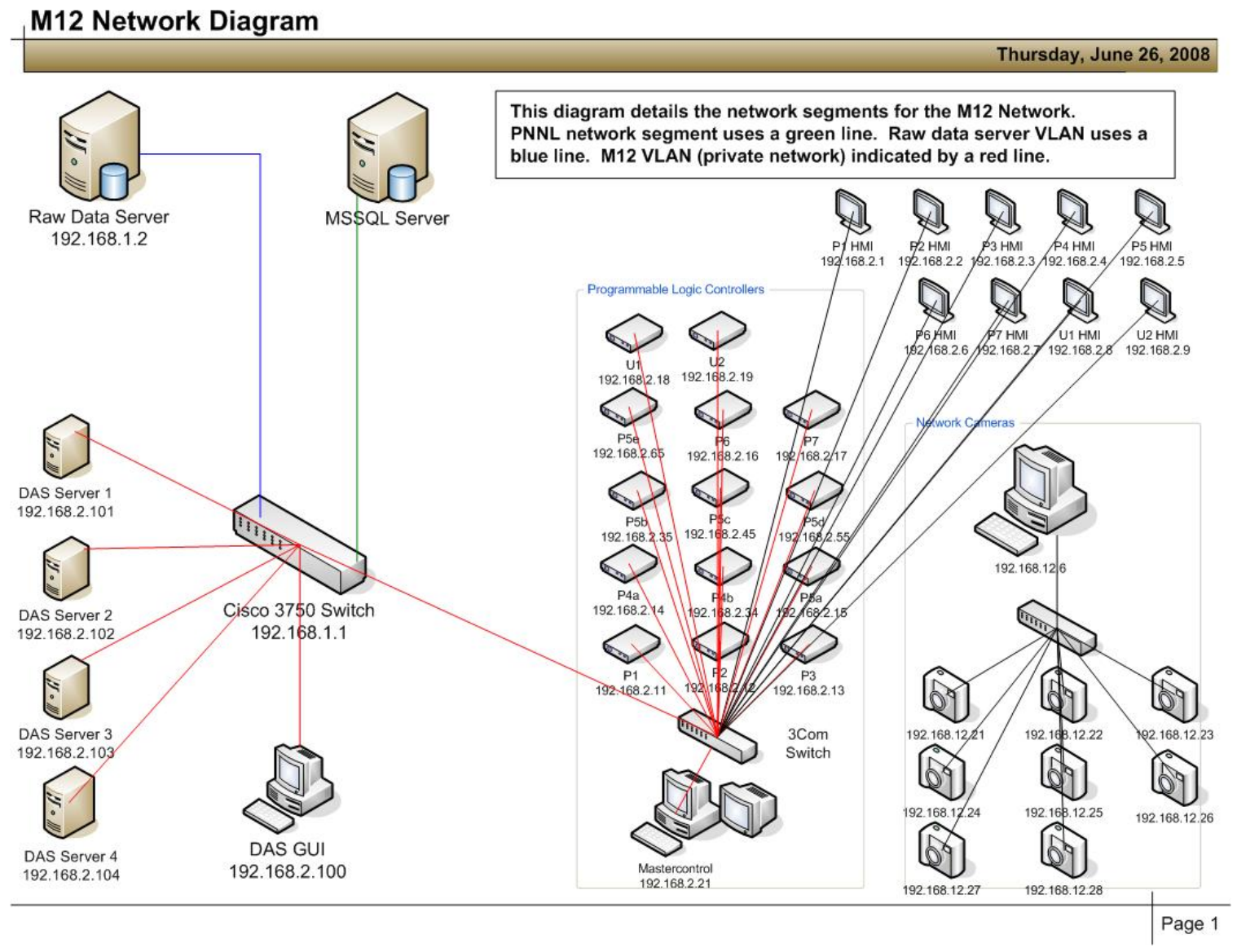

Figure 3.2. PEP Equipment

The GUI personal computer resides in the control room and is used to start and stop the data acquisition servers and set the recording rate. Each server that is located out in the high bay is independent of the others while collecting data. A global positioning system (GPS) receiver is used to provide a time stamp to each DAS server.

\subsubsection{Background}

The PEP data acquisition and control system was designed to be very robust and flexible in operation. While the same sensors are used for controlling the PEP and recording data, the sensor outputs are split into two loops to make certain that the DAS is completely separate from the control system. This 
requirement was put into the system to allow changes to the control system of the PEP to be performed without the rigorous verification and validation process that is required of any quality-affecting software program. This allowed many changes to the control system to be made during operation of the PEP system without affecting the quality of the data. The DAS and all related software did undergo PNNL software control procedures to make sure that the data meet quality standards.

From each signal splitter, one loop went to the control system, and the other went to the DAS. Four- to 20-ma outputs from all sensors were selected to make sure that the system was reliable and prevent long cable runs from introducing error in the signals. The data acquisition servers are manufactured by Microstar Laboratories, located in Bellevue, WA. Each server is capable of storing a large number of channels at a high sampling rate. For PEP, they have been set to a maximum sampling rate of $100 \mathrm{~Hz}$. Each server stores its information in a file local to that server. After data were collected, they were transferred to the raw data server and to the database server for analysis.

\subsubsection{Data Retrieval}

Over a terabyte of data were recorded by the four data acquisition servers during operations of the PEP during shakedown and Phase 1 testing. Those data are stored in binary files that are not human readable. Software was developed to provide analysts with an easy tool to retrieve the data they required by specifying the date/time, instruments, and sampling rate. This tool is composed of three main components: the data ingest server, the database itself, and the data retrieval tool. The data ingest server watches a specified directory on the database server, and when new files are put there, it uploads the data from that location into the database and transfers those data files into a directory where processed files are stored. The database itself uses Microsoft's Structured Query Language (SQL) server as a basis for the large database that stores all data and sensor information for the PEP. Finally, the data retrieval tool is a web-based application that allows staff to recover the data they need by specifying the time/date, instruments, and sampling rate necessary. The software produces a text file that can easily be imported into Excel or any other analysis package. This method provides a data file that meets quality standards for the PEP project.

Another method for retrieving data was commonly referred to the DAS widget. This tool is a simple application that allows users with detailed knowledge of the system to retrieve data if they do not have access to the web-based tool or if they need access to the data before the data have been imported into the database. This tool did not go through the software control procedure and does not provide data that meets quality standards for PEP. It will read a file from one server and produce a file with all the raw (ma) values from every instrument on that server. With that data and the information in the measurement and test equipment (M\&TE) list, staff can retrieve the data from all sensors in engineering units. It is more time consuming to retrieve data in this manner, but it allows data to be read in near real-time. This method was commonly used to provide near real-time data for PJM tuning.

\subsubsection{Data Storage}

All data recorded during operation of the PEP were first copied to the raw data server in PDL-W and then copied to the database server in the Laboratory Support Building (LSB). This procedure is detailed in the data-management plan and provides redundant storage of all data to confirm that data are not lost. 


\subsubsection{Pulse-Jet Mixing Operations}

The leaching vessels UFP-VSL-T02A and UFP-VSL-T01A and B were equipped with PJMs and several different operating programs to allow plant prototypic mixing, low-level operation, and additional data collection.

PJM mixing technology involves a pulse tube coupled with a jet nozzle. The nozzle end of the tube is immersed in the tank while periodic pressure, vacuum, and venting are supplied to the opposite end. Changing the applied pressure produces four operating phases for the pulse tube: 1) the drive mode, when pressure is applied, and the PJM tube discharges its contents at high velocity through the nozzle; 2) the vent mode, when the pressure is vented to the atmosphere, and the level inside the pulse tube and tank approach the same fill level; 3) the refill mode, when vacuum is applied to refill the pulse tube; and 4) the equilibration mode, when the pressure is vented to the atmosphere, and the pulse tube and tank approach the same fill level. The PJM system uses these operating phases in sequence to mix contents in the vessel.

PJM operations at PEP consisted of one of several control modes: Standard Mode (Regular), Standard Mode Short Cycle Mode, Standard Mode (Star Pattern-UFP-VSL-T02A only), and Simple Mode. However, PJM controls at PEP were not prototypic. Integrated Test A PJM operations used the control modes Standard Mode (Regular) and Simple Mode. PJM operating control modes used in PEP had the same basic cycle: Drive Phase, Vent Phase, and Vacuum Phase, followed by a Hold (or Vent) Phase. A timer set to the overall cycle time started at the beginning of each Drive Phase. A new Drive Phase began when either the cycle timer ran out, or the cycle entered the final Hold/Vent Phase, whichever was later. This condition existed to make sure the Vacuum Phase had completed before moving on to the next cycle.

The main difference between the Standard Mode and Simple Mode was the way each phase was handled. In Standard Mode operation, the Drive and Vent phases were time based while the Vacuum Phase was level based. The final Hold/Vent Phase filled the rest of the Cycle Time. In Simple Mode, the Vacuum Phase was also time-based.

\section{Standard Mode (Regular) Description}

Standard Mode operations in PEP controlled each PJM individually based on that PJM level instrument within one overall cycle time. Each cycle was controlled by five main variables: Cycle Time, Drive Time Set Point, Vent Time Set Point, L1 Level, and L2 Level. Additionally, each PJM could be enabled or disabled individually.

The entered Cycle Time controlled the peak-to-peak distance between cycles. However, if the value of the entered cycle time set point was shorter than the combined entered times for the PJM phases, the actual PJM cycle time would be the time it took the PJMs to complete the first three phases (Drive, Vent, and Vacuum) and the fourth phase (Hold) would be skipped.

The Drive Phase was controlled by the time entered as the Drive Time Set Point (corrected for temperature if enabled). Each PJM began the Drive Phase at the same instant but could have different lengths of time specified for driving each PJM. If any PJM did not finish within 30 seconds of the PJM 
with the shortest drive time, that PJM would be disabled (until it was re-enabled by the operator), and the cycle would continue to the next phase.

The Vent Phase was controlled by the Vent Time Set Point, which had no correction factors. Each PJM began the Vent Phase at the same instant, but could have different lengths of time specified for each PJM. If any PJM did not finish within 30 seconds of the PJM with the shortest vent time, that PJM would be disabled (until it was re-enabled by the operator), and the cycle would continue to the next phase. Generally, each Vent Time Set Point was set to a small number such as 500 milliseconds to smooth out the transition between the Drive and Vacuum Phases.

The Vacuum Phase was controlled by the L1 Level, which was set at the top of the desired PJM stroke. All PJMs began with the Vacuum Phase at the same instant, but each PJM L1 Level was set separately. If any PJM did not reach the L1 Level within 30 seconds of the first PJM to reach its L1 Level, that PJM was disabled (until it was re-enabled by the operator), and the cycle continued to the next phase. When tank temperatures were greater than $60^{\circ} \mathrm{C}$, and the tank level was above the PJM head, the Vacuum Phase was disabled, and each PJM vented to atmosphere until it reached the L1 Level set point. If the tank level was below the L1 Level, then the PJMs entered a control Short Cycle Mode. The Standard Mode in PEP also contained a Short Cycle Mode used during gravity refill operation when the tank level did not exceed the PJM head and during extremely low tank levels (to avoid overblowing). A Short Cycle in PEP consisted of a Drive Phase in which each enabled PJM drives for $20 \%$ of the Drive Time Set Point followed by a 10 -second Vent Phase.

The final phase in the control Standard Mode was a Hold Phase. All valves to the PJM were closed, causing the fluid level in each PJM to remain constant at the L1 Level.

Standard Mode operation in PEP also contained a temperature linearization variable that reduced the drive time to prevent overblows as the temperature increased - causing fluid viscosity changes. The variable was expressed as a slope, the percent of drive time decrease per degrees Celsius increase from $0^{\circ} \mathrm{C}$. This function was largely untested, but may have been enabled at some nominal slope during operations.

To prevent reaching the pump heel level in tank UFP-VSL-T02A in PEP, while still operating PJMs prototypically, UFP-VSL-T02A additionally could operate in Standard Mode (Star Pattern). The Star Pattern option followed the same rules as the regular PJM Standard Mode. The difference was that only two PJMs were enabled at a given time. When the operator set the Star Pattern button, the HMI, the center PJM, and one other PJM would follow through a set number of cycles. Then the outer PJM would be disabled, and a different outer PJM would be enabled for a set number of cycles. The pattern of enabling and disabling the five outer PJMs formed a five-point star.

\section{Simple Mode Description:}

As mentioned above, Simple Mode operated substantially the same as Standard Mode. Each cycle consisted of Drive, Vent, Vacuum, and Hold/Vent Phases. There were two main differences. The first difference was that all PJMs operated together as one unit. There were no individual levels used or individually enabling or disabling PJMs. The second difference was that each phase was controlled by a time. An operator set the Cycle Time, Drive Time Set Point (uncorrected), Vent Time Set Point, and Vacuum Time Set Point (uncorrected). Additionally, the operator could choose whether the last phase 
holds the PJM level or vents (returning the PJM level to the same as the tank level). There was an "uncorrected" target phase time entered by the operator, followed by a "corrected" time HMI display at the completion of a phase, indicating the actual time it took to complete the phase.

The Drive time had additional correction factors for temperature (same as in Standard Mode) and tank level. The tank level correction (if enabled) reduced the drive time according to the user-entered slope (\% drive time/inch) for every inch below the top of the PJM head (46 inches by default). For example, if the Drive Time Set Point was 10,000 milliseconds, the Drive Slope was 1\%/inch, and the tank level was at 36 inches, then the corrected drive time would be:

$$
(10,000 \text { milliseconds }) *\{1.00-[0.01 / \text { inch } *(46-36 \text { inches })]\}=9000 \text { milliseconds }
$$

The tank level used in the calculation was determined by the operator using either the tank bubbler, laser, or Drexelbrook probe.

The Vacuum time also contained a correction factor for tank level, but not for temperature. As the tank level decreased, the PJM fill level would decrease during the Vacuum Phase; therefore, a vacuum correction set point was implemented. The vacuum correction (if enabled) increased the vacuum time according to a user-entered vacuum slope (\% vacuum time/inch) for every inch the tank level was below 46 inches. For example, if the Vacuum Time Set Point was 10,000 milliseconds, the vacuum slope was $1 \% /$ inch, and the tank level was at 36 inches, then the corrected drive time would be:

$$
(10,000 \text { milliseconds }) *\{1.00+[0.01 / \text { inch } *(46-36 \text { inches })]\}=11,000 \text { milliseconds }
$$

The operator selectable Hold Phase was created to give the option to more closely resemble PJM Standard Mode operation. The Hold Phase prevented the PJM level from dropping after completing the Vacuum Phase. However, since PJM phase set points were time based and there was no level checking in the PJMs in Simple Mode, the PJM fill level could increase over successive cycles when using the Hold Phase - whether or not the drive or the vacuum time set points were decreased or increased, respectively. The successive increase in PJM level over time could result in fluid traveling all the way up into the hoses on top of the tank. If the Hold Phase was disabled, each PJM just vented to the tank level during the remainder of the PJM cycle.

\subsection{Testing Overview}

The following is a summary level overview of testing reported herein. Refer to the Test Instruction TI-WTP-PEP-065, hereafter referred to as TI, and the LRBs $(60230,60235,60269,60108,60229,60271$, and 60279) for additional details.

1. Transferred simulant from HLP-VSL-T22 to UFP-VSL-T01A (Batch 1) and added NaOH in-line.

2. Performed UFP-VSL-T01A leaching at $98^{\circ} \mathrm{C}$ for 16 hours and cooling using UFP-HX-T05A.

3. Completed initial transfer to UFP-VSL-T02A from UFP-VSL-T01A through UFP-HX-T05A.

4. Performed post-caustic-leach concentration from UFP-VSL-T01A contents (Batch 1) using UFP-FILT-T01A in UFP-VSL-T02A recirculation loop.

a. Filters were drained before solids concentration. 
5. Transferred simulant from HLP-VSL-T22 to UFP-VSL-T01B (Batch 2) and added NaOH in-line $(80 \%)$ and to the top of UFP-VSL-T01B $(20 \%)$.

6. Completed UFP-VSL-T01B leaching at $98^{\circ} \mathrm{C}$ for 16 hours and cooling using UFP-HX-T05B.

7. Performed post-caustic-leach concentration from UFP-VSL-T01B contents (Batch 2) using UFP-FILT-T01A in UFP-VSL-T02A recirculation loop.

8. Repeated HLP-VSL-T22 transfers to UFP-VSL-T01A/B, leaching and cooling in UFP-VSL-T01A/B, and post-caustic-leach concentration in UFP-VSL-T02A for a combined total of six batches (three UFP-VSL-T01A and three UFP-VSL-T01B batches).

a. Conducted backpulsing of UFP-FILT-T01A to enhance the filtration rate.

9. Evaluated bypass of the filter loop using a $\mathrm{CsBr}$ tracer.

10. Performed post-caustic-leach slurry wash using all five filters, UFP-FILT-T01A through -T05A.

11. Completed oxidative leaching in UFP-VSL-T02A at $25^{\circ} \mathrm{C}$ for 6 hours.

12. Washed and reconcentrated slurry in UFP-VSL-T02A using all five filters, UFP-FILT-T01A through -T05A.

13. Integrated Test A was put on "hold" due to UFP-VSL-T02A slurry air entrainment issues.

14. Demonstrated final solids concentration in UFP-VSL-T02A using all five filters, UFP-FILT-T01A through -T05A.

15. Completed the High-Solids Filter Test after completing Integrated Test B.

16. Repeated the $\mathrm{CsBr}$ tracer test after completing the High-Solids Filter Test.

\subsubsection{Test Preparation}

Several maintenance activities and changes were made to instrumentation and PEP systems before Integrated Test A. These modifications are summarized below. NOTE: The items below are not necessarily in chronological order:

- Pressure-relief modification work on the UFP-VSL-T02A recirculation loop was completed and approved by WTP on $1 / 29 / 09$. The modification work routed the vents from heat-exchanger valves PSV-0515 (UFP-HX-T02A) and PSV-0519 (UFP-HX-T03A) to vessel HLP-VSL-T27. The filter-loop modification also installed a new rupture disk at the discharge of the UFP-PMP-T43A pump, which also relieves to vessel HLP-VSL-T27. Two configuration control locks were added to HLP-VSL-T27 (from IW and UFP-VSL-T02A drain) to make sure that adequate capacity was available in the HLP-VSL-T27 vessel if the new rupture disk downstream of pump UFP-PMP-T43A failed. A new hardware interlock was also added to shut down the UFP-PMP-T42A and UFP-PMP-T43A pumps if the level in the HLP-VSL-T27 vessel increases above the new level probe. This hardware interlock made certain that HLP-VSL-T27 would not overfill if the rupture disk failed. Vessel HLP-VSL-T27 will no longer receive solids from UFP-VSL-T02A and will not be used for receipt of any material.

A lock remained on V05161, the isolation valve between UFP-HX-T03A and the low-point drain, until the PEP Hazards Analysis was revised - the initial Hazards Analysis did not evaluate operation of UFP-PMP-T42A and UFP-PMP-T43A pumps with the filter loop isolated from UFP-VSL-T02A.

o Software changes associated with pressure-relief filter-loop modification included adding the LS_1090 interlock for filter-loop overflow to vessel HLP-VSL-T27. If the HLP-VSL-T27 tank is 
full, pumps UFP-PMP-T42A/T43A would not start, and an alarm would show on the Alarm Server.

- Following the pressure relief modification described above, the UFP-VSL-T02A recirculation filter-loop in-service leak check was performed.

- The UFP-VSL-T02A recirculation loop return nozzle was repositioned because it was positioned in a nonprototypic location, resulting in the returning slurry impinging on the steam ring. Modifications to the return nozzle involved bending the nozzle in two places to redirect flow to the side of the steam ring (Operations Manager email, 07/20/09, 09:29).

- The laser in UFP-VSL-T02A was repositioned since it gave erroneous readings in previous testing efforts, and an air purge was installed.

- Level instruments in all the PJMs, Drexelbrooks, were replaced. The new Drexelbrooks had a Teflon coating, which is resistant to caustic, and included an inactive zone on the admittance probes. The modification was supposed to provide better control of PJM operation, but the probes still did not function reliably during leach operations. After installation, the Drexelbrooks were respanned.

o Software changes associated with this installment involved modifying the span of the level instruments in the three UFP vessels.

- An additional tank level instrument (Drexelbrook) was installed in UFP-VSL-T01A to provide additional tank level detection in the event that the bubblers plug and/or the laser performance becomes unreliable.

o Software and HMI changes associated with this installation include adding LT_0311 for the Drexelbrook and modifying HS-0323 to allow switching between laser, bubbler, and Drexelbrook for UFP-VSL-T01A level controls.

- Coriolis densitometer (CD) sampler stations in UFP-VSL-T01B and UFP-VSL-T02A vessels were repaired, and then in-service leak checks were performed on all CD sampler stations in the three UFP vessels.

- The DAS graphical user interface software was modified with an alarm to alert operators when the DAS is not responding properly.

- Indicators to all pump start/stop screens showing which interlocks are tripped (red indicates an interlock is engaged) were added to the HMI, making it easier for the HMI operator to see when a pump turns off unexpectedly.

- The simple mode PJM code was updated to include a vacuum time setpoint, current vacuum time indicator, vacuum slope input field for tank level compensation for tank levels below 46 inches (this field is inactive when tank levels are above 46 inches), and replacing the vacuum phase with the vent phase when tank temperatures exceed $60^{\circ} \mathrm{C}$.

The filters in the UFP-VSL-T02A recirculation loop were not cleaned before Integrated Test A.

\subsubsection{General Operations}

General operations through the course of Integrated Test A are listed as follows:

- Operation instructions, per Operations Manager, between 01/31/09 and 2/2/09 were to use the lasers in the UFP vessels for level and process controlling. Whenever a level measurement was questionable, such as the second or third in the case of UFP-VSL-T01A, another level instrument was 
used for comparison, and the most credible (knowing transfer volume or permeate mass removed coupled with the vessel volume vs. level correlation) was used.

- Stable level measurements were taken typically before and after transfers, concentrations, and washing to verify that the target tank level was met, or permeate mass was removed. Stable level measurements had recirculation pumps, spargers, and PJMs off and were then returned to the original configuration.

o The specific gravity was recorded any time a bubbler-level measurement was made to confirm level credibility since the bubblers plugged. When bubblers plugged, operators blew the lines with high pressure air ( $\sim 20 \mathrm{psig})$ and/or used small amounts of IW $(<200-\mathrm{mL})$ followed by high-pressure air to clear the lines.

- FRP-VSL-T01 and FEP-VSL-T01 vessel bubblers were turned off and were only turned on temporarily for completing shift rounds.

- Agitators were kept on for vessels containing simulant, the receipt vessels (FRP-VSL-T01, HLP-VSL-T22, and FEP-VSL-T01), and permeate vessels UFP-VSL-T62A and/or B as determined by the Operations Manager.

- The temperature setpoint on UFP-HX-T02A was adjusted as necessary to maintain the target run sheet temperature in the filter loop and then set to automatic mode.

- CD sampler stations were kept ON only when sampling was taking place, and then were turned off when not in use to minimize wear-and-tear on the sample pumps.

o Not all CD sample leg elevations were submerged for CD monitoring; therefore, affected sample legs were not completed on the $\mathrm{CD}$ reading datasheets.

o UFP-VSL-T01B CD sampler station monitoring (recording of temperature and density on datasheet) was not completed because of a nonconformance record (NCR) on the Coriolis flow meter. The two readings that were taken gave erroneous values; therefore, no more readings were taken thereafter.

- In-line caustic additions sent to the HLP-PMP-T21 discharge while transferring simulant from HLP-VSL-T22 to UFP-VSL-T01A/B were done per current operating procedures and not per the TI. The run sheet listed simulant and caustic volumes and addition rates separately; however, the flow meter downstream from the pump, FE-0119, only measures the combined flow rate (LRB 60108, pg. 109). A transfer rate for the simulant and caustic was computed and used as a target for each batch.

- On 2/1/09 (12:11, Operations Manager), an expedited change was approved on having the discharge valves for heat exchanger UFP-HX-T04A/B and UFP-HX-T05A/B open whether or not the heat exchangers are aligned or bypassed (LRB 60229, pg. 29).

- PJM drive times were adjusted after tuning parameters were reached to maintain steady pump operations in UFP-VSL-T02A.

o The PJM stroke was adjusted as necessary to avoid overblows (when the pulse tube discharges, all its contents but pressurized air are still applied, causing the air to come out of the bottom of the PJM) and/or to maintain filter-loop flow-rate targets per the run sheet. These adjustments gave stroke lengths outside the tolerance of the run sheet target.

o The PJM drive pressures had to be adjusted and tuned by the Lead Test Engineer between ambient and $98^{\circ} \mathrm{C}$ vessel temperatures. 
o (2/16/09 Test Director email). After post-oxidative-leach washing, the PJMs were tuned under standard mode, and standard mode was used unless the PJMs had unstable performance. Standard and simple mode PJM operation was used interchangeably as necessary to achieve stable PJM operations.

\subsection{Simulant}

PEP process testing was performed with a nonradioactive aqueous slurry of simulant waste chemicals and solids. The simulant composition and make-up recipe were provided by WTP as documented in Simulant Recommendation for Phase 1 Testing in the Pretreatment Engineering Platform. ${ }^{\text {(a) }}$ Aqueous chemical concentrations were within the ranges expected for waste feeds to the PTF. The hydroxide concentration was marginally one standard deviation lower than the average concentration expected in the feeds to the plant. The oxalate and phosphate components were at the lower end of the expected ranges, but the oxalate component was at the solubility limit, and the phosphate component was at or near the solubility limit. The solids components and blend were selected to obtain targeted solids mass loss (aluminum and chromium leaching and oxalate washing) and treatment time. The simulant was not selected to represent any particular Hanford tank waste type.

The simulant was blended from the components listed below. The basis for selecting the individual components and the comparison to actual waste behavior are provided where applicable in the indicated references:

- Boehmite (for Al) (Russell et al. 2009a)

- Gibbsite (for Al) (Russell et al. 2009b)

- Chromium oxyhydroxide (CrOOH) slurry (Rapko et al. 2007)

- Sodium oxalate

- Filtration simulant (Russell et al. 2009c)

- Supernate.

A separate chromium solids slurry simulant was prepared and added to the PEP process after post-caustic-leach washing (a nonprototypic addition) during the Shakedown/Functional Tests and Integrated Tests A and B. This approach was taken because laboratory-scale tests had shown that the high-temperature caustic-leaching step dissolved significant amounts of the $\mathrm{CrOOH}$ solids (Russell et al. 2009a). In Integrated Test D, the chromium solids component of the simulant was added during the simulant make-up process to demonstrate the PTF permanganate addition strategy.

Simulant was procured from NOAH Technologies Corporation (San Antonio, TX). Samples of each simulant batch were characterized to make sure that chemical and physical property requirements were met. Batches of the simulant were procured as follows:

- A 15-gallon trial batch of the blended simulant for laboratory testing to demonstrate the efficacy of the simulant fabrication procedure.

(a) P Sundar. 2008. Simulant Recommendation for Phase 1 Testing in the Pretreatment Engineering Platform. 24590-PTF-RT-08-006, Rev 0, Bechtel National, Inc., Richland, Washington. 
- A 250-gallon scale-up batch of the blended simulant to demonstrate scale-up of the simulant fabrication procedure to an intermediate scale.

- Batches 0, 1, and 2, each nominally 3500 gal, of blended simulant for the Shakedown/Functional Tests and Integrated Tests A and B. These batches did not contain the $\mathrm{CrOOH}$ component.

- Batch 3, nominally 1200 gal, for Integrated Test D. This batch contained the CrOOH solids component.

- The CrOOH solids slurry for the Shakedown/Functional Test and Integrated Tests A and B was obtained in two separate batches containing nominally 18 and $36 \mathrm{~kg}$ of $\mathrm{Cr}$ as $\mathrm{CrOOH}$.

\subsubsection{Sampling}

Four tests as described in the Pretreatment Engineering Platform (PEP) Testing (Phase I) Test Plan ${ }^{(a)}$ were conducted at the PEP over 5 months, which generated approximately 3300 samples, of which roughly 1400 were stored as archive samples. A significant number of the original intact samples and processed aliquots were analyzed for chemical composition, such as metals, anions, and hydroxide or physical properties, such as undissolved solids, density, and particle size. The Test Instruction for each test contained a sample collection and analysis table that summarized sampling events and the analysis to be performed on the collected samples. Appendix B provides a list of every sample collected in the Integrated Test $\mathrm{A}$ and the associated disposition. A list of samples taken and their purpose is provided in Appendix C. The actual time that samples were taken was recorded in the Test Instruction.

In general, slurry samples were collected using either the vessel's in-tank sampler or an in-line sampler. In-line samples were obtained from the slurry recirculation loop or transfer piping by drawing a side stream from the process flow as shown in Figure 3.3. To obtain a sample, the second valve was fully opened, and then the first valve was opened sufficiently to allow samples to be safely obtained. Samples were collected from the volume between the second and first valves while isolated from the process flow pipe. The vent valve and pressure gauge shown below, however, were not used for Integrated Test A sample collection. The sample line and valves were purged with at least three line volumes before each sampling event.

(a) GB Josephson, OP Bredt, JK Young, and DE Kurath. 2009. Test Plan for Pretreatment Engineering Platform (PEP) Testing (Phase I). TP-RPP-WTP-506, Rev. 0.4, Pacific Northwest National Laboratory, Richland, Washington. 


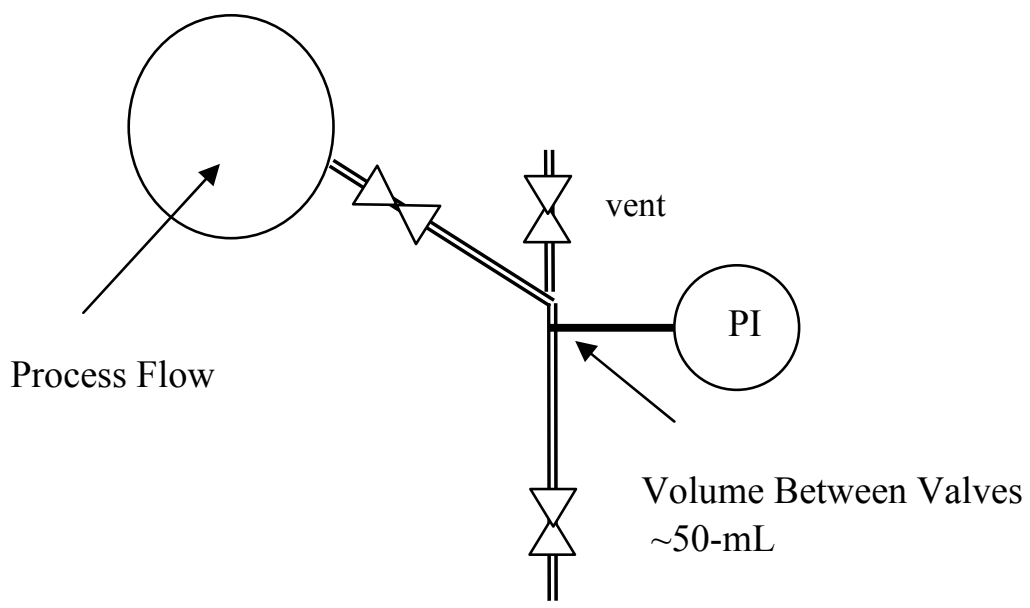

Figure 3.3. Simple In-Line Sample Valving

A schematic of the in-tank sampling system for UFP-VSL-T01A/B and UFP-VSL-T02A is shown in Figure 3.4. Samples were obtained with the sample loop in recirculation mode with slurry returned to the vessel. To obtain a sample, a valve was used to divert the entire flow to the sample bottle. The sampling valve and line were purged before each sample to minimize cross contamination with previous sampling events. Sample heights are provided in Appendix D.

Permeate (liquid) samples were taken from the permeate piping between the filter and the pulse pot. The sample line and valves were purged with at least three line volumes before each sampling event. 


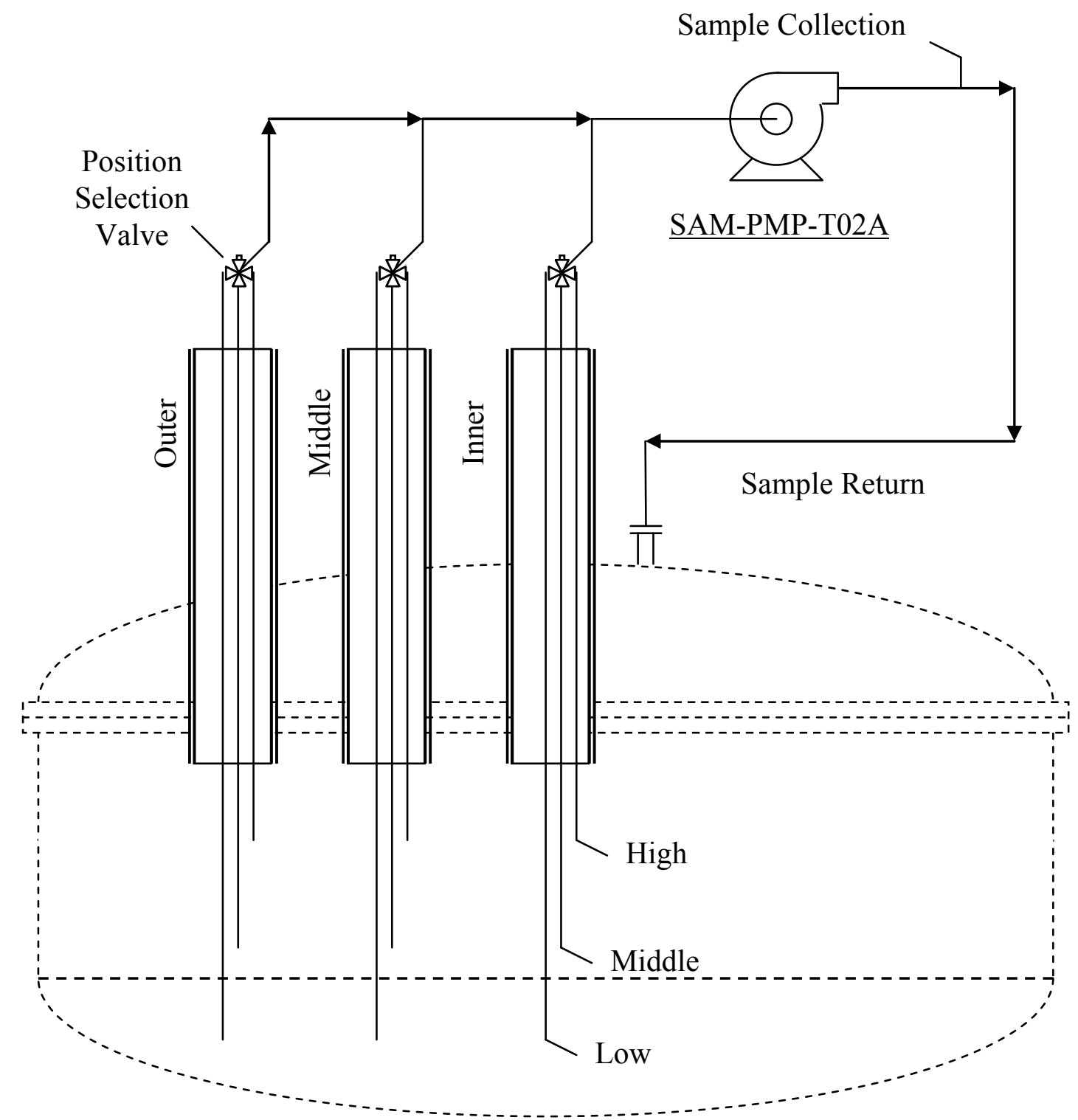

Figure 3.4. In-Tank Sampling, Showing the Three Radial Positions at Three Heights and Sampling Flow Loop

The organization responsible for the analysis is given in Table 3.3. 
Table 3.3. Sample Analysis Organizations

\begin{tabular}{lc}
\hline \multicolumn{1}{c}{ Analysis } & Organization \\
\hline Inductively coupled plasma (ICP) & \\
Ion chromatography (IC) & \\
Total inorganic carbon and total organic carbon & Southwest Research Institute \\
(TIC/TOC) & \\
Weight percent undissolved solids (UDS) & \\
Density & \\
\hline Heat capacity & \\
Shear strength & \\
Shear stress vs. shear rate & Pacific Northwest National Laboratory \\
Particle-size distribution & \\
X-ray diffraction & \\
Scanning electron microscope & \\
Raman & \\
Free hydroxide & \\
Weight Percent Undissolved Solids UDS ${ }^{(b)}$ & DOW Corning \\
\hline Gel Permeation chromatography (GPC) & \\
\hline (a) Guidelines for performing chemical, physical, and rheological properties measurements. \\
(b) Moisture analyzer method for determining UDS.
\end{tabular}

The following sections provide a description of the

- Sample naming convention

- Sample processing during PEP testing

- Analytes of interest

- Analytical methods

- Physical properties.

\subsubsection{Sample Naming Convention}

Every sample was given a unique sample name. The sample names were a composite of either six or seven separate descriptors. The descriptors are defined in Table 3.4, and the acronyms for each descriptor are defined in Table 3.5. The spacings between descriptors were separated by either an underscore symbol $\left(_{-}\right.$) or a space ( ). Sample naming examples are provided after Table 3.5. 
Table 3.4. Sample Naming Nomenclature Definitions

\begin{tabular}{|c|c|c|}
\hline Descriptor & Identification & Explanation \\
\hline Descriptor 1 & Test Name & $\begin{array}{l}\text { Refers to the Shakedown Test, Functional Test, Integrated Test A, } \\
\text { Integrated Test B, or Integrated Test D. }\end{array}$ \\
\hline Descriptor 2 & Location & $\begin{array}{l}\text { Identifies the location that the sample is being collected from based } \\
\text { on Table } 3.5 \text {. For example, a sample collected from a tank will } \\
\text { include the acronym of the tank followed by the location within the } \\
\text { tank. }\end{array}$ \\
\hline Descriptor 3 & Test Process Step & $\begin{array}{l}\text { Refers to the process step as identified in the sample collection and } \\
\text { analysis table in the governing Test Instruction. The test process } \\
\text { step includes an identifier for processes that are repeated during } \\
\text { testing. }\end{array}$ \\
\hline Descriptor 4 & Sequential Number & $\begin{array}{l}\text { Unique number identifier that increments by } 1 \text { each time a sample } \\
\text { was collected. All subsamples and separated samples generated } \\
\text { from the original sample had the same unique number identifier. }\end{array}$ \\
\hline Descriptor 5 & Sample Routing & $\begin{array}{l}\text { Refers to the sample destination, type of analysis, storage, or } \\
\text { archive. }\end{array}$ \\
\hline Descriptor 6 & Store code & Final destination code. \\
\hline Descriptor 7 & $\begin{array}{l}\text { Separated-sample } \\
\text { identifier }\end{array}$ & $\begin{array}{l}\text { Refers to the type of subsample; e.g., decantate, solids, rinse } \\
\text { solution. For a separated-sample, a descriptor of the matrix was } \\
\text { added after the sample routing identifier. }\end{array}$ \\
\hline
\end{tabular}

Table 3.5. Sample Naming Acronyms

\begin{tabular}{ll}
\hline Acronym & Definition \\
\hline Test Name (1 character) Descriptor 1 & \\
\hline $\mathrm{S}$ & Shakedown testing \\
$\mathrm{F}$ & Functional testing \\
$\mathrm{A}$ & First integrated test to be performed \\
$\mathrm{B}$ & Second integrated test to be performed \\
$\mathrm{C}$ & Third integrated test to be performed \\
$\mathrm{D}$ & Fourth integrated test to be performed \\
\hline Location (maximum of 5 characters) & Descriptor 2 \\
Vessels Name (3 characters) & \\
\hline T22 & \\
FRP & HLP-VSL-T22 \\
FEP & FRP-VSL-T01 \\
$01 \mathrm{~A}$ & FEP-VSL-T01 \\
$01 \mathrm{~B}$ & UFP-VSL-T01A \\
$02 \mathrm{~A}$ & UFP-VSL-T01B \\
T27 & UFP-VSL-T02A \\
$62 \mathrm{~A}$ & HLP-VSL-T27A \\
$62 \mathrm{~B}$ & UFP-VSL-T62A \\
\hline Vessel Sampling Location (2 characters) & UFP-VSL-T62B \\
\hline IH & \\
IM & Inner High \\
\hline IL & Inner Middle \\
MH & Inner Low \\
& Middle High \\
\hline
\end{tabular}


Table 3.5. Sample Naming Acronyms

\begin{tabular}{|c|c|}
\hline Acronym & Definition \\
\hline $\mathrm{MM}$ & Middle Middle \\
\hline ML & Middle Low \\
\hline $\mathrm{OH}$ & Outer High \\
\hline $\mathrm{OM}$ & Outer Middle \\
\hline OL & Outer Low \\
\hline RL & Recirculation line \\
\hline GT & Grab sample at top of a tank \\
\hline GM & Grab sample at middle of a tank \\
\hline GB & Grab sample at bottom of a tank \\
\hline \multicolumn{2}{|l|}{ In-Line/Transfer Sampling Locations (5 characters) } \\
\hline $\mathrm{T} 221 \mathrm{~A}$ & $\begin{array}{l}\text { In-line transfer from HLP-VSL-T22 to } \\
\text { UFP-VSL-T01A }\end{array}$ \\
\hline $01 \mathrm{~A} 2 \mathrm{~A}$ & $\begin{array}{l}\text { In-line transfer from UFP-VSL-T01A to } \\
\text { UFP-VSL-T02A }\end{array}$ \\
\hline $01 \mathrm{~B} 2 \mathrm{~A}$ & $\begin{array}{l}\text { In-line transfer from UFP-VSL-T01B to } \\
\text { UFP-VSL-T02A }\end{array}$ \\
\hline T221B & $\begin{array}{l}\text { In-line transfer from HLP-VSL-T22 to } \\
\text { UFP-VSL-T01B }\end{array}$ \\
\hline 000FL & Filter-loop in-line sample \\
\hline 00PF1 & Permeate filter number 1, UFP-FILT-T01A \\
\hline 00PF2 & Permeate filter number 2, UFP-FILT-T02A \\
\hline 00PF3 & Permeate filter number 3, UFP-FILT-T03A \\
\hline 00PF4 & Permeate filter number 4, UFP-FILT-T04A \\
\hline 00PF5 & Permeate filter number 5, UFP-FILT-T05A \\
\hline $00 \mathrm{C} 01$ & Downstream of CS-PMP-T01 \\
\hline $00 \mathrm{C} 02$ & Downstream of CS-PMP-T02 \\
\hline $00 \mathrm{C} 03$ & Downstream of CS-PMP-T03 \\
\hline \multicolumn{2}{|c|}{ Test Process Step (three characters ${ }^{(a)}$ plus two repetitive process characters ${ }^{(b)}$ ) Descriptor 3} \\
\hline (a) 001 through 032 (initial three characters) & As described in the guiding document such as \\
\hline $\begin{array}{l}\text { (b) A two-character field to identify any repetitive } \\
\text { process. }\end{array}$ & $\begin{array}{l}\text { Appendix A or B in TP-RPP-WTP-506 Rev.0 or the } \\
\text { governing Test Instruction. }\end{array}$ \\
\hline \multicolumn{2}{|l|}{ Repetitive Process ( 2 characters) } \\
\hline $\mathrm{XX}$ & No repeating process \\
\hline bT & $10^{\circ} \mathrm{C}$ below set-point temperature \\
\hline 00 & Time when set-point temperature reached (in hours) \\
\hline 01 through 16 & $\begin{array}{l}\text { Time after set-point temperature reached (in hours) or } \\
\text { cycle number, depending on the process step. }\end{array}$ \\
\hline \multicolumn{2}{|l|}{ Sequence Number (4 characters) Descriptor 4} \\
\hline 0001 through 9999 & $\begin{array}{l}\text { Sequential number incremented by } 1 \text { for replicate } \\
\text { samples }\end{array}$ \\
\hline \multicolumn{2}{|l|}{ Sample Routing ( 3 characters) Descriptor 5} \\
\hline ARC & Archive \\
\hline UDS & Undissolved solids \\
\hline XSP & XRD, SEM, and/or PSD \\
\hline AFA & Anti-foaming agent analysis \\
\hline SOX & Solid oxalate analysis \\
\hline
\end{tabular}


Table 3.5. Sample Naming Acronyms

\begin{tabular}{|c|c|}
\hline Acronym & Definition \\
\hline HTC & Heat capacity \\
\hline DEN & Density \\
\hline ICP & $\begin{array}{l}\text { Inductively coupled plasma optical emission } \\
\text { spectrometry (ICP) analysis }\end{array}$ \\
\hline RAM & Raman analysis \\
\hline RHE & Rheology (shear strength/shear stress) \\
\hline TDS & Total dissolved solids \\
\hline CUF & Cells Unit Filter (CUF)/parallel testing \\
\hline TFI & $\begin{array}{l}\text { Total inorganic carbon analysis (TIC)/free hydroxide } \\
\text { analysis (FOH)/Ion chromatography (IC) analysis }\end{array}$ \\
\hline ALK & Total alkalinity \\
\hline ADT & Acidity titration \\
\hline OST & Oxidation state titration \\
\hline OTR & Other \\
\hline SUP & Supernate, used when no Raman required \\
\hline \multicolumn{2}{|c|}{ Separated-samples ( 1 character) Descriptor 6} \\
\hline 0 & RTL-520 Store \\
\hline 1 & RTL-520 Archive \\
\hline 2 & SwRI \\
\hline 3 & RPL \\
\hline 4 & Applied Processing Engineering Laboratory (APEL) \\
\hline 5 & Dow Corning \\
\hline 6 & Other \\
\hline \multicolumn{2}{|c|}{ Separated-samples ( 1 character) Descriptor 7} \\
\hline $\mathrm{D}$ & Decanted supernatant (decantate) \\
\hline B & Bulk solids not rinsed \\
\hline $\mathrm{R}$ & Rinsate composite \\
\hline S & Rinsed solids \\
\hline $\mathrm{P}$ & Process, samples that were filtered/processed \\
\hline $\mathrm{F}$ & Filtrate \\
\hline
\end{tabular}

The sample names are constructed in the following manner:

\section{Descriptor1_Descriptor2_Descriptor3a_Descriptor3b_Descriptor4_Descriptor5_Descriptor6_ Descriptor7}

The following description is an example of sample naming for samples collected from HLP-VSL-T22 during Functional testing.

- The sample name for the first Functional Test grab slurry sample collected from the top of HLP-VSL-T22 during process step 1 that was to be archived would have been:

F_T22GT_001_XX_0001_ARC_1.

- For the same sample location and type described above, analyzed for PSD on a bulk solid that was not rinsed and ICP on the decantate, the original sample vial would have been labeled:

F_T22GM_001_XX_0009_XSP_4_B for the solid. 
- Since the solid phase always stayed in the original sample vial, and the liquid was decanted off into a new vial, then the new vial would have been labeled:

F_T22GM_001_XX_0009_DEN_0_D for the decantate.

- The decantate from the above sample container could have been subsampled for ICP and Raman; the ICP container would have been labeled as follows:

F_T22GM_001_XX_0009_ICP_2_D.

- The Raman container would have been labeled as follows:

F_T22GM_001_XX_0009_RAM_3_D.

\subsubsection{Sample Processing}

The samples were collected in pre-labeled sample containers that were prepared and staged within PDL-W based upon the Sample Collection and Analysis table in the governing Test Instruction. The required analysis determined the sample volume and sample collection container. Sample handling flow diagrams are given in Figure 3.5 through Figure 3.7 for samples collected and processed in PDL-W. Figure 3.6 is a continuation of Figure 3.5 and follows the handling of the wet centrifuged solids. Figure 3.7 indicates the sample flow for samples requiring UDS measurement. The letters "D," "B," "R," and "S," which are shown in the figures, were included in the sample names for phase-separated processed samples. The letter "D" was included in the decanted supernatant sample names. The letter "B" was included in the wet centrifuged solids sample names. The letter " $\mathrm{R}$ " was included in the sample name of the combined rinse solution. The letter " $S$ " was included in the sample names of the wet rinsed centrifuged solids. These figures represent two basic sample processing methods. One approach was used during non-leaching test steps, and another was used during leaching.

\subsubsection{Non-Leaching Samples}

Non-leaching samples were not always processed immediately after collection. Because samples might be collected at any time during testing, and testing was being performed 24 hours per day, non-leached samples collected after 0600 hours were typically processed and prepared for shipment by 0600 hours the morning after samples were collected. Non-leach samples used for the analyses listed below were shipped as collected in the container size specified and with the exception of the archive samples; tare and full sample weights were not required.

- Weight percent undissolved solids (UDS) (50-mL)

- Density $(50-\mathrm{mL})$

- Heat capacity (50-mL)

- Shear strength $(2 \times 1-\mathrm{L})$

- Shear stress vs. shear rate on original intact slurry (100-mL)

- Shear stress vs. shear rate on supernatant (100-mL)

- Total organic carbon on the slurry $(50-\mathrm{mL})$

- Inductively coupled plasma - Silicon $(50-\mathrm{mL})$

- Archive (50-mL). 
During a sampling event, if an intact slurry sample was collected for density analysis and another intact slurry sample was collected for weight percent UDS, then density measurements were performed on the supernatant in addition to the intact slurry submitted for density analysis. These density measurements were higher accuracy than density measurements obtained following the weight percent UDS method described in Bechtel procedure, Guidelines for Performing Chemical, Physical, and Rheological Properties Measurements (24590-WTP-GPG-RTD-001 Rev. 0). ${ }^{\text {(a) }}$

(a) GL Smith and K Prindiville. 2002. Guidelines for Performing Chemical, Physical, and Rheological Properties Measurements. 24590-WTP-GPG-RTD-001 Rev. 0, Bechtel National, Inc., Richland Washington. 


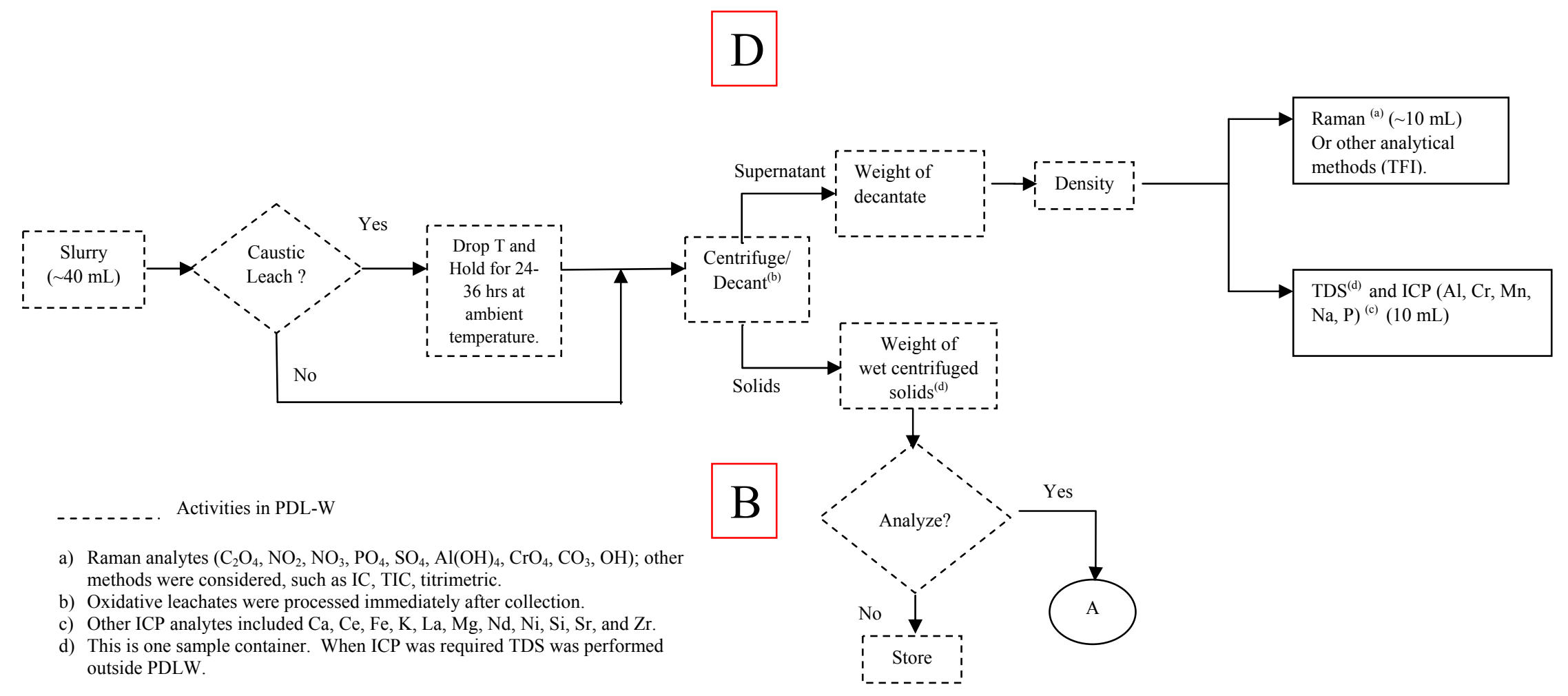

Figure 3.5. Sample Processing for Decantate Handling 


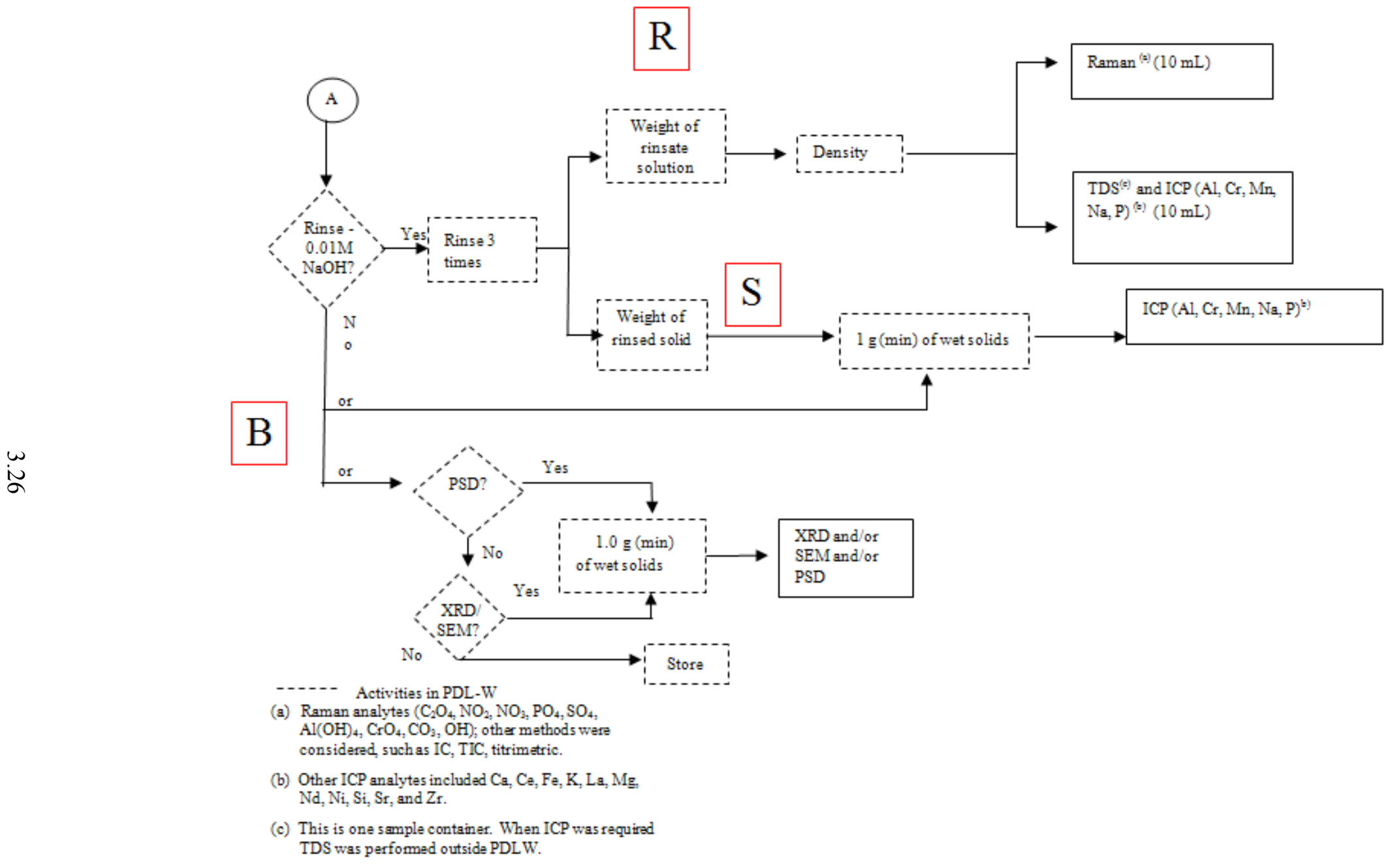

Figure 3.6. Sample Processing for Solids 


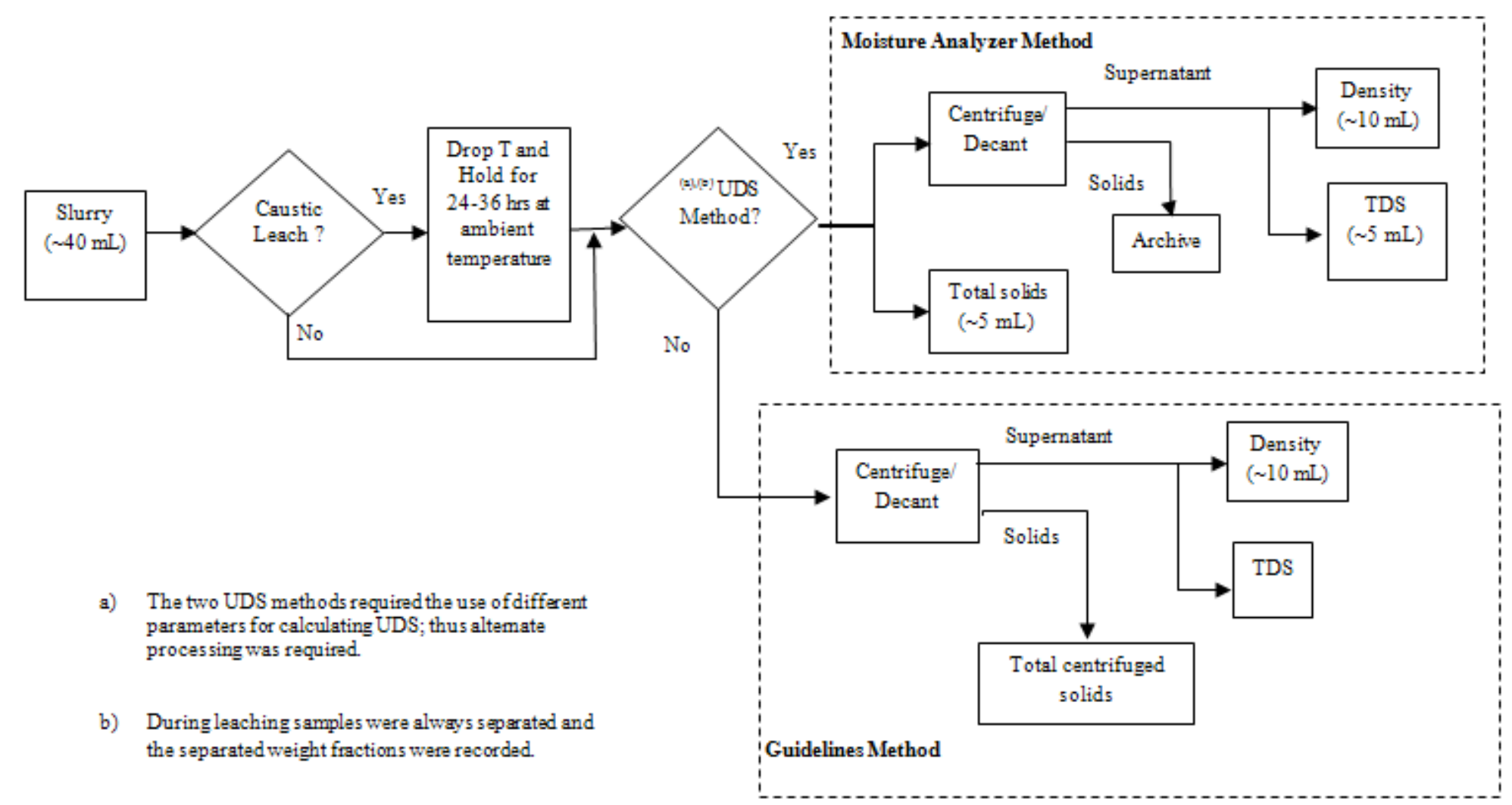

Figure 3.7. Sample Processing for UDS 
A single 50-mL sample was centrifuged and the supernatant decanted to allow multiple laboratories to perform several analyses simultaneously on the supernatant. To perform this phase separation, the original sample was centrifuged at $\sim 4500 \mathrm{G}$ with a swinging bucket rotor in PDL-W. The centrifuging time was initially set for 10 minutes. This centrifuging time was sufficient to cause phase separation during shakedown, Integrated Test A, and oxidative leaching. The actual centrifuging time is recorded on the sample bench sheets. After centrifuging, the supernatant was decanted and submitted for some or all the analyses listed below:

- ICP

- total dissolved solids

- density

- IC

- TOC

- Raman

- free hydroxide.

Not all these analyses were necessarily performed on every decanted supernatant. The required analysis was identified in the Sample Collection and Analysis table in the governing Test Instruction. Density was determined on the decanted supernatant at PDL-W for all samples being analyzed by Raman spectroscopy before the samples were transported for analysis. In addition, at times, density was determined by Southwest Research Institute (SwRI) before ICP analysis. The wet centrifuged solids from this same container were either submitted for ICP analysis, if it was required, or stored in the original sample collection container as excess. A tare weight (before filling) and gross weight (after filling) of the sample container were documented on sample bench sheets.

For Dow Corning to quantify the anti-foam agent (AFA) compounds by Gel Permeation Chromatography (GPC) in the solid and supernatant fractions of the slurry, two 50-mL slurry samples were collected and centrifuged at $4500 \mathrm{G}$ for 10 minutes, and the supernatant was decanted into two additional properly labeled 50-mL containers before shipping. Weighing empty and filled containers was not required.

For SwRI to quantify silicon by ICP in the AFA, a 50-mL slurry sample was collected and shipped as collected during non-leaching process steps. Once at SwRI, the slurry was centrifuged at $2200 \mathrm{G}$ for an hour, the supernatant was separated from the wet centrifuged solids, and then each phase was analyzed separately. During leaching, the phase separation occurred in PDL-W following the standard practice of centrifuging at $\sim 4500 \mathrm{G}$ for 10 minutes.

A single 50-mL container was filled for particle-size distribution (PSD), X-ray diffraction (XRD), and scanning electron microscopy (SEM). Initially, these samples were centrifuged. The supernatant was decanted and stored while the wet solids were submitted for analysis. Intact slurry samples were submitted after shakedown and Integrated Test A and initial Integrated Test B simulant characterization. If a phase-separated sample was submitted, then descriptor 7 was included in the sample name. If descriptor 7 was not in the name, then an intact sample was submitted for analysis. Weighing empty and filled containers was not required. 
For parallel CUF and bench-scale testing, varying amounts of either permeate or slurry samples were collected. A tare weight (before filling) and gross weight (after filling) of the sample container was recorded on the sample container. These samples were delivered to APEL by the next business day after sample collection.

\subsubsection{Leaching Samples}

The core critical analyses for leached samples were weight percent UDS, slurry density, inductively coupled plasma, ion chromatography, Raman, and free hydroxide analyses. Two 50-mL samples were collected for the UDS and density analyses. Another 50-mL sample was collected for the remaining core analyses.

- During caustic leaching, the original samples were immediately placed in a thermostatically controlled water bath at $20 \pm 2^{\circ} \mathrm{C}$ to decrease the sample temperature to $25 \pm 5^{\circ} \mathrm{C}$ after collection. Once the desired temperature was reached, which typically took less than 10 minutes for $50-\mathrm{mL}$ containers, the samples were allowed to sit for 24 hours in PDL-W. The density sample was shipped as collected. The two other samples to be used for analysis were centrifuged following the sample process described for non-leached samples.

- During oxidative leaching, sample processing began immediately after sample collection. The oxidative leach density sample was shipped as collected while the other two samples were phase separated immediately after collection by centrifuging and decanting the supernatant as described above for non-leached samples. To allow for consistency in comparing analytical results, the samples for ICP analysis collected immediately before adding $\mathrm{NaMnO}_{4}$ were phase separated immediately after collection. The oxidatively leached centrifuged solids from samples collected immediately before adding $\mathrm{NaMnO}_{4}$ and at the end of oxidative leaching for analysis were weighed and rinsed three times with $0.01-\mathrm{M} \mathrm{NaOH}$ solution. The actual quantity of rinse is recorded on sample bench sheets. The wet solids were vortexed three times to suspend and rinse the solids after each addition of $\mathrm{NaOH}$ solution. The rinsate was collected and weighed. The density of the rinsate was measured at PDL-W, and then it was split into subsamples for ICP and Raman analysis.

During leaching, additional 50-mL samples were collected for archiving. Caustic leached archived samples were also cooled and phase separated as described above before storage. The oxidatively leached archived samples were also immediately phase separated after collection as described above. The centrifuged oxidatively leached solids that went to storage were not rinsed.

\section{$\underline{\text { Undissolved Solids }}$}

The sample processing for UDS is given in Figure 3.7. The UDS was measured at PNNL only when results were needed the same day the sample was collected. A halogen moisture analyzer (HG63, Mettler Toledo) was used to determine the wt $\%$ UDS under procedure TPR-RPP-WTP-648. If results for multiple samples were required, then a modified version of the oven method outlined in Guidelines for Performing Chemical, Physical, and Rheological Properties Measurements (24590-WTP-GPG-RTD-001, Rev. 0) was used. ${ }^{\text {(a) }}$

(a) GL Smith and K Prindiville. 2002. Guidelines for Performing Chemical, Physical, and Rheological Properties Measurements. 24590-WTP-GPG-RTD-001 Rev. 0, Bechtel National, Inc., Richland Washington. 


\section{$\underline{\text { Supernatant Density }}$}

Density measurements were performed at PDL-W on samples that were analyzed by Raman. Density was measured in accordance with TPR-WTP-PEP-054, Determination of Density using Pycnometer or Graduated Cylinder. All other density values were analyzed externally by SwRI.

\subsubsection{Analytes of Interest}

The analytes of interest, analytical techniques, and required estimated quantitative limits were set for the analytical laboratories and are provided in Table 3.6. The limits included processing factors associated with preparation and analyses. For techniques where multiple analytes were measured, all analytes were requested to be reported. The preparative and analytical quality control (QC) requirements specified in Hanford Analytical Services Quality Assurance Requirements Documents (HASQARD), Rev. 2 were required to be applied to only the analytes of interest or target analytes. All other analytes were considered opportunistic, and QC failures for these analytes did not require corrective action as described in HASQARD, Rev. 2. For example, opportunistic analytes were expected to include (but are not limited to) As, B, Sn, and Zn. No additional analytes were introduced into the list of target analytes. 
Table 3.6. Estimated Quantitative Limits for Solids and Supernatants in Sample Loop Testing, PEP Operation, and Simulant Acceptance Samples

\begin{tabular}{|c|c|c|c|}
\hline Analyte & $\begin{array}{l}\text { Solids } \\
\mu \mathrm{g} / \mathrm{g}\end{array}$ & $\begin{array}{l}\text { Liquid solution } \\
\mu \mathrm{g} / \mathrm{mL}\end{array}$ & Analytical Technique \\
\hline $\mathrm{Al}$ & $4.0 \mathrm{E}+01$ & $5.0 \mathrm{E}+00$ & \multirow{17}{*}{ ICP-AES } \\
\hline $\mathrm{Ca}$ & $2.0 \mathrm{E}+01$ & $5.0 \mathrm{E}+00$ & \\
\hline $\mathrm{Ce}$ & $8.0 \mathrm{E}+00$ & $7.5 \mathrm{E}+01$ & \\
\hline $\mathrm{Cr}$ & $5.0 \mathrm{E}+00$ & $1.0 \mathrm{E}+00$ & \\
\hline $\mathrm{Fe}$ & $3.0 \mathrm{E}+02$ & $7.5 \mathrm{E}+01$ & \\
\hline K & $3.0 \mathrm{E}+01$ & $1.5 \mathrm{E}+01$ & \\
\hline $\mathrm{La}$ & $6.0 \mathrm{E}+00$ & $7.5 \mathrm{E}+01$ & \\
\hline $\mathrm{Mg}$ & $7.0 \mathrm{E}+00$ & $5.0 \mathrm{E}+00$ & \\
\hline $\mathrm{Mn}$ & $1.0 \mathrm{E}+01$ & $5.0 \mathrm{E}+00$ & \\
\hline $\mathrm{Na}$ & $3.0 \mathrm{E}+02$ & $7.5 \mathrm{E}+01$ & \\
\hline $\mathrm{Nd}$ & $1.0 \mathrm{E}+01$ & $5.0 \mathrm{E}+00$ & \\
\hline $\mathrm{Ni}$ & $2.0 \mathrm{E}+01$ & $3.0 \mathrm{E}+01$ & \\
\hline $\mathrm{P}$ & $5.0 \mathrm{E}+01$ & $1.0 \mathrm{E}+01$ & \\
\hline $\mathrm{Pb}$ & $3.0 \mathrm{E}+01$ & $7.5 \mathrm{E}+1$ & \\
\hline $\mathrm{Si}$ & $3.0 \mathrm{E}+03$ & $7.5 \mathrm{E}+01$ & \\
\hline $\mathrm{Sr}$ & $2.0 \mathrm{E}+01$ & $5.0 \mathrm{E}+00$ & \\
\hline $\mathrm{Zr}$ & $9.0 \mathrm{E}+01$ & $7.5 \mathrm{E}+01$ & \\
\hline $\mathrm{Cs}$ & N/A & $4 \mathrm{pg} / \mathrm{mL}$ & ICP-MS \\
\hline Chloride & $\mathrm{N} / \mathrm{A}$ & $1.2 \mathrm{E}+02$ & \multirow{6}{*}{$\begin{array}{l}\text { Ion Chromatography } \\
\text { (water-soluble species) }\end{array}$} \\
\hline Nitrite & N/A & $1.2 \mathrm{E}+02$ & \\
\hline Nitrate & N/A & $4.0 \mathrm{E}+01$ & \\
\hline Phosphate & N/A & $1.2 \mathrm{E}+02$ & \\
\hline Sulfate & N/A & $6.0 \mathrm{E}+01$ & \\
\hline Oxalate & N/A & $2.5 \mathrm{E}+02$ & \\
\hline Hydroxide & $\mathrm{N} / \mathrm{A}$ & $5 \mathrm{E}-03 \mathrm{M}$ & Titration \\
\hline Total inorganic carbon & N/A & $2.0 \mathrm{E}+02$ (as $\mathrm{C})$ & Coulometer \\
\hline Density & 0.9 to $1.7 \mathrm{gm} / \mathrm{mL}$ & 0.9 to $1.7 \mathrm{gm} / \mathrm{mL}$ & Gravimetric \\
\hline TDS & N/A & N/A & Gravimetric \\
\hline UDS & $\mathrm{N} / \mathrm{A}$ & $\mathrm{N} / \mathrm{A}$ & Gravimetric \\
\hline
\end{tabular}

\subsubsection{Analytical Methods}

This section describes the analytical methods used to determine the chemical composition of the PEP simulant samples collected during testing. Analytical results are provided in Section 5.

\subsubsection{Preparation for ICP or ICP-MS}

For the solutions, aliquots were diluted with hydrochloric acid and then analyzed. Dilutions were made with a representative aliquot of approximately 2 grams combined with $5-\mathrm{mL}$ of $50 \%$ hydrochloric acid and then diluted to a final volume of $50-\mathrm{mL}$ with DI water.

Two preparative techniques were used for solids. The first preparative technique was lithium metaborate/tetraborate fusion, referred to as prep method "80/20 Fusion." Aluminum, barium, cerium, 
chromium, iron, lanthanum, manganese, neodymium, silicon, and strontium were reported from the fusion. The second preparative method used concentrated nitric, perchloric, hydrofluoric, and hydrochloric acids in an open vessel and was referred to as the "Teflon" prep method. If residue remained from the "Teflon" method, then the residue was separated, dried, and fused using the lithium metaborate/tetraborate fusion technique. Both the "Teflon" and residue preparations were analyzed. The remaining metals were reported from either the "Teflon" digestion only or the combination of the two. If an analyte was detected in both the Teflon digestate and the residue fusions, then the reported result was the sum of the results obtained from the two preparations. These results were identified as "combined" on Sample Analysis Data Sheets while analytes reported from only the fusion were identified as " $80 / 20$ Fusion," and analytes reported solely from the acid digestions were identified as "Teflon" on the Sample Analysis Data Sheets. The preparative QC samples included a sample duplicate, preparation blank (PB), solid laboratory control samples (LCS) consisting of obsidian rock and basalt rock, and an aqueous laboratory control sample (LCS) and a matrix spike (MS) for "Teflon." The results are reported on an as-received or wet weight basis. The percent solids were determined at $105^{\circ} \mathrm{C}$ and reported on the Sample Analysis Data Sheets for all solids.

\subsubsection{Metals Analysis by ICP-AES}

All metals were determined by ICP-AES using SwRI procedure TAP01-0406-130 with the exception of cerium, cesium, lanthanum, and neodymium. The preparative QC samples (duplicate, PB, LCS, MS) were processed along with analytical workstation QC (initial and continuing calibration verifications, initial and continuing blanks, interference check samples, and post-digestion spikes).

\subsubsection{Metals Analysis by ICP-MS}

Cerium, cesium, lanthanum, and neodymium were determined by ICP-MS in accordance with SwRI procedure TAP01-0406-046. The preparative QC samples (duplicate, PB, LCS, MS) were processed along with analytical workstation QC (initial and continuing calibration verifications, initial and continuing blanks, interference check samples, and post-digestion spikes).

\subsubsection{Anions (IC)}

Decanted supernatant and rinsants were analyzed by IC for chloride, nitrate, nitrite phosphate, sulfate, and oxalate at SwRI in accordance with procedure SwRI TAP 01-0406-042. Approximately $0.25 \mathrm{~g}$ of the sample was diluted to $50-\mathrm{mL}$ using DIW. Since the dilution was performed by weight, the sample results are reported on a weight basis. The standard reporting by the laboratory is nitrate as N, nitrite as N, and phosphate as P. Shortly after PEP testing began, PNNL requested that results be reported as nitrate, nitrite, and phosphate and not as nitrogen or phosphorus. The IC analytical report narrative identifies conversion factors used to report as anions. QC samples generated at the analytical workstation included a sample replicate determination, preparation blank, LCS, and matrix spike (MS).

\subsubsection{Total Inorganic Carbon/Total Organic Carbon (TIC/TOC)}

The samples were analyzed for total carbon (TC) using a Dohrman DC-80 Carbon Analyzer in accordance with procedure SwRI TAP 01-0406-001. The liquids were directly injected, and the slurry was analyzed using the soil/sludge sampler. Another aliquot of the sample was acidified with sulfuric 
acid and sparged to remove inorganic carbon and then analyzed for TOC. The TIC is calculated from the difference in the TC and TOC results. All samples were analyzed in duplicate, and average results were reported when the relative percent differences (RPDs) were less than 20\%. If the RPD was greater than $20 \%$, then the sample was analyzed twice more, and the average of the quadruplicate analysis was reported. The liquids were corrected for density, and all sample results were reported on a weight basis. QC samples were generated at the analytical workstation and included a sample replicate determination, preparation blank, LCS, and MS.

\subsubsection{Hydroxide}

The free-hydroxide concentration was determined by potentiometric titration with standardized $\mathrm{HCl}$ according to procedure RPG-CMC-228, "Determination of Hydroxyl (OH-) and Alkalinity of Aqueous Solutions, Leachates, and Supernates and Operation of Brinkman 636 Auto-Titrator." The free hydroxide was defined as the first inflection point on the titration curve. QC samples were generated at the analytical workstation and included a sample replicate determination, preparation blank, and blank spike (BS).

\subsubsection{Raman}

Raman was used to quantify aluminate, carbonate, chromate, hydroxide, nitrate, nitrite, oxalate, phosphate, and sulfate following procedure RPG-CMC-240. If precipitate formed in the solution samples submitted for Raman before the analysis, then the samples were centrifuged, and aliquots of the liquid were pipetted and analyzed. Two sets of Raman results were reported for shakedown, Integrated Test A, and some of the Integrated Test B samples. The first set of results was generated using calibrations that were periodically adjusted to optimize performance of QC check samples. The second set of results was recalculated based on the original calibration parameters. The generation of these two sets of results and the discovery of the calibration adjustments are documented as RPP-WTP CAR, number 42708.1. Only Raman results from the recalculation are provided. As a result of using the original calibration parameters, an occasional QC sample falls outside of established performance limits. QC samples were generated at the analytical workstation and included a sample replicate determination, preparation blank, $\mathrm{BS}$, and MS.

\subsubsection{Preparation for Gel Permeation Chromatography (AFA components)}

Duplicate samples were collected to support this analysis. Both samples were centrifuged and phase separated at PDL-W before shipping. Once at the analytical laboratory, one of the two liquid fractions was mixed with toluene while the other was mixed with tetrahydrofuran (THF). Both were shaken for an hour and allowed to settle overnight. The upper organic layer was removed and allowed to concentrate. The toluene extract was dried at room temperature to completeness and brought to $3.0-\mathrm{mL}$ toluene. The THF extracts were concentrated to approximately $2-\mathrm{mL}$ at room temperature and then placed in an $80^{\circ} \mathrm{C}$ oven to dry to completeness and brought up to $3.0-\mathrm{mL}$ THF. One of the two solids fractions was extracted with $10-\mathrm{mL}$ of toluene while the other was extracted with $10.0-\mathrm{mL}$ of THF. In both cases, the solvent was added directly into the receipt vessel, and the initial sample and solvent were shaken for 2 hours. All solutions were filtered through $0.45-\mu \mathrm{m}$ PTFE syringe filters. The calibration was verified before samples were analyzed and after every 15 samples. 


\subsubsection{Gel Permeation Chromatography (AFA components)}

The toluene extract allowed polydimethylsiloxane (PDMS) to be analyzed by gel permeation chromatography (GPC) while the THF extract allowed polypropylene glycol (PPG) to be analyzed by GPC. The toluene extract was analyzed by a Waters 717 autosampler and a Waters 2410 differential refractometer. Two columns, a PLgel 5- $\mu \mathrm{m}$ guard column and a PLgel 5- $\mu \mathrm{m}$ Mixed-C column, were used for separation, and an HPLC grade toluene at a flow of $1.0-\mathrm{mL} /$ minute was used as the eluent. Both the columns and detector were heated to $45^{\circ} \mathrm{C}$. The THF extract was analyzed by a Waters 2695 Separation Module equipped with a vacuum degasser and a Waters 2410 differential refractometer. Two columns, a PLgel 5- $\mu \mathrm{m}$ guard column and a PLgel 5- $\mu \mathrm{m}$ Mixed-C column, were used for separation, and a certified grade THF at a flow of $1.0-\mathrm{mL} /$ minute was used as the eluent. Both the columns and detector were heated to $35^{\circ} \mathrm{C}$. The quantity of PDMS and PPG was used to determine the amount of AFA, Q2-3183A Antifoam, in the samples. However, SwRI had a back-up approach for tracing the fate of AFA by quantifying the Si in the liquid and solid fractions. SwRI received the intact slurry, separated the solid from the liquid, and analyzed each fraction for Si by ICP. Since the AFA is a proprietary composition belonging to Dow Corning, they performed the analytical work. The calibration was verified before samples were analyzed and after every 15 samples.

\subsubsection{Physical Properties}

This section describes the methods used to determine the physical properties of the PEP simulant samples, the crystal form and solids environment, density, wt $\%$ UDS, rheology, particle-size attributes, and heat capacity. A more detailed outline of the methods used in this section is given, where applicable, in the appropriate test data package supplied with the sample results for each characterization.

\subsubsection{Percent UDS and Density}

Weight percent UDS, wt $\%$ dissolved solids, bulk slurry density, and supernatant density were determined following 24590-WTP-GPG-RTD-001, Rev 0. Some steps in 24590-WTP-GPG-RTD-001, Rev $0,{ }^{(a)}$ were not performed because the results generated from these steps were not needed, or steps were slightly modified to reduce analysis time. These modifications are not believed to impact the final results.

Slurry sample UDS analysis was performed with the following modifications:

- Steps 4, 6, and 7 were omitted because the settling data were not required.

- Steps 8 and 9. Centrifuge the cones at $\sim 1000 \mathrm{G}$ for 1 hour. Record the volume of the total sample and the volume of centrifuged solids on the physical properties data sheet. After this, the WTP procedures required that the supernatant be decanted into a pre-weighed graduated cylinder to obtain the supernatant mass and volume. Rather than use a graduated cylinder, the volume of supernatant was calculated as the difference between the volume of the total sample and the volume of centrifuged solids.

(a) GL Smith and K Prindiville. 2002. Guidelines for Performing Chemical, Physical, and Rheological Properties Measurements. 24590-WTP-GPG-RTD-001 Rev. 0, Bechtel National, Inc., Richland Washington. 
- Step 11 requires decanting the centrifuged supernatant liquid to a pre-weighed graduated cylinder. Because of the high concentration of $\mathrm{NaOH}$ in some of the samples, some of the liquid was left in the cylinder. Rather than decant, the centrifuged supernatant liquid was pipetted to a preweighed glass Petri dish or vial, and the mass of the supernatant liquid was recorded.

- Step 12 was omitted because air-drying was not necessary.

Separated slurry samples UDS analysis was performed with the following modifications:

- Step 8 requires centrifuging at $\sim 1000 \mathrm{G}$ for 1 hour. Samples were centrifuged at $\sim 4500 \mathrm{G}$ for either 10 minutes or 1 hour. The centrifuge time is documented on the sample handling benchsheets in TDP-WTP-349.

- Wet solids (designated as "B") and decanted supernatants (designated as "D") were sent to SwRI along with empty vial and separated sample weights. These weights are also documented on the sample handing benchsheets in TDP-WTP-349.

- The wet solids were transferred to a tared container using DIW to completely remove the solids. The solids and DIW rinses were then oven dried to constant weight.

- The total weight of the supernatant was calculated from the sample-handling benchsheets. Since it was not feasible for the entire volume of supernatant to be oven dried, approximately $5-\mathrm{mL}$ was transferred to a Petri dish for drying.

- Density was determined on the supernatant liquid using a 5-mL volumetric flask.

\subsubsection{Slurry and Supernatant Density}

Three preweighed 5-mL Class A volumetric flasks were filled to the specified line with the liquid sample and reweighed. The density was calculated as the mass of the sample divided by the certified volume of the volumetric flask.

Slurry samples requiring a higher accuracy supernatant density than provided by the UDS analysis were subsampled and centrifuged for 1 hour at $1000 \mathrm{G}$, and the phase-separated supernatant was analyzed as described in the paragraph above.

\subsubsection{Rheological Measurements}

Rheological testing was conducted in accordance with RPL-COLLIOD-02, Measurement of Physical and Rheological Properties of Solutions, Slurries and Sludges. For the current study, two regions of tank waste flow behavior were considered: 1) incipient motion in settled tank waste solids (shear strength), and 2) non-elastic flow of tank waste slurries and supernates (flow curve).

\section{$\underline{\text { Shear-Strength Testing }}$}

For settled tank waste slurry solids, a finite stress must be applied before the material will begin to flow. The stress required to transition the material from elastic deformation to viscous flow is referred to as the shear strength, and its origin can be attributed to static and kinetic friction between individual particles and/or aggregates, the strength of the matrix supporting the coarse fraction (i.e., the interstitial fluid), and sludge cohesion arising from interparticle adhesive forces such as van der Waals forces. 
The shear strength was measured using the vane method. For the vane technique, the stress required to begin motion is determined by slowly rotating a vane immersed in the test sample's settled solids while continuously monitoring the resisting torque as a function of time. A material's static shear strength is then associated with the maximum torque measured during the transition from initial to steady-state vane rotation.

The maximum torque required for incipient motion is dependent on vane geometry. To account for vane-geometry effects, the shear strength is expressed in terms of the uniform and isotropic stress acting over the surface area of the cylinder of rotation swept out by the vane. The shear strength is related to the maximal torque during incipient motion according to Equation 3.1 (Barnes and Dzuy 2001):

$$
\tau_{S S}=\frac{M_{\max }}{4 \pi R^{3}\left(\frac{H}{2 R}+\frac{1}{3}\right)}
$$

Here, $\tau_{s s}$ is the shear strength $\left(\mathrm{N} / \mathrm{m}^{2}\right), M_{\max }$ is the maximum torque $(\mathrm{N} \cdot \mathrm{m})$, and $R$ and $H$ are the radius and height of the cylinder of rotation swept out by the vane $(\mathrm{m})$. Because the shear band observed upon slow rotation of the vane does not extend appreciably beyond the vane paddles, $R$ and $H$ are taken to be the dimensions of the vane itself.

The proximity of the vane to the sample container inner surfaces as well as the free surface of the settled solids can impact shear-strength results. As such, certain geometric constraints must be satisfied for the test to be considered independent of container geometry (Table 3.7).

Table 3.7. Vane Immersion Depth and Container Geometry Constraints for Shear-Strength Tests Using the Vane Technique

\begin{tabular}{lll}
\hline Constraint & Criterion & For $8 \times 16 \mathrm{~mm}(\mathrm{R} \times \mathrm{H})$ Vane \\
\hline Vane height to radius & $\mathrm{H}<7 \mathrm{R}$ & $\mathrm{H}<56 \mathrm{~mm}$ (Satisfied) \\
Container radius to vane radius & $\mathrm{R}_{\text {cont }}>2 \mathrm{R}$ & $\mathrm{R}_{\text {cont }}>16 \mathrm{~mm}$ \\
Immersion depth to vane height & $\mathrm{h}>\mathrm{H}$ & $\mathrm{h}>16 \mathrm{~mm}$ \\
$\begin{array}{l}\text { Separation between bottom of vane and } \\
\text { container floor }\left(\mathrm{h}_{\text {floor }}\right)\end{array}$ & $\mathrm{h}_{\text {floor }}>0.5 \mathrm{H}$ & $\mathrm{h}_{\text {floor }}>8 \mathrm{~mm}$ \\
\hline $\mathrm{R}=$ radius & & \\
$\mathrm{H}=$ height & & \\
\hline
\end{tabular}

\section{Flow-Curve Testing}

The non-elastic flow of tank waste slurries and supernates is characterized with rotational viscometry. The typical result of such testing is a set of flow-curve data, which show the stress response of a material to a range of applied rates-of-deformation. Specifically, flow-curve testing allows characterization of a material's shear stress, $\tau$, which is the response as a function of applied shear rate, $\dot{\gamma}$. Once measured, the flow-curve data can be interpreted with several constitutive equations for the viscous stress/rate-of-strain relationship. Such analysis allows the flow behavior over a broad range of conditions 
to be described with just a few rheological descriptors, such as viscosity, yield stress, consistency, and flow index.

A concentric cylinder rotational viscometer operated in controlled-rate mode was used for flow-curve testing of tank waste slurries and supernates. Rotational viscometers operate by placing a given volume of test sample into a measurement cup of known geometry. A cylindrical rotor attached to a torque sensor is then lowered into the sample until the slurry is even with, but does not cover, the top of the rotor. A single-point determination of a fluid's flow properties is made by spinning a rotor at a known rotational speed, $\Omega$, and measuring the resisting torque, $M$, acting on the rotor. The torque acting on the rotor can be directly related to the shear stress at the rotor using the equation,

$$
\tau=\frac{M}{2 \pi H R_{I}^{2}}
$$

The shear stress has units of force per area $\left(\mathrm{N} / \mathrm{m}^{2}\right)$. The rotational rate is related to the shear rate. However, calculating the fluid shear rate at the rotor is complicated by the fact that the shear rate depends on both the measurement-system geometry and the fluid rheological properties. For the simplest fluids (i.e., Newtonian fluids), the shear rate of the fluid at the rotor can be calculated given the geometry of the cup rotor shear by using the equation,

$$
\dot{\gamma}=\left(\frac{2 R_{O}^{2}}{R_{O}^{2}-R_{I}^{2}}\right) \Omega
$$

Here, the shear rate has units of inverse seconds $\left(\mathrm{s}^{-1}\right)$. Calculating the shear rate for materials showing more complex shear-stress versus shear-rate behavior (i.e., non-Newtonian fluids) requires estimates of yield stress and a degree of shear-thinning or shear-thickening. As the goal of rheological testing is to determine and quantify such behavior, these values are typically not known. This requirement can be circumvented by using a cup-and-rotor system with a small gap $(\sim 1 \mathrm{~mm})$ for fluid shear. For fluid flow in small-gap cup and rotor systems, shear-rate effects introduced by fluid properties are minimized such that Equation 3.3 provides an accurate determination of shear rate for non-Newtonian materials.

The resistance of a fluid to flow is often described in terms of the fluid's apparent viscosity, $\eta_{a p p}$, which is defined as the ratio of the shear stress to the shear rate:

$$
\eta_{\text {app }}=\frac{\tau}{\dot{\gamma}}
$$

For Newtonian fluids, the apparent viscosity is independent of the shear rate. For non-Newtonian fluids, the apparent viscosity will vary as a function of the shear rate. The unit of apparent viscosity is $\mathrm{Pa} \cdot \mathrm{s}$, although it is typically reported in the unit of centipoise (cP; where $1 \mathrm{cP}=1 \mathrm{mPa} \cdot \mathrm{s})$.

Flow-curve data are usually combined plots of $\tau$ and $\eta_{\text {app }}$ as a function of $\dot{\gamma}$. As stated above, flow-curve data can be interpreted with several constitutive equations (i.e., flow curves), allowing 
characterization of those data with just a few rheological descriptors. The behavior of tank waste sludges, slurries, and supernates can be described by four common flow-curve equations:

Newtonian-Newtonian fluids flow as a result of any applied stress and show constant viscosity over all shear conditions. The flow curve for Newtonian fluids is

$$
\tau=\eta \dot{\gamma}
$$

where $\eta$ is the Newtonian viscosity.

Ostwald (Power Law) - Power-law fluids flow as a result of any applied stress and have viscosities that either increase or decrease with increasing shear rate. They are described by,

$$
\tau=m \dot{\gamma}^{n}
$$

where $m$ is the power-law consistency index, and $n$ is the power-law index. Power-law fluids with $n<1$ are referred to as pseudoplastic (shear-thinning), whereas power-law fluids with $n>1$ are referred to as dilatant (shear-thickening).

Bingham Plastic - Bingham plastics are fluids that show finite yield points. A finite stress (i.e., the yield stress), must be exceeded before these types of materials flow. Once flow is initiated, the stress response of the material is Newtonian over the rest of the shear-rate range. Bingham plastics are described by

$$
\tau=\tau_{O}^{B}+k_{B} \dot{\gamma}
$$

where $\tau_{O}^{B}$ is the Bingham yield index, and $k_{B}$ is the Bingham consistency index.

Casson-Fluids that behave in accordance with a Casson model show a finite yield followed by pseudoplastic behavior. They are described by,

$$
(\tau)^{0.5}=\left(\tau_{o}^{C}\right)^{0.5}+\left(k_{C} \dot{\gamma}\right)^{0.5}
$$

where $\tau_{o}^{C}$ is the Casson yield index, and $k_{C}$ is the Casson consistency index. Although more limited in the types of flow behavior that it can describe relative to the Herschel-Bulkley equation, the Casson model is popular because it is capable of accurately describing many shear-thinning fluids and because units on the parameters are more physically meaningful (e.g., the consistency is in Pa·s versus Pa. ${ }^{n}$ for the Herschel-Bulkley model).

Power-law fluids, Bingham plastics, and Casson fluids are referred to as non-Newtonian fluids. Generally, liquids without internal and/or interconnected structures (such as tank waste supernatants) are Newtonian. Sludges and slurries are typically non-Newtonian, but their exact behavior depends on the concentration of solids and suspending phase chemistry. Sufficiently dilute slurries may show Newtonian behavior. 


\section{$\underline{\text { Rheology Instrumentation }}$}

Rheological characterization was accomplished using an Anton Parr Rheometer (MCR 301) for shear-rate measurements, and shear strength was performed on a Rotovisco RV20 Measuring System M equipped with an M5 measuring head and RC20 controller sold by HAAKE Mess-Technik GmbH u Co. (now the Thermo Electron Corporation). The M5 measuring head is a "Searle" type viscometer capable of producing rotational speeds up to $500 \mathrm{rpm}$ and measuring torques up to $0.049 \mathrm{~N} \cdot \mathrm{m}$. The minimum rotational speed and torque resolution achievable by this measuring head are $0.05 \mathrm{rpm}$ and $0.49 \mathrm{mN} \cdot \mathrm{m}$, respectively.

Calibration and analysis were performed according to TPR-WTP-PEP-049.

Specific measurement tools, such as cup-and-rotor assemblies and shear vanes, are attached to measure selected rheological properties. Shear-strength measurements employ an $8 \mathrm{~mm} \times 16 \mathrm{~mm}(\mathrm{R} \times \mathrm{H})$ shear vane tool. Flow-curve measurements employed an MV1 stainless steel measuring cup and rotor. The dimensions of the MV1 and vane measuring systems are listed in Table 3.8.

Table 3.8. Vane and Cup and Rotor Measuring System Dimensions

\begin{tabular}{lcccc}
\hline Measuring System & $\begin{array}{c}\text { Vane/Rotor Radius } \\
(\mathrm{mm})\end{array}$ & $\begin{array}{c}\text { Vane/Rotor Height } \\
(\mathrm{mm})\end{array}$ & $\begin{array}{c}\text { Container Radius } \\
(\mathrm{mm})\end{array}$ & $\begin{array}{c}\text { Gap Width } \\
(\mathrm{mm})\end{array}$ \\
\hline Vane Tool & 8 & 16 & $>16^{(\mathrm{a})}$ & $>8$ \\
MV1 & 20.04 & 60 & 21 & 0.96 \\
\hline
\end{tabular}

The temperature is controlled with a combination of the standard measuring system temperature jacket and a temperature-controlled recirculator. The jacket temperature is monitored using a Type-K thermocouple calibrated over $0^{\circ}$ to $100^{\circ} \mathrm{C}$ and connected to a calibrated multichannel temperature display. The temperature control is employed only for flow-curve measurements. Shear-strength measurements are carried out at ambient cell temperature.

The rheometer was controlled, and data were acquired with a remote computer connection using the RheoWin Pro Job Manager Software, Version 2.96. During measurement, the software automatically collects and converts rotor torque readings into shear stresses based on Equation 3.1 (for vane testing) or Equation 3.2 (for flow-curve testing). Likewise, the software also automatically converts the rotational rate readings into shear rates based on Equation 3.3.

\section{$\underline{\text { Rheology Materials and Methods }}$}

No sample treatment was performed before analysis with the exception of the mechanical agitation required to mix and subsample selected sample containers.

\section{Shear-Strength Testing}

Before testing, the simulant slurries that were provided for shear-strength testing were mixed thoroughly and subsequently allowed to settle for at least 48 to 72 hours. When possible, the shear strength was measured by immersing the $16-\times 16-\mathrm{mm}(\mathrm{D} \times \mathrm{H})$ vane tool to a depth of $15 \mathrm{~mm}$ into the settled solids. The vane was slowly rotated $(0.031 / \mathrm{sec})$ for 120 seconds. For the entire duration of 
rotation, the time, rotational rate, and vane torque were continuously monitored and recorded. At the end of the measurement, shear stress versus time data were parsed, and the maximum measured shear stress (i.e., the material's shear strength) was determined.

\section{Flow-Curve Testing}

Each flow curve was measured over an 11-minute period and split into three intervals. Over the first 5 minutes, the shear rate was smoothly increased from zero to $1000 \mathrm{~s}^{-1}$. For the next minute, the shear rate was held constant at $1000 \mathrm{~s}^{-1}$. For the final 5 minutes, the shear rate was smoothly reduced back to zero. During this time, the resisting torque and rotational rate were continuously monitored and recorded.

Results are provided in the test data packages listed below, with summary results provided in Appendix H.

- Integrated Tests A and B TDP-WTP-359

\subsubsection{Particle-Size Distribution}

Particle sizes were characterized according to procedure RPL-COLLOID-01, Rev. 1, Particle Size Analysis Using Malvern MS2000. This procedure uses a Mastersizer 2000 (Malvern Instruments, Inc., Southborough, MA 01772 USA) with a Hydro S wet dispersion accessory. Malvern lists the Mastersizer particle-size measurement range as nominally 0.02 to $2000-\mu \mathrm{m}$. The actual PSD measurement range is dependent on the accessory used as well as the properties of the solids being analyzed. The Malvern 2000 uses laser diffraction technology to define PSD.

The Hydro S wet-dispersion accessory consisted of a 150-mL dispersion unit coupled with a sample flow cell with a continuous variable and independent pump and stirrer and ultrasound. Flow, stirring rate, and sonication can be controlled and altered during measurement. PSD measurements can be made before, during, and after sonication, allowing the influence of each on the sample PSD to be determined. The primary measurement functions of the Malvern analyzer were controlled through Mastersizer 2000 software (Malvern Instruments, Ltd. Copyright 1998-2002). The properties applied to the test samples are summarized in Table 3.9.

The PSD measurements were conducted in either DIW or in a $0.01-\mathrm{M} \mathrm{NaOH}$ dispersion solution matrix, depending on the sample being analyzed. The sample dispersion was added drop-wise to the dispersion unit (while the pump and stirrer were active) until an obscuration in the range of 10 to $20 \%$ was reached. 
Table 3.9. Properties Applied to Group 8 Test Materials

\begin{tabular}{cc}
\hline Property & \\
\hline $\begin{array}{c}\text { Material selected for optical } \\
\text { properties }\end{array}$ & Ferric Oxide Hydroxide \\
Refractive Index (RI) & 2.94 \\
Absorption & 0 \\
Analysis mode & General purpose \\
Sensitivity & normal \\
Suspending Phase & Water/0.01-M NaOH \\
\hline
\end{tabular}

The size distributions of particles were measured under varying flow conditions before and after sonication. For each condition, multiple measurements of PSD were taken. The analyzer software then generated an average of these measurements. Both the individual measurement and average were saved to the analyzer data file.

\subsubsection{X-Ray Diffraction}

The sample mounts for XRD examination were prepared by first cleaning the solids. This procedure included centrifuging the solids into a pellet and decanting the solute. Fresh washing solution was added to the pellet, and it was resuspended. The pellets in solution were vortexed to reconstitute them into the solution, and the centrifuging procedure was repeated three times. Following the final centrifuging and decant, the remaining pellet was left to dry in a $105^{\circ} \mathrm{C}$ oven overnight. The pellet was then pulverized to a powder with a tungsten carbide milling chamber for 1 minute in the Angstrom shaker mill, mixed with an internal standard (rutile, $\mathrm{TiO}_{2}$, or alumina, $\mathrm{Al}_{2} \mathrm{O}_{3}$ ), milled for another 2 minutes to make sure that the two powders were a homogenous mixture, and then mounted into an off-axis, zero background, quartz sample holder. The XRD examination was conducted according to procedure APEL-PAD-V, Operation of Scintag Pad-V X-Ray Diffractometer. The XRD instrument used for these samples was the PNNL Scintag PAD V XRD (property number WD33356), located in Laboratory 102 in the APEL building. The data range for the sample was $5^{\circ}$ to $80^{\circ} 2 \theta$, with a step size of $0.02^{\circ} 2 \theta$ and count time of 2.0 seconds per step. Copper $\mathrm{K} \alpha \mathrm{X}$-rays were used. The X-ray tube operating conditions were $45 \mathrm{kV}$ and $40 \mathrm{~mA}$. Phase identification was done by use of the JADE search match routines (Version 6.0, Materials Data Inc.) with comparison to the International Centre for Diffraction Data (ICDD) database PDF-2, release 1999, which includes the Inorganic Crystal Structure Database (ICSD) maintained by Fachinformationszentrum (FIZ), Karlsruhe, Germany. The chemistry provided for Group 7, in order of decreasing concentration, was $\mathrm{Fe}, \mathrm{Na}, \mathrm{U}, \mathrm{P}, \mathrm{Ca}, \mathrm{Al}, \mathrm{Si}, \mathrm{Bi}, \mathrm{Sr}$, and $\mathrm{Mg}$. Phase identifications were first done without chemistry restrictions. Searches were restricted to the PDF and ICSD inorganic sections.

The pattern was also examined using RIQAS (release 4.0.0.26, 6/10/2002, Materials Data Inc.) Rietveld analysis software. The phases identified above were input into the analysis along with a polynomial background and an amorphous hump at $\sim 35^{\circ} 2 \theta$.

Details of the XRD analysis and results are provided in test data package, TDP-WTP-357, with summary results provided in Appendix $\mathrm{H}$. 


\subsubsection{Scanning Electron Microscope}

The final step just before the supernatant was decanted, the specimen was vortexed, and a small volume of slurry was drawn up using a pipette and placed on an aluminum stub within the same sample processing as above for the XRD sample preparation. The slurry was placed in an oven at $105^{\circ} \mathrm{C}$ to dry overnight. The sample was then coated with gold-palladium using a Polaron Range plasma sputter coater and analyzed with a JEOL SEM (property number WD30596) according to APEL-102-SEM, Scanning Electron Microscope Examination. Selected sample areas were evaluated by X-ray energy dispersive spectroscopy (EDS) for qualitative elemental composition.

Results are provided in the test data package, TDP-WTP-356, provided in Appendix H.

\subsubsection{Heat Capacity}

Approximately eight samples at key process steps were collected and analyzed for heat capacity during Integrated Tests A and B. Three nominal 30-mg subsamples were taken from each of these samples as they were vigorously stirred using a pipette with an enlarged tip to confirm that the slurry samples were representative. Each slurry sample was analyzed in triplicate, recognizing that obtaining a 30-mg representative sample is challenging.

A Perkin-Elmer DSC7 differential scanning calorimeter (DSC) was used to determine the heat capacity of each subsample at temperatures between $20^{\circ} \mathrm{C}$ and $95^{\circ} \mathrm{C}$. The DSC7 was temperature and enthalpy calibrated using a gallium standard (NIST SRM 2234) (m.p. 29.8 ${ }^{\circ} \mathrm{C}$ ) and/or an indium standard (NIST SRM 2232) (m.p. $156.6^{\circ} \mathrm{C}$ ).

The method used for heat-capacity measurement was as provided in the DSC7 operating manual. In this method, the heat capacity of the empty sample pan is measured to provide the "baseline"; the sample pan was a gold "volatile" sample pan designed to contain volatile samples such as the water in these PEP slurries. The heat capacity of a reference or standard material, such as sapphire or high-purity water, is then determined; the purity of these water standards was determined by measuring their electrical conductivity. Finally, the heat capacity of the sample is determined. The measured heat capacity of the sample is adjusted to remove the contribution of the empty pan. We further adjusted the reported heat capacity by applying a measured response factor for the high-purity water.

Optimally, the heat-capacity measurement approach would include analyzing a standard or reference material in the same container in which the sample was analyzed; however, because the analysis of these slurry samples required a hermetic seal, which cannot be broken and resealed, an equivalent gold volatile sample pan was used for the water. In addition, we characterized a sapphire standard to demonstrate instrument performance; the heat capacity of sapphire $\left(0.8 \mathrm{~J} / \mathrm{g}{ }^{\circ} \mathrm{C}\right)$ is significantly lower than that of the PEP slurries $(\sim 3.5 \mathrm{~J} / \mathrm{g})$ and does not provide an adequate calibration adjustment.

Results are provided in the heat capacity test data packages listed below and given in Appendix $\mathrm{H}$.

- Integrated Tests A and B TDP-WTP-368

- Integrated Test B TDP-WTP-372 


\subsection{Test Narration}

Caustic leaching in the ultrafiltration feed preparation vessels UFP-VSL-T01A and B was demonstrated in Integrated Test A using nonradioactive simulant. The subsequent sections summarize the process steps executed for Integrated Test A. Sampling events have been omitted from the Integrated Test A narrative for clarity, given the large number of sampling events and sample containers. A sample list is provided in Appendix B, while sampling events and analyses are included on the Integrated Test A Sampling Event Summary, Appendix C. For a more detailed time line of Integrated Test A, including sequential descriptions of testing problems, refer to Table E.1 in Appendix E, and/or the completed Test Instruction for Integrated Test A. Appendix J contains the Integrated Test A Operational Process Sheets that summarize the target parameters against the achieved test parameters. Values listed in Section 4 are generally not NQA-1 compliant and are for information only, as these values were taken off the HMI and were recorded in the Test Instruction and LRBs. NQA-1 data can be obtained from the DAS files provided separately on transportable hard drives, from the figures in this section, and the figures in Appendices F and G, which were plotted using NQA-1 compliant DAS data. Backpulsing, air entrainment issues, simulant degassing exercises, and level control will be discussed in Section 6 .

\subsection{Batch Preparation}

The Integrated Test A started on 1/31/2009 at approximately 04:30, and most process steps were completed approximately 21 days later on 2/21/09 at 02:27. The High-Solids Filter Test and the repeat of the Filter-Loop Bypass Test were conducted 1 month later starting on 3/20/09 at 21:10 and ending on $3 / 21 / 09$ at 17:14. The break was a result of troubleshooting problems with entrained air impacting the filter-loop flow rate. Figure 4.1 illustrates the start and completion times, duration, and schedule for Integrated Test A process steps.

PEP process testing was performed with a nonradioactive aqueous slurry of simulant waste solids and liquids that were stored in HLP-VSL-T22. Vessel HLP-VSL-T22 contained approximately 3000 gallons (76-in. tank elevation) of simulant at the start of the test. HLP-AGIT-T22 mixed the simulant at an agitator speed of $60 \mathrm{~Hz}$ before the transfers to UFP-VSL-T01A/B tanks. Before the first batch transfer, grab samples were collected from HLP-VSL-T22 from the middle and low elevations. However, the first attempt to collect the samples used an aluminum pipe, which partially dissolved (LRB 60108, pg. 110), yet analysis efforts revealed no impact to sampling results. Grab sampling was completed on 1/31/09 at 04:56. 


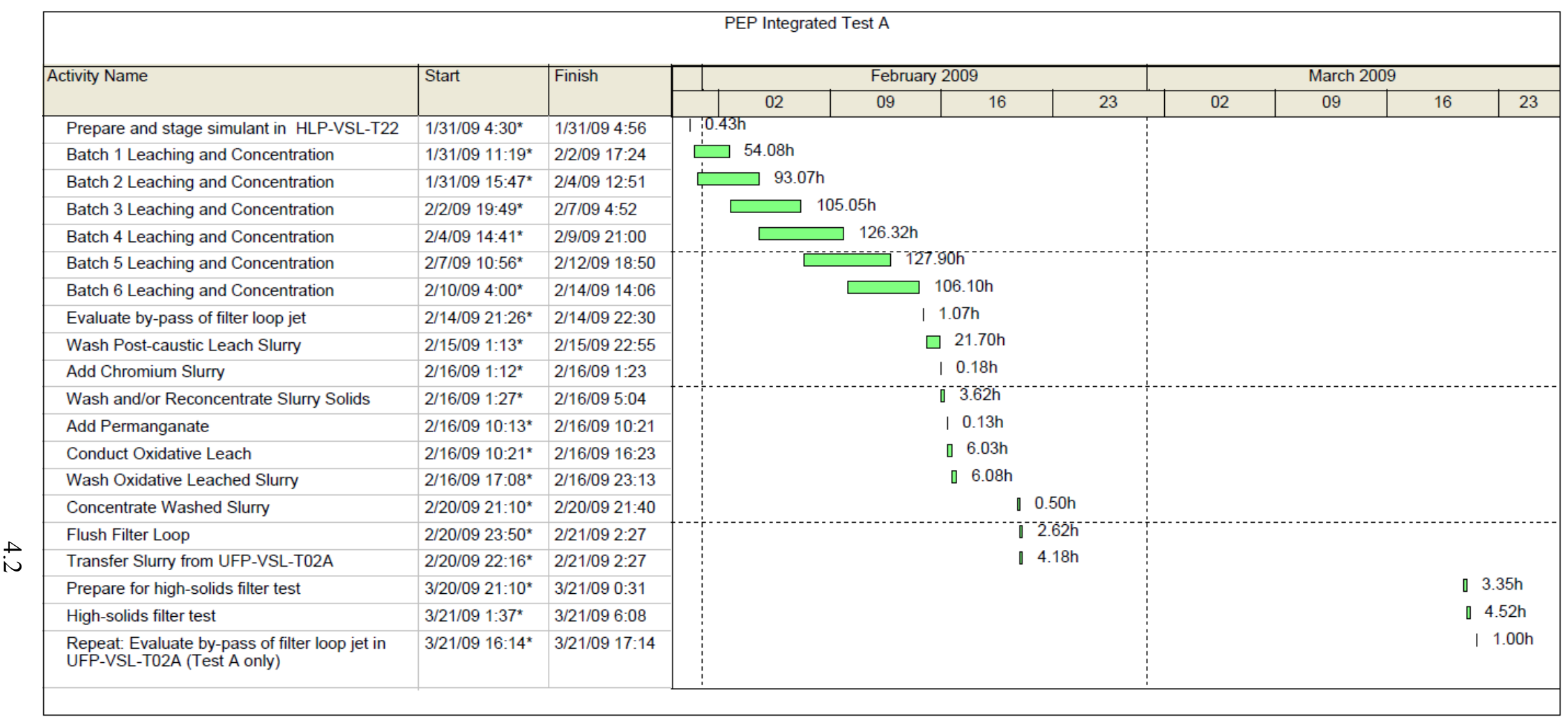

Figure 4.1. Integrated Test A Schedule of Completion 
Six UFP-VSL-T01 batches of simulant were leached, as prescribed by the run sheet in TI-WTP-PEP-065; three batches were processed in UFP-VSL-T01A, and three batches were processed in UFP-VSL-T01B. Simulant was transferred from HLP-VSL-T22 to UFP-VSL-T01A (three times; batches $1,3,5$ ) and B (three times; batches 2,4,6), concurrent with in-line caustic addition; however, a fraction of the caustic $(20 \%)$ was added to the top of the UFP-VSL-T01B batches. Caustic addition was split, in-line and to the top of the vessel, for UFP-VSL-T01B batches to demonstrate a proposed caustic control strategy that was based on slurry samples collected from UFP-VSL-T00001A/B. Integrated Test A caustic leaching operations demonstrated that if there was insufficient mixing in HLP-VSL-T22, causing an unknown aluminum solids inventory in UFP-VSL-T01B, operations could perform in-line caustic addition, then take samples from the UFP-VSL-T01B vessel to determine how much more caustic was needed for caustic leaching by adding it to the top of the vessel. Refer to Sections 9 and 14 in report WTP-RPT-197 for results on caustic additions made in-line versus on top of the vessel. Refer to Table 4.1 for transfer times for each of the six batches and Table 4.2 for the transfer volumes of simulant and 17.9-M caustic. Batches 1 and 2 transfers included $\sim 1872$ to $\sim 1877 \mathrm{~kg}$ of simulant and $\sim 637 \mathrm{~kg}$ of caustic, whereas the remaining batches had from $\sim 1469$ to $\sim 1662 \mathrm{~kg}$ of simulant and $\sim 621$ to $\sim 628 \mathrm{~kg}$ of caustic. AFA $(1000-\mathrm{mL})$ was added after PJMs were turned on during the transfers. PJM parameters were set to previously tuned parameters, and adjustments were made to achieve the target run parameter nozzle velocity of $4.8 \mathrm{~m} / \mathrm{s}$ and a PJM stroke length of $80 \%$. Refer to Section 5.3 for actual PJM peak average nozzle velocities and stroke lengths.

Table 4.1. Integrated Test A UFP Batch Transfer Times ${ }^{(a)}$

\begin{tabular}{|c|c|c|c|c|}
\hline Batch ID & Time Start & Time End & $\begin{array}{l}\text { PJMs Started } \\
\text { (Inches) }^{(\mathrm{b})}\end{array}$ & Transfer Time \\
\hline $\begin{array}{l}\text { Batch 1: HLP-VSL-T22 to } \\
\text { UFP-VSL-T01A }\end{array}$ & $1 / 31 / 09,11: 19$ & $1 / 31 / 09,11: 59$ & $40+$ & $\sim 45 \mathrm{~min}$ \\
\hline $\begin{array}{l}\text { Batch 2: HLP-VSL-T22 to } \\
\text { UFP-VSL-T01B }\end{array}$ & $1 / 31 / 09,15: 47$ & $1 / 31 / 09,17: 48$ & -- & $\sim 115 \mathrm{~min}$ \\
\hline $\begin{array}{l}\text { Batch 3: HLP-VSL-T22 to } \\
\text { UFP-VSL-T01A }\end{array}$ & $2 / 2 / 09,19: 49$ & $2 / 2 / 09,20: 20$ & 30 & $\sim 30$ min \\
\hline $\begin{array}{l}\text { Batch 4: HLP-VSL-T22 to } \\
\text { UFP-VSL-T01B }\end{array}$ & $2 / 4 / 09,14: 41$ & $2 / 4 / 09,15: 44$ & 53 & $\sim 60 \mathrm{~min}$ \\
\hline $\begin{array}{l}\text { Batch 5: HLP-VSL-T22 to } \\
\text { UFP-VSL-T01A }\end{array}$ & $2 / 7 / 09,10: 56$ & $2 / 7 / 09,11: 29$ & 42 & $\sim 30 \mathrm{~min}$ \\
\hline $\begin{array}{l}\text { Batch 6: HLP-VSL-T22 to } \\
\text { UFP-VSL-T01B }\end{array}$ & $2 / 10 / 09,04: 00$ & $2 / 10 / 09,06: 00$ & -- & $\sim 115$ min \\
\hline
\end{tabular}

(a) Data presented are based on HMI data.

(b) Note: Target Level to Start PJMs was 25 inches.

Temperature change was noted when caustic was added to the top of UFP-VSL-T01B. For Batch 6, the UFP-VSL-T01B temperature after caustic addition to the top of the vessel reached $\sim 65^{\circ} \mathrm{C}$; therefore, with approval, the vessel contents were cooled using the heat exchanger to meet the initial heatup temperature target. 
Table 4.2. Transfer Volumes Integrated Test A Batches ${ }^{(a)}$

\begin{tabular}{|c|c|c|c|c|c|}
\hline $\begin{array}{l}\text { Batch } \\
\text { No. }\end{array}$ & Vessel & Operation & Target Value & Units & Actual Value \\
\hline 1 & UFP-VSL-T01A & $\begin{array}{l}\text { Total Transfer } \\
\text { Volume }\end{array}$ & 501 & gal & 501.3 \\
\hline 1 & UFP-VSL-T01A & $\begin{array}{l}\text { In-line 17.9-M } \\
\text { Caustic Addition }\end{array}$ & 639 & $\mathrm{~kg}$ & 639 \\
\hline 2 & UFP-VSL-T01B & $\begin{array}{l}\text { Simulant Transfer } \\
\text { Volume }\end{array}$ & 479 & Gal & -- \\
\hline 2 & UFP-VSL-T01B & $\begin{array}{l}\text { 17.9-M Caustic } \\
\text { Addition }\end{array}$ & 511 & $\mathrm{~kg}$ & 511 \\
\hline 2 & UFP-VSL-T01B & $\begin{array}{l}\text { 17.9-M Caustic } \\
\text { Addition to the top } \\
\text { of the tank }\end{array}$ & 128 & $\mathrm{~kg}$ & 128.2 \\
\hline 3 & UFP-VSL-T01A & $\begin{array}{l}\text { Total Transfer } \\
\text { Volume }\end{array}$ & 426 & gal & 426 \\
\hline 3 & UFP-VSL-T01A & $\begin{array}{l}\text { In-line 17.9-M } \\
\text { Caustic Addition }\end{array}$ & 629 & $\mathrm{~kg}$ & 629 \\
\hline 4 & UFP-VSL-T01B & $\begin{array}{l}\text { Simulant Transfer } \\
\text { Volume }\end{array}$ & 393 & Gal & -- \\
\hline 4 & UFP-VSL-T01B & $\begin{array}{l}\text { 17.9-M Caustic } \\
\text { Addition }\end{array}$ & 504 & $\mathrm{~kg}$ & -- \\
\hline 4 & UFP-VSL-T01B & $\begin{array}{l}\text { 17.9-M Caustic } \\
\text { Addition to the top } \\
\text { of the tank }\end{array}$ & 126 & $\mathrm{~kg}$ & 126 \\
\hline 5 & UFP-VSL-T01A & $\begin{array}{l}\text { Total Transfer } \\
\text { Volume }\end{array}$ & 415 & gal & 415 \\
\hline 5 & UFP-VSL-T01A & $\begin{array}{l}\text { In-line 17.9-M } \\
\text { Caustic Addition }\end{array}$ & 625 & $\mathrm{~kg}$ & 625 \\
\hline 6 & UFP-VSL-T01B & $\begin{array}{l}\text { Simulant Transfer } \\
\text { Volume }\end{array}$ & 393 & Gal & 393 \\
\hline 6 & UFP-VSL-T01B & $\begin{array}{l}\text { 17.9-M Caustic } \\
\text { Addition }\end{array}$ & 499 & $\mathrm{~kg}$ & 499 \\
\hline 6 & UFP-VSL-T01B & $\begin{array}{l}\text { 17.9-M Caustic } \\
\text { Addition to the top } \\
\text { of the tank }\end{array}$ & 125 & $\mathrm{~kg}$ & -- \\
\hline
\end{tabular}

\subsection{Batch Heatup and Leach}

For each of the six batches, heating was conducted in two phases. Initial heating to a target temperature of $57^{\circ} \mathrm{C}$ (within 3 hours) used heat exchangers UFP-HX-T04A and B; heat exchangers UFP-HX-T05A and B were by-passed. Final heating to the temperature of $98^{\circ} \mathrm{C}$ (the target heat-up duration was 3.8 hours) was reached with direct steam injection. 
The PJM air supply lines had drain lines that diverted condensate formed in the air lines. PJMs switched from vacuum-fill to vent-fill upon reaching $60^{\circ} \mathrm{C}$, and PJM drain valves on the drain lines were opened when tank temperatures exceeded $65^{\circ} \mathrm{C}$ to avoid adding too much condensate to the vessel. At $90^{\circ} \mathrm{C}$, the ring air purge was adjusted to meet high-temperature flow-rate settings. PJMs were tuned to run sheet nozzle velocity targets when the final leaching temperature of $98{ }^{\circ} \mathrm{C}$ was reached. The target run parameter nozzle velocity was $4.8 \mathrm{~m} / \mathrm{s}$, and the PJM stroke length was $80 \%$ (refer to Section 5.3 for actual PJM peak average nozzle velocities and stroke lengths). Problems were noted with tuning the PJMs at this temperature (Batch 1). Increasing the drive pressure at the leach temperature would not produce higher PJM peak average nozzle velocities, as reported on the test instruction (TI). Generally, it was difficult to tune UFP-VSL-T01A/B PJMs at $98^{\circ} \mathrm{C}$ because level readings from the Drexelbrooks and lasers were not reliable at high temperatures.

The total leach-time target was 16 hours. During hours 1 through 15, Batches 1 through 6 had 2.9-L of IW added to the UFP-VSL-T01A/B vessels hourly to compensate for the low condensation accumulation rate. Table 4.3 summarizes heatup, leach times, and durations for each of the six batches.

Table 4.3. Start and Stop Times to Heat, and Caustic Leach Integrated Test A Batches ${ }^{(a)}$

\begin{tabular}{|c|c|c|c|c|c|c|}
\hline Batch \# & $\begin{array}{l}\text { Heat Start } \\
\text { Time }\end{array}$ & $\begin{array}{l}\text { Heat End } \\
\text { Time }\end{array}$ & $\begin{array}{c}\text { Heat } \\
\text { Duration }\end{array}$ & $\begin{array}{c}\text { Caustic } \\
\text { Leach Start } \\
\text { Time }\end{array}$ & $\begin{array}{c}\text { Caustic } \\
\text { Leach End } \\
\text { Time }\end{array}$ & $\begin{array}{c}\text { Duration } \\
\text { (hr) }\end{array}$ \\
\hline $\begin{array}{c}\text { Batch } 1 \\
\text { UFP-VSL-T01A }\end{array}$ & $\begin{array}{c}1 / 31 / 09 \\
14: 40\end{array}$ & $\begin{array}{c}1 / 31 / 09 \\
19: 40\end{array}$ & 5.65 & $\begin{array}{c}1 / 31 / 09 \\
19: 40\end{array}$ & $\begin{array}{c}2 / 1 / 09 \\
11: 40\end{array}$ & 16.55 \\
\hline $\begin{array}{c}\text { Batch } 2 \\
\text { UFP-VSL-T01B }\end{array}$ & $\begin{array}{c}1 / 31 / 09 \\
22: 07\end{array}$ & $\begin{array}{c}\text { 2/1/09 } \\
03: 20\end{array}$ & 5.22 & $\begin{array}{c}\text { 2/1/09, } \\
03: 20\end{array}$ & $\begin{array}{c}2 / 1 / 09 \\
19: 59\end{array}$ & 16.35 \\
\hline $\begin{array}{c}\text { Batch } 3 \\
\text { UFP-VSL-T01A }\end{array}$ & $\begin{array}{c}2 / 2 / 09 \\
21: 33\end{array}$ & $\begin{array}{c}2 / 3 / 09 \\
02: 45\end{array}$ & 5.20 & $\begin{array}{c}2 / 3 / 09 \\
02: 45\end{array}$ & $\begin{array}{c}2 / 3 / 09 \\
18: 45\end{array}$ & 16.00 \\
\hline $\begin{array}{c}\text { Batch } 4 \\
\text { UFP-VSL-T01B }\end{array}$ & $\begin{array}{c}2 / 4 / 09 \\
17: 43\end{array}$ & $\begin{array}{c}2 / 4 / 09 \\
22: 38\end{array}$ & 5.17 & $\begin{array}{c}2 / 4 / 09 \\
22: 38\end{array}$ & $\begin{array}{c}2 / 5 / 09 \\
14: 38\end{array}$ & 16.00 \\
\hline $\begin{array}{c}\text { Batch } 5 \\
\text { UFP-VSL-T01A }\end{array}$ & $\begin{array}{c}2 / 7 / 09 \\
12: 41\end{array}$ & $\begin{array}{c}2 / 7 / 09 \\
17: 28\end{array}$ & 4.78 & $\begin{array}{c}2 / 7 / 09 \\
17: 28\end{array}$ & $\begin{array}{c}2 / 8 / 09 \\
09: 29\end{array}$ & 16.02 \\
\hline $\begin{array}{c}\text { Batch } 6 \\
\text { UFP-VSL-T01B }\end{array}$ & $\begin{array}{c}2 / 10 / 09 \\
06: 00\end{array}$ & $\begin{array}{c}2 / 10 / 09 \\
12: 06\end{array}$ & 6.10 & $\begin{array}{c}2 / 10 / 09 \\
12: 06\end{array}$ & $\begin{array}{c}\text { 2/11/09 } \\
04: 05\end{array}$ & 15.98 \\
\hline
\end{tabular}

(a) Data presented are based on HMI data.

For the most part, the six batches were heated according to the Test Plan. However, as noted previously, Batch 6 UFP-VSL-T01B exceeded the initial heat-up temperature because of the higher-than-expected caustic addition rate; therefore, with Test Director guidance, the vessel contents were cooled using the heat exchanger to meet the initial heatup temperature run sheet target. On 1/31/09, 47 minutes of steam injection was inadvertently used to do the initial heating for UFP-VSL-T01B Batch 2 instead of using heat exchanger UFP-HX-T04B. Approximately 0.3 inches (per laser) of condensate was added during this time to UFP-VSL-T01B based on stable level measurements. Problems were noted with the automatic temperature controller during caustic leaching. While in automatic temperature control, leach temperatures reached $101.5^{\circ} \mathrm{C}(13: 30,2 / 1 / 09$, LRB 60229, pg. 32) for UFP-VSL-T01B Batch $2,103.8^{\circ} \mathrm{C}\left(17: 37,2 / 7 / 09\right.$, LRB 60229 , pg. 150) for UFP-VSL-T01A Batch 5, and $103^{\circ} \mathrm{C}(19: 35$, 
2/10/09, LRB 60230, pg. 21) for UFP-VSL-T01B Batch 6. The high temperature during UFP-VSL-T01B Batch 2 leaching may be due to a high-high level alarm that occurred $\sim 30$ minutes earlier that turned off the steam supply in UFP-VSL-T01B. The steam supply was turned back on immediately. However, the UFP-VSL-T01B PJMs were off since 13:05, 2/1/09; PJMs in UFP-VSL-T01B were turned on at 13:31, 2/1/09 (LRB 60229, pg. 33). With the UFP-VSL-T01B PJMs off, the prototypic RTD, located in the bottom region of Tank T01B, did not contact the steam heated slurry in the upper region of Tank T01B. Consequently, steam supply continued based on the cool temperature reading from the UFP-VSL-T01B prototypic RTD. Operators realized the caustic leach temperature target had been exceeded only when the UFP-VSL-T01B PJMs were turned back on and the slurry was mixed. The high temperature during UFP-VSL-T01A Batch 5 leaching may be due to the UFP-VSL-T01A PJMs locking up; no mixing was taking place (17:36, 2/7/2009, LRB 60229, pg. 150). UFP-VSL-T01A PJMs were turned back on at 17:40, and tank temperature starting decreasing. The cause for the high temperature during Batch 6 leaching is unclear since UFP-VSL-T01B PJMs were on. In addition, the controller would occasionally switch from auto to manual without operator action, resulting in unexpected temperature drops as low as $94^{\circ} \mathrm{C}$ (Batch 2). An unexpected shutoff for steam injection for Batch 4 dropped the leach temperature to $95.6^{\circ} \mathrm{C}$.

See Figure 4.2 through Figure 4.7 for batch heating and cooling temperature profiles.

\subsubsection{Cooling}

Six batches of simulant were leached and cooled between 02/01/09, 11:57, and 2/11/09, 07:10. Each of the batches was cooled to $60^{\circ} \mathrm{C}$ by recirculating tank contents through heat exchangers UFP-HX-T05A and $\mathrm{B}$ (aligned and bypassed as necessary to reach cooling curve targets); heat exchangers UFP-HX-T04A and B were bypassed. The exception was Batch 2. With approval, post-caustic-leach cooling in UFP-VSL-T01B to filtration temperature was accomplished by slowing the speed of pump UFP-PMP-T41B to 1.2 GPM instead of aligning and by-passing UFP-HX-T05B. The pump UFP-PMP-T41B speed was increased periodically to flush settled solids out of the recirculation line.

PJMs switched from vent-fill to vacuum-fill upon dropping to $60^{\circ} \mathrm{C}$, and the steam-ring air purge was adjusted to meet standard flow-rate settings. Refer to Table 4.4 for batch cool-down start and stop times. 
Table 4.4. Start/Stop Times to Partially Cool Integrated Test A Batches ${ }^{(a)}$

\begin{tabular}{cccc}
\hline Partially cool-down & Start Time & End Time & $\begin{array}{c}\text { Duration } \\
\text { (hr) }\end{array}$ \\
\hline $\begin{array}{c}\text { Batch 1 } \\
\text { UFP-VSL-T01A }\end{array}$ & $2 / 1 / 09,11: 57$ & $2 / 1 / 09,14: 59$ & 3.03 \\
$\begin{array}{c}\text { Batch 2 } \\
\text { UFP-VSL-T01B }\end{array}$ & $2 / 1 / 09,20: 15$ & $2 / 1 / 09,22: 56$ & 2.68 \\
$\begin{array}{c}\text { Batch 3 } \\
\text { UFP-VSL-T01A }\end{array}$ & $2 / 3 / 09,19: 10$ & $2 / 3 / 09,21: 55$ & 3.00 \\
$\begin{array}{c}\text { Batch 4 } \\
\text { UFP-VSL-T01B }\end{array}$ & $2 / 5 / 09,14: 45$ & $2 / 5 / 09,17: 30$ & 2.80 \\
$\begin{array}{c}\text { Batch 5 } \\
\text { UFP-VSL-T01A }\end{array}$ & $2 / 8 / 09,09: 29$ & $2 / 8 / 09,12: 33$ & 3.07 \\
$\begin{array}{c}\text { Batch 6 } \\
\text { UFP-VSL-T01B }\end{array}$ & $2 / 11 / 09,04: 25$ & $2 / 11 / 09,07: 10$ & 2.72 \\
\hline
\end{tabular}

(a) Data presented are based on HMI data.

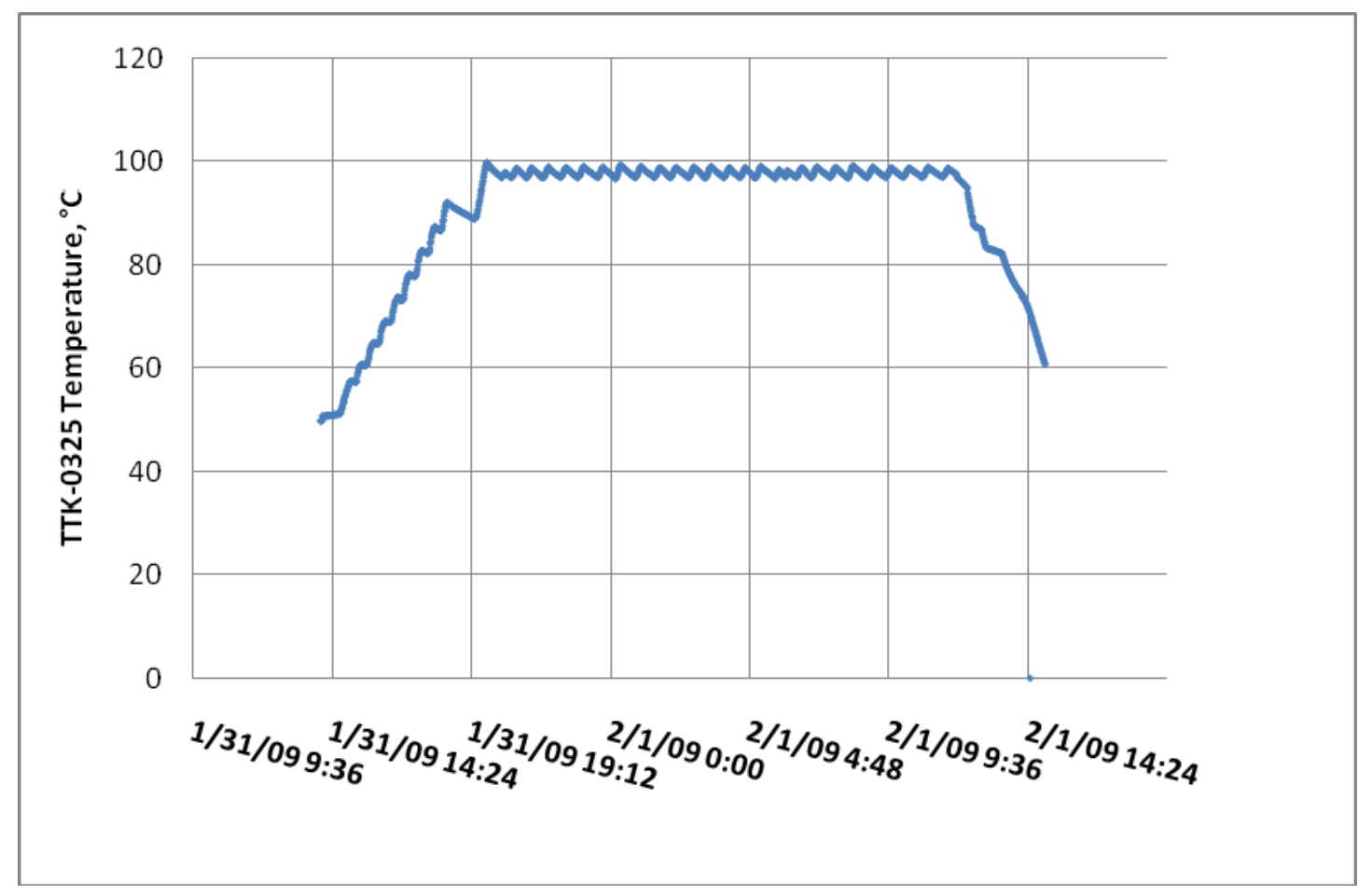

Figure 4.2. UFP-VSL-T01A Caustic Leach Batch 1 Heating and Partial Cooling 


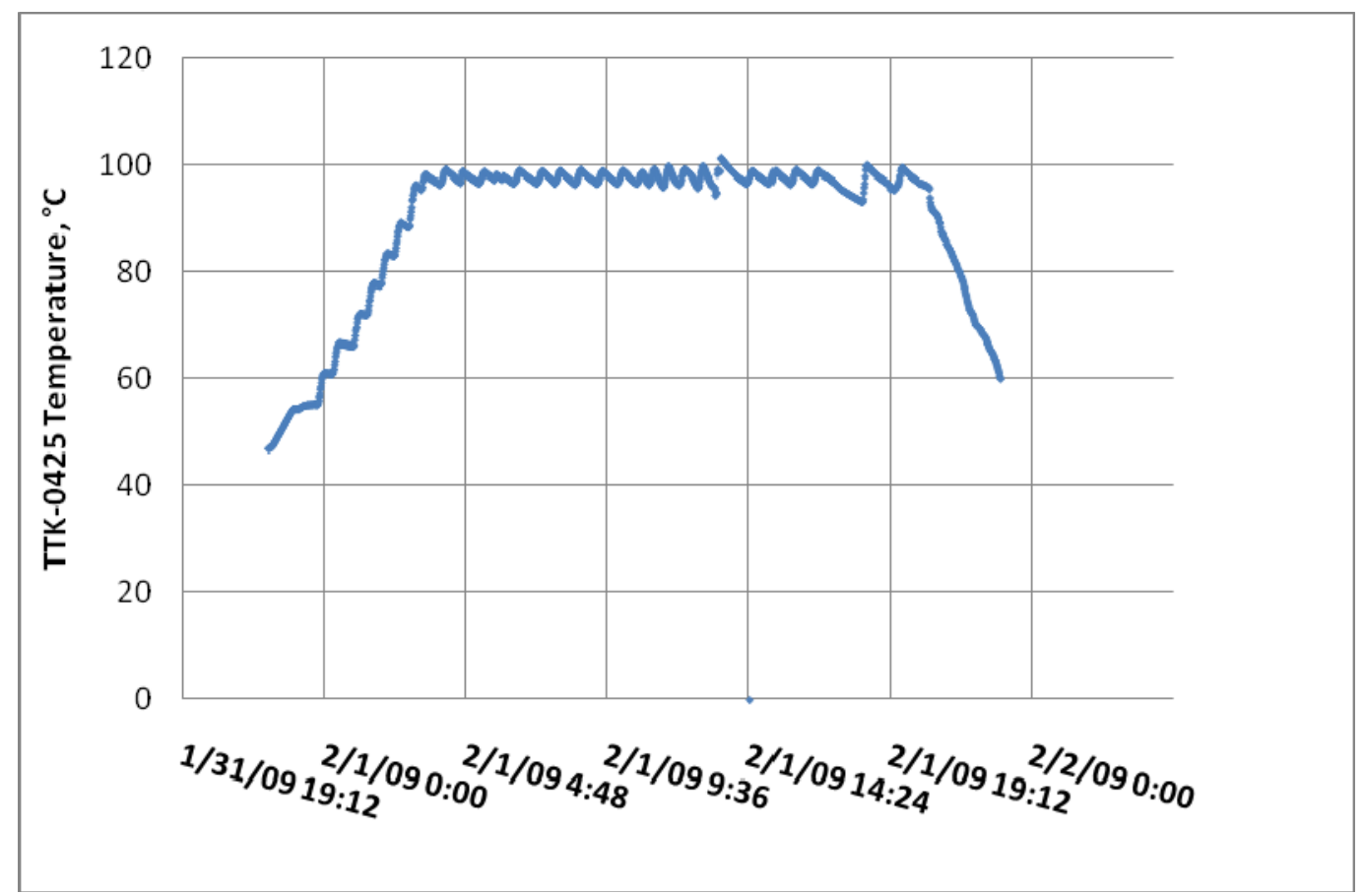

Figure 4.3. UFP-VSL-T01B Caustic Leach Batch 2 Heating and Partial Cooling

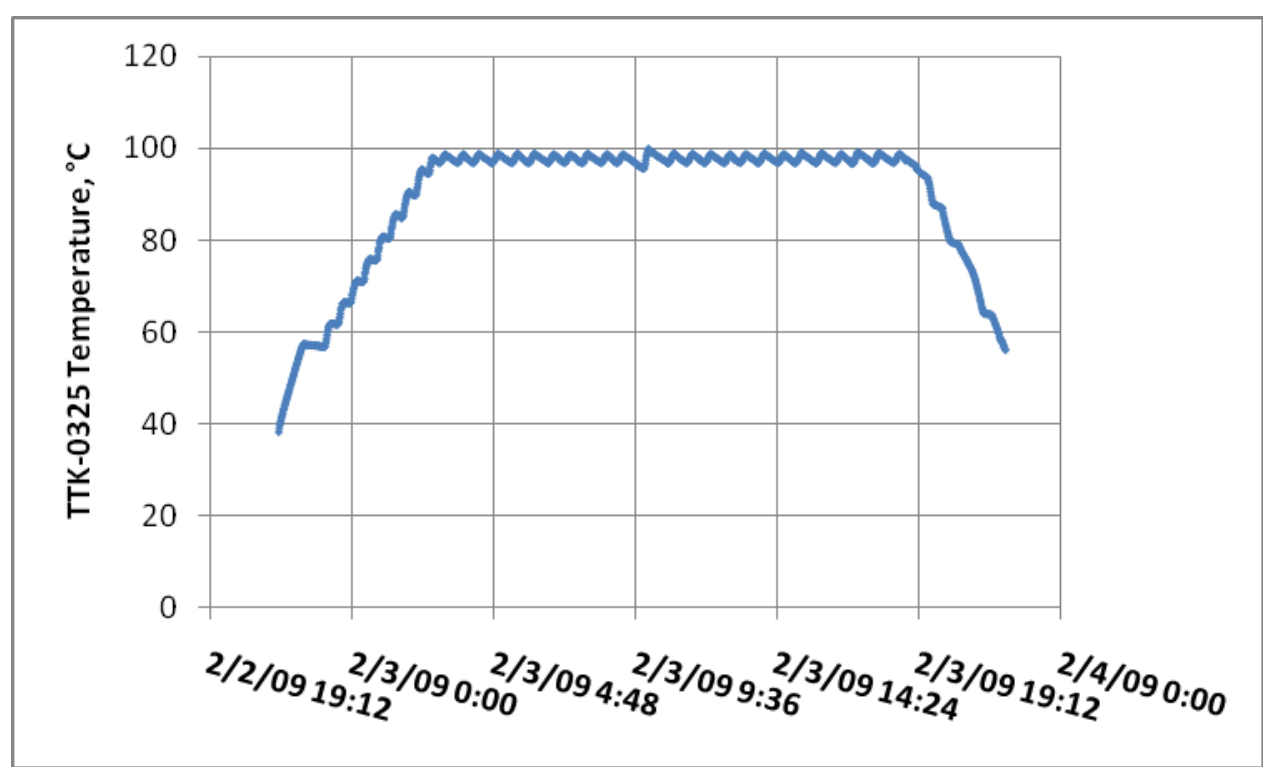

Figure 4.4. UFP-VSL-T01A Caustic Leach Batch 3 Heating and Partial Cooling 


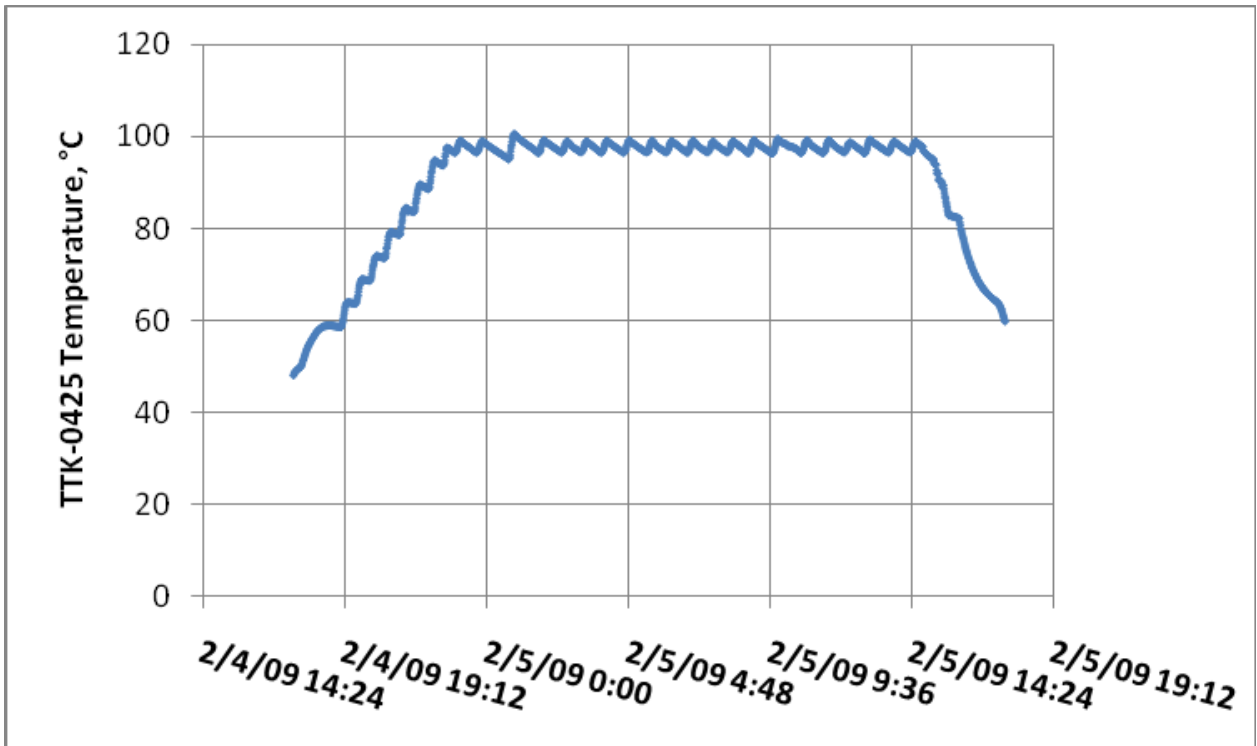

Figure 4.5. UFP-VSL-T01B Caustic Leach Batch 4 Heating and Partial Cooling

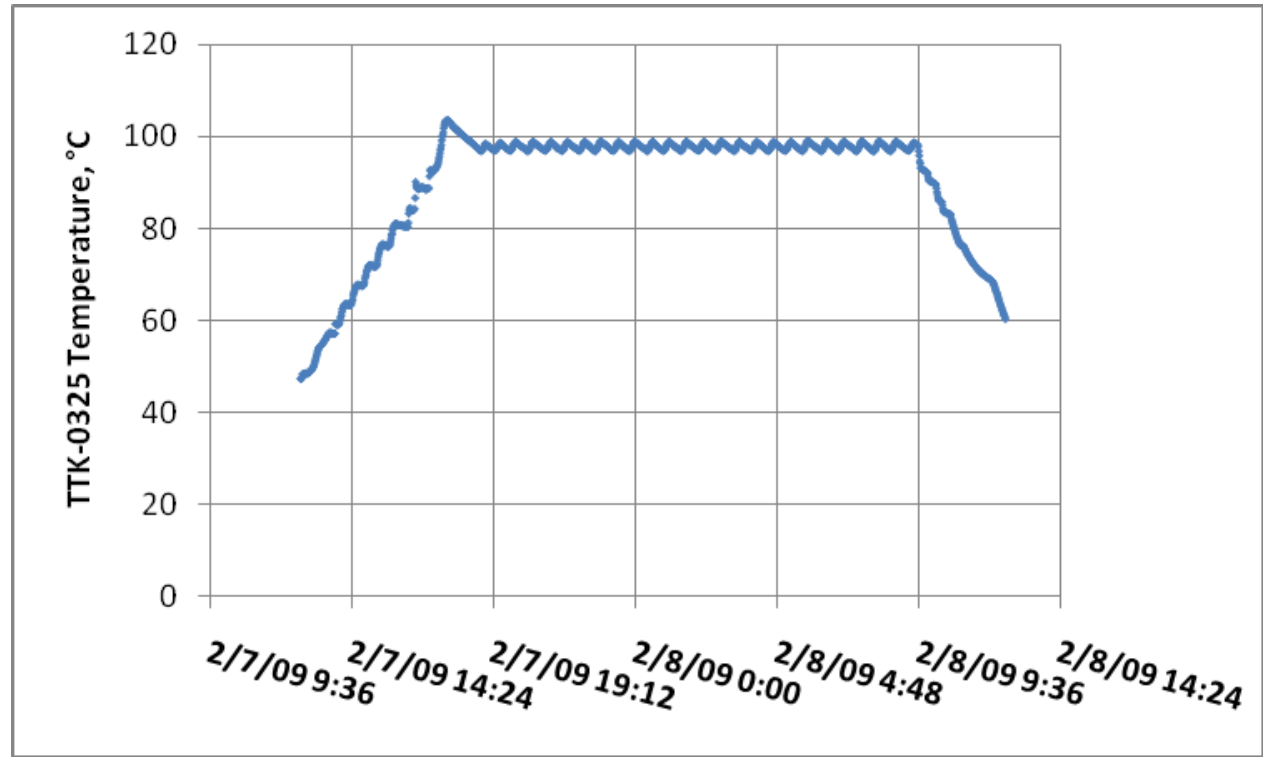

Figure 4.6. UFP-VSL-T01A Caustic Leach Batch 5 Heating and Partial Cooling 


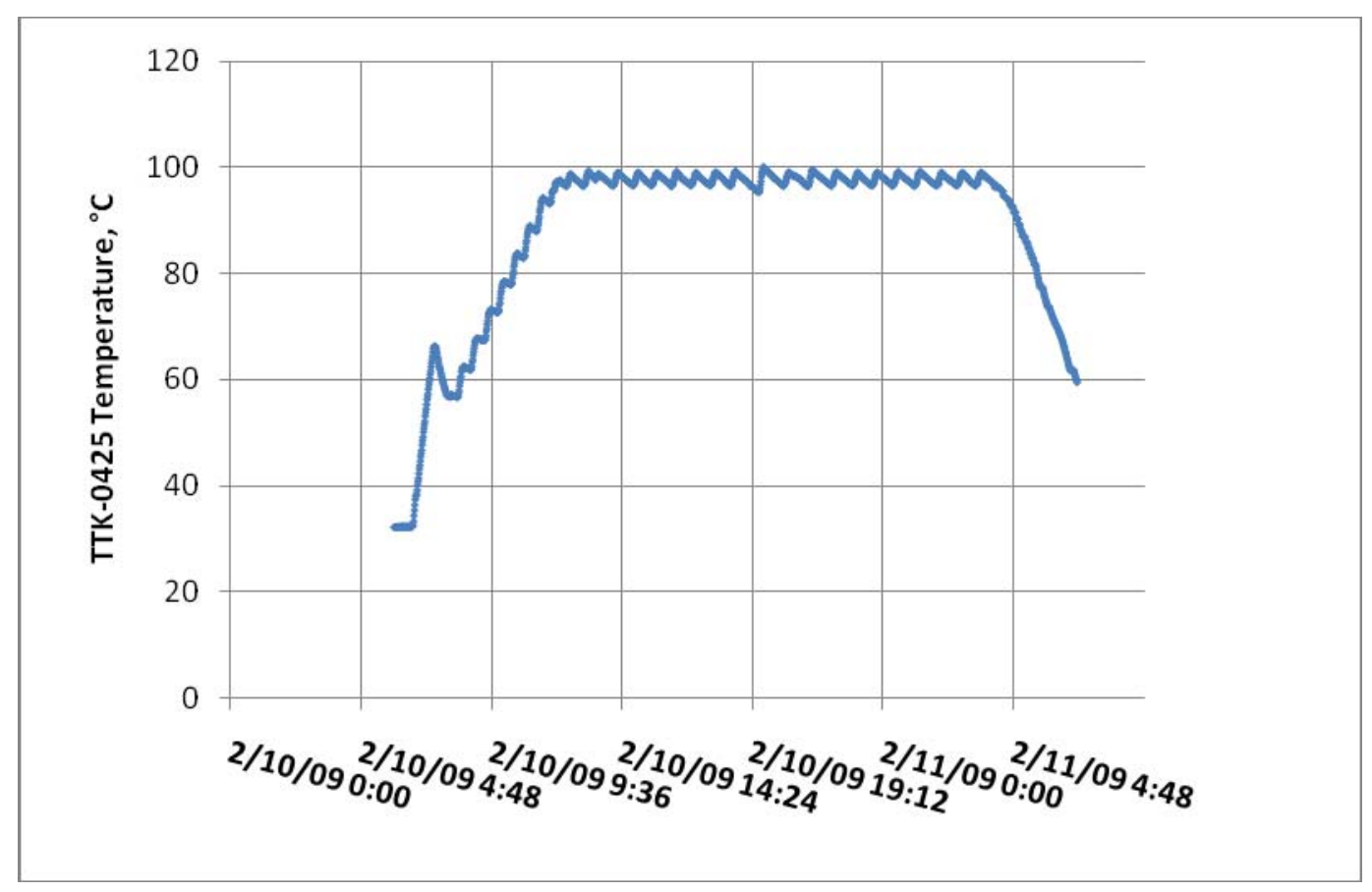

Figure 4.7. UFP-VSL-T01B Caustic Leach Batch 6 Heating and Partial Cooling

CD sampling during leaching was interrupted with CD sampling station pump problems. Consequently, the $\mathrm{CD}$ sampling station vacuum system was used to collect samples, or affected samples were delayed and collected during the next sample collection time (refer to Appendix E for test deviations). Other problems during leaching noted were the UFP-VSL-T01A/B PJMs overblowing. The level probes did not operate properly at various times during the operation, but specifically during or after heating. PJM drive times and drive pressures were frequently adjusted to stop the PJM overblows.

The laser and bubbler levels were used to monitor UFP-VSL-T01A/B tank levels during heating and caustic leaching and to monitor the UFP-VSL-T02A tank level during the automatic transfers from the leach tanks. Drexelbrooks in both UFP-VSL-T01A and UFP-VSL-T02A vessels were not working reliably for much of the test. Refer to Section 6.3 for level control and monitoring issues.

\subsection{Post-Caustic-Leach Solids Concentration}

Six leached batches were concentrated between 2/2/09, 01:34, and 2/14/09, 14:06. During solids concentration, caustic-leached feed was supplied by UFP-VSL-T01A and UFP-VSL-T01B alternately until a prototypic batch at $17-\mathrm{wt} \%$ UDS was achieved in UFP-VSL-T02A. This is to say that while one upfront UFP vessel was leaching, the other upfront UFP vessel was transferring batches to UFP-VSL-T02A.

The filters were cleaned during Shakedown/Functional Testing efforts; refer to WTP-RPT-190 for a description of filter cleaning exercises. Before the first transfer, ultrafilters were drained just before the concentration of solids was started; the shell side was not drained. The filter-loop draining was completed at 20:40,2/1/09. Then the filter loop was filled with simulant to bring the level down in 
UFP-VSL-T02A to operate and tune the PJMs to target run sheet parameters. The target run parameter nozzle velocity was $7.3 \mathrm{~m} / \mathrm{s}$, and the PJM stroke length was $80 \%$. Refer to Section 5.3 for actual PJM peak average nozzle velocity and stroke length.

Solids were concentrated in UFP-VSL-T02A using one filter bundle, UFP-FILT-T01A. The filter-loop configuration had heat exchanger UFP-HX-T02A aligned, and filters UFP-FILT-T02A through -T05A and heat exchanger UFP-HX-T03A were bypassed. When the contents of UFP-VSL-T02A reached 44.7 inches, one 11-gallon make-up addition from the feed preparation vessels (UFP-VSL-T01A/B) to the feed vessel (UFP-VSL-T02A) was automatically triggered by the program logic controller (PLC). Solids concentration was completed for each batch when the feed tank (UFP-VSL-T01A/B) had been emptied to the heel. The initial batch was transferred to UFP-VSL-T02A on $2 / 1 / 09,16: 34$. The solids concentration to $17-w t \%$ UDS was completed at 14:06 on 2/14/09. The concentration lasted 300 hours.

Slurry was transferred from UFP-VSL-T01A/B to UFP-VSL-T02A as needed to maintain a slurry level of 44.7 inches in UFP-VSL-T02A. The laser level was the preferred method for measuring level; however, the bubbler was also used when laser performance became unstable. Vessel recirculation valves V03125 and V04125, for UFP-VSL-T01A and B, respectively, were adjusted to obtain different flow rates for recirculation and batch transfers to UFP-VSL-T02A. During concentration, the temperature setpoint for heat exchanger UFP-HX-T02A was adjusted periodically to maintain the slurry at the target temperature of $25^{\circ} \mathrm{C}$.

Permeate was routed to UFP-VSL-T62B during solids concentration. Permeate samples were collected between the UFP-FILT-T01A filter bundle and the pulse pot at three evenly spaced times and inspected for particle breakthrough. No observations of particle breakthrough were noted.

Post-caustic-leach concentration filtering rates continued to decrease over time with the Filter T01A axial pressure drop increasing gradually. At 18:20, 2/4/09, the Test Director emailed that backpulsing would be applied through the course of filtration to improve permeate rates. Permeate flow rate after backpulsing was the same for all backpulse variations. See the backpulse description in Section 6.1. Figure 4.8 through Figure 4.35 show the filtration periods for each of the batches - where the prototypic UFP-VSL-T02A temperature was taken from TTK-0619. Flow meters upstream from Pump T42A and downstream from Pump T43A measure in gallons per minute; however axial velocity is calculated from the volume flow rate - where $15 \mathrm{ft} / \mathrm{s}$ correlates to 109 gallons per minute. The equation for TMP (average of inlet and outlet filter bundle pressure minus the shell side pressure) uses the inlet and outlet pressures for each filter bundle, where the outlet pressure of one filter bundle serves as the inlet pressure of the subsequent filter bundle. Because of this arrangement, TMP values for UFP-FILT-T02A and UFP-FILT-T05A are shown on Figures 4.8 through 4.35 even though only UFP-FILT-T01A was aligned. Refer to Section 8, Filtration, of report WTP-RPT-197 for TMP and temperature-corrected filter flux versus $\mathrm{wt} \%$ solids plots, along with permeate production rates. 


\section{Integrated Test A-Post-Caustic-Leach Concentration, Batch \#1}

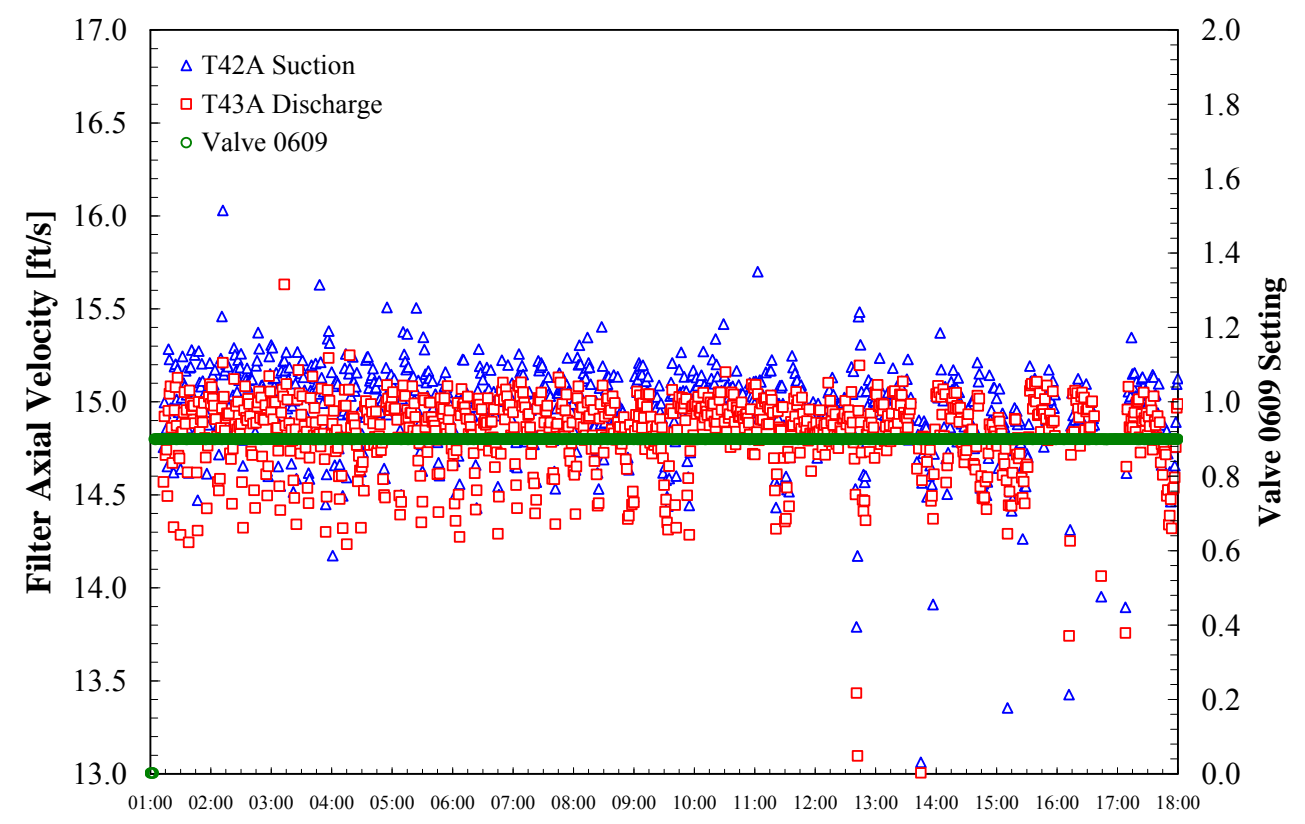

Time Stamp - 2/2/2009

Figure 4.8. Axial Velocity Based on Flow Measurements at the Suction to Pump T42A and the Discharge to Pump T43A for Post-Caustic-Leach Concentration, Batch \#1; Valve SV-0609 Setting (for information only) Scale 0 to 2 Correlates to $0 \%$ and $100 \%$ Open, Respectively

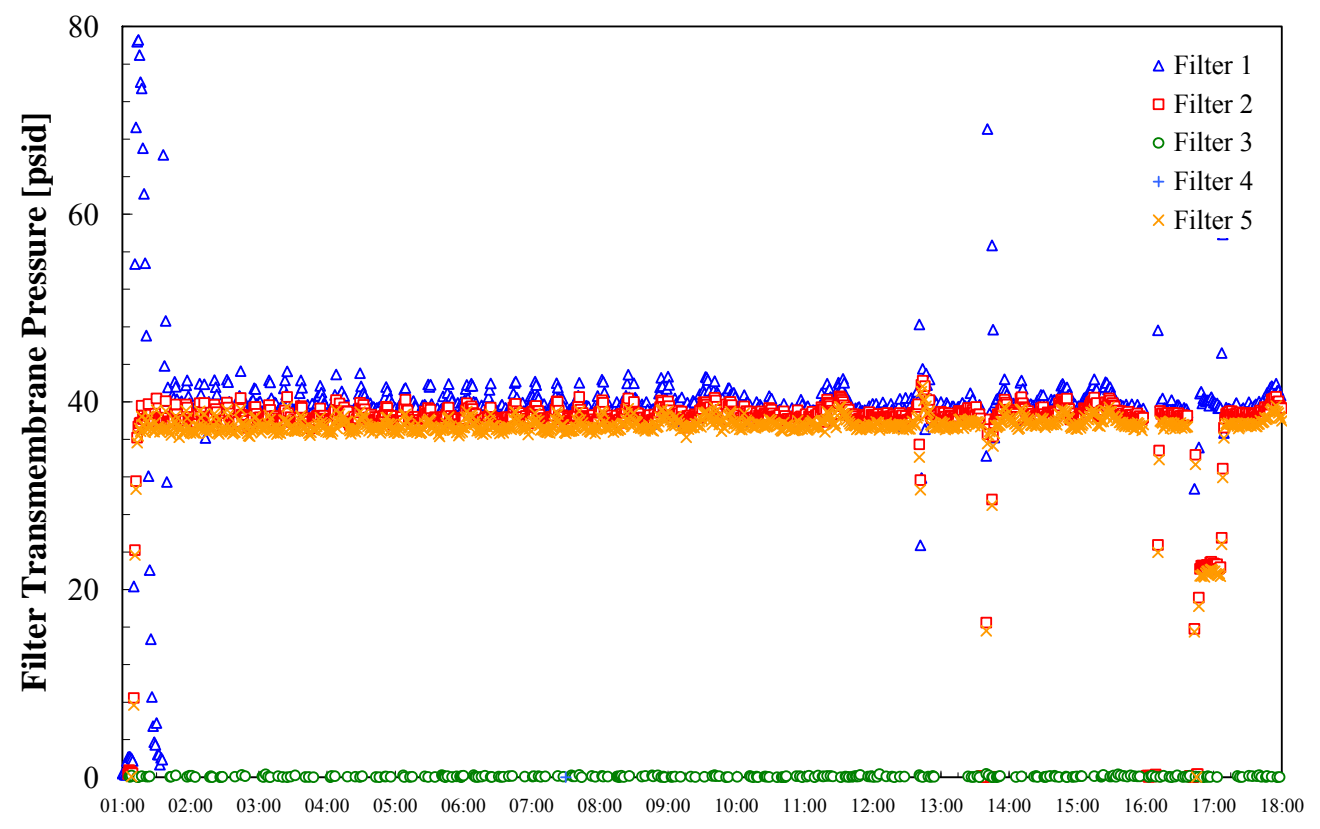

Time Stamp - 2/2/2009

Figure 4.9. TMP for Post-Caustic-Leach Concentration, Batch \#1 


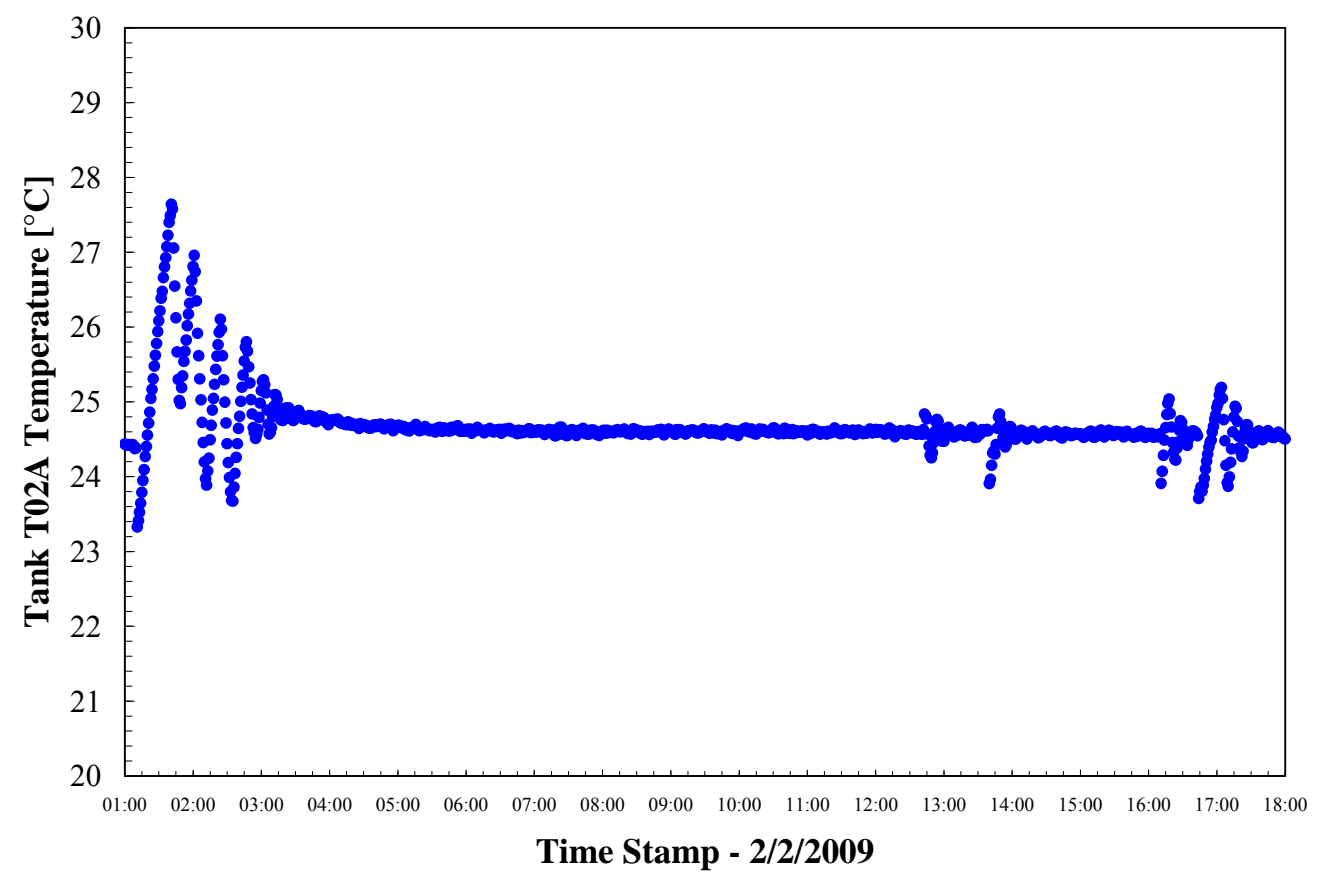

Figure 4.10. Tank T02A (TTK-0619) Temperature for Post-Caustic-Leach Concentration, Batch \#1

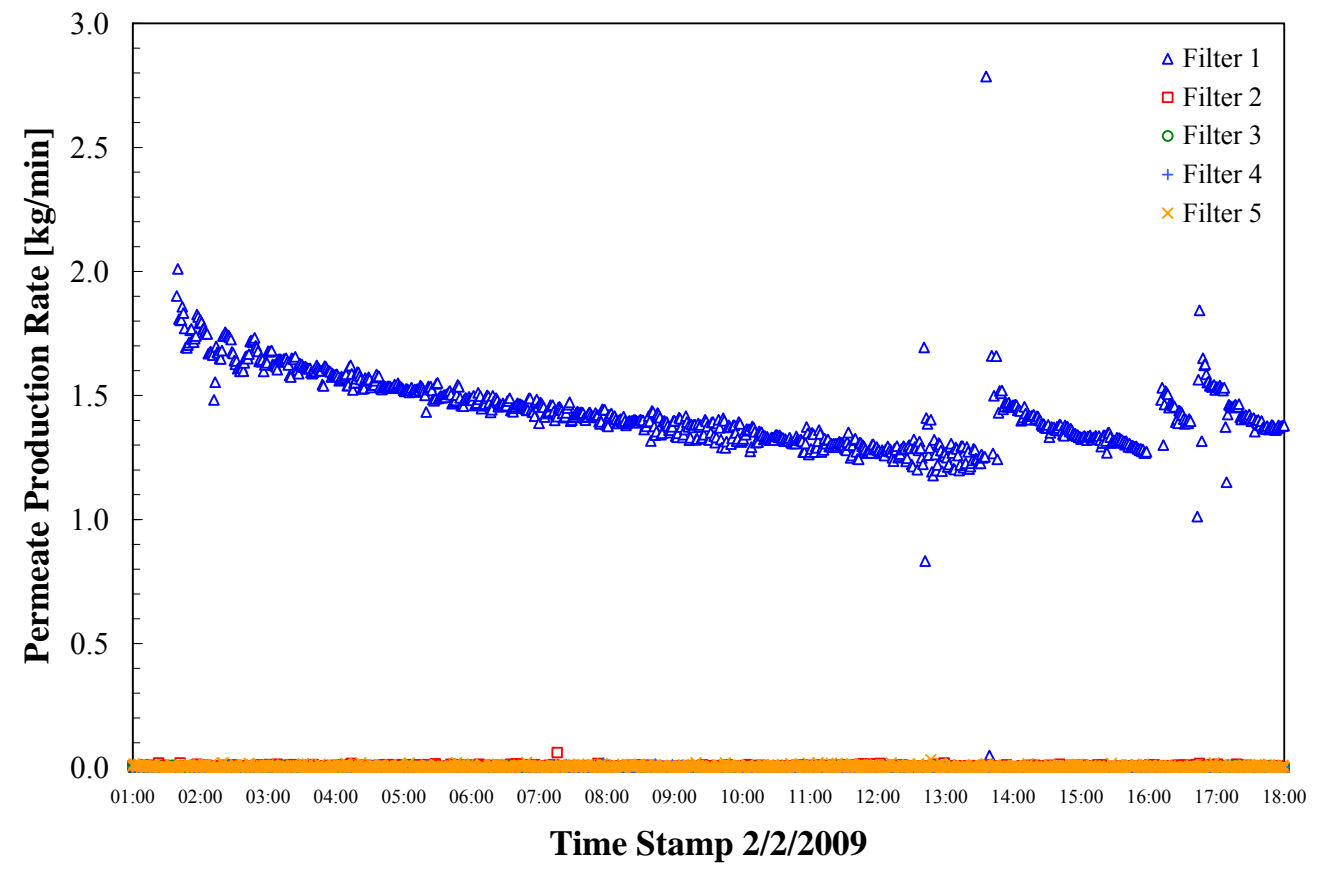

Figure 4.11. Permeate Production Rate for Post-Caustic-Leach Concentration, Batch \#1 


\section{Integrated Test A-Post-Caustic-Leach Concentration, Batch \#2}

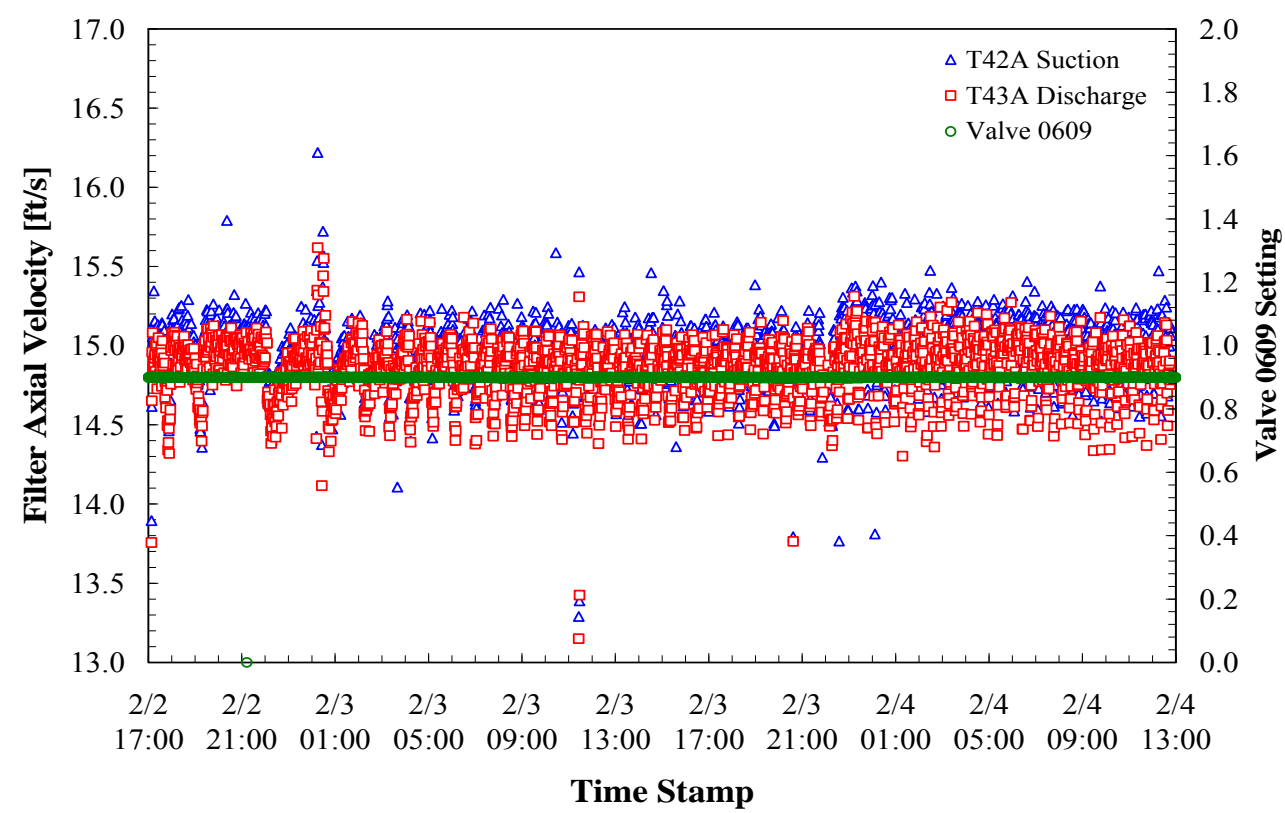

Figure 4.12. Axial Velocity Based on Flow Measurements at the Suction to Pump T42A and the Discharge to Pump T43A for Post-Caustic-Leach Concentration, Batch \#2; Valve SV-0609 Setting (for information only) Scale 0 to 2 Correlates to $0 \%$ and $100 \%$ Open, Respectively

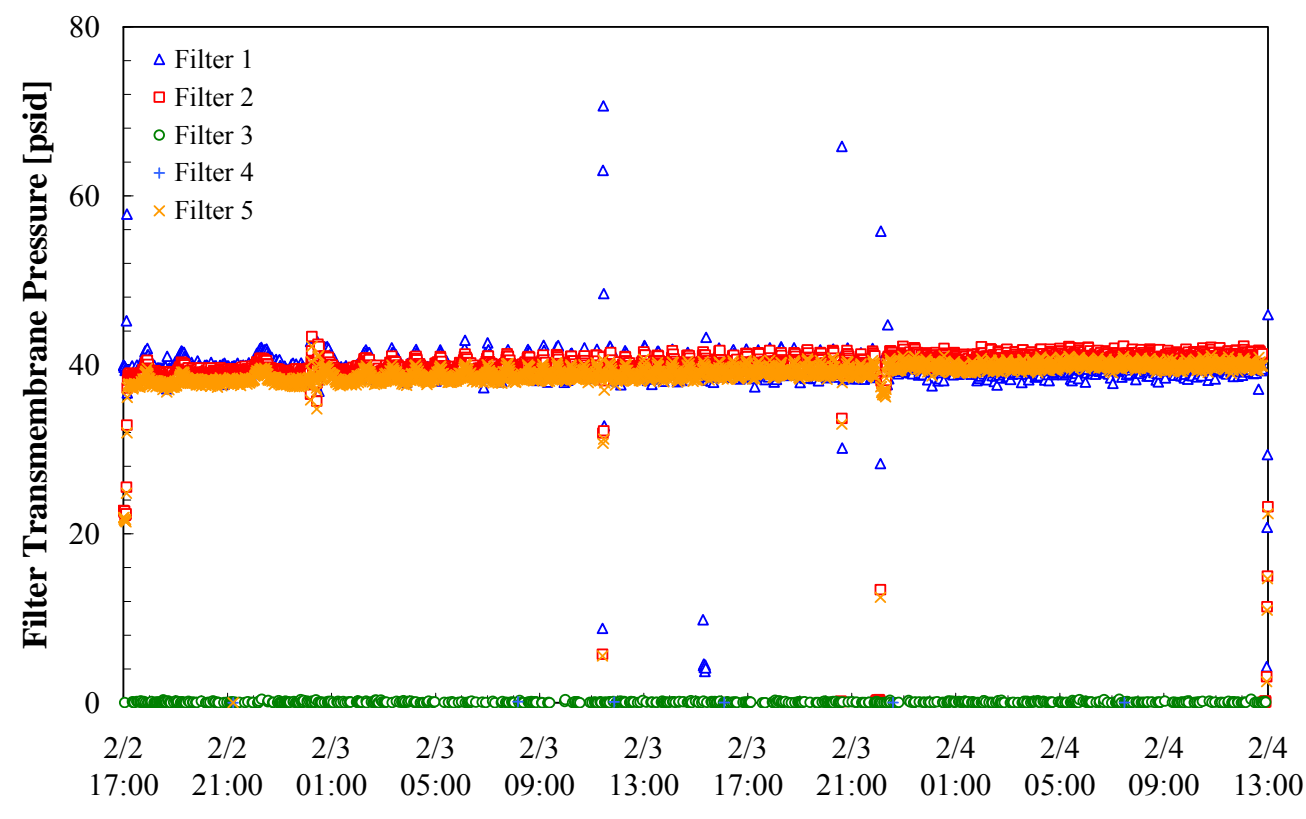

Time Stamp

Figure 4.13. TMP for Post-Caustic-Leach Concentration, Batch \#2 


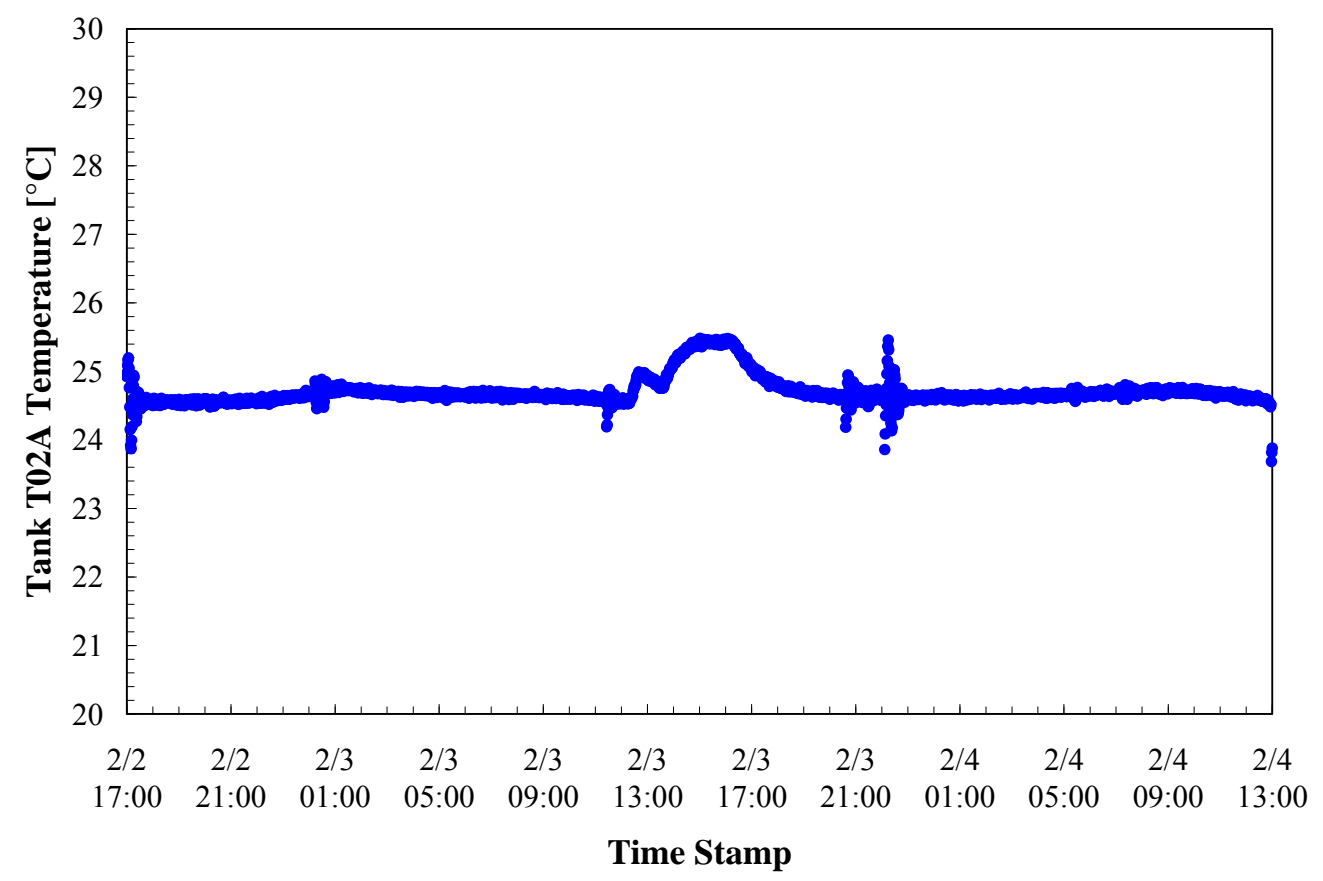

Figure 4.14. Tank T02A (TTK-0619) Temperature for Post-Caustic-Leach Concentration, Batch \#2

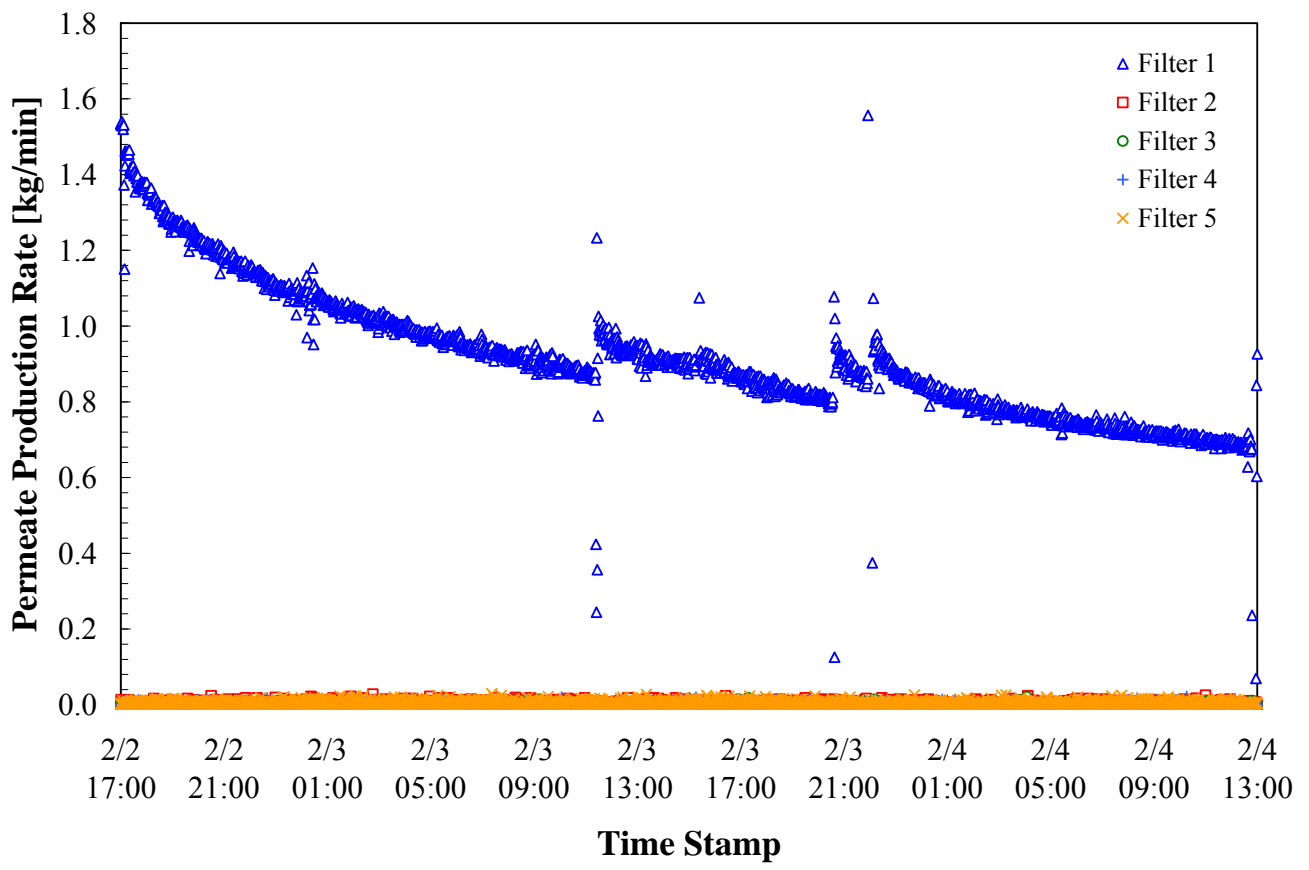

Figure 4.15. Permeate Production Rate for Post-Caustic-Leach Concentration, Batch \#2 


\section{Integrated Test A-Post-Caustic-Leach Concentration, Batch \#3}

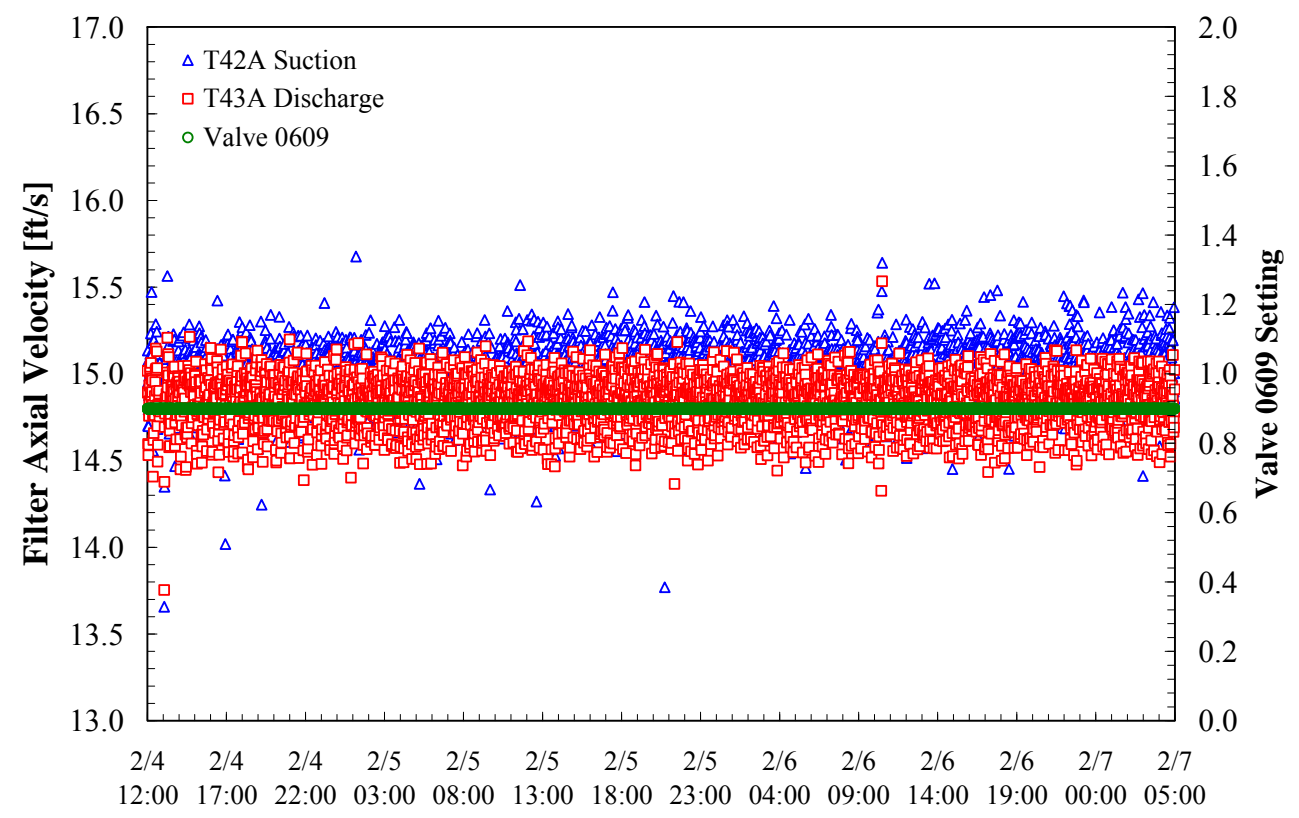

Time Stamp

Figure 4.16. Axial Velocity Based on Flow Measurements At the Suction to Pump T42A and the Discharge to Pump T43A for Post-Caustic-Leach Concentration, Batch \#3; Valve SV-0609 Setting (for information only) Scale 0 to 2 Correlates to $0 \%$ and $100 \%$ Open, Respectively

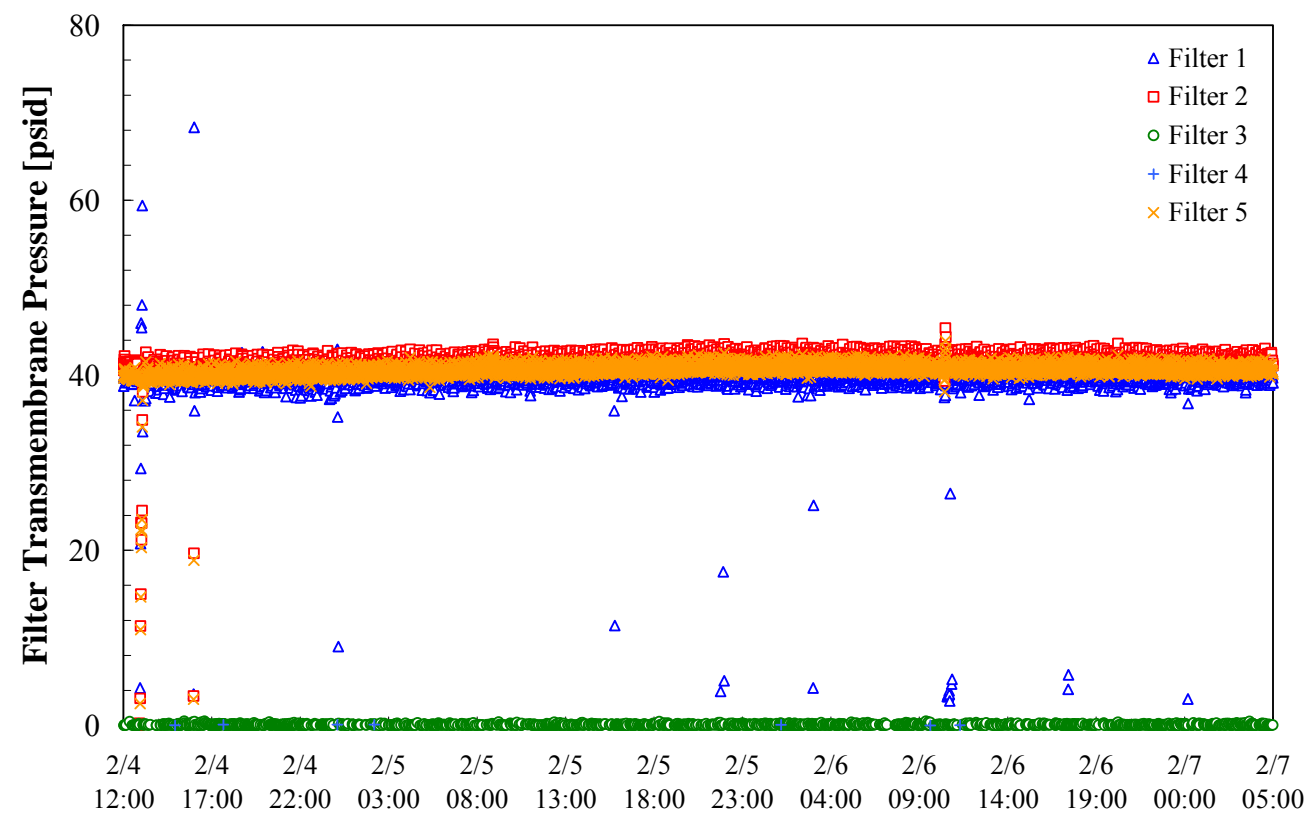

Time Stamp

Figure 4.17. TMP for Post-Caustic-Leach Concentration, Batch \#3 


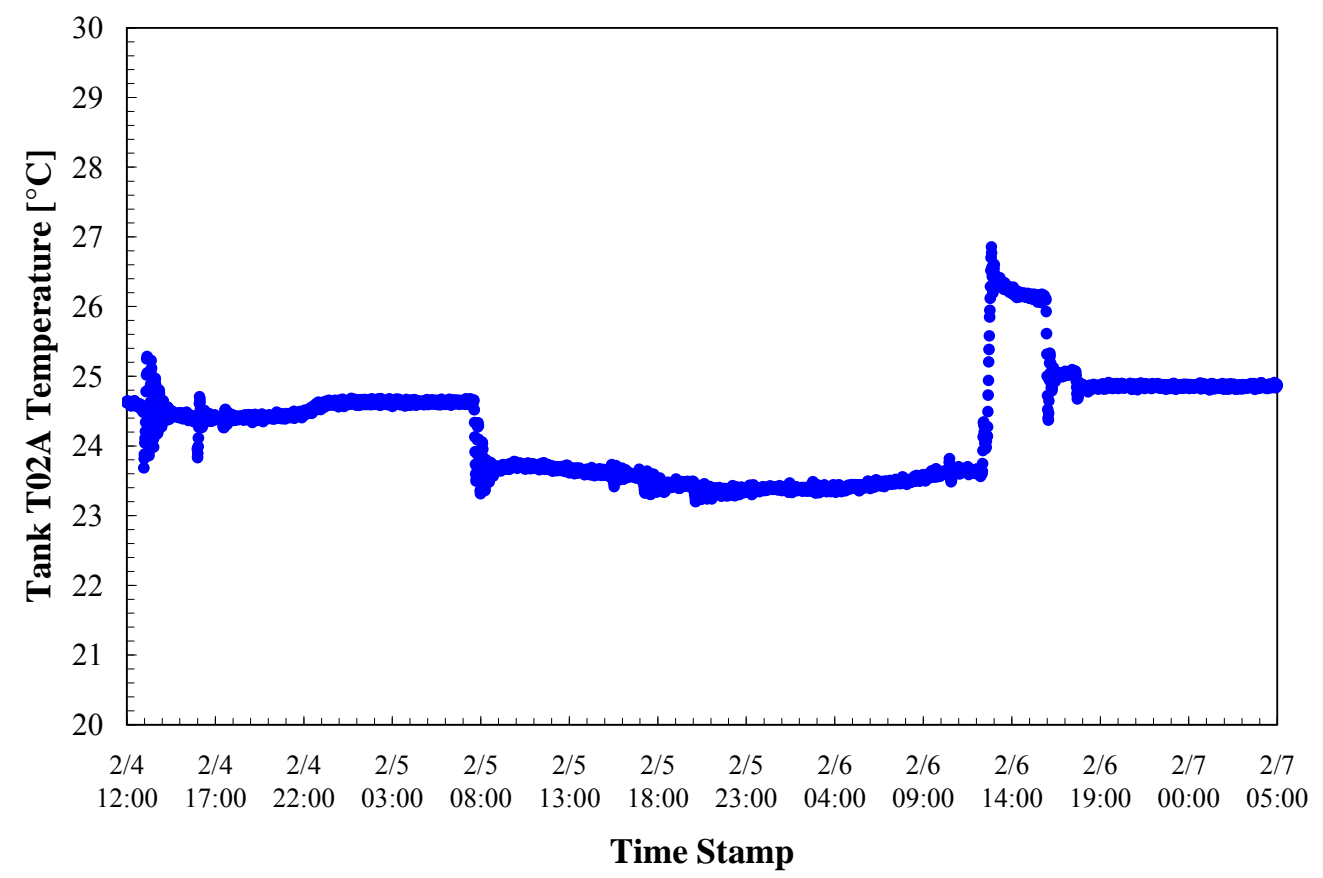

Figure 4.18. Tank T02A (TTK-0619) Temperature for Post-Caustic-Leach Concentration, Batch \#3

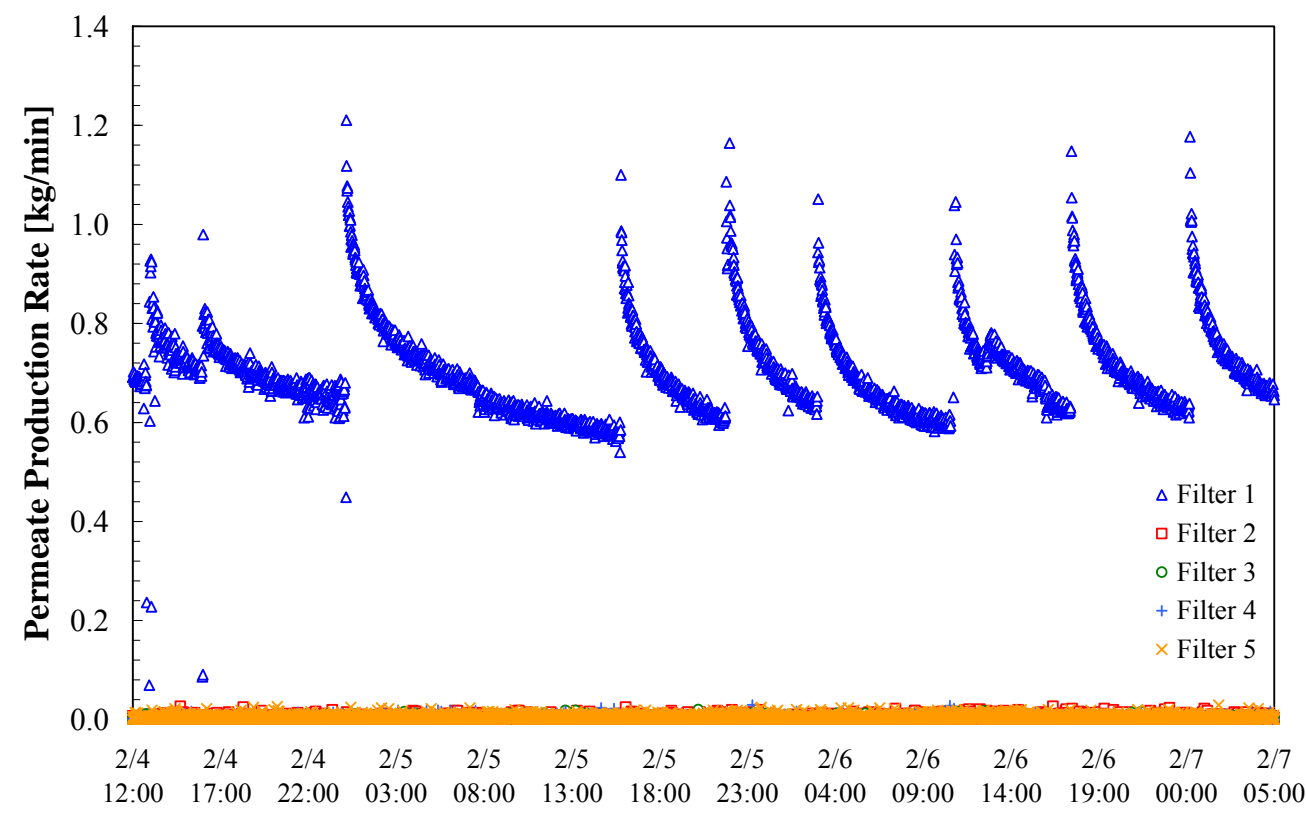

Time Stamp

Figure 4.19. Permeate Production Rate for Post-Caustic-Leach Concentration, Batch \#3 


\section{Integrated Test A-Post-Caustic-Leach Concentration, Batch \#4}

Figure 4.20 shows adjustments made to the filter-loop backpressure valve and pump speeds in response to pump vibration and permeate rate cycling from 10:00 to 21:00 on 2/8/09.

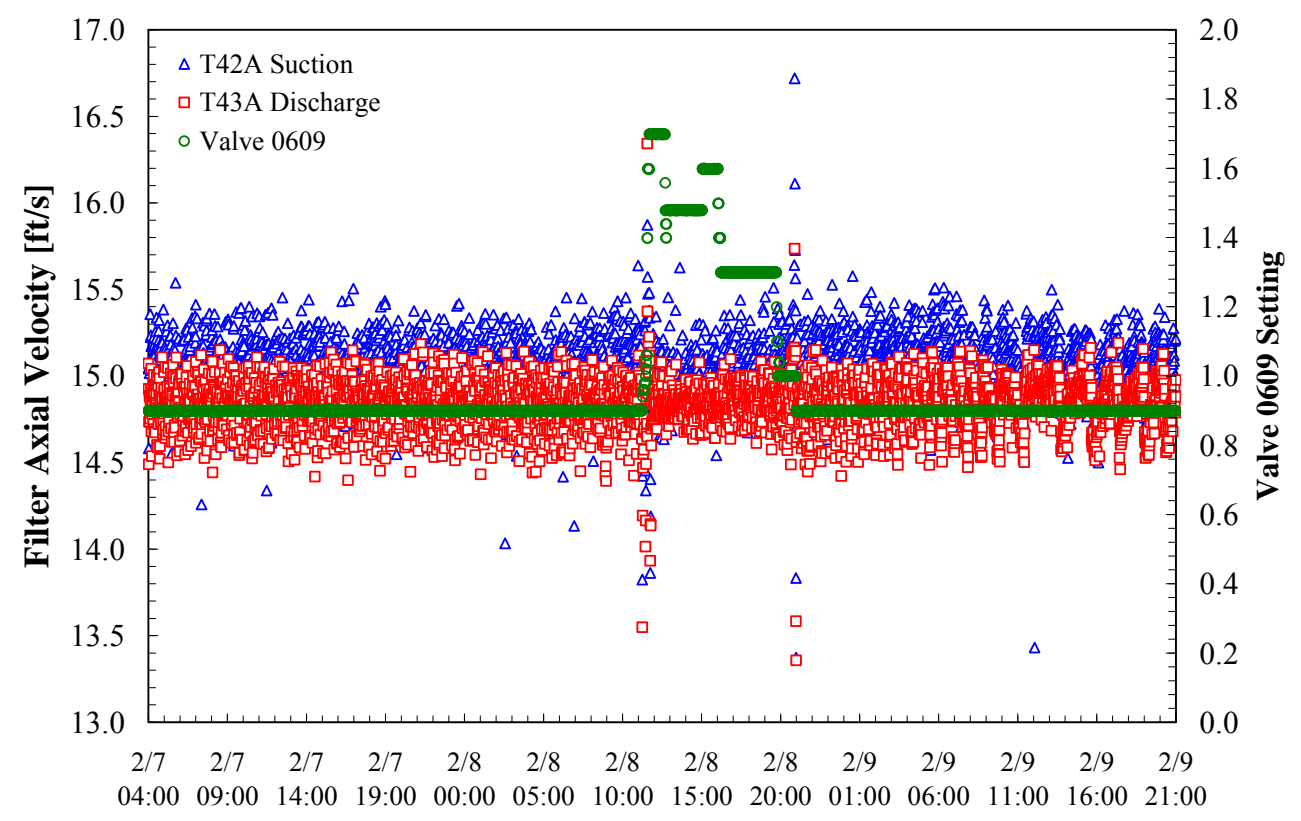

Time Stamp

Figure 4.20. Axial Velocity Based on Flow Measurements at the Suction to Pump T42A and the Discharge to Pump T43A for Post-Caustic-Leach Concentration, Batch \#4; Valve SV-0609 Setting (for information only) Scale 0 to 2 Correlates to $0 \%$ and $100 \%$ Open, Respectively 


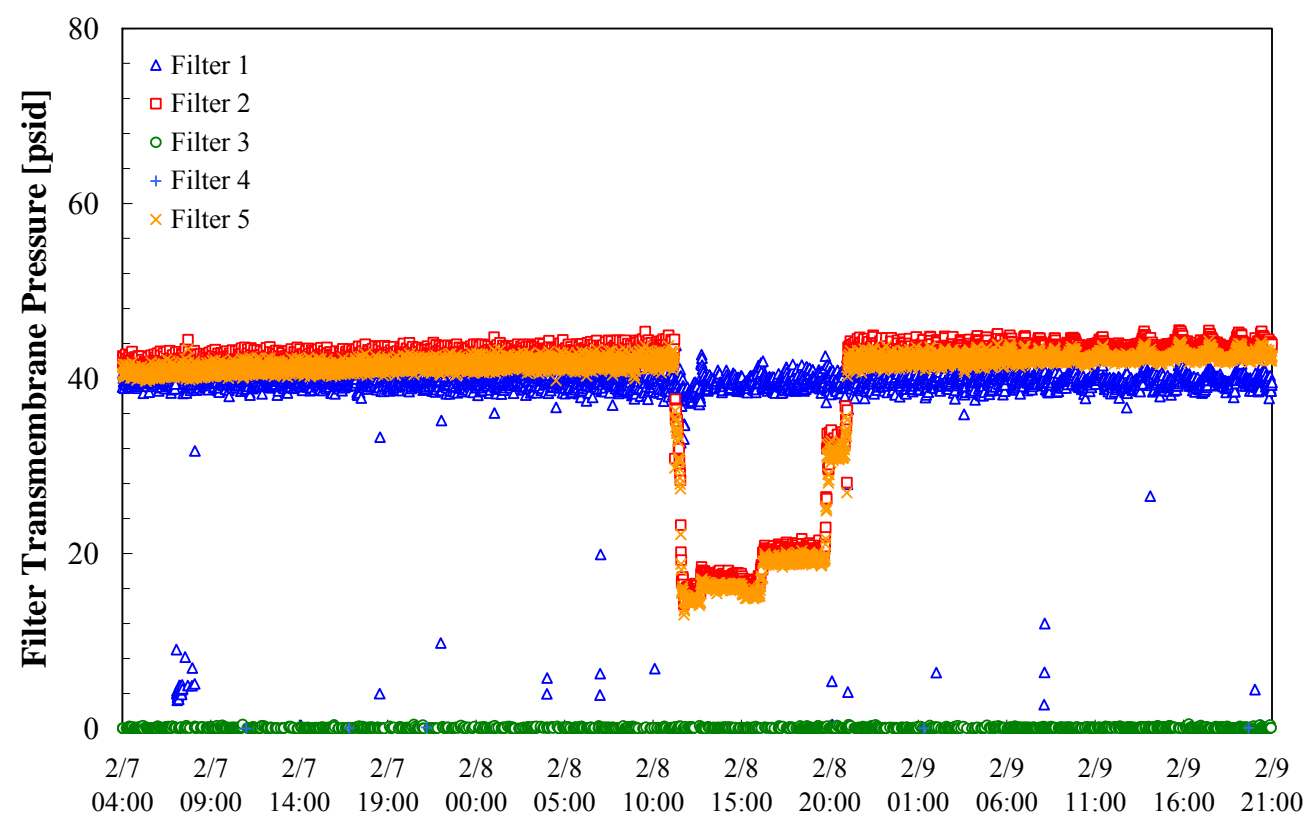

Time Stamp

Figure 4.21. TMP for Post-Caustic-Leach Concentration, Batch \#4

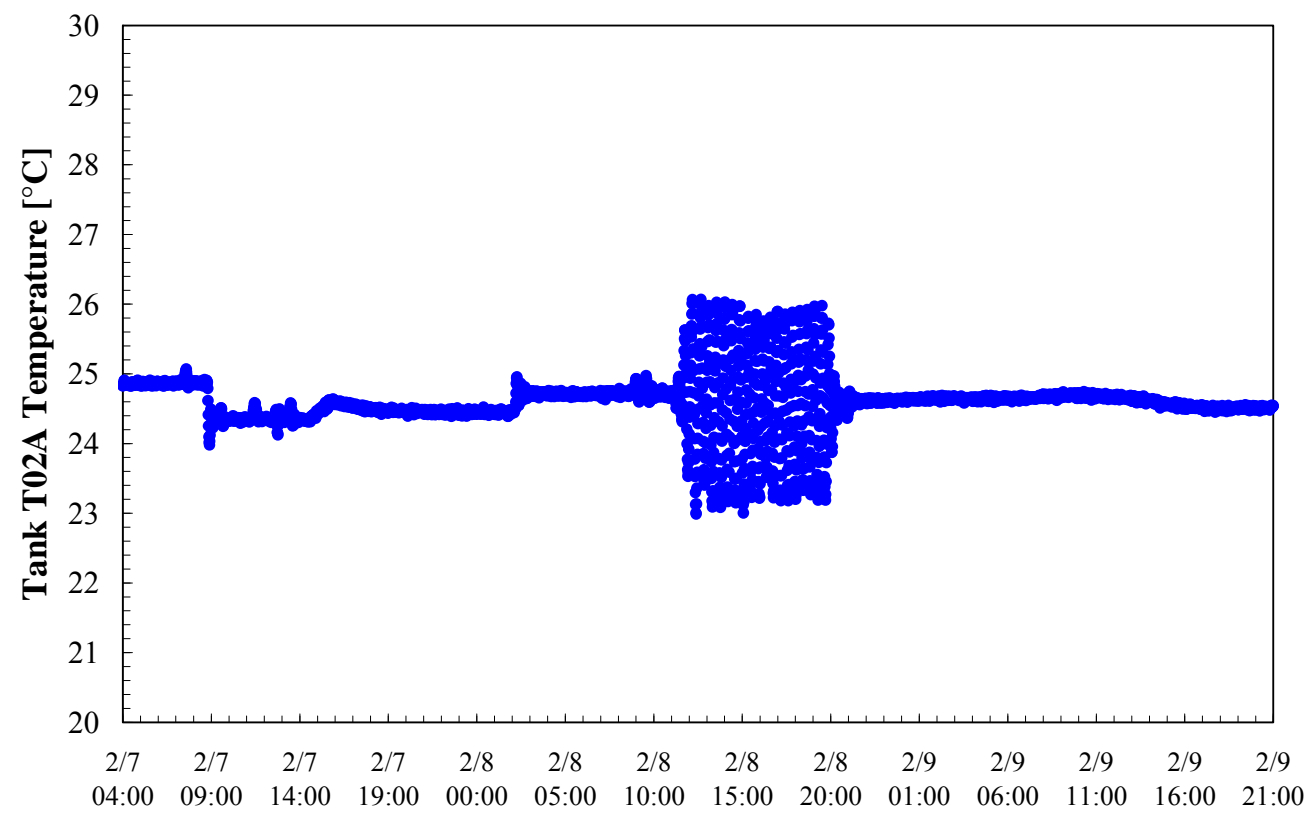

Time Stamp

Figure 4.22. Tank T02A (TTK-0619) Temperature for Post-Caustic-Leach Concentration, Batch \#4 


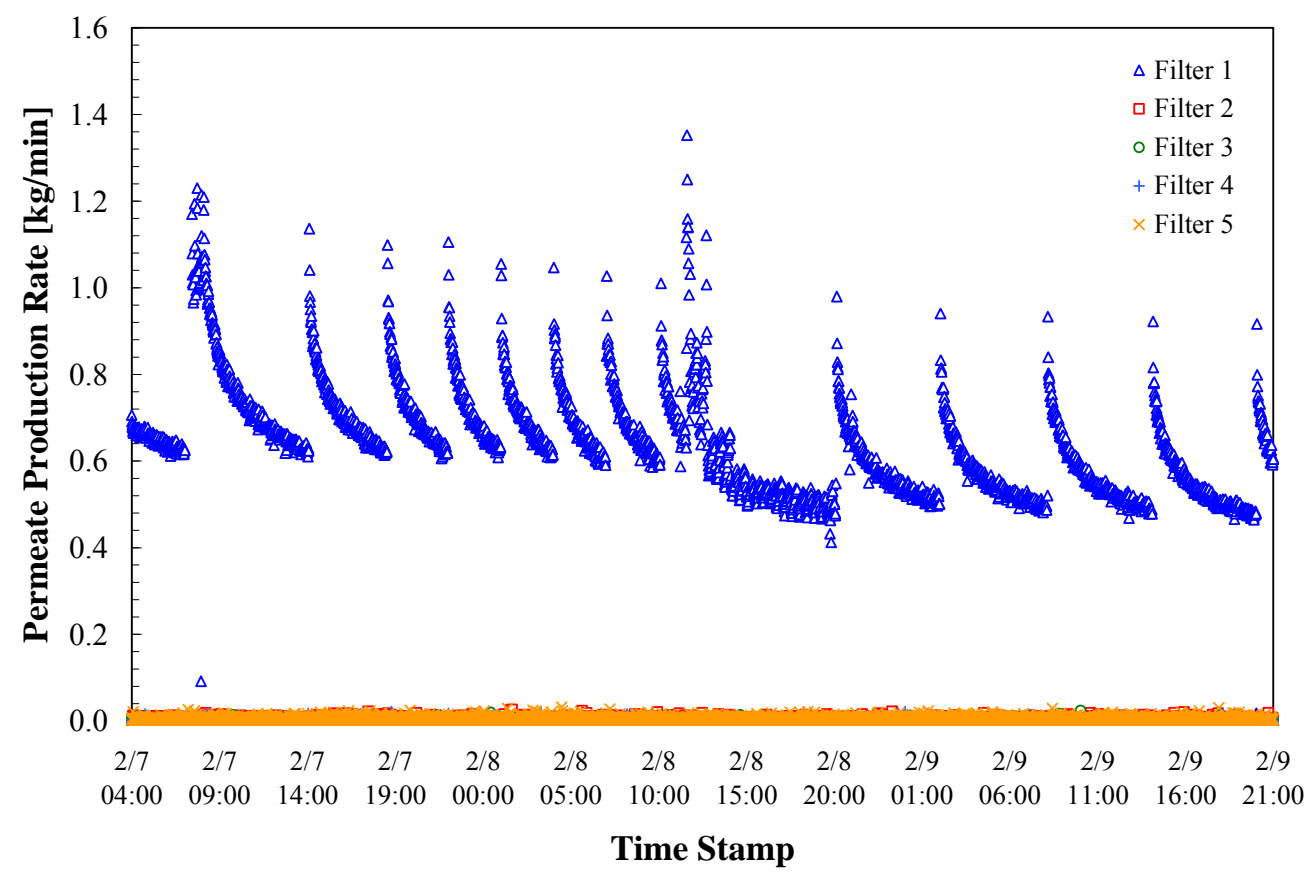

Figure 4.23. Permeate Production Rate for Post-Caustic-Leach Concentration, Batch \#4 


\section{Integrated Test A-Post-Caustic-Leach Concentration, Batch \#5}

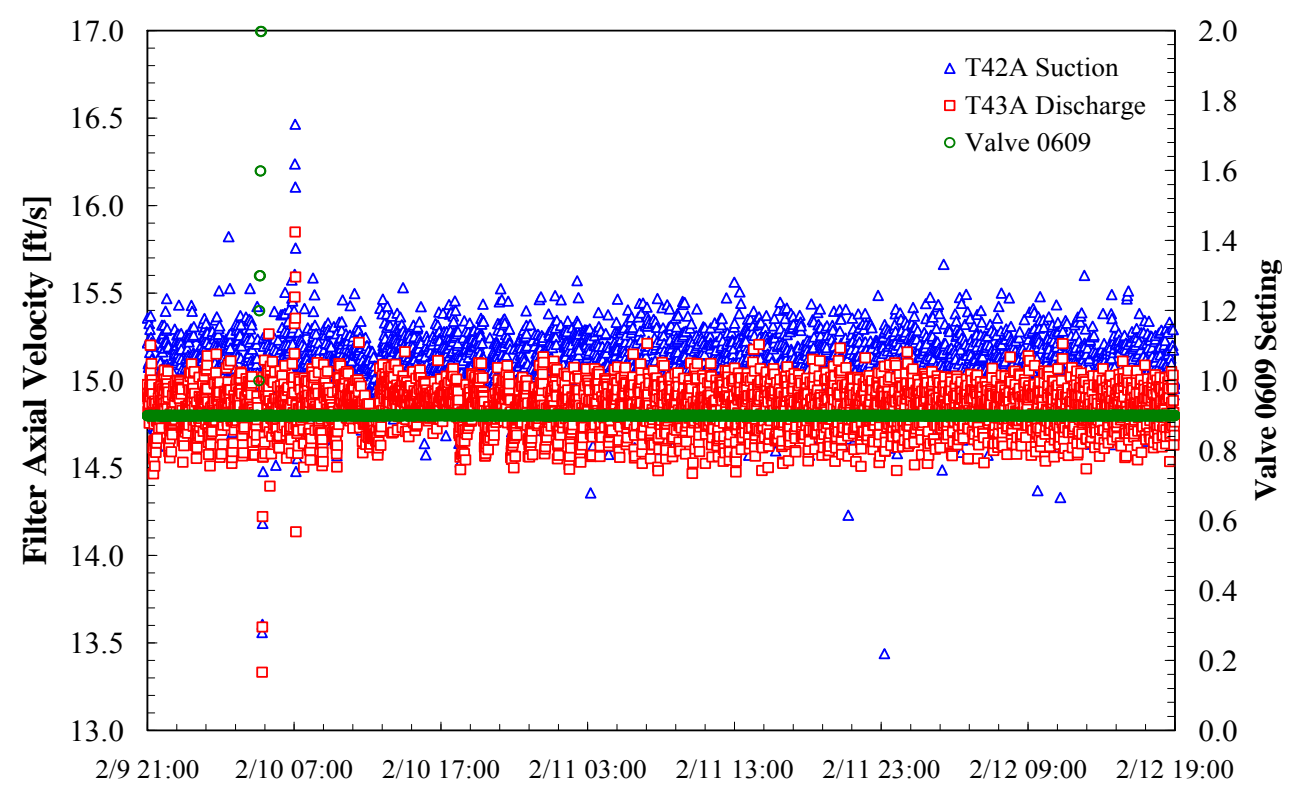

Time Stamp

Figure 4.24. Axial Velocity Based on Flow Measurements at the Suction to Pump T42A and the Discharge to Pump T43A for Post-Caustic-Leach Concentration, Batch \#5; Valve SV-0609 Setting (for information only) Scale 0 to 2 Correlates to $0 \%$ and $100 \%$ Open, Respectively

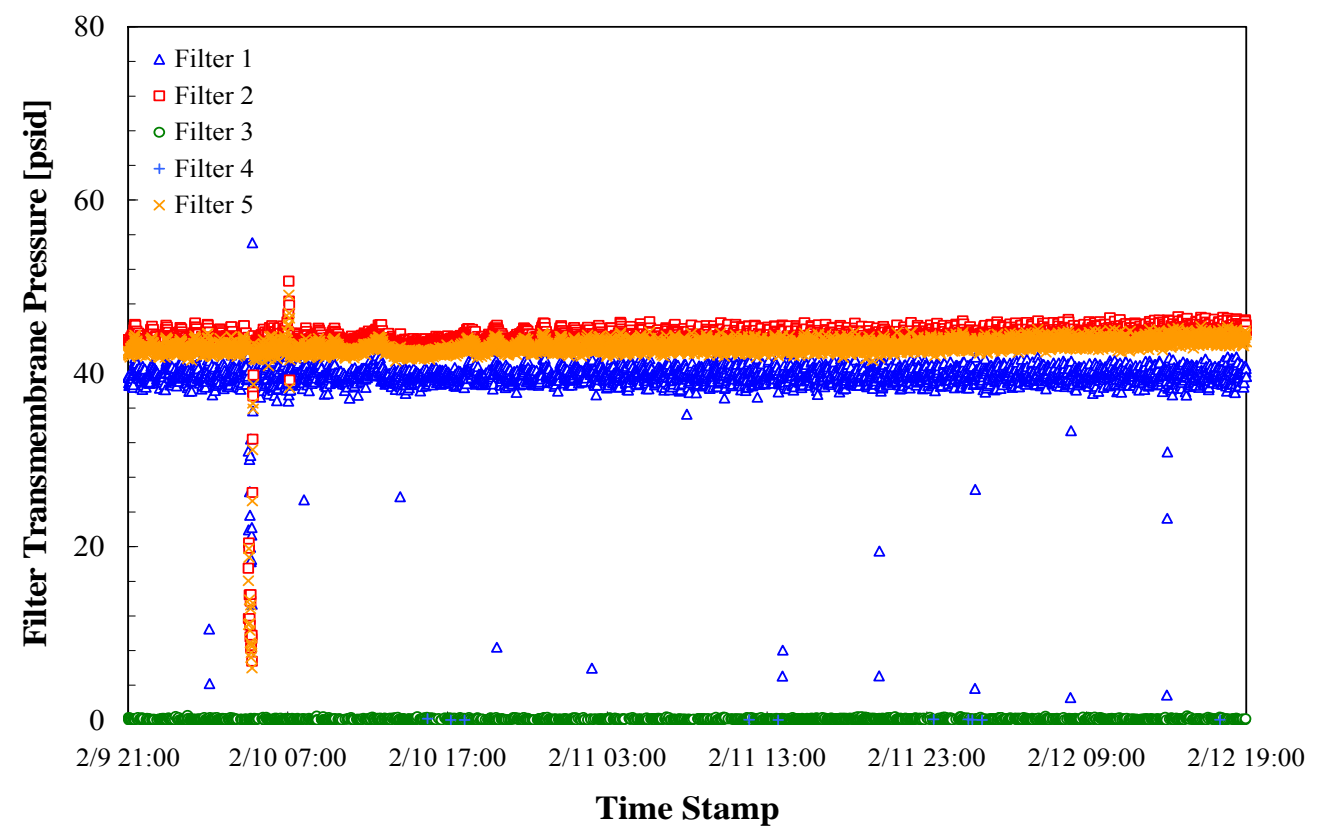

Figure 4.25. TMP for Post-Caustic-Leach Concentration, Batch \#5 


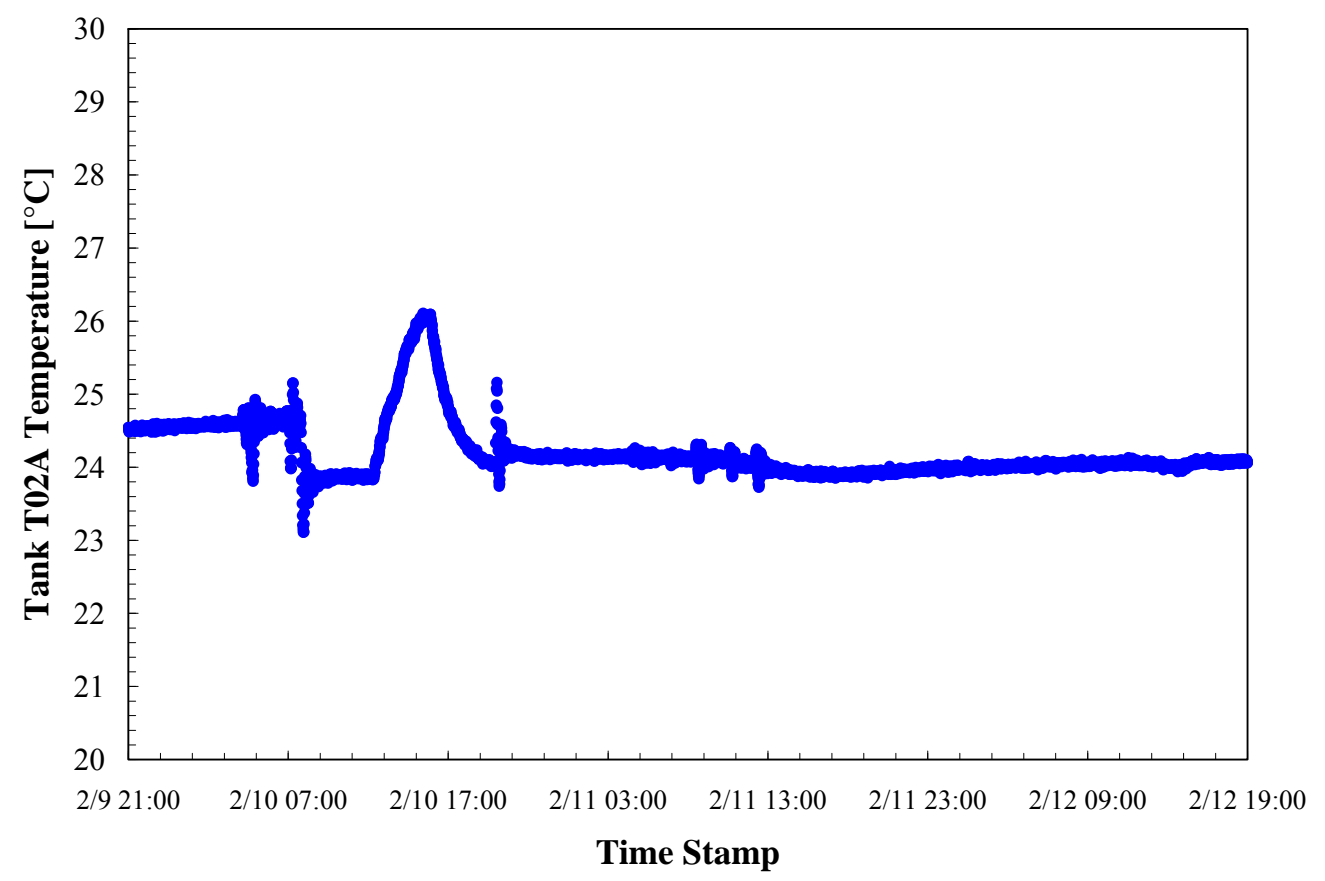

Figure 4.26. Tank T02A (TTK-0619) Temperature for Post-Caustic-Leach Concentration, Batch \#5

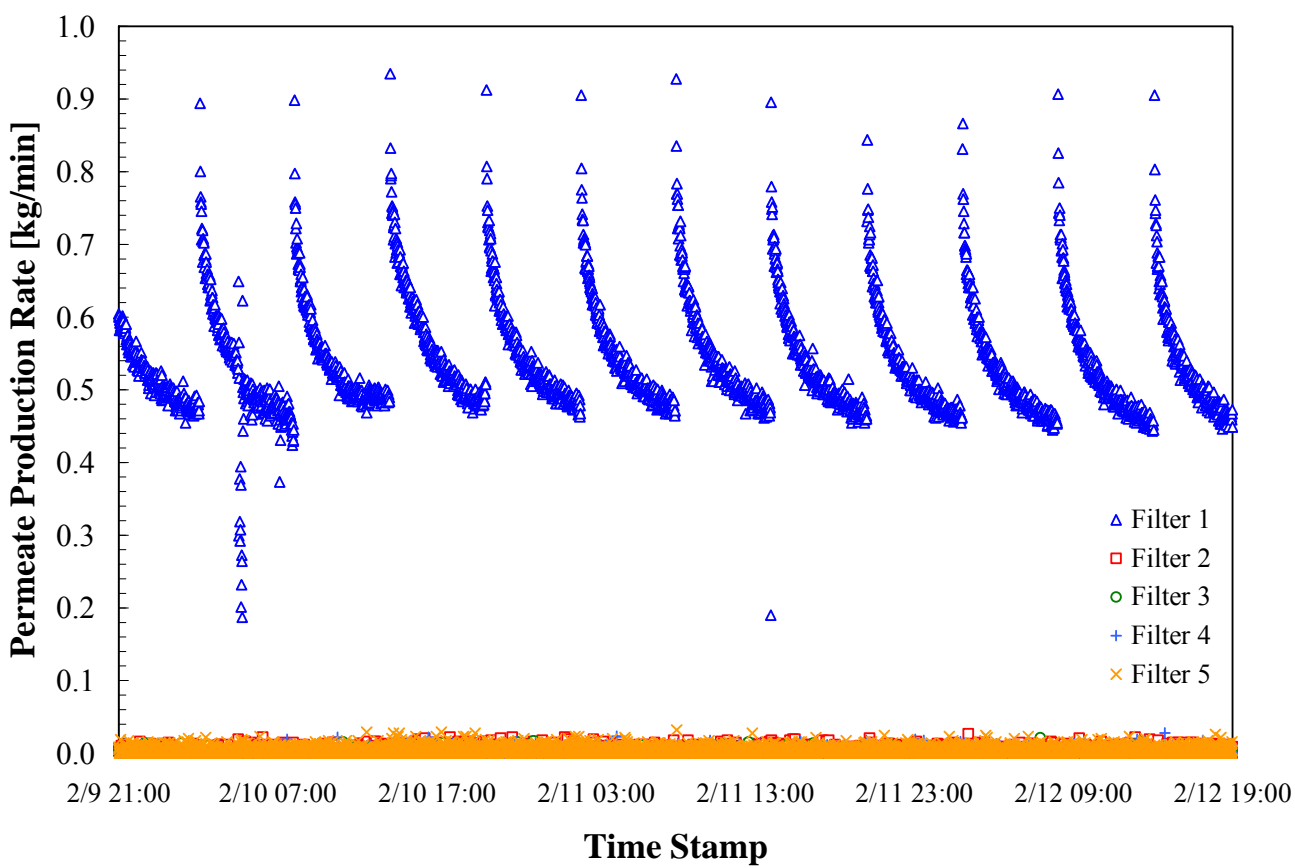

Figure 4.27. Permeate Production Rate for Post-Caustic-Leach Concentration, Batch \#5 


\section{Integrated Test A-Post-Caustic-Leach Concentration, Batch \#6}

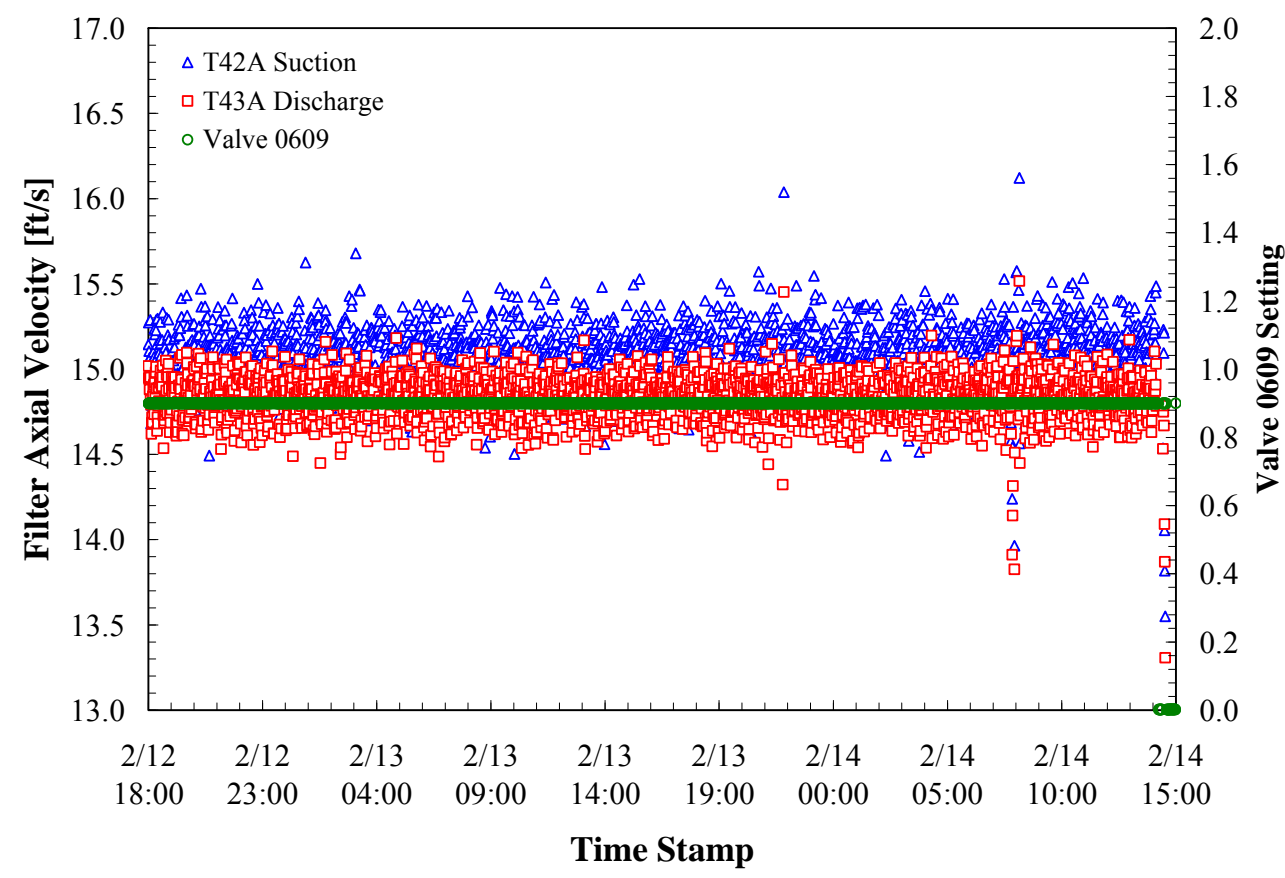

Figure 4.28. Axial Velocity Based on Flow Measurements at the Suction to Pump T42A and the Discharge to Pump T43A for Post-Caustic-Leach Concentration, Batch \#6; Valve SV-0609 Setting (for information only) Scale 0 to 2 Correlates to $0 \%$ and $100 \%$ Open, Respectively

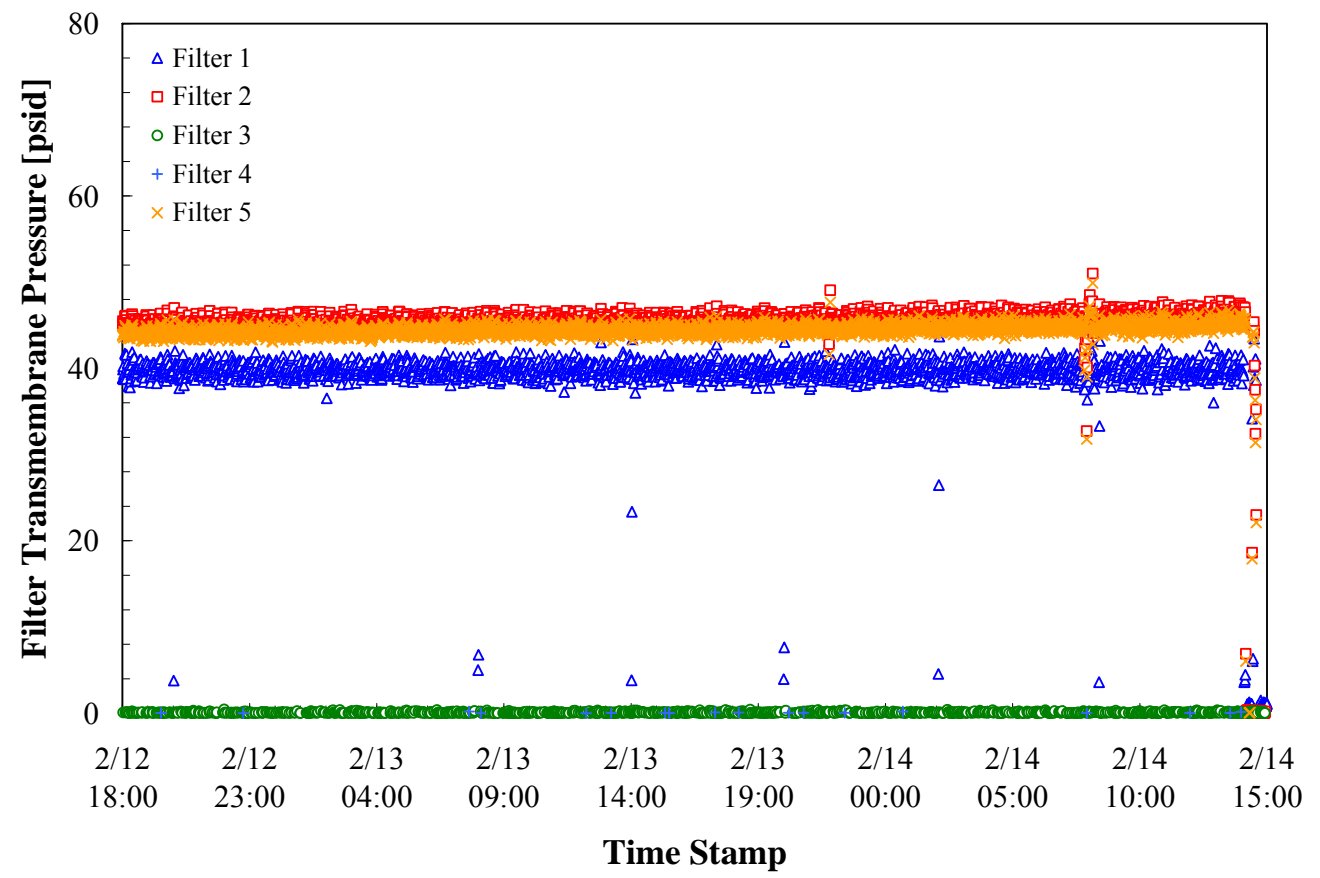

Figure 4.29. TMP for Post-Caustic-Leach Concentration, Batch \#6 


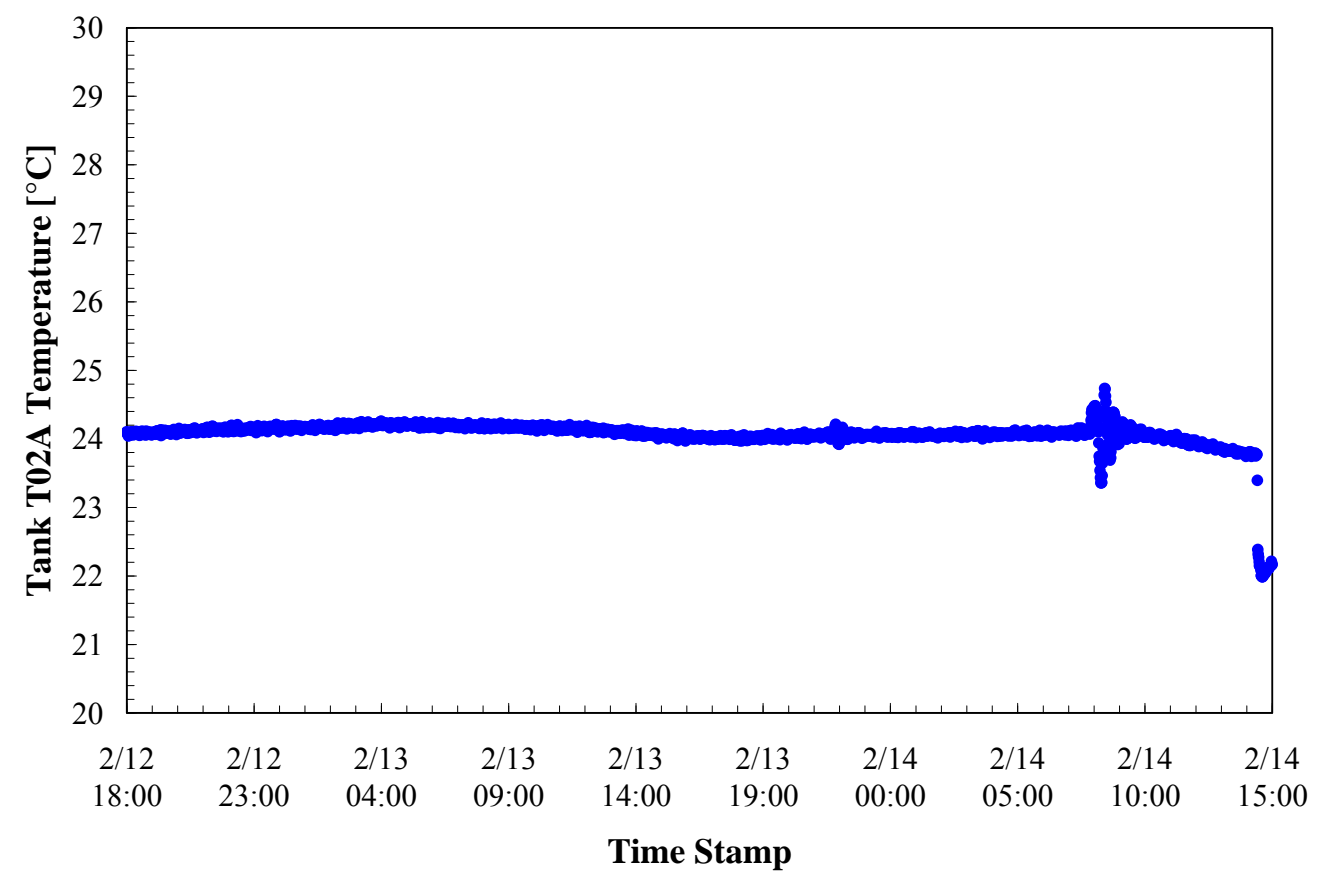

Figure 4.30. Tank T02A (TTK-0619) Temperature for Post-Caustic-Leach Concentration, Batch \#6

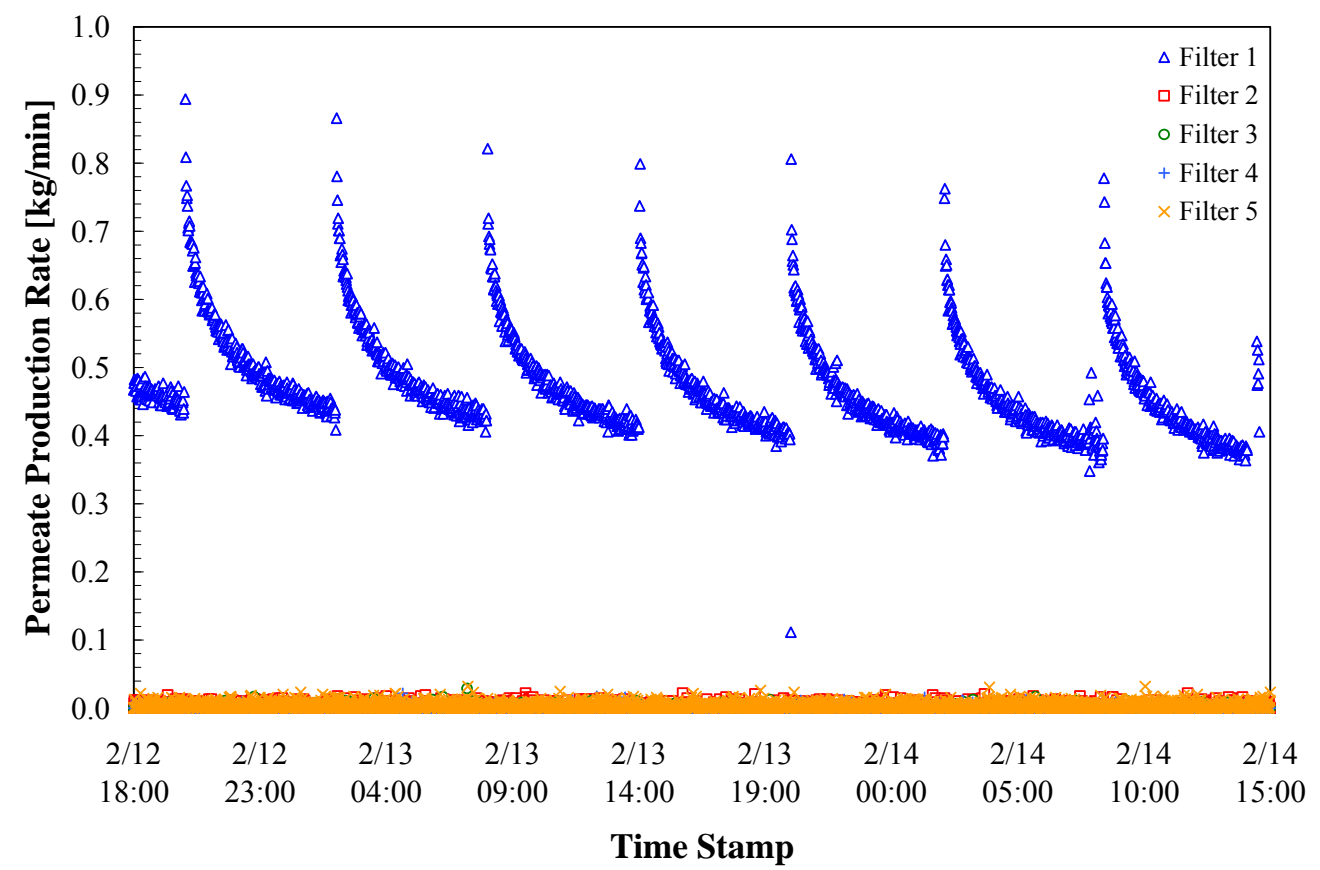

Figure 4.31. Permeate Production Rate for Post-Caustic-Leach Concentration, Batch \#6 


\section{Integrated Test A-Post-Caustic-Leach Concentration, All Batches}

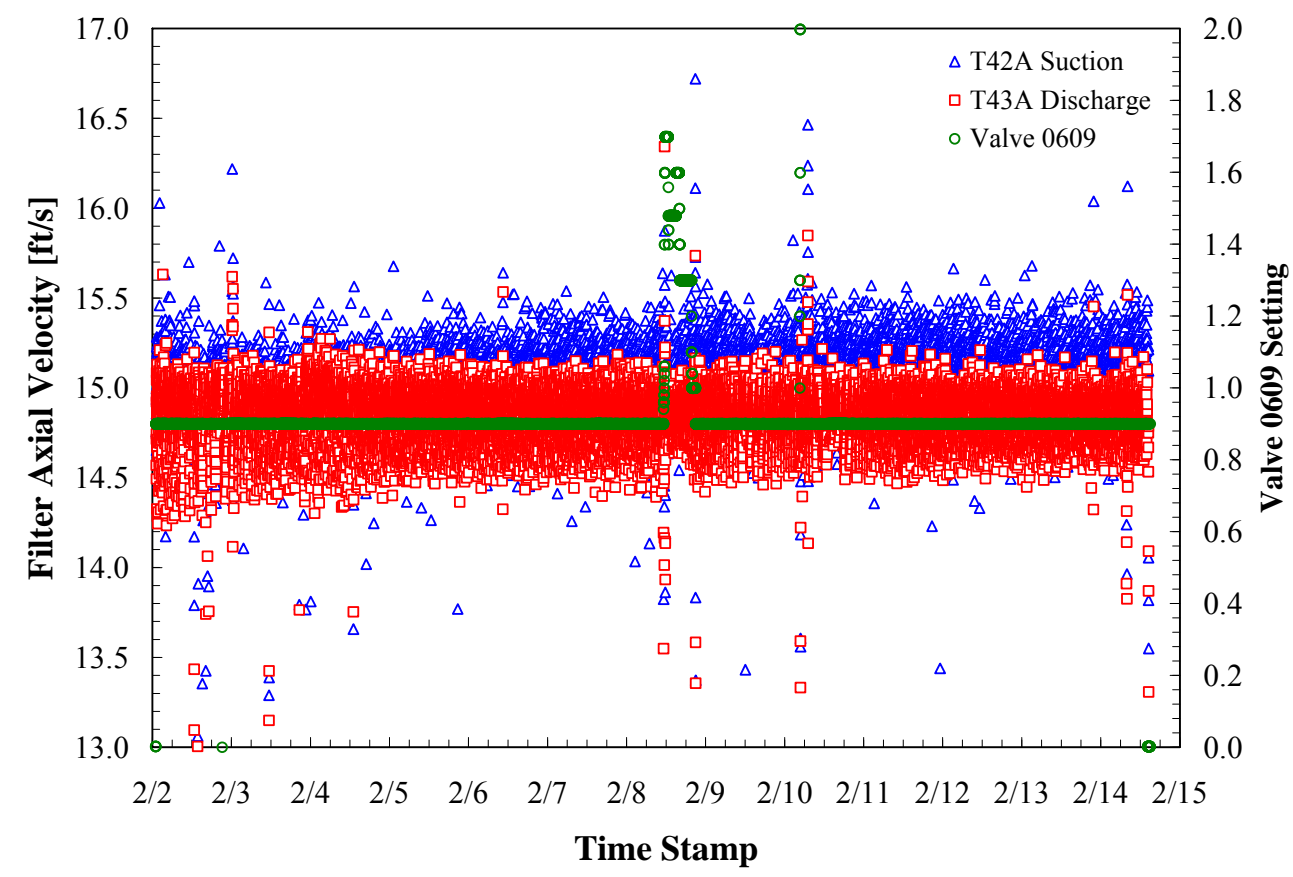

Figure 4.32. Axial Velocity Based on Flow Measurements at the Suction to Pump T42A and the Discharge to Pump T43A for Post-Caustic-Leach Concentration, All Batches; Valve SV-0609 Setting (for information only) Scale 0 to 2 Correlates to $0 \%$ and $100 \%$ Open, Respectively

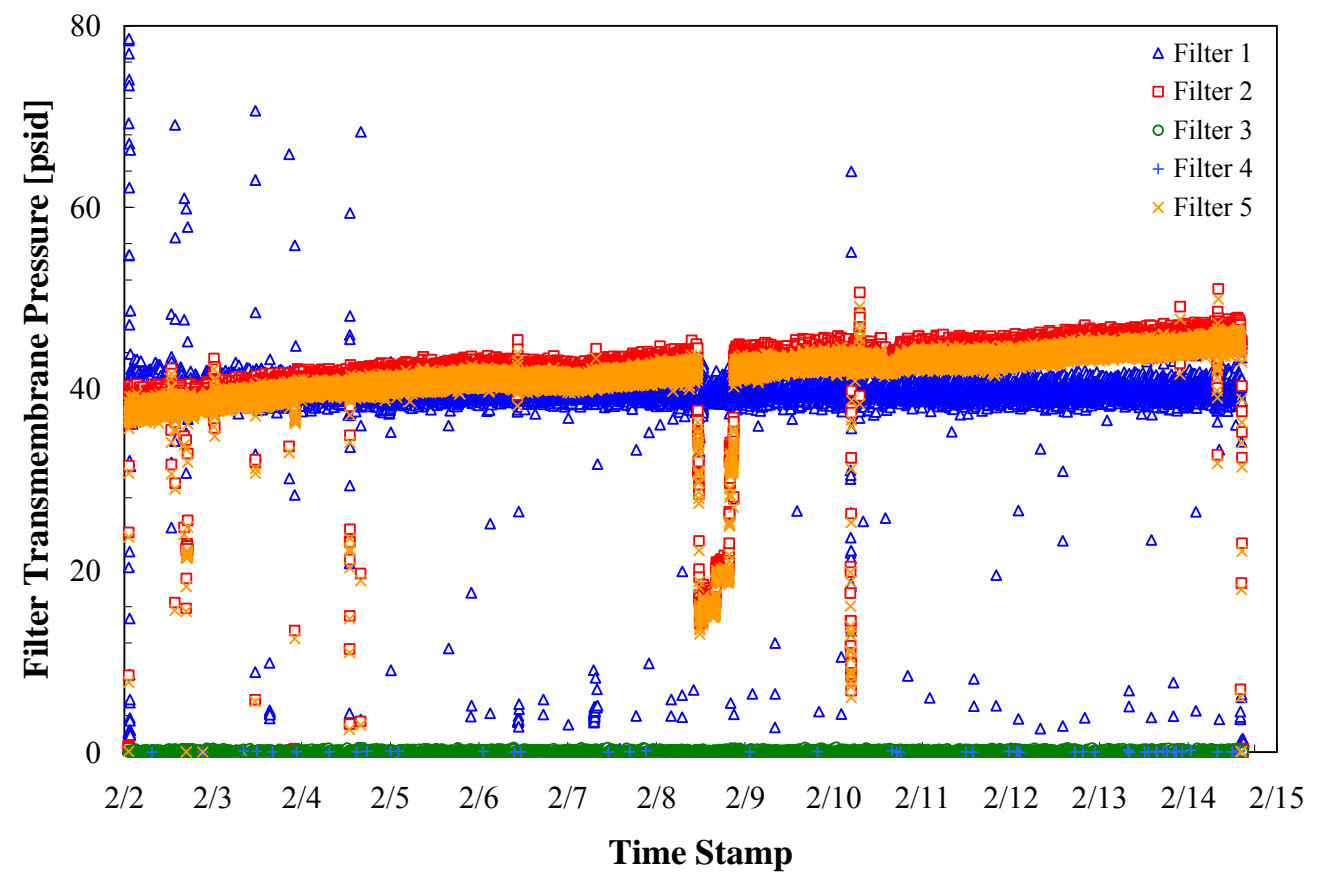

Figure 4.33. TMP for Post-Caustic-Leach Concentration, All Batches 


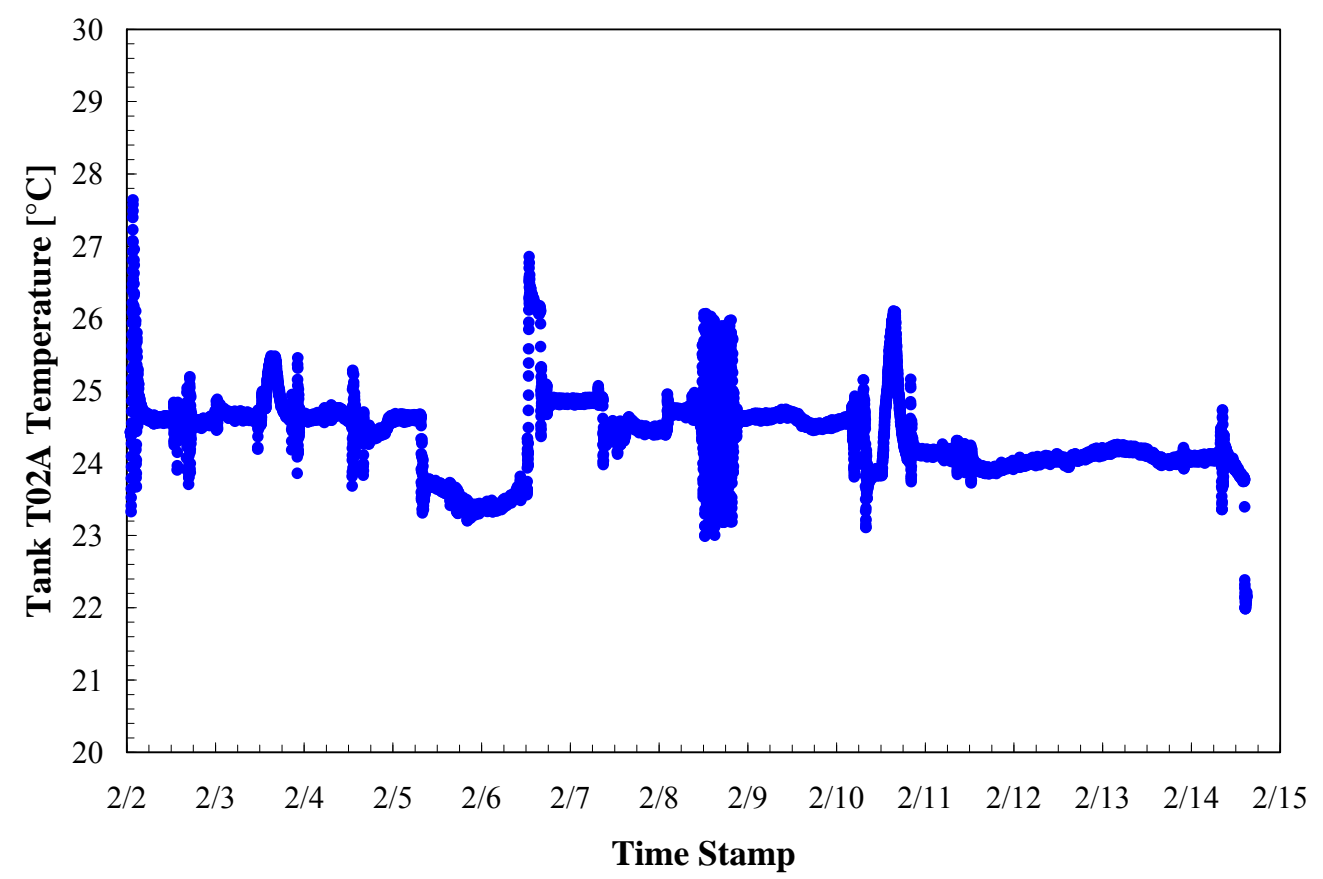

Figure 4.34. Tank T02A (TTK-0619) Temperature for Post-Caustic-Leach Concentration, All Batches

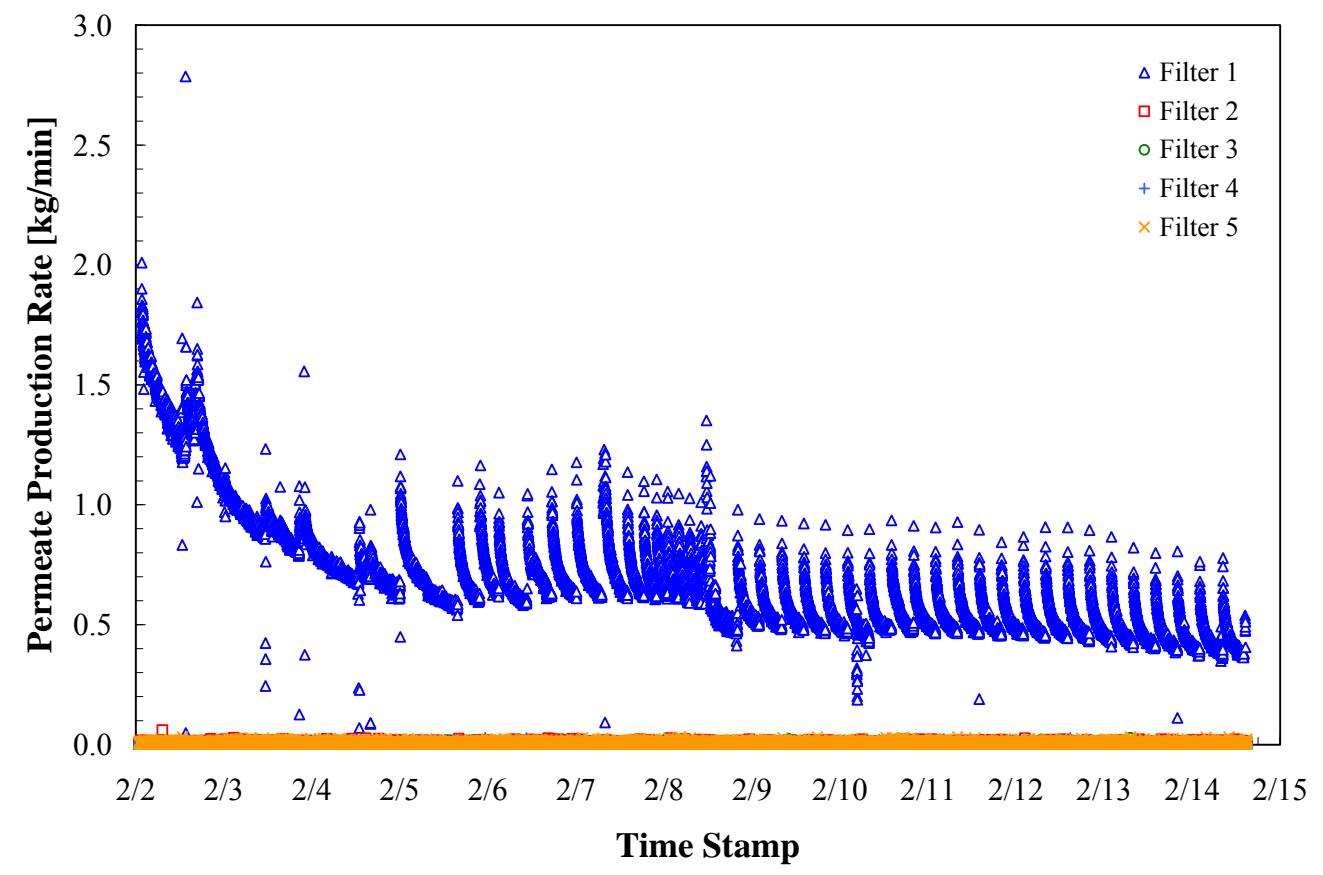

Figure 4.35. Permeate Production Rate for Post-Caustic-Leach Concentration, All Batches 
Even though filters UFP-FILT-T02A through -05A were bypassed during post-caustic-leach concentration, a drip leak from filters UFP-FILT-T02A and -T04A permeate lines to pulse pot UFP-PP-T02A was observed on 2/4/09 (LRB 60229, pg. 94 at 08:15). Apparently, the pulse pot UFP-PP-T02A manual isolation (VV70164) and the automatic valves were not isolating the air pressure source. An additional manual isolation valve from the air line was closed (VV70162) (LRB 60230, pg. 43). Permeate line sample gauges were left aligned to monitor line pressure for the remainder of the test.

At 13:26, 2/8/09 (LRB 60229, pg. 161), the permeate rate was cycling every $\sim 25$ minutes (LRB 60108, pg. 158). By 16:20, pump vibration was heard (LRB 60229, pg. 163) while UFP-VSL-T02A was at the trigger batch level of $\sim 45$ inches. Pump speed and backpressure (valve SV-0609) adjustments were made up until 21:00, 2/8/09, to alleviate pump vibration. By 21:02, the permeate rate was back to "normal" (LRB 60229, pg. 167). Figure 4.36 through Figure 4.38 show permeate, TMP, the filter-loop flow rate, and backpressure valve settings during the cycling. Refer to Section 6.2 for a discussion of filter-loop operation issues. Figure 4.38 shows the backpressure valve SV-0609 setting adjustments made during the permeate rate cycling in efforts to maintain the target filter-loop flow rate of $109 \mathrm{gpm}$.

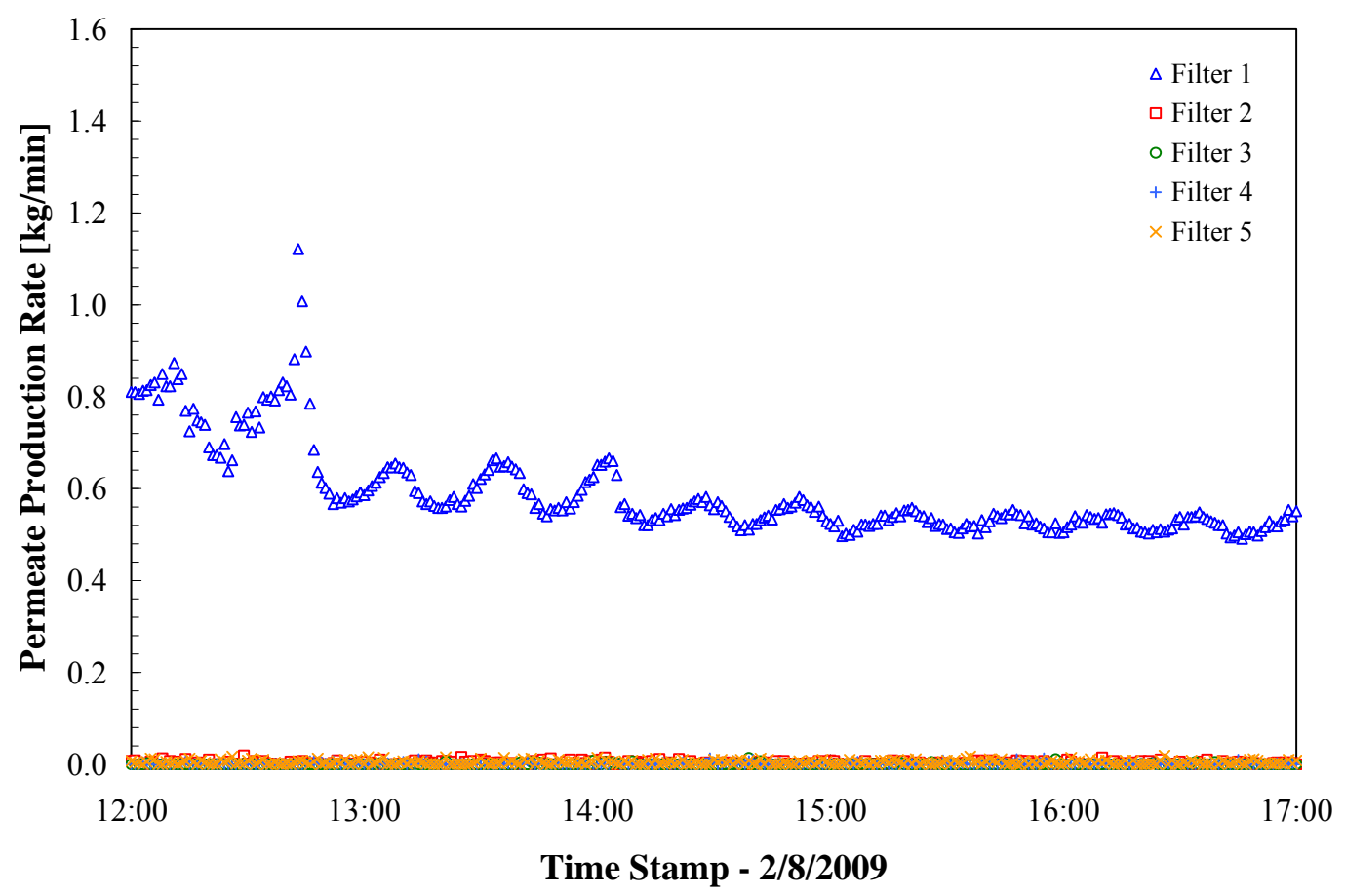

Figure 4.36. Permeate Production Rate During a Select Time Period in Integrated Test A 


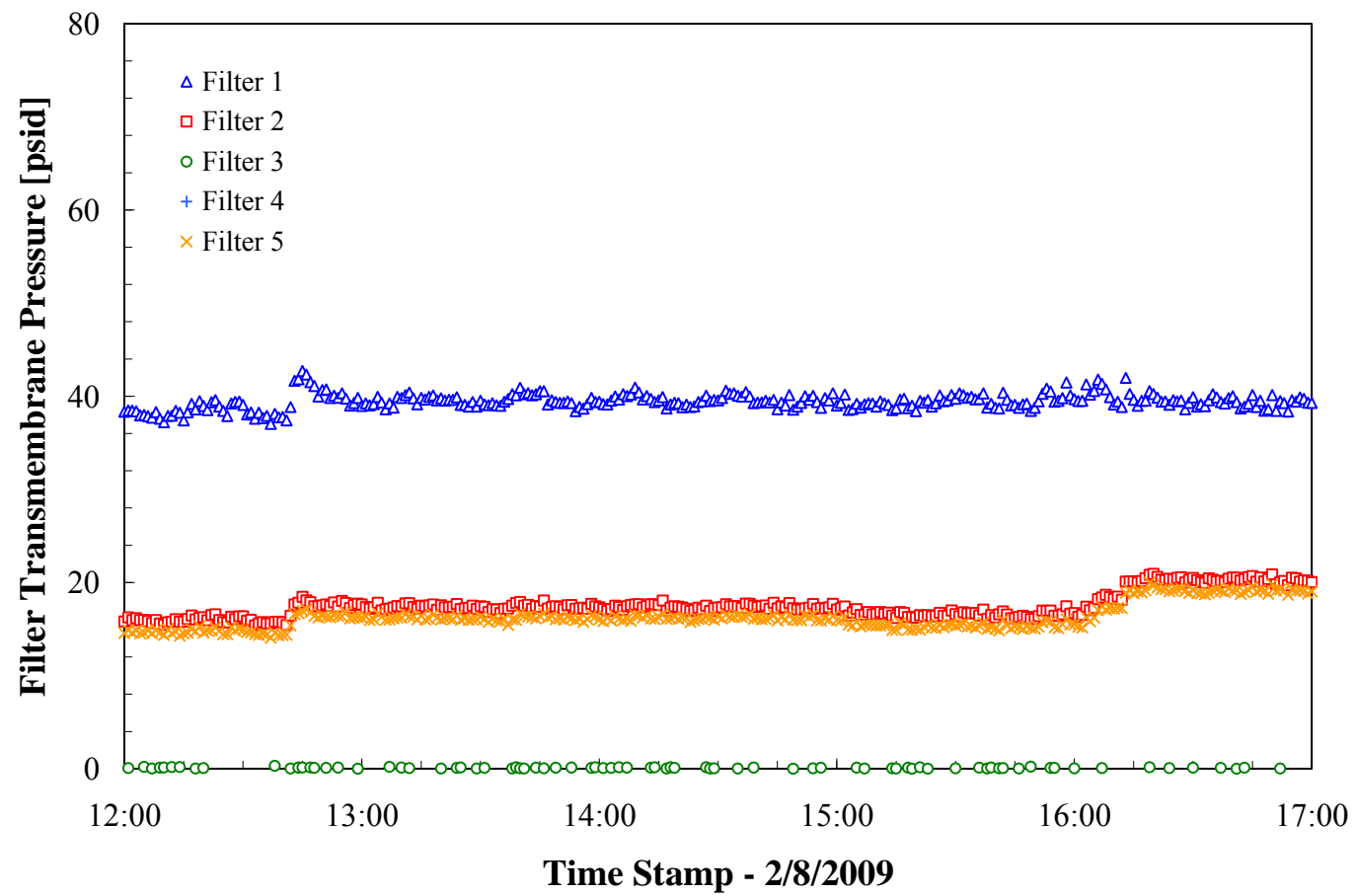

Figure 4.37. Filter TMP During a Select Time Period in Integrated Test A

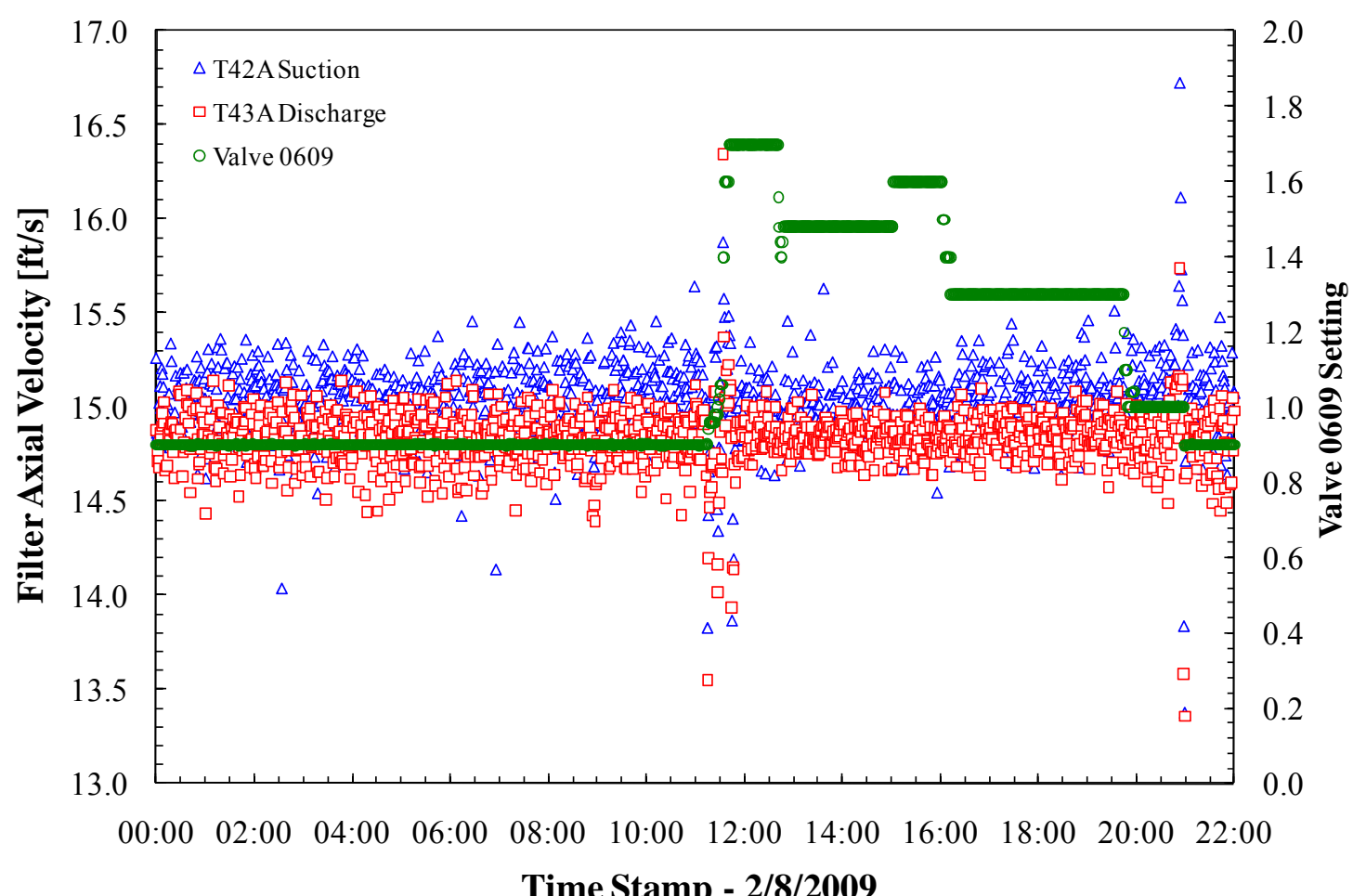

Figure 4.38. Slurry Flow Rate and Valve SV-0609 Setting During a Select Time Period in Integrated Test A; Valve SV-0609 Setting (for information only) Scale 0 to 2 Correlates to $0 \%$ and $100 \%$ Open, Respectively 
Spargers maintained target flow rates during solids concentration with periodic cleaning. At 05:00, 2/14/09 (LRB 60230, pg. 45), brown fluid was seen in the three steam-ring air purge rotameters, mostly in the UFP-VSL-T02A rotameter. The brown substance could have been simulant drawn into the sparger lines. Refer to Appendix $\mathrm{J}$ for more sparger operation information.

Occasionally, there were PJM overblow observations in UFP-VSL-T02A during solids concentration. Potential overblowing in UFP-VSL-T02A may have caused the recirculation pump vibration and unstable filter-loop flow rates caused by entrained air and indicated by a faulty flow meter located downstream of recirculation pump UFP-PMP-T43A (NCR 42317.1). Refer to Section 6.2 for a discussion of slurry air entrainment.

The 300 hours of post-caustic-leach concentration produced an 18 -wt $\%$ UDS - the target was $17-w t \%$ UDS. Refer to Section 5 for sample concentration results through the course of post-caustic-leach concentration in UFP-VSL-T02A. For a discussion on the dissolution of aluminum solids for Integrated Test A caustic-leach batches, refer to report WTP-RPT-197.

\subsection{Tracer Test}

The test to measure the bypass of the filter-loop return nozzle jet in UFP-VSL-T02A was conducted with a chemical tracer when solids concentration steps were completed on 2/14/09. After aligning the five filter bundles in preparation for the tracer test, the UFP-VSL-T02A level dropped to 47.1 inches, per the laser. With approval from the Test Director, 30 gallons of IW were added to compensate for the greater-than-expected tank level decrease. The PJMs were tuned to the target peak average velocity of $12 \mathrm{~m} / \mathrm{s}$ and a stroke length of $80 \%$. See Section 5.3 for actual PJM peak average nozzle velocity and stroke length.

On 2/14/09 at 21:26, the CsBr tracer was injected into the suction side of the recirculation pump UFP-PMP-T42A, and the tracer concentration in the loop and UFP-VSL-T02A was monitored via sample collections. The filter-loop flow rate was maintained at $90 \pm 5 \mathrm{gpm}$, as opposed to the run sheet target of $109 \pm 10$ gpm to minimize unstable filter loop pump(s) performance.

Since the target filter-loop flow rate was not achieved during the tracer test above, the tracer test was repeated after the High Solids Filter Test on 3/21/09. The steam-ring air purge and upper and lower air spargers were kept off during the repeat tracer test. The tracer test results suggest that the slurry was nearly completely mixed between 2 and 4 minutes, and overall, there was no bypass of the filter-loop return nozzle. Tracer test results are reported in the washing report, WTP-RPT-187.

\subsection{Post-Caustic-Leach Washing}

The post-caustic-leach slurry wash, using IW (0.01-M NaOH), started on 2/15/09 at 01:13 and ended at 22:55. The purpose of the post-caustic-leach slurry wash was to remove soluble solids. There were $100 \mathrm{IW}$ transfers with $41.6 \mathrm{~kg}$ per transfer. The target level in UFP-VSL-T02A to initiate wash water transfers was 44.5 inches. The wash was conducted using five ultrafilter bundles (all five filters aligned and heat exchanger UFP-HX-T02A aligned, but UFP-HX-T03A heat exchanger bypassed) with permeate routed to UFP-VSL-T62B. AFA was added after every $3^{\text {rd }}$ batch transfer to maintain a targeted AFA 
concentration of $350 \mathrm{ppm}$. However, filter-loop flow rate problems, caused by air entrainment (and consequently unstable UFP-PMP-T42A and T43A pump performance), were observed through the course of the post-caustic-leach concentration washing.

After the $12^{\text {th }} \mathrm{CD}$ sample event (wash \#56), per approval, the lower air spargers were turned off temporarily, and the steam-ring air purge rate was set to $0.07 \mathrm{~kg} / \mathrm{min}$ temporarily to improve the filter-loop flow rate. The steam ring air and lower and upper air spargers were turned off from 14:26 to 19:32 on 2/15/09 to minimize pump vibration. Refer to Figure 4.39 through Figure 4.42 for filtration activities during the post-caustic-leach concentration washing - where the prototypic UFP-VSL-T02A temperature was taken from TTK-0619. The number of IW batches required to reach the target of $0.25-\mathrm{M}$ free hydroxide following the aluminum leaching stage was 64 batches for Integrated Test A, compared to the WTP projections to reach the target of $0.25-\mathrm{M}$ free hydroxide of 64 batches. Post-caustic-leach washing efficiency is summarized in report WTP-RPT-187, however, an overall wash efficiency for Integrated Test A was 1.00. In addition, Section 8, Filtration, of report WTP-RPT-197 provides TMP and temperature-corrected filter flux versus $\mathrm{wt} \%$ solids plots - along with permeate production rates.

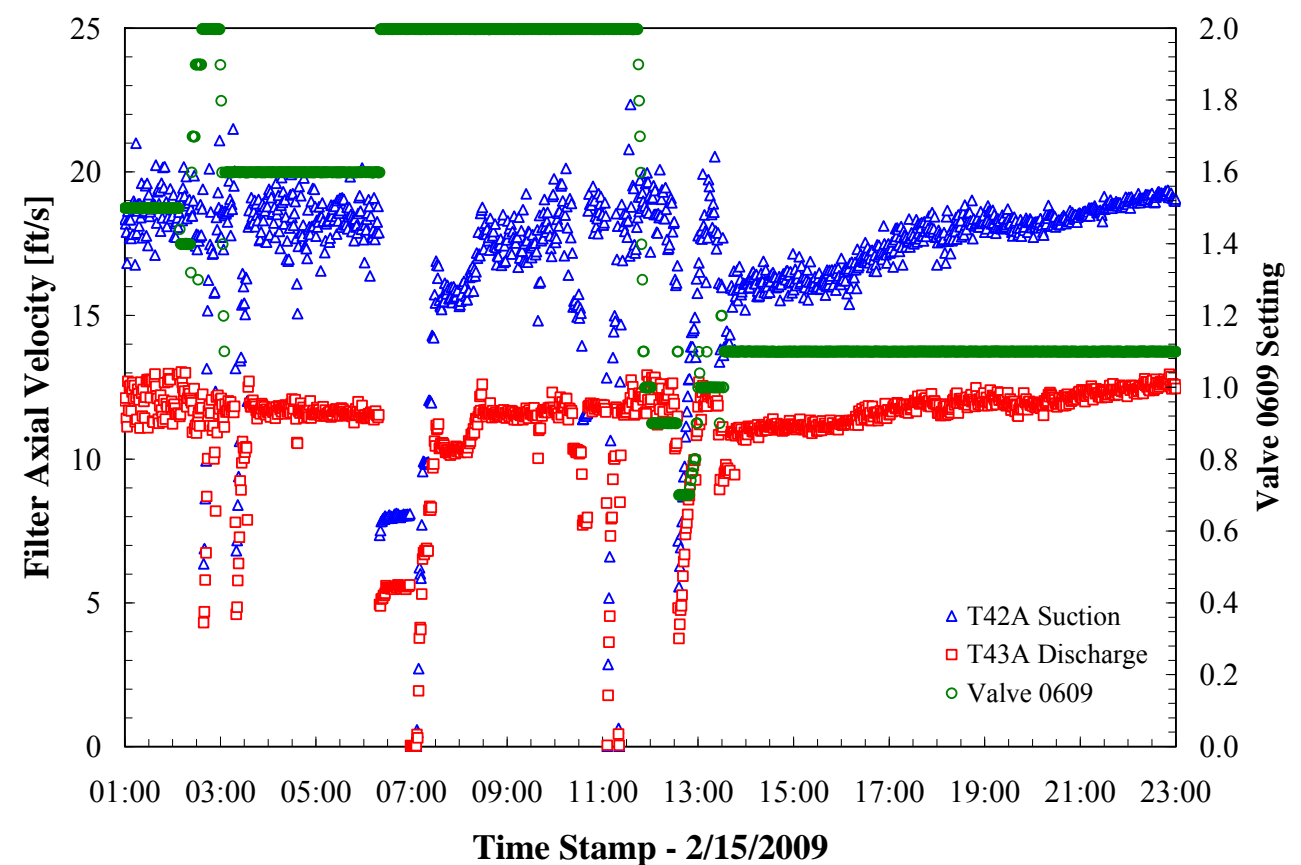

Figure 4.39. Axial Velocity Based on Flow Measurements at the Suction to Pump T42A and the Discharge to Pump T43A for Post-Caustic-Leach Slurry Wash; Valve SV-0609 Setting (for information only) Scale 0 to 2 Correlates to $0 \%$ and $100 \%$ Open, Respectively 


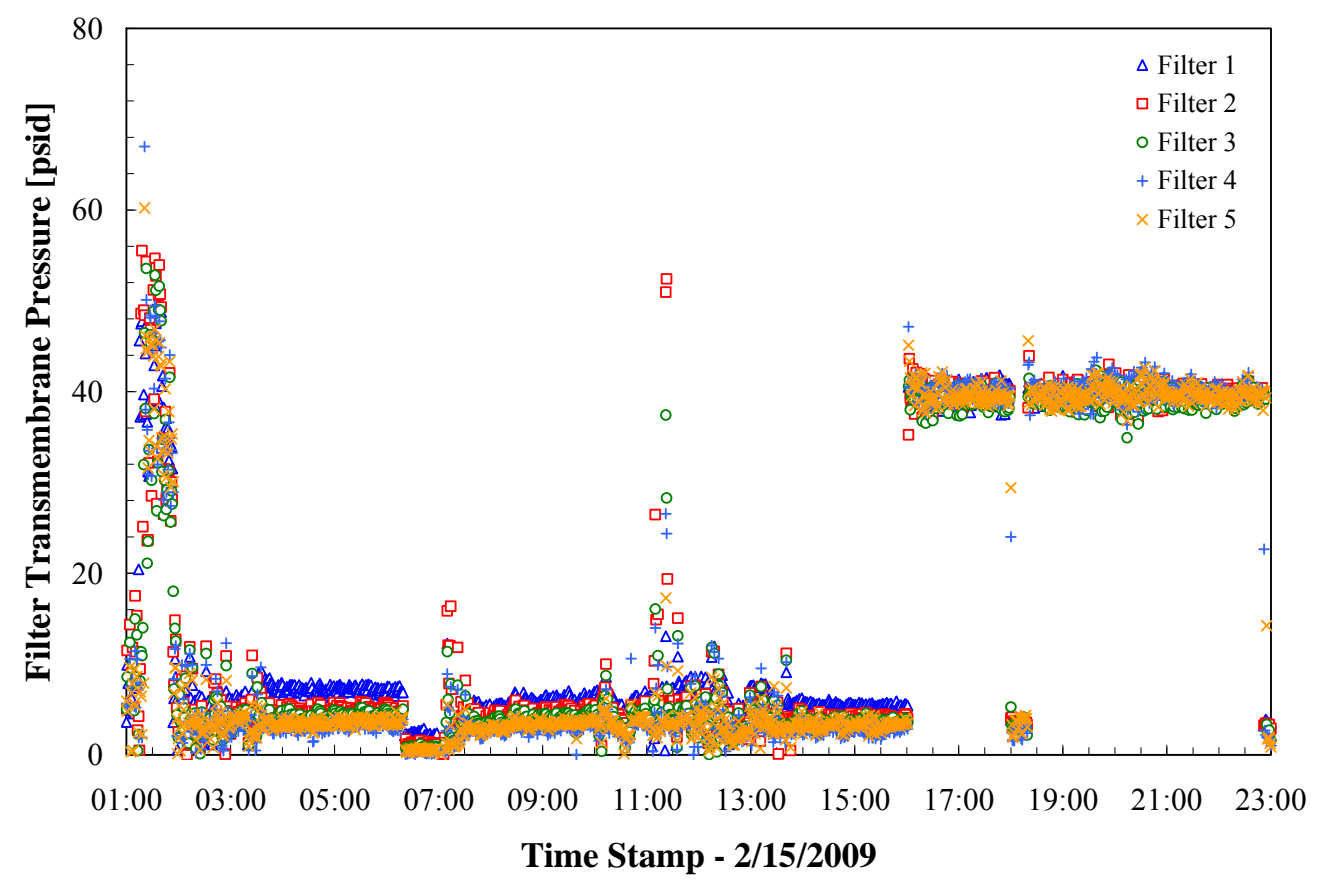

Figure 4.40. TMP for Post-Caustic-Leach Slurry Wash

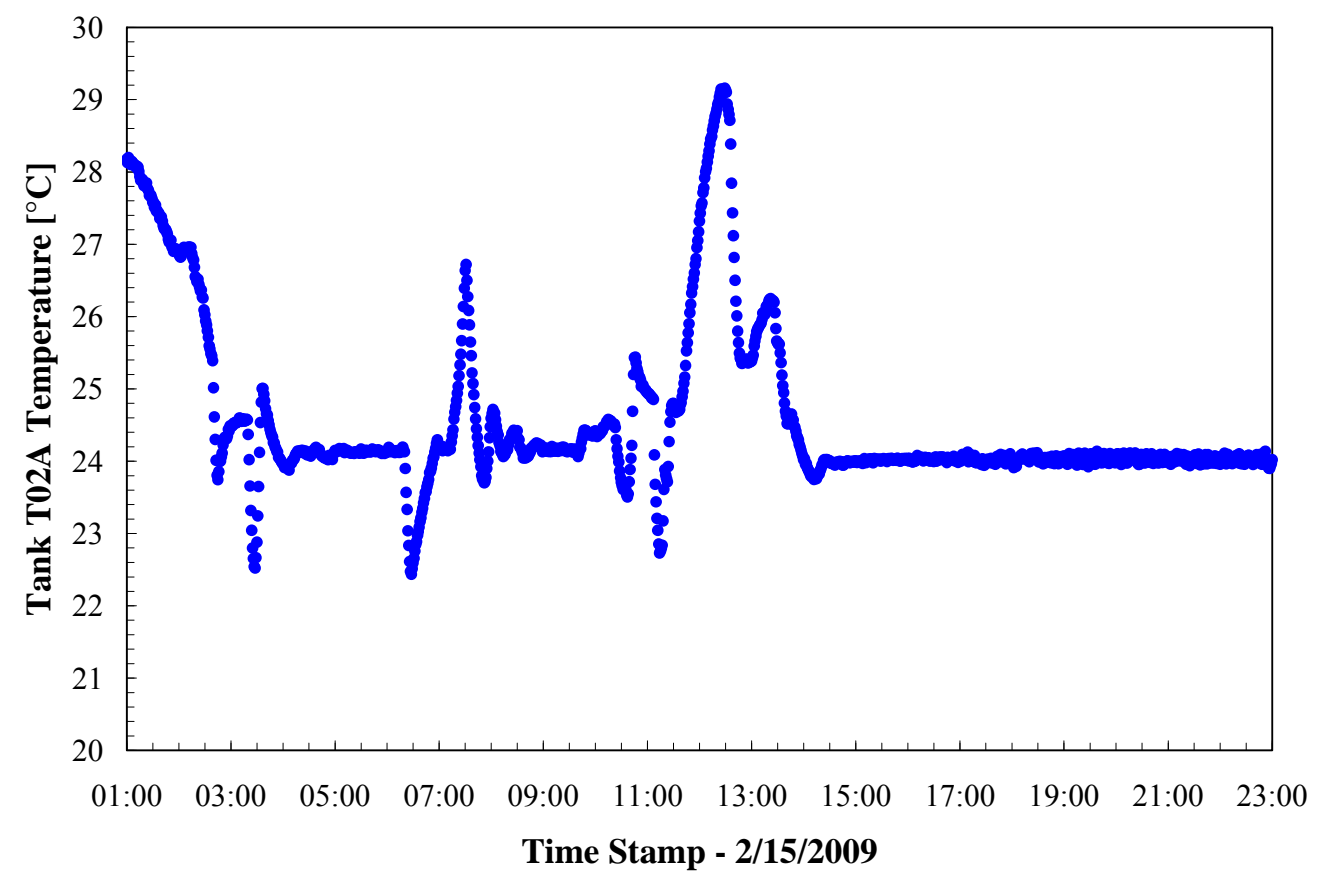

Figure 4.41. Tank T02A (TTK-0619) Temperature for Post-Caustic-Leach Slurry Wash 


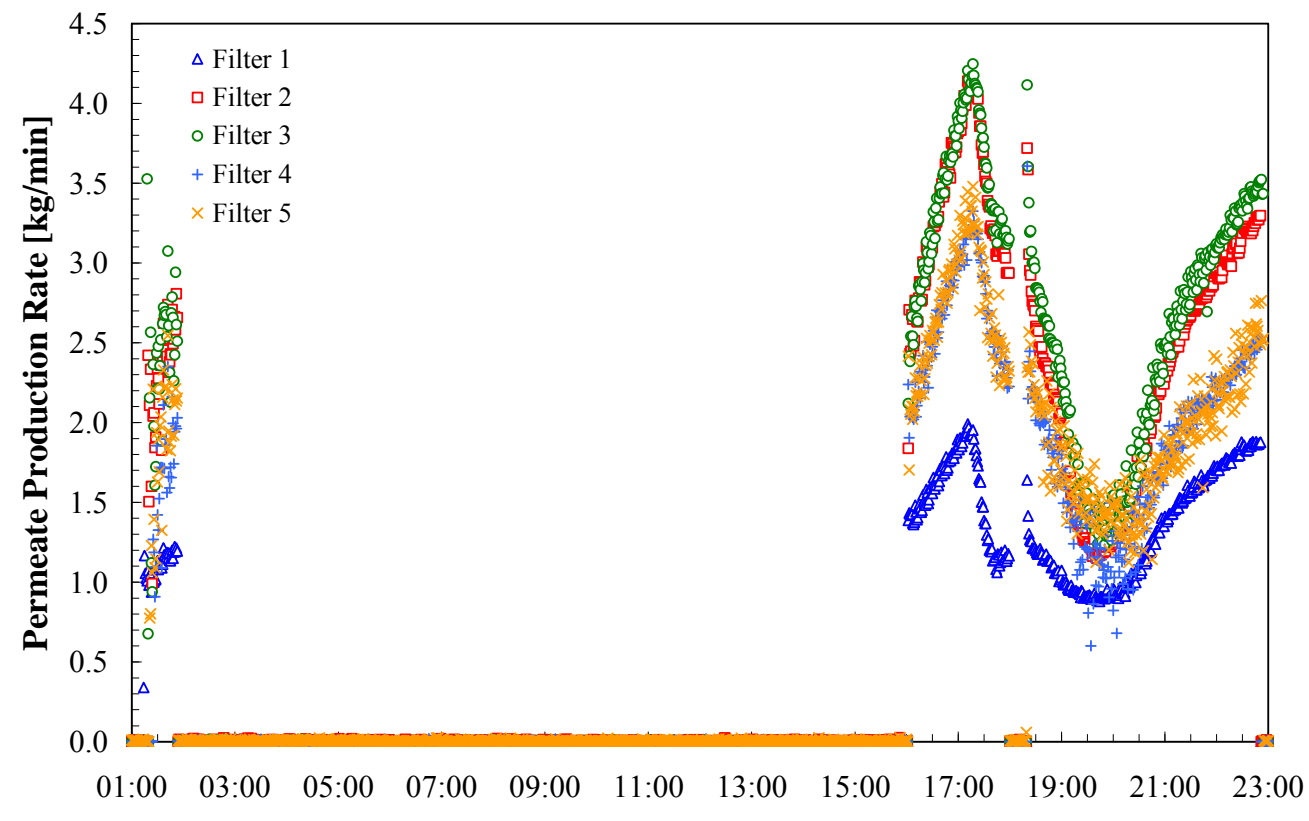

Time Stamp - 2/15/2009

Figure 4.42. Permeate Production Rate for Post-Caustic-Leach Slurry Wash

\subsection{Oxidative Leaching}

Chromium-bearing slurry and AFA were added to the washed slurry before the sodium permanganate on $2 / 16 / 09$. Chromium addition, although nonprototypic, was necessary because chromium in the simulant was found to oxidize to the $\mathrm{Cr}^{6+}$ state during the caustic leach.

Chromium slurry addition, 145 gallons, was started at 01:12 and ended at 01:23. Chromium solids were added (as slurry) in-line at the suction of recirculation pump UFP-PMP-T42A. The PJM nozzle velocity during this time was $\sim 13.6 \mathrm{~m} / \mathrm{s}$, the steam ring air was off, and the upper and lower air spargers were set at 0.01 and $0.04 \mathrm{~kg} / \mathrm{min}$, respectively. See Table 4.5 for the chromium oxyhydroxide slurry composition; however, only Batch 1 was used for Integrated Test A. 
Table 4.5. Batch 1 Chromium Slurry Composition for Integrated Test A

\begin{tabular}{|c|c|c|c|c|c|c|c|c|c|c|c|c|}
\hline \multirow{3}{*}{$\begin{array}{c}\text { Batch } \\
\text { Name } \\
\text { Sample ID } \\
\text { Parameter }\end{array}$} & \multirow{2}{*}{\multicolumn{3}{|c|}{$\begin{array}{l}\text { CrOOH Test Batch } 0 \\
\text { Cr-080623-250-SL } \\
\text { Total Slurry Fraction }\end{array}$}} & \multicolumn{6}{|c|}{$\begin{array}{l}\text { CrOOH Test Batch } 1 \\
\text { Cr-080729-2000-SL }\end{array}$} & \multirow{2}{*}{\multicolumn{3}{|c|}{$\begin{array}{c}\text { CrOOH Final Batch } \\
\text { Cr-080917-Final-SL } \\
\text { Total Slurry Fraction } \\
\end{array}$}} \\
\hline & & & & \multicolumn{3}{|c|}{ Total Slurry Fraction } & \multicolumn{3}{|c|}{ Liquid Fraction Only } & & & \\
\hline & $\mu \mathrm{g} / \mathrm{mL}$ & $\mathrm{Mol} / \mathrm{L}$ & $\%$ & $\mu \mathrm{g} / \mathrm{mL}$ & $\mathrm{Mol} / \mathrm{L}$ & $\%$ & $\mu \mathrm{g} / \mathrm{mL}$ & $\mathrm{Mol} / \mathrm{L}$ & $\%$ & $\mu \mathrm{g} / \mathrm{mL}$ & $\mathrm{Mol} / \mathrm{L}$ & $\%$ \\
\hline $\mathrm{Al}$ & $<3.5$ & 0.0001 & $<0.0002 \%$ & $<7.1$ & $<0.0003$ & $<0.0006 \%$ & 1.40 & 0.0001 & $0.0001 \%$ & 0.77 & 0.00003 & $0.0001 \%$ \\
\hline $\mathrm{B}$ & 14 & 0.0013 & $0.0010 \%$ & 4.15 & 0.0004 & $0.0003 \%$ & 1.80 & 0.0002 & $0.0002 \%$ & 3.3 & 0.0003 & $0.0003 \%$ \\
\hline $\mathrm{Ba}$ & 0.9 & 0.0000 & $0.0001 \%$ & 92.1 & 0.0007 & $0.0077 \%$ & 0.44 & 0.0000 & $0.0000 \%$ & 13.4 & 0.0001 & $0.0011 \%$ \\
\hline $\mathrm{Bi}$ & $<17.6$ & 0.0001 & $<0.0012 \%$ & $<3.5$ & $<0.0000$ & $<0.0003 \%$ & $<3$ & $<0.0000$ & $<0.0003 \%$ & $<1.5$ & $<0.0000$ & $<0.000 \%$ \\
\hline $\mathrm{Ca}$ & 44 & 0.0011 & $0.0030 \%$ & 12.5 & 0.0003 & $0.0010 \%$ & $<1.2$ & $<0.0000$ & $<0.0001 \%$ & 19.1 & 0.0005 & $0.0016 \%$ \\
\hline $\mathrm{Cd}$ & $<1.2$ & 0.0000 & $<0.0001 \%$ & 0.74 & 0.0000 & $0.0001 \%$ & 0.22 & 0.0000 & $0.0000 \%$ & 0.99 & 0.0000 & $0.0001 \%$ \\
\hline $\mathrm{Ce}$ & $<5.9$ & 0.0000 & $<0.0004 \%$ & $<1.2$ & $<0.0000$ & $<0.0001 \%$ & 1.00 & 0.0000 & $0.0001 \%$ & $<0.5$ & $<0.0000$ & $<0.000 \%$ \\
\hline $\mathrm{Cr}$ & 48,800 & 0.9385 & $3.3388 \%$ & 14,600 & 0.2808 & $1.2259 \%$ & 33.7 & 0.0006 & $0.0029 \%$ & 14,100 & 0.2712 & $1.1899 \%$ \\
\hline $\mathrm{Fe}$ & 23.3 & 0.0004 & $0.0016 \%$ & 5.58 & 0.0001 & $0.0005 \%$ & 0.37 & 0.0000 & $0.0000 \%$ & 7.0 & 0.0001 & $0.0006 \%$ \\
\hline $\mathrm{K}$ & 270 & 0.0069 & $0.0185 \%$ & 186 & 0.0047 & $0.0156 \%$ & 89.8 & 0.0023 & $0.0077 \%$ & 419 & 0.0107 & $0.0354 \%$ \\
\hline $\mathrm{La}$ & 2.3 & 0.0000 & $0.0002 \%$ & 0.56 & 0.0000 & $0.0000 \%$ & $<0.28$ & $<0.0000$ & $<0.0000 \%$ & 0.94 & 0.0000 & $0.0001 \%$ \\
\hline $\mathrm{Mg}$ & $<1.4$ & 0.0001 & $<0.0001 \%$ & 0.45 & 0.0000 & $0.0000 \%$ & $<0.23$ & $<0.0000$ & $<0.0000 \%$ & 0.72 & 0.0000 & $0.0001 \%$ \\
\hline $\mathrm{Mn}$ & 0.85 & 0.0000 & $0.0001 \%$ & 0.33 & 0.0000 & $0.0000 \%$ & 0.060 & 0.0000 & $0.0000 \%$ & 0.23 & 0.0000 & $0.0000 \%$ \\
\hline $\mathrm{Na}$ & 322,000 & 14.0062 & $22.031 \%$ & 90,550 & 3.9387 & $7.6029 \%$ & 99,100 & 4.3106 & $8.4701 \%$ & $<99,100$ & $<4.311$ & $<8.363 \%$ \\
\hline $\mathrm{Nd}$ & $<11.8$ & 0.0001 & $<0.0008 \%$ & $<2.4$ & $<0.0000$ & $<0.0002 \%$ & $<2$ & $<0.0000$ & $<0.0002 \%$ & $<1.0$ & $<0.0000$ & $<0.000 \%$ \\
\hline $\mathrm{Ni}$ & 10 & 0.0002 & $0.0007 \%$ & 0.68 & 0.0000 & $0.0001 \%$ & $<0.24$ & $<0.0000$ & $<0.0000 \%$ & 1.21 & 0.0000 & $0.0001 \%$ \\
\hline $\mathrm{P}$ & $<29.4$ & 0.0009 & $<0.0020 \%$ & $<6.1$ & $<0.0002$ & $<0.0005 \%$ & $<5$ & $<0.0002$ & $<0.0004 \%$ & $<2.5$ & $<0.0001$ & $<0.000 \%$ \\
\hline $\mathrm{Pb}$ & $<18.8$ & 0.0001 & $<0.0013 \%$ & $<3.8$ & $<0.0000$ & $<0.0003 \%$ & $<3.2$ & $<0.0000$ & $<0.0003 \%$ & $<1.6$ & $<0.0000$ & $<0.000 \%$ \\
\hline $\mathrm{S}$ & $<94.1$ & 0.0029 & $<0.0064 \%$ & 22 & 0.0007 & $0.0018 \%$ & 31.0 & 0.0010 & $0.0026 \%$ & 30 & 0.0009 & $0.0025 \%$ \\
\hline $\mathrm{Si}$ & 33.8 & 0.0012 & $0.0023 \%$ & 12.7 & 0.0005 & $0.0011 \%$ & 10.5 & 0.0004 & $0.0009 \%$ & 15 & 0.0005 & $0.0013 \%$ \\
\hline $\mathrm{Sr}$ & 0.13 & 0.0000 & $0.0000 \%$ & 168.5 & 0.0019 & $0.0141 \%$ & 0.054 & 0.0000 & $0.0000 \%$ & 30.6 & 0.0003 & $0.0026 \%$ \\
\hline Th & 90.7 & 0.0004 & $0.0062 \%$ & 27.8 & 0.0001 & $0.0023 \%$ & $<0.98$ & $<0.0000$ & $<0.0001 \%$ & 26.8 & 0.0001 & $0.0023 \%$ \\
\hline $\mathrm{Zn}$ & 11 & 0.0002 & $0.0008 \%$ & 3.60 & 0.0001 & $0.0003 \%$ & 1.60 & 0.0000 & $0.0001 \%$ & 9.43 & 0.0001 & $0.0008 \%$ \\
\hline $\mathrm{Zr}$ & $<0.6$ & 0.0000 & $<0.0000 \%$ & $<0.1$ & $<0.0000$ & $<0.0000 \%$ & $<0.11$ & $<0.0000$ & $<0.0000 \%$ & 0.073 & 0.0000 & $0.0000 \%$ \\
\hline
\end{tabular}


It was necessary to remove excess liquid introduced by chromium solids slurry with five ultrafilter bundles. After dewatering, additional solids washing was performed to remove the excess hydroxide included in the chromium solids slurry and to achieve a hydroxide concentration of $0.25-\mathrm{M} \mathrm{NaOH}$. The filter-loop flow rate decreased through the course of chromium washing; at 03:56, the filter-loop flow rate was $85 \mathrm{gpm}$ (103 gpm at 02:51 2/16/09 [TI pg. 202]). The run sheet target was $109 \pm 10 \mathrm{gpm}$ (TI, pg. 204). The steam ring air and upper and lower spargers were off during post-chromium slurry washing. Leaks in UFP-VSL-T02A PJMs 1807 and 1801 vacuum lines were identified; by 10:00, they were still leaking after tightening the connections, (2/16/09, LRB 60230, pg. 98). At 13:18 on 2/16/09 (LRB 60230, pg. 100), leaking simulant was observed coming out the tops of the PJMs in Tank T02A. This was caused by PJM overdraw when vacuum was applied too long and drew slurry up into the vacuum delivery piping. The process of turning the UFP-VSL-T02A PJMs off and then restarting them one by one was initiated, and the PJM parameter adjustments were made as necessary. Refer to Figure 4.43 through Figure 4.46 for filtration activities during the reconcentration and washing of the slurry after chromium addition-where the prototypic UFP-VSL-T02A temperature was taken from TTK-0619.

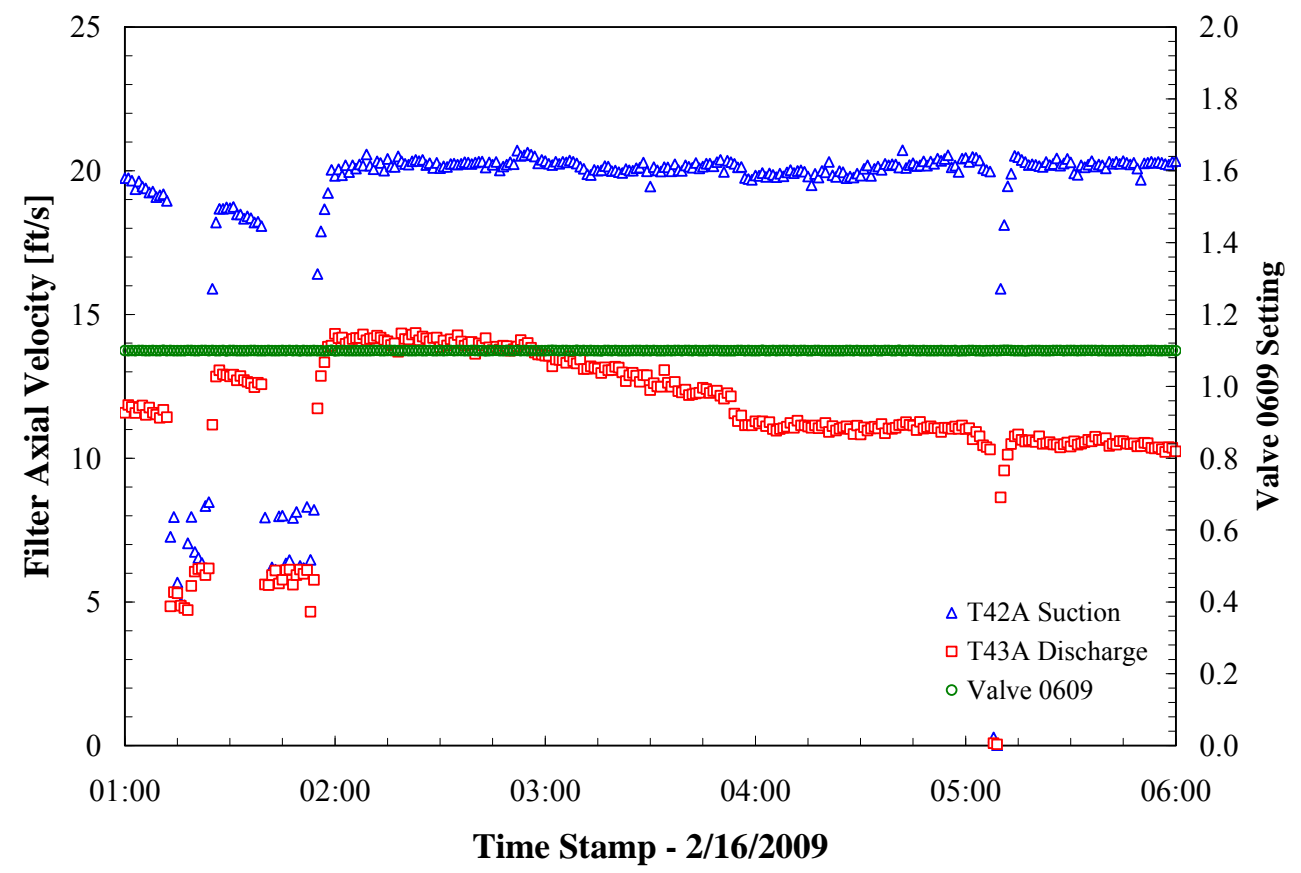

Figure 4.43. Axial Velocity Based on Flow Measurements at the Suction to Pump T42A and the Discharge to Pump T43A for the Reconcentration and Wash Operations Following Chromium Slurry Addition; Valve SV-0609 Setting (for information only) Scale 0 to 2 Correlates to $0 \%$ and $100 \%$ Open, Respectively 


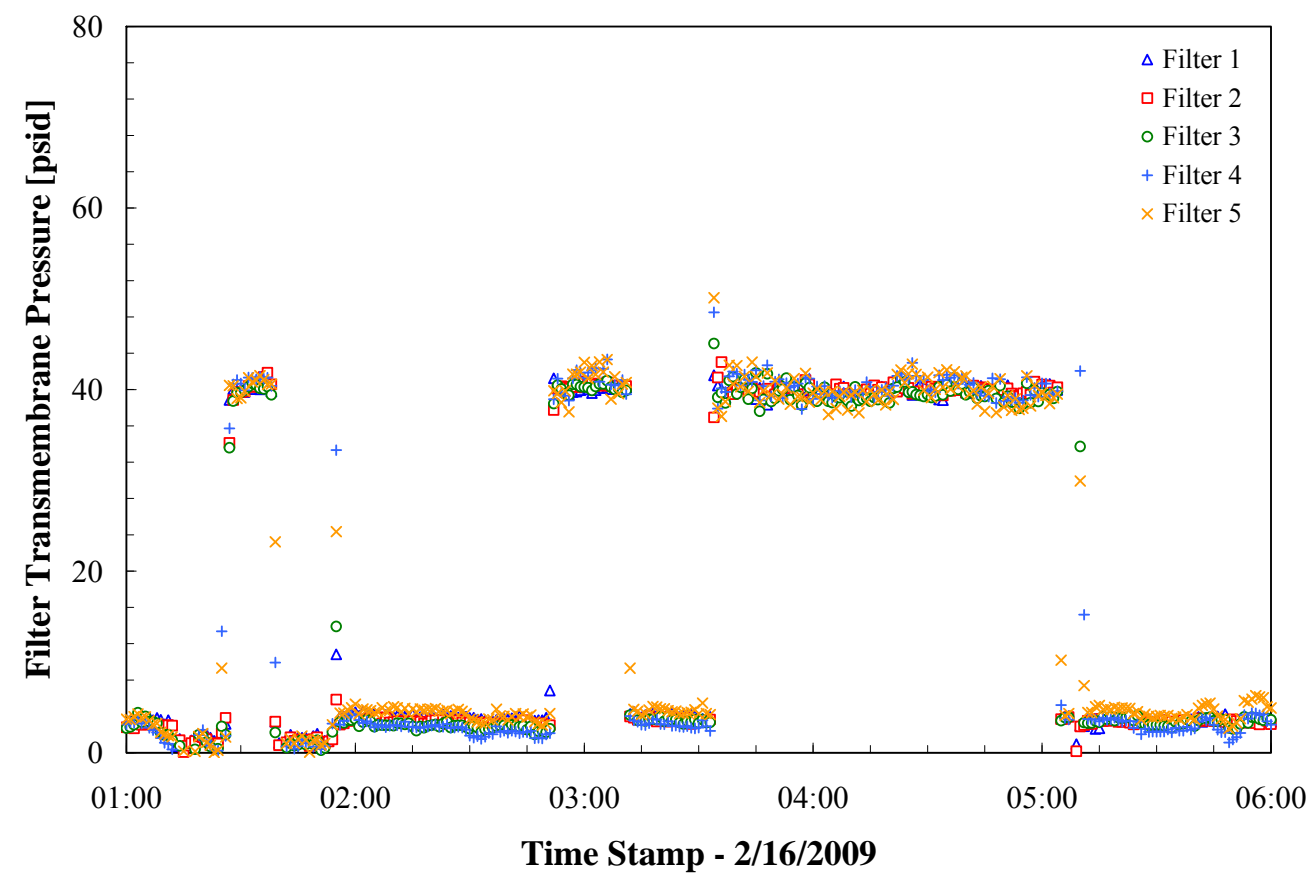

Figure 4.44. TMP for the Reconcentration and Wash Operations Following Chromium Slurry Addition

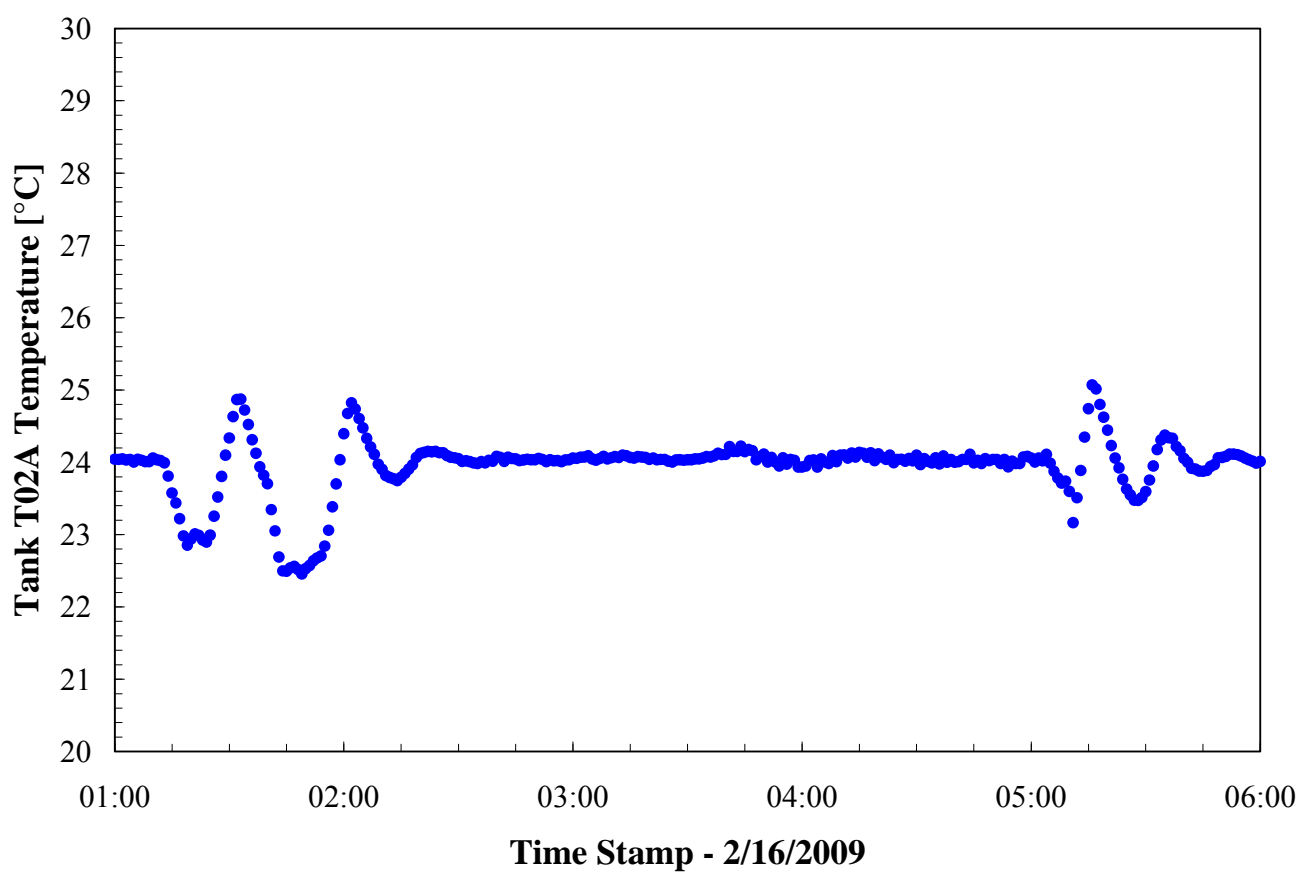

Figure 4.45. Tank T02A (TTK-0619) Temperature for the Reconcentration and Wash Operations Following Chromium Slurry Addition 


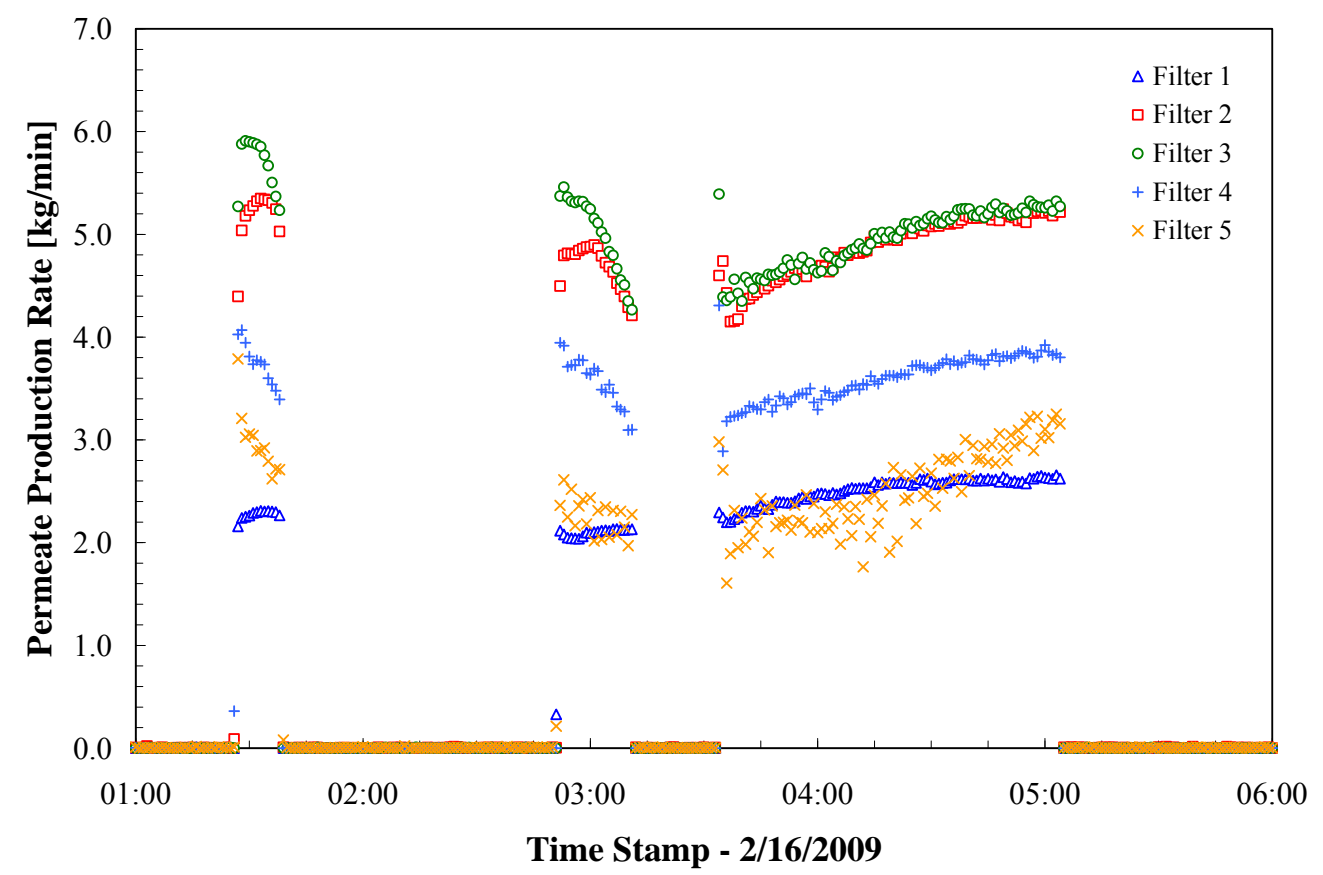

Figure 4.46. Permeate Production Rate for the Reconcentration and Wash Operations Following Chromium Slurry Addition

Permanganate additions started on 2/16/09 at 10:13 and ended at 10:21. The 1-M sodium permanganate addition, for a total of 37.2 gallons, was made to the suction side of pump UFP-PMP-T42A. Filter-loop flow rates increased (with no adjustment of the operating conditions) to the target flow rate during the sodium permanganate transfer, but declined soon after the transfer was complete.

Oxidative leaching started on 2/16/09 at 10:21 and ended at 16:23. The target filter-loop flow rate was 109 gpm; however, only $85 \mathrm{gpm}$ maximum was achieved momentarily because of air entrainment. The steam-ring air purge and upper air spargers were kept off, per guidance, as opposed to the original run sheet targets of 0.14 and 0.01 , respectively, in efforts to minimize entrained air in the filter loop. Although the lower air spargers were also supposed to be off to minimize entrained air in the filter loop, they were kept on at a rate of $\sim 0.04 \mathrm{~kg} / \mathrm{min}$. The target temperature of $25^{\circ} \mathrm{C}$ was maintained during the 6 hours of oxidative leaching; refer to Appendix G, "T02A temperatures" plots for specified times. The PJM nozzle velocity during oxidative leaching was $\sim 12.1 \mathrm{~m} / \mathrm{s}$. Refer to Section 5.3 for the PJM peak average velocity summary.

\subsection{Post-Oxidative Washing}

Post-oxidative-leach washing followed 6 hours of oxidative leaching. There were a total of 86 IW additions of $41.6 \mathrm{~kg}$ each. The steam-ring air purge and the upper and lower air spargers were kept off, per guidance, as opposed to the original run sheet targets of $0.14,0.01$, and $0.04 \mathrm{~kg} / \mathrm{min}$, respectively. Refer to Figure 4.47 through Figure 4.50 for filtration activities during post-oxidative-leach washing--where the prototypic UFP-VSL-T02A temperature was taken from TTK-0619. Refer to 
Section 8, Filtration, of report WTP-RPT-197 for TMP and temperature-corrected filter flux versus wt $\%$ solids plots - along with permeate production rates.

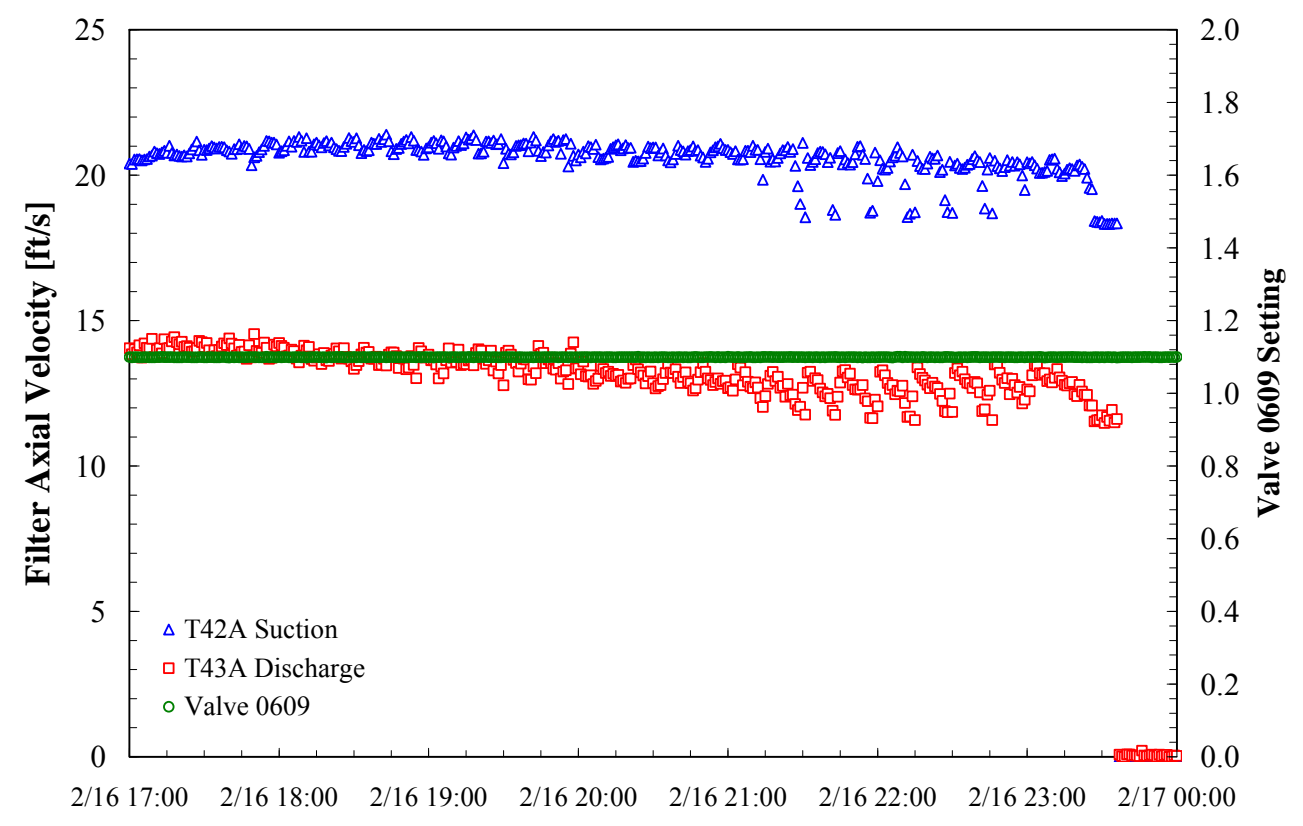

Time Stamp

Figure 4.47. Axial Velocity Based on Flow Measurements at the Suction to Pump T42A and the Discharge to Pump T43A for Post-Oxidative-Leach Slurry Wash; Valve SV-0609 Setting (for information only) Scale 0 to 2 Correlates to $0 \%$ and 100\% Open, Respectively

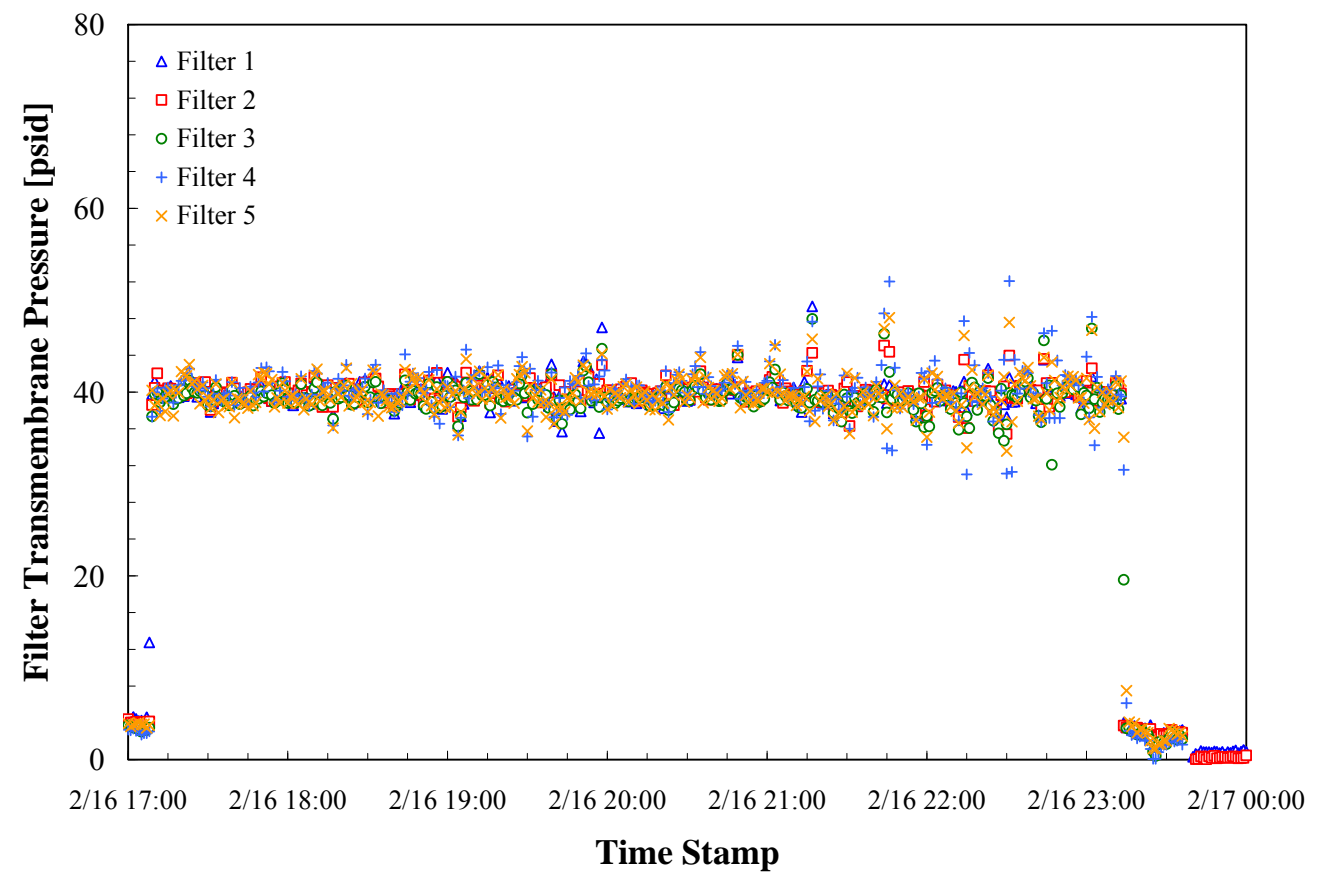

Figure 4.48. TMP for Post-Oxidative-Leach Slurry Wash 


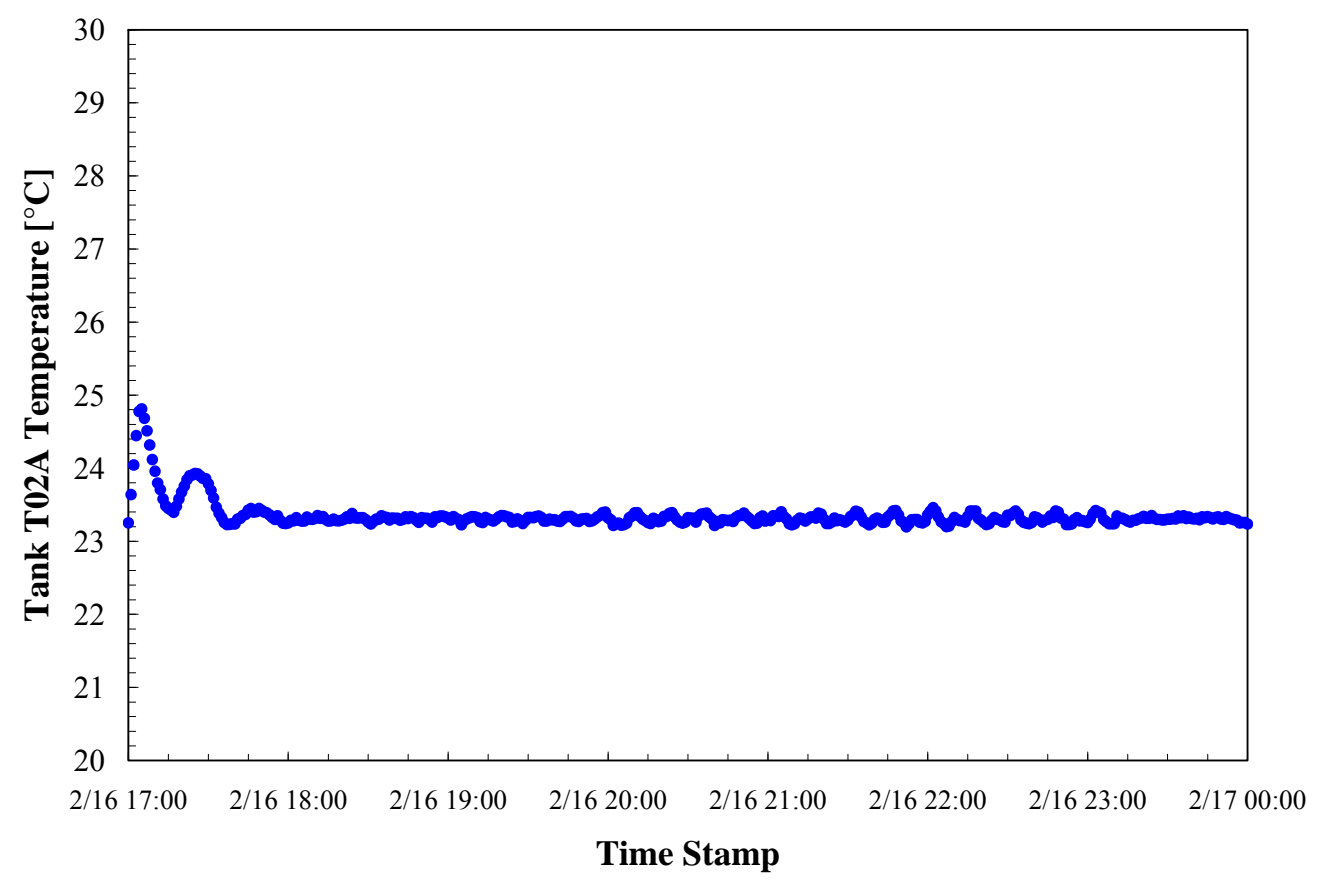

Figure 4.49. Tank T02A (TTK-0619) Temperature for Post-Oxidative-Leach Slurry Wash

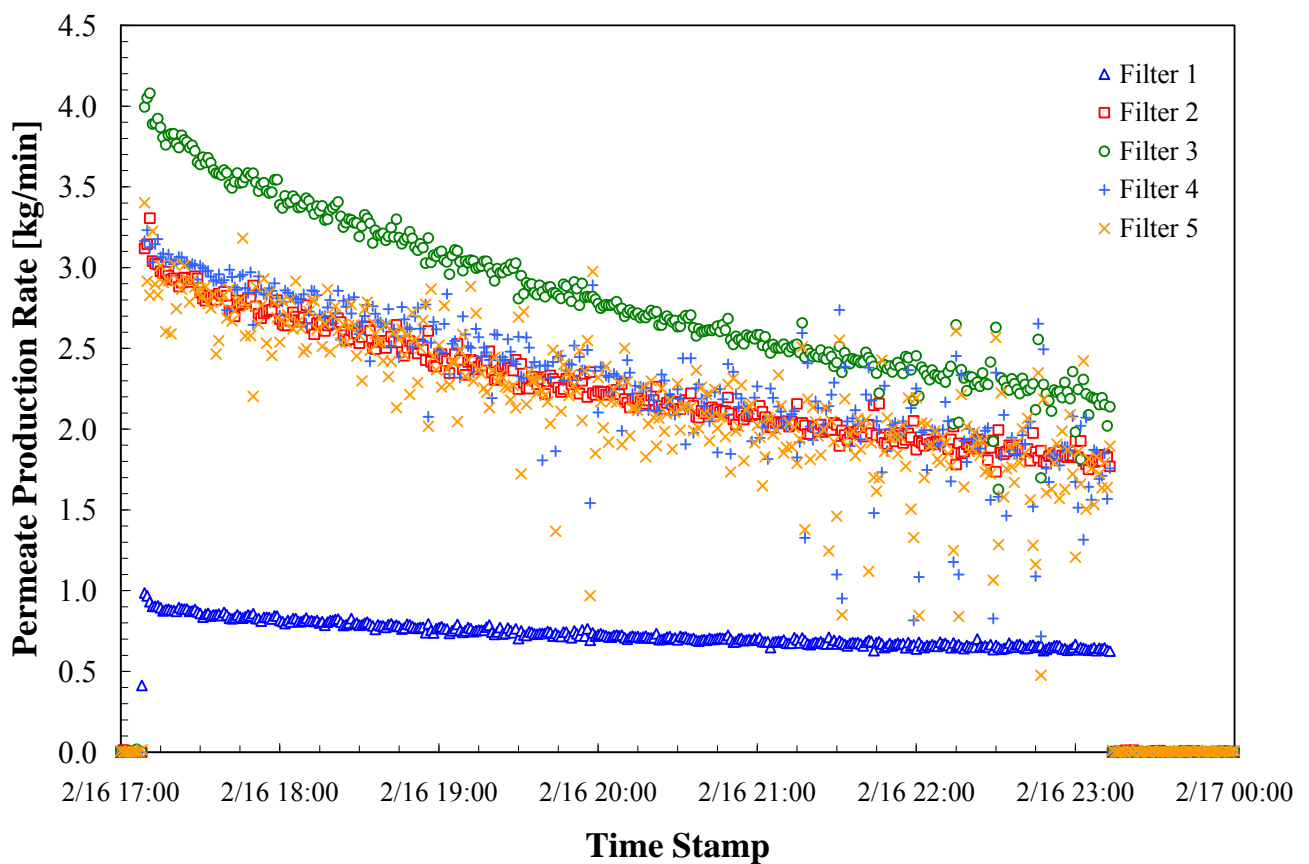

Figure 4.50. Permeate Production Rate for Post-Oxidative-Leach Slurry Wash 
Integrated Test A was put on hold after completing the post-oxidative-leach washing per the Test Director email 20:28 on 2/16/09. A list of tasks to perform after completing the wash was provided in the email. See discussion on the test suspension, Section 6.2.1.

\subsection{Final Concentration}

Integrated Test $A$ was restarted on the late afternoon of $2 / 20 / 09$. Final concentration to $20-w t \%$ was done using all five filters on 2/20/09 from 21:24 to 21:40. The stable level in UFP-VSL-T02A before concentration was 45.4 (laser). Bubblers were turned off, per guidance (TI pg. 215), for the final concentration. The steam-ring air purge and the upper and lower air spargers were kept off, per guidance (to manage air entrainment), as opposed to the original run sheet targets of $0.14,0.01$, and $0.04 \mathrm{~kg} / \mathrm{min}$, respectively. The stable level in UFP-VSL-T02A after concentration was 38.9 inches (laser). In addition, the maximum attainable filter-loop flow rate was $73 \mathrm{gpm}$ upstream from recirculation pump UFP-PMP-T42A (the flow rate on FI-0635, downstream from recirculation pump UFP-PMP-T43A, was higher) as opposed to the $109 \pm 10 \mathrm{gpm}$ run sheet target (TI Run Sheet). The flow meter performance was questionable at this time, as stated on the NCR list in Section 2. Refer to Figure 4.51 through Figure 4.54 for filtration activities during the final concentration - where the prototypic UFP-VSL-T02A temperature was taken from TTK-0619.

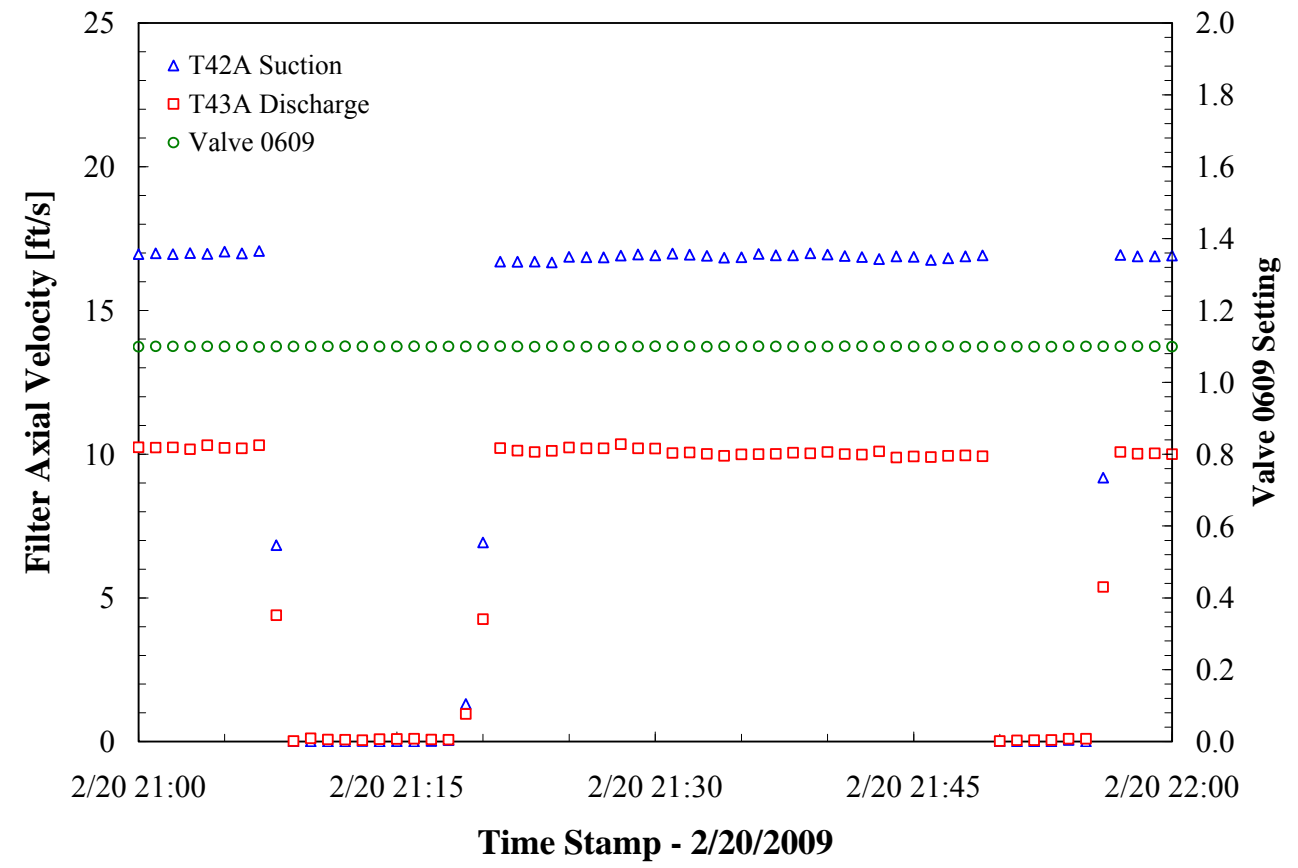

Figure 4.51. Axial Velocity Based on Flow Measurements at the Suction to Pump T42A and the Discharge to Pump T43A for Post-Oxidative-Leach Slurry Concentration; Valve SV-0609 Setting (for information only) Scale 0 to 2 Correlates to $0 \%$ and $100 \%$ Open, Respectively 


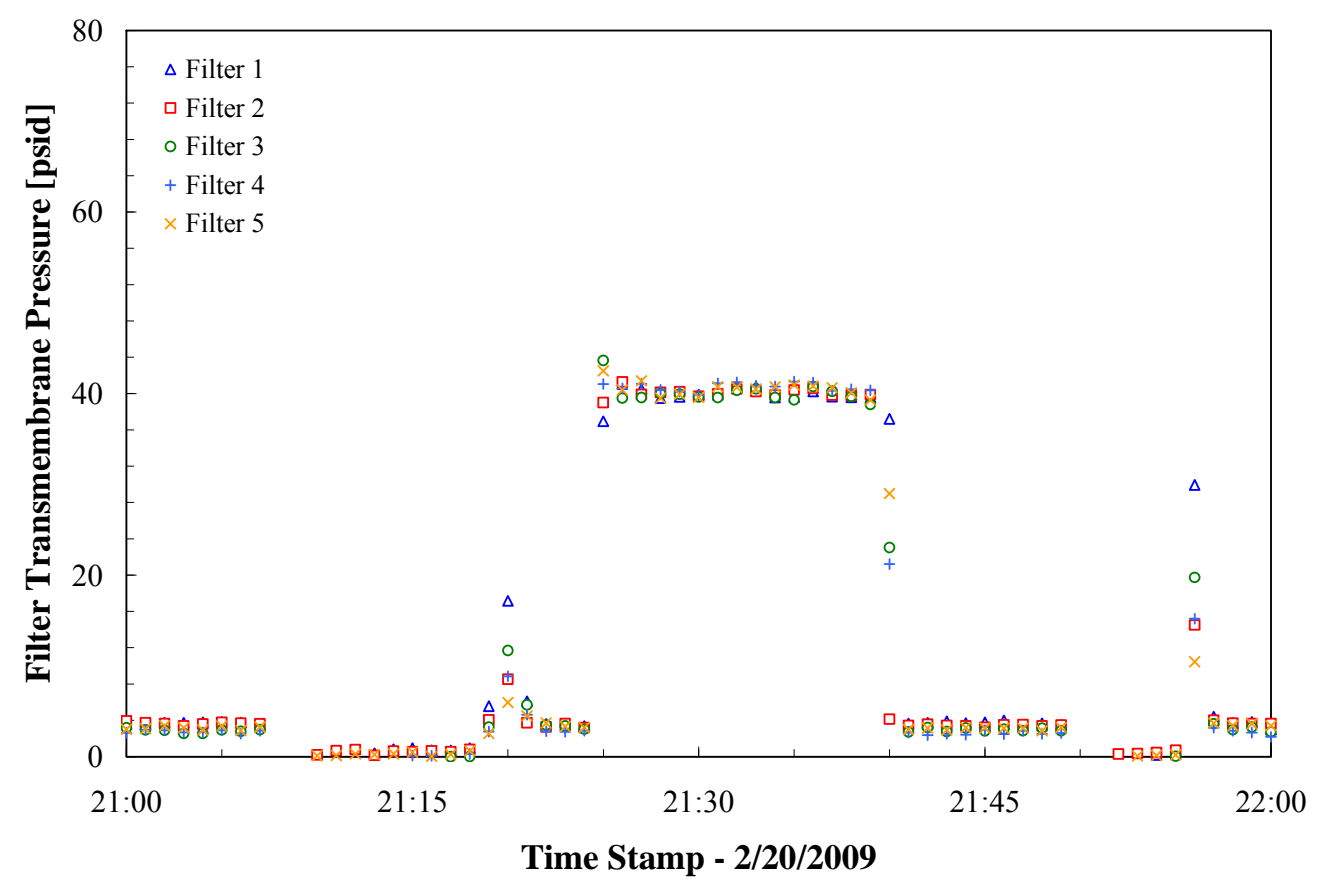

Figure 4.52. TMP for Post-Oxidative-Leach Slurry Concentration

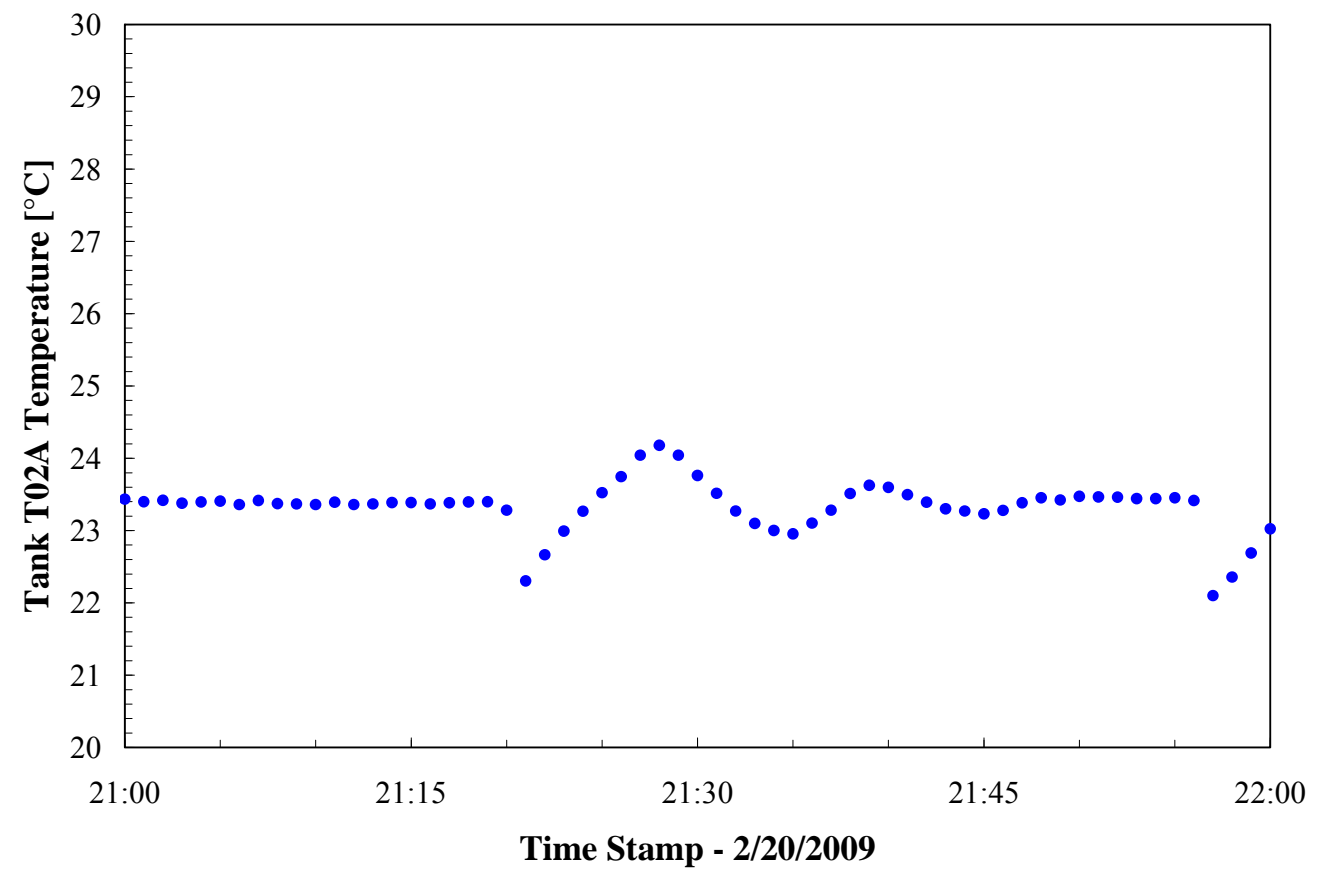

Figure 4.53. Tank T02A (TTK-0619) Temperature for Post-Oxidative-Leach Slurry Concentration 


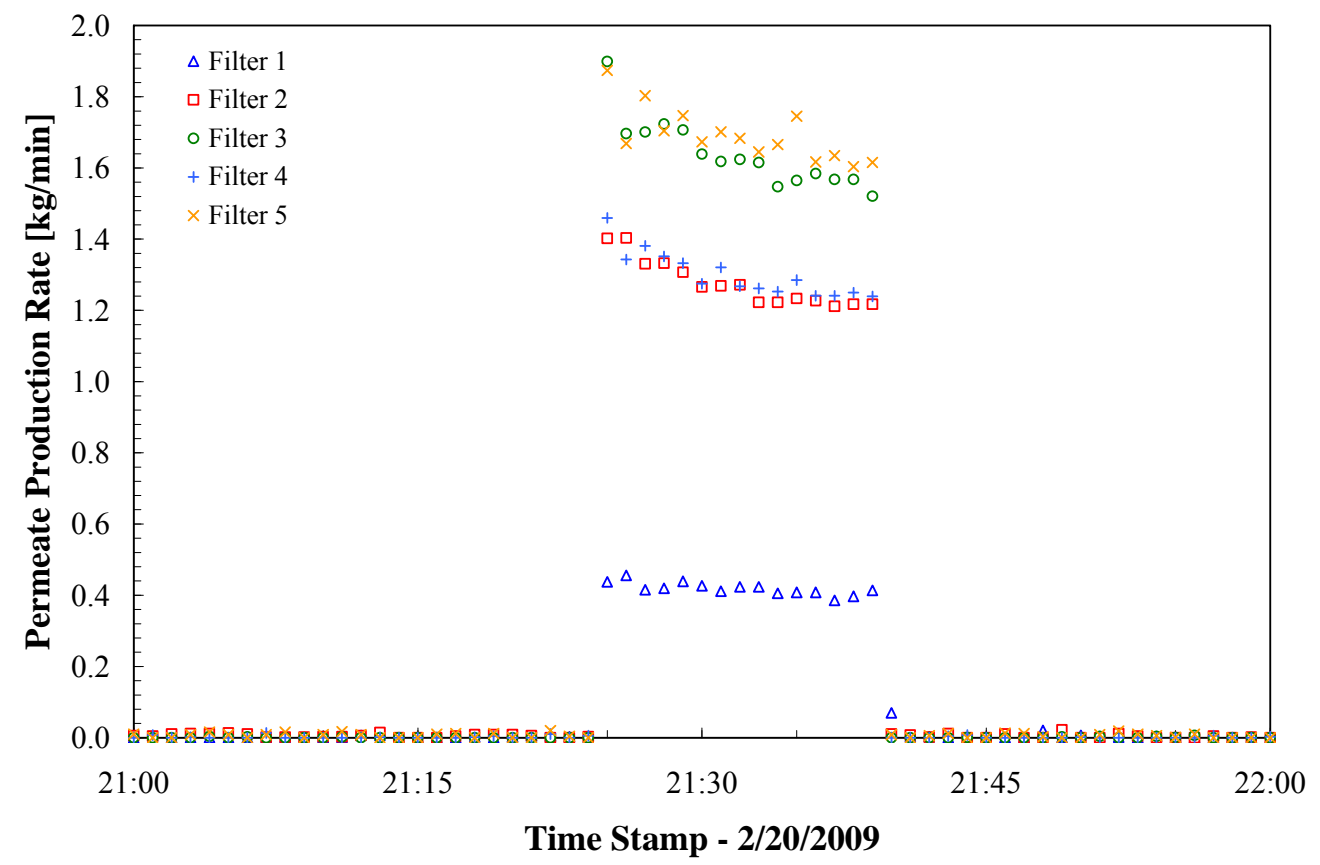

Figure 4.54. Permeate Production Rate for Post-Oxidative-Leach Slurry Concentration

Filter permeate and other flush solutions were transferred to permeate vessels UFP-VSL-T62A/B and/or totes. The High Solids Filter Test should have happened next, but due to the pump issues, it was postponed for a later date.

\subsection{Flush Filter Loop}

Simulant in the filter loop was drained directly to a tote without adding IW for later use: High Solids Filter Test, per Test Director guidance (TI pg. 225). Approximately 20 inches of simulant was also removed from UFP-VSL-T02A and put into totes to reach 20 inches in the vessel. Before test clean-up, a filter-loop study was performed on 2/20/09, 23:50, to evaluate how low the UFP-VSL-T02A tank level could reach, considering the filter-loop return nozzle location, while maintaining filter-loop flow rate targets. The filter-loop study indicated that the filter-loop flow rate dropped from 100 gpm at the 20-in. tank level to $83 \mathrm{gpm}$ at an 18.3-in. level per upstream flow meter FI-0623 upstream from UFP-PMP-T42A recirculation pump. The laser was noted as not reading below the 17.8-in. vessel level. The PJMs were turned off during the filter-loop study so that the camera quality would not be compromised. The camera showed the simulant return line exposed approximately 2 inches at the 18.3-in. vessel level (TI pg. 224). Then IW was transferred to UFP-VSL-T02A and circulated through the filter loop with recirculation pumps UFP-PMP-T42A and UFP-PMP-T43A to allow a representative in-line sample of the filter-loop contents to be collected.

As stated earlier, slurry from UFP-VSL-T02A and the filter loop was transferred to totes for later use. In efforts to retain as much concentrated slurry as possible from Integrated Test A, the simulant and IW slurry in UFP-VSL-T02A was also transferred into a tote. 


\subsection{High-Solids Filter Test}

The High-Solids Filter Test was to be done after performing the final concentration to 20 -wt $\%$, but due to the unstable filter-loop pump operations in previous testing processes, the High-Solids Filter Test was postponed until 3/20/09, 00:31, at the completion of Integrated Test B-caustic and oxidative leaching in UFP-VSL-T02A. The objective of the High-Solids Filter Test was to observe the solids concentration versus the filter flux.

Preparation for the High-Solids Filter Test started 3/20/09, 21:10 (LRB 60279, pg. 38), and ended 3/21/09, 00:31. Simulant from Integrated Test A stored in totes was added to UFP-VSL-T02A via transfer from UFP-VSL-T01B where it was mixed with PJM operation, and the steam ring was set to $0.20 \mathrm{~kg} / \mathrm{min}$ before it was sent to UFP-VSL-T02A to achieve the target solids concentration. The UFP-VSL-T02A stable level at this point was 51.4 inches (laser). UFP-VSL-T02A was then filled with IW via in-line to the UFP-PMP-T42A suction side to reach the target vessel level. The stable level in UFP-VSL-T02A after IW addition was 63.9 inches (laser). A calculated 86-mL of concentrated AFA was added to account for the volume of IW used to reach the tank level target per the run sheet.

The High-Solids Filter Test started on 3/21/09, 01:37, and ended 3/21/09, 06:08. Filters were configured to flow slurry through all five filters, but permeate was withdrawn only through the first filter, Filter T01A. The permeate rate through Filter T01A before backpulsing was $3.2 \mathrm{~kg} / \mathrm{min}$. After the permeate valve was opened, Filter T01A was backpulsed consecutively five times as quickly as possible while sending permeate back into UFP-VSL-T02A. Standard backpulse parameters were used: a deadband pressure of $5 \mathrm{psi}$, an overpressure of $40 \mathrm{psi}$, and a pulse pot level of initiation of 10 inches. After backpulsing was completed, the permeate rate through Filter T01A was $3.5 \mathrm{~kg} / \mathrm{min}$. Slurry in UFP-VSL-T02A was then concentrated using only the first filter bundle; permeate was sent to UFP-VSL-T62B. The steam-ring air purge and upper and lower air spargers were kept off, per guidance, as opposed to the original run sheet targets of $0.14,0.01$, and $0.04 \mathrm{~kg} / \mathrm{min}$, respectively. The UFP-VSL-T02A stable level after concentration was 22.8 inches (laser). The High-Solids Filter Test results are reported in the filtration report, WTP-RPT-185. Refer to Figure 4.55 through Figure 4.58 for filtration performance during the High-Solids Filter Test-where the prototypic UFP-VSL-T02A temperature was taken from TTK-0619.

The temperature target for UFP-VSL-T02A was exceeded towards the end of the High-Solids Filter Test. By 05:15, 3/21/09 (LRB 60279, pg. 43), the heat exchanger UFP-HX-T02A, TIC-0513, temperature setpoint was decreased to $20^{\circ} \mathrm{C}$ because the temperature in the tank was $26.4^{\circ} \mathrm{C}$, and the target was $23 \pm 2^{\circ} \mathrm{C}$. By 05:44, 3/21/09 (LRB 60279, pg. 43), the UFP-VSL-T02A heat exchanger temperature setpoint was set to manual operation in response to the increasing temperature in the UFP-VSL-T02A. This may have been influenced by the low level and high pump flow-rate operations. By 05:46, 3/21/09 (LRB 60271, pg. 4), the heat exchanger UFP-HX-T02A valve was set at 60\% (open) in response to a vessel temperature of $28.5^{\circ} \mathrm{C}$. Temperature readings from RTD thermowells were not reliable as indicated on NCR 42402.1.

After completing the High-Solids Filter Test, the cesium tracer test was repeated as noted previously in Section 4.4. 


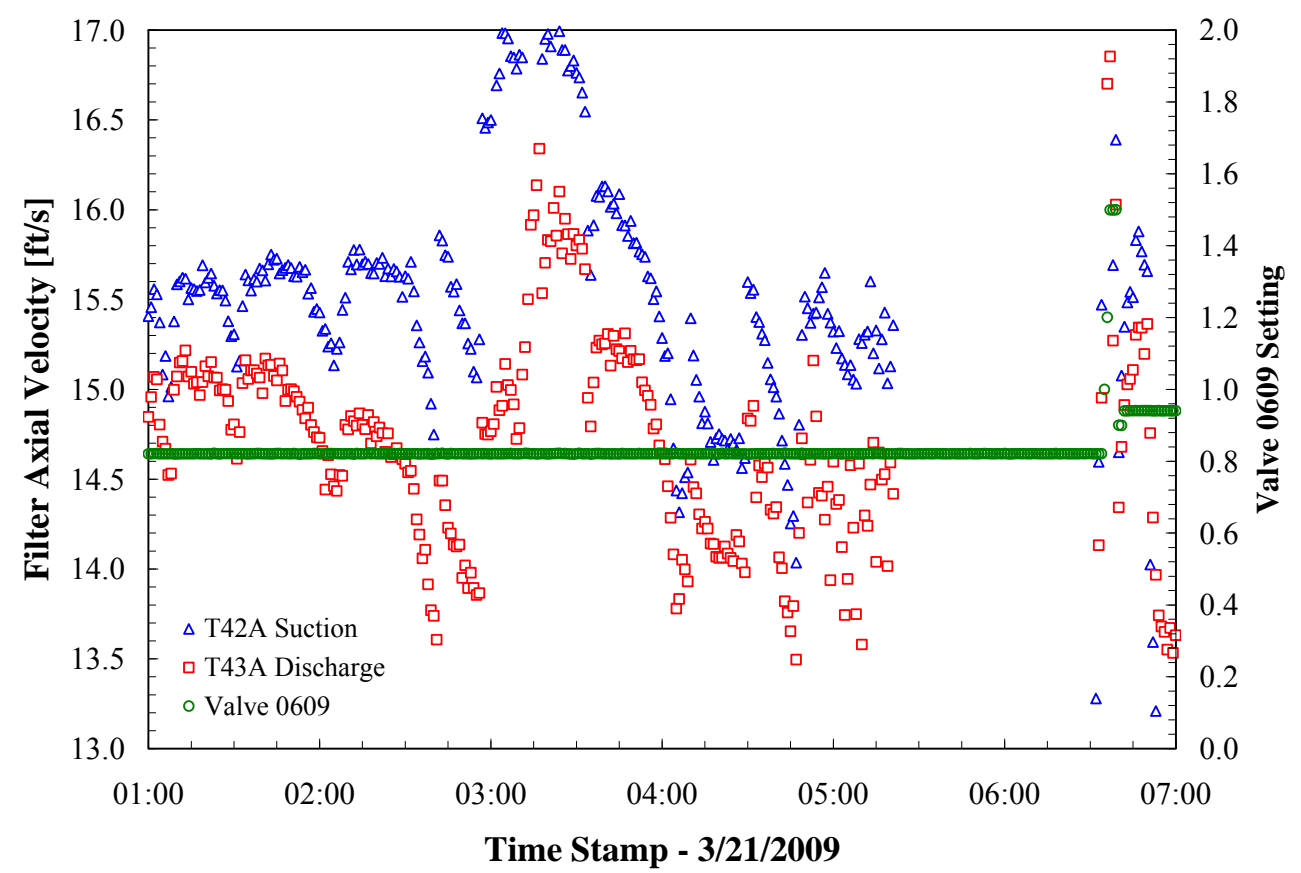

Figure 4.55. Axial Velocity Based on Flow Measurements at the Suction to Pump T42A and the Discharge to Pump T43A for the High-Solids Scaling Test Conducted on 3/21/2009; Valve SV-0609 Setting (for information only) Scale 0 to 2 Correlates to $0 \%$ and $100 \%$ Open, Respectively 


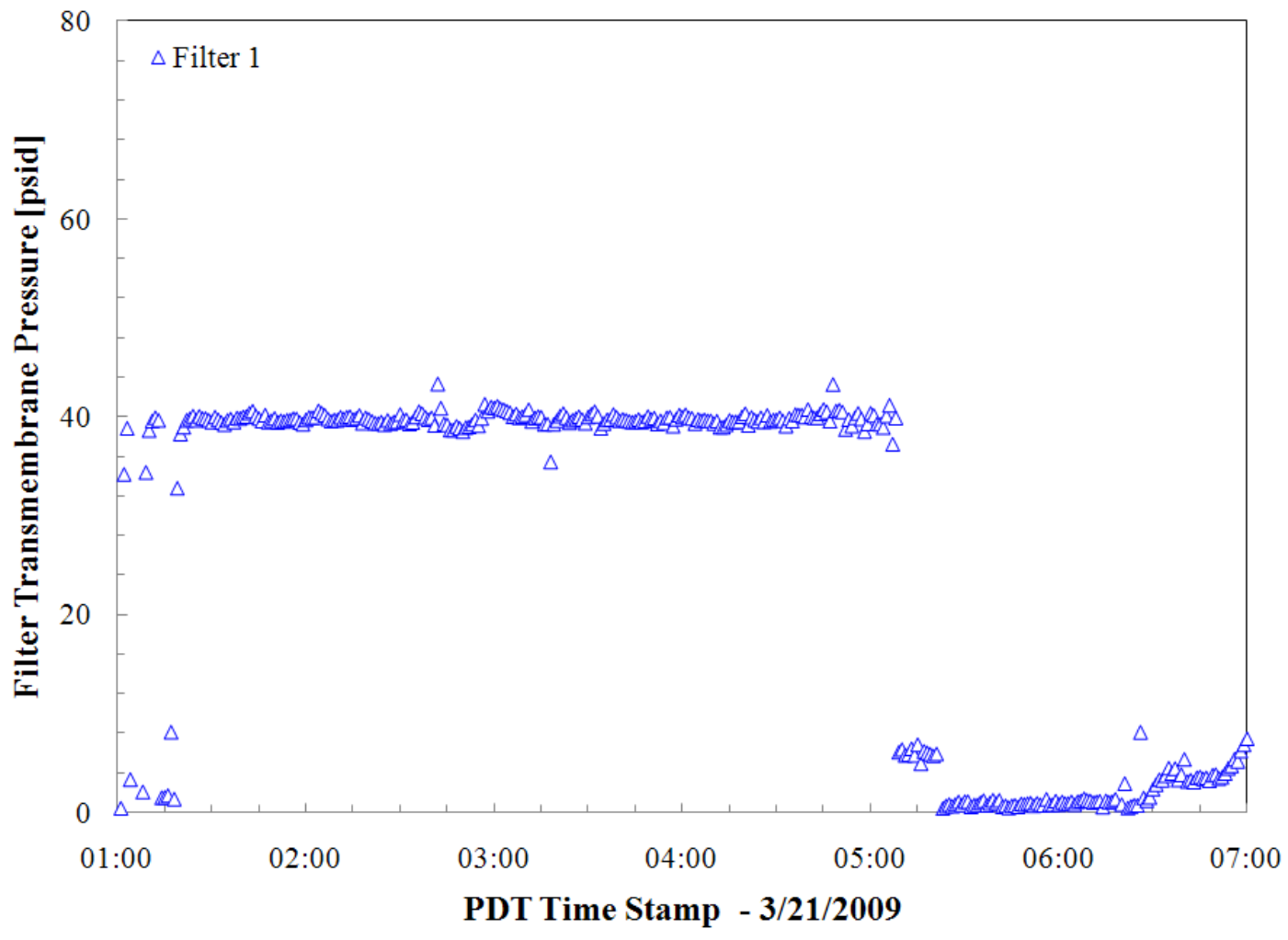

Figure 4.56. TMP for the High-Solids Scaling Test Conducted on 3/21/2009

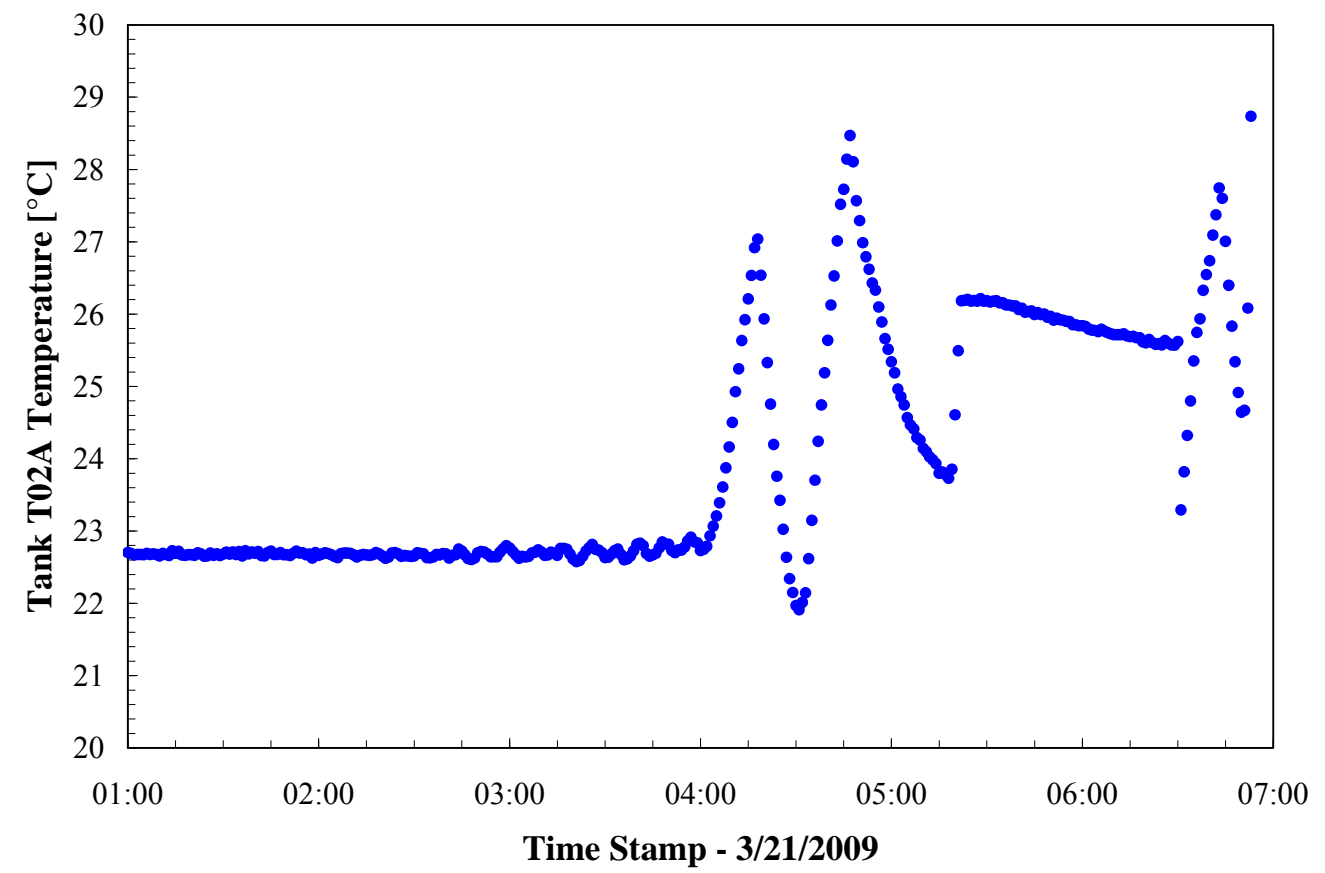

Figure 4.57. Tank T02A (TTK-0619) Temperature for the High-Solids Scaling Test Conducted on $3 / 21 / 2009$ 


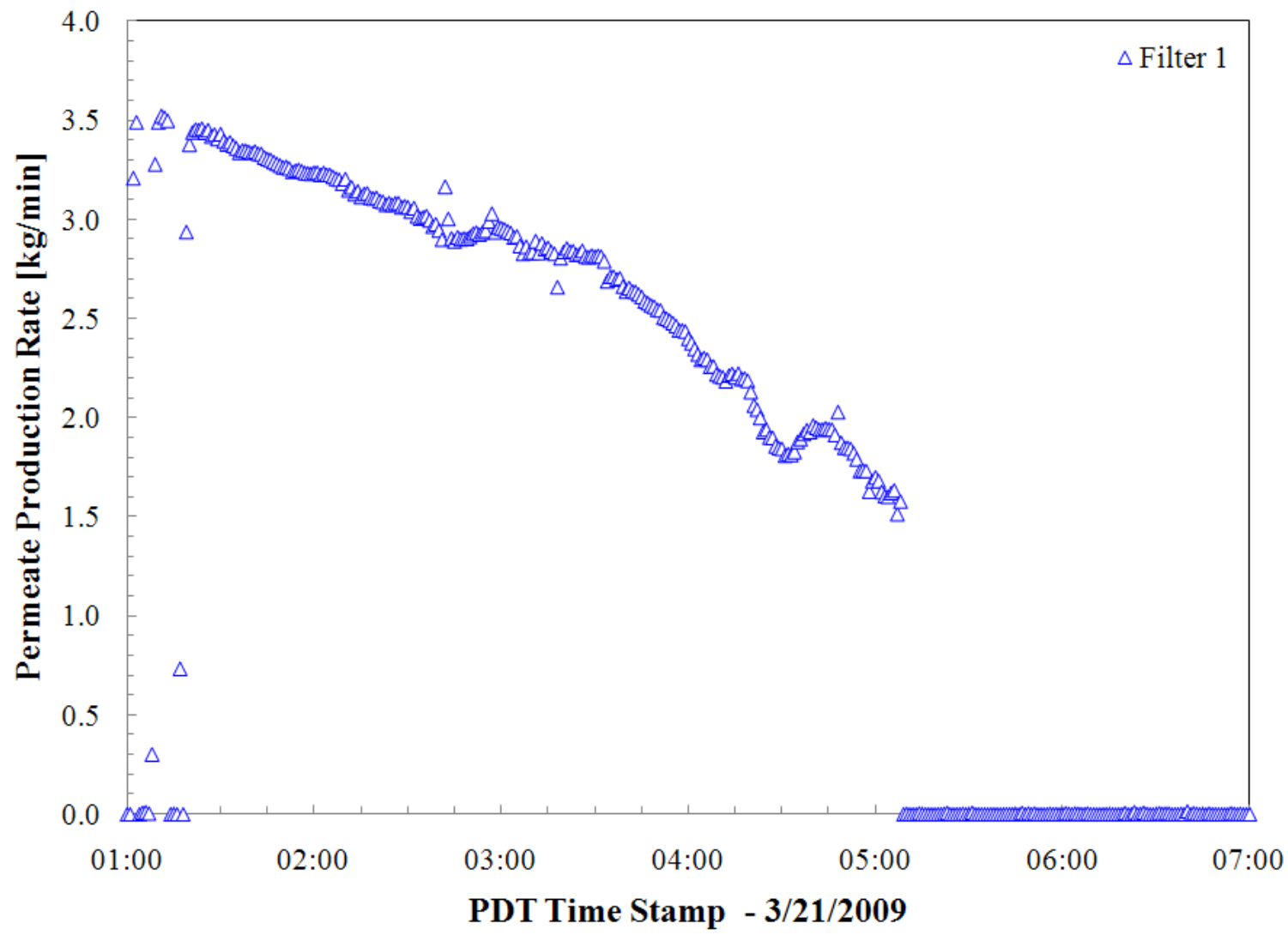

Figure 4.58. Permeate Production Rate for the High-Solids Scaling Test Conducted on 3/21/2009 



\subsection{Analytical Results}

Selected data obtained from the DAS during Integrated Test A have been plotted in 12-h intervals, and this is provided in Appendix G. Tables summarizing the results of sample measurements taken during testing are provided in Section 5.1 (analytical results) and Section 5.2 (physical-property results). The analysis of the analytical data is done in the final report, Sections 9, 10,11, and 12, of the Pretreatment Engineering Platform Phase 1 Final Test Report, WTP-RPT-197. PJM peak average velocity and stroke length, tuned iteratively to target values during testing, are provided in Section 5.3. Data, including figures, in this section are NQA-1 compliant.

\subsection{Analytical Data}

This section provides analytical summary tables for key processes. Reporting limits and uncertainties are in the final analytical reports submitted by each analytical laboratory. These reports are identified for each sample in Appendix B, which provides a list of all samples collected, the disposition of each sample, and references to final electronic analytical reports.

In many cases, such as during caustic and oxidative leaching, the slurry samples taken from PEP were analyzed after phase separation was performed. The wet centrifuged solids and the supernatant liquid from the samples were analyzed separately. In other cases, data are presented in a more raw form: the species concentration in the supernatant, the concentration in the wet centrifuged solids, and the masses of tare sample vial, vial plus total slurry, and vial plus wet centrifuged solids. The latter three masses can be used to calculate the mass fraction of wet centrifuged solids in the slurry, which in turn can be used in calculating the total species concentration in the original sample slurry. See Section A.5 in Appendix A of WTP-RPT-186, Rev 0, for more information on calculating the species concentration in the slurry.

Table 5.1 through Table 5.6 provide analytical results for samples collected during the Batch 1 through Batch 6 caustic leach in Tank T02A. The slurry density results were performed on intact slurry submitted to the analytical laboratory. The wt\% UDS, aluminum, and strontium slurry concentrations were calculated from samples phase-separated at PDL-W. The liquid analysis results are analytical results obtained from the phase-separated supernatants. Raman results, shown in brackets [ ], are provided as part of the liquid analyses and are presented in molar concentration (mole/liter).

Table 5.7 provides analytical results for samples collected during solids concentration of the caustic-leached slurry in Tank T02A. This concentration combined the leached solids from Batch 1 through 6 as described in Section 4. The wt $\%$ UDS and densities were obtained following analytical methods described in Section 3. An aliquot of the wet centrifuged solids was dried at $105^{\circ} \mathrm{C}$ to determine the percent solids that are reported with the reported analytes in the wet centrifuged solids.

Table 5.8 and Table 5.9 provide the cesium tracer analytical results for samples collected during Integrated Test A tracer tests using post-caustic-leached and concentrated slurry in Tank T02A. 
Table 5.1. Batch 1: Caustic Leach

\begin{tabular}{|c|c|c|c|c|c|c|c|c|c|c|c|c|c|}
\hline \multirow[b]{2}{*}{$\begin{array}{l}\text { Sample location: Tank } \\
\text { T02A, Condition: }\end{array}$} & \multicolumn{5}{|c|}{ Slurry Analyses } & \multicolumn{8}{|c|}{ Liquid Analyses } \\
\hline & $\begin{array}{l}\text { UDS } \\
\mathrm{Wt} \%\end{array}$ & $\begin{array}{l}\text { Slurry } \\
\text { Density } \\
\mathrm{g} / \mathrm{mL}^{(a)}\end{array}$ & $\begin{array}{r}\mathrm{Al} \\
\mu \mathrm{g} / \mathrm{g} \\
\end{array}$ & $\begin{array}{r}\mathrm{Sr} \\
\mu \mathrm{g} / \mathrm{g}\end{array}$ & $\begin{array}{r}\mathrm{Fe} \\
\mu \mathrm{g} / \mathrm{g} \\
\end{array}$ & $\begin{array}{l}\text { Liquid } \\
\text { Density } \\
\mathrm{g} / \mathrm{mL}^{(a)}\end{array}$ & $\begin{array}{c}\mathrm{Al} \\
\text { [Al(OH)4- } \\
\text { by } \\
\text { Raman] } \\
\mu \mathrm{g} / \mathrm{g} \\
{[\mathrm{M}]}\end{array}$ & $\begin{array}{c}\text { Free } \\
\text { OH- } \\
\text { [Raman] } \\
\mathrm{M} \\
{[\mathrm{M}]}\end{array}$ & $\begin{array}{c}\mathrm{C}_{2} \mathrm{O}_{4} \\
{[\mathrm{Raman}]} \\
\mu \mathrm{g} / \mathrm{g} \\
{[\mathrm{M}]}\end{array}$ & $\begin{array}{l}\mathrm{Cl}- \\
\mu \mathrm{g} / \mathrm{g} \\
\end{array}$ & $\begin{array}{c}\mathrm{NO}_{3} \\
\text { [Raman] } \\
\mu \mathrm{g} / \mathrm{g} \\
{[\mathrm{M}]}\end{array}$ & $\begin{array}{c}\mathrm{NO}_{2} \\
{[\text { Raman] }} \\
\mu \mathrm{g} / \mathrm{g} \\
{[\mathrm{M}]}\end{array}$ & $\begin{array}{c}\mathrm{SO}_{4} \\
\text { [Raman] } \\
\mu \mathrm{g} / \mathrm{g} \\
{[\mathrm{M}]}\end{array}$ \\
\hline After $\mathrm{NaOH}$ addition & 2.91 & 1.362 & 14468 & 32.7 & 3614 & 1.330 & $\begin{array}{c}4713 \\
{[0.263]}\end{array}$ & $\begin{array}{c}4.914 \\
{[5.153]}\end{array}$ & $\begin{array}{l}96.7 \\
{[\mathrm{n} / \mathrm{d}]}\end{array}$ & 799 & $\begin{array}{c}56966 \\
{[1.353]}\end{array}$ & $\begin{array}{c}13233 \\
{[0.429]}\end{array}$ & $\begin{array}{c}10266 \\
{[0.151]}\end{array}$ \\
\hline $88^{\circ} \mathrm{C}$ & 2.35 & 1.326 & - & - & - & 1.312 & $\begin{array}{c}6970 \\
{[0.354]}\end{array}$ & $\overline{[4.609]}$ & $\begin{array}{c}138 \\
{[<0.01]}\end{array}$ & 731 & $\begin{array}{c}52700 \\
{[1.240]}\end{array}$ & $\begin{array}{c}12200 \\
{[0.376]}\end{array}$ & $\begin{array}{c}9490 \\
{[0.139]}\end{array}$ \\
\hline $98^{\circ} \mathrm{C}$ - hour 0 & 2.38 & 1.323 & 13842 & 30.2 & 3289 & 1.308 & $\begin{array}{c}7220 \\
{[0.347]}\end{array}$ & $\begin{array}{c}4.328 \\
{[4.522]}\end{array}$ & $\begin{array}{l}172 \\
{[\mathrm{n} / \mathrm{d}]}\end{array}$ & 760 & $\begin{array}{c}52650 \\
{[1.216]}\end{array}$ & $\begin{array}{c}11900 \\
{[0.360]}\end{array}$ & $\begin{array}{c}9330 \\
{[0.137]}\end{array}$ \\
\hline $98^{\circ} \mathrm{C}$--hour 1 & 2.36 & 1.324 & - & 一 & - & 1.308 & $\begin{array}{c}6950 \\
{[0.348]}\end{array}$ & {$[\overline{4}-\overline{479}]$} & $\begin{array}{c}177 \\
{[<0.01]}\end{array}$ & 713 & $\begin{array}{c}51100 \\
{[1.194]}\end{array}$ & $\begin{array}{c}12100 \\
{[0.372]}\end{array}$ & $\begin{array}{c}9420 \\
{[0.134]}\end{array}$ \\
\hline $98^{\circ} \mathrm{C}$ - hour 2 & 2.36 & 1.324 & - & - & - & 1.308 & $\begin{array}{c}7390 \\
{[0.352]}\end{array}$ & {$[\overline{4.428}]$} & $\begin{array}{c}155 \\
{[<0.01]}\end{array}$ & 733 & $\begin{array}{c}51600 \\
{[1.190]}\end{array}$ & $\begin{array}{c}12100 \\
{[0.368]}\end{array}$ & $\begin{array}{c}9390 \\
{[0.133]}\end{array}$ \\
\hline $98^{\circ} \mathrm{C}$ - hour 4 & 2.37 & 1.318 & 13051 & 30.1 & 3300 & 1.305 & $\begin{array}{c}7270 \\
{[0.353]}\end{array}$ & {$[\overline{4} .373]$} & $\begin{array}{c}166 \\
{[<0.01]}\end{array}$ & 705 & $\begin{array}{c}50900 \\
{[1.172]}\end{array}$ & $\begin{array}{c}11900 \\
{[0.364]}\end{array}$ & $\begin{array}{c}9270 \\
{[0.131]}\end{array}$ \\
\hline $98^{\circ} \mathrm{C}-$ hour 8 & 2.20 & 1.306 & 13215 & 29.7 & 3223 & 1.296 & $\begin{array}{c}8050 \\
{[0.377]}\end{array}$ & $\begin{array}{c}4.185 \\
{[4.302]}\end{array}$ & $\begin{array}{c}249 \\
{[<0.01]}\end{array}$ & 731 & $\begin{array}{c}51000 \\
{[1.163]}\end{array}$ & $\begin{array}{c}11700 \\
{[0.363]}\end{array}$ & $\begin{array}{c}9190 \\
{[0.130]}\end{array}$ \\
\hline $98^{\circ} \mathrm{C}$-hour 10 & 2.15 & 1.323 & - & - & - & 1.291 & $\begin{array}{c}8000 \\
{[0.373]}\end{array}$ & {$[4.070]$} & $\begin{array}{c}187 \\
{[<0.01]}\end{array}$ & 692 & $\begin{array}{c}49400 \\
{[1.149]}\end{array}$ & $\begin{array}{c}11500 \\
{[0.348]}\end{array}$ & $\begin{array}{c}8990 \\
{[0.127]}\end{array}$ \\
\hline $98^{\circ} \mathrm{C}$-hour 12 & 2.18 & 1.316 & 13010 & 29.3 & 3214 & 1.286 & $\begin{array}{c}8410 \\
{[0.380]}\end{array}$ & $\begin{array}{c}3.956 \\
{[4.222]}\end{array}$ & $\begin{array}{c}251 \\
{[<0.01]}\end{array}$ & 721 & $\begin{array}{c}49400 \\
{[1.124]}\end{array}$ & $\begin{array}{c}11600 \\
{[0.356]}\end{array}$ & $\begin{array}{c}8910 \\
{[0.126]}\end{array}$ \\
\hline $98^{\circ} \mathrm{C}$-hour 14 & 2.17 & 1.298 & - & - & - & 1.291 & $\begin{array}{c}8190 \\
{[0.392]}\end{array}$ & {$[\overline{4.170]}$} & $\begin{array}{c}215 \\
{[<0.01]}\end{array}$ & 677 & $\begin{array}{c}48200 \\
{[1.120]}\end{array}$ & $\begin{array}{c}11300 \\
{[0.355]}\end{array}$ & $\begin{array}{c}8870 \\
{[0.125]}\end{array}$ \\
\hline $98^{\circ} \mathrm{C}$-hour 16 & 2.05 & 1.319 & 12751 & 28.9 & 3151 & 1.288 & $\begin{array}{c}8697 \\
{[0.393]}\end{array}$ & $\begin{array}{c}3.876 \\
{[4.095]}\end{array}$ & $\begin{array}{c}244 \\
{[\mathrm{n} / \mathrm{d}]}\end{array}$ & 703 & $\begin{array}{c}48533 \\
{[1.108]}\end{array}$ & $\begin{array}{c}11367 \\
{[0.351]}\end{array}$ & $\begin{array}{c}8787 \\
{[0.124]}\end{array}$ \\
\hline $\begin{array}{l}\text { After cool down and } \\
\text { transfer to } \\
\text { UFP-VSL-T02A }\end{array}$ & 2.14 & 1.303 & $12472^{(b)}$ & 28.9 & 3178 & 1.281 & {$[\overline{0.389}]$} & {$[\overline{4.024}]$} & {$[\overline{0.01]}$} & - & {$[\overline{1.101]}$} & {$[\overline{0.333}]$} & {$[\overline{0.123}]$} \\
\hline
\end{tabular}

(a) If high-accuracy density results are available, then they are provided. If high-accuracy density results are not available, then density results from the UDS are provided.

(b) Liquid Al fraction calculated from Raman data.

- Analysis not required in test plan.

Bold numbers are calculated values.

$\mathrm{Ca}, \mathrm{Mg}, \mathrm{Nd}$, and $\mathrm{PO}_{4}^{-}$are not provided in this table because they were not used in the caustic-leach data analysis.

Raman results are provided as bracketed value [ ].

M Molarity.

n/d Triplicate samples with analysis results below method reporting limit. 
Table 5.2. Batch 2: Caustic Leach

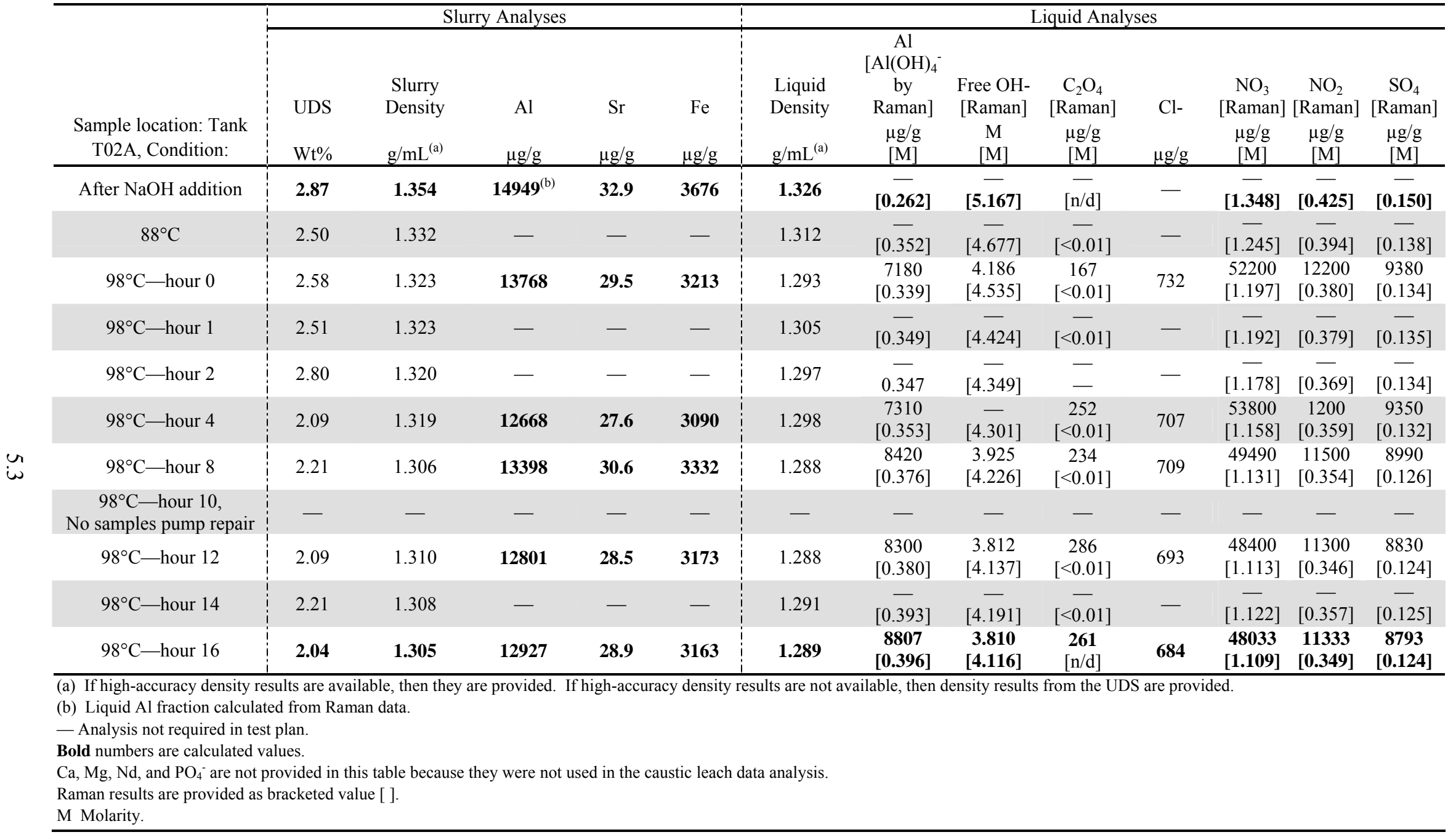


Table 5.3. Batch 3: Caustic Leach

\begin{tabular}{|c|c|c|c|c|c|c|c|c|c|c|c|c|c|}
\hline \multirow[b]{2}{*}{$\begin{array}{l}\text { Sample location: Tank } \\
\text { T02A, Condition: }\end{array}$} & \multicolumn{5}{|c|}{ Slurry Analyses } & \multicolumn{8}{|c|}{ Liquid Analyses } \\
\hline & $\begin{array}{l}\text { UDS } \\
\mathrm{Wt} \% \\
\end{array}$ & $\begin{array}{c}\text { Slurry } \\
\text { Density } \\
\mathrm{g} / \mathrm{mL}^{(\mathrm{a})} \\
\end{array}$ & $\begin{array}{c}\mathrm{Al} \\
\mu \mathrm{g} / \mathrm{g}\end{array}$ & $\begin{array}{c}\mathrm{Sr} \\
\mu \mathrm{g} / \mathrm{g} \\
\end{array}$ & $\begin{array}{c}\mathrm{Fe} \\
\mu \mathrm{g} / \mathrm{g} \\
\end{array}$ & $\begin{array}{l}\text { Liquid } \\
\text { Density } \\
\mathrm{g} / \mathrm{mL}^{(\mathrm{a})}\end{array}$ & $\begin{array}{c}\mathrm{Al} \\
{\left[\mathrm{Al}(\mathrm{OH})_{4}^{-}\right.} \\
\text {by Raman] } \\
\mu \mathrm{g} / \mathrm{g} \\
{[\mathrm{M}]} \\
\end{array}$ & $\begin{array}{c}\text { Free OH- } \\
\text { [Raman] } \\
\mathrm{M} \\
{[\mathrm{M}]} \\
\end{array}$ & $\begin{array}{c}\mathrm{C}_{2} \mathrm{O}_{4} \\
{[\mathrm{Raman}]} \\
\mu \mathrm{g} / \mathrm{g} \\
{[\mathrm{M}]}\end{array}$ & $\begin{array}{l}\mathrm{Cl}- \\
\mu \mathrm{g} / \mathrm{g}\end{array}$ & $\begin{array}{c}\mathrm{NO}_{3} \\
{[\mathrm{Raman}]} \\
\mu \mathrm{g} / \mathrm{g} \\
{[\mathrm{M}]} \\
\end{array}$ & $\begin{array}{c}\mathrm{NO}_{2} \\
\text { [Raman] } \\
\mu \mathrm{g} / \mathrm{g} \\
{[\mathrm{M}]} \\
\end{array}$ & $\begin{array}{c}\mathrm{SO}_{4} \\
\text { [Raman] } \\
\mu \mathrm{g} / \mathrm{g} \\
{[\mathrm{M}]} \\
\end{array}$ \\
\hline After $\mathrm{NaOH}$ addition & 2.83 & 1.356 & $14226^{(b)}$ & 31.3 & 3458 & 1.329 & {$[0.227]$} & [5.430] & {$[\overline{\mathrm{n} / \mathrm{d}}]$} & - & [1.297] & {$[0.411]$} & {$[0.145]$} \\
\hline $88^{\circ} \mathrm{C}$ & 2.78 & 1.334 & - & - & - & 1.315 & {$[\overline{0.347}]$} & {$[\overline{4.856]}$} & {$[\overline{0.01}]$} & - & [1.196] & {$[0.385]$} & [0.134] \\
\hline $98^{\circ} \mathrm{C}-$ hour 0 & 2.27 & 1.324 & 12293 & 28.4 & 3206 & 1.307 & $\begin{array}{c}6750 \\
{[0.343]}\end{array}$ & {$[\overline{4} \overline{751}]$} & $\begin{array}{c}181 \\
{[<0.01]}\end{array}$ & 690 & $\begin{array}{c}52600 \\
{[1.174]}\end{array}$ & $\begin{array}{c}11800 \\
{[0.373]}\end{array}$ & $\begin{array}{c}9200 \\
{[0.132]}\end{array}$ \\
\hline $98^{\circ} \mathrm{C}$ - hour 1 & 2.22 & 1.324 & - & - & - & 1.304 & {$[\overline{0.339}]$} & {$[\overline{4}-684]$} & {$[<-\overline{0.01}]$} & - & {$[\overline{1.157]}$} & {$[\overline{0.364]}$} & {$[\overline{0.129}]$} \\
\hline $98^{\circ} \mathrm{C}$-hour 2 & 2.10 & 1.322 & - & - & - & 1.304 & {$[\overline{0.346}]$} & {$[\overline{4} \overline{6} 88]$} & {$[<\overline{0.01}]$} & - & {$[\overline{1.152}]$} & {$[\overline{0.366}]$} & {$[\overline{0.129}]$} \\
\hline $98^{\circ} \mathrm{C}$--hour 4 & 2.18 & 1.323 & 13120 & 32.2 & 3519 & 1.295 & $\begin{array}{c}7210 \\
{[0.354]}\end{array}$ & {$\left[\begin{array}{c}- \\
{[497]}\end{array}\right.$} & $\begin{array}{c}232 \\
{[<0.01]}\end{array}$ & 671 & $\begin{array}{c}51300 \\
{[1.140]}\end{array}$ & $\begin{array}{c}11500 \\
{[0.362]}\end{array}$ & $\begin{array}{c}9020 \\
{[0.127]}\end{array}$ \\
\hline $98^{\circ} \mathrm{C}$-hour 8 & 2.10 & 1.312 & 12324 & 30.0 & 3140 & 1.297 & $\begin{array}{c}7600 \\
{[0.371]}\end{array}$ & {$[\overline{4.523]}$} & $\begin{array}{c}220 \\
{[<0.01]}\end{array}$ & 626 & $\begin{array}{l}47700 \\
{[1.111]}\end{array}$ & $\begin{array}{c}10800 \\
{[0.360]}\end{array}$ & $\begin{array}{c}8470 \\
{[0.124]}\end{array}$ \\
\hline $98^{\circ} \mathrm{C}$-hour 10 & 2.12 & 1.307 & - & - & - & 1.294 & {$[\overline{0.378]}$} & {$[\overline{4.448]}$} & {$[\overline{0.01]}$} & - & {$[\overline{1.097]}$} & {$[\overline{0.365}]$} & {$[\overline{0.124}]$} \\
\hline $98^{\circ} \mathrm{C}$-hour 12 & 1.87 & 1.307 & 12097 & 26.3 & 3077 & 1.296 & $\begin{array}{c}7920 \\
{[0.383]}\end{array}$ & {$[\overline{4.392}]$} & $\begin{array}{c}243 \\
{[<0.01]}\end{array}$ & 655 & $\begin{array}{c}49600 \\
{[1.087]}\end{array}$ & $\begin{array}{c}11100 \\
{[0.356]}\end{array}$ & $\begin{array}{c}11100 \\
{[0.122]}\end{array}$ \\
\hline $98^{\circ} \mathrm{C}$-hour 14 & 1.89 & 1.312 & - & - & - & 1.294 & {$[\overline{0.384}]$} & {$[\overline{4} \cdot \overline{288}]$} & {$[\overline{<0.01]}$} & - & {$[\overline{1.067]}$} & {$[\overline{0.348}]$} & {$[\overline{0.120}]$} \\
\hline $98^{\circ} \mathrm{C}$-hour 16 & 1.80 & 1.301 & 12018 & 26.2 & 3053 & 1.288 & $\begin{array}{c}8257 \\
{[0.393]}\end{array}$ & {$[4.316]$} & $\begin{array}{c}\mathbf{2 6 6} \\
{[\mathrm{n} / \mathrm{d}]}\end{array}$ & 645 & $\begin{array}{c}49100 \\
{[1.061]} \\
\end{array}$ & $\begin{array}{c}11000 \\
{[0.347]}\end{array}$ & $\begin{array}{c}8673 \\
{[0.119]} \\
\end{array}$ \\
\hline $\begin{array}{l}\text { (a) If high-accuracy density } \mathrm{r} \\
\text { (b) Liquid } \mathrm{Al} \text { fraction calcula } \\
\text { - Analysis not required in te } \\
\text { Bold numbers are calculated } \\
\mathrm{Ca}, \mathrm{Mg}, \mathrm{Nd} \text {, and } \mathrm{PO}_{4}^{-} \text {are not } \\
\text { Raman results are provided as } \\
\text { M Molarity. }\end{array}$ & edv & cause $t$ & 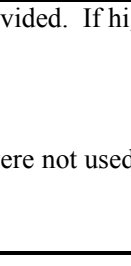 & & 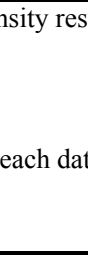 & ysis. & & & & & & & \\
\hline
\end{tabular}


Table 5.4. Batch 4: Caustic Leach

\begin{tabular}{|c|c|c|c|c|c|c|c|c|c|c|c|c|c|}
\hline \multirow[b]{2}{*}{$\begin{array}{l}\text { Sample location: Tank } \\
\text { T02A, Condition: }\end{array}$} & \multicolumn{5}{|c|}{ Slurry Analyses } & \multicolumn{8}{|c|}{ Liquid Analyses } \\
\hline & $\begin{array}{l}\text { UDS } \\
\mathrm{Wt} \%\end{array}$ & $\begin{array}{c}\text { Slurry } \\
\text { Density } \\
\text { g/mL }{ }^{(a)}\end{array}$ & $\begin{array}{c}\mathrm{Al} \\
\mu \mathrm{g} / \mathrm{g}\end{array}$ & $\begin{array}{c}\mathrm{Sr} \\
\mu \mathrm{g} / \mathrm{g}\end{array}$ & $\begin{array}{c}\mathrm{Fe} \\
\mu \mathrm{g} / \mathrm{g}\end{array}$ & $\begin{array}{l}\text { Liquid } \\
\text { Density } \\
\text { g/mL }{ }^{(a)}\end{array}$ & $\begin{array}{c}\mathrm{Al} \\
{\left[\mathrm{Al}(\mathrm{OH})_{4}^{-}\right.} \\
\text {by } \mathrm{Raman}] \\
\mu \mathrm{g} / \mathrm{g} \\
{[\mathrm{M}]}\end{array}$ & $\begin{array}{c}\text { Free OH } \\
\text { [Raman] } \\
\mathrm{M} \\
{[\mathrm{M}]}\end{array}$ & $\begin{array}{c}\mathrm{C}_{2} \mathrm{O}_{4} \\
\text { [Raman] } \\
\mu \mathrm{g} / \mathrm{g} \\
{[\mathrm{M}]}\end{array}$ & $\begin{array}{l}\mathrm{Cl}- \\
\mu \mathrm{g} / \mathrm{g}\end{array}$ & $\begin{array}{c}\mathrm{NO}_{3} \\
{[\mathrm{Raman}]} \\
\mu \mathrm{g} / \mathrm{g} \\
{[\mathrm{M}]}\end{array}$ & $\begin{array}{c}\mathrm{NO}_{2} \\
\text { [Raman] } \\
\mu \mathrm{g} / \mathrm{g} \\
{[\mathrm{M}]}\end{array}$ & $\begin{array}{c}\mathrm{SO}_{4} \\
\text { [Raman] } \\
\mu \mathrm{g} / \mathrm{g} \\
{[\mathrm{M}]}\end{array}$ \\
\hline After $\mathrm{NaOH}$ addition & 2.48 & 1.364 & $14012^{(b)}$ & 29.6 & 3378 & 1.341 & {$[\overline{-}$} & $\overline{[5.847]}$ & $\overline{[\mathrm{n} / \mathrm{d}]}$ & - & $\overline{-} \overline{1.259]}$ & $\overline{-} \overline{0.396}]$ & $\overline{[0.139]}$ \\
\hline $88^{\circ} \mathrm{C}$ & 2.18 & 1.347 & - & - & - & 1.321 & {$[\overline{0.340}]$} & {$[\overline{5.259}]$} & {$[\overline{0.01]}$} & - & $\overline{[1.185}]$ & $\overline{0.378]}$ & $\overline{[0.131]}$ \\
\hline $98^{\circ} \mathrm{C}$ - hour 0 & 2.12 & 1.334 & 12644 & 27.0 & 2974 & 1.315 & $\begin{array}{c}6820 \\
{[0.336]}\end{array}$ & {$[\overline{5.162}]$} & $\begin{array}{c}169 \\
{[<0.01]}\end{array}$ & 705 & $\begin{array}{c}52000 \\
{[1.156]}\end{array}$ & $\begin{array}{c}11600 \\
{[0.368]}\end{array}$ & $\begin{array}{c}8980 \\
{[0.128]}\end{array}$ \\
\hline $98^{\circ} \mathrm{C}$ - hour 1 & 2.12 & 1.340 & - & - & - & 1.317 & {$[\overline{0.344}]$} & {$[\overline{167]}$} & {$[\overline{0.01}]$} & - & $\overline{[1.151]}$ & {$[\overline{0.366}]$} & $\overline{[0.129}]$ \\
\hline $98^{\circ} \mathrm{C}$-hour 2 & 2.14 & 1.334 & - & - & - & 1.315 & $\overline{0.342}]$ & $\overline{[5.164]}$ & {$[\overline{0.01]}$} & - & $\overline{[1.132}]$ & {$[\overline{0.359}]$} & $\overline{[0.126]}$ \\
\hline $98^{\circ} \mathrm{C}$-hour 4 & 2.03 & 1.325 & 12368 & 27.4 & 3074 & 1.307 & $\begin{array}{c}7230 \\
{[0.363]}\end{array}$ & $\overline{[5.062}]$ & $\begin{array}{c}171 \\
{[<0.01]}\end{array}$ & 650 & $\begin{array}{c}48800 \\
{[1.142]}\end{array}$ & $\begin{array}{c}11000 \\
{[0.364]}\end{array}$ & $\begin{array}{c}8710 \\
{[0.127]}\end{array}$ \\
\hline $98^{\circ} \mathrm{C}$ - hour 8 & 1.88 & 1.327 & 12076 & 23.6 & 2688 & 1.305 & $\begin{array}{c}7740 \\
{[0.378]}\end{array}$ & {$[\overline{4} \overline{-} 919]$} & $\begin{array}{c}179 \\
{[<0.01]}\end{array}$ & 664 & $\begin{array}{c}51300 \\
{[1.119]}\end{array}$ & $\begin{array}{c}11400 \\
{[0.353]}\end{array}$ & $\begin{array}{c}9030 \\
{[0.125]}\end{array}$ \\
\hline $98^{\circ} \mathrm{C}$-hour 10 & 1.95 & 1.323 & - & - & - & 1.310 & {$[\overline{0.387}]$} & {$[\overline{4} \overline{890}]$} & {$[\overline{0.01}]$} & - & {$[\overline{1.098}]$} & {$[\overline{0.348}]$} & {$[\overline{0.123}]$} \\
\hline $98^{\circ} \mathrm{C}$-hour 12 & 1.92 & 1.315 & 12130 & 26.4 & 2955 & 1.305 & $\begin{array}{c}8150 \\
{[0.385]}\end{array}$ & {$[\overline{4.803}]$} & $\begin{array}{c}216 \\
{[<0.01]}\end{array}$ & 617 & $\begin{array}{c}46700 \\
{[1.085]}\end{array}$ & $\begin{array}{c}10500 \\
{[0.342]}\end{array}$ & $\begin{array}{c}8260 \\
{[0.122]}\end{array}$ \\
\hline $98^{\circ} \mathrm{C}$-hour 14 & 1.88 & 1.317 & - & - & - & 1.300 & {$[\overline{0.397]}$} & {$[\overline{-759}]$} & {$[\overline{0.01}]$} & - & $\overline{[1.075]}$ & {$[\overline{0.336}]$} & $\overline{[0.121]}$ \\
\hline $98^{\circ} \mathrm{C}$-hour 16 & 1.72 & 1.310 & 11778 & 26.8 & 3056 & 1.302 & $\begin{array}{c}8263 \\
{[0.402]} \\
\end{array}$ & {$[\overline{4.697]}$} & $\begin{array}{c}191 \\
{[\mathrm{n} / \mathrm{d}]}\end{array}$ & 635 & $\begin{array}{c}47867 \\
{[1.068]} \\
\end{array}$ & $\begin{array}{c}10767 \\
{[0.337]} \\
\end{array}$ & $\begin{array}{c}8423 \\
{[0.120]} \\
\end{array}$ \\
\hline $\begin{array}{l}\text { (a) If high-accuracy density } \\
\text { (b) Liquid } \mathrm{Al} \text { fraction calcul } \\
\text { - Analysis not required in } \mathrm{t} \\
\text { Bold numbers are calculated } \\
\mathrm{Ca}, \mathrm{Mg}, \mathrm{Nd} \text {, and } \mathrm{PO}_{4}^{-} \text {are no } \\
\text { Raman results are provided a } \\
\text { M Molarity. }\end{array}$ & $\begin{array}{l}\text { om Rai } \\
\text { ded in } t \\
\text { keted } v\end{array}$ & e because & ere not & the & leach & analysis. & & & & & & & \\
\hline
\end{tabular}


Table 5.5. Batch 5: Caustic Leach

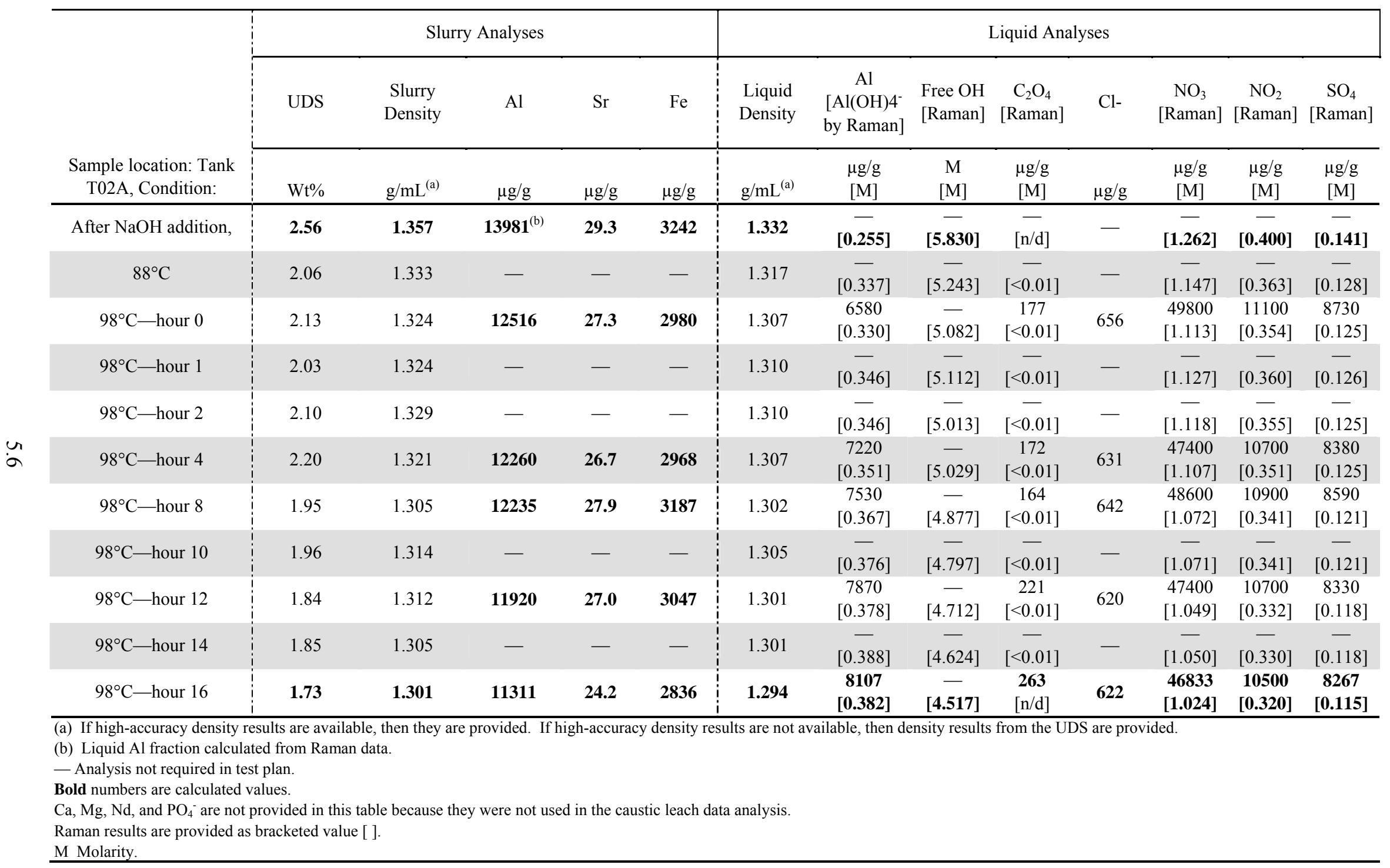


Table 5.6. Batch 6: Caustic Leach

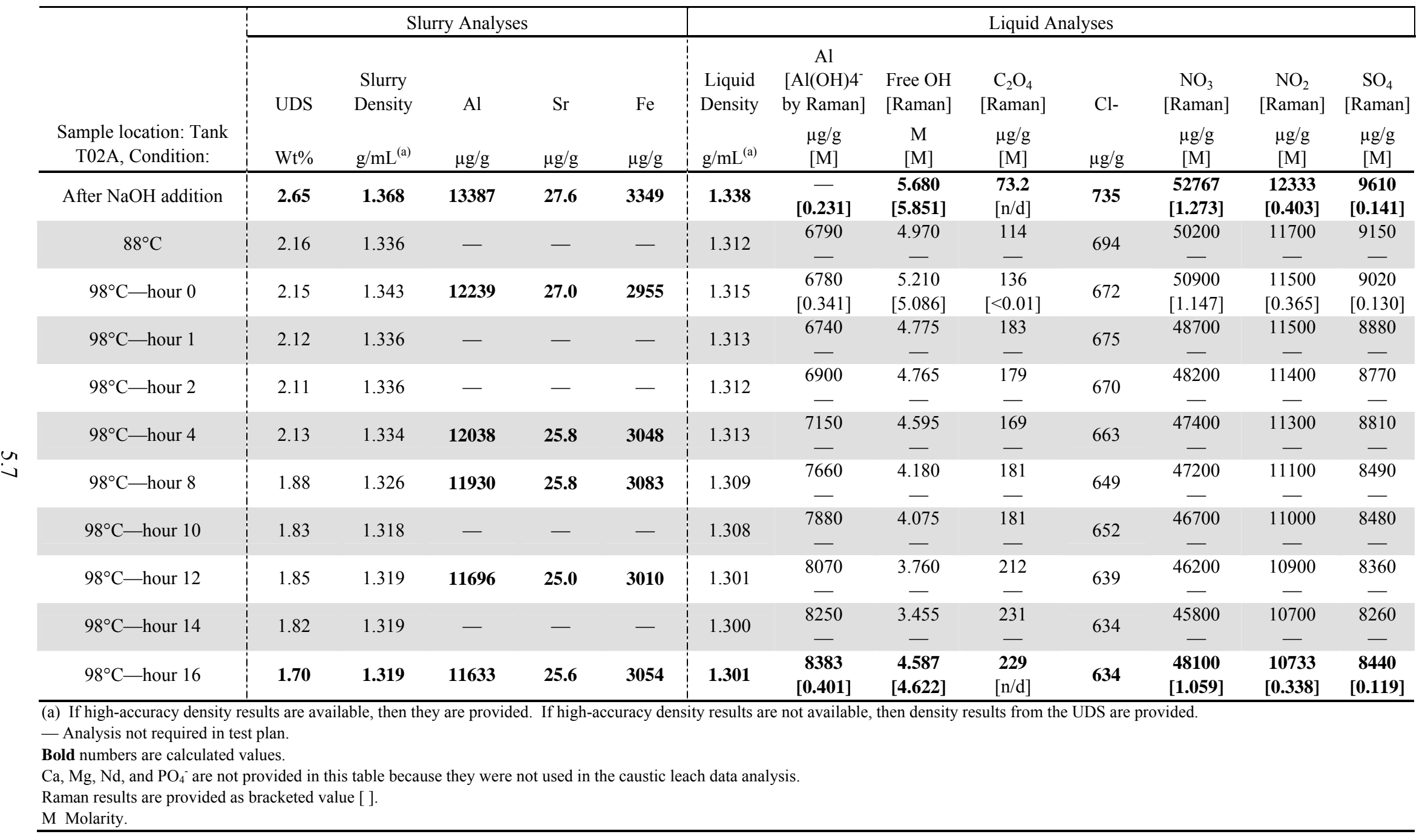


Table 5.7. Post-Caustic-Leach Concentration of Solids

\begin{tabular}{|c|c|c|c|c|c|}
\hline \multirow{3}{*}{$\begin{array}{l}\text { Sample location: Tank T02A, } \\
\text { Condition: }\end{array}$} & \multirow{2}{*}{\multicolumn{2}{|c|}{\begin{tabular}{|c|} 
Slurry \\
UDS Density
\end{tabular}}} & \multicolumn{2}{|c|}{ Liquid } & \multirow{3}{*}{$\begin{array}{c}\text { Wet Centrifuged Solids } \\
\mathrm{Si} \\
\mu \mathrm{g} / \mathrm{g}\end{array}$} \\
\hline & & & $\mathrm{Si}$ & Densityi & \\
\hline & $\mathrm{Wt} \%$ & $\mathrm{~g} / \mathrm{mL}$ & $\mu \mathrm{g} / \mathrm{g}$ & $\mathrm{g} / \mathrm{mL}$ & \\
\hline $\mathrm{T}=4$ hours & 2.54 & 1.299 & - & 1.255 & - \\
\hline $\mathrm{T}=8$ hours & 4.67 & 1.311 & - & $1.252 !$ & - \\
\hline $\mathrm{T}=32$ hours & 6.51 & 1.332 & - & 1.237 & - \\
\hline $\mathrm{T}=34$ hours & 6.06 & 1.334 & - & 1.257 & - \\
\hline $\mathrm{T}=56$ hours & 8.09 & 1.347 & - & 1.278 & - \\
\hline $\mathrm{T}=74$ hours & 9.06 & 1.373 & - & 1.313 & - \\
\hline $\mathrm{T}=94$ hours & 10.4 & 1.378 & - & 1.302 & - \\
\hline $\mathrm{T}=114$ hours & $\begin{array}{l}11.1 \\
11.1\end{array}$ & 1.388 & - & 1.294 & - \\
\hline $\mathrm{T}=134$ hours & 12.9 & 1.404 & - & 1.322 & - \\
\hline $\mathrm{T}=154$ hours & 13.0 & 1.418 & - & 1.320 & - \\
\hline $\mathrm{T}=184$ hours & $\begin{array}{l}14.5 \\
\end{array}$ & 1.431 & - & 1.314 & - \\
\hline $\mathrm{T}=204$ hours & 15.4 & 1.431 & - & 1.266 & - \\
\hline $\mathrm{T}=224$ hours & 14.5 & 1.423 & - & 1.300 & - \\
\hline $\mathrm{T}=244$ hours & 15.5 & 1.432 & - & 1.295 & - \\
\hline $\mathrm{T}=264$ hours & 16.1 & 1.457 & - & 1.303 & - \\
\hline $\mathrm{T}=265$ hours & 16.9 & 1.467 & - & 1.313 & - \\
\hline $\mathrm{T}=300$ hours & 17.4 & 1.467 & - & 1.302 & - \\
\hline After Concentration $^{(\mathrm{c})}$ & 18.3 & 1.399 & $68.2^{(a)}$ & 1.253 & $689^{(a, b)}$ \\
\hline
\end{tabular}

- Analysis not required in Test Plan.

(a) Original intact slurry submitted to analytical lab so phase separation was performed at analytical laboratory.

(b) $\%$ Solids $=61.91 \%$.

(c) Analytical results duplicated in Analysis at Key Process Steps table column heading Initial Slurry Composition for Post-Caustic-Leach Wash.

Table 5.8. Evaluation By-Pass of Filter-Loop Jet within Tank T02A on 02/14/09

\begin{tabular}{|c|c|c|c|}
\hline \multirow[b]{3}{*}{ TI Step } & \multirow[b]{3}{*}{ Condition: } & \multicolumn{2}{|r|}{ Liquid Analyses } \\
\hline & & $\begin{array}{c}\text { Filtration loop } \\
\text { Cs }\end{array}$ & $\begin{array}{c}\text { Outer Lower Port Tank T02A } \\
\text { Cs }\end{array}$ \\
\hline & & $\mu \mathrm{g} / \mathrm{g}$ & $\mu \mathrm{g} / \mathrm{g}$ \\
\hline & Before tracer addition & 0.00975 & - \\
\hline TI-65 12.1.4 & 1 min after tracer addition & 299 & 44.9 \\
\hline TI-65 12.1.4 & 2 min after tracer addition & 139 & 111 \\
\hline TI-65 12.1.4 & 4 min after tracer addition & 112 & 108 \\
\hline TI-65 12.1.4 & 8 min after tracer addition & 106 & 107 \\
\hline TI-65 12.1.4 & 16 min after tracer addition & 107 & 107 \\
\hline TI-65 12.1.4 & 30 min after tracer addition & 104 & 104 \\
\hline TI-65 12.1 .4 & 40 min after tracer addition & 103 & 103 \\
\hline TI-65 12.1.4 & 50 min after tracer addition & 101 & 102 \\
\hline TI-65 12.1.4 & 60 min after tracer addition & 101 & 101 \\
\hline
\end{tabular}


Table 5.9. Evaluation By-Pass of Filter-Loop Jet within Tank T02A on 3/21/09

\begin{tabular}{cc|cc} 
& & \multicolumn{2}{c}{ Liquid Analyses } \\
& & Cs & Outer Lower Port Tank T02A \\
TI Step & Condition: & $\mu \mathrm{g} / \mathrm{g}$ & $\mu \mathrm{g} / \mathrm{g}$ \\
\hline & Before tracer addition & 71.5 & - \\
TI-65 12.1.4 & 1 min after tracer addition & 112 & 65.3 \\
TI-65 12.1.4 & 2 min after tracer addition & 110 & 107 \\
TI-65 12.1.4 & 4 min after tracer addition & 107 & 106 \\
TI-65 12.1.4 & 8 min after tracer addition & 105 & 100 \\
TI-65 12.1.4 & 16 min after tracer addition & 102 & 104 \\
TI-65 12.1.4 & 30 min after tracer addition & 104 & 103 \\
TI-65 12.1.4 & 40 min after tracer addition & 98.6 & 104 \\
TI-65 12.1.4 & 50 min after tracer addition & 99.3 & $208^{(\mathrm{a})}$ \\
TI-65 12.1.4 & 60 min after tracer addition & 97.4 & $98^{(\mathrm{b})}$ \\
& & & 101 \\
\hline TI-65 12.1.4 & 70 min after tracer addition & - & \\
\hline - Analysis not required in Test Plan. & & \\
(a) Sample collected from UFP-VSL-T02A outer low CD port. & \\
(b) Sample collected from UFP-VSL-T02A outer middle CD port. &
\end{tabular}

Table 5.10 provides analytical results for samples collected during the post-caustic-leach washing in Tank T02A. Two separate slurry samples were collected for the wt $\%$ UDS and slurry density. The supernatant density is a higher accuracy density than can be provided by the UDS analysis. The silicon slurry concentrations were obtained by directed analysis of the slurry. The liquid analysis results are supernatant analytical results obtained from samples phase-separated in PDL-W. Raman results, shown in brackets [ ], are provided as part of the liquid analyses and presented in molar concentration (mole/liter).

Table 5.11 provides analytical results for samples collected during the oxidative leaching in Tank T02A. The slurry density results and silicon slurry concentrations were performed on intact slurry submitted to the analytical laboratory. The wt $\%$ UDS were calculated from samples phase separated at PDL-W. The liquid analyses results are supernatant analytical results obtained from samples phaseseparated at PDL-W. The solids analyses results are analytical results of rinsed solids obtained from sample phase separation and rinsing at PDL-W. The wet centrifuged solids were immediately rinsed after phase separation to remove the remaining supernatant from the wet solids in an attempt to interrupt further reaction. The rinsate results are analytical results of composited rinse solutions used during the rinsing of phase-separated solids. Raman results, shown in brackets, [ ], are provided as part of the liquid analyses and presented in molar concentration (mole/liter). An aliquot of the wet centrifuged solids was dried at $105^{\circ} \mathrm{C}$ to determine the percent solids that are reported with the reported analytes in the wet centrifuged solids. 
Table 5.10. Post-Caustic-Leach Wash

\begin{tabular}{|c|c|c|c|c|c|c|c|c|c|c|c|c|c|c|c|c|c|}
\hline \multirow{3}{*}{$\begin{array}{c}\text { Sample } \\
\text { location: Tank } \\
\text { T02A }\end{array}$} & \multicolumn{3}{|c|}{ Slurry Analyses } & \multicolumn{14}{|c|}{ Supernatant Analyses } \\
\hline & UDS & $\begin{array}{c}\text { Slurry } \\
\text { Density }^{(b)}\end{array}$ & $\mathrm{Si}$ & TDS & $\begin{array}{c}\text { Liquid } \\
\text { Density }\end{array}$ & $\begin{array}{c}\mathrm{Al} \\
{\left[\mathrm{Al}(\mathrm{OH})_{4}\right.} \\
\text { by Raman }]\end{array}$ & $\begin{array}{l}\text { Free } \mathrm{OH}^{-} \\
{[\mathrm{Raman}]}\end{array}$ & $\mathrm{Na}$ & $\begin{array}{c}\mathrm{Cr} \\
{\left[\mathrm{CrO}_{4}^{-2} \text { by }\right.} \\
\text { Raman] }\end{array}$ & $\begin{array}{c}\mathrm{C}_{2} \mathrm{O}_{4} \\
\text { [Raman] }\end{array}$ & $\begin{array}{c}\mathrm{NO}_{3} \\
\text { [Raman] }\end{array}$ & $\begin{array}{c}\mathrm{NO}_{2} \\
{[\text { Raman] }}\end{array}$ & $\begin{array}{c}\mathrm{SO}_{4} \\
{[\text { Raman] }}\end{array}$ & $\begin{array}{c}\mathrm{PO}_{4} \\
\text { [Raman] }\end{array}$ & $\mathrm{Cl}^{-}$ & PDMS & PPG \\
\hline & $\mathrm{Wt} \%$ & $\mathrm{~g} / \mathrm{mL}$ & $\mu \mathrm{g} / \mathrm{g}$ & $\mathrm{Wt} \%$ & $\mathrm{~g} / \mathrm{mL}$ & $\begin{array}{l}\mu \mathrm{g} / \mathrm{g} \\
{[\mathrm{M}]}\end{array}$ & $\begin{array}{l}\mathrm{M} \\
{[\mathrm{M}]}\end{array}$ & $\mu \mathrm{g} / \mathrm{g}$ & $\begin{array}{l}\mu \mathrm{g} / \mathrm{g} \\
{[\mathrm{M}]}\end{array}$ & $\begin{array}{l}\mu \mathrm{g} / \mathrm{g} \\
{[\mathrm{M}]}\end{array}$ & $\begin{array}{l}\mu \mathrm{g} / \mathrm{g} \\
{[\mathrm{M}]}\end{array}$ & $\begin{array}{l}\mu \mathrm{g} / \mathrm{g} \\
{[\mathrm{M}]}\end{array}$ & $\begin{array}{l}\mu \mathrm{g} / \mathrm{g} \\
{[\mathrm{M}]}\end{array}$ & $\begin{array}{l}\mu \mathrm{g} / \mathrm{g} \\
{[\mathrm{M}]}\end{array}$ & $\mu \mathrm{g} / \mathrm{g}$ & $\mathrm{mg} / \mathrm{g}$ & $\mathrm{mg} / \mathrm{g}$ \\
\hline After Wash 3 & - & - & - & - & - & $\begin{array}{c}6730 \\
{[0.298]}\end{array}$ & $\begin{array}{c}3.31 \\
{[3.448]}\end{array}$ & 101000 & $\begin{array}{c}2.56 \\
{[<0.001]}\end{array}$ & $\begin{array}{c}387 \\
{[<0.01]}\end{array}$ & $\begin{array}{c}35600 \\
{[0.770]}\end{array}$ & $\begin{array}{c}9240 \\
{[0.247]}\end{array}$ & $\begin{array}{c}6500 \\
{[0.087]}\end{array}$ & $\begin{array}{c}2580 \\
{[0.037]^{(\mathrm{e})}}\end{array}$ & 494 & - & - \\
\hline After Wash 6 & 18.9 & 1.351 & - & 22.7 & 1.208 & $\begin{array}{c}5790 \\
{[0.255]}\end{array}$ & $\begin{array}{c}2.84 \\
{[2.951]}\end{array}$ & 87800 & $\begin{array}{c}2.08 \\
{[<0.001]}\end{array}$ & $\begin{array}{c}577 \\
{[<0.01]}\end{array}$ & $\begin{array}{c}31300 \\
{[0.661]}\end{array}$ & $\begin{array}{c}8100 \\
{[0.211]}\end{array}$ & $\begin{array}{c}5840 \\
{[0.074]}\end{array}$ & $\begin{array}{c}2790 \\
{[0.045]^{(\mathrm{e})}}\end{array}$ & 432 & - & - \\
\hline After Wash $9 !$ & - & - & - & - & - & $\begin{array}{c}5190 \\
{[0.229]}\end{array}$ & $\begin{array}{c}2.52 \\
{[2.635]}\end{array}$ & 77700 & $\begin{array}{c}2.54 \\
{[<0.001]}\end{array}$ & $\begin{array}{c}763 \\
{[<0.01]}\end{array}$ & $\begin{array}{c}27900 \\
{[0.590]}\end{array}$ & $\begin{array}{c}7250 \\
{[0.187]}\end{array}$ & $\begin{array}{c}5100 \\
{[0.067]}\end{array}$ & $\begin{array}{c}3520 \\
{[0.054]^{(\mathrm{e})}}\end{array}$ & 384 & - & - \\
\hline After Wash $12 !$ & 18.9 & 1.305 & - & 18.6 & 1.168 & $\begin{array}{c}4560 \\
{[0.196]}\end{array}$ & $\begin{array}{c}2.21 \\
{[2.292]}\end{array}$ & 72300 & $\begin{array}{c}2.20 \\
{[<0.001]}\end{array}$ & $\begin{array}{c}1030 \\
{[<0.01]}\end{array}$ & $\begin{array}{c}24600 \\
{[0.506]}\end{array}$ & $\begin{array}{c}6350 \\
{[0.161]}\end{array}$ & $\begin{array}{c}4500 \\
{[0.057]}\end{array}$ & $\begin{array}{c}4030 \\
{[0.064]}\end{array}$ & 336 & - & - \\
\hline After Wash 15 & - & - & - & - & - & $\begin{array}{c}4050 \\
{[0.174]}\end{array}$ & $\begin{array}{c}2.04 \\
{[2.047]}\end{array}$ & 65800 & $\begin{array}{c}2.14 \\
{[<0.001]}\end{array}$ & $\begin{array}{c}1290 \\
{[<0.01]}\end{array}$ & $\begin{array}{l}22900 \\
{[0.447]}\end{array}$ & $\begin{array}{c}5700 \\
{[0.145]}\end{array}$ & $\begin{array}{c}4180 \\
{[0.050]}\end{array}$ & $\begin{array}{c}4950 \\
{[0.074]}\end{array}$ & 299 & - & - \\
\hline After Wash 19 & 19.2 & 1.261 & - & 14.3 & 1.133 & $\begin{array}{c}3290 \\
{[0.138]}\end{array}$ & $\begin{array}{c}1.59 \\
{[1.656]}\end{array}$ & 56700 & $\begin{array}{c}1.60 \\
{[<0.001]}\end{array}$ & $\begin{array}{c}1810 \\
{[<0.01]}\end{array}$ & $\begin{array}{c}18300 \\
{[0.363]}\end{array}$ & $\begin{array}{c}4660 \\
{[0.118]^{(\mathrm{e})}}\end{array}$ & $\begin{array}{c}3410 \\
{[0.041]}\end{array}$ & $\begin{array}{c}6210 \\
{[0.081]}\end{array}$ & 247 & - & - \\
\hline After Wash $24 !$ & - & - & - & - & - & $\begin{array}{c}2650 \\
{[0.107]}\end{array}$ & $\begin{array}{c}1.30 \\
{[1.363]}\end{array}$ & 48400 & $\begin{array}{c}1.48 \\
{[<0.001]}\end{array}$ & $\begin{array}{c}2490 \\
{[0.018]^{(\mathrm{e})}}\end{array}$ & $\begin{array}{c}16300 \\
{[0.289]}\end{array}$ & $\begin{array}{c}3770 \\
{[0.096]^{(\mathrm{e})}}\end{array}$ & $\begin{array}{c}2810 \\
{[0.032]}\end{array}$ & $\begin{array}{c}8210 \\
{[0.115]}\end{array}$ & 201 & - & - \\
\hline After Wash $30 !$ & 19.4 & 1.215 & - & 10.1 & 1.095 & $\begin{array}{c}2010 \\
{[0.078]}\end{array}$ & $\begin{array}{c}1.04 \\
{[1.019]}\end{array}$ & 38300 & $\begin{array}{c}1.12 \\
{[<0.001]}\end{array}$ & $\begin{array}{c}3560 \\
{[0.028]^{(\mathrm{e})}}\end{array}$ & $\begin{array}{c}12400 \\
{[0.217]}\end{array}$ & $\begin{array}{c}2810 \\
{[0.069]^{(\mathrm{e})}}\end{array}$ & $\begin{array}{c}2120 \\
{[0.024]}\end{array}$ & $\begin{array}{c}7480 \\
{[0.086]}\end{array}$ & 153 & - & - \\
\hline After Wash 37 & - & - & - & - & - & $\begin{array}{c}1570 \\
{[0.059]^{(\mathrm{f})}}\end{array}$ & $\begin{array}{c}0.79 \\
{[0.745]^{(\mathrm{f})}}\end{array}$ & 30900 & $\begin{array}{c}0.841 \\
{[<0.001]^{(f)}}\end{array}$ & $\begin{array}{c}5090 \\
{[0.060]^{(\mathrm{e}, \mathrm{f})}}\end{array}$ & $\begin{array}{c}9300 \\
{[0.171]^{(\mathrm{f})}}\end{array}$ & $\begin{array}{c}2070 \\
{[0.054]^{(\mathrm{e}, \mathrm{f})}}\end{array}$ & $\begin{array}{c}1590 \\
{[0.018]^{(\mathrm{f})}}\end{array}$ & $\begin{array}{c}5640 \\
{[0.071]^{(\mathrm{f})}}\end{array}$ & 114 & - & - \\
\hline After Wash $43 i$ & 19.2 & 1.206 & - & 6.61 & 1.051 & $\begin{array}{c}1250 \\
{[0.039]^{(e, f)}}\end{array}$ & $\begin{array}{c}0.58 \\
{[0.549]^{(e, f)}}\end{array}$ & 25400 & $\begin{array}{c}0.69 \\
{[<0.001]^{(\mathrm{f})}}\end{array}$ & $\begin{array}{c}6800 \\
{[0.077]^{(\mathrm{f})}}\end{array}$ & $\begin{array}{c}7060 \\
{[0.129]^{(\mathrm{f})}}\end{array}$ & $\begin{array}{c}1570 \\
{[0.034]^{(\mathrm{e}, \mathrm{f})}}\end{array}$ & $\begin{array}{c}1220 \\
{[0.013]^{(e, f)}}\end{array}$ & $\begin{array}{c}4360 \\
{[0.047]^{(e, f)}}\end{array}$ & 89.3 & - & - \\
\hline After Wash 49 & - & - & - & - & - & $\begin{array}{c}962 \\
{[0.032]^{(e, f)}}\end{array}$ & $\begin{array}{c}0.47 \\
{[0.423]^{(e, f)}}\end{array}$ & 21200 & $\begin{array}{c}<0.978 \\
{[<0.001]^{(\mathrm{f})}}\end{array}$ & $\begin{array}{c}8950 \\
{[0.095]^{(\mathrm{f})}}\end{array}$ & $\begin{array}{c}5390 \\
{[0.100]^{(\mathrm{f})}}\end{array}$ & $\begin{array}{c}1210 \\
{[0.025]^{(e, f)}}\end{array}$ & $\begin{array}{c}957 \\
{[0.010]^{(\mathrm{e}, \mathrm{f})}}\end{array}$ & $\begin{array}{c}3380 \\
{[0.039]^{(e, f)}}\end{array}$ & 70.4 & - & - \\
\hline After Wash 55 & 18.5 & 1.176 & - & 4.86 & 1.038 & $\begin{array}{c}676 \\
{[0.019]^{(\mathrm{e})}}\end{array}$ & $\begin{array}{c}0.30 \\
{[0.313]^{(\mathrm{e})}}\end{array}$ & 18300 & $\begin{array}{c}0.330 \\
{[<0.001]}\end{array}$ & $\begin{array}{c}10900 \\
{[0.111]}\end{array}$ & $\begin{array}{c}3880 \\
{[0.075]}\end{array}$ & $\begin{array}{c}892 \\
{[0.027]^{(\mathrm{e})}}\end{array}$ & $\begin{array}{c}700 \\
{[0.008]^{(\mathrm{e})}}\end{array}$ & $\begin{array}{c}2490 \\
{[0.027]^{(\mathrm{e})}}\end{array}$ & 56.5 & - & - \\
\hline After Wash 61 & - & - & - & - & - & $\begin{array}{c}529 \\
{[0.017]^{(\mathrm{e})}}\end{array}$ & $\begin{array}{c}0.28 \\
{[0.244]^{(\mathrm{e})}}\end{array}$ & 16400 & $\begin{array}{c}0.376 \\
{[<0.001]}\end{array}$ & $\begin{array}{c}12600 \\
{[0.132]}\end{array}$ & $\begin{array}{c}2970 \\
{[0.060]}\end{array}$ & $\begin{array}{c}690 \\
{[0.030]^{(\mathrm{e})}}\end{array}$ & $\begin{array}{c}548 \\
{[0.007]^{(\mathrm{e})}}\end{array}$ & $\begin{array}{c}1930 \\
{[0.019]^{(\mathrm{e})}}\end{array}$ & 41.8 & - & - \\
\hline After Wash 67 & 17.6 & 1.164 & - & 4.09 & 1.029 & $\begin{array}{c}391 \\
{[<0.01]}\end{array}$ & $\begin{array}{c}0.22 \\
{[0.182]^{(\mathrm{e})}}\end{array}$ & 14900 & $\begin{array}{c}<0.243 \\
{[<0.001]}\end{array}$ & $\begin{array}{c}14800 \\
{[0.159]}\end{array}$ & $\begin{array}{c}2270 \\
{[0.048]}\end{array}$ & $\begin{array}{c}540 \\
{[<0.03]}\end{array}$ & $\begin{array}{c}421 \\
{[0.005]^{(\mathrm{e})}}\end{array}$ & $\begin{array}{c}1510 \\
{[0.016]^{(\mathrm{e})}}\end{array}$ & 44 & - & - \\
\hline After Wash 73! & - & - & - & - & - & $\begin{array}{c}307 \\
{[<0.01]}\end{array}$ & $\begin{array}{c}0.16 \\
{[0.101]^{(\mathrm{e})}}\end{array}$ & 13700 & $\begin{array}{c}<0.248 \\
{[<0.001]}\end{array}$ & $\begin{array}{c}16100 \\
{[0.161]}\end{array}$ & $\begin{array}{c}1730 \\
{[0.040]}\end{array}$ & $\begin{array}{c}419 \\
{[<0.03]}\end{array}$ & $\begin{array}{c}324 \\
{[0.004]^{(\mathrm{e})}}\end{array}$ & $\begin{array}{c}1140 \\
{[<0.01]}\end{array}$ & 28.1 & - & - \\
\hline After Wash 79 & 17.1 & 1.135 & - & 3.01 & 1.029 & $\begin{array}{c}231 \\
{[<0.01]}\end{array}$ & $\begin{array}{c}0.12 \\
{[0.110]^{(\mathrm{e})}}\end{array}$ & 10500 & $\begin{array}{c}<0.247 \\
{[<0.001]}\end{array}$ & $\begin{array}{c}12100 \\
{[0.124]}\end{array}$ & $\begin{array}{c}1330 \\
{[0.033]}\end{array}$ & $\begin{array}{c}330 \\
{[<0.03]}\end{array}$ & $\begin{array}{c}247 \\
{[0.004]^{(\mathrm{e})}}\end{array}$ & $\begin{array}{c}878 \\
{[<0.01]}\end{array}$ & 22.7 & - & - \\
\hline After Wash 85 & - & - & - & - & - & $\begin{array}{c}188 \\
{[<0.01]}\end{array}$ & $\begin{array}{c}0.10 \\
{[0.092]^{(\mathrm{e})}}\end{array}$ & 8380 & $\begin{array}{c}<0.247 \\
{[<0.001]}\end{array}$ & $\begin{array}{c}9470 \\
{[0.100]}\end{array}$ & $\begin{array}{c}1040 \\
{[0.028]}\end{array}$ & $\begin{array}{c}263 \\
{[<0.03]}\end{array}$ & $\begin{array}{c}195 \\
{[0.003]^{(\mathrm{e})}}\end{array}$ & $\begin{array}{c}676 \\
{[<0.01]}\end{array}$ & 19.9 & - & - \\
\hline After Wash 90 & 17.2 & 1.131 & - & 1.88 & 1.020 & $\begin{array}{c}132 \\
{[<0.01]}\end{array}$ & $\begin{array}{c}0.09 \\
{[0.082]^{(\mathrm{e})}}\end{array}$ & 6820 & $\begin{array}{c}<0.249 \\
{[<0.001]}\end{array}$ & $\begin{array}{c}7620 \\
{[0.080]}\end{array}$ & $\begin{array}{c}840 \\
{[0.024]}\end{array}$ & $\begin{array}{c}222 \\
{[<0.03]}\end{array}$ & $\begin{array}{c}158 \\
{[0.002]^{(\mathrm{e})}}\end{array}$ & $\begin{array}{c}563 \\
{[<0.01]}\end{array}$ & 19.6 & - & - \\
\hline
\end{tabular}


Table 5.10. Post-Caustic-Leach Wash

\begin{tabular}{|c|c|c|c|c|c|c|c|c|c|c|c|c|c|c|c|c|}
\hline \multirow{3}{*}{$\begin{array}{c}\text { Sample } \\
\text { location: Tank } \\
\text { T02A }\end{array}$} & \multicolumn{3}{|c|}{ Slurry Analyses } & \multicolumn{13}{|c|}{ Supernatant Analyses } \\
\hline & UDS & $\begin{array}{c}\text { Slurry } \\
\text { Density }^{(\mathrm{b})}\end{array}$ & $\mathrm{Si}$ & TDS & $\begin{array}{c}\text { Liquid } \\
\text { Density }{ }^{(b)}\end{array}$ & $\begin{array}{c}\mathrm{Al} \\
{\left[\mathrm{Al}(\mathrm{OH})_{4}\right.} \\
\text { by Raman] }\end{array}$ & $\begin{array}{l}\text { Free } \mathrm{OH}^{-} \\
{[\text {Raman] }}\end{array}$ & $\mathrm{Na}$ & $\begin{array}{c}\mathrm{Cr} \\
{\left[\mathrm{CrO}_{4}^{-2} \text { by }\right.} \\
\text { Raman }]\end{array}$ & $\begin{array}{c}\mathrm{C}_{2} \mathrm{O}_{4} \\
{[\text { Raman] }}\end{array}$ & $\begin{array}{c}\mathrm{NO}_{3} \\
{[\text { Raman] }}\end{array}$ & $\begin{array}{c}\mathrm{NO}_{2} \\
\text { [Raman] }\end{array}$ & $\begin{array}{c}\mathrm{SO}_{4} \\
{[\text { Raman] }}\end{array}$ & $\begin{array}{c}\mathrm{PO}_{4} \\
\text { [Raman] }\end{array}$ & $\mathrm{Cl}^{-}$PDMS & PPG \\
\hline & $\mathrm{Wt} \%$ & $\mathrm{~g} / \mathrm{mL}$ & $\mu \mathrm{g} / \mathrm{g}$ & $\mathrm{Wt} \%$ & $\mathrm{~g} / \mathrm{mL}$ & $\begin{array}{l}\mu \mathrm{g} / \mathrm{g} \\
{[\mathrm{M}]}\end{array}$ & $\begin{array}{c}\mathrm{M} \\
{[\mathrm{M}]}\end{array}$ & $\mu \mathrm{g} / \mathrm{g}$ & $\begin{array}{l}\mu \mathrm{g} / \mathrm{g} \\
{[\mathrm{M}]}\end{array}$ & $\begin{array}{l}\mu \mathrm{g} / \mathrm{g} \\
{[\mathrm{M}]}\end{array}$ & $\begin{array}{l}\mu \mathrm{g} / \mathrm{g} \\
{[\mathrm{M}]}\end{array}$ & $\begin{array}{l}\mu \mathrm{g} / \mathrm{g} \\
{[\mathrm{M}]}\end{array}$ & $\begin{array}{l}\mu \mathrm{g} / \mathrm{g} \\
{[\mathrm{M}]}\end{array}$ & $\begin{array}{l}\mu \mathrm{g} / \mathrm{g} \\
{[\mathrm{M}]}\end{array}$ & $\mu \mathrm{g} / \mathrm{g} \quad \mathrm{mg} / \mathrm{g}$ & $\mathrm{mg} / \mathrm{g}$ \\
\hline After Wash 95 & - & - & - & - & - & $\begin{array}{c}140 \\
{[<0.01]}\end{array}$ & $\begin{array}{c}0.07 \\
{[<0.04]}\end{array}$ & 5550 & $\begin{array}{c}<0.244 \\
{[<0.001]}\end{array}$ & $\begin{array}{c}6070 \\
{[0.063]}\end{array}$ & $\begin{array}{c}686 \\
{[0.022]}\end{array}$ & $\begin{array}{c}184 \\
{[<0.03]}\end{array}$ & $\begin{array}{c}128 \\
{[0.002]^{(\mathrm{e})}}\end{array}$ & $\begin{array}{c}462 \\
{[<0.01]}\end{array}$ & 19.4 & - \\
\hline $\begin{array}{l}\text { After Final } \\
\text { Wash }^{(\mathrm{c})}\end{array}$ & 17.5 & 1.116 & $\begin{array}{c}\text { wet solid }^{(\mathrm{a})} \\
1400 \\
55.40 \% \\
\text { solids } \\
\text { liquid }^{(\mathrm{a})} \\
7.2\end{array}$ & $\begin{array}{l} \\
1.21 \\
\end{array}$ & 1.015 & $\begin{array}{c}107 \\
{[<0.01]}\end{array}$ & $\begin{array}{c}0.07 \\
{[<0.04]}\end{array}$ & 4590 & $\begin{array}{c}<0.247 \\
{[<0.001]}\end{array}$ & $\begin{array}{c}4940 \\
{[0.050]^{(\mathrm{e})}}\end{array}$ & $\begin{array}{c}589 \\
{[0.020]^{(\mathrm{e})}}\end{array}$ & $\begin{array}{c}160 \\
{[<0.03]}\end{array}$ & $\begin{array}{c}108 \\
{[0.002]^{(e)}}\end{array}$ & $\begin{array}{c}382 \\
{[<0.01]}\end{array}$ & $19.9 \mathrm{n} / \mathrm{d}^{(\mathrm{d})}$ & $\begin{array}{c}\text { SX } \\
\text { Failed }\end{array}$ \\
\hline
\end{tabular}

(a) Original intact slurry was submitted to the analytical laboratory, so phase separation was performed at the analytical laboratory.

(b) If high-accuracy density results are available, then they are provided. If high-accuracy density results are not available, then density results from the UDS are provided.

(c) Analytical results were duplicated in Analysis at Key Process Steps table, column heading Post-Caustic-Leach, Concentration and Wash Sample.

(d) Single sample non-detect with analysis results below method reporting limit.

(e) Detected result is qualitative. Result $>$ IDL but $<$ EQL (estimated quantitation limit).

(f) Solids observed in the sample before and after centrifuging - results are subject to large error.

二-Analysis not required in test plan.

Raman results are provided as bracketed value [].

M Molarity.

$\underline{\text { SX Failed - the organic phase failed to separate from the aqueous phase, and no results were obtained. }}$

Table 5.10 (contd)

\begin{tabular}{|c|c|c|c|c|c|c|}
\hline \multirow[b]{2}{*}{ Sample Location: Tank T02A } & \multicolumn{6}{|c|}{ Wet Centrifuged Solids } \\
\hline & $\begin{array}{c}\text { PDMS } \\
\mathrm{mg} / \mathrm{g}\end{array}$ & $\begin{array}{l}\mathrm{PPG} \\
\mathrm{mg} / \mathrm{g}\end{array}$ & $\begin{array}{c}\mathrm{Al} \\
\mu \mathrm{g} / \mathrm{g}\end{array}$ & $\begin{array}{c}\mathrm{Na} \\
\mu \mathrm{g} / \mathrm{g}\end{array}$ & $\begin{array}{c}\mathrm{P} \\
\mu \mathrm{g} / \mathrm{g}\end{array}$ & $\begin{array}{c}\mathrm{S} \\
\mu \mathrm{g} / \mathrm{g}\end{array}$ \\
\hline After Final Wash ${ }^{(\mathrm{c})}$ & 0.2 & 2.65 & 124000 & 11600 & 1880 & 48.6 \\
\hline
\end{tabular}


Table 5.11. Oxidative Leach

\begin{tabular}{|c|c|c|c|c|c|c|c|c|c|c|c|c|c|c|c|c|c|}
\hline \multirow[b]{2}{*}{$\begin{array}{l}\text { Sample location: Tank } \\
\text { T02A, Condition: }\end{array}$} & \multicolumn{2}{|c|}{ Slurry Analyses } & \multicolumn{15}{|c|}{ Liquid Analyses } \\
\hline & $\begin{array}{l}\text { UDS } \\
\mathrm{Wt} \%\end{array}$ & $\begin{array}{c}\text { Slurry } \\
\text { Density }^{(a)} \\
\text { g/mL } \\
\end{array}$ & $\begin{array}{c}\text { Liquid } \\
\text { Density }^{(\mathrm{a})} \\
\mathrm{g} / \mathrm{mL}\end{array}$ & $\begin{array}{c}\mathrm{Al} \\
{\left[\mathrm{Al}(\mathrm{OH})_{4}^{-}\right.} \\
\text {by } \mathrm{Raman}] \\
\mu \mathrm{g} / \mathrm{g} \\
{[\mathrm{M}]}\end{array}$ & $\begin{array}{c}\mathrm{Al} \text { (rinsate) } \\
{\left[\mathrm{Al}(\mathrm{OH})_{4}{ }^{-}\right.} \\
\text {by } \mathrm{Raman}] \\
\mu \mathrm{g} / \mathrm{g} \\
{[\mathrm{M}]}\end{array}$ & $\begin{array}{c}\mathrm{OH} \\
\text { [Free OH } \\
\text { by } \\
\text { Raman] } \\
\mathrm{M} \\
{[\mathrm{M}]} \\
\end{array}$ & $\begin{array}{c}\mathrm{Cr} \\
{\left[\mathrm{CrO}_{4}^{-2}\right.} \\
\text { by } \\
\text { Raman }] \\
\mu \mathrm{g} / \mathrm{g} \\
{[\mathrm{M}]} \\
\end{array}$ & $\begin{array}{c}\mathrm{Cr} \\
\text { (rinsate) } \\
{\left[\mathrm{CrO}_{4}^{-2}\right.} \\
\text { by } \\
\text { Raman }] \\
\mu \mathrm{g} / \mathrm{g} \\
{[\mathrm{M}]} \\
\end{array}$ & $\begin{array}{l}\mathrm{Mn} \\
\mu \mathrm{g} / \mathrm{g}\end{array}$ & $\begin{array}{c}\mathrm{Mn} \\
\text { (rinsate) } \\
\mu \mathrm{g} / \mathrm{g} \\
\end{array}$ & $\begin{array}{l}\text { PDMS } \\
\mathrm{mg} / \mathrm{g} \\
\end{array}$ & $\begin{array}{l}\text { PPG } \\
\mathrm{mg} / \mathrm{g}\end{array}$ & $\begin{array}{c}\mathrm{C}_{2} \mathrm{O}_{4} \\
{[\mathrm{Raman}]} \\
\mu \mathrm{g} / \mathrm{g} \\
{[\mathrm{M}]} \\
\end{array}$ & $\begin{array}{l}\mathrm{Cl}- \\
\mu \mathrm{g} / \mathrm{g} \\
\end{array}$ & $\begin{array}{c}\mathrm{NO}_{3} \\
\text { [Raman] } \\
\mu \mathrm{g} / \mathrm{g} \\
{[\mathrm{M}]} \\
\end{array}$ & $\begin{array}{c}\mathrm{NO}_{2} \\
\text { [Raman] } \\
\mu \mathrm{g} / \mathrm{g} \\
{[\mathrm{M}]} \\
\end{array}$ & $\begin{array}{c}\mathrm{SO}_{4} \\
\text { [Raman] } \\
\mu \mathrm{g} / \mathrm{g} \\
{[\mathrm{M}]} \\
\end{array}$ \\
\hline $\begin{array}{c}\text { Before } \mathrm{MnO}_{4} \\
\text { Addition }^{(\mathrm{b})}\end{array}$ & $\begin{array}{ll}18.7 \\
\end{array}$ & 1.147 & 1.035 & $\begin{array}{l}52.1 \\
{[\mathrm{n} / \mathrm{d}]}\end{array}$ & $\begin{array}{c}22.2^{(\mathrm{e})} \\
{[\mathrm{n} / \mathrm{d}]}\end{array}$ & $\begin{array}{c}0.26 \\
{[0.194]^{(d, e)}}\end{array}$ & $\begin{array}{l}10.4 \\
{[\mathrm{n} / \mathrm{d}]}\end{array}$ & $\begin{array}{l}14.2^{(\mathrm{e})} \\
{[\mathrm{n} / \mathrm{d}]}\end{array}$ & {$[\mathrm{n} / \mathrm{d}]$} & 0.625 & $\mathrm{n} / \mathrm{d}^{(\mathrm{f})}$ & $\begin{array}{c}\text { SX } \\
\text { Failed }\end{array}$ & $\begin{array}{c}644 \\
{[\mathrm{n} / \mathrm{d}]}\end{array}$ & 140 & $\begin{array}{c}3617 \\
{[0.054]^{(\mathrm{e})}}\end{array}$ & $\begin{array}{c}69.9 \\
{[0.029]^{(d, e)}}\end{array}$ & $\begin{array}{c}32.9 \\
{[0.001]^{(\mathrm{d}, \mathrm{e})}}\end{array}$ \\
\hline $\begin{array}{c}\text { After } \mathrm{MnO}_{4} \text { Addition- } \mathrm{Hr} 0 \\
\text { (5 minutes after } \mathrm{MnO}_{4} \\
\text { addition) }\end{array}$ & 16.3 & 1.148 & 1.030 & $\begin{array}{c}31.4 \\
{[<0.01]}\end{array}$ & - & $\begin{array}{c}0.05 \\
{[0.062]^{(\mathrm{d})}}\end{array}$ & $\begin{array}{c}7070 \\
{[0.134]}\end{array}$ & - & 0.225 & - & - & - & $\begin{array}{c}612 \\
{[<0.01]}\end{array}$ & 131 & $\begin{array}{c}3270 \\
{[0.064]}\end{array}$ & $\begin{array}{c}75.9 \\
{[<0.03]}\end{array}$ & $\begin{array}{c}148 \\
{[0.002]^{(\mathrm{d})}}\end{array}$ \\
\hline After $\mathrm{MnO}_{4}$ Addition- $\mathrm{Hr}$ & 16.7 & 1.155 & 1.025 & $\begin{array}{c}39.6 \\
{[<0.01]}\end{array}$ & - & $\begin{array}{c}0.08 \\
{[<0.04]}\end{array}$ & $\begin{array}{c}6990 \\
{[0.130]}\end{array}$ & - & 0.168 & - & - & - & $\begin{array}{c}630 \\
{[<0.01]}\end{array}$ & 136 & $\begin{array}{c}3360 \\
{[0.064]}\end{array}$ & $\begin{array}{c}77.9 \\
{[<0.03]}\end{array}$ & $\begin{array}{c}147 \\
{[0.002]^{(\mathrm{d})}}\end{array}$ \\
\hline After $\mathrm{MnO}_{4}$ Addition- $\mathrm{Hr} 2$ & $\begin{array}{ll}16.6 \\
16.6\end{array}$ & 1.149 & $\begin{array}{ll}1.026 \\
1\end{array}$ & $\begin{array}{c}44.6 \\
{[<0.01]}\end{array}$ & - & $\begin{array}{c}0.08 \\
{[<0.04]}\end{array}$ & $\begin{array}{c}6730 \\
{[0.124]}\end{array}$ & - & 0.740 & - & - & - & $\begin{array}{c}641 \\
{[<0.01]}\end{array}$ & 133 & $\begin{array}{c}3340 \\
{[0.064]}\end{array}$ & $\begin{array}{c}78.9 \\
{[<0.03]}\end{array}$ & $\begin{array}{c}140 \\
{[0.002]^{(\mathrm{d})}}\end{array}$ \\
\hline After $\mathrm{MnO}_{4}$ Addition- $\mathrm{Hr} 3$ & $\begin{array}{ll}16.7 \\
\end{array}$ & 1.150 & 1.024 & $\begin{array}{c}46.6 \\
{[<0.01]}\end{array}$ & - & $\begin{array}{c}0.08 \\
{[0.060]^{(\mathrm{d})}}\end{array}$ & $\begin{array}{c}6450 \\
{[0.120]}\end{array}$ & - & 0.682 & - & - & - & $\begin{array}{c}657 \\
{[<0.01]}\end{array}$ & 135 & $\begin{array}{c}3400 \\
{[0.065]}\end{array}$ & $\begin{array}{c}81.9 \\
{[<0.03]}\end{array}$ & $\begin{array}{c}135 \\
{[0.002]^{(\mathrm{d})}}\end{array}$ \\
\hline After $\mathrm{MnO}_{4}$ Addition- $\mathrm{Hr} 4$ & $\begin{array}{l}16.7 \\
16\end{array}$ & 1.146 & 1.023 & $\begin{array}{c}50.4 \\
{[<0.01]}\end{array}$ & - & $\begin{array}{c}0.09 \\
{[0.057]^{(\mathrm{d})}}\end{array}$ & $\begin{array}{c}6440 \\
{[0.119]}\end{array}$ & - & 0.629 & - & - & - & $\begin{array}{c}661 \\
{[<0.01]}\end{array}$ & 137 & $\begin{array}{c}3410 \\
{[0.066]}\end{array}$ & $\begin{array}{c}81 \\
{[<0.03]}\end{array}$ & $\begin{array}{c}134 \\
{[0.002]^{(\mathrm{d})}}\end{array}$ \\
\hline After $\mathrm{MnO}_{4}$ Addition- $\mathrm{Hr} 5$ & 16.7 & 1.148 & $\begin{array}{l}1.027 \\
\end{array}$ & $\begin{array}{c}51.4 \\
{[<0.01]}\end{array}$ & - & $\begin{array}{c}0.08 \\
{[0.086]^{(\mathrm{d})}}\end{array}$ & $\begin{array}{c}6270 \\
{[0.118]}\end{array}$ & - & 0.419 & - & - & - & $\begin{array}{c}674 \\
{[<0.01]}\end{array}$ & 139 & $\begin{array}{c}3470 \\
{[0.066]}\end{array}$ & $\begin{array}{c}81.2 \\
{[<0.03]}\end{array}$ & $\begin{array}{c}134 \\
{[0.002]^{(\mathrm{d})}}\end{array}$ \\
\hline $\begin{array}{c}\text { After } \mathrm{MnO}_{4} \text { Addition-Hr } 6 \\
\text { (Final after Oxidative } \\
\text { Leach) })^{(\mathrm{c})}\end{array}$ & 16.7 & 1.147 & 1.028 & $\begin{array}{l}52.4 \\
{[\mathrm{n} / \mathrm{d}]}\end{array}$ & $\begin{array}{l}20.9 \\
{[\mathrm{n} / \mathrm{d}]}\end{array}$ & $\begin{array}{c}0.08 \\
{[0.057]^{(\mathrm{d})}}\end{array}$ & $\begin{array}{c}6300 \\
{[0.117]}\end{array}$ & $\begin{array}{c}1413 \\
{[0.027]}\end{array}$ & 0.294 & 0.709 & $\mathrm{n} / \mathrm{d}^{(\mathrm{f})}$ & $\begin{array}{c}\text { SX } \\
\text { Failed }\end{array}$ & $\begin{array}{c}679 \\
{[\mathrm{n} / \mathrm{d}]}\end{array}$ & 142 & $\begin{array}{c}3490 \\
{[0.066]}\end{array}$ & $\begin{array}{l}81.3 \\
{[\mathrm{n} / \mathrm{d}]}\end{array}$ & $\begin{array}{c}137 \\
{[0.002]^{(\mathrm{d})}}\end{array}$ \\
\hline $\begin{array}{l}\text { (a) If high-accuracy density res } \\
\text { (b) Analytical results were dupl } \\
\text { (c) Analytical results were dupli } \\
\text { (d) Detected result is qualitative } \\
\text { (e) One value is significantly di } \\
\text { (f) Single sample non-detect wi } \\
\text { SX Failed - the organic phase f } \\
\text { [Raman] results are provided as } \\
\text { Bold numbers are calculated val } \\
\text { - Analysis not required by Tes }\end{array}$ & $\begin{array}{l}\text { licated in } \\
\text { e. Resul } \\
\text { liferent } f \\
\text { ith analy } \\
\text { failed to } \\
\text { brackete } \\
\text { lues. } \\
\text { st Plan. }\end{array}$ & $\begin{array}{l}\text { the Analys } \\
t>\text { IDL but } \\
\text { rom the oth } \\
\text { sis results be } \\
\text { separate fro } \\
\text { ed values []. }\end{array}$ & $\begin{array}{l}<\text { is at Key Pro } \\
<\text { EQL (estin } \\
\text { er two measu } \\
\text { elow method } \\
m \text { the aqueou }\end{array}$ & $\begin{array}{l}\text { mated quantita } \\
\text { ured values. } \\
\text { reporting lim } \\
\text { us phase, and r }\end{array}$ & $\begin{array}{l}\text { le column, heac } \\
\text { tion limit). } \\
\text { it. } \\
\text { no results were }\end{array}$ & obtained. & 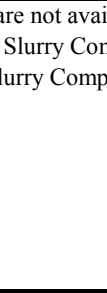 & 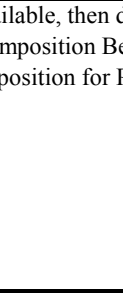 & 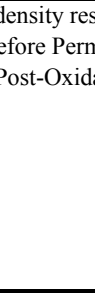 & 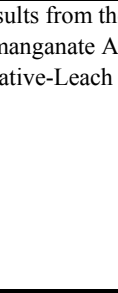 & 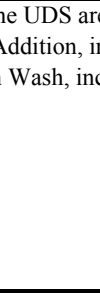 & & . & & data. & & \\
\hline
\end{tabular}


Table 5.11 (contd)

\begin{tabular}{|c|c|c|c|c|c|c|c|}
\hline \multirow[b]{2}{*}{$\begin{array}{l}\text { Sample location: Tank T02A, } \\
\text { Condition: }\end{array}$} & \multicolumn{7}{|c|}{ Rinsed Wet Centrifuged Solids } \\
\hline & $\begin{array}{c}\mathrm{Al} \\
\mu \mathrm{g} / \mathrm{g}\end{array}$ & $\begin{array}{c}\mathrm{Cr} \\
\mu \mathrm{g} / \mathrm{g}\end{array}$ & $\begin{array}{c}\mathrm{Sr} \\
\mu \mathrm{g} / \mathrm{g}\end{array}$ & $\begin{array}{c}\mathrm{Mn} \\
\mu \mathrm{g} / \mathrm{g}\end{array}$ & $\begin{array}{c}\mathrm{Si} \\
\mu \mathrm{g} / \mathrm{g}\end{array}$ & $\begin{array}{c}\text { PDMS } \\
\mathrm{mg} / \mathrm{g}\end{array}$ & $\begin{array}{l}\mathrm{PPG} \\
\mathrm{mg} / \mathrm{g}\end{array}$ \\
\hline $\begin{array}{l}\text { Before } \mathrm{MnO}_{4} \\
\text { Addition }^{(b)}\end{array}$ & 118000 & 20433 & 1143 & 20433 & $\begin{array}{c}\text { Wet centrifuge solids } 1570-\mu \mathrm{g} / \mathrm{g} \\
51.82 \% \text { solids } \\
\text { Liquid fraction } 70.2-\mu \mathrm{g} / \mathrm{g}^{(\mathrm{g})}\end{array}$ & 0.1 & 1.18 \\
\hline $\begin{array}{c}\text { After } \mathrm{MnO}_{4} \text { Addition- } \mathrm{Hr} 0 \\
\left(5 \text { minutes after } \mathrm{MnO}_{4} \text { addition) }\right.\end{array}$ & - & - & - & - & - & - & - \\
\hline After $\mathrm{MnO}_{4}$ Addition— $-\mathrm{Hr} 1$ & - & - & - & - & - & - & - \\
\hline After $\mathrm{MnO}_{4}$ Addition- $\mathrm{Hr} 2$ & - & - & - & - & - & - & - \\
\hline After $\mathrm{MnO}_{4}$ Addition— $\mathrm{Hr} 3$ & - & - & - & - & - & - & - \\
\hline After $\mathrm{MnO}_{4}$ Addition- $\mathrm{Hr} 4$ & - & - & - & - & - & - & - \\
\hline After $\mathrm{MnO}_{4}$ Addition- $\mathrm{Hr} 5$ & - & - & - & - & - & - & - \\
\hline $\begin{array}{l}\text { After } \mathrm{MnO}_{4} \text { Addition-Hr } 6 \text { (Final } \\
\text { after Oxidative Leach) }\end{array}$ & 122667 & 1170 & 1167 & 42100 & $\begin{array}{l}\text { Wet centrifuge solids } 1420-\mu \mathrm{g} / \mathrm{g} \\
50.61 \% \text { solids } \\
\text { Liquid fraction } 25.2-\mu \mathrm{g} / \mathrm{g}\end{array}$ & 0.2 & 2.25 \\
\hline \multicolumn{8}{|c|}{$\begin{array}{l}\text { Bold numbers are calculated values. } \\
\text { - Analysis not required by Test Plan. } \\
\text { (b) Analytical results were duplicated in the Analysis at Key Process Steps table column, heading Starting Slurry Composition Before Permanganate Addition, } \\
\text { including wt\% solids data. } \\
\text { (c) Analytical results were duplicated in the Analysis at Key Process Steps table, column heading Initial Slurry Composition for Post-Oxidative-Leach Wash, } \\
\text { including wt\% solids data. } \\
\text { (g) Original intact slurry was submitted to the analytical laboratory, so phase separation was performed at the analytical laboratory. }\end{array}$} \\
\hline
\end{tabular}


Table 5.12, Post Oxidative Leach Wash, provides analytical results for samples collected during the post-oxidative-leach washing in Tank T02A. Two separate slurry samples were collected for the weight percent UDS and slurry density. The supernatant density is a higher accuracy density than can be provided by the UDS analysis. The liquid analyses results are supernatant analytical results obtained from samples phase-separated in PDL-W. Raman results, shown in brackets, [ ], are provided as part of the liquid analyses and presented in molar concentration (mole/liter).

Table 5.13, Full Analysis, provides the concentration of key metal analytes, anions, total inorganic carbon (TIC), wt $\%$ UDS, and density at key process steps. Two separate slurry samples were collected for the wt $\%$ UDS and slurry density. The supernatant density is a higher accuracy density than can be provided by the UDS analysis. The concentrations of the metals and TIC in the slurry were calculated from samples phase-separated at PDL-W. The liquid analysis results are supernatant analytical results obtained from samples phase-separated in PDL-W. The wt $\%$ of wet centrifuged solids in the slurry can be used in calculating the total species concentration in the original samples slurry. See Section A.5 in Appendix A of WTP-RPT-186, Rev 0, for more information on calculating the species concentration in the slurry. An aliquot of the wet centrifuged solids was dried at $105^{\circ} \mathrm{C}$ to determine the percent solids that are reported with the reported analytes in the wet centrifuged solids.

Table 5.14, PEP Testing Reagent Tables (TDP-WTP-361), provides reagent analysis results. The reagent samples were shipped as collected. The analysis for contaminant metals and anions was performed on the initial samples. Contaminants were not detected in the $19-\mathrm{M} \mathrm{NaOH}$ above reporting limits with the exception of chloride present at $23.7-\mu \mathrm{g} / \mathrm{g}$, which is slightly above the reporting limit of $16.9-\mu \mathrm{g} / \mathrm{g}$. The 2- $\mathrm{M} \mathrm{HNO}_{3}$ showed multiple contaminants present. Several contaminants were detected in the $\mathrm{NaMnO}_{4}$ at a concentration slightly above reporting limits. Sulfate was present at over $800-\mu \mathrm{g} / \mathrm{g}$ in the $\mathrm{NaMnO}_{4}$. This sulfate concentration is much higher than its reporting limit. For Integrated Test A, the sample of interest is A 00C04 XXX XX 2610 OST 2. 
Table 5.12. Post-Oxidative-Leach Wash

\begin{tabular}{|c|c|c|c|c|c|c|c|c|c|c|c|c|c|c|}
\hline \multirow[b]{3}{*}{$\begin{array}{l}\text { Sample location: Tank } \\
\text { T02A, Condition: }\end{array}$} & \multicolumn{2}{|c|}{ Slurry Analyses } & \multicolumn{12}{|c|}{ Liquid Analyses } \\
\hline & UDS & $\begin{array}{c}\text { Slurry } \\
\text { Density }\end{array}$ & TDS & $\begin{array}{c}\text { Liquid } \\
\text { Density }\end{array}$ & $\begin{array}{c}\mathrm{Al} \\
{\left[\mathrm{Al}(\mathrm{OH})_{4} \text { by }\right.} \\
\text { Raman }]\end{array}$ & $\mathrm{Na}$ & $\begin{array}{c}\mathrm{Cr} \\
{\left[\mathrm{CrO}_{4}^{-2} \text { by }\right.} \\
\text { Raman }]\end{array}$ & $\mathrm{Sr}$ & $\begin{array}{c}\text { Free } \\
\text { OH- } \\
\text { [Raman] }\end{array}$ & $\begin{array}{c}\mathrm{NO}_{3} \\
\text { [Raman] }\end{array}$ & $\begin{array}{c}\mathrm{NO}_{2} \\
\text { [Raman] }\end{array}$ & $\begin{array}{c}\mathrm{SO}_{4} \\
{[\mathrm{Raman}]}\end{array}$ & $\begin{array}{c}\mathrm{C}_{2} \mathrm{O}_{4} \\
{[\text { Ramana }]}\end{array}$ & Cl- \\
\hline & $\mathrm{Wt} \%$ & $\mathrm{~g} / \mathrm{mL}$ & wt $\%$ & $\mathrm{~g} / \mathrm{mL}$ & $\begin{array}{l}\mu \mathrm{g} / \mathrm{g} \\
{[\mathrm{M}]}\end{array}$ & $\mu \mathrm{g} / \mathrm{g}$ & $\begin{array}{l}\mu \mathrm{g} / \mathrm{g} \\
{[\mathrm{M}]}\end{array}$ & $\mu \mathrm{g} / \mathrm{g}$ & $\begin{array}{l}\mathrm{M} \\
{[\mathrm{M}]}\end{array}$ & $\begin{array}{l}\mu \mathrm{g} / \mathrm{g} \\
{[\mathrm{M}]}\end{array}$ & $\begin{array}{l}\mu \mathrm{g} / \mathrm{g} \\
{[\mathrm{M}]}\end{array}$ & $\begin{array}{l}\mu \mathrm{g} / \mathrm{g} \\
{[\mathrm{M}]}\end{array}$ & $\begin{array}{l}\mu \mathrm{g} / \mathrm{g} \\
{[\mathrm{M}]}\end{array}$ & $\mu \mathrm{g} / \mathrm{g}$ \\
\hline After Wash 5 & 16.80 & 1.142 & 2.62 & 1.038 & $\begin{array}{c}45.9 \\
{[<0.01]}\end{array}$ & 7990 & $\begin{array}{c}5130 \\
{[0.094]}\end{array}$ & $<0.165$ & $\begin{array}{c}0.08 \\
{[<0.04]}\end{array}$ & $\begin{array}{c}2820 \\
{[0.057]}\end{array}$ & $\begin{array}{c}75.9 \\
{[<0.03]}\end{array}$ & $\begin{array}{c}110 \\
{[0.001]^{(\mathrm{c})}}\end{array}$ & $\begin{array}{c}544 \\
{[<0.01]}\end{array}$ & 114 \\
\hline After Wash 10 & - & - & - & - & $\begin{array}{c}39.7 \\
{[<0.01]}\end{array}$ & 6500 & $\begin{array}{c}4190 \\
{[0.076]}\end{array}$ & $<0.249$ & $\begin{array}{c}0.07 \\
{[<0.04]}\end{array}$ & $\begin{array}{c}2210 \\
{[0.048]}\end{array}$ & $\begin{array}{c}70 \\
{[<0.03]}\end{array}$ & $\begin{array}{c}94.7 \\
{[0.002]^{(\mathrm{c})}}\end{array}$ & $\begin{array}{c}447 \\
{[<0.01]}\end{array}$ & 92.9 \\
\hline After Wash 15 & 17.00 & 1.135 & 1.76 & 1.022 & $\begin{array}{c}33.9 \\
{[<0.01]}\end{array}$ & 5460 & $\begin{array}{c}3440 \\
{[0.062]}\end{array}$ & $<0.166$ & $\begin{array}{c}0.06 \\
{[<0.04]}\end{array}$ & $\begin{array}{c}1800 \\
{[0.041]}\end{array}$ & $\begin{array}{c}65.6 \\
{[<0.03]}\end{array}$ & $\begin{array}{c}72.7 \\
{[0.001]^{(\mathrm{c})}}\end{array}$ & $\begin{array}{c}369 \\
{[<0.01]}\end{array}$ & 73.4 \\
\hline After Wash 20 & - & - & $\vdots$ & - & $\begin{array}{c}28.1 \\
{[<0.01]}\end{array}$ & 4470 & $\begin{array}{c}2730 \\
{[0.050]}\end{array}$ & $<0.244$ & $\begin{array}{c}0.04 \\
{[<0.04]}\end{array}$ & $\begin{array}{c}1470 \\
{[0.035]}\end{array}$ & $\begin{array}{c}63.8 \\
{[0.027]^{(\mathrm{c})}}\end{array}$ & $\begin{array}{c}61.6 \\
{[<0.001]}\end{array}$ & $\begin{array}{c}295 \\
{[<0.01]}\end{array}$ & 64.5 \\
\hline After Wash 25 & 17.10 & 1.128 & 1.21 & 1.029 & $\begin{array}{c}21.9 \\
{[<0.01]}\end{array}$ & 3720 & $\begin{array}{c}2240 \\
{[0.041]}\end{array}$ & $<0.166$ & $\begin{array}{c}0.03 \\
{[<0.04]}\end{array}$ & $\begin{array}{c}1190 \\
{[0.031]}\end{array}$ & $\begin{array}{c}64.1 \\
{[0.029]^{(\mathfrak{c})}}\end{array}$ & $\begin{array}{c}46.9 \\
{[<0.001]}\end{array}$ & $\begin{array}{c}242 \\
{[<0.01]}\end{array}$ & 53.6 \\
\hline After Wash 30 & - & - & - & - & $\begin{array}{c}26.3 \\
{[<0.01]}\end{array}$ & 3240 & $\begin{array}{c}1870 \\
{[0.034]}\end{array}$ & $<0.250$ & $\begin{array}{c}0.05 \\
{[<0.04]}\end{array}$ & $\begin{array}{c}991 \\
{[0.027]}\end{array}$ & $\begin{array}{c}64.8 \\
{[<0.03]}\end{array}$ & $\begin{array}{c}43.2 \\
{[<0.001]}\end{array}$ & $\begin{array}{c}202 \\
{[<0.01]}\end{array}$ & 47.1 \\
\hline After Wash 35 & 17.30 & 1.147 & 0.83 & 0.996 & $\begin{array}{c}18.9 \\
{[<0.01]}\end{array}$ & 2730 & $\begin{array}{c}1540 \\
{[0.028]}\end{array}$ & $<0.166$ & $\begin{array}{c}0.03 \\
{[<0.04]}\end{array}$ & $\begin{array}{c}819 \\
{[0.024]}\end{array}$ & $\begin{array}{c}64.2 \\
{[0.025]^{(\mathrm{c})}}\end{array}$ & $\begin{array}{c}35.9 \\
{[<0.001]}\end{array}$ & $\begin{array}{c}164 \\
{[<0.01]}\end{array}$ & 38.4 \\
\hline After Wash 40 & - & 1.125 & - & 1.009 & $\begin{array}{c}17.3 \\
{[<0.01]}\end{array}$ & 2330 & $\begin{array}{c}1260 \\
{[0.023]}\end{array}$ & $<0.242$ & $\begin{array}{c}0.04 \\
{[<0.04]}\end{array}$ & $\begin{array}{c}687 \\
{[0.021]}\end{array}$ & $\begin{array}{c}63.7 \\
{[0.032]^{(\mathrm{c})}}\end{array}$ & $\begin{array}{c}27.3 \\
{[<0.001]}\end{array}$ & $\begin{array}{c}137 \\
{[<0.01]}\end{array}$ & 29.8 \\
\hline After Wash 48 & 17.40 & 1.144 & 0.56 & 0.989 & $\begin{array}{c}14.8 \\
{[<0.01]}\end{array}$ & 1830 & $\begin{array}{c}929 \\
{[0.017]}\end{array}$ & $<0.166$ & $\begin{array}{c}0.03 \\
{[<0.04]}\end{array}$ & $\begin{array}{c}509 \\
{[0.019]^{(\mathrm{c})}}\end{array}$ & $\begin{array}{c}64.8 \\
{[0.027]^{(\mathrm{c})}}\end{array}$ & $\begin{array}{c}23.9 \\
{[<0.001]}\end{array}$ & $\begin{array}{c}96.2 \\
{[<0.01]}\end{array}$ & 21.2 \\
\hline After Wash 54 & - & - & - & - & $\begin{array}{c}13.7 \\
{[<0.01]}\end{array}$ & 1580 & $\begin{array}{c}759 \\
{[0.013]}\end{array}$ & $<0.246$ & $\begin{array}{c}0.03 \\
{[<0.04]}\end{array}$ & $\begin{array}{c}428 \\
{[0.017]^{(\mathrm{c})}}\end{array}$ & $\begin{array}{c}64.4 \\
{[<0.03]}\end{array}$ & $\begin{array}{c}19.6 \\
{[<0.001]}\end{array}$ & $\begin{array}{c}80.1 \\
{[<0.01]}\end{array}$ & $<19.6$ \\
\hline After Wash 63 & 17.60 & 1.140 & 0.36 & 0.994 & $\begin{array}{c}11.2 \\
{[<0.01]}\end{array}$ & 1240 & $\begin{array}{c}540 \\
{[0.009]^{(\mathrm{c})}}\end{array}$ & $<0.166$ & $\begin{array}{c}0.02 \\
{[<0.04]}\end{array}$ & $\begin{array}{c}319 \\
{[0.015]^{(\mathrm{c})}}\end{array}$ & $\begin{array}{c}65.4 \\
{[<0.03]}\end{array}$ & $\begin{array}{c}19.9 \\
{[<0.001]}\end{array}$ & $\begin{array}{c}59.2 \\
{[<0.01]}\end{array}$ & $<19.9$ \\
\hline After Wash 72 & - & - & - & - & $\begin{array}{c}12.4 \\
{[<0.01]}\end{array}$ & 1010 & $\begin{array}{c}389 \\
{[0.007]^{(\mathrm{c})}}\end{array}$ & 0.279 & $\begin{array}{c}0.02 \\
{[<0.04]}\end{array}$ & $\begin{array}{c}257 \\
{[0.014]^{(\mathrm{c})}}\end{array}$ & $\begin{array}{c}65.1 \\
{[<0.03]}\end{array}$ & $\begin{array}{c}19.8 \\
{[<0.001]}\end{array}$ & $\begin{array}{c}40.3 \\
{[<0.01]}\end{array}$ & $<19.8$ \\
\hline After Wash 78 & 17.60 & 1.144 & 0.19 & 1.000 & $\begin{array}{c}10.8 \\
{[<0.01]}\end{array}$ & 884 & $\begin{array}{c}308 \\
{[0.005]^{(\mathrm{c})}}\end{array}$ & 0.508 & $\begin{array}{c}0.02 \\
{[<0.04]}\end{array}$ & $\begin{array}{c}220 \\
{[0.013]^{(\mathrm{c})}}\end{array}$ & $\begin{array}{c}65 \\
{[<0.03]}\end{array}$ & $\begin{array}{c}19.8 \\
{[<0.001]}\end{array}$ & $\begin{array}{c}32.3 \\
{[<0.01]}\end{array}$ & $<19.8$ \\
\hline After Wash 84 & - & - & - & - & $\begin{array}{c}11.4 \\
{[<0.01]}\end{array}$ & 806 & $\begin{array}{c}253 \\
{[0.004]^{(\mathrm{c})}}\end{array}$ & 0.773 & $\begin{array}{c}0.02 \\
{[<0.04]}\end{array}$ & $\begin{array}{c}189 \\
{[0.014]^{(\mathrm{c})}}\end{array}$ & $\begin{array}{c}63.1 \\
{[0.029]^{(\mathrm{c})}}\end{array}$ & $\begin{array}{c}19.2 \\
{[<0.001]}\end{array}$ & $\begin{array}{c}36.1 \\
{[<0.01]}\end{array}$ & $<19.2$ \\
\hline After Final Wash ${ }^{(b)}$ & 17.70 & 1.127 & 0.14 & 1.006 & $\begin{array}{c}11.4 \\
{[<0.01]}\end{array}$ & 801 & $\begin{array}{c}233 \\
{[0.004]^{(\mathrm{c})}}\end{array}$ & 0.868 & $\begin{array}{c}0.02 \\
{[<0.04]}\end{array}$ & $\begin{array}{c}180 \\
{[0.013]^{(\mathrm{c})}}\end{array}$ & $\begin{array}{c}64.1 \\
{[0.025]^{(\mathfrak{c})}}\end{array}$ & $\begin{array}{c}19.5 \\
{[<0.001]}\end{array}$ & $\begin{array}{c}36.3 \\
{[<0.01]}\end{array}$ & $<19.5$ \\
\hline
\end{tabular}

[Raman] results are provided as bracketed values [].

M Molarity.

(a) If high-accuracy density results are available, then they are provided. If high-accuracy density results are not available, then density results from the UDS are provided.

(b) Analytical results were duplicated in the Analysis at Key Process Steps table, column heading Post Oxidative Leach, Concentration, and Wash Sample.

(c) Detected result is qualitative. Result $>$ IDL but $<$ EQL (estimated quantitation limit). 
Table 5.13 (contd)

\begin{tabular}{|c|c|c|c|c|c|c|}
\hline \multirow[b]{2}{*}{ Analyte } & \multicolumn{2}{|c|}{$\begin{array}{l}\text { Initial Slurry Composition Before Permanganate } \\
\text { Addition }\end{array}$} & \multicolumn{2}{|c|}{$\begin{array}{l}\text { Initial Slurry Composition for Post Oxidative Leach } \\
\text { Wash }\end{array}$} & \multicolumn{2}{|c|}{$\begin{array}{c}\text { Post Oxidative Leach, Concentration, and Wash } \\
\text { Sample }\end{array}$} \\
\hline & $\begin{array}{c}\text { rinsed wet centrifuged } \\
\text { solids } \\
\mu \mathrm{g} / \mathrm{g}\end{array}$ & $\begin{array}{c}\text { Liquid Phase }^{(\mathrm{f})} \\
\mu \mathrm{g} / \mathrm{g}\end{array}$ & $\begin{array}{c}\text { rinsed wet centrifuged } \\
\text { solid } \\
\mu \mathrm{g} / \mathrm{g}\end{array}$ & $\begin{array}{l}\text { Liquid Phase }^{(\mathrm{f})} \\
\mu \mathrm{g} / \mathrm{g}\end{array}$ & $\begin{array}{l}\text { wet centrifuged solid } \\
\qquad \mu \mathrm{g} / \mathrm{g}\end{array}$ & $\begin{array}{l}\text { Liquid Phase } \\
\mu \mathrm{g} / \mathrm{g}\end{array}$ \\
\hline $\mathrm{Al}$ & $118000 \pm 4608$ & $52 \pm 2$ & $122667 \pm 4787$ & $52 \pm 2$ & - & $11.4 \pm 2.6$ \\
\hline $\mathrm{Ca}$ & $2937 \pm 128$ & $\mathrm{n} / \mathrm{d}$ & $2943 \pm 108$ & $\mathrm{n} / \mathrm{d}$ & - & $9.60 \pm 1.36$ \\
\hline $\mathrm{Ce}$ & $1157 \pm 45$ & $\mathrm{n} / \mathrm{d}$ & $1177 \pm 46$ & $0.0086 \pm 0.0019$ & - & $0.277 \pm 0.017$ \\
\hline $\mathrm{Cr}$ & $20433 \pm 796$ & $10.4 \pm 0.4$ & $1170 \pm 47$ & $6300 \pm 218$ & - & $233 \pm 14$ \\
\hline $\mathrm{Fe}$ & $95300 \pm 3724$ & $\mathrm{n} / \mathrm{d}$ & $97167 \pm 3795$ & $\mathrm{n} / \mathrm{d}$ & - & $27.3 \pm 3.0$ \\
\hline $\mathrm{K}$ & $31 \pm 8$ & $\mathrm{n} / \mathrm{d}$ & $107 \pm 9$ & $36.9 \pm 3.1$ & - & $<14.8 \pm 7.4$ \\
\hline $\mathrm{La}$ & $864 \pm 34$ & $\mathrm{n} / \mathrm{d}$ & $879 \pm 34$ & $0.0076 \pm 0.0019$ & - & $0.507 \pm 0.031$ \\
\hline $\mathrm{Mg}$ & $2013 \pm 81$ & $\mathrm{n} / \mathrm{d}$ & $2050 \pm 80$ & $\mathrm{n} / \mathrm{d}$ & - & $<4.95 \pm 2.48$ \\
\hline $\mathrm{Mn}$ & $20433 \pm 796$ & $\mathrm{n} / \mathrm{d}$ & $42100 \pm 1641$ & $0.294 \pm 0.049$ & - & $3.45 \pm 0.24$ \\
\hline $\mathrm{Na}$ & $10140 \pm 427$ & $7610 \pm 290$ & $11767 \pm 535$ & $9657 \pm 355$ & - & $801 \pm 57$ \\
\hline $\mathrm{Nd}$ & $2367 \pm 92$ & $0.0102 \pm 0.0019$ & $2407 \pm 94$ & $0.0224 \pm 0.0021$ & - & $1.81 \pm 0.11$ \\
\hline $\mathrm{Ni}$ & $2740 \pm 107$ & $\mathrm{n} / \mathrm{d}$ & $2790 \pm 109$ & $\mathrm{n} / \mathrm{d}$ & - & $0.718 \pm 0.251$ \\
\hline $\mathrm{P}$ & $927 \pm 36$ & $64.5 \pm 2.2$ & $993 \pm 39$ & $62.6 \pm 2.2$ & - & $23.2 \pm 1.5$ \\
\hline $\mathrm{Si}$ & $1733 \pm 127$ & $36.93 \pm 1.34$ & $1757 \pm 119$ & $28.07 \pm 1.05$ & - & $8.25 \pm 1.11$ \\
\hline $\mathrm{Sr}$ & $1143 \pm 45$ & $\mathrm{n} / \mathrm{d}$ & $1167 \pm 46$ & $\mathrm{n} / \mathrm{d}$ & - & $0.868 \pm 0.134$ \\
\hline $\mathrm{Zr}$ & $2580 \pm 101$ & $\mathrm{n} / \mathrm{d}$ & $2637 \pm 103$ & $\mathrm{n} / \mathrm{d}$ & - & $0.706 \pm 0.251$ \\
\hline $\mathrm{Cl}$ & - & $140 \pm 7$ & - & $142 \pm 7$ & - & $<19.5 \pm 9.8$ \\
\hline $\mathrm{NO}_{2}$ & - & $70 \pm 19$ & - & $81 \pm 19$ & - & $<64.1 \pm 32.1$ \\
\hline $\mathrm{NO}_{3}$ & - & $3283 \pm 128$ & - & $3490 \pm 123$ & - & $180 \pm 45$ \\
\hline $\mathrm{PO}_{4}$ & - & $201 \pm 19$ & - & $208 \pm 19$ & - & $81.5 \pm 30.2$ \\
\hline $\mathrm{SO}_{4}$ & - & $33 \pm 6$ & - & $137 \pm 7$ & - & $19.5 \pm 9.8$ \\
\hline $\mathrm{C}_{2} \mathrm{O}_{4}$ & - & $644 \pm 23$ & - & $679 \pm 24$ & - & $36.3 \pm 1.0$ \\
\hline Wt $\%$ Solids & 54.43 & - & 55.02 & - & - & - \\
\hline Free $\mathrm{OH}^{-}(\mu \mathrm{g} / \mathrm{mL})$ & - & $4.43 E+03$ & - & $1.38 E+03$ & - & $3.38 \mathrm{E}+02$ \\
\hline $\mathrm{TIC}^{(\mathrm{b})}$ & - & - & - & - & - & - \\
\hline PDMS (mg/g) & 0.1 & $\mathrm{n} / \mathrm{d}^{(\mathrm{c})}$ & 0.2 & $\mathrm{n} / \mathrm{d}^{(\mathrm{c})}$ & - & - \\
\hline PPG (mg/g) & 1.18 & SX Failed & 2.25 & SX Failed & - & - \\
\hline Density $(\mathrm{g} / \mathrm{mL})$ & - & $1.035 \pm 0.014$ & - & $1.028 \pm 0.009$ & - & $1.006 \pm 0.016$ \\
\hline $\mathrm{Wt} \% \mathrm{H}_{2} \mathrm{O}$ & - & - & - & - & - & - \\
\hline Slurry wt $\%$ UDS & $18.7 \pm 0.2$ & - & $16.7 \pm 0.1$ & - & $17.7 \pm 0.27$ & - \\
\hline Slurry Density $(\mathrm{g} / \mathrm{mL})$ & $1.147 \pm 0.010$ & - & $1.147 \pm 0.010$ & - & $1.127 \pm 0.018$ & - \\
\hline Weight percent Centrifuged Solids (\%) & 36.3 & - & 31.4 & - & - & - \\
\hline
\end{tabular}

\section{- Analysis not required in test plan.}

Bold numbers are calculated values.

$\mathrm{n} / \mathrm{d}$ Triplicate samples with analysis results below method reporting limit.

(f) Liquid phase data contain only results from the decanted supernate. No
(h) Si results from sample named "ICP" rather than sample named "AFA."

SX Failed - the organic phase failed to separate from the aqueous phase, and no results were obtained.

(b) TIC = Total Inorganic Carbon.

(c) Single sample non-detect with analysis results below method reporting limit. 
Table 5.14. PEP Testing Reagent Tables

\begin{tabular}{|c|c|c|c|c|c|c|c|c|c|c|c|c|c|c|c|c|c|c|}
\hline $\begin{array}{c}\text { Analysis } \\
\text { Date }\end{array}$ & $\begin{array}{c}\text { Free } \mathrm{OH}^{-} \\
\mathrm{M}\end{array}$ & $\begin{array}{l}\text { Density } \\
\mathrm{g} / \mathrm{mL}\end{array}$ & $\begin{array}{c}\mathrm{MnO}_{4}^{-} \\
\mathrm{M}\end{array}$ & $\begin{array}{l}\mathrm{C}_{2} \mathrm{O}_{4} \\
\mu \mathrm{g} / \mathrm{g}\end{array}$ & $\begin{array}{l}\mathrm{NO}_{3} \\
\mu \mathrm{g} / \mathrm{g}\end{array}$ & $\begin{array}{l}\mathrm{NO}_{2} \\
\mu \mathrm{g} / \mathrm{g}\end{array}$ & $\begin{array}{c}\mathrm{SO}_{4} \\
\mu \mathrm{g} / \mathrm{g}\end{array}$ & $\begin{array}{l}\mathrm{PO}_{4} \\
\mu \mathrm{g} / \mathrm{g}\end{array}$ & $\begin{array}{c}\mathrm{Al} \\
\mu \mathrm{g} / \mathrm{g}\end{array}$ & $\begin{array}{c}\mathrm{Ca} \\
\mu \mathrm{g} / \mathrm{g}\end{array}$ & $\begin{array}{c}\mathrm{Cr} \\
\mu \mathrm{g} / \mathrm{g}\end{array}$ & $\begin{array}{c}\mathrm{Fe} \\
\mu \mathrm{g} / \mathrm{g}\end{array}$ & $\begin{array}{c}\mathrm{Mg} \\
\mu \mathrm{g} / \mathrm{g}\end{array}$ & $\begin{array}{c}\mathrm{Mn} \\
\mu \mathrm{g} / \mathrm{g}\end{array}$ & $\begin{array}{c}\mathrm{Na} \\
\mu \mathrm{g} / \mathrm{g}\end{array}$ & $\begin{array}{c}\mathrm{Nd} \\
\mu \mathrm{g} / \mathrm{g}\end{array}$ & $\begin{array}{c}\mathrm{Sr} \\
\mu \mathrm{g} / \mathrm{g}\end{array}$ & $\begin{array}{c}\mathrm{Cl} \\
\mu \mathrm{g} / \mathrm{g}\end{array}$ \\
\hline \multicolumn{19}{|c|}{ PEP Testing 19-M NaOH Analysis } \\
\hline $1 / 15 / 2009$ & $\underset{\text { (a) }}{18.652 \mathrm{~N}}$ & 1.522 & - & $<19.5$ & $<86.4$ & $<64.2$ & $<19.5$ & $<59.7$ & $<9.60$ & $<4.70$ & $<0.960$ & $<9.60$ & $<4.70$ & $<0.960$ & 285000 & $<0.0188$ & $<0.960$ & $<19.5$ \\
\hline $1 / 30 / 2009$ & $18.6 \mathrm{~N}^{(\mathrm{a})}$ & 1.519 & - & $<16.9$ & $<74.7$ & $<55.5$ & $<16.9$ & $<51.6$ & $<9.94$ & $<9.94$ & $<0.994$ & $<19.9$ & $<9.94$ & $<0.994$ & 293000 & $<0.199$ & $<1.99$ & 23.7 \\
\hline $2 / 6 / 2009$ & 19.27 & - & - & - & - & - & - & - & - & - & - & - & - & - & - & - & - & - \\
\hline $2 / 12 / 2009$ & 19.22 & - & - & - & - & - & - & - & - & - & - & - & - & - & - & - & - & - \\
\hline $2 / 18 / 2009$ & 18.86 & - & - & - & - & - & - & - & - & - & - & - & - & - & - & - & - & - \\
\hline $2 / 18 / 2009$ & 18.61 & - & - & - & - & - & - & - & - & - & - & - & - & - & - & - & - & - \\
\hline $3 / 17 / 2009$ & 19.12 & - & - & - & - & - & - & - & - & - & - & - & - & - & - & - & - & - \\
\hline $3 / 18 / 2009$ & 18.95 & - & $-\overline{-}$ & - & $\overline{-}$ & - & - & $\ldots$ & - & - & - & - & - & - & -- & $-\overline{-}$ & $-\overline{-}$ & - \\
\hline \multicolumn{19}{|c|}{ PEP Testing 2-M $\mathrm{HNO}_{3}$ Analysis } \\
\hline $1 / 31 / 2009$ & - & - & - & 129 & 35300 & $<64.0$ & $<19.5$ & $<59.5$ & 1.16 & 21.2 & 0.298 & 4.53 & 0.503 & 0.198 & 4.99 & 0.0136 & $<0.0484$ & - \\
\hline \multicolumn{19}{|c|}{ PEP Testing $\mathrm{NaMnO}_{4}$ Analysis } \\
\hline $1 / 9 / 2009$ & - & 1.085 & 0.921 & $<19.4$ & $<85.8$ & $<63.7$ & 841 & $<59.3$ & $<4.60$ & $<4.60$ & 1.17 & $<4.60$ & $<4.60$ & 46800 & 19900 & $<0.0460$ & $<0.460$ & 21.2 \\
\hline $1 / 31 / 2009$ & - & 1.106 & 1.06 & $<18.4$ & 96 & $<60.6$ & 934 & $<56.3$ & $<4.59$ & $<4.59$ & $<0.918$ & $<9.18$ & $<4.59$ & 51500 & 22100 & $<0.0918$ & $<0.918$ & 28.5 \\
\hline $\begin{array}{l}\text { (a) Results } \\
- \text { Analysi } \\
\text { M Molarity }\end{array}$ & $\begin{array}{l}\text { reported } \\
\text { t requires }\end{array}$ & $\begin{array}{l}\text { Normality } \\
\text { Test Pla }\end{array}$ & & & & & & & & & & & & & & & & \\
\hline
\end{tabular}


Table 5.15 provides analytical results for samples collected during the end of the testing when filters were being flushed, and slurry was transferred out of Tank T02A. The wt\% UDS and densities were obtained following analytical methods described in Section 3 for slurry not phase-separated. The liquid analysis results are supernatant analytical results obtained from samples phase-separated in PDL-W. 
Table 5.15. Filter Flush Analytical Results

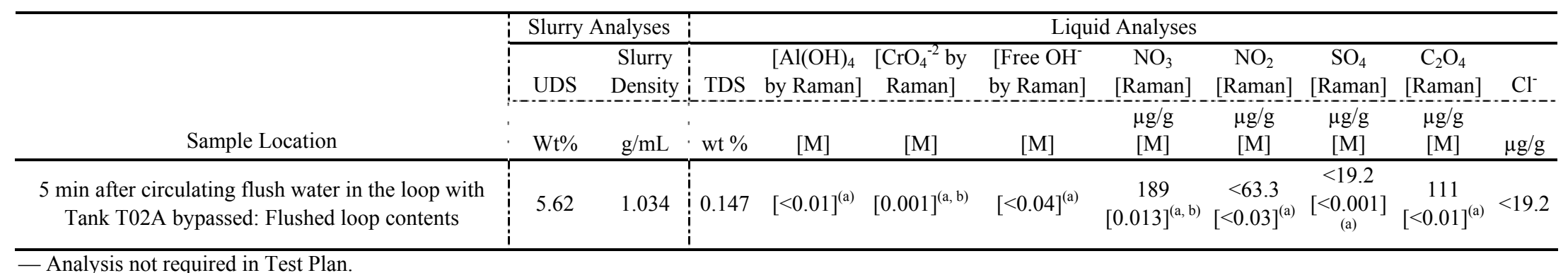

[Raman] results are provided as bracketed values [].

M Molarity.

(a) Solids observed in sample before and after centrifuging. Results are subject to larger error.

(b) Detected result is qualitative. Result $>$ IDL but $<$ EQL (estimated quantitation limit). 


\subsection{Physical Properties Data}

This section summarizes the heat capacity, PSD, rheology, XRD, and SEM results. The interested reader is referred to Appendix $\mathrm{H}$ for additional information.

\subsubsection{Heat Capacity}

Table 5.16, Heat-Capacity Measurements, provides a summary of the heat capacity data obtained at process steps of interest at both 40 and $80^{\circ} \mathrm{C}$. Heat capacity data over the temperature range 35 to $90^{\circ} \mathrm{C}$ for the samples listed can be found in Appendix $\mathrm{H}$.

Table 5.16. Heat-Capacity Measurements

\begin{tabular}{ccc}
\hline Sample location: Tank T01A & $\begin{array}{c}\text { Heat Capacity } \\
\mathrm{J} / \mathrm{g}{ }^{\circ} \mathrm{C} @ 40{ }^{\circ} \mathrm{C}\end{array}$ & $\begin{array}{c}\text { Heat Capacity } \\
\mathrm{J} / \mathrm{g}{ }^{\circ} \mathrm{C} @ 80^{\circ} \mathrm{C}\end{array}$ \\
\hline Initial slurry material & $3.32 \pm 0.02$ & $3.39 \pm 0.06$ \\
88C - Batch 1 & $3.35 \pm 0.09$ & $3.43 \pm 0.10$ \\
$988^{\circ} \mathrm{C}$-Start of Caustic Leach-Batch 1 & $3.48 \pm 0.01$ & $3.56 \pm 0.07$ \\
\hline
\end{tabular}

\subsubsection{Particle Size}

Table 5.17, Slurry Particle Size Distribution, provides the PSD data at key process steps before and after sonication. A summary of the run conditions, sample preparation, and sample analysis is given in Appendix H. 
Table 5.17. Slurry Particle-Size Distribution Results

\begin{tabular}{|c|c|c|c|c|c|c|}
\hline \multirow[b]{2}{*}{ Process Step Description } & \multicolumn{3}{|c|}{ Pre-Sonication (microns) } & \multicolumn{3}{|c|}{ Sonicated (microns) } \\
\hline & $\mathrm{d}(0.100)$ & $\mathrm{d}(0.500)$ & $\mathrm{d}(0.900)$ & $\mathrm{d}(0.100)$ & $\mathrm{d}(0.500)$ & $\mathrm{d}(0.900)$ \\
\hline Initial simulant characterization from HLP-VSL-T22 & 1.52 & 6.43 & 20.07 & 1.55 & 6.33 & 18.21 \\
\hline Post-caustic-leach concentration in Tank T02A - leached slurry & 0.65 & 1.88 & 38.20 & 0.55 & 1.35 & 18.04 \\
\hline \multirow{2}{*}{ Post-caustic-leach water wash - unwashed slurry ${ }^{(a)}$} & 0.63 & 1.10 & 1.96 & 0.58 & 0.99 & 1.74 \\
\hline & 0.57 & 1.20 & 2.68 & 0.50 & 0.93 & 1.73 \\
\hline Post-oxidative-leach concentration - final slurry & 0.66 & 1.41 & 3.25 & 0.49 & 1.14 & 4.54 \\
\hline
\end{tabular}




\subsubsection{Rheology}

Table 5.18, Integrated Test A Rheology Data, provides a summary of rheology measurements performed at key points throughout Integrated Test A. For simplicity, only Bingham yield stress and consistency fits for the down ramps have been reported here. A more extensive analysis of the rheology measurements taken are provided in Appendix $\mathrm{H}$ with flow curve analysis using both the Bingham and Casson models for both the up and down ramps. Table 5.19, Integrated Test A Shear-Strength Data, provides addition shear-strength measurements. 
Table 5.18. Integrated Test A Rheology Data Summary

\begin{tabular}{|c|c|c|c|c|c|c|}
\hline Process Test Step & Form & Measurement & $\begin{array}{c}\text { Shear Strength } \\
{[\mathrm{Pa}]^{(\mathrm{b})}}\end{array}$ & $\begin{array}{c}\text { Bingham } \\
\text { Yield } \\
{[\mathrm{Pa}]^{(\mathrm{a})}}\end{array}$ & $\begin{array}{c}\text { Bingham } \\
\text { Consistency } \\
{[\mathrm{mPa} \cdot \mathrm{s}]^{(\mathrm{a})}}\end{array}$ & $\begin{array}{l}\text { Supernate } \\
\text { Viscosity } \\
{[\mathrm{mPa} \cdot \mathrm{s}]^{(\mathrm{a})}}\end{array}$ \\
\hline Prior to transferring simulant from HLP-VSL-T22 to Tank T01A & Slurry & Flow Curve & - & - & - & $4.244 \mathrm{E}-03$ \\
\hline Prior to transferring simulant from HLP-VSL-T22 to Tank T01A & Slurry & Flow Curve & - & - & - & 4.241E-03 \\
\hline Prior to transferring simulant from HLP-VSL-T22 to Tank T01A & Slurry & Flow Curve & - & - & - & 4.199E-03 \\
\hline Tracer test -1 minute after tracer injection & Slurry & Flow Curve & - & 2.685 & 15.33 & - \\
\hline Tracer test -1 minute after tracer injection (repeat) & Slurry & Flow Curve & - & 9.516 & 35.56 & - \\
\hline Post-caustic-leach wash—initial slurry & Supernate & Flow Curve & - & - & - & 4.144 \\
\hline Post-caustic-leach wash-initial slurry & Slurry & Flow Curve & - & 2.769 & 14.84 & - \\
\hline Post-caustic-leach wash-intermediate slurry & Supernate & Flow Curve & - & - & - & 1.244 \\
\hline Post-caustic-leach wash_-intermediate slurry & Slurry & Flow Curve & - & 2.227 & 6.204 & - \\
\hline Post-caustic-leach wash-final slurry & Supernate & Flow Curve & - & - & - & 0.9893 \\
\hline Post-caustic-leach wash-final slurry & Slurry & Flow Curve & - & 2.115 & 5.037 & - \\
\hline Oxidative leach — before permanganate addition & Slurry & Shear Strength & $\begin{array}{l}7.623 \\
12.36\end{array}$ & - & - & - \\
\hline Oxidative leach — before permanganate addition & Slurry & Flow Curve & - & 5.751 & 6.576 & - \\
\hline Oxidative leach—final slurry & Supernate & Flow Curve & - & - & - & 1.021 \\
\hline Post-oxidative leach wash-intermediate slurry & Slurry & Flow Curve & - & 2.232 & 5.135 & - \\
\hline Post-oxidative leach wash-intermediate slurry & Supernate & Flow Curve & - & - & - & 0.9838 \\
\hline Post-oxidative leach wash—final slurry & Supernate & Flow Curve & - & - & - & 0.9693 \\
\hline Post-oxidative leach wash—final slurry & Slurry & Flow Curve & - & 1.219 & 4.838 & - \\
\hline Post-oxidative leach concentration-final slurry & Slurry & Shear Strength & $\begin{array}{c}8.51 \\
9.239\end{array}$ & - & - & - \\
\hline Post-oxidative leach concentration-final slurry & Slurry & Flow Curve & - & 4.515 & 6.121 & - \\
\hline Post-oxidative leach concentration-final slurry & Supernate & Flow Curve & - & $0^{(\mathrm{c})}$ & 0.9442 & - \\
\hline Prepare for high solids test & Supernate & Flow Curve & - & - & - & 0.999 \\
\hline
\end{tabular}

- Analysis not required in Test Plan.

(a) All results derived from flow curve analysis correspond to the down-ramp.

(b) Shear-strength measurements correspond to a $72 \mathrm{hr}$ gel time.

(c) Newtonian slurry treated as a Bingham Plastic with a zero yield stress. 
Table 5.19. Integrated Test A Shear-Strength Data

\begin{tabular}{|c|c|c|c|c|}
\hline & $\begin{array}{c}\text { Oxidative Leach } \\
\text { - before } \\
\text { permanganate } \\
\text { addition }\end{array}$ & $\begin{array}{c}\text { Oxidative } \\
\text { Leach - before } \\
\text { permanganate } \\
\text { addition }\end{array}$ & $\begin{array}{c}\text { Post-- } \\
\text { Oxidative } \\
\text { Leach } \\
\text { Concentration } \\
\text {--final slurry }\end{array}$ & $\begin{array}{c}\text { Post- } \\
\text { Oxidative } \\
\text { Leach } \\
\text { Concentration } \\
\text { - final slurry }\end{array}$ \\
\hline $\begin{array}{l}\text { Settling } \\
\text { Time } \\
\text { (hr) }\end{array}$ & $\begin{array}{l}\text { Maximum Shear } \\
\text { Stress }(\mathrm{Pa})\end{array}$ & $\begin{array}{c}\text { Maximum } \\
\text { Shear Stress } \\
(\mathrm{Pa})\end{array}$ & $\begin{array}{c}\text { Maximum } \\
\text { Shear Stress } \\
(\mathrm{Pa})\end{array}$ & $\begin{array}{c}\text { Maximum } \\
\text { Shear Stress } \\
(\mathrm{Pa})\end{array}$ \\
\hline 1 & 5.747 & 4.988 & 3.902 & 4.072 \\
\hline 2 & 5.374 & 4.930 & 4.091 & 4.221 \\
\hline 4 & 5.143 & 5.165 & 4.348 & 4.067 \\
\hline 8 & 4.953 & 5.280 & 4.270 & 4.605 \\
\hline 16 & 6.077 & 5.891 & 5.836 & 4.643 \\
\hline 32 & 6.158 & 6.540 & 5.538 & 6.639 \\
\hline 72 & 7.623 & 12.360 & 8.510 & 9.239 \\
\hline
\end{tabular}

\subsubsection{XRD}

The results of the XRD analysis of the initial simulant characterization samples taken before the commencement of Integrated Test A, including the XRD pattern of the simulant used for Integrated Test A, are provided in Appendix H.

\subsubsection{SEM}

Appendix H contains the SEM micrograph along with information on the sample preparation, analysis, and further results.

\subsection{Measured PJM Parameters}

Table 5.20 summarizes the actual PJM peak average nozzle velocities and stroke lengths achieved at various portions of the test. Data files collected for the PJM peak average velocities and stroke lengths were collected at a frequency of $10 \mathrm{~Hz}$. Figures 5.1 through 5.18 show the PJM peak average velocity curves during selected Integrated Test A operations. 
Table 5.20. PJM Parameters for Integrated Test A (TI-WTP-PEP-065)

\begin{tabular}{|c|c|c|c|c|c|c|c|c|c|c|}
\hline $\begin{array}{c}\text { Data Set } \\
\text { Date:Time } \\
\text { (mm/dd/yr) } \\
\text { (hr;min) }\end{array}$ & $\begin{array}{l}\text { TI Step \# } \\
\text { (TI p\#) }\end{array}$ & Vessel & $\begin{array}{l}\text { PJM } \\
\text { Operation } \\
\text { Mode }\end{array}$ & $\begin{array}{l}\text { Process Step } \\
\text { Description }\end{array}$ & $\begin{array}{c}\text { Target PJM } \\
\text { Nozzle } \\
\text { Velocity }(\mathrm{m} / \mathrm{s})\end{array}$ & $\begin{array}{l}\text { Actual PJM } \\
\text { Nozzle } \\
\text { Velocity } \\
(\mathrm{m} / \mathrm{s})\end{array}$ & $\begin{array}{l}\text { Target Stroke } \\
\text { Length } \\
\text { (in.) } \\
(\%)\end{array}$ & $\begin{array}{l}\text { Actual Stroke } \\
\text { Length } \\
\text { (in.) } \\
(\%)\end{array}$ & $\begin{array}{l}\text { Target PJM } \\
\text { Cycle Time } \\
(\mathrm{sec})\end{array}$ & $\begin{array}{l}\text { Actual PJM } \\
\text { Cycle Time } \\
(\mathrm{sec})\end{array}$ \\
\hline $\begin{array}{c}1 / 31 / 09 \\
12: 52-13: 13\end{array}$ & 7.1.2.5.1 (p67) & Tank T01A & Standard & $\begin{array}{c}\text { Start of caustic leach for } \\
\text { batch } 1\end{array}$ & $4.8 \pm 0.3$ & 5.3 & $\begin{array}{c}28.1 \pm 1.7 \\
80 \pm 5 \%\end{array}$ & $\begin{array}{l}29.8 \\
85 \%\end{array}$ & $35 \pm 1$ & 35.3 \\
\hline $\begin{array}{c}1 / 31 / 09 \\
20: 26-20: 55\end{array}$ & 7.1.3.14.2(p74) & Tank T01A & Simple & $\begin{array}{c}\text { Caustic leach } \\
@ 98^{\circ} \mathrm{C} \text { for batch } 1\end{array}$ & $4.8 \pm 0.3$ & 5.3 & $\begin{array}{l}28.1 \pm 1.7 \\
80 \pm 5 \%\end{array}$ & $\begin{array}{l}26.6 \\
76 \%\end{array}$ & $35 \pm 1$ & 35.3 \\
\hline $\begin{array}{l}2 / 1 / 09 \\
02: 56\end{array}$ & PJM tuning & Tank T01A & Simple & $\begin{array}{c}\text { Caustic leach } \\
\text { @ } 98^{\circ} \mathrm{C} \text { for batch } 1\end{array}$ & $4.8 \pm 0.3$ & 4.7 & $\begin{array}{l}28.1 \pm 1.7 \\
80 \pm 5 \%\end{array}$ & $\begin{array}{l}26.4 \\
75 \%\end{array}$ & $35 \pm 1$ & 35.3 \\
\hline $\begin{array}{l}2 / 1 / 09-2 / 2 / 09 \\
22: 01-01: 36\end{array}$ & 7.1.4.7.1 (p85) & Tank T02A & Standard & $\begin{array}{l}\text { Dewatering for } \\
\text { batch } 1\end{array}$ & $7.3 \pm 0.4$ & 7.6 & $\begin{array}{c}30.3 \pm 1.8 \\
80 \pm 5 \%\end{array}$ & $28.876 \%$ & $33 \pm 1$ & 33.2 \\
\hline $\begin{array}{c}1 / 31 / 09 \\
18: 20-19: 11\end{array}$ & 7.1.5.8.1 (p90) & Tank T01B & Standard & $\begin{array}{l}\text { Start of caustic leach for } \\
\text { batch } 2\end{array}$ & $4.8 \pm 0.3$ & 5.2 & $\begin{array}{l}28.1 \pm 1.7 \\
80 \pm 5 \%\end{array}$ & $\begin{array}{l}28.6 \\
82 \%\end{array}$ & $35 \pm 1$ & 35.1 \\
\hline $\begin{array}{c}\text { 2/1/09 } \\
\sim 04: 02\end{array}$ & 7.1.6.15.2 (p97) & Tank T01B & Simple & $\begin{array}{c}\text { Caustic leach } \\
@ 98^{\circ} \mathrm{C} \text { for batch } 2\end{array}$ & $4.8 \pm 0.3$ & 5.4 & $\begin{array}{l}28.1 \pm 1.7 \\
80 \pm 5 \%\end{array}$ & $\begin{array}{l}21.2 \\
60 \%\end{array}$ & $35 \pm 1$ & 35.1 \\
\hline $\begin{array}{c}2 / 1 / 09 \\
07: 51\end{array}$ & PJM tuning & Tank T01B & Simple & $\begin{array}{c}\text { Caustic leach } \\
\text { @ } 98^{\circ} \mathrm{C} \text { for batch } 2\end{array}$ & $4.8 \pm 0.3$ & 4.6 & $\begin{array}{l}28.1 \pm 1.7 \\
80 \pm 5 \%\end{array}$ & $\begin{array}{l}29.3 \\
84 \%\end{array}$ & $35 \pm 1$ & 35.1 \\
\hline $\begin{array}{c}2 / 2 / 09 \\
21: 13-21: 37\end{array}$ & 7.1.7.7.1 (p107) & Tank T01A & Standard & $\begin{array}{l}\text { Start of caustic leach for } \\
\text { batch } 3\end{array}$ & $4.8 \pm 0.3$ & 4.6 & $\begin{array}{c}28.1 \pm 1.7 \\
80 \pm 5 \%\end{array}$ & $\begin{array}{l}25.4 \\
72 \%\end{array}$ & $35 \pm 1$ & 35.3 \\
\hline $\begin{array}{c}2 / 3 / 09 \\
02: 54\end{array}$ & PJM tuning & Tank T01A & Simple & $\begin{array}{c}\text { Caustic leach } \\
@ 98^{\circ} \mathrm{C} \text { for batch } 3\end{array}$ & $4.8 \pm 0.3$ & 4.7 & $\begin{array}{l}28.1 \pm 1.7 \\
80 \pm 5 \%\end{array}$ & $\begin{array}{l}25.6 \\
73 \%\end{array}$ & $35 \pm 1$ & 35.3 \\
\hline $\begin{array}{c}2 / 3 / 09 \\
\sim 04: 40\end{array}$ & 7.1.7.23.2 (p115) & Tank T01A & Simple & $\begin{array}{c}\text { Caustic leach } \\
@ 98^{\circ} \mathrm{C} \text { for batch } 3\end{array}$ & $4.8 \pm 0.3$ & 4.5 & $\begin{array}{l}28.1 \pm 1.7 \\
80 \pm 5 \%\end{array}$ & $\begin{array}{l}26.7 \\
76 \%\end{array}$ & $35 \pm 1$ & 35.3 \\
\hline $\begin{array}{l}2 / 4 / 09 \\
\sim 16: 06\end{array}$ & 7.1.8.13.1 (p126) & Tank T01B & Standard & $\begin{array}{c}\text { Start of caustic leach for } \\
\text { batch } 4\end{array}$ & $4.8 \pm 0.3$ & 5.2 & $\begin{array}{c}28.1 \pm 1.7 \\
80 \pm 5 \%\end{array}$ & $\begin{array}{c}28 \\
80 \%\end{array}$ & $35 \pm 1$ & 35.1 \\
\hline $\begin{array}{c}2 / 5 / 09 \\
01: 43-02: 00\end{array}$ & 7.1.8.28.2 (p134) & Tank T01B & Simple & $\begin{array}{c}\text { Caustic leach } \\
@ 98^{\circ} \mathrm{C} \text { for batch } 4\end{array}$ & $4.8 \pm 0.3$ & 4.6 & $\begin{array}{l}28.1 \pm 1.7 \\
80 \pm 5 \%\end{array}$ & $\begin{array}{l}28.2 \\
80 \%\end{array}$ & $35 \pm 1$ & 35.1 \\
\hline $\begin{array}{c}2 / 7 / 09 \\
12: 02-12: 25\end{array}$ & 7.1.9.7.1 (p146) & Tank T01A & Standard & $\begin{array}{l}\text { Start of caustic leach for } \\
\text { batch } 5\end{array}$ & $4.8 \pm 0.3$ & 5.1 & $\begin{array}{c}28.1 \pm 1.7 \\
80 \pm 5 \%\end{array}$ & $\begin{array}{l}30.7 \\
87 \%\end{array}$ & $35 \pm 1$ & 35.3 \\
\hline $\begin{array}{c}2 / 7 / 09 \\
18: 49-19: 41\end{array}$ & 7.1.9.22.2(p154) & Tank T01A & Simple & $\begin{array}{c}\text { Caustic leach } \\
\text { @ } 98^{\circ} \mathrm{C} \text { for batch } 5\end{array}$ & $4.8 \pm 0.3$ & 4.8 & $\begin{array}{c}28.1 \pm 1.7 \\
80 \pm 5 \%\end{array}$ & $\begin{array}{l}27.1 \\
77 \%\end{array}$ & $35 \pm 1$ & 35.3 \\
\hline $\begin{array}{c}2 / 10 / 09 \\
06: 16-06: 44\end{array}$ & $\begin{array}{l}\text { 7.1.10.11.1 } \\
(\mathrm{p} 166)\end{array}$ & Tank T01B & Standard & $\begin{array}{l}\text { Start of caustic leach for } \\
\text { batch } 6\end{array}$ & $4.8 \pm 0.3$ & 4.6 & $\begin{array}{c}28.1 \pm 1.7 \\
80 \pm 5 \%\end{array}$ & $\begin{array}{l}28.2 \\
80 \%\end{array}$ & $35 \pm 1$ & 35.1 \\
\hline $\begin{array}{c}2 / 10 / 09 \\
13: 59-17: 15\end{array}$ & $\begin{array}{l}7.1 .10 .29 .2 \\
(\mathrm{p} 174-175)\end{array}$ & Tank T01B & Simple & $\begin{array}{c}\text { Caustic leach } \\
@ 98^{\circ} \mathrm{C} \text { for batch } 6\end{array}$ & $4.8 \pm 0.3$ & 4.0 & $\begin{array}{l}28.1 \pm 1.7 \\
80 \pm 5 \%\end{array}$ & $\begin{array}{l}21.7 \\
62 \%\end{array}$ & $35 \pm 1$ & 35.1 \\
\hline $\begin{array}{c}2 / 14 / 09 \\
19: 19\end{array}$ & PJM tuning & Tank T02A & Simple & $\begin{array}{l}\text { End of dewatering/ } \\
\text { start of tracer test }\end{array}$ & $12 \pm 0.6$ & 12.1 & $\begin{array}{c}30.3 \pm 1.8 \\
80 \pm 5 \%\end{array}$ & $\begin{array}{l}31.3 \\
83 \%\end{array}$ & $20 \pm 1$ & 20.8 \\
\hline $\begin{array}{c}2 / 14 / 09 \\
01: 45-19: 54\end{array}$ & $\begin{array}{l}\text { 7.1.12.1.3.1 } \\
\quad(\mathrm{p} 190)\end{array}$ & Tank T02A & Simple & $\begin{array}{l}\text { End of dewatering/ } \\
\text { start of tracer test }\end{array}$ & $12 \pm 0.6$ & 12 & $\begin{array}{c}30.3 \pm 1.8 \\
80 \pm 5 \%\end{array}$ & $\begin{array}{l}32.1 \\
85 \%\end{array}$ & $20 \pm 1$ & 20.7 \\
\hline
\end{tabular}


T01A at 13:05 on 1/ 31/ 09: Peak Average $=5.3(\mathrm{~m} / \mathrm{s})$

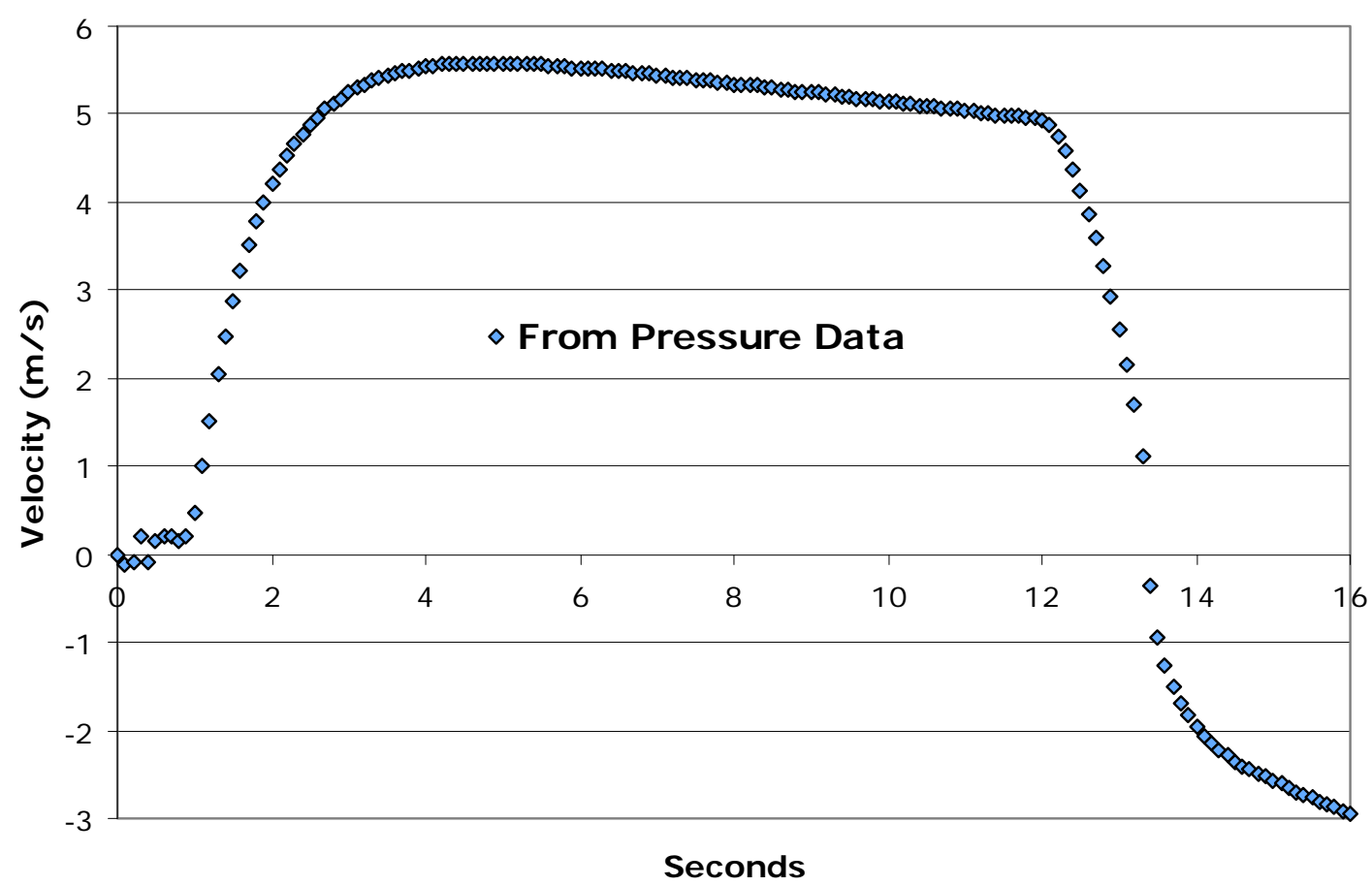

Figure 5.1. Tank T01A PJM Peak Average Velocity After Batch 1 Caustic Addition T01A at 20:48 on 1/31/ 09: Peak Average $=5.3(\mathrm{~m} / \mathrm{s})$

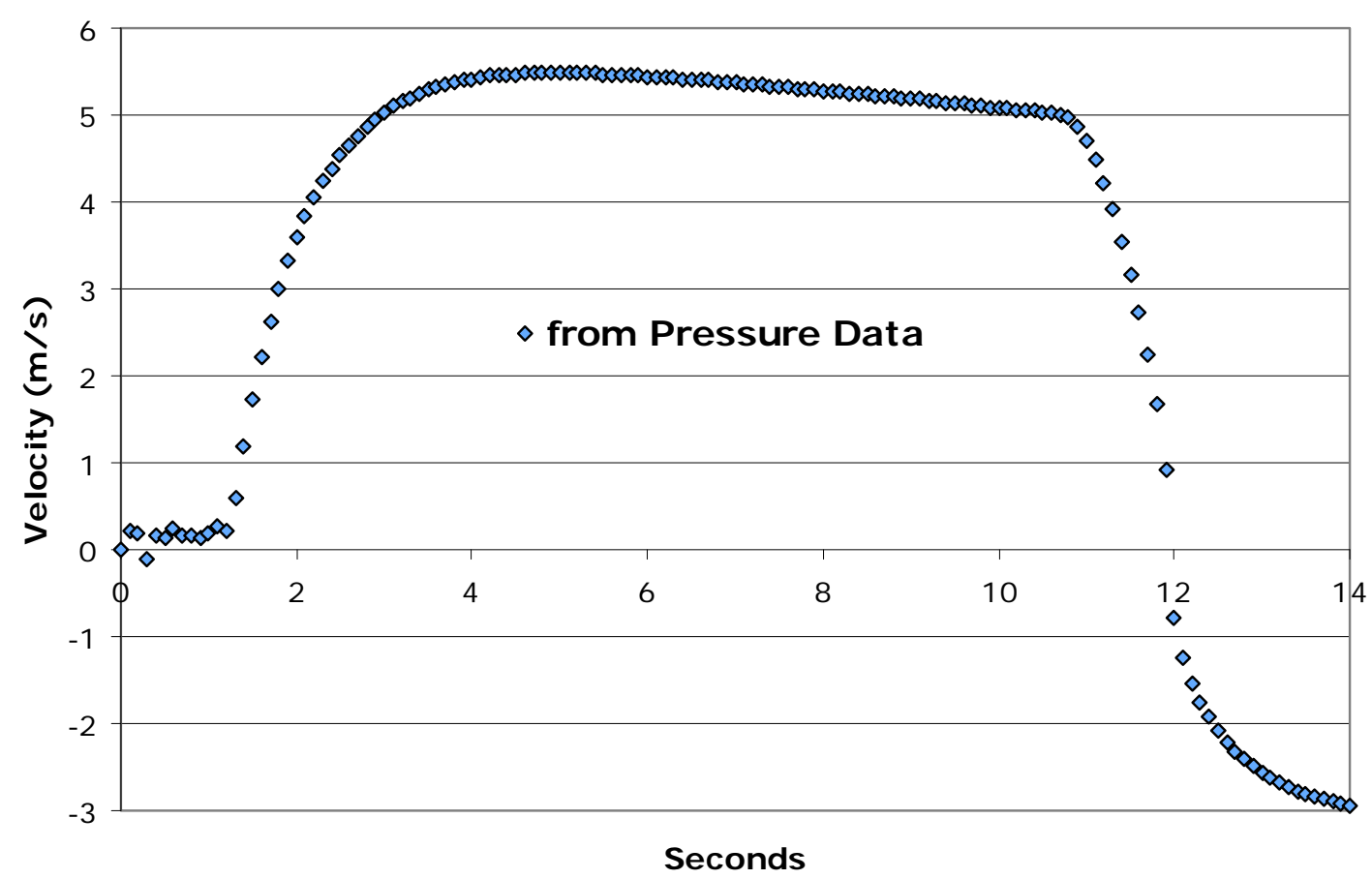

Figure 5.2. Tank T01A PJM Peak Average Velocity at the start of Batch 1 Caustic Leaching 
T01A at 02:56AM on 2/ 1/ 09: Peak Average $=4.7(\mathrm{~m} / \mathrm{s})$

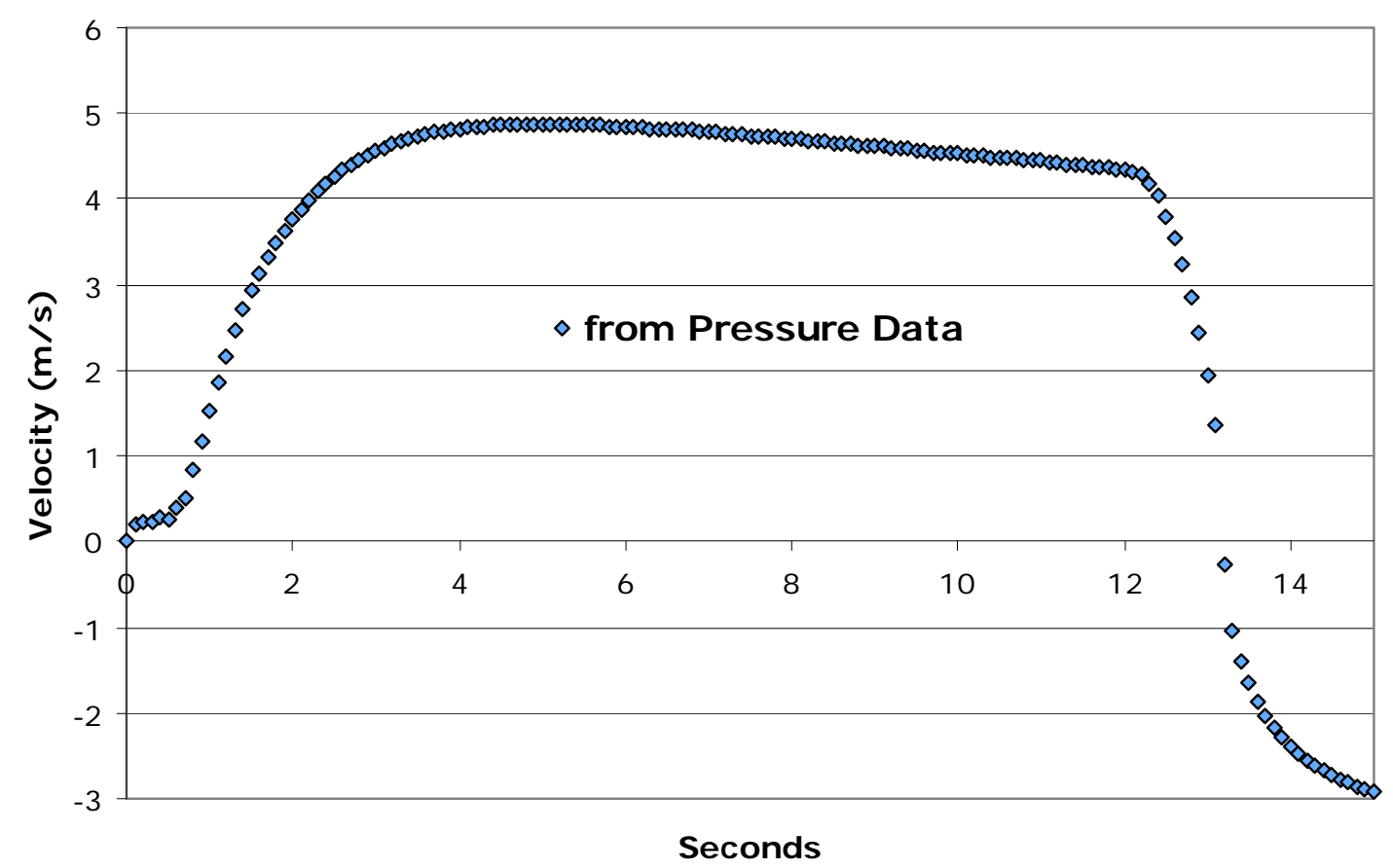

Figure 5.3. Tank T01A PJM Peak Average Velocity During Batch 1 Caustic Leaching

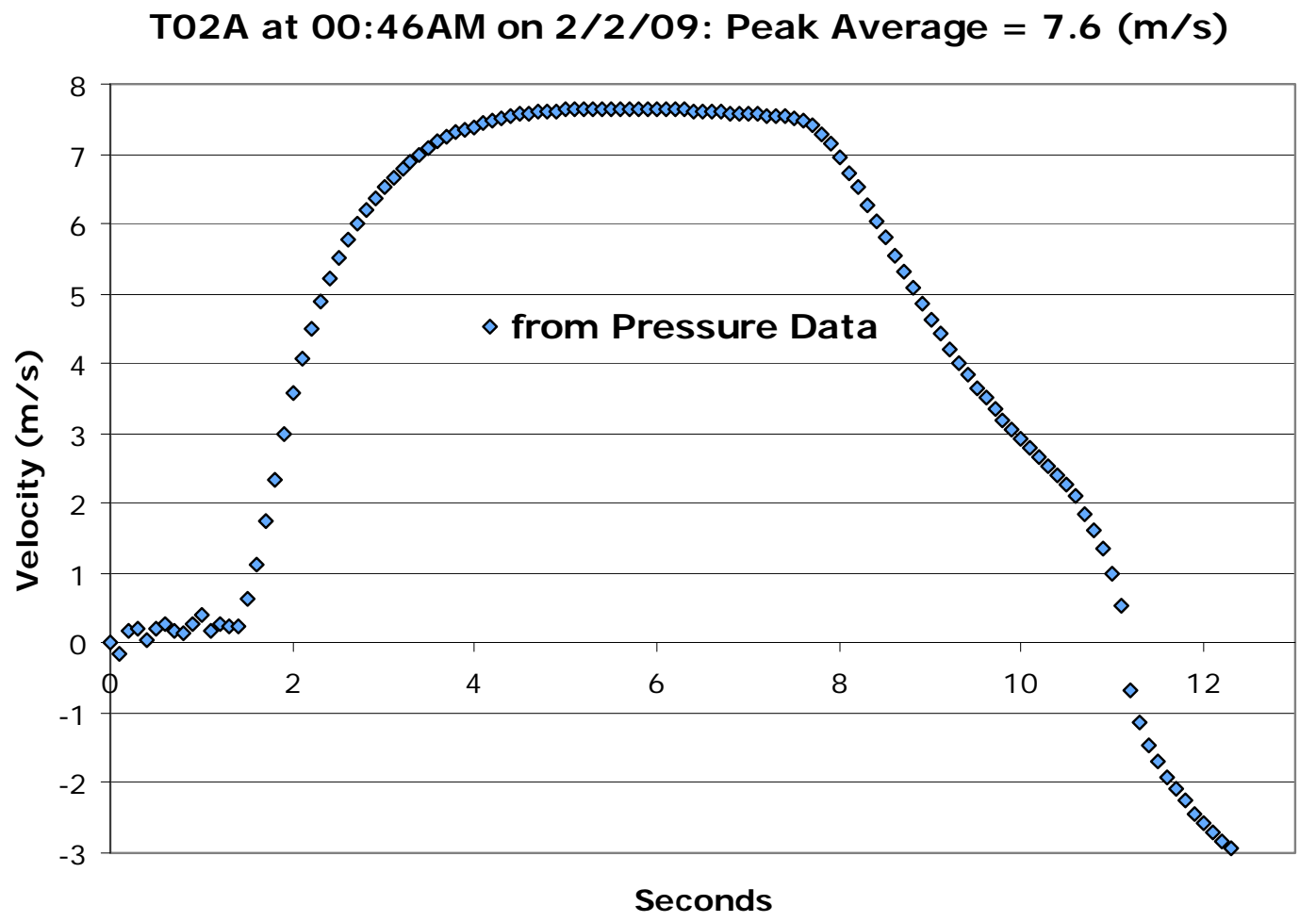

Figure 5.4. Tank T02A Peak Average Velocity After Initial Transfer from UFP-VSL-T01A 
T01B at 19:03 on 1/31/ 09: Peak Average $=5.2(\mathrm{~m} / \mathrm{s})$

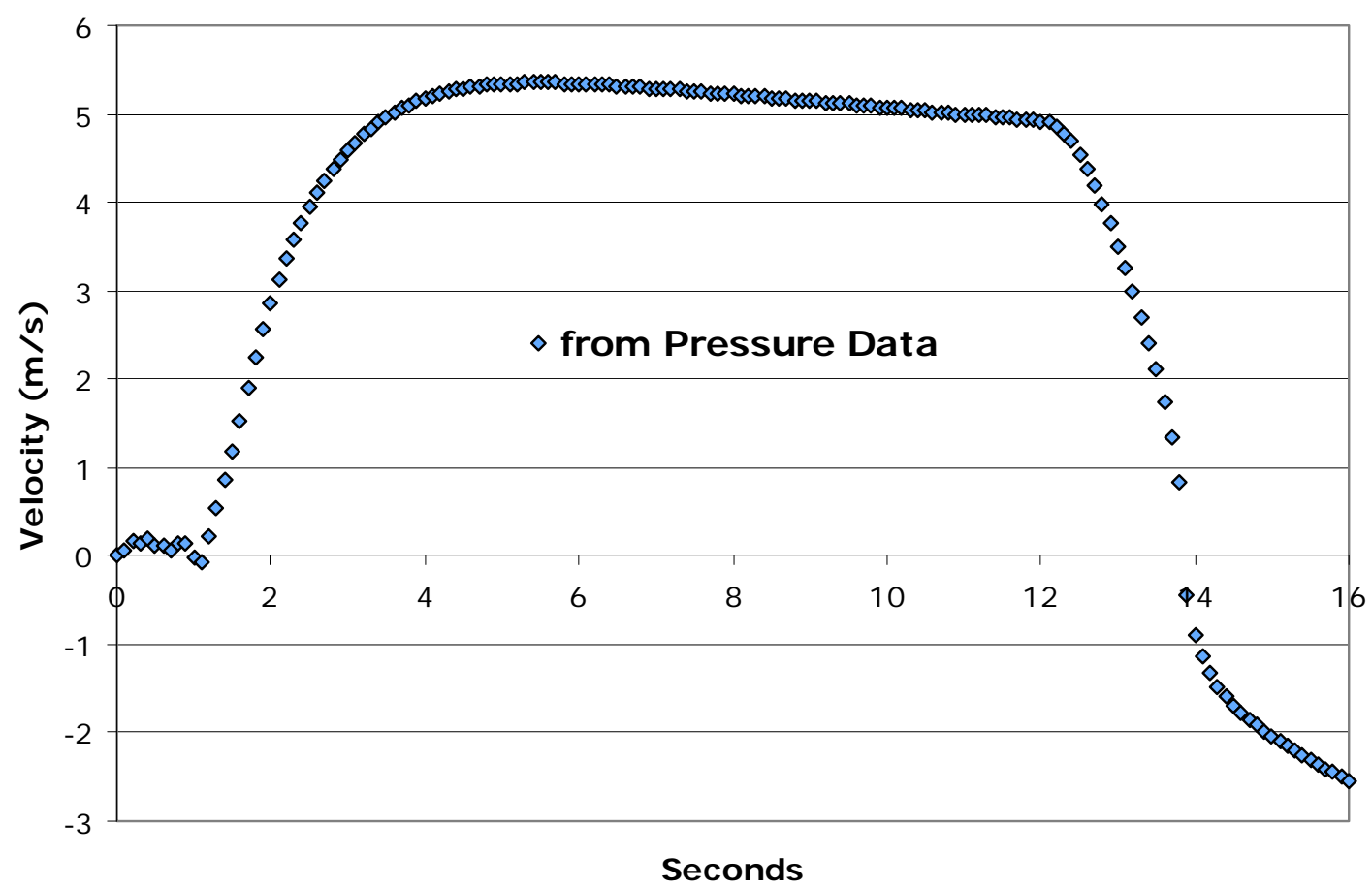

Figure 5.5. Tank T01B PJM Peak Average Velocity After Batch 2 Caustic Addition

T01B at 04:05AM on 2/ 1/ 09: Peak Average $=5.4(\mathrm{~m} / \mathrm{s})$

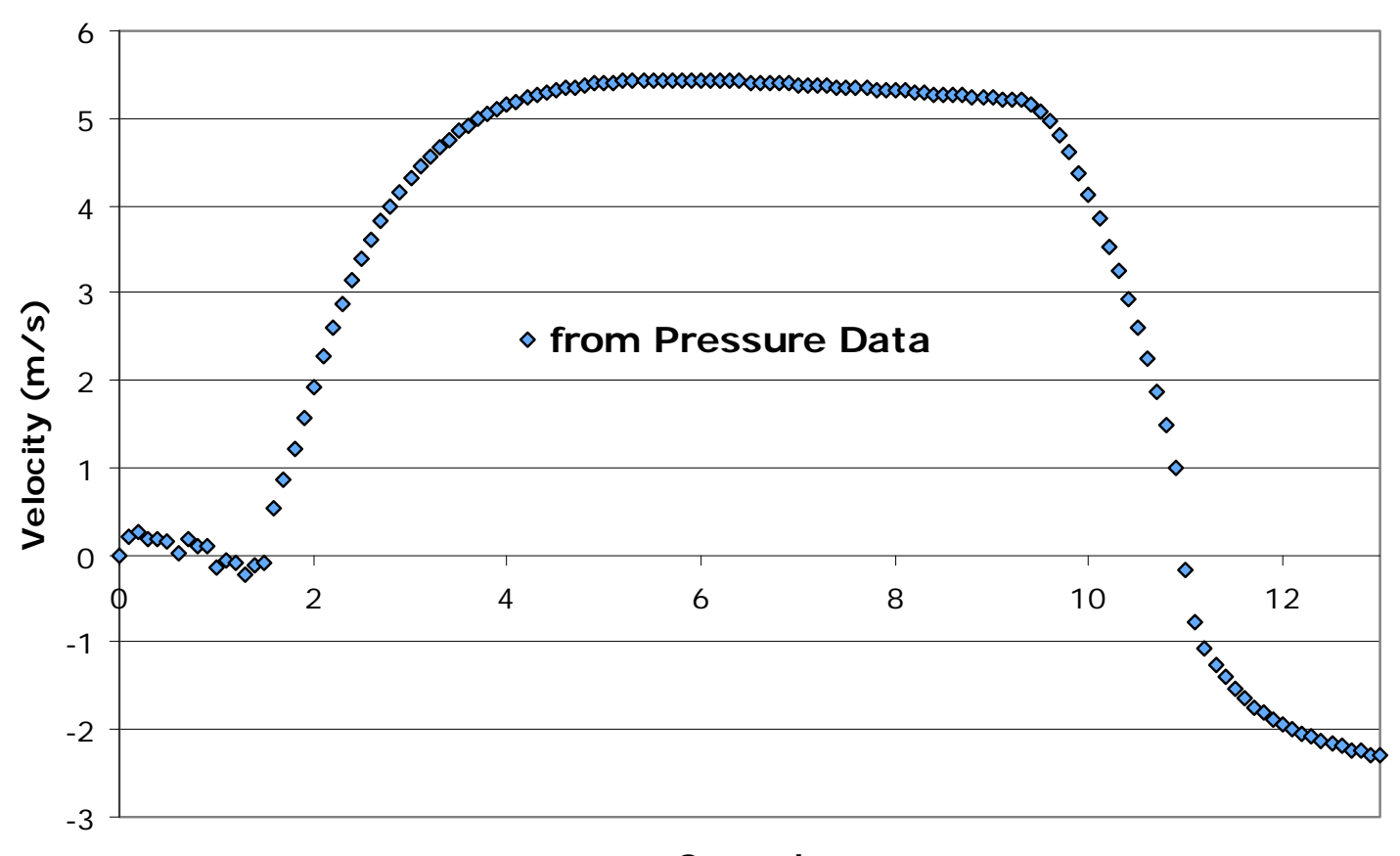

Seconds

Figure 5.6. Tank T01B PJM Peak Average Velocity at the start of Batch 2 Caustic Leach 


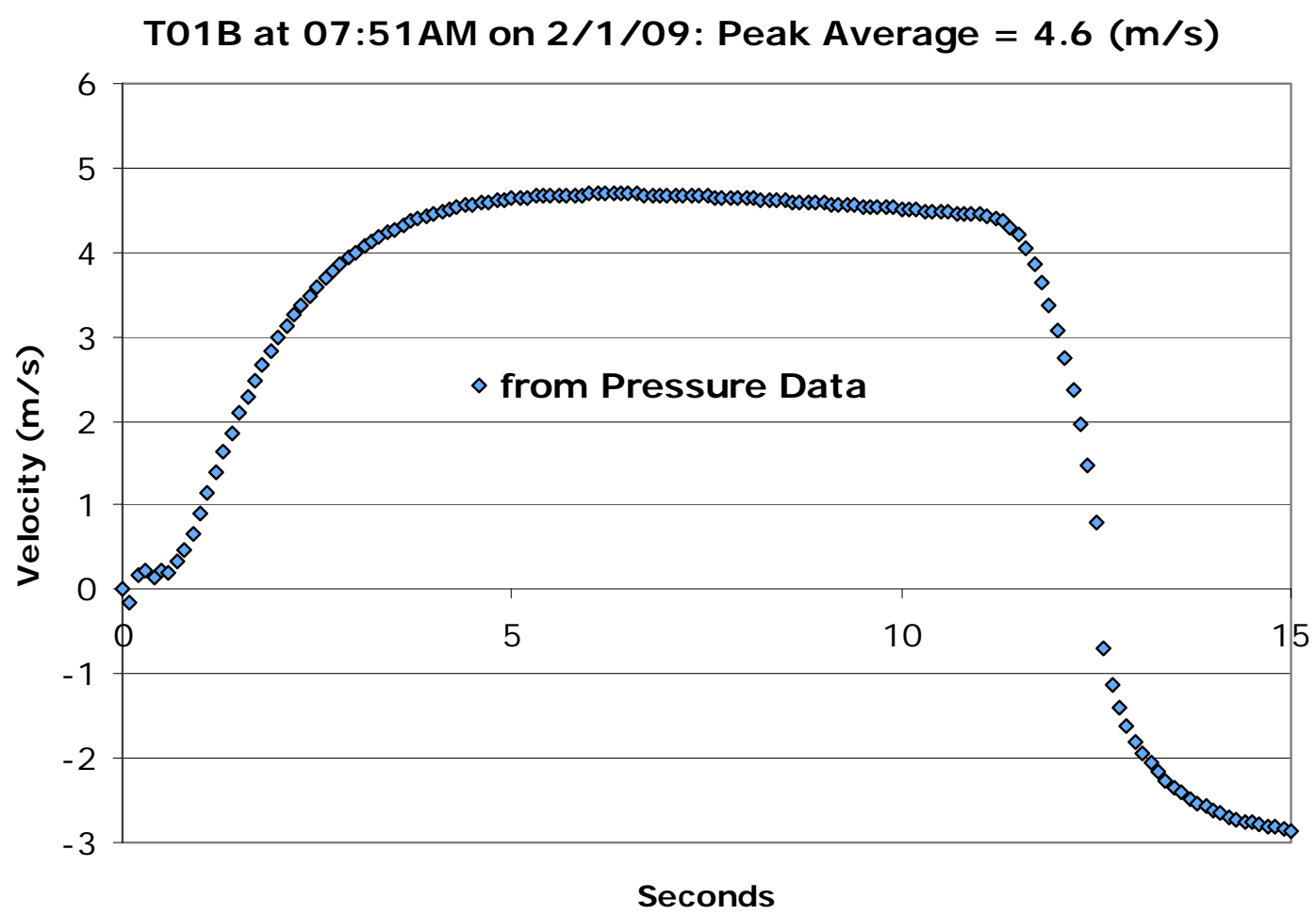

Figure 5.7. Tank T01B PJM Peak Average Velocity During Batch 2 Caustic Leach

T01A at 21:30 on 2/ 2/ 09: Peak Average $=4.6(\mathrm{~m} / \mathrm{s})$

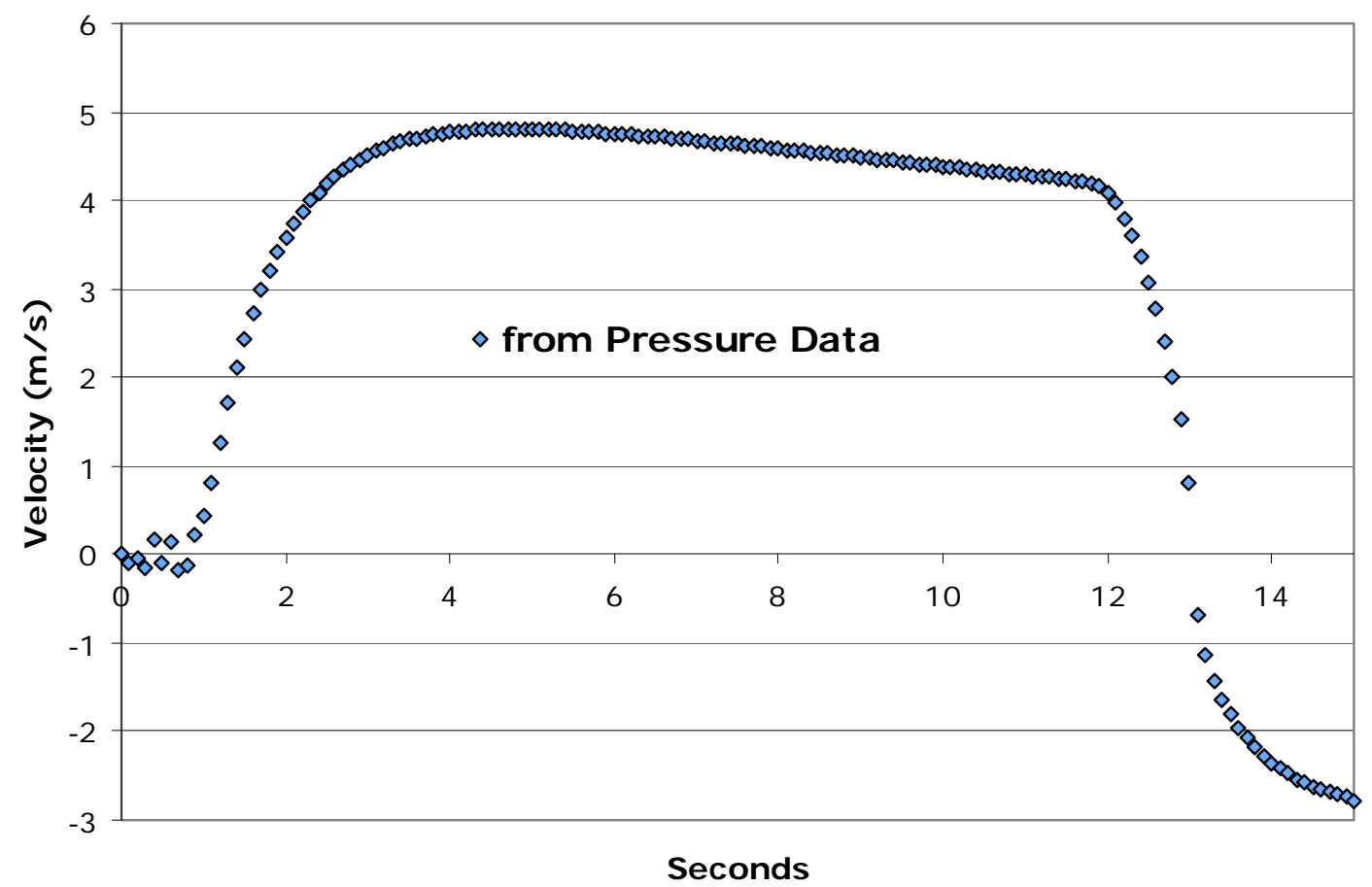

Figure 5.8. Tank T01A PJM Peak Average Velocity After Batch 3 Caustic Addition 


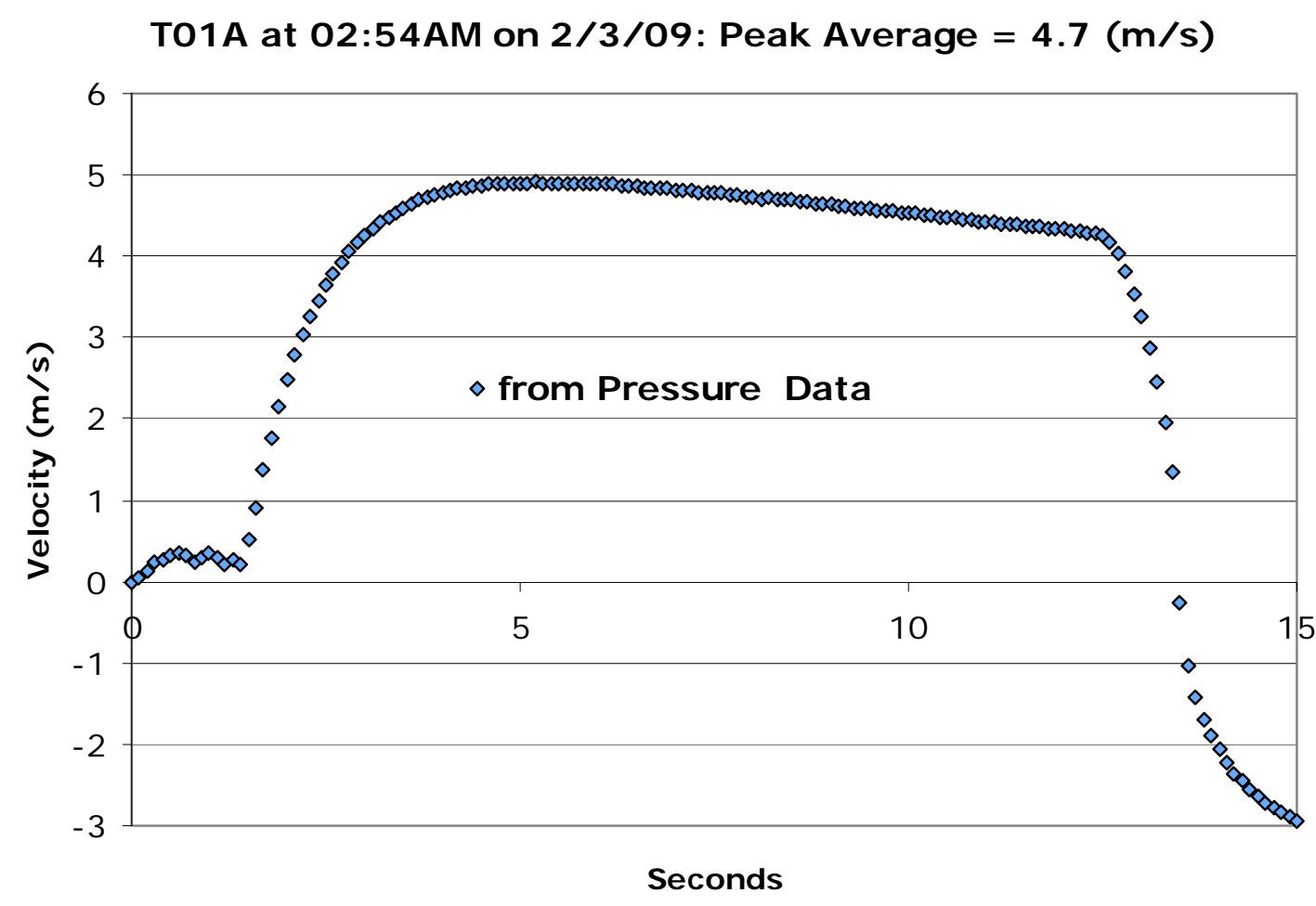

Figure 5.9. Tank T01A PJM Peak Average Velocity at the Start of Batch 3 Caustic Leach

T01A at 04:26AM on 2/ 3/ 09: Peak Average $=4.5(\mathrm{~m} / \mathrm{s})$

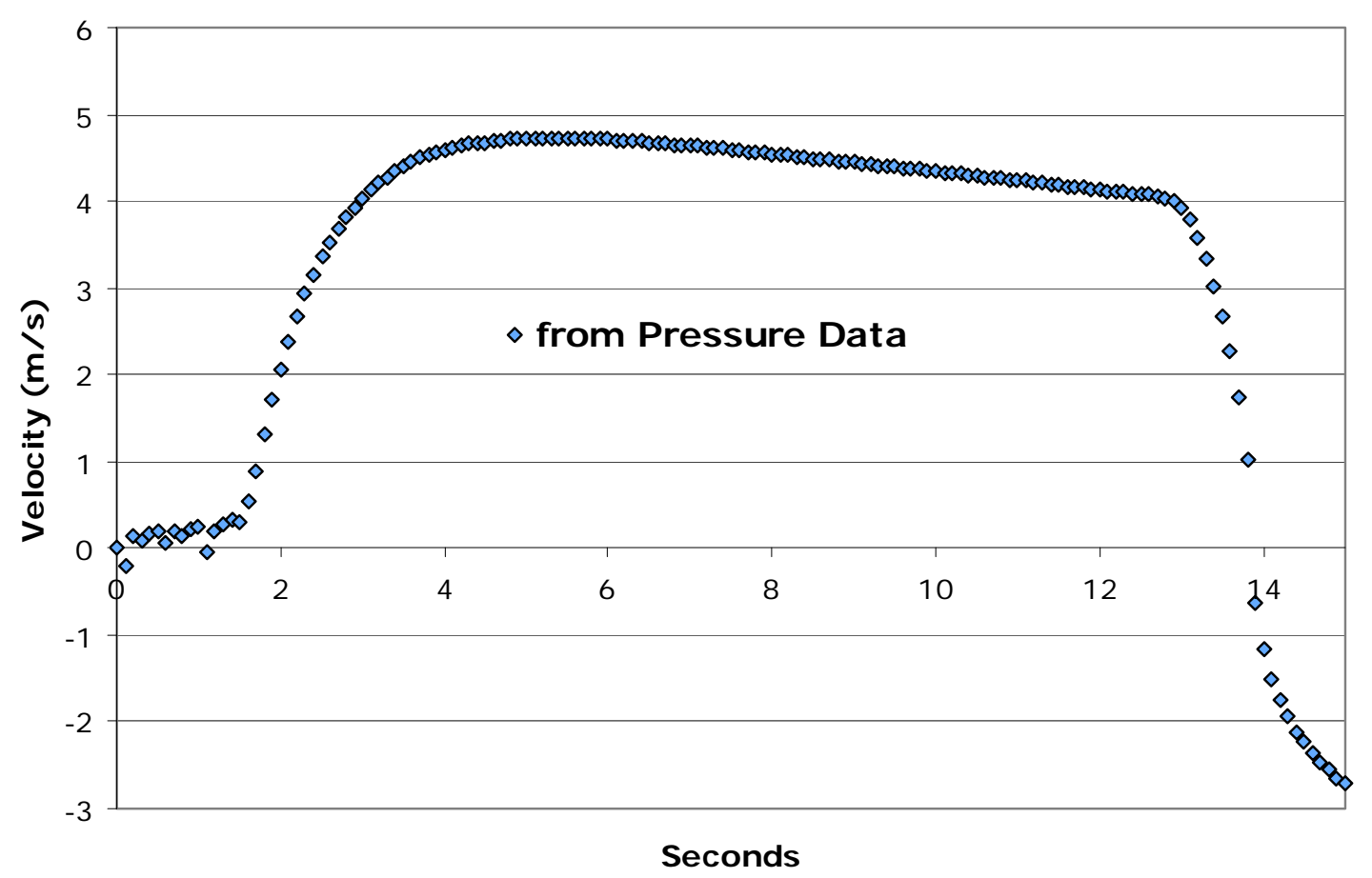

Figure 5.10. Tank T01A PJM Peak Average Velocity During Batch 3 Caustic Leach 
T01B at 18:30 on 2/ 4/ 09: Peak Average $=5.2(\mathrm{~m} / \mathrm{s})$

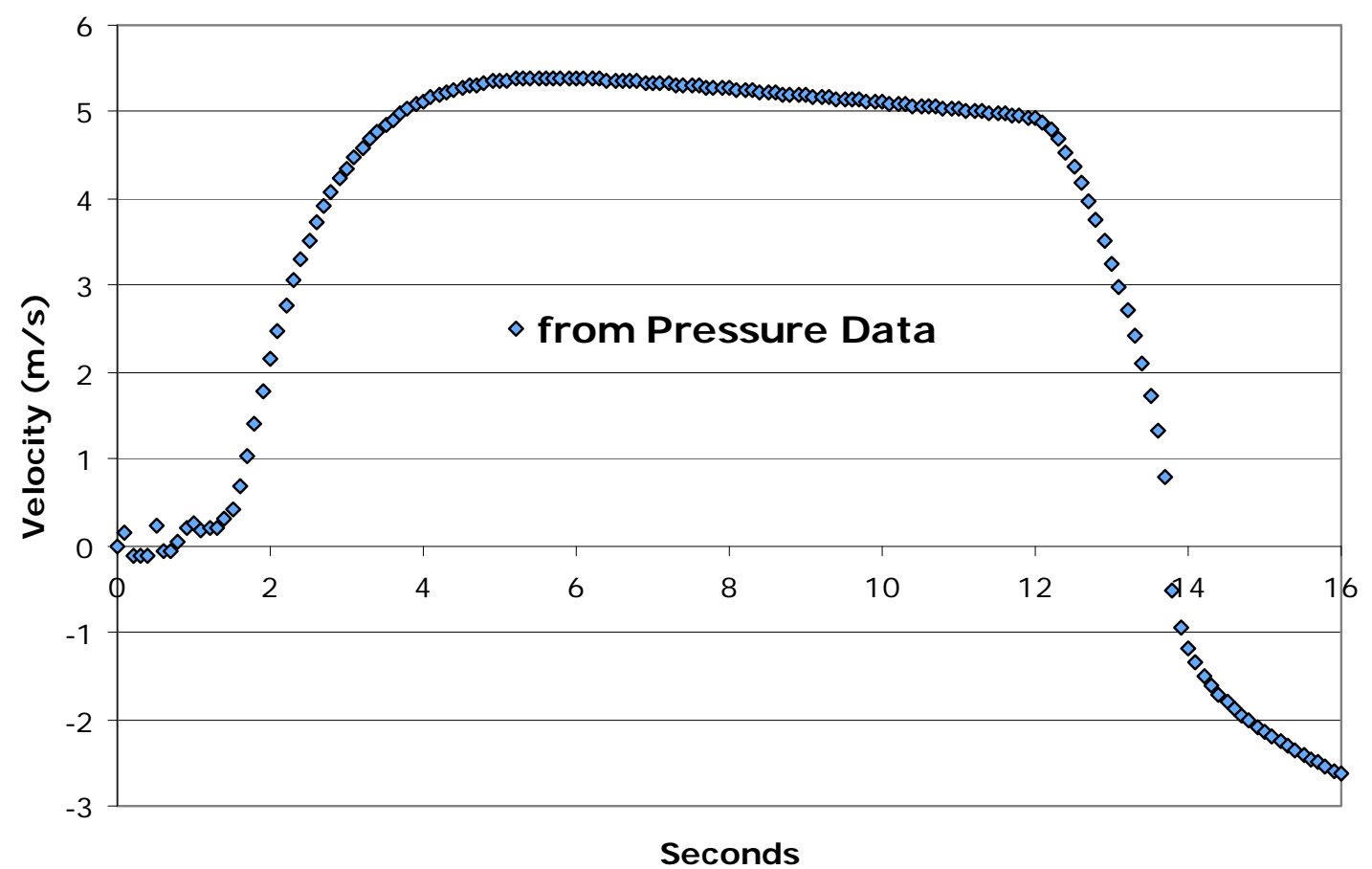

Figure 5.11. Tank T01B Peak Average Velocity Towards the End of Batch 4 Initial Heatup

T01B at 01:53AM on 2/ 5/ 09: Peak Average $=4.6(\mathrm{~m} / \mathrm{s})$

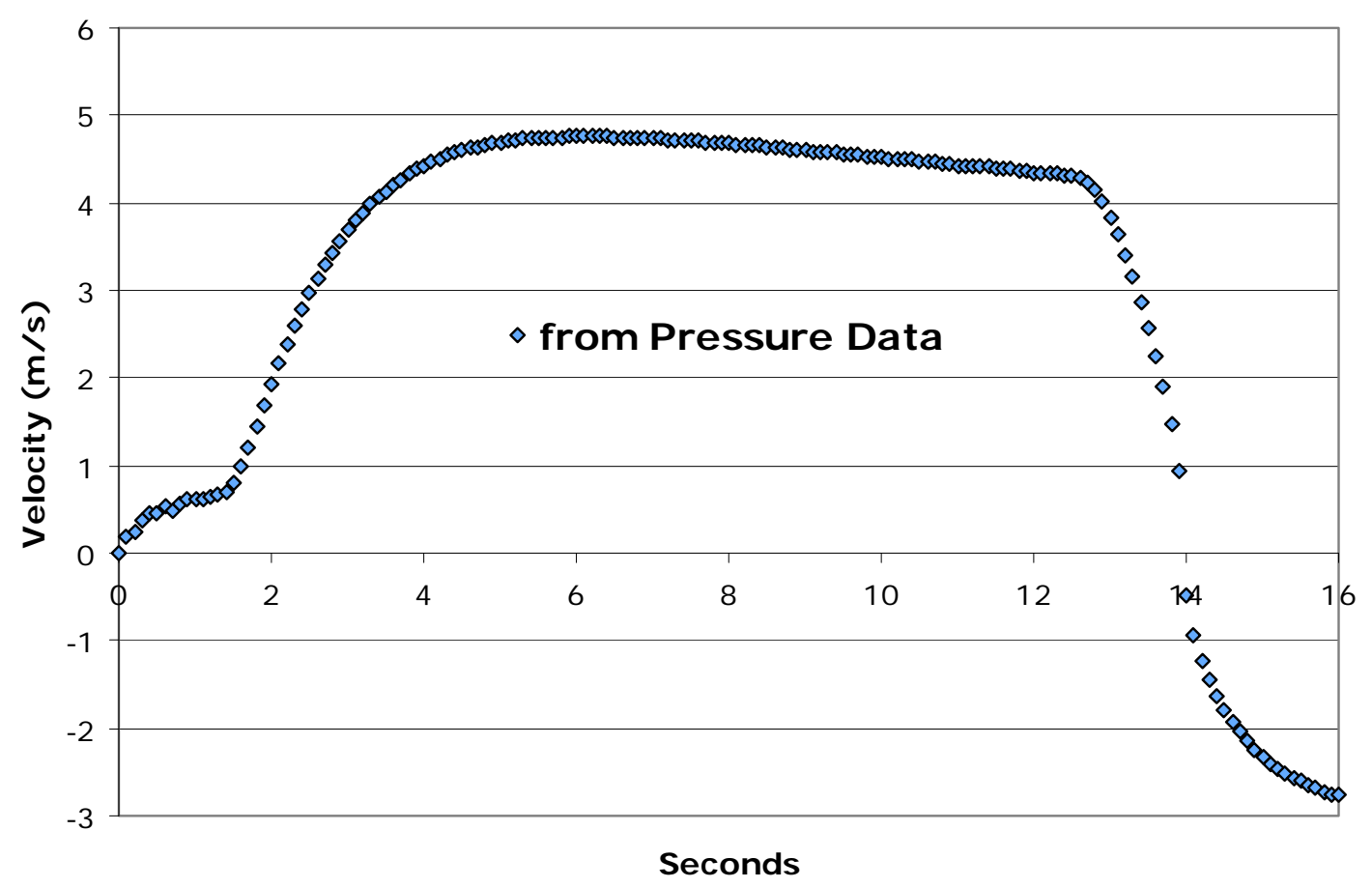

Figure 5.12. Tank T01B PJM Peak Average Velocity During Batch 4 Caustic Leach 


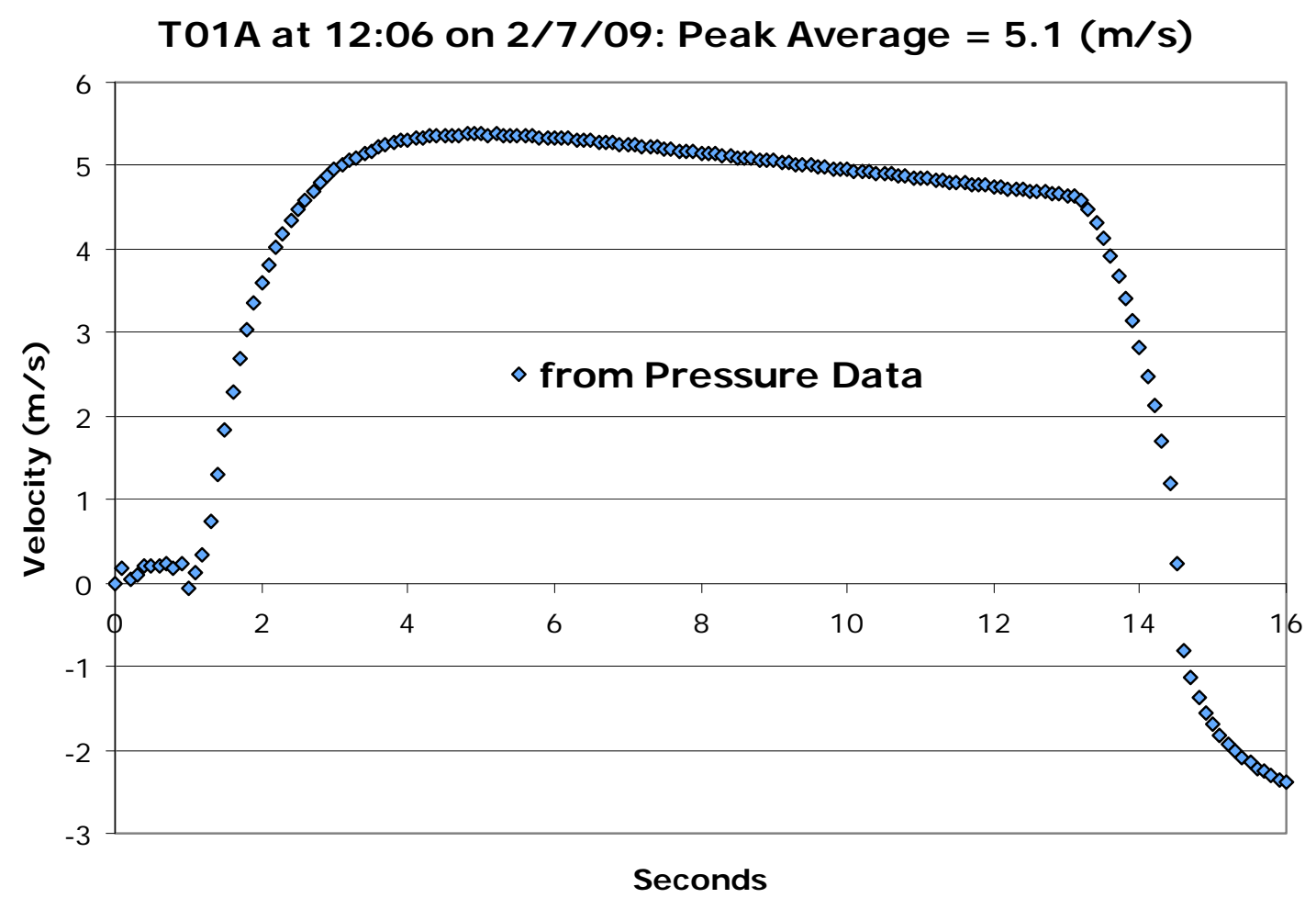

Figure 5.13. Tank T01A PJM Peak Average Velocity after Batch 5 Caustic Addition

T01A at 19:27 on 2/ 7/ 09: Peak Average $=4.8(\mathrm{~m} / \mathrm{s})$

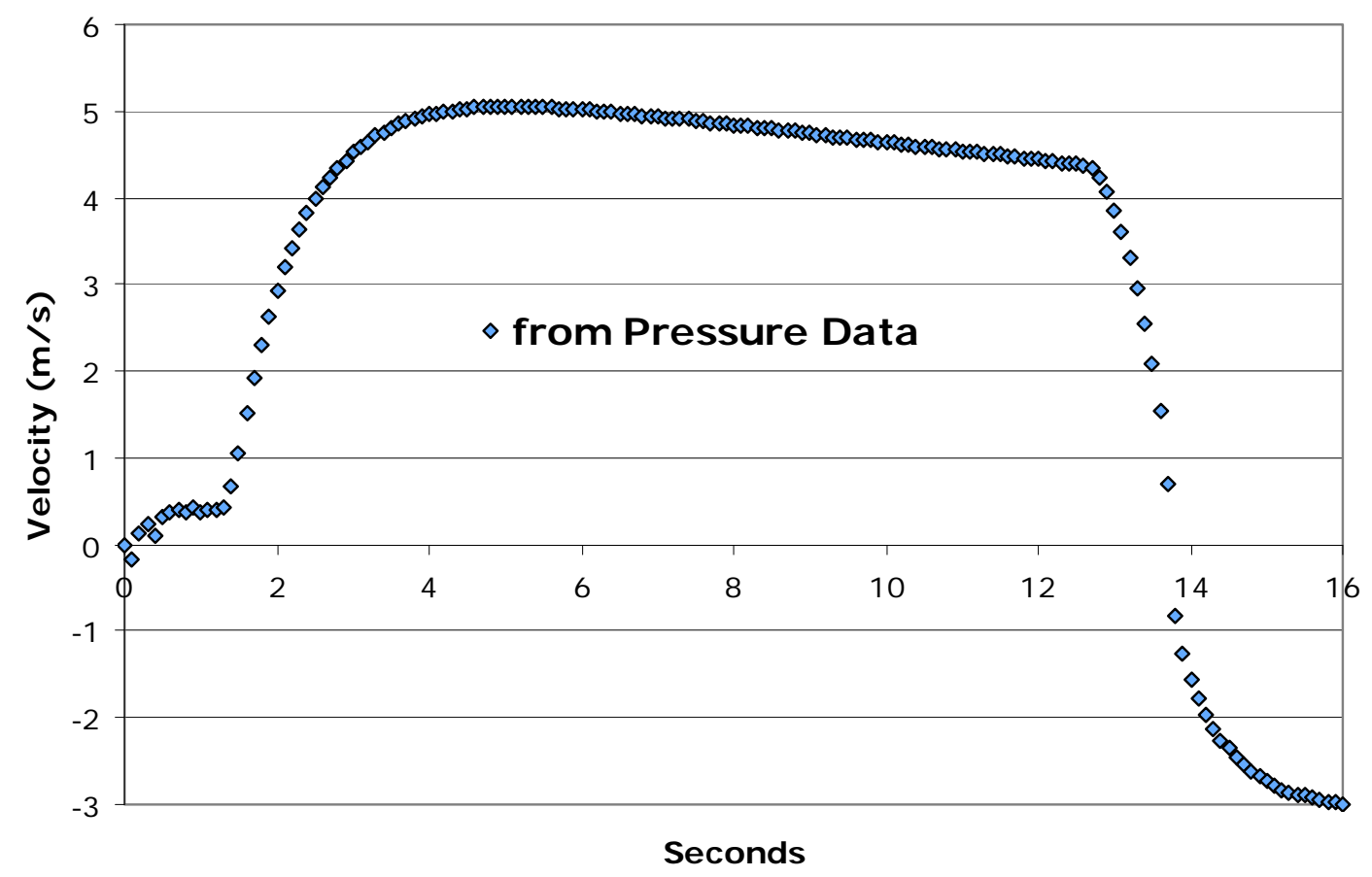

Figure 5.14. Tank T01A PJM Peak Average Velocity During Batch 5 Caustic Leaching 
T01B at 6:36AM on 2/ 10/ 09:Peak Average $=4.6(\mathrm{~m} / \mathrm{s})$

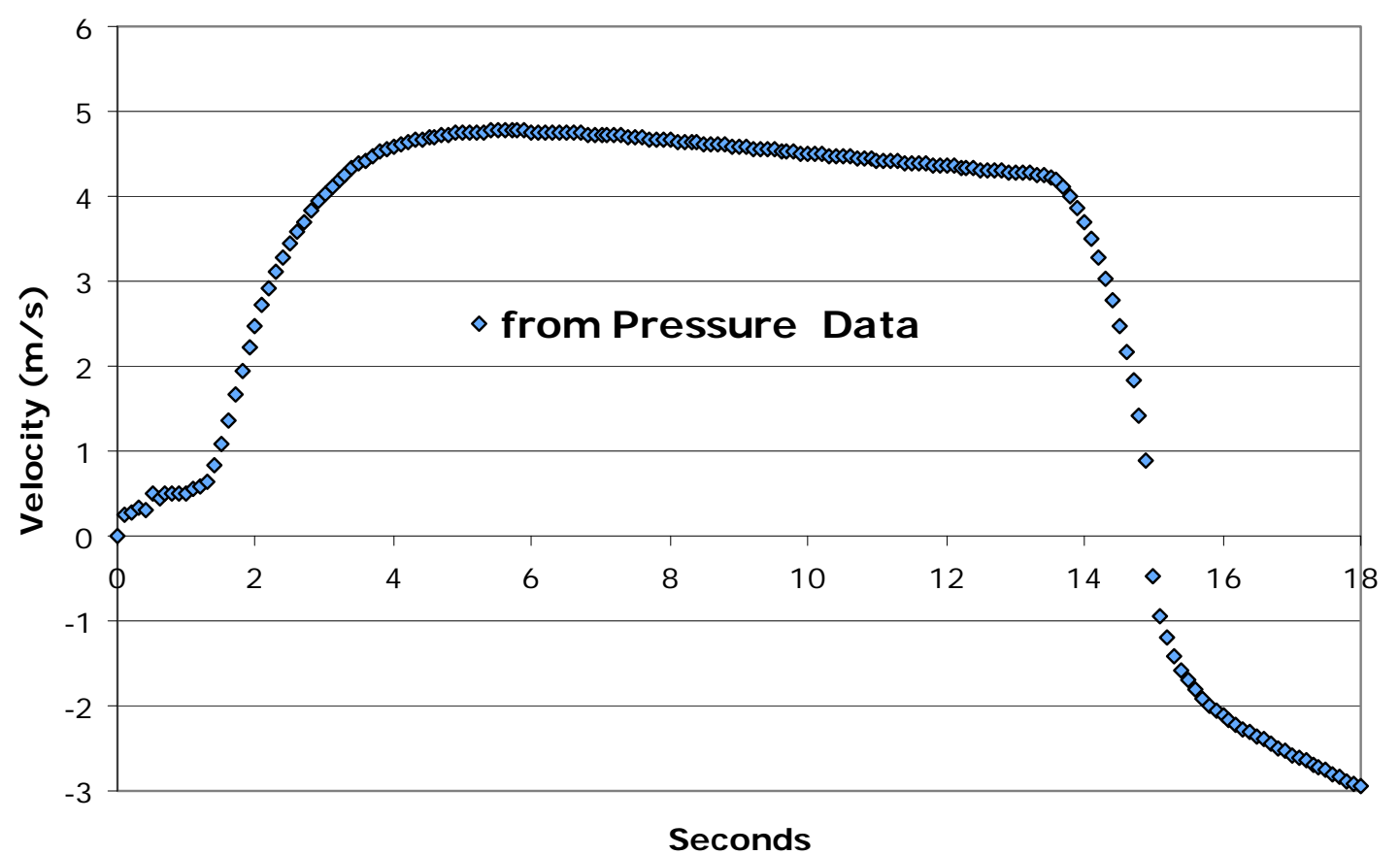

Figure 5.15. Tank T01B PJM Peak Average Velocity After Batch 6 Caustic Addition

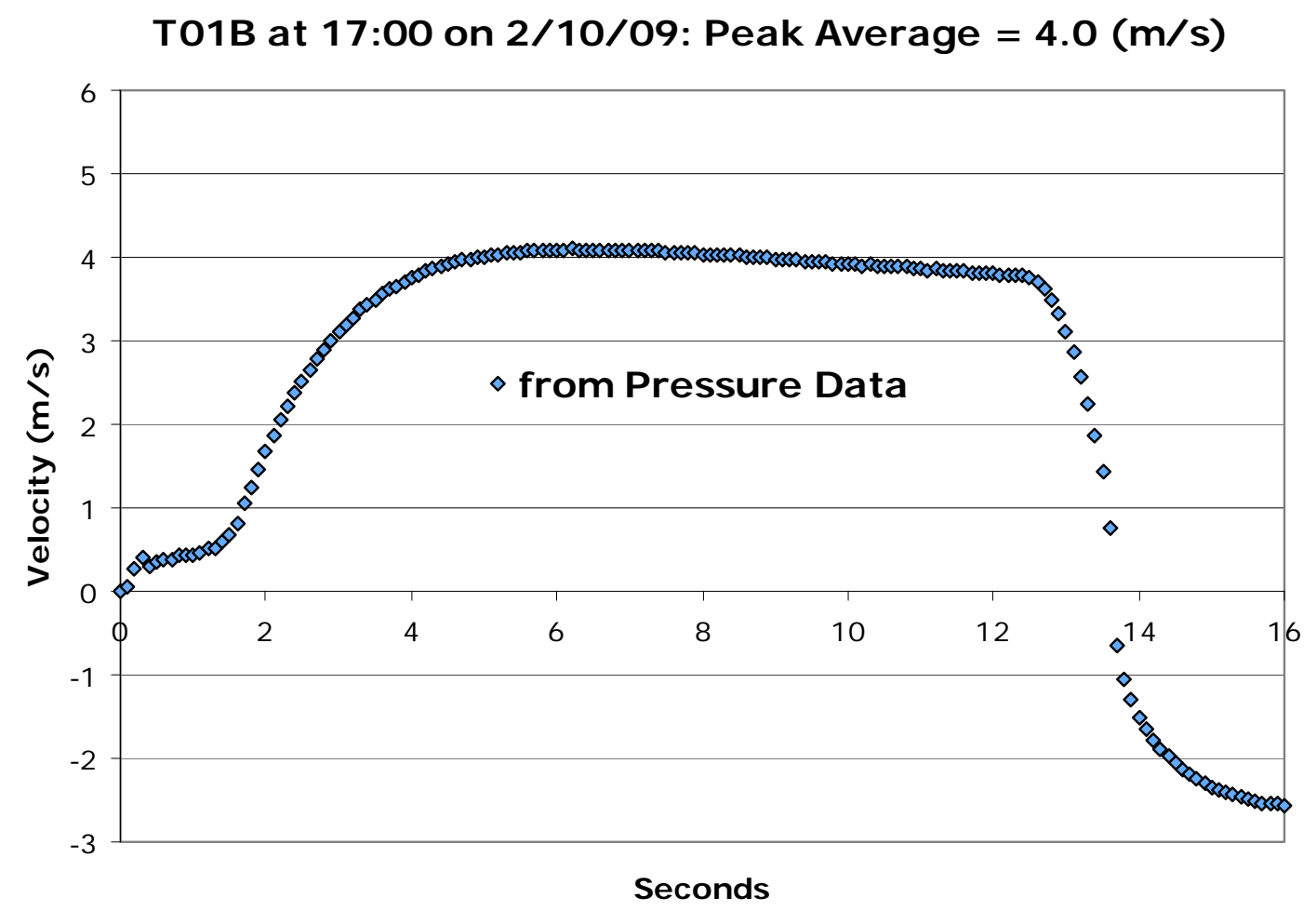

Figure 5.16. Tank T01B PJM Peak Average Velocity During Batch 6 Caustic Leaching 


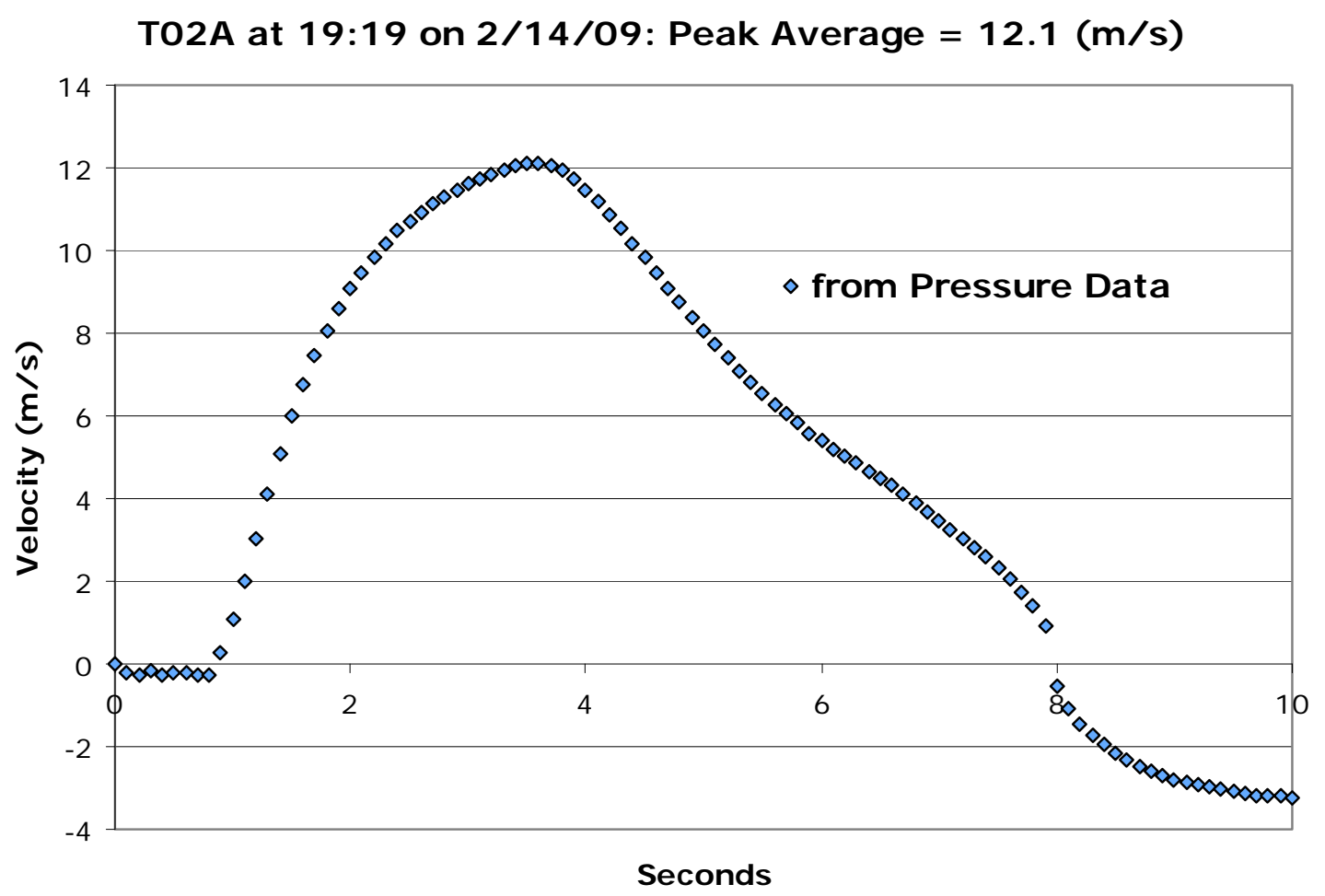

Figure 5.17. Tank T02A PJM Peak Average Velocity Before the Tracer Test

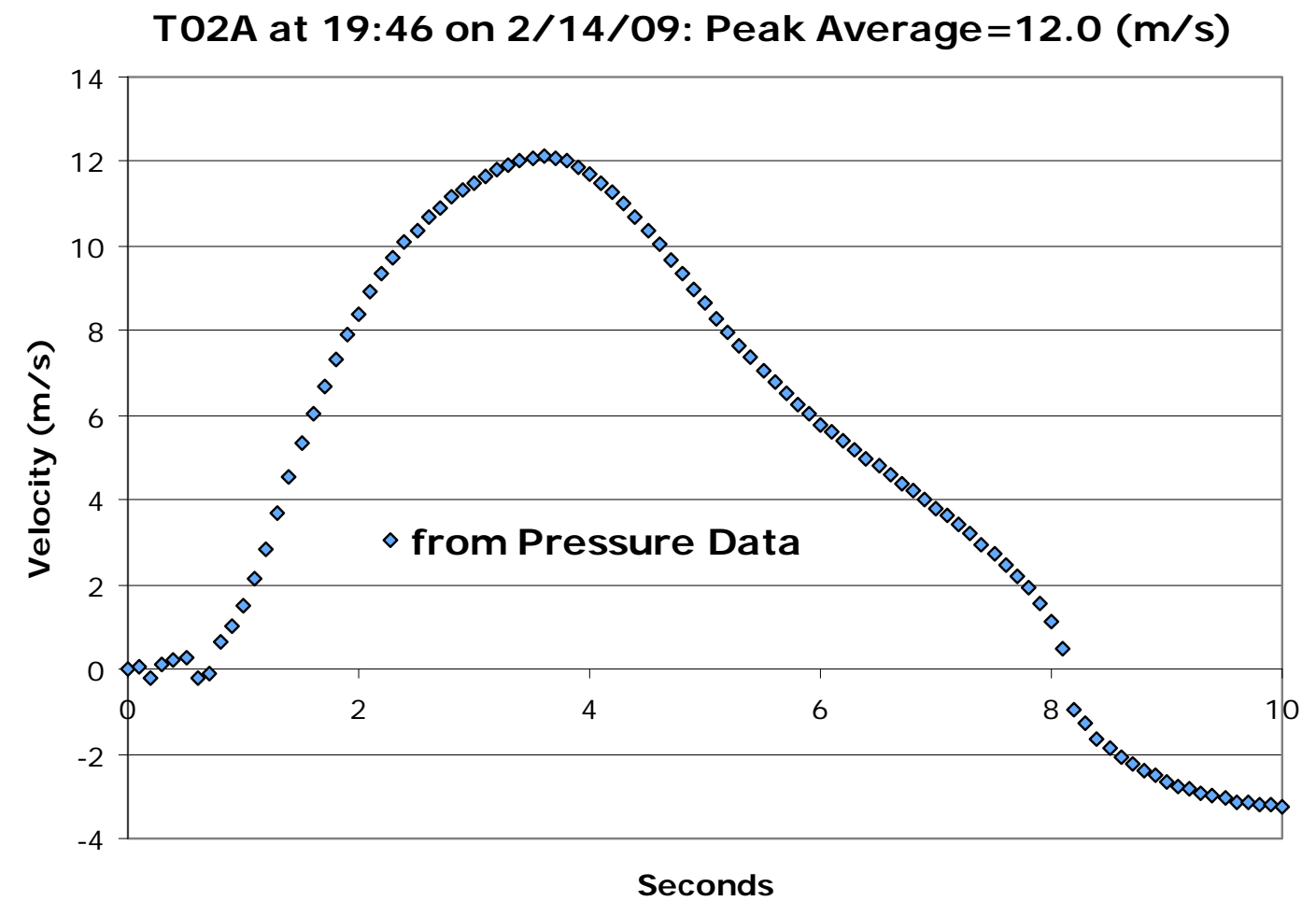

Figure 5.18. Tank T02A PJM Peak Average Velocity Before the Tracer Test 
The loss coefficients evaluated in the RPP-WTP PEP testing are presented in Table 5.21. The plots used for the evaluations of the loss coefficients are given in Figure 5.19 through Figure 5.22.

Table 5.21. Loss Coefficients Evaluated

\begin{tabular}{cccc}
\hline Tank & $\begin{array}{c}\text { Loss Coefficient for } \\
\text { Forward Flow }\end{array}$ & $\begin{array}{c}\text { Loss Coefficient for } \\
\text { Backward Flow }\end{array}$ & $\begin{array}{c}\text { Target Velocity } \\
(\mathrm{m} / \mathrm{s})\end{array}$ \\
\hline Tank T01A & 0.25 & 1.2 & 4.8 \\
Tank T01B & 0.38 & 1.3 & 4.8 \\
Tank T02A & 0.34 & 0.1 & 7.3 \\
Tank T02A & 0.1 & 0.1 & 12 \\
\hline
\end{tabular}

T01A: K_f $=0.25 \& K_{-} b=1.2$ for $4.9(\mathrm{~m} / \mathrm{s})$

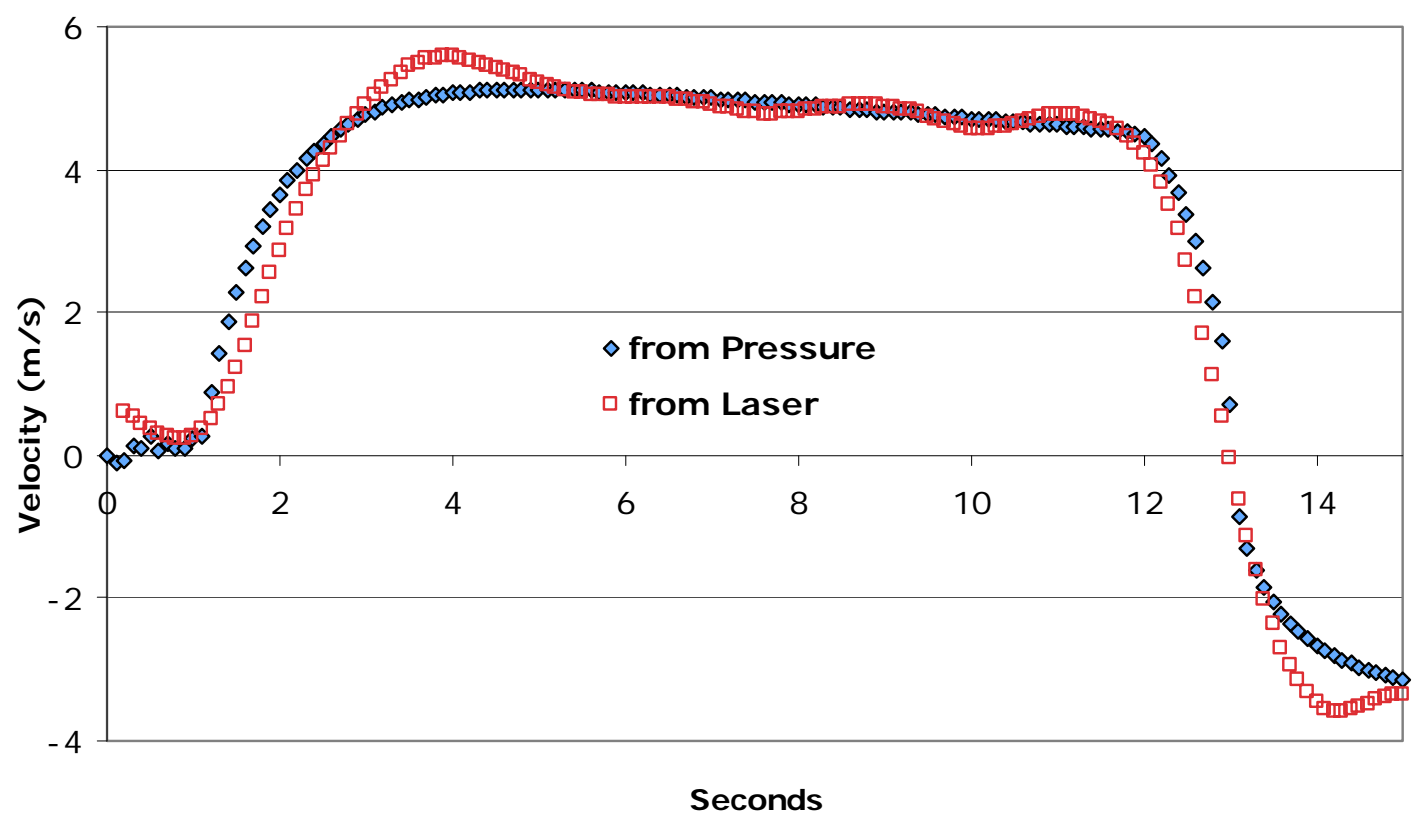

Figure 5.19. Nozzle Velocity Distributions Used to Evaluate the Loss Coefficients for Tank T01A Tank at the Peak Average Velocity of $4.9(\mathrm{~m} / \mathrm{s}): \mathrm{k}_{-} \mathrm{f}=0.25$ is the Loss Coefficient of the Forward Flow, and k_b $=1.2$ is the Loss Coefficient of the Backward Flow 


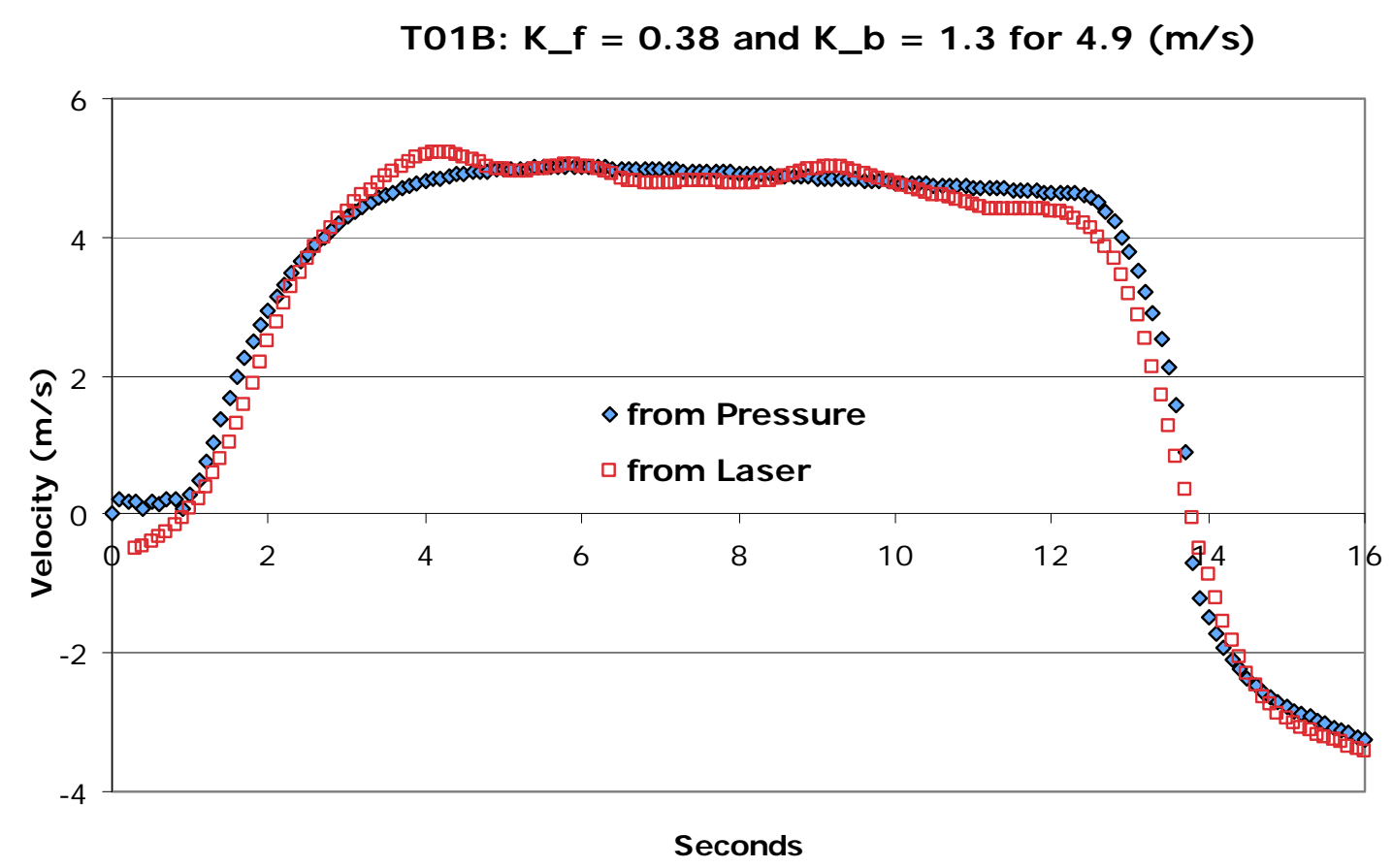

Figure 5.20. Nozzle Velocity Distributions Used to Evaluate the Loss Coefficients for Tank T01B Tank at the Peak Average Velocity of $4.9(\mathrm{~m} / \mathrm{s}): \mathrm{k} \_\mathrm{f}=0.38$ is the Loss Coefficient of the Forward Flow, and k_b $=1.3$ is the Loss Coefficient of the Backward Flow

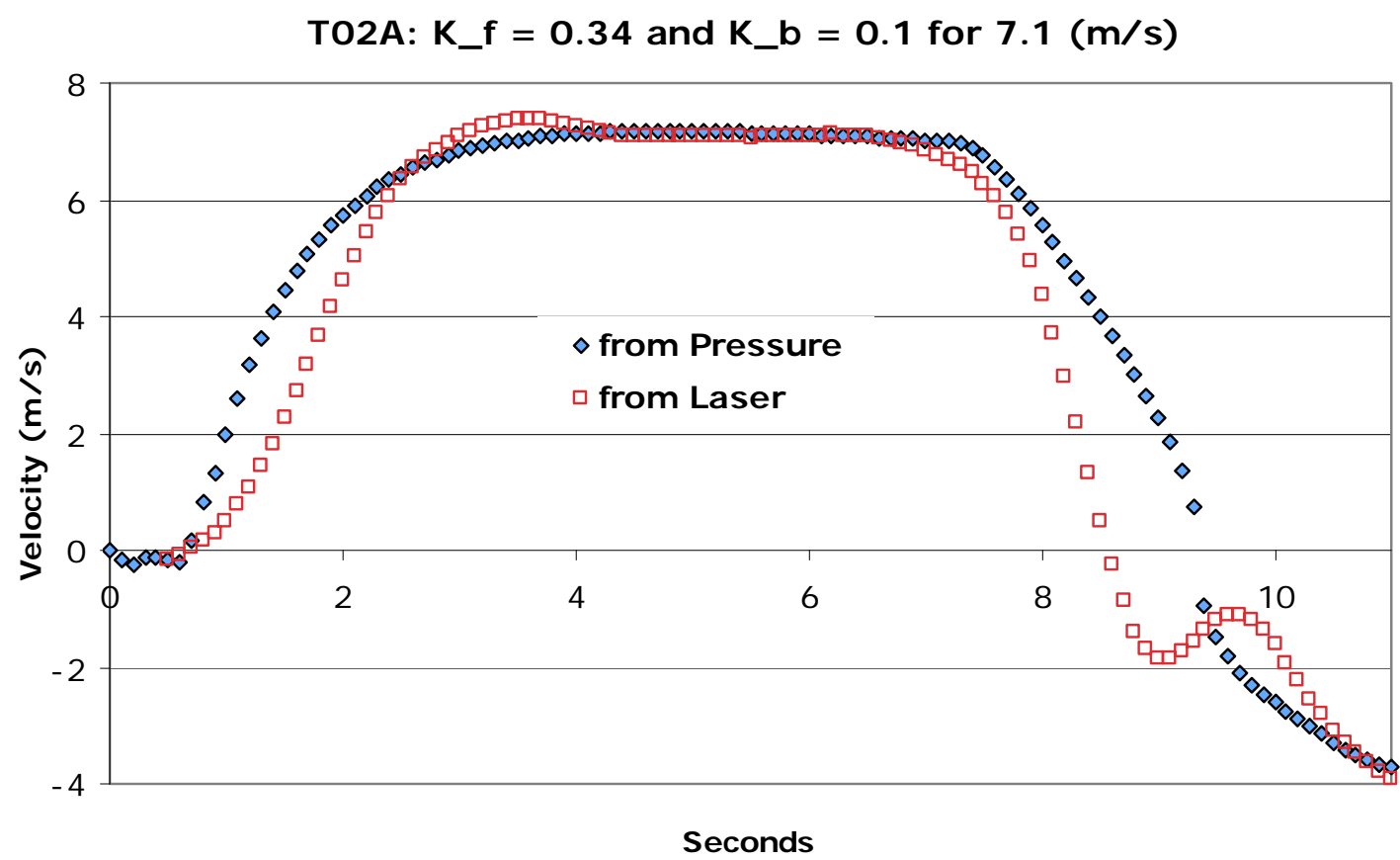

Figure 5.21. Nozzle Velocity Distributions Used to Evaluate the Loss Coefficients for Tank T02A Tank at the Peak Average Velocity of $7.1(\mathrm{~m} / \mathrm{s}): \mathrm{k} \_\mathrm{f}=0.34$ is the Loss Coefficient of the Forward Flow, and k_b $=0.1$ is the Loss Coefficient of the Backward Flow 


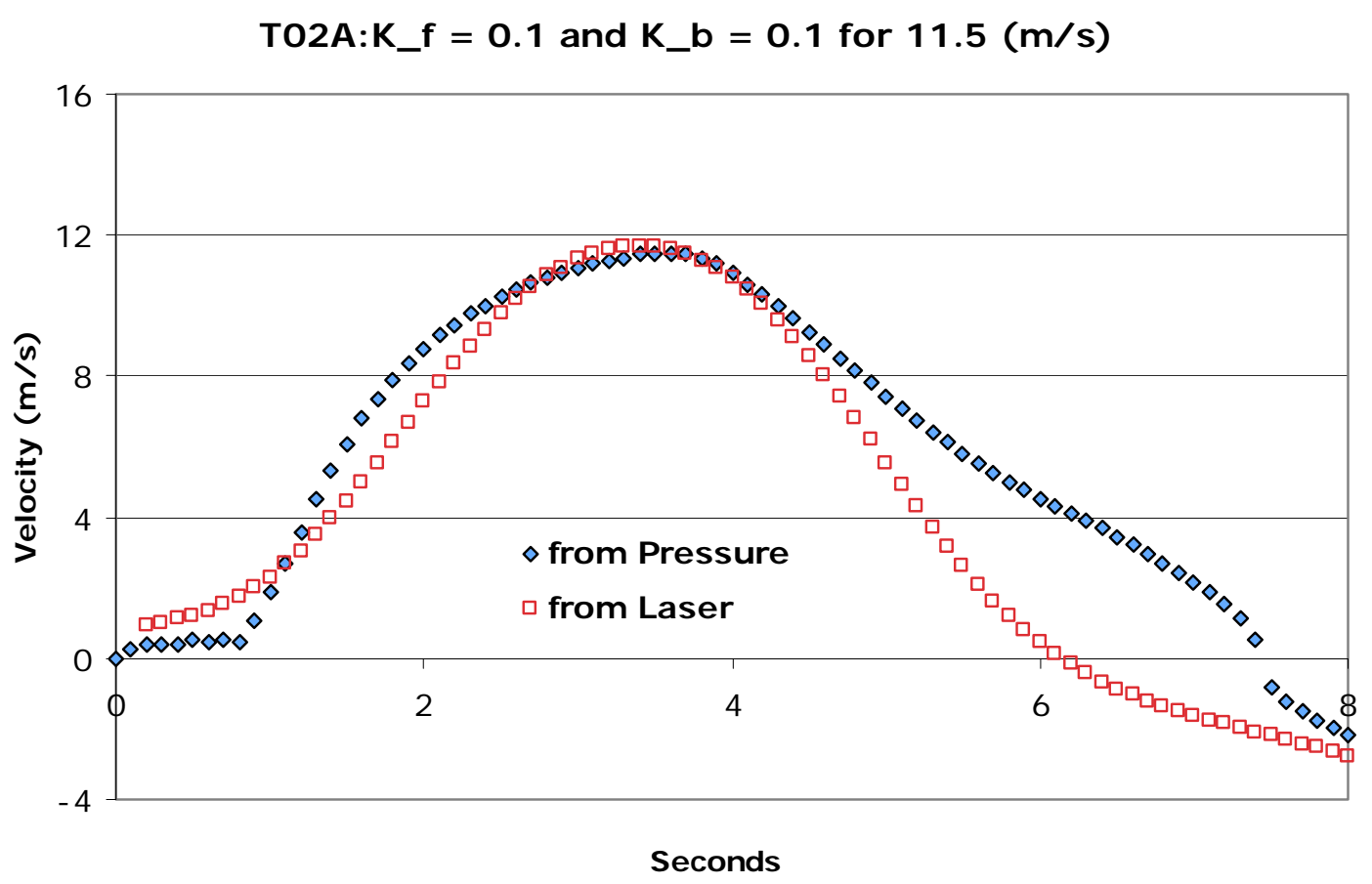

Figure 5.22. Nozzle Velocity Distributions Used to Evaluate the Loss Coefficients for Tank T02A Tank at the Peak Average Velocity of $11.5(\mathrm{~m} / \mathrm{s}): \mathrm{k} \_\mathrm{f}=0.1$ is the Loss Coefficient of the Forward Flow, and k_b $=0.1$ is the Loss Coefficient of the Backward Flow 


\subsection{Issues}

Subsequent sections discuss the difficulties encountered during Integrated Test A, their work-arounds addressed, and in some cases, the resolutions. Refer to Appendix E for a timeline of events. Data, including figures, in this section are NQA-1 compliant.

\subsection{Backpulse Description}

Backpulsing was applied through the course of post-caustic-leach filtration to increase the permeate production rate. Initially per email, 2/4/09, 18:20 (Test Director), backpulsing was to be done only if pumps UFP-PMP-T42A and T43A had been running continuously for at least 6 hours, and the permeate rate dropped to or below $0.70 \mathrm{~kg} / \mathrm{min}$ for at least 2 hours. Backpulse parameters were 40 psi overpressure (pressure above the average filter bundle tube side pressure), 5 psi deadband pressure (the pressure above the average filter bundle tube side pressure at which the backpulse is complete), and 10-in. backpulse initiation level (level drain setpoint). The first backpulse was performed on 2/5/09 at 00:07 (LRB 60108, pg. 130) using the standard backpulsing parameters noted above. A second backpulse was performed at 15:45 using the same backpulsing parameters (LRB 60229, pg. 121) per Test Director email, 2/5/09, $15: 13$.

By email (Test Director) 2/5/09, 17:14, backpulse parameters were changed, a number of consecutive backpulses were added, and backpulsing frequency was changed to every 12 hours. However, if the permeate rate dropped to $0.6 \mathrm{~kg} / \mathrm{min}$, the 6 - $\mathrm{hr}$ continuous pump operation for UFP-PMP-T42A and Pump T43A still applied. However, by 2/6/09, the 2/5/09 backpulse instructions were revised because pulse pot volume changes, as found when using a 40 psi overpressure, could not be doubled using overpressures of either 20 or 60 psi. Backpulsing instruction changes included having the permeate rate to be at or below $0.60 \mathrm{~kg} / \mathrm{min}$ before initiating a backpulse. While the deadband pressure was changed to achieve the target backpulse volume change in the pulse pot, the 10-in. level drain setpoint was kept the same for all backpulses.

Final backpulses performed were as shown in Table 6.1 (Test Director email, 2/6/09, 15:19).

Table 6.1. Initial Backpulses Performed During Post-Caustic-Leach Concentration

\begin{tabular}{|lllrl|}
\hline Reference & Date & Time & ID & Notes \\
\hline LRB 60108, pg. 142 & $2 / 5 / 09$ & $21: 45$ & 4 & Perform 2 backpulses: overpressure 40 psi, deadband pressure 5 psi \\
LRB 60108, pg. 144 & $2 / 6 / 09$ & $02: 59$ & 1 & Perform 1 backpulse: overpressure 20 psi, deadband pressure 5 psi \\
LRB 60108, pg. 145 & $2 / 6 / 09$ & $10: 45$ & 3 & Perform 2 backpulses: overpressure 20 psi, deadband pressure 5 psi \\
LRB 60229, pg. 136 & $2 / 6 / 09$ & $17: 24$ & 5a & Perform 1 backpulse: overpressure 60 psi, deadband pressure 20.7 psi \\
LRB 60108, pg. 149 & 2/7/09 & $00: 10$ & 5b & Perform 1 backpulse: overpressure 60 psi, deadband pressure $20.7 \mathrm{psi}$ \\
LRB 60229, pg. 142 & $2 / 7 / 09$ & $07: 11$ & 5c & Perform 5 backpulses: overpressure 60 psi, deadband pressure 20.7 psi \\
LRB 60229, pg. 148 & 2/7/09 & $14: 05$ & 5d & Perform 1 backpulse: overpressure 60 psi, deadband pressure 20.7 psi \\
LRB 60108, pg. 152 & 2/7/09 & 18:32 & 7 & Perform 1 backpulse: overpressure 75 psi, deadband pressure 32.5 psi \\
\hline All backpulses used a level drain setpoint of 10 inches, and multiple backpulses were done back-to-back. \\
\hline
\end{tabular}


Refer to Figure 6.1 through Figure 6.8 to view the pressure drop across Filter T01A, the TMP (the average of Filter T01A inlet and outlet pressures minus the shellside pressure), the permeate rate, and the pulse pot level for each of the backpulses, noted by the corresponding identification number (ID), in Table 6.1. The TMP is reported in pounds-per square-inch differential pressure (psid). The pulse pot levels shown in Figure 6.1 through Figure 6.8 are for information only since these instruments were not calibrated to NQA-1 requirements.

Per phone conversation 20:00 on 2/7/09 (LRB 60108, pg. 155), subsequent backpulses used the standard backpulse parameters.

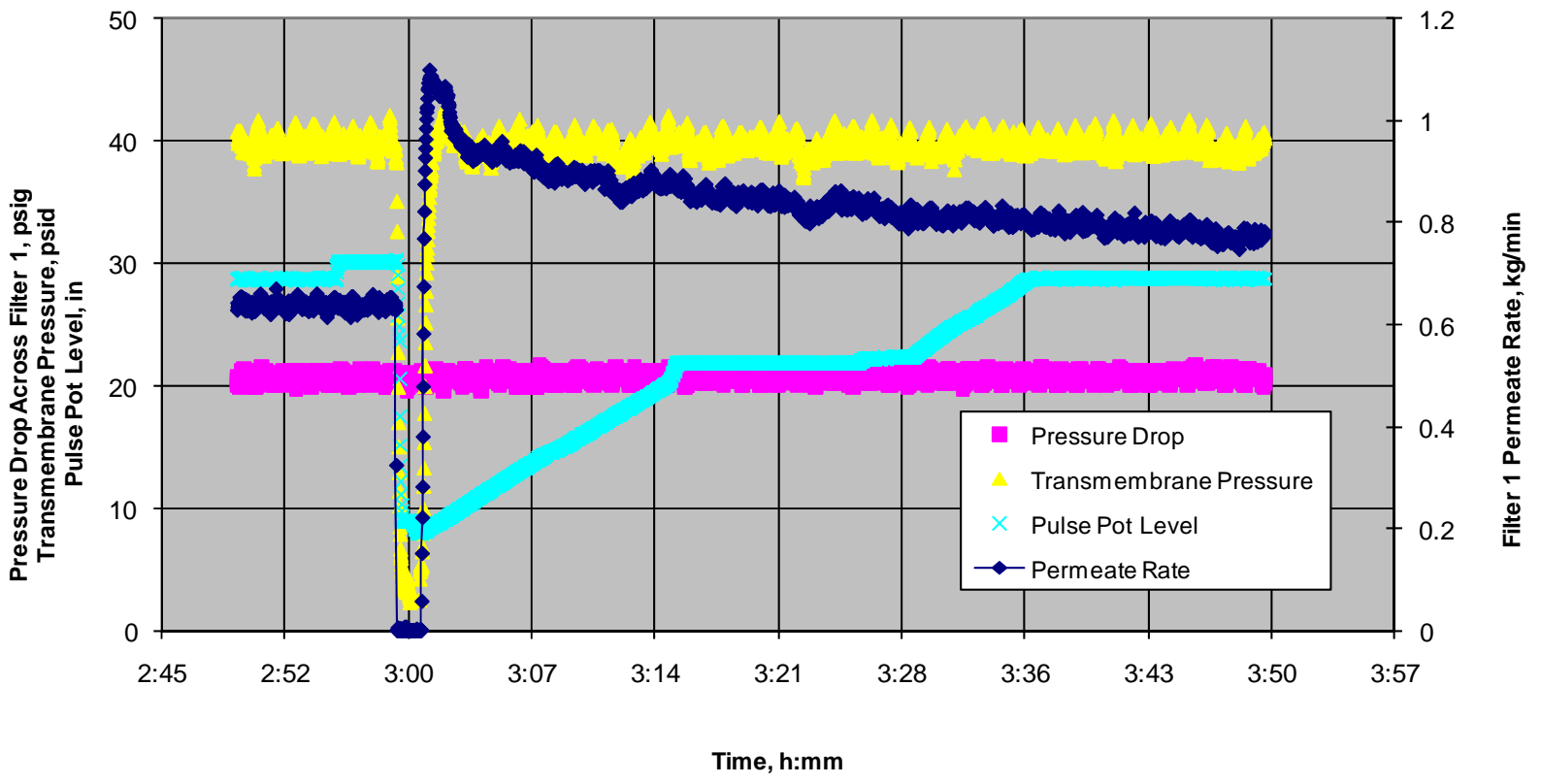

Figure 6.1. Filter T01A Backpulse (1) Using Deadband and Overpressures of 5 and 20 psi, 2/6/2009 


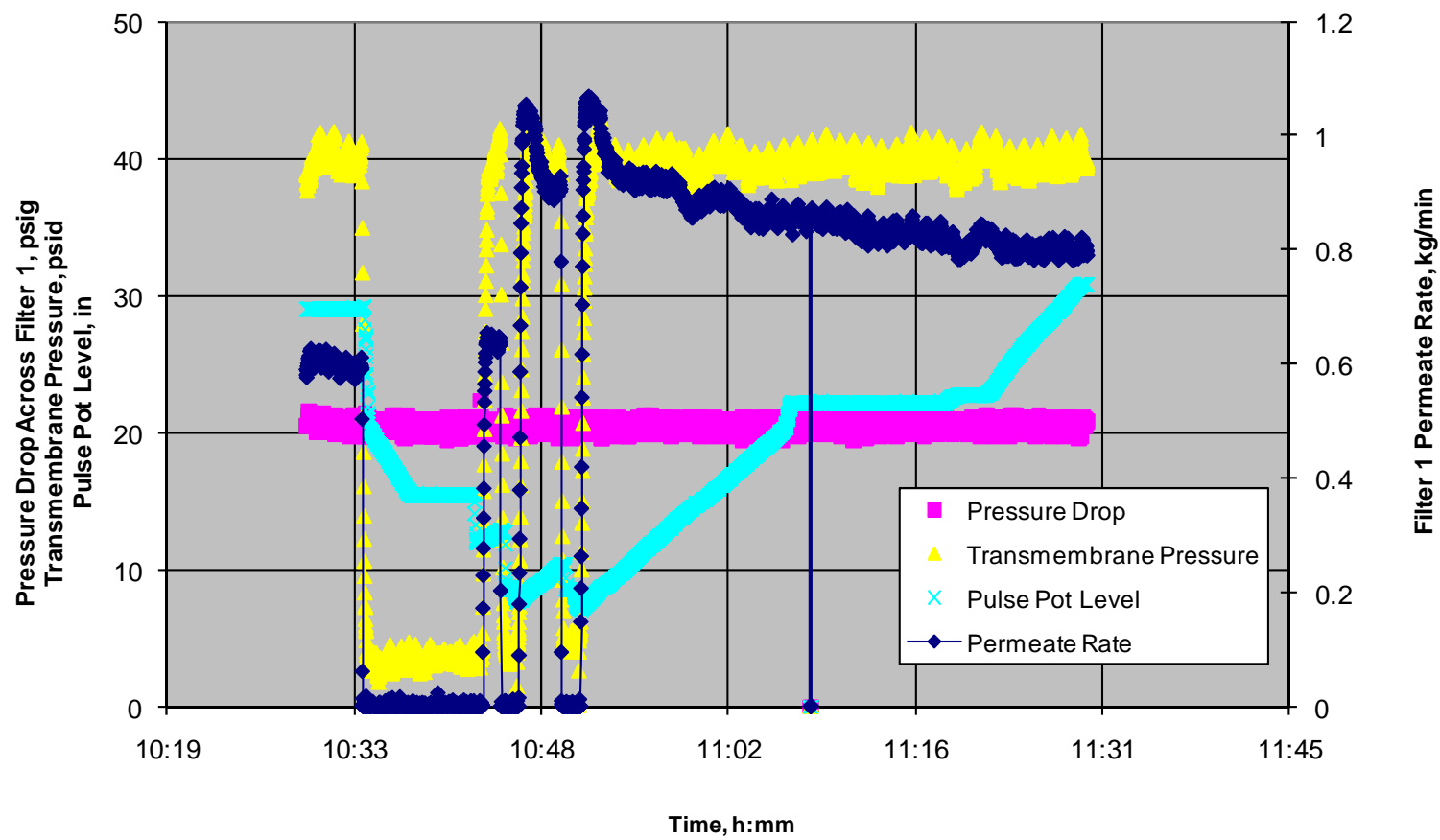

Figure 6.2. Two Consecutive Backpulses (3) on Filter T01A Backpulses Using Deadband and Overpressures of 5 and 20 psi, 2/6/2009

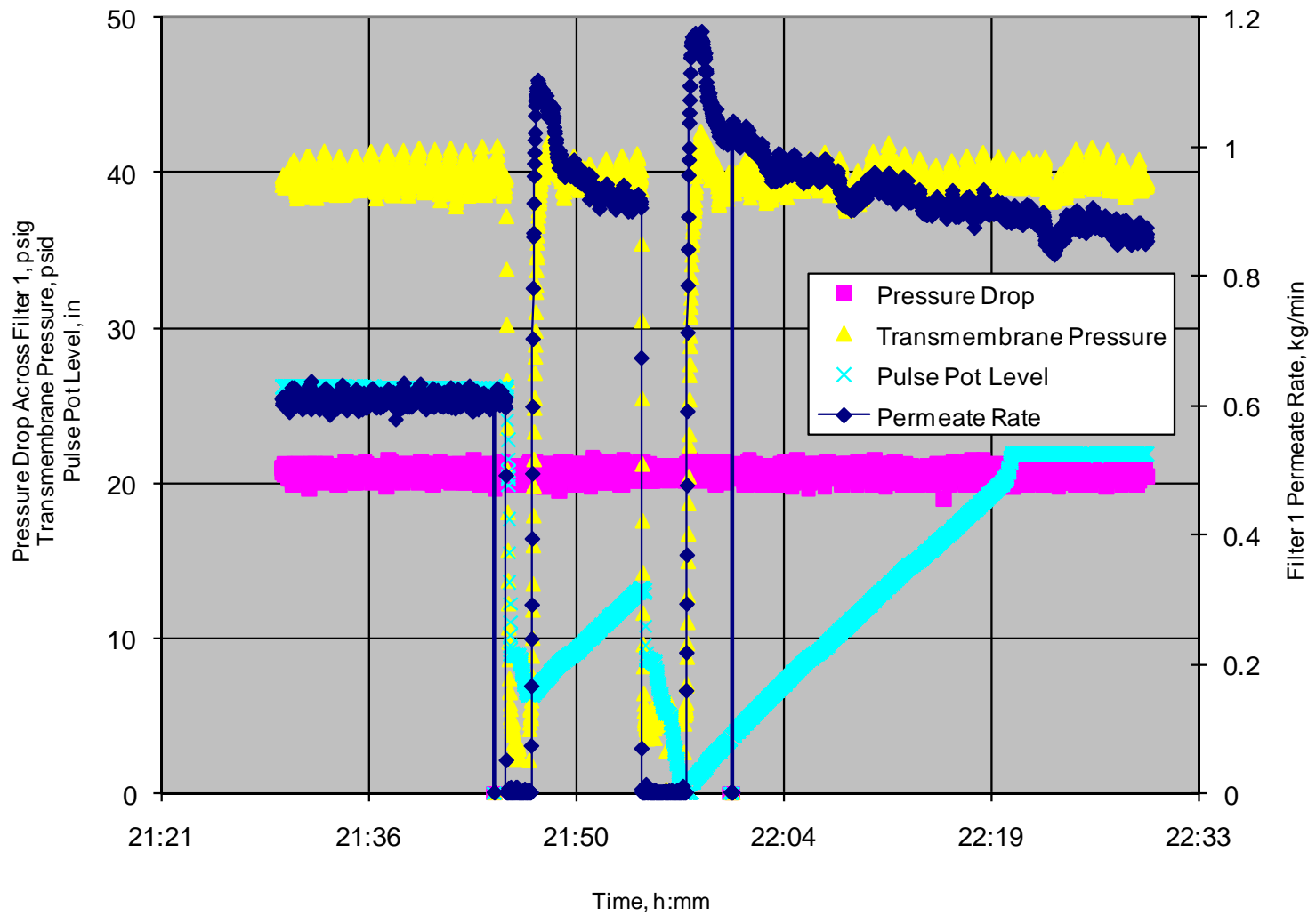

Figure 6.3. Two Consecutive Backpulses (4) on Filter T01A Using Deadband and Overpressures of 5 and $40 \mathrm{psi}, 2 / 5 / 2009$ 


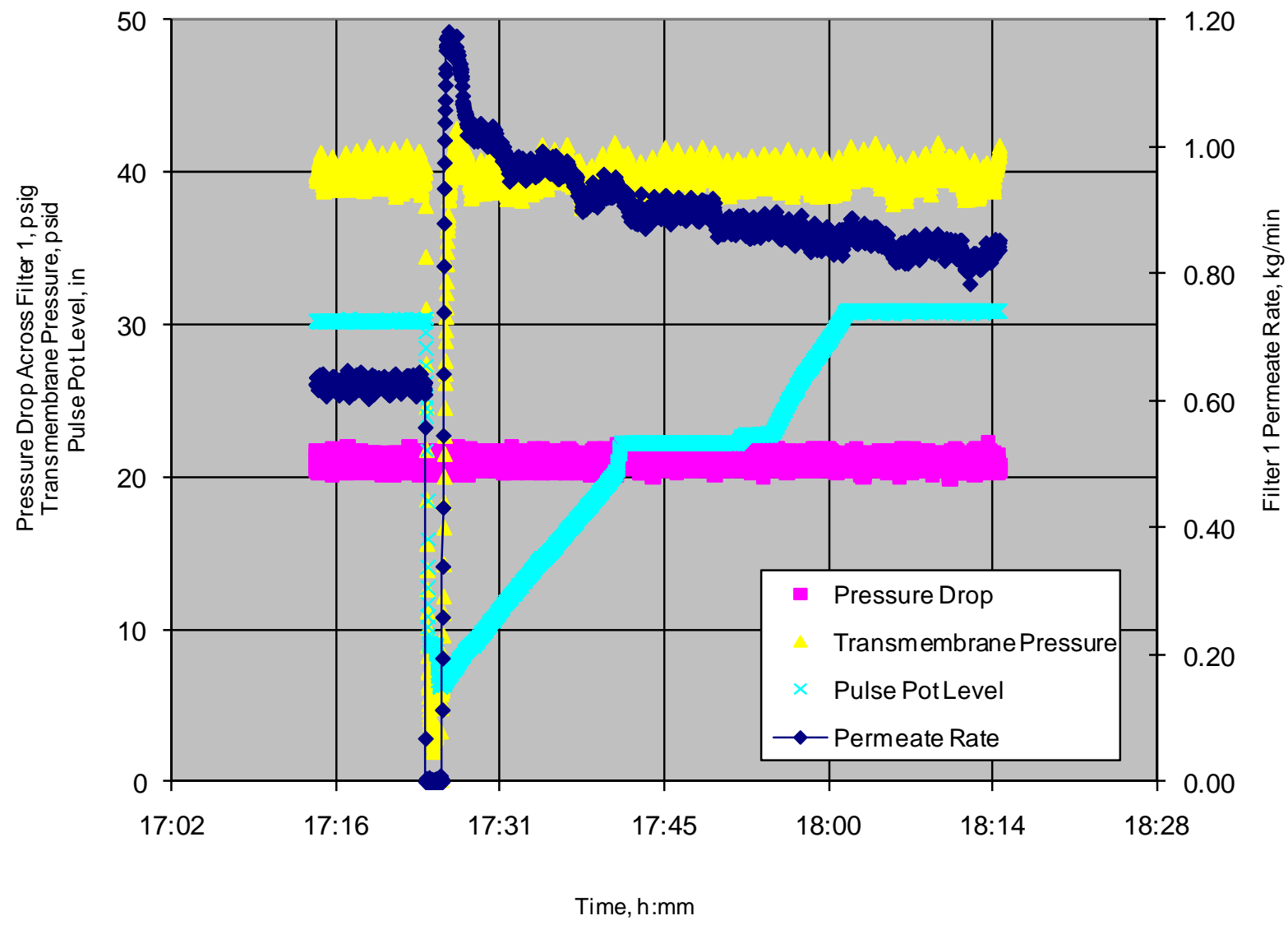

Figure 6.4. Filter T01A Backpulse (5a) Using Deadband and Overpressures of 20.7 and 60 psi, 2/6/2009 


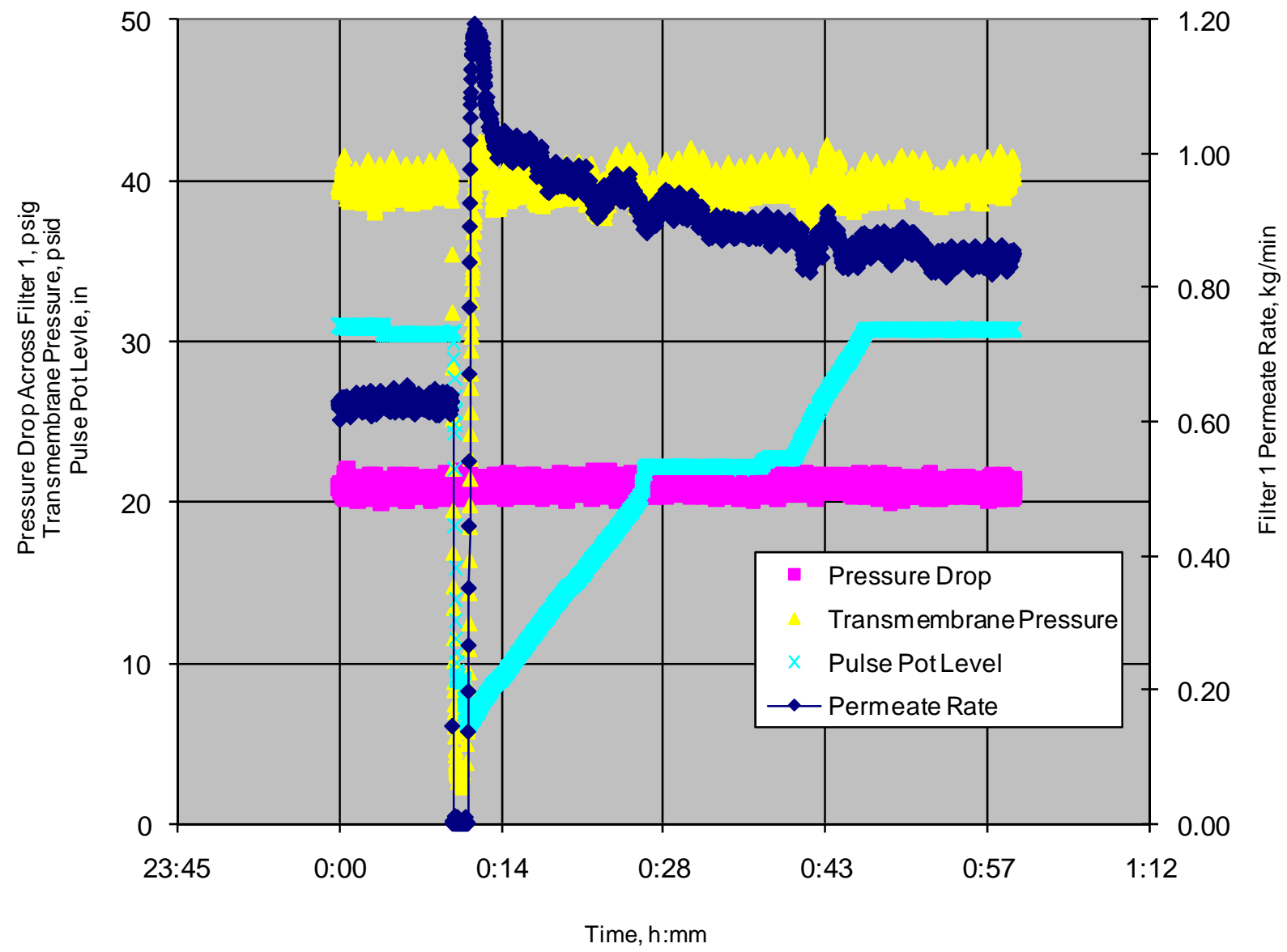

Figure 6.5. Filter T01A Backpulse (5b) Using Deadband and Overpressures of 20.7 and 60 psi, 2/7/2009 


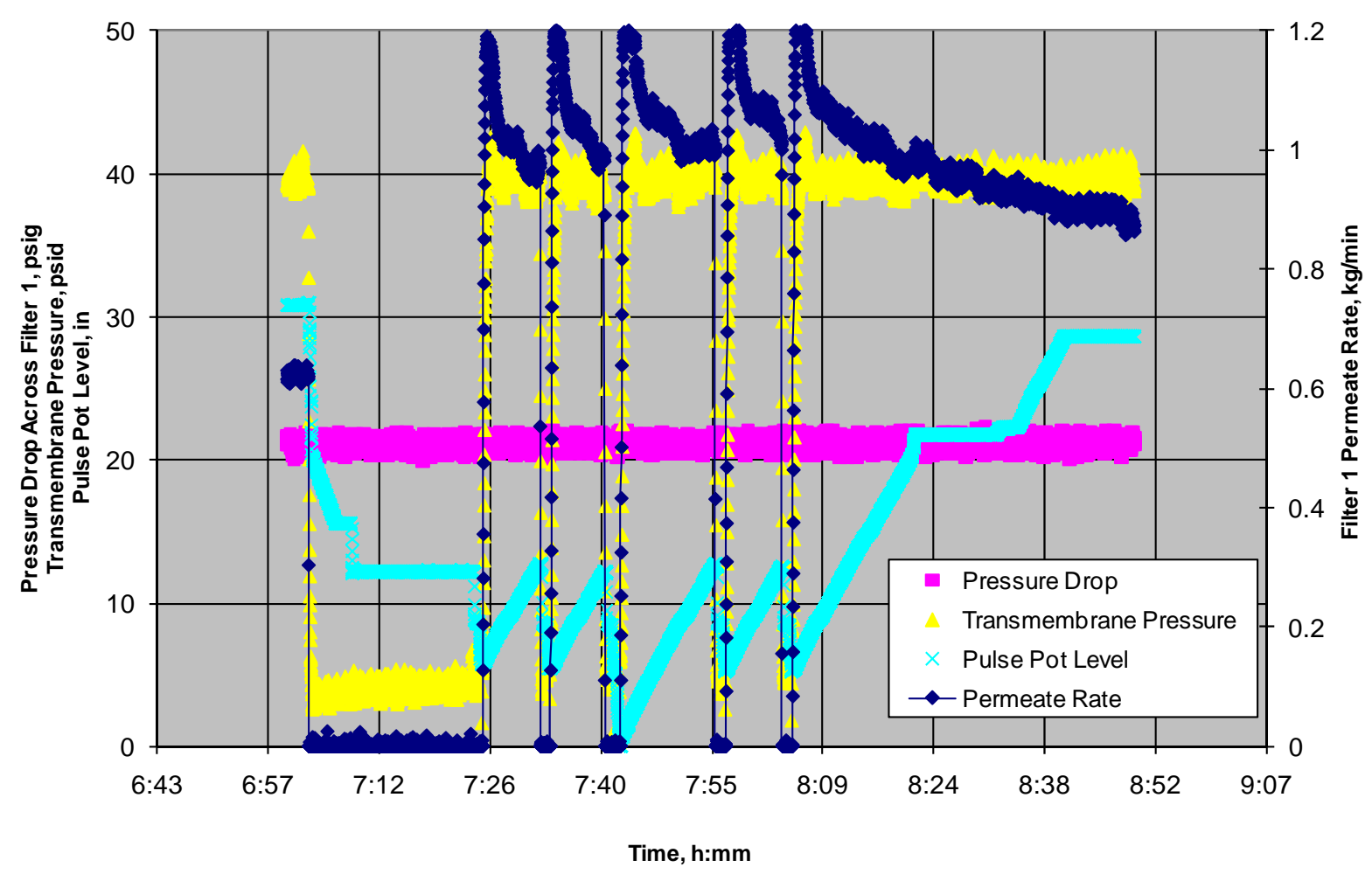

Figure 6.6. Five Consecutive Backpulses (5c) on Filter T01A Using Deadband and Overpressures of 20.7 and $60 \mathrm{psi}, 2 / 7 / 2009$ 


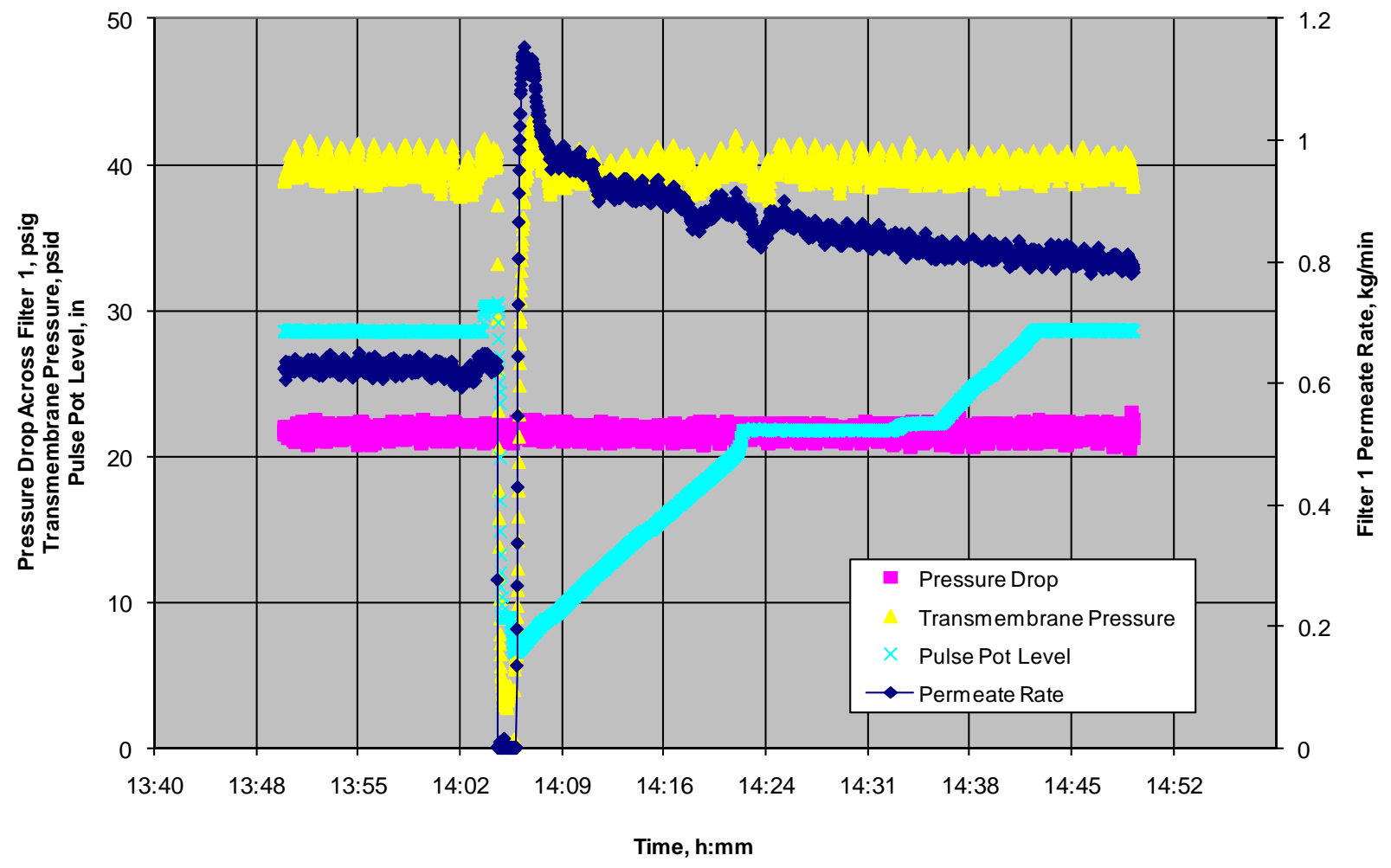

Figure 6.7. Filter T01A Backpulse (5d) Using Deadband and Overpressures of 20.7 and 60 psi, 2/7/2009 


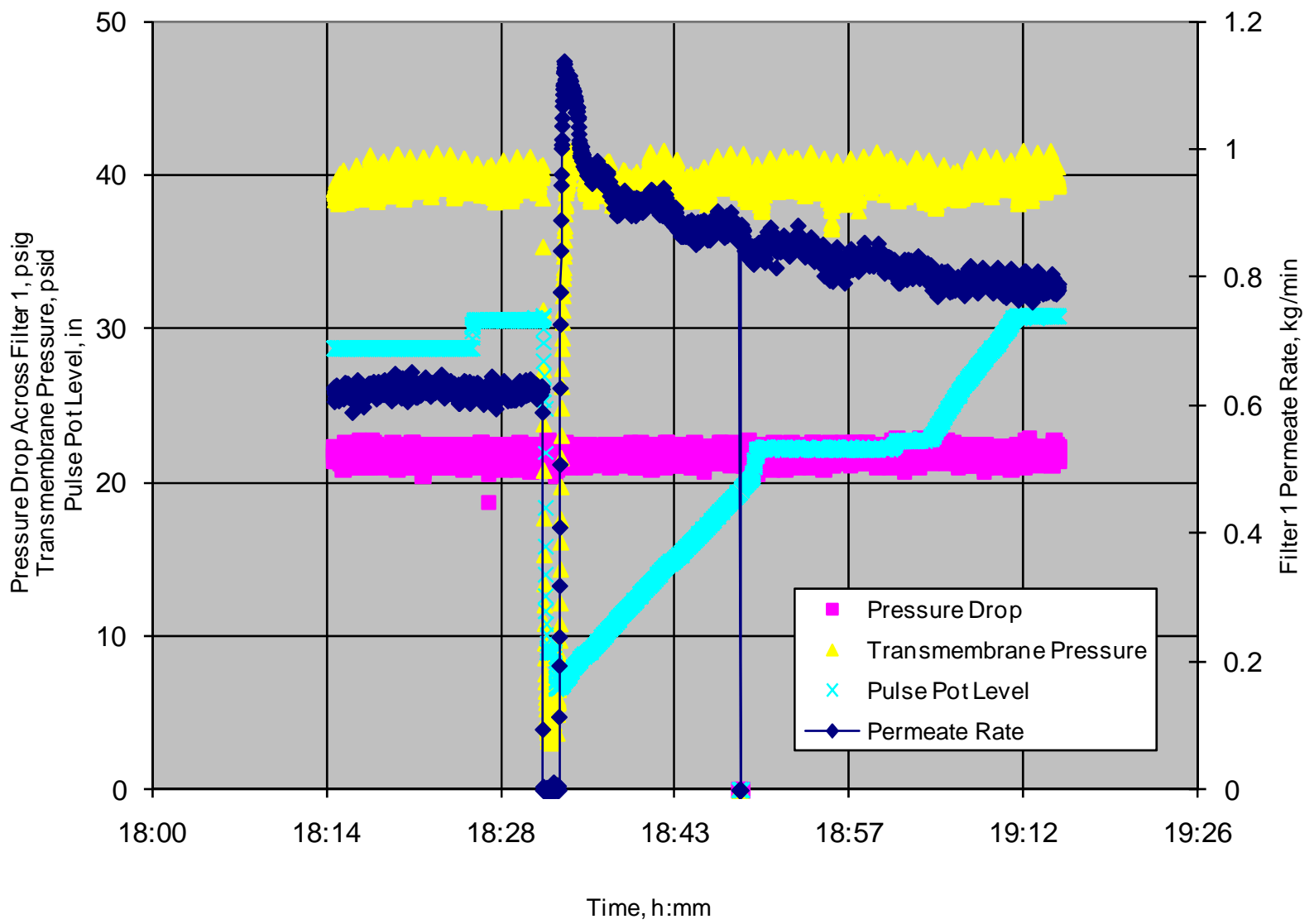

Figure 6.8. Filter T01A Backpulse (7) Using Deadband and Overpressures of 32.5 and 75 psi, 2/7/2009

The permeate performance after backpulsing was the same for all backpulse variations. The peak permeate after the backpulse was complete was $\sim 1.1 \pm 0.1 \mathrm{~kg} / \mathrm{min}$. However, permeate rates dropped below $1 \mathrm{~kg} / \mathrm{min}$ within 10 minutes.

On 2/5/09, 21:58 (LRB 60108, pgs. 142 and 143), two consecutive backpulses were executed. During the second backpulse, it was observed that there was a level decrease pause when the overpressure was being applied and the pulse pot level was decreasing. Then, the level decrease started up again until the pulse pot level indicated 0 inches. This backpulse "hiccup" was observed on several occasions, and the reasons for such behavior were neither identified nor understood. This automatic second-backpulse behavior occurred again on 2/8/09, 06:59 (LRB 60229, pg. 157) and 08:08, 2/9/09 (LRB 60230, pg. 3).

\subsection{Air Entrainment}

Vibration in the filter-loop pumps and increasing axial pressure drop across the filters was first observed during PJM tuning for non-Newtonian slurry in preparation for the tracer test $(2 / 14 / 09)$. Filter-loop flow rate and TMP unstable behavior were other symptoms of air entrainment that emerged at the start of post-caustic-leach washing (TI step 13.4.2). The tracer test was conducted at a tank level (laser) of 48.9 inches, per the most recent stable level measurement, and the post-caustic-leach washing was done at a tank level (laser) of 44.3 inches. Having checked that there were no leaks in the pump flanges and connections, such operational problems may have been caused by unintentionally introducing 
air sources into the filtration loop. Although there were PJM overblow observations noted for UFP-VSL-T02A during solids concentration, PJM pressure data examination did not provide clear confirmation of PJM overblows. The change in filter alignment, going from the alignment of UFP-FILT-T01A to the alignment of UFP-FILT-T02A through Filter T05A in preparation for the tracer test, may also have introduced air into the filtration loop. PJM tuning efforts to a higher velocity could also have resulted in PJM overblows. In addition to the operational problems, there was also a defective flow meter downstream from UFP-PMP-T43A as noted on NCR 42317.1. The air entrainment issue may have worsened when trying to increase the filter-loop flow rate based on the erroneous low value reported by the defective flow meter on the discharge line of UFP-PMP-T43A.

By 00:44, 2/15/09 (LRB 60230, pg. 59), post-caustic-leach washing proceeded with approval at a filter-loop flow rate of $88 \mathrm{gpm}$ (run sheet target was $109 \pm 10 \mathrm{gpm}$ ). This was in the midst of continuing unstable and below-target filter-loop flow rates, coupled with high axial pressure drops across most of the filters. Washing began at 01:13, 2/15/09 (LRB 60230, pg. 59), and TMPs were as high as 70 psi on Filters T02A and T03A intermittently at the beginning of washing.

Washing was suspended at 01:54, 2/15/09 (LRB 60230, pg. 60) because of unstable pump speed and TMPs.

Subsequent paragraphs will use instrument numbers associated with the UFP-VSL-T02A filter loop. Refer to Figure 6.9 for a layout of filter-loop instrument numbers. 


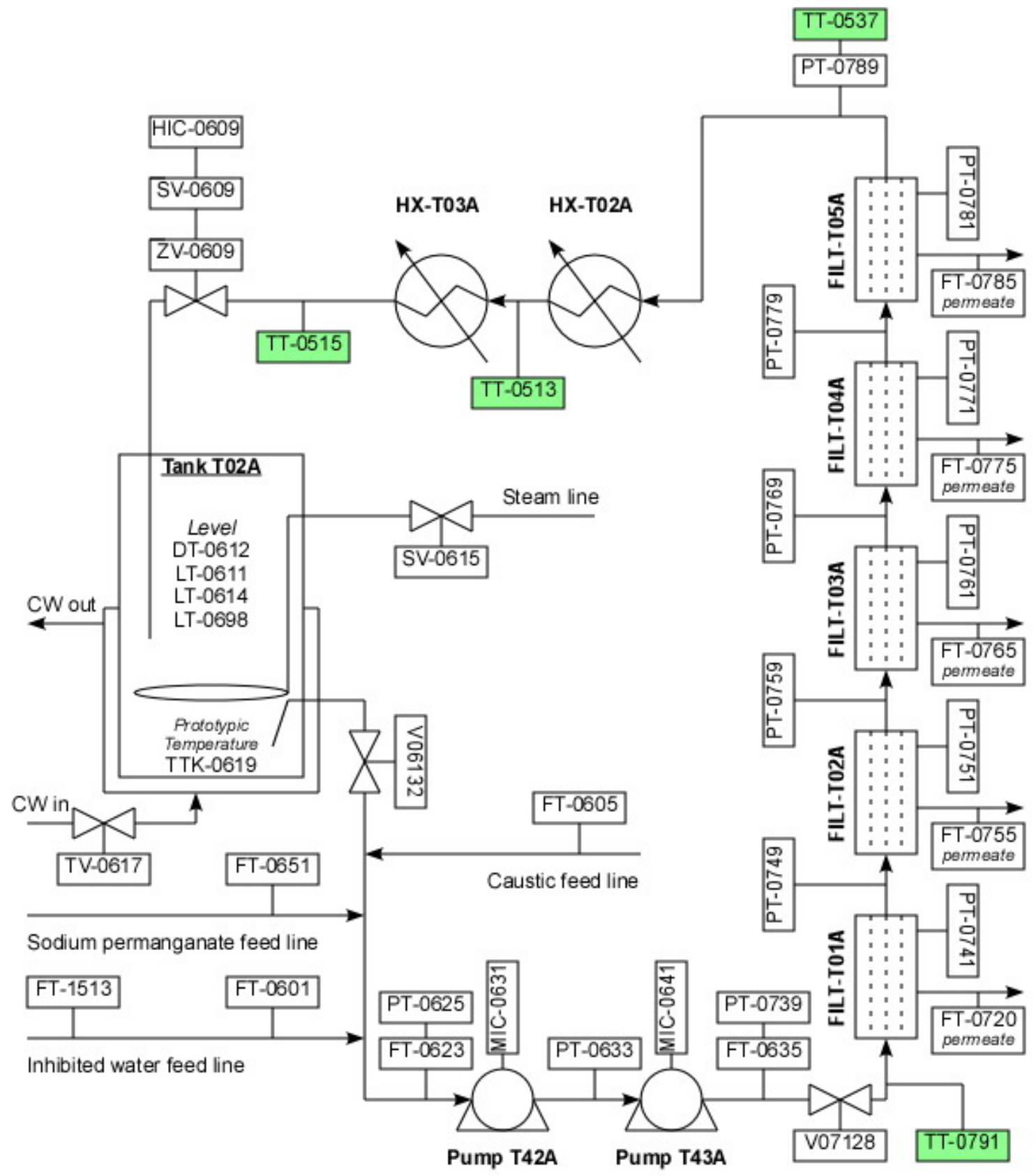

Figure 6.9. Schematic of UFP-VSL-T02A Loop and Filtration. Note that UFP-HX-T03A was bypassed during the washing operations of Integrated Test A. ${ }^{(a)}$

(a) $\mathrm{DT}=$ density transmitter, $\mathrm{HX}=$ heat exchanger, $\mathrm{LT}=$ level transmitter, $\mathrm{FT}=$ flow transmitter (includes the matching FE or Flow Element), PT = pressure transmitter (includes the matching PI or Pressure Instrument), $\mathrm{TT}=$ temperature transmitter (included the matching TE or Temperature Element), MIC = Motor Indicating Controller, V= Valve, SV = Solenoid Valve, ZV = Positioning Valve, and HIC = Human Interface Control. Instruments with Nonconformance Reports issued during testing are shaded. 
Filter-loop flow-rate investigations took place between 01:57, 2/15/09 (LRB 60230, pg. 60) and 14:26, 2/15/09 (LRB 60230, pg. 74). Several combinations of the following parameter settings were tried to achieve the target filter-loop flow rate:

- turning PJMs off, reducing steam-ring air flow rate, and turning lower spargers and PJMs off

- running with only one pump at a time (UFP-PMP-T42A and UFP-PMP-T43A) and ramping its speed incrementally with a fixed backpressure valve (SV-0609) position

- running both pumps at various speeds at a fixed backpressure valve position, etc.

Many setting combinations were tried to achieve the run sheet target flow rate of $109 \mathrm{gpm}$. The results were an $\sim 40$ gpm filter-loop flow rate difference between FT-0623 (upstream from UFP-PMP-T42A) and FT-0635 (downstream from UFP-PMP-T43A) and a maximum flow rate of $\sim 90$ gpm per FT-0635.

The 10:26, 2/15/09, 40-mL filter-loop sample was centrifuged to remove entrained air; this indicated a 3-mL level change (LRB 60230, pg. 73) or $\sim 7.5 \%$ air retention in slurry. It was also postulated that there could be loose connections and air leaks within the pump flanges and surrounding piping; however, per 14:07, 2/15/09 (LRB 60230, pg. 74), no indication of air leaks for flanges between UFP-VSL-T02A and UFP-PMP-T42A were found.

To determine the rate of entrained gas release at room temperature and atmospheric pressure, a 2-L graduated cylinder was filled with $1800-\mathrm{mL}$ of slurry, and a second graduated cylinder was filled with 53-mL of slurry (12:00, 2/15/09; LRB 60230, pg. 110). By 22:10 on 2/15/09, the stable level indicated a volume fraction change of $2.2 \%$ and $1.9 \%$ for the $2-\mathrm{L}$ and second graduated cylinders, respectively (the level was monitored until 03:40 on 2/16/09). A second experiment was done to see whether adding AFA would improve the gas release rate $(2 / 15 / 09,15: 11$; LRB 60230, pg. 111). Two graduated cylinders were filled with 50-mL simulant (density was $1.34 \mathrm{~g} / \mathrm{mL}$ ), one with AFA and the other without AFA. The results showed that AFA had no effect on the gas release rate- both achieved a $49.5-\mathrm{mL}$ stable level at the same time ( 2 hours and 39 minutes).

Between 16:02, 2/15/09 (LRB 60230, pg. 74) and 22:54, 2/15/09 (LRB 60230, pg. 88), post-caustic-leach washing commenced, per instruction indicated by the JTG (LRB 60235, pg. 9): proceed with whatever filter-loop flow rates that were achieved with upper and lower spargers, bubblers, and steam ring turned off (14:26, LRB 60230, pg. 74) and after waiting for $\sim 2$ hours. Filter-loop flow rates achieved were 116 gpm on FT-0623 and 78 gpm on FT-0635 (13:55, LRB 60230, pg. 74).

A camera put inside UFP-VSL-T02A showed vortexing of fluid, which may be likely the source contributing to air entrainment (23:52, 2/15/09, LRB 60230, pg. 88). Very little foam was observed (08:35, 2/16/09, LRB 60230, pg. 97). The vortex was around the filter loop return nozzle and appeared to draw the small quantity of foam present down into the slurry.

Pump Settings for the tracer were (LRB 60230, 56) as follows:

- $\mathrm{MC}-0631=95 \%$

- $\mathrm{MC}-0641=51 \%$ (manual)

- $\mathrm{HIC}-0609=60 \%$ 
- PI-0633=140 \pm 10 psi

- FT-0635=107 gpm (100 to 110 gpm).

Pump Settings for post-caustic-leach washing were (LRB 60230, pg. 74) as follows:

- $\mathrm{MC}-0631=85 \%$

- $\mathrm{MC}-0641=42 \%$

- $\mathrm{HIC}-0609=55 \%$

- $\mathrm{FT}-0623=116-120 \mathrm{gpm}$

- FT-0635=78-80 gpm.

The return line was submerged at the elevations for which the tracer and washing took place, and with no leaks in the pump flanges and connections, air entrainment must have been a result of the simulant retaining air introduced by bubblers, steam air purge, PJMs, and spargers. The air bubbles were apparently small enough that they could not overcome the shear strength of the simulant (the simulant at this point was concentrated to $\sim 17-\mathrm{wt} \%$, high solids material); thus, they did not reach the surface and were therefore retained in the simulant. Air retention could also have been influenced if the air bubbles attached themselves to solids in the slurry. With the presence of AFA, surface retention is decreased, which restricts coalescence, thus preventing bubbles from merging, growing, and escaping to the surface. Slurry retention of small air bubbles was also enhanced when slurry traveled through the pumps, which shear the existing bubbles into smaller bubbles, thus decreasing the chance that the bubbles will ever reach the surface. Similar behavior was observed in WTP-RPT-147, where Savannah River National Laboratory conducted slurry experiments using impellers for mixing (Stewart et al. 2007). The impellers sheared the gas bubbles, thus decreasing their size, which prevented them from reaching the surface. PNNL gas retention and release investigations also confirmed that gas retention is increased with decreasing yield stress slurries containing AFA (Stewart et al. 2008). Samples (\#2383 and \#2384) collected before sodium permanganate addition on 2/16/09 indicate a shear strength range of 7.6 to 12.4 $\mathrm{Pa}$, respectively, for a 72 hour settling time.

After chromium addition and before dewatering, higher filter-loop flow rates were achieved at the higher vessel level (01:57, 2/16/09, LRB 60230, pg. 90), and filter-loop flow rates dropped when dewatering was completed (03:30, 2/16/09, LRB 60230, pg. 92) due to the lower slurry level in Tank T02A. Another UFP-VSL-T02A recirculation loop sample $(39.5-\mathrm{mL})$ was taken and centrifuged down for 20 minutes $(4500 \mathrm{rpm})$; the final volume was $36.5-\mathrm{mL}$, indicating air entrainment of $\sim 7.6 \%(07: 05$, 2/16/09; LRB 60230, pg. 96).

Following the post-oxidative-leach washing, Integrated Test A was put on hold (midnight of 2/16/09). At the direction of the Test Director, a list of tasks were performed after completing the wash, however, only those associated with air entrainment are listed below:

- Chart filter-loop flow meters FT-0623 (pump UFP-PMP-T42A inlet) and 0635 (outlet of pump UFP-PMP-T43A), filter-loop pressure PT-0739 (inlet to filter UFP-FILT-T01A), and both pump speeds to understand the cause of low filter-loop flow rates and differences in flow meters.

- Retorque the mechanical connections from UFP-VSL-T02A to pump UFP-PMP-T42A in the event that there is a potential leak on the line. 


\subsubsection{Summary of Slurry Degassing and Filter-Loop Pump Exercises}

The following paragraphs summarize slurry degassing methods employed during Integrated Test A in efforts to degass the air entrained in the Tank T02A slurry.

The stable level measurement after the slurry was concentrated and washed was 54.6 inches at 23:40 and then 53.0 inches at 23:53 per laser (2/16/09, LRB 60230,pg. 116), which could suggest evidence of foam. PJMs 1801 and 1807 in UFP-VSL-T02A were back in service at 12:30, 2/17/09 (LRB 60230, pg. 118).

On 17:12, 2/17/09, the spargers, steam ring, and bubblers were off in UFP-VSL-T02A, while UFP-VSL-T02A was recirculating, and UFP-VSL-T02A PJM nozzle velocities were tuned to $12 \mathrm{~m} / \mathrm{s}$. Samples were collected from UFP-VSL-T02A at the request of the program manager (19:37 and 21:31, 2/17/09, LRB 60230, pg. 121).

A video was taken to capture the effects of turning the lower spargers on at $0.4 \mathrm{~kg} / \mathrm{min}$ and off during PJM operation, although image quality was poor and only got worse with time (LRB 60230,

pg. 123-124). At 22:20, 2/17/09 (LRB 60230, pg. 121), the lower spargers were turned on to $0.4 \mathrm{~kg} / \mathrm{min}$ :

\section{Subsequent Sparger operation indicates lower spargers set to $0.4 \mathrm{~kg} / \mathrm{min}$, and PJM operation indicates} $12 \mathrm{~m} / \mathrm{s}$ and $80 \%$ stroke (as tuned).

Flow before spargers:

- FT-0623 107 gpm

- FT-0635 78 gpm.

Flow after spargers turned on:

- FT-0623 75 gpm

- FT-0635 55 gpm.

A 5-in. tank level increase was seen with lower spargers ON (22:28, 2/17/09; LRB 60230, pg. 122).

The lower spargers were turned off at 00:10, 2/18/09 (LRB 60230, pg. 124), the filter-loop flow rate increased without making pump speed adjustments, and pump noise also stopped, pump vibration decreased, and a lower tank level was achieved. This improved filter loop pump performance indicates that some of the lower sparger air was being entrained in the pump intake.

Additional filter-loop flow rate exercises were conducted with PJMs ON and then with all sources (bubbler, steam ring, and spargers) off to see whether flow rates indicated by flow meters before UFP-PMP-T42A and after UFP-PMP-T43A converged at different pump speed settings and backpressure settings - 08:31 through 12:48, 2/18/98 (LRB 60230, pgs. 126-128). The difference between flow meters stayed the same, 30 to $40 \mathrm{gpm}$, regardless of pump settings or with the PJMs on or off. At 09:06, 2/18/09 (LRB 60230, pg. 128), two 40-mL samples and one 100-mL sample from the UFP-VSL-T02A recirculation loop were collected. These permeate samples had long spindle crystals at the time of collection they appeared to be oxalate (LRB 60235, pg. 30). 
Between 13:02 and 13:56, 2/18/09 (LRB 60230, pg. 129-130), exercises using a different number of spargers (total flow of $0.4 \mathrm{~kg} / \mathrm{min}$ ) were completed to see the effects on filter-loop flow rates. With the PJMs on, bubblers, upper spargers and steam ring air off, it was observed that turning three of the five lower spargers on (and when turning the other two of the five lower spargers on) caused the filter-loop flow rate to drop. When either three or the other two lower spargers were turned off, filter-loop flow rate increased without making pump setting adjustments (pump vibration and noise also decreased). The same result was seen when only the spargers on either side of Pump T42A inlet were turned on and off. The stable level per laser at 14:11 was 49.8 inches (LRB 60230, pg. 126-130). At 14:25, the laser level was 49.3 inches. After turning the pumps (UFP-PMP-T42A=65\%, UFP-PMP-T43A $=40 \%$, $\mathrm{HIC}=0609=55 \%$ ) and PJMs on, the stable level measurement at 15:15 was 49.1 inches per laser.

Figure 6.10 through Figure 6.18 show degassing operations listed in Table 6.2 through Table 6.5. Table 6.2 through Table 6.5 summarize mixing operations conducted to better understand air entrainment and air release in attempts to establish a gas-free simulant state.

NOTE: Mixing operations below indicate that lower spargers are set to $0.4 \mathrm{~kg} / \mathrm{min}$, the PJM operations are set to $12 \mathrm{~m} / \mathrm{s}$ and $80 \%$ stroke (as tuned), and the upper spargers and steam ring are OFF.

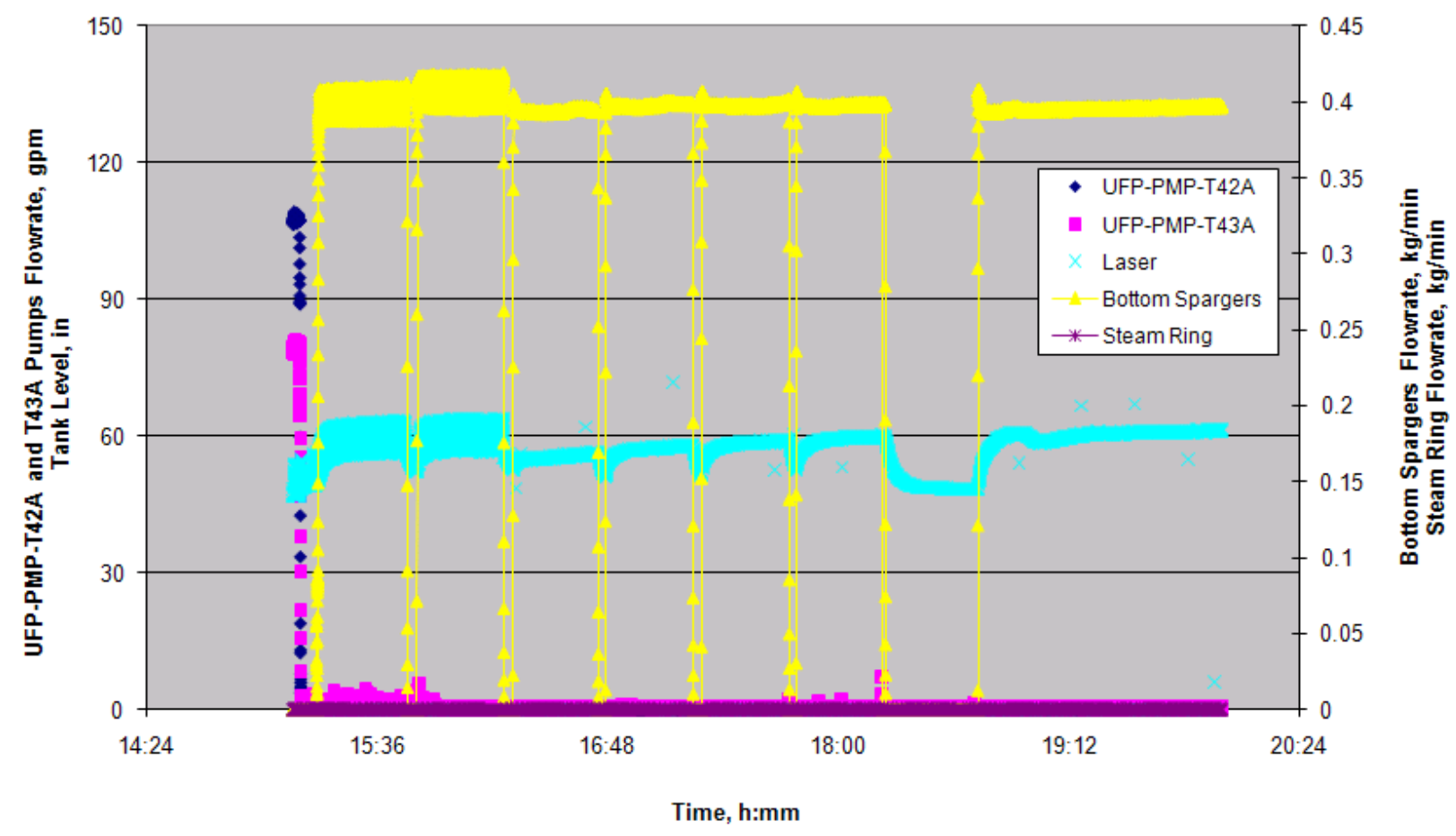

Figure 6.10. UFP-VSL-T02A Gas Accumulation and Gas Release Operations Using PJM and Lower Spargers, in Reference to Table 6.2 and Table 6.3 
Table 6.2. Gas Accumulation and Degassing Instructions per Guidance Section 1

\begin{tabular}{lccccccc}
\hline \multicolumn{7}{l}{ Section 1. Test to see how much gas accumulation in the slurry is achieved with PJM and sparger operation. } \\
\hline \multicolumn{1}{c}{ Operations } & PJMs & Spargers & Date & Time & LRB \# & Page \# & $\begin{array}{c}\text { UFP-VSL-T02A } \\
\text { Laser Level (in.) }\end{array}$ \\
\hline Stable Level Measurement & OFF & OFF & $2 / 18 / 09$ & $15: 15$ & 60230 & 133 & 49.1 \\
-- & ON & ON & $2 / 18 / 09$ & $15: 20$ & 60230 & 133 & Increasing tank levels \\
Stable Level Measurement & OFF & OFF & $2 / 18 / 09$ & $15: 48$ & 60230 & 133 & 52.5 \\
\hline
\end{tabular}

Table 6.3. Gas Accumulation and Degassing Instructions per Guidance Section 2

\begin{tabular}{|c|c|c|c|c|c|c|c|}
\hline Operations & PJMs & Spargers & Date & Time & LRB \# & Page \# & $\begin{array}{l}\text { UFP-VSL-T02A } \\
\text { Laser Level (in.) }\end{array}$ \\
\hline \multirow{2}{*}{ 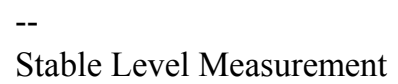 } & $\mathrm{ON}$ & $\mathrm{ON}$ & $2 / 18 / 09$ & $15: 49$ & 60230 & 133 & -- \\
\hline & OFF & OFF & $2 / 18 / 09$ & $16: 16$ & 60230 & 134 & -- \\
\hline \multirow{2}{*}{$\begin{array}{l}-- \\
-- \\
--\end{array}$} & OFF & OFF & 2/18/09 & $16: 18$ & 60230 & 134 & 53.3 \\
\hline & OFF & $\mathrm{ON}$ & 2/18/09 & $16: 19$ & 60230 & 134 & -- \\
\hline \multirow{2}{*}{$\begin{array}{l}\text { Stable Level Measurement } \\
\text {-- }\end{array}$} & OFF & OFF & 2/18/09 & $16: 46$ & 60230 & 134 & -- \\
\hline & OFF & OFF & 2/18/09 & $16: 48$ & 60230 & 134 & 51.3 \\
\hline \multirow{2}{*}{$\begin{array}{l}-- \\
\text { Stable Level Measurement }\end{array}$} & OFF & $\mathrm{ON}$ & $2 / 18 / 09$ & $16: 48$ & 60230 & 134 & -- \\
\hline & OFF & OFF & 2/18/09 & $17: 15$ & 60230 & 134 & -- \\
\hline \multirow{2}{*}{-- } & OFF & OFF & 2/18/09 & $17: 17$ & 60230 & 134 & 51.6 \\
\hline & OFF & ON & $2 / 18 / 09$ & $17: 17$ & 60230 & 134 & -- \\
\hline \multirow{2}{*}{$\begin{array}{l}\text { Stable Level Measurement } \\
\text {-- }\end{array}$} & OFF & OFF & $2 / 18 / 09$ & $17: 45$ & 60230 & 134 & -- \\
\hline & OFF & OFF & $2 / 18 / 09$ & $17: 47$ & 60230 & 134 & 52.9 \\
\hline \multirow{4}{*}{$\begin{array}{l}-- \\
\text { Stable Level Measurement } \\
-- \\
--\end{array}$} & OFF & ON & 2/18/09 & $17: 47$ & 60230 & 134 & -- \\
\hline & OFF & OFF & $2 / 18 / 09$ & $18: 15$ & 60230 & 134 & -- \\
\hline & OFF & OFF & 2/18/09 & $18: 44$ & 60230 & 134 & 48.4 \\
\hline & OFF & $\mathrm{ON}$ & $2 / 18 / 09$ & $18: 44$ & 60230 & 134 & -- \\
\hline
\end{tabular}


Table 6.4. Gas Accumulation and Degassing Instructions per Guidance

\begin{tabular}{lccccccc}
\hline \multicolumn{2}{c}{ Per Degassing Instructions provided on pg. 18 of LRB $60235 ;$ execution began at 20:17, 2/18/09. } \\
\hline \multicolumn{1}{c}{ Operations } & PJMs & Spargers & Date & Time & LRB \# & Page \# & UFP-VSL-T02A laser Level, (in.) \\
\hline 1) Stable Level Measurement & OFF & OFF & $2 / 18 / 09$ & $20: 17$ & 60230 & 134 & -- \\
-- & OFF & OFF & $2 / 18 / 09$ & $20: 47$ & 60230 & 134 & 48.7 \\
2)Pumps ON & ON & OFF & $2 / 18 / 09$ & $20: 56$ & 60230 & 135 & -- \\
3) Stable Level Measurement & OFF & OFF & $2 / 18 / 09$ & $22: 16$ & 60230 & 135 & -- \\
-- & OFF & OFF & $2 / 18 / 09$ & $22: 46$ & 60230 & 135 & 46.8 \\
4)-- & ON & ON & $2 / 18 / 09$ & $22: 46$ & 60230 & 135 & -- \\
5) Stable Level Measurement & OFF & OFF & $2 / 19 / 09$ & $00: 45$ & 60230 & 136 & -- \\
-- & OFF & OFF & $2 / 19 / 09$ & $01: 13$ & LT-0614 average & 47.6 \\
6) -- & ON & ON & $2 / 19 / 09$ & $01: 15$ & 60235 & 20 & -- \\
7) Stable Level Measurement & OFF & OFF & $2 / 19 / 09$ & $03: 15$ & 60235 & 20 & -- \\
-- & OFF & OFF & $2 / 19 / 09$ & $03: 45$ & 60235 & 21 & 48.1 \\
8) Pumps On & ON & OFF & $2 / 19 / 09$ & $03: 50$ & 60235 & 21 & -- \\
9) Stable Level Measurement & OFF & OFF & $2 / 19 / 09$ & $04: 20$ & 60235 & 21 & -- \\
-- & OFF & OFF & $2 / 19 / 09$ & $04: 50$ & 60235 & 21 & 46.5 \\
\hline
\end{tabular}

At 22:15, a recirculation loop sample was collected, and at 23:00, a slow leak at the flange where the hosing goes into the tank was observed on the south side of UFP-VSL-T02A. A pipe out of UFP-VSL-T02A was leaking chilled water, and a bucket was placed underneath for containment (2/18/09, LRB 60230, pg. 135).

An experiment was performed to assess the effect of dilution on the rate of degassing on 2/19/09. Three graduated cylinders contained $\sim 500-\mathrm{mL}$ of the following simulant dilutions: 1) $17-\mathrm{wt} \%$ simulant, 2) $17-w t \%$ simulant diluted to $10-w t \%$ and corresponding to $588-\mathrm{mL}$ of simulant with 412 of IW (capped and shaken and then poured into a graduated cylinder), and 3) a dilution of the existing volume of $17-w t \%$ simulant to the maximum operating level of UFP-VSL-T02A. This corresponded to $625-\mathrm{mL}$ of $17-\mathrm{wt} \%$ simulant and 375-mL of deionized water, capped and shaken and then poured into the graduated cylinder (LRB 60230, pg. 136 and 143). The total volume and settled solids volume of each graduated cylinder $( \pm 2-\mathrm{mL})$ was measured over a 7-hr period. It was observed that greater total volume and settled solids volume decreases occurred over time in the higher and lower weight percent samples, respectively. These results are shown in Table 6.5. 
Table 6.5. Sample Dilution vs. Degassing

\begin{tabular}{ccccccc}
\hline Hour & Graduated Cylinder A - 17-wt $\%$ & Graduated Cylinder B - 10-wt $\%$ & \multicolumn{2}{c}{ Graduated Cylinder C - 10.6-wt $\%$} \\
\hline $2 / 19 / 09$ & $\begin{array}{c}\text { Settled Solids } \\
\text { Volume }(\mathrm{mL})\end{array}$ & $\begin{array}{c}\text { Total Volume } \\
(\mathrm{mL})\end{array}$ & $\begin{array}{c}\text { Settled Solids } \\
\text { Volume }(\mathrm{mL})\end{array}$ & $\begin{array}{c}\text { Total Volume } \\
(\mathrm{mL})\end{array}$ & $\begin{array}{c}\text { Settled Solids } \\
\text { Volume }(\mathrm{mL})\end{array}$ & $\begin{array}{c}\text { Total Volume } \\
(\mathrm{mL})\end{array}$ \\
\hline Start & 440 & 455 & 440 & 490 & 440 & 490 \\
1 & 430 & 455 & 355 & 490 & 360 & 490 \\
2 & 425 & 450 & 335 & 490 & 340 & 490 \\
3 & 420 & 450 & 325 & 490 & 333 & 488 \\
4 & 420 & 450 & 317 & 490 & 325 & 488 \\
5 & 415 & 450 & 310 & 490 & 320 & 488 \\
6 & 412 & 450 & 305 & 490 & 315 & 488 \\
7 & 410 & 450 & 301 & 490 & 310 & 488 \\
\hline
\end{tabular}

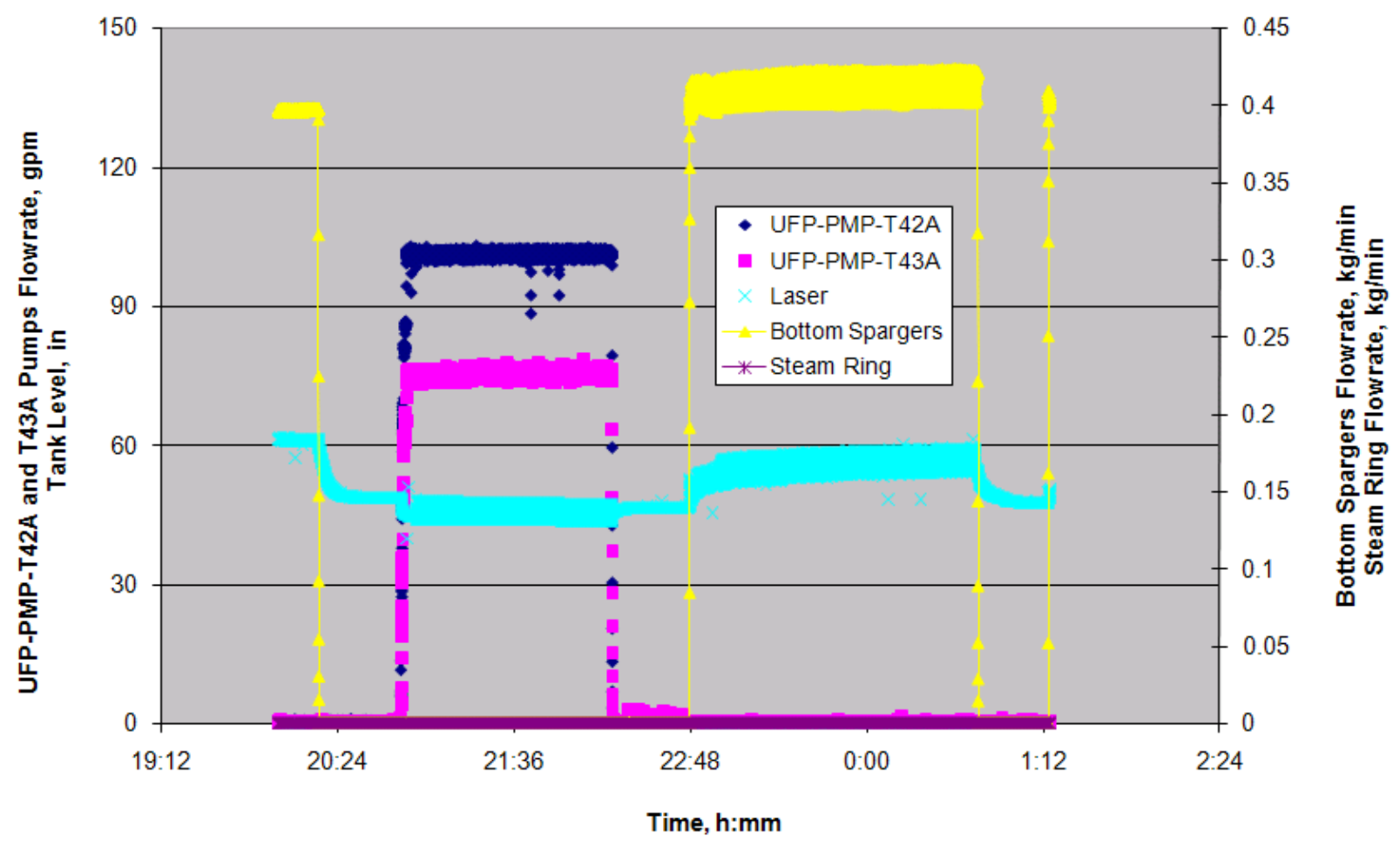

Figure 6.11. UFP-VSL-T02A Gas Release Operations, in Reference to Table 6.4, Steps 1 Through 5 


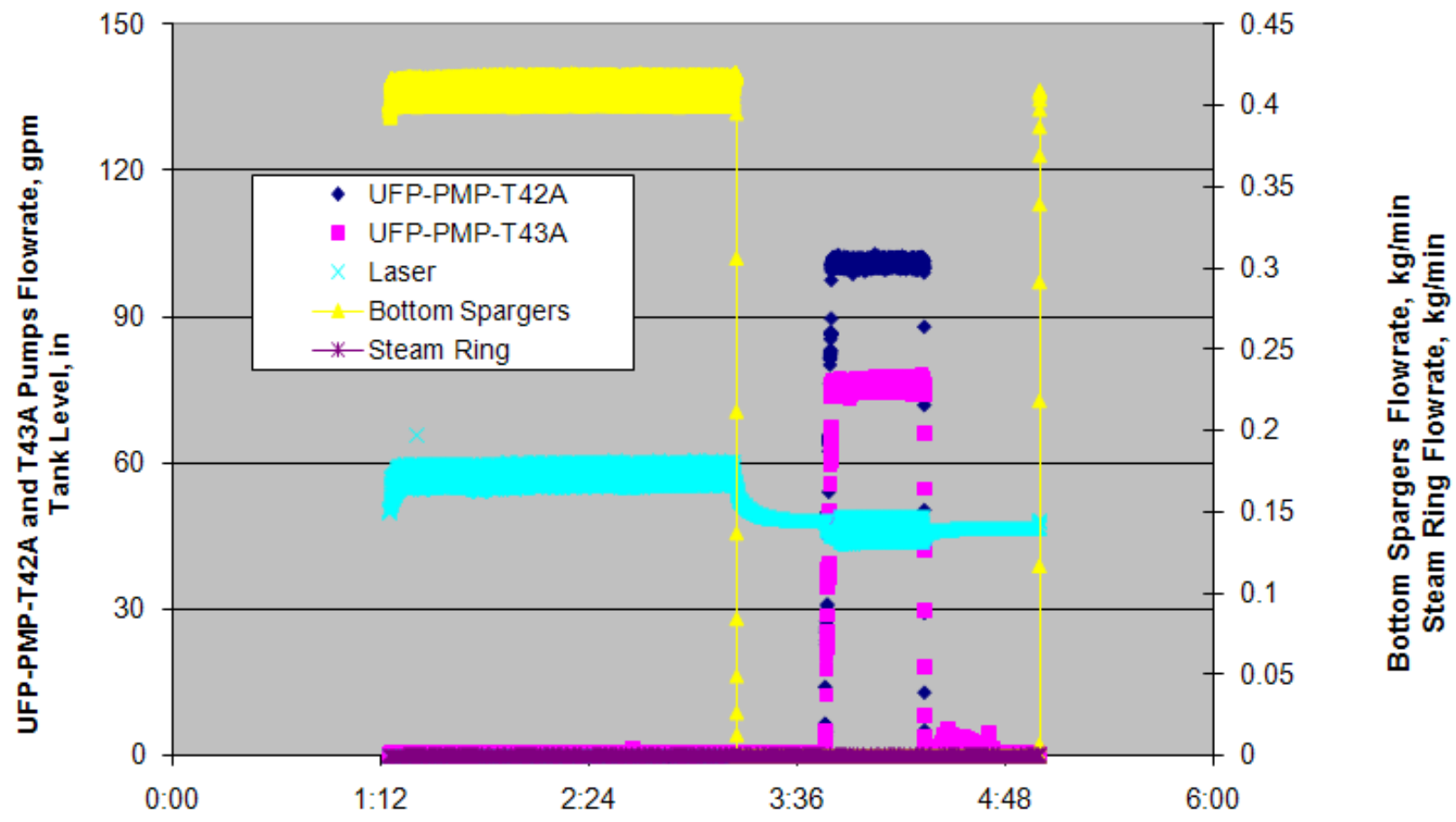

Time, h:mm

Figure 6.12. UFP-VSL-T02A Gas Release Operations, in Reference to Table 6.4, Steps 6 Through 9

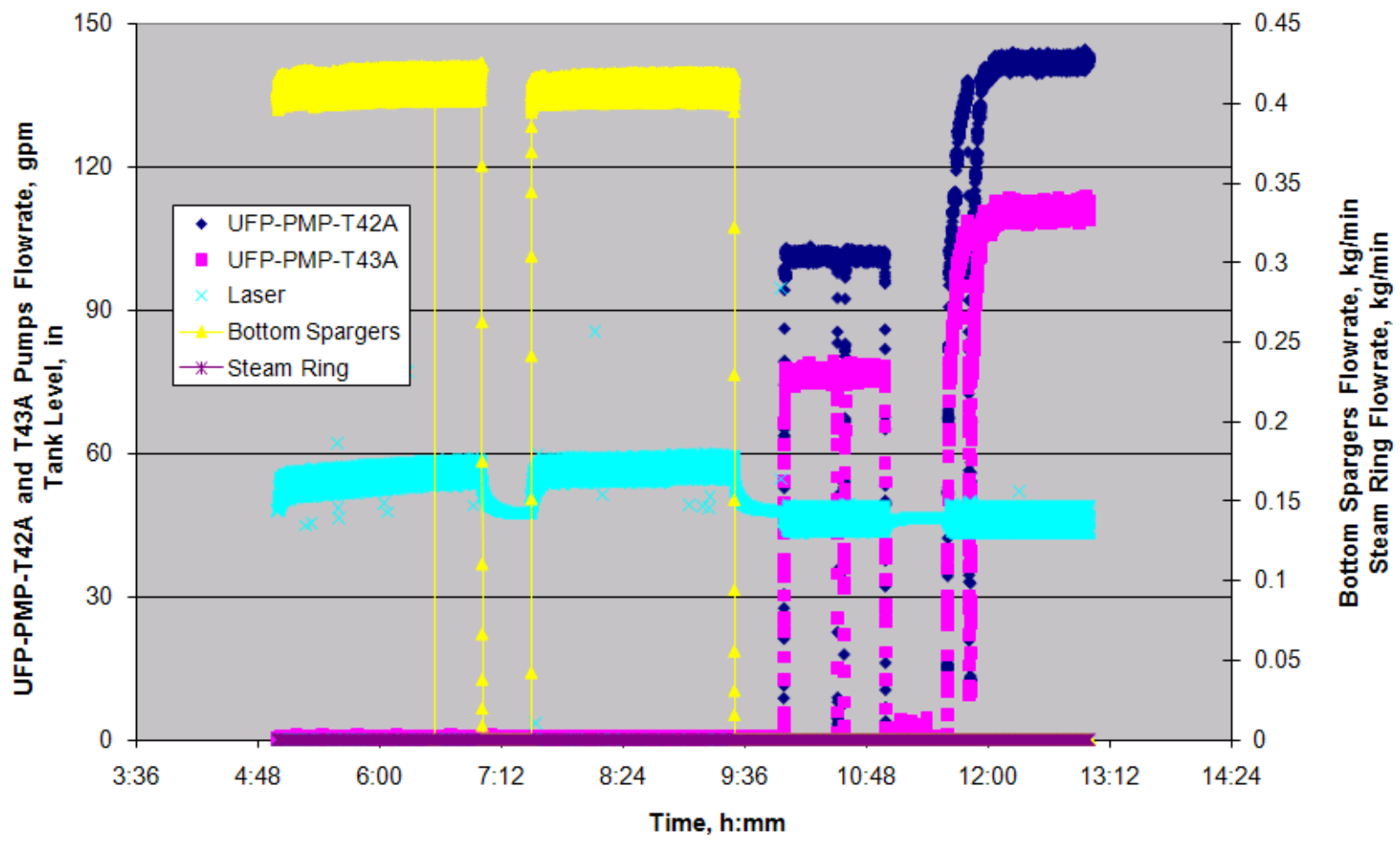

Figure 6.13. UFP-VSL-T02A Gas Release Operations, in Reference to Table 6.6 
Table 6.6. Degassing Instructions per Guidance, Part II

Per Degassing Instructions noted above (in Table 6.4), since last stable level is comparable to the smallest degassed level, repeat degassing sparger and PJM operations.

\begin{tabular}{|c|c|c|c|c|c|c|c|}
\hline Operations & PJMs & Spargers & Date & Time & LRB \# & Page \# & $\begin{array}{l}\text { UFP-VSL-T02A laser } \\
\text { Level, (in.) }\end{array}$ \\
\hline 4) -- & ON & $\mathrm{ON}$ & $2 / 19 / 09$ & 05:00 & 60235 & 21 & -- \\
\hline $\begin{array}{l}\text { 5) Stable Level } \\
\text { Measurement }\end{array}$ & OFF & OFF & $2 / 19 / 09$ & 07:00 & 60235 & 22 & -- \\
\hline-- & OFF & OFF & $2 / 19 / 09$ & $07: 30$ & 60235 & 22 & 47.5 \\
\hline 6) -- & $\mathrm{ON}$ & ON & $2 / 19 / 09$ & $07: 30$ & 60235 & 22 & -- \\
\hline $\begin{array}{l}\text { 7) Stable Level } \\
\text { Measurement }\end{array}$ & OFF & OFF & $2 / 19 / 09$ & 09:30 & 60235 & 22 & -- \\
\hline-- & OFF & OFF & $2 / 19 / 09$ & $10: 00$ & 60235 & 22 & 48.0 \\
\hline 8) Pumps On & $\mathrm{ON}$ & OFF & $2 / 19 / 09$ & $10: 00$ & 60235 & 22 & -- \\
\hline $\begin{array}{l}\text { 9) Stable Level } \\
\text { Measurement }\end{array}$ & OFF & OFF & $2 / 19 / 09$ & $10: 31$ & 60230 & 137 & -- \\
\hline 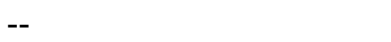 & OFF & OFF & $2 / 19 / 09$ & $10: 33$ & 60230 & 137 & 45.8 \\
\hline Pumps On & ON & OFF & $2 / 19 / 09$ & $10: 36$ & 60230 & 138 & -- \\
\hline Stable Level Measurement & OFF & OFF & $2 / 19 / 09$ & $11: 00$ & 60230 & 138 & -- \\
\hline-- & OFF & OFF & $2 / 19 / 09$ & $11: 30$ & 60230 & 138 & 46.5 \\
\hline
\end{tabular}

Between 11:36 and 13:45, 2/19/09 (LRB 60230, pgs. 138-140), PJMs and pumps were turned on; pump settings were adjusted to meet the target flow rate of $109 \mathrm{gpm}$. Stable level measurements at 13:30 still gave a 46.5-in. laser tank level. The pump settings were made at 13:45:

- $\mathrm{MC}-0631=71 \%$

- $\mathrm{MC}-0641=65 \%$

- $\mathrm{HIC}-0609=55 \%$

- FT-0635= 110 gpm

- $\mathrm{PI}-0739=\sim 142 \mathrm{psi}$

- PI-0789=55 psi

- PI-0625=-85 to -93 inches of water

- FT-0623=137 gpm.

Without making any adjustments to pump settings, a pressure drop was observed, and the flow on FT-0635 (downstream from UFP-PMP-T43A) increased to 130 gpm (LRB 60230, pg. 141). It seems as if a plug in the filter loop was released, but may have been attributed to a change in the quantity of air entrainment in the filter loop. After adjusting pump settings to bring the filter-loop level down to 109 gpm, the pump settings at 14:13 were as follows:

- $\mathrm{MC}-0631=71 \%$

- $\mathrm{MC}-0641=45 \%$

- HIC-0609=50\%

- FT-0635=109 gpm 
- PI-0739=110 psi

- PI-0789=50 psi

- PI-0625=-26 inches of water

- FT-0623=114 gpm.

(Note: there is no big difference between flow meters FT-0623 and FT-0635 at this time.)

UFP-VSL-T02A recirculation loop 40-mL samples were taken and centrifuged at the request of the Test Director at 16:15 and 16:32, 2/19/09 (LRB 60230, pg. 146). These samples had cloudy supernate. Similarly, there was an instance when permeate samples were taken and they too were cloudy, but white in appearance - like milk, but they cleared up in a few minutes. The cloudy appearance of the permeate samples indicates the presence of a large number of very small bubbles. After the samples were centrifuged, the supernate was a clear yellow color, and the sample volume decreased from 40 to $\sim 38$-mL, indicating a gas holdup of $\sim 6 \%$.

By 18:50, the PJM and pumps were on, and pumps had similar settings obtained at 14:13 (LRB 60230, pg. 148). The JTG met and decided to proceed with Integrated Test A using the steps described in Table 6.7 (per Test Director email 2/19/09, 7:02 PM). The steps in Table 6.7_were performed using PJM settings as is, even though the stroke did not meet the target - this was approved by the Test Director (19:11, LRB 60235, pg. 24).

NOTE: Mixing operations below indicate that upper spargers are OFF. 
Table 6.7. Attempts to Maintain Filter-Loop Target

\begin{tabular}{|c|c|c|c|}
\hline & & Started & Ended \\
\hline 1) & $\begin{array}{l}\text { With PJMs at current settings ( } \sim 12 \mathrm{~m} / \mathrm{s}, 80 \% \text { stroke, } 20 \mathrm{~s} \mathrm{cycle}) \text {, UFP-PMP-T42A at fixed } \\
\text { rpm, and UFP-PMP-T43A controlled to maintain } 109 \mathrm{gpm} \text { per FT-0635, turn lower air } \\
\text { spargers on "idle" flow rates }(0.04 \mathrm{~kg} / \mathrm{min} \text { total, } 13 \mathrm{scfh} / \mathrm{sparge} \text { tube) and run for } 4 \text { hours } \\
\text { with all other air sources OFF (zero air flow to steam-ring air purge and zero air flow to } \\
\text { level bubblers). If the filter-loop flow rate cannot be maintained at the target } 109 \text { gpm, } \\
\text { turn off the lower air spargers and degas the contents of UFP-VSL-T02A and the filter } \\
\text { loop as described below. Do NOT attempt to conduct step } 2 \text {. }\end{array}$ & $\begin{array}{l}18: 51, \\
2 / 19 / 09 \\
\text { (LRB 60230, } \\
\text { pg. 148) }\end{array}$ & $\begin{array}{l}23: 00,2 / 19 / 09 \\
(\mathrm{LRB} 60235 \\
\text { pg. 27) }\end{array}$ \\
\hline 2) & $\begin{array}{l}\text { If the flow rate remains at its targeted } 109 \text { gpm after the } 4 \text { hours in step } 1 \text {, take a stable } \\
\text { level measurement with spargers OFF, resume PJMs, resume pumping, resume the idle } \\
\text { sparge air flow rate, and turn ON the steam-ring air purge to } 0.16 \mathrm{~kg} / \mathrm{min} \text { (max). Run } \\
\text { these conditions for } 4 \text { hours. If the filter-loop flow rate cannot be maintained at the target } \\
109 \text { gpm, turn off the lower air spargers, and degas the contents of UFP-VSL-T02A and } \\
\text { the filter loop as described below. }\end{array}$ & $\begin{array}{l}23: 00 \\
2 / 19 / 09 \\
47.5 \text { inches } \\
\text { per laser }\end{array}$ & $\begin{array}{l}02: 49,2 / 20 / 09 \\
(\text { LRB } 60235 \\
\text { pg. 29) }\end{array}$ \\
\hline 3) & $\begin{array}{l}\text { If the flow rate remains at its targeted } 109 \mathrm{gpm} \text { after the } 4 \text { hours in step } 2 \text {, take a stable } \\
\text { level measurement with spargers and steam ring OFF. Proceed then to the final solids } \\
\text { concentration and subsequent steps of TI-065. }\end{array}$ & -- & -- \\
\hline \multicolumn{4}{|c|}{ Degas guidance: } \\
\hline A) & Spargers off, all mixing off, pumps off-record stable level. & $\begin{array}{l}03: 19 \\
2 / 20 / 09 \\
48.8 \text { inches }\end{array}$ & $03: 50,47.3 ”$ \\
\hline B) & Start spargers at full flow ( $0.4 \mathrm{~kg} / \mathrm{min}$ total flow), and start PJMs - run for 2 hours. & 03:57 & 06:09 \\
\hline C) & Stop spargers and run pumps at low flow for $10 \mathrm{~min}$ to circulate slurry in loop (PJMs on). & 06:09 & $06: 25$ \\
\hline D) & Stop pumps and run spargers at full flow for 2 hours (PJMs on). & $06: 25$ & 08:26 \\
\hline E) & Stop spargers - run PJMs for 0.5 hour, and then record stable level. & 08:26 & $\begin{array}{c}\text { 08:58, (LRB } \\
60235 \text {, pg. } 30) \\
09: 25, \\
48.8 \text { inches }\end{array}$ \\
\hline F) & $\begin{array}{l}\text { Start PJMs and loop pumps (no spargers) - attempt to bring filter-loop flow rate to } \\
109 \text { gpm. }\end{array}$ & $\begin{array}{c}\text { 09:28, } \\
\text { 2/20/09 } \\
\text { (LRB 60230, } \\
\text { pg. 157) }\end{array}$ & $\begin{array}{l}10: 34,2 / 20 / 09 \\
(\mathrm{LRB} 60230 \\
\quad \text { pg. } 158)\end{array}$ \\
\hline G) & If 109 gpm flow rate cannot be reached or maintained, repeat starting at step A. & -- & -- \\
\hline H) & $\begin{array}{l}\text { If filter-loop flow rate remains at target } 109 \text { gpm, proceed with TI-065 and the final solids } \\
\text { concentration step, etc. }\end{array}$ & -- & -- \\
\hline
\end{tabular}




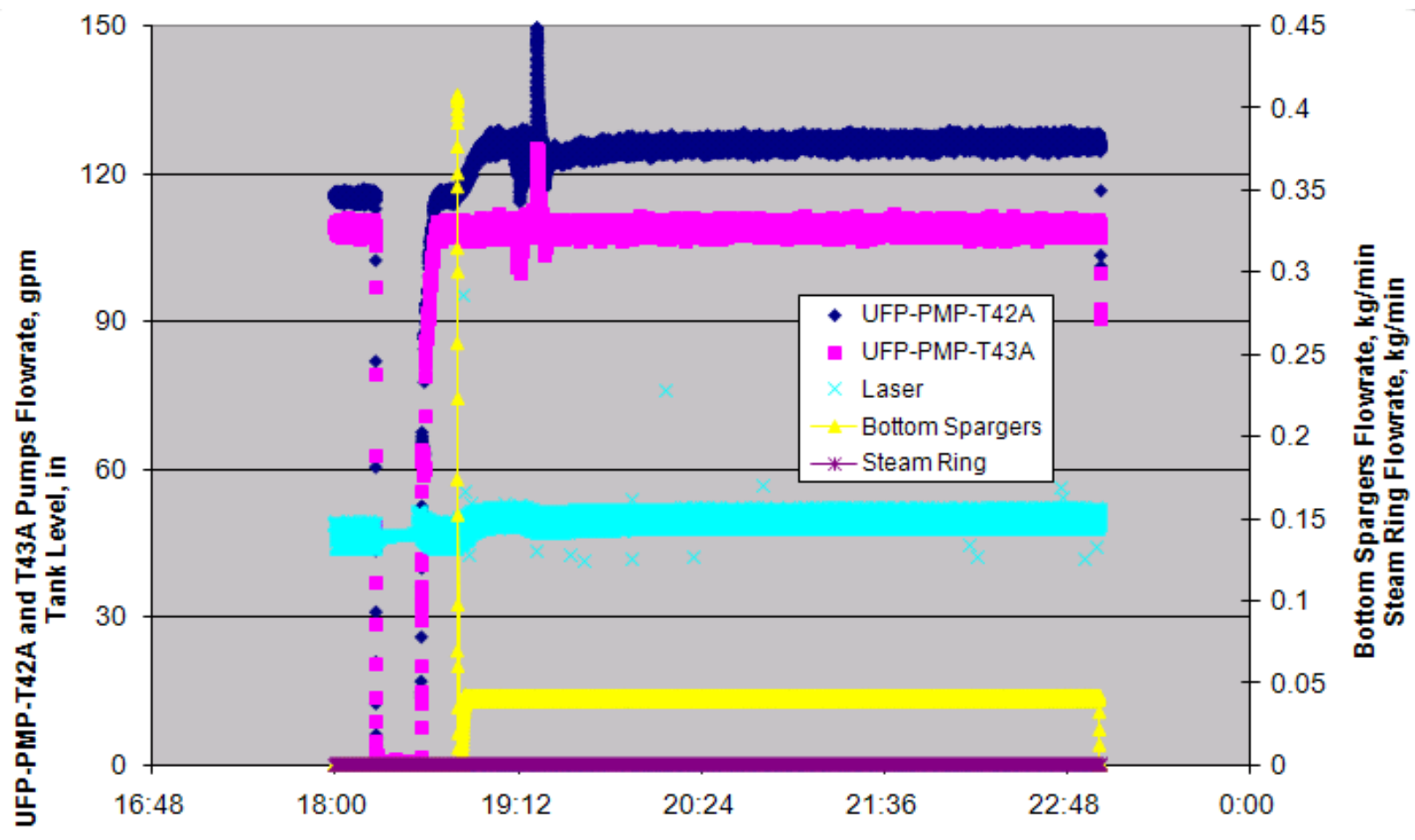

Time, h:mm

Figure 6.14. Operations for Restoring Target Filter-Loop Flow Rate, in Reference to Table 6.7, Step 1

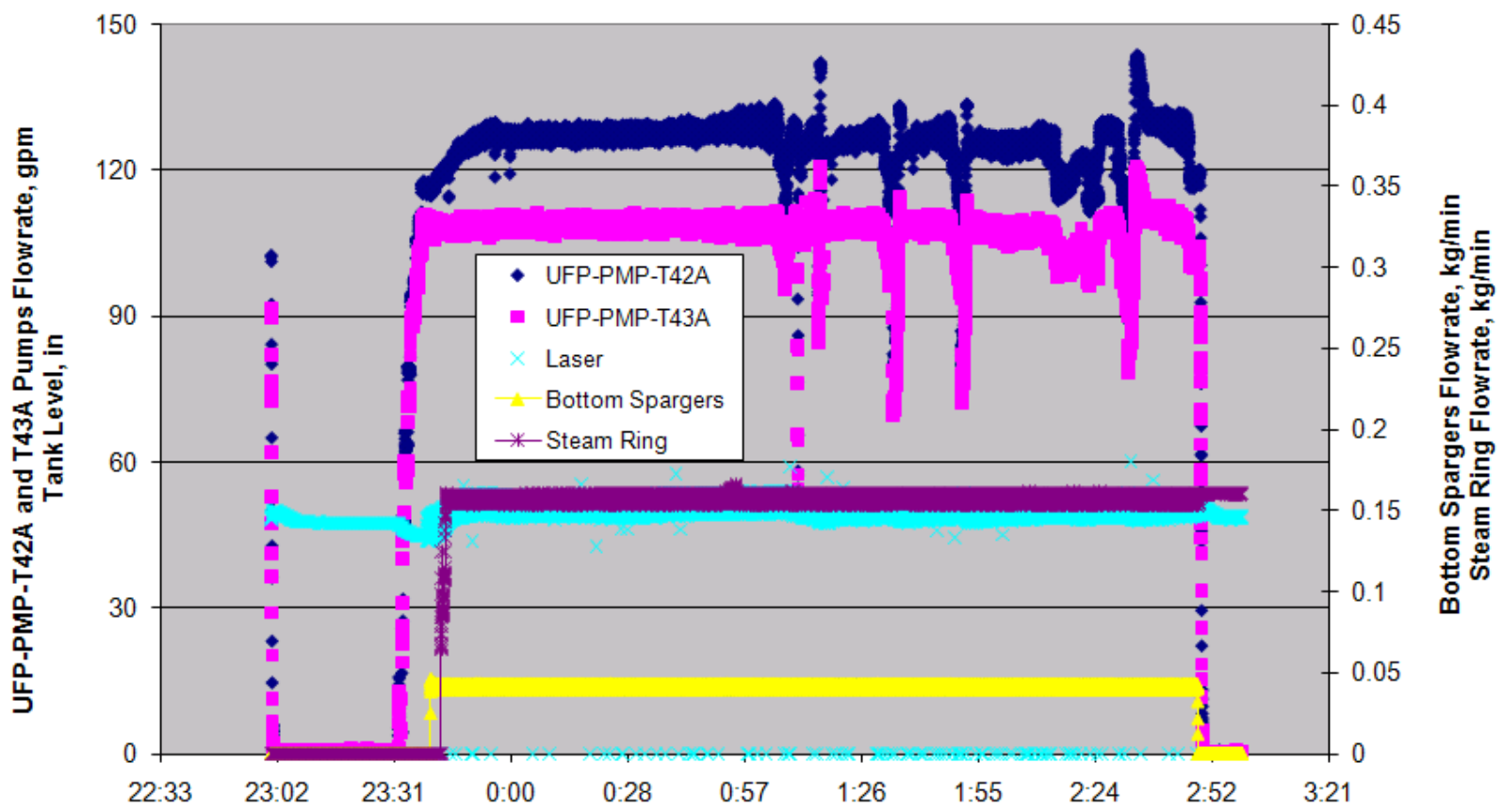

Time, h:mm

Figure 6.15. Operations for Restoring Target Filter-Loop Flow Rate, in Reference to Table 6.7, Step 2 


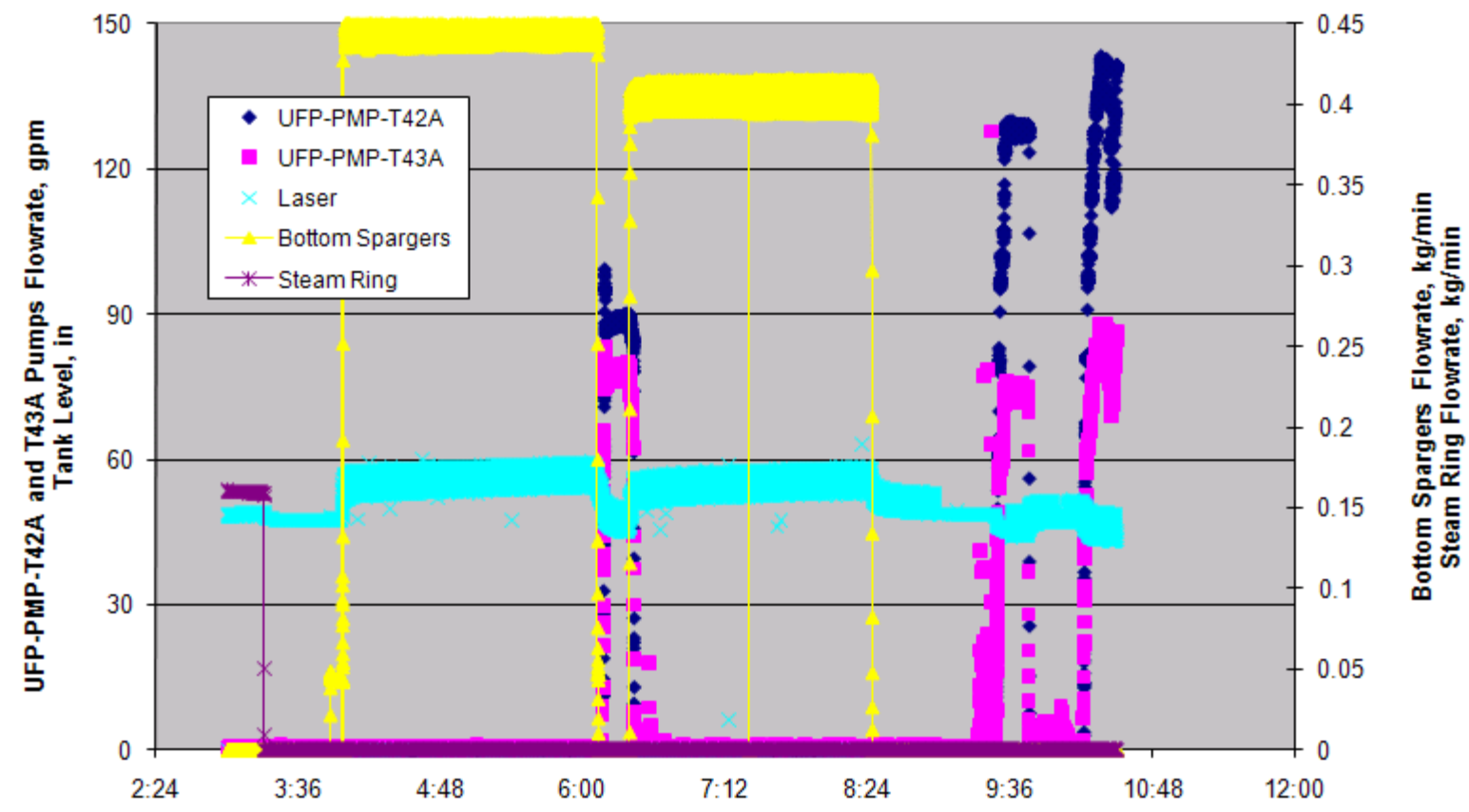

Time, h:mm

Figure 6.16. Operations for Restoring Target Filter-Loop Flow Rate, in Reference to Table 6.7, Steps A Through F

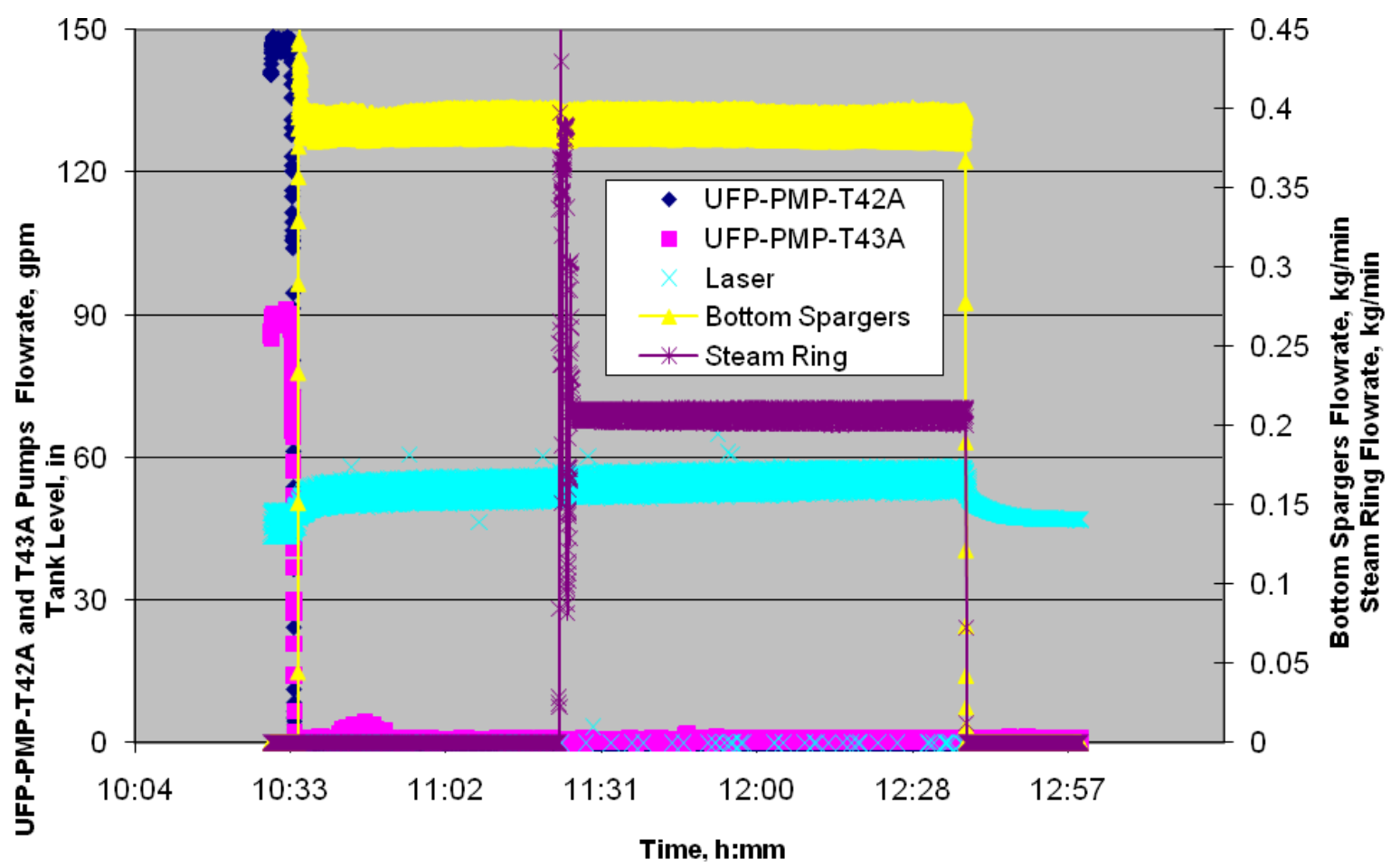

Figure 6.17. Operations for Restoring Target Filter-Loop Flow Rate, in Reference to Table 6.8, Steps 2 and 3 


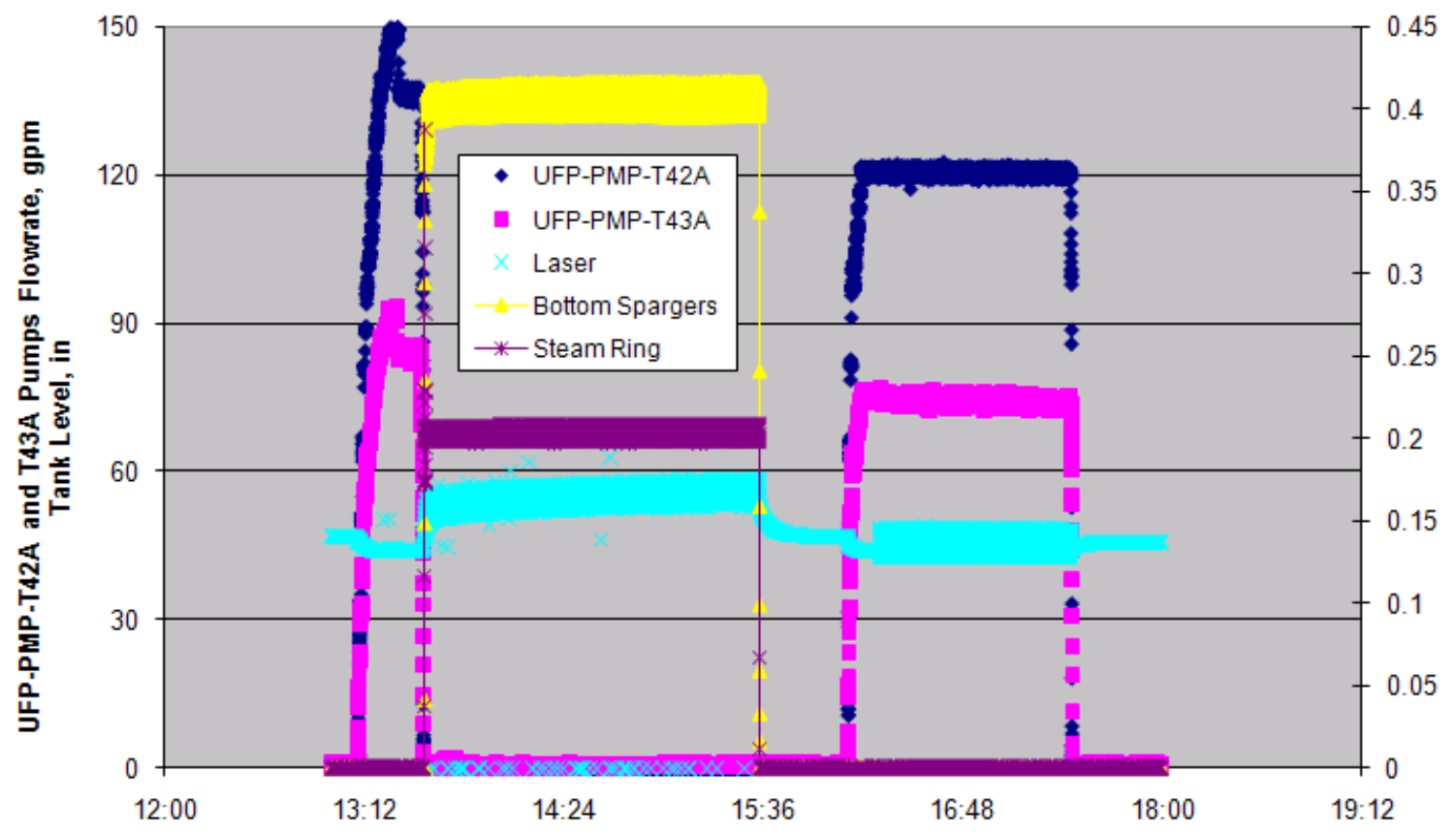

Time, h:mm

Figure 6.18. Operations for Restoring Target Filter-Loop Flow Rate, in Reference to Table 6.8, Steps 4 Through 7

Instructions above (Table 6.7) were superseded by the following Test Director email, 2/20/09 (LRB 60230, pg. 160):

NOTE: Mixing operations below indicate that upper spargers are OFF.

Table 6.8. Attempts to Maintain Target Filter-Loop Target, Part II

\begin{tabular}{|c|c|c|}
\hline Direction on Bubble/Pump Evaluation & Started & Ended \\
\hline 1) Spargers off, all mixing off, pumps off- - record stable level. & $\begin{array}{l}08: 58,2 / 20 / 09(\mathrm{LRB} \\
\quad 60230, \text { pg. } 156\end{array}$ & $09: 25,48.8^{\prime \prime}$ \\
\hline 2) Start spargers at full flow, and start PJMs - run for 2 hours. & $10: 35$ & $12: 35$ \\
\hline 3) Stop spargers and PJMs—let sit for 0.5 hours — record stable level. & $12: 35$ & 13:05, 46.9” \\
\hline Attempted to get target filter-loop flow rate—not successful. & $13: 10$ & $13: 30$ \\
\hline 4) Start spargers at full flow, and start PJMs - run for 2 hours. & $13: 33$ & $15: 34$ \\
\hline 5) Stop spargers and PJMs—let sit for 0.5 hours—record stable level. & $15: 34$ & $16: 00,46.9 ”$ \\
\hline 6) Start PJMs and loop pumps, spargers remain off-run for 1 hour. & 16:06 & $17: 27$ \\
\hline 7) Stop PJMs and loop pumps - let sit for 0.5 hours - record stable level. & $17: 27$ & $\begin{array}{l}17: 57,2 / 20 / 09,45.7 \\
(\text { LRB } 60230,163)\end{array}$ \\
\hline 8) Repeat this process. & -- & -- \\
\hline Steam ring @ $0.20 \mathrm{~kg} / \mathrm{min}$ anytime spargers are running & -- & -- \\
\hline $\begin{array}{l}\text { If after any step, the UFP-VSL-T02A level is }<47 \text { in. during stable level } \\
\text { measurement, attempt to bring pump flow to } 110 \mathrm{GPM} \text {. }\end{array}$ & -- & -- \\
\hline
\end{tabular}


More attempts were made to bring the filter-loop flow rate to the 109 gpm target; however, none were successful. On 2/20/2009 at19:22, facilities personnel tried verifying that flow meter FT-0635, downstream from UFP-PMP-T43A, was working within manufacturer's and PEP specifications. However, the flow meter FT-0635was found defective per NCR 42317.1. With approval, filtering was initiated having permeate going back in UFP-VSL-T02A. Backpulsing for each of the filters followed (2/20/09, LRB 60230, pg. 165).

With approval, staff then proceeded with the Test Instruction (2/20/09, LRB 60235, pg. 30).

Based on the flow rate divergence problems with flow meter FT-0635, its replacement was made after completing Integrated Test A on 2/26/09 (Operations Manager email, 16:55, 2/26/09) and Hanson email, 4/7/09, 17:25.

\subsubsection{Air Entrainment Operational Guidance and Recommendations}

Pump operation issues were minimized with the subsequent operating strategies exercised in Integrated Tests B (WTP-RPT-192) and D (WTP-RPT-193). Written guidance for UFP-VSL-T02A Mixing Systems Operation in the Presence of Air was provided as follows: ${ }^{\text {(a) }}$

In the event that filter-loop flow rates cannot be maintained at the targeted $109 \pm 10$ gpm using the pump-control guidance, and there is evidence that there is significant entrained air in the slurry, the following actions are to be taken.

\section{Tank T02A Systems Guidance}

1. Turn level bubbler off.

2. Turn steam-ring air purge off.

3. Allow 30 minutes to determine if the Tank T02A recirculation system recovers the desired flow rate. If flow is recovered, proceed with testing.

4. If flow does not recover, implement the initial degassing protocol listed below.

5. If flow recovers, proceed with testing.

6. If flow does not recover, turn off lower air spargers.

7. Allow 30 minutes to see if the system recovers flow. If flow is recovered, proceed with testing.

8. If flow does not recover, implement initial degassing protocol.

9. If flow recovers, proceed with testing.

10. If flow does not recover, contact the on-call Test Director.

11. Perform the extended degassing protocol listed below as needed to fully degas the system.

12. Reinitiate testing.

13. If flow rates cannot be maintained at the targeted $109 \pm 10 \mathrm{gpm}$, perform the initial degassing protocol.

(a) E-mail communication from R Burk, Bechtel, to SM Barnes, Bechtel, and GB Josephson and DE Kurath, PNNL, RE: Written Guidance for T02A Mixing Systems Operation in the Presence of Air, Filterloop Pump Operation, and PJM Operation During Integrated Test B. 3/11/09, 16:12. 
14. If flow does not recover, reduce the filter recirculation flow to $88 \pm 10$ gpm filter-loop flow rate (gives $\sim 12 \mathrm{ft} / \mathrm{s}$ axial velocity in the filter tubes).

15. If 88 gpm cannot be maintained, perform the extended degassing protocol as needed to fully degas Tank T02A.

16. Reinitiate testing.

17. If flow cannot be maintained at $109 \pm 10 \mathrm{gpm}$ or $88 \pm 10 \mathrm{gpm}$, continue testing with the filter recirculation loop flow rate reduced to $67 \pm 10 \mathrm{gpm}$. This filter-loop flow rate gives $\sim 9 \mathrm{ft} / \mathrm{s}$ axial velocity in the filter tubes and power/volume scaled mixing from the return nozzle jet).

18. If none of these actions are successful, begin raising the Tank T02A level by diluting with IW. Raise the tank level in 2-in. increments, allowing at least 30 min between IW additions.

$\underline{\text { Initial Degassing Protocol }}$

1. Turn off filter-loop pumps.

2. Take a stable level measurement.

3. Adjust lower air sparge flow rate to normal full flow $(0.40 \mathrm{~kg} / \mathrm{min}, \sim 2.4 \mathrm{scfm} / \mathrm{tube})$ and turn on PJMs.

4. Allow this configuration to run for $50 \mathrm{~min}$.

5. Turn off spargers and PJMs.

6. Wait for $10 \mathrm{~min}$.

7. Take a stable level measurement.

8. Turn pumps on and attempt to achieve target filter-loop flow rate.

Restore Tank T02A mixing systems per the stage of the Tank T02A systems operating guidance above where the initial degassing process was referenced.

\section{Extended Degassing Protocol}

1. Turn off filter-loop pumps and take a stable level measurement.

2. Adjust lower air sparge flow rate to normal full flow $(0.40 \mathrm{~kg} / \mathrm{min}, \sim 2.4 \mathrm{scfm} / \mathrm{tube})$ and turn on PJMs.

3. Allow this configuration to run for 4.5 hours.

4. Turn off spargers and PJMs.

5. Wait for 20 minutes.

6. Operate the filter-loop pumps for 10 minutes.

7. Take a stable level measurement.

8. Repeat steps 2 through 7 as needed to degas Tank T02A as determined by prior degassed level readings or by less than $5 \%$ gas volume holdup measured by centrifuging a slurry sample.

9. Turn pumps on, and attempt to achieve target filter-loop flow rate.

10. Restore Tank T02A mixing systems per the Tank T02A systems operating guidance above. 


\subsubsection{Recommendations}

Significant air entrainment was encountered during conduct of Integrated Test A. The air entrainment resulted in apparent cavitation (actual cavitation is the formation of gas bubbles due to low pressure at the pump impeller, resulting in decreased pump performance) of the filter loop pumps and led to reduced pumping capacity and flow rates through the filter loop. The series of events presented in previous sections of this report indicate the following:

- PJM overblows may have contributed to air entrainment in some cases. With the filter loop in operation, overblow bubbles could have been entrained into the pump in-take. Overblows were reported by the operations staff but this could not be confirmed with the available DAS data. Implementation of a robust control system to minimize PJM overblows, especially when the filter loop pumps are operating, is suggested as a means of mitigating the contribution of PJM overblows to the air entrainment issue.

- Air from the air spargers also appears to have contributed to the air entrainment issue. Separate tests indicate that the spargers closest to the pump intake and the return nozzle had the greatest impact. Suggested mitigating actions include reevaluating the location of the air sparge outlets and reducing the air flow to some or all of the air spargers.

- The bubbler level measuring device was located near the pump intake and may have contributed to the air entrainment issue. Although the air flow rate through the bubbler was relatively low the close proximity to the pump intake may have allowed the air to be pulled into the pump intake. It is suggested that the location of the bubbler level measuring instrument be evaluated.

- Air from the steam ring air purge may have been pulled into the pump intake.

- The partial failure of the flow meter (FT-0635) at the outlet of the filter loop pumps may have contributed to the magnitude of the air entrainment problem. At the completion of Integrated Test A this flow meter was found to be reading low. In attempting to achieve the target flow rate of 109 gpm, operators may have achieved a higher flow rate which would exacerbate the air entrainment from the filter loop return nozzle.

Based on the air entrainment operational issues encountered in Integrated Test A, future plant operations should avoid prolonged operations with low tank levels. The filter-loop return line in UFP-VSL-T02A was exposed at 20.3 inches (Section 4.9); therefore, operating at tank levels lower than this may have introduced air in the slurry during filter-loop recirculation. Bubblers and steam-ring and sparger settings only exacerbated the air entrainment at low levels. In addition, it would be prudent to investigate optimum PJM stroke and average peak velocities at different UFP-VSL-T02A tank levels and slurry properties in efforts to minimize, or prevent, air entrainment caused by PJM overblowing.

To expedite degassing efforts in plant operations, it is suggested to do the following:

- Operate spargers and PJMs with no pumps for 1 to 2 hours.

- Wait 30 minutes to 1 hour before taking a stable level measurement.

- Turn PJMs and filter-loop pumps ON for at least 30 minutes to degas. 
- Wait 30 minutes before taking a stable level measurement.

- Repeat PJM and pump operation until level stops decreasing.

\subsection{Level Instruments}

At several times during the test, there was no credible level instrument for status and level control in any of the ultrafeed vessels. Not having a reliable level instrument made filtering, filling a vessel to a target level, and auto batching confusing and problematic, not to mention the associated high- and low-level alarms interrupting steam injection, PJM, and pump operations. Please refer to Table E.1 in Appendix E for the sequential list of level instrumentation problems. The following paragraphs discuss the types of problems and work arounds, if any, for each of the level instruments: laser, bubbler, and Drexelbrook.

\subsubsection{Lasers}

Laser performance was unreliable throughout Integrated Test A efforts, but especially during the leaching and post-caustic-leach concentration. One of the symptoms of unstable laser level performance was high and low level spikes, which interrupted steam injection, PJM, and pump operation. When concentrating post-caustic-leach slurry, the UFP-VSL-T02A low-level spikes erroneously triggered batches from the feed vessel and/or shut down PJMs and filter-loop pumps:

- 15:58, 2/2/09 (LRB 60229, pg. 59). The $23^{\text {rd }}$ auto batch from UFP-VSL-T01A was triggered by an erroneous laser level in UFP-VSL-T02A. The low-low level alarm was triggered in UFP-VSL-T02A; therefore, pumps and PJMs were turned off.

- (LRB 60229, pg. 60) 16:52, 2/7/09 (LRB 60229, pg. 150). Two auto batches occurred within $\sim 35$ minutes of each other for unknown reason in UFP-VSL-T01B.

The UFP-VSL-T02A laser high-level spikes shut down PJMs and filter-loop pumps, and in one instance, this interrupted an autobatch transfer. Laser high-level spikes in vessels UFP-VSL-T01A and B interrupted steam injection, PJM, and pump operations, depending on the process at the time. Between 21:00 and 21:47, 2/10/09 (LRB 60230, pg. 22), high-level spikes in UFP-VSL-T01B caused steam injection, PJMs, and pumps in UFP-VSL-T01A/B to shut off - one of the instances was during an autobatch transfer from UFP-VSL-T01A to UFP-VSL-T02A in which the systems were restarted. Such autobatch transfer interruptions confused the operator because the batch counter does not distinguish between attempted batch transfers and completed batch transfers.

A second problem lasers encountered was level confusion as a result of foam and/or aerosol concentration in the vessel headspace. Simulant splashing, perhaps from overblowing or general PJM performance with high simulant levels in the tank levels, only exacerbated laser functionality at 01:14, 2/16/09 (LRB 60230, pg. 89). Turning the lower air spargers ON to idle flow rates at 00:50 increased the laser level from 51 to 54 inches, and foam seemed to appear; the filter pump speed was reduced to bring the laser level back to 51 inches. Aerosols, as indicated on emails between 2/4/09 and 2/5/09, caused the laser signal to stay stagnant ("flatline") in UFP-VSL-T02A - the error code associated with this behavior indicated that it was a weak signal (02:54, 2/3/09, LRB 60229, pg. 70). The UFP-VSL-T01B laser triggered level alarms, and then it stayed at a value of 55.5 inches. Consequently, the level control was switched to bubbler (09:55, 2/3/09, LRB 60229, pg. 78). The UFP-VSL-T01B laser no longer flatlined, but it still was not working. 
A couple of things were done to investigate laser performance. Observations indicate having a vent improves laser performance. The laser vent system plugs in UFP-VSL-T01B were removed to allow air flow past the lens (11:25, 2/1/09, LRB 60229, pg. 25). The sample port return on UFP-VSL-T01B was also opened temporarily to see if this would reduce the high-level spikes on the laser trend graph (00:41, 2/14/09, LRB 60235, pg. 3). As shown in Figure 6.19, stable laser levels were achieved with the UFP-VSL-T01B vent open - there were no high-level spikes on the laser trend plot.

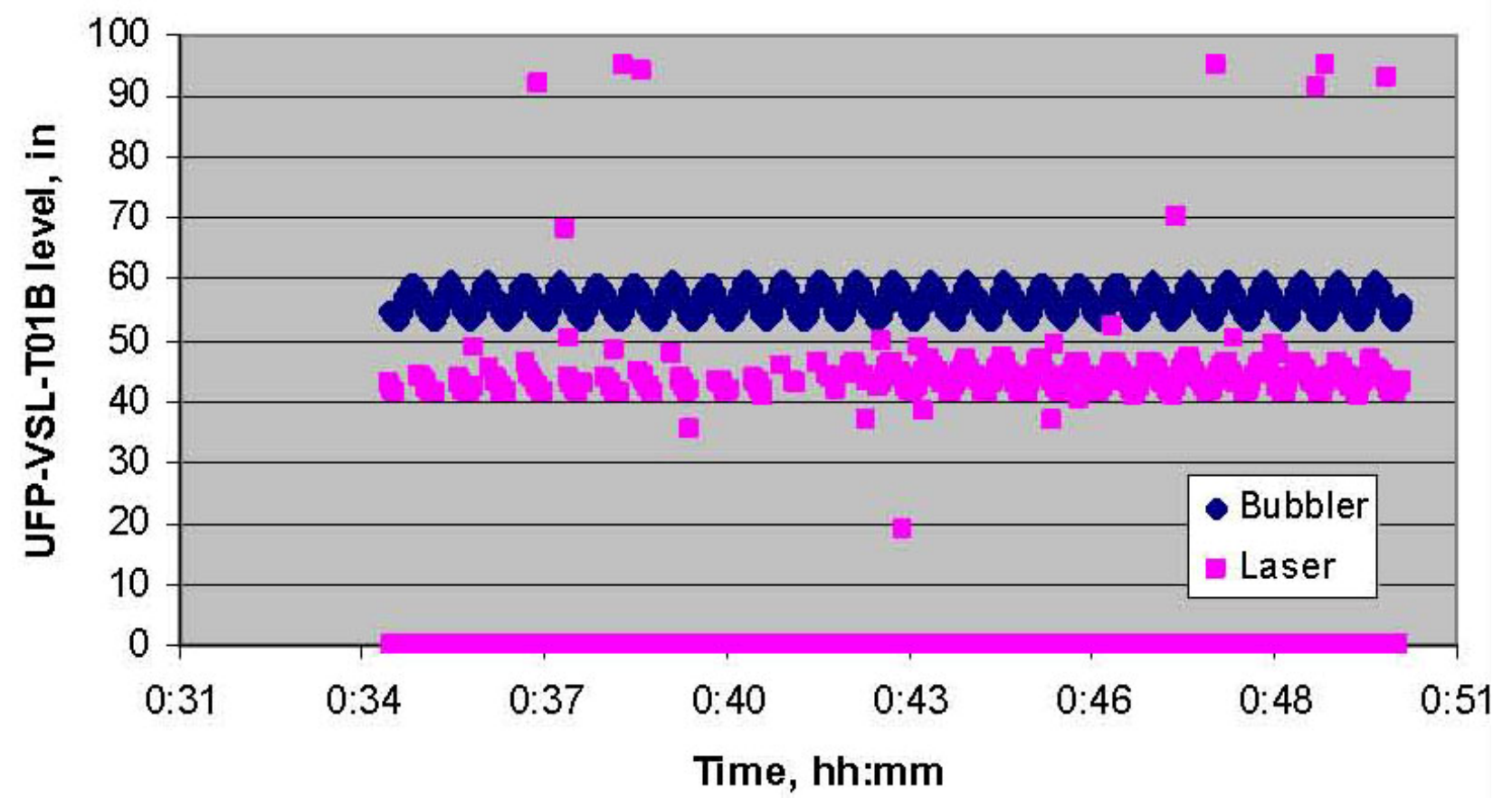

Figure 6.19. UFP-VSL-T01B Laser Performance Improved with Sample Port Open

With approval from testing and operations, the chemical addition vent flange was opened to improve laser performance in UFP-VSL-T02A — bleed air should flush vent space aerosols - refer to Figure 6.20 (13:48, 2/5/09, LRB 60229, pg. 119). By 13:52, UFP-VSL-T02A was changed to laser level control based on improved laser performance (09:28, 2/6/09, LRB 60229, pg. 130). On another occasion, the UFP-VSL-T02A funnel valve was opened fully to clear the headspace, which helped the laser read well. By 09:49, level control was back to the laser. 


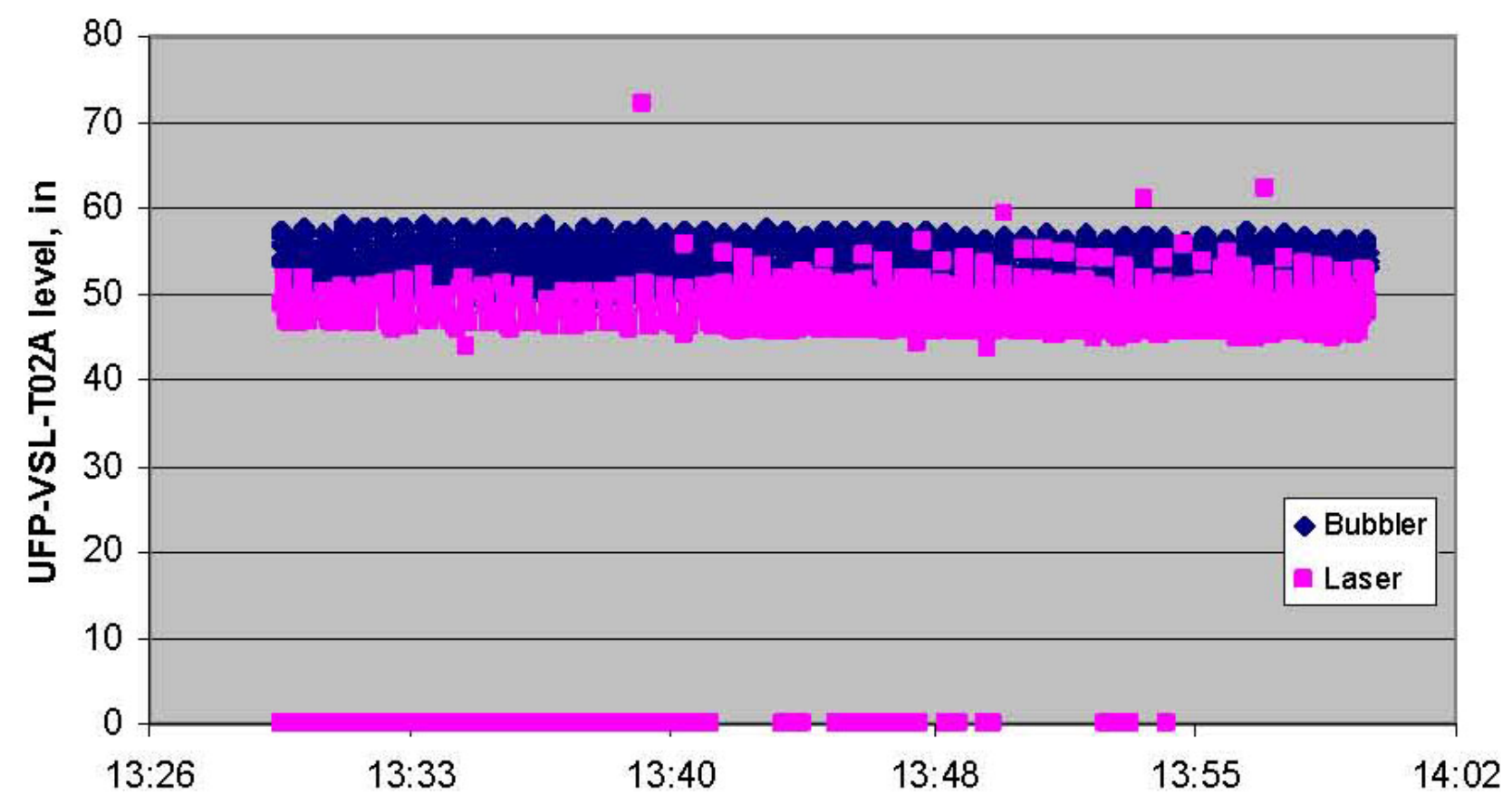

Time, hh:mm

Figure 6.20. UFP-VSL-T02A Laser Performance Improved After Opening Chemical Addition Vent Flange

Operators searched for alternative ways to improve laser performance (LRB 60229, pg. 117, 12:28). When the laser lenses were cleaned, simulant was found on them, and cleaning did not always bring the laser back into service. By 22:29, 2/3/09 (LRB 60229, pg. 92), the UFP-VSL-T01B lasers were stagnant. By 23:03, the instrument technicians cleaned the lens, but the laser still did not work (low return signal); foam may be to blame (LRB 60108, pg. 127). The steam air purge flow rate was increased to reduce the simulant build up on the laser lens while minimizing over-heating issues (Operations Manager Email, 7/20/2009, 09:29).

Also, lasers may not have functioned consistently because of the high possibility that the laser temperature specifications were exceeded during caustic leaching. Facilities personnel discovered that the lasers had a high temperature sensor alarming at $60^{\circ} \mathrm{C}$ (LRB 60229, pg. 25, 10:55). Although the temperature in the vessel headspace was not measured, this could explain why lasers in UFP-VSL-T01A and $\mathrm{B}$ did not work soon after reaching $88^{\circ} \mathrm{C}$ during caustic leaching. At 18:29, 01/31/09, operations were switched from laser to bubbler level control in UFP-VSL-T01A because the laser was reading 96 inches, and the temperature in the vessel was $\sim 88^{\circ} \mathrm{C}$ (LRB 60229, pg. 9). On 2/1/09, 02:37, the UFP-VSL-T01B level control was switched from the laser to the bubbler because the laser was reading 95 inches; the temperature in the vessel was $\sim 88^{\circ} \mathrm{C}$ (LRB 60229, pg. 14). Potentially, the temperature environment in the headspace exceeded the laser temperature specifications. 


\subsubsection{Bubblers}

Bubblers were susceptible to plugging on a regular basis. Blowing high-pressure air through the bubbler piping sometimes left the bubblers operational, and sometimes this was followed by water pressure (steam condensate and IW were used for bubbler cleaning). After the bubblers were successfully cleaned, the duration of functionality was unpredictable. Cleaning the bubblers was also problematic. High- and low-level spikes in the bubbler would interrupt PJM, steam injection, and pump operation if the bubbler was the level control instrument. For instance, at 19:00, 2/1/09, the UFP-VSL-T01B bubblers were cleaned because the specific gravity was reading $\sim 4$. During the cleaning, the UFP-VSL-T01A pump was tripped (LRB 60229, pg. 41). In time, the level control was switched to the laser before cleaning the bubblers to prevent equipment shutdowns. At other times, the bubblers were not cleaned if the laser was working well. When neither lasers nor bubblers worked, then the bubblers were cleaned. To assist bubbler credibility, samples were taken, and their density was measured to validate bubbler density readings.

Another bubbler issue only experienced in UFP-VSL-T02A was that their performance was influenced by filter pump (UFP-PMP-T42A and UFP-PMP-T43A) operations because of their physical location in the tank and PJM drive operations. When the filter-loop pumps were on, the flow from the pumps caused pressure changes at the bubblers. Consequently, the bubbler level and density readings were unstable, and erroneous at times, when the filter-loop pumps were on.

\subsubsection{Drexelbrook}

The Drexelbrook in UFP-VSL-T01A worked rather well (2/1/09, 09:02 through 09:08, LRB 60229, pg. 20). Level control in UFP-VSL-T01A was switched back and forth between Drexelbrook, bubbler, and laser because the noise in the bubbler level values caused high-level alarms in the vessel. Level control was kept the most stable with the Drexelbrook. The Drexelbrook level trend was smoother with the steam-ring air off than when it was on (LRB 60229, pg. 22-24). The PJM Drexelbrooks, on the other hand, were not quite as reliable (01:33, 2/12/09, LRB 60230, pg. 29). All PJM Drexelbrooks in UFP-VSL-T01A and UFP-VSL-T02A, except one in UFP-VSL-T01A, were flatlining. At times, the PJM stroke correlated well with the tank level change produced by the laser and/or bubbler. However, there were times when the PJM stroke per the Drexelbrooks was much shorter than the corresponding tank level change (14:58 on 2/1/09, LRB 60229, pg. 35). PJM probes 1601, 1603, and 1605 were not responding to the UFP-VSL-T01A PJM drive phase; they stayed at value 48 inches or 38 inches for 1605.

The 08:32 PJM probes in UFP-VSL-T02A were not working properly on 2/2/09 (LRB 60229, pg. 47), and PJM drive pressure increased without having made adjustments. While PJM operations may have been hindered by prolonged vacuum (pulling the simulant too high in the PJM tubes and air supply lines) and/or drive times (not driving the simulant far enough in the PJM tubes), operational problems could be associated with the simulant "coating," which is simulant exposure on Drexelbrook that permanently influenced its performance.

At times, there was no reliable level instrument (08:59, 2/2/09, LRB 60229, pg. 48). No PJM stroke was observed in UFP-VSL-T01A, and by 09:13, there were no credible level readings from any source in UFP-VSL-T01A (LRB 60229, pg. 49). Creative level control techniques were used in Integrated Test A given the issues with the laser and bubblers. Late on 2/2/09, the UFP-VSL-T01A laser was used for level control, although it was not reading correctly. To control autobatch transfers, it was made certain that 
batch transfers that were initiated at the proper tank level in UFP-VSL-T02A level control would be switched to the bubbler (21:20, 2/3/09, LRB 60229, pg. 90). Neither the UFP-VSL-T02A laser nor the bubbler was working. At 21:28, the bubbler was blown out, but with no success. While the bubbler was not accurate, its value was more stable than the laser values. When the estimated permeate had been collected, based on the permeate totalizer, the level control was then switched to the laser for triggering the next autobatch transfer. Once the transfer was complete, the level control was switched back to the bubbler.

In summary, obtaining credible level readings was a persistent problem in Integrated Test A. Having adequate venting in the vessel headspace appears to improve laser performance; it may also prevent splashing slurry on the lens. High-temperature application sensors should be selected, and perhaps it would be best to develop a laser self-cleaning system. It would be a good idea to use multiple sources for measuring levels. 


\subsection{Conclusions}

Integrated Test A demonstrated caustic leaching in the ultrafilter feed preparation vessels

UFP-VSL-T01A/B at $98^{\circ} \mathrm{C}$. While the outlined process steps in the Test Plan, TP-RPP-WTP-506, ${ }^{(a)}$ were accomplished, there were several operational difficulties throughout Integrated Test A.

Air entrainment produced unstable filter-loop pump performance. Slurry entrainment in the UFP-VSL-T02A filter loop could have resulted from the use of bubblers, steam ring, and spargers coupled with possible PJM overblowing as well as low-level tank operations that exposed the simulant return line. It is recommended that plant operations avoid prolonged low tank level operations and have an interlock shut down the filter-loop recirculation pumps when a PJM overblow is detected. Future PJM investigations should consider optimizing PJM stroke and average peak velocities at different UFP-VSL-T02A tank levels and slurry properties in efforts to minimize, or prevent, air entrainment caused by PJM overblowing.

Integrated Test A level issues demonstrate that having multiple sources for level indication and control was convenient. However, bubblers plugged frequently, and lasers flatlined on numerous occasions because of aerosols in the vessel headspace and/or exposure to high temperatures. Having adequate venting in the vessel headspace appears to improve laser performance; it may also prevent splashing slurry on the lens. Using laser self-cleaning systems could have improved the laser functionality, and lasers may have worked better if they had been made for high-temperature applications. Bubbler readings were also impacted by the flow from the PJMs and the filter-loop return nozzle because of the bubbler physical placement in UFP-VSL-T02A. In addition, the level indication in PJMs was also problematic, especially under high-temperature conditions.

Backpulsing of the filters was found to be effective for at least temporarily increasing filter permeate flow during post-caustic-leach concentration.

(a) G Josephson, O Bredt, J Young, and D Kurath. 2008. Test Plan: Pretreatment Engineering Platform (PEP) Testing (Phase I). TP-RPP-WTP-506, Rev. 0, Pacific Northwest National Laboratory, Richland, WA, 99352. 



\subsection{References}

Baldwin DL, PP Schonewill, JJ Toth, JL Huckaby, PW Eslinger, BD Hanson, DE Kurath, and MJ Minette. 2009. EFRT M12 Issue Resolution: Solids Washing. WTP-RPT-187, PNNL-18499, Pacific Northwest National Laboratory, Richland, Washington.

Barnes HA and NQ Dzuy. 2001. "Rotating vane rheometry - a review.” J. Non-Newtonian Fluid Mech. $1-14$.

Daniel RC, JM Billing, JR Bontha, CF Brown, PW Eslinger, BD Hanson, JL Huckaby, NK Karri, ML Kimura, DE Kurath, and MJ Minette. 2009. EFRT M12 Issue Resolution: Comparison of Filter Performance at PEP and CUF Scale. WTP-RPT-185, PNNL-18498, Pacific Northwest National Laboratory, Richland, Washington.

DOE/RL-96-68. 1998. Hanford Analytical Services Quality Assurance Requirements Documents (HASQARD), Revision 2. U.S. Department of Energy, Richland, Washington.

Josephson G, O Bredt, J Young, and D Kurath. 2008. Test Plan: Pretreatment Engineering Platform (PEP) Testing (Phase I). TP-RPP-WTP-506, Rev 0, Pacific Northwest National Laboratory, Richland, WA, 99352.

Geeting JGH, OP Bredt, CA Burns, EC Golovich, CE Guzman-Leong, GB Josephson, DE Kurath, GJ Sevigny, and RL Aaberg. 2009. Pretreatment Engineering Platform (PEP) Integrated Test B Run Report-Caustic and Oxidative Leaching in UFP-VSL-T02A. WTP-RPT-192, PNNL-18723, Pacific Northwest National Laboratory, Richland, Washington.

Josephson GB, JGH Geeting, OP Bredt, CA Burns, EC Golovich, CE Guzman-Leong, DE Kurath, and GH Sevigny. 2009. PEP Run Report for Simulant Shakedown/Functional Testing. WTP-RPT-190, PNNL-18823, Pacific Northwest National Laboratory, Richland, Washington.

Kuhn WL, ST Arm, JL Huckaby, DE Kurath, SD Rassat. 2008. Technical Basis for Scaling Relationships for the Pretreatment Engineering Platform. WTP-RPT-160, PNNL-16948, Pacific Northwest National Laboratory, Richland Washington.

Mahoney LA, SD Rassat, PW Eslinger, RL Aaberg, PM Aker, EC Golovich, BD Hanson, TS Hausmann, JL Huckaby, DE Kurath, MJ Minette, SK Sundaram, and ST Yokuda. 2009. EFRT Issue Resolution: Caustic Leach Rate Constants from PEP and Lab-Scale Tests. WTP-RPT-186, PNNL-18502, Pacific Northwest National Laboratory, Richland Washington.

Rapko BM, GJ Lumetta, JR Deschane, and RA Peterson. 2007. Process Development for Permanganate Addition During Oxidative Leaching of Hanford Tank Sludge Simulants. WTP-RPT-164, PNNL-16794, Pacific Northwest National Laboratory, Richland, Washington.

Rapko BM, CF Brown, PW Eslinger, MS Fountain, TS Hausmann, JL Huckaby, BD Hanson, DE Kurath, and MJ Minette. 2009. EFRT M12 Issue Resolution: Comparison of PEP and Bench-Scale Oxidative 
Leaching Results. WTP-RPT-188, PNNL-18500, Pacific Northwest National Laboratory, Richland, Washington.

Russell RL, RA Peterson, HD Smith, DE Rinehart, PM Aker, and EC Buck. 2009a. Development and Characterization of Boehmite Component Simulant. WTP-RPT-184, PNNL-18176, Pacific Northwest National Laboratory, Richland Washington.

Russell RL, RA Peterson, DE Rinehart, and HD Smith. 2009b. Development and Characterization of Gibbsite Component Simulant. WTP-RPT-176, PNNL-18013, Pacific Northwest National Laboratory, Richland Washington.

Russell RL, JM Billing, RA Peterson, DE Rinehart, and HD Smith. 2009c. Development and Demonstration of Ultrafiltration Simulants. WTP-RPT-183, PNNL-18090, Pacific Northwest National Laboratory, Richland Washington.

Sevigny GJ, OP Bredt, CA Burns, DE Kurath, JGH Geeting, EC Golovich, CE Guzman-Leong, and GB Josephson. 2009. PEP Integrated Test D Run Report-Caustic and Oxidative Leaching in UFP-VSL-T02A. WTP-RPT-193, PNNL-18741, Pacific Northwest National Laboratory, Richland, Washington.

Stewart CW, PA Meyer, MS Fountain, CE Guzman-Leong, SA Hartley-McBride, JL Huckaby, and BE Wells. 2007. Effect of Anti-Foam Agent on Gas Retention and Release Behavior in Simulated High-Level Waste. WTP-RPT-147, PNWD-3786, Battelle--Pacific Northwest Division, Richland, WA.

Stewart CW, CE Guzman-Leong, ST Arm, MG Butcher, EC Golovich, LK Jagoda, WR Park, RW Slaugh, Y Su, CF Wend, LA Mahoney, JM Alzheimer, JA Bailey, SK Cooley, DE Hurley, CD Johnson, LD Reid, HD Smith, BE Wells, and ST Yokuda. 2008. Results of Large-Scale Testing on Effects of Anti-Foam Agent on Gas Retention and Release. WTP-RPT-156, PNNL-17170, Pacific Northwest National Laboratory, Richland, WA.

Kuhn WL, ST Arm, JL Huckaby, DE Kurath, and SD Rassat. 2008. Scaling Relationships for the Pretreatment Engineering Platform. WTP-RPT-160, PNNL-16948, Pacific Northwest National Laboratory, Richland, Washington. 
Appendix A

Process Instrumentation Summary 



\section{Appendix A: Process Instrumentation Summary}

\section{Summary of Process Instrumentation}

\begin{tabular}{|c|c|c|c|c|c|c|}
\hline Component & Instrument ID & Min & Max & Units & $\begin{array}{c}\text { Tolerance } \\
(+/-)\end{array}$ & Notes \\
\hline \multicolumn{7}{|l|}{ Vessel T02A } \\
\hline T02A level (bubbler) & LT-0611 & 0.00 & 200.00 & in $\mathrm{H}_{2} \mathrm{O}$ & 0.15 & \\
\hline T02A density (bubbler) & DT-0612 & 0.00 & 200.00 & in $\mathrm{H}_{2} \mathrm{O}$ & 0.15 & \\
\hline T02A level (laser) & LT-0614 & -3.95 & 96.00 & in & 0.25 & $\begin{array}{l}\text { Calibration not finalized until } 03 / 24 / 2009 \text {, } \\
\text { but data using a previous calibration before } \\
\text { this date is within the instrument tolerance } \\
\text { (CCP-WTPSP-684). }\end{array}$ \\
\hline T02A prototypic temperature & TTK-0619 & 10.00 & 110.00 & $\mathrm{C}$ & 2 & \\
\hline \multicolumn{7}{|l|}{ T02A Temperature Array } \\
\hline $\mathrm{x}=\mathrm{A}, \mathrm{B}, \mathrm{D}, \mathrm{E}, \mathrm{F}, \mathrm{G}, \mathrm{H}, \mathrm{J}, \mathrm{K}, \mathrm{L}$ & TTx-0619 & 10.00 & 110.00 & $\mathrm{C}$ & 2 & \\
\hline $\mathrm{x}=\mathrm{A}, \mathrm{B}, \mathrm{D}, \mathrm{E}, \mathrm{F}, \mathrm{G}, \mathrm{H}, \mathrm{J}, \mathrm{K}, \mathrm{L}$ & TТx-0621 & 10.00 & 110.00 & $\mathrm{C}$ & 2 & \\
\hline$x=1-7$ & N39-x & 10.00 & 110.00 & $\mathrm{C}$ & 2 & \\
\hline$x=1-7$ & N41-X & 10.00 & 110.00 & $\mathrm{C}$ & 2 & \\
\hline$x=1-7$ & $\mathrm{~N} 42-\mathrm{x}$ & 10.00 & 110.00 & $\mathrm{C}$ & 2 & \\
\hline$x=1-7$ & N52-x & 10.00 & 110.00 & $\mathrm{C}$ & 2 & \\
\hline \multicolumn{7}{|l|}{ UFP-HX-T02A } \\
\hline UFP-HX-T02A cooling water flow & FT-0541 & 0.00 & 70.00 & gpm & 0.88 & \\
\hline UFP-HX-T02A cooling water inlet temperature & TT-0539 & 0.00 & 110.00 & $\mathrm{C}$ & 1.8 & \\
\hline UFP-HX-T02A cooling water outlet & TT-0540 & 0.00 & 110.00 & $\mathrm{C}$ & 1.8 & \\
\hline $\begin{array}{l}\text { UFP-HX-T02A CW ret. flow control valve } \\
\text { TV-0513 position }\end{array}$ & N/A & N/A & N/A & N/A & N/A & Not available. \\
\hline UFP-HX-T02A process delta pressure & PT-0535 & 5.50 & 155.50 & psig & 1 & $\begin{array}{l}\text { All Integrated Test A data "N/A" - instrument } \\
\text { not connected. See "PEP DAS2 M\&TE List } \\
\text { Rev 7.xls". }\end{array}$ \\
\hline UFP-HX-T02A process fluid inlet temperature & TT-0537 & 0.00 & 110.00 & $\mathrm{C}$ & 1.8 & For Information Only re: NCR 42402.1. \\
\hline UFP-HX-T02A process fluid outlet temperature & TT-0513 & 0.00 & 110.00 & $\mathrm{C}$ & 1.8 & For Information Only re: NCR 42402.1. \\
\hline \multicolumn{7}{|l|}{ Pumps T42A/T43A } \\
\hline Pump T42A discharge pressure (Pump T43A suction) & PT-0633 & 0.00 & 348.00 & psig & 0.26 & \\
\hline
\end{tabular}


Summary of Process Instrumentation

\begin{tabular}{|c|c|c|c|c|c|c|}
\hline Component & Instrument ID & Min & Max & Units & $\begin{array}{c}\text { Tolerance } \\
(+/-)\end{array}$ & Notes \\
\hline Pump T42A inlet flow & FT-0623 & 0.00 & 220.00 & gpm & 1.6 & \\
\hline Pump T42A speed & ST-0627 & 0.00 & 1785.00 & $\mathrm{rpm}$ & 4 & \\
\hline Pump T42A suction pressure & PT-0625 & -260.00 & 260.00 & in $\mathrm{H}_{2} \mathrm{O}$ & 0.4 & \\
\hline Pump T42A motor HP & JT-0629 & 0.00 & 100.00 & HP & 1.5 & For Information Only. \\
\hline Pump T43A outlet temperature & TT-0791 & 0.00 & 110.00 & $\mathrm{C}$ & 1.8 & $\begin{array}{l}\text { Should not be used as an indicator of } \\
\text { temperature re: NCR } 42402.1 \text {. }\end{array}$ \\
\hline Pump T43A discharge pressure & PT-0739 & 0.00 & 348.00 & psig & 0.26 & \\
\hline Pump T43A outlet flow & FT-0635 & 0.00 & 220.00 & gpm & 1.6 & $\begin{array}{l}\text { Suspect values reported between 02/14/2009 } \\
\text { and 02/27/2009 re: NCR 42317.1. }\end{array}$ \\
\hline Pump T43A speed & ST-0639 & 0.00 & 1785.00 & rpm & 4 & $\begin{array}{l}\text { Did not collect data for the beginning of } \\
\text { Integrated Test A; first data appears on } \\
02 / 09 / 2009 \text { at } 09: 11 \text { (see columns W and BX } \\
\text { in worksheet "Test A", which correspond to } \\
\text { the power meter and tachometer for Pump } \\
\text { T43A). }\end{array}$ \\
\hline Pump T43A motor HP & JT-0637 & 0.00 & 100.00 & HP & 1.5 & For Information Only. \\
\hline Flow control valve position & SV-0609 & 0.00 & 2.00 & Active & 1 & $\begin{array}{l}\text { For Information Only; when SV-0609 reads } 0 \\
\text { the valve is } 0 \% \text { open, } 2 \text { is } 100 \% \text { open. See } \\
\text { "PEP DAS2 M\&TE List Rev 7.xls". }\end{array}$ \\
\hline \multicolumn{7}{|l|}{ Pumps Air Entrainment Trouble Shoot } \\
\hline Air purge flow to steam sparger FT-1995 & FT-1995 & 0.00 & 0.73 & $\mathrm{~kg} / \mathrm{min}$ & 0.02 & $\begin{array}{l}\text { Accuracy revised at flow rates below } 0.090 \\
\mathrm{~kg} / \mathrm{min} \text { re: NCR } 38767.1(0.00045 \\
\mathrm{kg} / \mathrm{min} / \text { flow rate in } \mathrm{kg} / \mathrm{min} \times 100 \%)\end{array}$ \\
\hline Upper air sparger flow FT-1901 & FT-1901 & 0.00 & 0.73 & $\mathrm{~kg} / \mathrm{min}$ & 0.01 & $\begin{array}{l}\text { Accuracy revised at flow rates below } 0.090 \\
\mathrm{~kg} / \mathrm{min} \text { re: NCR } 38767.1(0.00045 \\
\mathrm{kg} / \mathrm{min} / \text { flow rate in } \mathrm{kg} / \mathrm{min} \times 100 \%)\end{array}$ \\
\hline Lower air sparger flow FT-1977 & FT-1977 & 0.00 & 0.73 & $\mathrm{~kg} / \mathrm{min}$ & 0.01 & $\begin{array}{l}\text { Correction required for data before } \\
01 / 05 / 2009 \text { re: NCR } 41090.1 \text {; accuracy } \\
\text { revised at flow rates below } 0.090 \mathrm{~kg} / \mathrm{min} \text { re: } \\
\text { NCR } 38767.1(0.00045 \mathrm{~kg} / \mathrm{min} / \text { flow rate in } \\
\mathrm{kg} / \mathrm{min} \times 100 \%) \text {. }\end{array}$ \\
\hline Instrument air to level and density bubbler & N/A & N/A & N/A & N/A & N/A & Not available. \\
\hline
\end{tabular}


Summary of Process Instrumentation

\begin{tabular}{|c|c|c|c|c|c|c|}
\hline Component & Instrument ID & Min & Max & Units & $\begin{array}{c}\text { Tolerance } \\
(+/-)\end{array}$ & Notes \\
\hline High pressure steam flow & FT-0645 & 0.00 & 33.43 & $\mathrm{cfm}$ & 0.41 & \\
\hline High pressure steam pressure & PT-0643 & 0.00 & 150.00 & psig & 0.11 & \\
\hline 100 psig utility air header flow & FT-0663 & 0.00 & 9.40 & $\mathrm{scfm}$ & 0.07 & \\
\hline Inhibited water header flow & FT-0667 & 0.00 & 26.00 & gpm & 0.2 & For Information Only re: NCR 38764.1. \\
\hline Inhibited water process water & FT-0601 & 0.00 & 189.27 & $\mathrm{~kg} / \mathrm{min}$ & 0.24 & \\
\hline 19M sodium hydroxide & FT-0605 & 0.00 & 75.71 & $\mathrm{~kg} / \mathrm{min}$ & 0.95 & \\
\hline $2 \mathrm{M}$ caustic flow & FT-0655 & 0.00 & 57.10 & $\mathrm{~kg} / \mathrm{min}$ & 0.71 & \\
\hline 2M nitric acid flow & FT-0659 & 0.00 & 15.00 & gpm & 0.11 & For Information Only re: NCR 38764.1. \\
\hline \multicolumn{7}{|l|}{ Filter UFP-FILT-T01A } \\
\hline Tubeside inlet temp. & TT-0791 & 0.00 & 110.00 & $\mathrm{C}$ & 1.8 & $\begin{array}{l}\text { Should not be used as an indicator of } \\
\text { temperature re: NCR } 42402.1 \text {. }\end{array}$ \\
\hline Tubeside inlet press. & PT-0739 & 0.00 & 348.00 & psig & 0.26 & \\
\hline Tubeside flow & FT-0635 & 0.00 & 220.00 & gpm & 1.6 & $\begin{array}{l}\text { Suspect values reported between 02/14/2009 } \\
\text { and 02/27/2009 re: NCR 42317.1. }\end{array}$ \\
\hline Shellside inlet press. & PT-0741 & 0.00 & 700.00 & psig & 0.53 & \\
\hline Shellside outlet press. & PI-0706 & N/A & N/A & N/A & N/A & Local gauge, not recorded. \\
\hline Shellside outlet flow & FT-0720 & 0.00 & 35.85 & $\mathrm{~kg} / \mathrm{min}$ & 0.45 & \\
\hline Transmembrane pressure TMP1 & N/A & N/A & N/A & N/A & N/A & $\begin{array}{l}\text { Via calculation: } \\
\text { (PT-0739+PT-0749)/2-PT-0741. }\end{array}$ \\
\hline \multicolumn{7}{|l|}{ Filter UFP-FILT-T02A } \\
\hline Tubeside inlet temp. & TT-0791 & 0.00 & 110.00 & $\mathrm{C}$ & 1.8 & $\begin{array}{l}\text { Should not be used as an indicator of } \\
\text { temperature re: NCR } 42402.1 \text {. }\end{array}$ \\
\hline Tubeside inlet press. & PT-0749 & 0.00 & 348.00 & psig & 0.26 & \\
\hline Tubeside flow & FT-0635 & 0.00 & 220.00 & gpm & 1.6 & $\begin{array}{l}\text { Suspect values reported between 02/14/2009 } \\
\text { and 02/27/2009 re: NCR 42317.1. }\end{array}$ \\
\hline Shellside inlet press. & PT-0751 & 0.00 & 700.00 & psig & 0.53 & \\
\hline Shellside outlet press. & PI-0708 & N/A & N/A & N/A & N/A & Local gauge, not recorded. \\
\hline Shellside flow & FT-0755 & 0.00 & 37.85 & $\mathrm{~kg} / \mathrm{min}$ & 0.05 & \\
\hline Transmembrane pressure TMP2 & N/A & N/A & N/A & N/A & N/A & $\begin{array}{l}\text { Via calculation: } \\
\text { (PT-0749+PT-0759)/2-PT-0751. }\end{array}$ \\
\hline
\end{tabular}


Summary of Process Instrumentation

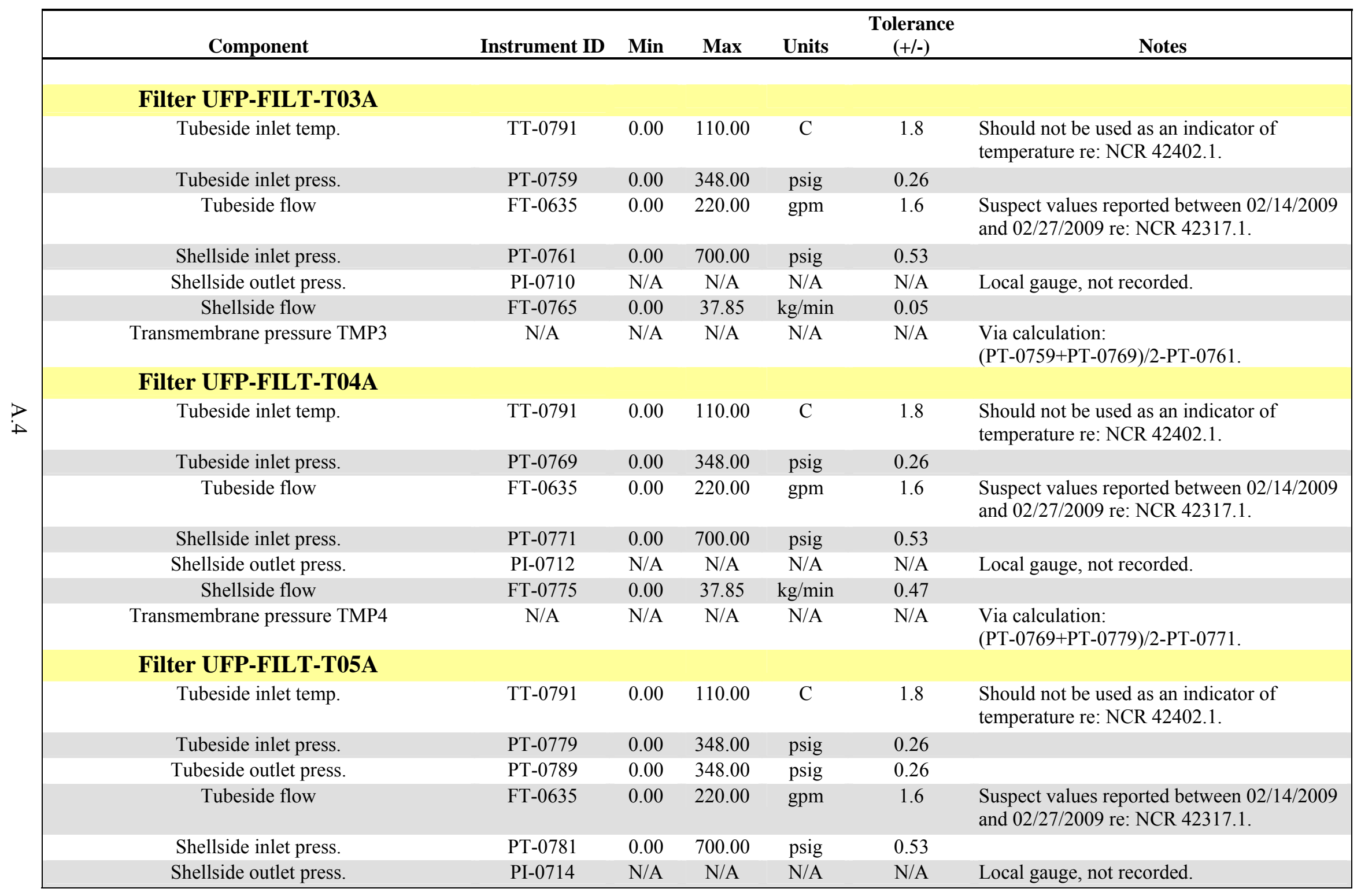


Summary of Process Instrumentation

\begin{tabular}{|c|c|c|c|c|c|c|}
\hline Component & Instrument ID & Min & Max & Units & $\begin{array}{c}\text { olerance } \\
(+/-)\end{array}$ & Notes \\
\hline Shellside flow & FT-0785 & 0.00 & 37.85 & $\mathrm{~kg} / \mathrm{min}$ & 0.24 & \\
\hline Transmembrane pressure TMP5 & N/A & N/A & N/A & N/A & $\mathrm{N} / \mathrm{A}$ & $\begin{array}{l}\text { Via calculation: } \\
\text { (PT-0779+PT-0789)/2-PT-0781. }\end{array}$ \\
\hline \multicolumn{7}{|l|}{ Pulsepot UFP-PP-T03A } \\
\hline Pulse valve status & PV-0743 & N/A & N/A & $\mathrm{N} / \mathrm{A}$ & N/A & Not available. \\
\hline Level & LT-0733 & 0.00 & 44.00 & inches & 0.1 & For Information Only. \\
\hline Permeate flow from Filter T01A & FT-0720 & 0.00 & 35.85 & $\mathrm{~kg} / \mathrm{min}$ & 0.45 & \\
\hline Inlet pressure & PT-0731 & 0.00 & 700.00 & psig & 0.53 & \\
\hline Outlet pressure & PT-0741 & 0.00 & 700.00 & psig & 0.53 & \\
\hline \multicolumn{7}{|l|}{ Pulsepot UFP-PP-T02A } \\
\hline Pulse valve status & PV-0753 & N/A & N/A & N/A & N/A & Not available. \\
\hline Level & LT-0725 & 0.00 & 44.00 & inches & 0.1 & For Information Only. \\
\hline Permeate flow from Filter T02A & FT-0755 & 0.00 & 37.85 & $\mathrm{~kg} / \mathrm{min}$ & 0.05 & \\
\hline Permeate flow from Filter T04A & FT-0775 & 0.00 & 37.85 & $\mathrm{~kg} / \mathrm{min}$ & 0.47 & \\
\hline Inlet pressure & PT-0723 & 0.00 & 700.00 & psig & 0.53 & \\
\hline Outlet pressure to Filter T02A & PT-0751 & 0.00 & 700.00 & psig & 0.53 & \\
\hline Outlet pressure to Filter T04A & PT-0771 & 0.00 & 700.00 & psig & 0.53 & \\
\hline \multicolumn{7}{|l|}{ Pulsepot UFP-PP-T01A } \\
\hline Pulse valve status & PV-0763 & N/A & $\mathrm{N} / \mathrm{A}$ & N/A & $\mathrm{N} / \mathrm{A}$ & Not available. \\
\hline Level & LT-0717 & 0.00 & 44.00 & inches & 0.1 & For Information Only. \\
\hline Permeate flow from Filter T03A & FT-0765 & 0.00 & 37.85 & $\mathrm{~kg} / \mathrm{min}$ & 0.05 & \\
\hline Permeate flow from Filter T05A & FT-0785 & 0.00 & 37.85 & $\mathrm{~kg} / \mathrm{min}$ & 0.24 & \\
\hline Inlet pressure & PT-0715 & 0.00 & 700.00 & psig & 0.53 & \\
\hline Outlet pressure to Filter T03A & PT-0761 & 0.00 & 700.00 & psig & 0.53 & \\
\hline Outlet pressure to Filter T05A & PT-0781 & 0.00 & 700.00 & psig & 0.53 & \\
\hline
\end{tabular}


Key components for measuring filtration performance at PEP include slurry and permeate flow meters, feed tank (Tank T02A) temperature sensors, and filter-loop pressure sensors. These sensors allow assessment of process conditions driving cross-flow filtration and of the performance of the filters in terms of permeate production. The process parameters of interest are 1) filter axial velocity (or slurry flow rate), 2) filter transmembrane and axial pressure drop, and 3) rate of permeate production. Permeate production is typically corrected for variations in slurry/supernate temperature (as well as variations in transmembrane pressure), and, as such, the process temperature is also of interest.

Table A.1 provides a summary of the PEP instrumentation used to assess slurry flow rates in the filtration loop. The two flow meters listed provide measurement of both suction and discharge flow rates from the circulation loop pumping system. Flow meter FT-0623 operates at the head pressure of the Tank T02A or lower, whereas flow meter FT-0635 operates at high pressure. Because of the pressure differential between flow meters, air entrained in the suction line is likely forced into solution on the discharge side. Some degree of air entrainment is expected from sparging of Tank T02A contents and slurry return. Because magnetic flow meters are sensitive to nonconductive phases like air, the reading on the discharge magnetic flow meter (FT-0635) is typically a few percent lower than that on the suction flow meter (FT-0623) for typical air entrainments observed during PEP runs. The divergence in flow meter readings may become severe if air entrainment becomes significant, but such behavior was not observed during the assessments of filter scaling effects.

Table A.1. PEP Instrumentation Used to Assess Slurry Flow Rates Through the Filtration Circulation Loop

\begin{tabular}{llc}
\hline Instrument ID & \multicolumn{1}{c}{ Description } & Units \\
\hline FT-0623 & $\begin{array}{l}\text { Instrument FT-0623 is a magnetic flow meter used to measure the volumetric } \\
\text { flow rate of slurry entering Pump T42A. It is located at the suction to Pump }\end{array}$ & gpm \\
& T42A. & gpm \\
FT-0635 & $\begin{array}{l}\text { Instrument FT-0635 is a magnetic flow meter used to measure the volumetric } \\
\text { flow rate of slurry exiting Pump T43A. It is located at the discharge to Pump } \\
\text { T43A. }\end{array}$ & \\
\hline
\end{tabular}

Determination of transmembrane and axial pressure differentials requires knowledge of the tubeside inlet/outlet pressures and of the shellside pressure for each filter bundle. Table A.2 provides a summary of the filter bundle instrumentation that provides this capability. 
Table A.2. PEP Filter Bundle Pressure Sensing Instrumentation

\begin{tabular}{|c|c|c|c|}
\hline Filter Bundle & Instrument ID & Description & Units \\
\hline \multirow[t]{3}{*}{1} & PT-0739 & $\begin{array}{l}\text { PT- } 0739 \text { provides measurement of the tubeside inlet pressure } \\
\text { for filter bundle } 1 \text {. It is located at the entrance to filter bundle } 1 \text {. }\end{array}$ & psig \\
\hline & PT-0749 & $\begin{array}{l}\text { PT- } 0749 \text { provides measurement of the tubeside outlet pressure } \\
\text { for filter bundle } 1 \text {. It is located at the entrance to filter bundle } 2 \text {. }\end{array}$ & psig \\
\hline & PT-0741 & $\begin{array}{l}\text { PT- } 0741 \text { provides measurement of the shellside pressure for } \\
\text { filter bundle } 1 \text {. }\end{array}$ & psig \\
\hline \multirow[t]{3}{*}{2} & PT-0749 & $\begin{array}{l}\text { PT- } 0749 \text { provides measurement of the tubeside inlet pressure } \\
\text { for filter bundle } 2 \text {. It is located at the entrance to filter bundle } 2 \text {. }\end{array}$ & psig \\
\hline & PT-0759 & $\begin{array}{l}\text { PT- } 0759 \text { provides measurement of the tubeside outlet pressure } \\
\text { for filter bundle } 2 \text {. It is located at the entrance to filter bundle } 3 \text {. }\end{array}$ & psig \\
\hline & PT-0751 & $\begin{array}{l}\text { PT- } 0751 \text { provides measurement of the shellside pressure for } \\
\text { filter bundle } 2 \text {. }\end{array}$ & psig \\
\hline \multirow[t]{3}{*}{3} & PT-0759 & $\begin{array}{l}\text { PT- } 0759 \text { provides measurement of the tubeside inlet pressure } \\
\text { for filter bundle } 3 \text {. It is located at the entrance to filter bundle } 3 \text {. }\end{array}$ & psig \\
\hline & PT-0769 & $\begin{array}{l}\text { PT- } 0769 \text { provides measurement of the tubeside outlet pressure } \\
\text { for filter bundle } 3 \text {. It is located at the entrance to filter bundle } 4 \text {. }\end{array}$ & psig \\
\hline & PT-0761 & $\begin{array}{l}\text { PT- } 0761 \text { provides measurement of the shellside pressure for } \\
\text { filter bundle } 3 \text {. }\end{array}$ & psig \\
\hline \multirow[t]{3}{*}{4} & PT-0769 & $\begin{array}{l}\text { PT- } 0769 \text { provides measurement of the tubeside inlet pressure } \\
\text { for filter bundle } 4 \text {. It is located at the entrance to filter bundle } 4 \text {. }\end{array}$ & psig \\
\hline & PT-0779 & $\begin{array}{l}\text { PT- } 0779 \text { provides measurement of the tubeside outlet pressure } \\
\text { for filter bundle } 4 \text {. It is located at the entrance to filter bundle } 5 \text {. }\end{array}$ & psig \\
\hline & PT-0771 & $\begin{array}{l}\text { PT- } 0771 \text { provides measurement of the shellside pressure for } \\
\text { filter bundle } 4 \text {. }\end{array}$ & psig \\
\hline \multirow[t]{3}{*}{5} & PT-0779 & $\begin{array}{l}\text { PT- } 0779 \text { provides measurement of the tubeside inlet pressure } \\
\text { for filter bundle } 5 \text {. It is located at the entrance to filter bundle } 5 \text {. }\end{array}$ & psig \\
\hline & PT-0789 & $\begin{array}{l}\text { PT- } 0779 \text { provides measurement of the tubeside outlet pressure } \\
\text { for filter bundle } 5 \text {. It is located at the exit from filter bundle } 5 \text {. }\end{array}$ & psig \\
\hline & PT-0781 & $\begin{array}{l}\text { PT- } 0781 \text { provides measurement of the shellside pressure for } \\
\text { filter bundle } 5 \text {. }\end{array}$ & psig \\
\hline
\end{tabular}

Permeate mass flow rates are measured by Coriolis flow meters. A summary of these instruments is provided in Table A.3.

Table A.3. PEP Coriolis Flow Meters for Permeate Production Rate Measurement

\begin{tabular}{|cllc|}
\hline Filter Bundle & Instrument ID & \multicolumn{1}{c}{ Description } & Units \\
\hline 1 & FT-0720 & $\begin{array}{l}\text { Coriolis flow meter FT-0720 measures permeate production rate } \\
\text { on filter bundle 1. }\end{array}$ & $\mathrm{kg} / \mathrm{min}$ \\
2 & FT-0755 & $\begin{array}{l}\text { Coriolis flow meter FT-0755 measures permeate production rate } \\
\text { on filter bundle 2. }\end{array}$ & $\mathrm{kg} / \mathrm{min}$ \\
3 & FT-0765 & $\begin{array}{l}\text { Coriolis flow meter FT-0765 measures permeate production rate } \\
\text { on filter bundle 3. }\end{array}$ & $\mathrm{kg} / \mathrm{min}$ \\
4 & FT-0775 & $\begin{array}{l}\text { Coriolis flow meter FT-0775 measures permeate production rate } \\
\text { on filter bundle 4. }\end{array}$ & $\mathrm{kg} / \mathrm{min}$ \\
5 & FT-0785 & $\begin{array}{l}\text { Coriolis flow meter FT-0785 measures permeate production rate } \\
\text { on filter bundle 5. }\end{array}$ & $\mathrm{kg} / \mathrm{min}$ \\
\hline
\end{tabular}


Corrections of permeate production rates for variations in process temperature are made against the temperature of Tank T02A. This vessel is fitted with an array of RTDs to determine the tank temperature profile. All permeate flux corrections were made using the prototypic temperature sensor TTK-0619. Other temperature sensors of interest are installed in thermowells located along the filter loop:

- TT-0791-indicates the inlet temperature to filter bundle 1

- TT-0537-indicates the outlet temperature from filter bundle 5

- TT-0513 - indicates the outlet temperature for UFP-HX-T02A

- TT-0515-indicates the outlet temperature for UFP-HX-T03A.

The energy required to pump the slurry also causes temperature rise in the filtration loop. Although the spiral plate heat exchanger (UFP-HX-T02A) removes this heat, temperature differentials (of a few degrees, depending on slurry thickness) still exist between the filtration loop and Tank T02A. While it would be more appropriate to correct filter flux using the filtration loop temperature, temperature sensors in the filtration loop appear to be subject to potential stagnation of the slurry at the sensor thermowells (see the results for the High-Solids Scaling Test in WTP-RPT-185). ${ }^{\text {(a) }}$ Because of these concerns, the tank temperature was selected as the temperature reference for filtration calculations.

The output signal from each of the PEP sensors listed in Table A.1 to Table A.3 was recorded by and stored in the PEP data acquisition system (DAS). The analog-to-digital conversion system has been calibrated to accurately convert the instrument signals, and they were stored in a read-only data file to confirm the integrity of the process data from each test. The recorded data were time stamped by the DAS system so that it could be matched to process data sheets and logbooks. The raw, stored instrument outputs were converted to engineering data with a data interrogation program, which was technically reviewed, validated, and verified according to QA-RPP-WTP-SCP, Software Control. For simplicity, the system for recording, storing, and converting data will be referred to as the PEP DAS.

(a) NCR 42402.1. 
Appendix B

Integrated Test A Sample List 



\section{Appendix B: Integrated Test A Sample List}

\section{Integrated Test A Sample List}

\begin{tabular}{|c|c|c|c|c|c|c|}
\hline TI\# & TI Step \# & TI Step Description & Sample ID & Analysis & Folder & Final Electronic Report File Name \\
\hline TI-065 & N/A & $\mathrm{n} / \mathrm{a}$ & A 01AML XXX XX 2805 UDS 4 & UDS & CCP-WTPSP-755 & CCP WTPSP 755 Int Test A UDS Data Analysis \\
\hline TI-065 & N/A & $\mathrm{n} / \mathrm{a}$ & A 02AML XXX XX 2806 UDS 4 & UDS & CCP-WTPSP-755 & CCP WTPSP 755 Int Test A UDS Data Analysis \\
\hline TI-065 & N/A & $\mathrm{n} / \mathrm{a}$ & A 02 ARL XXX XX 2840 UDS 2 & UDS & UDS R1 & UDS R1 Shake and Test A and Test B Step001 Date 08182009 \\
\hline TI-065 & $\mathrm{N} / \mathrm{A}$ & $\mathrm{n} / \mathrm{a}$ & A 02ARL XXX XX 2841 UDS 4 & UDS & CCP-WTPSP-755 & CCP WTPSP 755 Int Test A UDS Data Analysis \\
\hline TI-065 & N/A & $\mathrm{n} / \mathrm{a}$ & A 02ARL XXX XX 2842 RHE 4 & Rheology & TDP-WTP-359 & TDP WTP 359 Int Test A RHE Data on PEP Slurry \\
\hline TI-065 & N/A & Prepare and stage simulant & A T22GM 001 XX 1500 RAM 3 D & Raman & ASR 8324 & ASR 8324 RAM rev 1 \\
\hline TI-065 & N/A & Prepare and stage & A T22GM 001 XX 1501 UDS 2 & UDS & CCP-WTPSP-754 & CCP WTPSP 754 Shakedown UDS Data Analysis \\
\hline TI-065 & 1.1 .2 & Prepare and stage simulant & A T22GM 001 XX 2533 RAM 3 D & Raman & ASR 8342 & ASR 8342 RAM rev 1 \\
\hline TI-065 & 1.1 .2 & Pretest samples & A T22GM 001 XX 2532 UDS 2 & UDS & UDS R1 & UDS R1 Shake and Test A and Test B Step001 Date 08182009 \\
\hline TI-065 & 2.2 .2 & $\begin{array}{l}\text { Just prior to transfer simulant from HLP-VSL-T22 to UFP-T01 A \& } \\
\text { caustic addition }\end{array}$ & A T22GT 002 XX 1502 ARC 1 & Archive & & \\
\hline TI-065 & 2.2 .2 & $\begin{array}{l}\text { Just prior to transfer simulant from HLP-VSL-T22 to UFP-T01 A \& } \\
\text { caustic addition }\end{array}$ & A T22GM 002 XX 1503 ARC 1 & Archive & & \\
\hline TI-065 & 2.2.2 & $\begin{array}{l}\text { Just prior to transfer simulant from HLP-VSL-T22 to UFP-T01 A \& } \\
\text { caustic addition }\end{array}$ & A T22GB 002 XX 1504 ARC 1 & Archive & & \\
\hline TI-065 & 2.2 .2 & $\begin{array}{l}\text { Just prior to transfer simulant from HLP-VSL-T22 to UFP-T01 A \& } \\
\text { caustic addition }\end{array}$ & A T22GM 002 XX 1505 UDS 2 & UDS & UDS R1 & UDS R1 Shake and Test A and Test B Step001 Date 08182009 \\
\hline TI-065 & 2.2.2 & $\begin{array}{l}\text { Just prior to transfer simulant from HLP-VSL-T22 to UFP-T01 A \& } \\
\text { caustic addition (Batches } 1,3 \& 5 \text { ) }\end{array}$ & A T22GM 002 XX 1506 RHE 4 & Rheology & TDP-WTP-359 & TDP WTP 359 Int Test A RHE Data on PEP Slurry \\
\hline TI-065 & 2.2.2 & $\begin{array}{l}\text { Just prior to transfer simulant from HLP-VSL-T22 to UFP-T01 A \& } \\
\text { caustic addition (Batches } 1,3 \& 5 \text { ) }\end{array}$ & A T22GM 002 XX 1507 XSP 4 B & PSD & TDP-WTP-358 & TDP WTP 358 Int Test A PSD Data Analysis \\
\hline \multirow[t]{2}{*}{ TI-065 } & 2.2.2 & $\begin{array}{l}\text { Just prior to transfer simulant from HLP-VSL-T22 to UFP-T01 A \& } \\
\text { caustic addition (Batches } 1,3 \& 5 \text { ) }\end{array}$ & A T22GM 002 XX 1508 CUF 4 & Parallel Bench Test & & \\
\hline & 2.2 .2 & $\begin{array}{l}\text { Just prior to transfer simulant from HLP-VSL-T22 to UFP-T01 A \& } \\
\text { caustic addition (Batches } 1,3 \& 5 \text { ) }\end{array}$ & A T221A 002 XX 1509 ARC 1 & Archive & & \\
\hline TI-065 & 2.2 .2 & $\begin{array}{l}\text { Just prior to transfer simulant from HLP-VSL-T22 to UFP-T01 A \& } \\
\text { caustic addition (Batches } 1,3 \& 5 \text { ) }\end{array}$ & A T221A 002 XX 1510 ARC 1 & Archive & & \\
\hline TI-065 & 2.2 .2 & $\begin{array}{l}\text { Just prior to transfer simulant from HLP-VSL-T22 to UFP-T01 A \& } \\
\text { caustic addition (Batches } 1,3 \& 5 \text { ) }\end{array}$ & A T221A 002 XX 1511 ARC 1 & Archive & & \\
\hline TI-065 & 2.2 .2 & $\begin{array}{l}\text { Just prior to transfer simulant from HLP-VSL-T22 to UFP-T01 A \& } \\
\text { caustic addition (Batches } 1,3 \& 5 \text { ) }\end{array}$ & A T221A 002 XX 1512 ARC 1 & Archive & & \\
\hline TI-065 & 2.7 & Transfer from HLP-VSL-T22 to T01A ( 3 times - batches $1,3 \& 5$ ) & A 01 AIM 002 XX 1513 UDS 2 B & UDS & UDS R1 & UDS R1 Shake and Test A and Test B Step001 Date 08182009 \\
\hline
\end{tabular}


Integrated Test A Sample List

\begin{tabular}{|c|c|c|c|c|c|c|}
\hline TI\# & TI Step \# & TI Step Description & Sample ID & Analysis & Folder & Final Electronic Report File Name \\
\hline TI-065 & 2.7 & Transfer from HLP-VSL-T22 to T01A ( 3 times - batches $1,3 \& 5$ ) & A 01AIM 002 XX 1513 UDS 2 D & UDS & UDS R1 & UDS R1 Shake and Test A and Test B Step001 Date 08182009 \\
\hline TI-065 & 2.7 & Transfer from HLP-VSL-T22 to T01A ( 3 times - batches $1,3 \& 5$ ) & A 01 AIM 002 XX 1514 UDS 2 B & UDS & UDS R1 & UDS R1 Shake and Test A and Test B Step001 Date 08182009 \\
\hline TI-065 & 2.7 & Transfer from HLP-VSL-T22 to T01A ( 3 times - batches $1,3 \& 5$ ) & A 01AIM 002 XX 1514 UDS 2 D & UDS & UDS R1 & UDS R1 Shake and Test A and Test B Step001 Date 08182009 \\
\hline TI-065 & 2.7 & Transfer from HLP-VSL-T22 to T01A ( 3 times - batches $1,3 \& 5$ ) & A 01 AIM 002 XX 1515 UDS 2 B & UDS & UDS R1 & UDS R1 Shake and Test A and Test B Step001 Date 08182009 \\
\hline TI-065 & 2.7 & Transfer from HLP-VSL-T22 to T01A ( 3 times - batches $1,3 \& 5$ ) & A 01 AIM 002 XX 1515 UDS 2 D & UDS & UDS R1 & UDS R1 Shake and Test A and Test B Step001 Date 08182009 \\
\hline TI-065 & 2.7 & Transfer from HLP-VSL-T22 to T01A ( 3 times - batches $1,3 \& 5$ ) & A 01AIM 002 XX 1516 DEN 2 & Density Slurry & UDS R1 & UDS R1 Shake and Test A and Test B Step001 Date 08182009 \\
\hline TI-065 & 2.7 & Transfer from HLP-VSL-T22 to T01A ( 3 times - batches $1,3 \& 5$ ) & A 01 AIM 002 XX 1517 DEN 2 & Density Slurry & SWRI 090203-2 & 090203-2Density \\
\hline TI-065 & 2.7 & Transfer from HLP-VSL-T22 to T01A ( 3 times - batches $1,3 \& 5$ ) & A 01 AIM 002 XX 1518 DEN 2 & Density Slurry & SWRI 090203-2 & 090203-2Density \\
\hline TI-065 & 2.7 & Transfer from HLP-VSL-T22 to T01 A ( 3 times - batches $1,3 \& 5$ ) & A 01AIM 002 XX 1519 HTC 3 & Heat Capacity & Heat Capacity & TDP-WTP-368 \\
\hline TI-065 & 2.7 & Transfer from HLP-VSL-T22 to T01A ( 3 times - batches $1,3 \& 5$ ) & A 01 AIM 002 XX 1520 ICP 2 B & ICP Wet Solids & SWRI 090203-3 & 090203-3 and 090203-4 ICP and ICPMS \\
\hline TI-065 & 2.7 & Transfer from HLP-VSL-T22 to T01A ( 3 times - batches $1,3 \& 5$ ) & A 01AIM 002 XX 1520 ICP 2 D & ICP Supernate & SWRI 090219-22 & 090219-17 and 0902019-22 ICP and ICPMS \\
\hline TI-065 & 2.7 & Transfer from HLP-VSL-T22 to T01A ( 3 times - batches $1,3 \& 5$ ) & A 01 AIM 002 XX 1520 ICP 2 D & IC & SWRI 090306-12 & 090306-12IC \\
\hline TI-065 & 2.7 & Transfer from HLP-VSL-T22 to T01 A ( 3 times - batches $1,3 \& 5$ ) & A 01 AIM 002 XX 1520 RAM 3 D & Raman & ASR 8342 & ASR 8342 RAM rev 1 \\
\hline TI-065 & 2.7 & Transfer from HLP-VSL-T22 to UFP-VSL-T01A & A 01AIM 002 XX 1520 TFI 3 D & $\mathrm{OH}$ & ASR 8366 & ASR 8366_1 OH \\
\hline TI-065 & 2.7 & Transfer from HLP-VSL-T22 to T01A ( 3 times - batches $1,3 \& 5$ ) & A 01AIM 002 XX 1521 ICP 2 B & ICP Wet Solids & SWRI 090203-3 & 090203-3 and 090203-4 ICP and ICPMS \\
\hline TI-065 & 2.7 & Transfer from HLP-VSL-T22 to T01A ( 3 times - batches $1,3 \& 5$ ) & A 01AIM 002 XX 1521 ICP 2 D & ICP Supernate & SWRI 090219-22 & 090219-17 and 0902019-22 ICP and ICPMS \\
\hline TI-065 & 2.7 & Transfer from HLP-VSL-T22 to T01A ( 3 times - batches $1,3 \& 5$ ) & A 01AIM 002 XX 1521 ICP 2 D & IC & SWRI 090306-12 & 090306-12IC \\
\hline TI-065 & 2.7 & Transfer from HLP-VSL-T22 to T01 A ( 3 times - batches $1,3 \& 5$ ) & A 01 AIM 002 XX 1521 RAM 3 D & Raman & ASR 8342 & ASR 8342 RAM rev 1 \\
\hline TI-065 & 2.7 & Transfer from HLP-VSL-T22 to UFP-VSL-T01A & A 01AIM 002 XX 1521 TFI 3 D & $\mathrm{OH}$ & ASR 8366 & ASR 8366_1 OH \\
\hline TI-065 & 2.7 & Transfer from HLP-VSL-T22 to T01A ( 3 times - batches $1,3 \& 5$ ) & A 01 AIM 002 XX 1522 ICP 2 B & ICP Wet Solids & SWRI 090203-3 & 090203-3 and 090203-4 ICP and ICPMS \\
\hline TI-065 & 2.7 & Transfer from HLP-VSL-T22 to T01A ( 3 times - batches $1,3 \& 5$ ) & A 01AIM 002 XX 1522 ICP 2 D & ICP Supernate & SWRI 090219-22 & 090219-17 and 0902019-22 ICP and ICPMS \\
\hline TI-065 & 2.7 & Transfer from HLP-VSL-T22 to T01A ( 3 times - batches $1,3 \& 5$ ) & A 01 AIM 002 XX 1522 ICP 2 D & IC & SWRI 090306-12 & 090306-12IC \\
\hline TI-065 & 2.7 & Transfer from HLP-VSL-T22 to T01 A ( 3 times - batches $1,3 \& 5$ ) & A 01 AIM 002 XX 1522 RAM 3 D & Raman & ASR8342 & ASR 8342 RAM rev 1 \\
\hline TI-065 & 2.7 & Transfer from HLP-VSL-T22 to UFP-VSL-T01A & A 01 AIM 002 XX 1522 TFI 3 D & $\mathrm{OH}$ & ASR 8366 & ASR 8366_1 OH \\
\hline TI-065 & 2.7 & Transfer from HLP-VSL-T22 to T01A ( 3 times - batches $1,3 \& 5$ ) & A 01 AIM 002 XX 1523 CUF 4 & Parallel Bench Test & & \\
\hline TI-065 & 3.12 & Heat UFP-VSL-T01A to $88 \mathrm{C}$ & A 01 AIM 003 BT 1524 UDS 2 B & UDS & UDS R1 & UDS R1 Shake and Test A and Test B Step001 Date 08182009 \\
\hline TI-065 & 3.12 & Heat UFP-VSL-T01A to $88 \mathrm{C}$ & A 01 AIM 003 BT 1524 UDS 2 D & UDS & UDS R1 & UDS R1 Shake and Test A and Test B Step001 Date 08182009 \\
\hline TI-065 & 3.12 & Heat UFP-VSL-T01A to $88 \mathrm{C}$ & A 01AIM 003 BT 1525 DEN 2 & Density Slurry & SWRI 090203-2 & 090203-2Density \\
\hline TI-065 & 3.12 & Heat UFP-VSL-T01A to $88 \mathrm{C}$ & A 01AIM 003 BT 1526 HTC 3 & Heat Capacity & Heat Capacity & TDP-WTP-368 \\
\hline TI-065 & 3.12 & Heat UFP-VSL-T01A to $88 \mathrm{C}$ & A 01 AIM 003 BT 1527 ICP 2 D & ICP Supernate & SWRI 090219-22 & 090219-17 and 0902019-22 ICP and ICPMS \\
\hline TI-065 & 3.12 & Heat UFP-VSL-T01A to $88 \mathrm{C}$ & A $01 \mathrm{AIM} 003$ BT 1527 ICP 2 D & IC & SWRI 090306-12 & 090306-12IC \\
\hline TI-065 & 3.12 & Heat UFP-VSL-T01A to $88 \mathrm{C}$ & A 01 AIM 003 BT 1527 RAM 3 D & Raman & ASR 8342 & ASR 8342 RAM rev 1 \\
\hline TI-065 & 3.12 & Heat UFP-VSL-T01A to $88 \mathrm{C}$ & A 01AIH 003 BT 1528 ARC 1 B & Archive & & \\
\hline
\end{tabular}


Integrated Test A Sample List

\begin{tabular}{|c|c|c|c|c|c|c|}
\hline TI \# & TI Step \# & TI Step Description & Sample ID & Analysis & Folder & Final Electronic Report File Name \\
\hline TI-065 & 3.12 & Heat UFP-VSL-T01A to $88 \mathrm{C}$ & A $01 \mathrm{AIH} 003$ BT 1528 ARC 1 D & Archive & & \\
\hline TI-065 & 3.12 & Heat UFP-VSL-T01A to $88 \mathrm{C}$ & A 01AIL 003 BT 1529 ARC 1 B & Archive & & \\
\hline TI-065 & 3.12 & Heat UFP-VSL-T01A to $88 \mathrm{C}$ & A 01AIL 003 BT 1529 ARC 1 D & Archive & & \\
\hline TI-065 & 3.12 & Heat UFP-VSL-T01A to $88 \mathrm{C}$ & A $01 \mathrm{AMH} 003$ BT 1530 ARC 1 B & Archive & & \\
\hline TI-065 & 3.12 & Heat UFP-VSL-T01A to $88 \mathrm{C}$ & A $01 \mathrm{AMH} 003$ BT 1530 ARC 1 D & Archive & & \\
\hline TI-065 & 3.12 & Heat UFP-VSL-T01A to $88 \mathrm{C}$ & A $01 \mathrm{AMM} 003$ BT 1531 ARC 1 B & Archive & & \\
\hline TI-065 & 3.12 & Heat UFP-VSL-T01A to $88 \mathrm{C}$ & A $01 \mathrm{AMM} 003$ BT 1531 ARC 1 D & Archive & & \\
\hline TI-065 & 3.12 & Heat UFP-VSL-T01A to $88 \mathrm{C}$ & A 01AML 003 BT 1532 ARC 1 B & Archive & & \\
\hline TI-065 & 3.12 & Heat UFP-VSL-T01A to $88 \mathrm{C}$ & A 01AML 003 BT 1532 ARC 1 D & Archive & & \\
\hline TI-065 & 3.12 & Heat UFP-VSL-T01A to $88 \mathrm{C}$ & A $01 \mathrm{AOH} 003 \mathrm{BT} 1533 \mathrm{ARC} 1 \mathrm{~B}$ & Archive & & \\
\hline TI-065 & 3.12 & Heat UFP-VSL-T01A to $88 \mathrm{C}$ & A $01 \mathrm{AOH} 003$ BT 1533 ARC 1 D & Archive & & \\
\hline TI-065 & 3.12 & Heat UFP-VSL-T01A to $88 \mathrm{C}$ & A $01 \mathrm{AOM} 003$ BT 1534 ARC 1 B & Archive & & \\
\hline TI-065 & 3.12 & Heat UFP-VSL-T01A to $88 \mathrm{C}$ & A $01 \mathrm{AOM} 003 \mathrm{BT} 1534$ ARC 1 D & Archive & & \\
\hline TI-065 & 3.12 & Heat UFP-VSL-T01A to $88 \mathrm{C}$ & A 01AOL 003 BT 1535 ARC 1 B & Archive & & \\
\hline TI-065 & 3.12 & Heat UFP-VSL-T01A to $88 \mathrm{C}$ & A 01AOL 003 BT 1535 ARC 1 D & Archive & & \\
\hline TI-065 & 3.13 & Caustic leach & A 01 AIM 003001536 UDS 2 B & UDS & UDS R1 & UDS R1 Shake and Test A and Test B Step001 Date 08182009 \\
\hline TI-065 & 3.13 & Caustic leach & A 01 AIM 003001536 UDS 2 D & UDS & UDS R1 & UDS R1 Shake and Test A and Test B Step001 Date 08182009 \\
\hline TI-065 & 3.13 & Caustic leach & A 01 AIM 003001537 DEN 2 & Density Slurry & SWRI 090203-2 & 090203-2Density \\
\hline TI-065 & 3.13 & Caustic leach & A 01 AIM 003001538 HTC 3 & Heat Capacity & Heat Capacity & TDP-WTP-368 \\
\hline TI-065 & 3.13 & Caustic leach & A 01AIM 003001539 DEN 0 B & ICP Wet Solids & SWRI 090306-9 & 090306-9 ICP and ICPMS \\
\hline TI-065 & 3.13 & Caustic leach & A 01AIM 003001539 ICP 2 D & ICP Supernate & SWRI 090203-5 & 090203-5 and 090203-6 ICP and ICPMS \\
\hline TI-065 & 3.13 & Caustic leach & A 01 AIM 003001539 RAM 3 D & Raman & ASR8342 & ASR 8342 RAM rev 1 \\
\hline TI-065 & 3.13 & Caustic leach & A 01 AIM 003001539 TFI 2 D & IC & $\begin{array}{l}\text { SWRI 090203-21, } \\
\text { SWRI 090204-8 }\end{array}$ & 090203-21IC \\
\hline TI-065 & 3.13 & Caustic leach & A 01AIM 003001539 TFI 3 D & $\mathrm{OH}$ & ASR 8366 & ASR 8366 \\
\hline TI-065 & 3.13 & Caustic leach & A 01 AIM 003002614 UDS 2 B & UDS & UDS R1 & UDS R1 Shake and Test A and Test B Step001 Date 08182009 \\
\hline TI-065 & 3.13 & Caustic leach & A 01 AIM 003002614 UDS 2 D & UDS & UDS R1 & UDS R1 Shake and Test A and Test B Step001 Date 08182009 \\
\hline TI-065 & 3.13 & Caustic leach & A 01 AIM 003002615 UDS 2 B & UDS & UDS R1 & UDS R1 Shake and Test A and Test B Step001 Date 08182009 \\
\hline TI-065 & 3.13 & Caustic leach & A 01 AIM 003002615 UDS 2 D & UDS & UDS R1 & UDS R1 Shake and Test A and Test B Step001 Date 08182009 \\
\hline TI-065 & 3.13 & Caustic leach & A 01AIM 003002616 DEN 2 & Density Slurry & SWRI 090203-2 & 090203-2Density \\
\hline TI-065 & 3.13 & Caustic leach & A 01 AIM 003002617 DEN 2 & Density Slurry & SWRI 090203-2 & 090203-2Density \\
\hline TI-065 & 3.13 & Caustic leach & A 01AIM 003002618 DEN 0 B & ICP Wet Solids & SWRI 090306-9 & 090306-9 ICP and ICPMS \\
\hline TI-065 & 3.13 & Caustic leach & A 01 AIM 003002618 ICP 2 D & ICP Supernate & SWRI 090203-5 & 090203-5 and 090203-6 ICP and ICPMS \\
\hline
\end{tabular}


Integrated Test A Sample List

\begin{tabular}{|c|c|c|c|c|c|c|}
\hline TI \# & TI Step \# & TI Step Description & Sample ID & Analysis & Folder & Final Electronic Report File Name \\
\hline TI-065 & 3.13 & Caustic leach & A 01 AIM 003002618 RAM 3 D & Raman & ASR8342 & ASR 8342 RAM rev 1 \\
\hline TI-065 & 3.13 & Caustic leach & A 01AIM 003002618 TFI 2 D & IC & $\begin{array}{c}\text { SWRI 090203-21, } \\
\text { SWRI 090204-8 }\end{array}$ & 090203-21IC \\
\hline TI-065 & 3.13 & Caustic leach & A 01AIM 003002618 TFI 3 D & $\mathrm{OH}$ & ASR 8366 & ASR 8366_1 OH \\
\hline TI-065 & 3.13 & Caustic leach & A 01AIM 003002619 DEN 0 B & ICP Wet Solids & SWRI 090306-9 & 090306-9 ICP and ICPMS \\
\hline TI-065 & 3.13 & Caustic leach & A 01AIM 003002619 ICP 2 D & ICP Supernate & SWRI 090203-5 & $090203-5$ and $090203-6$ ICP and ICPMS \\
\hline TI-065 & 3.13 & Caustic leach & A 01 AIM 003002619 RAM 3 D & Raman & ASR8342 & ASR 8342 RAM rev 1 \\
\hline TI-065 & 3.13 & Caustic leach & A 01 AIM 003002619 TFI 2 D & IC & $\begin{array}{l}\text { SWRI 090203-21, } \\
\text { SWRI 090204-8 }\end{array}$ & 090203-21IC \\
\hline TI-065 & 3.13 & Caustic leach & A 01 AIM 003002619 TFI 3 D & $\mathrm{OH}$ & ASR 8366 & ASR 8366_1 OH \\
\hline TI-065 & 3.13 & Caustic leach & A $01 \mathrm{AIH} 003001540$ ARC $1 \mathrm{~B}$ & ICP Wet Solids & SWRI 090827 & $090827-7$ and $090827-8$ ICP and ICPMS \\
\hline TI-065 & 3.13 & Caustic leach & A 01 AIH 003001540 ARC 1 D & IC & SWRI 090827 & $090827-5$ and $0908027-6$ IC and TDS ${ }^{(1)}$ \\
\hline TI-065 & 3.13 & Caustic leach & A 01AIH 003001540 ARC 1 D & TDS & SWRI 090827 & $090827-5$ and $0908027-6$ IC and TDS ${ }^{(1)}$ \\
\hline TI-065 & 3.13 & Caustic leach & A $01 \mathrm{AMH} 003001542$ ARC $1 \mathrm{~B}$ & ICP Wet Solids & SWRI 090827 & $090827-7$ and $090827-8$ ICP and ICPMS \\
\hline TI-065 & 3.13 & Caustic leach & A 01AMH 003001542 ARC 1 D & IC & SWRI 090827 & $090827-5$ and $0908027-6$ IC and TDS ${ }^{(1)}$ \\
\hline TI-065 & 3.13 & Caustic leach & A $01 \mathrm{AMH} 003001542$ ARC $1 \mathrm{D}$ & TDS & SWRI 090827 & 090827-5 and 0908027-6 IC and TDS ${ }^{(1)}$ \\
\hline TI-065 & 3.13 & Caustic leach & A 01AML 003001544 ARC 1 B & ICP Wet Solids & SWRI 090827 & $090827-7$ and $090827-8$ ICP and ICPMS \\
\hline TI-065 & 3.13 & Caustic leach & A 01AML 003001544 ARC 1 D & $\mathrm{IC}$ & SWRI 090827 & $090827-5$ and $0908027-6$ IC and TDS ${ }^{(1)}$ \\
\hline TI-065 & 3.13 & Caustic leach & A 01 AML 003001544 ARC 1 D & TDS & SWRI 090827 & $090827-5$ and $0908027-6 \mathrm{IC}$ and TDS ${ }^{(1)}$ \\
\hline TI-065 & 3.13 & Caustic leach & A $01 \mathrm{AOM} 003001546$ ARC $1 \mathrm{~B}$ & ICP Wet Solids & SWRI 090827 & $090827-7$ and $090827-8$ ICP and ICPMS \\
\hline TI-065 & 3.13 & Caustic leach & A $01 \mathrm{AOM} 003001546$ ARC 1 D & $\mathrm{IC}$ & SWRI 090827 & $090827-5$ and $0908027-6$ IC and TDS ${ }^{(1)}$ \\
\hline TI-065 & 3.13 & Caustic leach & A $01 \mathrm{AOM} 003001546$ ARC $1 \mathrm{D}$ & TDS & SWRI 090827 & 090827-5 and 0908027-6 IC and TDS ${ }^{(1)}$ \\
\hline TI-065 & 3.13 & Caustic leach & A $01 \mathrm{AOL} 003001547$ ARC $1 \mathrm{~B}$ & ICP Wet Solids & SWRI 090827 & $090827-7$ and $090827-8$ ICP and ICPMS \\
\hline TI-065 & 3.13 & Caustic leach & A 01AOL 003001547 ARC 1 D & IC & SWRI 090827 & $090827-5$ and $0908027-6$ IC and TDS ${ }^{(1)}$ \\
\hline TI-065 & 3.13 & Caustic leach & A 01AOL 003001547 ARC 1 D & TDS & SWRI 090827 & $090827-5$ and $0908027-6$ IC and TDS ${ }^{(1)}$ \\
\hline TI-065 & 3.13 & Caustic leach & A 01AIL 003001541 ARC 1 B & ICP Wet Solids & SWRI 090827 & 090827-7 and 090827-8 ICP and ICPMS \\
\hline TI-065 & 3.13 & Caustic leach & A 01AIL 003001541 ARC 1 D & IC & SWRI 090827 & $090827-5$ and $0908027-6$ IC and TDS ${ }^{(1)}$ \\
\hline TI-065 & 3.13 & Caustic leach & A 01AIL 003001541 ARC 1 D & TDS & SWRI 090827 & $090827-5$ and $0908027-6$ IC and TDS ${ }^{(1)}$ \\
\hline TI-065 & 3.13 & Caustic leach & A 01 AMM 003001543 ARC 1 B & ICP Wet Solids & SWRI 090827 & $090827-7$ and $090827-8$ ICP and ICPMS \\
\hline TI-065 & 3.13 & Caustic leach & A 01 AMM 003001543 ARC 1 D & IC & SWRI 090827 & $090827-5$ and $0908027-6$ IC and TDS ${ }^{(1)}$ \\
\hline TI-065 & 3.13 & Caustic leach & A 01 AMM 003001543 ARC 1 D & TDS & SWRI 090827 & $090827-5$ and $0908027-6$ IC and TDS ${ }^{(1)}$ \\
\hline TI-065 & 3.13 & Caustic leach & A $01 \mathrm{AOH} 003001545$ ARC $1 \mathrm{~B}$ & ICP Wet Solids & SWRI 090827 & $090827-7$ and $090827-8$ ICP and ICPMS \\
\hline TI-065 & 3.13 & Caustic leach & A $01 \mathrm{AOH} 003001545$ ARC 1 D & IC & SWRI 090827 & $090827-5$ and $0908027-6$ IC and TDS ${ }^{(1)}$ \\
\hline
\end{tabular}


Integrated Test A Sample List

\begin{tabular}{|c|c|c|c|c|c|c|}
\hline TI\# & TI Step \# & TI Step Description & Sample ID & Analysis & Folder & Final Electronic Report File Name \\
\hline TI-065 & 3.13 & Caustic leach & A 01AOH 003001545 ARC 1 D & TDS & SWRI 090827 & 090827-5 and 0908027-6 IC and TDS ${ }^{(1)}$ \\
\hline TI-065 & 3.16 & Caustic leach & A 01AIM 003011548 UDS 2 B & UDS & UDS R1 & UDS R1 Shake and Test A and Test B Step001 Date 08182009 \\
\hline TI-065 & 3.16 & Caustic leach & A 01AIM 003011548 UDS 2 D & UDS & UDS R1 & UDS R1 Shake and Test A and Test B Step001 Date 08182009 \\
\hline TI-065 & 3.16 & Caustic leach & A 01 AIM 003011549 DEN 2 & Density Slurry & SWRI 090203-2 & 090203-2Density \\
\hline TI-065 & 3.16 & Caustic leach & A 01 AIM 003011550 ICP 2 D & ICP Supernate & SWRI 090219-22 & 090219-17 and 0902019-22 ICP and ICPMS \\
\hline TI-065 & 3.16 & Caustic leach & A 01 AIM 003011550 ICP 2 D & IC & SWRI 090306-12 & 090306-12IC \\
\hline TI-065 & 3.16 & Caustic leach & A 01 AIM 003011550 RAM 3 D & Raman & ASR8342 & ASR 8342 RAM rev 1 \\
\hline TI-065 & 3.16 & Caustic leach & A 01 AIM 003021551 UDS 2 B & UDS & UDS R1 & UDS R1 Shake and Test A and Test B Step001 Date 08182009 \\
\hline TI-065 & 3.16 & Caustic leach & A 01 AIM 003021551 UDS 2 D & UDS & UDS R1 & UDS R1 Shake and Test A and Test B Step001 Date 08182009 \\
\hline TI-065 & 3.16 & Caustic leach & A 01 AIM 003021552 DEN 2 & Density Slurry & SWRI 090203-2 & 090203-2Density \\
\hline TI-065 & 3.16 & Caustic leach & A 01AIM 003021553 ICP 2 D & ICP Supernate & SWRI 090219-22 & 090219-17 and 0902019-22 ICP and ICPMS \\
\hline TI-065 & 3.16 & Caustic leach & A 01 AIM 003021553 ICP 2 D & IC & SWRI 090306-12 & 090306-12IC \\
\hline TI-065 & 3.16 & Caustic leach & A 01 AIM 003021553 RAM 3 D & Raman & ASR 8342 & ASR 8342 RAM rev 1 \\
\hline TI-065 & 3.16 & Caustic leach & A 01 AIM 003031554 ARC 1 B & Archive & & \\
\hline TI-065 & 3.16 & Caustic leach & A 01 AIM 003031554 ARC 1 D & Archive & & \\
\hline TI-065 & 3.16 & Caustic leach & A 01 AIM 003041555 UDS 2 B & UDS & UDS R1 & UDS R1 Shake and Test A and Test B Step001 Date 08182009 \\
\hline TI-065 & 3.16 & Caustic leach & A 01AIM 003041555 UDS 2 D & UDS & UDS R1 & UDS R1 Shake and Test A and Test B Step001 Date 08182009 \\
\hline TI-065 & 3.16 & Caustic leach & A 01AIM 003041556 DEN 2 & Density Slurry & SWRI 090203-2 & 090203-2Density \\
\hline TI-065 & 3.16 & Caustic leach & A 01 AIM 003041557 ICP 2 B & ICP Wet Solids & SWRI 090203-3 & 090203-3 and 090203-4 ICP and ICPMS \\
\hline TI-065 & 3.16 & Caustic leach & A 01 AIM 003041557 ICP 2 D & ICP Supernate & SWRI 090219-22 & 090219-17 and 0902019-22 ICP and ICPMS \\
\hline TI-065 & 3.16 & Caustic leach & A 01 AIM 003041557 ICP 2 D & IC & SWRI 090306-12 & 090306-12IC \\
\hline TI-065 & 3.16 & Caustic leach & A 01 AIM 003041557 RAM 3 D & Raman & ASR 8342 & ASR 8342 RAM rev 1 \\
\hline TI-065 & 3.16 & Caustic leach & A 01AIL 003041559 ARC 1 B & Archive & & \\
\hline TI-065 & 3.16 & Caustic leach & A 01AIL 003041559 ARC 1 D & Archive & & \\
\hline TI-065 & 3.16 & Caustic leach & A $01 \mathrm{AMH} 003041560$ ARC $1 \mathrm{~B}$ & Archive & & \\
\hline TI-065 & 3.16 & Caustic leach & A $01 \mathrm{AMH} 003041560$ ARC $1 \mathrm{D}$ & Archive & & \\
\hline TI-065 & 3.16 & Caustic leach & A 01 AMM 003041561 ARC 1 B & Archive & & \\
\hline TI-065 & 3.16 & Caustic leach & A 01AMM 003041561 ARC 1 D & Archive & & \\
\hline TI-065 & 3.16 & Caustic leach & A 01 AML 003041562 ARC 1 B & Archive & & \\
\hline TI-065 & 3.16 & Caustic leach & A 01 AML 003041562 ARC 1 D & Archive & & \\
\hline TI-065 & 3.16 & Caustic leach & A $01 \mathrm{AOH} 003041563$ ARC $1 \mathrm{~B}$ & Archive & & \\
\hline TI-065 & 3.16 & Caustic leach & A $01 \mathrm{AOH} 003041563$ ARC 1 D & Archive & & \\
\hline TI-065 & 3.16 & Caustic leach & A $01 \mathrm{AOM} 003041564$ ARC $1 \mathrm{~B}$ & Archive & & \\
\hline
\end{tabular}


Integrated Test A Sample List

\begin{tabular}{|c|c|c|c|c|c|c|}
\hline TI \# & TI Step \# & TI Step Description & Sample ID & Analysis & Folder & Final Electronic Report File Name \\
\hline TI-065 & 3.16 & Caustic leach & A 01 AOM 003041564 ARC 1 D & Archive & & \\
\hline TI-065 & 3.16 & Caustic leach & A $01 \mathrm{AOL} 003041565$ ARC $1 \mathrm{~B}$ & Archive & & \\
\hline TI-065 & 3.16 & Caustic leach & A $01 \mathrm{AOL} 003041565$ ARC $1 \mathrm{D}$ & Archive & & \\
\hline TI-065 & 3.16 & Caustic leach & A 01AIM 003051566 ARC 1 B & Archive & & \\
\hline TI-065 & 3.16 & Caustic leach & A 01 AIM 003051566 ARC 1 D & Archive & & \\
\hline TI-065 & 3.16 & Caustic leach & A 01AIM 003061567 ARC 1 B & Archive & & \\
\hline TI-065 & 3.16 & Caustic leach & A 01AIM 003061567 ARC 1 D & Archive & & \\
\hline TI-065 & 3.16 & Caustic leach & A 01 AIM 003071568 ARC $1 \mathrm{~B}$ & Archive & & \\
\hline TI-065 & 3.16 & Caustic leach & A 01 AIM 003071568 ARC 1 D & Archive & & \\
\hline TI-065 & 3.16 & Caustic leach & A 01 AIM 003081569 UDS 2 B & UDS & UDS R1 & UDS R1 Shake and Test A and Test B Step001 Date 08182009 \\
\hline TI-065 & 3.16 & Caustic leach & A 01 AIM 003081569 UDS 2 D & UDS & UDS R1 & UDS R1 Shake and Test A and Test B Step001 Date 08182009 \\
\hline TI-065 & 3.16 & Caustic leach & A 01AIM 003081570 DEN 2 & Density Slurry & SWRI 090203-2 & 090203-2Density \\
\hline TI-065 & 3.16 & Caustic leach & A 01 AIM 003081571 ICP 2 B & ICP Wet Solids & SWRI 090203-3 & 090203-3 and 090203-4 ICP and ICPMS \\
\hline TI-065 & 3.16 & Caustic leach & A 01 AIM 003081571 ICP 2 D & ICP Supernate & SWRI 090203-5 & 090203-5 and 090203-6 ICP and ICPMS \\
\hline TI-065 & 3.16 & Caustic leach & A 01 AIM 003081571 RAM 3 D & Raman & ASR8342 & ASR 8342 RAM rev 1 \\
\hline TI-065 & 3.16 & Caustic leach & A 01AIM 003081571 RAM 3 D & $\mathrm{OH}$ & ASR 8342 & ASR 8342_1 OH \\
\hline TI-065 & 3.16 & Caustic leach & A 01AIM 003081571 TFI 2 D & IC & $\begin{array}{l}\text { SWRI 090203-21, } \\
\text { SWRI 090204-8 }\end{array}$ & 090203-21IC \\
\hline TI-065 & 3.16 & Caustic leach & A $01 \mathrm{AIH} 003081572$ ARC $1 \mathrm{~B}$ & Archive & & \\
\hline TI-065 & 3.16 & Caustic leach & A $01 \mathrm{AIH} 003081572$ ARC $1 \mathrm{D}$ & Archive & & \\
\hline TI-065 & 3.16 & Caustic leach & A 01 AIL 003081573 ARC 1 B & Archive & & \\
\hline TI-065 & 3.16 & Caustic leach & A 01AIL 003081573 ARC 1 D & Archive & & \\
\hline TI-065 & 3.16 & Caustic leach & A $01 \mathrm{AMH} 003081574$ ARC $1 \mathrm{~B}$ & Archive & & \\
\hline TI-065 & 3.16 & Caustic leach & A 01AMH 003081574 ARC 1 D & Archive & & \\
\hline TI-065 & 3.16 & Caustic leach & A 01 AMM 003081575 ARC 1 B & Archive & & \\
\hline TI-065 & 3.16 & Caustic leach & A $01 \mathrm{AMM} 003081575$ ARC $1 \mathrm{D}$ & Archive & & \\
\hline TI-065 & 3.16 & Caustic leach & A 01AML 003081576 ARC 1 B & Archive & & \\
\hline TI-065 & 3.16 & Caustic leach & A 01AML 003081576 ARC 1 D & Archive & & \\
\hline TI-065 & 3.16 & Caustic leach & A 01AOH 003081577 ARC 1 B & Archive & & \\
\hline TI-065 & 3.16 & Caustic leach & A 01AOH 003081577 ARC 1 D & Archive & & \\
\hline TI-065 & 3.16 & Caustic leach & A $01 \mathrm{AOM} 003081578$ ARC $1 \mathrm{~B}$ & Archive & & \\
\hline TI-065 & 3.16 & Caustic leach & A $01 \mathrm{AOM} 003081578$ ARC 1 D & Archive & & \\
\hline TI-065 & 3.16 & Caustic leach & A 01 AOL 003081579 ARC $1 \mathrm{~B}$ & Archive & & \\
\hline
\end{tabular}


Integrated Test A Sample List

\begin{tabular}{|c|c|c|c|c|c|c|}
\hline TI \# & TI Step \# & TI Step Description & Sample ID & Analysis & Folder & Final Electronic Report File Name \\
\hline TI-065 & 3.16 & Caustic leach & A 01AOL 003081579 ARC 1 D & Archive & & \\
\hline TI-065 & 3.16 & Caustic leach & A 01AIM 003091580 ARC 1 B & Archive & & \\
\hline TI-065 & 3.16 & Caustic leach & A 01AIM 003091580 ARC 1 D & Archive & & \\
\hline TI-065 & 3.16 & Caustic leach & A 01 AIM 003101581 UDS $2 \mathrm{~B}$ & UDS & UDS R1 & UDS R1 Shake and Test A and Test B Step001 Date 08182009 \\
\hline TI-065 & 3.16 & Caustic leach & A 01AIM 003101581 UDS 2 D & UDS & UDS R1 & UDS R1 Shake and Test A and Test B Step001 Date 08182009 \\
\hline TI-065 & 3.16 & Caustic leach & A 01AIM 003101582 DEN 2 & Density Slurry & SWRI 090203-2 & 090203-2Density \\
\hline TI-065 & 3.16 & Caustic leach & A 01AIM 003101583 ICP 2 D & ICP Supernate & SWRI 090219-22 & 090219-17 and 0902019-22 ICP and ICPMS \\
\hline TI-065 & 3.16 & Caustic leach & A 01AIM 003101583 ICP 2 D & IC & SWRI 090306-12 & 090306-12IC \\
\hline TI-065 & 3.16 & Caustic leach & A 01AIM 003101583 RAM 3 D & Raman & ASR8342 & ASR 8342 RAM rev 1 \\
\hline TI-065 & 3.16 & Caustic leach & A 01AIM 003111584 ARC 1 B & Archive & & \\
\hline TI-065 & 3.16 & Caustic leach & A 01AIM 003111584 ARC 1 D & Archive & & \\
\hline TI-065 & 3.16 & Caustic leach & A 01 AIM 003121585 UDS 2 B & UDS & UDS R1 & UDS R1 Shake and Test A and Test B Step001 Date 08182009 \\
\hline TI-065 & 3.16 & Caustic leach & A 01AIM 003121585 UDS 2 D & UDS & UDS R1 & UDS R1 Shake and Test A and Test B Step001 Date 08182009 \\
\hline TI-065 & 3.16 & Caustic leach & A 01 AIM 003121586 DEN 2 & Density Slurry & SWRI 090204-3 & 090204-3Density \\
\hline TI-065 & 3.16 & Caustic leach & A 01 AIM 003121587 ICP 2 B & ICP Wet Solids & SWRI 090204-6 & 090204 and 090205 ICP and ICPMS \\
\hline TI-065 & 3.16 & Caustic leach & A 01AIM 003121587 ICP 2 D & ICP Supernate & SWRI 090204-7 & 090204-7 and 090205-20 ICP and ICPMS \\
\hline TI-065 & 3.16 & Caustic leach & A 01AIM 003121587 RAM 3 D & Raman & ASR8343 & ASR 8343 RAM rev 1 \\
\hline TI-065 & 3.16 & Caustic leach & A 01AIM 003121587 RAM 3 D & $\mathrm{OH}$ & ASR 8343 & ASR 8343_1 OH \\
\hline TI-065 & 3.16 & Caustic leach & A 01 AIM 003121587 TFI 2 D & IC & $\begin{array}{l}\text { SWRI 090203-21, } \\
\text { SWRI 090204-8 }\end{array}$ & 090204-8 IC TIC and TOC \\
\hline TI-065 & 3.16 & Caustic leach & A 01AIH 003122532 ARC 1 D & Archive & & \\
\hline TI-065 & 3.16 & Caustic leach & A 01AIH 003122532 ARC 1 B & Archive & & \\
\hline TI-065 & 3.16 & Caustic leach & A 01AIL 003122533 ARC 1 B & Archive & & \\
\hline TI-065 & 3.16 & Caustic leach & A 01AIL 003122533 ARC 1 D & Archive & & \\
\hline TI-065 & 3.16 & Caustic leach & A 01AMH 003122534 ARC 1 B & Archive & & \\
\hline TI-065 & 3.16 & Caustic leach & A 01AMH 003122534 ARC 1 D & Archive & & \\
\hline TI-065 & 3.16 & Caustic leach & A 01 AMM 003121588 ARC 1 B & Archive & & \\
\hline TI-065 & 3.16 & Caustic leach & A 01AMM 003121588 ARC 1 D & Archive & & \\
\hline TI-065 & 3.16 & Caustic leach & A 01AML 003121589 ARC 1 B & Archive & & \\
\hline TI-065 & 3.16 & Caustic leach & A 01AML 003121589 ARC 1 D & Archive & & \\
\hline TI-065 & 3.16 & Caustic leach & A $01 \mathrm{AOH} 003121590$ ARC $1 \mathrm{~B}$ & Archive & & \\
\hline TI-065 & 3.16 & Caustic leach & A 01AOH 003121590 ARC 1 D & Archive & & \\
\hline TI-065 & 3.16 & Caustic leach & A $01 \mathrm{AOM} 003121591$ ARC $1 \mathrm{~B}$ & Archive & & \\
\hline
\end{tabular}


Integrated Test A Sample List

\begin{tabular}{|c|c|c|c|c|c|c|}
\hline TI \# & TI Step \# & TI Step Description & Sample ID & Analysis & Folder & Final Electronic Report File Name \\
\hline TI-065 & 3.16 & Caustic leach & A 01AOM 003121591 ARC 1 D & Archive & & \\
\hline TI-065 & 3.16 & Caustic leach & A 01AOL 003121592 ARC 1 B & Archive & & \\
\hline TI-065 & 3.16 & Caustic leach & A 01AOL 003121592 ARC 1 D & Archive & & \\
\hline TI-065 & 3.16 & Caustic leach & A 01 AIM 003141594 UDS 2 B & UDS & UDS R1 & UDS R1 Shake and Test A and Test B Step001 Date 08182009 \\
\hline TI-065 & 3.16 & Caustic leach & A 01 AIM 003141594 UDS 2 D & UDS & UDS R1 & UDS R1 Shake and Test A and Test B Step001 Date 08182009 \\
\hline TI-065 & 3.16 & Caustic leach & A 01AIM 003141595 DEN 2 & Density Slurry & SWRI 090204-3 & 090204-3Density \\
\hline TI-065 & 3.16 & Caustic leach & A 01AIM 003141596 ICP 2 D & ICP Supernate & SWRI 090219-22 & 090219-17 and 0902019-22 ICP and ICPMS \\
\hline TI-065 & 3.16 & Caustic leach & A 01AIM 003141596 ICP 2 D & IC & SWRI 090306-12 & 090306-12IC \\
\hline TI-065 & 3.16 & Caustic leach & A 01 AIM 003141596 RAM 3 D & Raman & ASR 8343 & ASR 8343 RAM rev 1 \\
\hline TI-065 & 3.16 & Caustic leach & A 01 AIM 003131593 ARC 1 B & Archive & & \\
\hline TI-065 & 3.16 & Caustic leach & A 01AIM 003131593 ARC 1 D & Archive & & \\
\hline TI-065 & 3.16 & Caustic leach & A 01AIM 003151597 ARC 1 B & Archive & & \\
\hline TI-065 & 3.16 & Caustic leach & A 01AIM 003151597 ARC 1 D & Archive & & \\
\hline TI-065 & 3.16 & Caustic leach & A 01 AIM 003161598 UDS 2 B & UDS & UDS R1 & UDS R1 Shake and Test A and Test B Step001 Date 08182009 \\
\hline TI-065 & 3.16 & Caustic leach & A 01 AIM 003161598 UDS 2 D & UDS & UDS R1 & UDS R1 Shake and Test A and Test B Step001 Date 08182009 \\
\hline TI-065 & 3.16 & Caustic leach & A 01 AIM 003161599 UDS 2 B & UDS & UDS R1 & UDS R1 Shake and Test A and Test B Step001 Date 08182009 \\
\hline TI-065 & 3.16 & Caustic leach & A 01 AIM 003161599 UDS 2 D & UDS & UDS R1 & UDS R1 Shake and Test A and Test B Step001 Date 08182009 \\
\hline TI-065 & 3.16 & Caustic leach & A 01 AIM 003161600 UDS $2 \mathrm{~B}$ & UDS & UDS R1 & UDS R1 Shake and Test A and Test B Step001 Date 08182009 \\
\hline TI-065 & 3.16 & Caustic leach & A 01AIM 003161600 UDS 2 D & UDS & UDS R1 & UDS R1 Shake and Test A and Test B Step001 Date 08182009 \\
\hline TI-065 & 3.16 & Caustic leach & A 01AIM 003161601 DEN 2 & Density Slurry & SWRI 090204-3 & 090204-3Density \\
\hline TI-065 & 3.16 & Caustic leach & A 01 AIM 003161602 DEN 2 & Density Slurry & SWRI 090204-3 & 090204-3Density \\
\hline TI-065 & 3.16 & Caustic leach & A 01 AIM 003161603 DEN 2 & Density Slurry & SWRI 090204-3 & 090204-3Density \\
\hline TI-065 & 3.16 & Caustic leach & A 01 AIM 003161604 ICP 2 B & ICP Wet Solids & SWRI 090204-6 & 090204 and 090205 ICP and ICPMS \\
\hline TI-065 & 3.16 & Caustic leach & A 01 AIM 003161604 ICP 2 D & ICP Supernate & SWRI 090204-7 & 090204-7 and 090205-20 ICP and ICPMS \\
\hline TI-065 & 3.16 & Caustic leach & A 01AIM 003161604 RAM 3 D & Raman & ASR8343 & ASR 8343 RAM rev 1 \\
\hline TI-065 & 3.16 & Caustic leach & A 01AIM 003161604 TFI 2 D & IC & $\begin{array}{l}\text { SWRI 090203-21, } \\
\text { SWRI 090204-8 }\end{array}$ & 090204-8 IC TIC and TOC \\
\hline TI-065 & 3.16 & Caustic leach & A 01AIM 003161604 TFI 3 D & $\mathrm{OH}$ & ASR 8366 & ASR 8366_1 OH \\
\hline TI-065 & 3.16 & Caustic leach & A 01 AIM 003161605 ICP 2 B & ICP Wet Solids & SWRI 090204-6 & 090204 and 090205 ICP and ICPMS \\
\hline TI-065 & 3.16 & Caustic leach & A 01AIM 003161605 ICP 2 D & ICP Supernate & SWRI 090204-7 & 090204-7 and 090205-20 ICP and ICPMS \\
\hline TI-065 & 3.16 & Caustic leach & A 01AIM 003161605 RAM 3 D & Raman & ASR8343 & ASR 8343 RAM rev 1 \\
\hline TI-065 & 3.16 & Caustic leach & A 01AIM 003161605 TFI 2 D & IC & $\begin{array}{l}\text { SWRI 090203-21, } \\
\text { SWRI 090204-8 }\end{array}$ & 090204-8 IC TIC and TOC \\
\hline
\end{tabular}


Integrated Test A Sample List

\begin{tabular}{|c|c|c|c|c|c|c|}
\hline TI\# & TI Step \# & TI Step Description & Sample ID & Analysis & Folder & Final Electronic Report File Name \\
\hline TI-065 & 3.16 & Caustic leach & A 01AIM 003161605 TFI 3 D & $\mathrm{OH}$ & ASR 8366 & ASR 8366_1 OH \\
\hline TI-065 & 3.16 & Caustic leach & A 01AIM 003161606 ICP 2 B & ICP Wet Solids & SWRI 090204-6 & 090204 and 090205 ICP and ICPMS \\
\hline TI-065 & 3.16 & Caustic leach & A 01 AIM 003161606 ICP 2 D & ICP Supernate & SWRI 090204-7 & 090204-7 and 090205-20 ICP and ICPMS \\
\hline TI-065 & 3.16 & Caustic leach & A 01 AIM 003161606 RAM 3 D & Raman & ASR 8343 & ASR 8343 RAM rev 1 \\
\hline TI-065 & 3.16 & Caustic leach & A 01AIM 003161606 TFI 2 D & IC & $\begin{array}{l}\text { SWRI 090203-21, } \\
\text { SWRI 090204-8 }\end{array}$ & 090204-8 IC TIC and TOC \\
\hline TI-065 & 3.16 & Caustic leach & A 01AIM 003161606 TFI 3 D & $\mathrm{OH}$ & ASR 8366 & ASR 8366_1 OH \\
\hline TI-065 & 3.16 & Caustic leach & A 01AIM 003161607 XSP 4 B & XRD & TDP-WTP-357 & TDP WTP 357 Int Test A XRD Data on PEP Slurry \\
\hline TI-065 & 3.16 & Caustic leach & A $01 \mathrm{AIH} 003161608$ ARC $1 \mathrm{~B}$ & Archive & & \\
\hline TI-065 & 3.16 & Caustic leach & A 01AIH 003161608 ARC 1 D & Archive & & \\
\hline TI-065 & 3.16 & Caustic leach & A 01AIL 003161609 ARC 1 B & Archive & & \\
\hline TI-065 & 3.16 & Caustic leach & A 01AIL 003161609 ARC 1 D & Archive & & \\
\hline TI-065 & 3.16 & Caustic leach & A 01AMH 003161610 ARC 1 B & Archive & & \\
\hline TI-065 & 3.16 & Caustic leach & A $01 \mathrm{AMH} 003161610$ ARC $1 \mathrm{D}$ & Archive & & \\
\hline TI-065 & 3.16 & Caustic leach & A 01AMM 003161611 ARC $1 \mathrm{~B}$ & Archive & & \\
\hline TI-065 & 3.16 & Caustic leach & A 01AMM 003161611 ARC 1 D & Archive & & \\
\hline TI-065 & 3.16 & Caustic leach & A 01AML 003161612 ARC 1 B & Archive & & \\
\hline TI-065 & 3.16 & Caustic leach & A 01AML 003161612 ARC 1 D & Archive & & \\
\hline TI-065 & 3.16 & Caustic leach & A $01 \mathrm{AOH} 003161613$ ARC $1 \mathrm{~B}$ & Archive & & \\
\hline TI-065 & 3.16 & Caustic leach & A $01 \mathrm{AOH} 003161613$ ARC $1 \mathrm{D}$ & Archive & & \\
\hline TI-065 & 3.16 & Caustic leach & A 01 AOM 003161614 ARC 1 B & Archive & & \\
\hline TI-065 & 3.16 & Caustic leach & A $01 \mathrm{AOM} 003161614$ ARC $1 \mathrm{D}$ & Archive & & \\
\hline TI-065 & 3.16 & Caustic leach & A 01AOL 003161615 ARC 1 B & Archive & & \\
\hline TI-065 & 3.16 & Caustic leach & A 01AOL 003161615 ARC 1 D & Archive & & \\
\hline TI-065 & 3.21 & Cool UFP-T01A contents with UFP-HX-T05A & A 01AIM 006 XX 1631 XSP 4 B & XRD & TDP-WTP-357 & TDP WTP 357 Int Test A XRD Data on PEP Slurry \\
\hline TI-065 & 4.3 & Initial transfer from T01A to T02A & A 01A2A 005 XX 1616 ARC 1 & Archive & & \\
\hline TI-065 & 4.3 & Initial transfer from $\mathrm{T} 01 \mathrm{~A}$ to $\mathrm{T} 02 \mathrm{~A}$ & A 01A2A 005 XX 1617 ARC 1 & Archive & & \\
\hline TI-065 & 4.5 & Initial transfer from $\mathrm{T} 01 \mathrm{~A}$ to $\mathrm{T} 02 \mathrm{~A}$ & A 02AML 005 XX 1618 UDS 2 & UDS & UDS R1 & UDS R1 Shake and Test A and Test B Step001 Date 08182009 \\
\hline TI-065 & 4.5 & Initial transfer from $\mathrm{T} 01 \mathrm{~A}$ to $\mathrm{T} 02 \mathrm{~A}$ & A 02AML 005 XX 1619 DEN 2 & Density Slurry & SWRI 090203-8 & 090203-8Density.rev 1 \\
\hline TI-065 & 4.5 & Initial transfer from $\mathrm{T} 01 \mathrm{~A}$ to $\mathrm{T} 02 \mathrm{~A}$ & A 02AML 005 XX 1619 DEN 2 & Density Supernate & SWRI 090203-8 & 090203-8Density.rev 1 \\
\hline TI-065 & 4.5 & Initial transfer from $\mathrm{T} 01 \mathrm{~A}$ to $\mathrm{T} 02 \mathrm{~A}$ & A 02AML 005 XX 1620 ICP 2 B & ICP Wet Solids & SWRI 090203-4 & 090203-3 and 090203-4 ICP and ICPMS \\
\hline TI-065 & 4.5 & Initial transfer from $\mathrm{T} 01 \mathrm{~A}$ to $\mathrm{T} 02 \mathrm{~A}$ & A 02 AML 005 XX 1620 RAM 3 D & Raman & ASR 8342 & ASR 8342 RAM rev 1 \\
\hline TI-065 & 4.5 & Initial transfer from $\mathrm{T} 01 \mathrm{~A}$ to $\mathrm{T} 02 \mathrm{~A}$ & A 02AIL 005 XX 1623 ARC 1 & Archive & & \\
\hline
\end{tabular}


Integrated Test A Sample List

\begin{tabular}{|c|c|c|c|c|c|c|}
\hline TI \# & TI Step \# & TI Step Description & Sample ID & Analysis & Folder & Final Electronic Report File Name \\
\hline TI-065 & 4.5 & Initial transfer from $\mathrm{T} 01 \mathrm{~A}$ to $\mathrm{T} 02 \mathrm{~A}$ & A $02 \mathrm{AMM} 005 \mathrm{XX} 1625$ ARC 1 & Archive & & \\
\hline TI-065 & 4.5 & Initial transfer from $\mathrm{T} 01 \mathrm{~A}$ to $\mathrm{T} 02 \mathrm{~A}$ & A $02 \mathrm{AOM} 005 \mathrm{XX} 1627$ ARC 1 & Archive & & \\
\hline TI-065 & 4.5 & Initial transfer from $\mathrm{T} 01 \mathrm{~A}$ to $\mathrm{T} 02 \mathrm{~A}$ & A 02AOL 005 XX 1628 ARC 1 & Archive & & \\
\hline TI-065 & 5.3 & Transfer from HLP-VSL-T22 to T01B ( 3 times - batches $2,4 \& 6$ ) & A T221B 008 XX 1663 ARC 1 & Archive & & \\
\hline TI-065 & 5.3 & Transfer from HLP-VSL-T22 to T01B ( 3 times - batches $2,4 \& 6$ ) & A T221B 008 XX 1664 ARC 1 & Archive & & \\
\hline TI-065 & 5.3 & Transfer from HLP-VSL-T22 to T01B ( 3 times - batches $2,4 \& 6$ ) & A T221B 008 XX 1665 ARC 1 & Archive & & \\
\hline TI-065 & 5.3 & Transfer from HLP-VSL-T22 to T01B ( 3 times - batches $2,4 \& 6$ ) & A T221B 008 XX 1666 ARC 1 & Archive & & \\
\hline TI-065 & 5.10 & Transfer from HLP-VSL-T22 to T01B ( 3 times - batches $2,4 \& 6$ ) & A 01 BIM 008 XX 1667 UDS 2 B & UDS & UDS R1 & UDS R1 Shake and Test A and Test B Step001 Date 08182009 \\
\hline TI-065 & 5.10 & Transfer from HLP-VSL-T22 to T01B ( 3 times - batches $2,4 \& 6$ ) & A 01BIM 008 XX 1667 UDS 2 D & UDS & UDS R1 & UDS R1 Shake and Test A and Test B Step001 Date 08182009 \\
\hline TI-065 & 5.10 & Transfer from HLP-VSL-T22 to T01B ( 3 times - batches $2,4 \& 6$ ) & A 01BIM 008 XX 1668 UDS 2 B & UDS & UDS R1 & UDS R1 Shake and Test A and Test B Step001 Date 08182009 \\
\hline TI-065 & 5.10 & Transfer from HLP-VSL-T22 to T01B ( 3 times - batches $2,4 \& 6$ ) & A 01BIM 008 XX 1668 UDS 2 D & UDS & UDS R1 & UDS R1 Shake and Test A and Test B Step001 Date 08182009 \\
\hline TI-065 & 5.10 & Transfer from HLP-VSL-T22 to T01B ( 3 times - batches $2,4 \& 6$ ) & A 01BIM 008 XX 1669 UDS 2 B & UDS & UDS R1 & UDS R1 Shake and Test A and Test B Step001 Date 08182009 \\
\hline TI-065 & 5.10 & Transfer from HLP-VSL-T22 to T01B ( 3 times - batches 2,4 \& 6) & A 01BIM 008 XX 1669 UDS 2 D & UDS & UDS R1 & UDS R1 Shake and Test A and Test B Step001 Date 08182009 \\
\hline TI-065 & 5.10 & Transfer from HLP-VSL-T22 to T01B ( 3 times - batches $2,4 \& 6$ ) & A 01BIM 008 XX 1670 DEN 2 & Density Slurry & SWRI 090203-8 & 090203-8Density.rev 1 \\
\hline TI-065 & 5.10 & Transfer from HLP-VSL-T22 to T01B ( 3 times - batches $2,4 \& 6$ ) & A 01BIM 008 XX 1671 DEN 2 & Density Slurry & SWRI 090203-8 & 090203-8Density.rev 1 \\
\hline TI-065 & 5.10 & Transfer from HLP-VSL-T22 to T01B ( 3 times - batches $2,4 \& 6$ ) & A 01BIM 008 XX 1672 DEN 2 & Density Slurry & SWRI 090203-8 & 090203-8Density.rev 1 \\
\hline TI-065 & 5.10 & Transfer from HLP-VSL-T22 to T01B ( 3 times - batches $2,4 \& 6$ ) & A 01BIM 008 XX 1673 ICP 2 B & ICP Wet Solids & SWRI 090203-4 & 090203-3 and 090203-4 ICP and ICPMS \\
\hline TI-065 & 5.10 & Transfer from HLP-VSL-T22 to T01B ( 3 times - batches $2,4 \& 6$ ) & A 01 BIM 008 XX 1673 RAM 3 D & Raman & ASR 8342 & ASR 8342 RAM rev 1 \\
\hline TI-065 & 5.10 & Transfer from HLP-VSL-T22 to T01B (3 times - batches 2,4 \& 6) & A 01BIM 008 XX 1674 ICP 2 B & ICP Wet Solids & SWRI 090203-4 & 090203-3 and 090203-4 ICP and ICPMS \\
\hline TI-065 & 5.10 & Transfer from HLP-VSL-T22 to T01B ( 3 times - batches $2,4 \& 6$ ) & A 01BIM 008 XX 1674 RAM 3 D & Raman & ASR8342 & ASR 8342 RAM rev 1 \\
\hline TI-065 & 5.10 & Transfer from HLP-VSL-T22 to T01B ( 3 times - batches $2,4 \& 6$ ) & A 01BIM 008 XX 1675 ICP 2 B & ICP Wet Solids & SWRI 090203-4 & 090203-3 and 090203-4 ICP and ICPMS \\
\hline TI-065 & 5.10 & Transfer from HLP-VSL-T22 to T01B ( 3 times - batches 2, 4 \& 6 ) & A 01 BIM 008 XX 1675 RAM 3 D & Raman & ASR8342 & ASR 8342 RAM rev 1 \\
\hline TI-065 & 6.13 & Heat UFP-VSL-T01B to $88 \mathrm{C}$ & A 01BIM 009 BT 1677 DEN 2 & Density Slurry & SWRI 090203-8 & 090203-8Density.rev 1 \\
\hline TI-065 & 6.13 & Heat UFP-VSL-T01B to $88 \mathrm{C}$ & A 01BIM 009 BT 1676 UDS 2 B & UDS & UDS R1 & UDS R1 Shake and Test A and Test B Step001 Date 08182009 \\
\hline TI-065 & 6.13 & Heat UFP-VSL-T01B to $88 \mathrm{C}$ & A 01BIM 009 BT 1676 UDS 2 D & UDS & UDS R1 & UDS R1 Shake and Test A and Test B Step001 Date 08182009 \\
\hline TI-065 & 6.13 & Heat UFP-VSL-T01B to $88 \mathrm{C}$ & A 01 BIM 009 BT 1678 RAM 3 D & Raman & ASR8342 & ASR 8342 RAM rev 1 \\
\hline TI-065 & 6.13 & Heat UFP-VSL-T01B to $88 \mathrm{C}$ & A 01BIL 009 BT 1679 ARC 1 B & Archive & & \\
\hline TI-065 & 6.13 & Heat UFP-VSL-T01B to $88 \mathrm{C}$ & A 01BIL 009 BT 1679 ARC 1 D & Archive & & \\
\hline TI-065 & 6.13 & Heat UFP-VSL-T01B to $88 \mathrm{C}$ & A 01BML 009 BT 1680 ARC 1 B & Archive & & \\
\hline TI-065 & 6.13 & Heat UFP-VSL-T01B to $88 \mathrm{C}$ & A 01BML 009 BT 1680 ARC 1 D & Archive & & \\
\hline TI-065 & 6.13 & Heat UFP-VSL-T01B to $88 \mathrm{C}$ & A 01BOL 009 BT 1681 ARC 1 B & Archive & & \\
\hline TI-065 & 6.13 & Heat UFP-VSL-T01B to $88 \mathrm{C}$ & A 01BOL 009 BT 1681 ARC 1 D & Archive & & \\
\hline TI-065 & 6.13 & Heat UFP-VSL-T01B to $88 \mathrm{C}$ & A 01BMM 009 BT 1682 ARC 1 B & Archive & & \\
\hline
\end{tabular}


Integrated Test A Sample List

\begin{tabular}{|c|c|c|c|c|c|c|}
\hline TI \# & TI Step \# & TI Step Description & Sample ID & Analysis & Folder & Final Electronic Report File Name \\
\hline TI-065 & 6.13 & Heat UFP-VSL-T01B to $88 \mathrm{C}$ & A 01BMM 009 BT 1682 ARC 1 D & Archive & & \\
\hline TI-065 & 6.13 & Heat UFP-VSL-T01B to $88 \mathrm{C}$ & A 01BOM 009 BT 1683 ARC 1 B & Archive & & \\
\hline TI-065 & 6.13 & Heat UFP-VSL-T01B to $88 \mathrm{C}$ & A 01BOM 009 BT 1683 ARC 1 D & Archive & & \\
\hline TI-065 & 6.15 & Caustic leach & A 01BIM 009001687 UDS 2 B & UDS & UDS R1 & UDS R1 Shake and Test A and Test B Step001 Date 08182009 \\
\hline TI-065 & 6.15 & Caustic leach & A 01BIM 009001687 UDS 2 D & UDS & UDS R1 & UDS R1 Shake and Test A and Test B Step001 Date 08182009 \\
\hline TI-065 & 6.15 & Caustic leach & A 01 BIM 009001688 DEN 2 & Density Slurry & SWRI 090203-8 & 090203-8Density.rev 1 \\
\hline TI-065 & 6.15 & Caustic leach & A 01BIM 009001689 DEN 0 B & ICP Wet Solids & SWRI 090625-24 & 090625-24 ICP and ICPMS \\
\hline TI-065 & 6.15 & Caustic leach & A 01BIM 009001689 ICP 2 D & ICP Supernate & SWRI 090203-6 & 090203-5 and 090203-6 ICP and ICPMS \\
\hline TI-065 & 6.15 & Caustic leach & A 01BIM 009001689 RAM 3 D & Raman & ASR8342 & ASR 8342 RAM rev 1 \\
\hline TI-065 & 6.16 & Caustic leach & A 01BIM 009001689 RAM 3 D & $\mathrm{OH}$ & ASR 8342 & ASR 8342_1 OH \\
\hline TI-065 & 6.16 & Caustic leach & A 01BIM 009001689 TFI 2 D & IC & $\begin{array}{l}\text { SWRI 090203-15, } \\
\text { SWRI 090204-15 }\end{array}$ & 090203-15IC \\
\hline TI-065 & 6.16 & Caustic leach & A 01BIM 009001689 DEN 0 B & ICP Wet Solids & 090625-24 & 090625-24 ICP and ICPMS \\
\hline TI-065 & 6.16 & Caustic leach & A 01BML 009001691 ARC 1 B & Archive & & \\
\hline TI-065 & 6.16 & Caustic leach & A 01BOL 009001692 ARC 1 B & Archive & & \\
\hline TI-065 & 6.16 & Caustic leach & A 01BMM 009001693 ARC 1 B & Archive & & \\
\hline TI-065 & 6.16 & Caustic leach & A 01BOM 009001694 ARC 1 B & Archive & & \\
\hline TI-065 & 6.16 & Caustic leach & A 01BMH 009001696 ARC 1 B & Archive & & \\
\hline TI-065 & 6.16 & Caustic leach & A 01BIL 009001690 ARC 1 B & Archive & & \\
\hline TI-065 & 6.16 & Caustic leach & A 01BIL 009001690 ARC 1 D & Archive & & \\
\hline TI-065 & 6.16 & Caustic leach & A 01BML 009001691 ARC 1 D & Archive & & \\
\hline TI-065 & 6.16 & Caustic leach & A 01BOL 009001692 ARC 1 D & Archive & & \\
\hline TI-065 & 6.16 & Caustic leach & A 01BMM 009001693 ARC 1 D & Archive & & \\
\hline TI-065 & 6.16 & Caustic leach & A 01BOM 009001694 ARC 1 D & Archive & & \\
\hline TI-065 & 6.16 & Caustic leach & A 01BMH 009001696 ARC 1 D & Archive & & \\
\hline TI-065 & 6.16 & Caustic leach & A 01BOH 009001697 ARC 1 B & Archive & & \\
\hline TI-065 & 6.16 & Caustic leach & A 01BOH 009001697 ARC 1 D & Archive & & \\
\hline TI-065 & 6.16 & Caustic leach & A 01BIM 009011698 UDS 2 B & UDS & UDS R1 & UDS R1 Shake and Test A and Test B Step001 Date 08182009 \\
\hline TI-065 & 6.16 & Caustic leach & A 01 BIM 009011698 UDS 2 D & UDS & UDS R1 & UDS R1 Shake and Test A and Test B Step001 Date 08182009 \\
\hline TI-065 & 6.16 & Caustic leach & A 01 BIM 009011699 DEN 2 & Density Slurry & SWRI 090203-8 & 090203-8Density.rev 1 \\
\hline TI-065 & 6.16 & Caustic leach & A 01 BIM 009011700 RAM 3 D & Raman & ASR8342 & ASR 8342 RAM rev 1 \\
\hline TI-065 & 6.16 & Caustic leach & A 01BIM 009021701 UDS 2 B & UDS & UDS R1 & UDS R1 Shake and Test A and Test B Step001 Date 08182009 \\
\hline TI-065 & 6.16 & Caustic leach & A 01BIM 009021701 UDS 2 D & UDS & UDS R1 & UDS R1 Shake and Test A and Test B Step001 Date 08182009 \\
\hline
\end{tabular}


Integrated Test A Sample List

\begin{tabular}{|c|c|c|c|c|c|c|}
\hline TI\# & TI Step \# & TI Step Description & Sample ID & Analysis & Folder & Final Electronic Report File Name \\
\hline TI-065 & 6.16 & Caustic leach & A 01BIM 009021702 DEN 2 & Density Slurry & SWRI 090203-8 & 090203-8Density.rev 1 \\
\hline TI-065 & 6.16 & Caustic leach & A 01BIM 009021703 RAM 3 D & Raman & ASR 8342 & ASR 8342 RAM rev 1 \\
\hline TI-065 & 6.16 & Caustic leach & A 01BIM 009031704 ARC 1 B & Archive & & \\
\hline TI-065 & 6.16 & Caustic leach & A 01BIM 009031704 ARC 1 D & Archive & & \\
\hline TI-065 & 6.16 & Caustic leach & A 01BIM 009041705 UDS 2 B & UDS & UDS R1 & UDS R1 Shake and Test A and Test B Step001 Date 08182009 \\
\hline TI-065 & 6.16 & Caustic leach & A 01 BIM 009041705 UDS 2 D & UDS & UDS R1 & UDS R1 Shake and Test A and Test B Step001 Date 08182009 \\
\hline TI-065 & 6.16 & Caustic leach & A 01BIM 009041706 DEN 2 & Density Slurry & SWRI 090203-8 & 090203-8Density.rev 1 \\
\hline TI-065 & 6.16 & Caustic leach & A 01BIM 009041707 DEN 0 D & ICP Supernate & SWRI 090625-25 & 090625-25 and 090625-26 ICP and ICPMS and IC \\
\hline TI-065 & 6.16 & Caustic leach & A 01BIM 009041707 DEN 0 D & IC & SWRI 090625-25 & 090625-25 and 090625-26 ICP and ICPMS and IC \\
\hline TI-065 & 6.16 & Caustic leach & A 01BIM 009041707 ICP 2 B & ICP Wet Solids & SWRI 090203-4 & 090203-3 and 090203-4 ICP and ICPMS \\
\hline TI-065 & 6.16 & Caustic leach & A 01BIM 009041707 RAM 3 D & Raman & ASR 8342 & ASR 8342 RAM rev 1 \\
\hline TI-065 & 6.16 & Caustic leach & A 01BOL 009041710 ARC 1 B & Archive & & \\
\hline TI-065 & 6.16 & Caustic leach & A 01BOL 009041710 ARC 1 D & Archive & & \\
\hline TI-065 & 6.16 & Caustic leach & A 01BMM 009041711 ARC 1 B & Archive & & \\
\hline TI-065 & 6.16 & Caustic leach & A 01BMM 009041711 ARC 1 D & Archive & & \\
\hline TI-065 & 6.16 & Caustic leach & A 01 BOM 009041712 ARC 1 B & Archive & & \\
\hline TI-065 & 6.16 & Caustic leach & A 01BOM 009041712 ARC 1 D & Archive & & \\
\hline TI-065 & 6.16 & Caustic leach & A 01BMH 009041714 ARC 1 B & Archive & & \\
\hline TI-065 & 6.16 & Caustic leach & A 01BMH 009041714 ARC 1 D & Archive & & \\
\hline TI-065 & 6.16 & Caustic leach & A 01BOH 009041715 ARC 1 B & Archive & & \\
\hline TI-065 & 6.16 & Caustic leach & A 01BOH 009041715 ARC 1 D & Archive & & \\
\hline TI-065 & 6.16 & Caustic leach & A 01BIL 009041708 ARC 1 B & Archive & & \\
\hline TI-065 & 6.16 & Caustic leach & A 01BIL 009041708 ARC 1 D & Archive & & \\
\hline TI-065 & 6.16 & Caustic leach & A 01BML 009041709 ARC 1 B & Archive & & \\
\hline TI-065 & 6.16 & Caustic leach & A 01BML 009041709 ARC 1 D & Archive & & \\
\hline TI-065 & 6.16 & Caustic leach & A 01BIM 009051716 ARC 1 D & Archive & & \\
\hline TI-065 & 6.16 & Caustic leach & A 01BIM 009051716 ARC 1 B & Archive & & \\
\hline TI-065 & 6.16 & Caustic leach & A 01BIM 009061717 ARC 1 B & Archive & & \\
\hline TI-065 & 6.16 & Caustic leach & A 01BIM 009061717 ARC 1 D & Archive & & \\
\hline TI-065 & 6.16 & Caustic leach & A 01BIM 009071718 ARC 1 B & Archive & & \\
\hline TI-065 & 6.16 & Caustic leach & A 01BIM 009071718 ARC 1 D & Archive & & \\
\hline TI-065 & 6.16 & Caustic leach & A 01BIM 009081719 UDS 2 B & UDS & UDS R1 & UDS R1 Shake and Test A and Test B Step001 Date 08182009 \\
\hline TI-065 & 6.16 & Caustic leach & A 01BIM 009081719 UDS 2 D & UDS & UDS R1 & UDS R1 Shake and Test A and Test B Step001 Date 08182009 \\
\hline
\end{tabular}


Integrated Test A Sample List

\begin{tabular}{|c|c|c|c|c|c|c|}
\hline TI \# & TI Step \# & TI Step Description & Sample ID & Analysis & Folder & Final Electronic Report File Name \\
\hline TI-065 & 6.16 & Caustic leach & A 01BIM 009081720 DEN 2 & Density Slurry & SWRI 090204-9 & 090204-9 Density \\
\hline TI-065 & 6.16 & Caustic leach & A 01BIM 009081721 ICP 2 B & ICP Wet Solids & SWRI 090204-19 & 090204 and 090205 ICP and ICPMS \\
\hline TI-065 & 6.16 & Caustic leach & A 01BIM 009081721 ICP 2 D & ICP Supernate & SWRI 090204-7 & 090204-7 and 090205-20 ICP and ICPMS \\
\hline TI-065 & 6.16 & Caustic leach & A 01BIM 009081721 RAM 3 D & Raman & ASR8343 & ASR 8343 RAM rev 1 \\
\hline TI-065 & 6.16 & Caustic leach & A 01BIM 009081721 RAM 3 D & $\mathrm{OH}$ & ASR 8343 & ASR 8343_1 OH \\
\hline TI-065 & 6.16 & Caustic leach & A 01BIM 009081721 TFI 2 D & IC & $\begin{array}{l}\text { SWRI 090203-15, } \\
\text { SWRI 090204-15 }\end{array}$ & 090204-15IC \\
\hline TI-065 & 6.16 & Caustic leach & A 01BIL 009081722 ARC 1 B & Archive & & \\
\hline TI-065 & 6.16 & Caustic leach & A 01BMM 009081725 ARC 1 B & Archive & & \\
\hline TI-065 & 6.16 & Caustic leach & A 01BOH 009081729 ARC 1 B & Archive & & \\
\hline TI-065 & 6.16 & Caustic leach & A 01BOM 009081726 ARC 1 B & Archive & & \\
\hline TI-065 & 6.16 & Caustic leach & A 01BML 009081723 ARC 1 B & Archive & & \\
\hline TI-065 & 6.16 & Caustic leach & A 01BMH 009081728 ARC 1 B & Archive & & \\
\hline TI-065 & 6.16 & Caustic leach & A 01BIH 009081727 ARC 1 B & Archive & & \\
\hline TI-065 & 6.16 & Caustic leach & A 01BOL 009081724 ARC 1 B & Archive & & \\
\hline TI-065 & 6.16 & Caustic leach & A 01BOL 009081724 ARC 1 D & Archive & & \\
\hline TI-065 & 6.16 & Caustic leach & A 01BOM 009081726 ARC 1 D & Archive & & \\
\hline TI-065 & 6.16 & Caustic leach & A 01BMM 009081725 ARC 1 D & Archive & & \\
\hline TI-065 & 6.16 & Caustic leach & A 01BOH 009081729 ARC 1 D & Archive & & \\
\hline TI-065 & 6.16 & Caustic leach & A 01BIL 009081722 ARC 1 D & Archive & & \\
\hline TI-065 & 6.16 & Caustic leach & A 01BML 009081723 ARC 1 D & Archive & & \\
\hline TI-065 & 6.16 & Caustic leach & A 01BIH 009081727 ARC 1 D & Archive & & \\
\hline TI-065 & 6.16 & Caustic leach & A 01BMH 009081728 ARC 1 D & Archive & & \\
\hline TI-065 & 6.16 & Caustic leach & A 01BIM 009111734 ARC 1 B & Archive & & \\
\hline TI-065 & 6.16 & Caustic leach & A 01BIM 009111734 ARC 1 D & Archive & & \\
\hline TI-065 & 6.16 & Caustic leach & A 01BIM 009121735 UDS 2 B & UDS & UDS R1 & UDS R1 Shake and Test A and Test B Step001 Date 08182009 \\
\hline TI-065 & 6.16 & Caustic leach & A 01BIM 009121735 UDS 2 D & UDS & UDS R1 & UDS R1 Shake and Test A and Test B Step001 Date 08182009 \\
\hline TI-065 & 6.16 & Caustic leach & A 01BIM 009121736 DEN 2 & Density Slurry & SWRI 090204-9 & 090204-9 Density \\
\hline TI-065 & 6.16 & Caustic leach & A 01BIM 009121737 ICP 2 B & ICP Wet Solids & SWRI 090204-19 & 090204 and 090205 ICP and ICPMS \\
\hline TI-065 & 6.16 & Caustic leach & A 01BIM 009121737 ICP 2 D & ICP Supernate & SWRI 090204-7 & 090204-7 and 090205-20 ICP and ICPMS \\
\hline TI-065 & 6.16 & Caustic leach & A 01BIM 009121737 RAM 3 D & Raman & ASR8343 & ASR 8343 RAM rev 1 \\
\hline TI-065 & 6.16 & Caustic leach & A 01BIM 009121737 RAM 3 D & $\mathrm{OH}$ & ASR 8343 & ASR 8343_1 OH \\
\hline
\end{tabular}


Integrated Test A Sample List

\begin{tabular}{|c|c|c|c|c|c|c|}
\hline TI \# & TI Step \# & TI Step Description & Sample ID & Analysis & Folder & Final Electronic Report File Name \\
\hline TI-065 & 6.16 & Caustic leach & A 01BIM 009121737 TFI 2 D & IC & $\begin{array}{l}\text { SWRI 090203-15, } \\
\text { SWRI 090204-15 }\end{array}$ & 090204-15IC \\
\hline TI-065 & 6.16 & Caustic leach & A 01BMM 009121741 ARC 1 B & Archive & & \\
\hline TI-065 & 6.16 & Caustic leach & A 01BMM 009121741 ARC 1 D & Archive & & \\
\hline TI-065 & 6.16 & Caustic leach & A 01BOH 009121745 ARC 1 B & Archive & & \\
\hline TI-065 & 6.16 & Caustic leach & A 01BOH 009121745 ARC 1 D & Archive & & \\
\hline TI-065 & 6.16 & Caustic leach & A 01BMH 009121744 ARC 1 B & Archive & & \\
\hline TI-065 & 6.16 & Caustic leach & A 01BMH 009121744 ARC 1 D & Archive & & \\
\hline TI-065 & 6.16 & Caustic leach & A 01BIH 009121743 ARC 1 B & Archive & & \\
\hline TI-065 & 6.16 & Caustic leach & A 01BIH 009121743 ARC 1 D & Archive & & \\
\hline TI-065 & 6.16 & Caustic leach & A 01BOM 009121742 ARC 1 B & Archive & & \\
\hline TI-065 & 6.16 & Caustic leach & A 01BOM 009121742 ARC 1 D & Archive & & \\
\hline TI-065 & 6.16 & Caustic leach & A 01BOL 009121740 ARC 1 B & Archive & & \\
\hline TI-065 & 6.16 & Caustic leach & A 01BOL 009121740 ARC 1 D & Archive & & \\
\hline TI-065 & 6.16 & Caustic leach & A 01BIL 009121738 ARC 1 B & Archive & & \\
\hline TI-065 & 6.16 & Caustic leach & A 01BIL 009121738 ARC 1 D & Archive & & \\
\hline TI-065 & 6.16 & Caustic leach & A 01BML 009121739 ARC 1 B & Archive & & \\
\hline TI-065 & 6.16 & Caustic leach & A 01BML 009121739 ARC 1 D & Archive & & \\
\hline TI-065 & 6.16 & Caustic leach & A 01BIM 009131746 ARC 1 B & Archive & & \\
\hline TI-065 & 6.16 & Caustic leach & A 01BIM 009131746 ARC 1 D & Archive & & \\
\hline TI-065 & 6.16 & Caustic leach & A 01BIM 009141747 UDS 2 B & UDS & UDS R1 & UDS R1 Shake and Test A and Test B Step001 Date 08182009 \\
\hline TI-065 & 6.16 & Caustic leach & A 01BIM 009141747 UDS 2 D & UDS & UDS R1 & UDS R1 Shake and Test A and Test B Step001 Date 08182009 \\
\hline TI-065 & 6.16 & Caustic leach & A 01BIM 009141748 DEN 2 & Density Slurry & SWRI 090204-9 & 090204-9 Density \\
\hline TI-065 & 6.16 & Caustic leach & A 01BIM 009141749 RAM 3 D & Raman & ASR8343 & ASR 8343 RAM rev 1 \\
\hline TI-065 & 6.16 & Caustic leach & A 01BIM 009151750 ARC 1 B & Archive & & \\
\hline TI-065 & 6.16 & Caustic leach & A 01BIM 009151750 ARC 1 D & Archive & & \\
\hline TI-065 & 6.16 & Caustic leach & A 01BIM 009161751 UDS 2 B & UDS & UDS R1 & UDS R1 Shake and Test A and Test B Step001 Date 08182009 \\
\hline TI-065 & 6.16 & Caustic leach & A 01BIM 009161751 UDS 2 D & UDS & UDS R1 & UDS R1 Shake and Test A and Test B Step001 Date 08182009 \\
\hline TI-065 & 6.16 & Caustic leach & A 01BIM 009161752 DEN 2 & Density Slurry & SWRI 090204-9 & 090204-9 Density \\
\hline TI-065 & 6.16 & Caustic leach & A 01BIM 009161753 ICP 2 B & ICP Wet Solids & SWRI 090204-19 & 090204 and 090205 ICP and ICPMS \\
\hline TI-065 & 6.16 & Caustic leach & A 01BIM 009161753 ICP 2 D & ICP Supernate & SWRI 090204-7 & 090204-7 and 090205-20 ICP and ICPMS \\
\hline TI-065 & 6.16 & Caustic leach & A 01 BIM 009161753 RAM 3 D & Raman & ASR 8343 & ASR 8343 RAM rev 1 \\
\hline TI-065 & 6.16 & Caustic leach & A 01 BIM 009161753 RAM 3 D & $\mathrm{OH}$ & ASR 8343 & ASR 8343_1 OH \\
\hline
\end{tabular}


Integrated Test A Sample List

\begin{tabular}{|c|c|c|c|c|c|c|}
\hline TI\# & TI Step \# & TI Step Description & Sample ID & Analysis & Folder & Final Electronic Report File Name \\
\hline TI-065 & 6.16 & Caustic leach & A 01BIM 009161753 TFI 2 D & IC & $\begin{array}{l}\text { SWRI 090203-15, } \\
\text { SWRI 090204-15 }\end{array}$ & 090204-15IC \\
\hline TI-065 & 6.16 & Caustic leach & A 01BIM 009161754 UDS 2 B & UDS & UDS R1 & UDS R1 Shake and Test A and Test B Step001 Date 08182009 \\
\hline TI-065 & 6.16 & Caustic leach & A 01BIM 009161754 UDS 2 D & UDS & UDS R1 & UDS R1 Shake and Test A and Test B Step001 Date 08182009 \\
\hline TI-065 & 6.16 & Caustic leach & A 01BIM 009161755 DEN 2 & Density Slurry & SWRI 090204-9 & 090204-9 Density \\
\hline TI-065 & 6.16 & Caustic leach & A 01BIM 009161756 ICP 2 B & ICP Wet Solids & SWRI 090204-19 & 090204 and 090205 ICP and ICPMS \\
\hline TI-065 & 6.16 & Caustic leach & A 01BIM 009161756 ICP 2 D & ICP Supernate & SWRI 090204-7 & 090204-7 and 090205-20 ICP and ICPMS \\
\hline TI-065 & 6.16 & Caustic leach & A 01BIM 009161756 RAM 3 D & Raman & ASR 8343 & ASR 8343 RAM rev 1 \\
\hline TI-065 & 6.16 & Caustic leach & A 01 BIM 009161756 RAM 3 D & $\mathrm{OH}$ & ASR 8343 & ASR 8343_1 OH \\
\hline TI-065 & 6.16 & Caustic leach & A 01BIM 009161756 TFI 2 D & IC & $\begin{array}{l}\text { SWRI 090203-15, } \\
\text { SWRI 090204-15 }\end{array}$ & 090204-15IC \\
\hline TI-065 & 6.16 & Caustic leach & A 01BIM 009161757 UDS 2 B & UDS & UDS R1 & UDS R1 Shake and Test A and Test B Step001 Date 08182009 \\
\hline TI-065 & 6.16 & Caustic leach & A 01BIM 009161757 UDS 2 D & UDS & UDS R1 & UDS R1 Shake and Test A and Test B Step001 Date 08182009 \\
\hline TI-065 & 6.16 & Caustic leach & A 01BIM 009161758 DEN 2 & Density Slurry & SWRI 090204-9 & 090204-9 Density \\
\hline TI-065 & 6.16 & Caustic leach & A 01BIM 009161759 ICP 2 B & ICP Wet Solids & SWRI 090204-19 & 090204 and 090205 ICP and ICPMS \\
\hline TI-065 & 6.16 & Caustic leach & A 01BIM 009161759 ICP 2 D & ICP Supernate & SWRI 090204-7 & 090204-7 and 090205-20 ICP and ICPMS \\
\hline TI-065 & 6.16 & Caustic leach & A 01BIM 009161759 RAM 3 D & Raman & ASR8343 & ASR 8343 RAM rev 1 \\
\hline TI-065 & 6.16 & Caustic leach & A 01BIM 009161759 RAM 3 D & $\mathrm{OH}$ & ASR 8343 & ASR 8343_1 OH \\
\hline TI-065 & 6.16 & Caustic leach & A 01BIM 009161759 TFI 2 D & IC & $\begin{array}{l}\text { SWRI 090203-15, } \\
\text { SWRI 090204-15 }\end{array}$ & 090204-15IC \\
\hline TI-065 & 6.16 & Caustic leach & A 01BOH 009161767 ARC 1 B & Archive & & \\
\hline TI-065 & 6.16 & Caustic leach & A 01BOH 009161767 ARC 1 D & Archive & & \\
\hline TI-065 & 6.16 & Caustic leach & A 01BMH 009161766 ARC 1 B & Archive & & \\
\hline TI-065 & 6.16 & Caustic leach & A 01BMH 009161766 ARC 1 D & Archive & & \\
\hline TI-065 & 6.16 & Caustic leach & A 01BIH 009161765 ARC 1 B & Archive & & \\
\hline TI-065 & 6.16 & Caustic leach & A 01BIH 009161765 ARC 1 D & Archive & & \\
\hline TI-065 & 6.16 & Caustic leach & A 01BOM 009161764 ARC $1 \mathrm{~B}$ & Archive & & \\
\hline TI-065 & 6.16 & Caustic leach & A 01BOM 009161764 ARC 1 D & Archive & & \\
\hline TI-065 & 6.16 & Caustic leach & A 01BMM 009161763 ARC 1 B & Archive & & \\
\hline TI-065 & 6.16 & Caustic leach & A 01BIL 009161760 ARC 1 B & Archive & & \\
\hline TI-065 & 6.16 & Caustic leach & A 01BML 009161761 ARC 1 B & Archive & & \\
\hline TI-065 & 6.16 & Caustic leach & A 01BOL 009161762 ARC 1 B & Archive & & \\
\hline TI-065 & 6.16 & Caustic leach & A 01BIL 009161760 ARC 1 D & Archive & & \\
\hline
\end{tabular}


Integrated Test A Sample List

\begin{tabular}{|c|c|c|c|c|c|c|}
\hline TI \# & TI Step \# & TI Step Description & Sample ID & Analysis & Folder & Final Electronic Report File Name \\
\hline TI-065 & 6.16 & Caustic leach & A 01BML 009161761 ARC 1 D & Archive & & \\
\hline TI-065 & 6.16 & Caustic leach & A 01BOL 009161762 ARC 1 D & Archive & & \\
\hline TI-065 & 6.16 & Caustic leach & A 01BMM 009161763 ARC 1 D & Archive & & \\
\hline TI-065 & 7.3 & $\begin{array}{l}\text { Just prior to transfer simulant from HLP-VSL-T22 to UFP-T01 A \& } \\
\text { caustic addition (Batches } 1,3 \& 5 \text { ) }\end{array}$ & A T22GM 13A XX 1771 UDS 2 & UDS & UDS R1 & UDS R1 Shake and Test A and Test B Step001 Date 08182009 \\
\hline TI-065 & 7.3 & $\begin{array}{l}\text { Just prior to transfer simulant from HLP-VSL-T22 to UFP-TO1 A \& } \\
\text { caustic addition (Batches } 1,3 \& 5 \text { ) }\end{array}$ & A T22GM 13A XX 1772 RHE 4 & Rheology & TDP-WTP-359 & TDP WTP 359 Int Test A RHE Data on PEP Slurry \\
\hline TI-065 & 7.3 & $\begin{array}{l}\text { Just prior to transfer simulant from HLP-VSL-T22 to UFP-TO1 A \& } \\
\text { caustic addition (Batches } 1,3 \& 5 \text { ) }\end{array}$ & A T22GM 13A XX 1773 XSP 4 B & Not Analyzed & & \\
\hline TI-065 & 7.3 & $\begin{array}{l}\text { Just prior to transfer simulant from HLP-VSL-T22 to UFP-T01 A \& } \\
\text { caustic addition (Batches } 1,3 \& 5 \text { ) }\end{array}$ & A T22GB 13A XX 1770 ARC 1 & Archive & & \\
\hline TI-065 & 7.3 & $\begin{array}{l}\text { Just prior to transfer simulant from HLP-VSL-T22 to UFP-T01 A \& } \\
\text { caustic addition (Batches } 1,3 \& 5 \text { ) }\end{array}$ & A T22GT 13A XX 1768 ARC 1 & Archive & & \\
\hline TI-065 & 7.3 & $\begin{array}{l}\text { Just prior to transfer simulant from HLP-VSL-T22 to UFP-T01 A \& } \\
\text { caustic addition (Batches } 1,3 \& 5 \text { ) }\end{array}$ & A T22GM 13A XX 1769 ARC 1 & Archive & & \\
\hline TI-065 & 7.9 & Transfer from HLP-VSL-T22 to T01A ( 3 times - batches $1,3 \& 5$ ) & A 01AIM 13A XX 1778 UDS 2 B & UDS & UDS R1 & UDS R1 Shake and Test A and Test B Step001 Date 08182009 \\
\hline TI-065 & 7.9 & Transfer from HLP-VSL-T22 to T01A ( 3 times - batches $1,3 \& 5$ ) & A 01AIM 13A XX 1778 UDS 2 D & UDS & UDS R1 & UDS R1 Shake and Test A and Test B Step001 Date 08182009 \\
\hline TI-065 & 7.9 & Transfer from HLP-VSL-T22 to T01A ( 3 times - batches $1,3 \& 5$ ) & A 01AIM 13A XX 1779 UDS 2 B & UDS & UDS R1 & UDS R1 Shake and Test A and Test B Step001 Date 08182009 \\
\hline TI-065 & 7.9 & Transfer from HLP-VSL-T22 to T01A ( 3 times - batches $1,3 \& 5$ ) & A 01AIM 13A XX 1779 UDS 2 D & UDS & UDS R1 & UDS R1 Shake and Test A and Test B Step001 Date 08182009 \\
\hline TI-065 & 7.9 & Transfer from HLP-VSL-T22 to T01A ( 3 times - batches $1,3 \& 5$ ) & A 01AIM 13A XX 1780 UDS 2 B & UDS & UDS R1 & UDS R1 Shake and Test A and Test B Step001 Date 08182009 \\
\hline TI-065 & 7.9 & Transfer from HLP-VSL-T22 to T01A ( 3 times - batches $1,3 \& 5$ ) & A 01AIM 13A XX 1780 UDS 2 D & UDS & UDS R1 & UDS R1 Shake and Test A and Test B Step001 Date 08182009 \\
\hline TI-065 & 7.9 & Transfer from HLP-VSL-T22 to T01A ( 3 times - batches $1,3 \& 5$ ) & A 01 AIM 13A XX 1781 DEN 2 & Density Slurry & SWRI 090205-4 & 090205Density \\
\hline TI-065 & 7.9 & Transfer from HLP-VSL-T22 to T01A ( 3 times - batches $1,3 \& 5$ ) & A 01 AIM 13A XX 1782 DEN 2 & Density Slurry & SWRI 090205-4 & 090205Density \\
\hline TI-065 & 7.9 & Transfer from HLP-VSL-T22 to T01A ( 3 times - batches $1,3 \& 5$ ) & A 01AIM 13A XX 1783 DEN 2 & Density Slurry & SWRI 090205-4 & 090205Density \\
\hline TI-065 & 7.9 & Transfer from HLP-VSL-T22 to T01A ( 3 times - batches $1,3 \& 5$ ) & A 01AIM 13A XX 1784 ICP 2 B & ICP Wet Solids & SWRI 090205-5 & 090204 and 090205 ICP and ICPMS \\
\hline TI-065 & 7.9 & Transfer from HLP-VSL-T22 to UFP-VLS-T01A & A 01AIM 13A XX 1784 RAM 3 D & Raman & ASR8345 & ASR 8345 RAM rev 1 \\
\hline TI-065 & 7.9 & Transfer from HLP-VSL-T22 to T01A ( 3 times - batches $1,3 \& 5$ ) & A 01AIM 13A XX 1785 ICP 2 B & ICP Wet Solids & SWRI 090205-5 & 090204 and 090205 ICP and ICPMS \\
\hline TI-065 & 7.9 & Transfer from HLP-VSL-T22 to UFP-VLS-T01A & A 01AIM 13A XX 1785 RAM 3 D & Raman & ASR8345 & ASR 8345 RAM rev 1 \\
\hline TI-065 & 7.9 & Transfer from HLP-VSL-T22 to T01A ( 3 times - batches $1,3 \& 5$ ) & A 01AIM 13A XX 1786 ICP 2 B & ICP Wet Solids & SWRI 090205-5 & 090204 and 090205 ICP and ICPMS \\
\hline TI-065 & 7.9 & Transfer from HLP-VSL-T22 to UFP-VLS-T01A & A 01AIM 13A XX 1786 RAM 3 D & Raman & ASR8345 & ASR 8345 RAM rev 1 \\
\hline TI-065 & 7.9 & Transfer from HLP-VSL-T22 to UFP-VLS-T01A & A T221A 13A XX 1777 ARC 1 & Archive & & \\
\hline TI-065 & 7.9 & Transfer from HLP-VSL-T22 to UFP-VLS-T01A & A T221A 13A XX 1774 ARC 1 & Archive & & \\
\hline TI-065 & 7.9 & Transfer from HLP-VSL-T22 to UFP-VLS-T01A & A T221A 13A XX 1775 ARC 1 & Archive & & \\
\hline TI-065 & 7.9 & Transfer from HLP-VSL-T22 to UFP-VLS-T01A & A T221A 13A XX 1776 ARC 1 & Archive & & \\
\hline
\end{tabular}


Integrated Test A Sample List

\begin{tabular}{|c|c|c|c|c|c|c|}
\hline TI\# & TI Step \# & TI Step Description & Sample ID & Analysis & Folder & Final Electronic Report File Name \\
\hline TI-065 & 7.21 & Heat UFP-VSL-T01A to $88 \mathrm{C}$ & A 01AIM 13A BT 1787 UDS 2 B & UDS & UDS R1 & UDS R1 Shake and Test A and Test B Step001 Date 08182009 \\
\hline TI-065 & 7.21 & Heat UFP-VSL-T01A to 88C & A 01AIM 13A BT 1787 UDS 2 D & UDS & UDS R1 & UDS R1 Shake and Test A and Test B Step001 Date 08182009 \\
\hline TI-065 & 7.21 & Heat UFP-VSL-T01A to $88 \mathrm{C}$ & A 01AIM 13A BT 1788 DEN 2 & Density Slurry & SWRI 090205-4 & 090205Density \\
\hline TI-065 & 7.21 & Heat UFP-VSL-T01A to $88^{\circ} \mathrm{C}$ & A 01AIM 13A BT 1789 RAM 3 D & Raman & ASR 8345 & ASR 8345 RAM rev 1 \\
\hline TI-065 & 7.21 & Heat UFP-VSL-T01A to $88^{\circ} \mathrm{C}$ & A 01AIH 13A BT 1790 ARC 1 B & Archive & & \\
\hline TI-065 & 7.21 & Heat UFP-VSL-T01A to $88^{\circ} \mathrm{C}$ & A 01AIH 13A BT 1790 ARC 1 D & Archive & & \\
\hline TI-065 & 7.21 & Heat UFP-VSL-T01A to $88^{\circ} \mathrm{C}$ & A 01AIL 13A BT 1791 ARC 1 B & Archive & & \\
\hline TI-065 & 7.21 & Heat UFP-VSL-T01A to $88^{\circ} \mathrm{C}$ & A 01AIL 13A BT 1791 ARC 1 D & Archive & & \\
\hline TI-065 & 7.21 & Heat UFP-VSL-T01A to $88^{\circ} \mathrm{C}$ & A 01AMH 13A BT 1792 ARC 1 B & Archive & & \\
\hline TI-065 & 7.21 & Heat UFP-VSL-T01A to $88^{\circ} \mathrm{C}$ & A 01AMH 13A BT 1792 ARC 1 D & Archive & & \\
\hline TI-065 & 7.21 & Heat UFP-VSL-T01A to $88^{\circ} \mathrm{C}$ & A 01AMM 13A BT 1793 ARC 1 B & Archive & & \\
\hline TI-065 & 7.21 & Heat UFP-VSL-T01A to $88^{\circ} \mathrm{C}$ & A 01AMM 13A BT 1793 ARC 1 D & Archive & & \\
\hline TI-065 & 7.21 & Heat UFP-VSL-T01A to $88^{\circ} \mathrm{C}$ & A 01AML 13A BT 1794 ARC 1 B & Archive & & \\
\hline TI-065 & 7.21 & Heat UFP-VSL-T01A to $88^{\circ} \mathrm{C}$ & A 01AML 13A BT 1794 ARC 1 D & Archive & & \\
\hline TI-065 & 7.21 & Heat UFP-VSL-T01A to $88^{\circ} \mathrm{C}$ & A 01AOH 13A BT 1795 ARC 1 B & Archive & & \\
\hline TI-065 & 7.21 & Heat UFP-VSL-T01A to $88^{\circ} \mathrm{C}$ & A 01AOH 13A BT 1795 ARC 1 D & Archive & & \\
\hline TI-065 & 7.21 & Heat UFP-VSL-T01A to $88^{\circ} \mathrm{C}$ & A 01AOM 13A BT 1796 ARC 1 B & Archive & & \\
\hline TI-065 & 7.21 & Heat UFP-VSL-T01A to $88^{\circ} \mathrm{C}$ & A 01AOM 13A BT 1796 ARC 1 D & Archive & & \\
\hline TI-065 & 7.21 & Heat UFP-VSL-T01A to $88^{\circ} \mathrm{C}$ & A 01AOL 13A BT 1797 ARC 1 B & Archive & & \\
\hline TI-065 & 7.21 & Heat UFP-VSL-T01A to $88^{\circ} \mathrm{C}$ & A 01AOL 13A BT 1797 ARC 1 D & Archive & & \\
\hline TI-065 & 7.22 & Caustic leach & A 01 AIM 13A 001798 UDS 2 B & UDS & UDS R1 & UDS R1 Shake and Test A and Test B Step001 Date 08182009 \\
\hline TI-065 & 7.22 & Caustic leach & A 01AIM 13A 001798 UDS 2 D & UDS & UDS R1 & UDS R1 Shake and Test A and Test B Step001 Date 08182009 \\
\hline TI-065 & 7.22 & Caustic leach & A 01AIM 13A 001799 DEN 2 & Density Slurry & SWRI 090205-4 & 090205Density \\
\hline TI-065 & 7.22 & Caustic leach & A 01 AIM $13 \mathrm{~A} 001800$ DEN 0 B & ICP Wet Solids & SWRI 090625-24 & 090625-24 ICP and ICPMS \\
\hline TI-065 & 7.22 & Caustic leach & A 01AIM 13A 001800 DEN 0 D & ICP Supernate & SWRI 090625-25 & 090625-25 and 090625-26 ICP and ICPMS and IC \\
\hline TI-065 & 7.22 & Caustic leach & A 01AIM 13A 001800 DEN 0 D & IC & SWRI 090625-25 & 090625-25 and 090625-26 ICP and ICPMS and IC \\
\hline TI-065 & 7.22 & Caustic leach & A 01AIM 13A 001800 RAM 3 D & Raman & ASR8345 & ASR 8345 RAM rev 1 \\
\hline TI-065 & 7.22 & Caustic leach & A 01AIL 13A 001802 ARC 1 B & Archive & & \\
\hline TI-065 & 7.22 & Caustic leach & A 01AIL 13A 001802 ARC 1 D & Archive & & \\
\hline TI-065 & 7.22 & Caustic leach & A 01AMH 13A 001803 ARC 1 D & Archive & & \\
\hline TI-065 & 7.22 & Caustic leach & A 01AMH 13A 001803 ARC 1 B & Archive & & \\
\hline TI-065 & 7.22 & Caustic leach & A 01AMM 13A 001804 ARC 1 B & Archive & & \\
\hline TI-065 & 7.22 & Caustic leach & A 01AMM 13A 001804 ARC 1 D & Archive & & \\
\hline
\end{tabular}


Integrated Test A Sample List

\begin{tabular}{|c|c|c|c|c|c|c|}
\hline TI \# & TI Step \# & TI Step Description & Sample ID & Analysis & Folder & Final Electronic Report File Name \\
\hline TI-065 & 7.22 & Caustic leach & A 01AML 13A 001805 ARC 1 B & Archive & & \\
\hline TI-065 & 7.22 & Caustic leach & A 01AML 13A 001805 ARC 1 D & Archive & & \\
\hline TI-065 & 7.22 & Caustic leach & A 01AOH 13A 001806 ARC $1 \mathrm{~B}$ & Archive & & \\
\hline TI-065 & 7.22 & Caustic leach & A 01AOH 13A 001806 ARC 1 D & Archive & & \\
\hline TI-065 & 7.22 & Caustic leach & A 01AOM 13A 001807 ARC 1 B & Archive & & \\
\hline TI-065 & 7.22 & Caustic leach & A 01AOM 13A 001807 ARC 1 D & Archive & & \\
\hline TI-065 & 7.22 & Caustic leach & A 01AOL 13A 001808 ARC 1 B & Archive & & \\
\hline TI-065 & 7.22 & Caustic leach & A 01AOL 13A 001808 ARC 1 D & Archive & & \\
\hline TI-065 & 7.25 & Caustic leach & A 01AIM 13A 011809 UDS 2 B & UDS & UDS R1 & UDS R1 Shake and Test A and Test B Step001 Date 08182009 \\
\hline TI-065 & 7.25 & Caustic leach & A 01AIM 13A 011809 UDS 2 D & UDS & UDS R1 & UDS R1 Shake and Test A and Test B Step001 Date 08182009 \\
\hline TI-065 & 7.25 & Caustic leach & A 01AIM 13A 011810 DEN 2 & Density Slurry & SWRI 090205-4 & 090205Density \\
\hline TI-065 & 7.25 & Caustic leach & A 01AIM 13A 011811 RAM 3 D & Raman & ASR8345 & ASR 8345 RAM rev 1 \\
\hline TI-065 & 7.25 & Caustic leach & A 01AIM 13A 021812 UDS 2 B & UDS & UDS R1 & UDS R1 Shake and Test A and Test B Step001 Date 08182009 \\
\hline TI-065 & 7.25 & Caustic leach & A 01AIM 13A 021812 UDS 2 D & UDS & UDS R1 & UDS R1 Shake and Test A and Test B Step001 Date 08182009 \\
\hline TI-065 & 7.25 & Caustic leach & A 01AIM 13A 021813 DEN 2 & Density Slurry & SWRI 090205-4 & 090205Density \\
\hline TI-065 & 7.25 & Caustic leach & A 01AIM 13A 021814 RAM 3 D & Raman & ASR8345 & ASR 8345 RAM rev 1 \\
\hline TI-065 & 7.25 & Caustic leach & A 01AIM 13A 031815 ARC 1 B & Archive & & \\
\hline TI-065 & 7.25 & Caustic leach & A 01AIM 13A 031815 ARC 1 D & Archive & & \\
\hline TI-065 & 7.25 & Caustic leach & A 01AIM 13A 041816 UDS 2 B & UDS & UDS R1 & UDS R1 Shake and Test A and Test B Step001 Date 08182009 \\
\hline TI-065 & 7.25 & Caustic leach & A 01AIM 13A 041816 UDS 2 D & UDS & UDS R1 & UDS R1 Shake and Test A and Test B Step001 Date 08182009 \\
\hline TI-065 & 7.25 & Caustic leach & A 01AIM 13A 041817 DEN 2 & Density Slurry & SWRI 090205-4 & 090205Density \\
\hline TI-065 & 7.25 & Caustic leach & A 01AIM 13A 041818 DEN 0 D & ICP Supernate & SWRI 090625-25 & 090625-25 and 090625-26 ICP and ICPMS and IC \\
\hline TI-065 & 7.25 & Caustic leach & A 01AIM 13A 041818 DEN 0 D & IC & SWRI 090625-25 & 090625-25 and 090625-26 ICP and ICPMS and IC \\
\hline TI-065 & 7.25 & Caustic leach & A 01AIM 13A 041818 ICP 2 B & ICP Wet Solids & SWRI 090205-5 & 090204 and 090205 ICP and ICPMS \\
\hline TI-065 & 7.25 & Caustic leach & A 01AIM 13A 041818 RAM 3 D & Raman & ASR8345 & ASR 8345 RAM rev 1 \\
\hline TI-065 & 7.25 & Caustic leach & A 01AOH 13A 041824 ARC 1 B & Archive & & \\
\hline TI-065 & 7.25 & Caustic leach & A 01AOH 13A 041824 ARC 1 D & Archive & & \\
\hline TI-065 & 7.25 & Caustic leach & A 01AOM 13A 041825 ARC 1 B & Archive & & \\
\hline TI-065 & 7.25 & Caustic leach & A 01AOM 13A 041825 ARC 1 D & Archive & & \\
\hline TI-065 & 7.25 & Caustic leach & A $01 \mathrm{AOL} 13 \mathrm{~A} 041826$ ARC $1 \mathrm{~B}$ & Archive & & \\
\hline TI-065 & 7.25 & Caustic leach & A 01AOL 13A 041826 ARC 1 D & Archive & & \\
\hline TI-065 & 7.25 & Caustic leach & A 01AIM 13A 051827 ARC 1 B & Archive & & \\
\hline TI-065 & 7.25 & Caustic leach & A 01AIM 13A 051827 ARC 1 D & Archive & & \\
\hline
\end{tabular}


Integrated Test A Sample List

\begin{tabular}{|c|c|c|c|c|c|c|}
\hline TI\# & TI Step \# & TI Step Description & Sample ID & Analysis & Folder & Final Electronic Report File Name \\
\hline TI-065 & 7.25 & Caustic leach & A 01AIH 13A 041819 ARC 1 B & Archive & & \\
\hline TI-065 & 7.25 & Caustic leach & A 01AIH 13A 041819 ARC 1 D & Archive & & \\
\hline TI-065 & 7.25 & Caustic leach & A 01AIL 13A 041820 ARC 1 B & Archive & & \\
\hline TI-065 & 7.25 & Caustic leach & A 01AIL 13A 041820 ARC 1 D & Archive & & \\
\hline TI-065 & 7.25 & Caustic leach & A 01AMH 13A 041821 ARC 1 B & Archive & & \\
\hline TI-065 & 7.25 & Caustic leach & A 01AMH 13A 041821 ARC 1 D & Archive & & \\
\hline TI-065 & 7.25 & Caustic leach & A 01AMM 13A 041822 ARC 1 B & Archive & & \\
\hline TI-065 & 7.25 & Caustic leach & A 01AMM 13A 041822 ARC 1 D & Archive & & \\
\hline TI-065 & 7.25 & Caustic leach & A 01AML 13A 041823 ARC 1 B & Archive & & \\
\hline TI-065 & 7.25 & Caustic leach & A 01AML 13A 041823 ARC 1 D & Archive & & \\
\hline TI-065 & 7.25 & Caustic leach & A 01 AIM 13A 061828 ARC 1 B & Archive & & \\
\hline TI-065 & 7.25 & Caustic leach & A 01AIM 13A 061828 ARC 1 D & Archive & & \\
\hline TI-065 & 7.25 & Caustic leach & A 01AIM 13A 071829 ARC 1 B & Archive & & \\
\hline TI-065 & 7.25 & Caustic leach & A 01AIM 13A 071829 ARC 1 D & Archive & & \\
\hline TI-065 & 7.25 & Caustic leach & A 01AIM 13A 081830 UDS 2 B & UDS & UDS R1 & UDS R1 Shake and Test A and Test B Step001 Date 08182009 \\
\hline TI-065 & 7.25 & Caustic leach & A 01AIM 13A 081830 UDS 2 D & UDS & UDS R1 & UDS R1 Shake and Test A and Test B Step001 Date 08182009 \\
\hline TI-065 & 7.25 & Caustic leach & A 01AIM 13A 081831 DEN 2 & Density Slurry & SWRI 090206-2 & 090206Density \\
\hline TI-065 & 7.25 & Caustic leach & A 01AIM 13A 081832 DEN 0 D & ICP Supernate & SWRI 090625-25 & 090625-25 and 090625-26 ICP and ICPMS and IC \\
\hline TI-065 & 7.25 & Caustic leach & A 01AIM 13A 081832 DEN 0 D & $\mathrm{IC}$ & SWRI 090625-25 & 090625-25 and 090625-26 ICP and ICPMS and IC \\
\hline TI-065 & 7.25 & Caustic leach & A 01AIM 13A 081832 ICP 2 B & ICP Wet Solids & SWRI 090206-4 & 090204-16 and 090206-4 and 090210-14 and 090210-15 ICP and ICPMS \\
\hline TI-065 & 7.25 & Caustic leach & A 01AIM 13A 081832 RAM 3 D & Raman & ASR 8347 & ASR 8347 RAM rev 1 \\
\hline TI-065 & 7.25 & Caustic leach & A 01AIH 13A 081833 ARC 1 B & Archive & & \\
\hline TI-065 & 7.25 & Caustic leach & A 01AIL 13A 081834 ARC 1 B & Archive & & \\
\hline TI-065 & 7.25 & Caustic leach & A 01AMH 13A 081835 ARC 1 B & Archive & & \\
\hline TI-065 & 7.25 & Caustic leach & A 01AMM 13A 081836 ARC 1 B & Archive & & \\
\hline TI-065 & 7.25 & Caustic leach & A 01AML 13A 081837 ARC 1 B & Archive & & \\
\hline TI-065 & 7.25 & Caustic leach & A $01 \mathrm{AOH} 13 \mathrm{~A} 081838$ ARC $1 \mathrm{~B}$ & Archive & & \\
\hline TI-065 & 7.25 & Caustic leach & A 01AOM 13A 081839 ARC 1 B & Archive & & \\
\hline TI-065 & 7.25 & Caustic leach & A $01 \mathrm{AOL} 13 \mathrm{~A} 081840 \mathrm{ARC} 1 \mathrm{~B}$ & Archive & & \\
\hline TI-065 & 7.25 & Caustic leach & A 01AIM 13A 091841 ARC 1 B & Archive & & \\
\hline TI-065 & 7.25 & Caustic leach & A 01AIH 13A 081833 ARC 1 D & Archive & & \\
\hline TI-065 & 7.25 & Caustic leach & A 01AIL 13A 081834 ARC 1 D & Archive & & \\
\hline TI-065 & 7.25 & Caustic leach & A 01AMH 13A 081835 ARC 1 D & Archive & & \\
\hline
\end{tabular}


Integrated Test A Sample List

\begin{tabular}{|c|c|c|c|c|c|c|}
\hline TI \# & TI Step \# & TI Step Description & Sample ID & Analysis & Folder & Final Electronic Report File Name \\
\hline TI-065 & 7.25 & Caustic leach & A 01AMM 13A 081836 ARC 1 D & Archive & & \\
\hline TI-065 & 7.25 & Caustic leach & A 01AML 13A 081837 ARC 1 D & Archive & & \\
\hline TI-065 & 7.25 & Caustic leach & A 01AOH 13A 081838 ARC $1 \mathrm{D}$ & Archive & & \\
\hline TI-065 & 7.25 & Caustic leach & A 01AOM 13A 081839 ARC 1 D & Archive & & \\
\hline TI-065 & 7.25 & Caustic leach & A 01AOL 13A 081840 ARC 1 D & Archive & & \\
\hline TI-065 & 7.25 & Caustic leach & A 01AIM 13A 091841 ARC $1 \mathrm{D}$ & Archive & & \\
\hline TI-065 & 7.25 & Caustic leach & A 01AIM 13A 101842 UDS 2 B & UDS & UDS R1 & UDS R1 Shake and Test A and Test B Step001 Date 08182009 \\
\hline TI-065 & 7.25 & Caustic leach & A 01AIM 13A 101842 UDS 2 D & UDS & UDS R1 & UDS R1 Shake and Test A and Test B Step001 Date 08182009 \\
\hline TI-065 & 7.25 & Caustic leach & A 01AIM 13A 101843 DEN 2 & Density Slurry & SWRI 090206-2 & 090206Density \\
\hline TI-065 & 7.25 & Caustic leach & A 01AIM 13A 101844 RAM 3 D & Raman & ASR 8347 & ASR 8347 RAM rev 1 \\
\hline TI-065 & 7.25 & Caustic leach & A 01AIM 13A 111845 ARC 1 B & Archive & & \\
\hline TI-065 & 7.25 & Caustic leach & A 01AIM 13A 111845 ARC 1 D & Archive & & \\
\hline TI-065 & 7.25 & Caustic leach & A 01AIM 13A 121846 UDS 2 B & UDS & UDS R1 & UDS R1 Shake and Test A and Test B Step001 Date 08182009 \\
\hline TI-065 & 7.25 & Caustic leach & A 01AIM 13A 121846 UDS 2 D & UDS & UDS R1 & UDS R1 Shake and Test A and Test B Step001 Date 08182009 \\
\hline TI-065 & 7.25 & Caustic leach & A 01AIM 13A 121847 DEN 2 & Density Slurry & SWRI 090206-2 & 090206Density \\
\hline TI-065 & 7.25 & Caustic leach & A 01AIM 13A 121848 DEN 0 D & ICP Supernate & SWRI 090625-25 & 090625-25 and 090625-26 ICP and ICPMS and IC \\
\hline TI-065 & 7.25 & Caustic leach & A 01AIM 13A 121848 DEN 0 D & IC & SWRI 090625-25 & 090625-25 and 090625-26 ICP and ICPMS and IC \\
\hline TI-065 & 7.25 & Caustic leach & A 01AIM 13A 121848 ICP 2 B & ICP Wet Solids & SWRI 090206-4 & 090204-16 and 090206-4 and 090210-14 and 090210-15 ICP and ICPMS \\
\hline TI-065 & 7.25 & Caustic leach & A 01AIM 13A 121848 RAM 3 D & Raman & ASR8347 & ASR 8347 RAM rev 1 \\
\hline TI-065 & 7.25 & Caustic leach & A 01AIH 13A 121849 ARC 1 B & Archive & & \\
\hline TI-065 & 7.25 & Caustic leach & A 01AIH 13A 121849 ARC 1 D & Archive & & \\
\hline TI-065 & 7.25 & Caustic leach & A 01AIL 13A 121850 ARC 1 B & Archive & & \\
\hline TI-065 & 7.25 & Caustic leach & A 01AIL 13A 121850 ARC 1 D & Archive & & \\
\hline TI-065 & 7.25 & Caustic leach & A 01AMH 13A 121851 ARC 1 B & Archive & & \\
\hline TI-065 & 7.25 & Caustic leach & A 01AMH 13A 121851 ARC 1 D & Archive & & \\
\hline TI-065 & 7.25 & Caustic leach & A 01AMM 13A 121852 ARC 1 B & Archive & & \\
\hline TI-065 & 7.25 & Caustic leach & A 01AMM 13A 121852 ARC 1 D & Archive & & \\
\hline TI-065 & 7.25 & Caustic leach & A 01AML 13A 121853 ARC 1 B & Archive & & \\
\hline TI-065 & 7.25 & Caustic leach & A 01AML 13A 121853 ARC 1 D & Archive & & \\
\hline TI-065 & 7.25 & Caustic leach & A $01 \mathrm{AOH} 13 \mathrm{~A} 121854$ ARC $1 \mathrm{~B}$ & Archive & & \\
\hline TI-065 & 7.25 & Caustic leach & A $01 \mathrm{AOH} 13 \mathrm{~A} 121854$ ARC $1 \mathrm{D}$ & Archive & & \\
\hline TI-065 & 7.25 & Caustic leach & A 01AOM 13A 121855 ARC 1 B & Archive & & \\
\hline TI-065 & 7.25 & Caustic leach & A 01AOM 13A 121855 ARC 1 D & Archive & & \\
\hline
\end{tabular}


Integrated Test A Sample List

\begin{tabular}{|c|c|c|c|c|c|c|}
\hline TI \# & TI Step \# & TI Step Description & Sample ID & Analysis & Folder & Final Electronic Report File Name \\
\hline TI-065 & 7.25 & Caustic leach & A 01AOL 13A 121856 ARC 1 B & Archive & & \\
\hline TI-065 & 7.25 & Caustic leach & A 01AOL 13A 121856 ARC 1 D & Archive & & \\
\hline TI-065 & 7.25 & Caustic leach & A 01AIM 13A 131857 ARC 1 B & Archive & & \\
\hline TI-065 & 7.25 & Caustic leach & A 01AIM 13A 131857 ARC 1 D & Archive & & \\
\hline TI-065 & 7.25 & Caustic leach & A 01AIM 13A 141858 UDS 2 B & UDS & UDS R1 & UDS R1 Shake and Test A and Test B Step001 Date 08182009 \\
\hline TI-065 & 7.25 & Caustic leach & A 01AIM 13A 141858 UDS 2 D & UDS & UDS R1 & UDS R1 Shake and Test A and Test B Step001 Date 08182009 \\
\hline TI-065 & 7.25 & Caustic leach & A 01AIM 13A 141859 DEN 2 & Density Slurry & SWRI 090206-2 & 090206Density \\
\hline TI-065 & 7.25 & Caustic leach & A 01AIM 13A 141860 RAM 3 D & Raman & ASR8347 & ASR 8347 RAM rev 1 \\
\hline TI-065 & 7.25 & Caustic leach & A 01AIM 13A 151861 ARC 1 B & Archive & & \\
\hline TI-065 & 7.25 & Caustic leach & A 01AIM 13A 151861 ARC 1 D & Archive & & \\
\hline TI-065 & 7.25 & Caustic leach & A 01AIM 13A 161862 UDS 2 B & UDS & UDS R1 & UDS R1 Shake and Test A and Test B Step001 Date 08182009 \\
\hline TI-065 & 7.25 & Caustic leach & A 01AIM 13A 161862 UDS 2 D & UDS & UDS R1 & UDS R1 Shake and Test A and Test B Step001 Date 08182009 \\
\hline TI-065 & 7.25 & Caustic leach & A 01 AIM 13A 161863 UDS 2 B & UDS & UDS R1 & UDS R1 Shake and Test A and Test B Step001 Date 08182009 \\
\hline TI-065 & 7.25 & Caustic leach & A 01AIM 13A 161863 UDS 2 D & UDS & UDS R1 & UDS R1 Shake and Test A and Test B Step001 Date 08182009 \\
\hline TI-065 & 7.25 & Caustic leach & A 01AIM 13A 161864 UDS 2 B & UDS & UDS R1 & UDS R1 Shake and Test A and Test B Step001 Date 08182009 \\
\hline TI-065 & 7.25 & Caustic leach & A 01AIM 13A 161864 UDS 2 D & UDS & UDS R1 & UDS R1 Shake and Test A and Test B Step001 Date 08182009 \\
\hline TI-065 & 7.25 & Caustic leach & A 01AIM 13A 161865 DEN 2 & Density Slurry & SWRI 090206-2 & 090206Density \\
\hline TI-065 & 7.25 & Caustic leach & A 01AIM 13A 161866 DEN 2 & Density Slurry & SWRI 090206-2 & 090206Density \\
\hline TI-065 & 7.25 & Caustic leach & A 01AIM 13A 161867 DEN 2 & Density Slurry & SWRI 090206-2 & 090206Density \\
\hline TI-065 & 7.25 & Caustic leach & A 01AIM 13A 161868 DEN 0 D & ICP Supernate & SWRI 090625-25 & 090625-25 and 090625-26 ICP and ICPMS and IC \\
\hline TI-065 & 7.25 & Caustic leach & A 01AIM 13A 161868 DEN 0 D & IC & SWRI 090625-25 & 090625-25 and 090625-26 ICP and ICPMS and IC \\
\hline TI-065 & 7.25 & Caustic leach & A 01AIM 13A 161868 ICP 2 B & ICP Wet Solids & SWRI 090206-4 & 090204-16 and 090206-4 and 090210-14 and 090210-15 ICP and ICPMS \\
\hline TI-065 & 7.25 & Caustic leach & A 01AIM 13A 161868 RAM 3 D & Raman & ASR8347 & ASR 8347 RAM rev 1 \\
\hline TI-065 & 7.25 & Caustic leach & A 01AIM 13A 161869 DEN 0 D & ICP Supernate & SWRI 090625-25 & 090625-25 and 090625-26 ICP and ICPMS and IC \\
\hline TI-065 & 7.25 & Caustic leach & A 01AIM 13A 161869 DEN 0 D & $\mathrm{IC}$ & SWRI 090625-25 & 090625-25 and 090625-26 ICP and ICPMS and IC \\
\hline TI-065 & 7.25 & Caustic leach & A 01AIM 13A 161869 ICP 2 B & ICP Wet Solids & SWRI 090206-4 & 090204-16 and 090206-4 and 090210-14 and 090210-15 ICP and ICPMS \\
\hline TI-065 & 7.25 & Caustic leach & A 01AIM 13A 161869 RAM 3 D & Raman & ASR8347 & ASR 8347 RAM rev 1 \\
\hline TI-065 & 7.25 & Caustic leach & A 01AIM 13A 161870 DEN $0 \mathrm{D}$ & ICP Supernate & SWRI 090625-25 & 090625-25 and 090625-26 ICP and ICPMS and IC \\
\hline TI-065 & 7.25 & Caustic leach & A 01AIM 13A 161870 DEN 0 D & IC & SWRI 090625-25 & 090625-25 and 090625-26 ICP and ICPMS and IC \\
\hline TI-065 & 7.25 & Caustic leach & A 01AIM 13A 161870 ICP 2 B & ICP Wet Solids & SWRI 090206-4 & 090204-16 and 090206-4 and 090210-14 and 090210-15 ICP and ICPMS \\
\hline TI-065 & 7.25 & Caustic leach & A 01AIM 13A 161870 RAM 3 D & Raman & ASR8347 & ASR 8347 RAM rev 1 \\
\hline TI-065 & 7.25 & Caustic leach & A 01AIM 13A 161871 XSP 4 B & $\mathrm{XRD}$ & TDP-WTP-357 & TDP WTP 357 Int Test A XRD Data on PEP Slurry \\
\hline TI-065 & 7.25 & Caustic leach & A 01AIH 13A 161872 ARC 1 B & Archive & & \\
\hline
\end{tabular}


Integrated Test A Sample List

\begin{tabular}{|c|c|c|c|c|c|c|}
\hline TI \# & TI Step \# & TI Step Description & Sample ID & Analysis & Folder & Final Electronic Report File Name \\
\hline TI-065 & 7.25 & Caustic leach & A 01AIH 13A 161872 ARC 1 D & Archive & & \\
\hline TI-065 & 7.25 & Caustic leach & A 01AIL 13A 161873 ARC 1 B & Archive & & \\
\hline TI-065 & 7.25 & Caustic leach & A 01AIL 13A 161873 ARC 1 D & Archive & & \\
\hline TI-065 & 7.25 & Caustic leach & A 01AMH 13A 161874 ARC 1 B & Archive & & \\
\hline TI-065 & 7.25 & Caustic leach & A 01AMH 13A 161874 ARC 1 D & Archive & & \\
\hline TI-065 & 7.25 & Caustic leach & A 01AMM 13A 161875 ARC 1 B & Archive & & \\
\hline TI-065 & 7.25 & Caustic leach & A 01AMM 13A 161875 ARC 1 D & Archive & & \\
\hline TI-065 & 7.25 & Caustic leach & A 01AML 13A 161876 ARC 1 B & Archive & & \\
\hline TI-065 & 7.25 & Caustic leach & A 01AML 13A 161876 ARC $1 \mathrm{D}$ & Archive & & \\
\hline TI-065 & 7.25 & Caustic leach & A $01 \mathrm{AOH} 13 \mathrm{~A} 161877$ ARC $1 \mathrm{~B}$ & Archive & & \\
\hline TI-065 & 7.25 & Caustic leach & A 01AOH 13A 161877 ARC 1 D & Archive & & \\
\hline TI-065 & 7.25 & Caustic leach & A 01AOM 13A 161878 ARC 1 B & Archive & & \\
\hline TI-065 & 7.25 & Caustic leach & A 01AOM 13A 161878 ARC 1 D & Archive & & \\
\hline TI-065 & 7.25 & Caustic leach & A 01AOL 13A 161879 ARC 1 B & Archive & & \\
\hline TI-065 & 7.25 & Caustic leach & A 01AOL 13A 161879 ARC 1 D & Archive & & \\
\hline TI-065 & 7.33 & Cool UFP-T01A contents with UFP-HX-T05A & A 01 AIM 13A XX 1880 XSP 4 B & $\mathrm{XRD}$ & TDP-WTP-357 & TDP WTP 357 Int Test A XRD Data on PEP Slurry \\
\hline TI-065 & 8.14 & Transfer from HLP-VSL-T22 to T01B ( 3 times - batches 2, 4 \& 6) & A 01BIM 13B XX 1916 UDS 2 B & UDS & UDS R1 & UDS R1 Shake and Test A and Test B Step001 Date 08182009 \\
\hline TI-065 & 8.14 & Transfer from HLP-VSL-T22 to T01B ( 3 times - batches $2,4 \&$ 6) & A 01BIM 13B XX 1916 UDS 2 D & UDS & UDS R1 & UDS R1 Shake and Test A and Test B Step001 Date 08182009 \\
\hline TI-065 & 8.14 & Transfer from HLP-VSL-T22 to T01B ( 3 times - batches 2, 4 \& 6) & A 01BIM 13B XX 1917 UDS 2 B & UDS & UDS R1 & UDS R1 Shake and Test A and Test B Step001 Date 08182009 \\
\hline TI-065 & 8.14 & Transfer from HLP-VSL-T22 to T01B ( 3 times - batches $2,4 \& 6$ ) & A 01BIM 13B XX 1917 UDS 2 D & UDS & UDS R1 & UDS R1 Shake and Test A and Test B Step001 Date 08182009 \\
\hline TI-065 & 8.14 & Transfer from HLP-VSL-T22 to T01B ( 3 times - batches 2, $4 \& 6$ ) & A 01BIM 13B XX 1918 UDS 2 B & UDS & UDS R1 & UDS R1 Shake and Test A and Test B Step001 Date 08182009 \\
\hline TI-065 & 8.14 & Transfer from HLP-VSL-T22 to T01B ( 3 times - batches $2,4 \&$ 6) & A 01BIM 13B XX 1918 UDS 2 D & UDS & UDS R1 & UDS R1 Shake and Test A and Test B Step001 Date 08182009 \\
\hline TI-065 & 8.14 & Transfer from HLP-VSL-T22 to T01B ( 3 times - batches $2,4 \& 6$ ) & A 01BIM 13B XX 1919 DEN 2 & Density Slurry & SWRI 090207-2 & 090207-2 DEN \\
\hline TI-065 & 8.14 & Transfer from HLP-VSL-T22 to T01B ( 3 times - batches $2,4 \&$ 6) & A 01BIM 13B XX 1919 DEN 2 & Density Supernate & SWRI 090207-2 & 090207-2 DEN \\
\hline TI-065 & 8.14 & Transfer from HLP-VSL-T22 to T01B ( 3 times - batches $2,4 \& 6$ ) & A 01BIM 13B XX 1920 DEN 2 & Density Slurry & SWRI $090207-2$ & 090207-2 DEN \\
\hline TI-065 & 8.14 & Transfer from HLP-VSL-T22 to T01B ( 3 times - batches 2, 4 \& 6) & A 01BIM 13B XX 1920 DEN 2 & Density Supernate & SWRI 090207-2 & 090207-2 DEN \\
\hline TI-065 & 8.14 & Transfer from HLP-VSL-T22 to T01B ( 3 times - batches 2, $4 \& 6$ ) & A 01BIM 13B XX 1921 DEN 2 & Density Slurry & SWRI 090207-2 & 090207-2 DEN \\
\hline TI-065 & 8.14 & Transfer from HLP-VSL-T22 to T01B ( 3 times - batches $2,4 \& 6$ ) & A 01BIM 13B XX 1921 DEN 2 & Density Supernate & SWRI 090207-2 & 090207-2 DEN \\
\hline TI-065 & 8.14 & Transfer from HLP-VSL-T22 to T01B ( 3 times - batches $2,4 \& 6$ ) & A 01BIM 13B XX 1922 ICP 2 B & ICP Wet Solids & SWRI 090207-3 & 090207-3 ICP and ICPMS \\
\hline TI-065 & 8.14 & Transfer from HLP-VSL-T22 to UFP-VLS-T01B & A 01BIM 13B XX 1922 RAM 3 D & Raman & ASR 8350 & ASR 8350 RAM rev 1 \\
\hline TI-065 & 8.14 & Transfer from HLP-VSL-T22 to T01B ( 3 times - batches 2, 4 \& 6) & A 01BIM 13B XX 1923 ICP 2 B & ICP Wet Solids & SWRI 090207-3 & 090207-3 ICP and ICPMS \\
\hline TI-065 & 8.14 & Transfer from HLP-VSL-T22 to UFP-VLS-T01B & A 01BIM 13B XX 1923 RAM 3 D & Raman & ASR 8350 & ASR 8350 RAM rev 1 \\
\hline TI-065 & 8.14 & Transfer from HLP-VSL-T22 to T01B ( 3 times - batches $2,4 \&$ 6) & A 01BIM 13B XX 1924 ICP 2 B & ICP Wet Solids & SWRI 090207-3 & 090207-3 ICP and ICPMS \\
\hline
\end{tabular}


Integrated Test A Sample List

\begin{tabular}{|c|c|c|c|c|c|c|}
\hline TI \# & TI Step \# & TI Step Description & Sample ID & Analysis & Folder & Final Electronic Report File Name \\
\hline TI-065 & 8.14 & Transfer from HLP-VSL-T22 to UFP-VLS-T01B & A 01BIM 13B XX 1924 RAM 3 D & Raman & ASR8350 & ASR 8350 RAM rev 1 \\
\hline TI-065 & 8.14 & Transfer from HLP-VSL-T22 to UFP-VLS-T01B & A T221B 13B XX 1912 ARC 1 & Archive & & \\
\hline TI-065 & 8.14 & Transfer from HLP-VSL-T22 to UFP-VLS-T01B & A T221B 13B XX 1913 ARC 1 & Archive & & \\
\hline TI-065 & 8.14 & Transfer from HLP-VSL-T22 to UFP-VLS-T01B & A T221B 13B XX 1914 ARC 1 & Archive & & \\
\hline TI-065 & 8.14 & Transfer from HLP-VSL-T22 to UFP-VLS-T01B & A T221B 13B XX 1915 ARC 1 & Archive & & \\
\hline TI-065 & 8.26 & Heat UFP-VSL-T01B to $88 \mathrm{C}$ & A 01BIM 13B BT 1925 UDS 2 B & UDS & UDS R1 & UDS R1 Shake and Test A and Test B Step001 Date 08182009 \\
\hline TI-065 & 8.26 & Heat UFP-VSL-T01B to $88 \mathrm{C}$ & A 01BIM 13B BT 1925 UDS 2 D & UDS & UDS R1 & UDS R1 Shake and Test A and Test B Step001 Date 08182009 \\
\hline TI-065 & 8.26 & Heat UFP-VSL-T01B to $88 \mathrm{C}$ & A 01BIM 13B BT 1926 DEN 2 & Density Slurry & SWRI 090207-2 & 090207-2 DEN \\
\hline TI-065 & 8.26 & Heat UFP-VSL-T01B to $88 \mathrm{C}$ & A 01BIM 13B BT 1926 DEN 2 & Density Supernate & SWRI 090207-2 & 090207-2 DEN \\
\hline TI-065 & 8.26 & Heat UFP-VSL-T01B to $88 \mathrm{C}$ & A 01BIM 13B BT 1927 RAM 3 D & Raman & ASR 8350 & ASR 8350 RAM rev 1 \\
\hline TI-065 & 8.26 & Heat UFP-VSL-T01B to $88 \mathrm{C}$ & A 01BIL 13B BT 1928 ARC 1 B & Archive & & \\
\hline TI-065 & 8.26 & Heat UFP-VSL-T01B to $88 \mathrm{C}$ & A 01BIL 13B BT 1928 ARC 1 D & Archive & & \\
\hline TI-065 & 8.26 & Heat UFP-VSL-T01B to $88 \mathrm{C}$ & A 01BML 13B BT 1929 ARC 1 B & Archive & & \\
\hline TI-065 & 8.26 & Heat UFP-VSL-T01B to $88 \mathrm{C}$ & A 01BML 13B BT 1929 ARC 1 D & Archive & & \\
\hline TI-065 & 8.26 & Heat UFP-VSL-T01B to $88 \mathrm{C}$ & A 01BOL 13B BT 1930 ARC 1 B & Archive & & \\
\hline TI-065 & 8.26 & Heat UFP-VSL-T01B to 88C & A 01BOL 13B BT 1930 ARC 1 D & Archive & & \\
\hline TI-065 & 8.26 & Heat UFP-VSL-T01B to $88 \mathrm{C}$ & A 01BMM 13B BT 1931 ARC 1 B & Archive & & \\
\hline TI-065 & 8.26 & Heat UFP-VSL-T01B to $88 \mathrm{C}$ & A 01BMM 13B BT 1931 ARC 1 D & Archive & & \\
\hline TI-065 & 8.26 & Heat UFP-VSL-T01B to $88 \mathrm{C}$ & A 01BOM 13B BT 1932 ARC 1 B & Archive & & \\
\hline TI-065 & 8.26 & Heat UFP-VSL-T01B to 88C & A 01BOM 13B BT 1932 ARC 1 D & Archive & & \\
\hline TI-065 & 8.26 & Heat UFP-VSL-T01B to $88 \mathrm{C}$ & A 01BMH 13B BT 1934 ARC 1 B & Archive & & \\
\hline TI-065 & 8.26 & Heat UFP-VSL-T01B to $88 \mathrm{C}$ & A 01BMH 13B BT 1934 ARC 1 D & Archive & & \\
\hline TI-065 & 8.26 & Heat UFP-VSL-T01B to $88 \mathrm{C}$ & A 01BOH 13B BT 1935 ARC 1 B & Archive & & \\
\hline TI-065 & 8.26 & Heat UFP-VSL-T01B to $88 \mathrm{C}$ & A 01BOH 13B BT 1935 ARC 1 D & Archive & & \\
\hline TI-065 & 8.28 & Caustic leach & A 01BIM 13B 001936 UDS 2 B & UDS & UDS R1 & UDS R1 Shake and Test A and Test B Step001 Date 08182009 \\
\hline TI-065 & 8.28 & Caustic leach & A 01BIM 13B 001936 UDS 2 D & UDS & UDS R1 & UDS R1 Shake and Test A and Test B Step001 Date 08182009 \\
\hline TI-065 & 8.28 & Caustic leach & A 01BIM 13B 001937 DEN 2 & Density Slurry & SWRI 090207-2 & 090207-2 DEN \\
\hline TI-065 & 8.28 & Caustic leach & A 01BIM 13B 001937 DEN 2 & Density Supernate & SWRI 090207-2 & 090207-2 DEN \\
\hline TI-065 & 8.28 & Caustic leach & A 01BIM 13B 001938 DEN 0 B & ICP Wet Solids & SWRI 090625-24 & 090625-24 ICP and ICPMS \\
\hline TI-065 & 8.28 & Caustic leach & A 01BIM 13B 001938 DEN 0 D & ICP Supernate & SWRI 090625-25 & 090625-25 and 090625-26 ICP and ICPMS and IC \\
\hline TI-065 & 8.28 & Caustic leach & A 01BIM 13B 001938 DEN 0 D & IC & SWRI 090625-25 & 090625-25 and 090625-26 ICP and ICPMS and IC \\
\hline TI-065 & 8.28 & Caustic leach & A 01BIM 13B 001938 RAM 3 D & Raman & ASR 8350 & ASR 8350 RAM rev 1 \\
\hline TI-065 & 8.28 & Caustic leach & A 01BIL 13B 001939 ARC 1 B & Archive & & \\
\hline
\end{tabular}


Integrated Test A Sample List

\begin{tabular}{|c|c|c|c|c|c|c|}
\hline TI \# & TI Step \# & TI Step Description & Sample ID & Analysis & Folder & Final Electronic Report File Name \\
\hline TI-065 & 8.28 & Caustic leach & A 01BIL 13B 001939 ARC 1 D & Archive & & \\
\hline TI-065 & 8.28 & Caustic leach & A 01BML 13B 001940 ARC 1 B & Archive & & \\
\hline TI-065 & 8.28 & Caustic leach & A 01BML 13B 001940 ARC 1 D & Archive & & \\
\hline TI-065 & 8.28 & Caustic leach & A 01BOL 13B 001941 ARC 1 B & Archive & & \\
\hline TI-065 & 8.28 & Caustic leach & A 01BOL 13B 001941 ARC 1 D & Archive & & \\
\hline TI-065 & 8.28 & Caustic leach & A 01BMM 13B 001942 ARC 1 B & Archive & & \\
\hline TI-065 & 8.28 & Caustic leach & A 01BMM 13B 001942 ARC 1 D & Archive & & \\
\hline TI-065 & 8.28 & Caustic leach & A 01BOM 13B 001943 ARC 1 B & Archive & & \\
\hline TI-065 & 8.28 & Caustic leach & A 01BOM 13B 001943 ARC 1 D & Archive & & \\
\hline TI-065 & 8.28 & Caustic leach & A 01BMH 13B 001945 ARC 1 B & Archive & & \\
\hline TI-065 & 8.28 & Caustic leach & A 01BMH 13B 001945 ARC 1 D & Archive & & \\
\hline TI-065 & 8.28 & Caustic leach & A 01BOH 13B 001946 ARC 1 B & Archive & & \\
\hline TI-065 & 8.28 & Caustic leach & A 01BOH 13B 001946 ARC 1 D & Archive & & \\
\hline TI-065 & 8.28 & Caustic leach & A 01BIM 13B 011947 UDS 2 B & UDS & UDS R1 & UDS R1 Shake and Test A and Test B Step001 Date 08182009 \\
\hline TI-065 & 8.28 & Caustic leach & A 01BIM 13B 011947 UDS 2 D & UDS & UDS R1 & UDS R1 Shake and Test A and Test B Step001 Date 08182009 \\
\hline TI-065 & 8.28 & Caustic leach & A 01BIM 13B 011948 DEN 2 & Density Slurry & SWRI 090207-2 & 090207-2 DEN \\
\hline TI-065 & 8.28 & Caustic leach & A 01BIM 13B 011948 DEN 2 & Density Supernate & SWRI 090207-2 & 090207-2 DEN \\
\hline TI-065 & 8.28 & Caustic leach & A 01BIM 13B 011949 RAM 3 D & Raman & ASR8350 & ASR 8350 RAM rev 1 \\
\hline TI-065 & 8.28 & Caustic leach & A 01BIM 13B 021950 UDS 2 B & UDS & UDS R1 & UDS R1 Shake and Test A and Test B Step001 Date 08182009 \\
\hline TI-065 & 8.28 & Caustic leach & A 01BIM 13B 021950 UDS 2 D & UDS & UDS R1 & UDS R1 Shake and Test A and Test B Step001 Date 08182009 \\
\hline TI-065 & 8.28 & Caustic leach & A 01BIM 13B 021951 DEN 2 & Density Slurry & SWRI 090207-2 & 090207-2 DEN \\
\hline TI-065 & 8.28 & Caustic leach & A 01BIM 13B 021951 DEN 2 & Density Supernate & SWRI 090207-2 & 090207-2 DEN \\
\hline TI-065 & 8.28 & Caustic leach & A 01BIM 13B 021952 RAM 3 D & Raman & ASR8350 & ASR 8350 RAM rev 1 \\
\hline TI-065 & 8.28 & Caustic leach & A 01BIM 13B 031953 ARC 1 B & Archive & & \\
\hline TI-065 & 8.28 & Caustic leach & A 01BIM 13B 031953 ARC 1 D & Archive & & \\
\hline TI-065 & 8.28 & Caustic leach & A 01BIM 13B 031953 ARC 1 B & Archive & & \\
\hline TI-065 & 8.28 & Caustic leach & A 01BIM 13B 031953 ARC 1 D & Archive & & \\
\hline TI-065 & 8.28 & Caustic leach & A 01BIM 13B 041954 UDS 2 B & UDS & UDS R1 & UDS R1 Shake and Test A and Test B Step001 Date 08182009 \\
\hline TI-065 & 8.28 & Caustic leach & A 01BIM 13B 041954 UDS 2 D & UDS & UDS R1 & UDS R1 Shake and Test A and Test B Step001 Date 08182009 \\
\hline TI-065 & 8.28 & Caustic leach & A 01BIM 13B 041955 DEN 2 & Density Slurry & SWRI 090207-2 & 090207-2 DEN \\
\hline TI-065 & 8.28 & Caustic leach & A 01BIM 13B 041955 DEN 2 & Density Supernate & SWRI 090207-2 & 090207-2 DEN \\
\hline TI-065 & 8.28 & Caustic leach & A 01BIM 13B 041956 DEN 0 D & ICP Supernate & SWRI 090625-25 & 090625-25 and 090625-26 ICP and ICPMS and IC \\
\hline TI-065 & 8.28 & Caustic leach & A 01BIM 13B 041956 DEN 0 D & $\mathrm{IC}$ & SWRI 090625-25 & 090625-25 and 090625-26 ICP and ICPMS and IC \\
\hline
\end{tabular}


Integrated Test A Sample List

\begin{tabular}{|c|c|c|c|c|c|c|}
\hline TI \# & TI Step \# & TI Step Description & Sample ID & Analysis & Folder & Final Electronic Report File Name \\
\hline TI-065 & 8.28 & Caustic leach & A 01BIM 13B 041956 ICP 2 B & ICP Wet Solids & SWRI 090207-3 & 090207-3 ICP and ICPMS \\
\hline TI-065 & 8.28 & Caustic leach & A 01BIM 13B 041956 RAM 3 D & Raman & ASR8350 & ASR 8350 RAM rev 1 \\
\hline TI-065 & 8.28 & Caustic leach & A 01BIL 13B 041957 ARC 1 B & Archive & & \\
\hline TI-065 & 8.28 & Caustic leach & A 01BIL 13B 041957 ARC 1 D & Archive & & \\
\hline TI-065 & 8.28 & Caustic leach & A 01BML 13B 041958 ARC 1 B & Archive & & \\
\hline TI-065 & 8.28 & Caustic leach & A 01BML 13B 041958 ARC 1 D & Archive & & \\
\hline TI-065 & 8.28 & Caustic leach & A 01BOL 13B 041959 ARC 1 B & Archive & & \\
\hline TI-065 & 8.28 & Caustic leach & A 01BOL 13B 041959 ARC 1 D & Archive & & \\
\hline TI-065 & 8.28 & Caustic leach & A 01BMM 13B 041960 ARC 1 B & Archive & & \\
\hline TI-065 & 8.28 & Caustic leach & A 01BMM 13B 041960 ARC 1 D & Archive & & \\
\hline TI-065 & 8.28 & Caustic leach & A 01BOM 13B 041961 ARC 1 B & Archive & & \\
\hline TI-065 & 8.28 & Caustic leach & A 01BOM 13B 041961 ARC 1 D & Archive & & \\
\hline TI-065 & 8.28 & Caustic leach & A 01BMH 13B 041963 ARC 1 B & Archive & & \\
\hline TI-065 & 8.28 & Caustic leach & A 01BMH 13B 041963 ARC 1 D & Archive & & \\
\hline TI-065 & 8.28 & Caustic leach & A 01BOH 13B 041964 ARC 1 B & Archive & & \\
\hline TI-065 & 8.28 & Caustic leach & A 01BOH 13B 041964 ARC 1 D & Archive & & \\
\hline TI-065 & 8.28 & Caustic leach & A 01BIM 13B 051965 ARC 1 B & Archive & & \\
\hline TI-065 & 8.28 & Caustic leach & A 01BIM 13B 051965 ARC 1 D & Archive & & \\
\hline TI-065 & 8.28 & Caustic leach & A 01BIM 13B 061966 ARC 1 B & Archive & & \\
\hline TI-065 & 8.28 & Caustic leach & A 01BIM 13B 061966 ARC 1 D & Archive & & \\
\hline TI-065 & 8.28 & Caustic leach & A 01BIM 13B 071967 ARC 1 B & Archive & & \\
\hline TI-065 & 8.28 & Caustic leach & A 01BIM 13B 071967 ARC 1 D & Archive & & \\
\hline TI-065 & 8.28 & Caustic leach & A 01BIM 13B 081968 UDS 2 B & UDS & UDS R1 & UDS R1 Shake and Test A and Test B Step001 Date 08182009 \\
\hline TI-065 & 8.28 & Caustic leach & A 01BIM 13B 081968 UDS 2 D & UDS & UDS R1 & UDS R1 Shake and Test A and Test B Step001 Date 08182009 \\
\hline TI-065 & 8.28 & Caustic leach & A 01BIM 13B 081969 DEN 2 & Density Slurry & SWRI 090207-2 & 090207-2 DEN \\
\hline TI-065 & 8.28 & Caustic leach & A 01BIM 13B 081969 DEN 2 & Density Supernate & SWRI 090207-2 & 090207-2 DEN \\
\hline TI-065 & 8.28 & Caustic leach & A 01BIM 13B 081970 RAM 3 D & Raman & ASR 8350 & ASR 8350 RAM rev 1 \\
\hline TI-065 & 8.28 & Caustic leach & A 01BIM 13B 081970 DEN 0 D & ICP Supernate & SWRI 090625-25 & 090625-25 and 090625-26 ICP and ICPMS and IC \\
\hline TI-065 & 8.28 & Caustic leach & A 01BIM 13B 081970 DEN 0 D & IC & SWRI 090625-25 & 090625-25 and 090625-26 ICP and ICPMS and IC \\
\hline TI-065 & 8.28 & Caustic leach & A 01BIM 13B 081970 ICP 2 B & ICP Wet Solids & SWRI 090207-3 & 090207-3 ICP and ICPMS \\
\hline TI-065 & 8.28 & Caustic leach & A 01BOH 13B 081978 ARC 1 B & Archive & & \\
\hline TI-065 & 8.28 & Caustic leach & A 01BOH 13B 081978 ARC 1 D & Archive & & \\
\hline TI-065 & 8.28 & Caustic leach & A 01BML 13B 081972 ARC 1 D & Archive & & \\
\hline
\end{tabular}


Integrated Test A Sample List

\begin{tabular}{|c|c|c|c|c|c|c|}
\hline TI\# & TI Step \# & TI Step Description & Sample ID & Analysis & Folder & Final Electronic Report File Name \\
\hline TI-065 & 8.28 & Caustic leach & A 01BML 13B 081972 ARC 1 B & Archive & & \\
\hline TI-065 & 8.28 & Caustic leach & A 01BMM 13B 081974 ARC 1 B & Archive & & \\
\hline TI-065 & 8.28 & Caustic leach & A 01BMM 13B 081974 ARC 1 D & Archive & & \\
\hline TI-065 & 8.28 & Caustic leach & A 01BIL 13B 081971 ARC 1 D & Archive & & \\
\hline TI-065 & 8.28 & Caustic leach & A 01BIL 13B 081971 ARC 1 B & Archive & & \\
\hline TI-065 & 8.28 & Caustic leach & A 01BOL 13B 081973 ARC 1 B & Archive & & \\
\hline TI-065 & 8.28 & Caustic leach & A 01BOL 13B 081973 ARC 1 D & Archive & & \\
\hline TI-065 & 8.28 & Caustic leach & A 01BOM 13B 081975 ARC 1 B & Archive & & \\
\hline TI-065 & 8.28 & Caustic leach & A 01BOM 13B 081975 ARC 1 D & Archive & & \\
\hline TI-065 & 8.28 & Caustic leach & A 01BMH 13B 081977 ARC 1 B & Archive & & \\
\hline TI-065 & 8.28 & Caustic leach & A 01BMH 13B 081977 ARC 1 D & Archive & & \\
\hline TI-065 & 8.28 & Caustic leach & A 01BIM 13B 091979 ARC 1 B & Archive & & \\
\hline TI-065 & 8.28 & Caustic leach & A 01BIM 13B 091979 ARC 1 D & Archive & & \\
\hline TI-065 & 8.28 & Caustic leach & A 01BIM 13B 101980 UDS 2 B & UDS & UDS R1 & UDS R1 Shake and Test A and Test B Step001 Date 08182009 \\
\hline TI-065 & 8.28 & Caustic leach & A 01BIM 13B 101980 UDS 2 D & UDS & UDS R1 & UDS R1 Shake and Test A and Test B Step001 Date 08182009 \\
\hline TI-065 & 8.28 & Caustic leach & A 01BIM 13B 101981 DEN 2 & Density Slurry & SWRI 090207-2 & 090207-2 DEN \\
\hline TI-065 & 8.28 & Caustic leach & A 01BIM 13B 101981 DEN 2 & Density Supernate & SWRI 090207-2 & 090207-2 DEN \\
\hline TI-065 & 8.28 & Caustic leach & A 01BIM 13B 101982 RAM 3 D & Raman & ASR 8352 & ASR 8352 RAM rev 1 \\
\hline TI-065 & 8.28 & Caustic leach & A 01BIM 13B 111983 ARC 1 B & Archive & & \\
\hline TI-065 & 8.28 & Caustic leach & A 01BIM 13B 111983 ARC 1 D & Archive & & \\
\hline TI-065 & 8.28 & Caustic leach & A 01BIM 13B 121984 UDS 2 B & UDS & UDS R1 & UDS R1 Shake and Test A and Test B Step001 Date 08182009 \\
\hline TI-065 & 8.28 & Caustic leach & A 01BIM 13B 121984 UDS 2 D & UDS & UDS R1 & UDS R1 Shake and Test A and Test B Step001 Date 08182009 \\
\hline TI-065 & 8.28 & Caustic leach & A 01BIM 13B 121985 DEN 2 & Density Slurry & SWRI 090210-16 & 090210-16 DEN \\
\hline TI-065 & 8.28 & Caustic leach & A 01BIM 13B 121986 DEN 0 D & ICP Supernate & SWRI 090625-25 & 090625-25 and 090625-26 ICP and ICPMS and IC \\
\hline TI-065 & 8.28 & Caustic leach & A 01BIM 13B 121986 DEN 0 D & IC & SWRI 090625-25 & 090625-25 and 090625-26 ICP and ICPMS and IC \\
\hline TI-065 & 8.28 & Caustic leach & A 01BIM 13B 121986 ICP 2 B & ICP Wet Solids & SWRI 090210-15 & 090204-16 and 090206-4 and 090210-14 and 090210-15 ICP and ICPMS \\
\hline TI-065 & 8.28 & Caustic leach & A 01BIM 13B 121986 RAM 3 D & Raman & ASR8352 & ASR 8352 RAM rev 1 \\
\hline TI-065 & 8.28 & Caustic leach & A 01BIL 13B 121987 ARC 1 B & Archive & & \\
\hline TI-065 & 8.28 & Caustic leach & A 01BIL 13B 121987 ARC 1 D & Archive & & \\
\hline TI-065 & 8.28 & Caustic leach & A 01BML 13B 121988 ARC 1 B & Archive & & \\
\hline TI-065 & 8.28 & Caustic leach & A 01BOL 13B 121989 ARC 1 D & Archive & & \\
\hline TI-065 & 8.28 & Caustic leach & A 01BMM 13B 121990 ARC 1 B & Archive & & \\
\hline TI-065 & 8.28 & Caustic leach & A 01BOM 13B 121991 ARC 1 B & Archive & & \\
\hline
\end{tabular}


Integrated Test A Sample List

\begin{tabular}{|c|c|c|c|c|c|c|}
\hline TI\# & TI Step \# & TI Step Description & Sample ID & Analysis & Folder & Final Electronic Report File Name \\
\hline TI-065 & 8.28 & Caustic leach & A 01BMM 13B 121990 ARC 1 D & Archive & & \\
\hline TI-065 & 8.28 & Caustic leach & A 01BOM 13B 121991 ARC 1 D & Archive & & \\
\hline TI-065 & 8.28 & Caustic leach & A 01BMH 13B 121993 ARC 1 B & Archive & & \\
\hline TI-065 & 8.28 & Caustic leach & A 01BMH 13B 121993 ARC 1 D & Archive & & \\
\hline TI-065 & 8.28 & Caustic leach & A 01BOH 13B 121994 ARC 1 B & Archive & & \\
\hline TI-065 & 8.28 & Caustic leach & A 01BOH 13B 121994 ARC 1 D & Archive & & \\
\hline TI-065 & 8.28 & Caustic leach & A 01BML 13B 121988 ARC 1 D & Archive & & \\
\hline TI-065 & 8.28 & Caustic leach & A 01BOL 13B 121989 ARC 1 B & Archive & & \\
\hline TI-065 & 8.28 & Caustic leach & A 01BIM 13B 131995 ARC 1 B & Archive & & \\
\hline TI-065 & 8.28 & Caustic leach & A 01BIM 13B 131995 ARC 1 D & Archive & & \\
\hline TI-065 & 8.28 & Caustic leach & A 01BIM 13B 141996 UDS 2 B & UDS & UDS R1 & UDS R1 Shake and Test A and Test B Step001 Date 08182009 \\
\hline TI-065 & 8.28 & Caustic leach & A 01BIM 13B 141996 UDS 2 D & UDS & UDS R1 & UDS R1 Shake and Test A and Test B Step001 Date 08182009 \\
\hline TI-065 & 8.28 & Caustic leach & A 01BIM 13B 141997 DEN 2 & Density Slurry & SWRI 090210-16 & 090210-16 DEN \\
\hline TI-065 & 8.28 & Caustic leach & A 01BIM 13B 141998 RAM 3 D & Raman & ASR 8352 & ASR 8352 RAM rev 1 \\
\hline TI-065 & 8.28 & Caustic leach & A 01BIM 13B 151999 ARC 1 B & Archive & & \\
\hline TI-065 & 8.28 & Caustic leach & A 01BIM 13B 151999 ARC 1 D & Archive & & \\
\hline TI-065 & 8.28 & Caustic leach & A 01BIM 13B 162002 RAM 3 D & Raman & ASR8352 & ASR 8352 RAM rev 1 \\
\hline TI-065 & 8.28 & Caustic leach & A 01BIM 13B 162005 RAM 3 D & Raman & ASR 8352 & ASR 8352 RAM rev 1 \\
\hline TI-065 & 8.28 & Caustic leach & A 01BIM 13B 162008 RAM 3 D & Raman & ASR8352 & ASR 8352 RAM rev 1 \\
\hline TI-065 & 8.28 & Caustic leach & A 01BIM 13B 162000 UDS 2 B & UDS & UDS R1 & UDS R1 Shake and Test A and Test B Step001 Date 08182009 \\
\hline TI-065 & 8.28 & Caustic leach & A 01BIM 13B 162000 UDS 2 D & UDS & UDS R1 & UDS R1 Shake and Test A and Test B Step001 Date 08182009 \\
\hline TI-065 & 8.28 & Caustic leach & A 01BIM 13B 162001 DEN 2 & Density Slurry & SWRI 090210-16 & 090210-16 DEN \\
\hline TI-065 & 8.28 & Caustic leach & A 01BIM 13B 162002 DEN 0 D & ICP Supernate & SWRI 090625-26 & 090625-25 and 090625-26 ICP and ICPMS and IC \\
\hline TI-065 & 8.28 & Caustic leach & A 01BIM 13B 162002 DEN 0 D & $\mathrm{IC}$ & SWRI 090625-26 & 090625-25 and 090625-26 ICP and ICPMS and IC \\
\hline TI-065 & 8.28 & Caustic leach & A 01BIM 13B 162002 ICP 2 B & ICP Wet Solids & SWRI 090210-15 & 090204-16 and 090206-4 and 090210-14 and 090210-15 ICP and ICPMS \\
\hline TI-065 & 8.28 & Caustic leach & A 01BIM 13B 162003 UDS 2 B & UDS & UDS R1 & UDS R1 Shake and Test A and Test B Step001 Date 08182009 \\
\hline TI-065 & 8.28 & Caustic leach & A 01BIM 13B 162003 UDS 2 D & UDS & UDS R1 & UDS R1 Shake and Test A and Test B Step001 Date 08182009 \\
\hline TI-065 & 8.28 & Caustic leach & A 01BIM 13B 162004 DEN 2 & Density Slurry & SWRI 090210-16 & 090210-16 DEN \\
\hline TI-065 & 8.28 & Caustic leach & A 01BIM 13B 162005 DEN 0 D & ICP Supernate & SWRI 090625-26 & 090625-25 and 090625-26 ICP and ICPMS and IC \\
\hline TI-065 & 8.28 & Caustic leach & A 01BIM 13B 162005 DEN 0 D & $\mathrm{IC}$ & SWRI 090625-26 & 090625-25 and 090625-26 ICP and ICPMS and IC \\
\hline TI-065 & 8.28 & Caustic leach & A 01BIM 13B 162005 ICP 2 B & ICP Wet Solids & SWRI 090210-15 & 090204-16 and 090206-4 and 090210-14 and 090210-15 ICP and ICPMS \\
\hline TI-065 & 8.28 & Caustic leach & A 01BIM 13B 162006 UDS 2 B & UDS & UDS R1 & UDS R1 Shake and Test A and Test B Step001 Date 08182009 \\
\hline TI-065 & 8.28 & Caustic leach & A 01BIM 13B 162006 UDS 2 D & UDS & UDS R1 & UDS R1 Shake and Test A and Test B Step001 Date 08182009 \\
\hline
\end{tabular}


Integrated Test A Sample List

\begin{tabular}{|c|c|c|c|c|c|c|}
\hline TI\# & TI Step \# & TI Step Description & Sample ID & Analysis & Folder & Final Electronic Report File Name \\
\hline TI-065 & 8.28 & Caustic leach & A 01BIM 13B 162007 DEN 2 & Density Slurry & SWRI 090210-16 & 090210-16 DEN \\
\hline TI-065 & 8.28 & Caustic leach & A 01BIM 13B 162008 DEN 0 D & ICP Supernate & SWRI 090625-26 & 090625-25 and 090625-26 ICP and ICPMS and IC \\
\hline TI-065 & 8.28 & Caustic leach & A 01BIM 13B 162008 DEN 0 D & IC & SWRI 090625-26 & 090625-25 and 090625-26 ICP and ICPMS and IC \\
\hline TI-065 & 8.28 & Caustic leach & A 01BIM 13B 162008 ICP 2 B & ICP Wet Solids & SWRI 090210-15 & $090204-16$ and $090206-4$ and $090210-14$ and $090210-15$ ICP and ICPMS \\
\hline TI-065 & 8.28 & Caustic leach & A 01BIL 13B 162009 ARC 1 B & Archive & & \\
\hline TI-065 & 8.28 & Caustic leach & A 01BIL 13B 162009 ARC 1 D & Archive & & \\
\hline TI-065 & 8.28 & Caustic leach & A 01BML 13B 162010 ARC 1 B & Archive & & \\
\hline TI-065 & 8.28 & Caustic leach & A 01BML 13B 162010 ARC 1 D & Archive & & \\
\hline TI-065 & 8.28 & Caustic leach & A 01BOL 13B 162011 ARC 1 B & Archive & & \\
\hline TI-065 & 8.28 & Caustic leach & A 01BOL 13B 162011 ARC 1 D & Archive & & \\
\hline TI-065 & 8.28 & Caustic leach & A 01BMM 13B 162012 ARC 1 B & Archive & & \\
\hline TI-065 & 8.28 & Caustic leach & A 01BMM 13B 162012 ARC 1 D & Archive & & \\
\hline TI-065 & 8.28 & Caustic leach & A 01BOM 13B 162013 ARC 1 B & Archive & & \\
\hline TI-065 & 8.28 & Caustic leach & A 01BOM 13B 162013 ARC 1 D & Archive & & \\
\hline TI-065 & 8.28 & Caustic leach & A 01BMH 13B 162015 ARC 1 B & Archive & & \\
\hline TI-065 & 8.28 & Caustic leach & A 01BMH 13B 162015 ARC 1 D & Archive & & \\
\hline TI-065 & 8.28 & Caustic leach & A 01BOH 13B 162016 ARC 1 B & Archive & & \\
\hline TI-065 & 8.28 & Caustic leach & A 01BOH 13B 162016 ARC 1 D & Archive & & \\
\hline TI-065 & 9.3 & $\begin{array}{l}\text { Just prior to transfer simulant from HLP-VSL-T22 to UFP-T01 A \& } \\
\text { caustic addition (Batches } 1,3 \& 5 \text { ) }\end{array}$ & A T22GM 13C XX 2020 UDS 2 & UDS & UDS R1 & UDS R1 Shake and Test A and Test B Step001 Date 08182009 \\
\hline TI-065 & 9.3 & $\begin{array}{l}\text { Just prior to transfer simulant from HLP-VSL-T22 to UFP-TO1 A \& } \\
\text { caustic addition (Batches } 1,3 \text { \& 5) }\end{array}$ & A T22GM 13C XX 2021 RHE 4 & Rheology & TDP-WTP-359 & TDP WTP 359 Int Test A RHE Data on PEP Slurry \\
\hline TI-065 & 9.3 & $\begin{array}{l}\text { Just prior to transfer simulant from HLP-VSL-T22 to UFP-TO1 A \& } \\
\text { caustic addition (Batches } 1,3 \& 5 \text { ) }\end{array}$ & A T22GM 13C XX 2022 XSP 4 B & Not Analyzed & & \\
\hline TI-065 & 9.3 & $\begin{array}{l}\text { Just prior to transfer simulant from HLP-VSL-T22 to UFP-TO1 A \& } \\
\text { caustic addition (Batches } 1,3 \& 5 \text { ) }\end{array}$ & A T22GM 13C XX 2023 OTR 6 & Vijay & & \\
\hline TI-065 & 9.3 & $\begin{array}{l}\text { Just prior to transfer simulant from HLP-VSL-T22 to UFP-TO1 A \& } \\
\text { caustic addition (Batches } 1,3 \& 5 \text { ) }\end{array}$ & A T221A 13C XX 2024 ARC 1 & Archive & & \\
\hline TI-065 & 9.3 & $\begin{array}{l}\text { Just prior to transfer simulant from HLP-VSL-T22 to UFP-TO1 A \& } \\
\text { caustic addition (Batches } 1,3 \& 5 \text { ) }\end{array}$ & A T221A 13C XX 2025 ARC 1 & Archive & & \\
\hline TI-065 & 9.3 & $\begin{array}{l}\text { Just prior to transfer simulant from HLP-VSL-T22 to UFP-TO1 A \& } \\
\text { caustic addition (Batches } 1,3 \& 5 \text { ) }\end{array}$ & A T221A 13C XX 2026 ARC 1 & Archive & & \\
\hline TI-065 & 9.3 & $\begin{array}{l}\text { Just prior to transfer simulant from HLP-VSL-T22 to UFP-TO1 A \& } \\
\text { caustic addition (Batches } 1,3 \text { \& 5) }\end{array}$ & A T221A 13C XX 2027 ARC 1 & Archive & & \\
\hline TI-065 & 9.9 & Transfer from HLP-VSL-T22 to T01 A ( 3 times - batches $1,3 \& 5$ ) & A 01AIM 13C XX 2028 UDS 2 B & UDS & UDS R1 & UDS R1 Shake and Test A and Test B Step001 Date 08182009 \\
\hline
\end{tabular}


Integrated Test A Sample List

\begin{tabular}{|c|c|c|c|c|c|c|}
\hline TI\# & TI Step \# & TI Step Description & Sample ID & Analysis & Folder & Final Electronic Report File Name \\
\hline TI-065 & 9.9 & Transfer from HLP-VSL-T22 to T01 A (3 times - batches $1,3 \& 5$ ) & A 01AIM 13C XX 2028 UDS 2 D & UDS & UDS R1 & UDS R1 Shake and Test A and Test B Step001 Date 08182009 \\
\hline TI-065 & 9.9 & Transfer from HLP-VSL-T22 to T01 A ( 3 times - batches $1,3 \& 5$ ) & A 01AIM 13C XX 2029 UDS 2 B & UDS & UDS R1 & UDS R1 Shake and Test A and Test B Step001 Date 08182009 \\
\hline TI-065 & 9.9 & Transfer from HLP-VSL-T22 to T01 A (3 times - batches 1,3 \& 5) & A 01 AIM 13C XX 2029 UDS 2 D & UDS & UDS R1 & UDS R1 Shake and Test A and Test B Step001 Date 08182009 \\
\hline TI-065 & 9.9 & Transfer from HLP-VSL-T22 to T01 A (3 times - batches $1,3 \& 5$ ) & A 01AIM 13C XX 2030 UDS 2 B & UDS & UDS R1 & UDS R1 Shake and Test A and Test B Step001 Date 08182009 \\
\hline TI-065 & 9.9 & Transfer from HLP-VSL-T22 to T01 A (3 times - batches $1,3 \& 5$ ) & A 01AIM 13C XX 2030 UDS 2 D & UDS & UDS R1 & UDS R1 Shake and Test A and Test B Step001 Date 08182009 \\
\hline TI-065 & 9.9 & Transfer from HLP-VSL-T22 to T01 A ( 3 times - batches $1,3 \& 5$ ) & A 01AIM 13C XX 2031 DEN 2 & Density Slurry & SWRI 090210-12 & 090210-12 DEN \\
\hline TI-065 & 9.9 & Transfer from HLP-VSL-T22 to T01 A (3 times - batches $1,3 \& 5$ ) & A 01AIM 13C XX 2032 DEN 2 & Density Slurry & SWRI 090210-12 & 090210-12 DEN \\
\hline TI-065 & 9.9 & Transfer from HLP-VSL-T22 to T01 A (3 times - batches $1,3 \& 5$ ) & A 01AIM 13C XX 2033 DEN 2 & Density Slurry & SWRI 090210-12 & 090210-12 DEN \\
\hline TI-065 & 9.9 & Transfer from HLP-VSL-T22 to T01 A (3 times - batches 1,3 \& 5) & A 01 AIM 13C XX 2034 ICP 2 B & ICP Wet Solids & SWRI 090210-14 & 090204-16 and 090206-4 and 090210-14 and 090210-15 ICP and ICPMS \\
\hline TI-065 & 9.9 & Transfer from HLP-VSL-T22 to UFP-VLS-T01A & A 01 AIM 13C XX 2034 RAM 3 D & Raman & ASR8352 & ASR 8352 RAM rev 1 \\
\hline TI-065 & 9.9 & Transfer from HLP-VSL-T22 to T01 A (3 times - batches 1,3 \& 5) & A 01AIM 13C XX 2035 ICP 2 B & ICP Wet Solids & SWRI 090210-14 & 090204-16 and 090206-4 and 090210-14 and 090210-15 ICP and ICPMS \\
\hline TI-065 & 9.9 & Transfer from HLP-VSL-T22 to UFP-VLS-T01A & A 01AIM 13C XX 2035 RAM 3 D & Raman & ASR8352 & ASR 8352 RAM rev 1 \\
\hline TI-065 & 9.9 & Transfer from HLP-VSL-T22 to T01 A ( 3 times - batches $1,3 \& 5$ ) & A 01 AIM 13C XX 2036 ICP 2 B & ICP Wet Solids & SWRI 090210-14 & $090204-16$ and $090206-4$ and $090210-14$ and $090210-15$ ICP and ICPMS \\
\hline TI-065 & 9.9 & Transfer from HLP-VSL-T22 to UFP-VLS-T01A & A 01AIM 13C XX 2036 RAM 3 D & Raman & ASR 8352 & ASR 8352 RAM rev 1 \\
\hline TI-065 & 9.9 & Transfer from HLP-VSL-T22 to T01A (3 times - batches 1,3 \& 5) & A 01AIM 13C XX 2760 CUF 4 & Parallel Bench Test & & \\
\hline TI-065 & 9.20 & Heat UFP-VSL-T01A to $88 \mathrm{C}$ & A 01AIM 13C BT 2038 UDS 2 B & UDS & SWRI 090210-13 & UDS R1 Shake and Test A and Test B Step001 Date 08182009 \\
\hline TI-065 & 9.20 & Heat UFP-VSL-T01A to $88 \mathrm{C}$ & A 01AIM 13C BT 2038 UDS 2 D & UDS & SWRI 090210-13 & UDS R1 Shake and Test A and Test B Step001 Date 08182009 \\
\hline TI-065 & 9.20 & Heat UFP-VSL-T01A to $88 \mathrm{C}$ & A 01AIM 13C BT 2039 DEN 2 & Density Slurry & SWRI 090210-12 & 090210-12 DEN \\
\hline TI-065 & 9.20 & Heat UFP-VSL-T01A to $88^{\circ} \mathrm{C}$ & A 01AIM 13C BT 2040 RAM 3 D & Raman & ASR8352 & ASR 8352 RAM rev 1 \\
\hline TI-065 & 9.20 & Heat UFP-VSL-T01A to $88^{\circ} \mathrm{C}$ & A 01AIL 13C BT 2042 ARC 1 B & Archive & & \\
\hline TI-065 & 9.20 & Heat UFP-VSL-T01A to $88^{\circ} \mathrm{C}$ & A 01AMH 13C BT 2043 ARC 1 B & Archive & & \\
\hline TI-065 & 9.20 & Heat UFP-VSL-T01A to $88^{\circ} \mathrm{C}$ & A 01AMM 13C BT 2044 ARC 1 B & Archive & & \\
\hline TI-065 & 9.20 & Heat UFP-VSL-T01A to $88^{\circ} \mathrm{C}$ & A 01AML 13C BT 2045 ARC 1 B & Archive & & \\
\hline TI-065 & 9.20 & Heat UFP-VSL-T01A to $88^{\circ} \mathrm{C}$ & A 01AOH 13C BT 2046 ARC 1 B & Archive & & \\
\hline TI-065 & 9.20 & Heat UFP-VSL-T01A to $88^{\circ} \mathrm{C}$ & A 01AOM 13C BT 2047 ARC 1 B & Archive & & \\
\hline TI-065 & 9.20 & Heat UFP-VSL-T01A to $88^{\circ} \mathrm{C}$ & A 01AOL 13C BT 2048 ARC 1 B & Archive & & \\
\hline TI-065 & 9.21 & Caustic leach & A 01AIM 13C 002049 UDS 2 B & UDS & UDS R1 & UDS R1 Shake and Test A and Test B Step001 Date 08182009 \\
\hline TI-065 & 9.21 & Caustic leach & A 01AIM 13C 002049 UDS 2 D & UDS & UDS R1 & UDS R1 Shake and Test A and Test B Step001 Date 08182009 \\
\hline TI-065 & 9.21 & Caustic leach & A 01 AIM 13C 002050 DEN 2 & Density Slurry & SWRI 090210-12 & 090210-12 DEN \\
\hline TI-065 & 9.21 & Caustic leach & A 01AIM 13C 002051 DEN 0 B & ICP Wet Solids & SWRI 090625-24 & 090625-24 ICP and ICPMS \\
\hline TI-065 & 9.21 & Caustic leach & A 01AIM 13C 002051 DEN 0 D & ICP Supernate & SWRI 090625-25 & 090625-25 and 090625-26 ICP and ICPMS and IC \\
\hline TI-065 & 9.21 & Caustic leach & A 01AIM 13C 002051 DEN 0 D & IC & SWRI 090625-25 & 090625-25 and 090625-26 ICP and ICPMS and IC \\
\hline TI-065 & 9.24 & Caustic leach & A 01AIM 13C 002051 RAM 3 D & Raman & ASR8352 & ASR 8352 RAM rev 1 \\
\hline
\end{tabular}


Integrated Test A Sample List

\begin{tabular}{|c|c|c|c|c|c|c|}
\hline TI \# & TI Step \# & TI Step Description & Sample ID & Analysis & Folder & Final Electronic Report File Name \\
\hline TI-065 & 9.24 & Caustic leach & A 01AIL 13C 002053 ARC 1 B & Archive & & \\
\hline TI-065 & 9.24 & Caustic leach & A $01 \mathrm{AMH} 13 \mathrm{C} 002054$ ARC $1 \mathrm{~B}$ & Archive & & \\
\hline TI-065 & 9.24 & Caustic leach & A 01AMM 13C 002055 ARC 1 B & Archive & & \\
\hline TI-065 & 9.24 & Caustic leach & A 01AML 13C 002056 ARC 1 B & Archive & & \\
\hline TI-065 & 9.24 & Caustic leach & A $01 \mathrm{AOH} 13 \mathrm{C} 002057 \mathrm{ARC} 1 \mathrm{~B}$ & Archive & & \\
\hline TI-065 & 9.24 & Caustic leach & A 01AOM 13C 002058 ARC 1 B & Archive & & \\
\hline TI-065 & 9.24 & Caustic leach & A 01AOL 13C 002059 ARC 1 B & Archive & & \\
\hline TI-065 & 9.24 & Caustic leach & A 01AIM 13C 012060 UDS 2 B & UDS & UDS R1 & UDS R1 Shake and Test A and Test B Step001 Date 08182009 \\
\hline TI-065 & 9.24 & Caustic leach & A 01AIM 13C 012060 UDS 2 D & UDS & UDS R1 & UDS R1 Shake and Test A and Test B Step001 Date 08182009 \\
\hline TI-065 & 9.24 & Caustic leach & A 01AIM 13C 012061 DEN 2 & Density Slurry & SWRI 090210-12 & 090210-12 DEN \\
\hline TI-065 & 9.24 & Caustic leach & A 01 AIM 13C 012062 RAM 3 D & Raman & ASR 8352 & ASR 8352 RAM rev 1 \\
\hline TI-065 & 9.24 & Caustic leach & A 01AIM 13C 022063 UDS 2 B & UDS & UDS R1 & UDS R1 Shake and Test A and Test B Step001 Date 08182009 \\
\hline TI-065 & 9.24 & Caustic leach & A 01 AIM 13C 022063 UDS 2 D & UDS & UDS R1 & UDS R1 Shake and Test A and Test B Step001 Date 08182009 \\
\hline TI-065 & 9.24 & Caustic leach & A 01AIM 13C 022065 DEN 2 & Density Slurry & SWRI 090210-12 & 090210-12 DEN \\
\hline TI-065 & 9.24 & Caustic leach & A 01AIM 13C 022066 RAM 3 D & Raman & ASR8352 & ASR 8352 RAM rev 1 \\
\hline TI-065 & 9.24 & Caustic leach & A 01AIM 13C 032067 ARC 1 B & Archive & & \\
\hline TI-065 & 9.24 & Caustic leach & A 01AIM 13C 032067 ARC 1 D & Archive & & \\
\hline TI-065 & 9.24 & Caustic leach & A 01AIM 13C 042068 UDS 2 B & UDS & UDS R1 & UDS R1 Shake and Test A and Test B Step001 Date 08182009 \\
\hline TI-065 & 9.24 & Caustic leach & A 01AIM 13C 042068 UDS 2 D & UDS & UDS R1 & UDS R1 Shake and Test A and Test B Step001 Date 08182009 \\
\hline TI-065 & 9.24 & Caustic leach & A 01AIM 13C 042069 DEN 2 & Density Slurry & SWRI 090210-12 & 090210-12 DEN \\
\hline TI-065 & 9.24 & Caustic leach & A 01AIM 13C 042070 DEN 0 D & ICP Supernate & SWRI 090625-25 & 090625-25 and 090625-26 ICP and ICPMS and IC \\
\hline TI-065 & 9.24 & Caustic leach & A 01AIM 13C 042070 DEN 0 D & IC & SWRI 090625-25 & 090625-25 and 090625-26 ICP and ICPMS and IC \\
\hline TI-065 & 9.24 & Caustic leach & A 01AIM 13C 042070 ICP 2 B & ICP Wet Solids & SWRI 090210-14 & 090204-16 and 090206-4 and 090210-14 and 090210-15 ICP and ICPMS \\
\hline TI-065 & 9.24 & Caustic leach & A 01AIM 13C 042070 RAM 3 D & Raman & ASR8352 & ASR 8352 RAM rev 1 \\
\hline TI-065 & 9.24 & Caustic leach & A 01AIL 13C 042072 ARC 1 B & Archive & & \\
\hline TI-065 & 9.24 & Caustic leach & A 01AIL 13C 042072 ARC 1 D & Archive & & \\
\hline TI-065 & 9.24 & Caustic leach & A 01AMH 13C 042073 ARC 1 B & Archive & & \\
\hline TI-065 & 9.24 & Caustic leach & A 01AMH 13C 042073 ARC 1 D & Archive & & \\
\hline TI-065 & 9.24 & Caustic leach & A 01AMM 13C 042074 ARC 1 B & Archive & & \\
\hline TI-065 & 9.24 & Caustic leach & A 01AMM 13C 042074 ARC 1 D & Archive & & \\
\hline TI-065 & 9.24 & Caustic leach & A 01AML 13C 042075 ARC 1 B & Archive & & \\
\hline TI-065 & 9.24 & Caustic leach & A 01AML 13C 042075 ARC 1 D & Archive & & \\
\hline TI-065 & 9.24 & Caustic leach & A 01AOH 13C 042076 ARC 1 B & Archive & & \\
\hline
\end{tabular}


Integrated Test A Sample List

\begin{tabular}{|c|c|c|c|c|c|c|}
\hline TI \# & TI Step \# & TI Step Description & Sample ID & Analysis & Folder & Final Electronic Report File Name \\
\hline TI-065 & 9.24 & Caustic leach & A 01AOH 13C 042076 ARC 1 D & Archive & & \\
\hline TI-065 & 9.24 & Caustic leach & A 01AOM 13C 042077 ARC 1 B & Archive & & \\
\hline TI-065 & 9.24 & Caustic leach & A 01AOM 13C 042077 ARC 1 D & Archive & & \\
\hline TI-065 & 9.24 & Caustic leach & A 01AOL 13C 042078 ARC 1 B & Archive & & \\
\hline TI-065 & 9.24 & Caustic leach & A 01AOL 13C 042078 ARC 1 D & Archive & & \\
\hline TI-065 & 9.24 & Caustic leach & A 01AIM 13C 052079 ARC 1 B & Archive & & \\
\hline TI-065 & 9.24 & Caustic leach & A 01AIM 13C 052079 ARC 1 D & Archive & & \\
\hline TI-065 & 9.24 & Caustic leach & A 01AIM 13C 062080 ARC 1 B & Archive & & \\
\hline TI-065 & 9.24 & Caustic leach & A 01AIM 13C 062080 ARC 1 D & Archive & & \\
\hline TI-065 & 9.24 & Caustic leach & A 01AIM 13C 072081 ARC 1 B & Archive & & \\
\hline TI-065 & 9.24 & Caustic leach & A 01AIM 13C 072081 ARC 1 D & Archive & & \\
\hline TI-065 & 9.24 & Caustic leach & A 01AIM 13C 082082 UDS 2 B & UDS & UDS R1 & UDS R1 Shake and Test A and Test B Step001 Date 08182009 \\
\hline TI-065 & 9.24 & Caustic leach & A 01AIM 13C 082082 UDS 2 D & UDS & UDS R1 & UDS R1 Shake and Test A and Test B Step001 Date 08182009 \\
\hline TI-065 & 9.24 & Caustic leach & A 01AIM 13C 082083 DEN 2 & Density Slurry & SWRI 090210-12 & 090210-12 DEN \\
\hline TI-065 & 9.24 & Caustic leach & A 01AIM 13C 082084 DEN 0 D & ICP Supernate & SWRI 090625-25 & 090625-25 and 090625-26 ICP and ICPMS and IC \\
\hline TI-065 & 9.24 & Caustic leach & A 01AIM 13C 082084 DEN 0 D & IC & SWRI 090625-25 & 090625-25 and 090625-26 ICP and ICPMS and IC \\
\hline TI-065 & 9.24 & Caustic leach & A 01AIM 13C 082084 ICP 2 B & ICP Wet Solids & SWRI 090210-14 & 090204-16 and 090206-4 and 090210-14 and 090210-15 ICP and ICPMS \\
\hline TI-065 & 9.24 & Caustic leach & A 01AIM 13C 082084 RAM 3 D & Raman & ASR8352 & ASR 8352 RAM rev 1 \\
\hline TI-065 & 9.24 & Caustic leach & A 01AMM 13C 082088 ARC 1 B & Archive & & \\
\hline TI-065 & 9.24 & Caustic leach & A 01AMM 13C 082088 ARC 1 D & Archive & & \\
\hline TI-065 & 9.24 & Caustic leach & A 01AML 13C 082089 ARC 1 B & Archive & & \\
\hline TI-065 & 9.24 & Caustic leach & A 01AML 13C 082089 ARC 1 D & Archive & & \\
\hline TI-065 & 9.24 & Caustic leach & A $01 \mathrm{AOH} 13 \mathrm{C} 082090 \mathrm{ARC} 1 \mathrm{~B}$ & Archive & & \\
\hline TI-065 & 9.24 & Caustic leach & A $01 \mathrm{AOH} 13 \mathrm{C} 082090$ ARC $1 \mathrm{D}$ & Archive & & \\
\hline TI-065 & 9.24 & Caustic leach & A 01AOM 13C 082091 ARC 1 B & Archive & & \\
\hline TI-065 & 9.24 & Caustic leach & A 01AOM 13C 082091 ARC 1 D & Archive & & \\
\hline TI-065 & 9.24 & Caustic leach & A 01AOL 13C 082092 ARC 1 B & Archive & & \\
\hline TI-065 & 9.24 & Caustic leach & A 01AOL 13C 082092 ARC 1 D & Archive & & \\
\hline TI-065 & 9.24 & Caustic leach & A 01AIL 13C 082086 ARC 1 D & Archive & & \\
\hline TI-065 & 9.24 & Caustic leach & A 01AMH 13C 082087 ARC 1 D & Archive & & \\
\hline TI-065 & 9.24 & Caustic leach & A 01AIL 13C 082086 ARC 1 B & Archive & & \\
\hline TI-065 & 9.24 & Caustic leach & A 01AMH 13C 082087 ARC 1 B & Archive & & \\
\hline TI-065 & 9.24 & Caustic leach & A 01AIM 13C 092093 ARC 1 B & Archive & & \\
\hline
\end{tabular}


Integrated Test A Sample List

\begin{tabular}{|c|c|c|c|c|c|c|}
\hline TI \# & TI Step \# & TI Step Description & Sample ID & Analysis & Folder & Final Electronic Report File Name \\
\hline TI-065 & 9.24 & Caustic leach & A 01AIM 13C 092093 ARC 1 D & Archive & & \\
\hline TI-065 & 9.24 & Caustic leach & A 01AIM 13C 102094 UDS 2 B & UDS & UDS R1 & UDS R1 Shake and Test A and Test B Step001 Date 08182009 \\
\hline TI-065 & 9.24 & Caustic leach & A 01AIM 13C 102094 UDS 2 D & UDS & UDS R1 & UDS R1 Shake and Test A and Test B Step001 Date 08182009 \\
\hline TI-065 & 9.24 & Caustic leach & A 01AIM 13C 102095 DEN 2 & Density Slurry & SWRI 090210-12 & 090210-12 DEN \\
\hline TI-065 & 9.24 & Caustic leach & A 01AIM 13C 102096 RAM 3 D & Raman & ASR8352 & ASR 8352 RAM rev 1 \\
\hline TI-065 & 9.24 & Caustic leach & A 01AIM 13C 112097 ARC 1 B & Archive & & \\
\hline TI-065 & 9.24 & Caustic leach & A 01AIM 13C 112097 ARC 1 D & Archive & & \\
\hline TI-065 & 9.24 & Caustic leach & A 01AIM 13C 122098 UDS 2 B & UDS & UDS R1 & UDS R1 Shake and Test A and Test B Step001 Date 08182009 \\
\hline TI-065 & 9.24 & Caustic leach & A 01AIM 13C 122098 UDS 2 D & UDS & UDS R1 & UDS R1 Shake and Test A and Test B Step001 Date 08182009 \\
\hline TI-065 & 9.24 & Caustic leach & A 01AIM 13C 122099 DEN 2 & Density Slurry & SWRI 090210-12 & 090210-12 DEN \\
\hline TI-065 & 9.24 & Caustic leach & A 01AIM 13C 122100 DEN 0 D & ICP Supernate & SWRI 090625-25 & 090625-25 and 090625-26 ICP and ICPMS and IC \\
\hline TI-065 & 9.24 & Caustic leach & A 01AIM 13C 122100 DEN 0 D & IC & SWRI 090625-25 & 090625-25 and 090625-26 ICP and ICPMS and IC \\
\hline TI-065 & 9.24 & Caustic leach & A 01AIM 13C 122100 ICP 2 B & ICP Wet Solids & SWRI 090210-14 & 090204-16 and 090206-4 and 090210-14 and 090210-15 ICP and ICPMS \\
\hline TI-065 & 9.24 & Caustic leach & A 01AIM 13C 122100 RAM 3 D & Raman & ASR8352 & ASR 8352 RAM rev 1 \\
\hline TI-065 & 9.24 & Caustic leach & A 01AMM 13C 122105 ARC 1 B & Archive & & \\
\hline TI-065 & 9.24 & Caustic leach & A 01AMM 13C 122105 ARC 1 D & Archive & & \\
\hline TI-065 & 9.24 & Caustic leach & A 01AMH 13C 122104 ARC 1 B & Archive & & \\
\hline TI-065 & 9.24 & Caustic leach & A 01AMH 13C 122104 ARC 1 D & Archive & & \\
\hline TI-065 & 9.24 & Caustic leach & A $01 \mathrm{AOH} 13 \mathrm{C} 122107$ ARC $1 \mathrm{~B}$ & Archive & & \\
\hline TI-065 & 9.24 & Caustic leach & A 01AML 13C 122106 ARC $1 \mathrm{~B}$ & Archive & & \\
\hline TI-065 & 9.24 & Caustic leach & A 01AOH 13C 122107 ARC 1 D & Archive & & \\
\hline TI-065 & 9.24 & Caustic leach & A 01AML 13C 122106 ARC 1 D & Archive & & \\
\hline TI-065 & 9.24 & Caustic leach & A 01AOL 13C 122109 ARC 1 B & Archive & & \\
\hline TI-065 & 9.24 & Caustic leach & A 01AOL 13C 122109 ARC 1 D & Archive & & \\
\hline TI-065 & 9.24 & Caustic leach & A 01AOM 13C 122108 ARC 1 B & Archive & & \\
\hline TI-065 & 9.24 & Caustic leach & A 01AOM 13C 122108 ARC 1 D & Archive & & \\
\hline TI-065 & 9.24 & Caustic leach & A 01AIL 13C 122103 ARC 1 B & Archive & & \\
\hline TI-065 & 9.24 & Caustic leach & A 01AIL 13C 122103 ARC 1 D & Archive & & \\
\hline TI-065 & 9.24 & Caustic leach & A 01AIM 13C 132110 ARC 1 B & Archive & & \\
\hline TI-065 & 9.24 & Caustic leach & A 01AIM 13C 132110 ARC 1 D & Archive & & \\
\hline TI-065 & 9.24 & Caustic leach & A 01AIM 13C 142111 UDS 2 B & UDS & UDS R1 & UDS R1 Shake and Test A and Test B Step001 Date 08182009 \\
\hline TI-065 & 9.24 & Caustic leach & A 01AIM 13C 142111 UDS 2 D & UDS & UDS R1 & UDS R1 Shake and Test A and Test B Step001 Date 08182009 \\
\hline TI-065 & 9.24 & Caustic leach & A 01AIM 13C 142112 DEN 2 & Density Slurry & SWRI 090210-12 & 090210-12 DEN \\
\hline
\end{tabular}


Integrated Test A Sample List

\begin{tabular}{|c|c|c|c|c|c|c|}
\hline TI \# & TI Step \# & TI Step Description & Sample ID & Analysis & Folder & Final Electronic Report File Name \\
\hline TI-065 & 9.24 & Caustic leach & A 01AIM 13C 142113 RAM 3 D & Raman & ASR8352 & ASR 8352 RAM rev 1 \\
\hline TI-065 & 9.24 & Caustic leach & A 01AIM 13C 152114 ARC 1 B & Archive & & \\
\hline TI-065 & 9.24 & Caustic leach & A 01AIM 13C 152114 ARC 1 D & Archive & & \\
\hline TI-065 & 9.24 & Caustic leach & A 01AIM 13C 162115 UDS 2 B & UDS & UDS R1 & UDS R1 Shake and Test A and Test B Step001 Date 08182009 \\
\hline TI-065 & 9.24 & Caustic leach & A 01AIM 13C 162115 UDS 2 D & UDS & UDS R1 & UDS R1 Shake and Test A and Test B Step001 Date 08182009 \\
\hline TI-065 & 9.24 & Caustic leach & A 01AIM 13C 162116 UDS 2 B & UDS & UDS R1 & UDS R1 Shake and Test A and Test B Step001 Date 08182009 \\
\hline TI-065 & 9.24 & Caustic leach & A 01AIM 13C 162116 UDS 2 D & UDS & UDS R1 & UDS R1 Shake and Test A and Test B Step001 Date 08182009 \\
\hline TI-065 & 9.24 & Caustic leach & A 01AIM 13C 162117 UDS 2 B & UDS & UDS R1 & UDS R1 Shake and Test A and Test B Step001 Date 08182009 \\
\hline TI-065 & 9.24 & Caustic leach & A 01AIM 13C 162117 UDS 2 D & UDS & UDS R1 & UDS R1 Shake and Test A and Test B Step001 Date 08182009 \\
\hline TI-065 & 9.24 & Caustic leach & A 01AIM 13C 162118 DEN 2 & Density Slurry & SWRI 090211-10 & 090211-10 DEN \\
\hline TI-065 & 9.24 & Caustic leach & A 01AIM 13C 162119 DEN 2 & Density Slurry & SWRI 090211-10 & 090211-10 DEN \\
\hline TI-065 & 9.24 & Caustic leach & A 01AIM 13C 162120 DEN 2 & Density Slurry & SWRI 090211-10 & 090211-10 DEN \\
\hline TI-065 & 9.24 & Caustic leach & A 01AIM 13C 162121 DEN 0 D & ICP Supernate & SWRI 090625-25 & 090625-25 and 090625-26 ICP and ICPMS and IC \\
\hline TI-065 & 9.24 & Caustic leach & A 01AIM 13C 162121 DEN 0 D & $\mathrm{IC}$ & SWRI 090625-25 & $090625-25$ and $090625-26$ ICP and ICPMS and IC \\
\hline TI-065 & 9.24 & Caustic leach & A 01AIM 13C 162121 ICP 2 B & ICP Wet Solids & SWRI 090211-11 & 090211-11 090213-10 ICP and ICPMS \\
\hline TI-065 & 9.24 & Caustic leach & A 01AIM 13C 162121 RAM 3 D & Raman & ASR8357 & ASR 8357 RAM rev 1 \\
\hline TI-065 & 9.24 & Caustic leach & A 01AIM 13C 162122 DEN 0 D & ICP Supernate & SWRI 090625-25 & 090625-25 and 090625-26 ICP and ICPMS and IC \\
\hline TI-065 & 9.24 & Caustic leach & A 01AIM 13C 162122 DEN 0 D & IC & SWRI 090625-25 & 090625-25 and 090625-26 ICP and ICPMS and IC \\
\hline TI-065 & 9.24 & Caustic leach & A 01AIM 13C 162122 ICP 2 B & ICP Wet Solids & SWRI 090211-11 & 090211-11 090213-10 ICP and ICPMS \\
\hline TI-065 & 9.24 & Caustic leach & A 01 AIM 13C 162122 RAM 3 D & Raman & ASR8357 & ASR 8357 RAM rev 1 \\
\hline TI-065 & 9.24 & Caustic leach & A 01 AIM 13C 162123 DEN 0 D & ICP Supernate & SWRI 090625-25 & 090625-25 and 090625-26 ICP and ICPMS and IC \\
\hline TI-065 & 9.24 & Caustic leach & A 01AIM 13C 162123 DEN 0 D & IC & SWRI 090625-25 & 090625-25 and 090625-26 ICP and ICPMS and IC \\
\hline TI-065 & 9.24 & Caustic leach & A 01AIM 13C 162123 ICP 2 B & ICP Wet Solids & SWRI 090211-11 & 090211-11 090213-10 ICP and ICPMS \\
\hline TI-065 & 9.24 & Caustic leach & A 01AIM 13C 162123 RAM 3 D & Raman & ASR8357 & ASR 8357 RAM rev 1 \\
\hline TI-065 & 9.24 & Caustic leach & A 01AIM 13C 162124 XSP 4 B & $\mathrm{XRD}$ & TDP-WTP-357 & TDP WTP 357 Int Test A XRD Data on PEP Slurry \\
\hline TI-065 & 9.24 & Caustic leach & A T22GM 13C 162037 OTR 6 & Vijay & & \\
\hline TI-065 & 9.24 & Caustic leach & A 01AMH 13C 162127 ARC 1 B & Archive & & \\
\hline TI-065 & 9.24 & Caustic leach & A 01AIL 13C 162126 ARC 1 B & Archive & & \\
\hline TI-065 & 9.24 & Caustic leach & A 01AIL 13C 162126 ARC 1 D & Archive & & \\
\hline TI-065 & 9.24 & Caustic leach & A $01 \mathrm{AMM} 13 \mathrm{C} 162128$ ARC $1 \mathrm{~B}$ & Archive & & \\
\hline TI-065 & 9.24 & Caustic leach & A 01AMM 13C 162128 ARC 1 D & Archive & & \\
\hline TI-065 & 9.24 & Caustic leach & A 01AML 13C 162129 ARC 1 B & Archive & & \\
\hline TI-065 & 9.24 & Caustic leach & A 01AML 13C 162129 ARC 1 D & Archive & & \\
\hline
\end{tabular}


Integrated Test A Sample List

\begin{tabular}{|c|c|c|c|c|c|c|}
\hline TI \# & TI Step \# & TI Step Description & Sample ID & Analysis & Folder & Final Electronic Report File Name \\
\hline TI-065 & 9.24 & Caustic leach & A $01 \mathrm{AOH} 13 \mathrm{C} 162130 \mathrm{ARC} 1 \mathrm{~B}$ & Archive & & \\
\hline TI-065 & 9.24 & Caustic leach & A 01AOH 13C 162130 ARC 1 D & Archive & & \\
\hline TI-065 & 9.24 & Caustic leach & A $01 \mathrm{AOM} 13 \mathrm{C} 162132 \mathrm{ARC} 1 \mathrm{~B}$ & Archive & & \\
\hline TI-065 & 9.24 & Caustic leach & A 01AOM 13C 162132 ARC 1 D & Archive & & \\
\hline TI-065 & 9.24 & Caustic leach & A 01AOL 13C 162133 ARC 1 B & Archive & & \\
\hline TI-065 & 9.24 & Caustic leach & A 01AOL 13C 162133 ARC 1 D & Archive & & \\
\hline TI-065 & 9.24 & Caustic leach & A 01AMH 13C 162127 ARC 1 D & Archive & & \\
\hline TI-065 & 9.32 & Cool UFP-T01A contents with UFP-HX-T05A & A 01 AIM 13 C XX 2134 XSP 4 B & XRD & TDP-WTP-357 & TDP WTP 357 Int Test A XRD Data on PEP Slurry \\
\hline TI-065 & 10.13 & Transfer from HLP-VSL-T22 to T01B ( 3 times - batches $2,4 \& 6$ ) & A 01BIM 13D XX 2170 UDS 2 B & UDS & UDS R1 & UDS R1 Shake and Test A and Test B Step001 Date 08182009 \\
\hline TI-065 & 10.13 & Transfer from HLP-VSL-T22 to T01B ( 3 times - batches $2,4 \& 6$ ) & A 01BIM 13D XX 2170 UDS 2 D & UDS & UDS R1 & UDS R1 Shake and Test A and Test B Step001 Date 08182009 \\
\hline TI-065 & 10.13 & Transfer from HLP-VSL-T22 to T01B ( 3 times - batches $2,4 \& 6$ ) & A 01BIM 13D XX 2171 UDS 2 B & UDS & UDS R1 & UDS R1 Shake and Test A and Test B Step001 Date 08182009 \\
\hline TI-065 & 10.13 & Transfer from HLP-VSL-T22 to T01B ( 3 times - batches $2,4 \& 6$ ) & A 01BIM 13D XX 2171 UDS 2 D & UDS & UDS R1 & UDS R1 Shake and Test A and Test B Step001 Date 08182009 \\
\hline TI-065 & 10.13 & Transfer from HLP-VSL-T22 to T01B ( 3 times - batches 2, 4 \& 6) & A 01BIM 13D XX 2172 UDS 2 B & UDS & UDS R1 & UDS R1 Shake and Test A and Test B Step001 Date 08182009 \\
\hline TI-065 & 10.13 & Transfer from HLP-VSL-T22 to T01B (3 times - batches $2,4 \& 6$ ) & A 01BIM 13D XX 2172 UDS 2 D & UDS & UDS R1 & UDS R1 Shake and Test A and Test B Step001 Date 08182009 \\
\hline TI-065 & 10.13 & Transfer from HLP-VSL-T22 to T01B ( 3 times - batches $2,4 \& 6$ ) & A 01BIM 13D XX 2173 DEN 2 & Density Slurry & SWRI 090213-8 & 090213-8 DEN slurry \\
\hline TI-065 & 10.13 & Transfer from HLP-VSL-T22 to T01B ( 3 times - batches $2,4 \& 6$ ) & A 01BIM 13D XX 2174 DEN 2 & Density Slurry & SWRI 090213-8 & 090213-8 DEN slurry \\
\hline TI-065 & 10.13 & Transfer from HLP-VSL-T22 to T01B ( 3 times - batches $2,4 \& 6$ ) & A 01BIM 13D XX 2175 DEN 2 & Density Slurry & SWRI 090213-8 & 090213-8 DEN slurry \\
\hline TI-065 & 10.13 & Transfer from HLP-VSL-T22 to T01B (3 times - batches $2,4 \& 6$ ) & A 01BIM 13D XX 2176 ICP 2 B & ICP Wet Solids & SWRI 090213-10 & 090211-11 090213-10 ICP and ICPMS \\
\hline TI-065 & 10.13 & Transfer from HLP-VSL-T22 to T01B ( 3 times - batches $2,4 \& 6$ ) & A 01BIM 13D XX 2176 ICP 2 D & IC & UDS R1 & 090216-13 and 090219-19 IC and ICP and ICPMS \\
\hline TI-065 & 10.13 & Transfer from HLP-VSL-T22 to T01B (3 times - batches $2,4 \& 6$ ) & A 01BIM 13D XX 2176 ICP 2 D & ICP Supernate & UDS R1 & 090216-13 and 090219-19 IC and ICP and ICPMS \\
\hline TI-065 & 10.13 & Transfer from HLP-VSL-T22 to UFP-VLS-T01B & A 01BIM 13D XX 2176 RAM 3 D & Raman & ASR8357 & ASR 8357 RAM rev 1 \\
\hline TI-065 & 10.13 & Transfer from HLP-VSL-T22 to UFP-VLS-T01B & A 01BIM 13D XX 2176 TFI 3 D & $\mathrm{OH}$ & ASR 8358 & ASR $8358 \mathrm{OH}$ \\
\hline TI-065 & 10.13 & Transfer from HLP-VSL-T22 to T01B ( 3 times - batches $2,4 \& 6$ ) & A 01BIM 13D XX 2177 ICP 2 B & ICP Wet Solids & SWRI 090213-10 & 090211-11 090213-10 ICP and ICPMS \\
\hline TI-065 & 10.13 & Transfer from HLP-VSL-T22 to T01B ( 3 times - batches 2, 4 \& 6) & A 01BIM 13D XX 2177 ICP 2 D & IC & UDS R1 & 090216-13 and 090219-19 IC and ICP and ICPMS \\
\hline TI-065 & 10.13 & Transfer from HLP-VSL-T22 to T01B ( 3 times - batches $2,4 \& 6$ ) & A 01BIM 13D XX 2177 ICP 2 D & ICP Supernate & UDS R1 & 090216-13 and 090219-19 IC and ICP and ICPMS \\
\hline TI-065 & 10.13 & Transfer from HLP-VSL-T22 to UFP-VLS-T01B & A 01BIM 13D XX 2177 RAM 3 D & Raman & ASR 8357 & ASR 8357 RAM rev 1 \\
\hline TI-065 & 10.13 & Transfer from HLP-VSL-T22 to UFP-VLS-T01B & A 01BIM 13D XX 2177 TFI 3 D & $\mathrm{OH}$ & ASR 8358 & ASR $8358 \mathrm{OH}$ \\
\hline TI-065 & 10.13 & Transfer from HLP-VSL-T22 to T01B ( 3 times - batches $2,4 \& 6$ ) & A 01BIM 13D XX 2178 ICP 2 B & ICP Wet Solids & SWRI 090213-10 & 090211-11 090213-10 ICP and ICPMS \\
\hline TI-065 & 10.13 & Transfer from HLP-VSL-T22 to T01B ( 3 times - batches $2,4 \& 6$ ) & A 01BIM 13D XX 2178 ICP 2 D & IC & UDS R1 & 090216-13 and 090219-19 IC and ICP and ICPMS \\
\hline TI-065 & 10.13 & Transfer from HLP-VSL-T22 to T01B ( 3 times - batches $2,4 \& 6$ ) & A 01BIM 13D XX 2178 ICP 2 D & ICP Supernate & UDS R1 & 090216-13 and 090219-19 IC and ICP and ICPMS \\
\hline TI-065 & 10.13 & Transfer from HLP-VSL-T22 to UFP-VLS-T01B & A 01BIM 13D XX 2178 RAM 3 D & Raman & ASR8357 & ASR 8357 RAM rev 1 \\
\hline TI-065 & 10.13 & Transfer from HLP-VSL-T22 to UFP-VLS-T01B & A 01BIM 13D XX 2178 TFI 3 D & $\mathrm{OH}$ & ASR 8358 & ASR $8358 \mathrm{OH}$ \\
\hline TI-065 & 10.13 & Transfer from HLP-VSL-T22 to UFP-VLS-T01B & A T221B 13D XX 2166 ARC 1 & Archive & & \\
\hline
\end{tabular}


Integrated Test A Sample List

\begin{tabular}{|c|c|c|c|c|c|c|}
\hline TI \# & TI Step \# & TI Step Description & Sample ID & Analysis & Folder & Final Electronic Report File Name \\
\hline TI-065 & 10.13 & Transfer from HLP-VSL-T22 to UFP-VLS-T01B & A T221B 13D XX 2167 ARC 1 & Archive & & \\
\hline TI-065 & 10.13 & Transfer from HLP-VSL-T22 to UFP-VLS-T01B & A T221B 13D XX 2168 ARC 1 & Archive & & \\
\hline TI-065 & 10.13 & Transfer from HLP-VSL-T22 to UFP-VLS-T01B & A T221B 13D XX 2169 ARC 1 & Archive & & \\
\hline TI-065 & 10.27 & Heat UFP-VSL-T01A to $88^{\circ} \mathrm{C}$ & A 01BIM 13D BT 2179 UDS 2 B & UDS & UDS R1 & UDS R1 Shake and Test A and Test B Step001 Date 08182009 \\
\hline TI-065 & 10.27 & Heat UFP-VSL-T01A to $88^{\circ} \mathrm{C}$ & A 01BIM 13D BT 2179 UDS 2 D & UDS & UDS R1 & UDS R1 Shake and Test A and Test B Step001 Date 08182009 \\
\hline TI-065 & 10.27 & Heat UFP-VSL-T01A to $88^{\circ} \mathrm{C}$ & A 01BIM 13D BT 2180 DEN 2 & Density Slurry & SWRI 090213-8 & 090213-8 DEN slurry \\
\hline TI-065 & 10.27 & Heat UFP-VSL-T01A to $88^{\circ} \mathrm{C}$ & A 01BIM 13D BT 2181 TFI 3 D & $\mathrm{OH}$ & ASR8358 & ASR $8358 \mathrm{OH}$ \\
\hline TI-065 & 10.27 & Heat UFP-VSL-T01A to $88^{\circ} \mathrm{C}$ & A 01BIL 13D BT 2182 ARC 1 B & Archive & & \\
\hline TI-065 & 10.27 & Heat UFP-VSL-T01A to $88^{\circ} \mathrm{C}$ & A 01BIL 13D BT 2182 ARC 1 D & Archive & & \\
\hline TI-065 & 10.27 & Heat UFP-VSL-T01A to $88^{\circ} \mathrm{C}$ & A 01BML 13D BT 2183 ARC 1 B & Archive & & \\
\hline TI-065 & 10.27 & Heat UFP-VSL-T01A to $88^{\circ} \mathrm{C}$ & A 01BML 13D BT 2183 ARC 1 D & Archive & & \\
\hline TI-065 & 10.27 & Heat UFP-VSL-T01A to $88^{\circ} \mathrm{C}$ & A 01BOL 13D BT 2184 ARC 1 B & Archive & & \\
\hline TI-065 & 10.27 & Heat UFP-VSL-T01A to $88^{\circ} \mathrm{C}$ & A 01BOL 13D BT 2184 ARC 1 D & Archive & & \\
\hline TI-065 & 10.27 & Heat UFP-VSL-T01A to $88^{\circ} \mathrm{C}$ & A 01BMM 13D BT 2185 ARC 1 B & Archive & & \\
\hline TI-065 & 10.27 & Heat UFP-VSL-T01A to $88^{\circ} \mathrm{C}$ & A 01BMM 13D BT 2185 ARC 1 D & Archive & & \\
\hline TI-065 & 10.27 & Heat UFP-VSL-T01A to $88^{\circ} \mathrm{C}$ & A 01BOM 13D BT 2186 ARC 1 B & Archive & & \\
\hline TI-065 & 10.27 & Heat UFP-VSL-T01A to $88^{\circ} \mathrm{C}$ & A 01BOM 13D BT 2186 ARC 1 D & Archive & & \\
\hline TI-065 & 10.27 & Heat UFP-VSL-T01A to $88^{\circ} \mathrm{C}$ & A 01BMH 13D BT 2188 ARC 1 B & Archive & & \\
\hline TI-065 & 10.27 & Heat UFP-VSL-T01A to $88^{\circ} \mathrm{C}$ & A 01BMH 13D BT 2188 ARC 1 D & Archive & & \\
\hline TI-065 & 10.27 & Heat UFP-VSL-T01A to $88^{\circ} \mathrm{C}$ & A 01BOH 13D BT 2189 ARC 1 B & Archive & & \\
\hline TI-065 & 10.27 & Heat UFP-VSL-T01A to $88^{\circ} \mathrm{C}$ & A 01BOH 13D BT 2189 ARC 1 D & Archive & & \\
\hline TI-065 & 10.29 & Caustic leach & A 01BIM 13D 002190 UDS 2 B & UDS & UDS R1 & UDS R1 Shake and Test A and Test B Step001 Date 08182009 \\
\hline TI-065 & 10.29 & Caustic leach & A 01BIM 13D 002190 UDS 2 D & UDS & UDS R1 & UDS R1 Shake and Test A and Test B Step001 Date 08182009 \\
\hline TI-065 & 10.29 & Caustic leach & A 01BIM 13D 002191 DEN 2 & Density Slurry & SWRI 090213-8 & 090213-8 DEN slurry \\
\hline TI-065 & 10.29 & Caustic leach & A 01BIM 13D 002192 DEN 0 D & ICP Supernate & SWRI 090625-26 & 090625-25 and 090625-26 ICP and ICPMS and IC \\
\hline TI-065 & 10.29 & Caustic leach & A 01BIM 13D 002192 DEN 0 D & IC & SWRI 090625-26 & 090625-25 and 090625-26 ICP and ICPMS and IC \\
\hline TI-065 & 10.29 & Caustic leach & A 01BIM 13D 002192 RAM 3 D & Raman & ASR 8362 & ASR 8362 RAM rev 1 \\
\hline TI-065 & 10.29 & Caustic leach & A 01BIM 13D 002192 RAM 3 D & $\mathrm{OH}$ & ASR 8362 & ASR $8362 \_1$ OH \\
\hline TI-065 & 10.29 & Caustic leach & A 01BIM 13D 002192 DEN 0 B & ICP Wet Solids & SWRI 090625-24 & 090625-24 ICP and ICPMS \\
\hline TI-065 & 10.29 & Caustic leach & A 01BIL 13D 002193 ARC 1 B & Archive & & \\
\hline TI-065 & 10.29 & Caustic leach & A 01BIL 13D 002193 ARC 1 D & Archive & & \\
\hline TI-065 & 10.29 & Caustic leach & A 01BML 13D 002194 ARC 1 B & Archive & & \\
\hline TI-065 & 10.29 & Caustic leach & A 01BML 13D 002194 ARC 1 D & Archive & & \\
\hline
\end{tabular}


Integrated Test A Sample List

\begin{tabular}{|c|c|c|c|c|c|c|}
\hline TI \# & TI Step \# & TI Step Description & Sample ID & Analysis & Folder & Final Electronic Report File Name \\
\hline TI-065 & 10.29 & Caustic leach & A 01BOL 13D 002195 ARC 1 B & Archive & & \\
\hline TI-065 & 10.29 & Caustic leach & A 01BOL 13D 002195 ARC 1 D & Archive & & \\
\hline TI-065 & 10.29 & Caustic leach & A 01BMM 13D 002196 ARC 1 B & Archive & & \\
\hline TI-065 & 10.29 & Caustic leach & A 01BMM 13D 002196 ARC 1 D & Archive & & \\
\hline TI-065 & 10.29 & Caustic leach & A 01BOM 13D 002197 ARC 1 B & Archive & & \\
\hline TI-065 & 10.29 & Caustic leach & A 01BOM 13D 002197 ARC 1 D & Archive & & \\
\hline TI-065 & 10.29 & Caustic leach & A 01BMH 13D 002199 ARC 1 B & Archive & & \\
\hline TI-065 & 10.29 & Caustic leach & A 01BMH 13D 002199 ARC 1 D & Archive & & \\
\hline TI-065 & 10.29 & Caustic leach & A 01BOH 13D 002200 ARC 1 B & Archive & & \\
\hline TI-065 & 10.29 & Caustic leach & A 01BOH 13D 002200 ARC 1 D & Archive & & \\
\hline TI-065 & 10.29 & Caustic leach & A 01BIM 13D 012201 UDS 2 B & UDS & UDS R1 & UDS R1 Shake and Test A and Test B Step001 Date 08182009 \\
\hline TI-065 & 10.29 & Caustic leach & A 01BIM 13D 012201 UDS 2 D & UDS & UDS R1 & UDS R1 Shake and Test A and Test B Step001 Date 08182009 \\
\hline TI-065 & 10.29 & Caustic leach & A 01BIM 13D 012202 DEN 2 & Density Slurry & SWRI 090213-8 & 090213-8 DEN slurry \\
\hline TI-065 & 10.29 & Caustic leach & A 01BIM 13D 012203 RAM 3 D & IC & SWRI 090213-11 & 090213-11 090225-17 090225-22 IC and ICP and ICPMS \\
\hline TI-065 & 10.29 & Caustic leach & A 01BIM 13D 012203 TFI 3 D & $\mathrm{OH}$ & ASR 8358 & ASR $8358 \mathrm{OH}$ \\
\hline TI-065 & 10.29 & Caustic leach & A 01BIM 13D 022204 UDS 2 B & UDS & UDS R1 & UDS R1 Shake and Test A and Test B Step001 Date 08182009 \\
\hline TI-065 & 10.29 & Caustic leach & A 01BIM 13D 022204 UDS 2 D & UDS & UDS R1 & UDS R1 Shake and Test A and Test B Step001 Date 08182009 \\
\hline TI-065 & 10.29 & Caustic leach & A 01BIM 13D 022205 DEN 2 & Density Slurry & SWRI 090213-8 & 090213-8 DEN slurry \\
\hline TI-065 & 10.29 & Caustic leach & A 01BIM 13D 022206 RAM 3 D & IC & SWRI 090213-11 & 090213-11 090225-17 090225-22 IC and ICP and ICPMS \\
\hline TI-065 & 10.29 & Caustic leach & A 01BIM 13D 022206 TFI 3 D & $\mathrm{OH}$ & ASR8358 & ASR $8358 \mathrm{OH}$ \\
\hline TI-065 & 10.29 & Caustic leach & A 01BIM 13D 032207 ARC 1 B & Archive & & \\
\hline TI-065 & 10.29 & Caustic leach & A 01BIM 13D 032207 ARC 1 D & Archive & & \\
\hline TI-065 & 10.29 & Caustic leach & A 01BIM 13D 042208 UDS 2 B & UDS & UDS R1 & UDS R1 Shake and Test A and Test B Step001 Date 08182009 \\
\hline TI-065 & 10.29 & Caustic leach & A 01BIM 13D 042208 UDS 2 D & UDS & UDS R1 & UDS R1 Shake and Test A and Test B Step001 Date 08182009 \\
\hline TI-065 & 10.29 & Caustic leach & A 01BIM 13D 042209 DEN 2 & Density Slurry & SWRI 090213-8 & 090213-8 DEN slurry \\
\hline TI-065 & 10.29 & Caustic leach & A 01BIM 13D 042210 ICP 2 B & ICP Wet Solids & SWRI 090213-10 & 090211-11 090213-10 ICP and ICPMS \\
\hline TI-065 & 10.29 & Caustic leach & A 01BIM 13D 042210 RAM 3 D & IC & SWRI 090213-11 & 090213-11 090225-17 090225-22 IC and ICP and ICPMS \\
\hline TI-065 & 10.29 & Caustic leach & A 01BIM 13D 042210 TFI 3 D & $\mathrm{OH}$ & ASR8358 & ASR $8358 \mathrm{OH}$ \\
\hline TI-065 & 10.29 & Caustic leach & A 01BIL 13D 042211 ARC 1 B & Archive & & \\
\hline TI-065 & 10.29 & Caustic leach & A 01BIL 13D 042211 ARC 1 D & Archive & & \\
\hline TI-065 & 10.29 & Caustic leach & A 01BML 13D 042212 ARC 1 B & Archive & & \\
\hline TI-065 & 10.29 & Caustic leach & A 01BML 13D 042212 ARC 1 D & Archive & & \\
\hline
\end{tabular}


Integrated Test A Sample List

\begin{tabular}{|c|c|c|c|c|c|c|}
\hline TI \# & TI Step \# & TI Step Description & Sample ID & Analysis & Folder & Final Electronic Report File Name \\
\hline TI-065 & 10.29 & Caustic leach & A 01BOL 13D 042213 ARC 1 B & Archive & & \\
\hline TI-065 & 10.29 & Caustic leach & A 01BOL 13D 042213 ARC 1 D & Archive & & \\
\hline TI-065 & 10.29 & Caustic leach & A 01BMM 13D 042214 ARC 1 B & Archive & & \\
\hline TI-065 & 10.29 & Caustic leach & A 01BMM 13D 042214 ARC 1 D & Archive & & \\
\hline TI-065 & 10.29 & Caustic leach & A 01BOM 13D 042215 ARC 1 B & Archive & & \\
\hline TI-065 & 10.29 & Caustic leach & A 01BOM 13D 042215 ARC 1 D & Archive & & \\
\hline TI-065 & 10.29 & Caustic leach & A 01BMH 13D 042217 ARC 1 B & Archive & & \\
\hline TI-065 & 10.29 & Caustic leach & A 01BMH 13D 042217 ARC 1 D & Archive & & \\
\hline TI-065 & 10.29 & Caustic leach & A 01BOH 13D 042218 ARC 1 B & Archive & & \\
\hline TI-065 & 10.29 & Caustic leach & A 01BOH 13D 042218 ARC 1 D & Archive & & \\
\hline TI-065 & 10.29 & Caustic leach & A 01BIM 13D 052219 ARC 1 B & Archive & & \\
\hline TI-065 & 10.29 & Caustic leach & A 01BIM 13D 052219 ARC 1 D & Archive & & \\
\hline TI-065 & 10.29 & Caustic leach & A 01BIM 13D 062220 ARC 1 B & Archive & & \\
\hline TI-065 & 10.29 & Caustic leach & A 01BIM 13D 062220 ARC 1 D & Archive & & \\
\hline TI-065 & 10.29 & Caustic leach & A 01BIM 13D 072221 ARC 1 B & Archive & & \\
\hline TI-065 & 10.29 & Caustic leach & A 01BIM 13D 072221 ARC 1 D & Archive & & \\
\hline TI-065 & 10.29 & Caustic leach & A 01BIM 13D 082222 UDS 2 B & UDS & UDS R1 & UDS R1 Shake and Test A and Test B Step001 Date 08182009 \\
\hline TI-065 & 10.29 & Caustic leach & A 01BIM 13D 082222 UDS 2 D & UDS & UDS R1 & UDS R1 Shake and Test A and Test B Step001 Date 08182009 \\
\hline TI-065 & 10.29 & Caustic leach & A 01BIM 13D 082223 DEN 2 & Density Slurry & SWRI 090213-8 & 090213-8 DEN slurry \\
\hline TI-065 & 10.29 & Caustic leach & A 01BIM 13D 082224 ICP 2 B & ICP Wet Solids & SWRI 090213-10 & 090211-11 090213-10 ICP and ICPMS \\
\hline TI-065 & 10.29 & Caustic leach & A 01BIM 13D 082224 RAM 3 D & IC & SWRI 090213-11 & 090213-11 090225-17 090225-22 IC and ICP and ICPMS \\
\hline TI-065 & 10.29 & Caustic leach & A 01BIM 13D 082224 TFI 3 D & $\mathrm{OH}$ & ASR8358 & ASR $8358 \mathrm{OH}$ \\
\hline TI-065 & 10.29 & Caustic leach & A 01BIL 13D 082225 ARC 1 B & Archive & & \\
\hline TI-065 & 10.29 & Caustic leach & A 01BIL 13D 082225 ARC 1 D & Archive & & \\
\hline TI-065 & 10.29 & Caustic leach & A 01BML 13D 082226 ARC 1 B & Archive & & \\
\hline TI-065 & 10.29 & Caustic leach & A 01BML 13D 082226 ARC 1 D & Archive & & \\
\hline TI-065 & 10.29 & Caustic leach & A 01BOL 13D 082227 ARC 1 B & Archive & & \\
\hline TI-065 & 10.29 & Caustic leach & A 01BOL 13D 082227 ARC 1 D & Archive & & \\
\hline TI-065 & 10.29 & Caustic leach & A 01BMM 13D 082228 ARC 1 B & Archive & & \\
\hline TI-065 & 10.29 & Caustic leach & A 01BMM 13D 082228 ARC 1 D & Archive & & \\
\hline TI-065 & 10.29 & Caustic leach & A 01BOM 13D 082229 ARC 1 B & Archive & & \\
\hline TI-065 & 10.29 & Caustic leach & A 01BOM 13D 082229 ARC 1 D & Archive & & \\
\hline TI-065 & 10.29 & Caustic leach & A 01BMH 13D 082231 ARC 1 B & Archive & & \\
\hline
\end{tabular}


Integrated Test A Sample List

\begin{tabular}{|c|c|c|c|c|c|c|}
\hline TI \# & TI Step \# & TI Step Description & Sample ID & Analysis & Folder & Final Electronic Report File Name \\
\hline TI-065 & 10.29 & Caustic leach & A 01BMH 13D 082231 ARC 1 D & Archive & & \\
\hline TI-065 & 10.29 & Caustic leach & A 01BOH 13D 082232 ARC 1 B & Archive & & \\
\hline TI-065 & 10.29 & Caustic leach & A 01BOH 13D 082232 ARC 1 D & Archive & & \\
\hline TI-065 & 10.29 & Caustic leach & A 01BIM 13D 092233 ARC 1 B & Archive & & \\
\hline TI-065 & 10.29 & Caustic leach & A 01BIM 13D 092233 ARC 1 D & Archive & & \\
\hline TI-065 & 10.29 & Caustic leach & A 01BIM 13D 102234 UDS 2 B & UDS & UDS R1 & UDS R1 Shake and Test A and Test B Step001 Date 08182009 \\
\hline TI-065 & 10.29 & Caustic leach & A 01BIM 13D 102234 UDS 2 D & UDS & UDS R1 & UDS R1 Shake and Test A and Test B Step001 Date 08182009 \\
\hline TI-065 & 10.29 & Caustic leach & A 01BIM 13D 102235 DEN 2 & Density Slurry & SWRI 090213-8 & 090213-8 DEN slurry \\
\hline TI-065 & 10.29 & Caustic leach & A 01BIM 13D 102236 RAM 3 D & IC & SWRI 090213-11 & 090213-11 090225-17 090225-22 IC and ICP and ICPMS \\
\hline TI-065 & 10.29 & Caustic leach & A 01BIM 13D 102236 TFI 3 D & $\mathrm{OH}$ & ASR8358 & ASR $8358 \mathrm{OH}$ \\
\hline TI-065 & 10.29 & Caustic leach & A 01BIM 13D 112237 ARC 1 B & Archive & & \\
\hline TI-065 & 10.29 & Caustic leach & A 01BIM 13D 112237 ARC 1 D & Archive & & \\
\hline TI-065 & 10.29 & Caustic leach & A 01BIM 13D 122238 UDS 2 B & UDS & UDS R1 & UDS R1 Shake and Test A and Test B Step001 Date 08182009 \\
\hline TI-065 & 10.29 & Caustic leach & A 01BIM 13D 122238 UDS 2 D & UDS & UDS R1 & UDS R1 Shake and Test A and Test B Step001 Date 08182009 \\
\hline TI-065 & 10.29 & Caustic leach & A 01BIM 13D 122239 DEN 2 & Density Slurry & SWRI 090213-8 & 090213-8 DEN slurry \\
\hline TI-065 & 10.29 & Caustic leach & A 01BIM 13D 122240 ICP 2 B & ICP Wet Solids & SWRI 090213-10 & 090211-11 090213-10 ICP and ICPMS \\
\hline TI-065 & 10.29 & Caustic leach & A 01BIM 13D 122240 RAM 3 D & IC & SWRI 090213-11 & 090213-11 090225-17 090225-22 IC and ICP and ICPMS \\
\hline TI-065 & 10.29 & Caustic leach & A 01BIM 13D 122240 TFI 3 D & $\mathrm{OH}$ & ASR 8358 & ASR $8358 \mathrm{OH}$ \\
\hline TI-065 & 10.29 & Caustic leach & A 01BIL 13D 122241 ARC 1 B & Archive & & \\
\hline TI-065 & 10.29 & Caustic leach & A 01BIL 13D 122241 ARC 1 D & Archive & & \\
\hline TI-065 & 10.29 & Caustic leach & A 01BML 13D 122242 ARC 1 B & Archive & & \\
\hline TI-065 & 10.29 & Caustic leach & A 01BML 13D 122242 ARC 1 D & Archive & & \\
\hline TI-065 & 10.29 & Caustic leach & A 01BOL 13D 122243 ARC 1 B & Archive & & \\
\hline TI-065 & 10.29 & Caustic leach & A 01BOL 13D 122243 ARC 1 D & Archive & & \\
\hline TI-065 & 10.29 & Caustic leach & A 01BMM 13D 122244 ARC 1 B & Archive & & \\
\hline TI-065 & 10.29 & Caustic leach & A 01BMM 13D 122244 ARC 1 D & Archive & & \\
\hline TI-065 & 10.29 & Caustic leach & A 01BOM 13D 122245 ARC 1 B & Archive & & \\
\hline TI-065 & 10.29 & Caustic leach & A 01BOM 13D 122245 ARC 1 D & Archive & & \\
\hline TI-065 & 10.29 & Caustic leach & A 01BMH 13D 122247 ARC 1 B & Archive & & \\
\hline TI-065 & 10.29 & Caustic leach & A 01BMH 13D 122247 ARC 1 D & Archive & & \\
\hline TI-065 & 10.29 & Caustic leach & A 01BOH 13D 122248 ARC 1 B & Archive & & \\
\hline TI-065 & 10.29 & Caustic leach & A 01BOH 13D 122248 ARC 1 D & Archive & & \\
\hline TI-065 & 10.29 & Caustic leach & A 01BIM 13D 132249 ARC 1 B & Archive & & \\
\hline
\end{tabular}


Integrated Test A Sample List

\begin{tabular}{|c|c|c|c|c|c|c|}
\hline TI\# & TI Step \# & TI Step Description & Sample ID & Analysis & Folder & Final Electronic Report File Name \\
\hline TI-065 & 10.29 & Caustic leach & A 01BIM 13D 132249 ARC 1 D & Archive & & \\
\hline TI-065 & 10.29 & Caustic leach & A 01BIM 13D 142250 UDS 2 B & UDS & UDS R1 & UDS R1 Shake and Test A and Test B Step001 Date 08182009 \\
\hline TI-065 & 10.29 & Caustic leach & A 01BIM 13D 142250 UDS 2 D & UDS & UDS R1 & UDS R1 Shake and Test A and Test B Step001 Date 08182009 \\
\hline TI-065 & 10.29 & Caustic leach & A 01BIM 13D 142251 DEN 2 & Density Slurry & SWRI 090213-8 & 090213-8 DEN slurry \\
\hline TI-065 & 10.29 & Caustic leach & A 01BIM 13D 142252 RAM 3 D & IC & SWRI 090213-11 & 090213-11 090225-17 090225-22 IC and ICP and ICPMS \\
\hline TI-065 & 10.29 & Caustic leach & A 01BIM 13D 142252 TFI 3 D & $\mathrm{OH}$ & ASR 8358 & ASR $8358 \mathrm{OH}$ \\
\hline TI-065 & 10.29 & Caustic leach & A 01BIM 13D 152253 ARC 1 B & Archive & & \\
\hline TI-065 & 10.29 & Caustic leach & A 01BIM 13D 152253 ARC 1 D & Archive & & \\
\hline TI-065 & 10.29 & Caustic leach & A 01BIM 13D 162256 RAM 3 D & Raman & ASR 8362 & ASR 8362 RAM rev 1 \\
\hline TI-065 & 10.29 & Caustic leach & A 01BIM 13D 162256 RAM 3 D & $\mathrm{OH}$ & ASR 8362 & ASR $8362 \_1 \mathrm{OH}$ \\
\hline TI-065 & 10.29 & Caustic leach & A 01BIM 13D 162259 RAM 3 D & Raman & ASR 8362 & ASR 8362 RAM rev 1 \\
\hline TI-065 & 10.29 & Caustic leach & A 01BIM 13D 162259 RAM 3 D & $\mathrm{OH}$ & ASR 8362 & ASR 8362_1 OH \\
\hline TI-065 & 10.29 & Caustic leach & A 01BIM 13D 162262 RAM 3 D & Raman & ASR 8362 & ASR 8362 RAM rev 1 \\
\hline TI-065 & 10.29 & Caustic leach & A 01BIM 13D 162262 RAM 3 D & $\mathrm{OH}$ & ASR 8362 & ASR $8362 \_1 \mathrm{OH}$ \\
\hline TI-065 & 10.29 & Caustic leach & A 01BIM 13D 162254 UDS 2 B & UDS & UDS R1 & UDS R1 Shake and Test A and Test B Step001 Date 08182009 \\
\hline TI-065 & 10.29 & Caustic leach & A 01BIM 13D 162254 UDS 2 D & UDS & UDS R1 & UDS R1 Shake and Test A and Test B Step001 Date 08182009 \\
\hline TI-065 & 10.29 & Caustic leach & A 01BIM 13D 162255 DEN 2 & Density Slurry & SWRI 090213-8 & 090213-8 DEN slurry \\
\hline TI-065 & 10.29 & Caustic leach & A 01BIM 13D 162256 DEN 0 D & ICP Supernate & SWRI 090625-26 & 090625-25 and 090625-26 ICP and ICPMS and IC \\
\hline TI-065 & 10.29 & Caustic leach & A 01BIM 13D 162256 DEN 0 D & IC & SWRI 090625-26 & 090625-25 and 090625-26 ICP and ICPMS and IC \\
\hline TI-065 & 10.29 & Caustic leach & A 01BIM 13D 162256 ICP 2 B & ICP Wet Solids & SWRI 090213-10 & 090211-11 090213-10 ICP and ICPMS \\
\hline TI-065 & 10.29 & Caustic leach & A 01BIM 13D 162257 UDS 2 B & UDS & UDS R1 & UDS R1 Shake and Test A and Test B Step001 Date 08182009 \\
\hline TI-065 & 10.29 & Caustic leach & A 01BIM 13D 162257 UDS 2 D & UDS & UDS R1 & UDS R1 Shake and Test A and Test B Step001 Date 08182009 \\
\hline TI-065 & 10.29 & Caustic leach & A 01BIM 13D 162258 DEN 2 & Density Slurry & SWRI 090213-8 & 090213-8 DEN slurry \\
\hline TI-065 & 10.29 & Caustic leach & A 01BIM 13D 162259 DEN 0 D & ICP Supernate & SWRI 090625-26 & 090625-25 and 090625-26 ICP and ICPMS and IC \\
\hline TI-065 & 10.29 & Caustic leach & A 01BIM 13D 162259 DEN 0 D & IC & SWRI 090625-26 & 090625-25 and 090625-26 ICP and ICPMS and IC \\
\hline TI-065 & 10.29 & Caustic leach & A 01BIM 13D 162259 ICP 2 B & ICP Wet Solids & SWRI 090213-10 & 090211-11 090213-10 ICP and ICPMS \\
\hline TI-065 & 10.29 & Caustic leach & A 01BIM 13D 162260 UDS 2 B & UDS & UDS R1 & UDS R1 Shake and Test A and Test B Step001 Date 08182009 \\
\hline TI-065 & 10.29 & Caustic leach & A 01BIM 13D 162260 UDS 2 D & UDS & UDS R1 & UDS R1 Shake and Test A and Test B Step001 Date 08182009 \\
\hline TI-065 & 10.29 & Caustic leach & A 01BIM 13D 162261 DEN 2 & Density Slurry & SWRI 090213-8 & 090213-8 DEN slurry \\
\hline TI-065 & 10.29 & Caustic leach & A 01BIM 13D 162262 DEN 0 D & ICP Supernate & SWRI 090625-26 & 090625-25 and 090625-26 ICP and ICPMS and IC \\
\hline TI-065 & 10.29 & Caustic leach & A 01BIM 13D 162262 DEN 0 D & IC & SWRI 090625-26 & 090625-25 and 090625-26 ICP and ICPMS and IC \\
\hline TI-065 & 10.29 & Caustic leach & A 01BIM 13D 162262 ICP 2 B & ICP Wet Solids & SWRI 090213-10 & 090211-11 090213-10 ICP and ICPMS \\
\hline TI-065 & 10.29 & Caustic leach & A 01BIL 13D 162263 ARC 1 B & Archive & & \\
\hline
\end{tabular}


Integrated Test A Sample List

\begin{tabular}{|c|c|c|c|c|c|c|}
\hline TI\# & TI Step \# & TI Step Description & Sample ID & Analysis & Folder & Final Electronic Report File Name \\
\hline TI-065 & 10.29 & Caustic leach & A 01BIL 13D 162263 ARC 1 D & Archive & & \\
\hline TI-065 & 10.29 & Caustic leach & A 01BML 13D 162264 ARC 1 B & Archive & & \\
\hline TI-065 & 10.29 & Caustic leach & A 01BML 13D 162264 ARC 1 D & Archive & & \\
\hline TI-065 & 10.29 & Caustic leach & A 01BOL 13D 162265 ARC 1 B & Archive & & \\
\hline TI-065 & 10.29 & Caustic leach & A 01BOL 13D 162265 ARC 1 D & Archive & & \\
\hline TI-065 & 10.29 & Caustic leach & A 01BOM 13D 162267 ARC 1 B & Archive & & \\
\hline TI-065 & 10.29 & Caustic leach & A 01BOM 13D 162267 ARC 1 D & Archive & & \\
\hline TI-065 & 10.29 & Caustic leach & A 01BMH 13D 162269 ARC 1 B & Archive & & \\
\hline TI-065 & 10.29 & Caustic leach & A 01BMH 13D 162269 ARC 1 D & Archive & & \\
\hline TI-065 & 10.29 & Caustic leach & A 01BOH 13D 162270 ARC 1 B & Archive & & \\
\hline TI-065 & 10.29 & Caustic leach & A 01BOH 13D 162270 ARC 1 D & Archive & & \\
\hline TI-065 & 10.29 & Caustic leach & A 01BMM 13D 162266 ARC 1 B & Archive & & \\
\hline TI-065 & 10.29 & Caustic leach & A 01BMM 13D 162266 ARC 1 D & Archive & & \\
\hline TI-065 & 11.6 & no description listed & A 00PF1 007 XX 1661 ARC 4 & Precipitation Study & & \\
\hline TI-065 & 11.6 & no description listed & A 00PF1 007 XX 1662 ARC 4 & Precipitation Study & & \\
\hline TI-065 & 11.6 & Post-caustic-leach concentration & A 00PF1 007 XX 2758 CUF 4 & Precipitation Study & & \\
\hline TI-065 & 11.6 & Post-caustic-leach concentration & A 00PF1 007 XX 2759 CUF 4 & Precipitation Study & & \\
\hline TI-065 & 11.6 & $\begin{array}{l}\text { Post-caustic-leach concentrate solids in UFP-VSL-T02A from } \\
\text { UFP-VSL-T01A/B (dewatering) }\end{array}$ & A 02AML 007 XX 1632 UDS 2 & Not Analyzed & & \\
\hline TI-065 & 11.6 & $\begin{array}{l}\text { Post-caustic-leach concentrate solids in UFP-VSL-T02A from } \\
\text { UFP-VSL-T01A/B (dewatering) }\end{array}$ & A 02 AML 007 XX 1633 UDS 2 & Not Analyzed & & \\
\hline TI-065 & 11.6 & $\begin{array}{l}\text { Post-caustic-leach concentrate solids in UFP-VSL-T02A from } \\
\text { UFP-VSL-T01A/B (dewatering) }\end{array}$ & A 02AML 007 XX 1634 UDS 2 & UDS & UDS R1 & UDS R1 Shake and Test A and Test B Step001 Date 08182009 \\
\hline TI-065 & 11.6 & $\begin{array}{l}\text { Post-caustic-leach concentrate solids in UFP-VSL-T02A from } \\
\text { UFP-VSL-T01A/B (dewatering) }\end{array}$ & A 02AML 007 XX 1635 UDS 2 & Not Analyzed & & \\
\hline TI-065 & 11.6 & $\begin{array}{l}\text { Post-caustic-leach concentrate solids in UFP-VSL-T02A from } \\
\text { UFP-VSL-T01A/B (dewatering) }\end{array}$ & A 02AML 007 XX 1636 UDS 2 & Not Analyzed & & \\
\hline TI-065 & 11.6 & $\begin{array}{l}\text { Post-caustic-leach concentrate solids in UFP-VSL-T02A from } \\
\text { UFP-VSL-T01A/B (dewatering) }\end{array}$ & A 02AML 007 XX 1637 UDS 2 & Not Analyzed & & \\
\hline TI-065 & 11.6 & $\begin{array}{l}\text { Post-caustic-leach concentrate solids in UFP-VSL-T02A from } \\
\text { UFP-VSL-T01A/B (dewatering) }\end{array}$ & A 02 AML 007 XX 1638 UDS 2 & Not Analyzed & & \\
\hline TI-065 & 11.6 & $\begin{array}{l}\text { Post-caustic-leach concentrate solids in UFP-VSL-T02A from } \\
\text { UFP-VSL-T01A/B (dewatering) }\end{array}$ & A 02 AML 007 XX 1639 UDS 2 & Not Analyzed & & \\
\hline TI-065 & 11.6 & $\begin{array}{l}\text { Post-caustic-leach concentrate solids in UFP-VSL-T02A from } \\
\text { UFP-VSL-T01A/B (dewatering) }\end{array}$ & A 02AML 007 XX 1640 UDS 2 & Not Analyzed & & \\
\hline
\end{tabular}


Integrated Test A Sample List

\begin{tabular}{|c|c|c|c|c|c|c|}
\hline TI \# & TI Step \# & TI Step Description & Sample ID & Analysis & Folder & Final Electronic Report File Name \\
\hline TI-065 & 11.6 & $\begin{array}{l}\text { Post-caustic-leach concentrate solids in UFP-VSL-T02A from } \\
\text { UFP-VSL-T01A/B (dewatering) }\end{array}$ & A 02AML 007 XX 1641 UDS 2 & UDS & UDS R1 & UDS R1 Shake and Test A and Test B Step001 Date 08182009 \\
\hline TI-065 & 11.6 & $\begin{array}{l}\text { Post-caustic-leach concentrate solids in UFP-VSL-T02A from } \\
\text { UFP-VSL-T01A/B (dewatering) }\end{array}$ & A 02AML 007 XX 1642 UDS 2 & Not Analyzed & & \\
\hline TI-065 & 11.6 & $\begin{array}{l}\text { Post-caustic-leach concentrate solids in UFP-VSL-T02A from } \\
\text { UFP-VSL-T01A/B (dewatering) }\end{array}$ & A 02AML 007 XX 1643 UDS 2 & Not Analyzed & & \\
\hline TI-065 & 11.6 & $\begin{array}{l}\text { Post-caustic-leach concentrate solids in UFP-VSL-T02A from } \\
\text { UFP-VSL-T01A/B (dewatering) }\end{array}$ & A 02AML 007 XX 1644 UDS 2 & Not Analyzed & & \\
\hline TI-065 & 11.6 & $\begin{array}{l}\text { Post-caustic-leach concentrate solids in UFP-VSL-T02A from } \\
\text { UFP-VSL-T01A/B (dewatering) }\end{array}$ & A 02AML 007 XX 1645 UDS 2 & Not Analyzed & & \\
\hline TI-065 & 11.6 & $\begin{array}{l}\text { Post-caustic-leach concentrate solids in UFP-VSL-T02A from } \\
\text { UFP-VSL-T01A/B (dewatering) }\end{array}$ & A 02AML 007 XX 1646 UDS 2 & Not Analyzed & & \\
\hline TI-065 & 11.6 & $\begin{array}{l}\text { Post-caustic-leach concentrate solids in UFP-VSL-T02A from } \\
\text { UFP-VSL-T01A/B (dewatering) }\end{array}$ & A 02AML 007 XX 1647 UDS 2 & Not Analyzed & & \\
\hline TI-065 & 11.6 & $\begin{array}{l}\text { Post-caustic-leach concentrate solids in UFP-VSL-T02A from } \\
\text { UFP-VSL-T01A/B (dewatering) }\end{array}$ & A 02AML 007 XX 1648 UDS 2 & UDS & UDS R1 & UDS R1 Shake and Test A and Test B Step001 Date 08182009 \\
\hline TI-065 & 11.6 & $\begin{array}{l}\text { Post-caustic-leach concentrate solids in UFP-VSL-T02A from } \\
\text { UFP-VSL-T01A/B (dewatering) }\end{array}$ & A 02AML 007 XX 1649 UDS 2 & UDS & UDS R1 & UDS R1 Shake and Test A and Test B Step001 Date 08182009 \\
\hline TI-065 & 11.6 & $\begin{array}{l}\text { Post-caustic-leach concentrate solids in UFP-VSL-T02A from } \\
\text { UFP-VSL-T01A/B (dewatering) }\end{array}$ & A 02AML 007 XX 1650 UDS 2 & Not Analyzed & & \\
\hline TI-065 & 11.6 & $\begin{array}{l}\text { Post-caustic-leach concentrate solids in UFP-VSL-T02A from } \\
\text { UFP-VSL-T01A/B (dewatering) }\end{array}$ & A 02AML 007 XX 1651 UDS 2 & Not Analyzed & & \\
\hline TI-065 & 11.6 & $\begin{array}{l}\text { Post-caustic-leach concentrate solids in UFP-VSL-T02A from } \\
\text { UFP-VSL-T01A/B (dewatering) }\end{array}$ & A 02AML 007 XX 1652 UDS 2 & Not Analyzed & & \\
\hline TI-065 & 11.6 & $\begin{array}{l}\text { Post-caustic-leach concentrate solids in UFP-VSL-T02A from } \\
\text { UFP-VSL-T01A/B (dewatering) }\end{array}$ & A 02AML 007 XX 1653 UDS 2 & Not Analyzed & & \\
\hline TI-065 & 11.6 & $\begin{array}{l}\text { Post-caustic-leach concentrate solids in UFP-VSL-T02A from } \\
\text { UFP-VSL-T01A/B (dewatering) }\end{array}$ & A 02AML 007 XX 1654 UDS 2 & Not Analyzed & & \\
\hline TI-065 & 11.6 & $\begin{array}{l}\text { Post-caustic-leach concentrate solids in UFP-VSL-T02A from } \\
\text { UFP-VSL-T01A/B (dewatering) }\end{array}$ & A 02AML 007 XX 1655 UDS 2 & Not Analyzed & & \\
\hline TI-065 & 11.6 & $\begin{array}{l}\text { Post-caustic-leach concentrate solids in UFP-VSL-T02A from } \\
\text { UFP-VSL-T01A/B (dewatering) }\end{array}$ & A 02AML 007 XX 1656 UDS 2 & Not Analyzed & & \\
\hline TI-065 & 11.6 & $\begin{array}{l}\text { Post-caustic-leach concentrate solids in UFP-VSL-T02A from } \\
\text { UFP-VSL-T01A/B (dewatering) }\end{array}$ & A 02AML 007 XX 1657 UDS 2 & Not Analyzed & & \\
\hline TI-065 & 11.6 & $\begin{array}{l}\text { Post-caustic-leach concentrate solids in UFP-VSL-T02A from } \\
\text { UFP-VSL-T01A/B (dewatering) }\end{array}$ & A 02AML 007 XX 2544 UDS 2 & Not Analyzed & & \\
\hline TI-065 & 11.6 & $\begin{array}{l}\text { Post-caustic-leach concentrate solids in UFP-VSL-T02A from } \\
\text { UFP-VSL-T01A/B (dewatering) }\end{array}$ & A 02AML 007 XX 2545 UDS 2 & Not Analyzed & & \\
\hline
\end{tabular}


Integrated Test A Sample List

\begin{tabular}{|c|c|c|c|c|c|c|}
\hline TI \# & TI Step \# & TI Step Description & Sample ID & Analysis & Folder & Final Electronic Report File Name \\
\hline TI-065 & 11.6 & $\begin{array}{l}\text { Post-caustic-leach concentrate solids in UFP-VSL-T02A from } \\
\text { UFP-VSL-T01A/B (dewatering) }\end{array}$ & A 02AML 007 XX 2546 UDS 2 & UDS & UDS R1 & UDS R1 Shake and Test A and Test B Step001 Date 08182009 \\
\hline TI-065 & 11.6 & $\begin{array}{l}\text { Post-caustic-leach concentrate solids in UFP-VSL-T02A from } \\
\text { UFP-VSL-T01A/B (dewatering) }\end{array}$ & A 02AML 007 XX 2547 UDS 2 & Not Analyzed & & \\
\hline TI-065 & 11.6 & $\begin{array}{l}\text { Post-caustic-leach concentrate solids in UFP-VSL-T02A from } \\
\text { UFP-VSL-T01A/B (dewatering) }\end{array}$ & A 02AML 007 XX 2548 UDS 2 & Not Analyzed & & \\
\hline TI-065 & 11.6 & $\begin{array}{l}\text { Post-caustic-leach concentrate solids in UFP-VSL-T02A from } \\
\text { UFP-VSL-T01A/B (dewatering) }\end{array}$ & A 02AML 007 XX 2549 UDS 2 & Not Analyzed & & \\
\hline TI-065 & 11.6 & $\begin{array}{l}\text { Post-caustic-leach concentrate solids in UFP-VSL-T02A from } \\
\text { UFP-VSL-T01A/B (dewatering) }\end{array}$ & A 02 AML 007 XX 2550 UDS 2 & Not Analyzed & & \\
\hline TI-065 & 11.6 & $\begin{array}{l}\text { Post-caustic-leach concentrate solids in UFP-VSL-T02A from } \\
\text { UFP-VSL-T01A/B (dewatering) }\end{array}$ & A 02AML 007 XX 2551 UDS 2 & Not Analyzed & & \\
\hline TI-065 & 11.6 & $\begin{array}{l}\text { Post-caustic-leach concentrate solids in UFP-VSL-T02A from } \\
\text { UFP-VSL-T01A/B (dewatering) }\end{array}$ & A 02 AML 007 XX 2552 UDS 2 & Not Analyzed & & \\
\hline TI-065 & 11.6 & $\begin{array}{l}\text { Post-caustic-leach concentrate solids in UFP-VSL-T02A from } \\
\text { UFP-VSL-T01A/B (dewatering) }\end{array}$ & A 02AML 007 XX 2553 UDS 2 & Not Analyzed & & \\
\hline TI-065 & 11.6 & no description listed & A 00PF1 007 XX 2554 ARC 4 & Precipitation Study & & \\
\hline TI-065 & 11.6 & no description listed & A 00PF1 007 XX 2555 ARC 4 & Precipitation Study & & \\
\hline TI-065 & 11.6 & $\begin{array}{l}\text { Post-caustic-leach concentrate solids in UFP-VSL-T02A from } \\
\text { UFP-VSL-T01A/B (dewatering) }\end{array}$ & A 02AML 007 XX 2641 UDS 2 & Not Analyzed & & \\
\hline TI-065 & 11.6 & $\begin{array}{l}\text { Post-caustic-leach concentrate solids in UFP-VSL-T02A from } \\
\text { UFP-VSL-T01A/B (dewatering) }\end{array}$ & A 02AML 007 XX 2642 UDS 2 & UDS & UDS R1 & UDS R1 Shake and Test A and Test B Step001 Date 08182009 \\
\hline TI-065 & 11.6 & $\begin{array}{l}\text { Post-caustic-leach concentrate solids in UFP-VSL-T02A from } \\
\text { UFP-VSL-T01A/B (dewatering) }\end{array}$ & A 02AML 007 XX 2643 UDS 2 & Not Analyzed & & \\
\hline TI-065 & 11.6 & $\begin{array}{l}\text { Post-caustic-leach concentrate solids in UFP-VSL-T02A from } \\
\text { UFP-VSL-T01A/B (dewatering) }\end{array}$ & A 02AML 007 XX 2644 UDS 2 & Not Analyzed & & \\
\hline TI-065 & 11.6 & $\begin{array}{l}\text { Post-caustic-leach concentrate solids in UFP-VSL-T02A from } \\
\text { UFP-VSL-T01A/B (dewatering) }\end{array}$ & A 02AML 007 XX 2645 UDS 2 & Not Analyzed & & \\
\hline TI-065 & 11.6 & $\begin{array}{l}\text { Post-caustic-leach concentrate solids in UFP-VSL-T02A from } \\
\text { UFP-VSL-T01A/B (dewatering) }\end{array}$ & A 02AML 007 XX 2646 UDS 2 & Not Analyzed & & \\
\hline TI-065 & 11.6 & $\begin{array}{l}\text { Post-caustic-leach concentrate solids in UFP-VSL-T02A from } \\
\text { UFP-VSL-T01A/B (dewatering) }\end{array}$ & A 02AML 007 XX 2647 UDS 2 & Not Analyzed & & \\
\hline TI-065 & 11.6 & $\begin{array}{l}\text { Post-caustic-leach concentrate solids in UFP-VSL-T02A from } \\
\text { UFP-VSL-T01A/B (dewatering) }\end{array}$ & A 02AML 007 XX 2648 UDS 2 & Not Analyzed & & \\
\hline TI-065 & 11.6 & $\begin{array}{l}\text { Post-caustic-leach concentrate solids in UFP-VSL-T02A from } \\
\text { UFP-VSL-T01A/B (dewatering) }\end{array}$ & A 02AML 007 XX 2649 UDS 2 & Not Analyzed & & \\
\hline TI-065 & 11.6 & $\begin{array}{l}\text { Post-caustic-leach concentrate solids in UFP-VSL-T02A from } \\
\text { UFP-VSL-T01A/B (dewatering) }\end{array}$ & A 02AML 007 XX 2650 UDS 2 & Not Analyzed & & \\
\hline
\end{tabular}


Integrated Test A Sample List

\begin{tabular}{|c|c|c|c|c|c|c|}
\hline TI \# & TI Step \# & TI Step Description & Sample ID & Analysis & Folder & Final Electronic Report File Name \\
\hline TI-065 & 11.6 & $\begin{array}{l}\text { Post-caustic-leach concentrate solids in UFP-VSL-T02A from } \\
\text { UFP-VSL-T01A/B (dewatering) }\end{array}$ & A 02AML 007 XX 2651 UDS 2 & Not Analyzed & & \\
\hline TI-065 & 11.6 & $\begin{array}{l}\text { Post-caustic-leach concentrate solids in UFP-VSL-T02A from } \\
\text { UFP-VSL-T01A/B (dewatering) }\end{array}$ & A 02AML 007 XX 2652 UDS 2 & UDS & UDS R1 & UDS R1 Shake and Test A and Test B Step001 Date 08182009 \\
\hline TI-065 & 11.6 & $\begin{array}{l}\text { Post-caustic-leach concentrate solids in UFP-VSL-T02A from } \\
\text { UFP-VSL-T01A/B (dewatering) }\end{array}$ & A 02 AML 007 XX 2653 UDS 2 & Not Analyzed & & \\
\hline TI-065 & 11.6 & $\begin{array}{l}\text { Post-caustic-leach concentrate solids in UFP-VSL-T02A from } \\
\text { UFP-VSL-T01A/B (dewatering) }\end{array}$ & A 02AML 007 XX 2654 UDS 2 & Not Analyzed & & \\
\hline TI-065 & 11.6 & $\begin{array}{l}\text { Post-caustic-leach concentrate solids in UFP-VSL-T02A from } \\
\text { UFP-VSL-T01A/B (dewatering) }\end{array}$ & A 02AML 007 XX 2655 UDS 2 & Not Analyzed & & \\
\hline TI-065 & 11.6 & $\begin{array}{l}\text { Post-caustic-leach concentrate solids in UFP-VSL-T02A from } \\
\text { UFP-VSL-T01A/B (dewatering) }\end{array}$ & A 02AML 007 XX 2656 UDS 2 & Not Analyzed & & \\
\hline TI-065 & 11.6 & $\begin{array}{l}\text { Post-caustic-leach concentrate solids in UFP-VSL-T02A from } \\
\text { UFP-VSL-T01A/B (dewatering) }\end{array}$ & A 02AML 007 XX 2657 UDS 2 & Not Analyzed & & \\
\hline TI-065 & 11.6 & $\begin{array}{l}\text { Post-caustic-leach concentrate solids in UFP-VSL-T02A from } \\
\text { UFP-VSL-T01A/B (dewatering) }\end{array}$ & A 02AML 007 XX 2658 UDS 2 & Not Analyzed & & \\
\hline TI-065 & 11.6 & $\begin{array}{l}\text { Post-caustic-leach concentrate solids in UFP-VSL-T02A from } \\
\text { UFP-VSL-T01A/B (dewatering) }\end{array}$ & A 02AML 007 XX 2659 UDS 2 & Not Analyzed & & \\
\hline TI-065 & 11.6 & $\begin{array}{l}\text { Post-caustic-leach concentrate solids in UFP-VSL-T02A from } \\
\text { UFP-VSL-T01A/B (dewatering) }\end{array}$ & A 02AML 007 XX 2660 UDS 2 & Not Analyzed & & \\
\hline TI-065 & 11.6 & $\begin{array}{l}\text { Post-caustic-leach concentrate solids in UFP-VSL-T02A from } \\
\text { UFP-VSL-T01A/B (dewatering) }\end{array}$ & A 02AML 007 XX 2661 UDS 2 & Not Analyzed & & \\
\hline TI-065 & 11.6 & $\begin{array}{l}\text { Post-caustic-leach concentrate solids in UFP-VSL-T02A from } \\
\text { UFP-VSL-T01A/B (dewatering) }\end{array}$ & A 02 AML 007 XX 2662 UDS 2 & UDS & UDS R1 & UDS R1 Shake and Test A and Test B Step001 Date 08182009 \\
\hline TI-065 & 11.6 & $\begin{array}{l}\text { Post-caustic-leach concentrate solids in UFP-VSL-T02A from } \\
\text { UFP-VSL-T01A/B (dewatering) }\end{array}$ & A 02AML 007 XX 2663 UDS 2 & Not Analyzed & & \\
\hline TI-065 & 11.6 & $\begin{array}{l}\text { Post-caustic-leach concentrate solids in UFP-VSL-T02A from } \\
\text { UFP-VSL-T01A/B (dewatering) }\end{array}$ & A 02AML 007 XX 2664 UDS 2 & Not Analyzed & & \\
\hline TI-065 & 11.6 & $\begin{array}{l}\text { Post-caustic-leach concentrate solids in UFP-VSL-T02A from } \\
\text { UFP-VSL-T01A/B (dewatering) }\end{array}$ & A 02AML 007 XX 2665 UDS 2 & Not Analyzed & & \\
\hline TI-065 & 11.6 & $\begin{array}{l}\text { Post-caustic-leach concentrate solids in UFP-VSL-T02A from } \\
\text { UFP-VSL-T01A/B (dewatering) }\end{array}$ & A 02AML 007 XX 2666 UDS 2 & Not Analyzed & & \\
\hline TI-065 & 11.6 & $\begin{array}{l}\text { Post-caustic-leach concentrate solids in UFP-VSL-T02A from } \\
\text { UFP-VSL-T01A/B (dewatering) }\end{array}$ & A 02AML 007 XX 2667 UDS 2 & Not Analyzed & & \\
\hline TI-065 & 11.6 & $\begin{array}{l}\text { Post-caustic-leach concentrate solids in UFP-VSL-T02A from } \\
\text { UFP-VSL-T01A/B (dewatering) }\end{array}$ & A 02 AML 007 XX 2668 UDS 2 & Not Analyzed & & \\
\hline TI-065 & 11.6 & $\begin{array}{l}\text { Post-caustic-leach concentrate solids in UFP-VSL-T02A from } \\
\text { UFP-VSL-T01A/B (dewatering) }\end{array}$ & A 02AML 007 XX 2669 UDS 2 & Not Analyzed & & \\
\hline
\end{tabular}


Integrated Test A Sample List

\begin{tabular}{|c|c|c|c|c|c|c|}
\hline TI \# & TI Step \# & TI Step Description & Sample ID & Analysis & Folder & Final Electronic Report File Name \\
\hline TI-065 & 11.6 & $\begin{array}{l}\text { Post-caustic-leach concentrate solids in UFP-VSL-T02A from } \\
\text { UFP-VSL-T01A/B (dewatering) }\end{array}$ & A 02AML 007 XX 2670 UDS 2 & Not Analyzed & & \\
\hline TI-065 & 11.6 & $\begin{array}{l}\text { Post-caustic-leach concentrate solids in UFP-VSL-T02A from } \\
\text { UFP-VSL-T01A/B (dewatering) }\end{array}$ & A 02AML 007 XX 2671 UDS 2 & Not Analyzed & & \\
\hline TI-065 & 11.6 & $\begin{array}{l}\text { Post-caustic-leach concentrate solids in UFP-VSL-T02A from } \\
\text { UFP-VSL-T01A/B (dewatering) }\end{array}$ & A 02AML 007 XX 2672 UDS 2 & UDS & UDS R1 & UDS R1 Shake and Test A and Test B Step001 Date 08182009 \\
\hline TI-065 & 11.6 & $\begin{array}{l}\text { Post-caustic-leach concentrate solids in UFP-VSL-T02A from } \\
\text { UFP-VSL-T01A/B (dewatering) }\end{array}$ & A 02AML 007 XX 2673 UDS 2 & Not Analyzed & & \\
\hline TI-065 & 11.6 & $\begin{array}{l}\text { Post-caustic-leach concentrate solids in UFP-VSL-T02A from } \\
\text { UFP-VSL-T01A/B (dewatering) }\end{array}$ & A 02AML 007 XX 2674 UDS 2 & Not Analyzed & & \\
\hline TI-065 & 11.6 & $\begin{array}{l}\text { Post-caustic-leach concentrate solids in UFP-VSL-T02A from } \\
\text { UFP-VSL-T01A/B (dewatering) }\end{array}$ & A 02AML 007 XX 2675 UDS 2 & Not Analyzed & & \\
\hline TI-065 & 11.6 & $\begin{array}{l}\text { Post-caustic-leach concentrate solids in UFP-VSL-T02A from } \\
\text { UFP-VSL-T01A/B (dewatering) }\end{array}$ & A 02AML 007 XX 2676 UDS 2 & Not Analyzed & & \\
\hline TI-065 & 11.6 & $\begin{array}{l}\text { Post-caustic-leach concentrate solids in UFP-VSL-T02A from } \\
\text { UFP-VSL-T01A/B (dewatering) }\end{array}$ & A 02AML 007 XX 2677 UDS 2 & Not Analyzed & & \\
\hline TI-065 & 11.6 & $\begin{array}{l}\text { Post-caustic-leach concentrate solids in UFP-VSL-T02A from } \\
\text { UFP-VSL-T01A/B (dewatering) }\end{array}$ & A 02AML 007 XX 2678 UDS 2 & Not Analyzed & & \\
\hline TI-065 & 11.6 & $\begin{array}{l}\text { Post-caustic-leach concentrate solids in UFP-VSL-T02A from } \\
\text { UFP-VSL-T01A/B (dewatering) }\end{array}$ & A 02AML 007 XX 2679 UDS 2 & Not Analyzed & & \\
\hline TI-065 & 11.6 & $\begin{array}{l}\text { Post-caustic-leach concentrate solids in UFP-VSL-T02A from } \\
\text { UFP-VSL-T01A/B (dewatering) }\end{array}$ & A 02AML 007 XX 2680 UDS 2 & Not Analyzed & & \\
\hline TI-065 & 11.6 & $\begin{array}{l}\text { Post-caustic-leach concentrate solids in UFP-VSL-T02A from } \\
\text { UFP-VSL-T01A/B (dewatering) }\end{array}$ & A 02 AML 007 XX 2681 UDS 2 & Not Analyzed & & \\
\hline TI-065 & 11.6 & $\begin{array}{l}\text { Post-caustic-leach concentrate solids in UFP-VSL-T02A from } \\
\text { UFP-VSL-T01A/B (dewatering) }\end{array}$ & A 02AML 007 XX 2682 UDS 2 & UDS & UDS R1 & UDS R1 Shake and Test A and Test B Step001 Date 08182009 \\
\hline TI-065 & 11.6 & $\begin{array}{l}\text { Post-caustic-leach concentrate solids in UFP-VSL-T02A from } \\
\text { UFP-VSL-T01A/B (dewatering) }\end{array}$ & A 02AML 007 XX 2683 UDS 2 & Not Analyzed & & \\
\hline TI-065 & 11.6 & $\begin{array}{l}\text { Post-caustic-leach concentrate solids in UFP-VSL-T02A from } \\
\text { UFP-VSL-T01A/B (dewatering) }\end{array}$ & A 02AML 007 XX 2684 UDS 2 & Rheology & TDP-WTP-359 & TDP WTP 359 Int Test A RHE Data on PEP Slurry \\
\hline TI-065 & 11.6 & $\begin{array}{l}\text { Post-caustic-leach concentrate solids in UFP-VSL-T02A from } \\
\text { UFP-VSL-T01A/B (dewatering) }\end{array}$ & A 02AML 007 XX 2685 UDS 2 & Not Analyzed & & \\
\hline TI-065 & 11.6 & $\begin{array}{l}\text { Post-caustic-leach concentrate solids in UFP-VSL-T02A from } \\
\text { UFP-VSL-T01A/B (dewatering) }\end{array}$ & A 02AML 007 XX 2686 UDS 2 & Not Analyzed & & \\
\hline TI-065 & 11.6 & $\begin{array}{l}\text { Post-caustic-leach concentrate solids in UFP-VSL-T02A from } \\
\text { UFP-VSL-T01A/B (dewatering) }\end{array}$ & A 02AML 007 XX 2687 UDS 2 & Not Analyzed & & \\
\hline TI-065 & 11.6 & $\begin{array}{l}\text { Post-caustic-leach concentrate solids in UFP-VSL-T02A from } \\
\text { UFP-VSL-T01A/B (dewatering) }\end{array}$ & A 02AML 007 XX 2688 UDS 2 & Not Analyzed & & \\
\hline
\end{tabular}


Integrated Test A Sample List

\begin{tabular}{|c|c|c|c|c|c|c|}
\hline TI \# & TI Step \# & TI Step Description & Sample ID & Analysis & Folder & Final Electronic Report File Name \\
\hline TI-065 & 11.6 & $\begin{array}{l}\text { Post-caustic-leach concentrate solids in UFP-VSL-T02A from } \\
\text { UFP-VSL-T01A/B (dewatering) }\end{array}$ & A 02AML 007 XX 2689 UDS 2 & Not Analyzed & & \\
\hline TI-065 & 11.6 & $\begin{array}{l}\text { Post-caustic-leach concentrate solids in UFP-VSL-T02A from } \\
\text { UFP-VSL-T01A/B (dewatering) }\end{array}$ & A 02AML 007 XX 2690 UDS 2 & Not Analyzed & & \\
\hline TI-065 & 11.6 & $\begin{array}{l}\text { Post-caustic-leach concentrate solids in UFP-VSL-T02A from } \\
\text { UFP-VSL-T01A/B (dewatering) }\end{array}$ & A 02AML 007 XX 2691 UDS 2 & Not Analyzed & & \\
\hline TI-065 & 11.6 & $\begin{array}{l}\text { Post-caustic-leach concentrate solids in UFP-VSL-T02A from } \\
\text { UFP-VSL-T01A/B (dewatering) }\end{array}$ & A 02AML 007 XX 2692 UDS 2 & UDS & CCP-WTPSP-755 & CCP WTPSP 755 Int Test A UDS Data Analysis \\
\hline TI-065 & 11.6 & $\begin{array}{l}\text { Post-caustic-leach concentrate solids in UFP-VSL-T02A from } \\
\text { UFP-VSL-T01A/B (dewatering) }\end{array}$ & A 02 AML 007 XX 2693 UDS 2 & UDS & CCP-WTPSP-755 & CCP WTPSP 755 Int Test A UDS Data Analysis \\
\hline TI-065 & 11.6 & $\begin{array}{l}\text { Post-caustic-leach concentrate solids in UFP-VSL-T02A from } \\
\text { UFP-VSL-T01A/B (dewatering) }\end{array}$ & A 02AML 007 XX 2694 UDS 2 & PSD & TDP-WTP-358 & TDP WTP 358 Int Test A PSD Data Analysis \\
\hline TI-065 & 11.6 & $\begin{array}{l}\text { Post-caustic-leach concentrate solids in UFP-VSL-T02A from } \\
\text { UFP-VSL-T01A/B (dewatering) }\end{array}$ & A 00PF1 007 -suspension fluid & PSD & TDP-WTP-358 & TDP WTP 358 Int Test A PSD Data Analysis \\
\hline TI-065 & 11.6 & $\begin{array}{l}\text { Post-caustic-leach concentrate solids in UFP-VSL-T02A from } \\
\text { UFP-VSL-T01A/B (dewatering) }\end{array}$ & A 02AML 007 XX 2695 UDS 2 & Not Analyzed & & \\
\hline TI-065 & 11.6 & $\begin{array}{l}\text { Post-caustic-leach concentrate solids in UFP-VSL-T02A from } \\
\text { UFP-VSL-T01A/B (dewatering) }\end{array}$ & A 02AML 007 XX 2696 UDS 2 & Not Analyzed & & \\
\hline TI-065 & 11.6 & $\begin{array}{l}\text { Post-caustic-leach concentrate solids in UFP-VSL-T02A from } \\
\text { UFP-VSL-T01A/B (dewatering) }\end{array}$ & A 02AML 007 XX 2697 UDS 2 & UDS & UDS R1 & UDS R1 Shake and Test A and Test B Step001 Date 08182009 \\
\hline TI-065 & 11.6 & $\begin{array}{l}\text { Post-caustic-leach concentrate solids in UFP-VSL-T02A from } \\
\text { UFP-VSL-T01A/B (dewatering) }\end{array}$ & A 02AML 007 XX 2698 UDS 2 & Not Analyzed & & \\
\hline TI-065 & 11.6 & $\begin{array}{l}\text { Post-caustic-leach concentrate solids in UFP-VSL-T02A from } \\
\text { UFP-VSL-T01A/B (dewatering) }\end{array}$ & A 02AML 007 XX 2699 UDS 2 & Not Analyzed & & \\
\hline TI-065 & 11.6 & $\begin{array}{l}\text { Post-caustic-leach concentrate solids in UFP-VSL-T02A from } \\
\text { UFP-VSL-T01A/B (dewatering) }\end{array}$ & A 02AML 007 XX 2700 UDS 2 & Not Analyzed & & \\
\hline TI-065 & 11.6 & $\begin{array}{l}\text { Post-caustic-leach concentrate solids in UFP-VSL-T02A from } \\
\text { UFP-VSL-T01A/B (dewatering) }\end{array}$ & A 02 AML 007 XX 2701 UDS 2 & Not Analyzed & & \\
\hline TI-065 & 11.6 & $\begin{array}{l}\text { Post-caustic-leach concentrate solids in UFP-VSL-T02A from } \\
\text { UFP-VSL-T01A/B (dewatering) }\end{array}$ & A 02AML 007 XX 2702 UDS 2 & Not Analyzed & & \\
\hline TI-065 & 11.6 & $\begin{array}{l}\text { Post-caustic-leach concentrate solids in UFP-VSL-T02A from } \\
\text { UFP-VSL-T01A/B (dewatering) }\end{array}$ & A 02 AML 007 XX 2703 UDS 2 & Not Analyzed & & \\
\hline TI-065 & 11.6 & $\begin{array}{l}\text { Post-caustic-leach concentrate solids in UFP-VSL-T02A from } \\
\text { UFP-VSL-T01A/B (dewatering) }\end{array}$ & A 02 AML 007 XX 2704 UDS 2 & Not Analyzed & & \\
\hline TI-065 & 11.6 & $\begin{array}{l}\text { Post-caustic-leach concentrate solids in UFP-VSL-T02A from } \\
\text { UFP-VSL-T01A/B (dewatering) }\end{array}$ & A 02 AML 007 XX 2705 UDS 2 & Not Analyzed & & \\
\hline TI-065 & 11.6 & $\begin{array}{l}\text { Post-caustic-leach concentrate solids in UFP-VSL-T02A from } \\
\text { UFP-VSL-T01A/B (dewatering) }\end{array}$ & A 02AML 007 XX 2706 UDS 2 & Not Analyzed & & \\
\hline
\end{tabular}


Integrated Test A Sample List

\begin{tabular}{|c|c|c|c|c|c|c|}
\hline TI \# & TI Step \# & TI Step Description & Sample ID & Analysis & Folder & Final Electronic Report File Name \\
\hline TI-065 & 11.6 & $\begin{array}{l}\text { Post-caustic-leach concentrate solids in UFP-VSL-T02A from } \\
\text { UFP-VSL-T01A/B (dewatering) }\end{array}$ & A 02AML 007 XX 2707 UDS 2 & UDS & UDS R1 & UDS R1 Shake and Test A and Test B Step001 Date 08182009 \\
\hline TI-065 & 11.6 & $\begin{array}{l}\text { Post-caustic-leach concentrate solids in UFP-VSL-T02A from } \\
\text { UFP-VSL-T01A/B (dewatering) }\end{array}$ & A 02AML 007 XX 2708 UDS 2 & Not Analyzed & & \\
\hline TI-065 & 11.6 & $\begin{array}{l}\text { Post-caustic-leach concentrate solids in UFP-VSL-T02A from } \\
\text { UFP-VSL-T01A/B (dewatering) }\end{array}$ & A 02AML 007 XX 2709 UDS 2 & Not Analyzed & & \\
\hline TI-065 & 11.6 & $\begin{array}{l}\text { Post-caustic-leach concentrate solids in UFP-VSL-T02A from } \\
\text { UFP-VSL-T01A/B (dewatering) }\end{array}$ & A 02AML 007 XX 2710 UDS 2 & Not Analyzed & & \\
\hline TI-065 & 11.6 & $\begin{array}{l}\text { Post-caustic-leach concentrate solids in UFP-VSL-T02A from } \\
\text { UFP-VSL-T01A/B (dewatering) }\end{array}$ & A 02AML 007 XX 2711 UDS 2 & Not Analyzed & & \\
\hline TI-065 & 11.6 & $\begin{array}{l}\text { Post-caustic-leach concentrate solids in UFP-VSL-T02A from } \\
\text { UFP-VSL-T01A/B (dewatering) }\end{array}$ & A 02 AML $007 \mathrm{XX} 2712$ UDS 2 & Not Analyzed & & \\
\hline TI-065 & 11.6 & $\begin{array}{l}\text { Post-caustic-leach concentrate solids in UFP-VSL-T02A from } \\
\text { UFP-VSL-T01A/B (dewatering) }\end{array}$ & A 02AML 007 XX 2713 UDS 2 & Not Analyzed & & \\
\hline TI-065 & 11.6 & $\begin{array}{l}\text { Post-caustic-leach concentrate solids in UFP-VSL-T02A from } \\
\text { UFP-VSL-T01A/B (dewatering) }\end{array}$ & A 02 AML 007 XX 2714 UDS 2 & Not Analyzed & & \\
\hline TI-065 & 11.6 & $\begin{array}{l}\text { Post-caustic-leach concentrate solids in UFP-VSL-T02A from } \\
\text { UFP-VSL-T01A/B (dewatering) }\end{array}$ & A 02AML 007 XX 2715 UDS 2 & Not Analyzed & & \\
\hline TI-065 & 11.6 & $\begin{array}{l}\text { Post-caustic-leach concentrate solids in UFP-VSL-T02A from } \\
\text { UFP-VSL-T01A/B (dewatering) }\end{array}$ & A 02 AML 007 XX 2716 UDS 2 & Not Analyzed & & \\
\hline TI-065 & 11.6 & $\begin{array}{l}\text { Post-caustic-leach concentrate solids in UFP-VSL-T02A from } \\
\text { UFP-VSL-T01A/B (dewatering) }\end{array}$ & A 02AML 007 XX 2717 UDS 2 & UDS & UDS R1 & UDS R1 Shake and Test A and Test B Step001 Date 08182009 \\
\hline TI-065 & 11.6 & $\begin{array}{l}\text { Post-caustic-leach concentrate solids in UFP-VSL-T02A from } \\
\text { UFP-VSL-T01A/B (dewatering) }\end{array}$ & A 02AML 007 XX 2718 UDS 2 & Not Analyzed & & \\
\hline TI-065 & 11.6 & $\begin{array}{l}\text { Post-caustic-leach concentrate solids in UFP-VSL-T02A from } \\
\text { UFP-VSL-T01A/B (dewatering) }\end{array}$ & A 02AML 007 XX 2719 UDS 2 & Not Analyzed & & \\
\hline TI-065 & 11.6 & $\begin{array}{l}\text { Post-caustic-leach concentrate solids in UFP-VSL-T02A from } \\
\text { UFP-VSL-T01A/B (dewatering) }\end{array}$ & A 02AML 007 XX 2720 UDS 2 & Not Analyzed & & \\
\hline TI-065 & 11.6 & $\begin{array}{l}\text { Post-caustic-leach concentrate solids in UFP-VSL-T02A from } \\
\text { UFP-VSL-T01A/B (dewatering) }\end{array}$ & A 02AML 007 XX 2721 UDS 2 & Not Analyzed & & \\
\hline TI-065 & 11.6 & $\begin{array}{l}\text { Post-caustic-leach concentrate solids in UFP-VSL-T02A from } \\
\text { UFP-VSL-T01A/B (dewatering) }\end{array}$ & A 02AML 007 XX 2722 UDS 2 & Not Analyzed & & \\
\hline TI-065 & 11.6 & $\begin{array}{l}\text { Post-caustic-leach concentrate solids in UFP-VSL-T02A from } \\
\text { UFP-VSL-T01A/B (dewatering) }\end{array}$ & A 02AML 007 XX 2723 UDS 2 & Not Analyzed & & \\
\hline TI-065 & 11.6 & $\begin{array}{l}\text { Post-caustic-leach concentrate solids in UFP-VSL-T02A from } \\
\text { UFP-VSL-T01A/B (dewatering) }\end{array}$ & A 02AML 007 XX 2724 UDS 2 & Not Analyzed & & \\
\hline TI-065 & 11.6 & $\begin{array}{l}\text { Post-caustic-leach concentrate solids in UFP-VSL-T02A from } \\
\text { UFP-VSL-T01A/B (dewatering) }\end{array}$ & A 02AML 007 XX 2725 UDS 2 & Not Analyzed & & \\
\hline
\end{tabular}


Integrated Test A Sample List

\begin{tabular}{|c|c|c|c|c|c|c|}
\hline TI \# & TI Step \# & TI Step Description & Sample ID & Analysis & Folder & Final Electronic Report File Name \\
\hline TI-065 & 11.6 & $\begin{array}{l}\text { Post-caustic-leach concentrate solids in UFP-VSL-T02A from } \\
\text { UFP-VSL-T01A/B (dewatering) }\end{array}$ & A 02AML 007 XX 2737 UDS 2 & Not Analyzed & & \\
\hline TI-065 & 11.6 & $\begin{array}{l}\text { Post-caustic-leach concentrate solids in UFP-VSL-T02A from } \\
\text { UFP-VSL-T01A/B (dewatering) }\end{array}$ & A 02AML 007 XX 2738 UDS 2 & UDS & UDS R1 & UDS R1 Shake and Test A and Test B Step001 Date 08182009 \\
\hline TI-065 & 11.6 & $\begin{array}{l}\text { Post-caustic-leach concentrate solids in UFP-VSL-T02A from } \\
\text { UFP-VSL-T01A/B (dewatering) }\end{array}$ & A 02 AML 007 XX 2739 UDS 2 & Not Analyzed & & \\
\hline TI-065 & 11.6 & $\begin{array}{l}\text { Post-caustic-leach concentrate solids in UFP-VSL-T02A from } \\
\text { UFP-VSL-T01A/B (dewatering) }\end{array}$ & A 02AML 007 XX 2740 UDS 2 & Not Analyzed & & \\
\hline TI-065 & 11.6 & $\begin{array}{l}\text { Post-caustic-leach concentrate solids in UFP-VSL-T02A from } \\
\text { UFP-VSL-T01A/B (dewatering) }\end{array}$ & A 02AML 007 XX 2741 UDS 2 & Not Analyzed & & \\
\hline TI-065 & 11.6 & $\begin{array}{l}\text { Post-caustic-leach concentrate solids in UFP-VSL-T02A from } \\
\text { UFP-VSL-T01A/B (dewatering) }\end{array}$ & A 02AML 007 XX 2742 UDS 2 & Not Analyzed & & \\
\hline TI-065 & 11.6 & $\begin{array}{l}\text { Post-caustic-leach concentrate solids in UFP-VSL-T02A from } \\
\text { UFP-VSL-T01A/B (dewatering) }\end{array}$ & A 02AML 007 XX 2743 UDS 2 & Not Analyzed & & \\
\hline TI-065 & 11.6 & $\begin{array}{l}\text { Post-caustic-leach concentrate solids in UFP-VSL-T02A from } \\
\text { UFP-VSL-T01A/B (dewatering) }\end{array}$ & A 02AML 007 XX 2744 UDS 2 & Not Analyzed & & \\
\hline TI-065 & 11.6 & $\begin{array}{l}\text { Post-caustic-leach concentrate solids in UFP-VSL-T02A from } \\
\text { UFP-VSL-T01A/B (dewatering) }\end{array}$ & A 02AML 007 XX 2745 UDS 2 & Not Analyzed & & \\
\hline TI-065 & 11.6 & $\begin{array}{l}\text { Post-caustic-leach concentrate solids in UFP-VSL-T02A from } \\
\text { UFP-VSL-T01A/B (dewatering) }\end{array}$ & A 02AML 007 XX 2746 UDS 2 & Not Analyzed & & \\
\hline TI-065 & 11.6 & $\begin{array}{l}\text { Post-caustic-leach concentrate solids in UFP-VSL-T02A from } \\
\text { UFP-VSL-T01A/B (dewatering) }\end{array}$ & A 02AML 007 XX 2747 UDS 2 & Not Analyzed & & \\
\hline TI-065 & 11.6 & $\begin{array}{l}\text { Post-caustic-leach concentrate solids in UFP-VSL-T02A from } \\
\text { UFP-VSL-T01A/B (dewatering) }\end{array}$ & A 02 AML 007 XX 2748 UDS 2 & UDS & UDS R1 & UDS R1 Shake and Test A and Test B Step001 Date 08182009 \\
\hline TI-065 & 11.6 & $\begin{array}{l}\text { Post-caustic-leach concentrate solids in UFP-VSL-T02A from } \\
\text { UFP-VSL-T01A/B (dewatering) }\end{array}$ & A 02AML 007 XX 2749 UDS 2 & Not Analyzed & & \\
\hline TI-065 & 11.6 & $\begin{array}{l}\text { Post-caustic-leach concentrate solids in UFP-VSL-T02A from } \\
\text { UFP-VSL-T01A/B (dewatering) }\end{array}$ & A 02 AML 007 XX 2750 UDS 2 & Not Analyzed & & \\
\hline TI-065 & 11.6 & $\begin{array}{l}\text { Post-caustic-leach concentrate solids in UFP-VSL-T02A from } \\
\text { UFP-VSL-T01A/B (dewatering) }\end{array}$ & A 02AML 007 XX 2751 UDS 2 & Not Analyzed & & \\
\hline TI-065 & 11.6 & $\begin{array}{l}\text { Post-caustic-leach concentrate solids in UFP-VSL-T02A from } \\
\text { UFP-VSL-T01A/B (dewatering) }\end{array}$ & A 02AML 007 XX 2752 UDS 2 & Not Analyzed & & \\
\hline TI-065 & 11.6 & $\begin{array}{l}\text { Post-caustic-leach concentrate solids in UFP-VSL-T02A from } \\
\text { UFP-VSL-T01A/B (dewatering) }\end{array}$ & A 02 AML $007 \mathrm{XX} 2753$ UDS 2 & Not Analyzed & & \\
\hline TI-065 & 11.6 & $\begin{array}{l}\text { Post-caustic-leach concentrate solids in UFP-VSL-T02A from } \\
\text { UFP-VSL-T01A/B (dewatering) }\end{array}$ & A 02 AML 007 XX 2754 UDS 2 & Not Analyzed & & \\
\hline TI-065 & 11.6 & $\begin{array}{l}\text { Post-caustic-leach concentrate solids in UFP-VSL-T02A from } \\
\text { UFP-VSL-T01A/B (dewatering) }\end{array}$ & A 02AML 007 XX 2755 UDS 2 & Not Analyzed & & \\
\hline
\end{tabular}


Integrated Test A Sample List

\begin{tabular}{|c|c|c|c|c|c|c|}
\hline TI\# & TI Step \# & TI Step Description & Sample ID & Analysis & Folder & Final Electronic Report File Name \\
\hline TI-065 & 11.6 & $\begin{array}{l}\text { Post-caustic-leach concentrate solids in UFP-VSL-T02A from } \\
\text { UFP-VSL-T01A/B (dewatering) }\end{array}$ & A 02AML 007 XX 2756 UDS 2 & Not Analyzed & & \\
\hline TI-065 & 11.6 & $\begin{array}{l}\text { Post-caustic-leach concentrate solids in UFP-VSL-T02A from } \\
\text { UFP-VSL-T01A/B (dewatering) }\end{array}$ & A 02AML 007 XX 2764 UDS 2 & Not Analyzed & & \\
\hline TI-065 & 11.6 & $\begin{array}{l}\text { Post-caustic-leach concentrate solids in UFP-VSL-T02A from } \\
\text { UFP-VSL-T01A/B (dewatering) }\end{array}$ & A 02AML 007 XX 2765 UDS 2 & UDS & UDS R1 & UDS R1 Shake and Test A and Test B Step001 Date 08182009 \\
\hline TI-065 & 11.6 & $\begin{array}{l}\text { Post-caustic-leach concentrate solids in UFP-VSL-T02A from } \\
\text { UFP-VSL-T01A/B (dewatering) }\end{array}$ & A 02AML 007 XX 2766 UDS 2 & Not Analyzed & & \\
\hline TI-065 & 11.6 & $\begin{array}{l}\text { Post-caustic-leach concentrate solids in UFP-VSL-T02A from } \\
\text { UFP-VSL-T01A/B (dewatering) }\end{array}$ & A 02AML 007 XX 2767 UDS 2 & Not Analyzed & & \\
\hline TI-065 & 11.6 & $\begin{array}{l}\text { Post-caustic-leach concentrate solids in UFP-VSL-T02A from } \\
\text { UFP-VSL-T01A/B (dewatering) }\end{array}$ & A 02 AML 007 XX 2768 UDS 2 & Not Analyzed & & \\
\hline TI-065 & 11.6 & $\begin{array}{l}\text { Post-caustic-leach concentrate solids in UFP-VSL-T02A from } \\
\text { UFP-VSL-T01A/B (dewatering) }\end{array}$ & A 02AML 007 XX 2769 UDS 2 & Not Analyzed & & \\
\hline TI-065 & 11.6 & $\begin{array}{l}\text { Post-caustic-leach concentrate solids in UFP-VSL-T02A from } \\
\text { UFP-VSL-T01A/B (dewatering) }\end{array}$ & A 02AML 007 XX 2770 UDS 2 & Not Analyzed & & \\
\hline TI-065 & 11.6 & $\begin{array}{l}\text { Post-caustic-leach concentrate solids in UFP-VSL-T02A from } \\
\text { UFP-VSL-T01A/B (dewatering) }\end{array}$ & A 02AML 007 XX 2771 UDS 2 & Not Analyzed & & \\
\hline TI-065 & 11.6 & $\begin{array}{l}\text { Post-caustic-leach concentrate solids in UFP-VSL-T02A from } \\
\text { UFP-VSL-T01A/B (dewatering) }\end{array}$ & A 02AML 007 XX 2772 UDS 2 & Not Analyzed & & \\
\hline TI-065 & 11.6 & $\begin{array}{l}\text { Post-caustic-leach concentrate solids in UFP-VSL-T02A from } \\
\text { UFP-VSL-T01A/B (dewatering) }\end{array}$ & A 02AML 007 XX 2773 UDS 2 & UDS & UDS R1 & UDS R1 Shake and Test A and Test B Step001 Date 08182009 \\
\hline TI-065 & $\mathrm{n} / \mathrm{a}$ & $\mathrm{n} / \mathrm{a}$ & A 000FL 007 XX 2813 RHE 4 & Rheology & TDP-WTP-359 & TDP WTP 359 Int Test A RHE Data on PEP Slurry \\
\hline TI-065 & 11.6 & $\begin{array}{l}\text { Post-caustic-leach concentrate solids in UFP-VSL-T02A from } \\
\text { UFP-VSL-T01A/B (dewatering) }\end{array}$ & A 00PF1 007 XX 1658 ARC 1 & Archive & & \\
\hline TI-065 & 11.6 & $\begin{array}{l}\text { Post-caustic-leach concentrate solids in UFP-VSL-T02A from } \\
\text { UFP-VSL-T01A/B (dewatering) }\end{array}$ & A 00PF1 007 XX 1659 ARC 1 & Archive & & \\
\hline TI-065 & 11.6 & $\begin{array}{l}\text { Post-caustic-leach concentrate solids in UFP-VSL-T02A from } \\
\text { UFP-VSL-T01A/B (dewatering) }\end{array}$ & A 00PF1 007 XX 1660 ARC 1 & Archive & & \\
\hline TI-065 & 11.6 & $\begin{array}{l}\text { Post-caustic-leach concentrate solids in UFP-VSL-T02A from } \\
\text { UFP-VSL-T01A/B (dewatering) }\end{array}$ & A 00PF1 007 XX 2727 ARC 1 & Archive & & \\
\hline TI-065 & 11.6 & $\begin{array}{l}\text { Post-caustic-leach concentrate solids in UFP-VSL-T02A from } \\
\text { UFP-VSL-T01A/B (dewatering) }\end{array}$ & A 00PF1 007 XX 2728 ARC 1 & Archive & & \\
\hline TI-065 & 11.6 & $\begin{array}{l}\text { Post-caustic-leach concentrate solids in UFP-VSL-T02A from } \\
\text { UFP-VSL-T01A/B (dewatering) }\end{array}$ & A 00PF1 007 XX 2729 ARC 1 & Archive & & \\
\hline TI-065 & 11.6 & $\begin{array}{l}\text { Post-caustic-leach concentrate solids in UFP-VSL-T02A from } \\
\text { UFP-VSL-T01A/B (dewatering) }\end{array}$ & A 00PF1 007 XX 2730 ARC 1 & Archive & & \\
\hline TI-065 & 11.6 & $\begin{array}{l}\text { Post-caustic-leach concentrate solids in UFP-VSL-T02A from } \\
\text { UFP-VSL-T01A/B (dewatering) }\end{array}$ & A 00PF1 007 XX 2731 ARC 1 & Archive & & \\
\hline
\end{tabular}


Integrated Test A Sample List

\begin{tabular}{|c|c|c|c|c|c|c|}
\hline TI \# & TI Step \# & TI Step Description & Sample ID & Analysis & Folder & Final Electronic Report File Name \\
\hline TI-065 & 11.6 & $\begin{array}{l}\text { Post-caustic-leach concentrate solids in UFP-VSL-T02A from } \\
\text { UFP-VSL-T01A/B (dewatering) }\end{array}$ & A 00 PF 1007 XX 2732 ARC 1 & Archive & & \\
\hline TI-065 & 11.6 & $\begin{array}{l}\text { Post-caustic-leach concentrate solids in UFP-VSL-T02A from } \\
\text { UFP-VSL-T01A/B (dewatering) }\end{array}$ & A 00 PF1 007 XX 2733 ARC 1 & Archive & & \\
\hline TI-065 & 12.1.4 & Evaluate by-pass of filter loop jet within UFP-VSL-T02A & A 000FL 014012272 ICP 2 D & ICP Supernate & SWRI 090217-16 & 090217-16 ICP and ICPMS \\
\hline TI-065 & 12.1.4 & Evaluate by-pass of filter loop jet within UFP-VSL-T02A & A 000FL 014016002 ICP 2 D & ICP Supernate & SWRI 090324-34 & $090320-15$ and $090324-33$ and $090324-34$ and $090324-35$ ICP and ICPMS \\
\hline TI-065 & 12.1.4 & Evaluate by-pass of filter loop jet within UFP-VSL-T02A & A 000FL 014022273 ICP 2 D & ICP Supernate & SWRI 090217-16 & 090217-16 ICP and ICPMS \\
\hline TI-065 & 12.1.4 & Evaluate by-pass of filter loop jet within UFP-VSL-T02A & A 000FL 014026003 ICP 2 D & ICP Supernate & SWRI 090324-34 & 090320-15 and 090324-33 and 090324-34 and 090324-35 ICP and ICPMS \\
\hline TI-065 & 12.1.4 & Evaluate by-pass of filter loop jet within UFP-VSL-T02A & A 000FL 014042274 ICP 2 D & ICP Supernate & SWRI 090217-16 & 090217-16 ICP and ICPMS \\
\hline TI-065 & 12.1.4 & Evaluate by-pass of filter loop jet within UFP-VSL-T02A & A 000FL 014046004 ICP 2 D & ICP Supernate & SWRI 090324-34 & 090320-15 and 090324-33 and 090324-34 and 090324-35 ICP and ICPMS \\
\hline TI-065 & 12.1.4 & Evaluate by-pass of filter loop jet within UFP-VSL-T02A & A 000FL 014082275 ICP 2 D & ICP Supernate & SWRI 090217-16 & 090217-16 ICP and ICPMS \\
\hline TI-065 & 12.1.4 & Evaluate by-pass of filter loop jet within UFP-VSL-T02A & A 000FL 014086005 ICP 2 D & ICP Supernate & SWRI 090324-34 & 090320-15 and 090324-33 and 090324-34 and 090324-35 ICP and ICPMS \\
\hline TI-065 & 12.1.4 & Evaluate by-pass of filter loop jet within UFP-VSL-T02A & A 000FL 014162276 ICP 2 D & ICP Supernate & SWRI 090217-16 & 090217-16 ICP and ICPMS \\
\hline TI-065 & 12.1.4 & Evaluate by-pass of filter loop jet within UFP-VSL-T02A & A 000FL 014166006 ICP 2 D & ICP Supernate & SWRI 090324-34 & 090320-15 and 090324-33 and 090324-34 and 090324-35 ICP and ICPMS \\
\hline TI-065 & 12.1.4 & Evaluate by-pass of filter loop jet within UFP-VSL-T02A & A 000FL 014302277 ICP 2 D & ICP Supernate & SWRI 090217-16 & 090217-16 ICP and ICPMS \\
\hline TI-065 & 12.1.4 & Evaluate by-pass of filter loop jet within UFP-VSL-T02A & A 000FL 014306007 ICP 2 D & ICP Supernate & SWRI 090324-34 & 090320-15 and 090324-33 and 090324-34 and 090324-35 ICP and ICPMS \\
\hline TI-065 & 12.1.4 & Evaluate by-pass of filter loop jet within UFP-VSL-T02A & A 000FL 014402278 ICP 2 D & ICP Supernate & SWRI 090217-16 & 090217-16 ICP and ICPMS \\
\hline TI-065 & 12.1.4 & Evaluate by-pass of filter loop jet within UFP-VSL-T02A & A 000FL 014406008 ICP 2 D & ICP Supernate & SWRI 090324-34 & 090320-15 and 090324-33 and 090324-34 and 090324-35 ICP and ICPMS \\
\hline TI-065 & 12.1.4 & Evaluate by-pass of filter loop jet within UFP-VSL-T02A & A 000FL 014502279 ICP 2 D & ICP Supernate & SWRI 090217-16 & 090217-16 ICP and ICPMS \\
\hline TI-065 & 12.1.4 & Evaluate by-pass of filter loop jet within UFP-VSL-T02A & A 000FL 014506009 ICP 2 D & ICP Supernate & SWRI 090324-34 & 090320-15 and 090324-33 and 090324-34 and 090324-35 ICP and ICPMS \\
\hline TI-065 & 12.1.4 & Evaluate by-pass of filter loop jet within UFP-VSL-T02A & A 000FL 014602280 ICP 2 D & ICP Supernate & SWRI 090217-16 & 090217-16 ICP and ICPMS \\
\hline TI-065 & 12.1.4 & Evaluate by-pass of filter loop jet within UFP-VSL-T02A & A 000FL 014606010 ICP 2 D & ICP Supernate & SWRI 090324-34 & 090320-15 and 090324-33 and 090324-34 and 090324-35 ICP and ICPMS \\
\hline TI-065 & 12.1.4 & Evaluate by-pass of filter loop jet within UFP-VSL-T02A & A 000FL 014 BT 2271 ICP 2 D & ICP Supernate & SWRI 090217-16 & 090217-16 ICP and ICPMS \\
\hline TI-065 & 12.1.4 & Evaluate by-pass of filter loop jet within UFP-VSL-T02A & A 000FL 014 BT 6001 ICP 2 D & ICP Supernate & SWRI 090324-34 & $090320-15$ and $090324-33$ and $090324-34$ and $090324-35$ ICP and ICPMS \\
\hline TI-065 & 12.1.4 & Evaluate by-pass of filter loop jet within UFP-VSL-T02A & A 02AOL 014012281 RHE 4 & Rheology & TDP-WTP-359 & TDP WTP 359 Int Test A RHE Data on PEP Slurry \\
\hline TI-065 & 12.1.4 & Evaluate by-pass of filter loop jet within UFP-VSL-T02A & A $02 A O L ~ 014012282$ ICP 2 D & ICP Supernate & SWRI 090217-16 & 090217-16 ICP and ICPMS \\
\hline TI-065 & 12.1.4 & Evaluate by-pass of filter loop jet within UFP-VSL-T02A & A 02AOL 014016011 RHE 4 & Rheology & TDP WTP 363 & TDP WTP 363 Int Test B RHE Data on PEP slurry \\
\hline TI-065 & 12.1.4 & Evaluate by-pass of filter loop jet within UFP-VSL-T02A & A 02 AOL 014016011 RHE 4 & Rheology & TDP-WTP-363 & TDP WTP 363 Int Test B RHE Data on PEP slurry \\
\hline TI-065 & 12.1.4 & Evaluate by-pass of filter loop jet within UFP-VSL-T02A & A 02AOL 014016012 ICP 2 D & ICP Supernate & SWRI 090324-34 & 090320-15 and 090324-33 and 090324-34 and 090324-35 ICP and ICPMS \\
\hline TI-065 & 12.1.4 & Evaluate by-pass of filter loop jet within UFP-VSL-T02A & A $02 \mathrm{AOL} 014022283$ ICP $2 \mathrm{D}$ & ICP Supernate & SWRI 090217-16 & 090217-16 ICP and ICPMS \\
\hline TI-065 & 12.1.4 & Evaluate by-pass of filter loop jet within UFP-VSL-T02A & A 02AOL 014026013 ICP 2 D & ICP Supernate & SWRI 090324-34 & 090320-15 and 090324-33 and 090324-34 and 090324-35 ICP and ICPMS \\
\hline TI-065 & 12.1.4 & Evaluate by-pass of filter loop jet within UFP-VSL-T02A & A $02 \mathrm{AOL} 014042284$ ICP $2 \mathrm{D}$ & ICP Supernate & SWRI 090217-16 & 090217-16 ICP and ICPMS \\
\hline TI-065 & 12.1.4 & Evaluate by-pass of filter loop jet within UFP-VSL-T02A & A $02 \mathrm{AOL} 014046014$ ICP $2 \mathrm{D}$ & ICP Supernate & SWRI 090324-34 & $090320-15$ and $090324-33$ and $090324-34$ and $090324-35$ ICP and ICPMS \\
\hline
\end{tabular}


Integrated Test A Sample List

\begin{tabular}{|c|c|c|c|c|c|c|}
\hline TI \# & TI Step \# & TI Step Description & Sample ID & Analysis & Folder & Final Electronic Report File Name \\
\hline TI-065 & 12.1 .4 & Evaluate by-pass of filter loop jet within UFP-VSL-T02A & A 02AOL 014082285 ICP 2 D & ICP Supernate & SWRI 090217-16 & 090217-16 ICP and ICPMS \\
\hline TI-065 & 12.1.4 & Evaluate by-pass of filter loop jet within UFP-VSL-T02A & A 02AOL 014086015 ICP 2 D & ICP Supernate & SWRI 090324-34 & 090320-15 and 090324-33 and 090324-34 and 090324-35 ICP and ICPMS \\
\hline TI-065 & 12.1.4 & Evaluate by-pass of filter loop jet within UFP-VSL-T02A & A 02AOL 014162286 ICP 2 D & ICP Supernate & SWRI 090217-16 & 090217-16 ICP and ICPMS \\
\hline TI-065 & 12.1.4 & Evaluate by-pass of filter loop jet within UFP-VSL-T02A & A 02AOL 014166016 ICP 2 D & ICP Supernate & SWRI 090324-34 & 090320-15 and 090324-33 and 090324-34 and 090324-35 ICP and ICPMS \\
\hline TI-065 & 12.1.4 & Evaluate by-pass of filter loop jet within UFP-VSL-T02A & A 02AOL 014302287 ICP 2 D & ICP Supernate & SWRI 090217-16 & 090217-16 ICP and ICPMS \\
\hline TI-065 & 12.1 .4 & Evaluate by-pass of filter loop jet within UFP-VSL-T02A & A 02AOL 014306017 ICP 2 D & ICP Supernate & SWRI 090324-34 & $090320-15$ and $090324-33$ and $090324-34$ and $090324-35$ ICP and ICPMS \\
\hline TI-065 & 12.1.4 & Evaluate by-pass of filter loop jet within UFP-VSL-T02A & A 02AOL 014402288 ICP 2 D & ICP Supernate & SWRI 090217-16 & 090217-16 ICP and ICPMS \\
\hline TI-065 & 12.1.4 & Evaluate by-pass of filter loop jet within UFP-VSL-T02A & A 02AOL 014406018 ICP 2 D & ICP Supernate & SWRI 090324-34 & 090320-15 and 090324-33 and 090324-34 and 090324-35 ICP and ICPMS \\
\hline TI-065 & 12.1.4 & Evaluate by-pass of filter loop jet within UFP-VSL-T02A & A 02AOL 014502289 ICP 2 D & ICP Supernate & SWRI 090217-16 & 090217-16 ICP and ICPMS \\
\hline TI-065 & 12.1.4 & Evaluate by-pass of filter loop jet within UFP-VSL-T02A & A 02AOL 014506019 ICP 2 D & ICP Supernate & SWRI 090324-35 & 090320-15 and 090324-33 and 090324-34 and 090324-35 ICP and ICPMS \\
\hline TI-065 & 12.1.4 & Evaluate by-pass of filter loop jet within UFP-VSL-T02A & A 02AOL 014602290 ICP 2 D & ICP Supernate & SWRI 090217-16 & 090217-16 ICP and ICPMS \\
\hline TI-065 & 12.1 .4 & Evaluate by-pass of filter loop jet within UFP-VSL-T02A & A 02AOL 014606020 ICP 2 D & ICP Supernate & SWRI 090324-35 & $090320-15$ and $090324-33$ and $090324-34$ and $090324-35$ ICP and ICPMS \\
\hline TI-065 & 12.1.4 & Evaluate by-pass of filter loop jet within UFP-VSL-T02A & A 02AOL 014706022 ICP 2 D & ICP Supernate & SWRI 090324-34 & $090320-15$ and $090324-33$ and $090324-34$ and $090324-35$ ICP and ICPMS \\
\hline TI-065 & 12.1.4 & Evaluate by-pass of filter loop jet within UFP-VSL-T02A & A 02AOM 014606021 ICP 2 D & ICP Supernate & SWRI 090324-34 & $090320-15$ and $090324-33$ and $090324-34$ and $090324-35$ ICP and ICPMS \\
\hline TI-065 & 12.1.4 & Evaluate by-pass of filter loop jet within UFP-VSL-T02A & A 0CSBR 014 XX 6000 ICP 2 & ICP Supernate & SWRI 090324-35 & $090320-15$ and $090324-33$ and $090324-34$ and $090324-35$ ICP and ICPMS \\
\hline TI-065 & $\mathrm{n} / \mathrm{a}$ & $\mathrm{n} / \mathrm{a}$ & A 000FL 014 XX 2814 RHE 4 & Rheology & TDP-WTP-359 & TDP WTP 359 Int Test A RHE Data on PEP Slurry \\
\hline TI-065 & 13.1 & Post-caustic-leach water wash & A 02AOL 015 XX 2291 UDS 2 & UDS & UDS R1 & UDS R1 Shake and Test A and Test B Step001 Date 08182009 \\
\hline TI-065 & 13.1 & Post-caustic-leach water wash & A 02AOL 015 XX 2293 RHE 4 & Rheology & TDP-WTP-359 & TDP WTP 359 Int Test A RHE Data on PEP Slurry \\
\hline TI-065 & 13.1 & Post-caustic-leach water wash & A 02AOL 015 XX 2294 RHE 4 & Rheology & TDP-WTP-359 & TDP WTP 359 Int Test A RHE Data on PEP Slurry \\
\hline TI-065 & 13.1 & Post-caustic-leach water wash & A $02 \mathrm{AOL} 015$ XX 2295 ICP 2 B & ICP Wet Solids & SWRI 090217-14 & 090217-14 090219-18 090219-19 ICP and ICPMS \\
\hline TI-065 & 13.1 & Post-caustic-leach water wash & A 02AOL 015 XX 2295 ICP 2 D & ICP Supernate & SWRI 090217-13 & 090217-13 and 090219-11 ICP and ICPMS \\
\hline TI-065 & 13.1 & Post-caustic-leach slurry wash & A 02AOL 015 XX 2295 RAM 3 D & Raman & ASR 8362 & ASR 8362 RAM rev 1 \\
\hline TI-065 & 13.1 & Post-caustic-leach water wash & A 02AOL 015 XX 2295 TFI 2 D & IC & SWRI 090217-15, & 090217-15IC Rev 1 \\
\hline TI-065 & 13.1 & Post-caustic-leach slurry wash & A 02AOL 015 XX 2295 TFI 3 D & $\mathrm{OH}$ & ASR 8363 & ASR8363 OH \\
\hline TI-065 & 13.1 & Post-caustic-leach water wash & A 02AOL 015 XX 2296 SOX 2 & TIC TOC & SWRI 090217-12 & 090217-12 TIC TOC \\
\hline TI-065 & 13.1 & Post-caustic-leach water wash & A 02 AOL 015 XX 2297 AFA 2 & ICP Wet Solids & SWRI 090217-11 & 090217-11 ICP and ICPMS \\
\hline TI-065 & 13.1 & Post-caustic-leach water wash & A 02AOL 015 XX 2297 AFA 2 & ICP Supernate & SWRI 090217-11 & 090217-11 ICP and ICPMS \\
\hline TI-65 & 13.1 & Post-caustic-leach water wash & A 02AOL 015 XX 2298 AFA 5 B & THF Wet Solids & 48837 & PNNL 48837 Report.pdf \\
\hline TI-65 & 13.1 & Post-caustic-leach water wash & A 02AOL 015 XX 2298 AFA 5 D & THF Supernate & 48837 & PNNL 48837 Report.pdf \\
\hline TI-65 & 13.1 & Post-caustic-leach water wash & A 02AOL 015 XX 2299 AFA 5 D & Toluene Supernate & 48837 & PNNL 48837 Report.pdf \\
\hline TI-65 & 13.1 & Post-caustic-leach water wash & A 02AOL 015 XX 2299 AFA 5 B & Toluene Wet Solids & 48837 & PNNL 48837 Report.pdf \\
\hline TI-065 & 13.1 & Post-caustic-leach water wash & A 02AOL 015 XX 2809 XSP 0 & PSD & TDP-WTP-358 & TDP WTP 358 Int Test A PSD Data Analysis \\
\hline TI-065 & 13.1 & Post-caustic-leach water wash & A 02AOL 015 XX 2811 XSP 0 & PSD & TDP-WTP-358 & TDP WTP 358 Int Test A PSD Data Analysis \\
\hline
\end{tabular}


Integrated Test A Sample List

\begin{tabular}{|c|c|c|c|c|c|c|}
\hline TI \# & TI Step \# & TI Step Description & Sample ID & Analysis & Folder & Final Electronic Report File Name \\
\hline TI-065 & 13.4 .3 & Post-caustic-leach water wash & A $02 \mathrm{AOL} 015012300 \mathrm{ICP} 2 \mathrm{D}$ & ICP Supernate & SWRI 090217-13 & 090217-13 and 090219-11 ICP and ICPMS \\
\hline TI-065 & 13.4 .3 & Post-caustic-leach water wash & A 02AOL 015012300 RAM 3 D & Raman & ASR 8362 & ASR 8362 RAM rev 1 \\
\hline TI-065 & 13.4 .3 & Post-caustic-leach water wash & A 02AOL 015012300 TFI 2 D & IC & SWRI 090217-15 & 090217-15IC Rev 1 \\
\hline TI-065 & 13.4.3 & Post-caustic-leach water wash & A 02AOL 015012300 TFI 3 D & $\mathrm{OH}$ & ASR 8363 & ASR $8363 \mathrm{OH}$ \\
\hline TI-065 & 13.4.3 & Post-caustic-leach water wash & A 02 AOL 015022301 UDS 2 & UDS & UDS R1 & UDS R1 Shake and Test A and Test B Step001 Date 08182009 \\
\hline TI-065 & 13.4 .3 & Post-caustic-leach water wash & A 02AOL 015022302 DEN 2 & Density & SWRI 090217-10 & 090217-10 DEN \\
\hline TI-065 & 13.4 .3 & Post-caustic-leach water wash & A 02 AOL 015022302 DEN 2 & Density Supernate & SWRI 090217-10 & 090217-10 liquidDEN \\
\hline TI-065 & 13.4 .3 & Post-caustic-leach water wash & A $02 A O L 015022303$ ICP 2 D & ICP Supernate & SWRI 090217-13 & 090217-13 and 090219-11 ICP and ICPMS \\
\hline TI-065 & 13.4 .3 & Post-caustic-leach water wash & A 02AOL 015022303 RAM 3 D & Raman & ASR 8362 & ASR 8362 RAM rev 1 \\
\hline TI-065 & 13.4 .3 & Post-caustic-leach water wash & A $02 \mathrm{AOL} 015022303$ TFI $2 \mathrm{D}$ & IC & SWRI 090217-15 & 090217-15IC Rev 1 \\
\hline TI-065 & 13.4 .3 & Post-caustic-leach water wash & A 02AOL 015022303 TFI 3 D & $\mathrm{OH}$ & ASR 8363 & ASR8363 OH \\
\hline TI-065 & 13.4 .3 & Post-caustic-leach water wash & A 02AOL 015032304 ICP 2 D & ICP Supernate & SWRI 090217-13 & 090217-13 and 090219-11 ICP and ICPMS \\
\hline TI-065 & 13.4.3 & Post-caustic-leach water wash & A 02AOL 015032304 RAM 3 D & Raman & ASR 8362 & ASR 8362 RAM rev 1 \\
\hline TI-065 & 13.4 .3 & Post-caustic-leach water wash & A 02AOL 015032304 TFI 2 D & IC & SWRI 090217-15 & 090217-15IC Rev 1 \\
\hline TI-065 & 13.4 .3 & Post-caustic-leach water wash & A 02AOL 015032304 TFI 3 D & $\mathrm{OH}$ & ASR 8363 & ASR8363 OH \\
\hline TI-065 & 13.4.3 & Post-caustic-leach water wash & A $02 A O L ~ 015042305$ UDS 2 & UDS & UDS R1 & UDS R1 Shake and Test A and Test B Step001 Date 08182009 \\
\hline TI-065 & 13.4 .3 & Post-caustic-leach water wash & A 02AOL 015042306 DEN 2 & Density & SWRI 090217-10 & 090217-10 DEN \\
\hline TI-065 & 13.4 .3 & Post-caustic-leach water wash & A 02AOL 015042306 DEN 2 & Density Supernate & SWRI 090217-10 & 090217-10 liquidDEN \\
\hline TI-065 & 13.4 .3 & Post-caustic-leach water wash & A 02AOL 015042307 ICP 2 D & ICP Supernate & SWRI 090217-13 & 090217-13 and 090219-11 ICP and ICPMS \\
\hline TI-065 & 13.4 .3 & Post-caustic-leach water wash & A 02AOL 015042307 RAM 3 D & Raman & ASR 8362 & ASR 8362 RAM rev 1 \\
\hline TI-065 & 13.4 .3 & Post-caustic-leach water wash & A 02AOL 015042307 TFI 2 D & IC & SWRI 090217-15 & 090217-15IC Rev 1 \\
\hline TI-065 & 13.4 .3 & Post-caustic-leach water wash & A 02AOL 015042307 TFI 3 D & $\mathrm{OH}$ & ASR 8363 & ASR8363 OH \\
\hline TI-065 & 13.4 .3 & Post-caustic-leach water wash & A 02AOL 015052308 ICP 2 D & ICP Supernate & SWRI 090217-13 & 090217-13 and 090219-11 ICP and ICPMS \\
\hline TI-065 & 13.4 .3 & Post-caustic-leach water wash & A 02AOL 015052308 RAM 3 D & Raman & ASR 8362 & ASR 8362 RAM rev 1 \\
\hline TI-065 & 13.4 .3 & Post-caustic-leach water wash & A 02AOL 015052308 TFI 2 D & IC & SWRI 090217-15 & 090217-15IC Rev 1 \\
\hline TI-065 & 13.4 .3 & Post-caustic-leach water wash & A 02AOL 015052308 TFI 3 D & $\mathrm{OH}$ & ASR 8363 & ASR8363 OH \\
\hline TI-065 & 13.4.3 & Post-caustic-leach water wash & A 02AOL 015062309 UDS 2 & UDS & UDS R1 & UDS R1 Shake and Test A and Test B Step001 Date 08182009 \\
\hline TI-065 & 13.4 .3 & Post-caustic-leach water wash & A 02AOL 015062310 DEN 2 & Density & SWRI 090217-10 & 090217-10 DEN \\
\hline TI-065 & 13.4 .3 & Post-caustic-leach water wash & A 02AOL 015062310 DEN 2 & Density Supernate & SWRI 090217-10 & 090217-10 liquidDEN \\
\hline TI-065 & 13.4 .3 & Post-caustic-leach water wash & A $02 \mathrm{AOL} 015062311$ ICP $2 \mathrm{D}$ & ICP Supernate & SWRI 090217-13 & 090217-13 and 090219-11 ICP and ICPMS \\
\hline TI-065 & 13.4 .3 & Post-caustic-leach water wash & A 02AOL 015062311 RAM 3 D & Raman & ASR 8362 & ASR 8362 RAM rev 1 \\
\hline TI-065 & 13.4 .3 & Post-caustic-leach water wash & A 02AOL 015062311 TFI 2 D & IC & SWRI 090217-15 & 090217-15IC Rev 1 \\
\hline TI-065 & 13.4 .3 & Post-caustic-leach water wash & A 02AOL 015062311 TFI 3 D & $\mathrm{OH}$ & ASR 8363 & ASR8363 OH \\
\hline
\end{tabular}


Integrated Test A Sample List

\begin{tabular}{|c|c|c|c|c|c|c|}
\hline TI \# & TI Step \# & TI Step Description & Sample ID & Analysis & Folder & Final Electronic Report File Name \\
\hline TI-065 & 13.4 .3 & Post-caustic-leach water wash & A 02AOL 015072312 ICP 2 D & ICP Supernate & SWRI 090217-13 & 090217-13 and 090219-11 ICP and ICPMS \\
\hline TI-065 & 13.4.3 & Post-caustic-leach water wash & A 02AOL 015072312 RAM 3 D & Raman & ASR 8362 & ASR 8362 RAM rev 1 \\
\hline TI-065 & 13.4.3 & Post-caustic-leach water wash & A 02AOL 015072312 TFI 2 D & IC & SWRI 090217-15 & 090217-15IC Rev 1 \\
\hline TI-065 & 13.4.3 & Post-caustic-leach water wash & A 02AOL 015072312 TFI 3 D & $\mathrm{OH}$ & ASR 8363 & ASR8363 OH \\
\hline TI-065 & 13.4.3 & Post-caustic-leach water wash & A 02 AOL 015082313 UDS 2 & UDS & UDS R1 & UDS R1 Shake and Test A and Test B Step001 Date 08182009 \\
\hline TI-065 & 13.4.3 & Post-caustic-leach water wash & A 02AOL 015082314 DEN 2 & Density & SWRI 090217-10 & 090217-10 DEN \\
\hline TI-065 & 13.4.3 & Post-caustic-leach water wash & A $02 \mathrm{AOL} 015082314$ DEN 2 & Density Supernate & SWRI 090217-10 & 090217-10 liquidDEN \\
\hline TI-065 & 13.4.3 & Post-caustic-leach water wash & A 02AOL 015082315 ICP 2 D & ICP Supernate & SWRI 090217-13 & 090217-13 and 090219-11 ICP and ICPMS \\
\hline TI-065 & 13.4.3 & Post-caustic-leach water wash & A $02 A O L ~ 015082315$ RAM 3 D & Raman & ASR 8362 & ASR 8362 RAM rev 1 \\
\hline TI-065 & 13.4.3 & Post-caustic-leach water wash & A 02AOL 015082315 TFI 2 D & IC & SWRI 090217-15 & 090217-15IC Rev 1 \\
\hline TI-065 & 13.4.3 & Post-caustic-leach water wash & A 02AOL 015082315 TFI 3 D & $\mathrm{OH}$ & ASR 8363 & ASR8363 OH \\
\hline TI-065 & 13.4.3 & Post-caustic-leach water wash & A $02 A O L ~ 015092316$ ICP 2 D & ICP Supernate & SWRI 090219-22 & 090219-17 and 0902019-22 ICP and ICPMS \\
\hline TI-065 & 13.4.3 & Post-caustic-leach water wash & A 02AOL 015092316 RAM 3 D & Raman & ASR 8365 & ASR 8365.01 RAM rev 1 \\
\hline TI-065 & 13.4.3 & Post-caustic-leach water wash & A 02AOL 015092316 TFI 2 D & IC & $\begin{array}{l}\text { SWRI 090219-7, } \\
\text { SWRI 090219-24, } \\
\text { SWRI 090219-27 }\end{array}$ & 090219-24IC \\
\hline TI-065 & 13.4.3 & Post-caustic-leach water wash & A 02AOL 015092316 TFI 3 D & $\mathrm{OH}$ & ASR 8366 & ASR 8366_1 OH \\
\hline TI-065 & 13.4 .3 & Post-caustic-leach water wash & A 02 AOL 015102317 UDS 2 & UDS & UDS R1 & UDS R1 Shake and Test A and Test B Step001 Date 08182009 \\
\hline TI-065 & 13.4.3 & Post-caustic-leach water wash & A 02AOL 015102318 DEN 2 & Density & SWRI 090217-10 & 090217-10 DEN \\
\hline TI-065 & 13.4.3 & Post-caustic-leach water wash & A 02AOL 015102318 DEN 2 & Density Supernate & SWRI 090217-10 & 090217-10 liquidDEN \\
\hline TI-065 & 13.4 .3 & Post-caustic-leach water wash & A 02AOL 015102319 RHE 4 & Rheology & TDP-WTP-359 & TDP WTP 359 Int Test A RHE Data on PEP Slurry \\
\hline TI-065 & 13.4.3 & Post-caustic-leach water wash & A 02AOL 015102320 RHE 4 & Rheology & TDP-WTP-359 & TDP WTP 359 Int Test A RHE Data on PEP Slurry \\
\hline TI-065 & 13.4.3 & Post-caustic-leach water wash & A 02AOL 015102321 ICP 2 D & ICP Supernate & SWRI 090219-22 & 090219-17 and 0902019-22 ICP and ICPMS \\
\hline TI-065 & 13.4.3 & Post-caustic-leach water wash & A 02AOL 015102321 RAM 3 D & Raman & ASR 8365 & ASR 8365.01 RAM rev 1 \\
\hline TI-065 & 13.4.3 & Post-caustic-leach water wash & A 02AOL 015102321 TFI 2 D & IC & $\begin{array}{l}\text { SWRI 090219-7, } \\
\text { SWRI 090219-24, } \\
\text { SWRI 090219-27 }\end{array}$ & 090219-24IC \\
\hline TI-065 & 13.4.3 & Post-caustic-leach water wash & A 02AOL 015102321 TFI 3 D & $\mathrm{OH}$ & ASR 8366 & ASR 8366_1 OH \\
\hline TI-065 & 13.4.3 & Post-caustic-leach water wash & A 02AOL 015112322 ICP 2 D & ICP Supernate & SWRI 090219-23 & 090219-12 and 090219-23 TDS and ICP \\
\hline TI-065 & 13.4 .3 & Post-caustic-leach water wash & A 02AOL 015112322 RAM 3 D & Raman & ASR 8365 & ASR 8365.01 RAM rev 1 \\
\hline TI-065 & 13.4 .3 & Post-caustic-leach water wash & A 02AOL 015112322 TFI 2 D & IC & $\begin{array}{l}\text { SWRI 090219-7, } \\
\text { SWRI 090219-24, } \\
\text { SWRI 090219-27 }\end{array}$ & 090219-24IC \\
\hline TI-065 & 13.4.3 & Post-caustic-leach water wash & A 02AOL 015112322 TFI 3 D & $\mathrm{OH}$ & ASR 8366 & ASR 8366_1 OH \\
\hline
\end{tabular}


Integrated Test A Sample List

\begin{tabular}{|c|c|c|c|c|c|c|}
\hline TI \# & TI Step \# & TI Step Description & Sample ID & Analysis & Folder & Final Electronic Report File Name \\
\hline TI-065 & 13.4 .3 & Post-caustic-leach water wash & A 02AOL 015122323 UDS 2 & UDS & UDS R1 & UDS R1 Shake and Test A and Test B Step001 Date 08182009 \\
\hline TI-065 & 13.4.3 & Post-caustic-leach water wash & A 02AOL 015122324 DEN 2 & Density & SWRI 090217-10 & 090217-10 DEN \\
\hline TI-065 & 13.4 .3 & Post-caustic-leach water wash & A 02AOL 015122324 DEN 2 & Density Supernate & SWRI 090217-10 & 090217-10 liquidDEN \\
\hline TI-065 & 13.4 .3 & Post-caustic-leach water wash & A 02AOL 015122325 ICP 2 D & ICP Supernate & SWRI 090217-13 & 090217-13 and 090219-11 ICP and ICPMS \\
\hline TI-065 & 13.4 .3 & Post-caustic-leach water wash & A 02AOL 015122325 RAM 3 D & Raman & ASR 8362 & ASR 8362 RAM rev 1 \\
\hline TI-065 & 13.4 .3 & Post-caustic-leach water wash & A 02AOL 015122325 TFI 2 D & $\mathrm{IC}$ & SWRI 090217-15 & 090217-15IC Rev 1 \\
\hline TI-065 & 13.4 .3 & Post-caustic-leach water wash & A $02 \mathrm{AOL} 015122325$ TFI $3 \mathrm{D}$ & $\mathrm{OH}$ & ASR 8363 & ASR8363 OH \\
\hline TI-065 & 13.4 .3 & Post-caustic-leach water wash & A 02AOL 015132326 ICP 2 D & ICP Supernate & SWRI 090217-13 & 090217-13 and 090219-11 ICP and ICPMS \\
\hline TI-065 & 13.4 .3 & Post-caustic-leach water wash & A 02AOL 015132326 RAM 3 D & Raman & ASR 8362 & ASR 8362 RAM rev 1 \\
\hline TI-065 & 13.4 .3 & Post-caustic-leach water wash & A 02AOL 015132326 TFI 2 D & $\mathrm{IC}$ & SWRI 090217-15 & 090217-15IC Rev 1 \\
\hline TI-065 & 13.4 .3 & Post-caustic-leach water wash & A 02AOL 015132326 TFI 3 D & $\mathrm{OH}$ & ASR 8363 & ASR8363 OH \\
\hline TI-065 & 13.4 .3 & Post-caustic-leach water wash & A 02AOL 015142327 UDS 2 & UDS & UDS R1 & UDS R1 Shake and Test A and Test B Step001 Date 08182009 \\
\hline TI-065 & 13.4 .3 & Post-caustic-leach water wash & A $02 \mathrm{AOL} 015142328 \mathrm{DEN} 2$ & Density & SWRI 090217-10 & 090217-10 DEN \\
\hline TI-065 & 13.4 .3 & Post-caustic-leach water wash & A 02AOL 015142328 DEN 2 & Density Supernate & SWRI 090217-10 & 090217-10 liquidDEN \\
\hline TI-065 & 13.4 .3 & Post-caustic-leach water wash & A 02AOL 015142329 ICP 2 D & ICP Supernate & SWRI 090217-13 & 090217-13 and 090219-11 ICP and ICPMS \\
\hline TI-065 & 13.4 .3 & Post-caustic-leach water wash & A 02AOL 015142329 RAM 3 D & Raman & ASR 8362 & ASR 8362 RAM rev 1 \\
\hline TI-065 & 13.4 .3 & Post-caustic-leach water wash & A 02AOL 015142329 TFI 2 D & IC & SWRI 090217-15 & 090217-15IC Rev 1 \\
\hline TI-065 & 13.4.3 & Post-caustic-leach water wash & A 02AOL 015142329 TFI 3 D & $\mathrm{OH}$ & ASR 8363 & ASR8363 OH \\
\hline TI-065 & 13.4 .3 & Post-caustic-leach water wash & A 02AOL 015152330 ICP 2 D & ICP Supernate & SWRI 090217-13 & 090217-13 and 090219-11 ICP and ICPMS \\
\hline TI-065 & 13.4 .3 & Post-caustic-leach water wash & A 02AOL 015152330 RAM 3 D & Raman & ASR 8362 & ASR 8362 RAM rev 1 \\
\hline TI-065 & 13.4 .3 & Post-caustic-leach water wash & A 02AOL 015152330 TFI 2 D & IC & SWRI 090217-15 & 090217-15IC Rev 1 \\
\hline TI-065 & 13.4 .3 & Post-caustic-leach water wash & A 02AOL 015152330 TFI 3 D & $\mathrm{OH}$ & ASR 8363 & ASR8363 OH \\
\hline TI-065 & 13.4 .3 & Post-caustic-leach water wash & A $02 \mathrm{AOL} 015162331$ UDS 2 & UDS & UDS R1 & UDS R1 Shake and Test A and Test B Step001 Date 08182009 \\
\hline TI-065 & 13.4 .3 & Post-caustic-leach water wash & A 02AOL 015162332 DEN 2 & Density & SWRI 090217-10 & 090217-10 DEN \\
\hline TI-065 & 13.4 .3 & Post-caustic-leach water wash & A 02AOL 015162332 DEN 2 & Density Supernate & SWRI 090217-10 & 090217-10 liquidDEN \\
\hline TI-065 & 13.4 .3 & Post-caustic-leach water wash & A 02AOL 015162333 ICP 2 D & ICP Supernate & SWRI 090217-13 & 090217-13 and 090219-11 ICP and ICPMS \\
\hline TI-065 & 13.4 .3 & Post-caustic-leach water wash & A 02AOL 015162333 RAM 3 D & Raman & ASR 8362 & ASR 8362 RAM rev 1 \\
\hline TI-065 & 13.4 .3 & Post-caustic-leach water wash & A 02AOL 015162333 TFI 2 D & IC & SWRI 090217-15 & 090217-15IC Rev 1 \\
\hline TI-065 & 13.4 .3 & Post-caustic-leach water wash & A 02AOL 015162333 TFI 3 D & $\mathrm{OH}$ & ASR 8363 & ASR8363 OH \\
\hline TI-065 & 13.4.3 & Post-caustic-leach water wash & A 02AOL 015172334 ICP 2 D & ICP Supernate & SWRI 090217-13 & 090217-13 and 090219-11 ICP and ICPMS \\
\hline TI-065 & 13.4 .3 & Post-caustic-leach water wash & A 02AOL 015172334 RAM 3 D & Raman & ASR 8362 & ASR 8362 RAM rev 1 \\
\hline TI-065 & 13.4 .3 & Post-caustic-leach water wash & A 02AOL 015172334 TFI 2 D & IC & SWRI 090217-15 & 090217-15IC Rev 1 \\
\hline TI-065 & 13.4.3 & Post-caustic-leach water wash & A 02AOL 015172334 TFI 3 D & $\mathrm{OH}$ & ASR 8363 & ASR8363 OH \\
\hline
\end{tabular}


Integrated Test A Sample List

\begin{tabular}{|c|c|c|c|c|c|c|}
\hline TI \# & TI Step \# & TI Step Description & Sample ID & Analysis & Folder & Final Electronic Report File Name \\
\hline TI-065 & 13.4 .3 & Post-caustic-leach water wash & A 02AOL 015182335 UDS 2 & UDS & UDS R1 & UDS R1 Shake and Test A and Test B Step001 Date 08182009 \\
\hline TI-065 & 13.4 .3 & Post-caustic-leach water wash & A 02AOL 015182336 DEN 2 & Density & SWRI 090217-10 & 090217-10 DEN \\
\hline TI-065 & 13.4 .3 & Post-caustic-leach water wash & A 02AOL 015182336 DEN 2 & Density Supernate & SWRI 090217-10 & 090217-10 liquidDEN \\
\hline TI-065 & 13.4 .3 & Post-caustic-leach water wash & A 02AOL 015182337 ICP 2 D & ICP Supernate & SWRI 090217-13 & 090217-13 and 090219-11 ICP and ICPMS \\
\hline TI-065 & 13.4 .3 & Post-caustic-leach water wash & A 02AOL 015182337 RAM 3 D & Raman & ASR 8362 & ASR 8362 RAM rev 1 \\
\hline TI-065 & 13.4 .3 & Post-caustic-leach water wash & A 02AOL 015182337 TFI 2 D & IC & SWRI 090217-15 & 090217-15IC Rev 1 \\
\hline TI-065 & 13.4 .3 & Post-caustic-leach water wash & A 02AOL 015182337 TFI 3 D & $\mathrm{OH}$ & ASR 8363 & ASR8363 OH \\
\hline TI-065 & 13.4 .3 & Post-caustic-leach water wash & A 02AOL 015192338 ICP 2 D & ICP Supernate & SWRI 090217-13 & 090217-13 and 090219-11 ICP and ICPMS \\
\hline TI-065 & 13.4 .3 & Post-caustic-leach water wash & A 02AOL 015192338 RAM 3 D & Raman & ASR 8362 & ASR 8362 RAM rev 1 \\
\hline TI-065 & 13.4 .3 & Post-caustic-leach water wash & A 02AOL 015192338 TFI 2 D & IC & SWRI 090217-15 & 090217-15IC Rev 1 \\
\hline TI-065 & 13.4 .3 & Post-caustic-leach water wash & A 02AOL 015192338 TFI 3 D & $\mathrm{OH}$ & ASR 8363 & ASR8363 OH \\
\hline TI-065 & 13.4 .3 & Post-caustic-leach water wash & A 00PF1 015 XX 2339 ARC 4 & Precipitation Study & & \\
\hline TI-065 & 13.4 .3 & Post-caustic-leach water wash & A 00PF1 015 XX 2340 ARC 4 & Precipitation Study & & \\
\hline TI-065 & 13.4 .3 & Post-caustic-leach water wash & A 00PF1 015 XX 2341 ARC 4 & Precipitation Study & & \\
\hline TI-065 & 13.4 .3 & Post-caustic-leach water wash & A 00PF1 015 XX 2342 ARC 4 & Precipitation Study & & \\
\hline TI-065 & 13.4 .3 & Post-caustic-leach water wash & A 00 PF1 015 XX 2343 ARC 4 & Precipitation Study & & \\
\hline TI-065 & 13.4 .3 & Post-caustic-leach water wash & A 00 PF1 015 XX 2344 ARC 4 & Precipitation Study & & \\
\hline TI-065 & 13.4 .3 & Post-caustic-leach water wash & A 00PF1 015 XX 2345 ARC 4 & Precipitation Study & & \\
\hline TI-065 & 13.4 .3 & Post-caustic-leach water wash & A 00PF1 015 XX 2346 ARC 4 & Precipitation Study & & \\
\hline TI-065 & 13.4 .3 & Post-caustic-leach water wash & A 00PF1 015 XX 2347 ARC 4 & Precipitation Study & & \\
\hline TI-065 & 13.4 .3 & Post-caustic-leach water wash & A 00PF1 015 XX 2348 ARC 4 & Precipitation Study & & \\
\hline TI-065 & 13.4 .3 & Post-caustic-leach water wash & A 00PF1 015 XX 2349 ARC 4 & Precipitation Study & & \\
\hline TI-065 & 13.4 .3 & Post-caustic-leach water wash & A 00PF1 015 XX 2350 ARC 4 & Precipitation Study & & \\
\hline TI-065 & 13.4 .3 & Post-caustic-leach water wash & A 00PF1 015 XX 2351 ARC 4 & Precipitation Study & & \\
\hline TI-065 & 13.4 .3 & Post-caustic-leach water wash & A 00PF1 015 XX 2352 ARC 4 & Precipitation Study & & \\
\hline TI-065 & 13.4 .3 & Post-caustic-leach water wash & A 00PF1 015 XX 2353 ARC 4 & Precipitation Study & & \\
\hline TI-065 & 13.4 .3 & Post-caustic-leach water wash & A 00PF1 015 XX 2354 ARC 4 & Precipitation Study & & \\
\hline TI-065 & 13.4 .3 & Post-caustic-leach water wash & A 00PF1 015 XX 2355 ARC 4 & Precipitation Study & & \\
\hline TI-065 & 13.4 .3 & Post-caustic-leach water wash & A 00 PF 1015 XX 2356 ARC 4 & Precipitation Study & & \\
\hline TI-065 & 13.4 .3 & Post-caustic-leach water wash & A 00PF1 015 XX 2357 ARC 4 & Precipitation Study & & \\
\hline TI-065 & 13.4 .3 & Post-caustic-leach water wash & A 00PF1 015 XX 2358 ARC 4 & Precipitation Study & & \\
\hline TI-065 & 13.4 .3 & Post-caustic-leach water wash & A 00PF1 015 XX 2359 ARC 4 & Precipitation Study & & \\
\hline TI-065 & 13.4 .3 & Post-caustic-leach water wash & A 00PF1 015 XX 2360 ARC 4 & Precipitation Study & & \\
\hline
\end{tabular}


Integrated Test A Sample List

\begin{tabular}{|c|c|c|c|c|c|c|}
\hline TI \# & TI Step \# & TI Step Description & Sample ID & Analysis & Folder & Final Electronic Report File Name \\
\hline TI-065 & 13.4.3 & Post-caustic-leach water wash & A 00PF1 015 XX 2361 ARC 4 & Precipitation Study & & \\
\hline TI-065 & 13.4.3 & Post-caustic-leach water wash & A 00PF1 015 XX 2362 ARC 4 & Precipitation Study & & \\
\hline TI-065 & 13.4.3 & Post-caustic-leach water wash & A 00PF 1015 XX 2363 ARC 4 & Precipitation Study & & \\
\hline TI-065 & 13.4 .3 & Post-caustic-leach water wash & A 00PF1 015 XX 2364 ARC 4 & Precipitation Study & & \\
\hline TI-065 & 13.4 .3 & Post-caustic-leach water wash & A 00PF1 015 XX 2365 ARC 4 & Precipitation Study & & \\
\hline TI-065 & 13.4.3 & Post-caustic-leach water wash & A 00PF1 015 XX 2366 ARC 4 & Precipitation Study & & \\
\hline TI-065 & 13.4.3 & Post-caustic-leach water wash & A 00PF1 015 XX 2367 ARC 4 & Precipitation Study & & \\
\hline TI-065 & 13.4.3 & Post-caustic-leach water wash & A 00PF1 015 XX 2368 ARC 4 & Precipitation Study & & \\
\hline TI-065 & 13.4.3 & Post-caustic-leach water wash & A 00PF1 015 XX 2369 ARC 4 & Precipitation Study & & \\
\hline TI-065 & 13.4 .3 & Post-caustic-leach water wash & A 00PF1 015 XX 2370 ARC 4 & Precipitation Study & & \\
\hline TI-065 & 13.4.3 & Post-caustic-leach water wash & A 00PF1 015 XX 2371 ARC 4 & Precipitation Study & & \\
\hline TI-065 & 13.4.3 & Post-caustic-leach water wash & A 00PF1 015 XX 2372 ARC 4 & Precipitation Study & & \\
\hline TI-065 & 13.4 .3 & Post-caustic-leach water wash & A 00PF1 015 XX 2373 ARC 4 & Precipitation Study & & \\
\hline TI-065 & 13.4 .3 & Post-caustic-leach water wash & A 00PF1 015 XX 2374 ARC 4 & Precipitation Study & & \\
\hline TI-065 & 13.4.3 & Post-caustic-leach water wash & A 00PF 1015 XX 2375 ARC 4 & Precipitation Study & & \\
\hline TI-065 & 13.4.3 & Post-caustic-leach water wash & A 00PF1 015 XX 2376 ARC 4 & Precipitation Study & & \\
\hline TI-065 & 13.4.3 & Post-caustic-leach water wash & A 00PF1 015 XX 2556 ARC 4 & Precipitation Study & & \\
\hline TI-065 & 13.4 .3 & Post-caustic-leach water wash & A 00PF1 015 XX 2557 ARC 4 & Precipitation Study & & \\
\hline TI-065 & 13.4 .3 & Post-caustic-leach water wash & A 00PF1 015 XX 2558 ARC 4 & Precipitation Study & & \\
\hline TI-065 & 13.4 .3 & Post-caustic-leach water wash & A 00PF1 015 XX 2559 ARC 4 & Precipitation Study & & \\
\hline TI-065 & 13.4 .3 & Post-caustic-leach water wash & A 00PF1 015 XX 2560 ARC 4 & Precipitation Study & & \\
\hline TI-065 & 13.4 .3 & Post-caustic-leach water wash & A 00PF1 015 XX 2561 ARC 4 & Precipitation Study & & \\
\hline TI-065 & 13.4 .3 & Post-caustic-leach water wash & A 00PF1 015 XX 2562 ARC 4 & Precipitation Study & & \\
\hline TI-065 & 13.4 .3 & Post-caustic-leach water wash & A 00PF1 015 XX 2563 ARC 4 & Precipitation Study & & \\
\hline TI-065 & 13.4.3 & Post-caustic-leach water wash & A 00PF1 015 XX 2564 ARC 4 & Precipitation Study & & \\
\hline TI-065 & 13.4 .3 & Post-caustic-leach water wash & A 00PF1 015 XX 2565 ARC 4 & Precipitation Study & & \\
\hline TI-065 & 13.4 .3 & Post-caustic-leach water wash & A 00PF1 015 XX 2566 ARC 4 & Precipitation Study & & \\
\hline TI-065 & 13.4 .3 & Post-caustic-leach water wash & A 00PF1 015 XX 2567 ARC 4 & Precipitation Study & & \\
\hline TI-065 & 13.4.3 & Post-caustic-leach water wash & A 00PF1 015 XX 2568 ARC 4 & Precipitation Study & & \\
\hline TI-065 & 13.4 .3 & Post-caustic-leach water wash & A 00PF1 015 XX 2569 ARC 4 & Precipitation Study & & \\
\hline TI-065 & 13.4 .3 & Post-caustic-leach water wash & A 00PF1 015 XX 2570 ARC 4 & Precipitation Study & & \\
\hline TI-065 & 13.4 .3 & Post-caustic-leach water wash & A 00PF1 015 XX 2571 ARC 4 & Precipitation Study & & \\
\hline TI-065 & 13.4 .3 & Post-caustic-leach water wash & A 00PF 1015 XX 2572 ARC 4 & Precipitation Study & & \\
\hline
\end{tabular}


Integrated Test A Sample List

\begin{tabular}{|c|c|c|c|c|c|c|}
\hline TI \# & TI Step \# & TI Step Description & Sample ID & Analysis & Folder & Final Electronic Report File Name \\
\hline TI-065 & 13.4 .3 & Post-caustic-leach water wash & A 00PF1 015 XX 2573 ARC 4 & Precipitation Study & & \\
\hline TI-065 & 13.4 .3 & Post-caustic-leach water wash & A 00PF1 015 XX 2574 ARC 4 & Precipitation Study & & \\
\hline TI-065 & 13.4 .3 & Post-caustic-leach water wash & A 00PF1 015 XX 2575 ARC 4 & Precipitation Study & & \\
\hline TI-065 & 13.4 .3 & Post-caustic-leach water wash & A 00PF1 015 XX 2576 ARC 4 & Precipitation Study & & \\
\hline TI-065 & 13.4 .3 & Post-caustic-leach water wash & A 00PF1 015 XX 2577 ARC 4 & Precipitation Study & & \\
\hline TI-065 & 13.4 .3 & Post-caustic-leach water wash & A 00PF1 015 XX 2578 ARC 4 & Precipitation Study & & \\
\hline TI-065 & 13.4 .3 & Post-caustic-leach water wash & A 00PF1 015 XX 2579 ARC 4 & Precipitation Study & & \\
\hline TI-065 & 13.4 .3 & Post-caustic-leach water wash & A 00PF1 015 XX 2580 ARC 4 & Precipitation Study & & \\
\hline TI-065 & 13.4 .3 & Post-caustic-leach water wash & A 00PF1 015 XX 2581 ARC 4 & Precipitation Study & & \\
\hline TI-065 & 13.4 .3 & Post-caustic-leach water wash & A 00PF1 015 XX 2582 ARC 4 & Precipitation Study & & \\
\hline TI-065 & 13.4 .3 & Post-caustic-leach water wash & A 00PF1 015 XX 2583 ARC 4 & Precipitation Study & & \\
\hline TI-065 & 13.4 .3 & Post-caustic-leach water wash & A 00PF1 015 XX 2584 ARC 4 & Precipitation Study & & \\
\hline TI-065 & 13.4 .3 & Post-caustic-leach water wash & A 00PF1 015 XX 2585 ARC 4 & Precipitation Study & & \\
\hline TI-065 & 13.4 .3 & Post-caustic-leach water wash & A 00PF1 015 XX 2586 ARC 4 & Precipitation Study & & \\
\hline TI-065 & 13.4 .3 & Post-caustic-leach water wash & A 00 PF1 015 XX 2587 ARC 4 & Precipitation Study & & \\
\hline TI-065 & 13.4 .3 & Post-caustic-leach water wash & A 00PF 1015 XX 2588 ARC 4 & Precipitation Study & & \\
\hline TI-065 & 13.4 .3 & Post-caustic-leach water wash & A 00PF1 015 XX 2589 ARC 4 & Precipitation Study & & \\
\hline TI-065 & 13.4 .3 & Post-caustic-leach water wash & A 00PF1 015 XX 2590 ARC 4 & Precipitation Study & & \\
\hline TI-065 & 13.4 .3 & Post-caustic-leach water wash & A 00PF1 015 XX 2591 ARC 4 & Precipitation Study & & \\
\hline TI-065 & 13.4 .3 & Post-caustic-leach water wash & A 00PF1 015 XX 2592 ARC 4 & Precipitation Study & & \\
\hline TI-065 & 13.4 .3 & Post-caustic-leach water wash & A 00PF1 015 XX 2593 ARC 4 & Precipitation Study & & \\
\hline TI-065 & 13.4 .3 & Post-caustic-leach water wash & A 00PF1 015 XX 2594 ARC 4 & Precipitation Study & & \\
\hline TI-065 & 13.4 .3 & Post-caustic-leach water wash & A 00PF1 015 XX 2595 ARC 4 & Precipitation Study & & \\
\hline TI-065 & 13.4 .3 & Post-caustic-leach water wash & A 00PF1 015 XX 2596 ARC 4 & Precipitation Study & & \\
\hline TI-065 & 13.4 .3 & Post-caustic-leach water wash & A 00PF1 015 XX 2597 ARC 4 & Precipitation Study & & \\
\hline TI-065 & 13.4 .3 & Post-caustic-leach water wash & A 00PF1 015 XX 2620 ARC 4 & Precipitation Study & & \\
\hline TI-065 & 13.4 .3 & Post-caustic-leach water wash & A 00PF1 015 XX 2621 ARC 4 & Precipitation Study & & \\
\hline TI-065 & 13.4 .3 & Post-caustic-leach water wash & A 00PF1 015 XX 2622 ARC 4 & Precipitation Study & & \\
\hline TI-065 & 13.4 .3 & Post-caustic-leach water wash & A 00PF1 015 XX 2623 ARC 4 & Precipitation Study & & \\
\hline TI-065 & 13.4 .3 & Post-caustic-leach water wash & A 00PF1 015 XX 2624 ARC 4 & Precipitation Study & & \\
\hline TI-065 & 13.4 .3 & Post-caustic-leach water wash & A 00PF1 015 XX 2625 ARC 4 & Precipitation Study & & \\
\hline TI-065 & 13.4 .3 & Post-caustic-leach water wash & A 00PF1 015 XX 2626 ARC 4 & Precipitation Study & & \\
\hline TI-065 & 13.4 .3 & Post-caustic-leach water wash & A 00PF1 015 XX 2627 ARC 4 & Precipitation Study & & \\
\hline
\end{tabular}


Integrated Test A Sample List

\begin{tabular}{|c|c|c|c|c|c|c|}
\hline TI \# & TI Step \# & TI Step Description & Sample ID & Analysis & Folder & Final Electronic Report File Name \\
\hline TI-065 & 13.4 .3 & Post-caustic-leach water wash & A 00PF1 015 XX 2628 ARC 4 & Precipitation Study & & \\
\hline TI-065 & 13.4 .3 & Post-caustic-leach water wash & A 00PF1 015 XX 2629 ARC 4 & Precipitation Study & & \\
\hline TI-065 & 13.4 .3 & Post-caustic-leach water wash & A 00PF 1015 XX 2630 ARC 4 & Precipitation Study & & \\
\hline TI-065 & 13.4 .3 & Post-caustic-leach water wash & A 00PF1 015 XX 2631 ARC 4 & Precipitation Study & & \\
\hline TI-065 & 13.4 .3 & Post-caustic-leach water wash & A 00PF 1015 XX 2632 ARC 4 & Precipitation Study & & \\
\hline TI-065 & 13.4 .3 & Post-caustic-leach water wash & A 00PF1 015 XX 2633 ARC 4 & Precipitation Study & & \\
\hline TI-065 & 13.4 .3 & Post-caustic-leach water wash & A 00PF1 015 XX 2634 ARC 4 & Precipitation Study & & \\
\hline TI-065 & 13.4 .3 & Post-caustic-leach water wash & A 00PF1 015 XX 2635 ARC 4 & Precipitation Study & & \\
\hline TI-065 & 13.4.3 & Post-caustic-leach water wash & A 00PF1 015 XX 2636 ARC 4 & Precipitation Study & & \\
\hline TI-065 & 13.4 .3 & Post-caustic-leach water wash & A 00PF1 015 XX 2637 ARC 4 & Precipitation Study & & \\
\hline TI-065 & 13.4 .3 & Post-caustic-leach water wash & A 00PF1 015 XX 2638 ARC 4 & Precipitation Study & & \\
\hline TI-065 & 13.4 .3 & Post-caustic-leach water wash & A 00PF1 015 XX 2639 ARC 4 & Precipitation Study & & \\
\hline TI-065 & 13.4 .3 & Post-caustic-leach water wash & A 02AOL 015 XX 2292 DEN 2 & Density & SWRI 090217-10 & 090217-10 DEN \\
\hline TI-065 & 13.4 .3 & Post-caustic-leach water wash & A $02 \mathrm{AOL} 015$ XX 2292 DEN 2 & Density Supernate & SWRI 090217-10 & 090217-10 liquidDEN \\
\hline TI-065 & 13.4 .4 & End of post-caustic-leach slurry wash & A 02 AOL 015 XX 2535 UDS 2 & UDS & UDS R1 & UDS R1 Shake and Test A and Test B Step001 Date 08182009 \\
\hline TI-065 & 13.4 .4 & End of post-caustic-leach slurry wash & A $02 \mathrm{AOL} 015 \mathrm{XX} 2536 \mathrm{DEN} 2$ & Density & SWRI 090217-10 & 090217-10 DEN \\
\hline TI-065 & 13.4.4 & End of post-caustic-leach slurry wash & A $02 \mathrm{AOL} 015 \mathrm{XX} 2536 \mathrm{DEN} 2$ & Density Supernate & SWRI 090217-10 & 090217-10 liquidDEN \\
\hline TI-065 & 13.4 .4 & End of post-caustic-leach slurry wash & A $02 \mathrm{AOL} 015 \mathrm{XX} 2537$ ICP $2 \mathrm{~B}$ & ICP Wet Solids & SWRI 090217-14 & 090217-14 090219-18 090219-19 ICP and ICPMS \\
\hline TI-065 & 13.4.4 & End of post-caustic-leach slurry wash & A $02 \mathrm{AOL} 015$ XX 2537 ICP 2 D & ICP Supernate & SWRI 090217-13 & 090217-13 and 090219-11 ICP and ICPMS \\
\hline TI-065 & 13.4.4 & End of post-caustic-leach slurry wash & A $02 A O L ~ 015$ XX 2537 RAM 3 D & Raman & ASR 8362 & ASR 8362 RAM rev 1 \\
\hline TI-065 & 13.4.4 & End of post-caustic-leach slurry wash & A 02AOL 015 XX 2537 TFI 2 D & IC & SWRI 090217-15 & 090217-15IC Rev 1 \\
\hline TI-065 & 13.4 .4 & End of post-caustic-leach slurry wash & A 02AOL 015 XX 2537 TFI 3 D & $\mathrm{OH}$ & ASR 8363 & ASR8363 OH \\
\hline TI-065 & 13.4.4 & End of post-caustic-leach slurry wash & A $02 \mathrm{AOL} 015 \mathrm{XX} 2538$ SOX 2 & TIC TOC & SWRI 090217-12 & 090217-12 TIC TOC \\
\hline TI-065 & 13.4.4 & End of post-caustic-leach slurry wash & A $02 \mathrm{AOL} 015 \mathrm{XX} 2539$ AFA 2 & ICP Wet Solids & SWRI 090217-11 & 090217-11 ICP and ICPMS \\
\hline TI-065 & 13.4.4 & End of post-caustic-leach slurry wash & A 02AOL 015 XX 2539 AFA 2 & ICP Supernate & SWRI 090217-11 & 090217-11 ICP and ICPMS \\
\hline TI-065 & 13.4 .4 & End post-caustic-leach water wash & A $02 \mathrm{AOL} 015 \mathrm{XX} 2540 \mathrm{AFA} 5 \mathrm{~B}$ & THF Wet Solids & 48837 & PNNL 48837 Report.pdf \\
\hline TI-065 & 13.4.4 & End post-caustic-leach water wash & A $02 \mathrm{AOL} 015 \mathrm{XX} 2540$ AFA $5 \mathrm{D}$ & THF Supernate & 48837 & PNNL 48837 Report.pdf \\
\hline TI-065 & 13.4 .4 & End post-caustic-leach water wash & A $02 \mathrm{AOL} 015 \mathrm{XX} 2541$ AFA 5 B & Toluene Wet Solids & 48837 & PNNL 48837 Report.pdf \\
\hline TI-065 & 13.4.4 & End post-caustic-leach water wash & A 02AOL 015 XX 2541 AFA 5 D & Toluene Supernate & 48837 & PNNL 48837 Report.pdf \\
\hline TI-065 & 13.4.4 & End of post-caustic-leach slurry wash & A 02AOL 015 XX 2542 RHE 4 & Rheology & TDP-WTP-359 & TDP WTP 359 Int Test A RHE Data on PEP Slurry \\
\hline TI-065 & 13.4.4 & End of post-caustic-leach slurry wash & A 02AOL 015 XX 2543 RHE 4 & Rheology & TDP-WTP-359 & TDP WTP 359 Int Test A RHE Data on PEP Slurry \\
\hline TI-065 & $\mathrm{n} / \mathrm{a}$ & $\mathrm{n} / \mathrm{a}$ & A 000FL 015 XX 2812 RHE 4 & Rheology & TDP-WTP-359 & TDP WTP 359 Int Test A RHE Data on PEP Slurry \\
\hline
\end{tabular}


Integrated Test A Sample List

\begin{tabular}{|c|c|c|c|c|c|c|}
\hline TI \# & TI Step \# & TI Step Description & Sample ID & Analysis & Folder & Final Electronic Report File Name \\
\hline TI-065 & $\begin{array}{c}14.11 \text { and } \\
14.12\end{array}$ & Before permanganate addition & A 02 AOL 018 XX 2377 UDS 2 & UDS & UDS R1 & UDS R1 Shake and Test A and Test B Step001 Date 08182009 \\
\hline TI-065 & $\begin{array}{c}14.11 \text { and } \\
14.12\end{array}$ & Before permanganate addition & A $02 \mathrm{AOL} 018 \mathrm{XX} 2378$ UDS 2 & UDS & UDS R1 & UDS R1 Shake and Test A and Test B Step001 Date 08182009 \\
\hline TI-065 & $\begin{array}{c}14.11 \text { and } \\
14.12\end{array}$ & Before permanganate addition & A 02AOL 018 XX 2379 UDS 2 & UDS & UDS R1 & UDS R1 Shake and Test A and Test B Step001 Date 08182009 \\
\hline TI-065 & $\begin{array}{c}14.11 \text { and } \\
14.12\end{array}$ & Before permanganate addition & A 02AOL 018 XX 2380 DEN 2 & Density Slurry & SWRI 090219-10 & 090219-10 DEN \\
\hline TI-065 & $\begin{array}{c}14.11 \text { and } \\
14.12\end{array}$ & Before permanganate addition & A $02 \mathrm{AOL} 018 \mathrm{XX} 2381$ DEN 2 & Density Slurry & SWRI 090219-10 & 090219-10 DEN \\
\hline TI-065 & $\begin{array}{c}14.11 \text { and } \\
14.12\end{array}$ & Before permanganate addition & A 02AOL 018 XX 2382 DEN 2 & Density Slurry & SWRI 090219-10 & 090219-10 DEN \\
\hline TI-065 & $\begin{array}{c}14.11 \text { and } \\
14.12\end{array}$ & Before permanganate addition & A 02AOL 018 XX 2383 RHE 4 & Rheology & TDP-WTP-359 & TDP WTP 359 Int Test A RHE Data on PEP Slurry \\
\hline TI-065 & $\begin{array}{c}14.11 \text { and } \\
14.12\end{array}$ & Before permanganate addition & A 02AOL 018 XX 2384 RHE 4 & Rheology & TDP-WTP-359 & TDP WTP 359 Int Test A RHE Data on PEP Slurry \\
\hline TI-065 & $\begin{array}{c}14.11 \text { and } \\
14.12\end{array}$ & Before permanganate addition & A 02AOL 018 XX 2385 RHE 4 & Rheology & TDP-WTP-359 & TDP WTP 359 Int Test A RHE Data on PEP Slurry \\
\hline TI-065 & $\begin{array}{c}14.11 \text { and } \\
14.12\end{array}$ & Before permanganate addition & A 02AOL 018 XX 2386 ICP 2 D & ICP Supernate & SWRI 090219-11 & 090217-13 and 090219-11 ICP and ICPMS \\
\hline TI-065 & $\begin{array}{c}14.11 \text { and } \\
14.12\end{array}$ & Before permanganate addition & A 02AOL 018 XX 2386 ICP 2 R & ICP Rinsate & SWRI 090219-17 & 090219-17 and 0902019-22 ICP and ICPMS \\
\hline TI-065 & $\begin{array}{c}14.11 \text { and } \\
14.12\end{array}$ & Before permanganate addition & A $02 \mathrm{AOL} 018$ XX 2386 ICP $2 \mathrm{~S}$ & ICP Wet Solids & SWRI 090219-18 & 090217-14 090219-18 090219-19 ICP and ICPMS \\
\hline TI-065 & $\begin{array}{c}14.11 \text { and } \\
14.12\end{array}$ & Before permanganate addition & A $02 \mathrm{AOL} 018 \mathrm{XX} 2386$ TFI 2 D & IC & SWRI 090219-6 & 090219-6IC \\
\hline TI-065 & $\begin{array}{c}14.11 \text { and } \\
14.12\end{array}$ & Before permanganate addition & A 02AOL 018 XX 2386 RAM 3 D & Raman & ASR 8362 & ASR 8362 RAM rev 1 \\
\hline TI-065 & $\begin{array}{c}14.11 \text { and } \\
14.12\end{array}$ & Before permanganate addition & A 02AOL 018 XX 2386 RAM 3 R & Raman & ASR 8362 & ASR 8362 RAM rev 1 \\
\hline TI-065 & $\begin{array}{c}14.11 \text { and } \\
14.12\end{array}$ & Before permanganate addition & A $02 \mathrm{AOL} 018 \mathrm{XX} 2386$ TFI 3 D & $\mathrm{OH}$ & ASR 8363 & ASR8363 OH \\
\hline TI-065 & $\begin{array}{c}14.11 \text { and } \\
14.12\end{array}$ & Before permanganate addition & A $02 \mathrm{AOL} 018$ XX 2387 ICP 2 D & ICP Supernate & SWRI 090219-11 & 090217-13 and 090219-11 ICP and ICPMS \\
\hline TI-065 & $\begin{array}{c}14.11 \text { and } \\
14.12\end{array}$ & Before permanganate addition & A 02AOL 018 XX 2387 ICP 2 R & ICP Rinsate & SWRI 090219-17 & 090219-17 and 0902019-22 ICP and ICPMS \\
\hline
\end{tabular}


Integrated Test A Sample List

\begin{tabular}{|c|c|c|c|c|c|c|}
\hline TI \# & TI Step \# & TI Step Description & Sample ID & Analysis & Folder & Final Electronic Report File Name \\
\hline TI-065 & $\begin{array}{c}14.11 \text { and } \\
14.12\end{array}$ & Before permanganate addition & A 02AOL 018 XX 2387 ICP $2 \mathrm{~S}$ & ICP Wet Solids & SWRI 090219-18 & 090217-14 090219-18 090219-19 ICP and ICPMS \\
\hline TI-065 & $\begin{array}{c}14.11 \text { and } \\
14.12\end{array}$ & Before permanganate addition & A $02 \mathrm{AOL} 018 \mathrm{XX} 2387$ TFI $2 \mathrm{D}$ & IC & SWRI 090219-6 & 090219-6IC \\
\hline TI-065 & $\begin{array}{c}14.11 \text { and } \\
14.12\end{array}$ & Before permanganate addition & A 02AOL 018 XX 2387 RAM 3 D & Raman & ASR 8362 & ASR 8362 RAM rev 1 \\
\hline TI-065 & $\begin{array}{c}14.11 \text { and } \\
14.12\end{array}$ & Before permanganate addition & A 02AOL 018 XX 2387 RAM 3 R & Raman & ASR 8362 & ASR 8362 RAM rev 1 \\
\hline TI-065 & $\begin{array}{c}14.11 \text { and } \\
14.12\end{array}$ & Before permanganate addition & A 02AOL 018 XX 2387 TFI 3 D & $\mathrm{OH}$ & ASR 8363 & ASR8363 OH \\
\hline TI-065 & $\begin{array}{c}14.11 \text { and } \\
14.12\end{array}$ & Before permanganate addition & A 02AOL 018 XX 2388 ICP 2 D & ICP Supernate & SWRI 090219-11 & 090217-13 and 090219-11 ICP and ICPMS \\
\hline TI-065 & $\begin{array}{c}14.11 \text { and } \\
14.12\end{array}$ & Before permanganate addition & A $02 \mathrm{AOL} 018 \mathrm{XX} 2388 \mathrm{ICP} 2 \mathrm{R}$ & ICP Rinsate & SWRI 090219-17 & 090219-17 and 0902019-22 ICP and ICPMS \\
\hline TI-065 & $\begin{array}{c}14.11 \text { and } \\
14.12\end{array}$ & Before permanganate addition & A $02 \mathrm{AOL} 018$ XX 2388 ICP $2 \mathrm{~S}$ & ICP Wet Solids & SWRI 090219-18 & 090217-14 090219-18 090219-19 ICP and ICPMS \\
\hline TI-065 & $\begin{array}{c}14.11 \text { and } \\
14.12\end{array}$ & Before permanganate addition & A 02AOL 018 XX 2388 TFI 2 D & IC & SWRI 090219-6 & 090219-6IC \\
\hline TI-065 & $\begin{array}{c}14.11 \text { and } \\
14.12\end{array}$ & Before permanganate addition & A 02AOL 018 XX 2388 RAM 3 D & Raman & ASR 8362 & ASR 8362 RAM rev 1 \\
\hline TI-065 & $\begin{array}{c}14.11 \text { and } \\
14.12\end{array}$ & Before permanganate addition & A 02AOL 018 XX 2388 RAM 3 R & Raman & ASR 8362 & ASR 8362 RAM rev 1 \\
\hline TI-065 & $\begin{array}{c}14.11 \text { and } \\
14.12\end{array}$ & Before permanganate addition & A 02AOL 018 XX 2388 TFI 3 D & $\mathrm{OH}$ & ASR 8363 & ASR8363 OH \\
\hline TI-065 & $\begin{array}{c}14.11 \text { and } \\
14.12\end{array}$ & Before permanganate addition & A $02 \mathrm{AOL} 018 \mathrm{XX} 2389$ AFA 2 & ICP Supernate & SWRI 090219-19 & 090216-13 and 090219-19 IC and ICP and ICPMS \\
\hline TI-065 & $\begin{array}{c}14.11 \text { and } \\
14.12\end{array}$ & Before permanganate addition & A $02 \mathrm{AOL} 018 \mathrm{XX} 2389$ AFA 2 & ICP Wet Solids & SWRI 090219-19 & 090217-14 090219-18 090219-19 ICP and ICPMS \\
\hline TI- 65 & $\begin{array}{c}14.11 \text { and } \\
14.12\end{array}$ & Permanganate addition & A 02AOL 018 XX 2390 AFA 5 B & THF Wet Solids & 48880 & PNNL 48880 Report.pdf \\
\hline TI- 65 & $\begin{array}{c}14.11 \text { and } \\
14.12\end{array}$ & Permanganate addition & A $02 \mathrm{AOL} 018 \mathrm{XX} 2390 \mathrm{AFA} 5 \mathrm{D}$ & THF Supernate & 48880 & PNNL 48880 Report.pdf \\
\hline TI- 65 & $\begin{array}{c}14.11 \text { and } \\
14.12\end{array}$ & Permanganate addition & A 02AOL 018 XX 2391 AFA 5 B & Toluene Wet Solids & 48880 & PNNL 48880 Report.pdf \\
\hline TI-65 & $\begin{array}{c}14.11 \text { and } \\
14.12\end{array}$ & Permanganate addition & A 02AOL 018 XX 2391 AFA 5 D & Toluene Supernate & 48880 & PNNL 48880 Report.pdf \\
\hline TI-065 & $\begin{array}{c}14.11 \text { and } \\
14.12\end{array}$ & Before permanganate addition & A $02 \mathrm{AOL} 018 \mathrm{XX} 2392$ CUF 4 & Parallel Bench Test & & \\
\hline TI-065 & 14.14 & Oxidative leach $5 \mathrm{~min}$ after $\mathrm{KMnO} 4$ & A 02AOL 018 XX 2393 UDS 2 B & UDS & UDS R1 & UDS R1 Shake and Test A and Test B Step001 Date 0818200 \\
\hline
\end{tabular}


Integrated Test A Sample List

\begin{tabular}{|c|c|c|c|c|c|c|}
\hline TI\# & TI Step \# & TI Step Description & Sample ID & Analysis & Folder & Final Electronic Report File Name \\
\hline TI-065 & 14.14 & Oxidative leach $5 \mathrm{~min}$ after $\mathrm{KMnO} 4$ & A 02AOL 018 XX 2393 UDS 2 D & UDS & UDS R1 & UDS R1 Shake and Test A and Test B Step001 Date 08182009 \\
\hline TI-065 & 14.14 & Oxidative leach $5 \mathrm{~min}$ after $\mathrm{KMnO} 4$ & A 02AOL 018 XX 2394 DEN 2 & Density Slurry & SWRI 090219-10 & 090219-10 DEN \\
\hline TI-065 & 14.14 & Oxidative leach $5 \mathrm{~min}$ after $\mathrm{KMnO} 4$ & A 02 AOL 018 XX 2395 ICP 2 D & ICP Supernate & SWRI 090219-11 & 090217-13 and 090219-11 ICP and ICPMS \\
\hline TI-065 & 14.14 & Oxidative leach 5 min after $\mathrm{KMnO} 4$ & A 02AOL 018 XX 2395 RAM 3 D & Raman & ASR 8362 & ASR 8362 RAM rev 1 \\
\hline TI-065 & 14.14 & Oxidative leach $5 \mathrm{~min}$ after $\mathrm{KMnO} 4$ & A 02AOL 018 XX 2395 TFI 2 D & IC & SWRI 090219-6 & 090219-6IC \\
\hline TI-065 & 14.14 & Oxidative leach $5 \mathrm{~min}$ after $\mathrm{KMnO} 4$ & A 02AOL 018 XX 2395 TFI 3 D & $\mathrm{OH}$ & ASR 8363 & ASR8363 OH \\
\hline TI-065 & 14.14 & Oxidative leach & A 02AOL 019012396 UDS 2 B & UDS & UDS R1 & UDS R1 Shake and Test A and Test B Step001 Date 08182009 \\
\hline TI-065 & 14.14 & Oxidative leach & A 02AOL 019012396 UDS 2 D & UDS & UDS R1 & UDS R1 Shake and Test A and Test B Step001 Date 08182009 \\
\hline TI-065 & 14.14 & Oxidative leach & A 02AOL 019012397 DEN 2 & Density Slurry & SWRI 090219-10 & 090219-10 DEN \\
\hline TI-065 & 14.14 & Oxidative leach & A 02AOL 019012398 ICP 2 D & ICP Supernate & SWRI 090219-11 & 090217-13 and 090219-11 ICP and ICPMS \\
\hline TI-065 & 14.14 & Oxidative leach & A 02AOL 019012398 RAM 3 D & Raman & ASR 8362 & ASR 8362 RAM rev 1 \\
\hline TI-065 & 14.14 & Oxidative leach & A 02AOL 019012398 TFI 2 D & $\mathrm{IC}$ & SWRI 090219-6 & 090219-6IC \\
\hline TI-065 & 14.14 & Oxidative leach & A 02AOL 019012398 TFI 3 D & $\mathrm{OH}$ & ASR 8363 & ASR8363 OH \\
\hline TI-065 & 14.14 & Oxidative leach & A 02AOL 019022399 UDS 2 B & UDS & UDS R1 & UDS R1 Shake and Test A and Test B Step001 Date 08182009 \\
\hline TI-065 & 14.14 & Oxidative leach & A 02AOL 019022399 UDS 2 D & UDS & UDS R1 & UDS R1 Shake and Test A and Test B Step001 Date 08182009 \\
\hline TI-065 & 14.14 & Oxidative leach & A 02AOL 019022400 DEN 2 & Density Slurry & SWRI 090219-10 & 090219-10 DEN \\
\hline TI-065 & 14.14 & Oxidative leach & A 02AOL 019022401 ICP 2 D & ICP Supernate & SWRI 090219-11 & 090217-13 and 090219-11 ICP and ICPMS \\
\hline TI-065 & 14.14 & Oxidative leach & A 02AOL 019022401 RAM 3 D & Raman & ASR 8362 & ASR 8362 RAM rev 1 \\
\hline TI-065 & 14.14 & Oxidative leach & A 02AOL 019022401 TFI 2 D & IC & SWRI 090219-6 & 090219-6IC \\
\hline TI-065 & 14.14 & Oxidative leach & A 02AOL 019022401 TFI 3 D & $\mathrm{OH}$ & ASR 8363 & ASR8363 OH \\
\hline TI-065 & 14.14 & Oxidative leach & A 02AOL 019032402 UDS 2 B & UDS & UDS R1 & UDS R1 Shake and Test A and Test B Step001 Date 08182009 \\
\hline TI-065 & 14.14 & Oxidative leach & A 02AOL 019032402 UDS 2 D & UDS & UDS R1 & UDS R1 Shake and Test A and Test B Step001 Date 08182009 \\
\hline TI-065 & 14.14 & Oxidative leach & A 02AOL 019032403 DEN 2 & Density Slurry & SWRI 090219-10 & 090219-10 DEN \\
\hline TI-065 & 14.14 & Oxidative leach & A 02AOL 019032404 ICP 2 D & ICP Supernate & SWRI 090219-11 & 090217-13 and 090219-11 ICP and ICPMS \\
\hline TI-065 & 14.14 & Oxidative leach & A 02AOL 019032404 RAM 3 D & Raman & ASR 8362 & ASR 8362 RAM rev 1 \\
\hline TI-065 & 14.14 & Oxidative leach & A 02AOL 019032404 TFI 2 D & IC & SWRI 090219-6 & 090219-6IC \\
\hline TI-065 & 14.14 & Oxidative leach & A 02AOL 019032404 TFI 3 D & $\mathrm{OH}$ & ASR 8363 & ASR8363 OH \\
\hline TI-065 & 14.14 & Oxidative leach & A 02AOL 019042405 UDS 2 B & UDS & UDS R1 & UDS R1 Shake and Test A and Test B Step001 Date 08182009 \\
\hline TI-065 & 14.14 & Oxidative leach & A 02AOL 019042405 UDS 2 D & UDS & UDS R1 & UDS R1 Shake and Test A and Test B Step001 Date 08182009 \\
\hline TI-065 & 14.14 & Oxidative leach & A 02AOL 019042406 DEN 2 & Density Slurry & SWRI 090219-10 & 090219-10 DEN \\
\hline TI-065 & 14.14 & Oxidative leach & A 02AOL 019042407 ICP 2 D & ICP Supernate & SWRI 090219-11 & 090217-13 and 090219-11 ICP and ICPMS \\
\hline TI-065 & 14.14 & Oxidative leach & A 02AOL 019042407 RAM 3 D & Raman & ASR 8362 & ASR 8362 RAM rev 1 \\
\hline TI-065 & 14.14 & Oxidative leach & A 02AOL 019042407 TFI 2 D & IC & SWRI 090219-6 & 090219-6IC \\
\hline
\end{tabular}


Integrated Test A Sample List

\begin{tabular}{|c|c|c|c|c|c|c|}
\hline TI\# & TI Step \# & TI Step Description & Sample ID & Analysis & Folder & Final Electronic Report File Name \\
\hline TI-065 & 14.14 & Oxidative leach & A 02AOL 019042407 TFI 3 D & $\mathrm{OH}$ & ASR 8363 & ASR8363 OH \\
\hline TI-065 & 14.14 & Oxidative leach & A 02AOL 019052408 UDS 2 B & UDS & UDS R1 & UDS R1 Shake and Test A and Test B Step001 Date 08182009 \\
\hline TI-065 & 14.14 & Oxidative leach & A 02AOL 019052408 UDS 2 D & UDS & UDS R1 & UDS R1 Shake and Test A and Test B Step001 Date 08182009 \\
\hline TI-065 & 14.14 & Oxidative leach & A 02AOL 019052409 DEN 2 & Density Slurry & SWRI 090219-10 & 090219-10 DEN \\
\hline TI-065 & 14.14 & Oxidative leach & A 02AOL 019052410 ICP 2 D & ICP Supernate & SWRI 090219-11 & 090217-13 and 090219-11 ICP and ICPMS \\
\hline TI-065 & 14.14 & Oxidative leach & A 02AOL 019052410 RAM 3 D & Raman & ASR 8362 & ASR 8362 RAM rev 1 \\
\hline TI-065 & 14.14 & Oxidative leach & A 02AOL 019052410 TFI 2 D & IC & SWRI 090219-6 & 090219-6IC \\
\hline TI-065 & 14.14 & Oxidative leach & A 02AOL 019052410 TFI 3 D & $\mathrm{OH}$ & ASR 8363 & ASR8363 OH \\
\hline TI-065 & 14.14 & Oxidative leach & A 02AOL 019062411 UDS 2 B & UDS & UDS R1 & UDS R1 Shake and Test A and Test B Step001 Date 08182009 \\
\hline TI-065 & 14.14 & Oxidative leach & A 02AOL 019062411 UDS 2 D & UDS & UDS R1 & UDS R1 Shake and Test A and Test B Step001 Date 08182009 \\
\hline TI-065 & 14.14 & Oxidative leach & A 02AOL 019062412 UDS 2 B & UDS & UDS R1 & UDS R1 Shake and Test A and Test B Step001 Date 08182009 \\
\hline TI-065 & 14.14 & Oxidative leach & A 02AOL 019062412 UDS 2 D & UDS & UDS R1 & UDS R1 Shake and Test A and Test B Step001 Date 08182009 \\
\hline TI-065 & 14.14 & Oxidative leach & A 02AOL 019062413 UDS 2 B & UDS & UDS R1 & UDS R1 Shake and Test A and Test B Step001 Date 08182009 \\
\hline TI-065 & 14.14 & Oxidative leach & A 02AOL 019062413 UDS 2 D & UDS & UDS R1 & UDS R1 Shake and Test A and Test B Step001 Date 08182009 \\
\hline TI-065 & 14.14 & Oxidative leach & A 02AOL 019062414 DEN 2 & Density Slurry & SWRI 090219-10 & 090219-10 DEN \\
\hline TI-065 & 14.14 & Oxidative leach & A 02AOL 019062415 DEN 2 & Density Slurry & SWRI 090219-10 & 090219-10 DEN \\
\hline TI-065 & 14.14 & Oxidative leach & A 02AOL 019062416 DEN 2 & Density Slurry & SWRI 090219-10 & 090219-10 DEN \\
\hline TI-065 & 14.14 & Oxidative leach & A 02AOL 019062417 RHE 4 & Rheology & TDP-WTP-359 & TDP WTP 359 Int Test A RHE Data on PEP Slurry \\
\hline TI-065 & 14.14 & Oxidative leach & A 02AOL 019062418 ICP 2 D & ICP Supernate & SWRI 090219-11 & 090217-13 and 090219-11 ICP and ICPMS \\
\hline TI-065 & 14.14 & Oxidative leach & A $02 \mathrm{AOL} 019062418$ ICP $2 \mathrm{R}$ & ICP Rinsate & SWRI 090219-17 & 090219-17 and 0902019-22 ICP and ICPMS \\
\hline TI-065 & 14.14 & Oxidative leach & A 02AOL 019062418 ICP $2 \mathrm{~S}$ & ICP Wet Solids & SWRI 090219-18 & 090217-14 090219-18 090219-19 ICP and ICPMS \\
\hline TI-065 & 14.14 & Oxidative leach & A 02AOL 019062418 RAM 3 D & Raman & ASR 8362 & ASR 8362 RAM rev 1 \\
\hline TI-065 & 14.14 & Oxidative leach & A 02AOL 019062418 RAM 3 R & Raman & ASR 8362 & ASR 8362 RAM rev 1 \\
\hline TI-065 & 14.14 & Oxidative leach & A 02AOL 019062418 TFI 2 D & IC & SWRI 090219-6 & 090219-6IC \\
\hline TI-065 & 14.14 & Oxidative leach & A 02AOL 019062418 TFI 3 D & $\mathrm{OH}$ & ASR 8363 & ASR $8363 \mathrm{OH}$ \\
\hline TI-065 & 14.14 & Oxidative leach & A 02AOL 019062419 ICP 2 D & ICP Supernate & SWRI 090219-11 & 090217-13 and 090219-11 ICP and ICPMS \\
\hline TI-065 & 14.14 & Oxidative leach & A $02 \mathrm{AOL} 019062419$ ICP $2 \mathrm{R}$ & ICP Rinsate & SWRI 090219-17 & 090219-17 and 0902019-22 ICP and ICPMS \\
\hline TI-065 & 14.14 & Oxidative leach & A $02 \mathrm{AOL} 019062419$ ICP $2 \mathrm{~S}$ & ICP Wet Solids & SWRI 090219-18 & 090217-14 090219-18 090219-19 ICP and ICPMS \\
\hline TI-065 & 14.14 & Oxidative leach & A 02AOL 019062419 RAM 3 D & Raman & ASR 8362 & ASR 8362 RAM rev 1 \\
\hline TI-065 & 14.14 & Oxidative leach & A 02AOL 019062419 RAM 3 R & Raman & ASR 8362 & ASR 8362 RAM rev 1 \\
\hline TI-065 & 14.14 & Oxidative leach & A 02AOL 019062419 TFI 2 D & IC & SWRI 090219-6 & 090219-6IC \\
\hline TI-065 & 14.14 & Oxidative leach & A 02AOL 019062419 TFI 3 D & $\mathrm{OH}$ & ASR 8363 & ASR $8363 \mathrm{OH}$ \\
\hline TI-065 & 14.14 & Oxidative leach & A $02 A O L ~ 019062420$ ICP 2 D & ICP Supernate & SWRI 090219-11 & 090217-13 and 090219-11 ICP and ICPMS \\
\hline
\end{tabular}


Integrated Test A Sample List

\begin{tabular}{|c|c|c|c|c|c|c|}
\hline TI\# & TI Step \# & TI Step Description & Sample ID & Analysis & Folder & Final Electronic Report File Name \\
\hline TI-065 & 14.14 & Oxidative leach & A 02AOL 019062420 ICP 2 R & ICP Rinsate & SWRI 090219-17 & 090219-17 and 0902019-22 ICP and ICPMS \\
\hline TI-065 & 14.14 & Oxidative leach & A $02 A O L ~ 019062420$ ICP $2 \mathrm{~S}$ & ICP Supernate & SWRI 090219-18 & 090217-14 090219-18 090219-19 ICP and ICPMS \\
\hline TI-065 & 14.14 & Oxidative leach & A 02AOL 019062420 RAM 3 D & Raman & ASR 8362 & ASR 8362 RAM rev 1 \\
\hline TI-065 & 14.14 & Oxidative leach & A 02AOL 019062420 RAM 3 R & Raman & ASR 8362 & ASR 8362 RAM rev 1 \\
\hline TI-065 & 14.14 & Oxidative leach & A 02AOL 019062420 TFI 2 D & IC & SWRI 090219-6 & 090219-6IC \\
\hline TI-065 & 14.14 & Oxidative leach & A 02AOL 019062420 TFI 3 D & $\mathrm{OH}$ & ASR 8363 & ASR8363 OH \\
\hline TI-65 & 14.14 & Oxidative leach & A $02 \mathrm{AOL} 019062421$ AFA $5 \mathrm{~B}$ & THF Wet Solids & 48880 & PNNL 48880 Report.pdf \\
\hline TI-65 & 14.14 & Oxidative leach & A 02AOL 019062421 AFA 5 D & THF Supernate & 48880 & PNNL 48880 Report.pdf \\
\hline TI- 65 & 14.14 & Oxidative leach & A $02 \mathrm{AOL} 019062422$ AFA 5 B & Toluene Wet Solids & 48880 & PNNL 48880 Report.pdf \\
\hline TI-65 & 14.14 & Oxidative leach & A 02AOL 019062422 AFA 5 D & Toluene Supernate & 48880 & PNNL 48880 Report.pdf \\
\hline TI-065 & 14.14 & Oxidative leach & A 02AOL 019062423 AFA 5 B & ICP Wet Solids & SWRI 090225-17 & 090225-17 and 090225-21 ICP and ICPMS \\
\hline TI-065 & 14.14 & Oxidative leach & A 02AOL 019062423 AFA 5 D & ICP Supernate & SWRI 090225-17 & 090213-11 090225-17 090225-22 IC and ICP and ICPMS \\
\hline TI-065 & 15.3 .5 & Post-oxidative-leach wash & A $02 A O L 020012424$ UDS 2 & UDS & UDS R1 & UDS R1 Shake and Test A and Test B Step001 Date 08182009 \\
\hline TI-065 & 15.3.5 & Post-oxidative-leach wash & A 02AOL 020012425 ICP 2 D & ICP Supernate & SWRI 090219-11 & 090217-13 and 090219-11 ICP and ICPMS \\
\hline TI-065 & 15.3.5 & Post-oxidative-leach wash & A $02 \mathrm{AOL} 020012425$ RAM $3 \mathrm{D}$ & Raman & ASR 8362 & ASR 8362 RAM rev 1 \\
\hline TI-065 & 15.3.5 & Post-oxidative-leach wash & A 02AOL 020012425 TFI 2 D & IC & SWRI 090219-6 & 090219-6IC \\
\hline TI-065 & 15.3 .5 & Post-oxidative-leach wash & A 02AOL 020012425 TFI 3 D & $\mathrm{OH}$ & ASR 8363 & ASR8363 OH \\
\hline TI-065 & 15.3 .5 & Post-oxidative-leach wash & A $02 \mathrm{AOL} 020022426$ ICP 2 D & ICP Supernate & SWRI 090219-12 & 090219-12 and 090219-23 TDS and ICP \\
\hline TI-065 & 15.3 .5 & Post-oxidative-leach wash & A 02AOL 020022426 ICP 2 D & TDS & SWRI 090219-12 & 090219-12 and 090219-23 TDS and ICP \\
\hline TI-065 & 15.3 .5 & Post-oxidative-leach wash & A 02AOL 020022426 RAM 3 D & Raman & ASR 8362 & ASR 8362 RAM rev 1 \\
\hline TI-065 & 15.3.5 & Post-oxidative-leach wash & A $02 \mathrm{AOL} 020022426$ TFI $2 \mathrm{D}$ & IC & SWRI 090219-6 & 090219-6IC \\
\hline TI-065 & 15.3 .5 & Post-oxidative-leach wash & A 02AOL 020022426 TFI 3 D & $\mathrm{OH}$ & ASR 8363 & ASR8363 OH \\
\hline TI-065 & 15.3.5 & Post-oxidative-leach wash & A 02AOL 020032427 UDS 2 & UDS & UDS R1 & UDS R1 Shake and Test A and Test B Step001 Date 08182009 \\
\hline TI-065 & 15.3.5 & Post-oxidative-leach wash & A 02AOL 020032428 ICP 2 D & ICP Supernate & SWRI 090219-11 & 090217-13 and 090219-11 ICP and ICPMS \\
\hline TI-065 & 15.3 .5 & Post-oxidative-leach wash & A 02AOL 020032428 RAM 3 D & Raman & ASR 8362 & ASR 8362 RAM rev 1 \\
\hline TI-065 & 15.3 .5 & Post-oxidative-leach wash & A 02AOL 020032428 TFI 2 D & IC & SWRI 090219-6 & 090219-6IC \\
\hline TI-065 & 15.3.5 & Post-oxidative-leach wash & A 02AOL 020032428 TFI 3 D & $\mathrm{OH}$ & ASR 8363 & ASR8363 OH \\
\hline TI-065 & 15.3 .5 & Post-oxidative-leach wash & A 02AOL 020042429 ICP 2 D & ICP Supernate, & SWRI 090219-12 & 090219-12 and 090219-23 TDS and ICP \\
\hline TI-065 & 15.3 .5 & Post-oxidative-leach wash & A 02AOL 020042429 ICP 2 D & TDS & SWRI 090219-12 & 090219-12 and 090219-23 TDS and ICP \\
\hline TI-065 & 15.3.5 & Post-oxidative-leach wash & A 02AOL 020042429 RAM 3 D & Raman & ASR 8362 & ASR 8362 RAM rev 1 \\
\hline TI-065 & 15.3 .5 & Post-oxidative-leach wash & A 02AOL 020042429 TFI 2 D & IC & SWRI 090219-6 & 090219-6IC \\
\hline TI-065 & 15.3 .5 & Post-oxidative-leach wash & A 02AOL 020042429 TFI 3 D & $\mathrm{OH}$ & ASR 8363 & ASR8363 OH \\
\hline TI-065 & 15.3.5 & Post-oxidative-leach wash & A 02AOL 020052430 UDS 2 & UDS & UDS R1 & UDS R1 Shake and Test A and Test B Step001 Date 08182009 \\
\hline
\end{tabular}


Integrated Test A Sample List

\begin{tabular}{|c|c|c|c|c|c|c|}
\hline TI \# & TI Step \# & TI Step Description & Sample ID & Analysis & Folder & Final Electronic Report File Name \\
\hline TI-065 & 15.3 .5 & Post-oxidative-leach wash & A $02 \mathrm{AOL} 020052431$ ICP $2 \mathrm{D}$ & ICP Supernate & SWRI 090219-11 & 090217-13 and 090219-11 ICP and ICPMS \\
\hline TI-065 & 15.3 .5 & Post-oxidative-leach wash & A 02AOL 020052431 RAM 3 D & Raman & ASR 8362 & ASR 8362 RAM rev 1 \\
\hline TI-065 & 15.3.5 & Post-oxidative-leach wash & A 02AOL 020052431 TFI 2 D & IC & SWRI 090219-6 & 090219-6IC \\
\hline TI-065 & 15.3.5 & Post-oxidative-leach wash & A 02AOL 020052431 TFI 3 D & $\mathrm{OH}$ & ASR 8363 & ASR $8363 \mathrm{OH}$ \\
\hline TI-065 & 15.3 .5 & Post-oxidative-leach wash & A 02AOL 020062432 ICP 2 D & ICP Supernate, TDS & $\begin{array}{l}\text { SWRI 090219-11; } \\
\text { SWRI 090219-12 }\end{array}$ & 090219-12 and 090219-23 TDS and ICP \\
\hline TI-065 & 15.3.5 & Post-oxidative-leach wash & A 02AOL 020062432 RAM 3 D & Raman & ASR 8362 & ASR 8362 RAM rev 1 \\
\hline TI-065 & 15.3.5 & Post-oxidative-leach wash & A $02 A O L ~ 020062432$ TFI 2 D & IC & SWRI 090219-6 & 090219-6IC \\
\hline TI-065 & 15.3 .5 & Post-oxidative-leach wash & A 02AOL 020062432 TFI 3 D & $\mathrm{OH}$ & ASR 8363 & ASR $8363 \mathrm{OH}$ \\
\hline TI-065 & 15.3.5 & Post-oxidative-leach wash & A 02AOL 020072433 UDS 2 & UDS & UDS R1 & UDS R1 Shake and Test A and Test B Step001 Date 08182009 \\
\hline TI-065 & 15.3 .5 & Post-oxidative-leach wash & A 02AOL 020072434 ICP 2 D & ICP Supernate & SWRI 090219-11 & 090217-13 and 090219-11 ICP and ICPMS \\
\hline TI-065 & 15.3.5 & Post-oxidative-leach wash & A 02AOL 020072434 RAM 3 D & Raman & ASR 8362 & ASR 8362 RAM rev 1 \\
\hline TI-065 & 15.3 .5 & Post-oxidative-leach wash & A 02AOL 020072434 TFI 2 D & IC & SWRI 090219-6 & 090219-6IC \\
\hline TI-065 & 15.3 .5 & Post-oxidative-leach wash & A 02AOL 020072434 TFI 3 D & $\mathrm{OH}$ & ASR 8363 & ASR $8363 \mathrm{OH}$ \\
\hline TI-065 & 15.3.5 & Post-oxidative-leach wash & A $02 \mathrm{AOL} 020082435$ DEN 2 & Density Slurry & SWRI 090219-10 & 090219-10 DEN \\
\hline TI-065 & 15.3 .5 & Post-oxidative-leach wash & A $02 \mathrm{AOL} 020082435$ DEN 2 & Density Supernate & SWRI 090219-10 & 090219-10 liquidDEN \\
\hline TI-065 & 15.3.5 & Post-oxidative-leach wash & A $02 \mathrm{AOL} 020082436$ RHE 4 & Rheology & TDP-WTP-359 & TDP WTP 359 Int Test A RHE Data on PEP Slurry \\
\hline TI-065 & 15.3 .5 & Post-oxidative-leach wash & A 02AOL 020082437 RHE 4 & Rheology & TDP-WTP-359 & TDP WTP 359 Int Test A RHE Data on PEP Slurry \\
\hline TI-065 & 15.3.5 & Post-oxidative-leach wash & A $02 \mathrm{AOL} 020082438$ ICP 2 D & ICP Supernate & SWRI 090219-12 & 090219-12 and 090219-23 TDS and ICP \\
\hline TI-065 & 15.3.5 & Post-oxidative-leach wash & A $02 \mathrm{AOL} 020082438$ ICP 2 D & TDS & SWRI 090219-12 & 090219-12 and 090219-23 TDS and ICP \\
\hline TI-065 & 15.3 .5 & Post-oxidative-leach wash & A 02AOL 020082438 RAM 3 D & Raman & ASR 8362 & ASR 8362 RAM rev 1 \\
\hline TI-065 & 15.3 .5 & Post-oxidative-leach wash & A 02AOL 020082438 TFI 2 D & IC & $\begin{array}{l}\text { SWRI 090219-7, } \\
\text { SWRI 090219-24, } \\
\text { SWRI 090219-27 }\end{array}$ & 090219-7IC \\
\hline TI-065 & 15.3 .5 & Post-oxidative-leach wash & A 02AOL 020082438 TFI 3 D & $\mathrm{OH}$ & ASR 8363 & ASR8363 OH \\
\hline TI-065 & 15.3.5 & Post-oxidative-leach wash & A 02 AOL 020092439 UDS 2 & UDS & UDS R1 & UDS R1 Shake and Test A and Test B Step001 Date 08182009 \\
\hline TI-065 & 15.3.5 & Post-oxidative-leach wash & A 02AOL 020092440 ICP 2 D & ICP Supernate & SWRI 090219-11 & 090217-13 and 090219-11 ICP and ICPMS \\
\hline TI-065 & 15.3.5 & Post-oxidative-leach wash & A 02AOL 020092440 RAM 3 D & Raman & ASR 8362 & ASR 8362 RAM rev 1 \\
\hline TI-065 & 15.3 .5 & Post-oxidative-leach wash & A 02AOL 020092440 TFI 2 D & IC & $\begin{array}{l}\text { SWRI 090219-7, } \\
\text { SWRI 090219-24, } \\
\text { SWRI 090219-27 }\end{array}$ & 090219-7IC \\
\hline TI-065 & 15.3 .5 & Post-oxidative-leach wash & A 02AOL 020092440 TFI 3 D & $\mathrm{OH}$ & ASR 8363 & ASR8363 OH \\
\hline TI-065 & 15.3.5 & Post-oxidative-leach wash & A 02AOL 020102441 ICP 2 D & ICP Supernate, & SWRI 090219-12 & 090219-12 and 090219-23 TDS and ICP \\
\hline TI-065 & 15.3.5 & Post-oxidative-leach wash & A 02AOL 020102441 ICP 2 D & TDS & SWRI 090219-12 & 090219-12 and 090219-23 TDS and ICP \\
\hline
\end{tabular}


Integrated Test A Sample List

\begin{tabular}{|c|c|c|c|c|c|c|}
\hline TI \# & TI Step \# & TI Step Description & Sample ID & Analysis & Folder & Final Electronic Report File Name \\
\hline TI-065 & 15.3 .5 & Post-oxidative-leach wash & A 02AOL 020102441 RAM 3 D & Raman & ASR 8362 & ASR 8362 RAM rev 1 \\
\hline TI-065 & 15.3 .5 & Post-oxidative-leach wash & A 02AOL 020102441 TFI 2 D & IC & $\begin{array}{l}\text { SWRI 090219-7, } \\
\text { SWRI 090219-24, } \\
\text { SWRI 090219-27 }\end{array}$ & 090219-7IC \\
\hline TI-065 & 15.3 .5 & Post-oxidative-leach wash & A 02AOL 020102441 TFI 3 D & $\mathrm{OH}$ & ASR 8363 & ASR8363 OH \\
\hline TI-065 & 15.3 .5 & Post-oxidative-leach wash & A 02AOL 020112442 UDS 2 & UDS & UDS R1 & UDS R1 Shake and Test A and Test B Step001 Date 08182009 \\
\hline TI-065 & 15.3.5 & Post-oxidative-leach wash & A 02AOL 020112443 ICP 2 D & ICP Supernate & SWRI 090219-11 & 090217-13 and 090219-11 ICP and ICPMS \\
\hline TI-065 & 15.3 .5 & Post-oxidative-leach wash & A 02AOL 020112443 RAM 3 D & Raman & ASR 8362 & ASR 8362 RAM rev 1 \\
\hline TI-065 & 15.3 .5 & Post-oxidative-leach wash & A 02AOL 020112443 TFI 2 D & $\mathrm{IC}$ & $\begin{array}{l}\text { SWRI 090219-7, } \\
\text { SWRI 090219-24, } \\
\text { SWRI 090219-27 }\end{array}$ & 090219-7IC \\
\hline TI-065 & 15.3 .5 & Post-oxidative-leach wash & A 02AOL 020112443 TFI 3 D & $\mathrm{OH}$ & ASR 8363 & ASR8363 OH \\
\hline TI-065 & 15.3.5 & Post-oxidative-leach wash & A 02AOL 020122444 ICP 2 D & ICP Supernate & SWRI 090219-12 & 090219-12 and 090219-23 TDS and ICP \\
\hline TI-065 & 15.3 .5 & Post-oxidative-leach wash & A 02AOL 020122444 ICP 2 D & TDS & SWRI 090219-12 & 090219-12 and 090219-23 TDS and ICP \\
\hline TI-065 & 15.3 .5 & Post-oxidative-leach wash & A 02AOL 020122444 RAM 3 D & Raman & ASR 8362 & ASR 8362 RAM rev 1 \\
\hline TI-065 & 15.3.5 & Post-oxidative-leach wash & A 02AOL 020122444 TFI 2 D & IC & $\begin{array}{l}\text { SWRI 090219-7, } \\
\text { SWRI 090219-24, } \\
\text { SWRI 090219-27 }\end{array}$ & 090219-7IC \\
\hline TI-065 & 15.3.5 & Post-oxidative-leach wash & A 02AOL 020122444 TFI 3 D & $\mathrm{OH}$ & ASR 8363 & ASR8363 OH \\
\hline TI-065 & 15.3 .5 & Post-oxidative-leach wash & A 02AOL 020132445 UDS 2 & UDS & UDS R1 & UDS R1 Shake and Test A and Test B Step001 Date 08182009 \\
\hline TI-065 & 15.3.5 & Post-oxidative-leach wash & A 02AOL 020132446 ICP 2 D & ICP Supernate & SWRI 090219-11 & 090217-13 and 090219-11 ICP and ICPMS \\
\hline TI-065 & 15.3 .5 & Post-oxidative-leach wash & A 02AOL 020132446 RAM 3 D & Raman & ASR 8362 & ASR 8362 RAM rev 1 \\
\hline TI-065 & 15.3.5 & Post-oxidative-leach wash & A 02AOL 020132446 TFI 2 D & IC & $\begin{array}{l}\text { SWRI 090219-7, } \\
\text { SWRI 090219-24, } \\
\text { SWRI 090219-27 }\end{array}$ & 090219-7IC \\
\hline TI-065 & 15.3 .5 & Post-oxidative-leach wash & A 02AOL 020132446 TFI 3 D & $\mathrm{OH}$ & ASR 8363 & ASR8363 OH \\
\hline TI-065 & 15.3 .5 & Post-oxidative-leach wash & A 02AOL 020132448 UDS 2 & UDS & UDS R1 & UDS R1 Shake and Test A and Test B Step001 Date 08182009 \\
\hline TI-065 & 15.3 .5 & Post-oxidative-leach wash & A 02AOL 020142447 ICP 2 D & ICP Supernate & SWRI 090219-12 & 090219-12 and 090219-23 TDS and ICP \\
\hline TI-065 & 15.3.5 & Post-oxidative-leach wash & A 02AOL 020142447 ICP 2 D & TDS & SWRI 090219-12 & 090219-12 and 090219-23 TDS and ICP \\
\hline TI-065 & 15.3.5 & Post-oxidative-leach wash & A 02AOL 020142447 RAM 3 D & Raman & ASR 8362 & ASR 8362 RAM rev 1 \\
\hline TI-065 & 15.3.5 & Post-oxidative-leach wash & A 02AOL 020142447 TFI 2 D & IC & $\begin{array}{l}\text { SWRI 090219-7, } \\
\text { SWRI 090219-24, } \\
\text { SWRI 090219-27 }\end{array}$ & 090219-7IC \\
\hline TI-065 & 15.3 .5 & Post-oxidative-leach wash & A 02AOL 020142447 TFI 3 D & $\mathrm{OH}$ & ASR 8363 & ASR8363 OH \\
\hline TI-065 & 15.3.6 & Post-oxidative-leach wash & A $02 \mathrm{AOL} 020 \mathrm{XX} 2452$ ICP $2 \mathrm{D}$ & ICP Supernate & SWRI 090219-12 & 090219-12 and 090219-23 TDS and ICP \\
\hline TI-065 & 15.3.6 & Post-oxidative-leach wash & A 02AOL 020 XX 2452 ICP 2 D & TDS & SWRI 090219-12 & 090219-12 and 090219-23 TDS and ICP \\
\hline
\end{tabular}


Integrated Test A Sample List

\begin{tabular}{|c|c|c|c|c|c|c|}
\hline TI \# & TI Step \# & TI Step Description & Sample ID & Analysis & Folder & Final Electronic Report File Name \\
\hline TI-065 & 15.3.6 & Post-oxidative-leach wash & A 02AOL 020 XX 2449 DEN 2 & Density Slurry & SWRI 090219-10 & 090219-10 DEN \\
\hline TI-065 & 15.3.6 & Post-oxidative-leach wash & A 02AOL 020 XX 2449 DEN 2 & Density Supernate & SWRI 090219-10 & 090219-10 liquidDEN \\
\hline TI-065 & 15.3.6 & Post-oxidative-leach wash & A 02AOL 020 XX 2450 RHE 4 & Rheology & TDP-WTP-359 & TDP WTP 359 Int Test A RHE Data on PEP Slurry \\
\hline TI-065 & 15.3.6 & Post-oxidative-leach wash & A 02AOL 020 XX 2451 RHE 4 & Rheology & TDP-WTP-359 & TDP WTP 359 Int Test A RHE Data on PEP Slurry \\
\hline TI-065 & 15.3.6 & Post-oxidative-leach wash- $5 \mathrm{~min}$ after permeate valves closed & A 02 AOL 020 XX 2452 RAM 3 D & Raman & ASR 8362 & ASR 8362 RAM rev 1 \\
\hline TI-065 & 15.3.6 & Post-oxidative-leach wash & A 02AOL 020 XX 2452 TFI 2 D & IC & $\begin{array}{l}\text { SWRI 090219-7, } \\
\text { SWRI 090219-24, } \\
\text { SWRI 090219-27 }\end{array}$ & 090219-7IC \\
\hline TI-065 & 15.3.6 & Post-oxidative-leach wash- $5 \mathrm{~min}$ after permeate valves closed & A $02 \mathrm{AOL} 020 \mathrm{XX} 2452$ TFI $3 \mathrm{D}$ & $\mathrm{OH}$ & ASR 8363 & ASR8363 OH \\
\hline TI-065 & 15.3.6 & Post-oxidative-leach concentration & A 02AML 021 XX 2453 UDS 2 & UDS & UDS R1 & UDS R1 Shake and Test A and Test B Step001 Date 08182009 \\
\hline TI-065 & 15.3.6 & Post-oxidative-leach concentration & A 02AML 021 XX 2454 DEN 2 & Density Slurry & SWRI 090225-19 & 090225-19 Density Liquid \\
\hline TI-065 & 15.3.6 & Post-oxidative-leach concentration & A 02AML 021 XX 2454 DEN 2 & Density Supernate & SWRI 090225-19 & 090225-19 Density Liquid \\
\hline TI-065 & 15.3.6 & Post-oxidative-leach concentration & A 02AML 021 XX 2455 RHE 4 & Rheology & TDP-WTP-359 & TDP WTP 359 Int Test A RHE Data on PEP Slurry \\
\hline TI-065 & 15.3.6 & Post-oxidative-leach concentration & A 02AML 021 XX 2456 RHE 4 & Rheology & TDP-WTP-359 & TDP WTP 359 Int Test A RHE Data on PEP Slurry \\
\hline TI-065 & 15.3.6 & Post-oxidative-leach concentration & A 02AML 021 XX 2457 RHE 4 & Rheology & TDP-WTP-359 & TDP WTP 359 Int Test A RHE Data on PEP Slurry \\
\hline TI-065 & 15.3.6 & Post-oxidative-leach concentration & A 02AML 021 XX 2458 RHE 4 & Rheology & TDP-WTP-359 & TDP WTP 359 Int Test A RHE Data on PEP Slurry \\
\hline TI-065 & 15.3.6 & Post-oxidative-leach concentration & A 02AML 021 XX 2459 ICP 2 B & ICP Wet Solids & SWRI 090225-21 & 090225-17 and 090225-21 ICP and ICPMS \\
\hline TI-065 & 15.3.6 & Post-oxidative-leach concentration & A 02AML 021 XX 2459 ICP 2 D & ICP Supernate & SWRI 090225-22 & 090213-11 090225-17 090225-22 IC and ICP and ICPMS \\
\hline TI-065 & 15.3.6 & Post-oxidative-leach concentration & A 02AML 021 XX 2459 TFI 2 D & IC & SWRI 090225-20 & 090225-20IC \\
\hline TI-065 & 15.3.6 & Post-oxidative-leach concentration & A 02AML 021 XX 2459 RAM 3 D & Raman & ASR 8365 & ASR 8365.01 RAM rev 1 \\
\hline TI-065 & 15.3.6 & Post-oxidative-leach concentration & A 02AML 021 XX 2459 TFI 3 D & $\mathrm{OH}$ & ASR 8366 & ASR 8366_1 OH \\
\hline TI-065 & 15.3.6 & Post-oxidative-leach concentration & A 02AML 021 XX 2460 XSP 4 & PSD & TDP-WTP-358 & TDP WTP 358 Int Test A PSD Data Analysis \\
\hline TI-065 & 15.3 .6 & Post-oxidative-leach concentration & A 02AML 021 XX 2460 XSP 4 & XRD & TDP-WTP-357 & TDP WTP 357 Int Test A XRD Data on PEP Slurry \\
\hline TI-065 & 15.3.6 & Post-oxidative-leach concentration & A 02AML 021 XX 2460 XSP 4 & SEM & TDP-WTP-356 & TDP WTP 356 Int Test A SEM Data Analysis \\
\hline TI-065 & 15.3.6 & Post-oxidative-leach concentration & A 02AML 021 XX 2461 AFA 2 & ICP Supernate & SWRI 090225-17 & 090213-11 090225-17 090225-22 IC and ICP and ICPMS \\
\hline TI-065 & 15.3.6 & Post-oxidative-leach concentration & A 02AML 021 XX 2461 AFA 2 & ICP Wet Solids & SWRI 090225-17 & 090225-17 and 090225-21 ICP and ICPMS \\
\hline TI-65 & 15.3.6 & Post-oxidative-leach concentration & A 02AML 021 XX 2462 AFA 5 B & Toluene Wet Solids & 49000 & PNNL 49000 Report.pdf \\
\hline TI-65 & 15.3.6 & Post-oxidative-leach concentration & A 02AML 021 XX 2462 AFA 5 D & Toluene Supernate & 49000 & PNNL 49000 Report.pdf \\
\hline TI-65 & 15.3.6 & Post-oxidative-leach concentration & A 02AML 021 XX 2463 AFA 5 B & THF Wet Solids & 49000 & PNNL 49000 Report.pdf \\
\hline TI- 65 & 15.3.6 & Post-oxidative-leach concentration & A 02AML 021 XX 2463 AFA 5 D & THF Supernate & 49000 & PNNL 49000 Report.pdf \\
\hline TI-065 & 16.8 & Prepare for high solids filter test & A 02 AML 022 XX 2464 UDS 2 & UDS & SWRI 090324-29 & 090324-29UDS R1 \\
\hline TI-065 & 16.8 & Prepare for high solids filter test & A 02AML 022 XX 2465 RHE 4 & Rheology & TDP WTP 363 & TDP WTP 363 Int Test B RHE Data on PEP slurry \\
\hline TI-065 & 16.8 & Prepare for high solids filter test & A 02AML 022 XX 2465 RHE 4 & Rheology & TDP-WTP-363 & TDP WTP 363 Int Test B RHE Data on PEP slurry \\
\hline
\end{tabular}


Integrated Test A Sample List

\begin{tabular}{|c|c|c|c|c|c|c|}
\hline TI \# & TI Step \# & TI Step Description & Sample ID & Analysis & Folder & Final Electronic Report File Name \\
\hline TI-065 & 16.8 & Prepare for high solids filter test & A 02AML 022 XX 2467 CUF 4 & CUF & & \\
\hline TI-065 & 16.13 & High-solids filter test & A 02 AML 023 XX 2468 UDS 2 & UDS & SWRI 090324-30 & 090324-30UDS R1 \\
\hline TI-065 & 16.13 & High-solids filter test & A 02AML 023 XX 2469 UDS 2 & UDS & SWRI 090324-29 & 090324-29UDS R1 \\
\hline TI-065 & 16.13 & High-solids filter test & A 02 AML 023 XX 2470 UDS 2 & UDS & SWRI 090324-29 & 090324-29UDS R1 \\
\hline TI-065 & 16.15 & High-solids filter test & A 02AML 023 XX 2471 UDS 2 & UDS & SWRI 090324-29 & 090324-29UDS R1 \\
\hline TI-065 & 16.15 & High-solids filter test & A 02AML 023 XX 2472 UDS 2 & UDS & SWRI 090324-29 & 090324-29UDS R1 \\
\hline TI-065 & 16.15 & High-solids filter test & A 02AML 023 XX 2473 UDS 2 & UDS & SWRI 090324-29 & 090324-29UDS R1 \\
\hline TI-065 & 16.15 & High-solids filter test & A 02AML 023 XX 2474 UDS 2 & UDS & SWRI 090324-29 & 090324-29UDS R1 \\
\hline TI-065 & 16.15 & High-solids filter test & A 02AML 023 XX 2475 UDS 2 & UDS & SWRI 090324-29 & 090324-29UDS R1 \\
\hline TI-065 & 16.15 & High-solids filter test & A 02AML 023 XX 2476 UDS 2 & UDS & SWRI 090324-29 & 090324-29UDS R1 \\
\hline TI-065 & 16.15 & High-solids filter test & A 02AML 023 XX 2477 UDS 2 & UDS & SWRI 090324-29 & 090324-29UDS R1 \\
\hline TI-065 & 16.15 & High-solids filter test & A 02 AML 023 XX 2478 UDS 2 & UDS & SWRI 090324-29 & 090324-29UDS R1 \\
\hline TI-065 & 16.15 & High-solids filter test & A 02AML 023 XX 2479 UDS 2 & UDS & SWRI 090324-29 & 090324-29UDS R1 \\
\hline TI-065 & 16.15 & High-solids filter test & A 02 AML 023 XX 2480 UDS 2 & UDS & SWRI 090324-29 & 090324-29UDS R1 \\
\hline TI-065 & 16.15 & High-solids filter test & A 02 AML 023 XX 2481 UDS 2 & UDS & SWRI 090324-29 & 090324-29UDS R1 \\
\hline TI-065 & 16.15 & High-solids filter test & A 02AML 023 XX 2482 UDS 2 & UDS & SWRI 090324-29 & 090324-29UDS R1 \\
\hline TI-065 & 16.15 & High-solids filter test & A 02AML 023 XX 2483 UDS 2 & UDS & SWRI 090324-29 & 090324-29UDS R1 \\
\hline TI-065 & 16.15 & High-solids filter test & A 02 AML 023 XX 2484 UDS 2 & UDS & SWRI 090324-29 & 090324-29UDS R1 \\
\hline TI-065 & 16.15 & High-solids filter test & A 02AML 023 XX 2485 UDS 2 & UDS & SWRI 090324-29 & 090324-29UDS R1 \\
\hline TI-065 & 16.15 & High-solids filter test & A 02 AML 023 XX 2486 UDS 2 & UDS & SWRI 090324-30 & 090324-30UDS R1 \\
\hline TI-065 & 16.18 & High-solids filter test & A 02AML 023 XX 2488 UDS 2 & UDS & SWRI 090324-30 & 090324-30UDS R1 \\
\hline TI-065 & 16.18 & High-solids filter test & A 02AML 023 XX 2489 UDS 2 & UDS & SWRI 090324-30 & 090324-30UDS R1 \\
\hline TI-065 & 16.18 & High-solids filter test & A 02 AML 023 XX 2490 UDS 2 & UDS & SWRI 090324-30 & 090324-30UDS R1 \\
\hline TI-065 & 17.2 & Flush filter loop & A 000FL 024 XX 2514 UDS 2 & UDS & UDS R1 & UDS R1 Shake and Test A and Test B Step001 Date 08182009 \\
\hline TI-065 & 17.2 & Flush filter loop & A 000FL 024 XX 2515 RAM 3 D & Raman & ASR 8365 & ASR 8365.01 RAM rev 1 \\
\hline TI-065 & 17.2 & Flush filter loop & A 000FL 024 XX 2515 TFI 2 D & IC & SWRI 090225-20 & 090225-20IC \\
\hline
\end{tabular}


Integrated Test A Sample List

\begin{tabular}{l}
\hline TI \# TI Step \# $\quad$ TI Step Description \\
(1) Pending final report receipt. \\
"Archive" indicates samples were sent to storage/archive in RTL or sent out of PDL-W for additional studies. \\
TI Step \# have been adjusted to allow samples collected in a single sampling event to listed together. \\
Density slurry - analysis performed on slurry. \\
Density supernate - analysis performed on supernate. \\
Free hydroxide (OH). \\
Inductively coupled plasma supernate (ICP supernate) ICP performed on the supernate. \\
Inductively coupled plasma wet solids (ICP wet solids) ICP performed on wet solids. \\
Ion chromatography (IC). \\
Particle size distribution (PSD). \\
Rheology is a general term used to describe either Shear strength or Shear stress vs. shear rate analysis - actual analytical report specify specific analysis. \\
Scanning electron microscope (SEM). \\
THF Wet Solids, THF Supernate, Toluene Wet Solids, Toluene Supernate are the solvent extraction performed on either the supernate or wet solid prior to analysis by Gel Permeation chromatography (GPC). \\
Total inorganic carbon and total organic carbon (TIC/TOC). \\
Weight Percent Undissolved Solids (UDS).
\end{tabular}

Weight Percent Undisol tolids (UDS).

X-ray diffraction $(\mathrm{XRD})$ 

Appendix C

\section{Sampling Event Summary}





\section{Appendix C: Sampling Event Summary}

\section{ESPS Analytical Matrix (2/3/09), Integrated Test A Sampling Event Summary}

The test instruction for Integrated Test A used the 1/27/09 version of the sample matrix. However, several changes followed sample matrix 1/27/09. The sampling matrix below summarizes the actual number of samples planned for

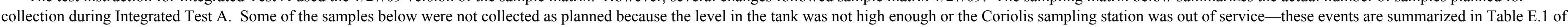
Appendix E.

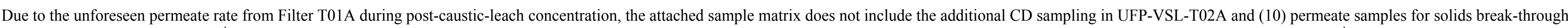

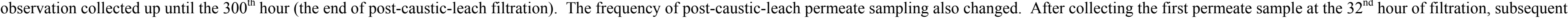
permeate samples were taken 24 hours thereafter. These changes affected process step B.1.7.

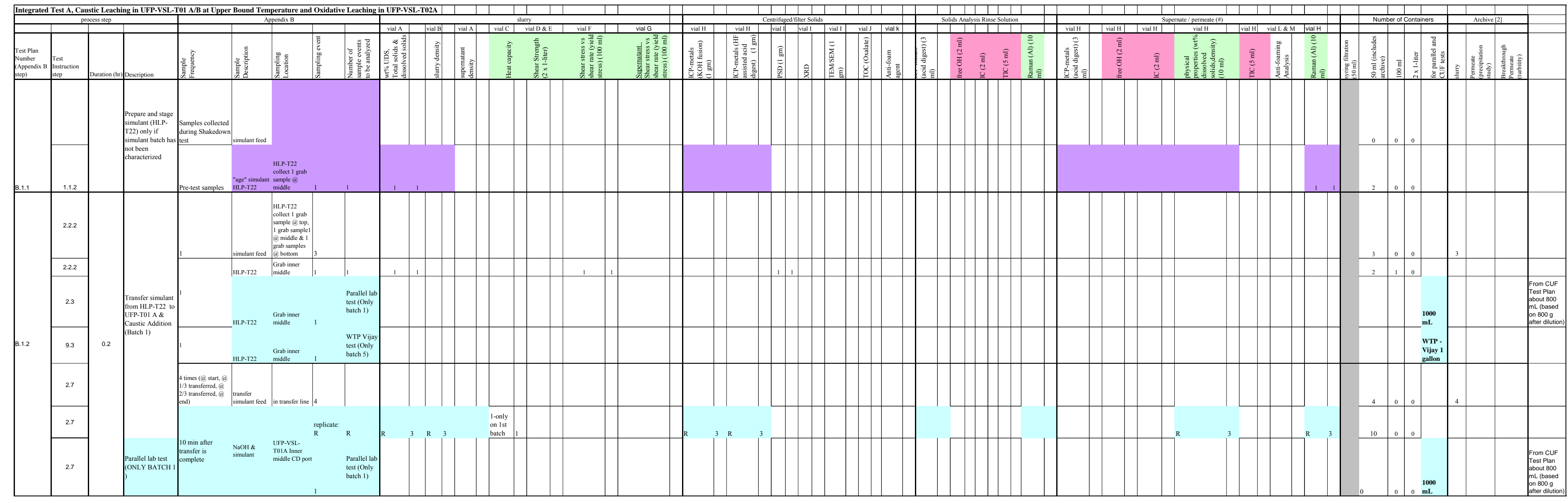


Integrated Test A Sampling Event Summary

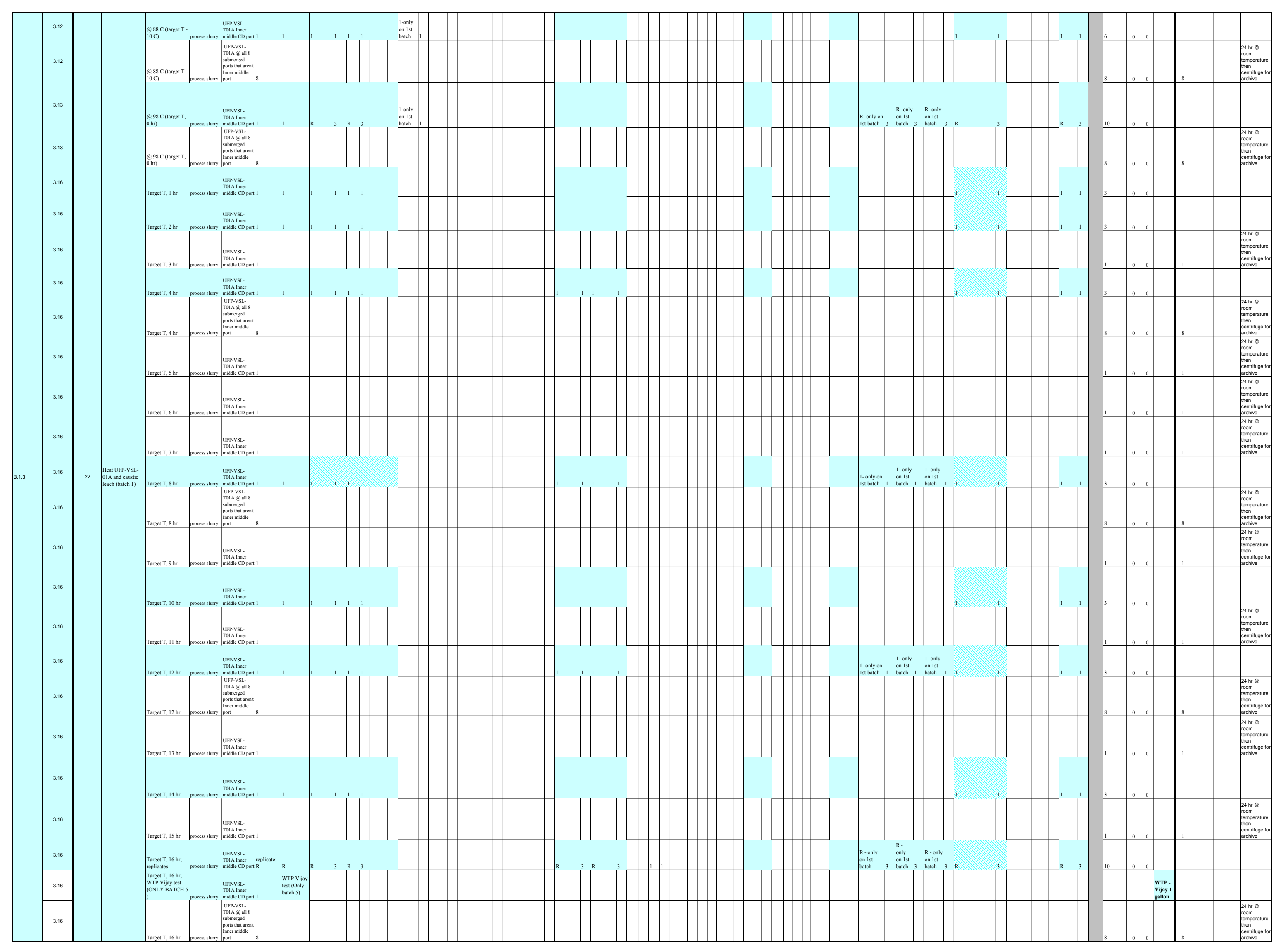


Integrated Test A Sampling Event Summary

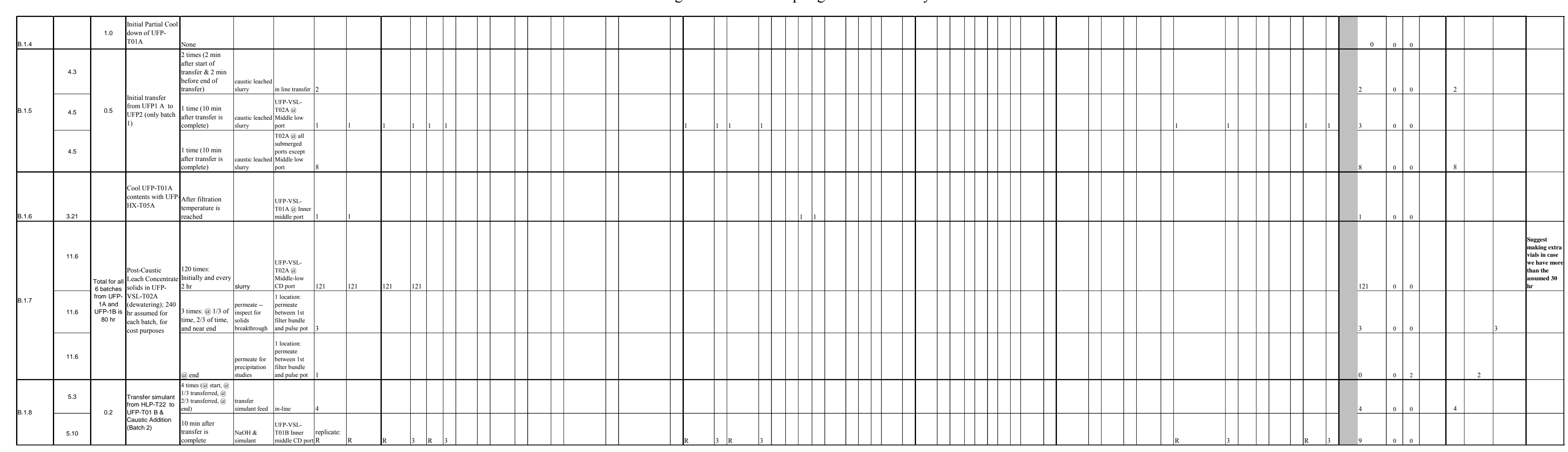




\section{Integrated Test A Sampling Event Summary}

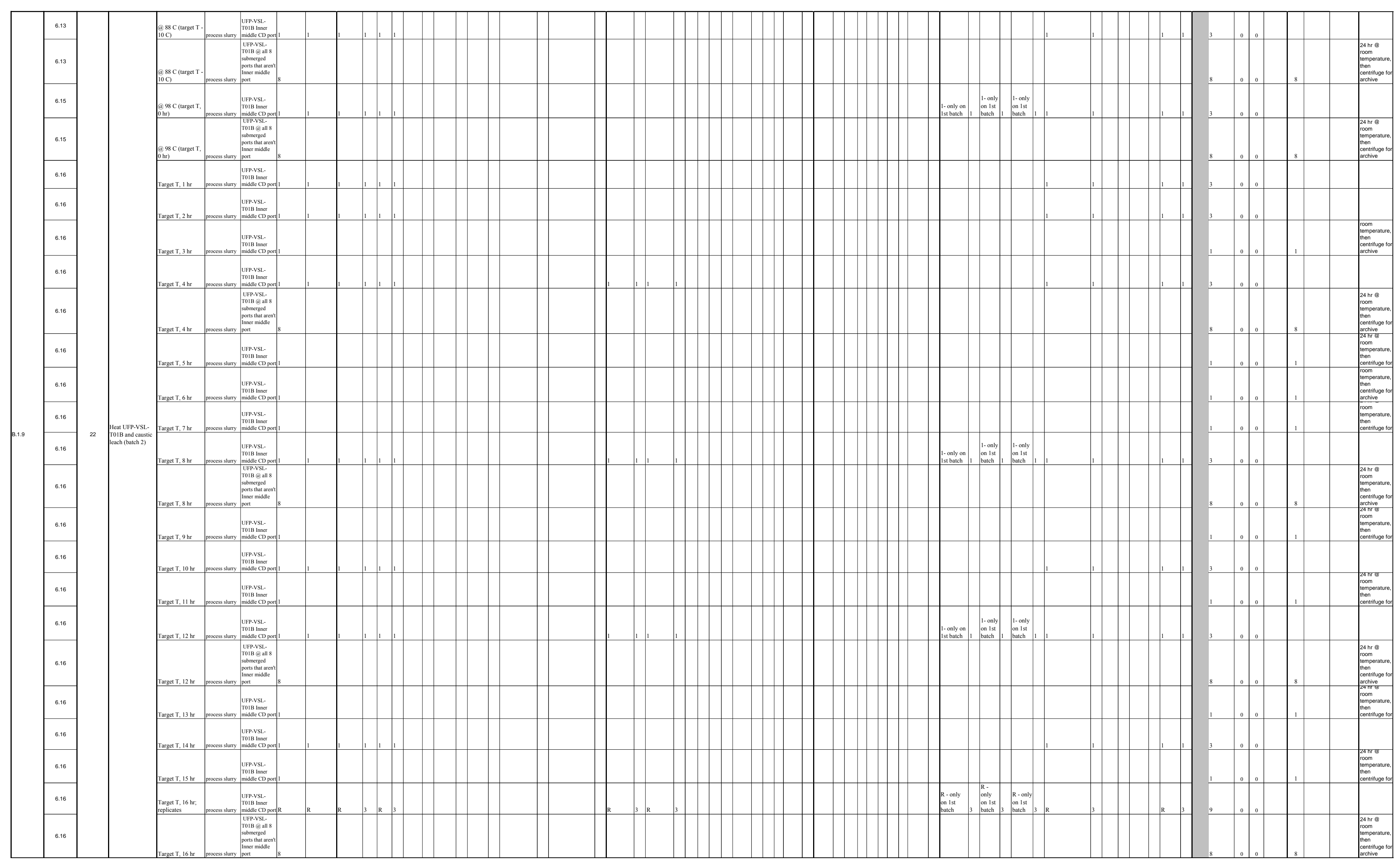


Integrated Test A Sampling Event Summary

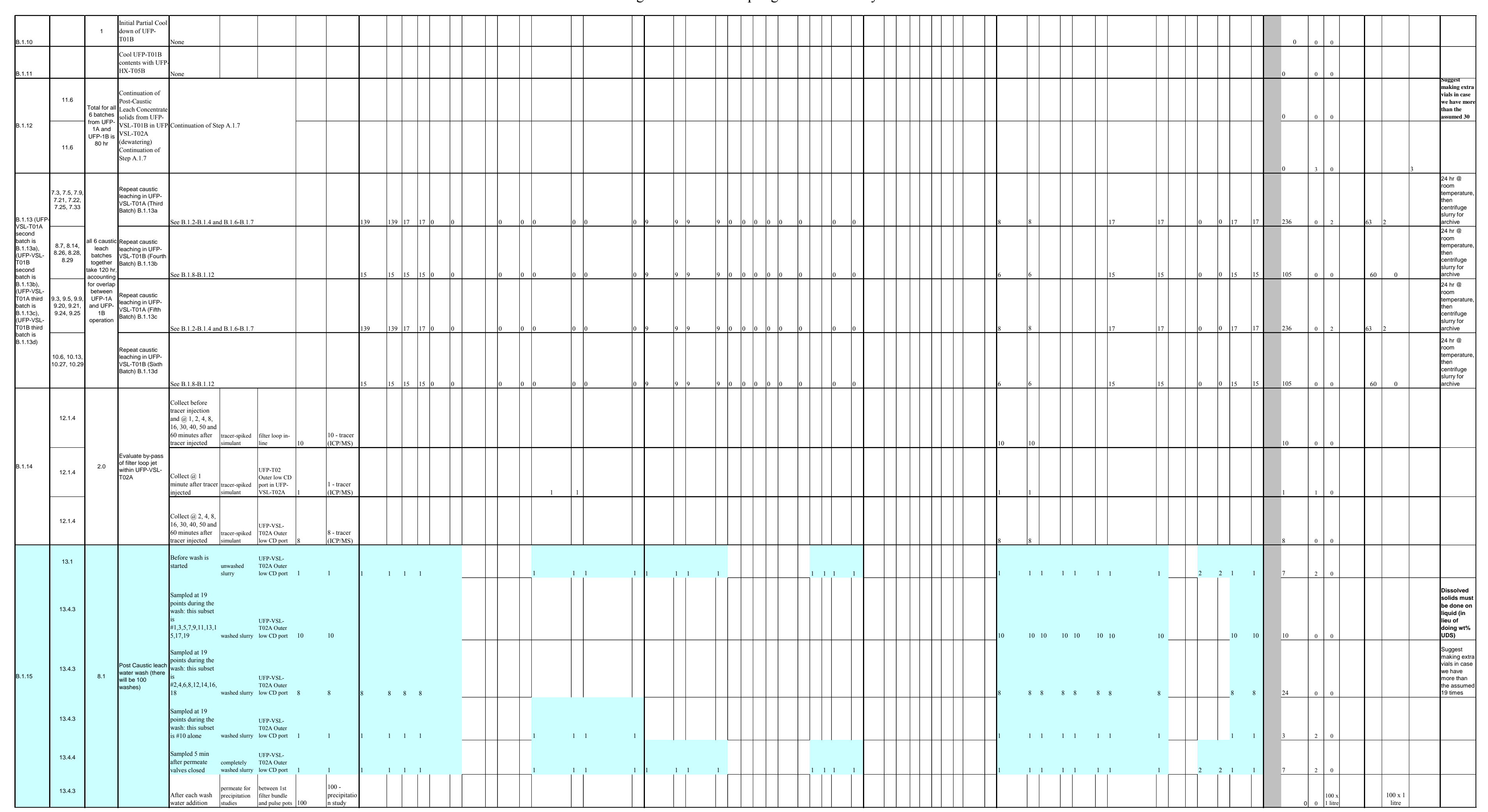


Integrated Test A Sampling Event Summary

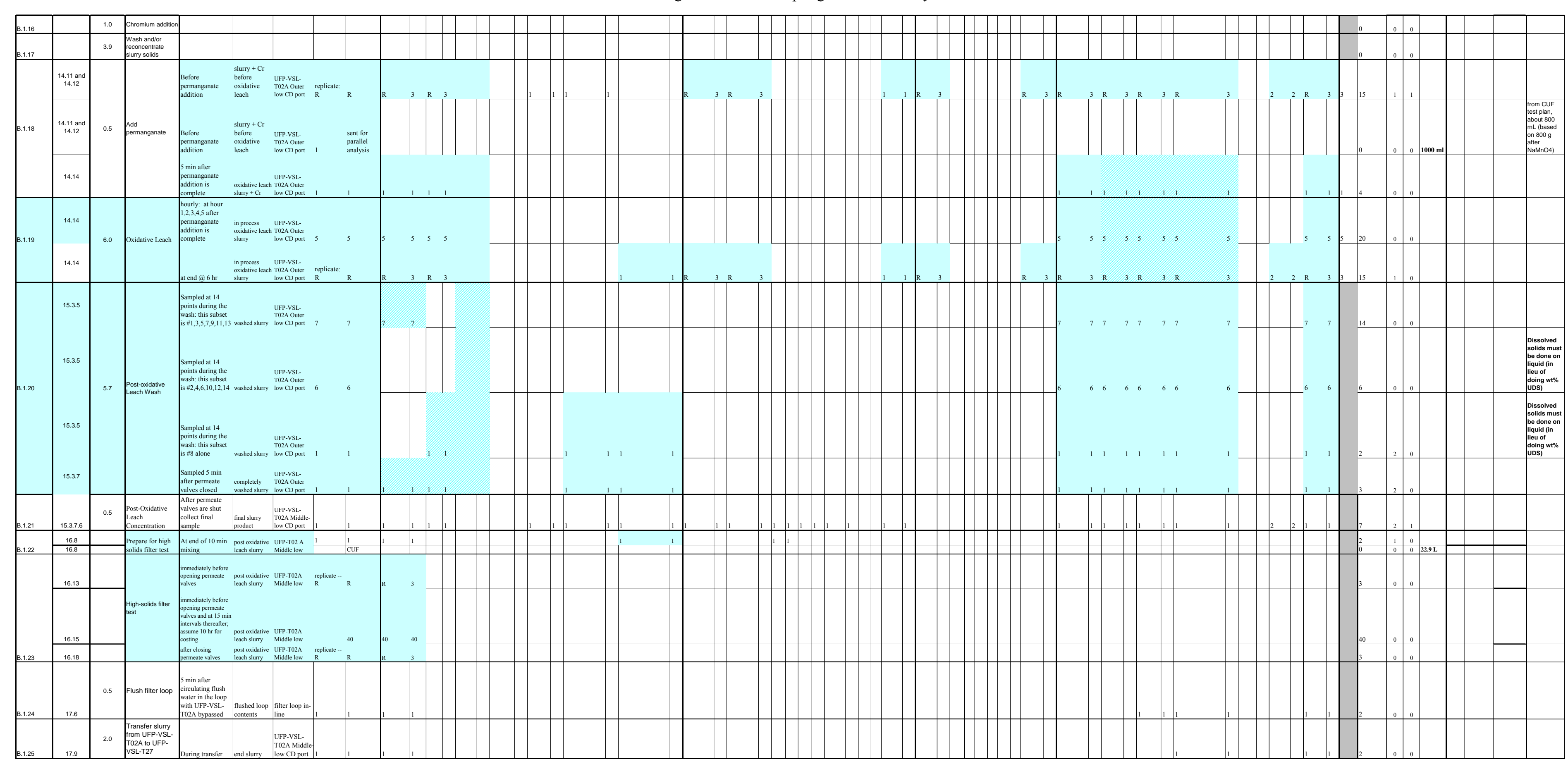


Integrated Test A Sampling Event Summary

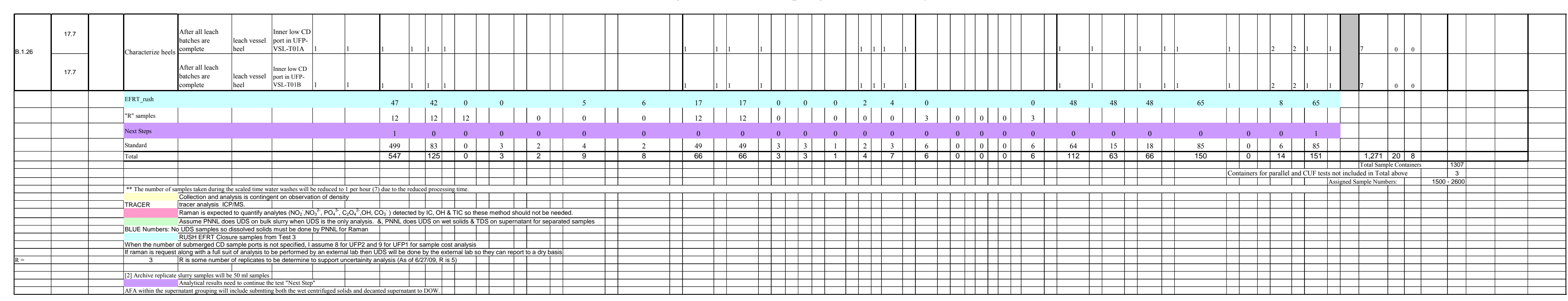

NOTES:

Leach samples were cooled immediately to prevent further reaction of aluminum in the slurry. 

Appendix D

\section{Coriolis Sample Elevations}





\section{Appendix D: Coriolis Sample Elevations}

\begin{tabular}{|c|c|c|c|}
\hline Date: & $12 / 31 / 2008$ & Project No.: & $<53032>$ \\
\hline To: & Dean Kurath & Internal & File/LB \\
\hline From: & $\begin{array}{l}\text { Matt Fountain } \\
\text { Gary Josephson }\end{array}$ & Distribution: & \\
\hline Subject: & Coriolis Sampler Elevations & & \\
\hline
\end{tabular}

'As installed' elevations of the Coriolis samplers were estimated by bubbling air through each sampler leg and measuring the bubbler pressure on the tank's lower bubbler leg calibrated pressure transmitter.

Method: After recording the pressure of the lowest bubbler leg the bubbler tubing was disconnected from the air supply and the supply attached to the Coriolis sampler stand via the low point drain and connecting Teflon tubing with Swagelock fittings. Air was bubbled through the tubing to each sampler leg and the pressure recorded. In UFP-1A and UFP-1B the PJMs were operating and the "lowest" pressure was recorded, when the PJMs were filled. Levels in UFP-1A, UFP-1B, and UFP-2A were measured on LT-0323, LT-0423, and LT-0611, respectively.

The measured pressures were compared with the measured differences in tubing lengths between the bottom-middle and middle-upper to make the final estimate sample elevations. Measured tubing differences were considered absolute and measured elevations were adjusted as much as an inch so that the offsets matched measured lengths.

Below are listed the "expected" sampler elevations based upon initial design and the results of this study:

\begin{tabular}{|c|l|l|l|l|l|l|}
\hline Vessel & \multicolumn{3}{|c|}{ Initial design elevation } & \multicolumn{3}{c|}{ "as installed" sample tube elevation } \\
\hline UFP-1A & Low & Middle & High & Low & Middle & High \\
\hline Inner & 2 & 33 & 64 & 11 & 33 & 64 \\
\hline Middle & 11 & 33 & 55 & 9 & 31 & 53 \\
\hline Outer & 11 & 33 & 55 & 9 & 31 & 53 \\
\hline UFP-1B & & & & & & \\
\hline Inner & 2 & 33 & 64 & 10 & 32 & 63 \\
\hline Middle & 11 & 33 & 55 & 6 & 28 & 51 \\
\hline Outer & 11 & 33 & 55 & 6 & 51 & 29 \\
\hline UFP-2A & & & & & & \\
\hline Inner & 50 & 57 & 64 & 51 & 58 & 65 \\
\hline Middle & 2 " off & 33 & 55 & 6 & 34 & 56 \\
\hline bottom & & 33 & 55 & 11 & 33 & 56 \\
\hline
\end{tabular}

Conclusions: The 'as installed' sampler levels agree quite well with the design with the following exceptions: 
- The UFP-1B outer/middle and outer/high sampling tubing has been switched. (An NCR is being written and the tubing needs to be corrected and the correction date recorded. Samples taken until then are incorrect.)

- The inner low sampler tubes were cut shorter than originally anticipated. Instead of sampling very close (2") from the bottom of the vessel the sample is 10 "-11" off the bottom.

- The middle and outer samplers in UFP-1B were installed about 4"-5" lower than initially intended.

The design of the UFP-2A middle/low position was designed to be 2 inches off the vessel bottom at the middle radial location. With a 2:1 elliptical head the elevation rise for the middle sampling location is estimated to be $\sim 3.5$ " so the measured 6 " elevation from the center tank bottom is actually very close. 
Appendix E

Integrated Test A Timeline 



\section{Appendix E: Integrated Test A Timeline}

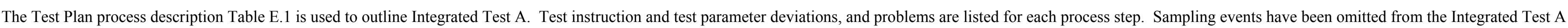

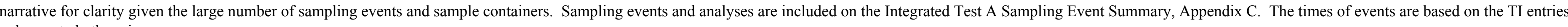
unless noted otherwise.

Table E.1. Integrated Test A - Caustic Leaching in UFP-VSL-T01A/B and Oxidative Leaching in UFP-VSL-T02A

\begin{tabular}{|c|c|c|c|c|c|}
\hline Test Step & Test Step & Description/Purpose & Key Process Conditions & Sampling & Test Deviations and Problems \\
\hline B.1.1 & $\begin{array}{c}\text { Prepare and stage simulant } \\
\text { in } \\
\text { HLP-VSL-T22 } \\
\text { Completed 1/31/09, 04:56 } \\
\end{array}$ & $\begin{array}{l}\text { Prepare sufficient quantity of Phase } 1 \text { simulant } \\
\text { in HLP-VSL-T22 to complete tests. } \\
\text { Characterize simulant for specification of all } \\
\text { simulant-dependent process parameters (e.g., } \\
\text { batch volumes, caustic and permanganate } \\
\text { additions). } \\
\text { Note: This characterization may have been } \\
\text { completed at the start of functional testing. }\end{array}$ & $\begin{array}{l}\text { HLP-VSL-T22 agitator speed set so vessel } \\
\text { is well mixed; recirculate the tank with the } \\
\text { pump. }\end{array}$ & 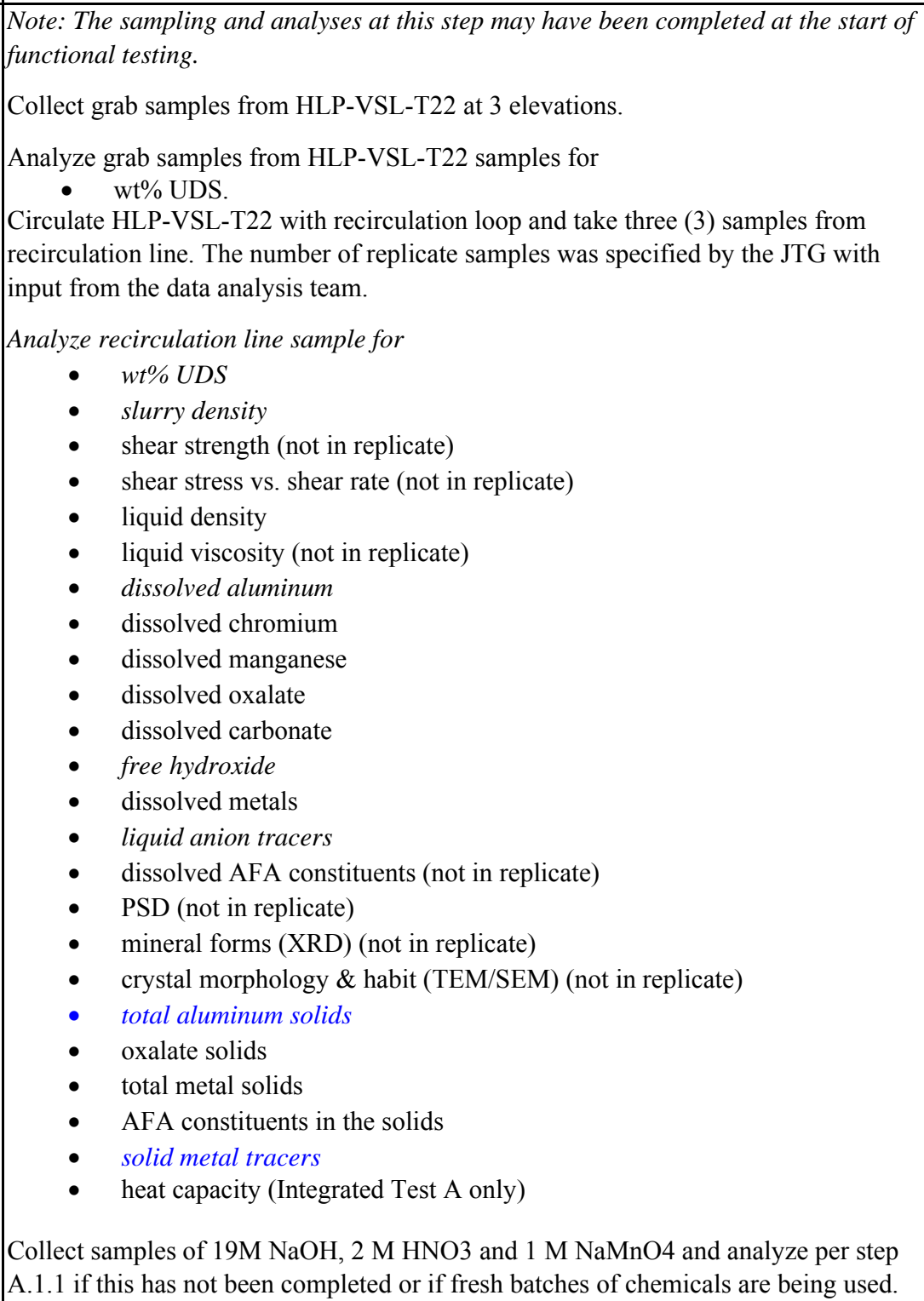 & $\begin{array}{l}\text { The portable sampling station was cannibalized, it had no pump. In efforts } \\
\text { to collect pretest samples, an aluminum pipe was inserted in HLP-VSL-T22 } \\
\text { and visual observations indicated the pipe had partially dissolved. (LRB } \\
60108, \text { pg. 110) Associated Operations Shift Supervisor spoke to operators } \\
\text { to prevent this from happening again and notifications were sent to team } \\
\text { about the affected samples numbers from the event. } \\
\\
\text { Vacuum was used to collect the } 2-40 \mathrm{~mL} \text { pretest samples, by collecting } \\
500-\mathrm{mL} \text { in a 1-Liter container. The 1-Liter was shaken, then an aliquot was } \\
\text { poured into 1-40 mL containers, the "shake and aliquot" method was } \\
\text { repeated for the second } 40 \text {-mL sample. (TI pg. } 64 \text { ). }\end{array}$ \\
\hline
\end{tabular}




\begin{tabular}{|c|c|c|c|c|c|}
\hline Test Step & Test Step & Description/Purpose & Key Process Conditions & Sampling & Test Deviations and Problems \\
\hline B.1.2 & $\begin{array}{c}\text { Transfer } \\
\text { simulant from } \\
\text { HLP-VSL-T22 } \\
\text { to } \\
\text { UFP-VSL-T01A } \\
\text { and add } \\
\text { NaOH in-line } \\
\text { Started } 1 / 31 / 09,11: 19 \\
\text { Ended } 1 / 31 / 09 \\
11: 59\end{array}$ & $\begin{array}{l}\text { Transfer prototypic quantity of simulant from } \\
\text { HLP-VSL-T22 to UFP-VSL-T01A. } \\
\text { Add anti-foam agent directly to UFP-VSL- } \\
\text { T01A. } \\
\text { Add NaOH via in-line addition. }\end{array}$ & $\begin{array}{l}\text { UFP-VSL-T01A empty (rinsed and } \\
\text { drained) or with heel characterized in } \\
\text { previous integrated process test. } \\
\text { HLP-VSL-T22 agitator speed set so vessel } \\
\text { is well mixed. } \\
\text { Set transfer rate to ensure turbulent flow in } \\
\text { transfer pipes. } \\
\text { UFP-VSL-T01A PJM velocities and cycle } \\
\text { times match PTF PJM power/volume. }\end{array}$ & $\begin{array}{l}\text { Collect grab samples using the portable CD sampler from } 3 \text { elevations in HLP-VSL- } \\
\text { T22 just before transfer is initiated and archive test samples. Collect } 3 \text { grab samples } \\
\text { from the middle elevation in HLP-VSL-T22. For the first batch of Integrated Test A } \\
\text { collect a } 1 \text { L grab sample using the portable CD sampler prior to initiating the transfer } \\
\text { to UFP-VSL-T01A. } \\
\text { Analyze the } 3 \text { grab samples obtained at the middle elevation for } \\
\text { wt\% UDS } \\
\text { shear stress vs. shear rate } \\
\text { Archive remaining grab samples from HLP-VSL-T22. } \\
\text { Collect in-line samples at the start of the transfer, at } 2 \text { times spaced evenly during the } \\
\text { transfer, and near the end of the transfer. } \\
\text { Archive in-line samples. } \\
\text { Record density in UFP-VSL-T01A at the inner-low CD sample port. } \\
\text { For the first batch of Integrated Test A, collect sample for parallel lab testing } \\
\text { from inner CD sample port. For all batches and both tests, collect } 3 \text { samples for } \\
\text { characterization from inner CD port } 10 \text { min after transfer is complete. } \\
\text { Transport inner CD port parallel lab testing sample to APEL. } \\
\text { Analyze inner CD port characterization samples for } \\
\text { - wt\% UDS } \\
\text { - slurry density } \\
\text { - liquid density } \\
\text { - fissolved aluminum } \\
\text { free hydroxide } \\
\text { liquid anion tracers } \\
\text { total aluminum solids } \\
\text { - solid metal tracers } \\
\text { heat capacity (Integrated Test A only).(not in replicate) }\end{array}$ & $\begin{array}{l}\text { Grab Sampling just prior to transferring from HLP-VSL-T22 to } \\
\text { UFP-VSL-T01A only collected samples from middle and low elevations, } \\
\text { the high grab sample could not be collected with instrumentation used (TI). } \\
\text { Changes were made to the target transfer rates for the simulant and caustic } \\
\text { addition because the HLP-VSL-T22 flowmeter, FQI-0119, reports a } \\
\text { combined transfer rate for both the simulant and caustic, where as the test } \\
\text { instruction treated these independently. TI and LRB 60108, pg. 109. } \\
\text { Batch 1, UFP-VSL-T01A, PJMs were turned on at a tank level of 40+ } \\
\text { inches during the transfer from HLP-VSL-T22 to UFP-VSL-T01A while } \\
\text { the Run Sheet stated to do this at a maximum tank level of } 25 \text { inches (TI: } \\
\text { Run Sheet). }\end{array}$ \\
\hline B.1.3 & $\begin{array}{c}\text { Heat } \\
\text { UFP-VSL-T01A } \\
\text { and caustic leach } \\
\text { Initial Heatup: } \\
\text { Started 1/31/09, } \\
\text { 14:40 } \\
\text { Ended 1/31/09, } 15: 01 \\
\text { Final Heatup: } \\
\text { Started 1/31/09, } \\
\text { 15:30 } \\
\text { Ended 1/31/09, 19:40 } \\
\text { Caustic Leach: } \\
\text { Started 1/31/09, 19:40 } \\
\text { Ended 2/01/09, } \\
\text { 11:40 }\end{array}$ & $\begin{array}{l}\text { Heat UFP-VSL-T01A using steam injection. } \\
\text { Demonstrate caustic leaching in UFP-VSL- } \\
\text { T01A and obtain process performance data for } \\
\text { 4.5-scale facility. } \\
\text { Demonstrate vessel temperature instrumentation } \\
\text { and control strategy. } \\
\text { Demonstrate operation of UPF-VSL-T01A PJMs } \\
\text { at elevated temperatures. }\end{array}$ & $\begin{array}{l}\text { Heat and blend at plant-time. } \\
\text { Integrated Test A leach at } 98^{\circ} \mathrm{C} \text { for } 16 \mathrm{hr} \text {. } \\
\text { UFP-VSL-T01A PJM velocities and cycle } \\
\text { times match PTF PJM power/volume. }\end{array}$ & $\begin{array}{l}\text { Record density in UFP-VSL-T01A at inner CD sample point during heat-up to within } \\
10^{\circ} \mathrm{C} \text { of the leaching temperature and when not collecting samples at other ports. } \\
\text { Collect samples from inner } \mathrm{CD} \text { sample port when the vessel is within } 10^{\circ} \mathrm{C} \text { of the } \\
\text { leaching set point temperature, when the vessel reaches the leaching set point } \\
\text { temperature (time }=0 \text { ), and at } 1 \text {-hr intervals until the end of the leaching period. } \\
\text { For the first batch of Integrated Test A, collect triplicate samples at time } 0 \text {. } \\
\text { Analyze the inner } \mathrm{CD} \text { port samples collected when the vessel is within } 10^{\circ} \mathrm{C} \text { of } \\
\text { the leaching setpoint temperature, at times } 0,1,2,4,8,10,12,14, \text { and } 16 \mathrm{hr} \text { for } \\
\text { - w\% UDS } \\
\text { - slurry density } \\
\text { liquid density } \\
\text { dissolved aluminum } \\
\text { - lique hydroxide } \\
\text { - qeat capion tracers } \\
\text { vessel is within } 10^{\circ} \mathrm{C} \text { of leaching setpoint and first sample taken after } \\
\text { temperature setpoint is reached) (not in replicate). } \\
\text { For samples taken at times } 4,8,12 \text {, and } 16 \mathrm{hr} \text {, analyze for } \\
\text { - total aluminum solids }\end{array}$ & $\begin{array}{l}\text { At 18:29, 01/31/09, switched from bubbler to laser level control in UFP- } \\
\text { VSL-T01A because laser was reading } 96 \text { inches, temperature in vessel is } \\
-88^{\circ} \mathrm{C} \text { (LRB 60229, pg. } 9 \text { ). } \\
\text { Stable level measurement taken at the end of initial heat for Batch } 1 \text { UFP- } \\
\text { VSL-T01A had vessel isolation valve V03129 open- deviation to TI. It was } \\
\text { closed } ~ 50 \text { minutes prior to reaching final target leach temperature per Run } \\
\text { Sheet (18:50 on 01/31/09). (TI). } \\
\text { Steam controller TIC-0325 shut itself off, was put back onto automatic } \\
\text { mode at } 19: 00,1 / 31 / 09 \text { (LRB } 60108, \text { pg. } 112 \text { ). } \\
\text { Level at end of heatup was } 63.1, \text { versus Run Sheet target of } 61 \pm 2 \text { in (TI: } \\
\text { Run Sheet). } \\
\text { Batch } 1 \text { UFP-VSL-T01A PJM tuning at } 98^{\circ} \mathrm{C} \text { was difficult because drive } \\
\text { pressure was not stable. Drive pressure and time setpoint were adjusted }\end{array}$ \\
\hline
\end{tabular}




\begin{tabular}{|c|c|c|c|c|c|}
\hline Test Step & $\begin{array}{l}\text { Test Step } \\
\end{array}$ & Description/Purpose & Key Process Conditions & Sampling & $\begin{array}{c}\text { Test Deviations and Problems } \\
\end{array}$ \\
\hline & & & & $\begin{array}{l}\text { solid metal tracers } \\
\text { Samples at final time should be obtained in triplicate (3) and all samples } \\
\text { analyzed as indicated. Only samples from the first batch of Integrated Test A are } \\
\text { to be analyzed with a rapid TAT for resolution of the EFRT issues. } \\
\text { Analyze last solids sample (not in replicate) to determine boehmite and gibbsite in Al } \\
\text { solid phase, XRD } \\
\text { Archive separated liquid and solid fractions of remaining inner CD port samples. } \\
\text { Collect samples from each submerged CD sample port when vessel is within } 10^{\circ} \mathrm{C} \text { of } \\
\text { the eeaching setpoint temperature, when vessel reaches the leaching setpoint } \\
\text { temperature, and at 4-hr intervals after vessel reaches the leaching temperature until } \\
\text { leaching is finished. } \\
\text { Archive separated liquid and solid fractions of CD port samples. }\end{array}$ & $\begin{array}{l}\text { until Run Sheet PJM nozzle velocity were met (21:13 on 01/31/09). (TI). } \\
\text { 2/1/09, 09:02 through 09:08 (LRB 60229, pg. 20), level control in UFP- } \\
\text { VSL-T1A was switched back and forth between Drexelbrook, bubbler and } \\
\text { laser because the noise in the bubblers level values caused highhl-evel alarms } \\
\text { in the vessel. Level control was kept with the Drexelbrook, the most stable. } \\
\text { The Drexelbrook level trend was smoother with the steam-ring air off, then } \\
\text { when it was on (LRB 60229, pg. 22-24). } \\
\end{array}$ \\
\hline B.1.4 & \begin{tabular}{|c} 
Partially cool-down slurry \\
in \\
UFP-VSL-T01A \\
Started $2 / 1 / 09$ \\
$11: 57$ \\
Ended $2 / 1 / 109$ \\
$14: 59$ \\
\end{tabular} & $\begin{array}{l}\text { Cool-down of UFP-VSL-T01A contents to } \\
\text { quench the caustic leaching process. }\end{array}$ & \begin{tabular}{|l|} 
Recirculate slurry in UFP-VSL-T01A \\
through external chiller (UFP-HX-T05A) to \\
reduce vessel contents to $60^{\circ} \mathrm{C}$. \\
UFP-VSL-T01A PJM velocities and cycle \\
times match PTF PJM power/volume.
\end{tabular} & None. & $\begin{array}{l}\text { 13:00 on 2/1/09 (LRB 60229, pg. 32) UFP-VSL-T01A pump was turned } \\
\text { back on after it was tripped by a high-high level alarm in UFP-VSL-T01B. } \\
\text { 14:58 on 2/1/09 (LRB 60229, pg. 35) PJM probes } 1601,1603 \text {, and } 1605 \\
\text { were not responding to the PJM drive phase, they stayed at value } 48 \text { inches } \\
\text { or } 38 \text { inches for } 1605 \text {. }\end{array}$ \\
\hline B.1.5 & $\begin{array}{c}\text { Transfer leached } \\
\text { slurry from } \\
\text { UFP-VSL-T01A } \\
\text { to } \\
\text { UFP-VSL-T02A } \\
\text { Started 2/1/09, } \\
\text { 15:54 } \\
\text { Ended 2/1/09, } \\
\text { 16:34 }\end{array}$ & $\begin{array}{l}\text { Demonstrate batch transfer of feed from UFP- } \\
\text { VSL-T01A to UFP-VSL-T02A. } \\
\text { During initial transfer to fill UFP-VSL-T02A } \\
\text { cool to filtration temperature using UFP-HX- } \\
\text { T05A. }\end{array}$ & \begin{tabular}{|l|} 
UFP-VSL-T02A empty (rinsed and \\
drained) or with heel characterized in \\
previous integrated process test. \\
Transfer at scale-time. \\
UFP-VSL-T01A PJM velocities and cycle \\
times match PTF PJM power/volume. \\
UFP-VSL-T02A PJM velocities and cycle \\
times match PTF PJM power/volume.
\end{tabular} & \begin{tabular}{|l} 
Collect in-line transfer samples 2 min after start of transfer and 2 min before end of \\
transfer. \\
Archive in-line transfer samples. \\
Record density at lowest center CD sampling port in UFP-VSL-T01A. \\
Record density at lowest middle-radius CD sampling port in UFP-VSL-T02A. \\
Collect sample from each submerged CD port in UFP-VSL-T02A 10 min after \\
transfer is complete. \\
Analyze the inner CD port sample for \\
wt\% UDS \\
slurry density \\
- liquid density \\
disolved aluminum \\
free hydroxide \\
liquid anion tracers \\
total aluminum solids \\
Archive separated liquid and solid fractions of remaining CD port samples.
\end{tabular} & 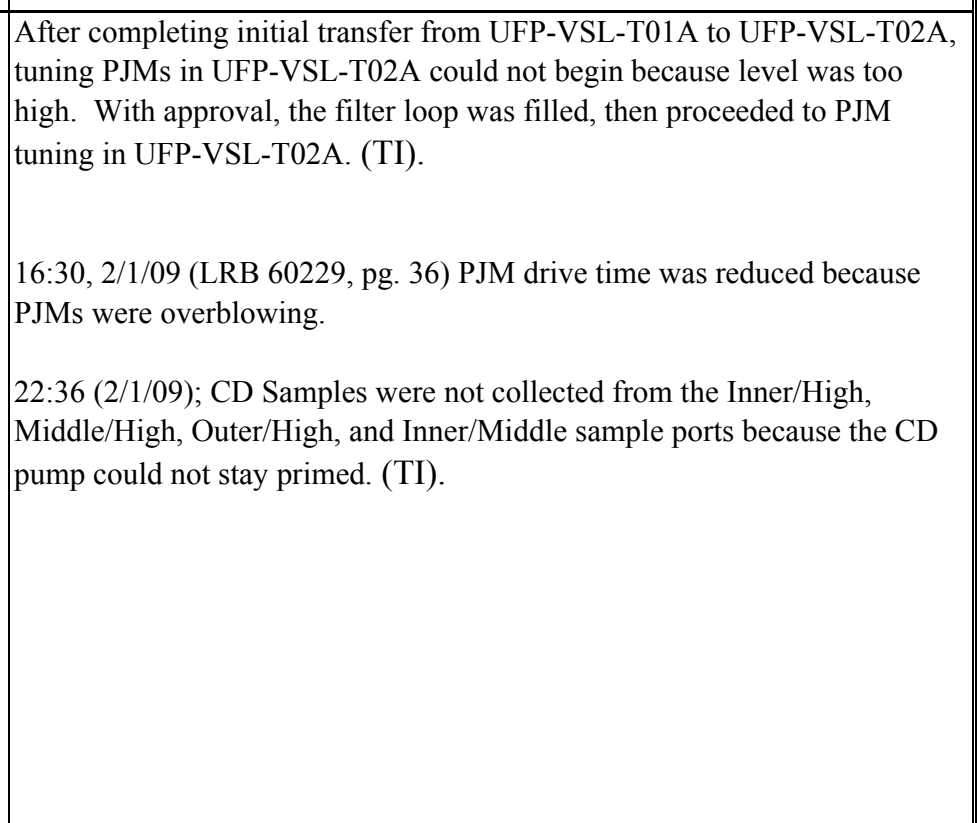 \\
\hline B.1.6 & $\begin{array}{c}\text { Cool slurry in } \\
\text { UFP-VSL-T01A with } \\
\text { UFP-HX-T05A } \\
\text { Started 2/1/09, } \\
\text { 15:01 } \\
\text { Ended 2/1/09, } \\
\text { 15:15 }\end{array}$ & $\begin{array}{l}\text { Recirculate remaining slurry in UFP-VSL-T01A } \\
\text { through UFP-HX-T05A to cool to the filtration } \\
\text { temperature. } \\
\text { Determine if aluminum re-precipitates as } \\
\text { gibbsite. }\end{array}$ & $\begin{array}{l}\text { Recirculate at scale-time. } \\
\text { UFP-VSL-T01A PJM velocities and cycle } \\
\text { times match PTF PJM power/volume. }\end{array}$ & $\begin{array}{l}\text { Sample inner CD port and separate phases. Analyze solids phase to determine } \\
\text { boehmite and gibbsite in Al solid phase, XRD. }\end{array}$ & $\begin{array}{l}\text { 19:00, 2/1/09, UFP-VSL-T01B bubblers were cleaned because specific } \\
\text { gravity was reading } \sim \text {, during the cleaning, UFP-VSL-T01A pump was } \\
\text { tripped, then it was restarted (LRB 60229, pg. 41). } \\
\text { 21:55, 2/1/09 (LRB 60229, pg. 43), UFP-VSL-T01A PJM drive pressures } \\
\text { for } 1601 \text { and } 1603 \text { were lower than other drive pressures. }\end{array}$ \\
\hline
\end{tabular}




\begin{tabular}{|c|c|c|c|c|c|}
\hline Test Step & Test Step & \begin{tabular}{|c|} 
Description/Purpose \\
\end{tabular} & \begin{tabular}{|l|} 
Key Process Conditions \\
\end{tabular} & \begin{tabular}{|c|} 
Sampling \\
\end{tabular} & \begin{tabular}{|c|} 
Test Deviations and Problems \\
\end{tabular} \\
\hline B.1.7 & $\begin{array}{l}\text { Concentrate solids in } \\
\text { UFP-VSL-T02A } \\
\text { Started 2/2/09, } \\
\text { 01:34 (TI pg. 186) } \\
\text { Ended 2/2/09, } \\
\text { 17:24 }\end{array}$ & $\begin{array}{l}\text { Concentrate solids in UFP-VSL-T02A. Transfer } \\
11 \text { gal batches of slurry from UFP-VSL-T01A to } \\
\text { UFP-VSL-TO2A as needed to maintain the level } \\
\text { in UFP-VSL-T02A. This step is complete when } \\
\text { UFP-VSL-T01A has been emptied to its heel. } \\
\text { Demonstrate control strategy for make-up } \\
\text { additions from the UFP-VSL-T01A/B B UFP- } \\
\text { VSL-T02A during solids concentration. } \\
\text { Steps B.1.8 - B.1.11 should be initiated and } \\
\text { performed so that step B.1.12 can be started as } \\
\text { soon as step B.1.7 is complete. }\end{array}$ & $\begin{array}{l}\text { Ultrafilters preconditioned and flushed; } \\
\text { drained just before concentration of solids } \\
\text { is started. } \\
\text { Concentrate at plant-time. } \\
\text { One 10-ft ultrafilter bundle. } \\
\text { UFP-VSL-T01A PJM velocities and cycle } \\
\text { times match PTF PJM power/volume. } \\
\text { UFP-VSL-T01B PJM velocities and cycle } \\
\text { times match PTF PJM power/volume. } \\
\text { UFP-VSL-T02A PJM velocities and cycle } \\
\text { times match PTF PJM power/volume. } \\
\text { Route permeate to UFP-VSL-T62B. }\end{array}$ & $\begin{array}{l}\text { Record density in UFP-VSL-T02A at a middle CD sampling location during } \\
\text { concentration. } \\
\text { Collect permeate samples between each filter bundle and the pulse pots at } 3 \text { evenly } \\
\text { spaced times and inspect for particle break-through. } \\
\text { Collect samples from the middle-low CD sample port initially and every } 2 \mathrm{hr} \text {. } \\
\text { Analyze the CD samples for } \\
\text { wt\% UDS. } \\
\text { Record density at all CD sample locations every } 2 \mathrm{hr} \text {. } \\
\text { Collect permeate sample from the permeate sample port between the first filter bundle } \\
\text { near end of solids concentration step. } \\
\text { Submit permeate sample for precipitation studies. }\end{array}$ & 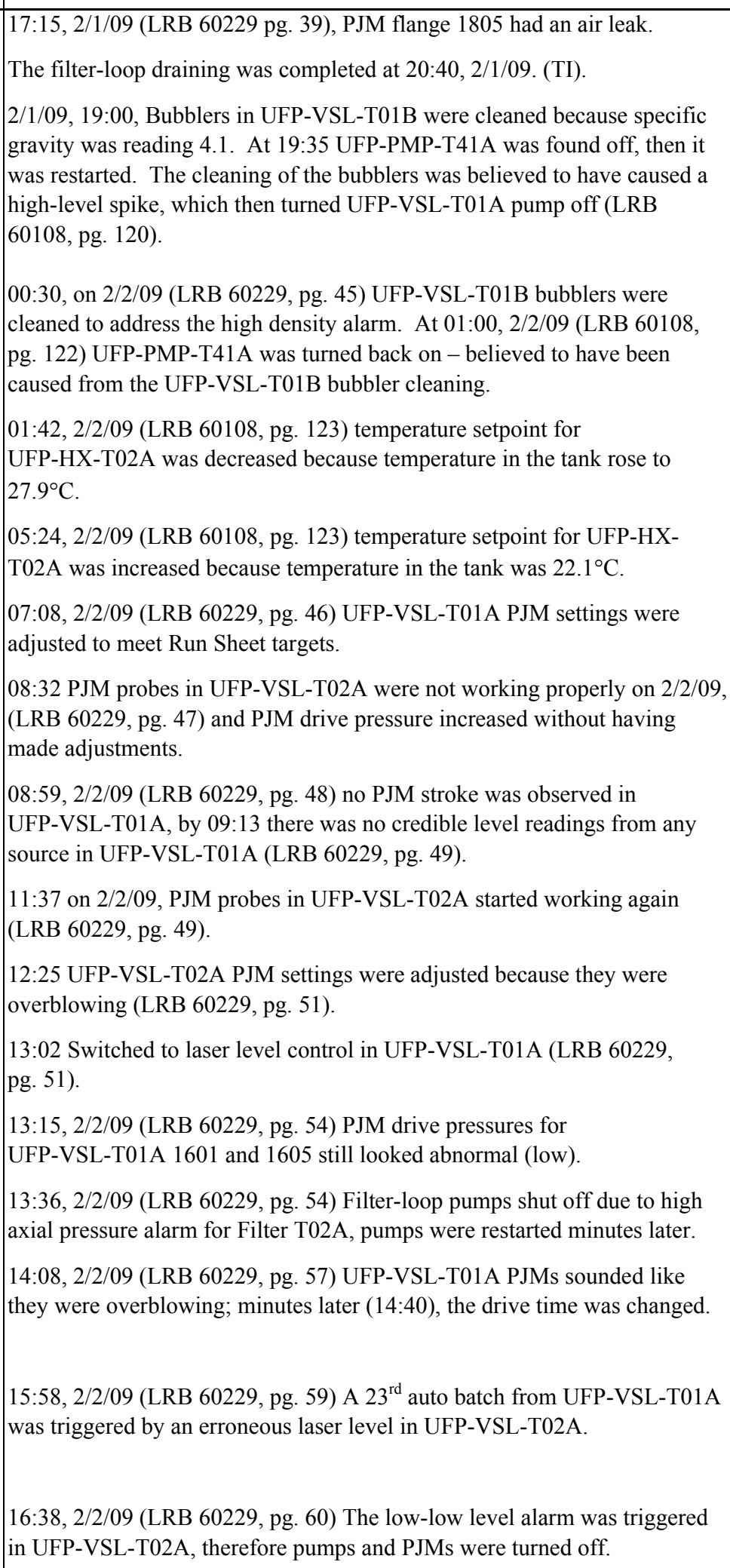 \\
\hline
\end{tabular}




\begin{tabular}{|c|c|c|c|c|c|}
\hline Test Step & $\begin{array}{l}\text { Test Step } \\
\end{array}$ & Description/Purpose & Key Process Conditions & $\begin{array}{l}\text { Sampling } \\
\end{array}$ & Test Deviations and Problems \\
\hline & & & & & $\begin{array}{l}\text { On 2/2/09, between 16:00 and 16:50 (LRB 60229, pg. 60) UFP-VSL-T02A } \\
\text { level was unstable, causing low-low level alarms, which then caused the } \\
\text { pumps and PJMs to turn off, which then triggered high transmembrane } \\
\text { pressure (TMP) alarms- the TMP would decrease once pumps were } \\
\text { restarted. Low-low level alarms would occur whenever filter-loop flow rate } \\
\text { exceeded } 83 \text { GPM (16:50, } 2 / 2 / 09, \text { LRB } 60229 \text {, pg. . } 62 \text { ). Auto batch } \\
\text { operations were suspended until conditions stabilized. } \\
\text { 17:24, 2/2/09 (LRB 60229, pg. } 62 \text { ). In response to the low low level alarms } \\
\text { in UFP-VSL-T02A, the low-low interlock delay was changed to } 3 \text { seconds } \\
\text { in all three UFP vessels, and the laser out of range filter low to } 0 \text { inches. } \\
\text { Late } 2 / 2 / 09 \text { UFP-VSL-T01A laser was used for level control although it } \\
\text { was not reading correctly. PJMs were turned off in UFP-VSL-T01A } \\
\text { because of overblowing, level in tank was } ~ 14 \text { inches per laser. (LRB } \\
60108 \text {, pg. 124). }\end{array}$ \\
\hline B.1.8 & $\begin{array}{c}\text { Transfer } \\
\text { simulant from } \\
\text { HLP-VSL-T22 } \\
\text { to } \\
\text { UFP-VSL-T01B } \\
\text { and add } \\
\text { NaOH in-line } \\
\text { Started } 1 / 31 / 09 \\
\text { 15:47 } \\
\text { Ended 1/31/09, } \\
\text { 17:48-includes caustic } \\
\text { addition made to the top } \\
\text { of the vessel. }\end{array}$ & \begin{tabular}{|l|} 
Transfer prototypic quantity of simulant from \\
HLP-VSL-T22 to UFP-VSL-T01B. \\
Add anti-foam agent directly to UFP-VSL- \\
T01B. \\
Add $80 \%$ of prototypic $19 \mathrm{M}$ NaOH via in-line \\
addition. Add remaining $20 \%$ of caustic directly \\
to UFP-VSL-T01B. \\
\end{tabular} & 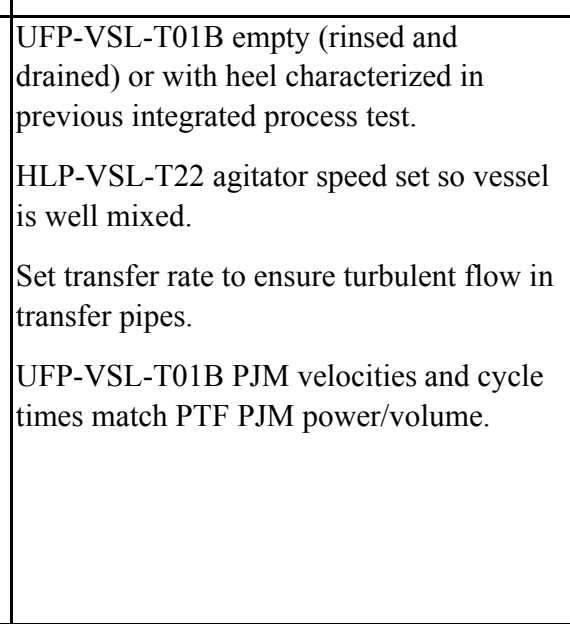 & $\begin{array}{l}\text { Collect in-line samples at the start of the transfer, at } 2 \text { times spaced evenly during the } \\
\text { transfer, and near the end of the transfer. } \\
\text { Archive in-line samples. } \\
\text { Record density in UFP-VSL-T01A at all submerged CD sample points. Collect } \\
\text { sample from inner CD port } 10 \text { min after transfer is complete. Analyze inner CD port } \\
\text { sample for } \\
\text { - wt\% UDS } \\
\text { - slurry density } \\
\text { - liquid density } \\
\text { dissolved aluminum } \\
\text { free hydroxide } \\
\text { - tiquid anion tracers } \\
\text { tolal aluminum solids }\end{array}$ & $\begin{array}{l}\text { Changes were made to the target transfer rates for the simulant and caustic } \\
\text { addition because the HLP-VSL-T22 flowmeter, FQI-0119, reports a } \\
\text { combined transfer rate for both the simulant and caustic, where as the test } \\
\text { instruction treated these independently. (TI). }\end{array}$ \\
\hline B.1.9 & $\begin{array}{c}\text { Heat } \\
\text { UFP-VSL-T01B } \\
\text { and caustic leach } \\
\text { Initial Heatup: } \\
\text { Started 1/3/1/109, } \\
22: 07 \\
\text { Ended 1/31/09, } \\
\text { 23:15 } \\
\text { Final Heatup: } \\
\text { Started 1/31/09, } \\
23: 35 \\
\text { Ended 2/1/09, } \\
03: 20 \\
\text { Caustic Leach: } \\
\text { Started 2/1/09, } \\
03: 20 \\
\text { Ended 2/1/09, } \\
\text { 19:59 }\end{array}$ & $\begin{array}{l}\text { Heat UFP-VSL-T01B using steam injection and } \\
\text { add remaining 20\% of prototypic } 19 \mathrm{M} \mathrm{NaOH} \\
\text { directly to UFP-VSL-T01B. } \\
\text { Demonstrate caustic leaching in UFP-VSL- } \\
\text { T01B and obtain process performance data for } \\
\text { 4.5-scale facility. }\end{array}$ & $\begin{array}{l}\text { Heat and mix at plant-time. } \\
\text { Integrated Test A leach at } 98^{\circ} \mathrm{C} \text { for } 16 \mathrm{hr} . \\
\text { UFP-VSL-T01B PJM velocities and cycle } \\
\text { times match PTF PJM power/volume. }\end{array}$ & $\begin{array}{l}\text { Record density in UFP-VSL-T01B at inner CD sample point during heat-up to within } \\
10^{\circ} \mathrm{C} \text { of the leaching temperature and when not collecting samples at other ports. } \\
\text { Collect samples from inner CD sample port when the vessel is within } 10^{\circ} \mathrm{C} \text { of the } \\
\text { leaching setpoint temperature, when the vessel reaches the leaching setpoint } \\
\text { temperature (time }=0 \text { ), and at } 1 \text {-hr intervals until the end of the leaching period. } \\
\text { Analyze the inner CD port samples collected when the vessel is within } 10^{\circ} \mathrm{C} \text { of the } \\
\text { leaching setpoint temperature, at times } 0,1,2,4,8,10,12,14 \text { and } 16 \mathrm{hr} \text { for } \\
\text { wt } \mathrm{UDS} \\
\text { - slurry density } \\
\text { liquid density } \\
\text { dissolved aluminum } \\
\text { - free hydroxide } \\
\text { liquid anion tracers. } \\
\text { For samples taken at times } 4,8,12 \text {, and } 16 \mathrm{hr} \text {, analyze for } \\
\text { - total aluminum solids } \\
\text { solid metal tracers } \\
\text { Samples at final time should be obtained in triplicate (3) and all samples analyzed as } \\
\text { indicated. }\end{array}$ & $\begin{array}{l}\text { On 1/31/09, steam injection was inadvertently used to do the initial heating } \\
\text { for UFP-VSL-T01B Batch 2, instead of using UFP-HX-T04B. Forty-seven } \\
\text { minutes of steam injection were completed, and luckily there was not more } \\
\text { than } 0.3 \text { inches (per laser) of condensation added during this time based on } \\
\text { stable level measurements. UFP-HX-T04B was used to reach the target } \\
\text { initial heat-up temperature per the Run Sheet (22:54 on 1/31/09, LRB } \\
60108 \text {, pg. 117). } \\
\text { 2/1/109, 00:50 and at 02:43, Steam controller TIC-0425 shut itself off. It } \\
\text { was restarted and ramp time revised as appropriate (LRB 60108, pg. 118). } \\
\text { CD samples at } 10^{\circ} \mathrm{C} \text { below final heat leach temperature were only taken } \\
\text { from the low and middle elevations since the high elevation samples could } \\
\text { not keep the CD sample pump primed- could have been influenced by } \\
\text { steam injection valve fully open (02:31 on 2/1/09). (TI). } \\
\text { 2/1/09, 02:37, UFP-VSL-T01B level control was switched from laser to } \\
\text { bubbler, because laser was reading } 95 \text { inches, temperature in vessel is } \\
\text { - } 88^{\circ} \mathrm{C} \text { (LRB 60229, pg. 14). }\end{array}$ \\
\hline
\end{tabular}




\begin{tabular}{|c|c|c|c|c|c|}
\hline Test Step & $\begin{array}{l}\text { Test Step } \\
\end{array}$ & Description/Purpose & Key Process Conditions & $\begin{array}{l}\text { Sampling } \\
\end{array}$ & \begin{tabular}{|c|} 
Test Deviations and Problems \\
\end{tabular} \\
\hline & & & & $\begin{array}{l}\text { Archive separated liquid and solid fractions of remaining inner } \mathrm{CD} \text { port samples. } \\
\text { Collect samples from each submerged CD sample port when vessel is within } 10^{\circ} \mathrm{C} \text { of } \\
\text { the leaching setpoint temperature, when vessel reaches the leaching setpoint } \\
\text { temperature, and at 4-hr intervals after vessel reaches the leaching temperature until } \\
\text { leaching is finished. } \\
\text { Archive separated liquid and solid fractions of CD port samples. }\end{array}$ & 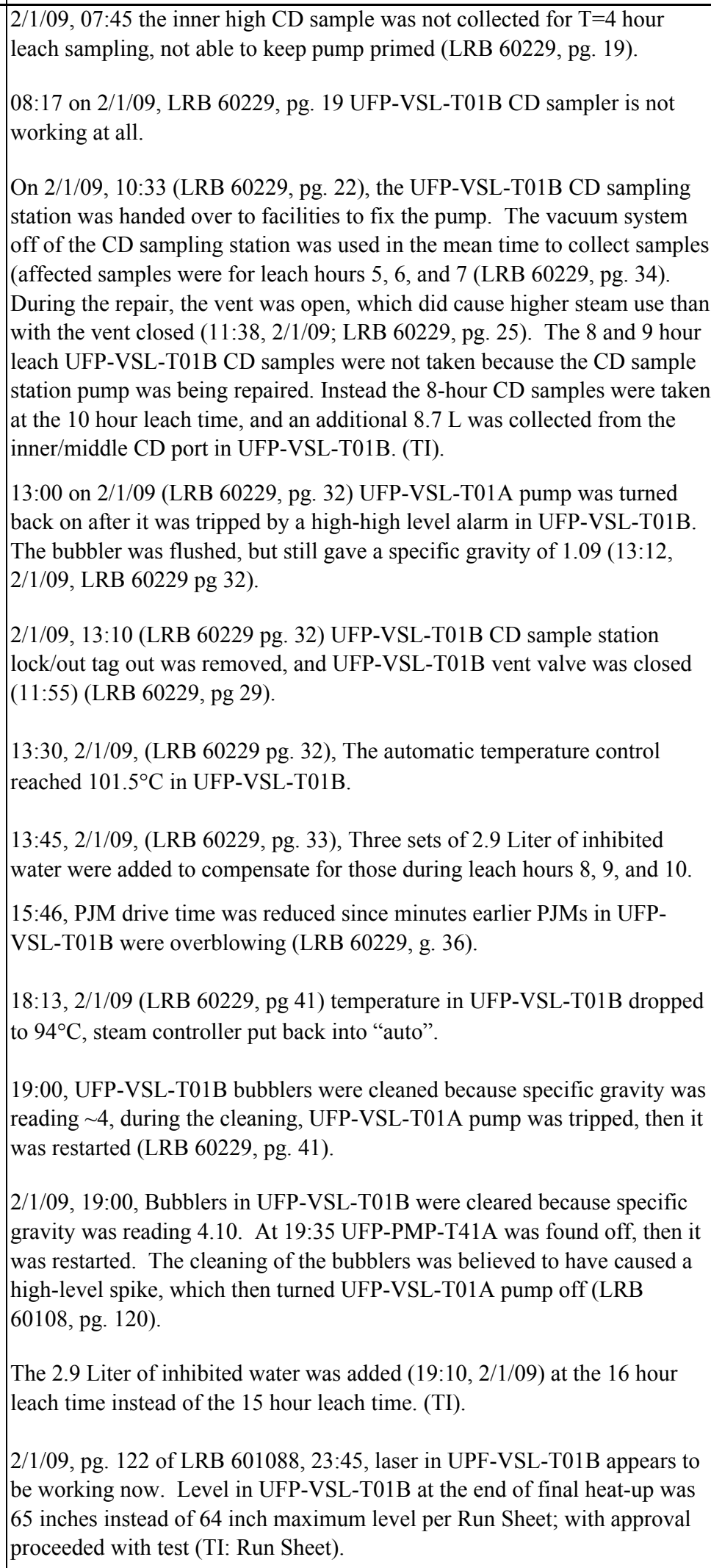 \\
\hline
\end{tabular}




\begin{tabular}{|c|c|c|c|c|c|}
\hline Test Step & Test Step & \begin{tabular}{|c|} 
Description/Purpose \\
\end{tabular} & Key Process Conditions & Sampling & \begin{tabular}{|c|} 
Test Deviations and Problems \\
\end{tabular} \\
\hline B.1.10 & $\begin{array}{c}\text { Initial Partial Cool Down } \\
\text { in } \\
\text { UFP-VSL-T01B } \\
\text { Started 2/1/09, } \\
20.15 \\
\text { Ended 2/1/09, } \\
22: 56\end{array}$ & $\begin{array}{l}\text { Cool-down of UFP-VSL-T01B contents to } \\
\text { quench the caustic leaching process. }\end{array}$ & \begin{tabular}{|l|} 
Recirculate slurry in UFP-VSL-T01B \\
through external chiller (UFP-HX-T05B) to \\
reduce vessel contents to $60^{\circ} \mathrm{C}$. \\
UFP-VSL-T01B PJM velocities and cycle \\
times match PTF PJM power/volume.
\end{tabular} & None. & $\begin{array}{l}\text { With approval, post-caustic-leach cooling in UFP-VSL-T01B batch } 2 \\
\text { cooling to filtration temperature was accomplished by slowing the speed of } \\
\text { pump UFP-PMP-T41B to } 1.2 \text { GPM instead of aligning and by-passing } \\
\text { UFP-HX-T05B, per test instruction, as needed to meet cooling curve target. } \\
\text { Pump UFP-PMP-T41B speed was increased periodically to flush out settled } \\
\text { solids. (TI, pg. 103). } \\
\text { Per LRB 60229, pg. 43, UFP-VSL-T01B PJMs were left off until 20:45, } \\
\text { after taking a stable level measurement at 20:13. }\end{array}$ \\
\hline B.1.11 & $\begin{array}{c}\text { Cool slurry in } \\
\text { UFP-VSL-T01B with } \\
\text { UFP-HX-T05B } \\
\text { Completed 2/3/08, } \\
\text { 23:08 }\end{array}$ & $\begin{array}{l}\text { Recirculate slurry from UFP-VSL-T01A through } \\
\text { UFP-HX-T05A to cool to the filtration } \\
\text { temperature. }\end{array}$ & $\begin{array}{l}\text { Recirculate at scale-time. } \\
\text { UFP-VSL-T01B PJM velocities and cycle } \\
\text { times match PTF PJM power/volume. }\end{array}$ & None. & $\begin{array}{l}\text { 00:30, on 2/2/09 (LRB 60229, pg. 45) UFP-VSL-T01B bubblers were } \\
\text { cleaned to address the high density alarm. At 01:00, } 2 / 2 / 09 \text { (LRB } 60108 \text {, } \\
\text { pg. 122) UFP-PMP-T41B was turned back on, was thought to have turned } \\
\text { off from the bubbler cleaning. } \\
\text { 04:00 on 2/2/09 (LRB 60229, pg. 46) UFP-VSL-T01B bubblers were } \\
\text { cleaned again. }\end{array}$ \\
\hline B.1.12 & $\begin{array}{c}\text { Concentrate solids in } \\
\text { UFP-VSL-T02A } \\
\text { Started 2/2/09, } \\
17: 24 \\
\text { Ended 2/4/09, } \\
\text { 12:51 }\end{array}$ & $\begin{array}{l}\text { Continuation of Step B.1.7, but with post- } \\
\text { caustic-leach feed from UFP-VSL-T01B. } \\
\text { Caustic leached feed to be supplied by UFP- } \\
\text { VSL-T } 1 \text {-1A and UFP-VSL-T01B alternately } \\
\text { until a prototypic batch at 17 wt\% UDS is } \\
\text { achieved in UFP-VSL-T02A. } \\
\text { Demonstrate control strategy for make-up } \\
\text { additions from the feed preparation vessels } \\
\text { (UFP-VSL-T01AB/B to the feed vessel (UFP- } \\
\text { VSL-T02A) during solids concentration. } \\
\text { Demonstrate ultrafiltration end points. }\end{array}$ & \begin{tabular}{|l|} 
Concentrate at plant-time. \\
One 10-ft ultrafilter bundle. \\
UFP-VSL-T01A PJM velocities and cycle \\
times match PTF PJM power/volume. \\
UFP-VSL-T01B PJM velocities and cycle \\
times match PTF PJM power/volume. \\
UFP-VSL-T02A PJM velocities and cycle \\
times match PTF PJM power/volume. \\
Route permeate to UFP-VSL-T62B.
\end{tabular} & 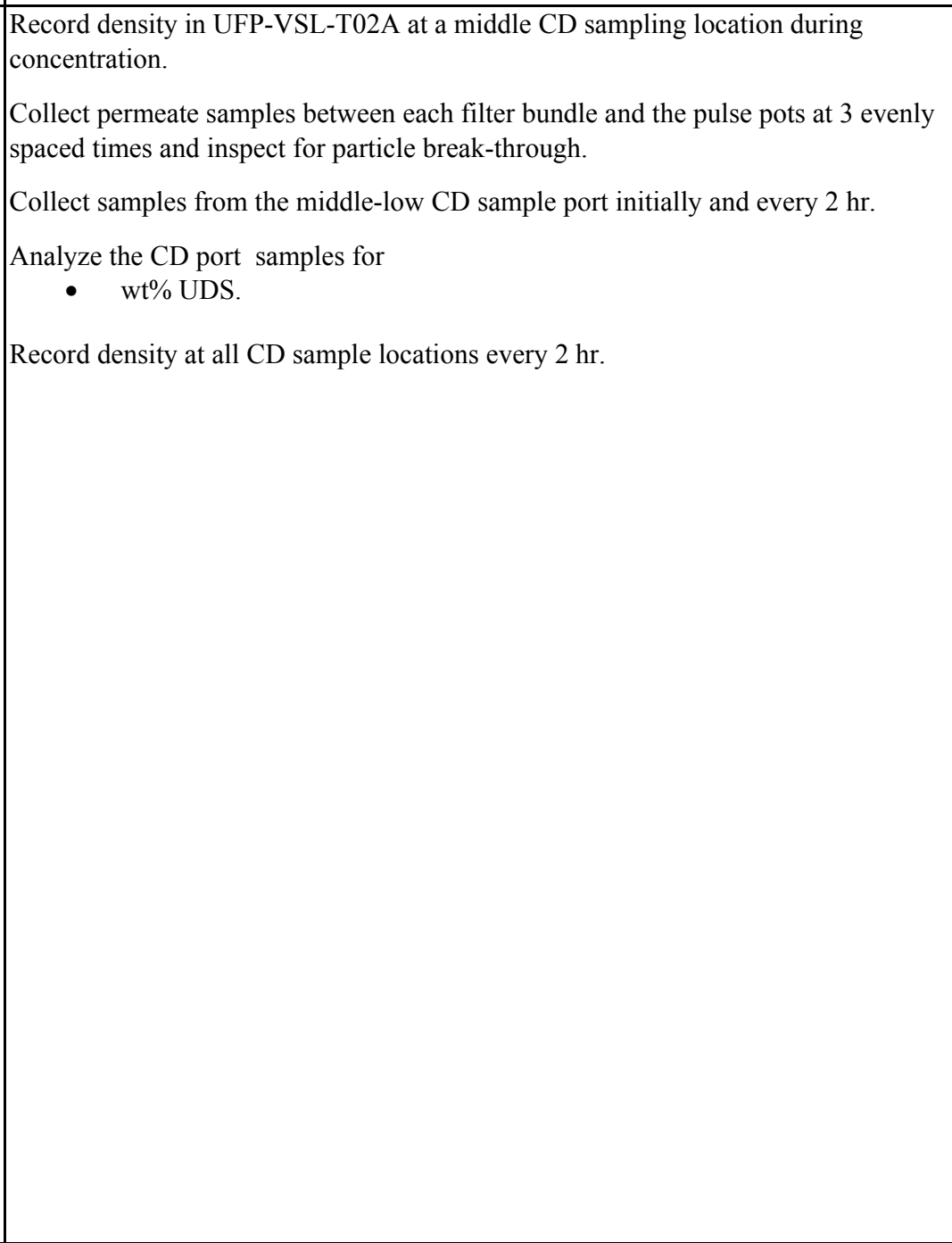 & 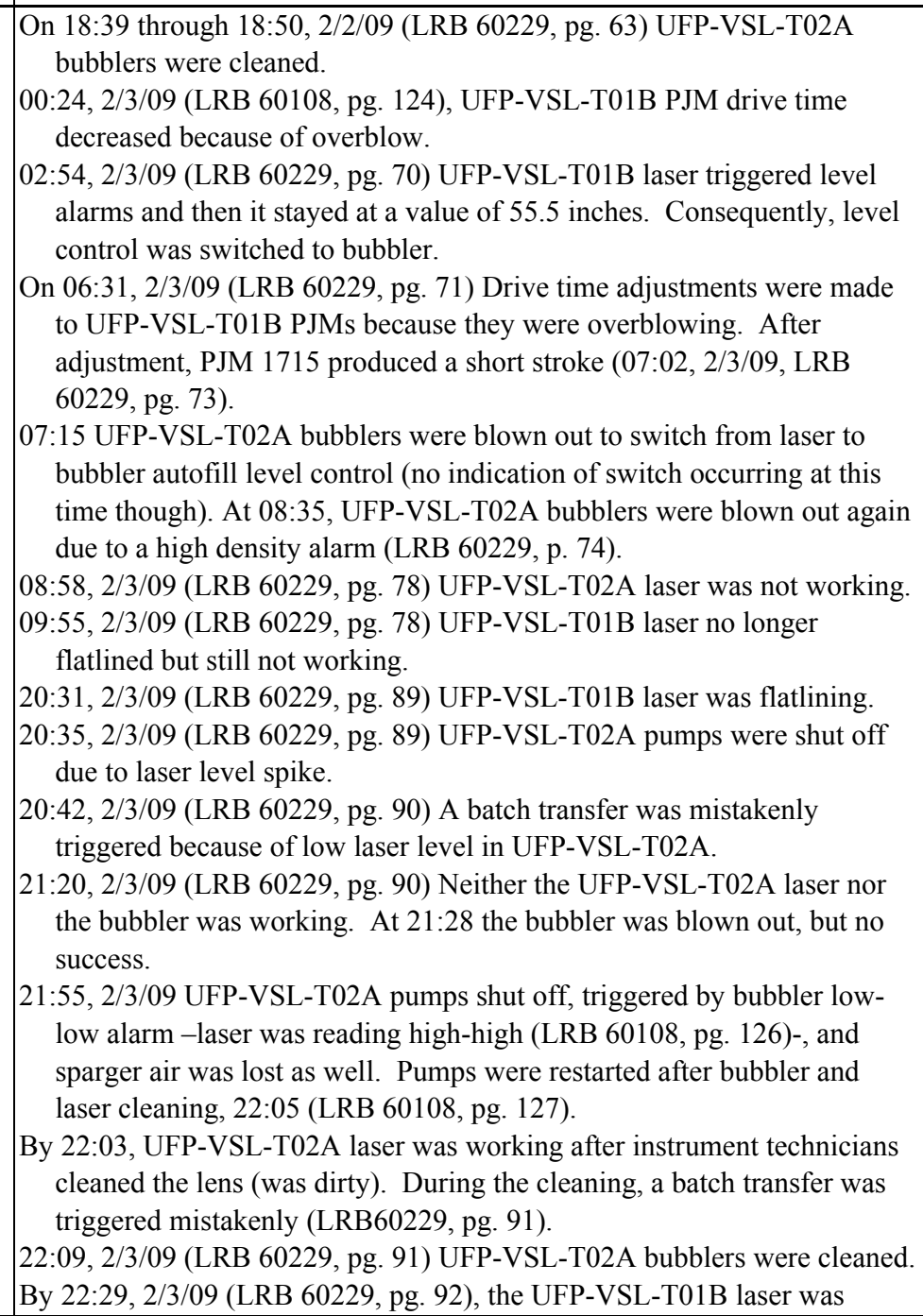 \\
\hline
\end{tabular}




\begin{tabular}{|c|c|c|c|c|c|}
\hline Test Step & Test Step & Description/Purpose & Key Process Conditions & Sampling & \begin{tabular}{|c|} 
Test Deviations and Problems \\
\end{tabular} \\
\hline & & & & & $\begin{array}{l}\text { stagnant, by 23:03, instrument technicians cleaned the lens, but the laser } \\
\text { still did not work (low return signal)-foam may be to blame (LRB } \\
\text { 60108, pg. 127). The level control was the bubbler. } \\
08: 00,2 / 4 / 09 \text { (LRB } 60108 \text {, pg. } 129 \text { ) The batch transfer counter read } 34 \\
\text { batches although it was accidentally reset after making } \sim 10 \text { transfers. }\end{array}$ \\
\hline B.1.13 & $\begin{array}{l}\text { Repeat caustic leaching in } \\
\text { UFP-VSL-T01A and } \\
\text { UFP-VSL-T01B }\end{array}$ & $\begin{array}{l}\text { Repeat steps B.1.2 - B.1.4 and B.1.6 - B.1.12 } \\
\text { until objective of step B.1.12 (prototypic batch } \\
\text { volume of } 17 \text { wt\% solids in UFP-VSL-T02A) is } \\
\text { achieved. }\end{array}$ & As above. & As above. & See added rows below for subsequent batch descriptions \\
\hline $\begin{array}{c}\text { B.1.13 } \\
\\
\text { Repeat } \\
\text { caustic } \\
\text { leaching in } \\
\text { UFP-VSL- } \\
\text { T01A and } \\
\text { UFP-VSL- } \\
\text { T01B }\end{array}$ & 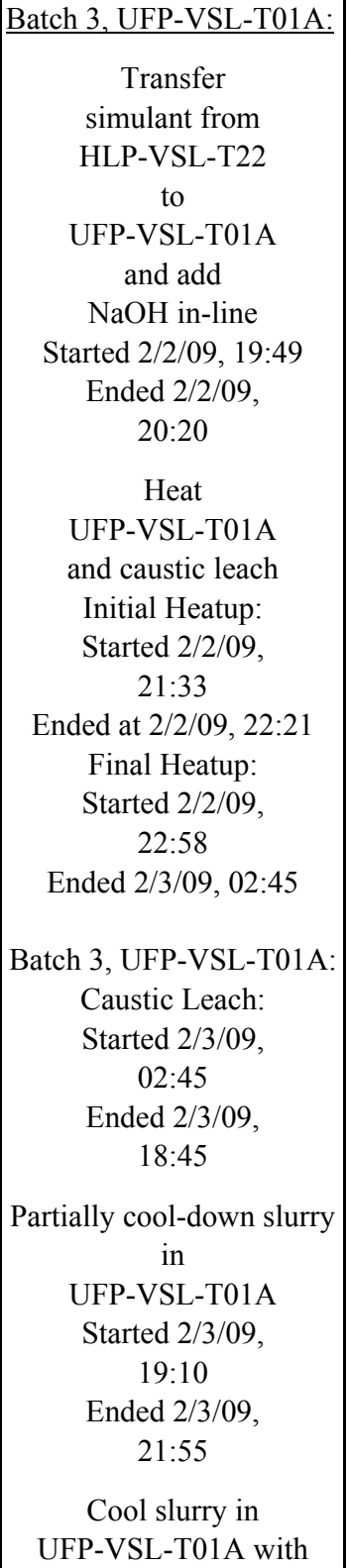 & \begin{tabular}{|l|} 
Repeat steps B.1.2 - B.1.4 and B.1.6 - B.1.1. \\
until objective of step B.1.12 (prototypic batch \\
volume of 17 wt\% solids in UFP-VSL-T02A) is \\
achieved.
\end{tabular} & -- & -- & 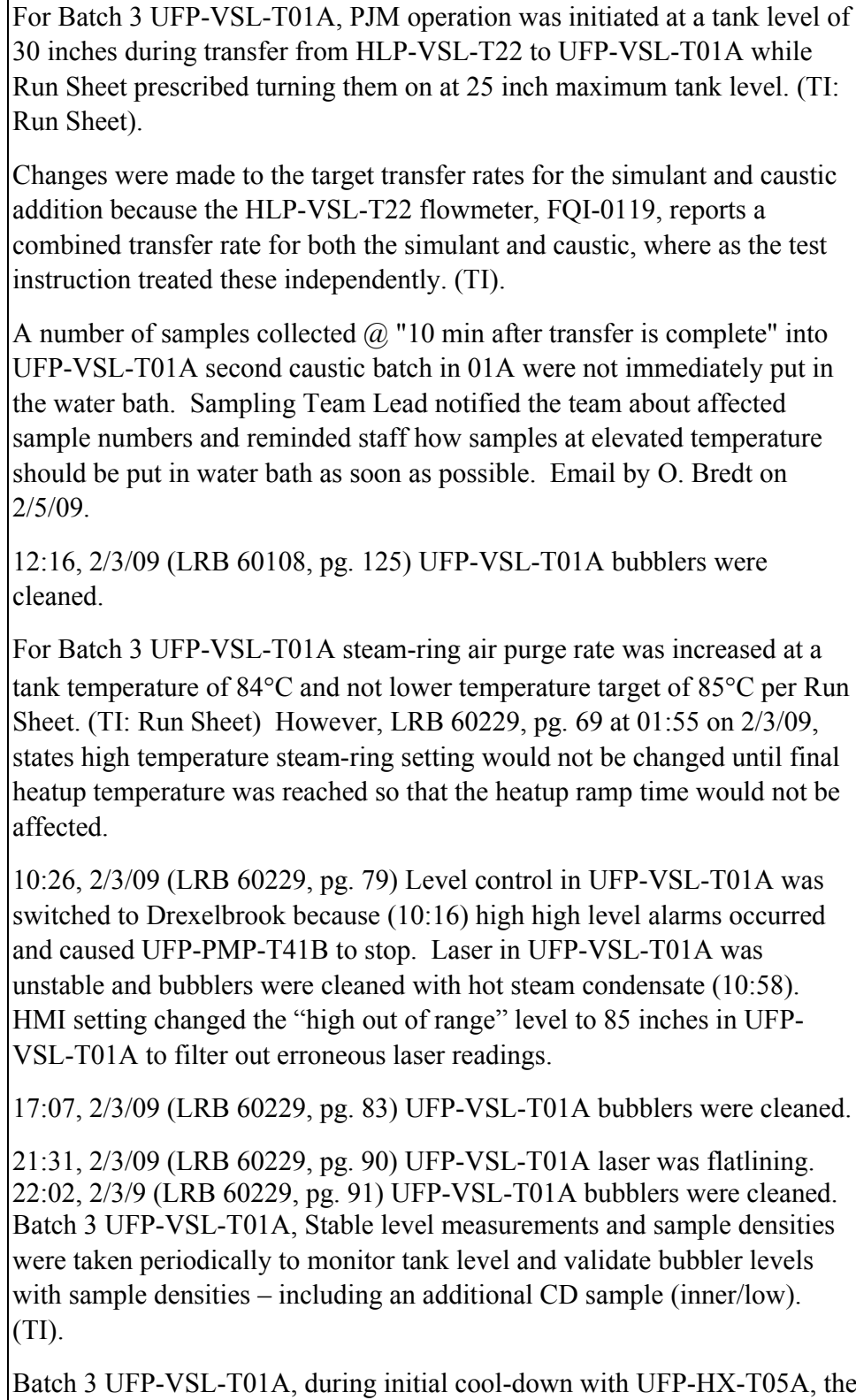 \\
\hline
\end{tabular}




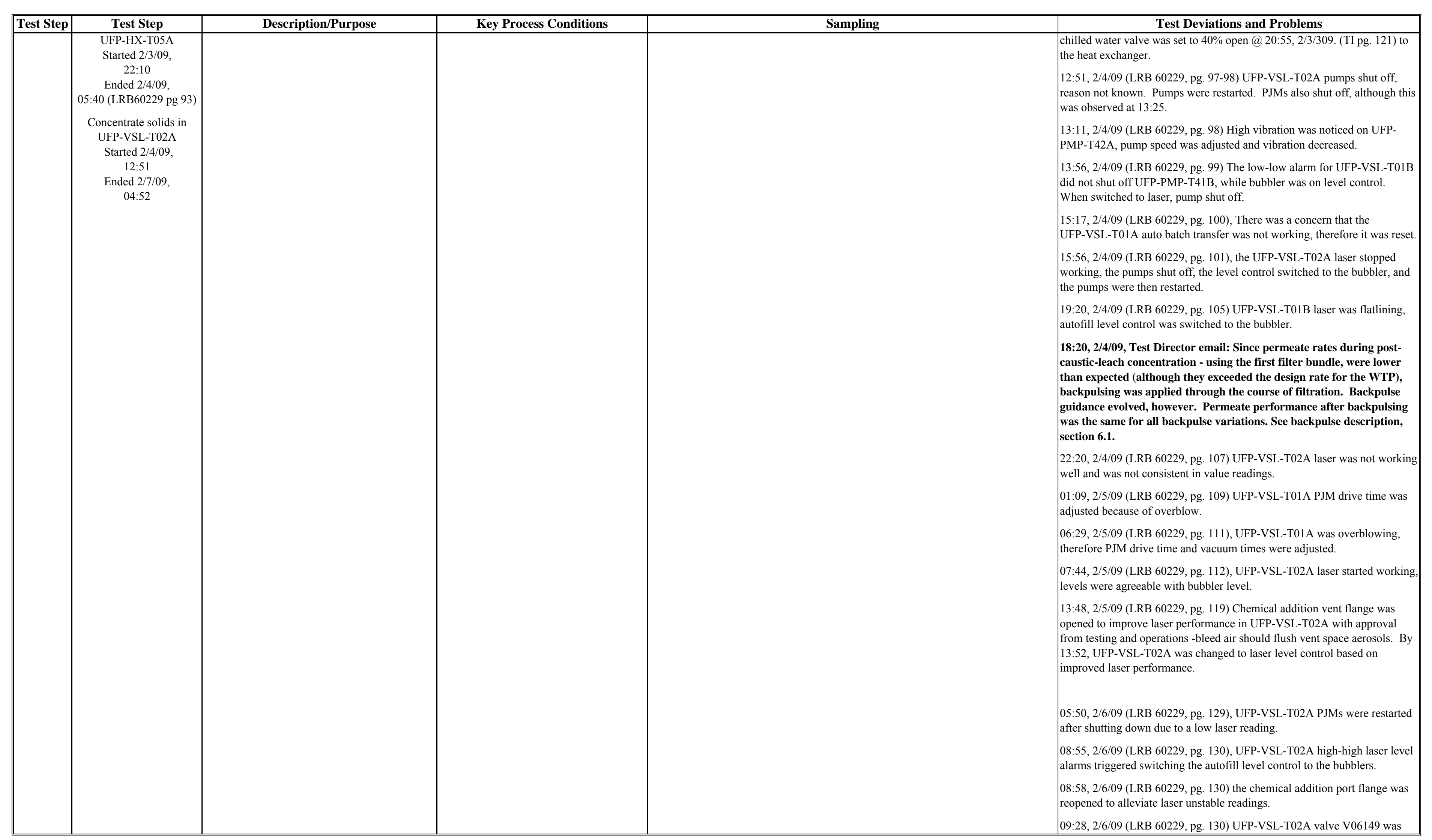




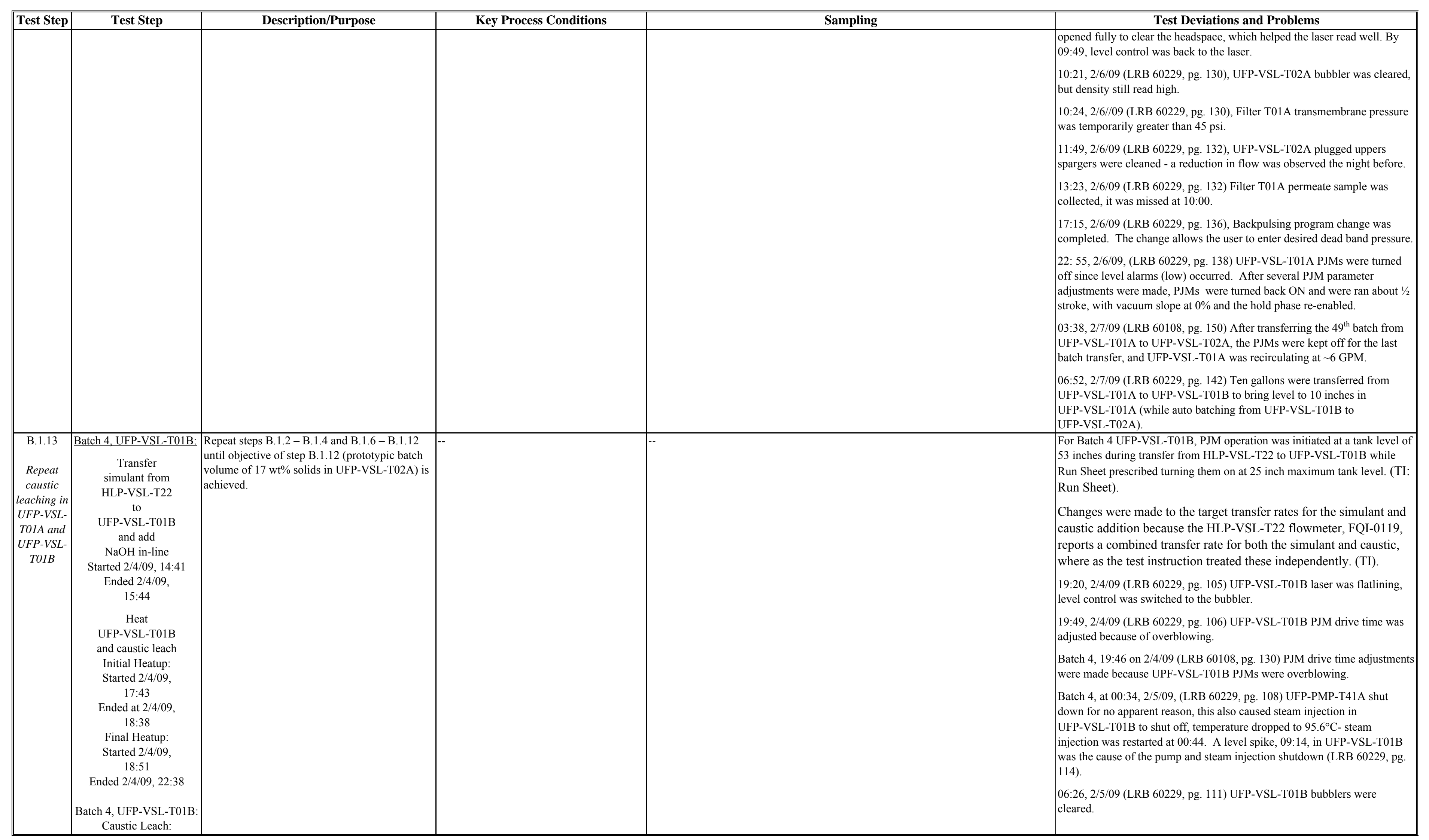




\begin{tabular}{|c|c|c|c|c|c|}
\hline Test Step & \begin{tabular}{c|} 
Test Step \\
\end{tabular} & Description/Purpose & Key Process Conditions & Sampling & \begin{tabular}{|c|} 
Test Deviations and Problems \\
\end{tabular} \\
\hline & \begin{tabular}{|c|} 
Started 2/4/09, \\
$22: 38$ \\
Ended 2/5/09, \\
14:38 \\
Initial Partial Cool Down \\
in \\
UFP-VSL-T01B \\
Started 2/5/09, \\
14445 \\
Ended 2/5/09, \\
$17: 30$ \\
Cool slurry in \\
UFP-VSL-T01B with \\
UFP-HX-T05B \\
Completed 2/5/08, \\
23:55 (LRB 60229 \\
pg 128) \\
Concentrate solids in \\
UFP-VSL-T02A \\
Started 2/7/09, \\
04.52 \\
Ended 2/9/09, \\
$21: 00$ \\
21:00
\end{tabular} & & & & 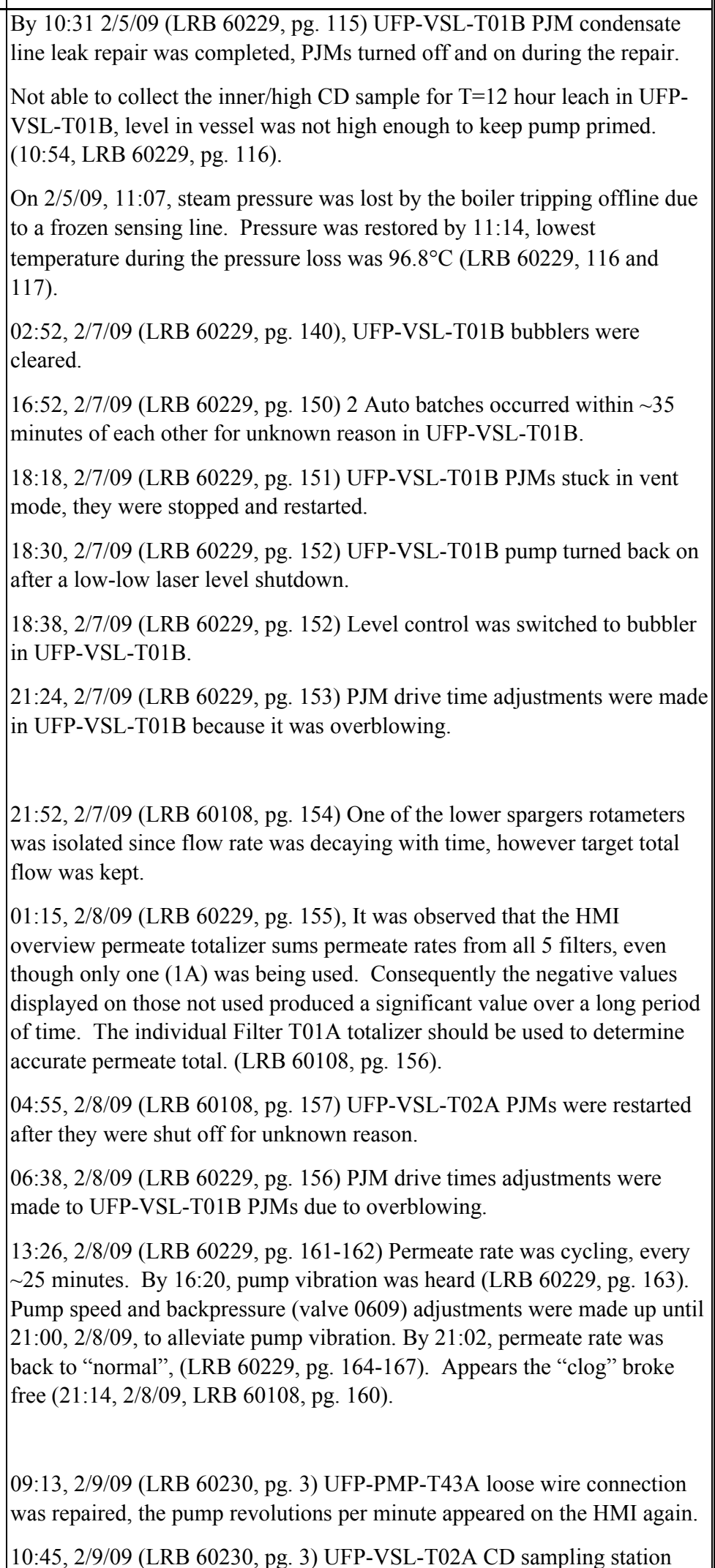 \\
\hline
\end{tabular}




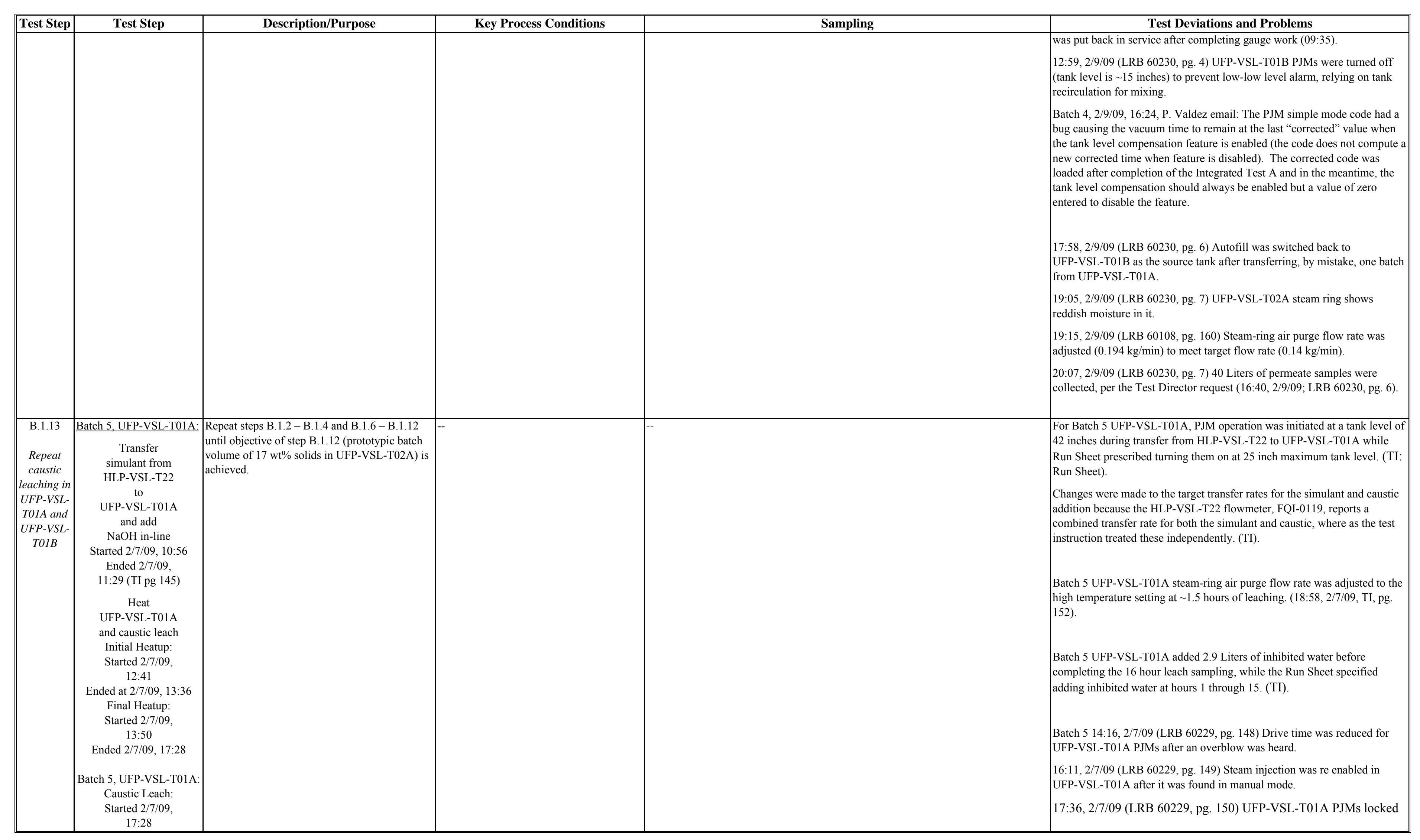




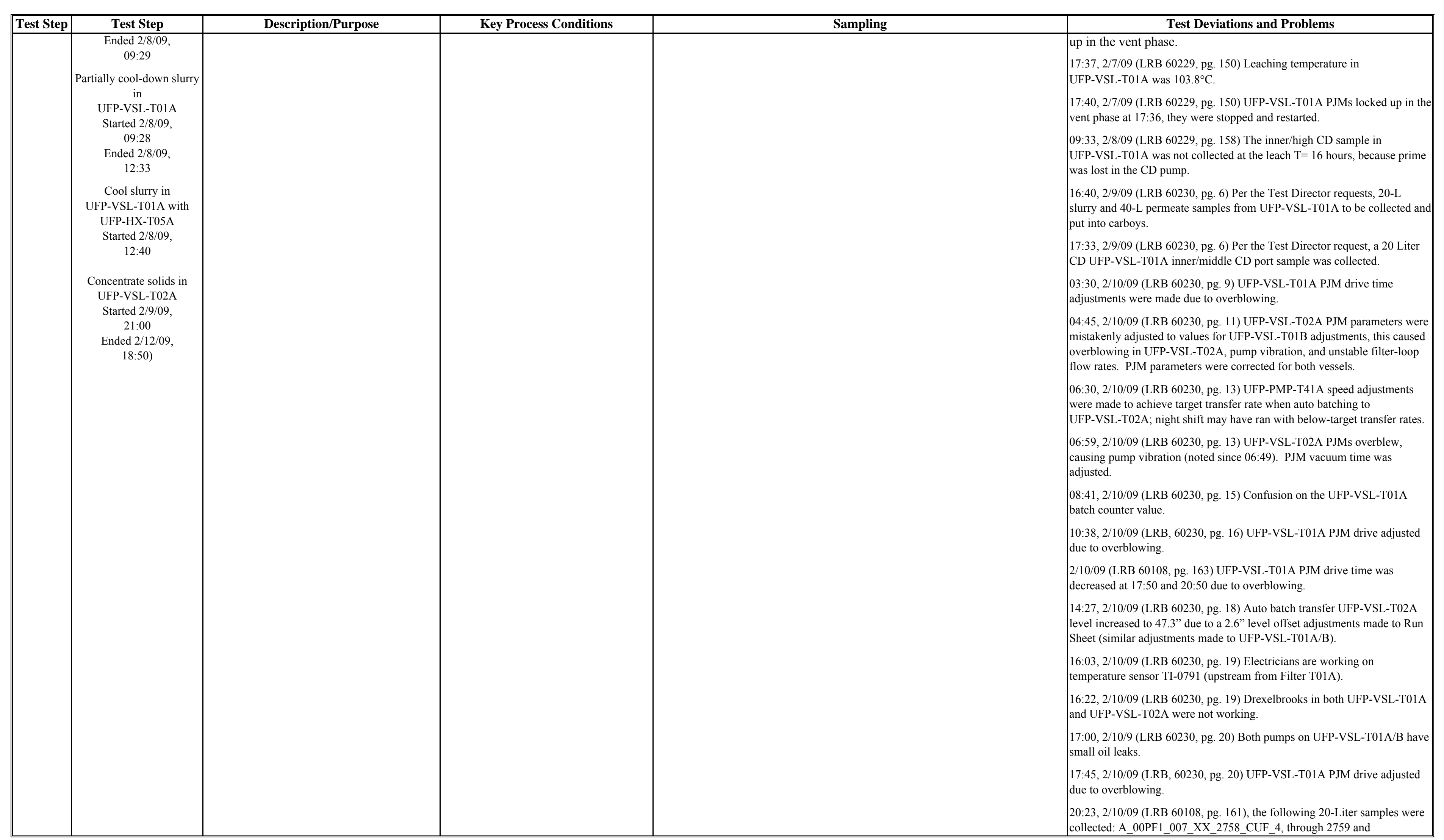




\begin{tabular}{|c|c|c|c|c|c|}
\hline Test Step & $\begin{array}{l}\text { Test Step } \\
\end{array}$ & Description/Purpose & \begin{tabular}{|l|} 
Key Process Conditions \\
\end{tabular} & $\begin{array}{l}\text { Sampling } \\
\end{array}$ & \begin{tabular}{|c|} 
Test Deviations and Problems \\
\end{tabular} \\
\hline & & & & & 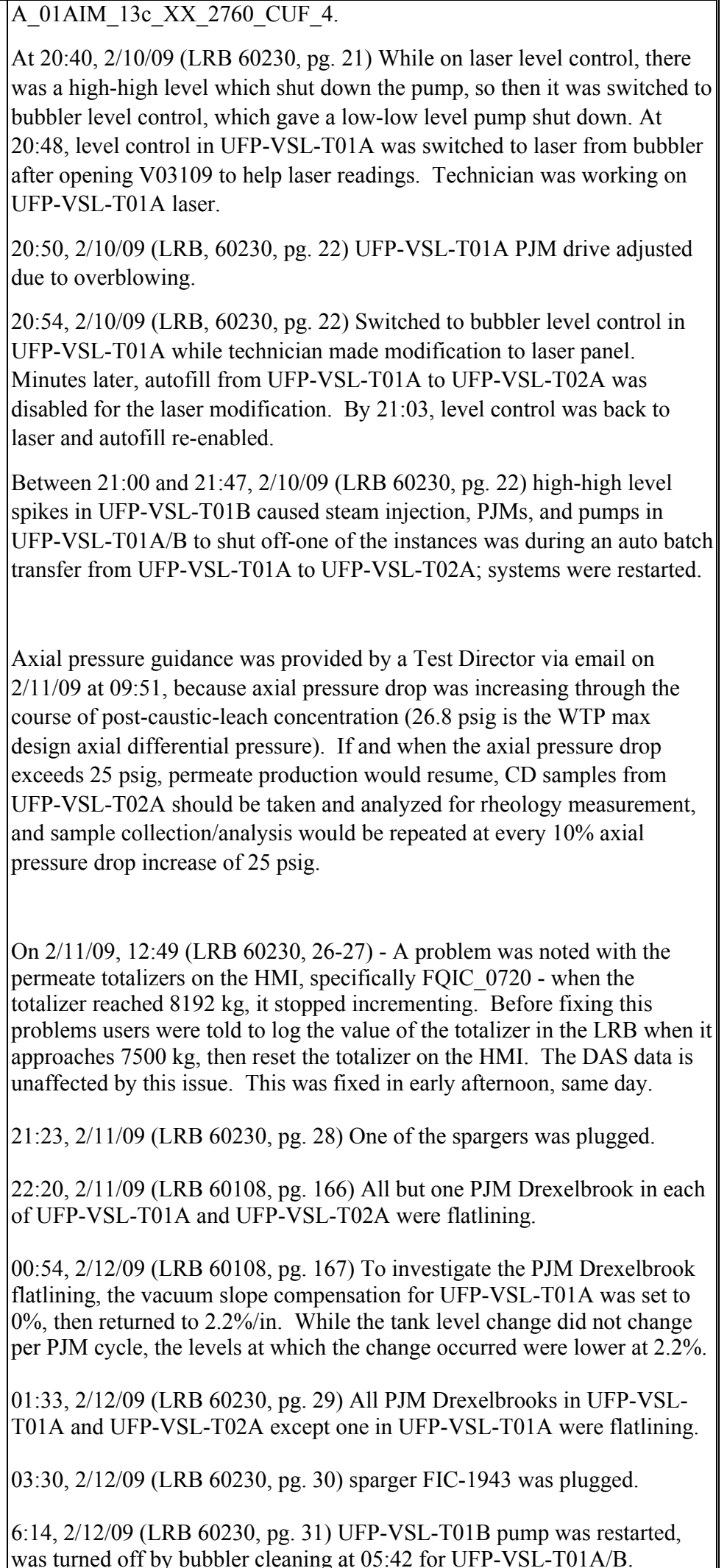 \\
\hline
\end{tabular}




\begin{tabular}{|c|c|c|c|c|c|}
\hline Test Step & Test Step & Description/Purpose & Key Process Conditions & $\begin{array}{l}\text { Sampling } \\
\end{array}$ & $\begin{array}{l}\text { Test Deviations and Problems } \\
\end{array}$ \\
\hline & & & & & 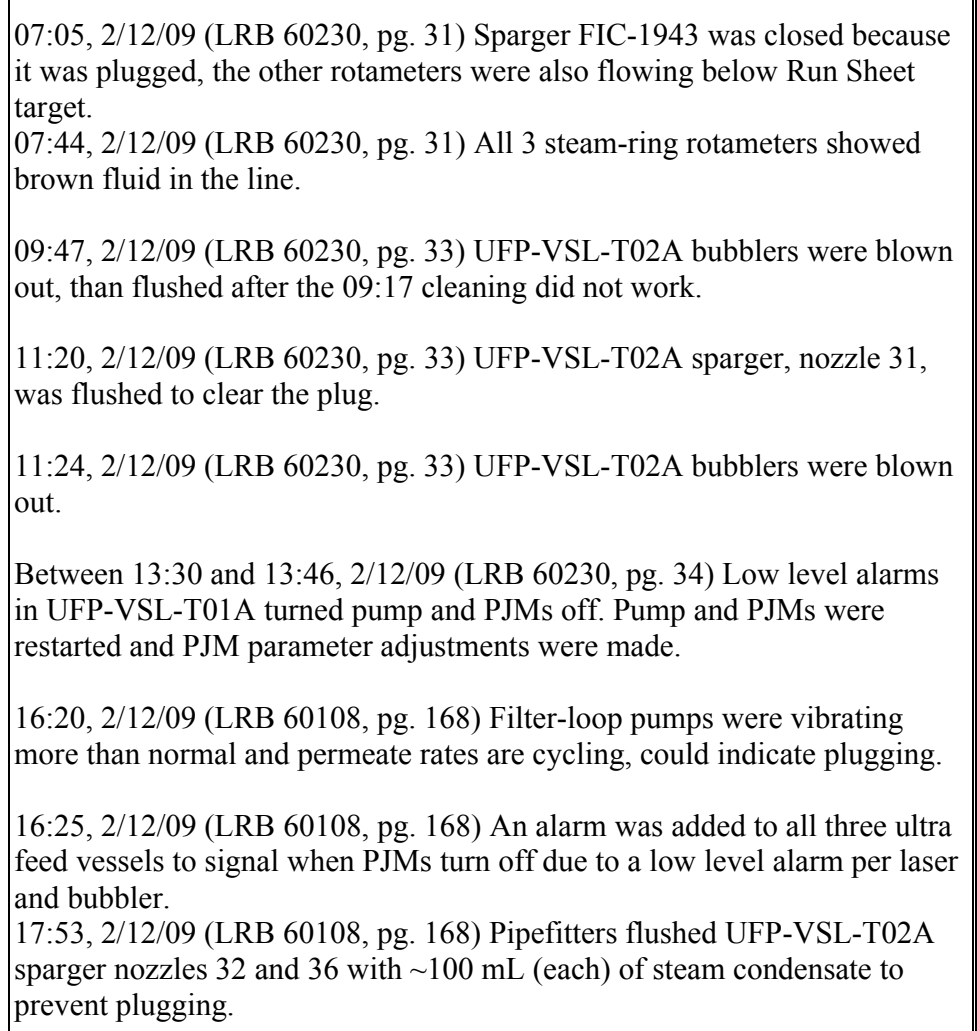 \\
\hline $\begin{array}{c}\text { B.1.13 } \\
\text { Repeat } \\
\text { caustic } \\
\text { leaching in } \\
\text { UFP-VSL- } \\
\text { T01A and } \\
\text { UFP-VSL- } \\
\text { T01B }\end{array}$ & \begin{tabular}{|c} 
Batch 6, UFP-VSL-T01B: \\
Transfer \\
simulant from \\
HLP-VSL-T22 \\
to \\
UFP-VSL-T01B \\
and add \\
NaOH in-line \\
Started 2/10/09, 04:00 \\
Ended 2/10/09, \\
$06: 00$ \\
Heat \\
UFP-VSL-T01B \\
and caustic leach \\
Initial Heatup: \\
NA $\rightarrow$ \\
See deviations \\
Final Heatup: \\
Started 2/10/09, \\
08:12 \\
Ended 2/10/09, 12:06 \\
Batch 6, UFP-VSL-T01B:
\end{tabular} & $\begin{array}{l}\text { Repeat steps B.1.2 - B.1.1.4 and B.1.6 - B.1.12 } \\
\text { untit objective of step B.1.12 (prototypic batch } \\
\text { volume of } 17 \text { wt solids in UFP-VSL-T02A) is } \\
\text { achieved. }\end{array}$ & & & 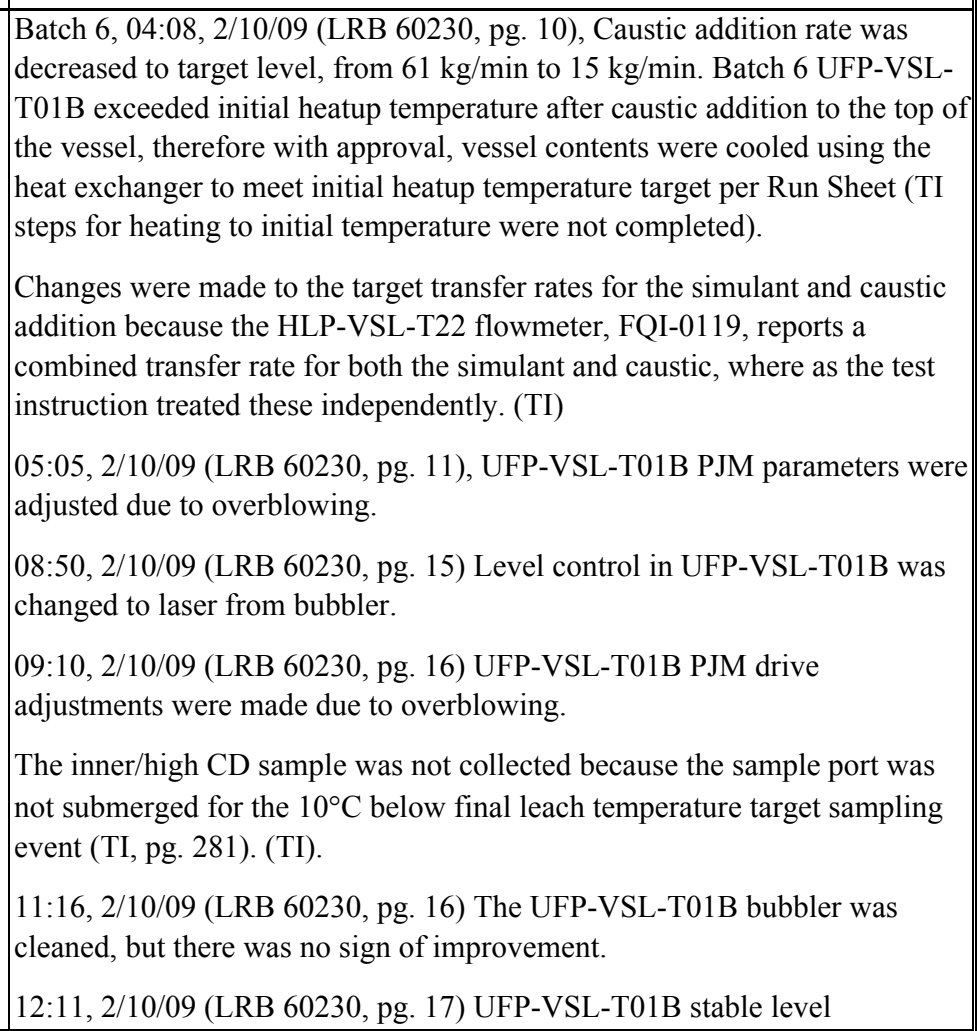 \\
\hline
\end{tabular}




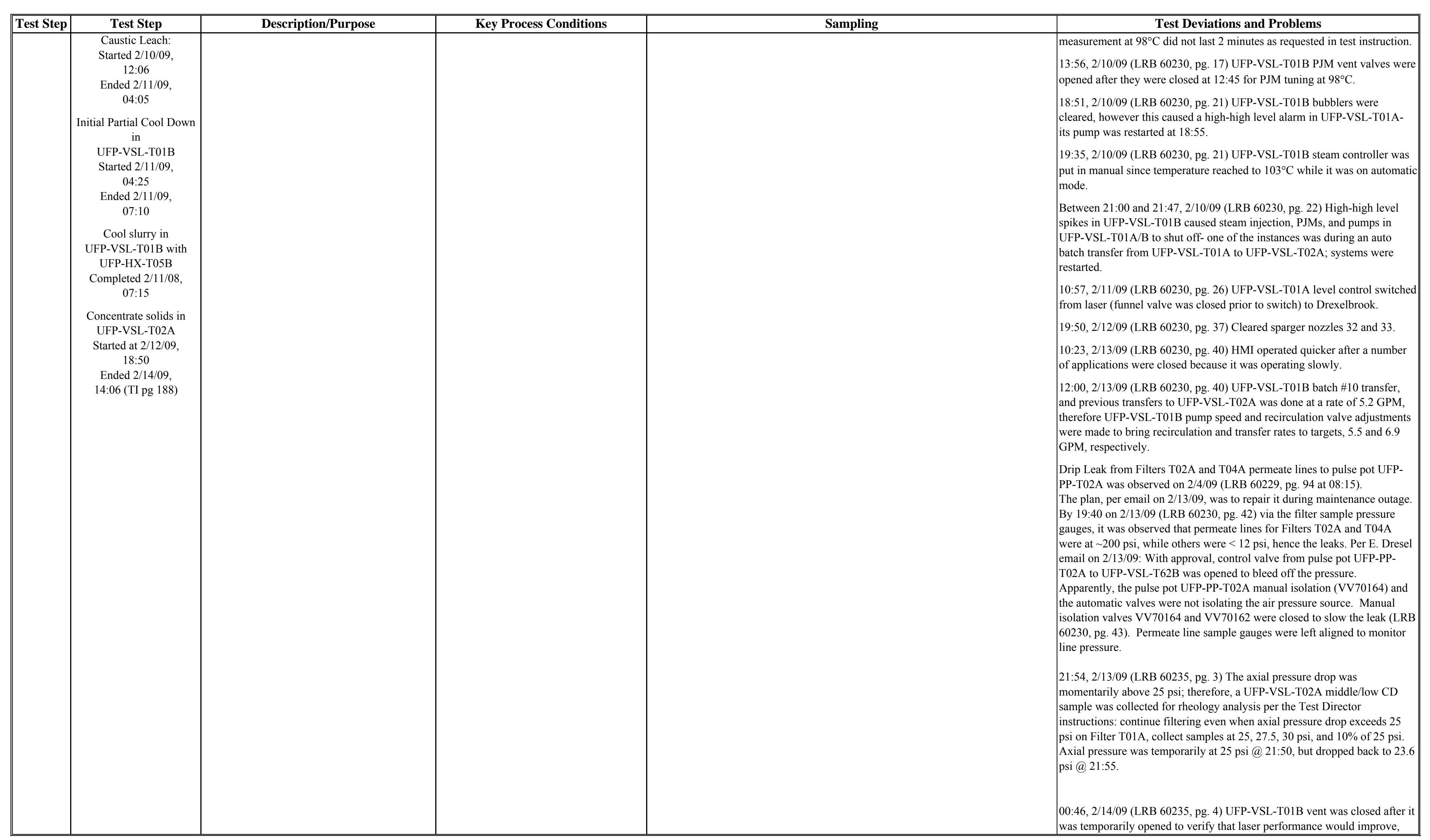




\begin{tabular}{|c|c|c|c|c|c|}
\hline Test Step & Test Step & Description/Purpose & Key Process Conditions & Sampling & \begin{tabular}{|c|} 
Test Deviations and Problems \\
\end{tabular} \\
\hline & & & & & $\begin{array}{l}\text { laser was alarming on high levels, this stopped with vent open- } \\
\text { UFP-VSL-T01B on bubbler level-control (LRB 60230, pg. 44). } \\
\text { 01:28, 2/14/09 (LRB 60230, pg. 45) UFP-VSL-T02A upper spargers were } \\
\text { cleaned successfully. } \\
\text { 05:00, 2/14/09 (LRB 60230, pg. 45) Brown fluid is seen in the } 3 \text { steam-ring } \\
\text { air purge rotameters, mostly in the UFP-VSL-T02A rotameter. } \\
\text { 07:54 2/14/09 (LRB 60230, pg. 46) UFP-VSL-T02A bubblers were } \\
\text { cleaned, filter-loop pump settings were changed to minimize vibration. } \\
\text { Between 08:00 and 08:17, 2/14/09 (LRB 60230, pg. } 46 \text {-47) } \\
\text { UFP-VSL-T02A PJM and pump settings were adjusted to address potential } \\
\text { overblow concerns causing instable filter-loop pump operations. However } \\
\text { the 07:54 bubbler cleaning was believed to have caused for pump vibration. }\end{array}$ \\
\hline B.1.14 & \begin{tabular}{c|} 
Evaluate \\
by-pass of filter-loop jet in \\
UFP-VSL-T02A \\
(Integrated Test A only) \\
Started 2/14/09, \\
$21: 26$ \\
Ended 2/14/09, \\
$22: 30$ \\
\\
\end{tabular} & 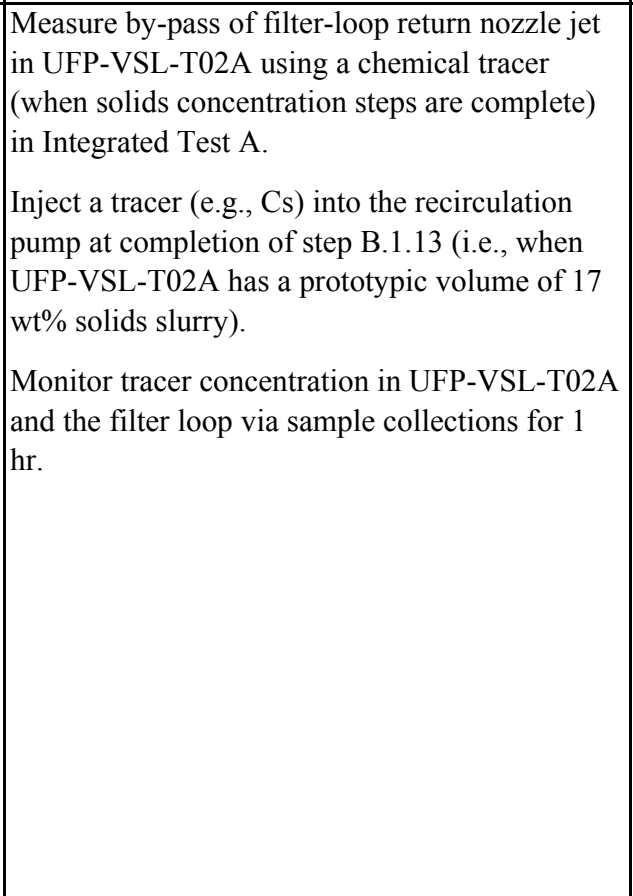 & $\begin{array}{l}\text { Permeate valves closed. } \\
\text { Mix at scale-time. } \\
\text { Five ultrafilter bundles. } \\
\text { UFP-VSL-T02A PJM velocities match PTF } \\
\text { PJM velocities, and cycle time is } 1 / 4.5 \text { that } \\
\text { of PTF. } \\
\end{array}$ & $\begin{array}{l}\text { Integrated Test A only: } \\
\text { Collect filter-loop in-line samples before tracer injection and at } \sim 1,2,4,8,16,30 \text {, } \\
40,50 \text { and } 60 \text { min after tracer injection. } \\
\text { Analyze filter-loop in-line samples for } \\
\quad \text { tracer. } \\
\text { Collect CD outer bottom port sample in UFP-VSL-T02A at } 1,2,4,8,16,30,40,50 \\
\text { and } 60 \text { min after tracer injection. } \\
\text { Analyze initial CD port sample for } \\
- \text { shear stress vs. shear rate. } \\
\text { Analyze all CD samples for } \\
-\quad \text { tracer. }\end{array}$ & 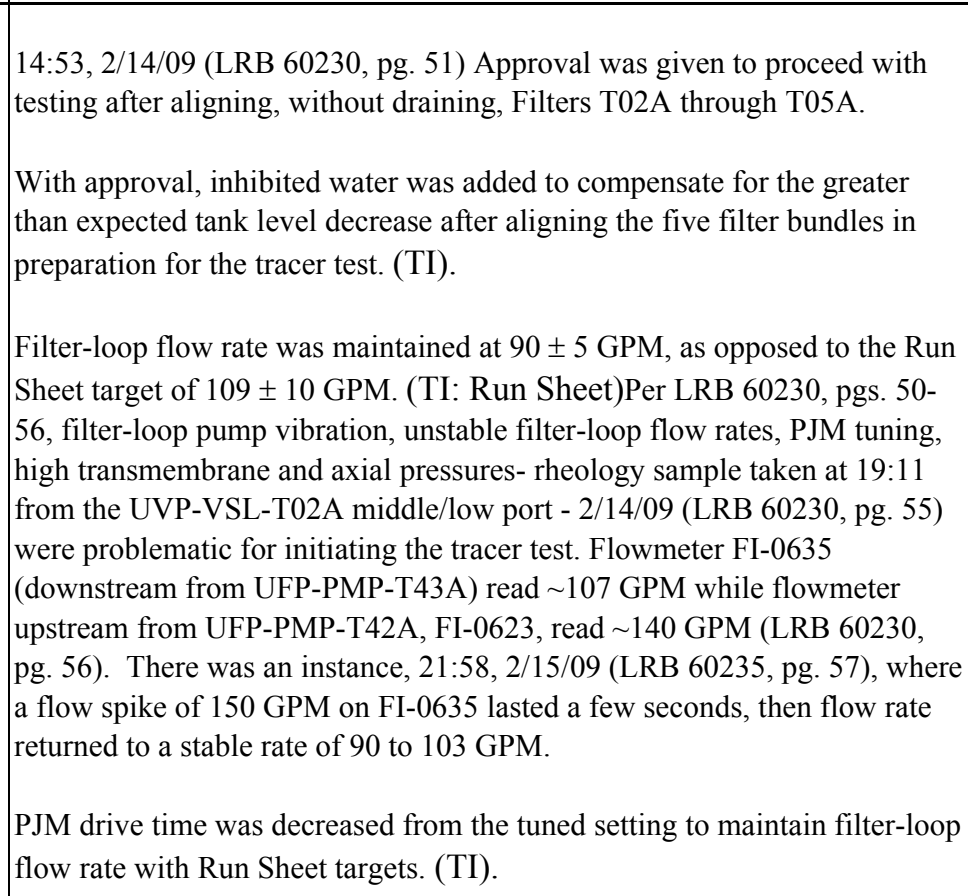 \\
\hline & \begin{tabular}{|c|} 
Repeat: \\
Evaluate \\
by-pass of filter-loop jet in \\
UFP-VSL-T02A \\
(Integrated Test A only) \\
Started 3/121/09, \\
16:14 \\
Ended 3/21/09, \\
$17: 14$ \\
\end{tabular} & - & -- & -- & $\begin{array}{l}\text { Since target filter-loop flow rate was not achieved during the tracer test } \\
\text { above, the tracer test was repeated after the High Solids Filter Test was } \\
\text { completed on } 2 / 21 / 09 \text {. } \\
\text { Steam-ring air purge, upper and lower air spargers were kept off, per } \\
\text { Guidance, as opposed to original Run Sheet targets of } 0.14,0.01 \text {, and } 0.04 \\
\mathrm{~kg} / \text { min, respectively. (TI: Run Sheet). } \\
\text { Two spare samples were collected. An outer/middle CD sample was } \\
\text { collected at } 17: 16 \text {, then an outer/low CD sample was collected at 17:29- } \\
\text { after } 10 \text { minutes of lower sparger operations at } 0.4 \mathrm{~kg} / \text { min. (LRB } 60271 \text {, } \\
\text { pg. 16). }\end{array}$ \\
\hline B.1.15 & $\begin{array}{c}\text { Post-caustic-leach slurry } \\
\text { wash } \\
\text { Started } 2 / 15 / 09\end{array}$ & $\begin{array}{l}\text { Demonstrate post-caustic-leach slurry wash to } \\
\text { remove soluble solids and achieve targeted }\end{array}$ & $\begin{array}{l}\text { Wash at scale-time. } \\
\text { Five ultrafilter bundles. }\end{array}$ & $\begin{array}{l}\text { Collect outer-radius lowest CD port sample in UFP-VSL-T02A before wash is } \\
\text { started (initial sample). }\end{array}$ & $\begin{array}{l}\text { 23:13, 2/14/09 (LRB 60230, pg. 57) UFP-VSL-T02A bubblers were } \\
\text { cleaned, but not successfully. }\end{array}$ \\
\hline
\end{tabular}




\begin{tabular}{|c|c|c|c|c|c|}
\hline Test Step & Test Step & \begin{tabular}{|l|} 
Description/Purpose \\
\end{tabular} & Key Process Conditions & Sampling & \begin{tabular}{|c|} 
Test Deviations and Problems \\
\end{tabular} \\
\hline & $\begin{array}{l}01: 13 \\
\text { Ended 2/15/09 } \\
22: 55\end{array}$ & $\begin{array}{l}\text { hydroxide concentration. } \\
\text { Measure filter flux and axial pressure drop } \\
\text { across filter bundles as functions of solids } \\
\text { content. } \\
\text { Monitor distribution of oxalate and dissolved } \\
\text { species to establish washing efficiencies. } \\
\text { Monitor ultrafiltration leachates for precipitation } \\
\text { of solids. }\end{array}$ & $\begin{array}{l}\text { UFP-VSL-T02A PJM velocities match PTF } \\
\text { PJM velocities, and cycle time is } 1 / 4.5 \text { that } \\
\text { of PTF. } \\
\text { Route permeate to UFP-VSL-T62B until } \\
\text { sodium ion concentration in permeate is } \\
\text { estimated to be } 3.5 \mathrm{M}, \text { route remaining } \\
\text { permeate to UFP-VSL-T62A/B. } \\
\text { Close permeate valves at end of wash. }\end{array}$ & 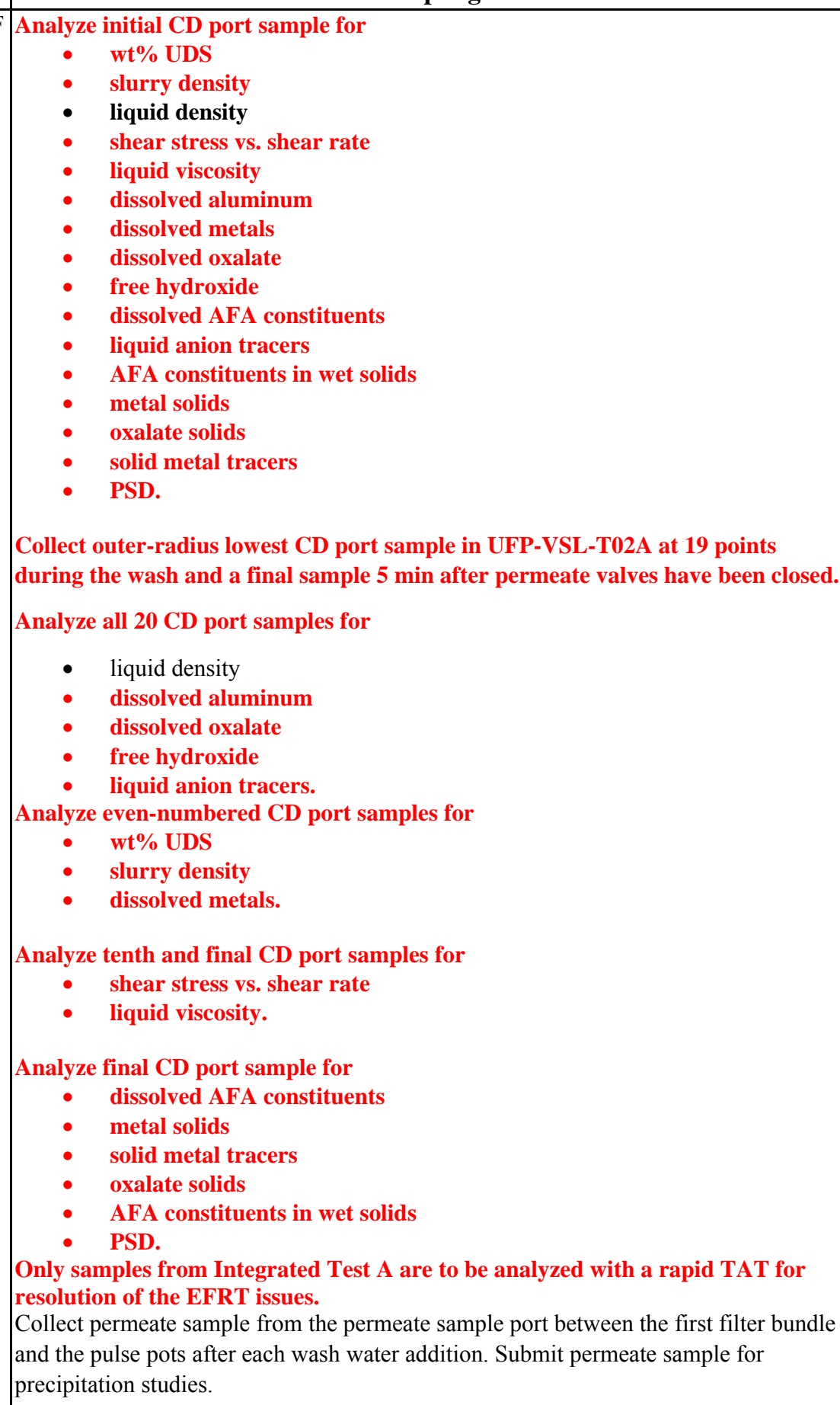 & 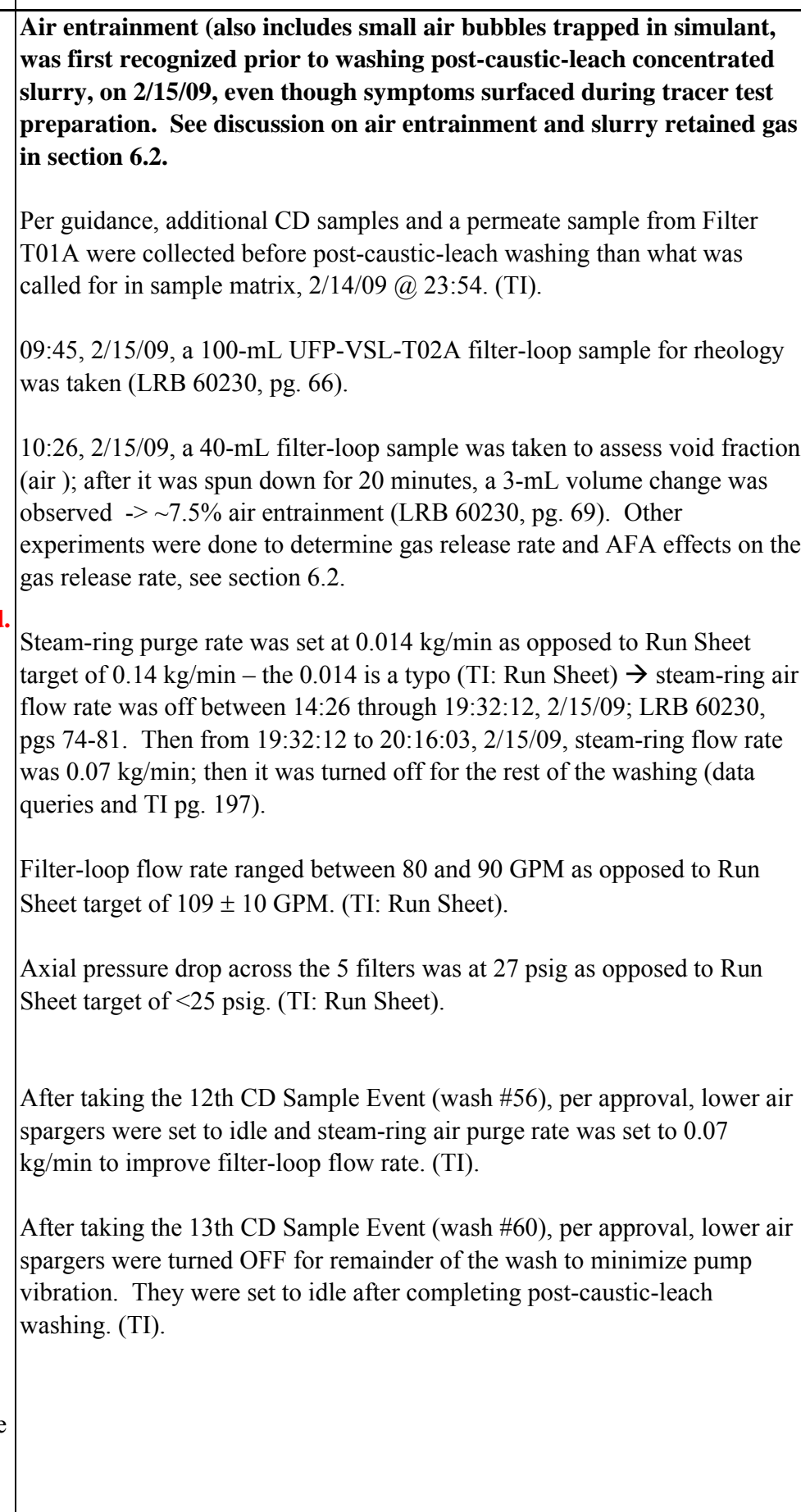 \\
\hline B.1.16 & \begin{tabular}{c|} 
Chromium slurry addition \\
Started $2 / 16 / 09$ \\
$01: 12$ \\
Ended $2 / 16 / 09$ \\
$01: 23$
\end{tabular} & $\begin{array}{l}\text { Add chromium solids (as a slurry) in-line at } \\
\text { suction of UFP-PMP-T42A. }\end{array}$ & \begin{tabular}{|l} 
Non-prototypic. \\
Permeate valves closed. \\
No change in mixing or pumping \\
parameters from Step B.1.11.
\end{tabular} & None. & $\begin{array}{l}\text { 01:14, 2/16/09 (LRB 60230, pg. 89) Turning lower air spargers ON to idle } \\
\text { flow rates at 00:50, increased laser level from } 51 \text { to } 54 \text { inches, appeared to } \\
\text { be foam; filter pump speed was reduced to bring laser level back to } 51 \\
\text { inches. }\end{array}$ \\
\hline
\end{tabular}




\begin{tabular}{|c|c|c|c|c|c|}
\hline Test Step & Test Step & Description/Purpose & Key Process Conditions & Sampling & \begin{tabular}{|c|} 
Test Deviations and Problems \\
\end{tabular} \\
\hline & & & & & $\begin{array}{l}\text { PJM nozzle velocity was } 13.6 \mathrm{~m} / \mathrm{s} \text { as opposed to Run Sheet target of } 12 \pm \\
0.6 \mathrm{~m} / \mathrm{s} \text {. (TI: Run Sheet). } \\
\text { Steam-ring air purge rate was OFF, per Guidance, as opposed to original } \\
\text { Run Sheet target of } 0.14 \mathrm{~kg} / \mathrm{min} \text {. (TI: Run Sheet). }\end{array}$ \\
\hline B.1.17 & $\begin{array}{c}\text { Wash and/or reconcentrate } \\
\text { slurry solids } \\
\text { Started 2/16/09, 01:27 } \\
\text { Ended } 2 / 16 / 09 \\
05: 04\end{array}$ & $\begin{array}{l}\begin{array}{l}\text { Wash and/or reconcentrate slurry, if necessary, } \\
\text { to remove excess liquid introduced by chromium } \\
\text { solids slurry. }\end{array} \\
\end{array}$ & $\begin{array}{l}\text { Non-prototypic. } \\
\text { Concentrate and wash with } 5 \text { ultrafilter } \\
\text { bundles. }\end{array}$ & None. & 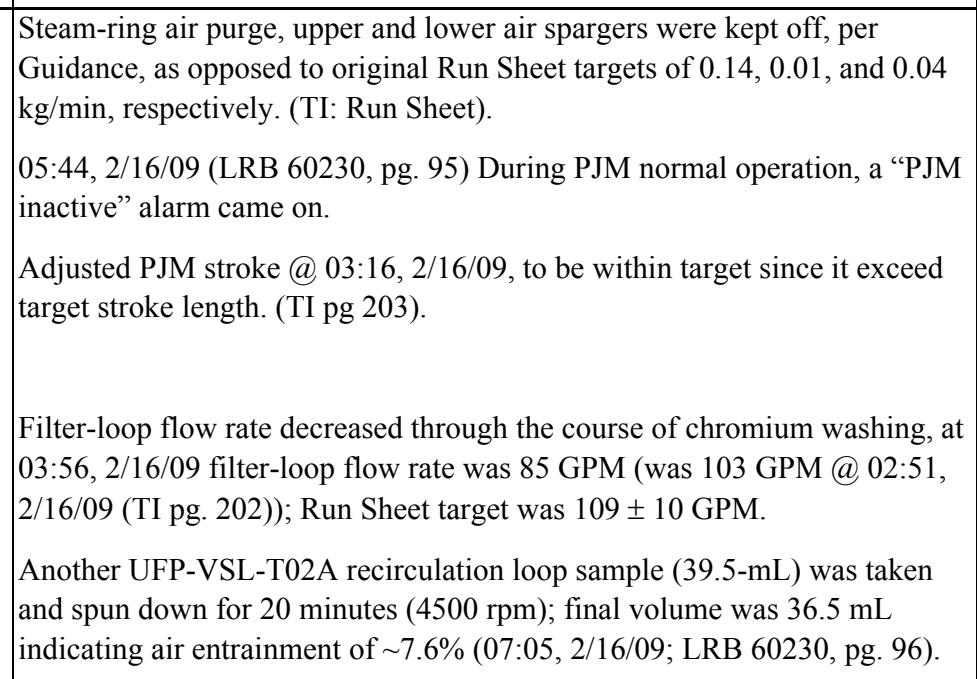 \\
\hline B.1.18 & $\begin{array}{l}\text { Add permanganate } \\
\text { Started 2/16/109, } 10: 13 \\
\text { Ended 2/16/09, 10:21 }\end{array}$ & $\begin{array}{l}\begin{array}{l}\text { Demonstrate in-line addition of oxidative leach } \\
\text { chemicals. }\end{array} \\
\end{array}$ & $\begin{array}{l}\text { Add chemicals at scale-time. } \\
\text { UFP-VSL-T02A PJM velocities match PTF } \\
\text { PJM velocities, and cycle time is } 1 / 4.5 \text { that } \\
\text { of PTF. }\end{array}$ & 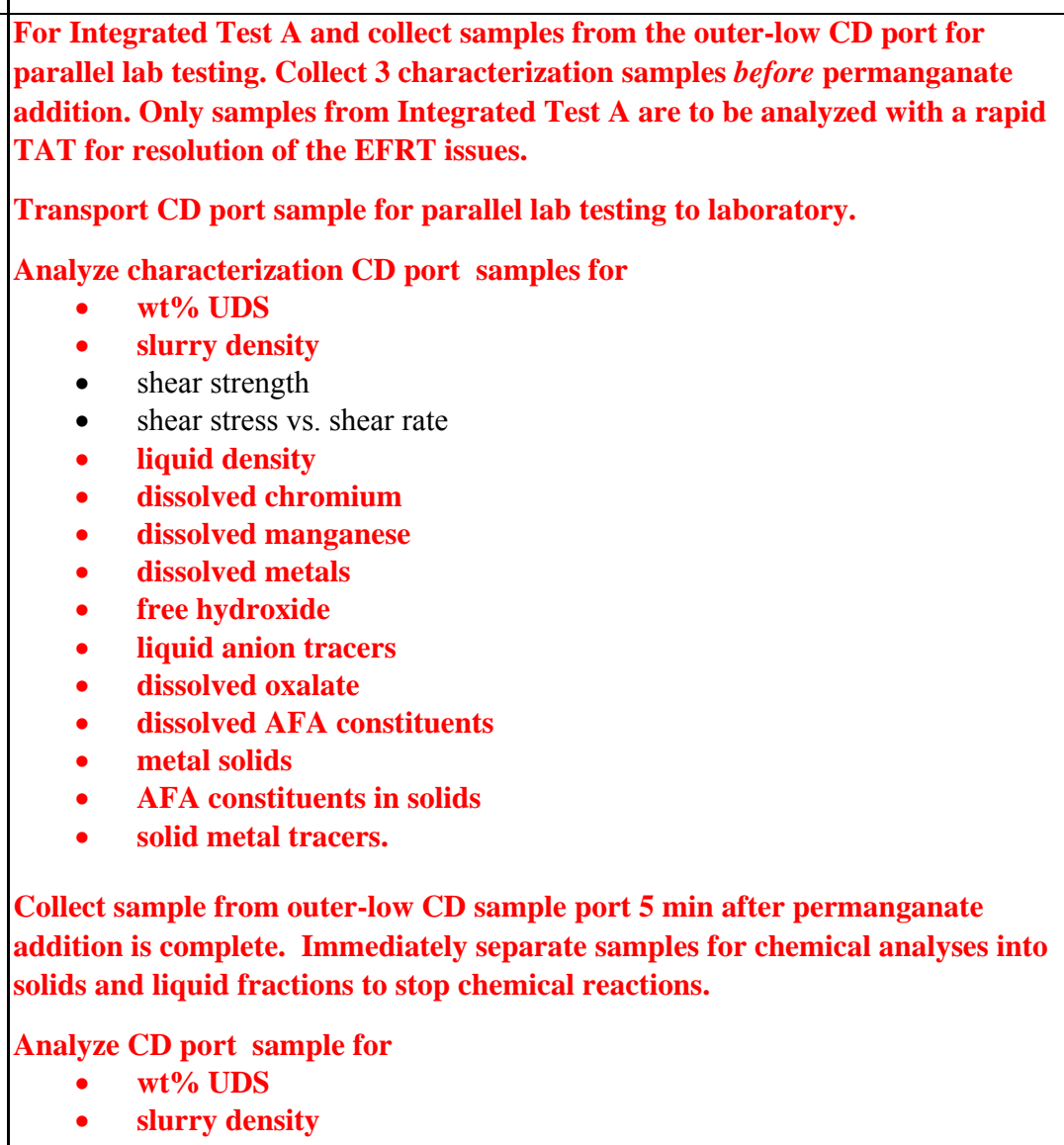 & $\begin{array}{l}\text { Steam-ring air purge, upper and lower air spargers were supposed to be off, } \\
\text { per Guidance, as opposed to original Run Sheet targets of 0.14, } 0.01 \text {, and } \\
0.04 \mathrm{~kg} / \mathrm{min} \text {, respectively. (TI: Run Sheet)However: Lower sparger flow } \\
\text { rate was ON at the Run Sheet target of } 0.04 \mathrm{~kg} / \mathrm{min}, 08: 302 / 16 / 09 \text { (TI pg. } \\
\text { 206). } \\
\text { In addition, maximum attainable filter-loop flow rate was } 85 \mathrm{GPM} \text {, opposed } \\
\text { to the } 109 \pm 10 \mathrm{GPM} \text { Run Sheet target. (TI: Run Sheet). } \\
\text { 09:10, 2/16/09 (LRB 60230, pg. } 97 \text { ) Sampling folks noted great difficulty is } \\
\text { redispersing the centrifuged solids after decanting the supernate. The } \\
\text { vortex mixer alone was not getting the job done. An ultrasonic bath was } \\
\text { considered, but more forcible manual mixing along with vortex mixing } \\
\text { solved the problem. } \\
\\
\text { Leaks on UFP-VSL-T02A PJMs } 1807 \text { and } 1801 \text { vacuum lines were } \\
\text { identified, by 10:00, they were still leaking after tightening the connections, } \\
\text { (2/16/09, LRB 60230, pg. } 98 \text { ). }\end{array}$ \\
\hline
\end{tabular}




\begin{tabular}{|c|c|c|c|c|c|}
\hline \begin{tabular}{|l|} 
Test Step \\
\end{tabular} & Test Step & Description/Purpose & Key Process Conditions & $\begin{array}{ll} & \text { liquid density } \\
\text { - } & \text { dissolved chromium } \\
\text { - } & \text { dissolved manganese } \\
\text { - } & \text { liquid anion tracers. }\end{array}$ & Test Deviations and Problems \\
\hline B.1.19 & $\begin{array}{c}\text { Oxidative } \\
\text { Leach } \\
\text { Started } 2 / 11 / 69,10: 21 \\
\text { Ended 2/16/09, 16:23 }\end{array}$ & $\begin{array}{l}\text { Demonstrate oxidative leaching and establish } \\
\text { performance of Phase } 1 \text { simulant in PEP. }\end{array}$ & $\begin{array}{l}\text { Leach and mix at plant-time. } \\
\text { UFP-VSL-T02A PJM velocities match PTF } \\
\text { PJM velocities, and cycle time is } 1 / 4.5 \text { that } \\
\text { of PTF. } \\
\text { UFP-PMP-T42A and UFP-PMP-T43A } \\
\text { flow rate at } 1 / 4.5^{5} \text { that of PTF. } \\
\end{array}$ & 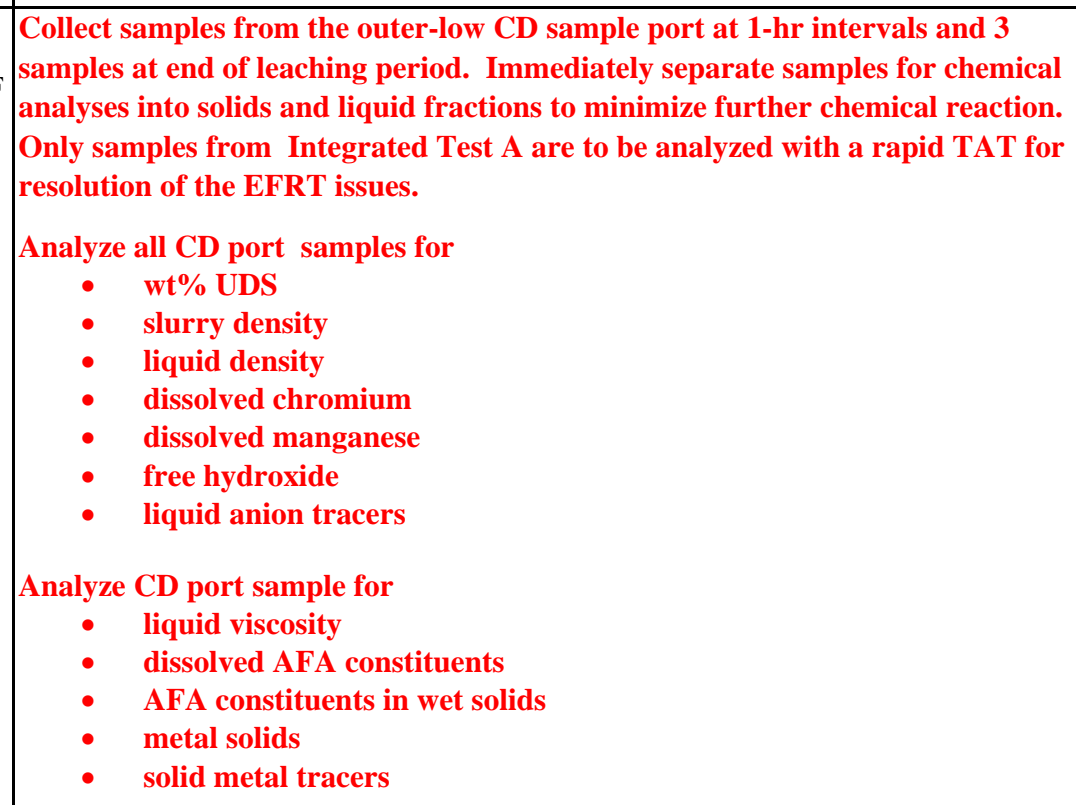 & 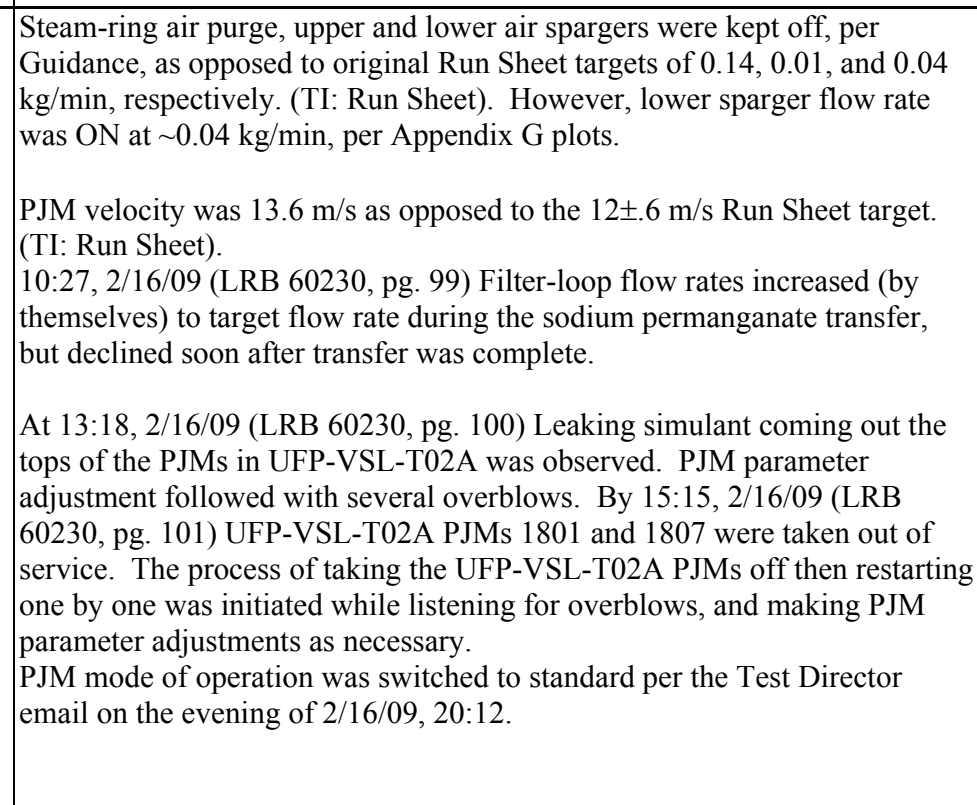 \\
\hline B.1.20 & $\begin{array}{c}\text { Wash oxidatively leached } \\
\text { slurry } \\
\text { Started 2/16/09, 17:08 } \\
\text { Ended 2/16/09, 23:13 }\end{array}$ & $\begin{array}{l}\text { Demonstrate post-oxidative leach slurry wash. } \\
\text { Monitor permeate for precipitation of solids. }\end{array}$ & $\begin{array}{l}\text { Wash at scale-time. } \\
\text { Five ultrafilter bundles. } \\
\text { UFP-VSL-T02A PJM velocities match PTF } \\
\text { PJM velocities, and cycle time is } 1 / 4.5 \text { that } \\
\text { of PTF. } \\
\text { Route permeate to UFP-VSL-T62A/B. }\end{array}$ & 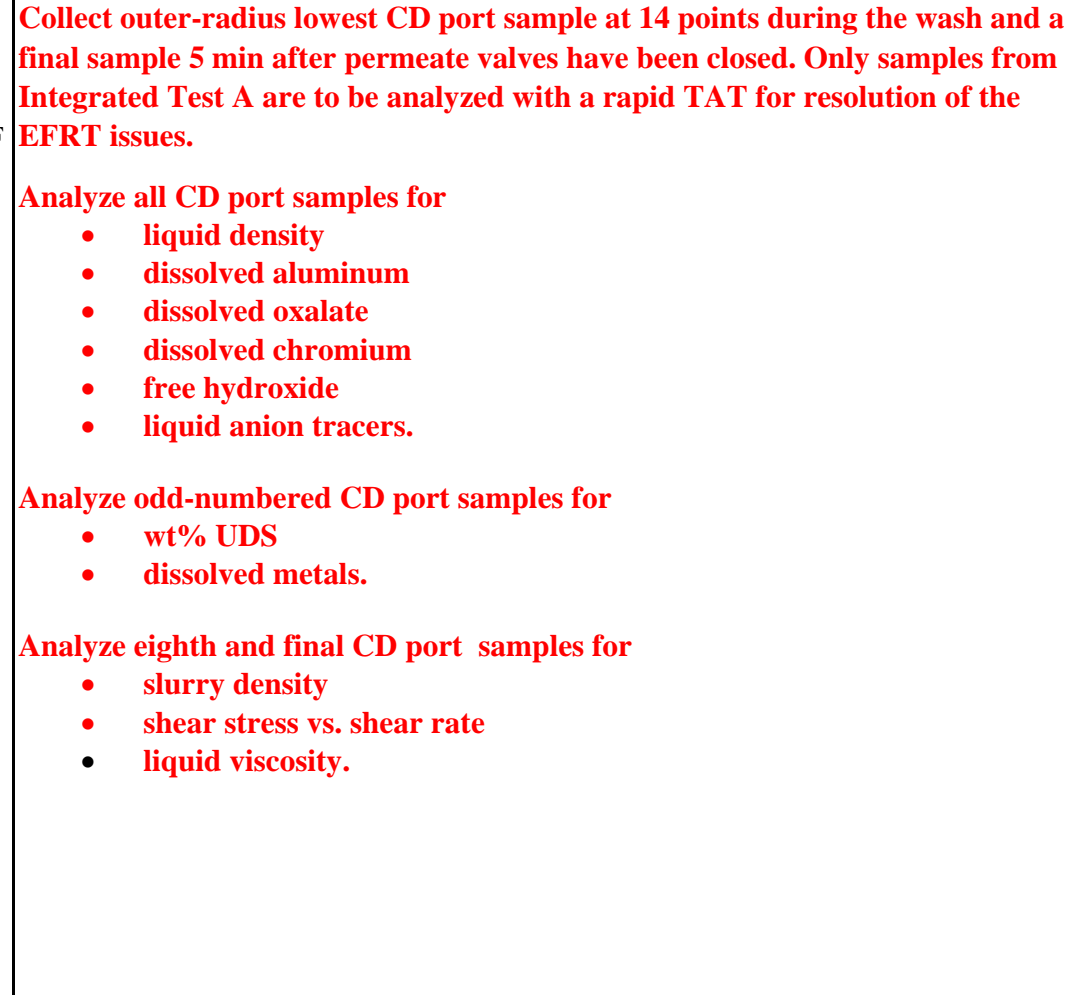 & 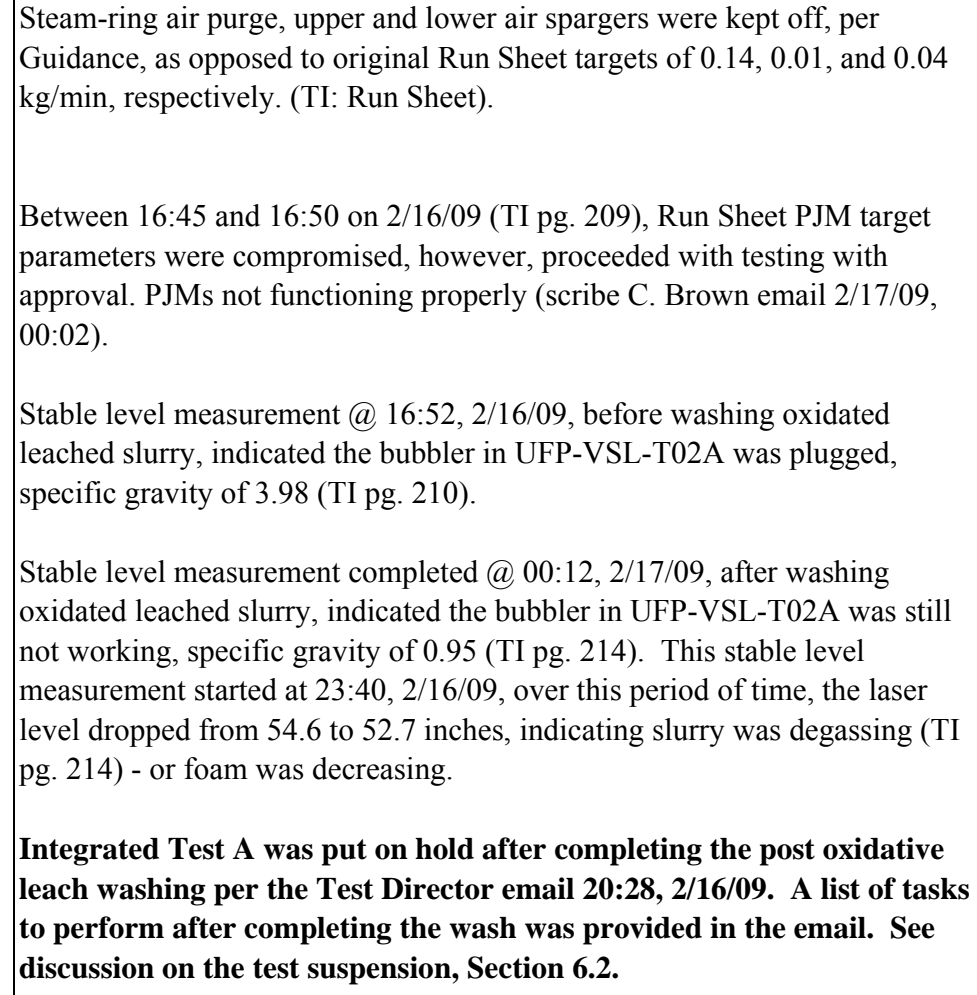 \\
\hline
\end{tabular}




\begin{tabular}{|c|c|c|c|c|c|}
\hline Test Step & Test Step & Description/Purpose & Key Process Conditions & Sampling & Test Deviations and Problems \\
\hline B.1.21 & $\begin{array}{c}\text { Concentrate washed slurry } \\
\text { Started 2/20/09, 21:10 } \\
\text { Ended 2/20/09, } \\
21: 40\end{array}$ & $\begin{array}{l}\text { Demonstrate final solids concentration to } 20 \\
\text { wt\% UDS. } \\
\text { Demonstrate ultrafiltration end points. } \\
\text { Monitor permeate for precipitation of solids. }\end{array}$ & \begin{tabular}{|l|} 
Concentrate at scale-time. \\
Five ultrafilter bundles. \\
UFP-VSL-T02A PJM velocities match PTF \\
PJM velocities, and cycle time is $1 / 4.5$ that \\
of PTF. \\
Route permeate to UFP-VSL-T62A/B.
\end{tabular} & 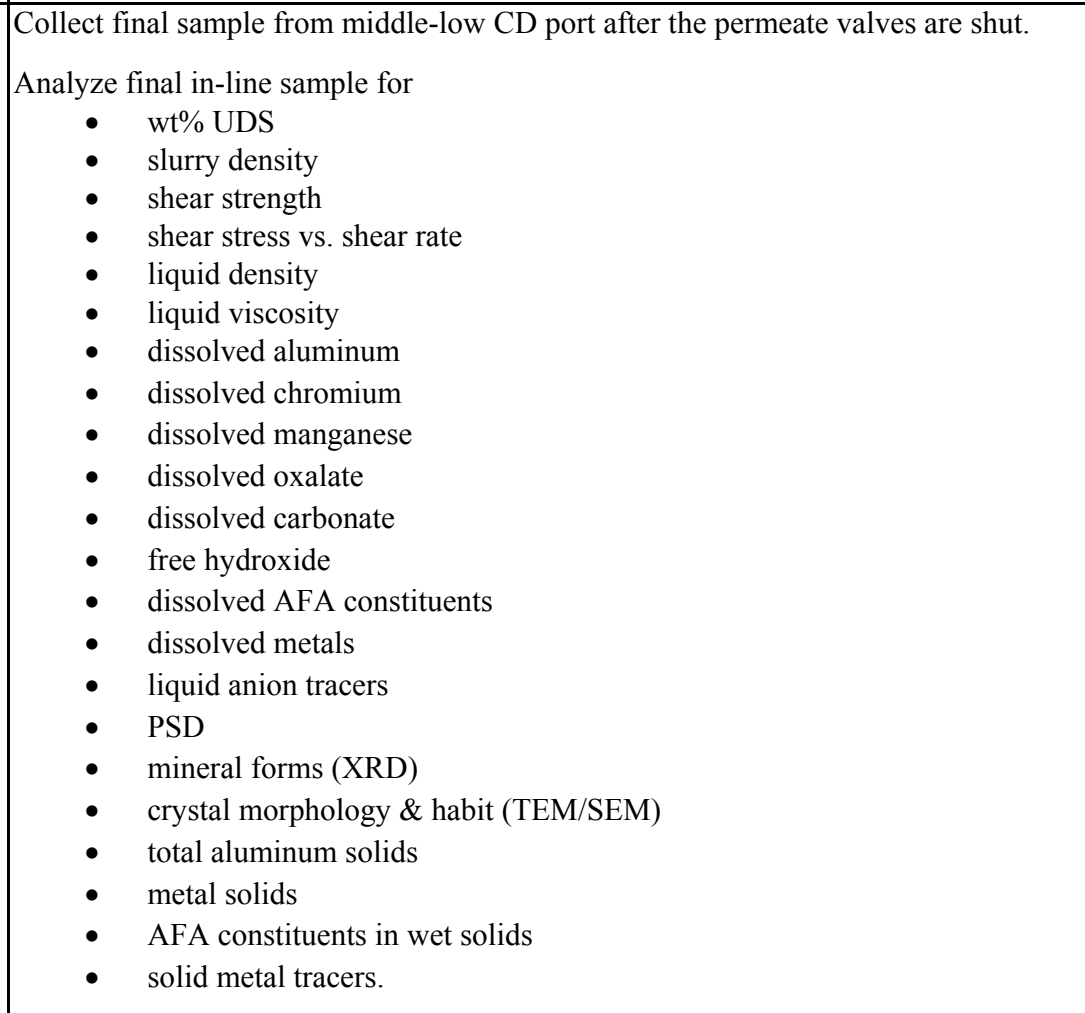 & $\begin{array}{l}\text { Restarted Integrated Test A on the evening of 2/20/09. } \\
\text { Took stable level measurement before commencing final concentration. } \\
\text { Bubblers were turned off, per guidance (TI pg. 215). } \\
\text { Steam-ring air purge, upper and lower air spargers were kept off, per } \\
\text { Guidance, as opposed to original Run Sheet targets of } 0.14,0.01 \text {, and } 0.04 \\
\text { kg/min, respectively. (TI: Run Sheet). } \\
\text { In addition, maximum attainable filter-loop flow rate was } 73 \mathrm{GPM} \\
\text { upstream from UFP-PMP-T42A (flow rate on FI-0635, downstream from } \\
\text { UFP-PMP-T43A was higher) as opposed to the } 109 \pm 10 \mathrm{GPM} \text { Run Sheet } \\
\text { target. (TI: Run Sheet) Flowmeter performance is questionable at this } \\
\text { time. }\end{array}$ \\
\hline B.1.22 & $\begin{array}{c}\text { Prepare for } \\
\text { high-solids filter test } \\
\text { Started 3/20/09, 21:10, } \\
\text { (LRB 60279, pg. 38) } \\
\text { Ended 3/21/09, 00:31 }\end{array}$ & $\begin{array}{l}\text { Add inhibited water in-line to suction of UFP- } \\
\text { PMPP-T42A untit slurry level in UFP-VSL-T02A } \\
\text { is at the 'High Mixing Volume' level. } \\
\text { Mix UFP-VSL-T02A with filter-loop jet for } 10 \\
\text { min. }\end{array}$ & $\begin{array}{l}\text { UFP-VSL-T02A PJM velocities match PTF } \\
\text { PJM velocities, and cycle time is } 1 / 4.5 \text { that } \\
\text { of PTF. }\end{array}$ & $\begin{array}{l}\text { Collect samples from the middle-low CD sample port for parallel CUF test and } \\
\text { characterization at end of } 10 \text { min mixing period. } \\
\text { Transport filter-loop sample for CUF to APEL. } \\
\text { Analyze characterization filter-loop in-line sample for } \\
\text { wt\% UDS } \\
\text { liquid viscosity }\end{array}$ & 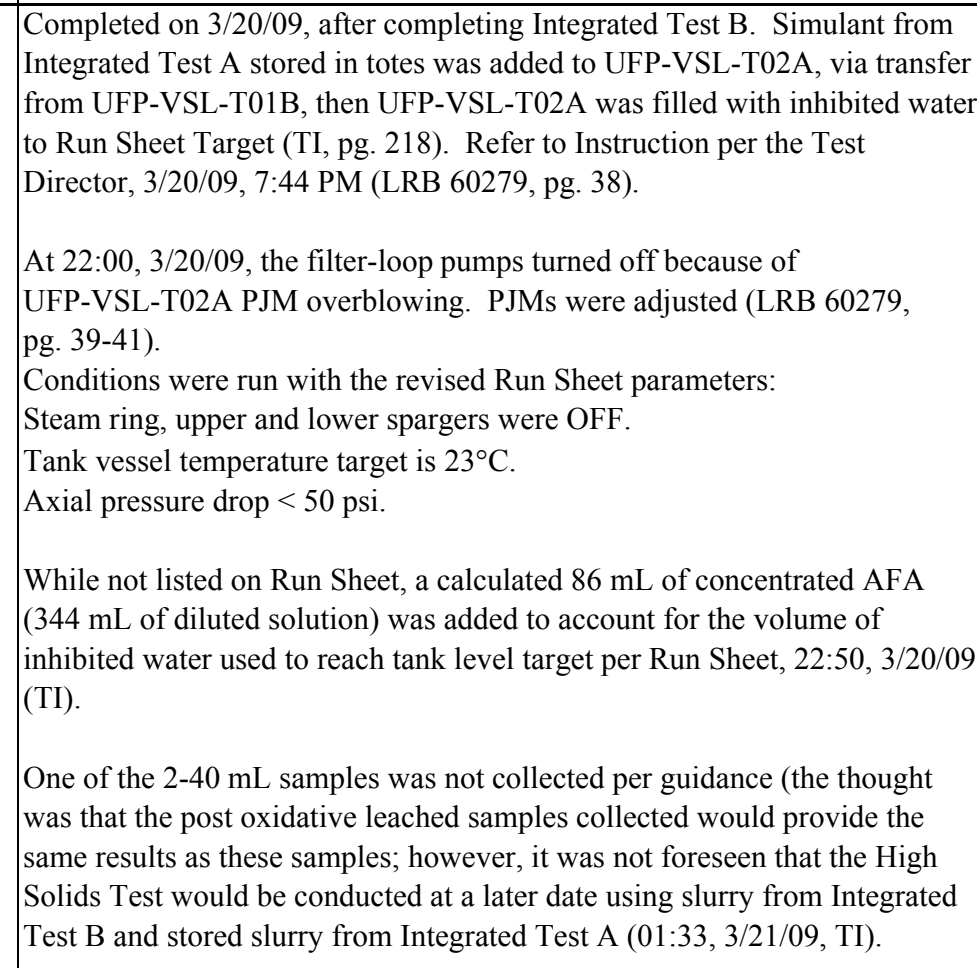 \\
\hline B.1.23 & $\begin{array}{l}\text { High-solids filter test } \\
\text { Started 3/21/09, 01:37, } \\
\text { Ended 3/21/09, 06:08 }\end{array}$ & $\begin{array}{l}\text { Configure filters to flow slurry through all } 5 \\
\text { filters. Concentrate slurry in UPF-VSL-To2A to } \\
20 \text { wt } \% \text { solids using only the first filter bundle. }\end{array}$ & $\mid \begin{array}{l}\text { Filter-loop flow through all filter bundles. } \\
\text { Withdraw permeate from first filter bundle }\end{array}$ & $\begin{array}{l}\text { Collect three (3) samples from the middle-low CD sample port after backpulsing is } \\
\text { complete. After the permeate flow has been routed to T-62A/B begin taking samples } \\
\text { from the middle-low CD sample port and at } 15 \text { min intervals thereafter. The final }\end{array}$ & $\begin{array}{l}\text { Steam-ring air purge, upper and lower air spargers were kept off, per } \\
\text { Guidance, as opposed to original Run Sheet targets of } 0.14,0.01 \text {, and } 0.04\end{array}$ \\
\hline
\end{tabular}




\begin{tabular}{|c|c|c|c|c|c|}
\hline Test Step & $\begin{array}{l}\text { Test Step } \\
\end{array}$ & $\begin{array}{c}\text { Description/Purpose } \\
\end{array}$ & Key Process Conditions & \begin{tabular}{|c|} 
Sampling \\
\end{tabular} & $\begin{array}{lc}\text { Test Deviations and Problems } \\
\end{array}$ \\
\hline & & $\begin{array}{l}\text { After the permeate valves are opened, backpulse } \\
\text { Filter T01A five times as rapidly as possible. } \\
\text { Record permeate flow from first filter bundle as } \\
\text { a function of time. } \\
\text { Collect in-line slury samples to establish wt } \% \\
\text { UDD periodically during solids concentration } \\
\text { process. }\end{array}$ & \begin{tabular}{|l|} 
only. \\
TMP 40 psid. \\
Filter-loop flow 109 GPM $(15 \mathrm{ft} / \mathrm{sec})$. \\
Permeate flow routed to UFP-VSL-T62A \\
or B. \\
UFP-VSL-T02A PJM velocities match PTF \\
PJM velocities, and cycle time is $1 / 4.5$ that \\
of PTF.
\end{tabular} & $\begin{array}{l}\text { samples are to be taken and analyzed in triplicate (3). } \\
\text { Analyze CD sample port samples for wt } \% \text { UDS. }\end{array}$ & $\begin{array}{l}\text { kg/min, respectively. (TI: Run Sheet). } \\
05: 15,3 / 21 / 09 \text { (LRB } 60279 \text {, pg. } 43 \text { ) Heat exchanger, UFP-HX-T02A, TIC- } \\
0513 \text { temperature setpoint is decreased to } 20^{\circ} \mathrm{C} \text { because temperature in the } \\
\text { tank is } 26.4^{\circ} \mathrm{C} \text {, target is } 23 \pm 2{ }^{\circ} \mathrm{C} \text {. } \\
05: 44,3 / 21 / 09 \text { (LRB } 60279 \text {, pg. } 43 \text { ) Heat exchanger, UFP-HX-T02A, TIC- } \\
0513 \text { temperature setpoint is set to manual operation, valve open }=28 \% \\
\text { because temperature is increasing fast (low level at high pump flow rate). } \\
05: 46,3 / 21 / 09 \text { (LRB } 60271 \text {, pg. } 4 \text { ) Heat exchanger, UFP-HX-T02A valve is } \\
\text { set to open }=60 \% \text {, temperature in the vessel is } 28.5^{\circ} \mathrm{C} \text { is }\end{array}$ \\
\hline B.1.24 & \begin{tabular}{|c|} 
Flush filter loop \\
Started 2/20/09, 22:16, \\
(LRB 60230, pg. 167) \\
Completed 2/21/09, 02:27 \\
\\
\\
\end{tabular} & $\begin{array}{l}\text { Flush slurry in filter loop back into UFP-VSL- } \\
\text { T02A with inhibited water. } \\
\text { Sample UFP-VSL-T02A to determine if filter } \\
\text { flush resulted in the addition of significant } \\
\text { amounts of flush water. } \\
\text { Configure filter loop to by-pass UFP-VSL-T02A } \\
\text { and circulate flush water with UFP-PMP-T42A } \\
\text { andor UFP-PMP-T43A to allow collection of a } \\
\text { representative in-line sample of the filter-loop } \\
\text { contents. }\end{array}$ & $\begin{array}{l}\text { Flush at scale-time. } \\
\text { UFP-VSL-T02A PJM velocities match PTF } \\
\text { PJM velocities, and cycle time is } 1 / 4.5 \text { that } \\
\text { of PTF. }\end{array}$ & 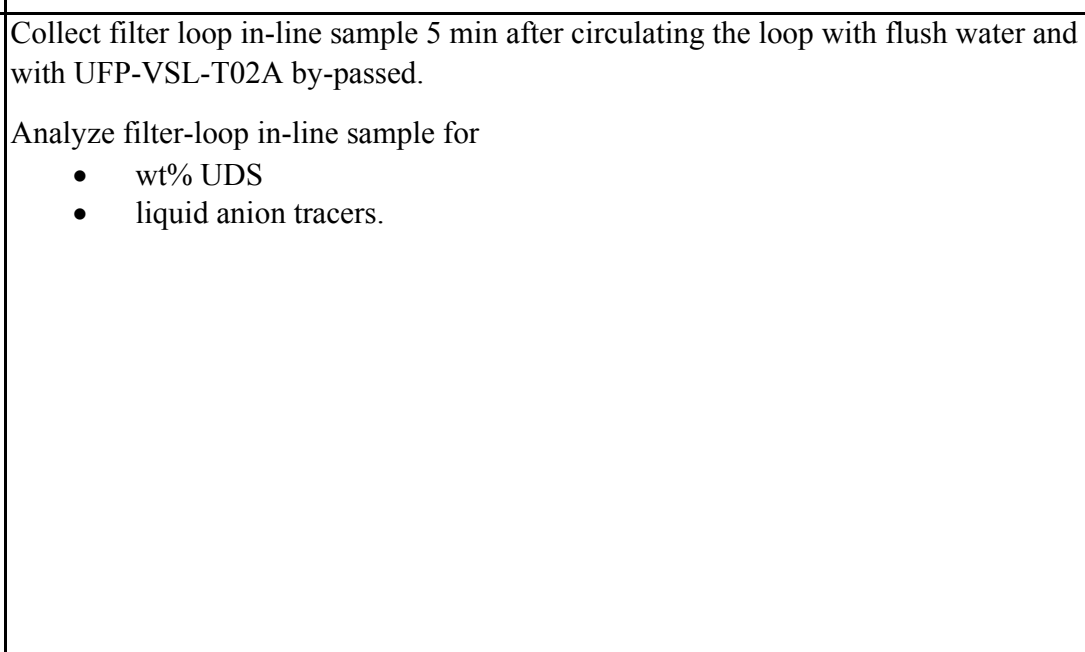 & 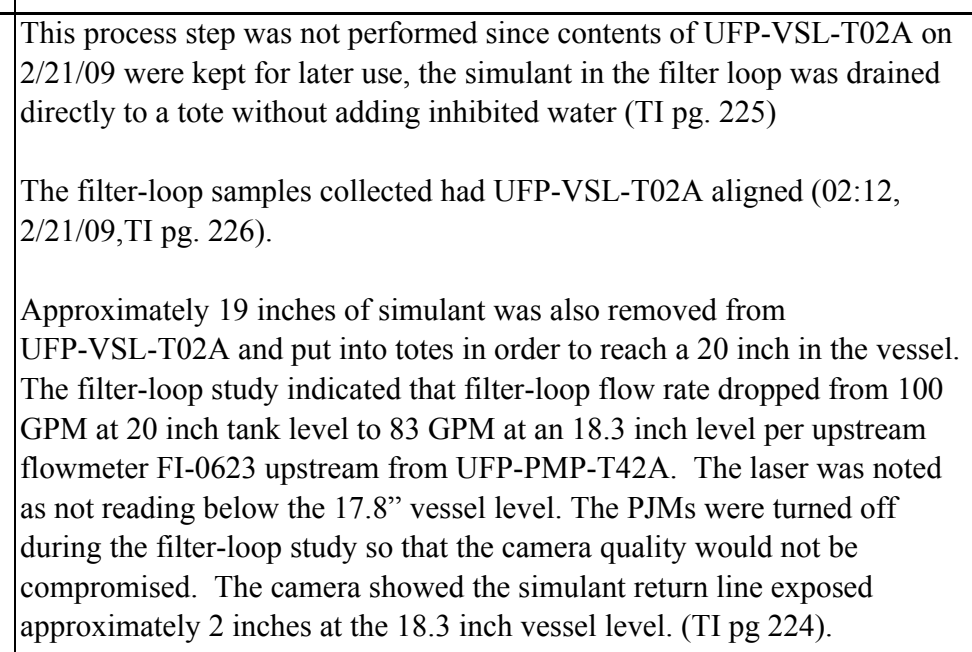 \\
\hline B.1.25 & \begin{tabular}{|c|} 
Transfer slurry from \\
UUP-VSL-T2A to \\
UFP-VSL-62A/B or totes \\
as directed by the WTP \\
test director \\
Completed 2/21/09, 02:27
\end{tabular} & $\begin{array}{l} \\
\text { Transfer slurry from UFP-VSL-T02A to } \\
\text { UFP-VSL-62A/B or totes using a portable heel } \\
\text { pump. }\end{array}$ & Per procedure. & $\begin{array}{l}\text { Collect sample from middle-low CD sample port prior to transfer. } \\
\text { Analyze in-line sample for } \\
\begin{array}{c}\text { wt\% UDS } \\
\text { liquid anion tracers. }\end{array}\end{array}$ & CD samples were not collected (TI pg 303). \\
\hline
\end{tabular}




\section{Appendix F}

Sparger Operations 



\section{Appendix F: Sparger Operations}

In addition to PJMs, air spargers were used in UFP-VSL-T02A to help with the mixing and suspension of solids. For Integrated Test A, target flow rates were idle ones, $0.01 \mathrm{~kg} / \mathrm{min}$ (total flow) for the lower spargers and $0.04 \mathrm{~kg} / \mathrm{min}$ (total) for the upper. While the data in this appendix are for information only, the figures are NQA-1 compliant.

UFP-VSL-T02A sparger air flow rates deviated from the target values in some instances during the tests. The upper spargers lost air flows on two occasions during dewatering of leached slurry. In both cases, the sparger nozzles were found plugged. The air flow rate came back to the target value after the spargers were cleaned.

1. The upper spargers air flow rate started fluctuating $\sim 06: 30,2 / 5 / 09$ and lost air flow from $\sim 06: 30$ to $\sim 12: 00,2 / 6 / 09$. The upper spargers were cleaned at 11:49, 2/6/09.

2. The upper spargers air flow rate started fluctuating $\sim 04: 00,2 / 12 / 09$ and lost air flow from 22:00, 2/13 to $03: 30,2 / 14 / 09$. The upper spargers were cleaned at 01:28, 2/14/09.

Sparging nozzles were clogged or plugged thus causing the lower sparger air flow rates lower than the target values. The spargers were cleaned occasionally to maintain the air flow rates within the target ranges. Air flow rates spiked during cleaning. Spargers were turned off per test instruction to take stable level measurements in UFP-VSL-T02A. On many occasions, sparging air was shut off for troubleshooting of abnormal operations, i.e., slurry air entrainments.

1. 21:57, 2/3/09. UFP-VSL-T02A pumps shut off, triggered by bubbler low-low alarm, and sparger air was lost as well. Spargers were restarted after cleaning of bubbler and laser 22:05.

2. $21: 52,2 / 7 / 09$. One of the lower sparger rotameters was isolated since flow rate was decaying with time. However, target total flow was kept.

3. $21: 23,2 / 11 / 09$. One of the spargers was plugged.

4. 03:30, 2/12/09. Sparger, nozzle 31 was plugged and the associated flow meter FIC-1943 was closed at $07: 05,2 / 12 / 09$.

5. 11:20, 2/12/09. Sparger nozzle 31, was flushed to clear the plug.

6. 19:50, 2/12/09. Cleared sparger nozzles 32 and 33.

7. 10:20, 2/15/09. After taking the 13th CD Sample Event (wash \#60), per approval, lower air spargers were turned OFF for remainder of the wash to minimize pump vibration. They were set to idle after completing post-caustic-leach washing. (TI).

8. Between 01:57, 2/15/09 and 14:26, 2/15/09, lower spargers were turned on and off several times for filter-loop flow-rate investigations.

9. 00:50, 2/16/09. Turned lower air spargers ON to idle flow rates to see effect on pumps.

10. 13:02, 2/18/09. Exercises using different number of spargers (total flow of $0.4 \mathrm{~kg} / \mathrm{min}$ ) to see effects on filter-loop flow rate.

11. 15:20, 2/18/09 to $15: 34,2 / 20 / 09$. Lower spargers were on and off many times, per Run Sheet, during slurry degassing and filter-loop pump exercises. 


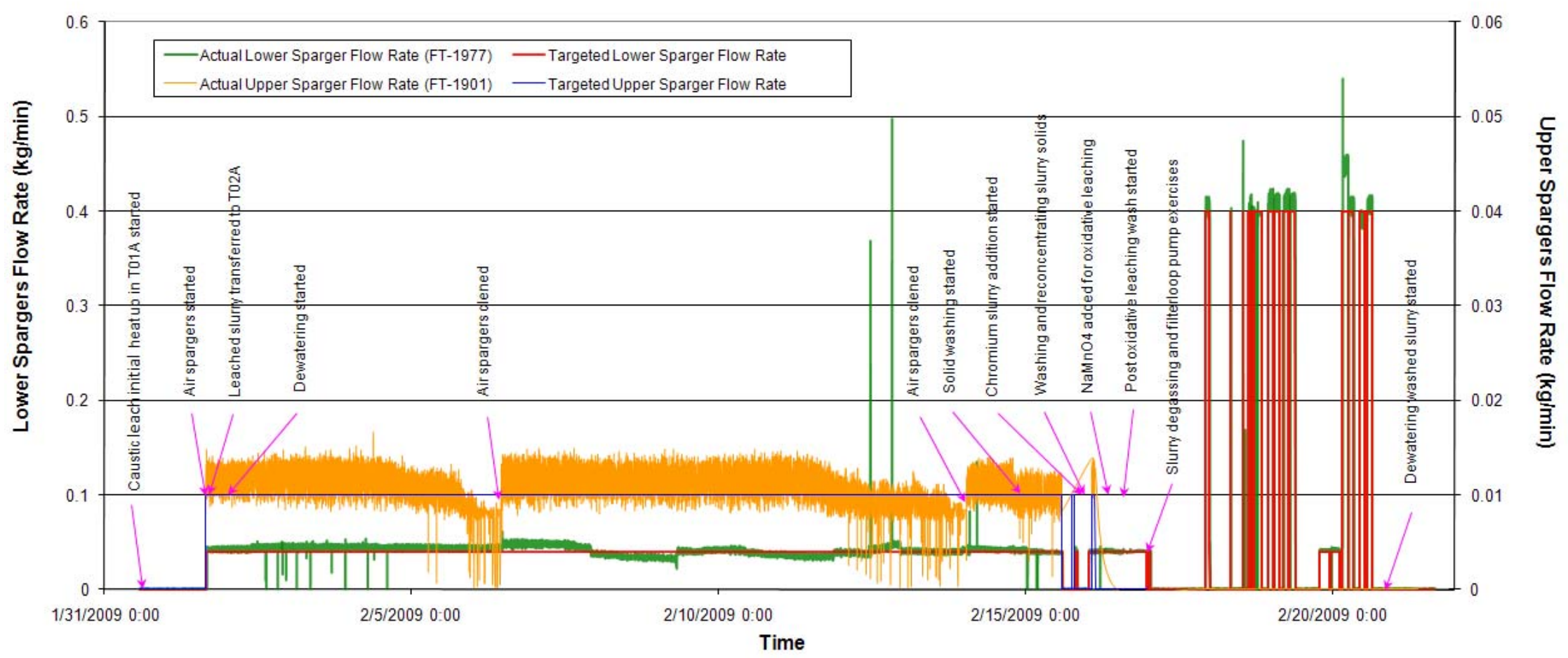




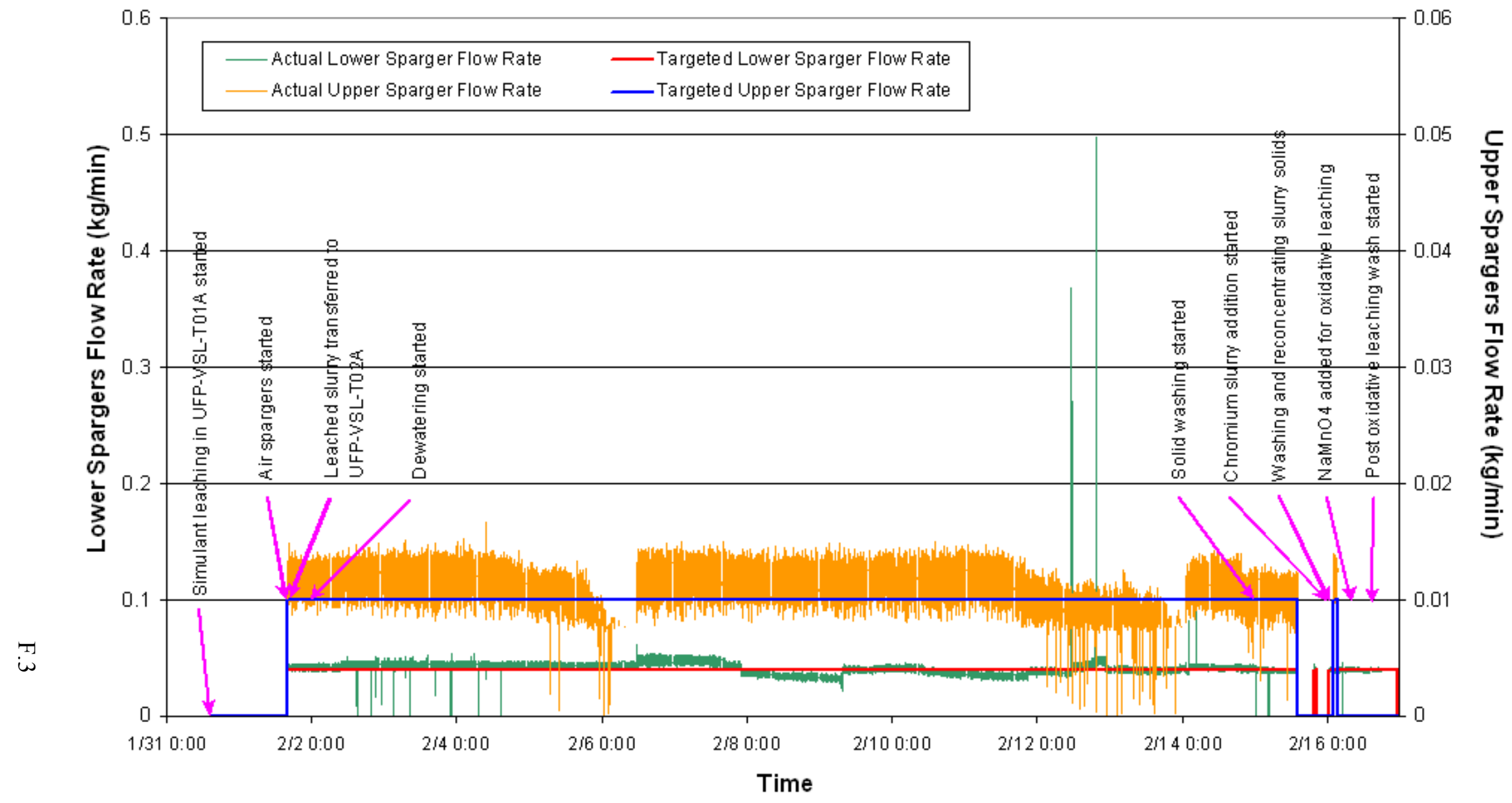

Figure F.2. Integrated Test A Air Sparge Flow Rates between 1/31/2009 and 2/16/2009 


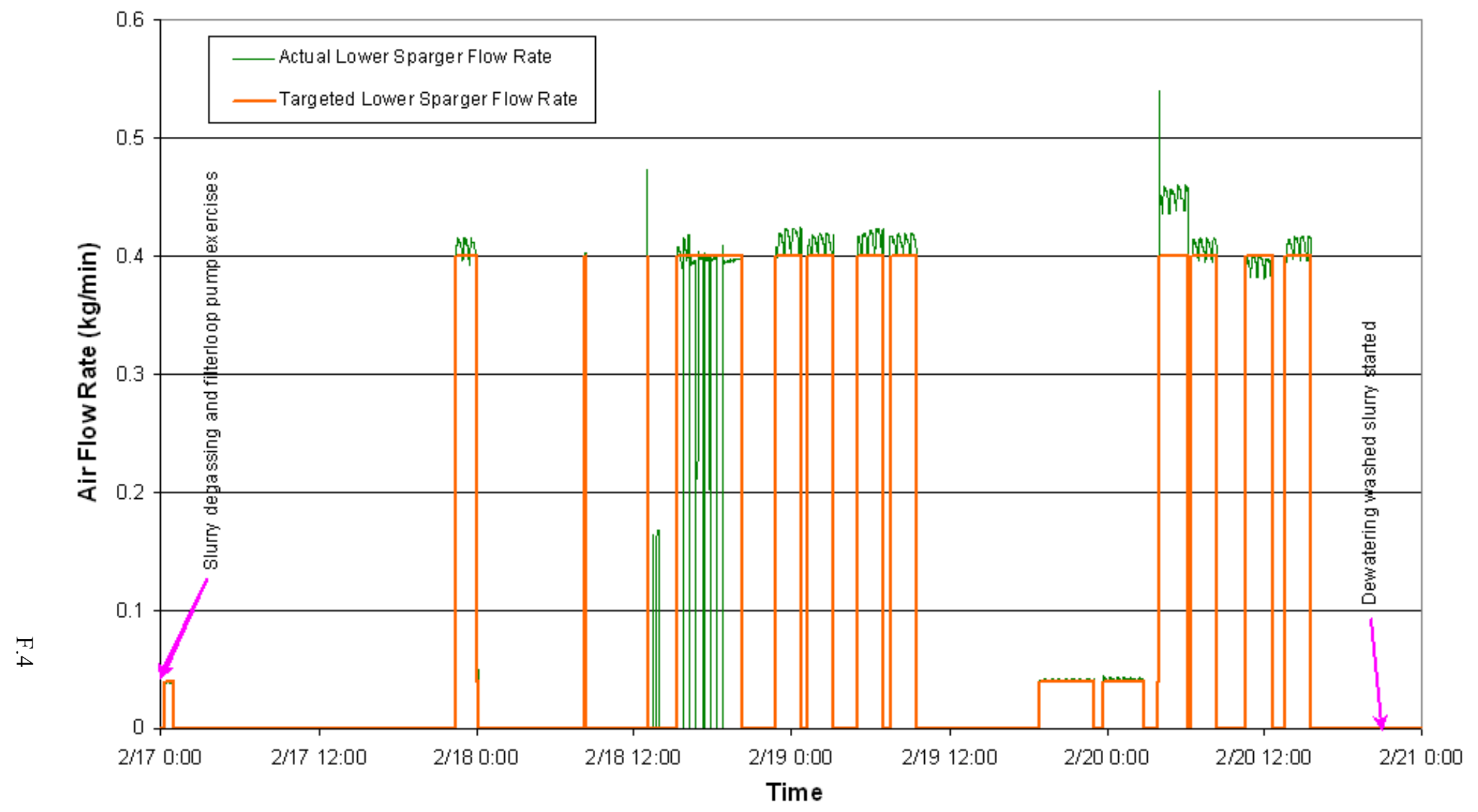

Figure F.3. Integrated Test A Air Sparge Flow Rates between 2/17/2009 and 2/21/2009 
Appendix G

\section{2-Hour Plots}





\section{Appendix G: Integrated Test A Data Plots}

\section{1/31/09 00:00 - 01/31/09 12:00}

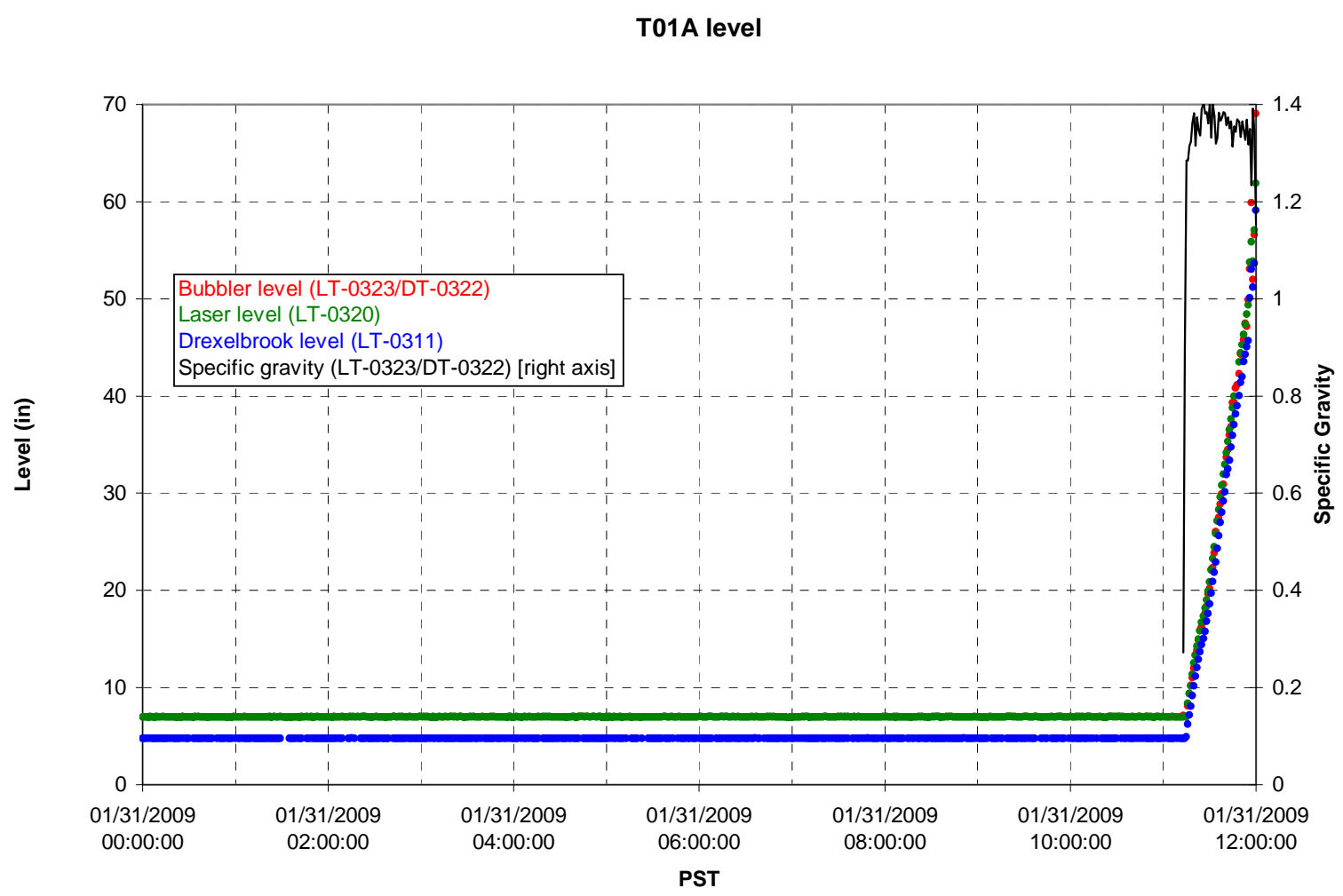


T01A temperatures

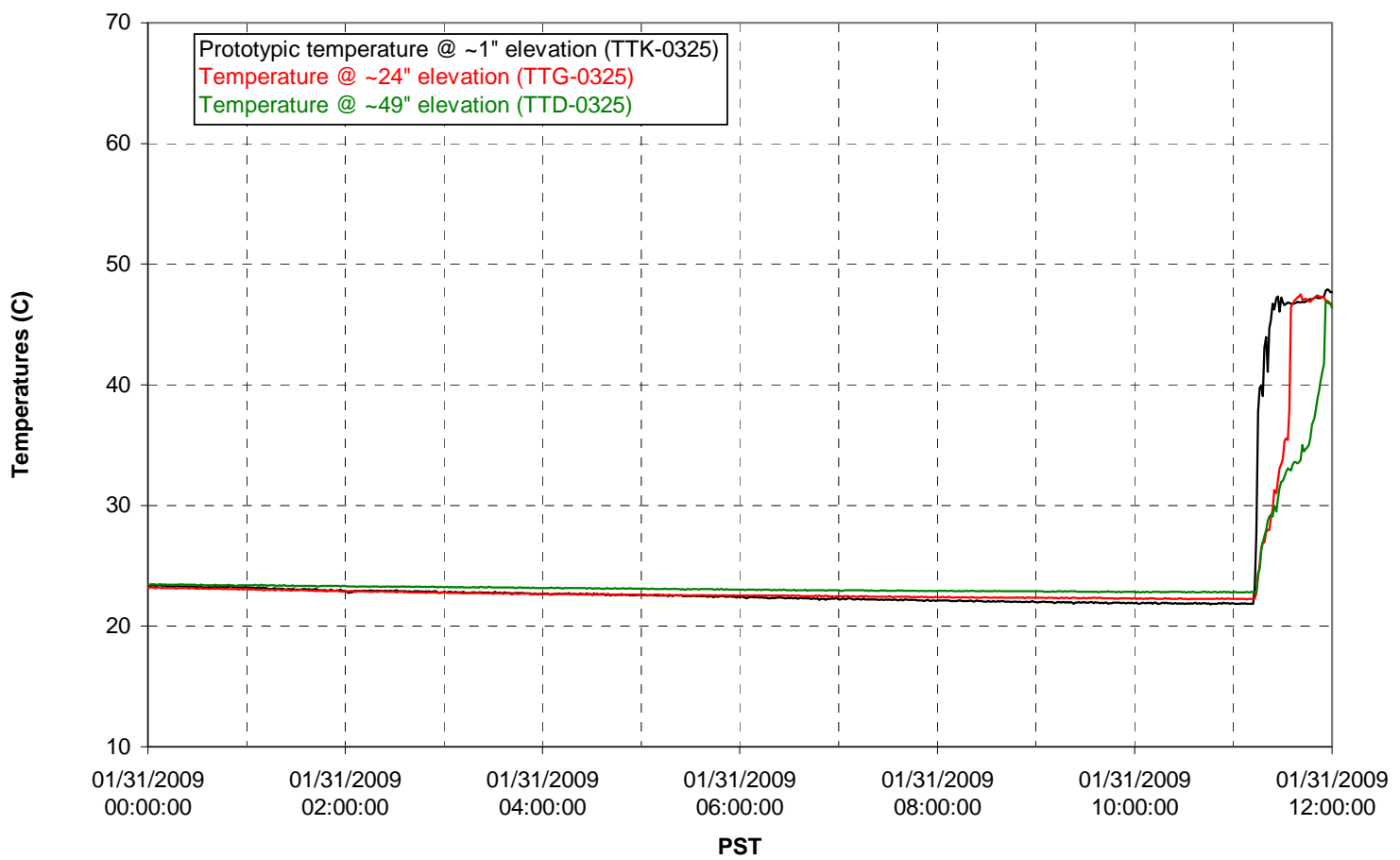

T01B level

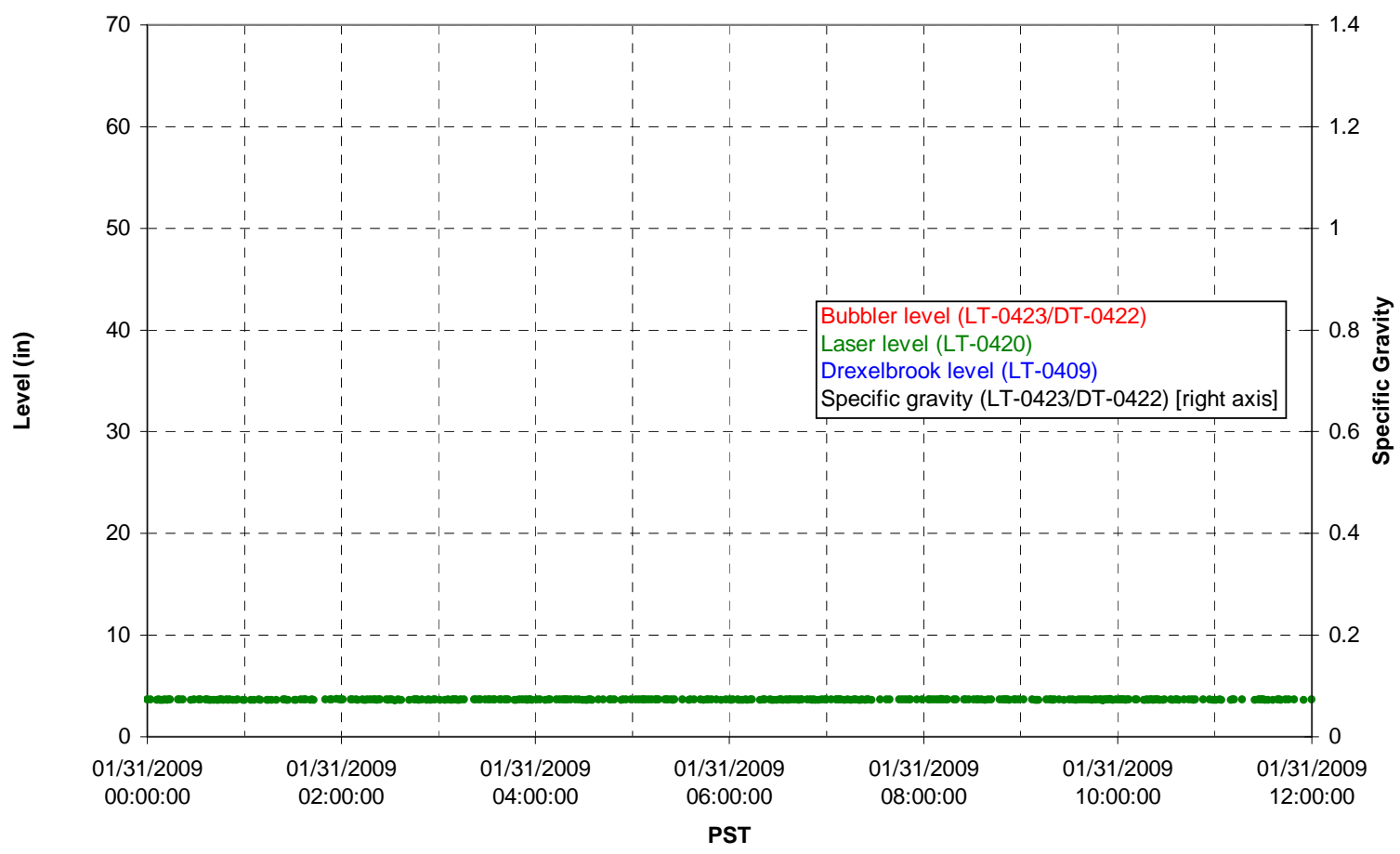

G.2 
T01B temperatures

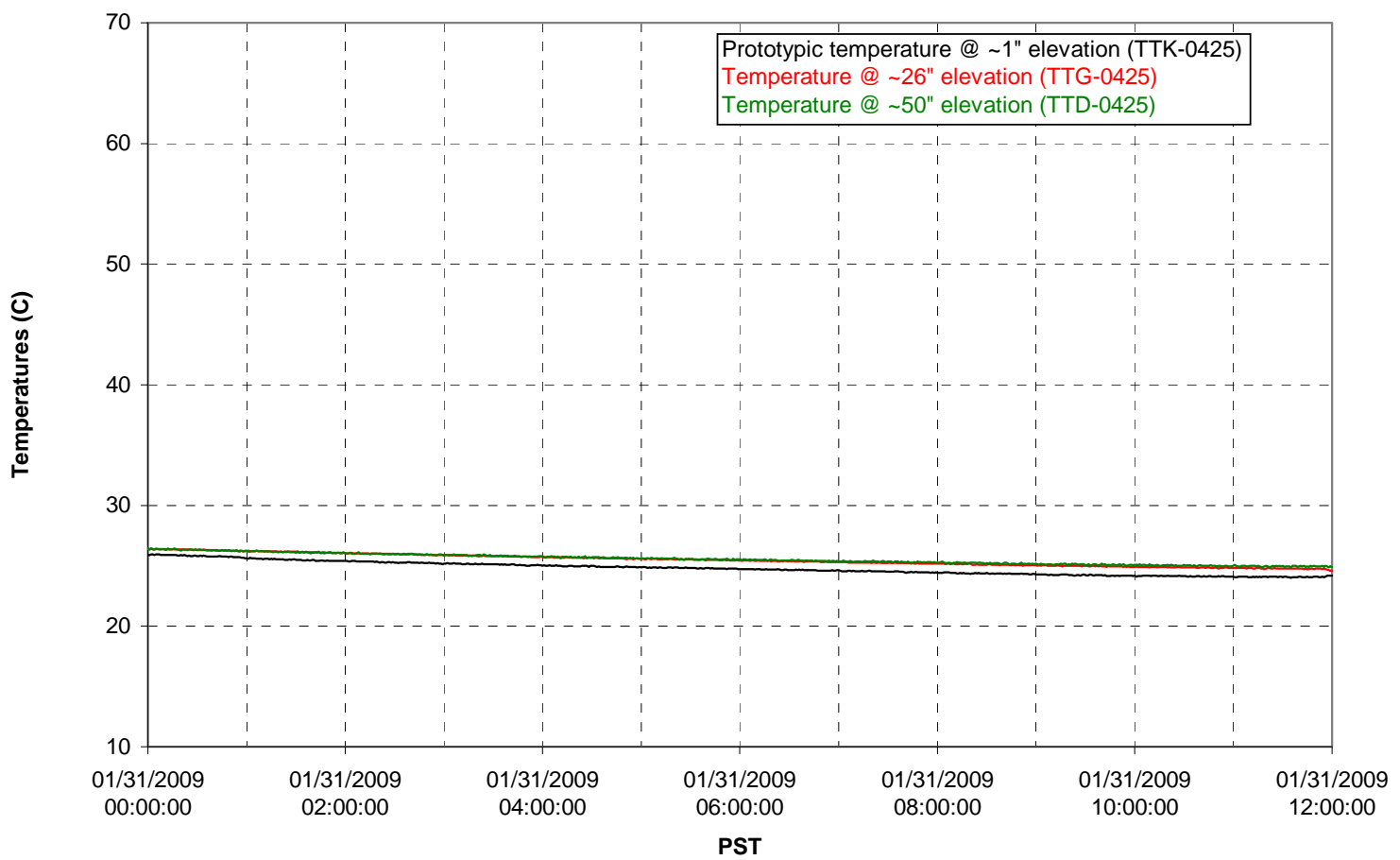

T02A level

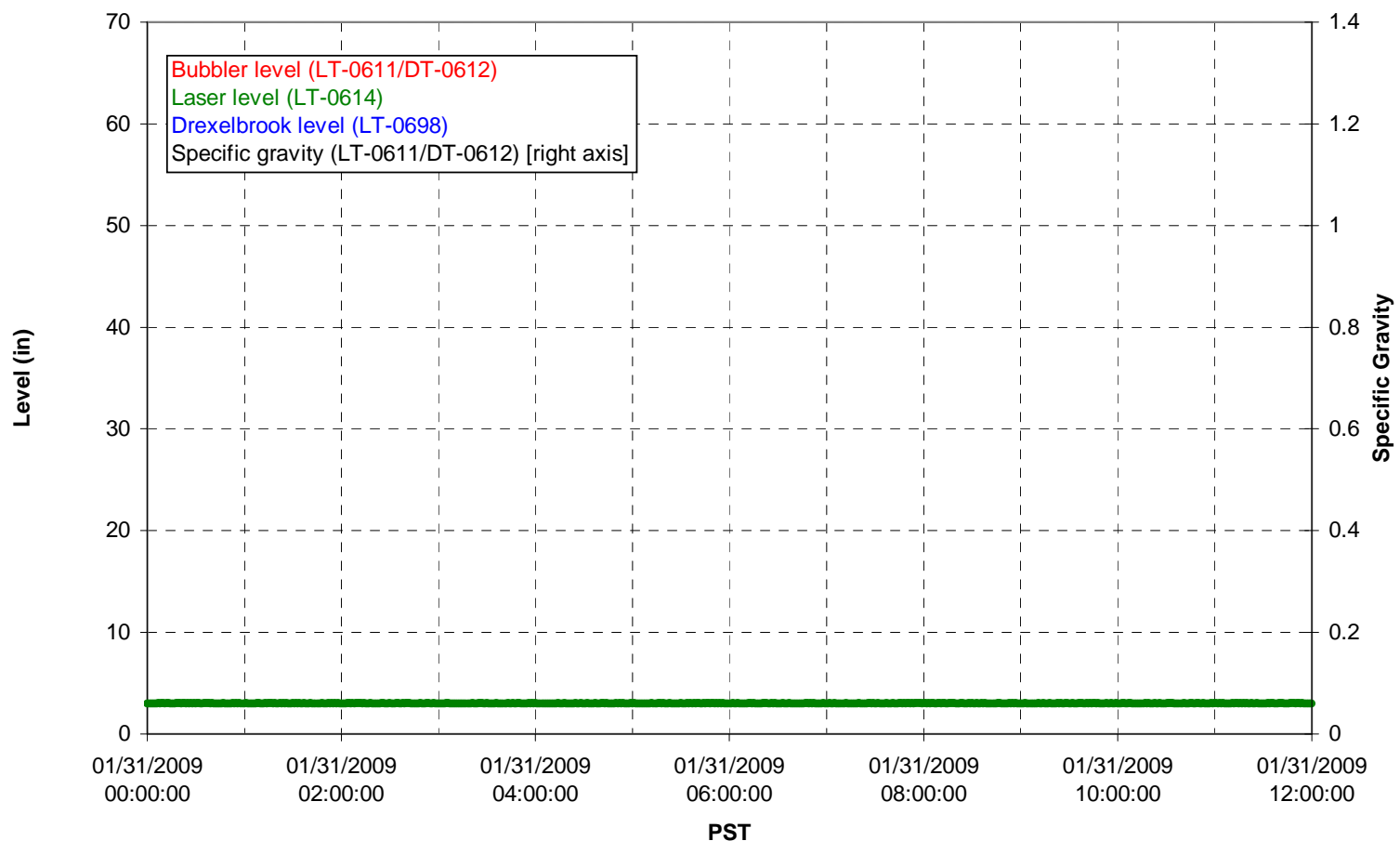

G.3 
T02A temperatures

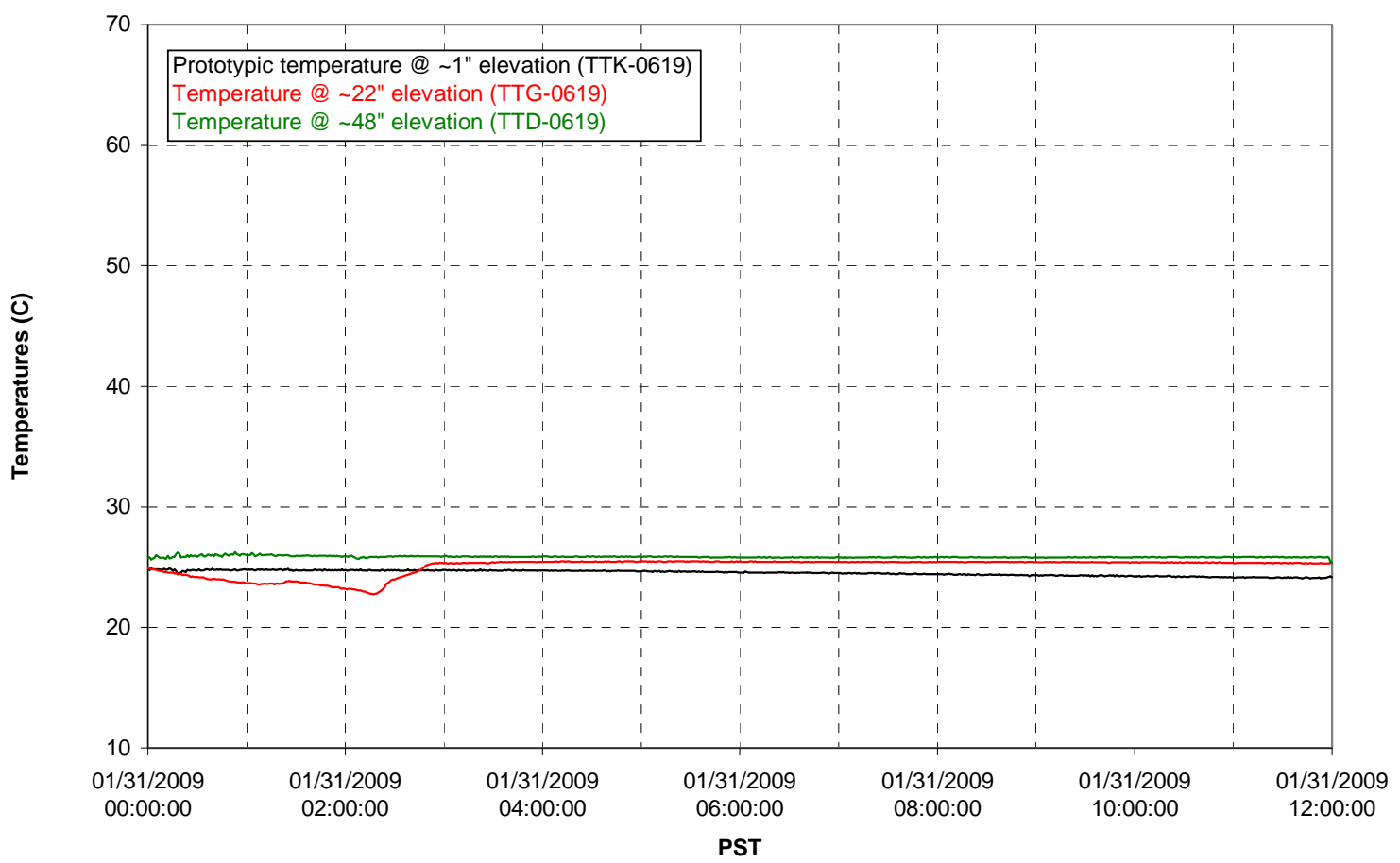

T02A and filter loop temperatures

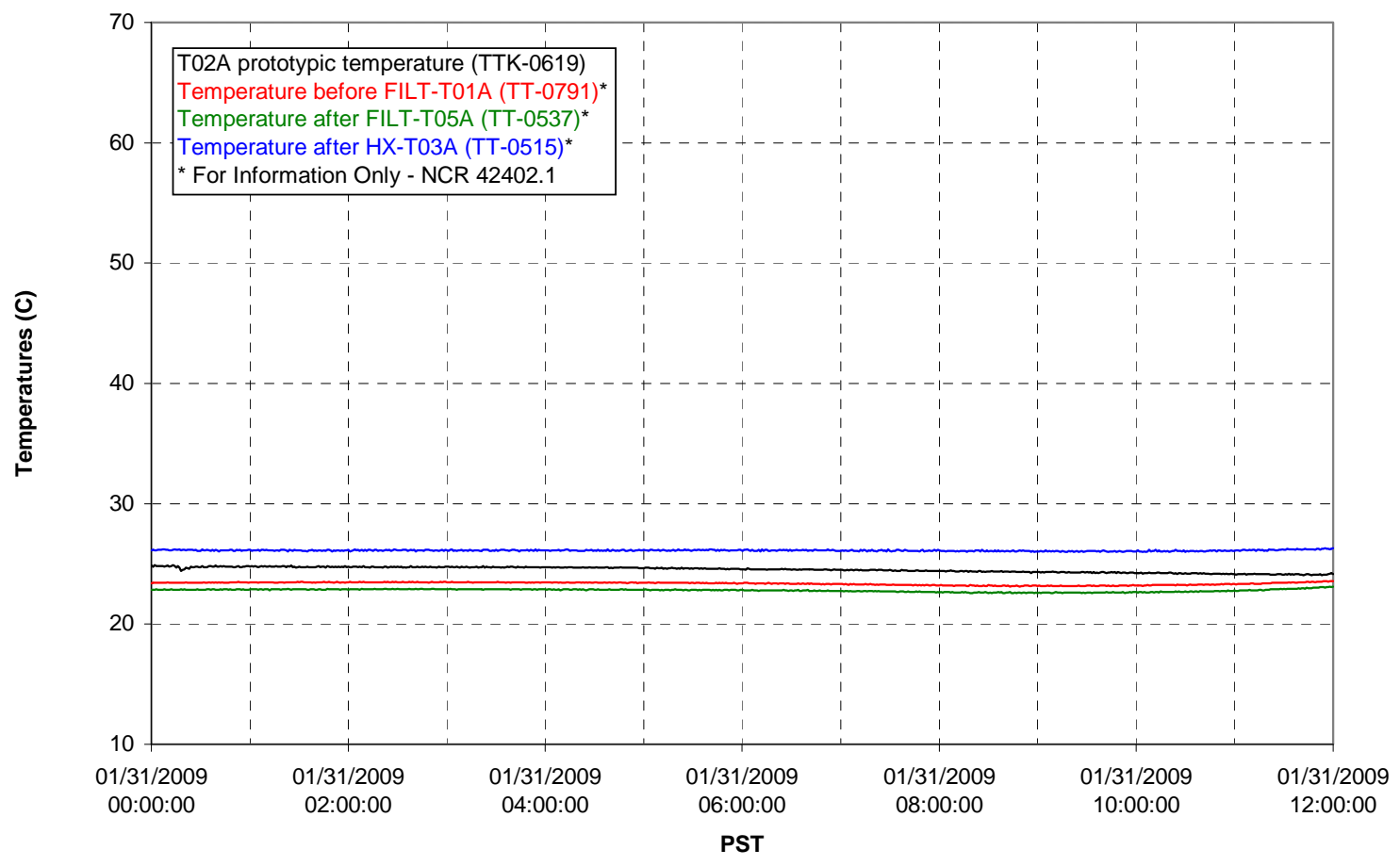

G.4 
Pump Pressures and Flow

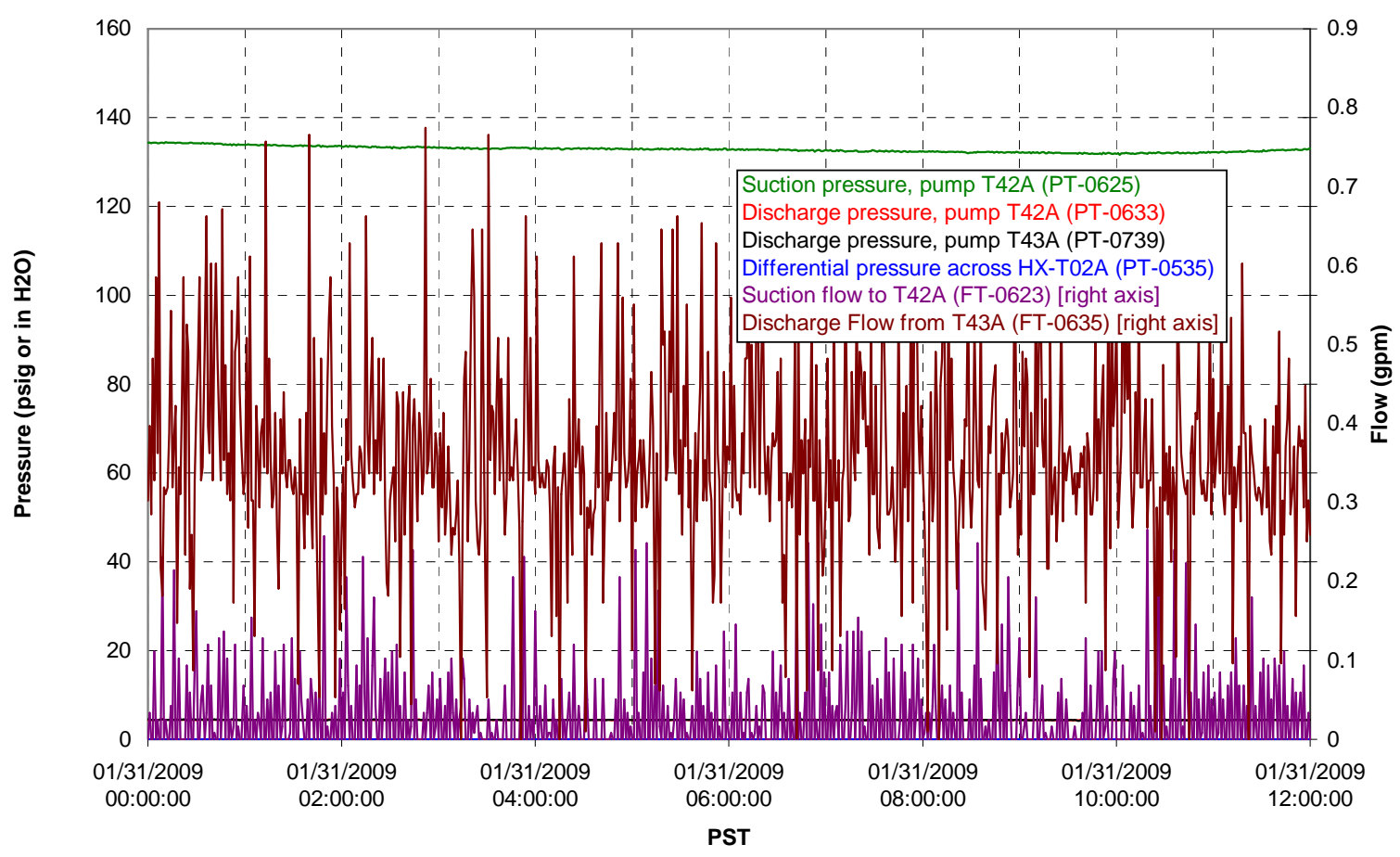

Axial pressure drop

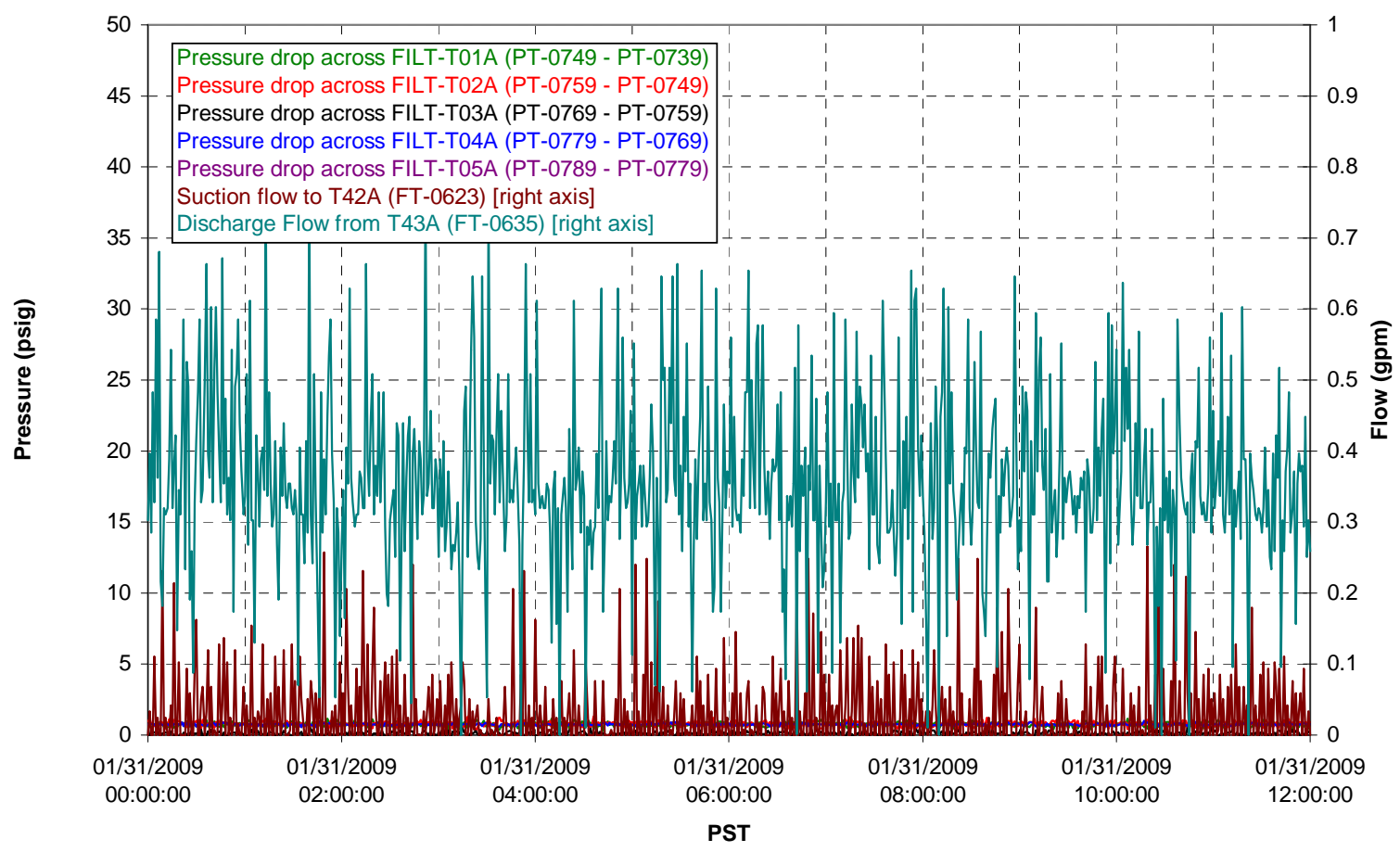


Permeate flow rates

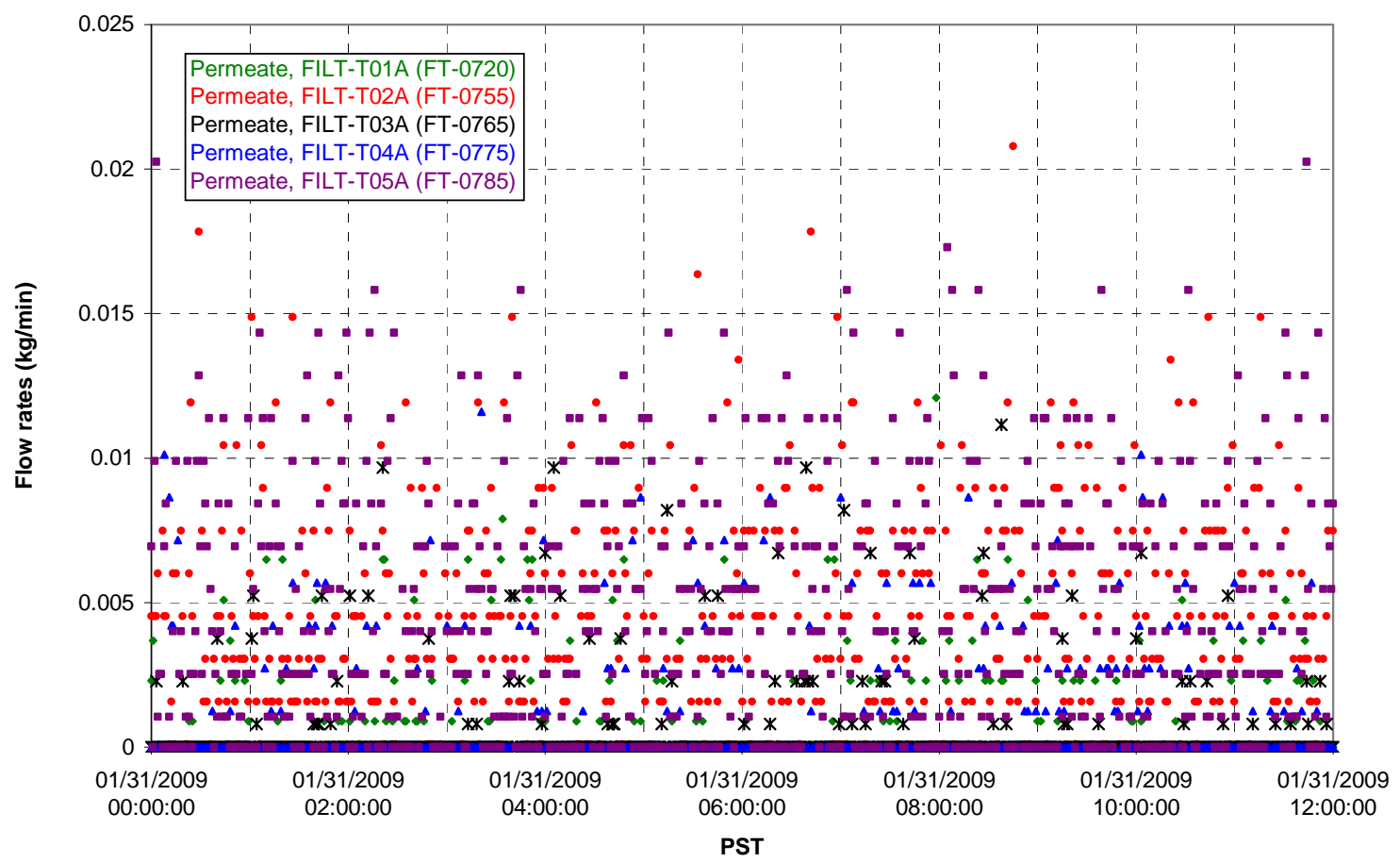

T02A Inner Temperature Tree

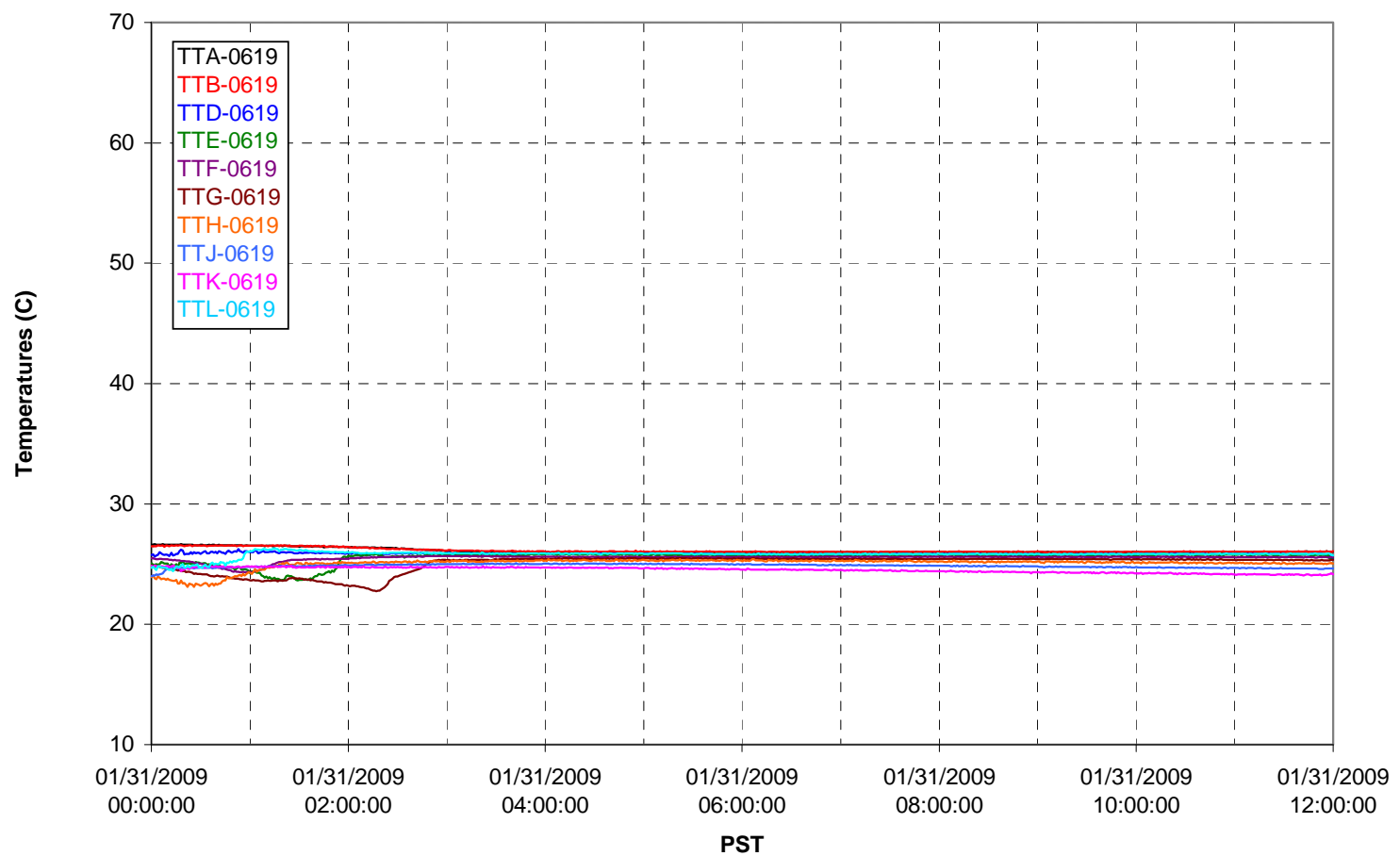


T02A Outer Temperature Tree

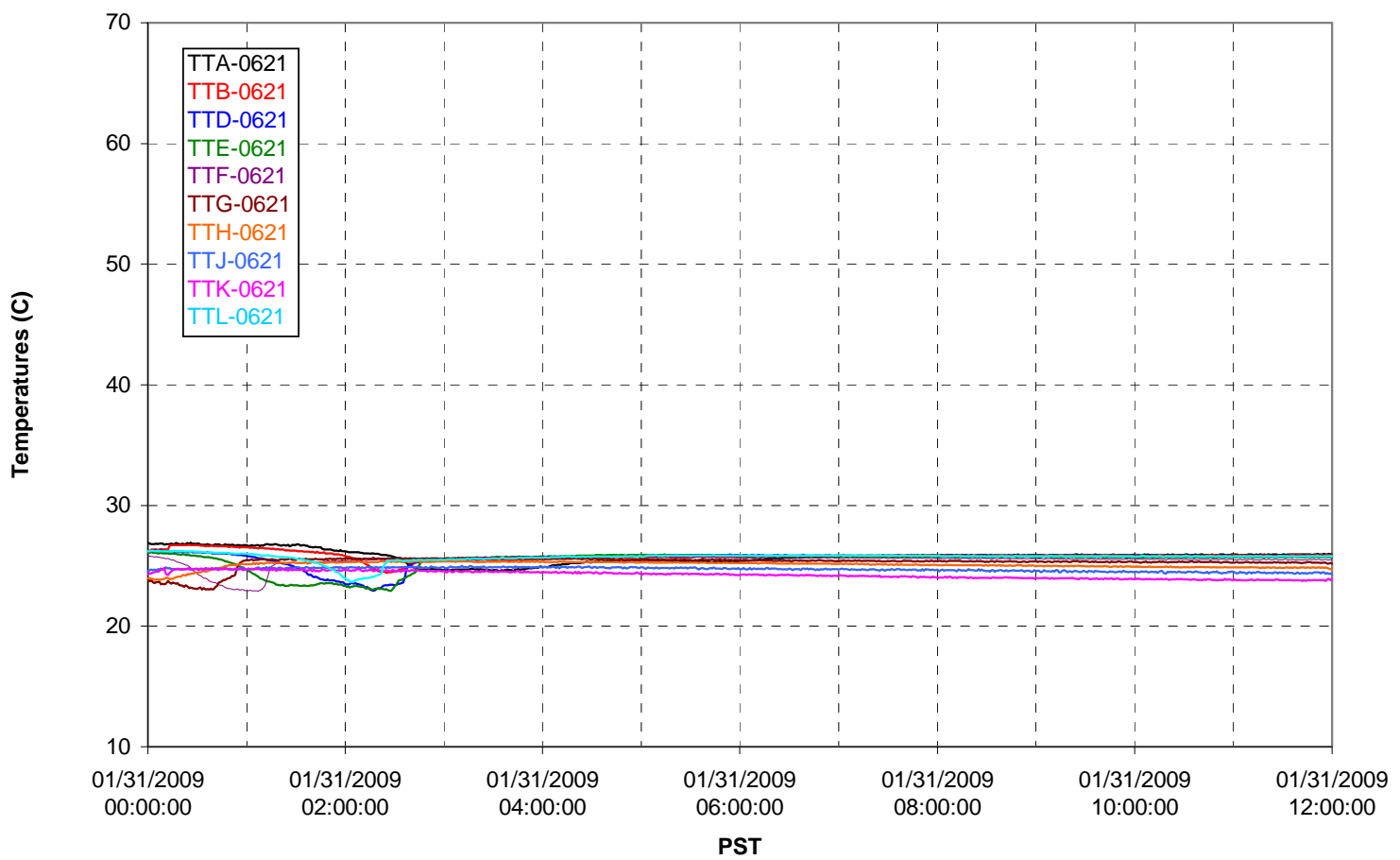

T02A temperatures

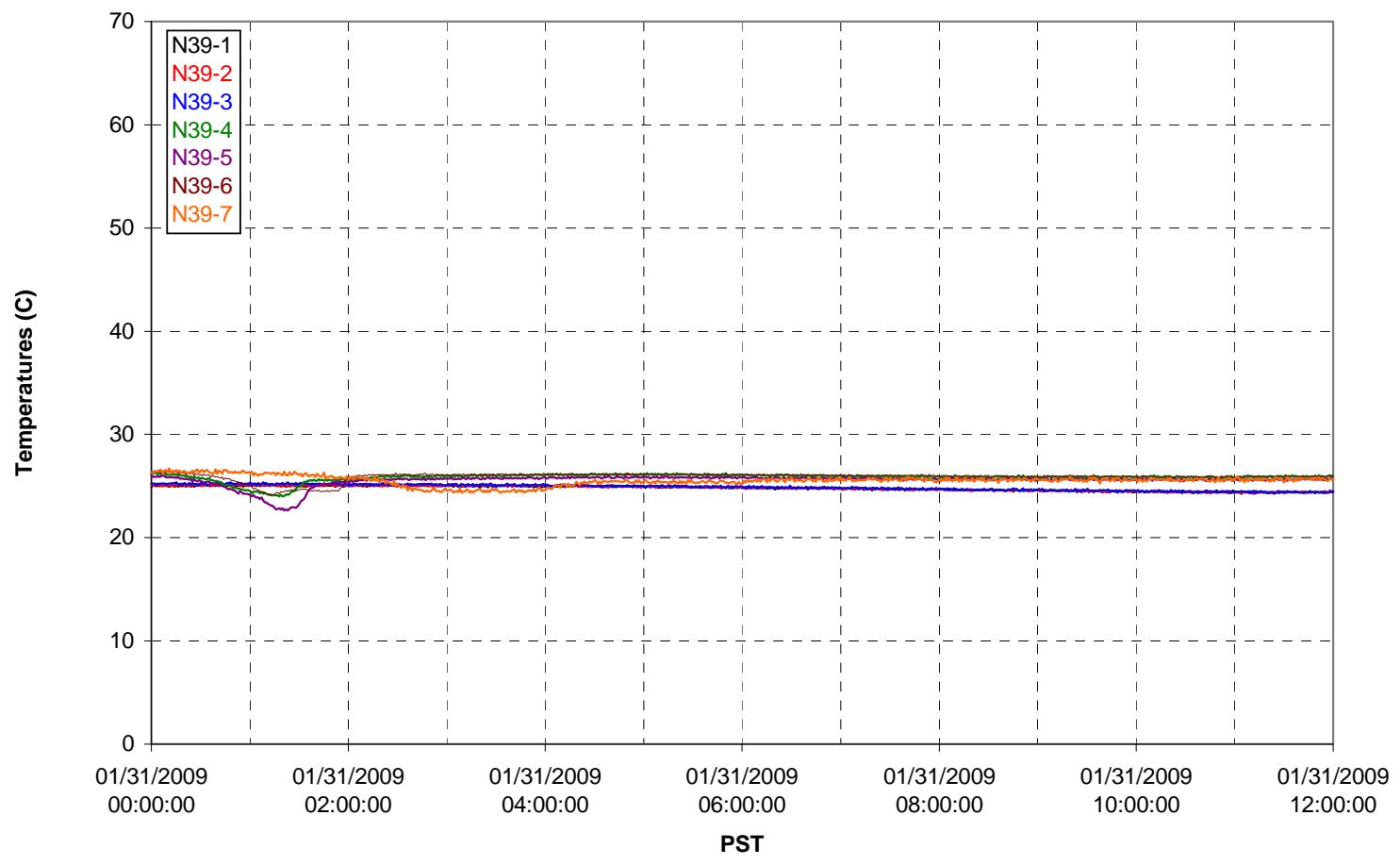

G.7 
T02A temperatures

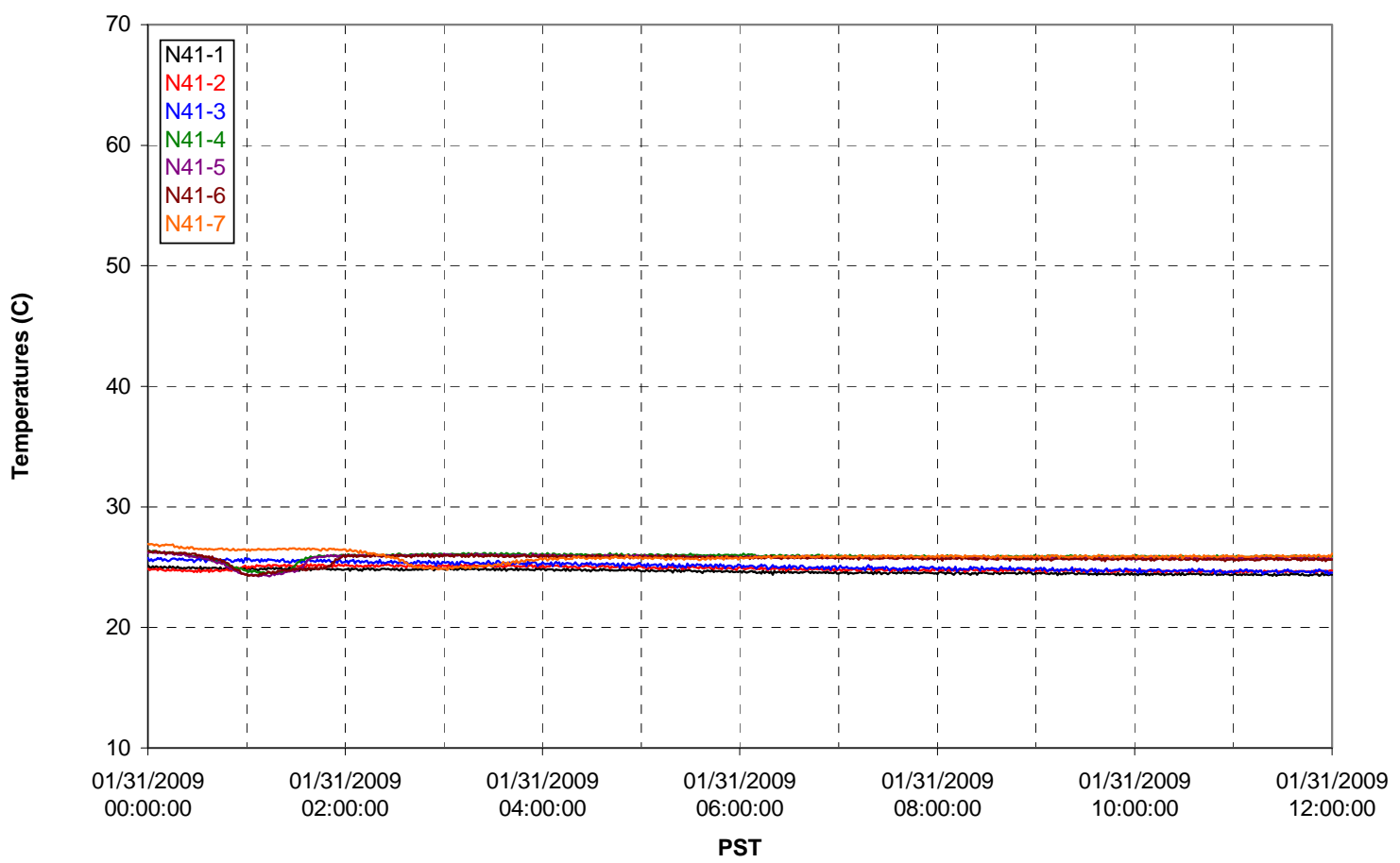

T02A temperatures

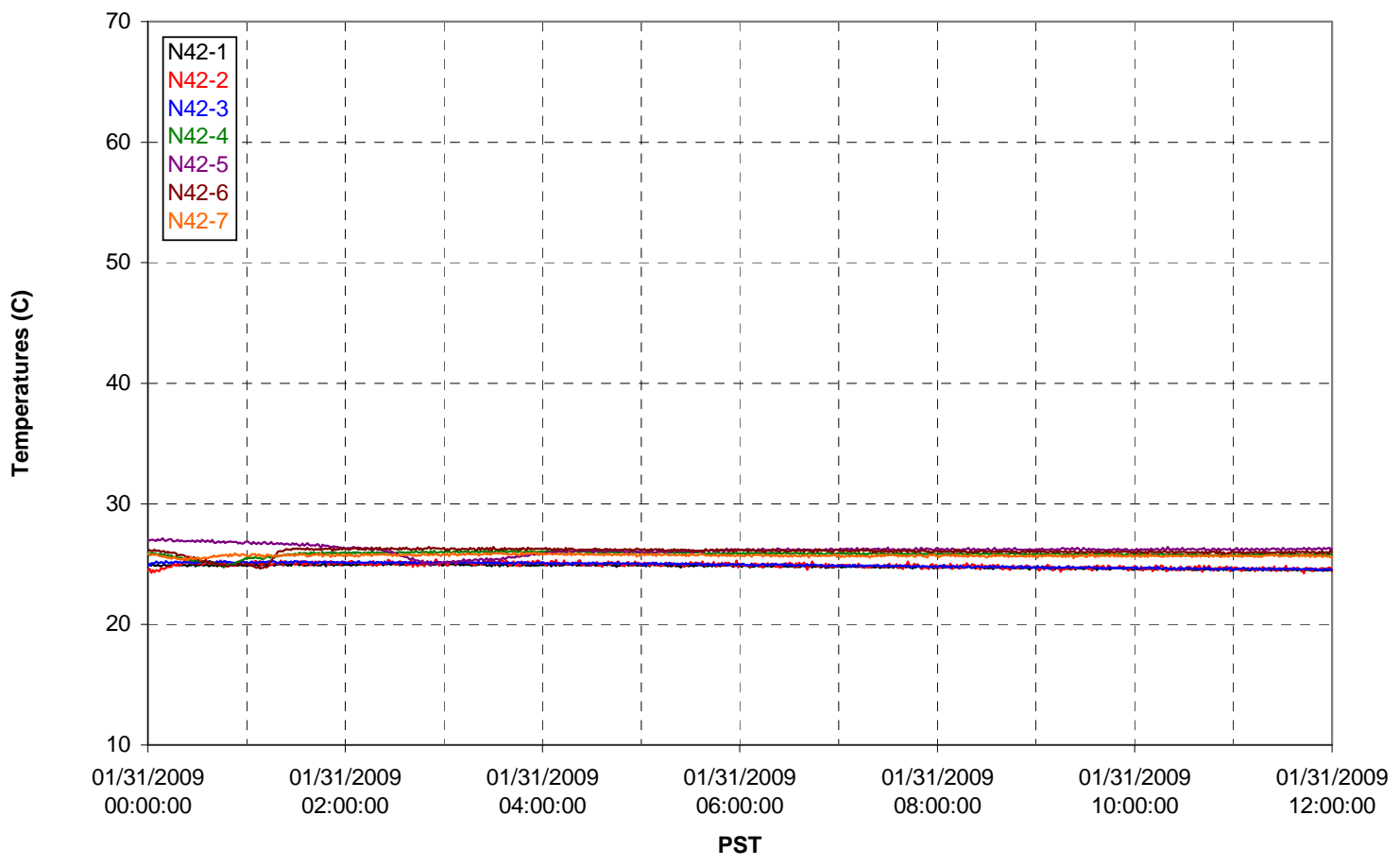

G.8 
T02A temperatures

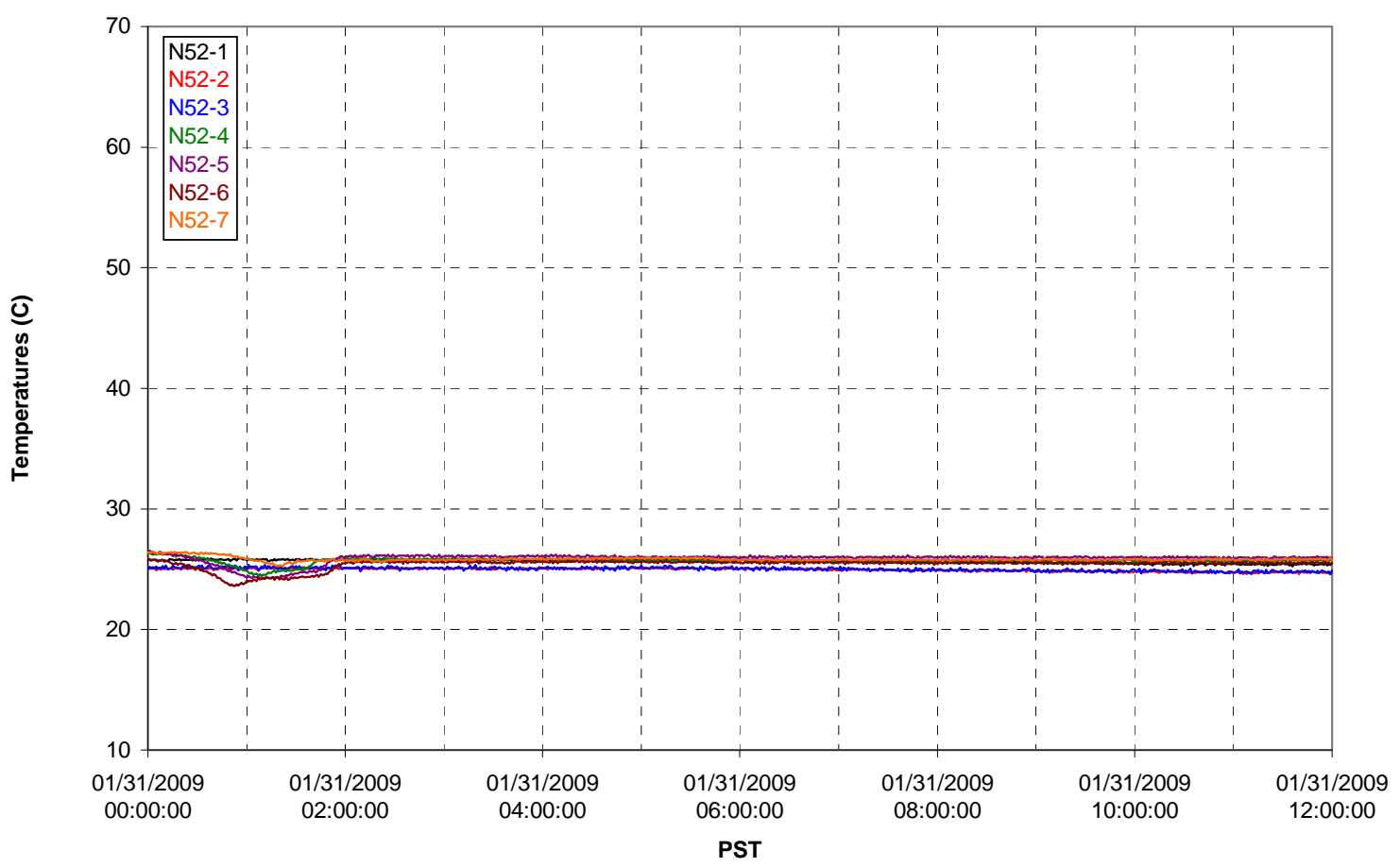

T02A Heating and Cooling

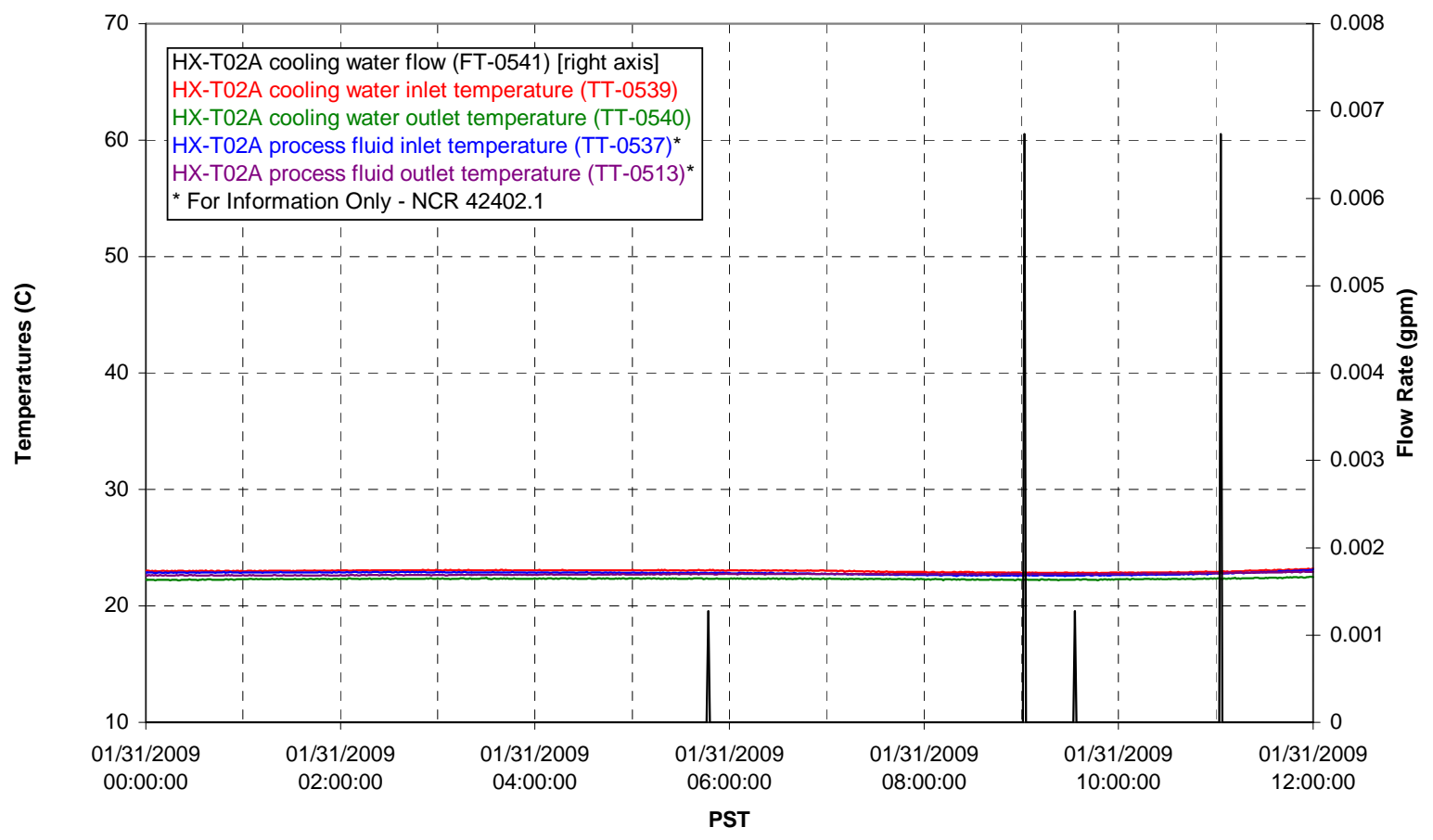

G.9 
Pump Operation

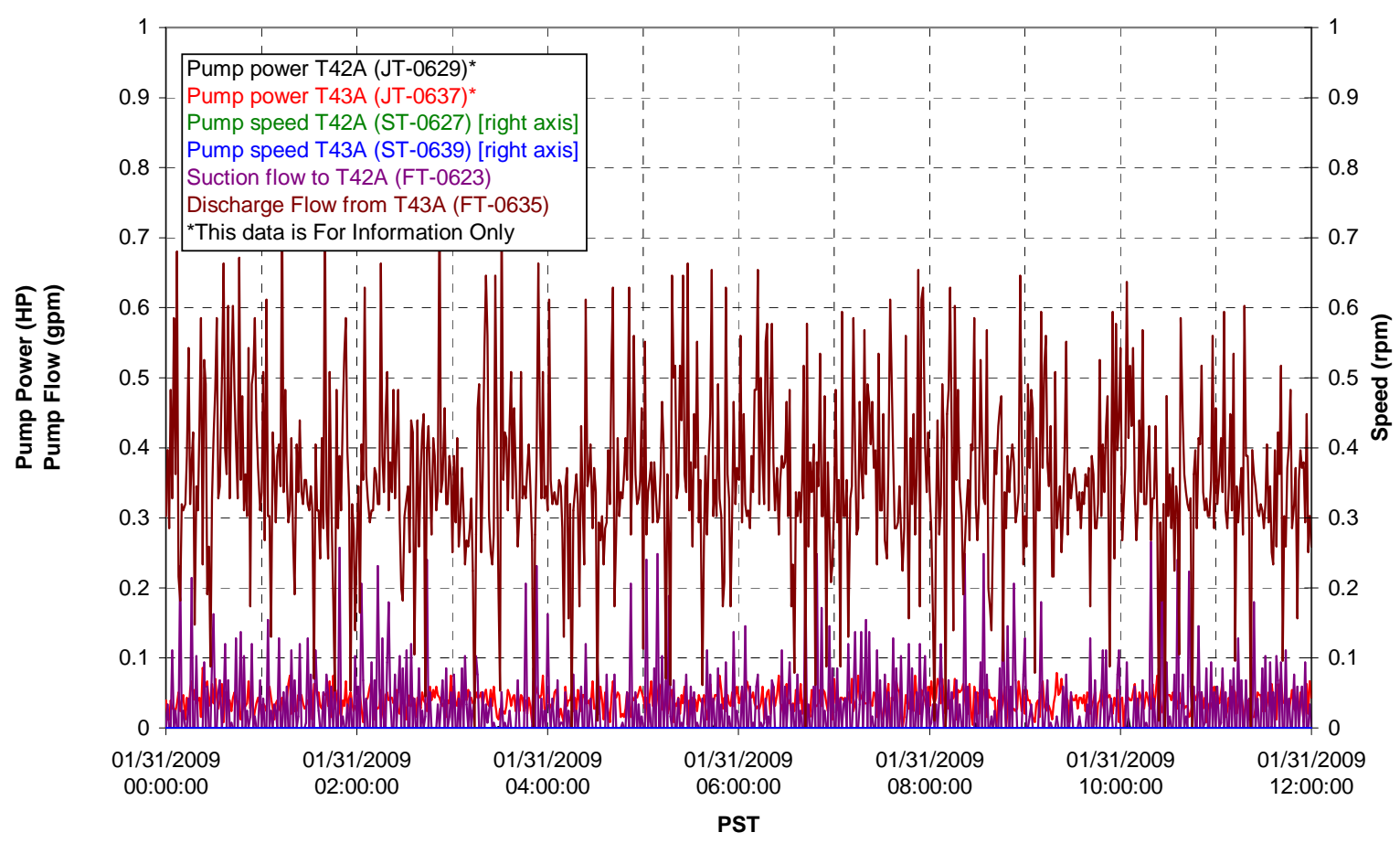

Pulsepot UFP-PP-T01A

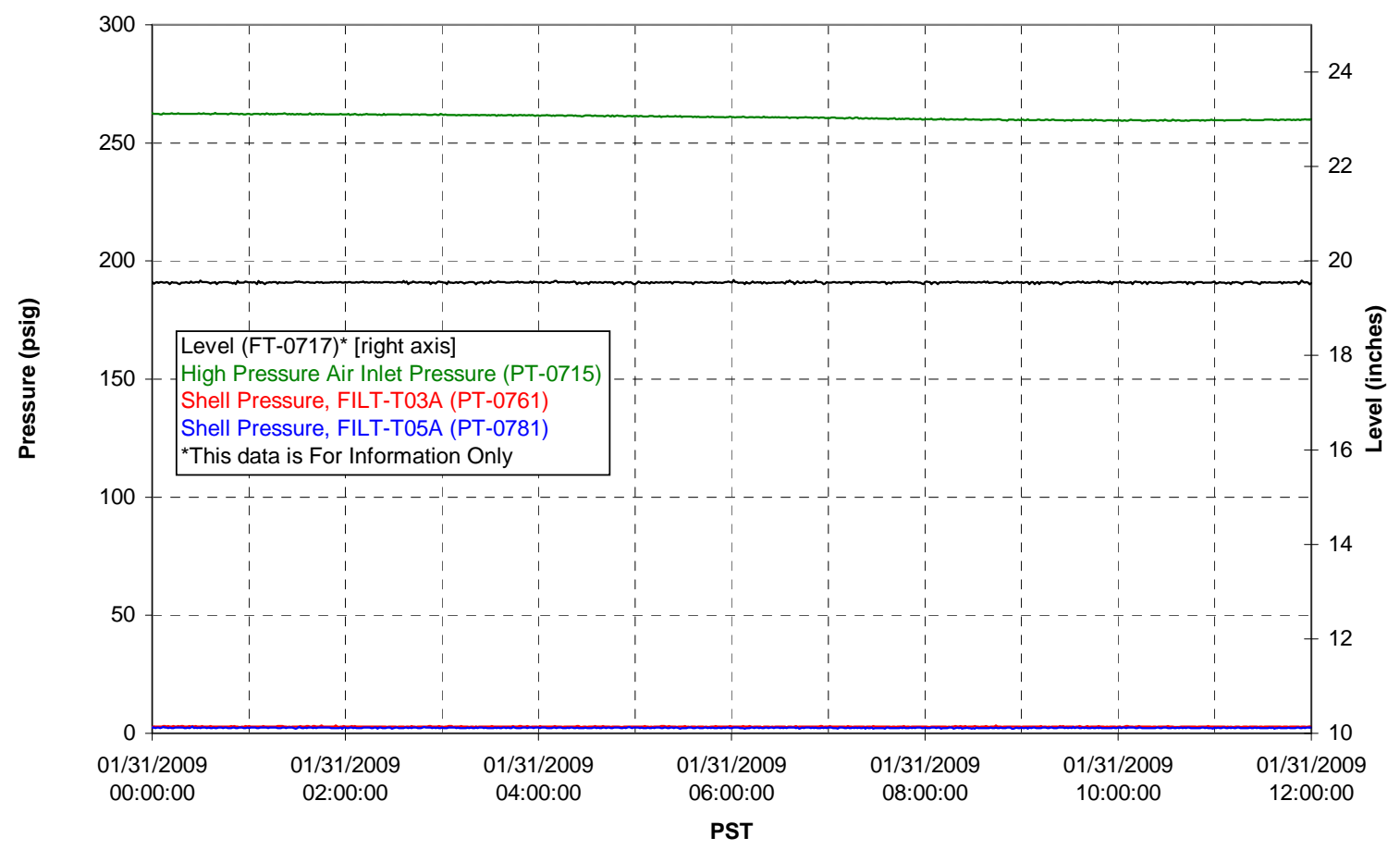


Pulsepot UFP-PP-T02A

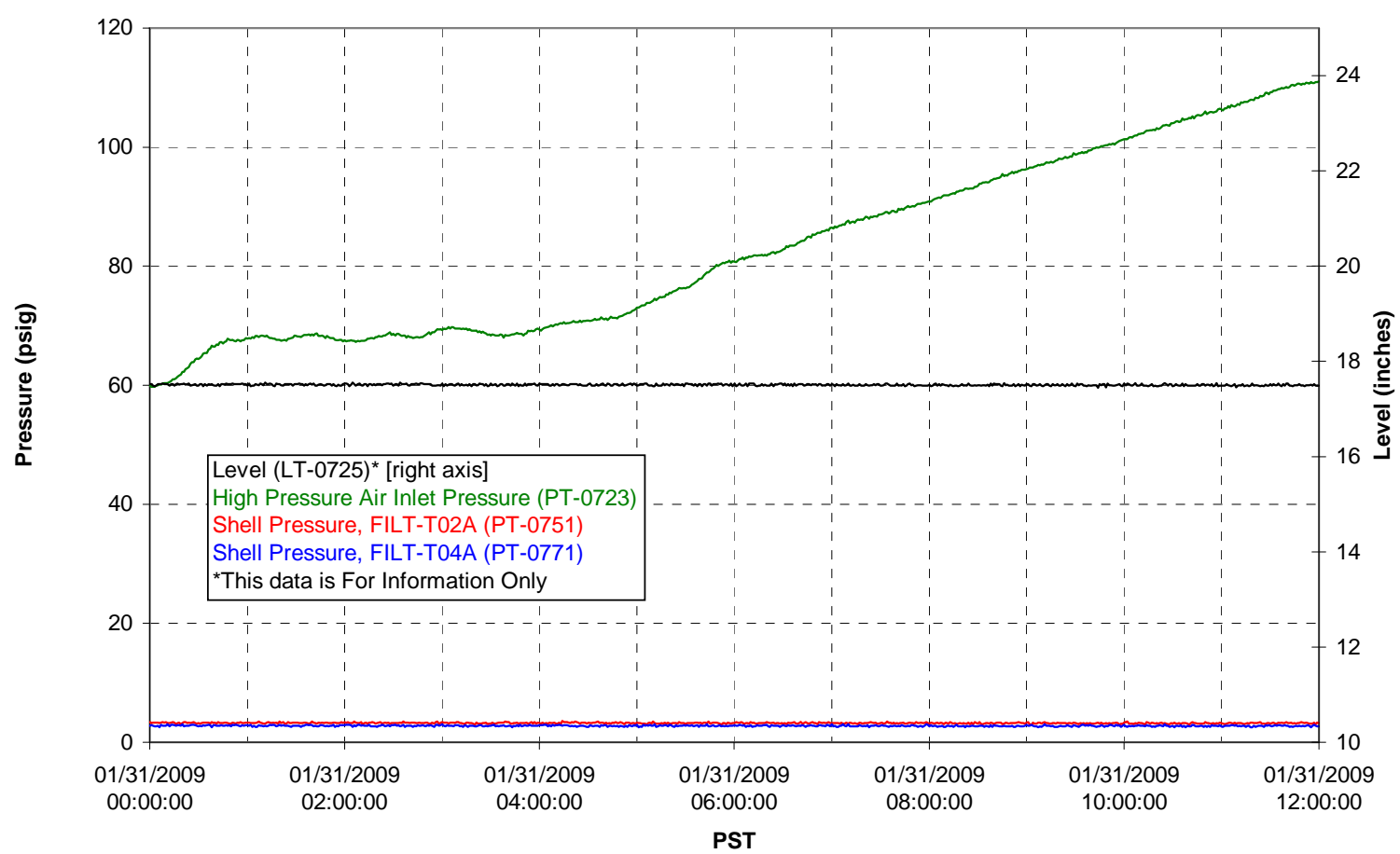

Pulsepot UFP-PP-T03A

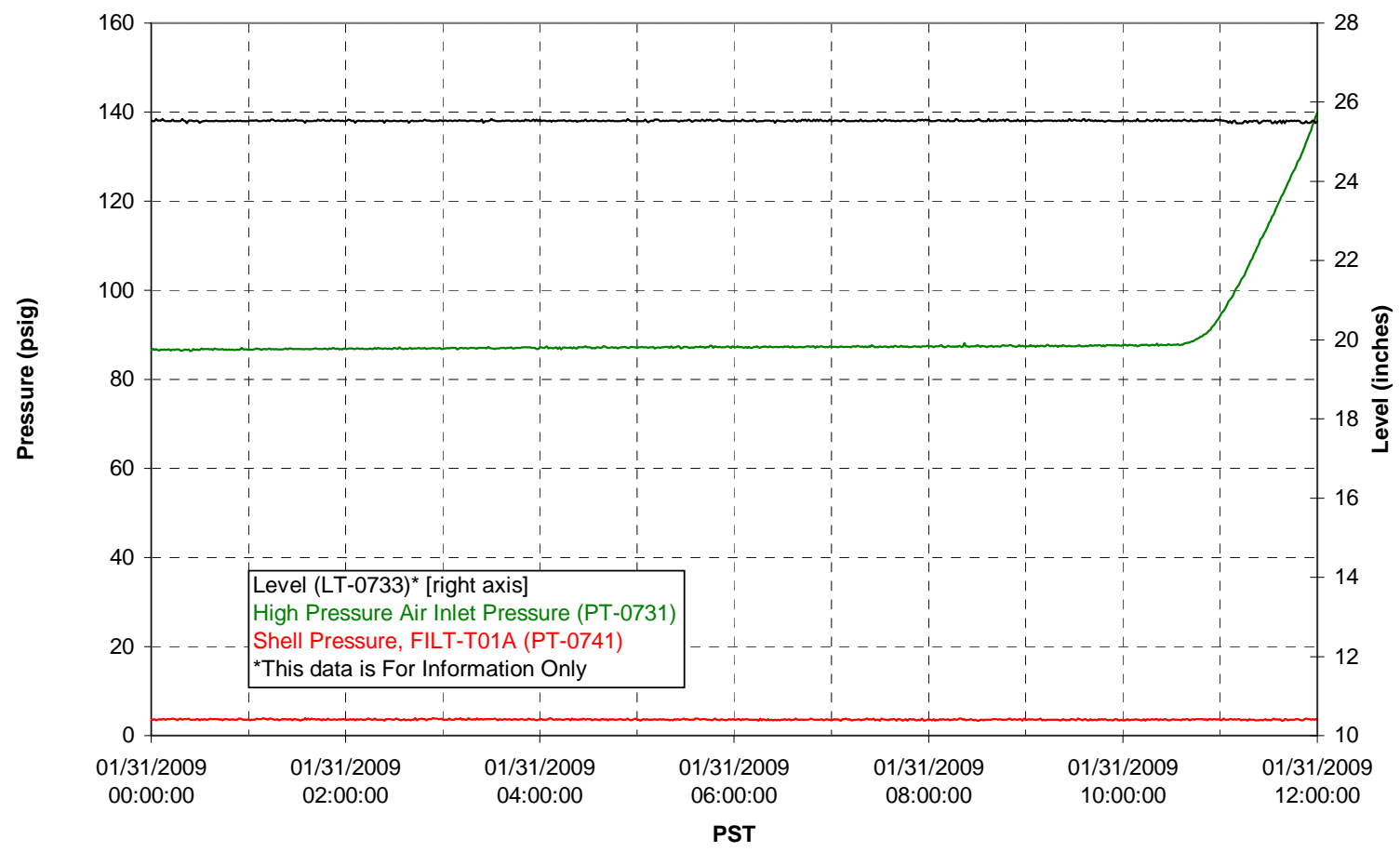

G.11 
Pulsepot Levels

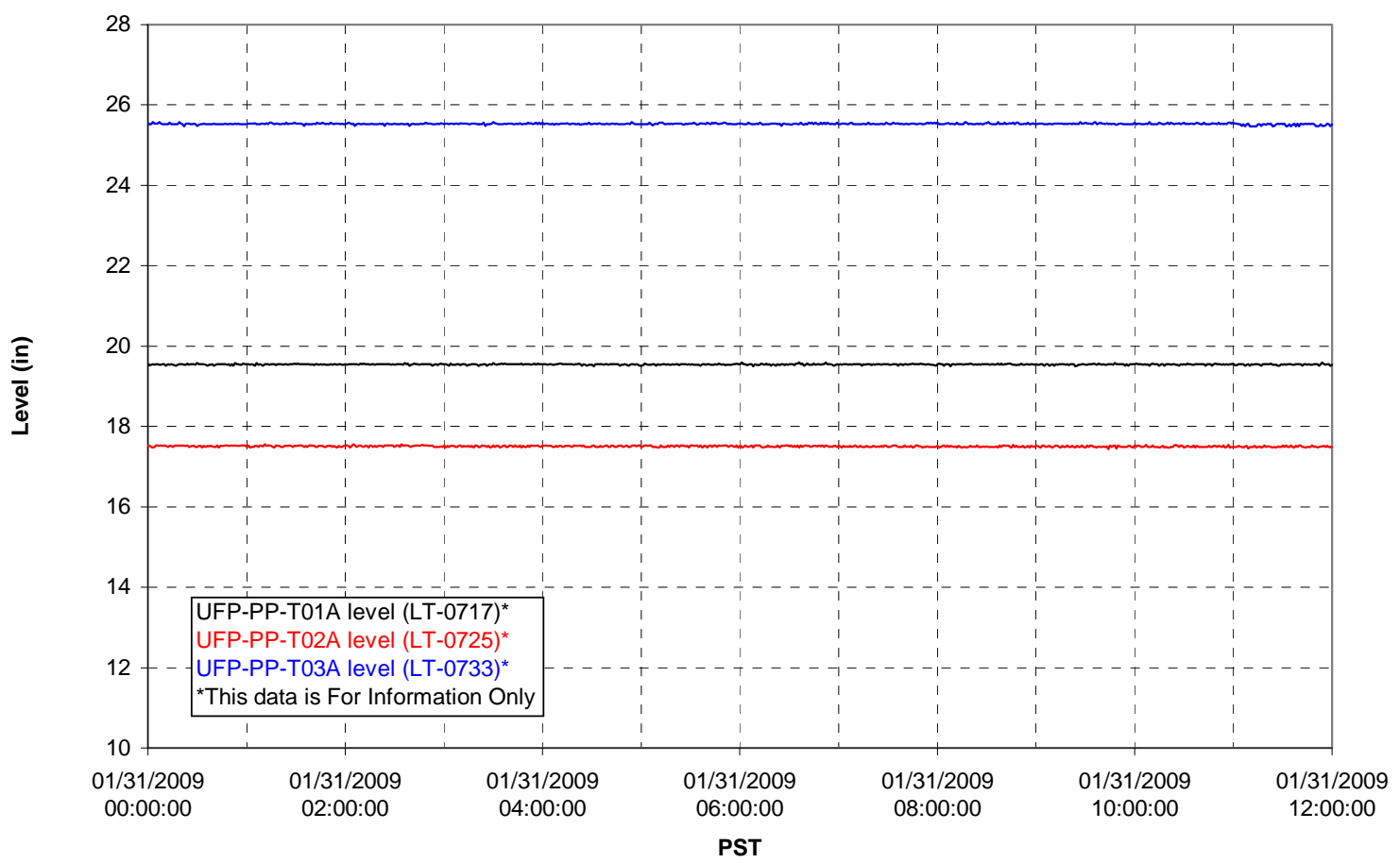

Filter UFP-FILT-T01A

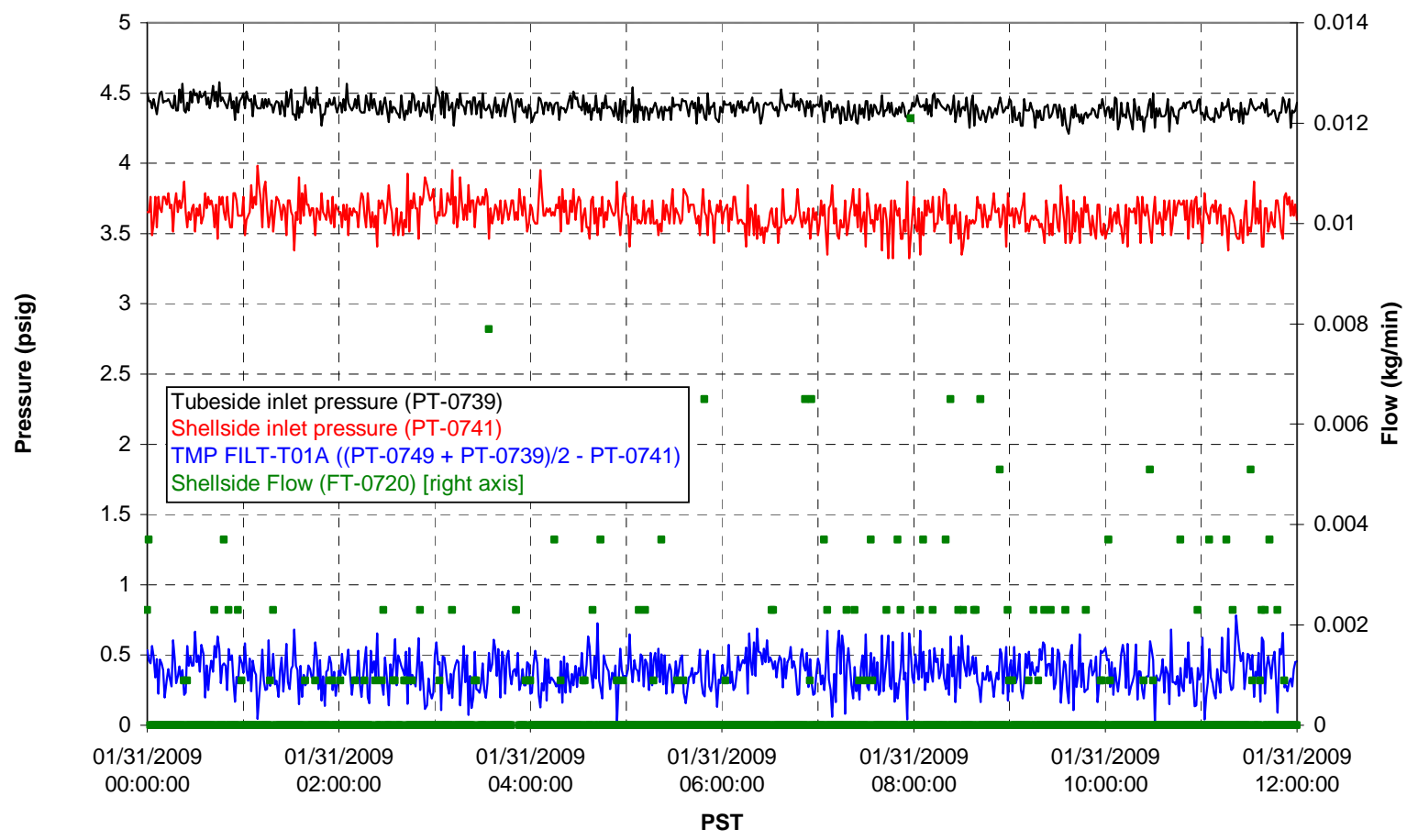


Filter UFP-FILT-T02A

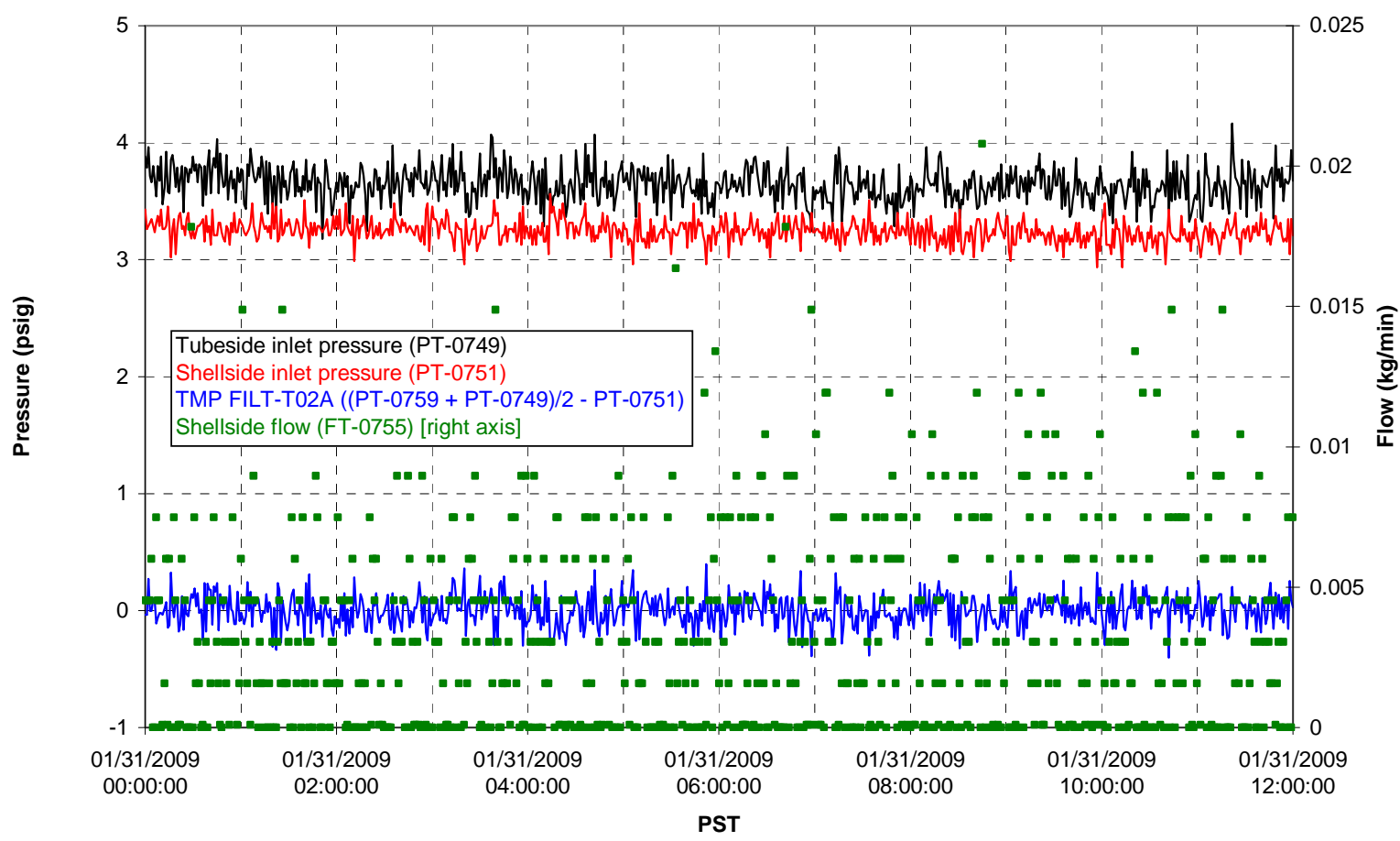

Filter UFP-FILT-T03A

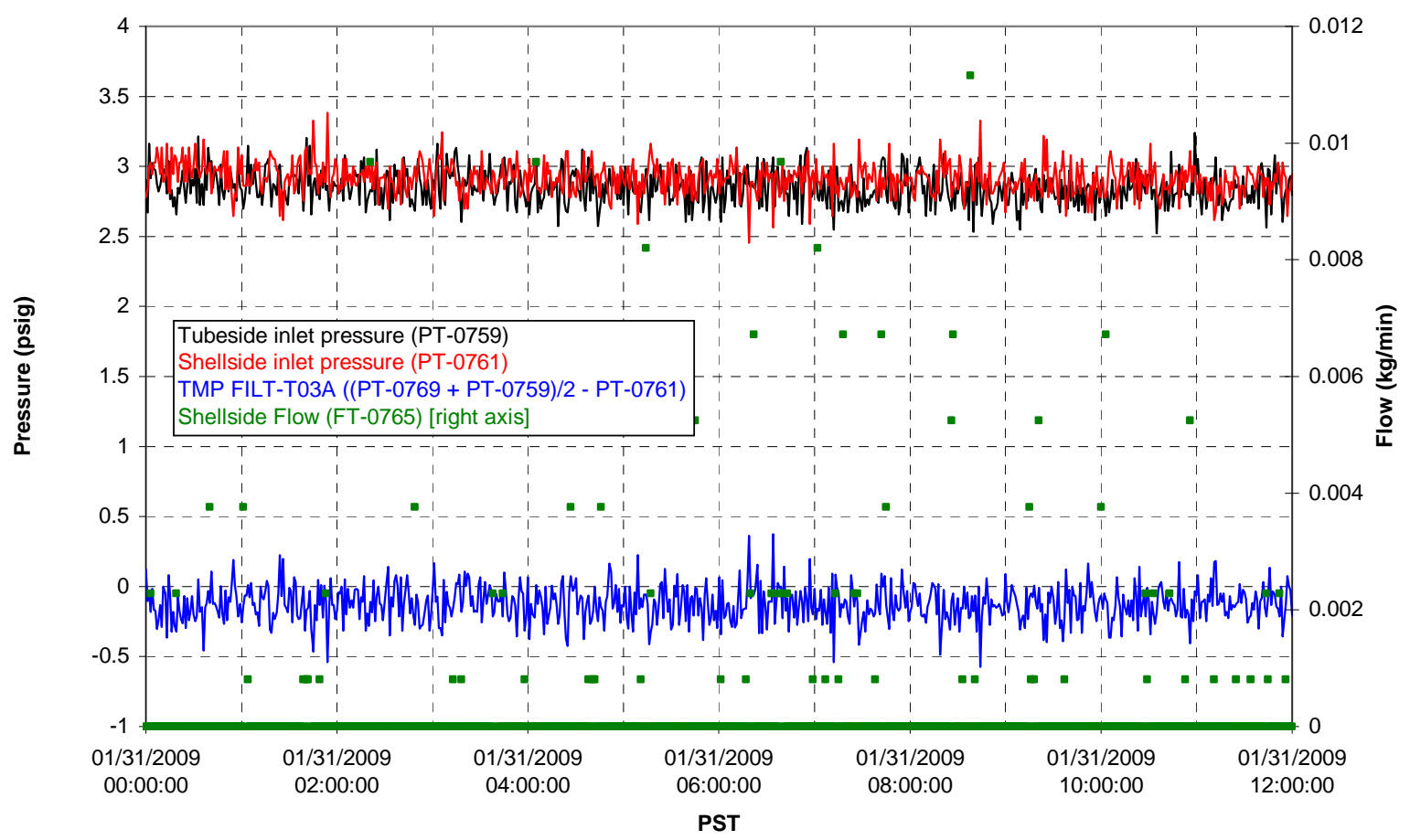


Filter UFP-FILT-T04A

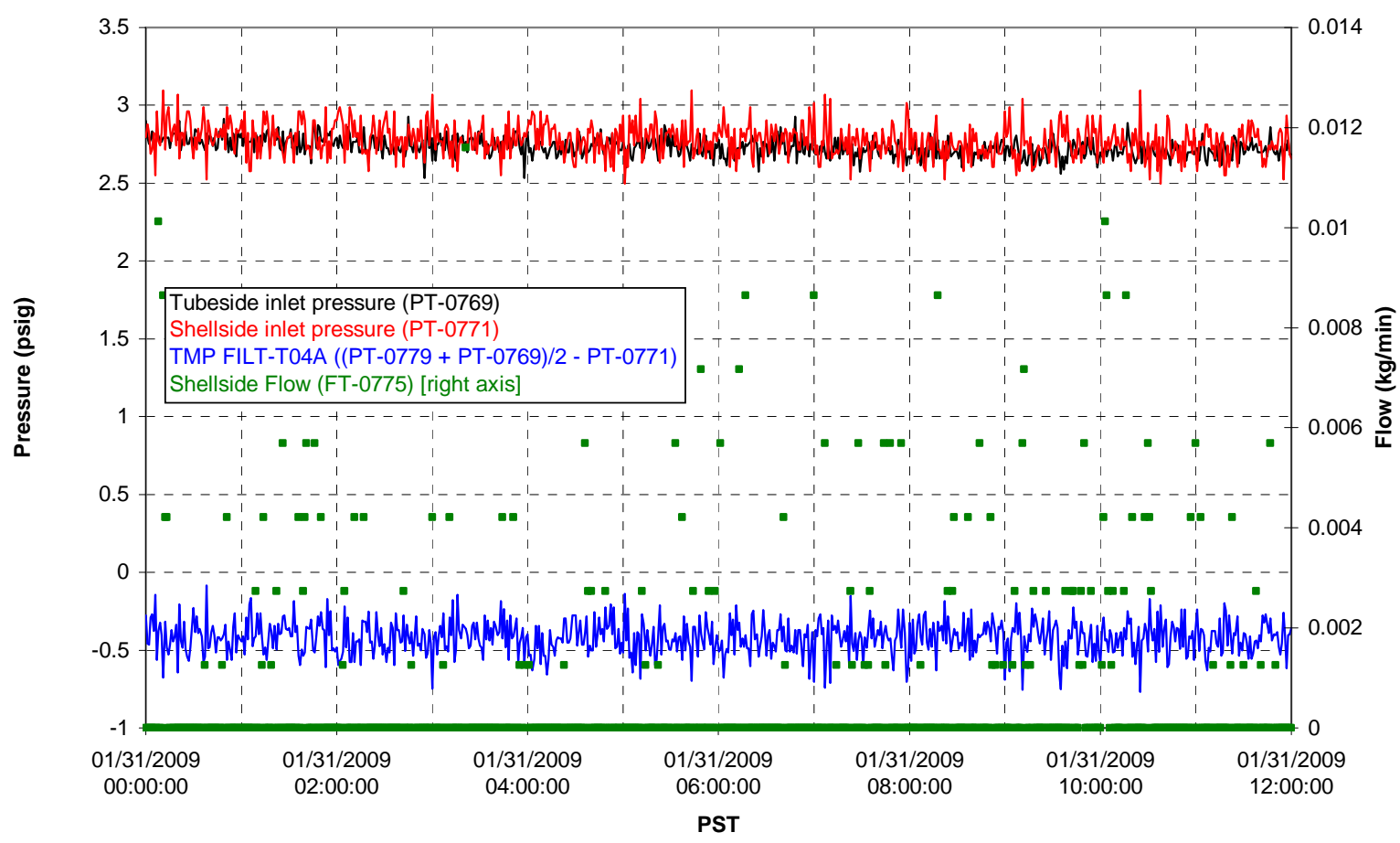

Filter UFP-FILT-T05A

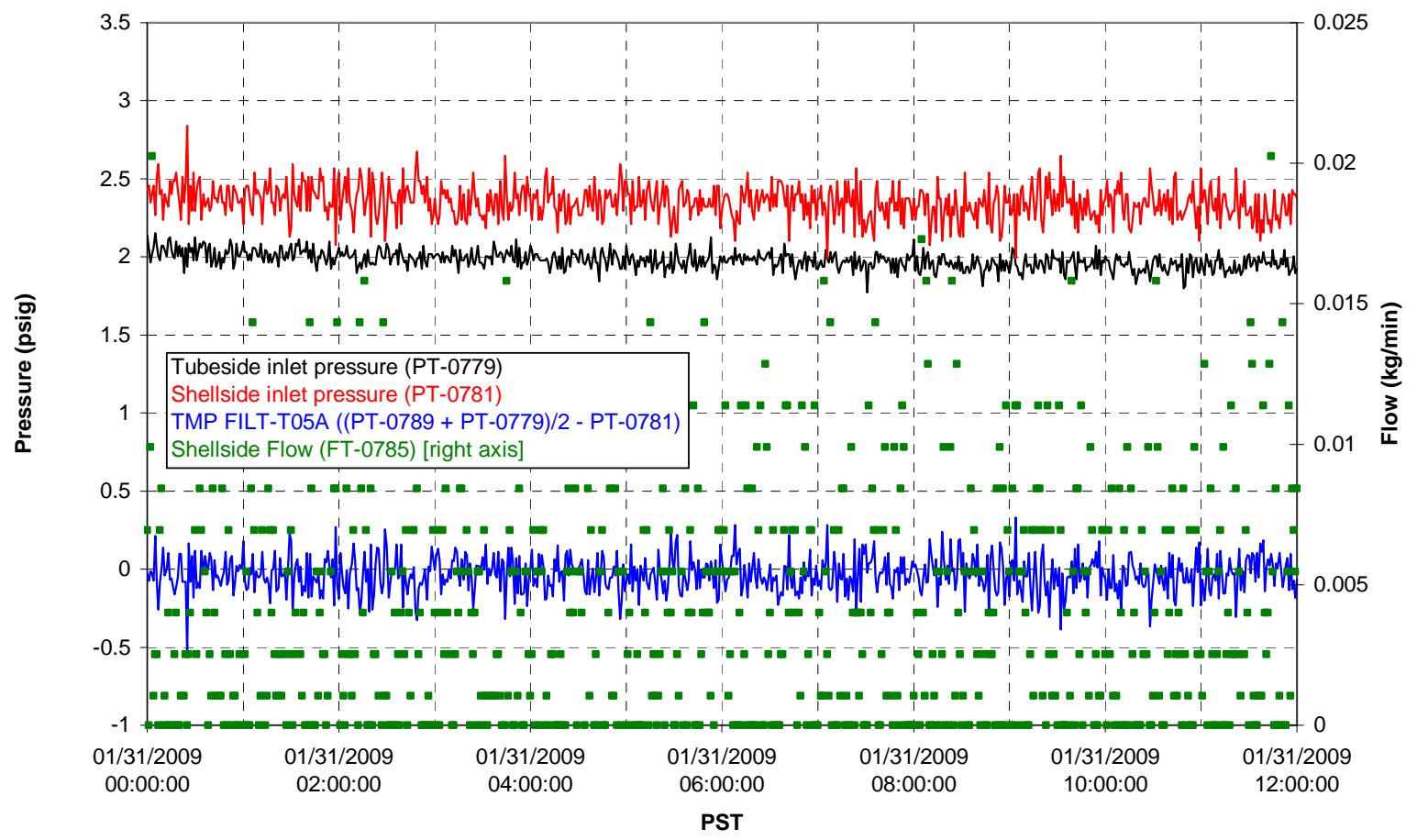



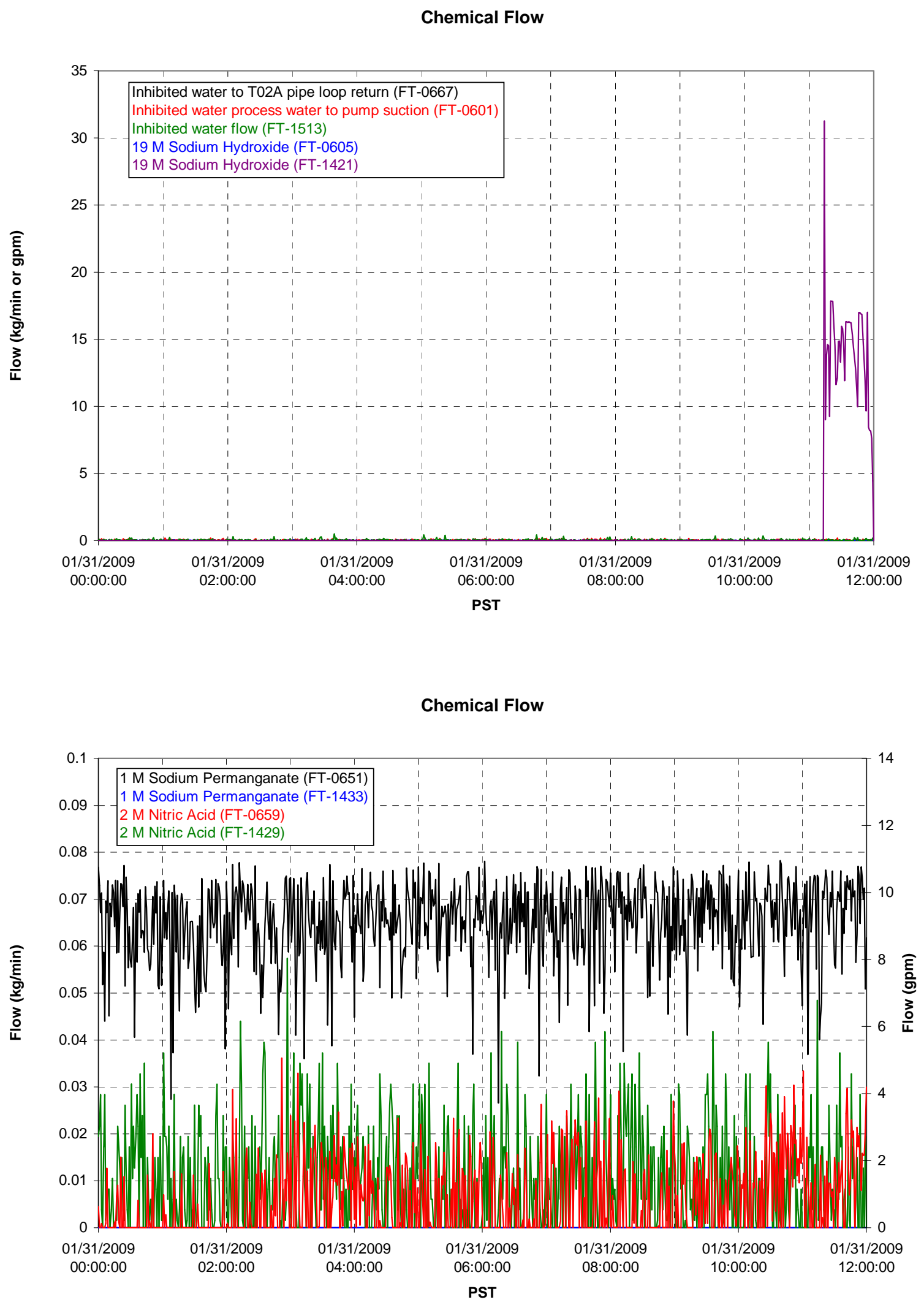
Air Flows

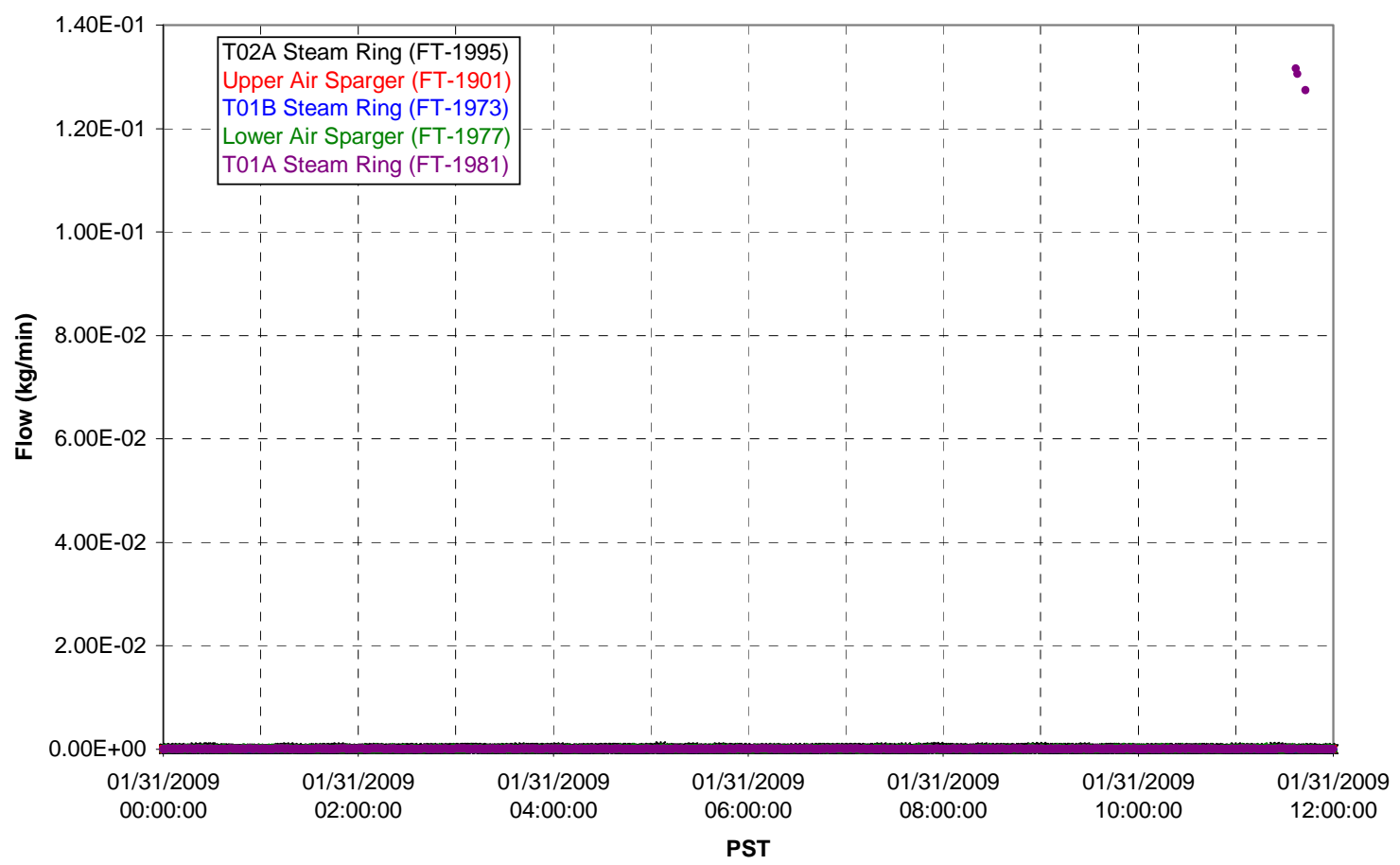

T02A Steam

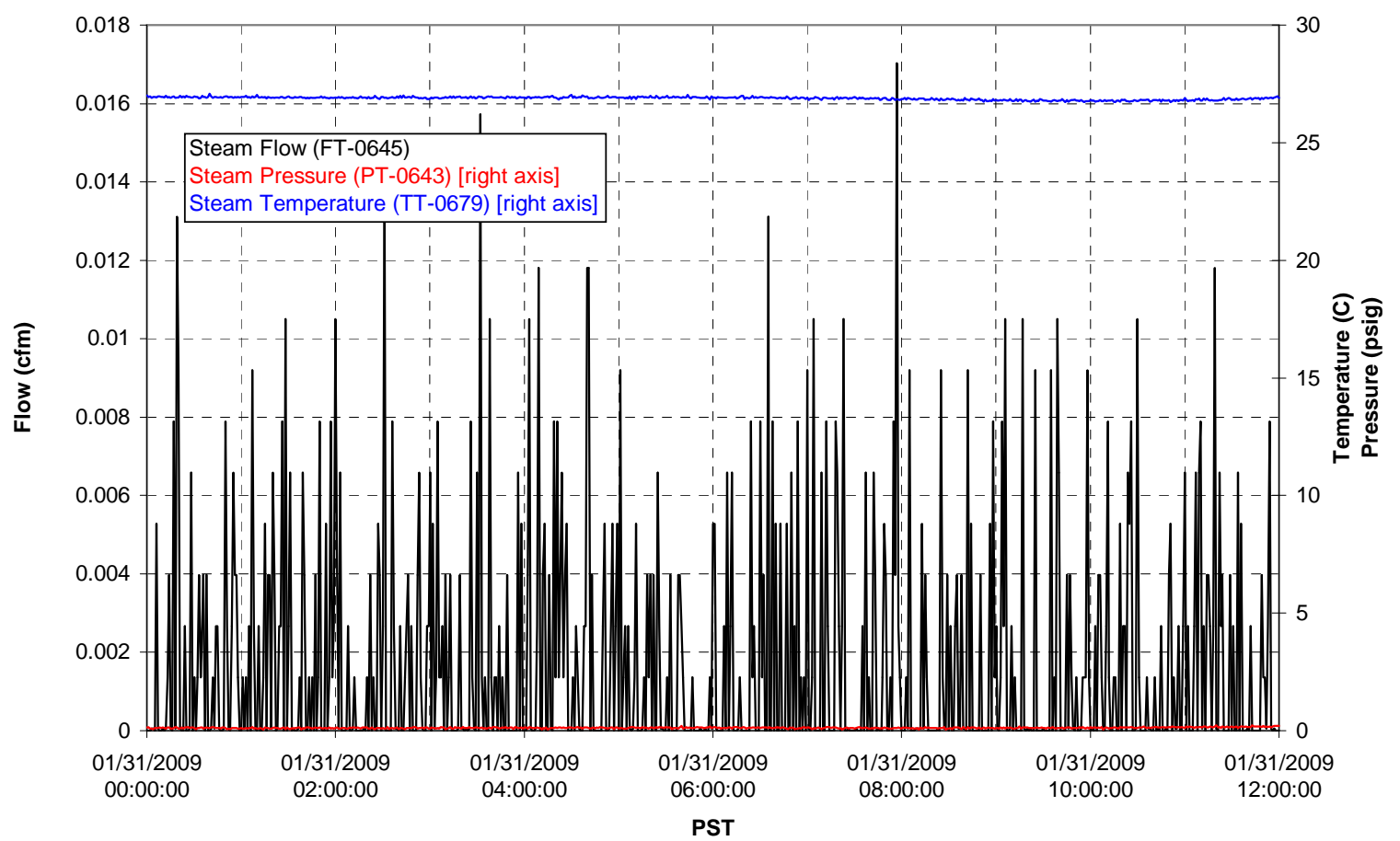


T01A Steam

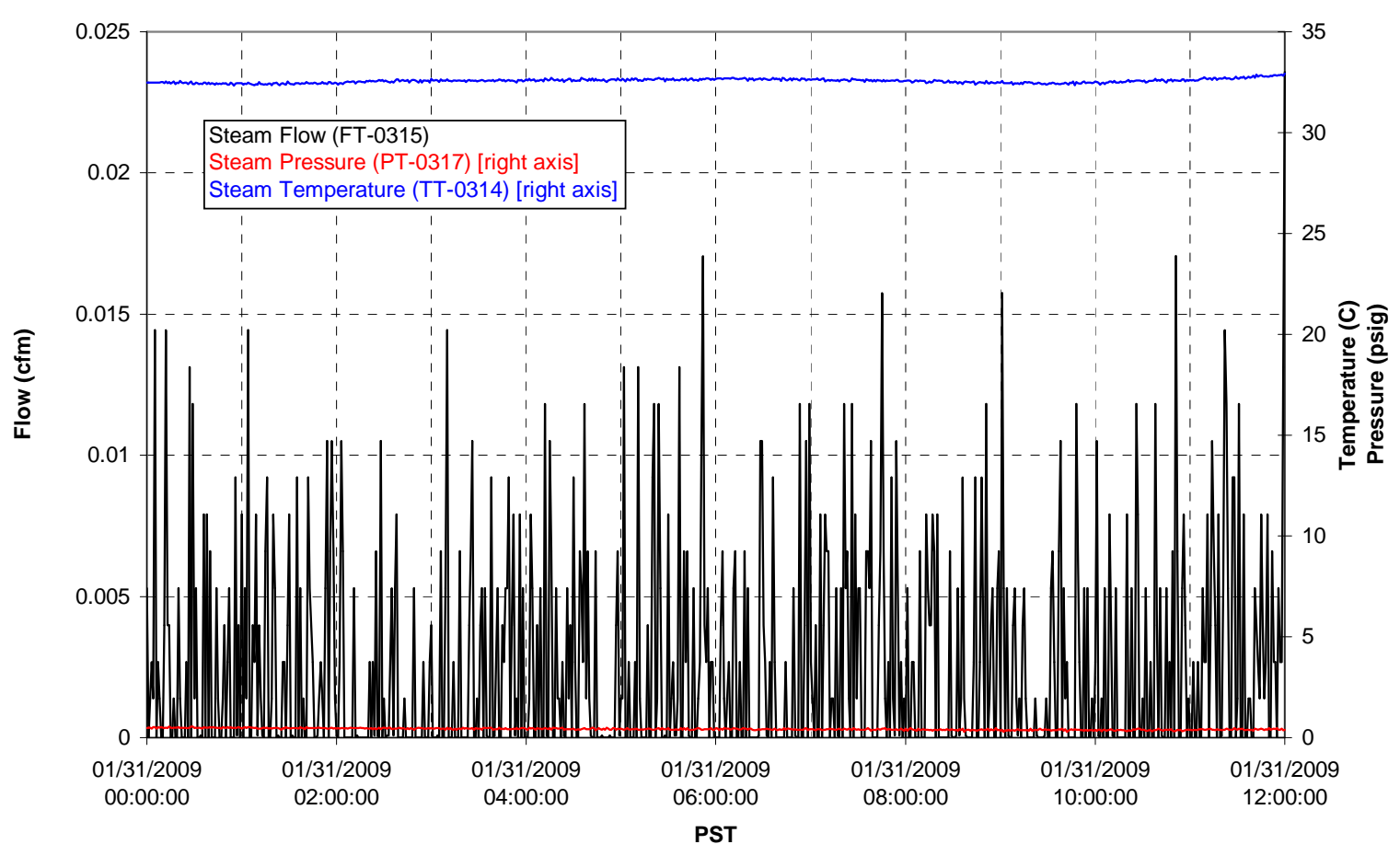

T01B Steam

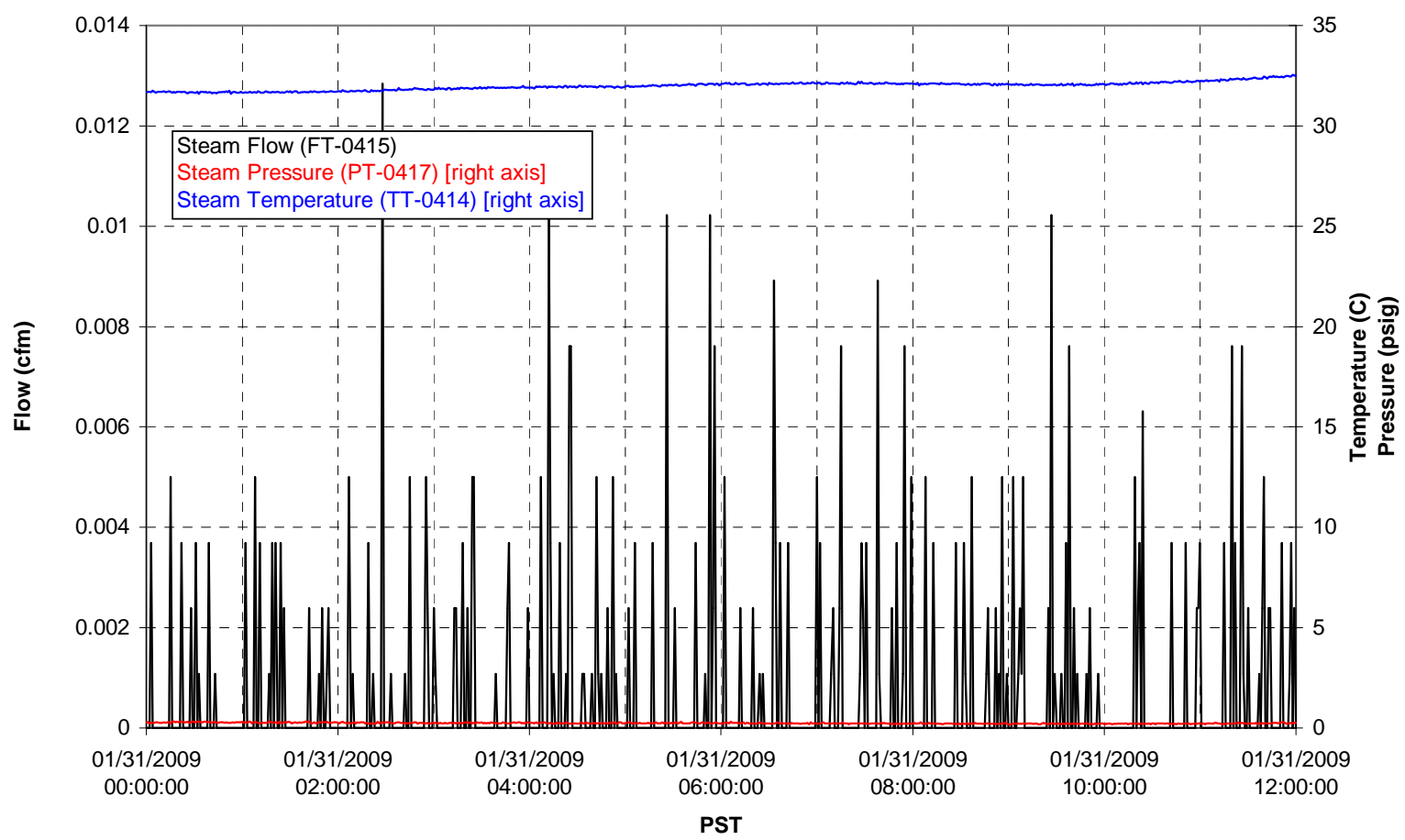




$$
\text { G.18 }
$$




\section{Integrated Test A Data Plots 01/31/09 12:00 - 02/01/09 00:00}


T01A level

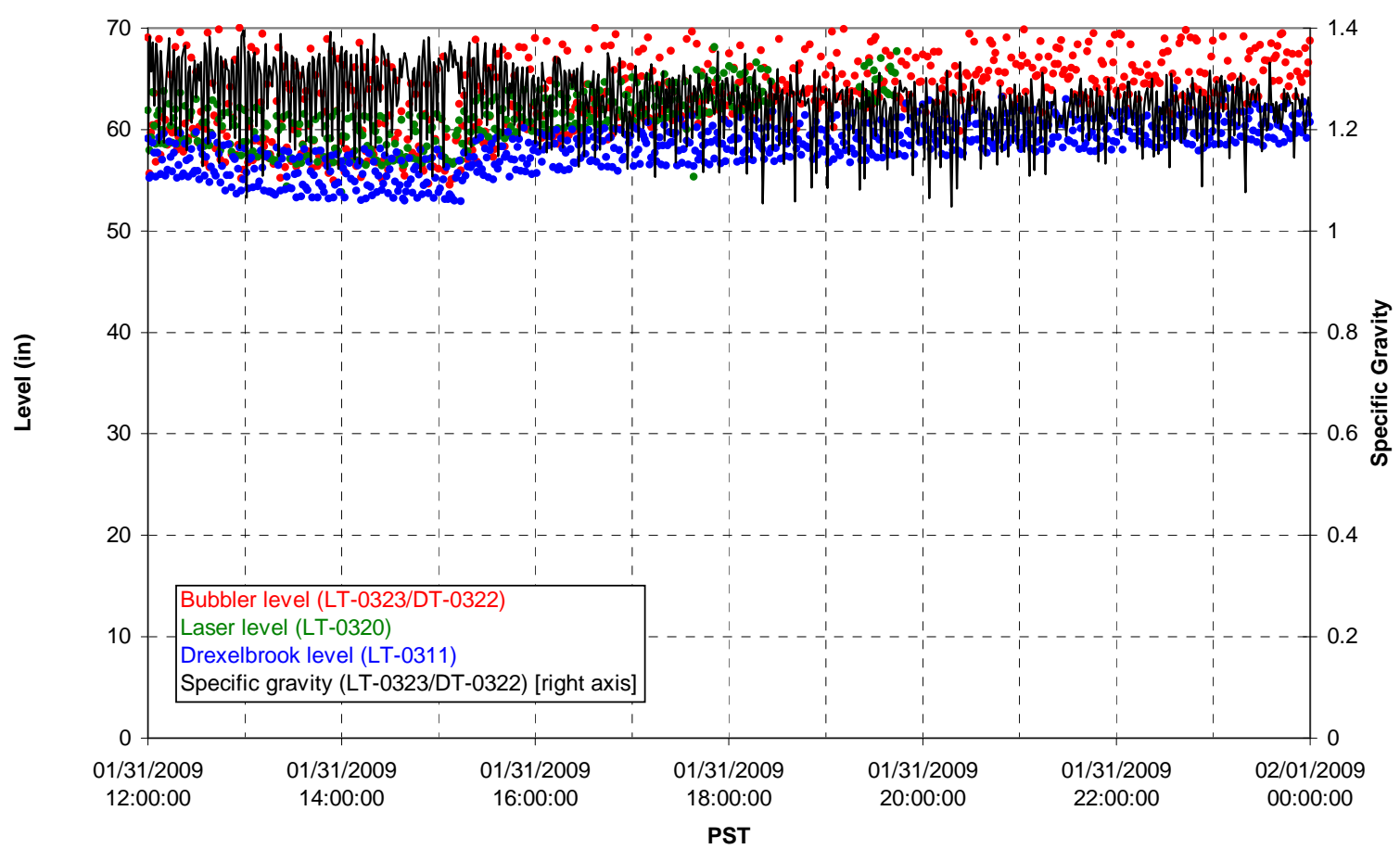

T01A temperatures

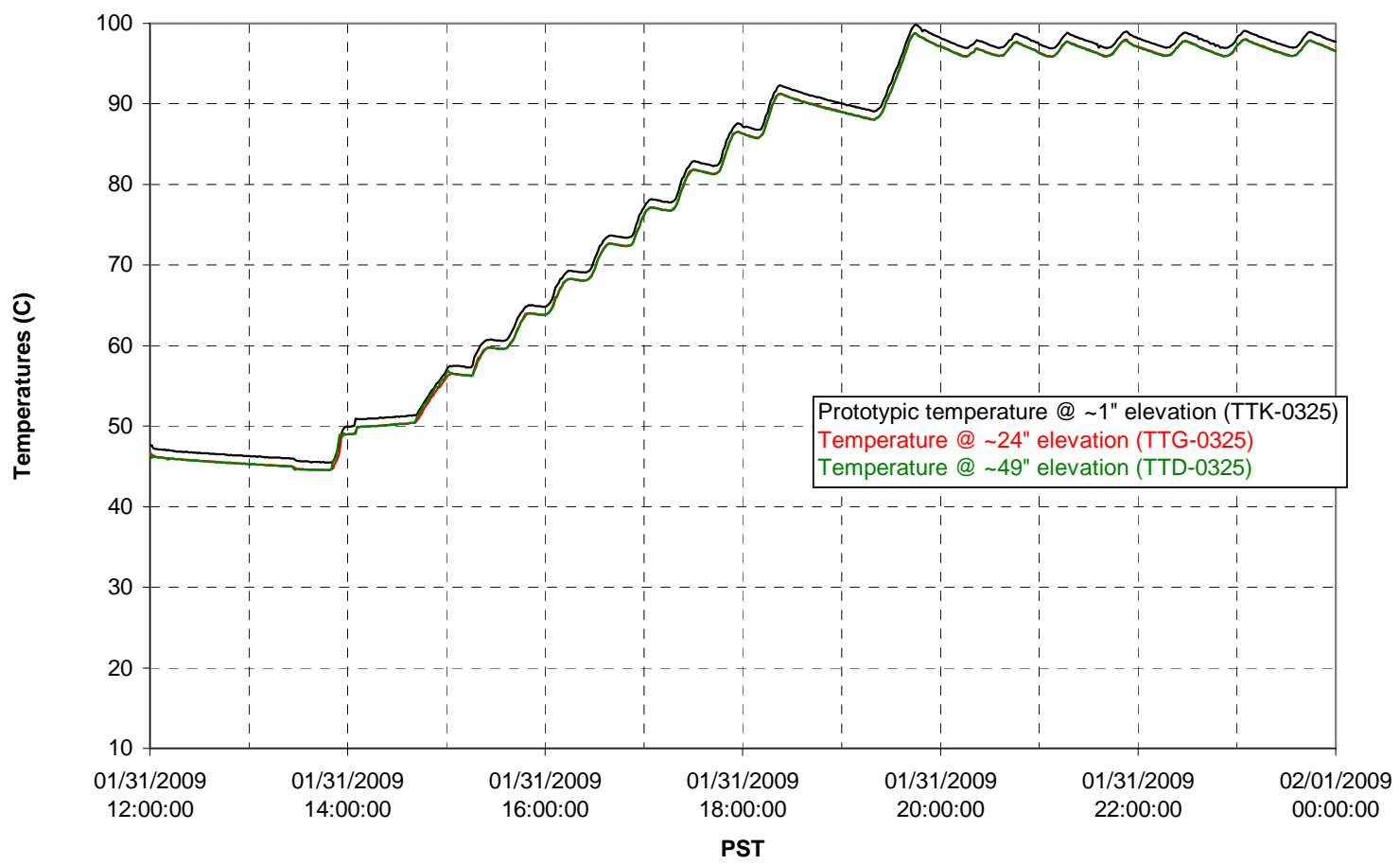


T01B level

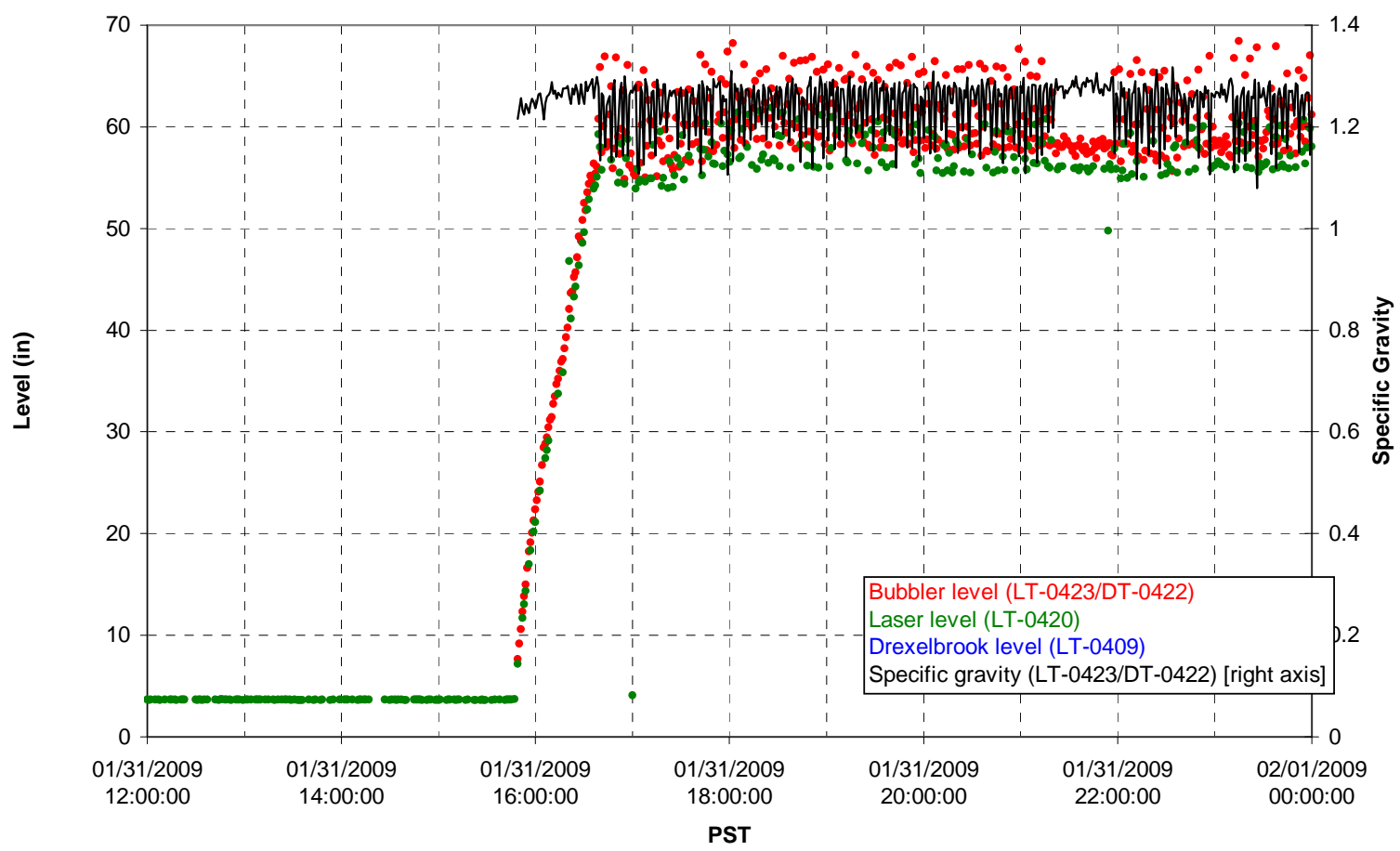

T01B temperatures

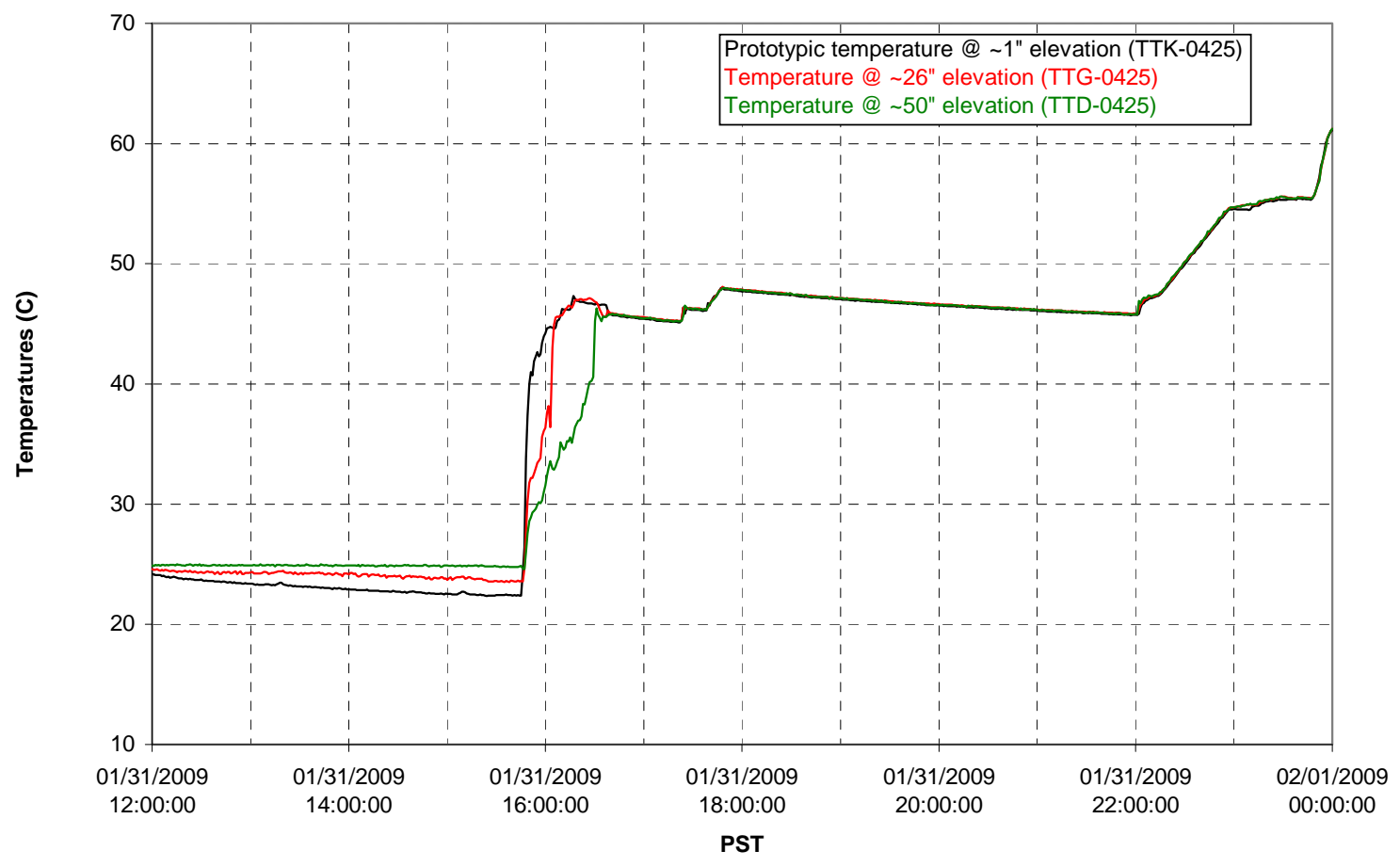


T02A level

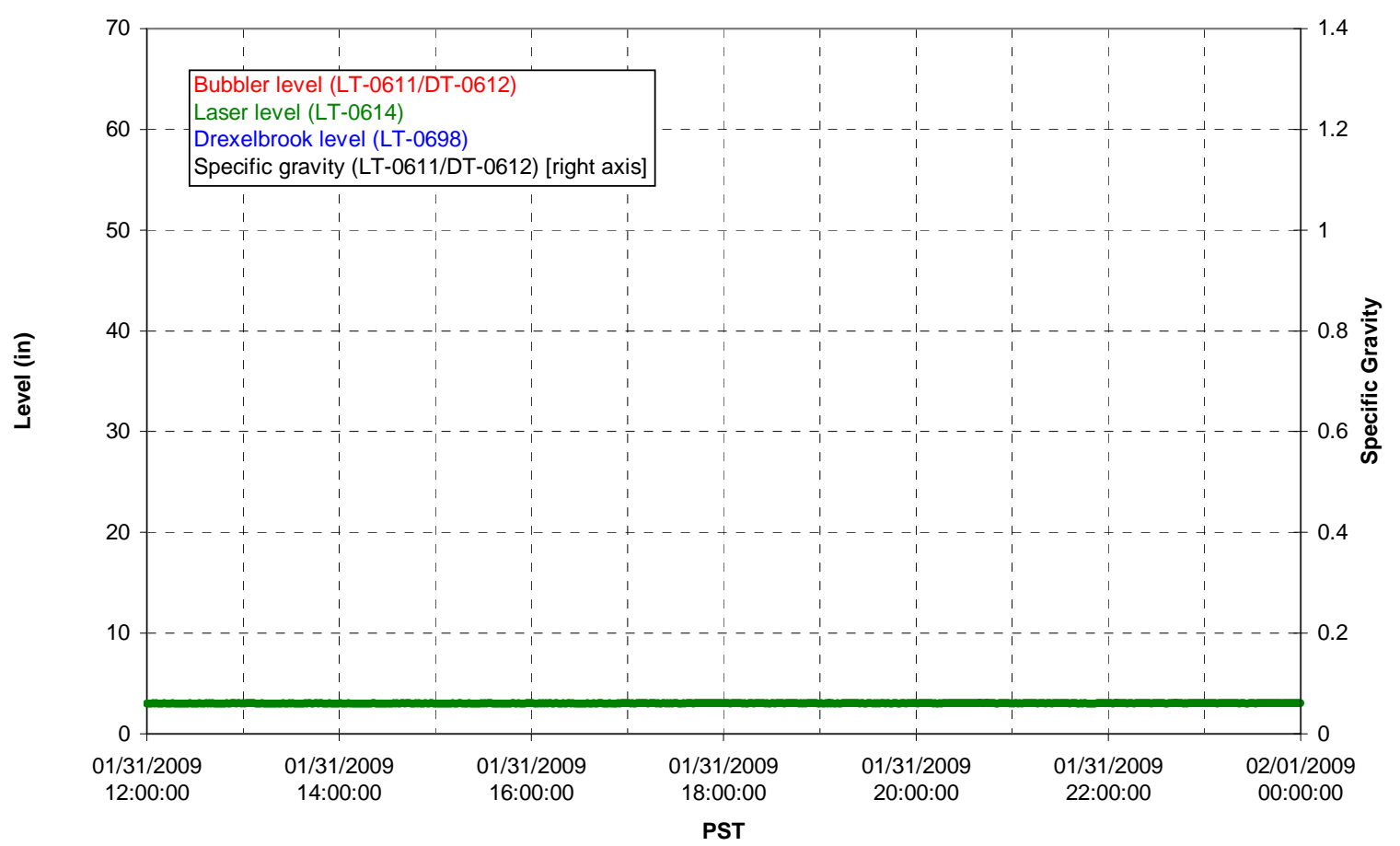

T02A temperatures

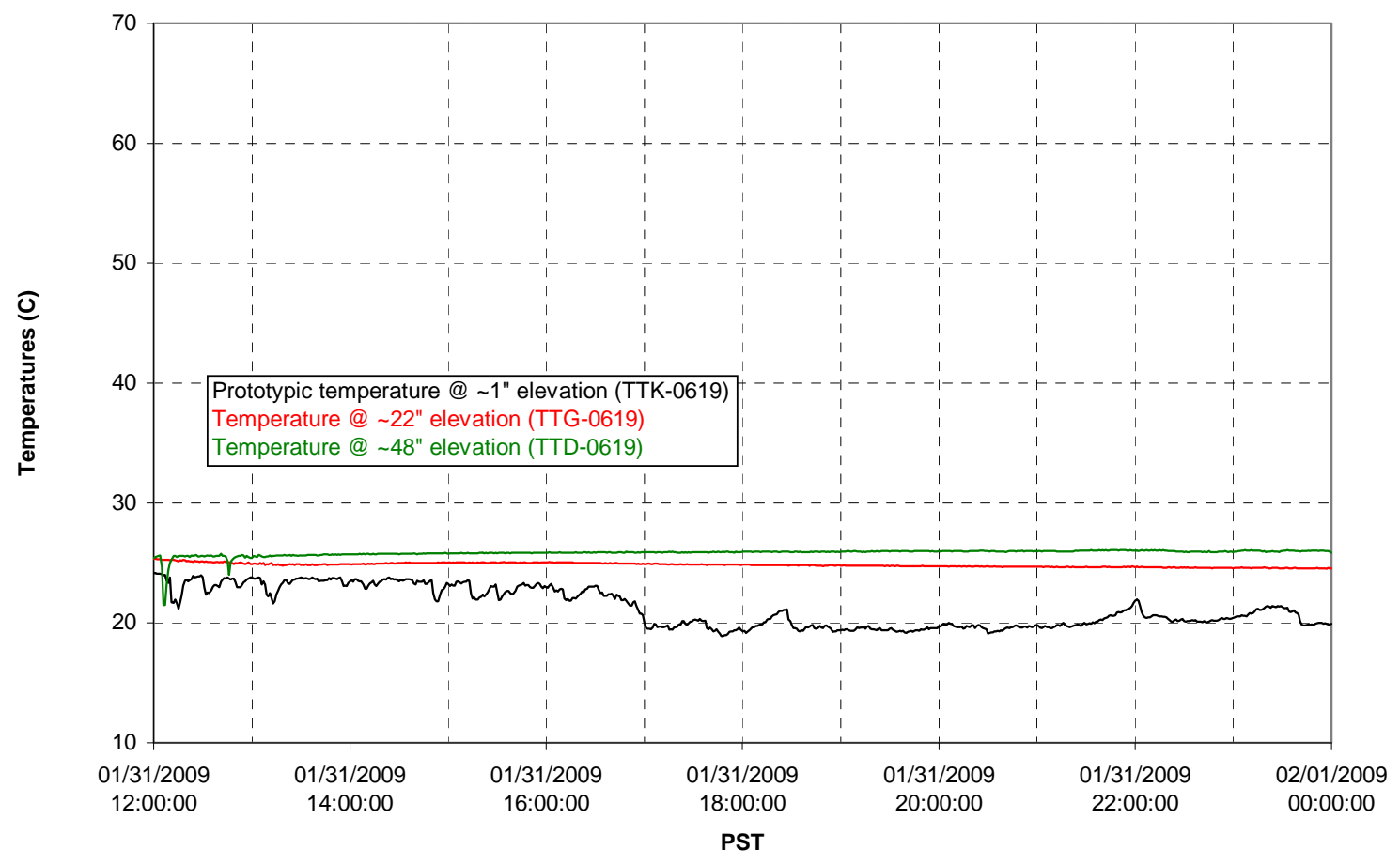


T02A and filter loop temperatures

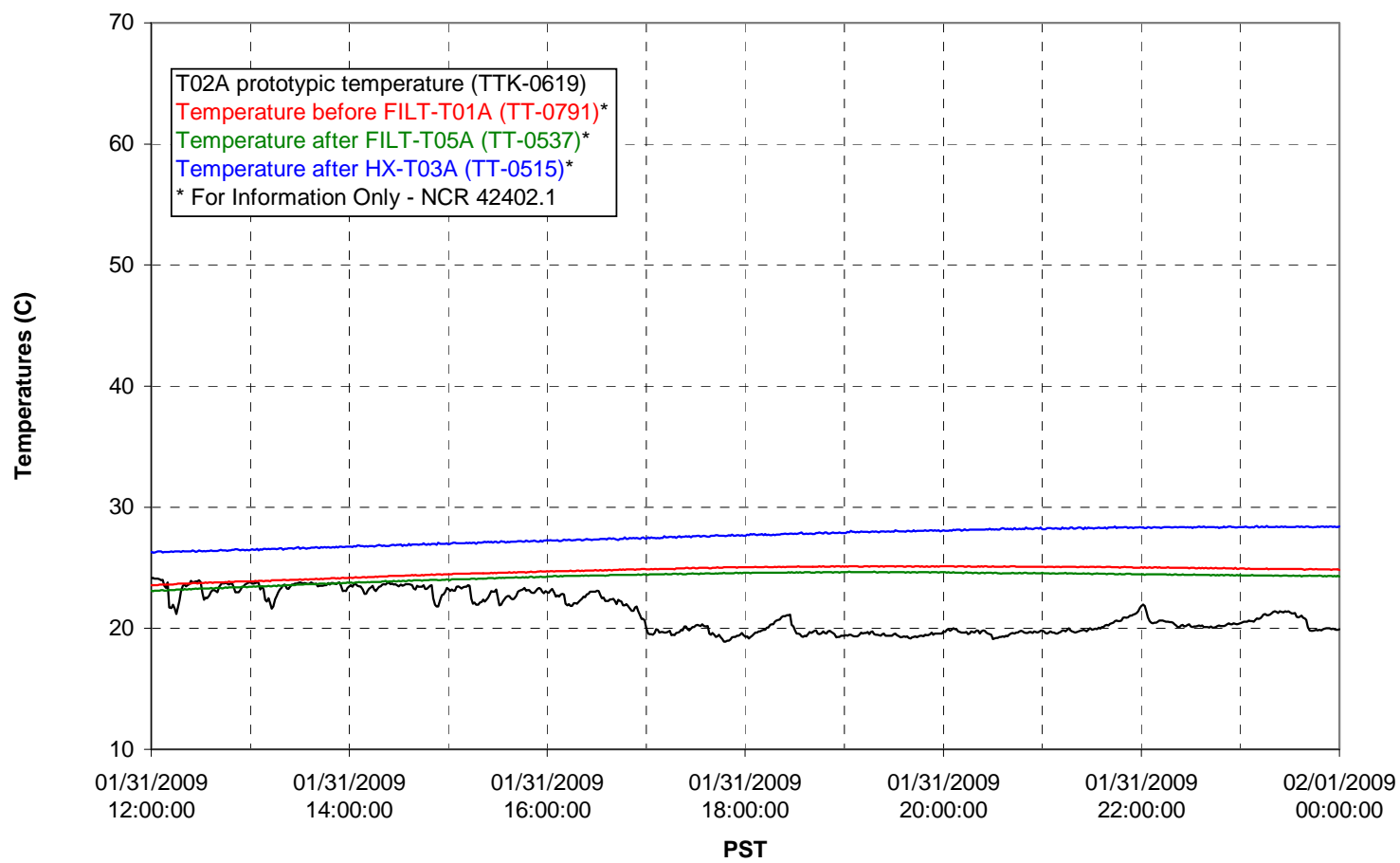

Pump Pressures and Flow

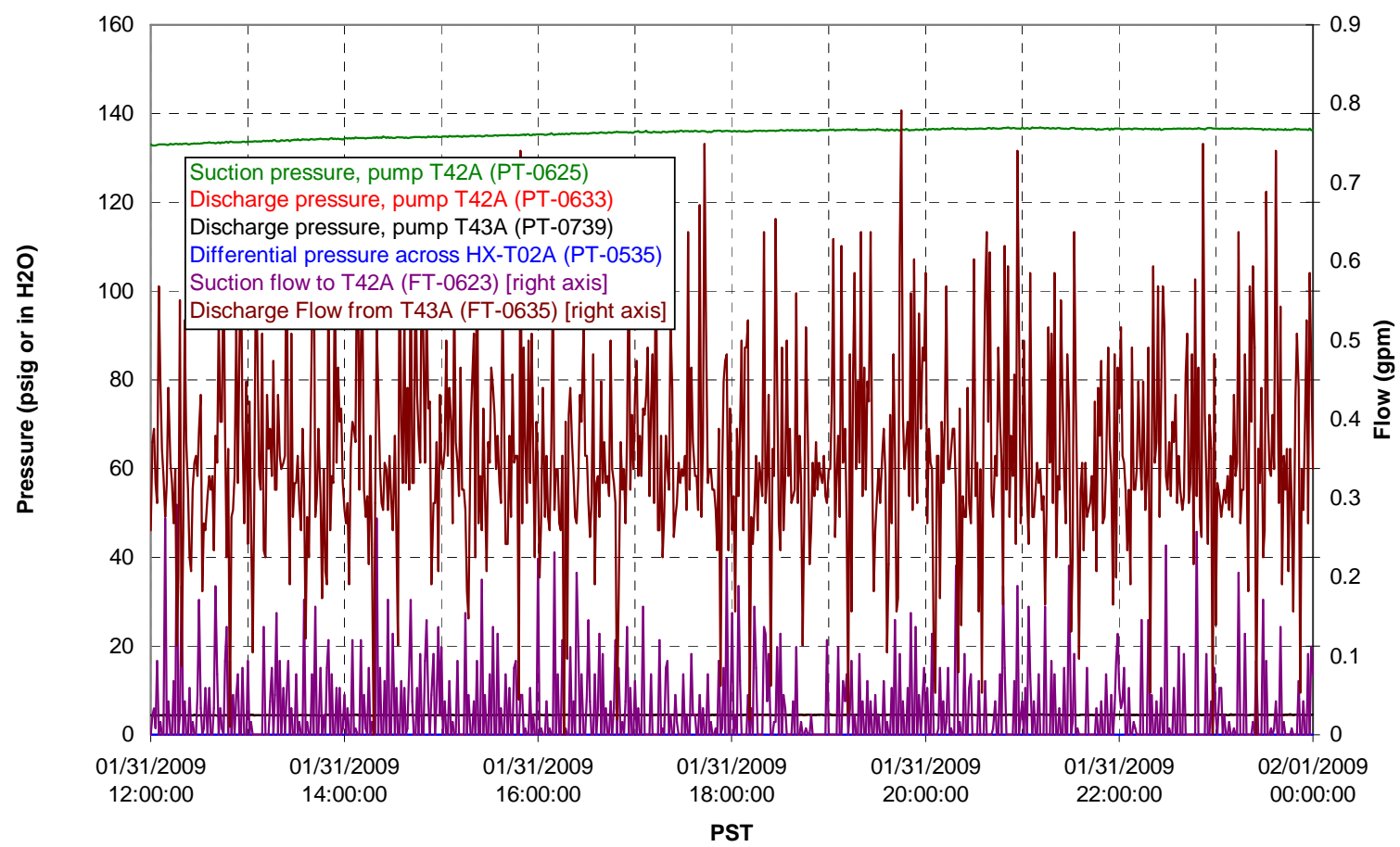


Axial pressure drop

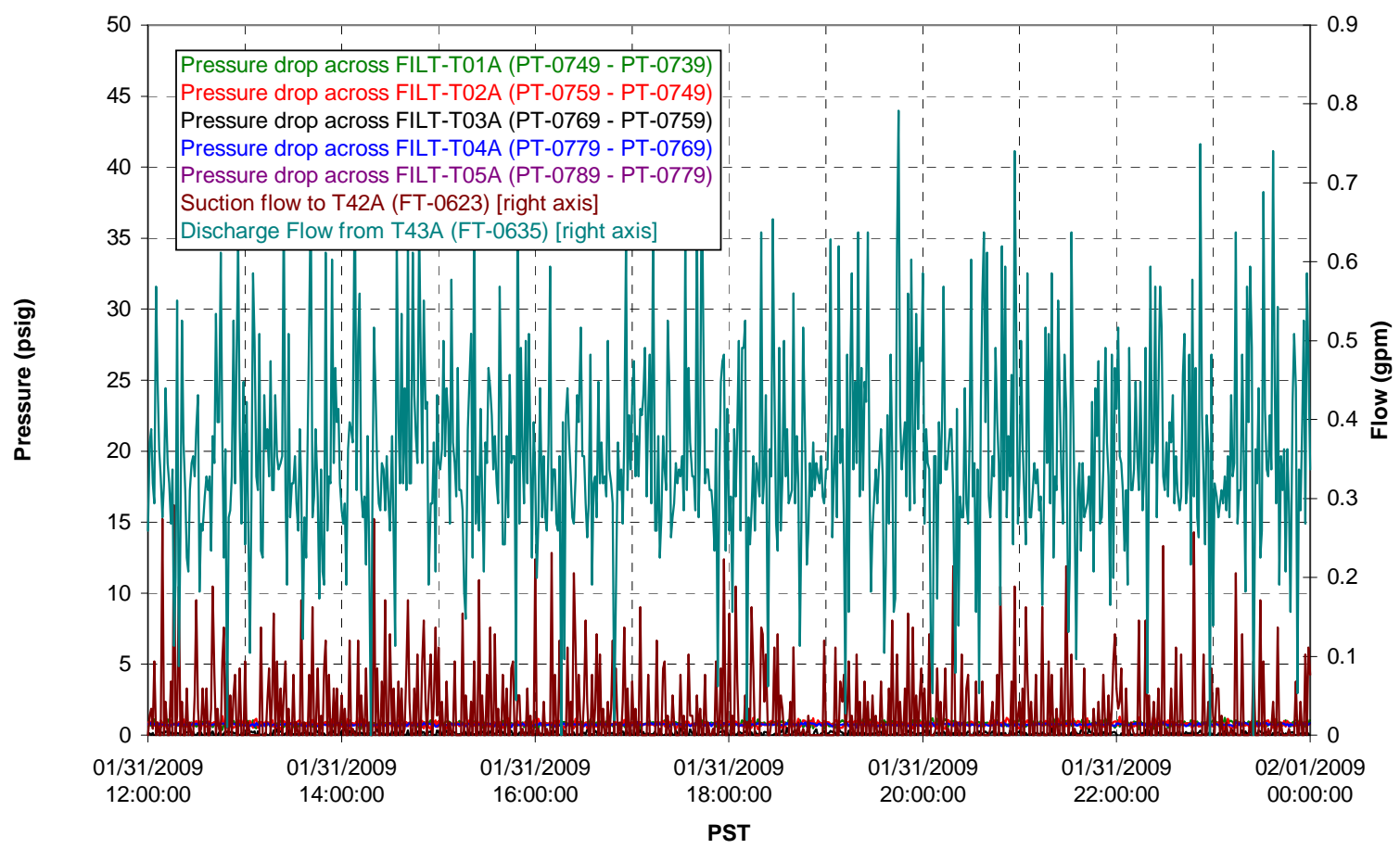

Permeate flow rates

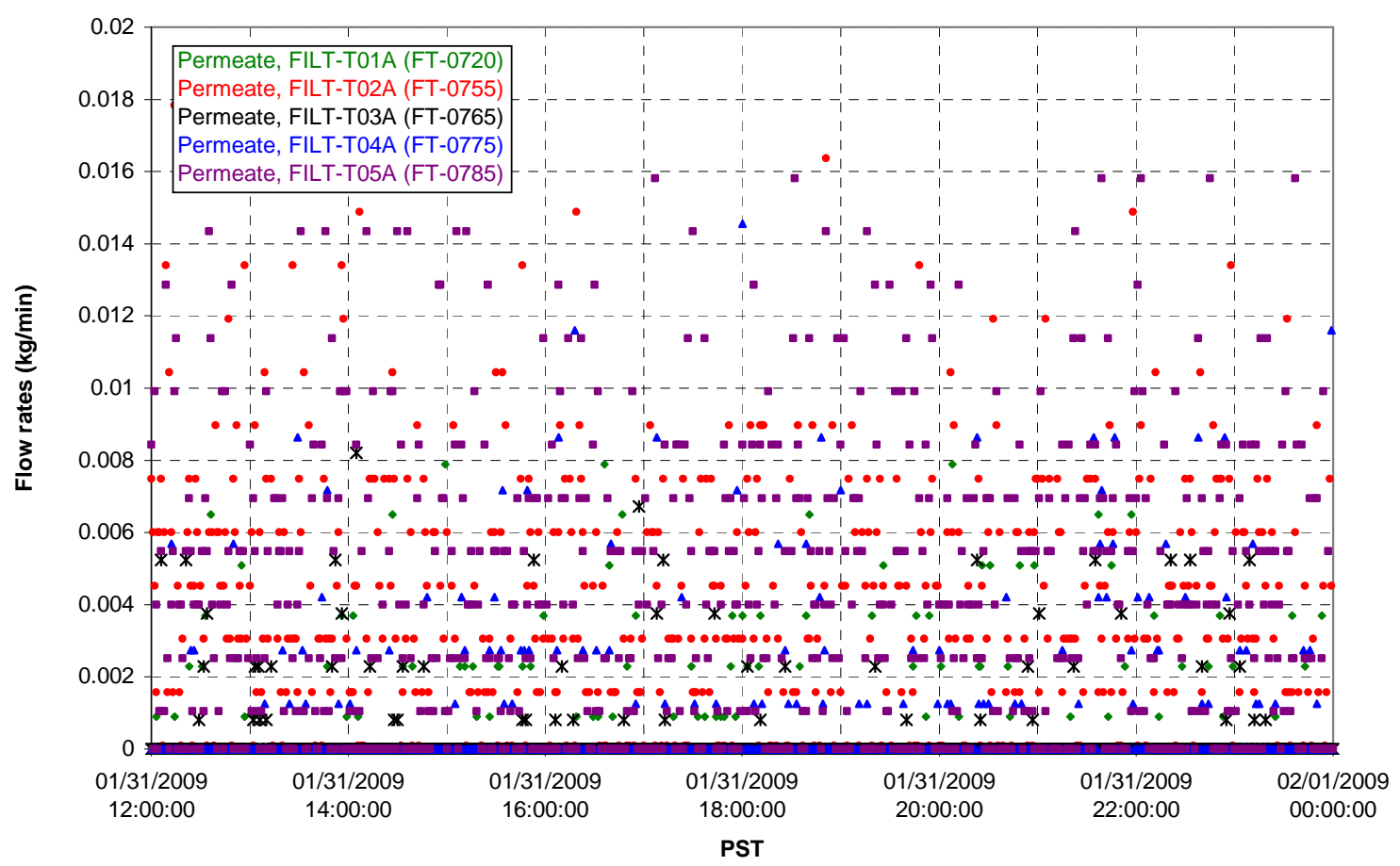


T02A Inner Temperature Tree

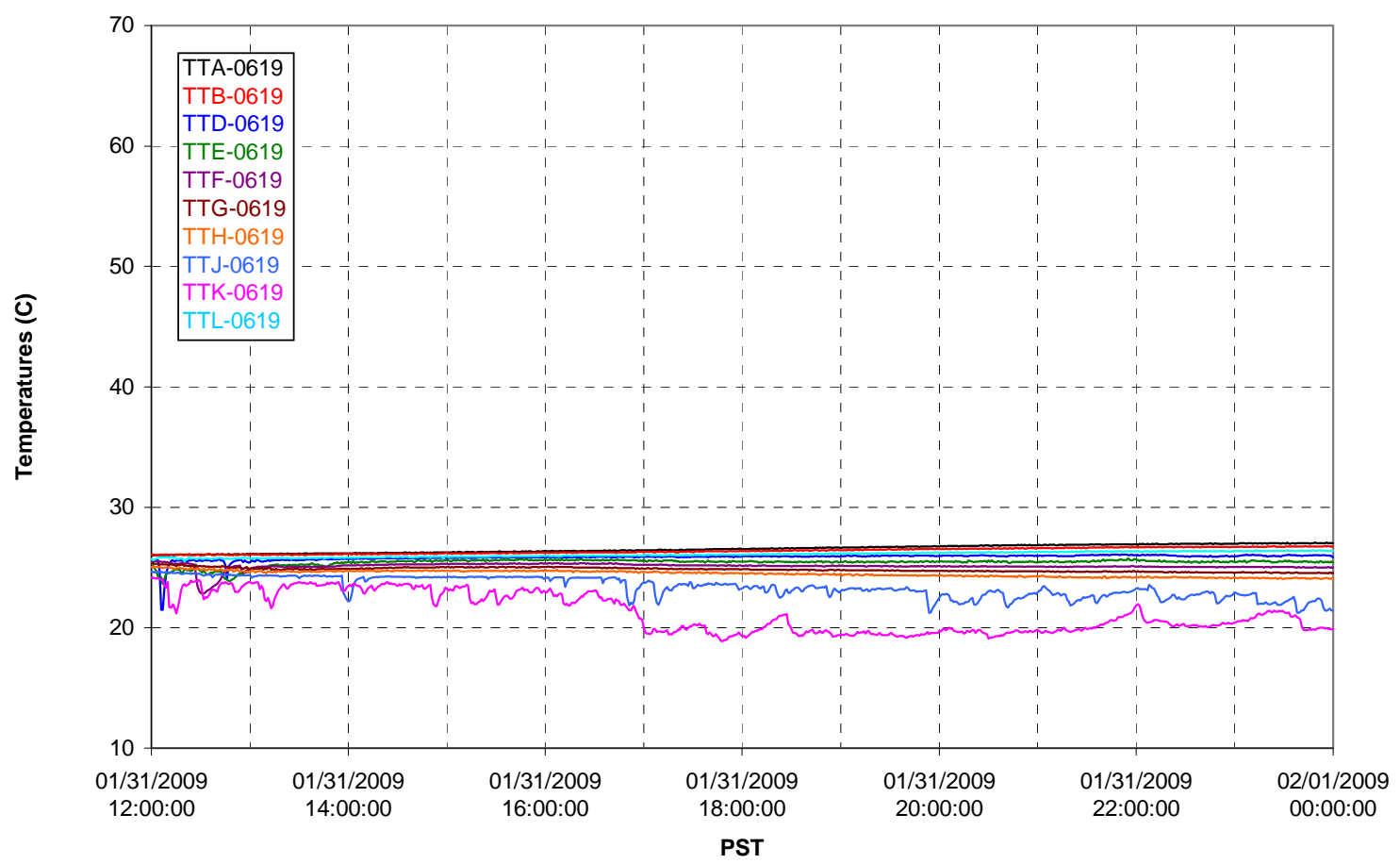

T02A Outer Temperature Tree

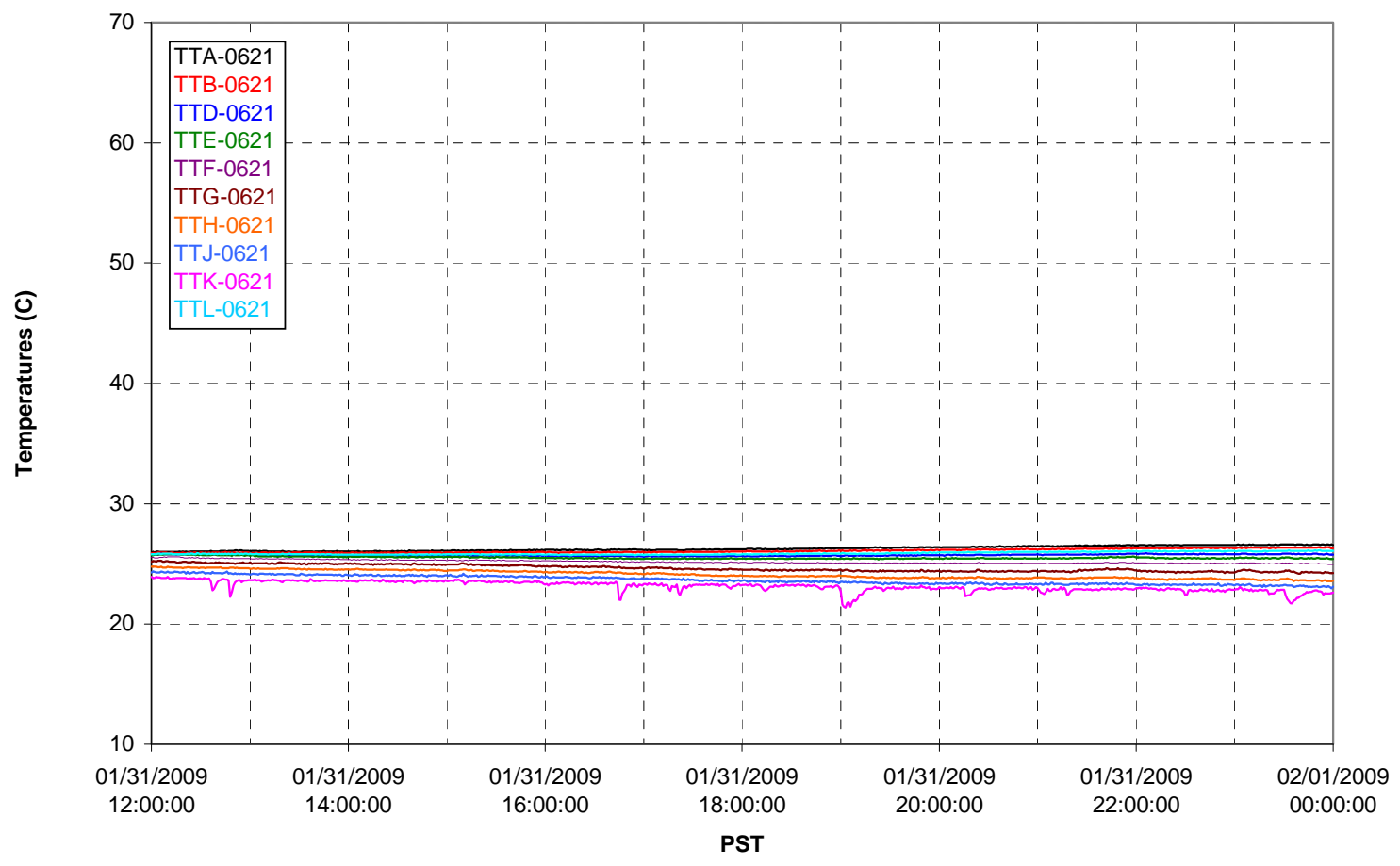

G.25 
T02A temperatures

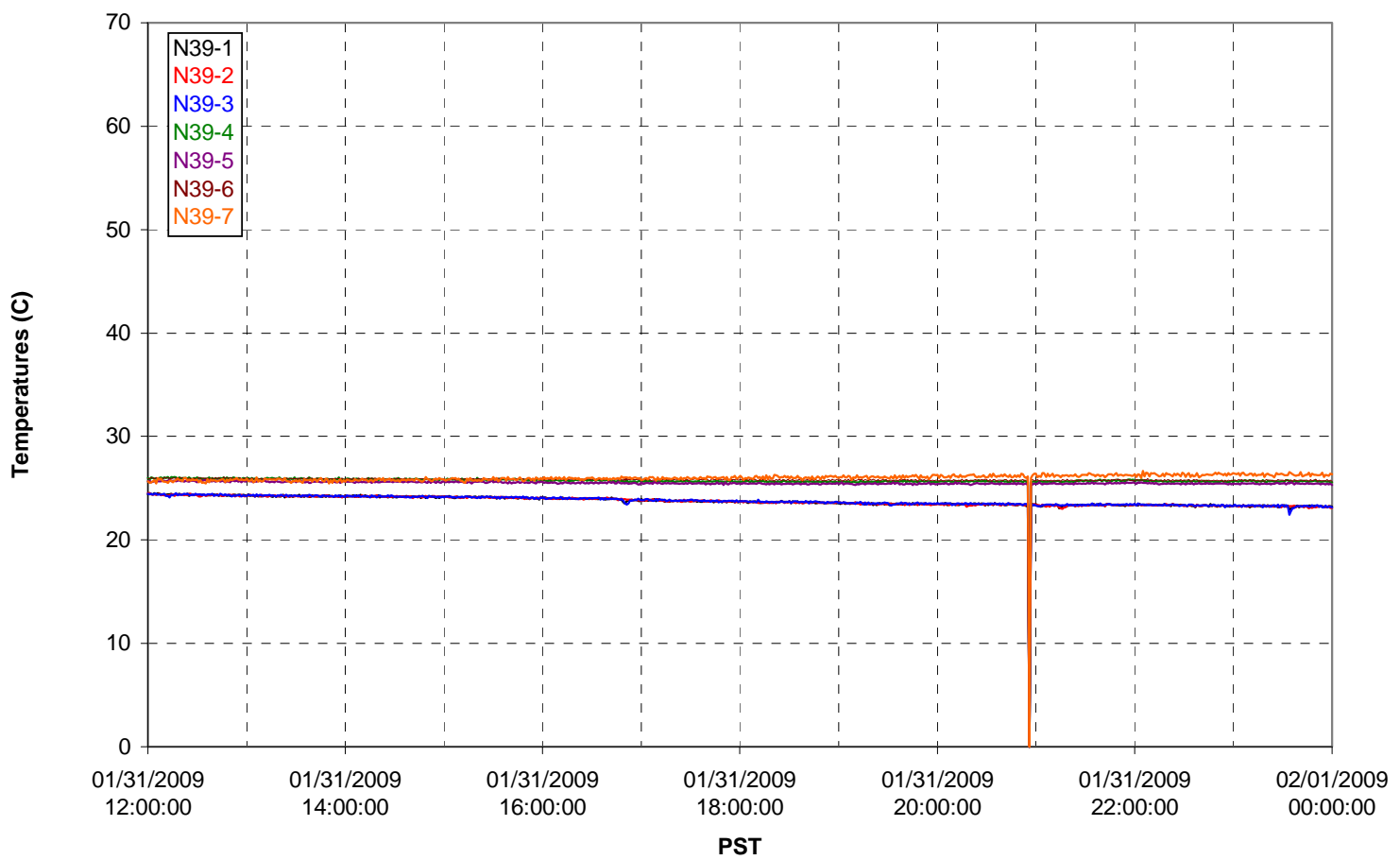

T02A temperatures

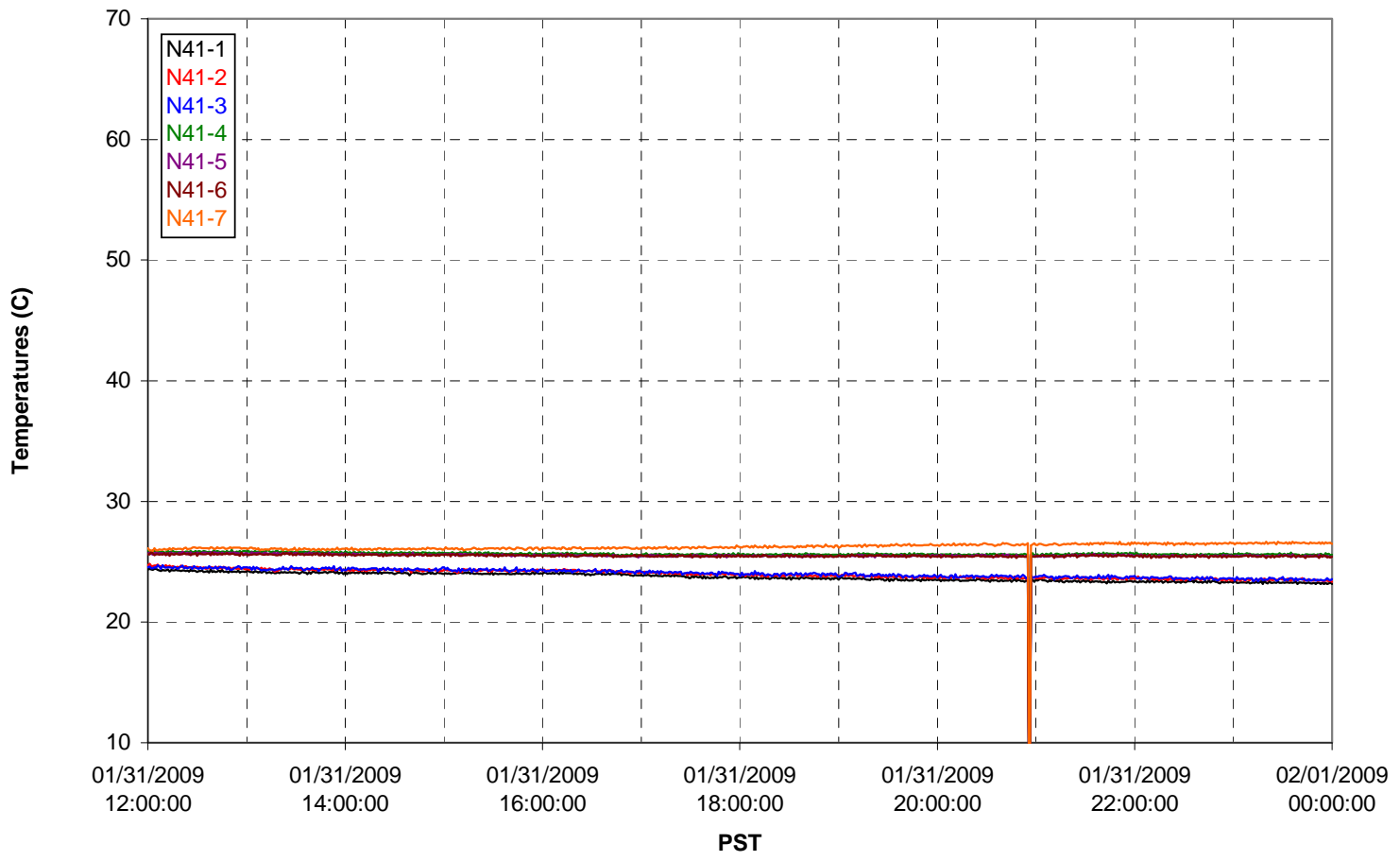


T02A temperatures

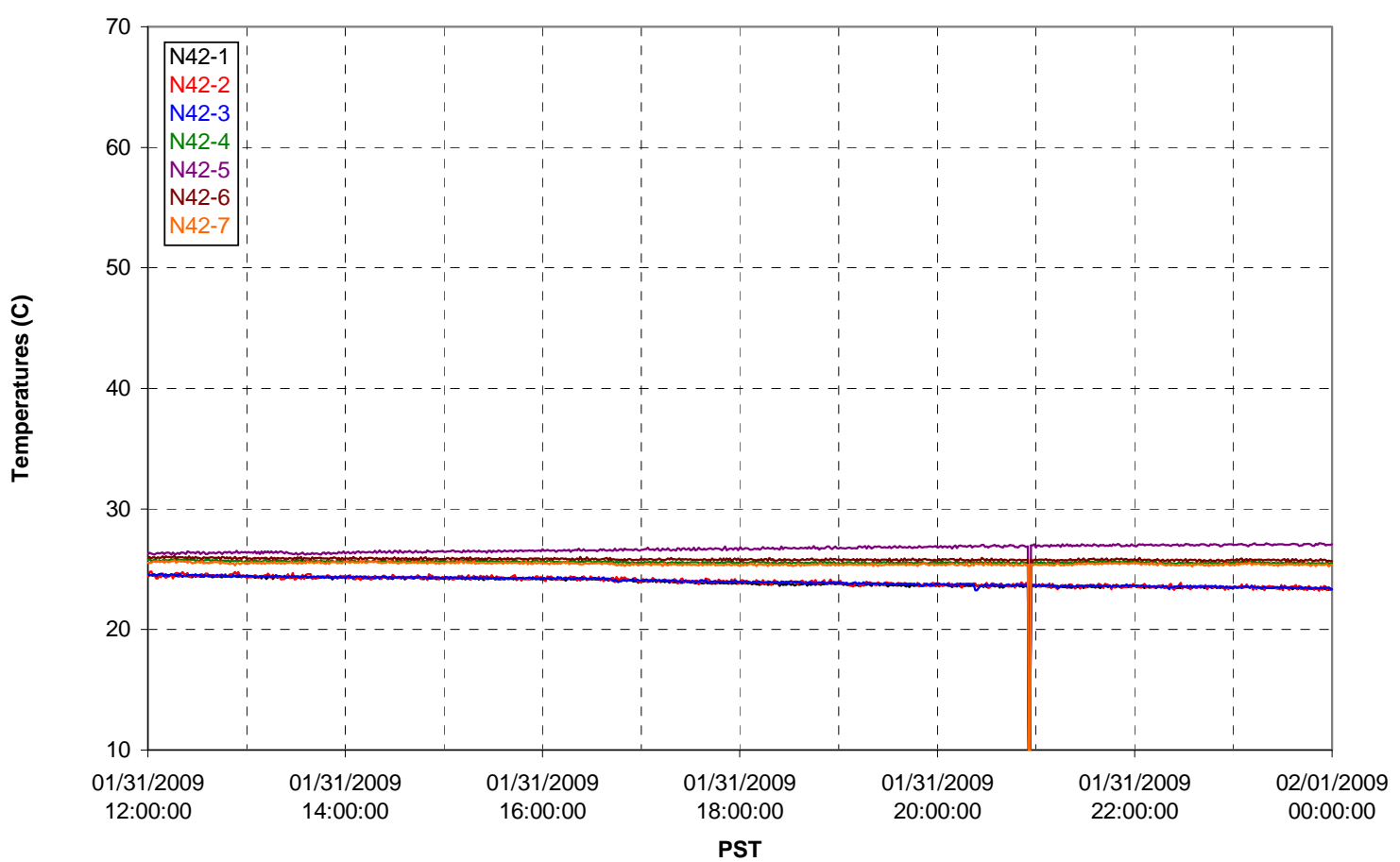

T02A temperatures

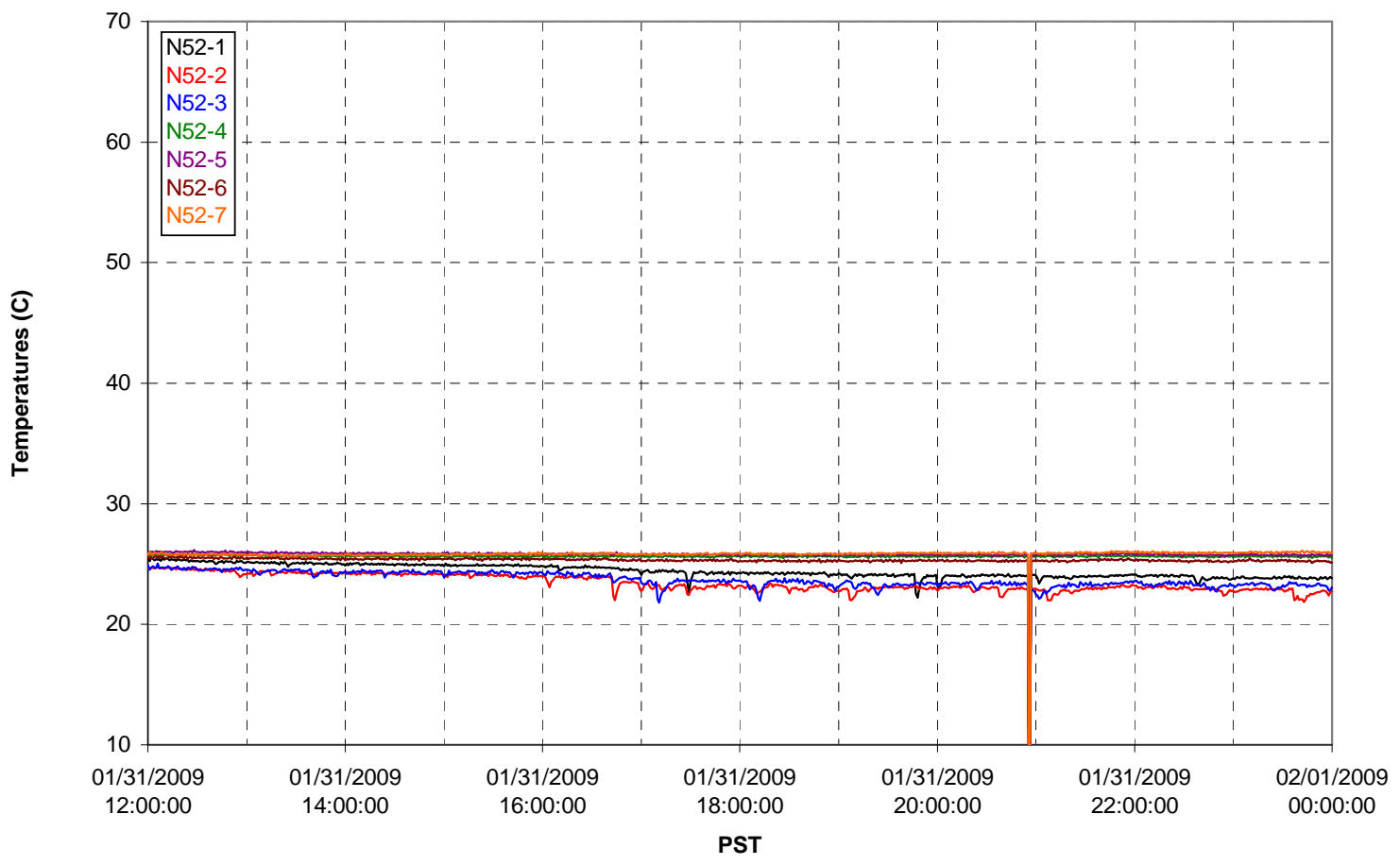


T02A Heating and Cooling

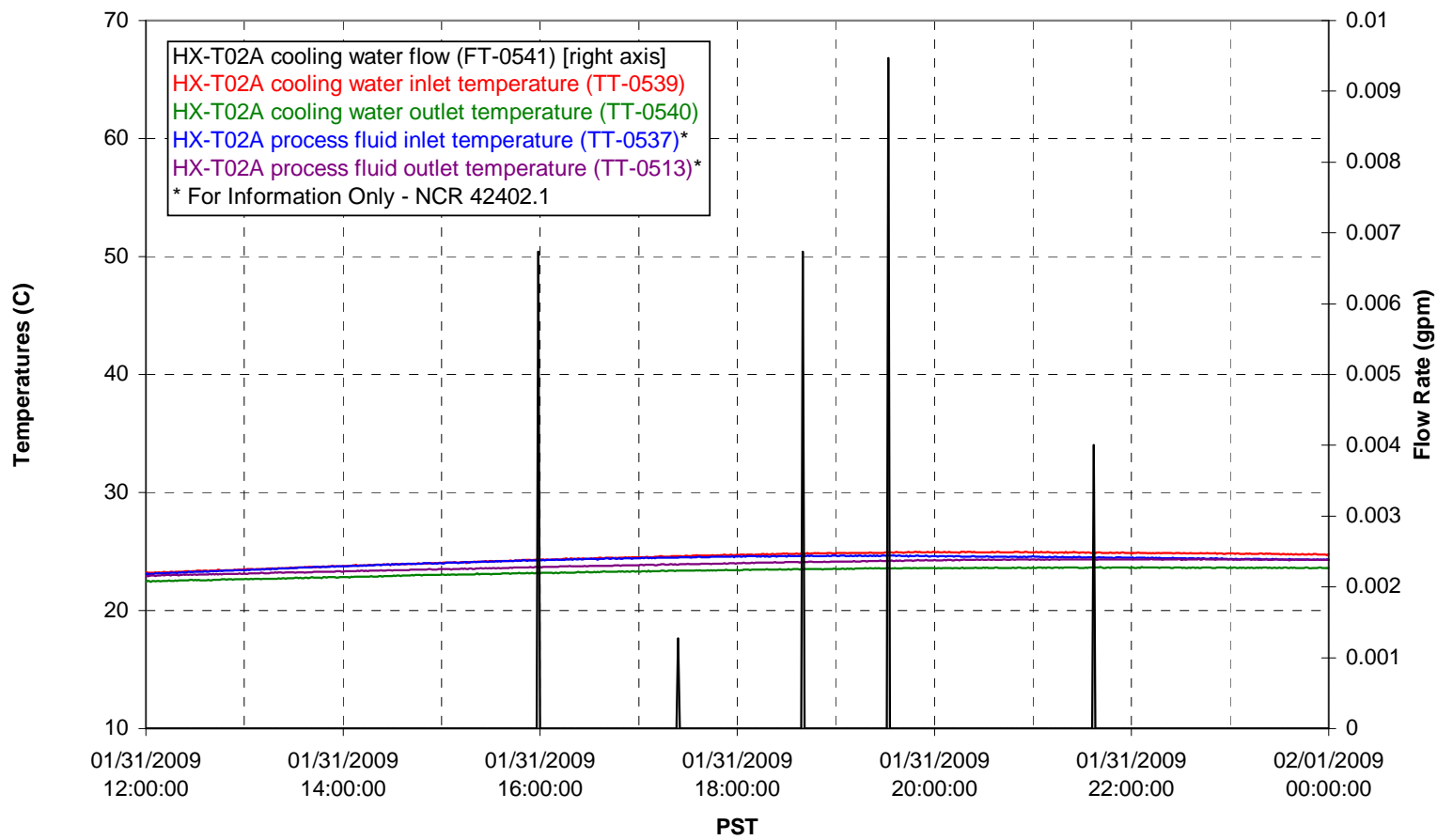

Pump Operation

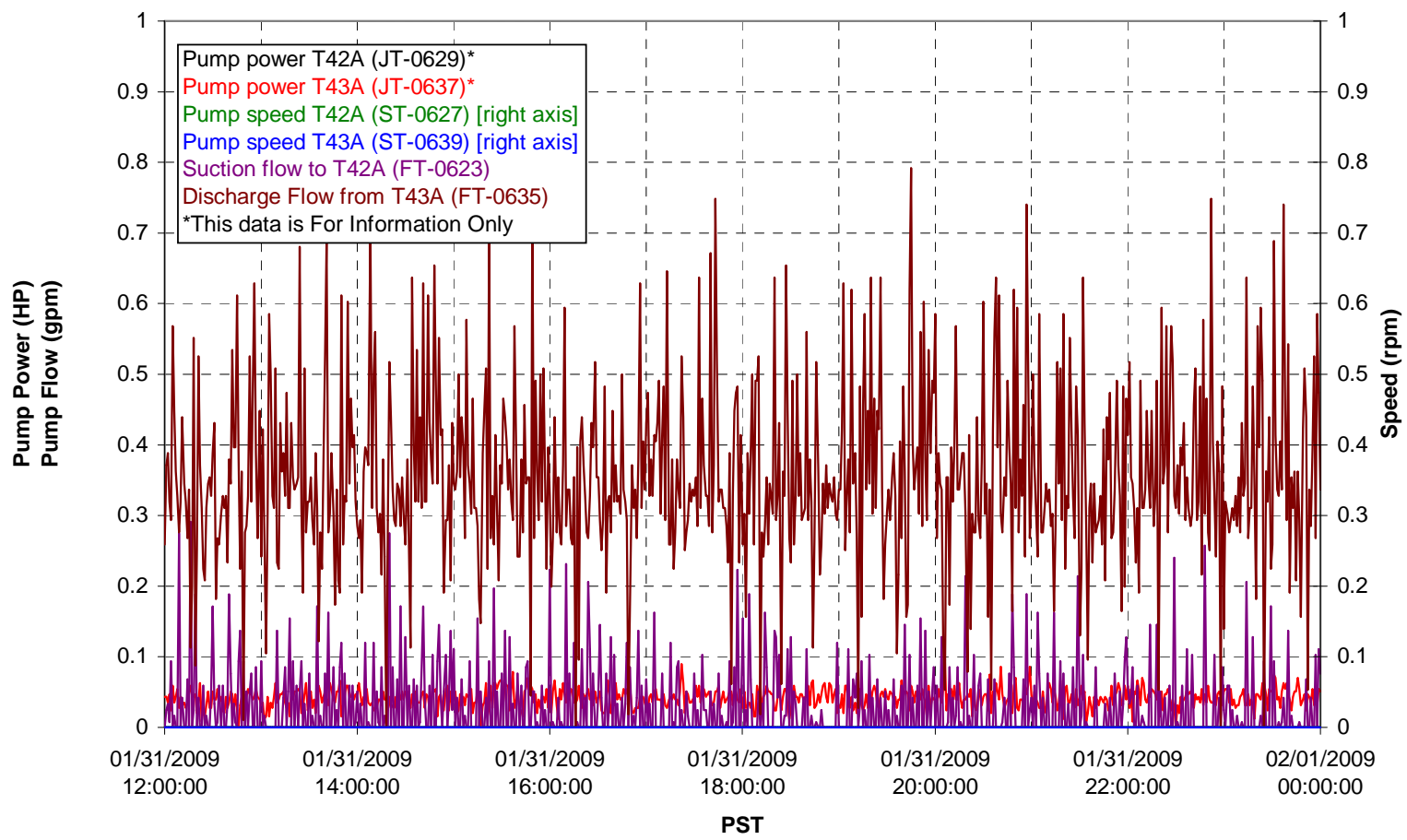


Pulsepot UFP-PP-T01A

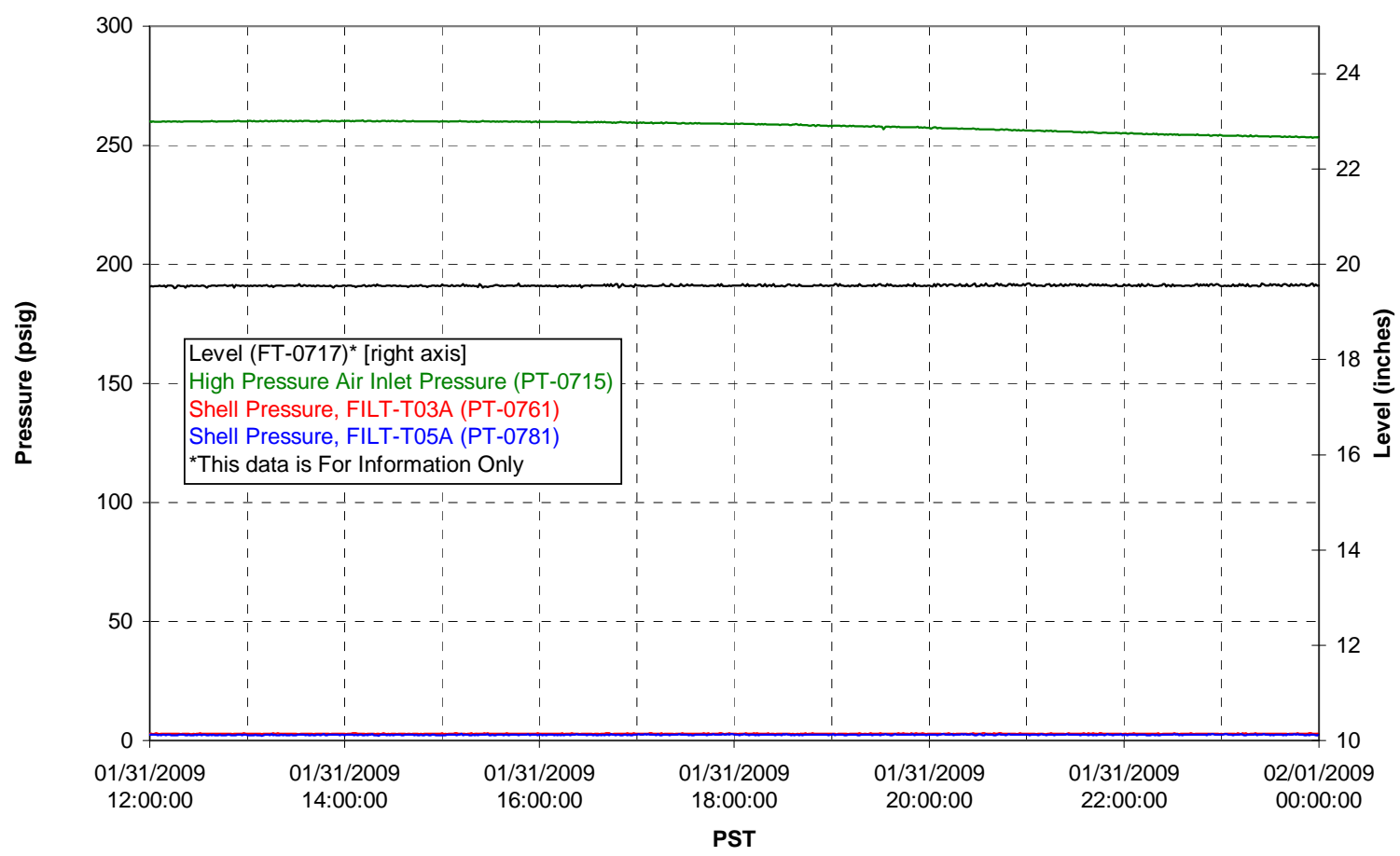

Pulsepot UFP-PP-T02A

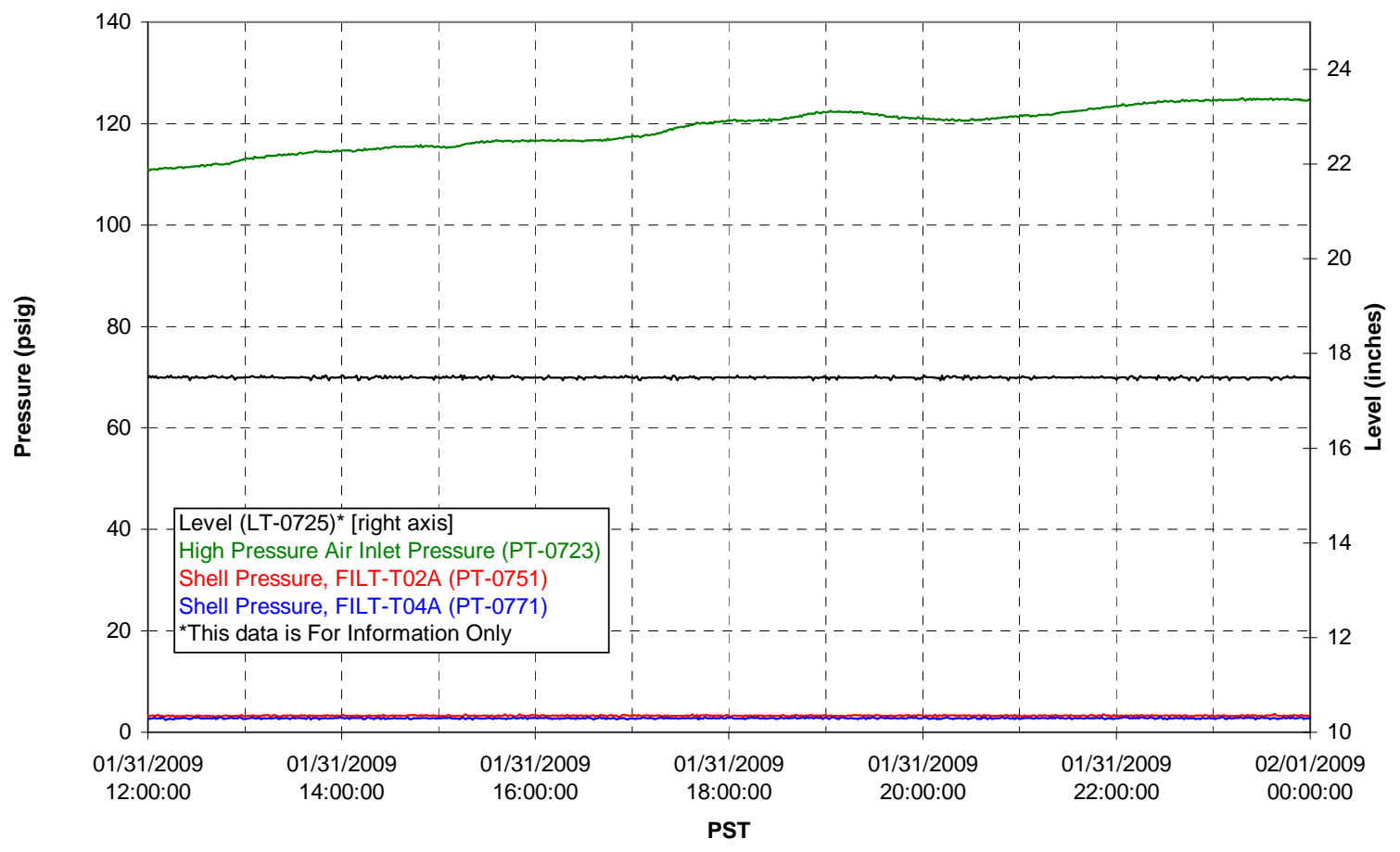


Pulsepot UFP-PP-T03A

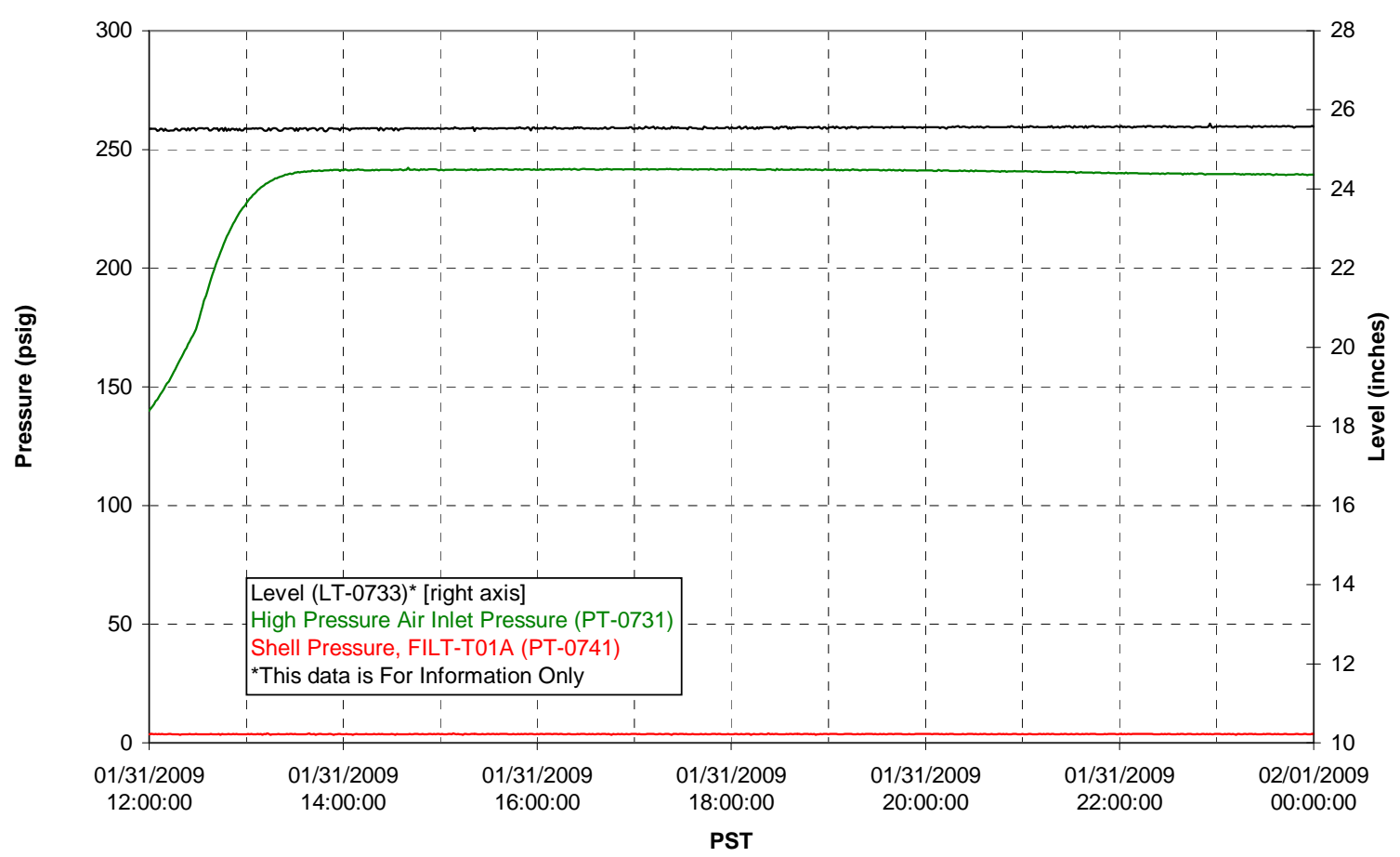

Pulsepot Levels

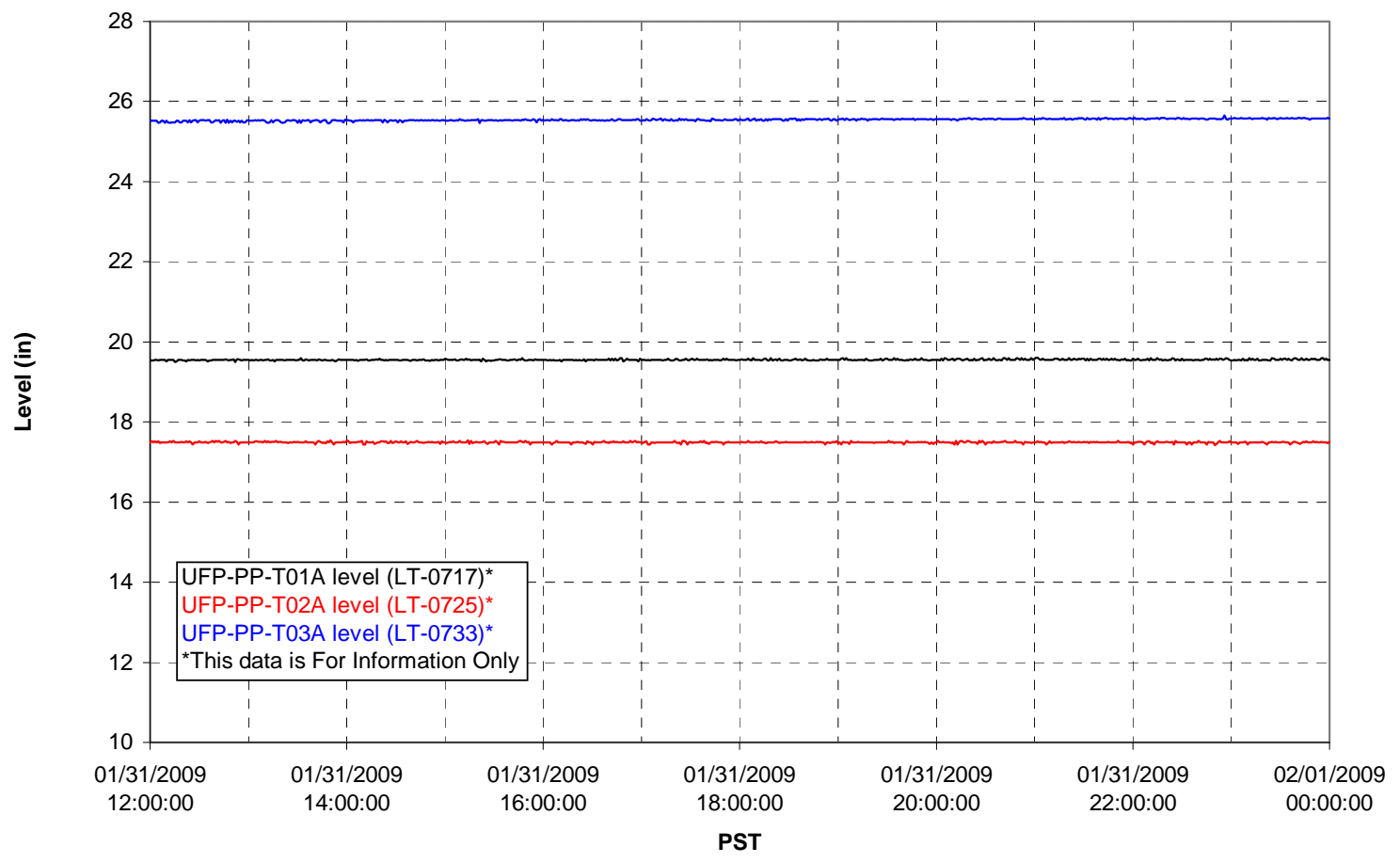


Filter UFP-FILT-T01A

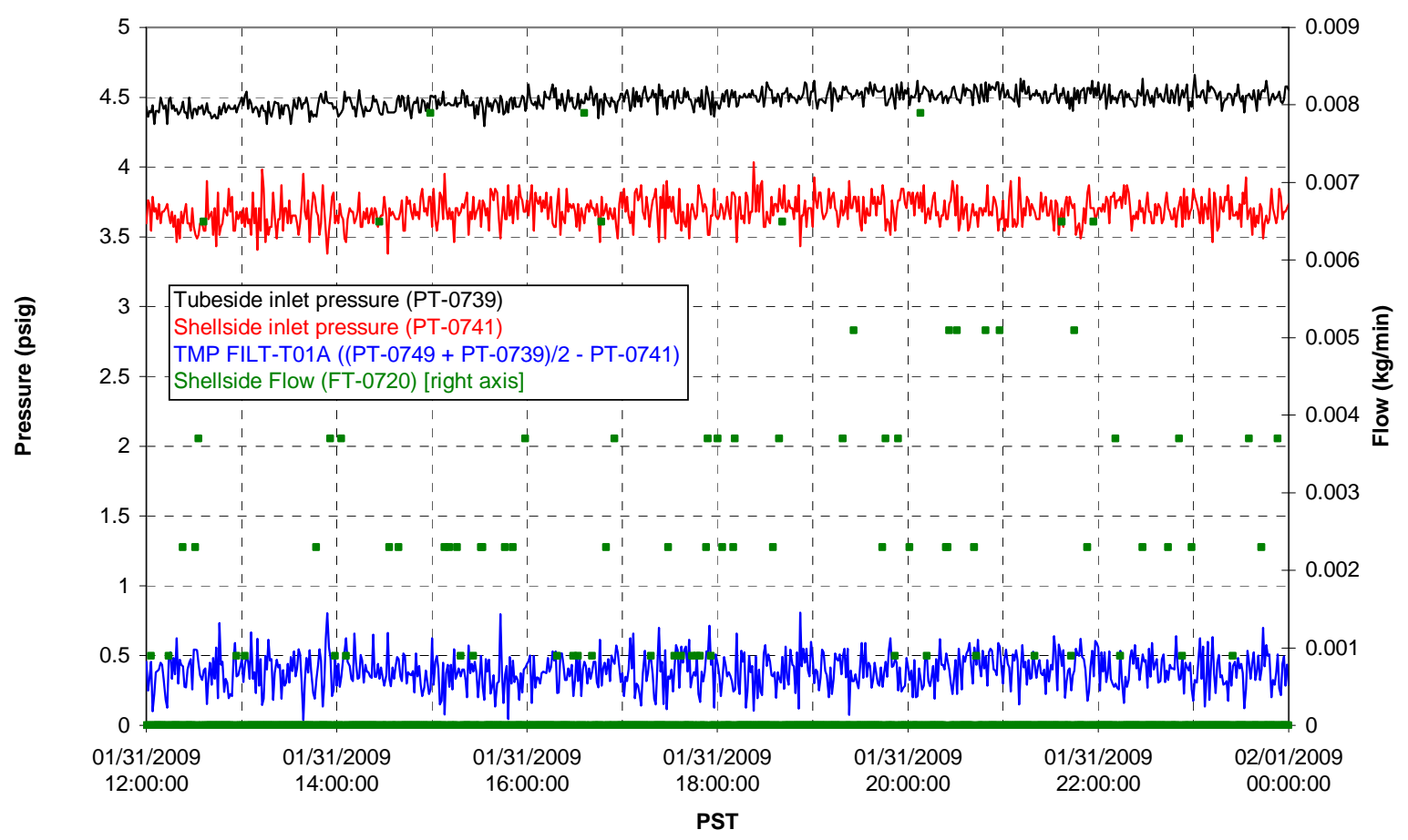

Filter UFP-FILT-T02A

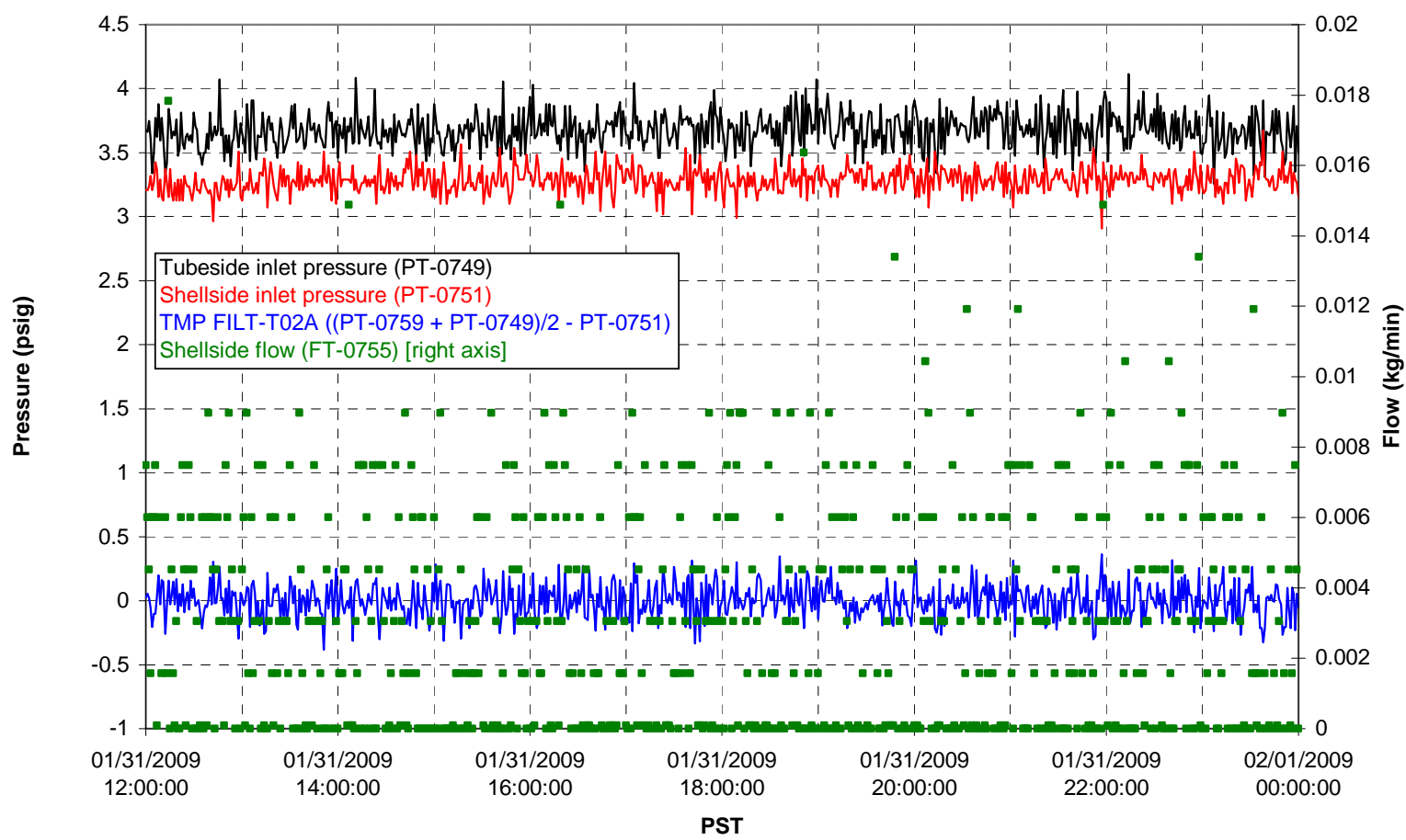


Filter UFP-FILT-T03A

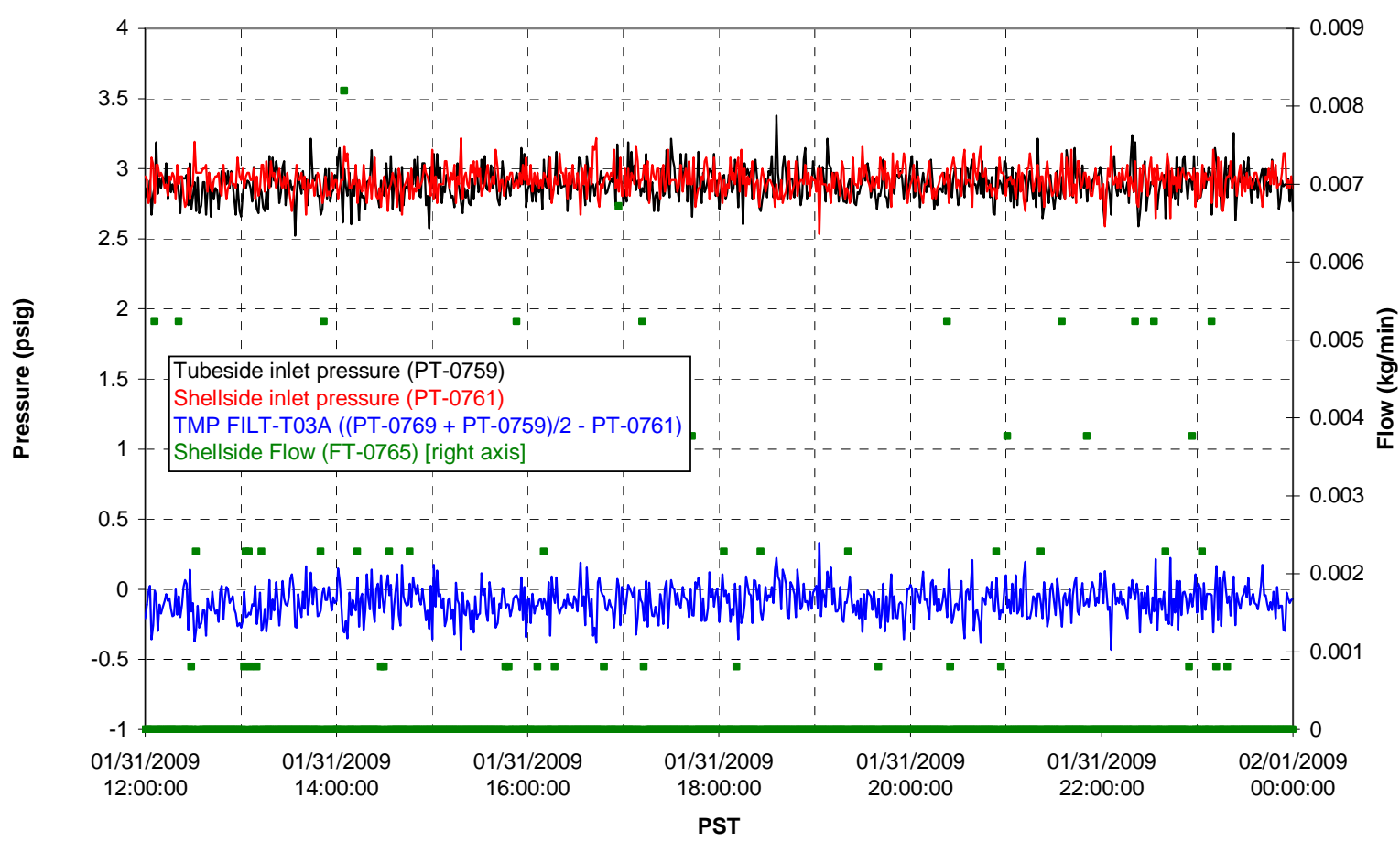

Filter UFP-FILT-T04A

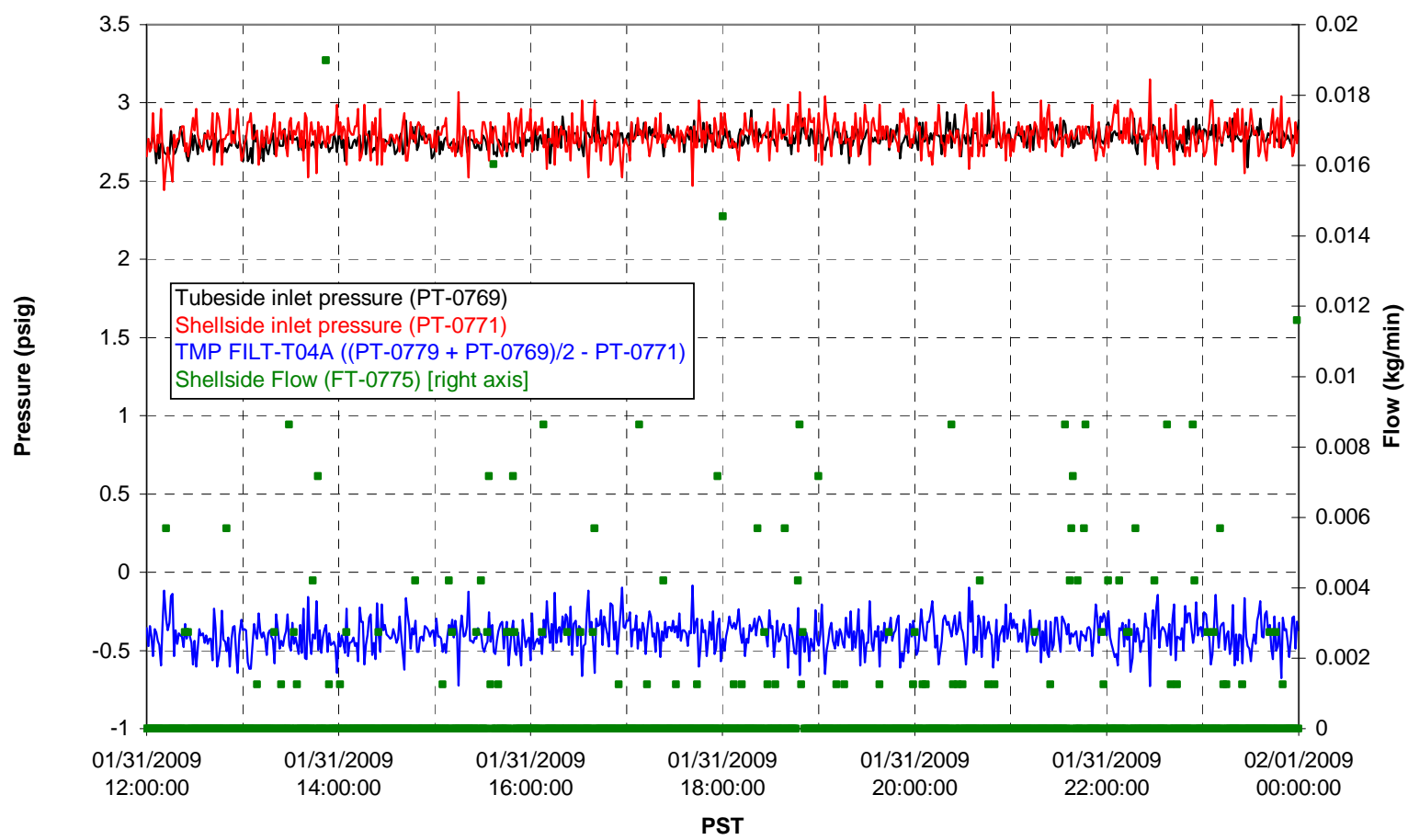


Filter UFP-FILT-T05A

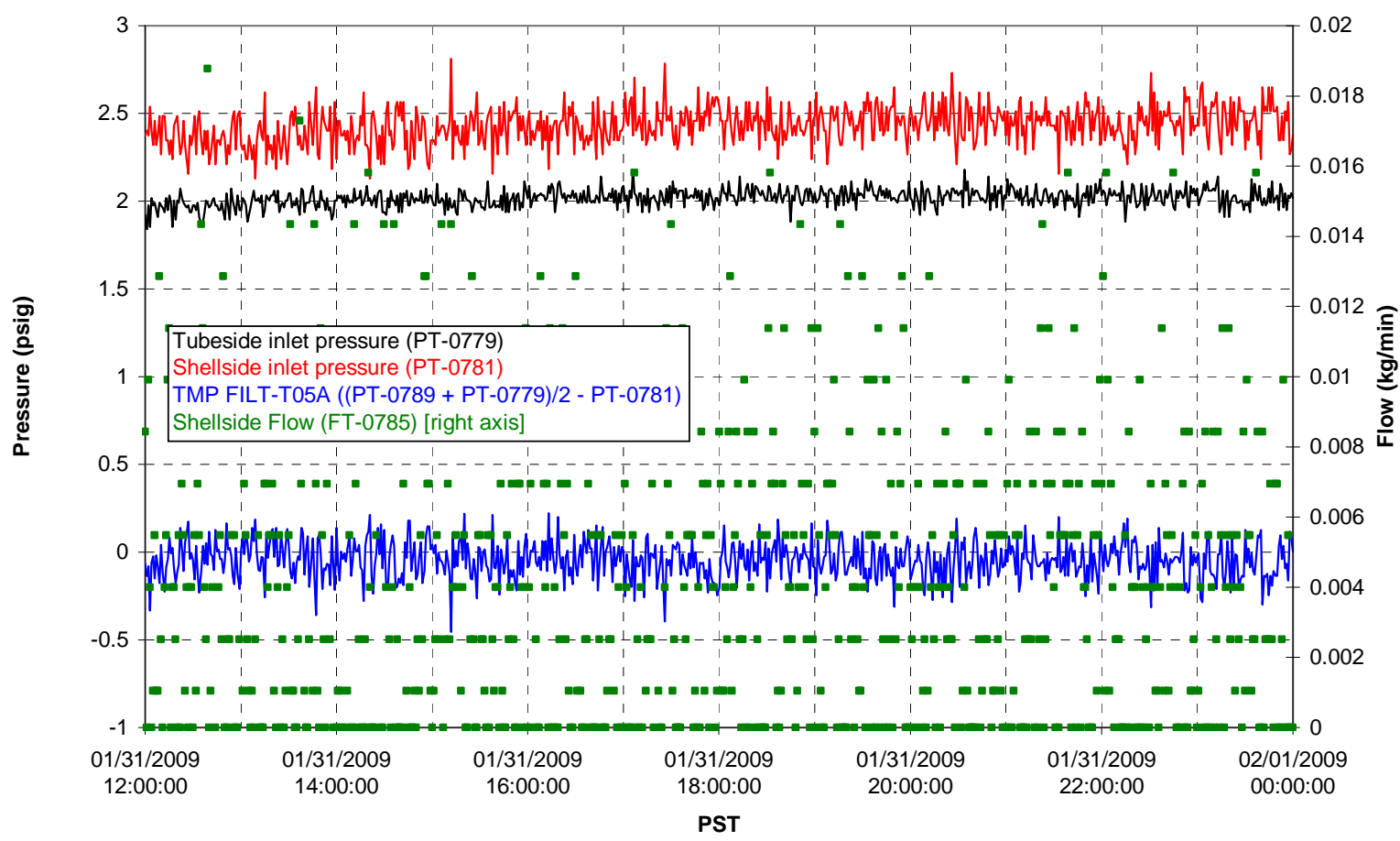

Chemical Flow

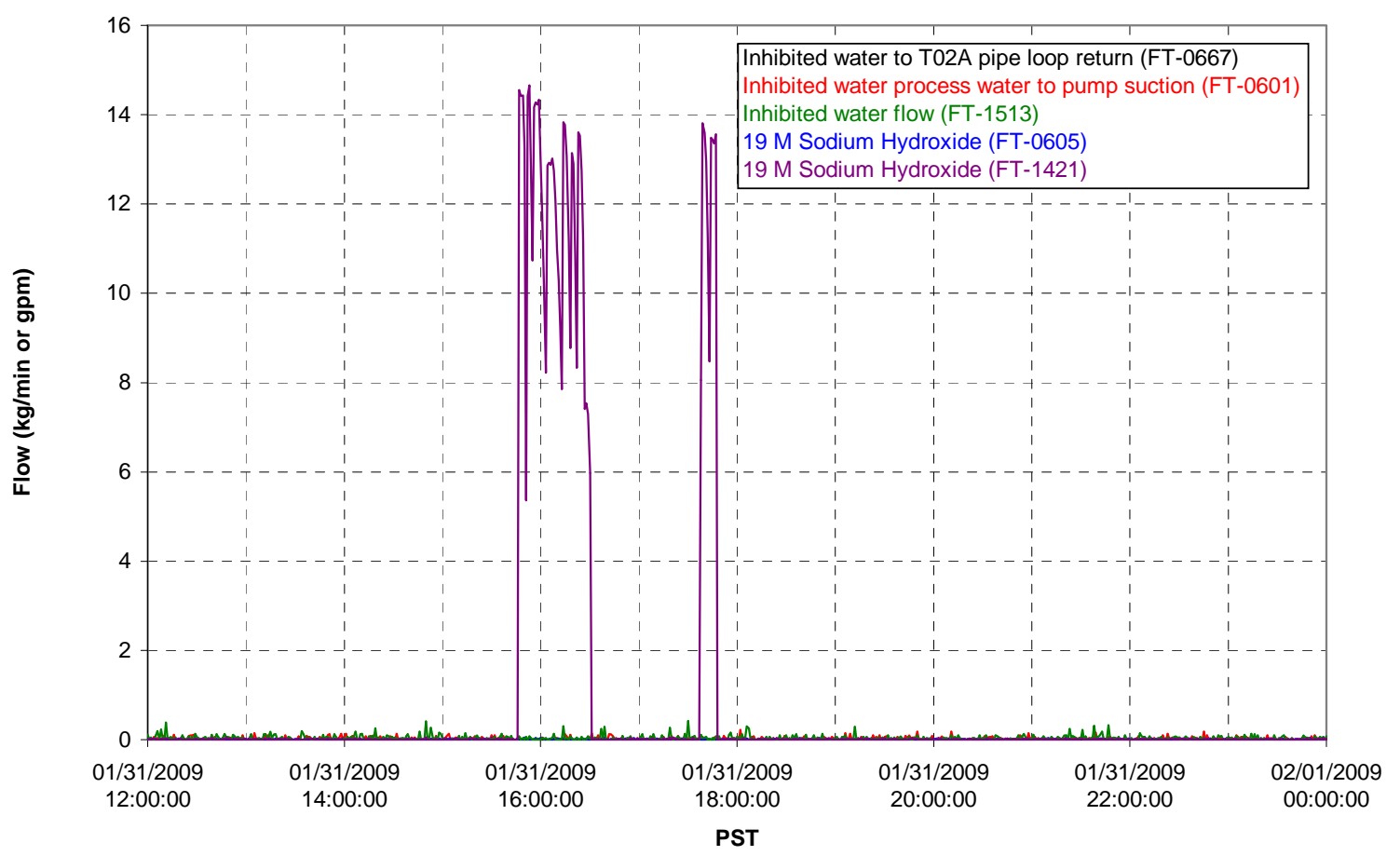




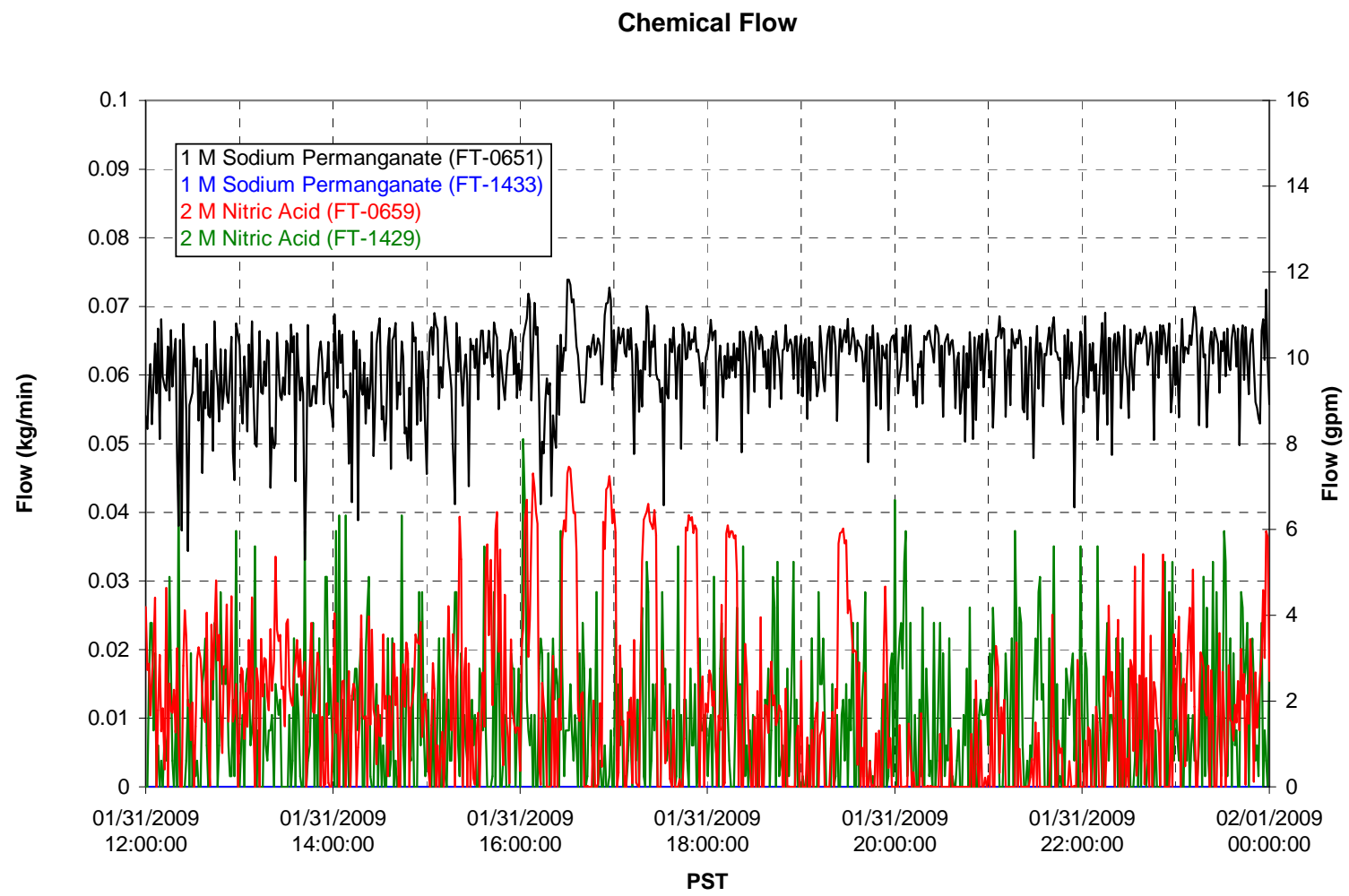

Air Flows

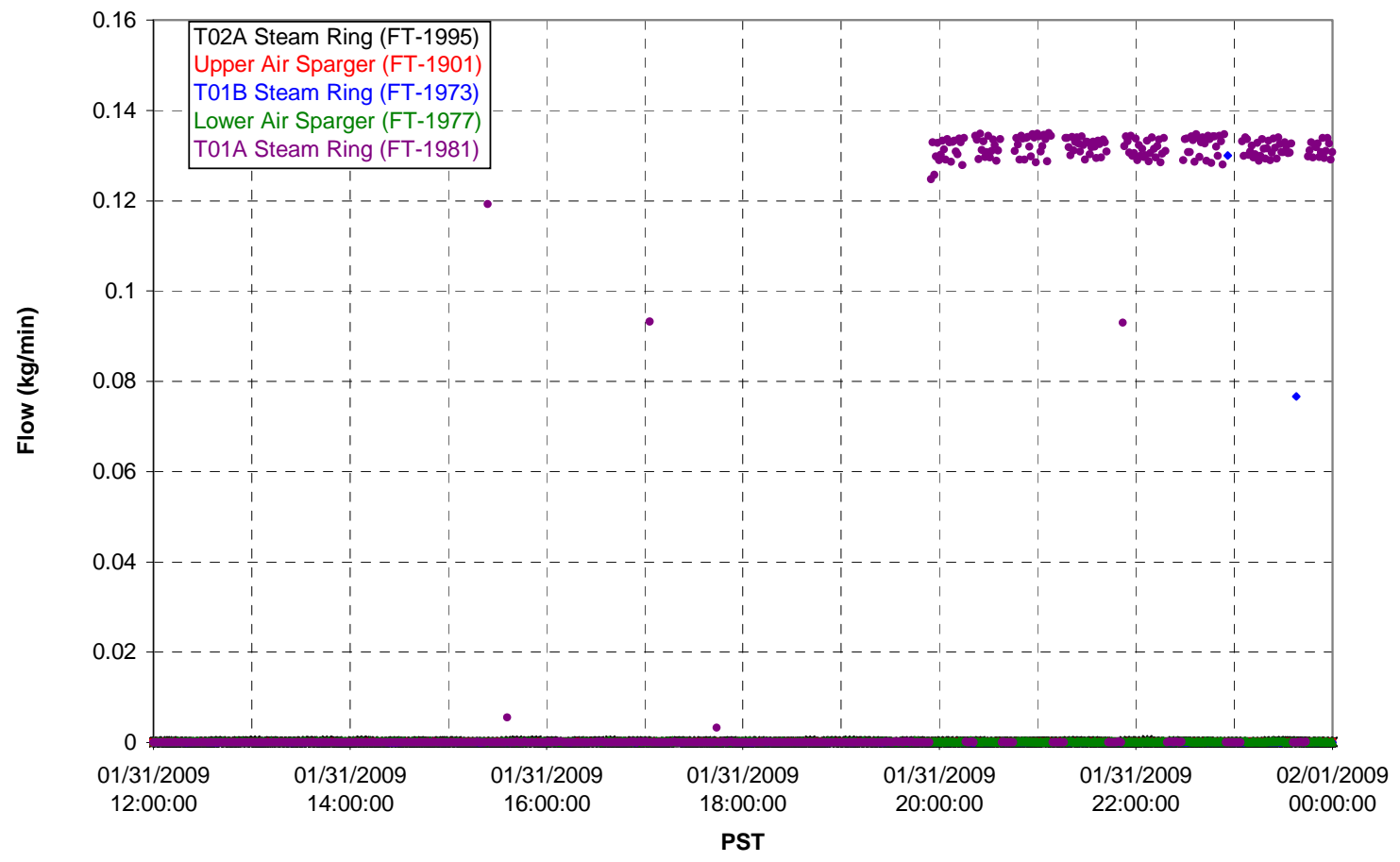


T02A Steam

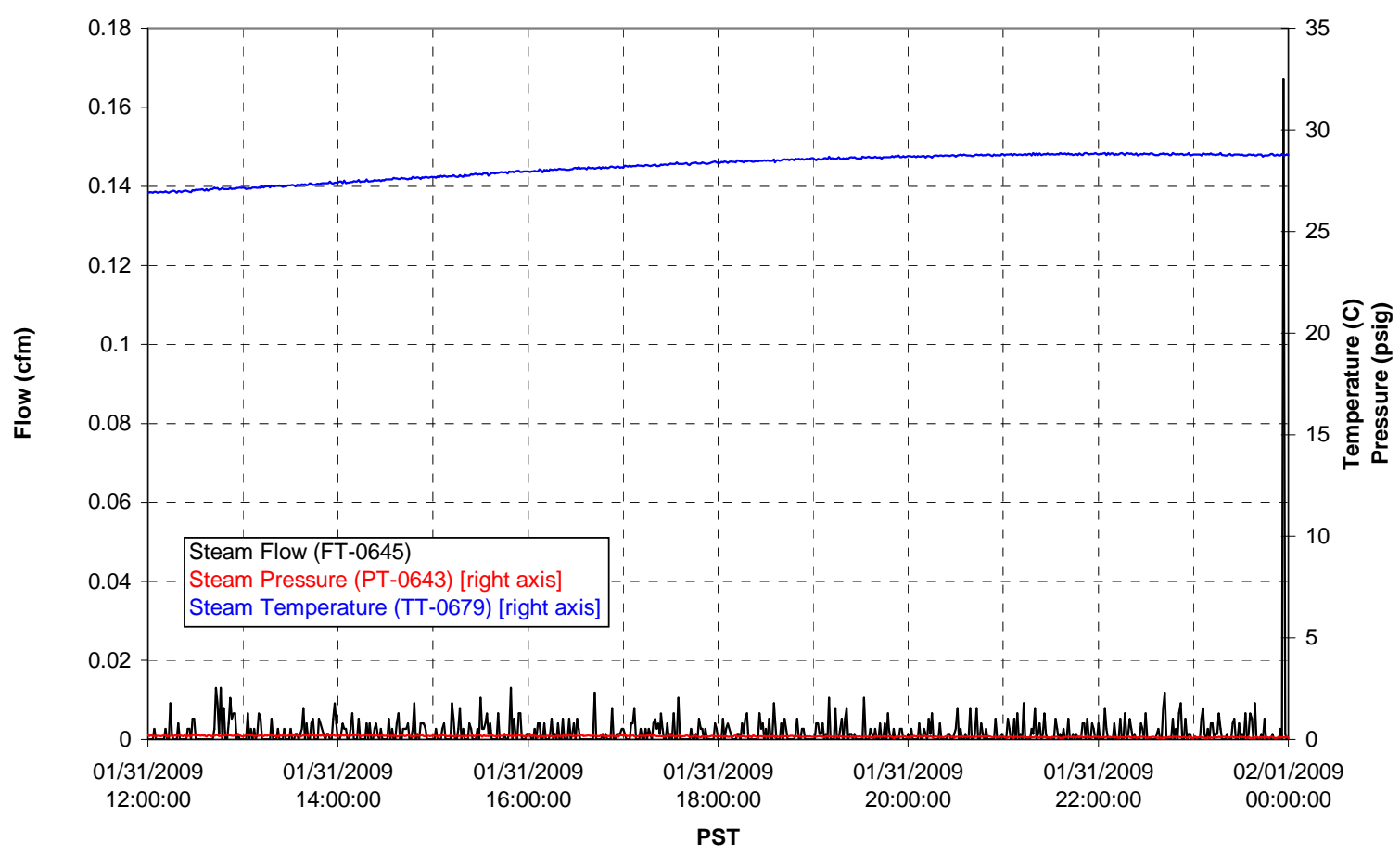

T01A Steam

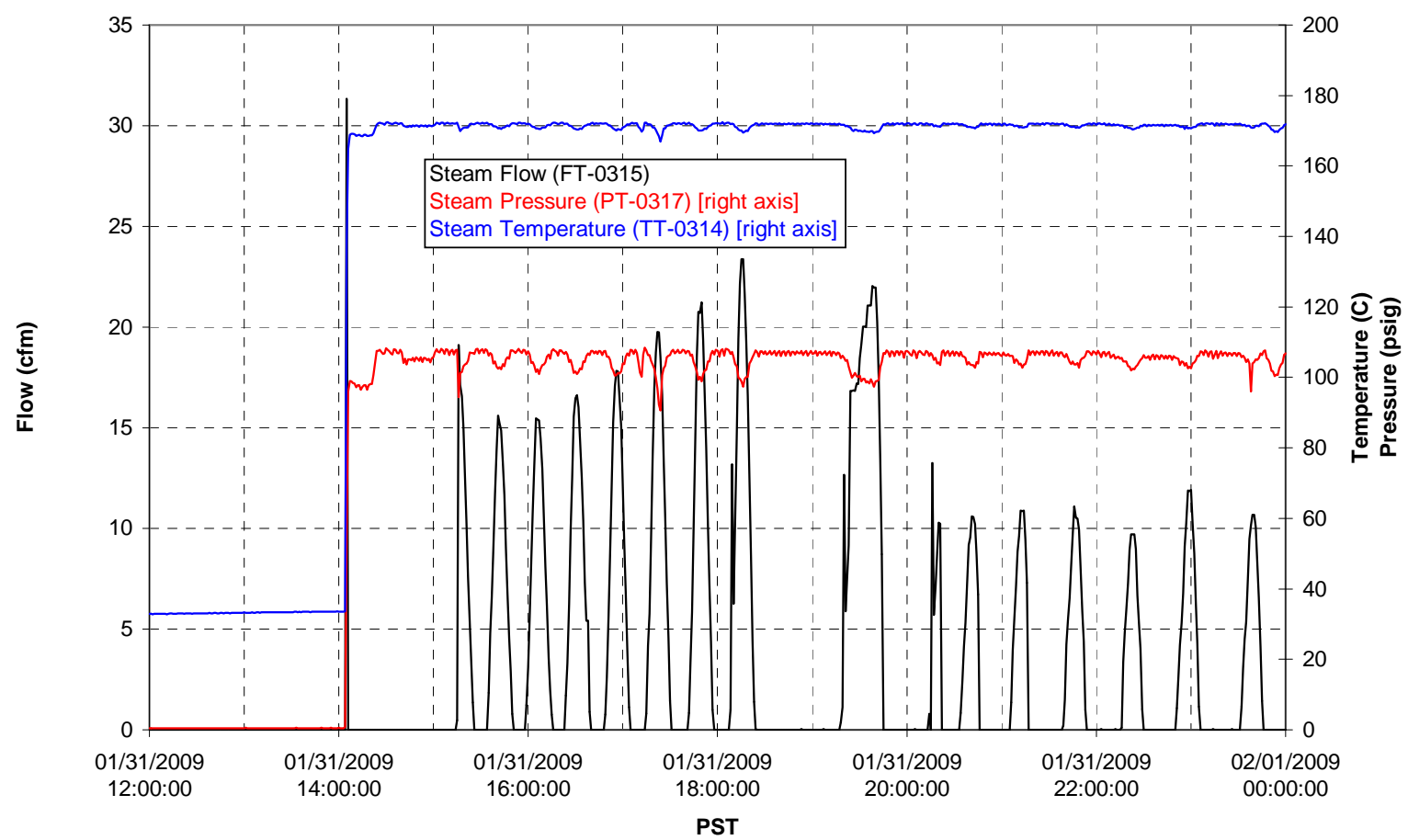


T01B Steam

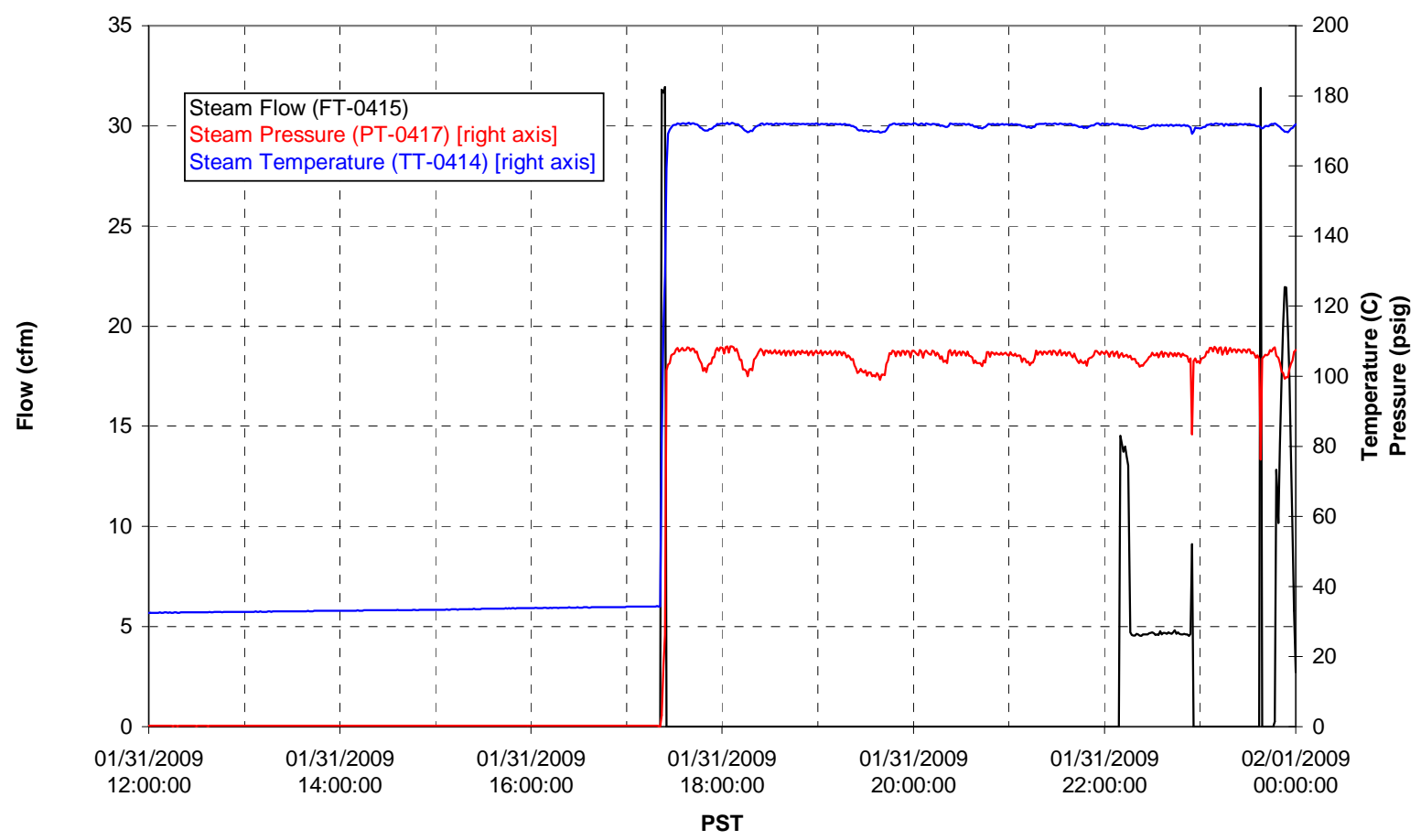




\section{Integrated Test A Data Plots 02/01/09 00:00 - 02/01/09 12:00}


T01A level

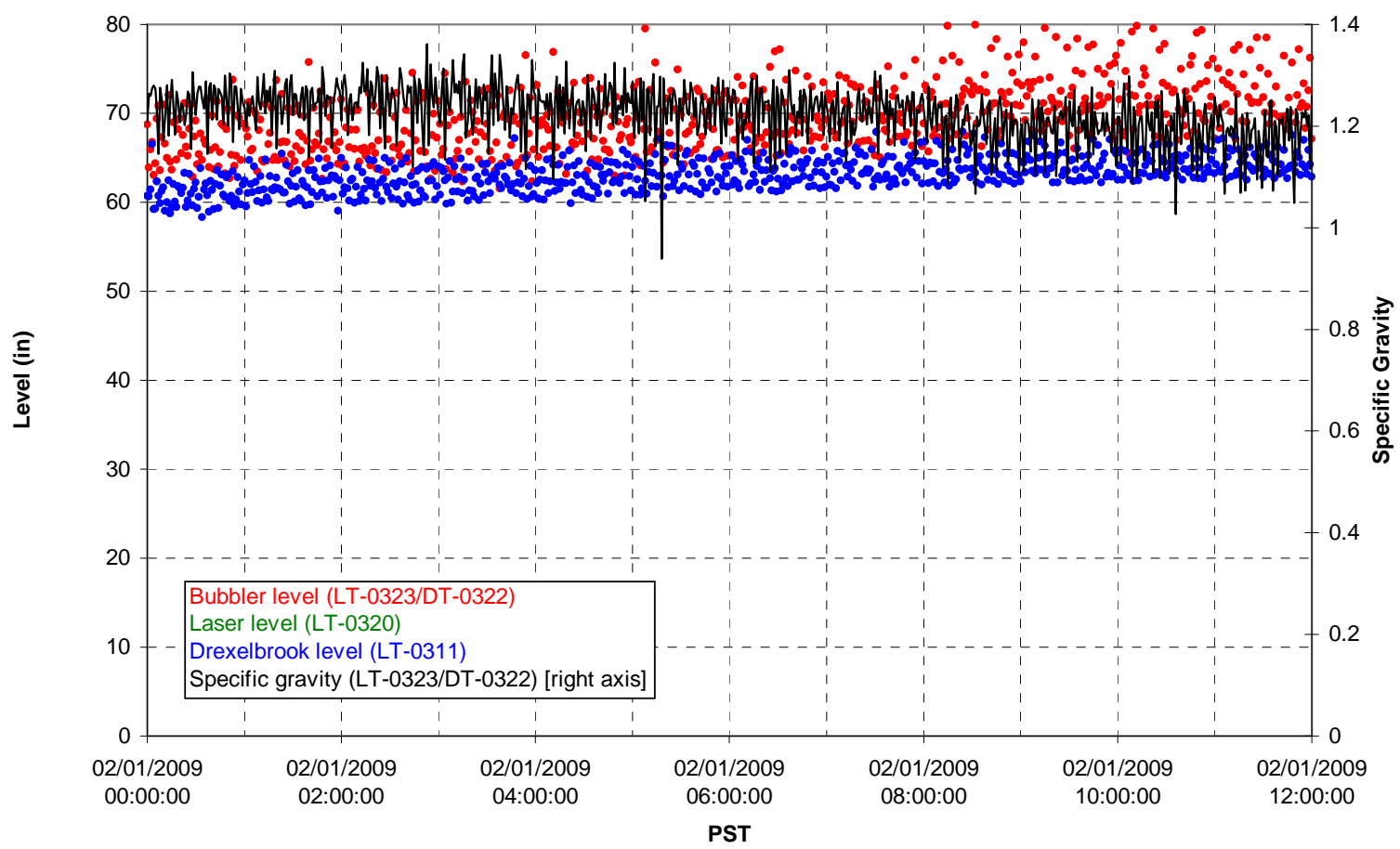

T01A temperatures

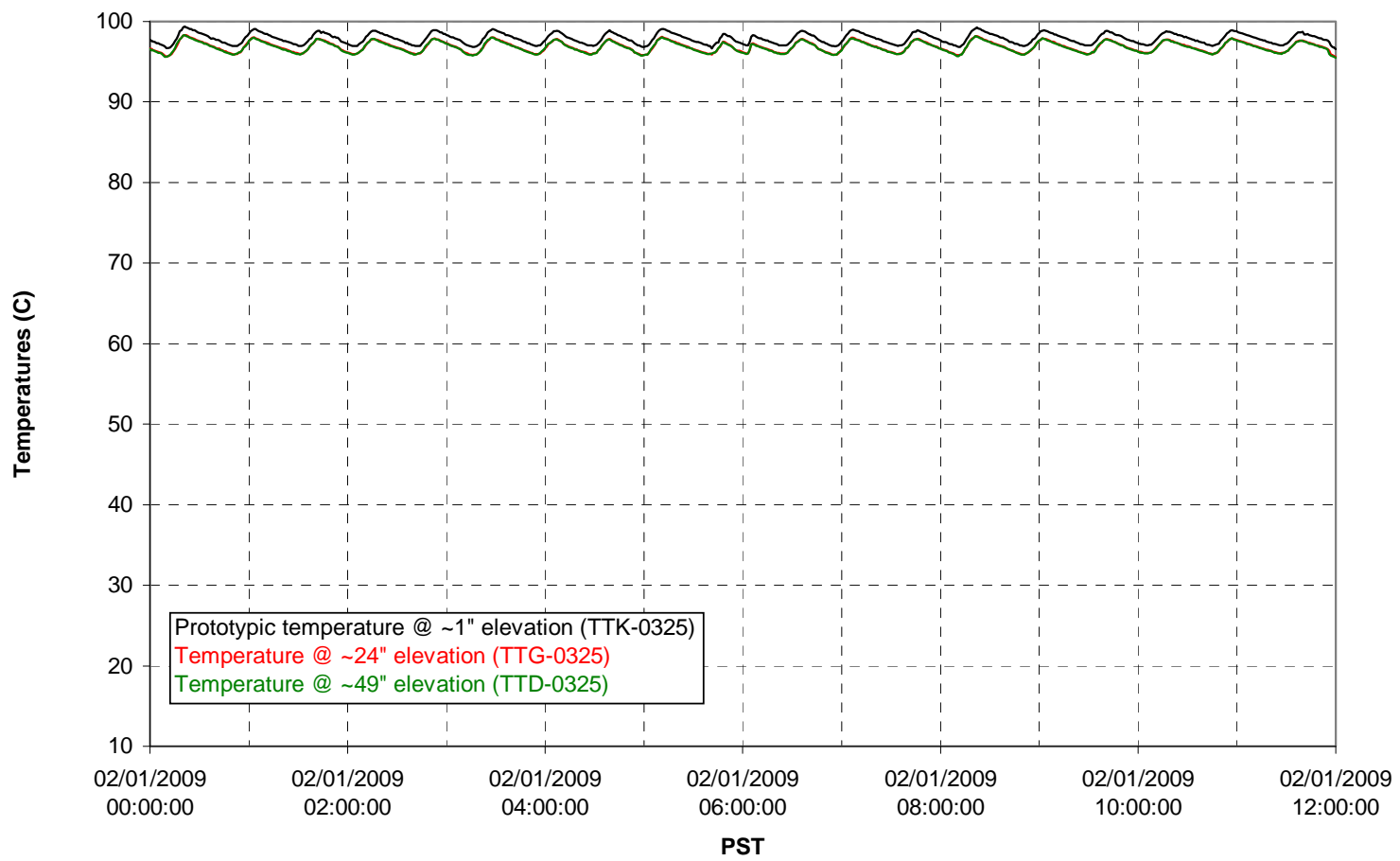


T01B level

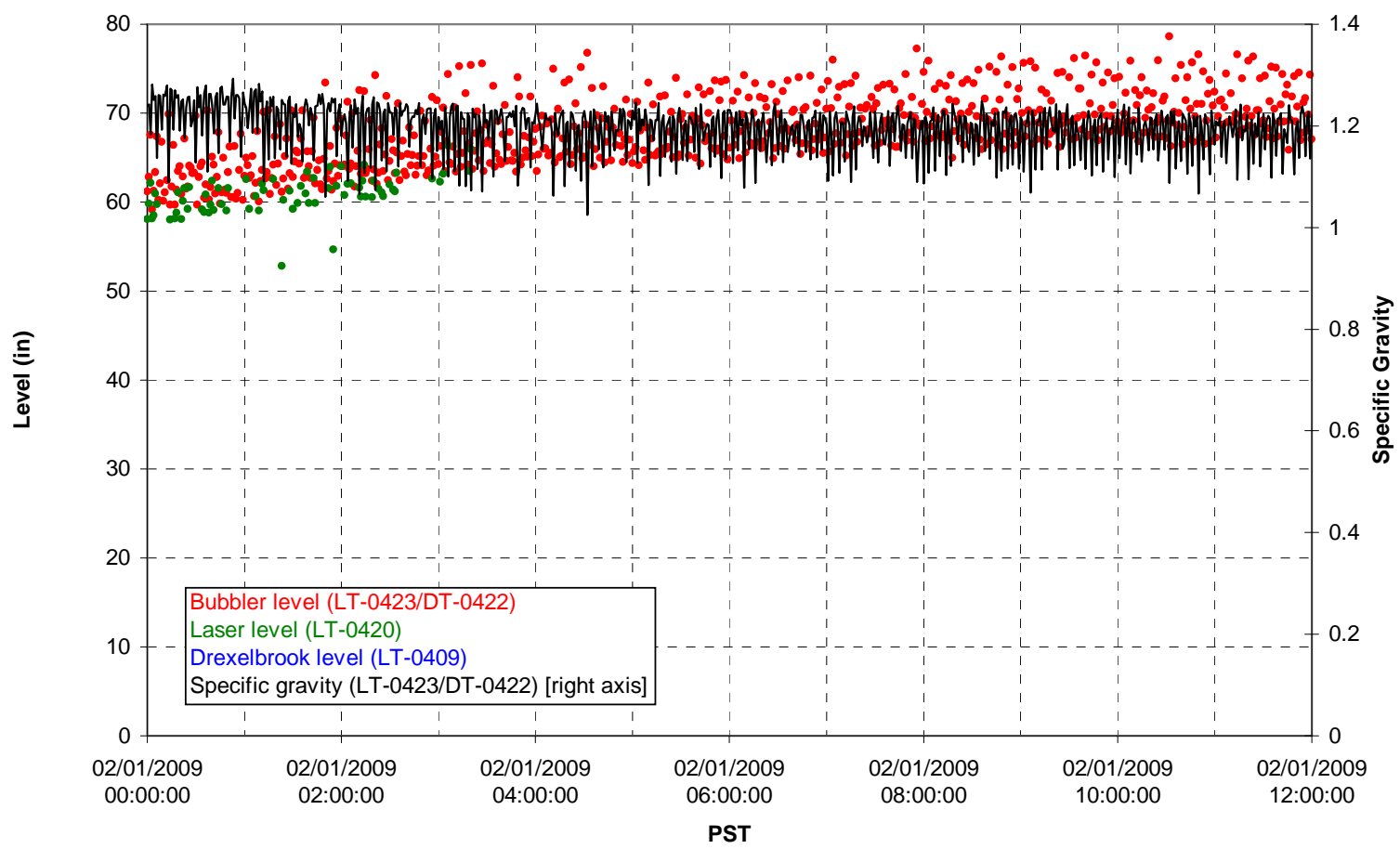

T01B temperatures

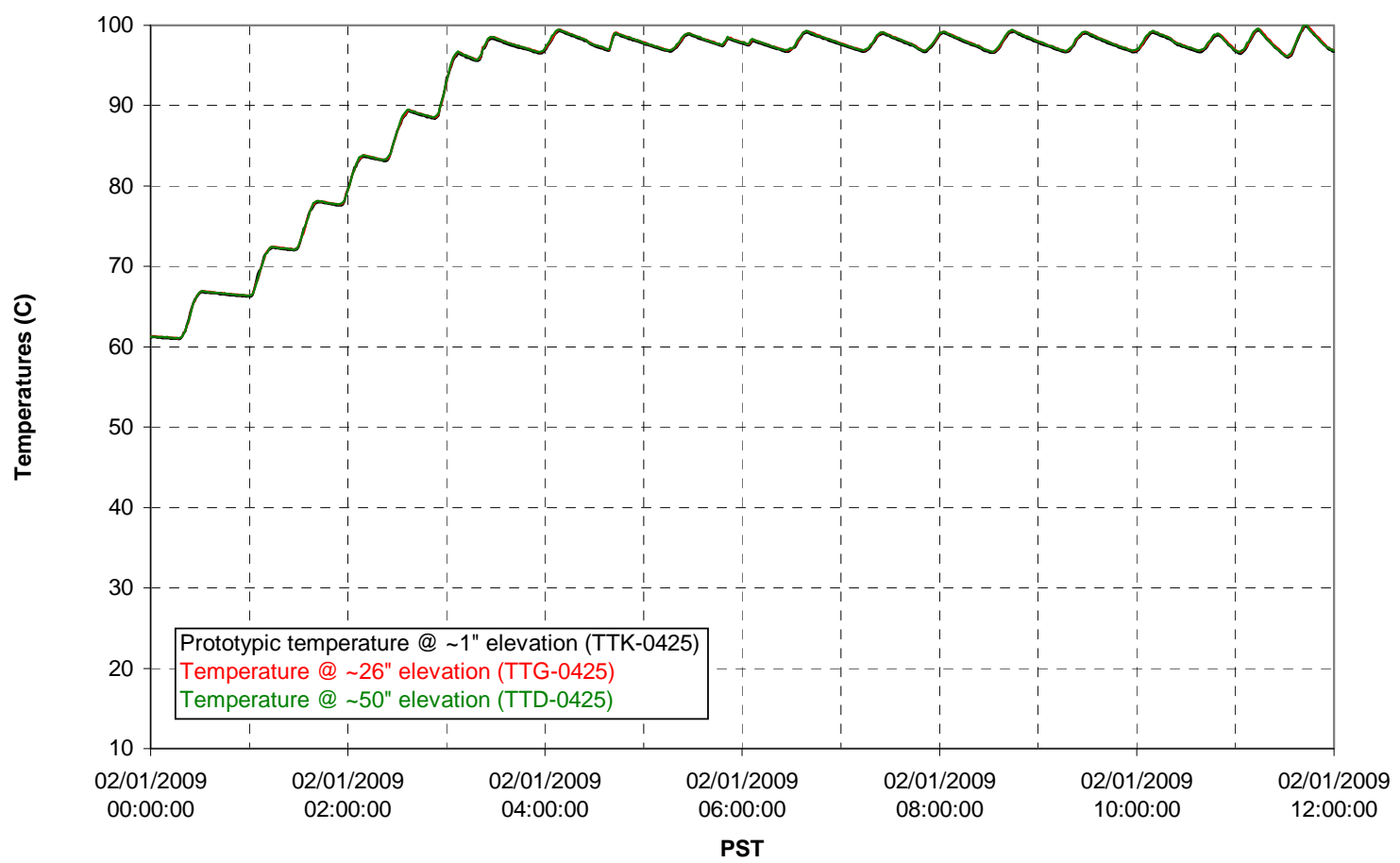


T02A level

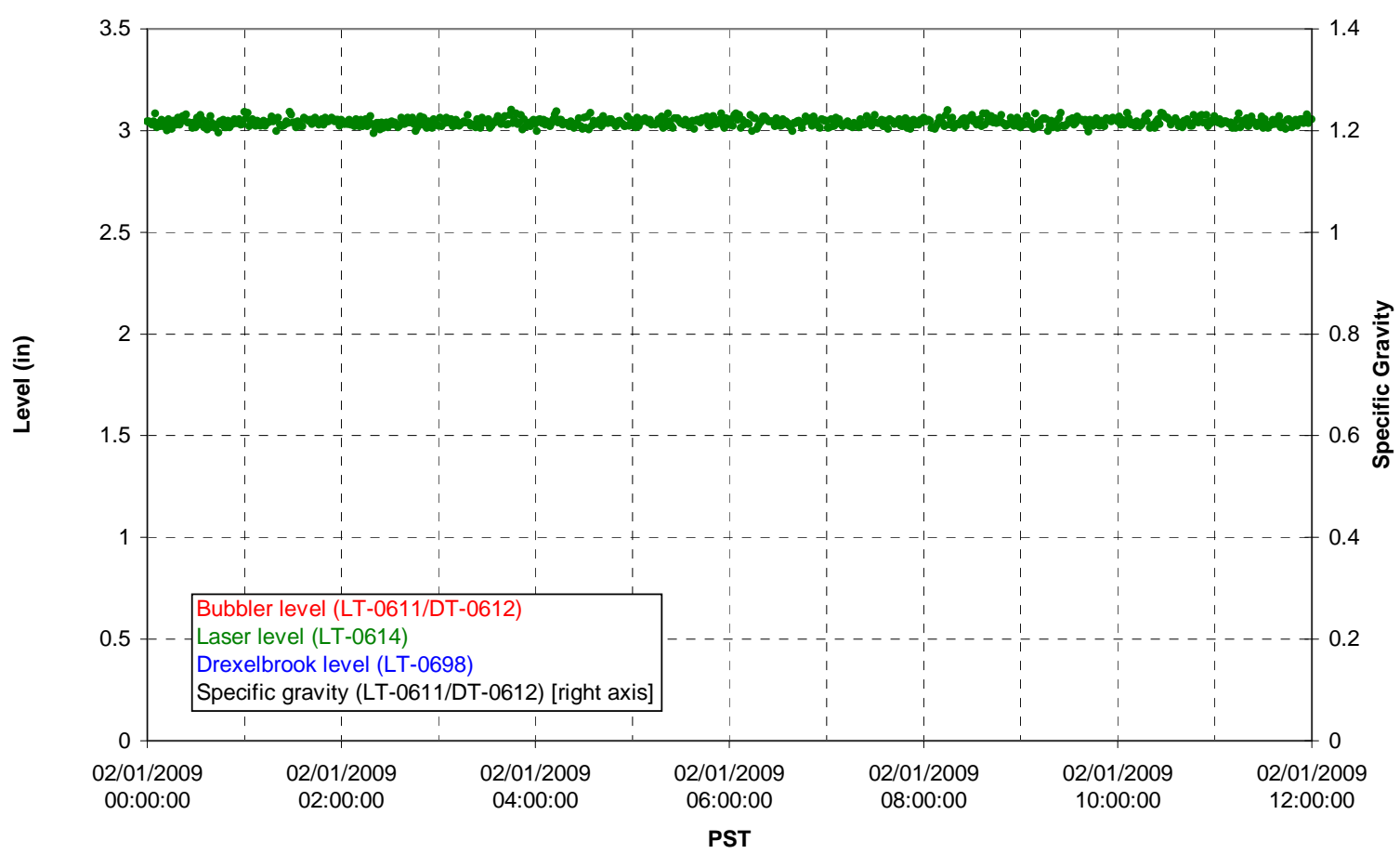

T02A temperatures

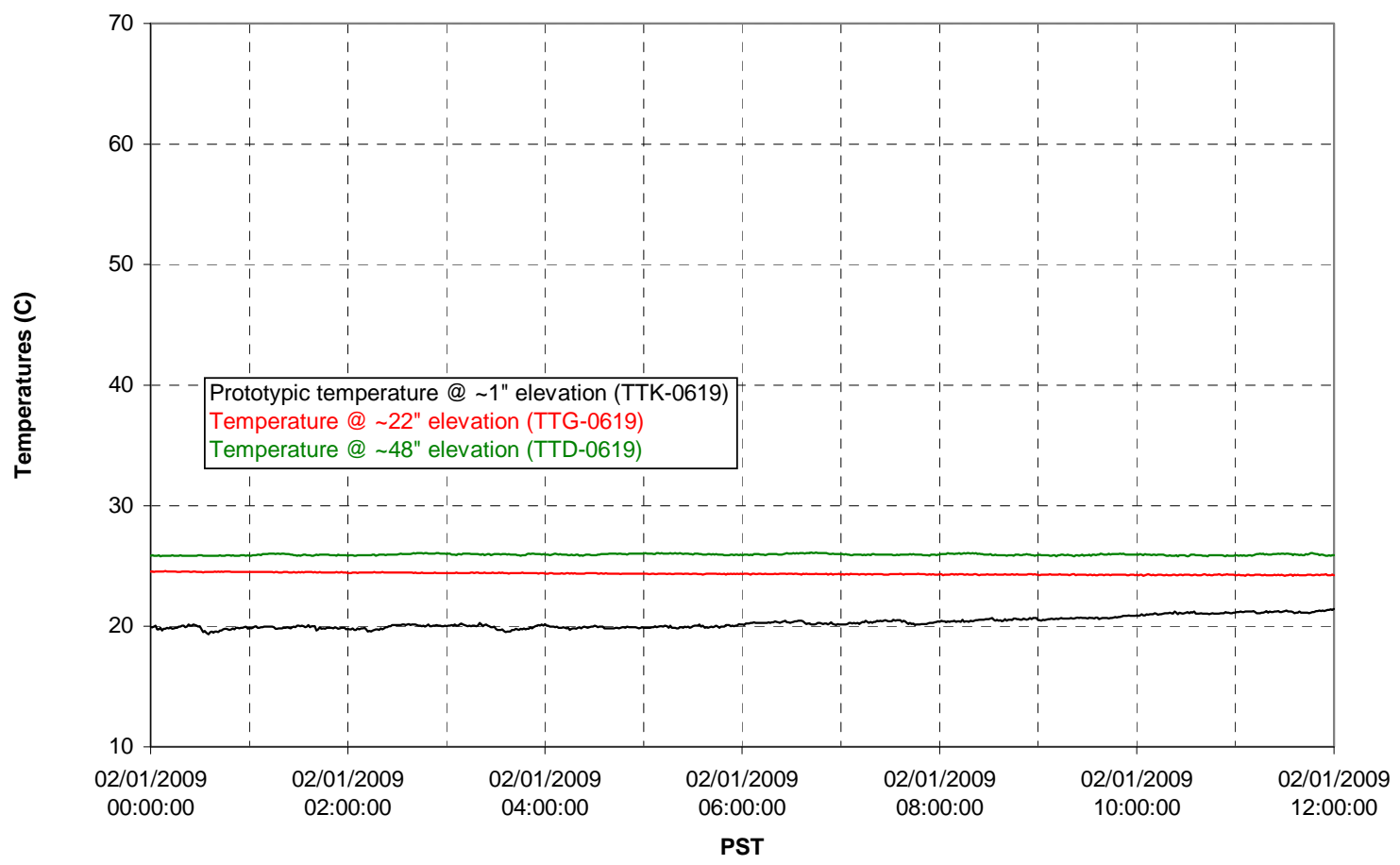


T02A and filter loop temperatures

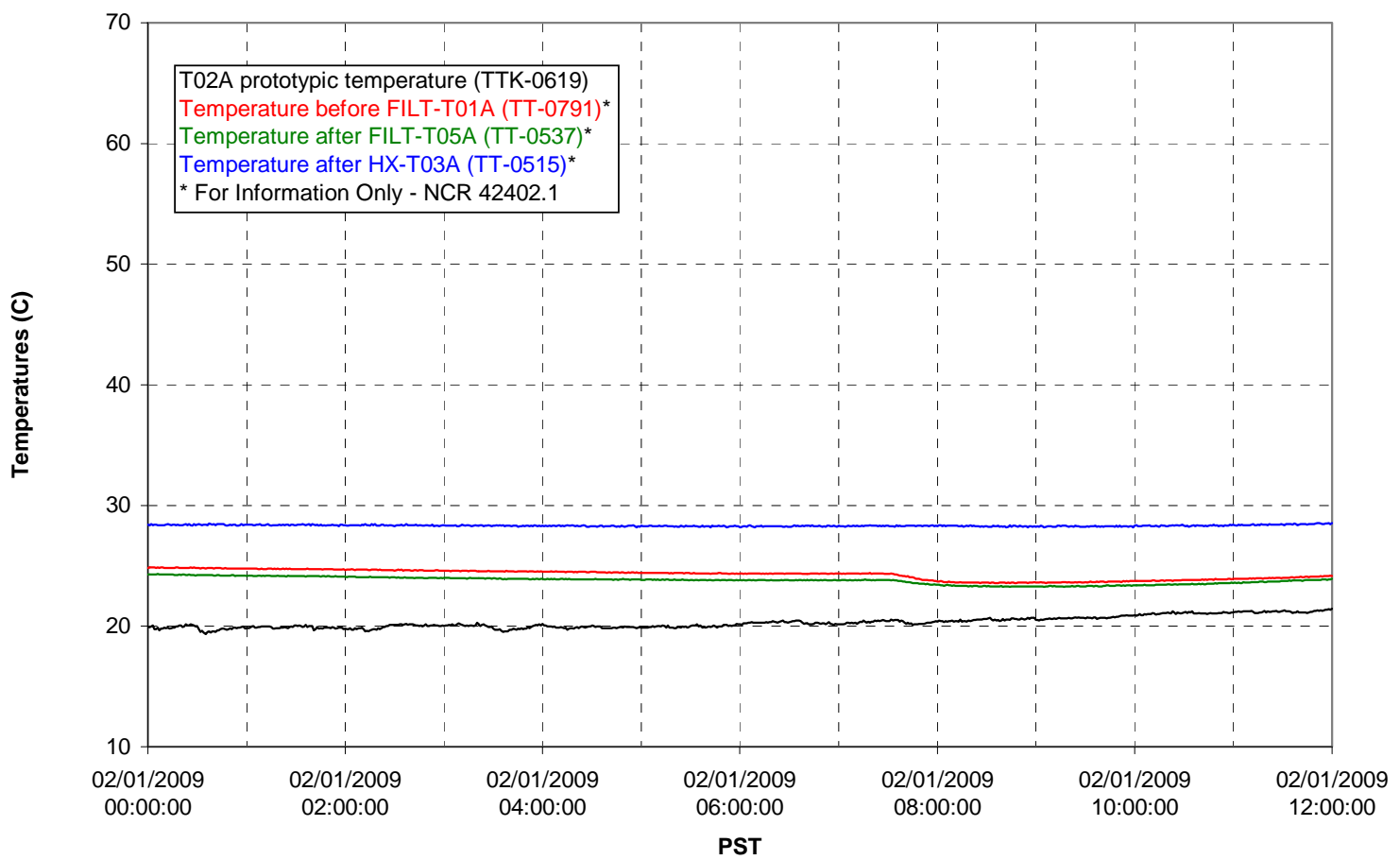

Pump Pressures and Flow

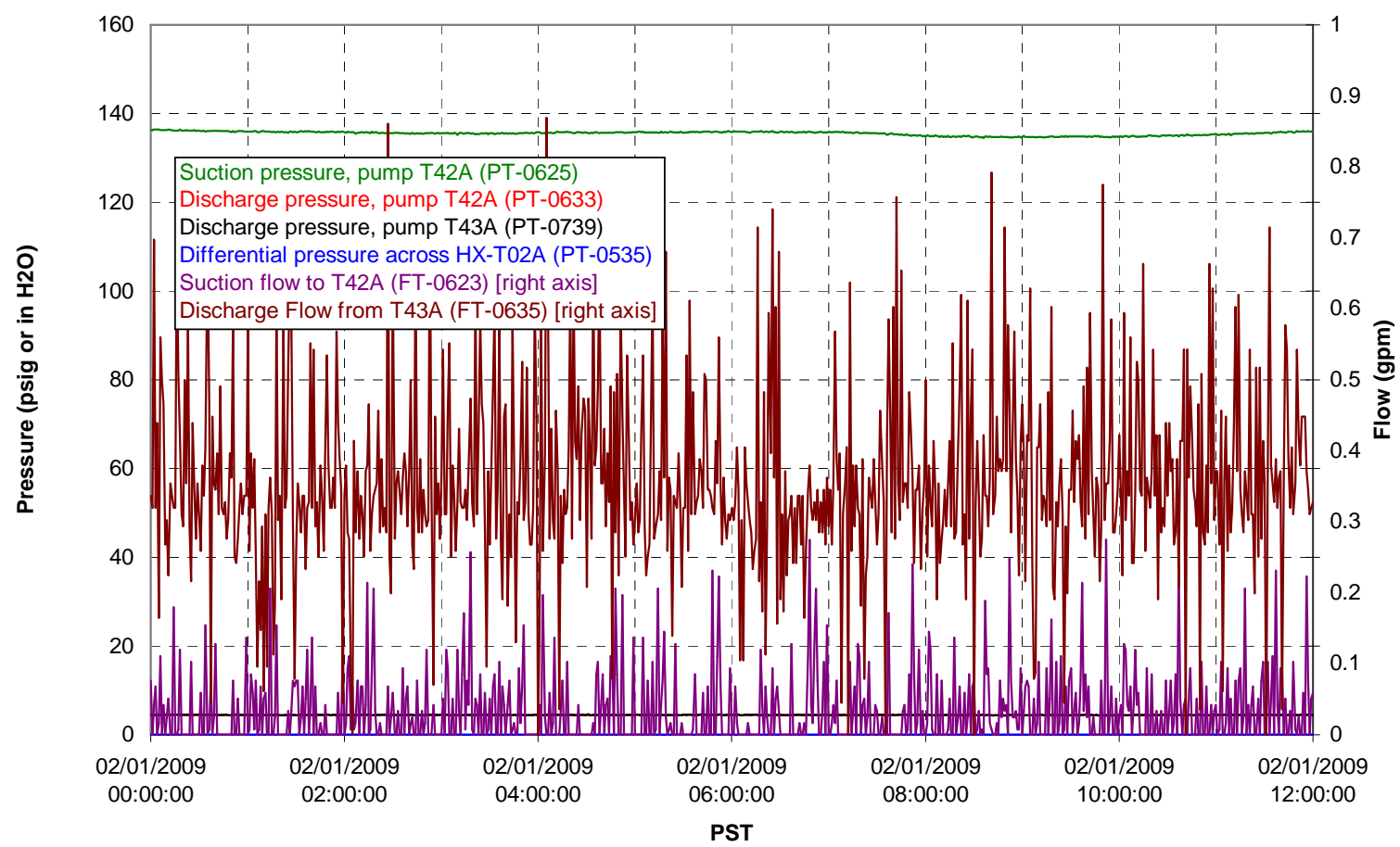


Axial pressure drop

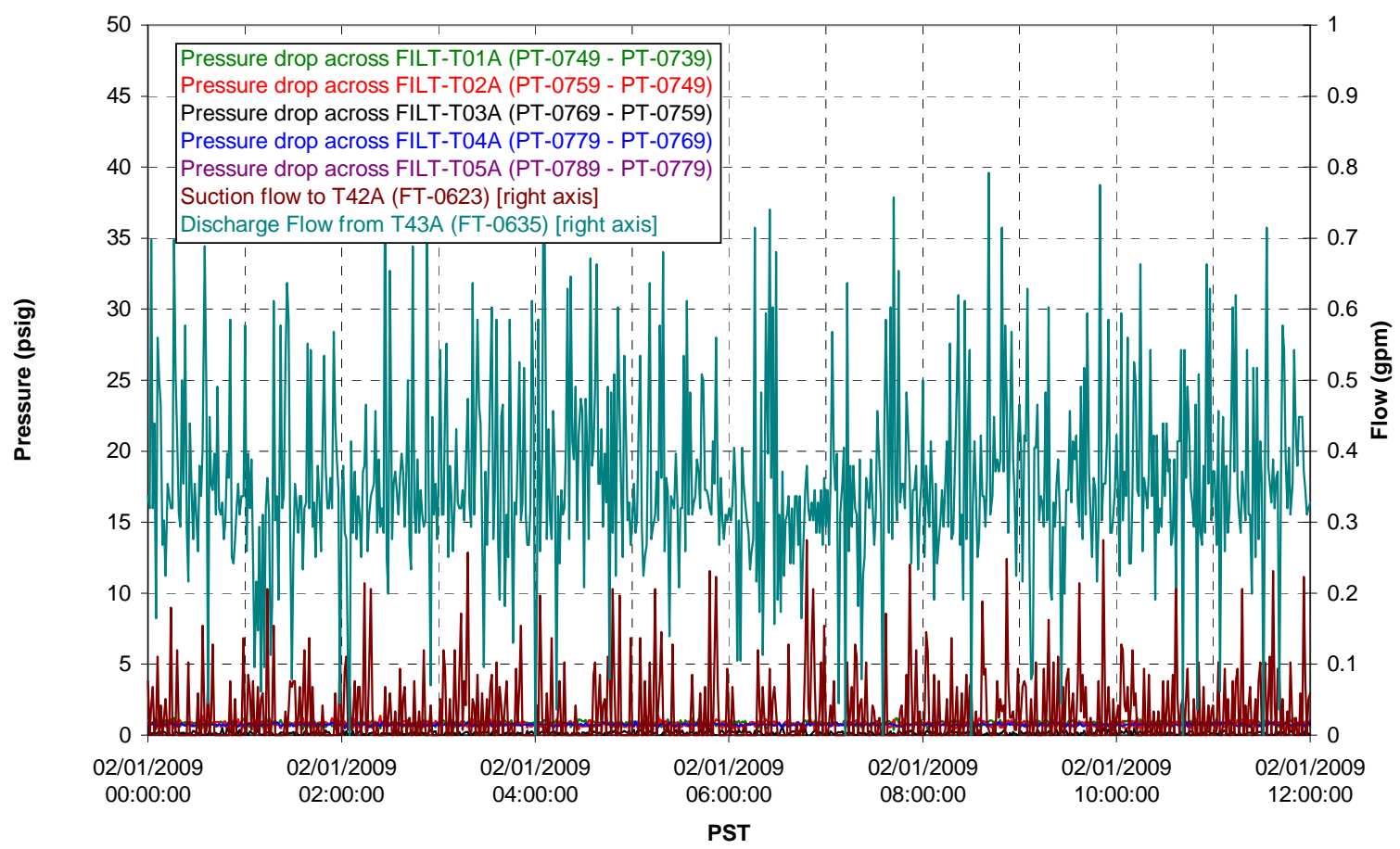

Permeate flow rates

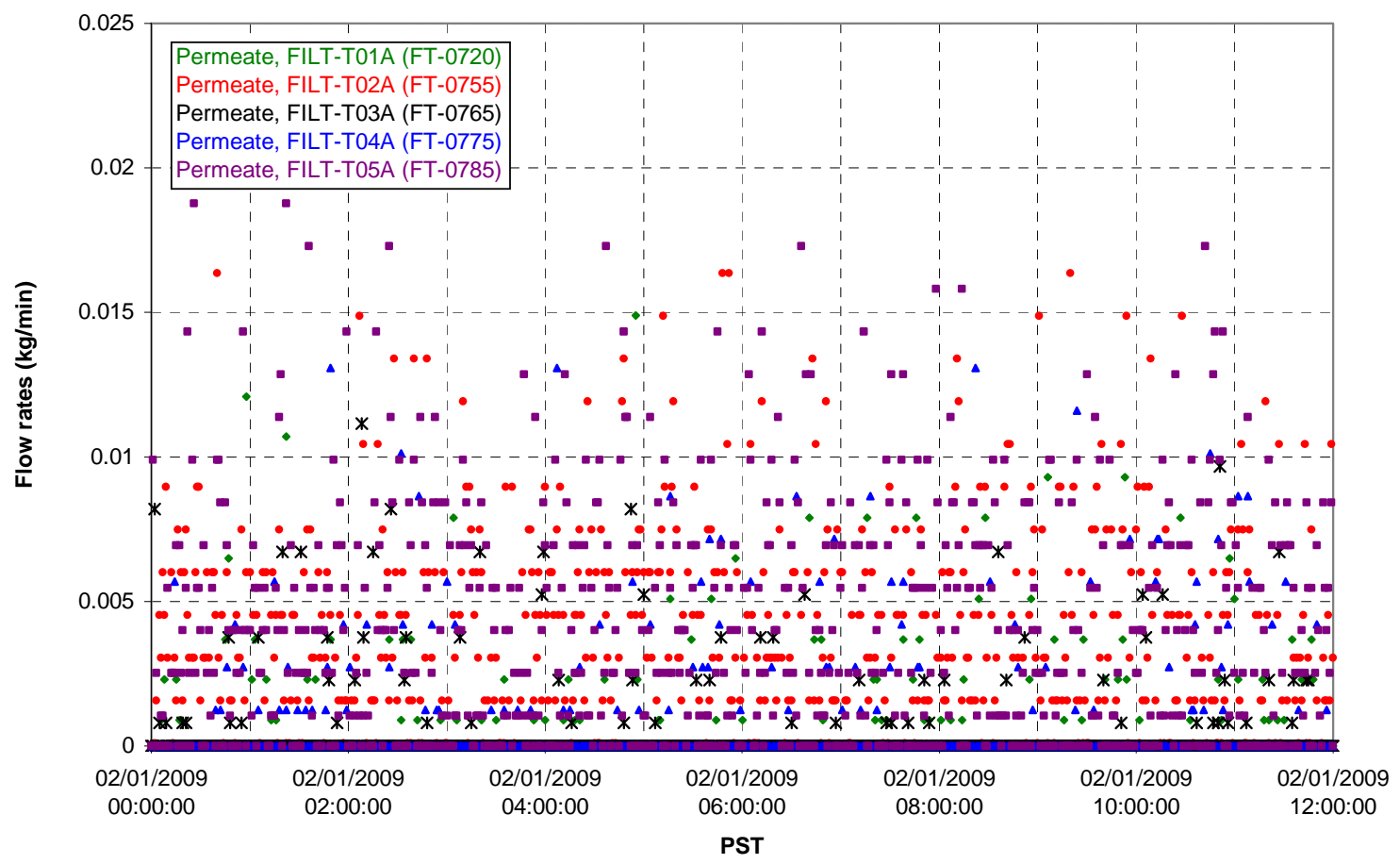


T02A Inner Temperature Tree

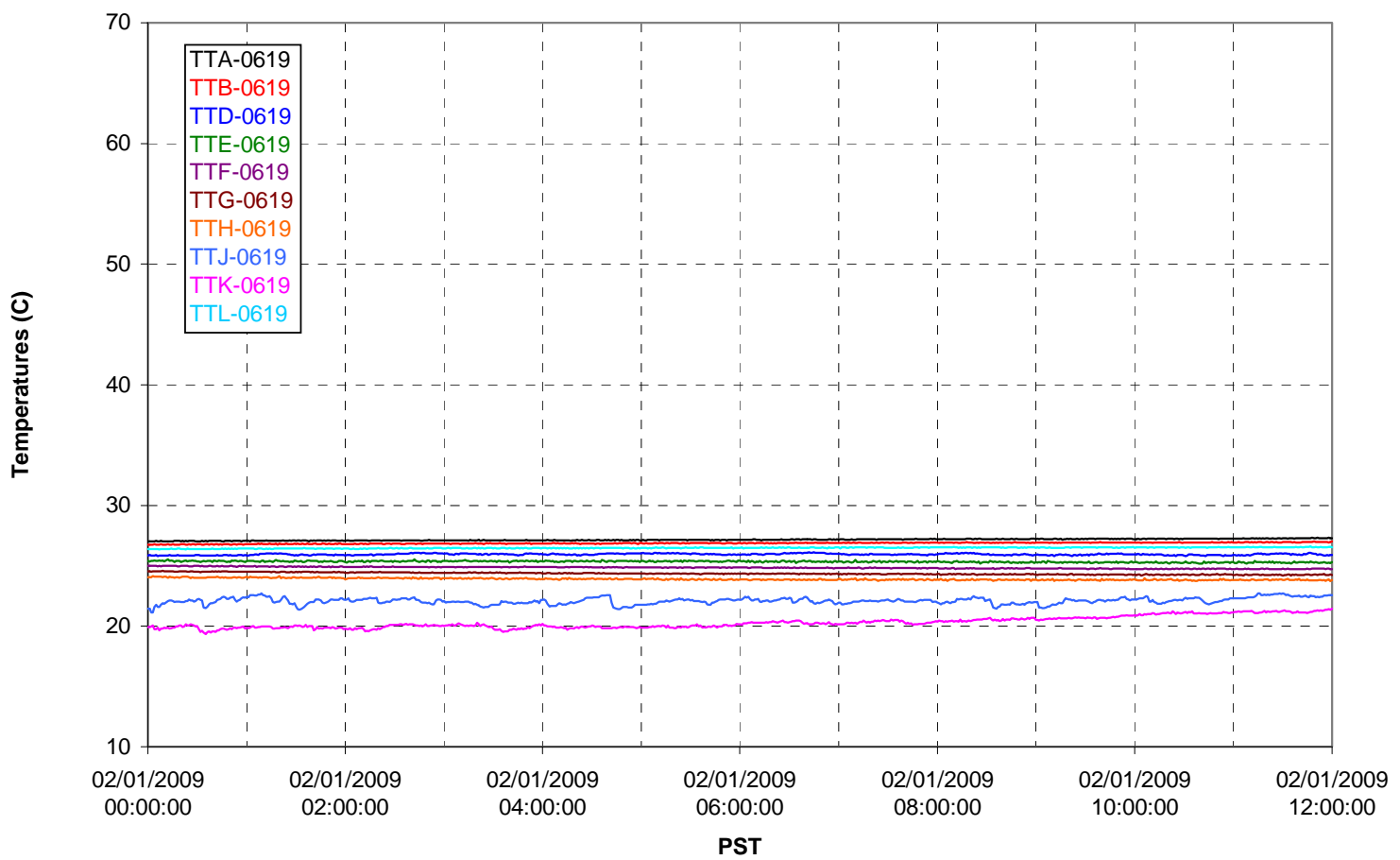

T02A Outer Temperature Tree

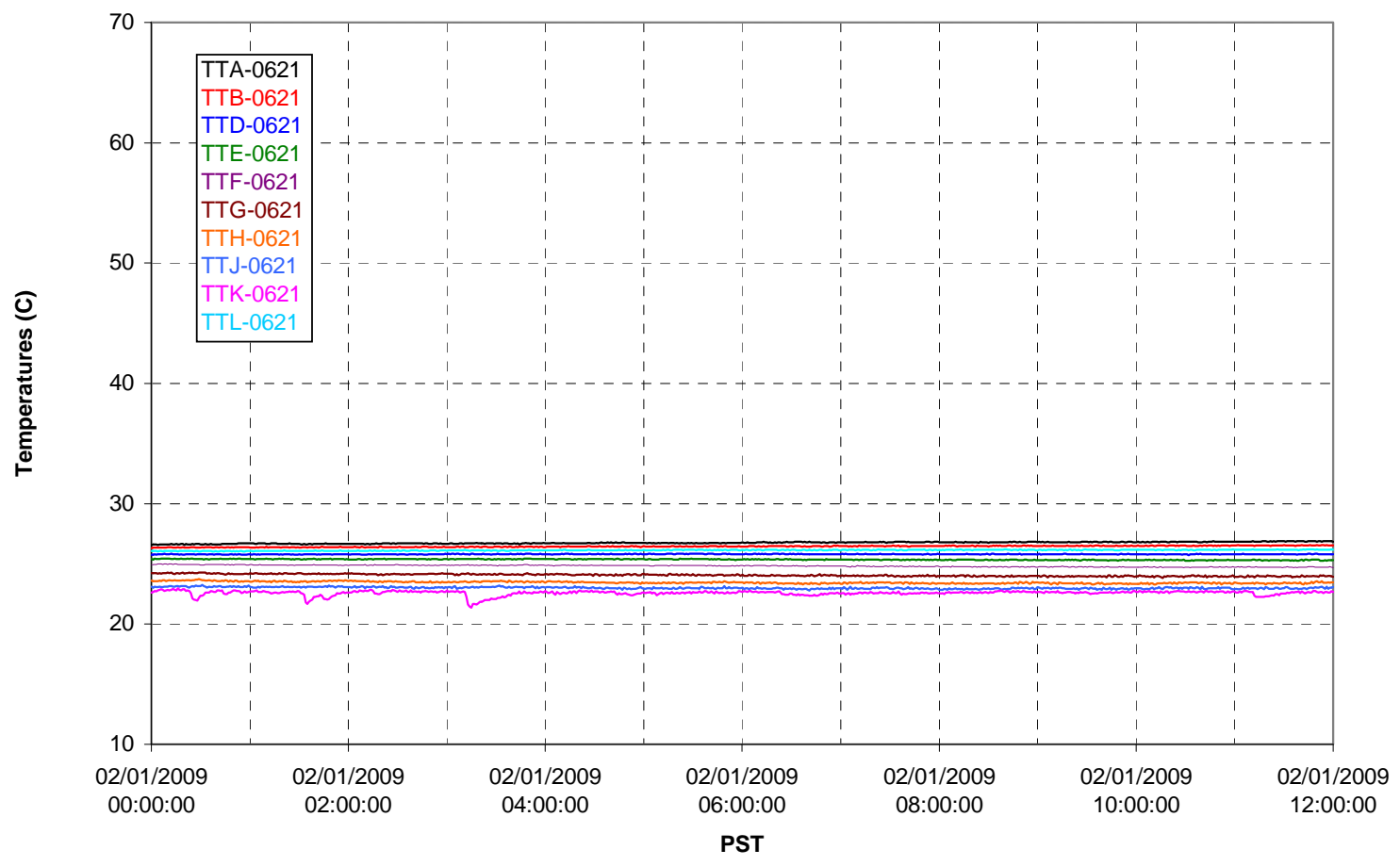


T02A temperatures

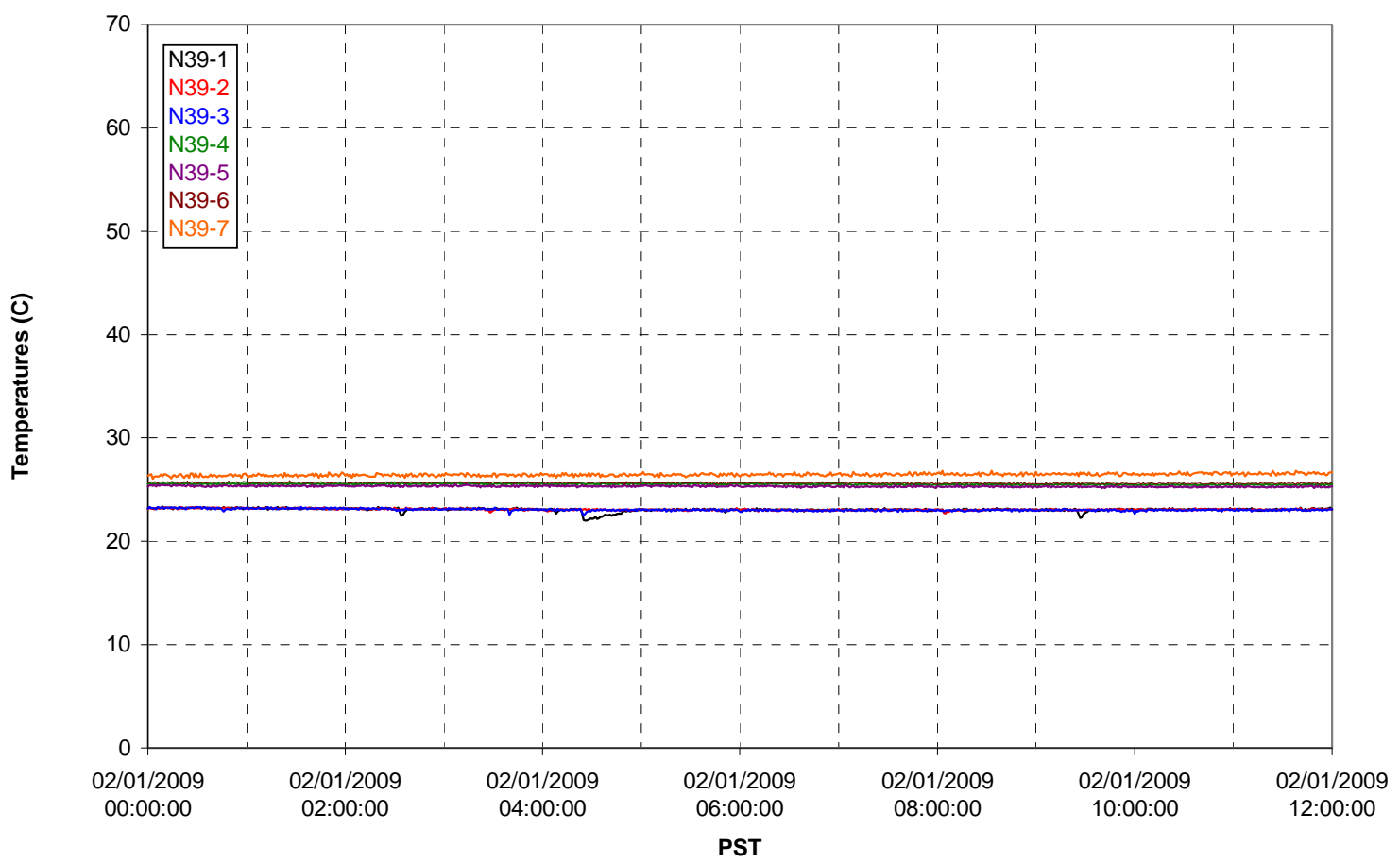

T02A temperatures

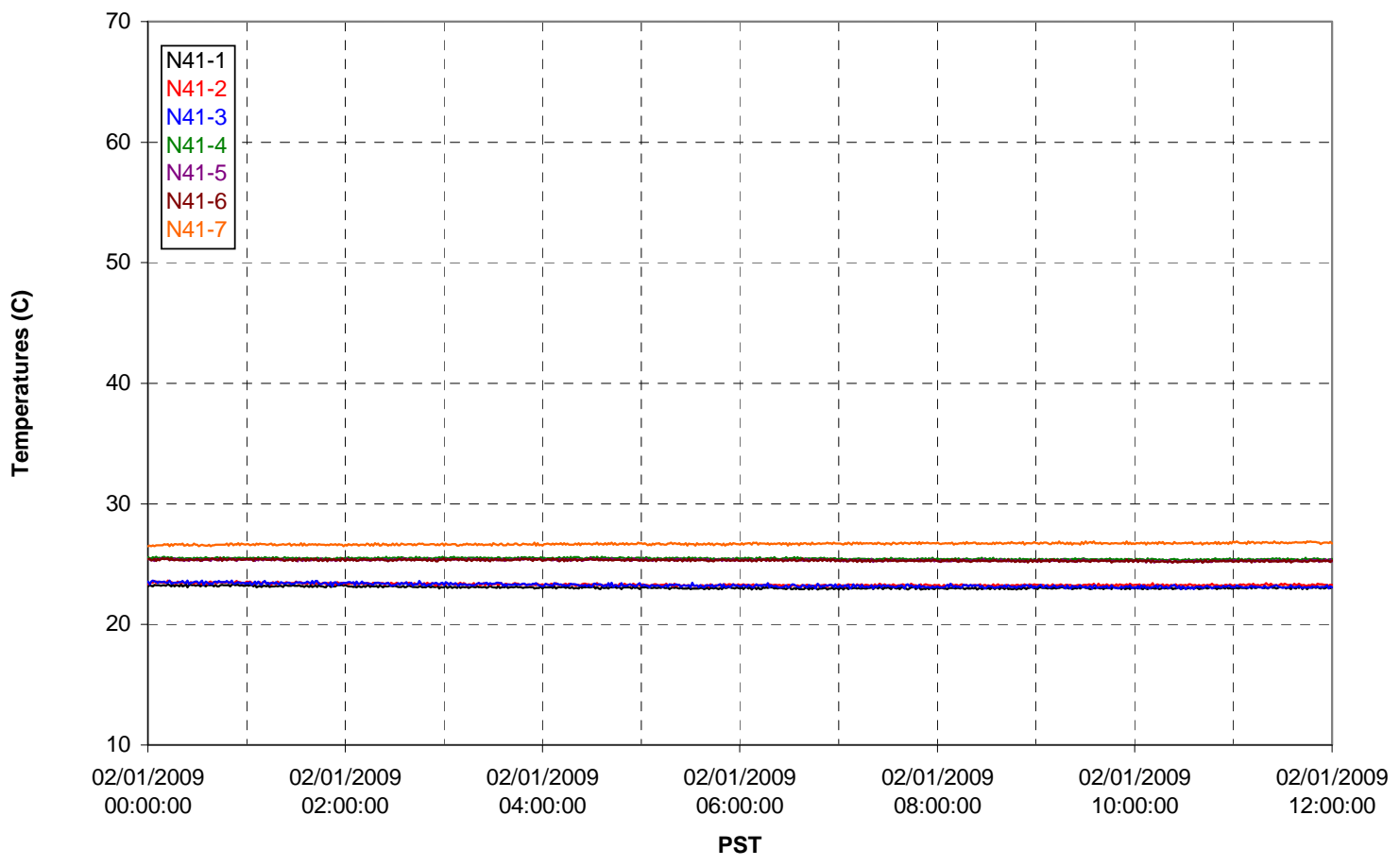


T02A temperatures

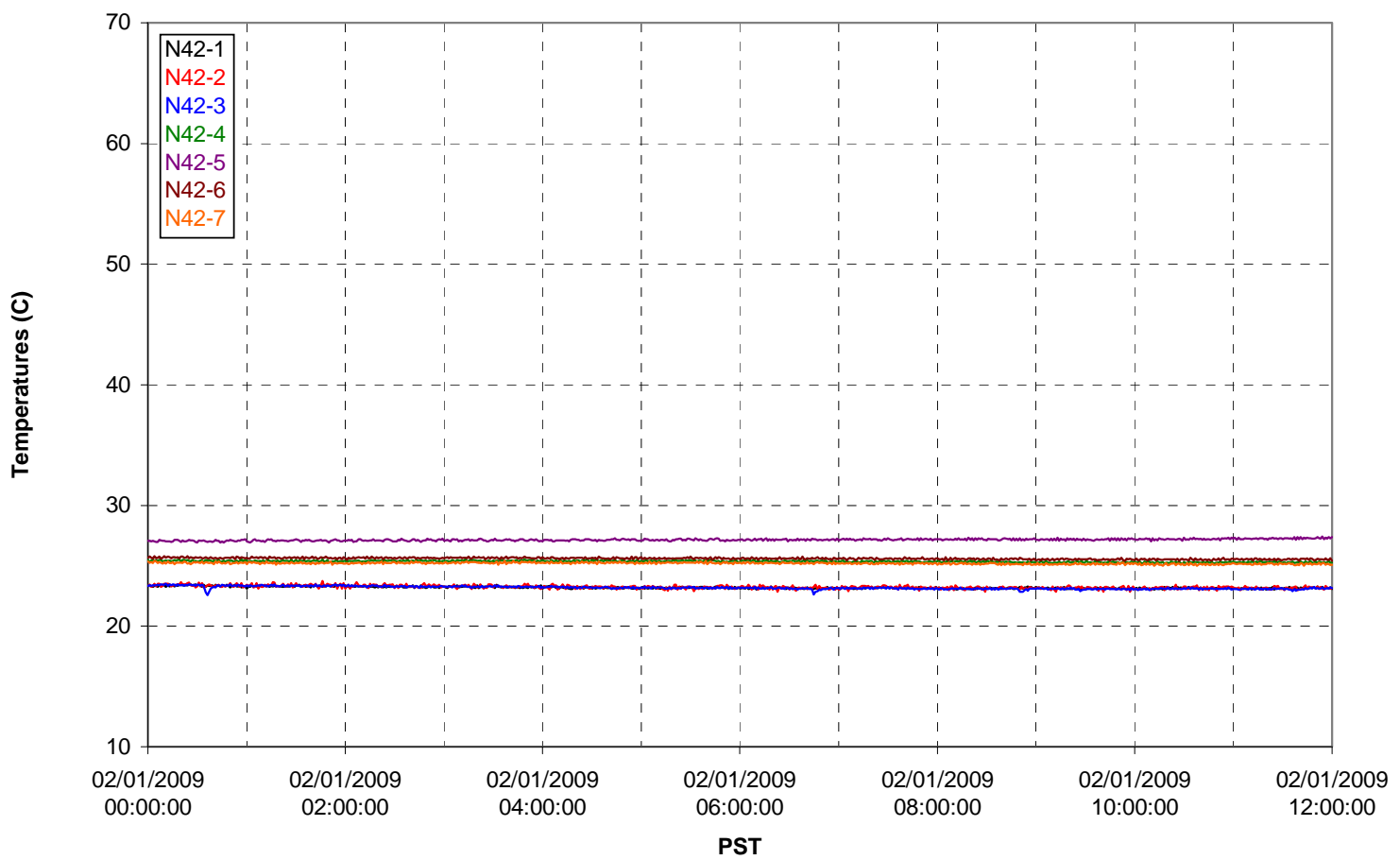

T02A temperatures

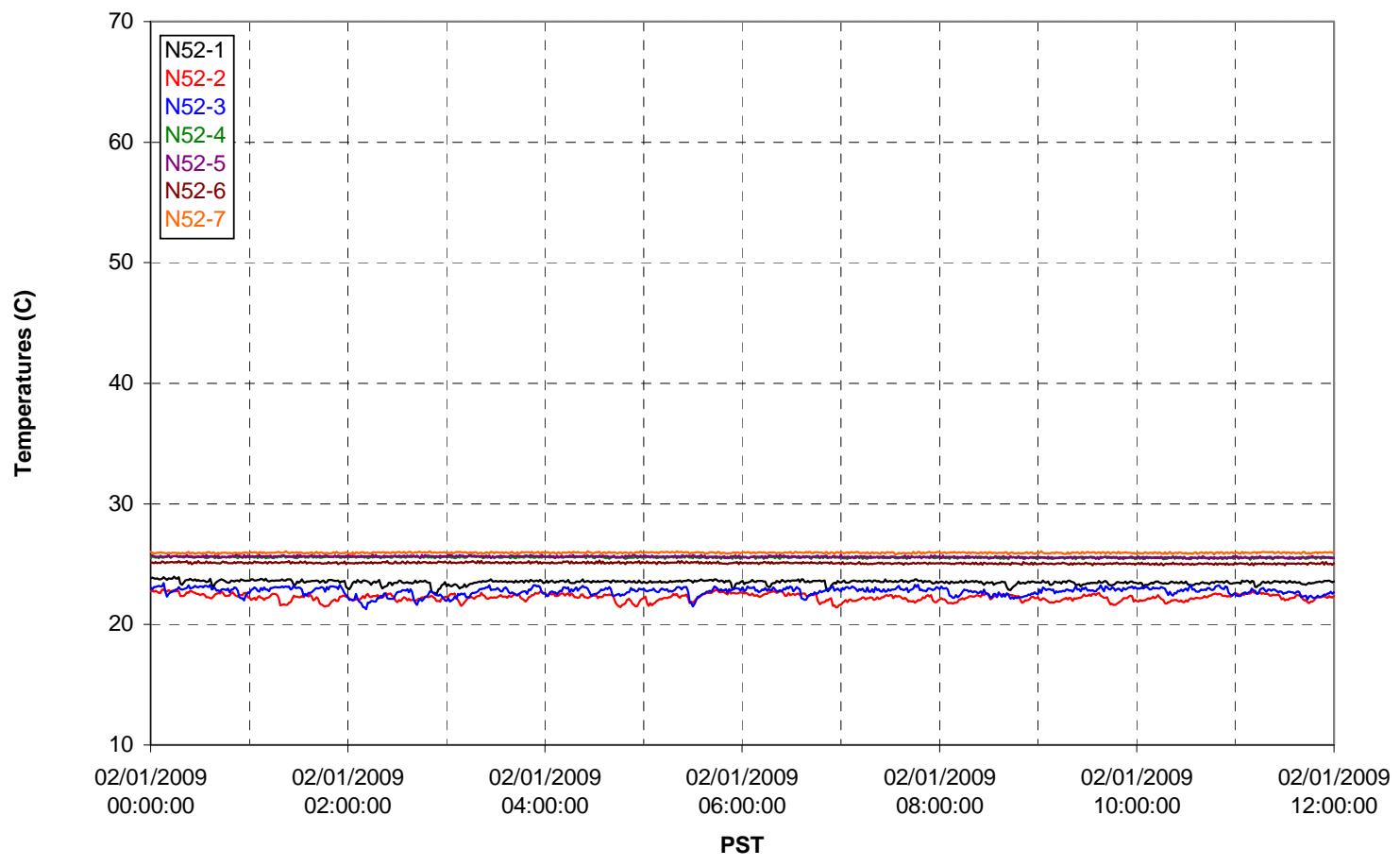


T02A Heating and Cooling

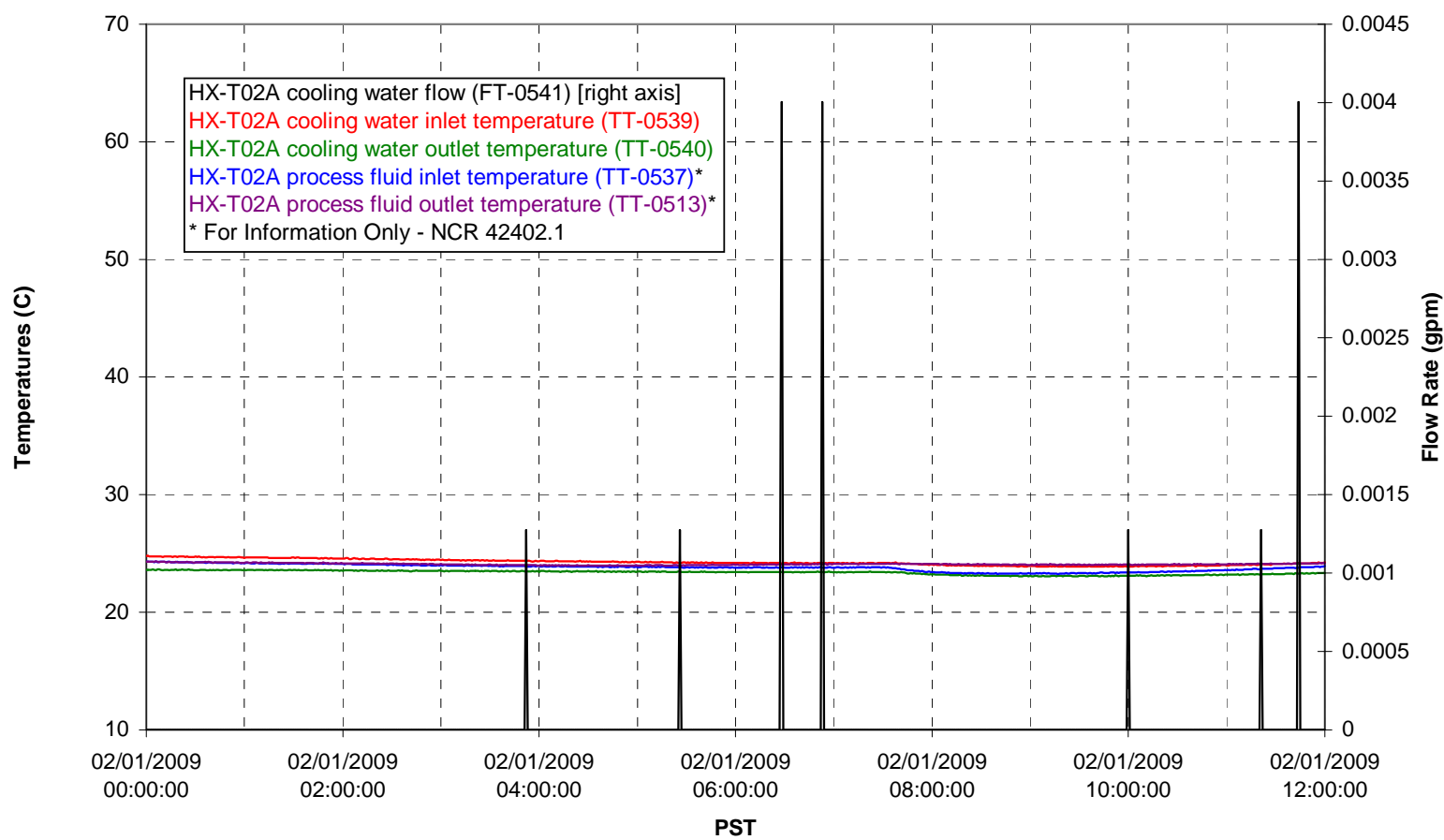

Pump Operation

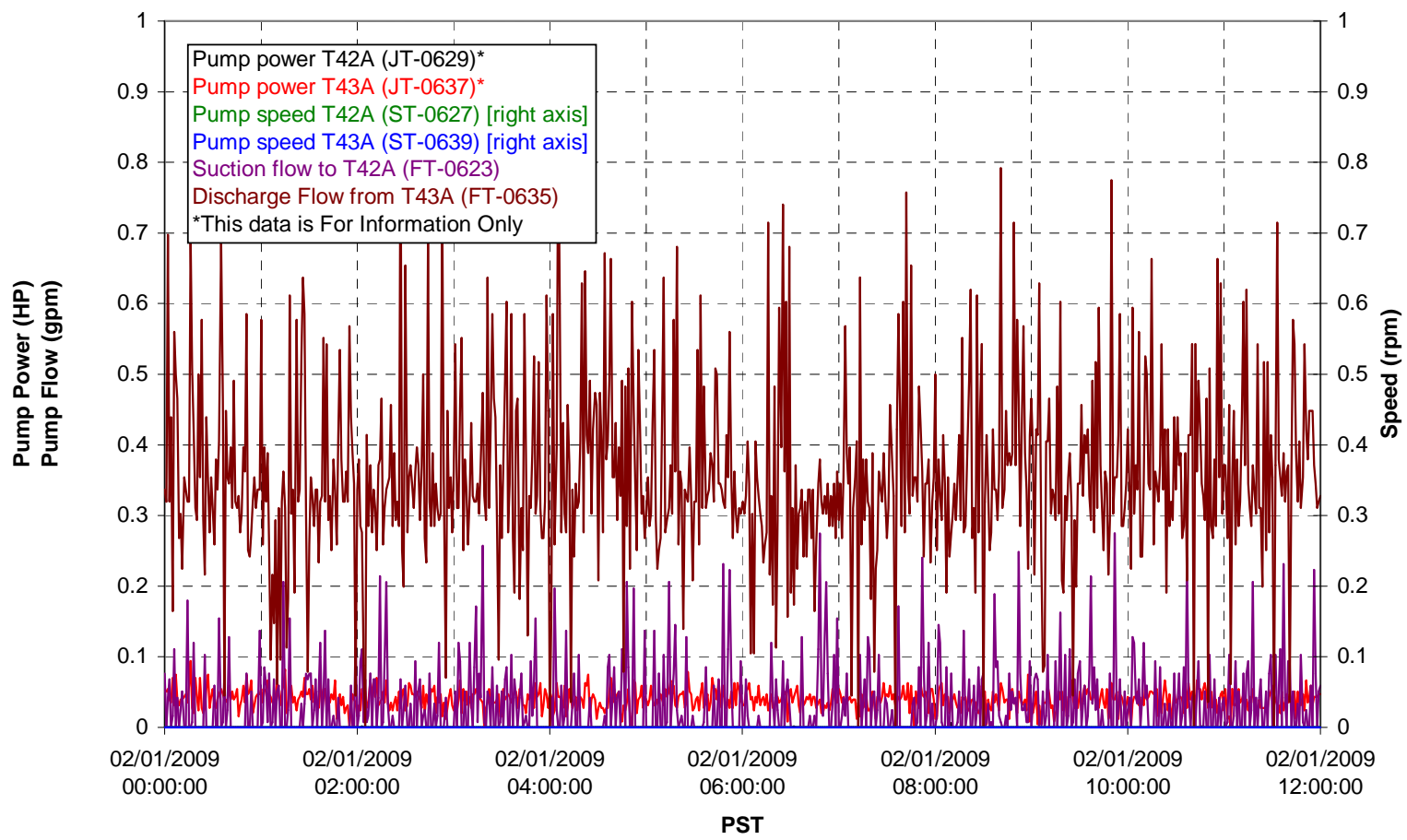


Pulsepot UFP-PP-T01A

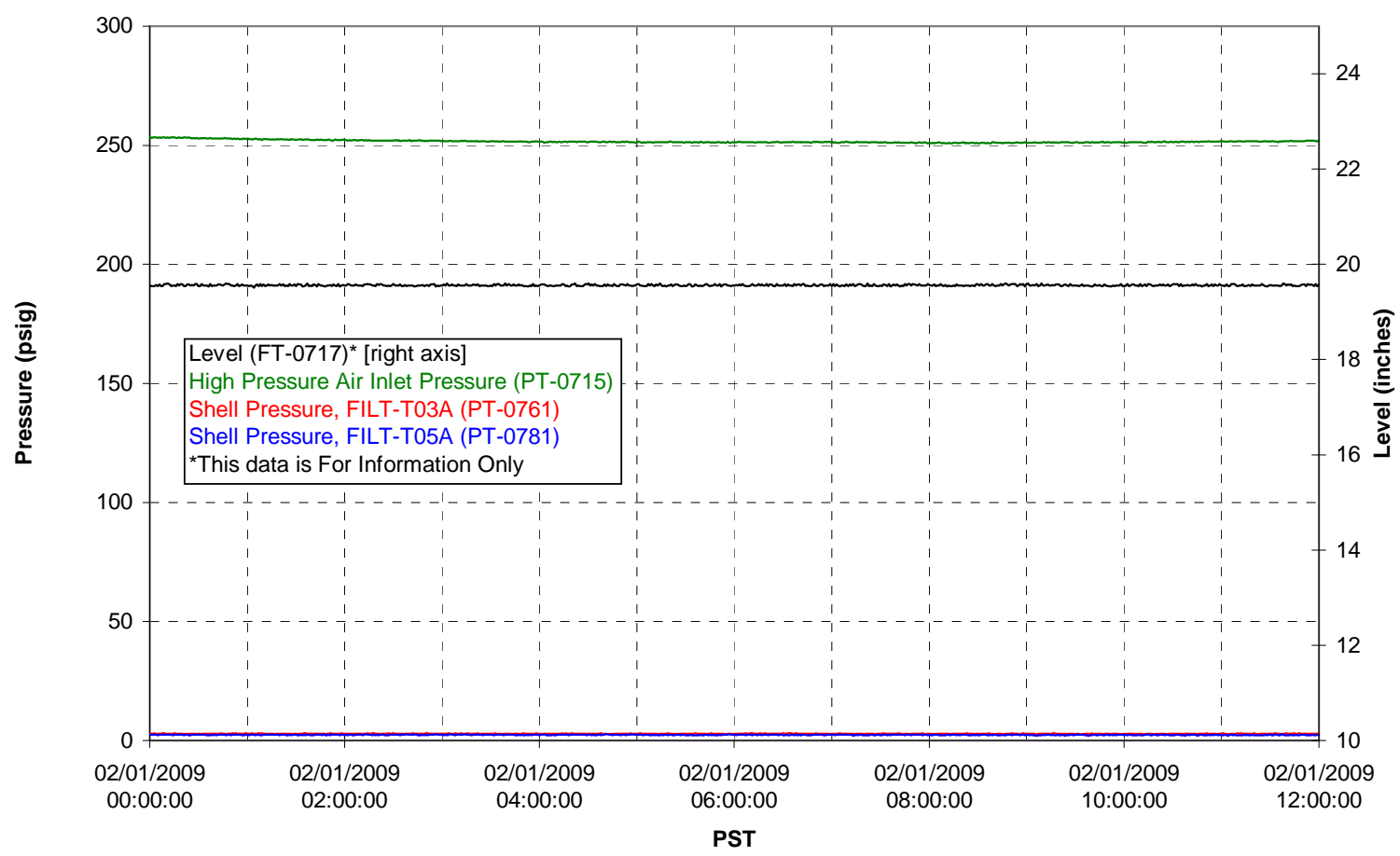

Pulsepot UFP-PP-T02A

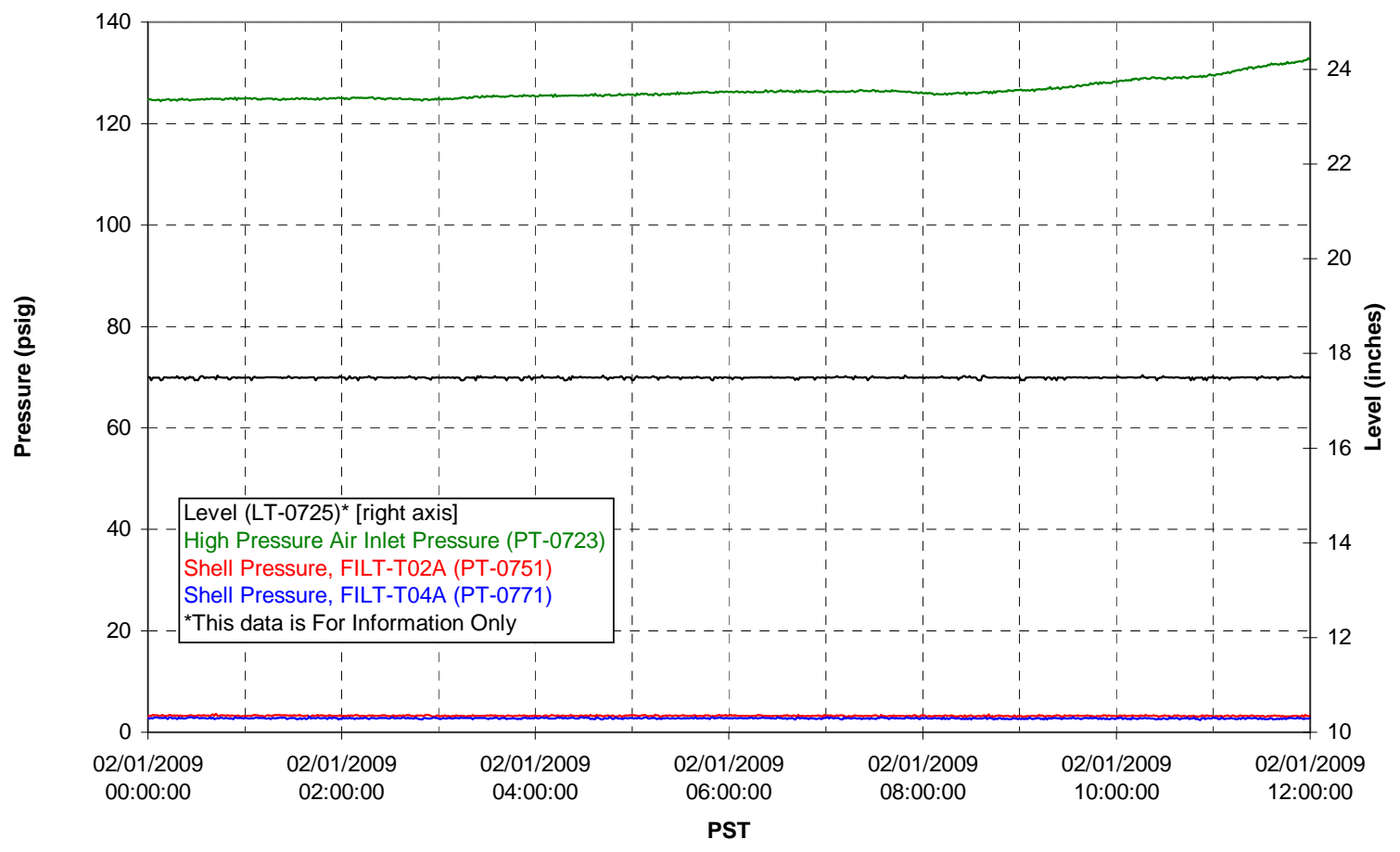


Pulsepot UFP-PP-T03A

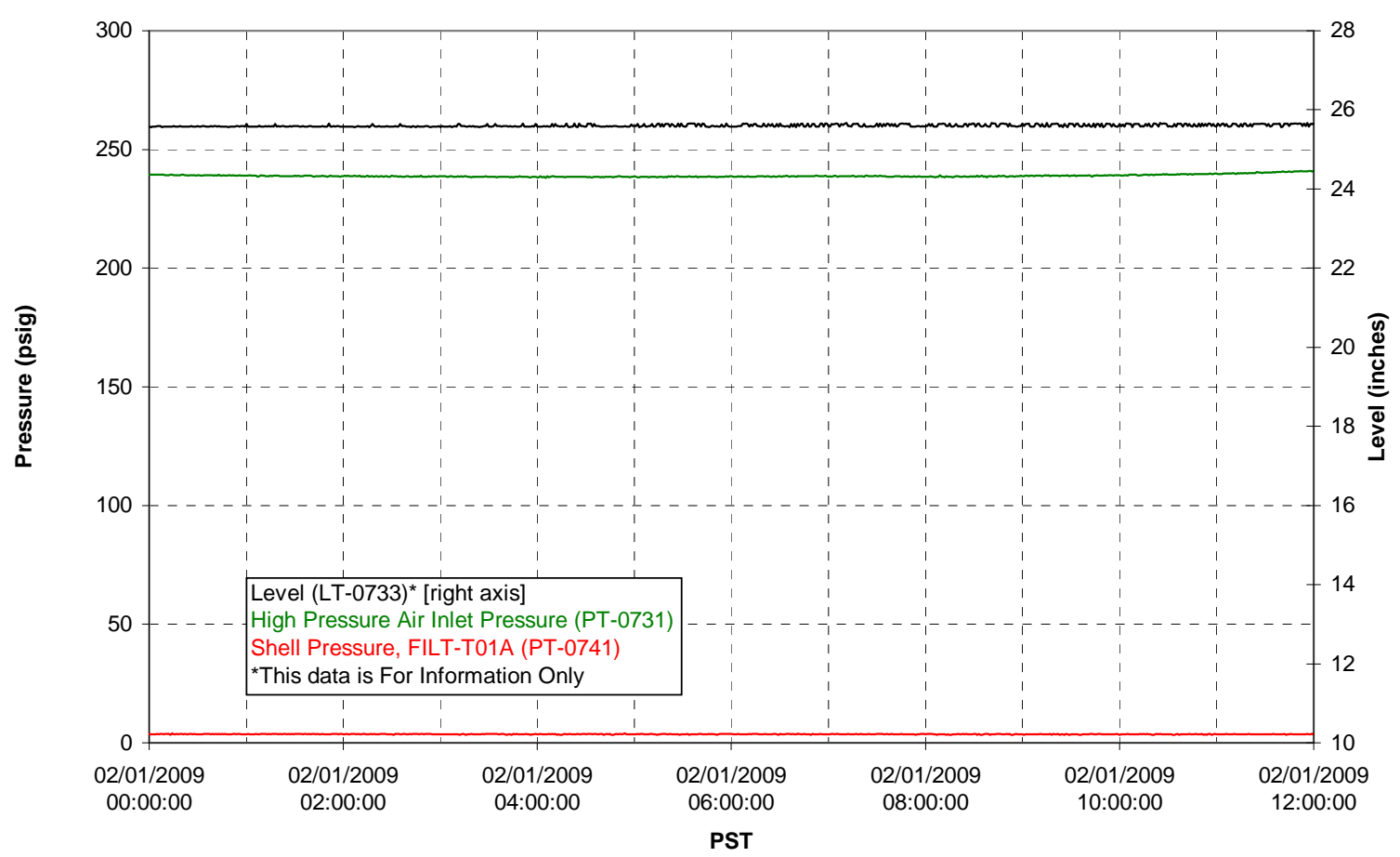

Pulsepot Levels

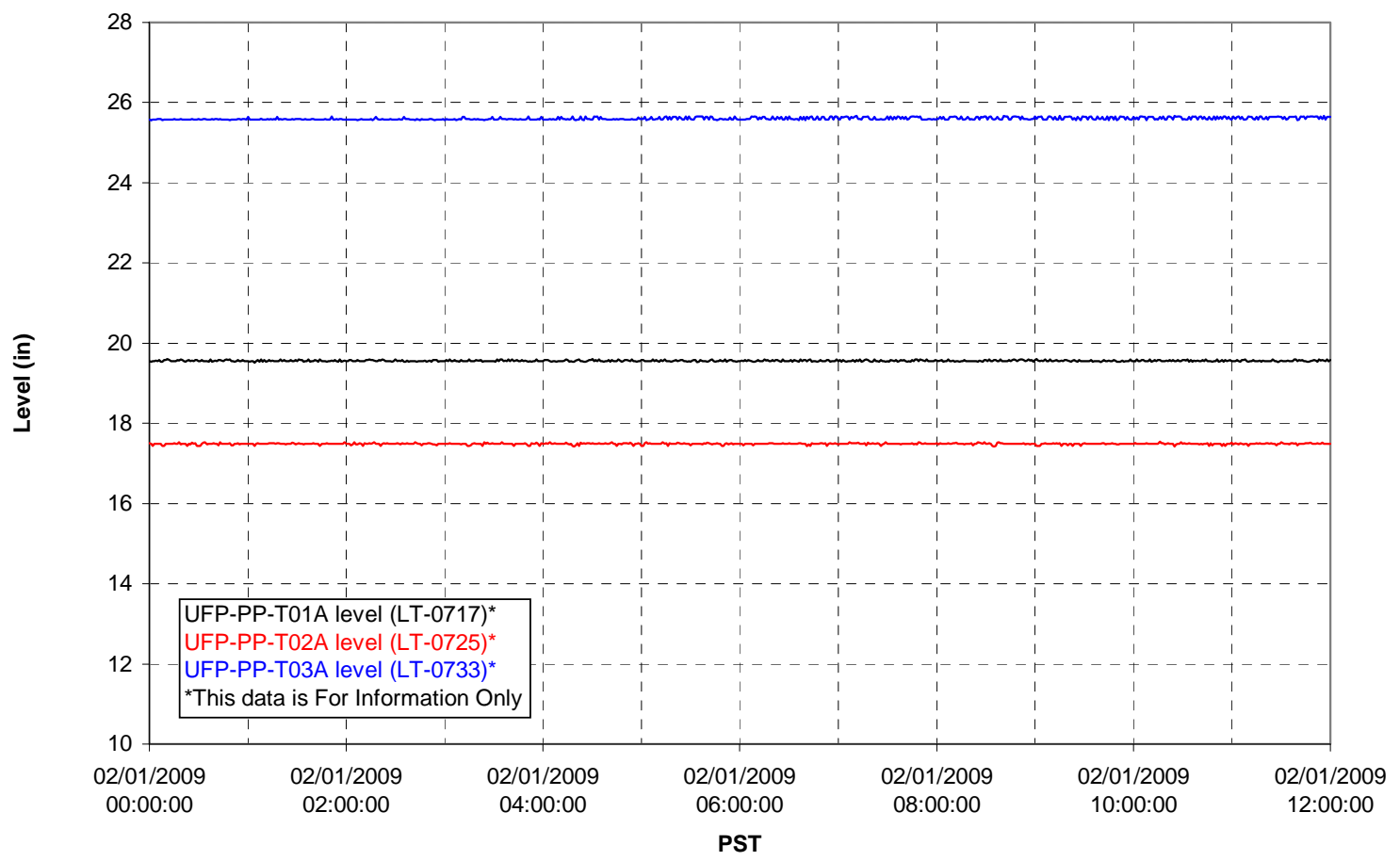


Filter UFP-FILT-T01A

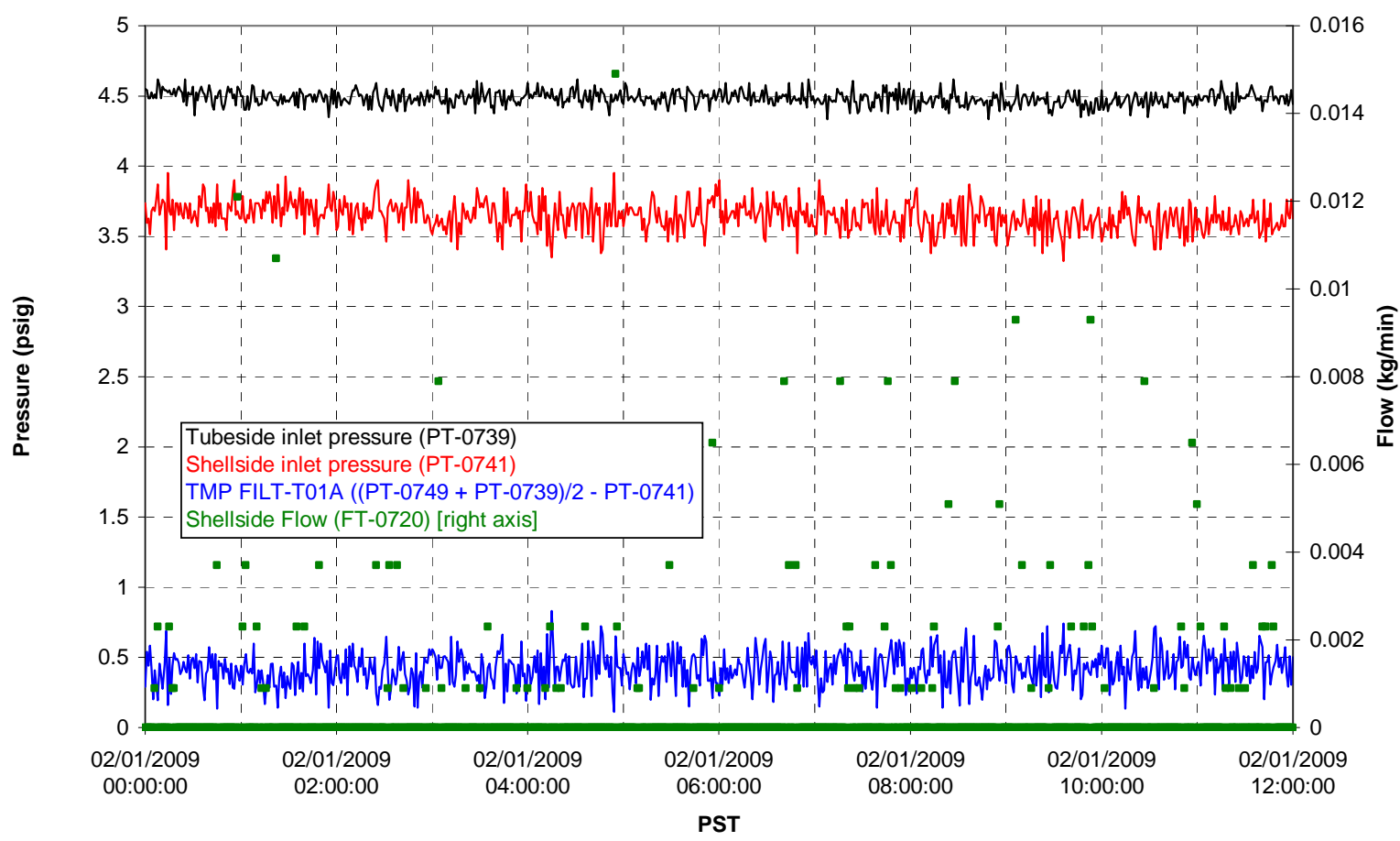

Filter UFP-FILT-T02A

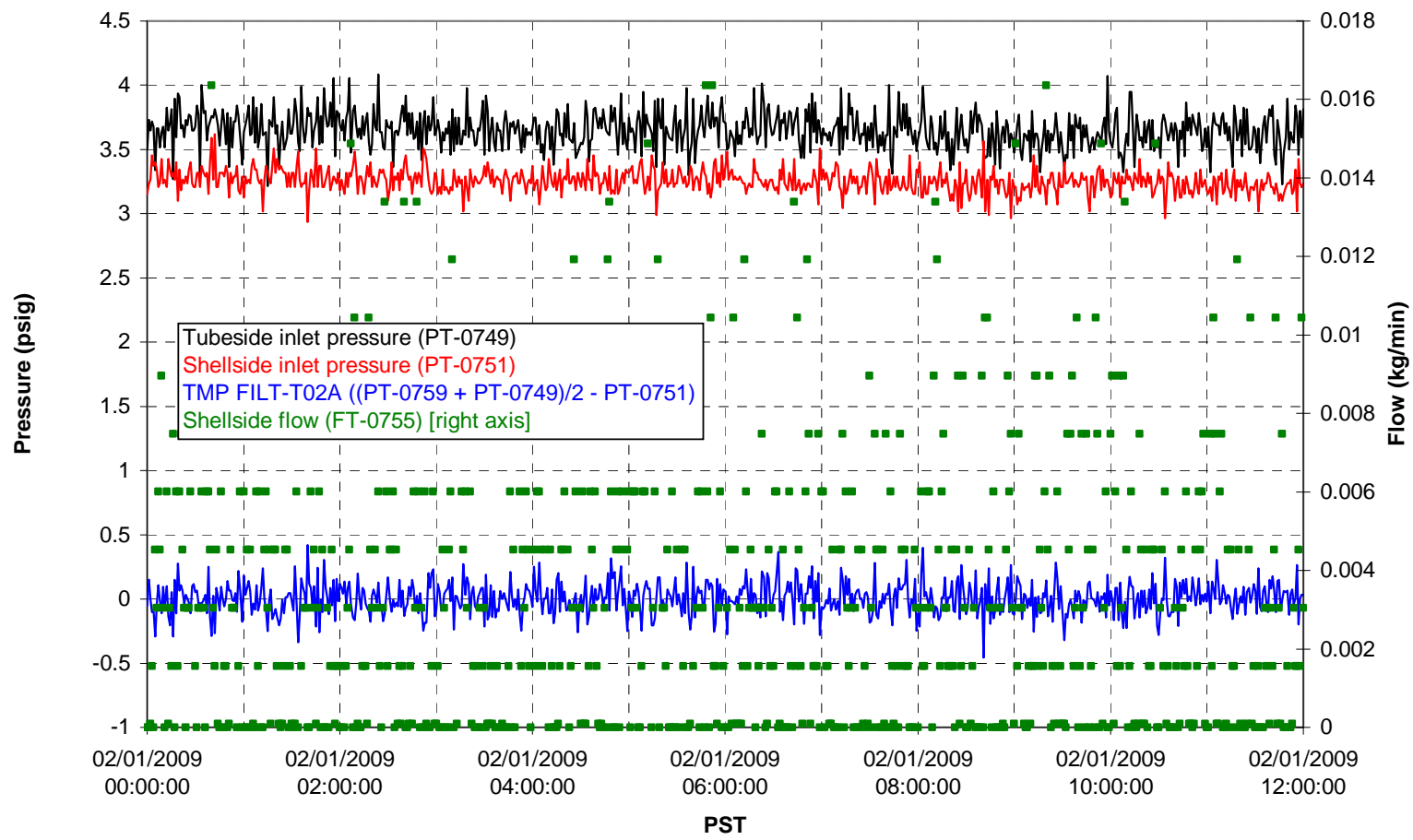


Filter UFP-FILT-T03A

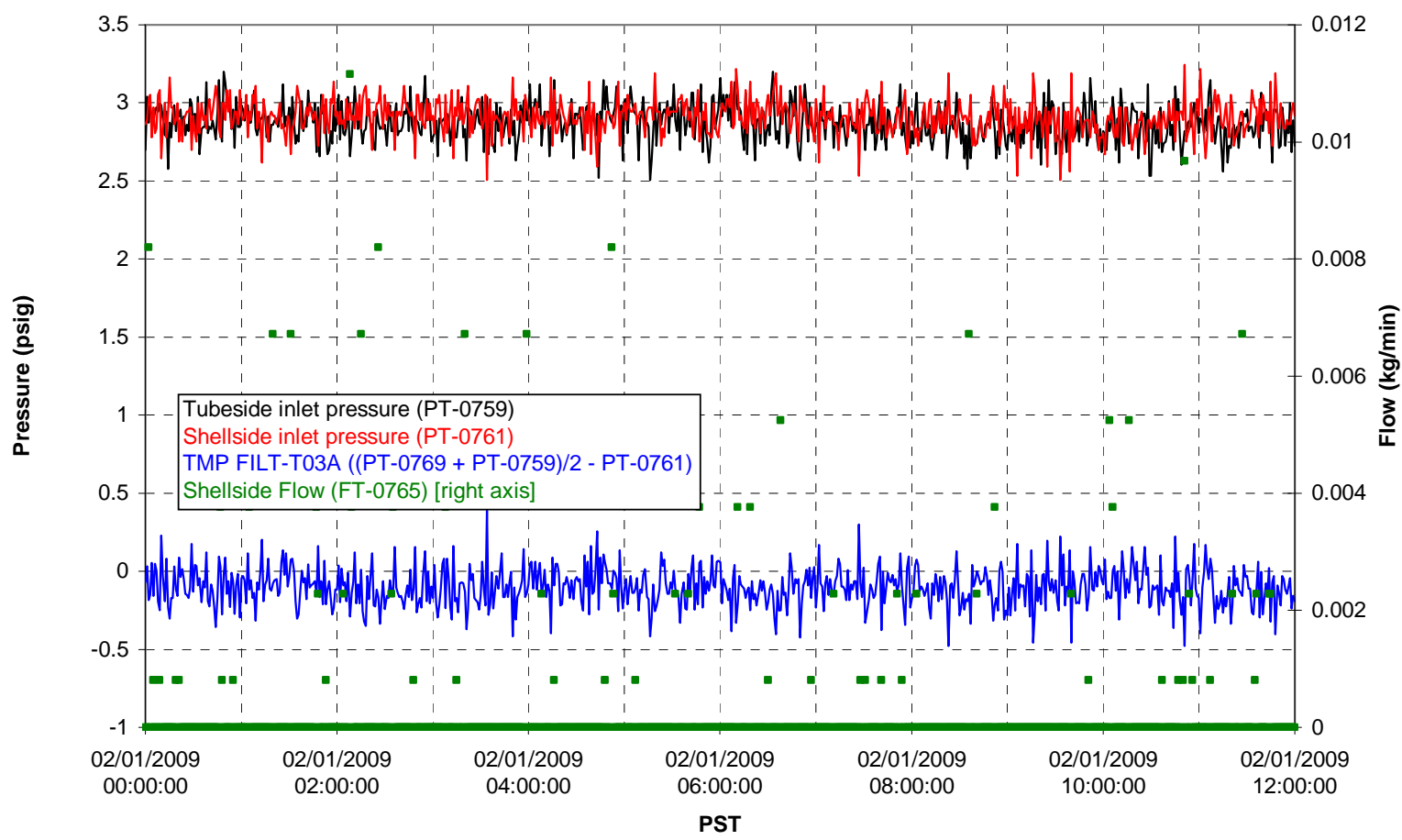

Filter UFP-FILT-T04A

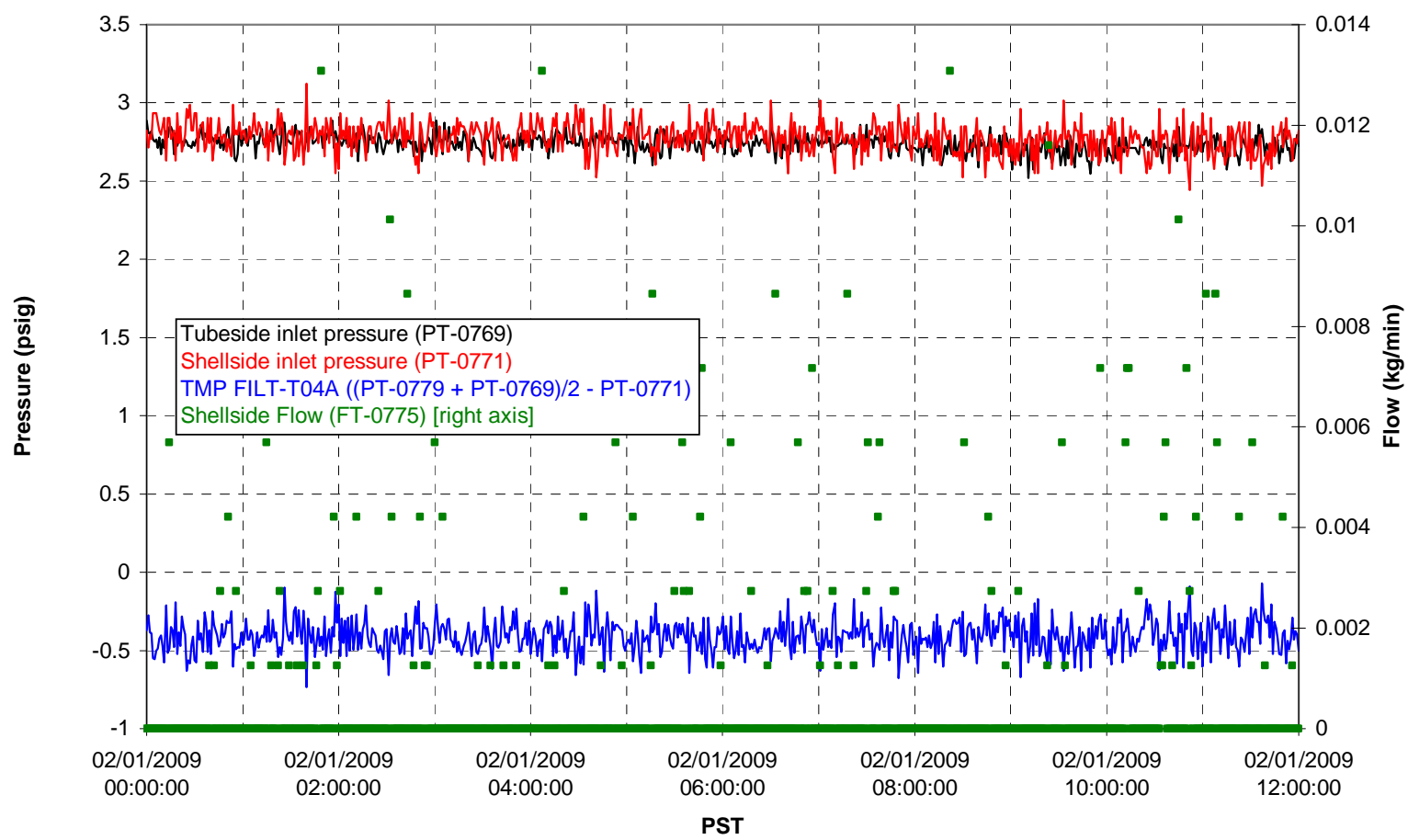


Filter UFP-FILT-T05A

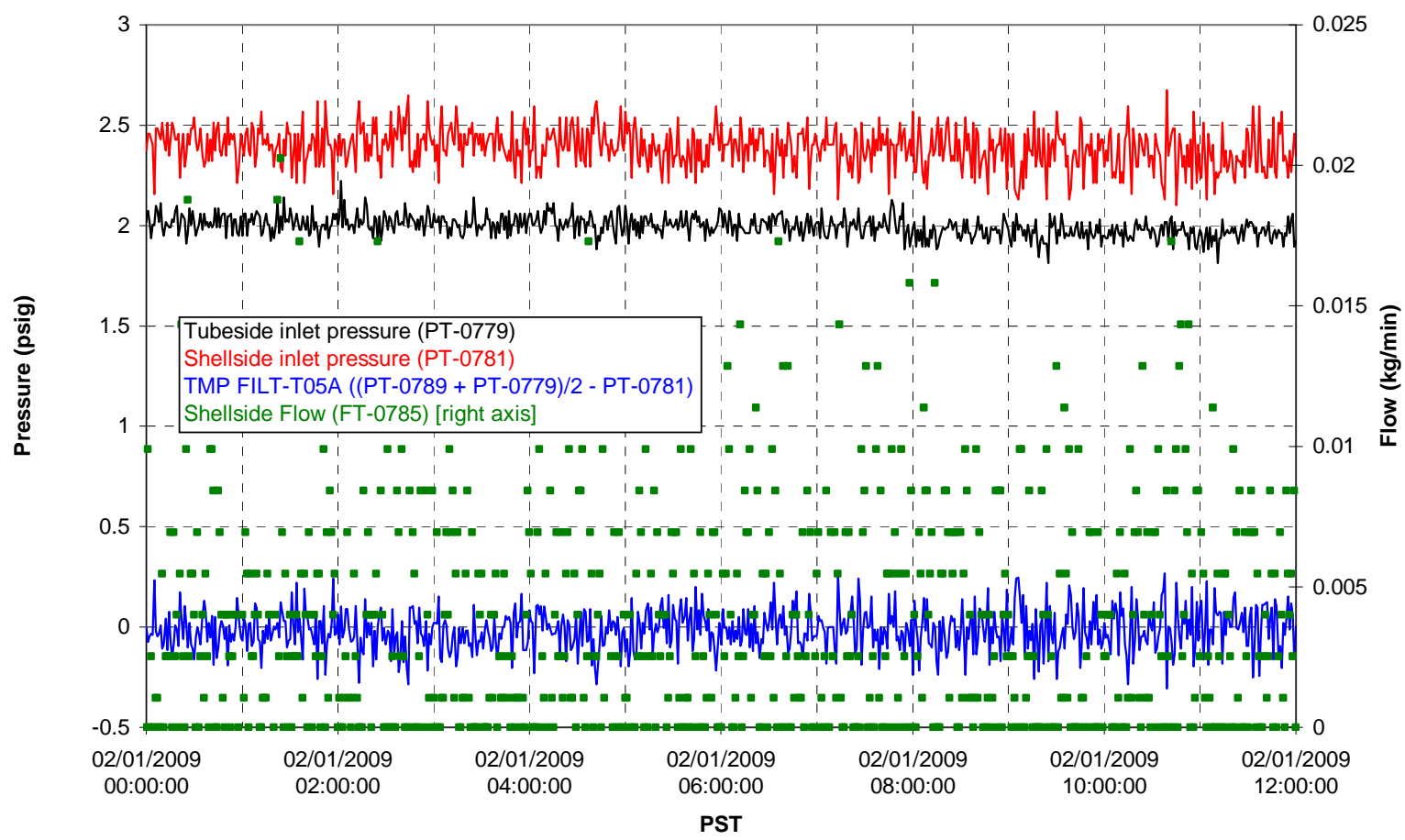

Chemical Flow

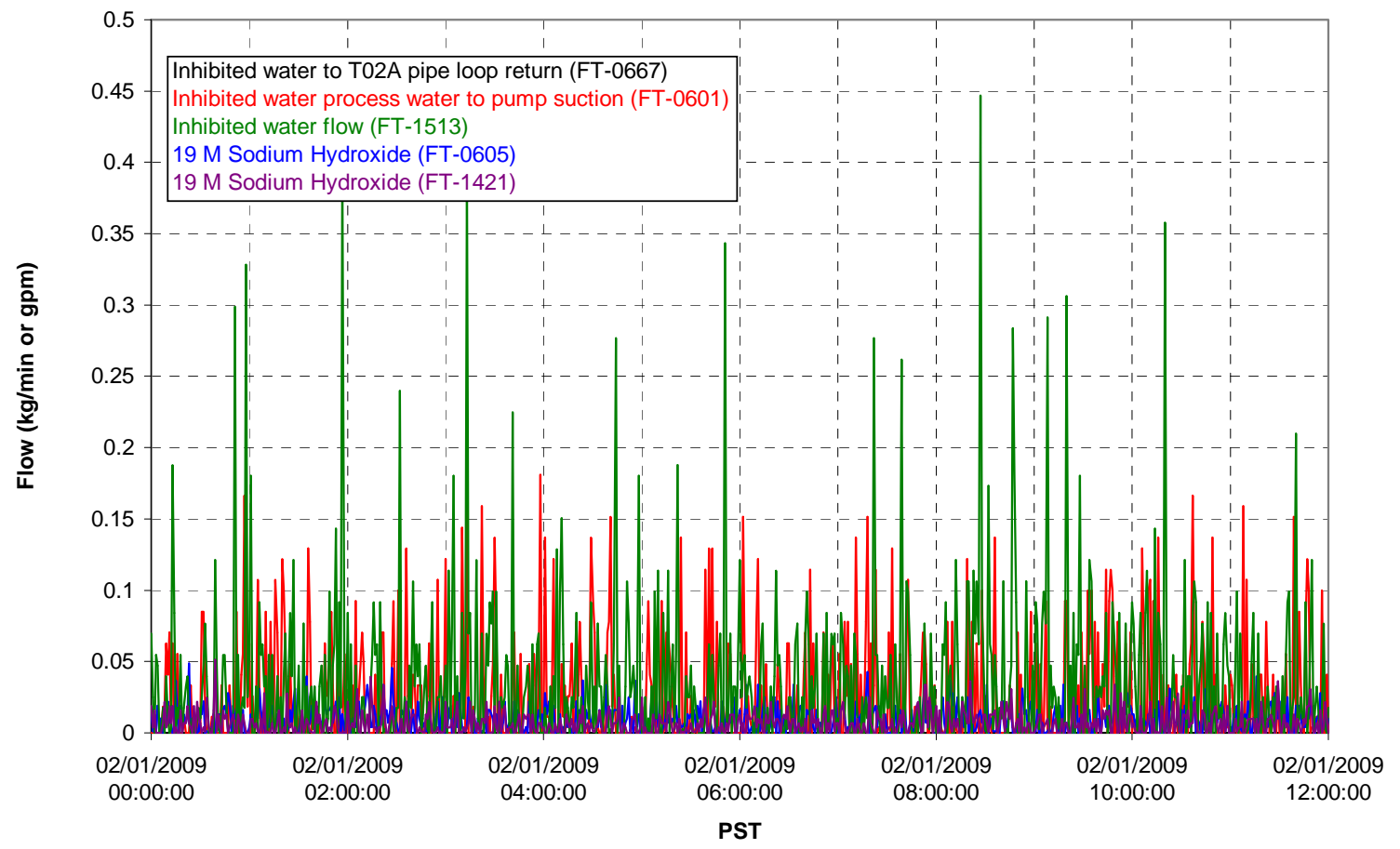




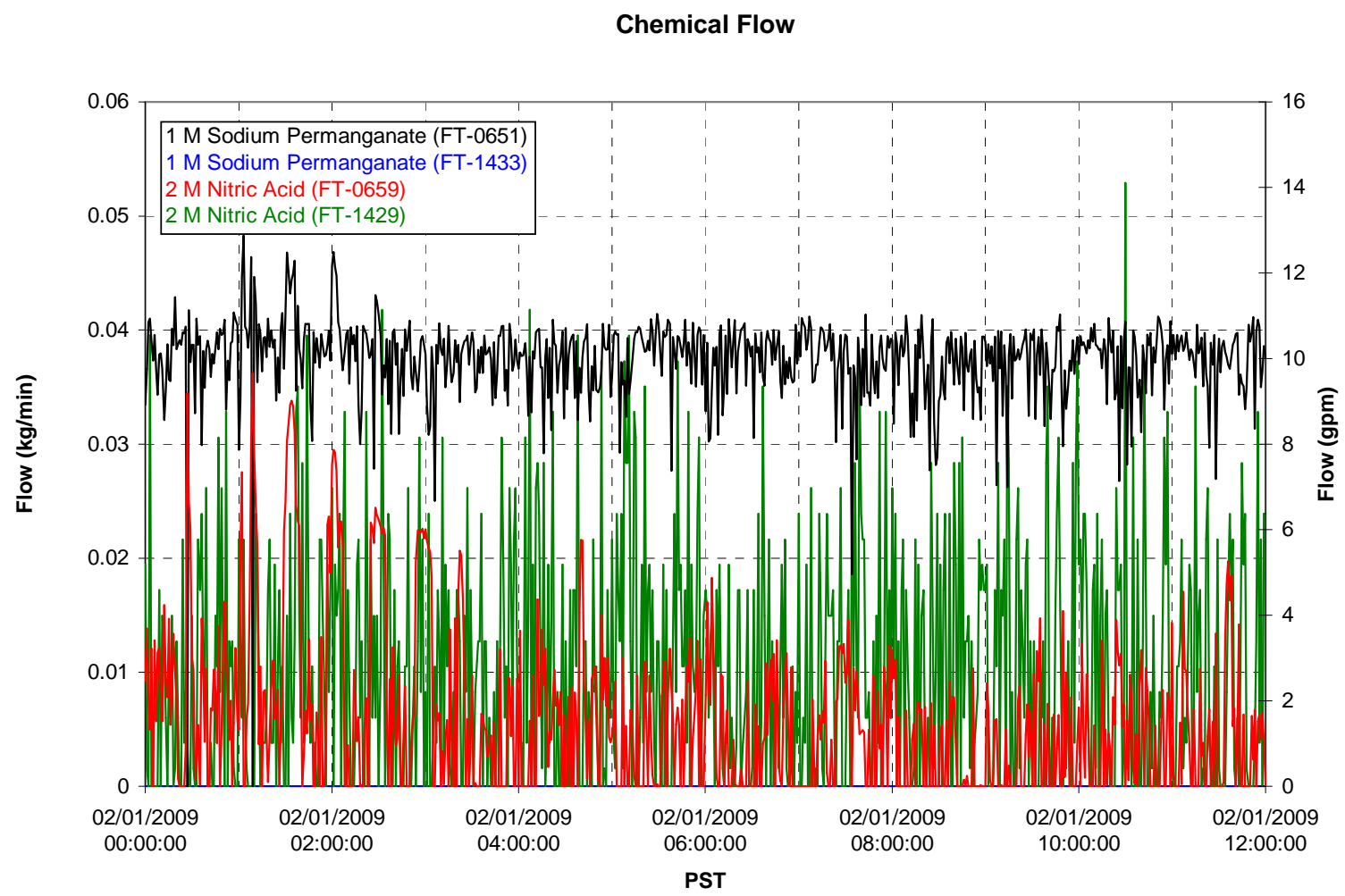

Air Flows

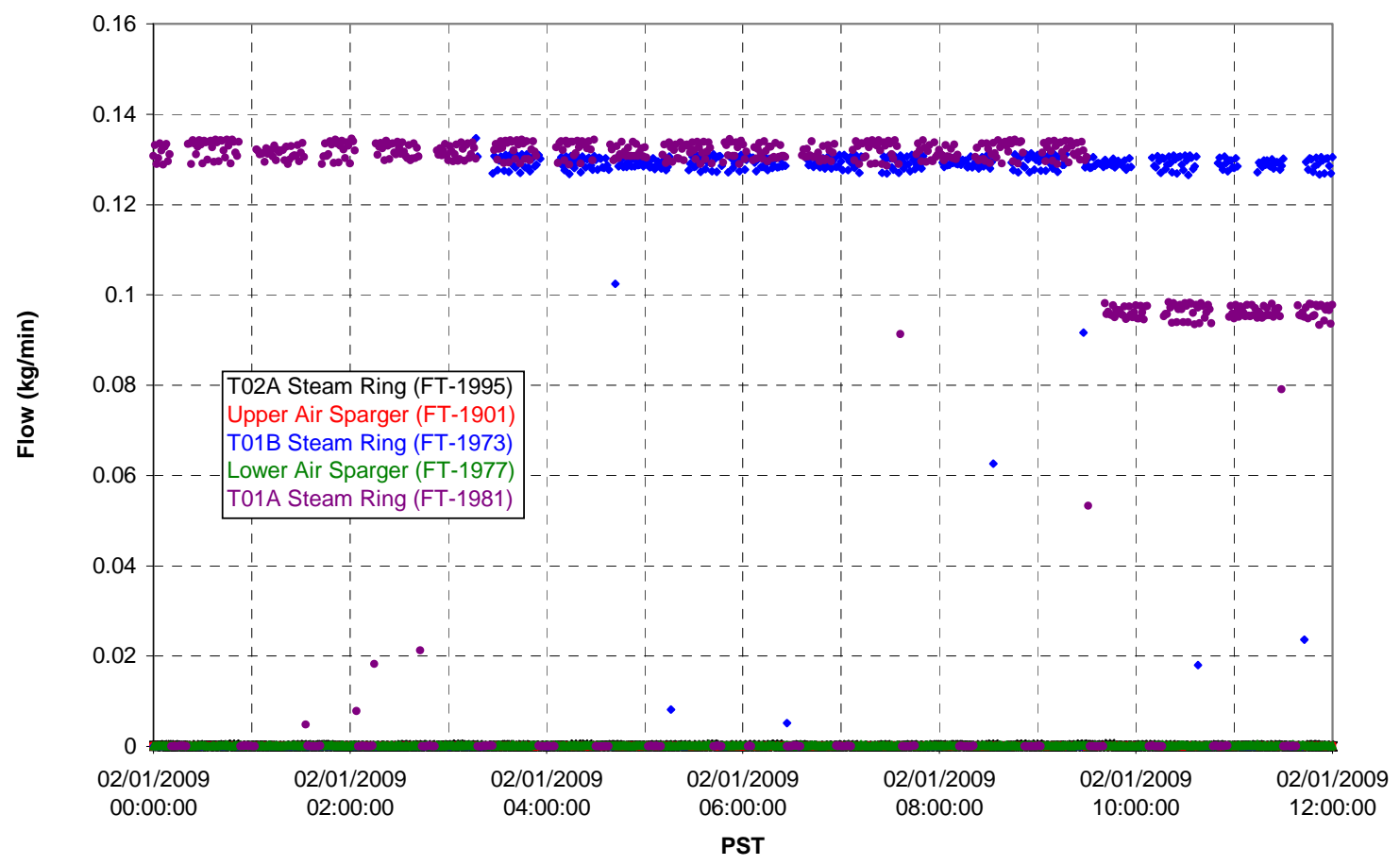


T02A Steam

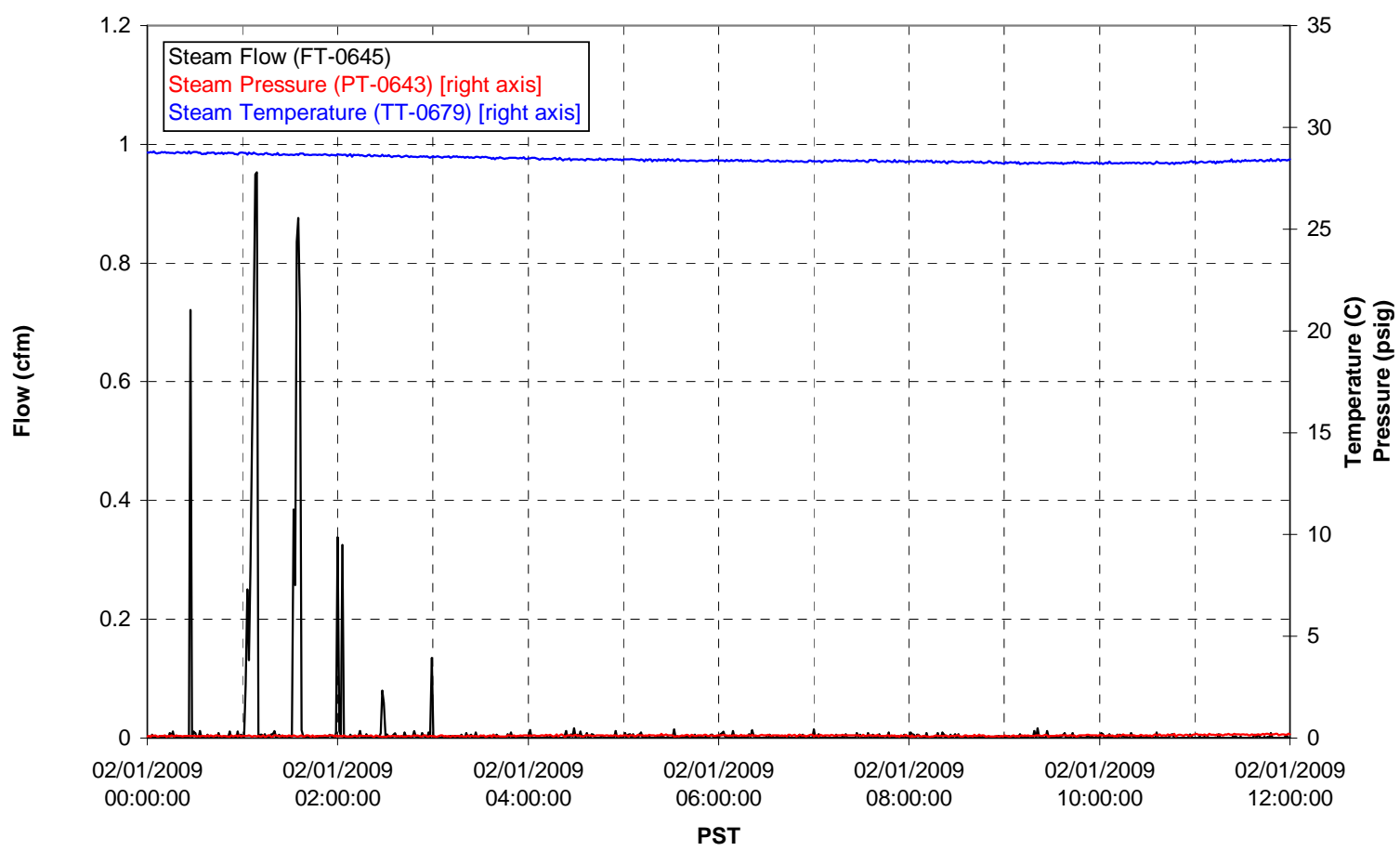

T01A Steam

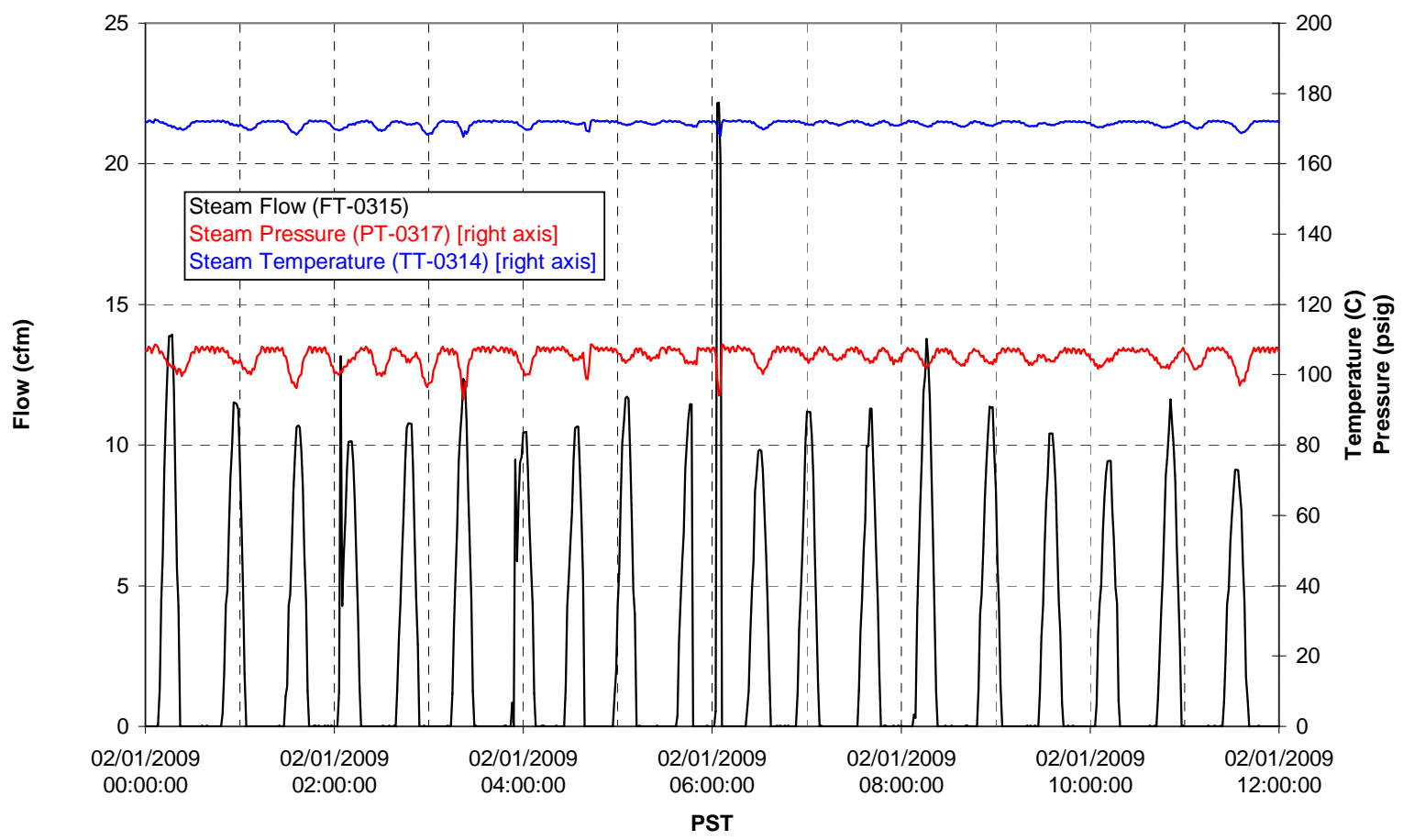


T01B Steam

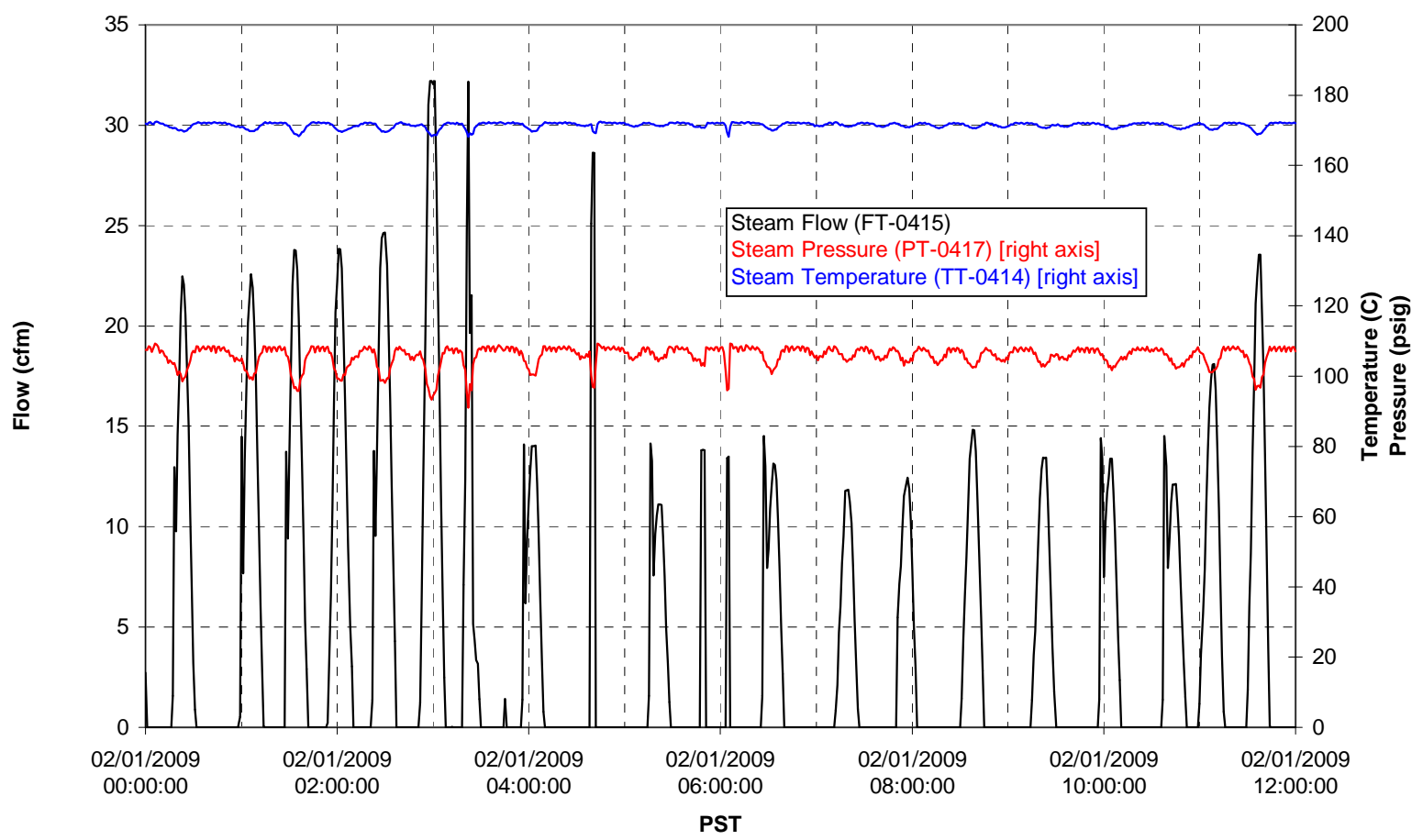




\section{Integrated Test A Data Plots 02/01/09 12:00 - 02/02/09 00:00}


T01A level

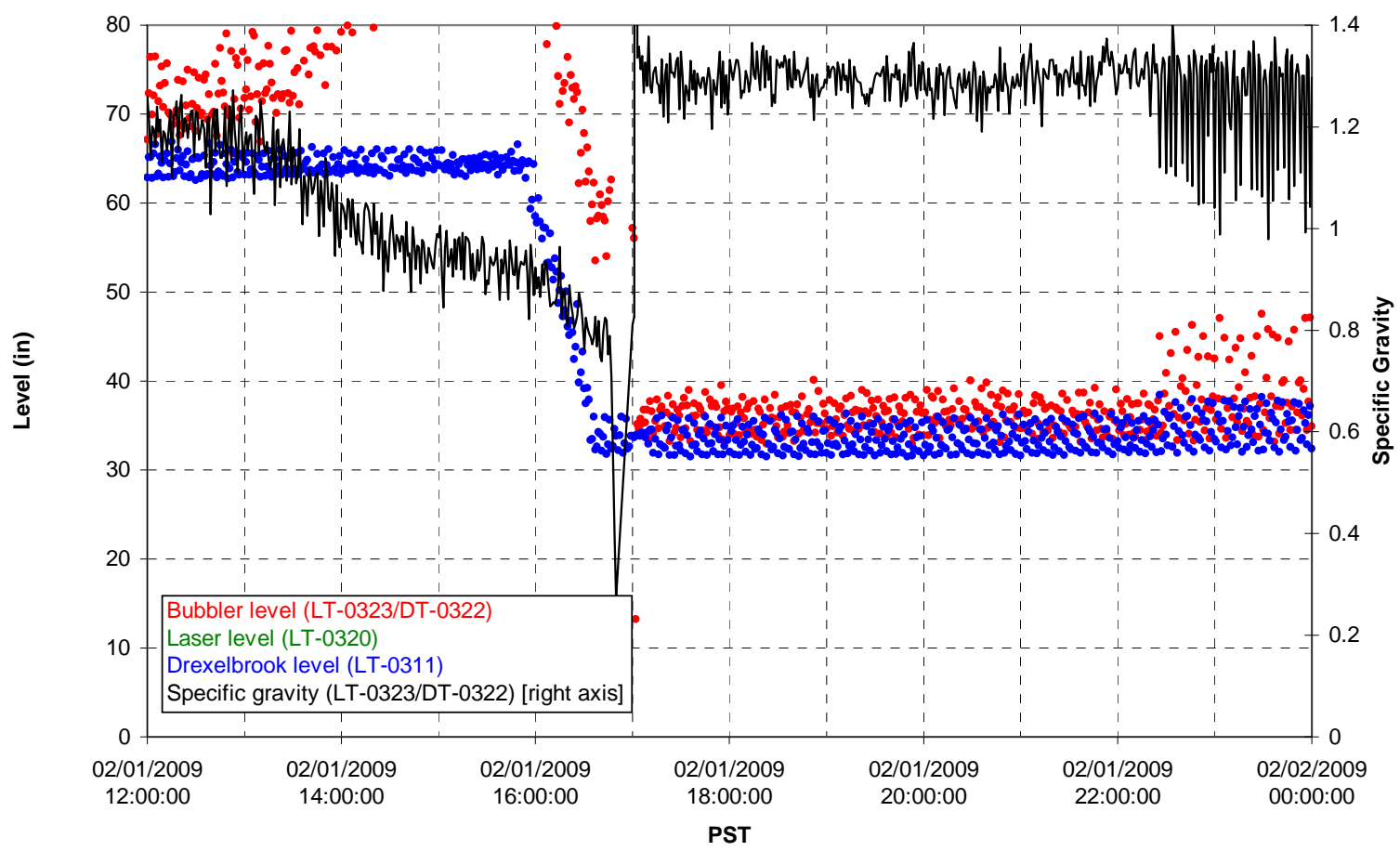

T01A temperatures

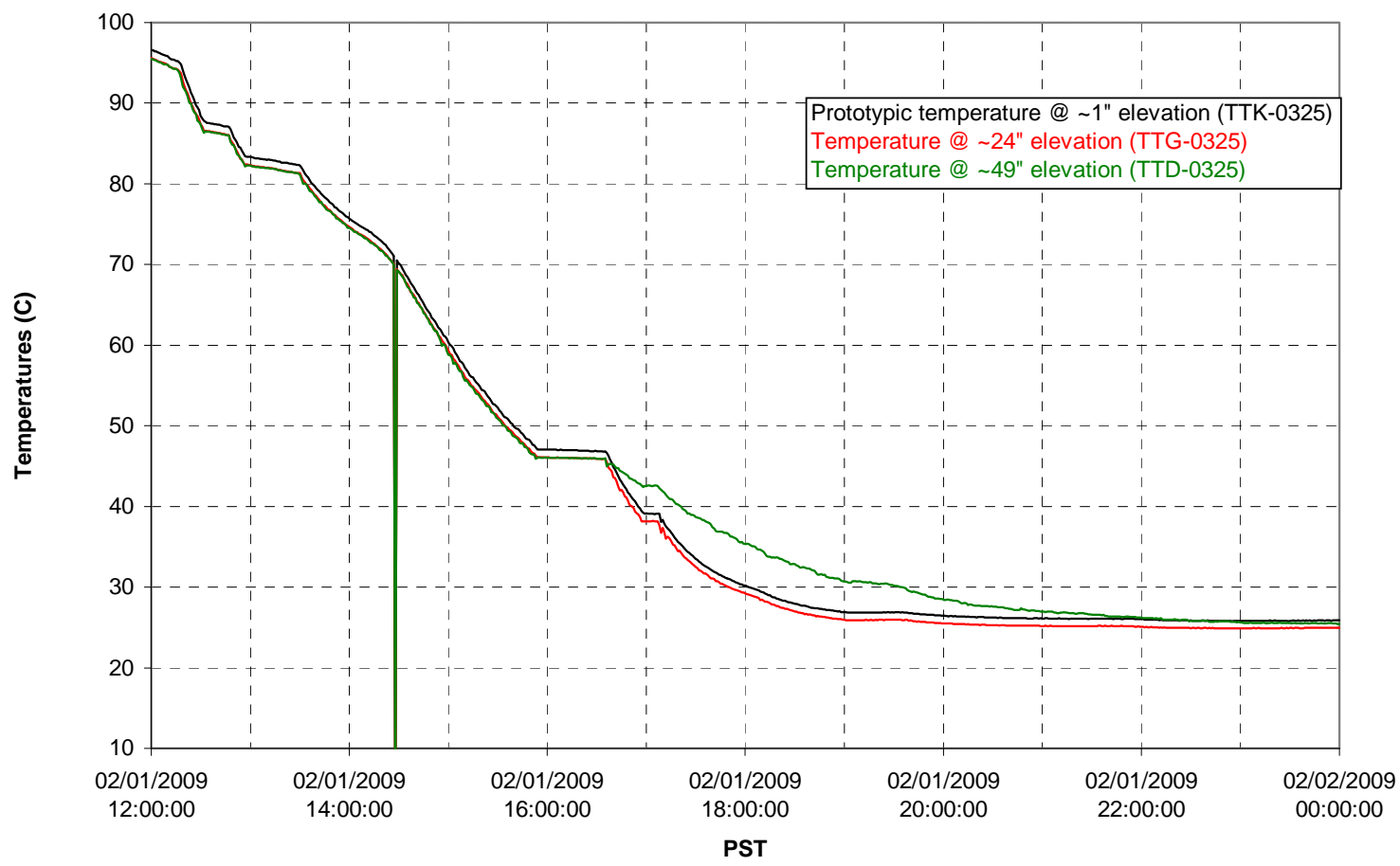


T01B level

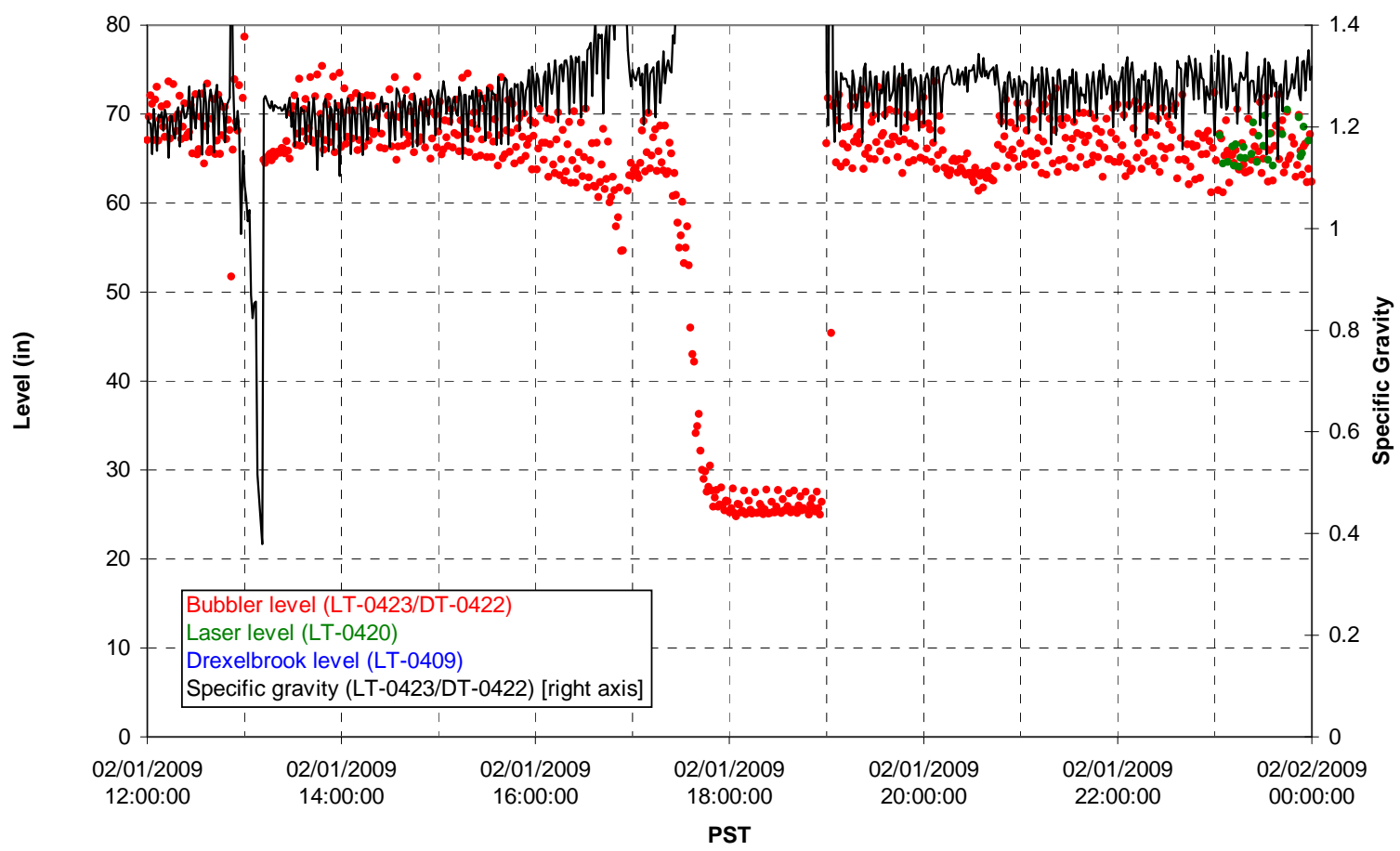

T01B temperatures

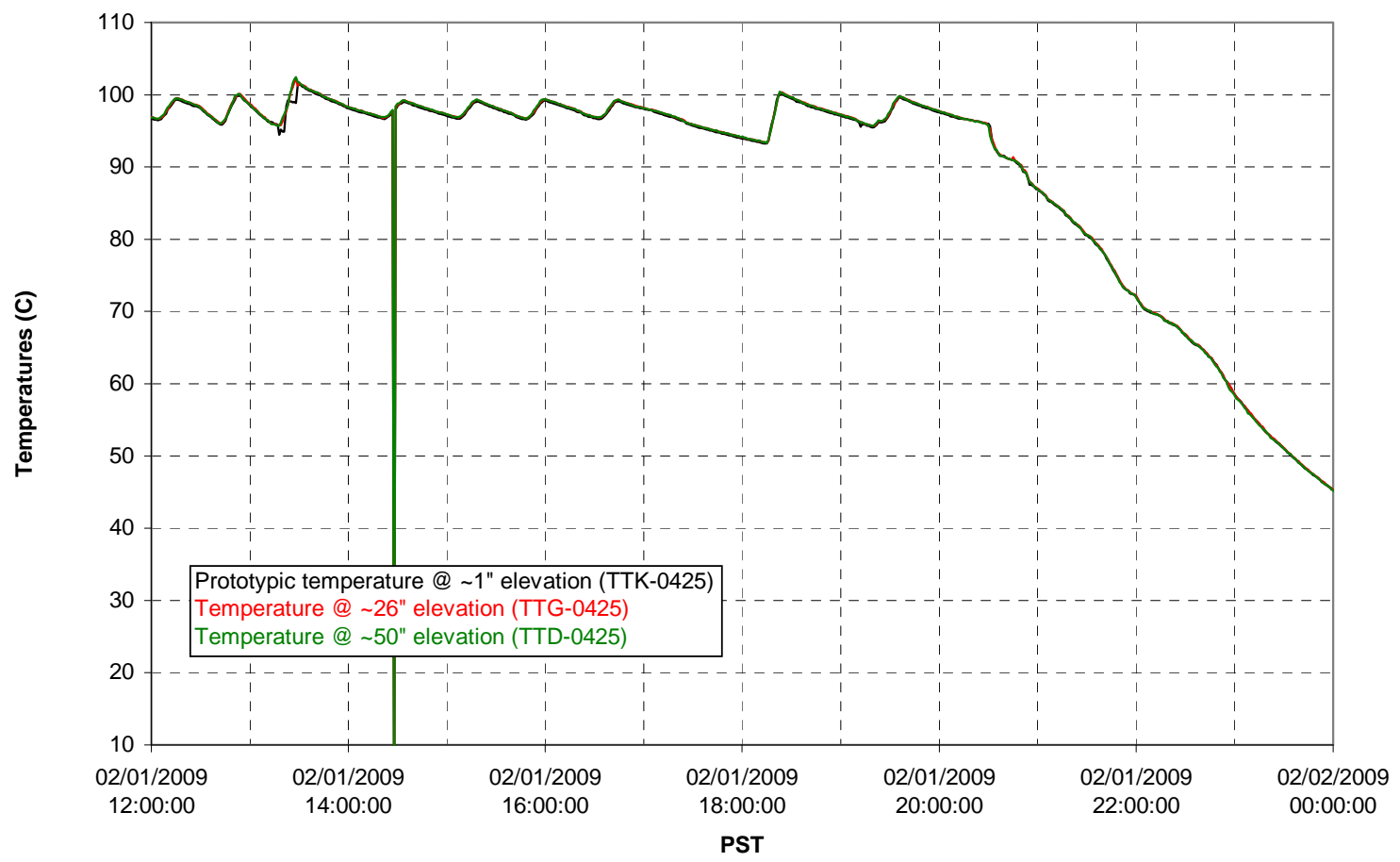


T02A level

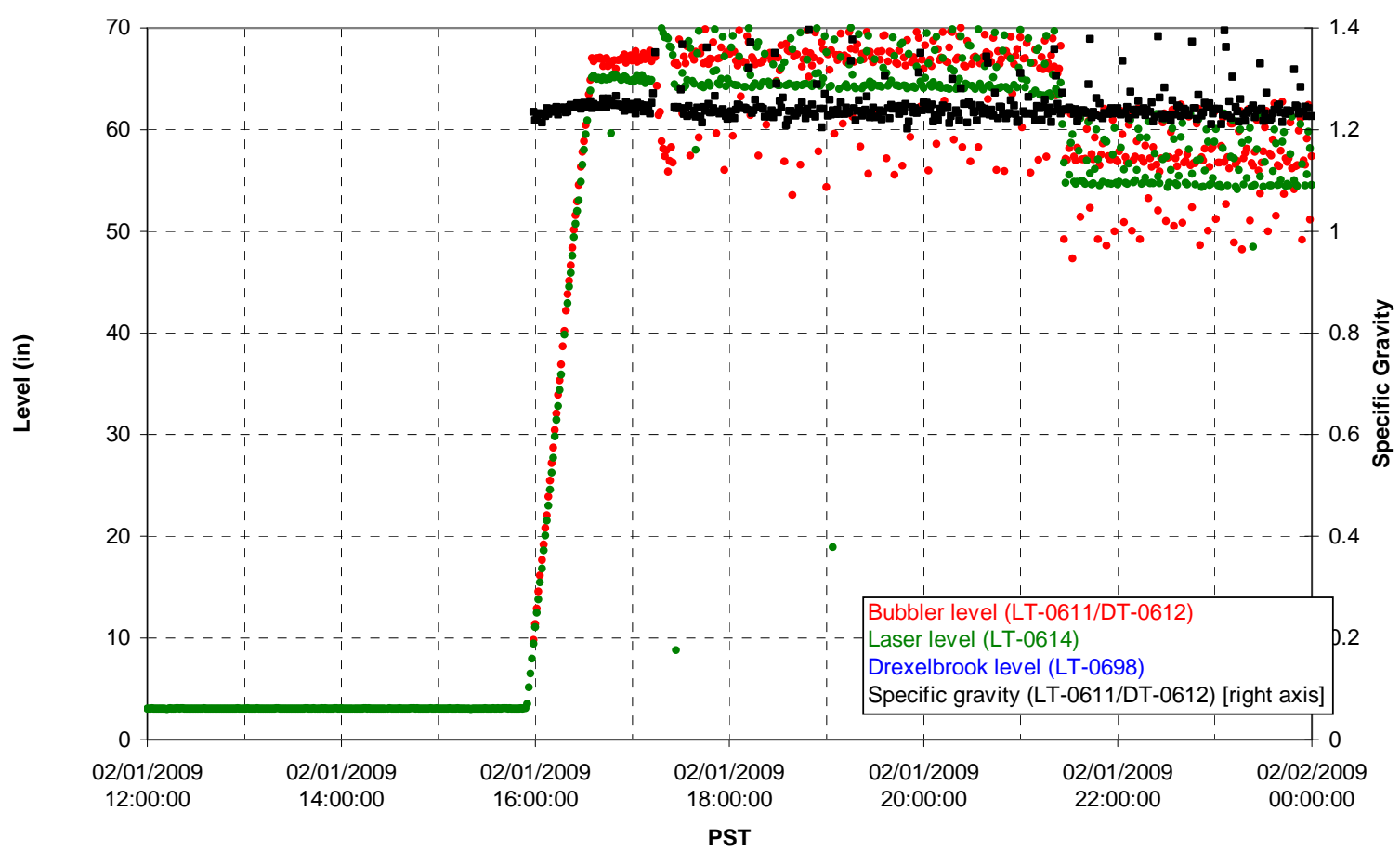

T02A temperatures

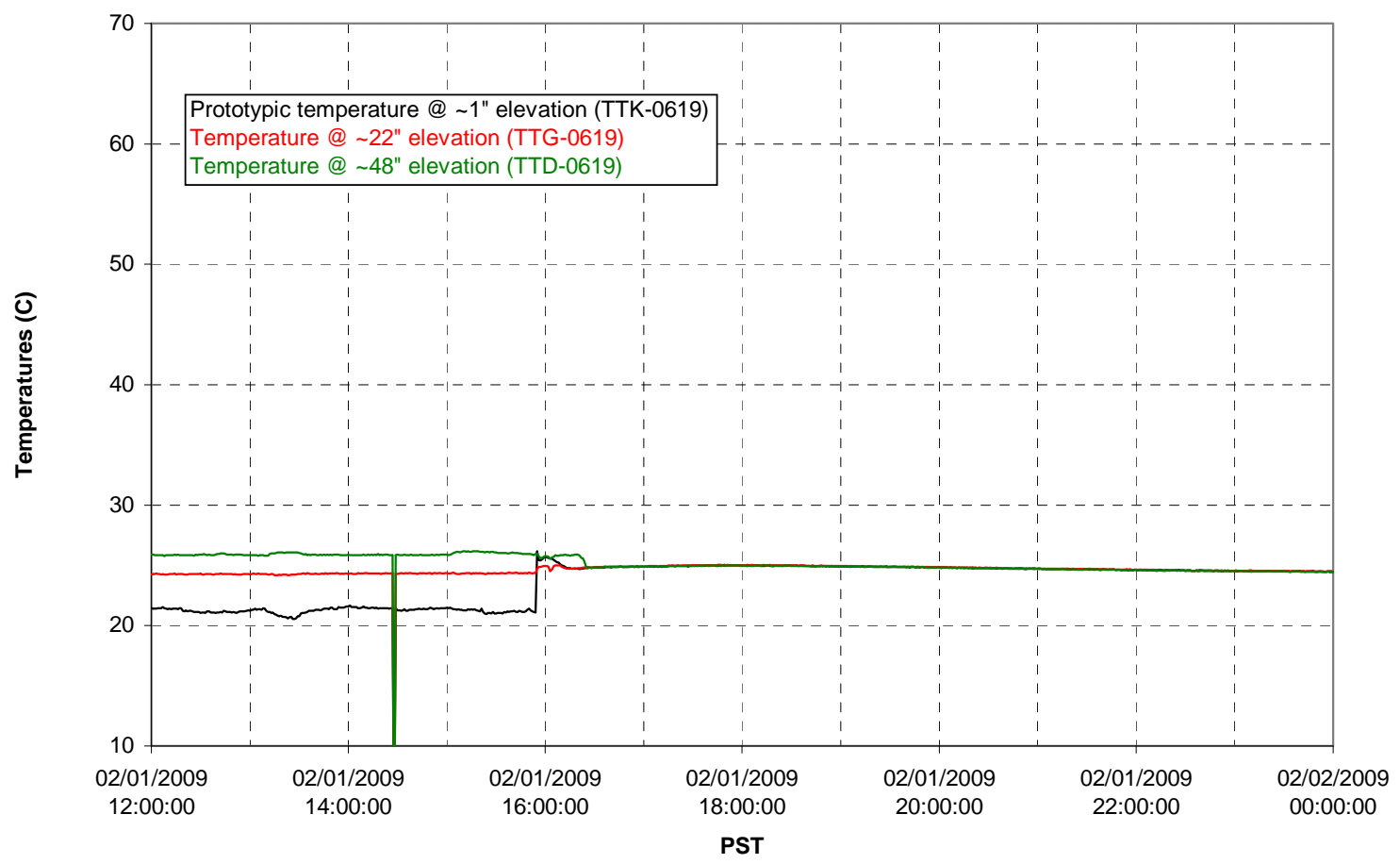


T02A and filter loop temperatures

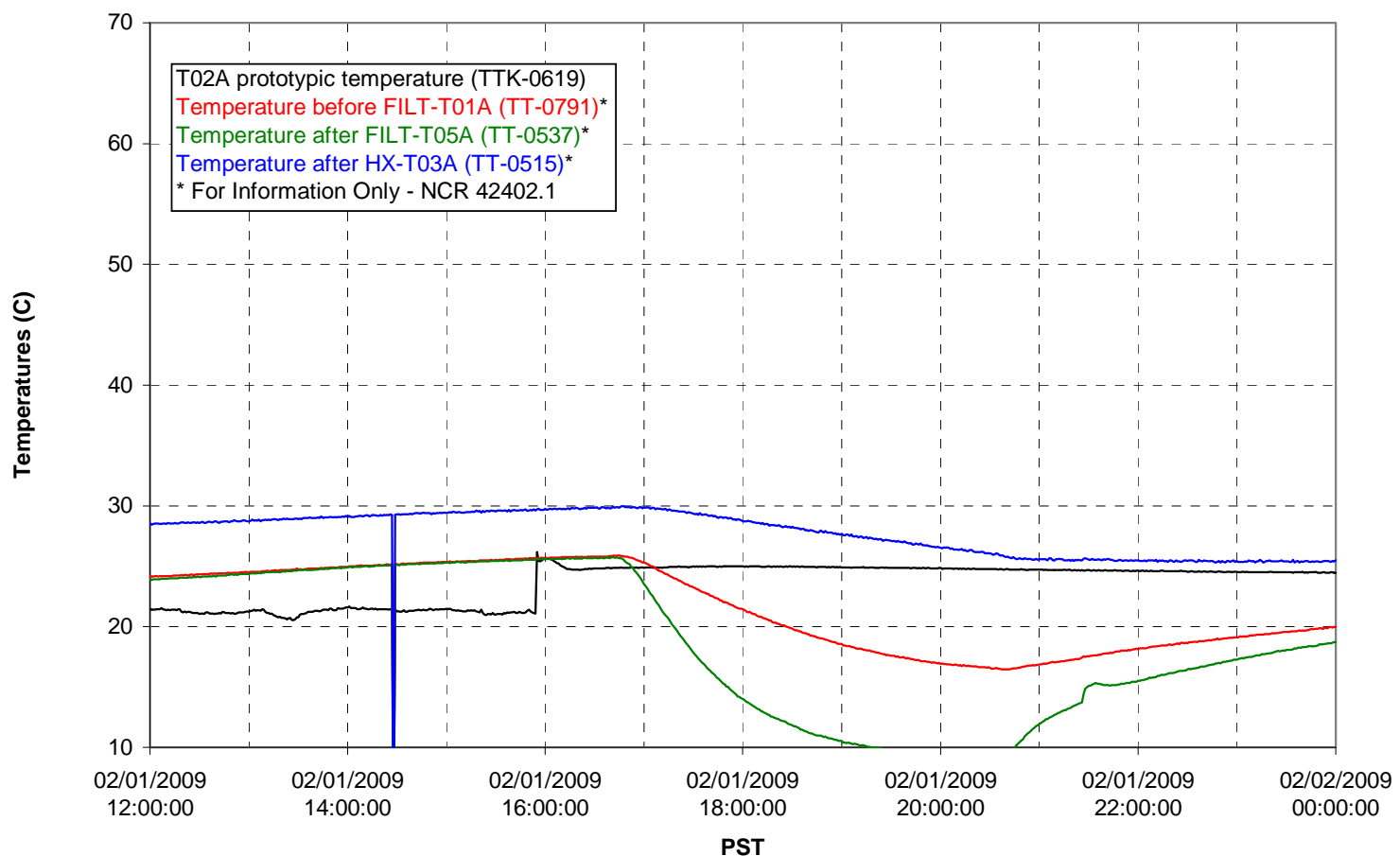

Pump Pressures and Flow

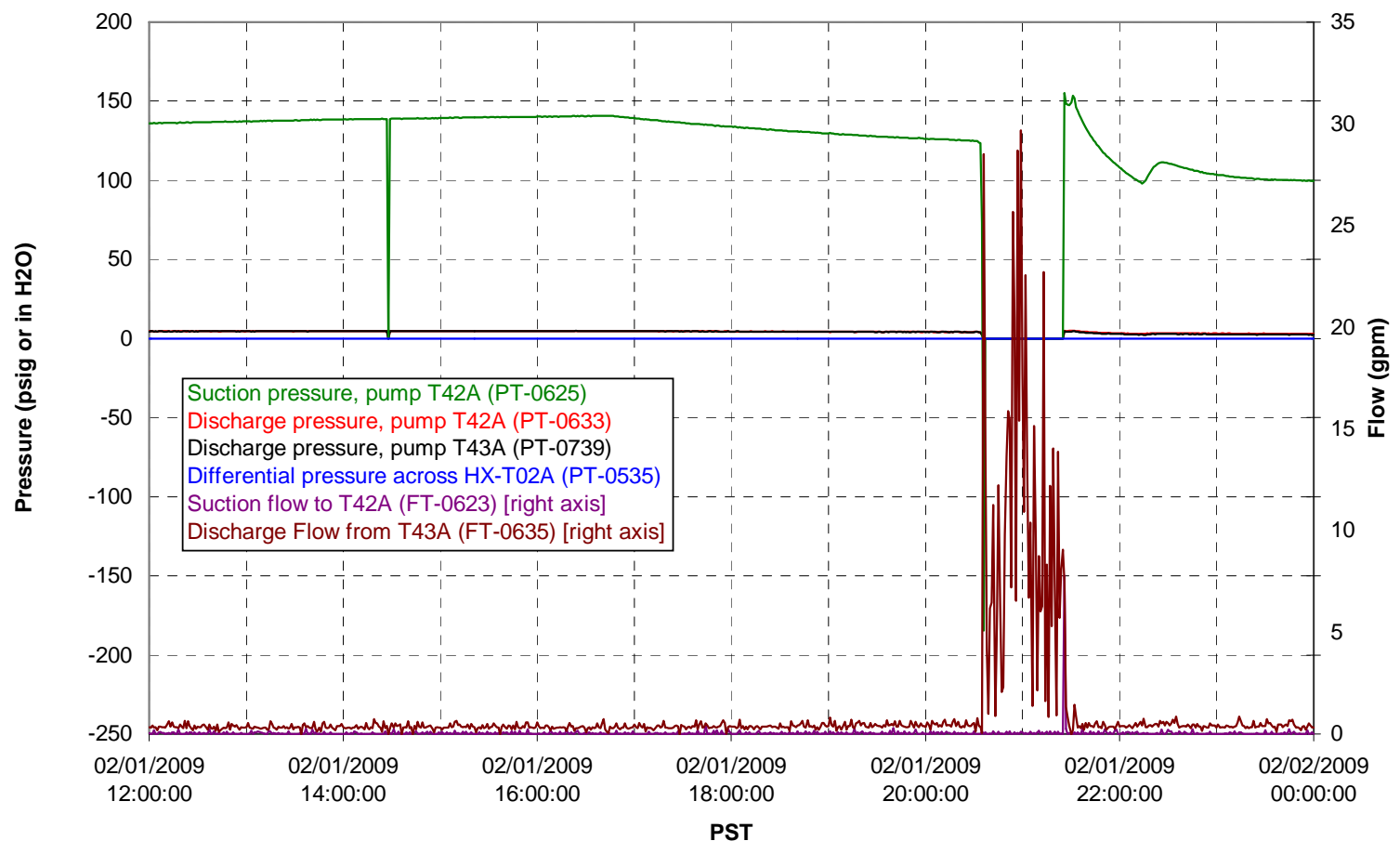


Axial pressure drop

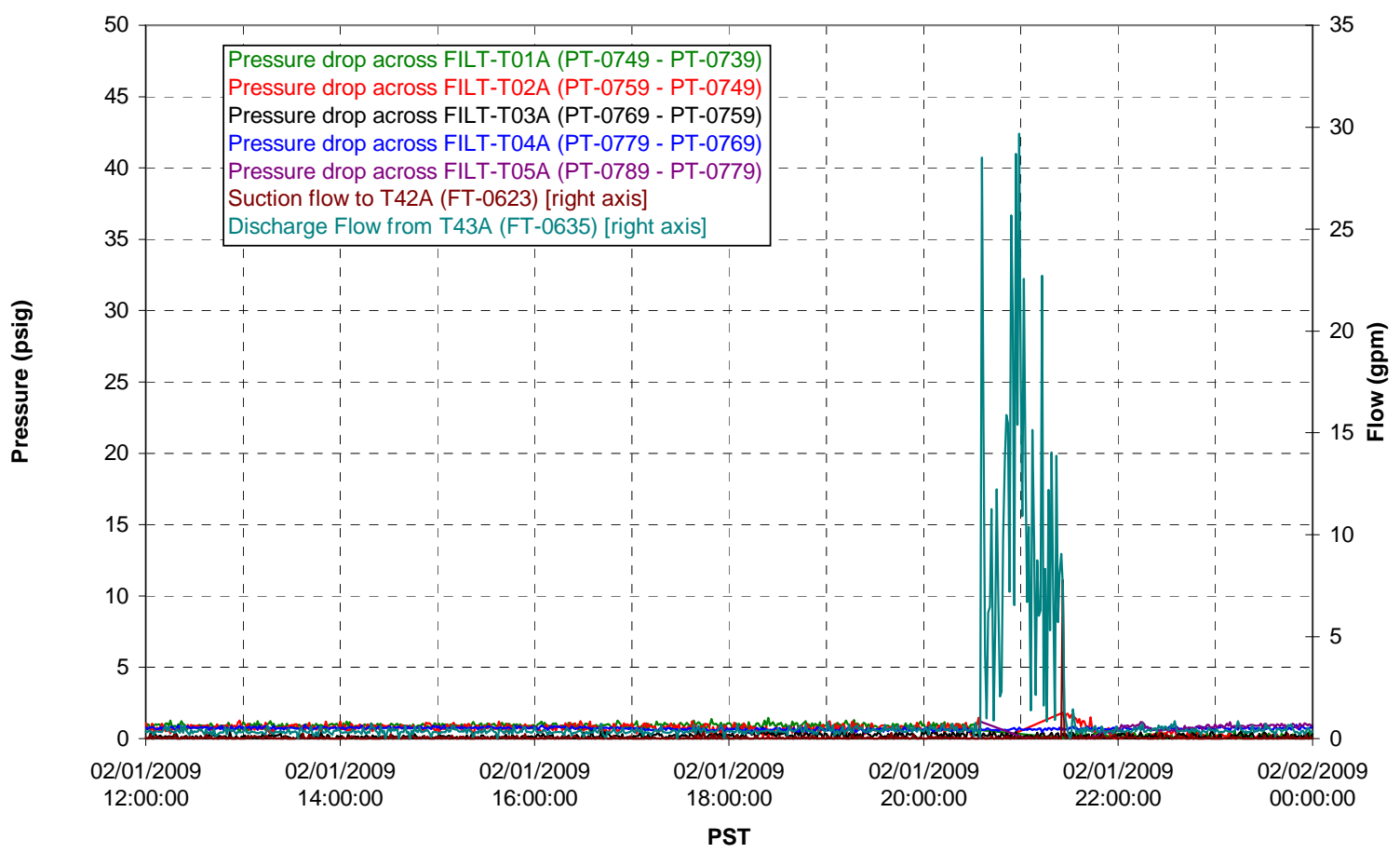

Permeate flow rates

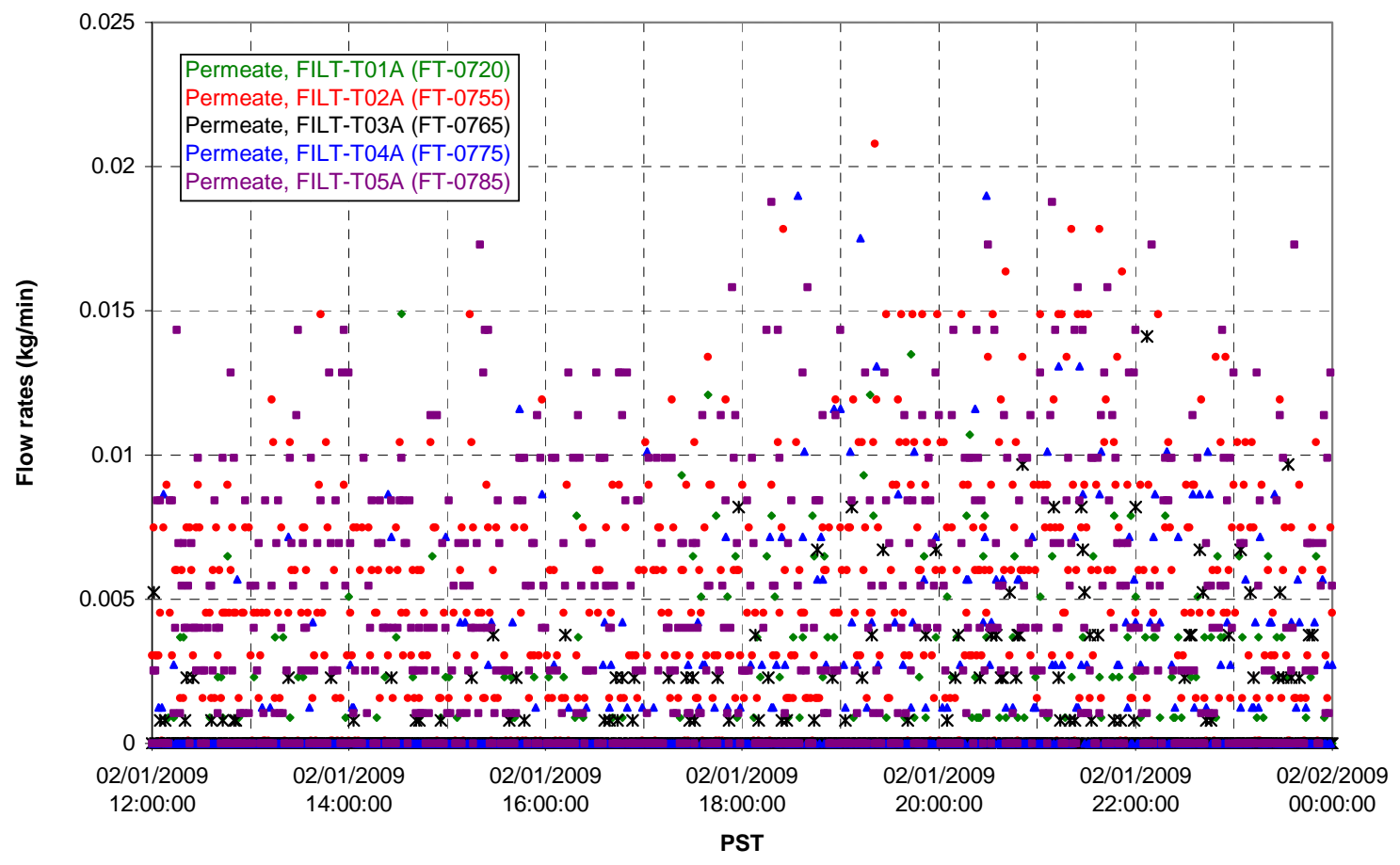


T02A Inner Temperature Tree

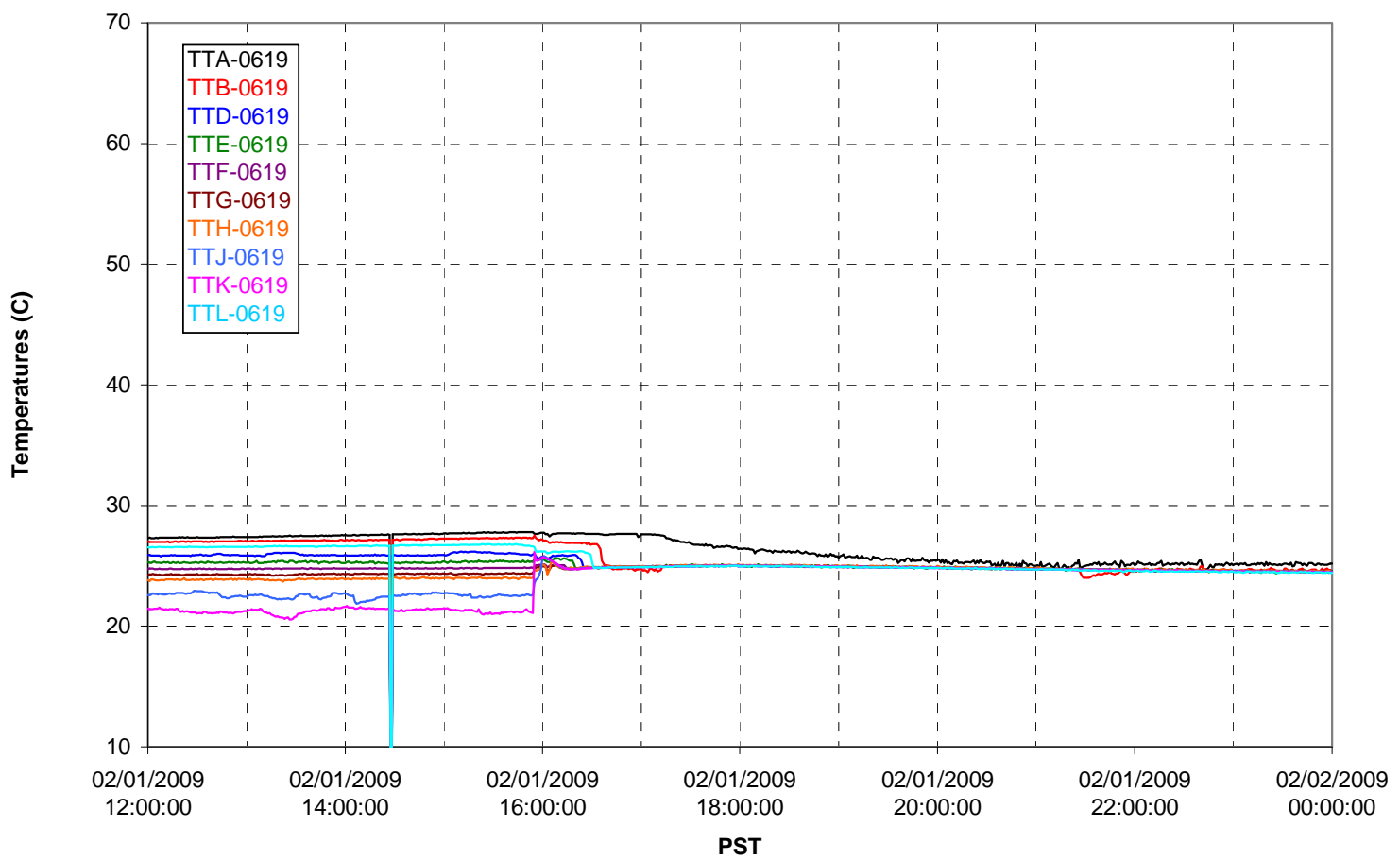

T02A Outer Temperature Tree

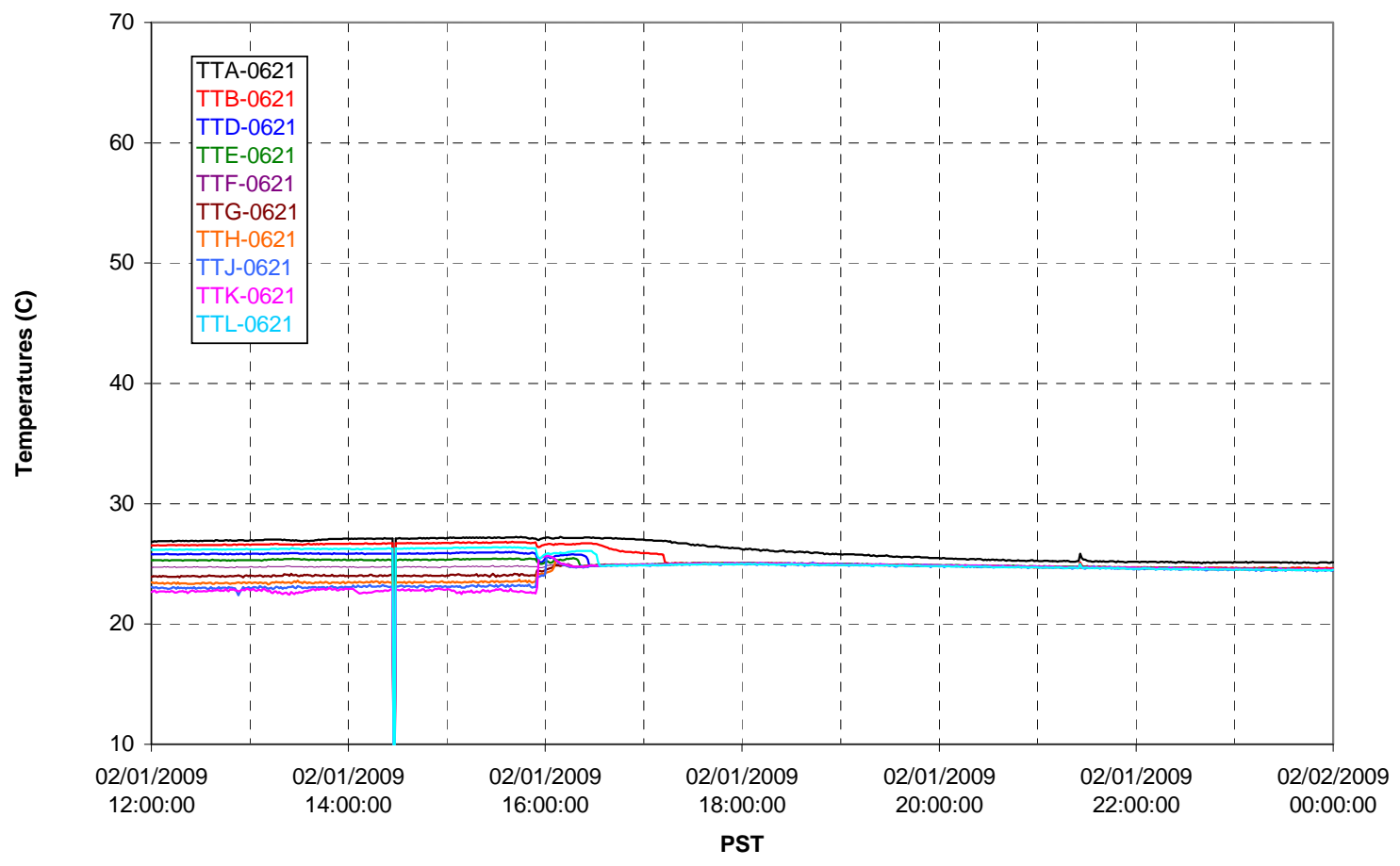


T02A temperatures

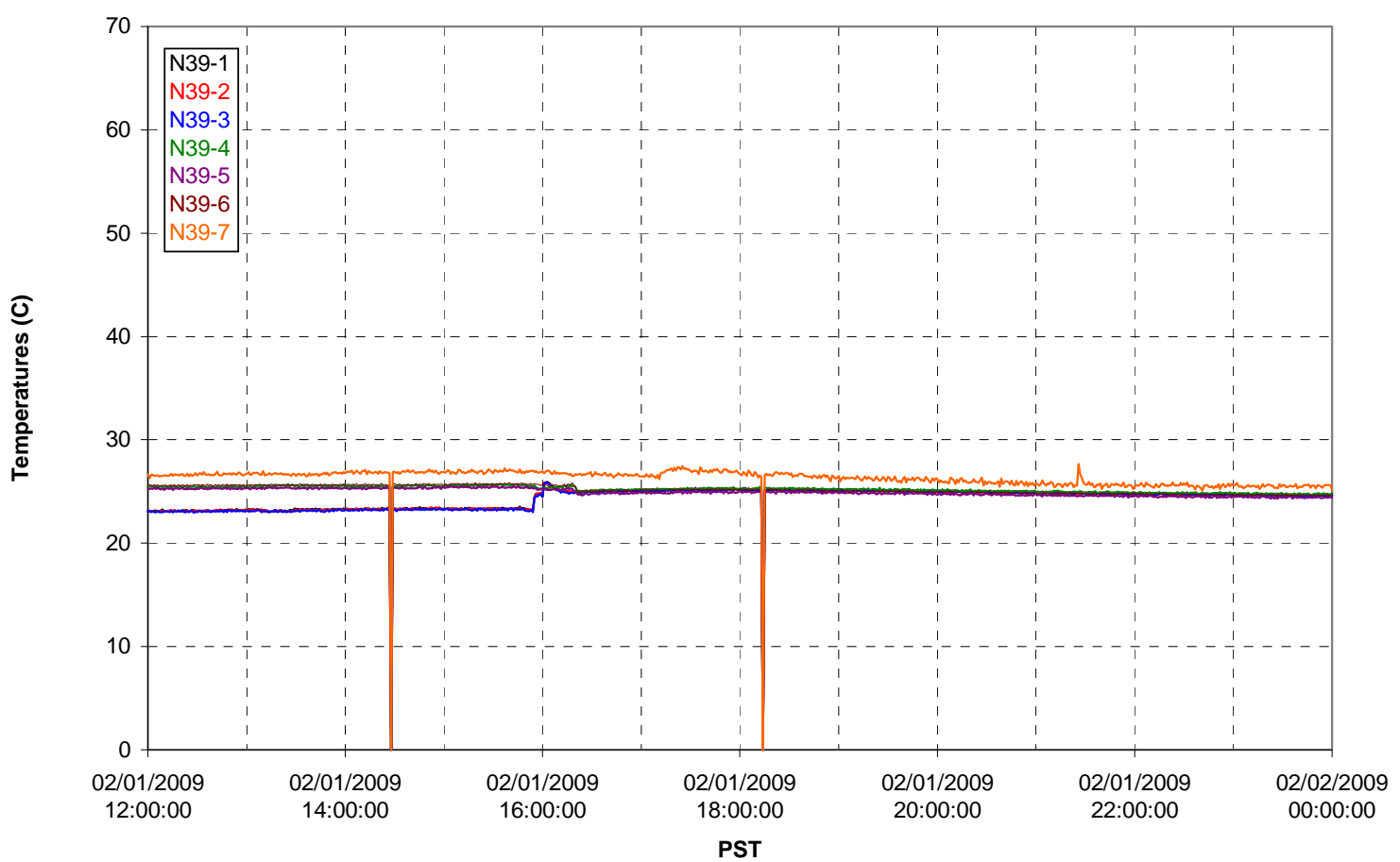

T02A temperatures

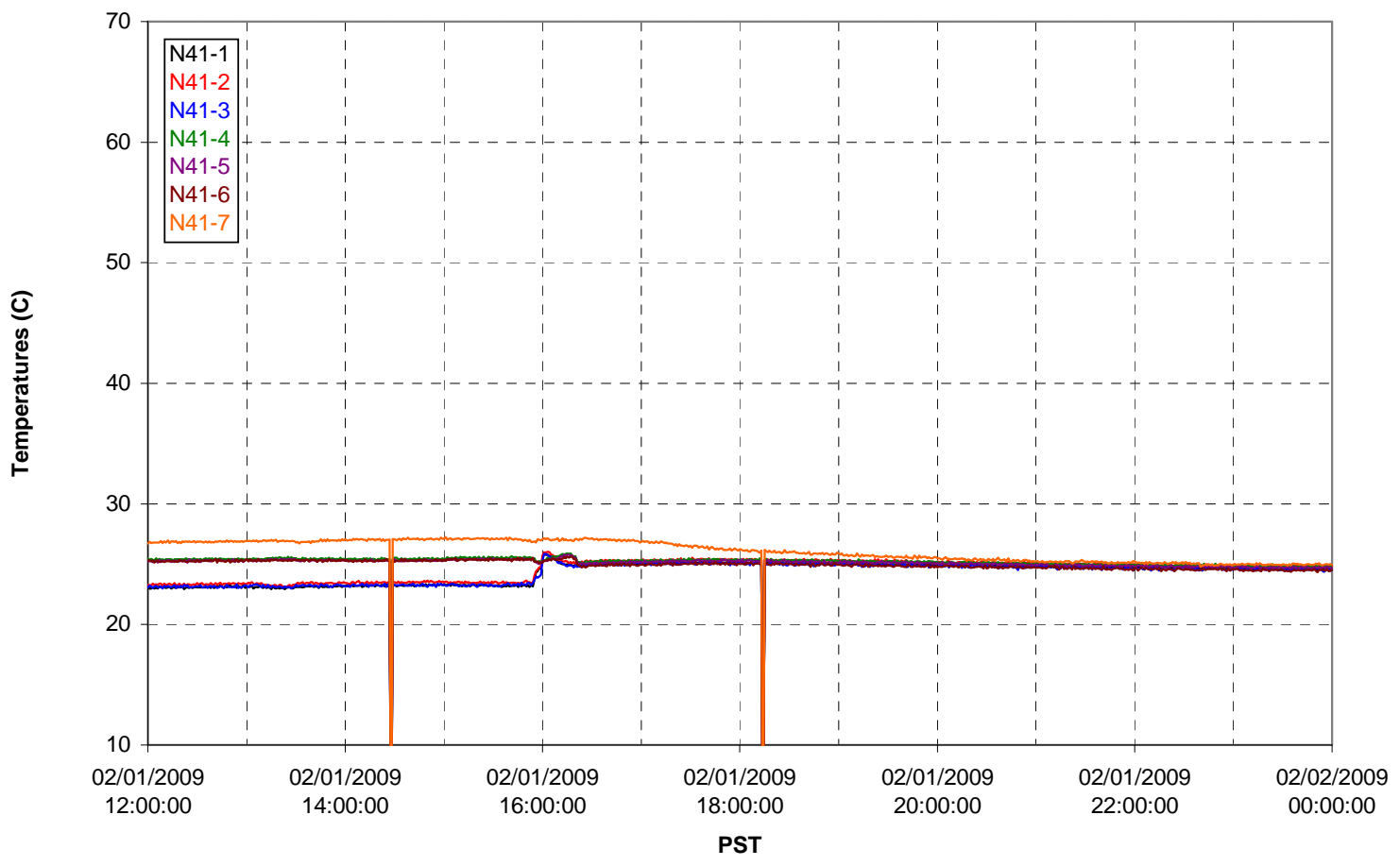


T02A temperatures

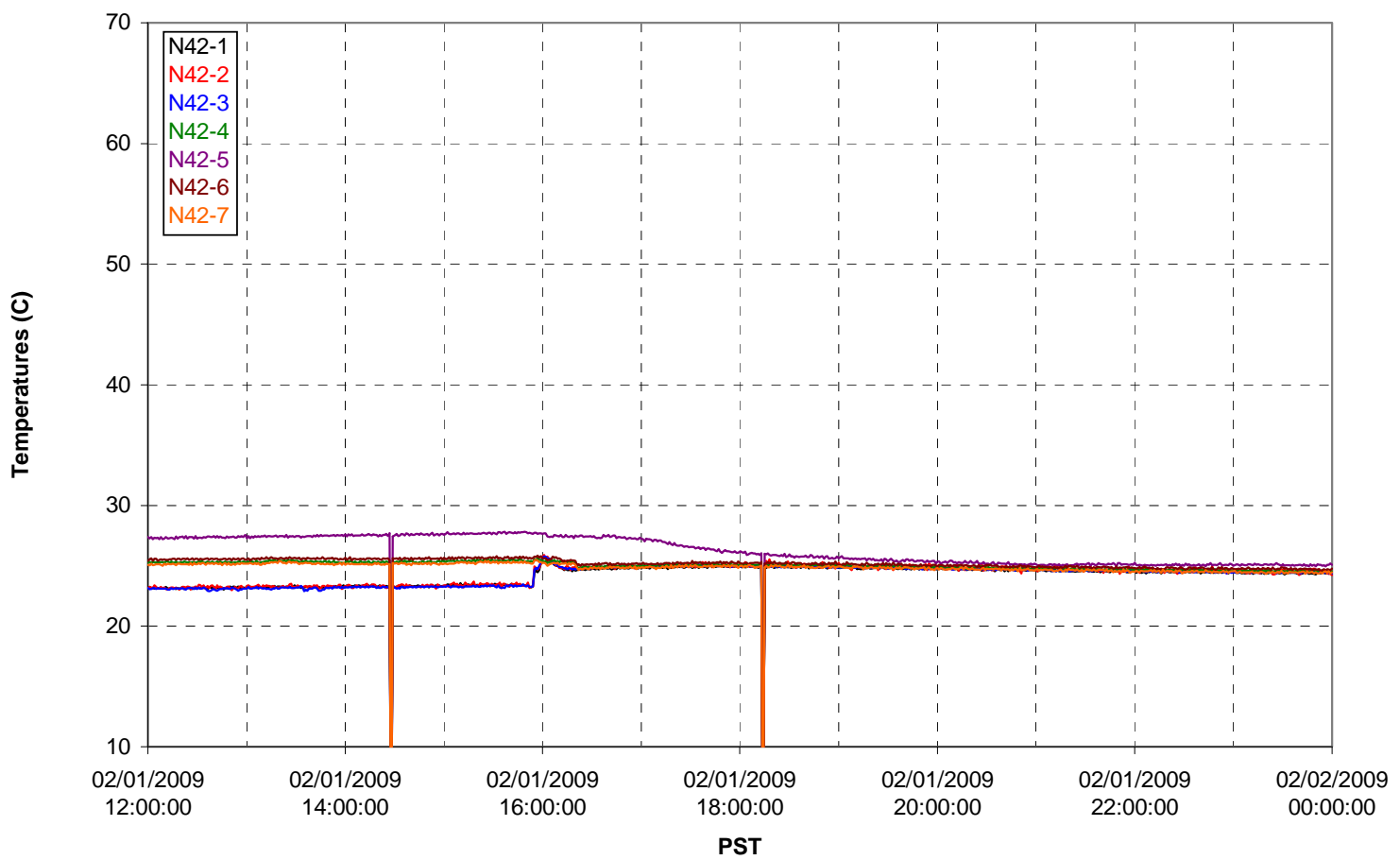

T02A temperatures

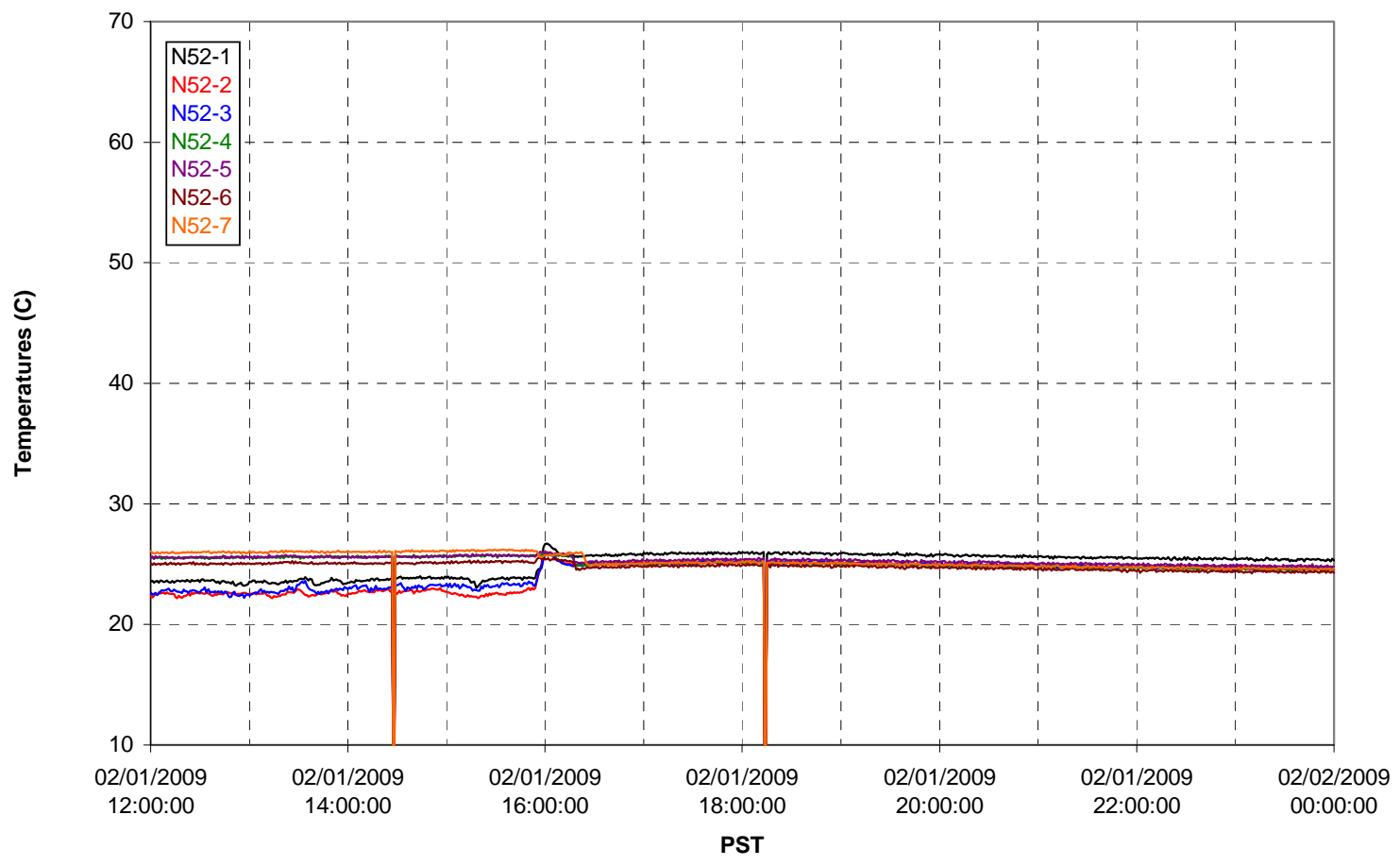


T02A Heating and Cooling

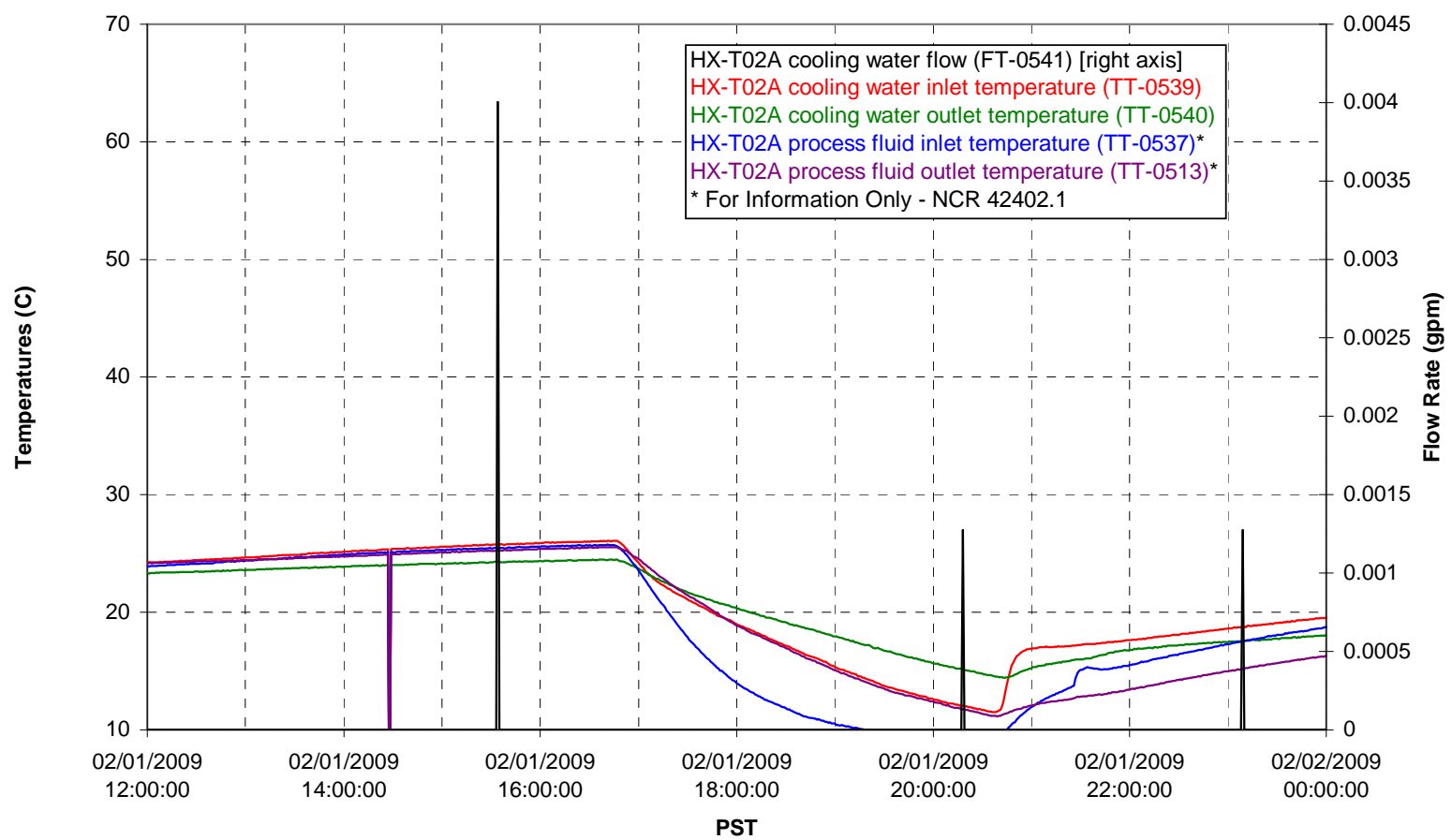

Pump Operation

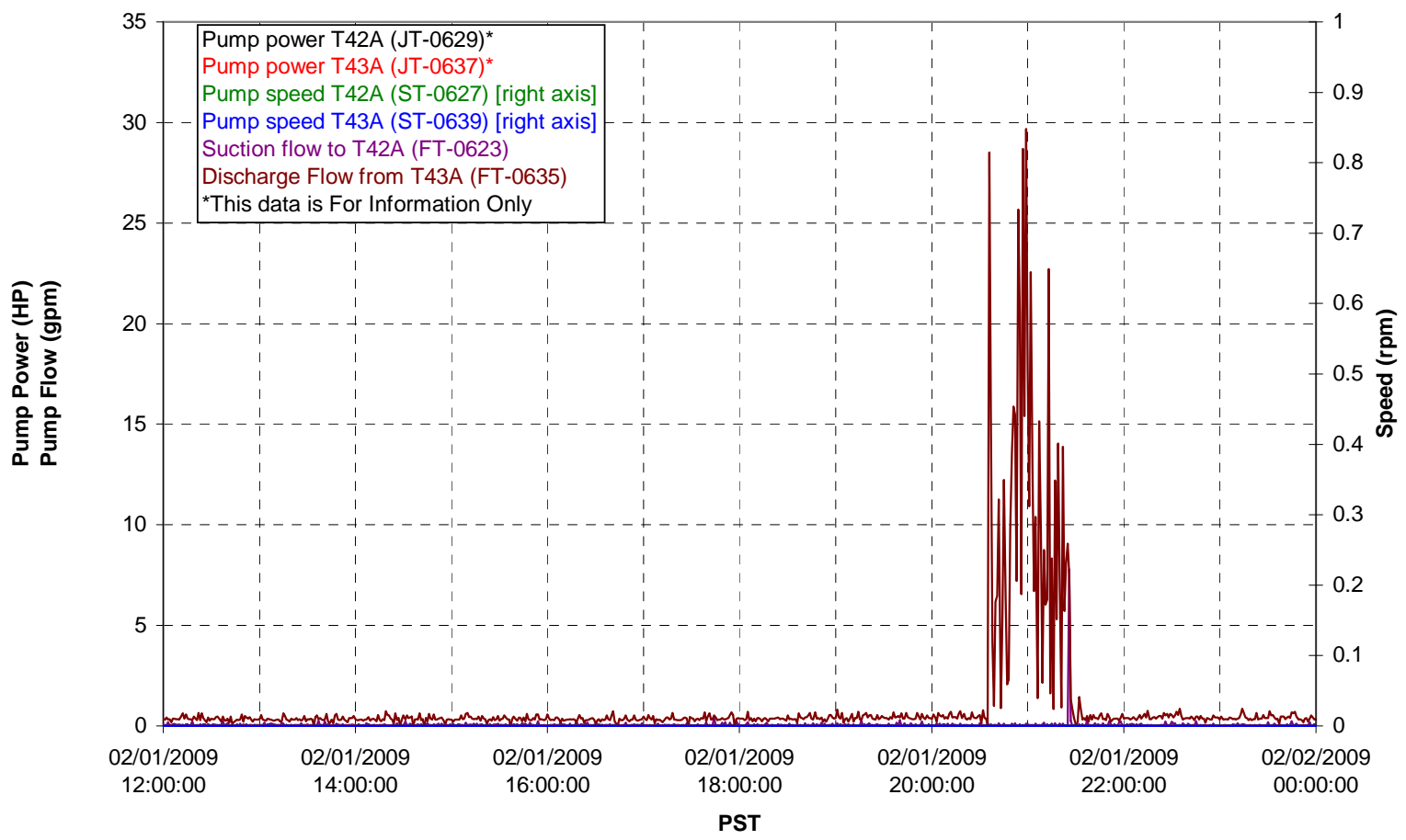


Pulsepot UFP-PP-T01A

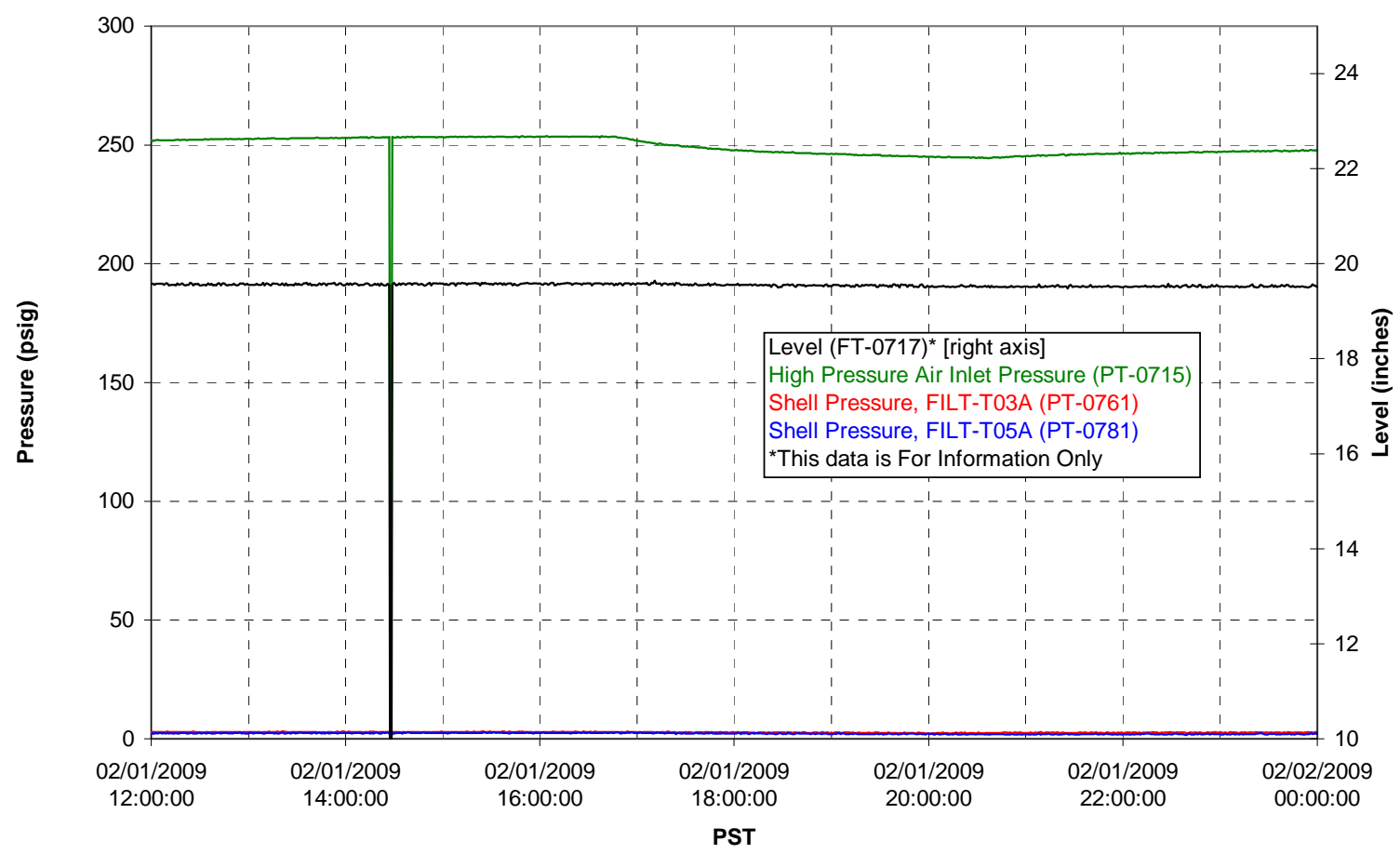

Pulsepot UFP-PP-T02A

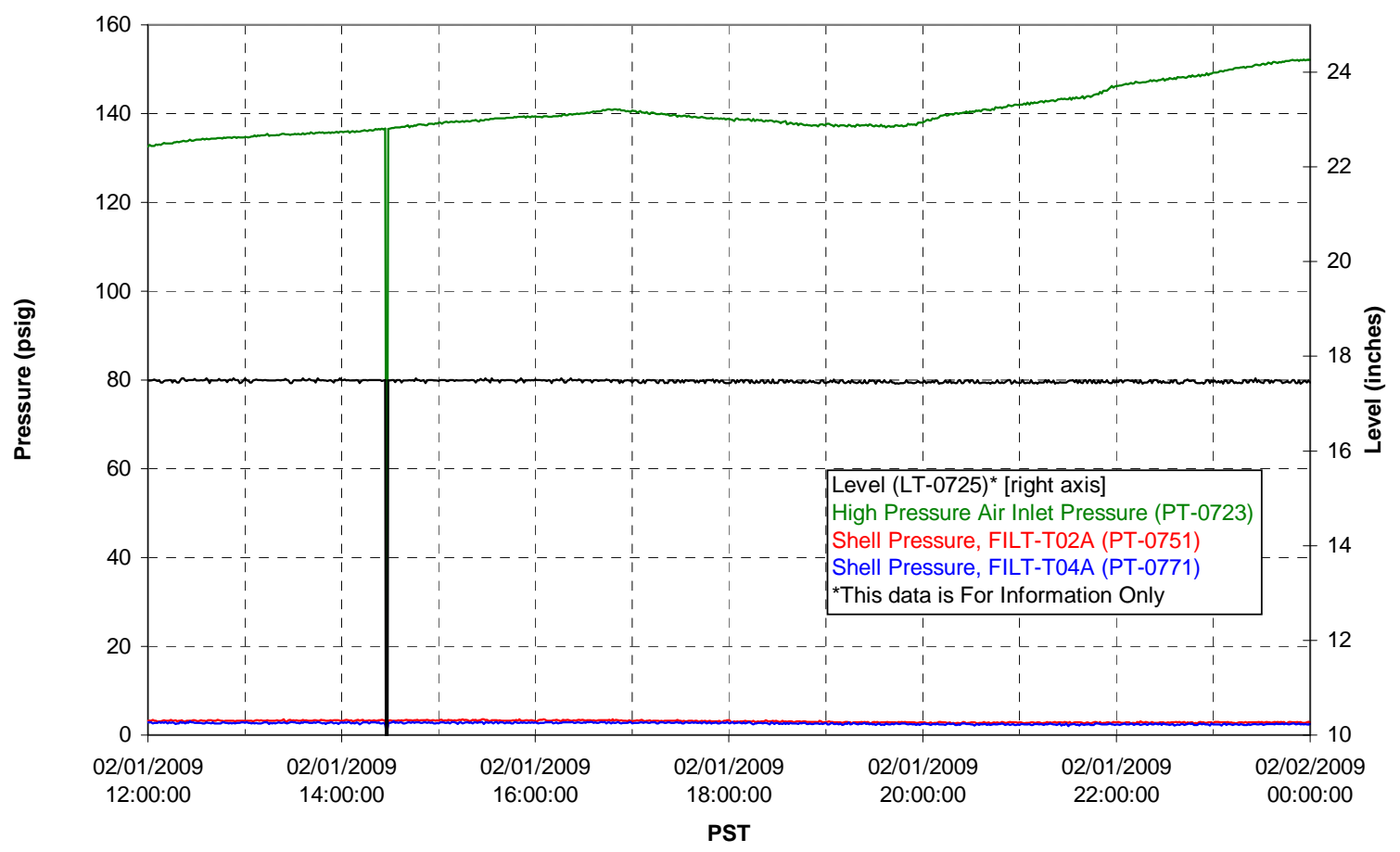


Pulsepot UFP-PP-T03A

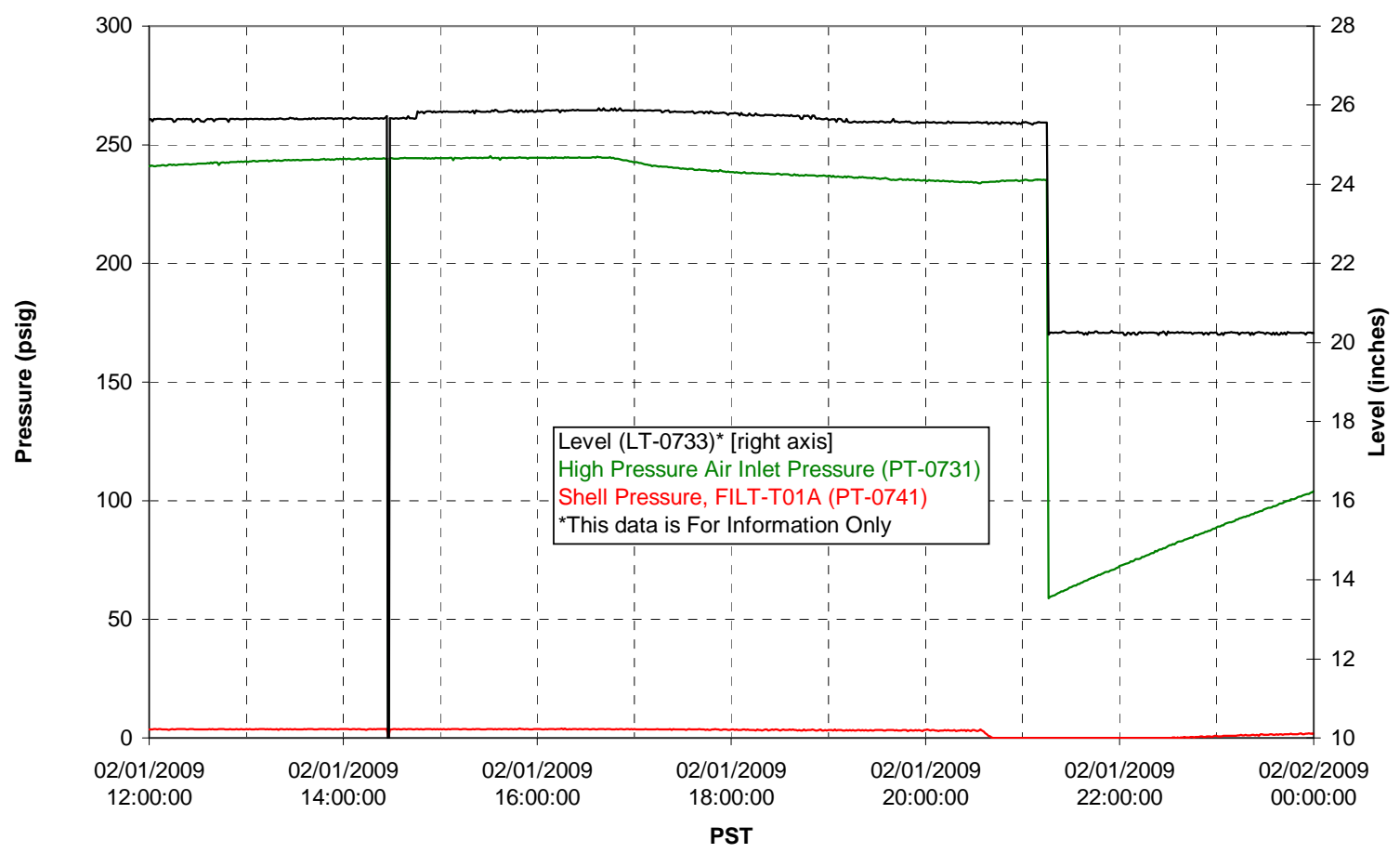

Pulsepot Levels

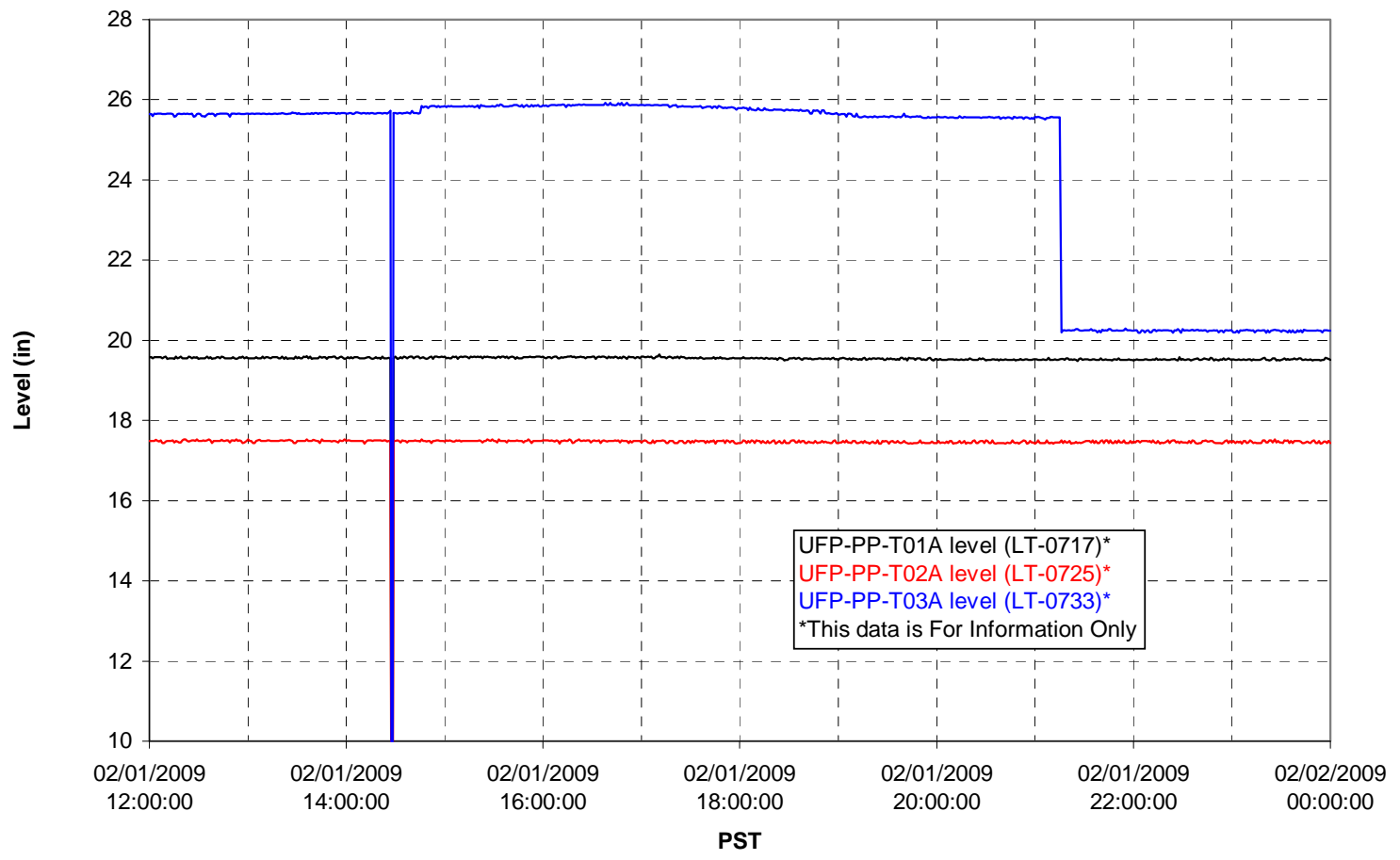


Filter UFP-FILT-T01A

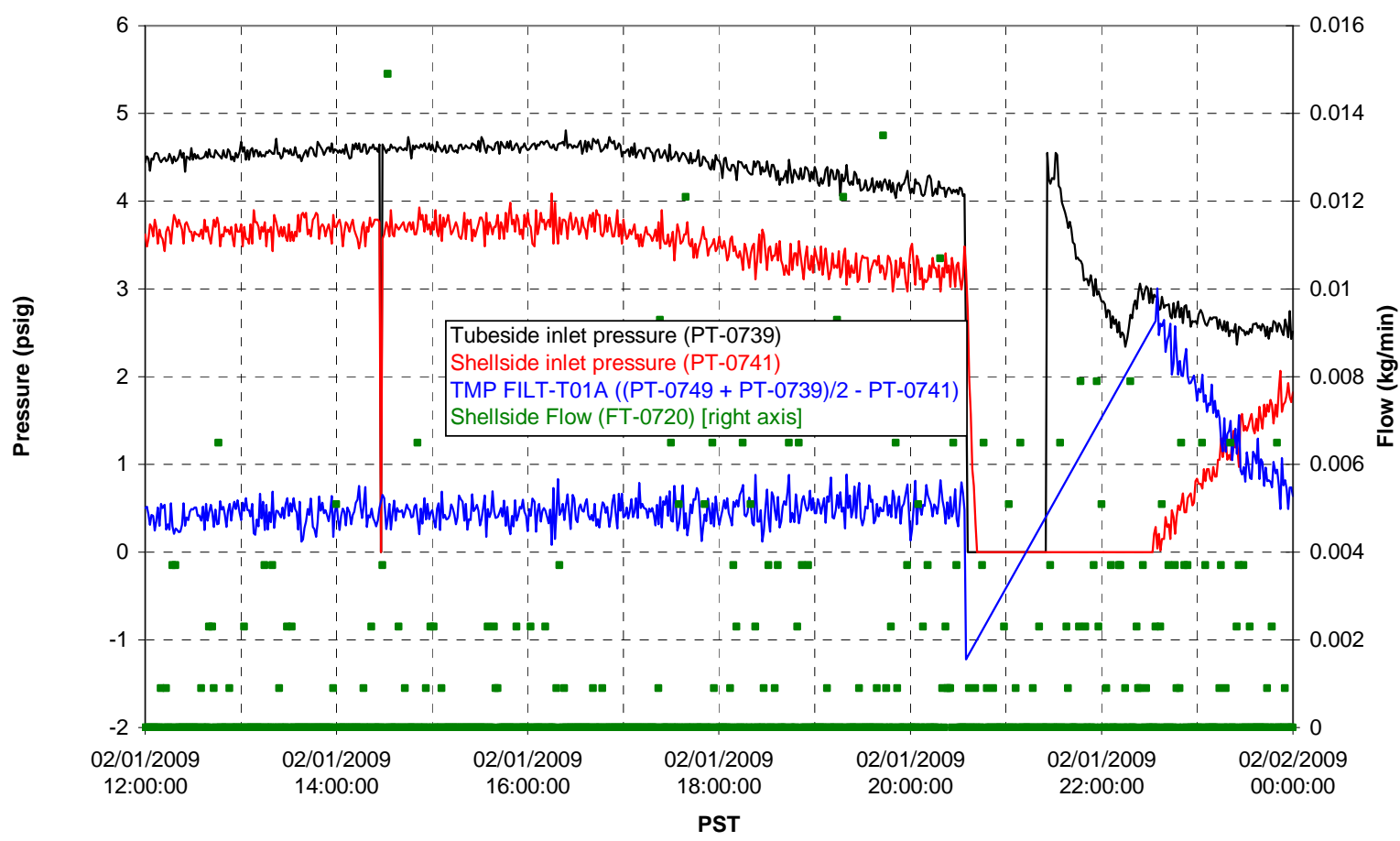

Filter UFP-FILT-T02A

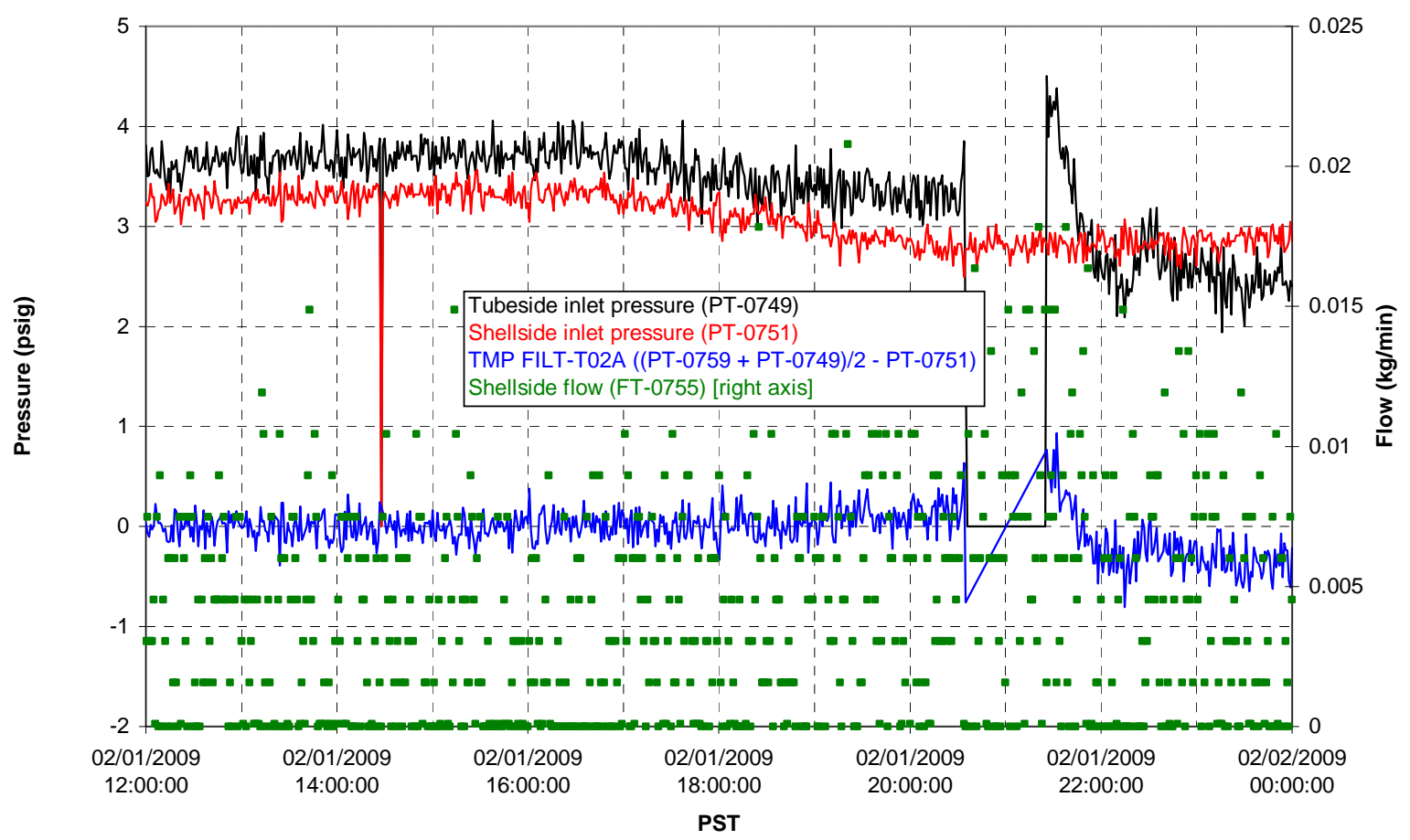


Filter UFP-FILT-T03A

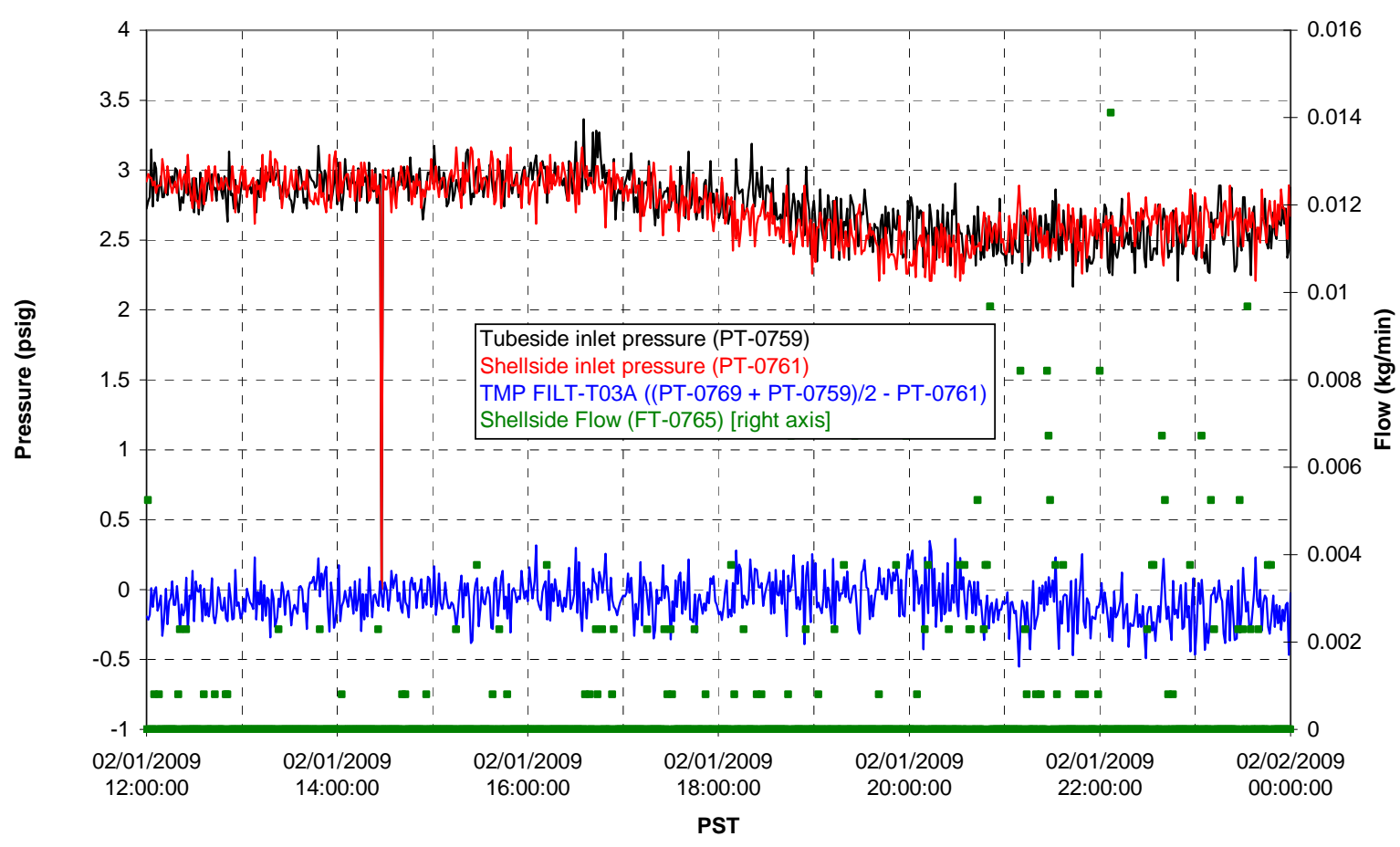

Filter UFP-FILT-T04A

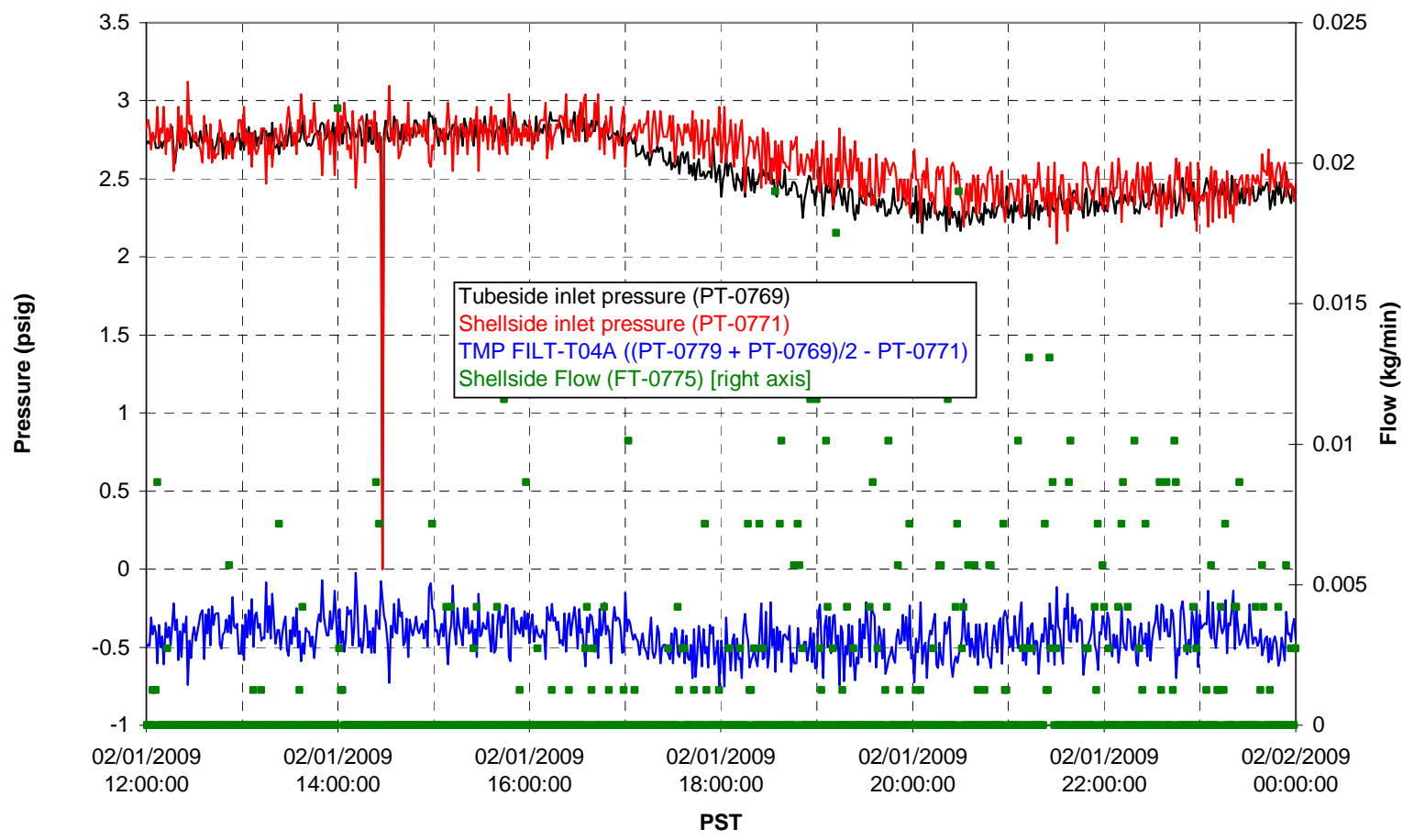


Filter UFP-FILT-T05A

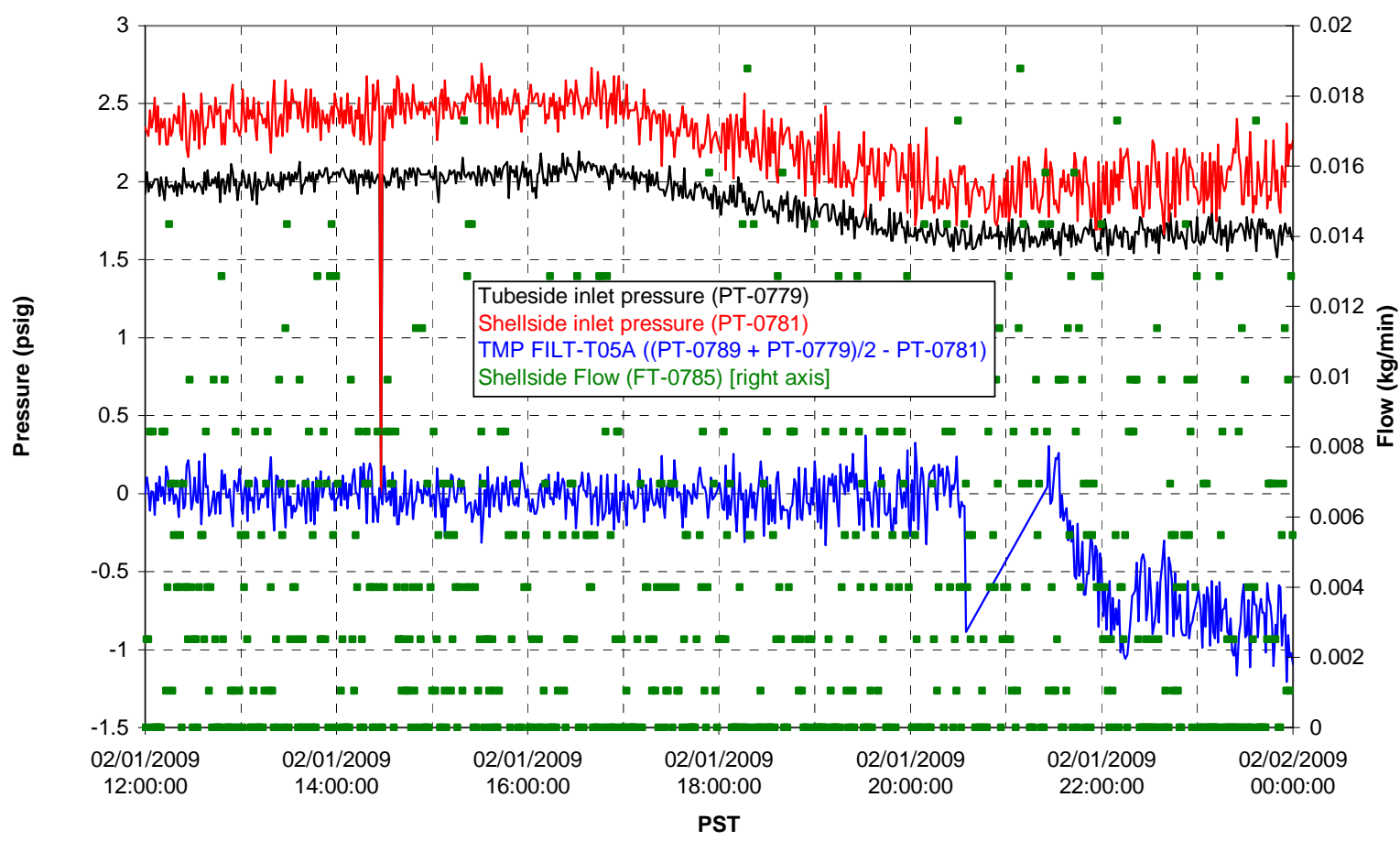

Chemical Flow

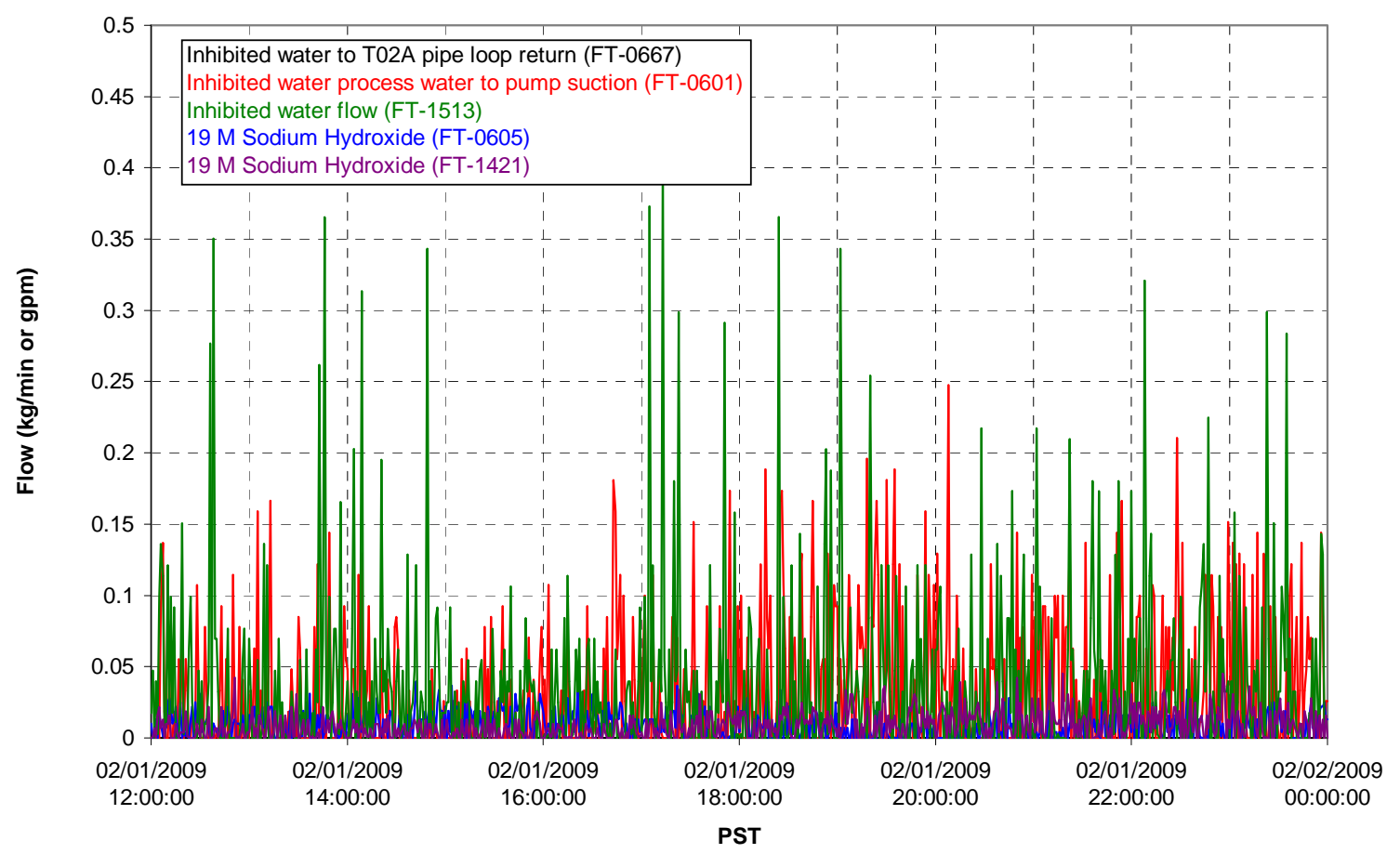




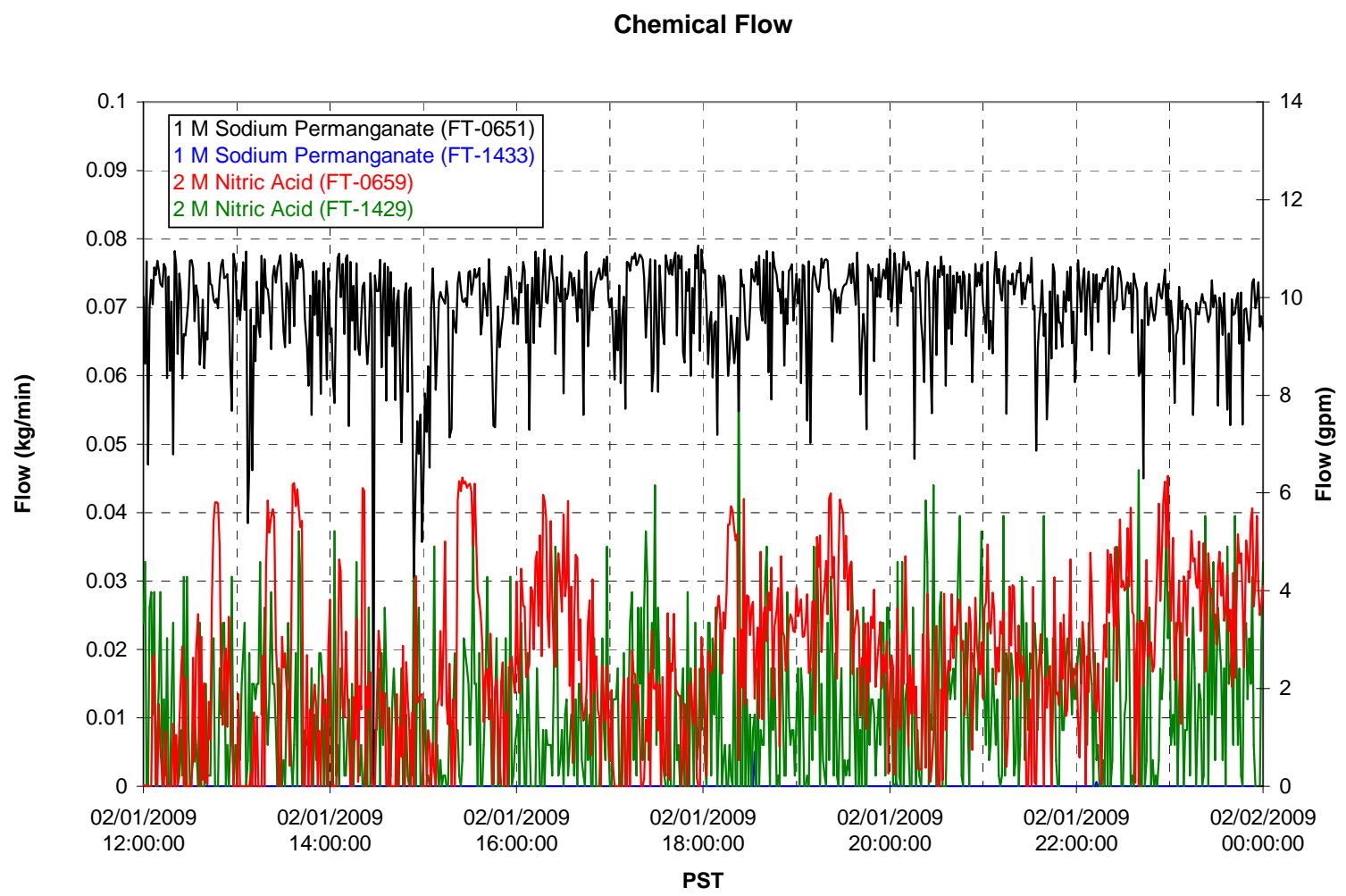

Air Flows

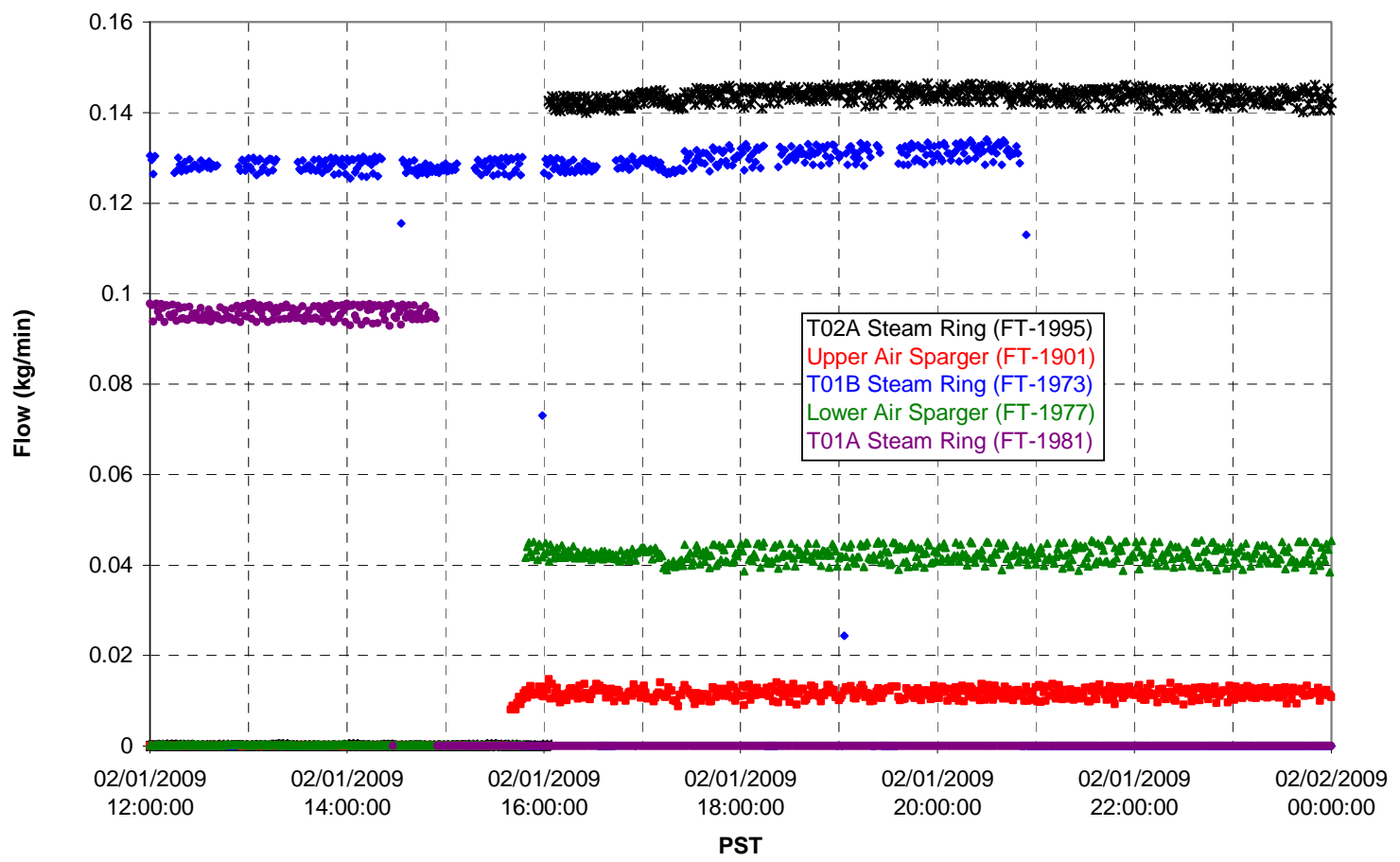


T02A Steam

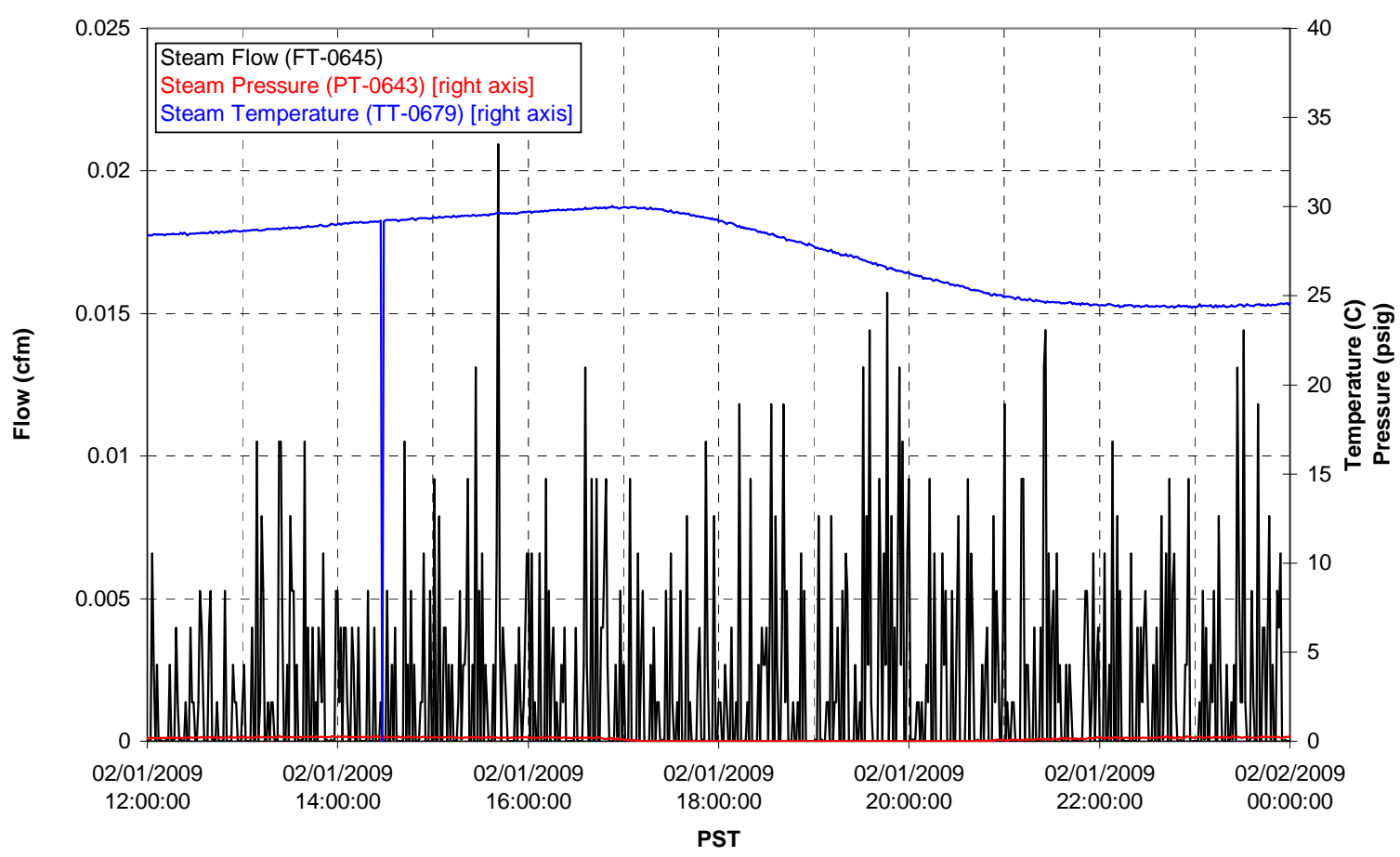

T01A Steam

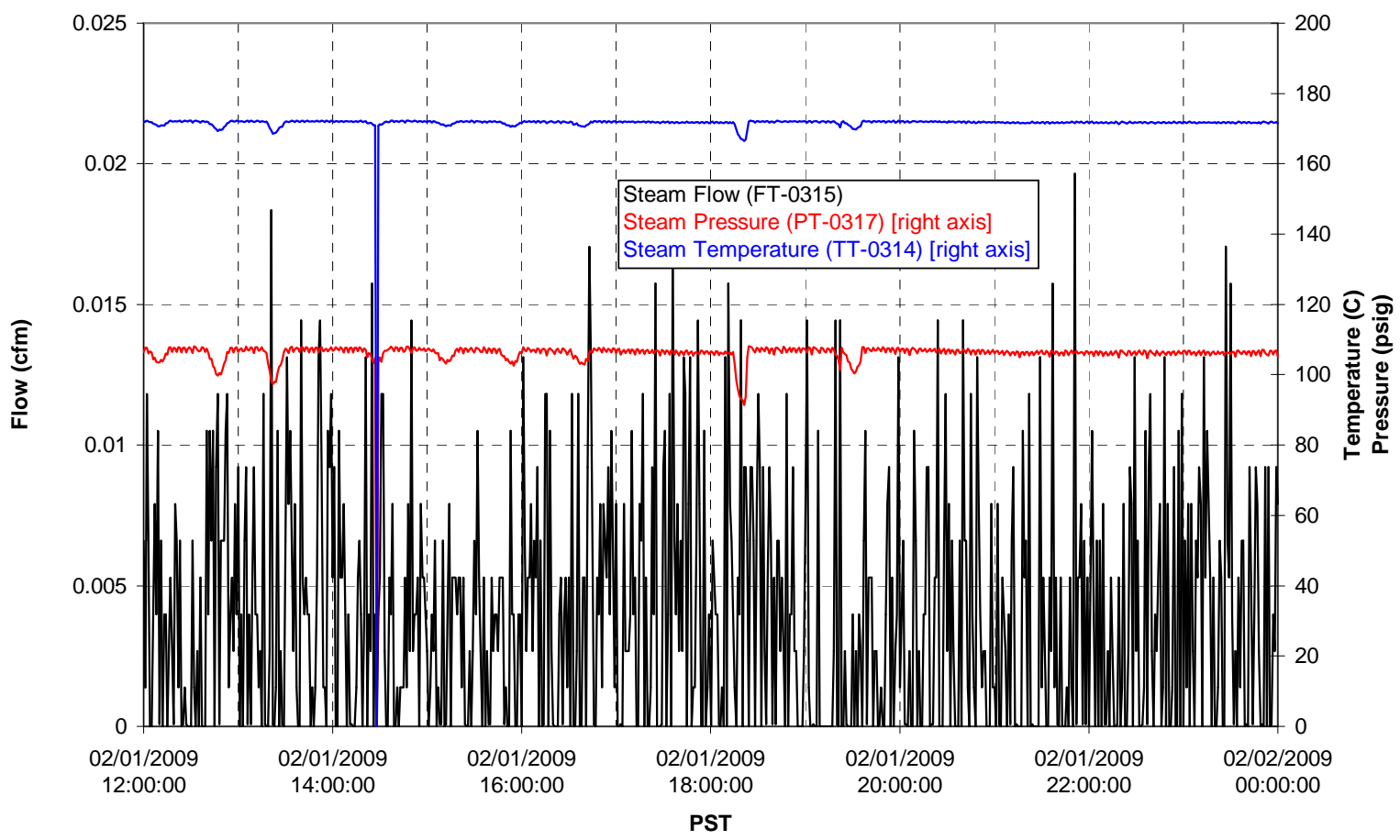


T01B Steam

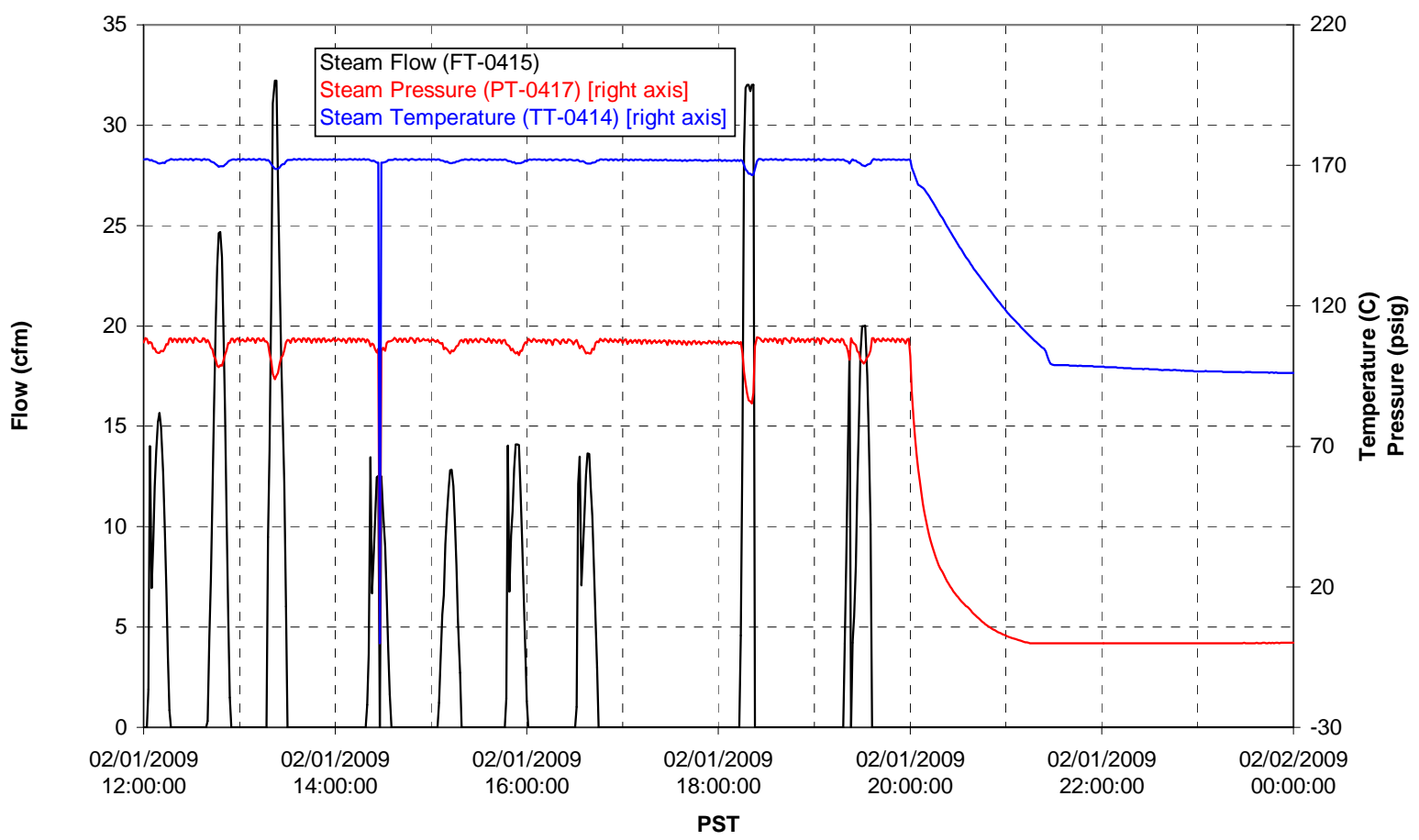




\section{Integrated Test A Data Plots 02/02/09 00:00 - 02/02/09 12:00}


T01A level

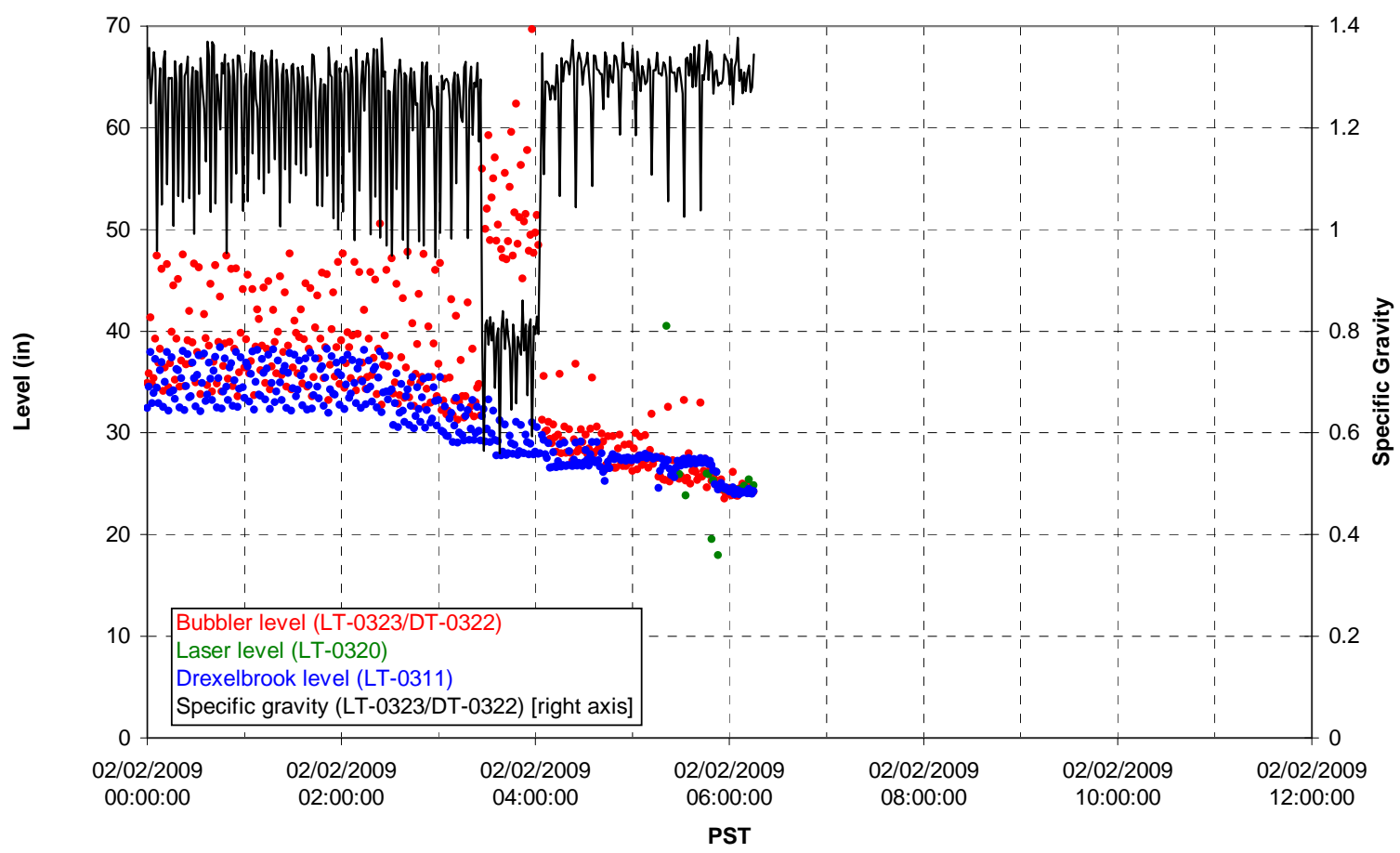

T01A temperatures

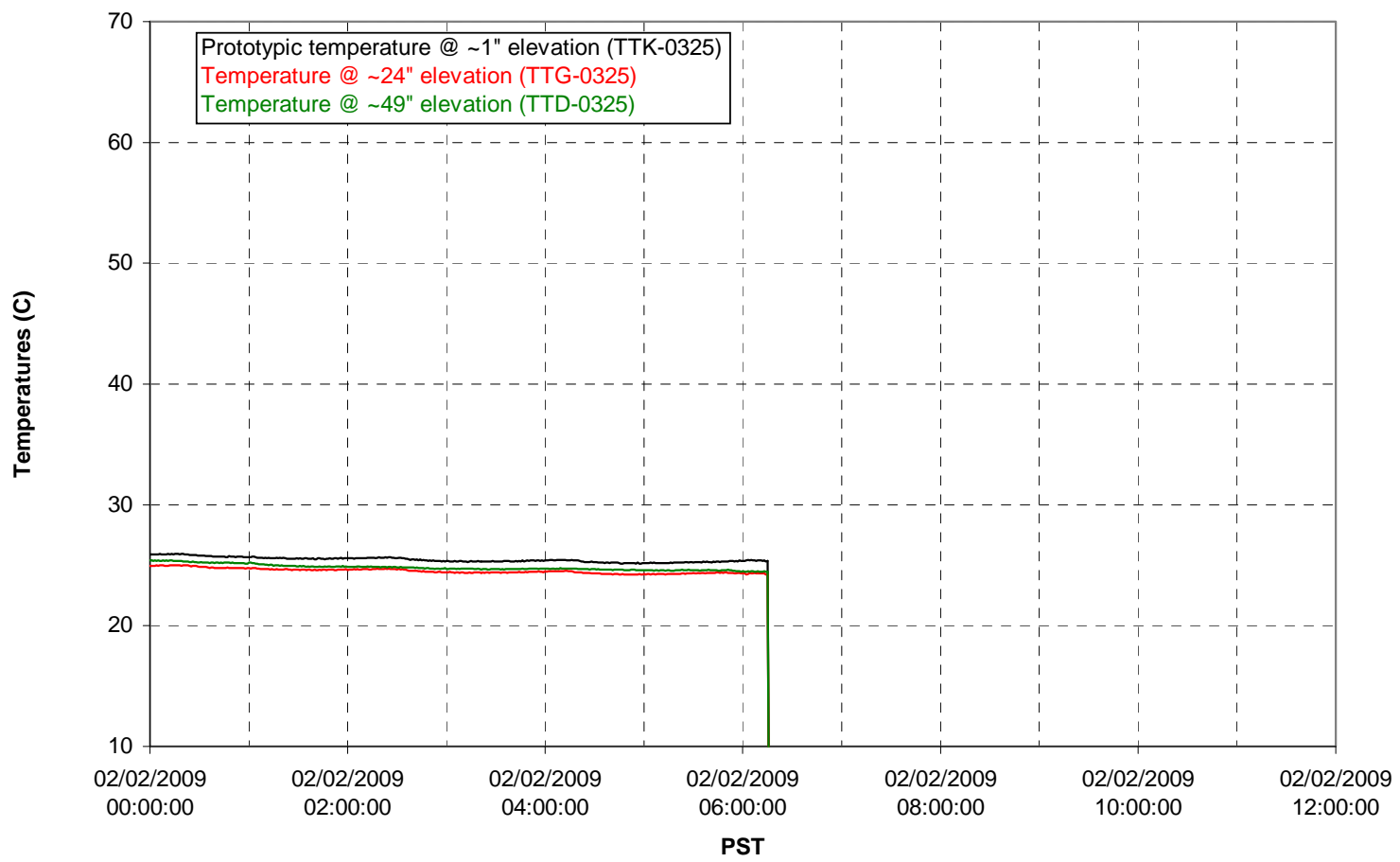


T01B level

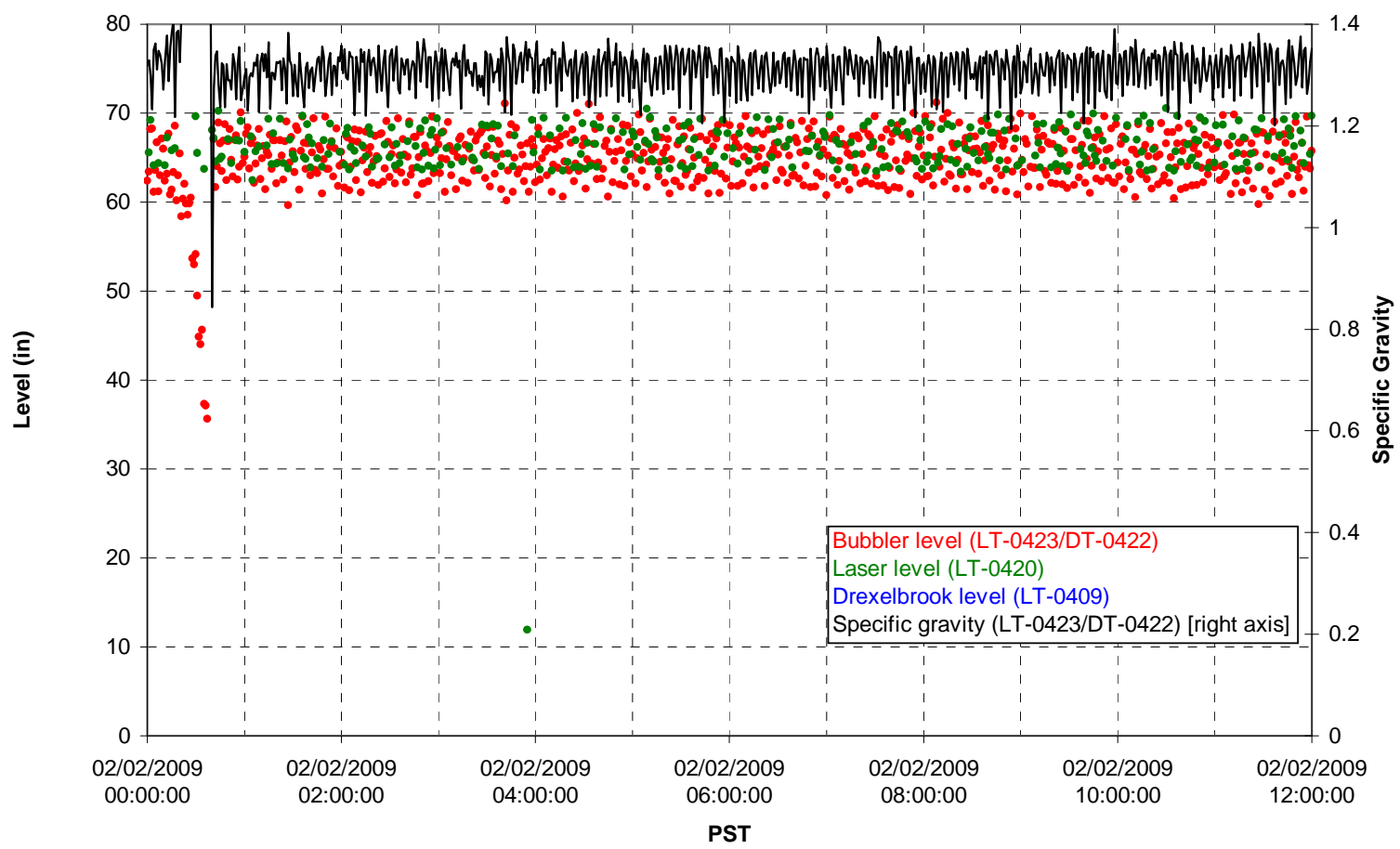

T01B temperatures

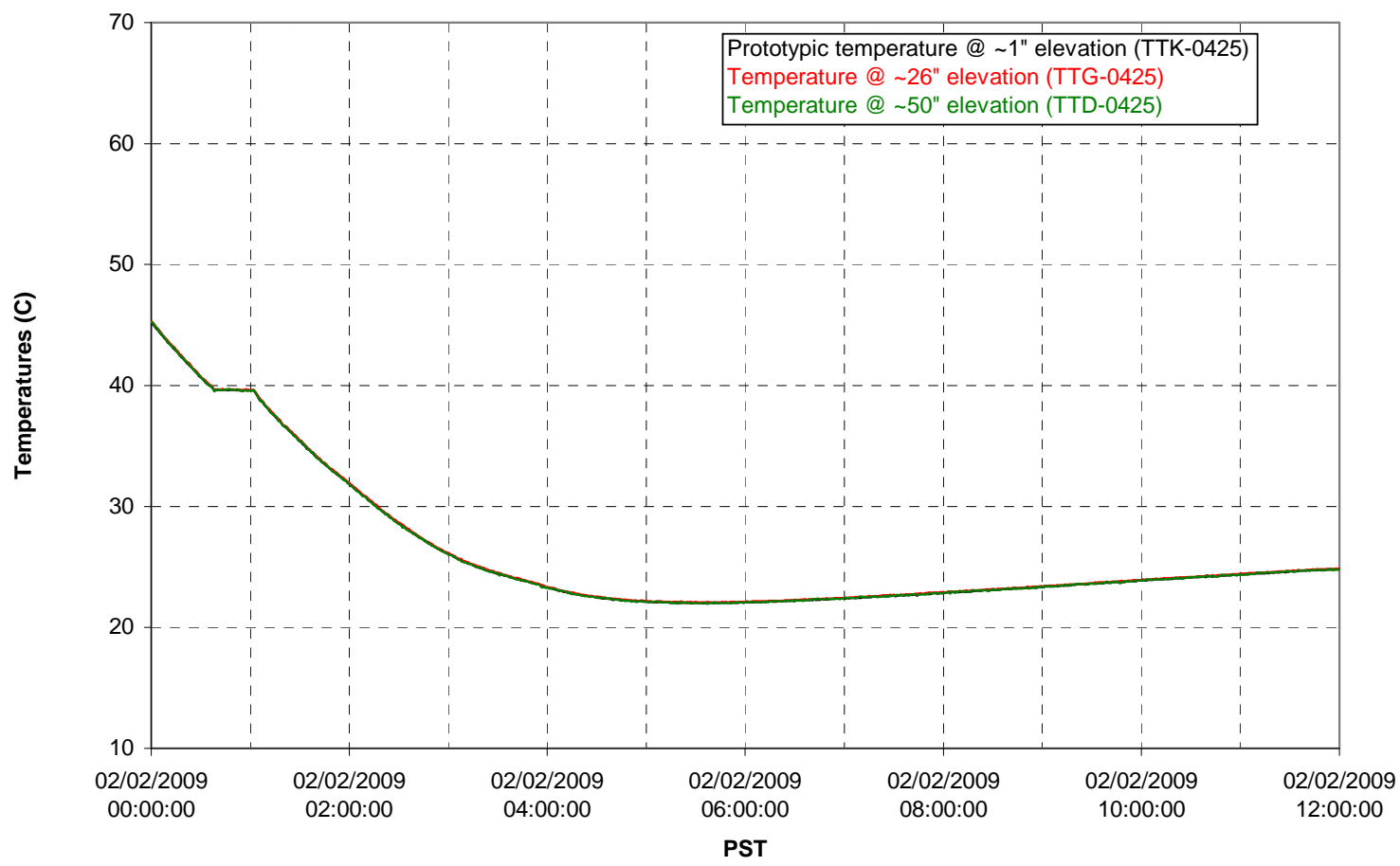

G.75 
T02A level

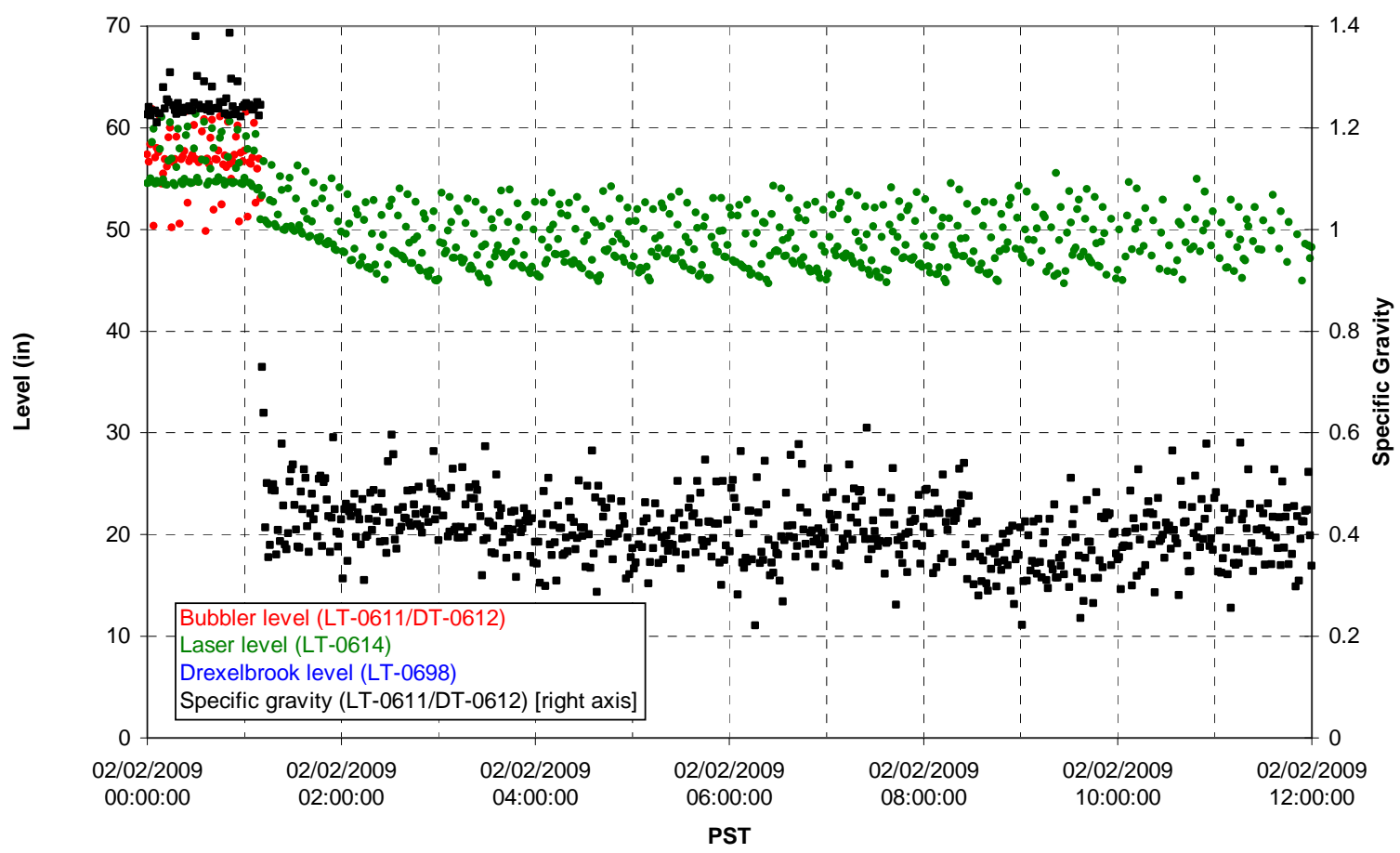

T02A temperatures

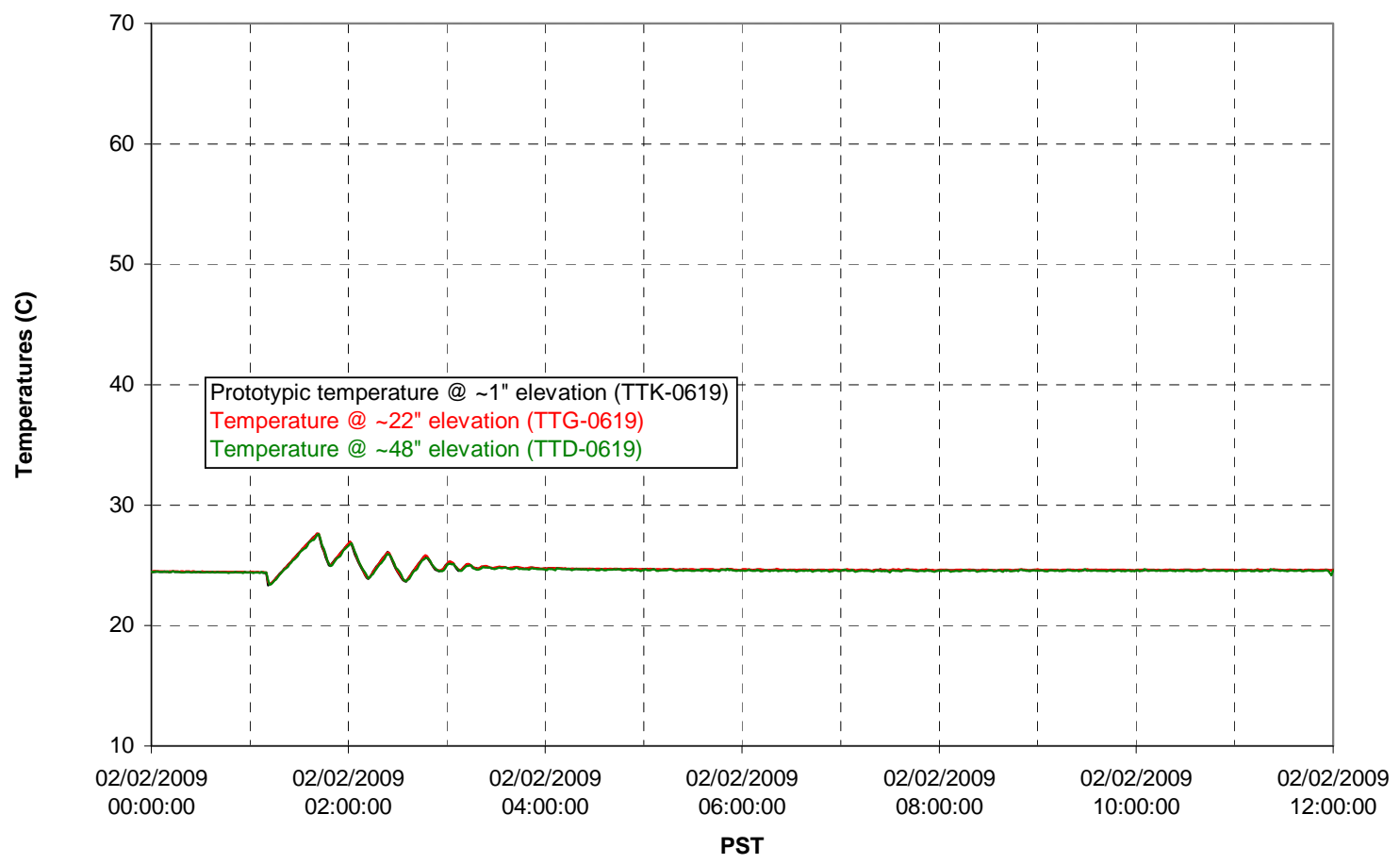

G.76 
T02A and filter loop temperatures

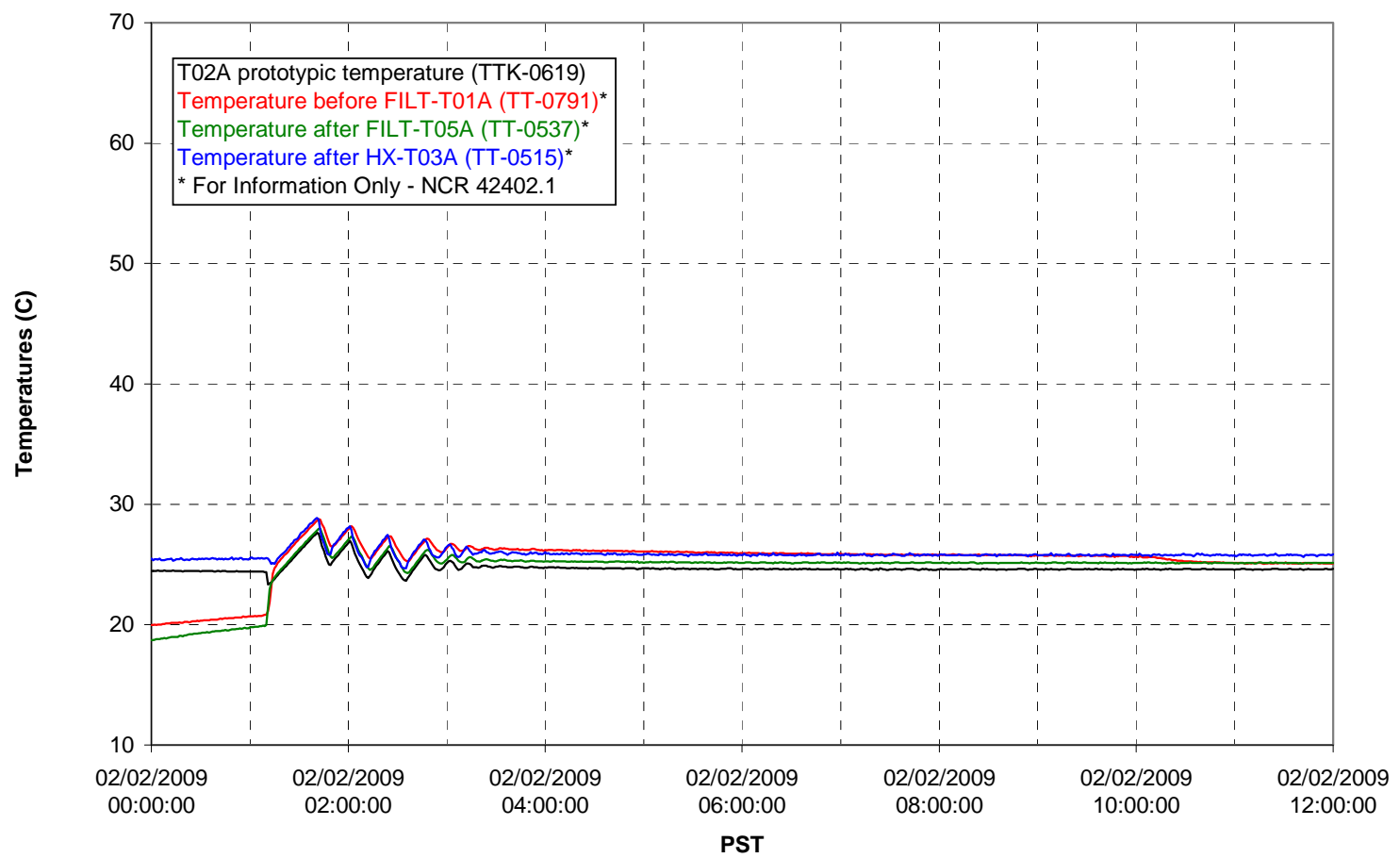

Pump Pressures and Flow

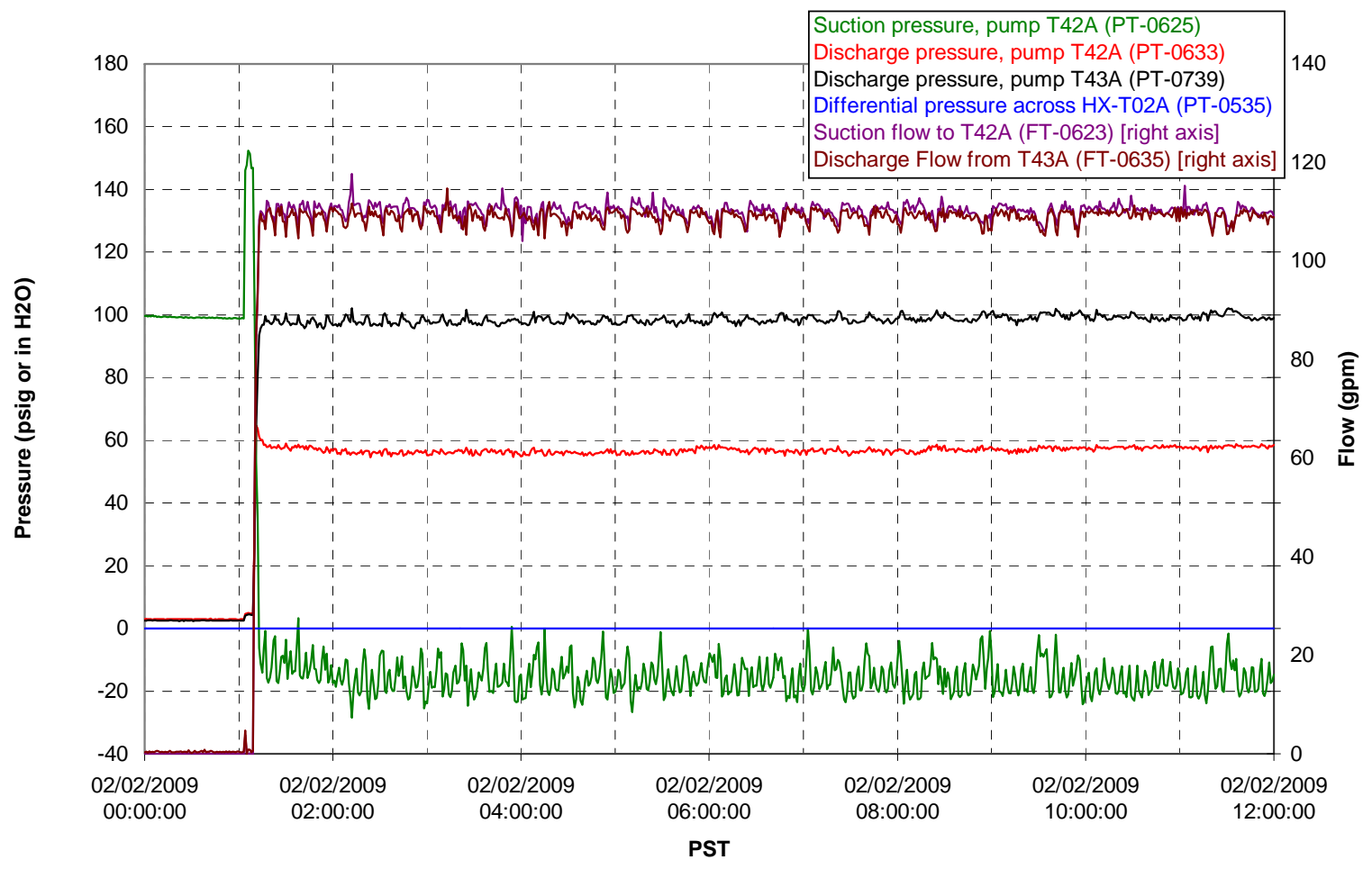


Axial pressure drop

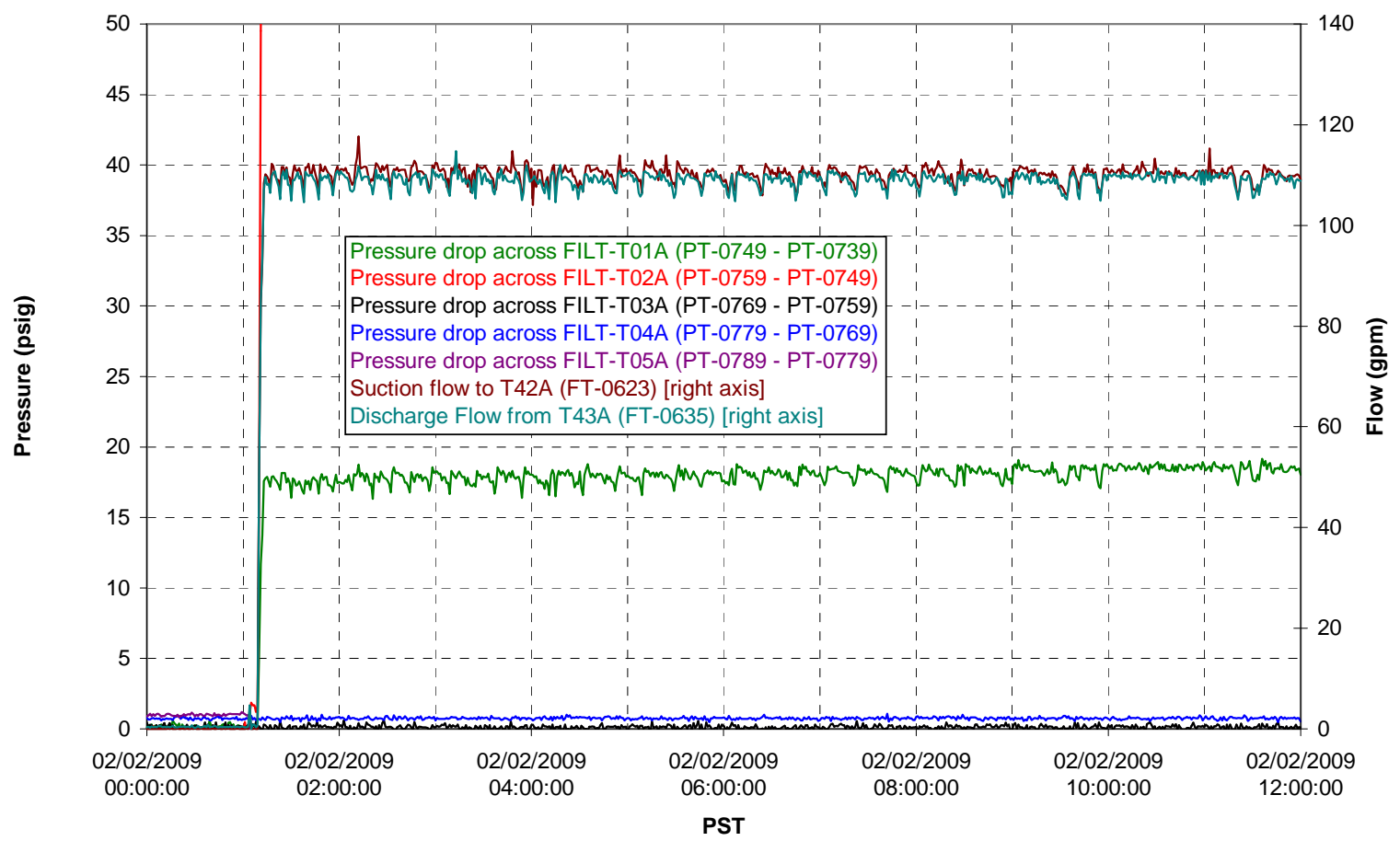

Permeate flow rates

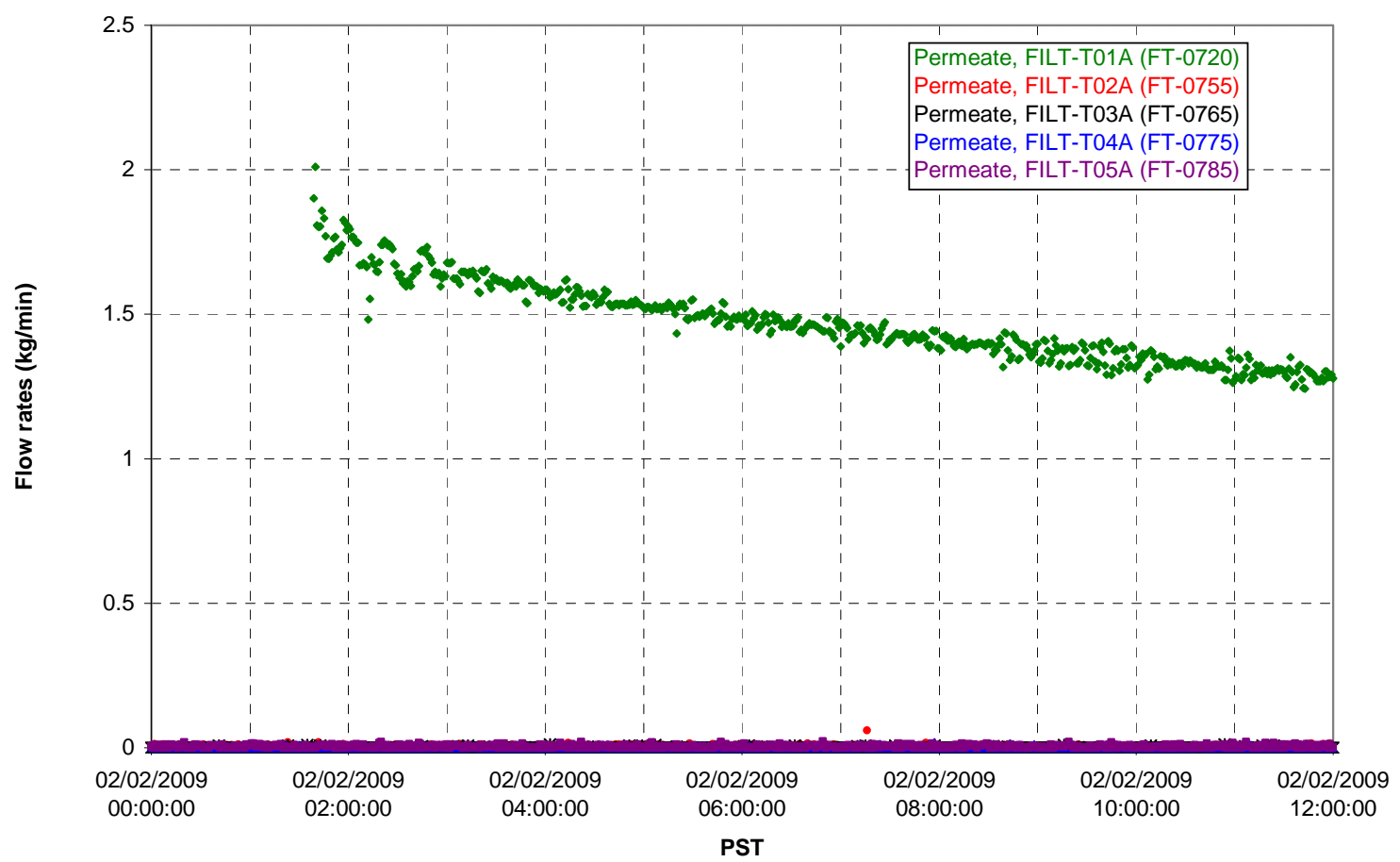

G.78 
T02A Inner Temperature Tree

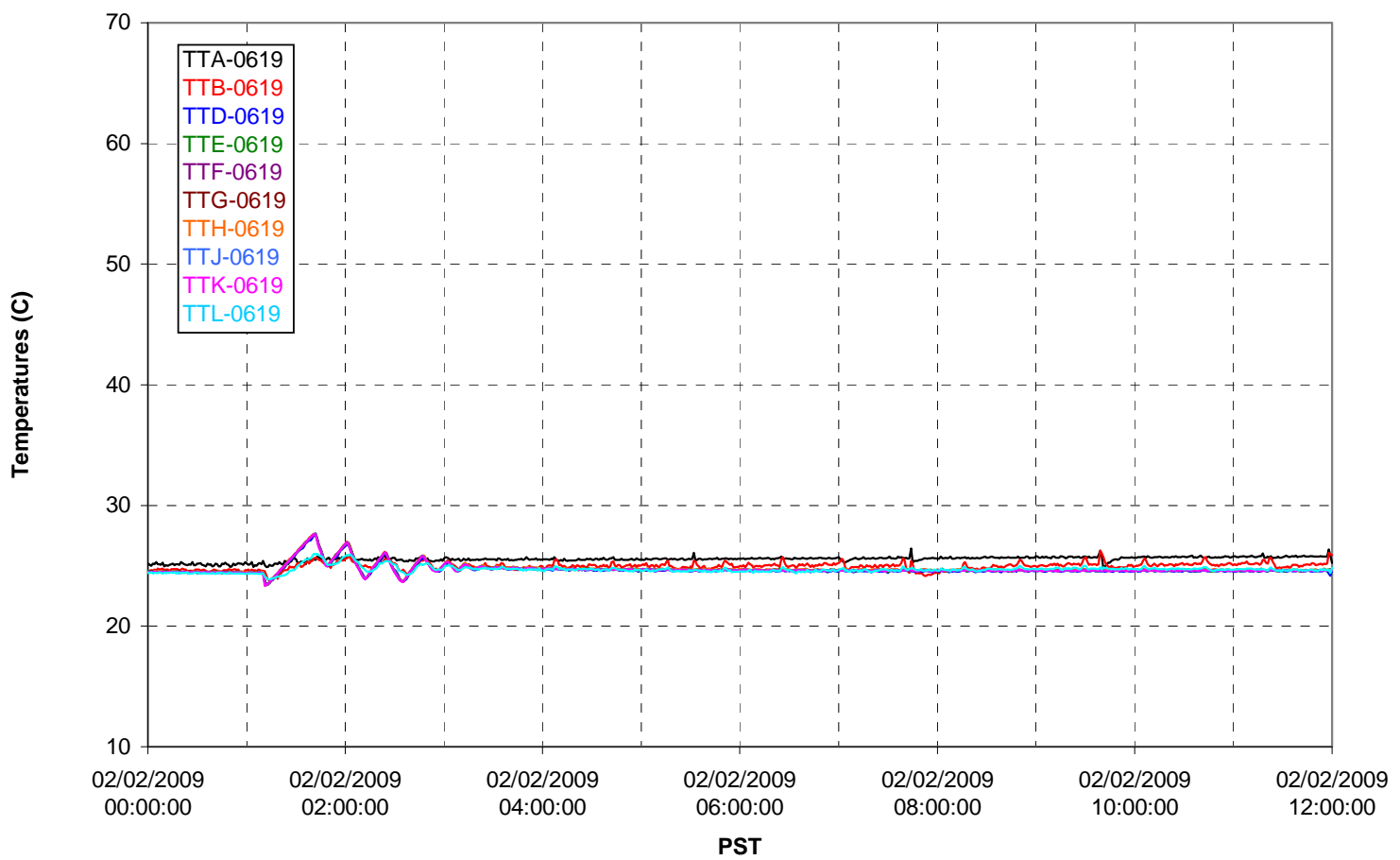

T02A Outer Temperature Tree

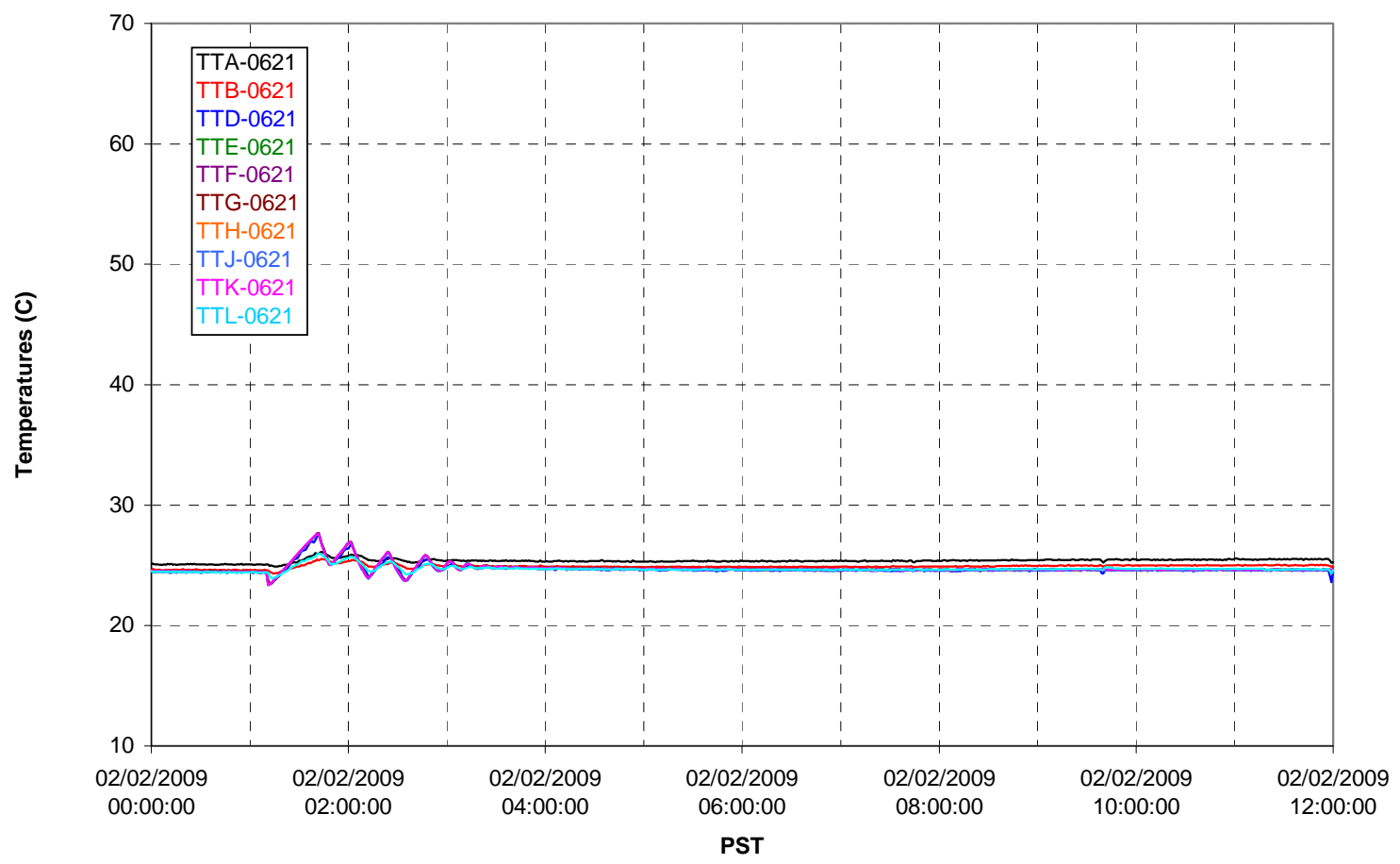


T02A temperatures

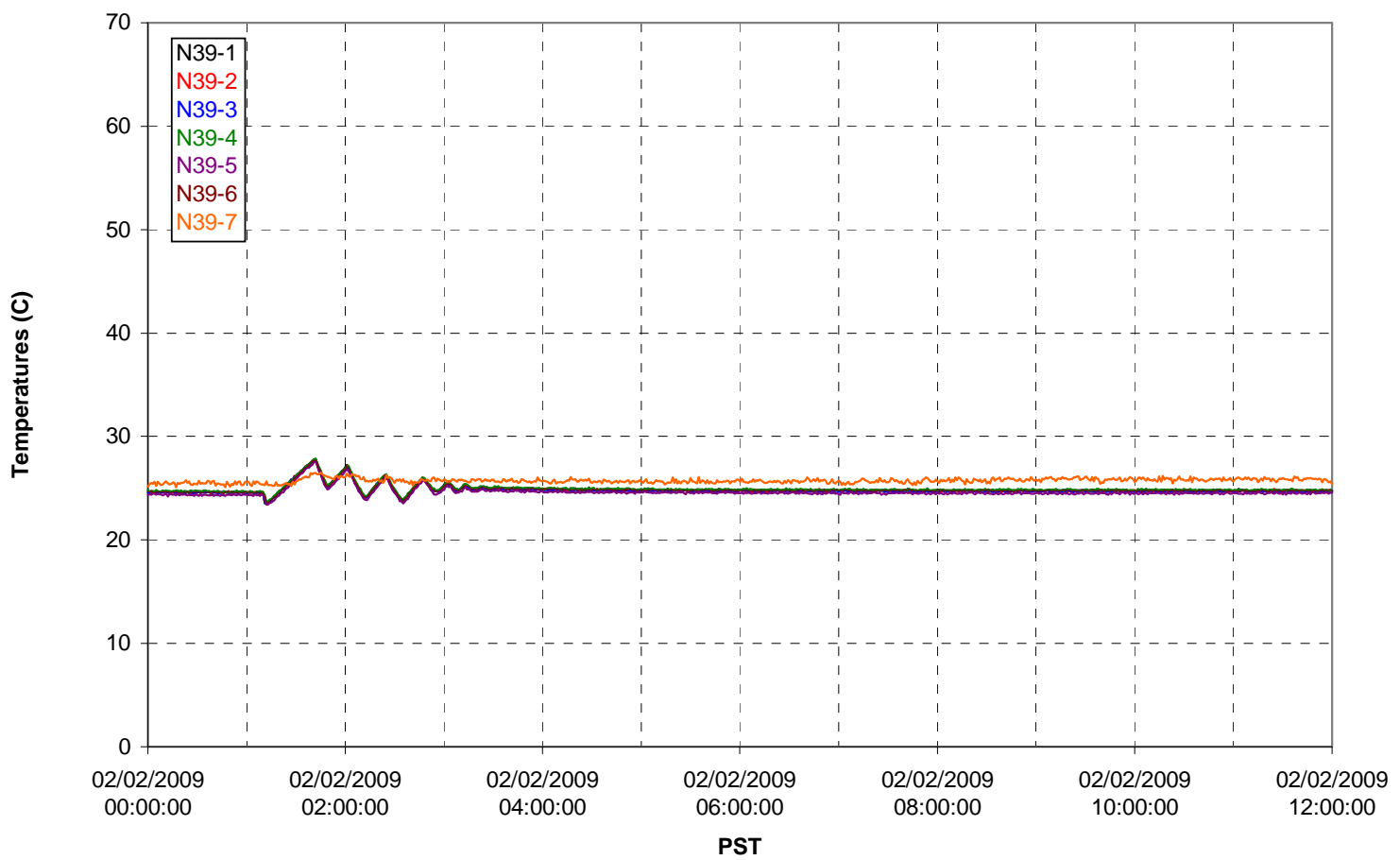

T02A temperatures

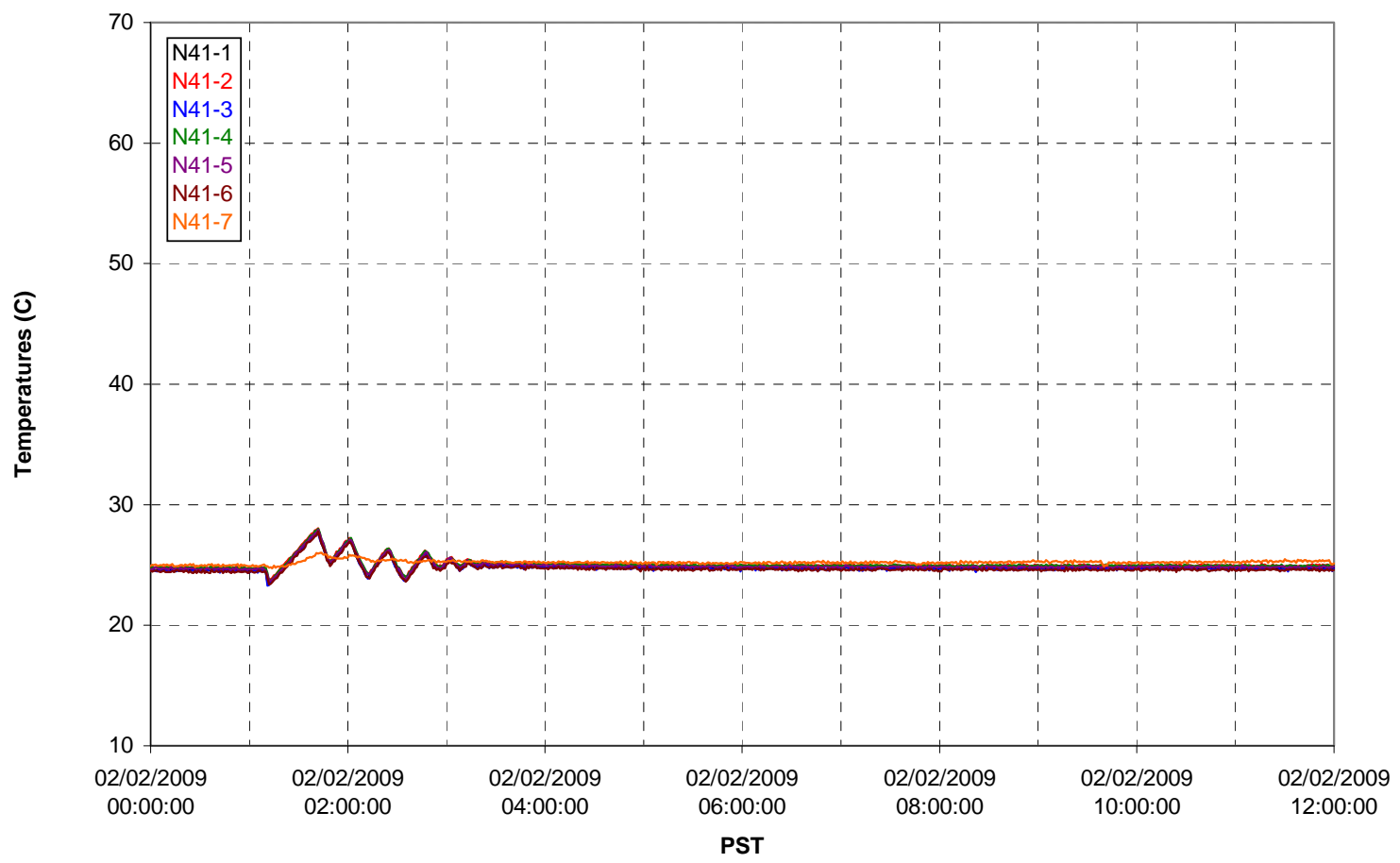


T02A temperatures

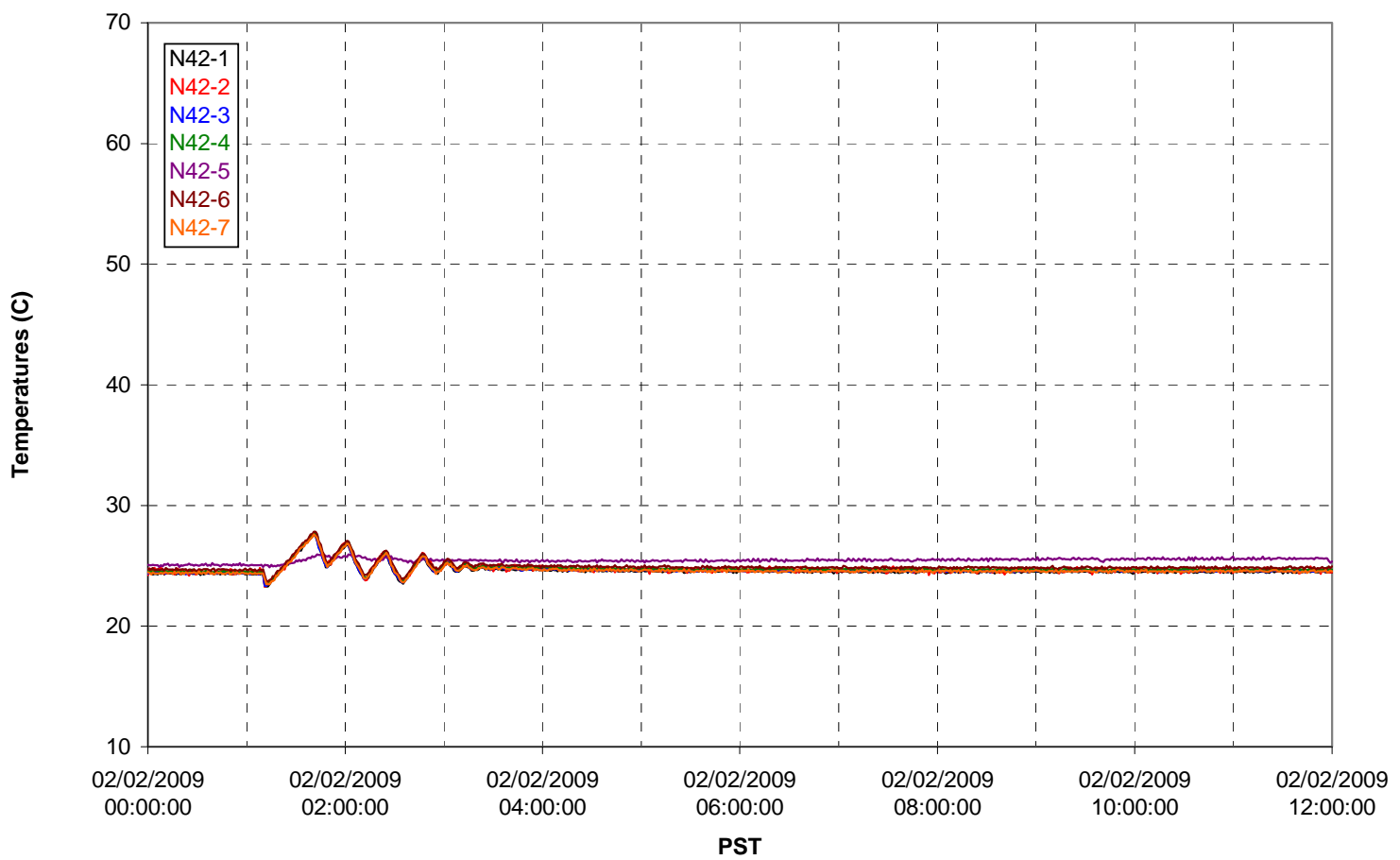

T02A temperatures

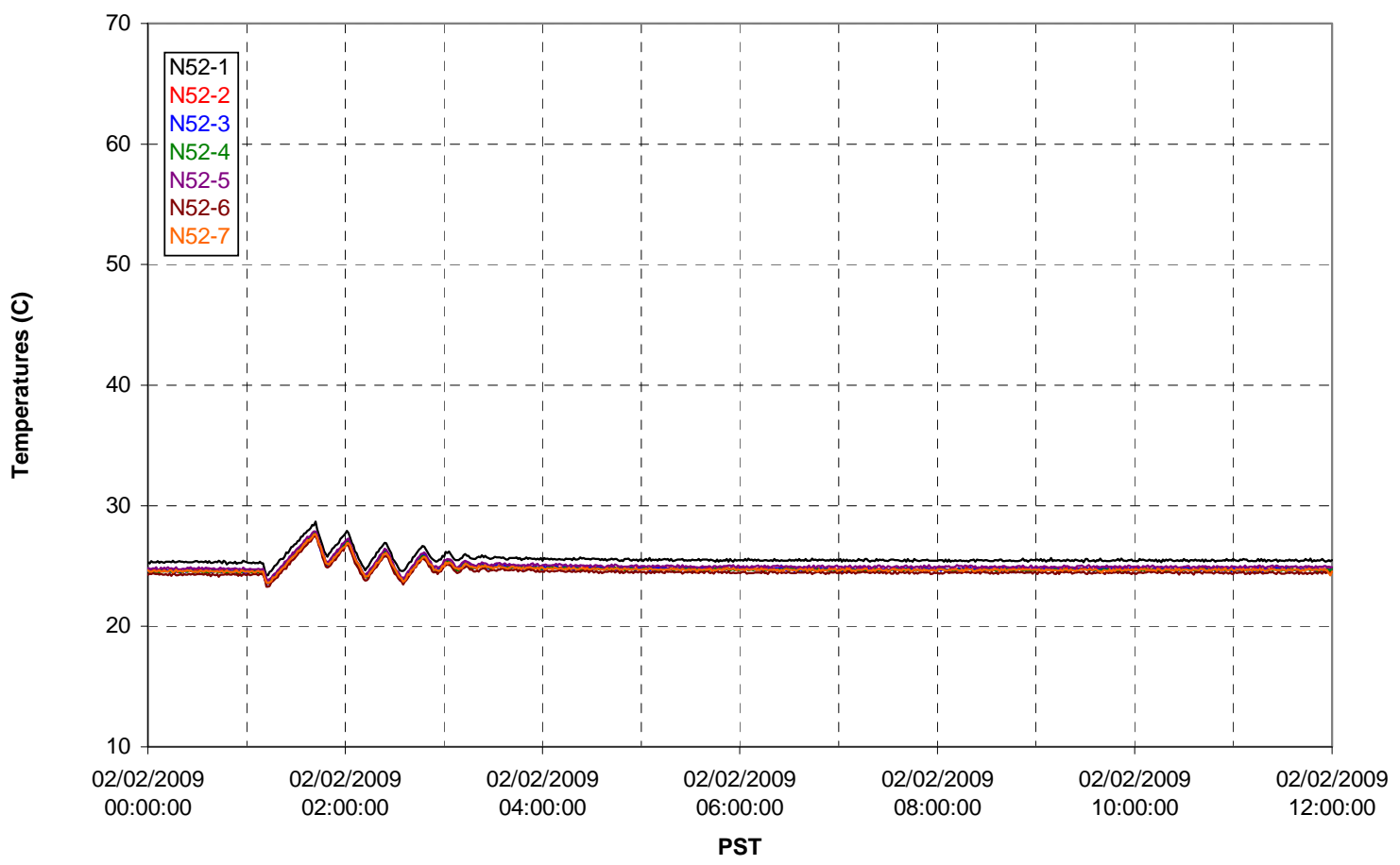


T02A Heating and Cooling

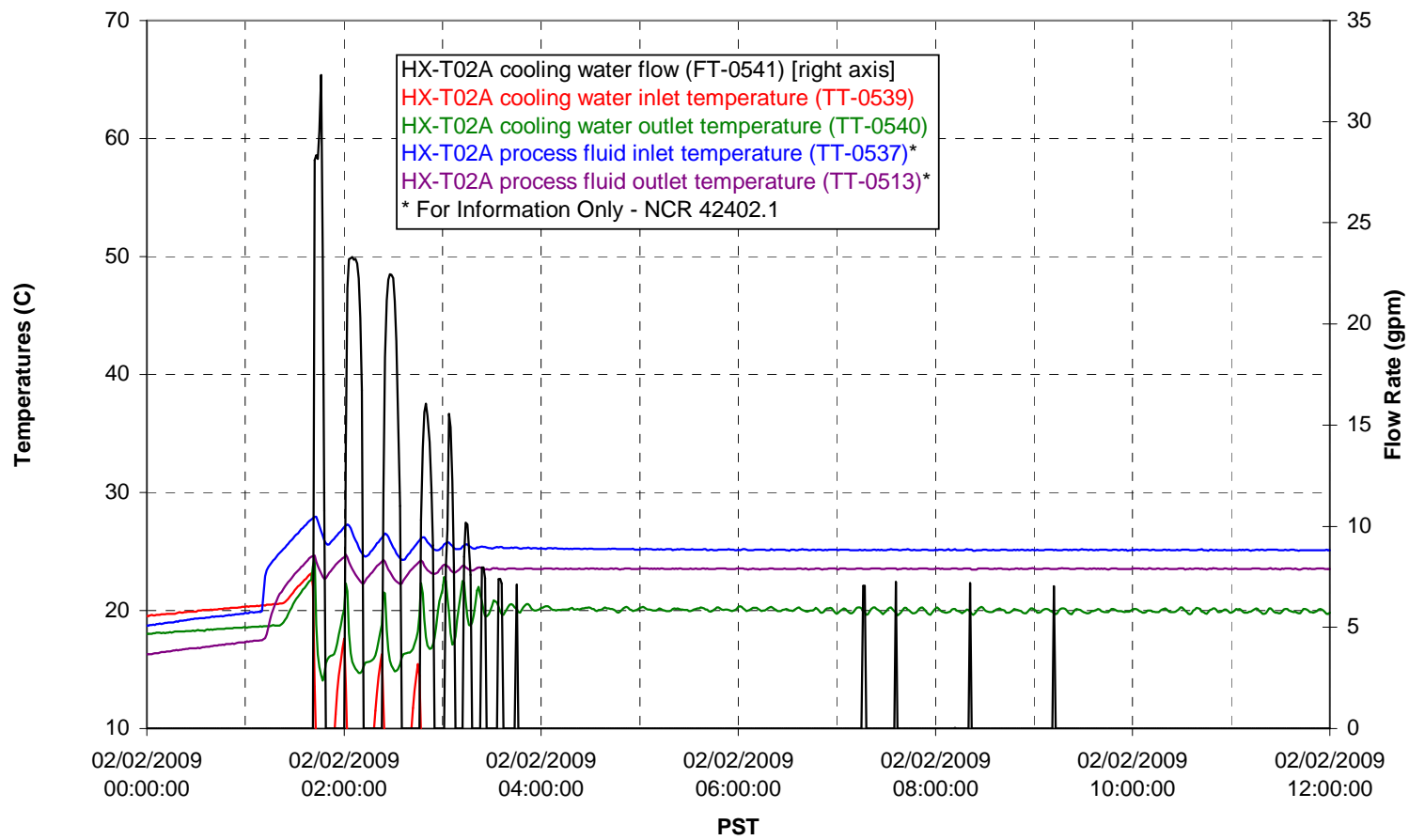

Pump Operation

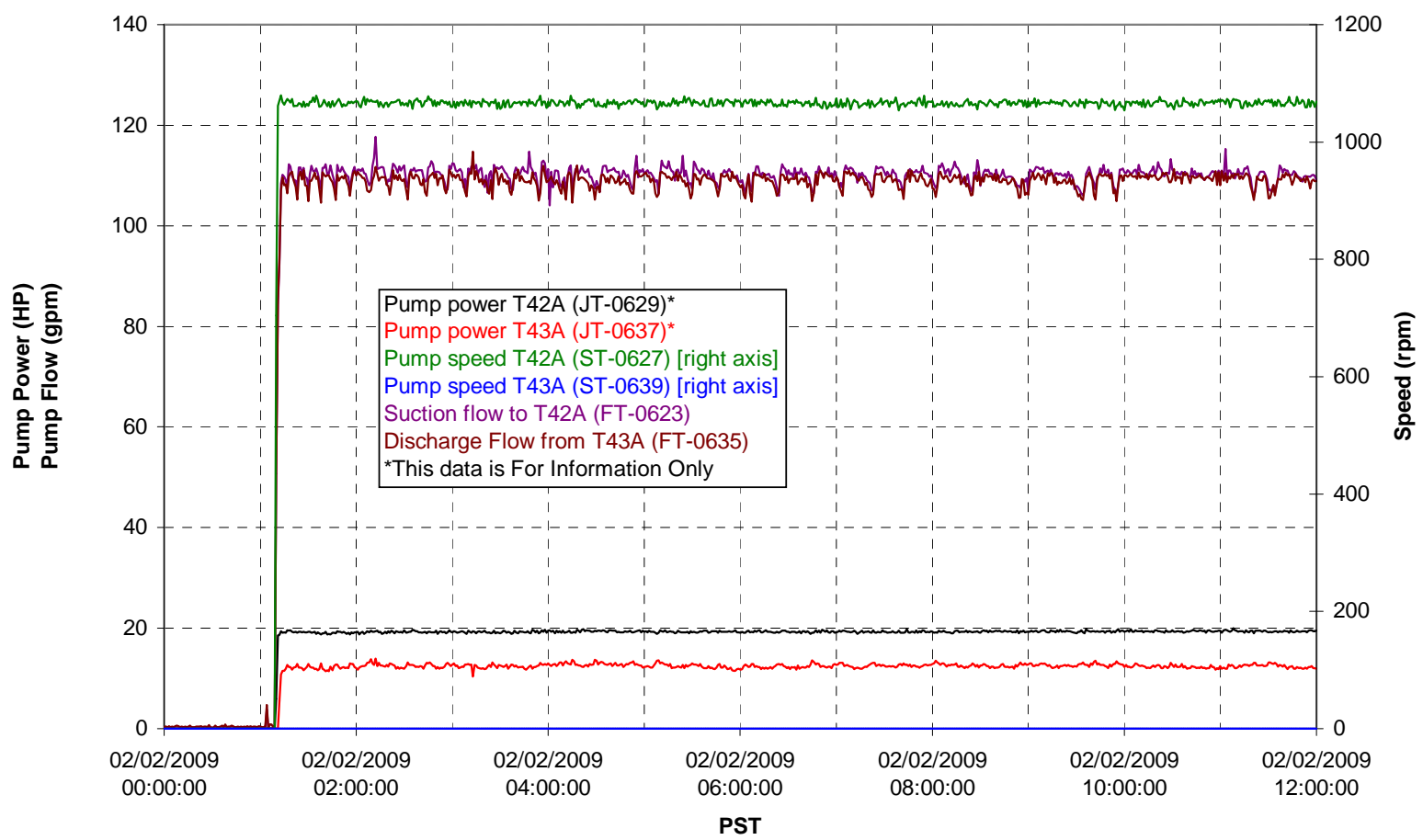


Pulsepot UFP-PP-T01A

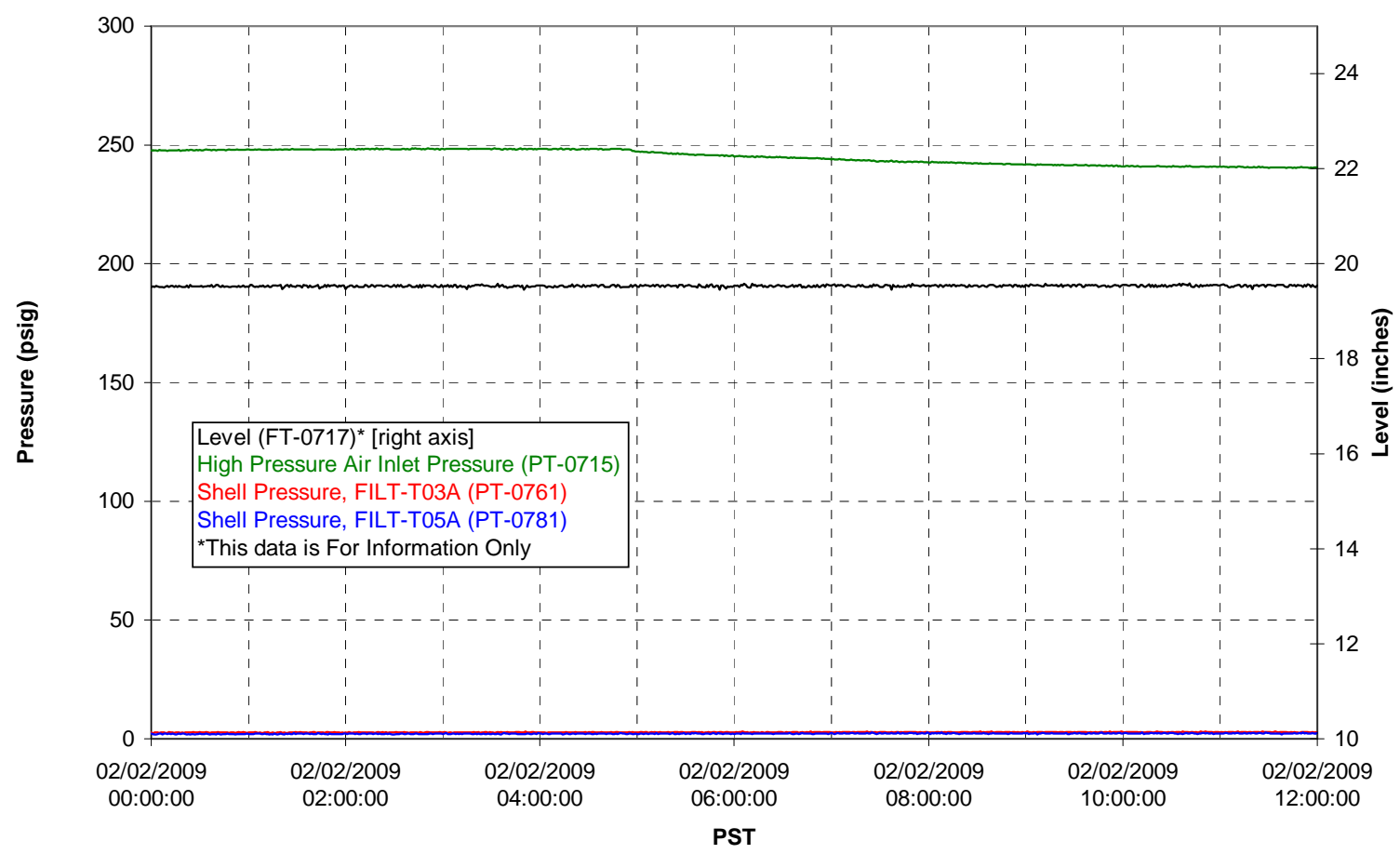

Pulsepot UFP-PP-T02A

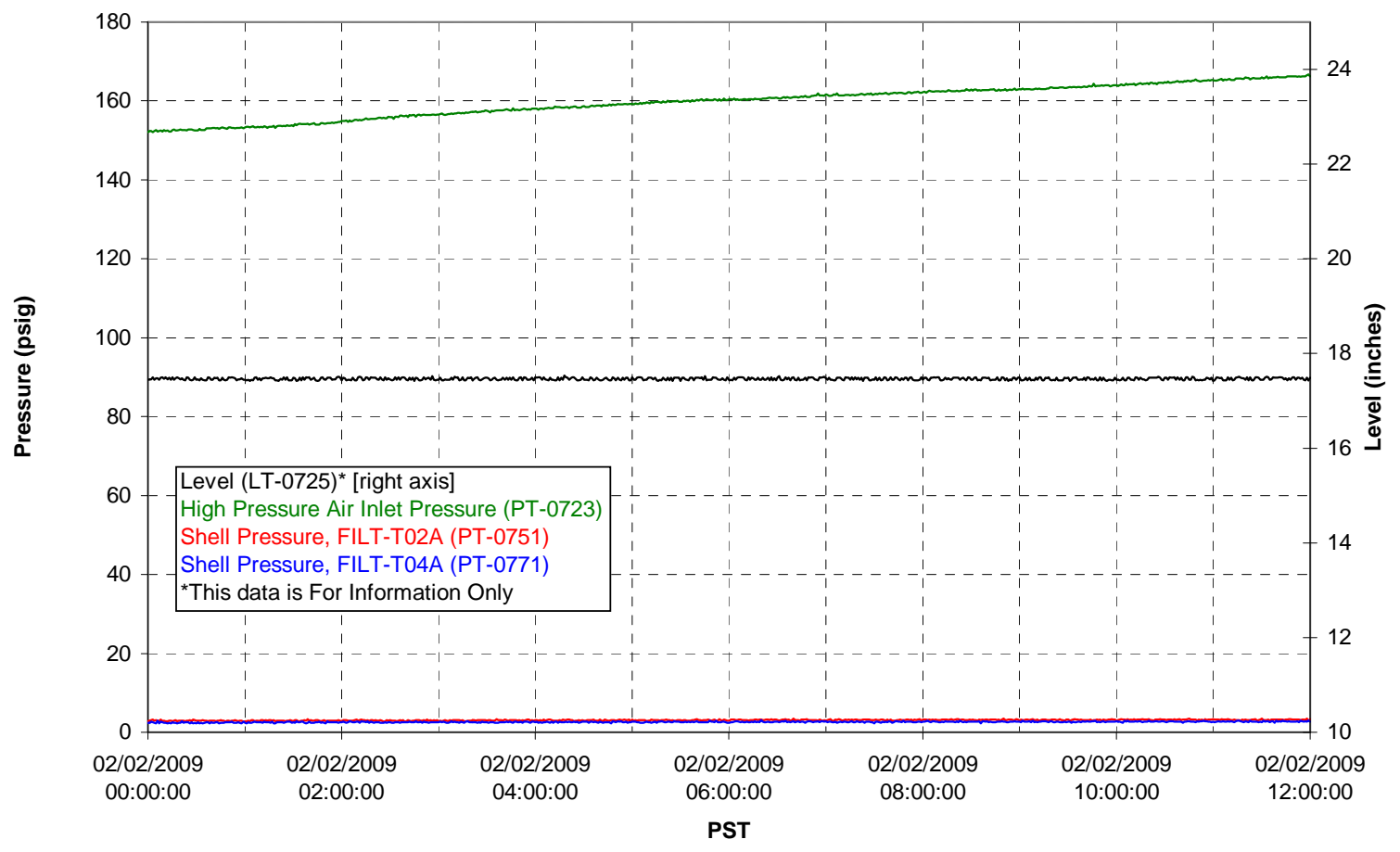


Pulsepot UFP-PP-T03A

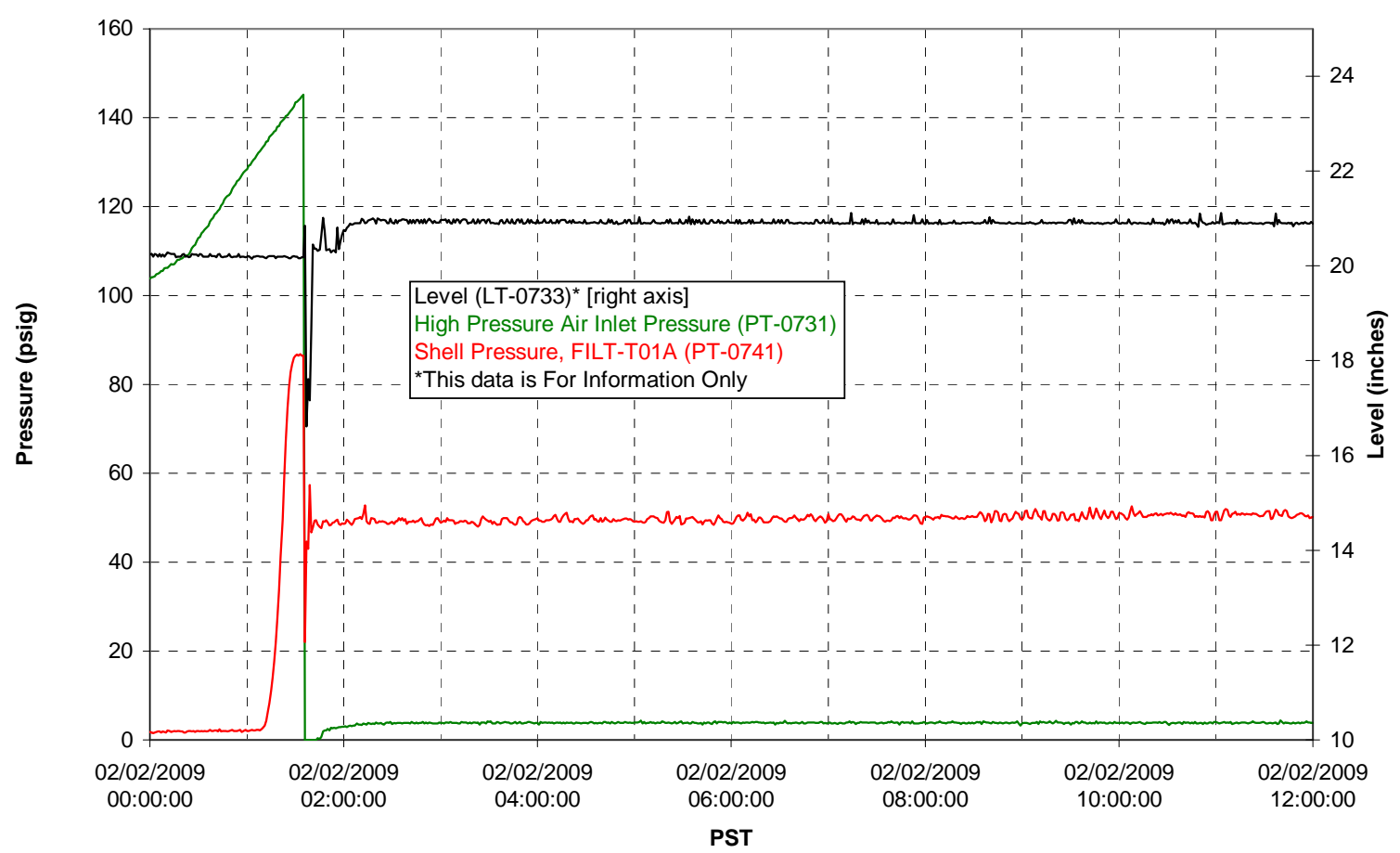

Pulsepot Levels

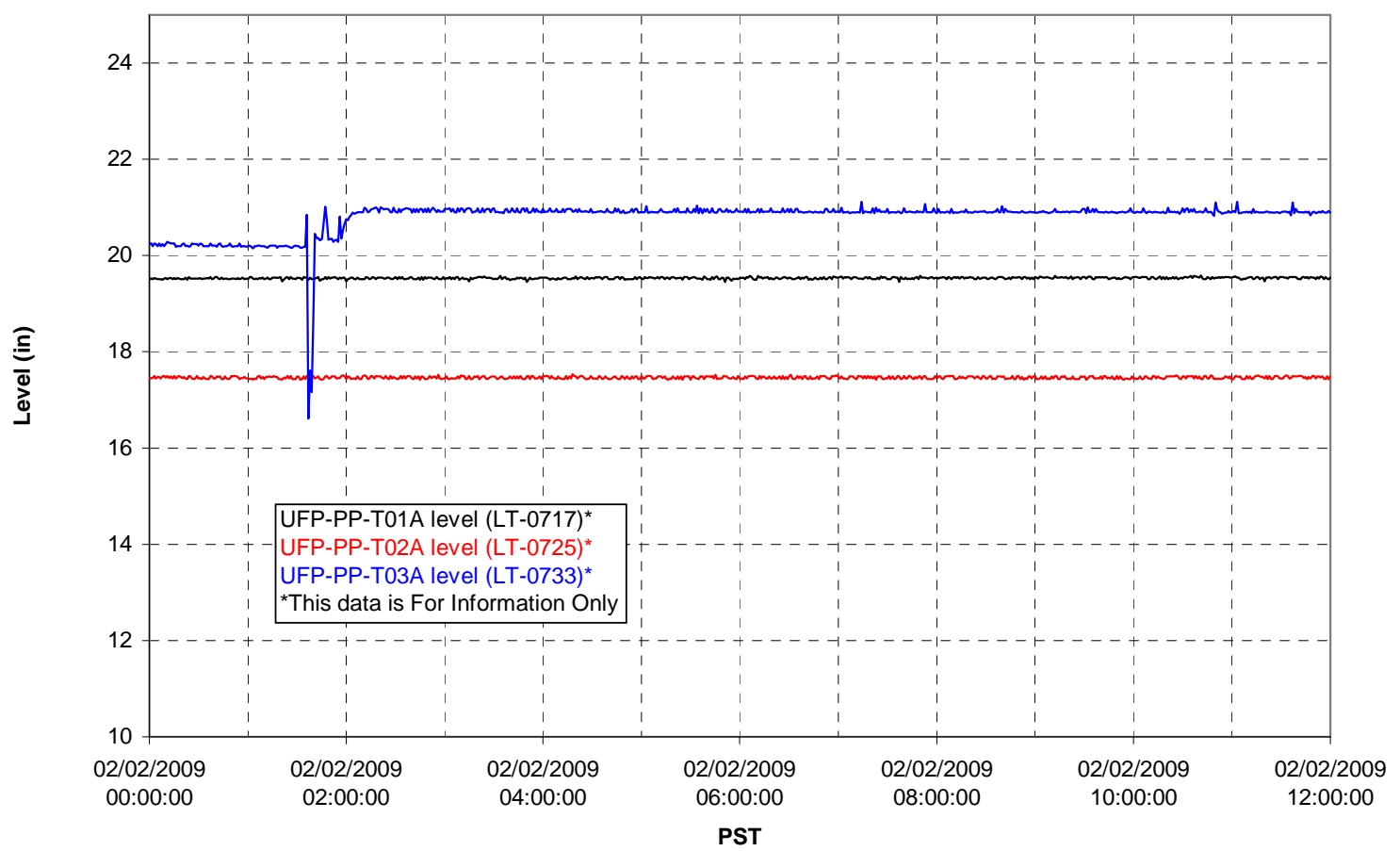


Filter UFP-FILT-T01A

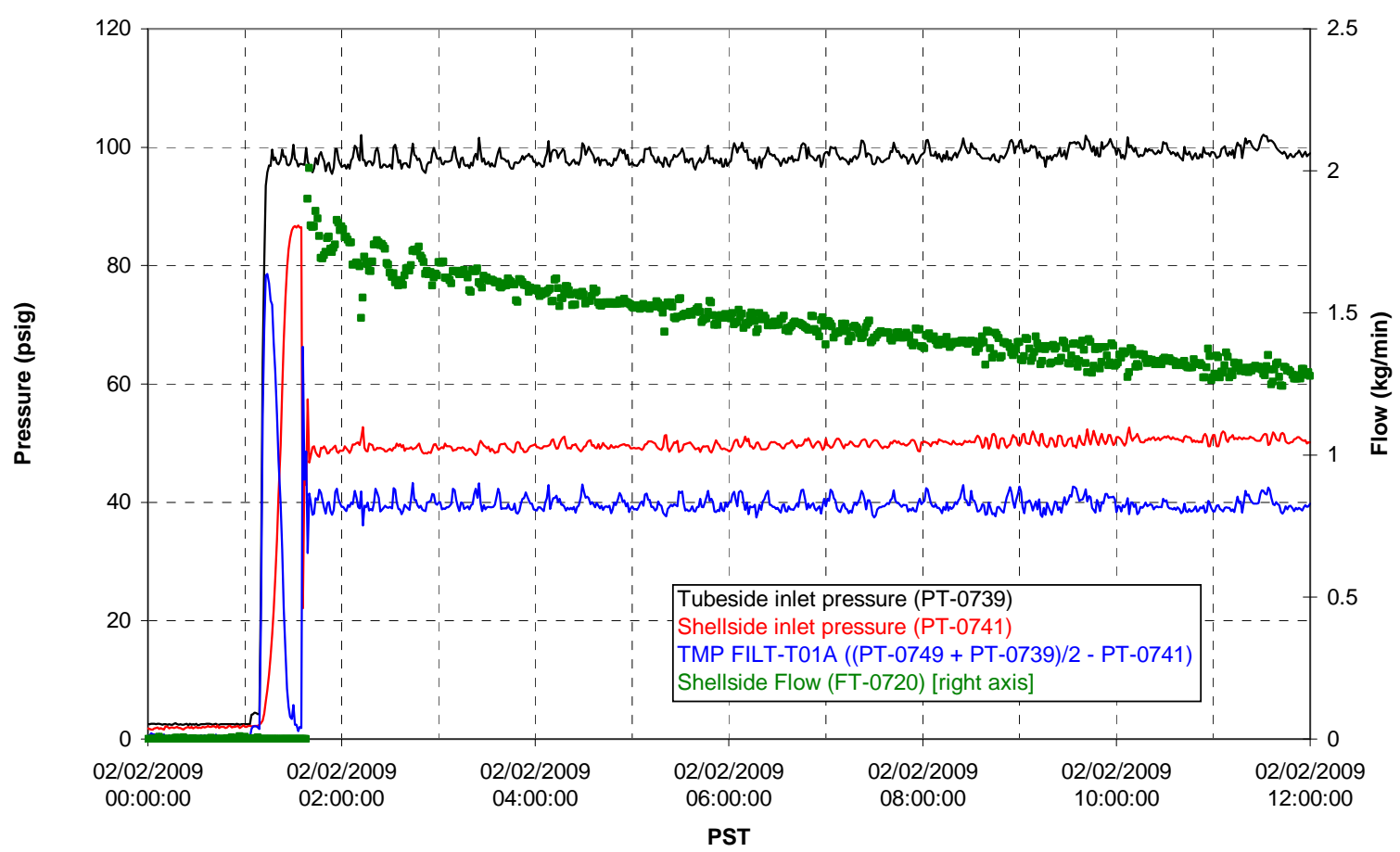

Filter UFP-FILT-T02A

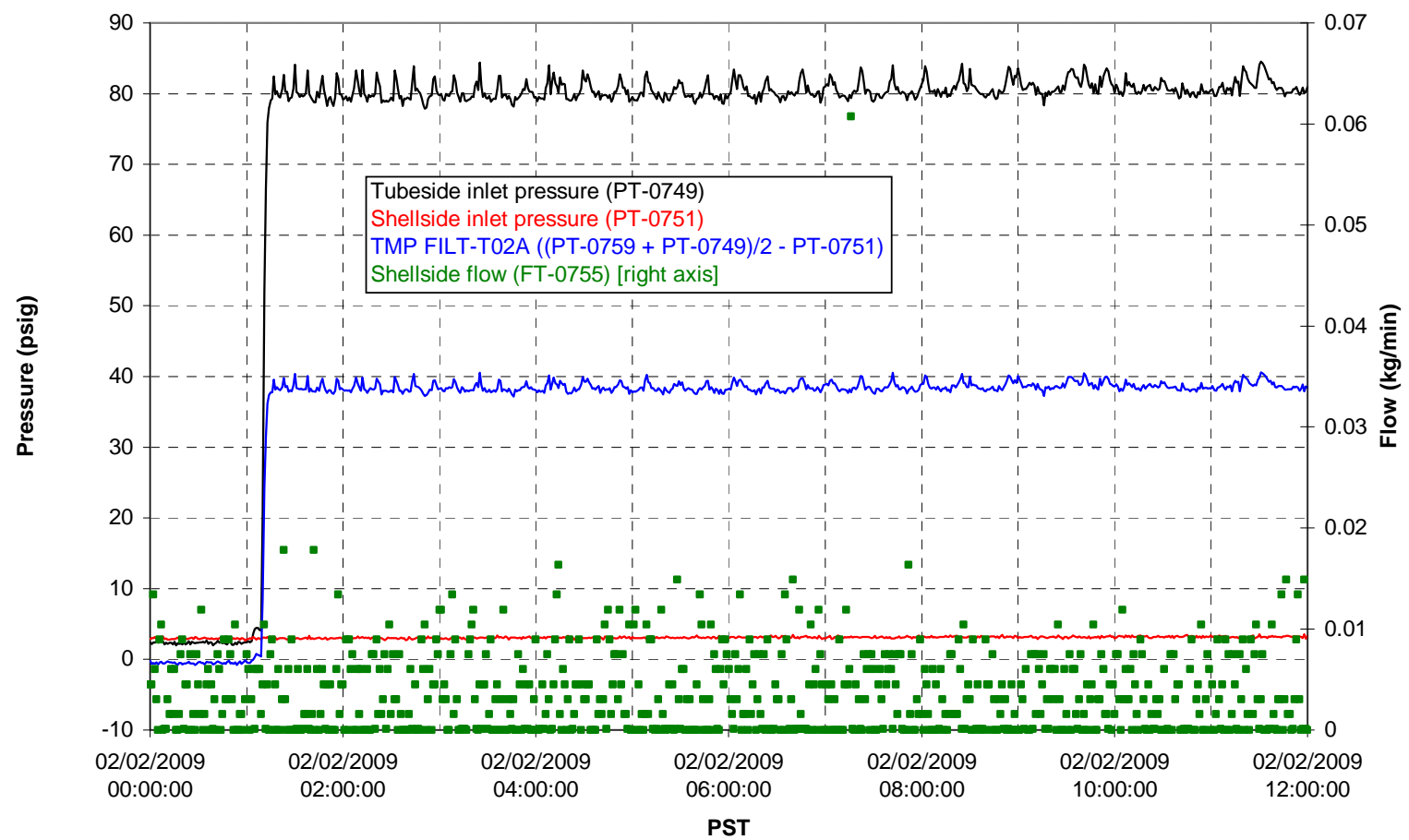


Filter UFP-FILT-T03A

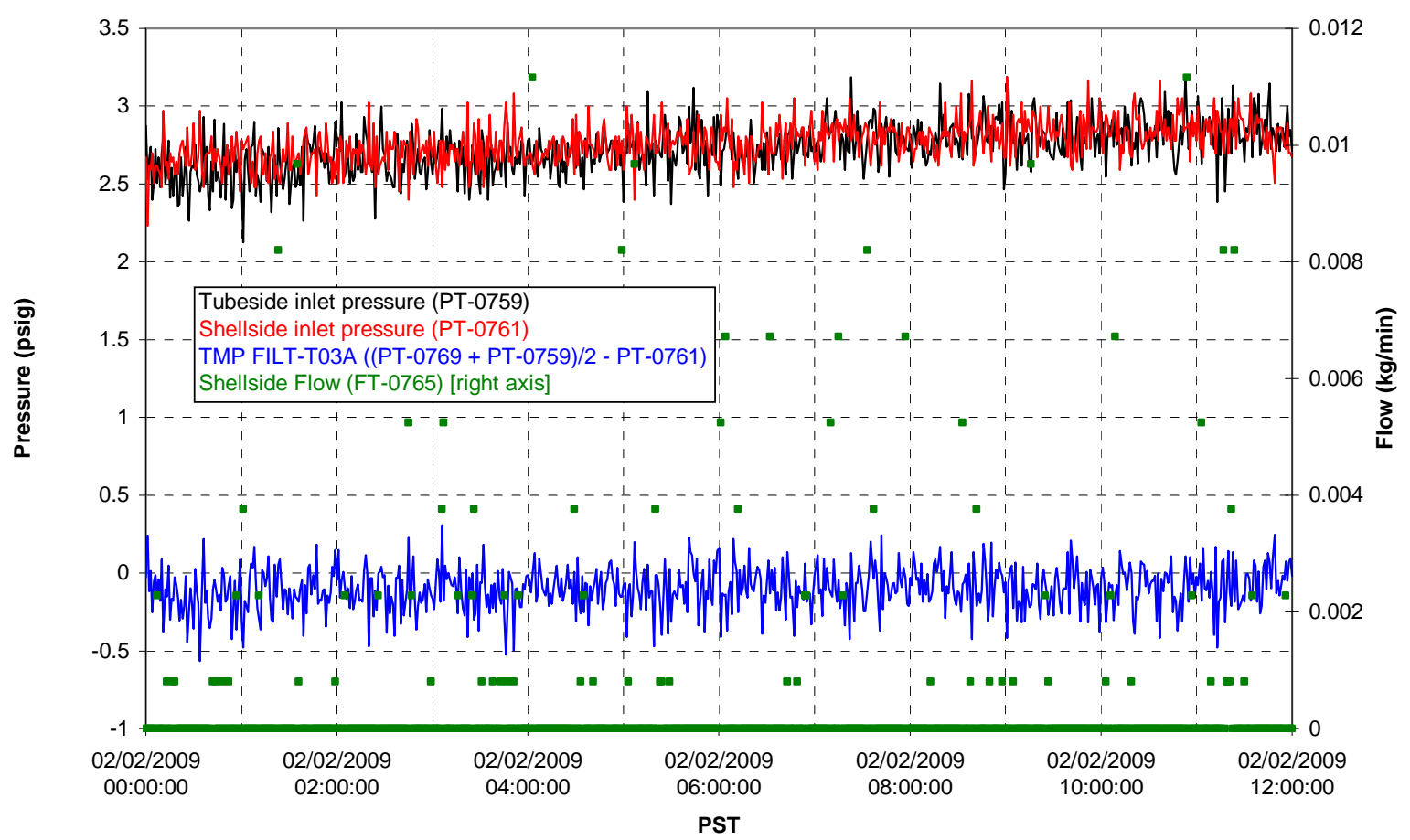

Filter UFP-FILT-T04A

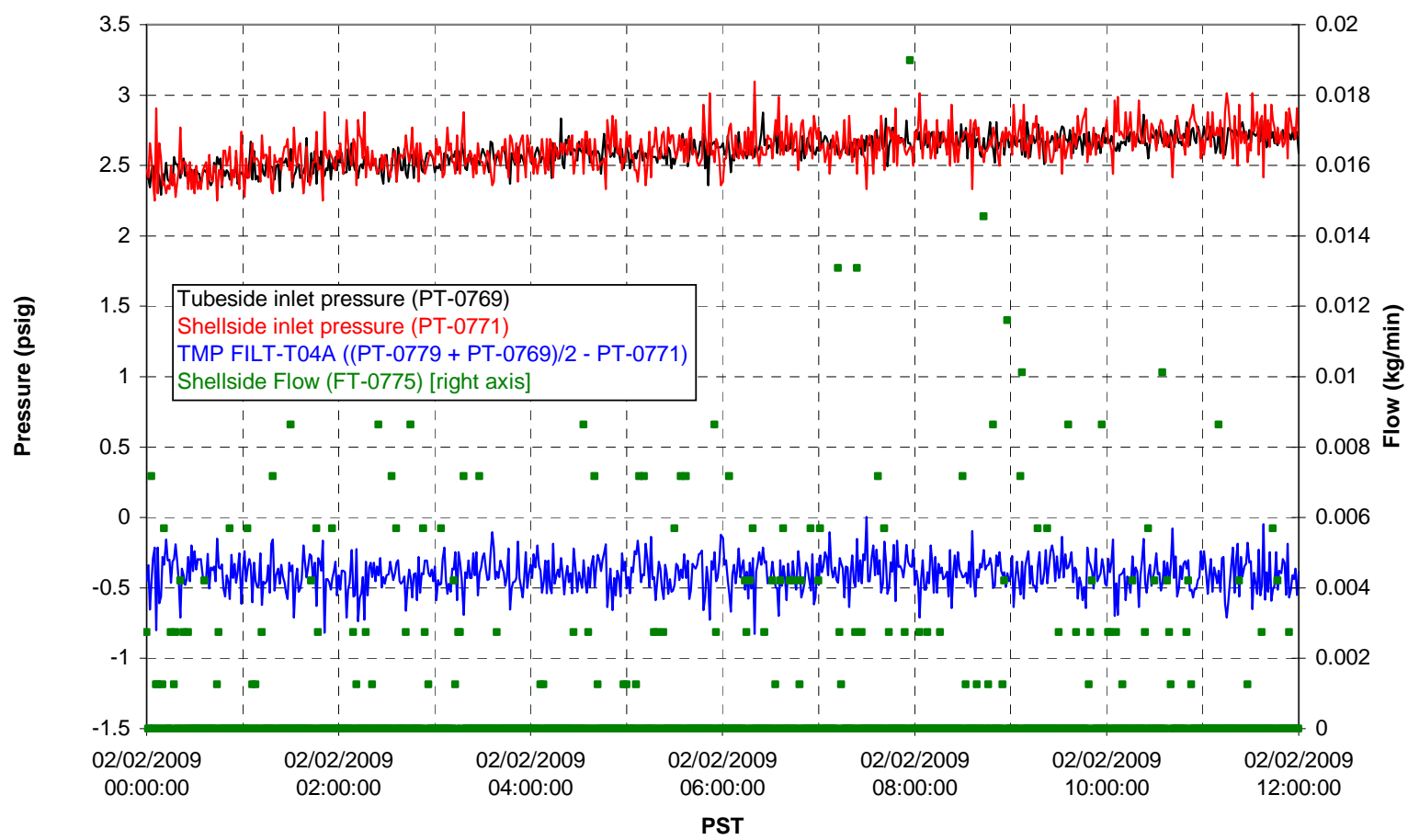


Filter UFP-FILT-T05A

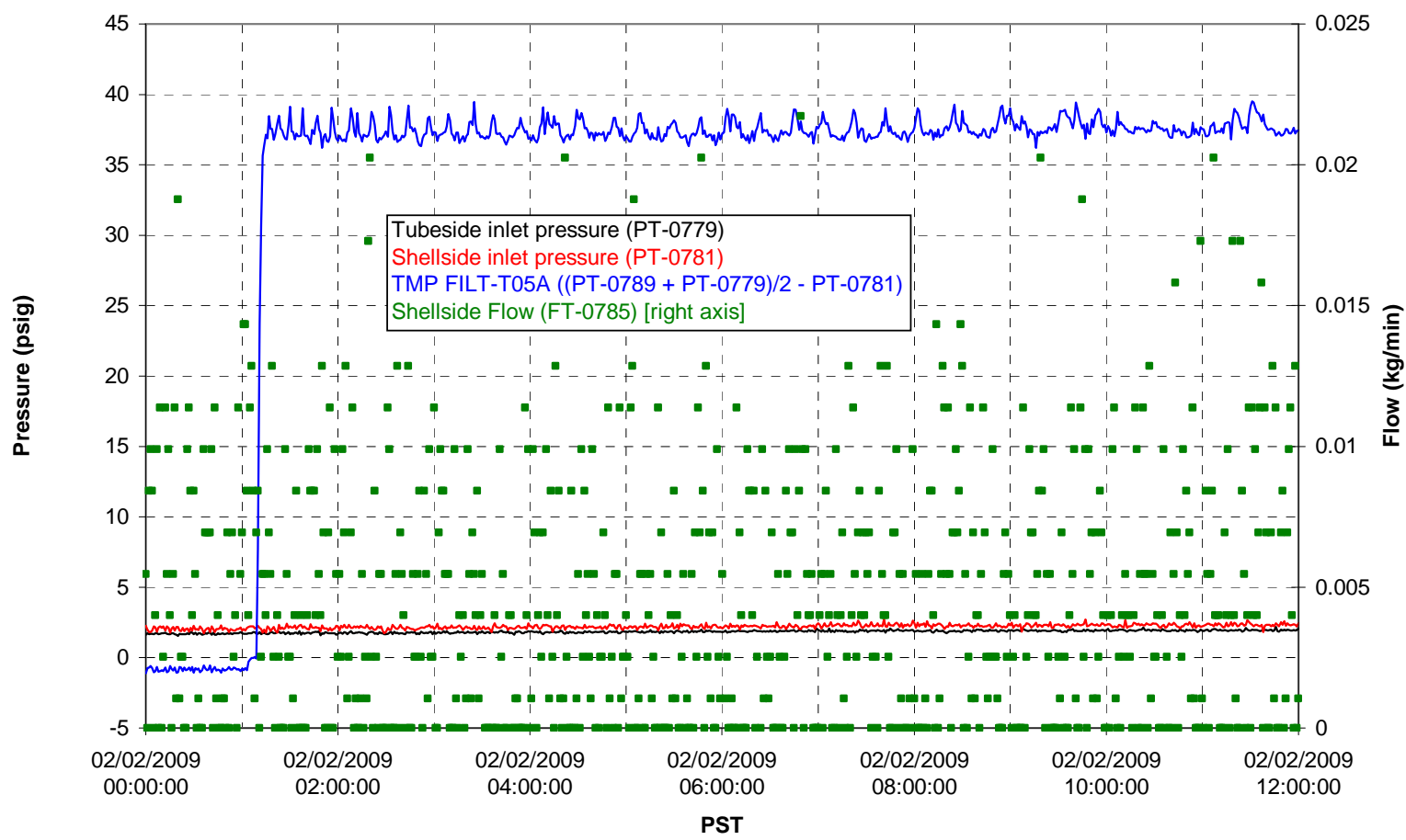

Chemical Flow

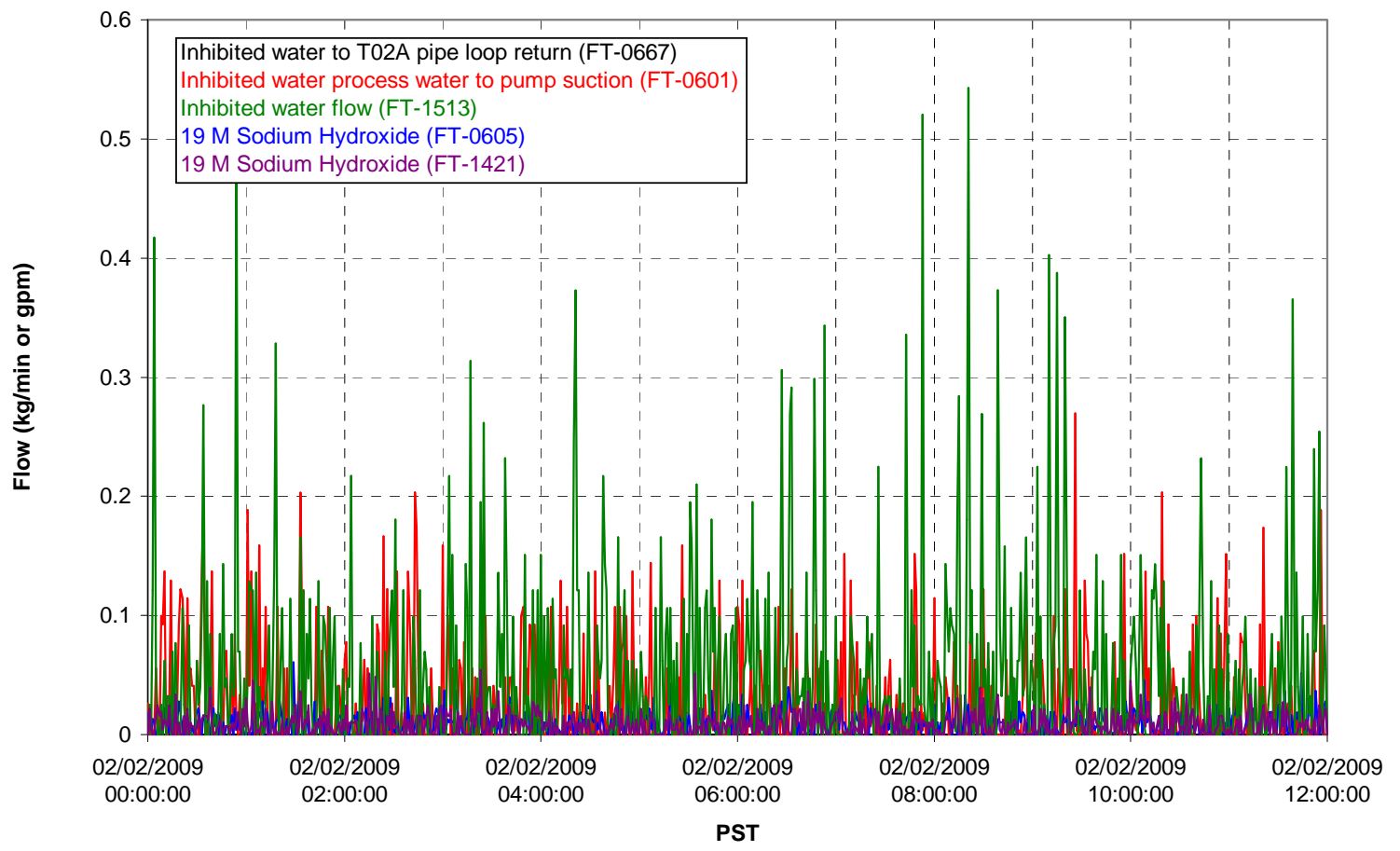




\section{Chemical Flow}

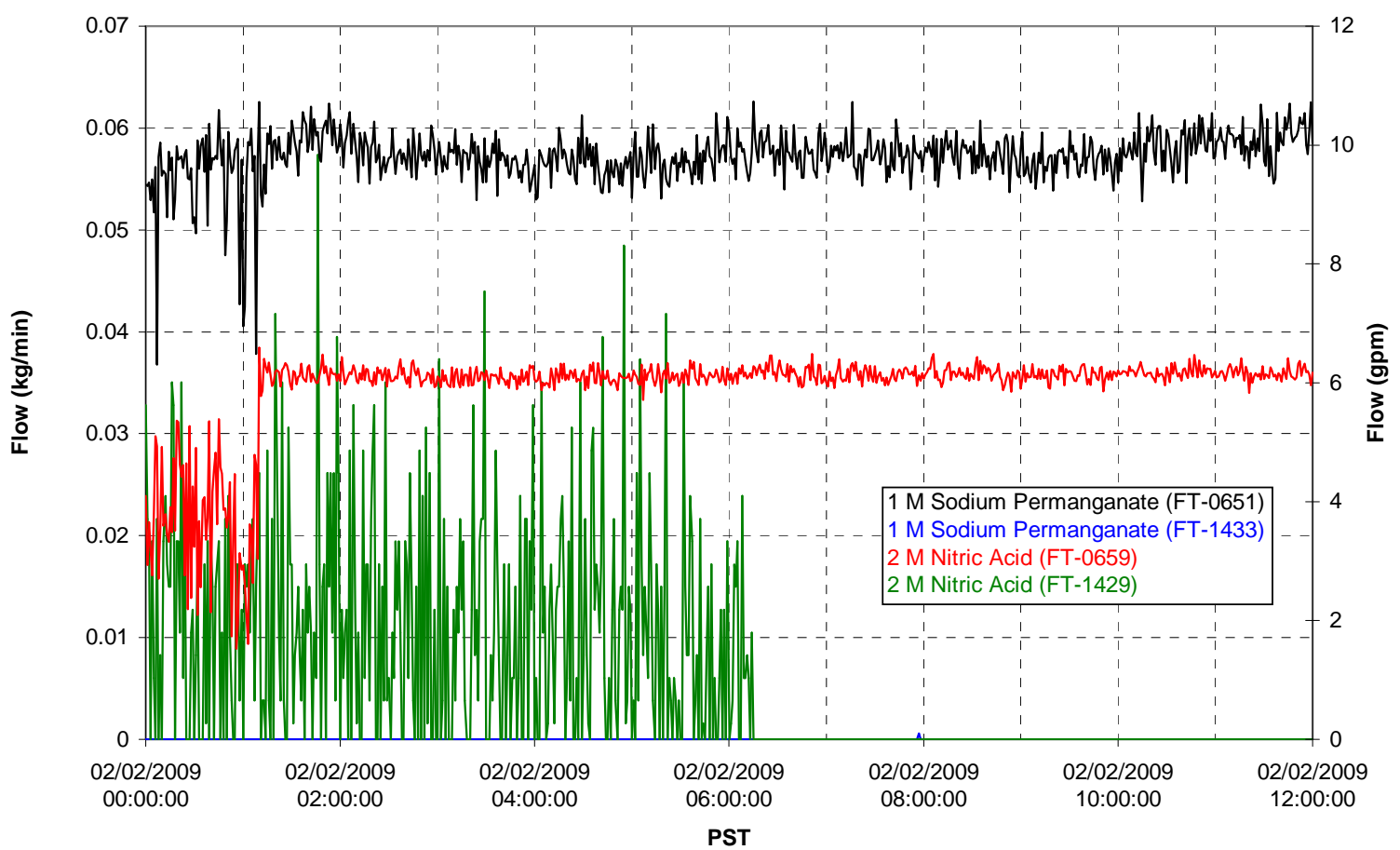

Air Flows

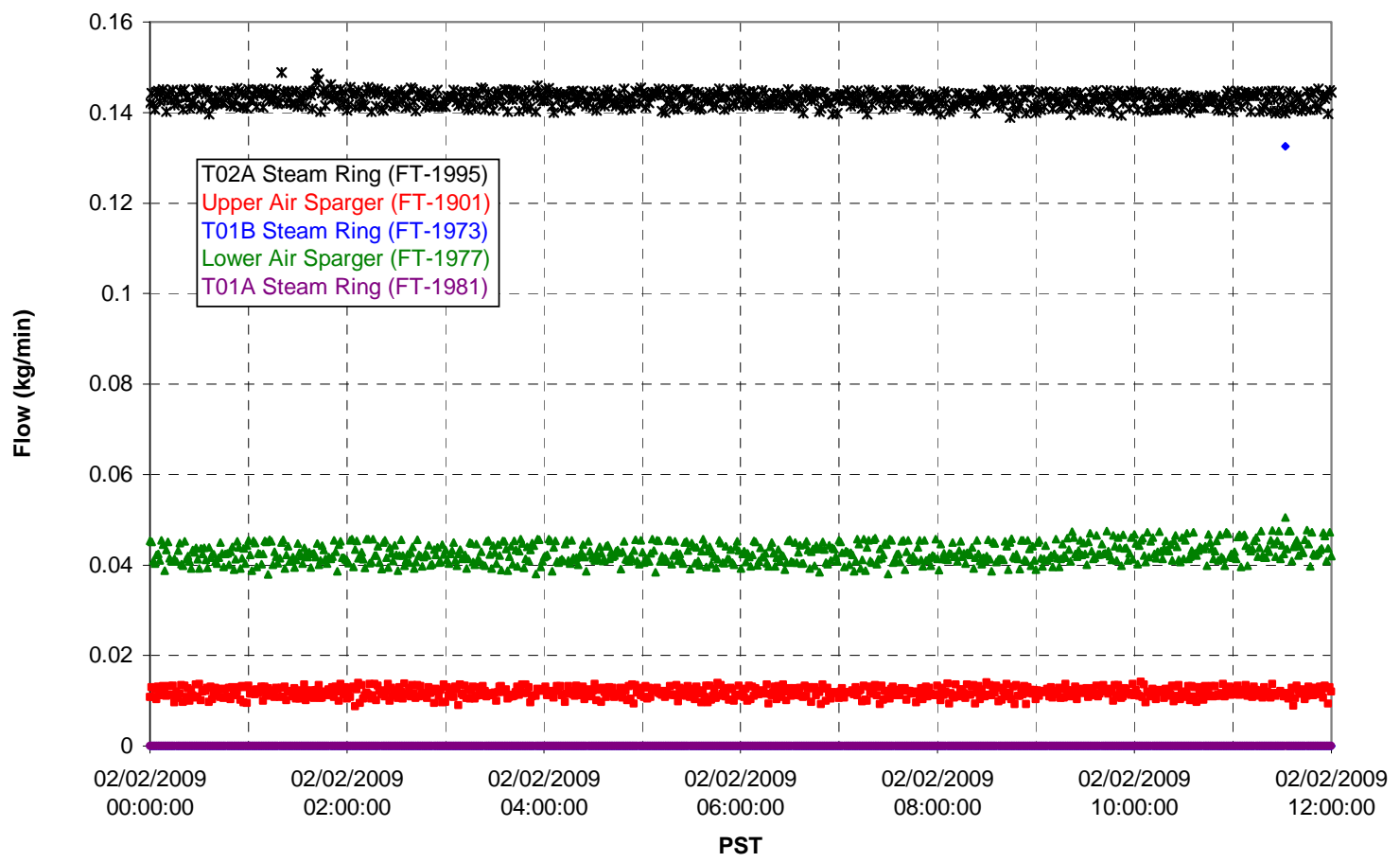


T02A Steam

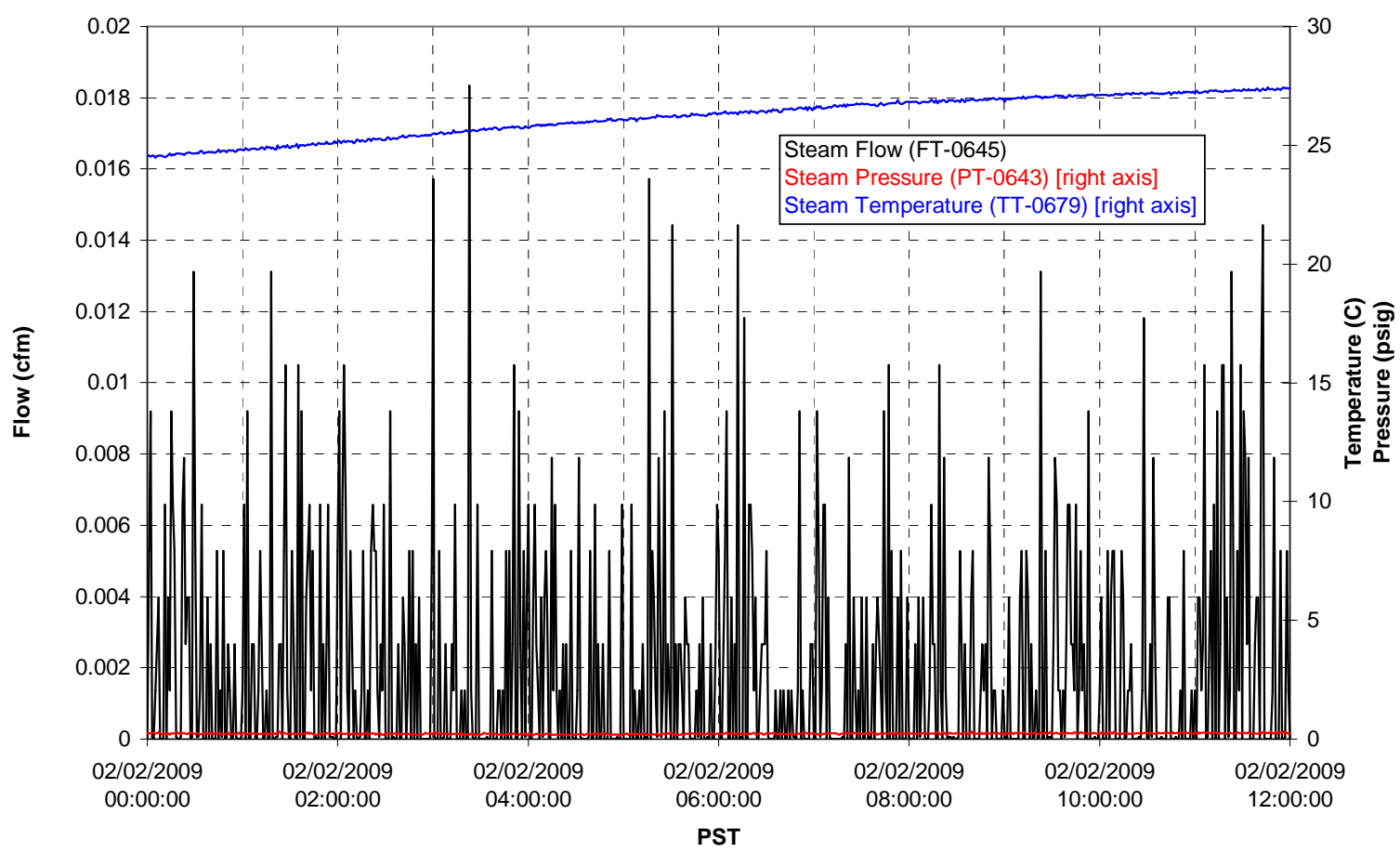

T01A Steam

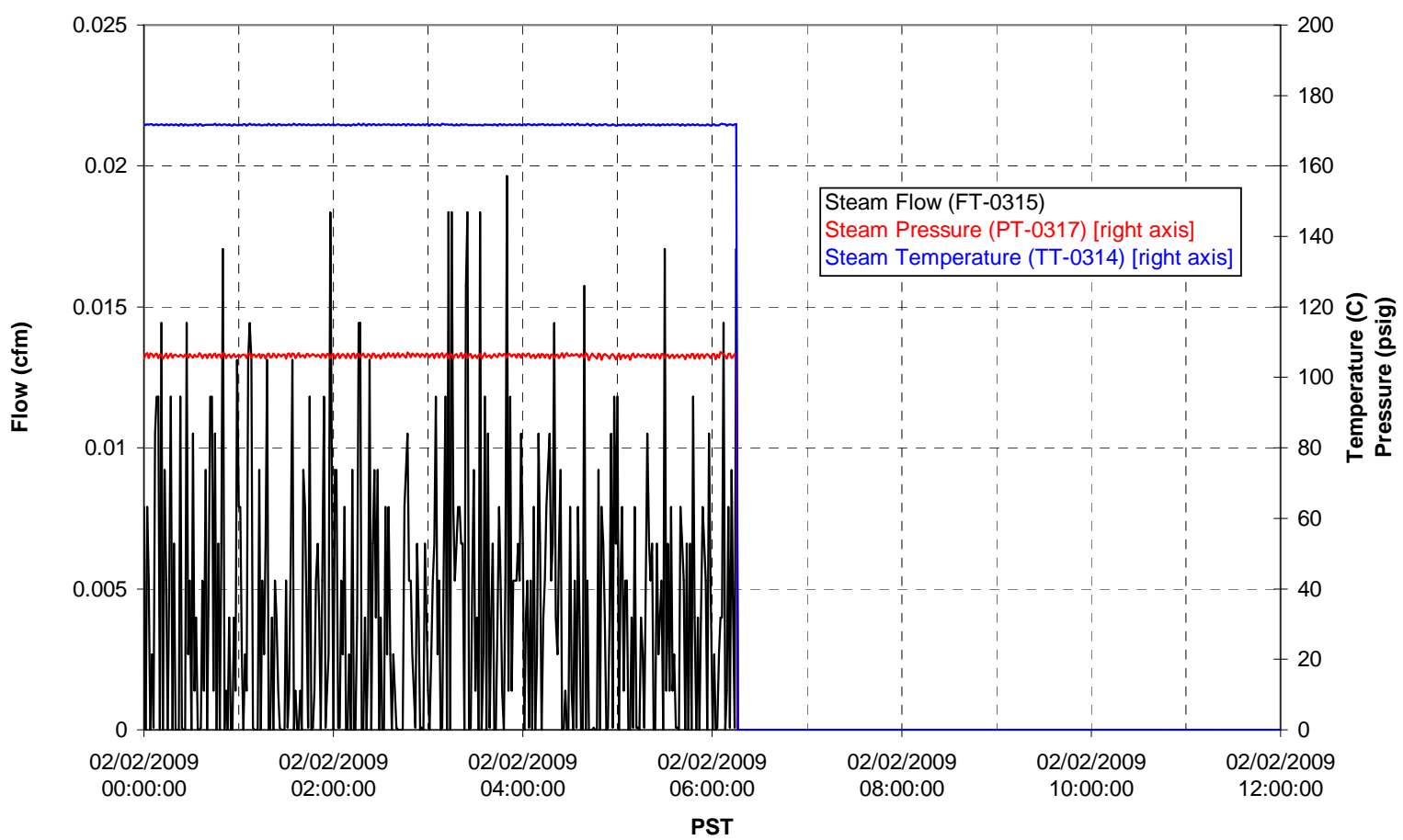


T01B Steam

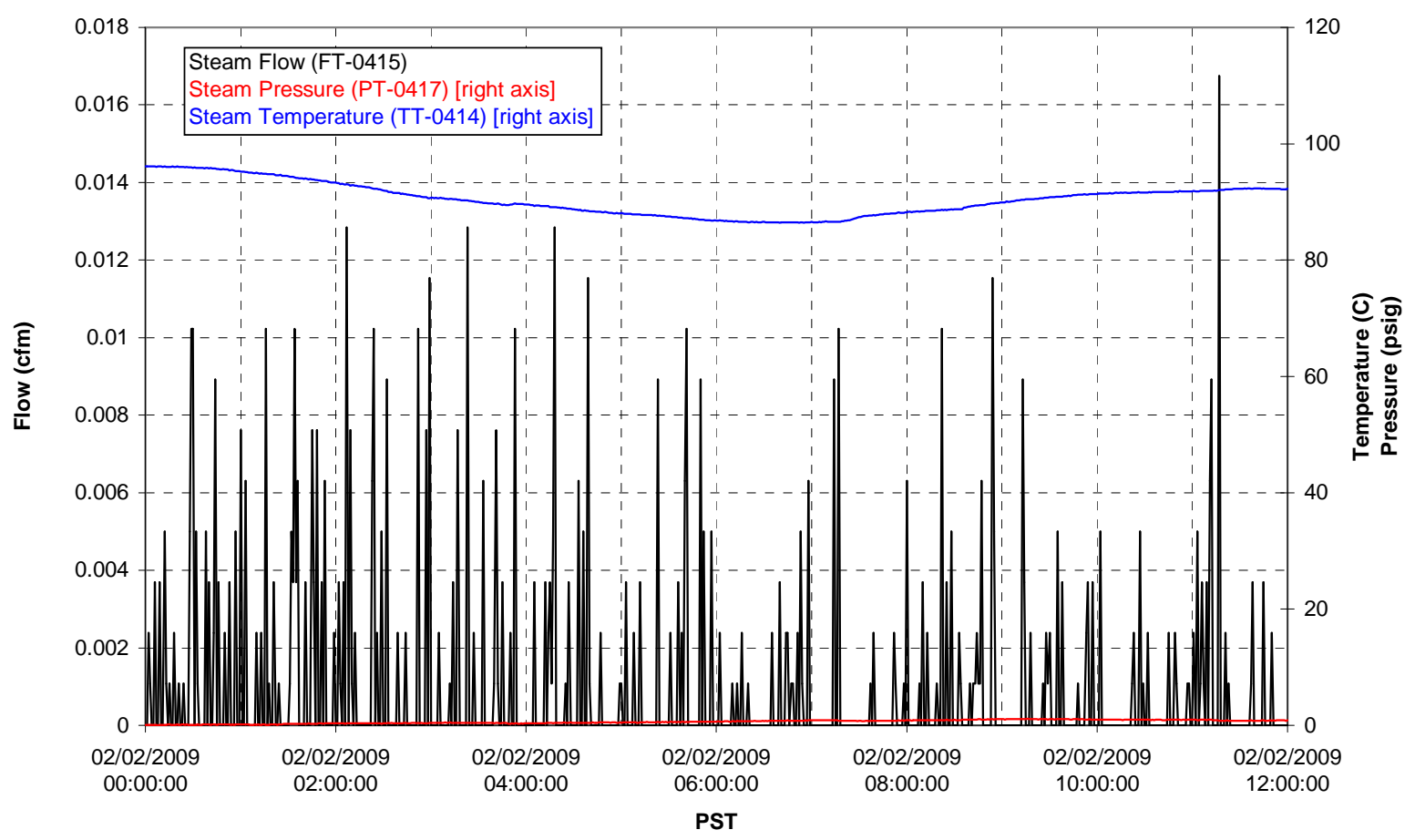




\section{Integrated Test A Data Plots 02/02/09 12:00 - 02/03/09 00:00}


T01A level

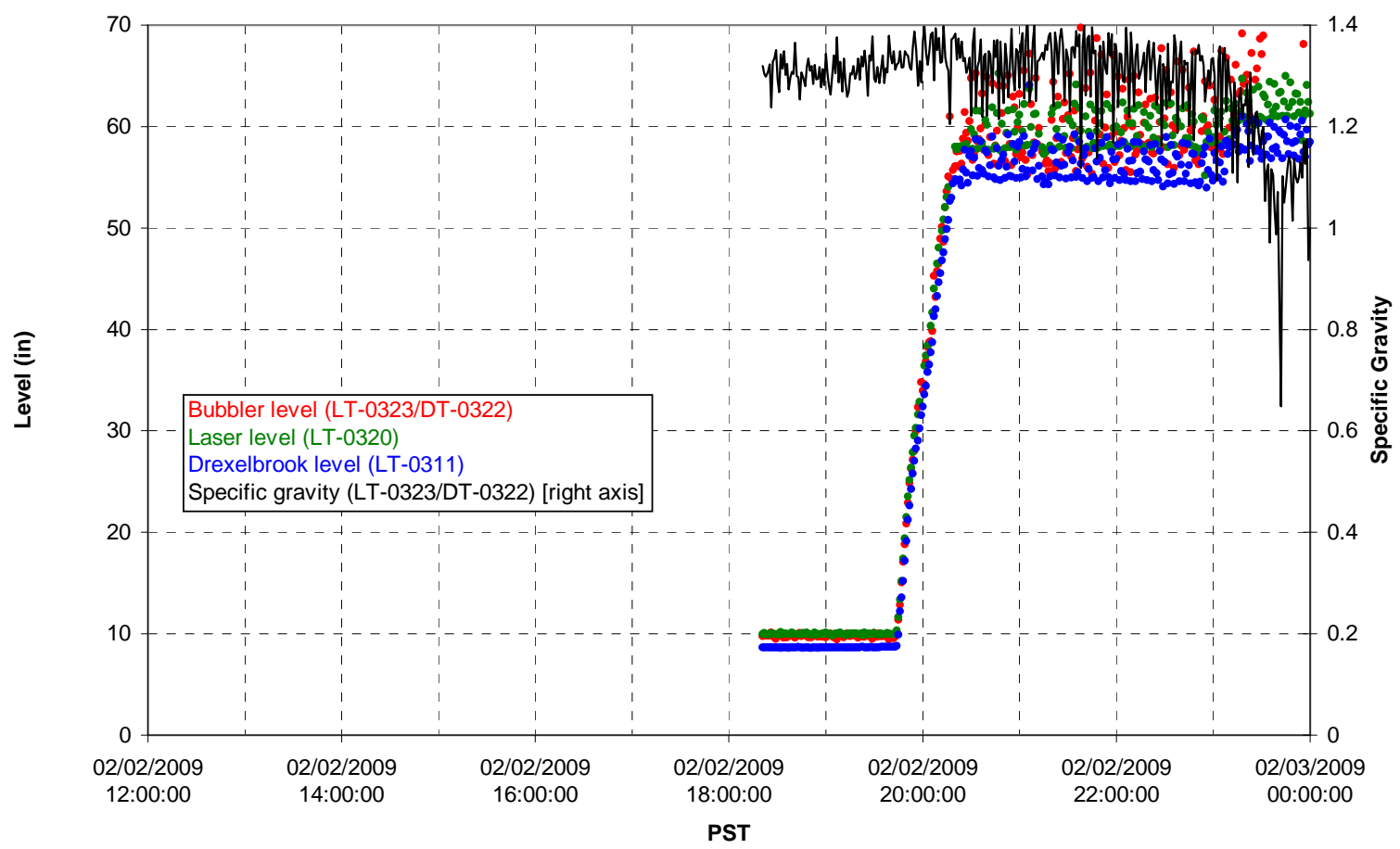

T01A temperatures

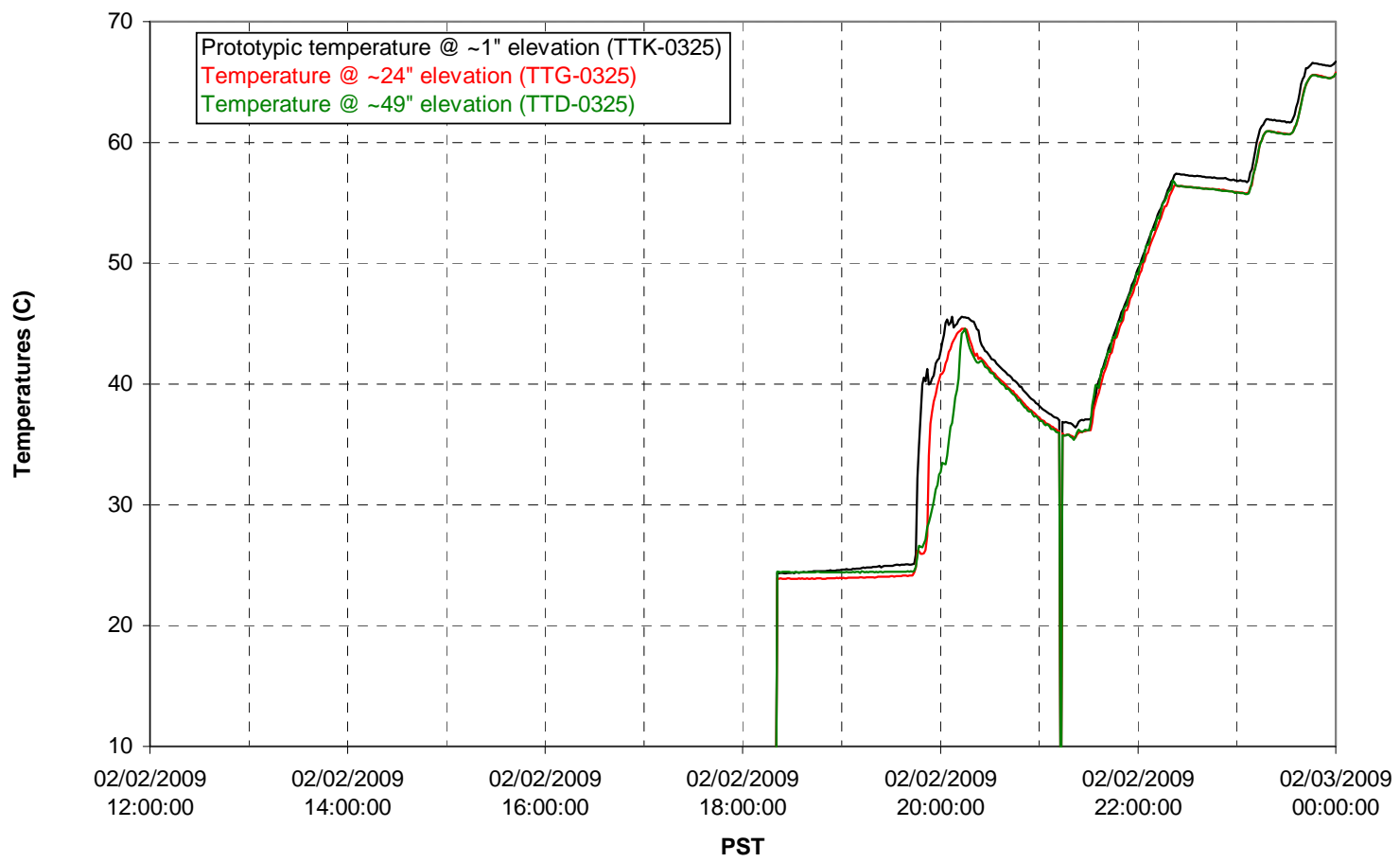


T01B level

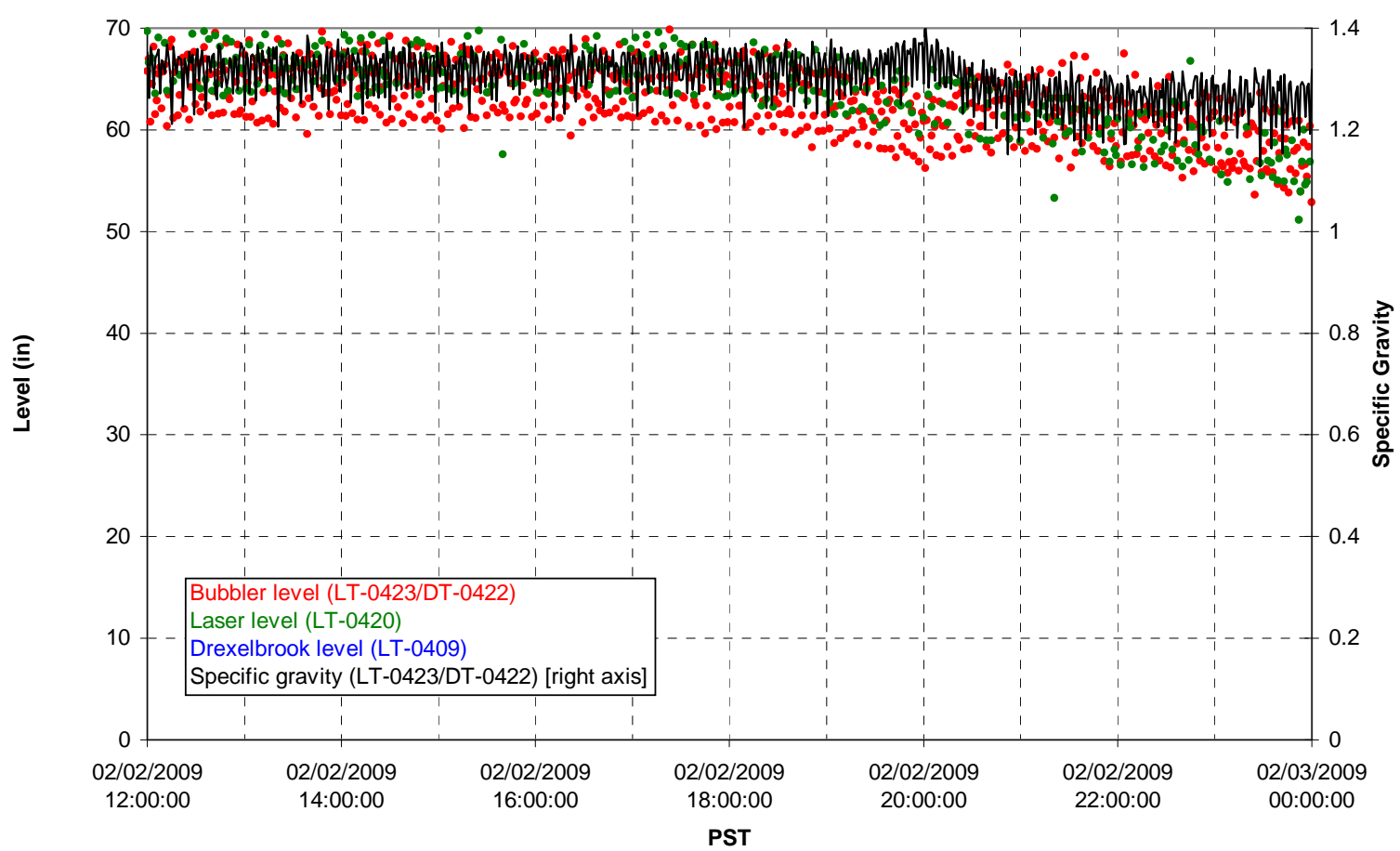

T01B temperatures

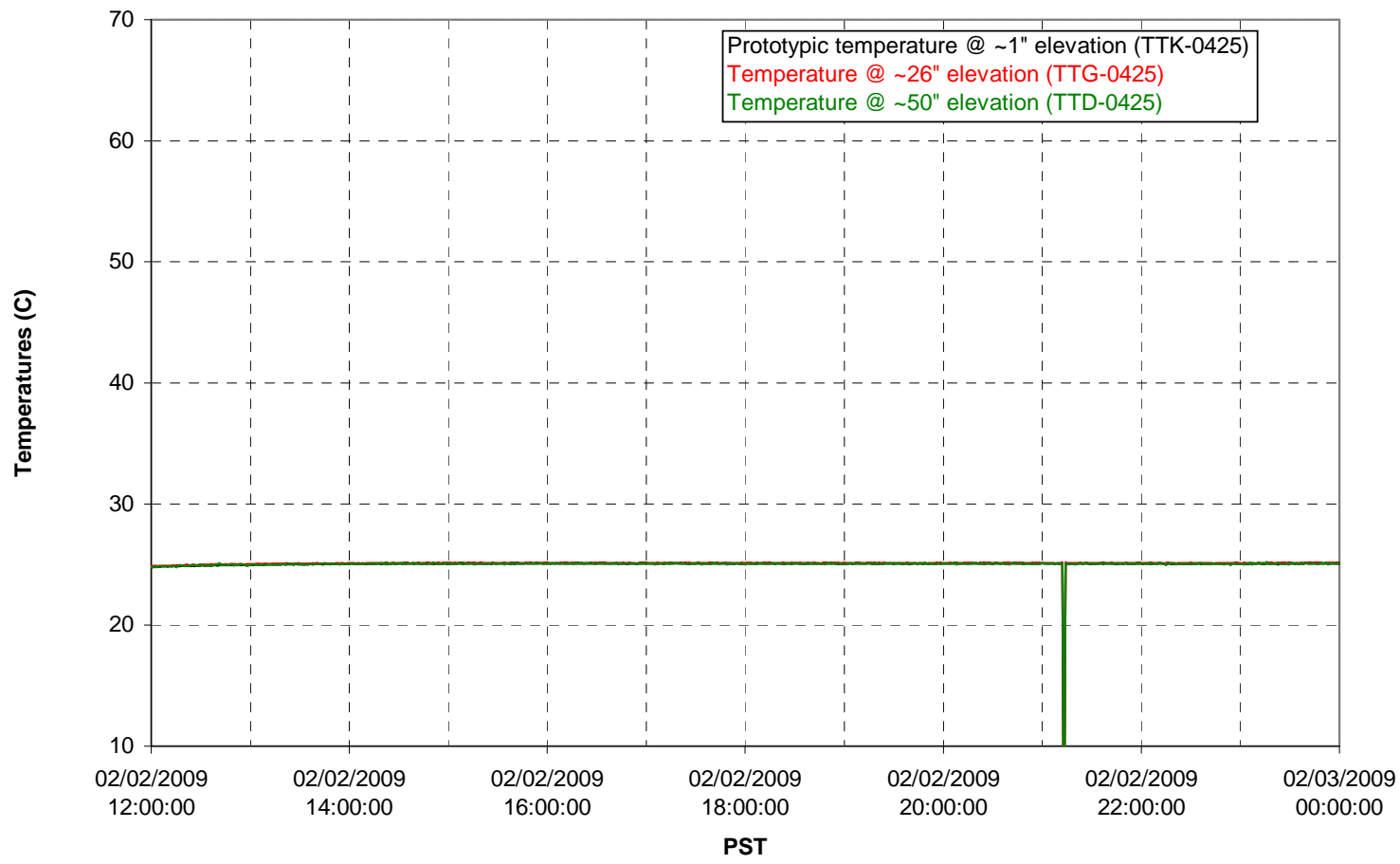


T02A level

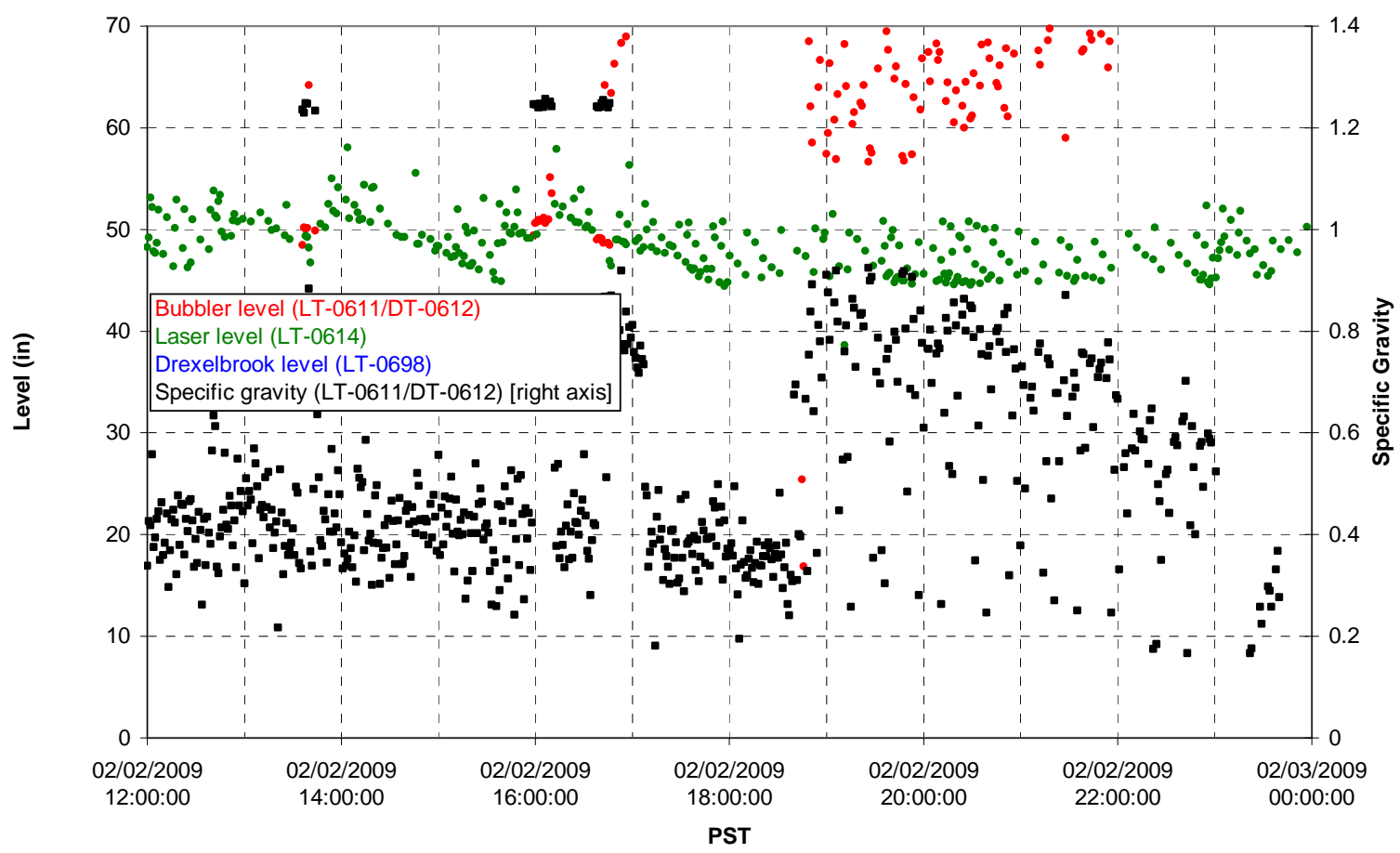

T02A temperatures

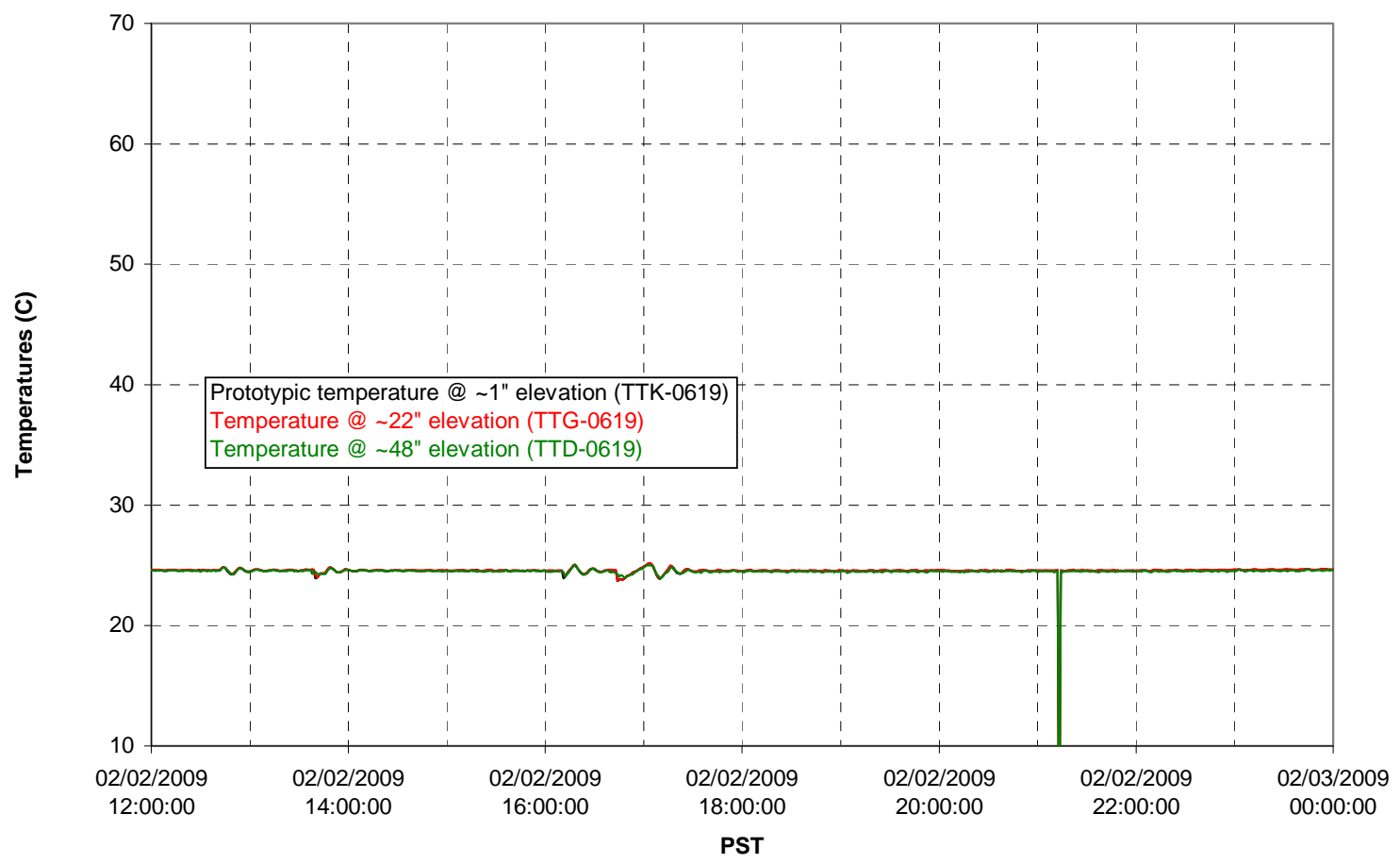


T02A and filter loop temperatures

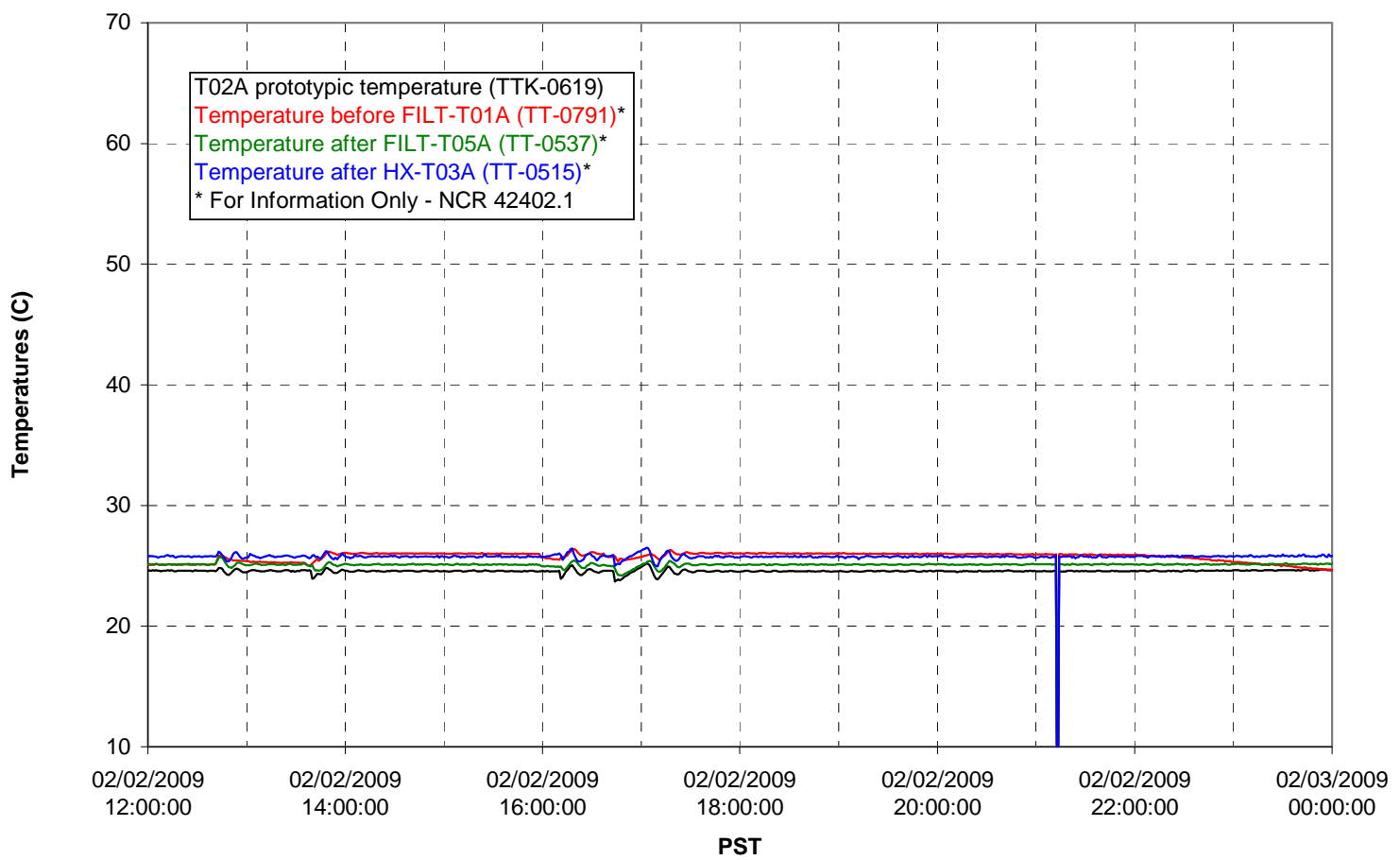

Pump Pressures and Flow

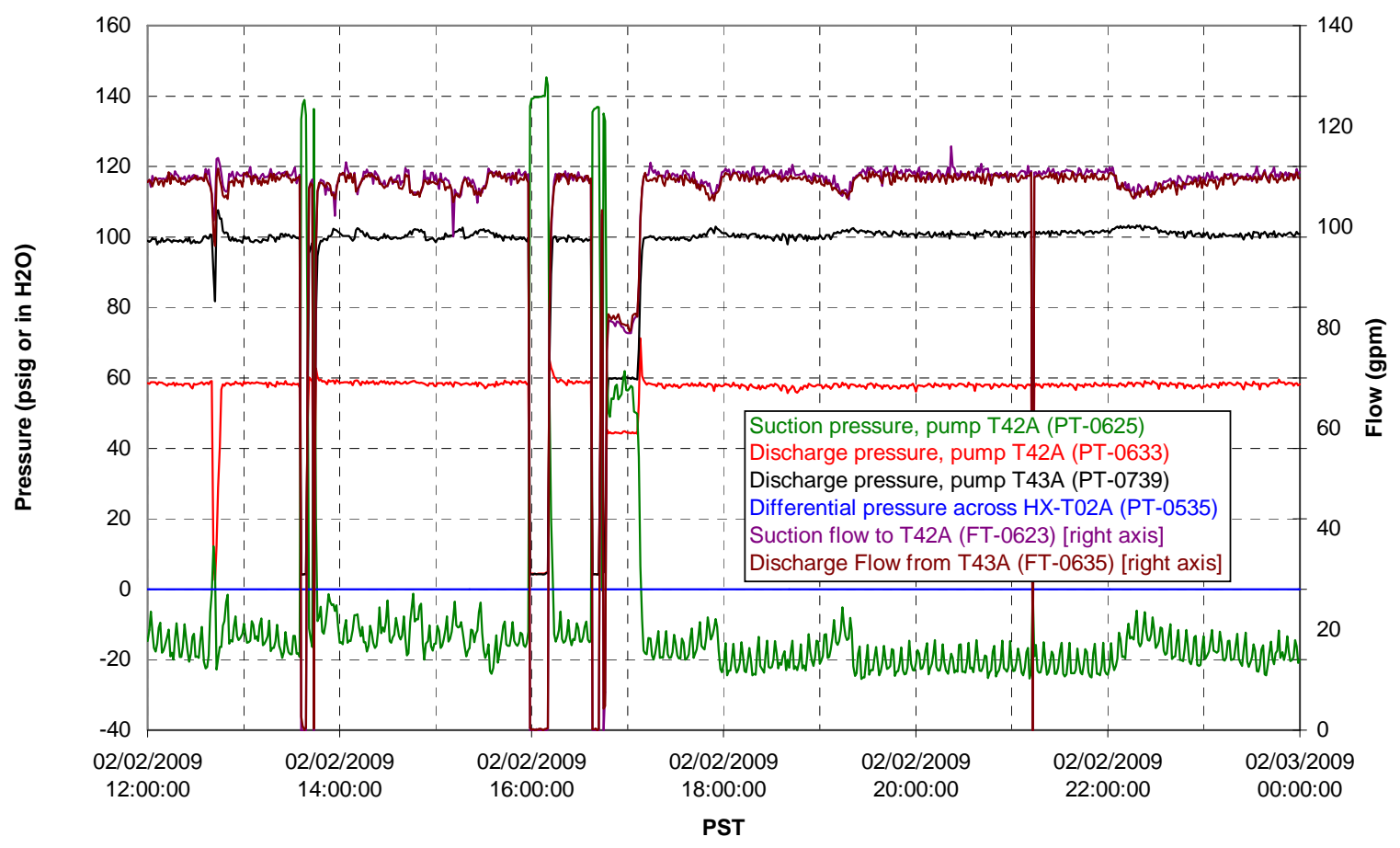


Axial pressure drop

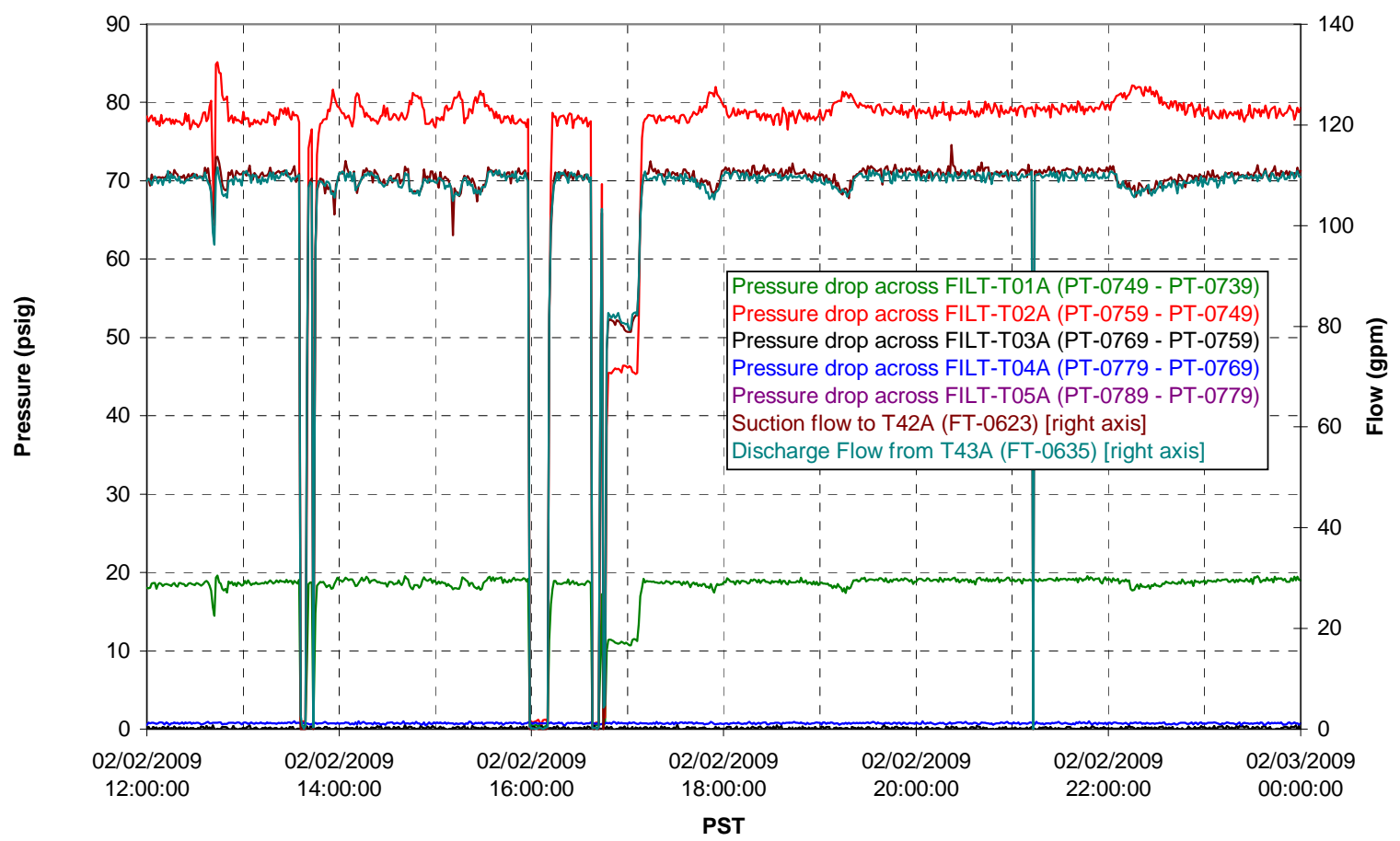

Permeate flow rates

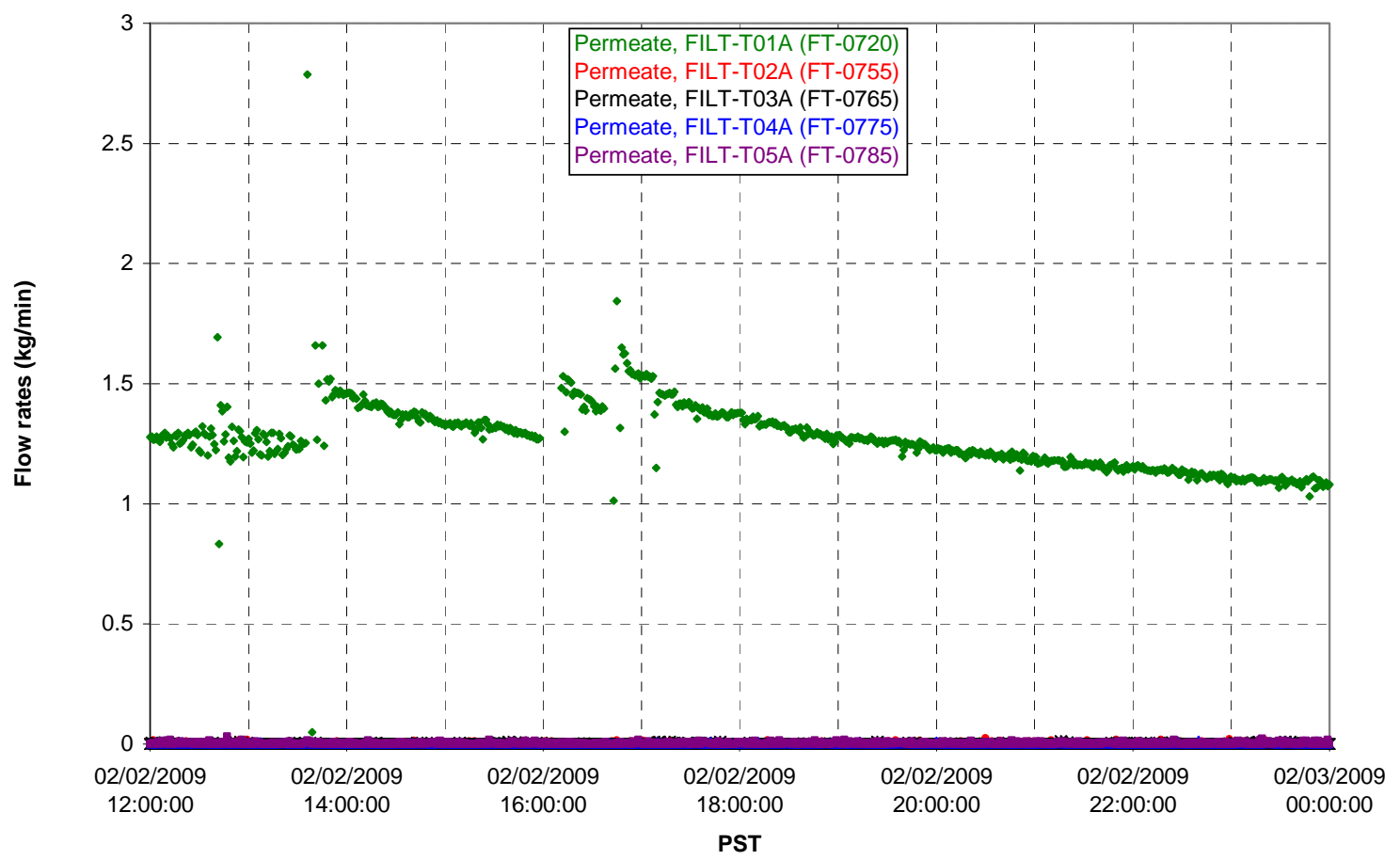


T02A Inner Temperature Tree

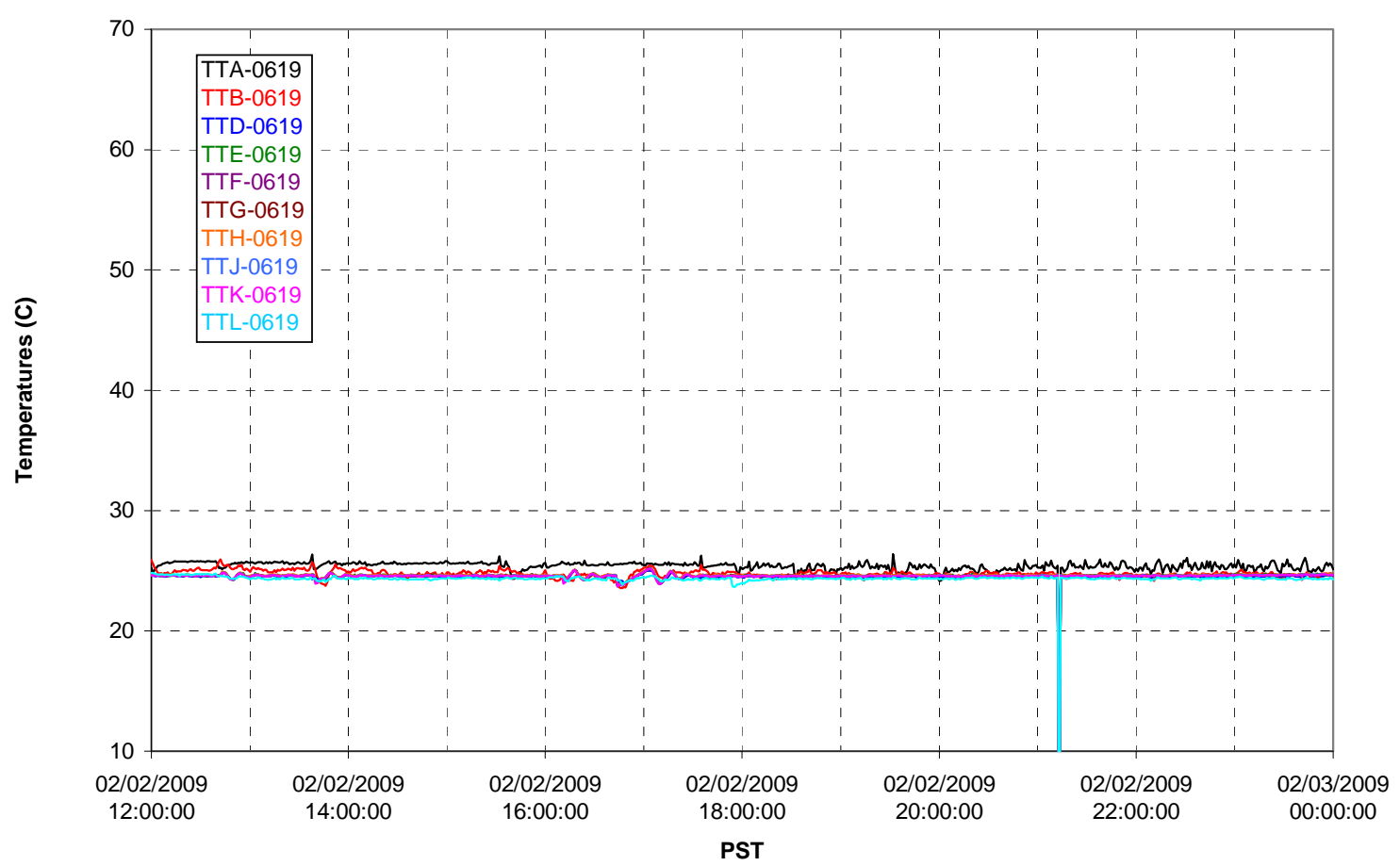

T02A Outer Temperature Tree

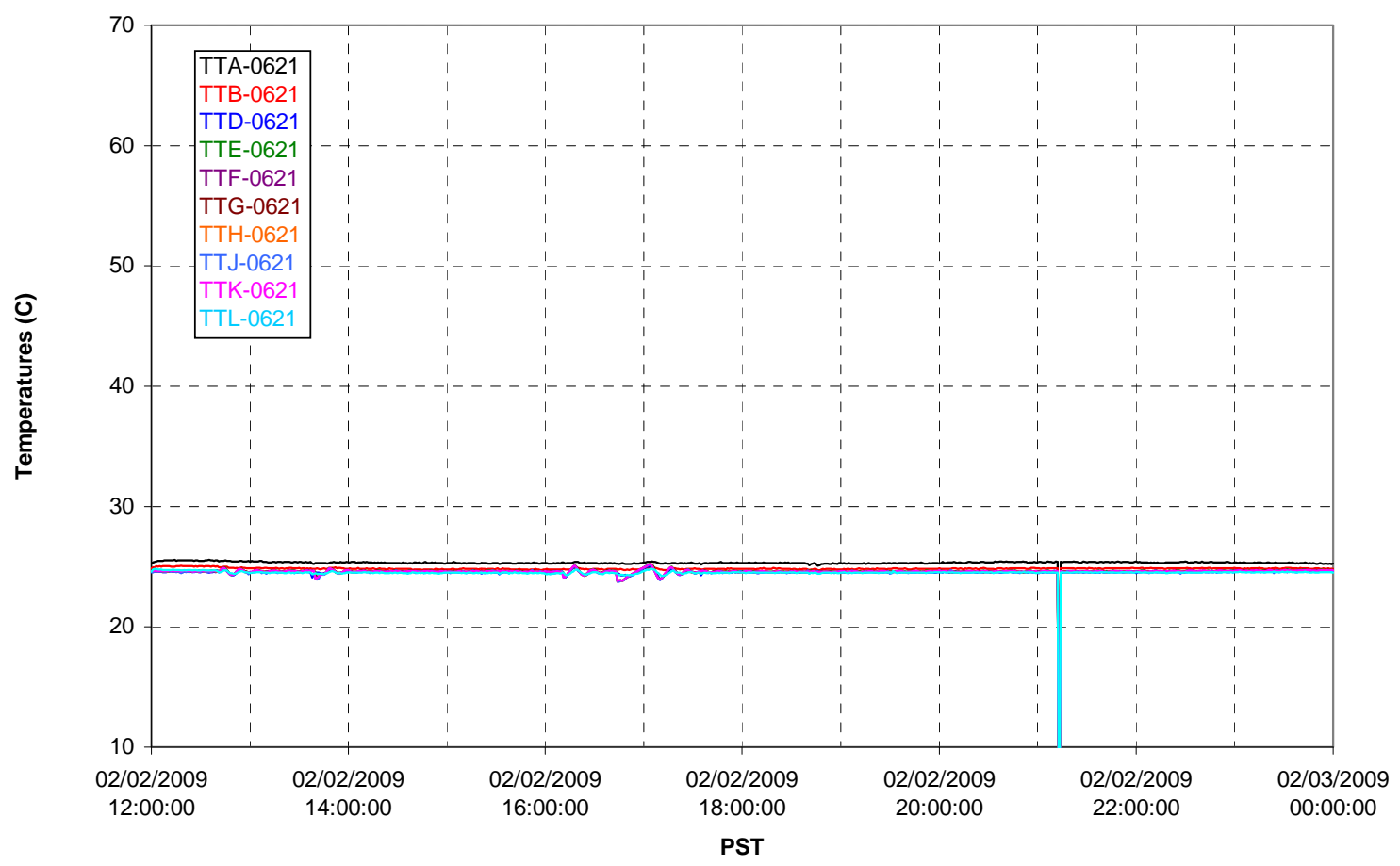


T02A temperatures

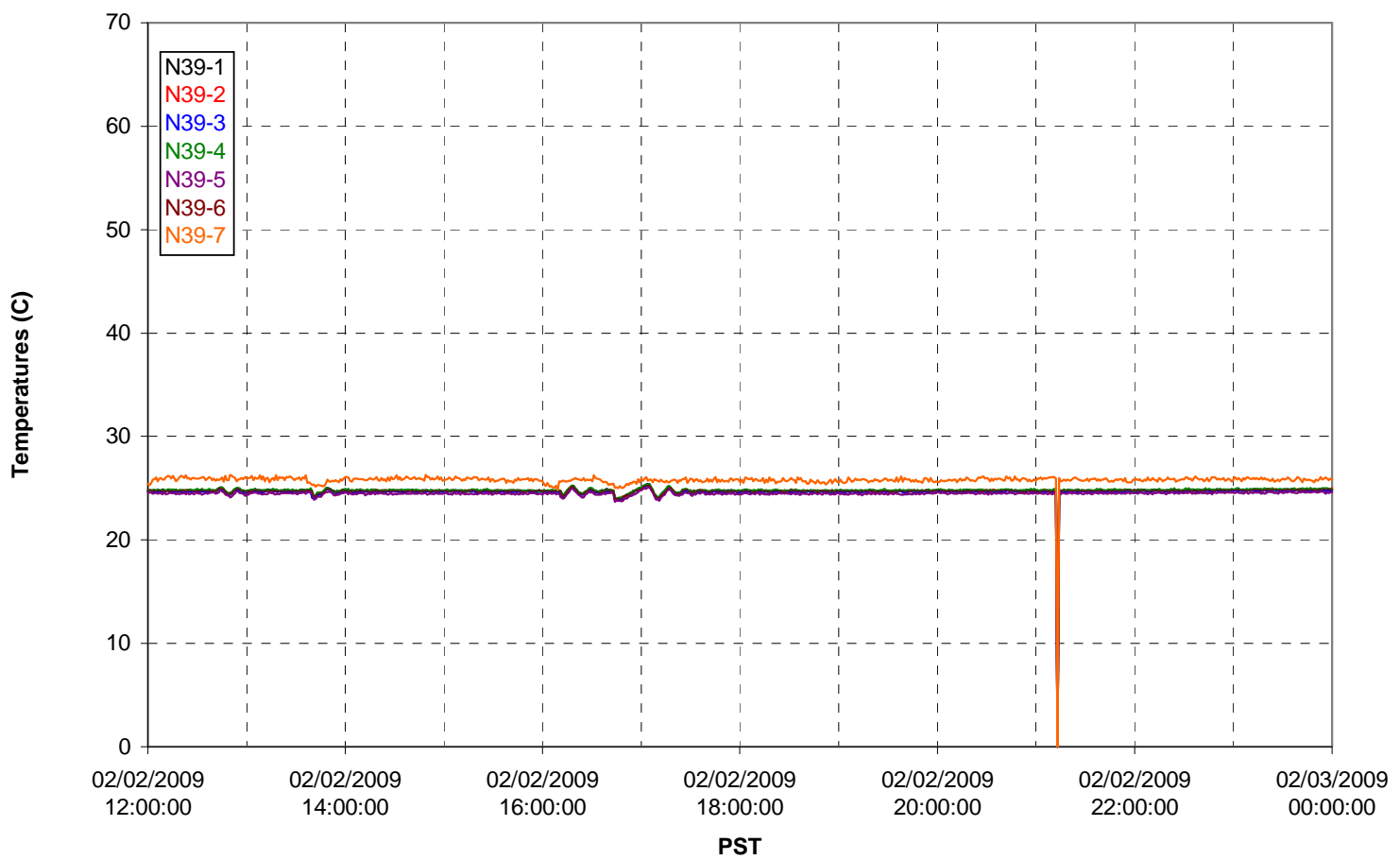

T02A temperatures

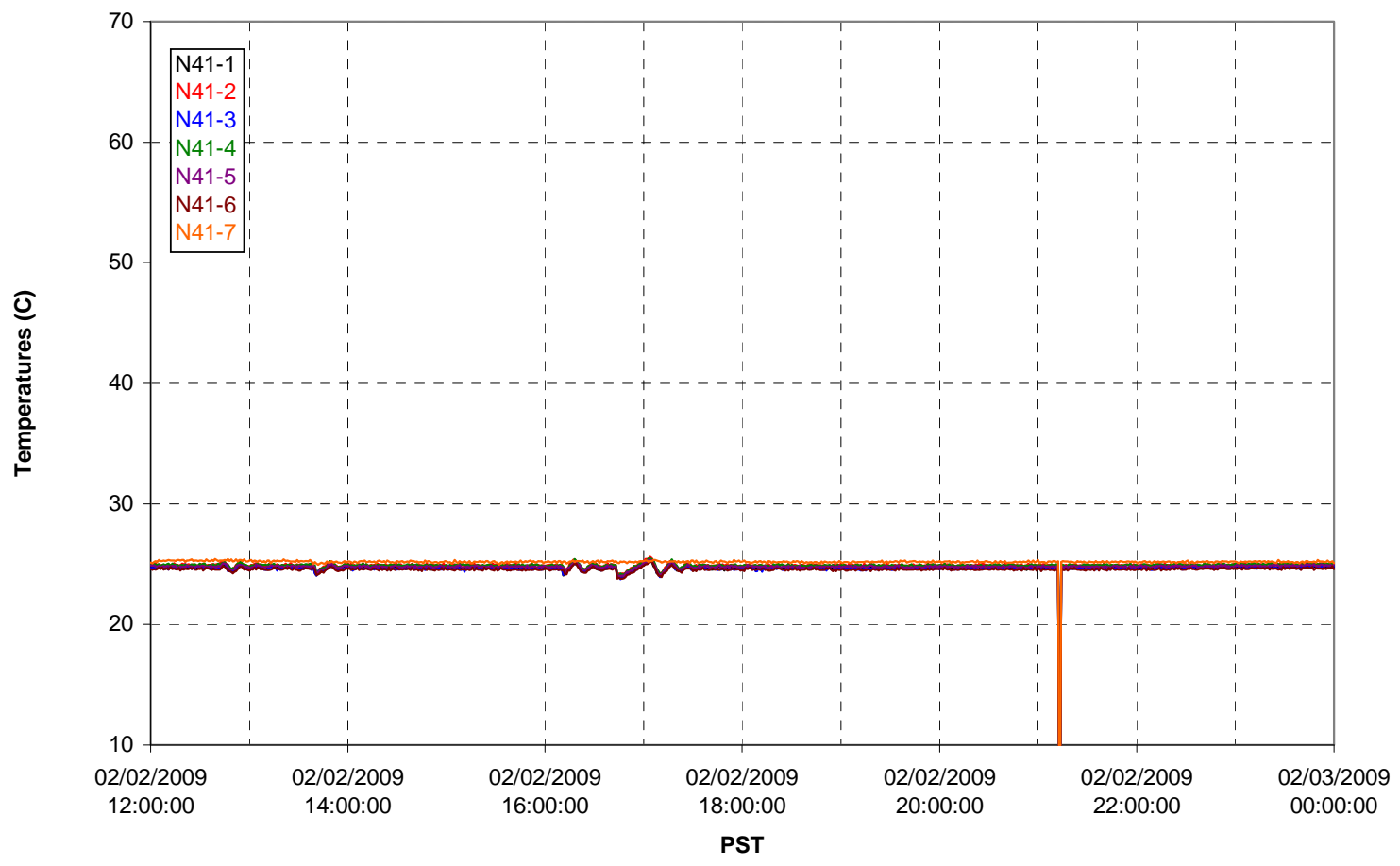


T02A temperatures

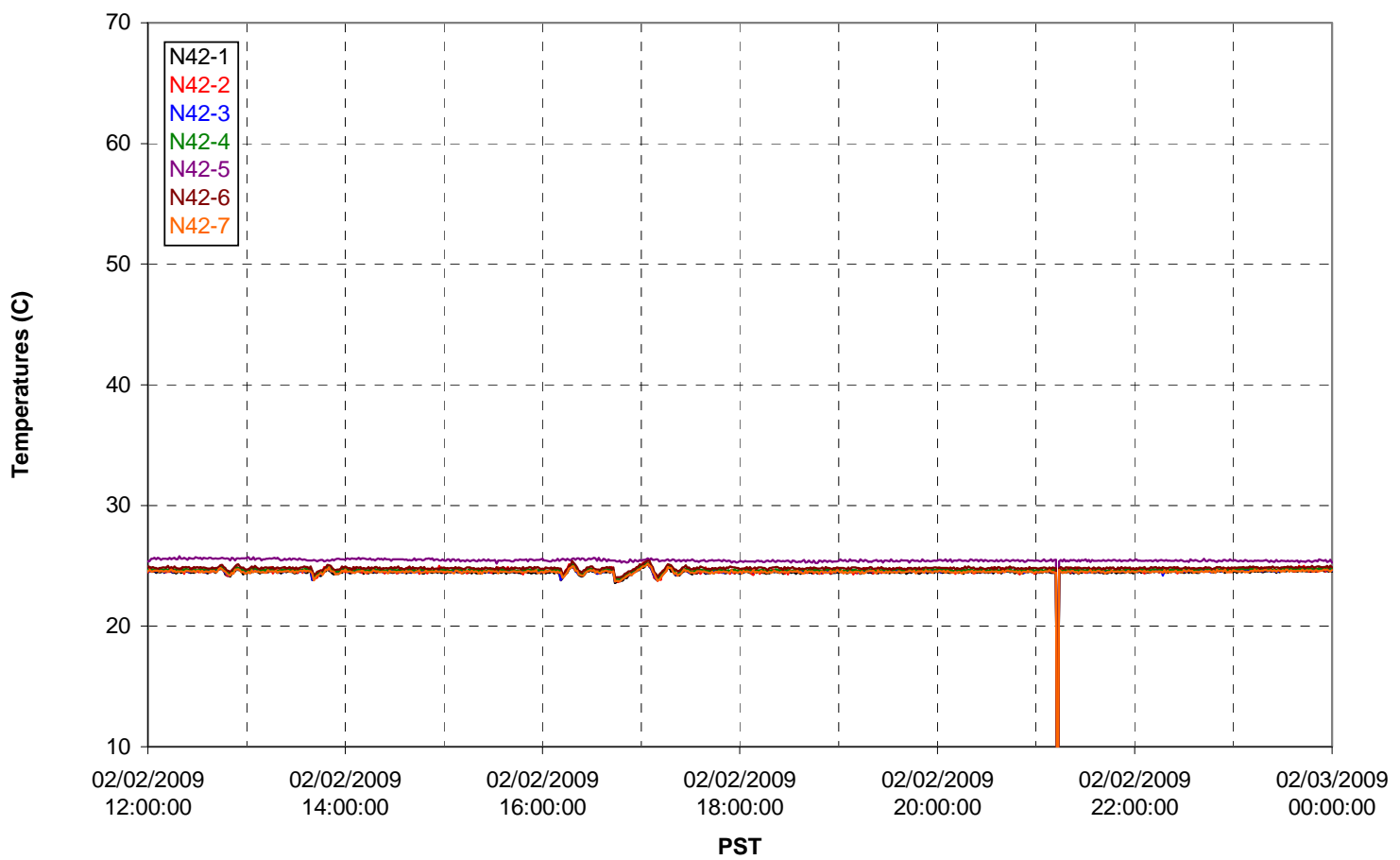

T02A temperatures

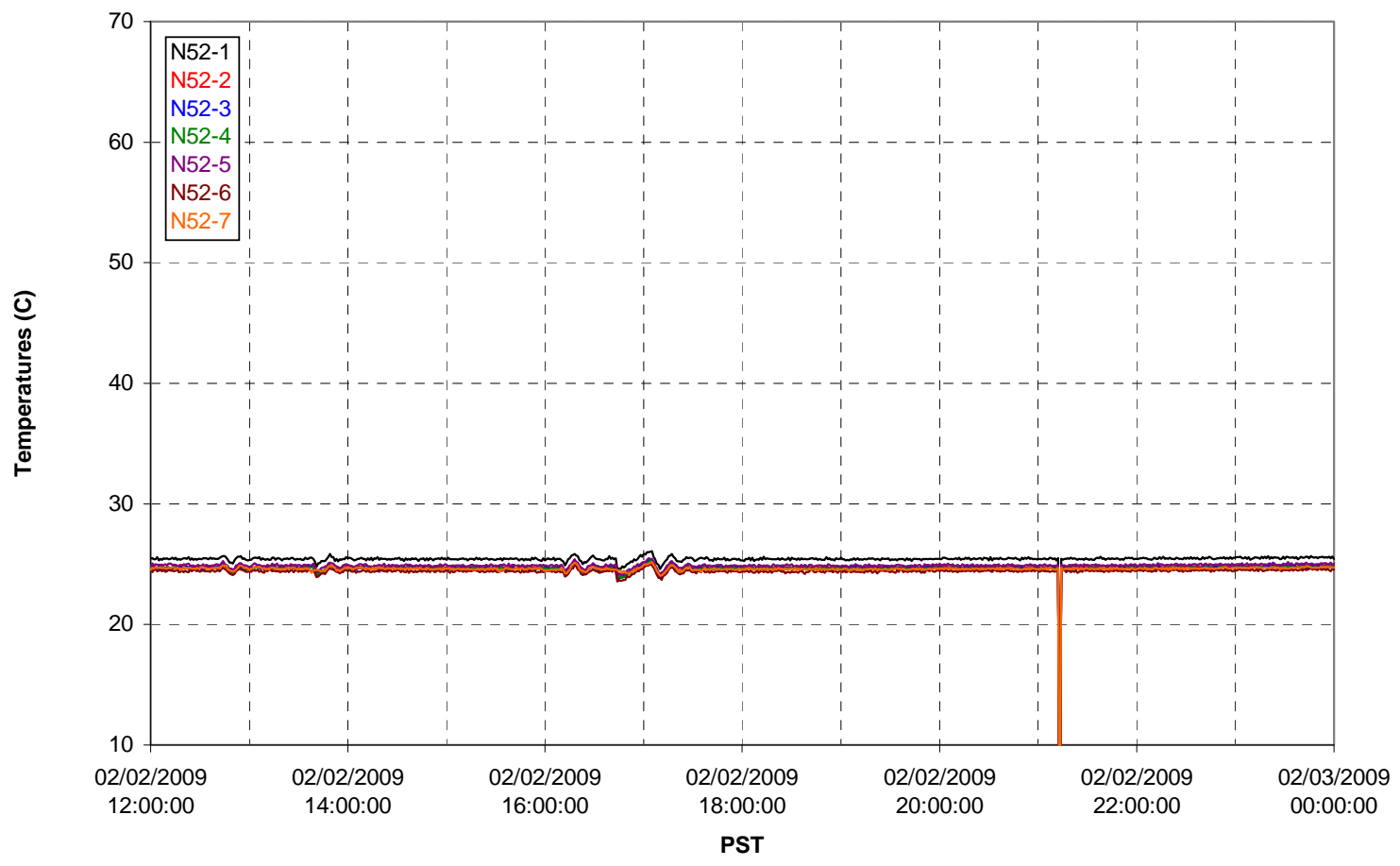


T02A Heating and Cooling

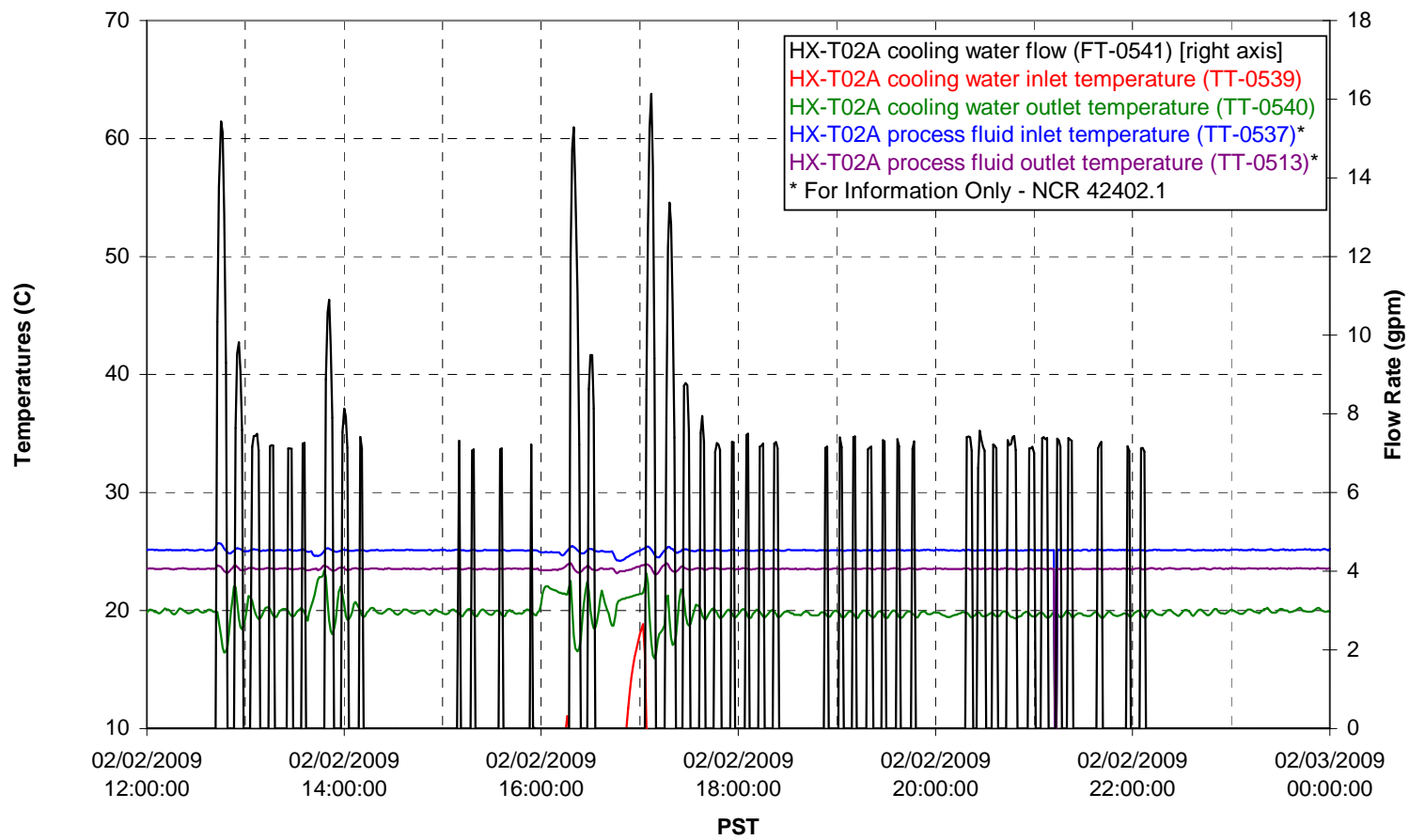

Pump Operation

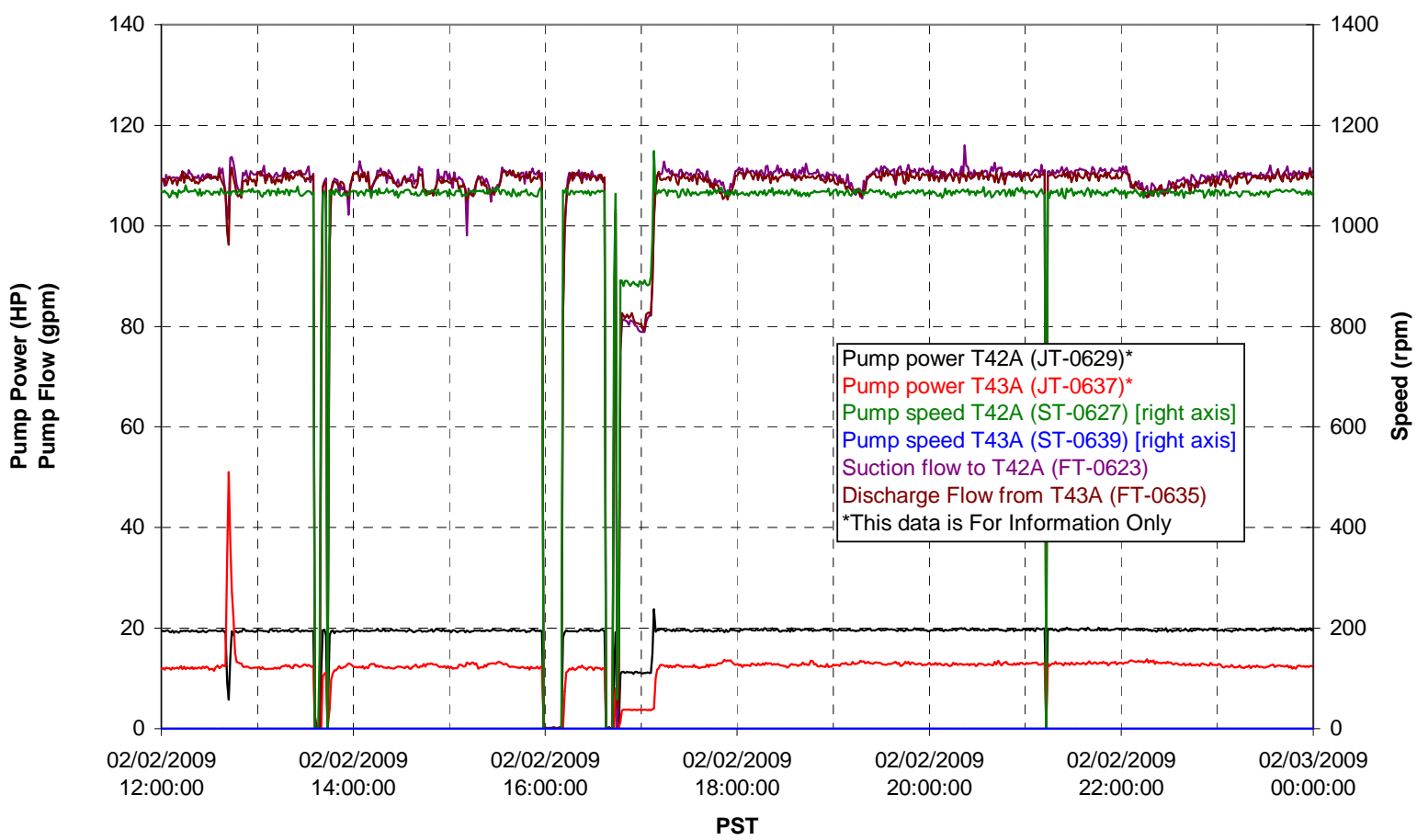


Pulsepot UFP-PP-T01A

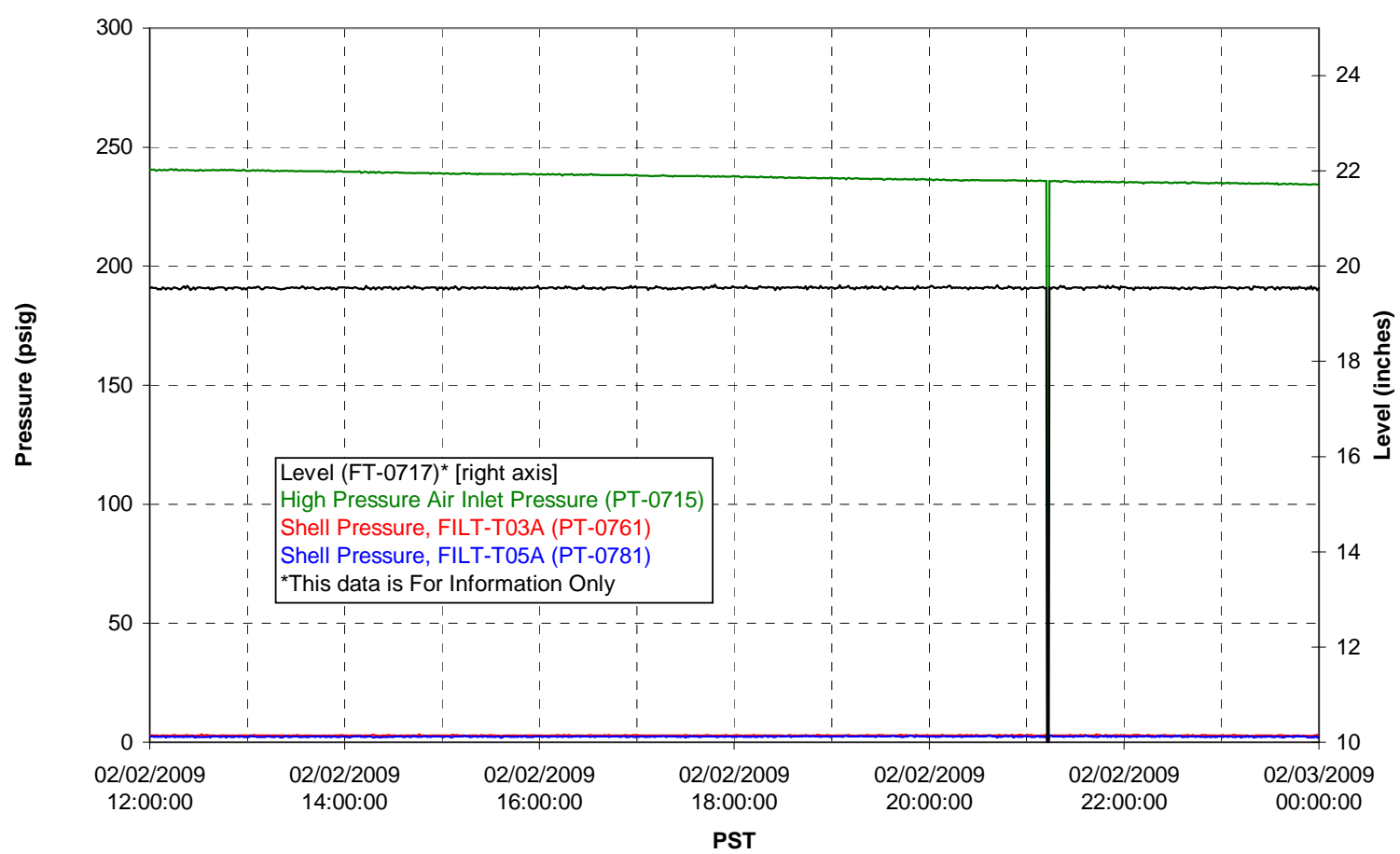

Pulsepot UFP-PP-T02A

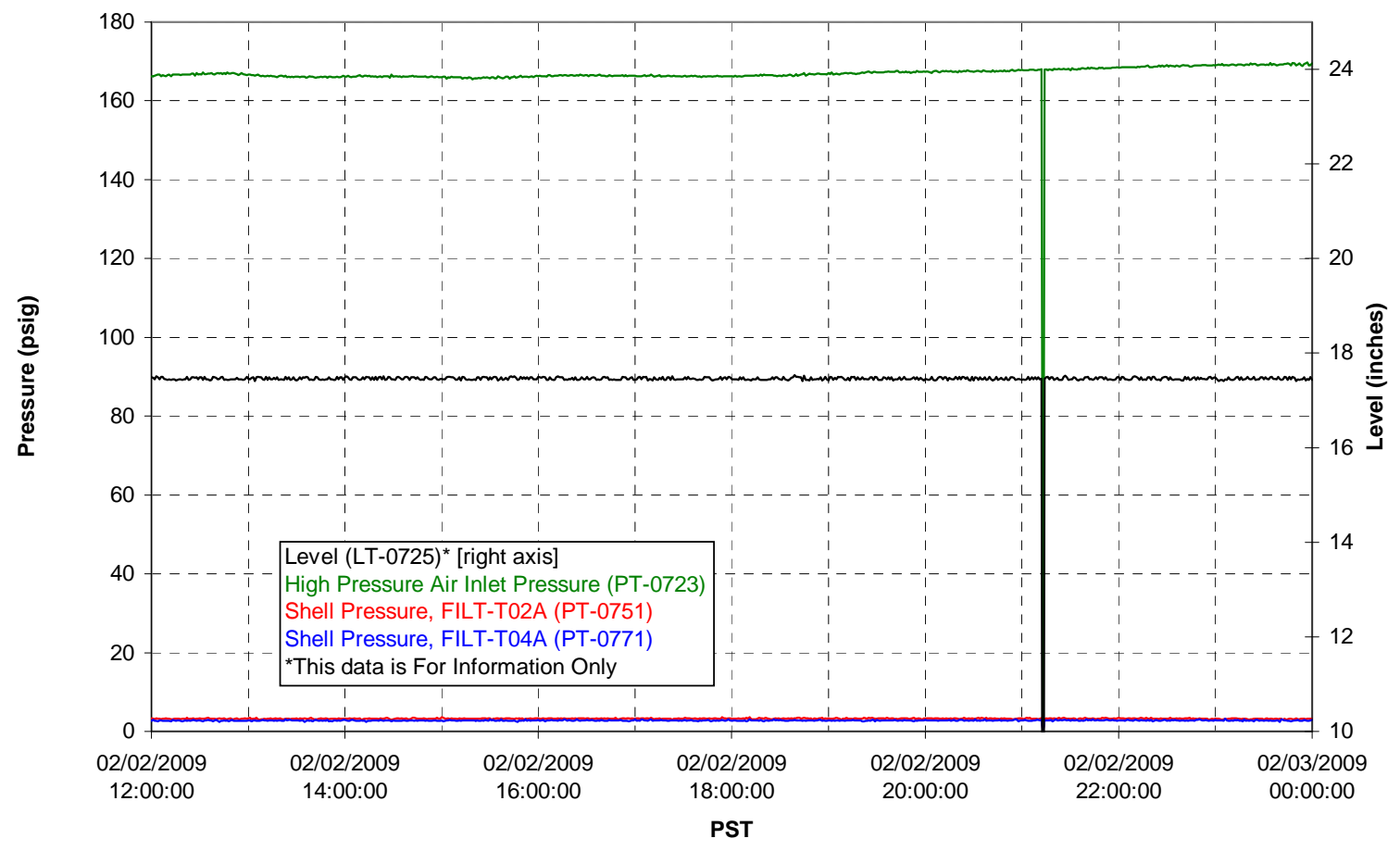

G.101 
Pulsepot UFP-PP-T03A

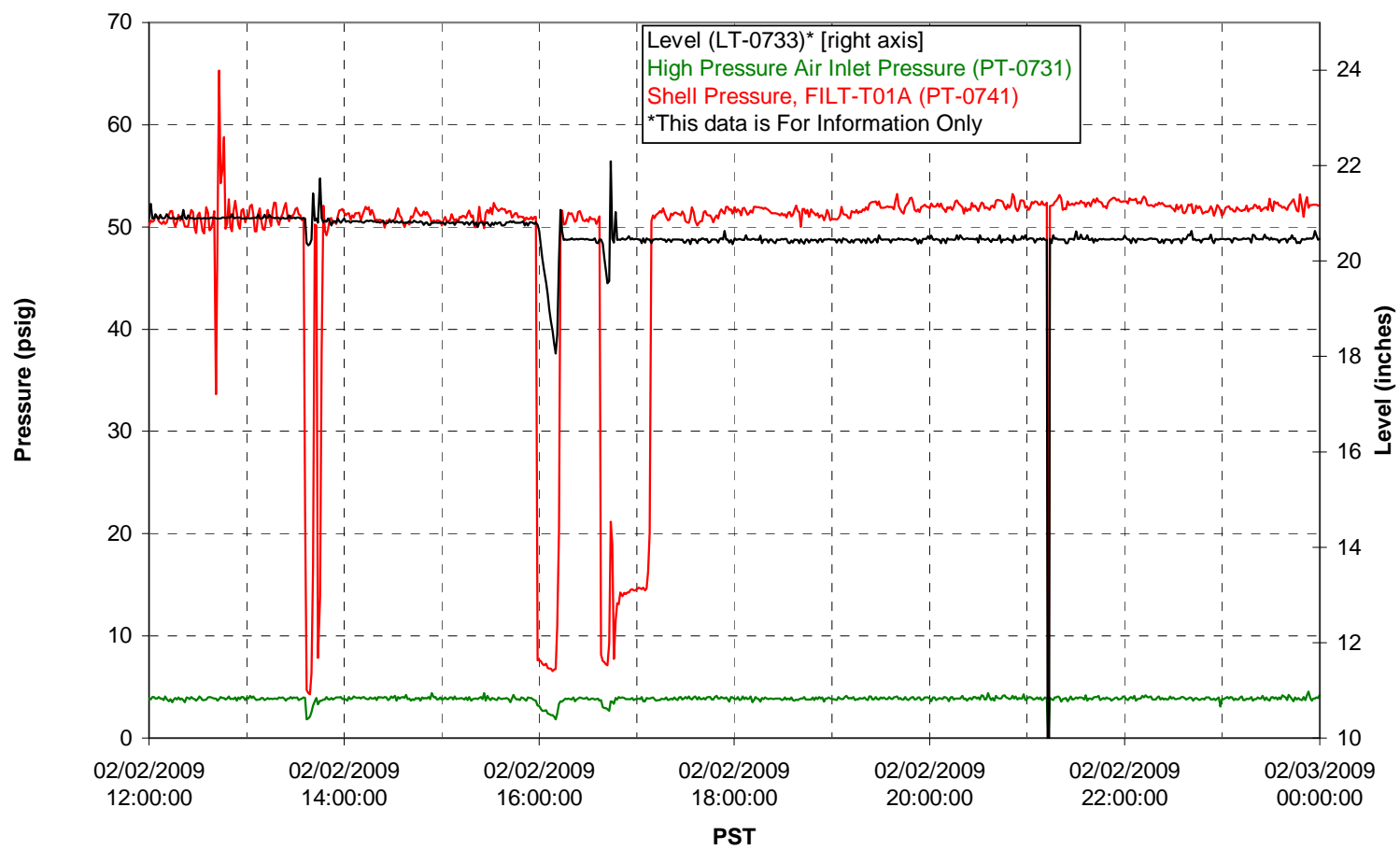

Pulsepot Levels

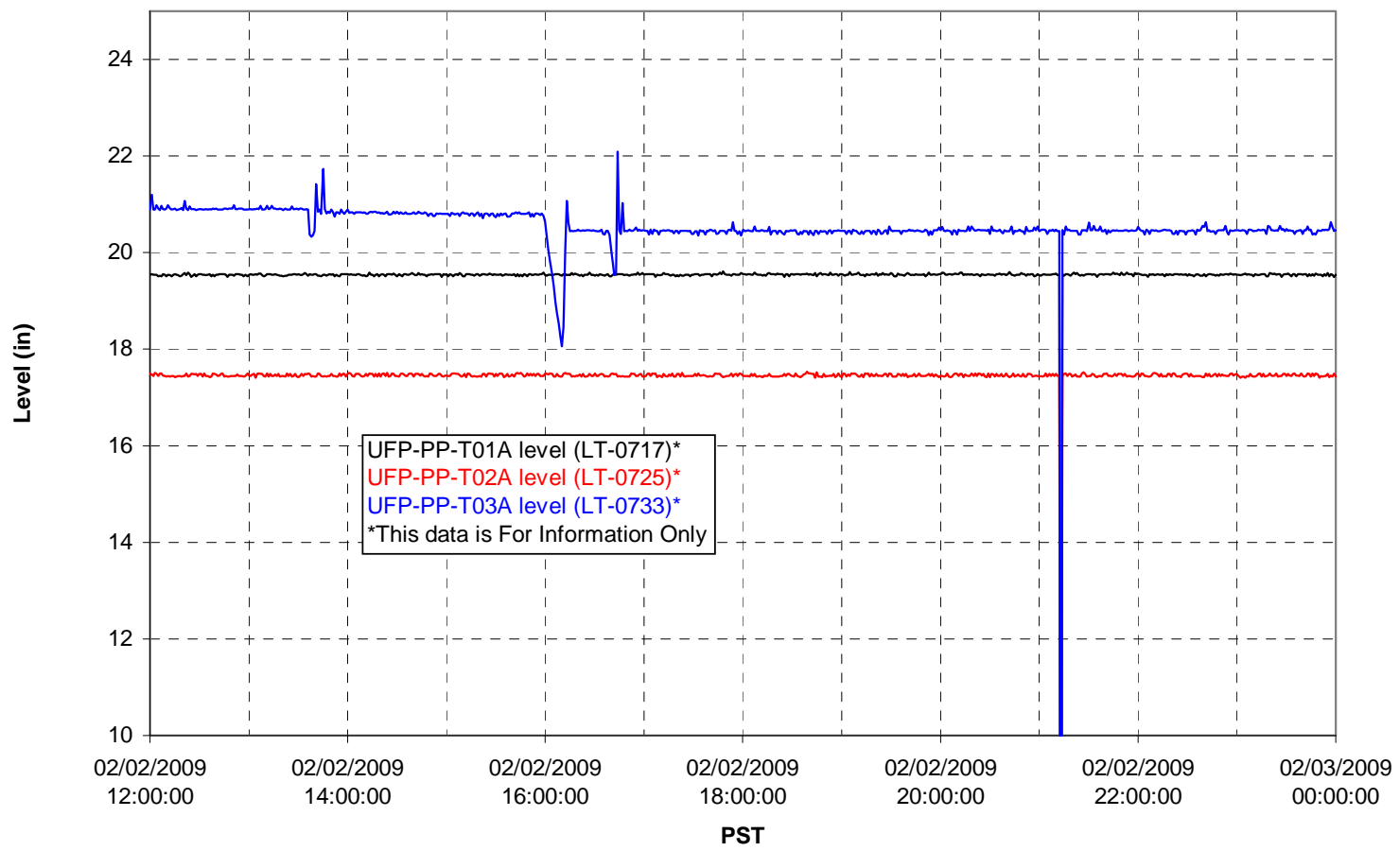

G.102 
Filter UFP-FILT-T01A

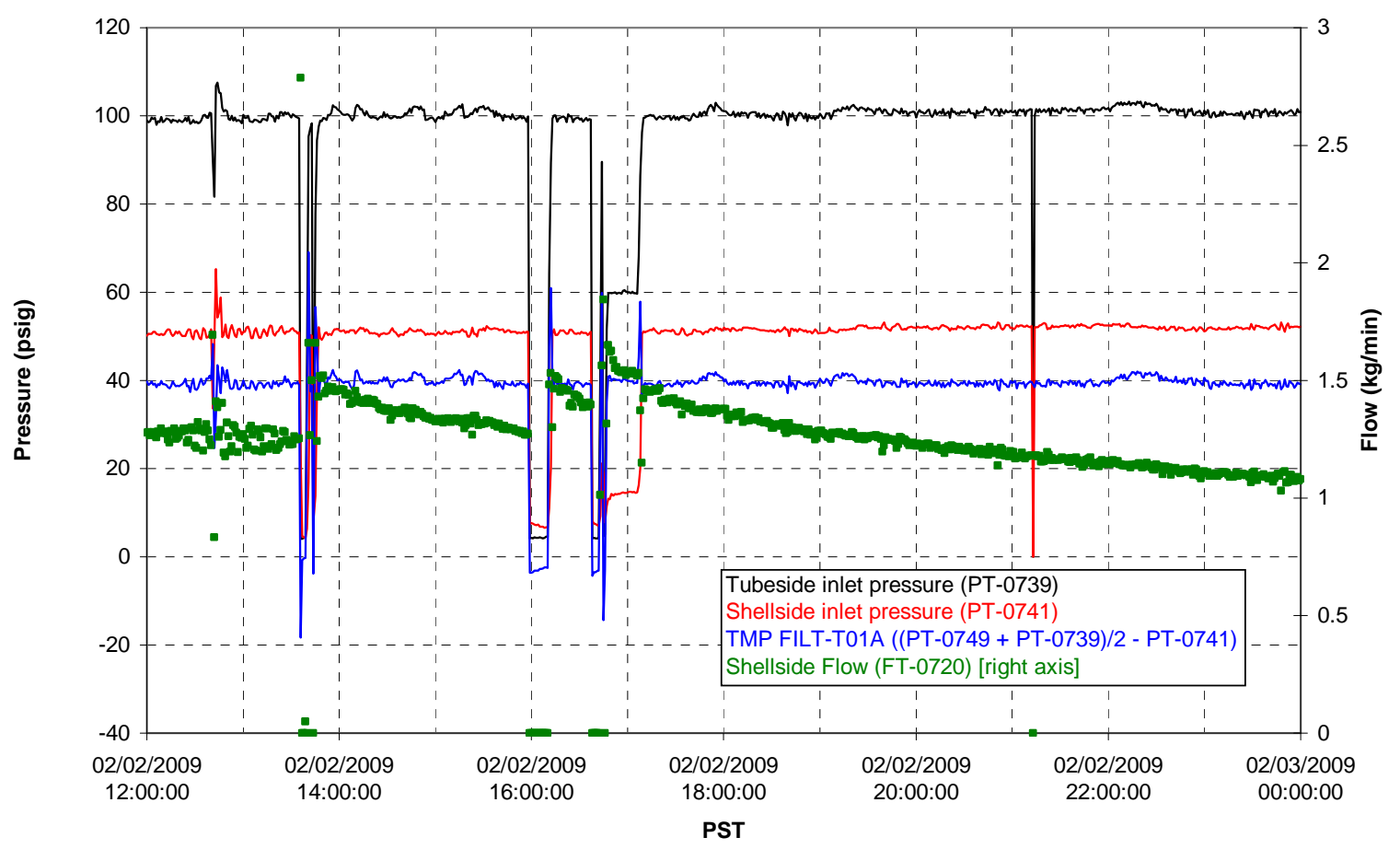

Filter UFP-FILT-T02A

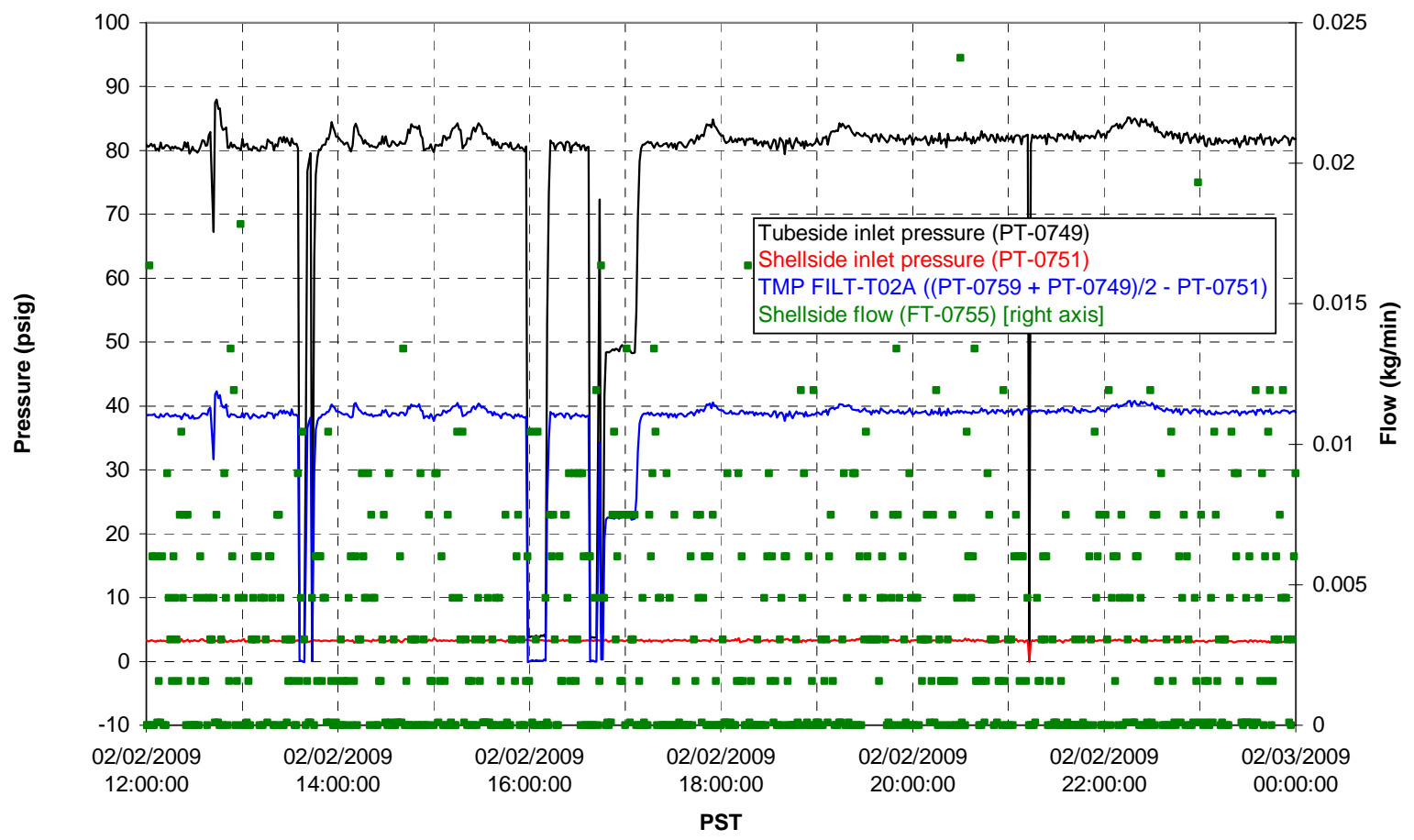

G.103 
Filter UFP-FILT-T03A

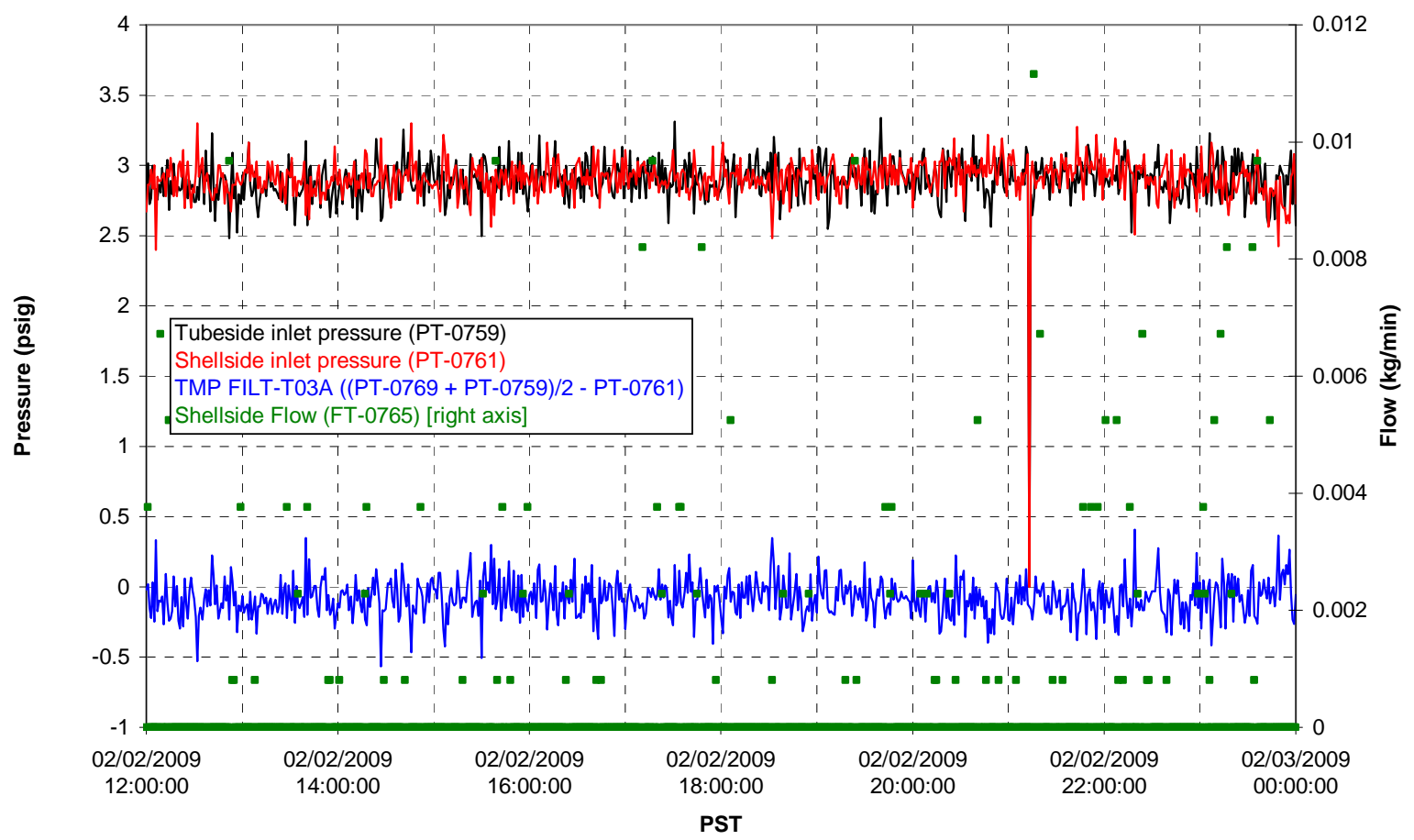

Filter UFP-FILT-T04A

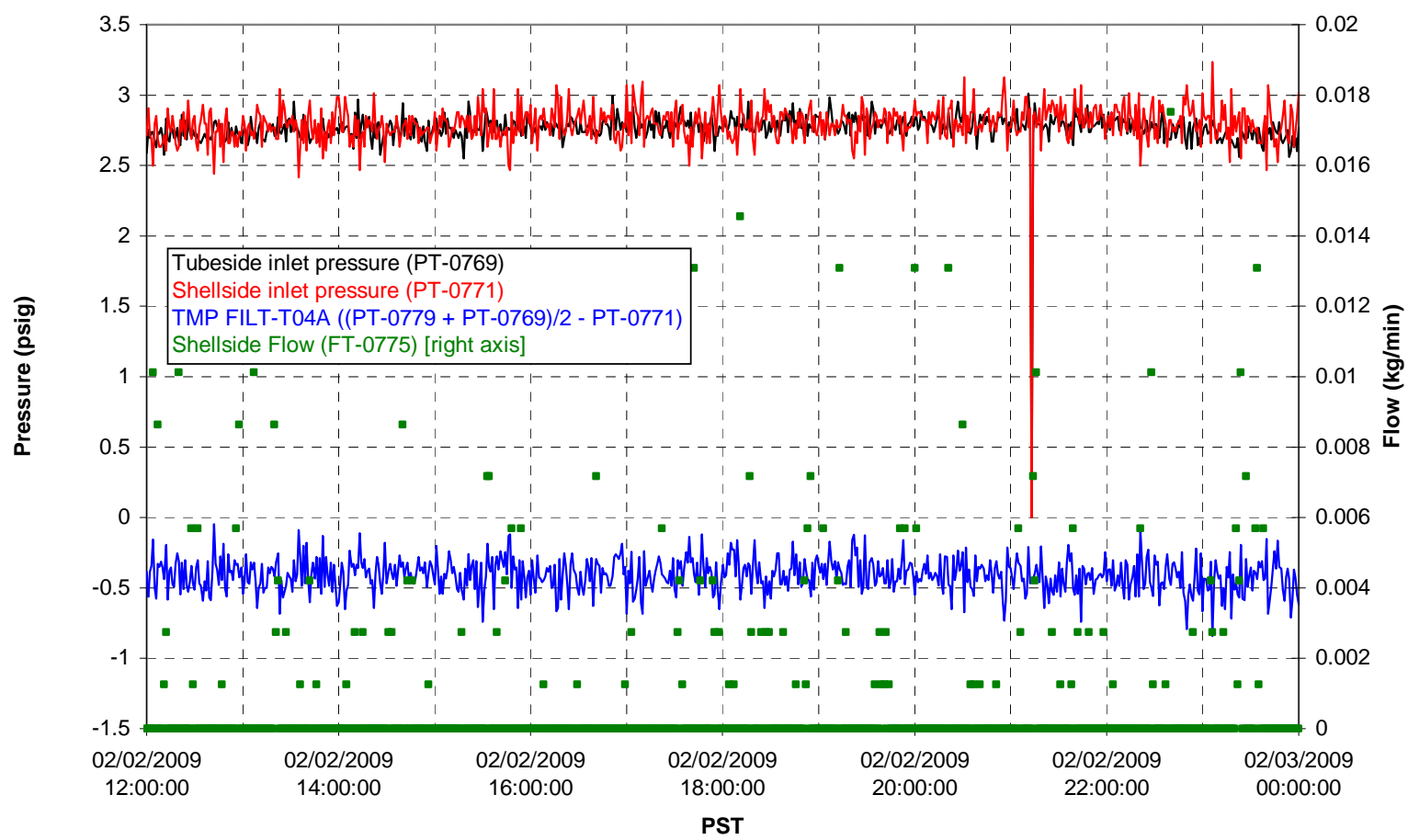


Filter UFP-FILT-T04A

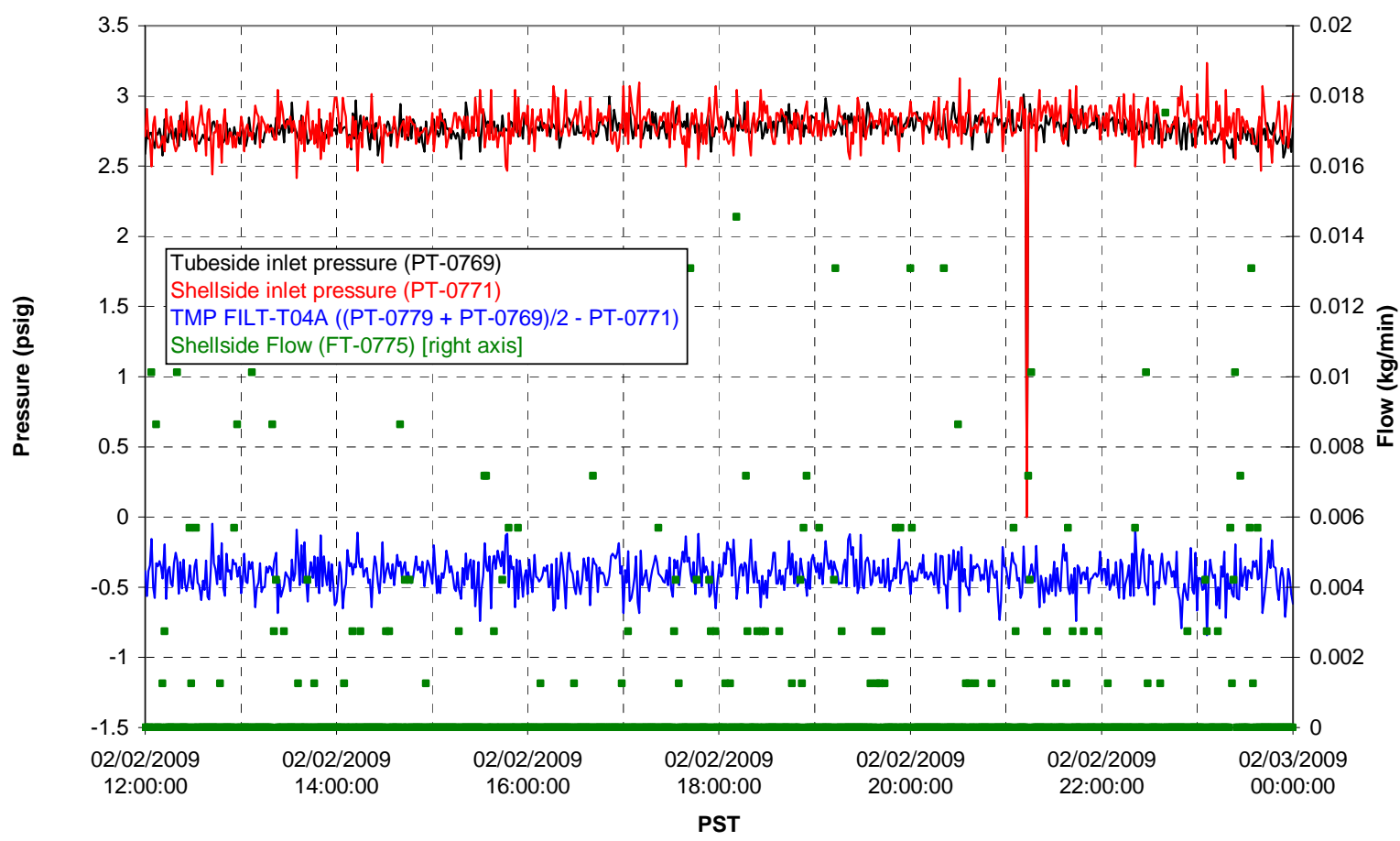

Chemical Flow

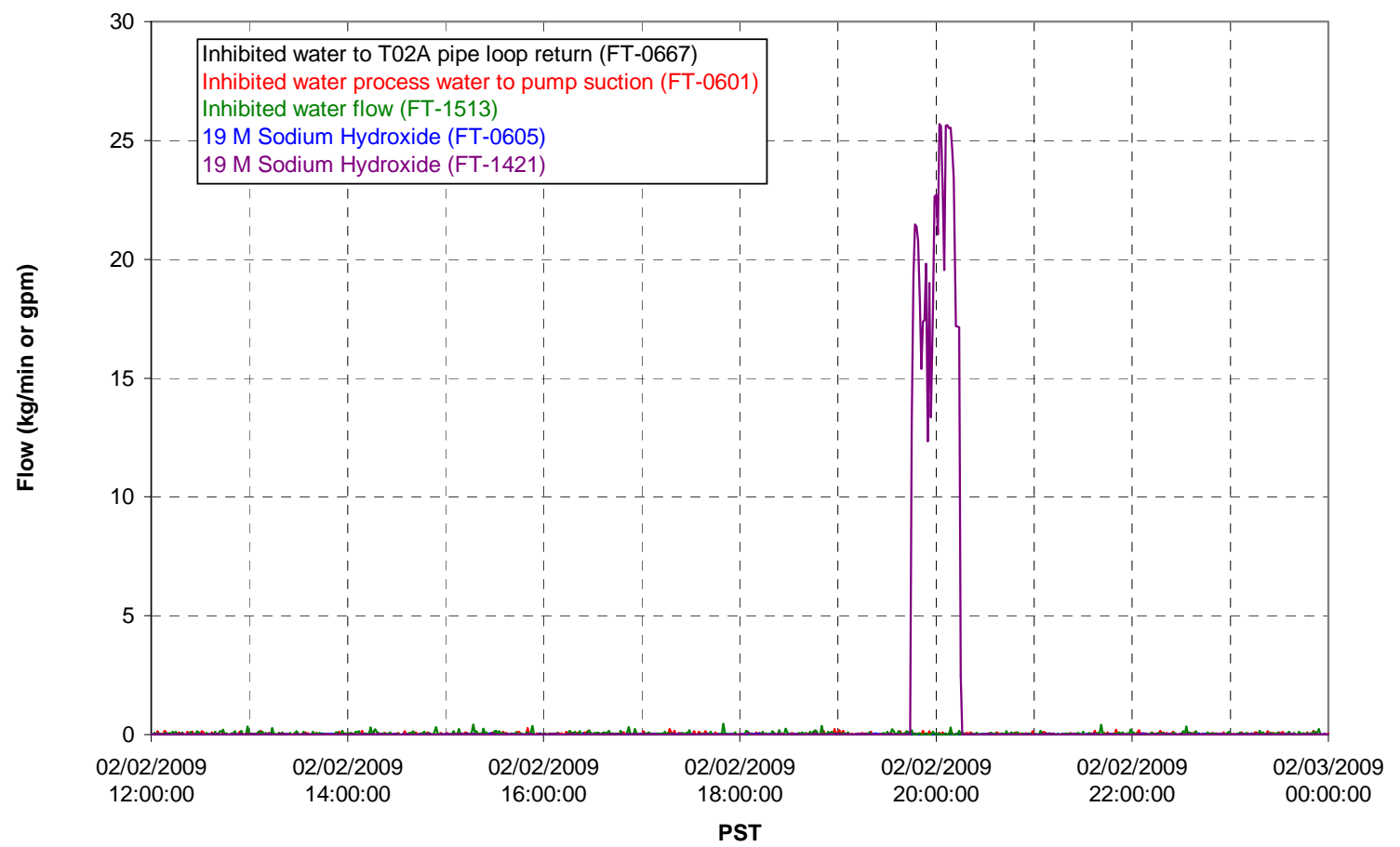


Chemical Flow

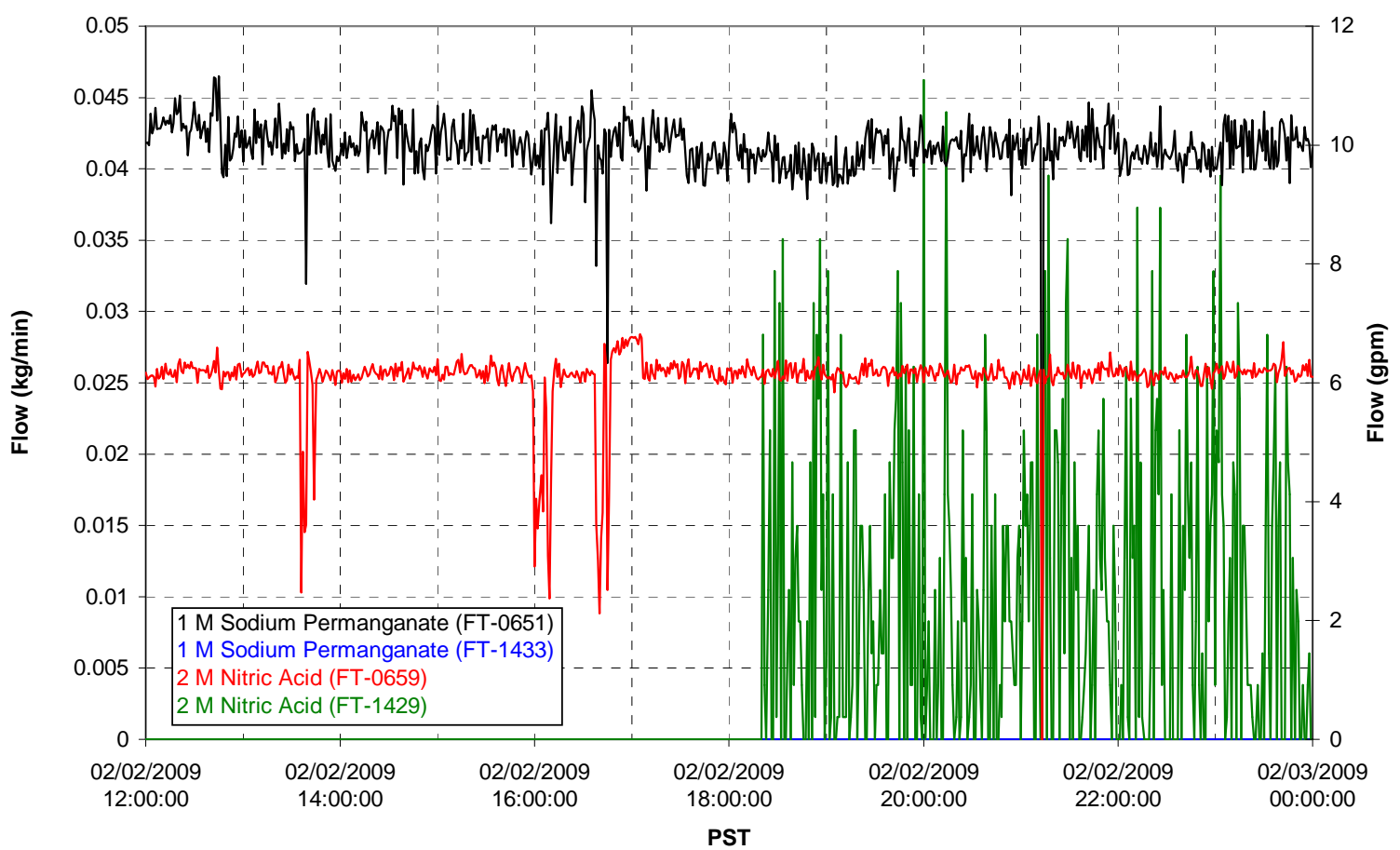

Air Flows

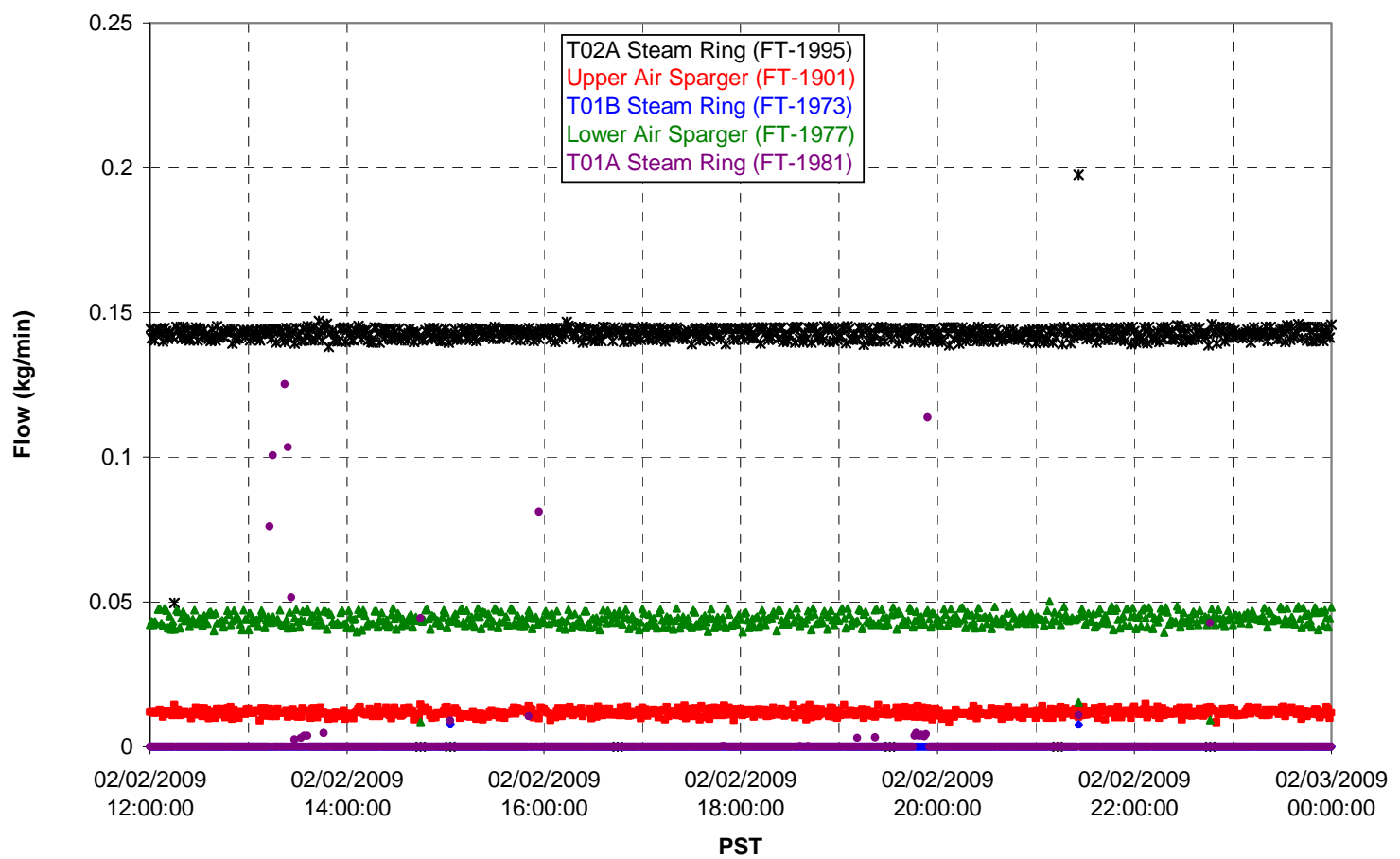

G.106 
T02A Steam

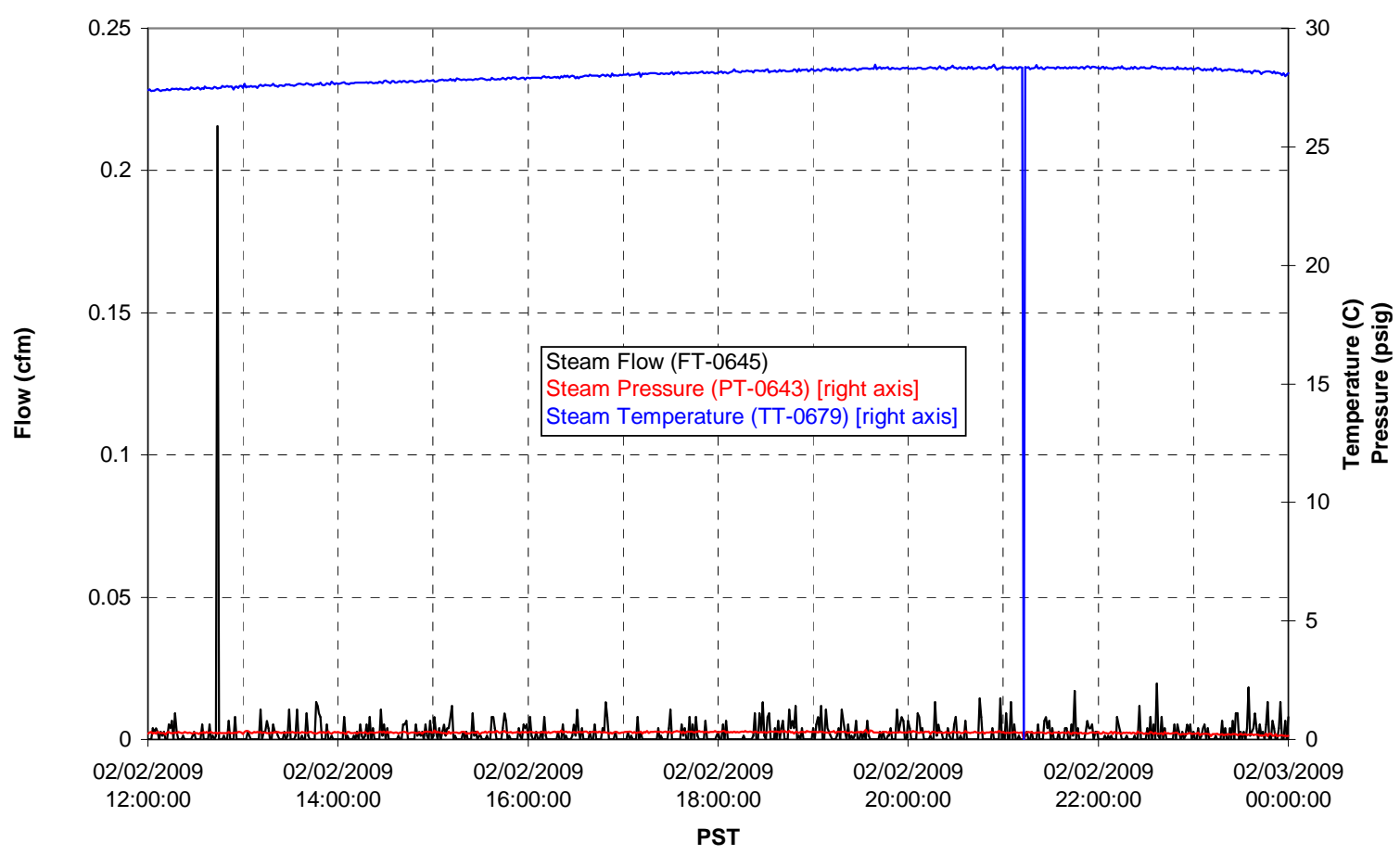

T01A Steam

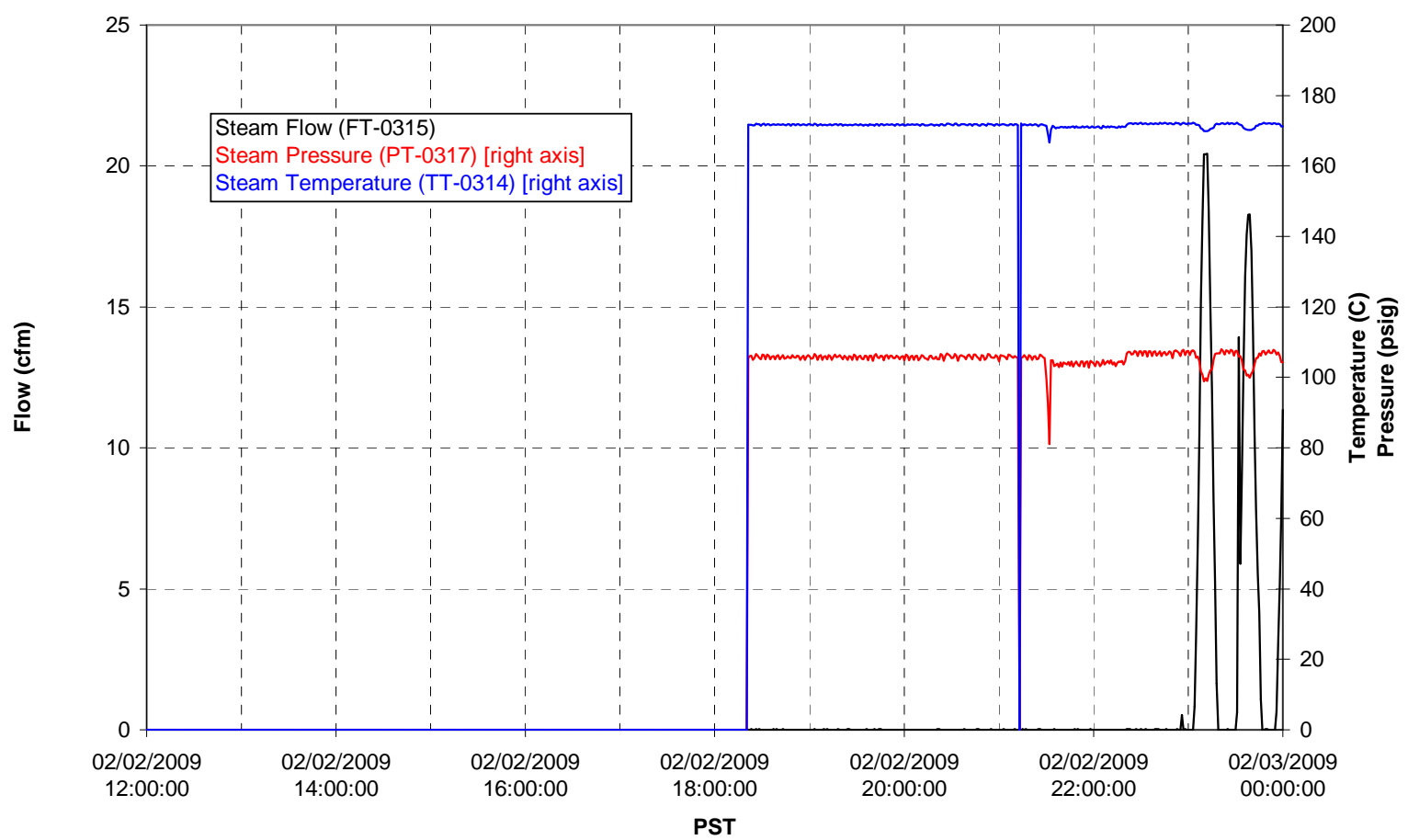

G.107 
T01B Steam

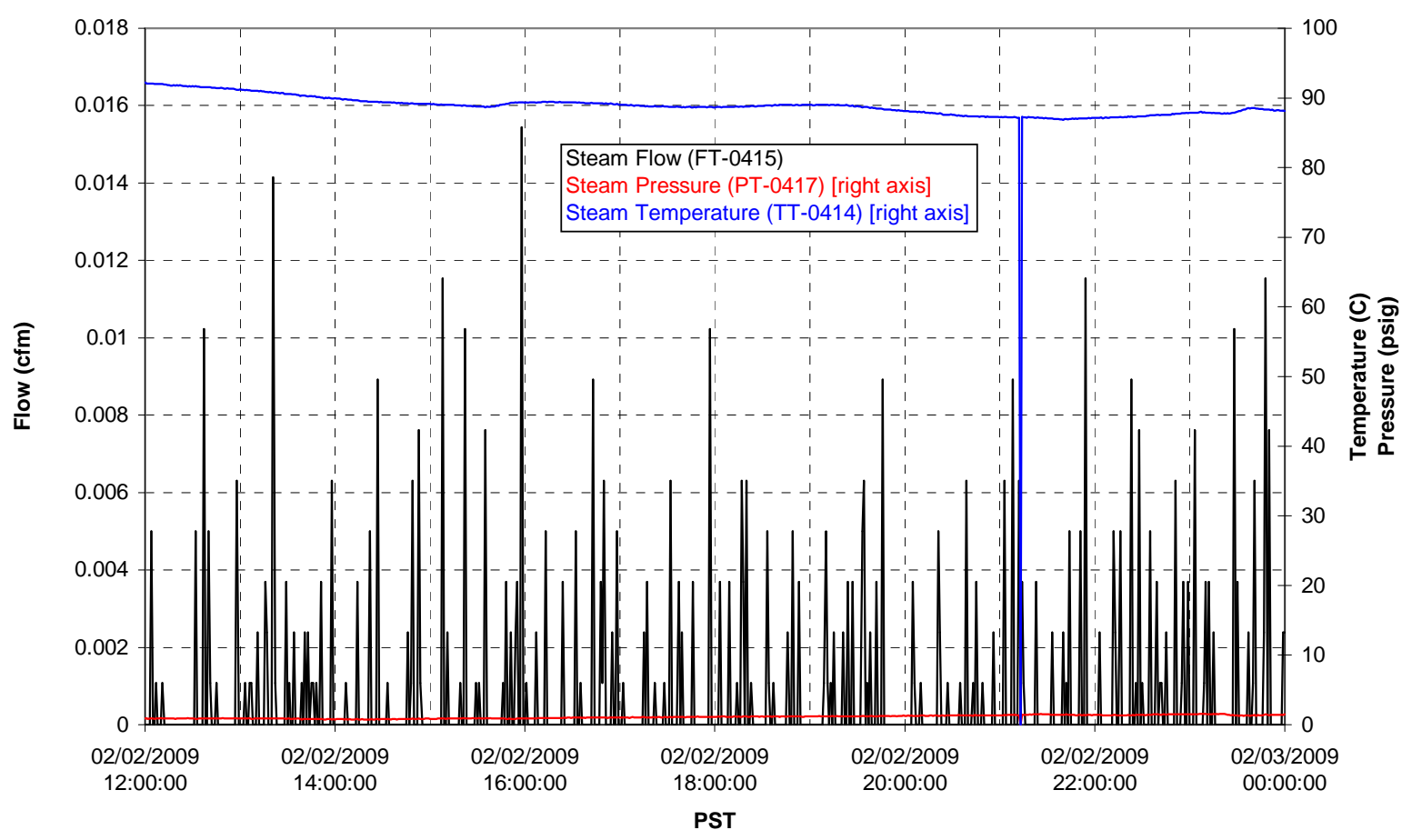




\section{Integrated Test A Data Plots 02/03/09 00:00 - 02/03/09 12:00}


T01A level

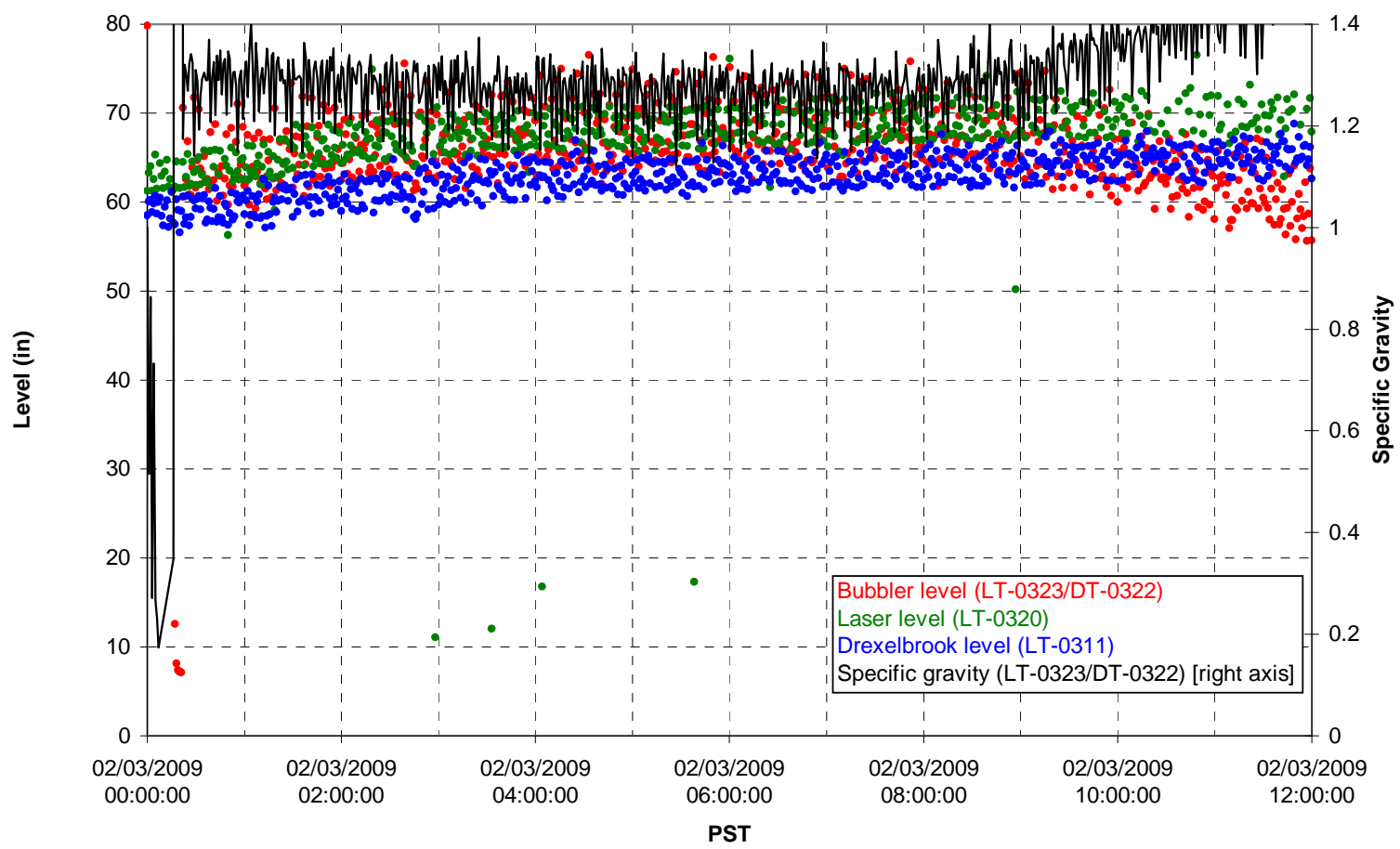

T01A temperatures

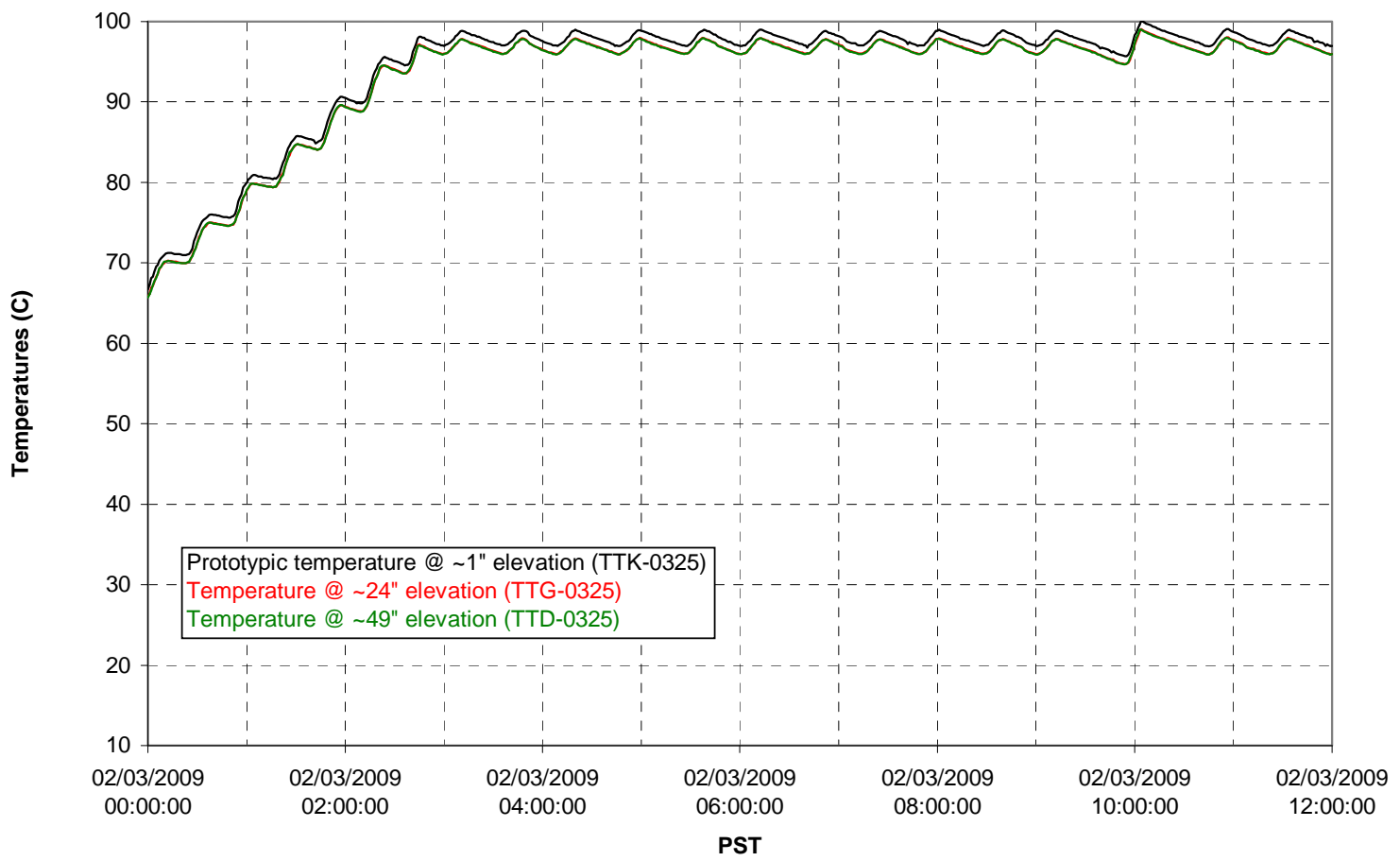


T01B level

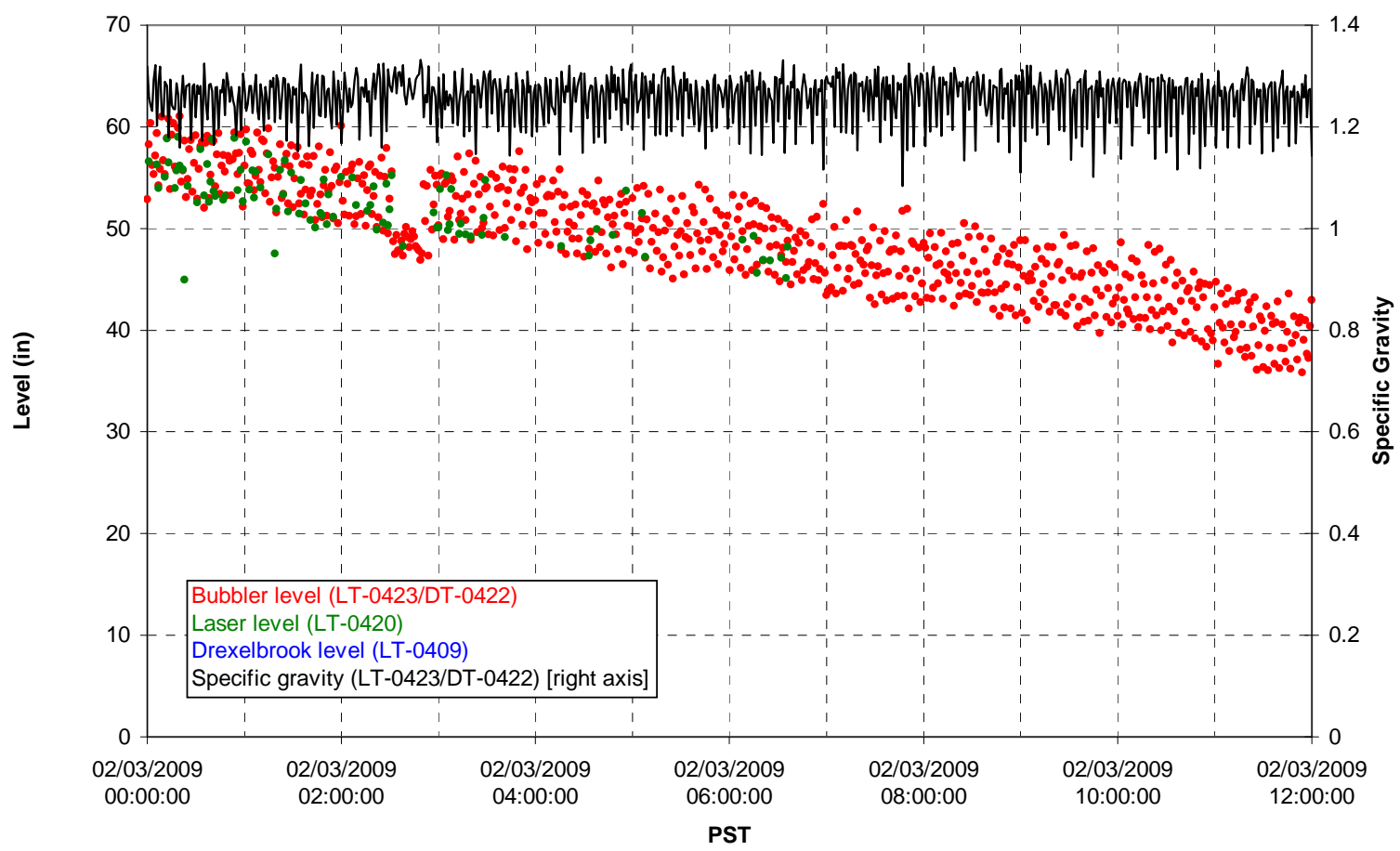

T01B temperatures

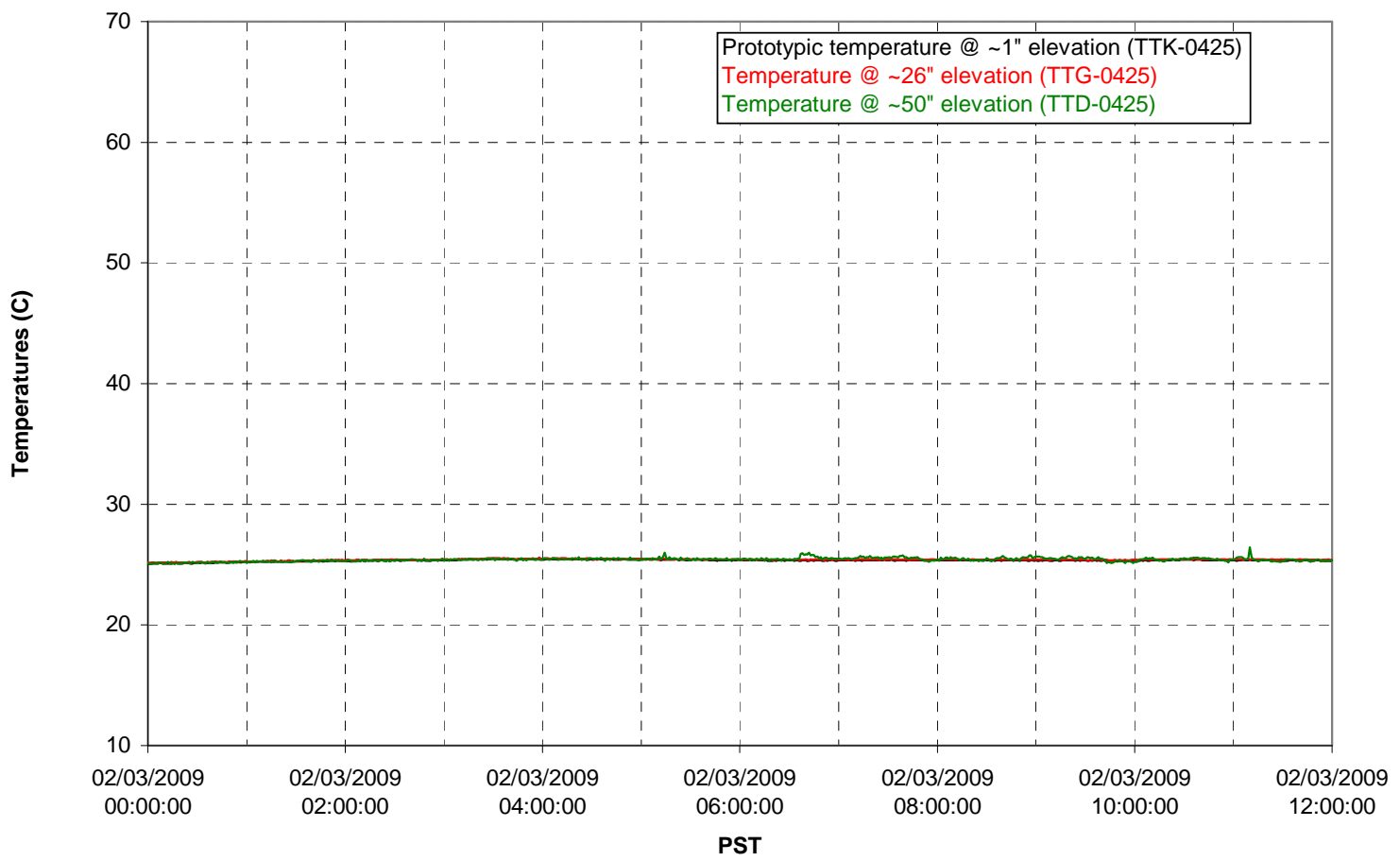


T02A level

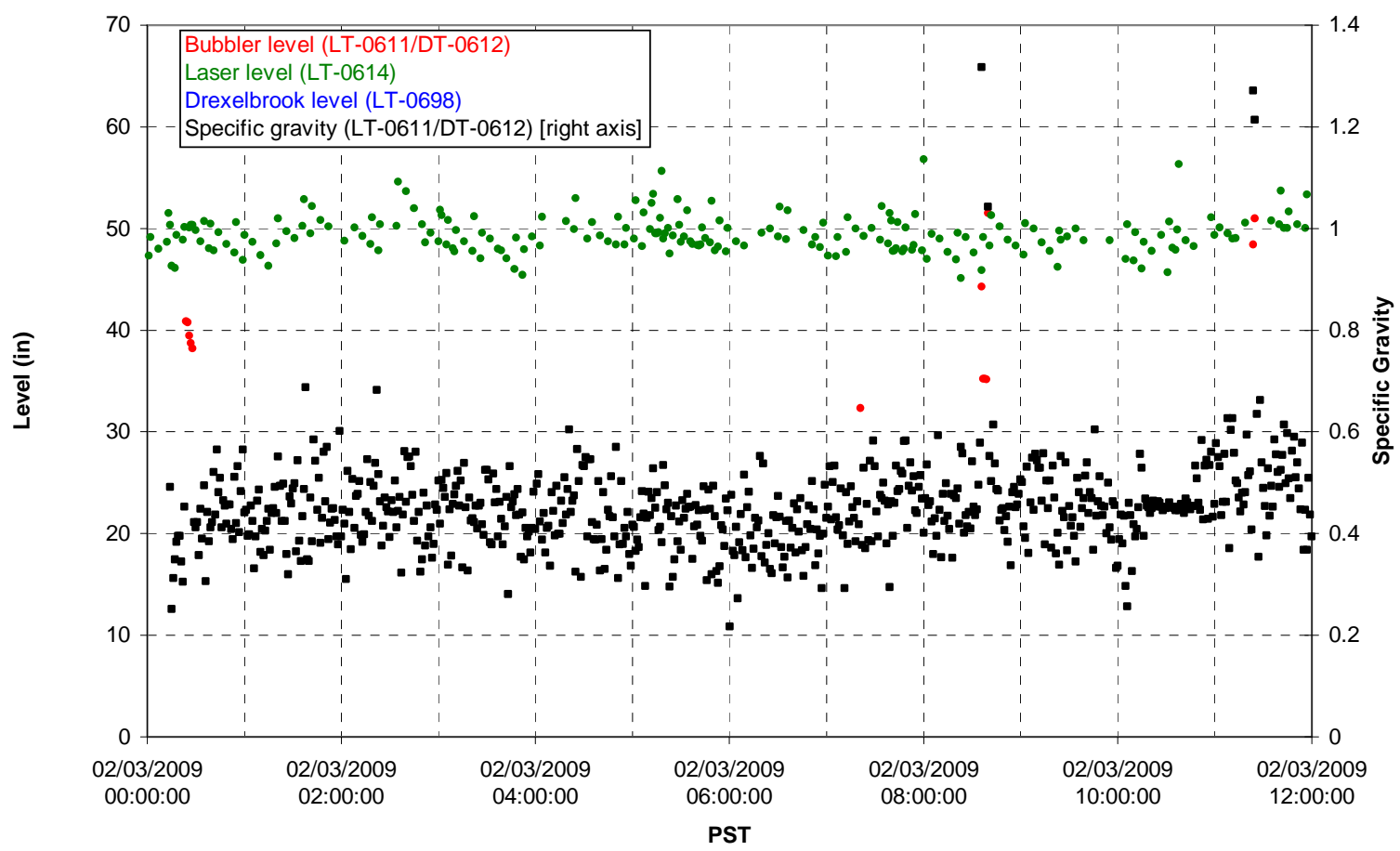

T02A temperatures

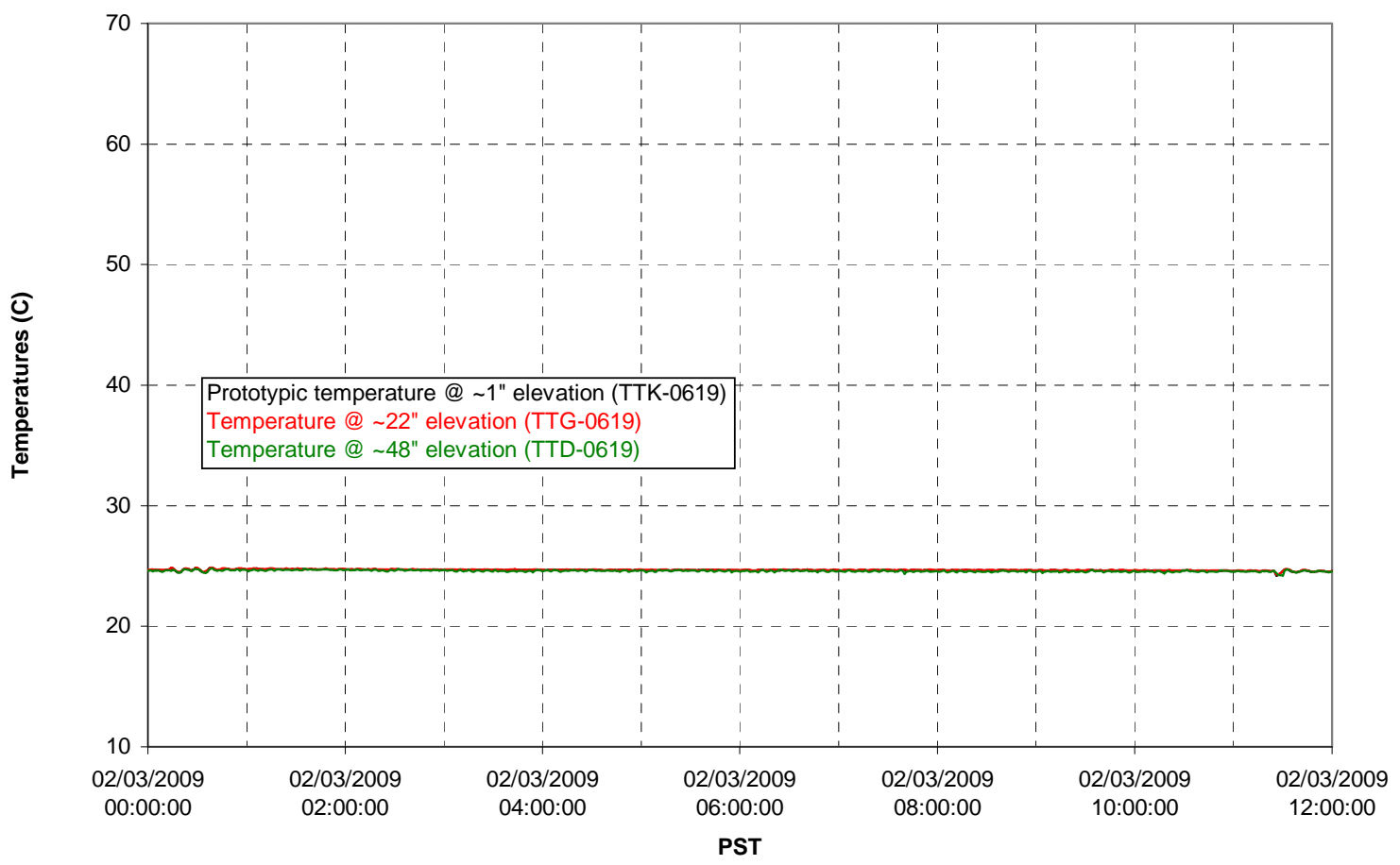

G.112 
T02A and filter loop temperatures

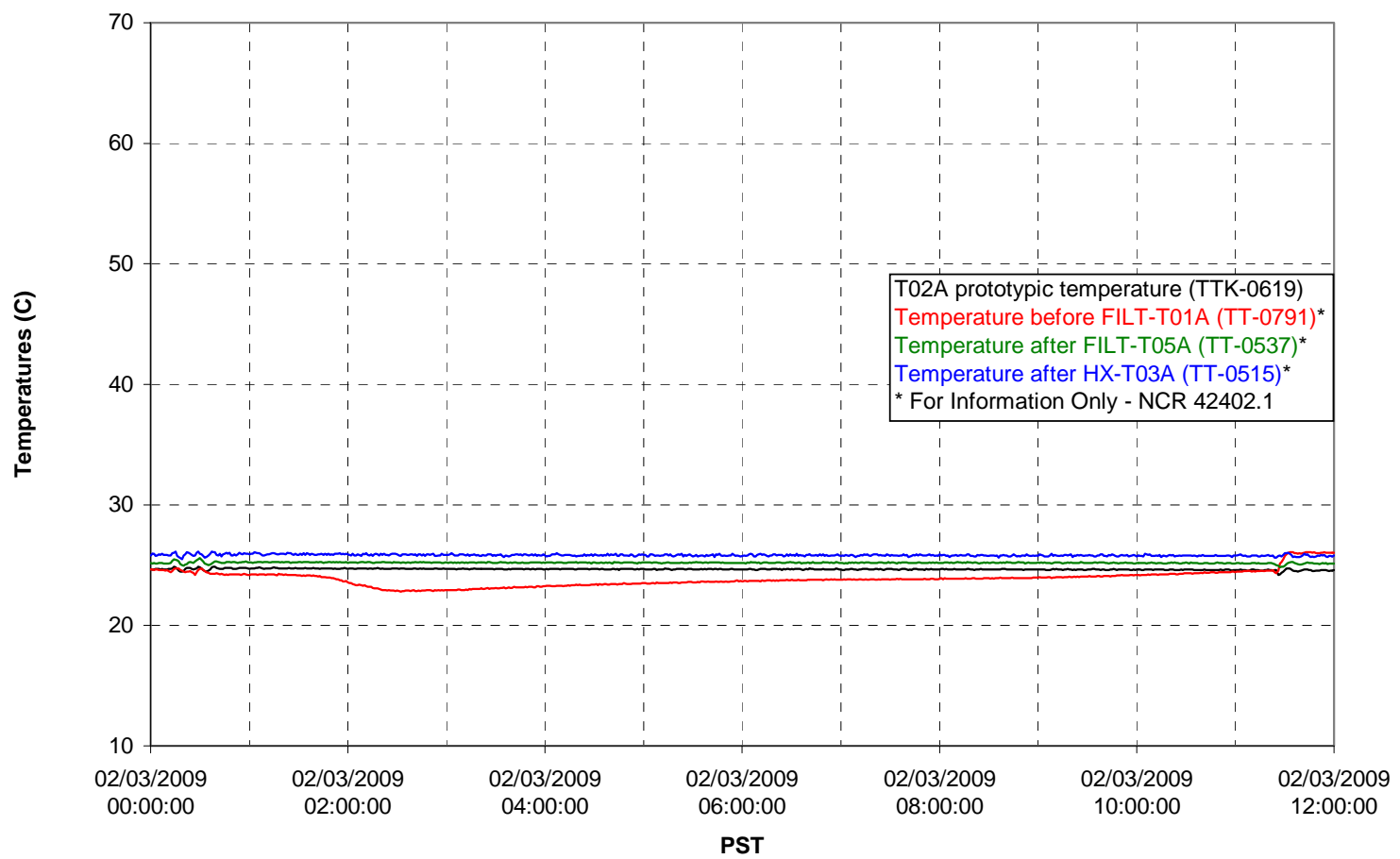

Pump Pressures and Flow

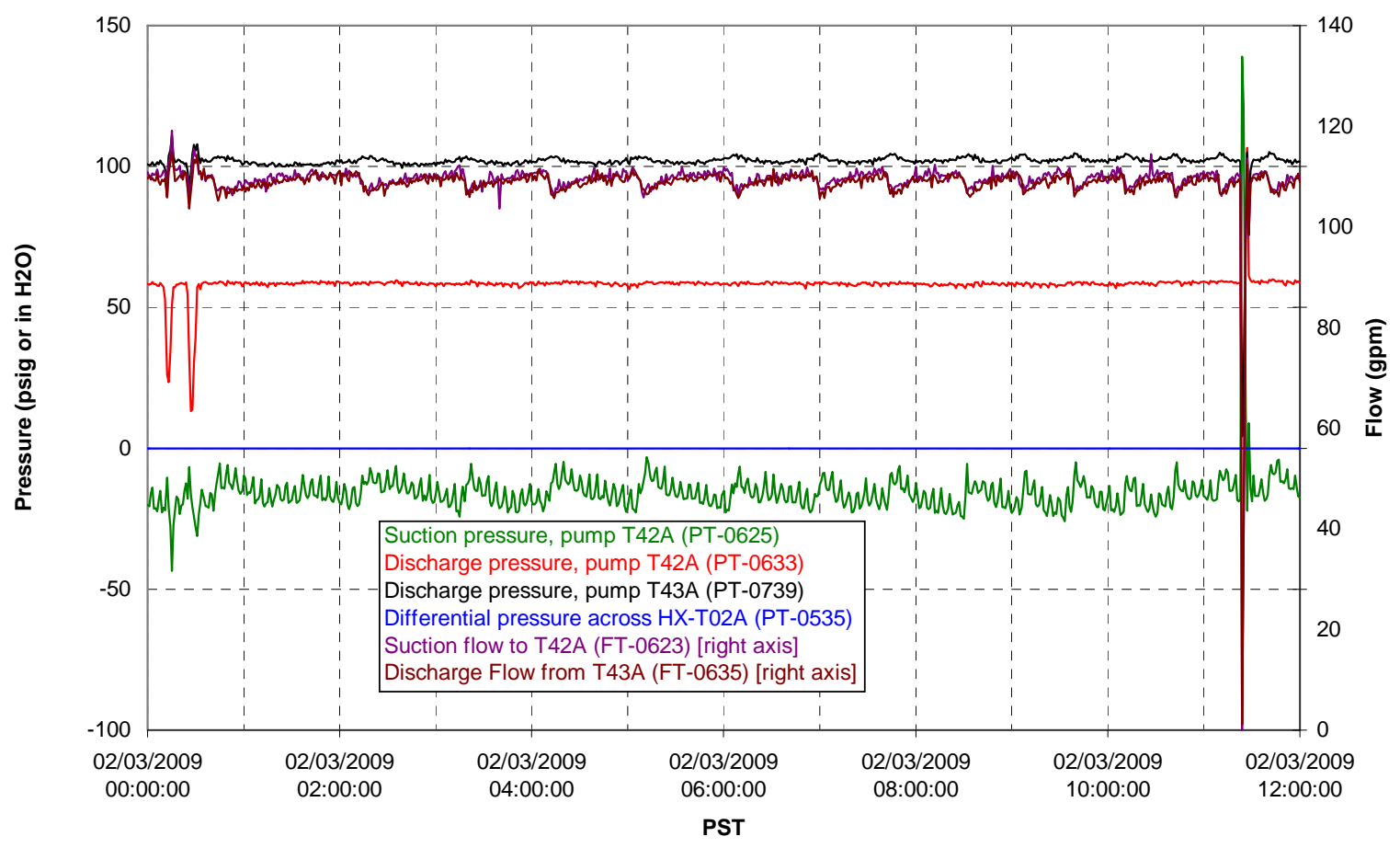

G.113 
Axial pressure drop

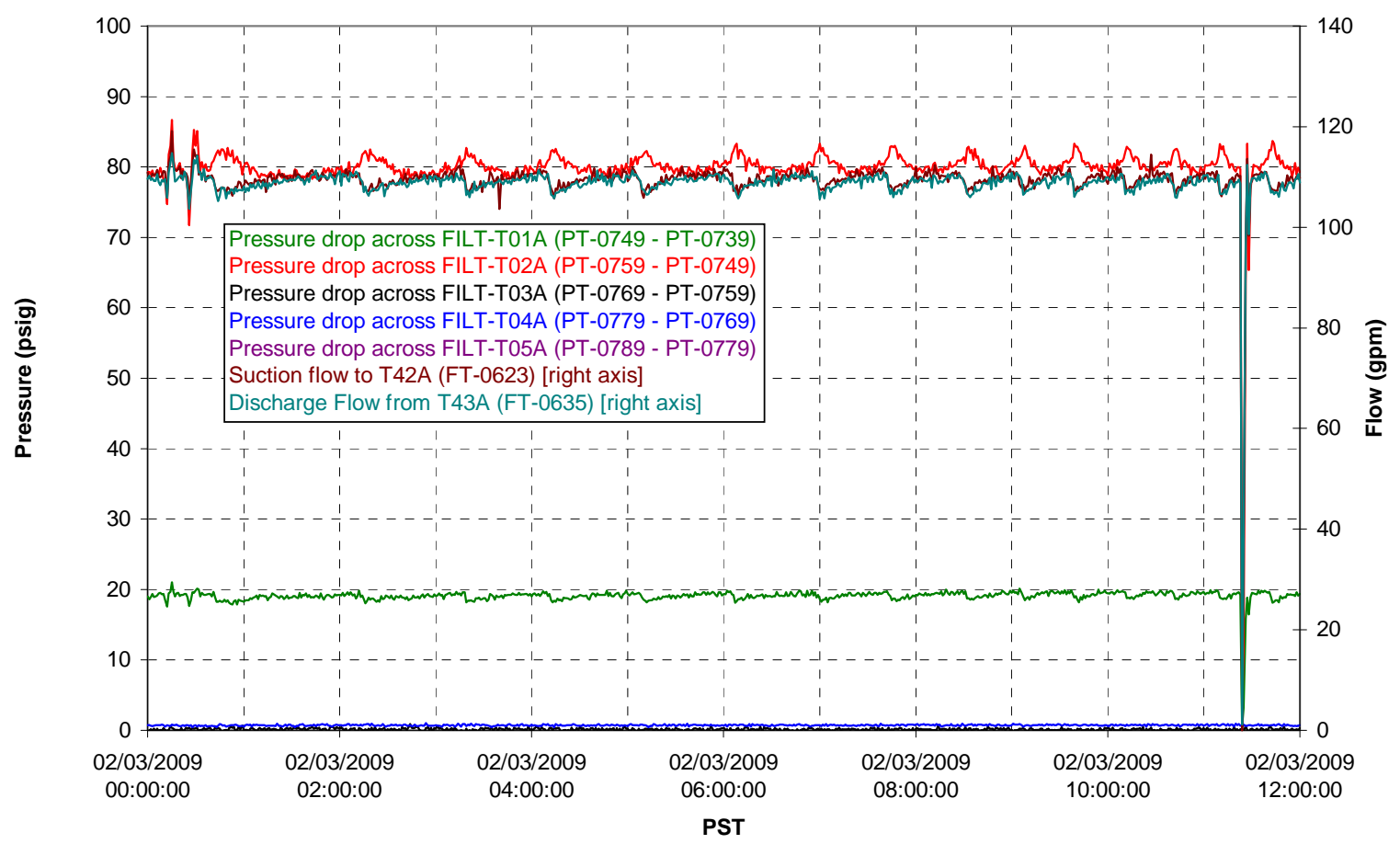

Permeate flow rates

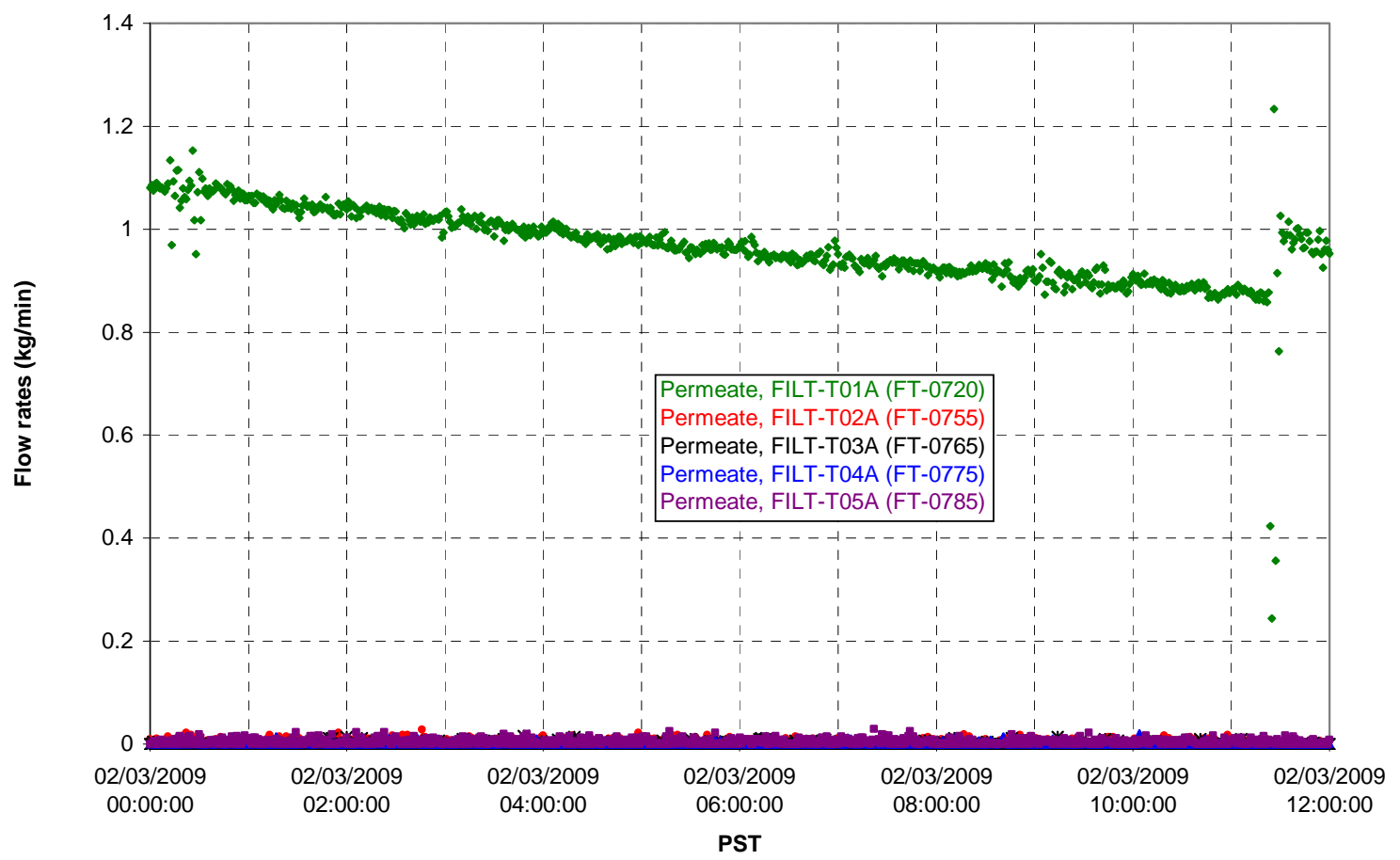

G. 114 
T02A Inner Temperature Tree

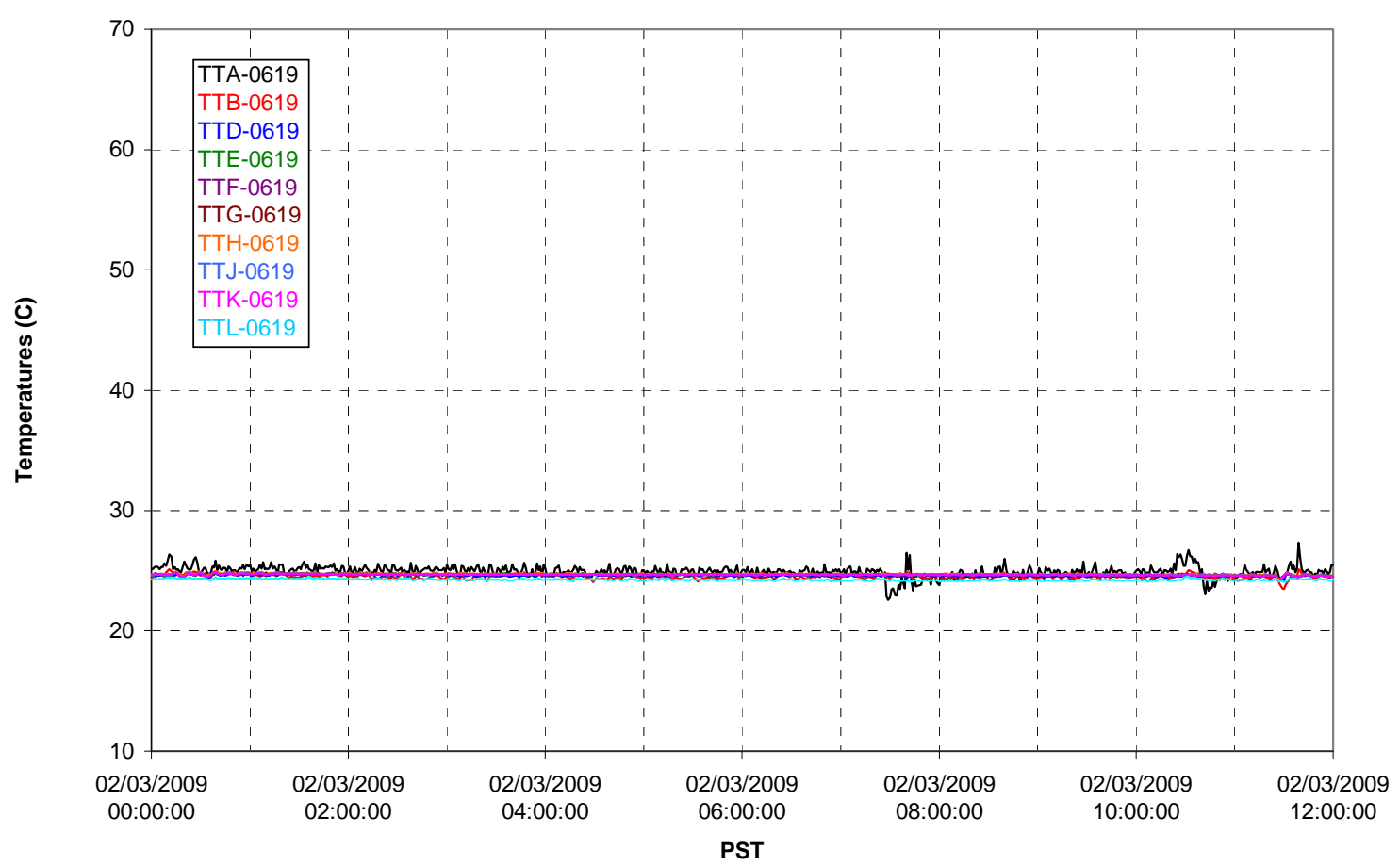

T02A Outer Temperature Tree

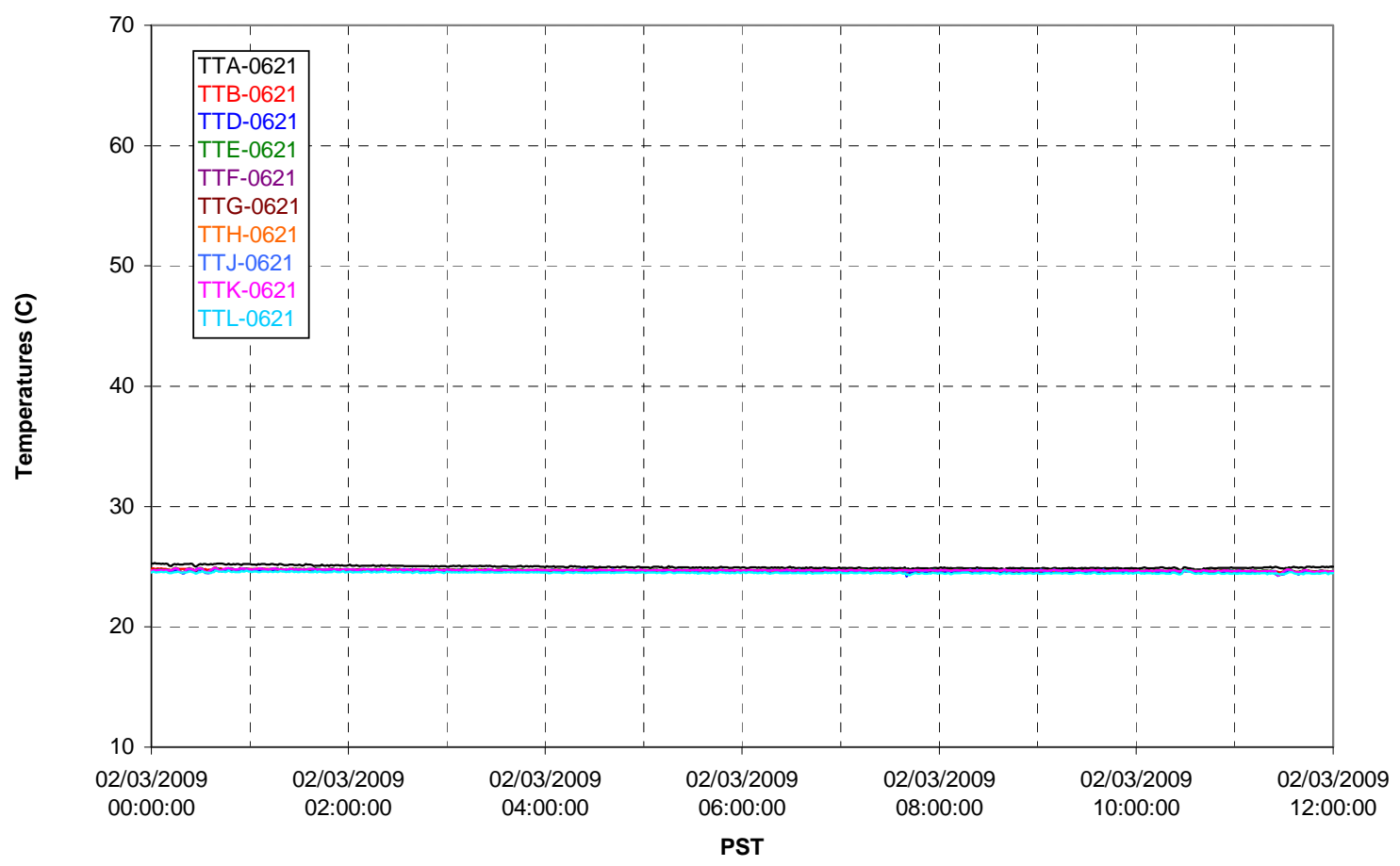

G. 115 
T02A temperatures

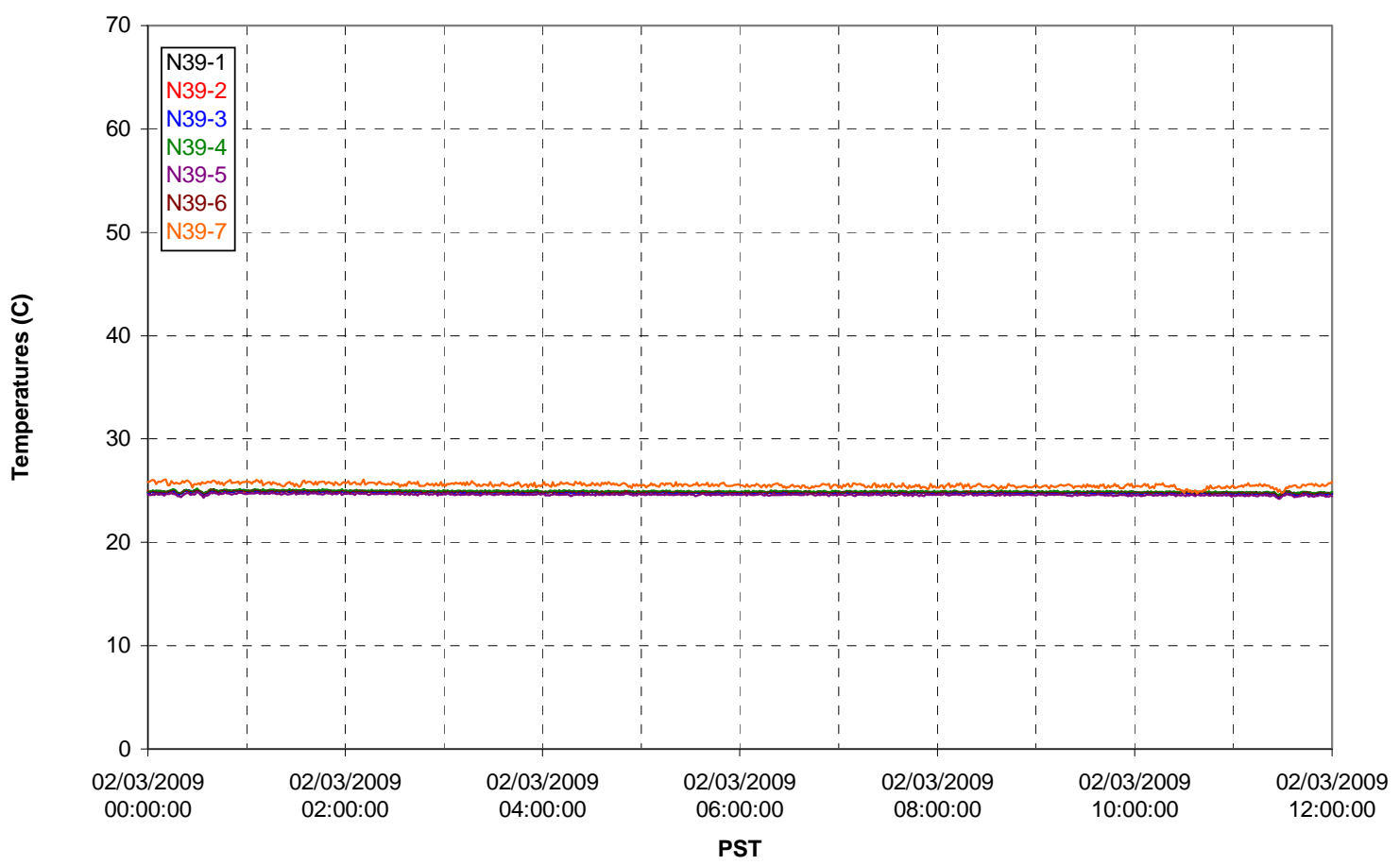

T02A temperatures

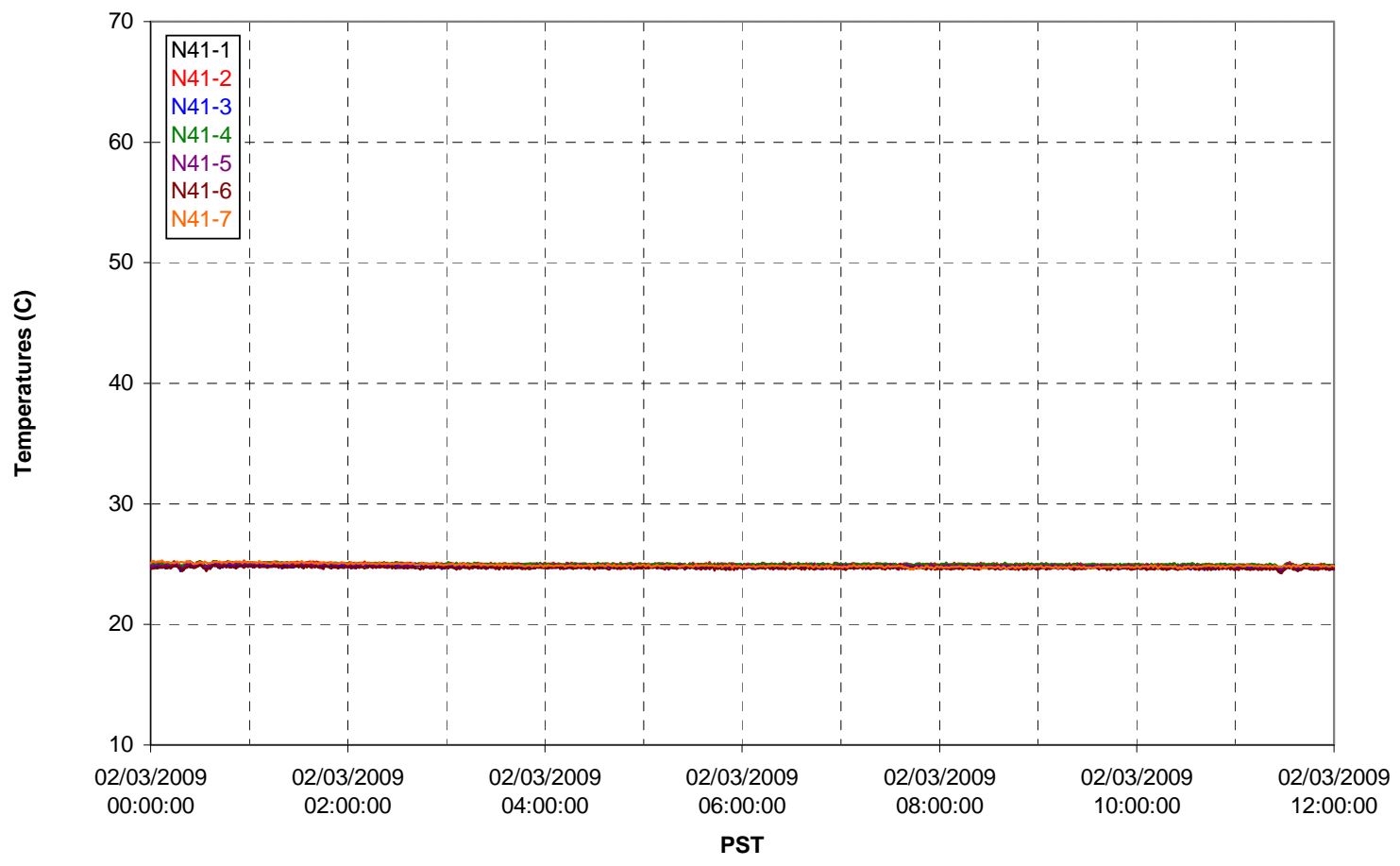

G.116 
T02A temperatures

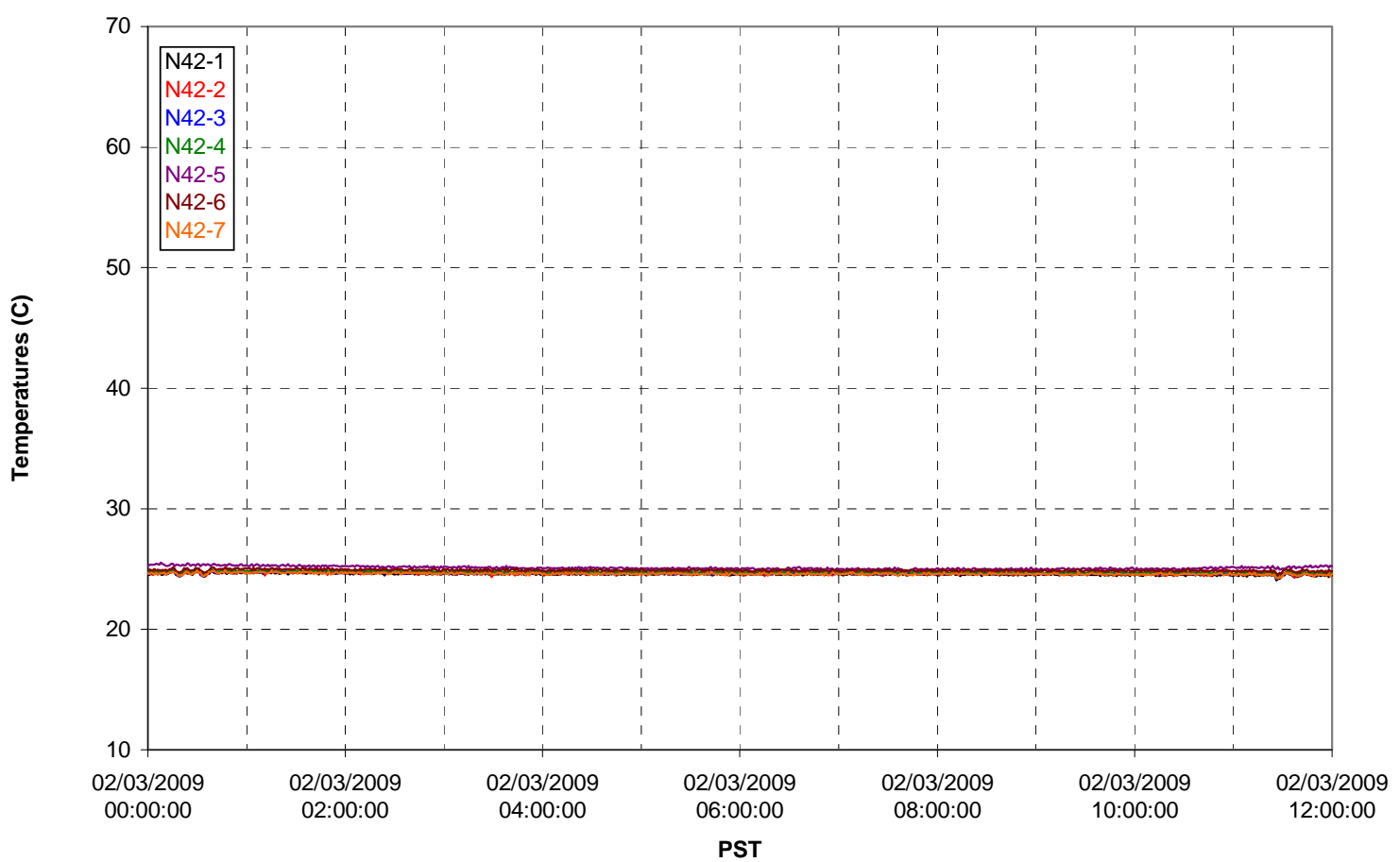

T02A temperatures

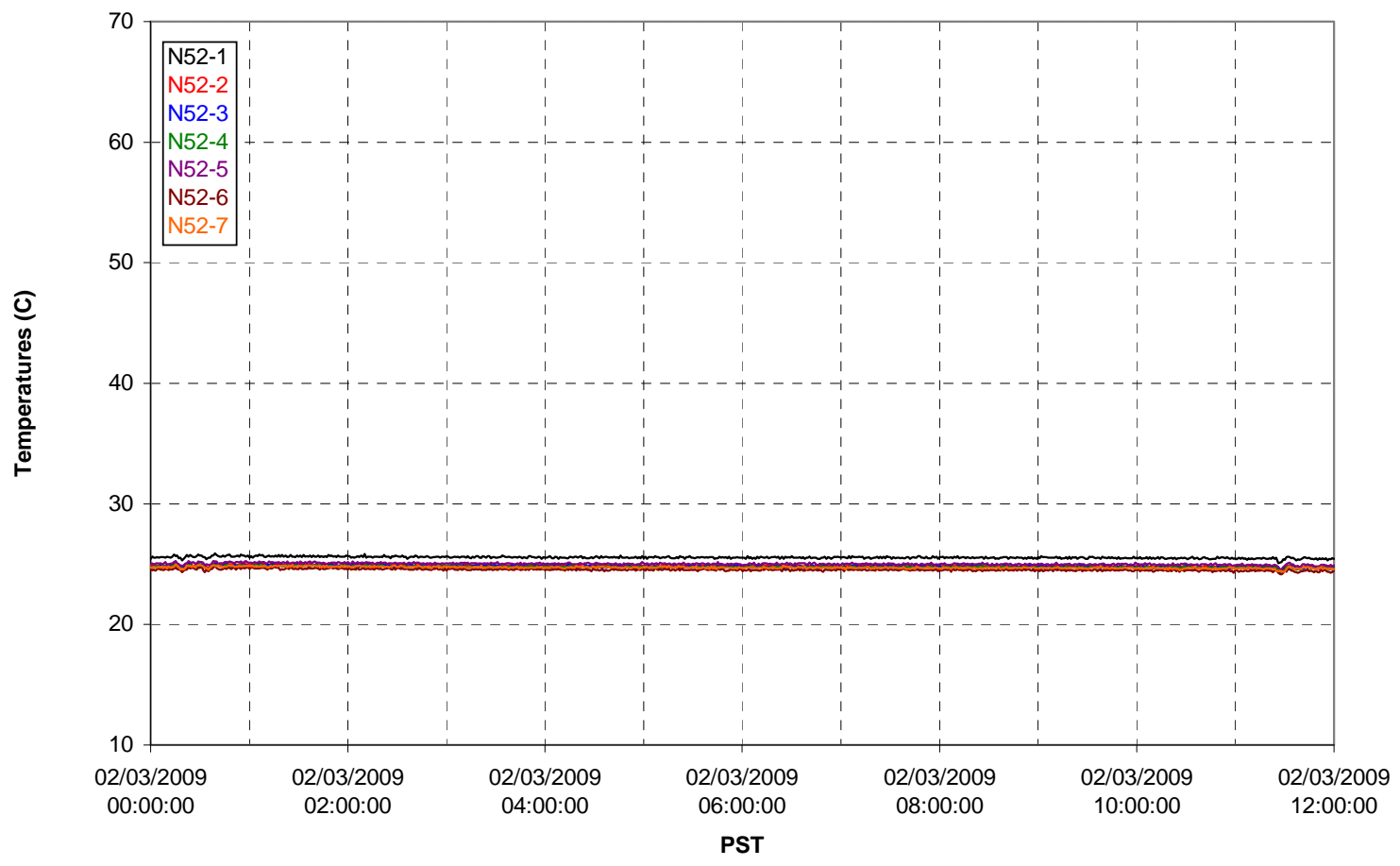

G.117 
T02A Heating and Cooling

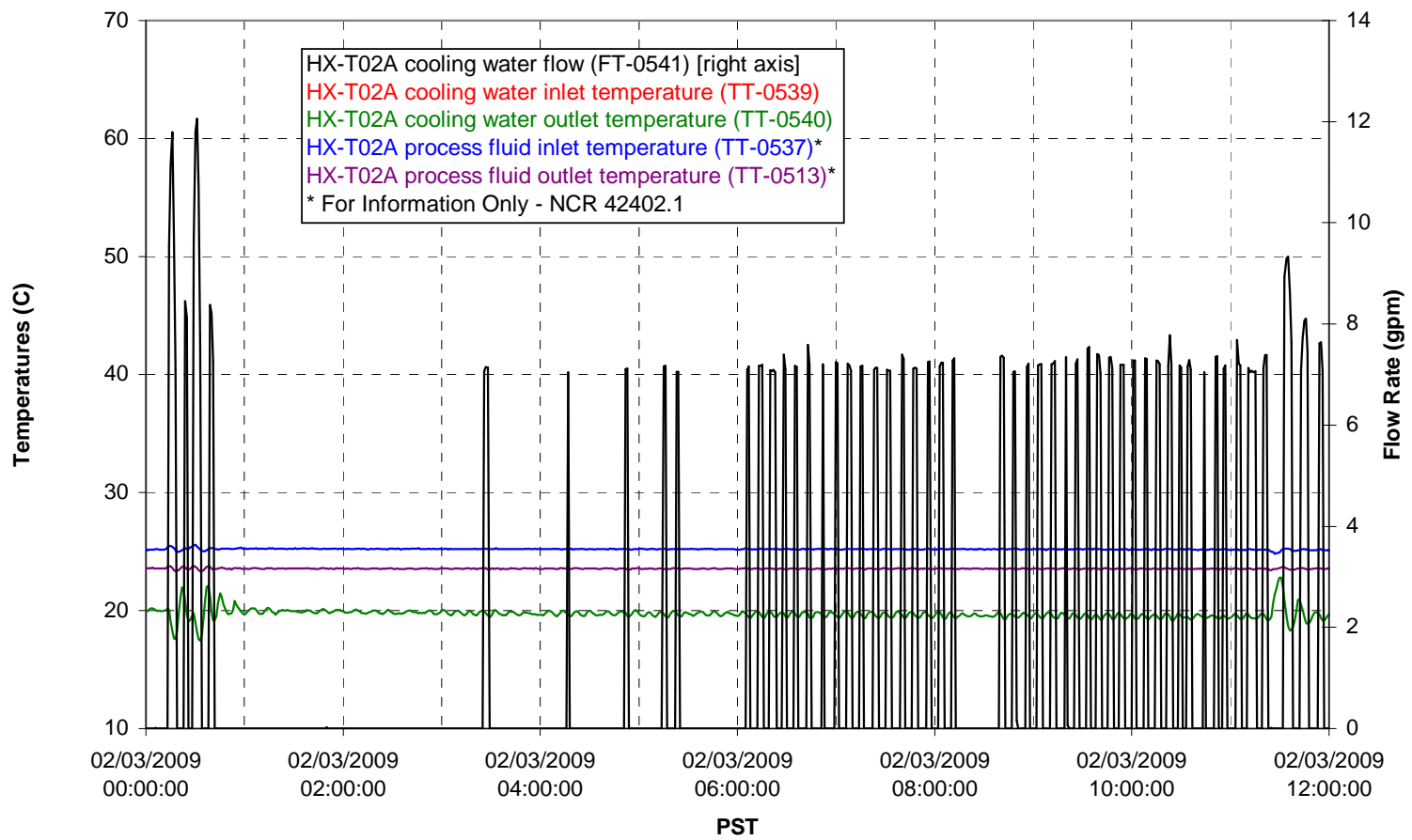

Pump Operation

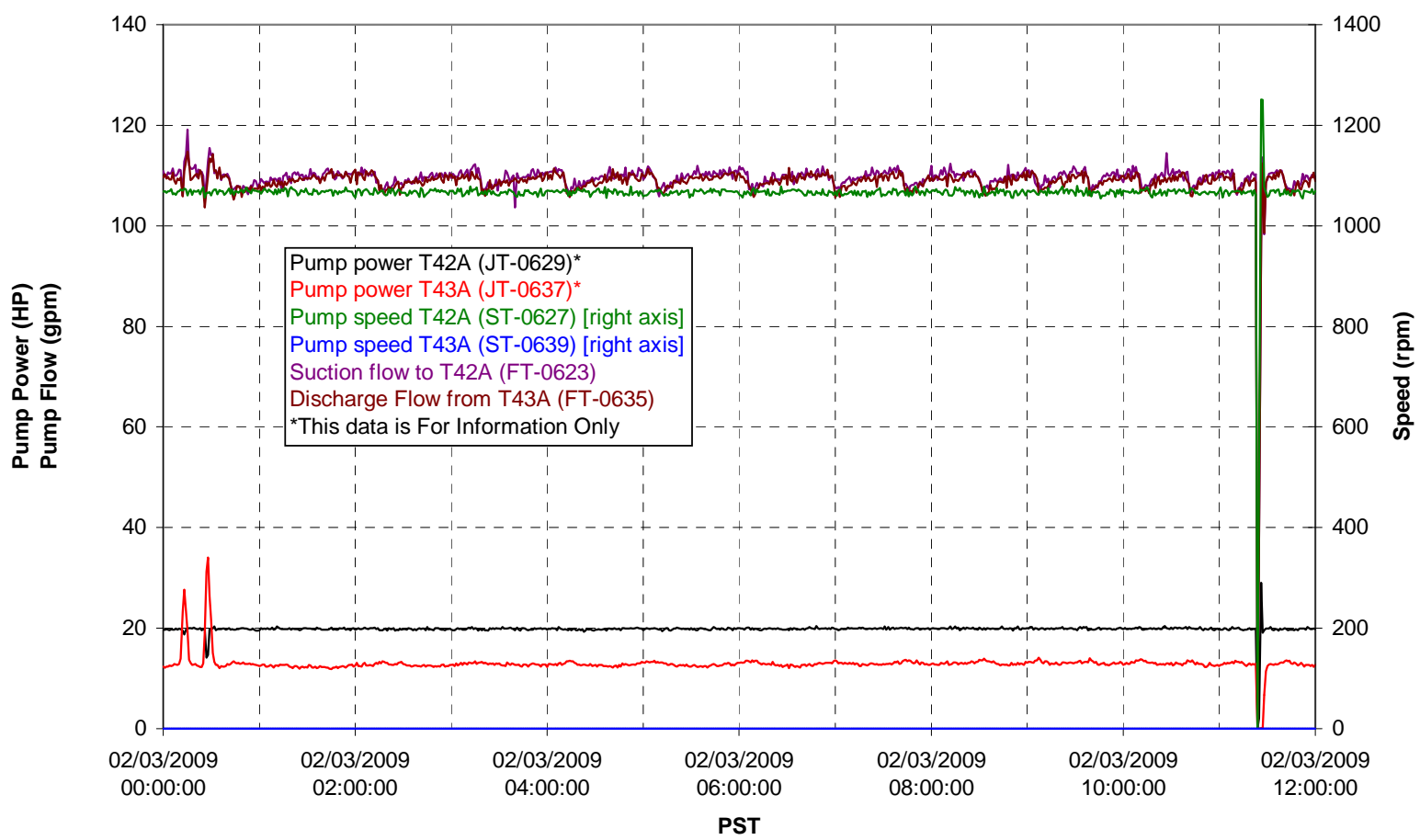


Pulsepot UFP-PP-T01A

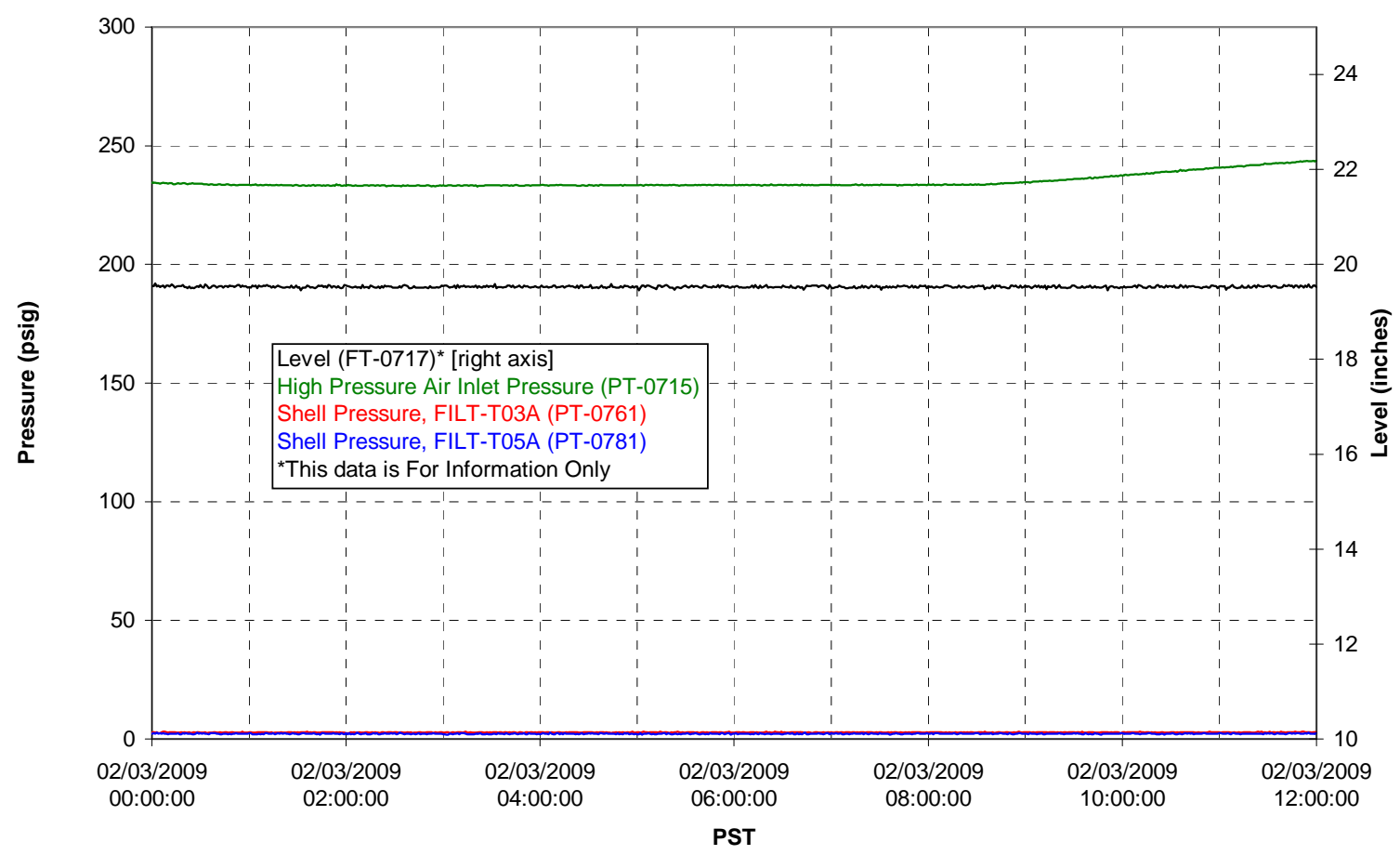

Pulsepot UFP-PP-T02A

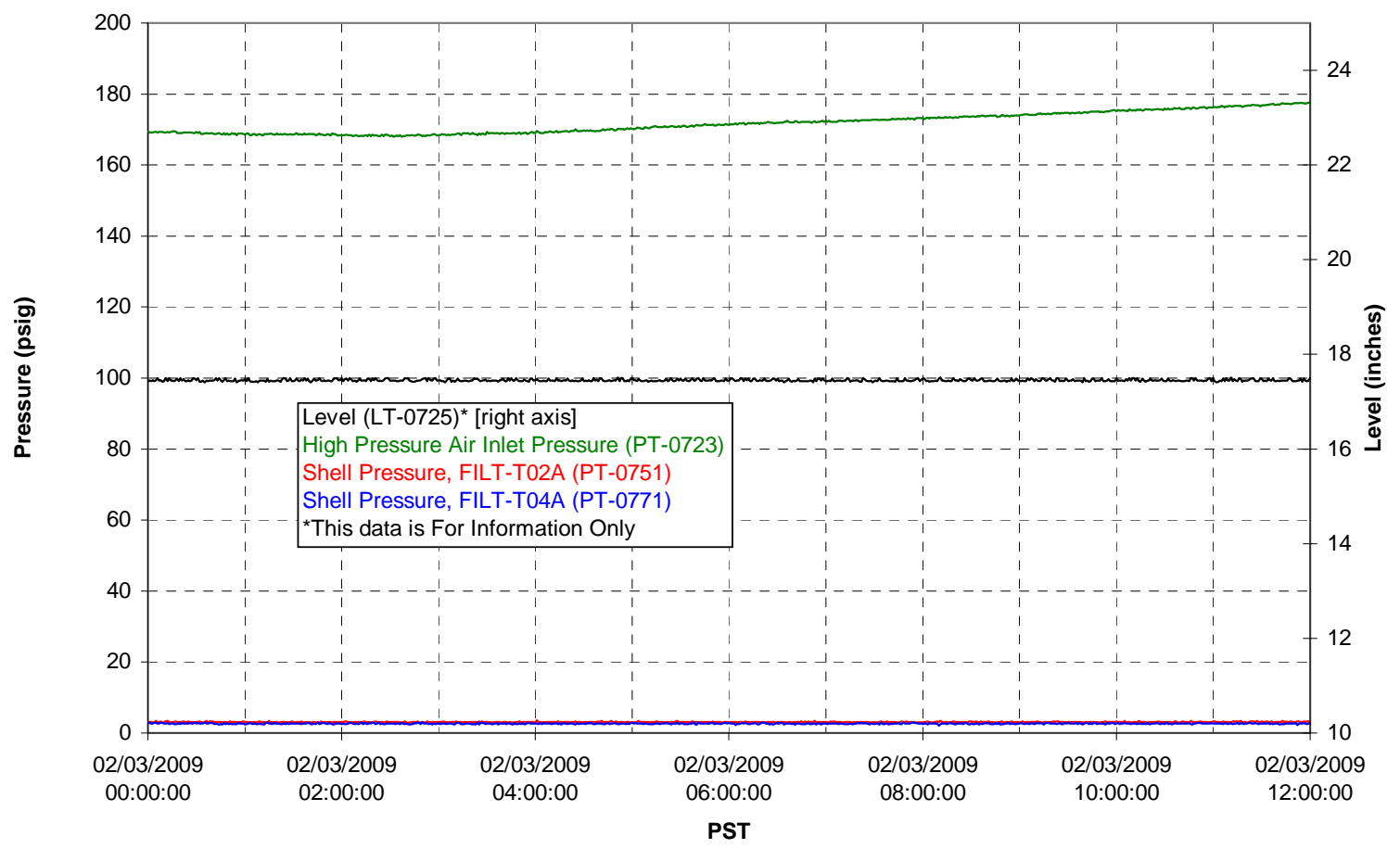

G.119 
Pulsepot UFP-PP-T03A

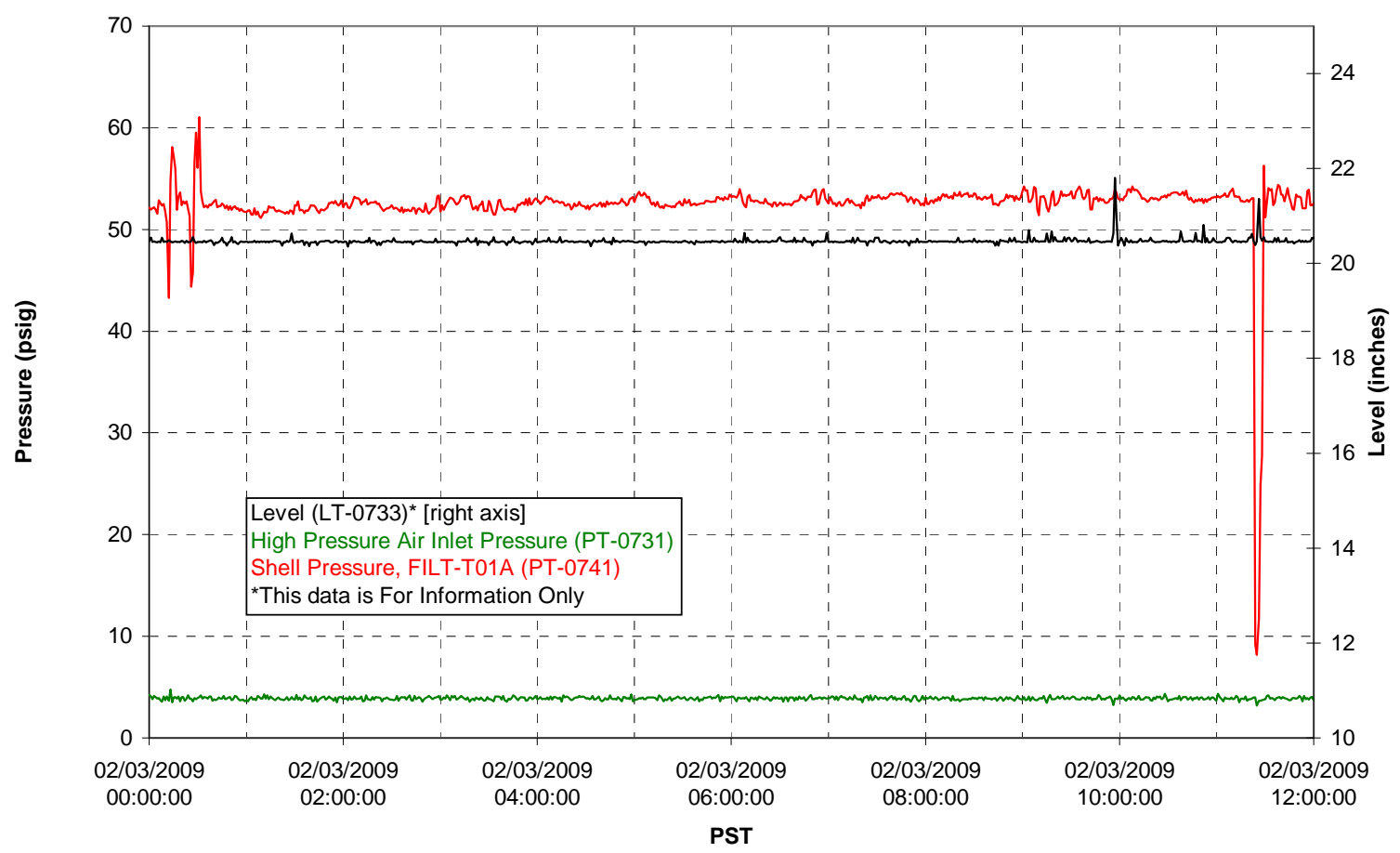

Pulsepot Levels

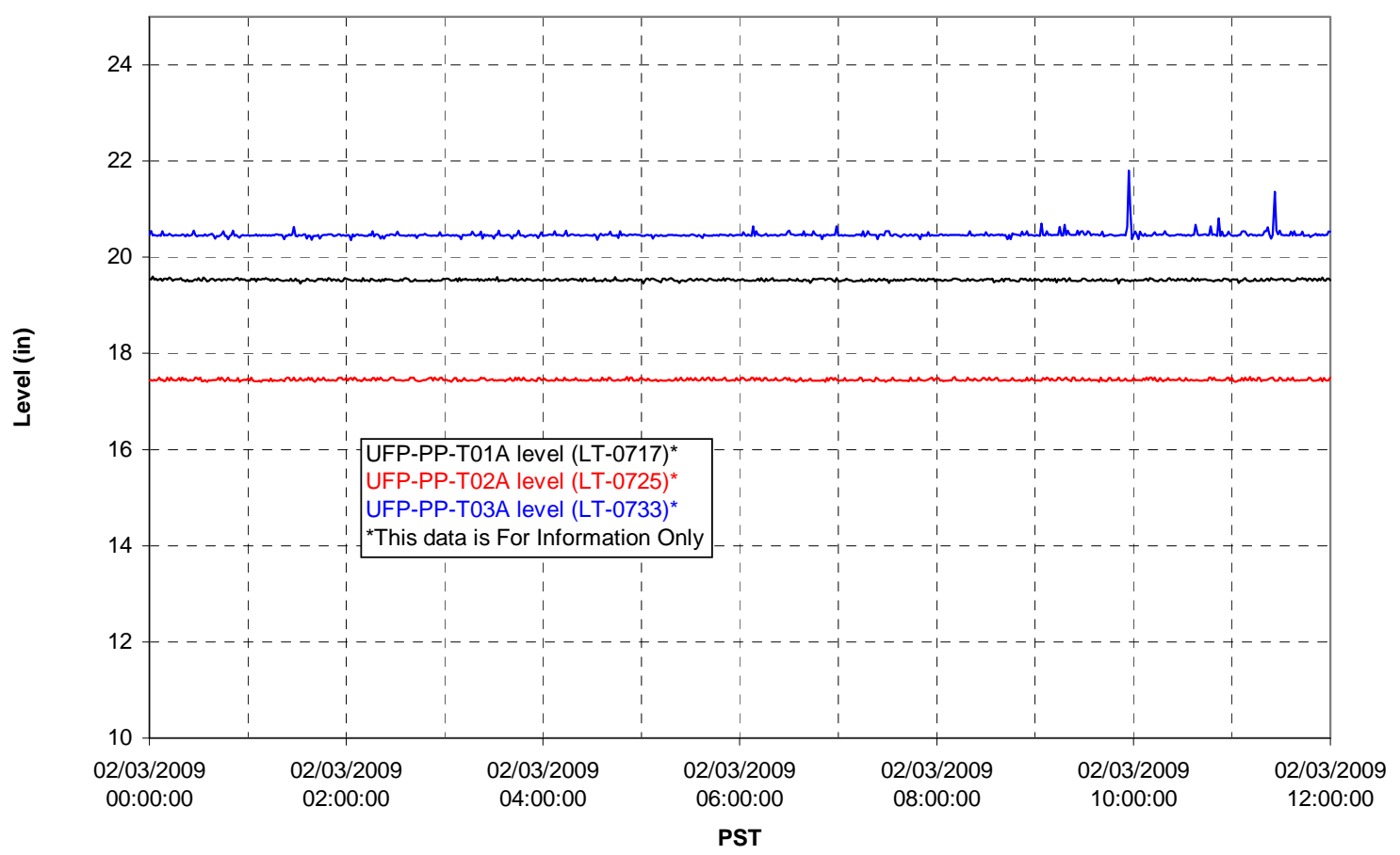

G. 120 
Filter UFP-FILT-T01A

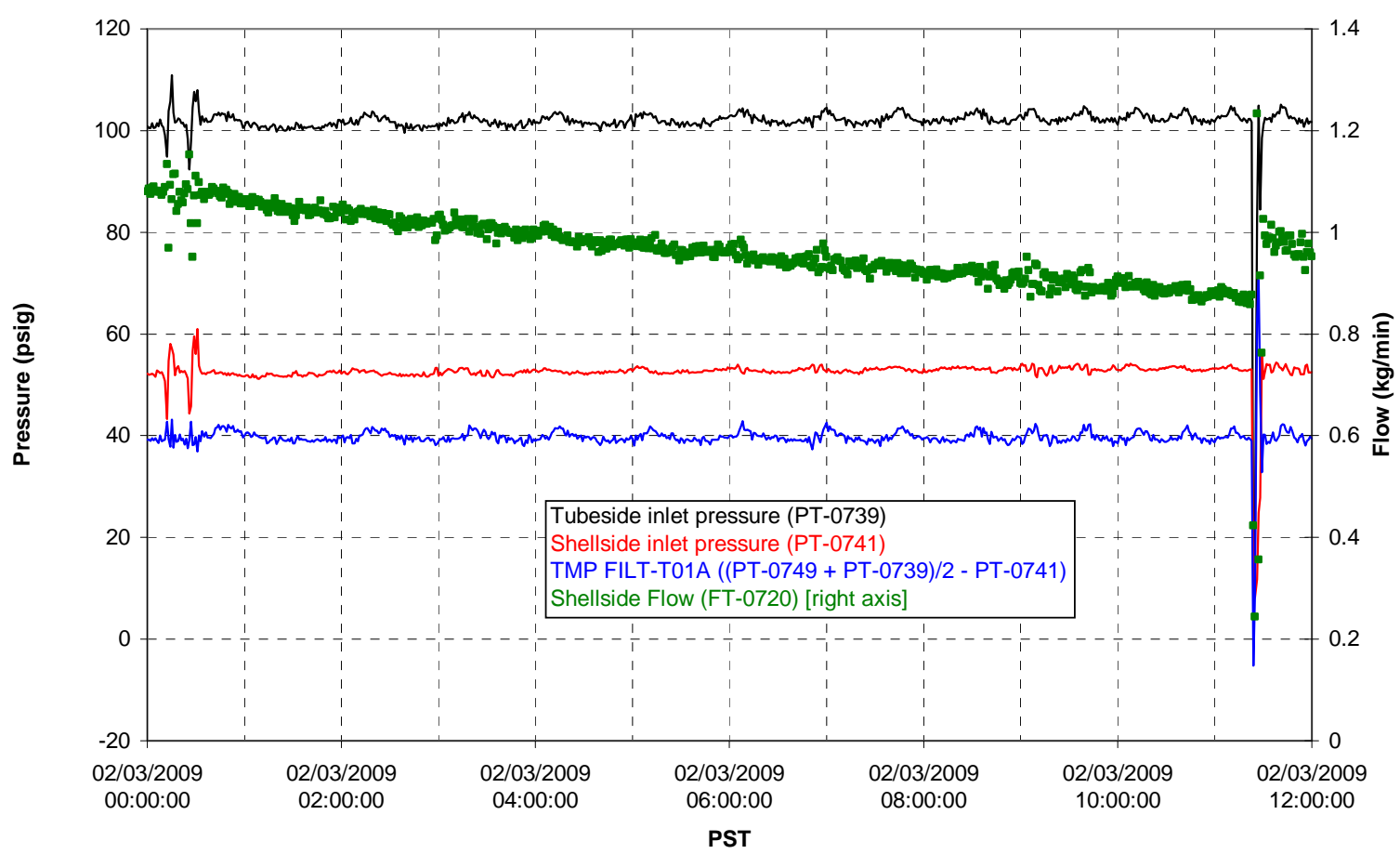

Filter UFP-FILT-T02A

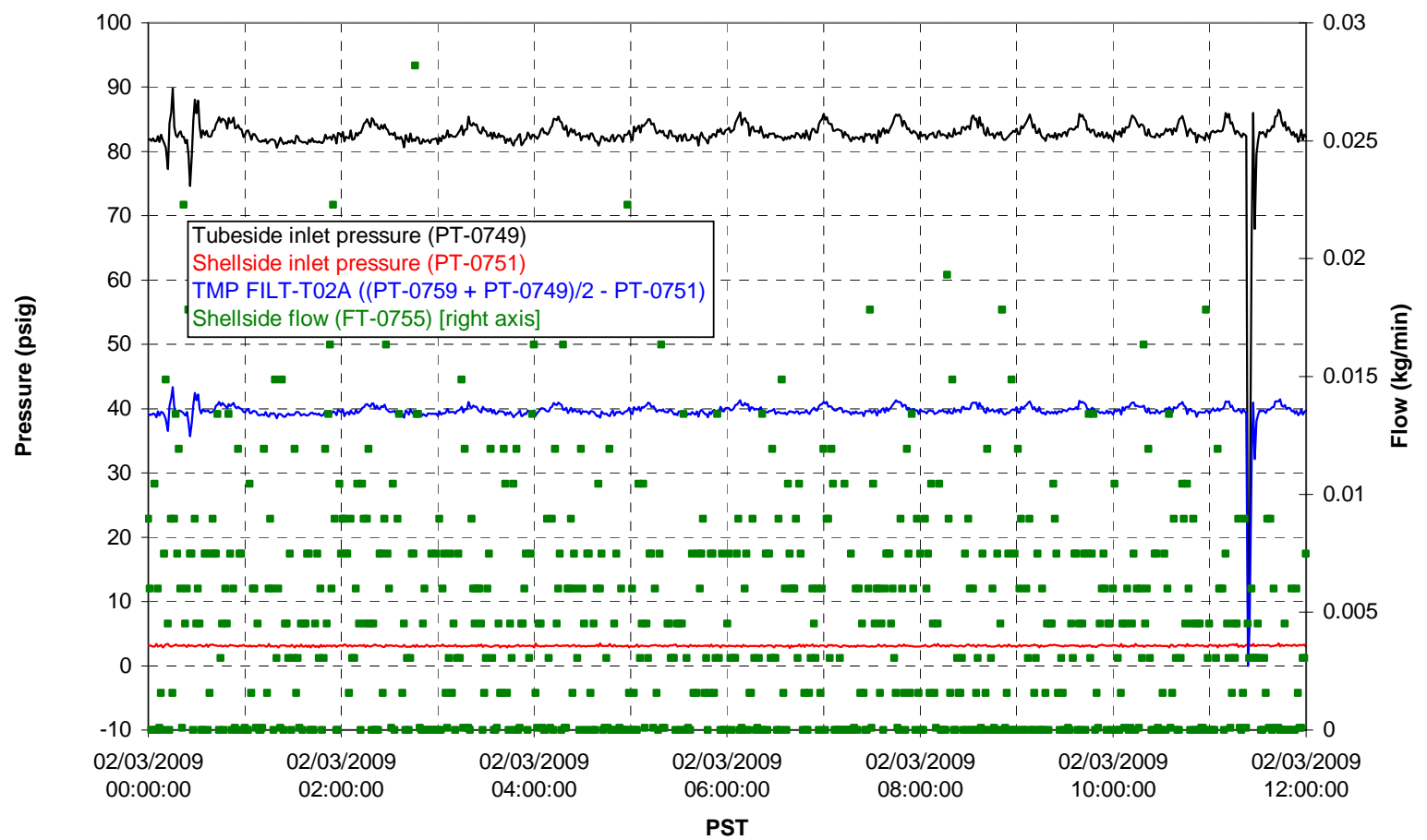


Filter UFP-FILT-T03A

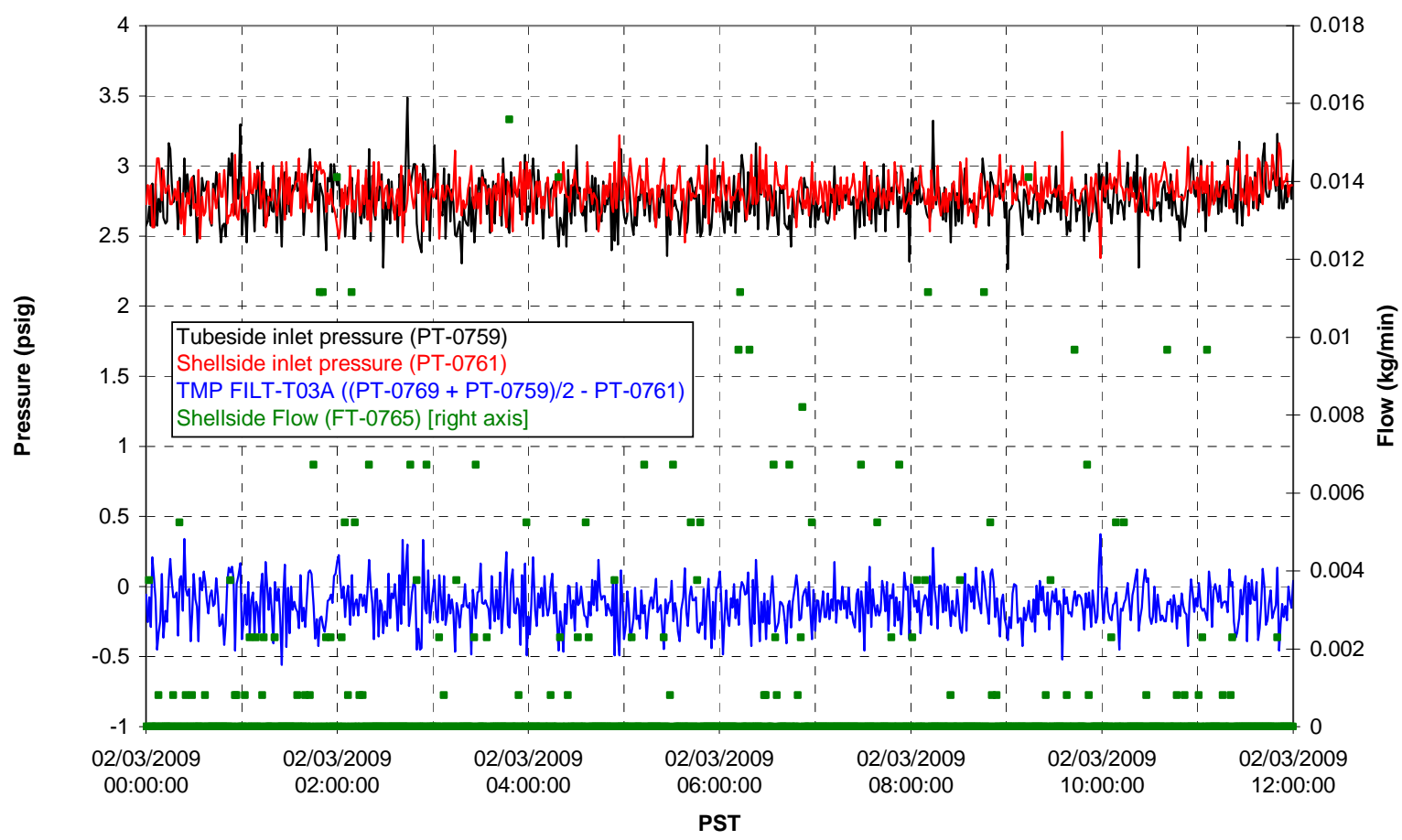

Filter UFP-FILT-T04A

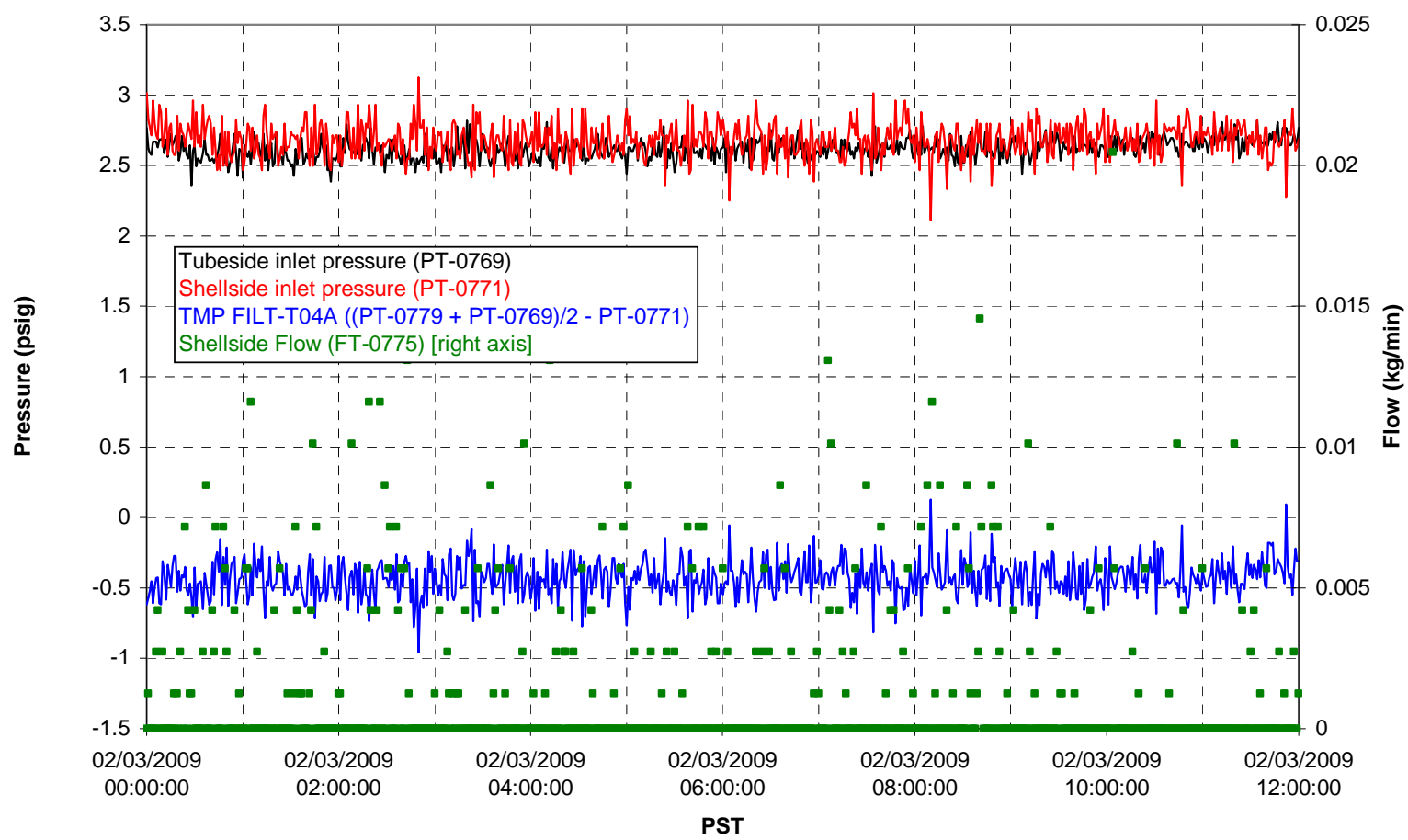

G.122 
Filter UFP-FILT-T05A

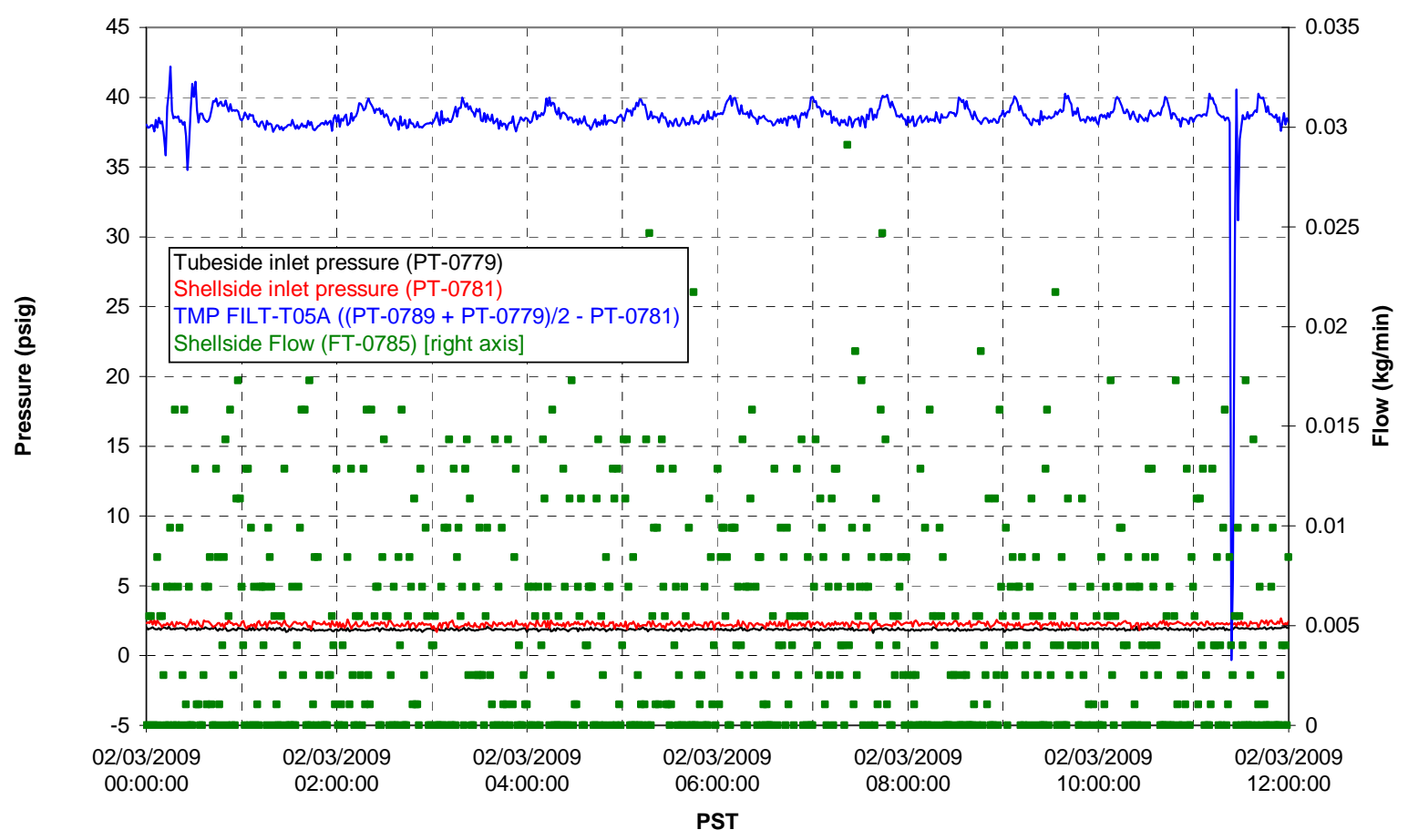

Chemical Flow

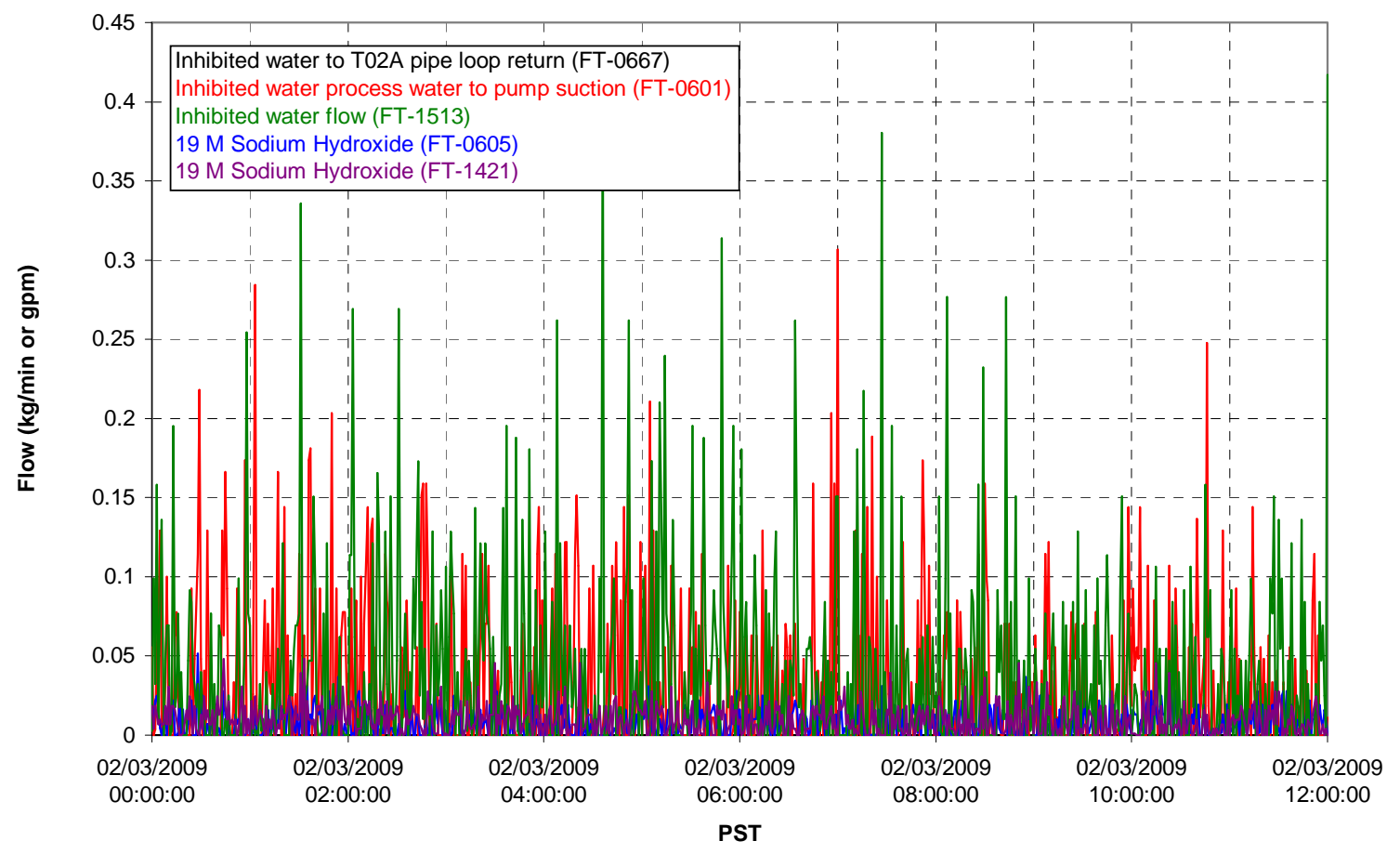

G.123 
Chemical Flow

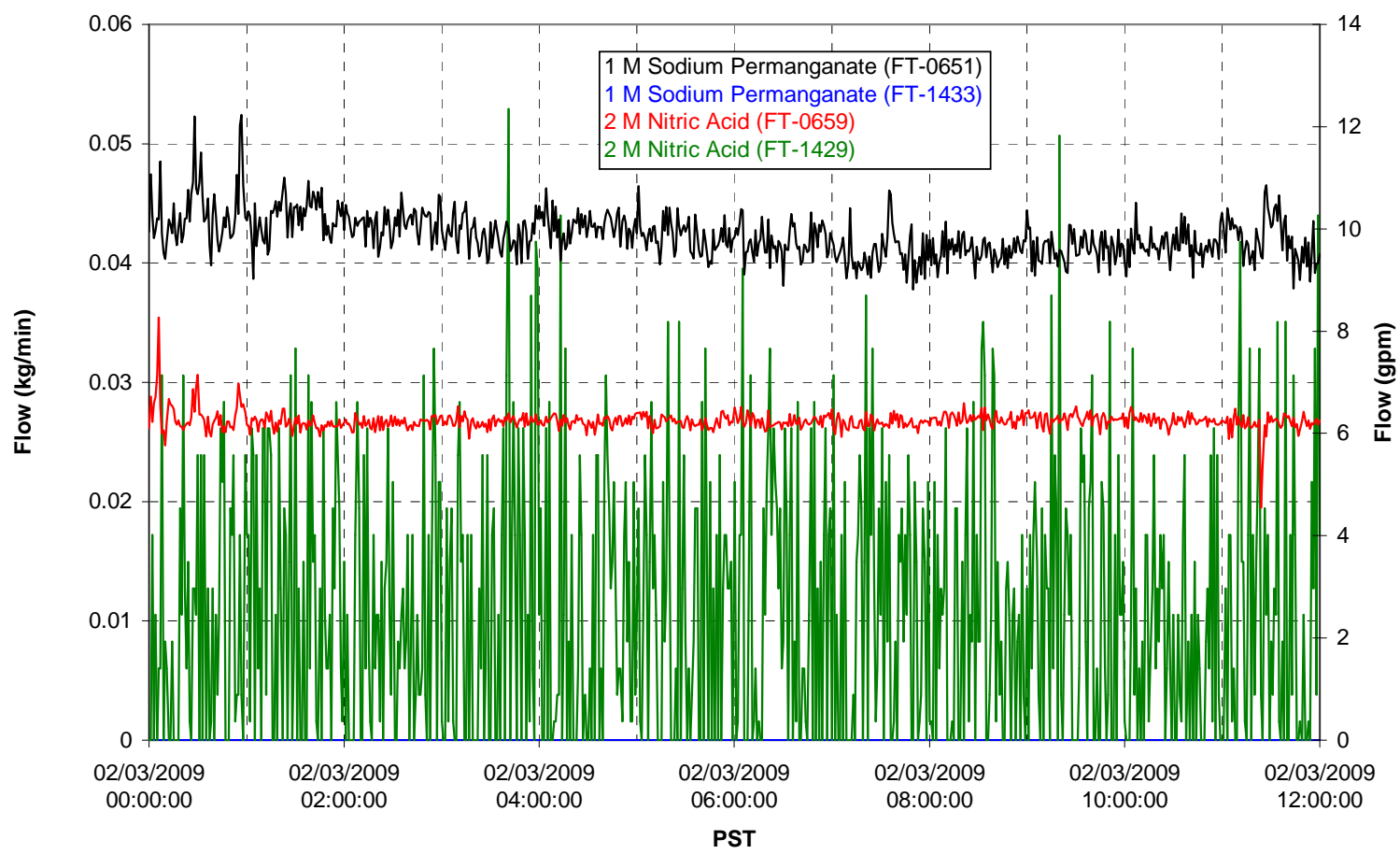

Air Flows

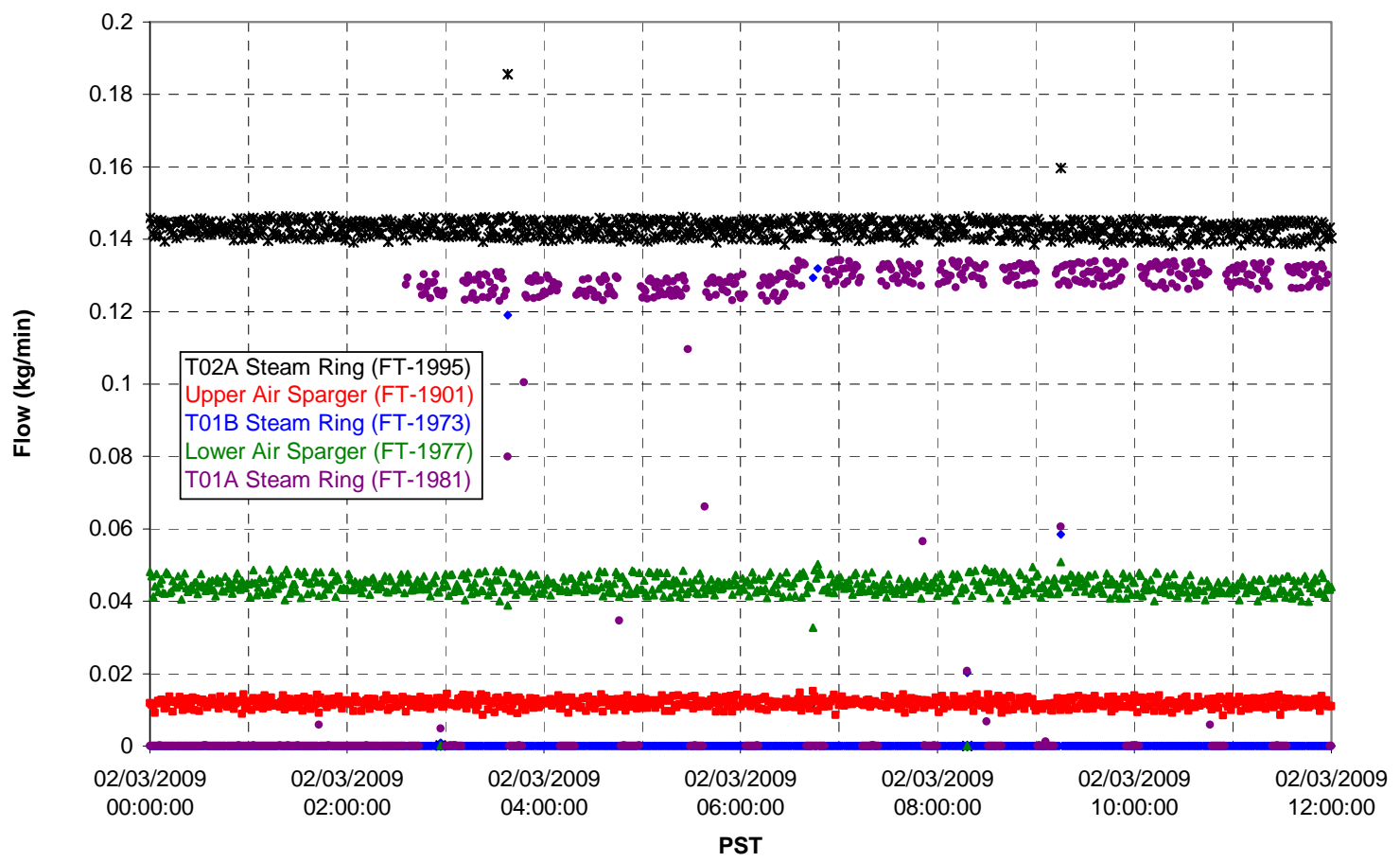


T02A Steam

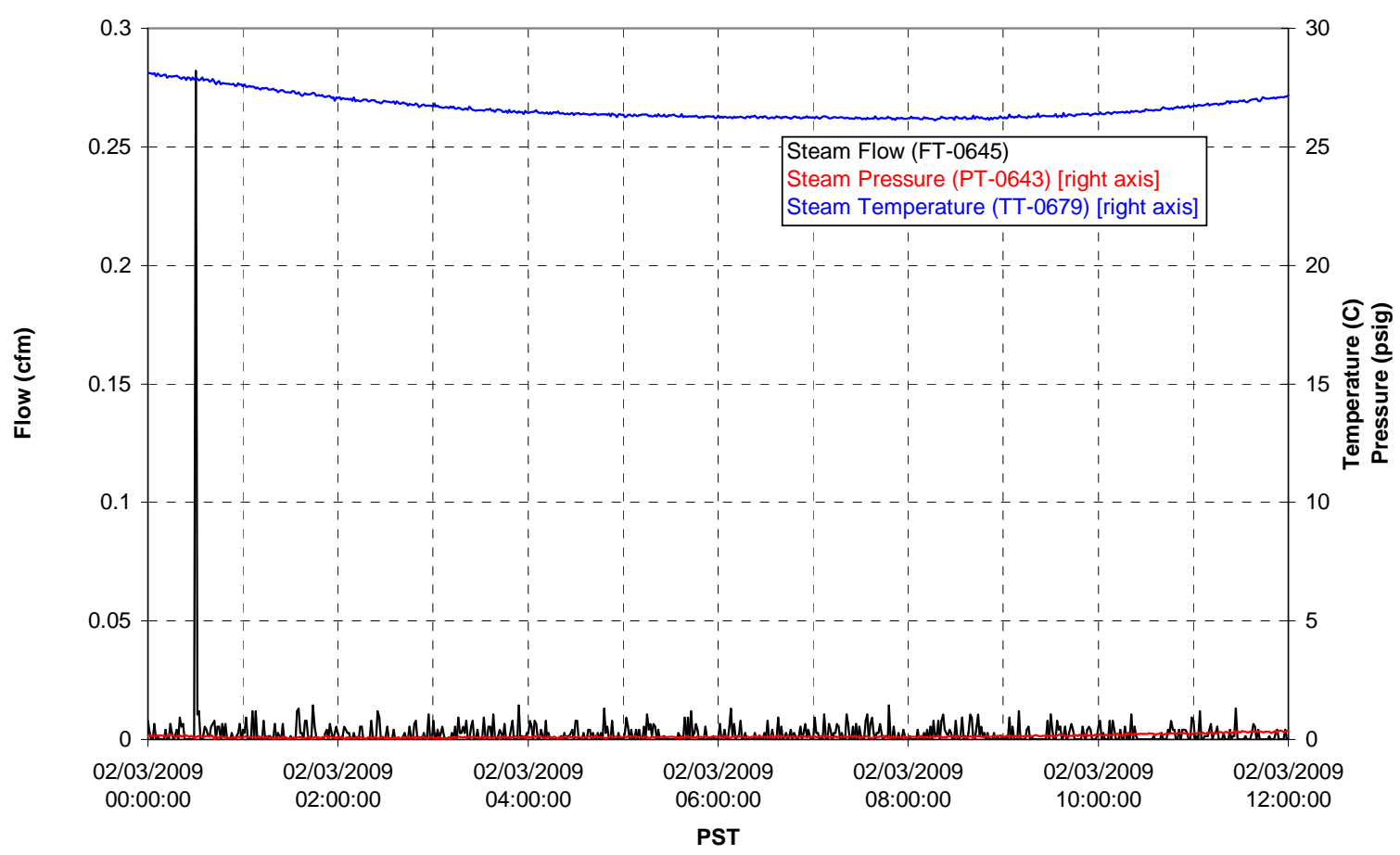

T01A Steam

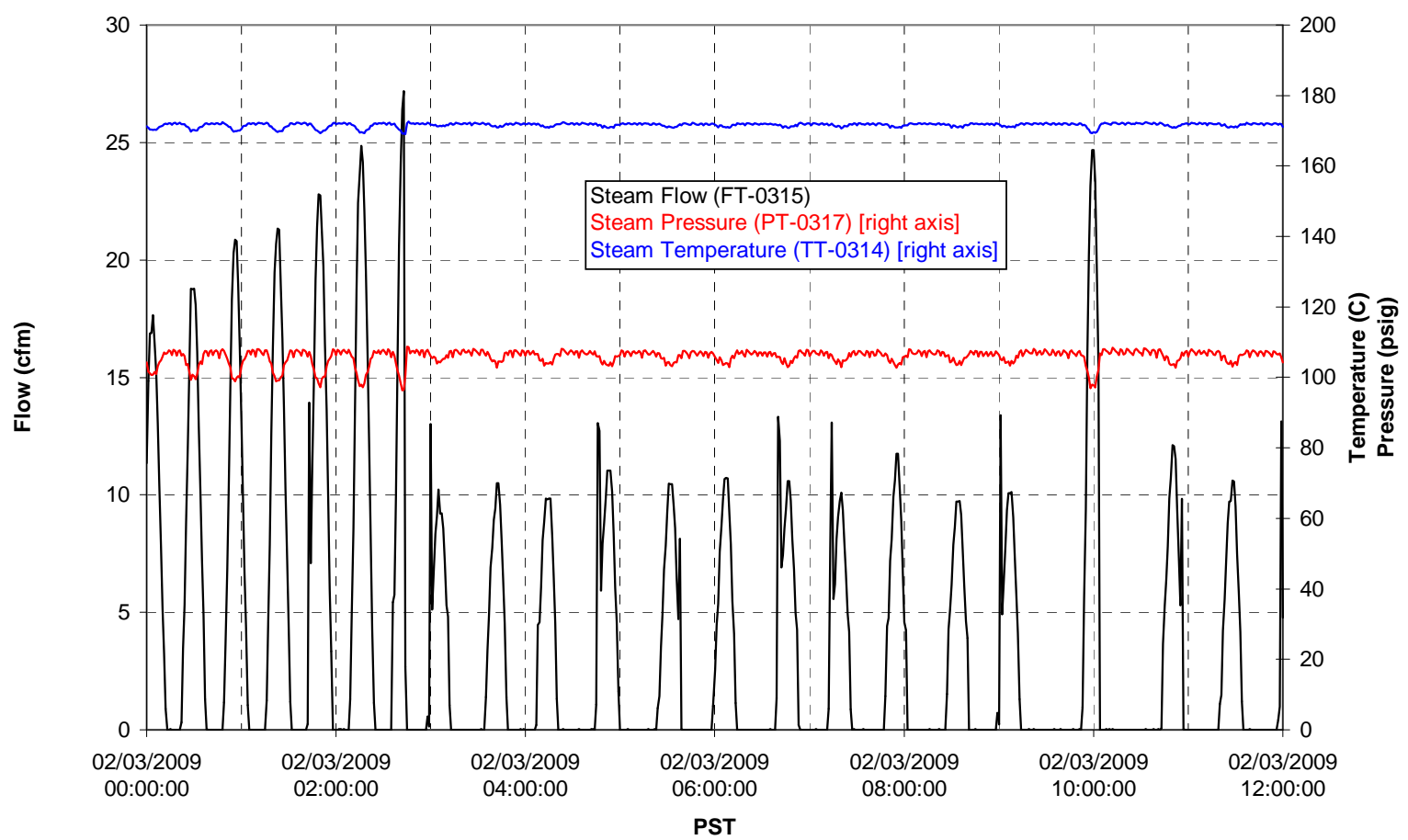

G. 125 
T01B Steam

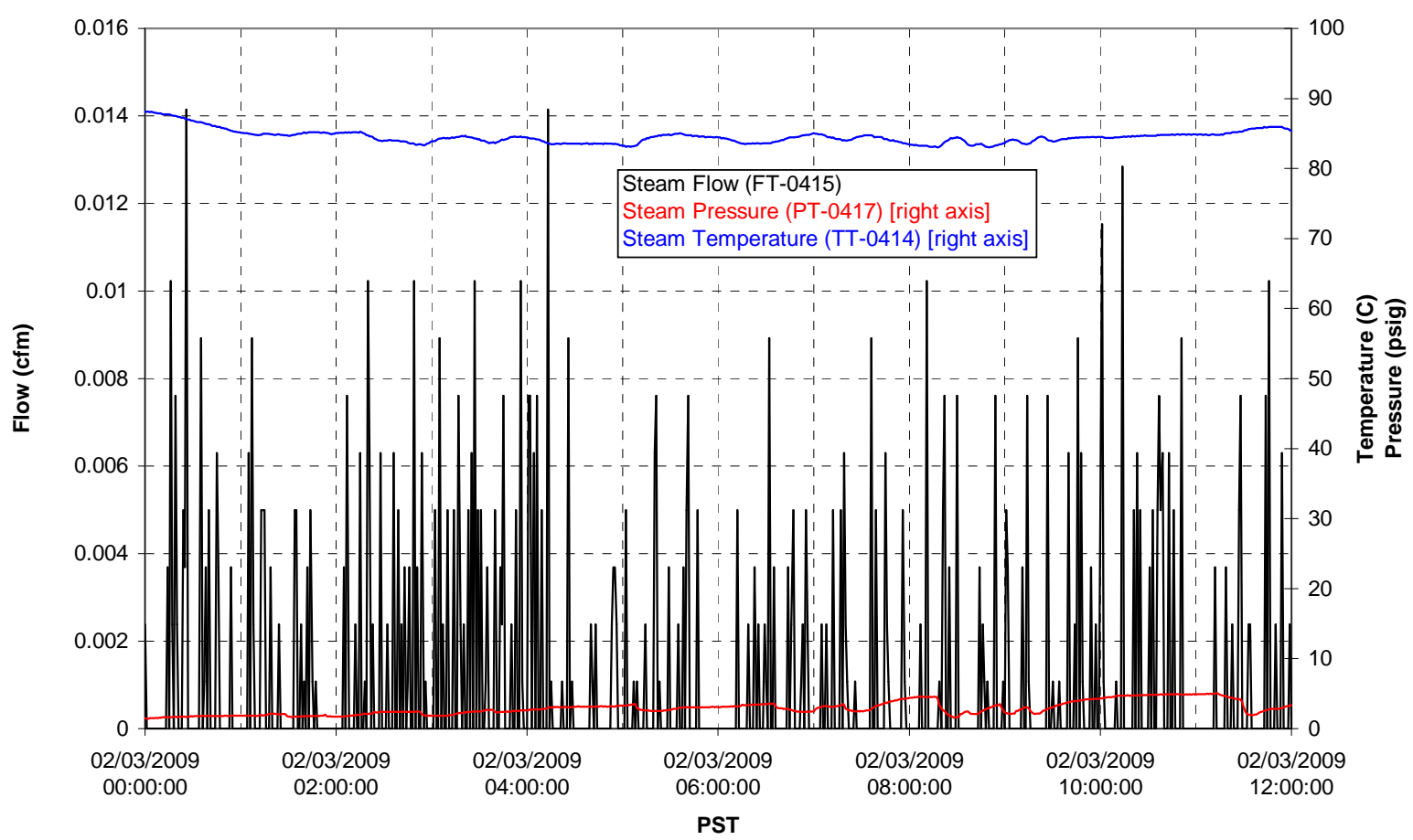




\section{Integrated Test A Data Plots 02/03/09 12:00 - 02/04/09 00:00}


T01A level

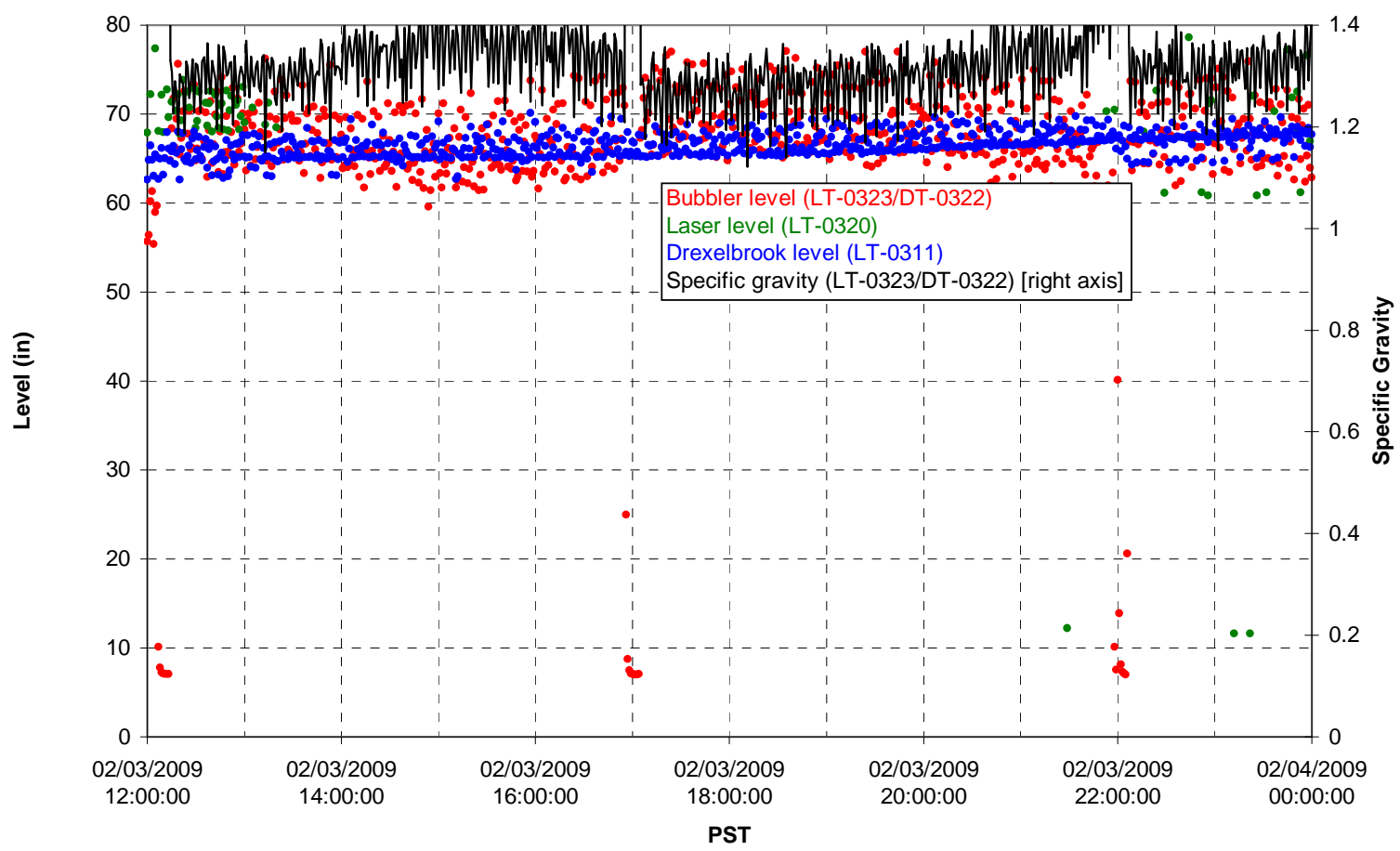

T01A temperatures

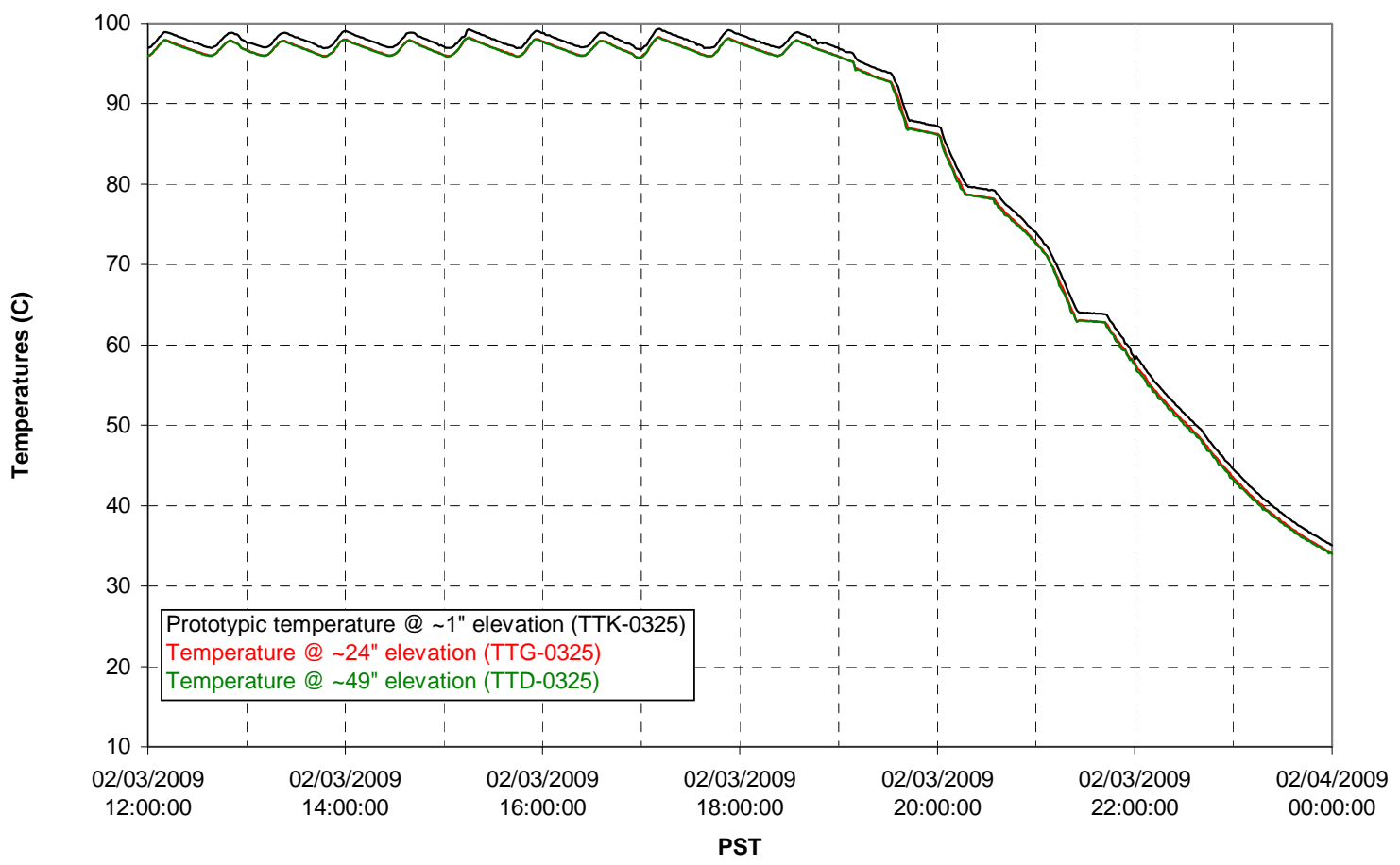


T01B level

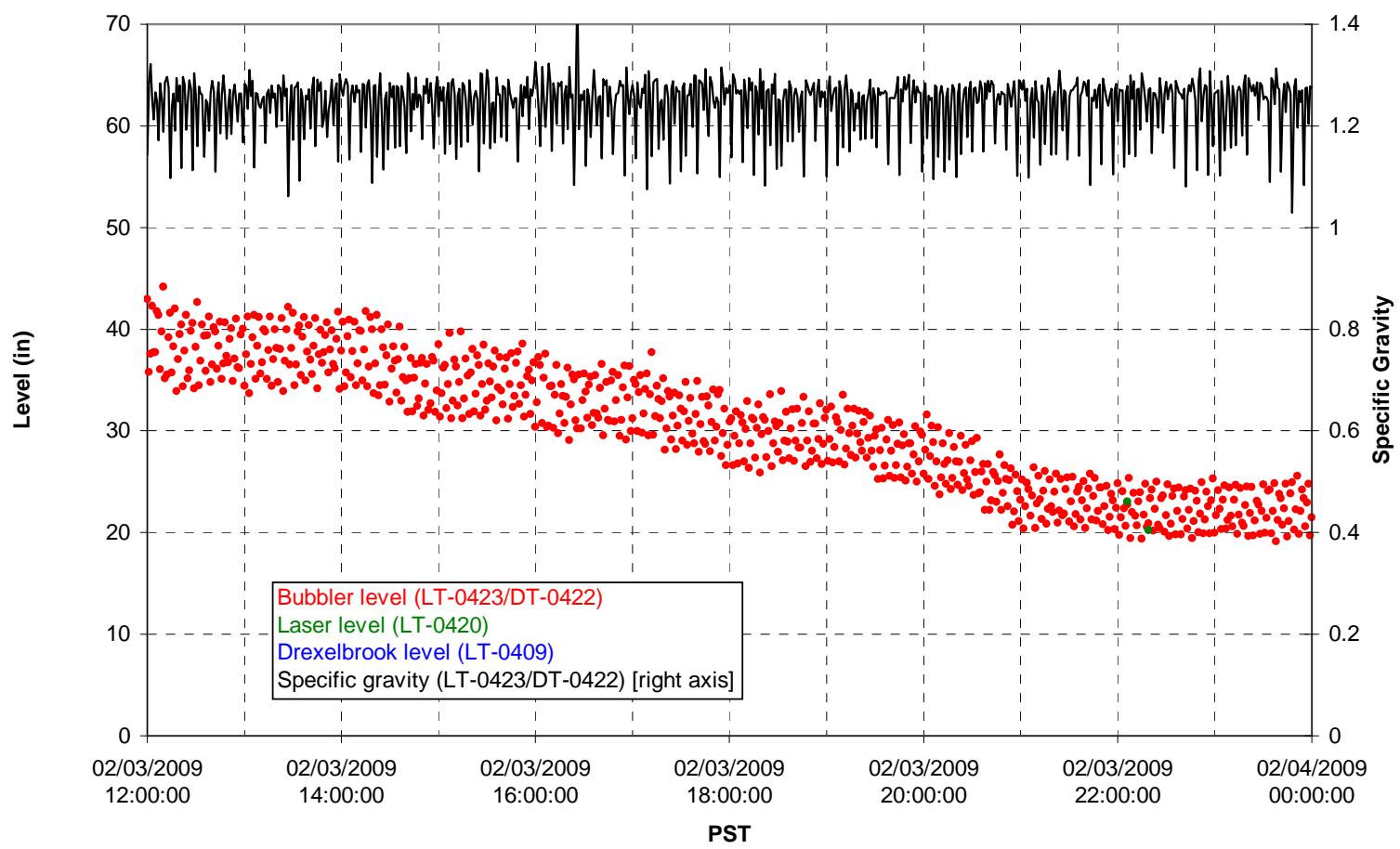

T01B temperatures

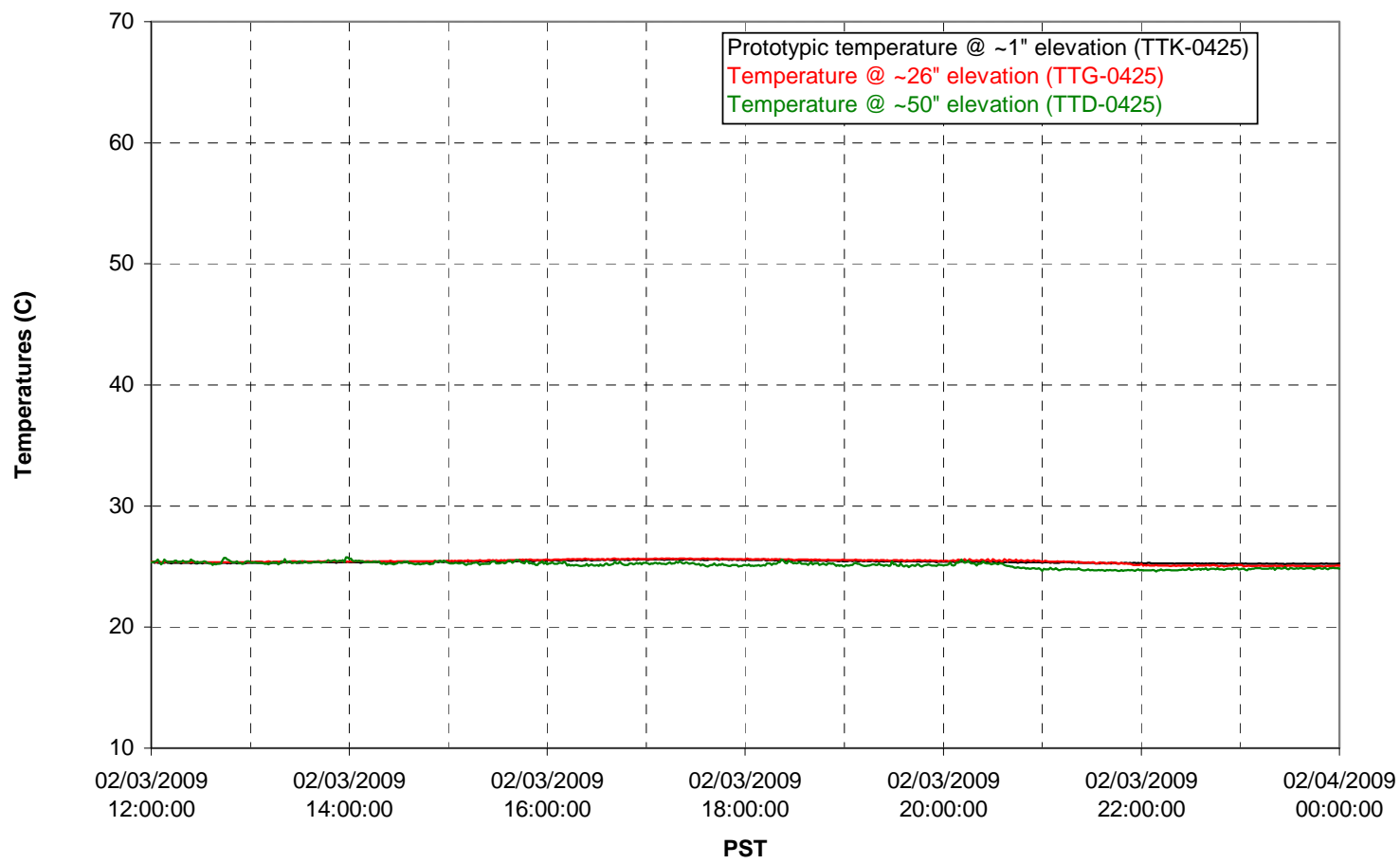

G.129 
T02A level

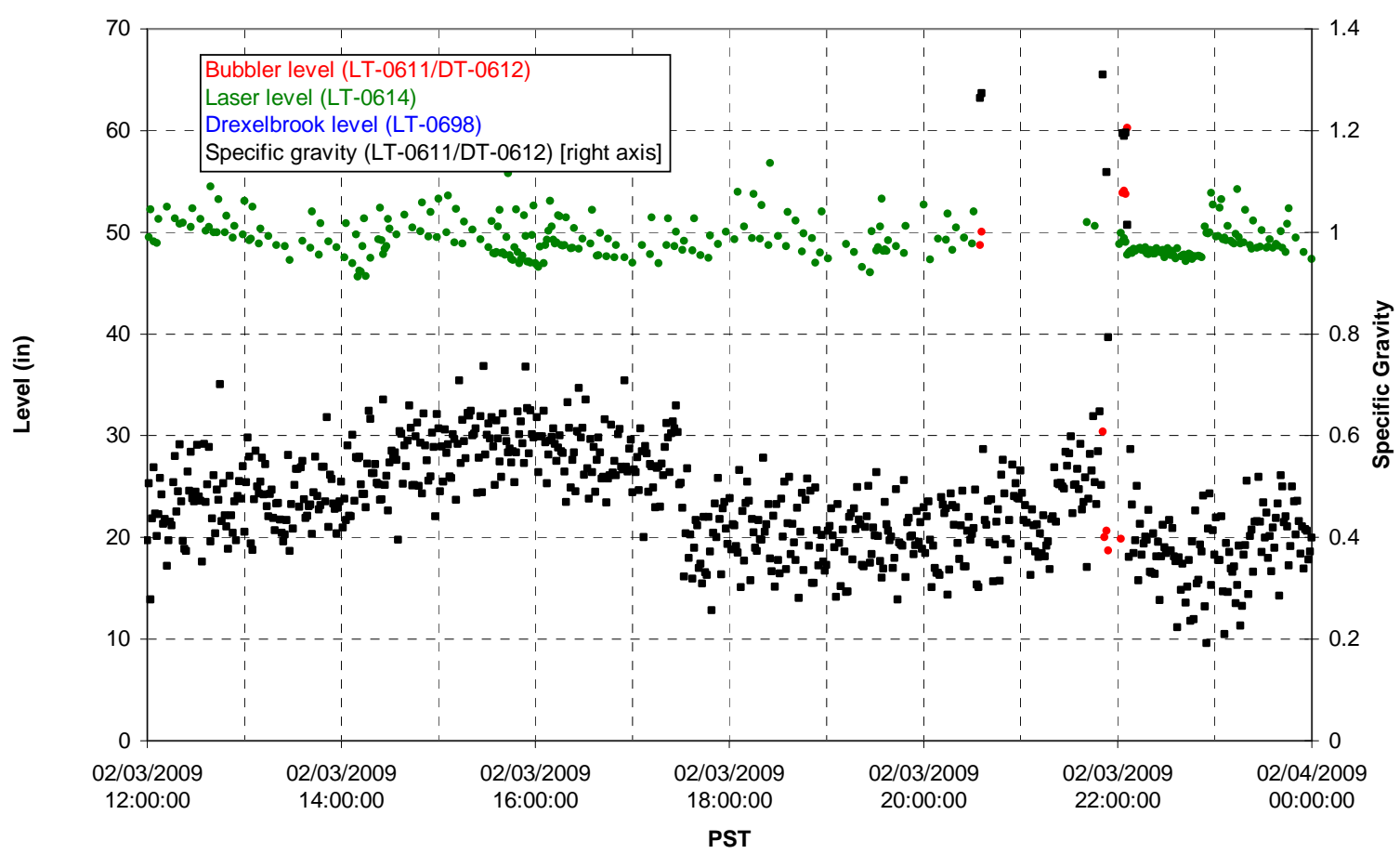

T02A temperatures

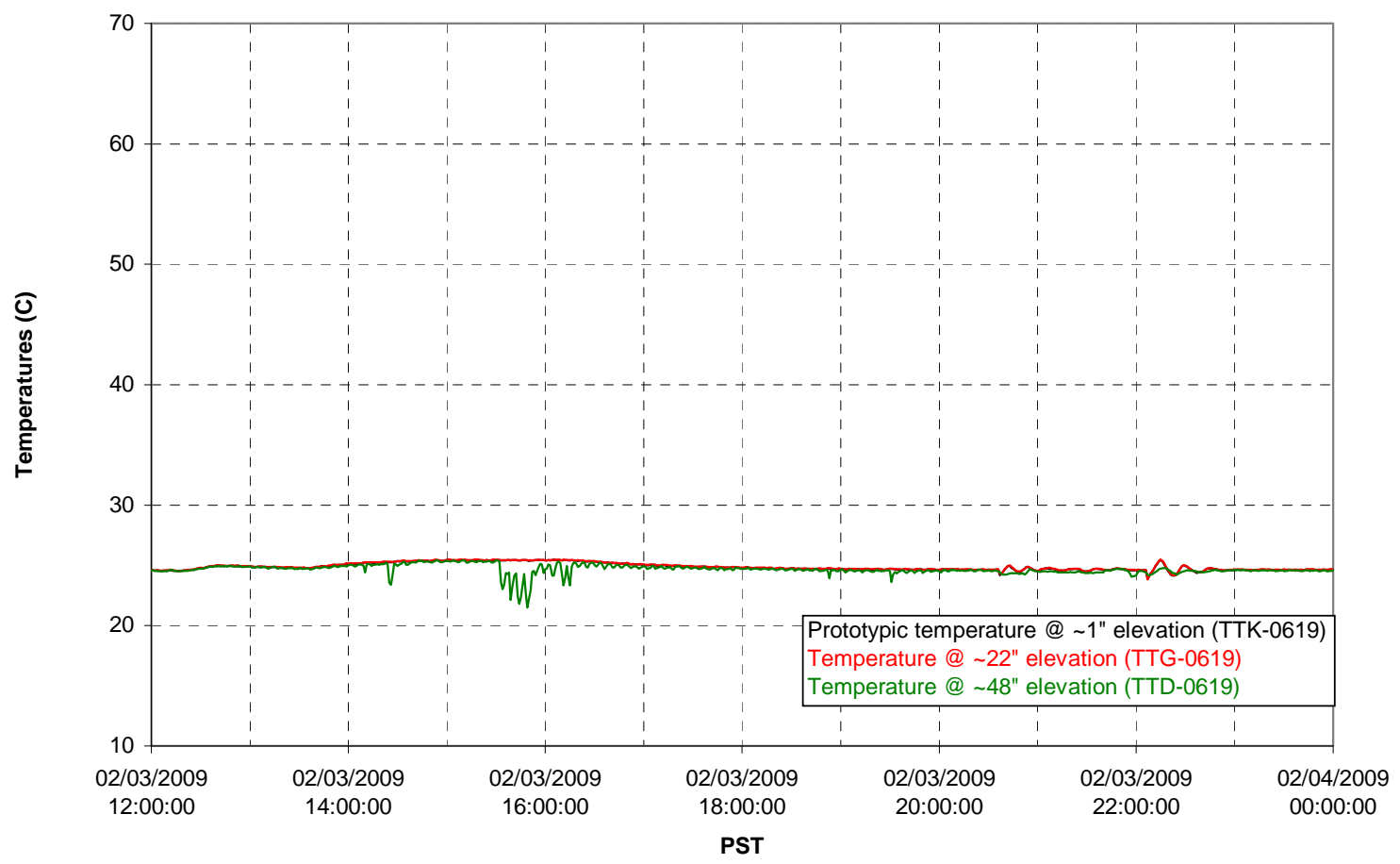

G.130 
T02A and filter loop temperatures

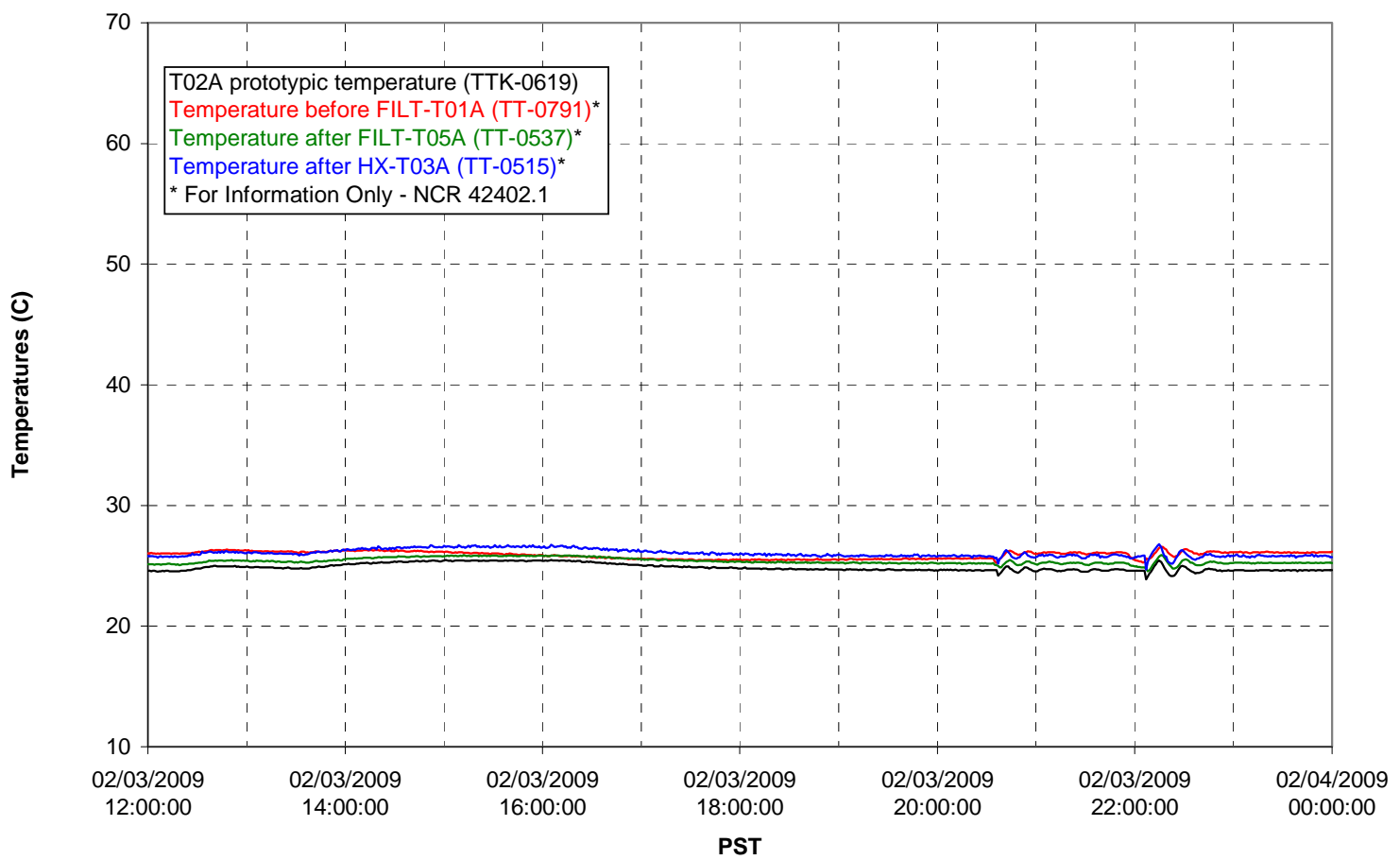

Pump Pressures and Flow

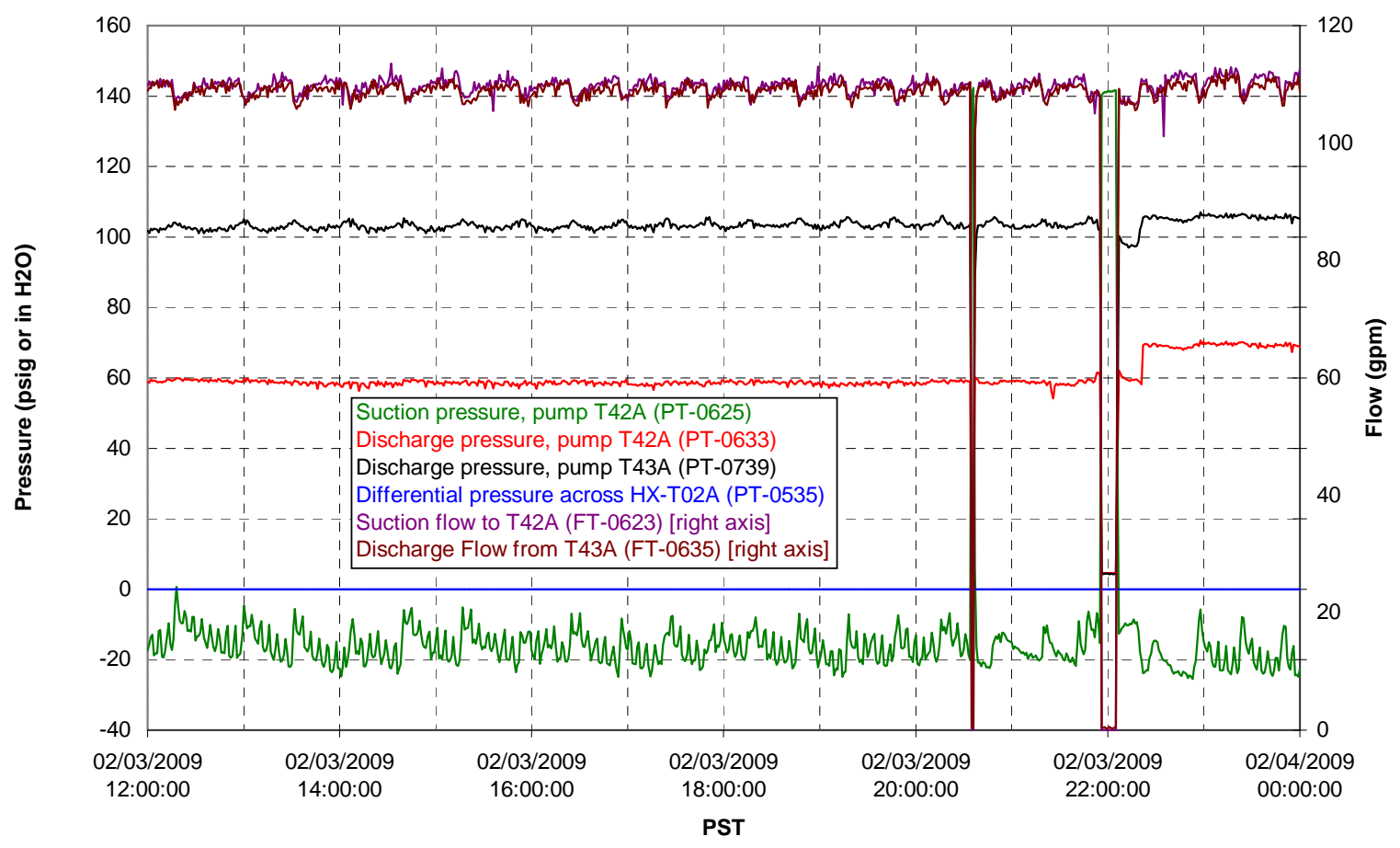




\section{Axial pressure drop}

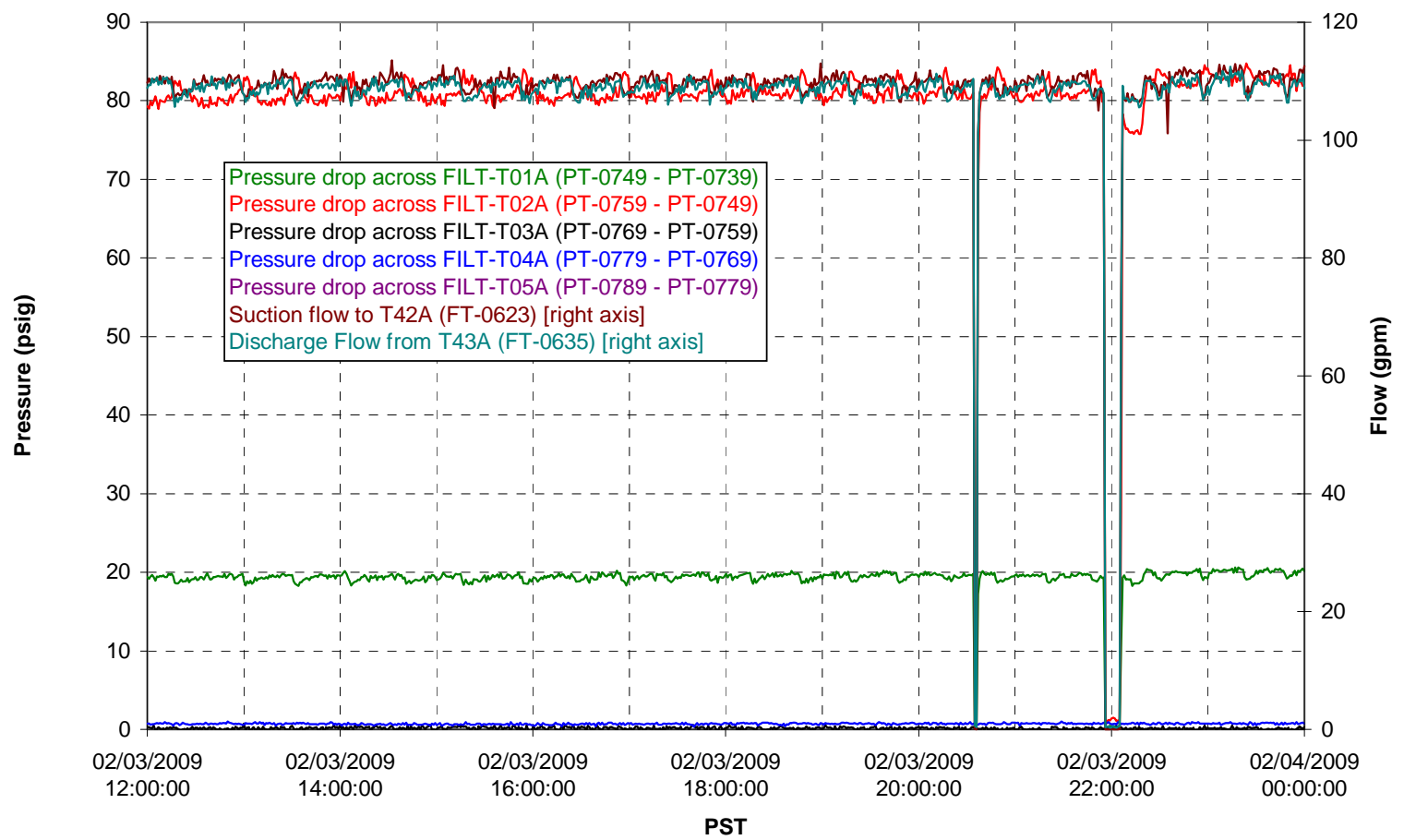

Permeate flow rates

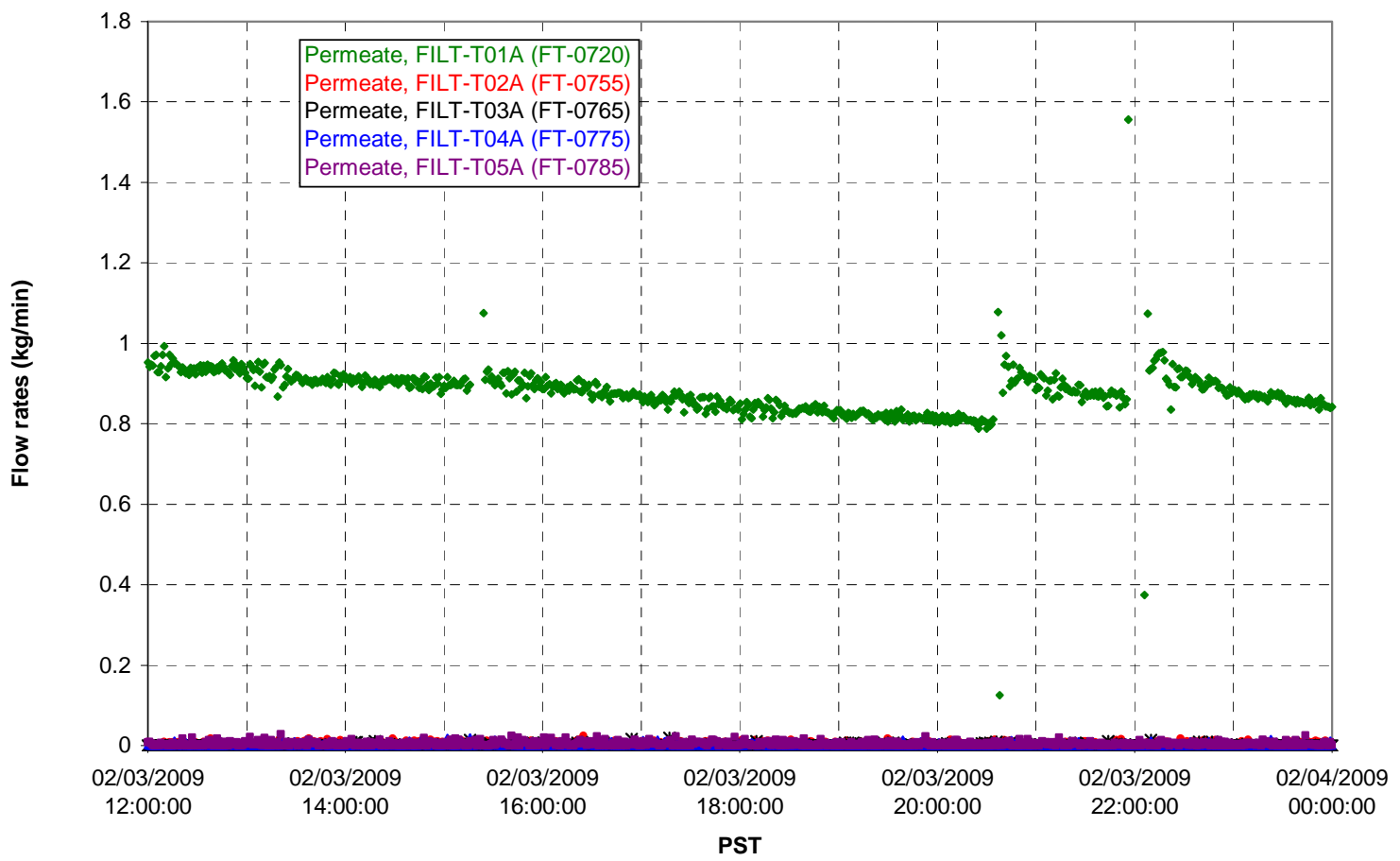

G.132 
T02A Inner Temperature Tree

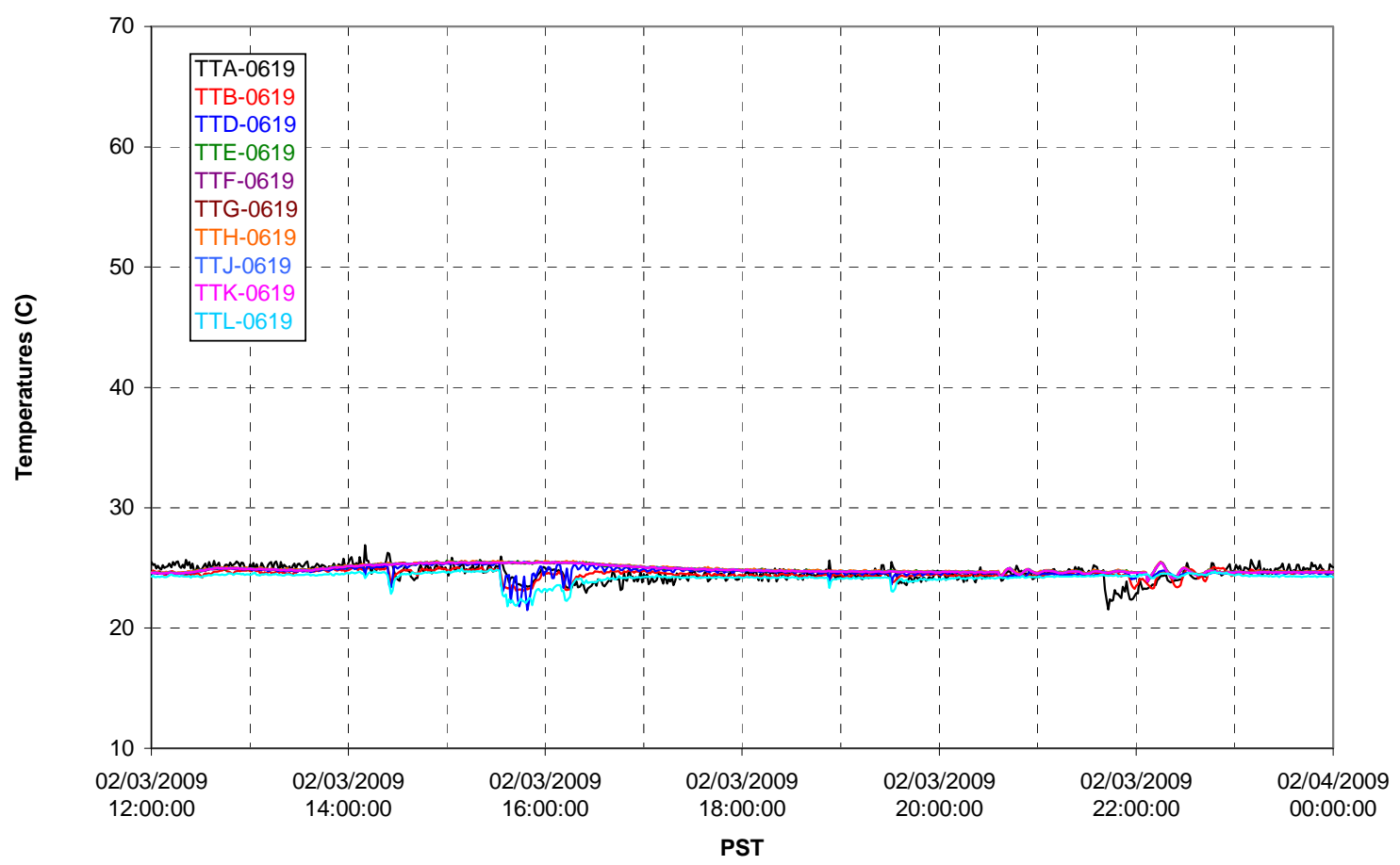

T02A Outer Temperature Tree

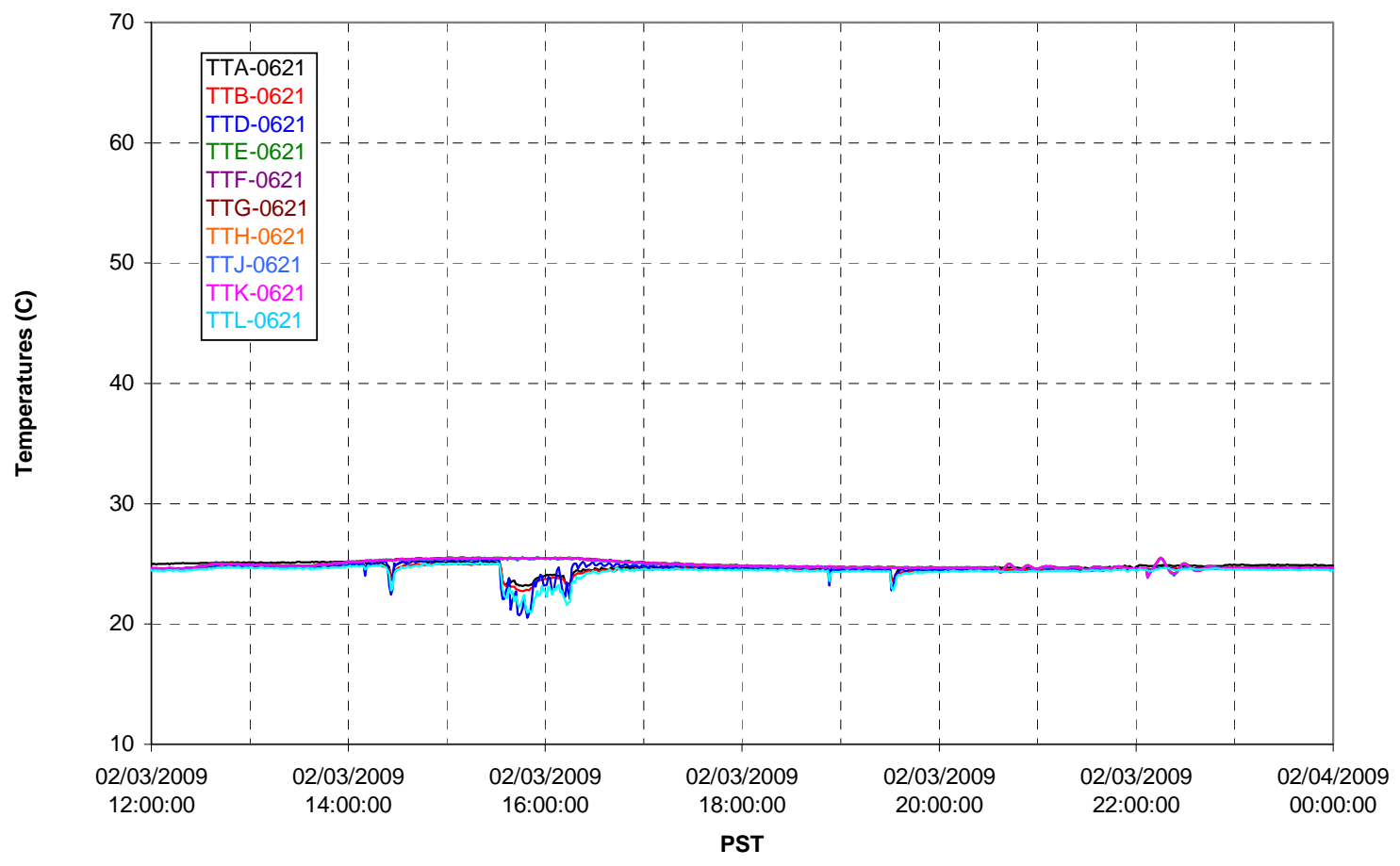

G.133 
T02A temperatures

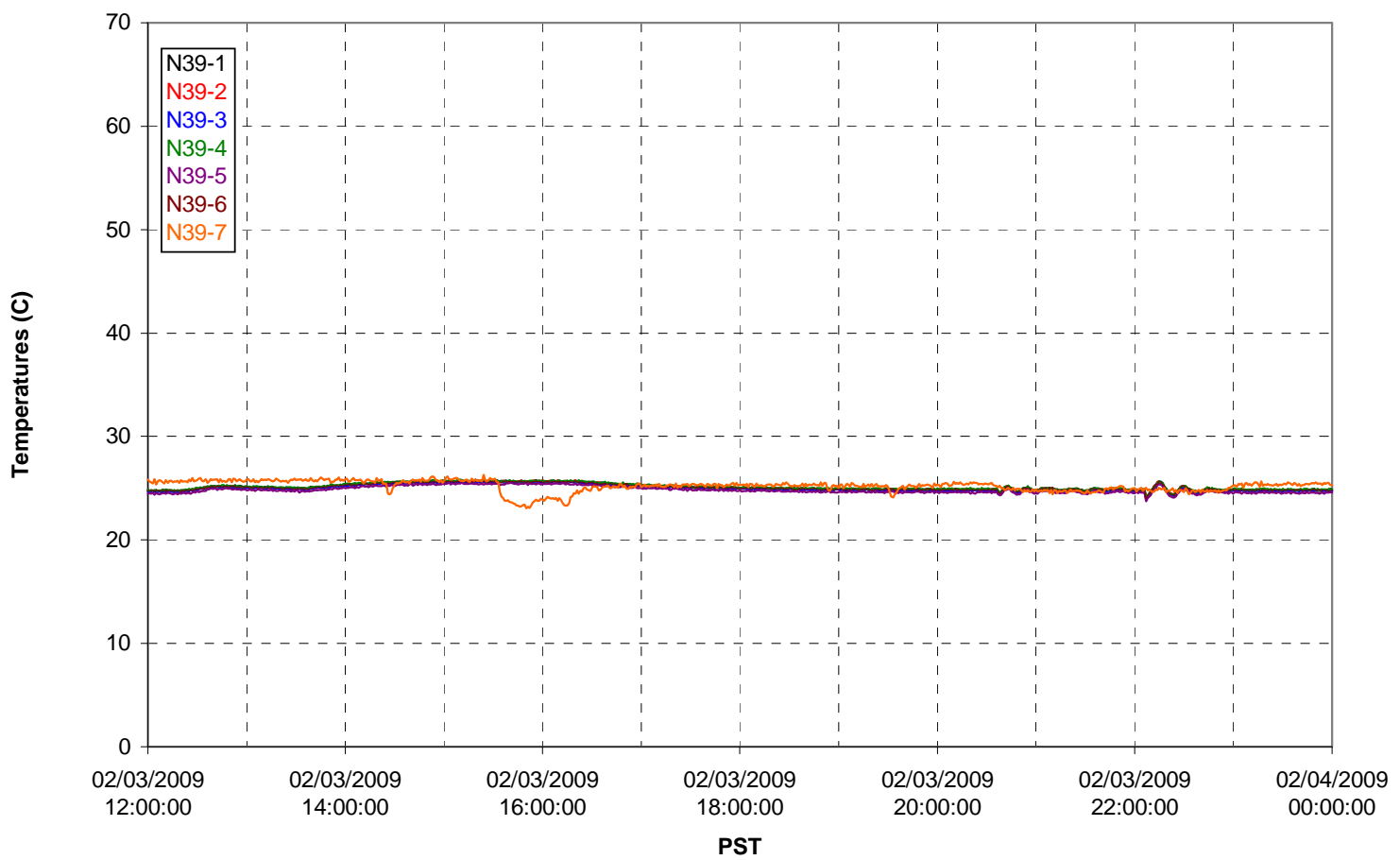

T02A temperatures

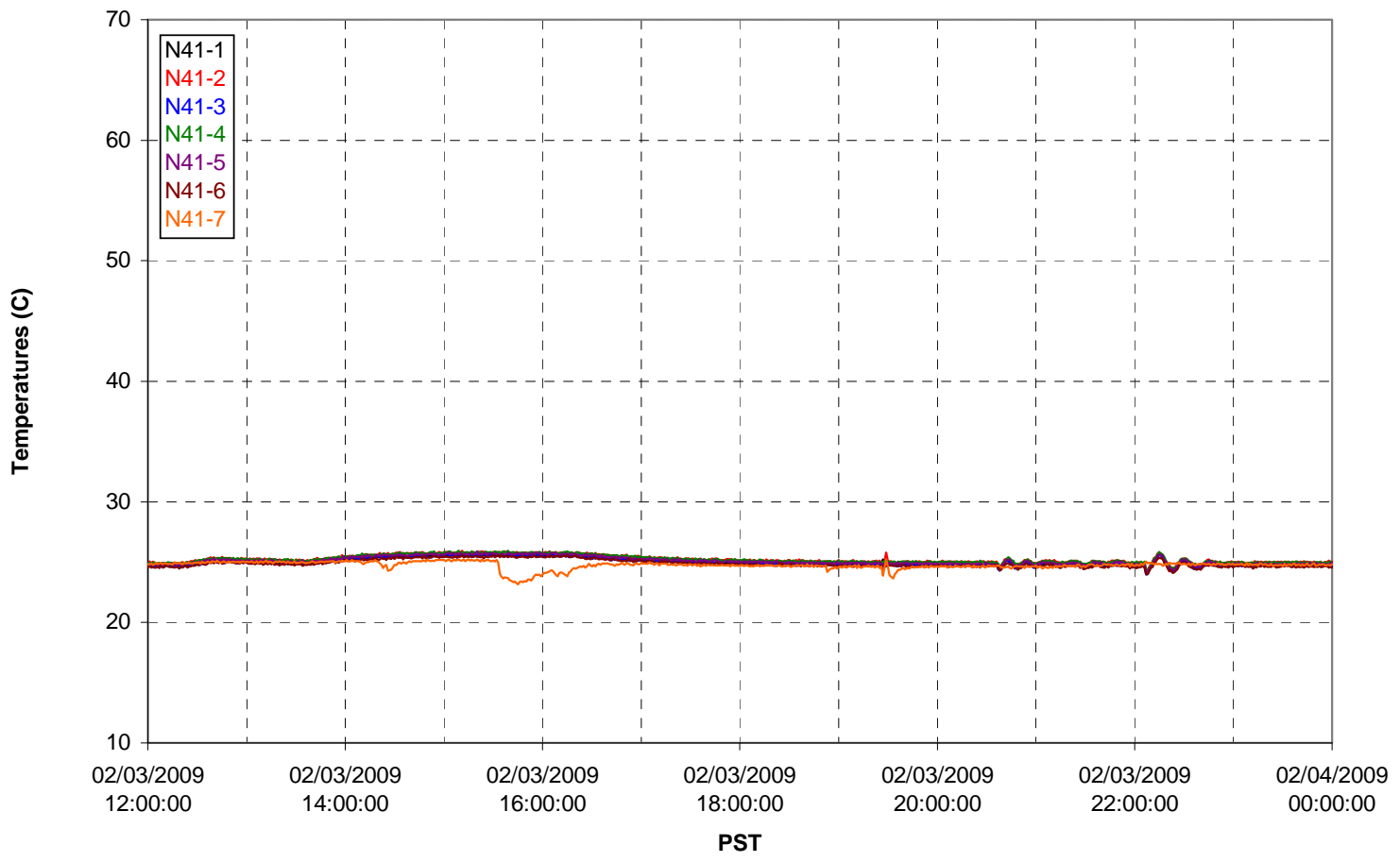

G.134 
T02A temperatures

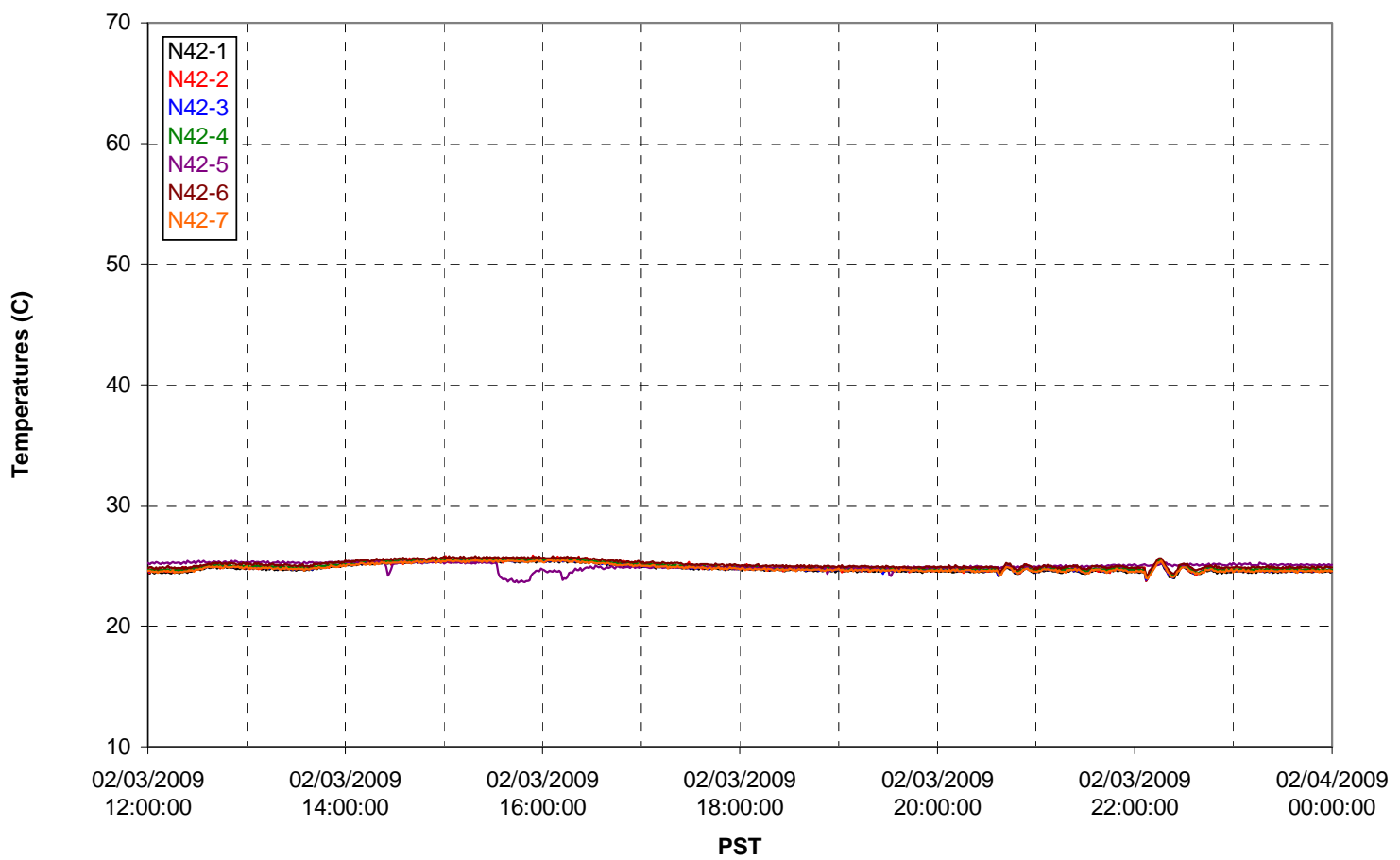

T02A temperatures

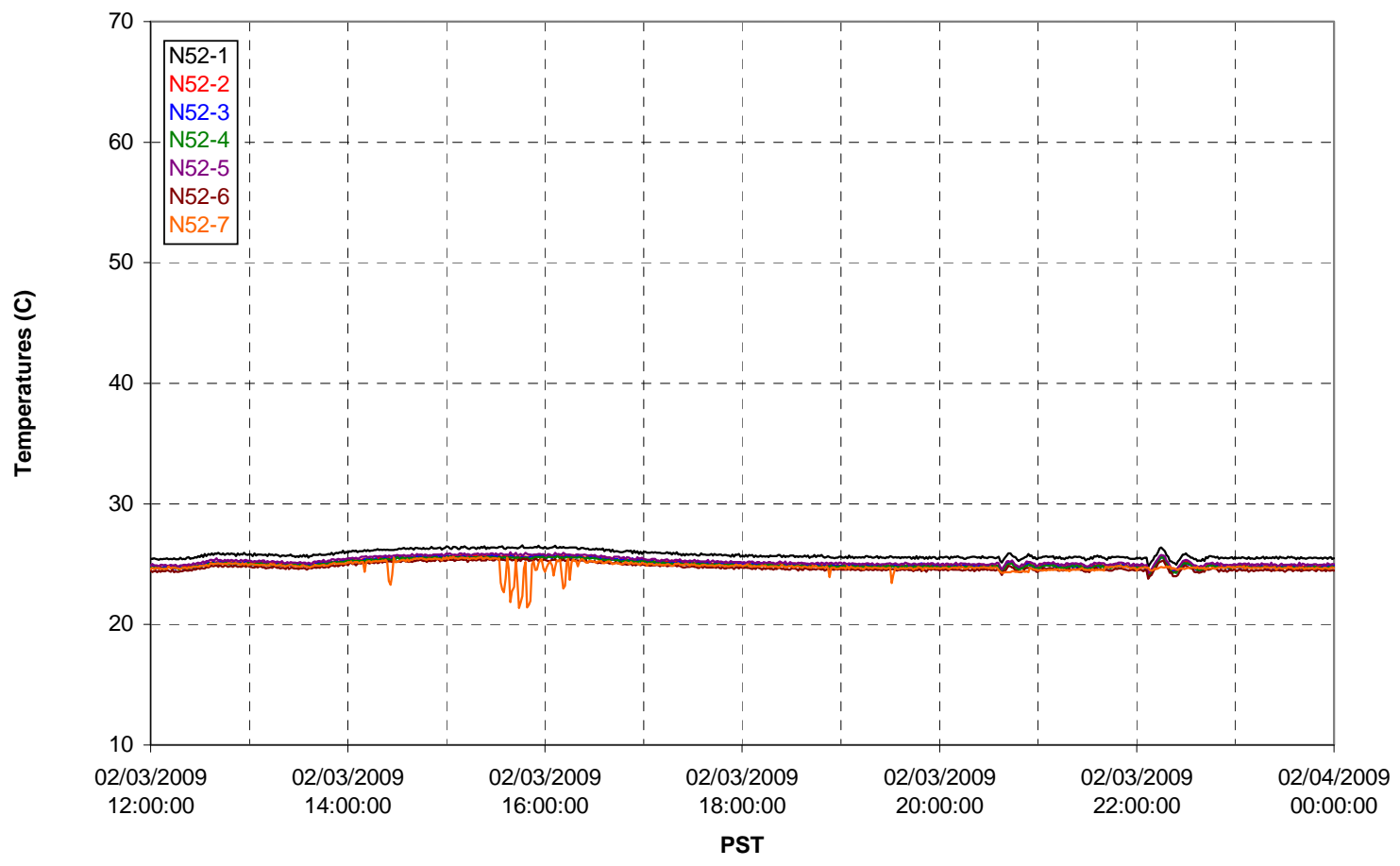

G.135 
T02A Heating and Cooling

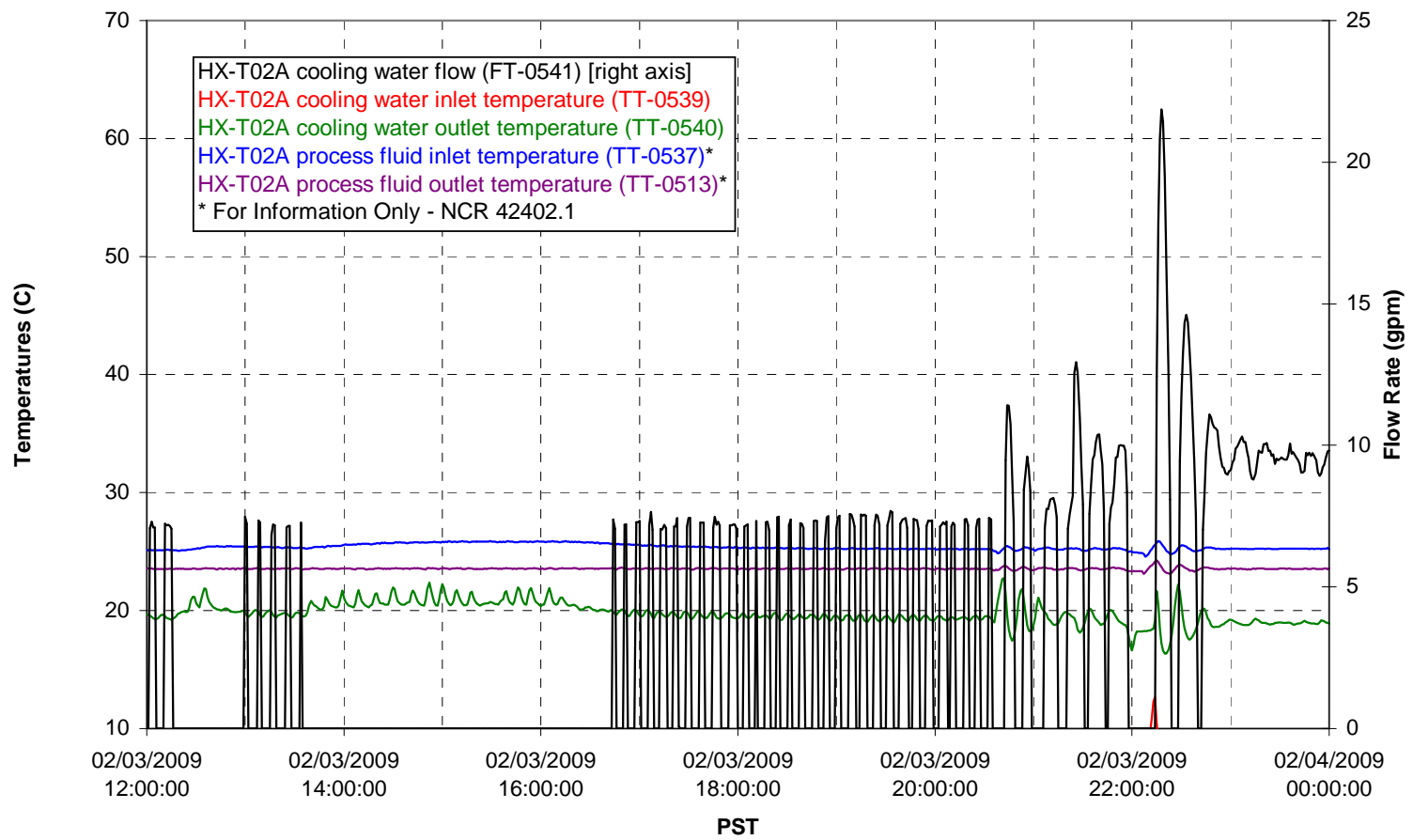

Pump Operation

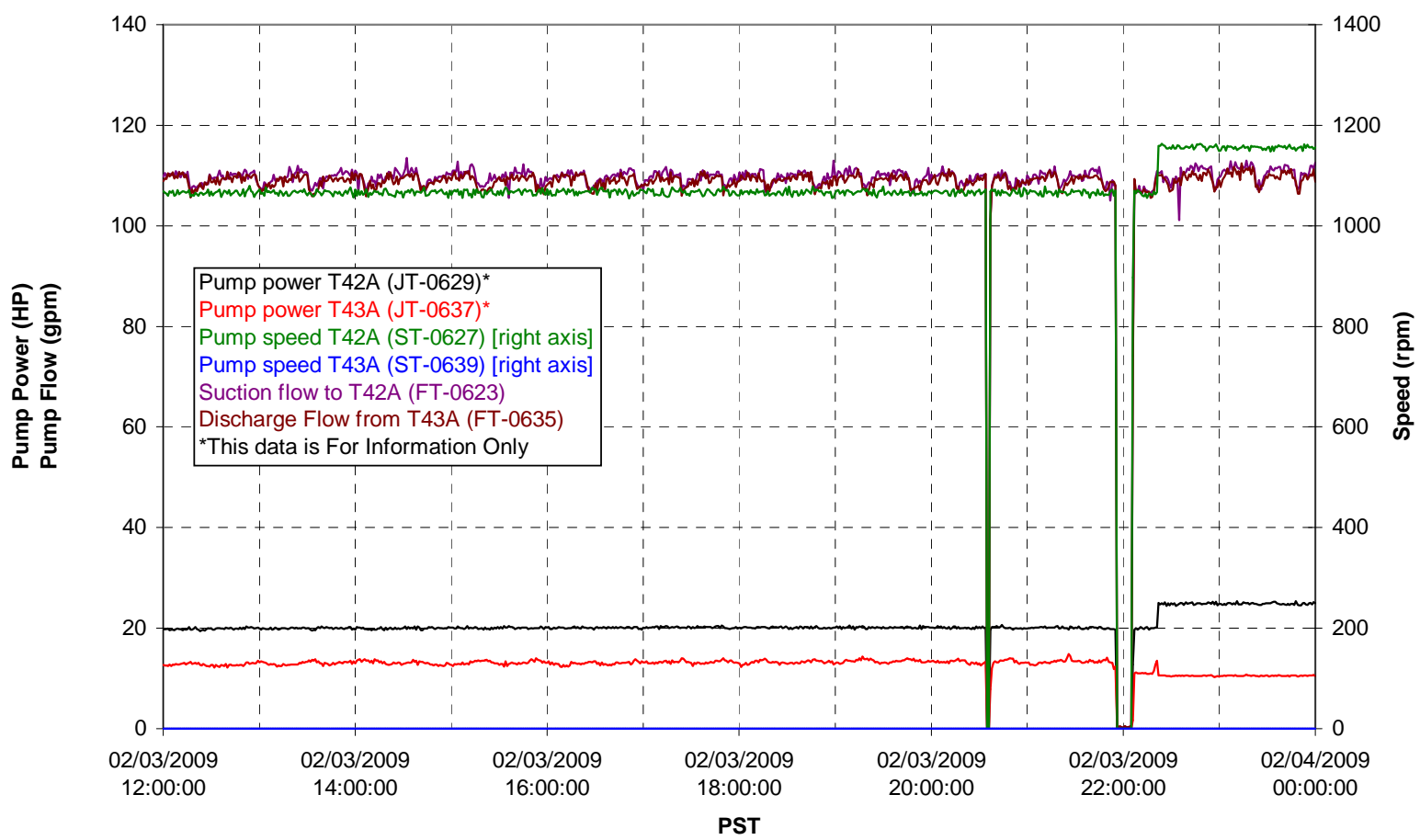


Pulsepot UFP-PP-T01A

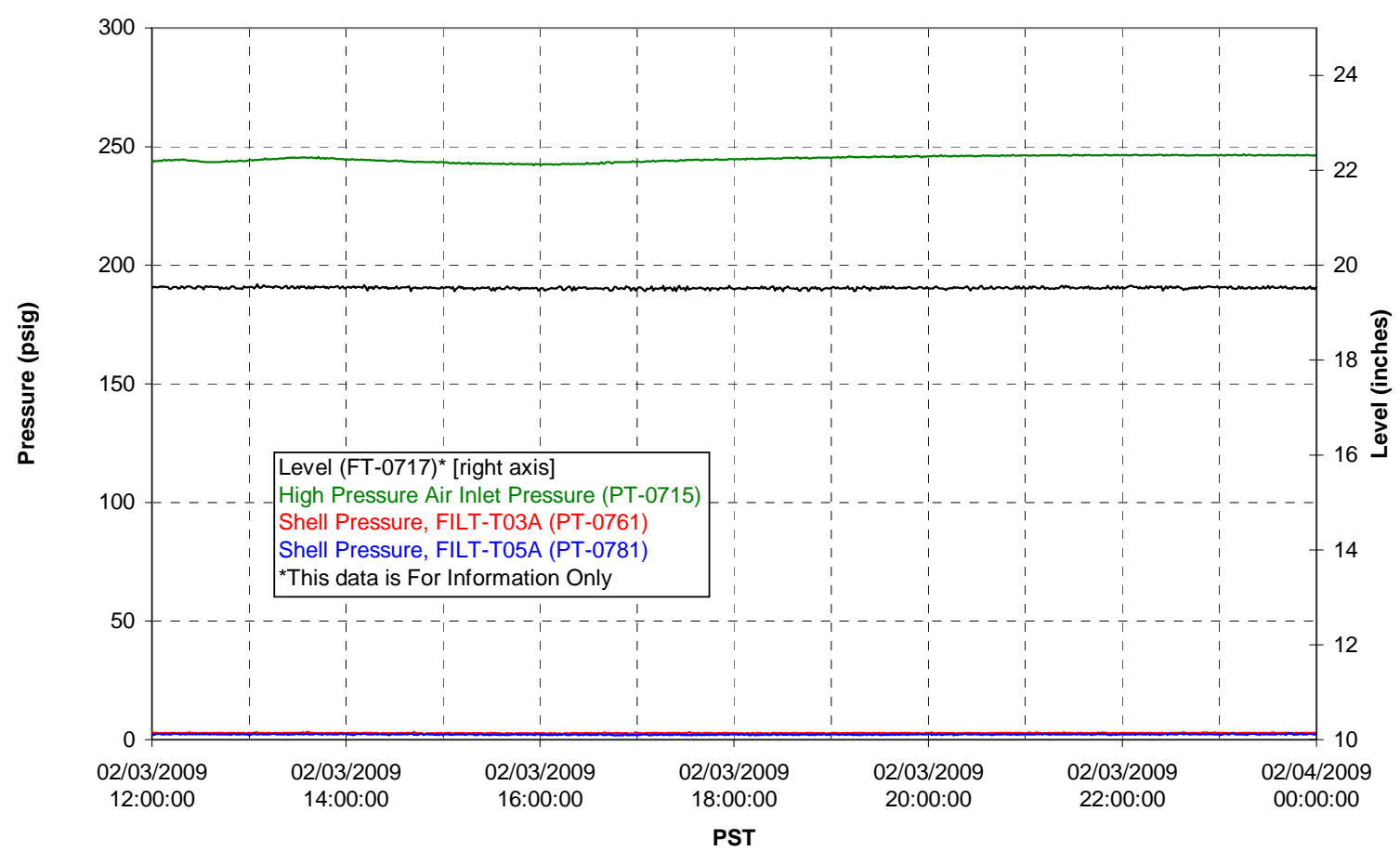

Pulsepot UFP-PP-T02A

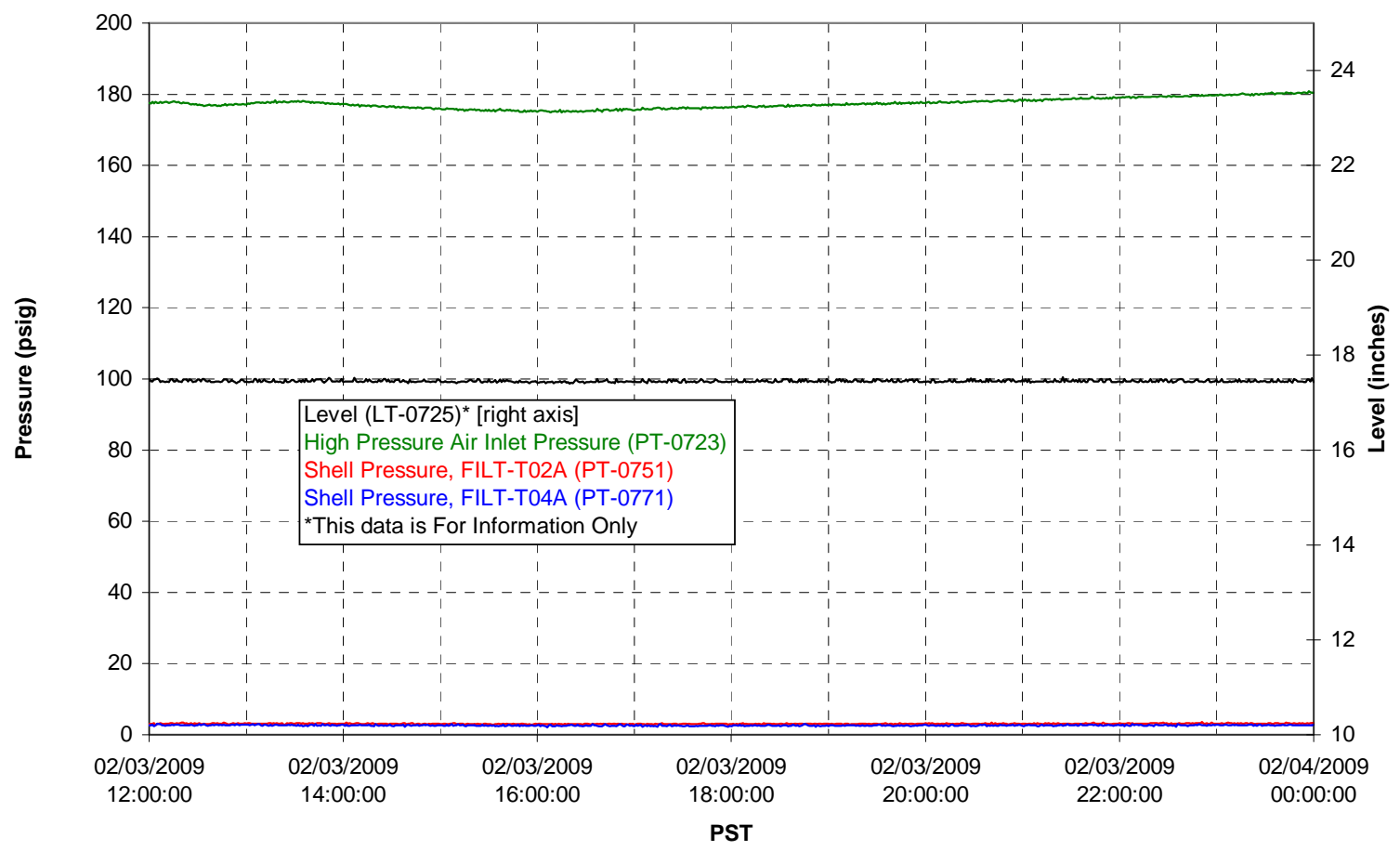

G.137 
Pulsepot UFP-PP-T03A

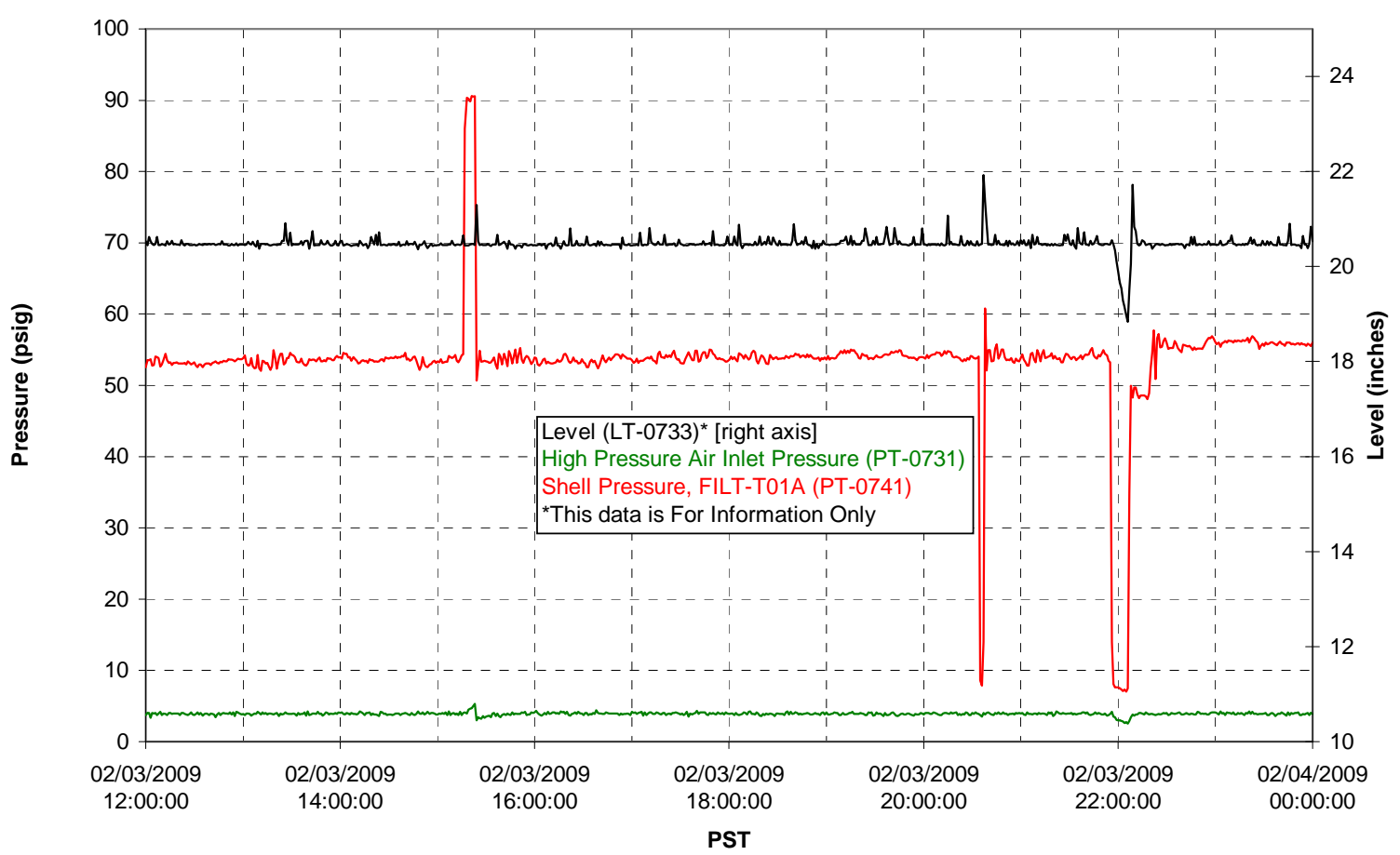

Pulsepot Levels

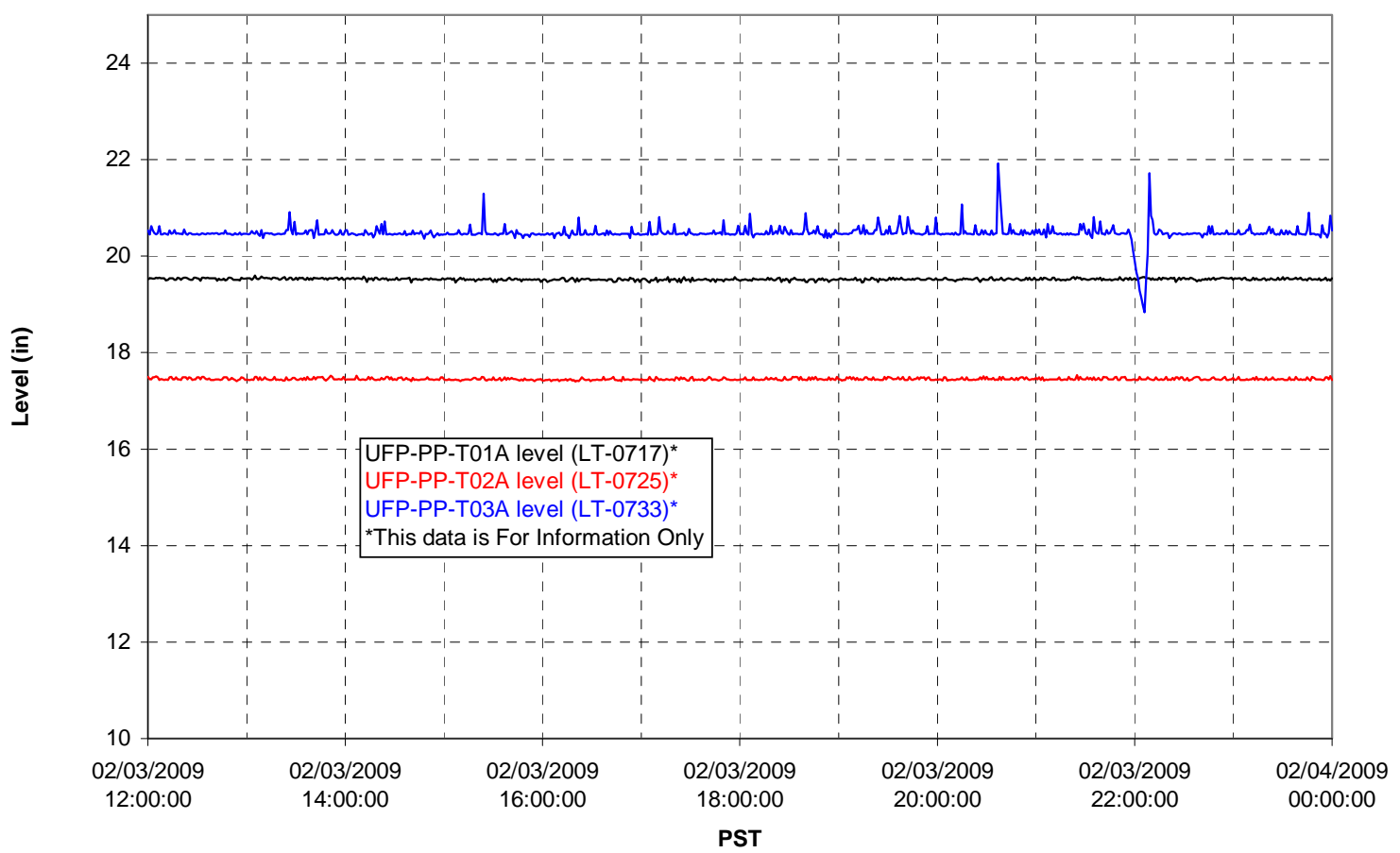

G.138 
Filter UFP-FILT-T01A

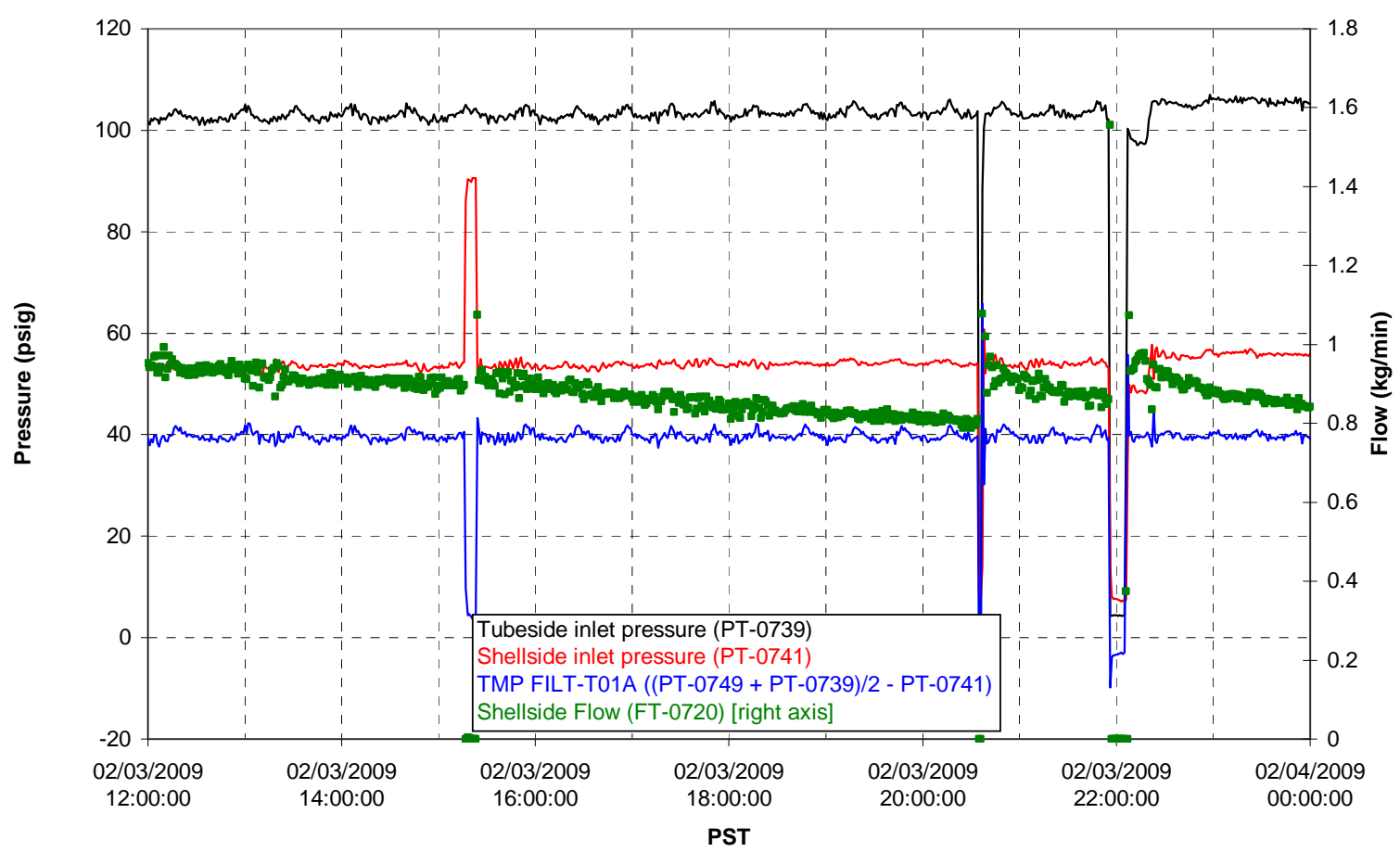

Filter UFP-FILT-T02A

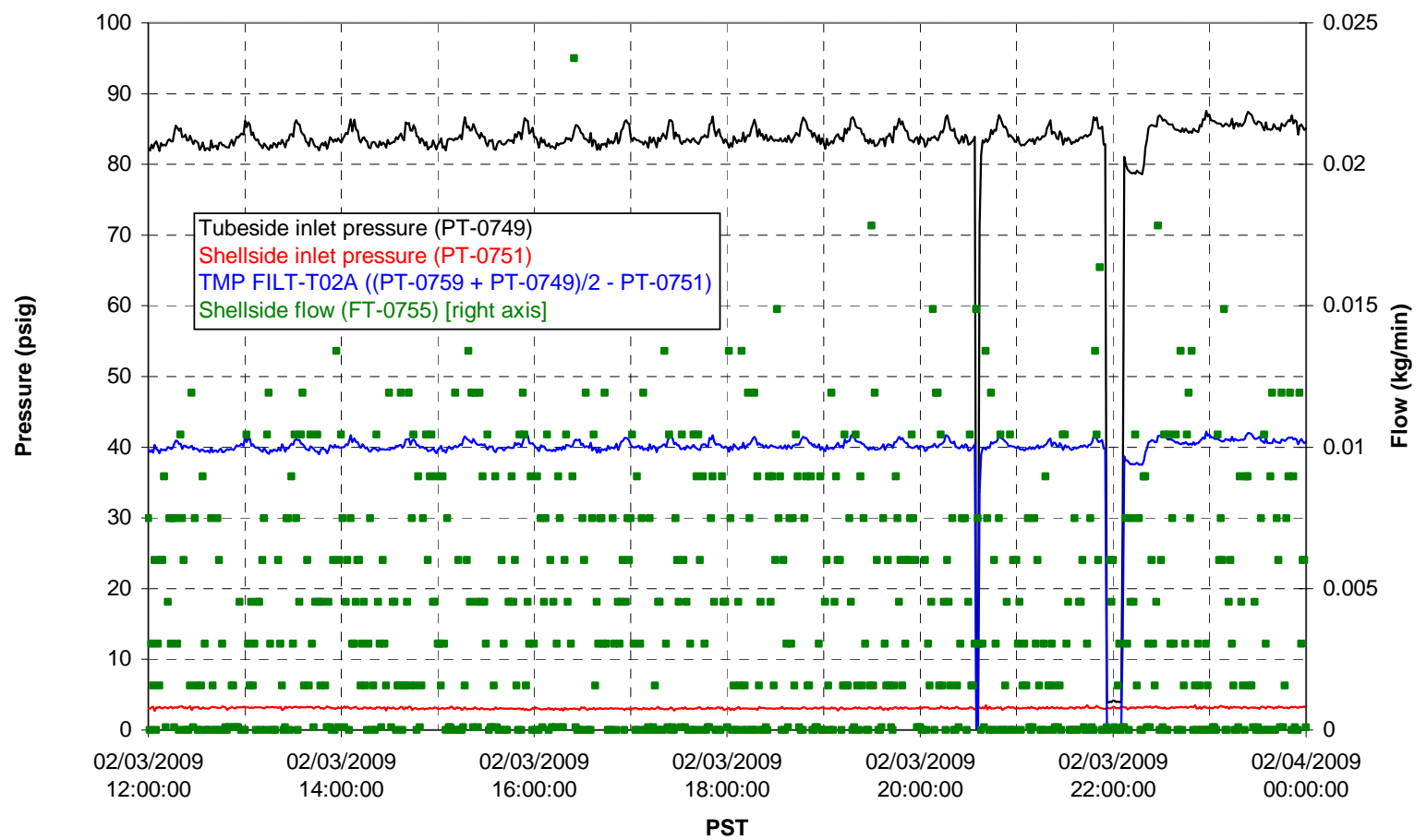

G.139 
Filter UFP-FILT-T03A

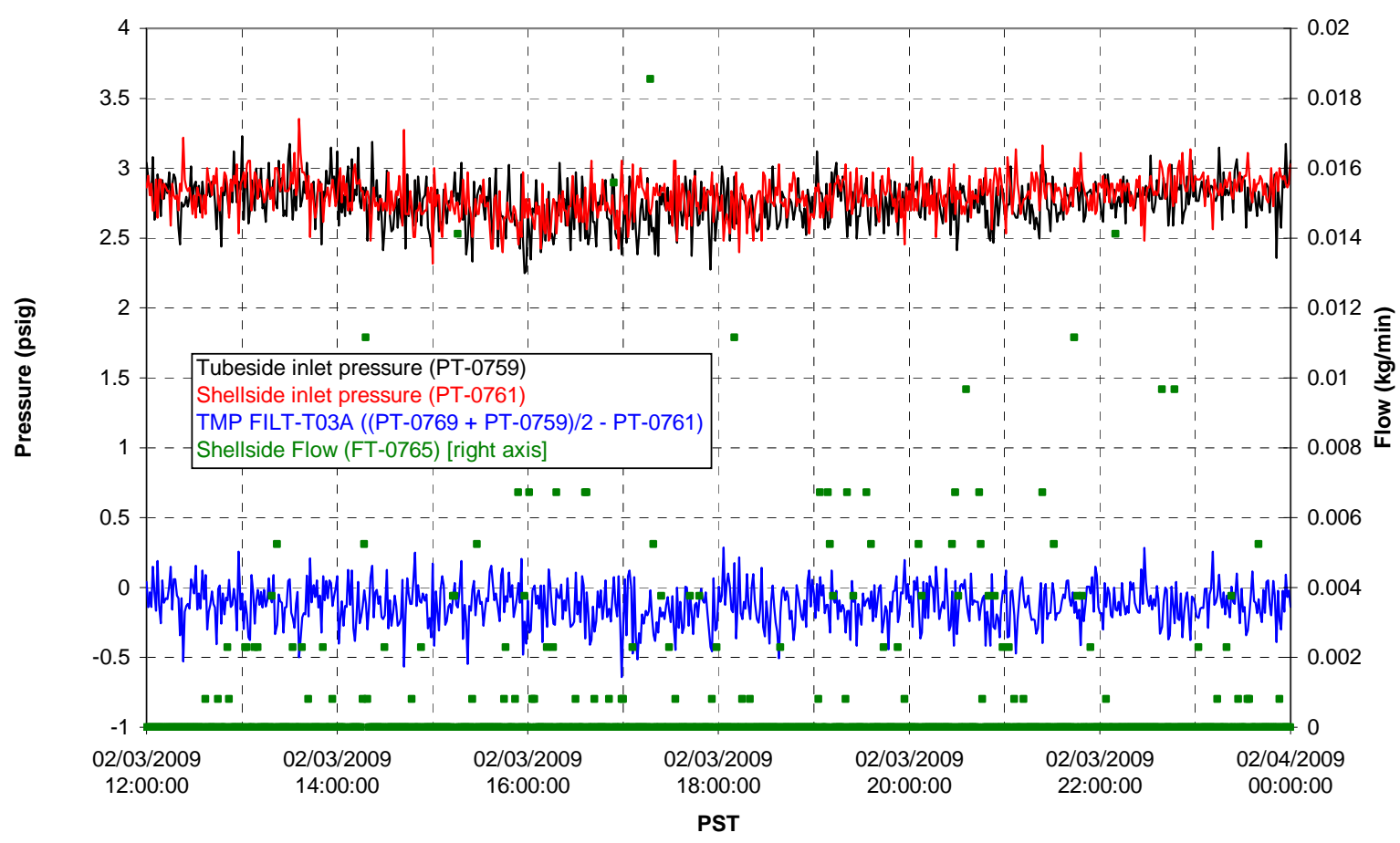

Filter UFP-FILT-T04A

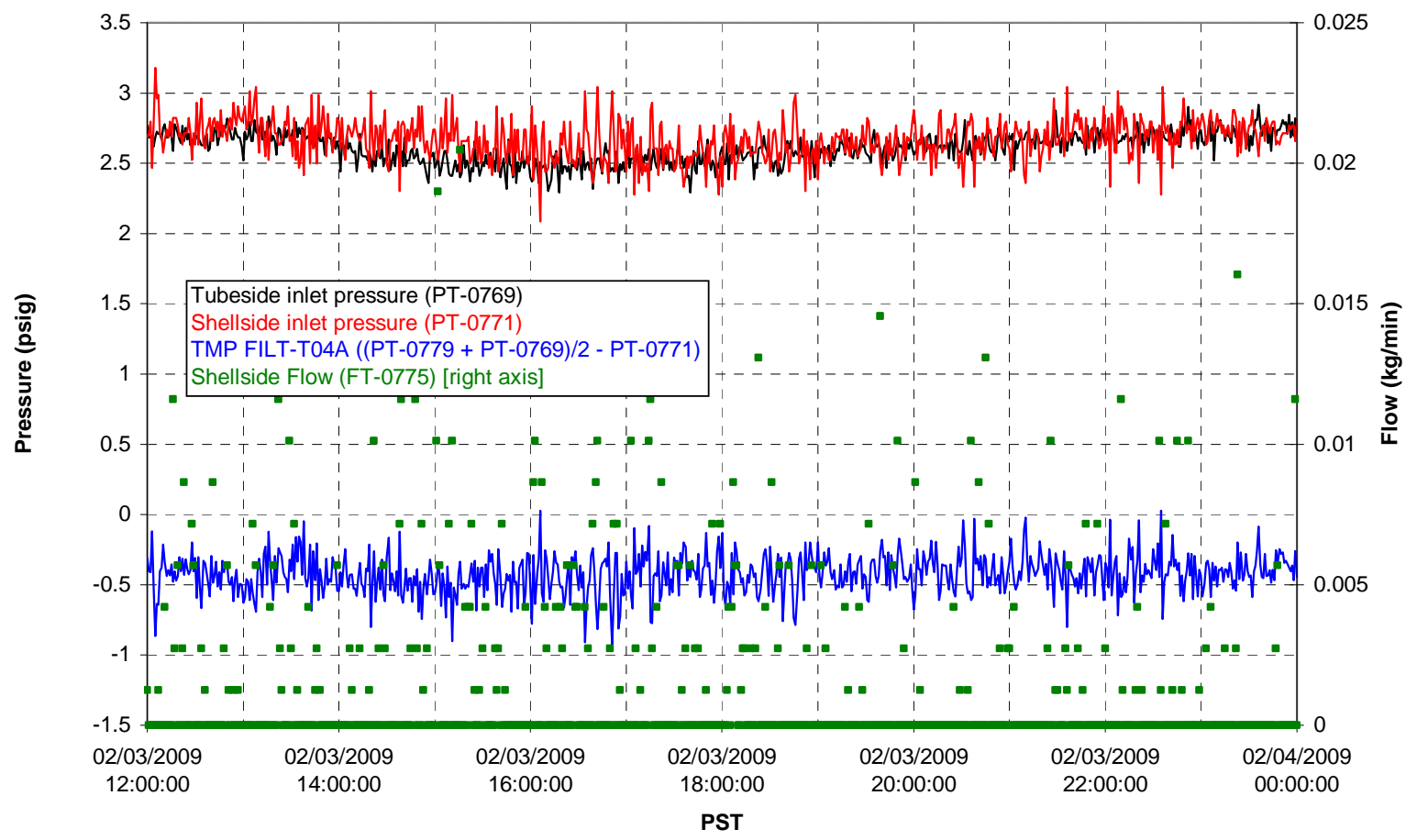

G.140 
Filter UFP-FILT-T05A

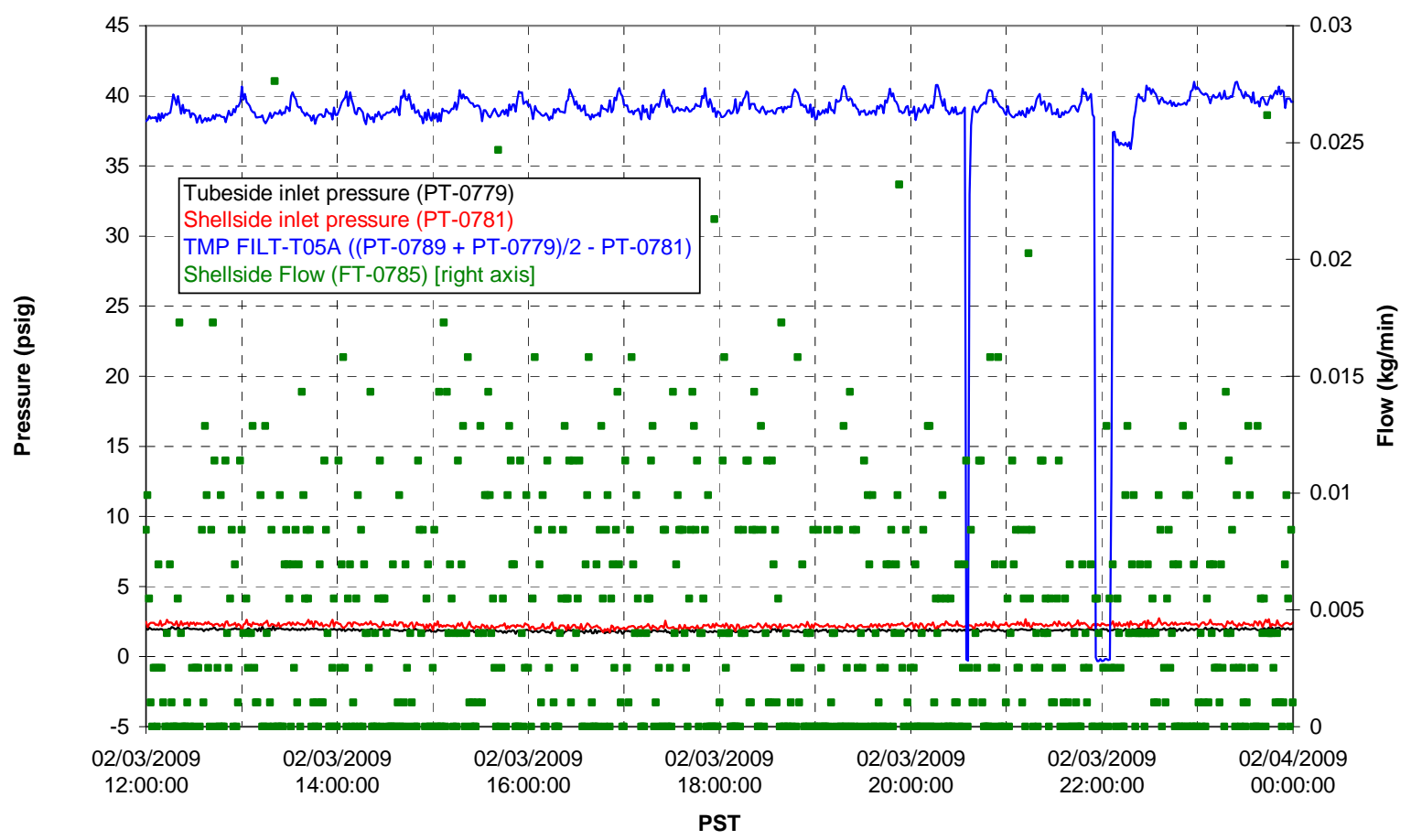

Chemical Flow

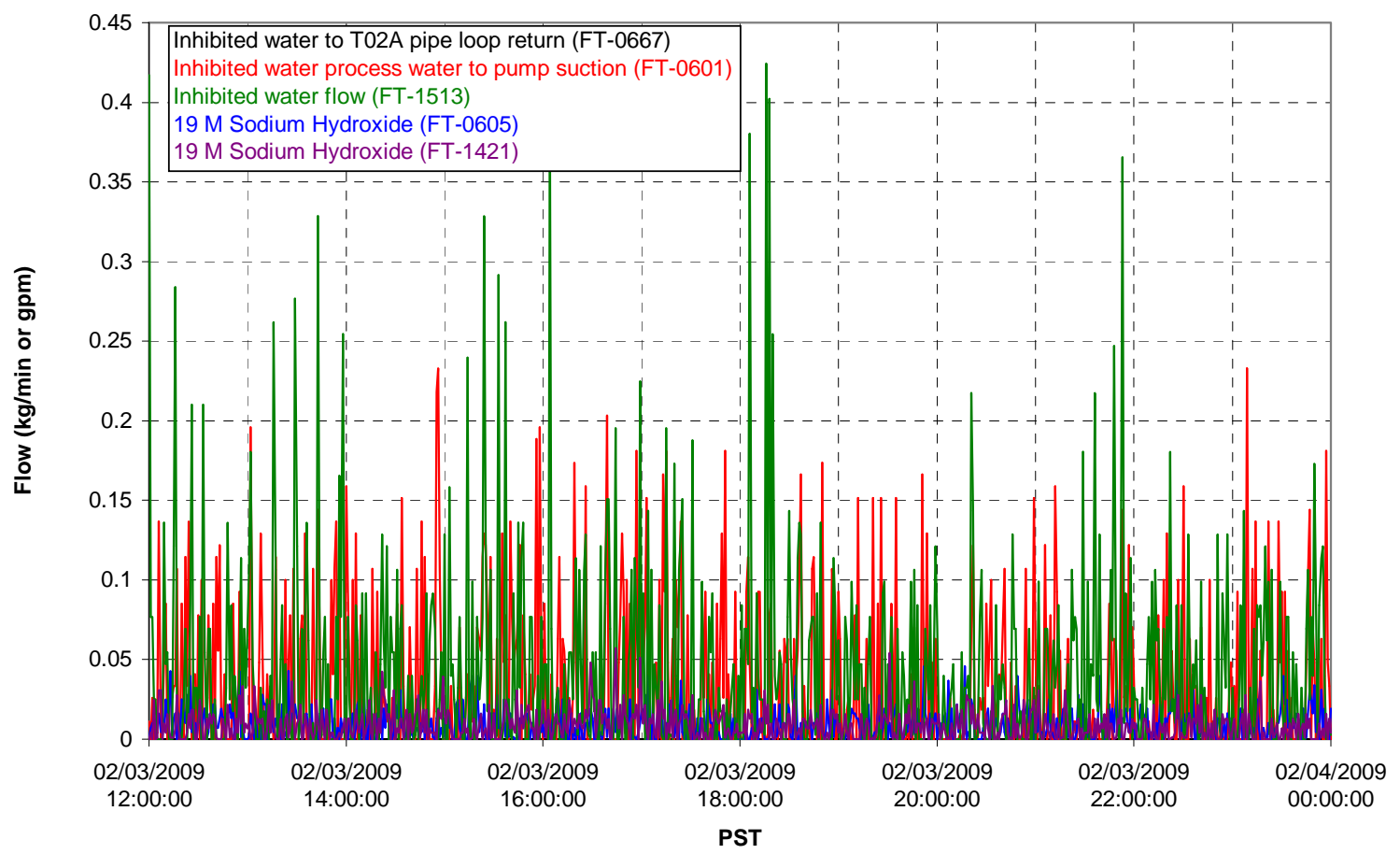


Chemical Flow

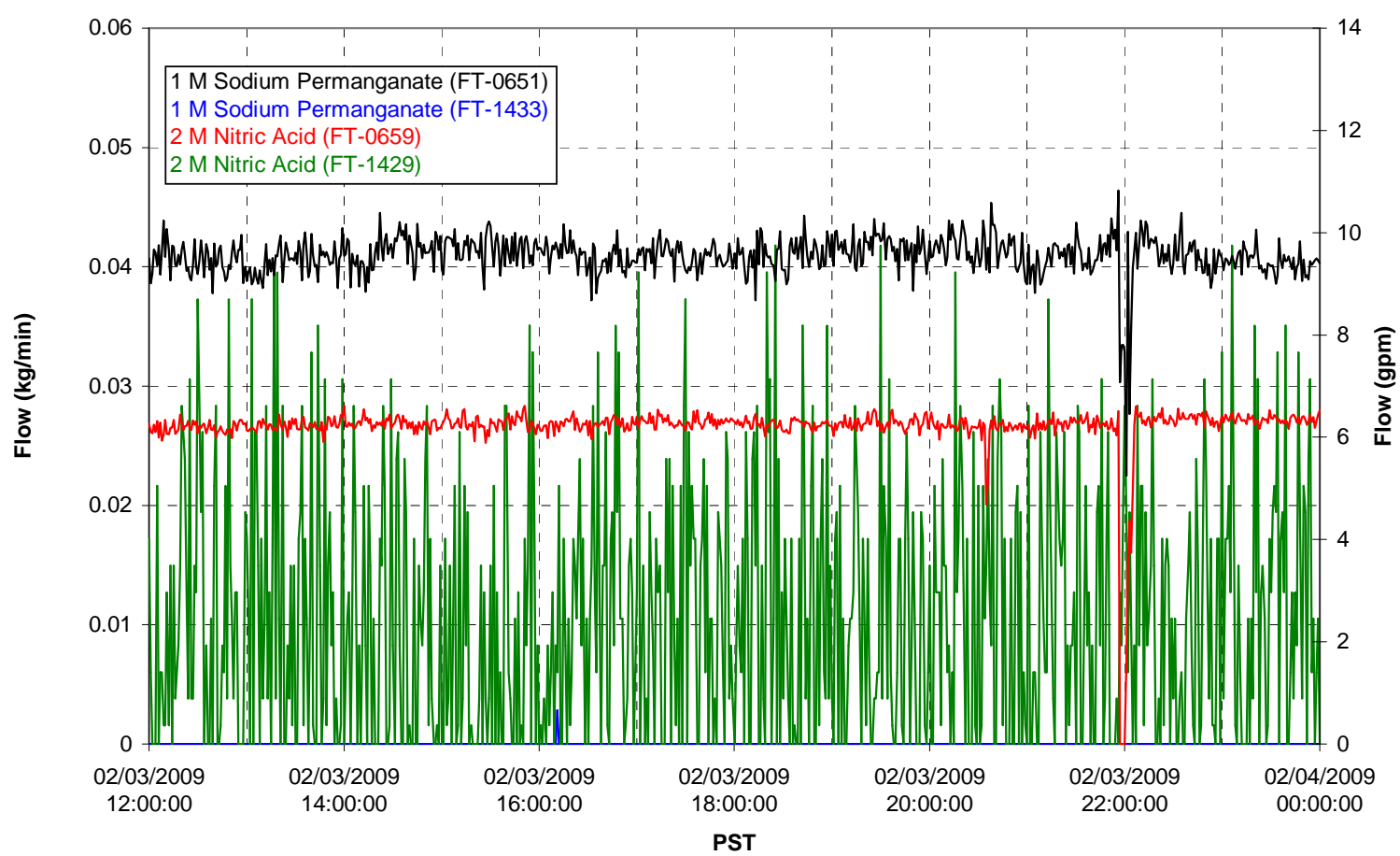

Air Flows

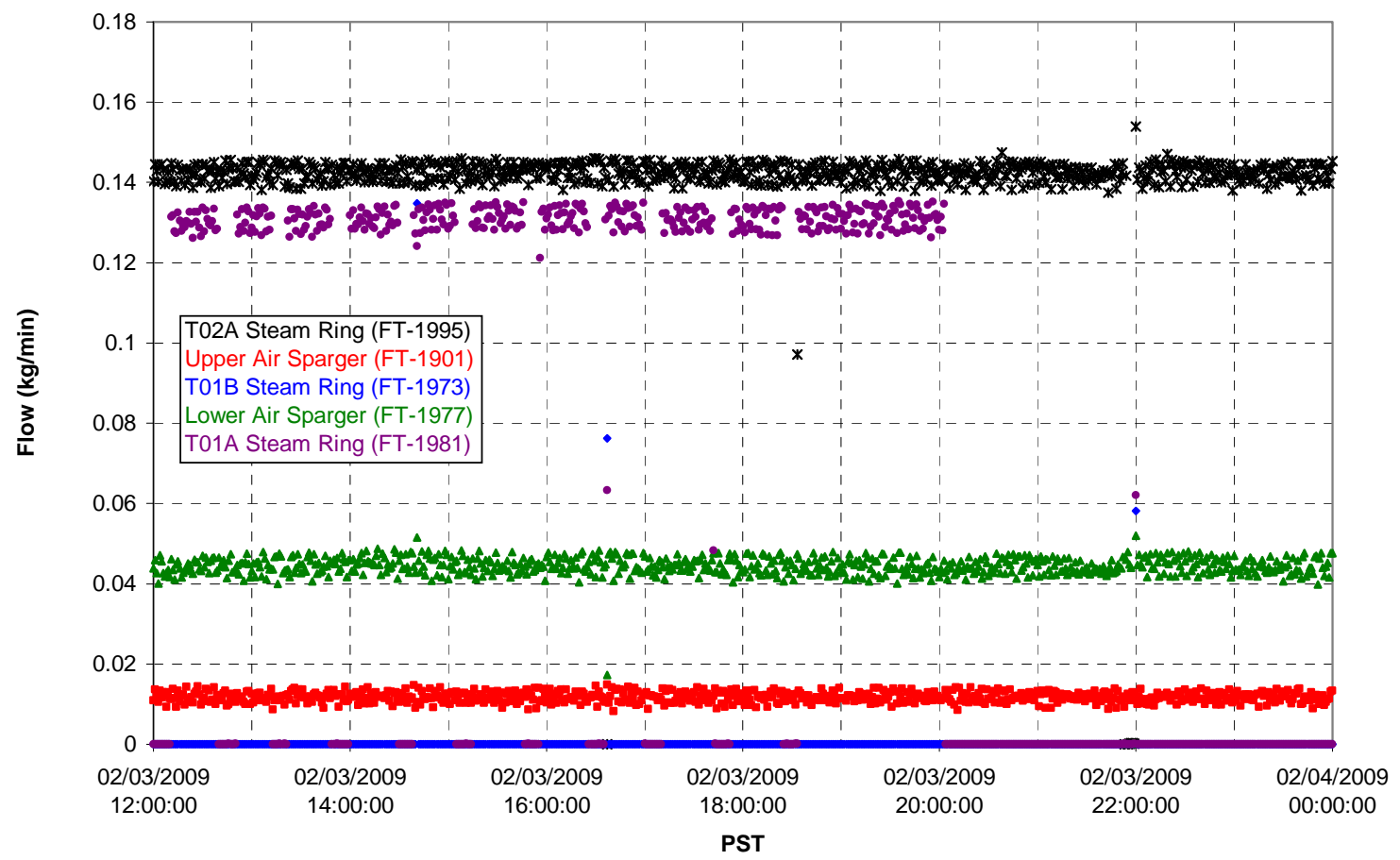


T02A Steam

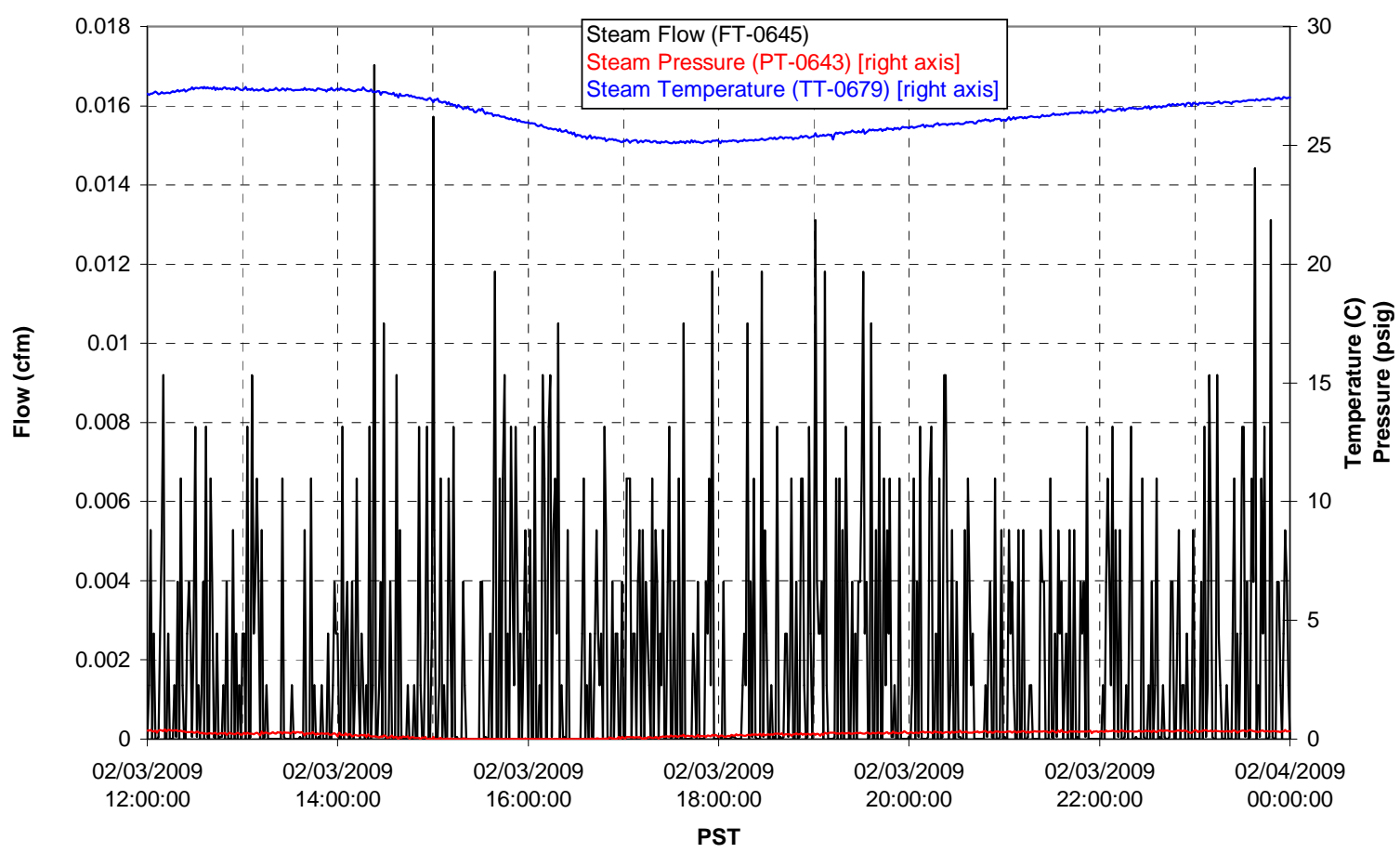

T01A Steam

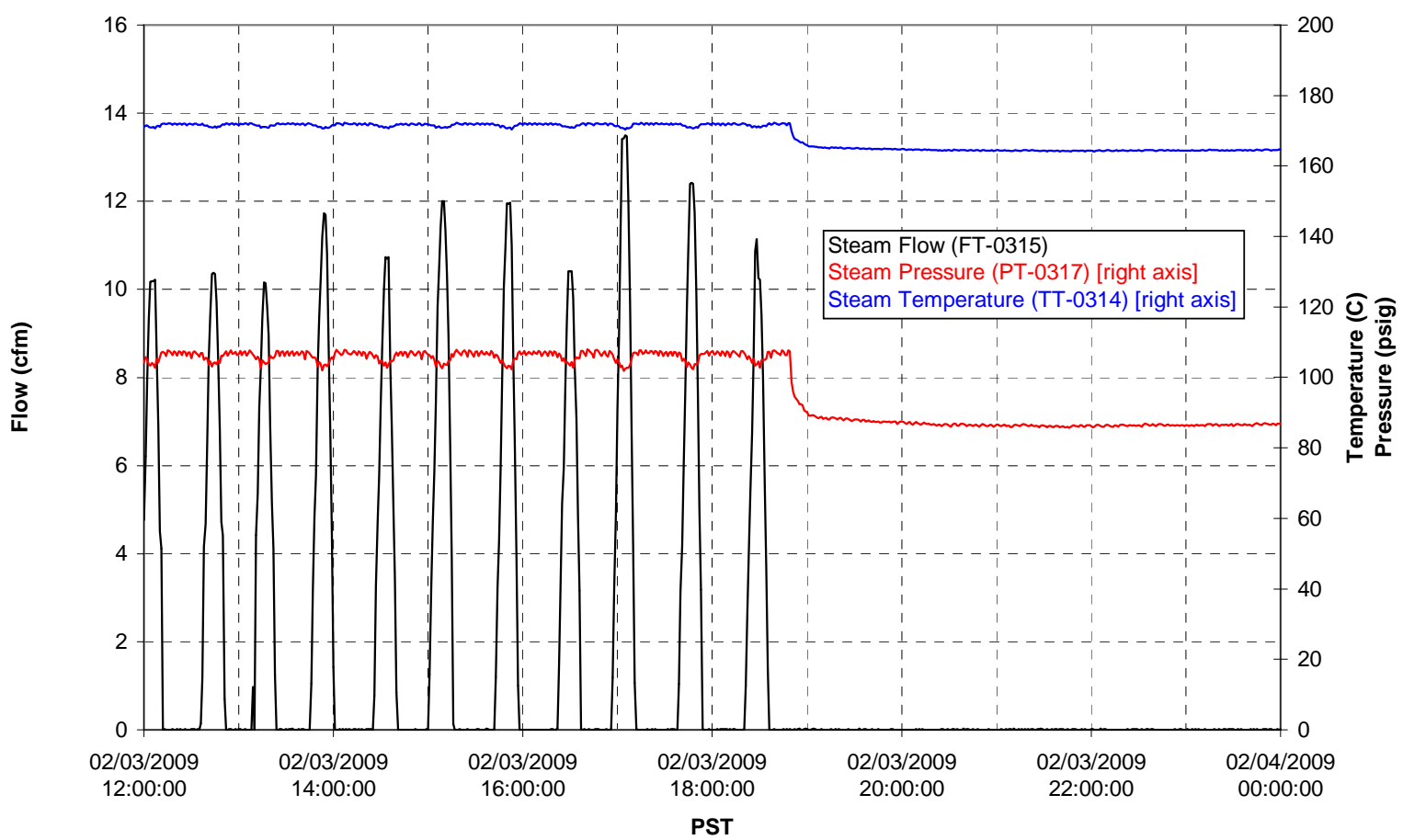


T01B Steam

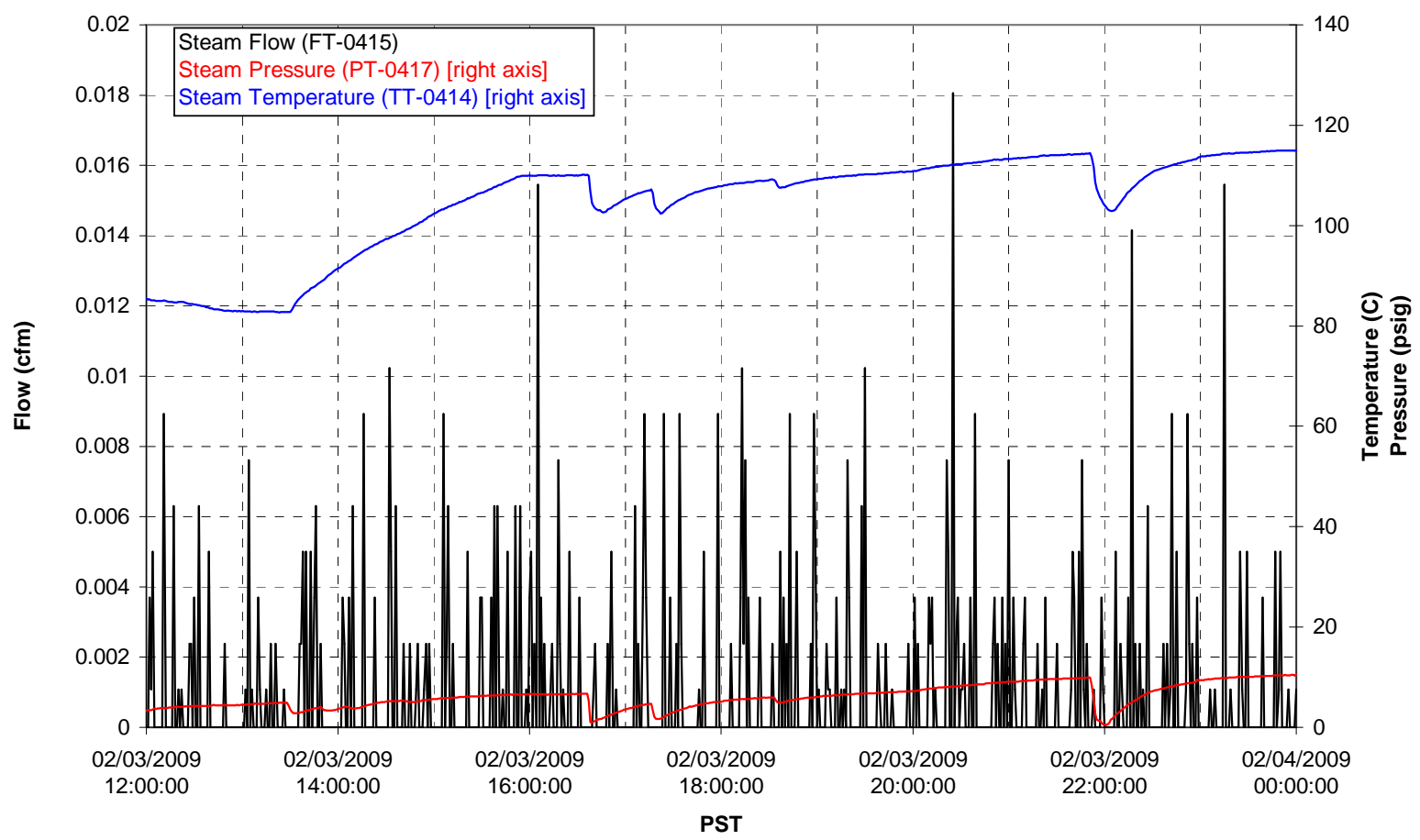




\section{Integrated Test A Data Plots 02/04/09 00:00 - 02/04/09 12:00}


T01A level

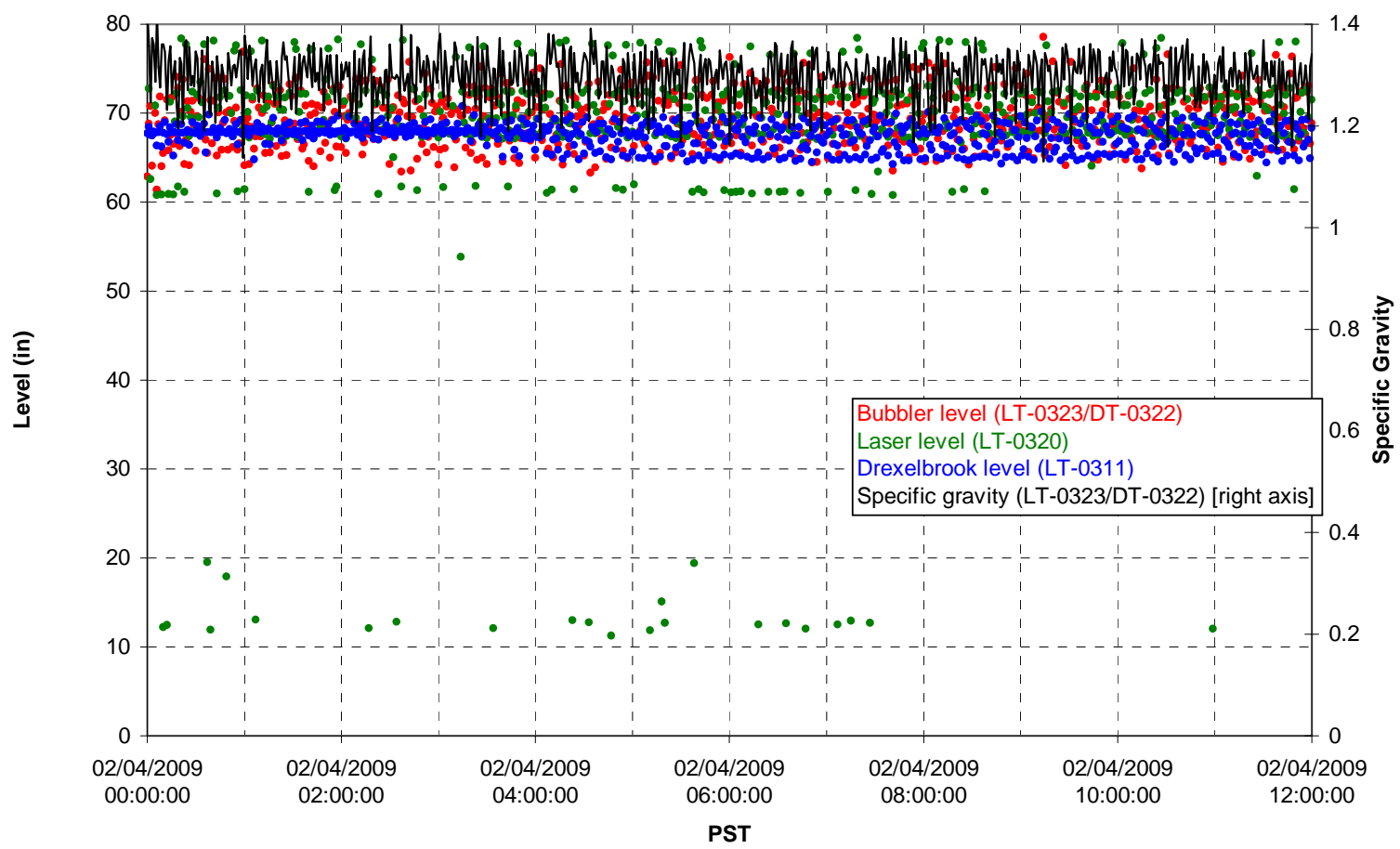

T01A temperatures

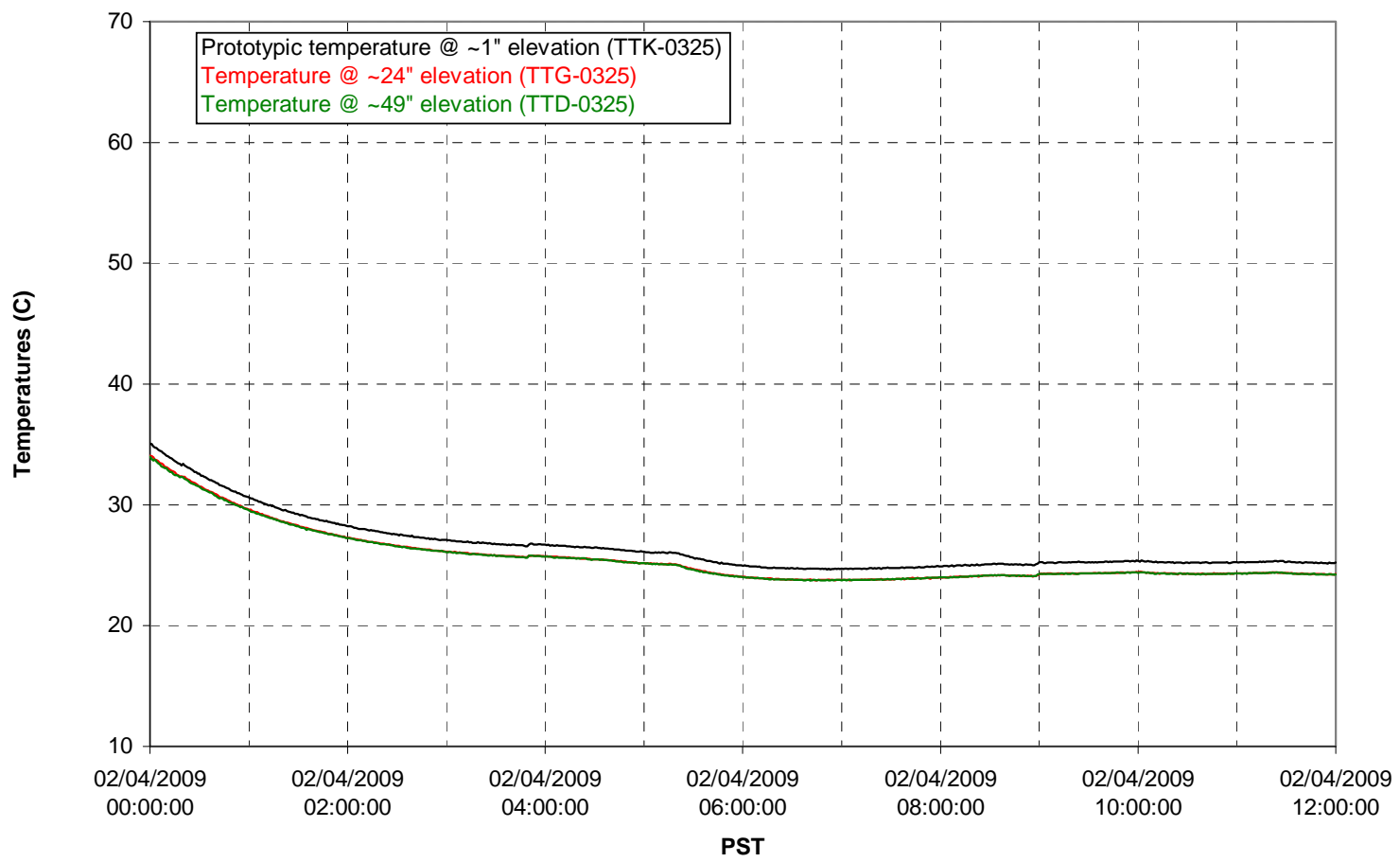


T01B level

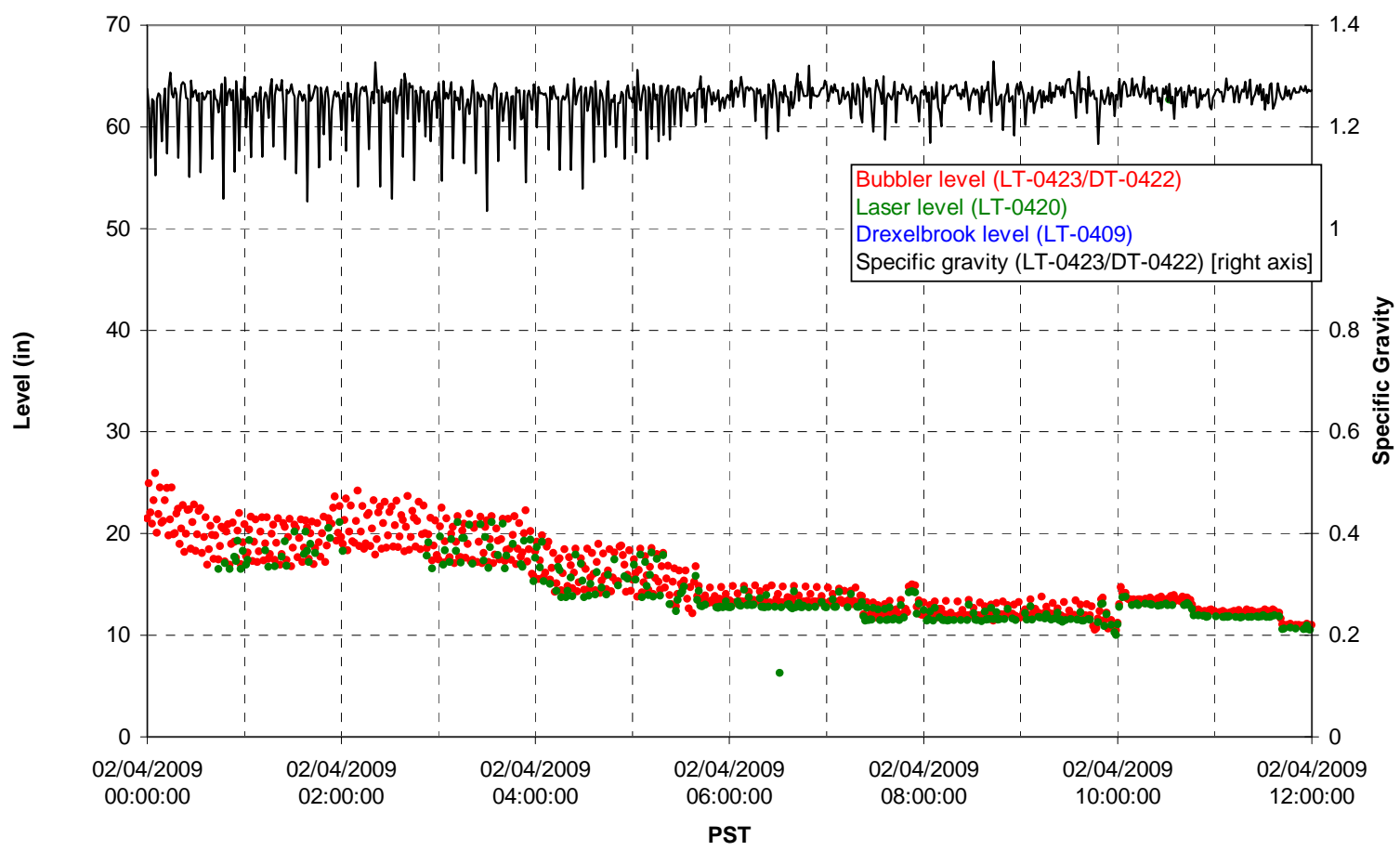

T01B temperatures

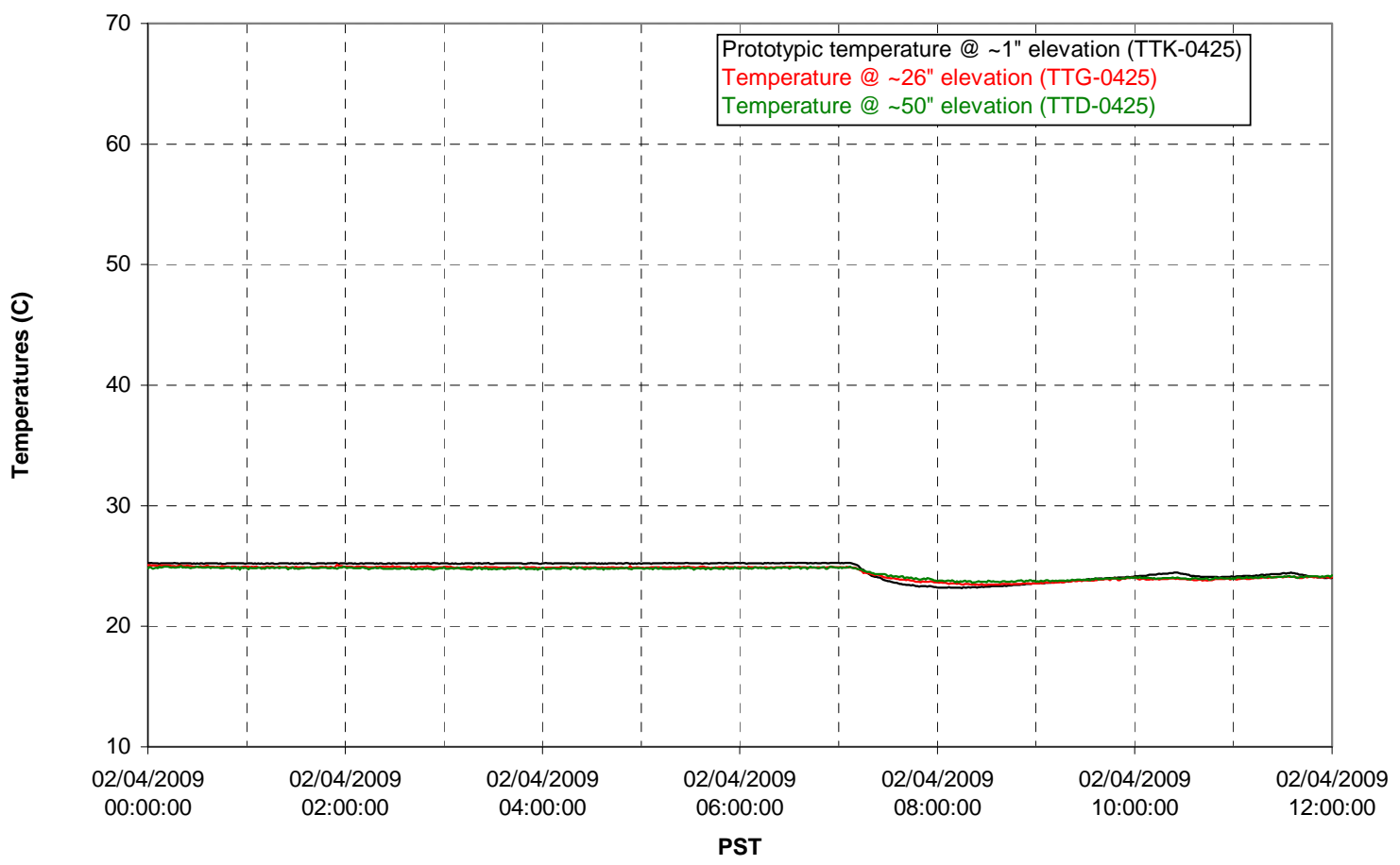


T02A level

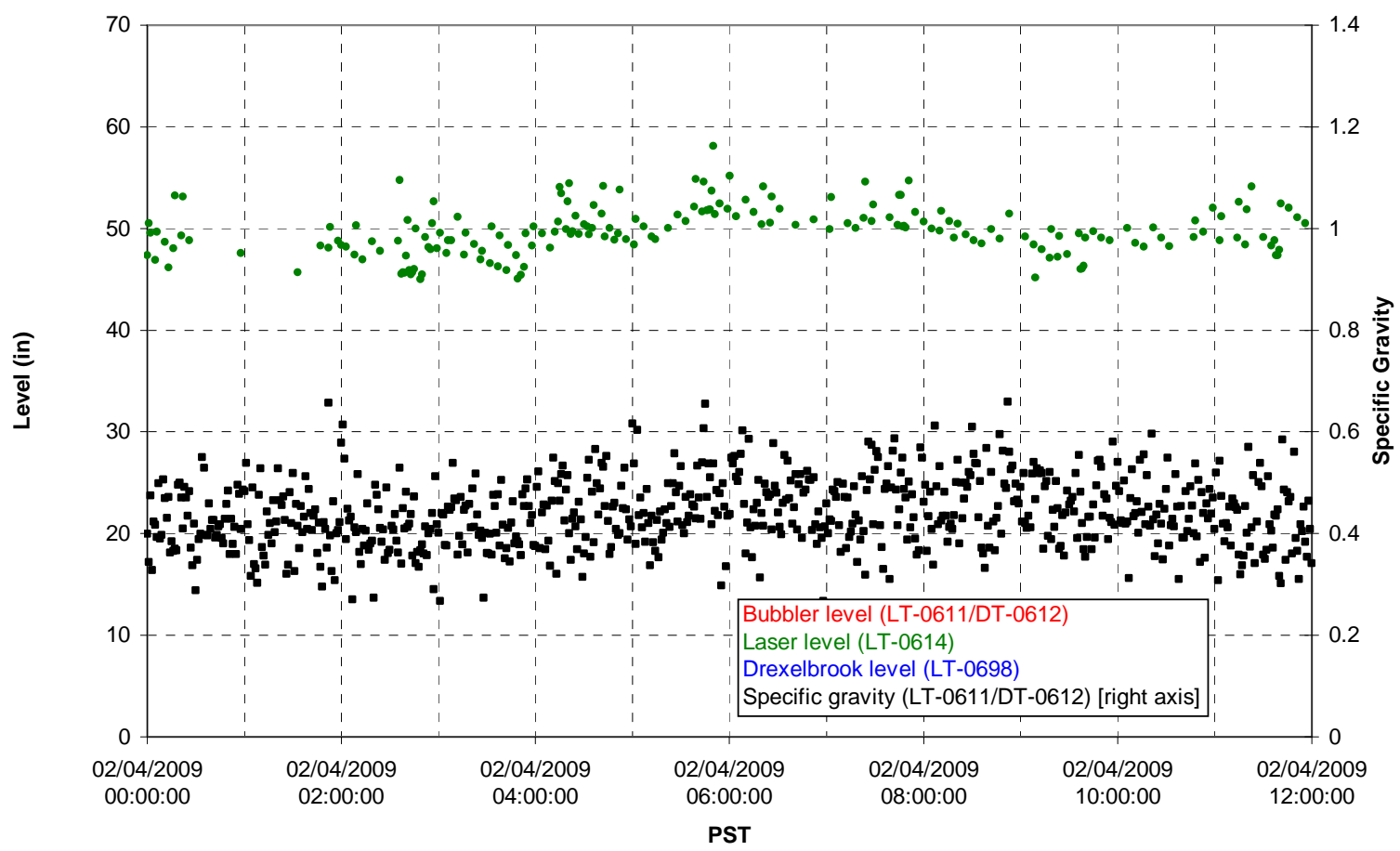

T02A temperatures

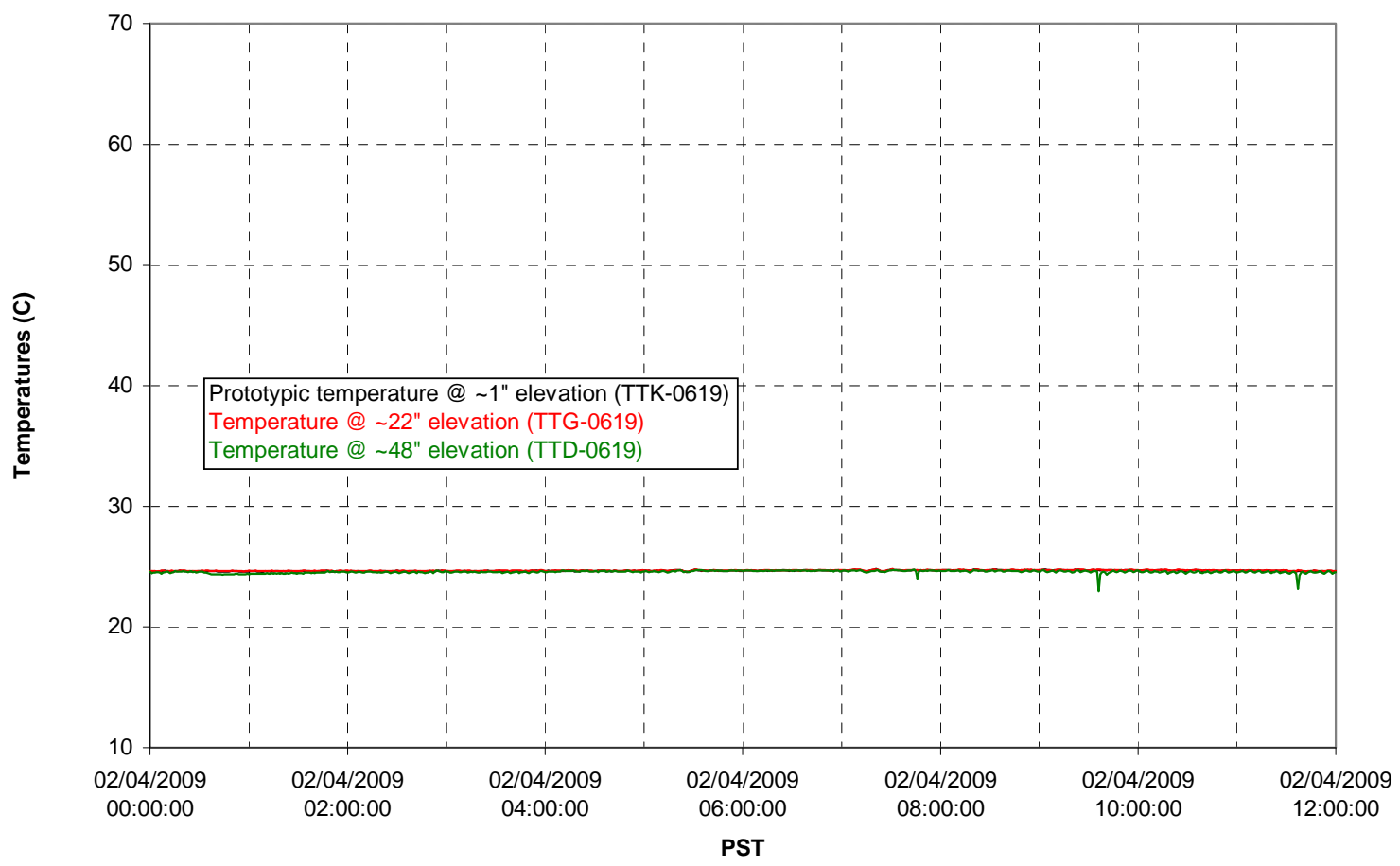

G.148 
T02A and filter loop temperatures

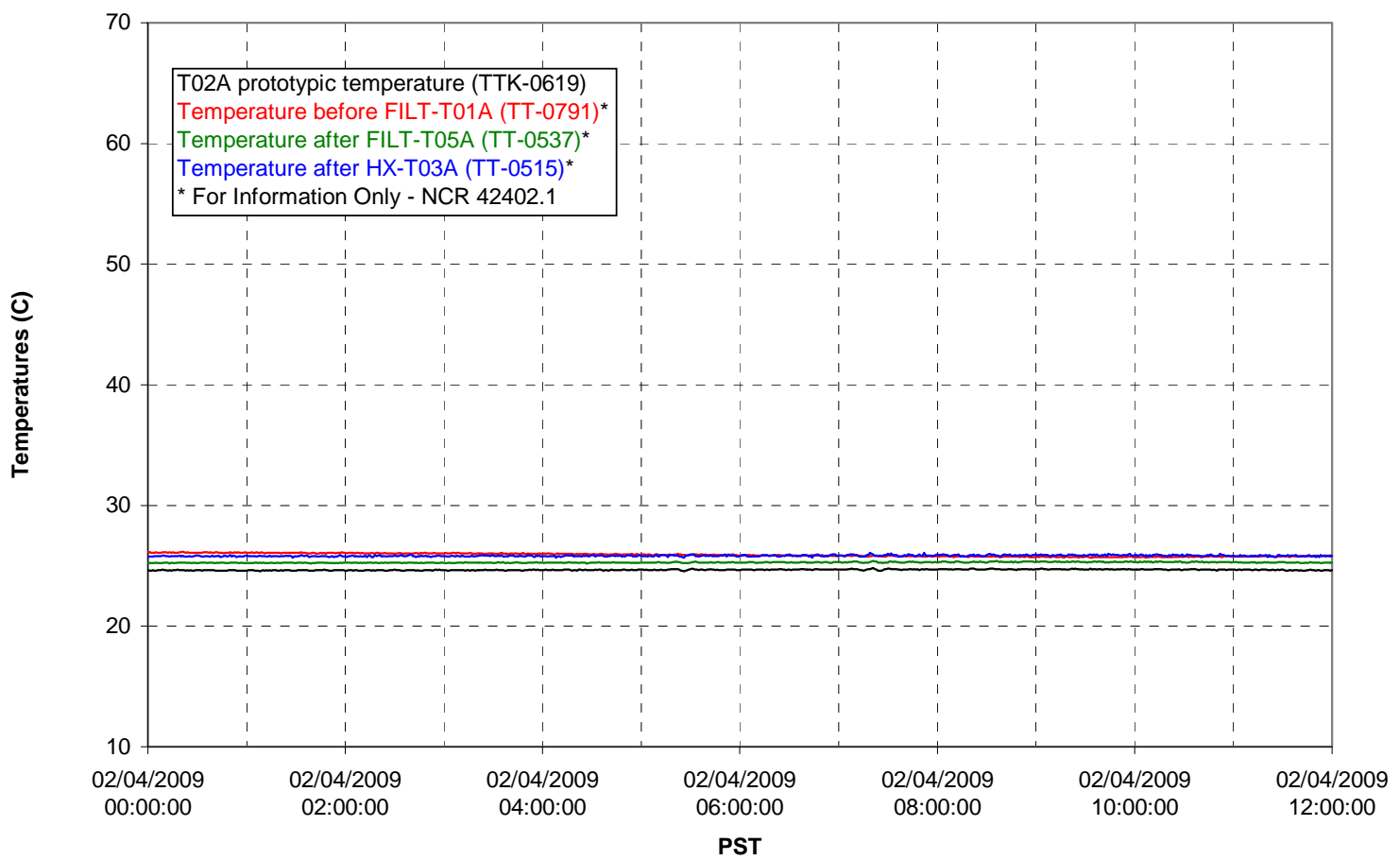

Pump Pressures and Flow

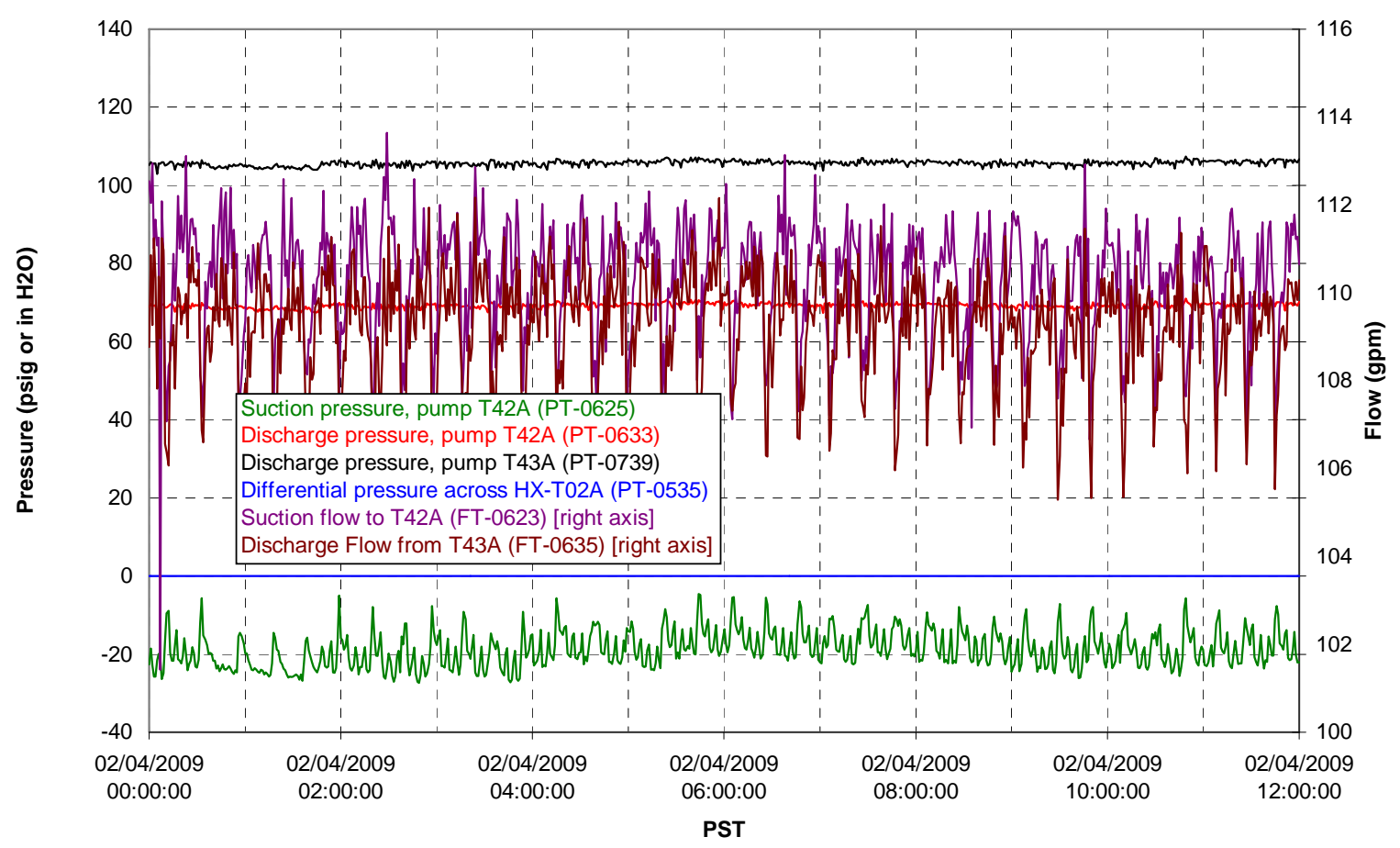


Axial pressure drop

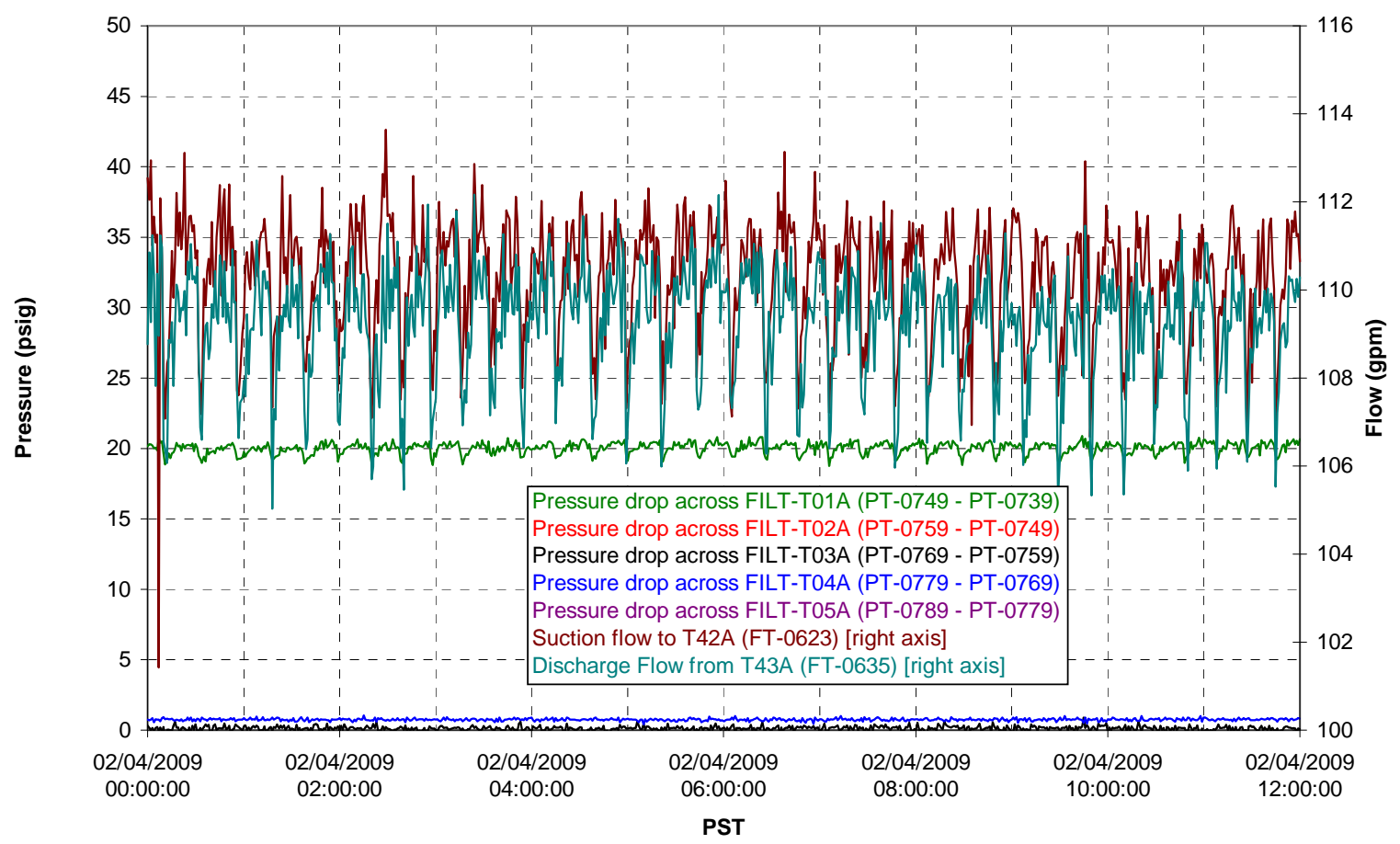

Permeate flow rates

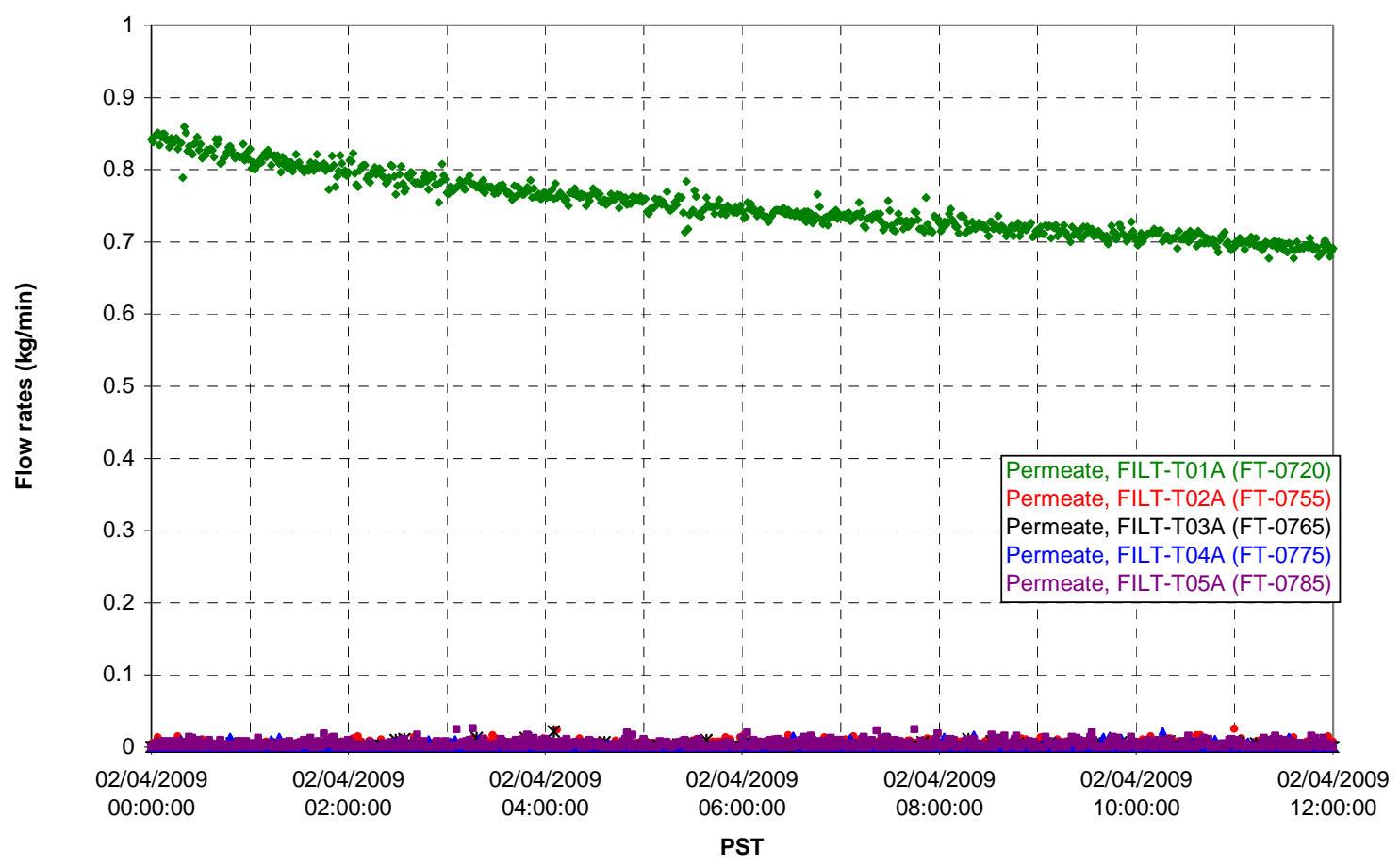

G.150 
T02A Inner Temperature Tree

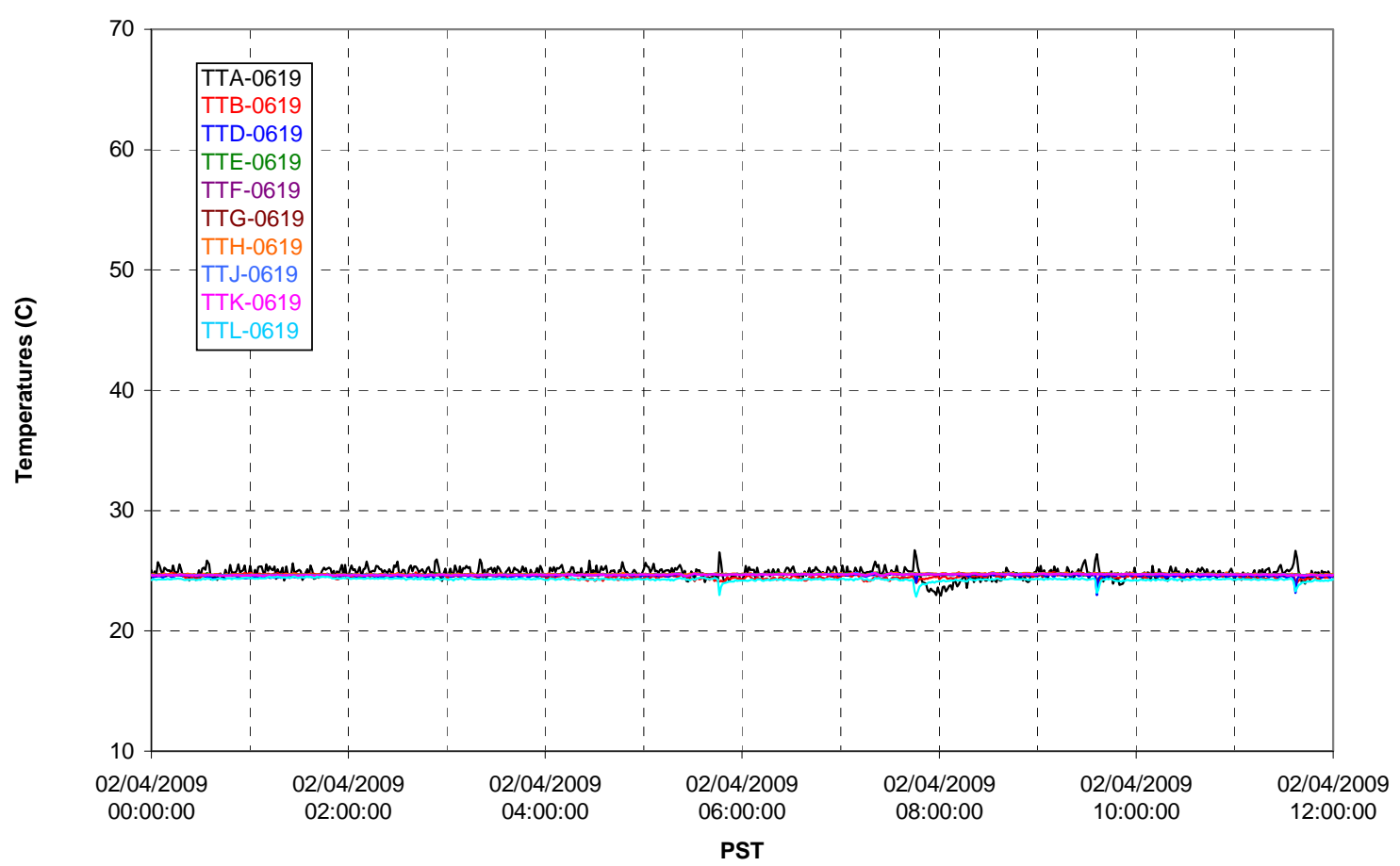

T02A Outer Temperature Tree

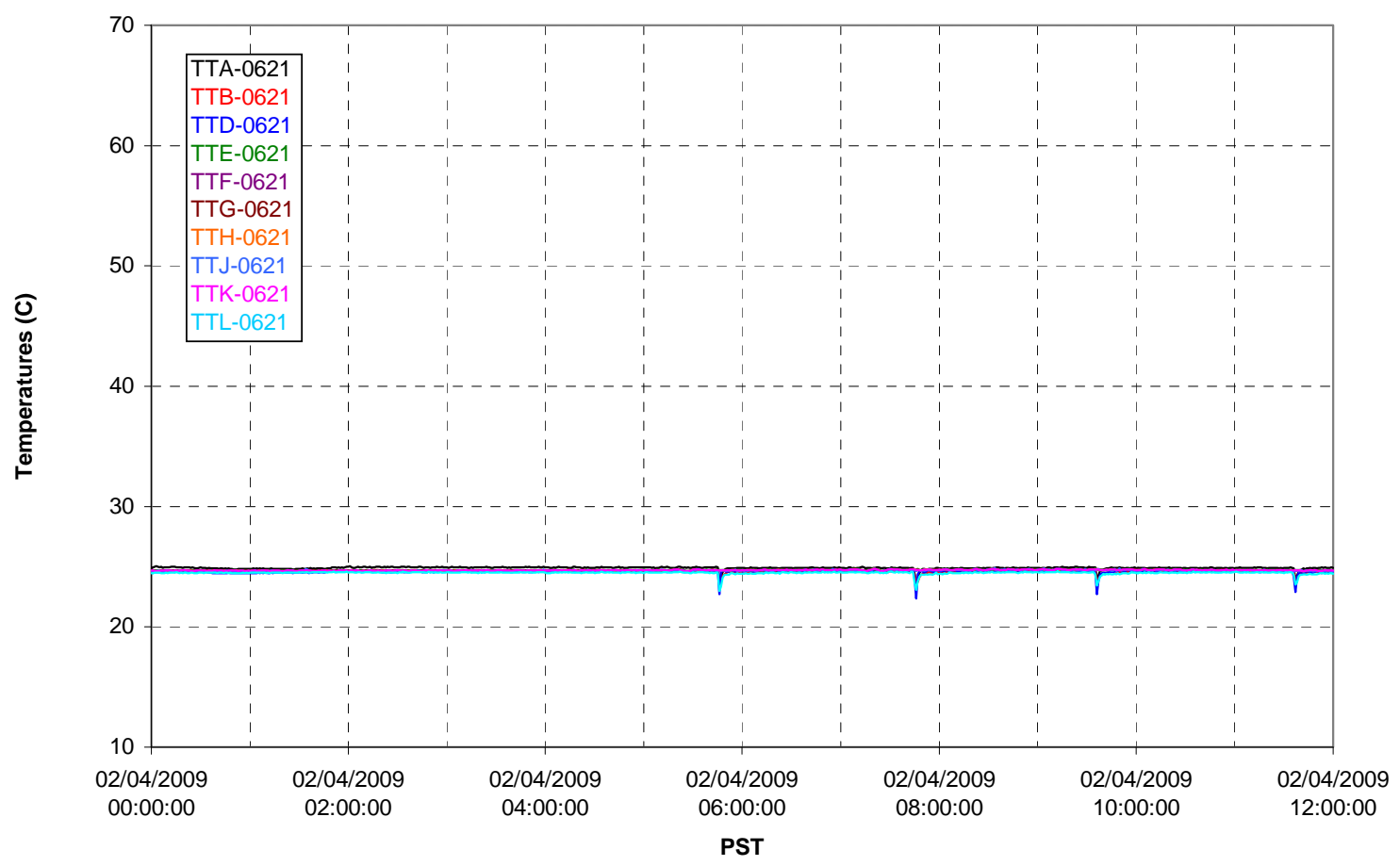

G.151 
T02A temperatures

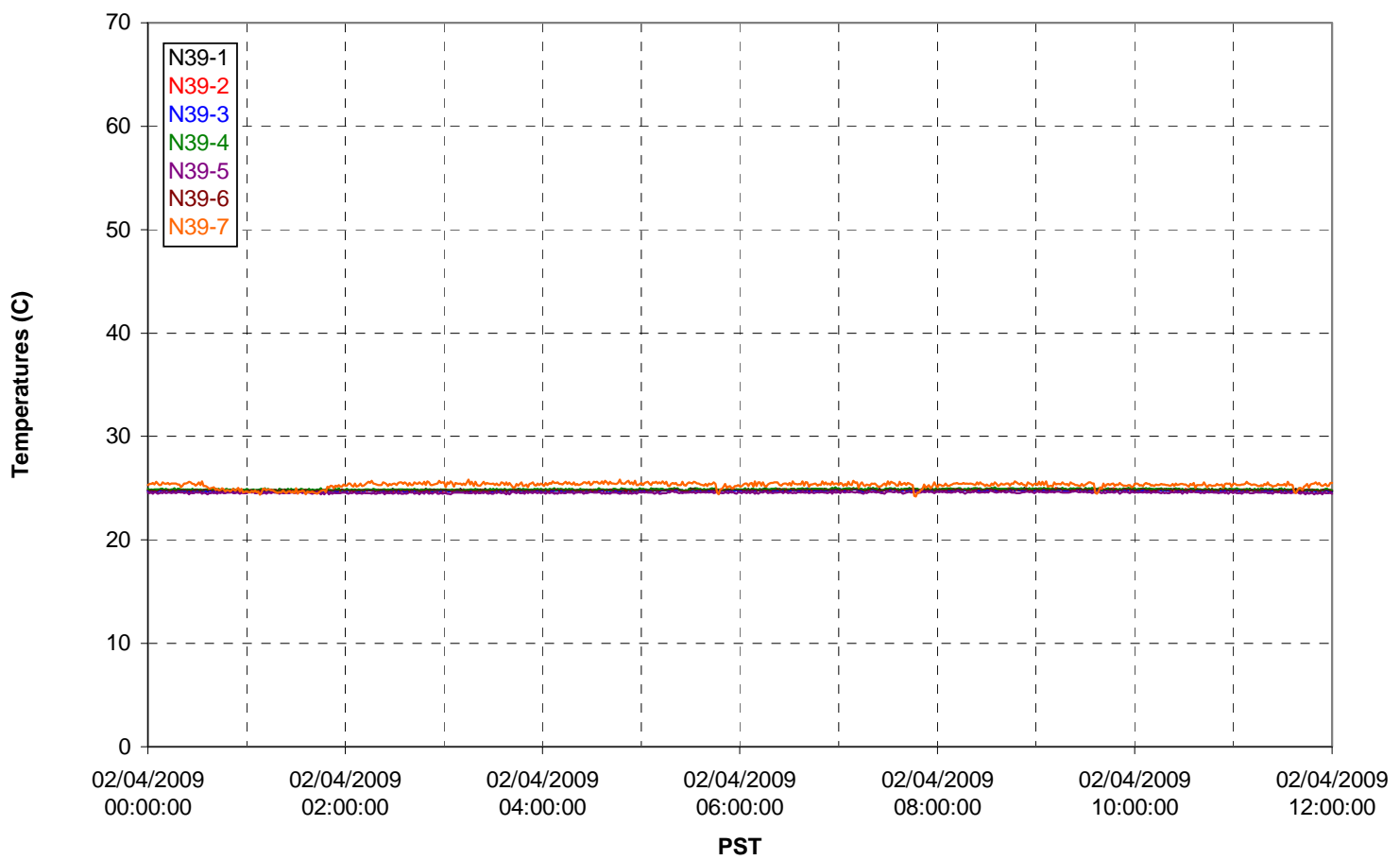

T02A temperatures

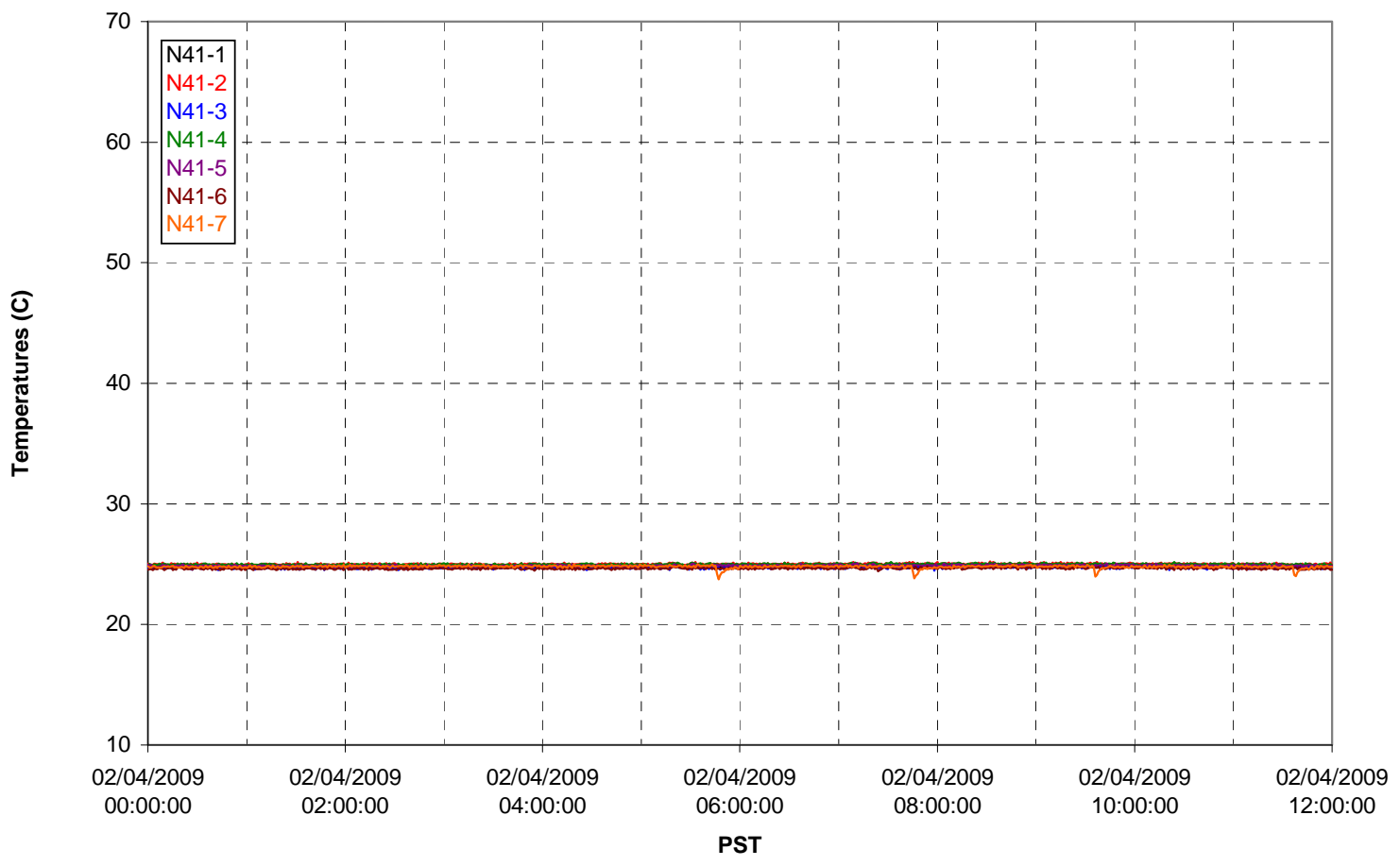

G.152 
T02A temperatures

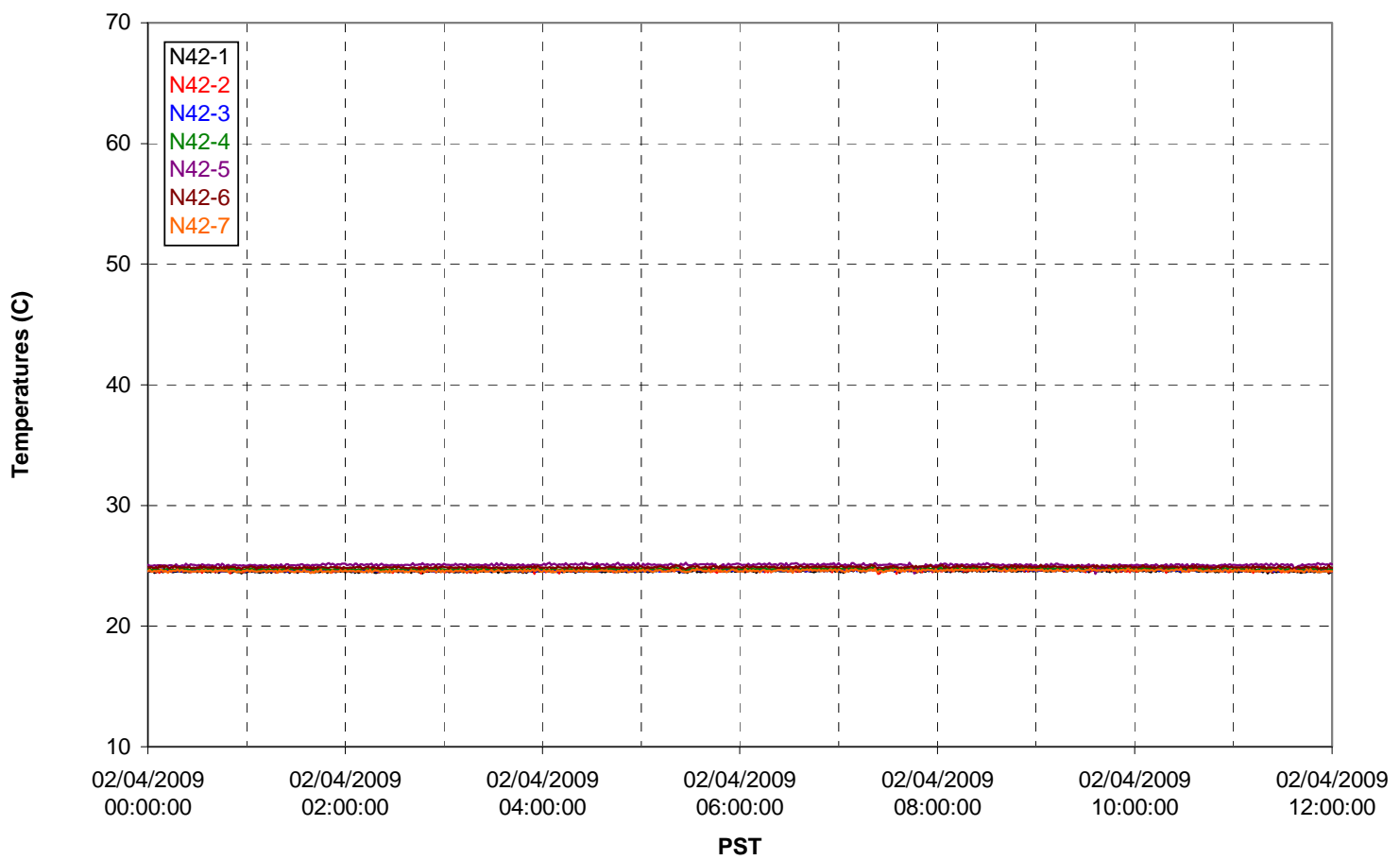

T02A temperatures

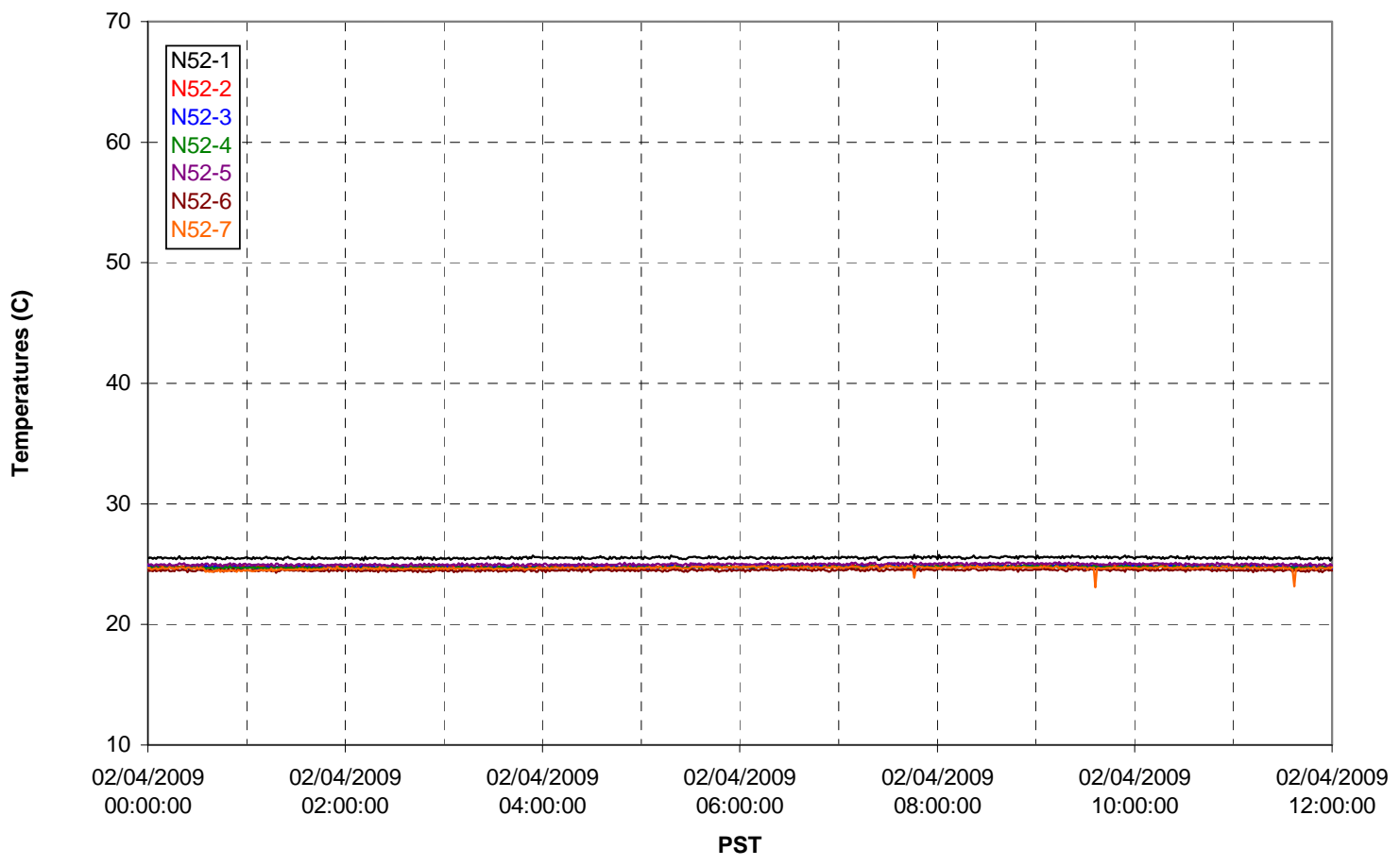

G.153 
T02A Heating and Cooling

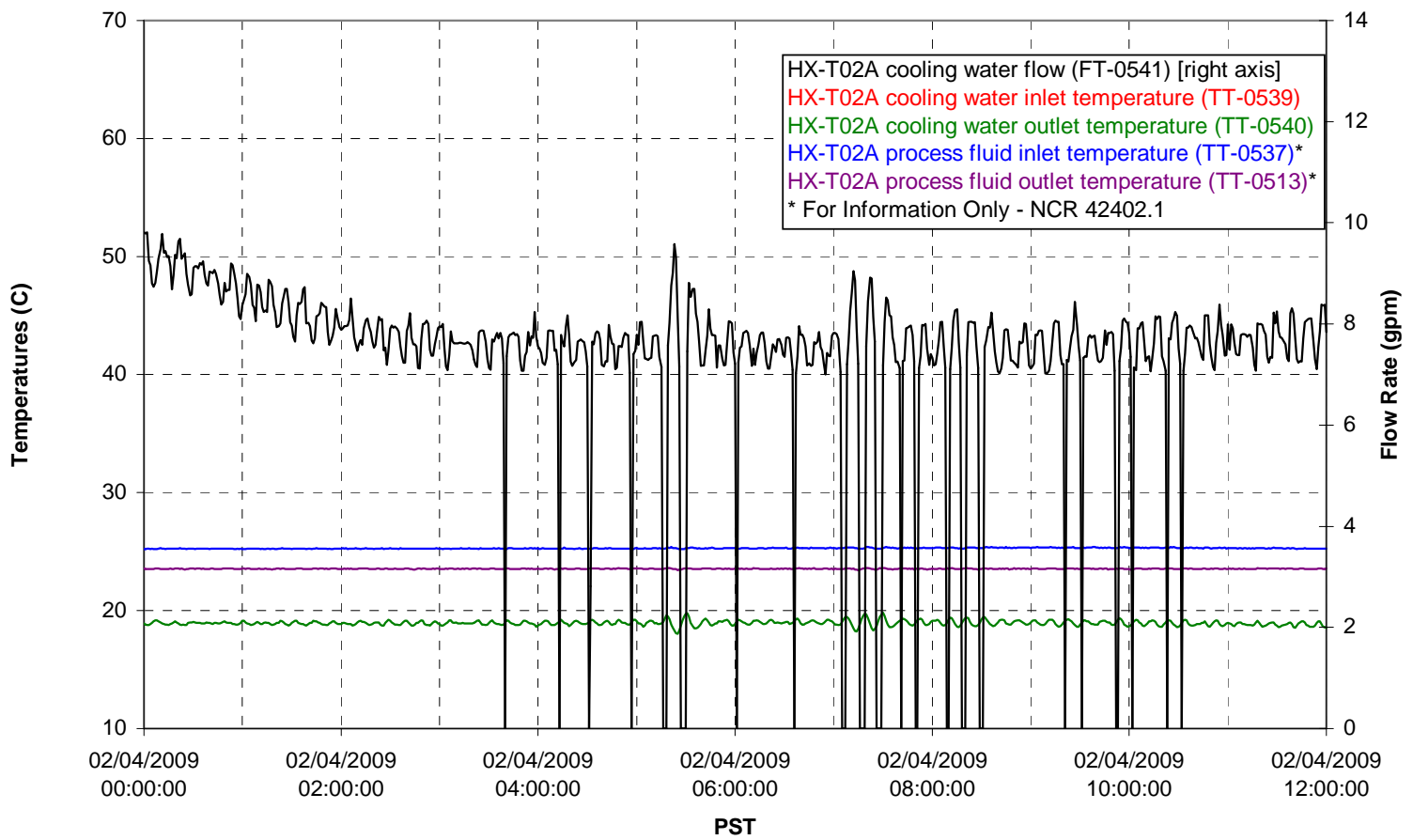

Pump Operation

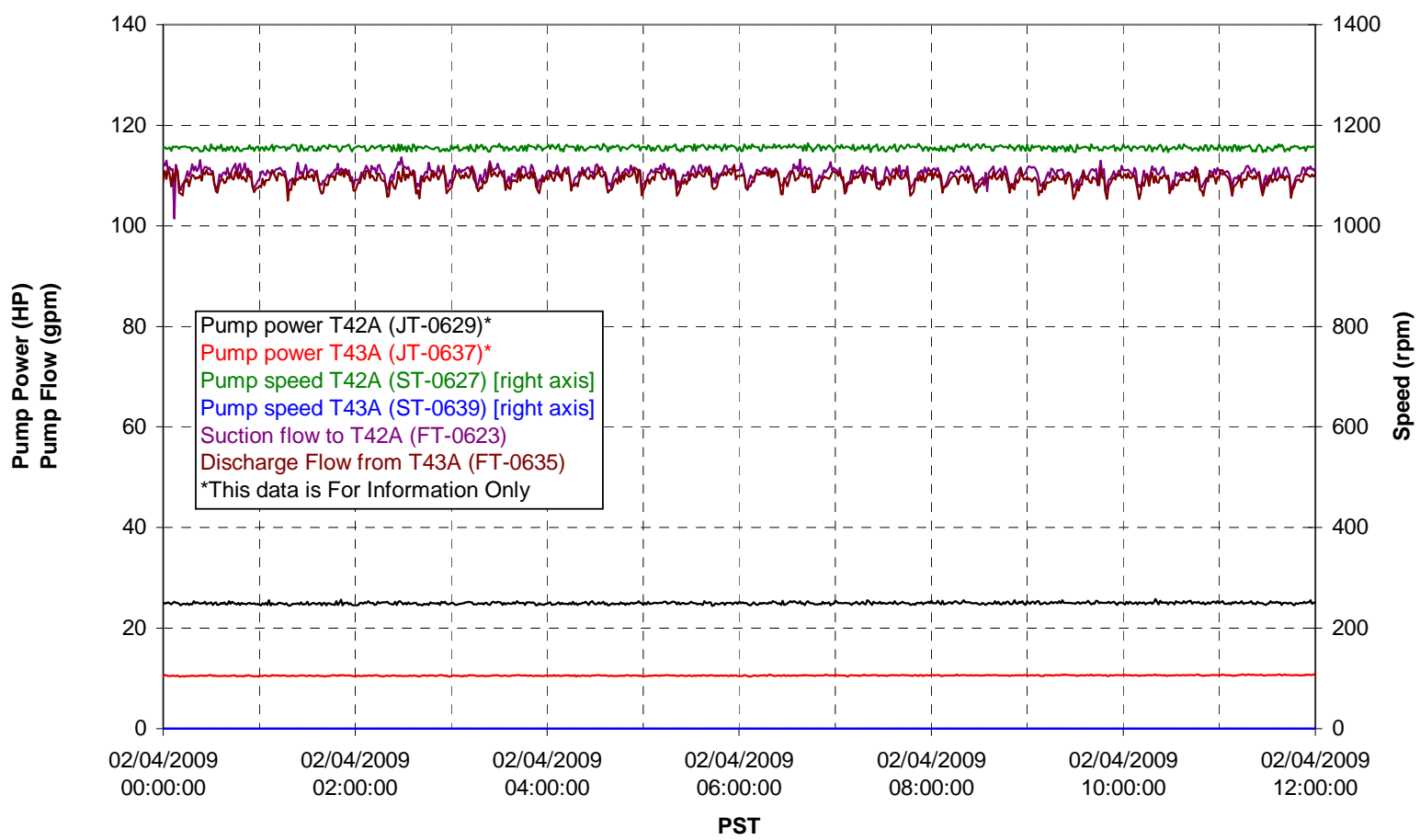

G.154 
Pulsepot UFP-PP-T01A

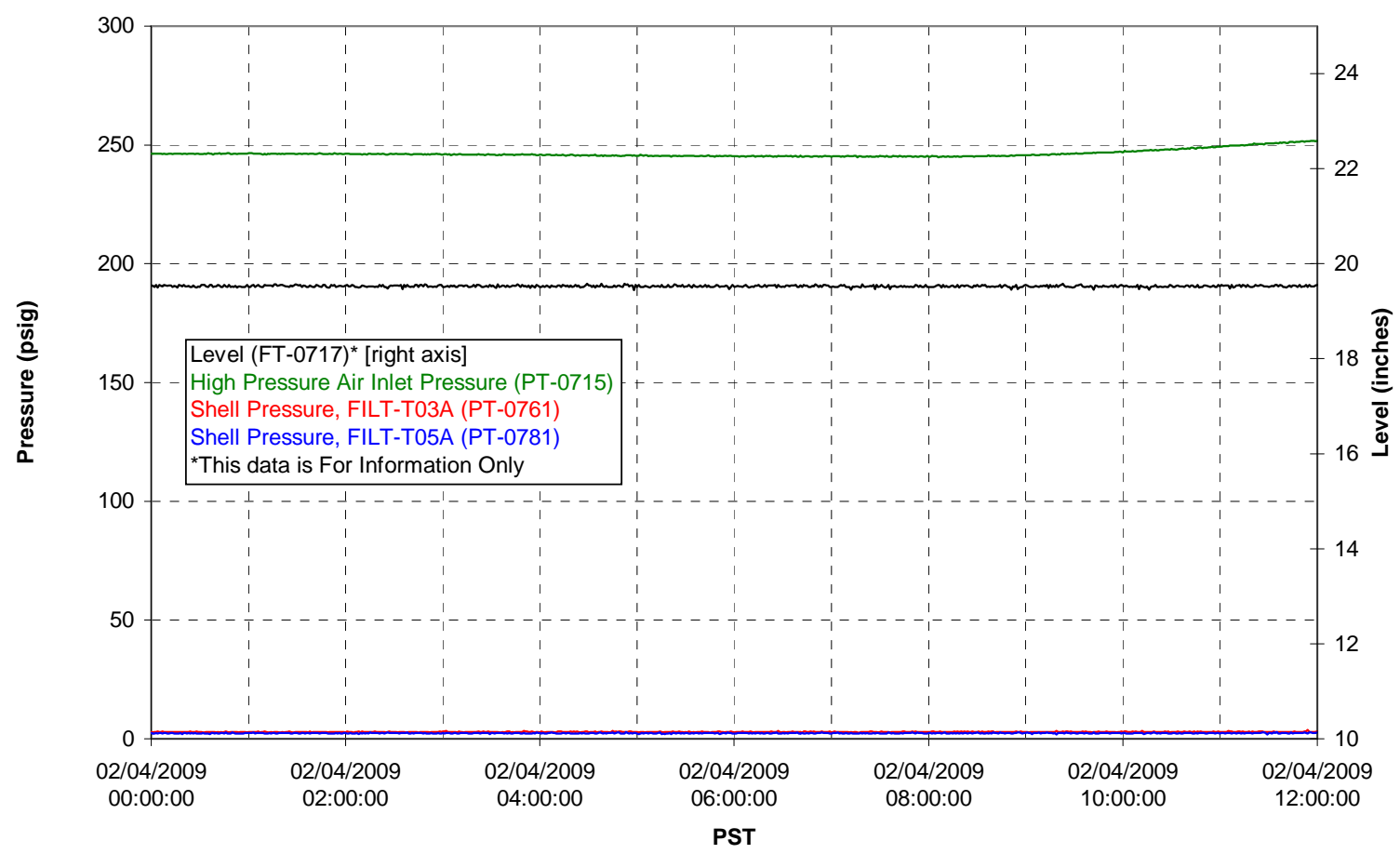

Pulsepot UFP-PP-T02A

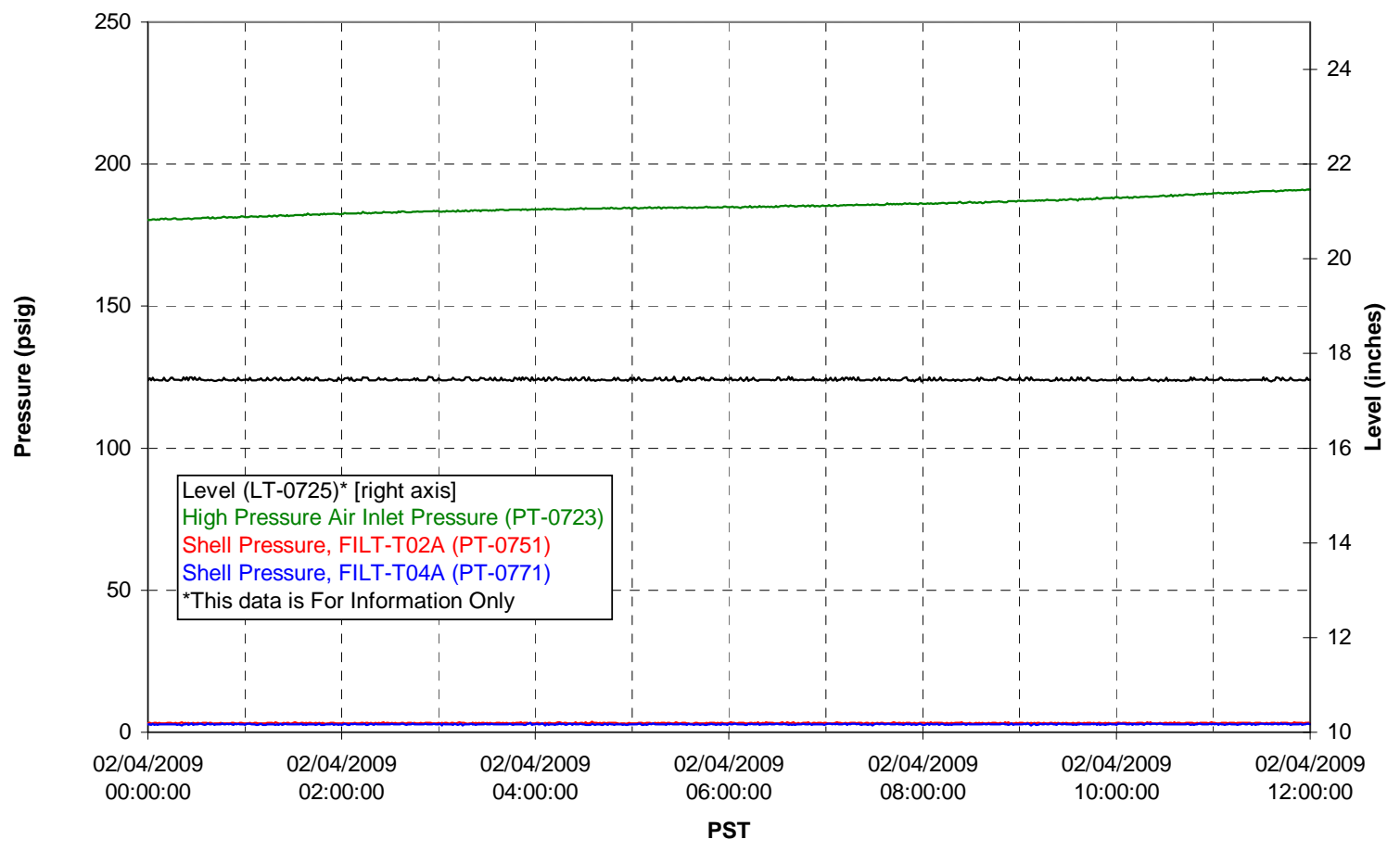

G. 155 
Pulsepot UFP-PP-T03A

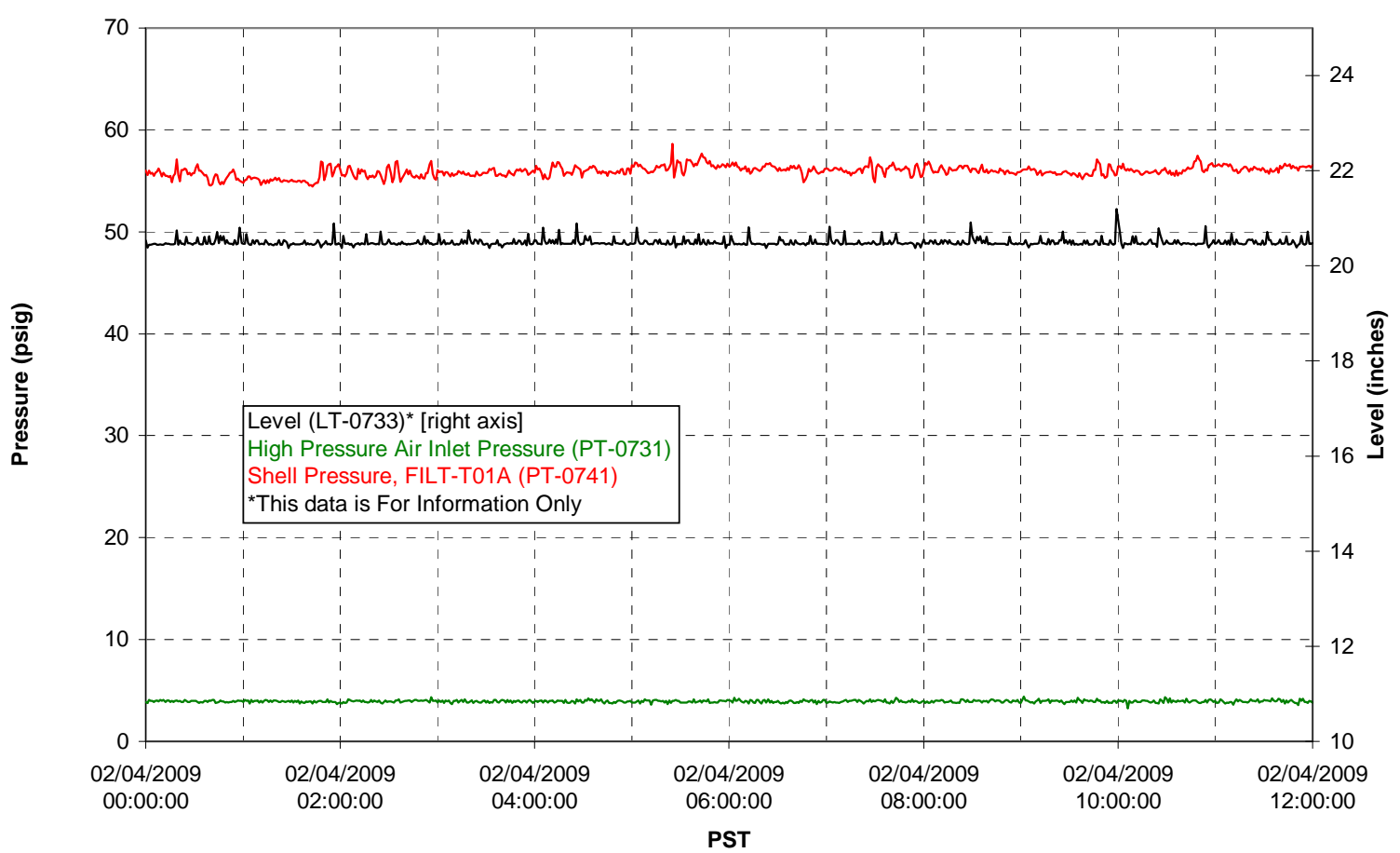

Pulsepot Levels

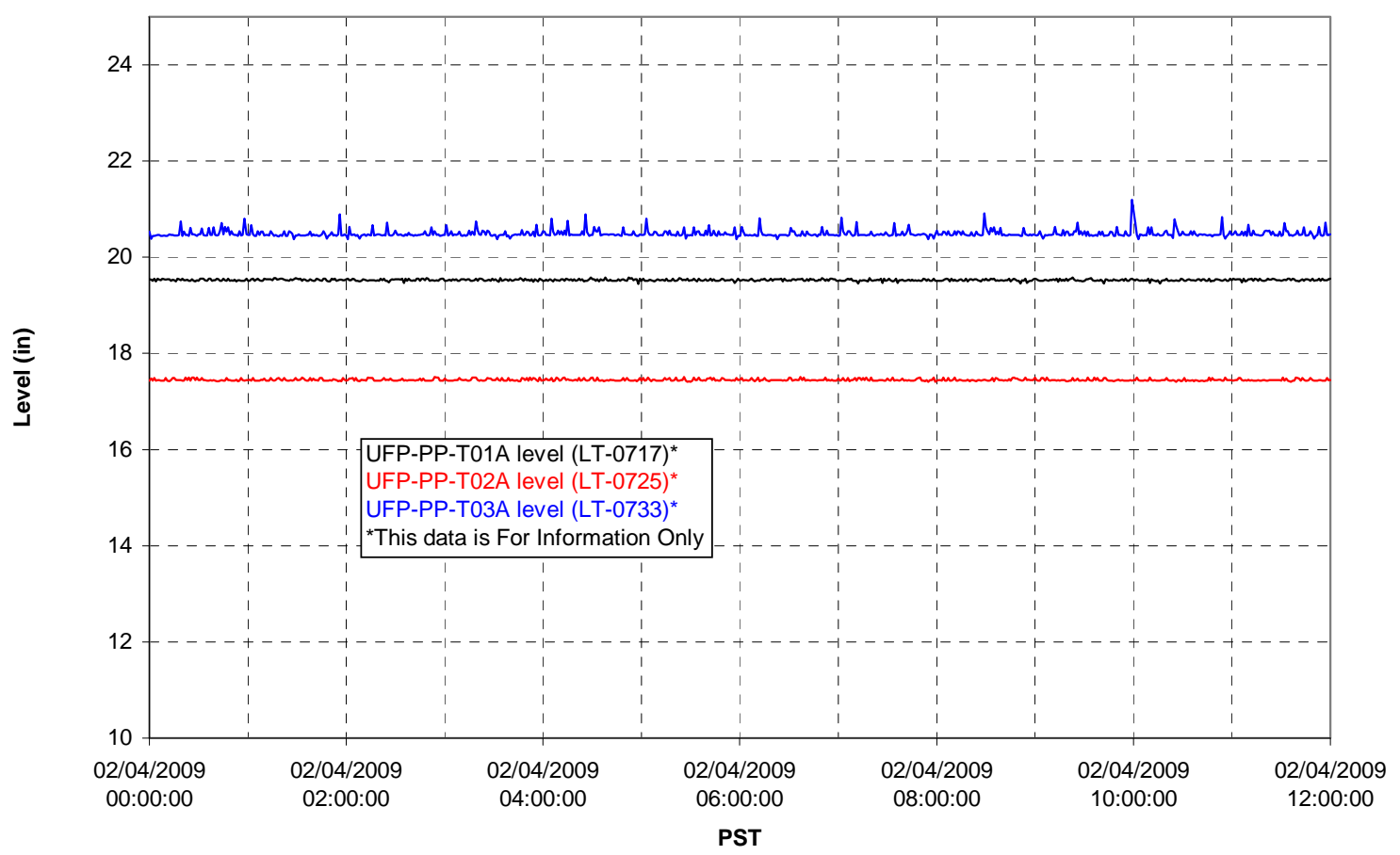

G.156 
Filter UFP-FILT-T01A

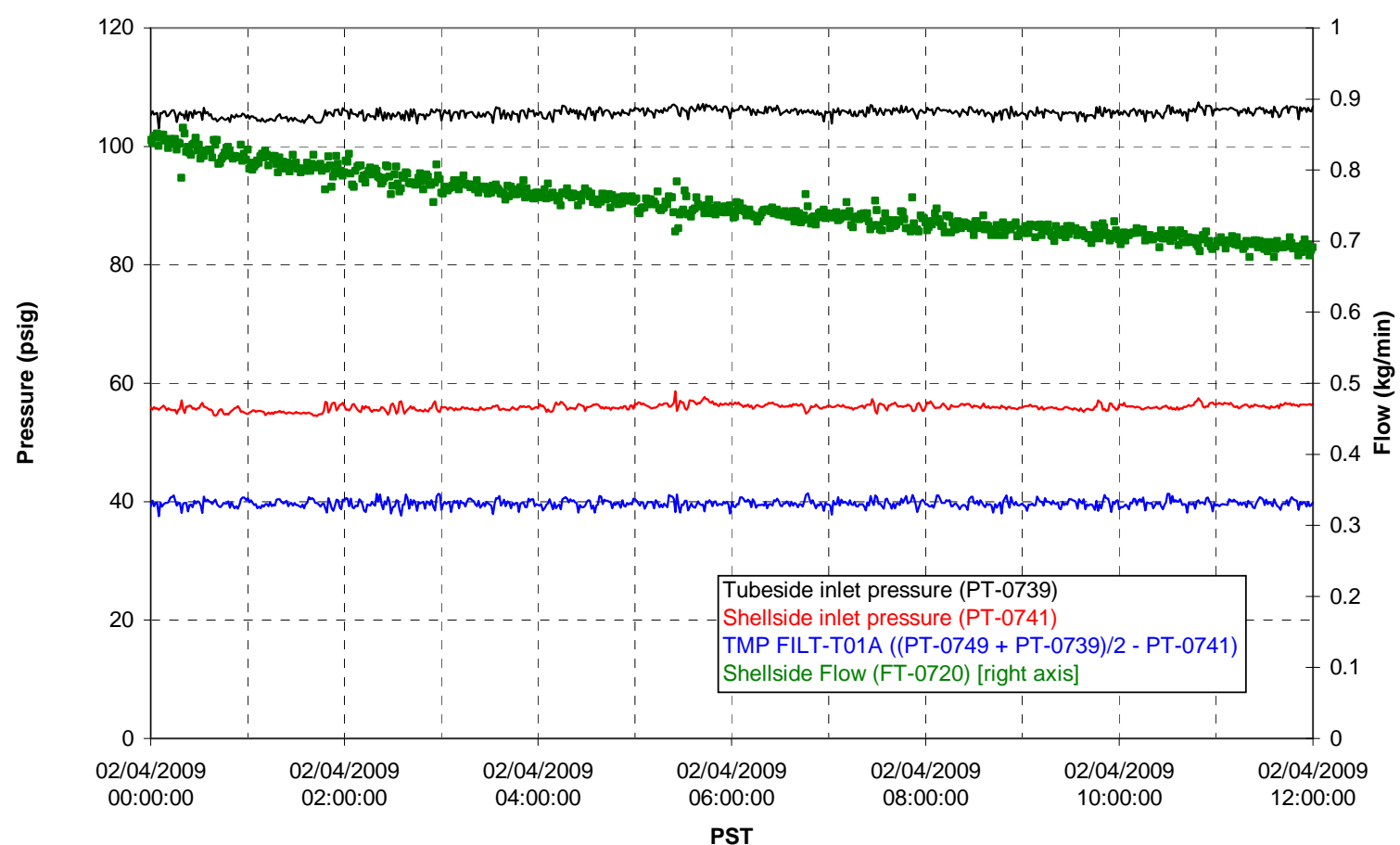

Filter UFP-FILT-T02A

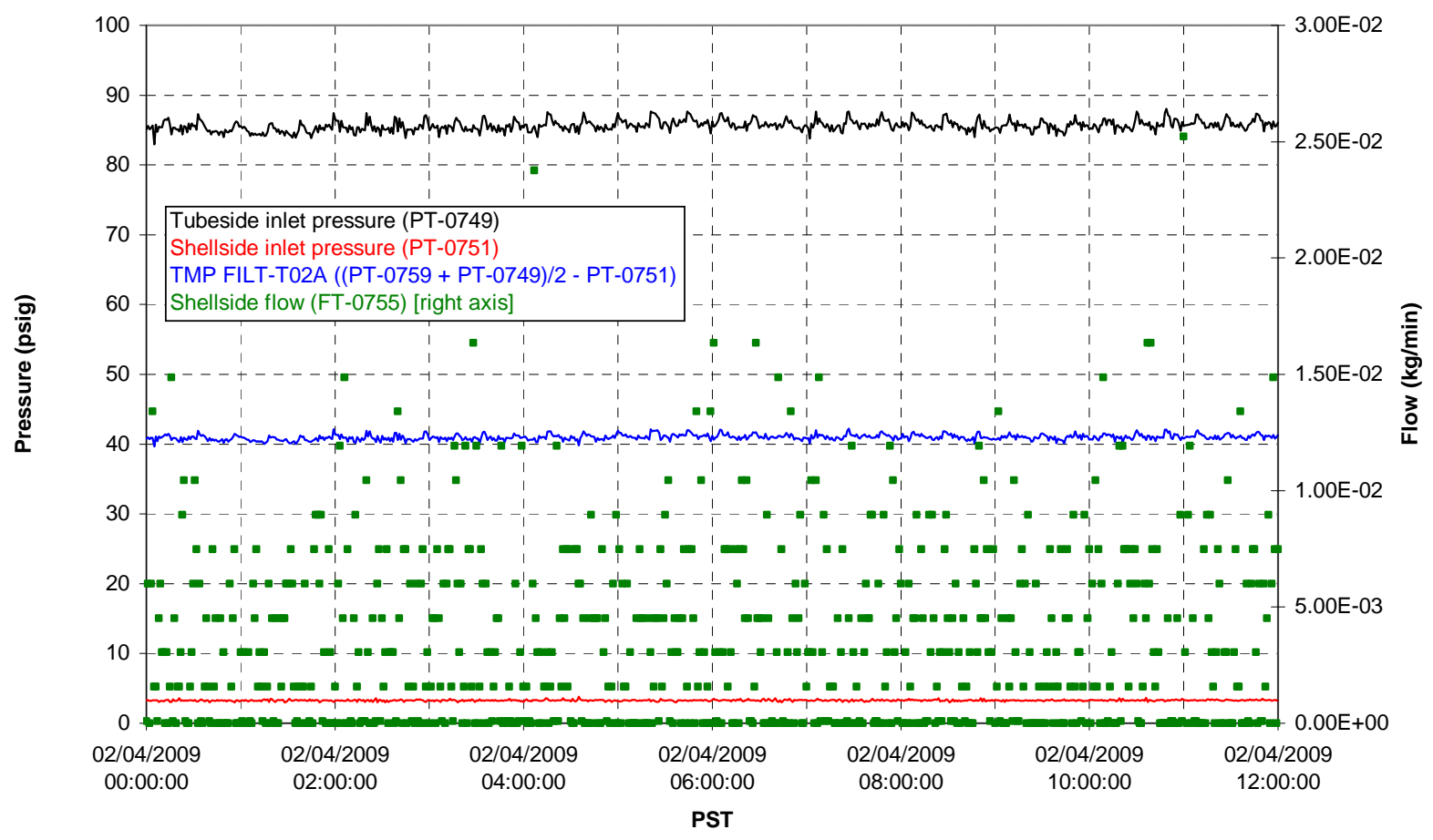


Filter UFP-FILT-T02A

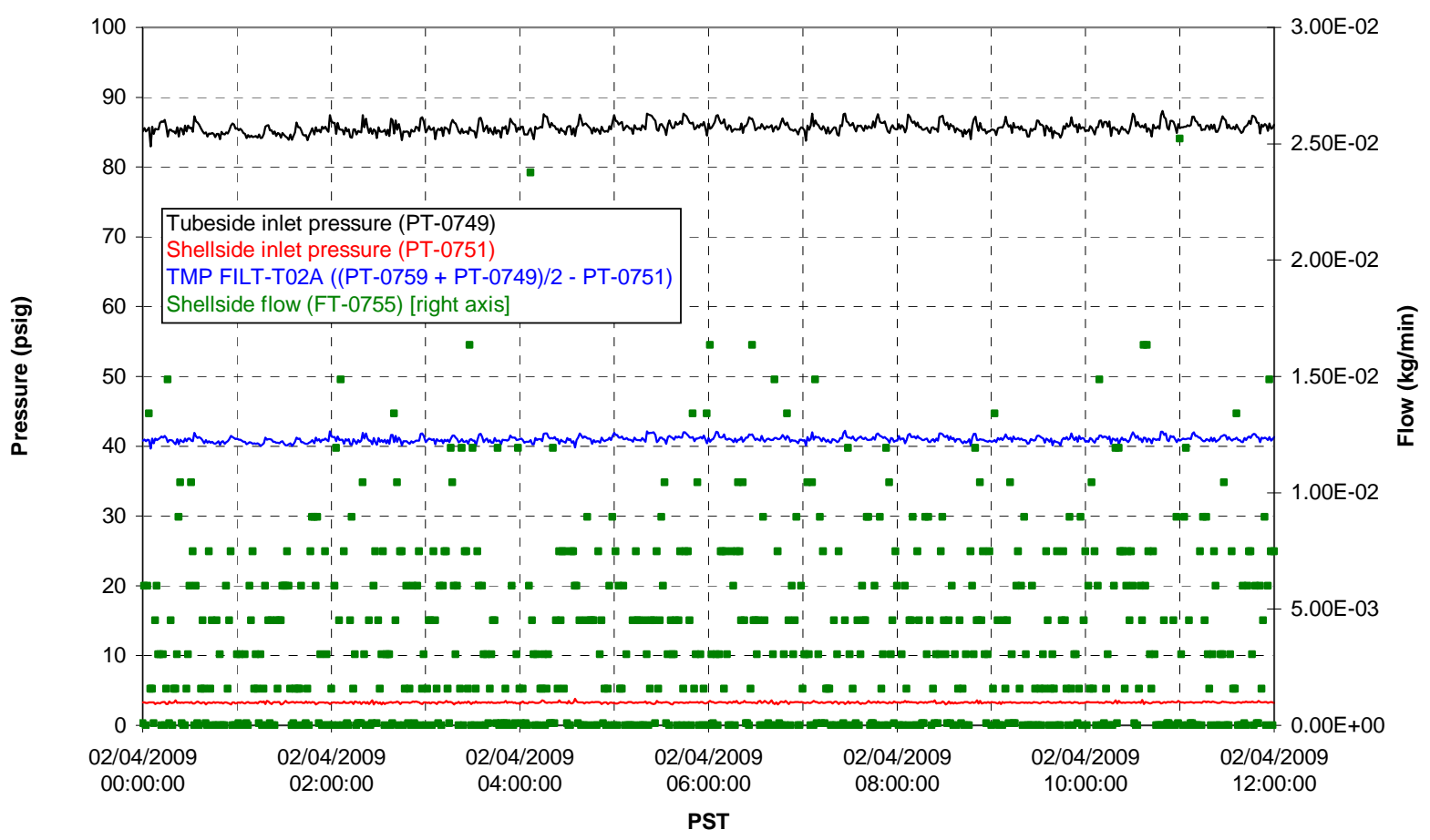

Filter UFP-FILT-T04A

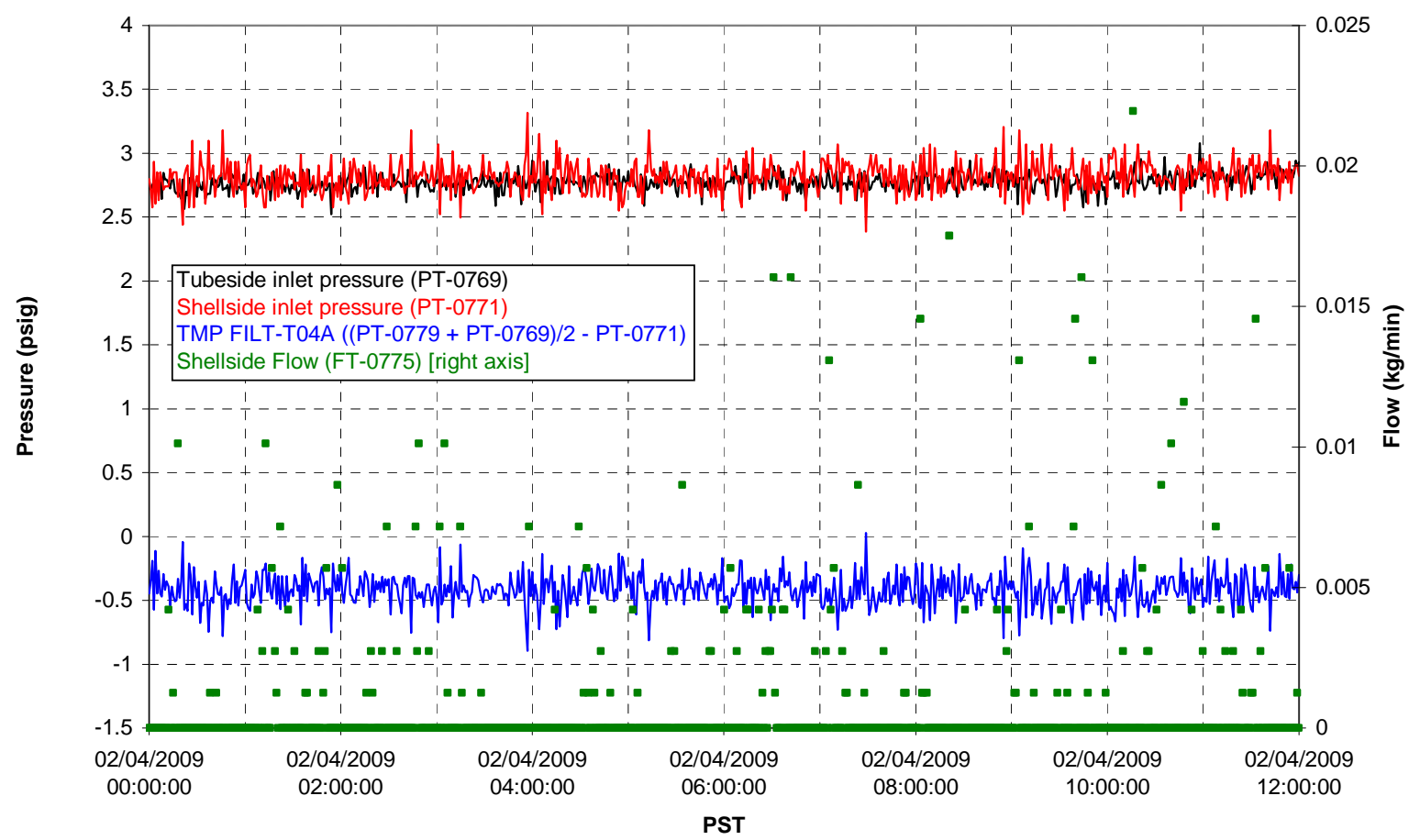


Filter UFP-FILT-T05A

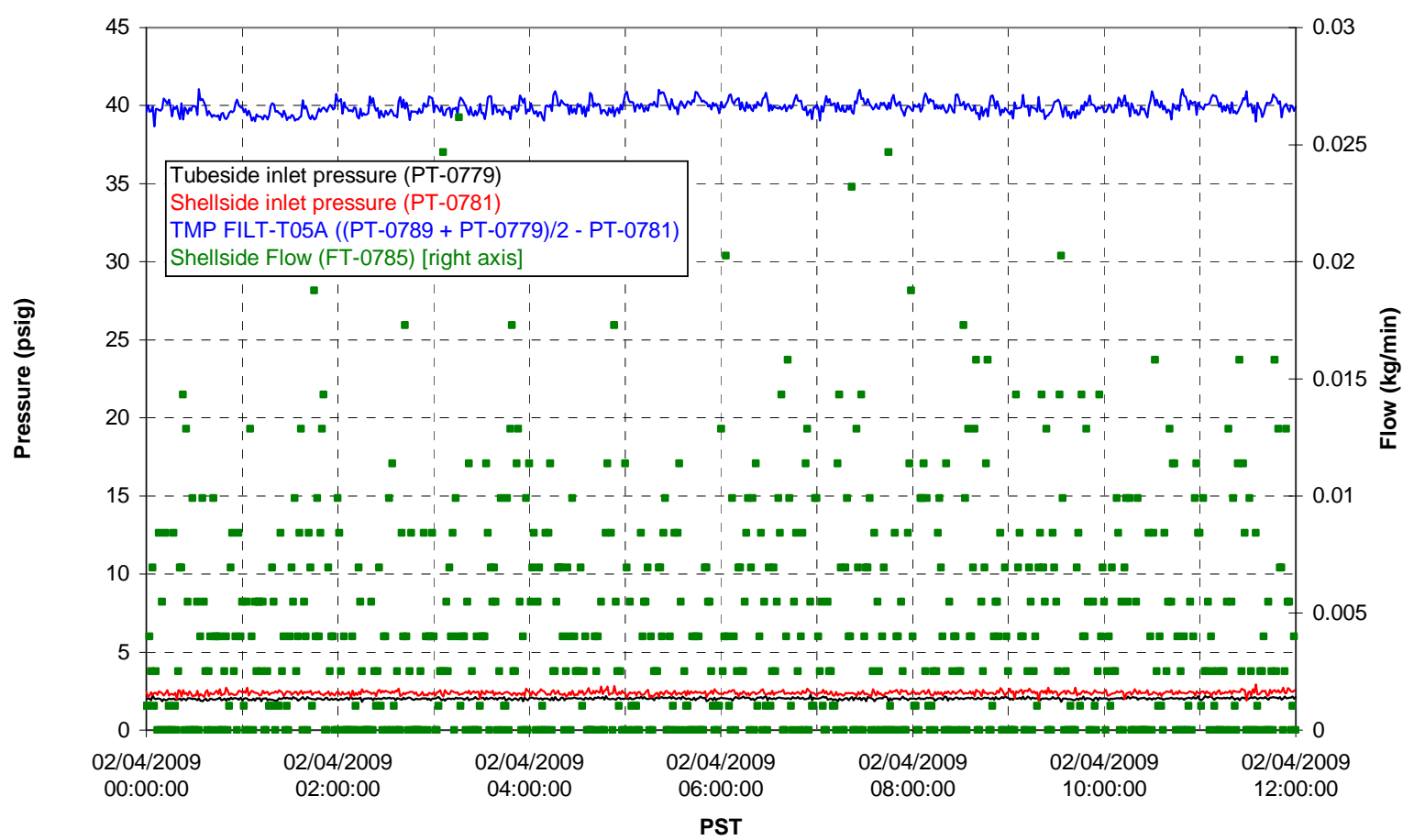

Chemical Flow

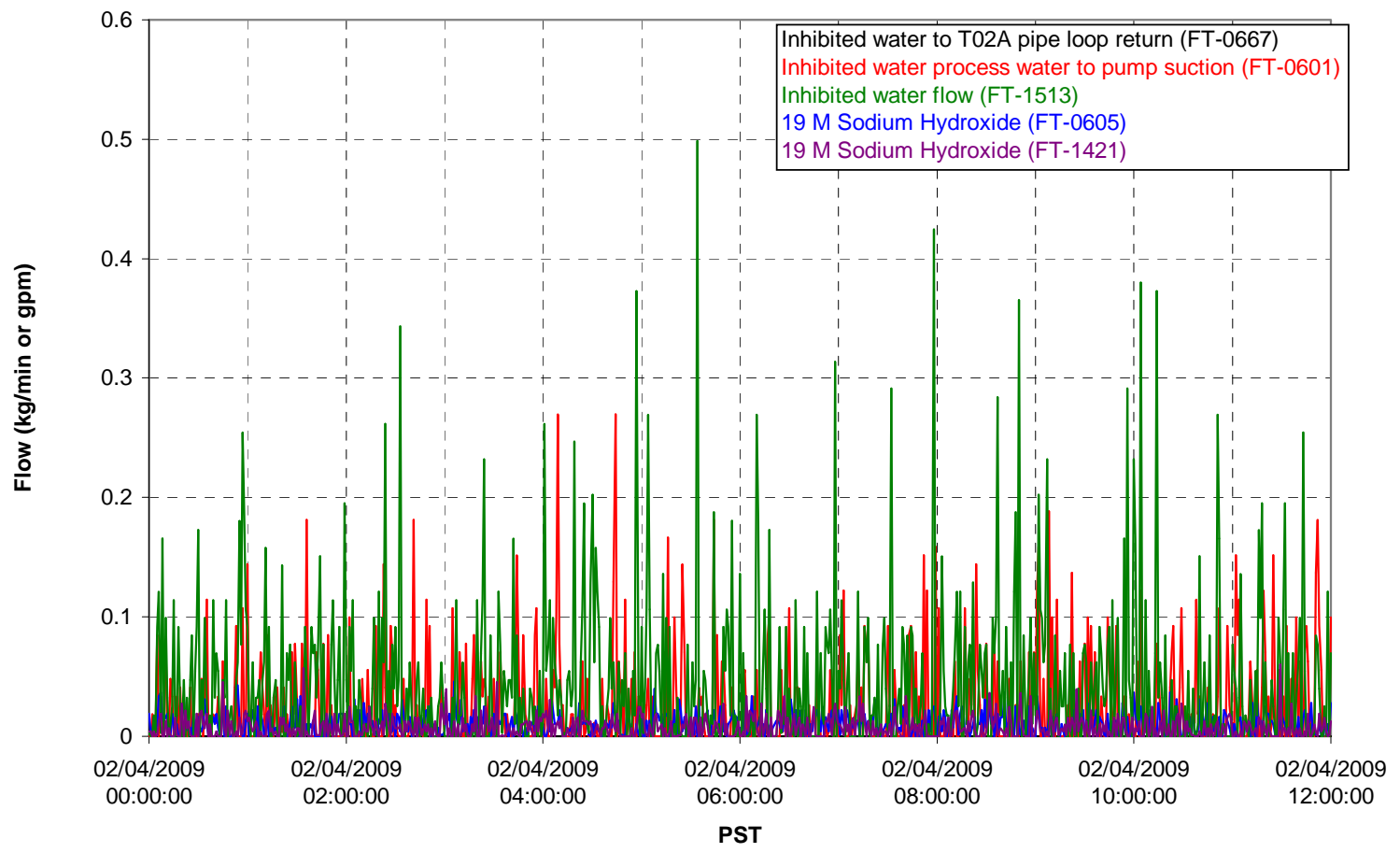

G.159 
Chemical Flow

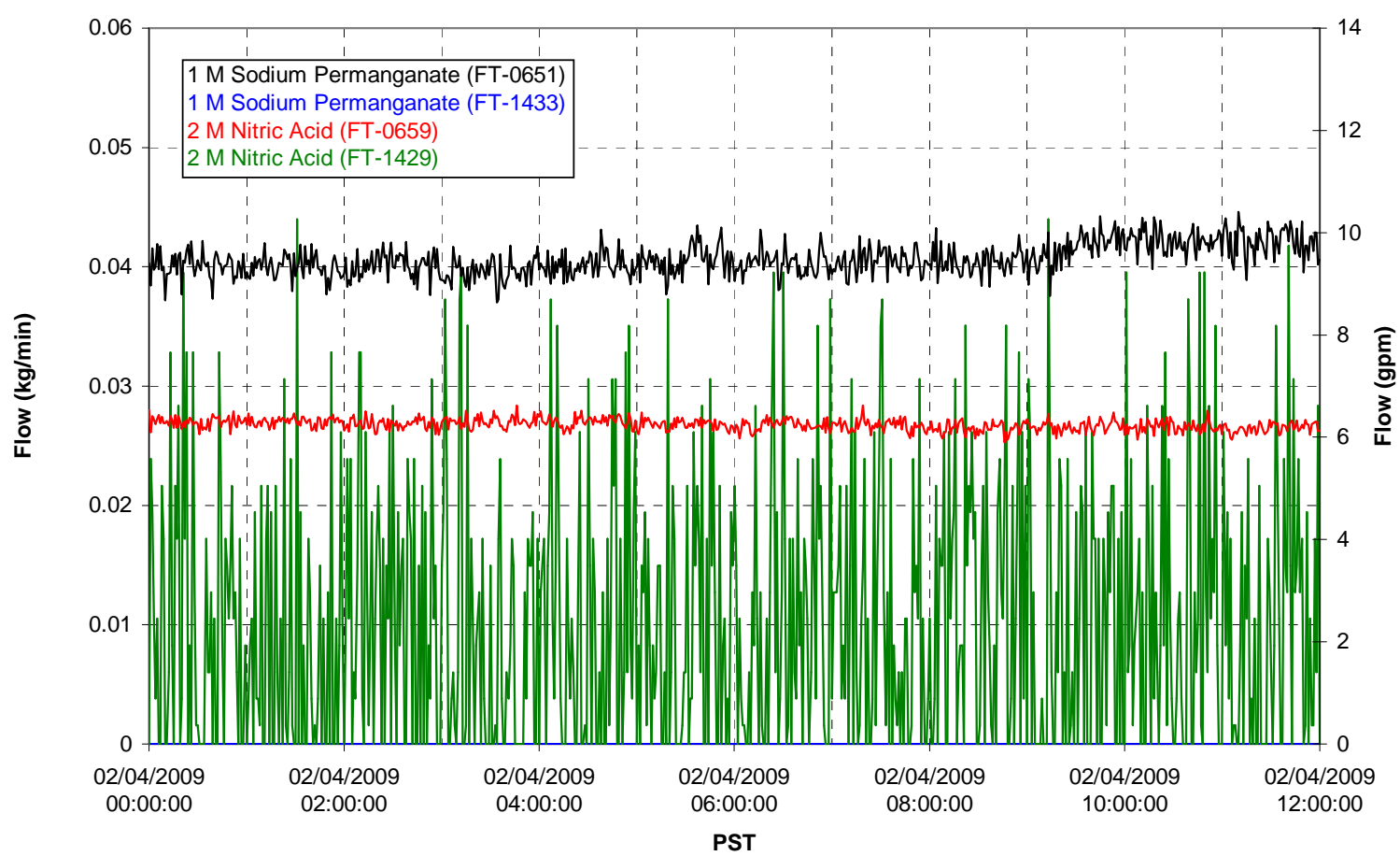

Air Flows

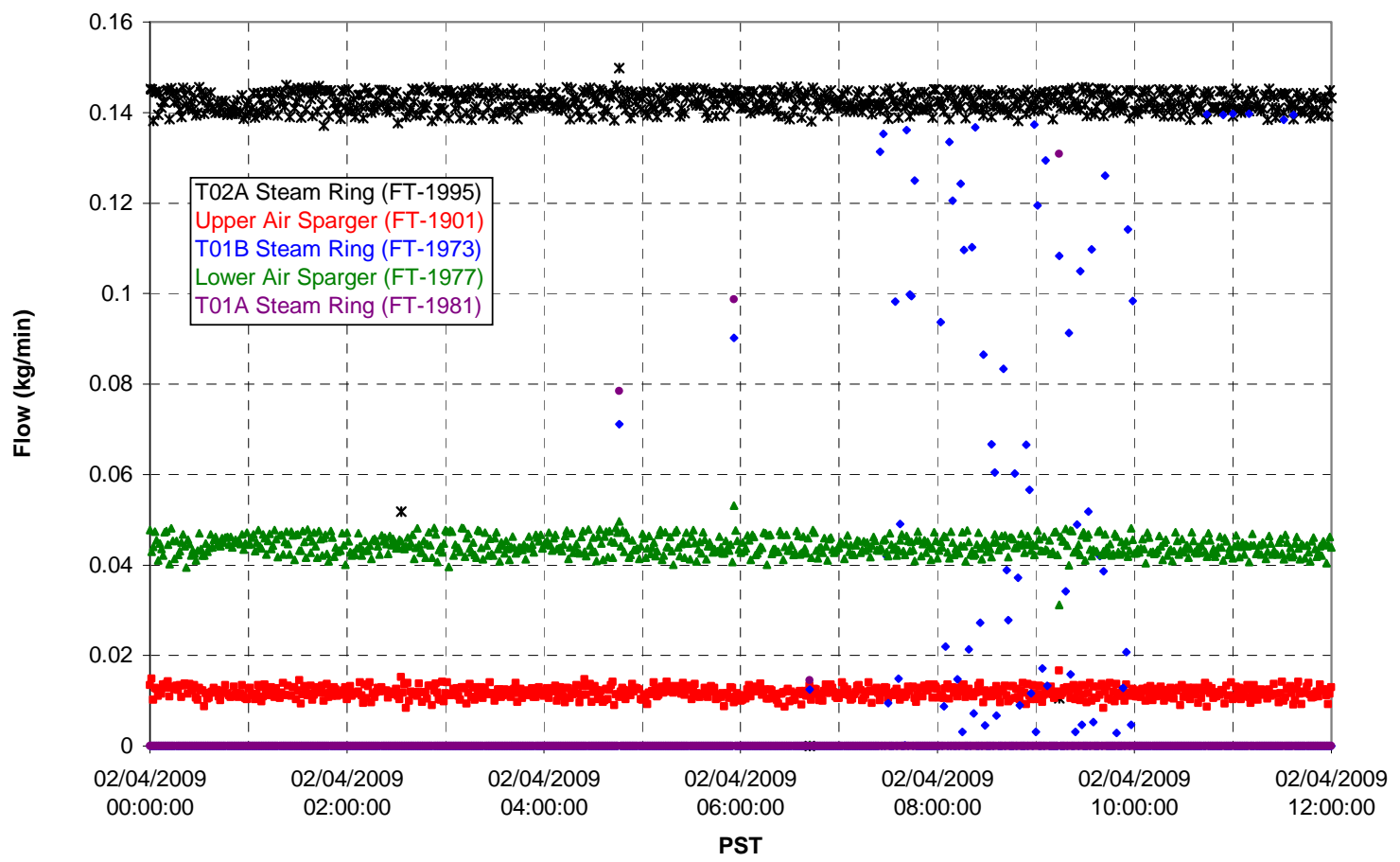

G.160 
T02A Steam

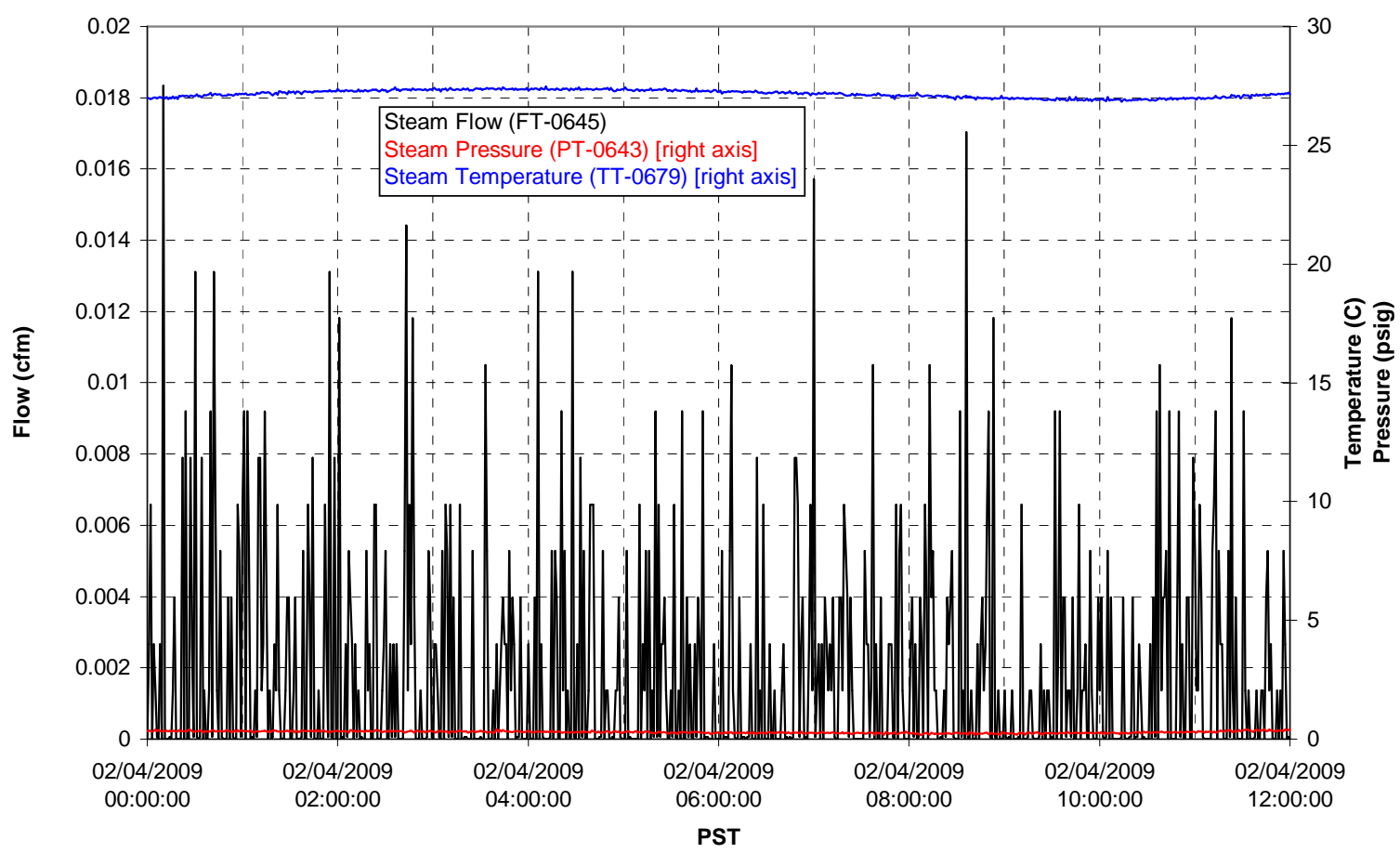

T01A Steam

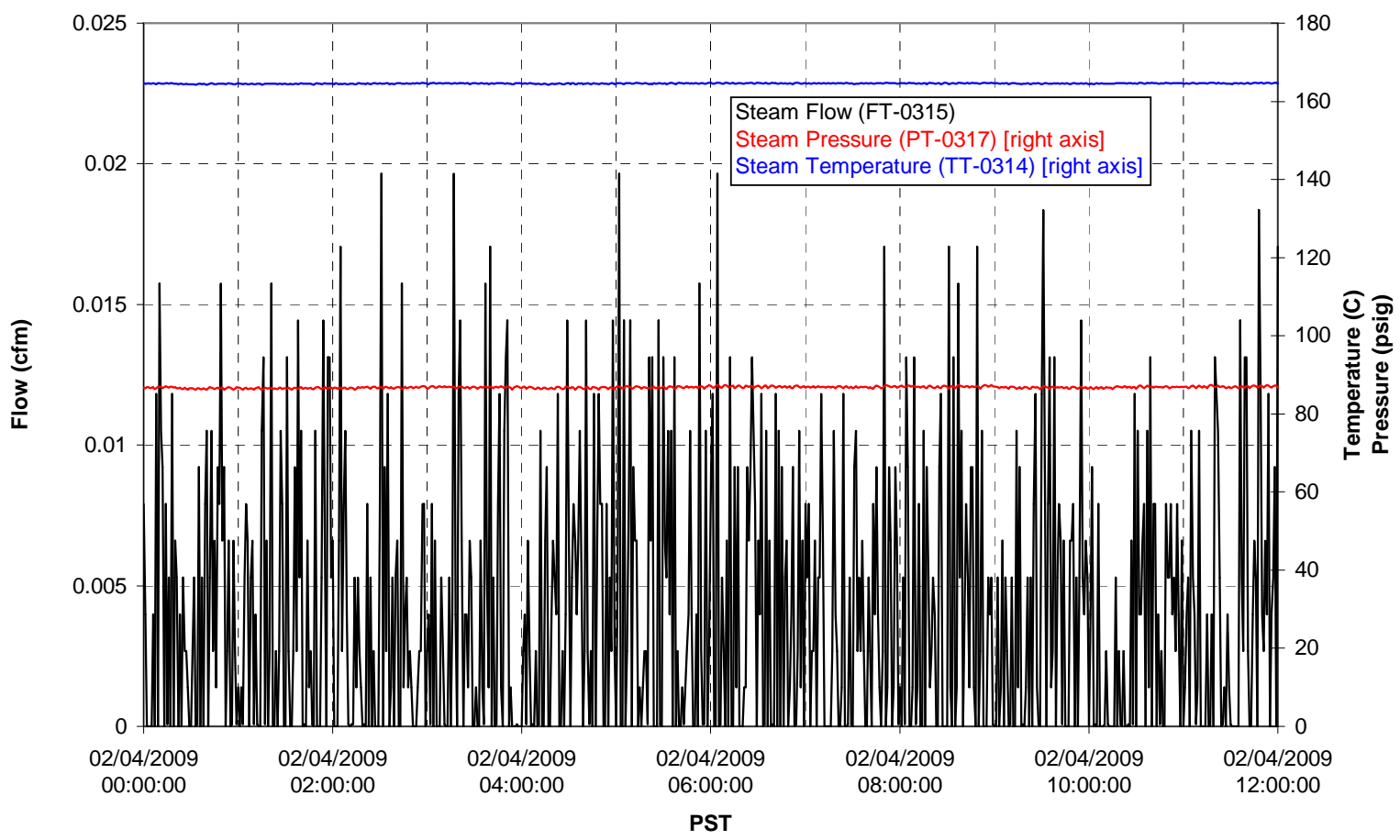


T01B Steam

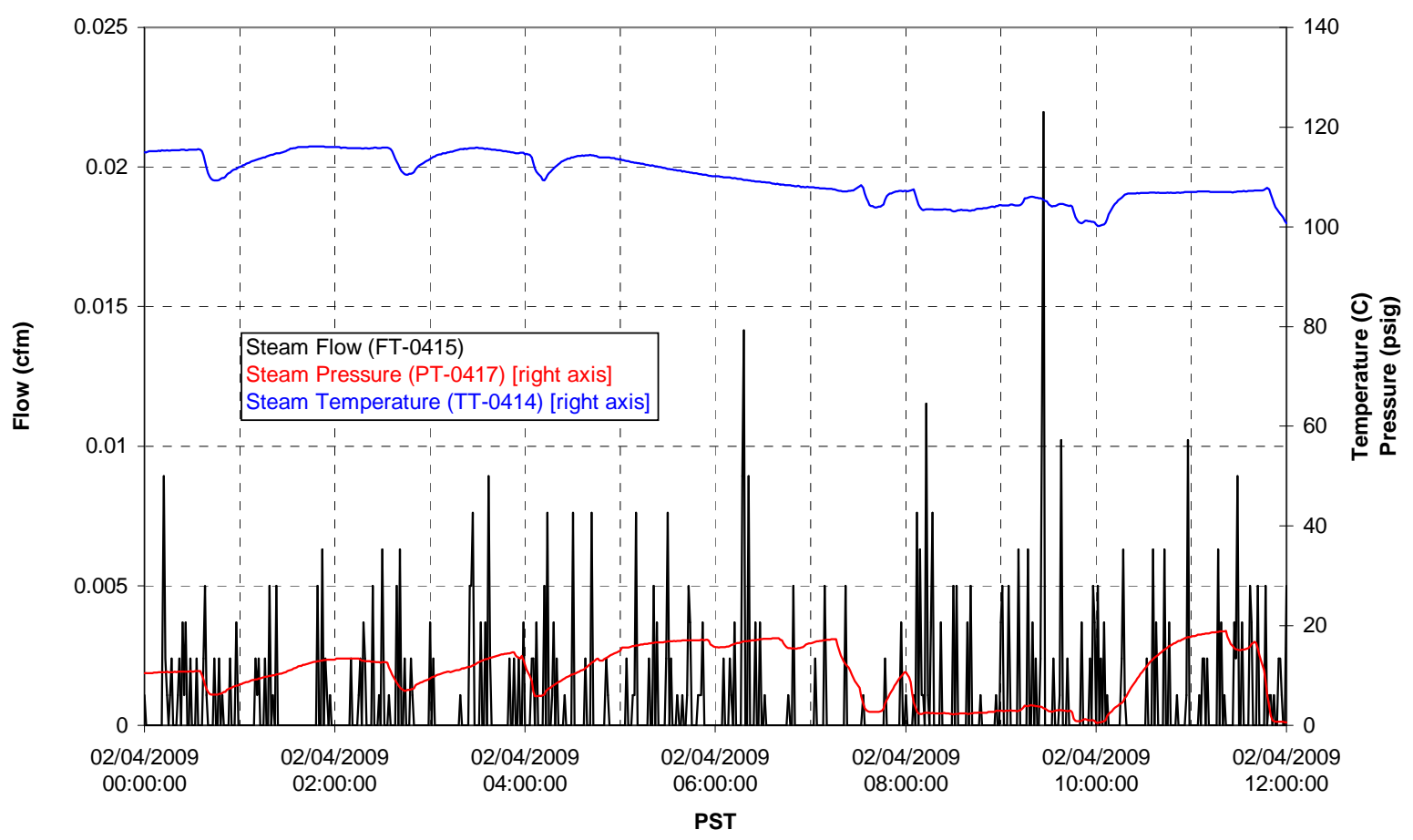




\section{Integrated Test A Data Plots 02/04/09 12:00 - 02/05/09 00:00}


T01A level

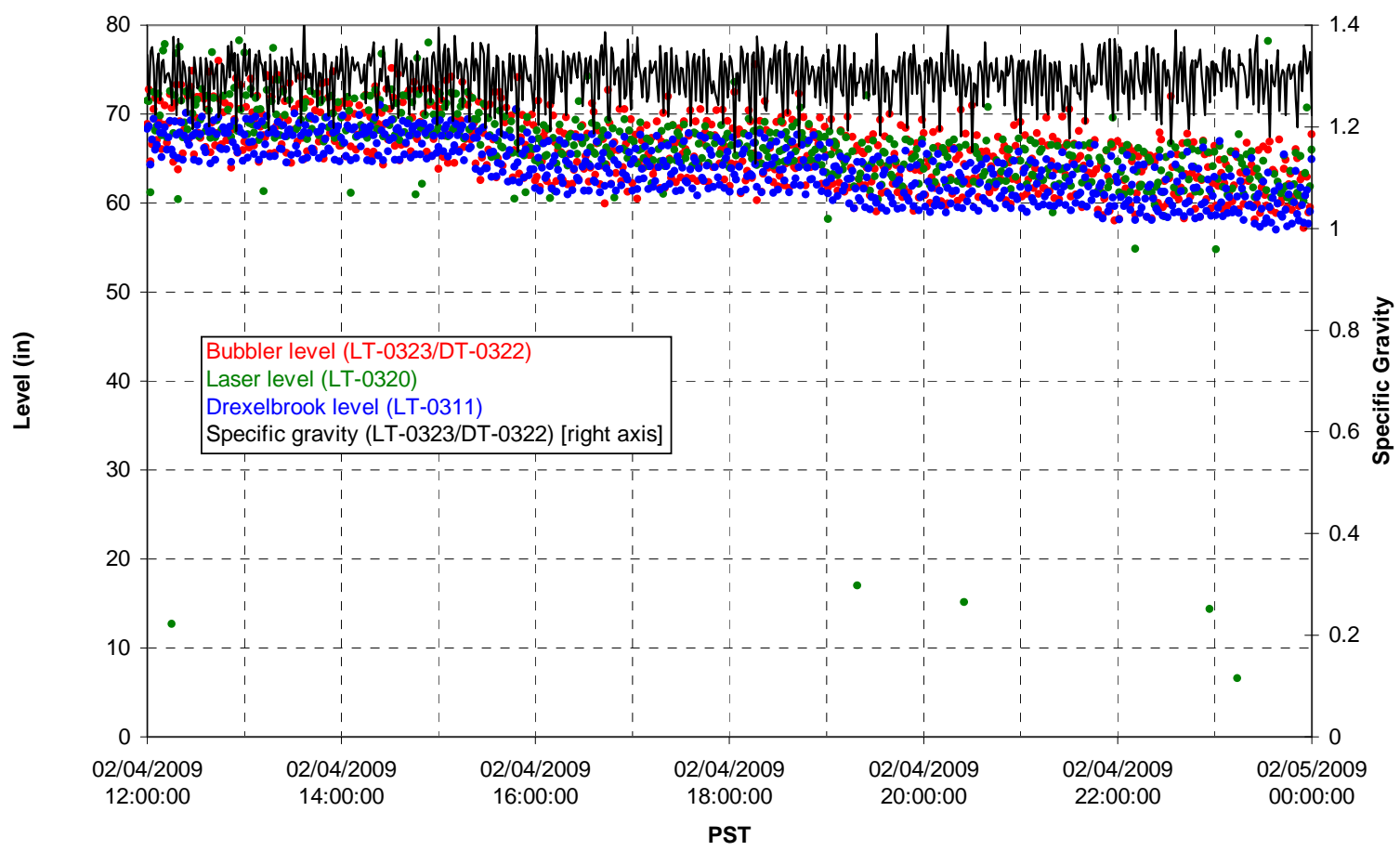

T01A temperatures

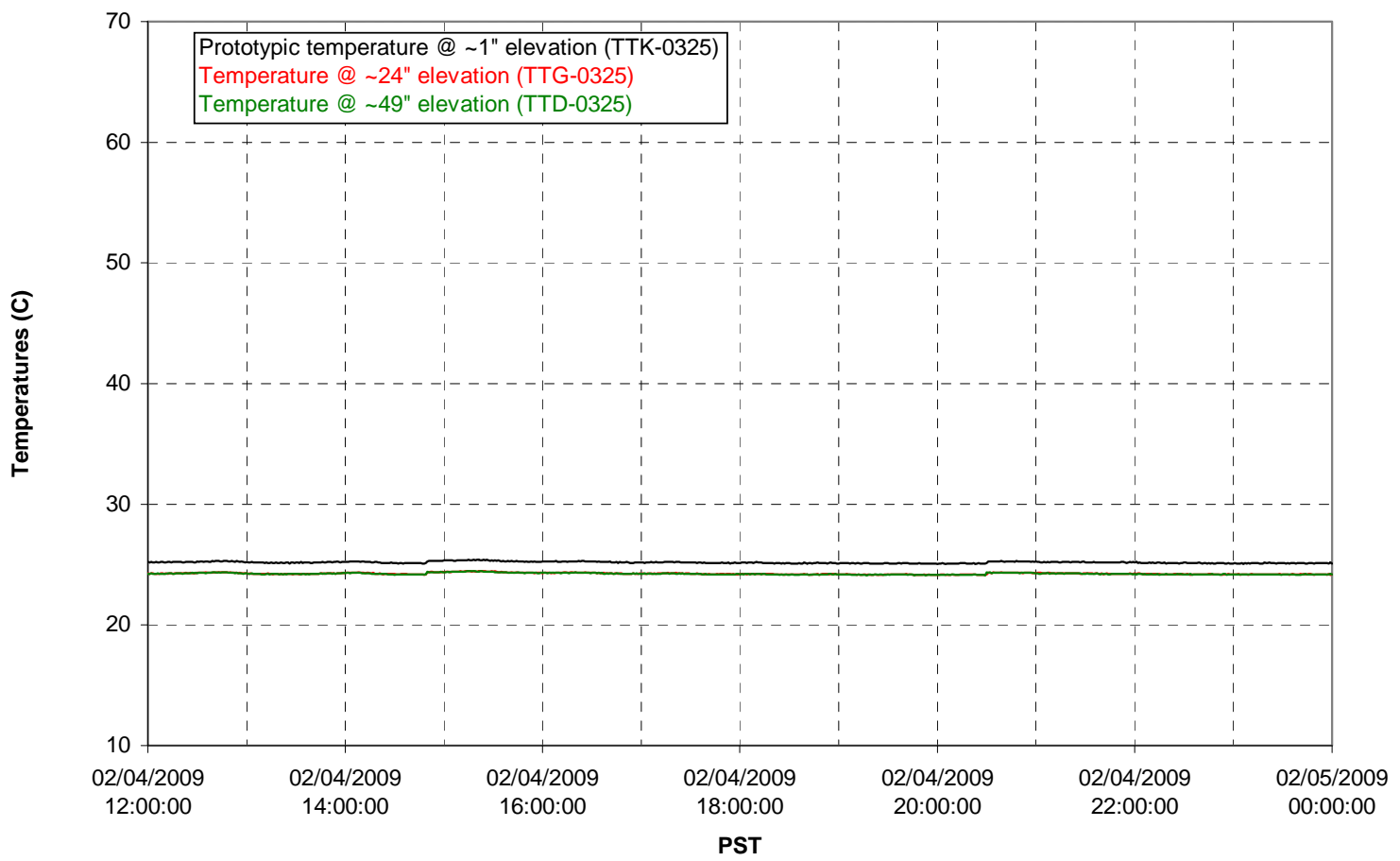


T01B level

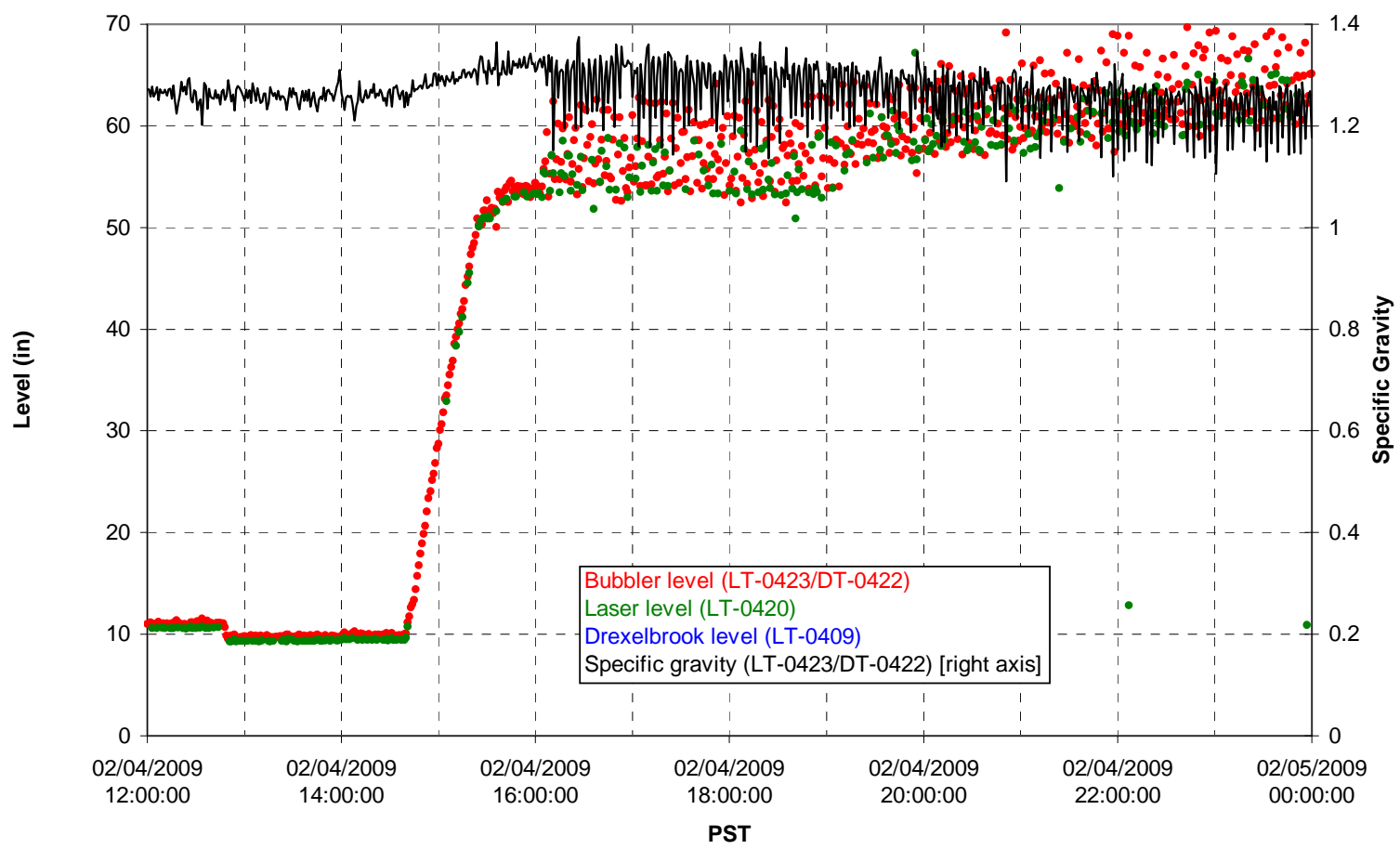

T01B temperatures

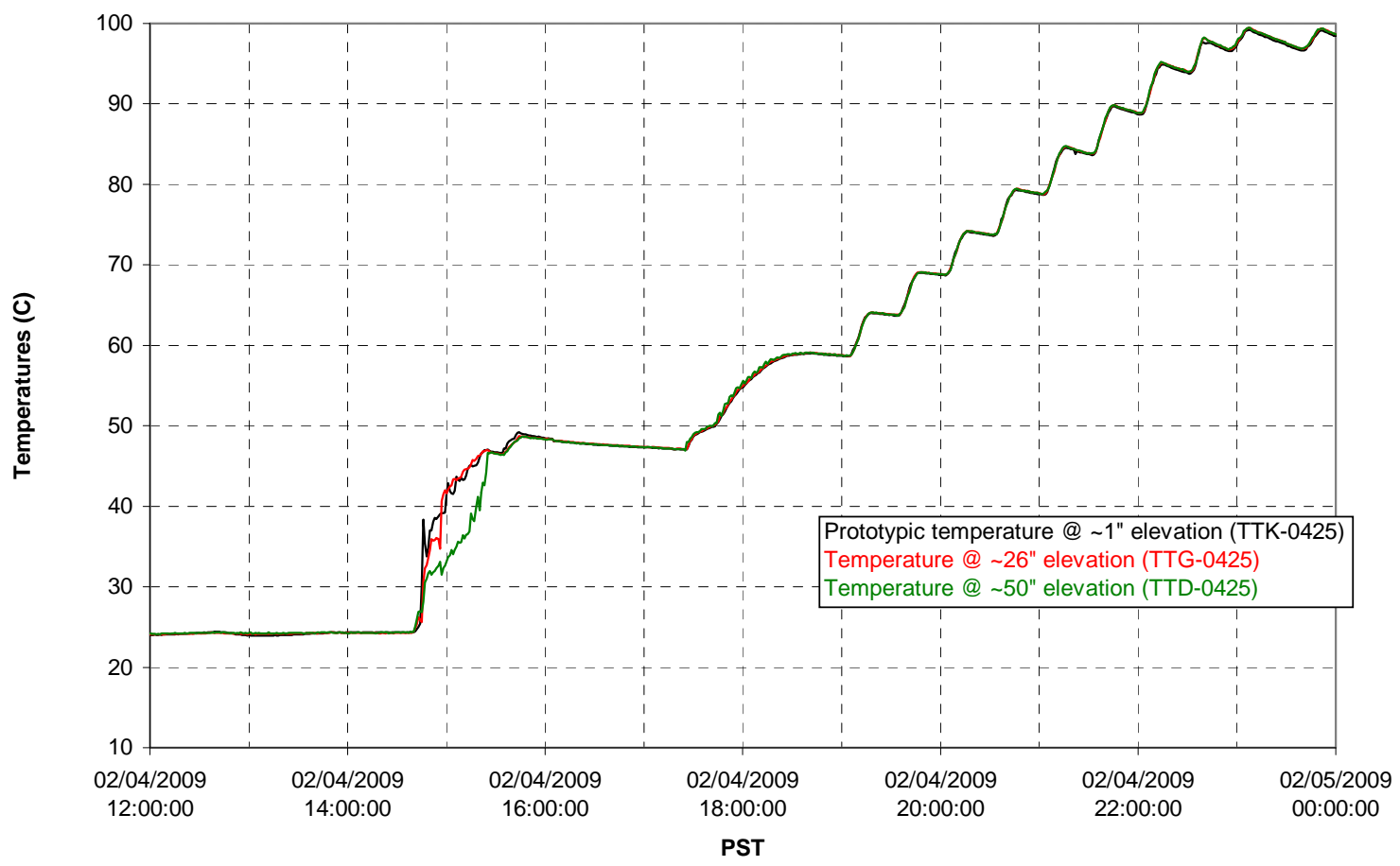


T02A level

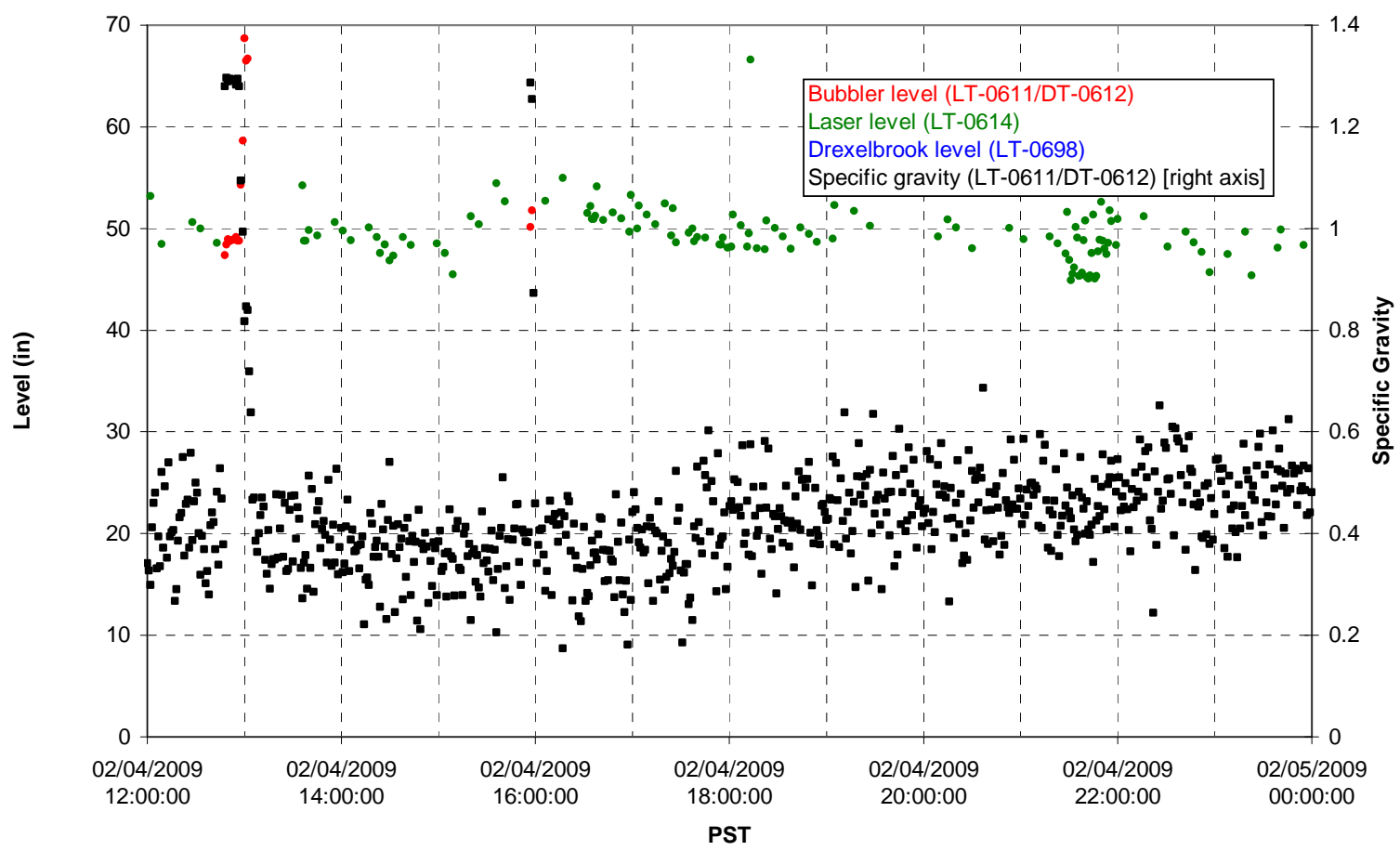

T02A temperatures

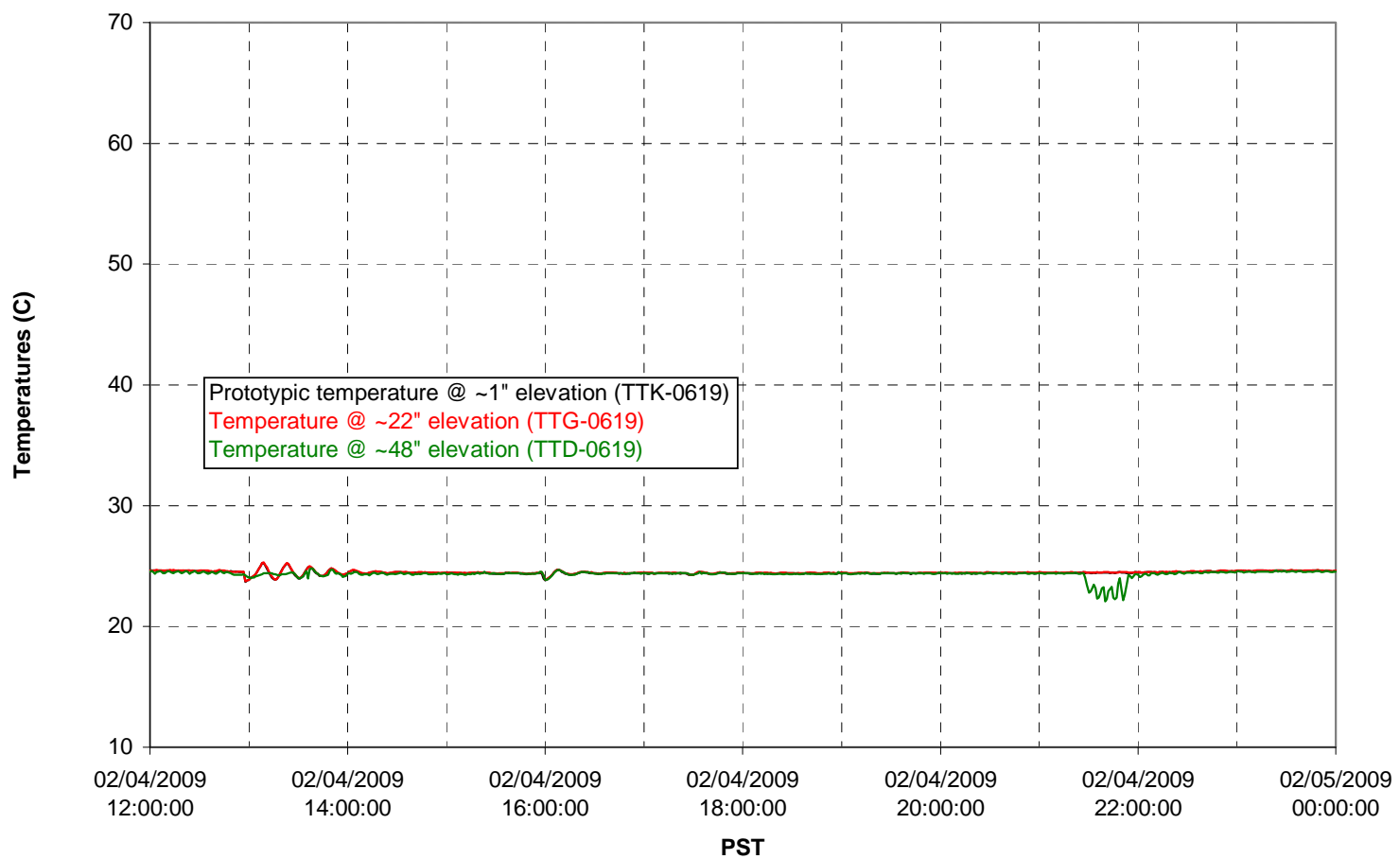

G.166 
T02A and filter loop temperatures

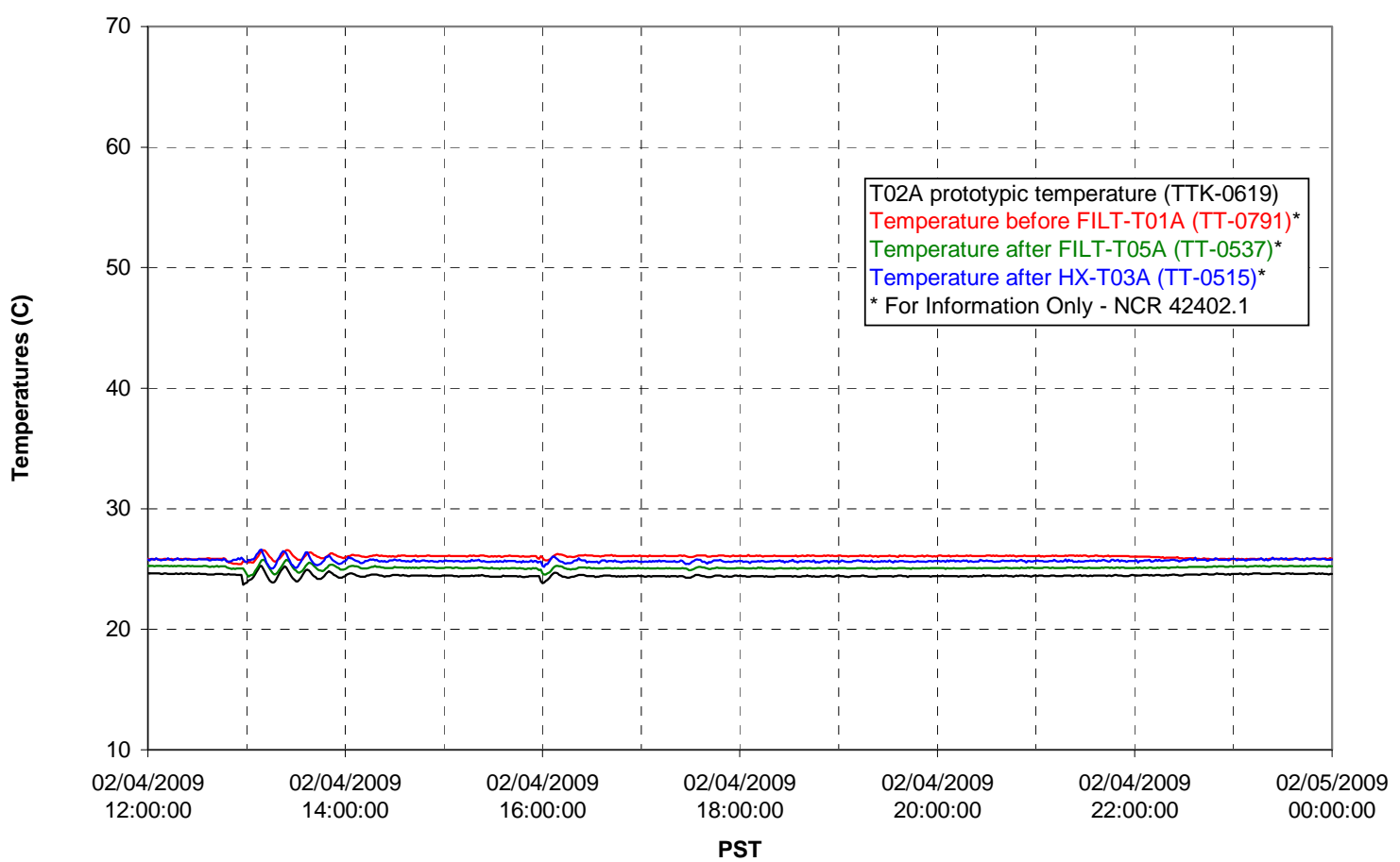

Pump Pressures and Flow

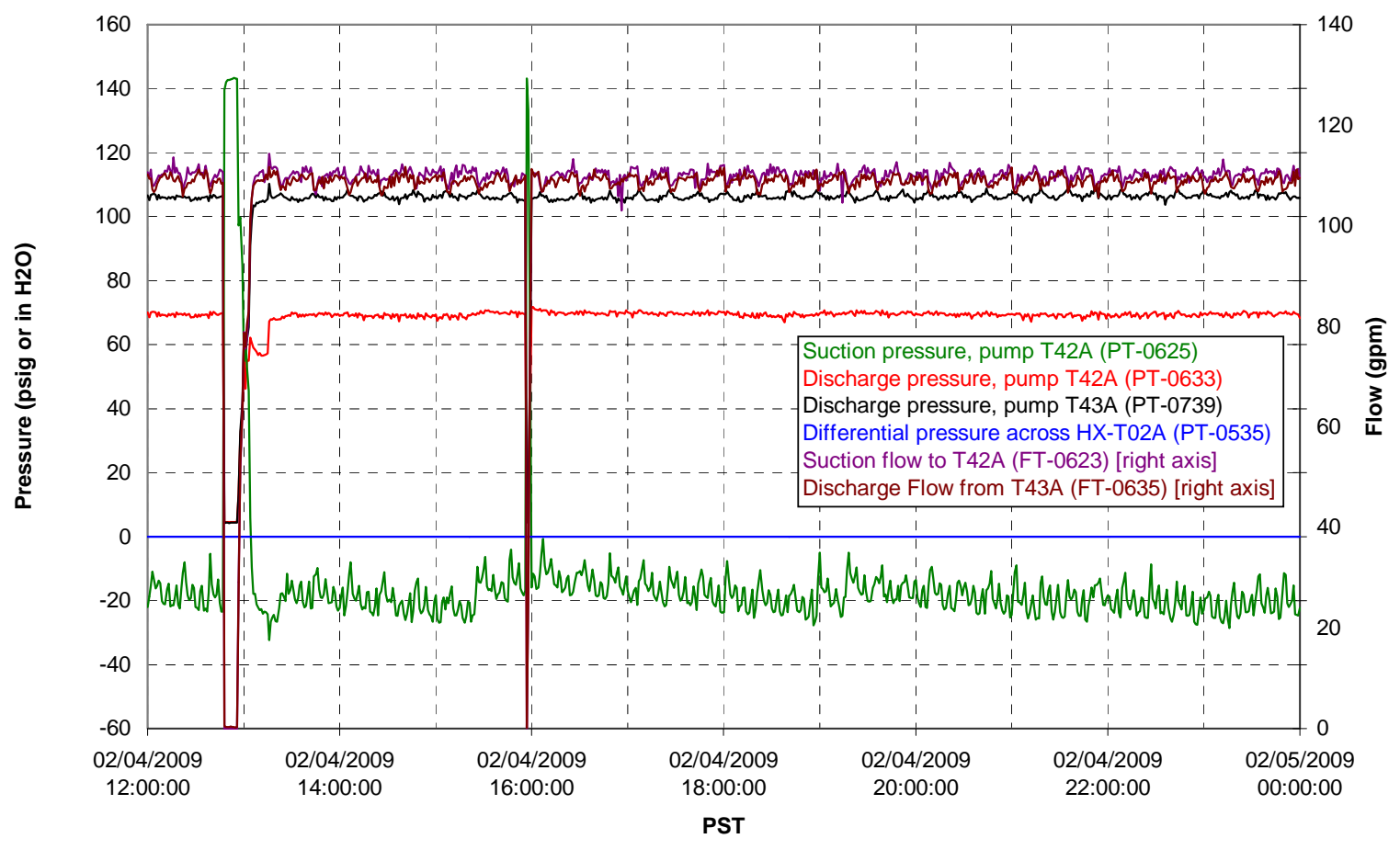


Axial pressure drop

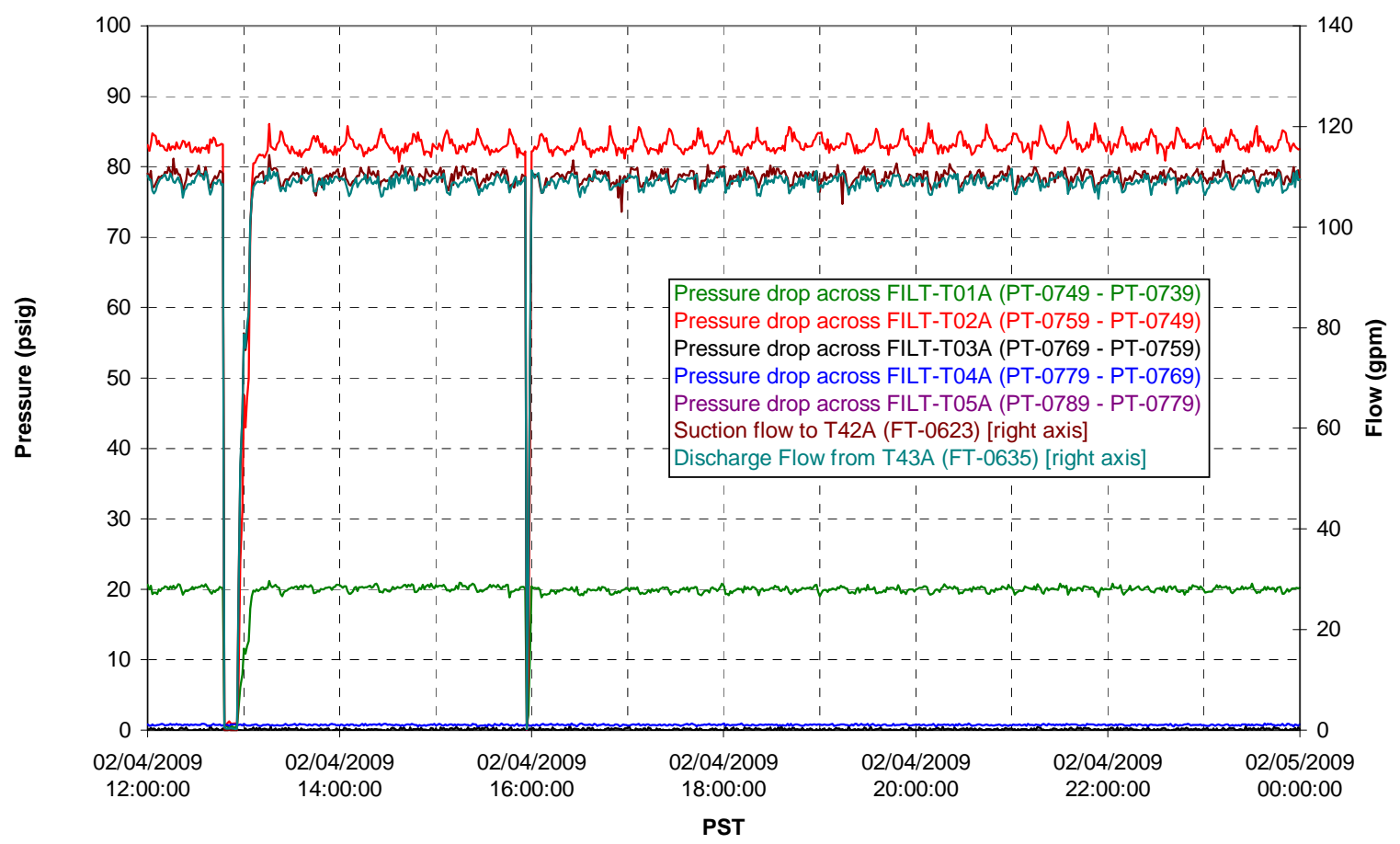

Permeate flow rates

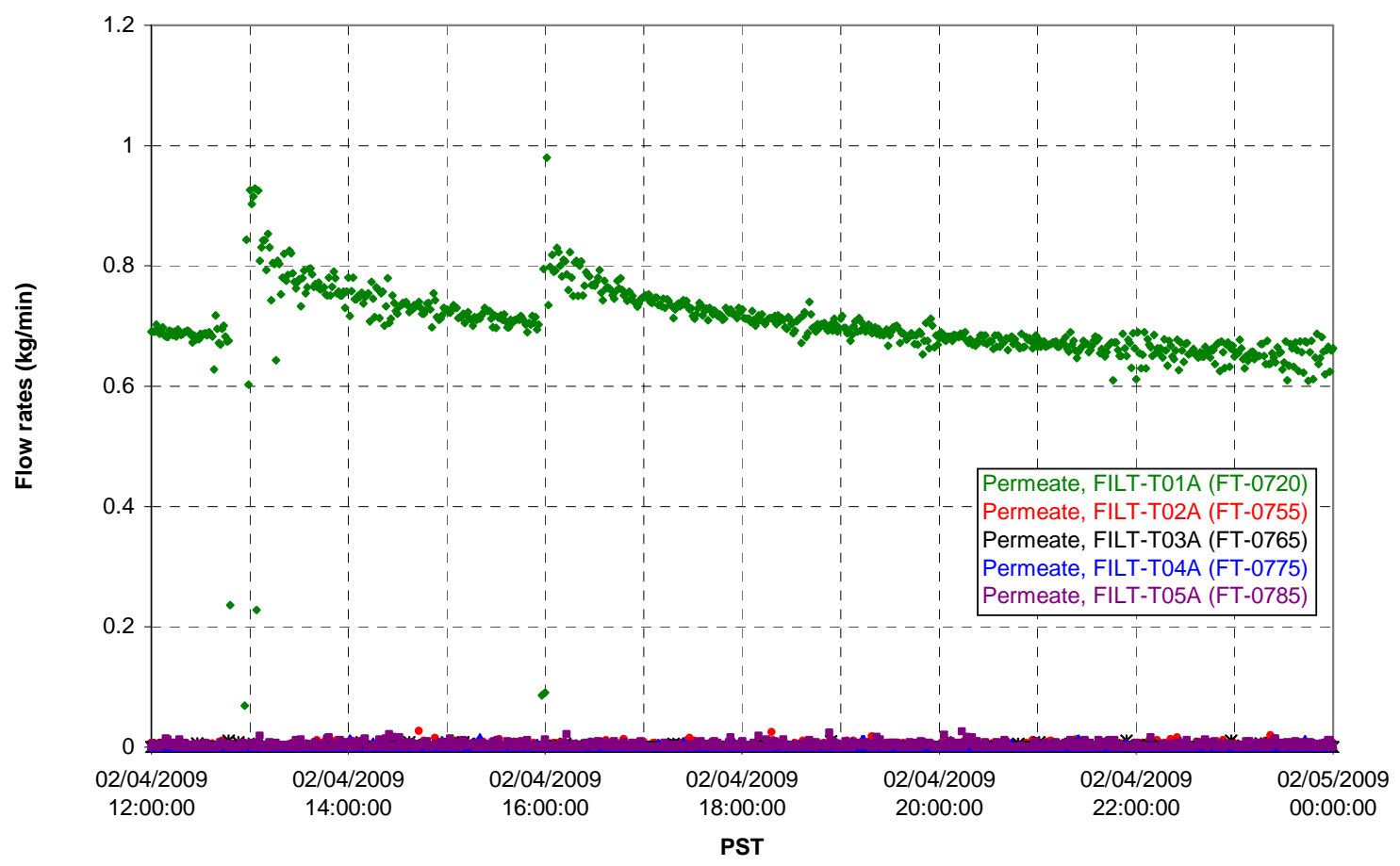


T02A Inner Temperature Tree

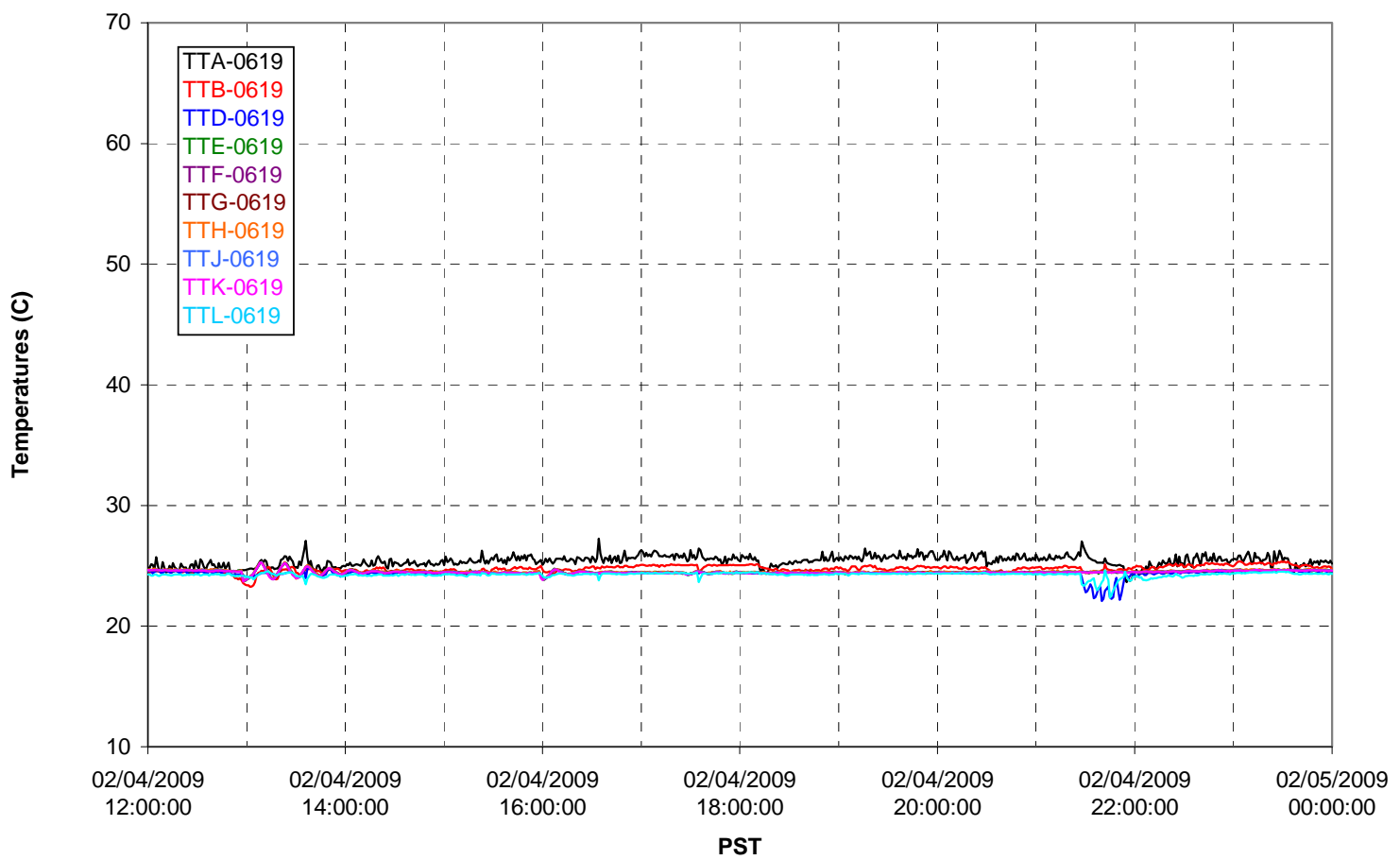

T02A Outer Temperature Tree

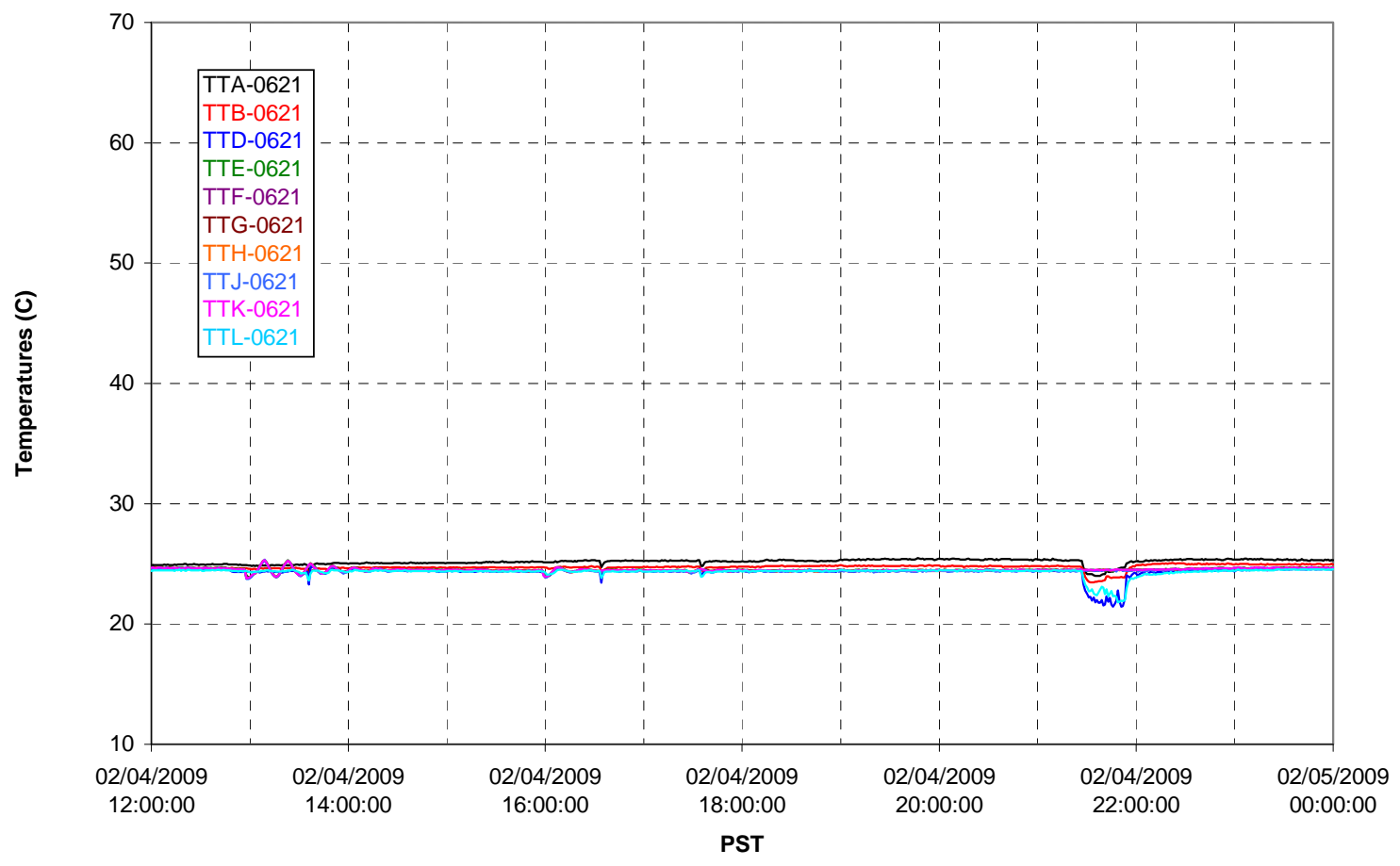


T02A temperatures

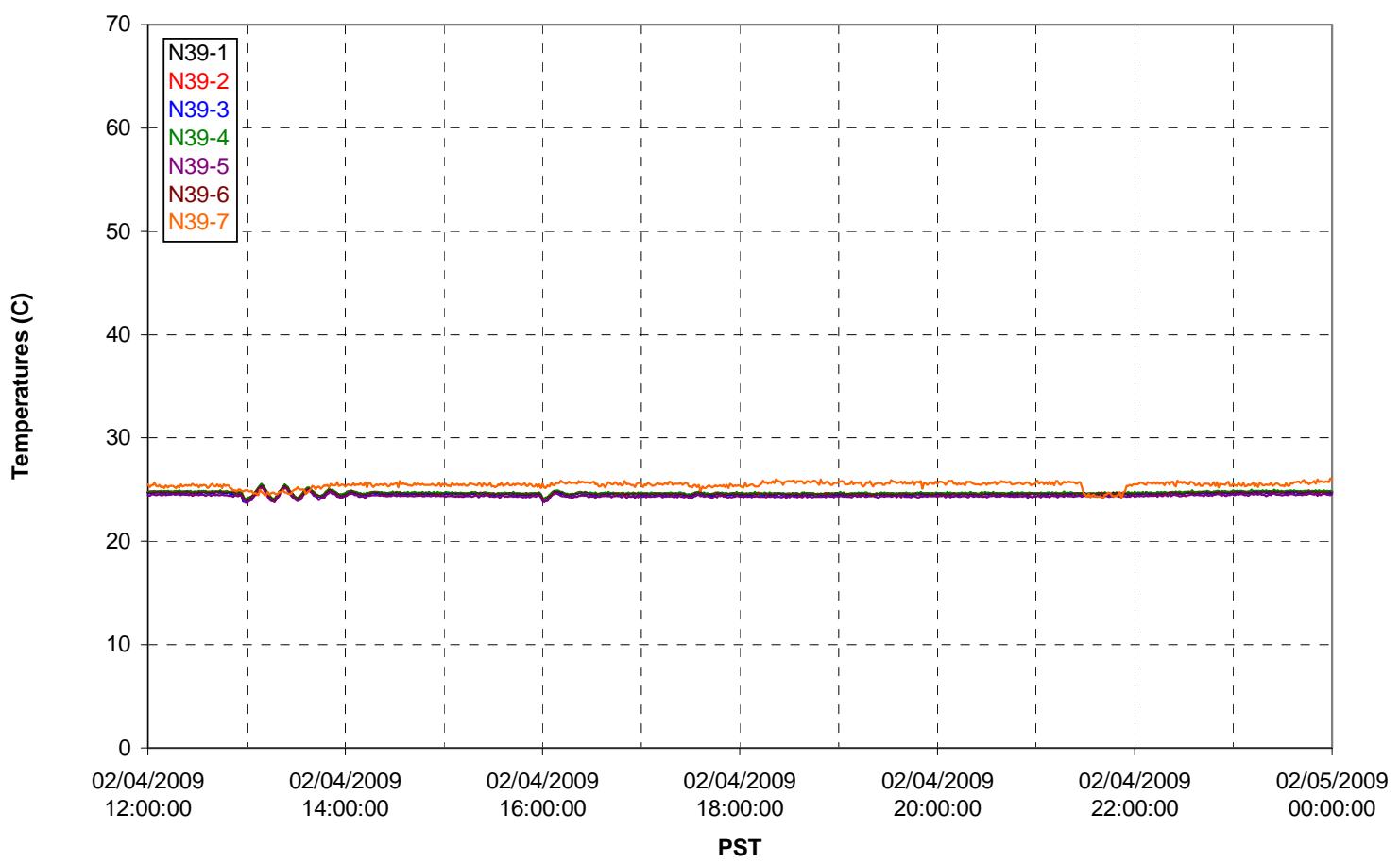

T02A temperatures

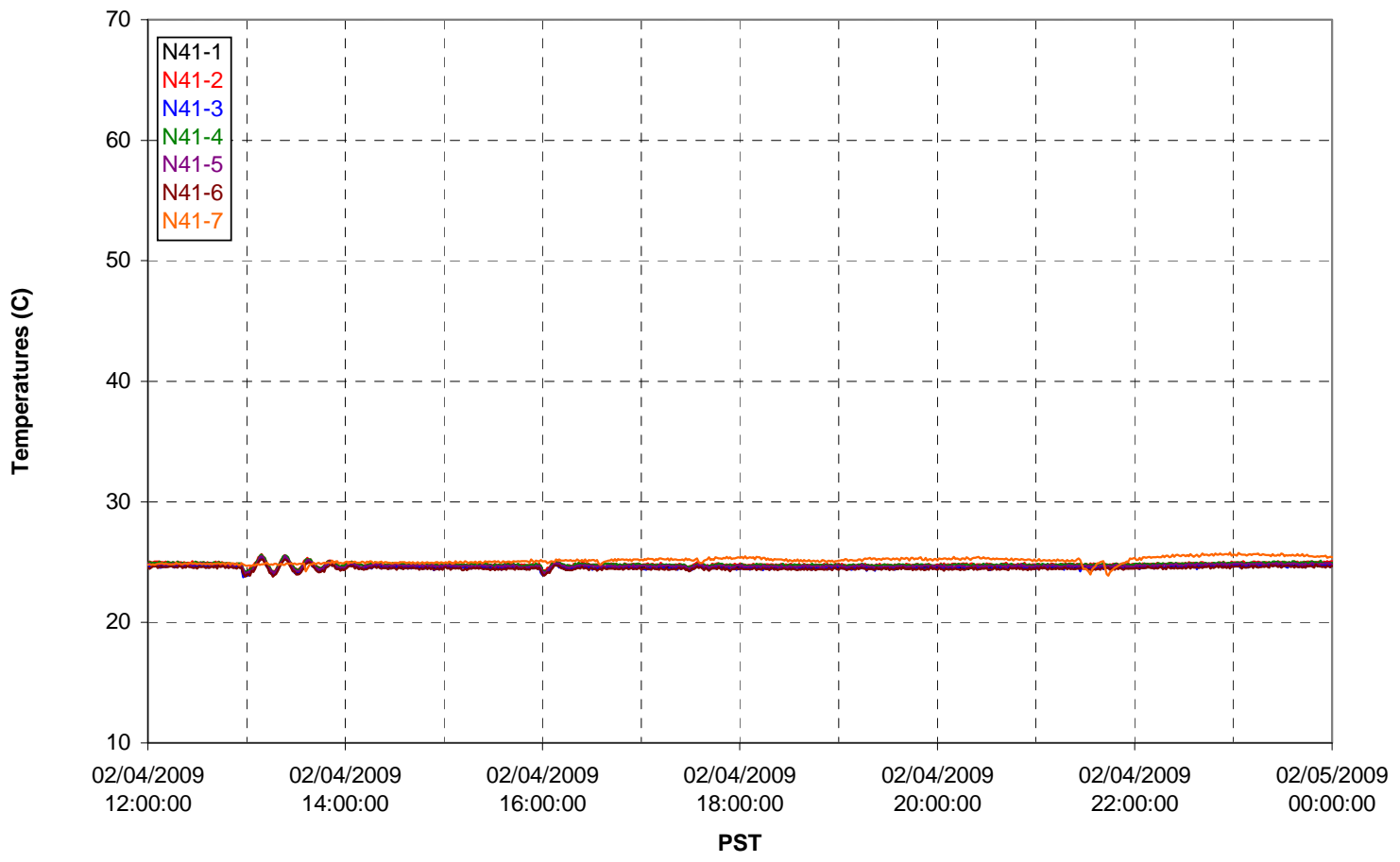

G.170 
T02A temperatures

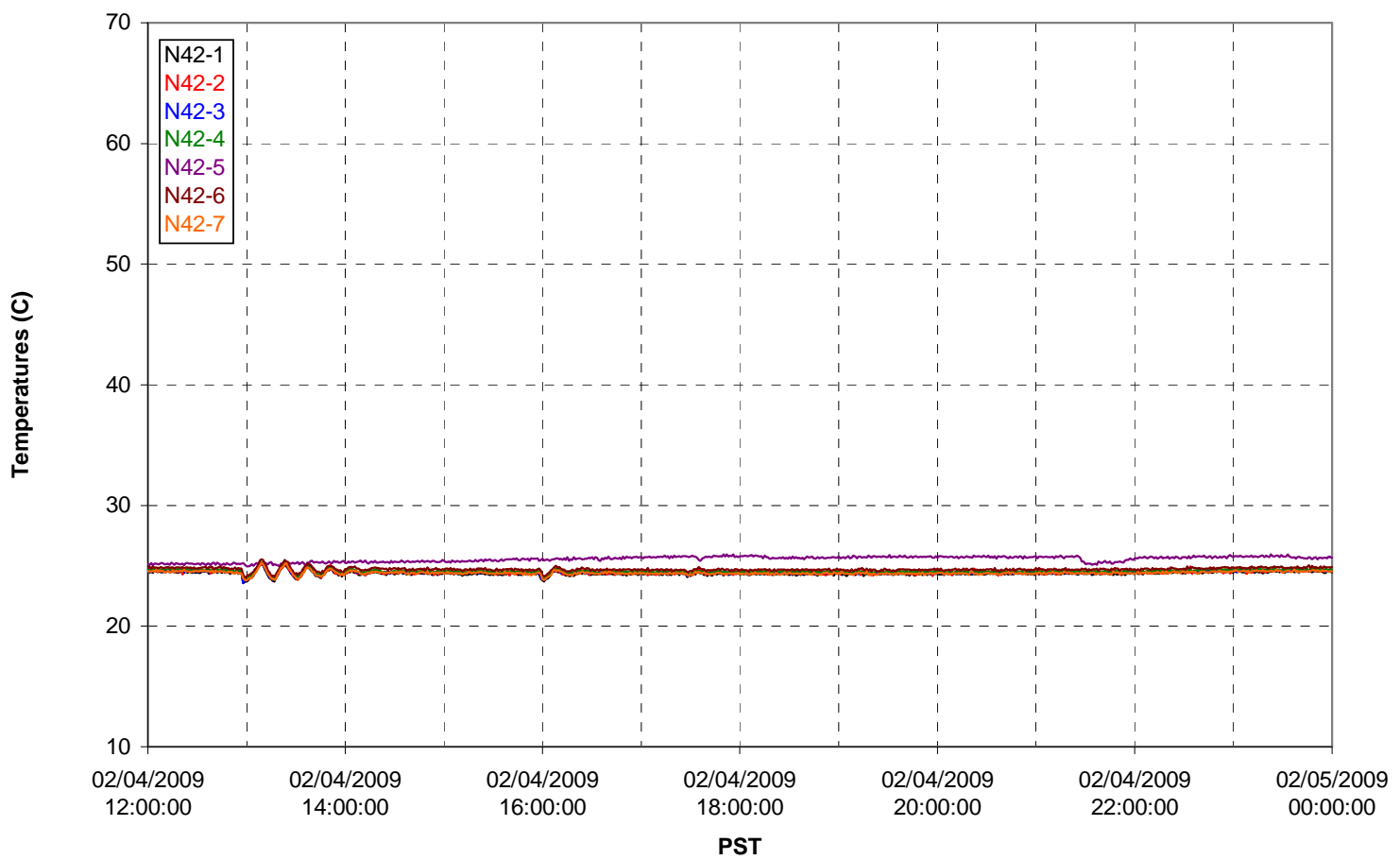

T02A temperatures

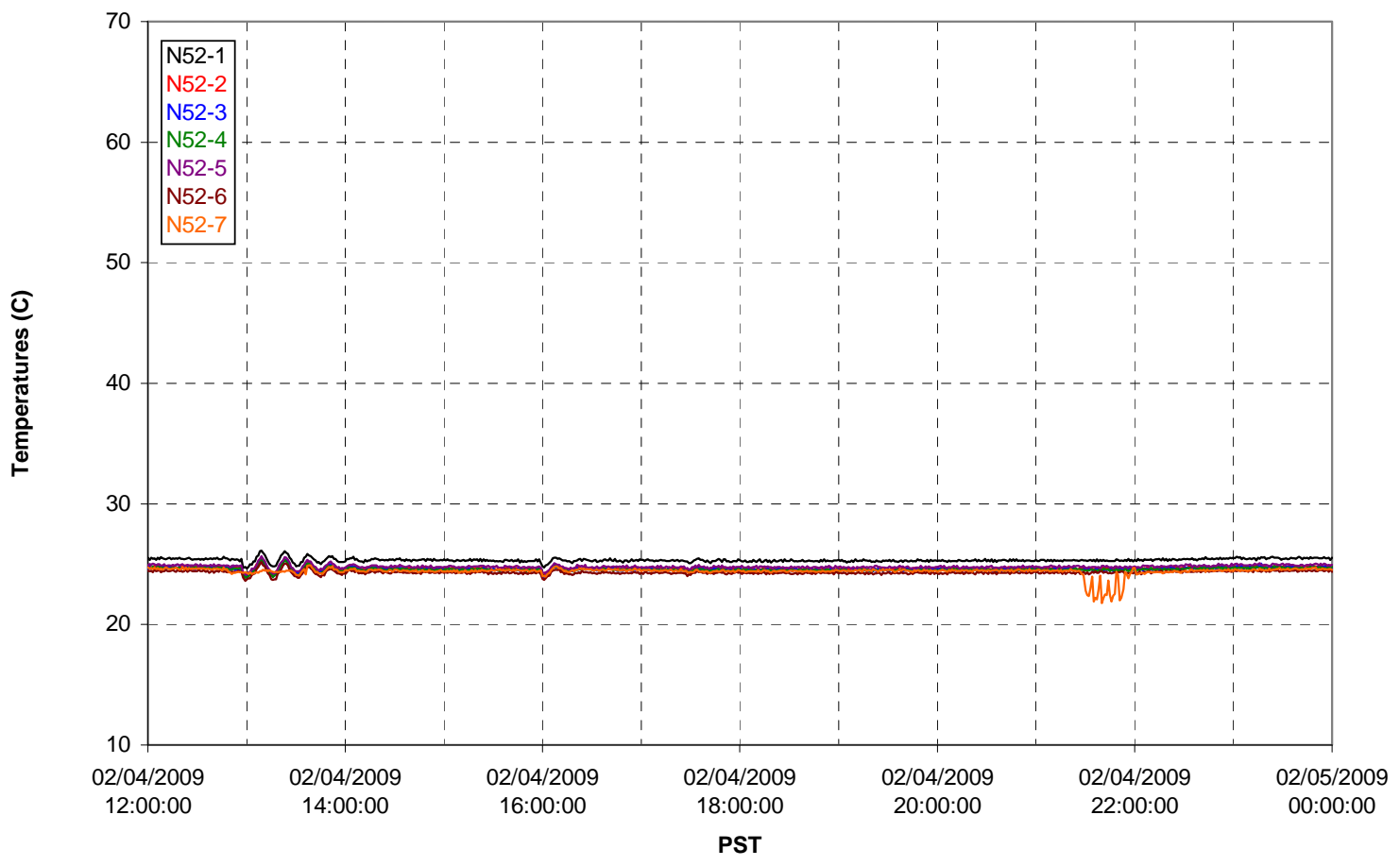

G.171 
T02A Heating and Cooling

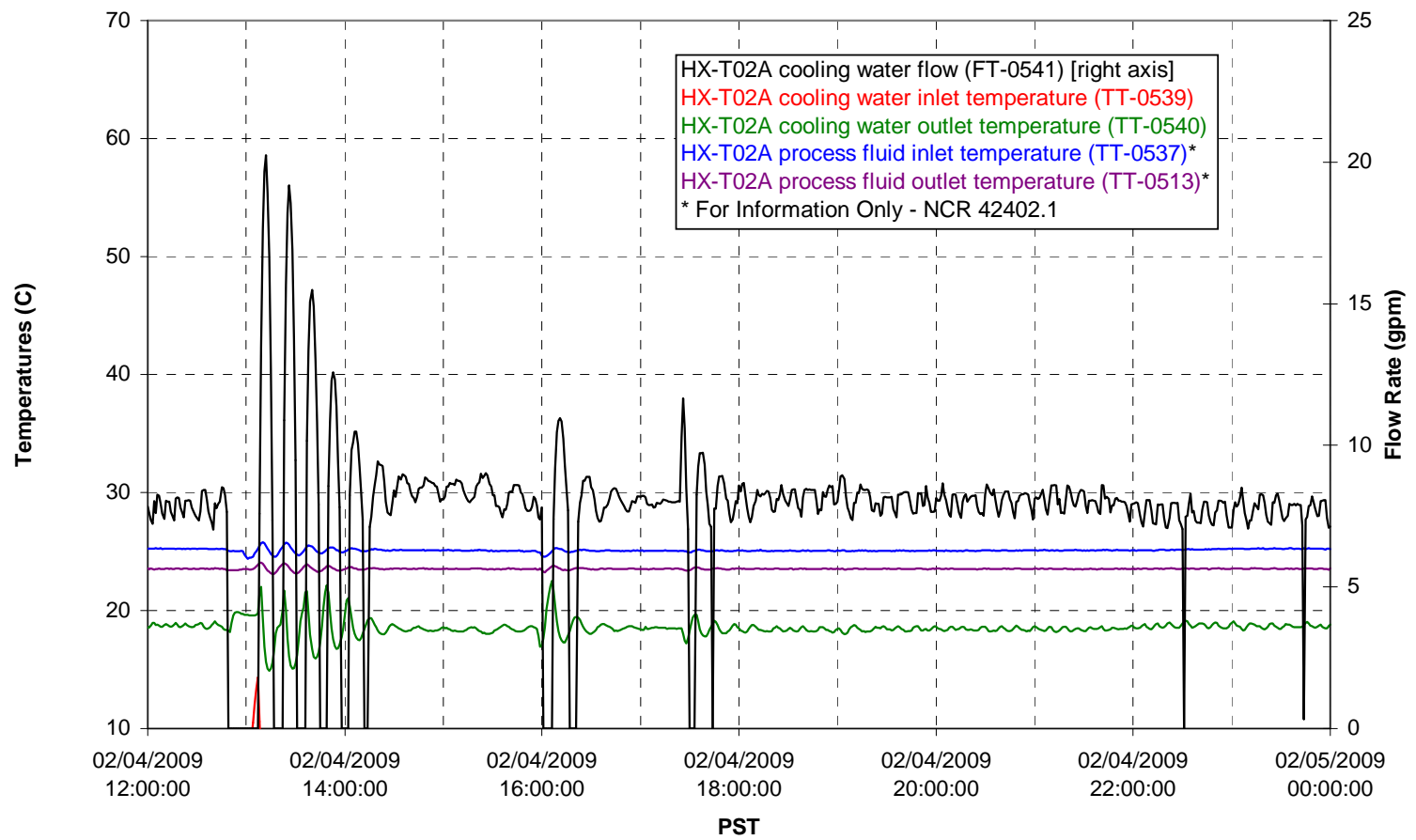

Pump Operation

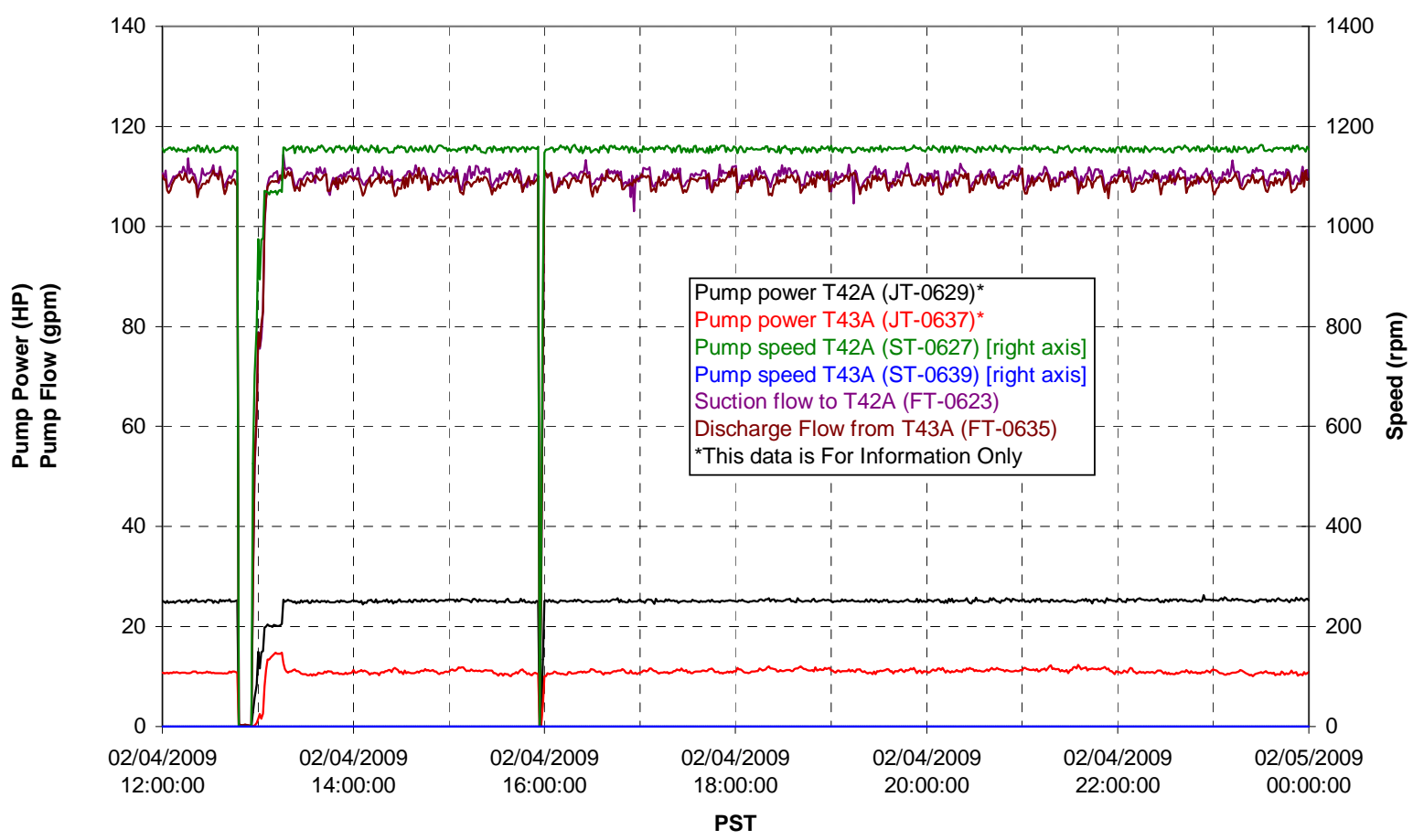

G.172 
Pulsepot UFP-PP-T01A

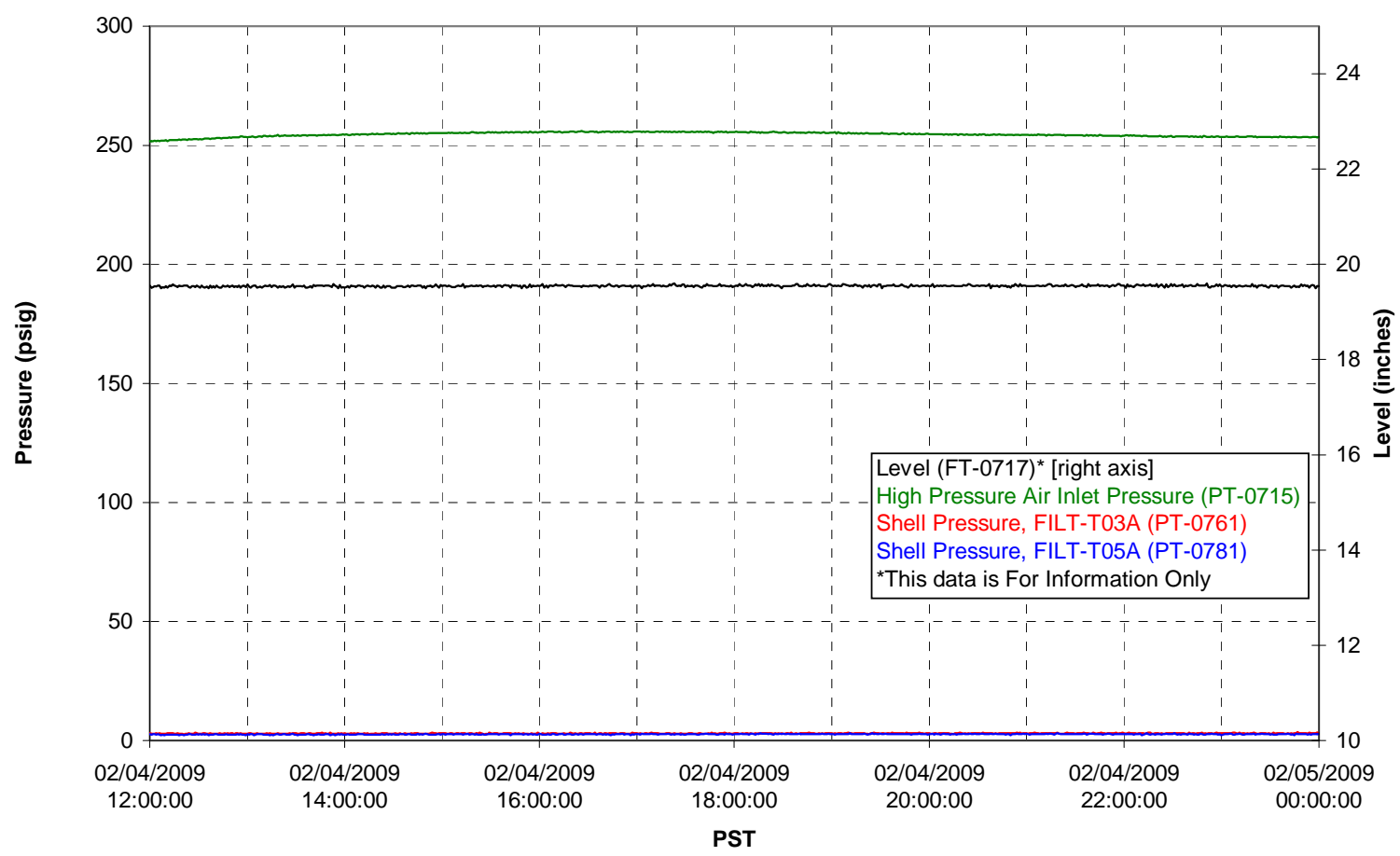

Pulsepot UFP-PP-T02A

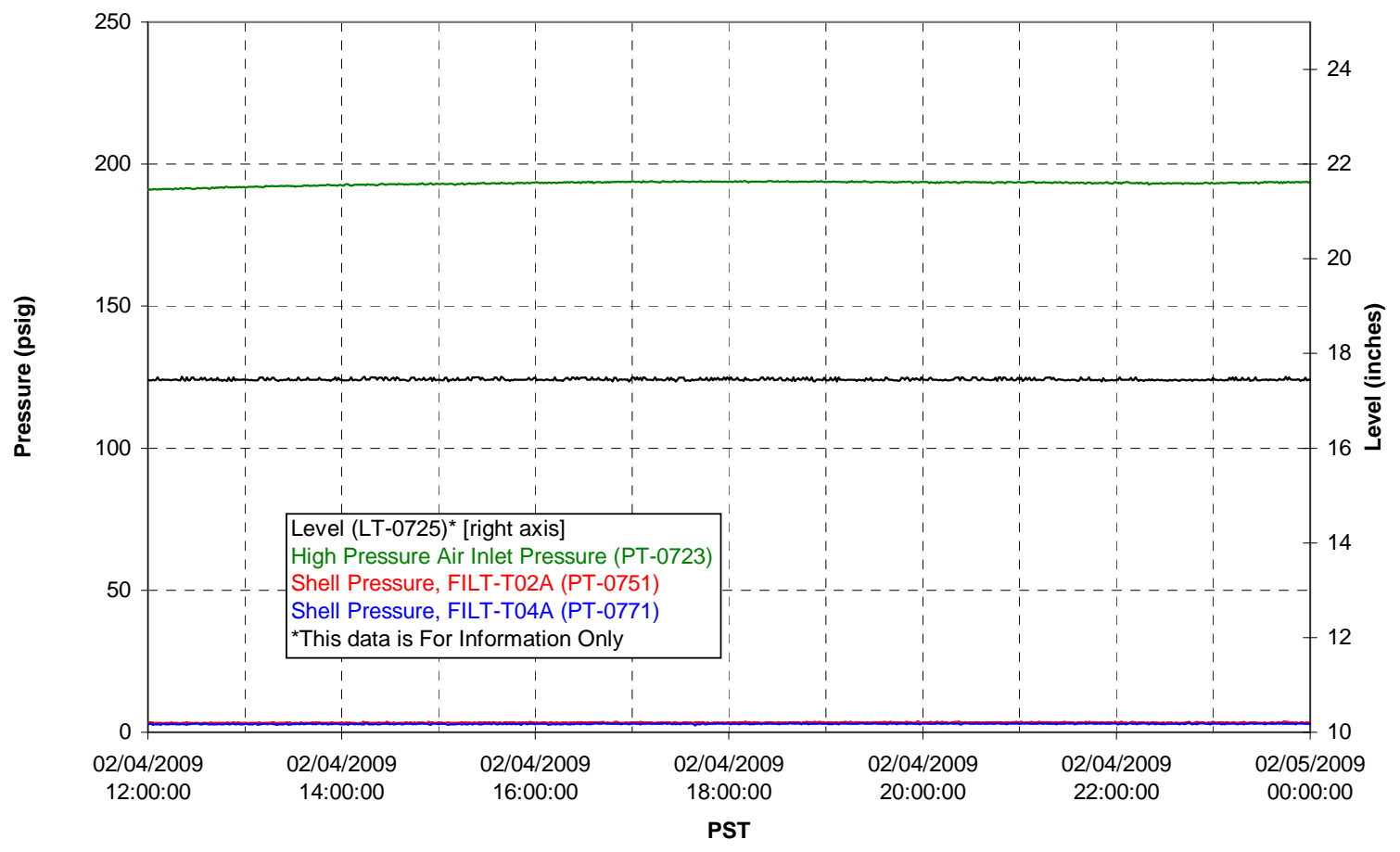

G.173 
Pulsepot UFP-PP-T03A

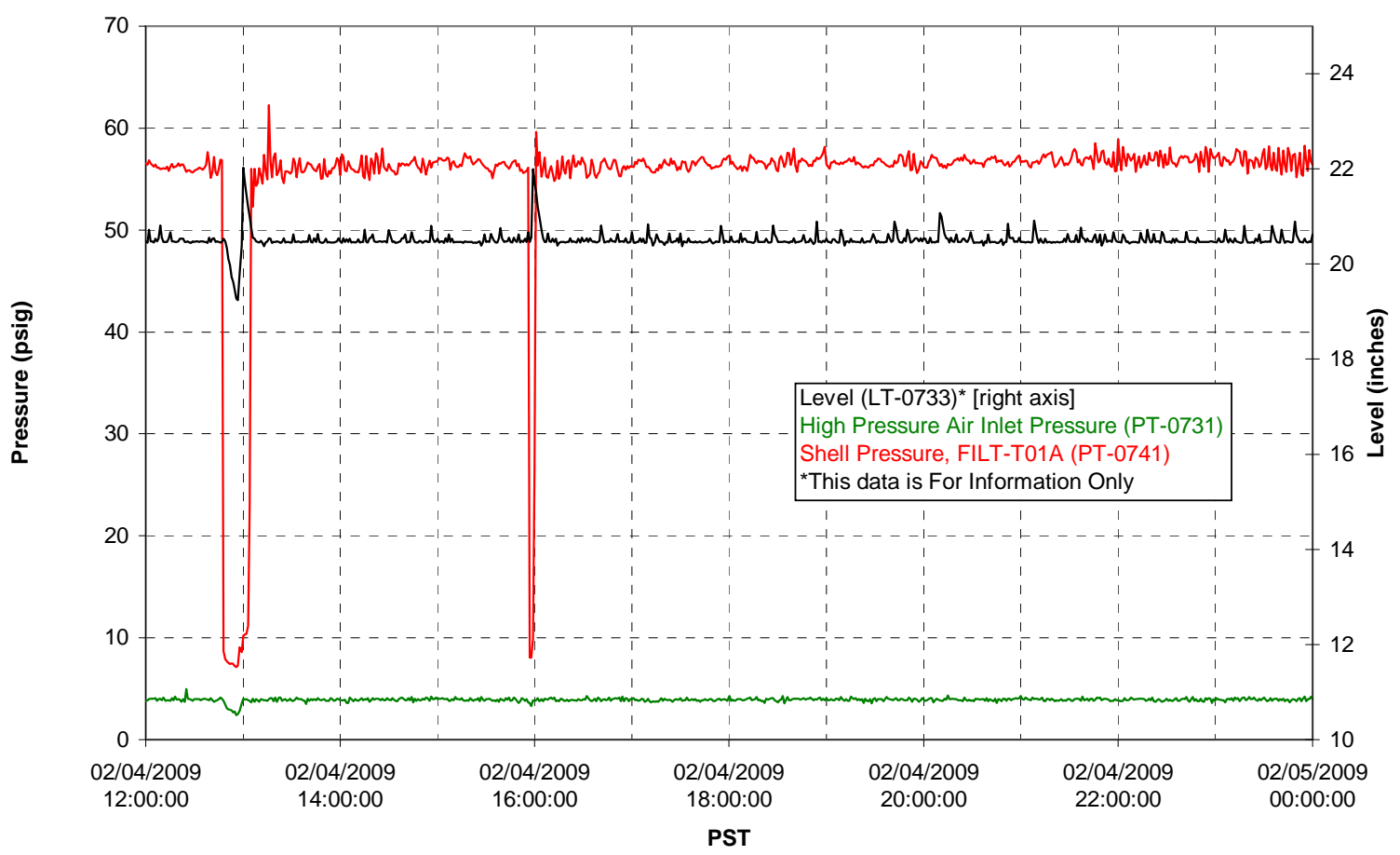

Pulsepot Levels

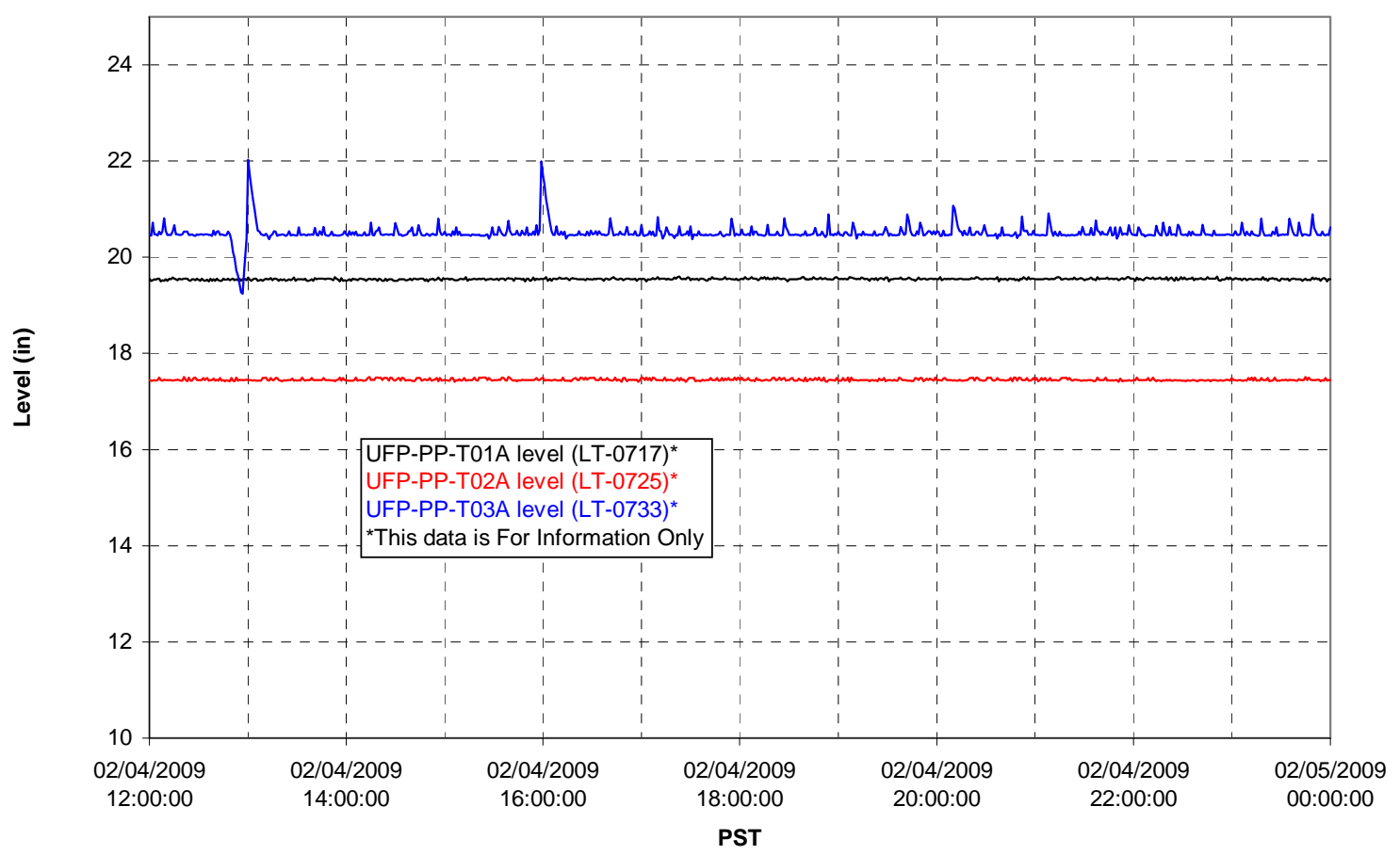


Filter UFP-FILT-T01A

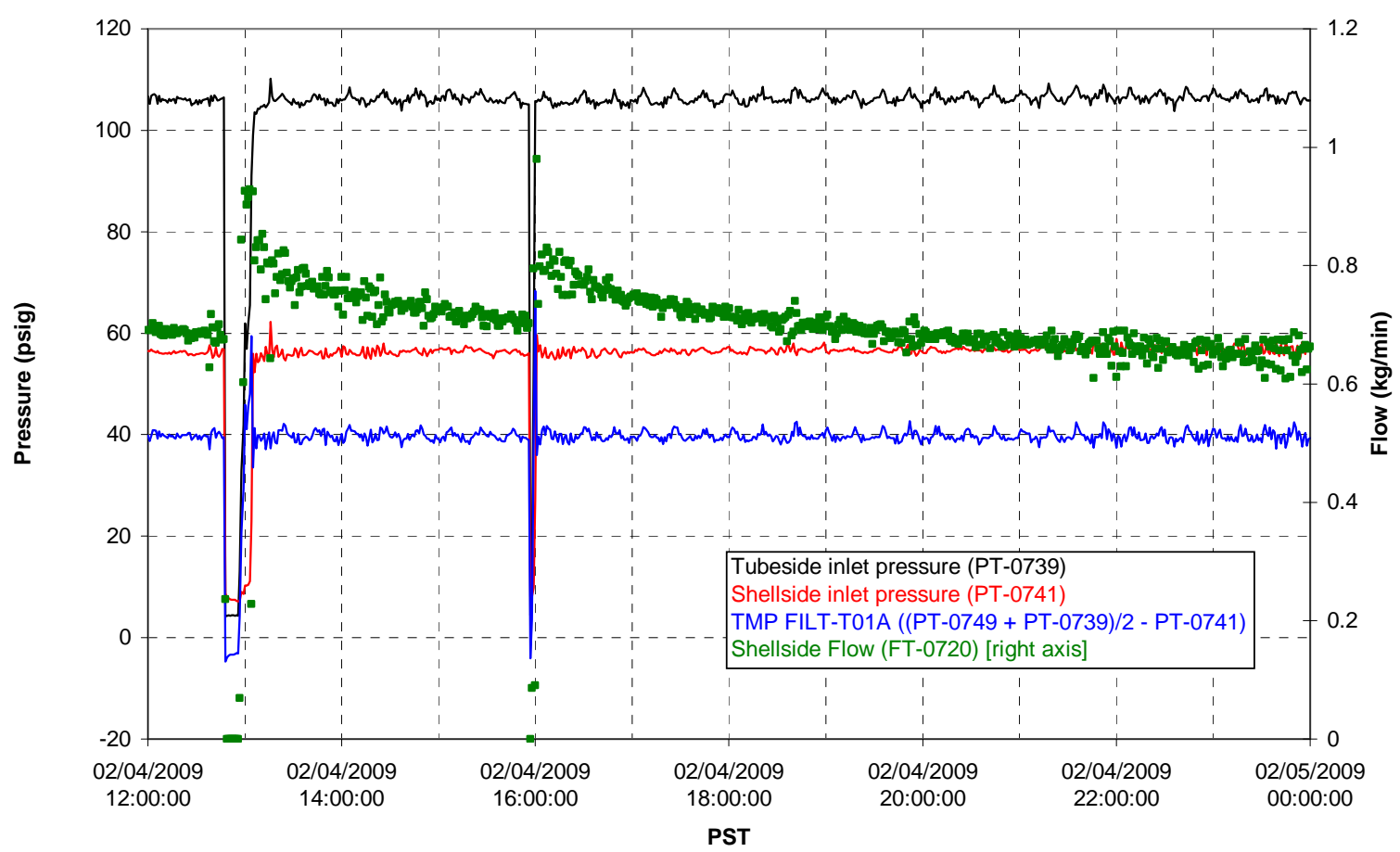

Filter UFP-FILT-T02A

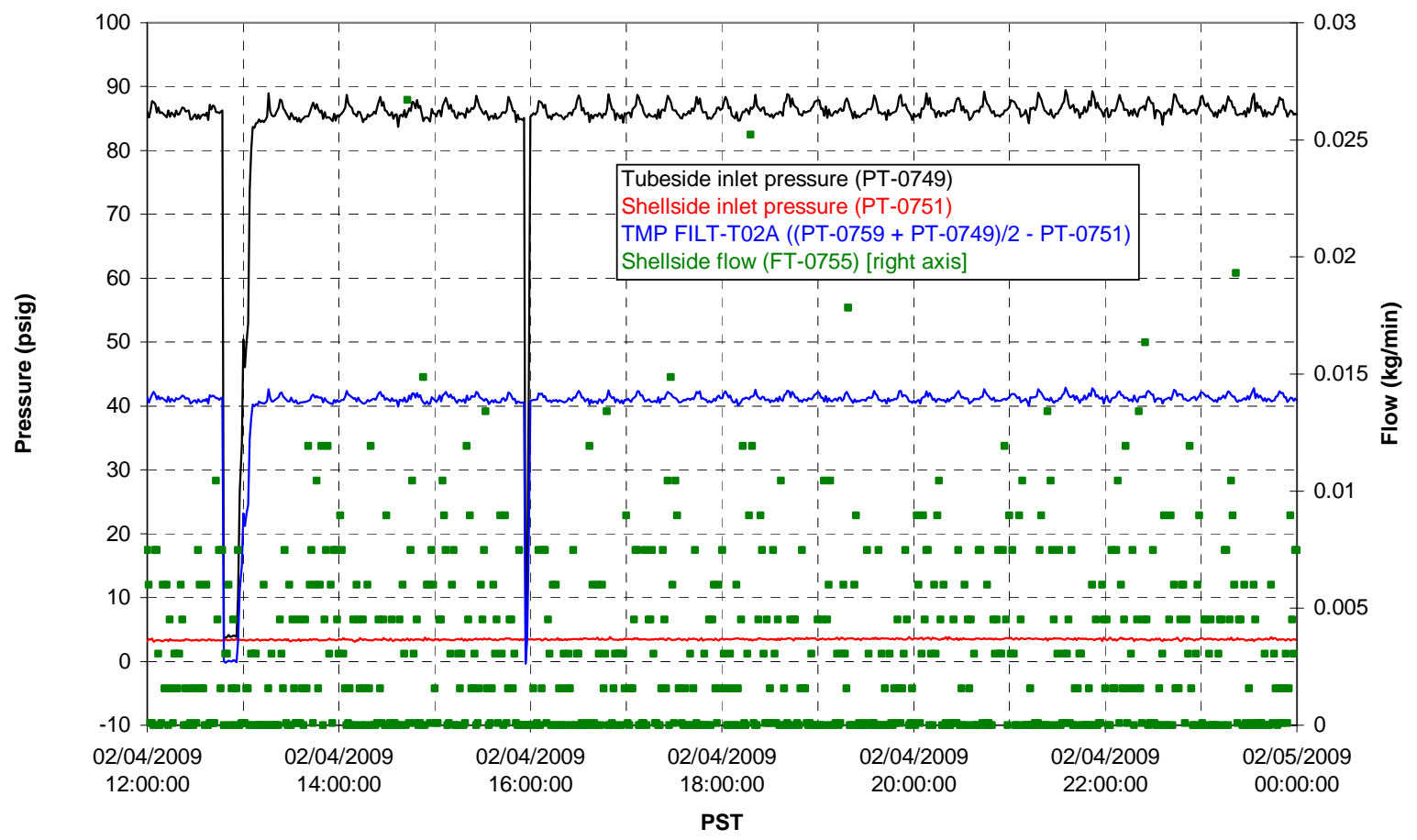

G.175 
Filter UFP-FILT-T03A

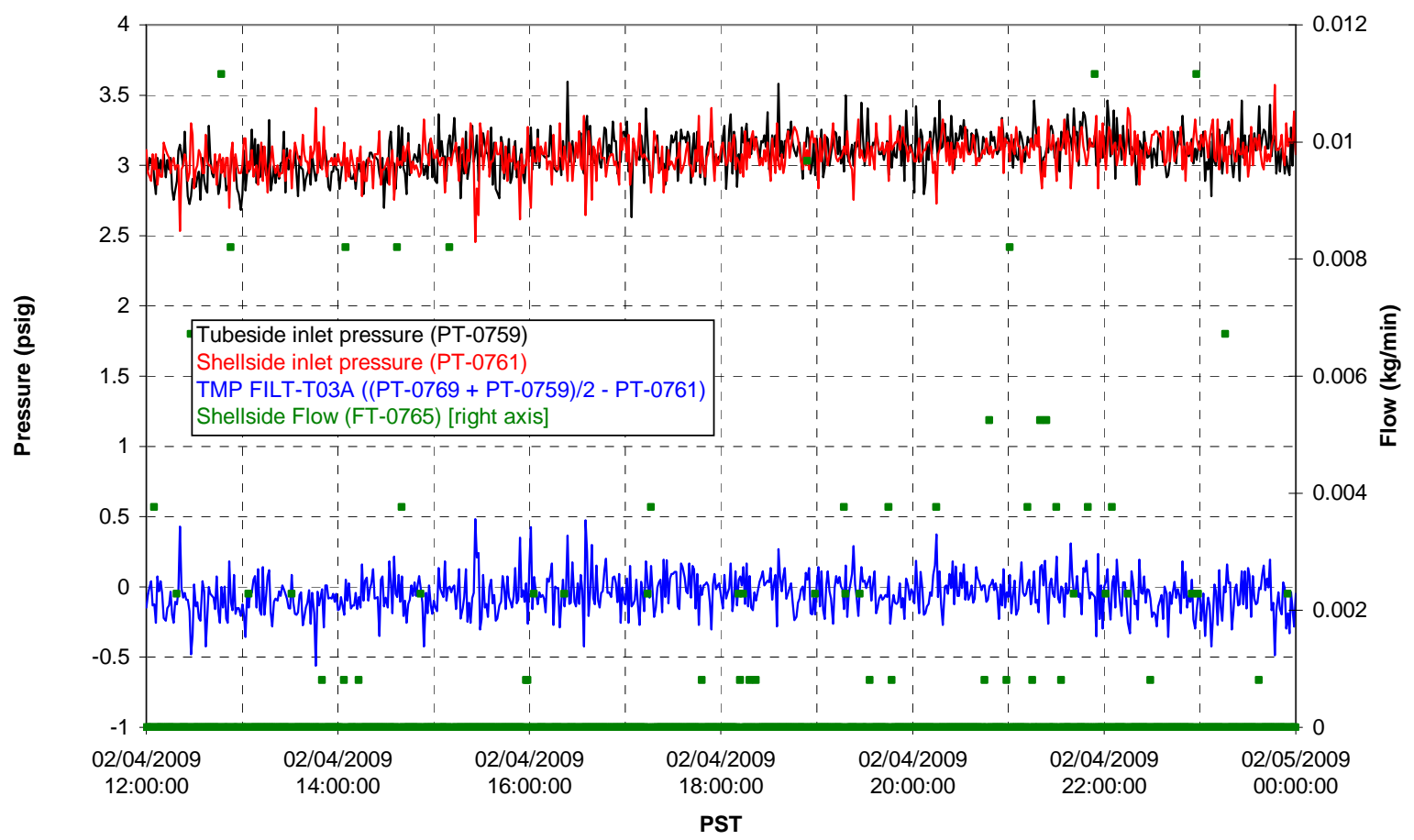

Filter UFP-FILT-T04A

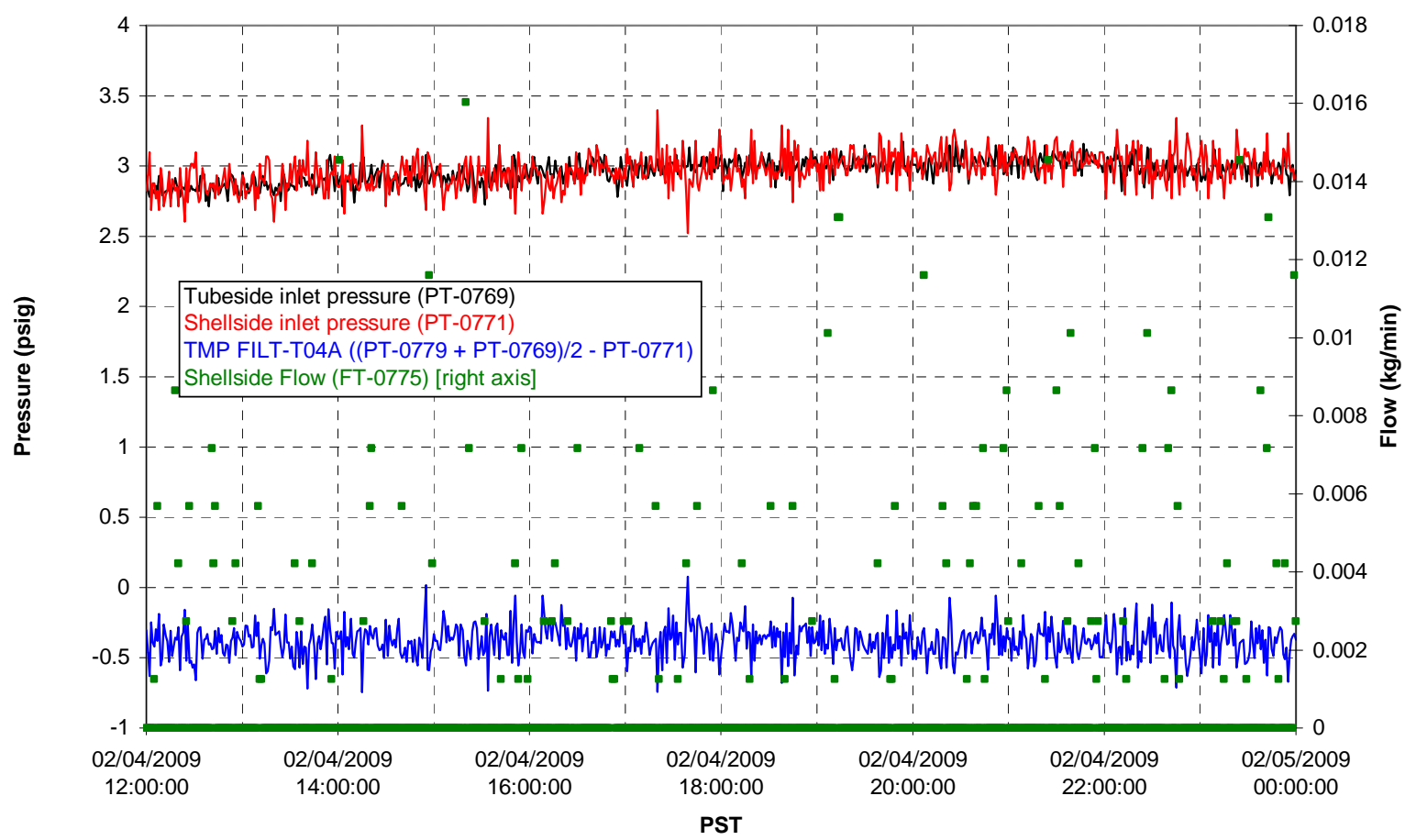


Filter UFP-FILT-T05A

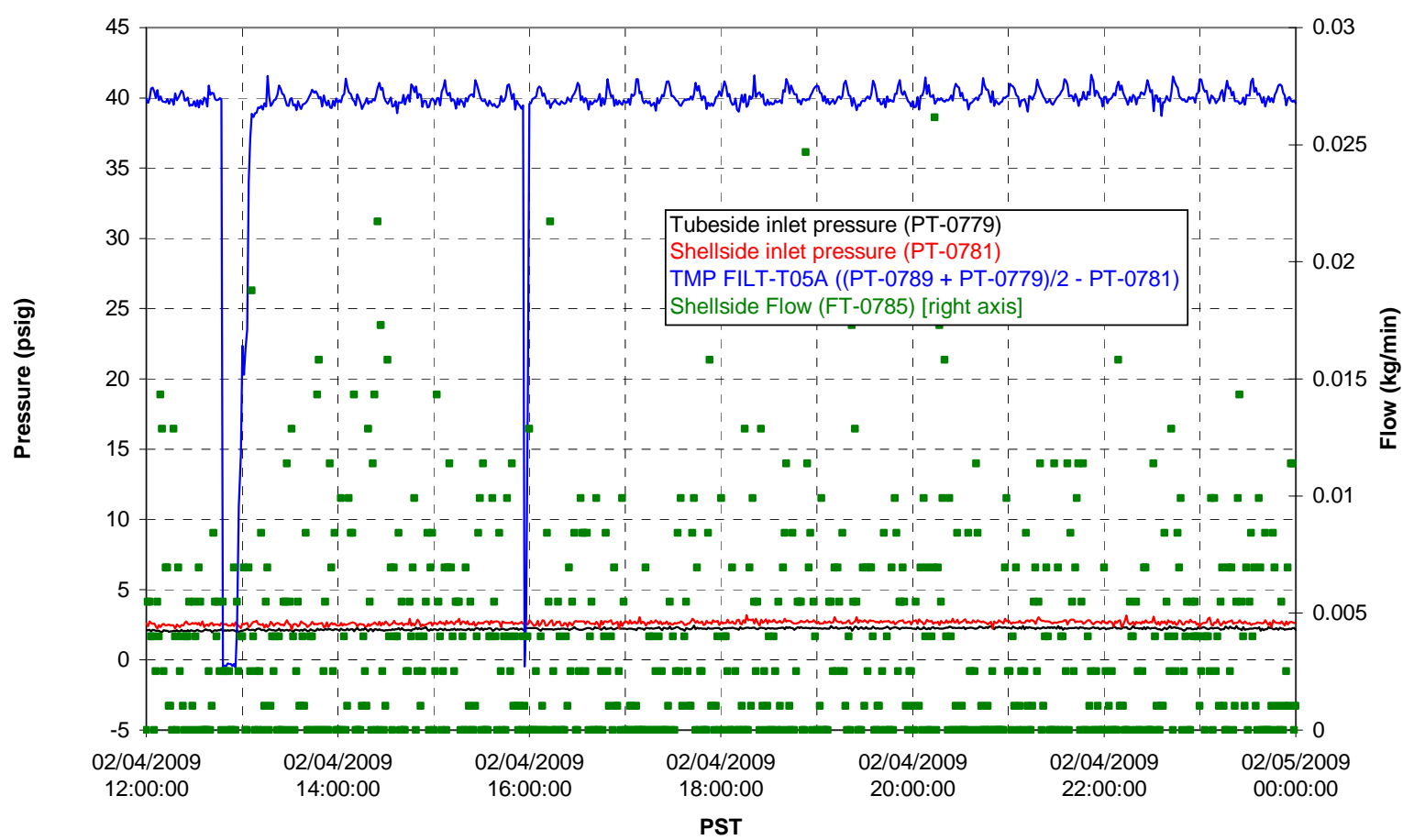

Chemical Flow

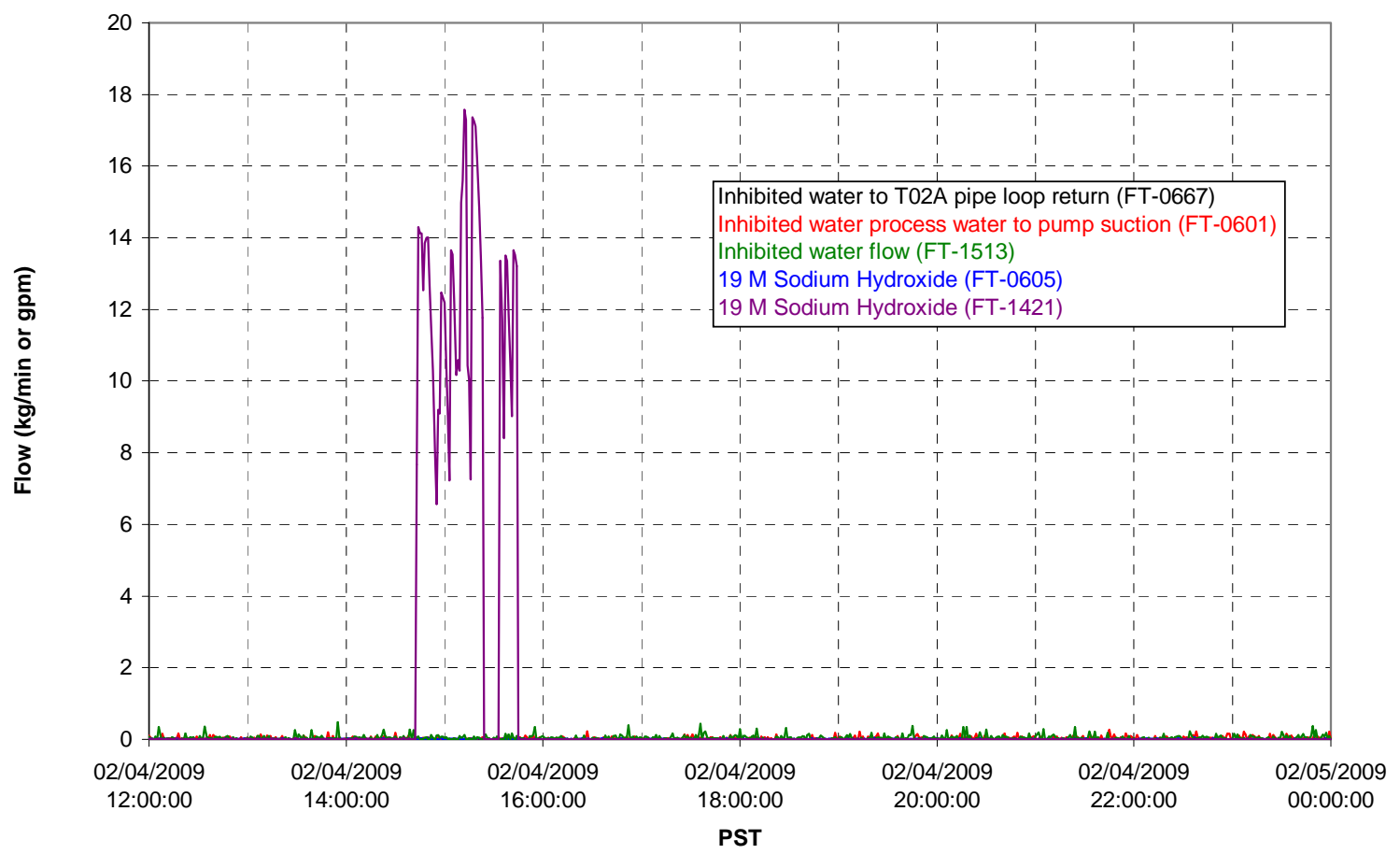

G. 177 


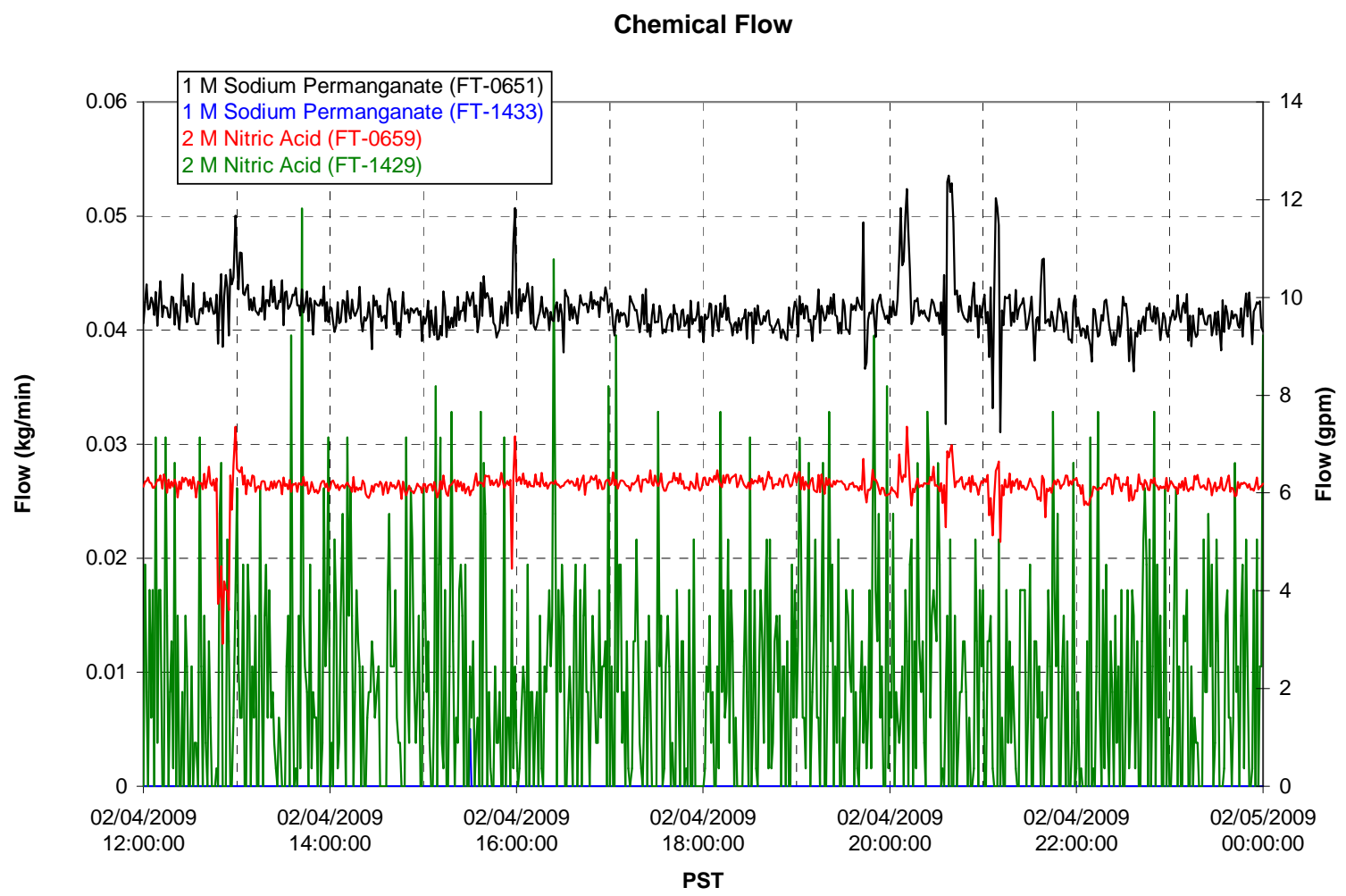

Air Flows

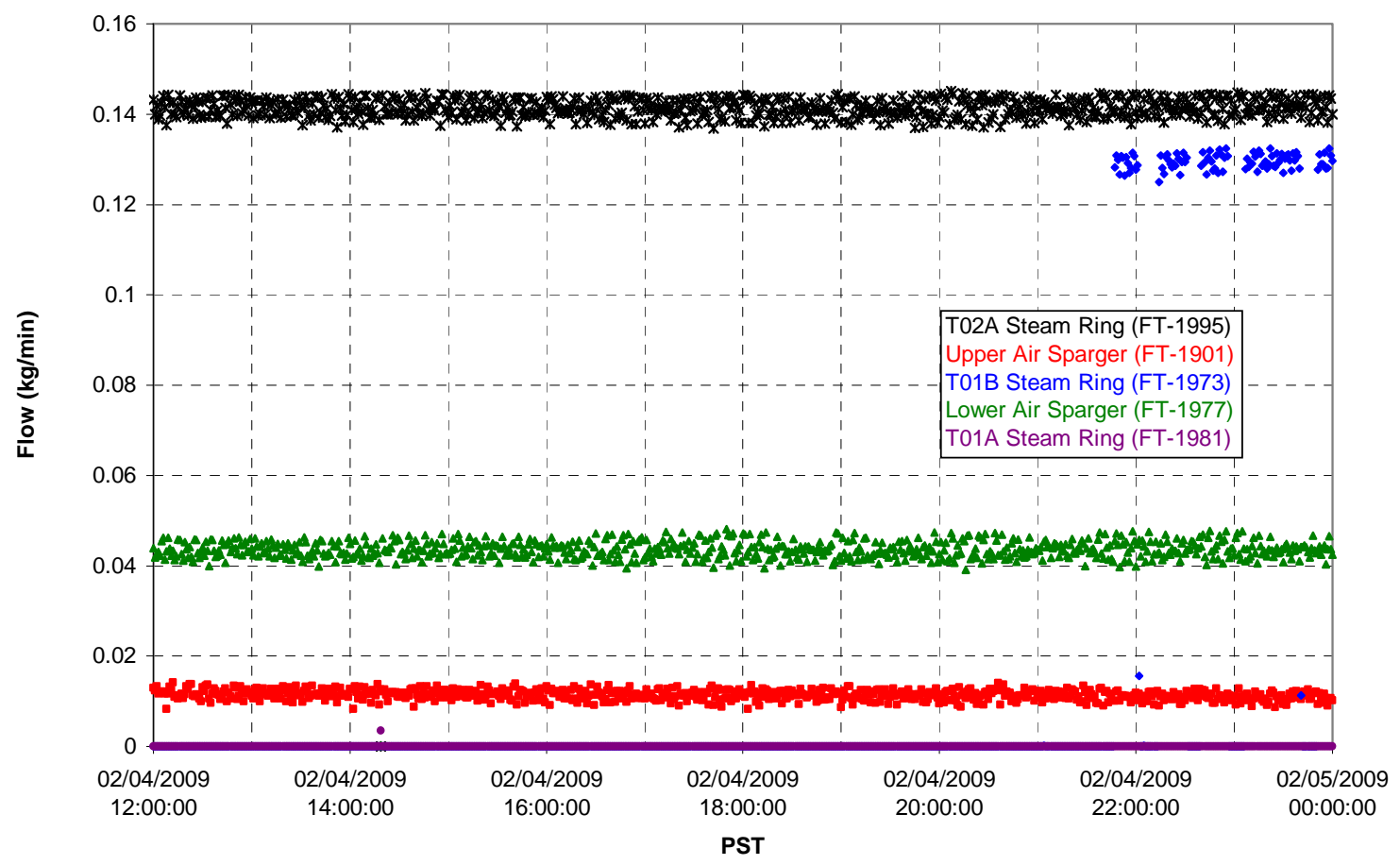


T02A Steam

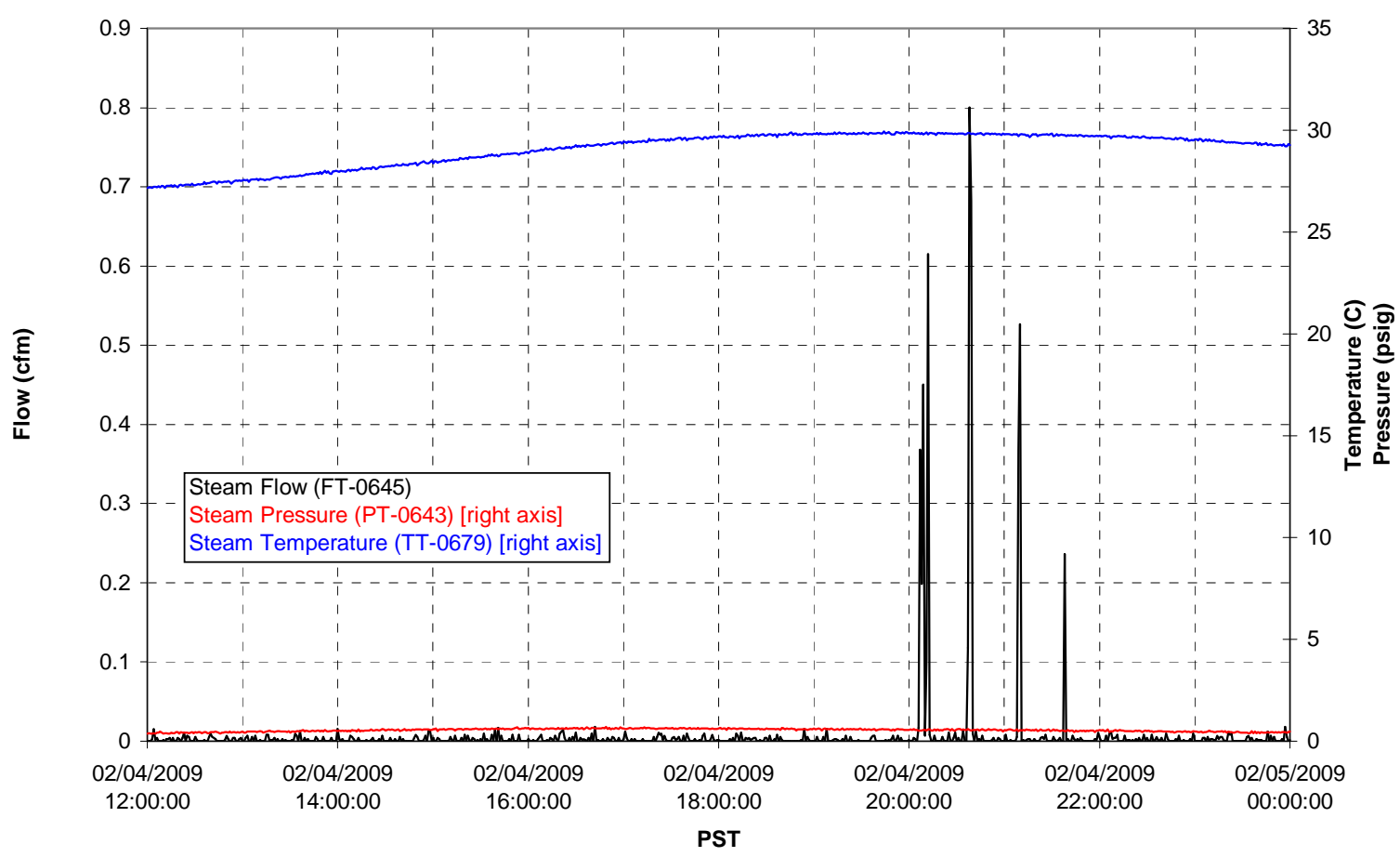

T01A Steam

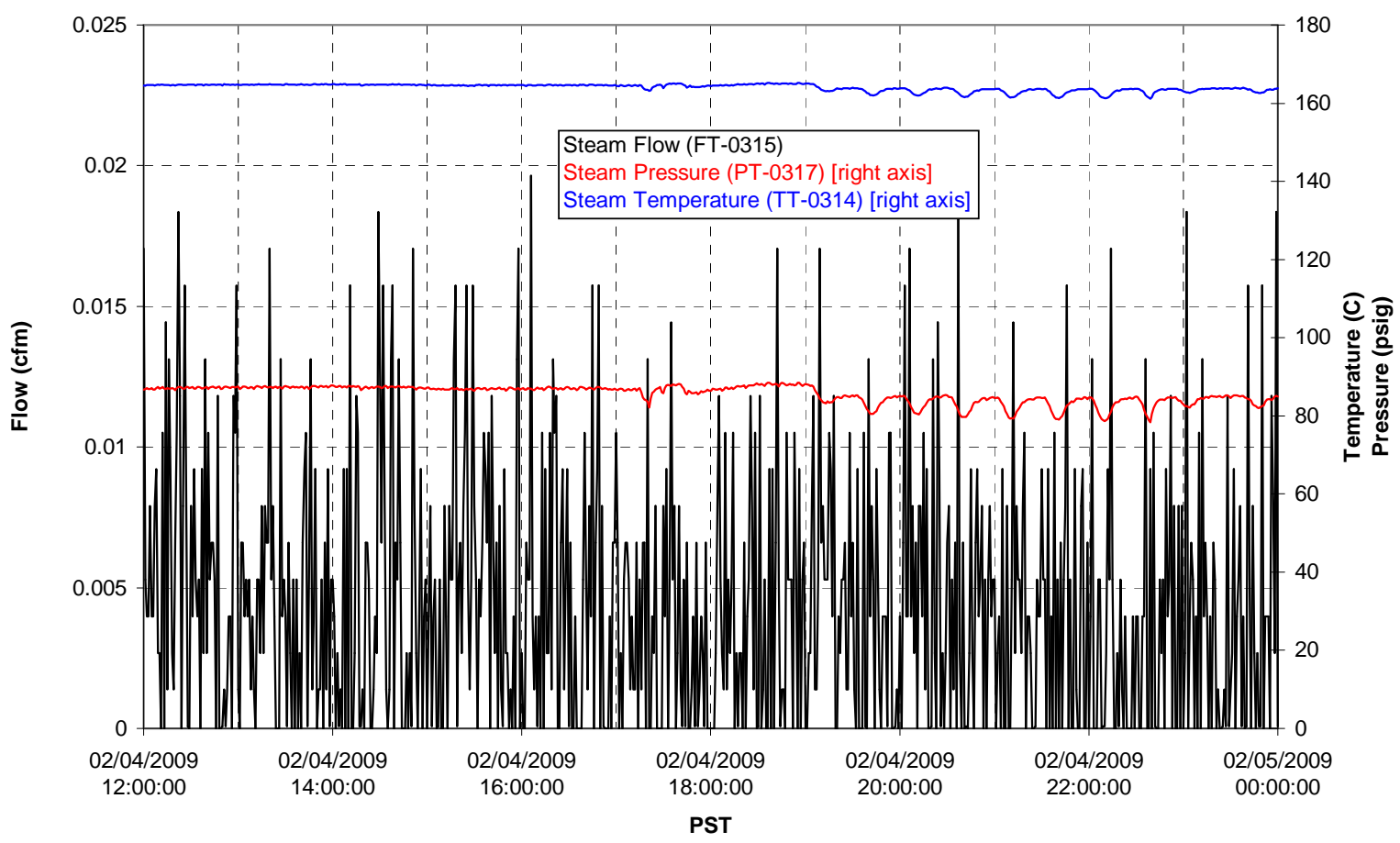


T01B Steam

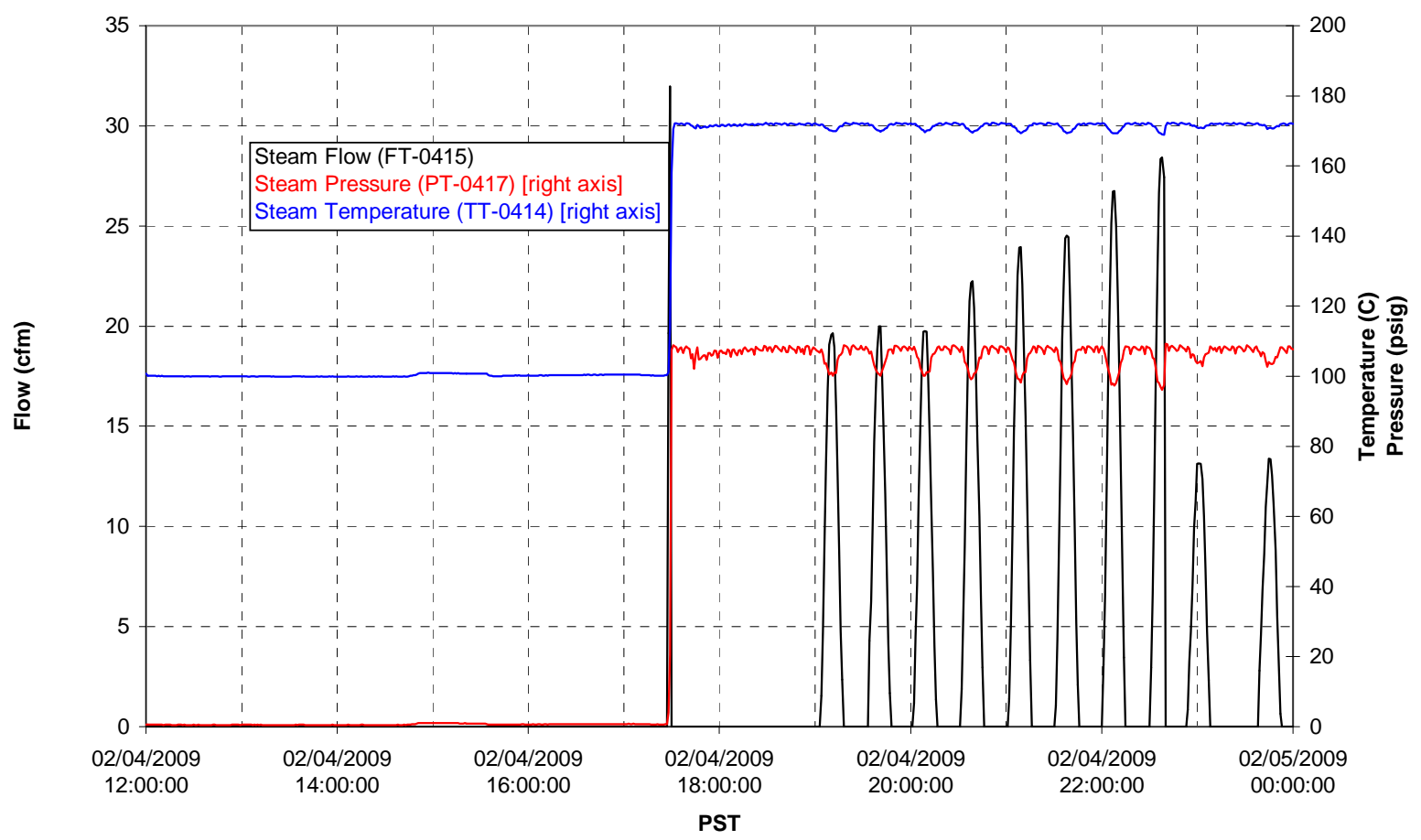




\section{Integrated Test A Data Plots 02/05/09 00:00 - 02/05/09 12:00}


T01A level

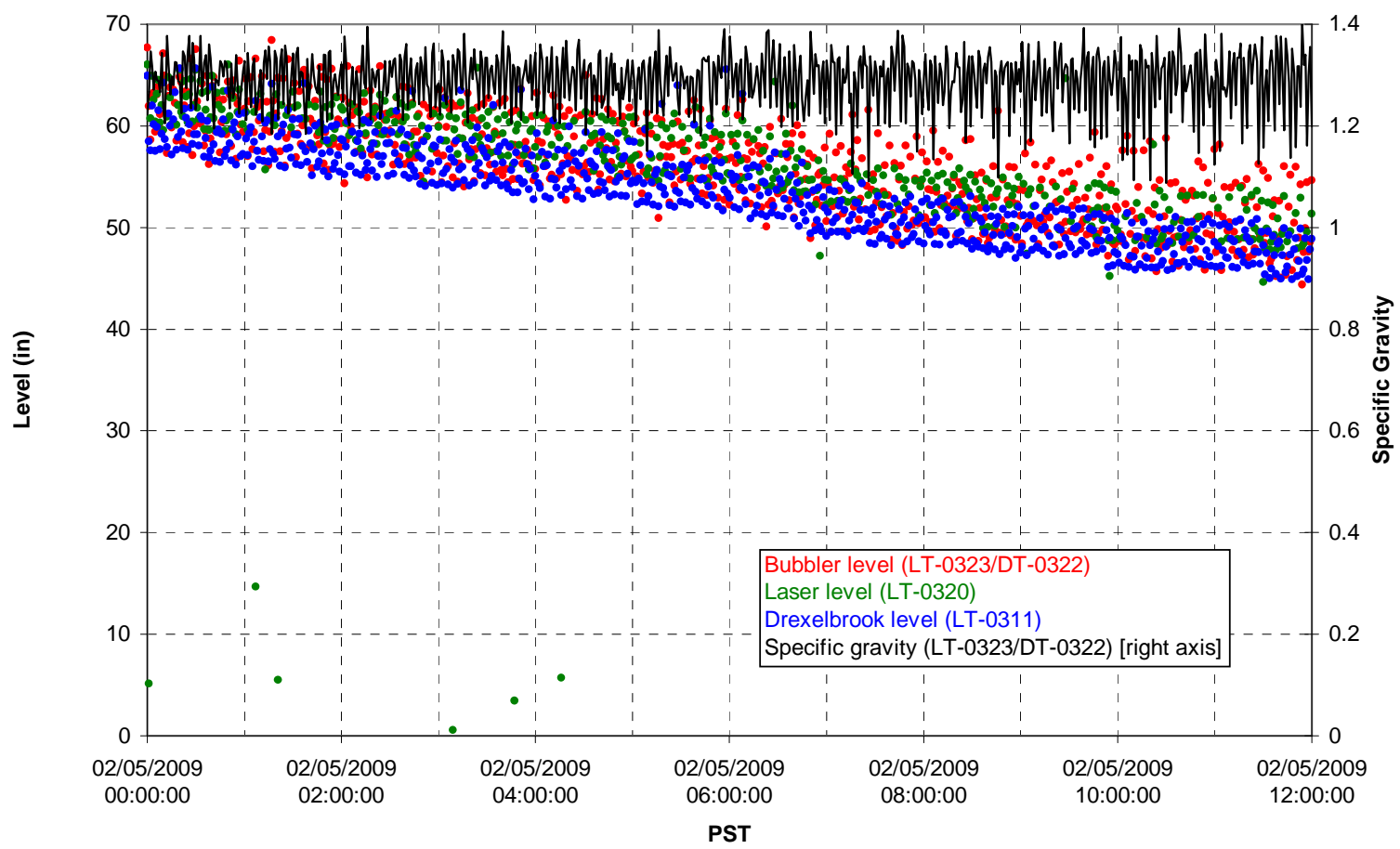

T01A temperatures

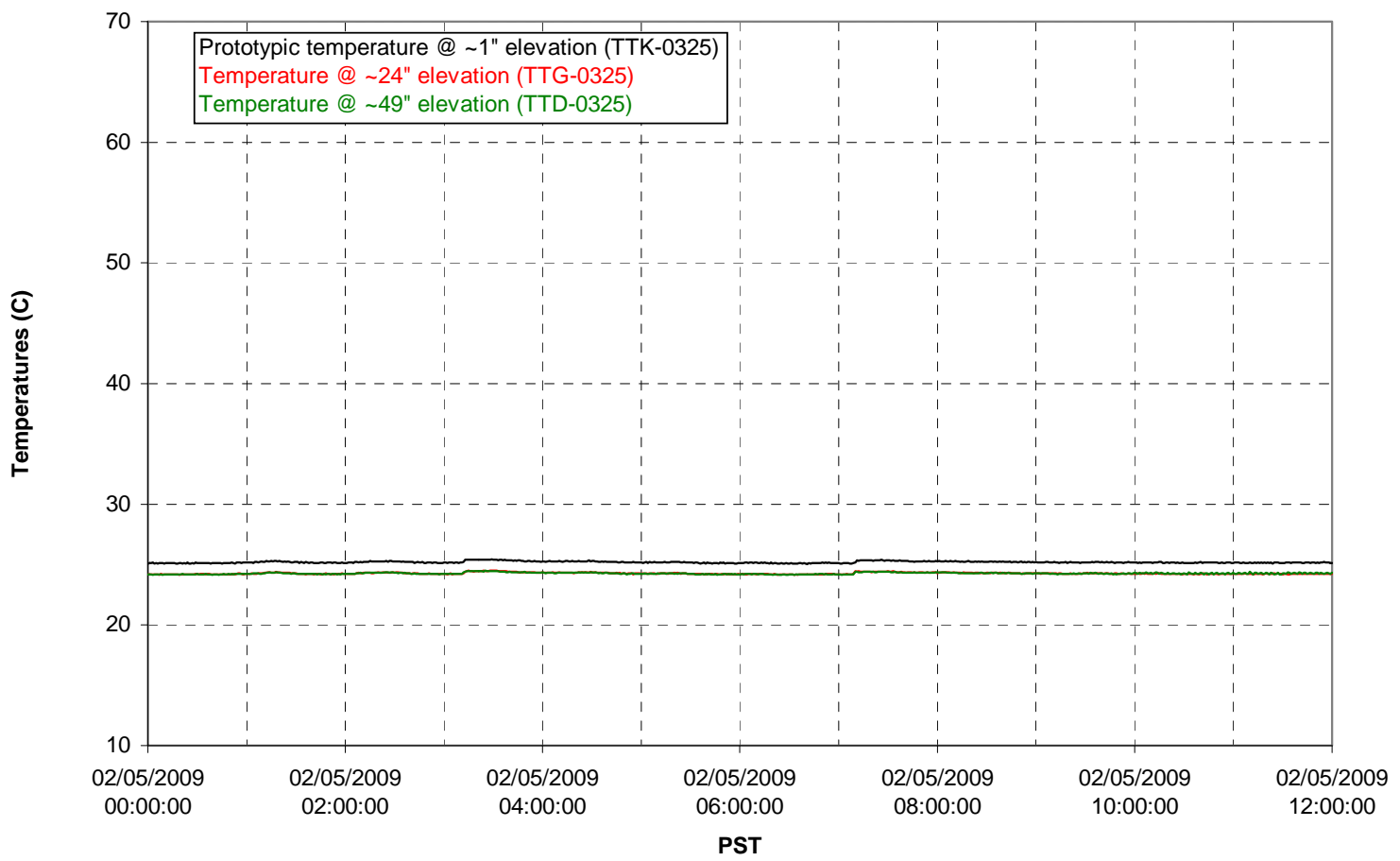


T01B level

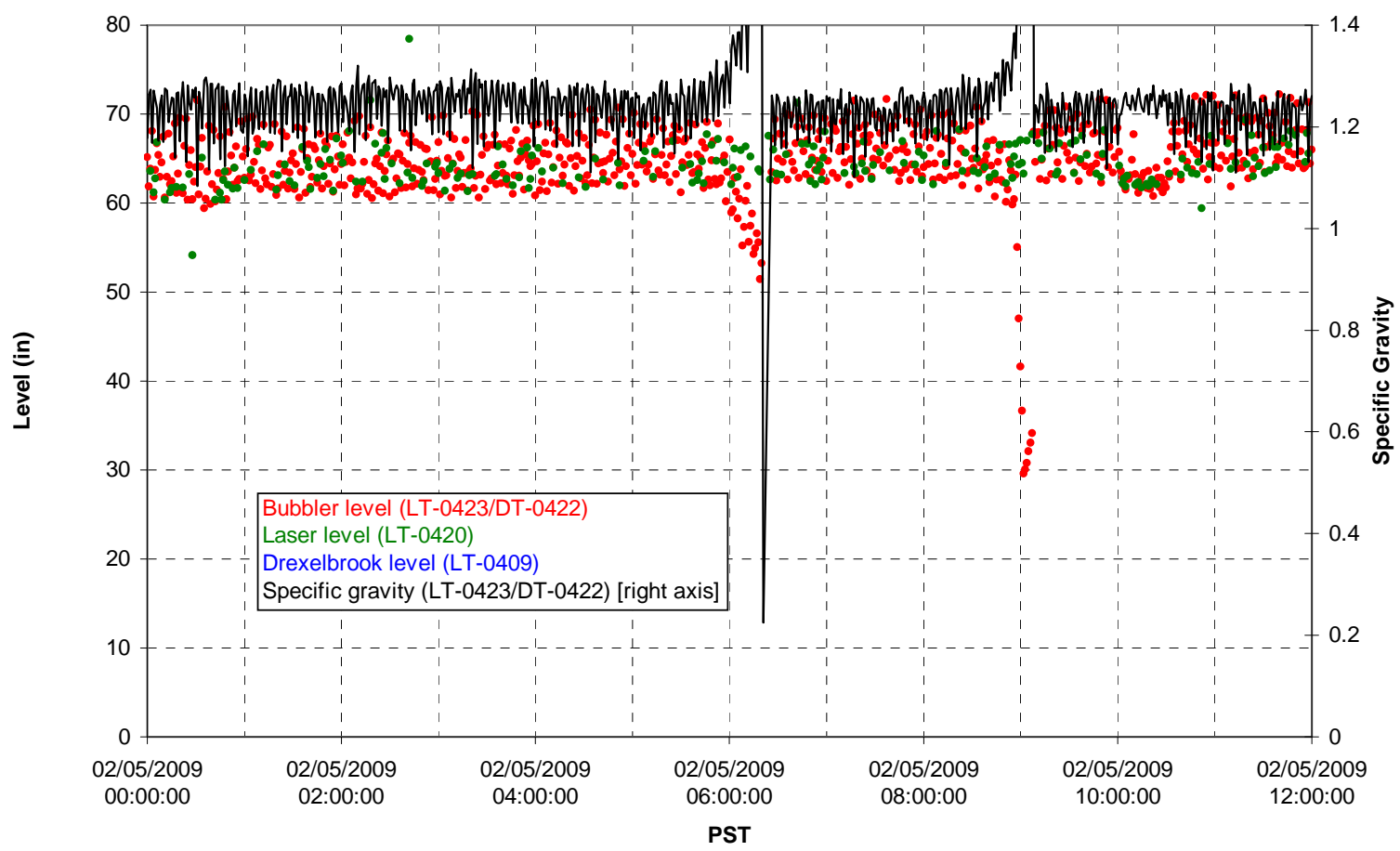

T01B temperatures

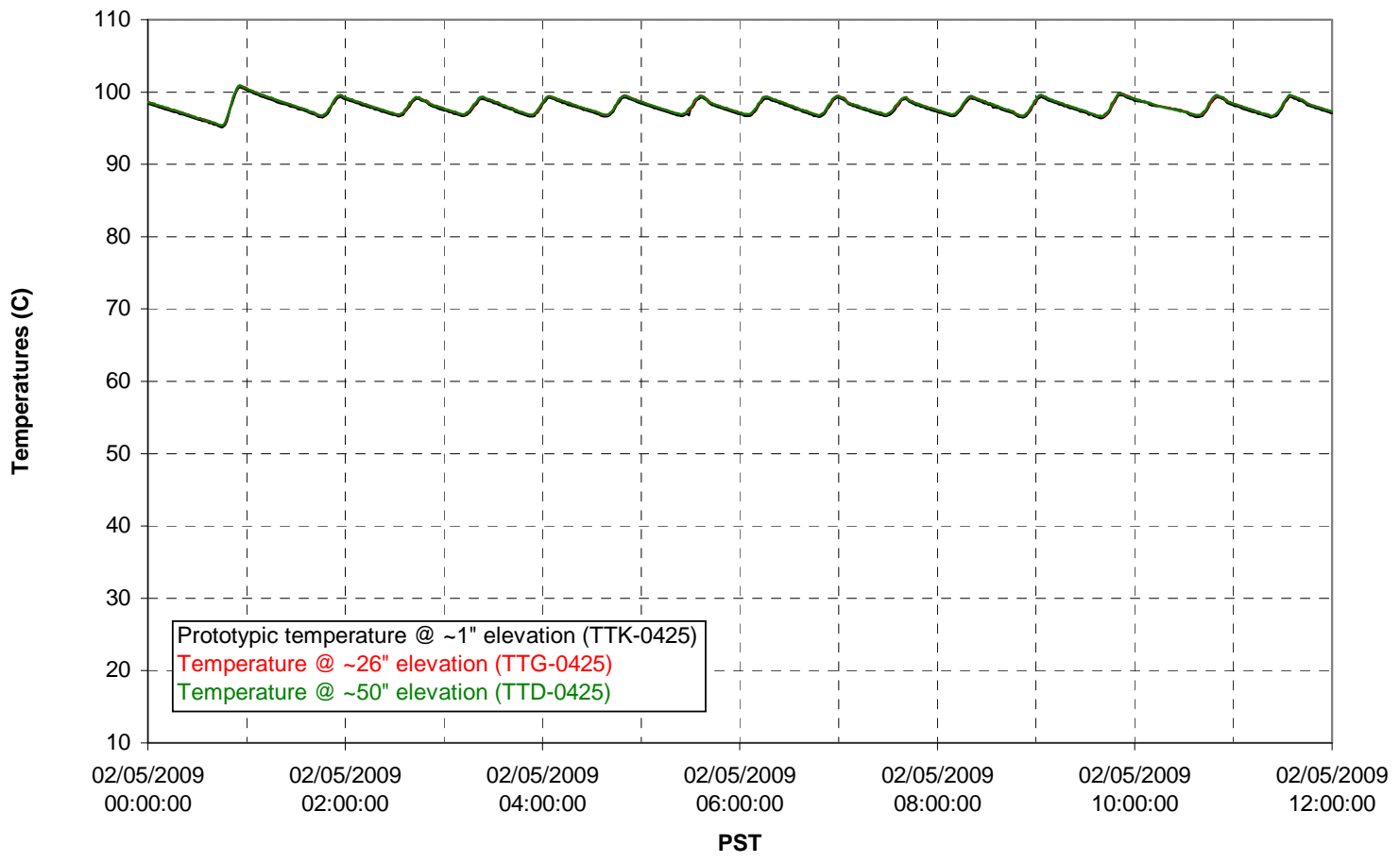

G.183 
T02A level

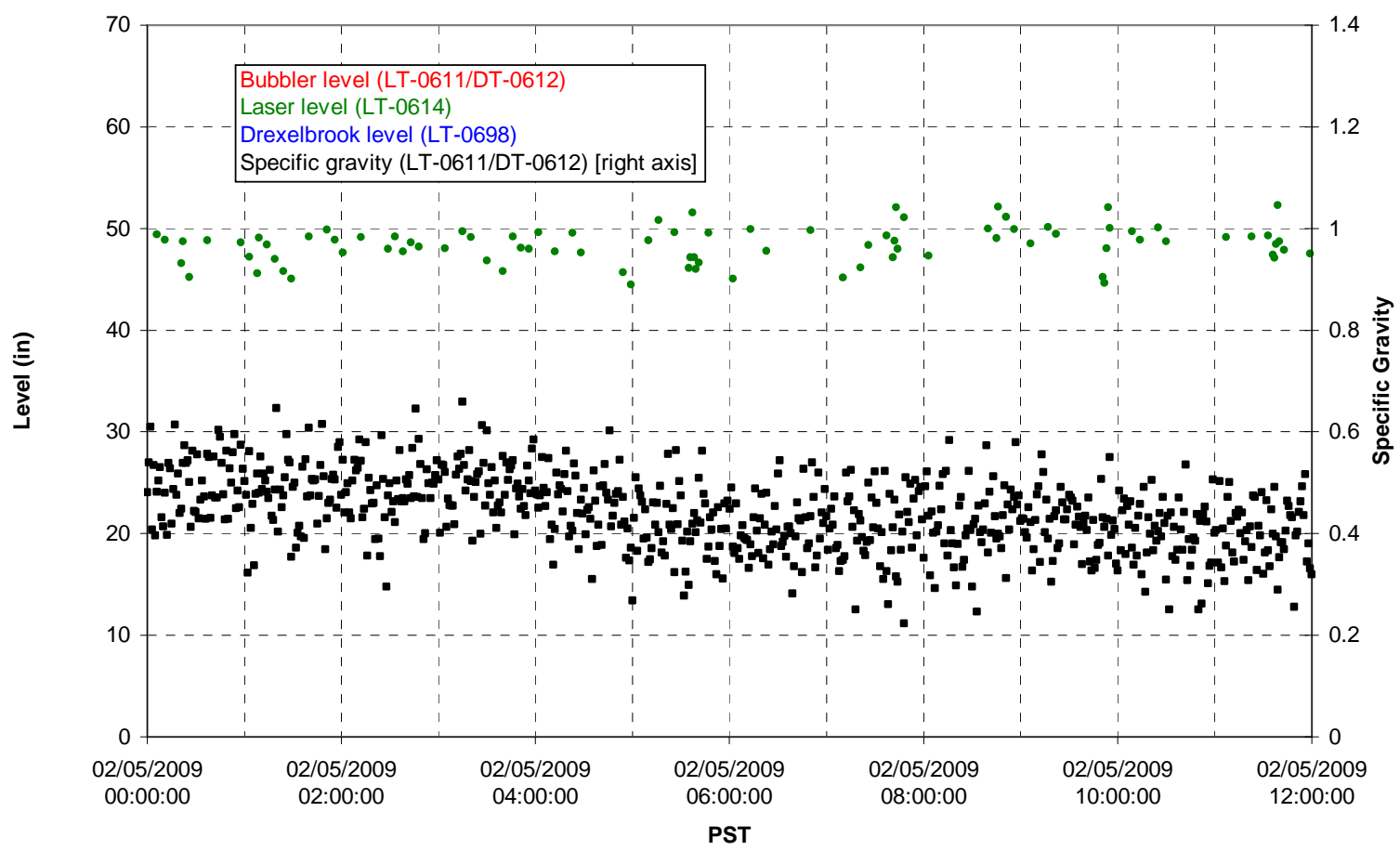

T02A temperatures

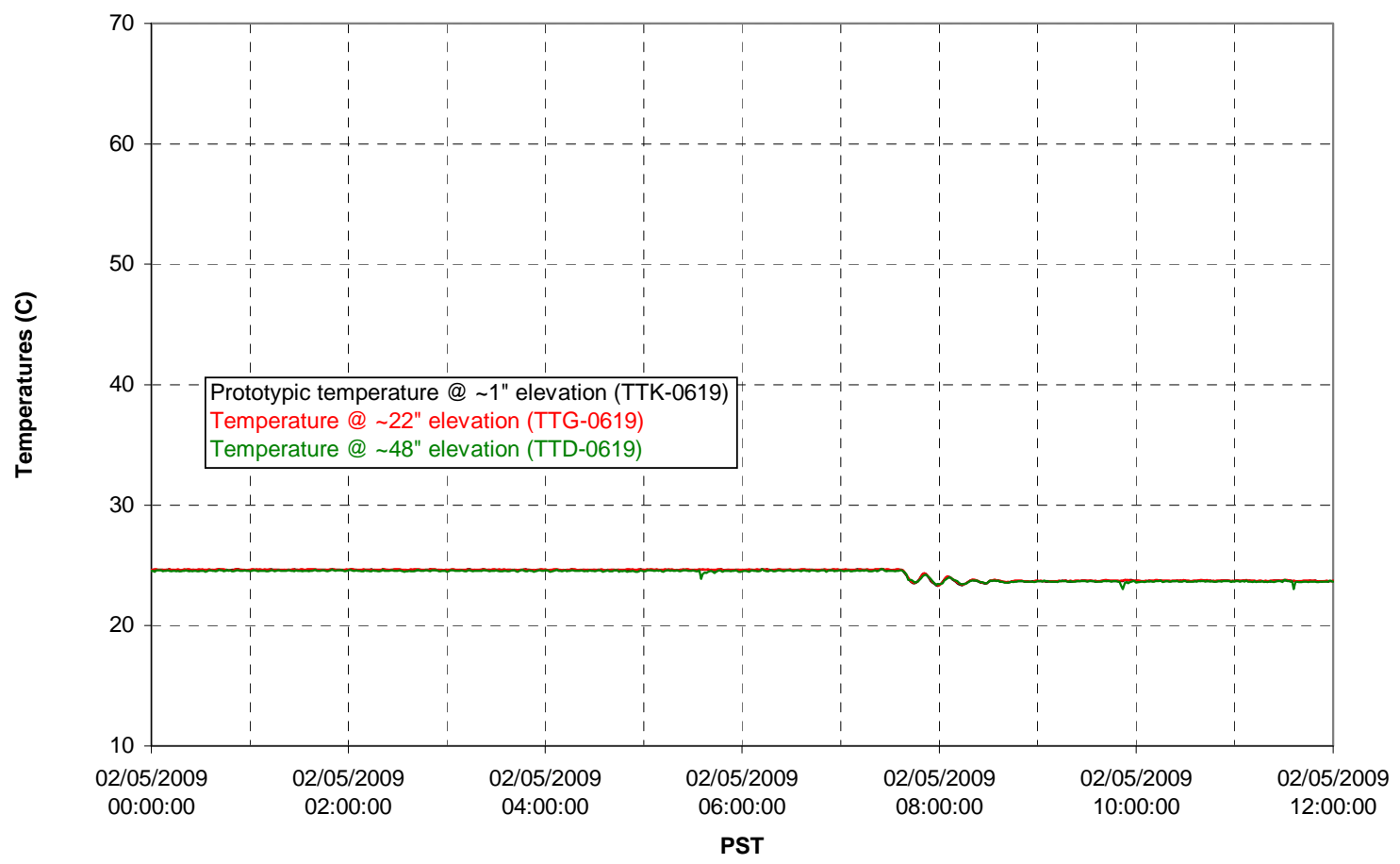

G.184 
T02A and filter loop temperatures
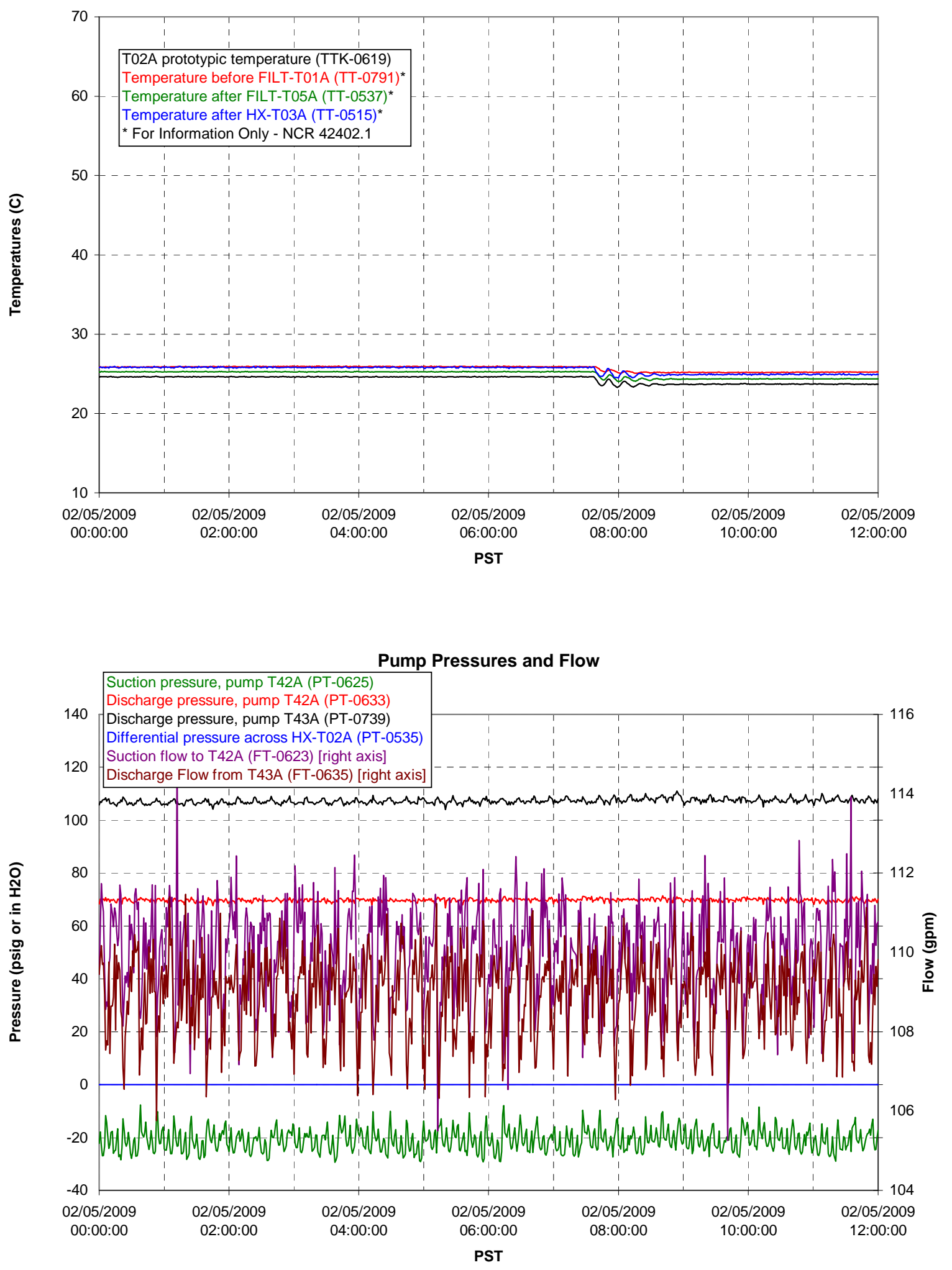

G. 185 


\section{Axial pressure drop}

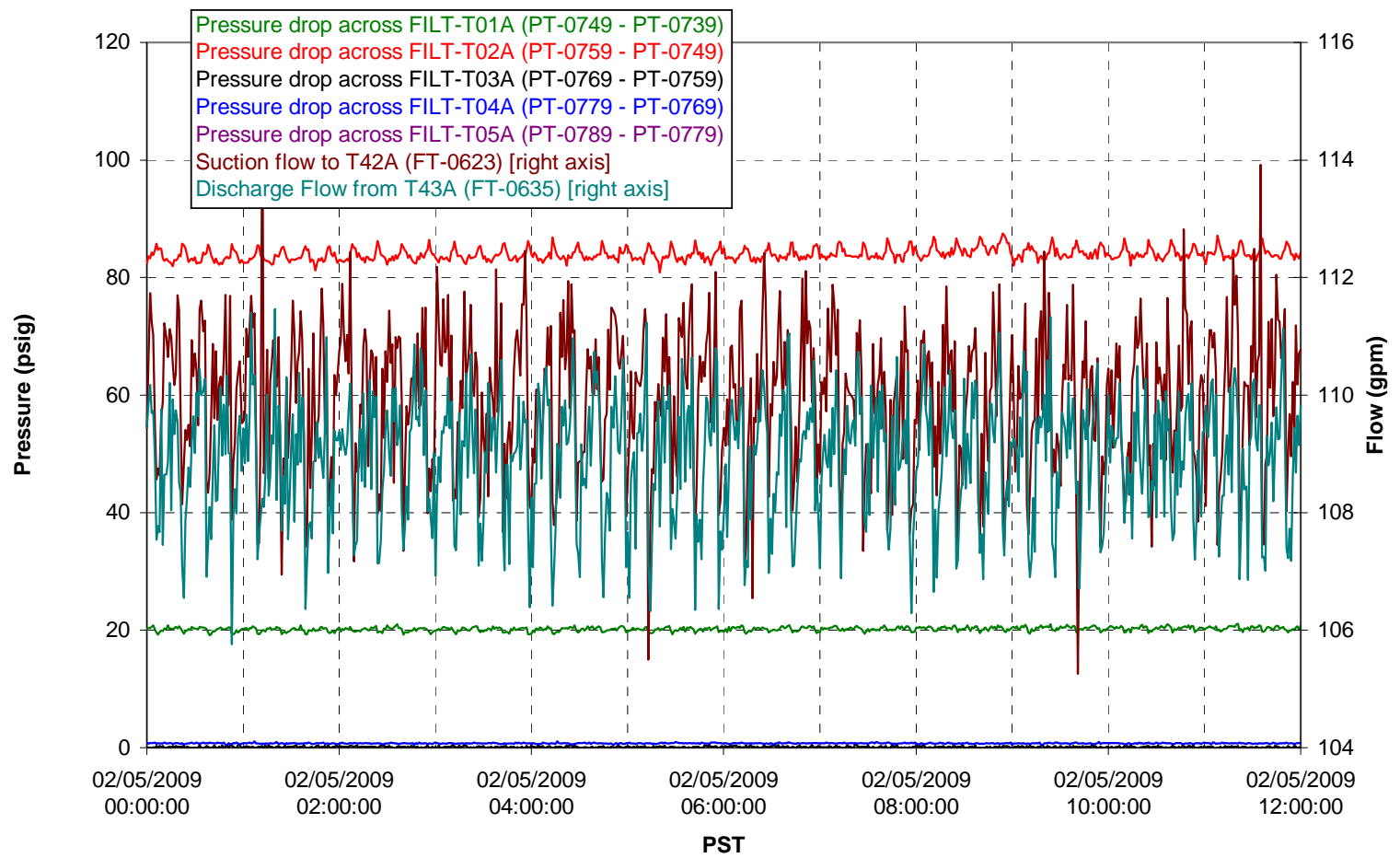

Permeate flow rates

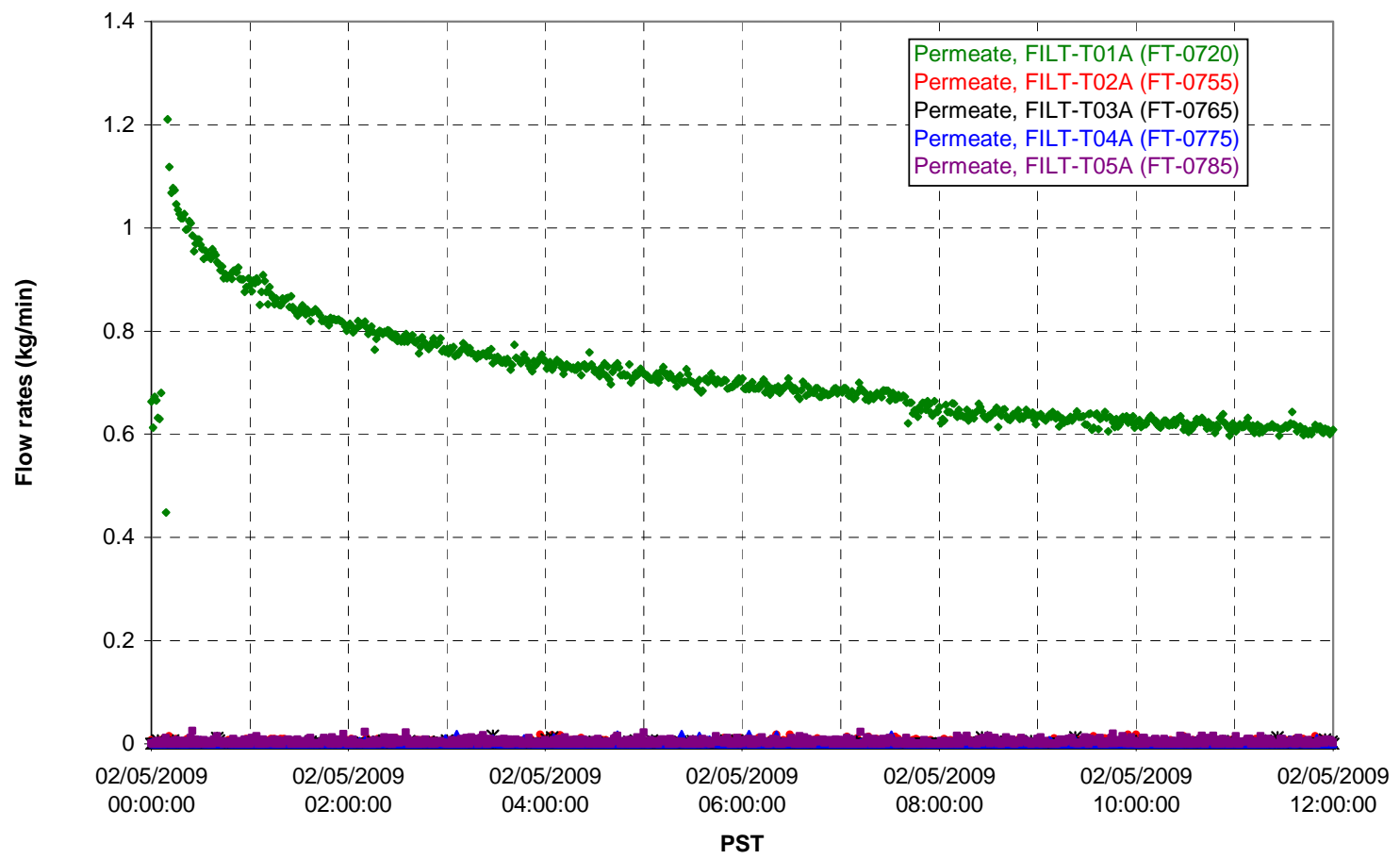


T02A Inner Temperature Tree

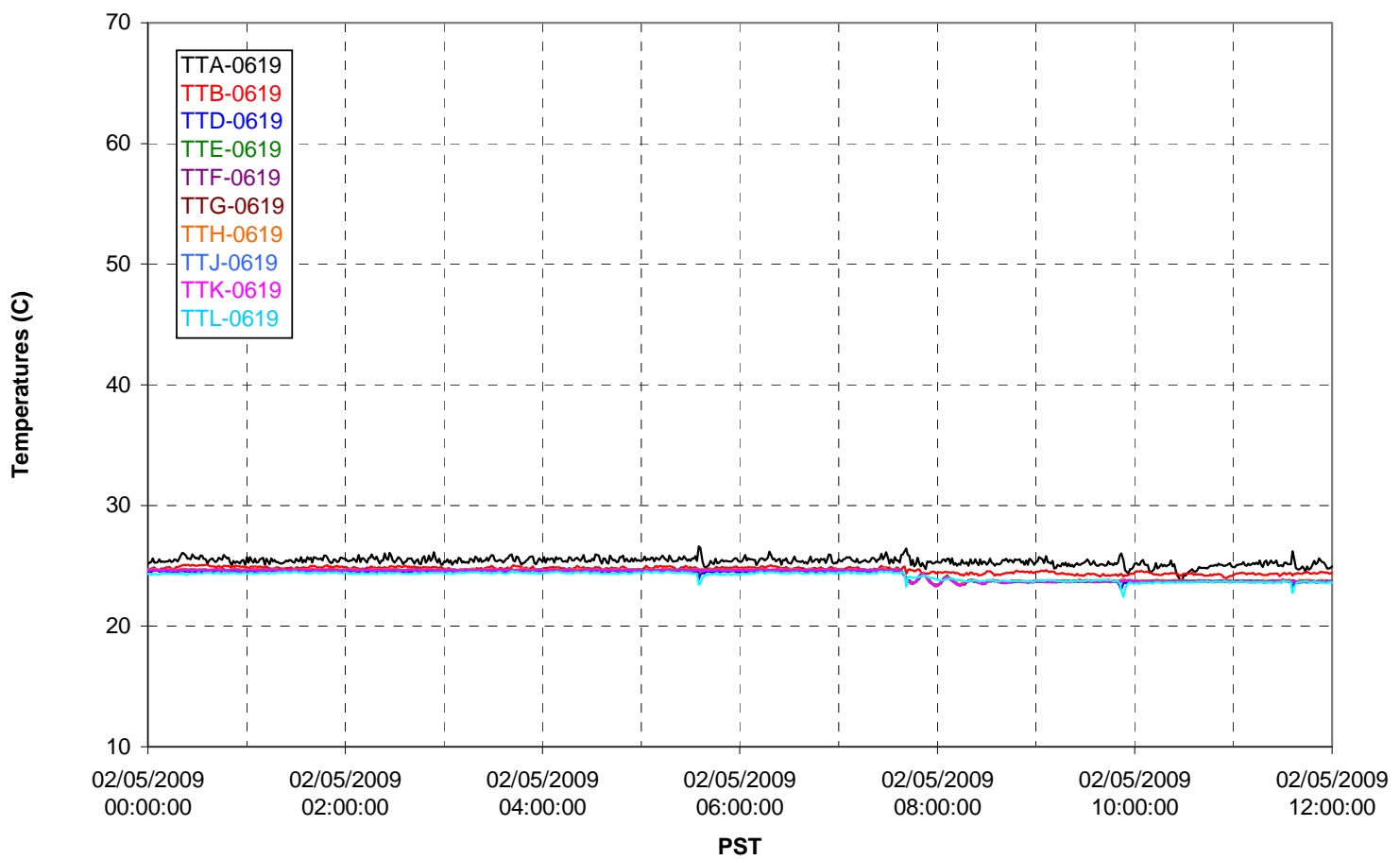

T02A Outer Temperature Tree

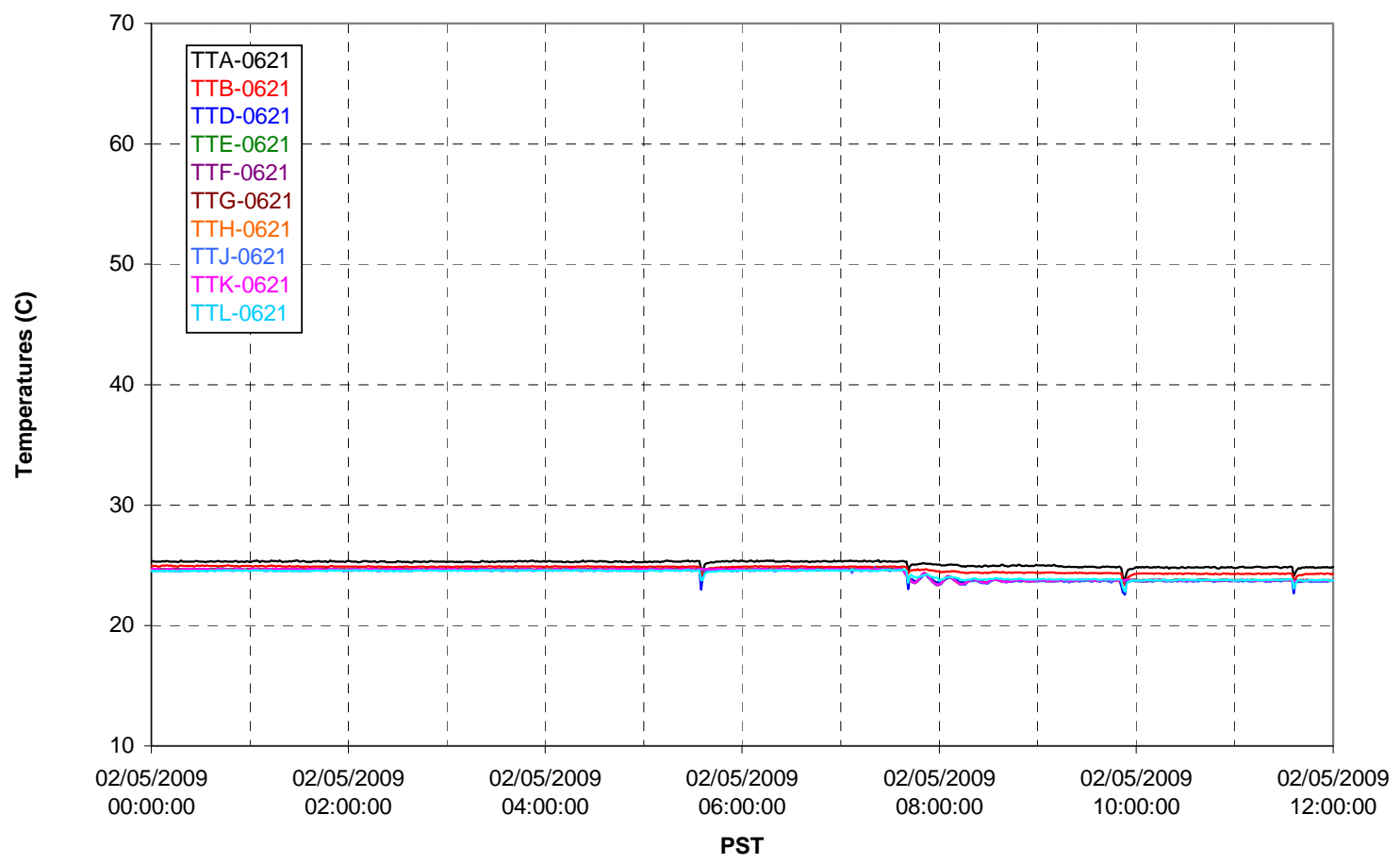

G.187 
T02A temperatures

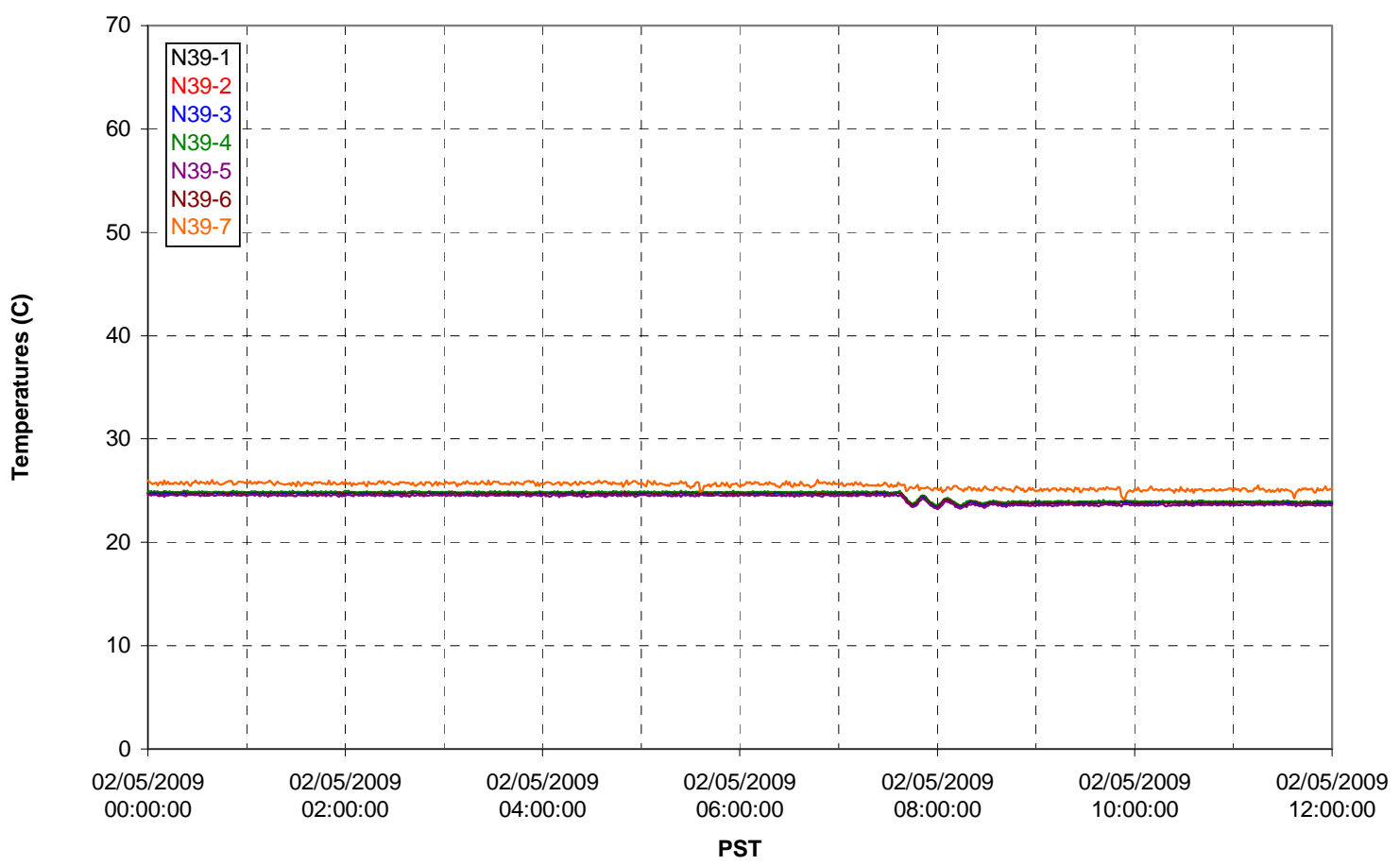

T02A temperatures

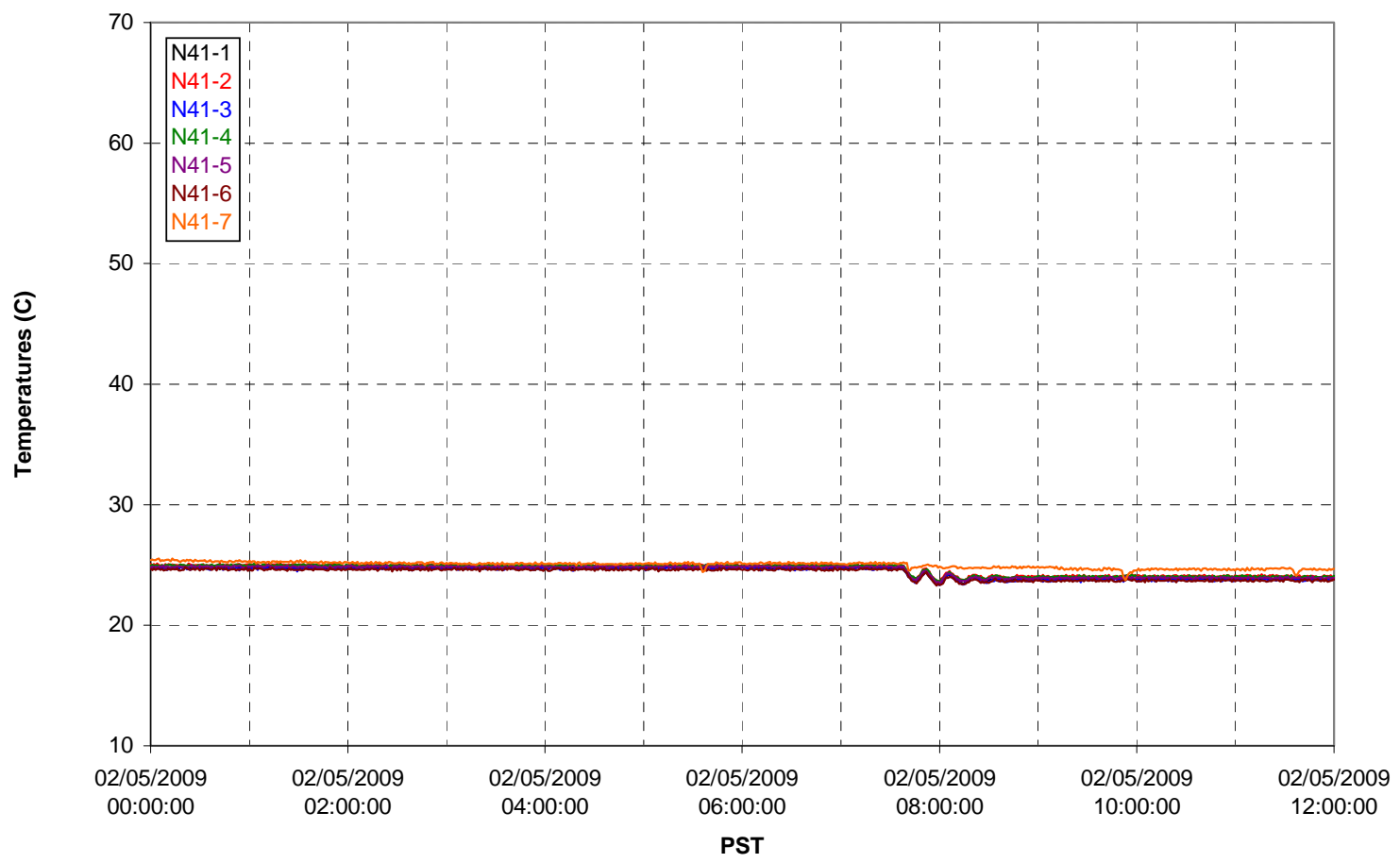

G.188 
T02A temperatures

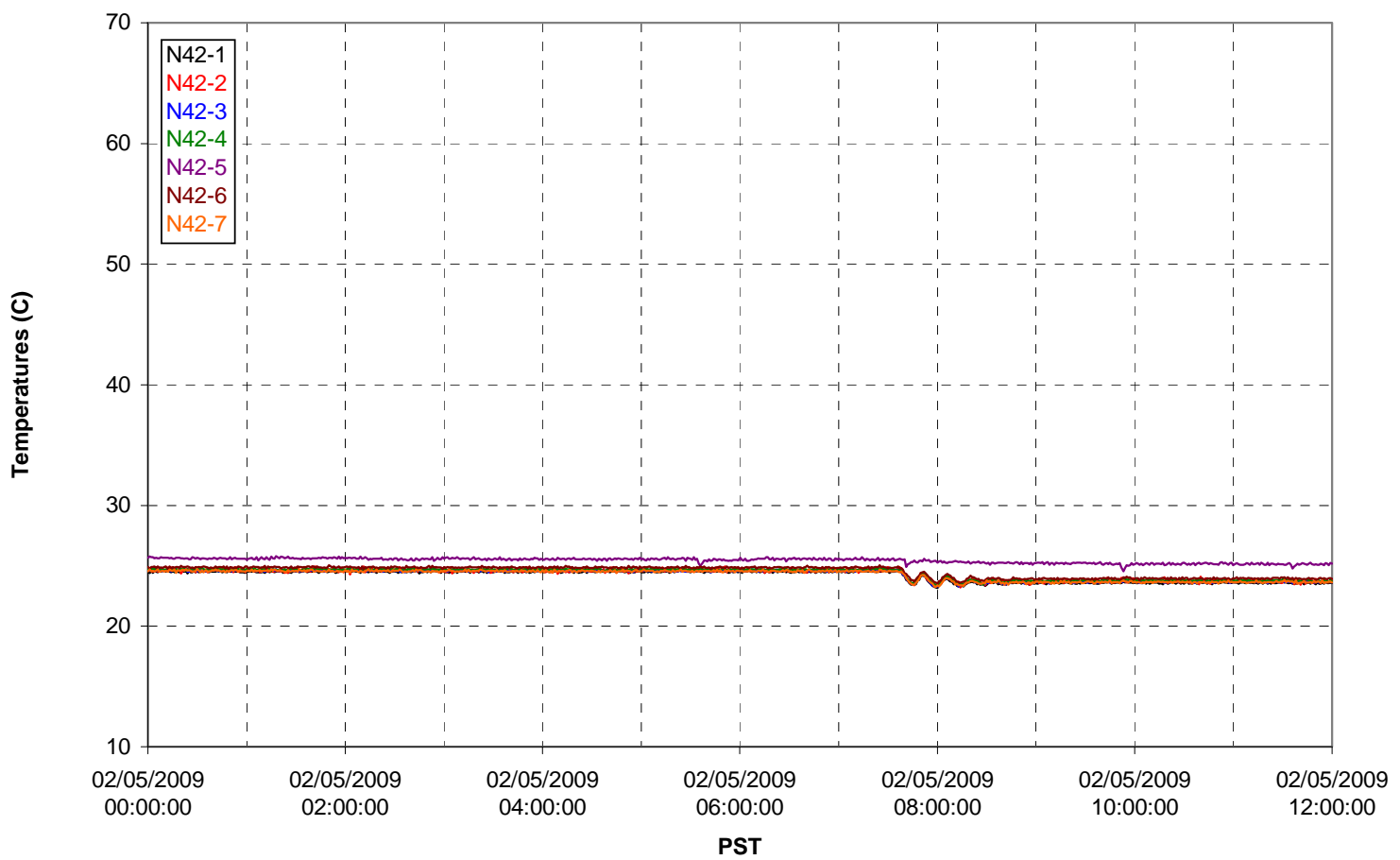

T02A temperatures

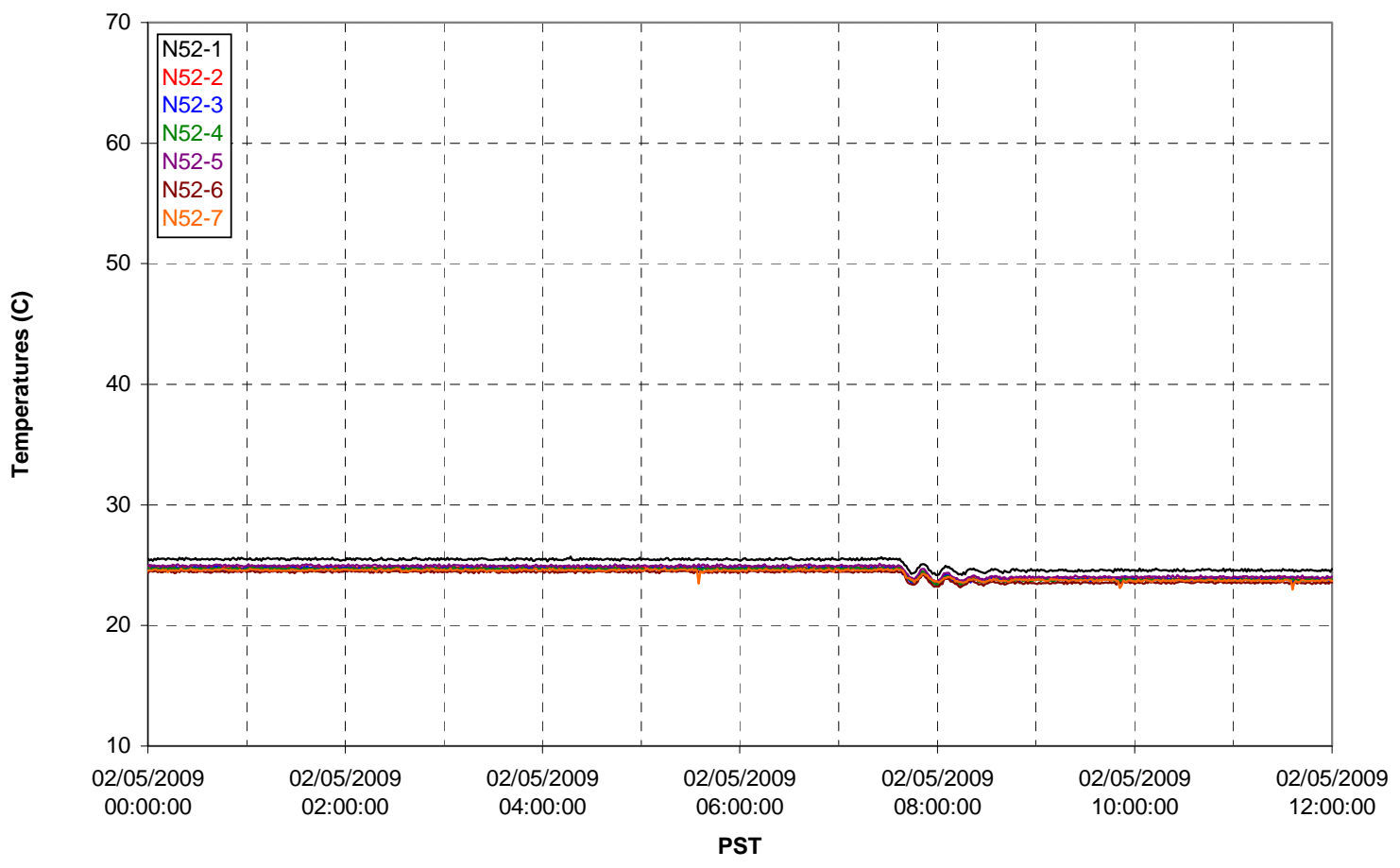

G.189 
T02A Heating and Cooling

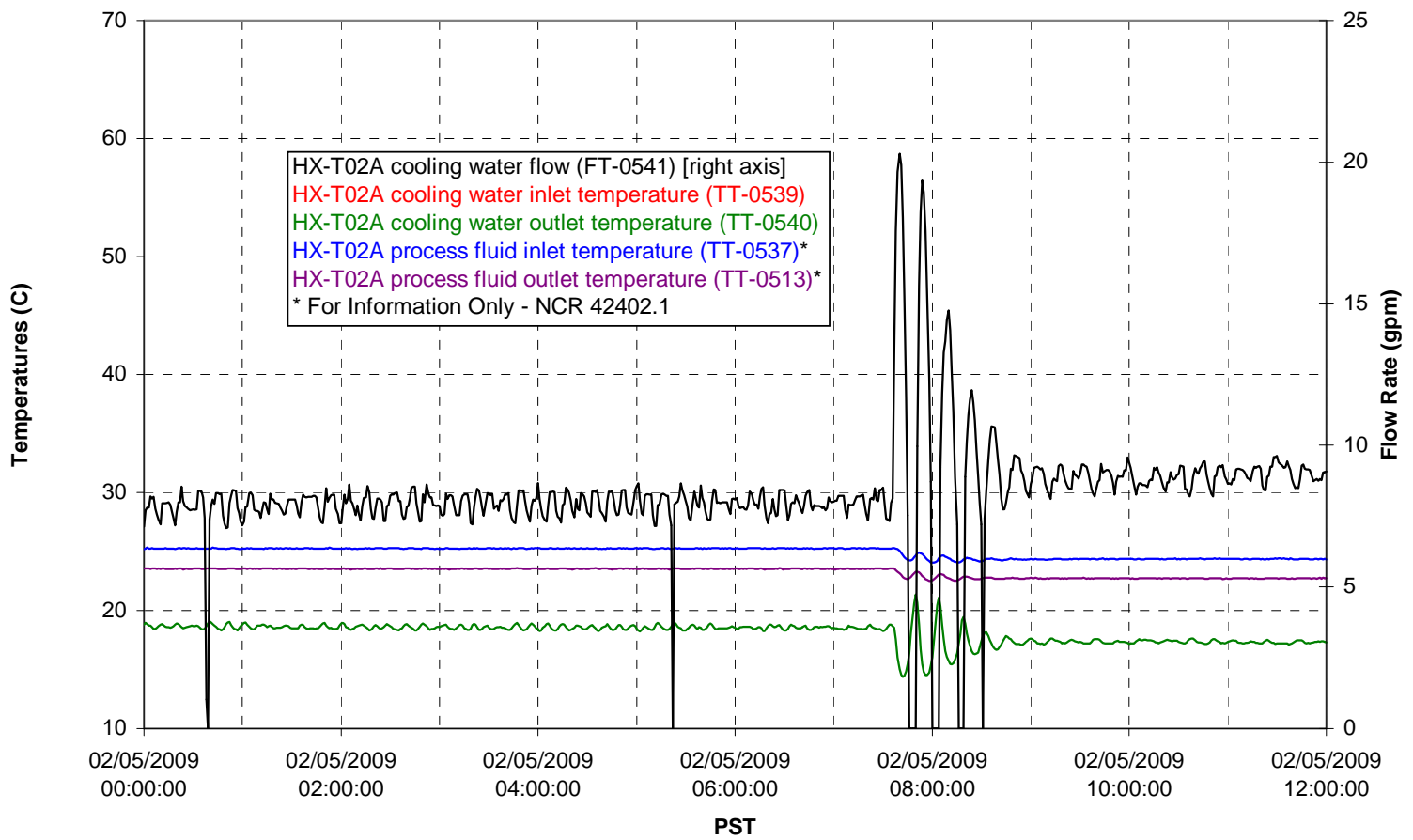

Pump Operation

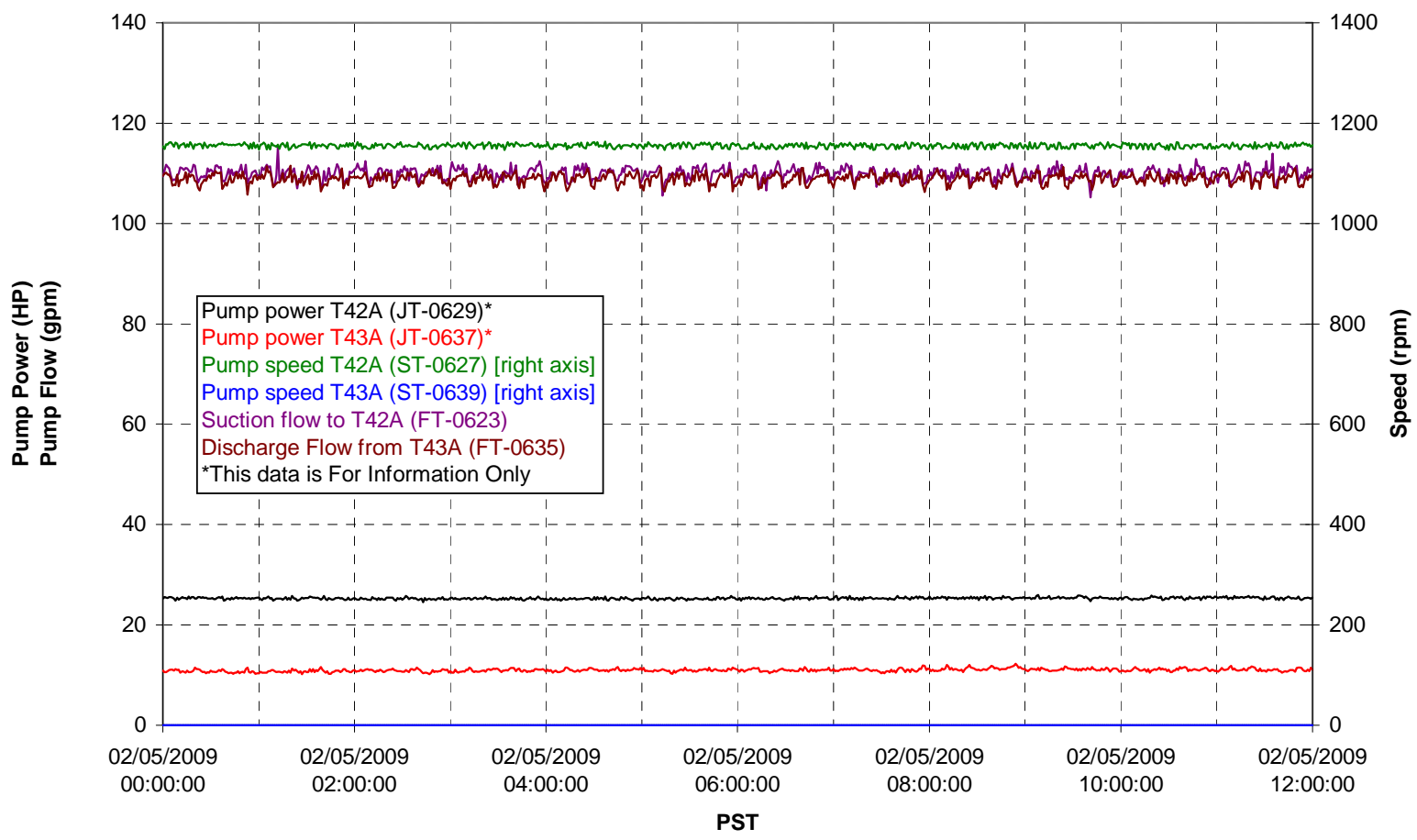

G.190 
Pulsepot UFP-PP-T01A

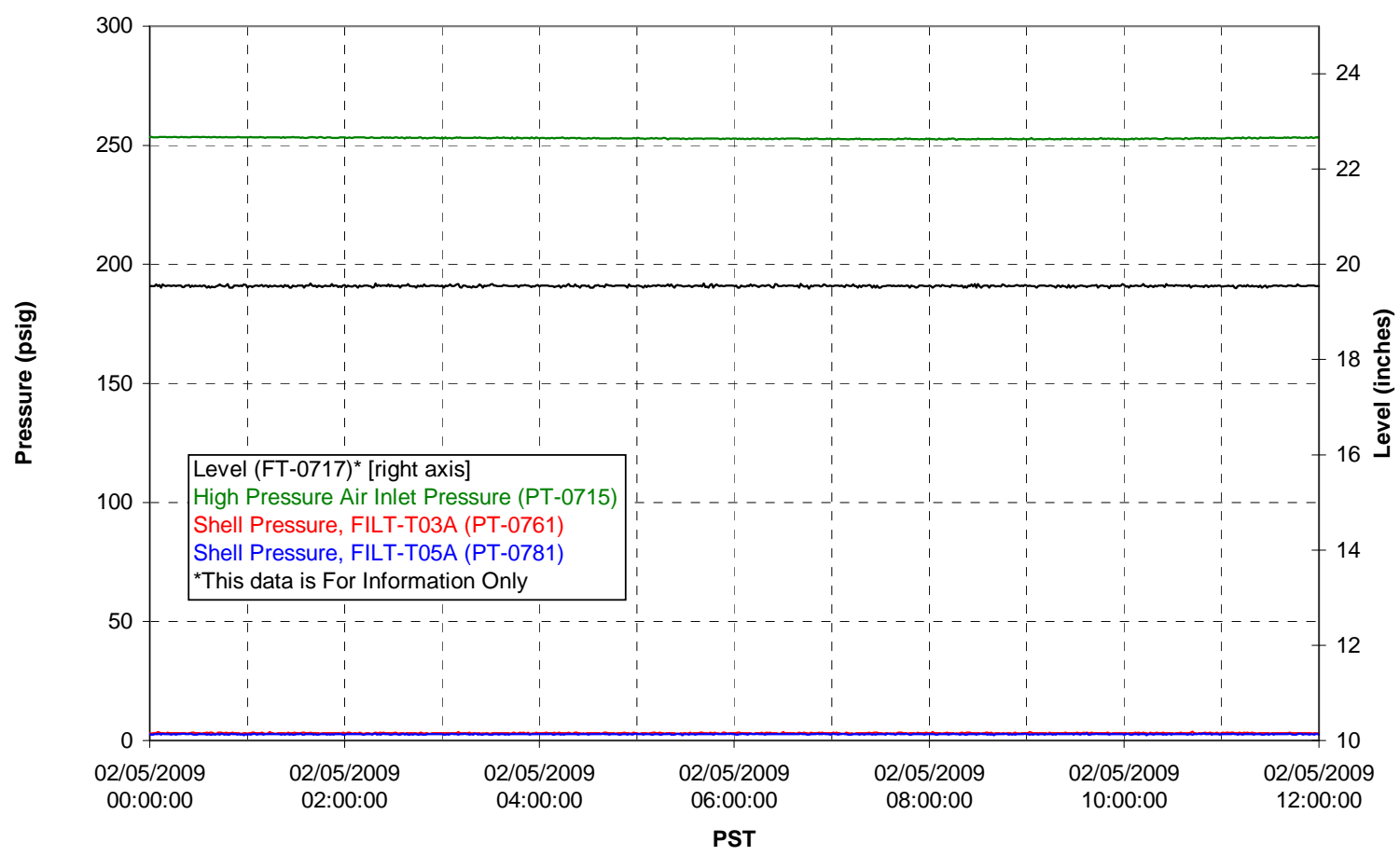

Pulsepot UFP-PP-T02A

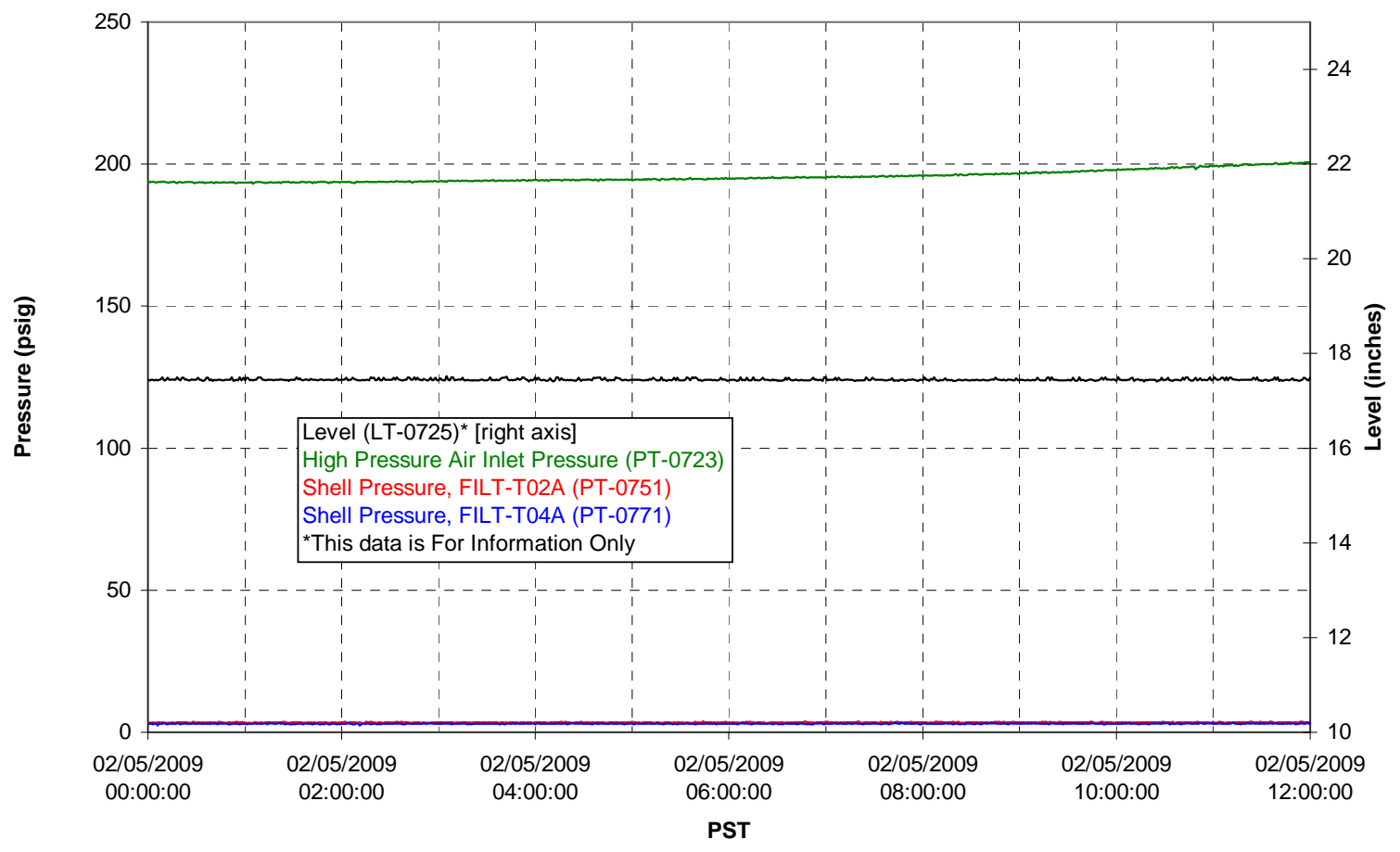

G.191 
Pulsepot UFP-PP-T03A

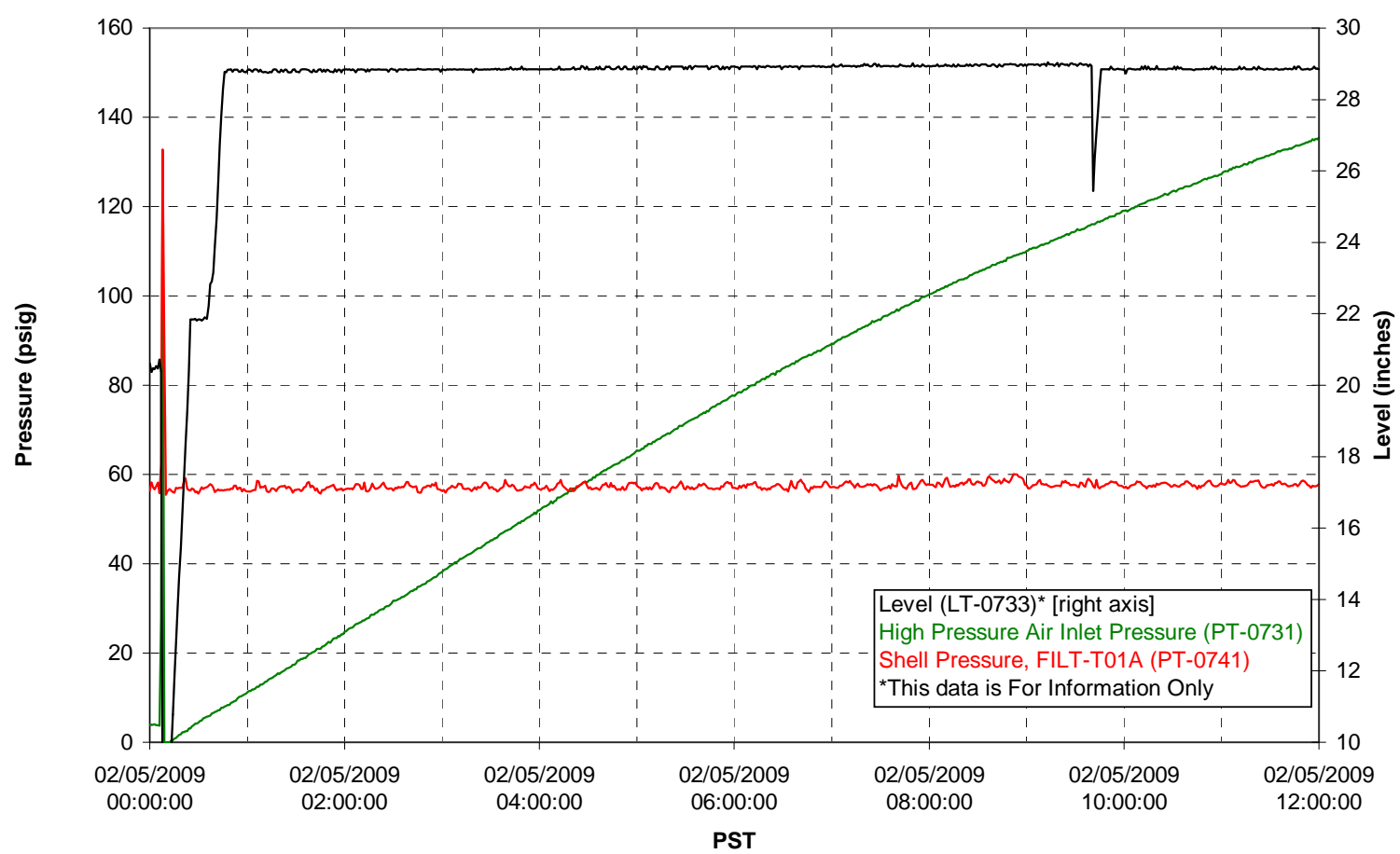

Pulsepot Levels

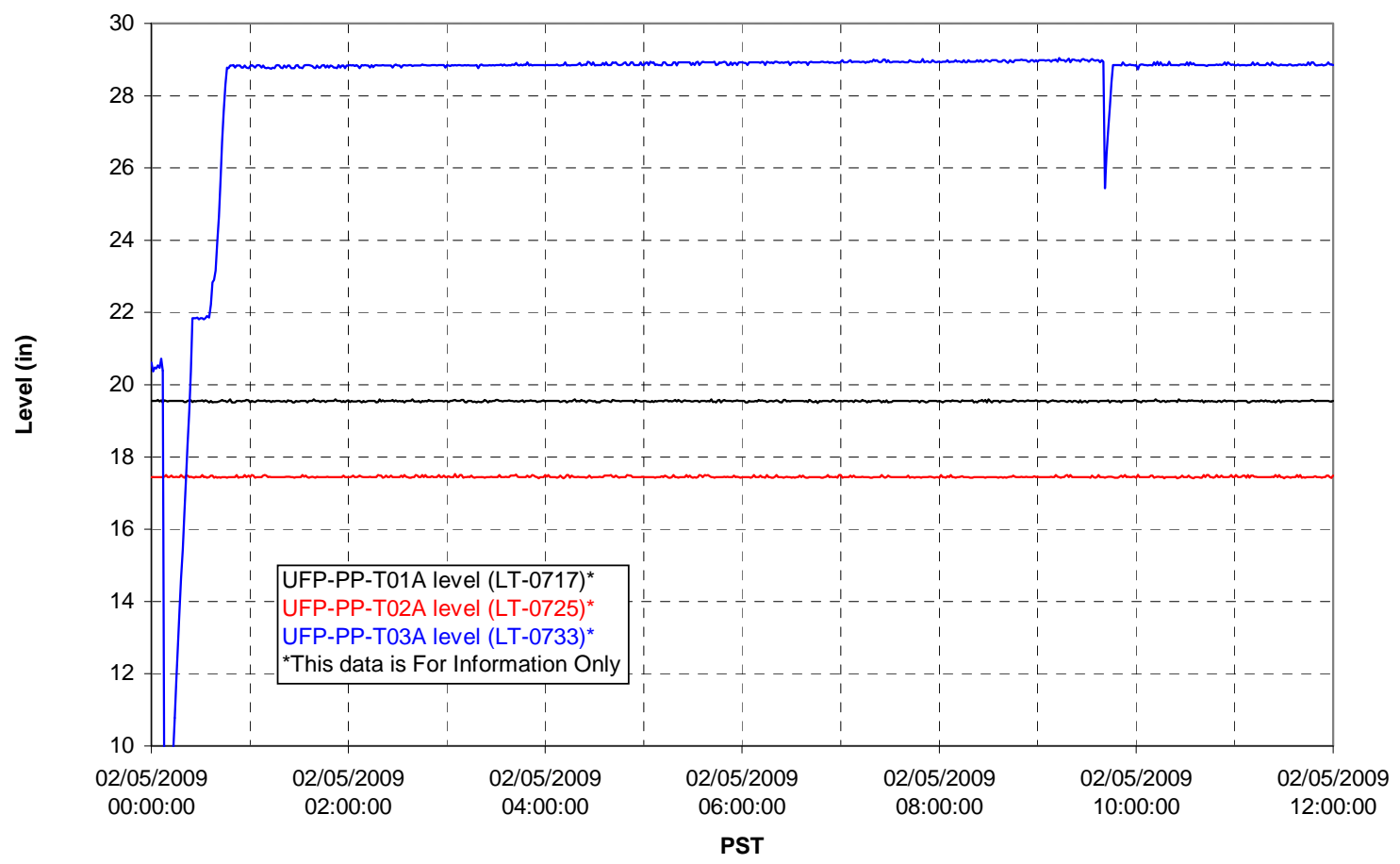

G.192 
Filter UFP-FILT-T01A

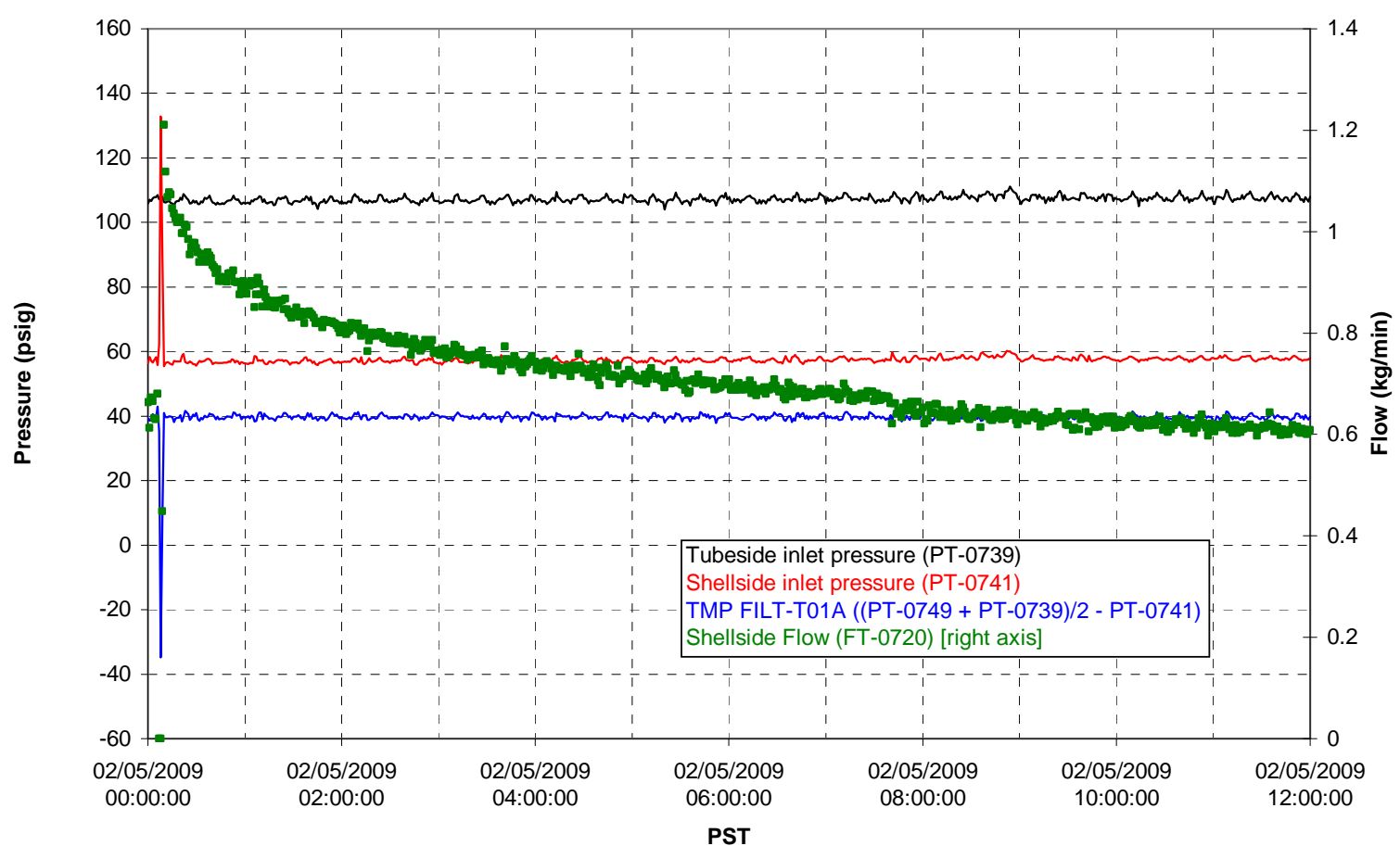

Filter UFP-FILT-T02A

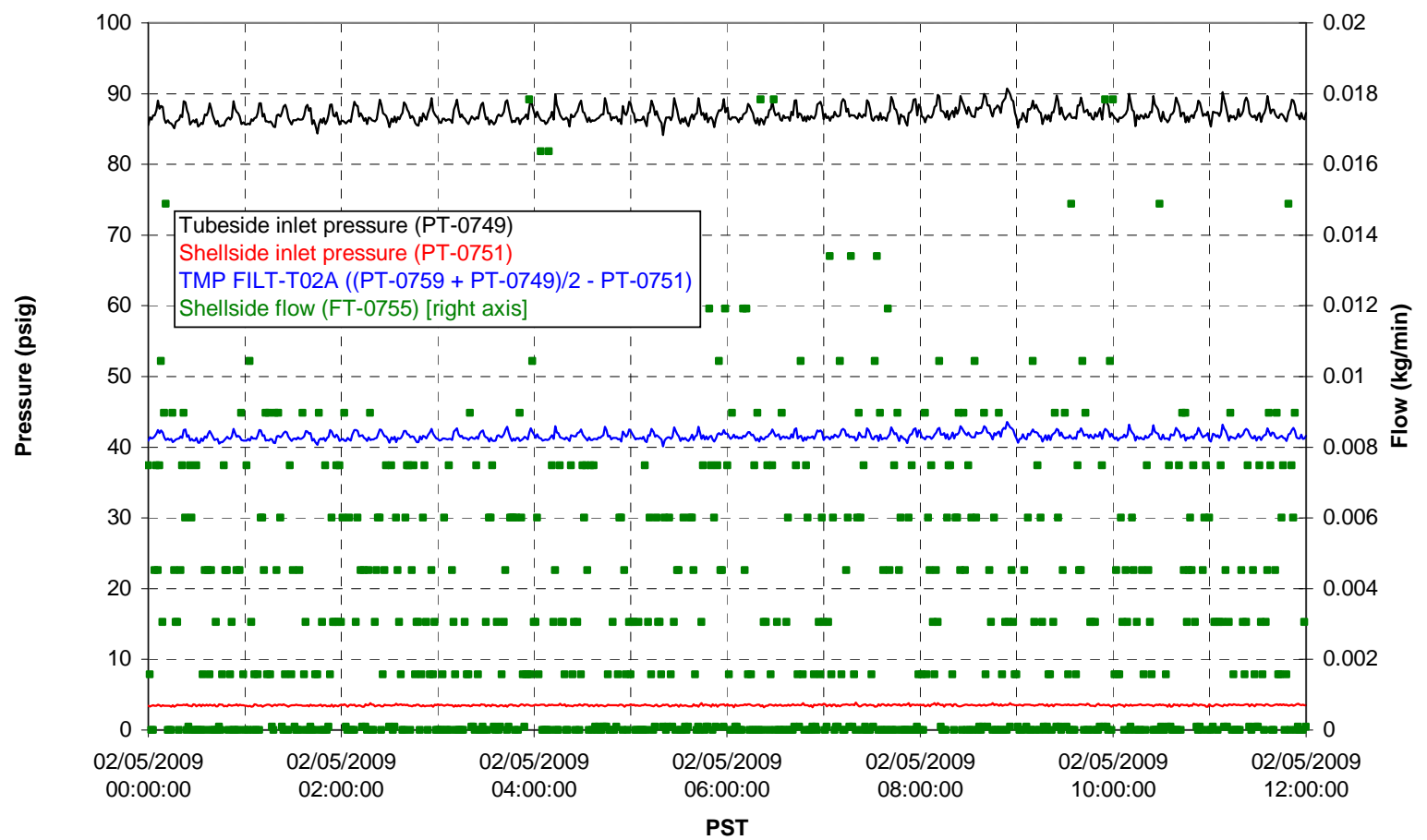

G.193 
Filter UFP-FILT-T03A

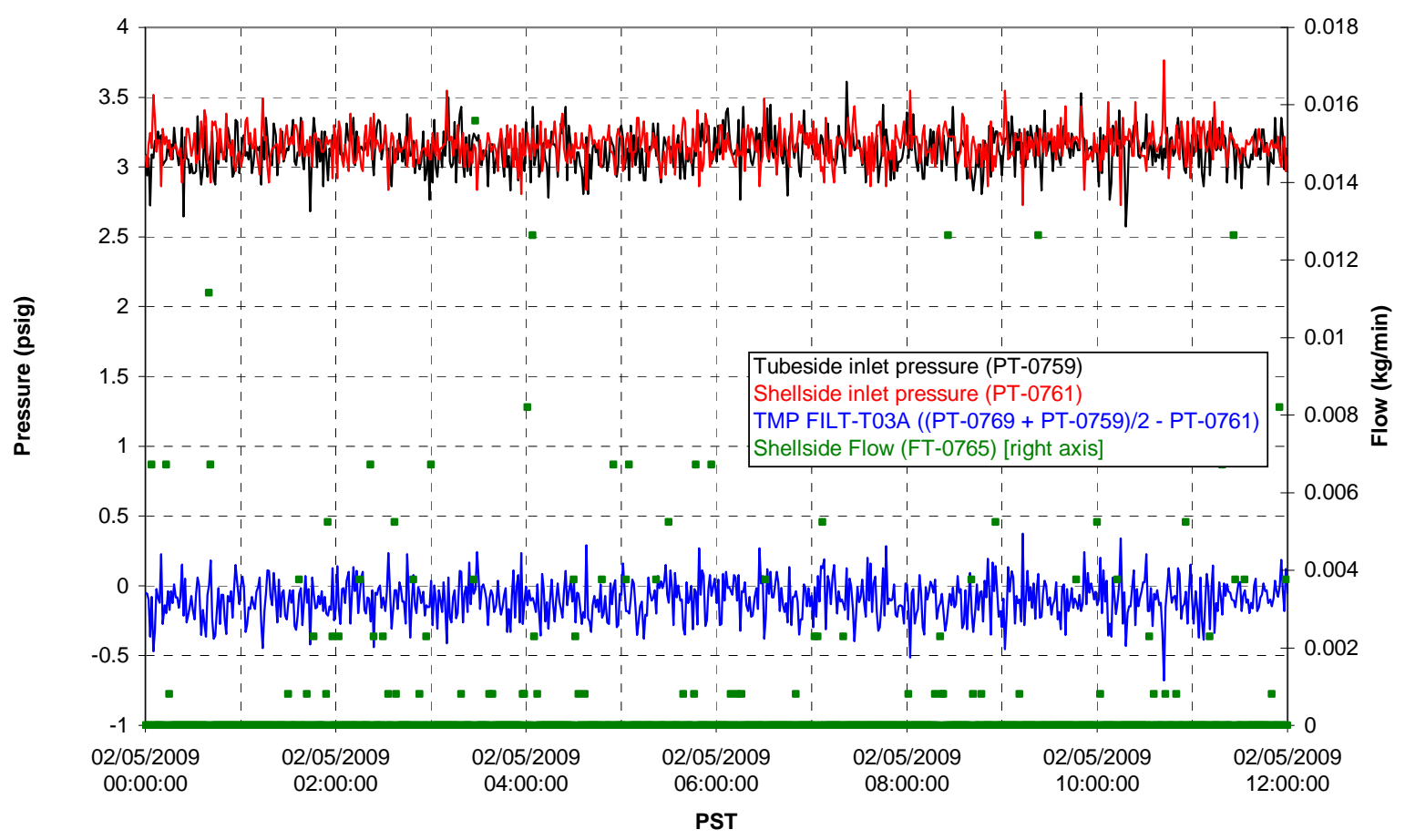

Filter UFP-FILT-T04A

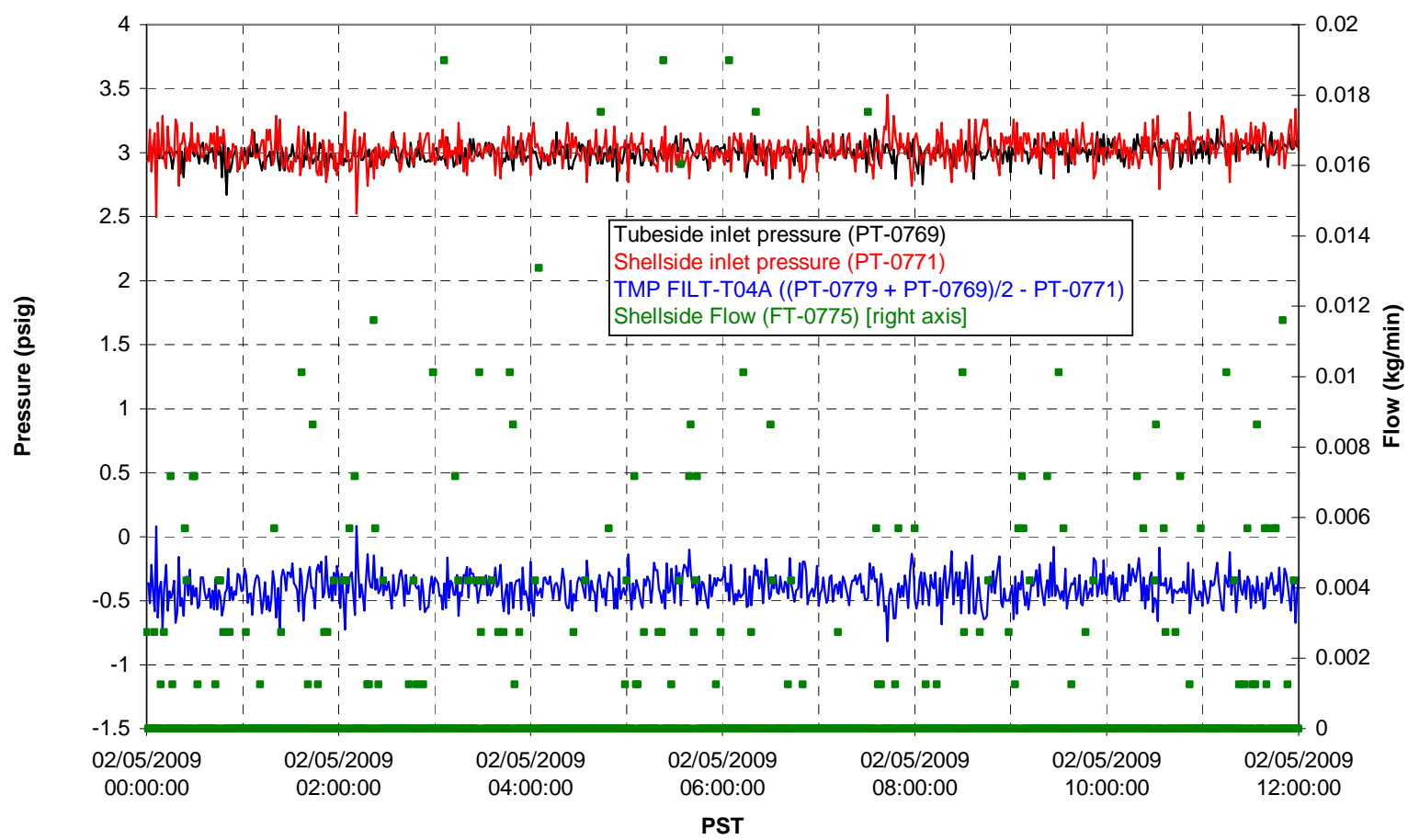


Filter UFP-FILT-T05A

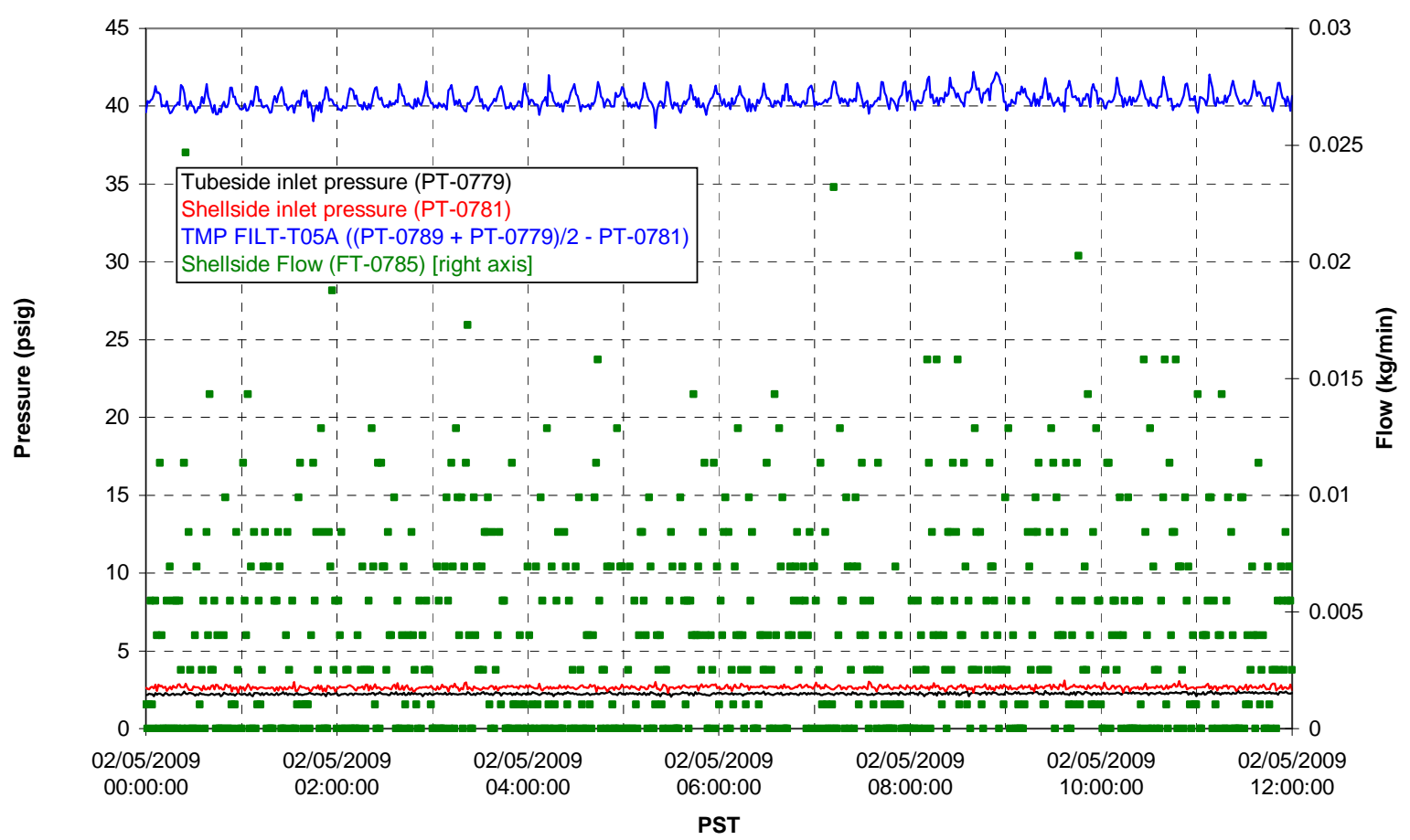

Chemical Flow

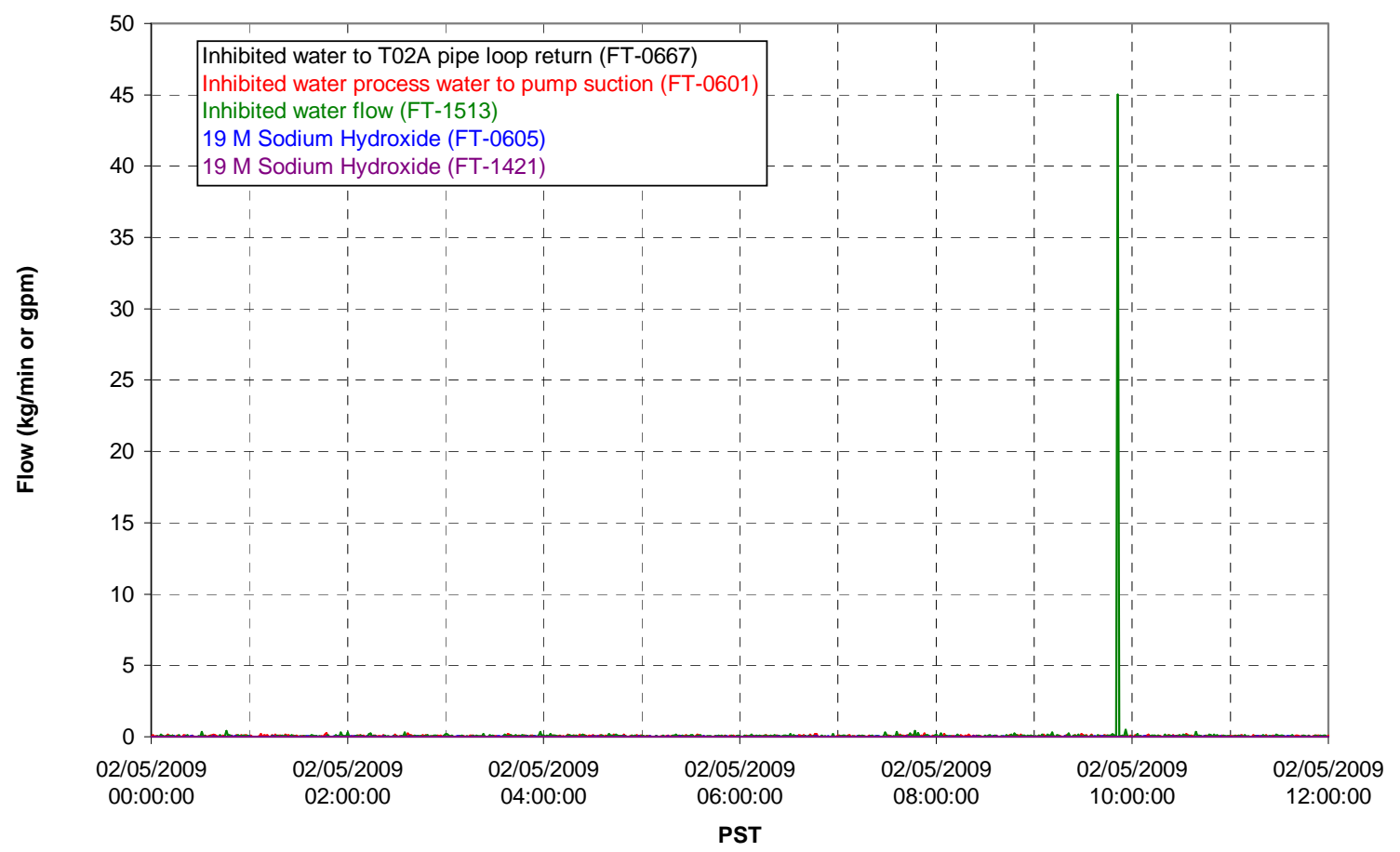

G.195 


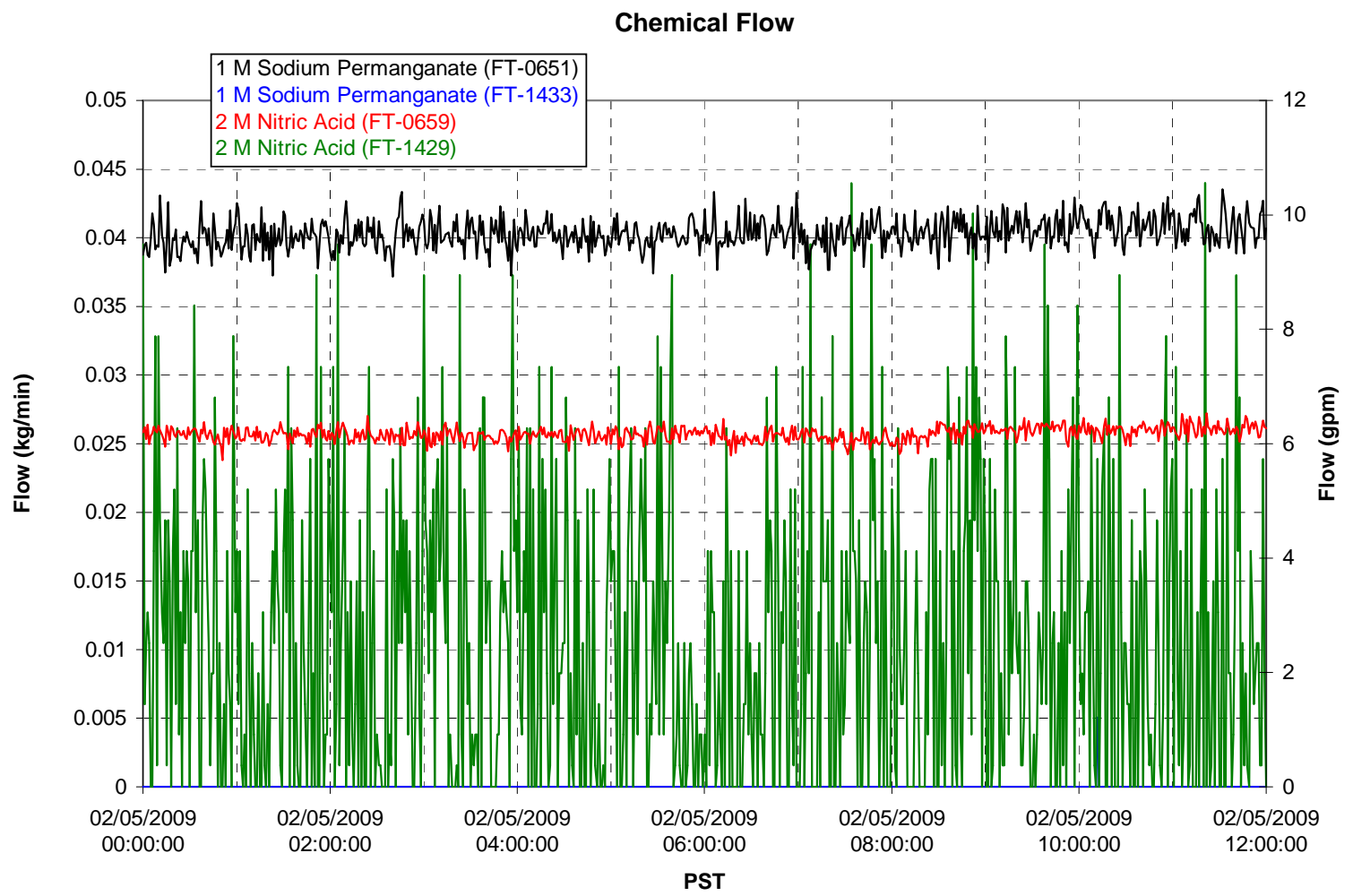

Air Flows

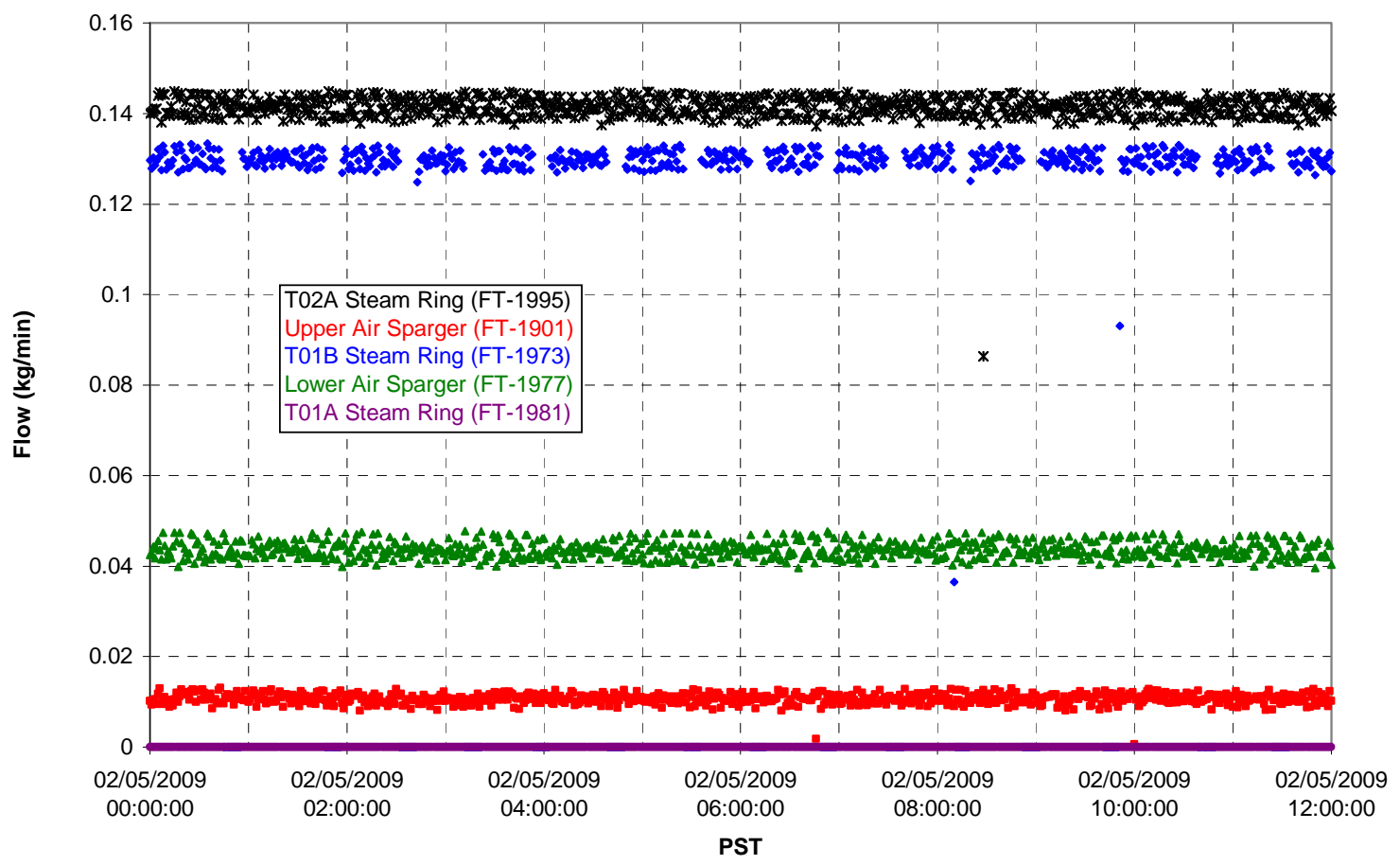

G.196 
T02A Steam

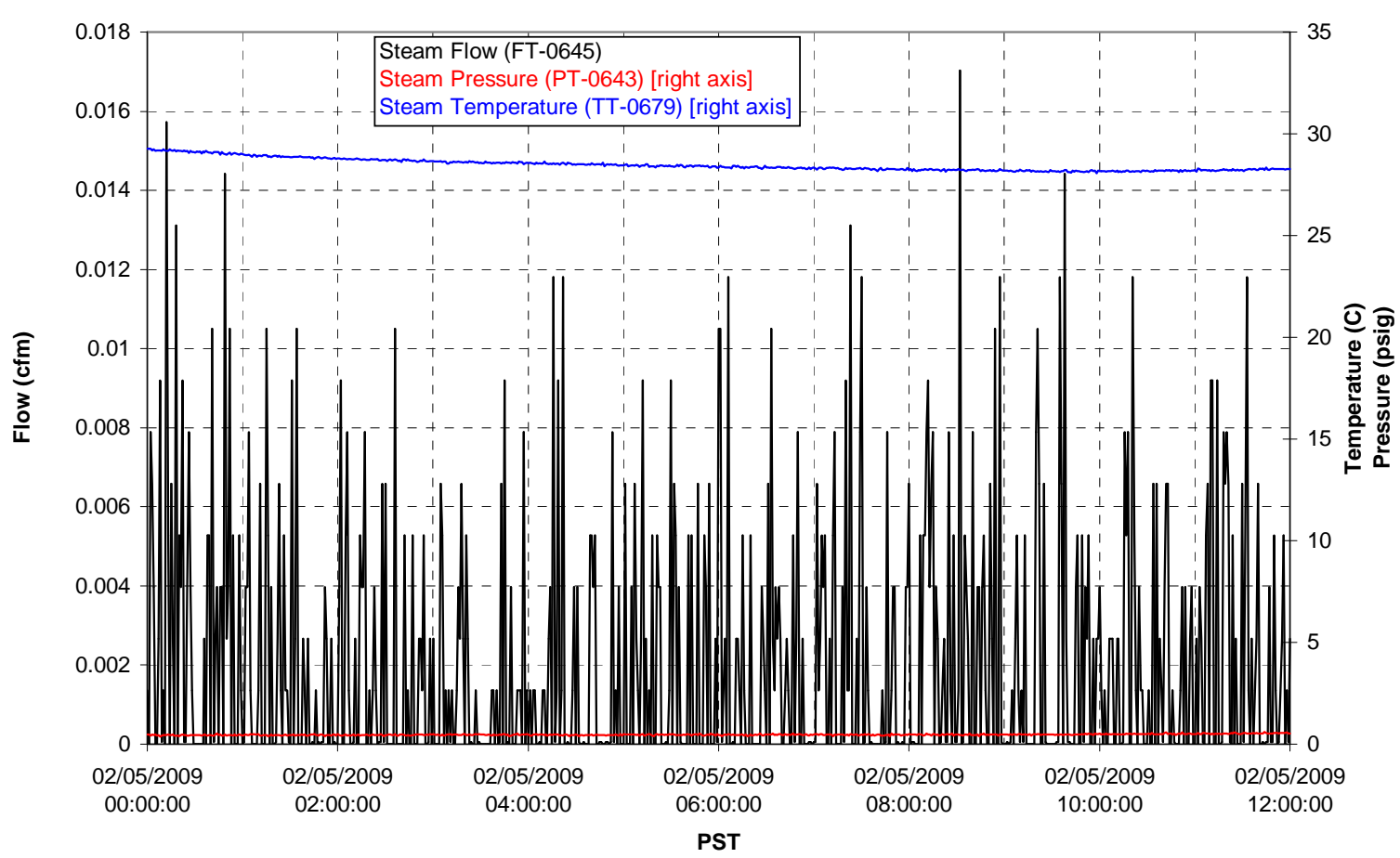

T01A Steam

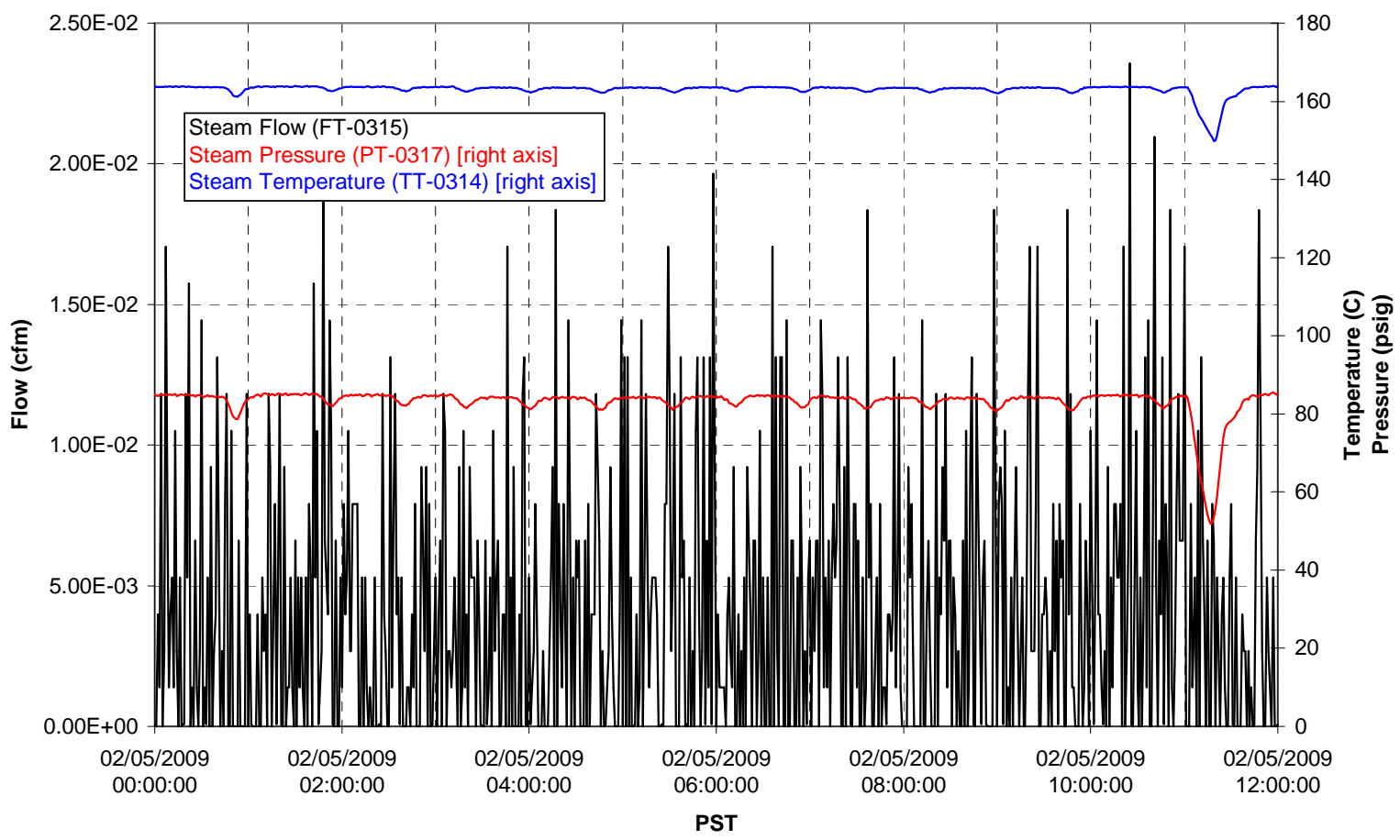


T01B Steam

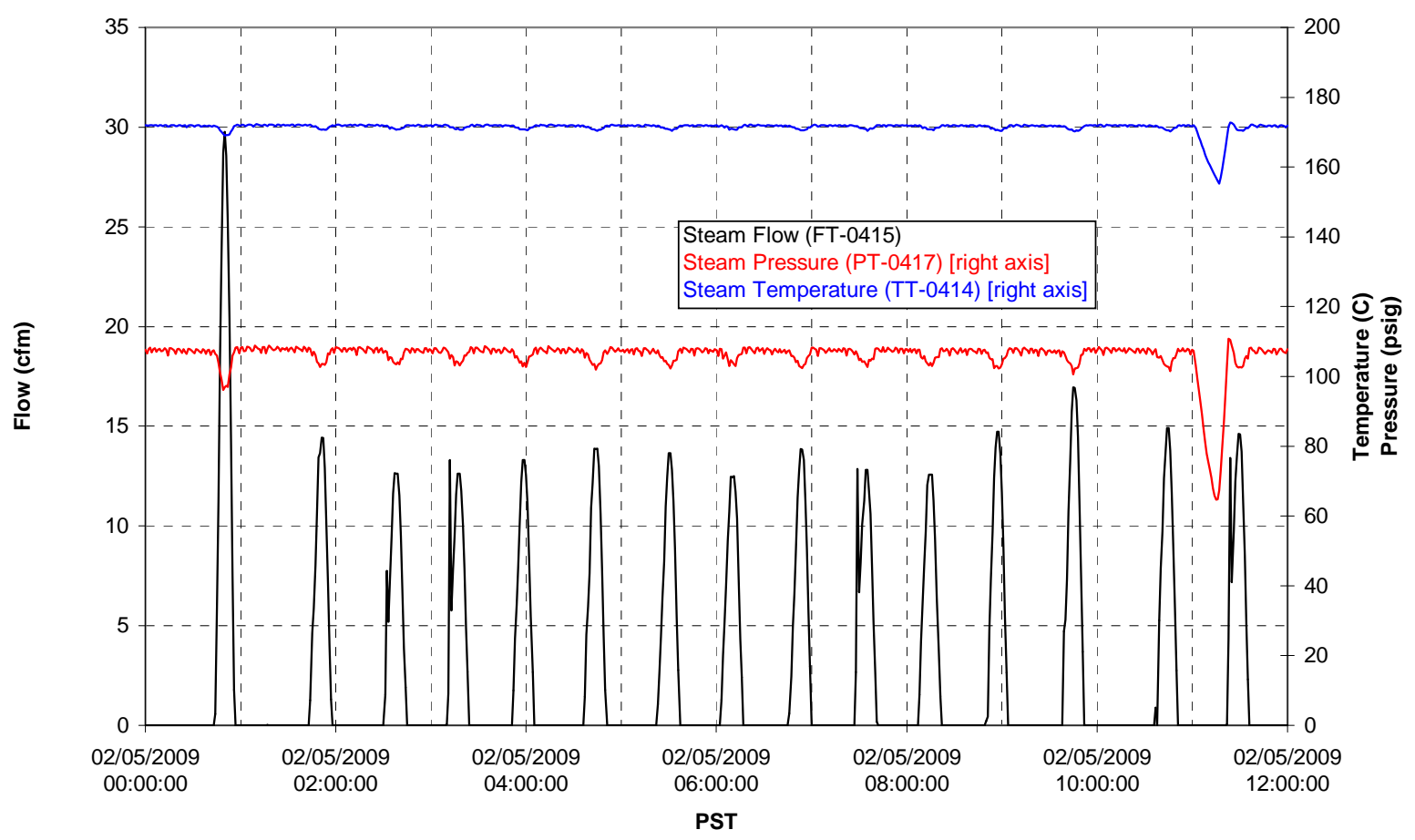




\section{Integrated Test A Data Plots 02/05/09 12:00 - 02/06/09 00:00}


T01A level

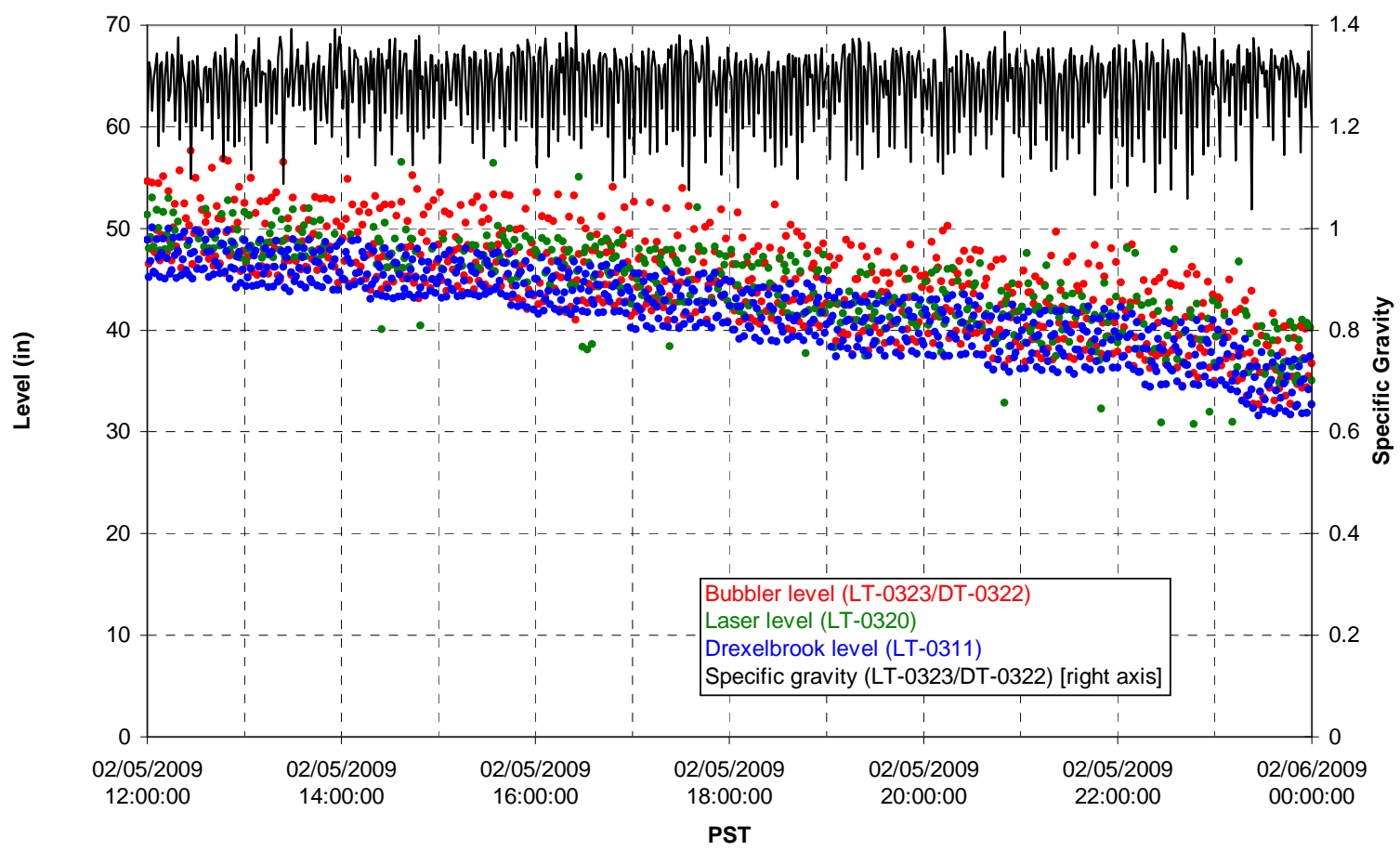

T01A temperatures

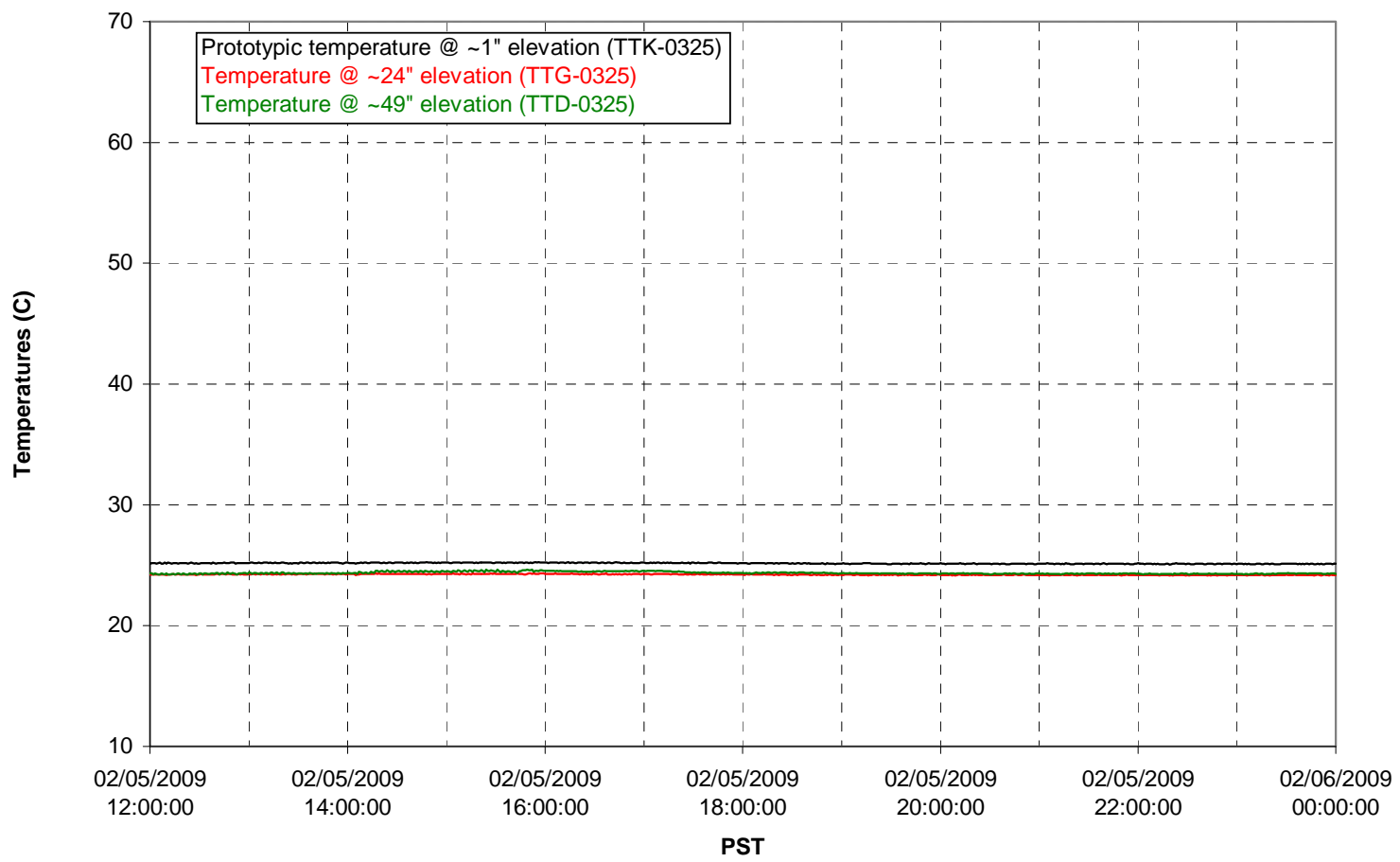


T01B level

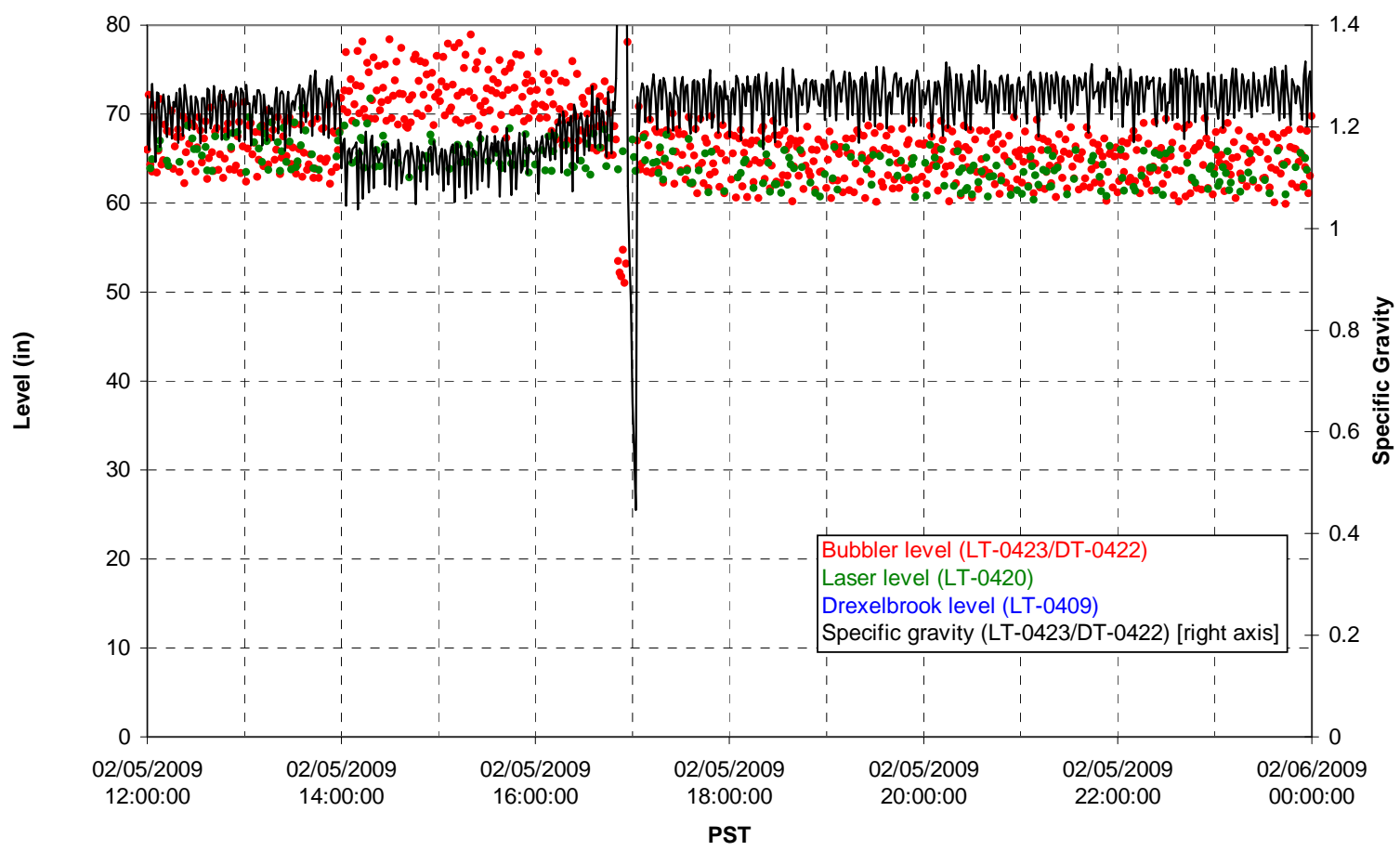

T01B temperatures

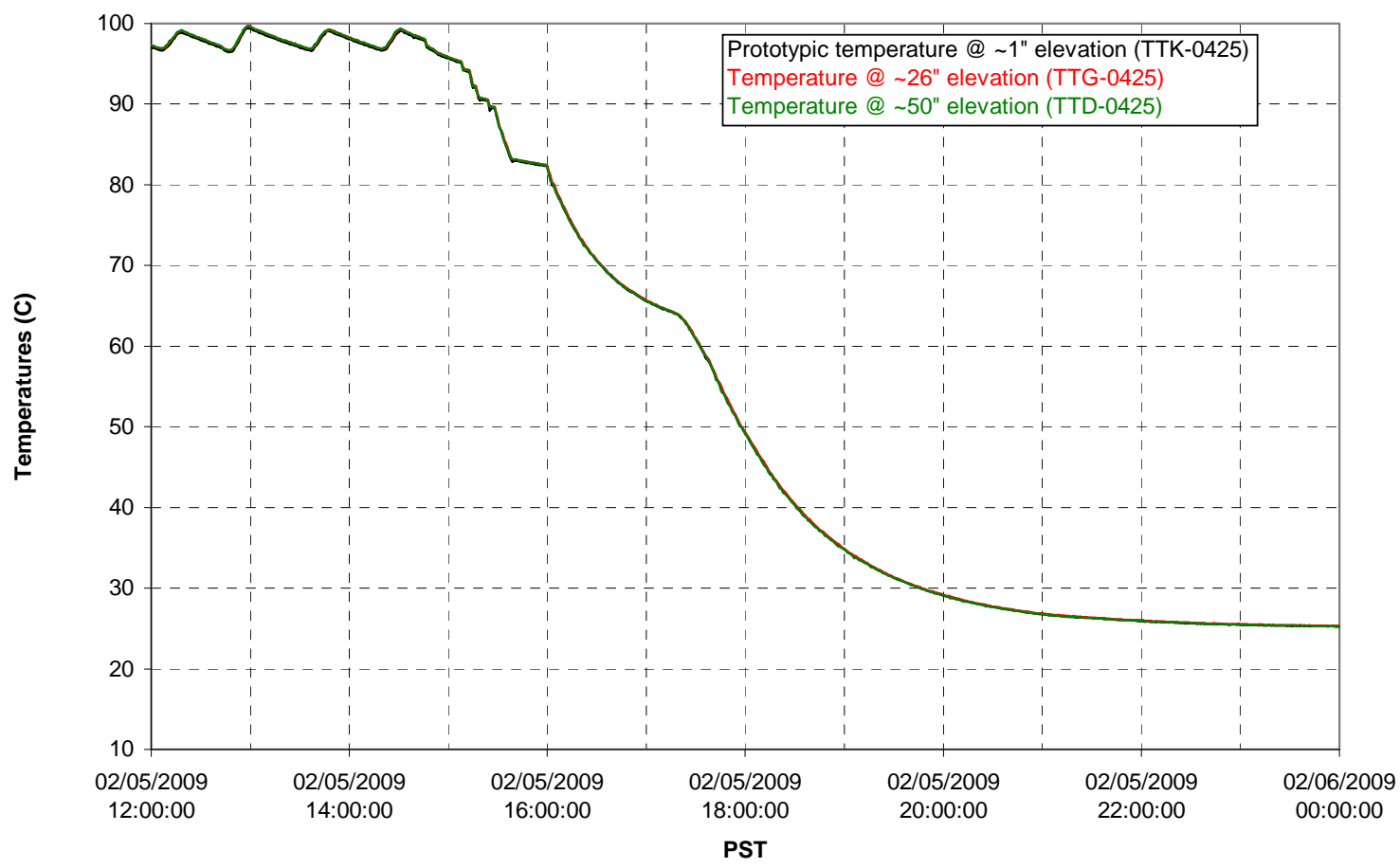


T02A level

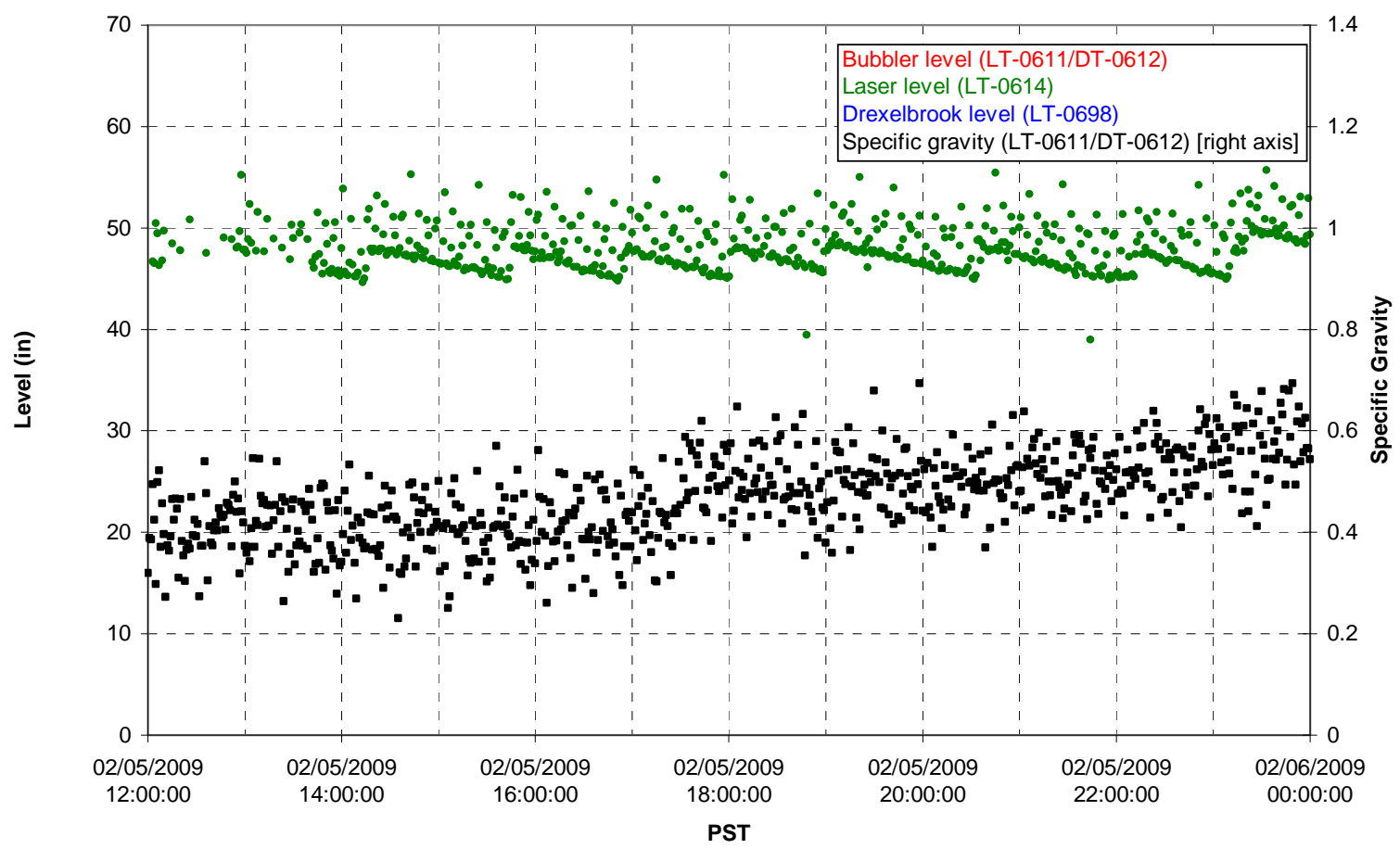

T02A temperatures

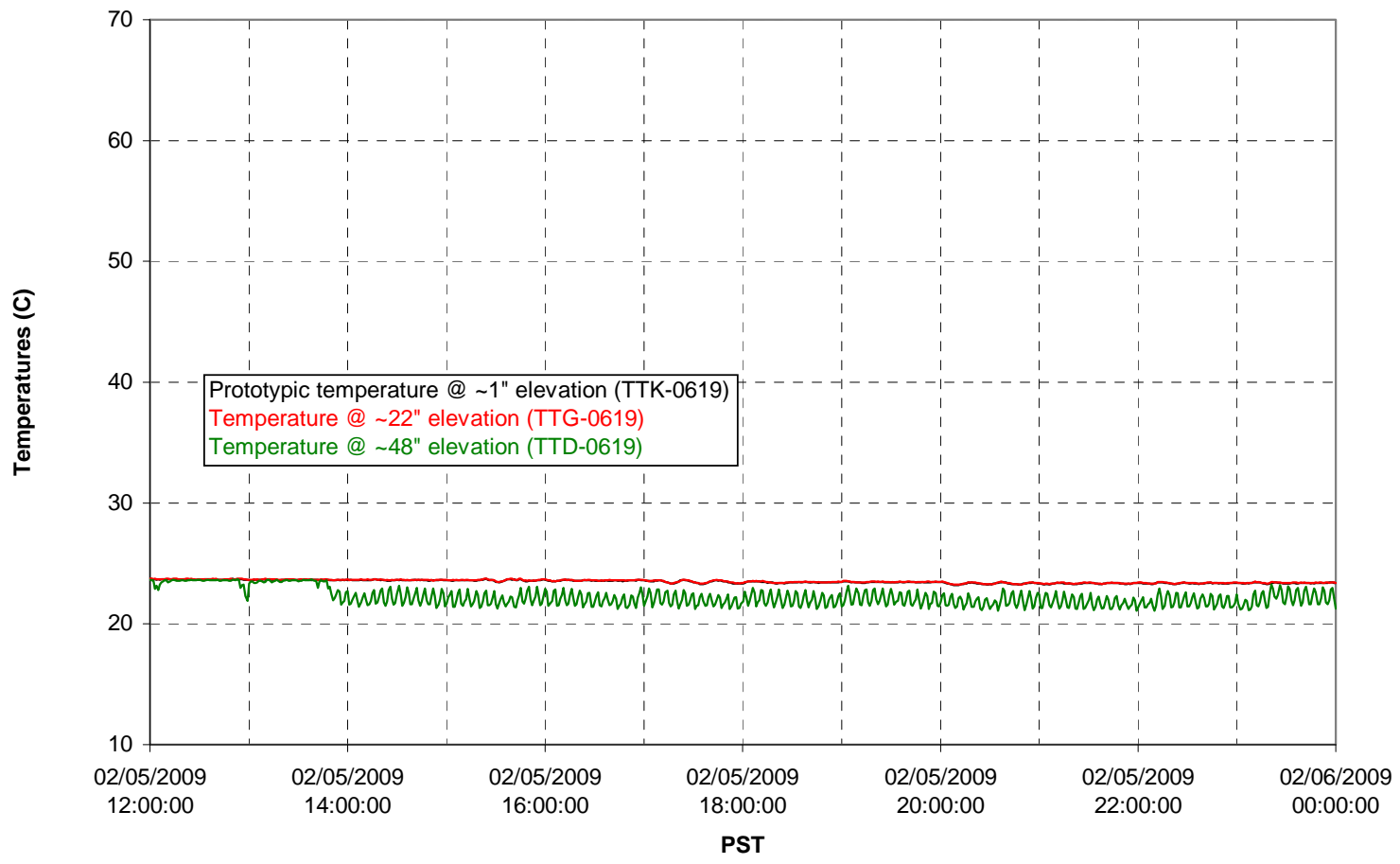

G.202 
T02A and filter loop temperatures

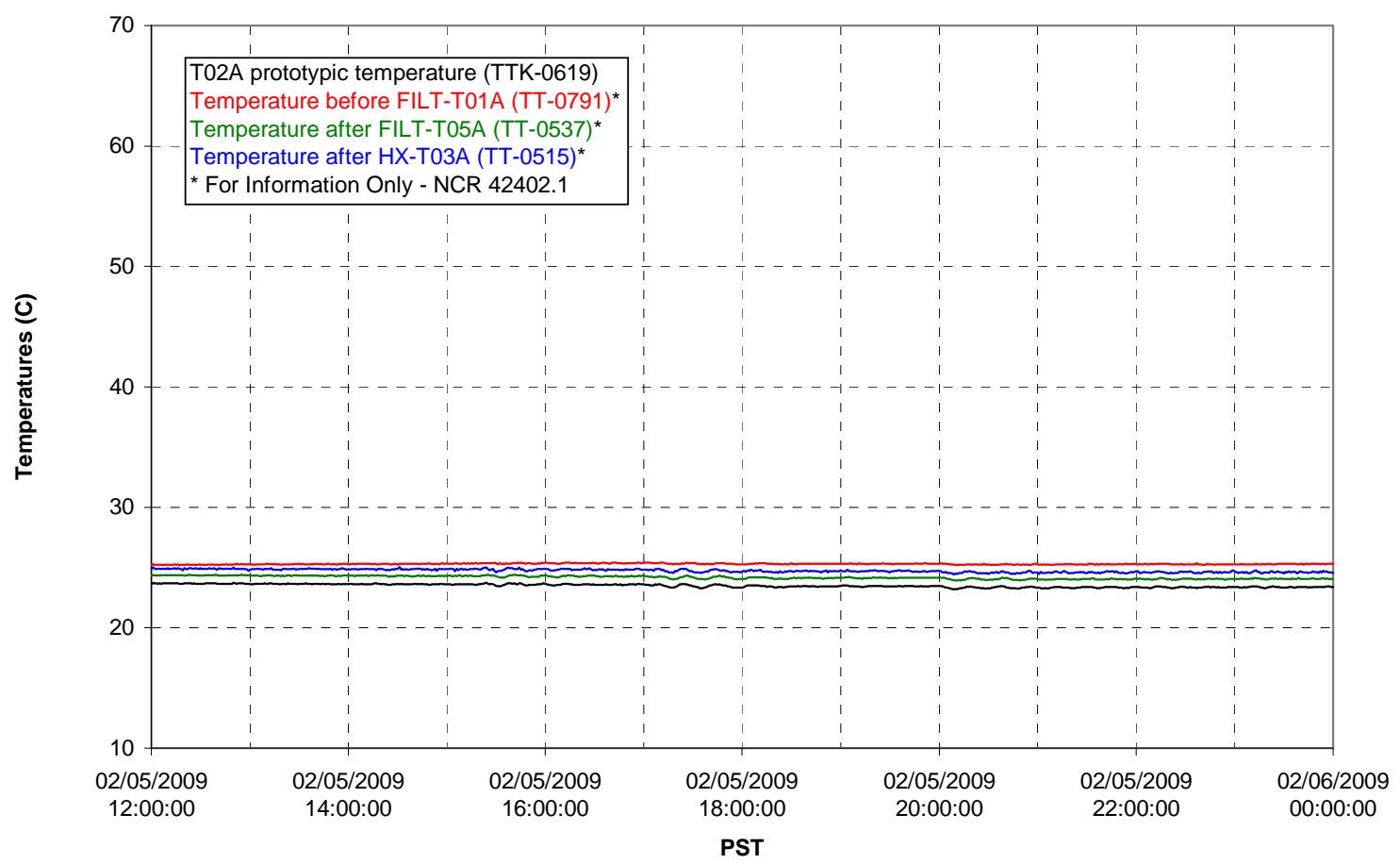

Pump Pressures and Flow

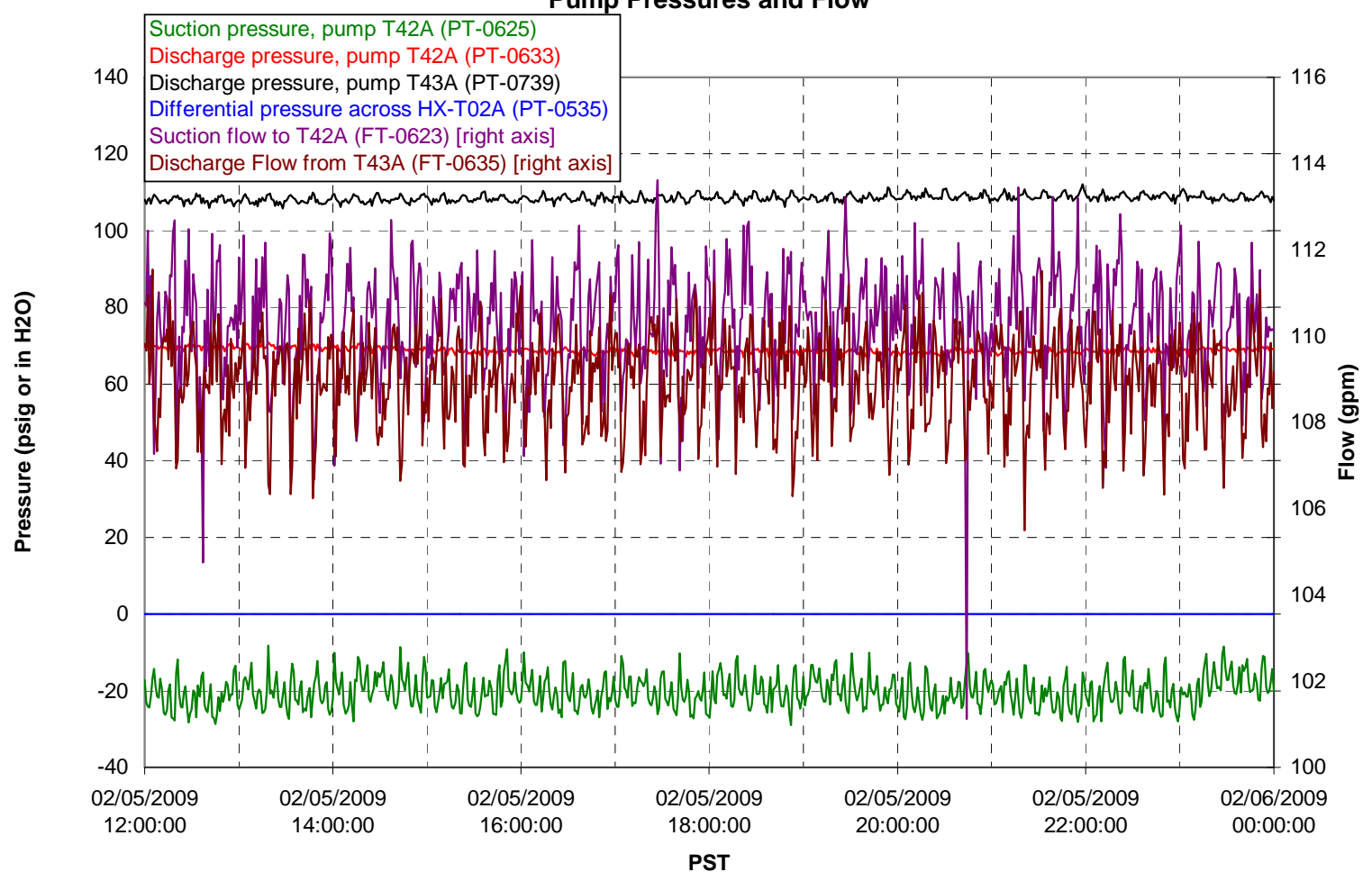


Axial pressure drop

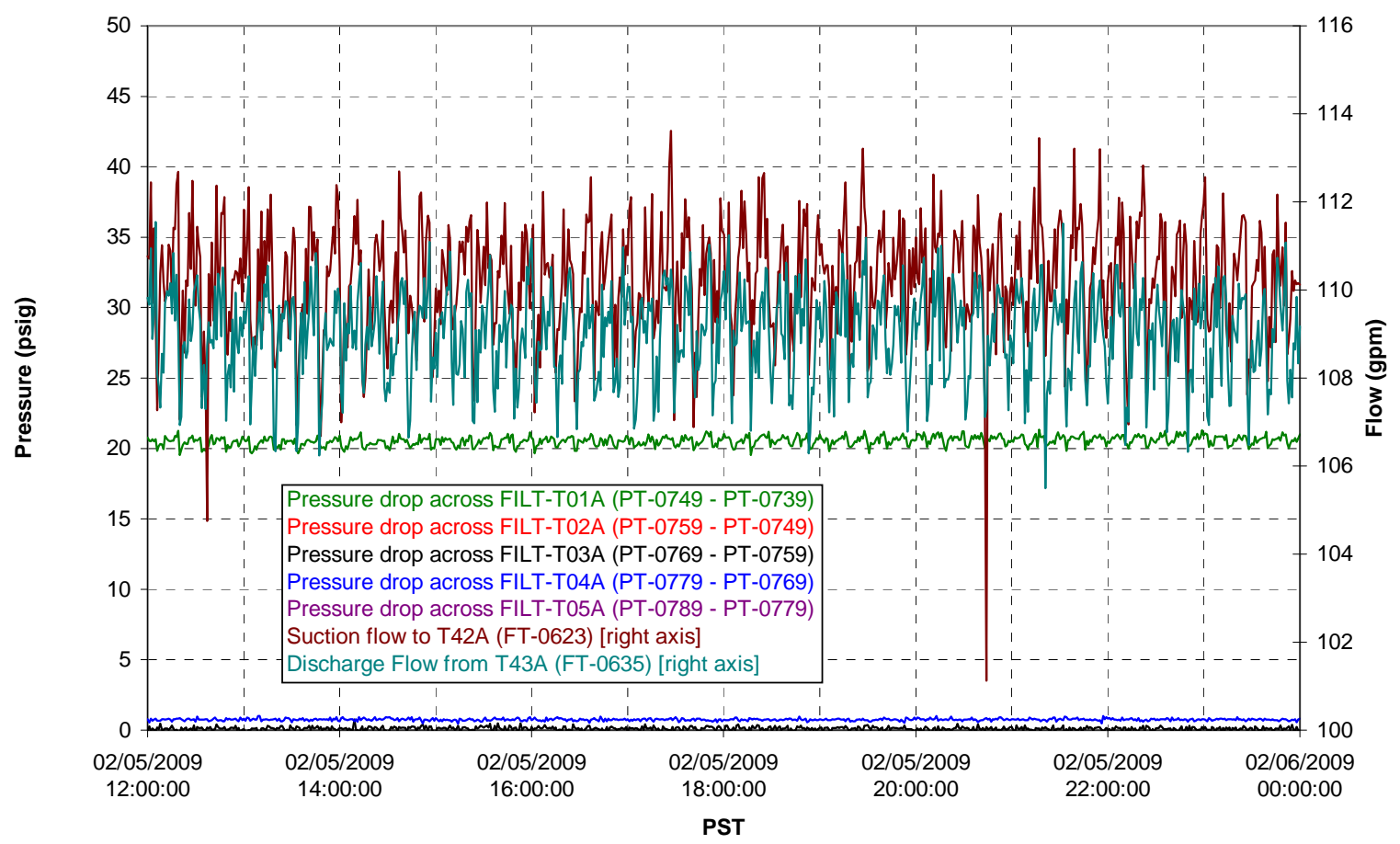

Permeate flow rates

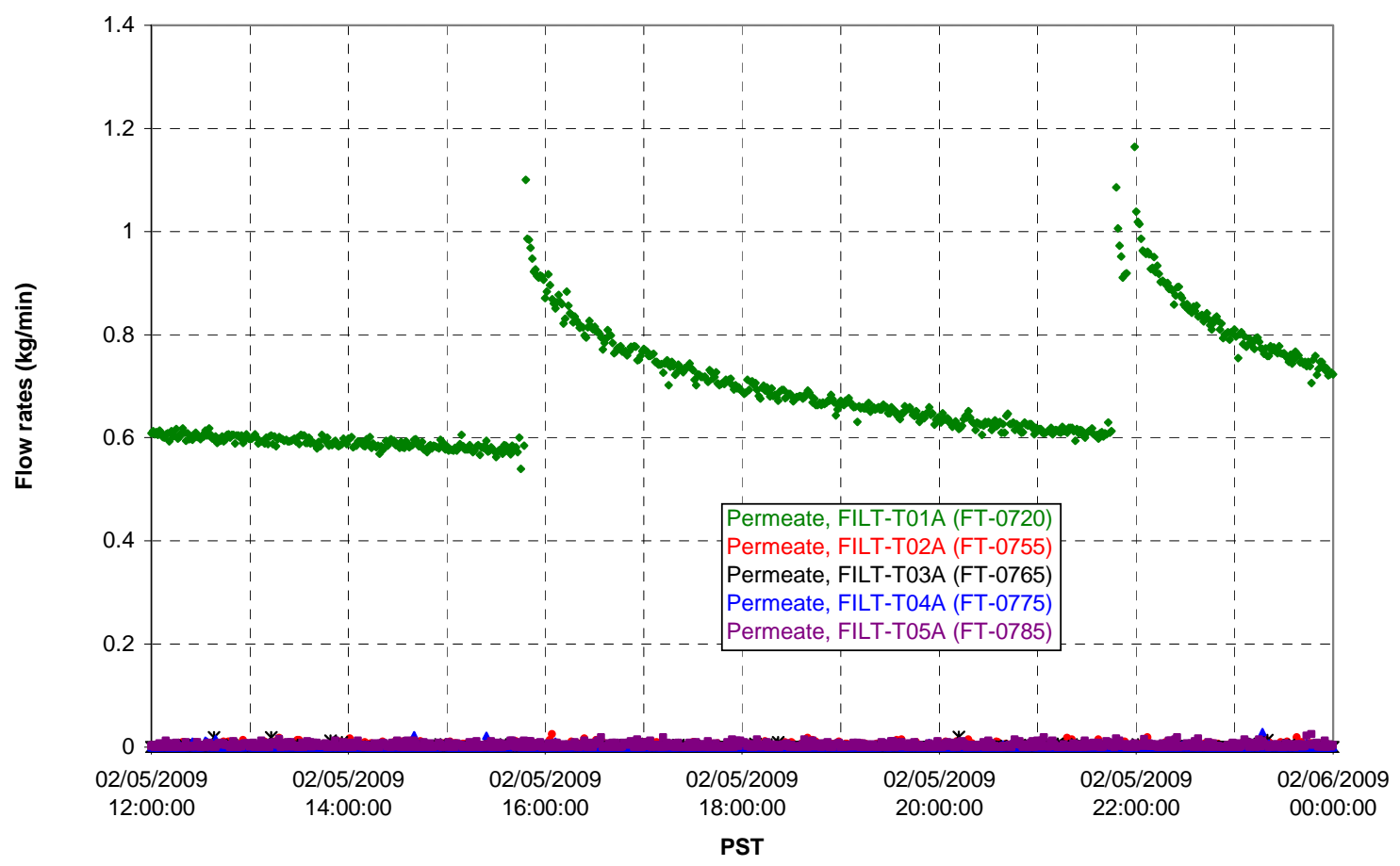


T02A Inner Temperature Tree

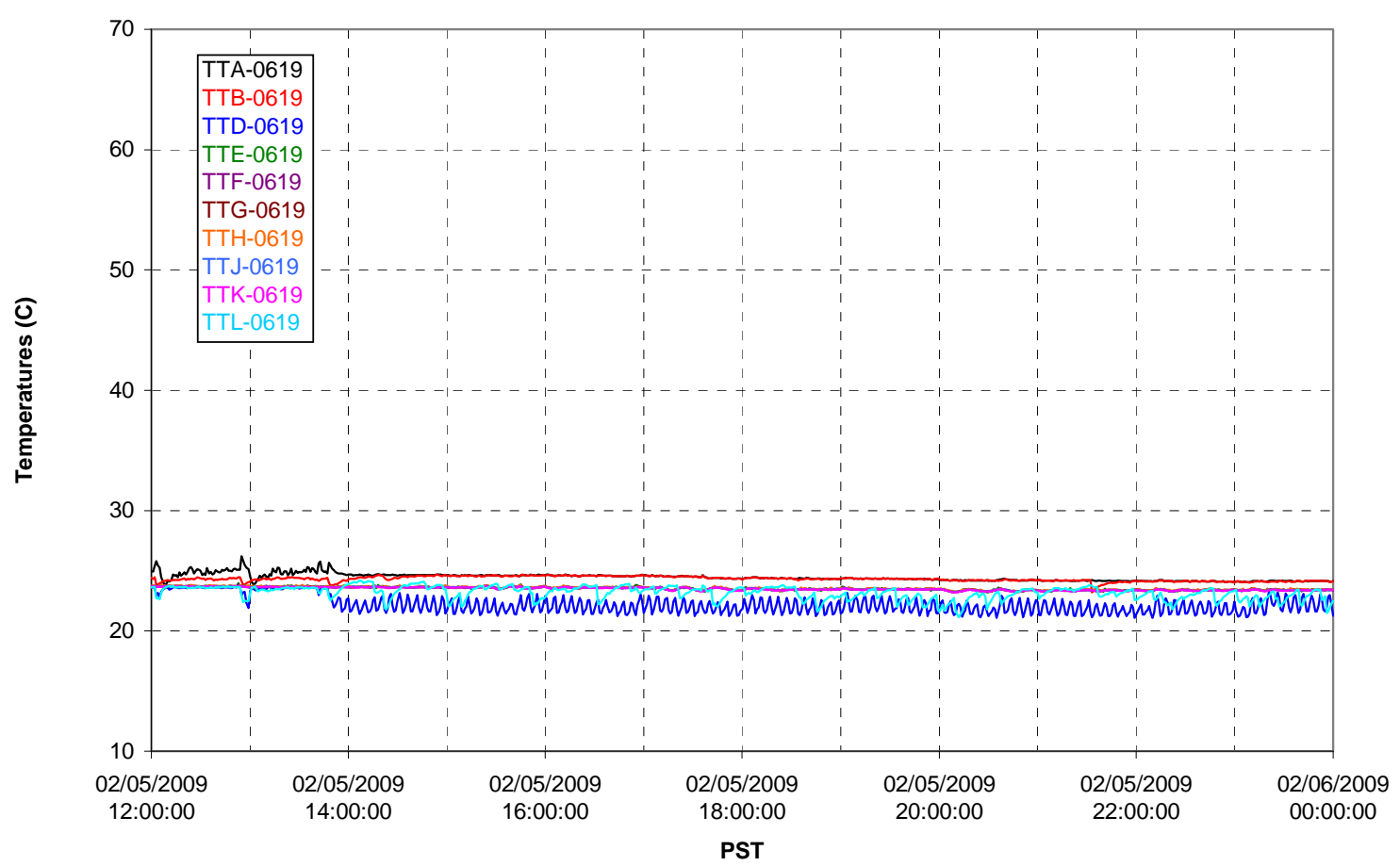

T02A Outer Temperature Tree

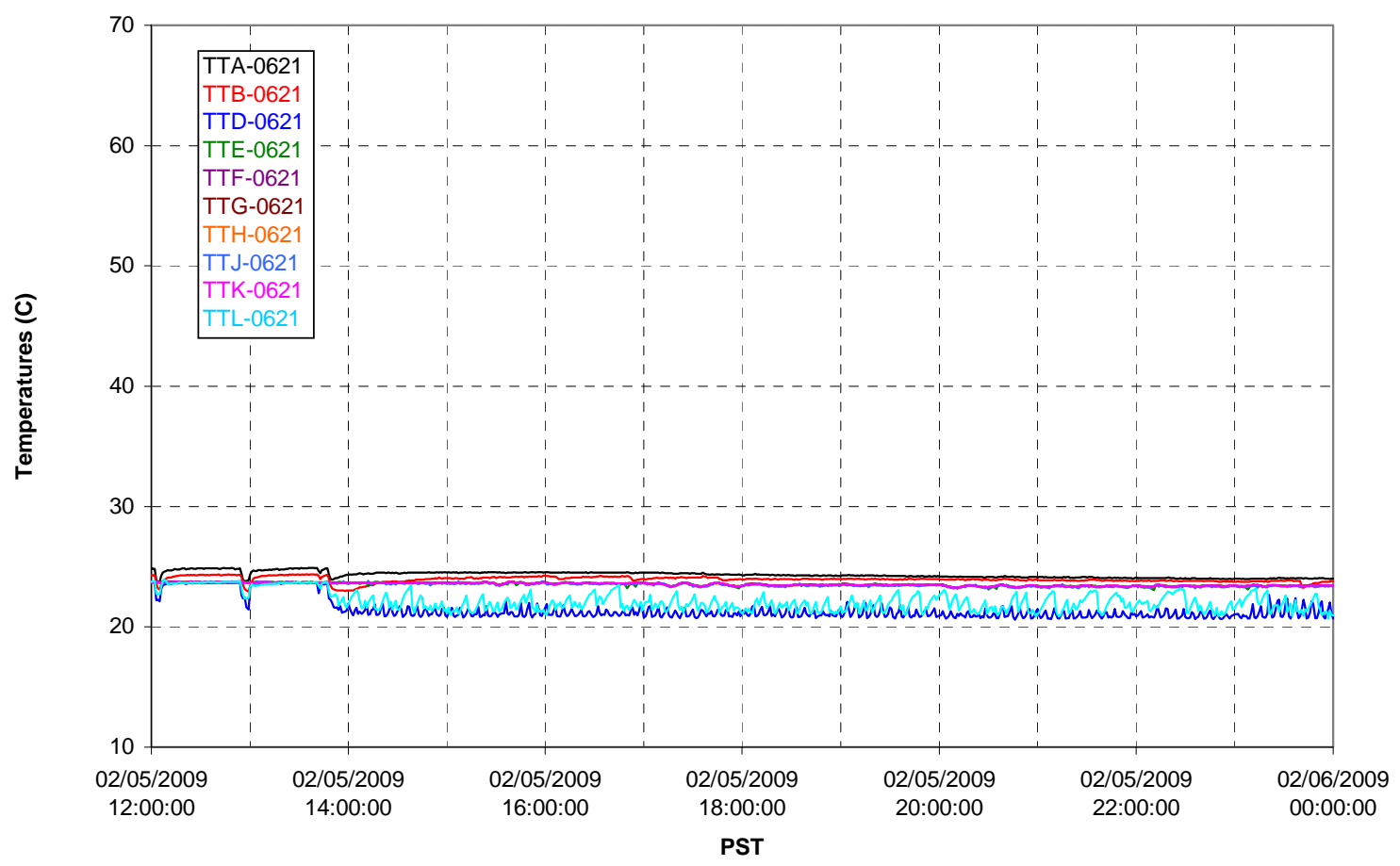


T02A temperatures

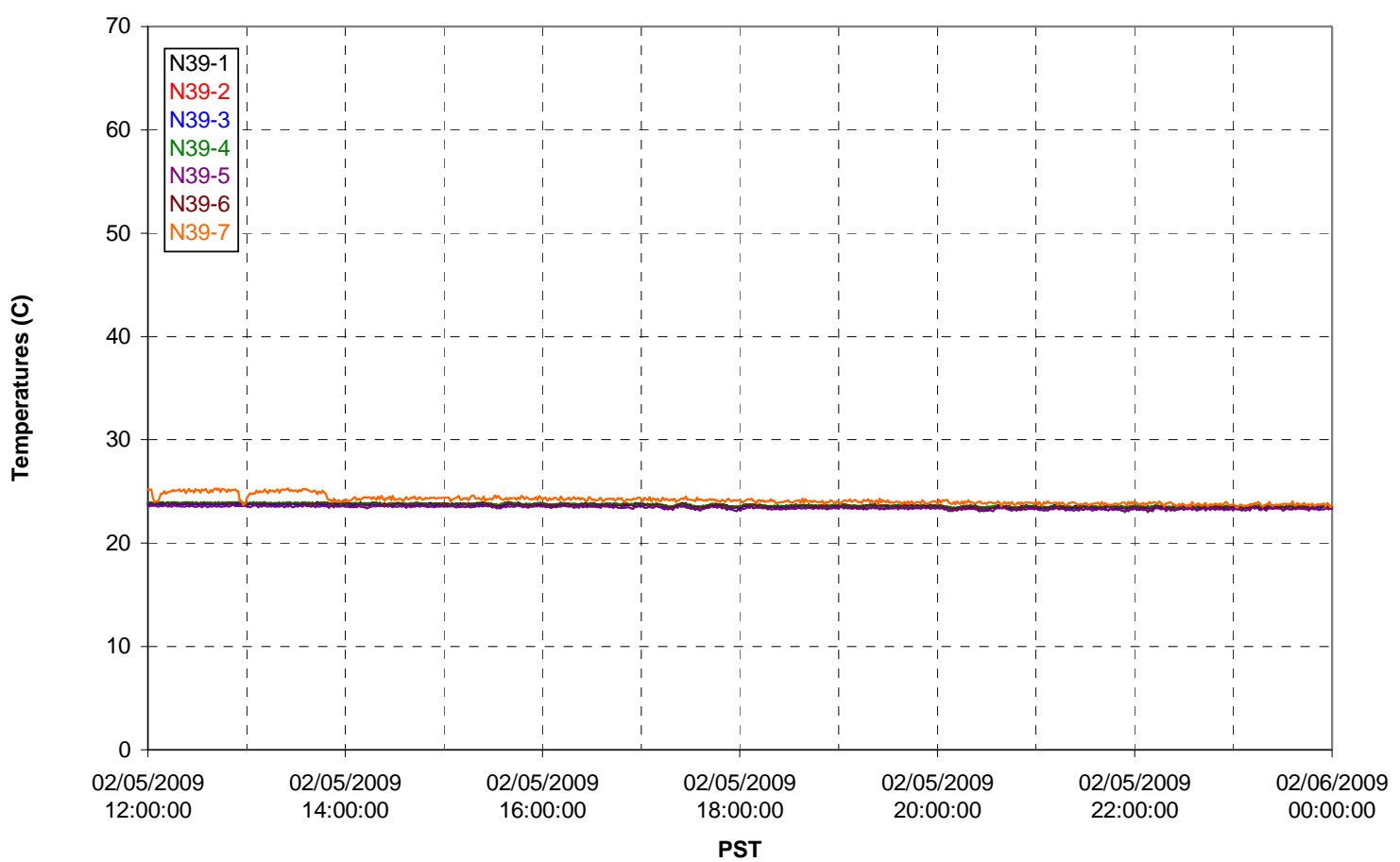

T02A temperatures

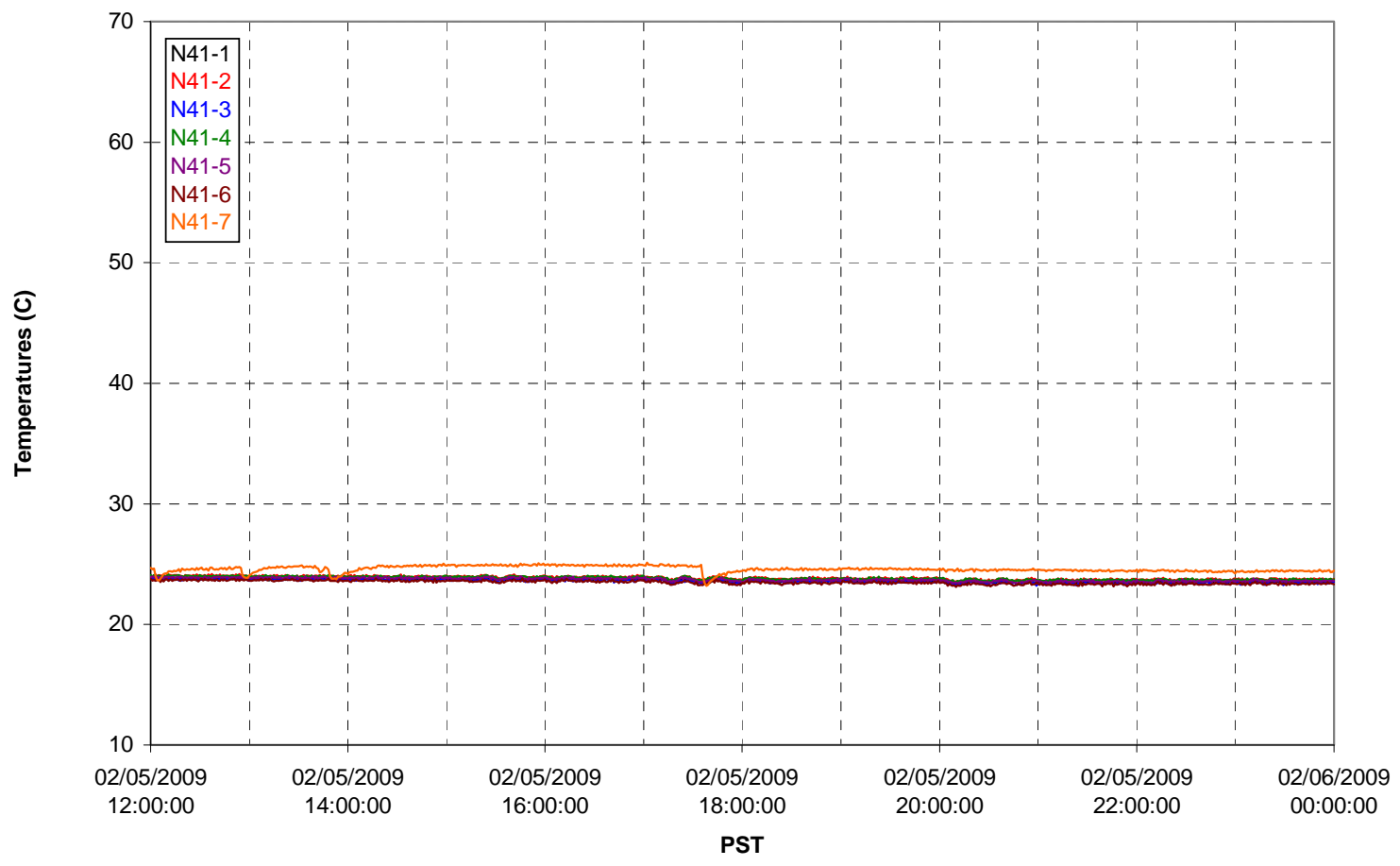


T02A temperatures

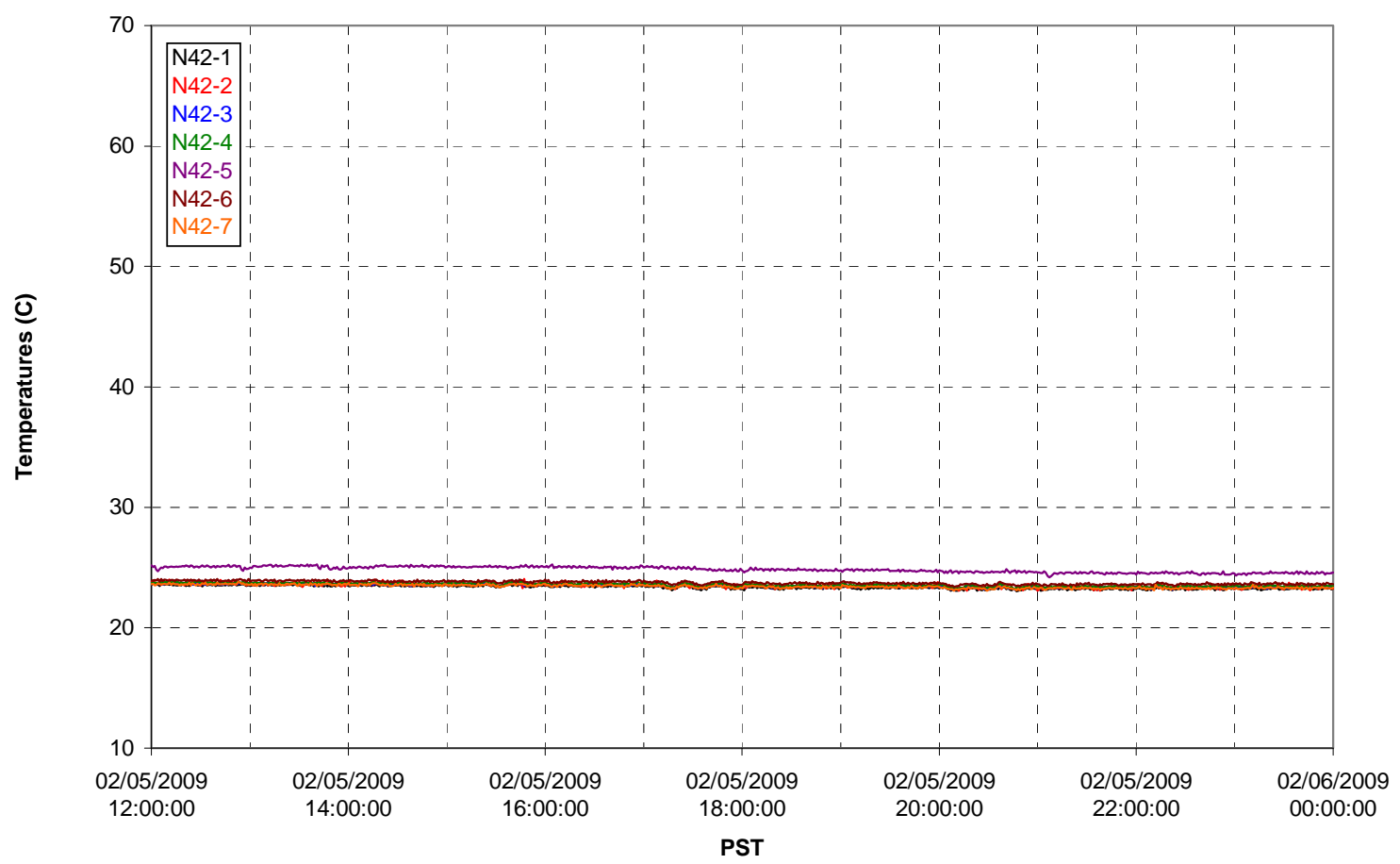

T02A temperatures

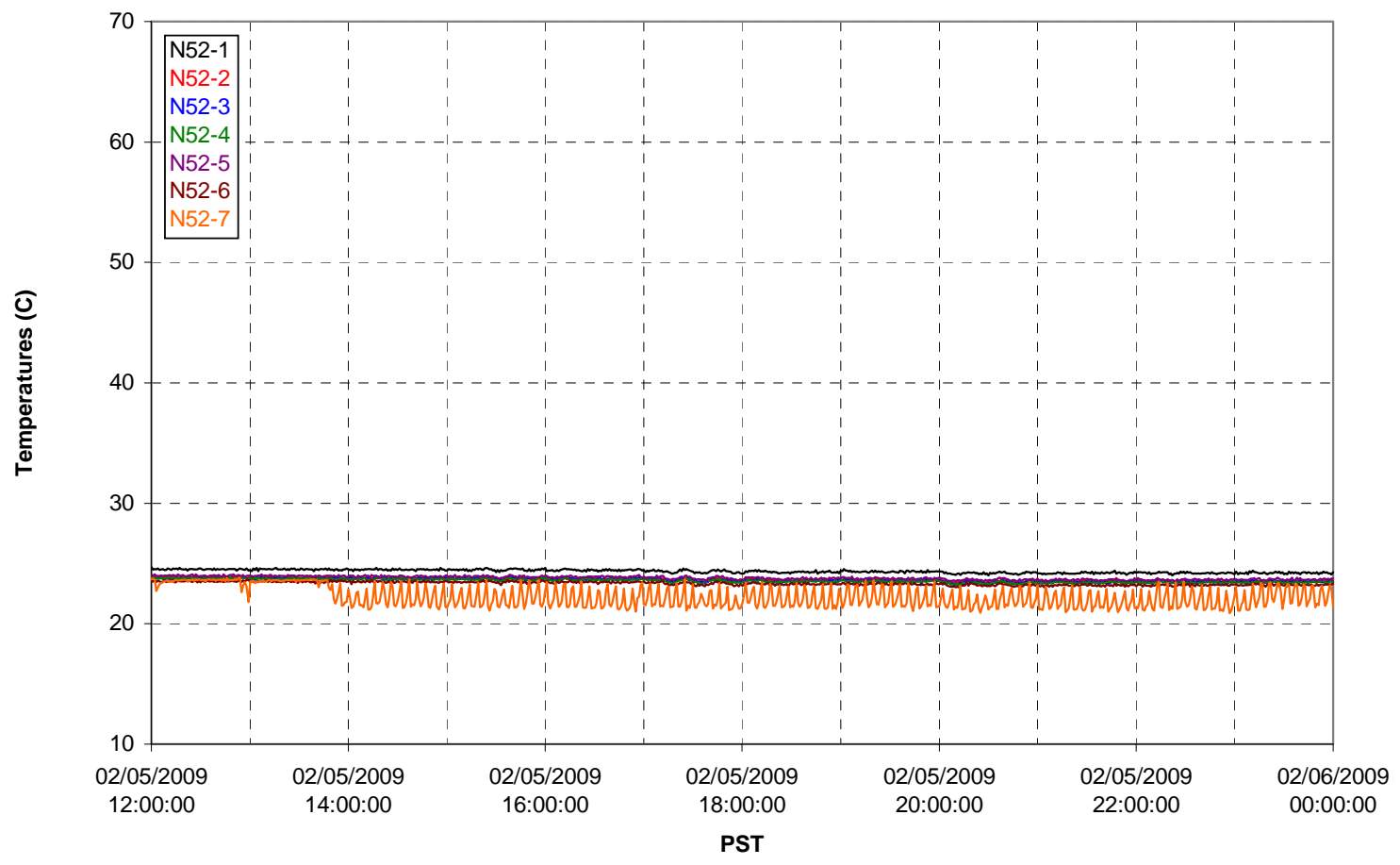


T02A Heating and Cooling

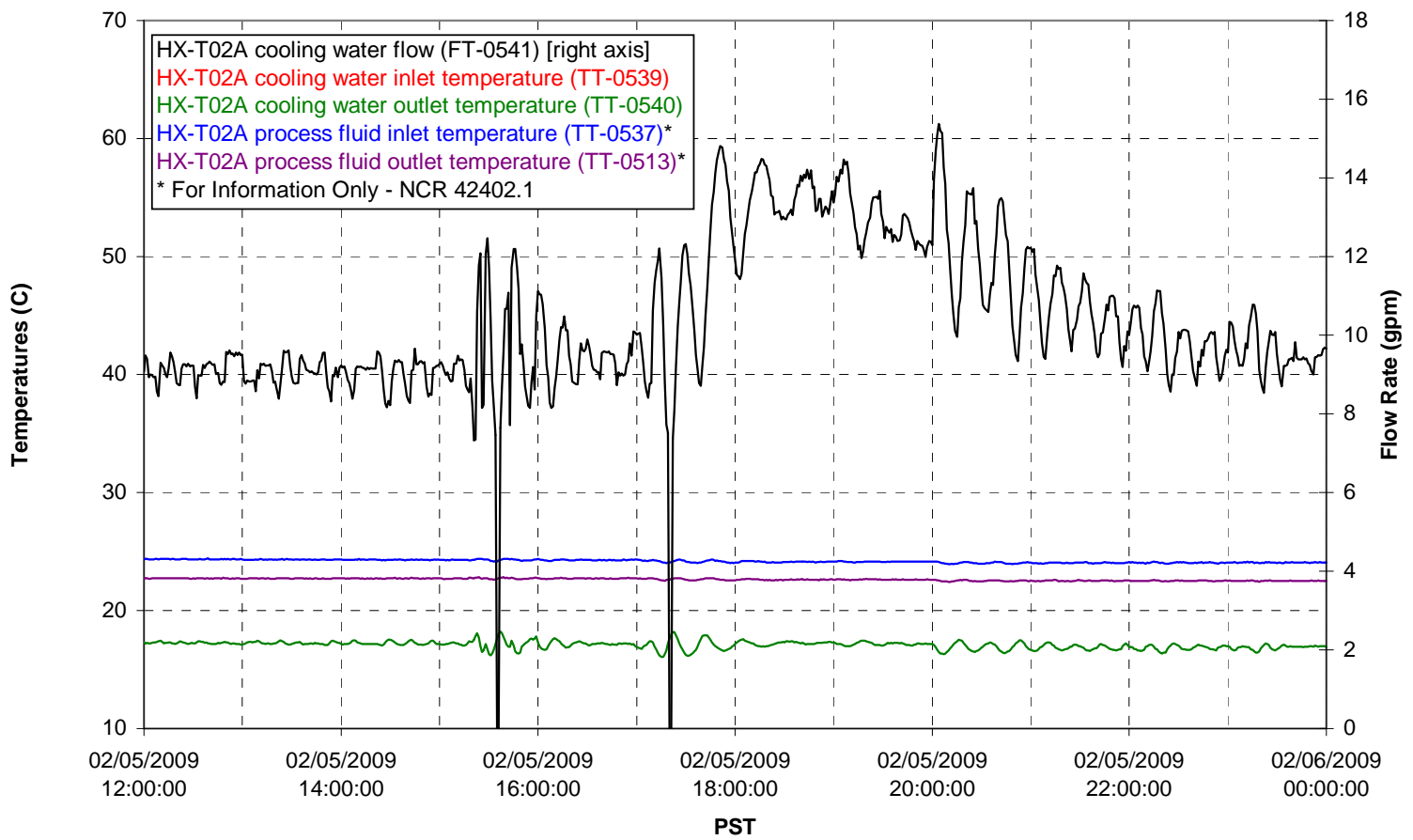

Pump Operation

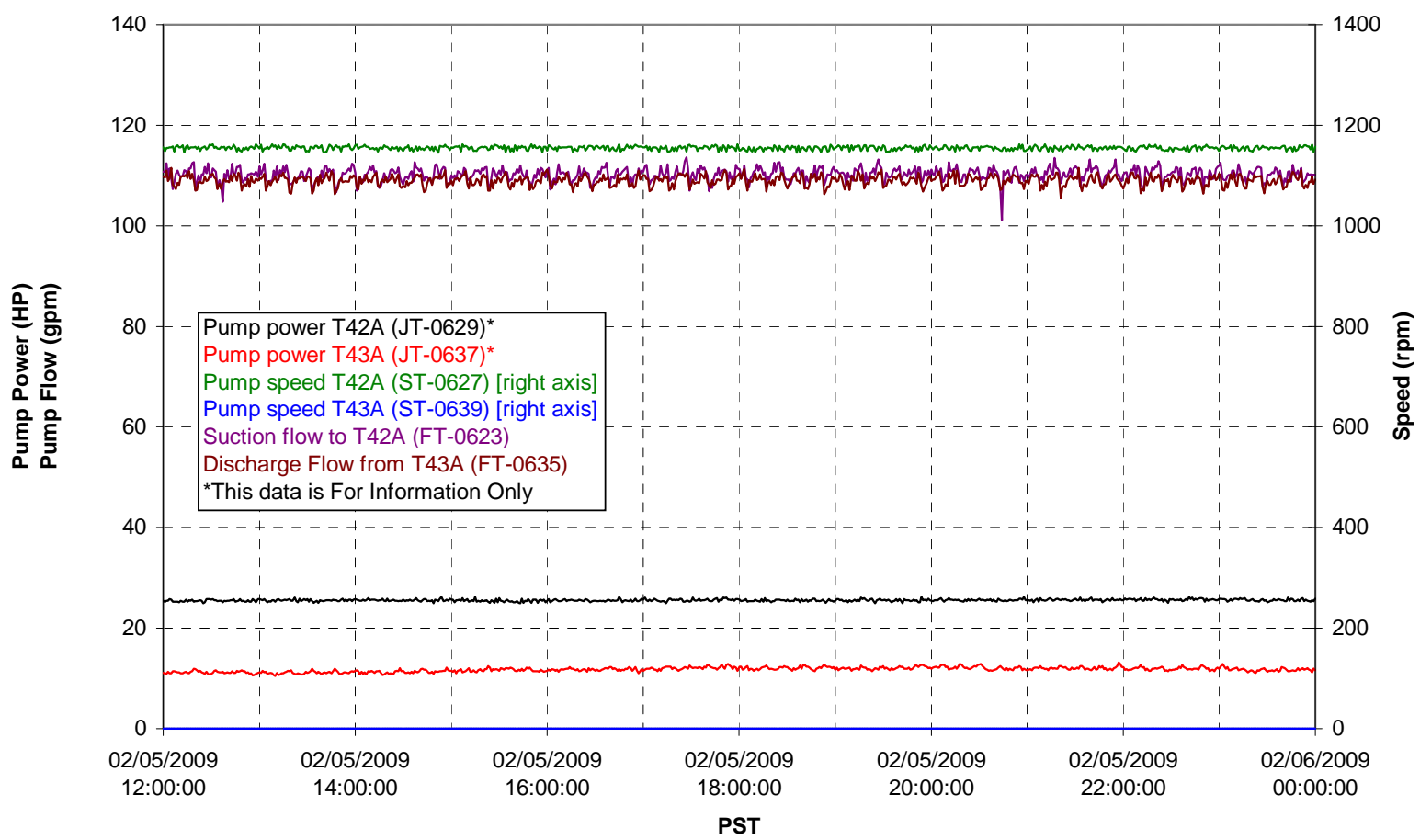


Pulsepot UFP-PP-T01A

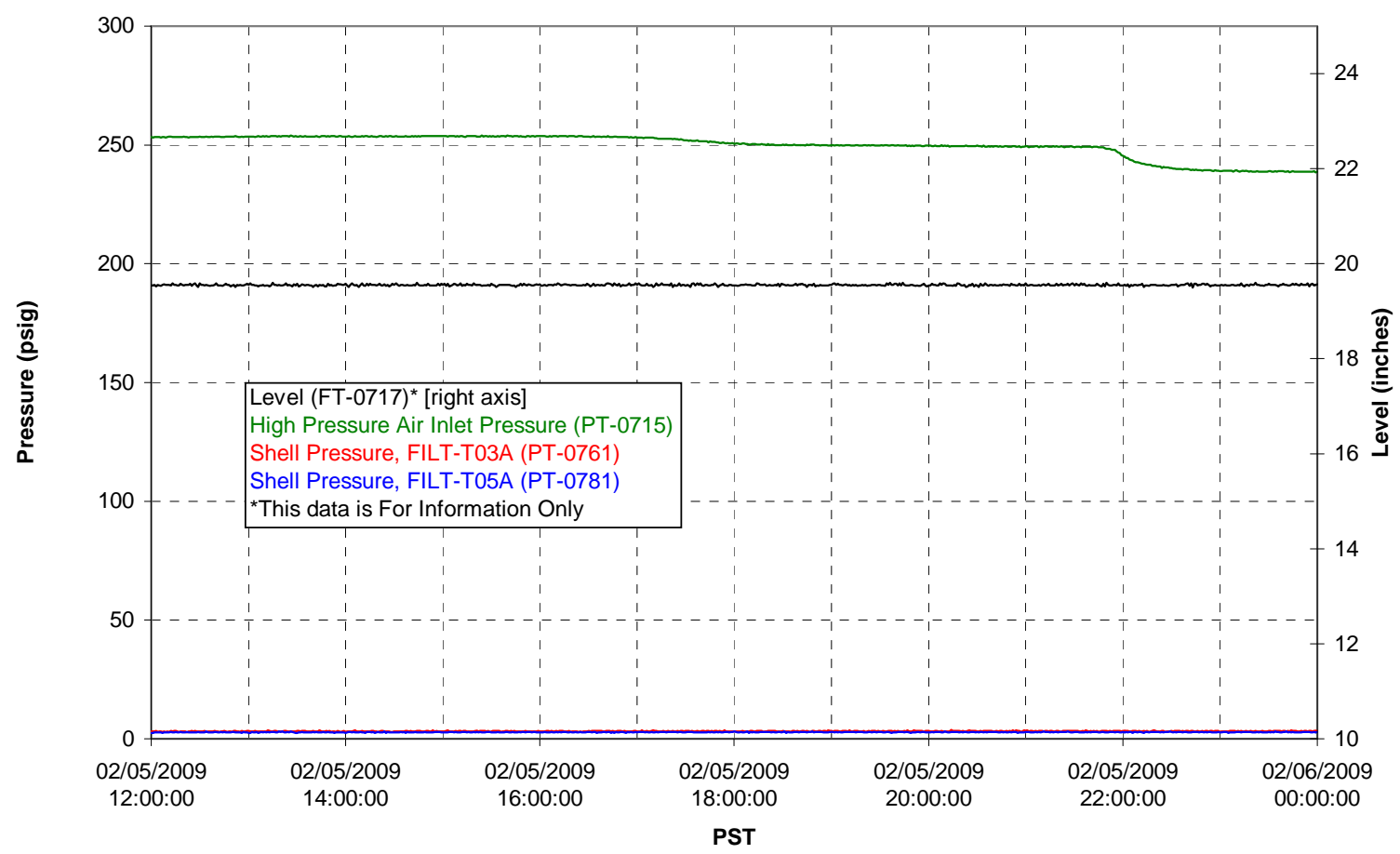

Pulsepot UFP-PP-T02A

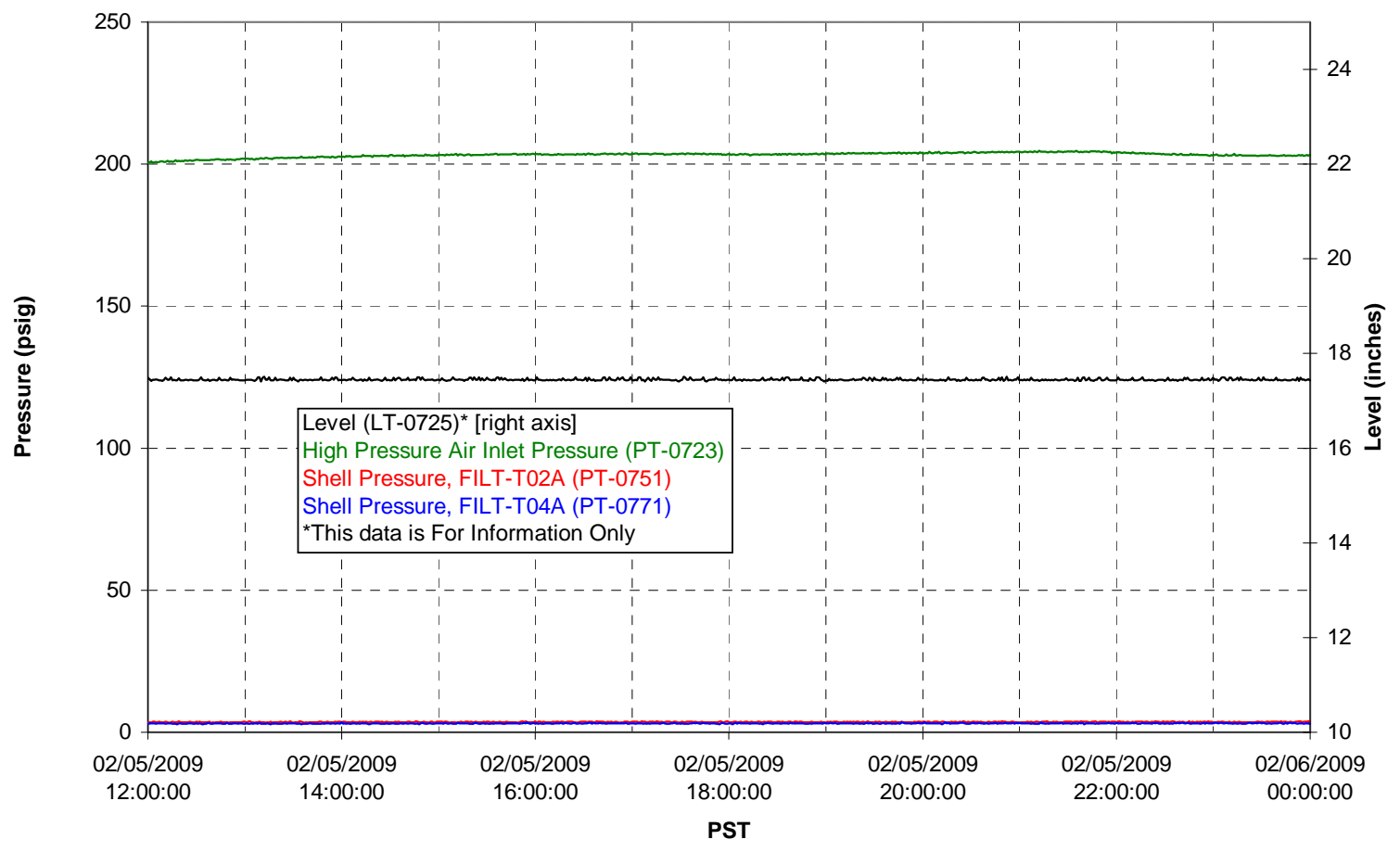


Pulsepot UFP-PP-T03A

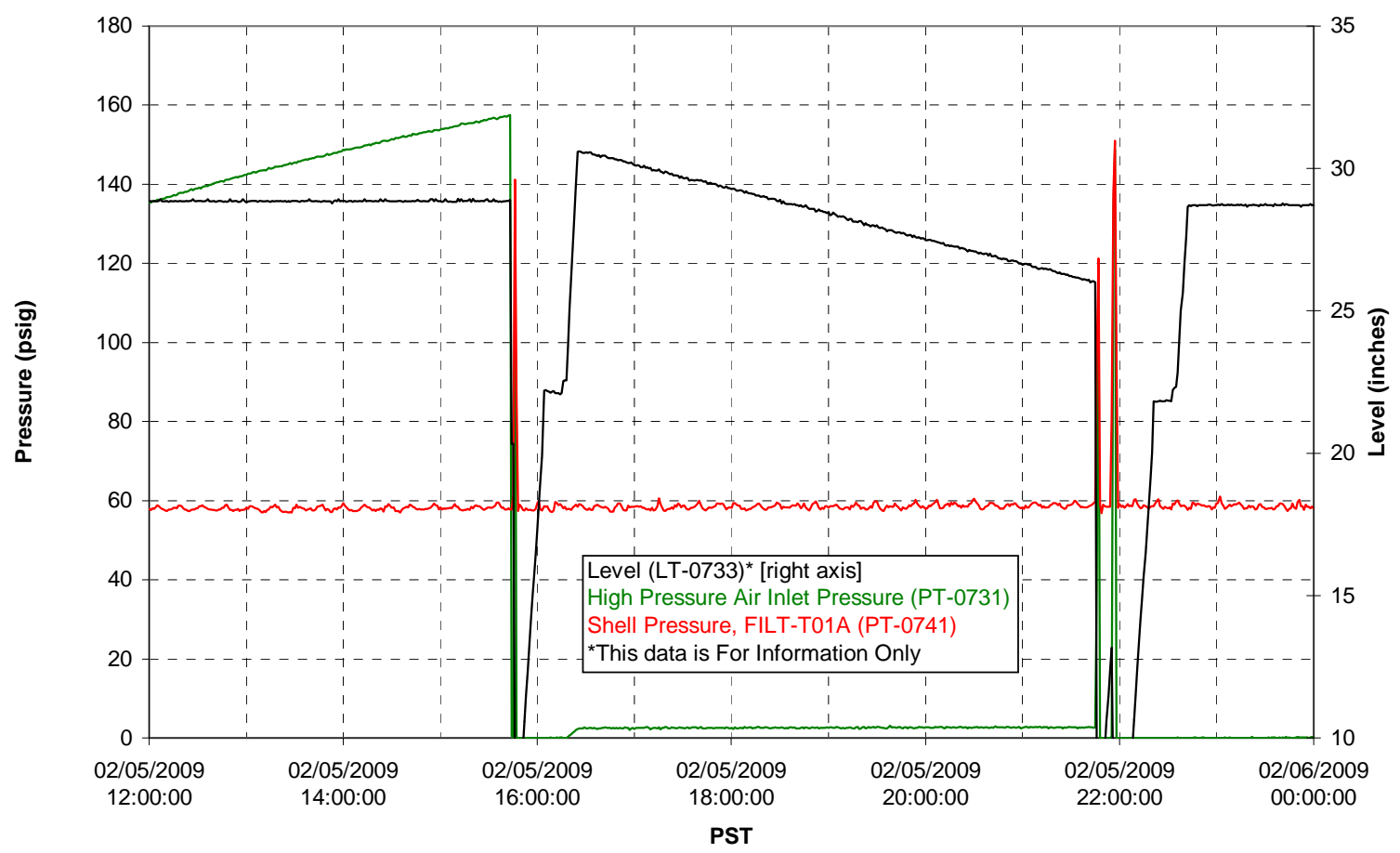

Pulsepot Levels

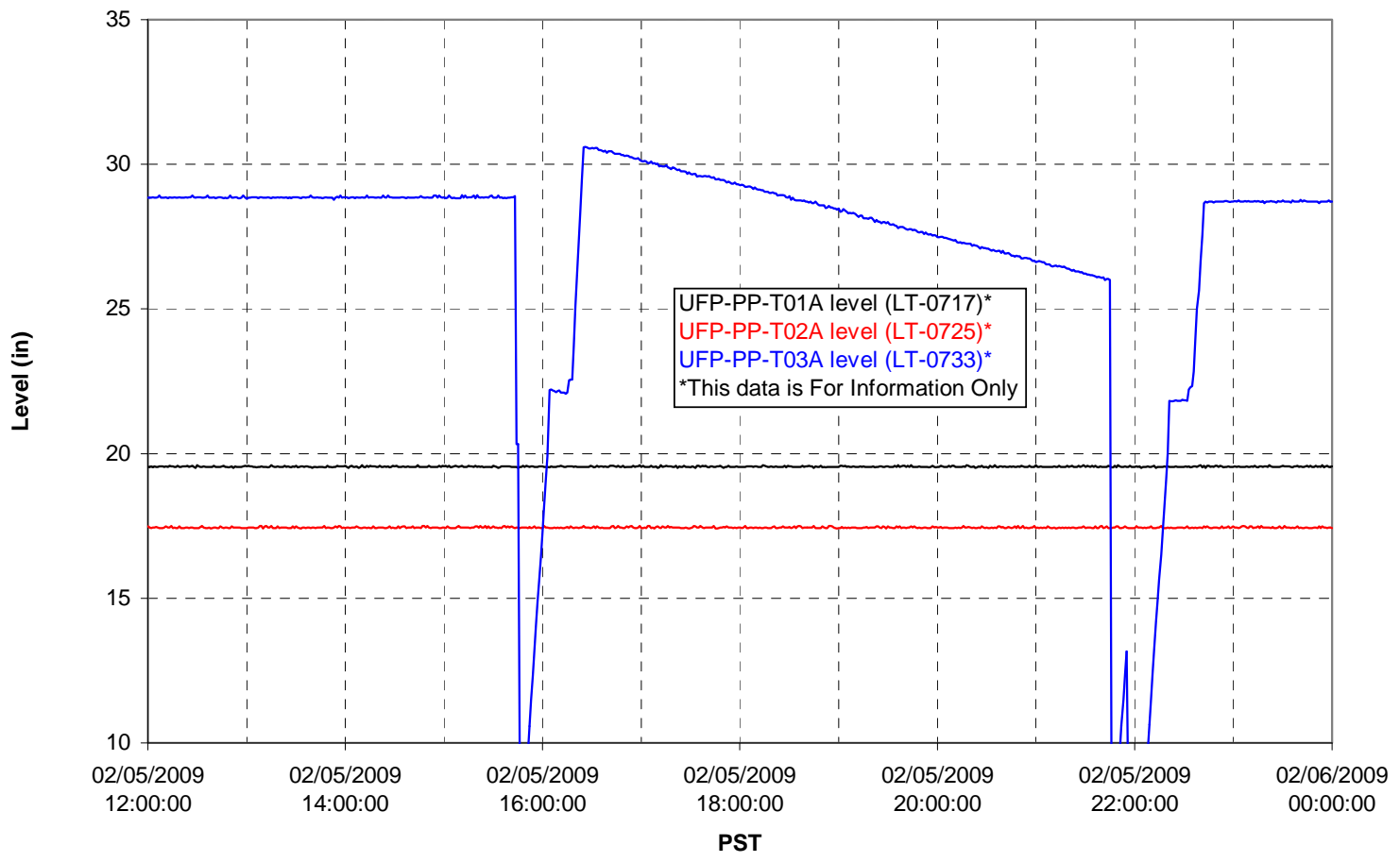


Filter UFP-FILT-T01A

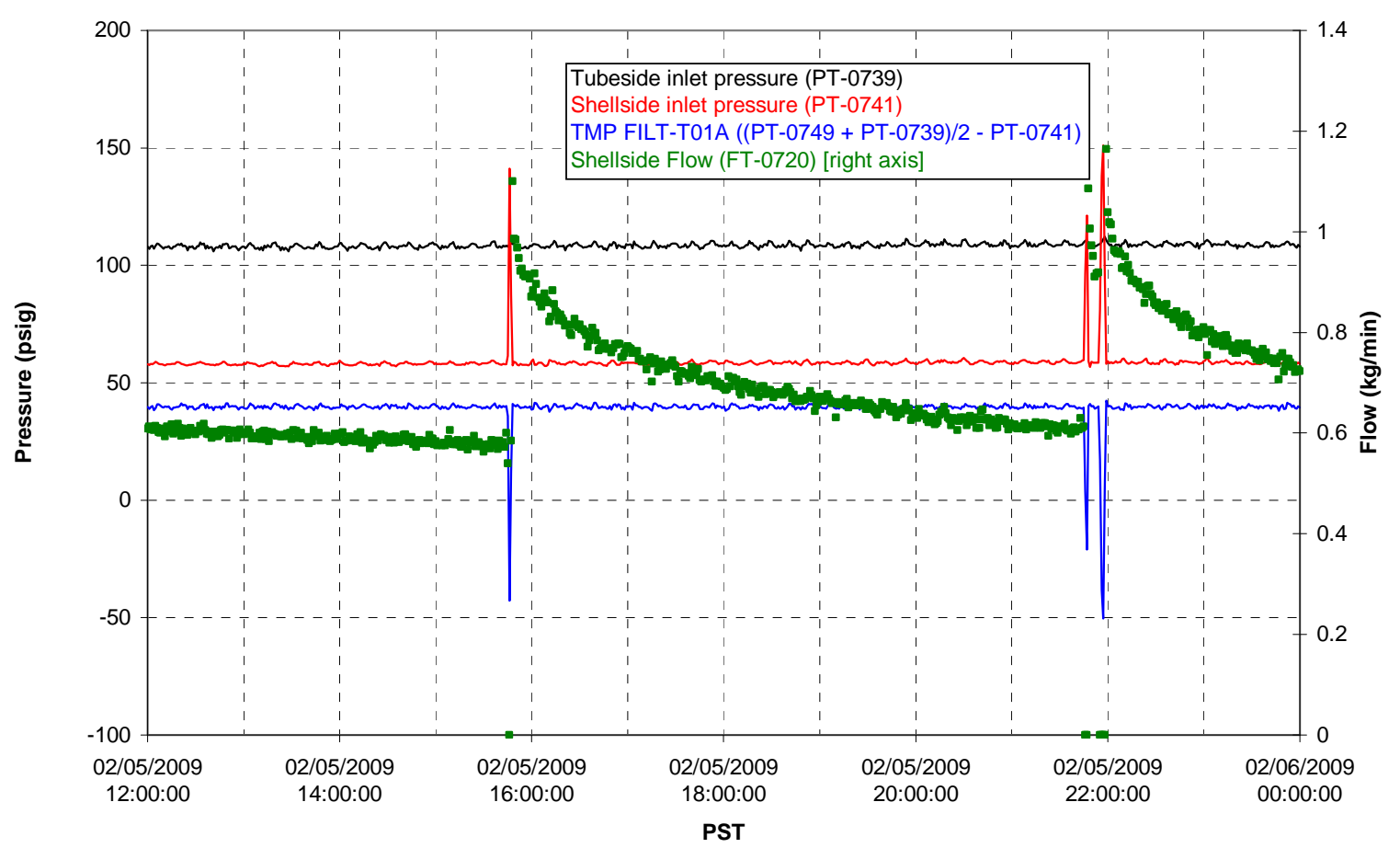

Filter UFP-FILT-T02A

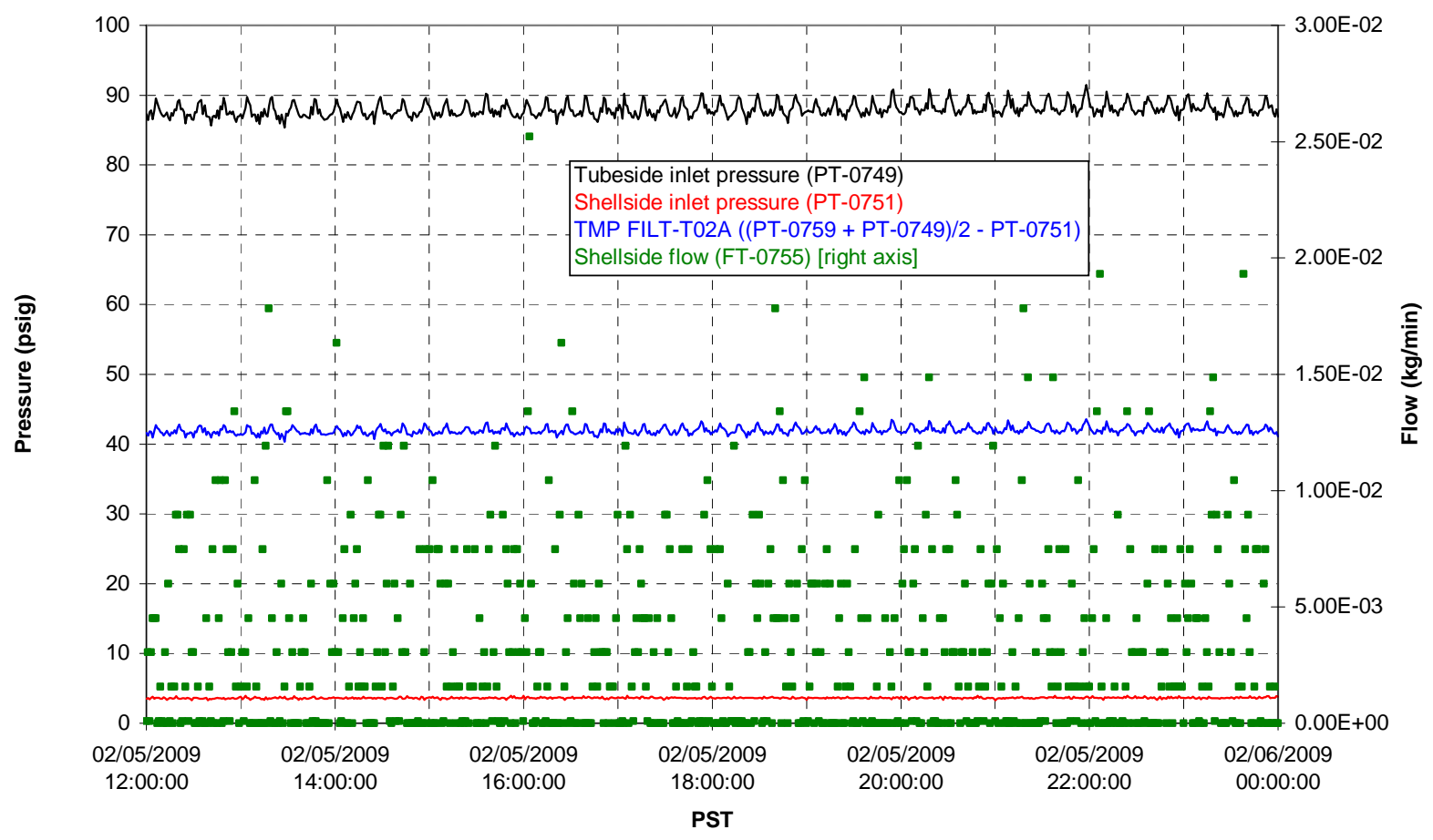


Filter UFP-FILT-T03A

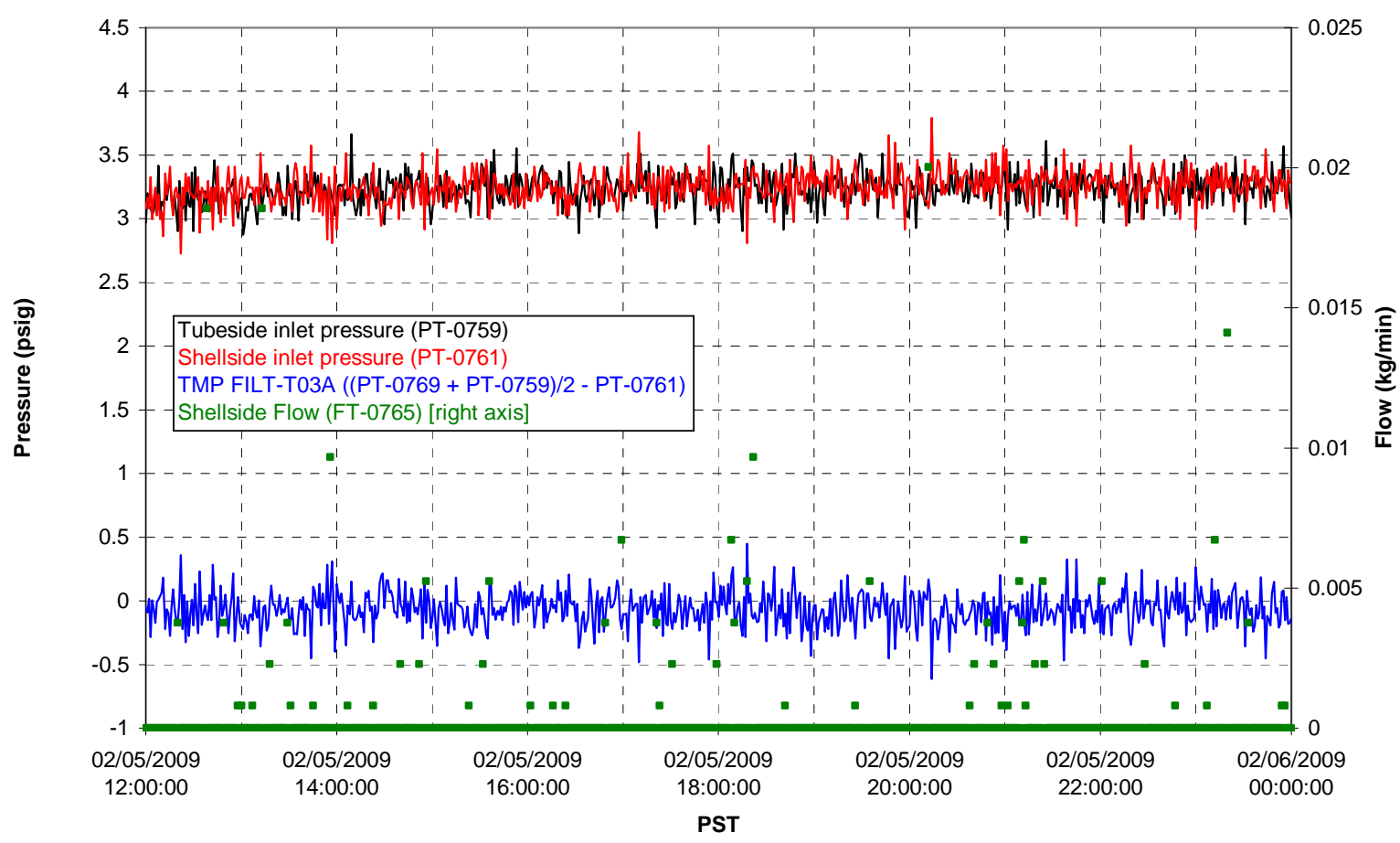

Filter UFP-FILT-T04A

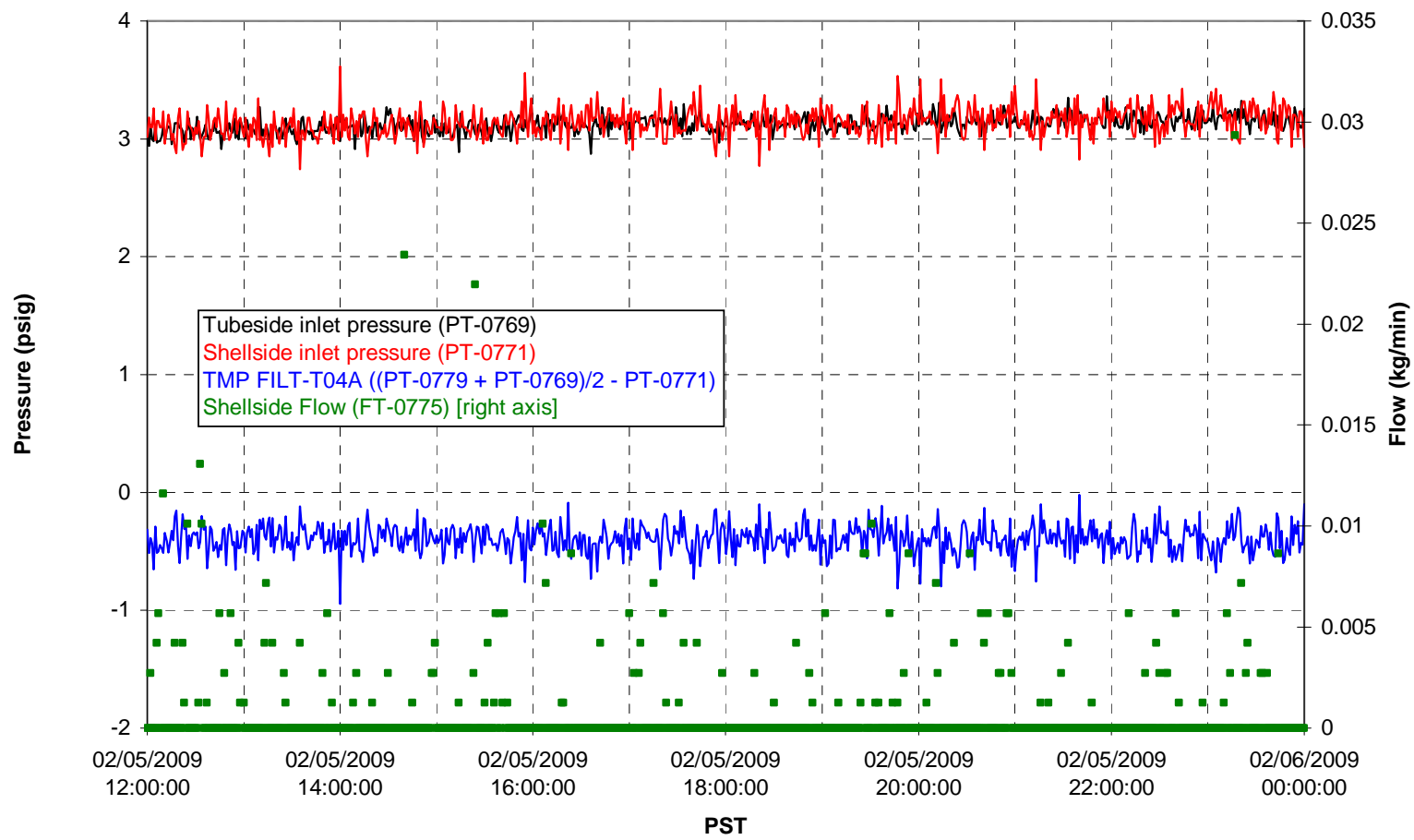


Filter UFP-FILT-T05A

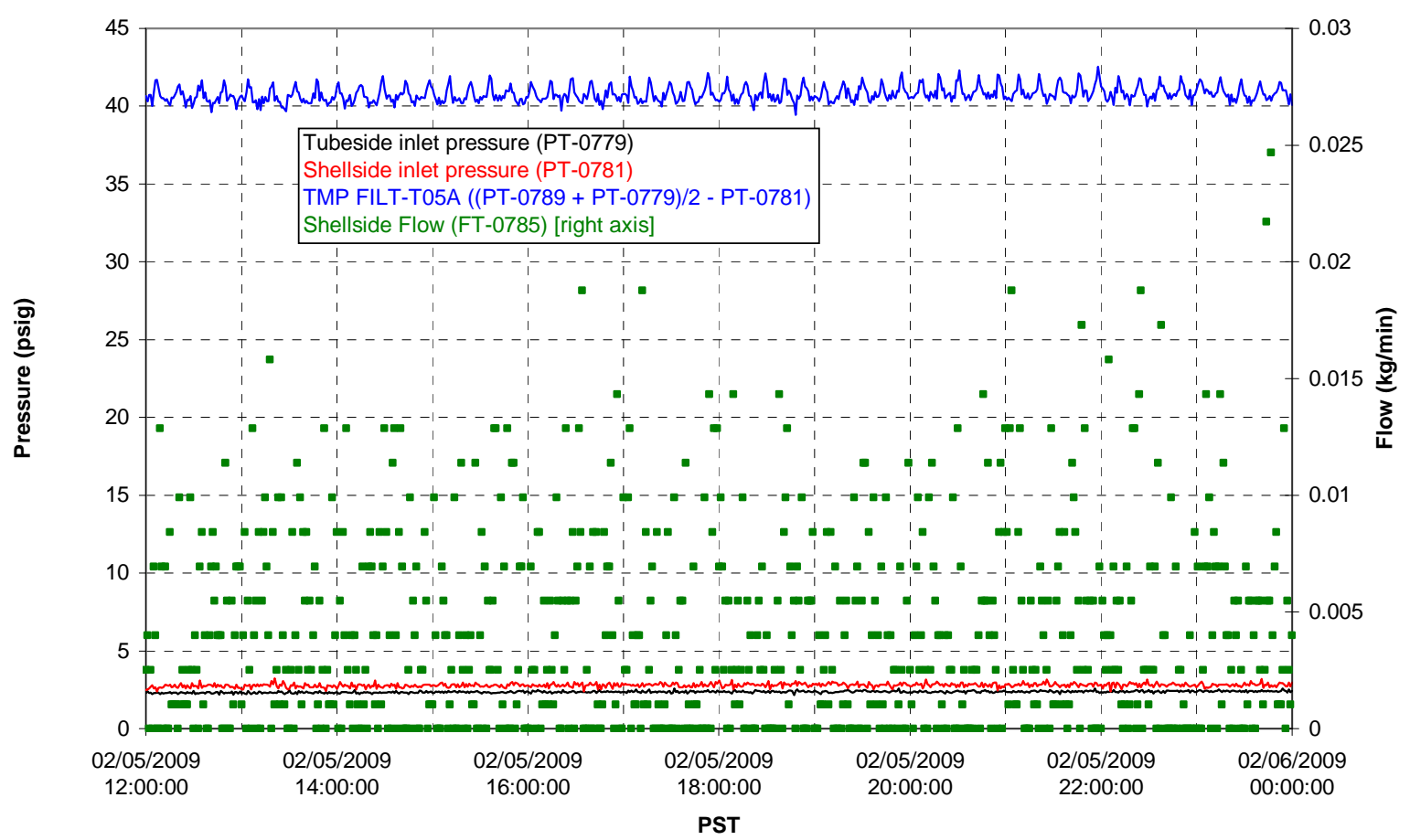

Chemical Flow

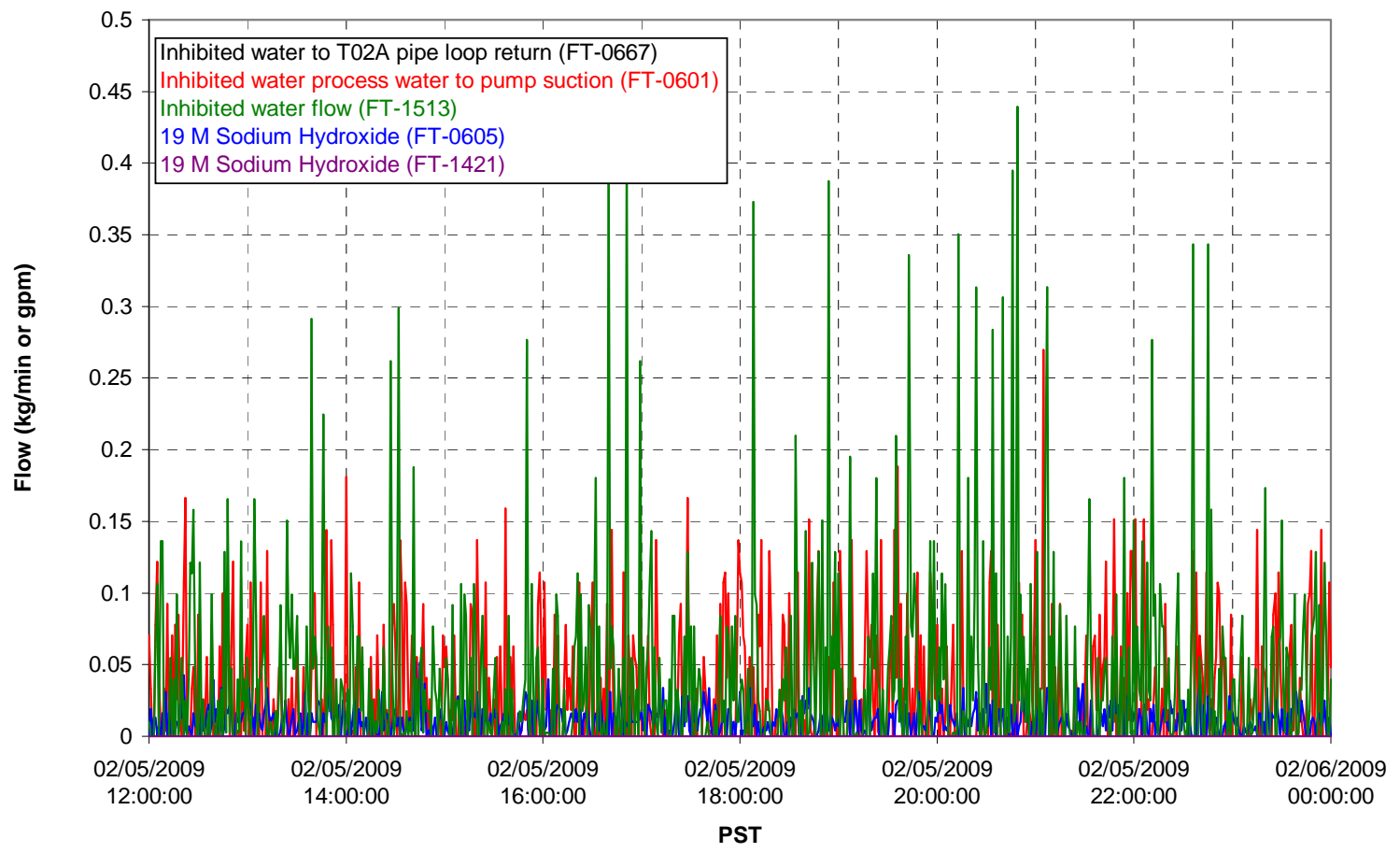


Chemical Flow

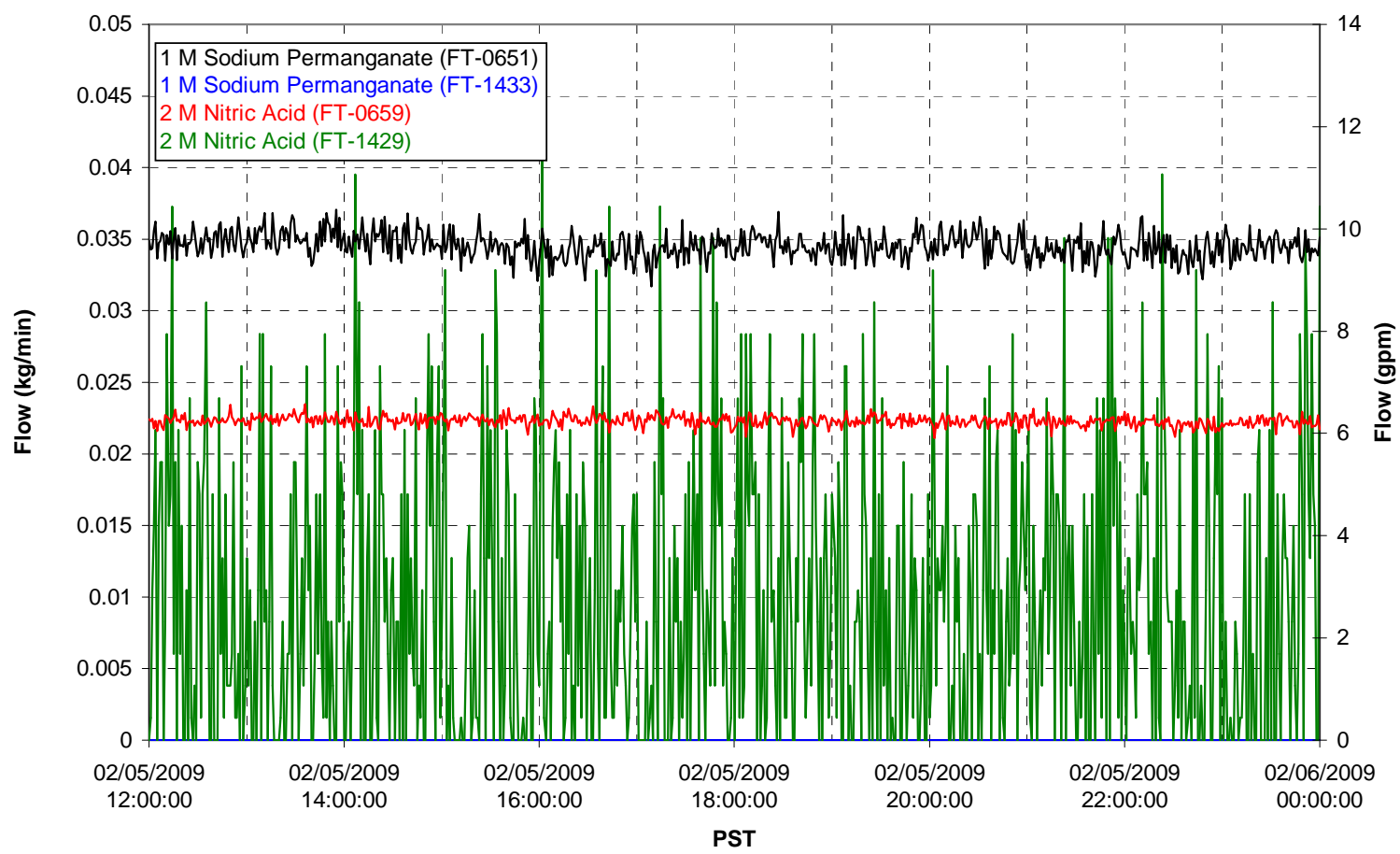

Air Flows

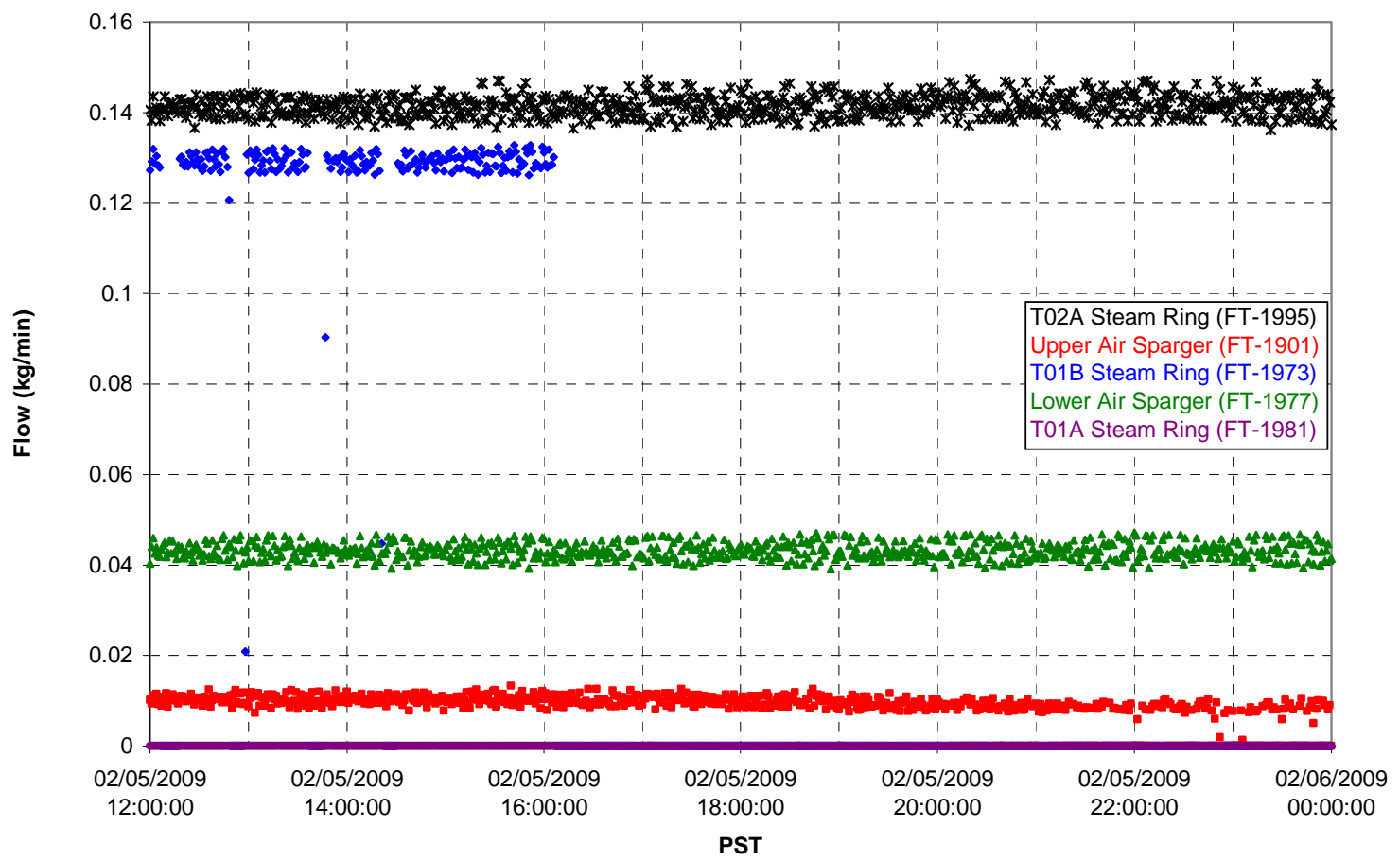


T02A Steam

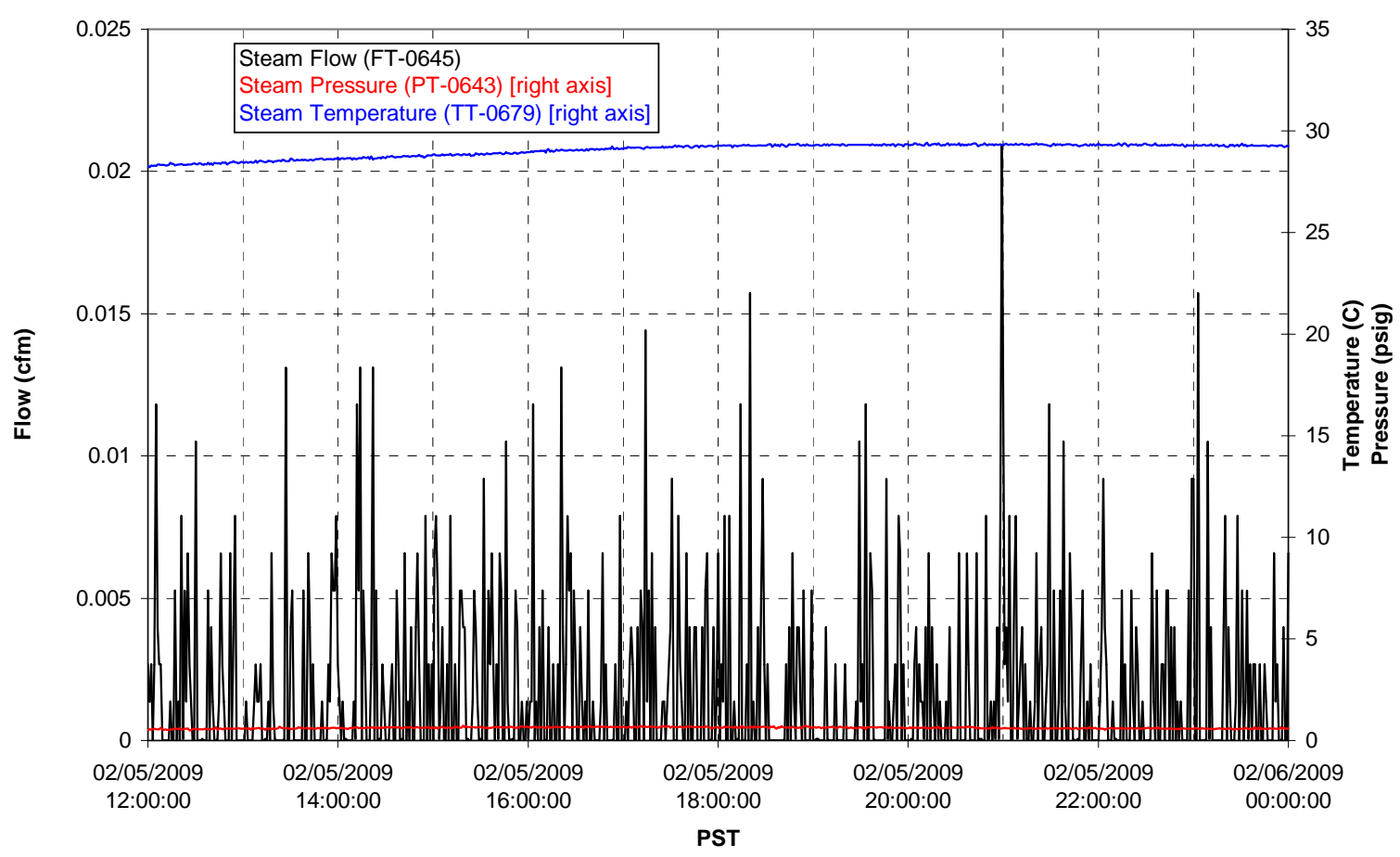

T01A Steam

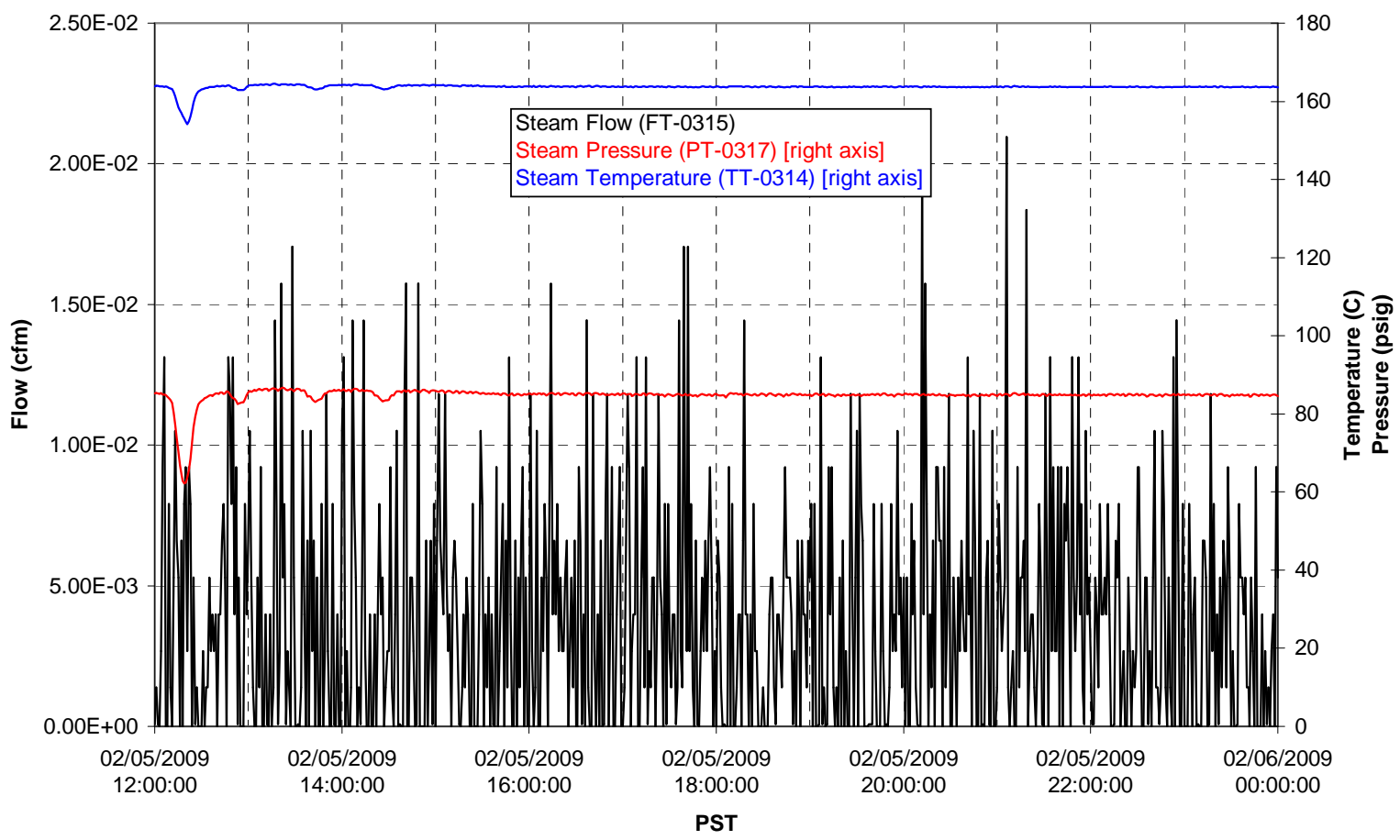


T01B Steam

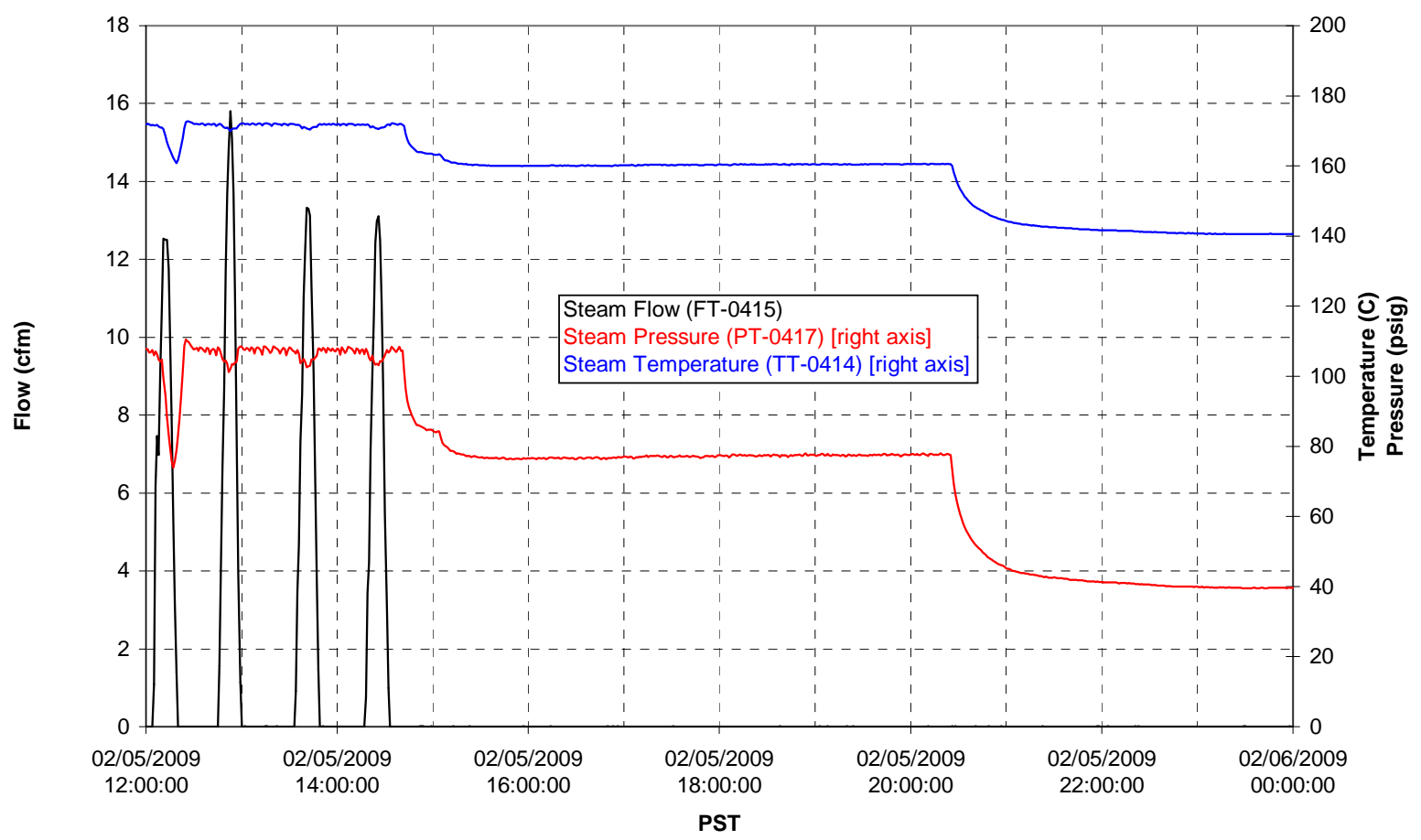




\section{Integrated Test A Data Plots 02/06/09 00:00 - 02/06/09 12:00}


T01A level

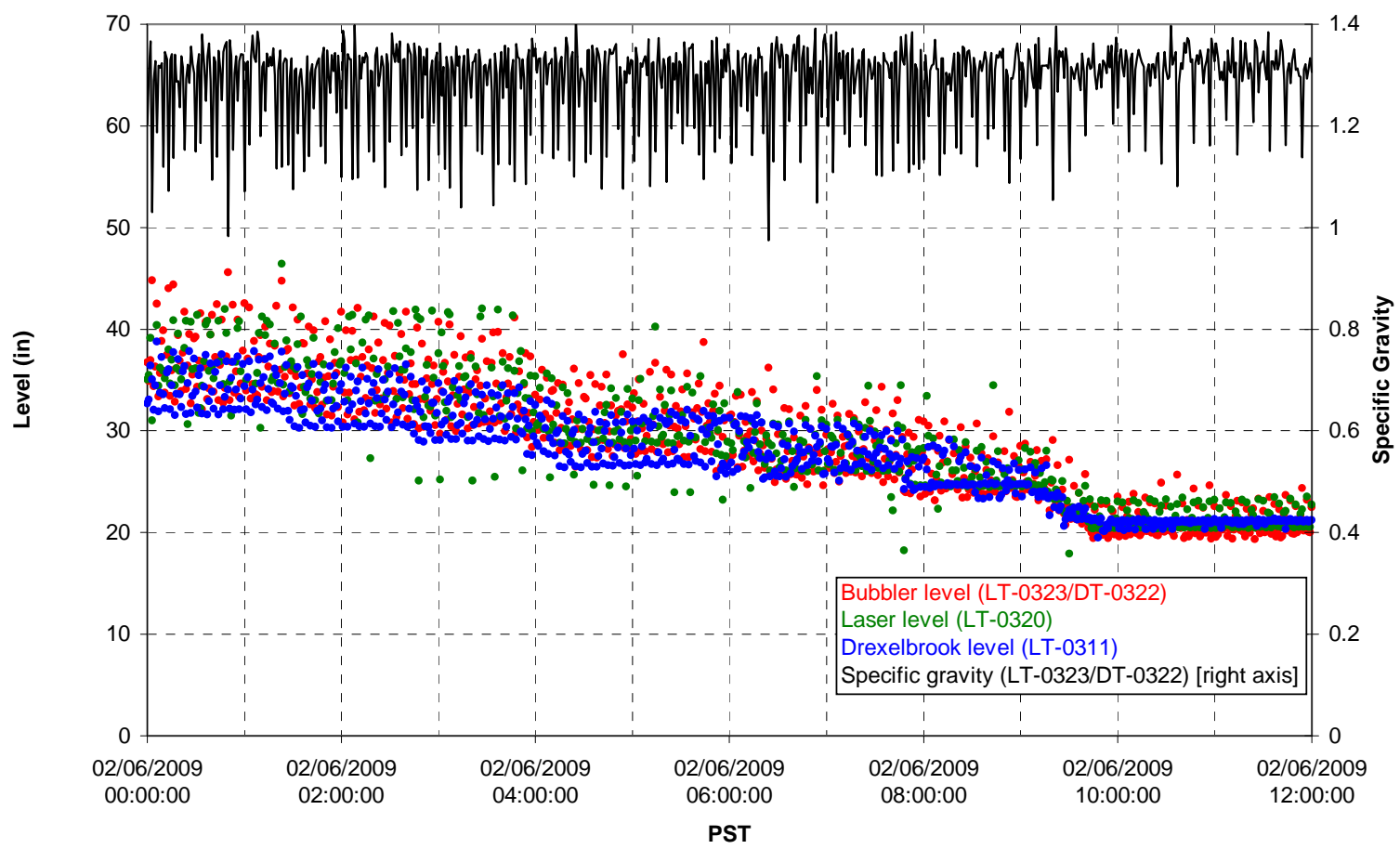

T01A temperatures

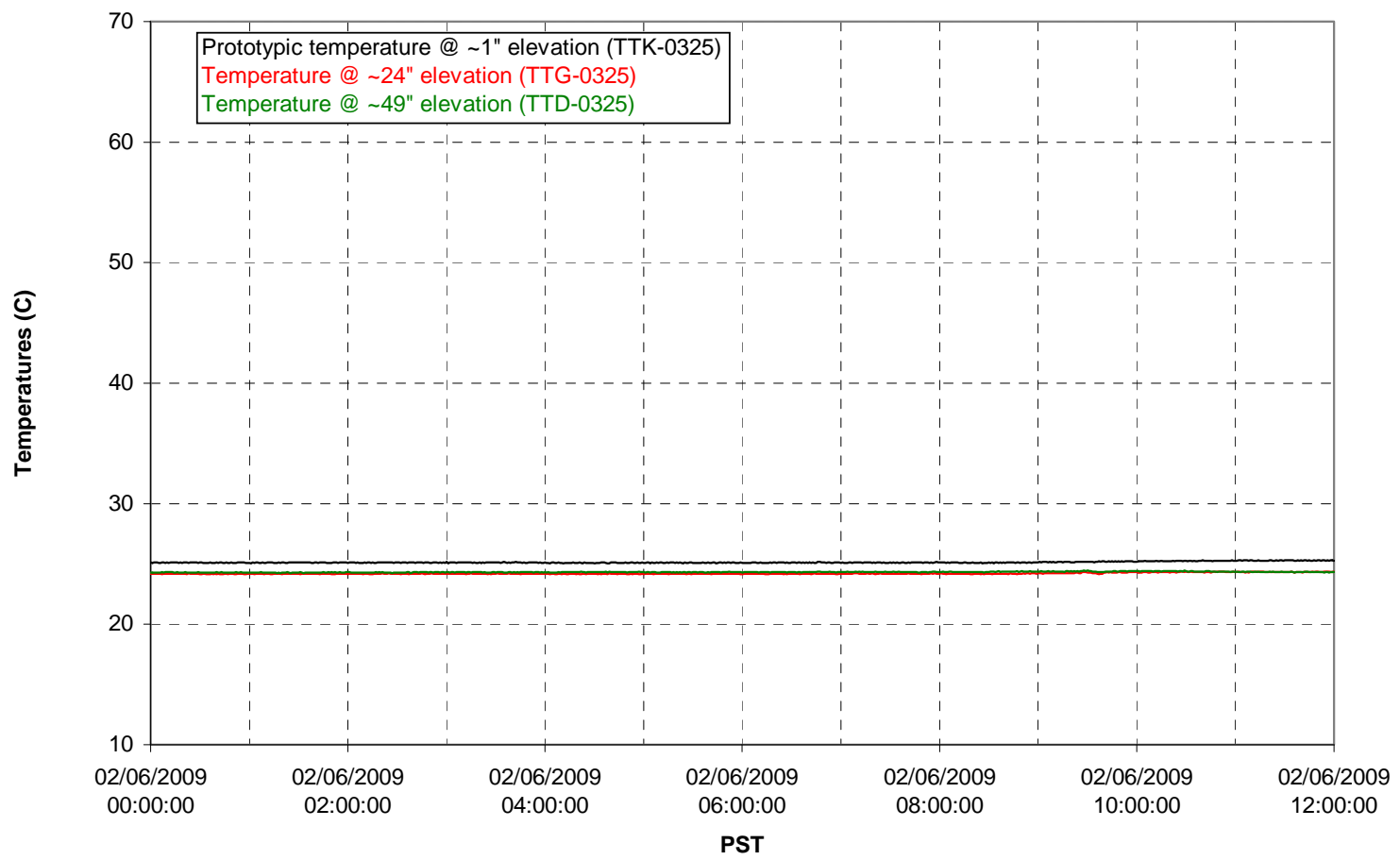


T01B level

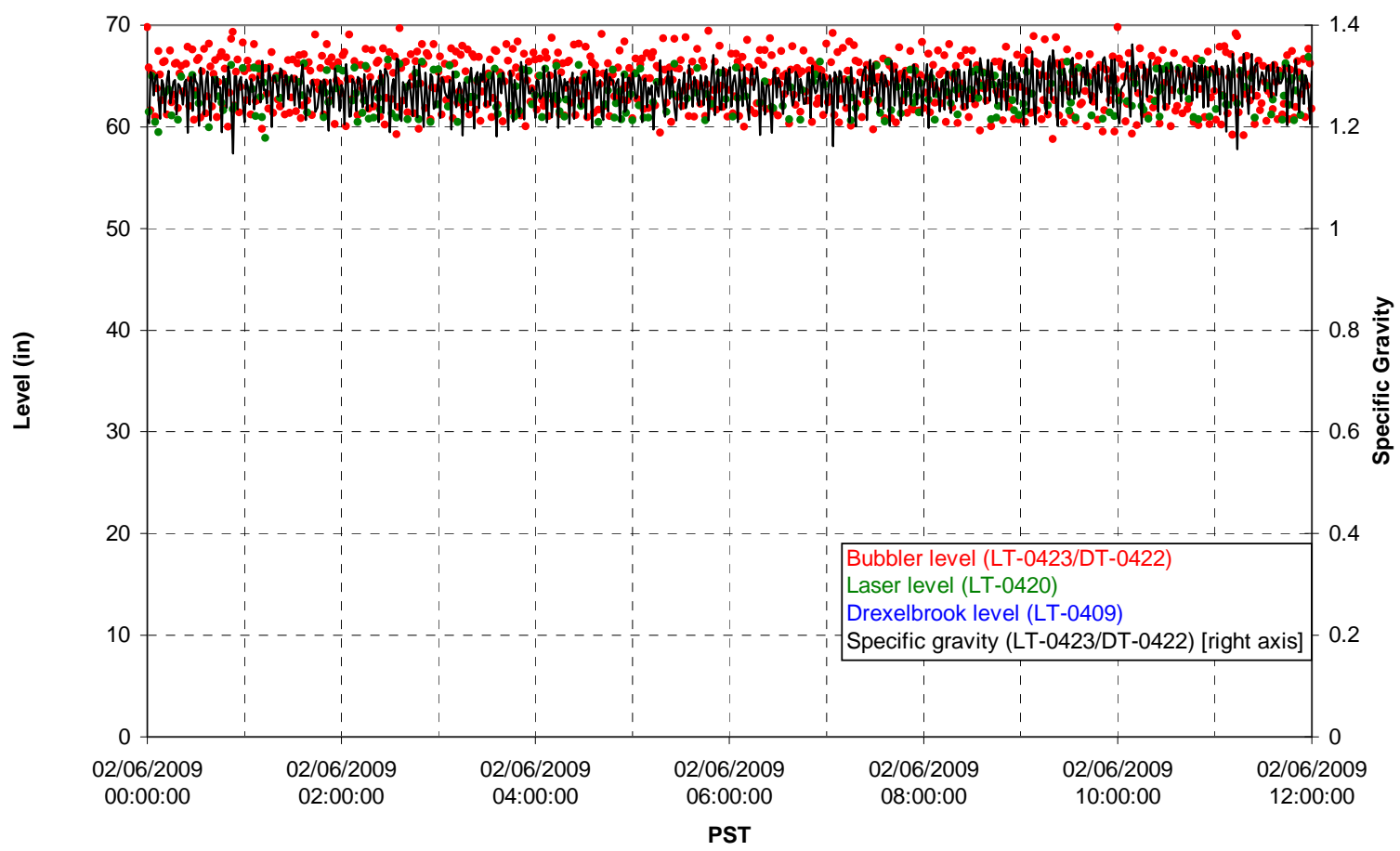

T01B temperatures

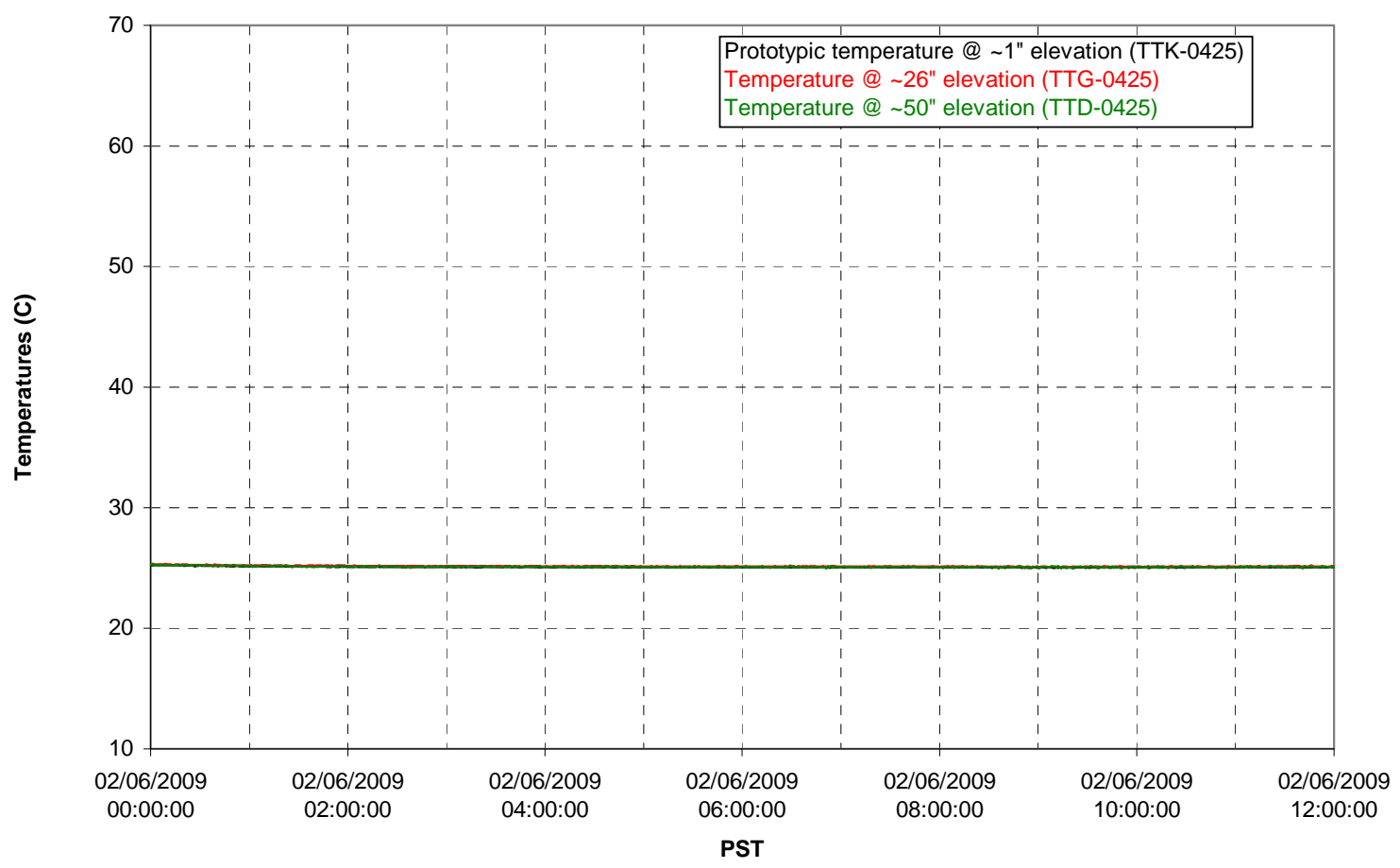


T02A level

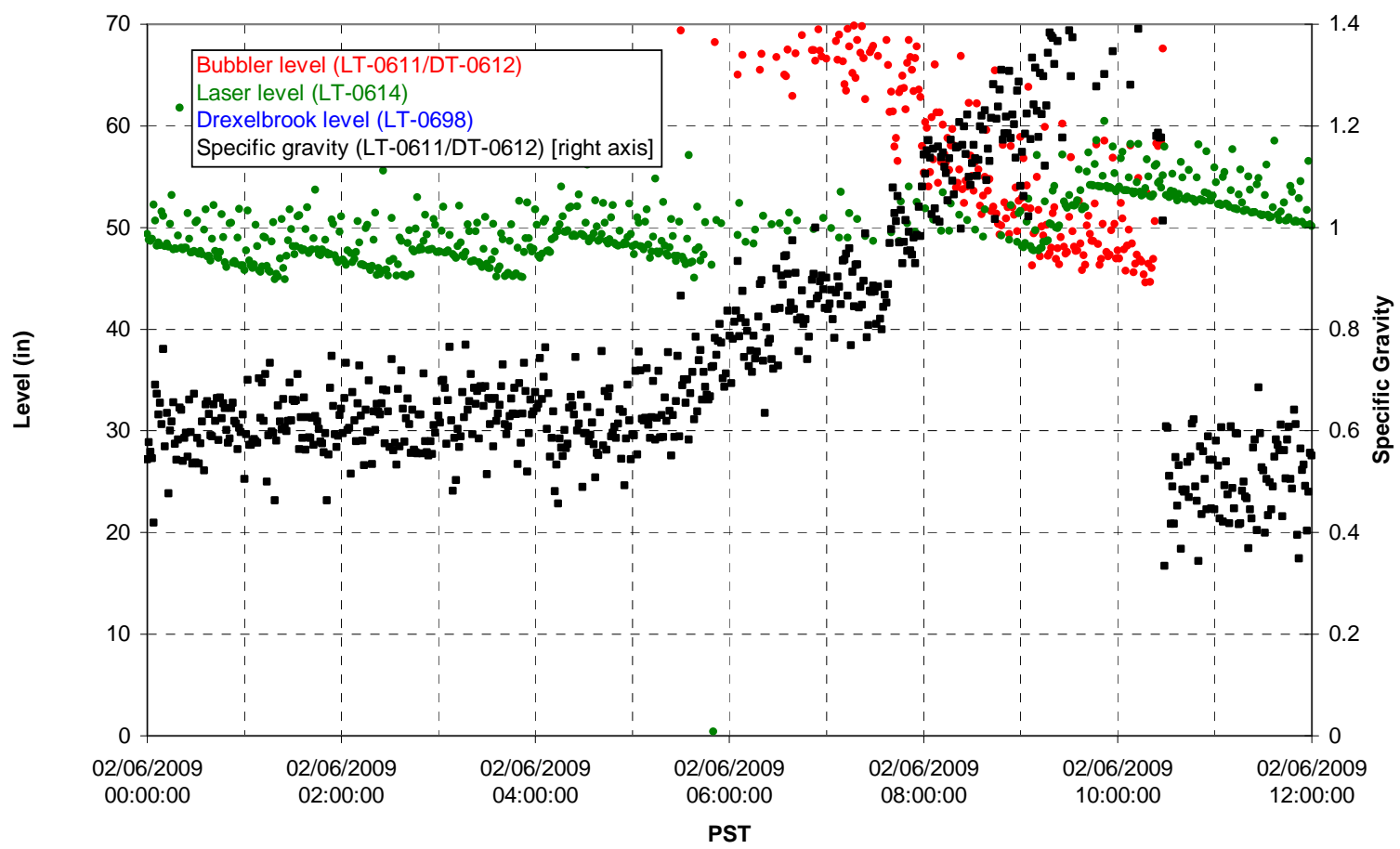

T02A temperatures

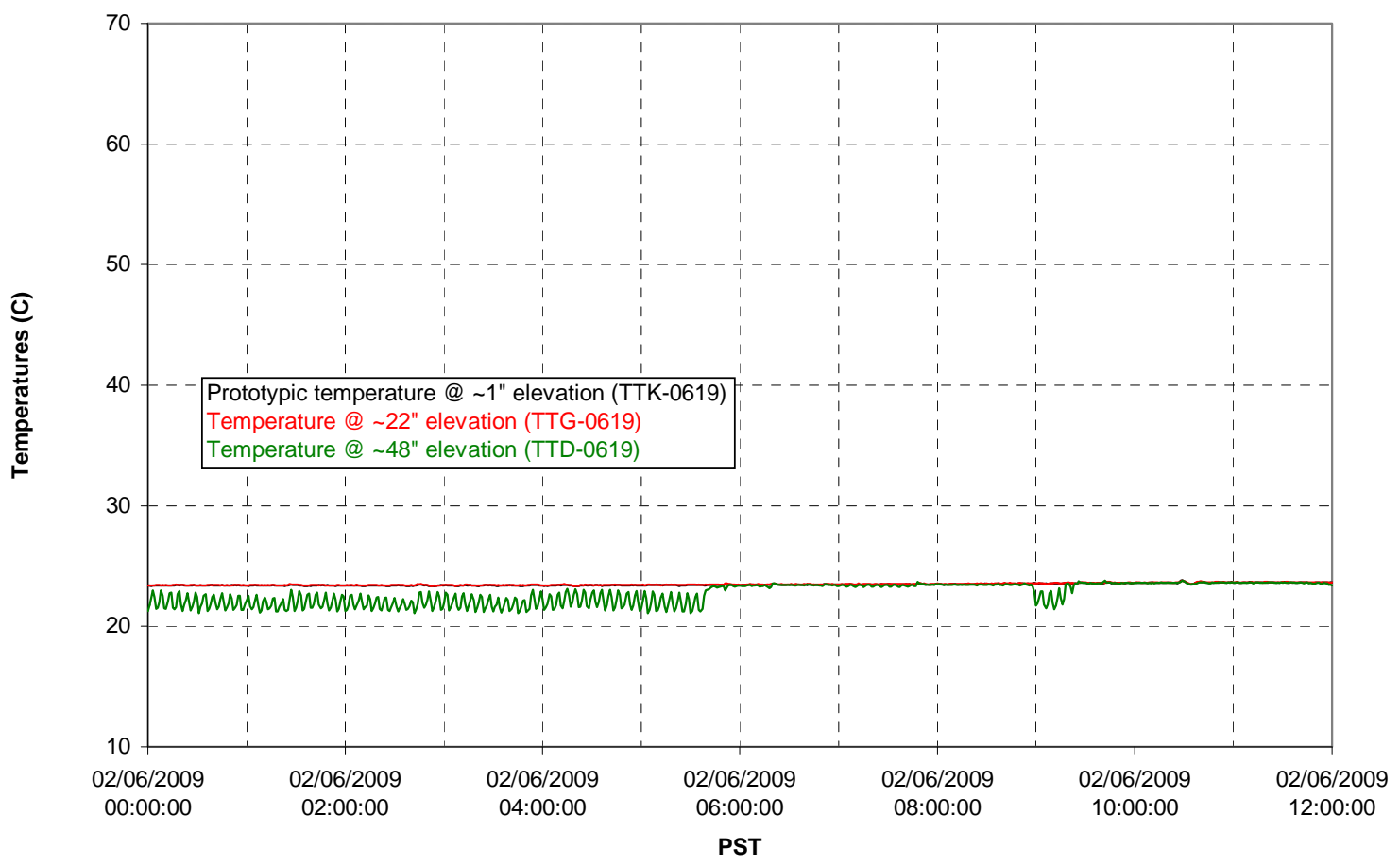

G. 220 
T02A and filter loop temperatures

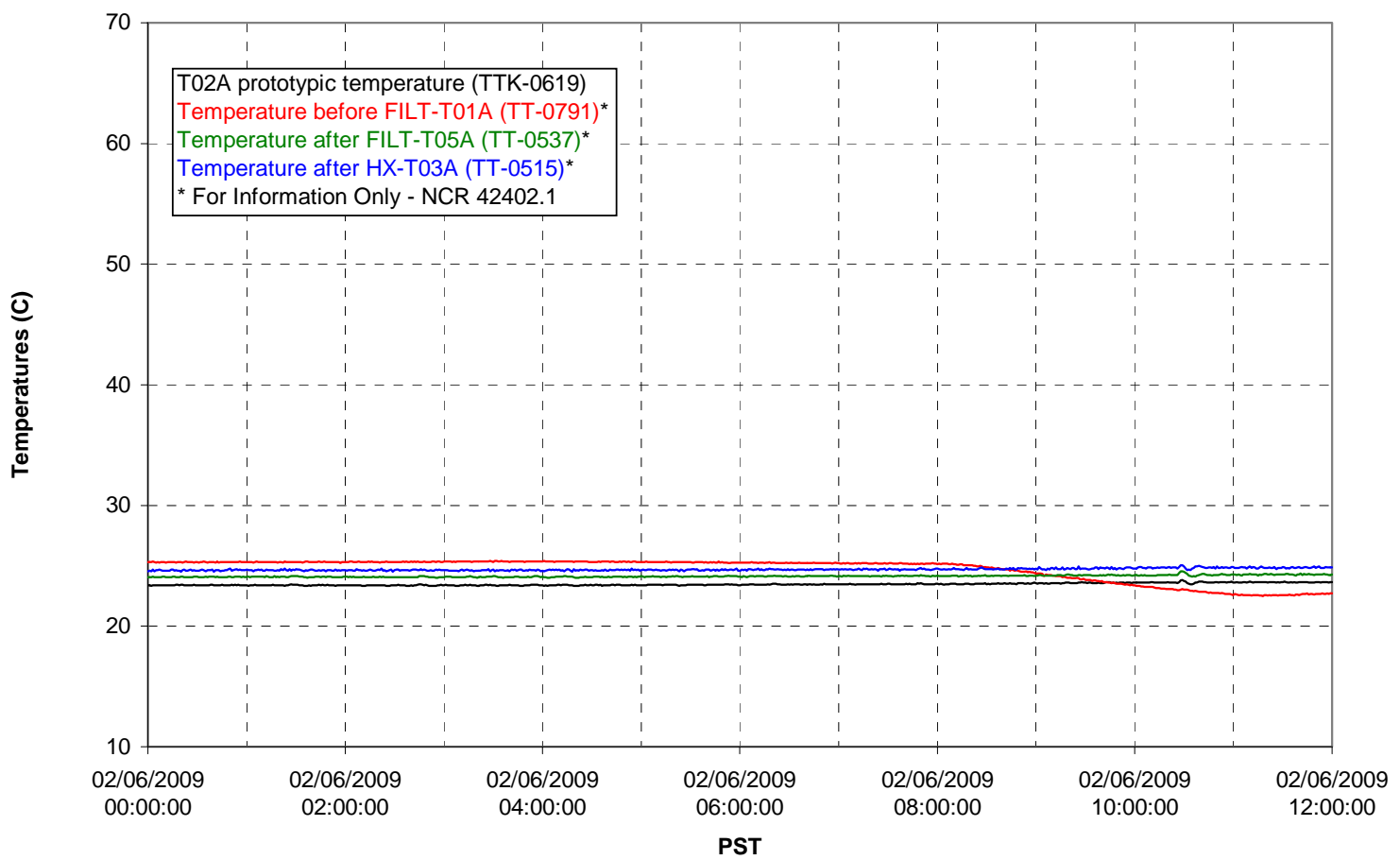

Pump Pressures and Flow

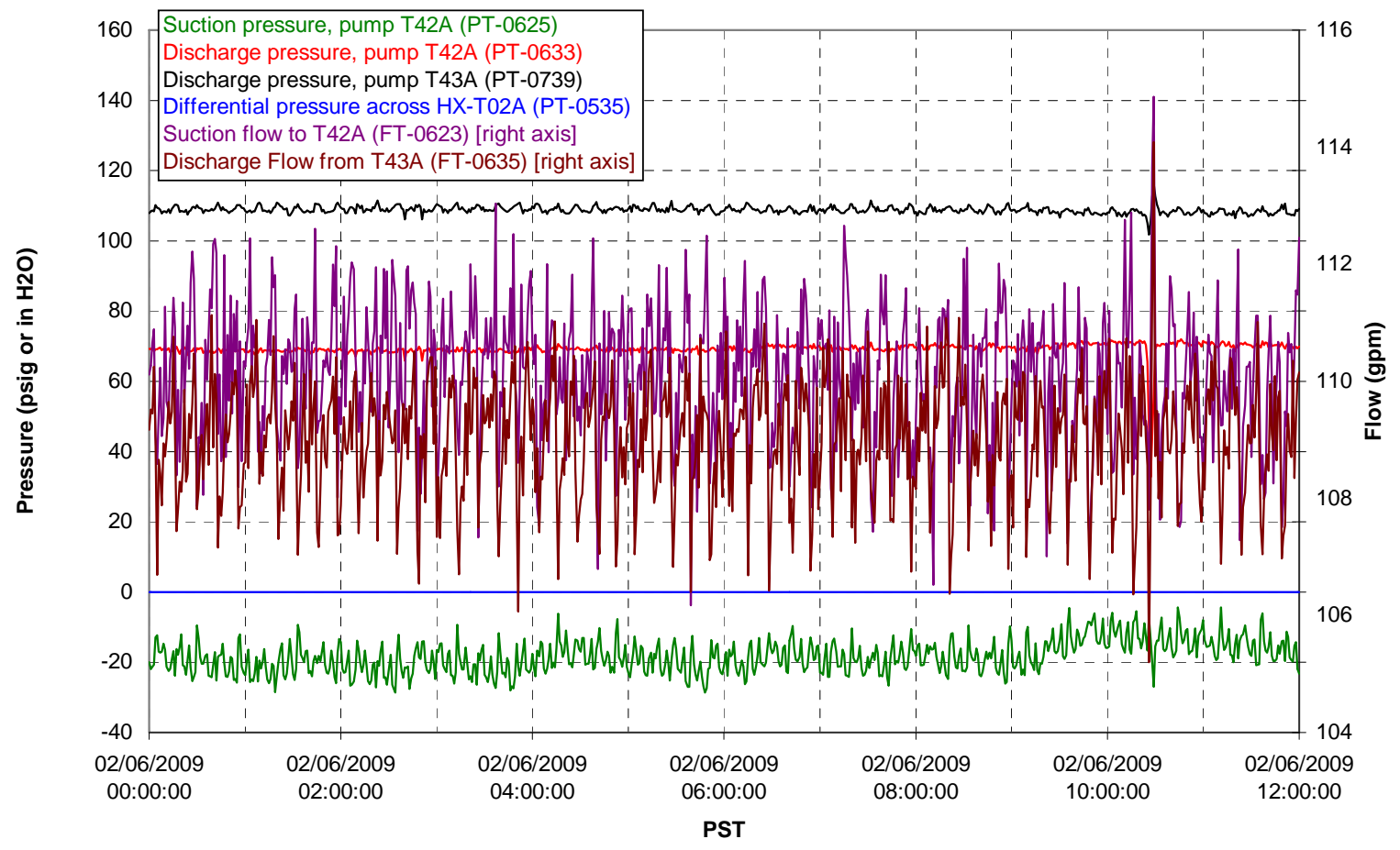


Axial pressure drop

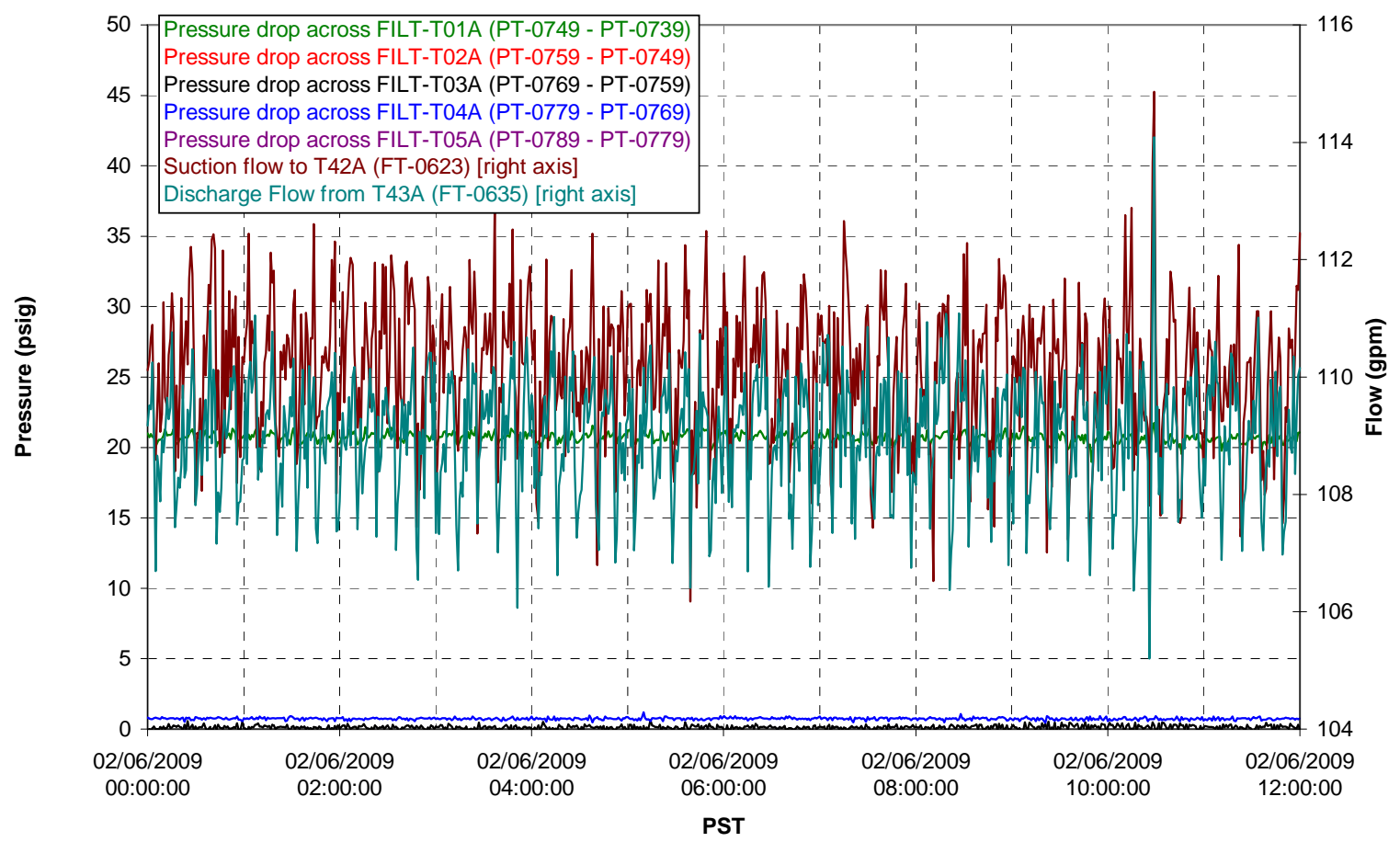

Permeate flow rates

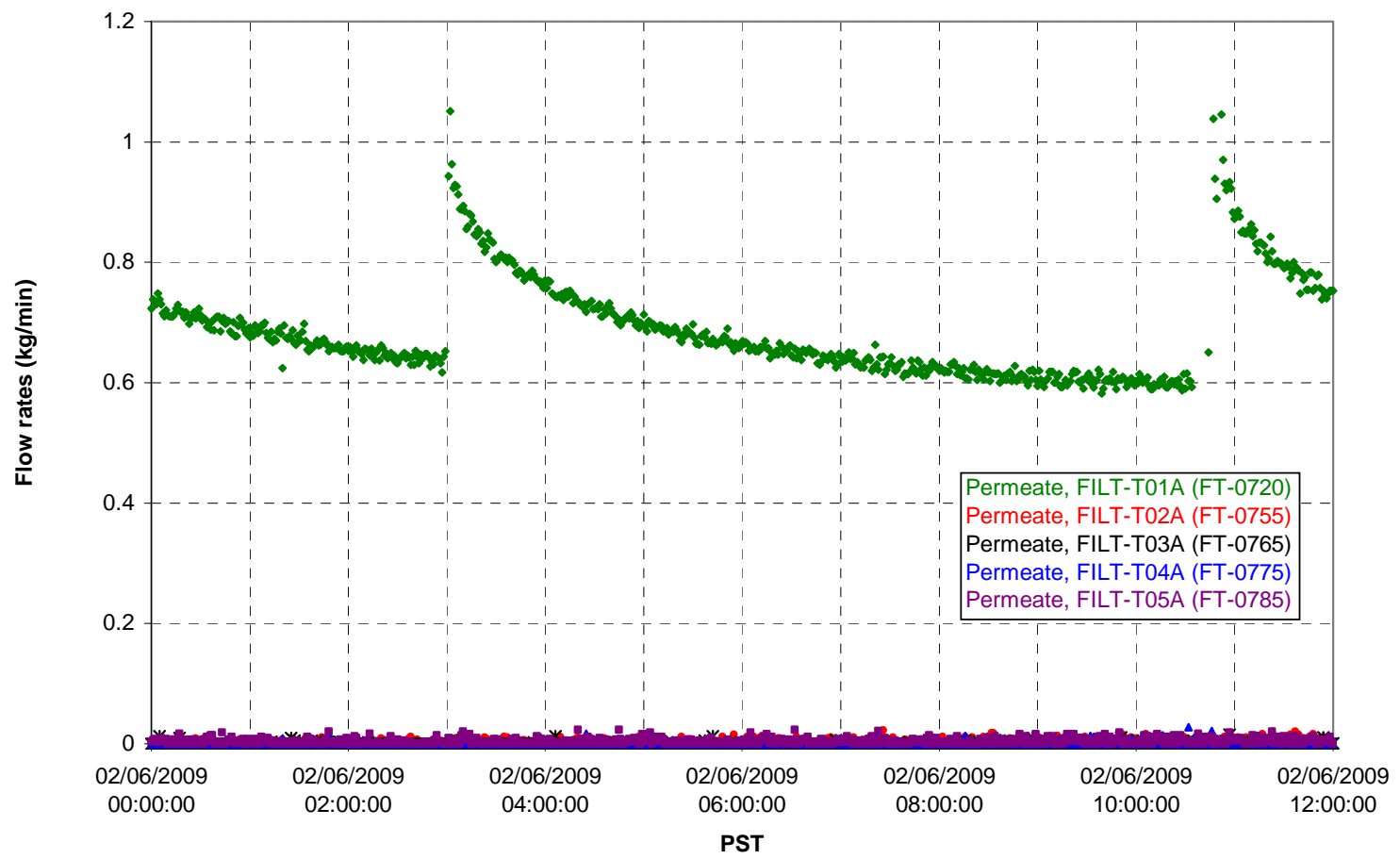


T02A Inner Temperature Tree

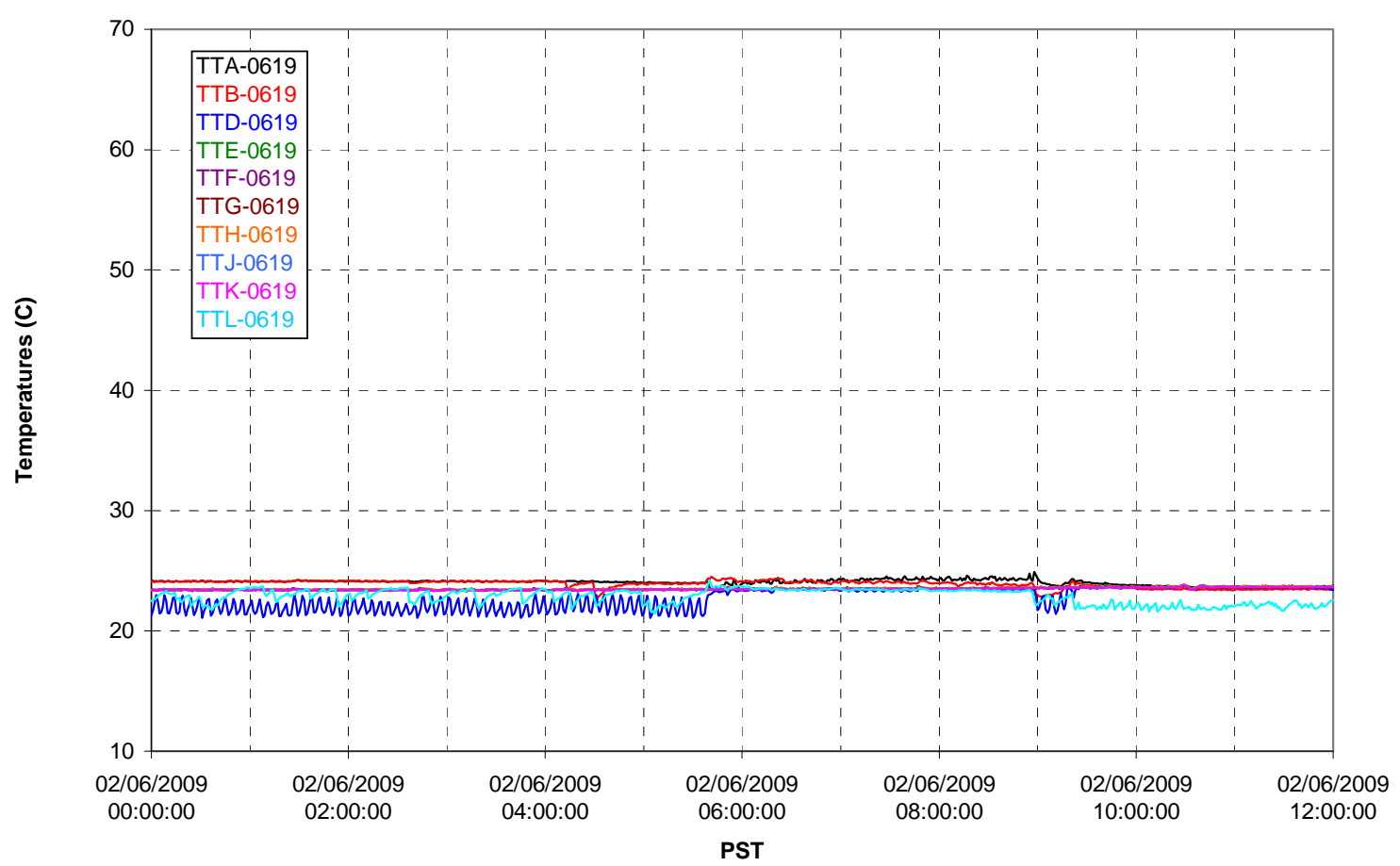

T02A Outer Temperature Tree

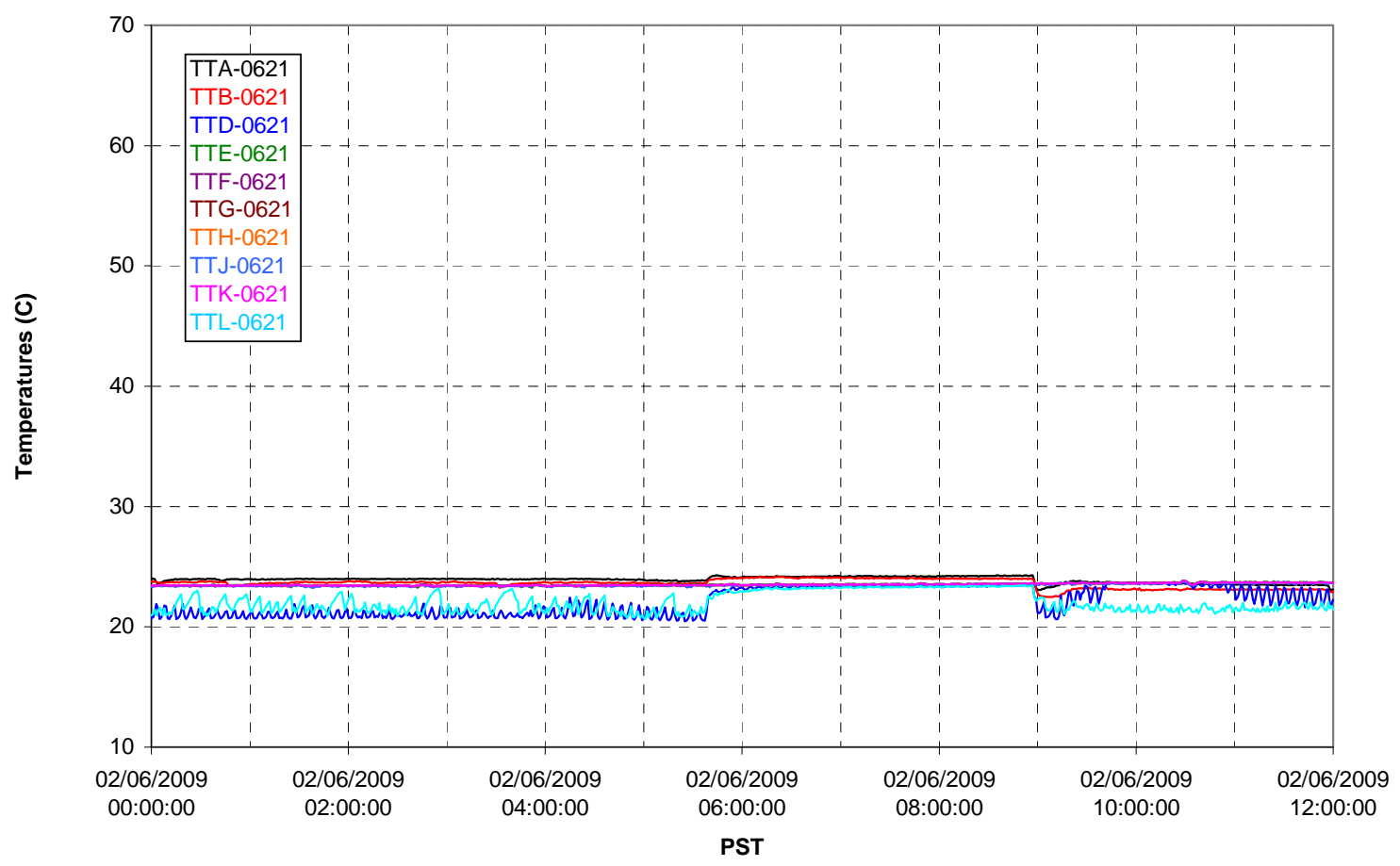


T02A temperatures

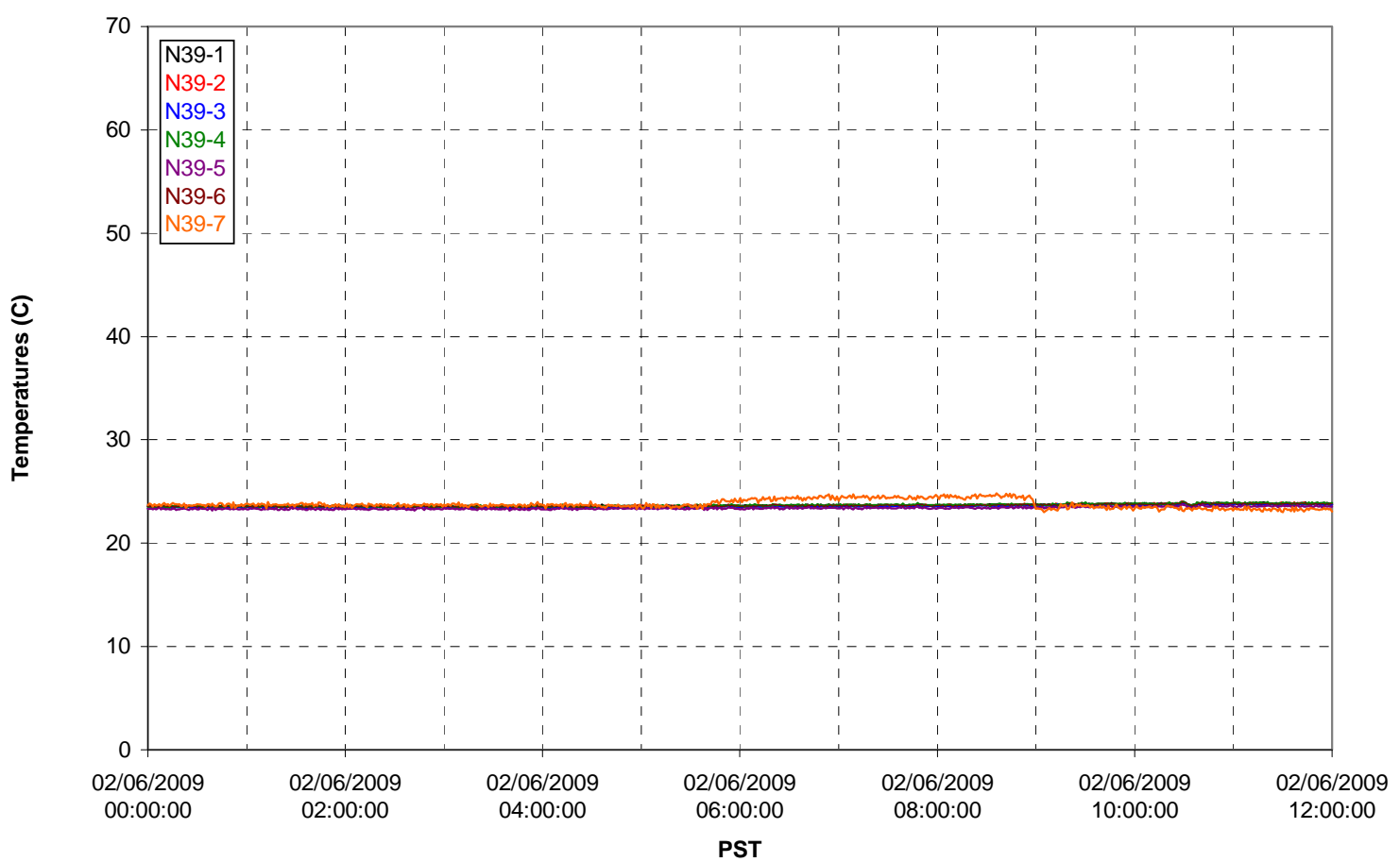

T02A temperatures

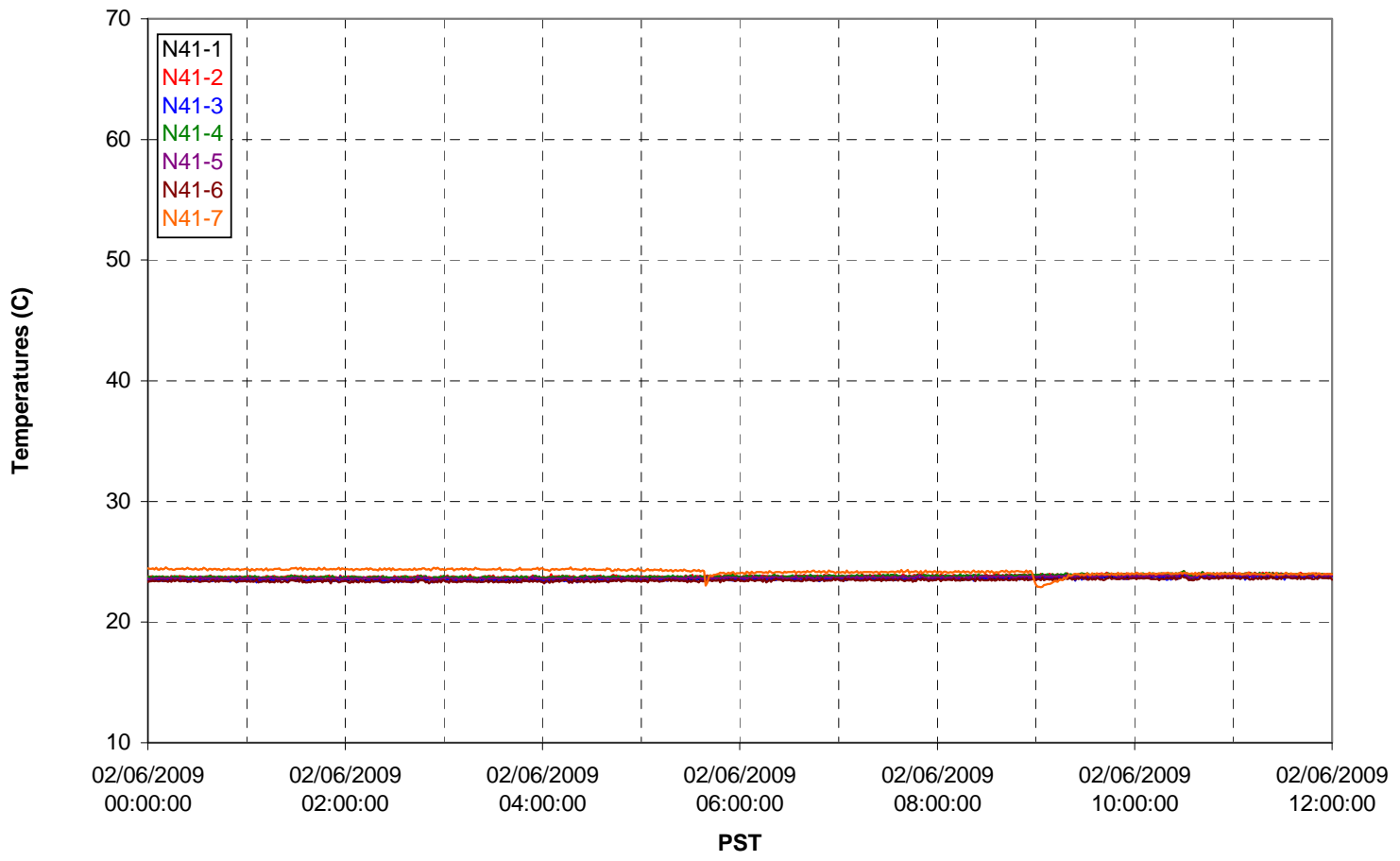


T02A temperatures

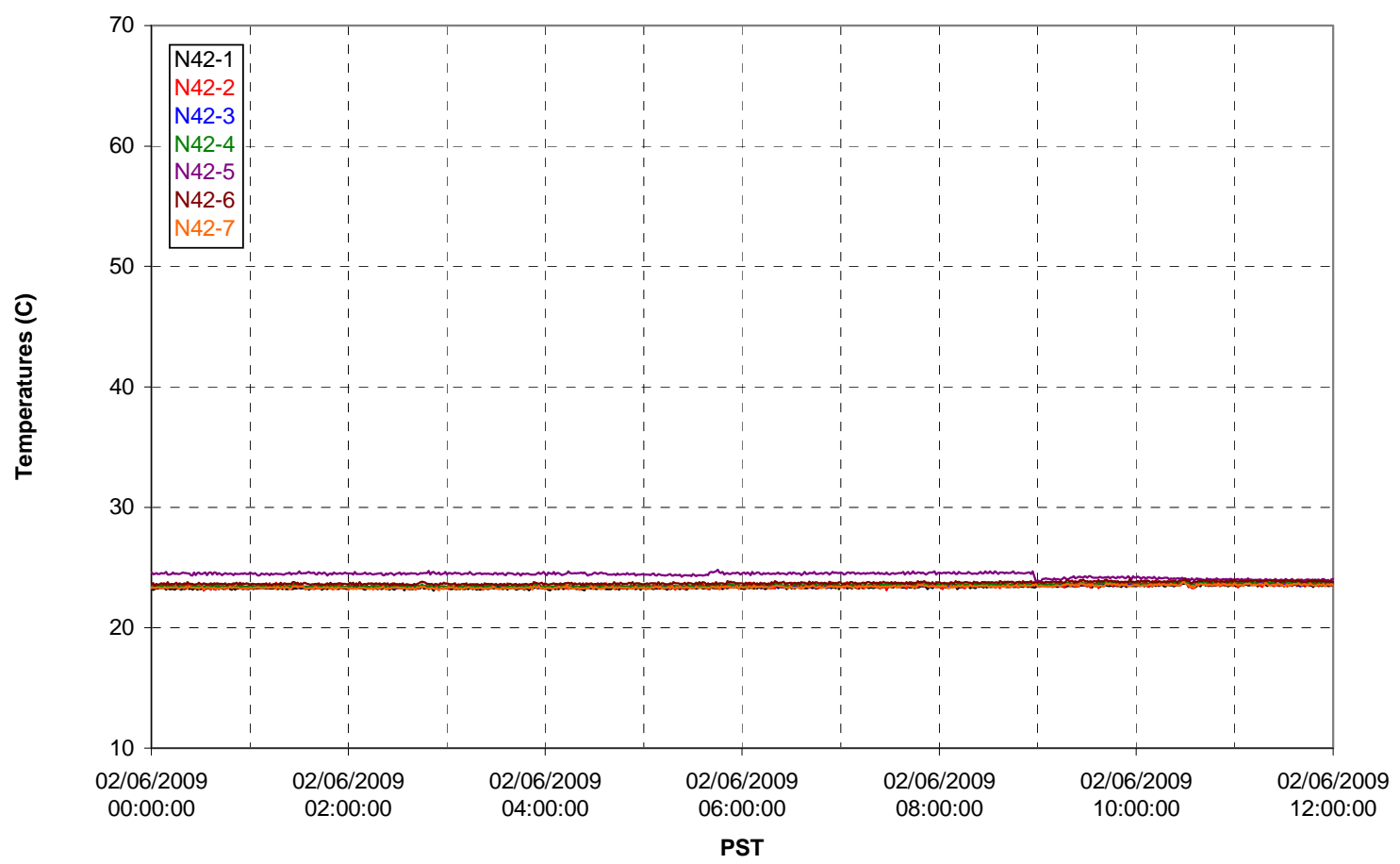

T02A temperatures

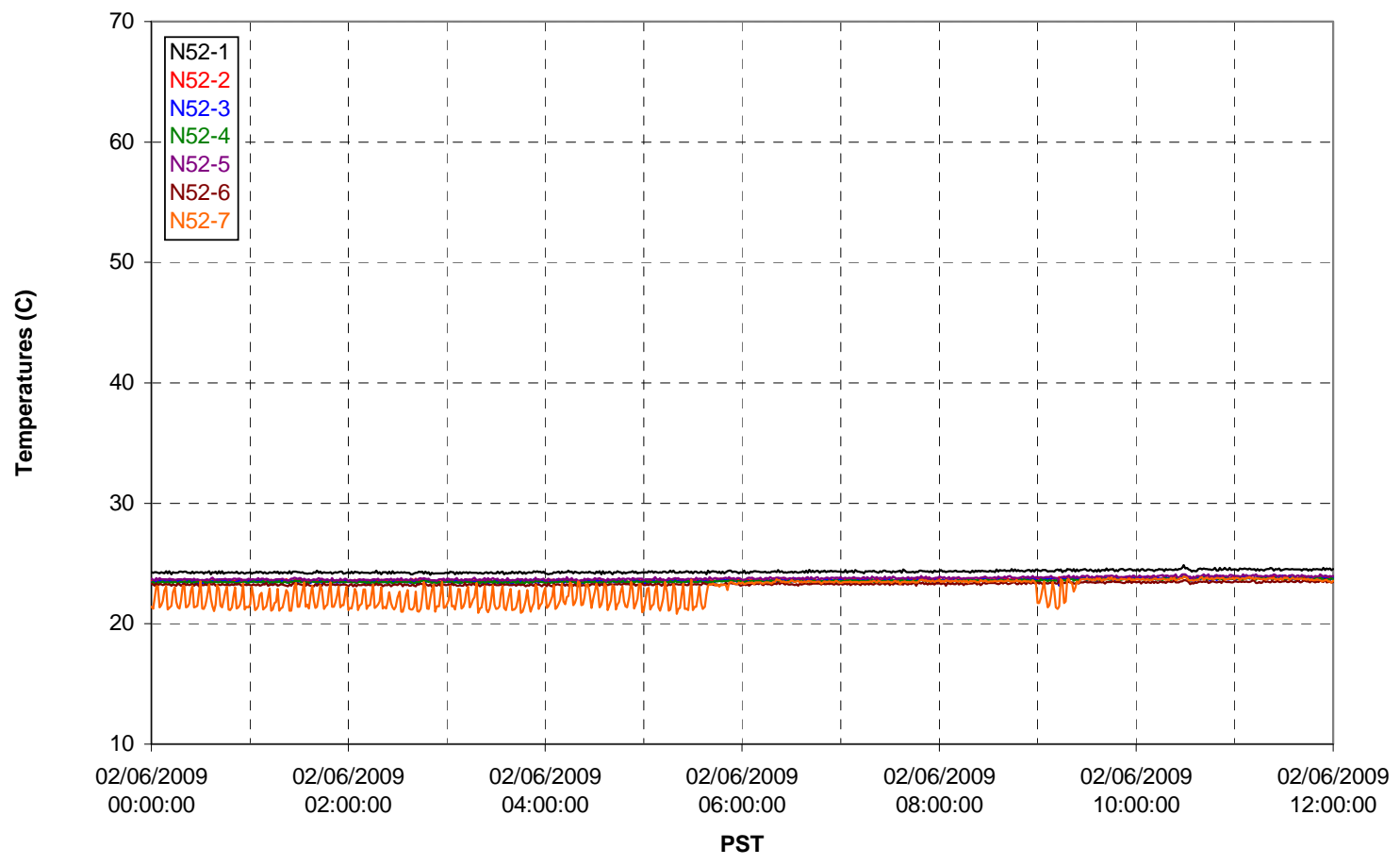


T02A Heating and Cooling

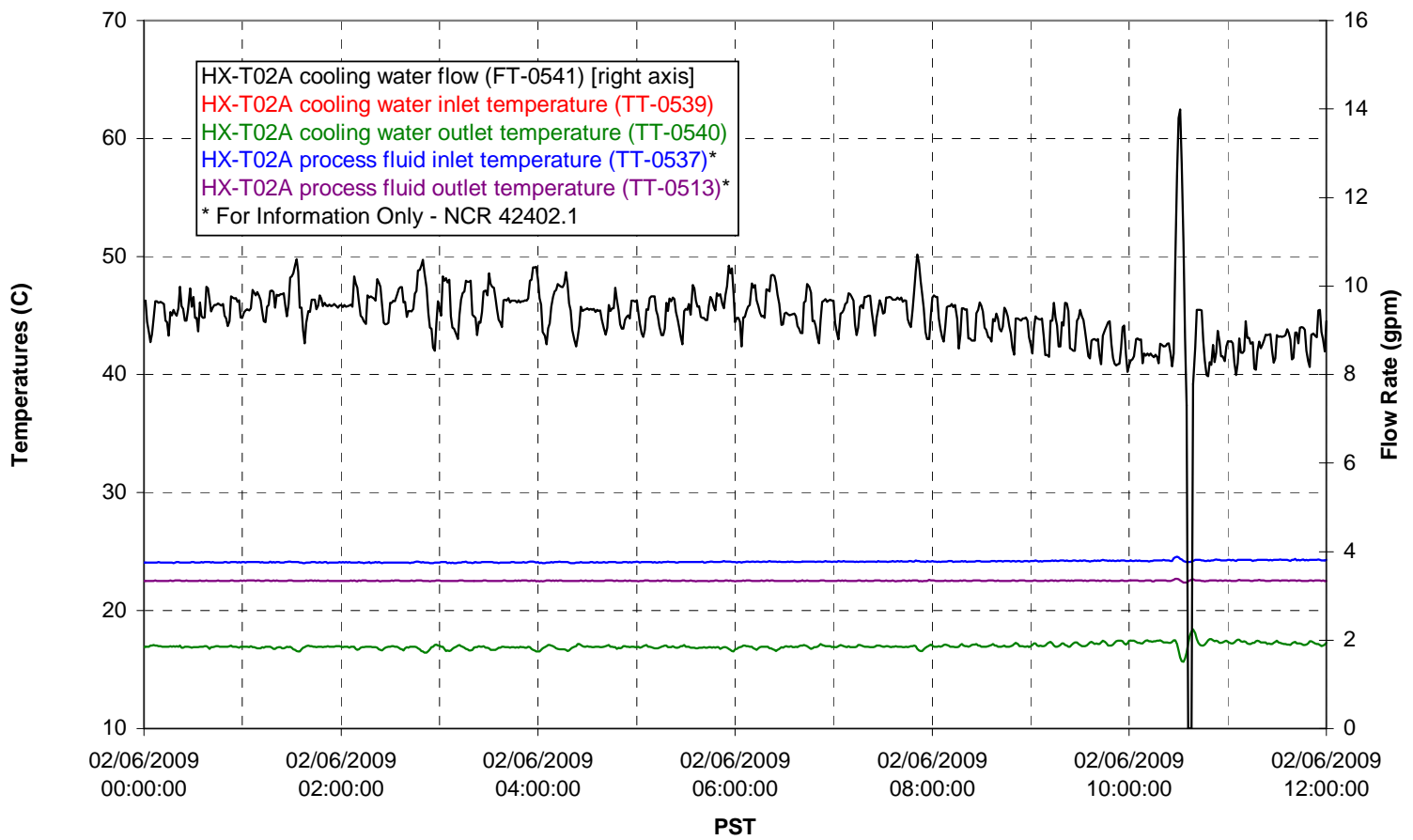

Pump Operation

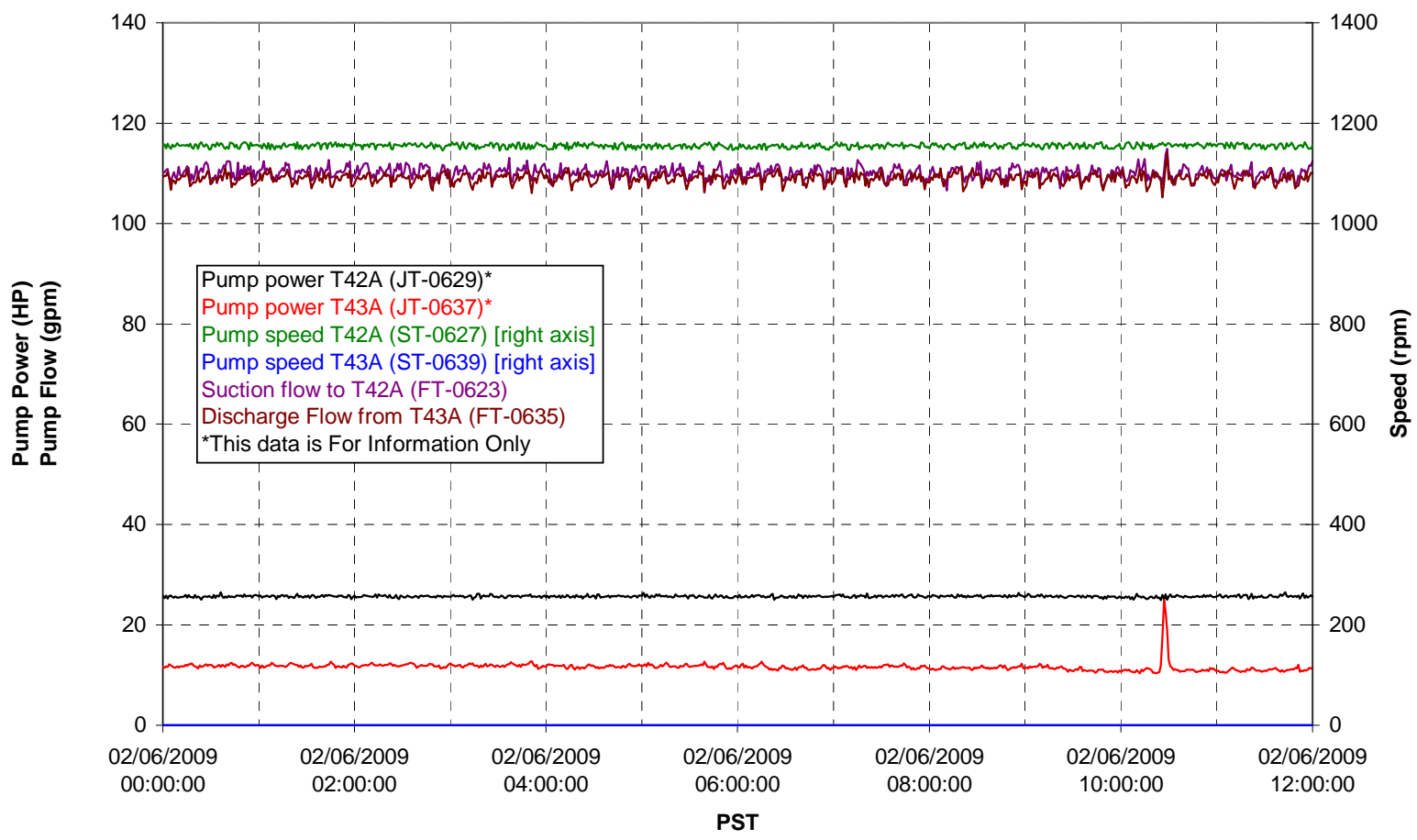

G.226 
Pulsepot UFP-PP-T01A

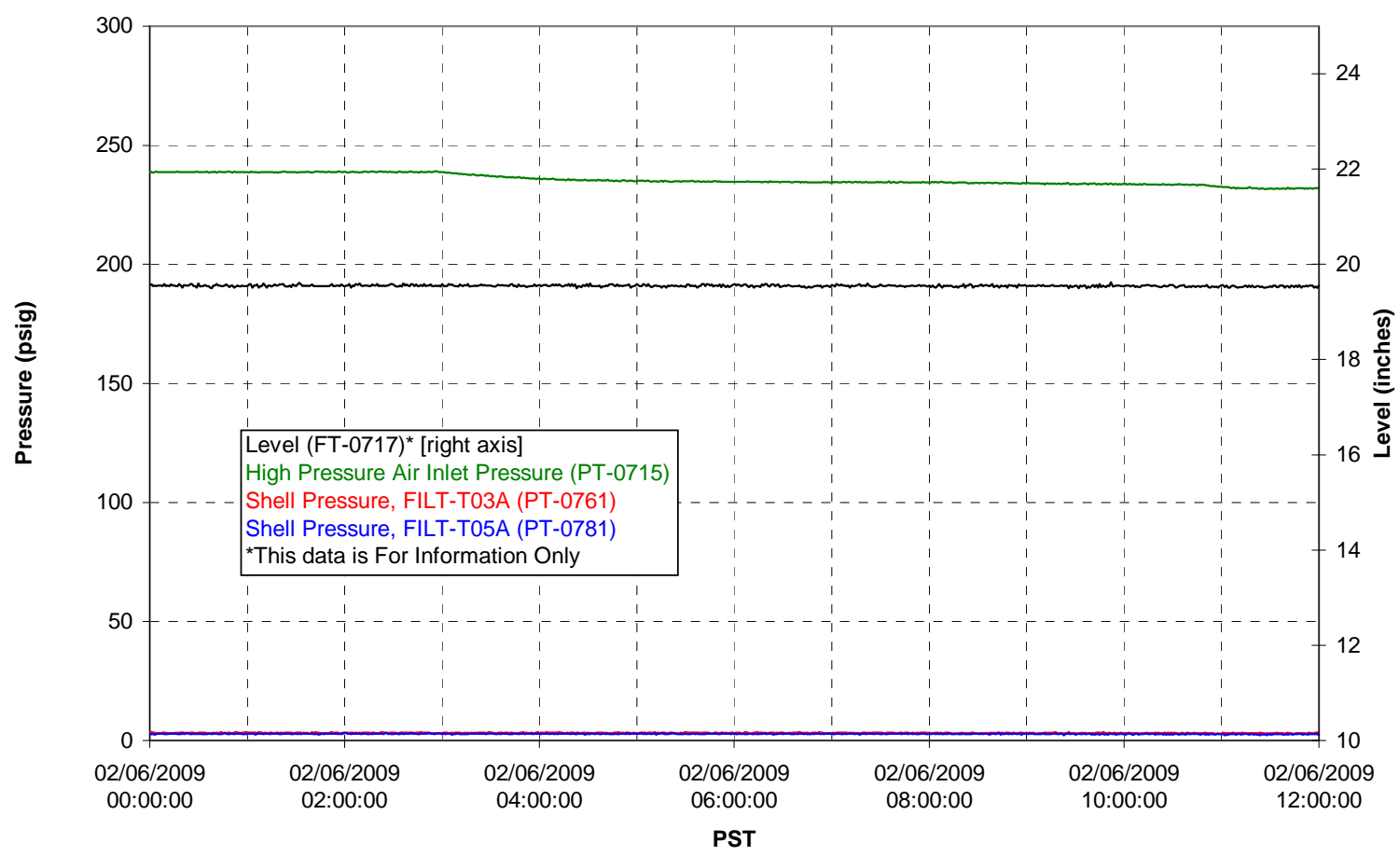

Pulsepot UFP-PP-T02A

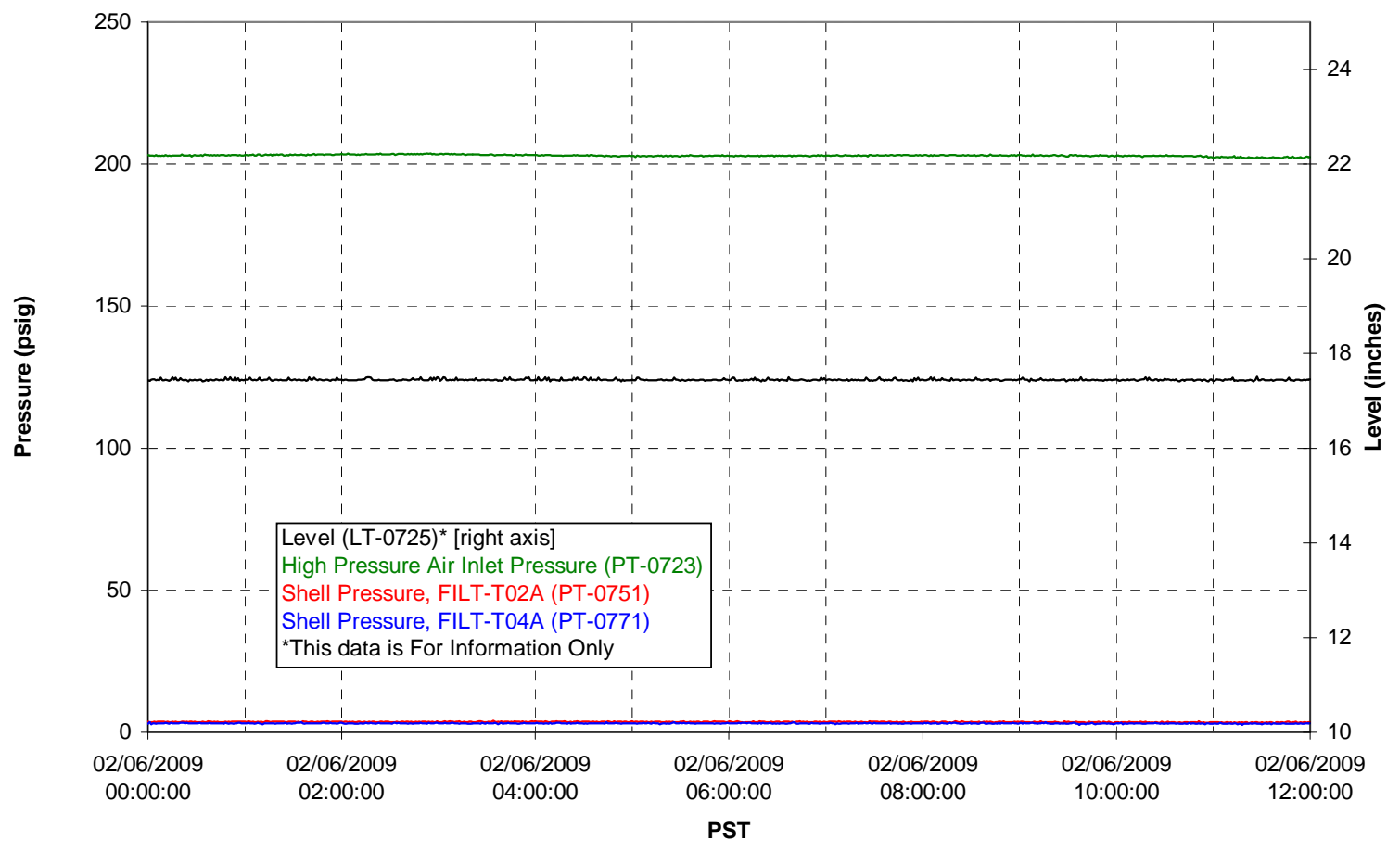


Pulsepot UFP-PP-T03A

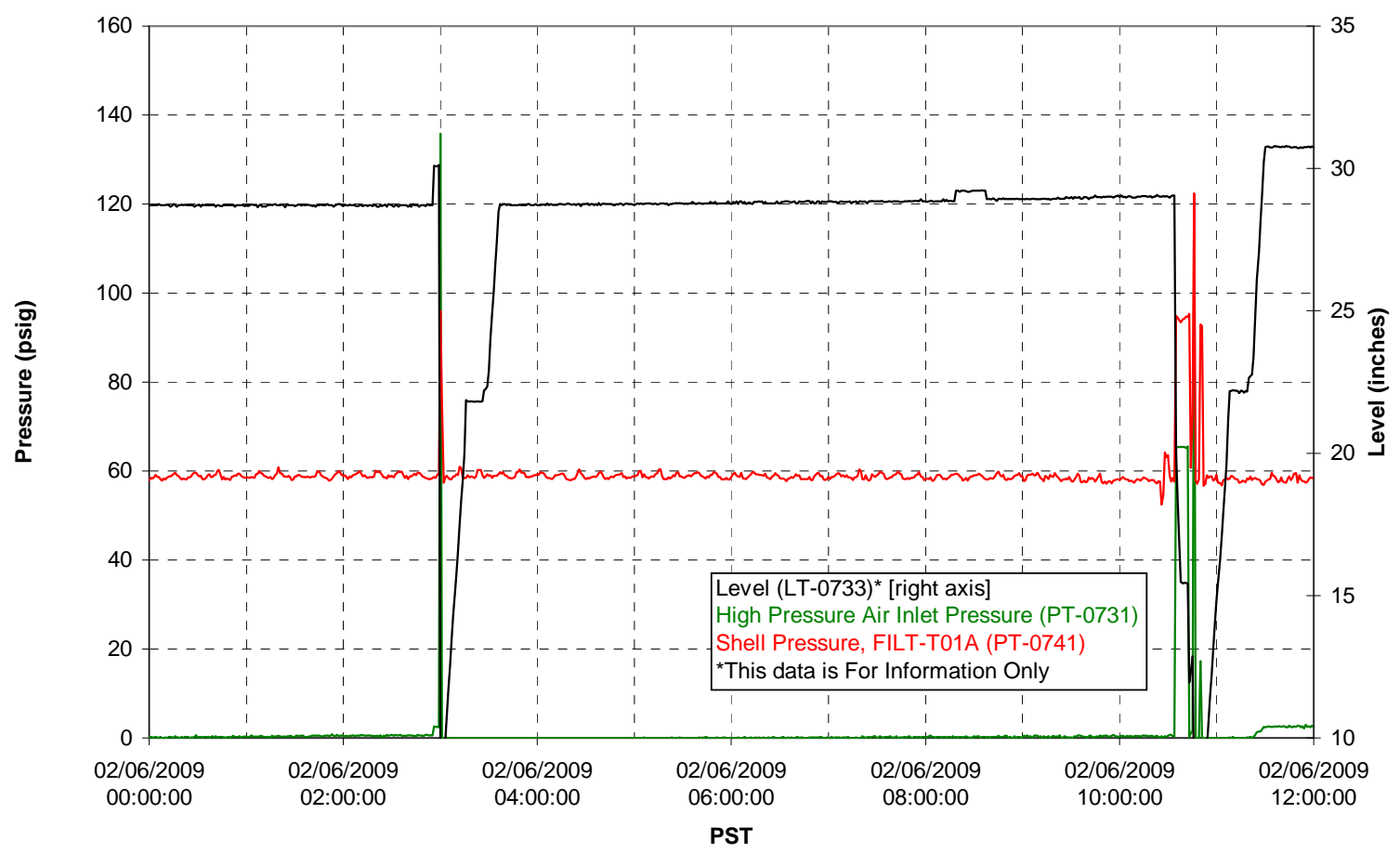

Pulsepot Levels

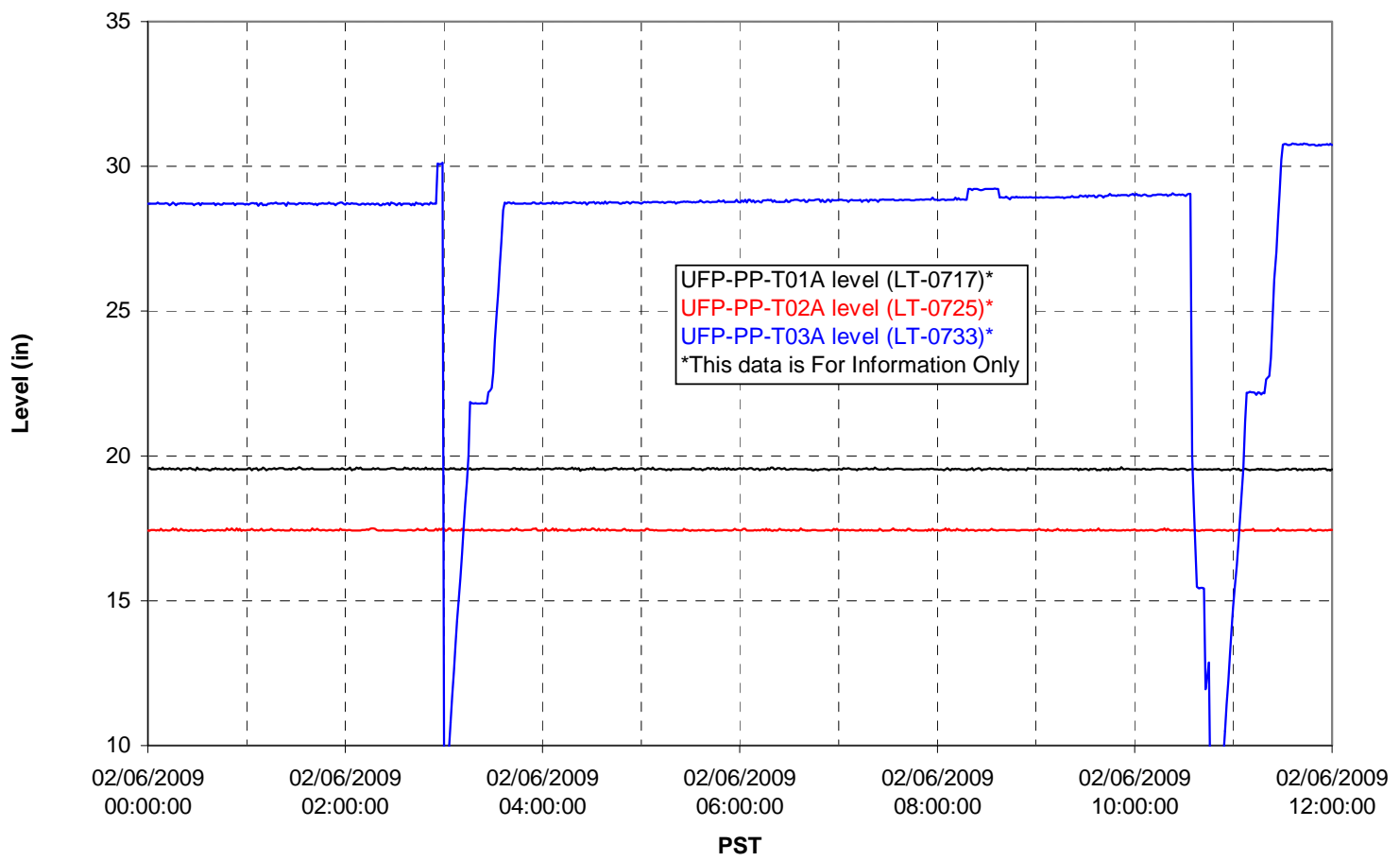


Filter UFP-FILT-T01A

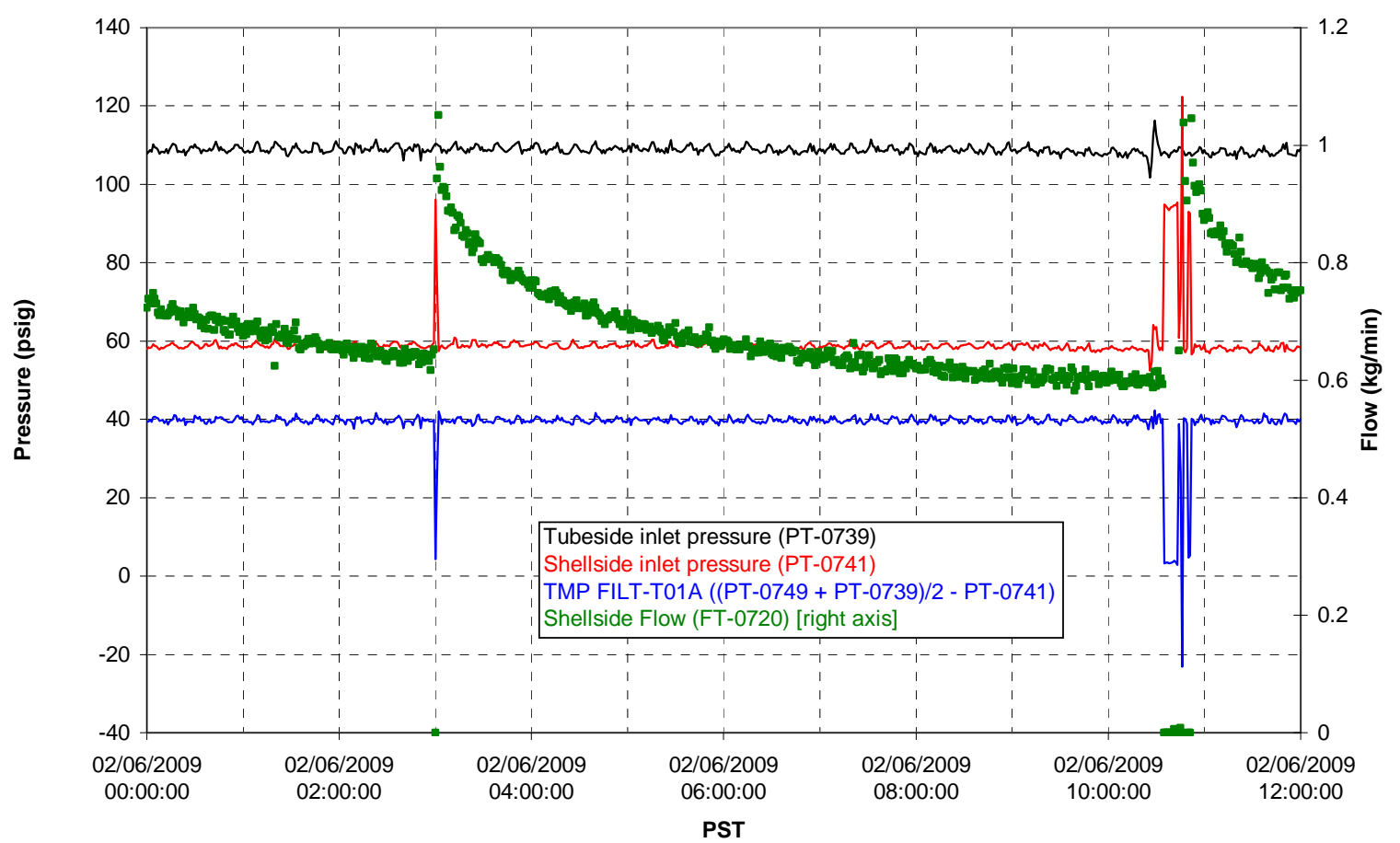

Filter UFP-FILT-T02A

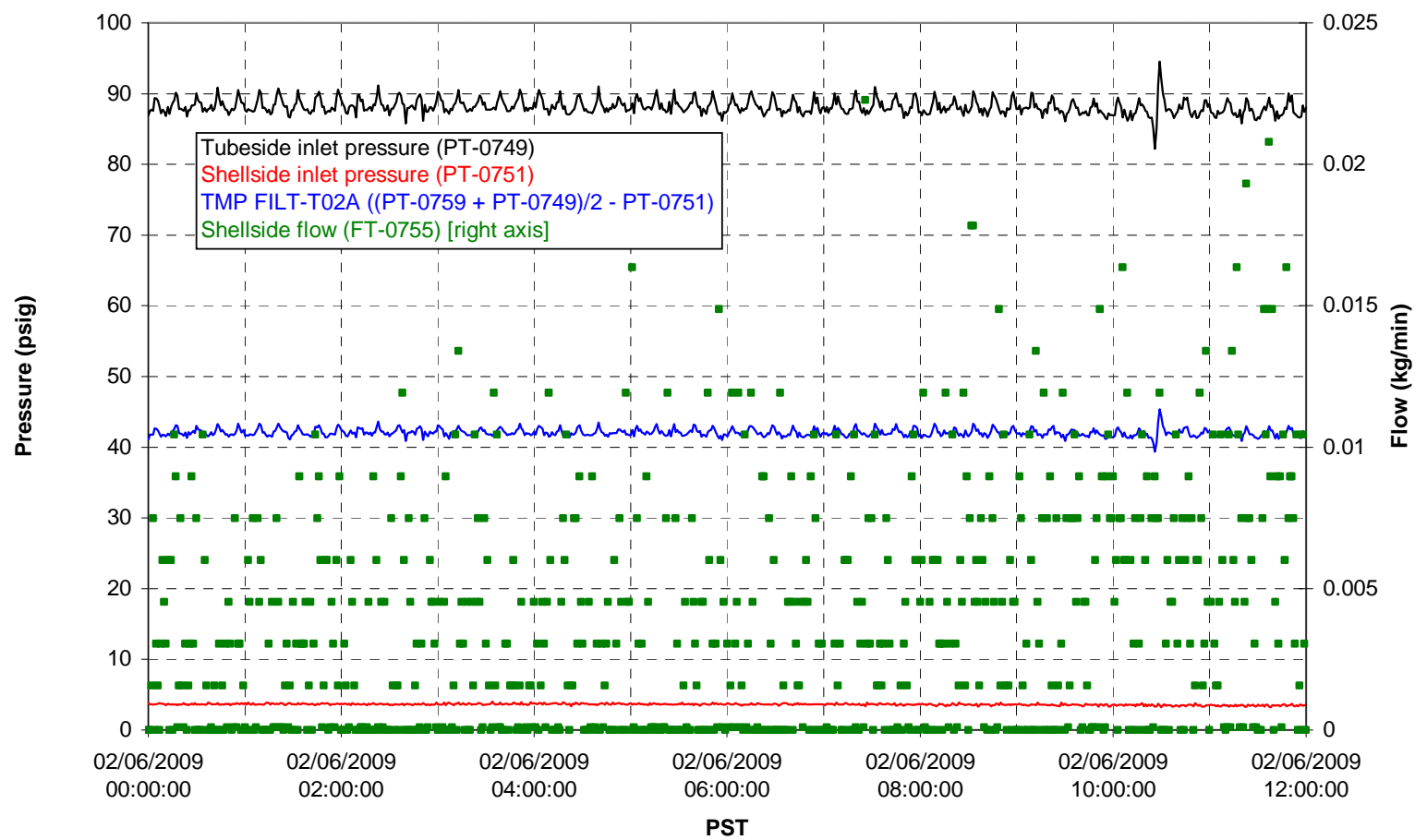


Filter UFP-FILT-T03A

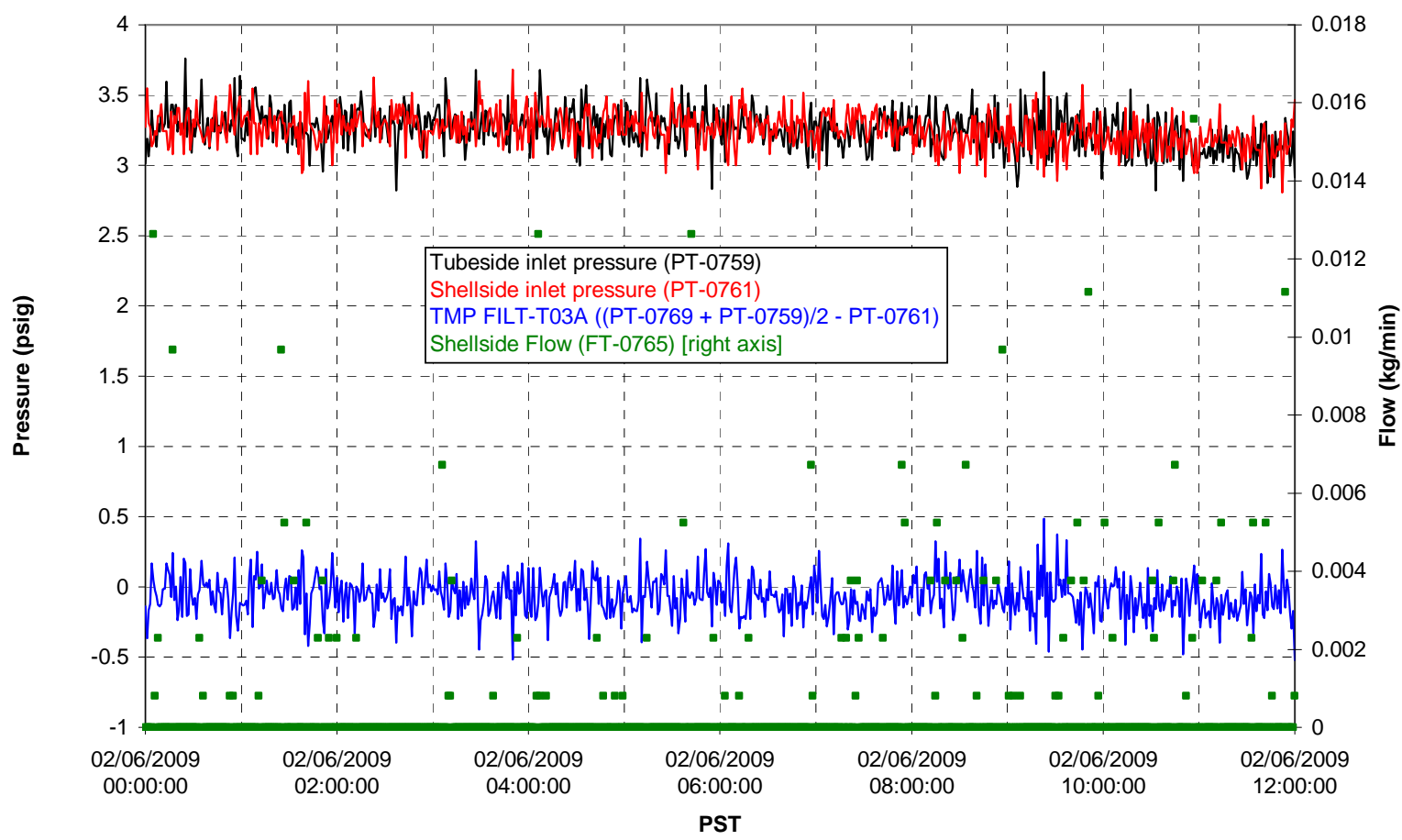

Filter UFP-FILT-T04A

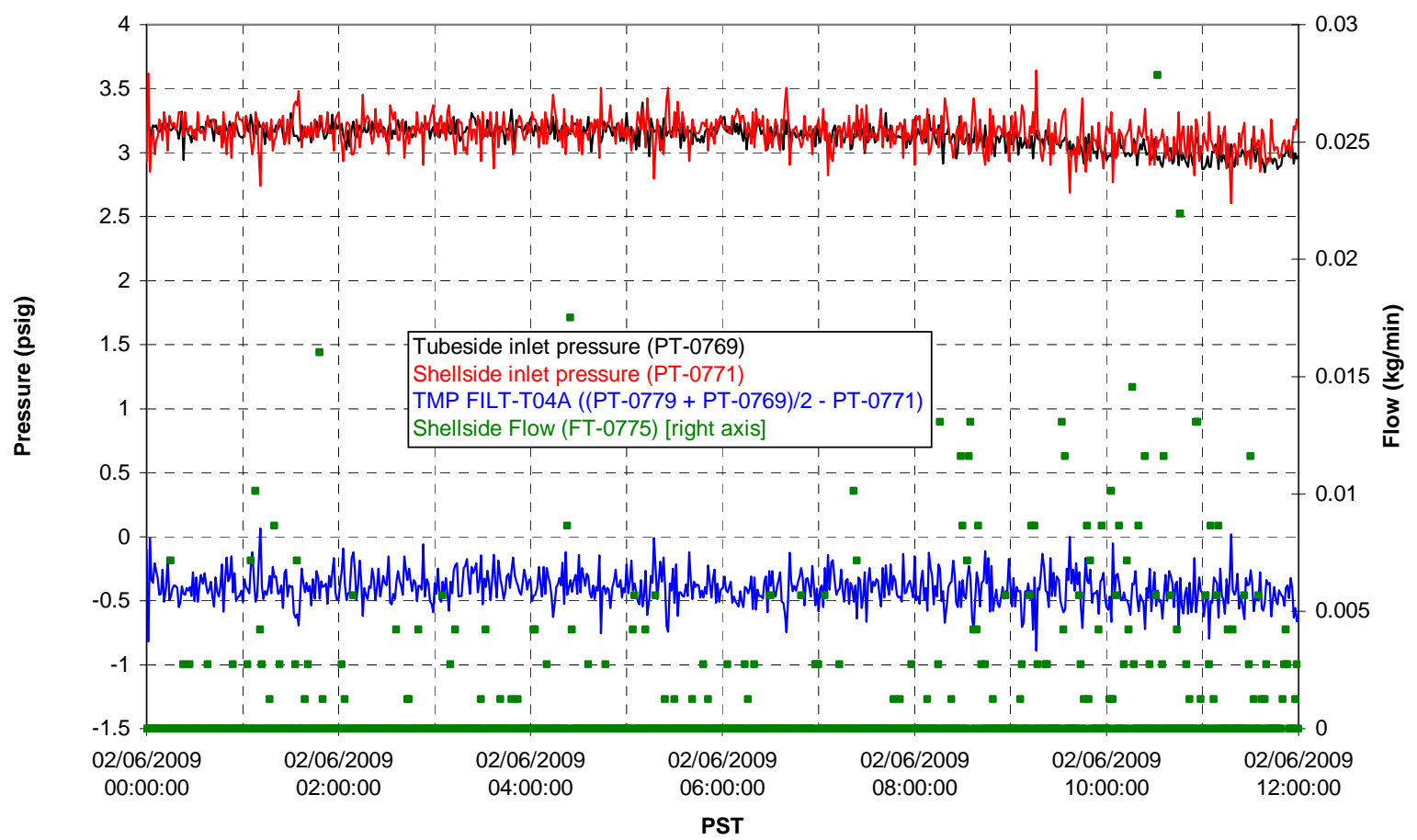


Filter UFP-FILT-T05A

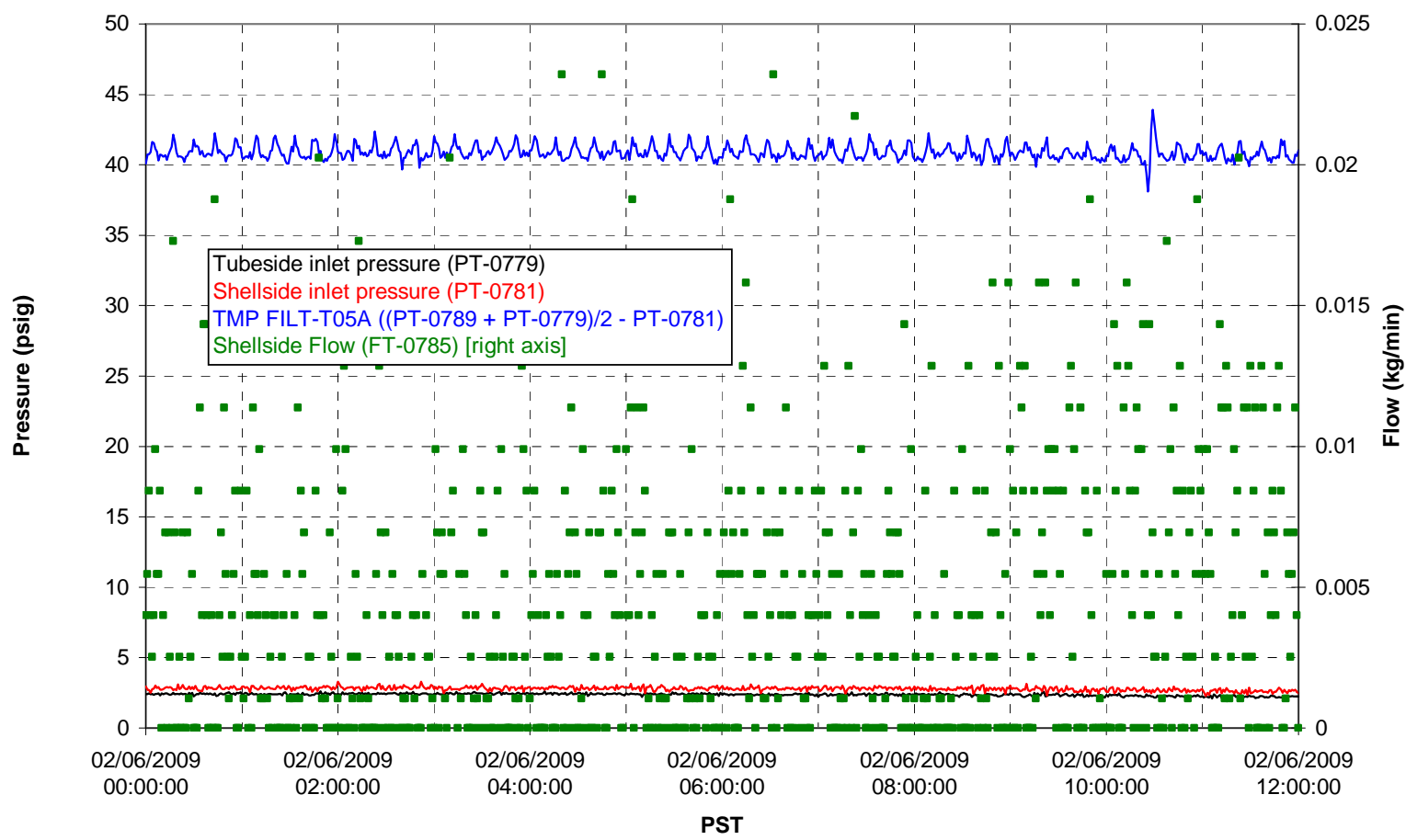

Chemical Flow

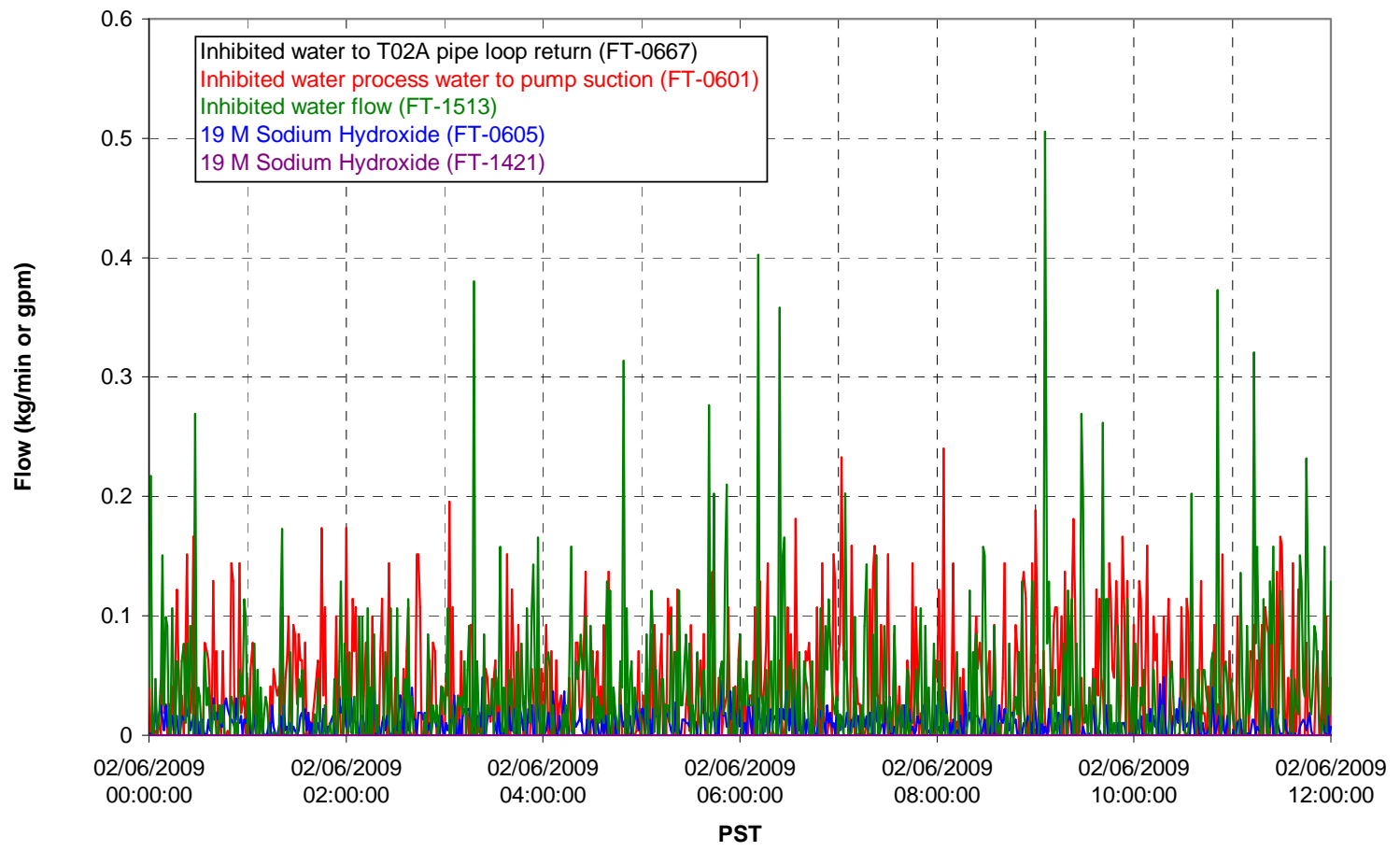


Chemical Flow

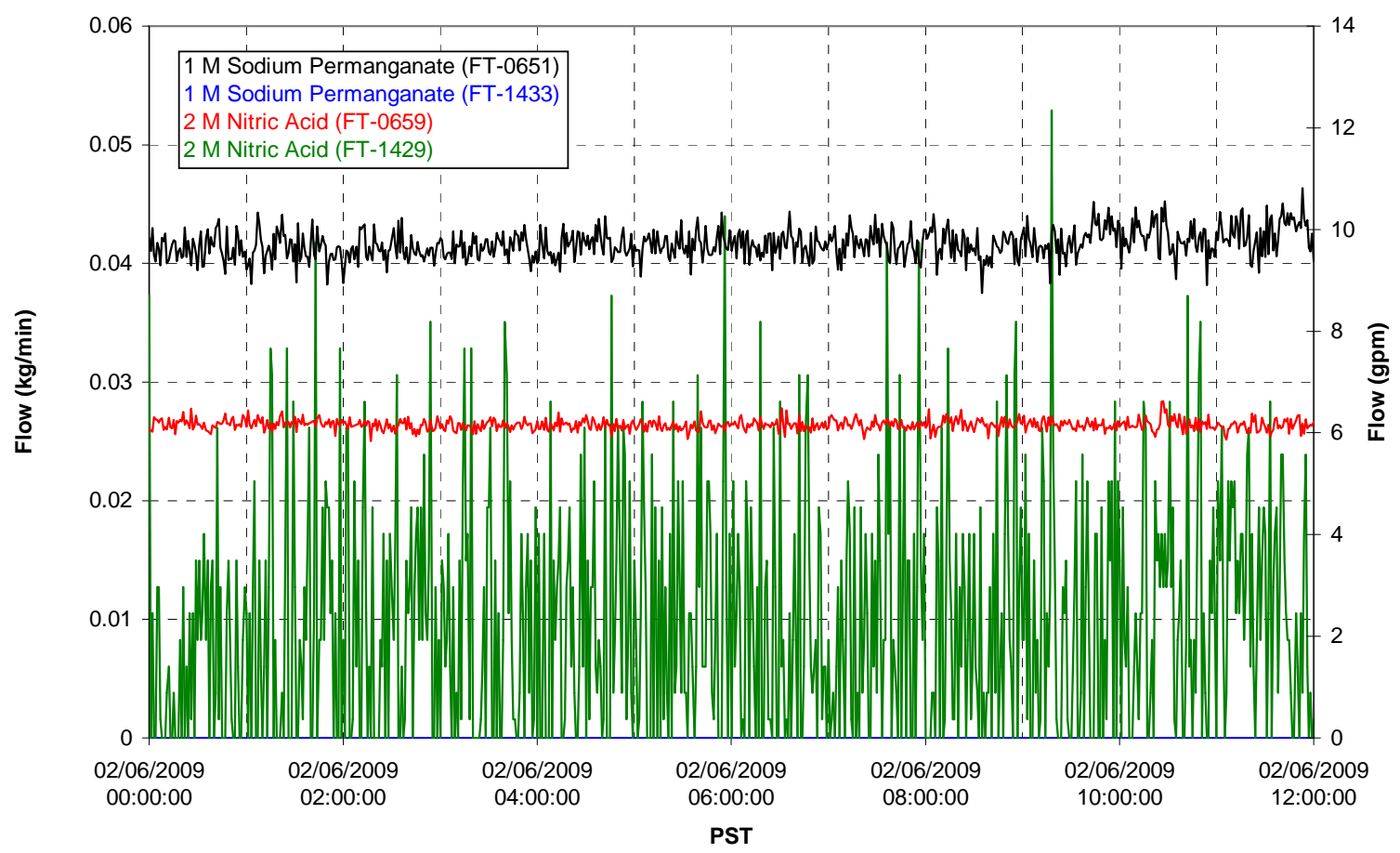

Air Flows

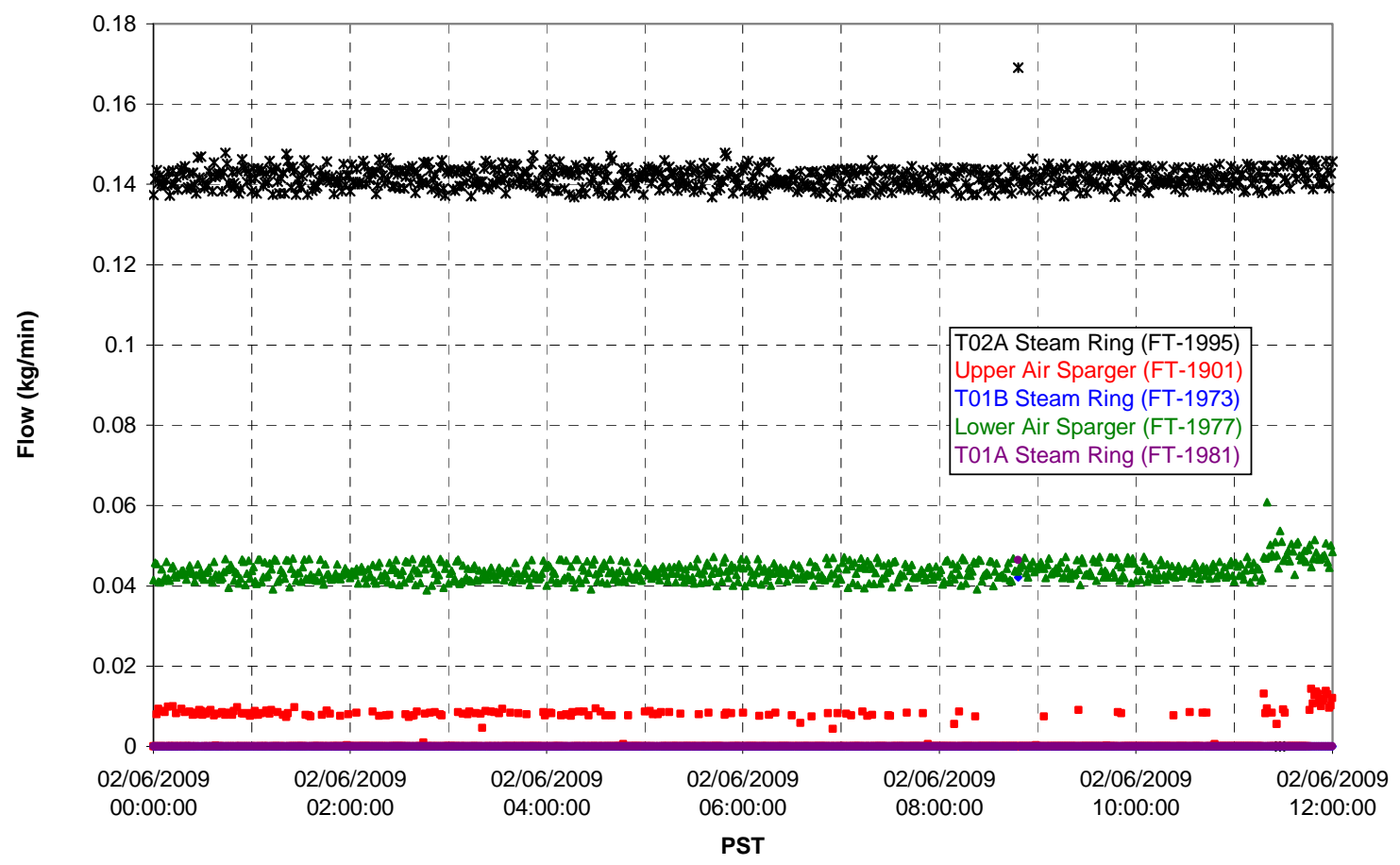


T02A Steam

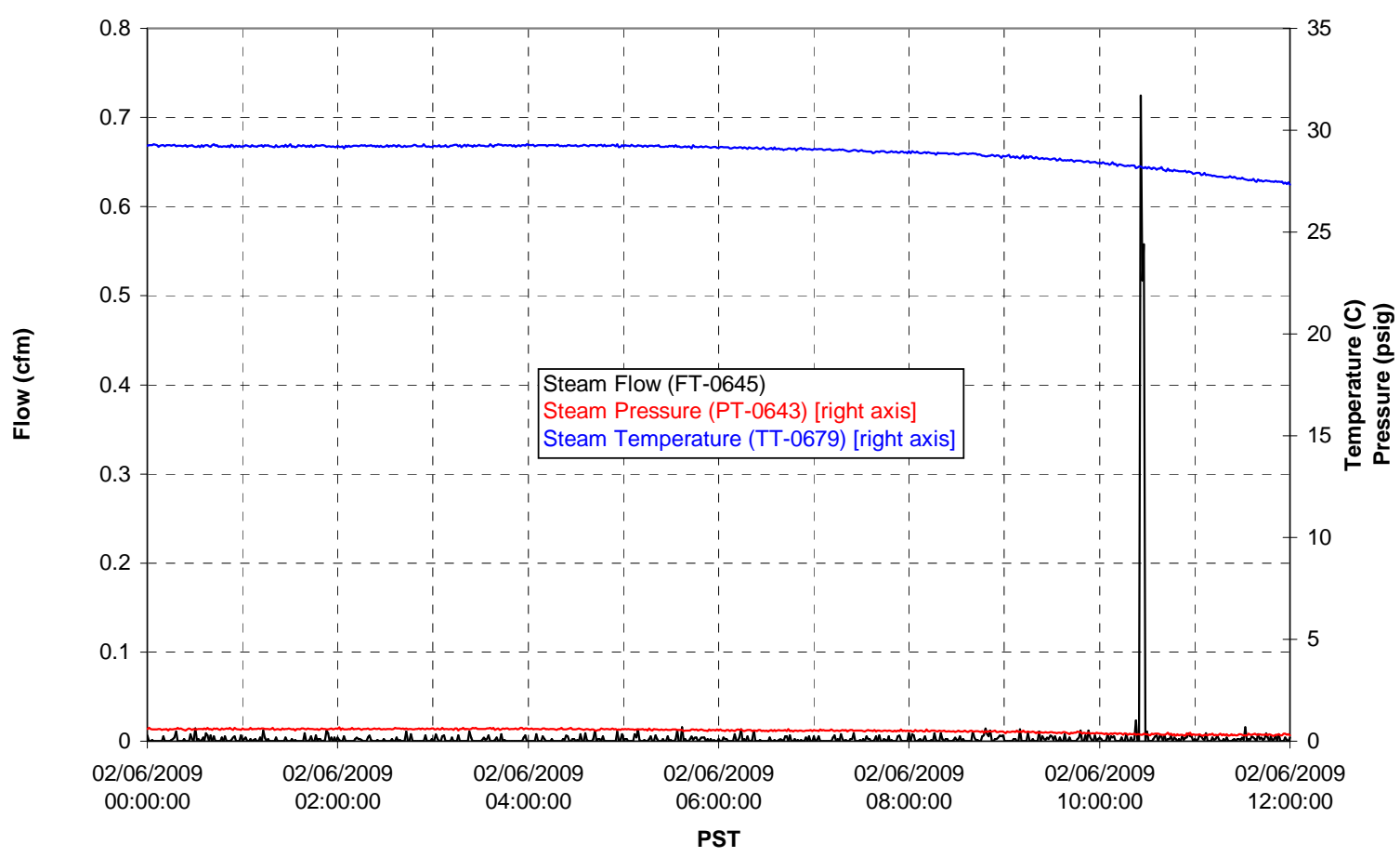

T01A Steam

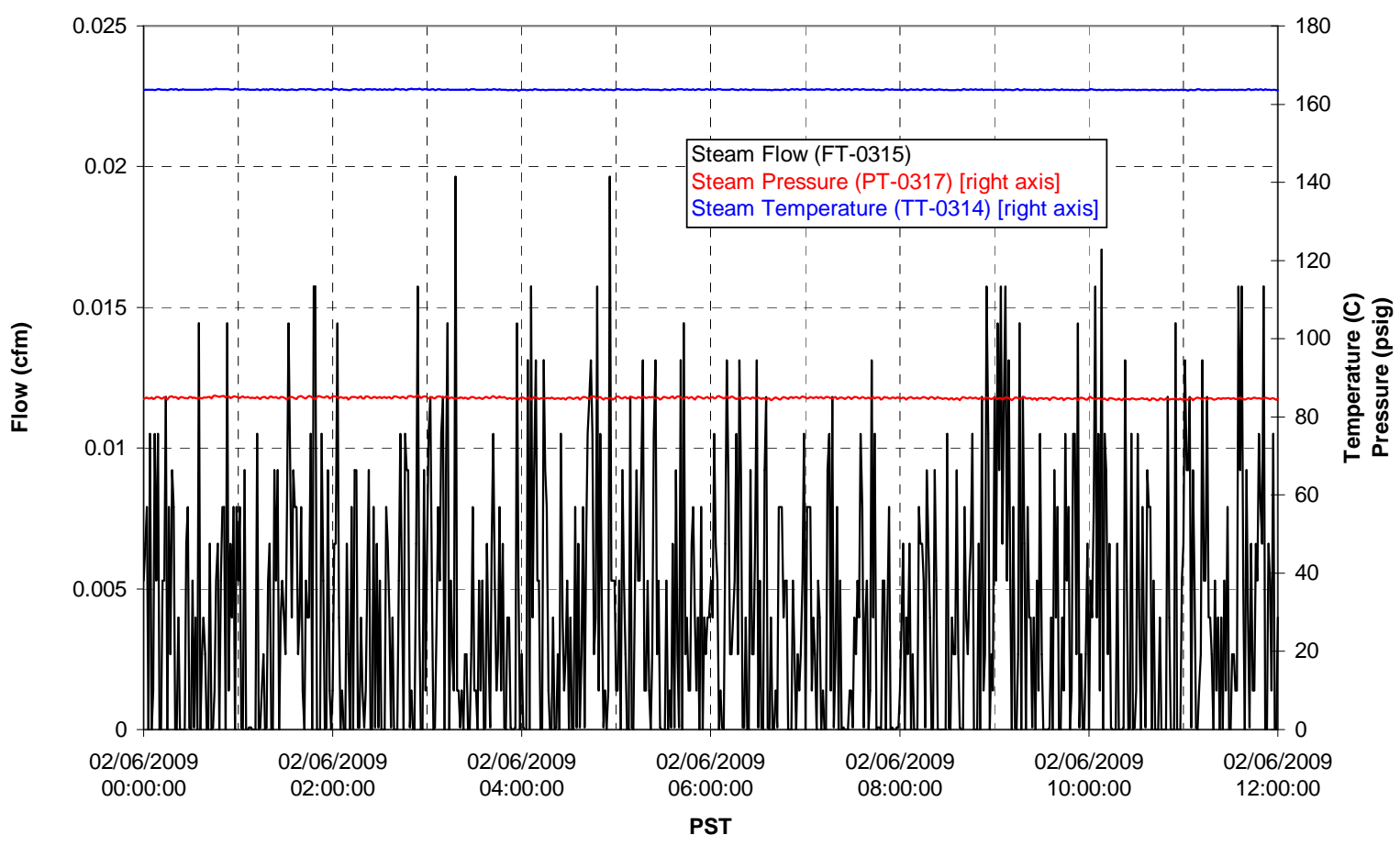


T01B Steam

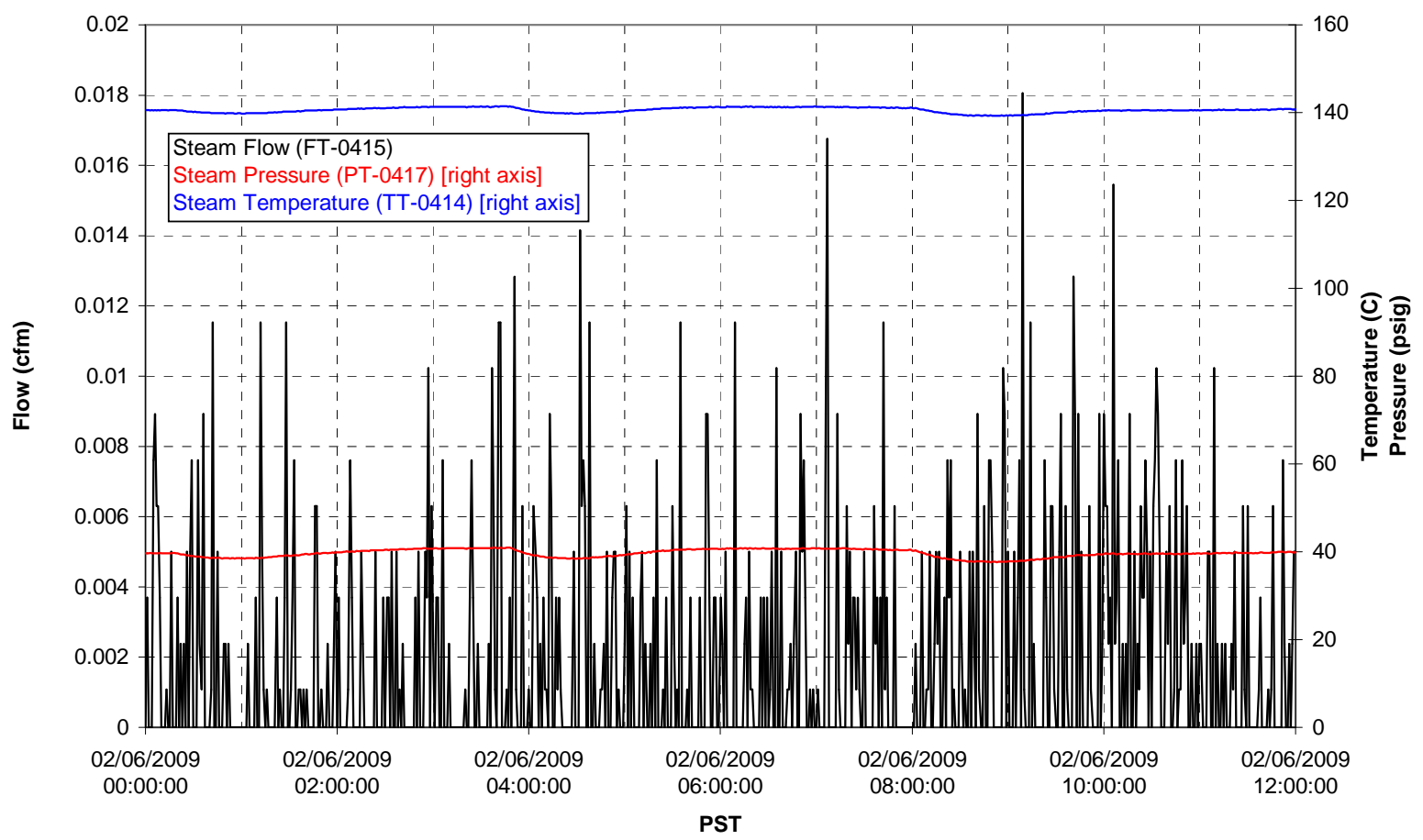




\section{Integrated Test A Data Plots 02/06/09 12:00 - 02/07/09 00:00}


T01A level

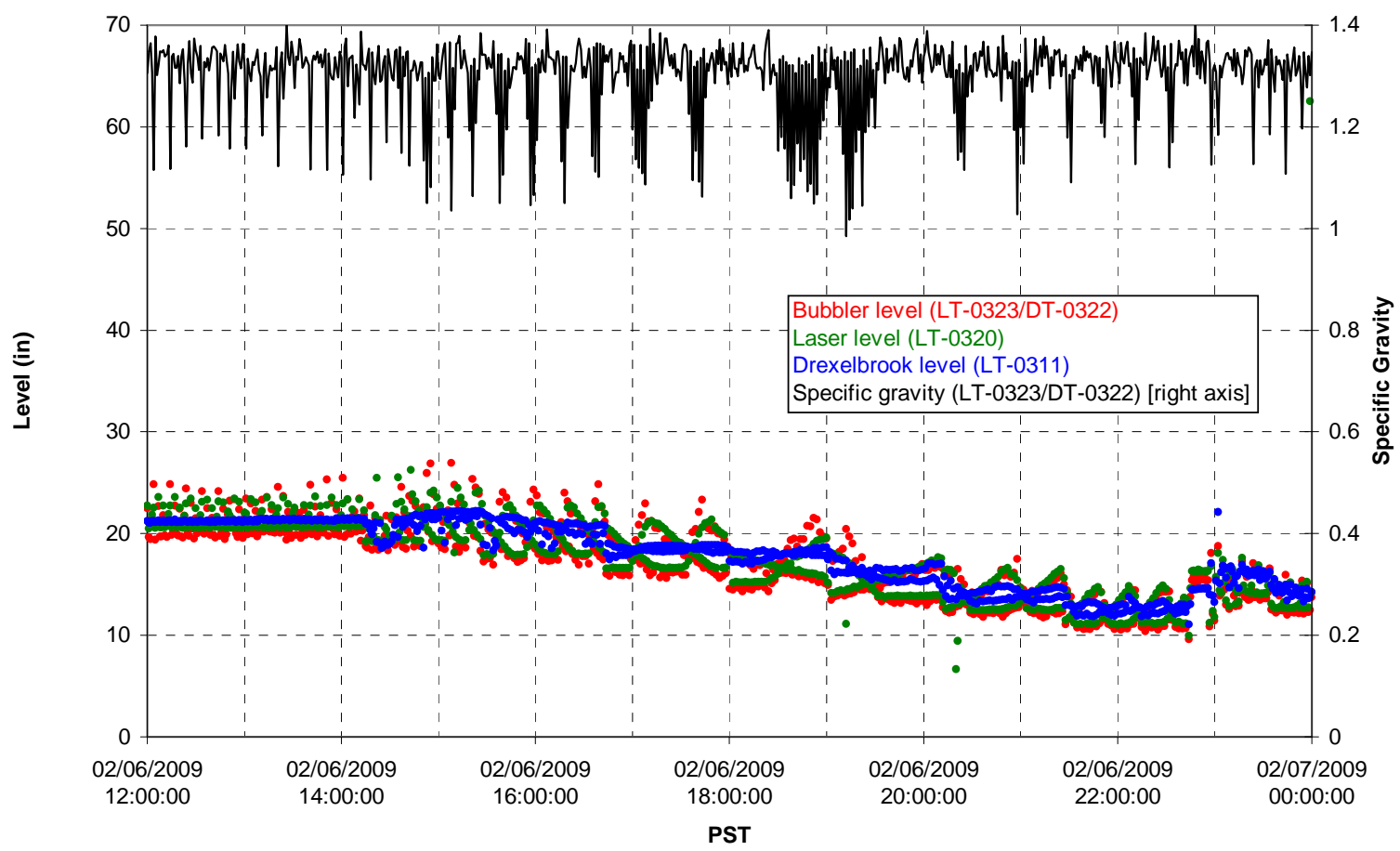

T01A temperatures

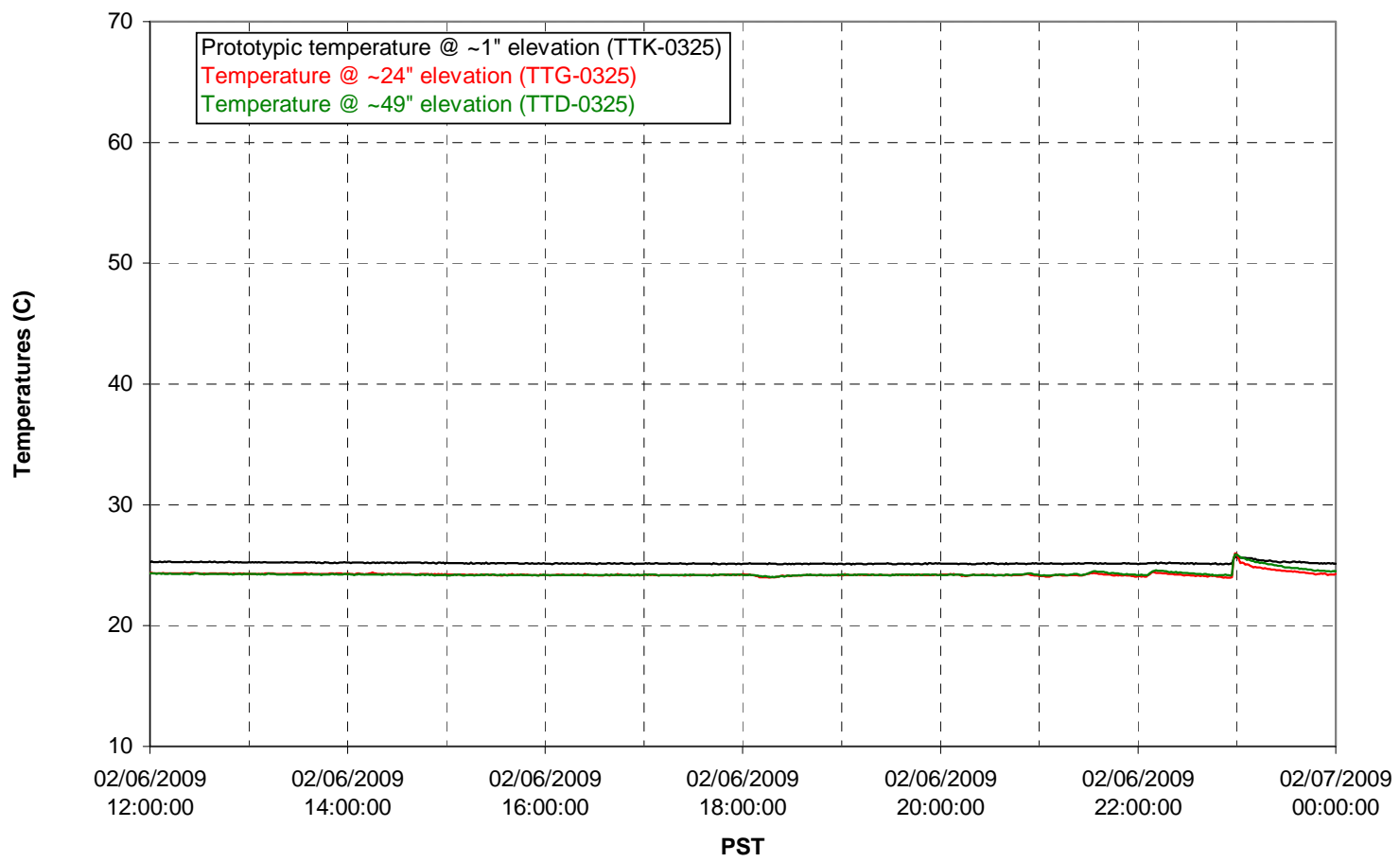


T01B level

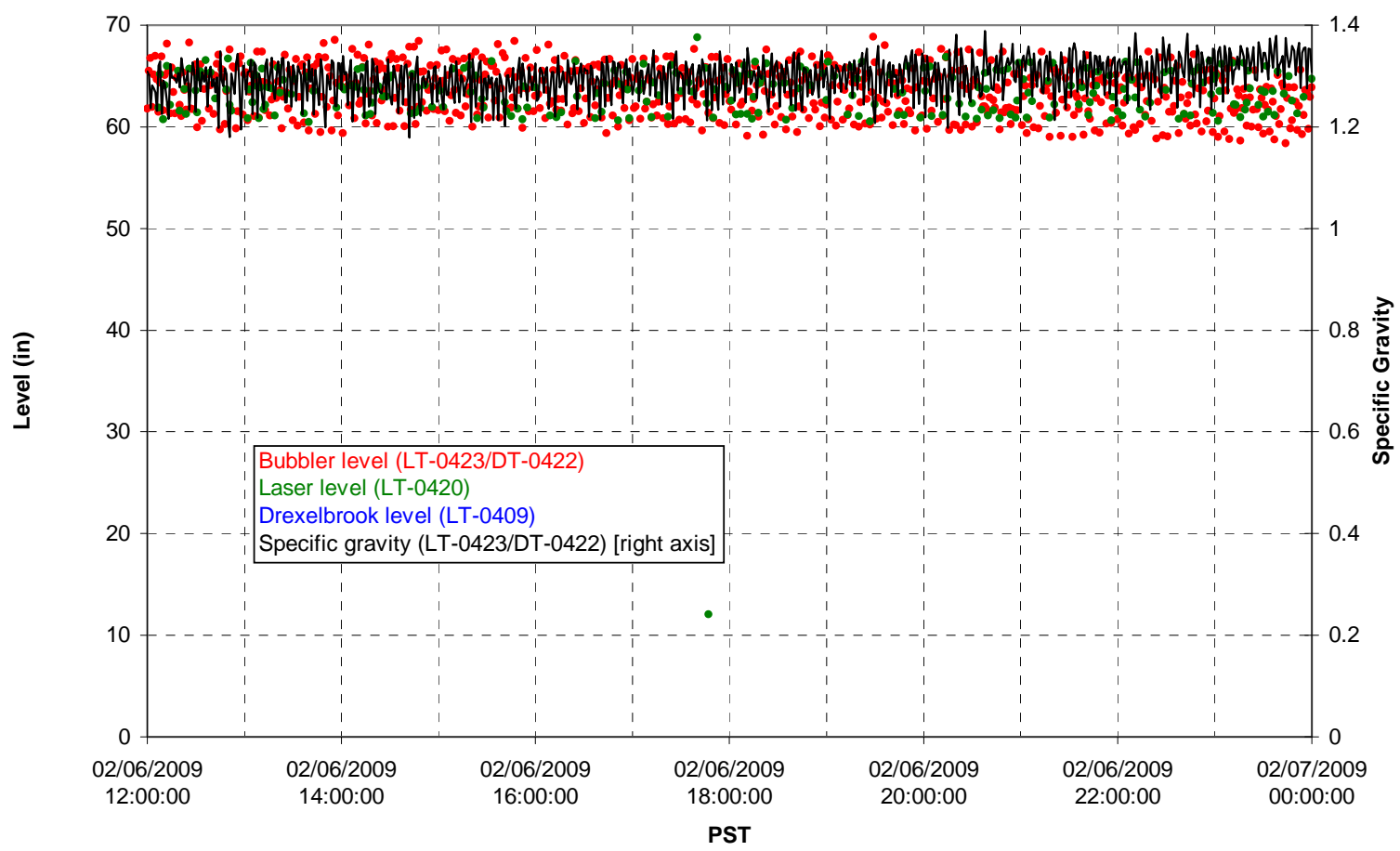

T01B temperatures

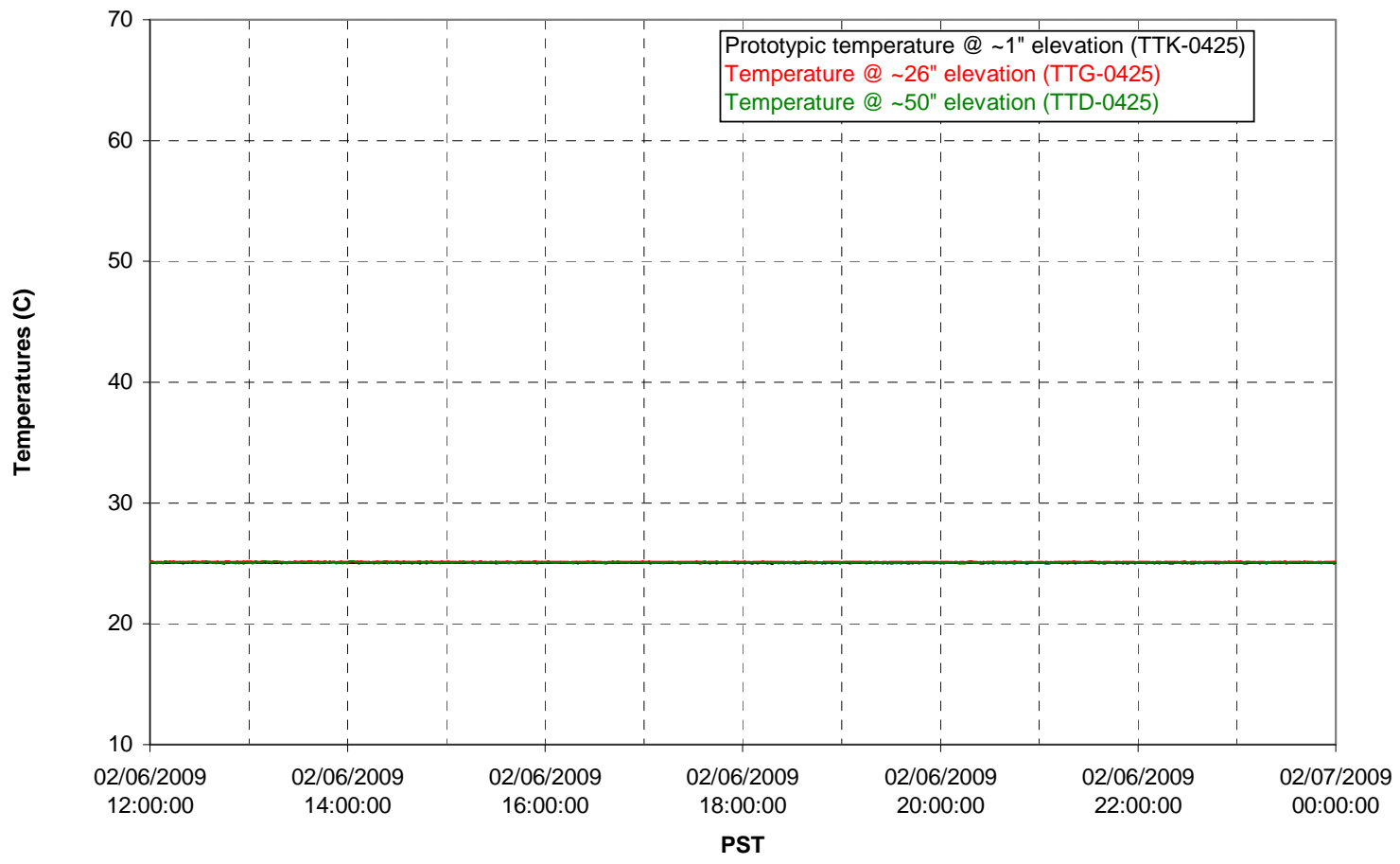


T02A level

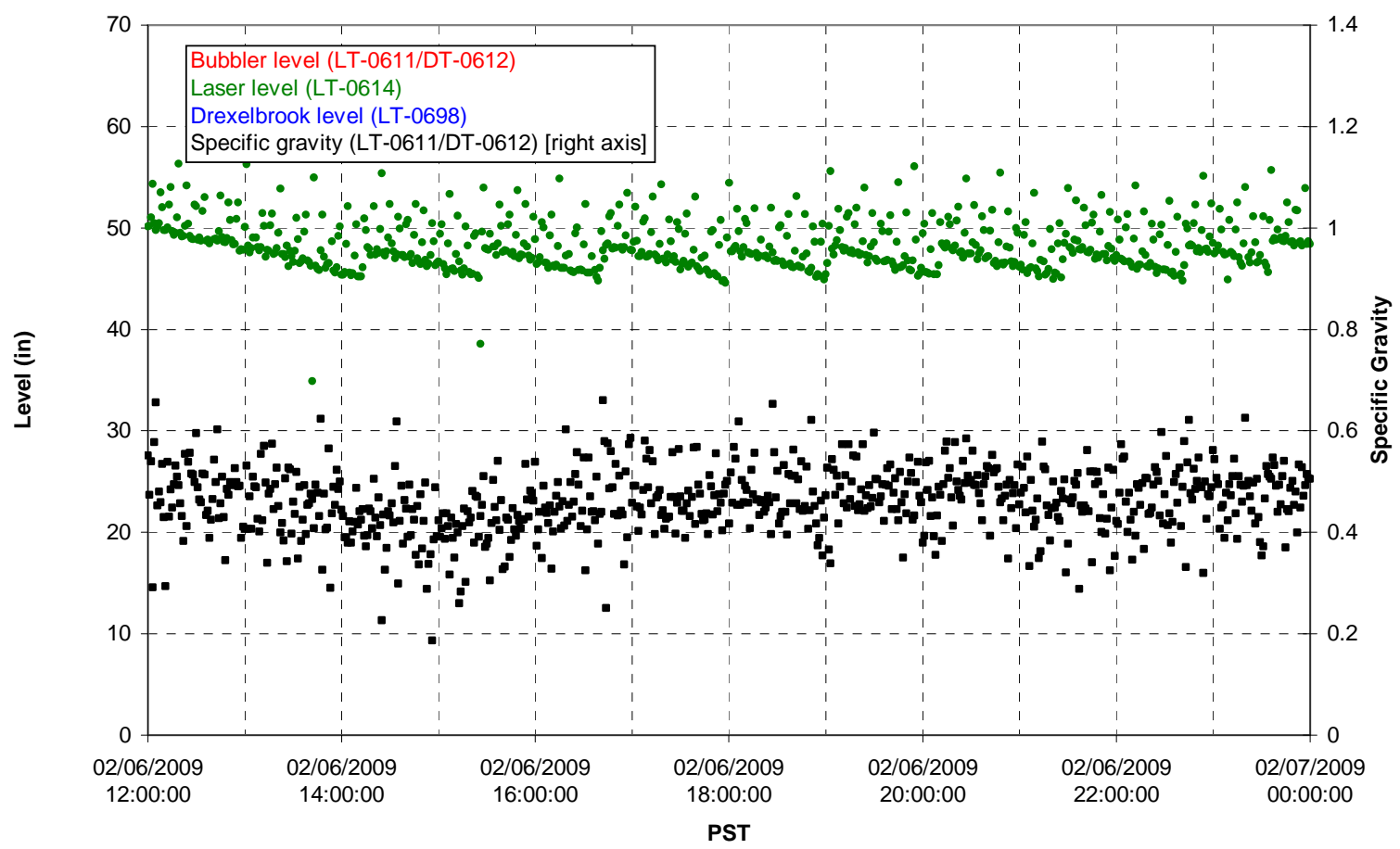

T02A temperatures

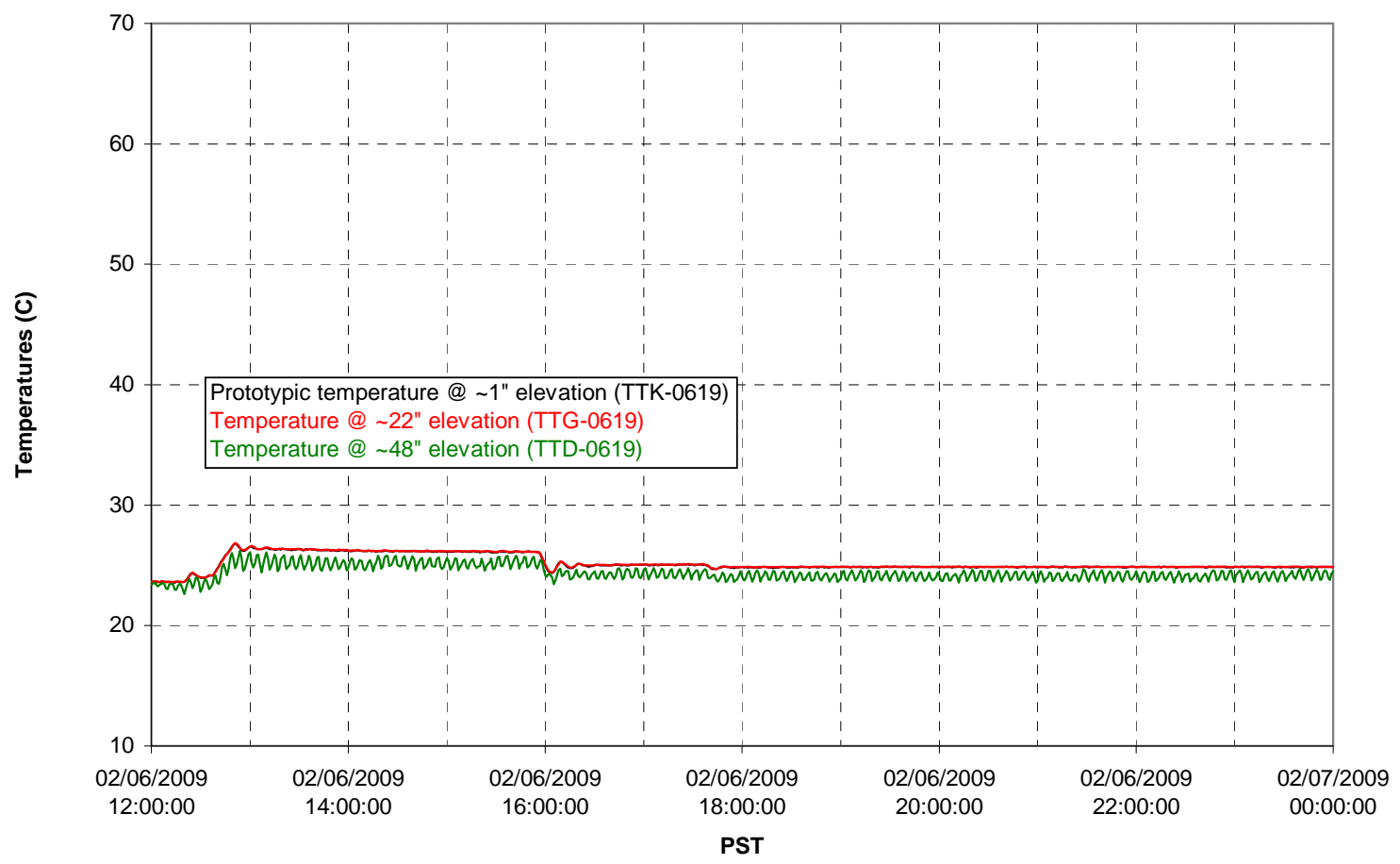


T02A and filter loop temperatures

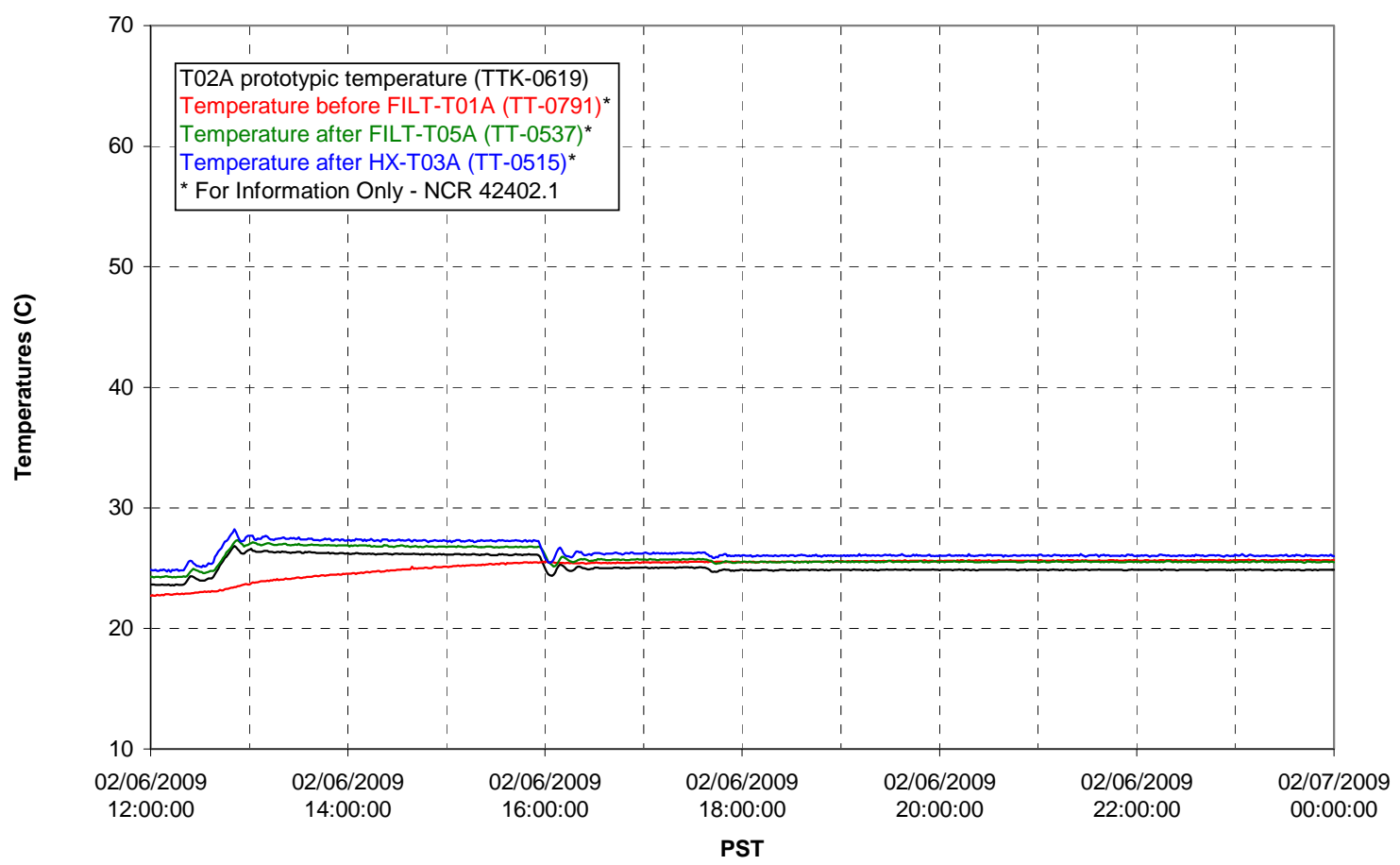

Pump Pressures and Flow

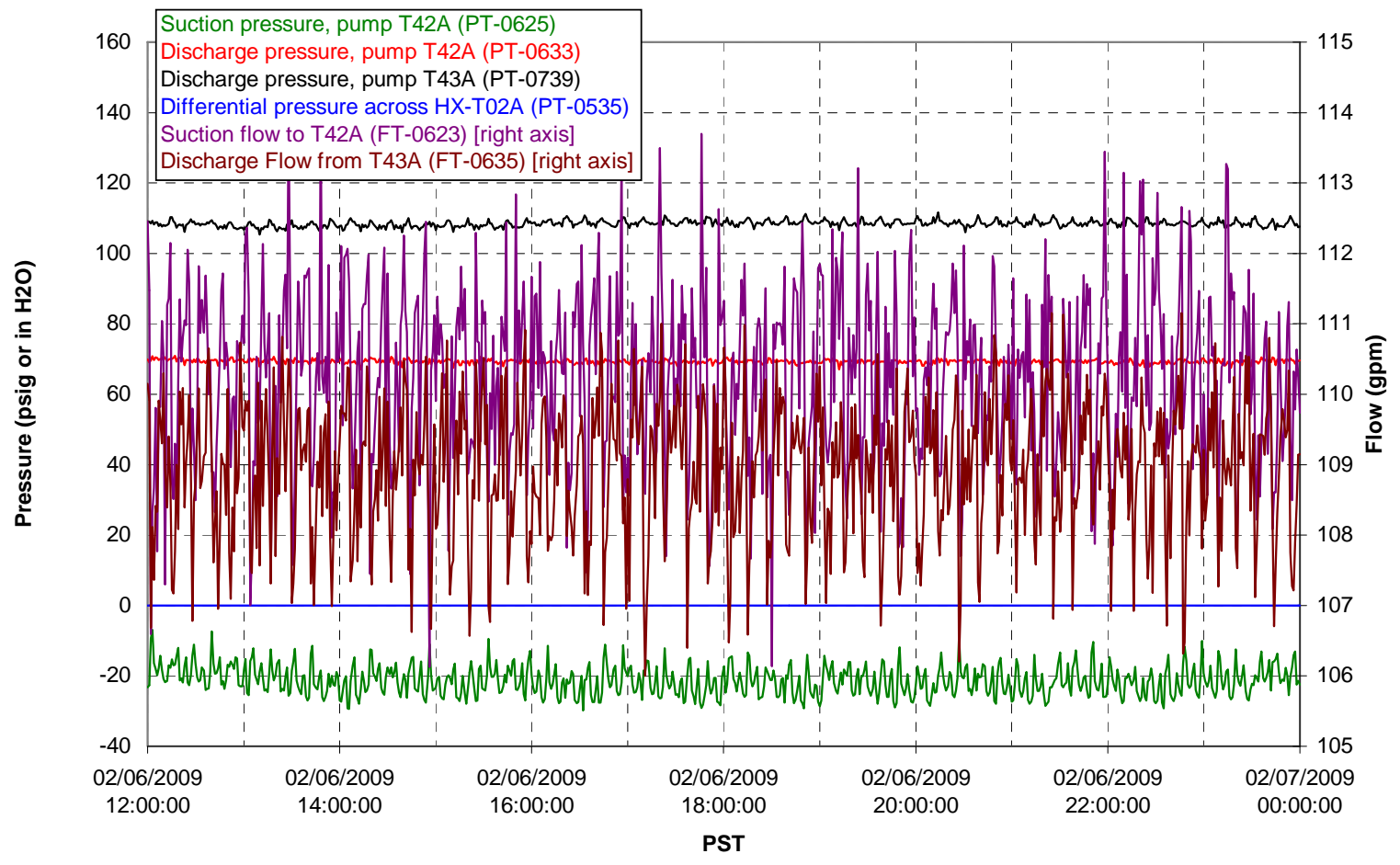




\section{Axial pressure drop}

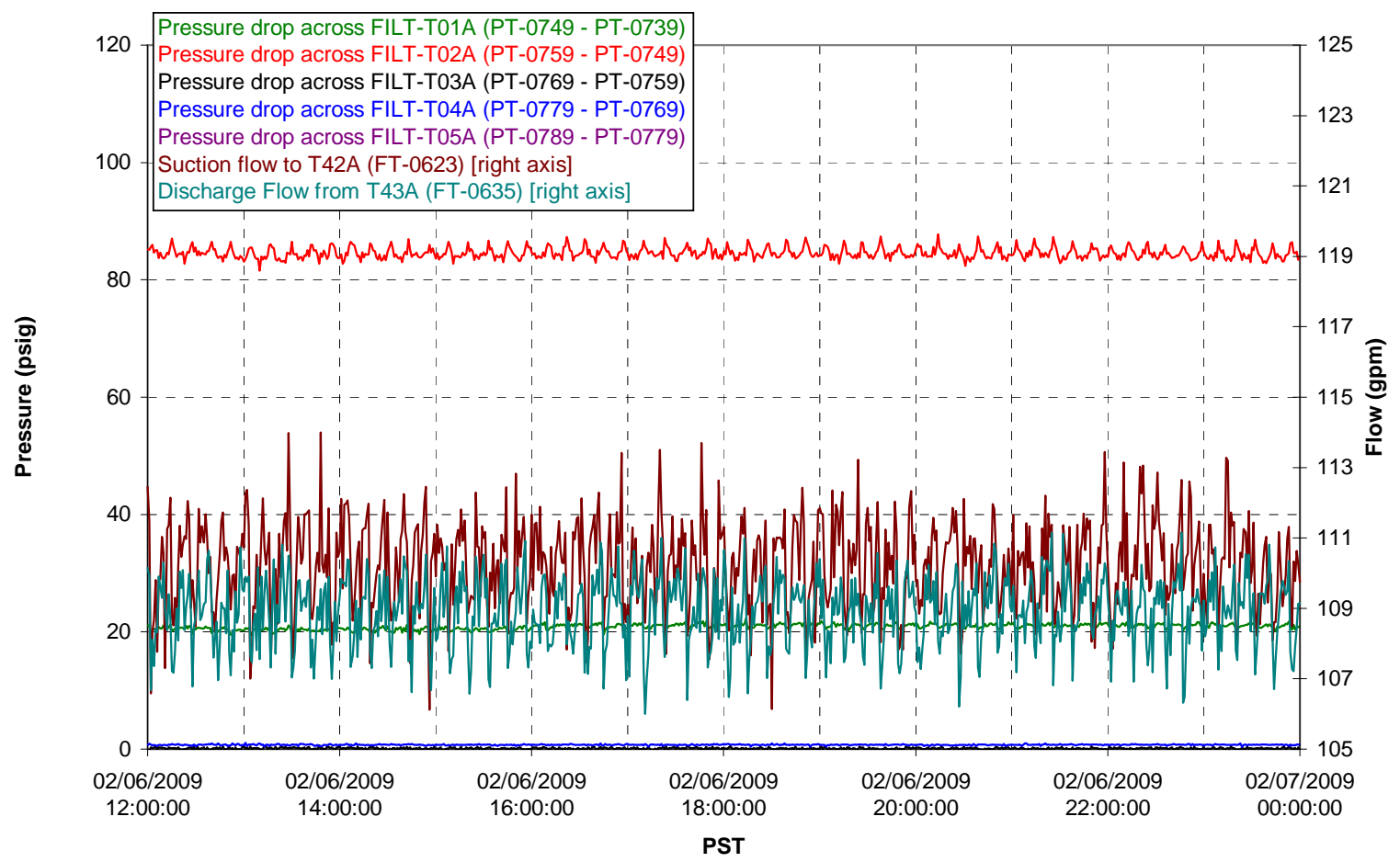

Permeate flow rates

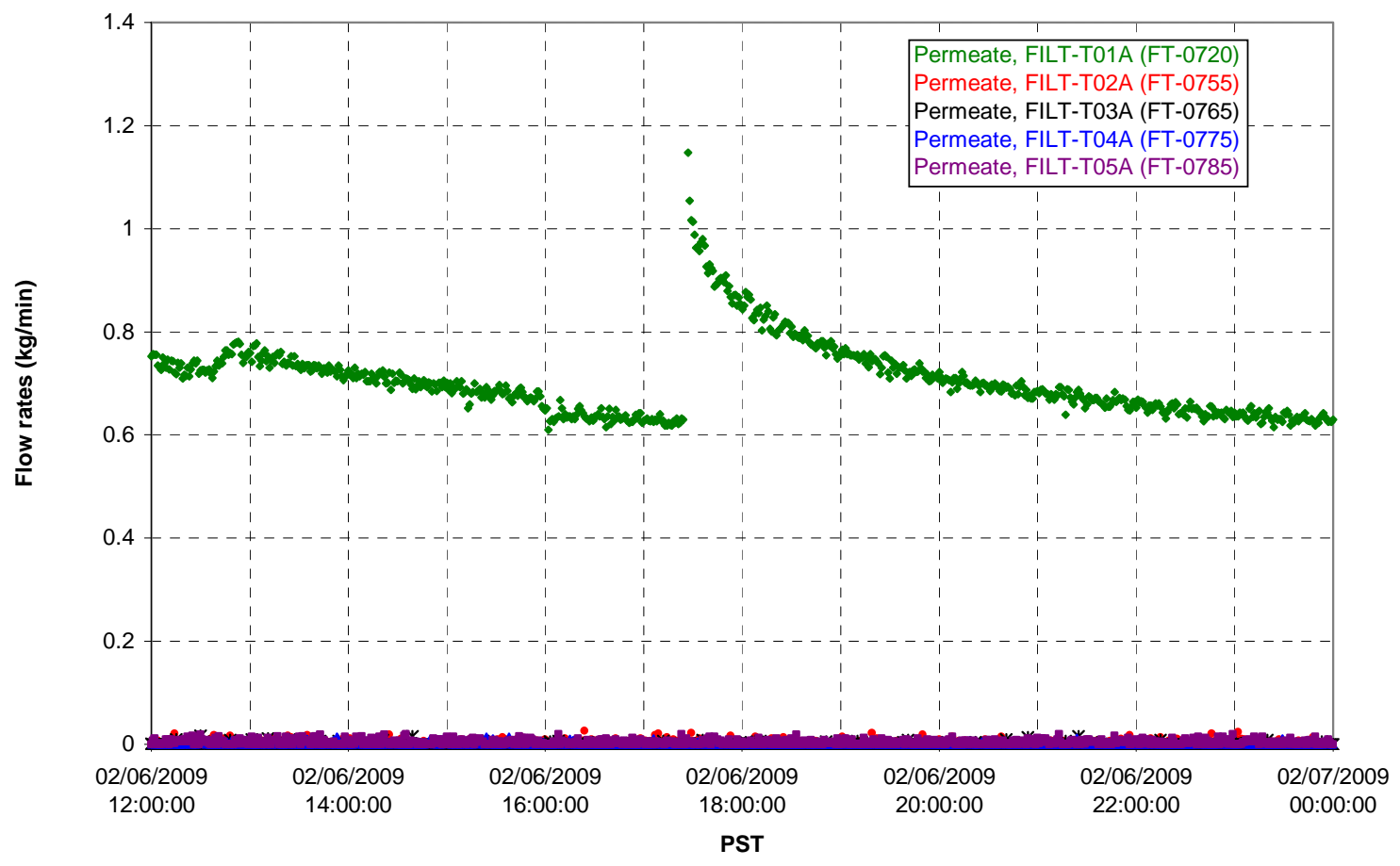


T02A Inner Temperature Tree

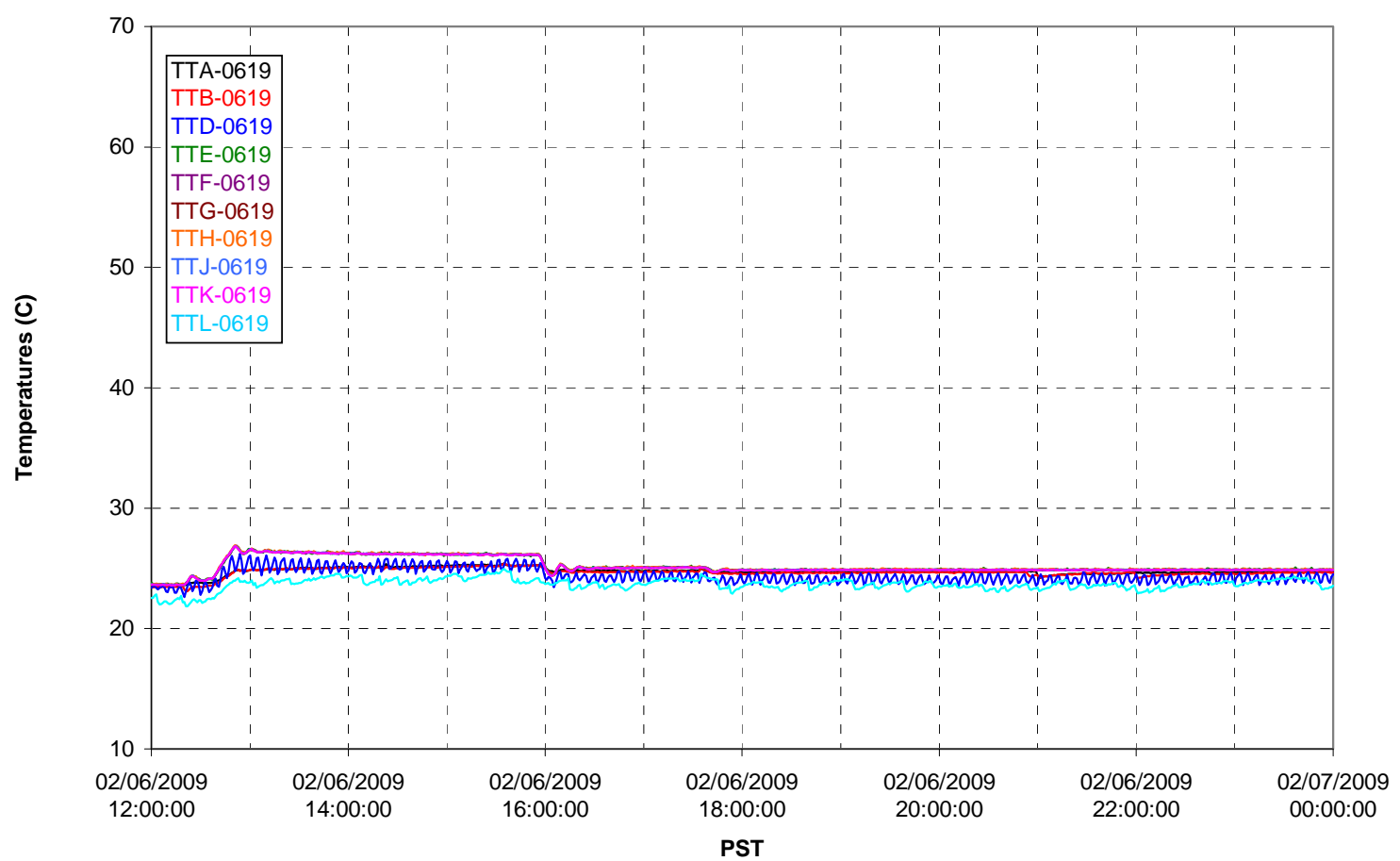

T02A Outer Temperature Tree

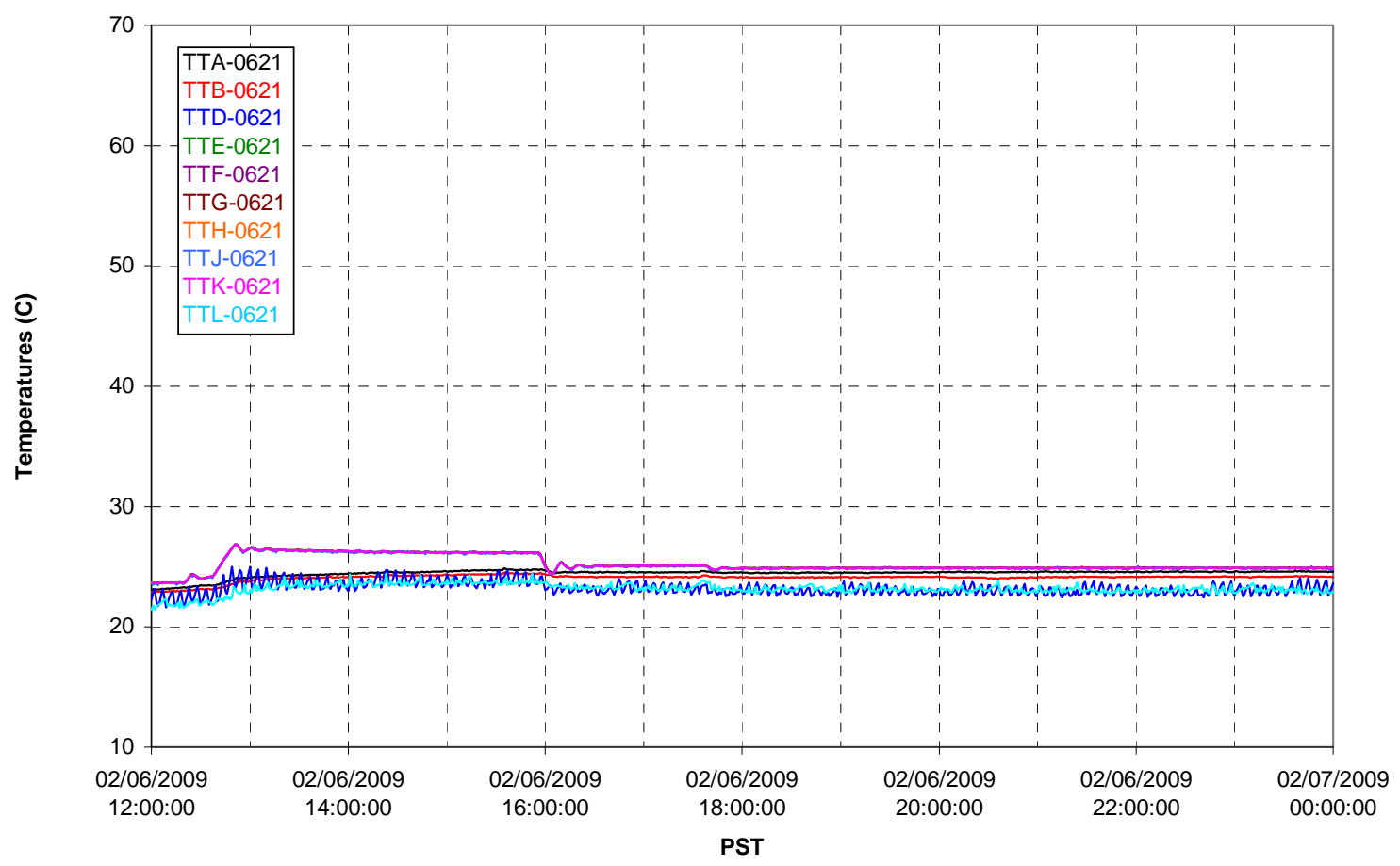


T02A temperatures

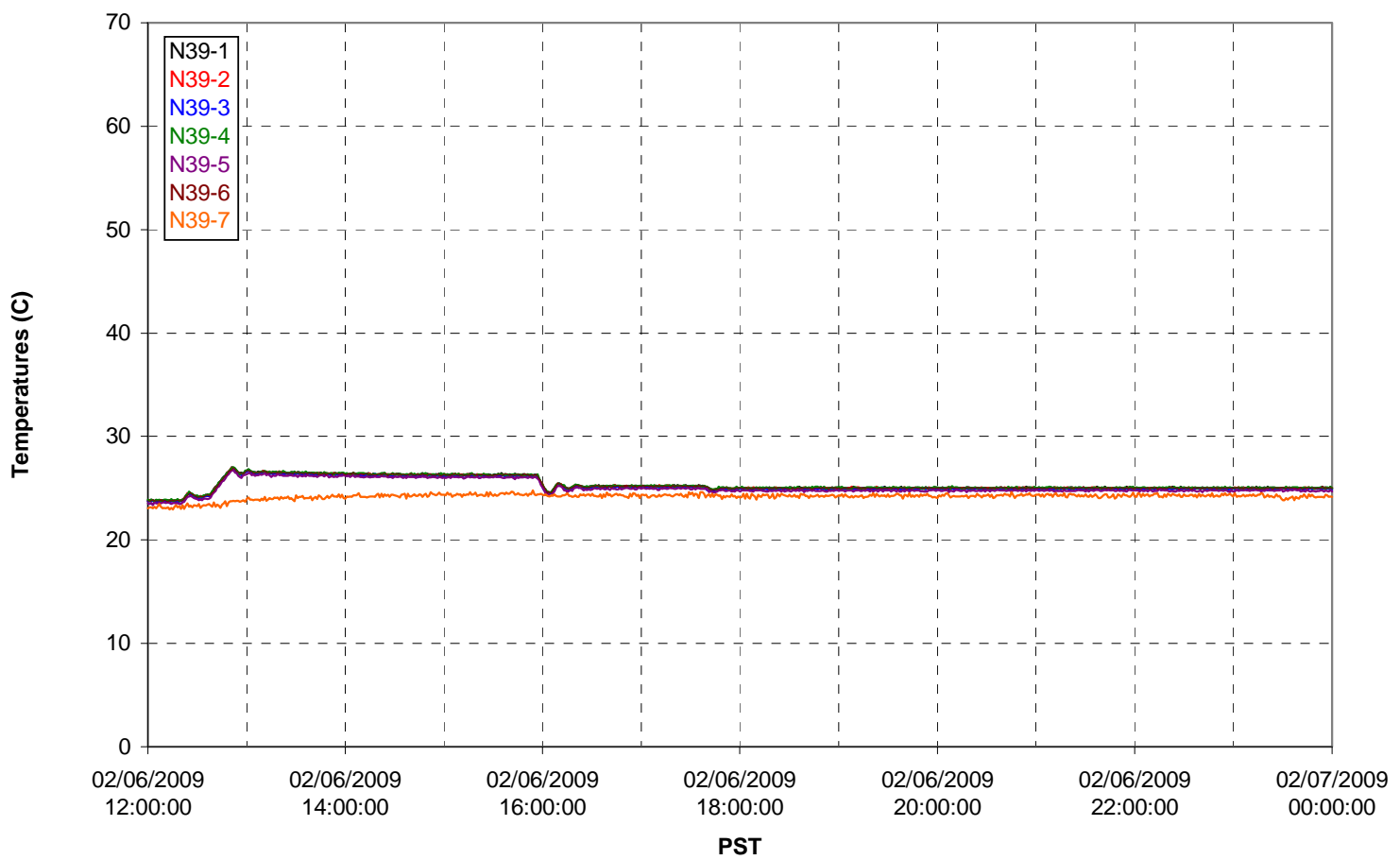

T02A temperatures

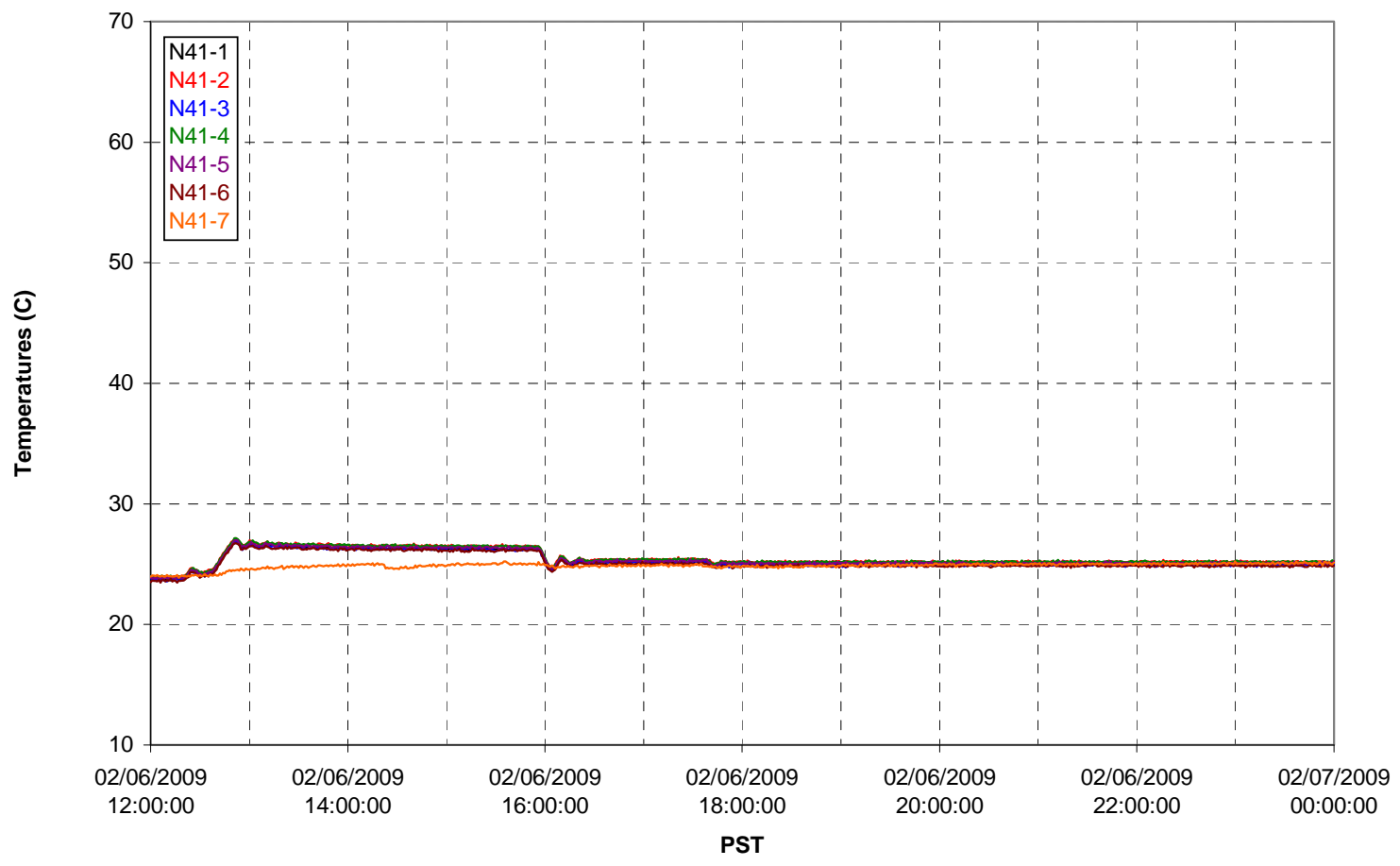


T02A temperatures

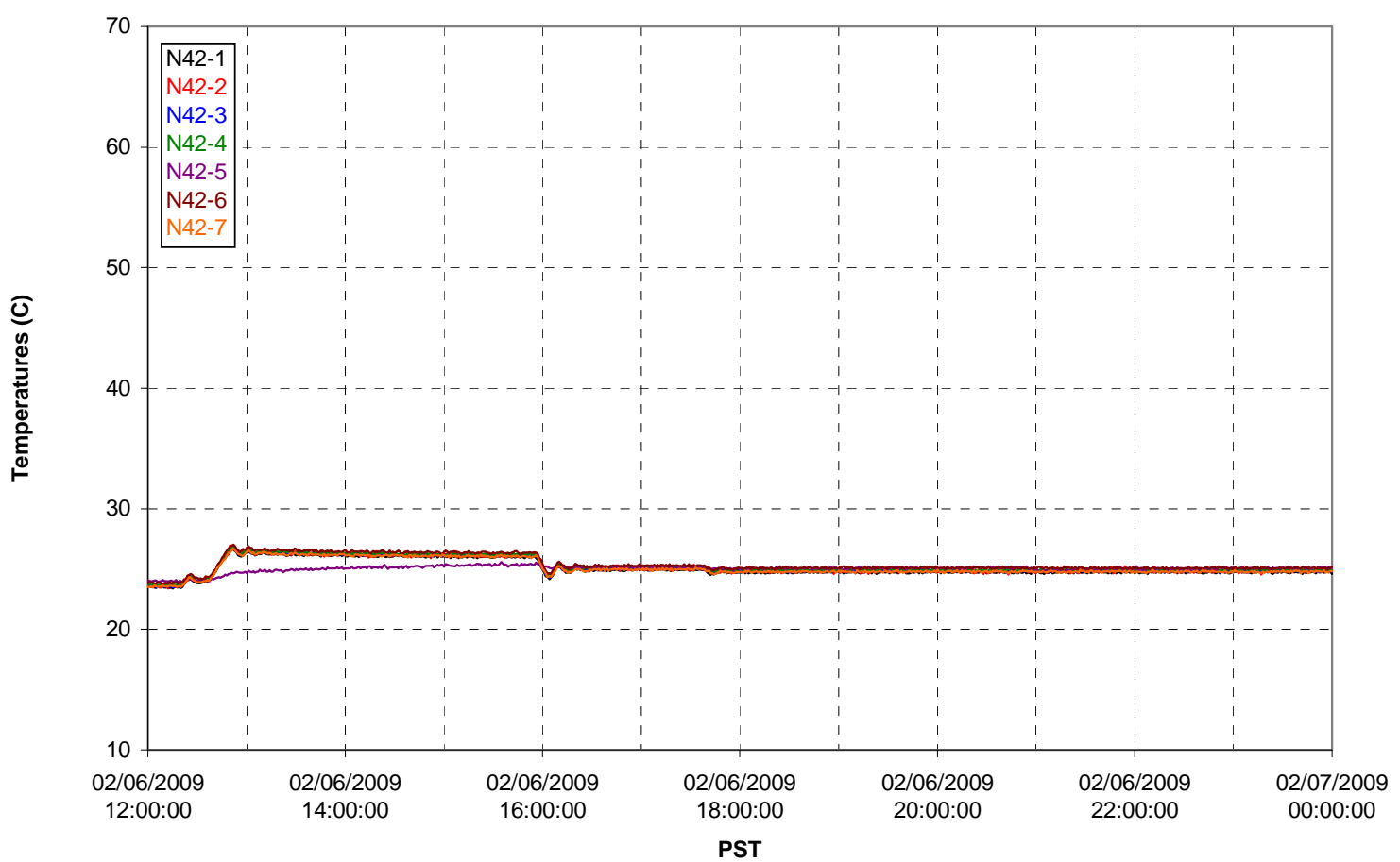

T02A temperatures

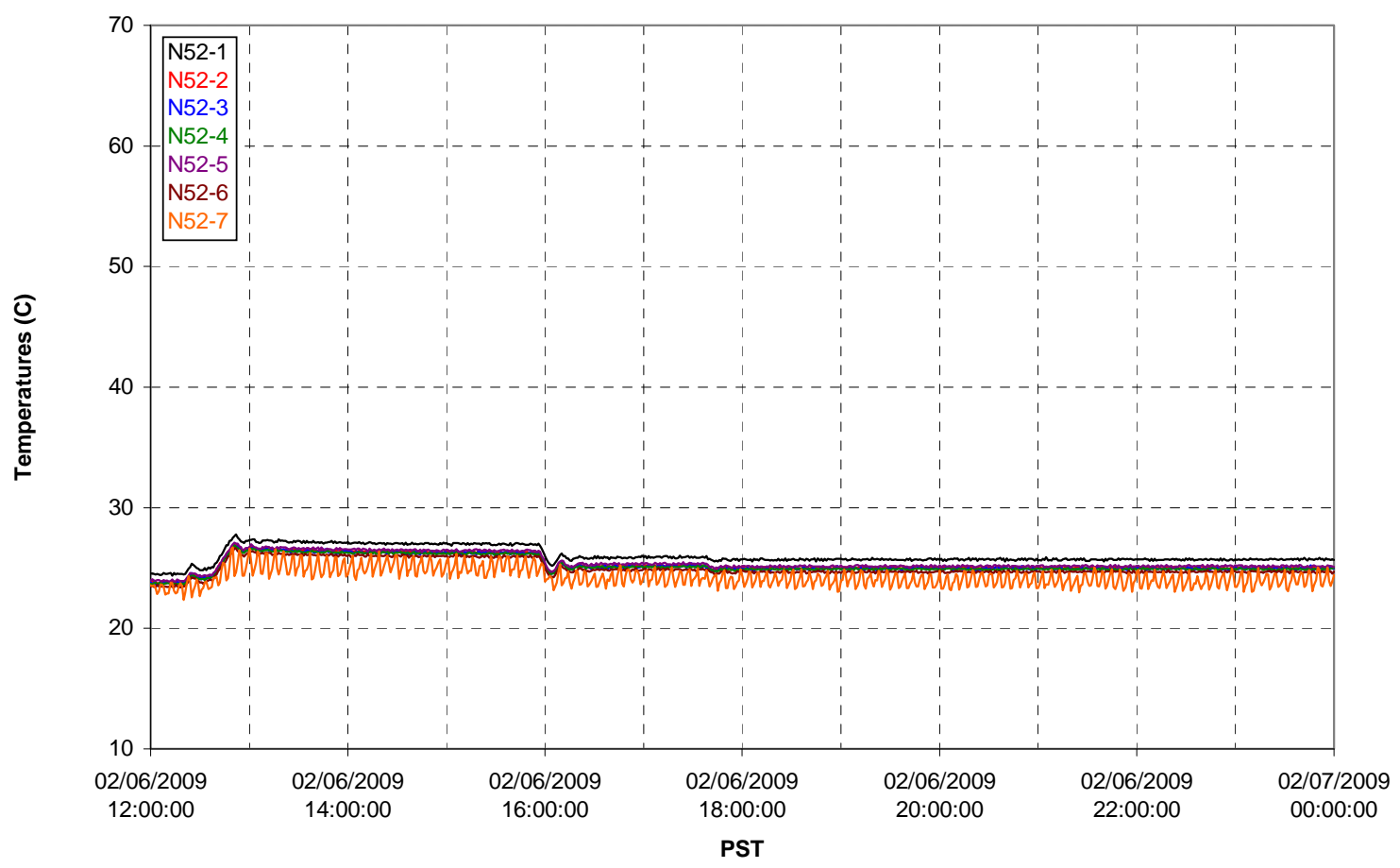


T02A Heating and Cooling

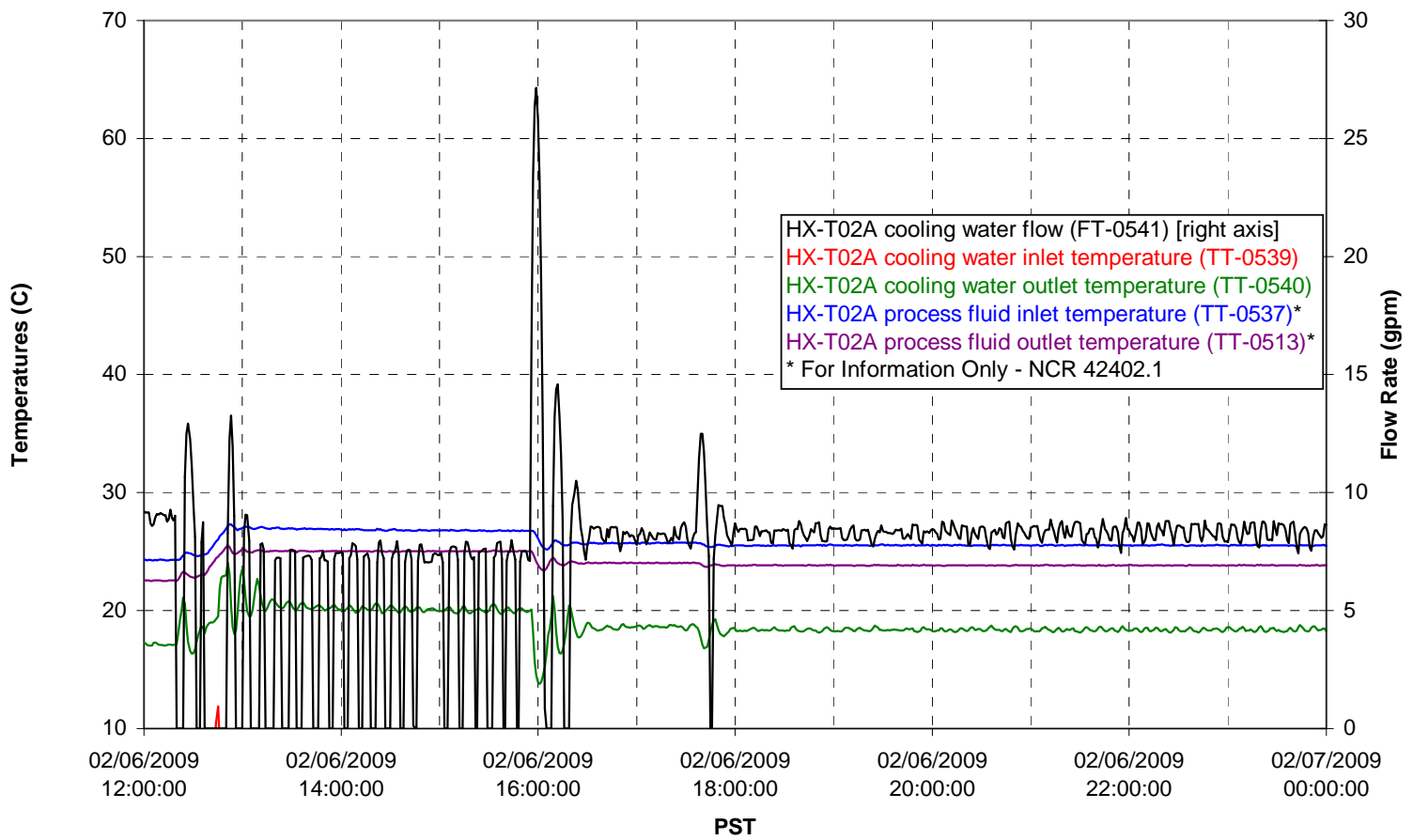

Pump Operation

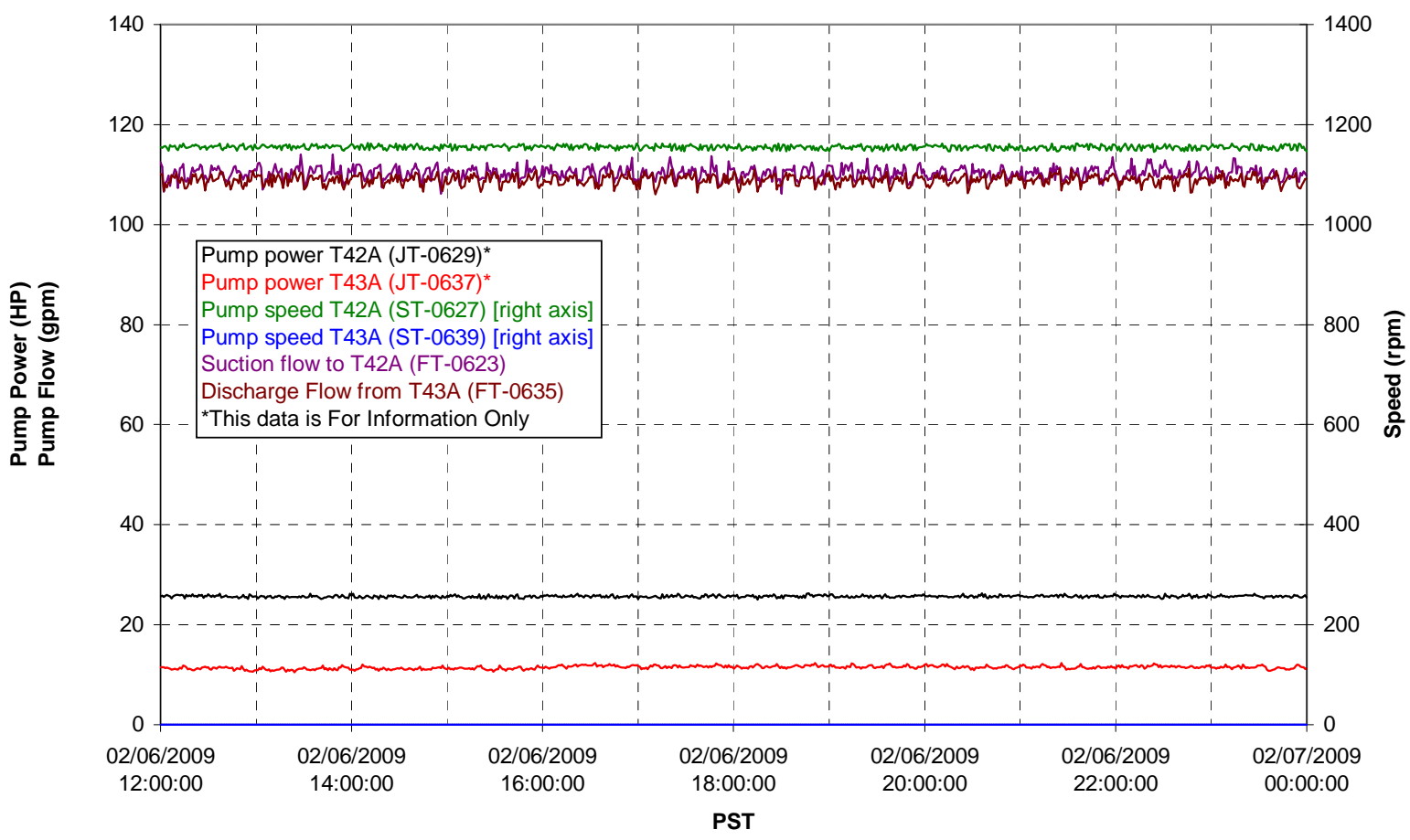


Pulsepot UFP-PP-T01A

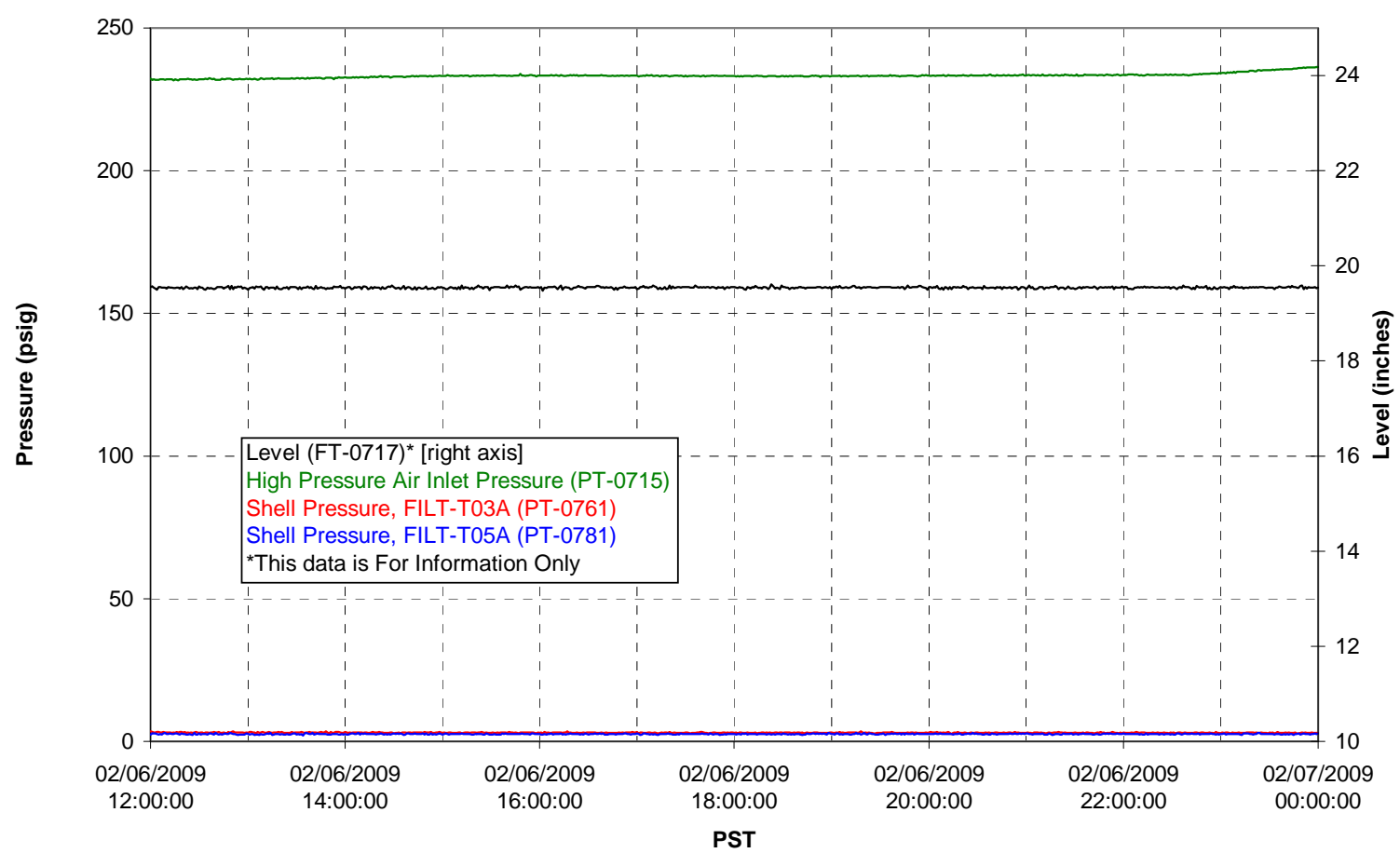

Pulsepot UFP-PP-T02A

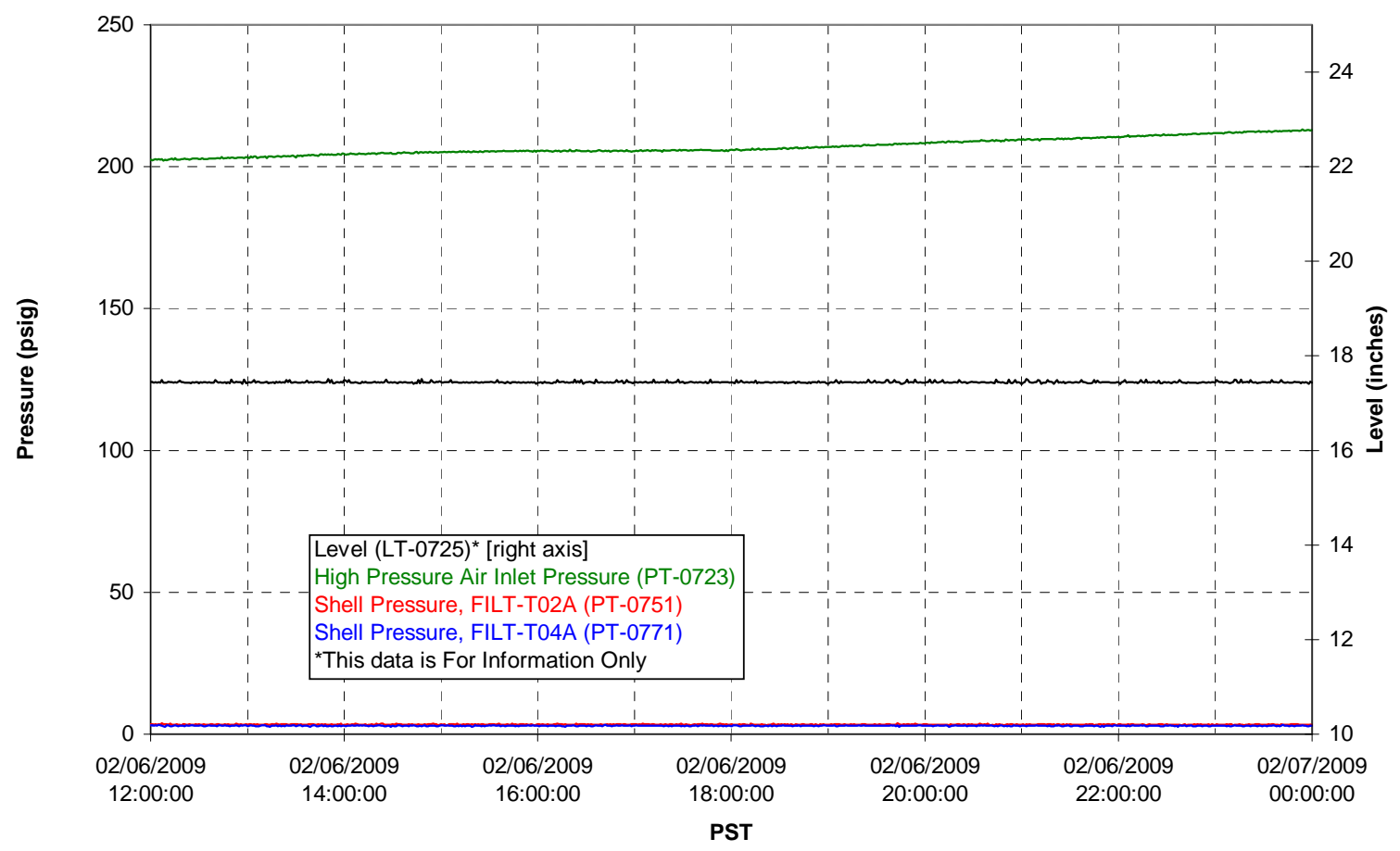


Pulsepot UFP-PP-T03A

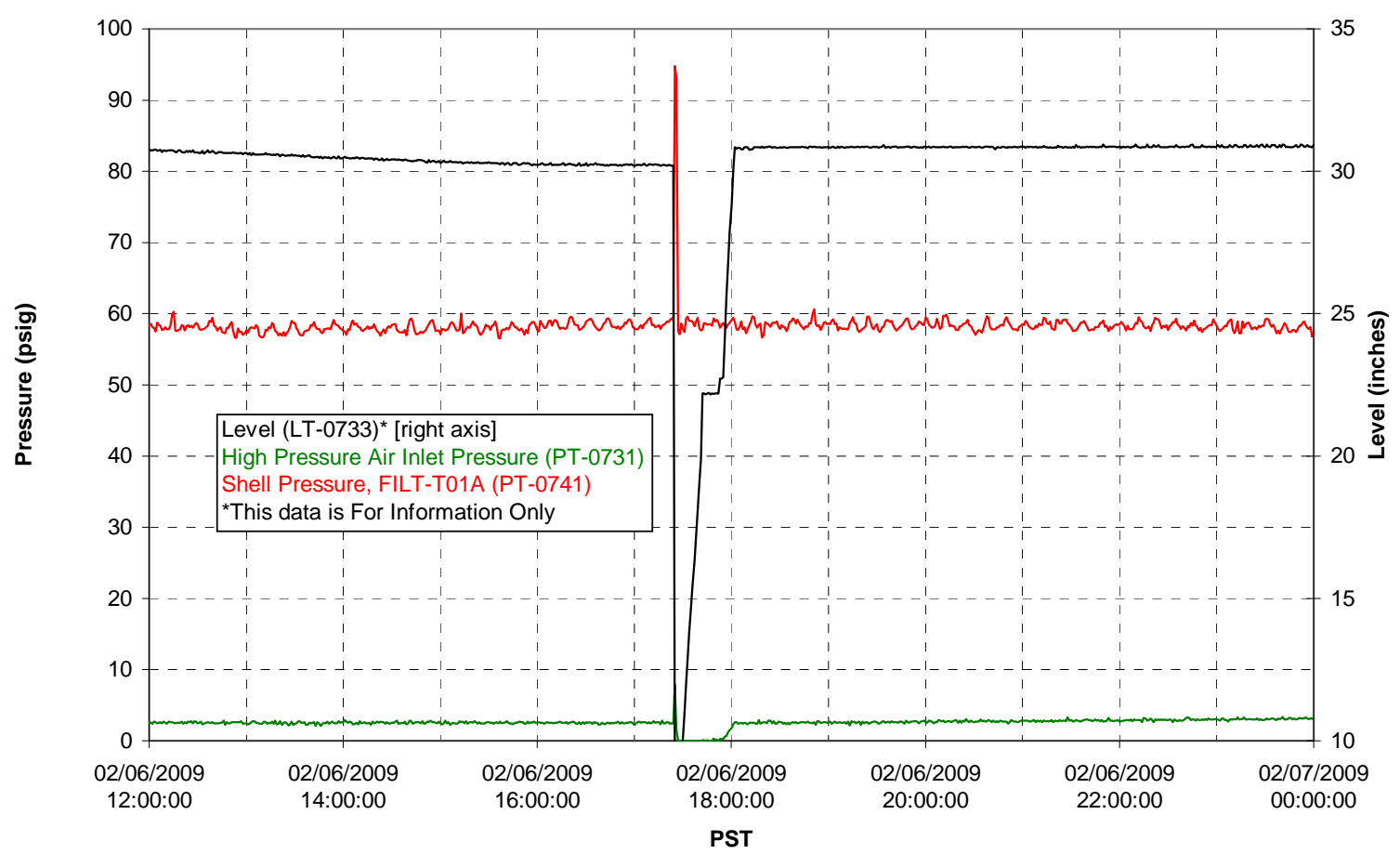

Pulsepot Levels

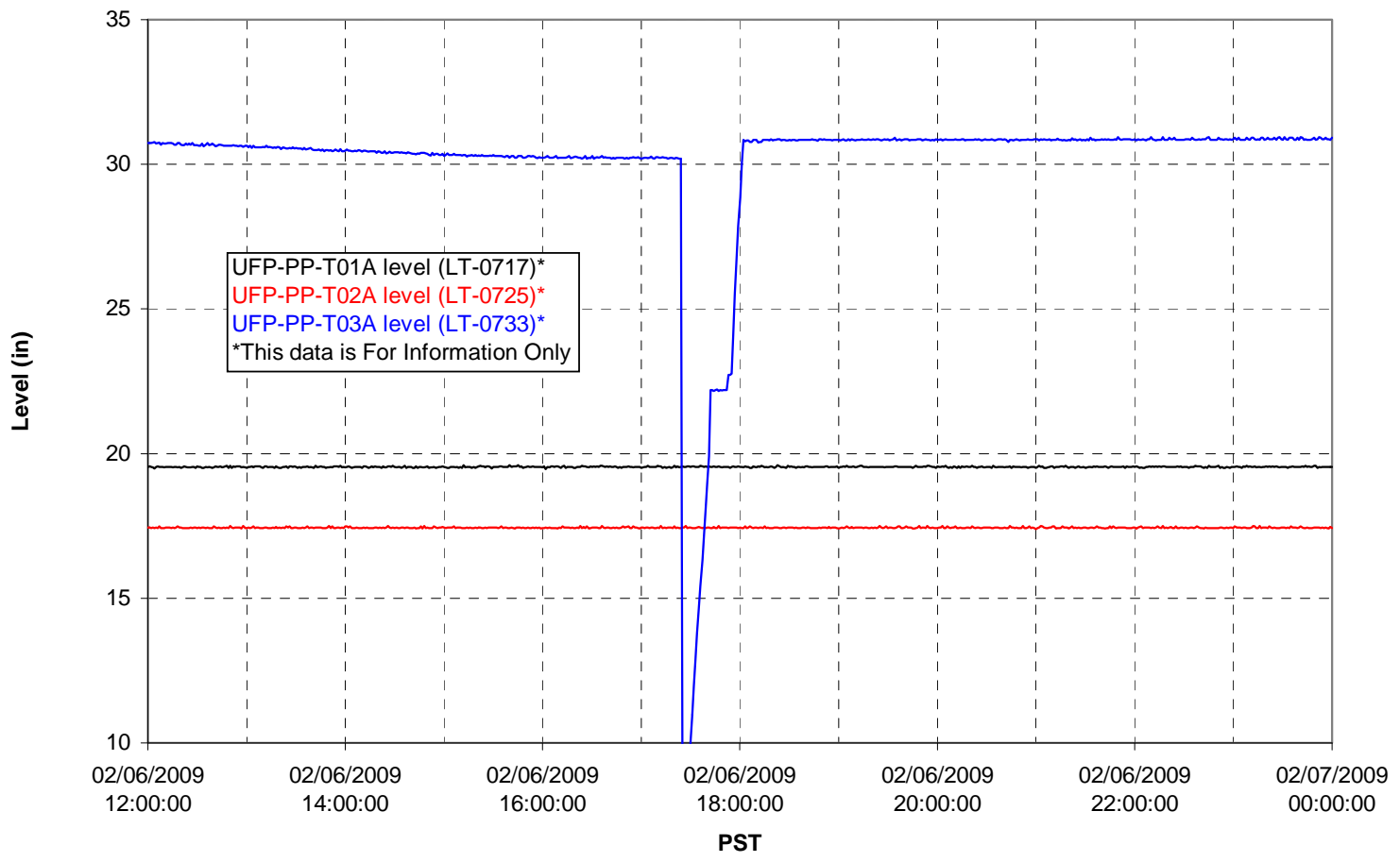


Filter UFP-FILT-T01A

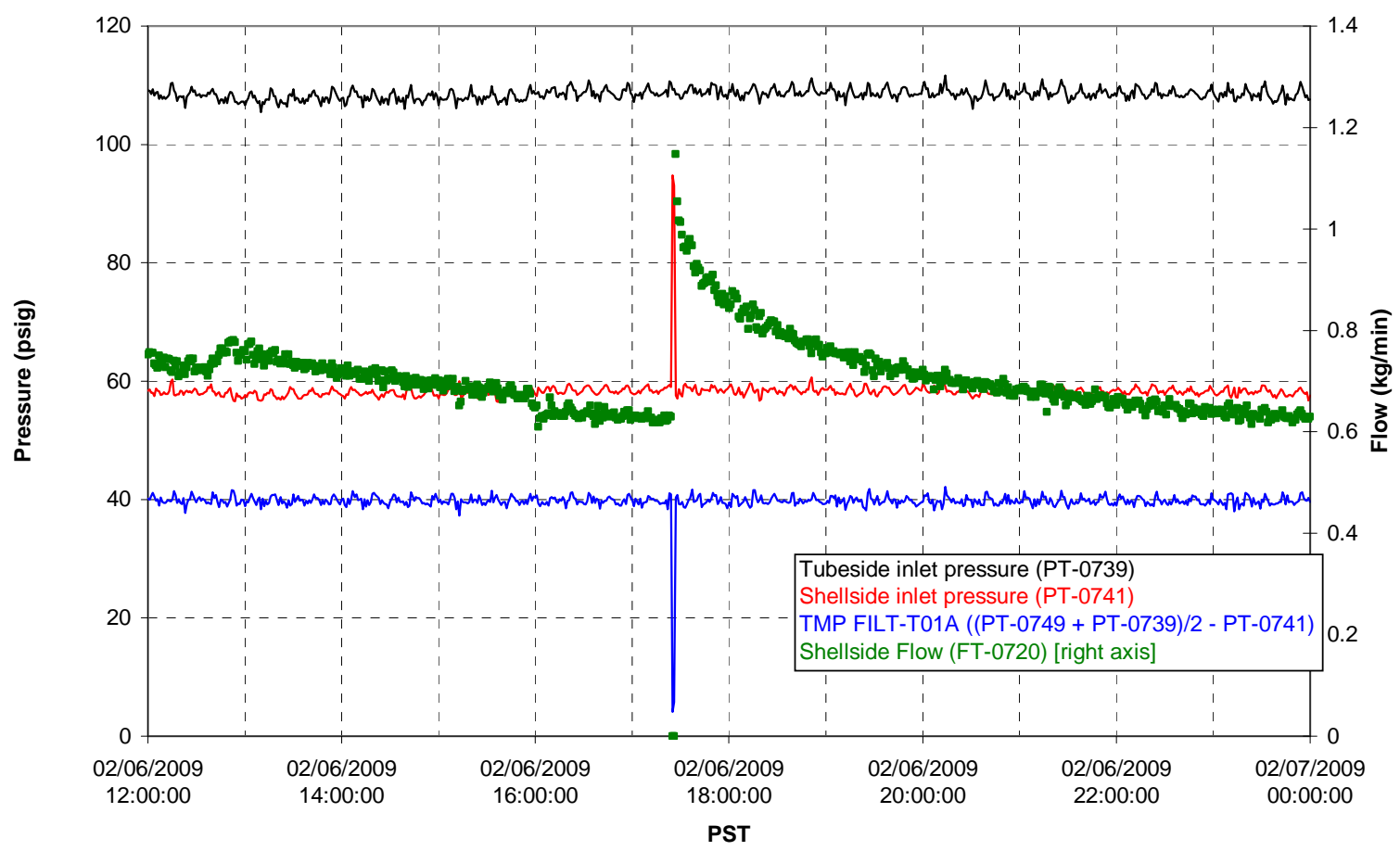

Filter UFP-FILT-T02A

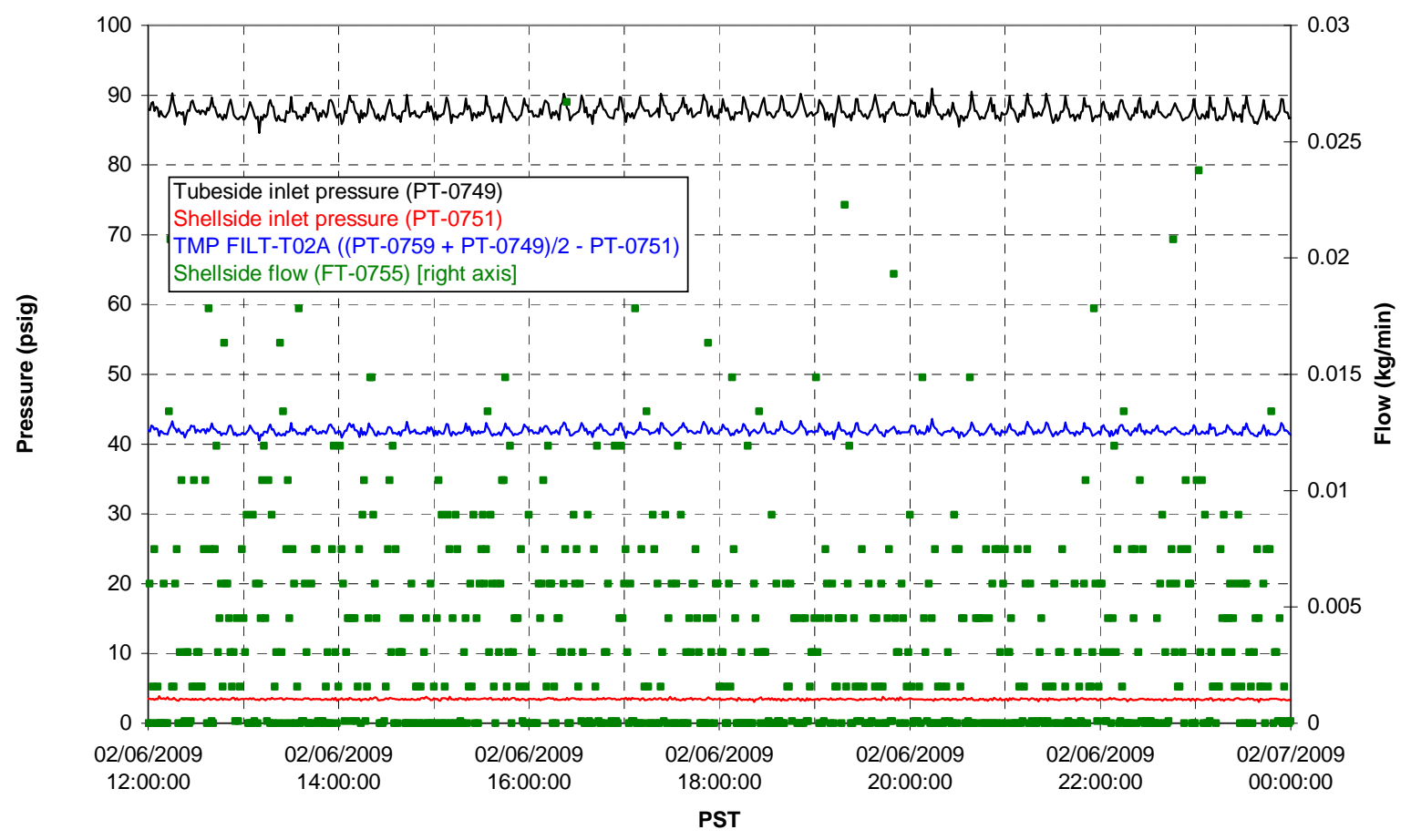


Filter UFP-FILT-T03A

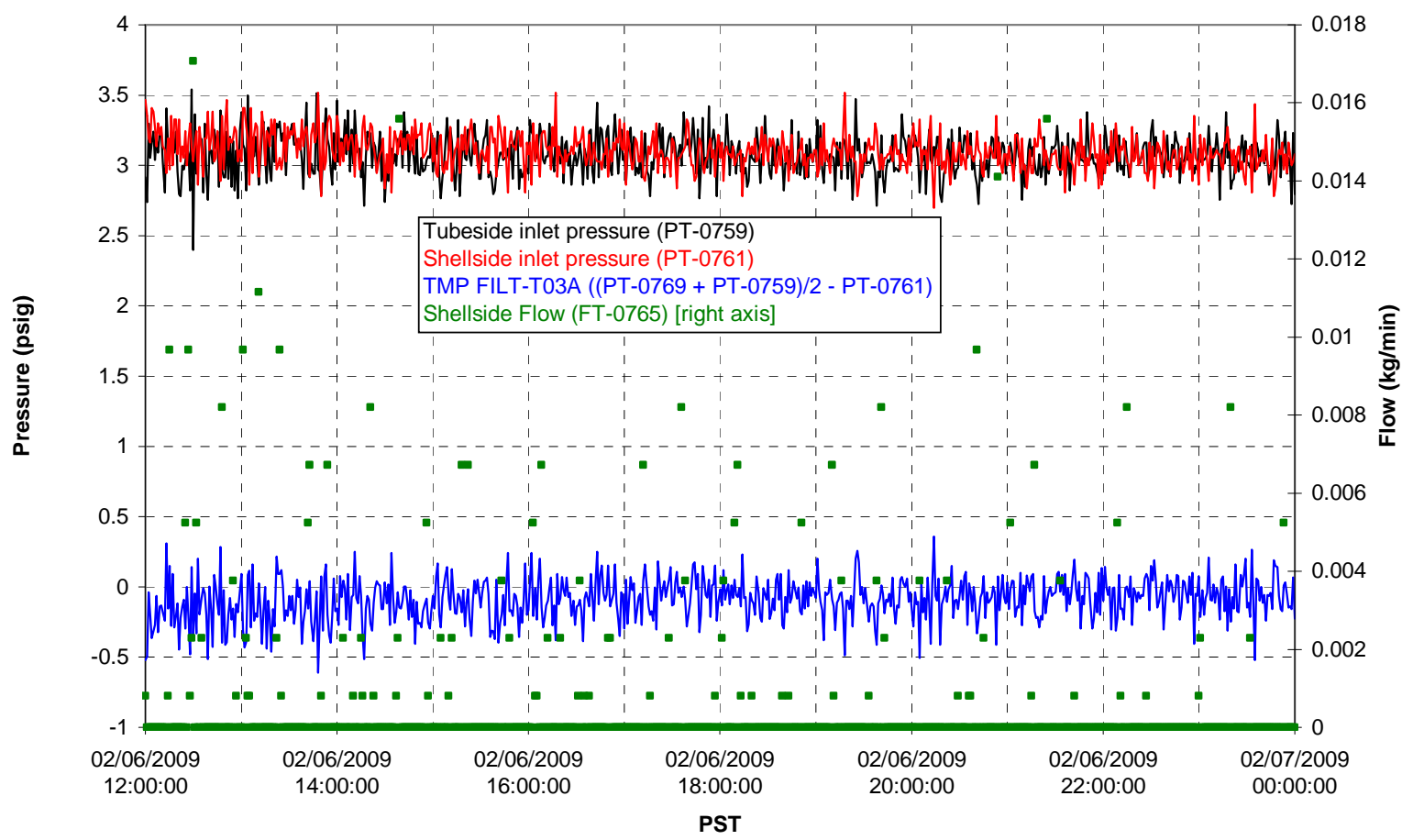

Filter UFP-FILT-T04A

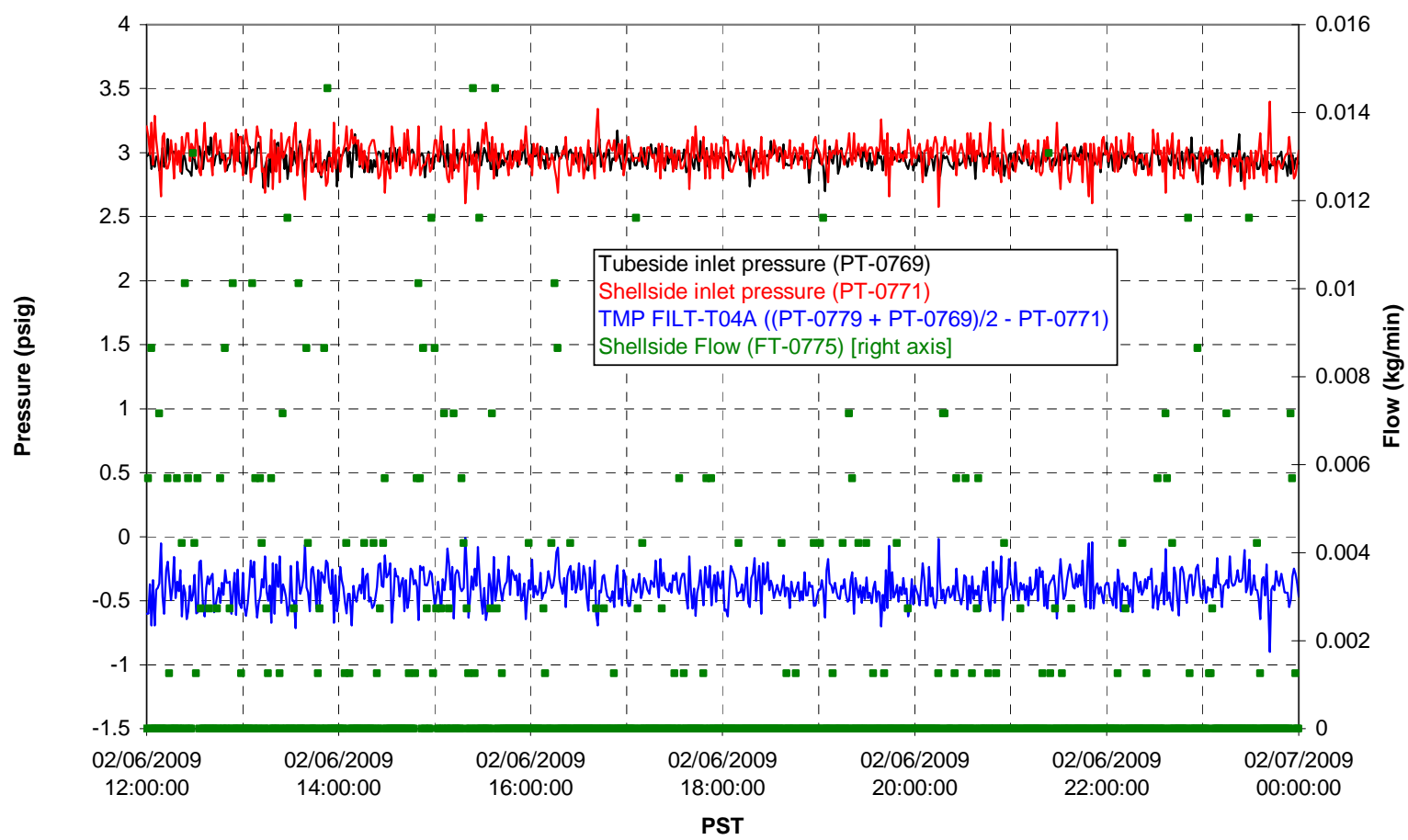


Filter UFP-FILT-T05A

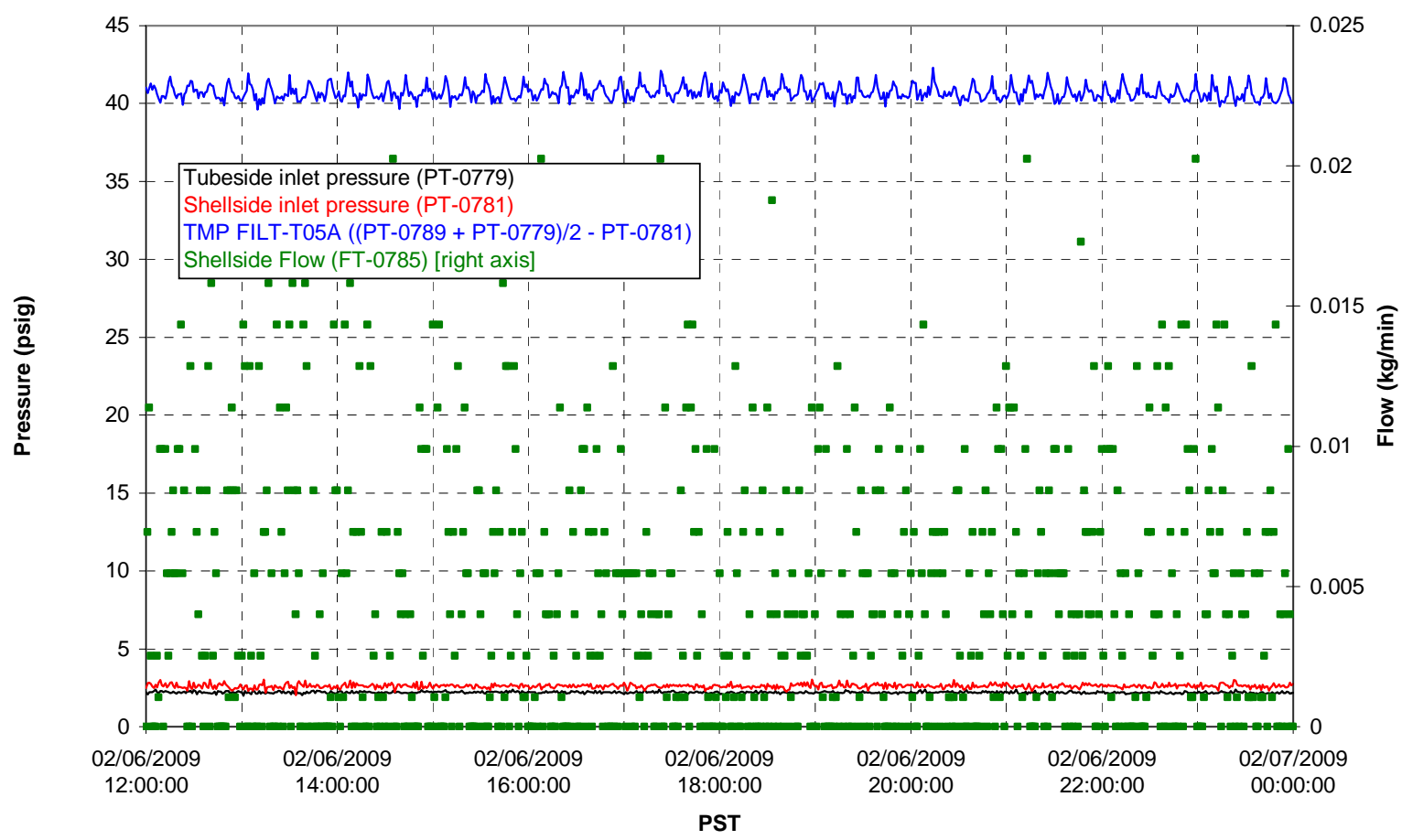

Chemical Flow

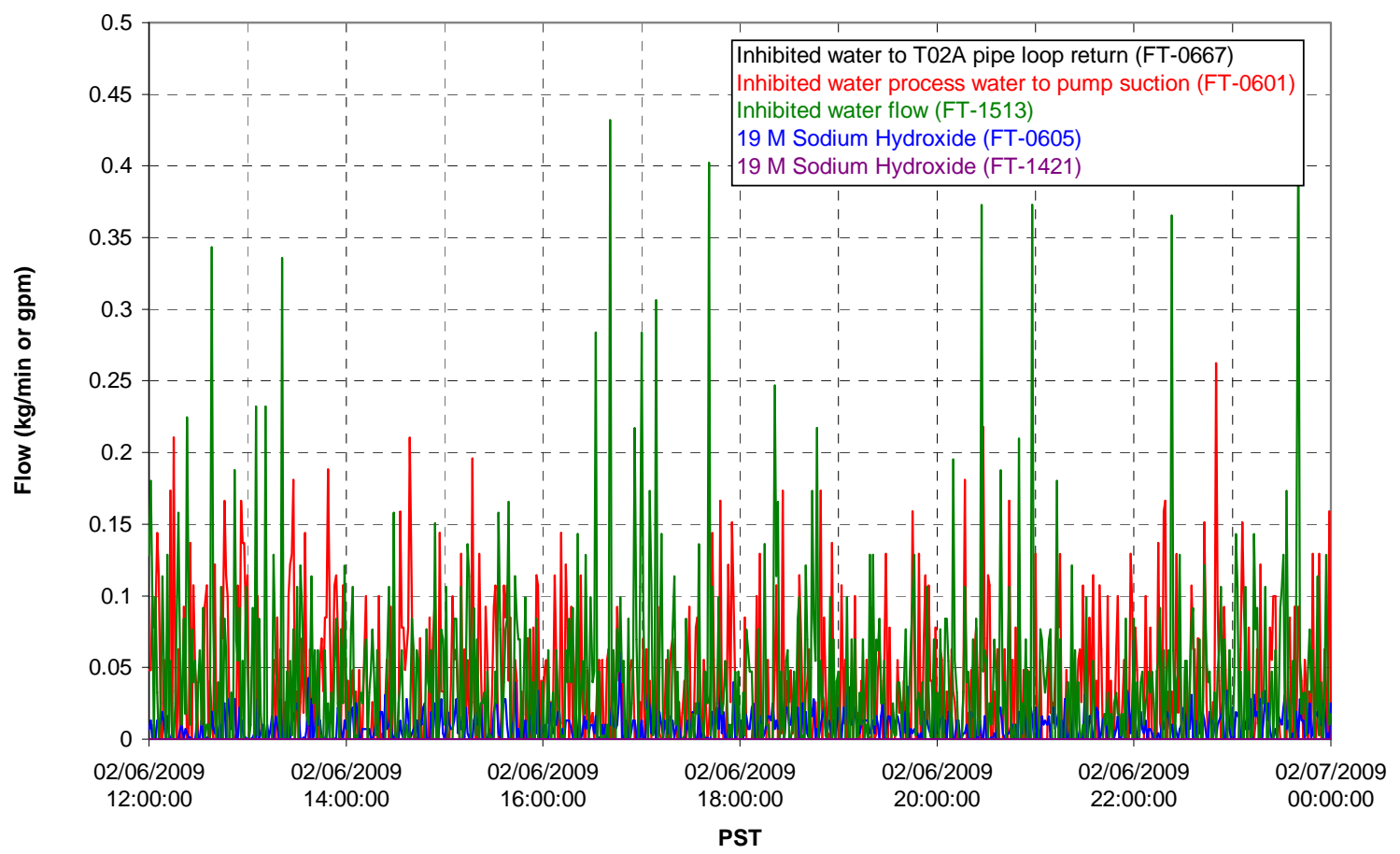


Chemical Flow

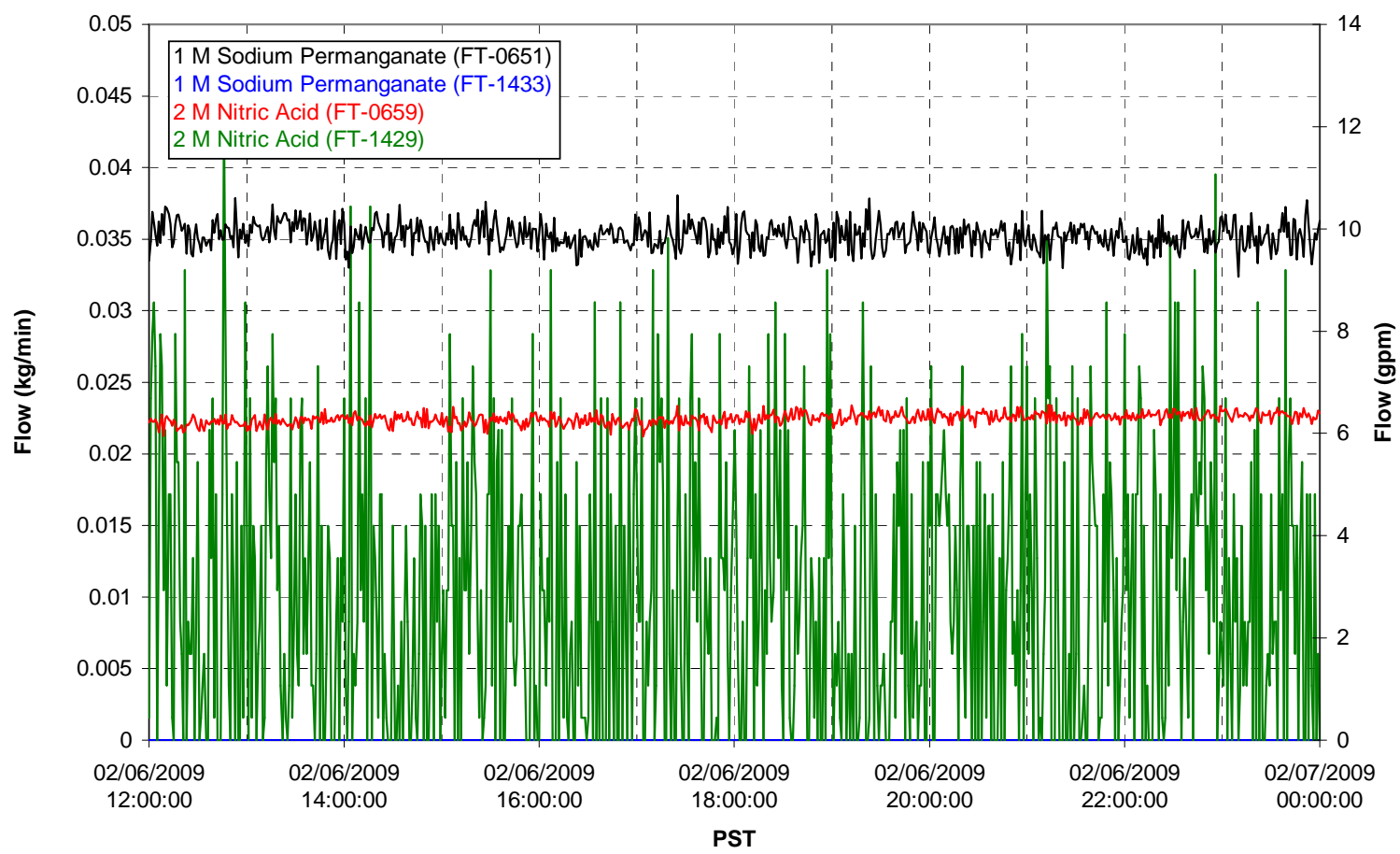

Air Flows

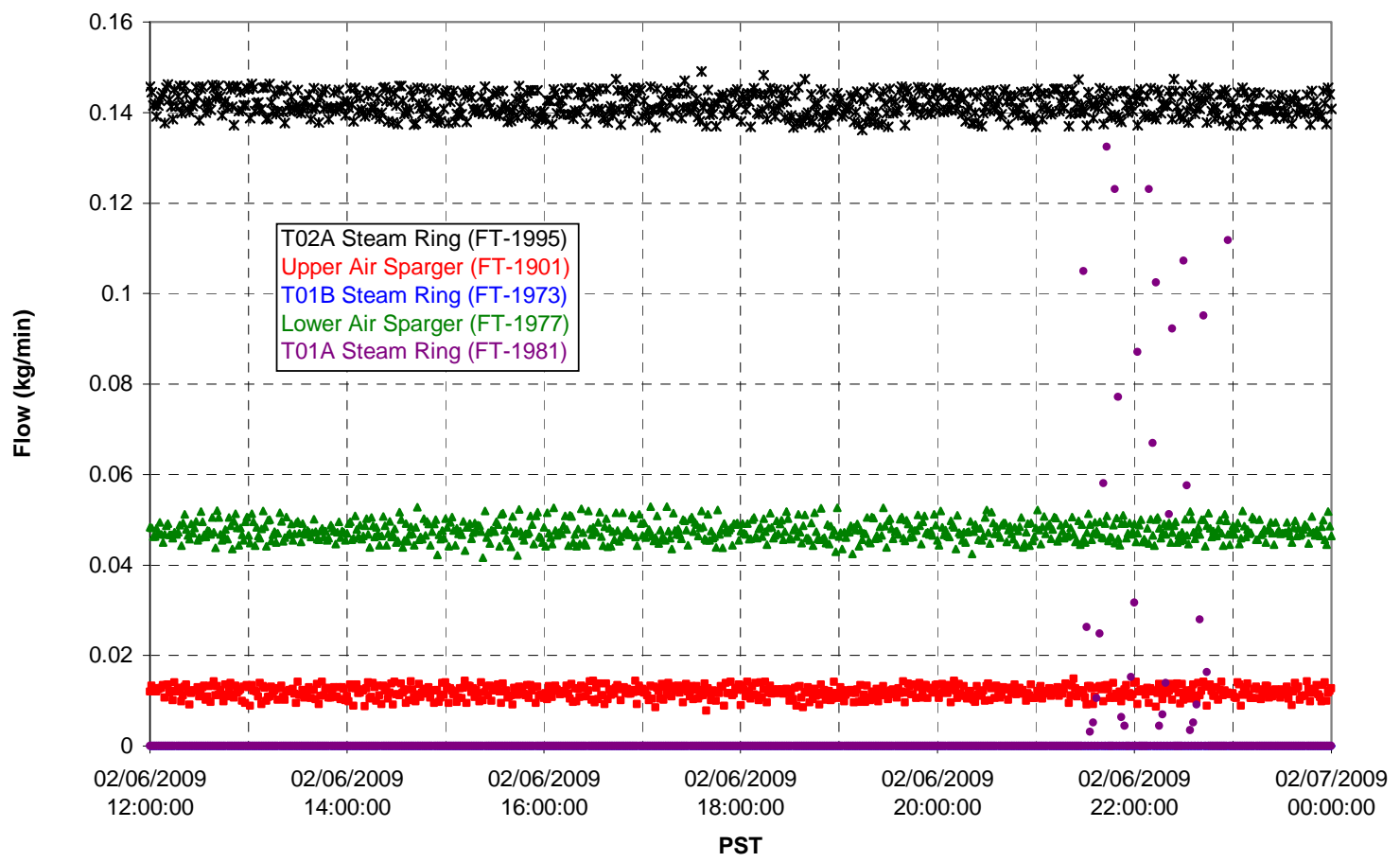


T02A Steam

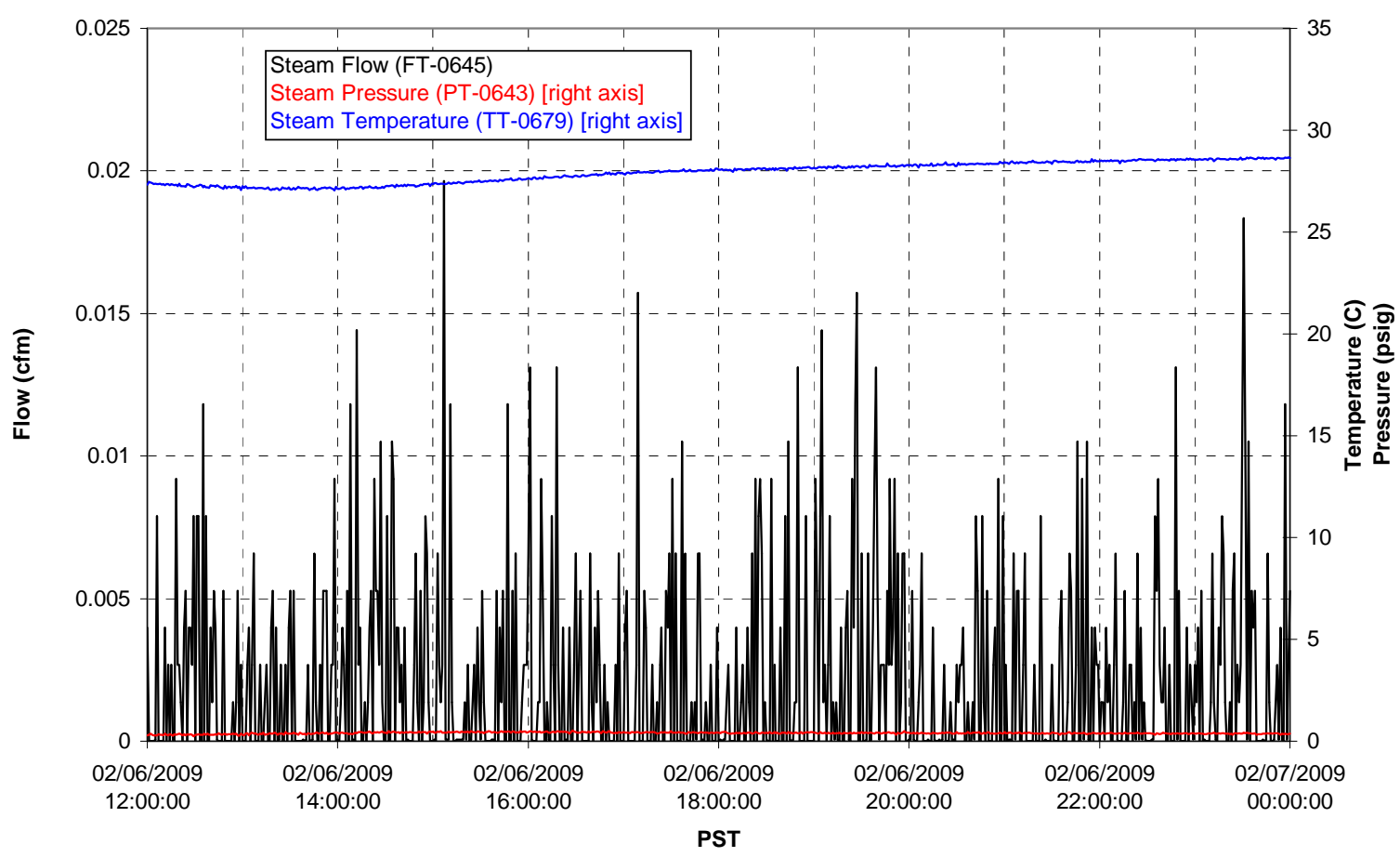

T01A Steam

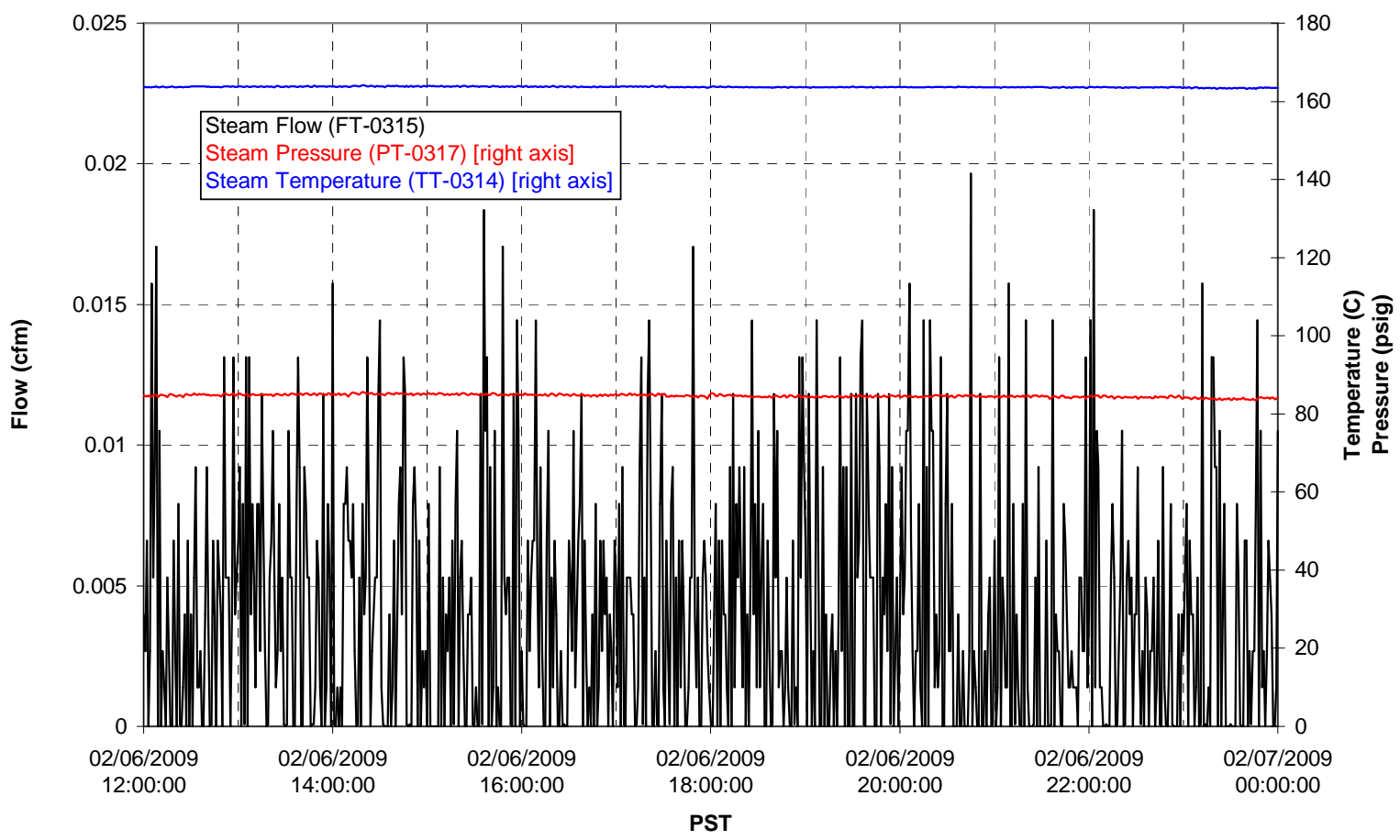


T01B Steam

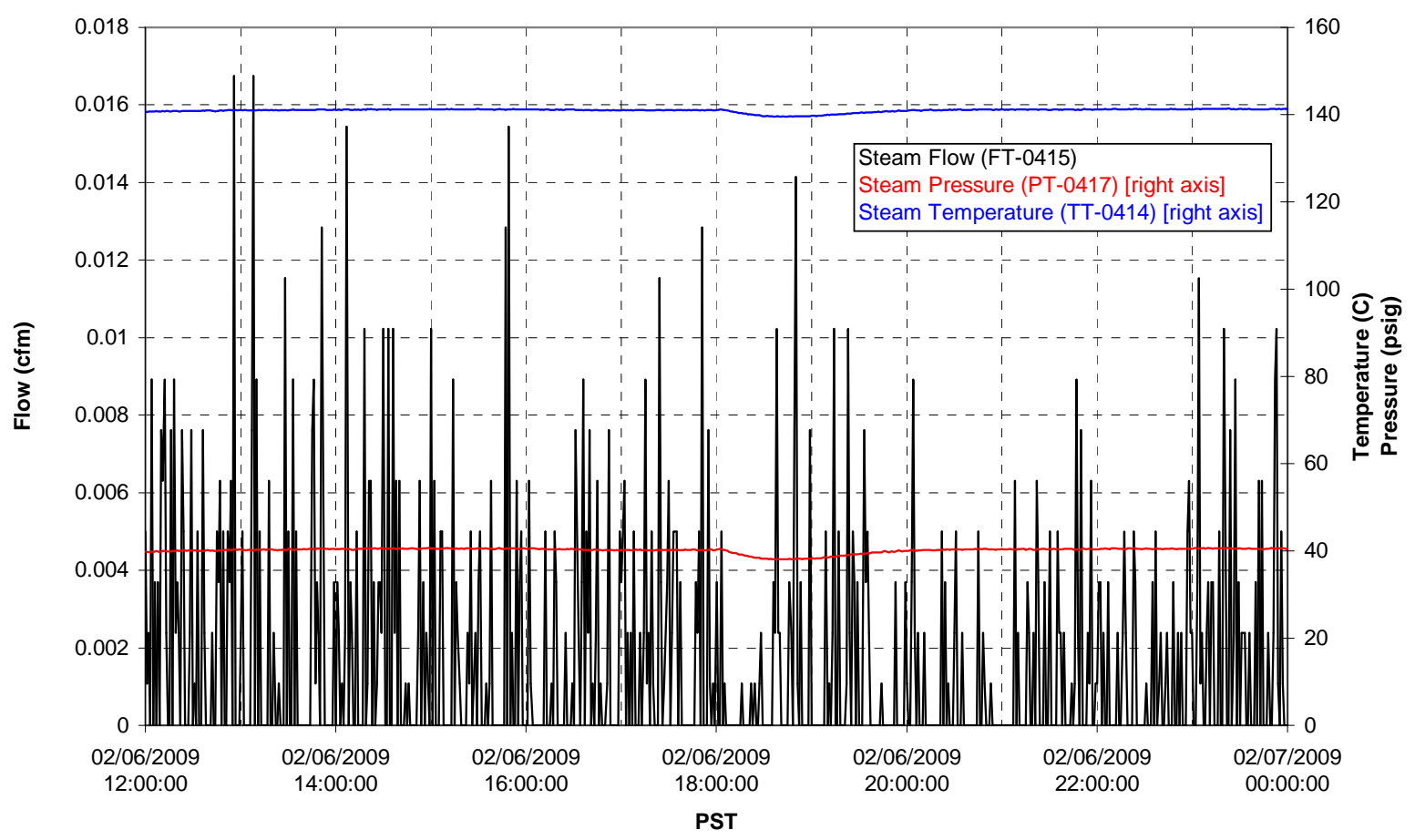




\section{Integrated Test A Data Plots 02/07/09 00:00 - 02/07/09 12:00}


T01A level

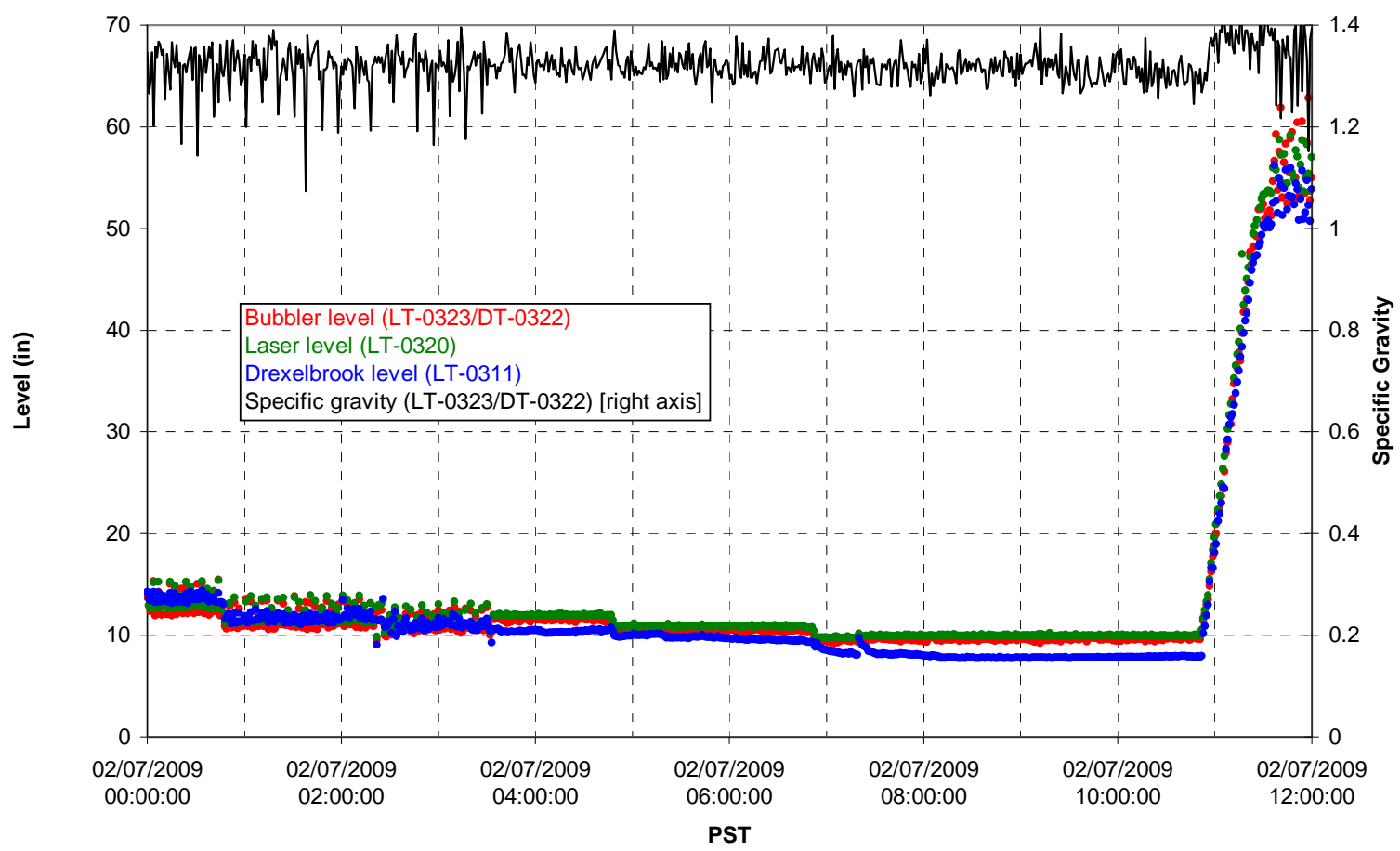

T01A temperatures

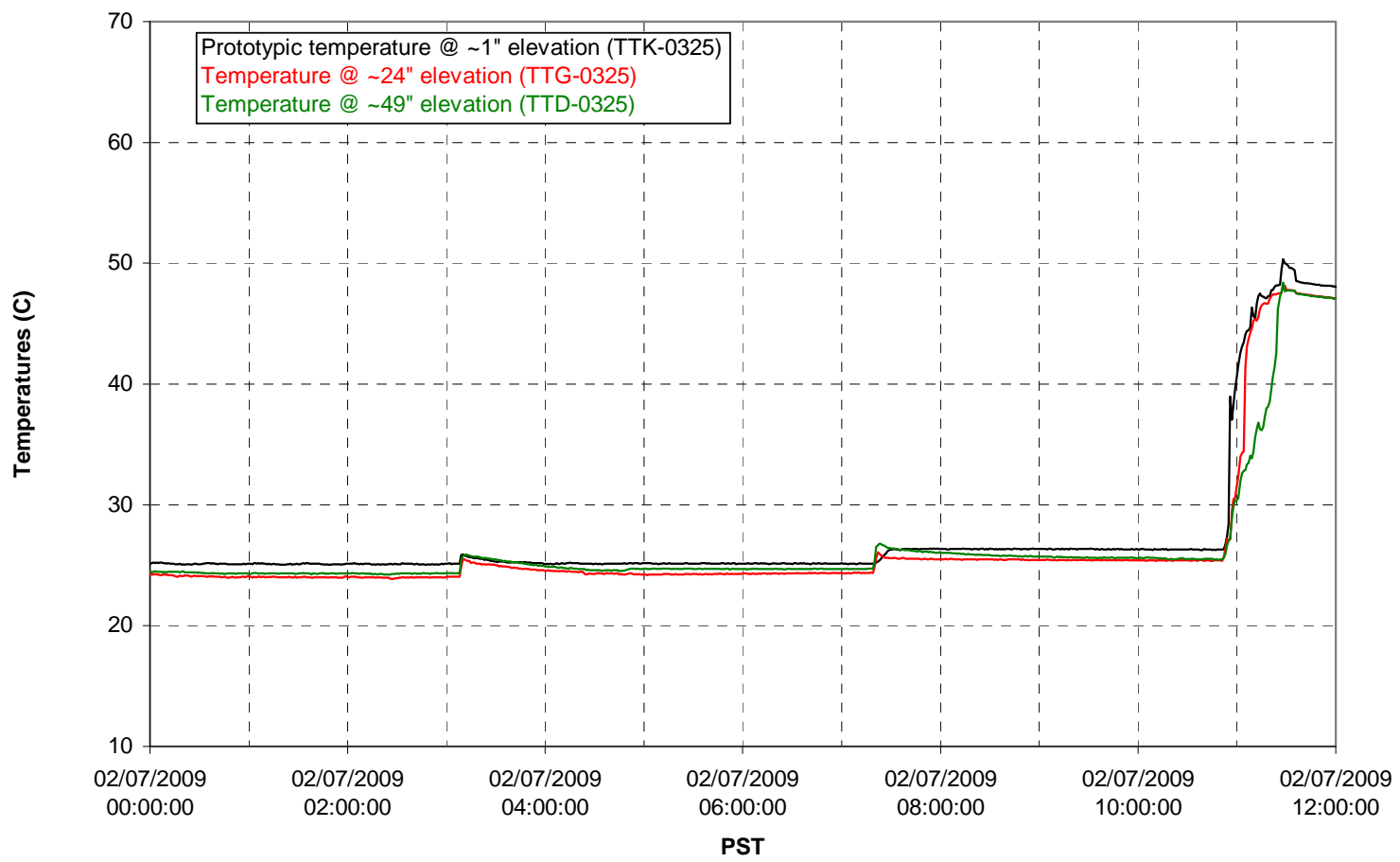


T01B level

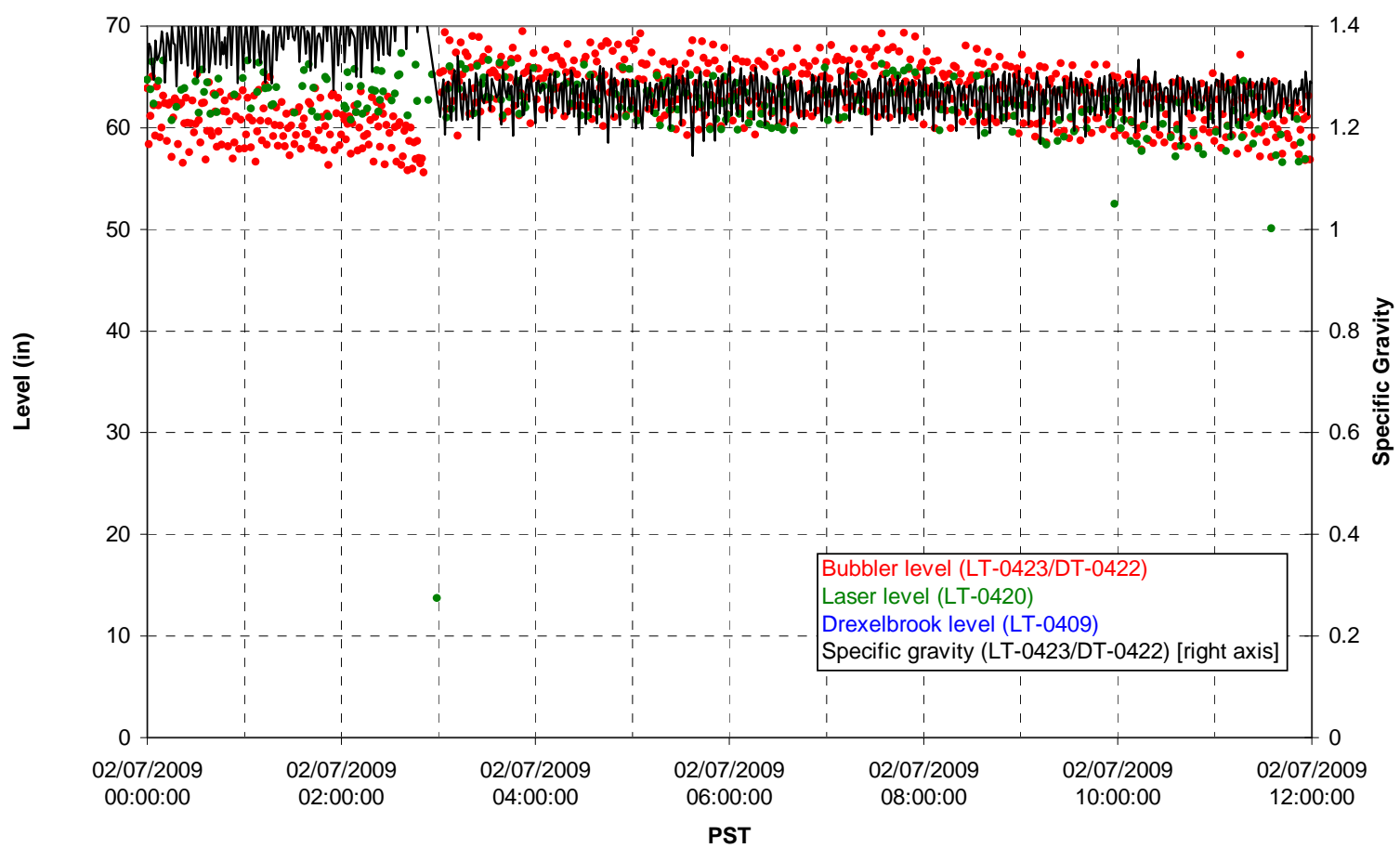

T01B temperatures

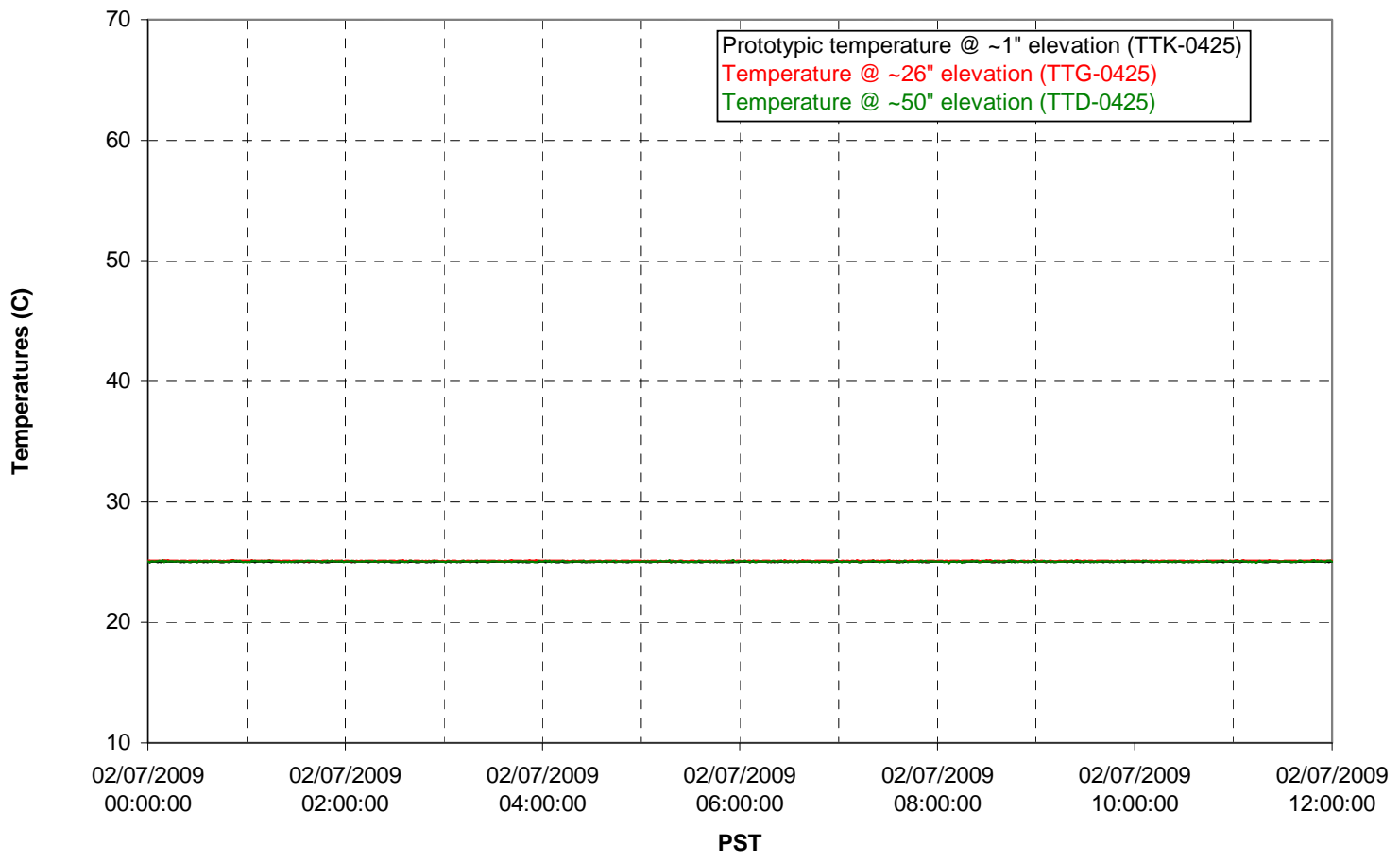


T02A level

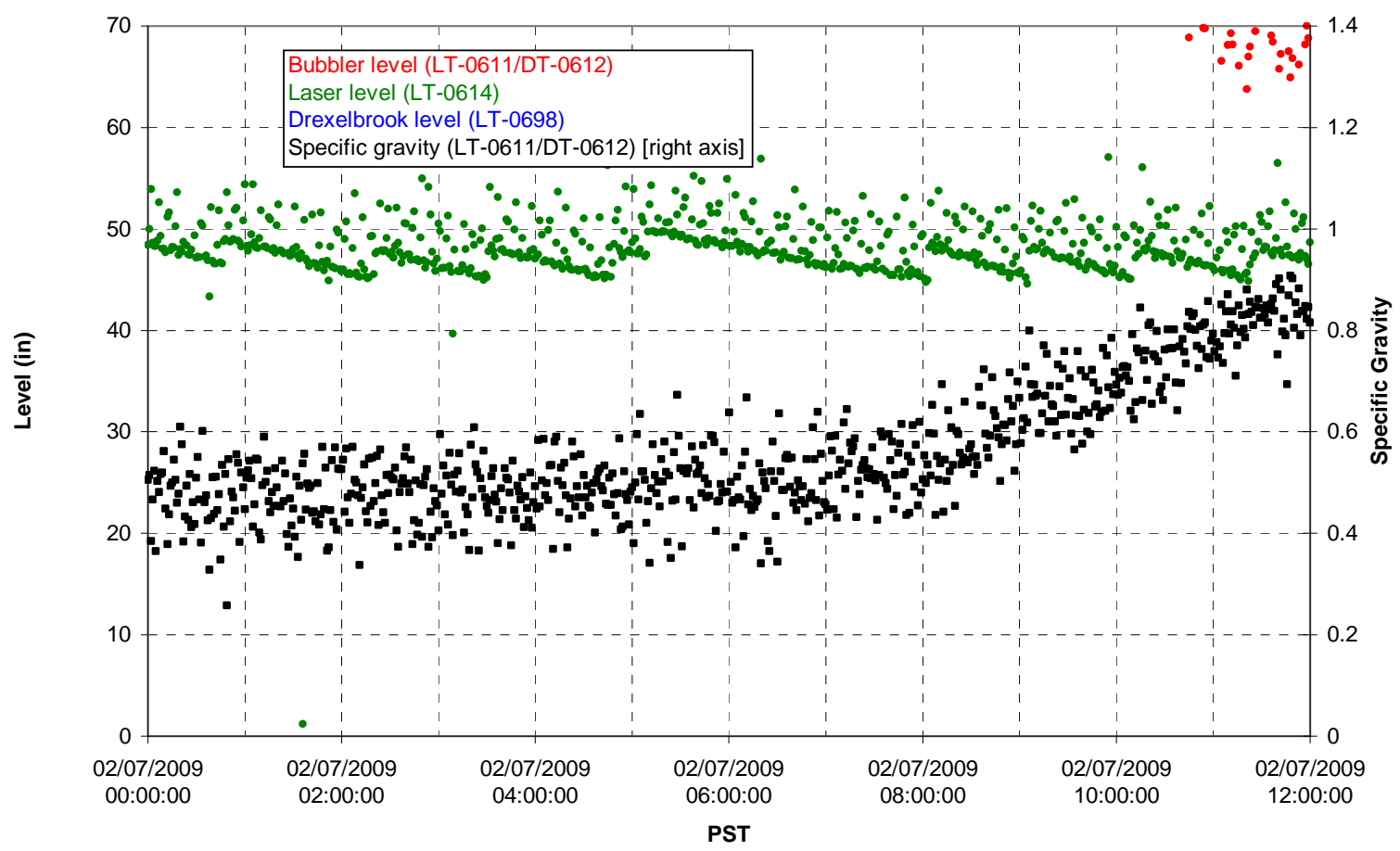

T02A temperatures

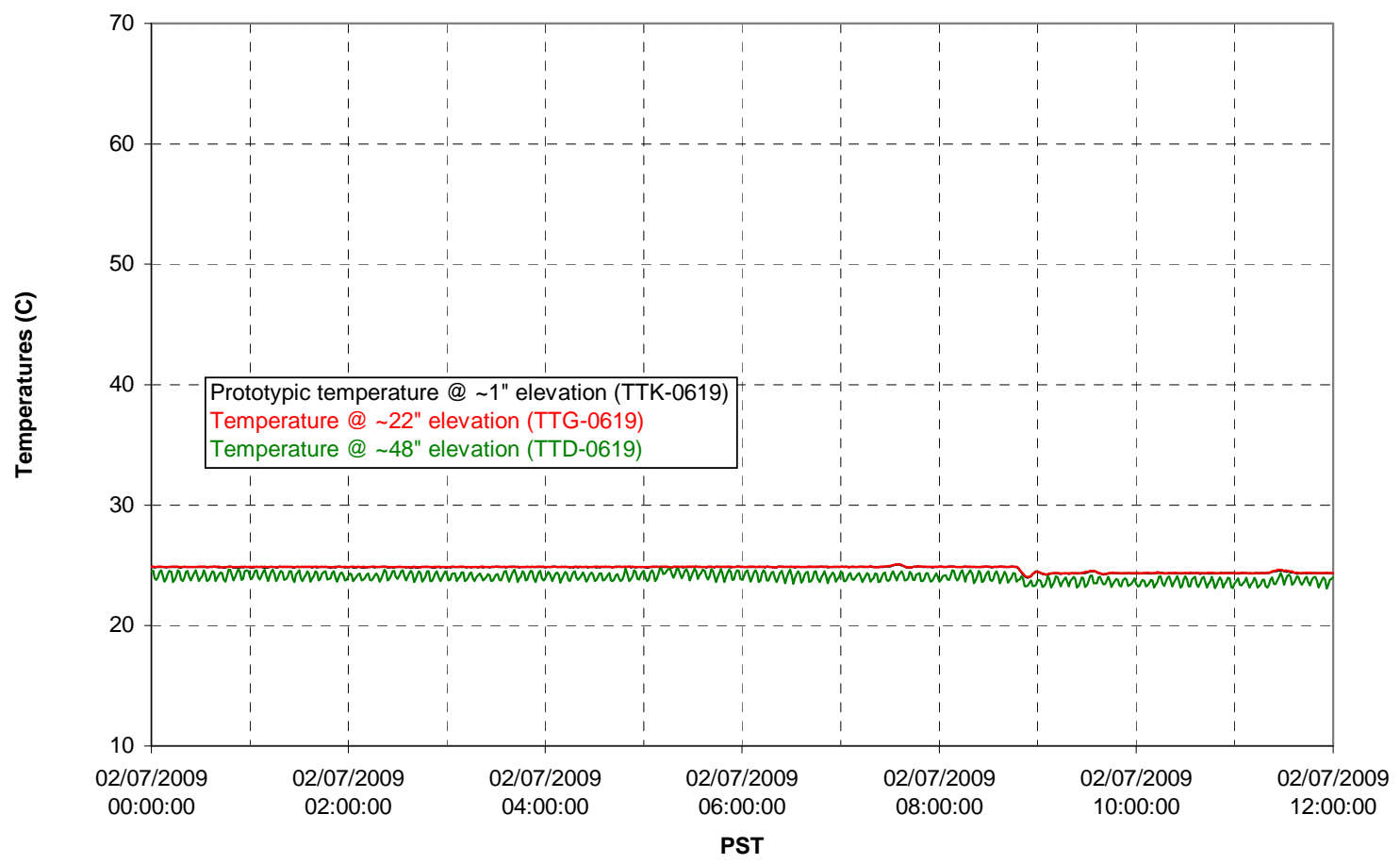


T02A and filter loop temperatures

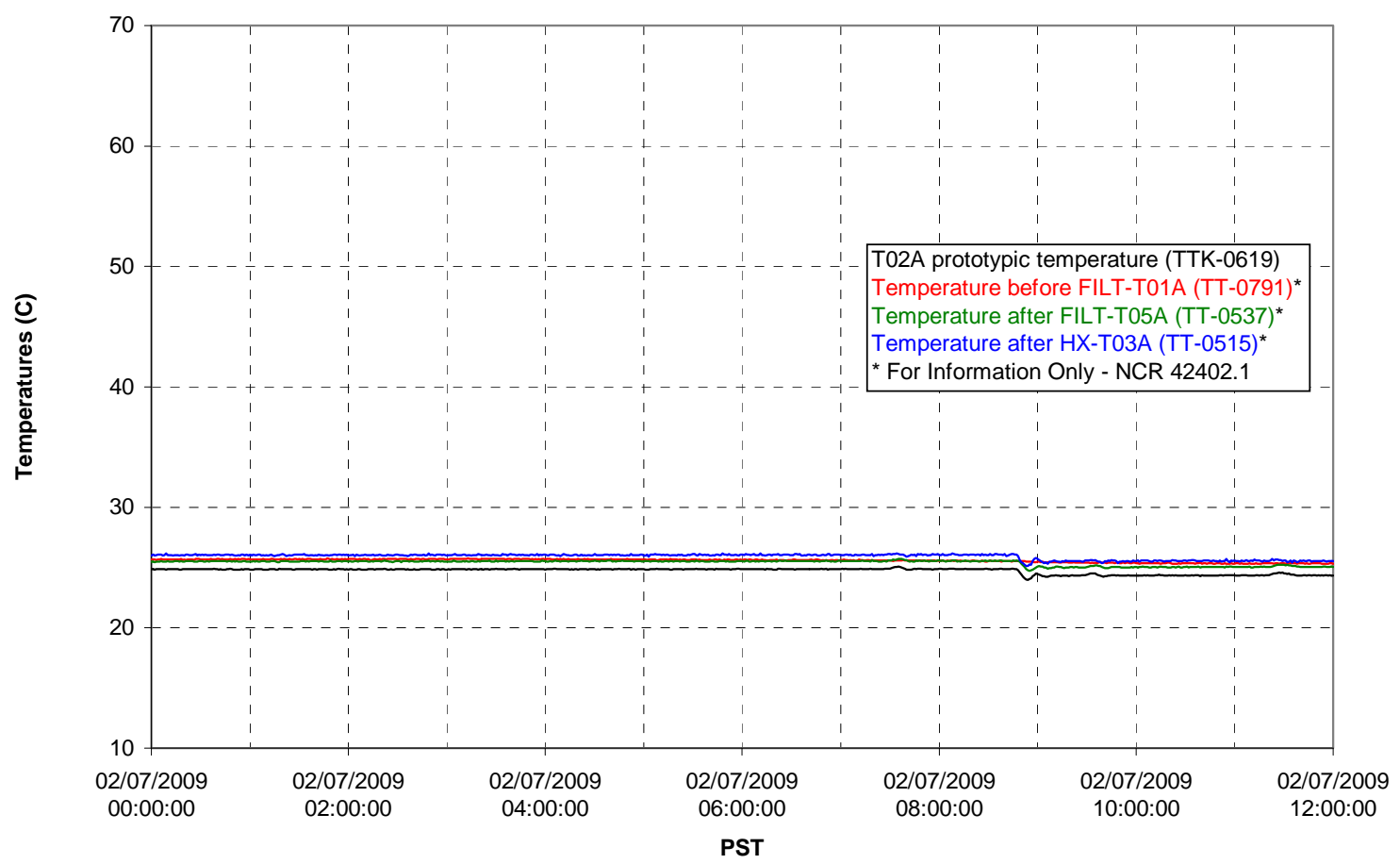

Pump Pressures and Flow

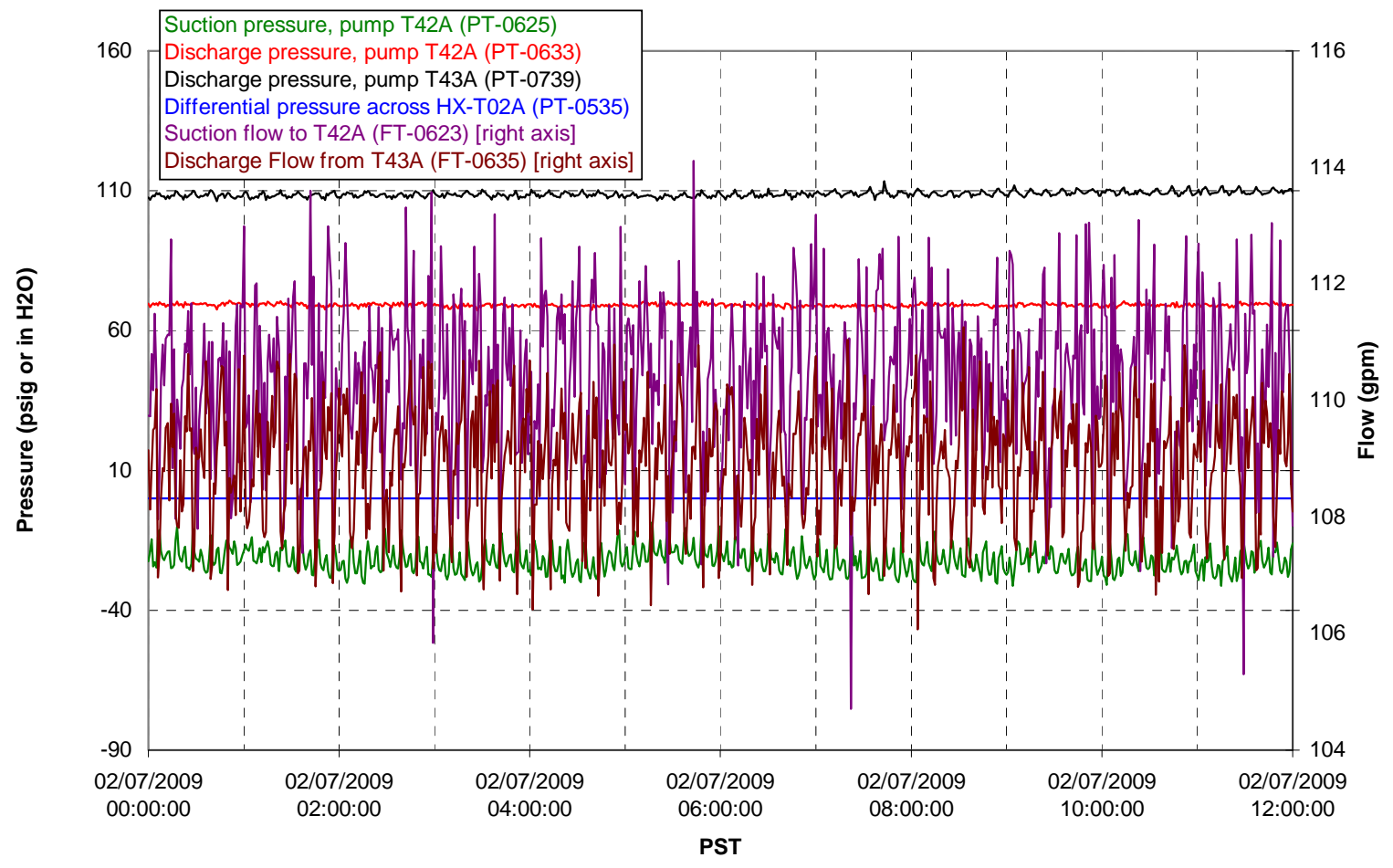


Axial pressure drop

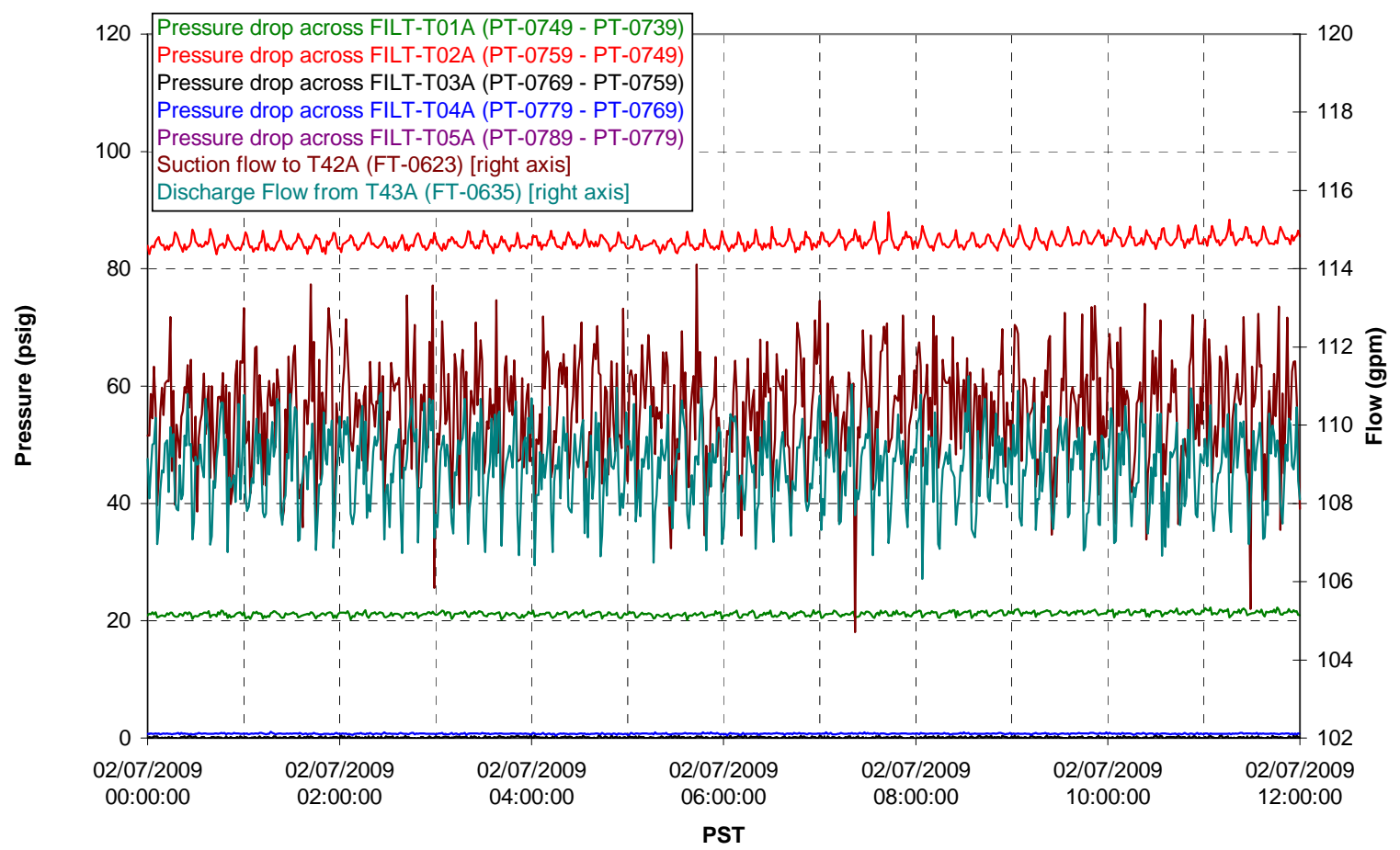

Permeate flow rates

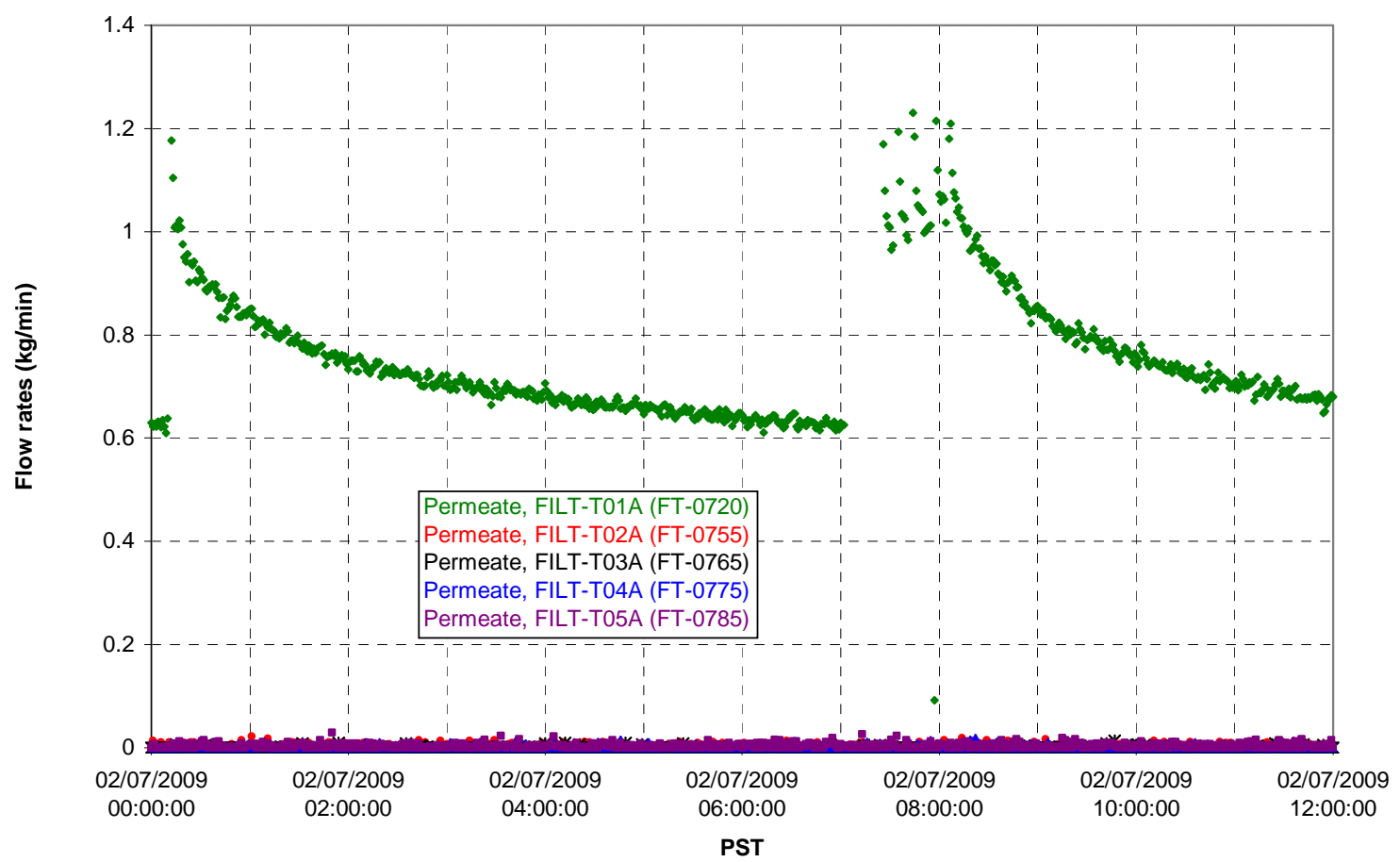


T02A Inner Temperature Tree

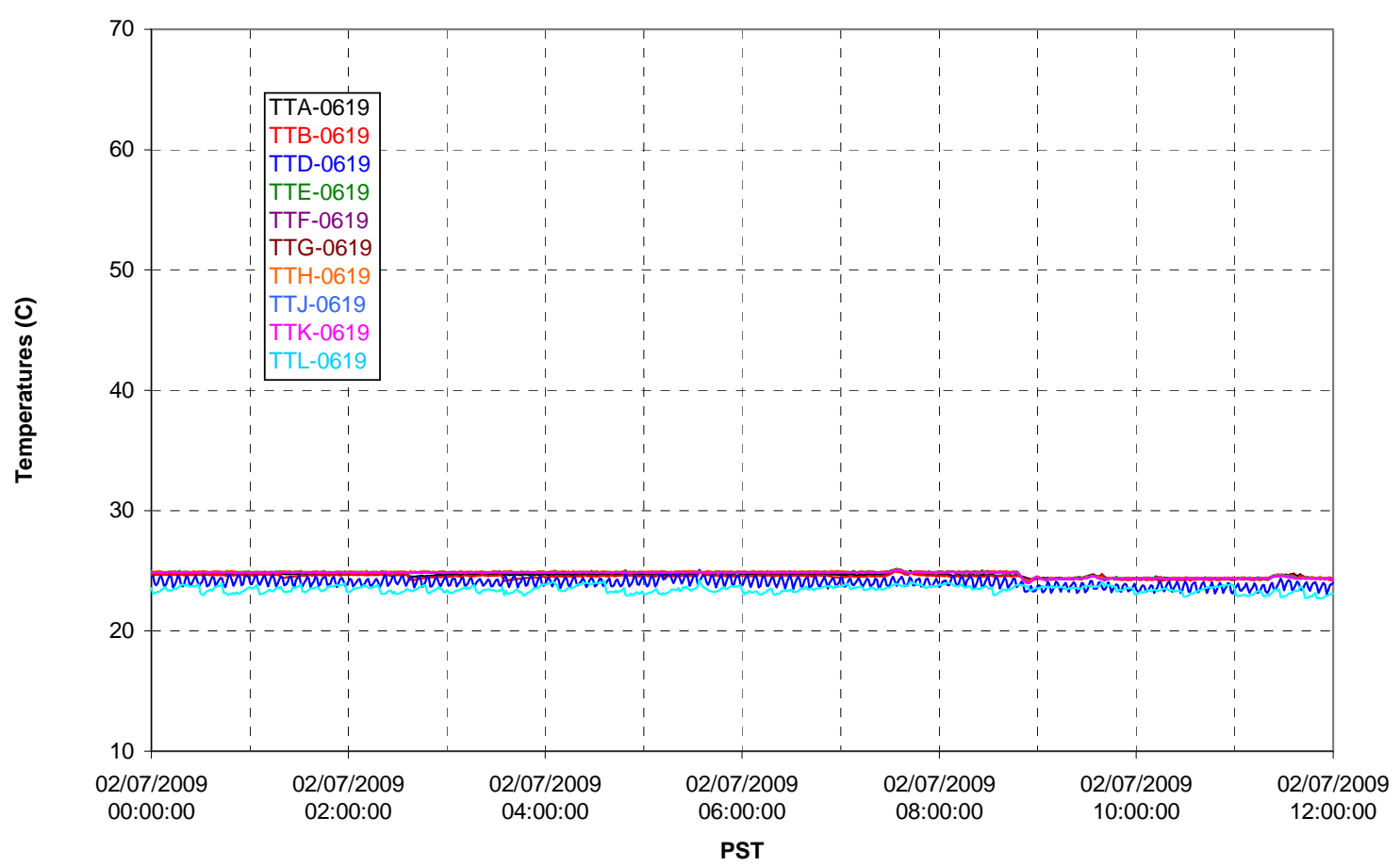

T02A Outer Temperature Tree

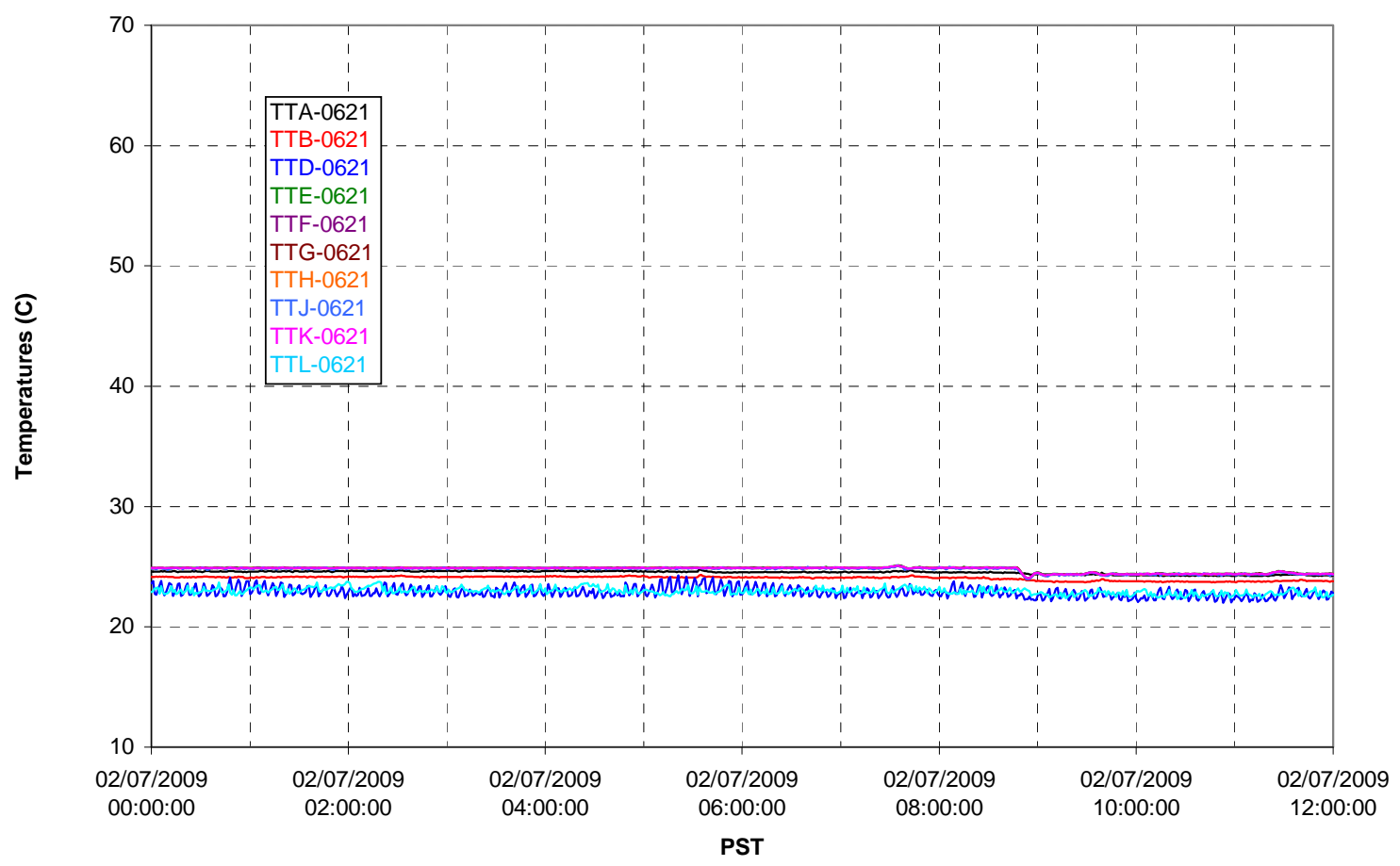


T02A temperatures

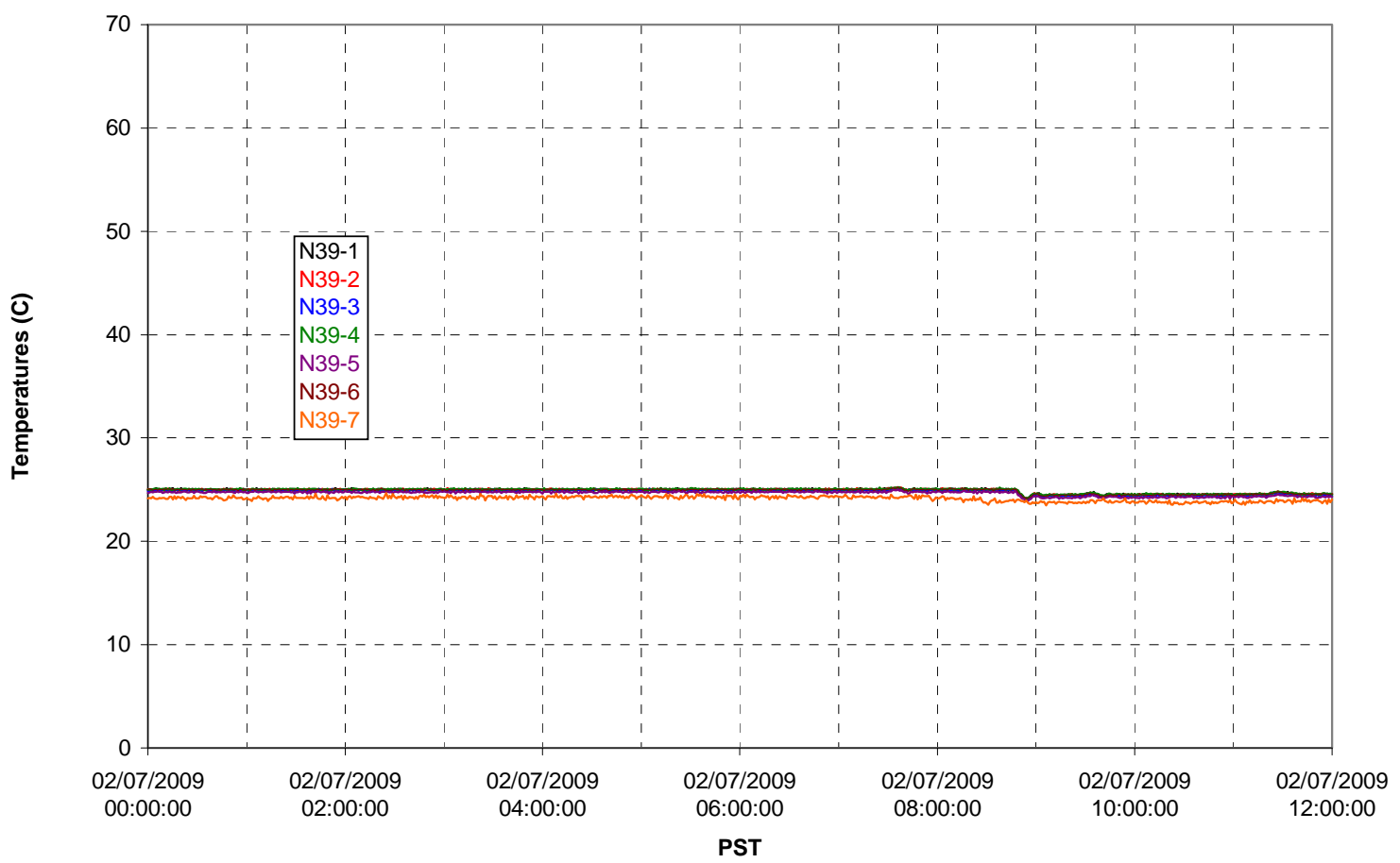

T02A temperatures

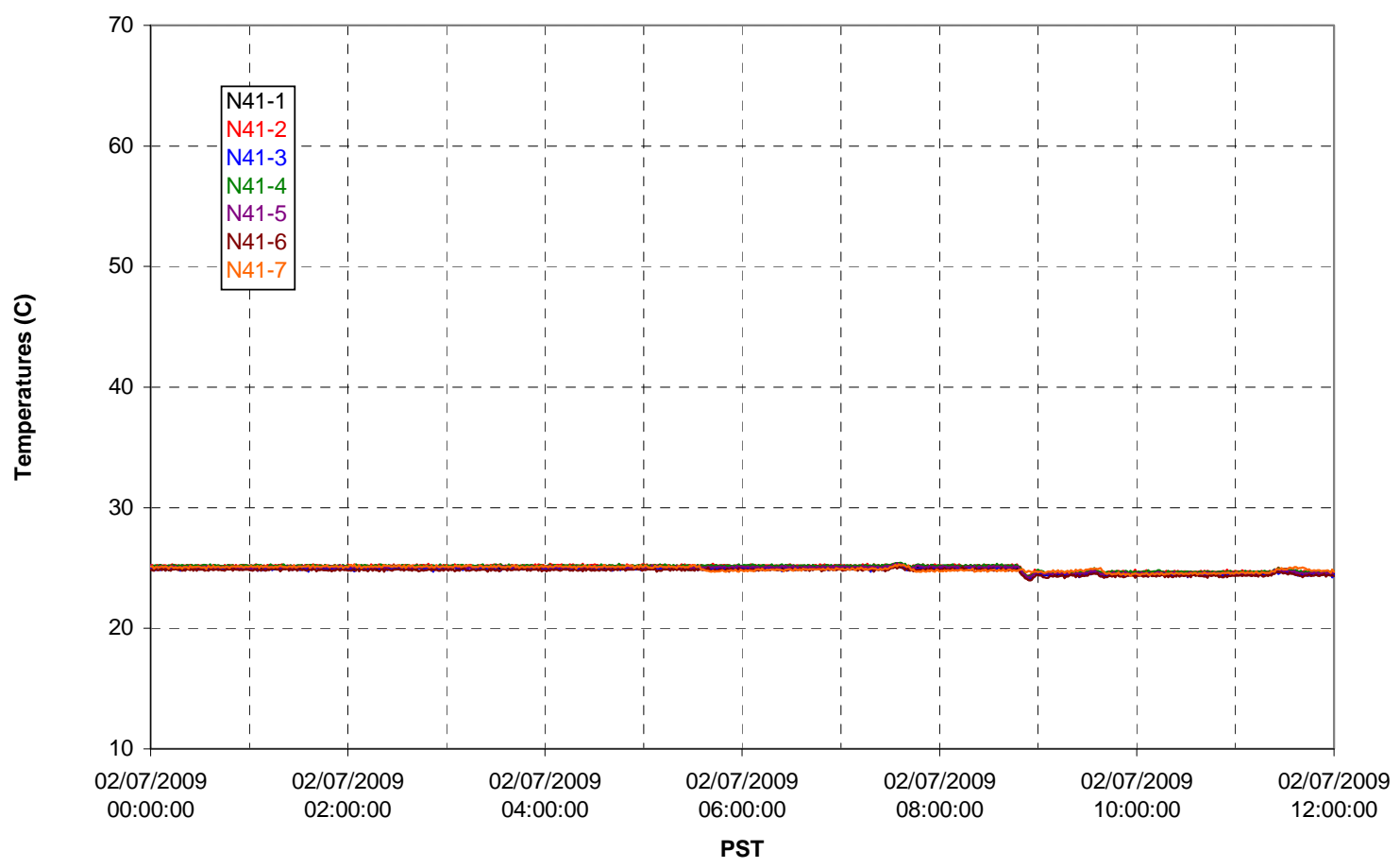


T02A temperatures

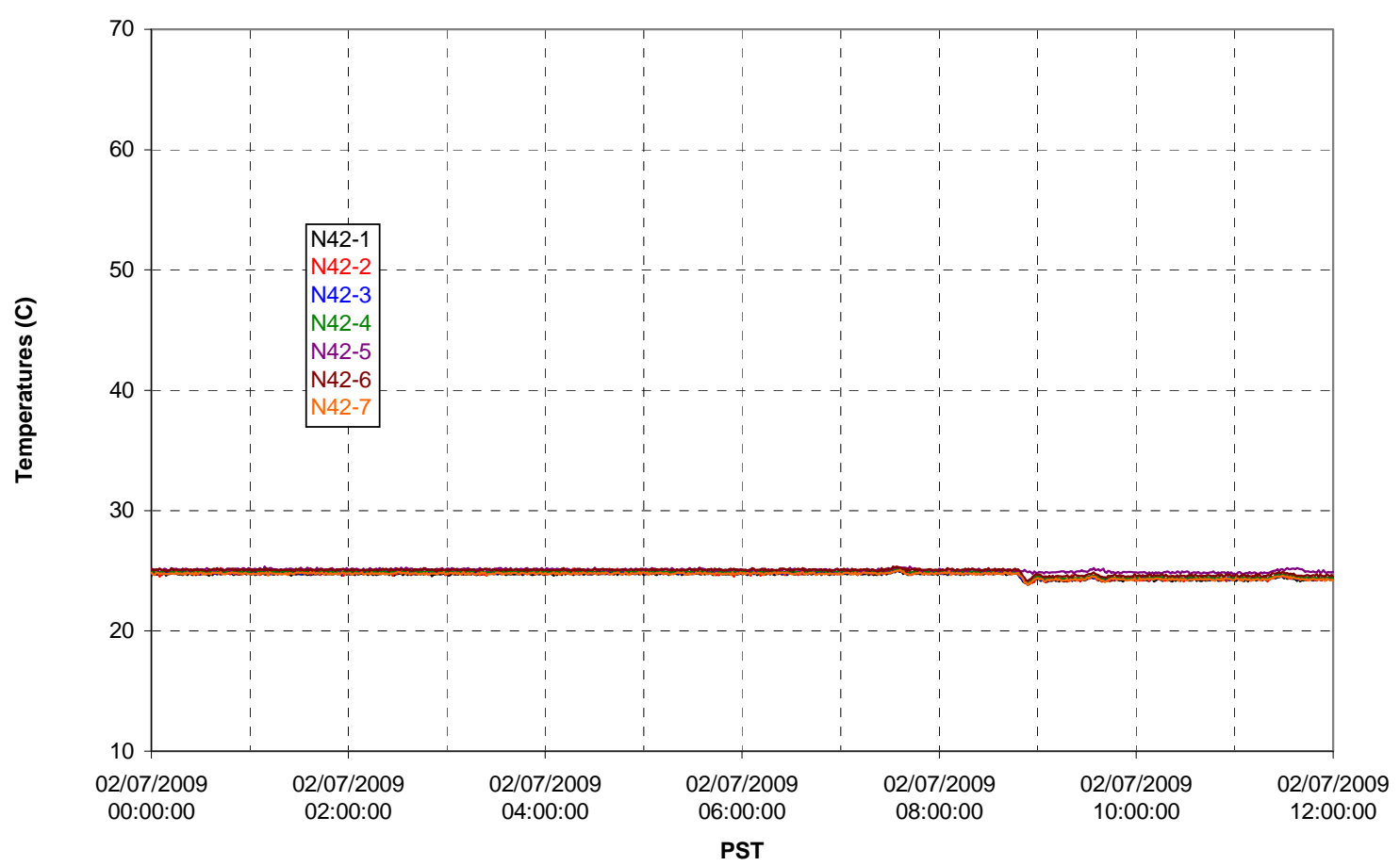

T02A temperatures

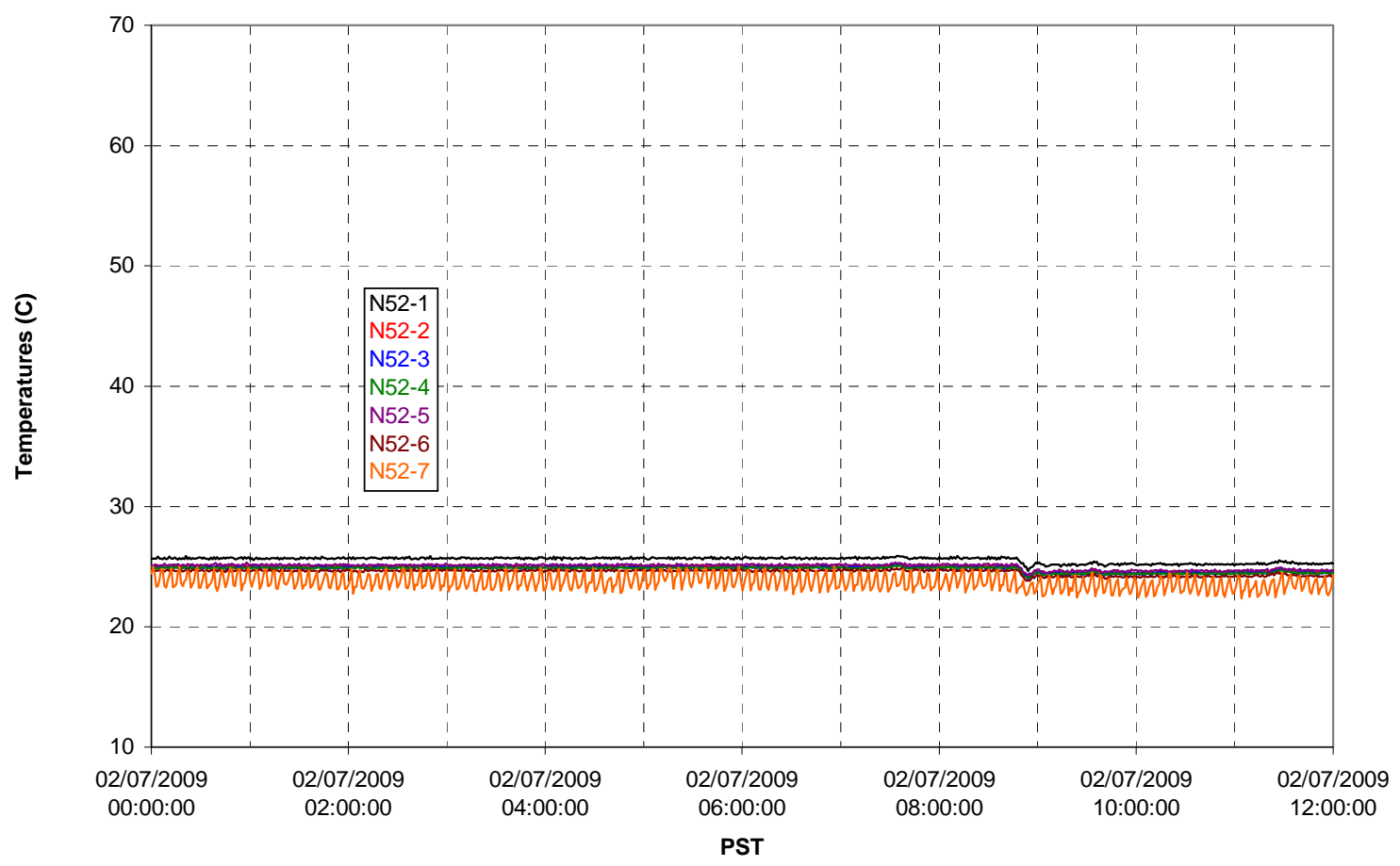


T02A Heating and Cooling

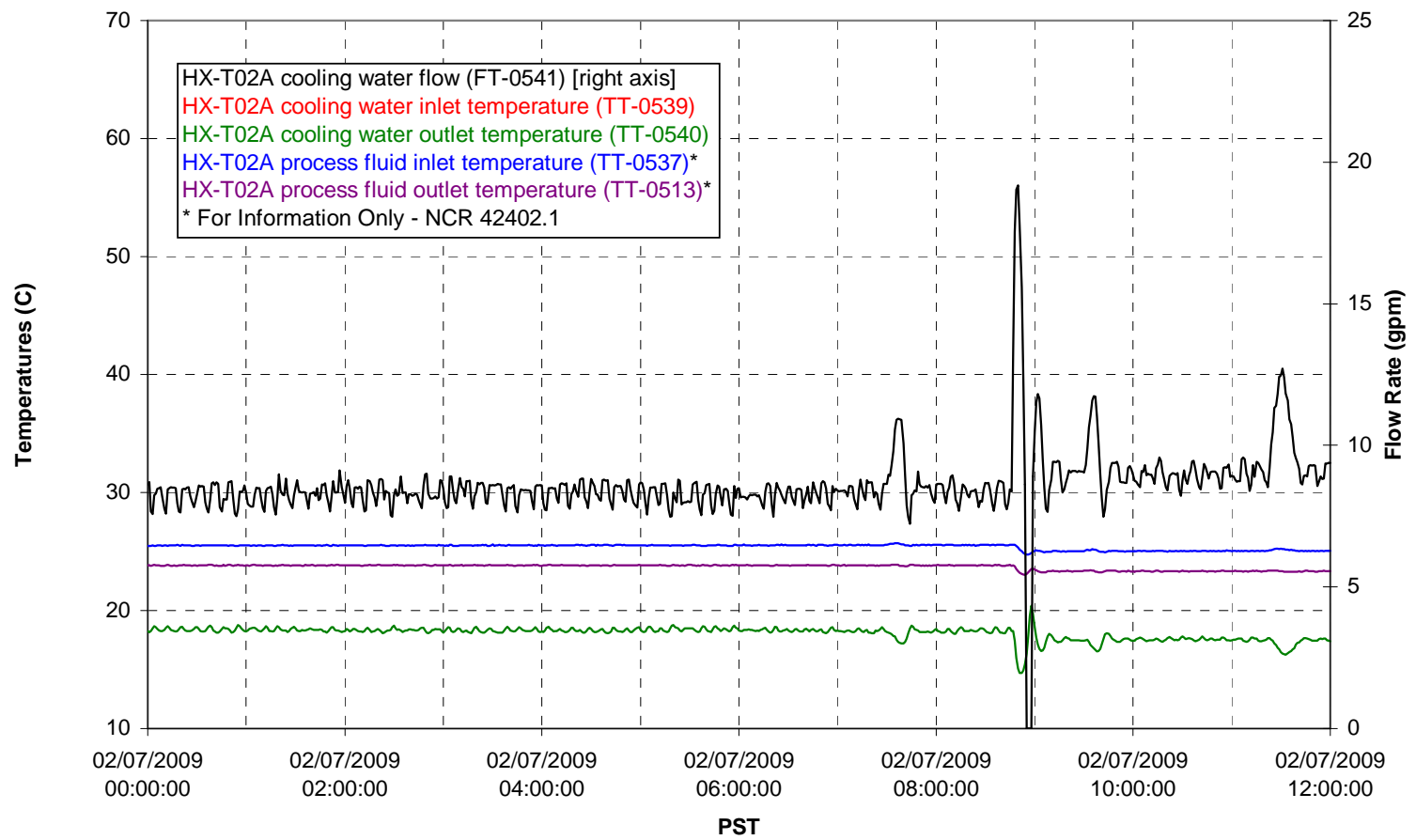

Pump Operation

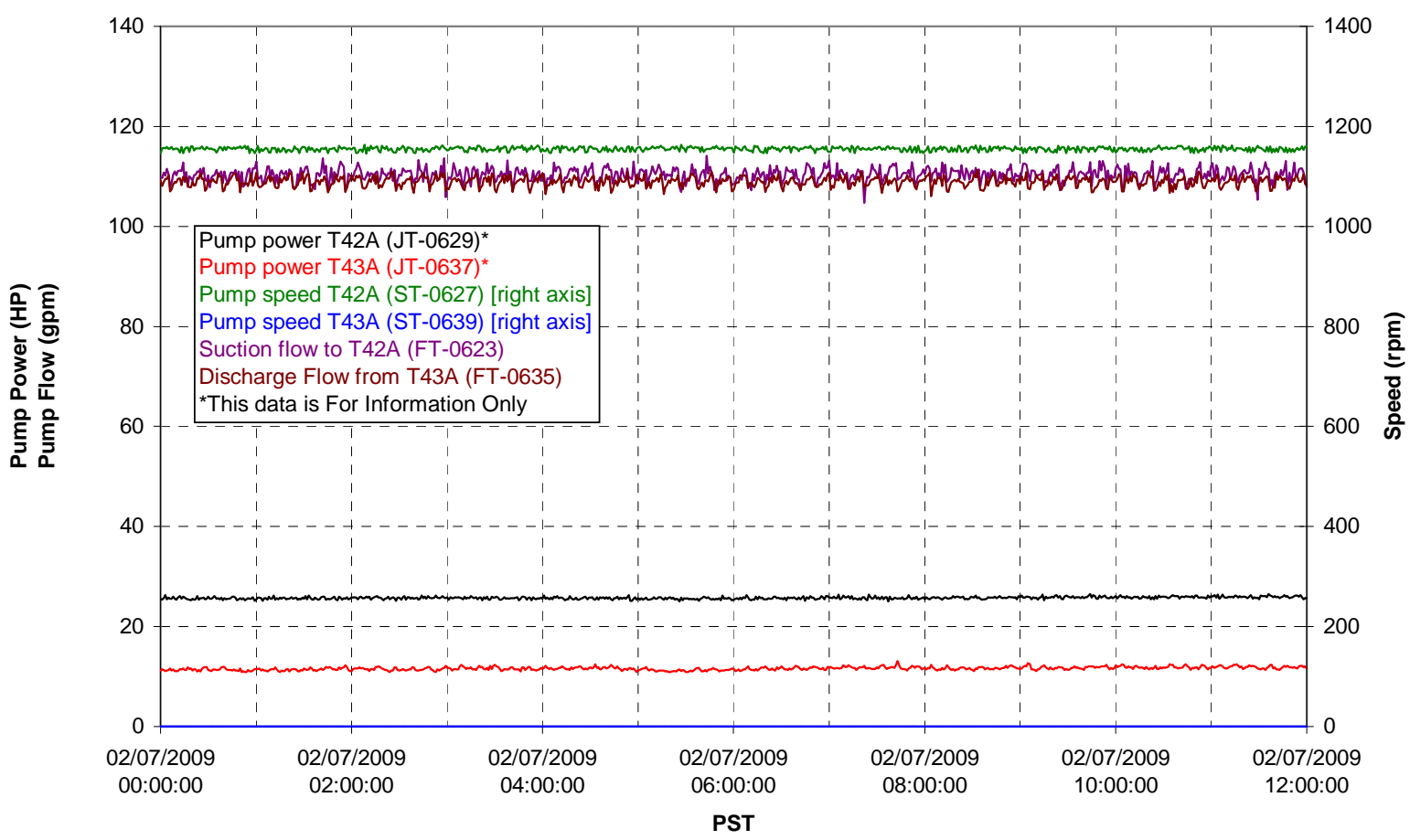


Pulsepot UFP-PP-T01A

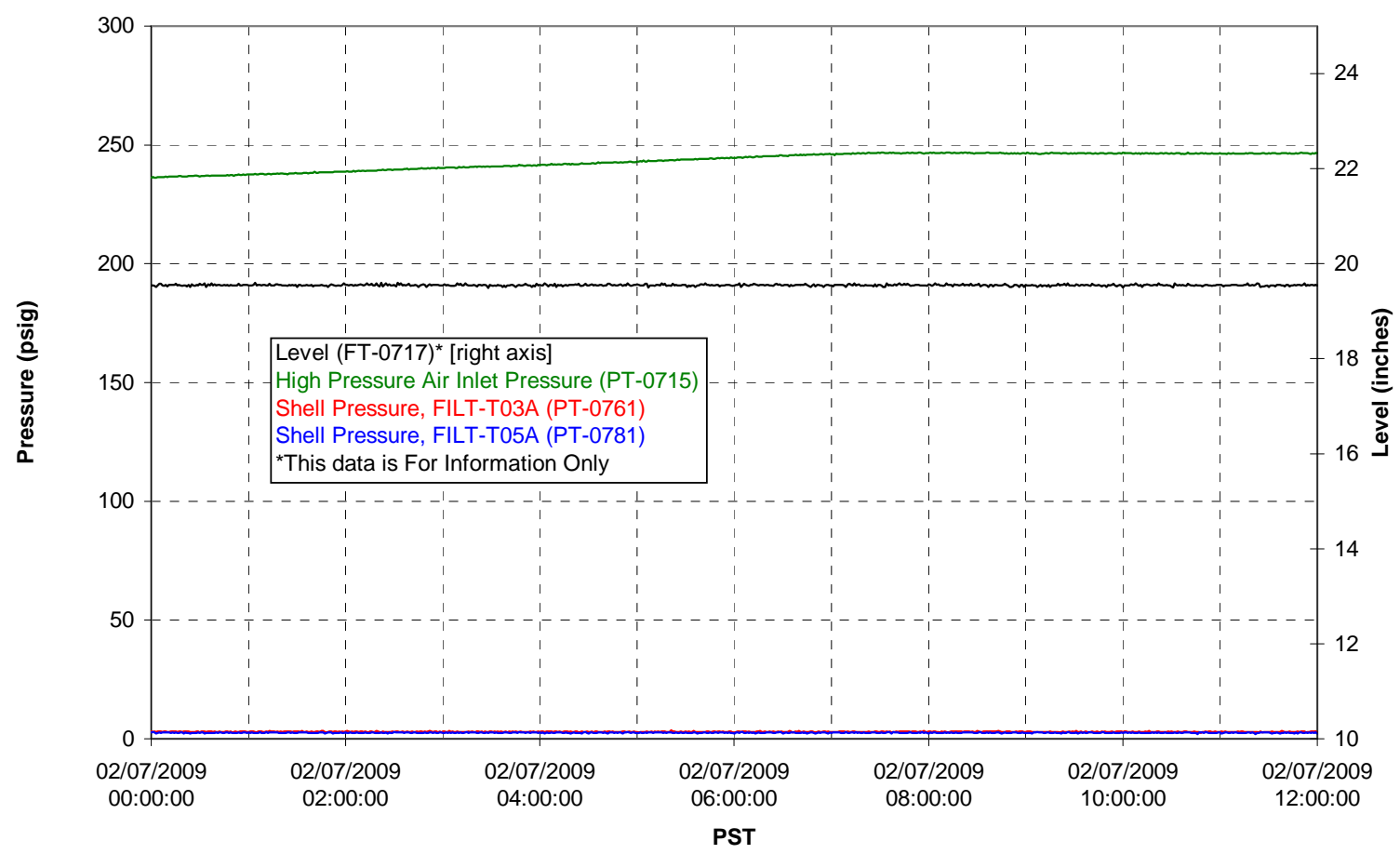

Pulsepot UFP-PP-T02A

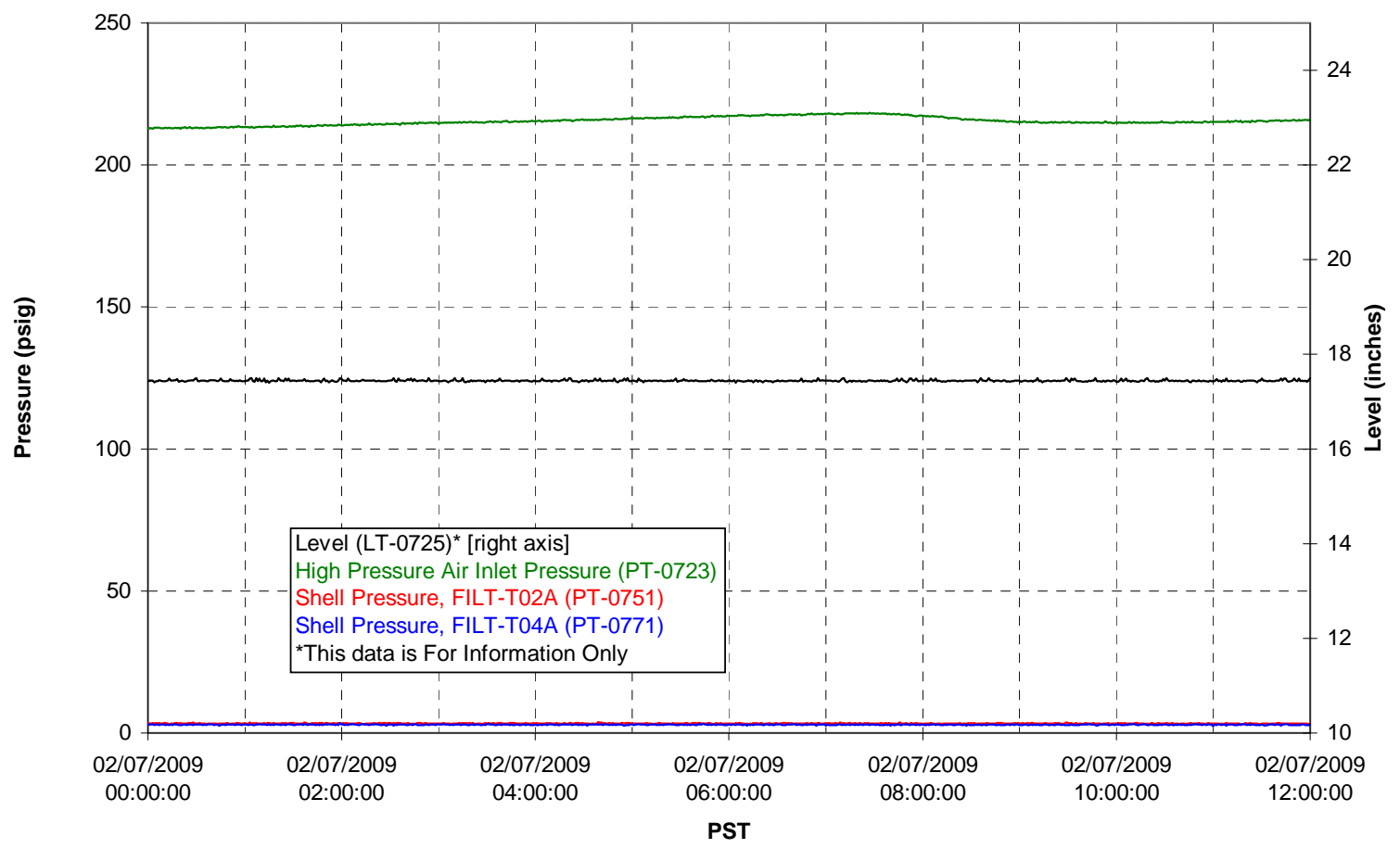


Pulsepot UFP-PP-T03A

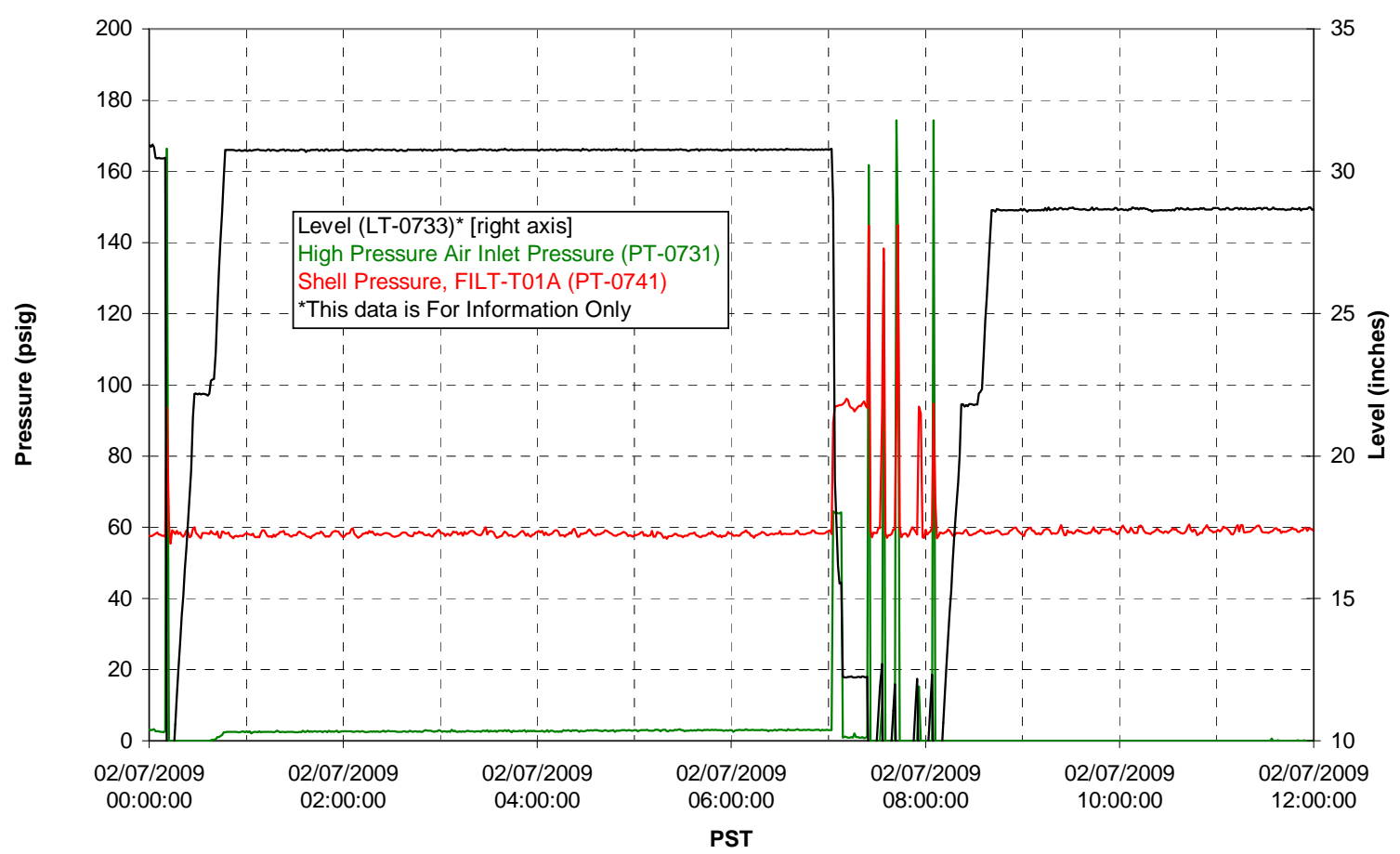

Pulsepot Levels

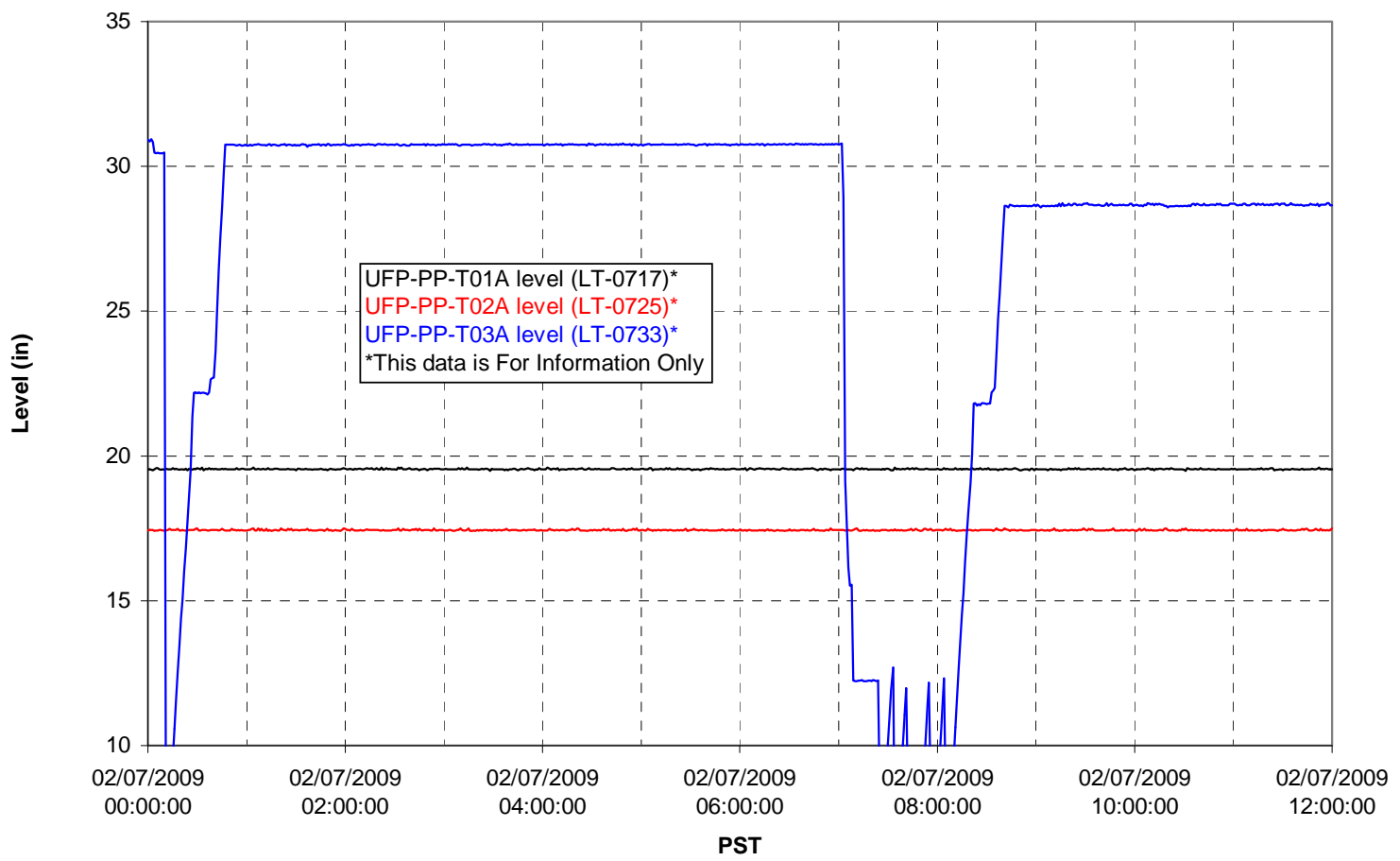


Filter UFP-FILT-T01A

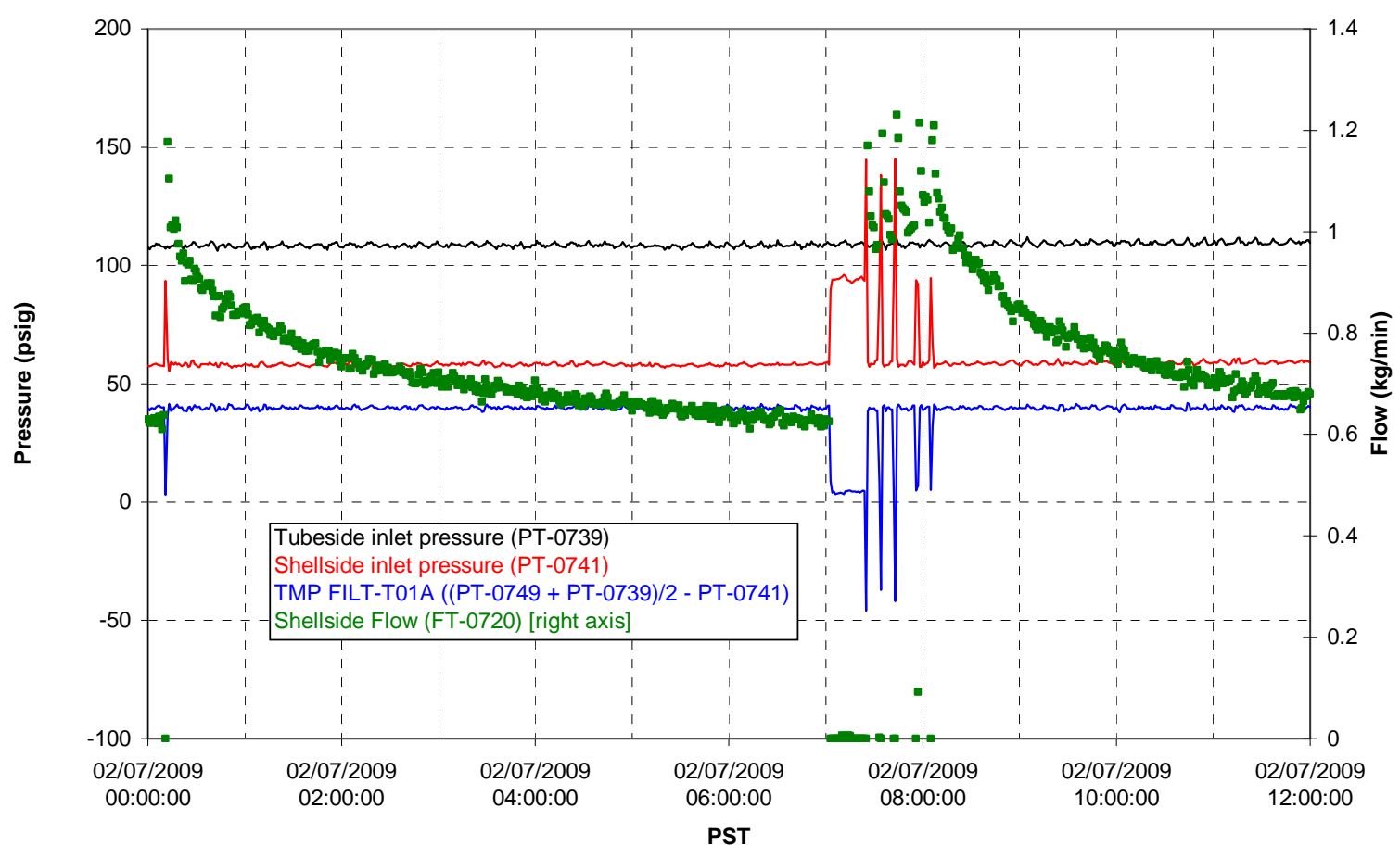

Filter UFP-FILT-T02A

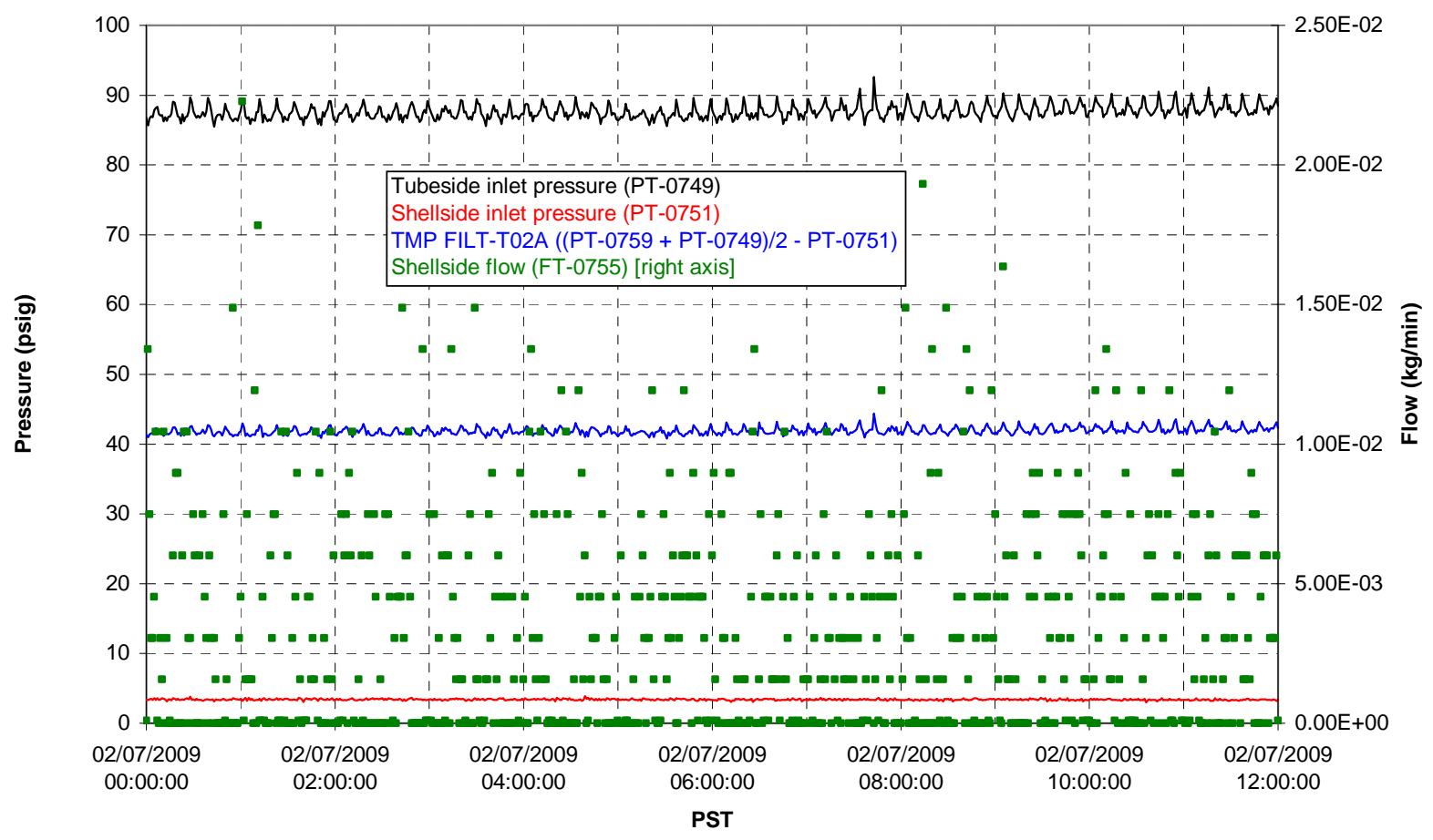


Filter UFP-FILT-T03A

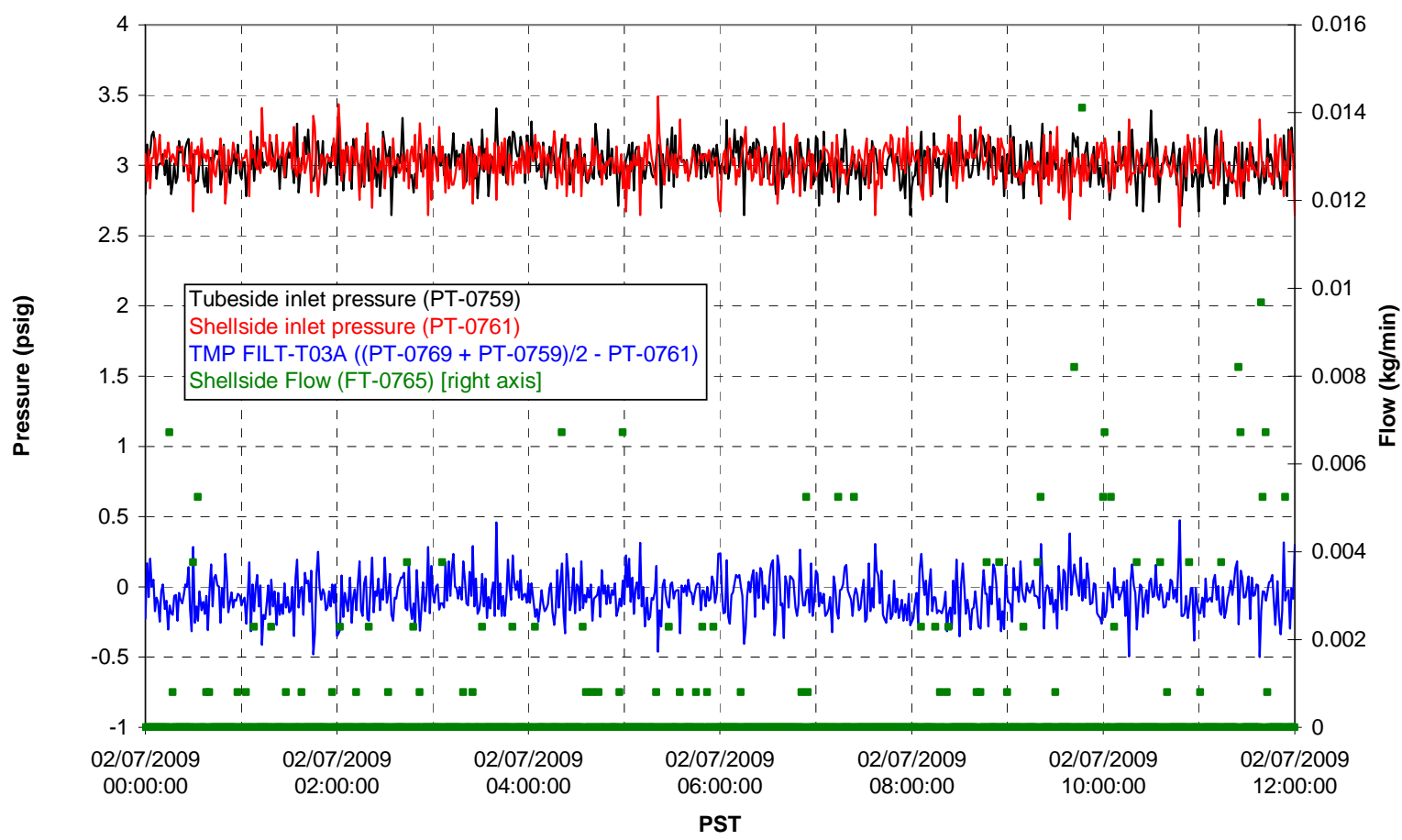

Filter UFP-FILT-T04A

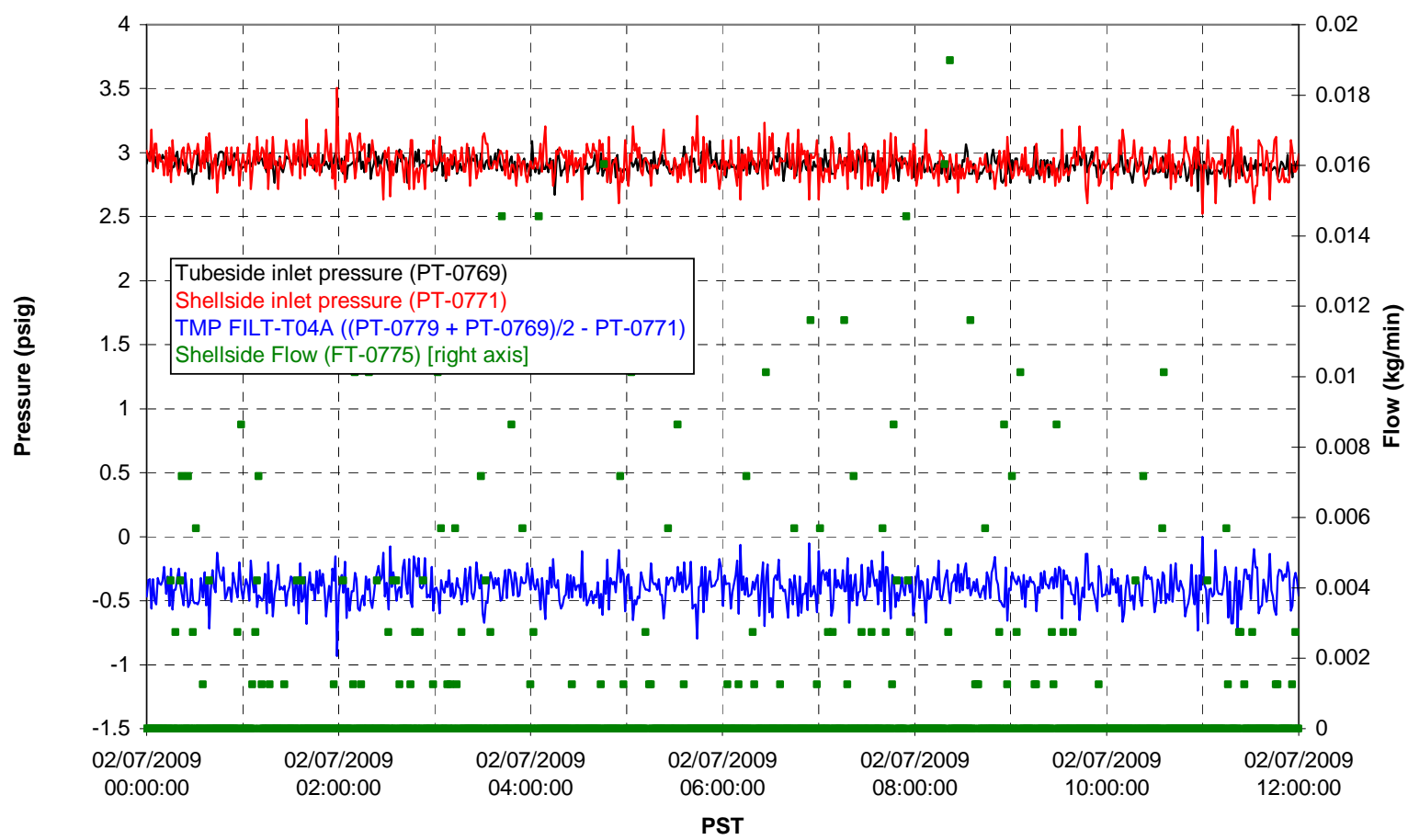


Filter UFP-FILT-T05A

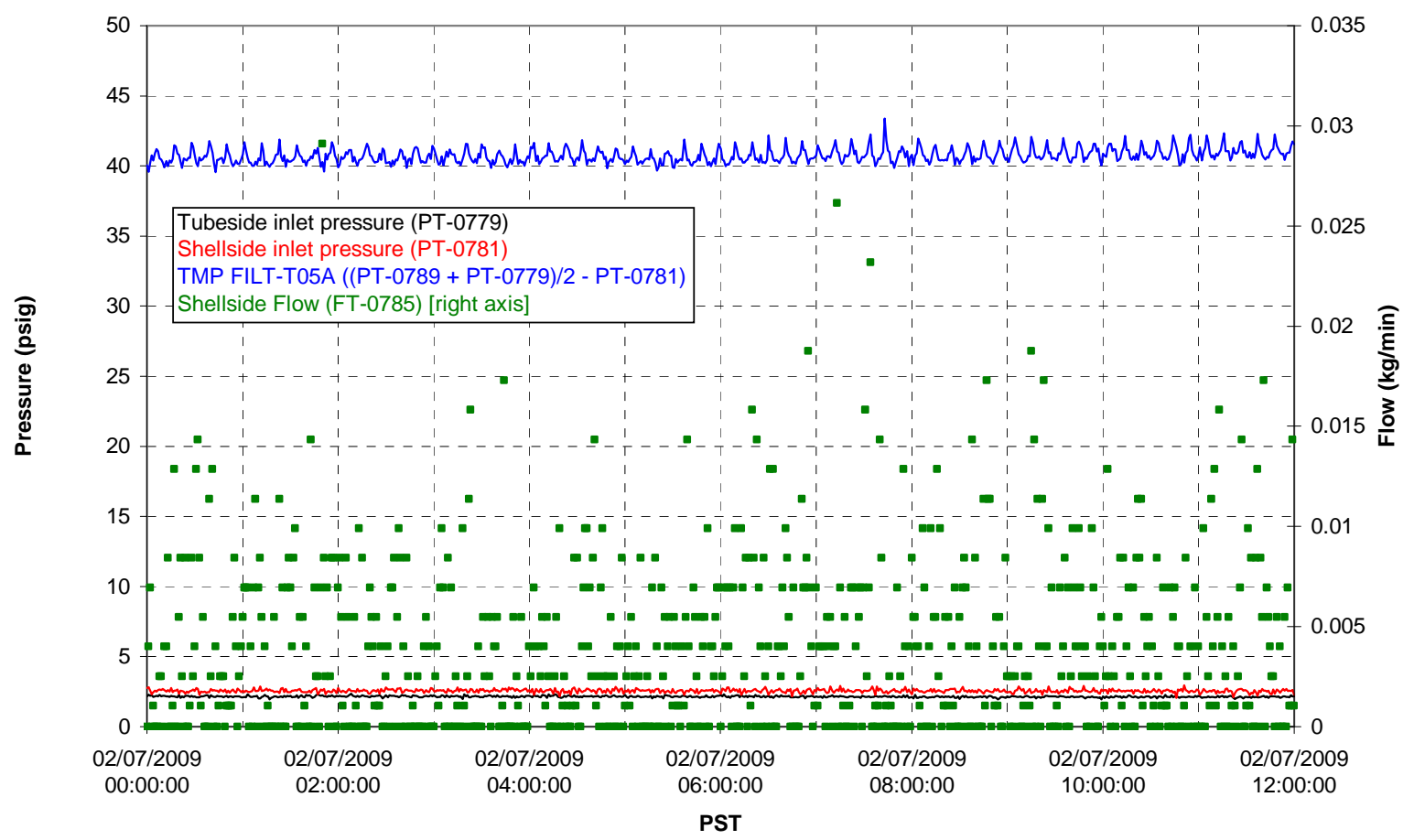

Chemical Flow

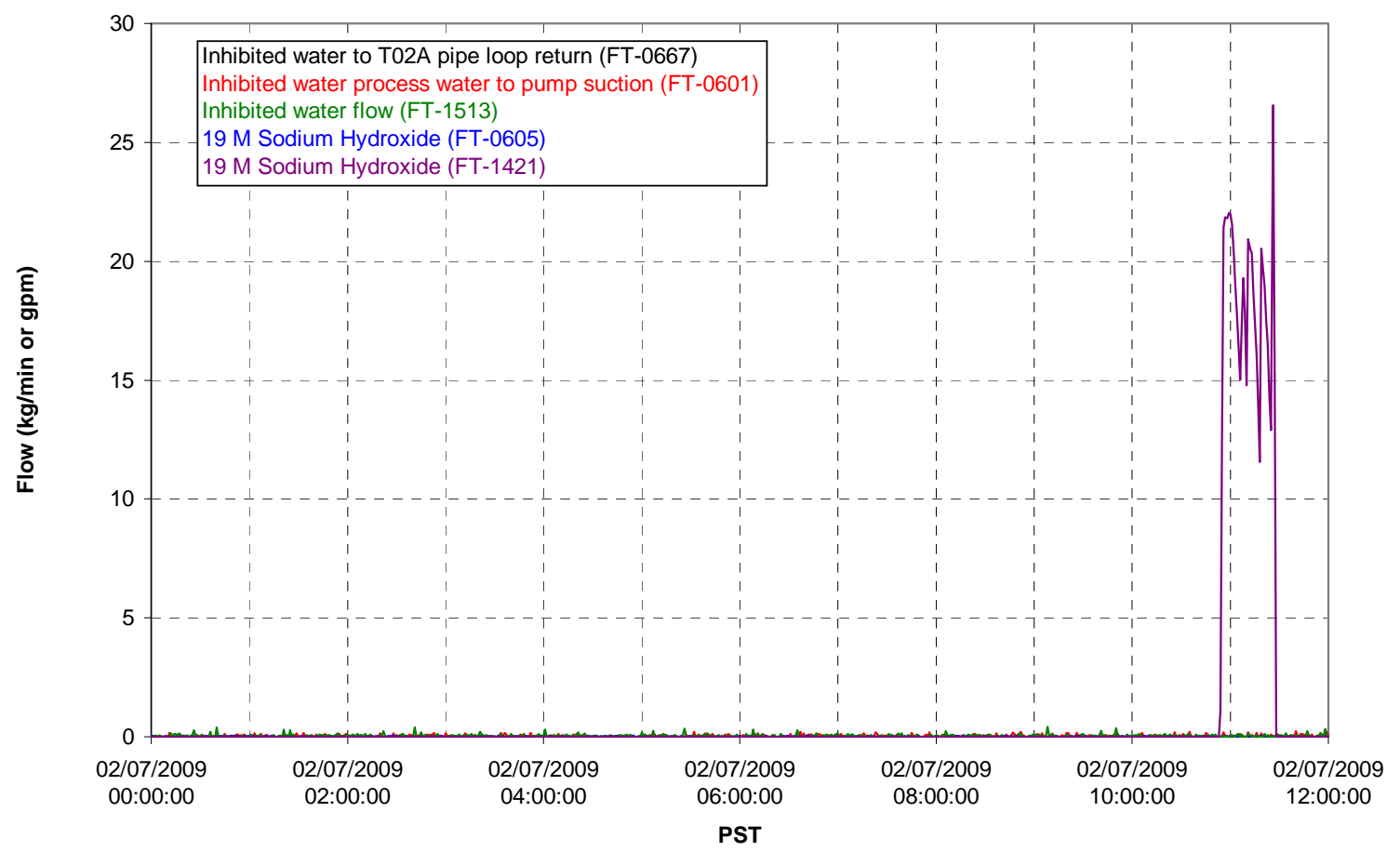


Chemical Flow

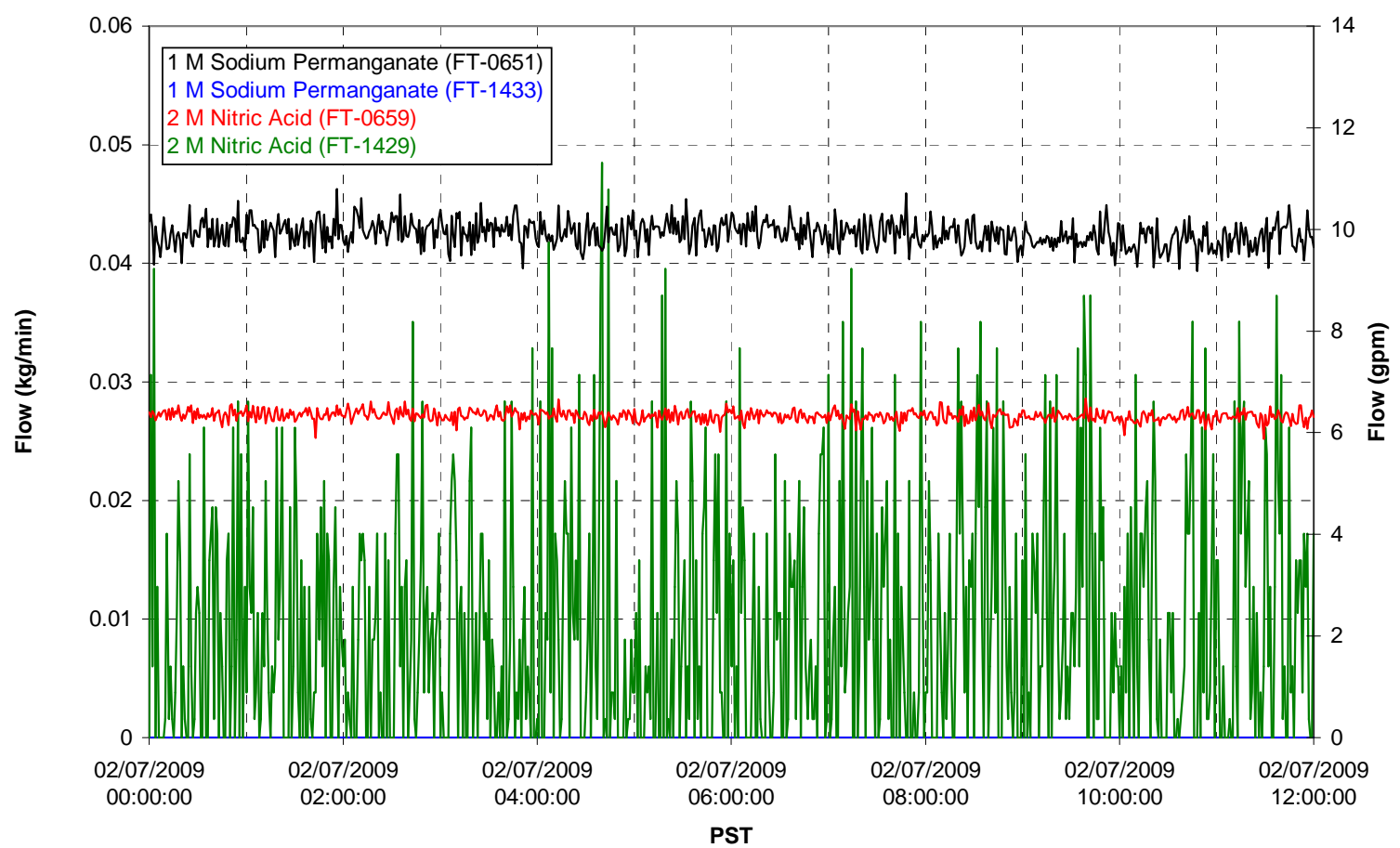

Air Flows

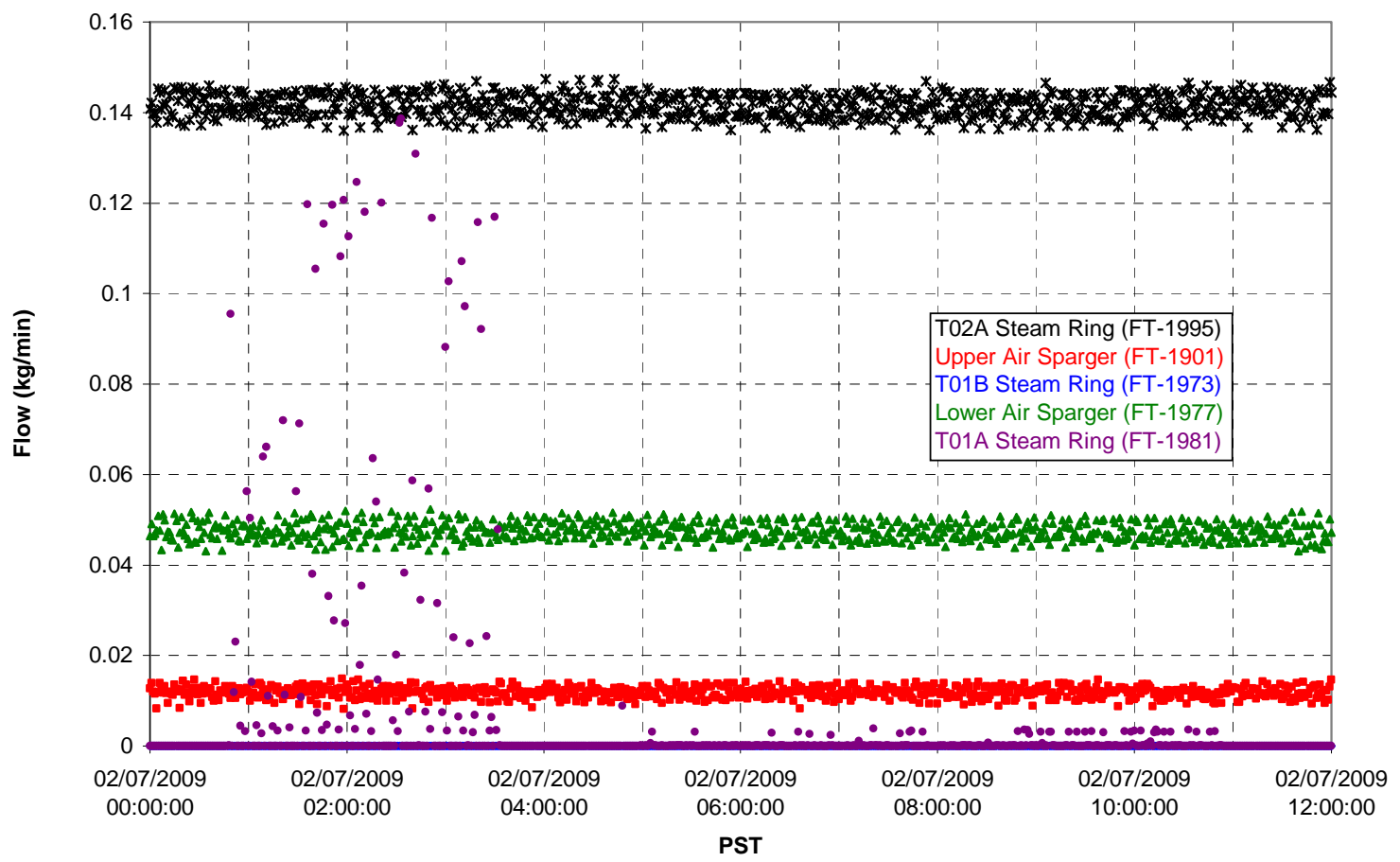


T02A Steam

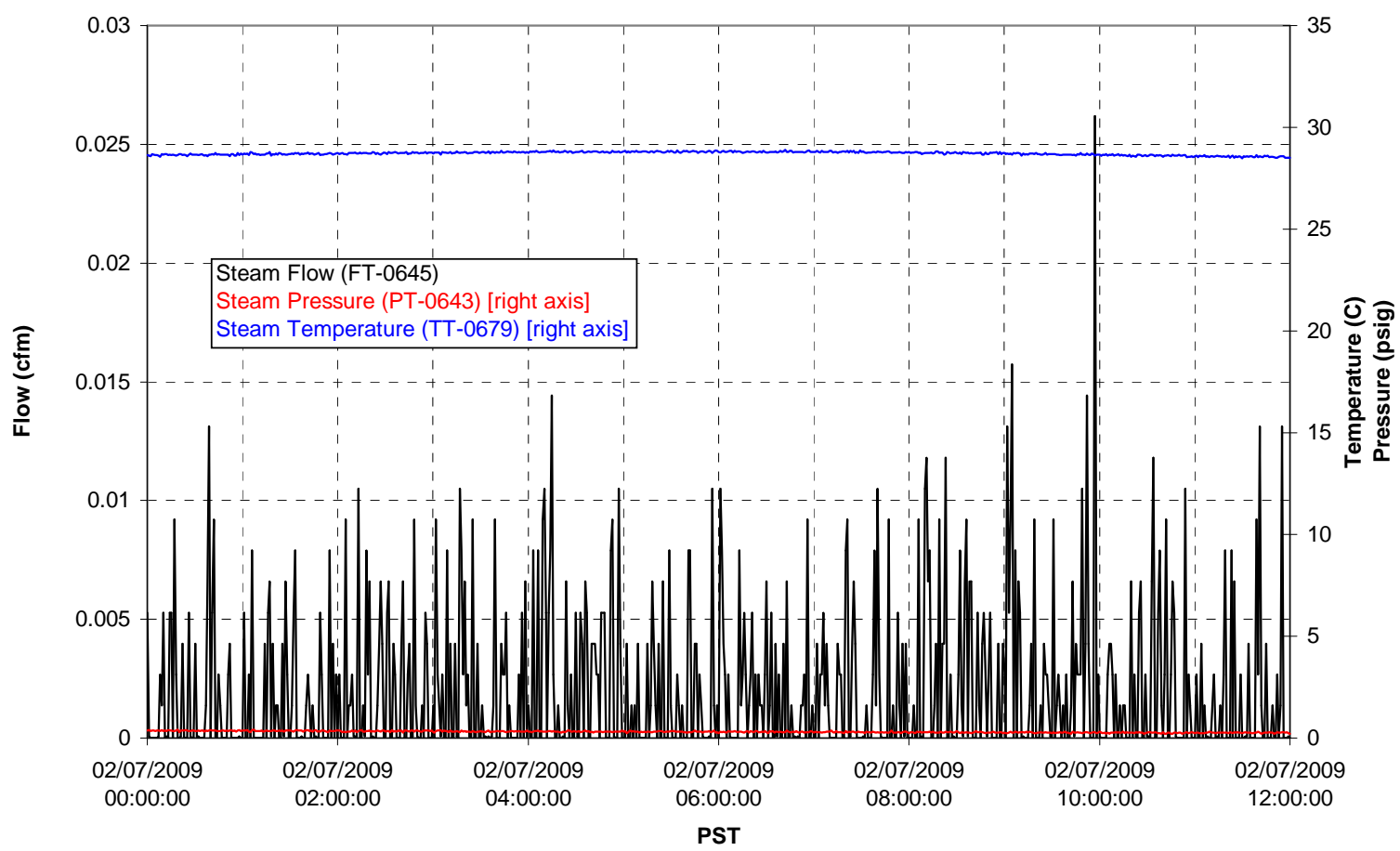

T01A Steam

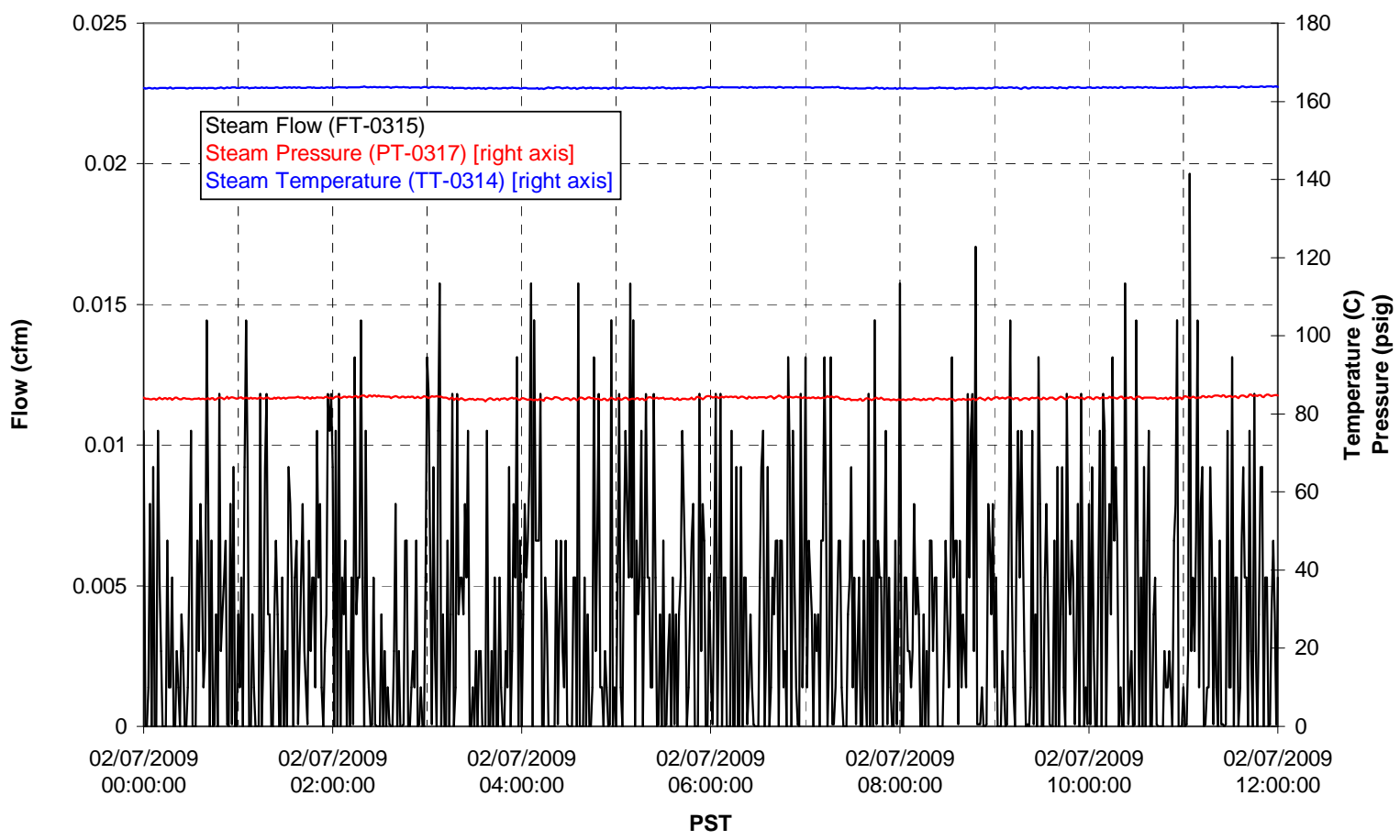


T01B Steam

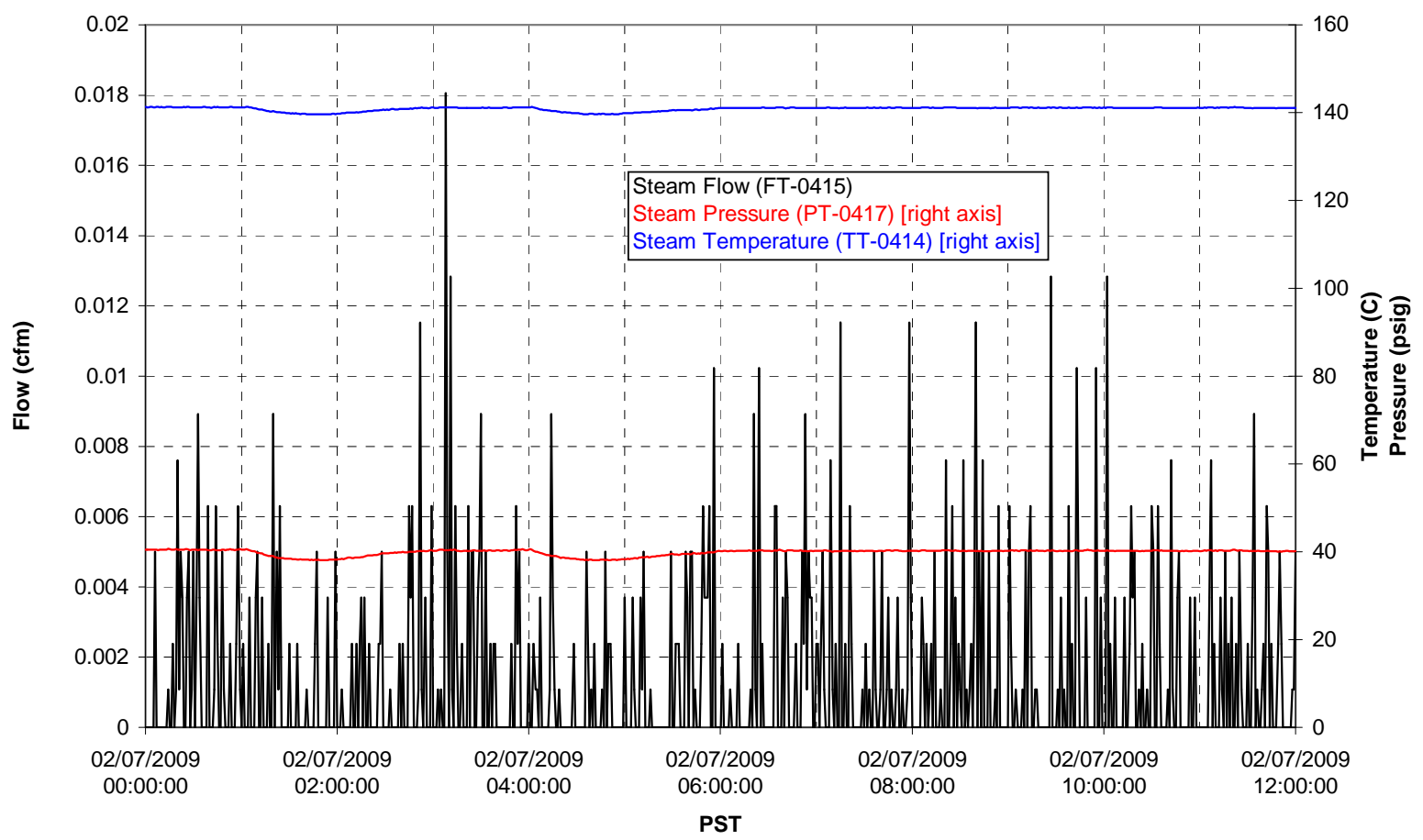




\section{Integrated Test A Data Plots 02/07/09 12:00 - 02/08/09 00:00}


T01A level

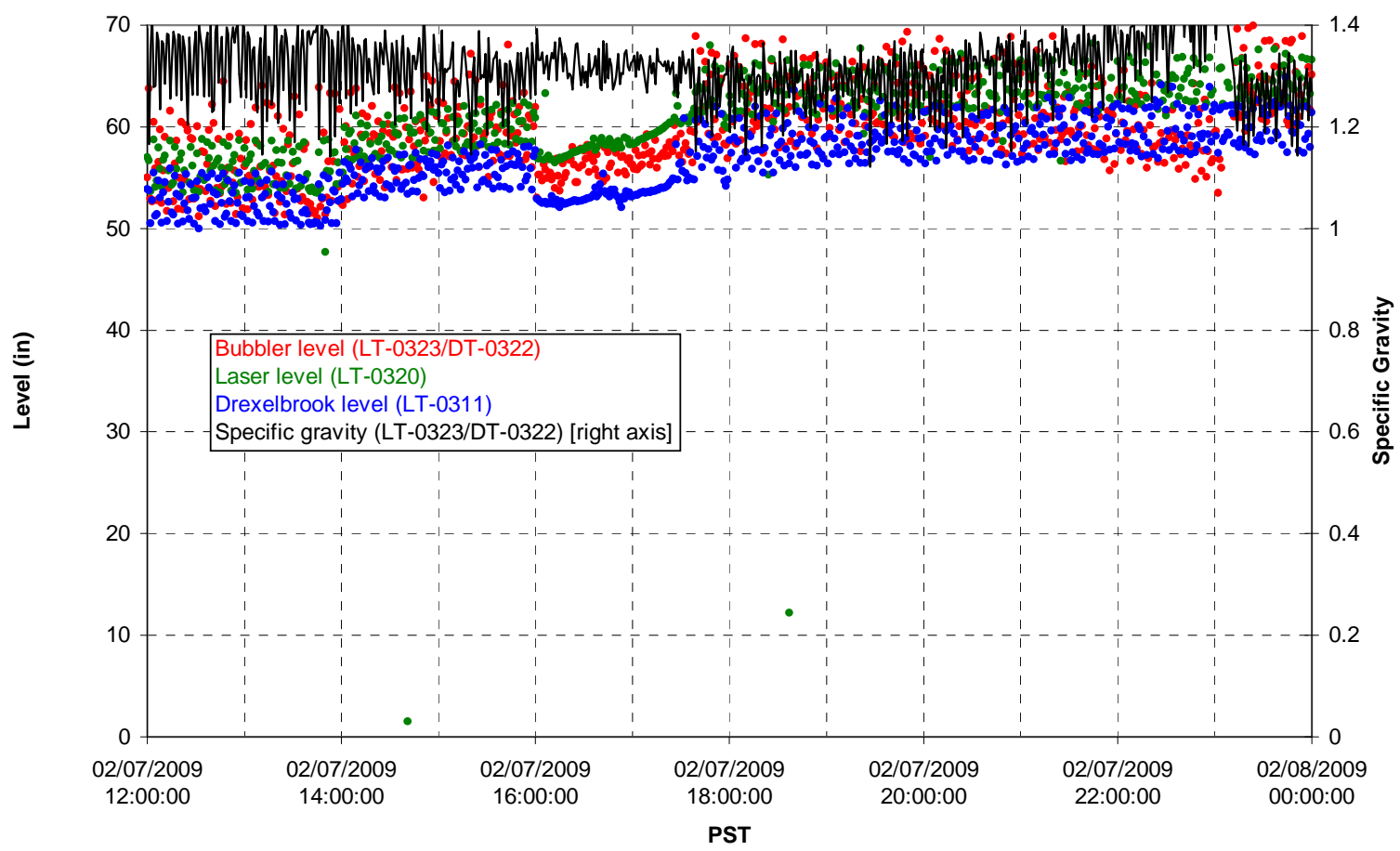

T01A temperatures

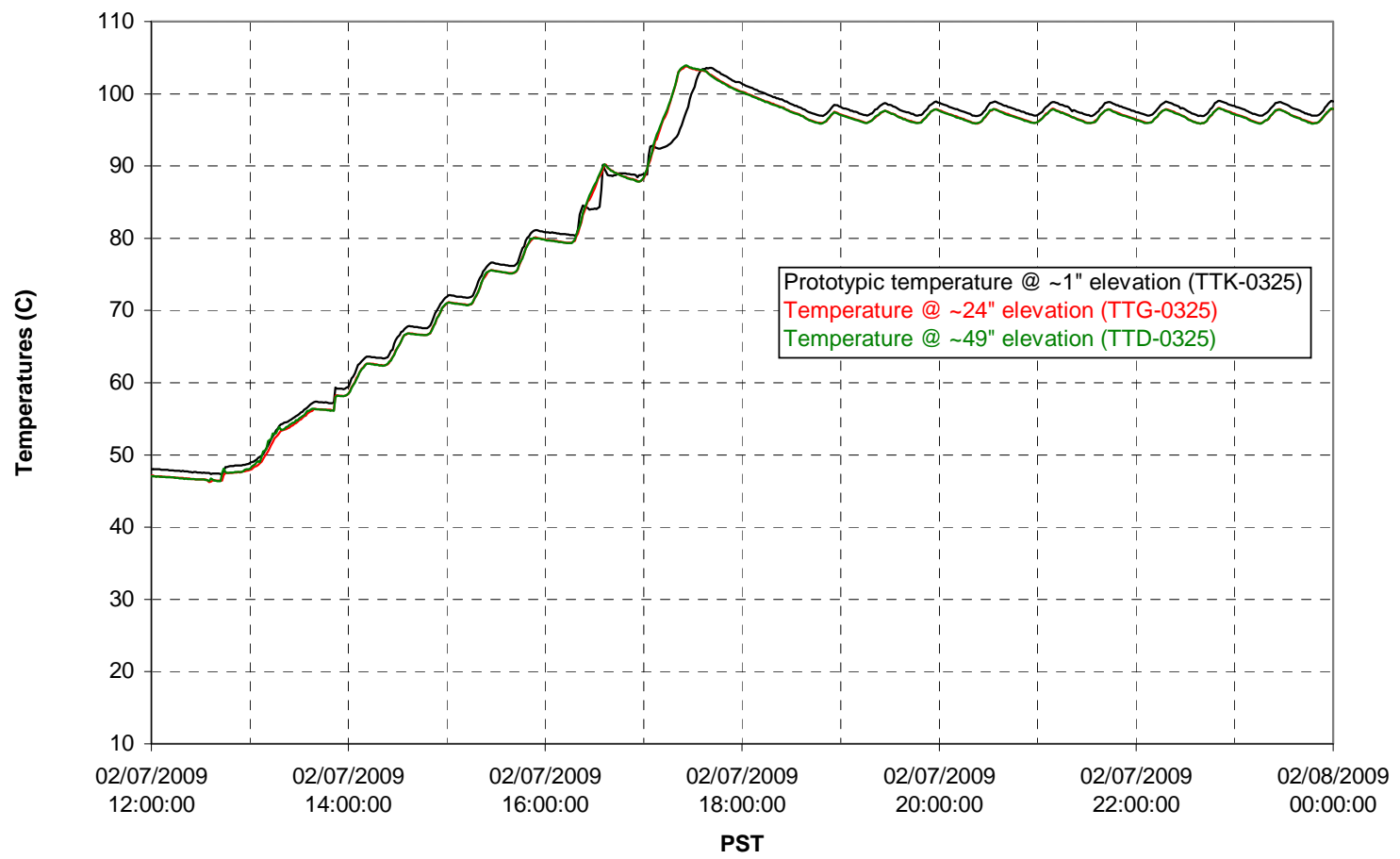


T01B level

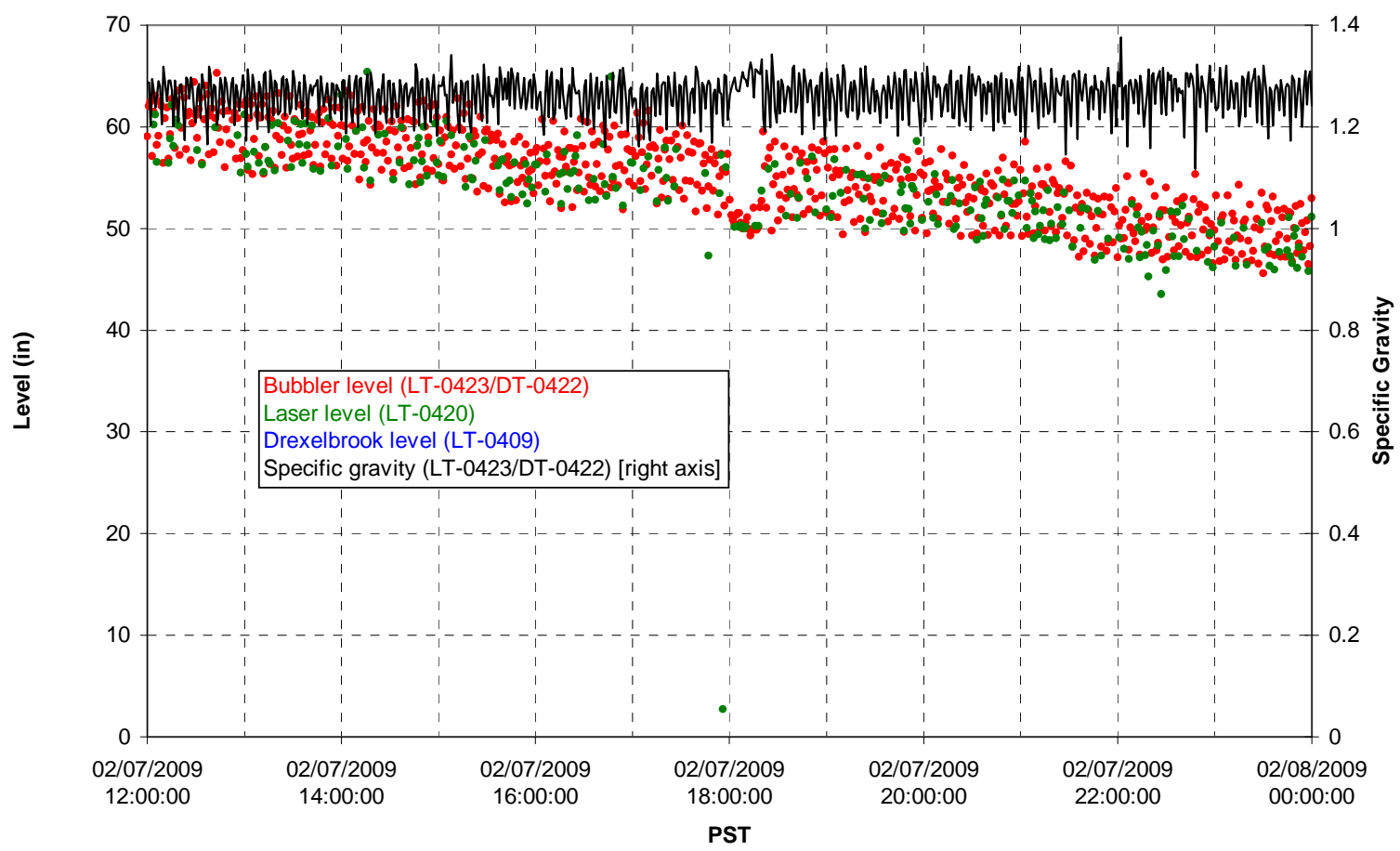

T01B temperatures

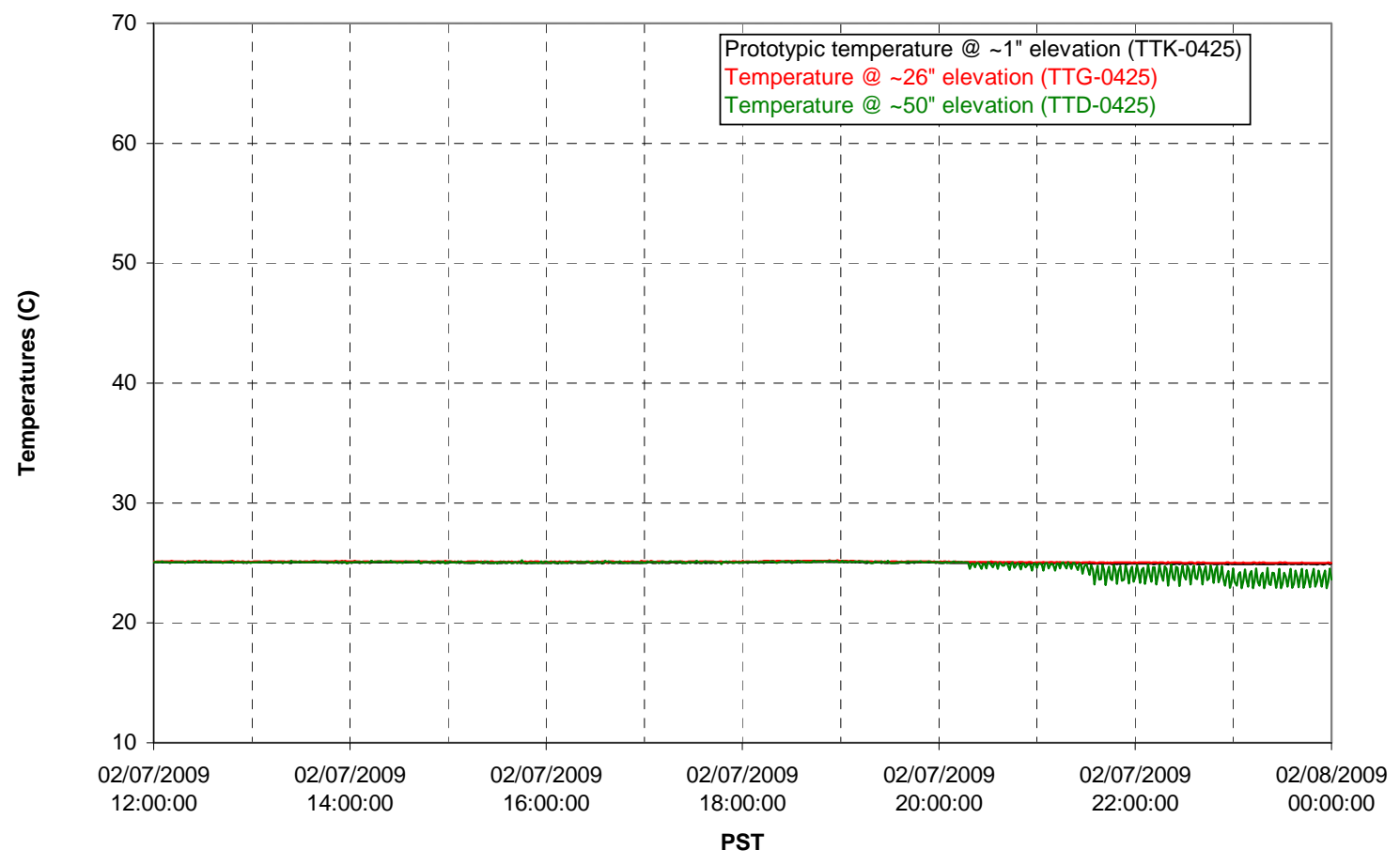


T02A level

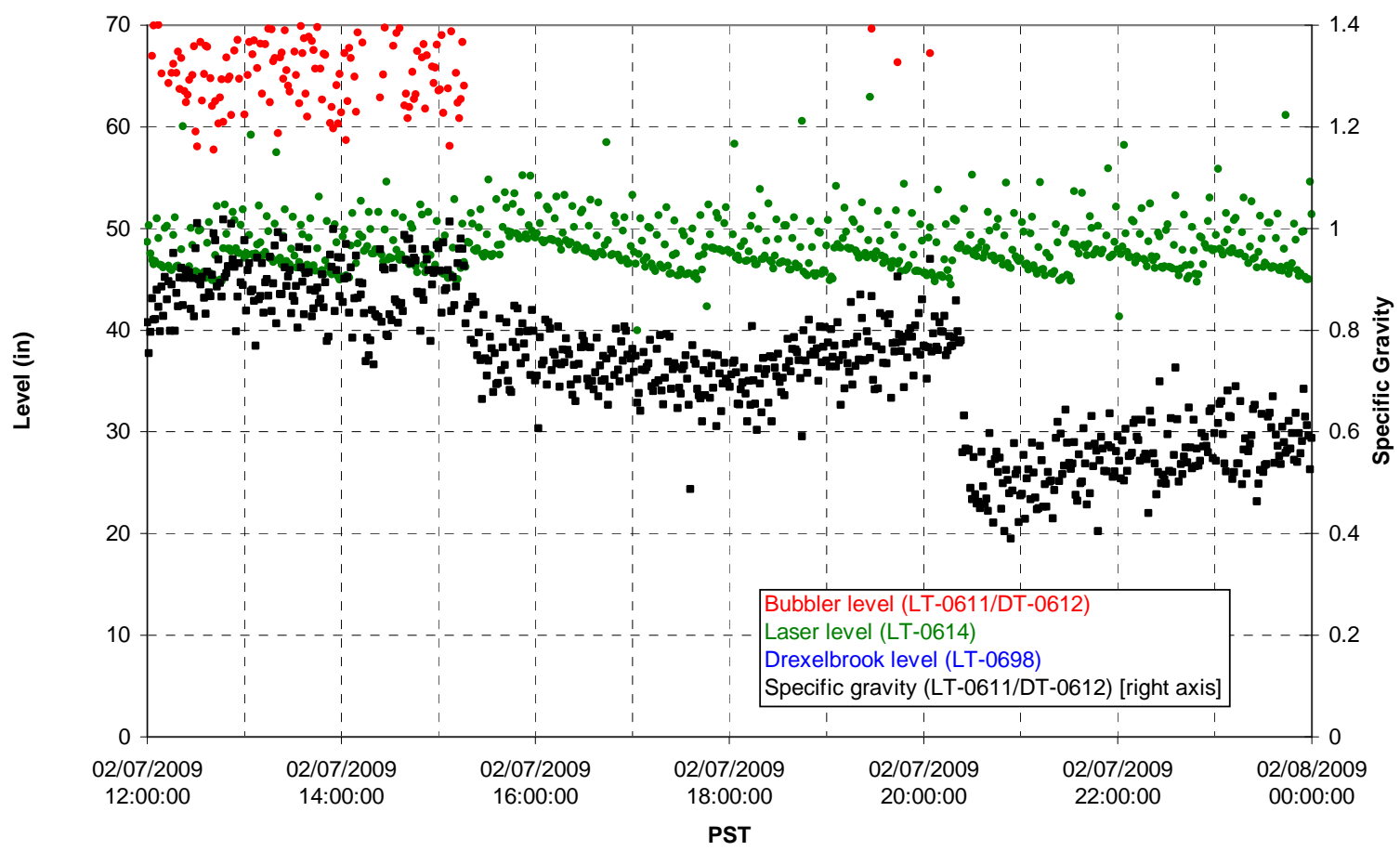

T02A temperatures

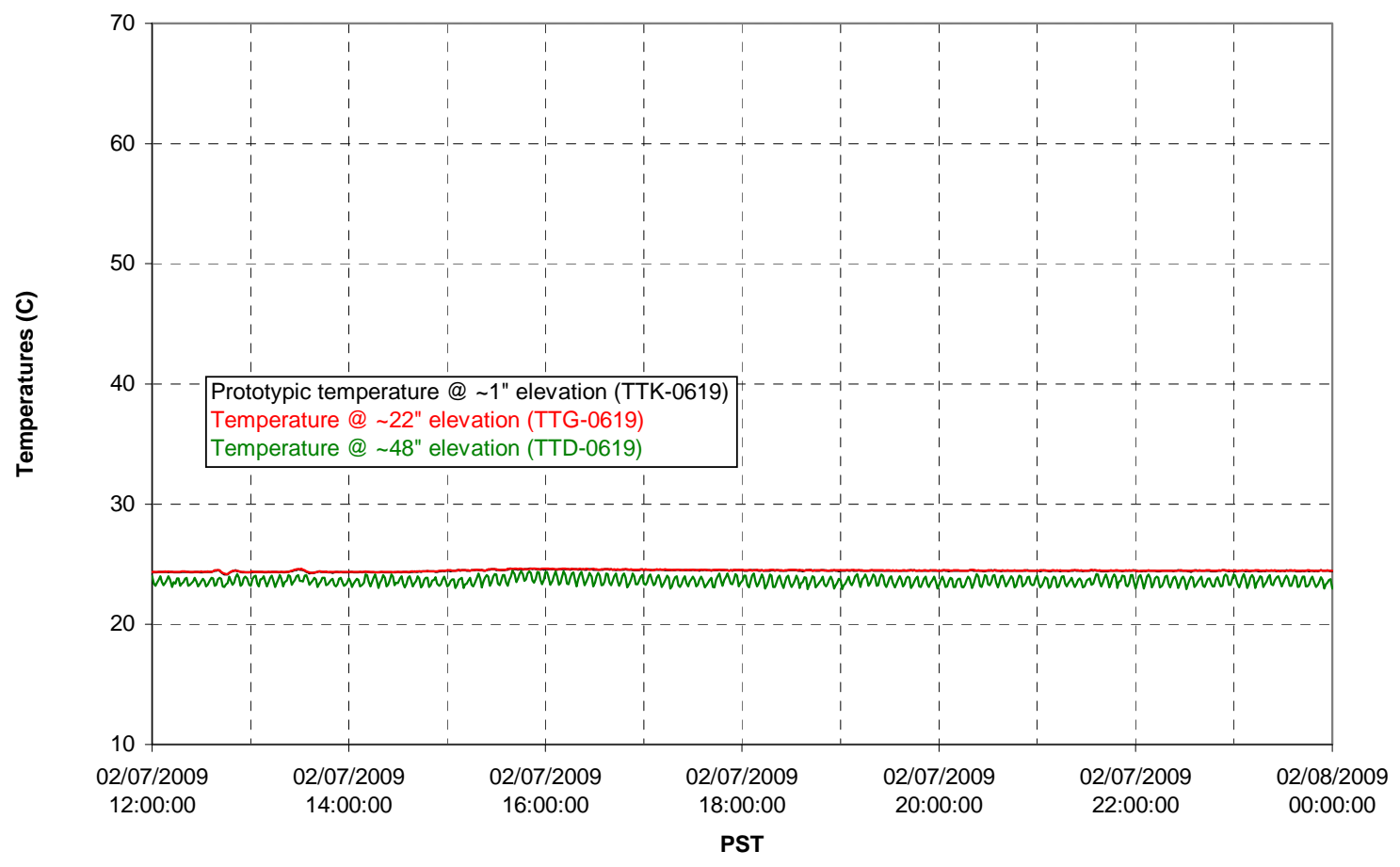

G.274 
T02A and filter loop temperatures
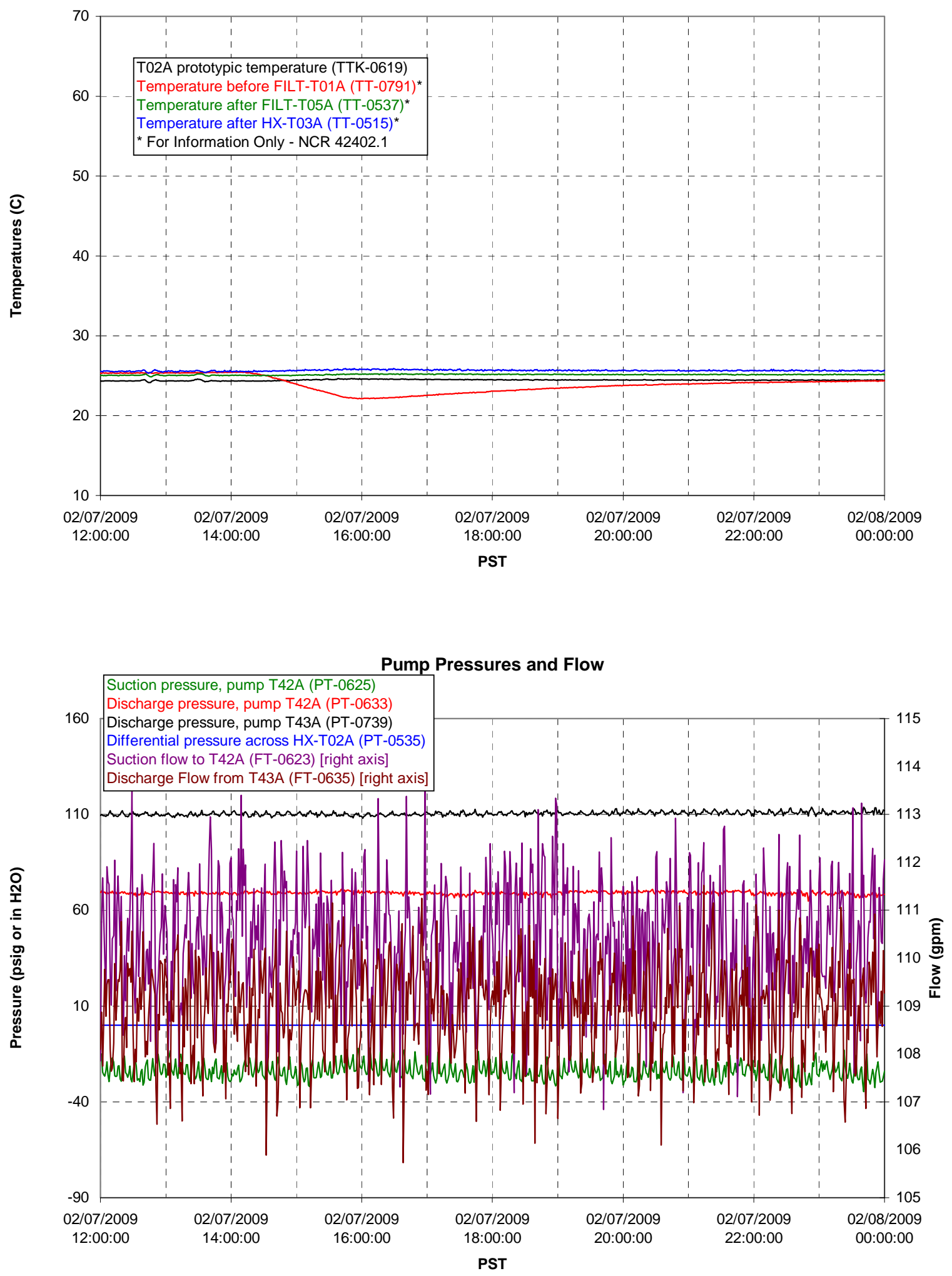


\section{Axial pressure drop}

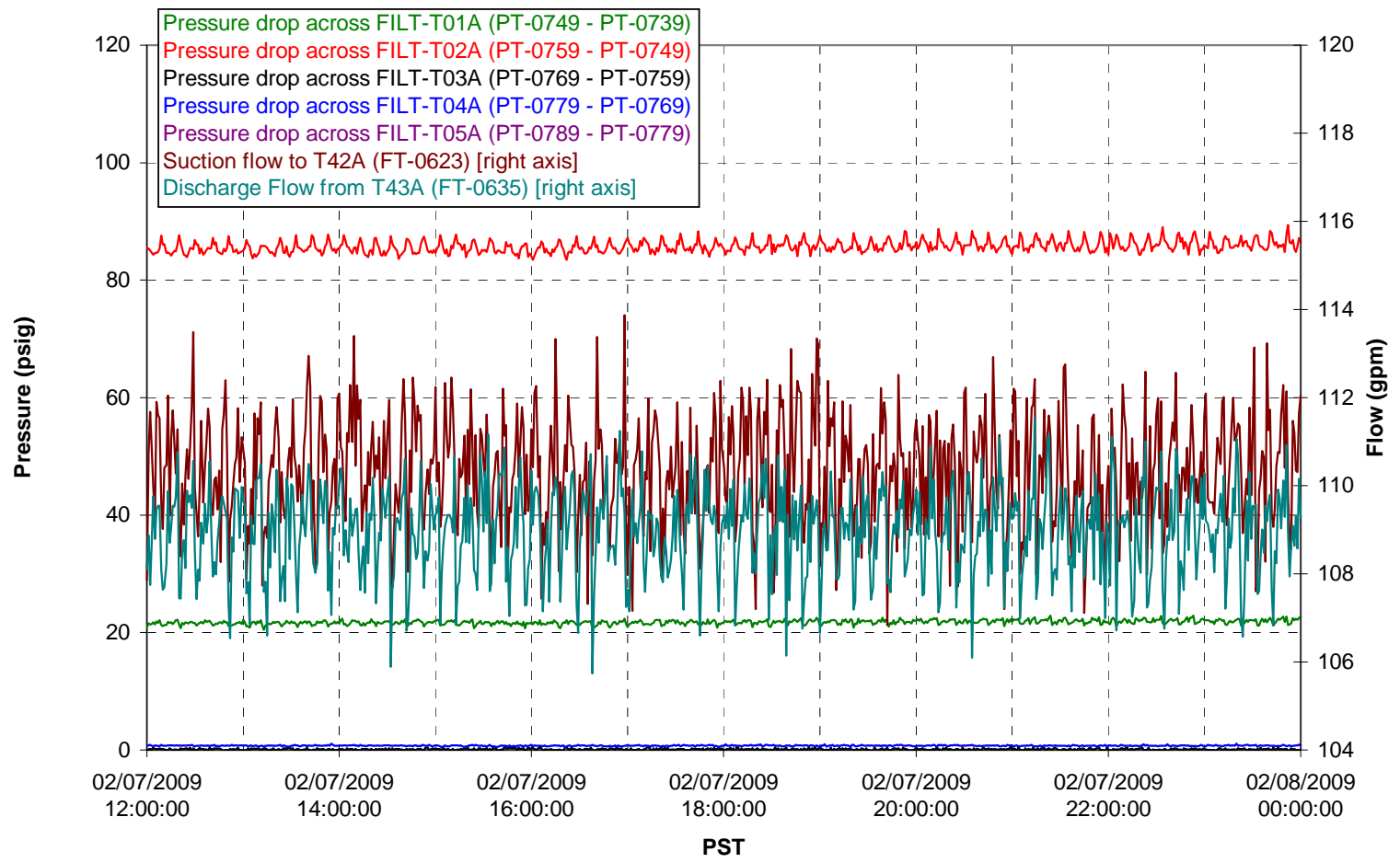

Permeate flow rates

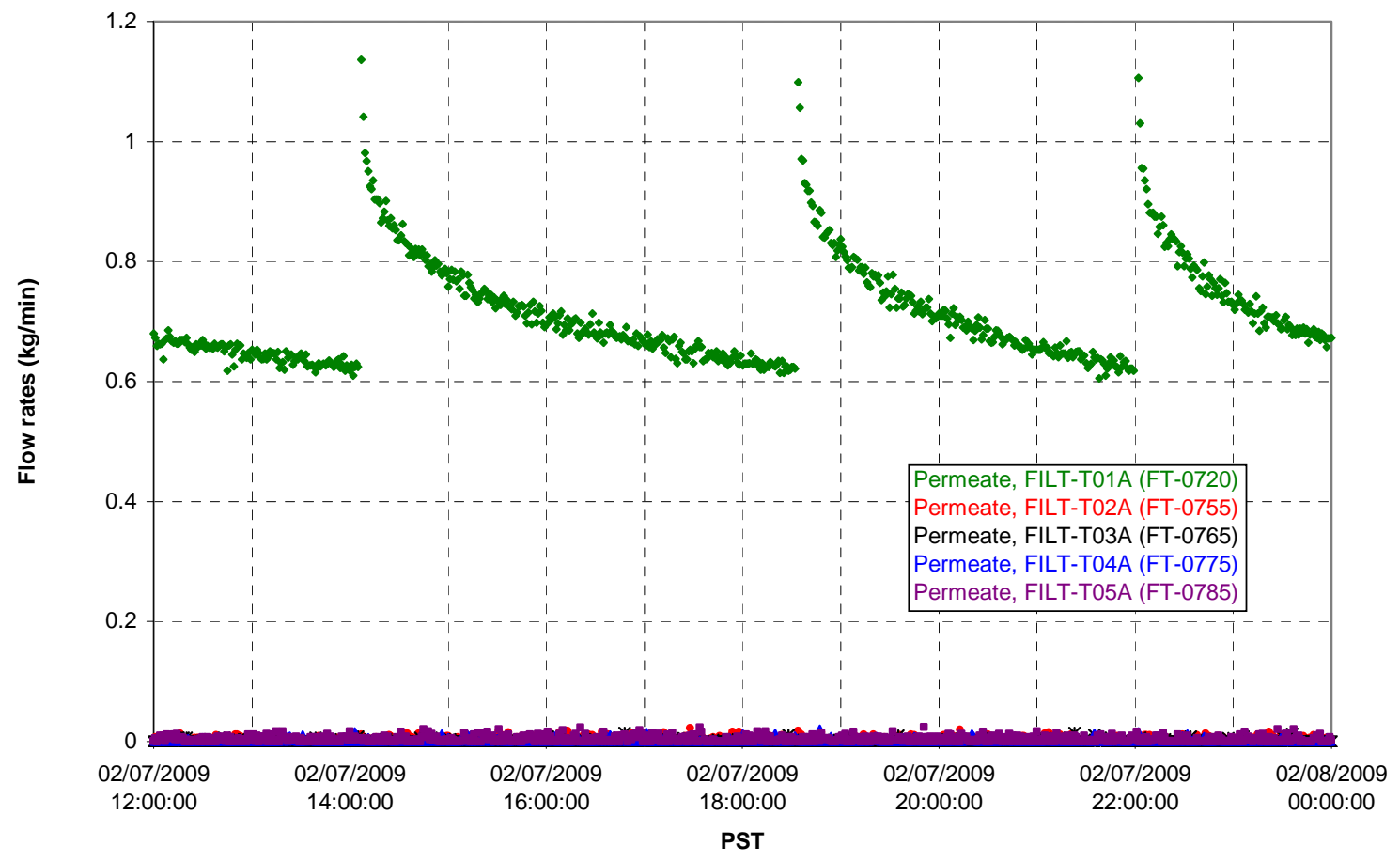


T02A Inner Temperature Tree

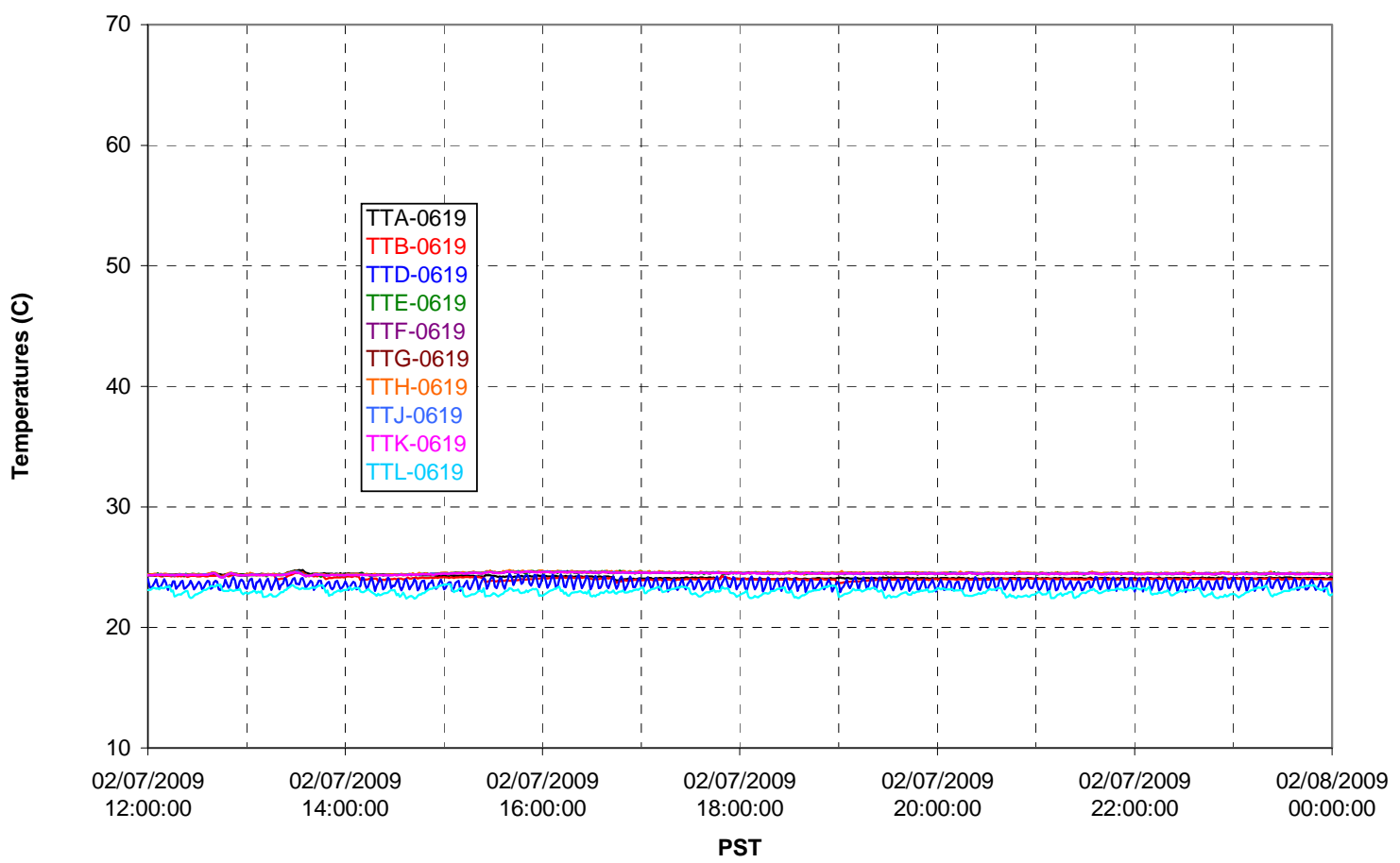

T02A Outer Temperature Tree

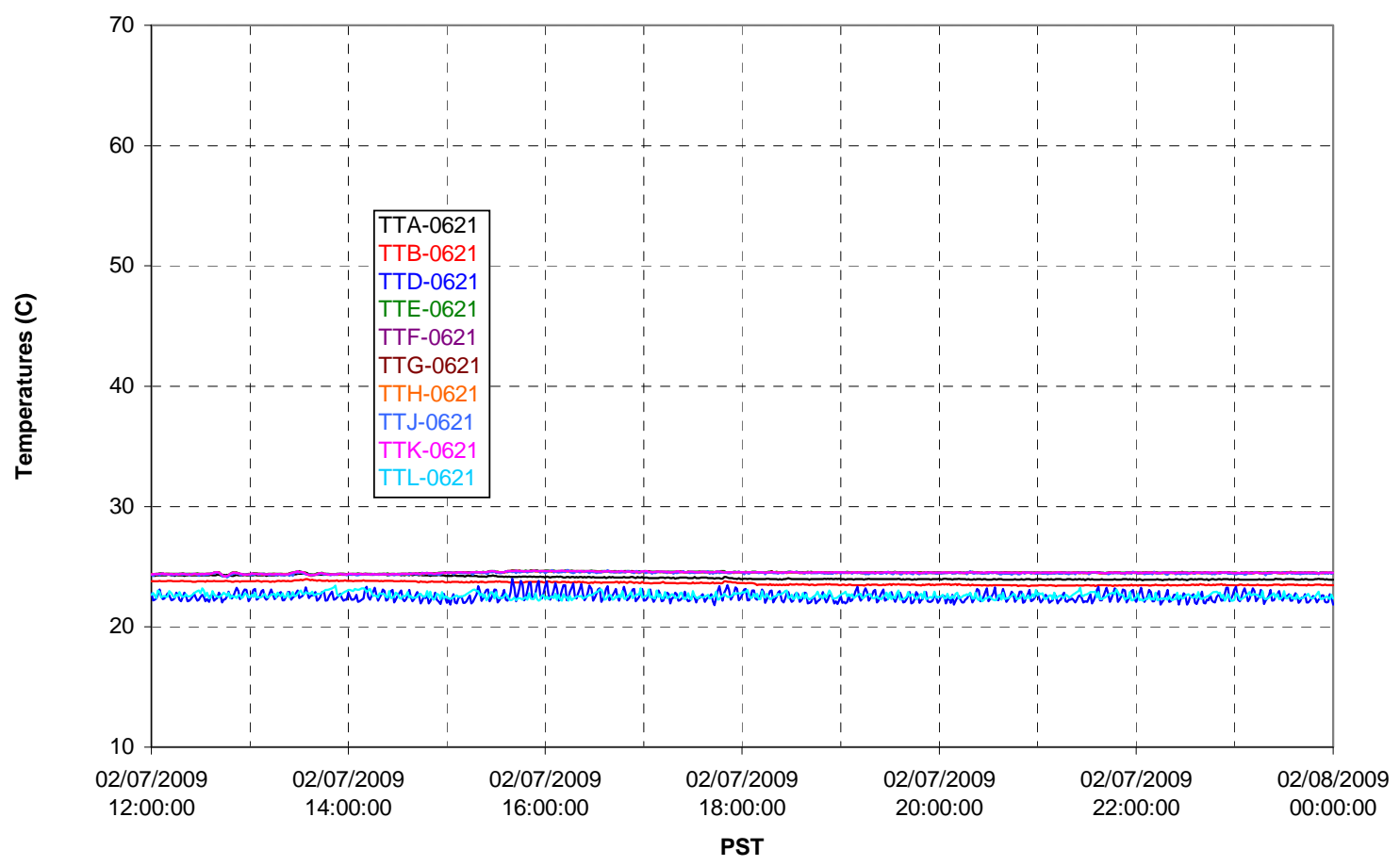


T02A temperatures

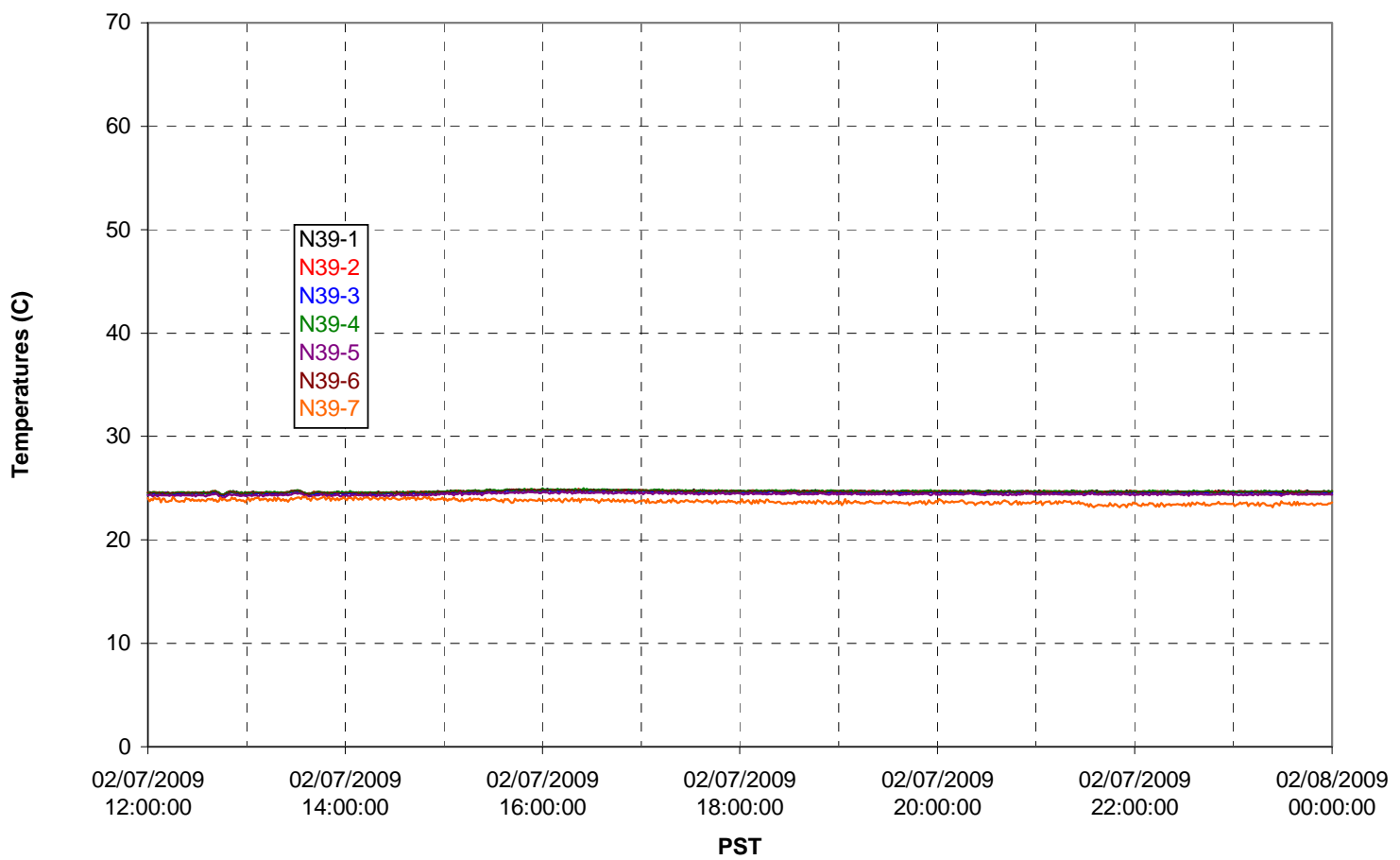

T02A temperatures

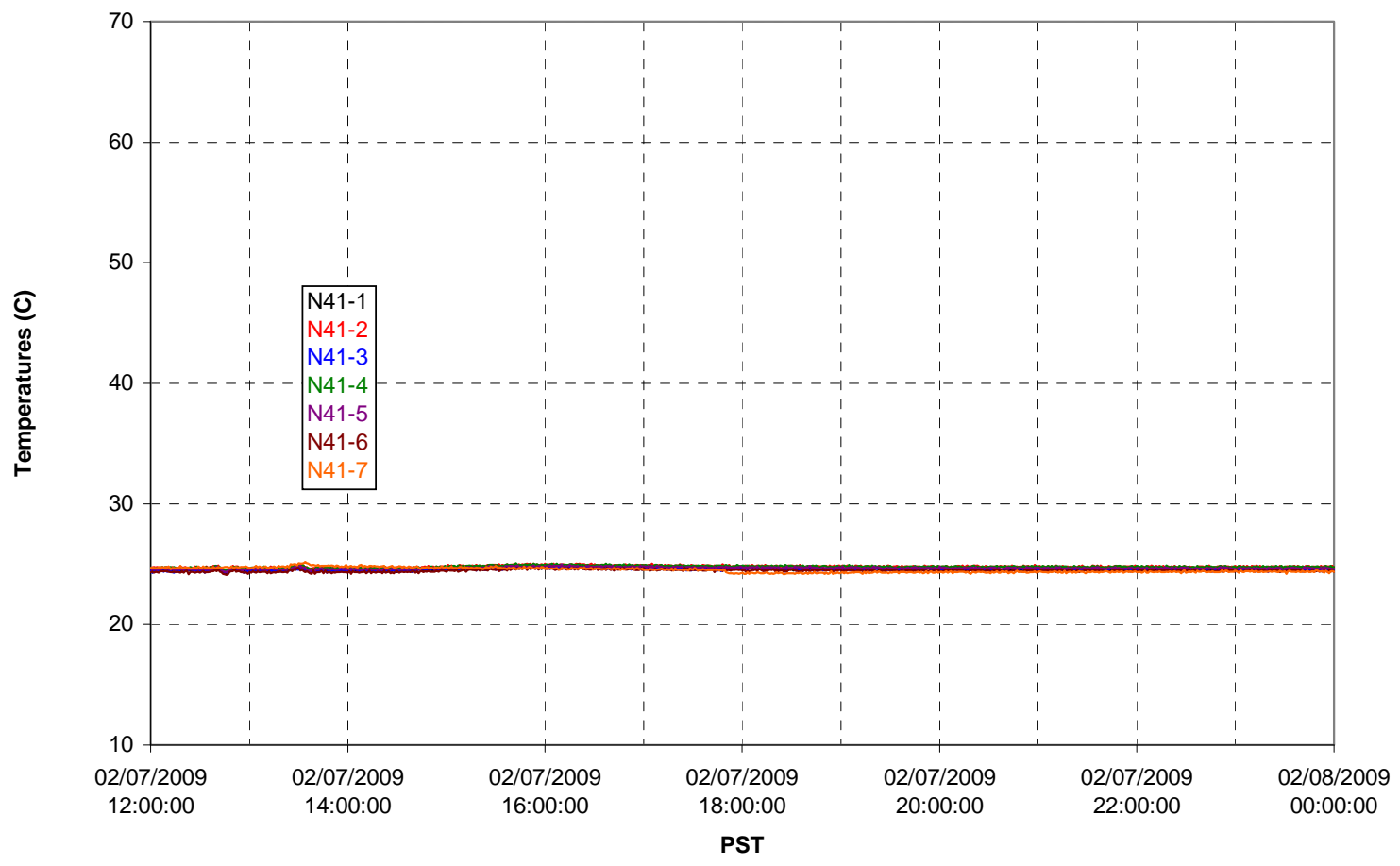


T02A temperatures

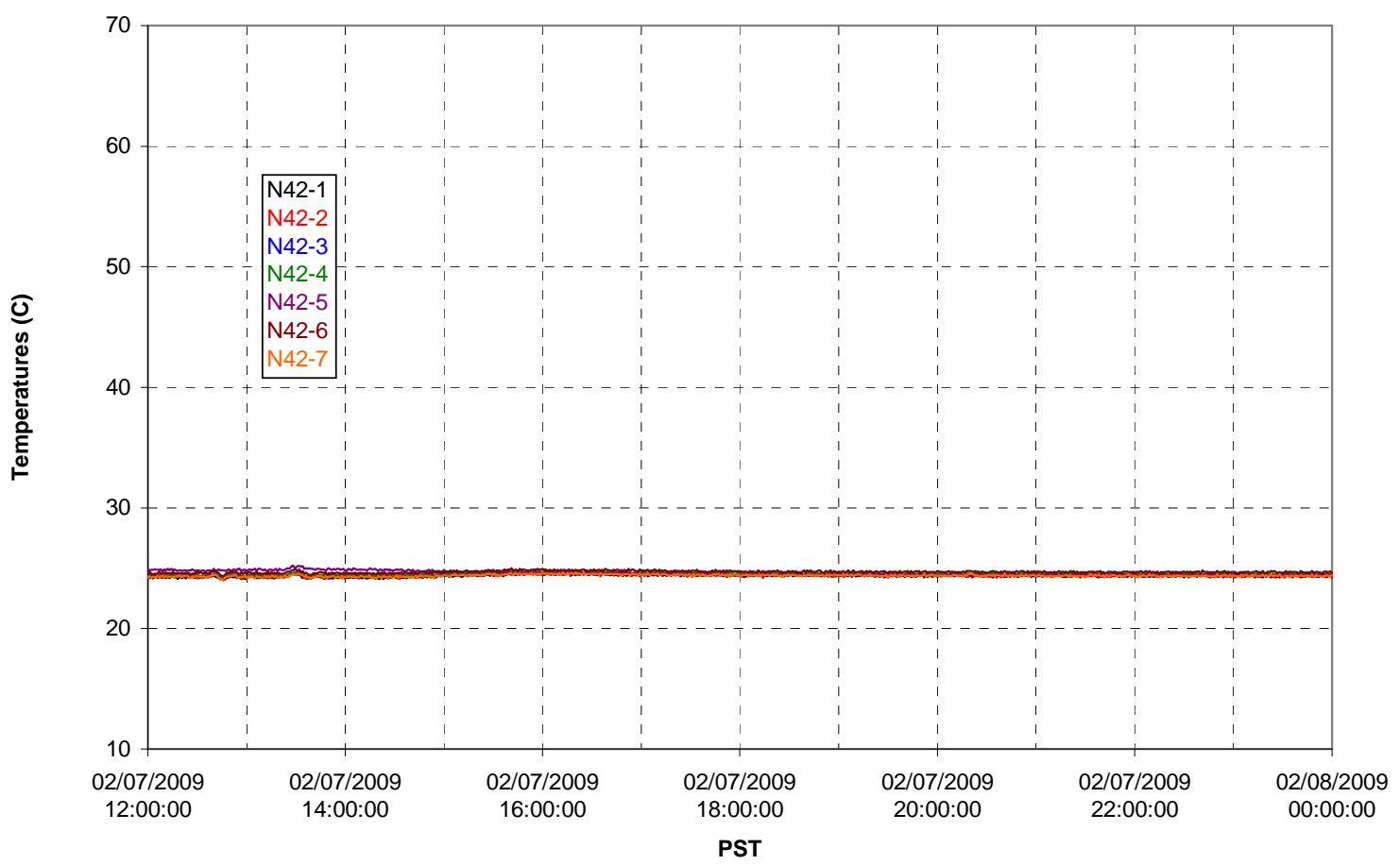

T02A temperatures

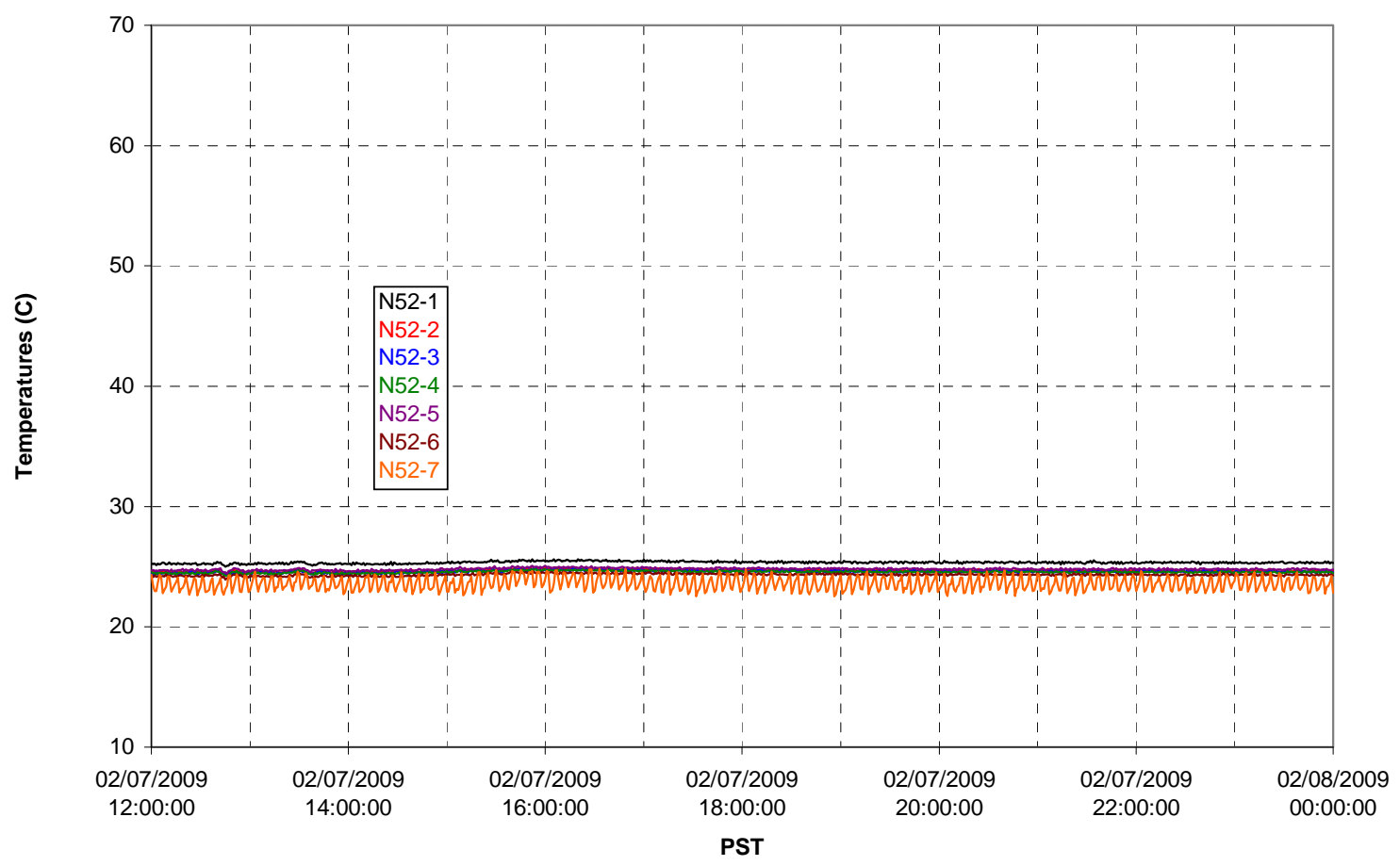


T02A Heating and Cooling

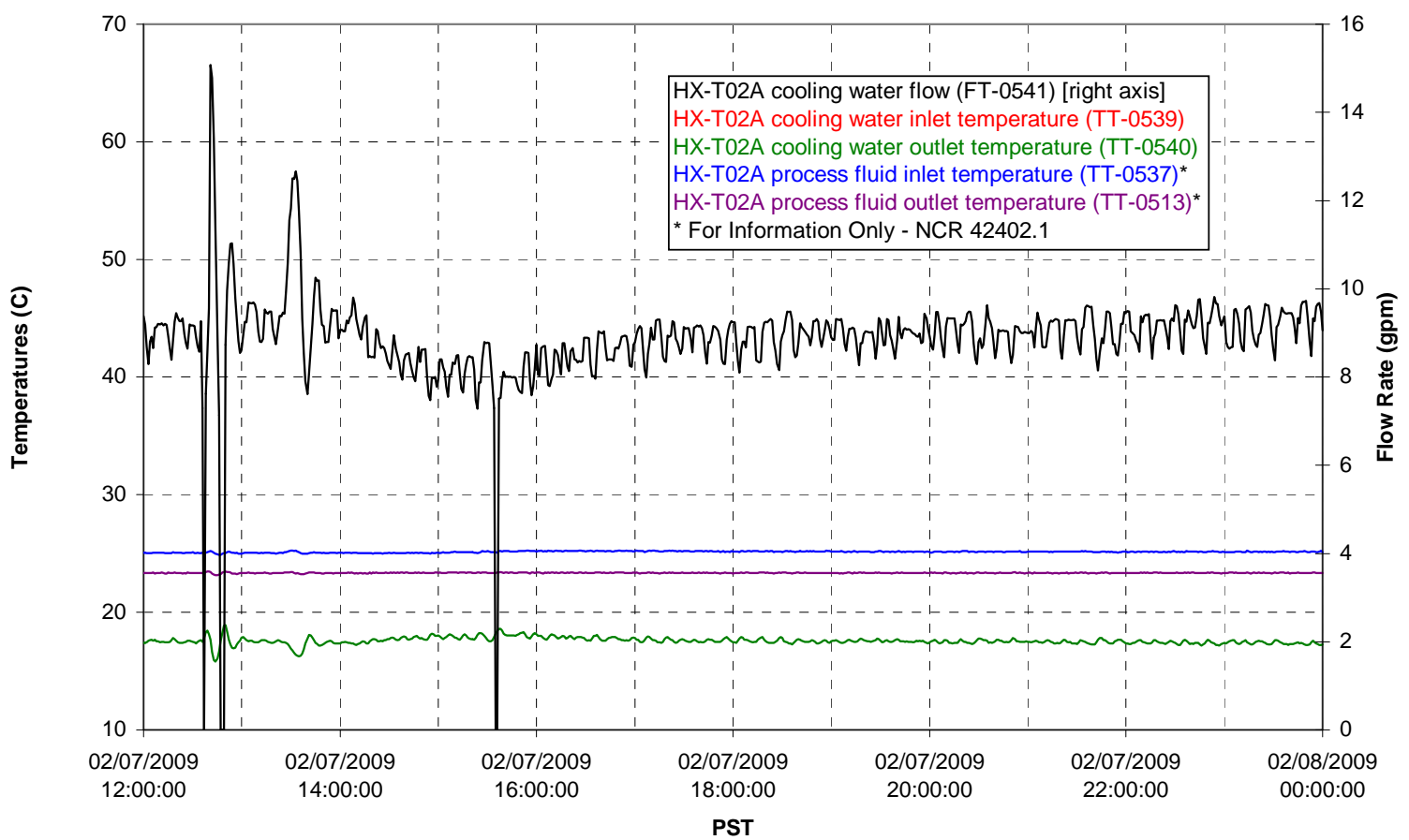

Pump Operation

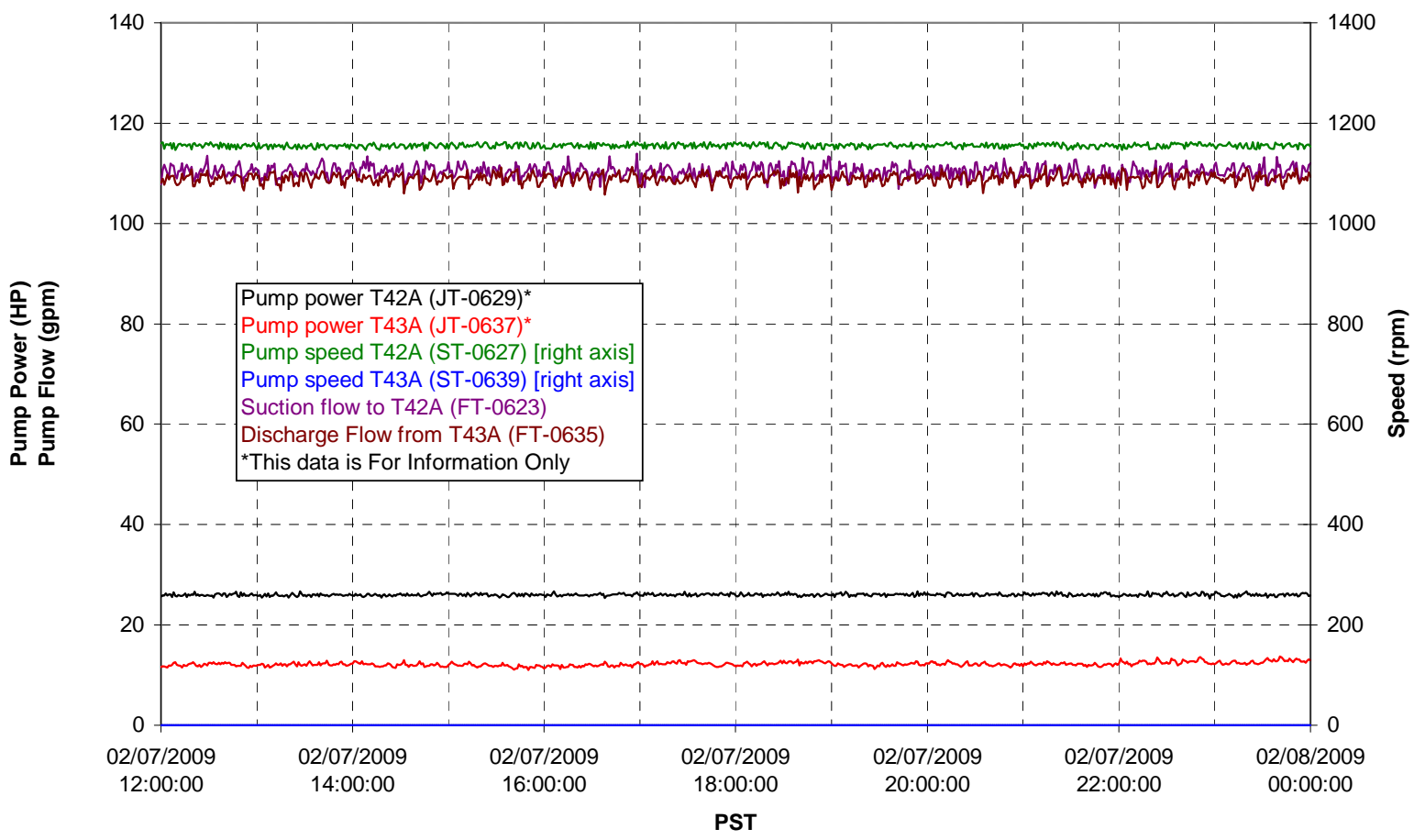


Pulsepot UFP-PP-T01A

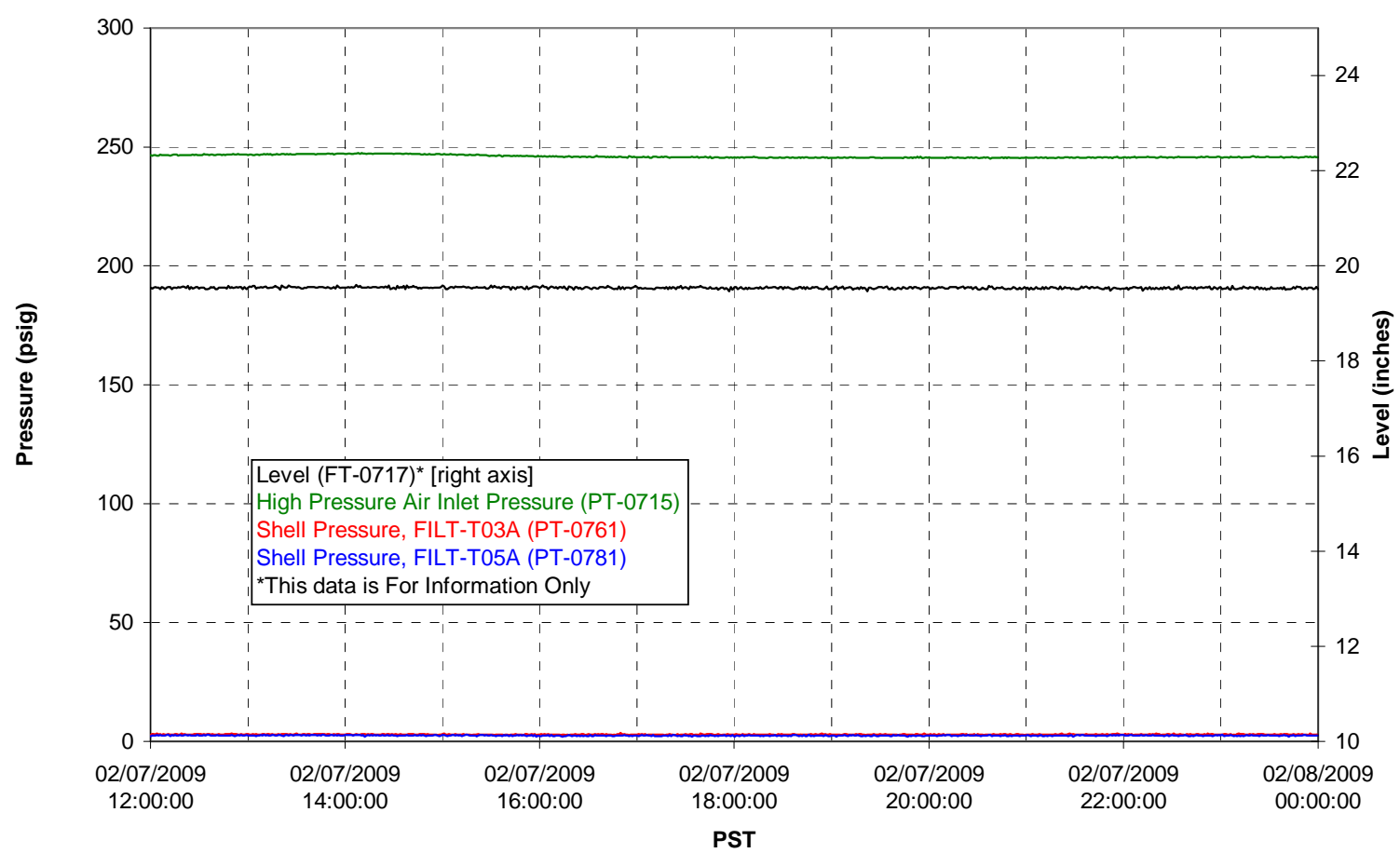

Pulsepot UFP-PP-T02A

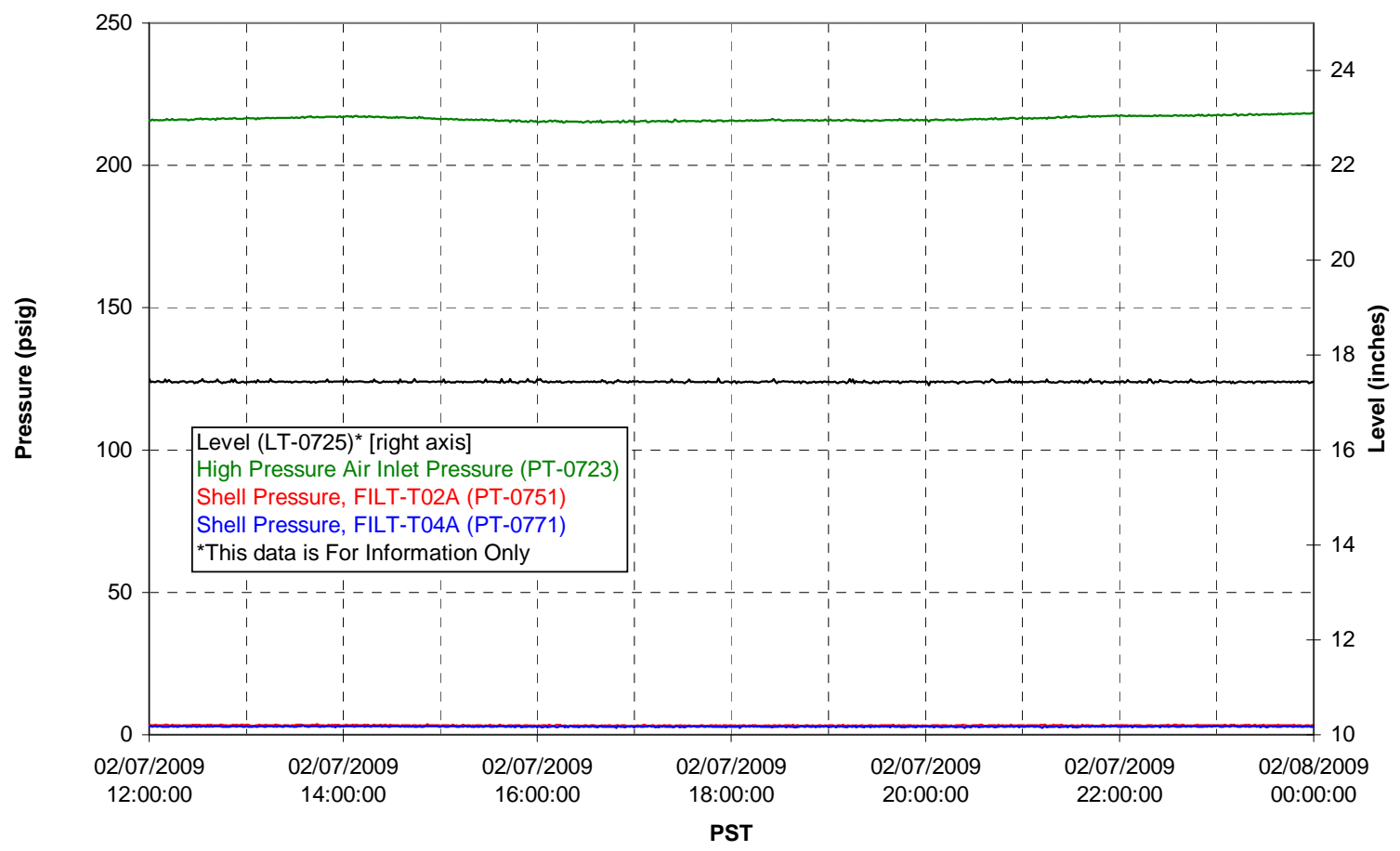


Pulsepot UFP-PP-T03A

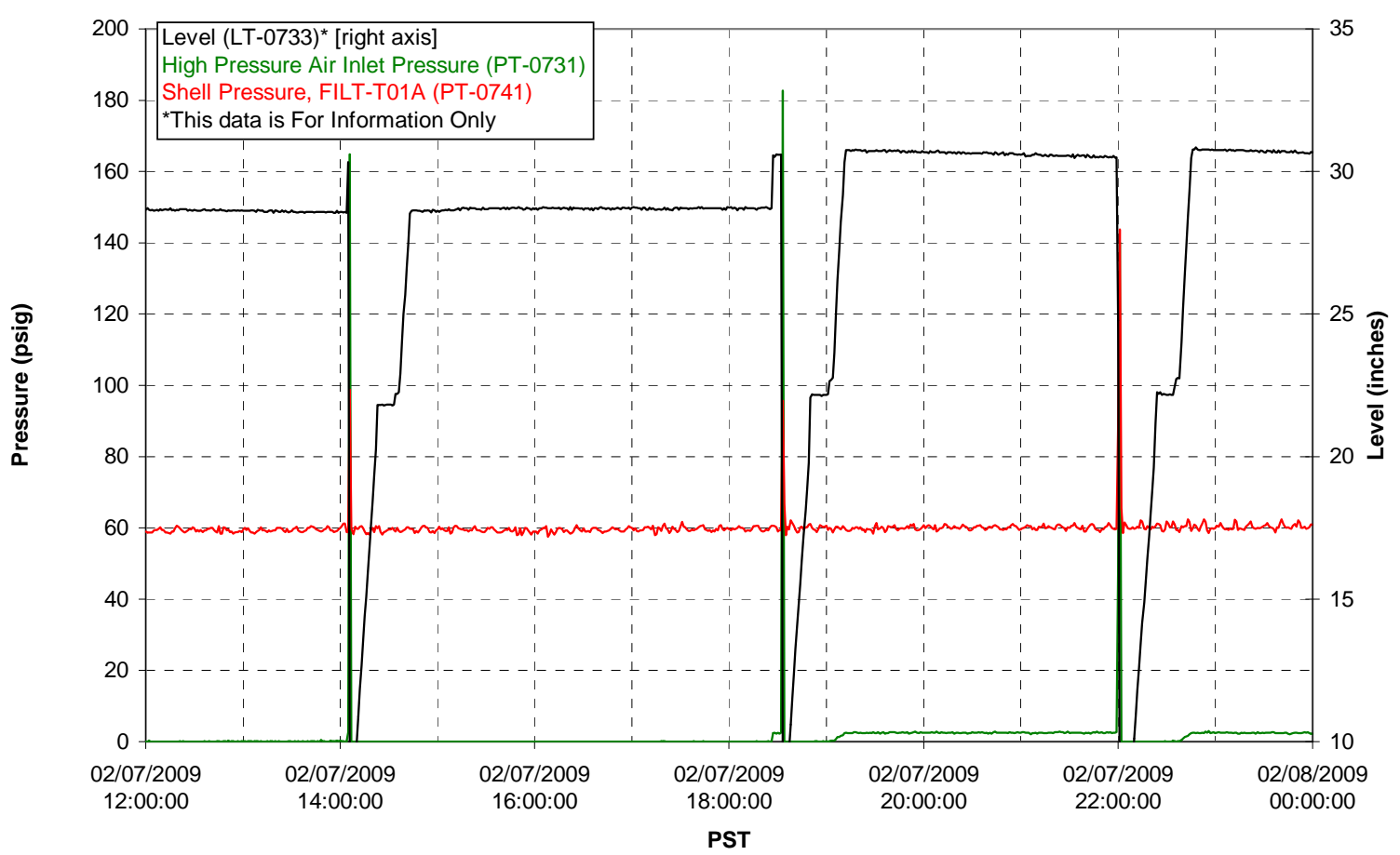

Pulsepot Levels

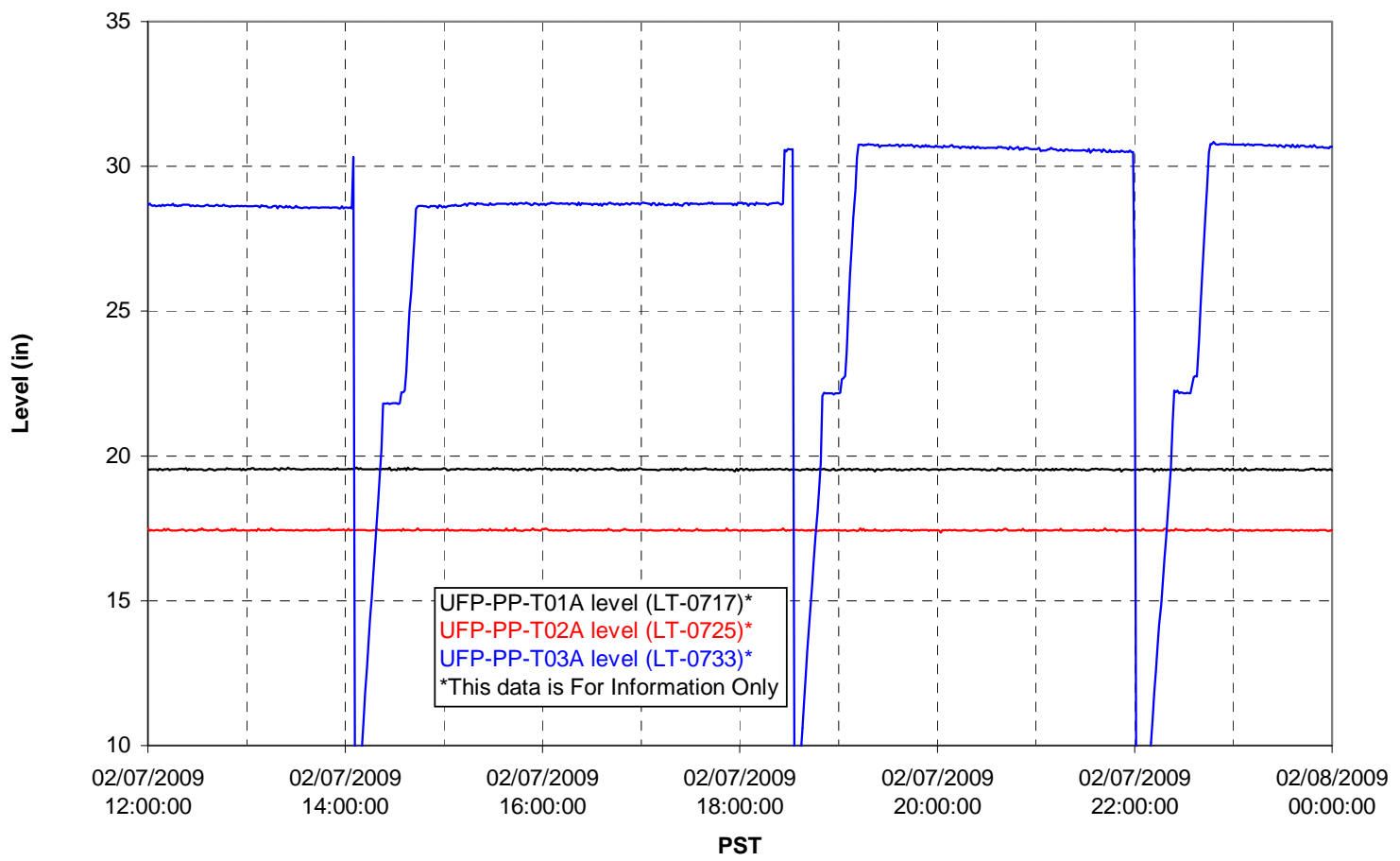


Filter UFP-FILT-T01A

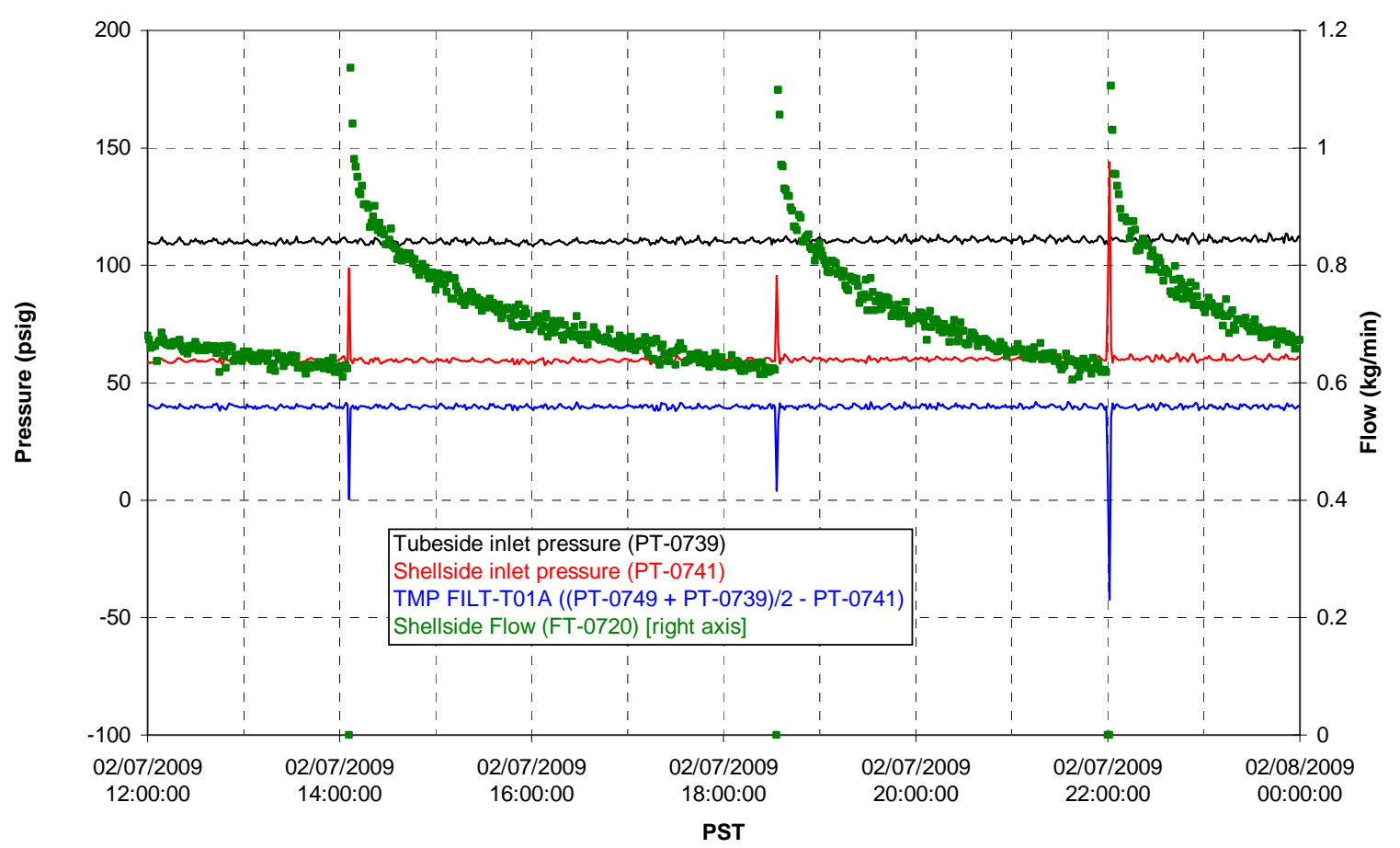

Filter UFP-FILT-T02A

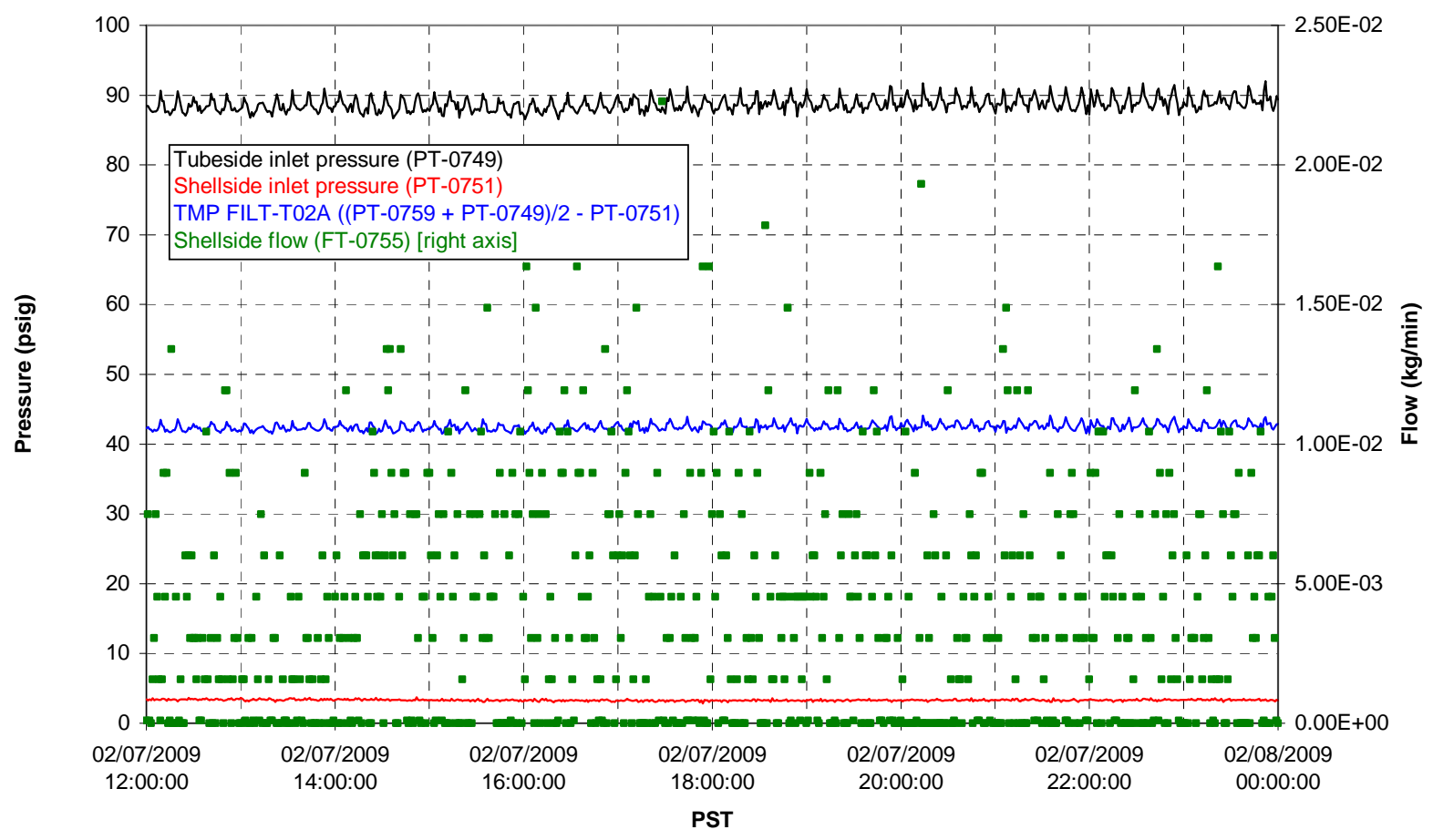


Filter UFP-FILT-T03A

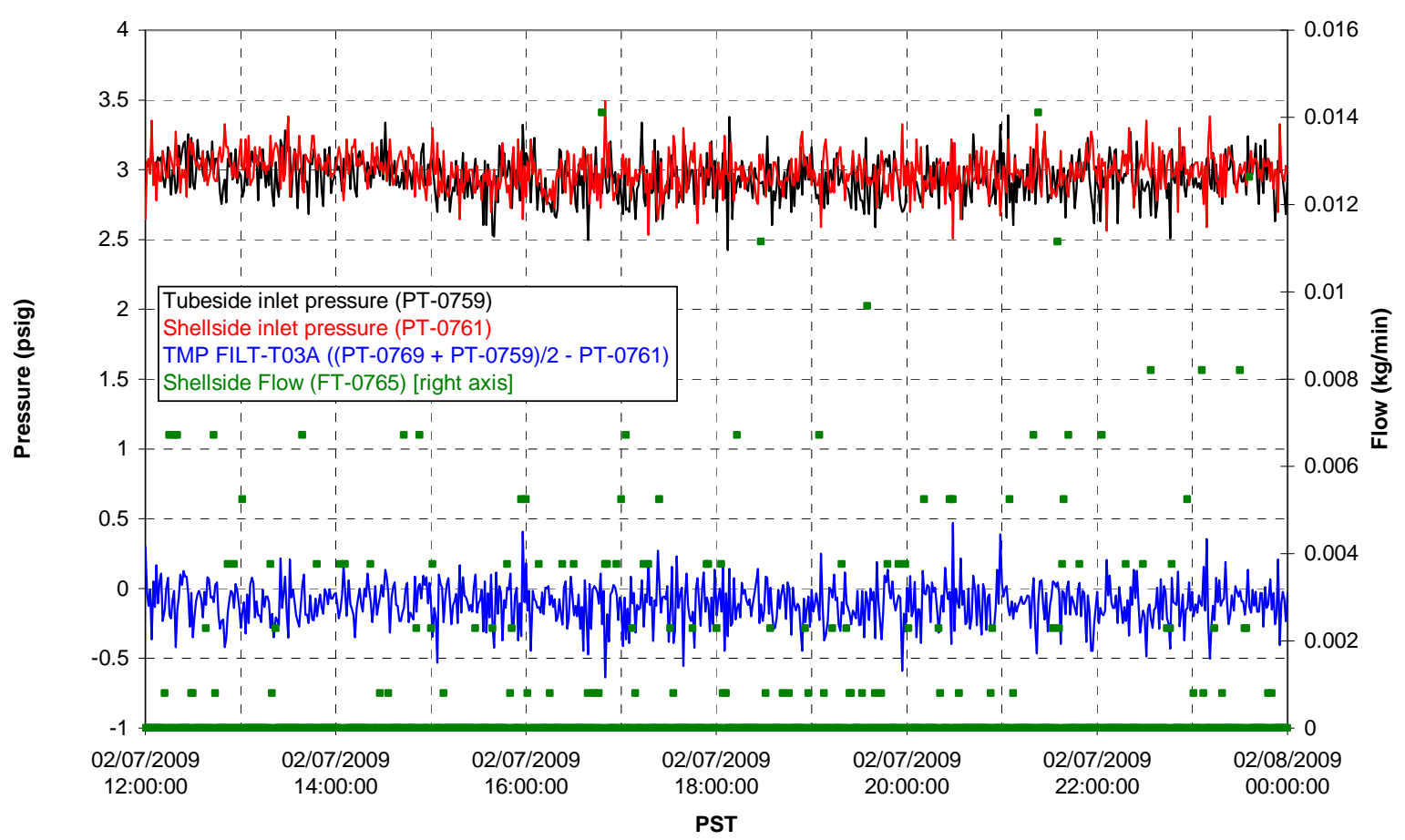

Filter UFP-FILT-T04A

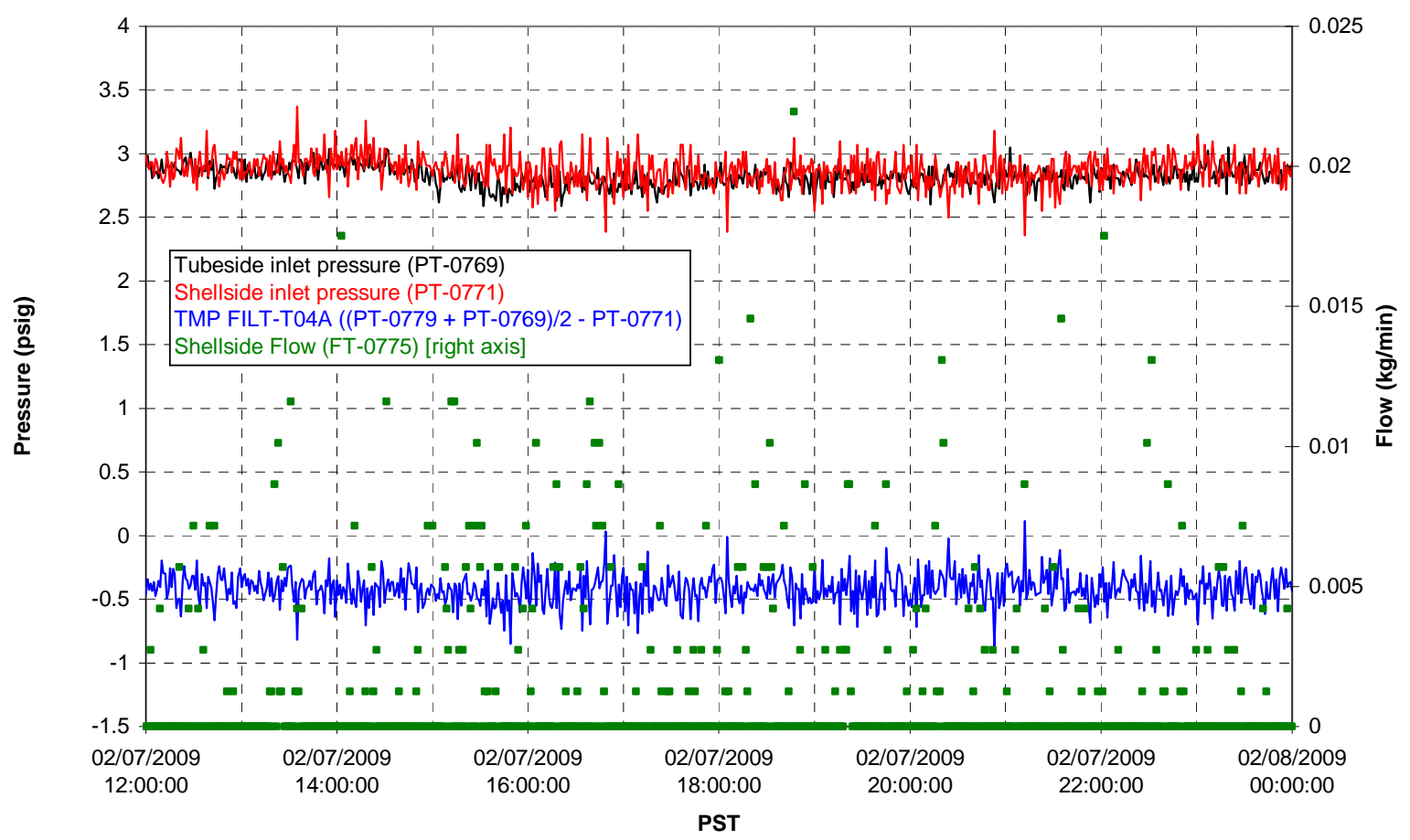


Filter UFP-FILT-T05A

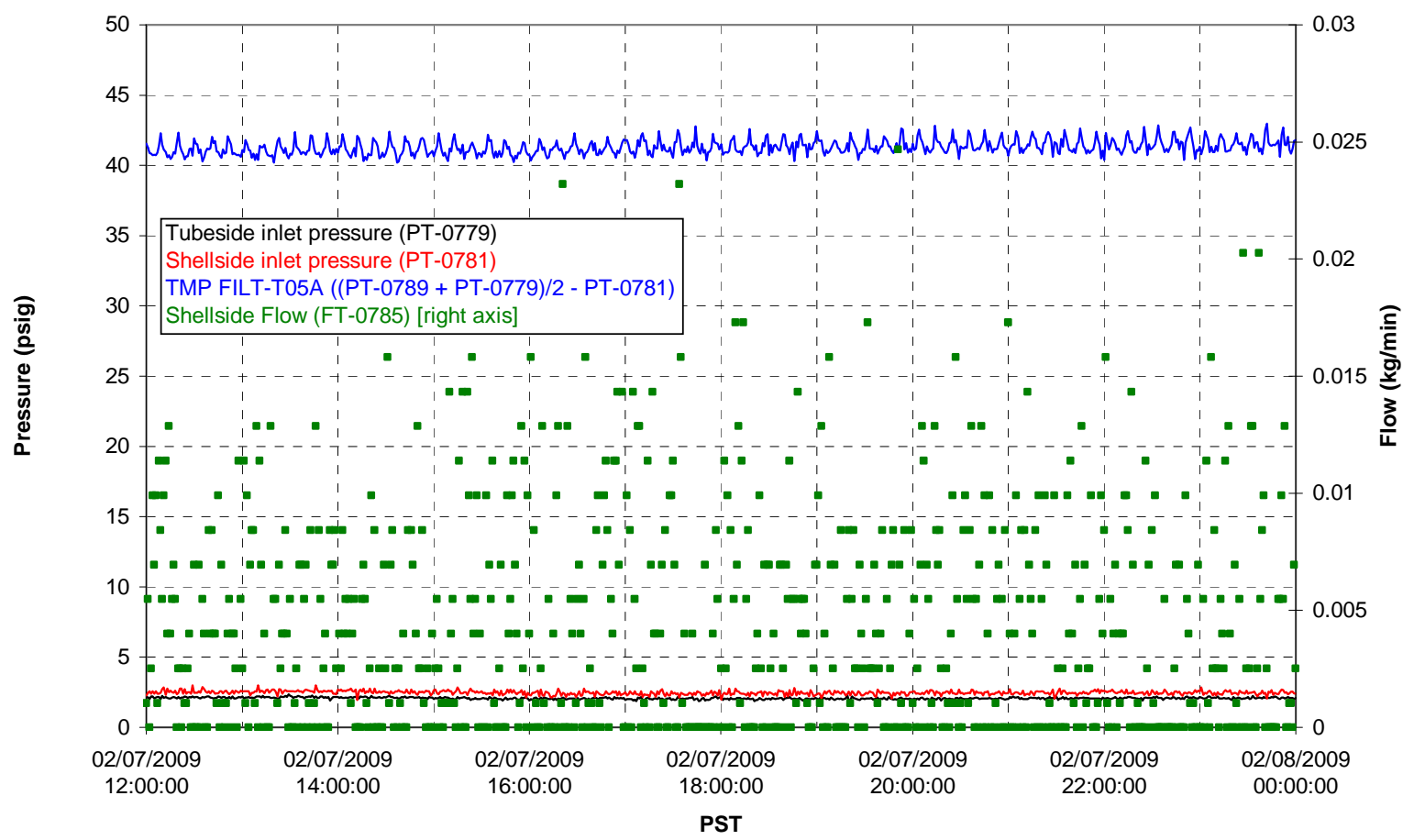

Chemical Flow

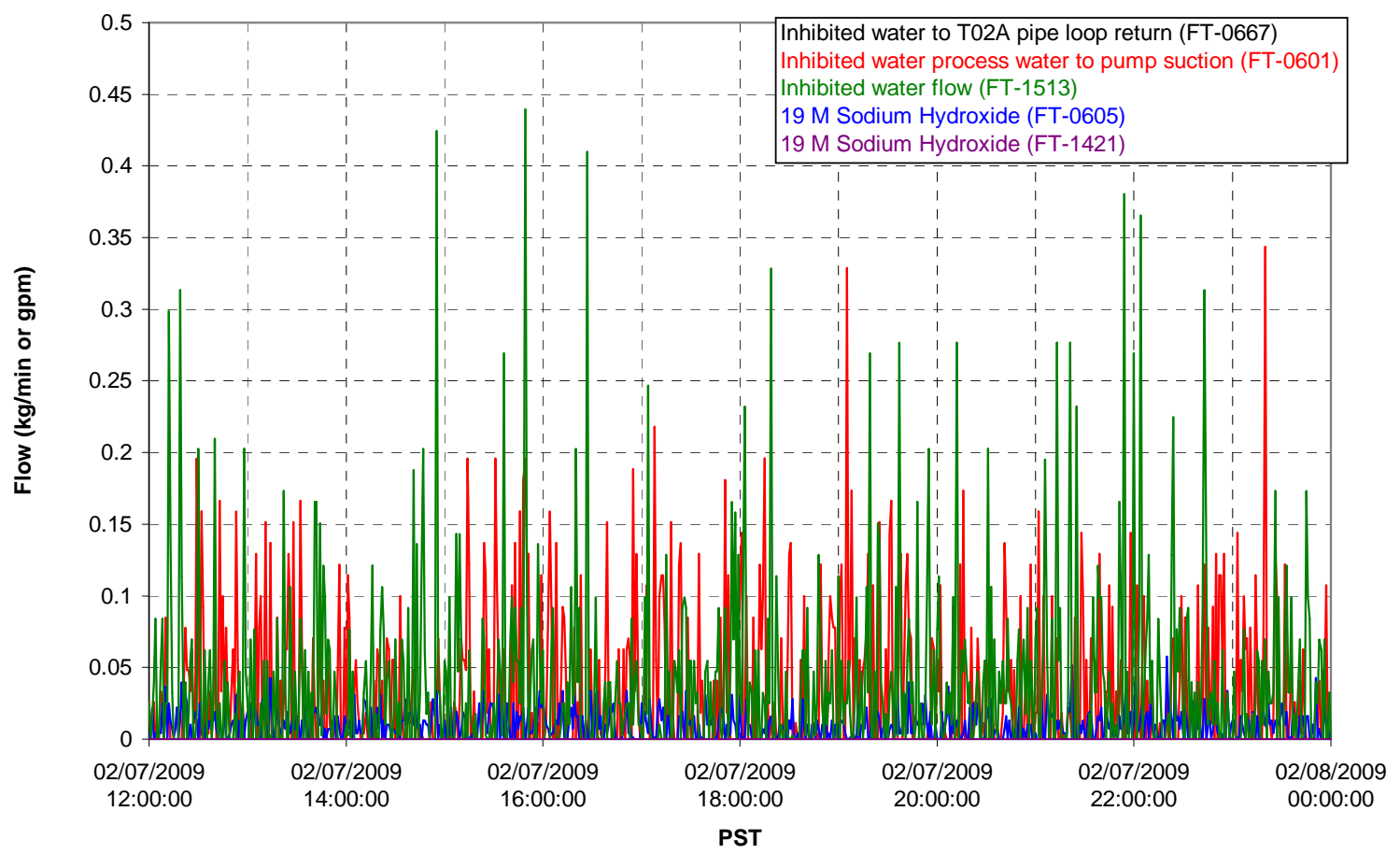


Chemical Flow

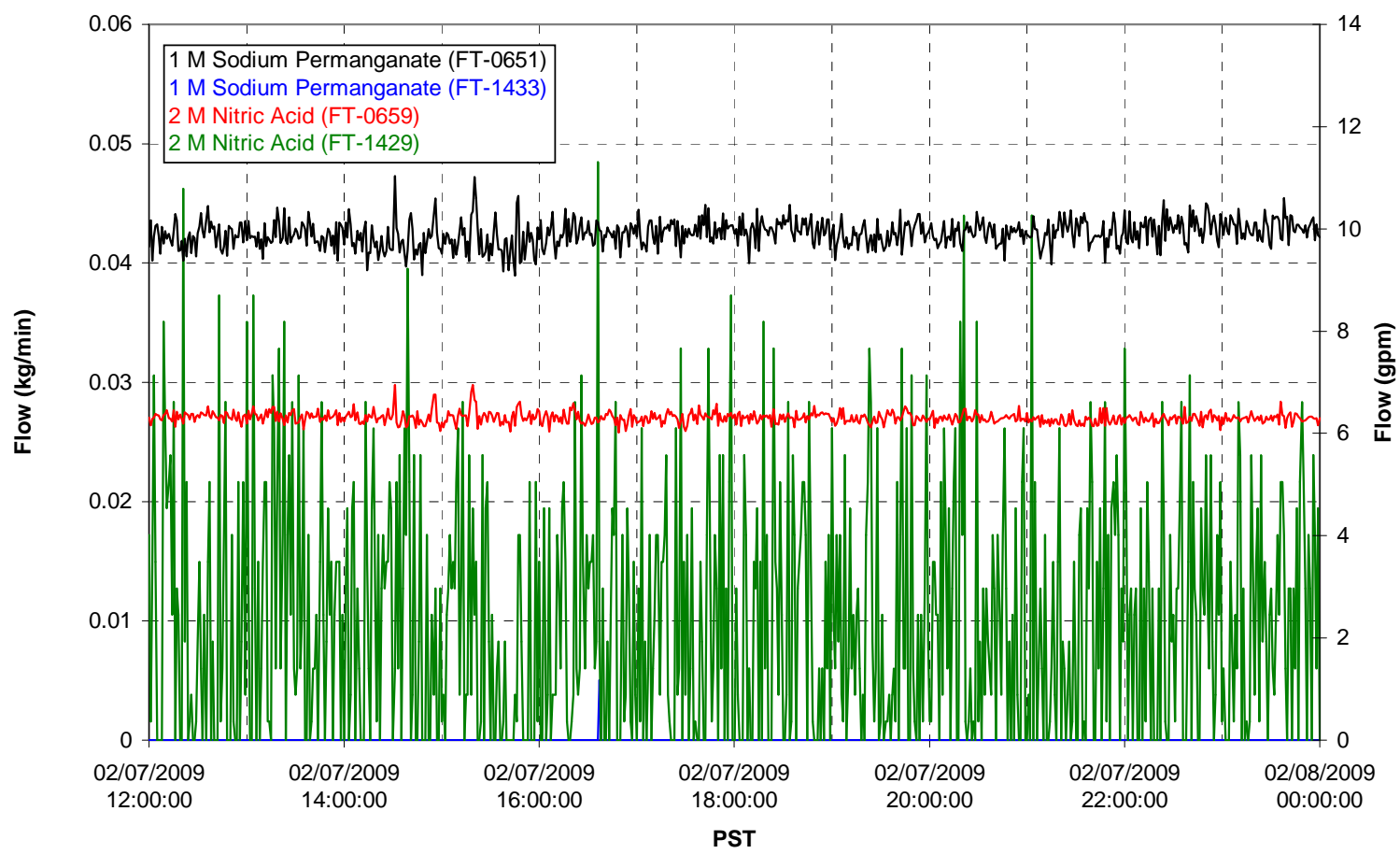

Air Flows

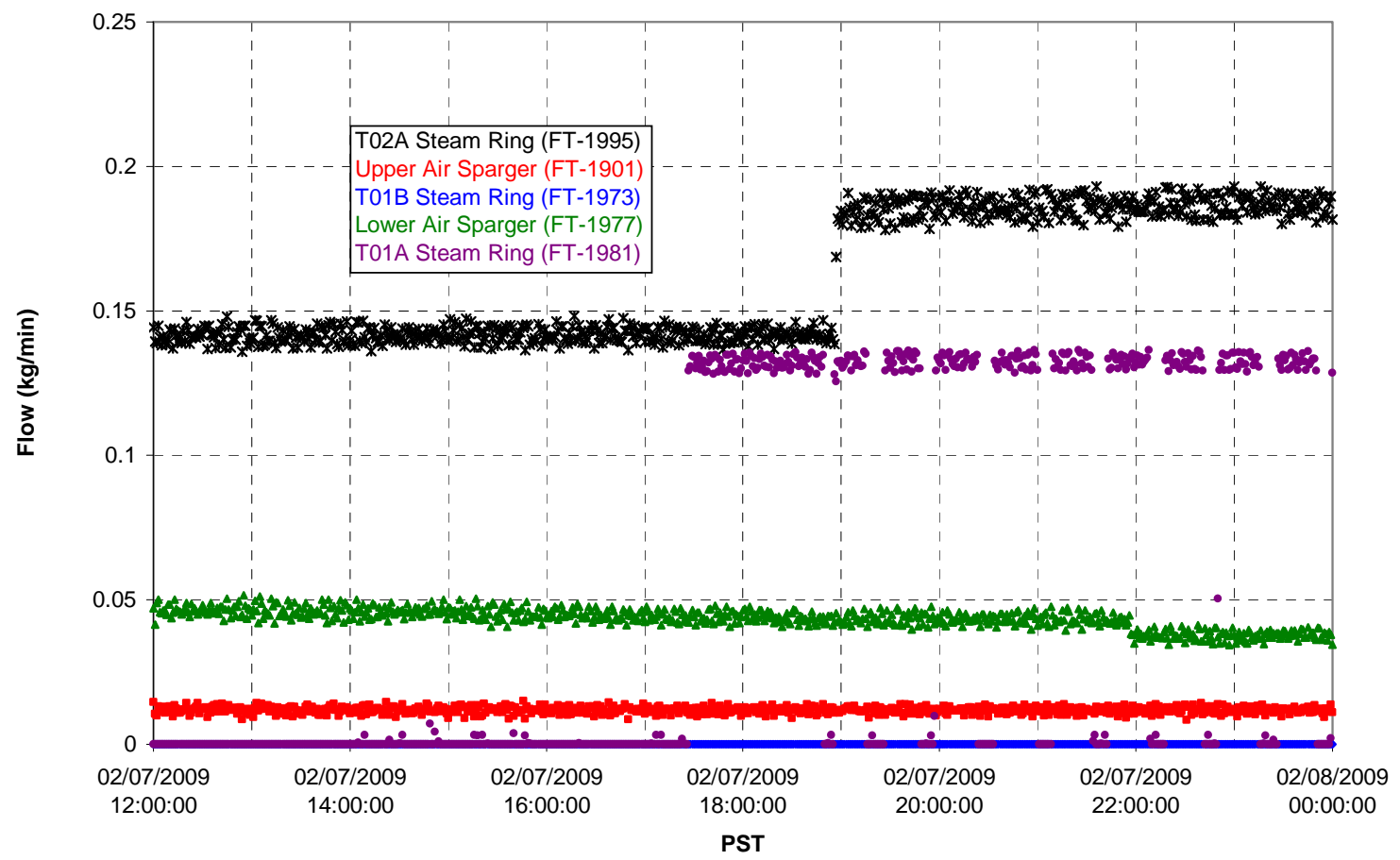


T02A Steam

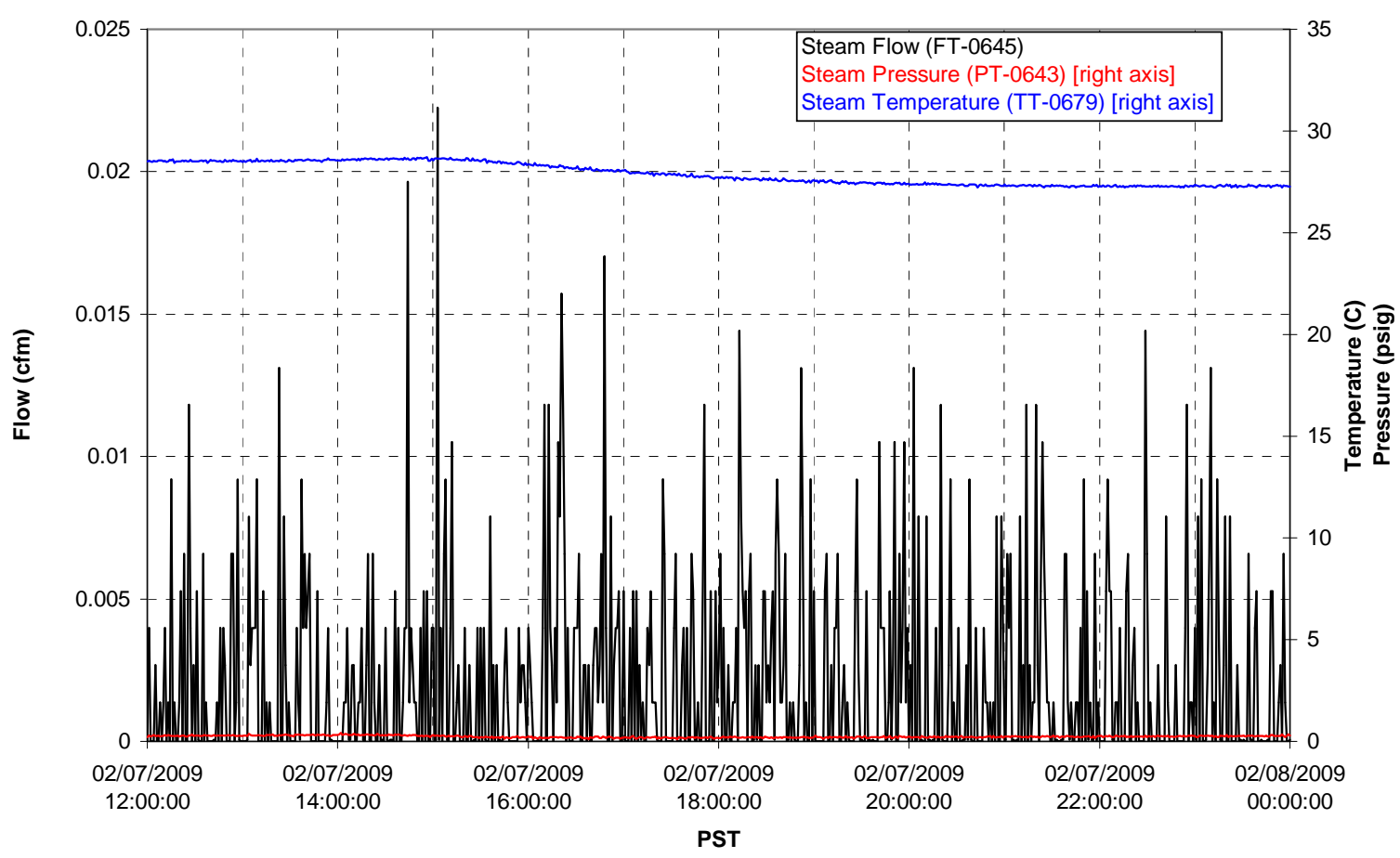

T01A Steam

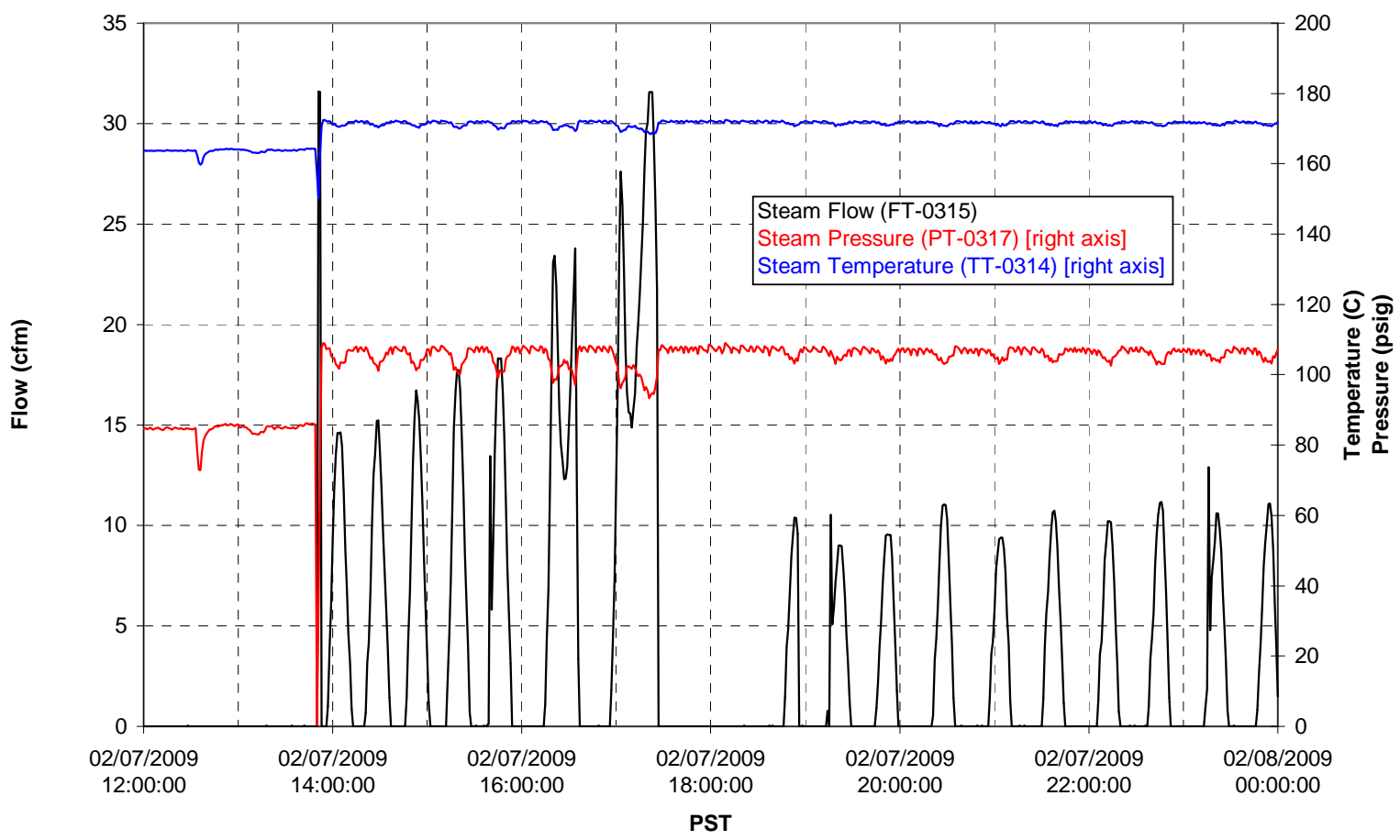


T01B Steam

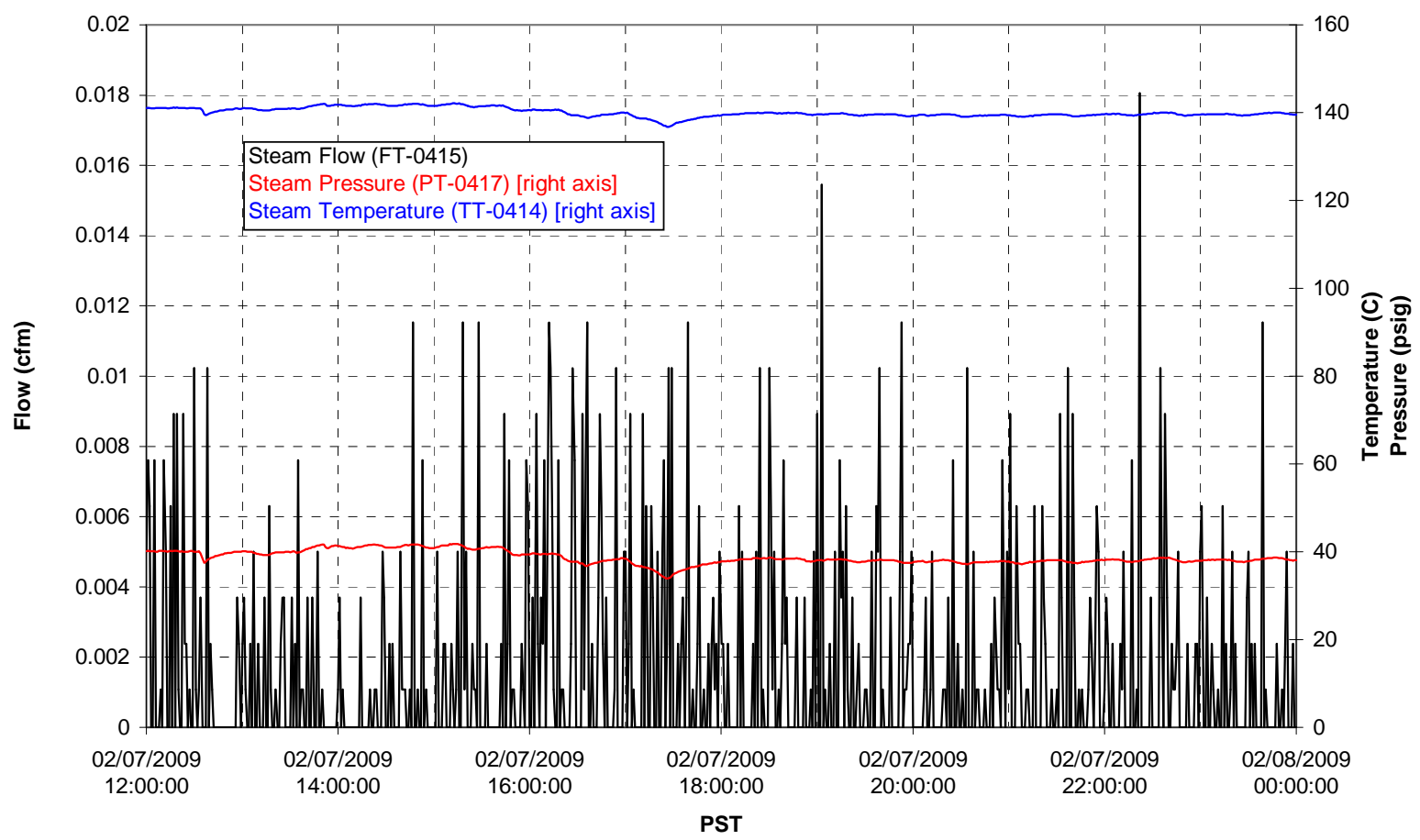




\section{Integrated Test A Data Plots 02/08/09 00:00 - 02/08/09 12:00}


T01A level

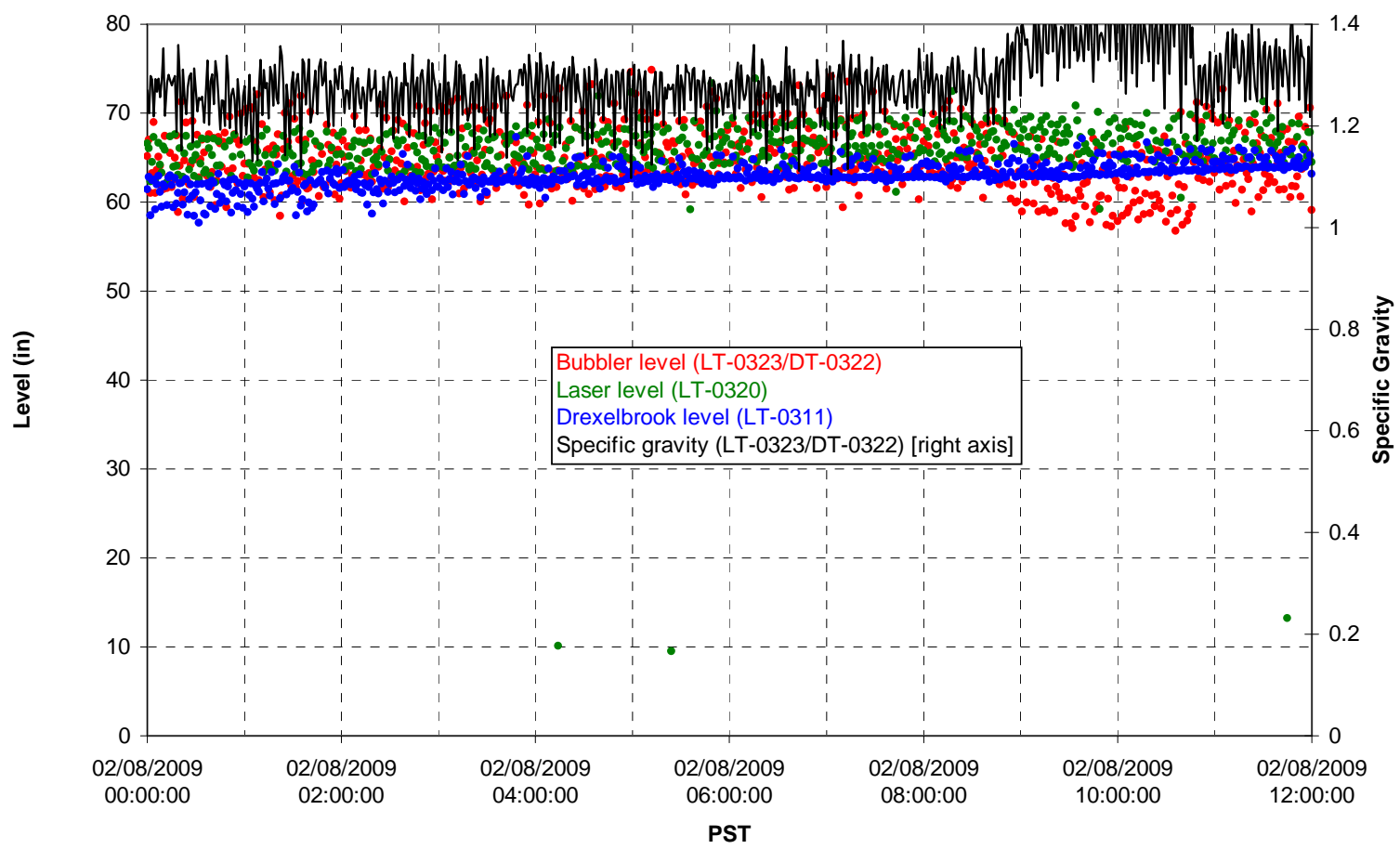

T01A temperatures

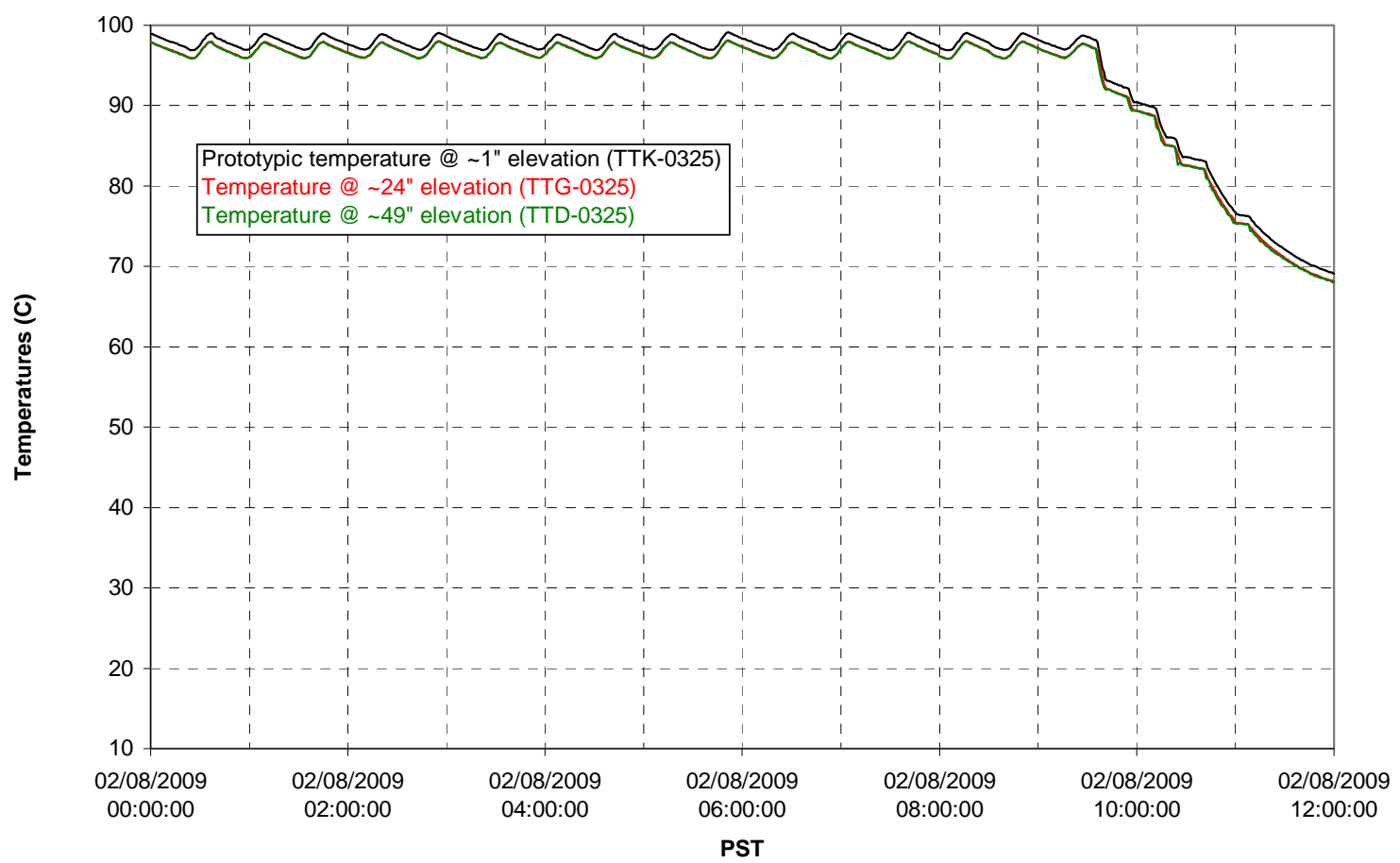


T01B level

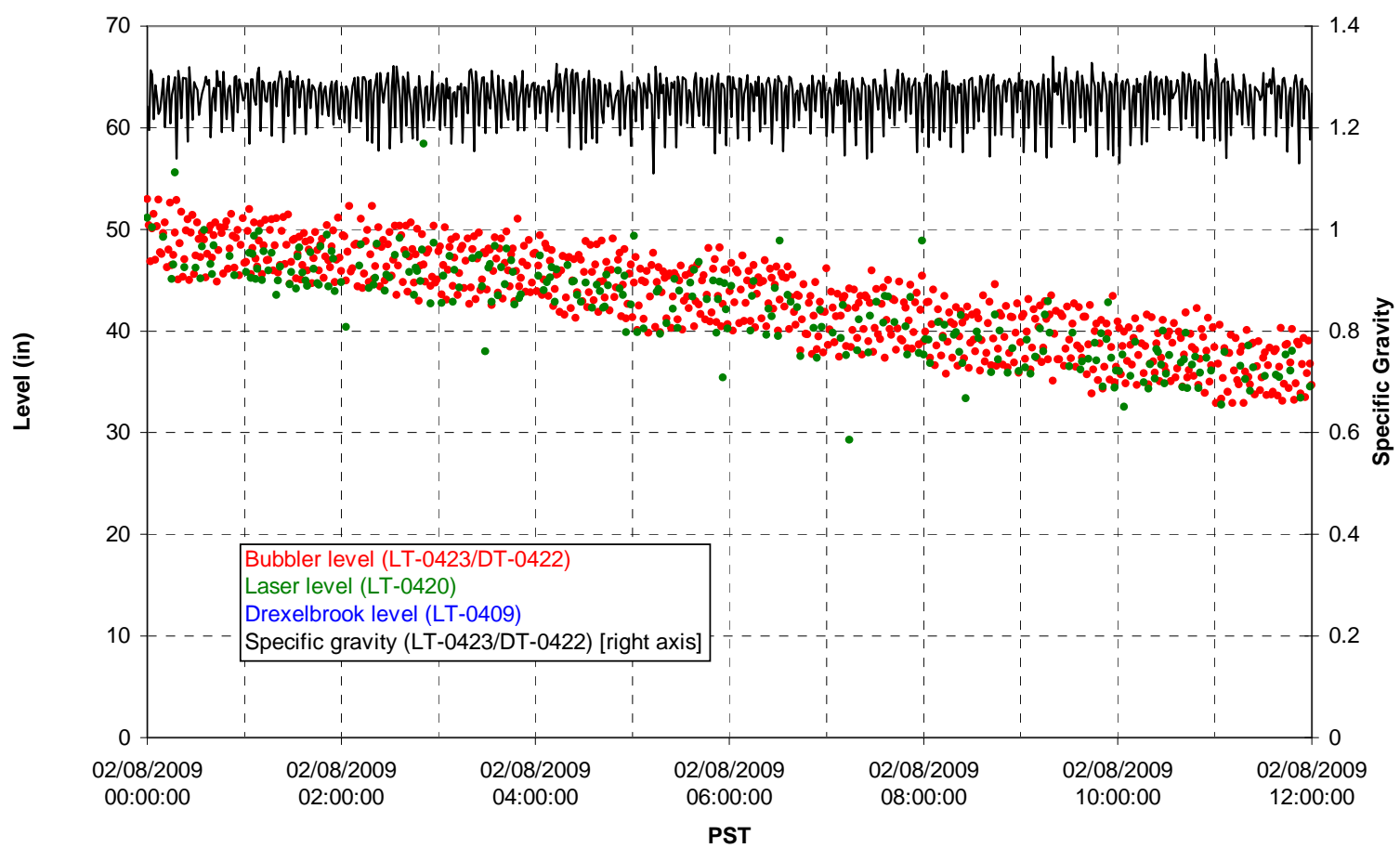

T01B temperatures

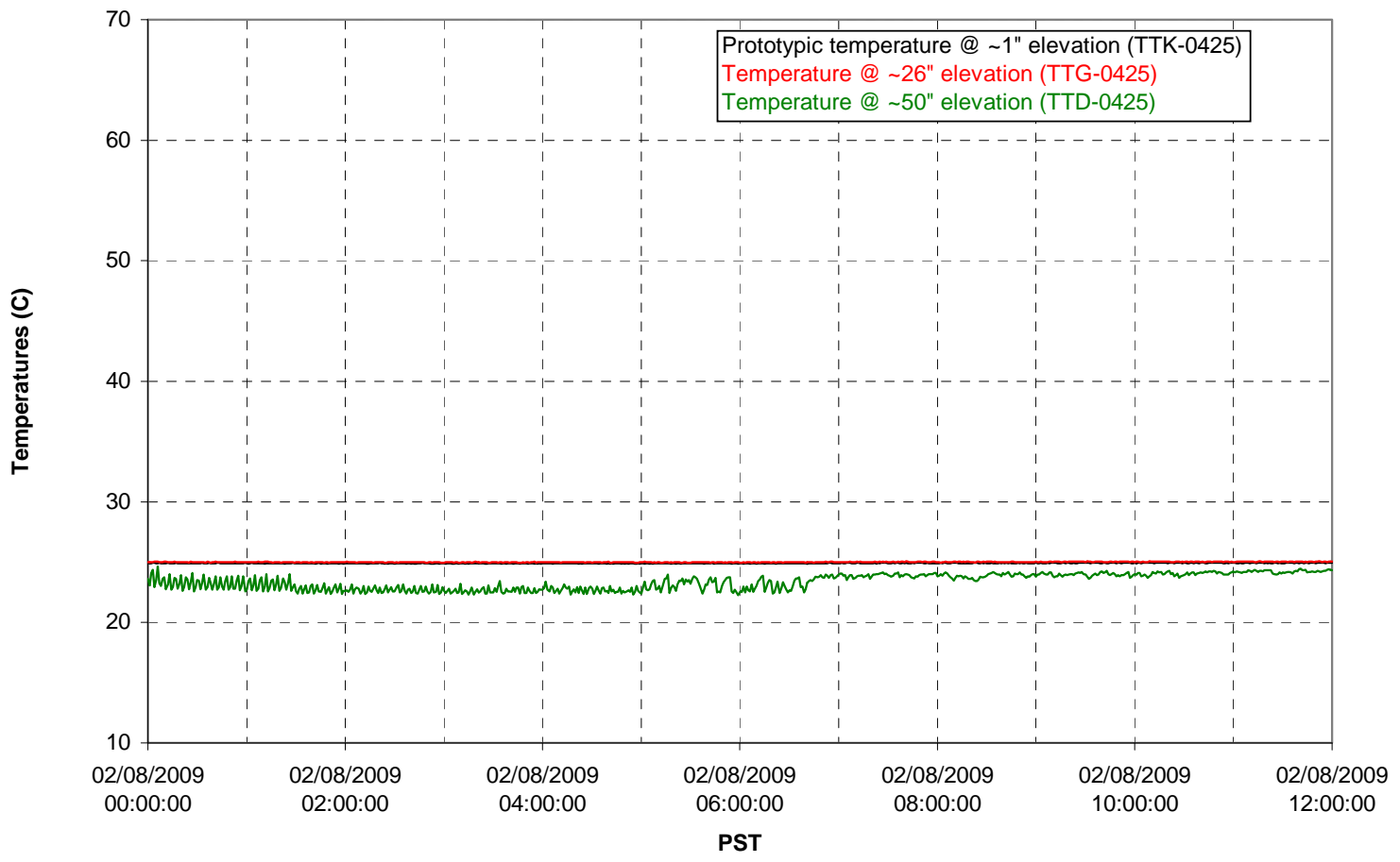


T02A level

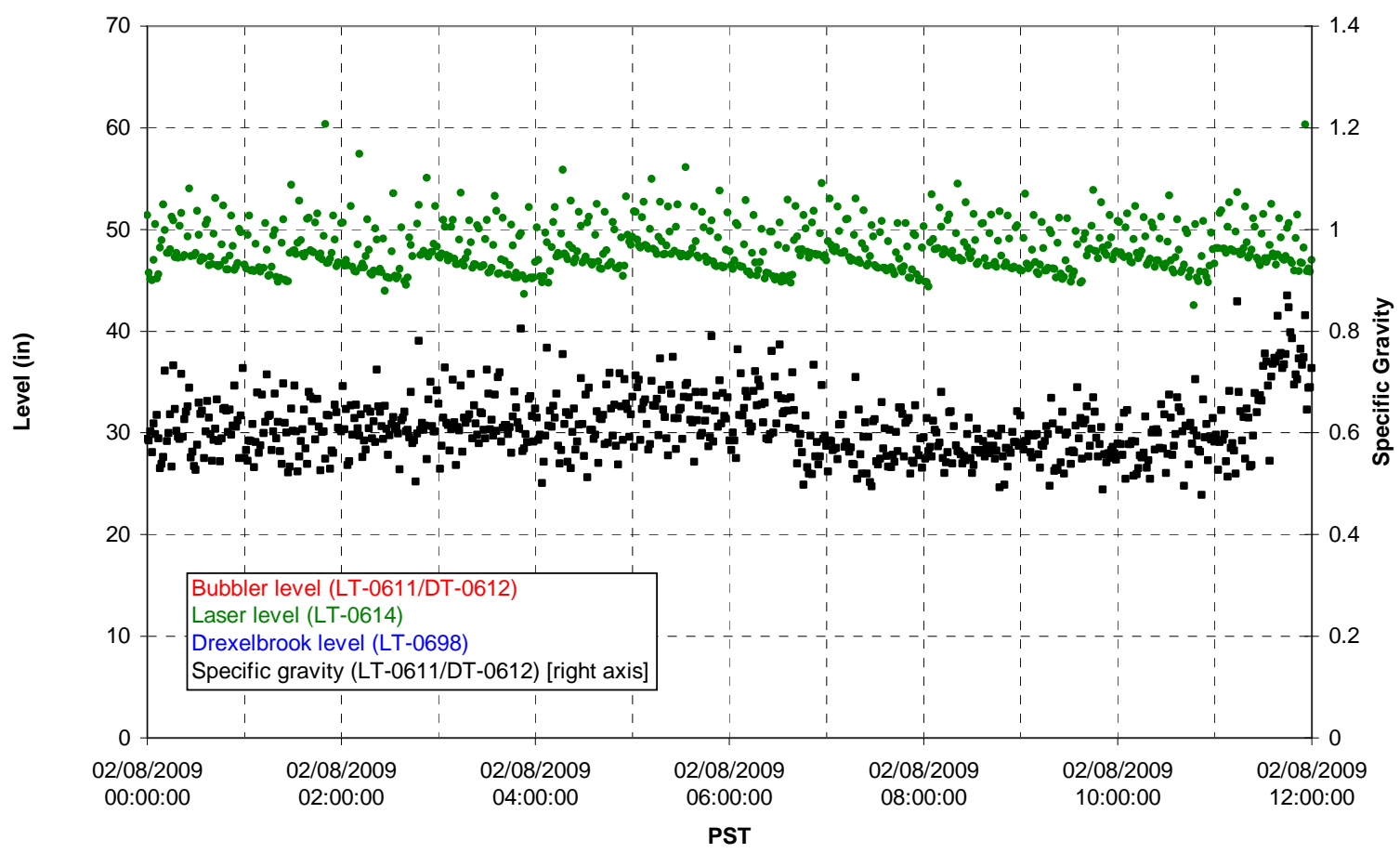

T02A temperatures

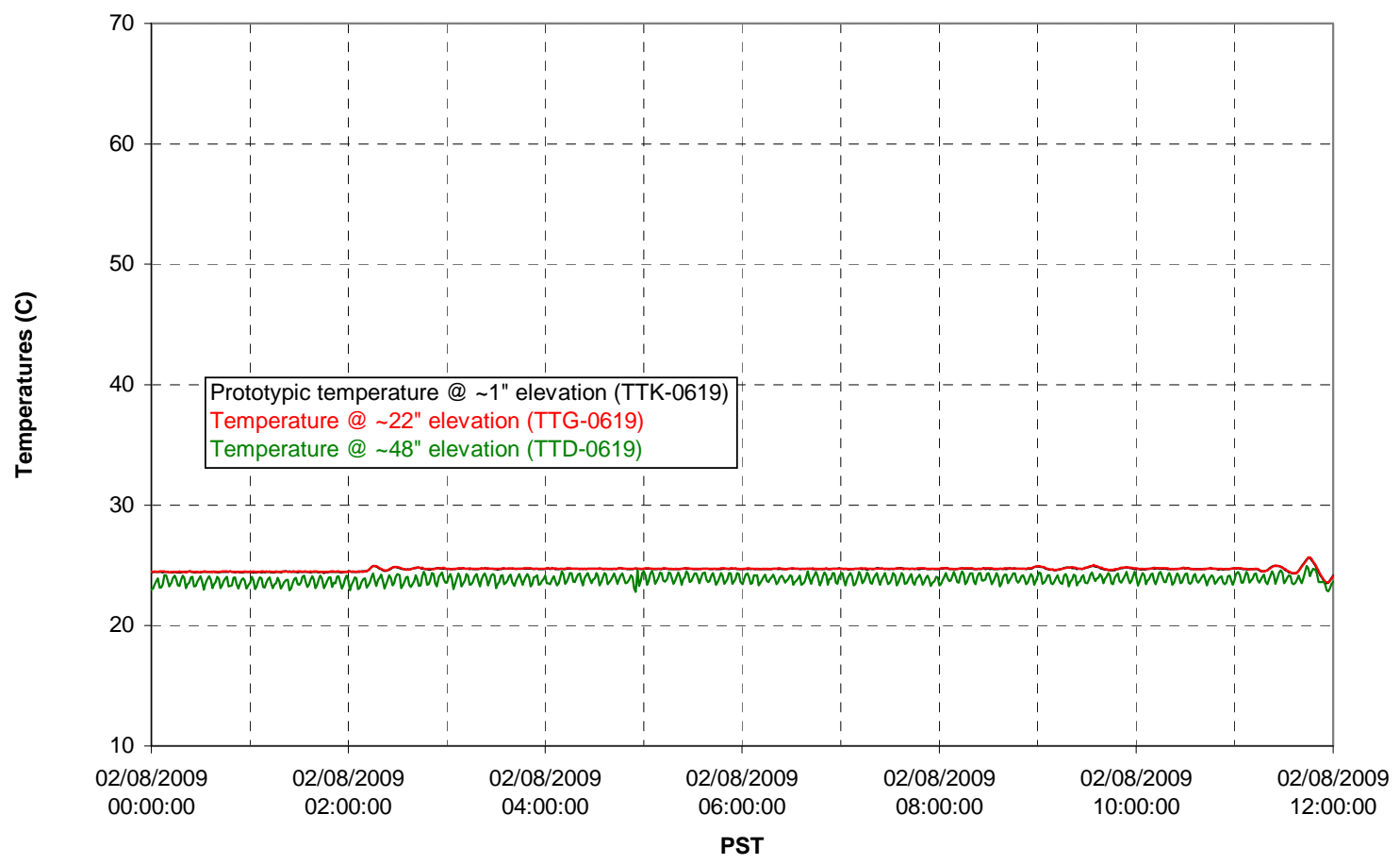

G.292 
T02A and filter loop temperatures

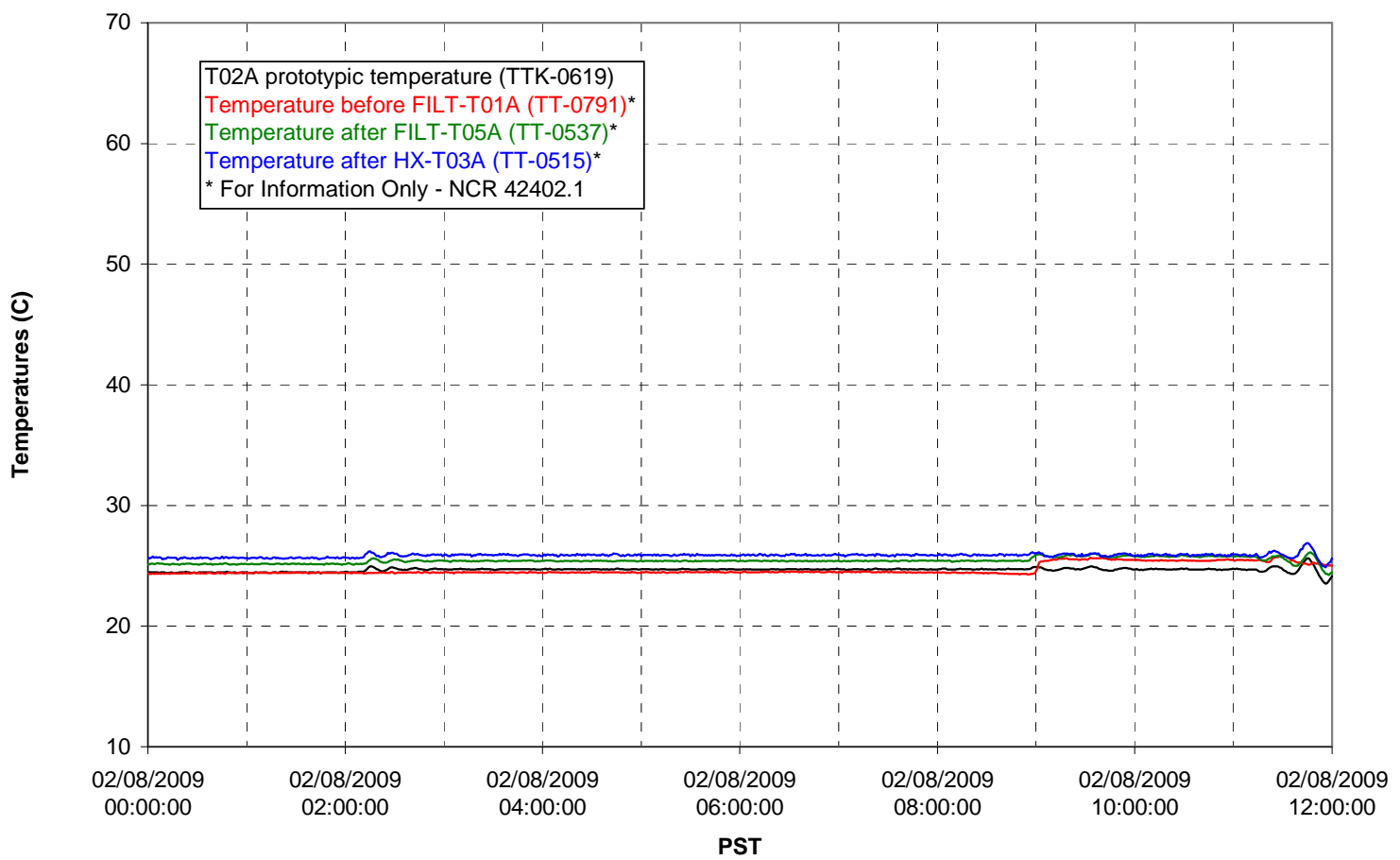

Pump Pressures and Flow

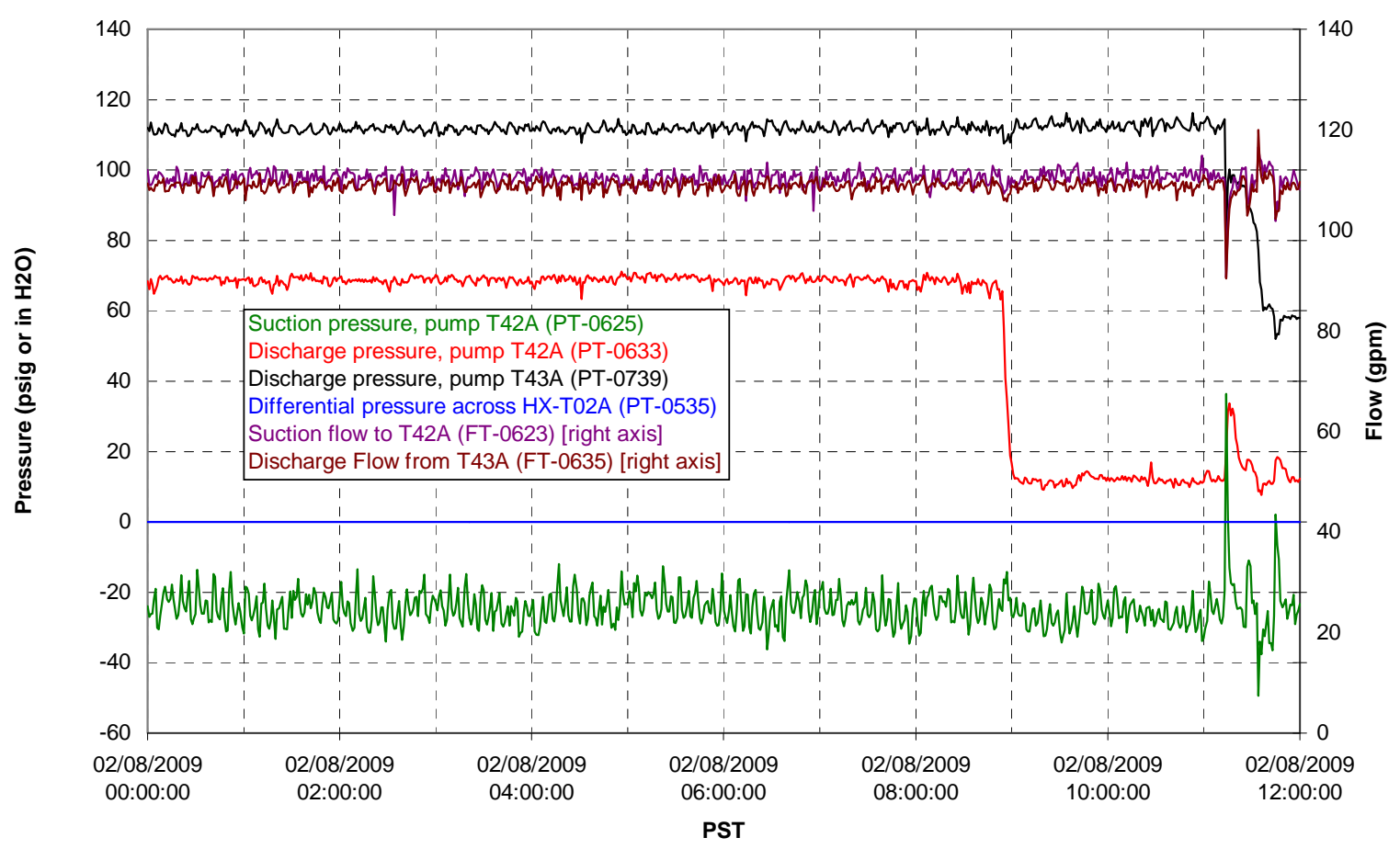


Axial pressure drop

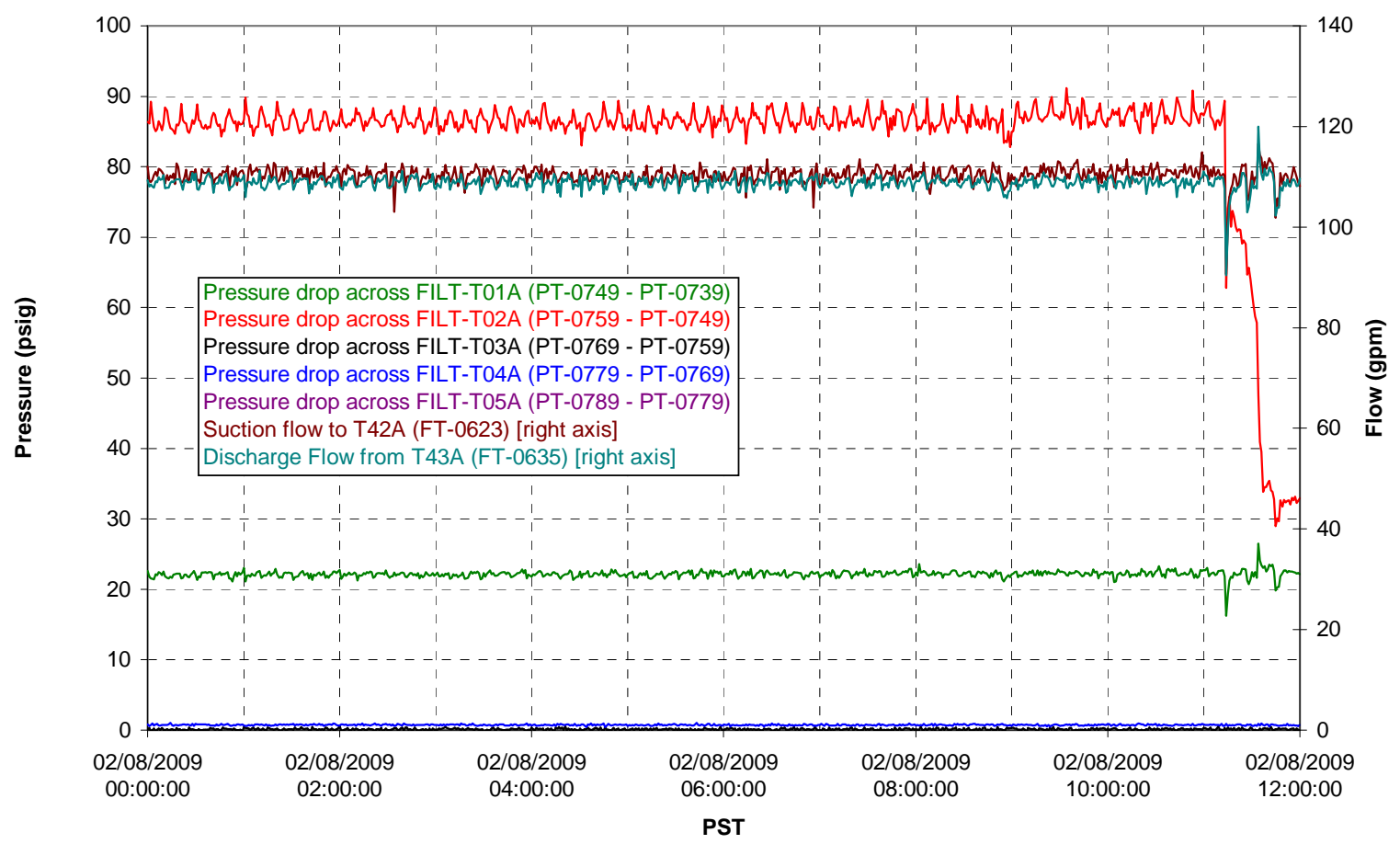

Permeate flow rates

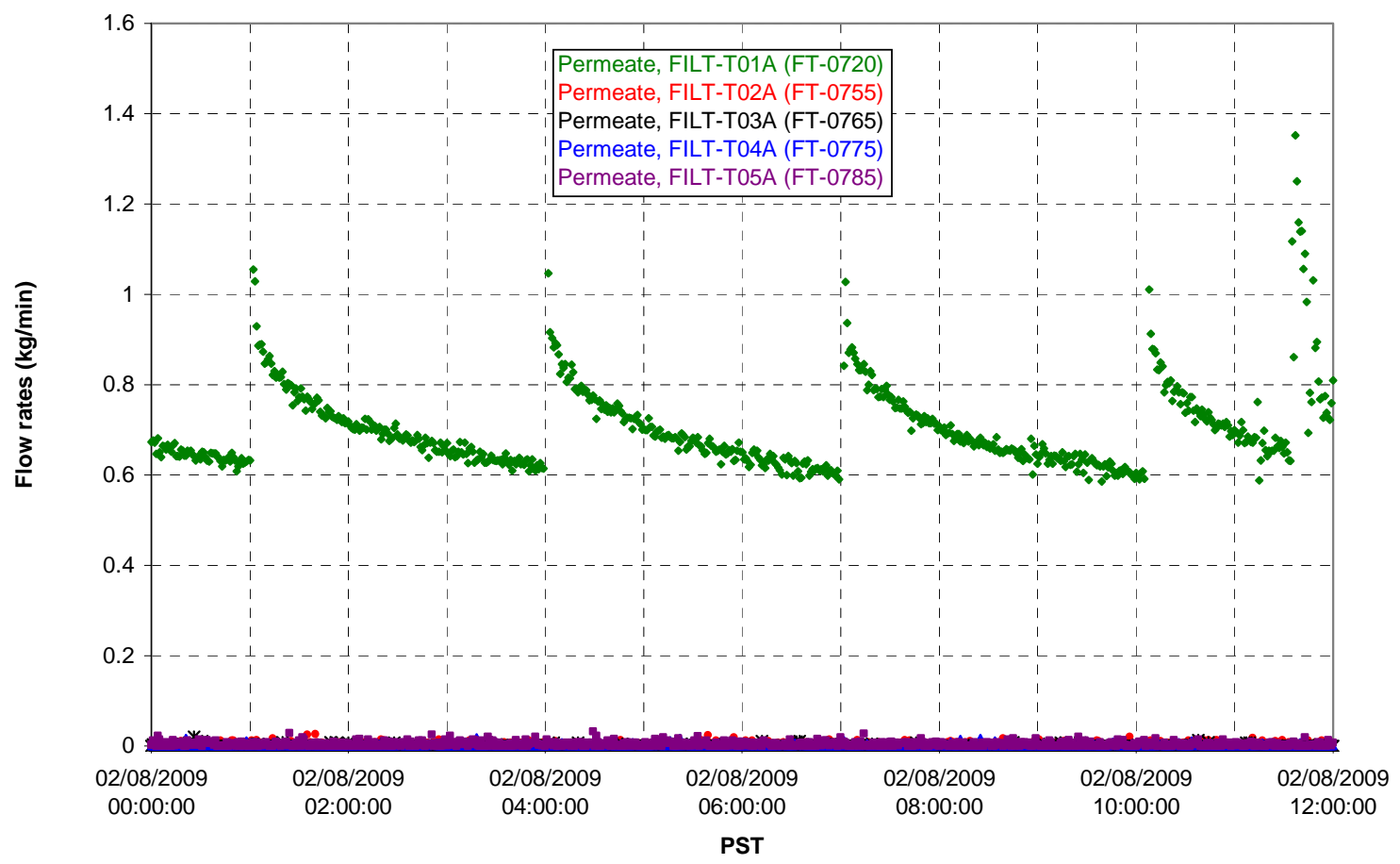


T02A Inner Temperature Tree

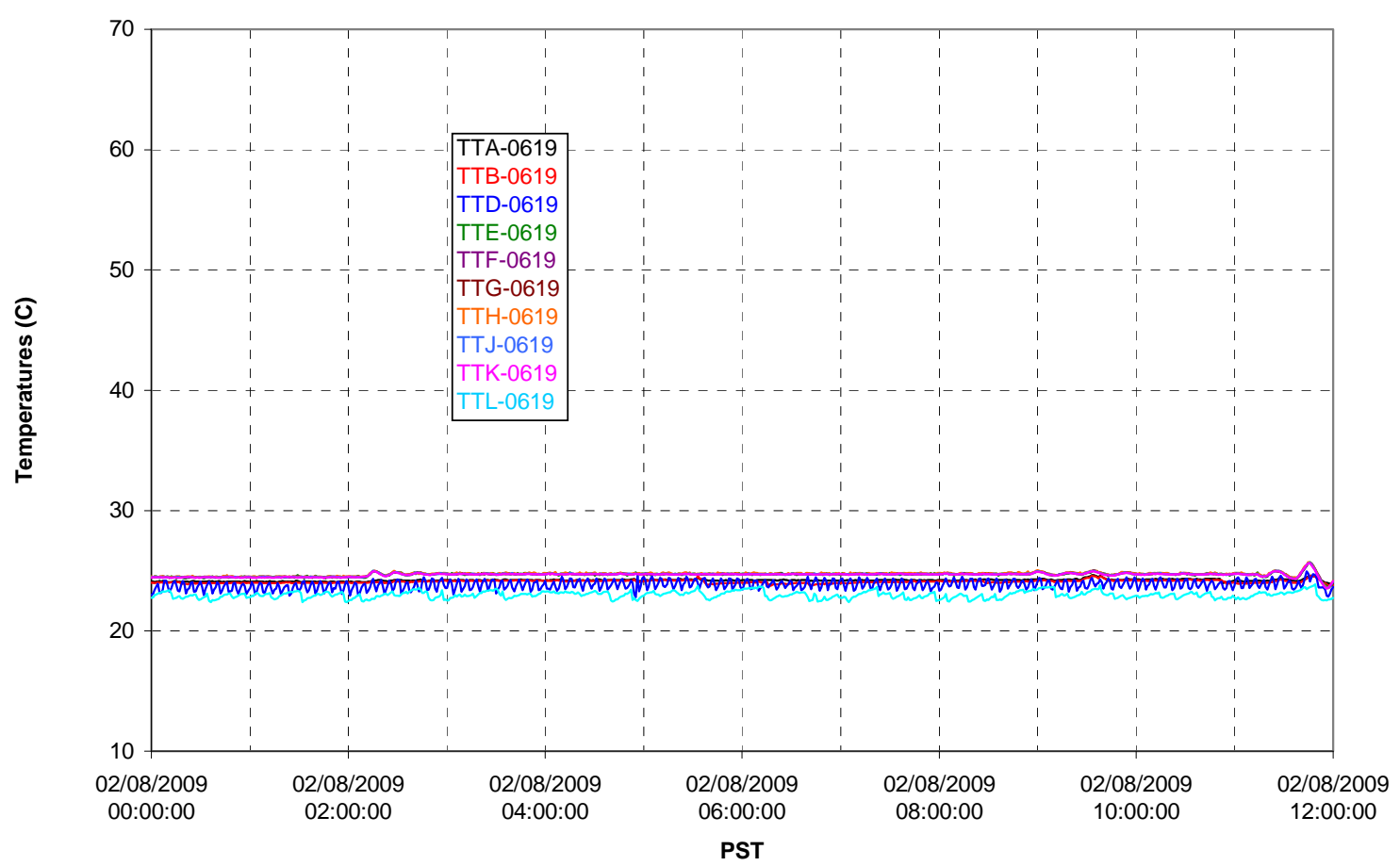

T02A Outer Temperature Tree

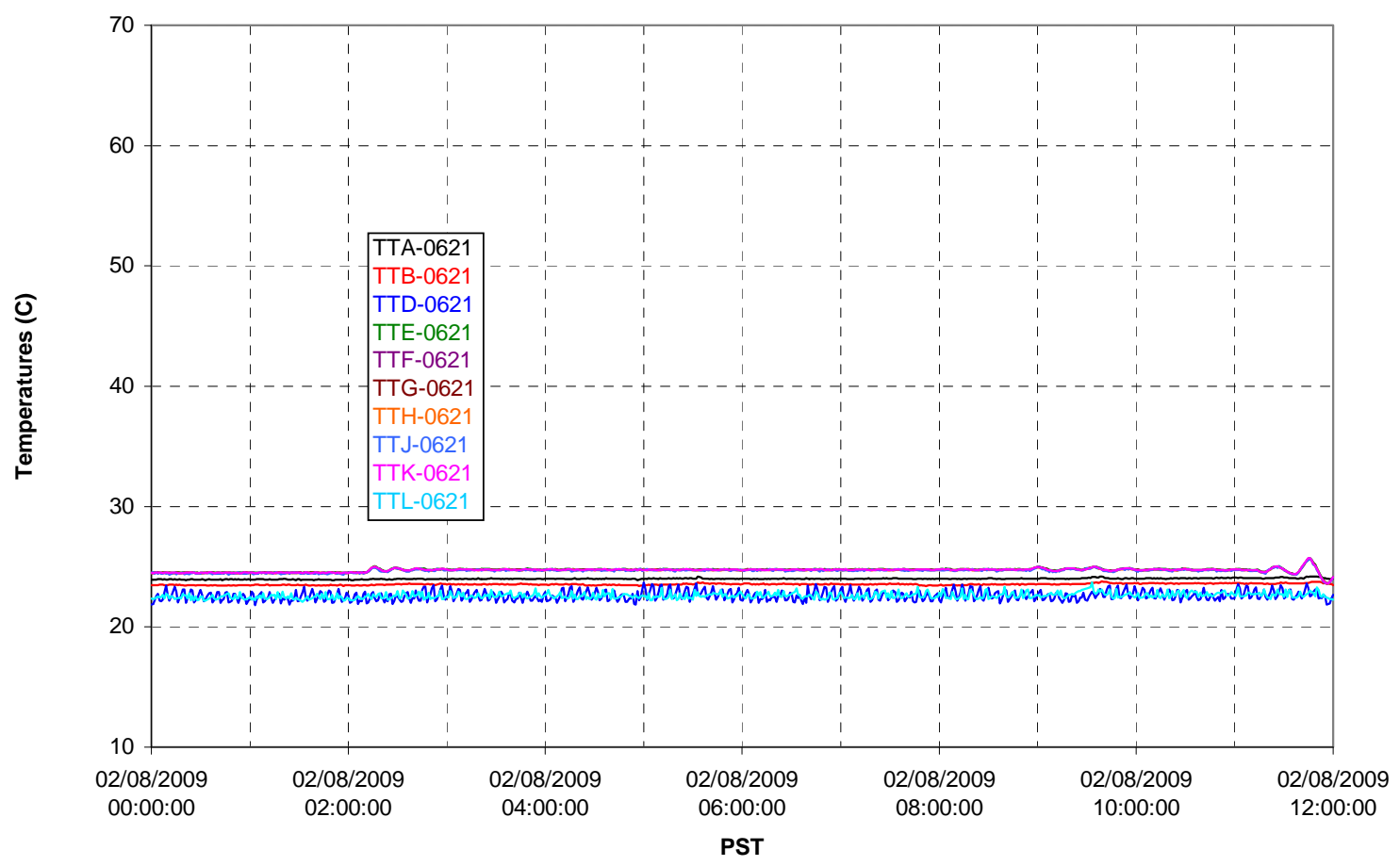


T02A temperatures

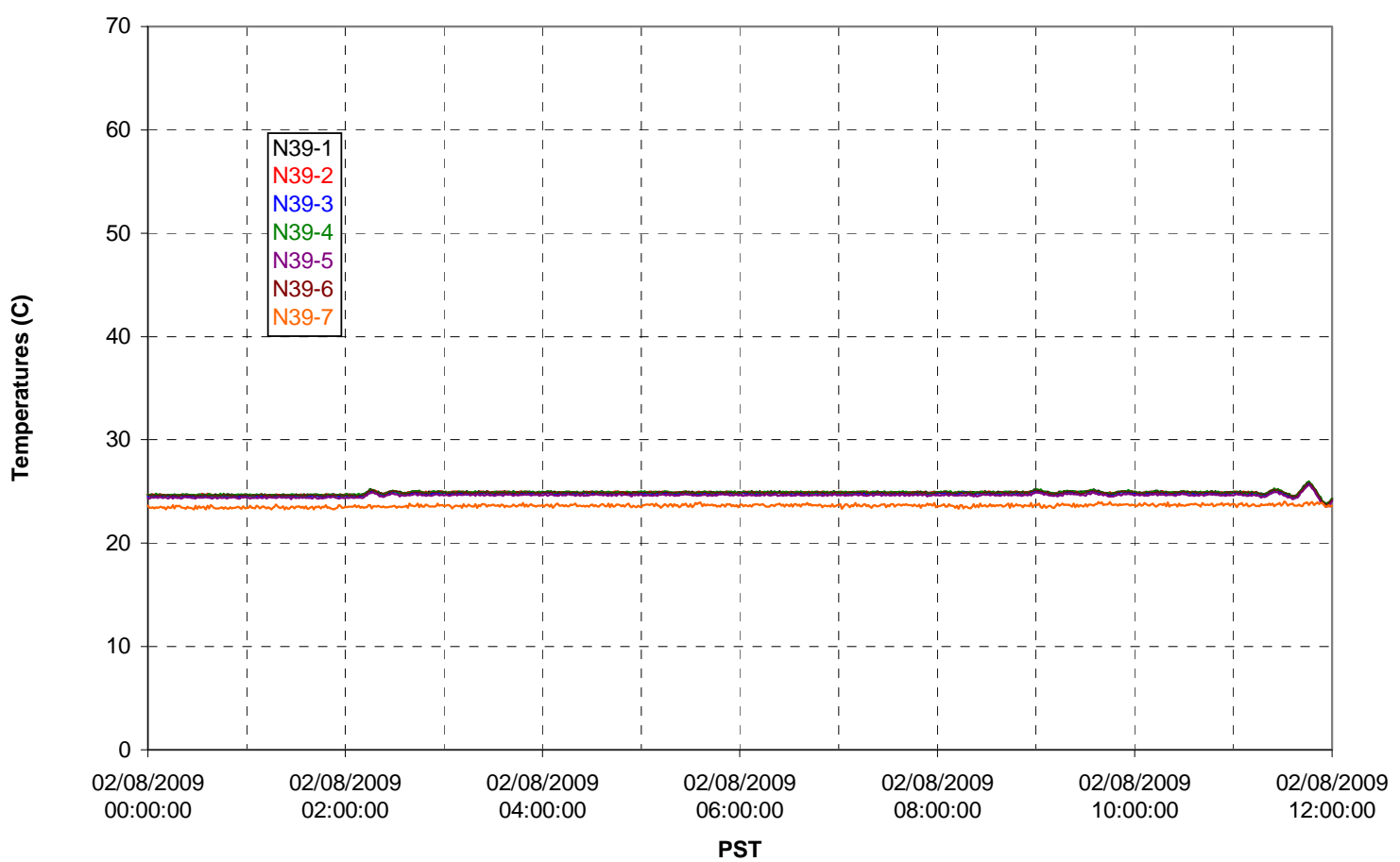

T02A temperatures

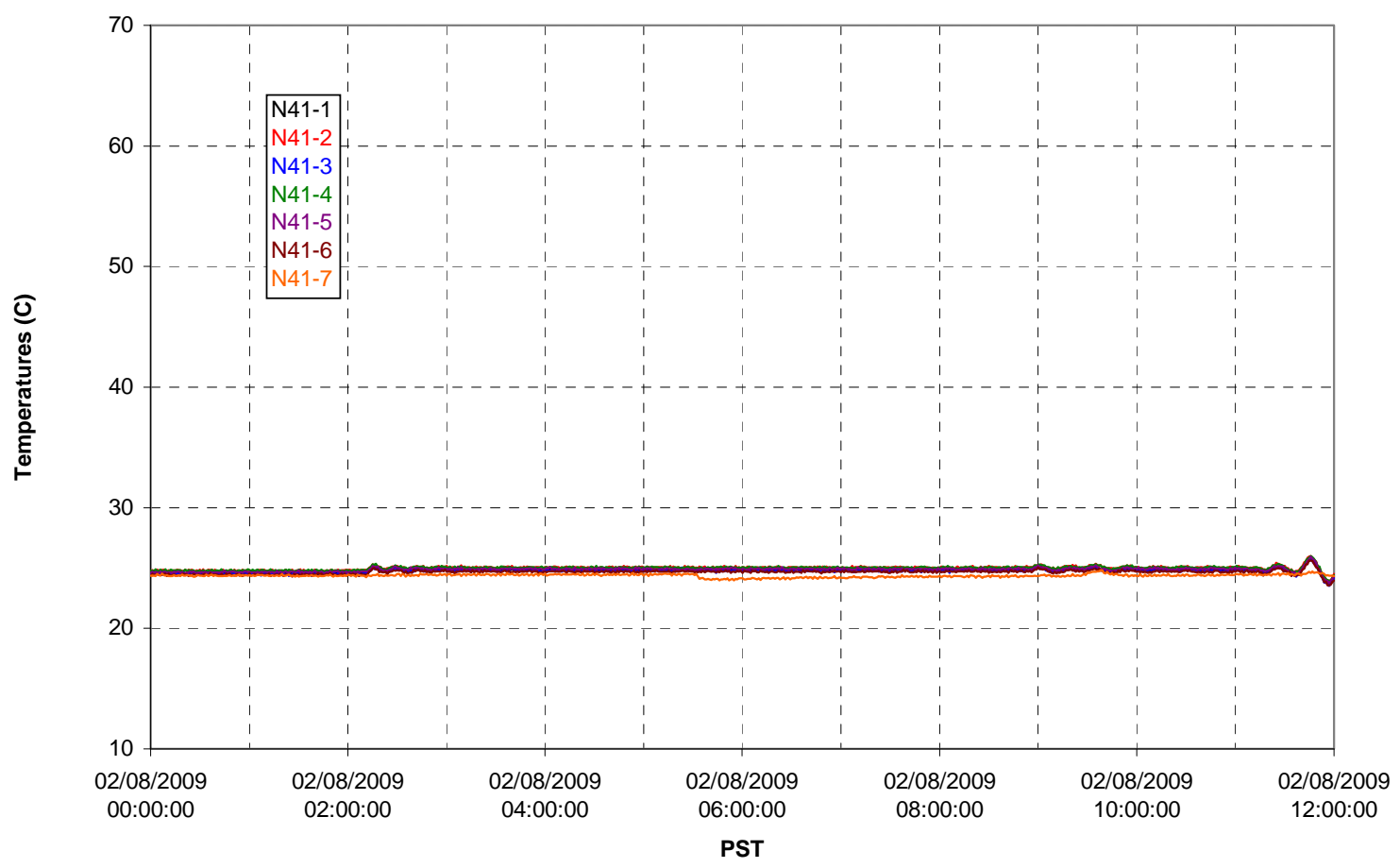


T02A temperatures

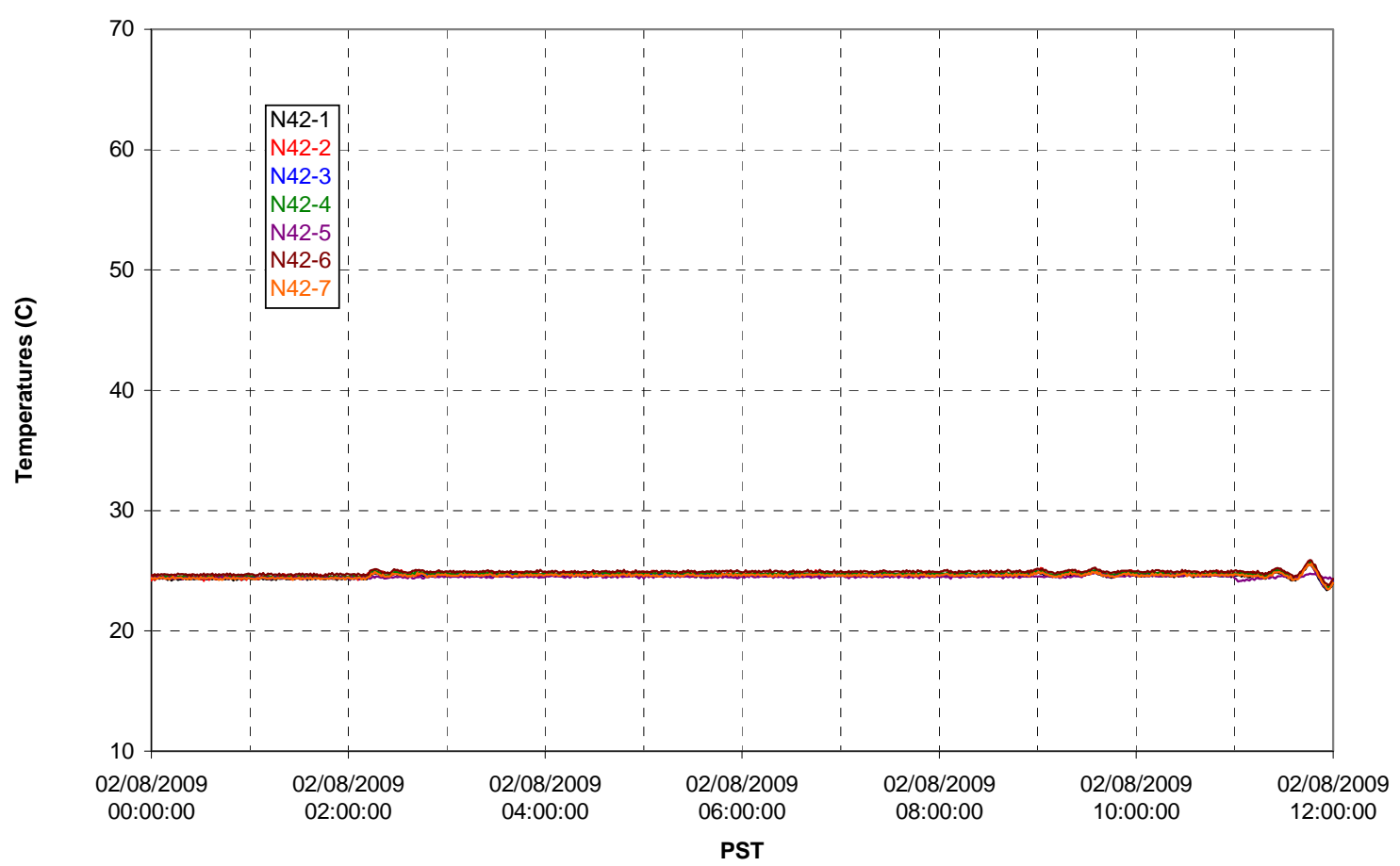

T02A temperatures

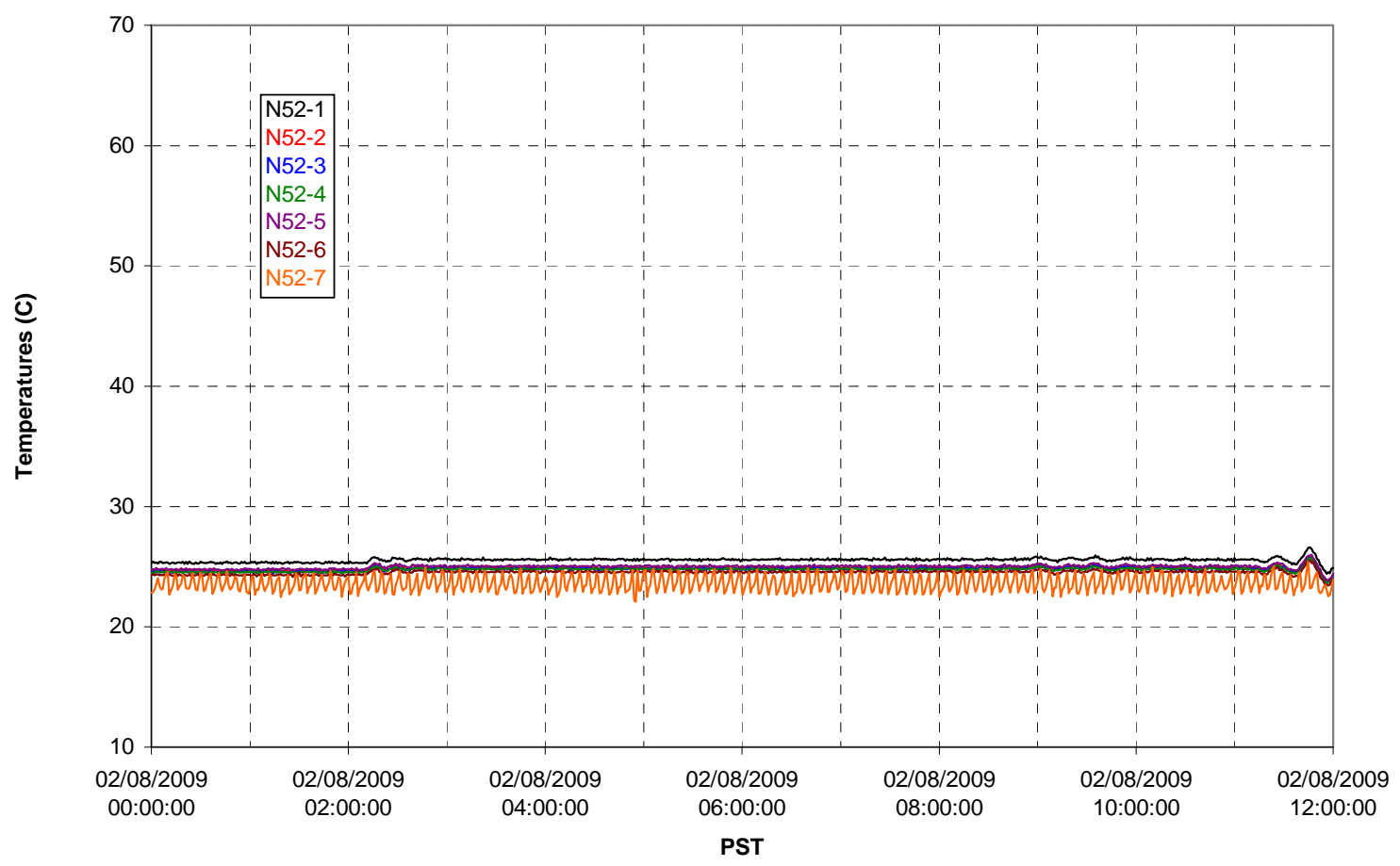


T02A Heating and Cooling

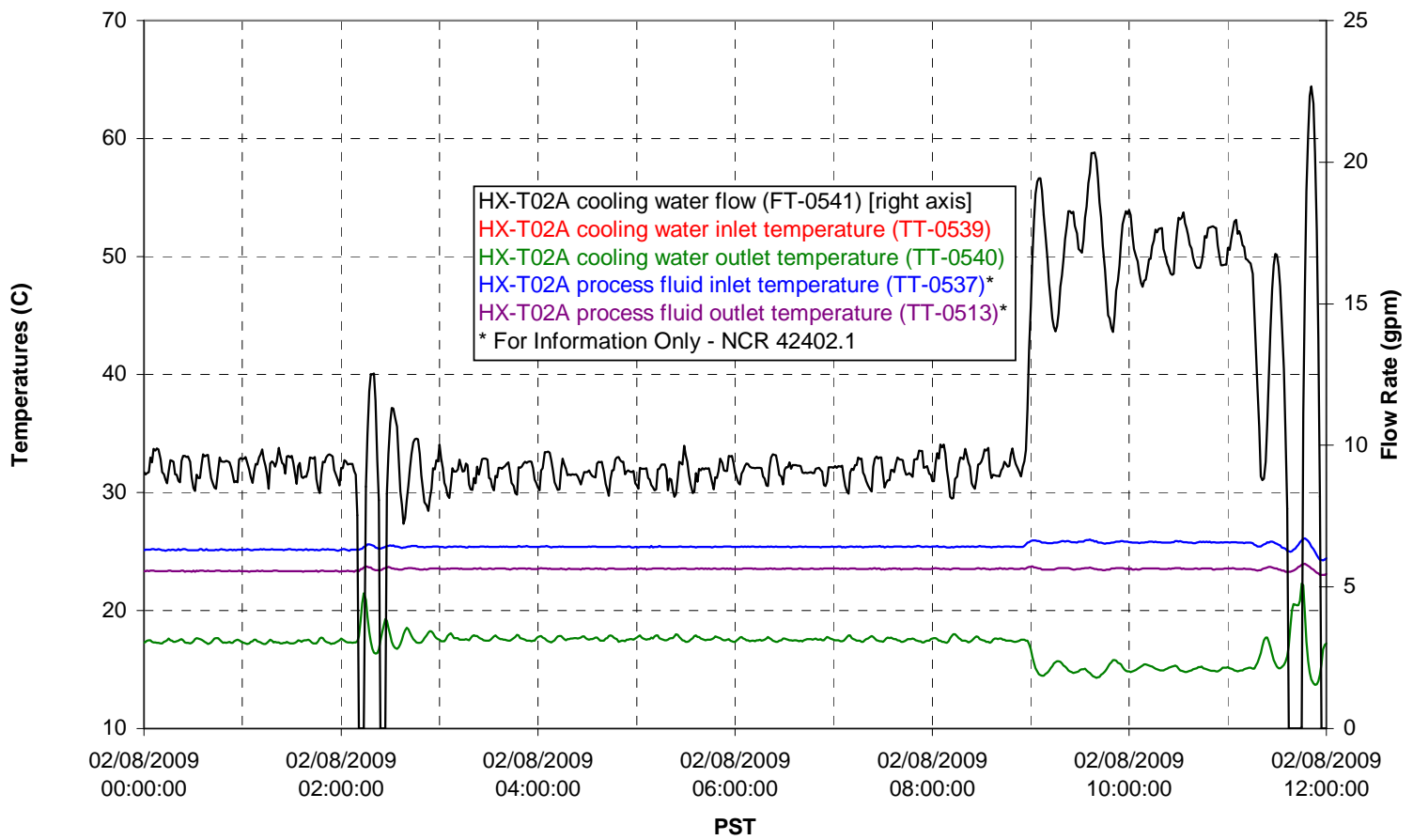

Pump Operation

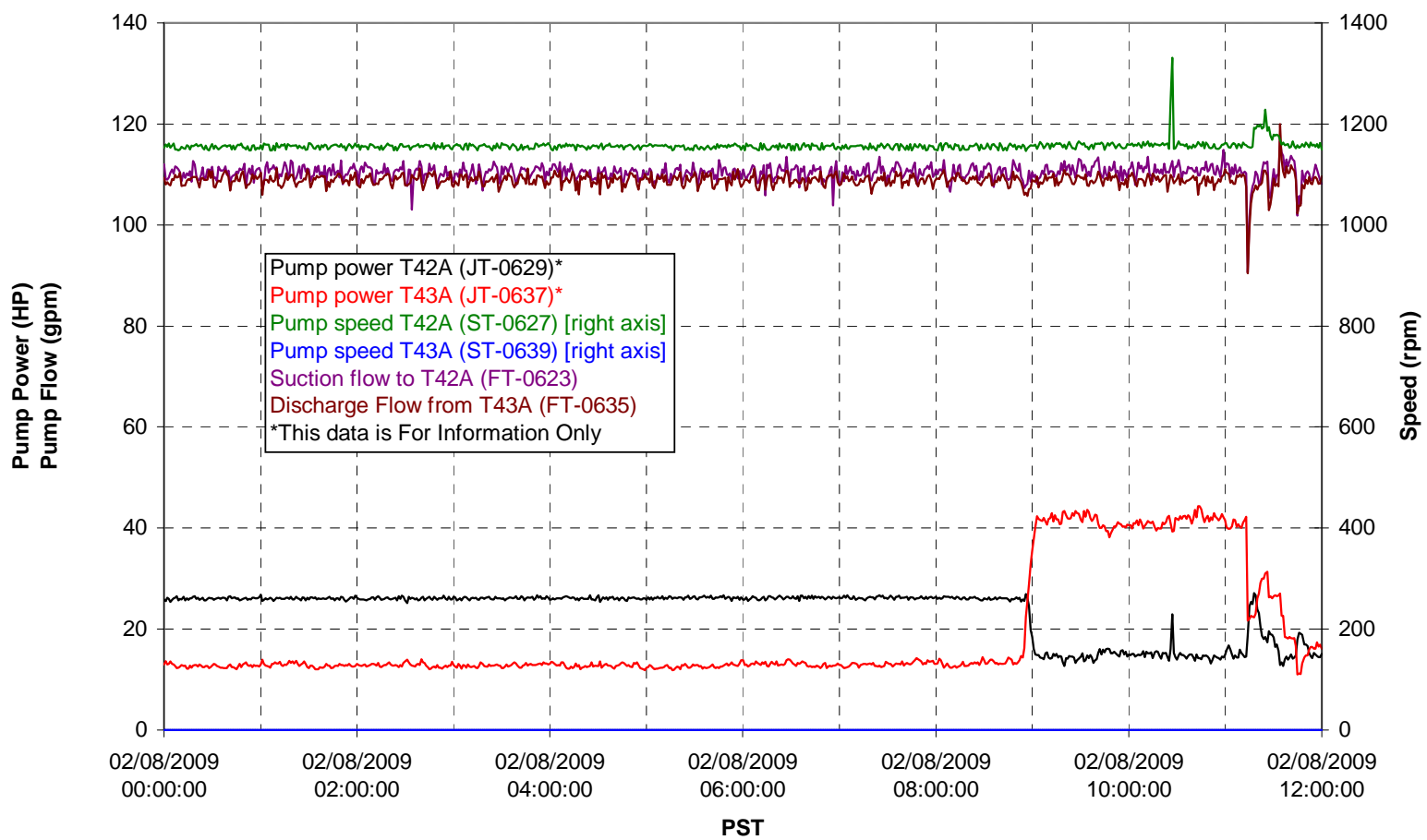


Pulsepot UFP-PP-T01A

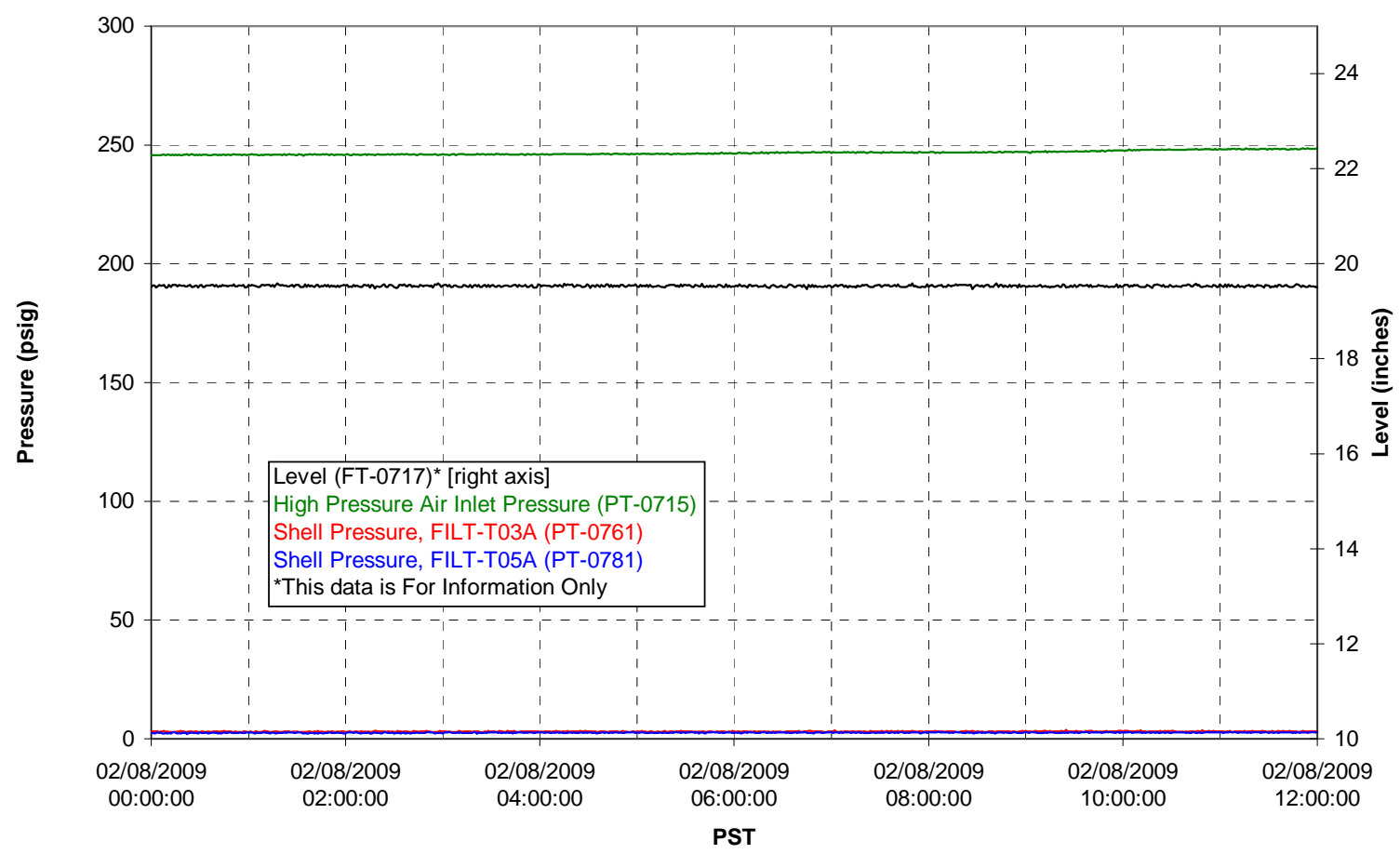

Pulsepot UFP-PP-T02A

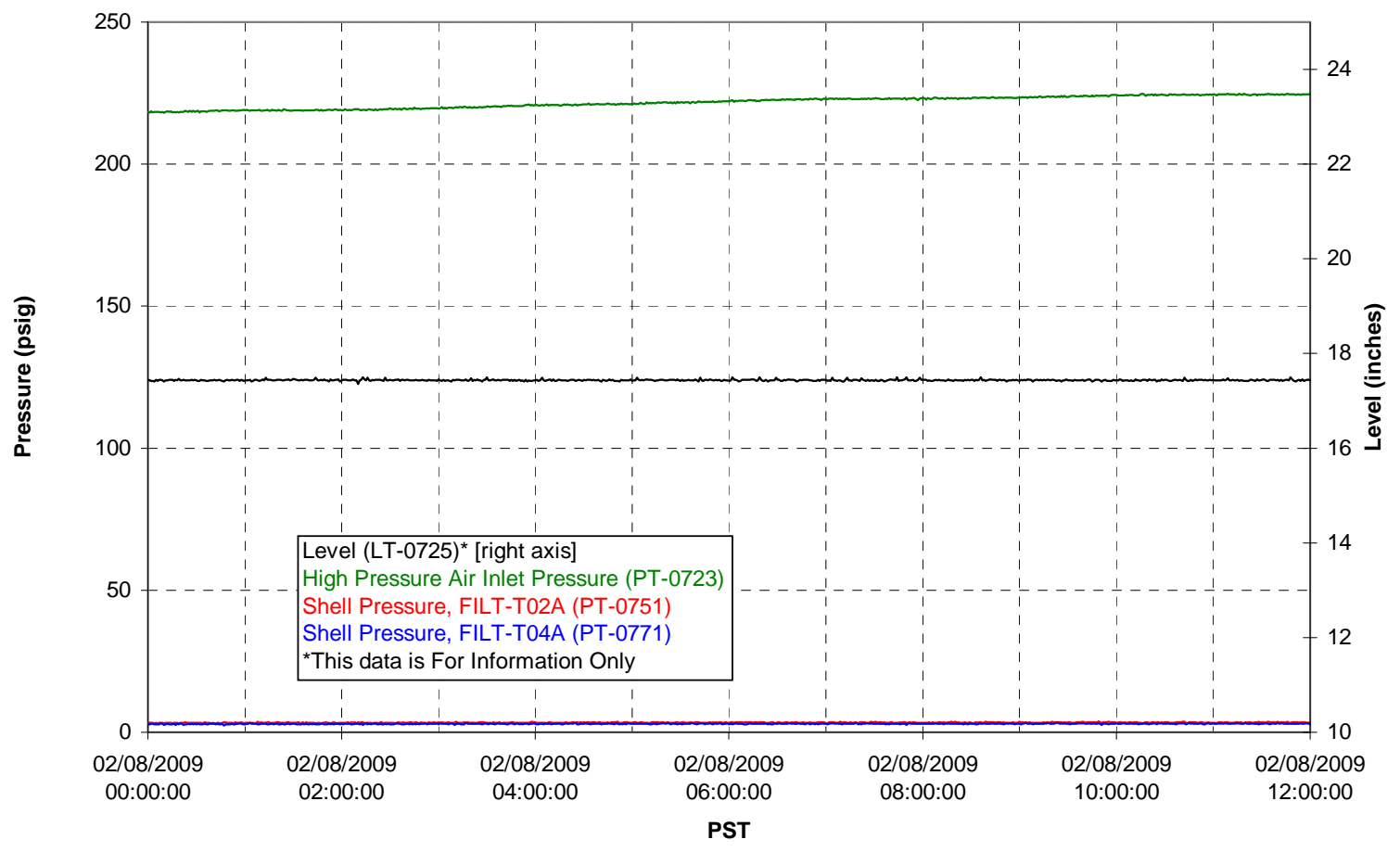


Pulsepot UFP-PP-T03A

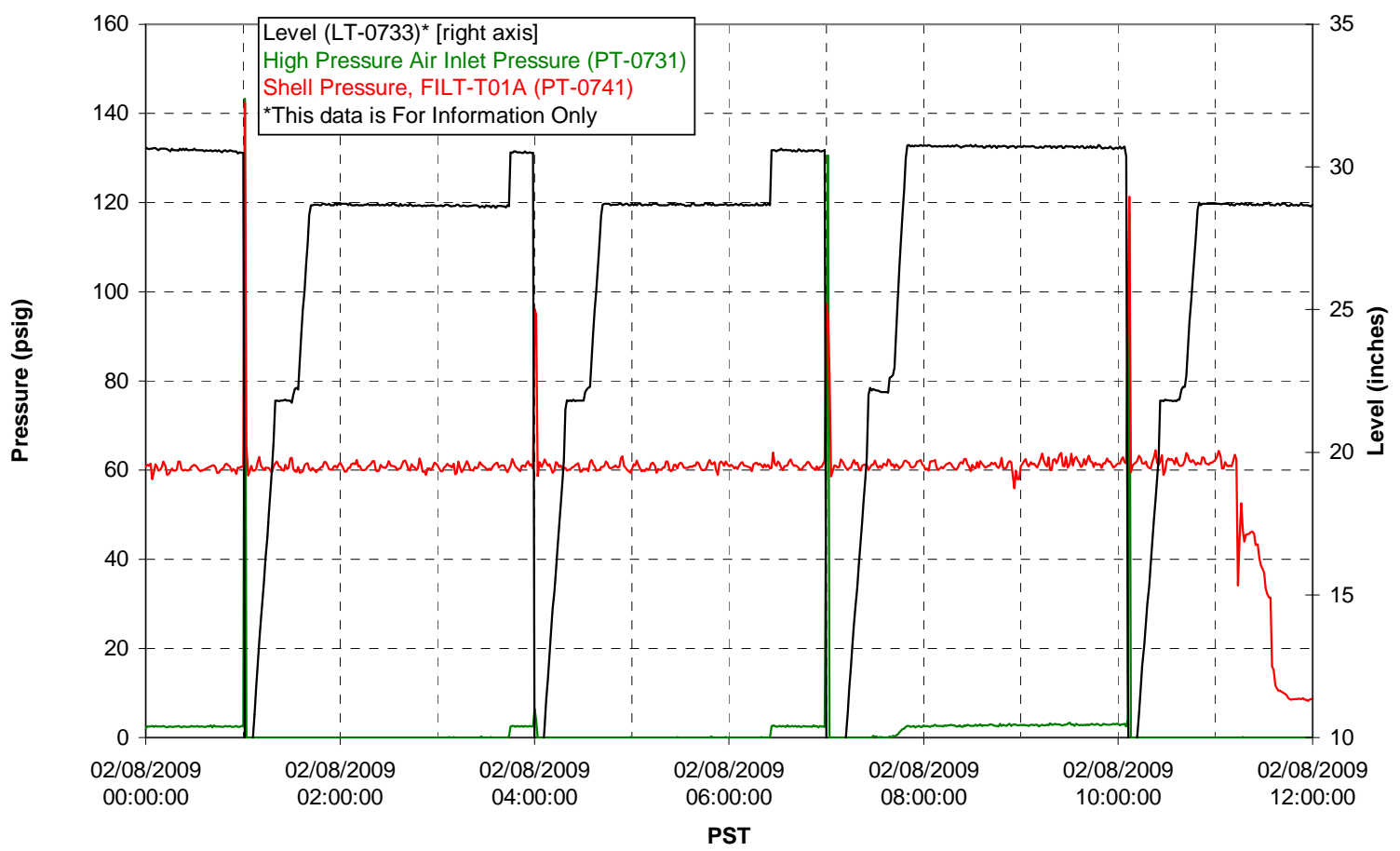

Pulsepot Levels

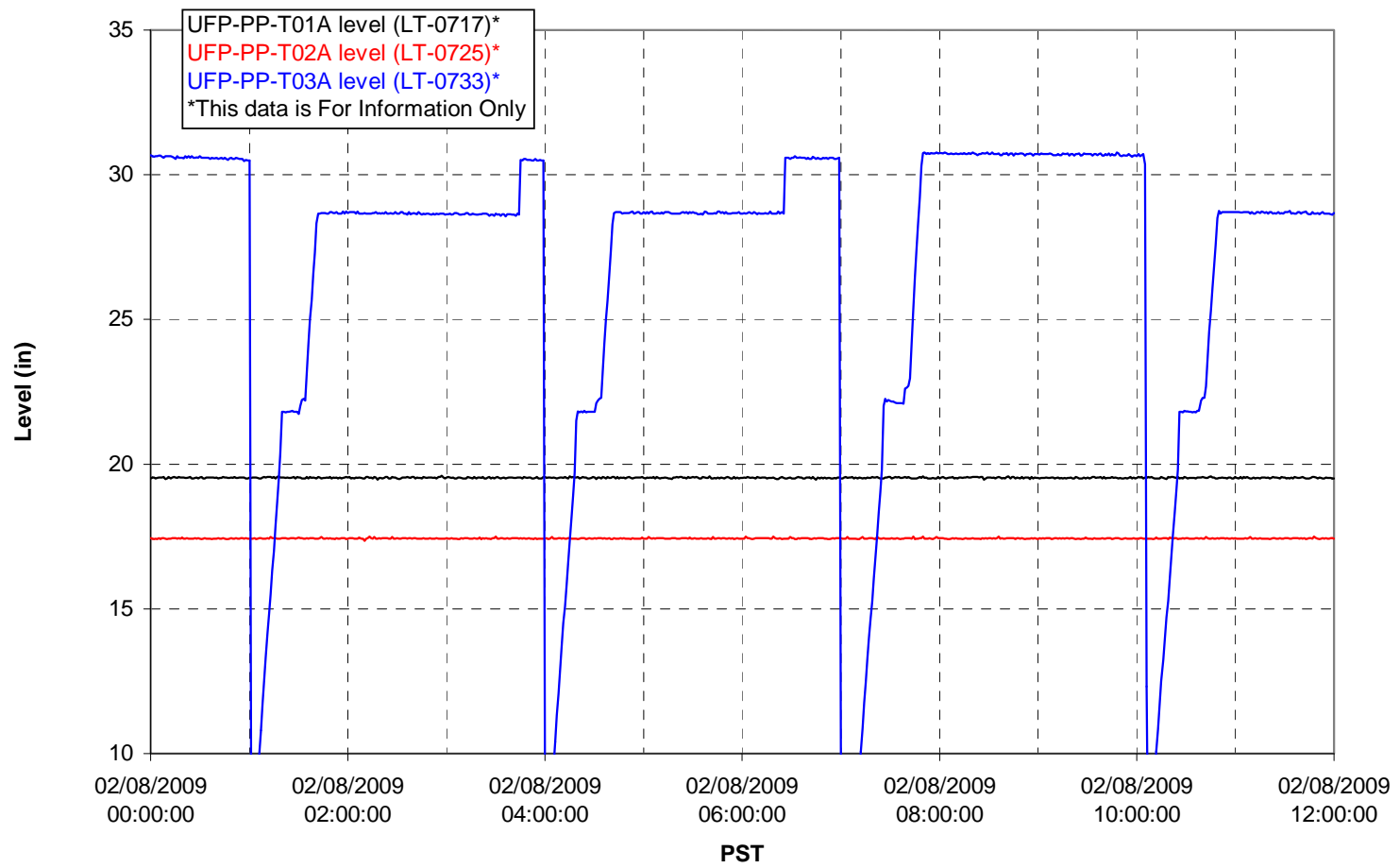


Filter UFP-FILT-T01A

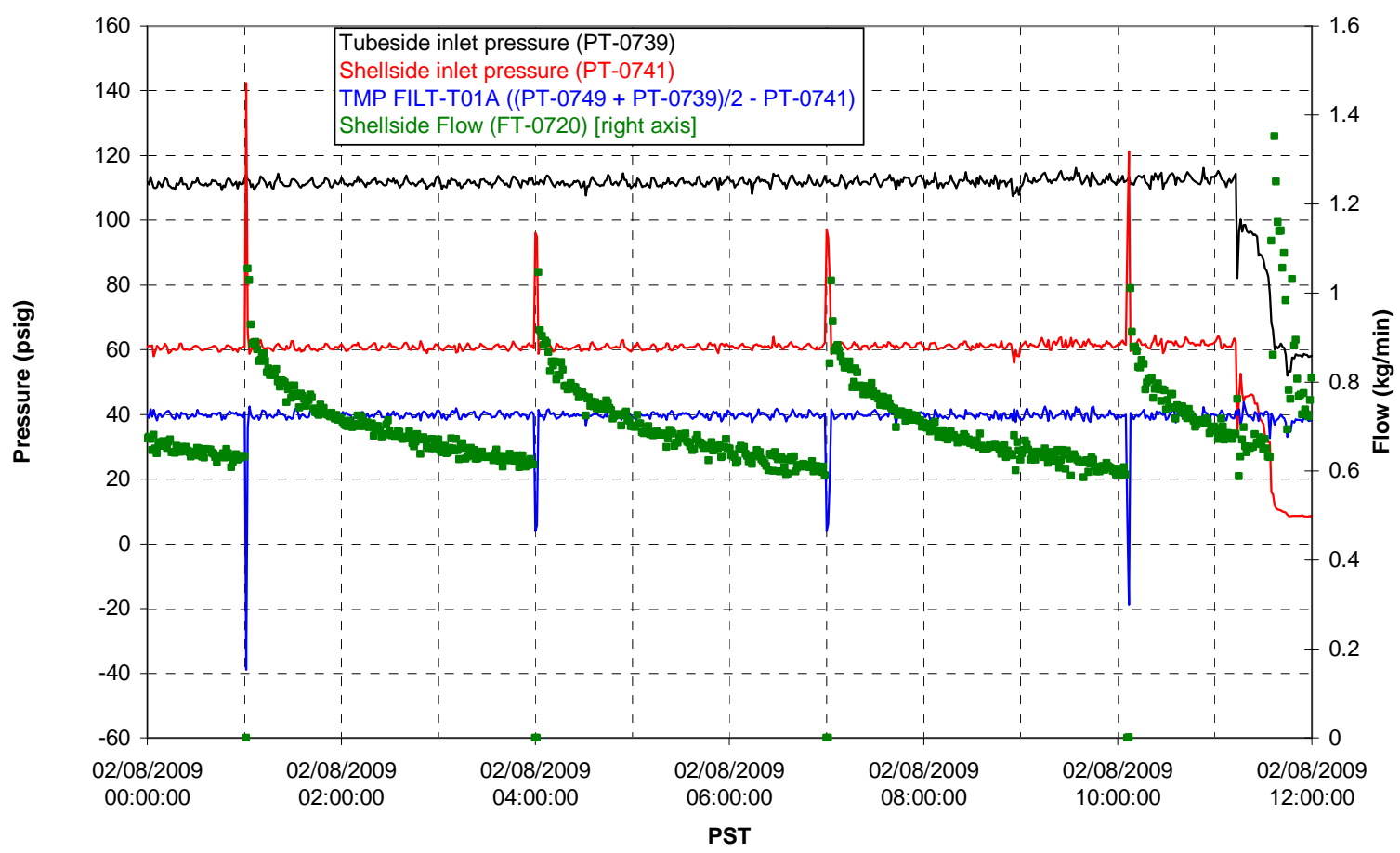

Filter UFP-FILT-T02A

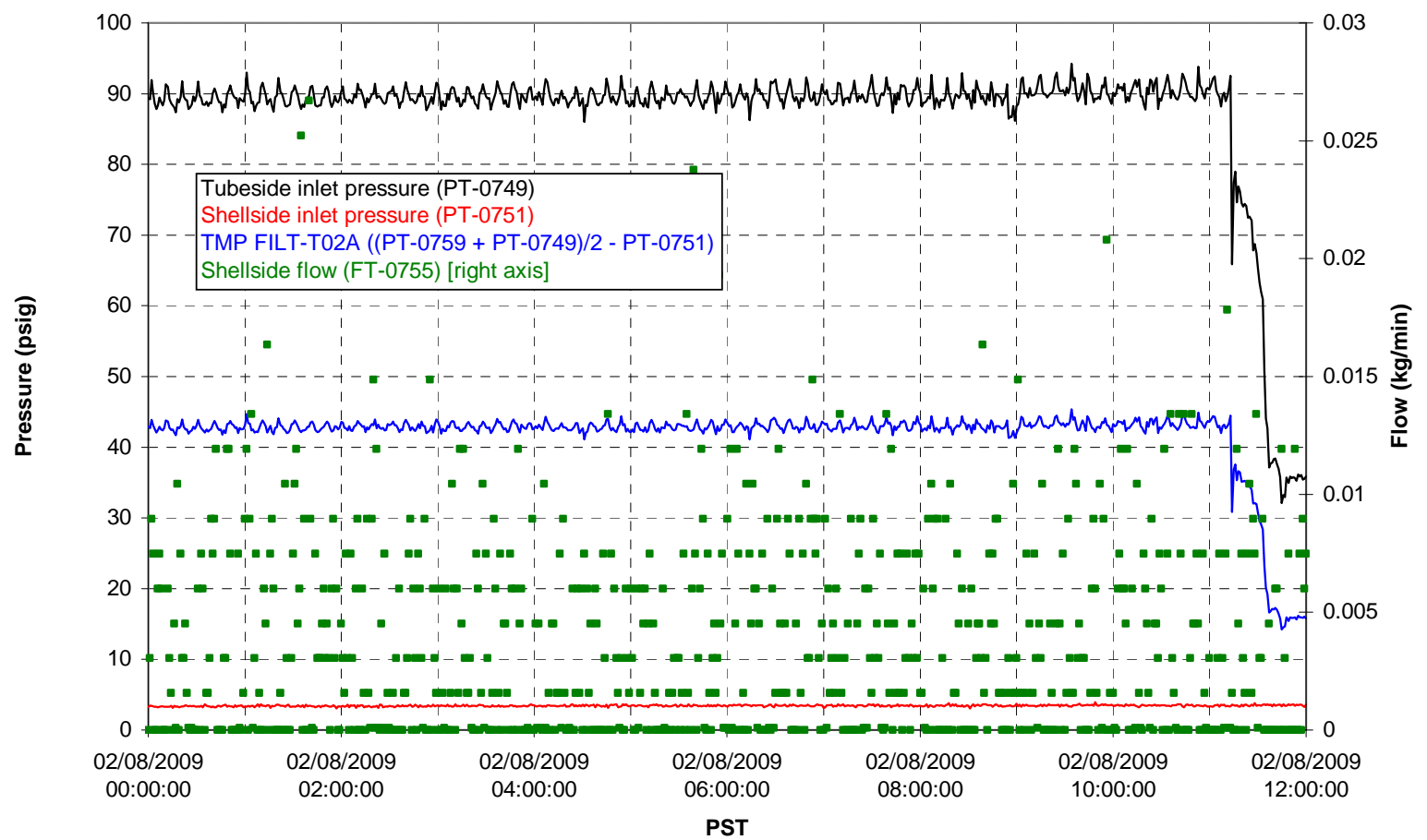


Filter UFP-FILT-T03A

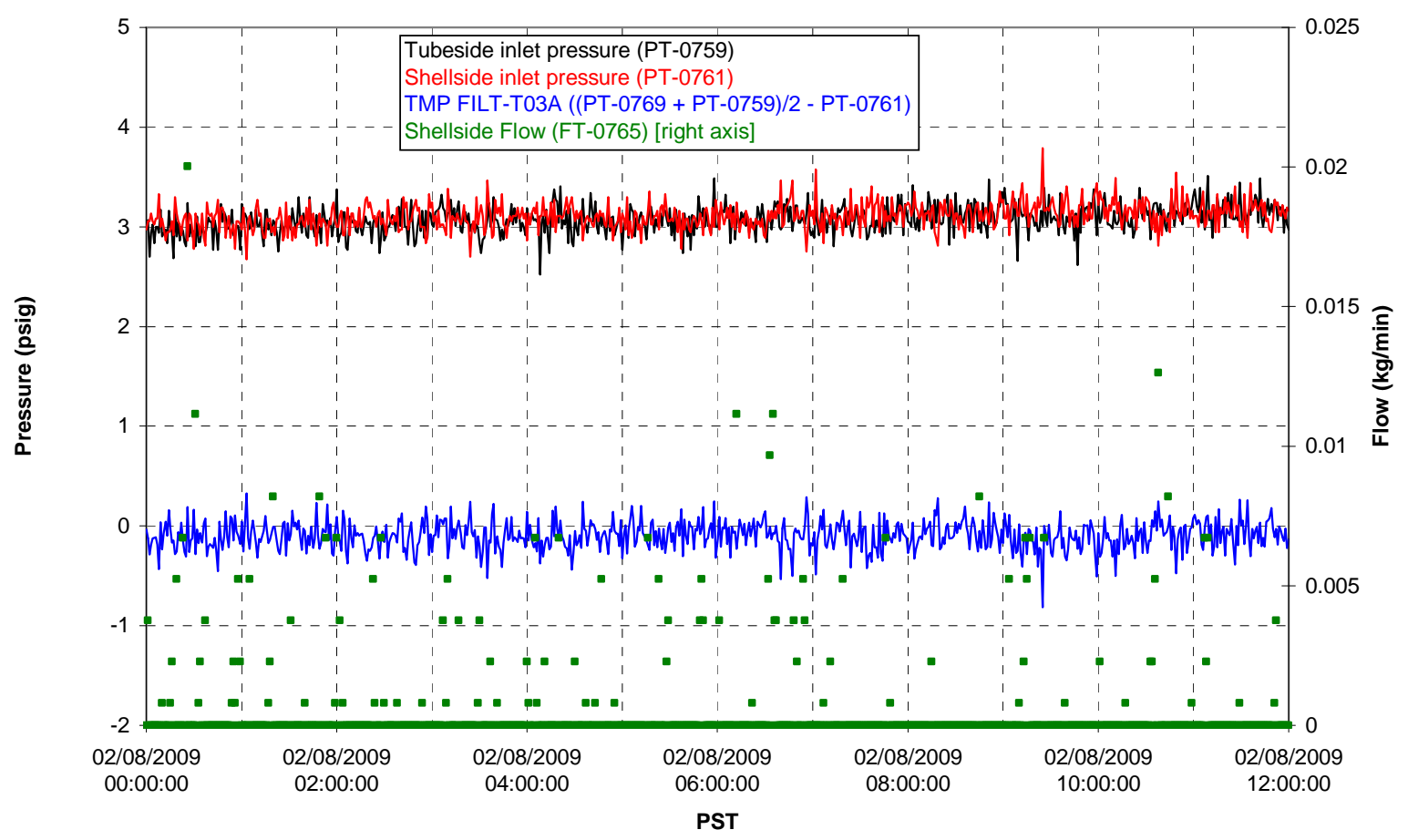

Filter UFP-FILT-T04A

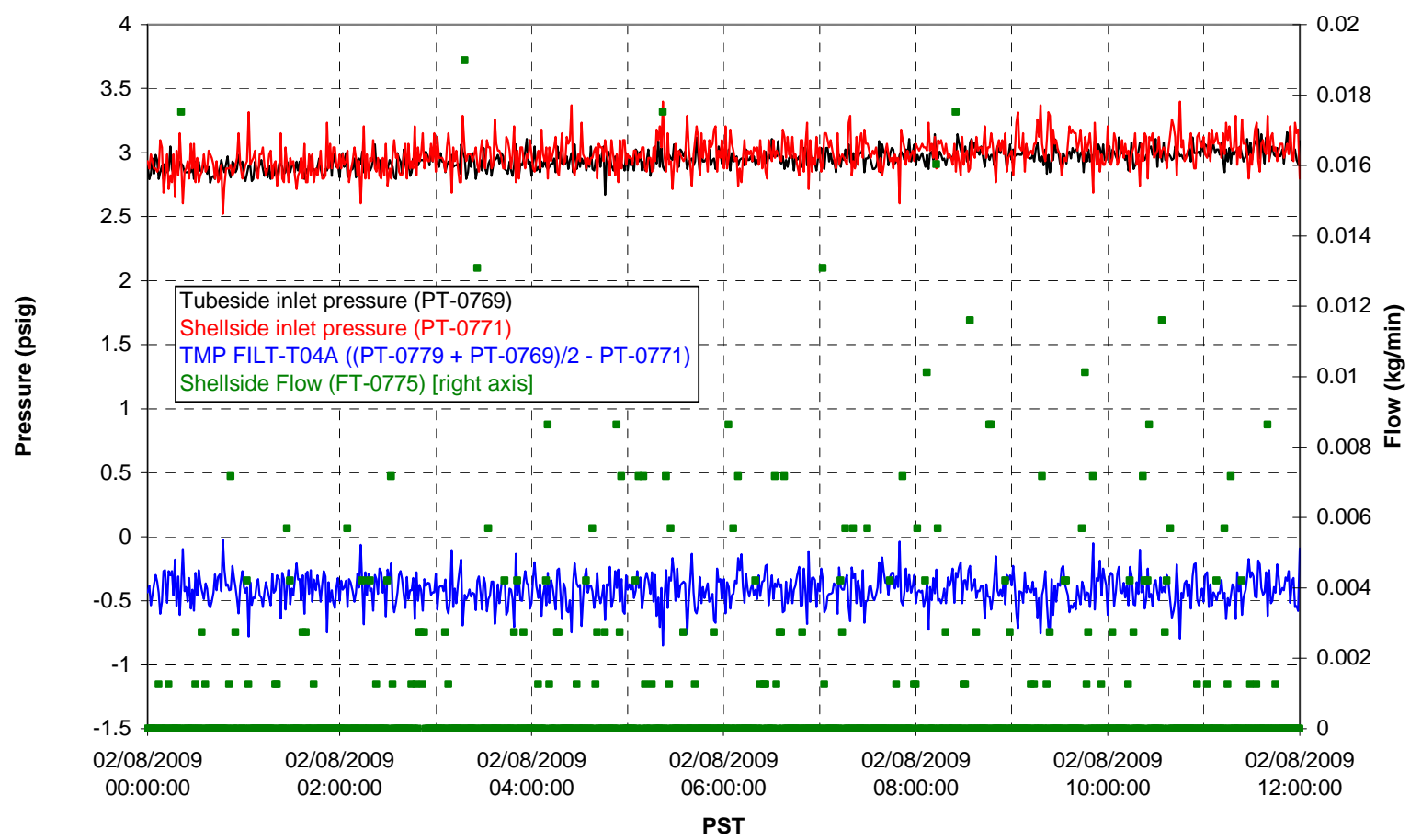


Filter UFP-FILT-T05A

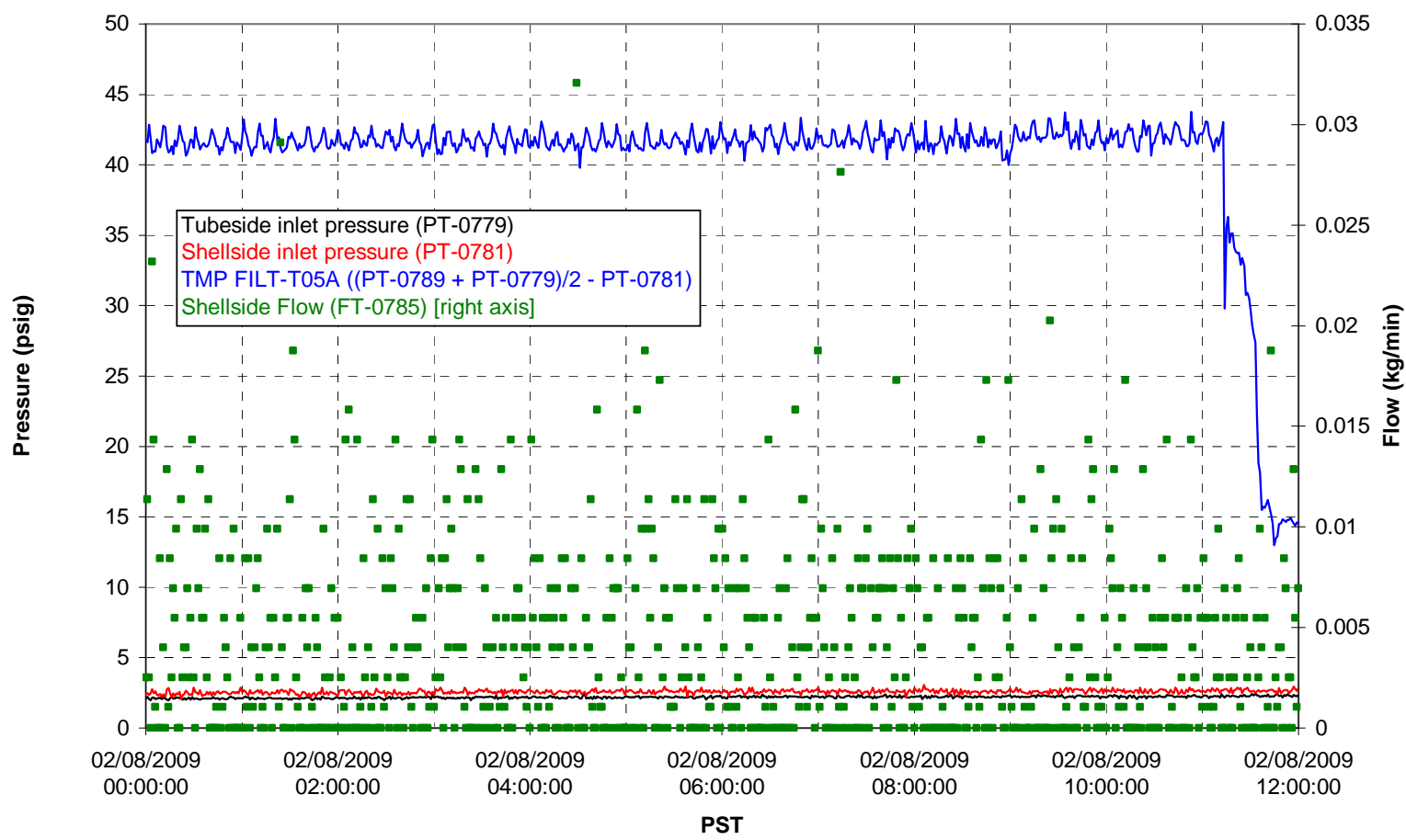

Chemical Flow

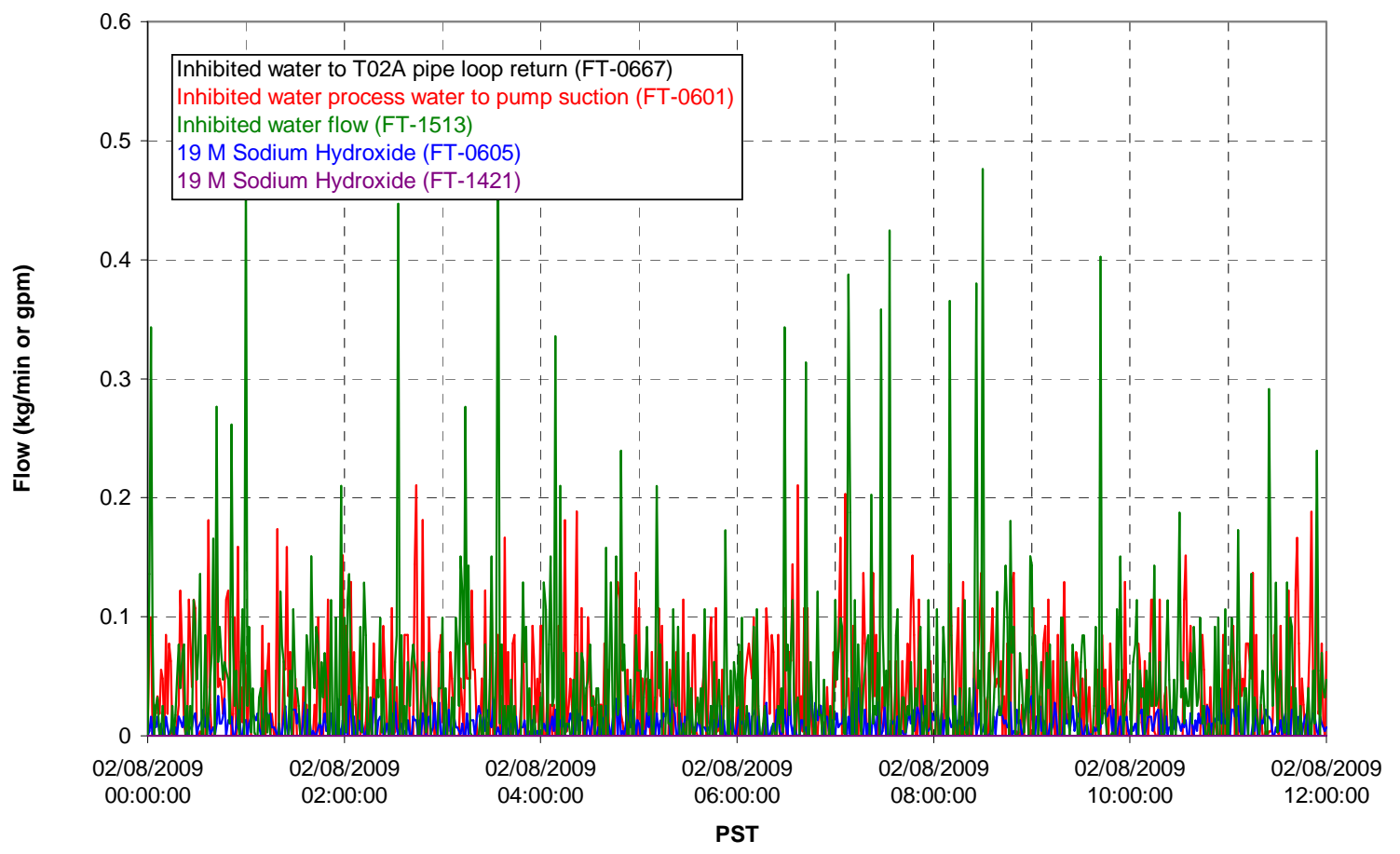




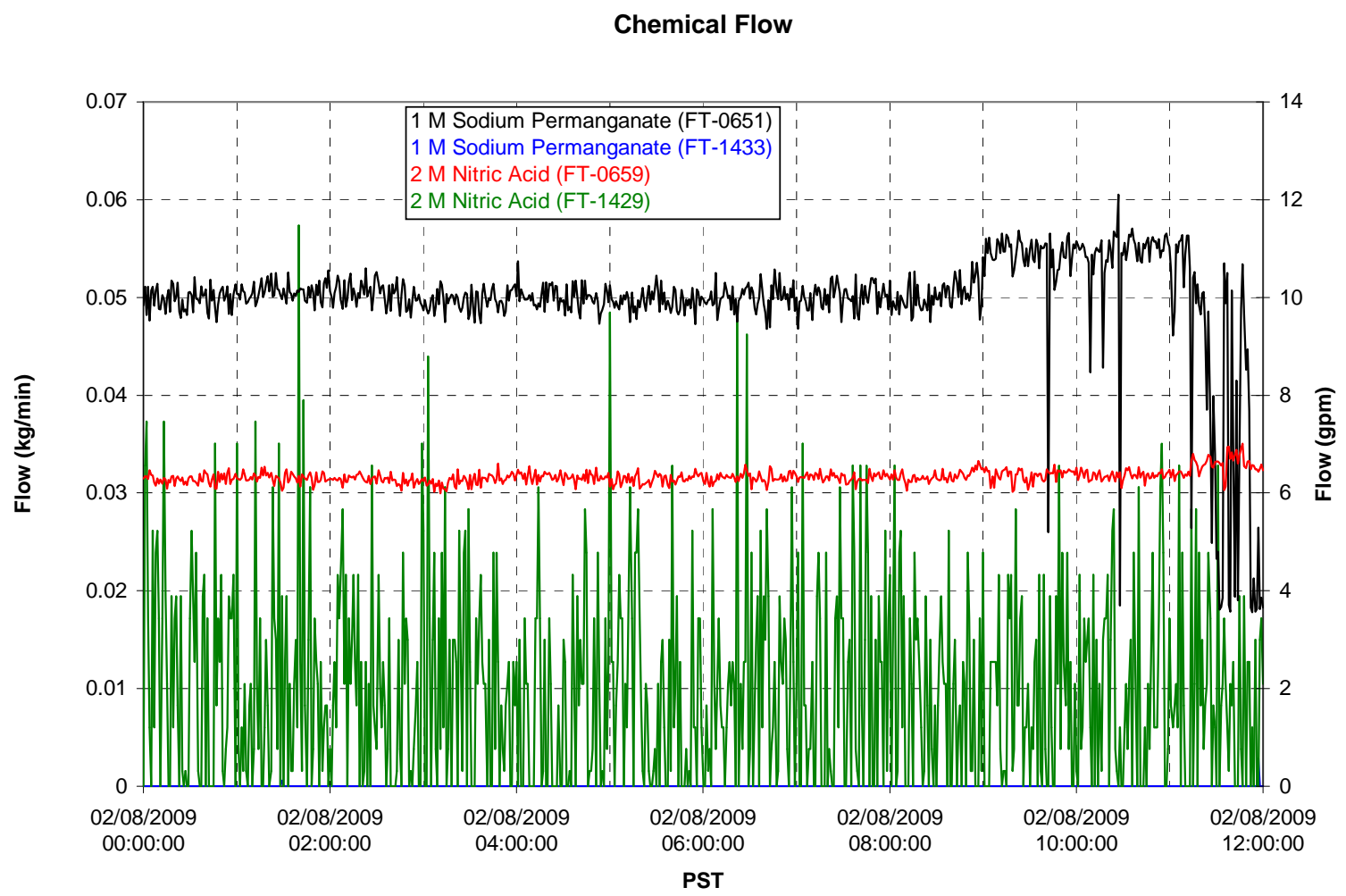

Air Flows

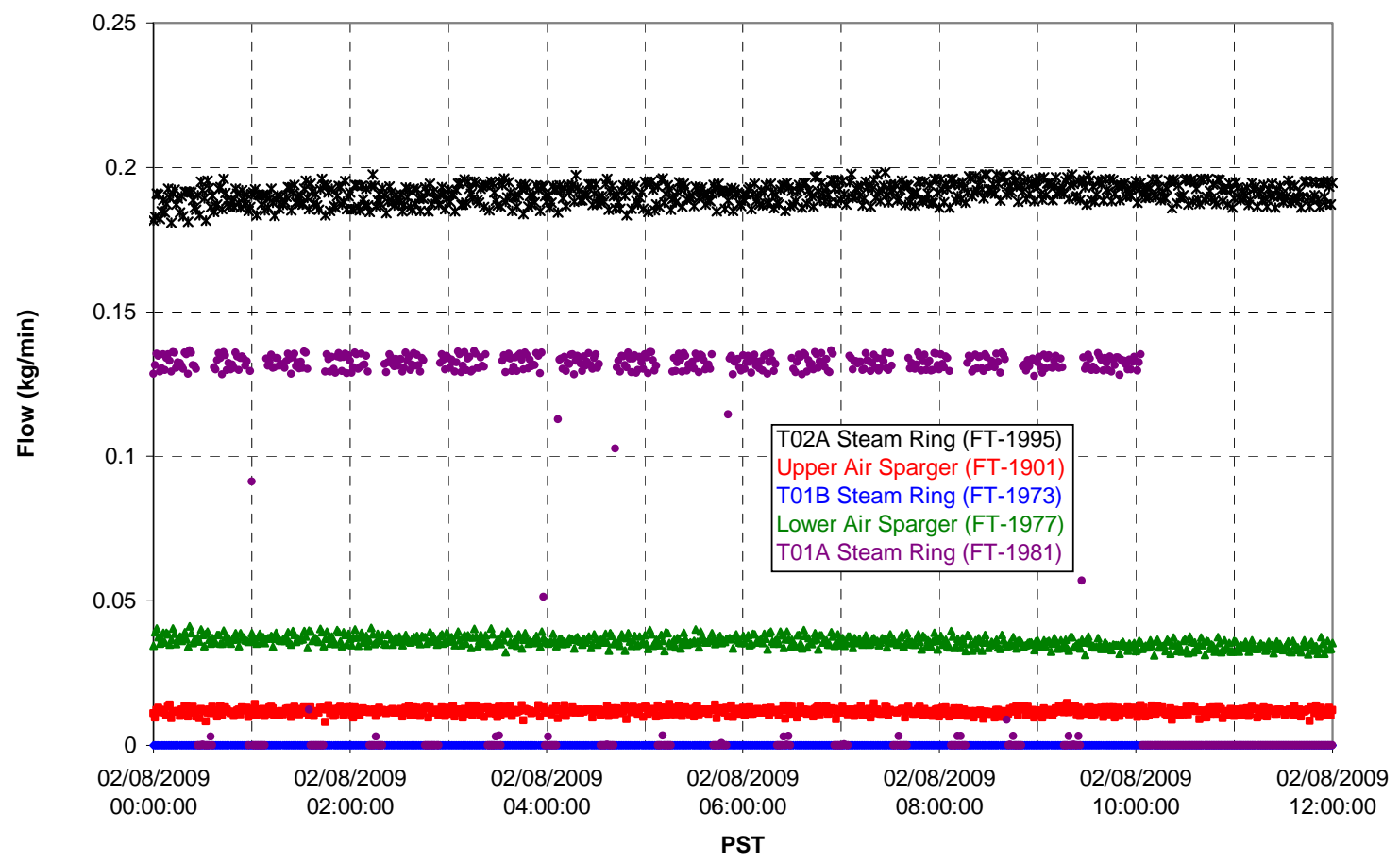


T02A Steam

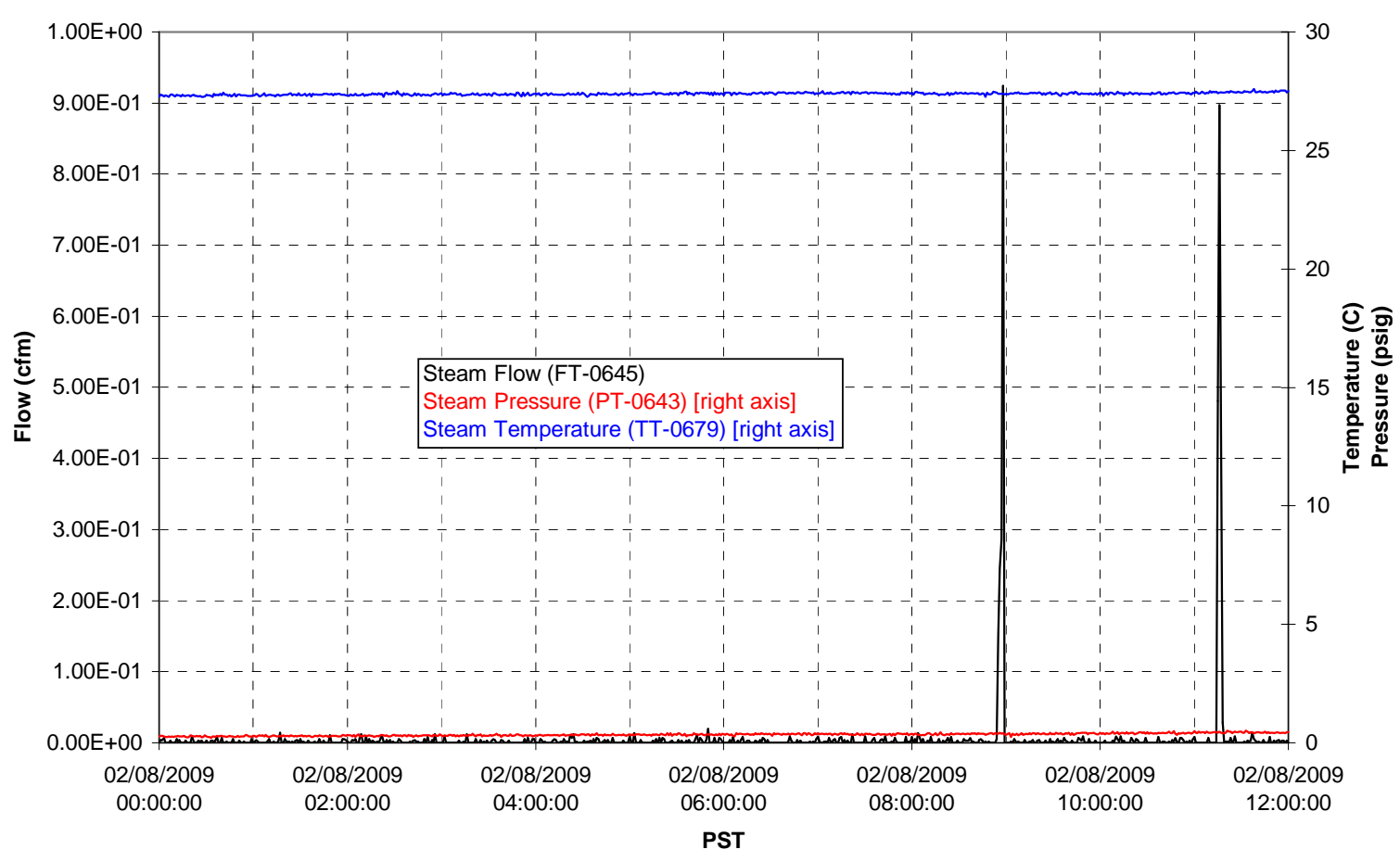

T01A Steam

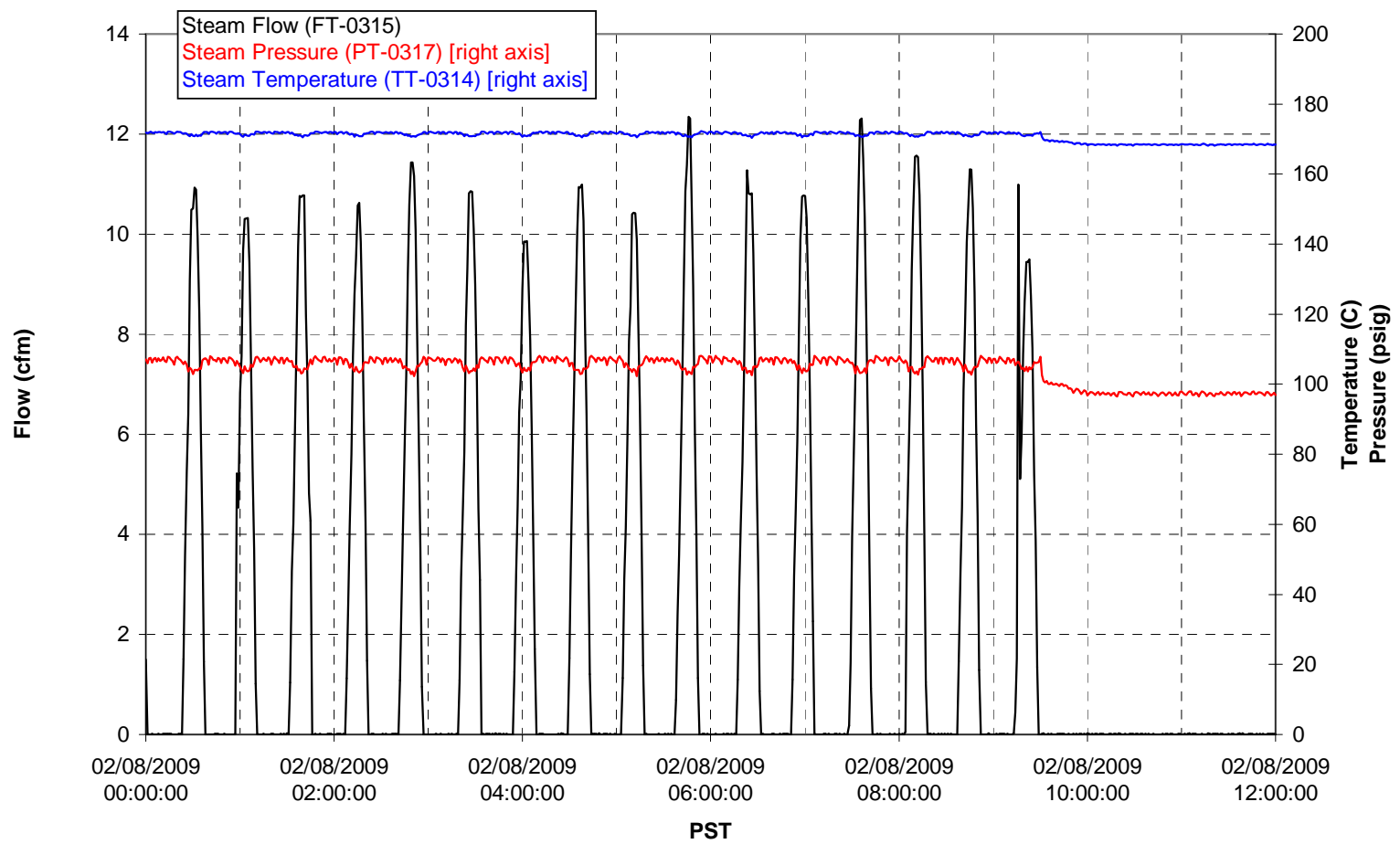


T01B Steam

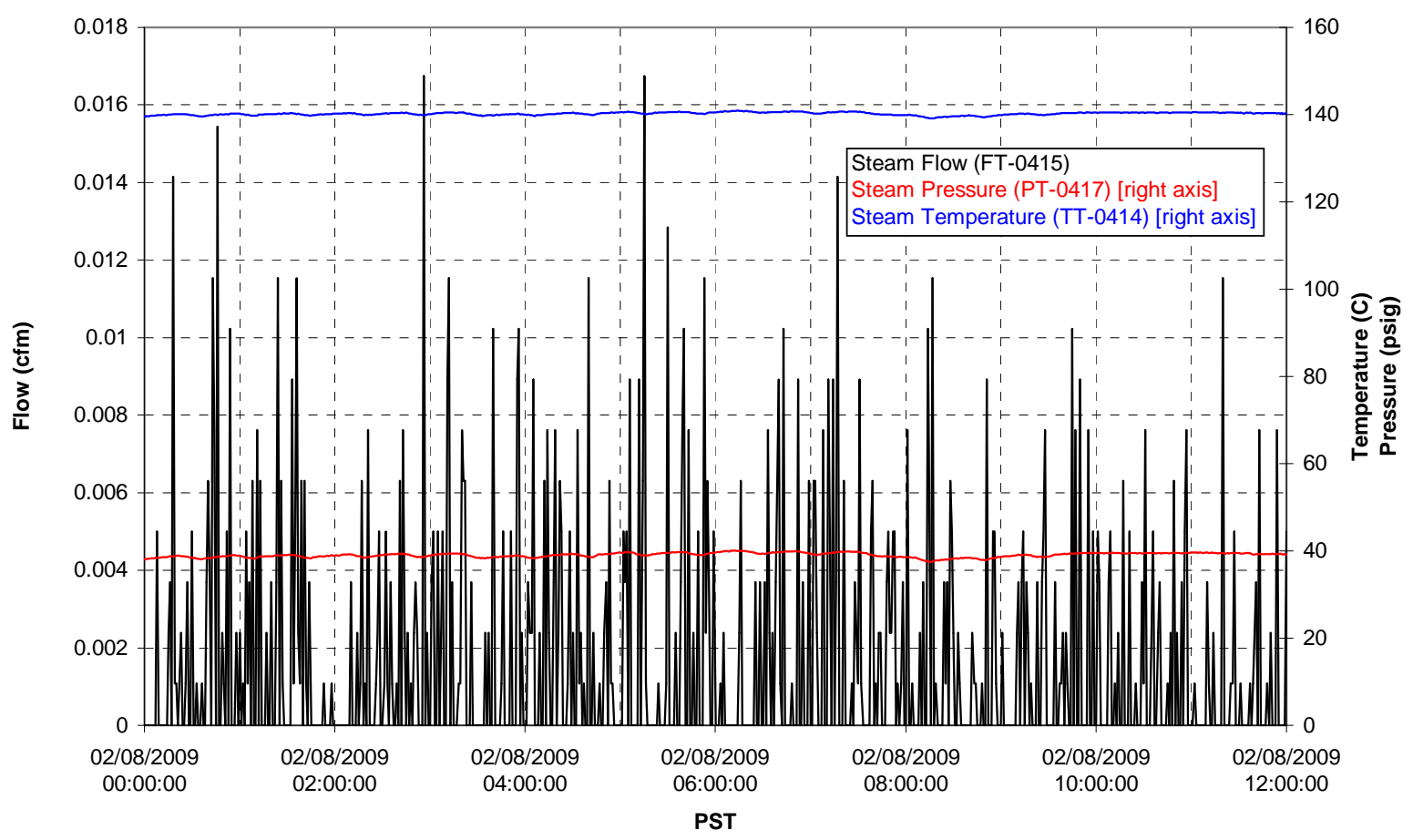




\section{Integrated Test A Data Plots 02/08/09 12:00 - 02/09/09 00:00}


T01A level

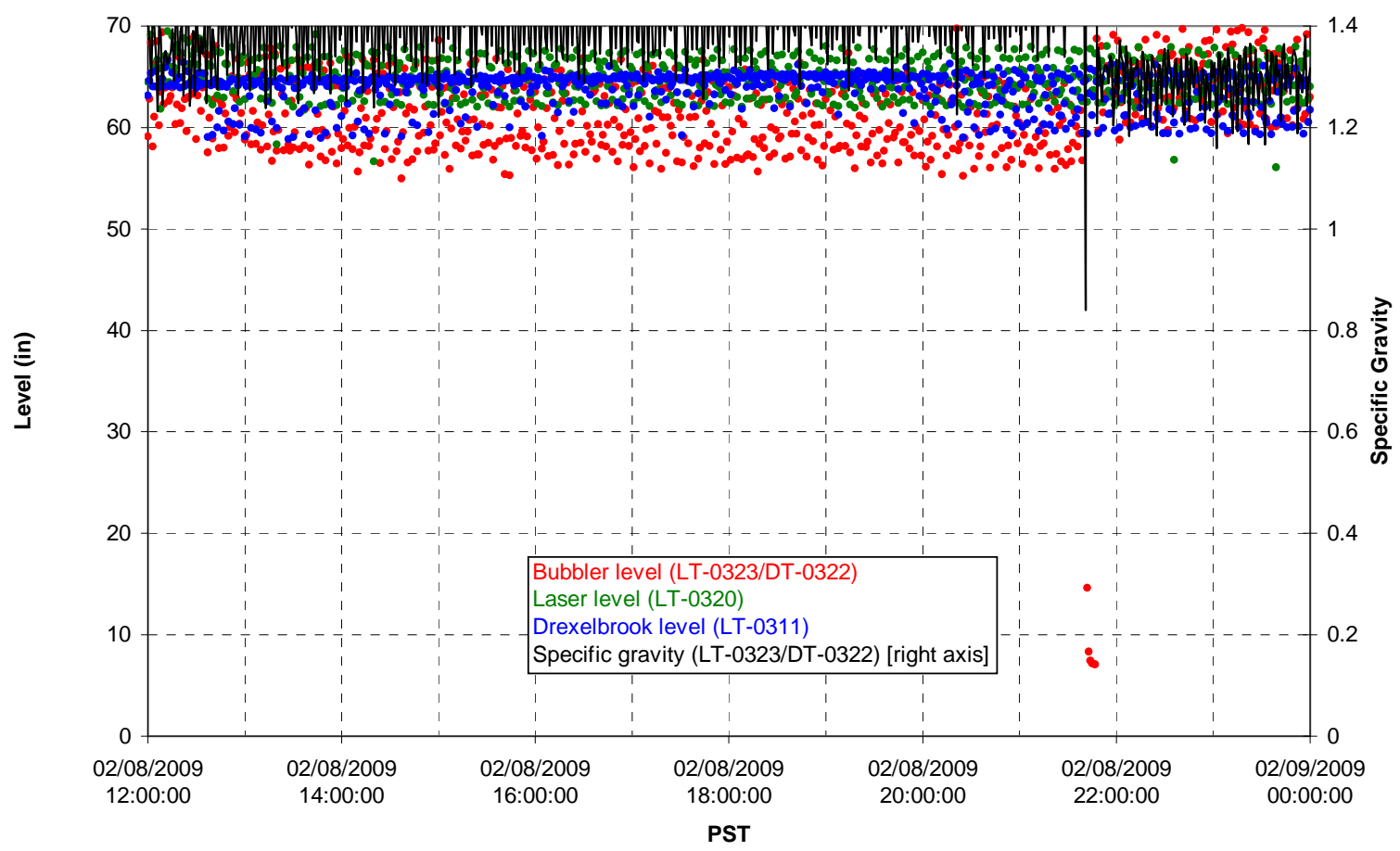

T01A temperatures

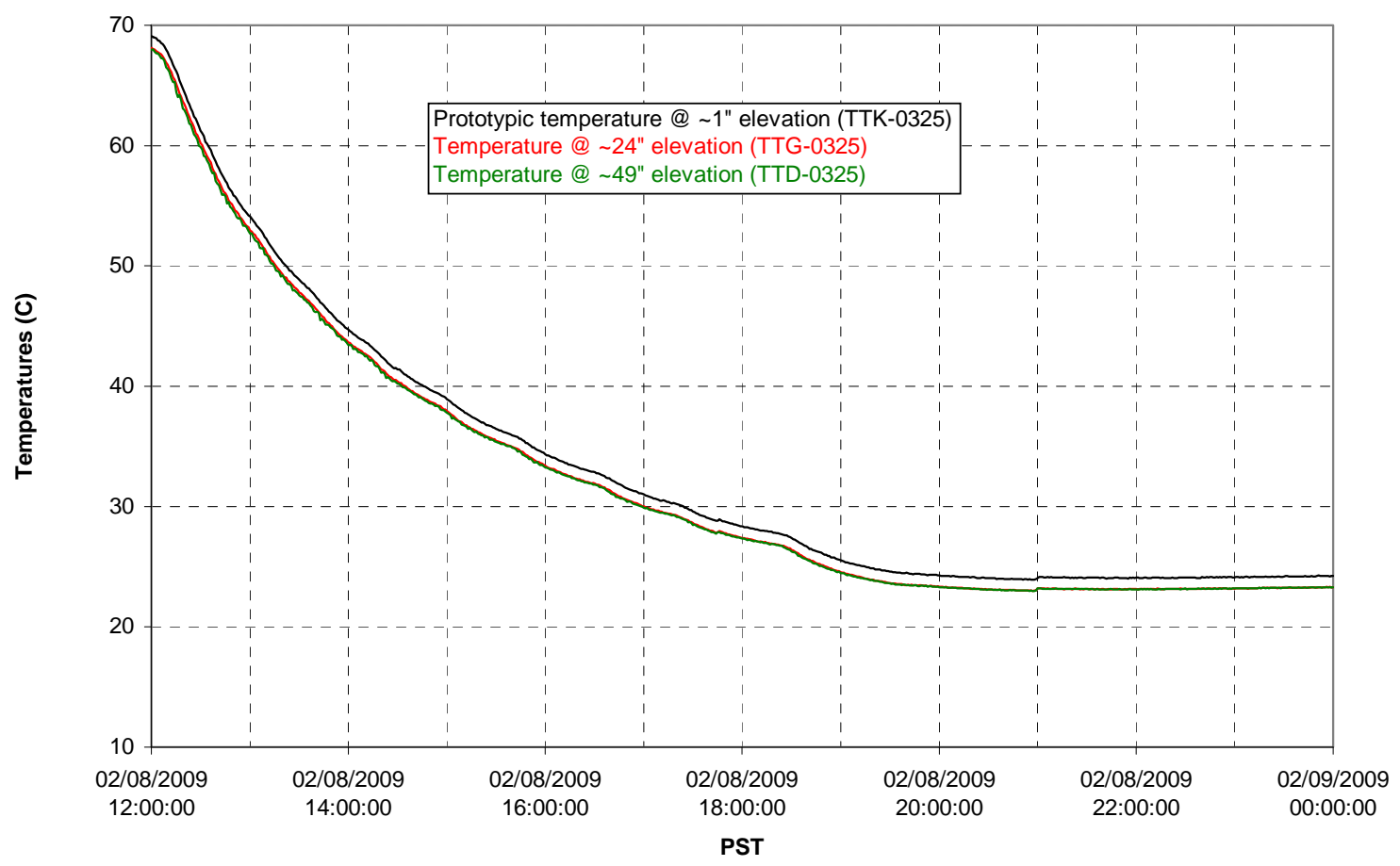


T01B level

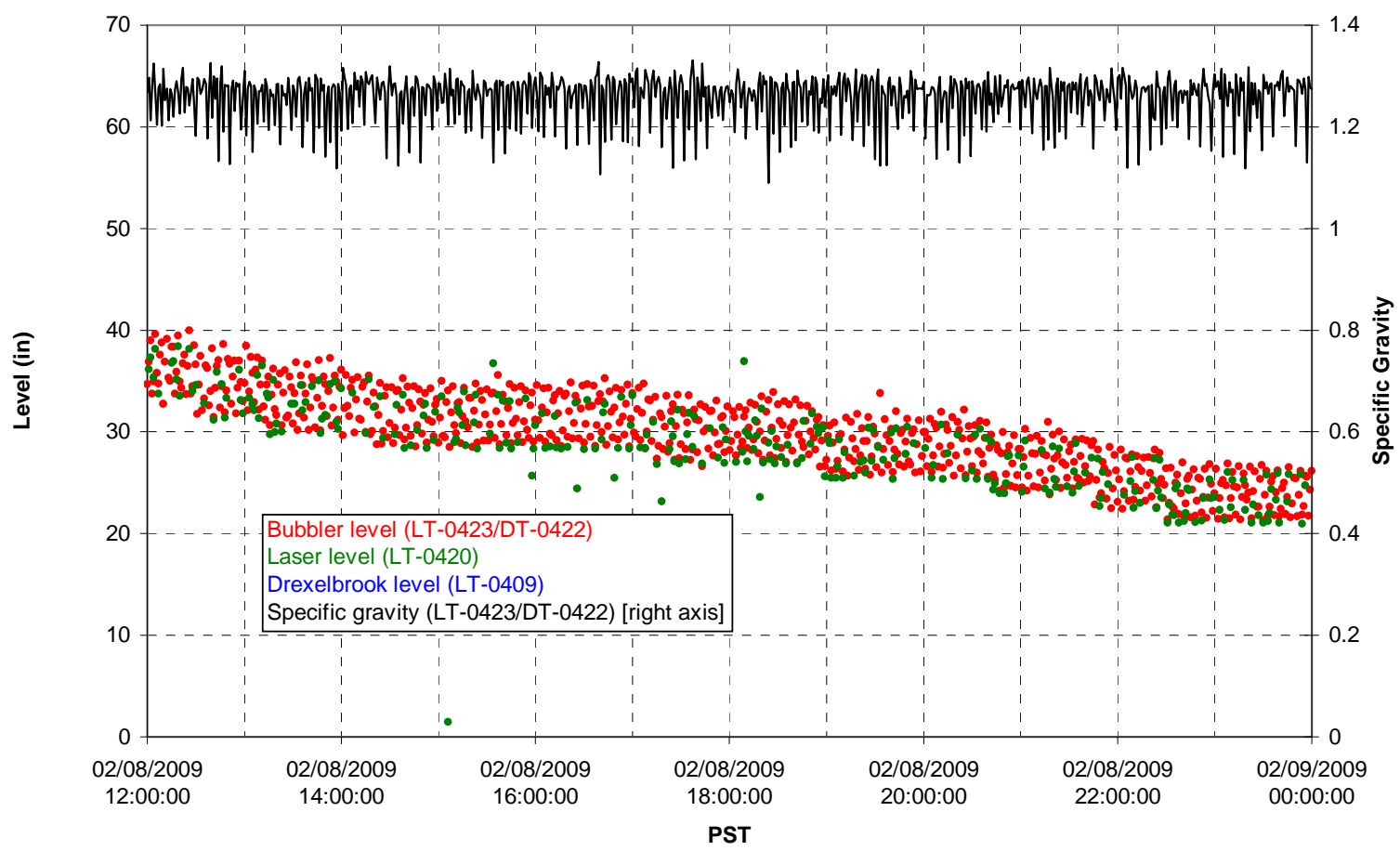

T01B temperatures

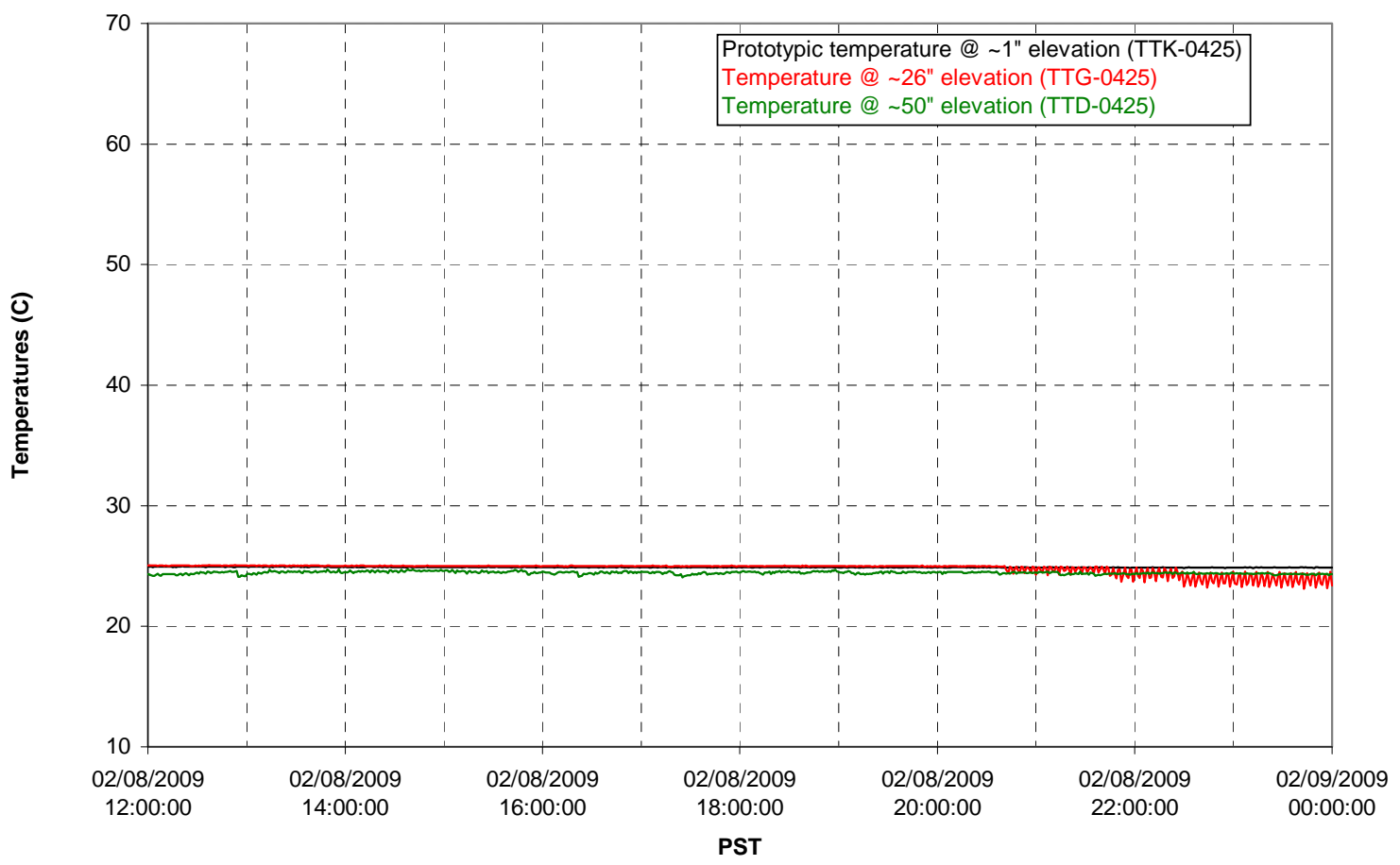


T02A level

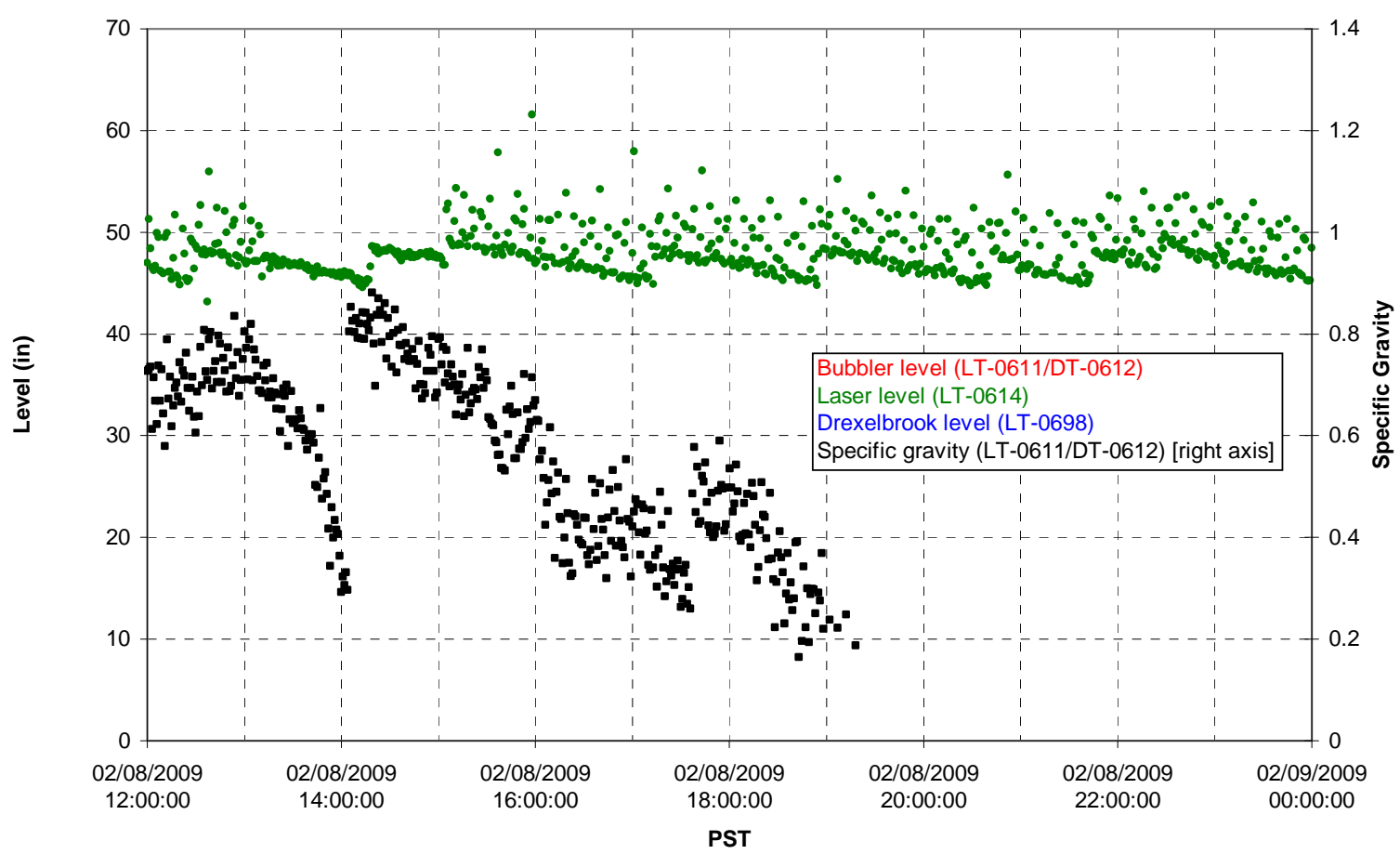

T02A temperatures

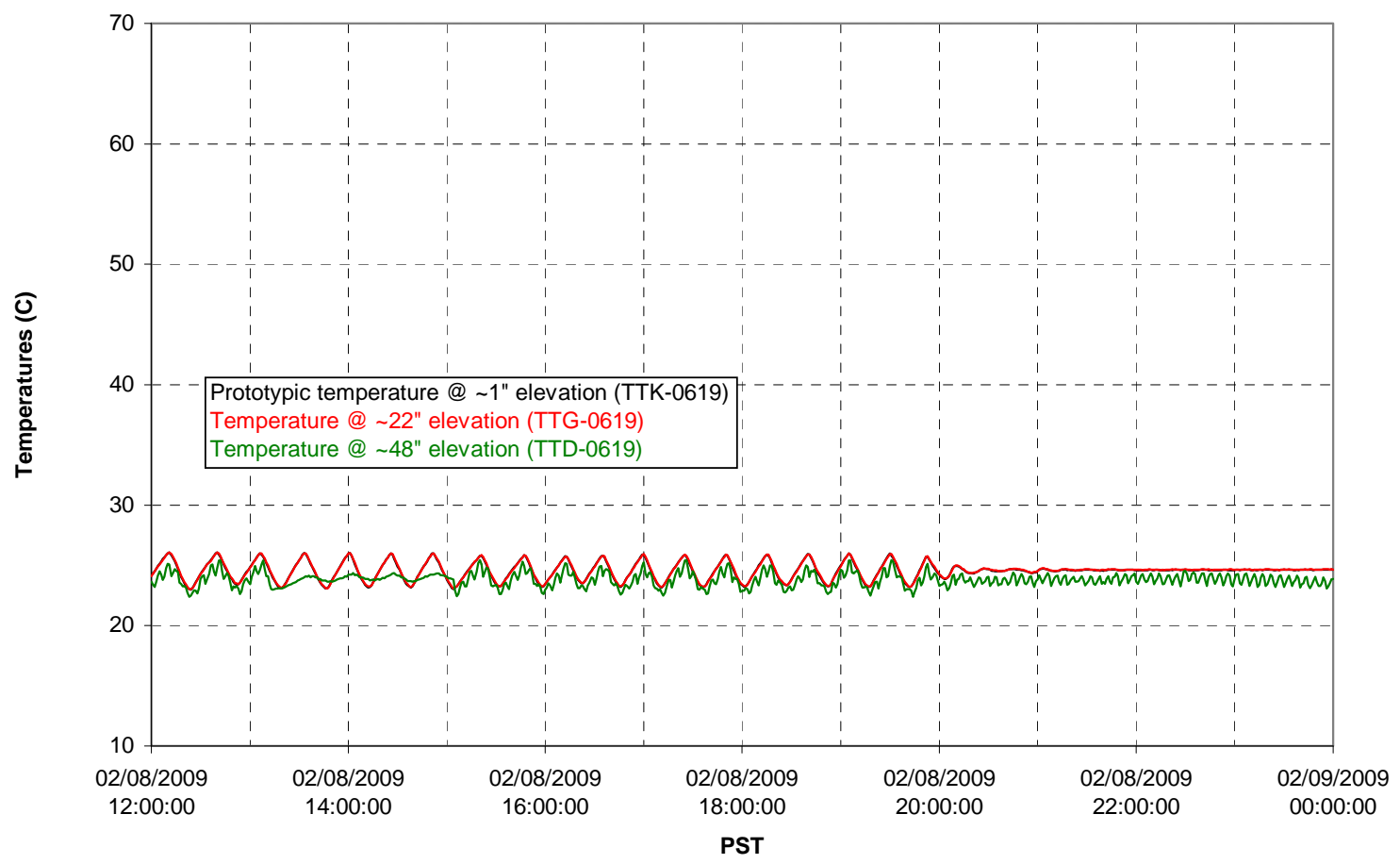


T02A and filter loop temperatures
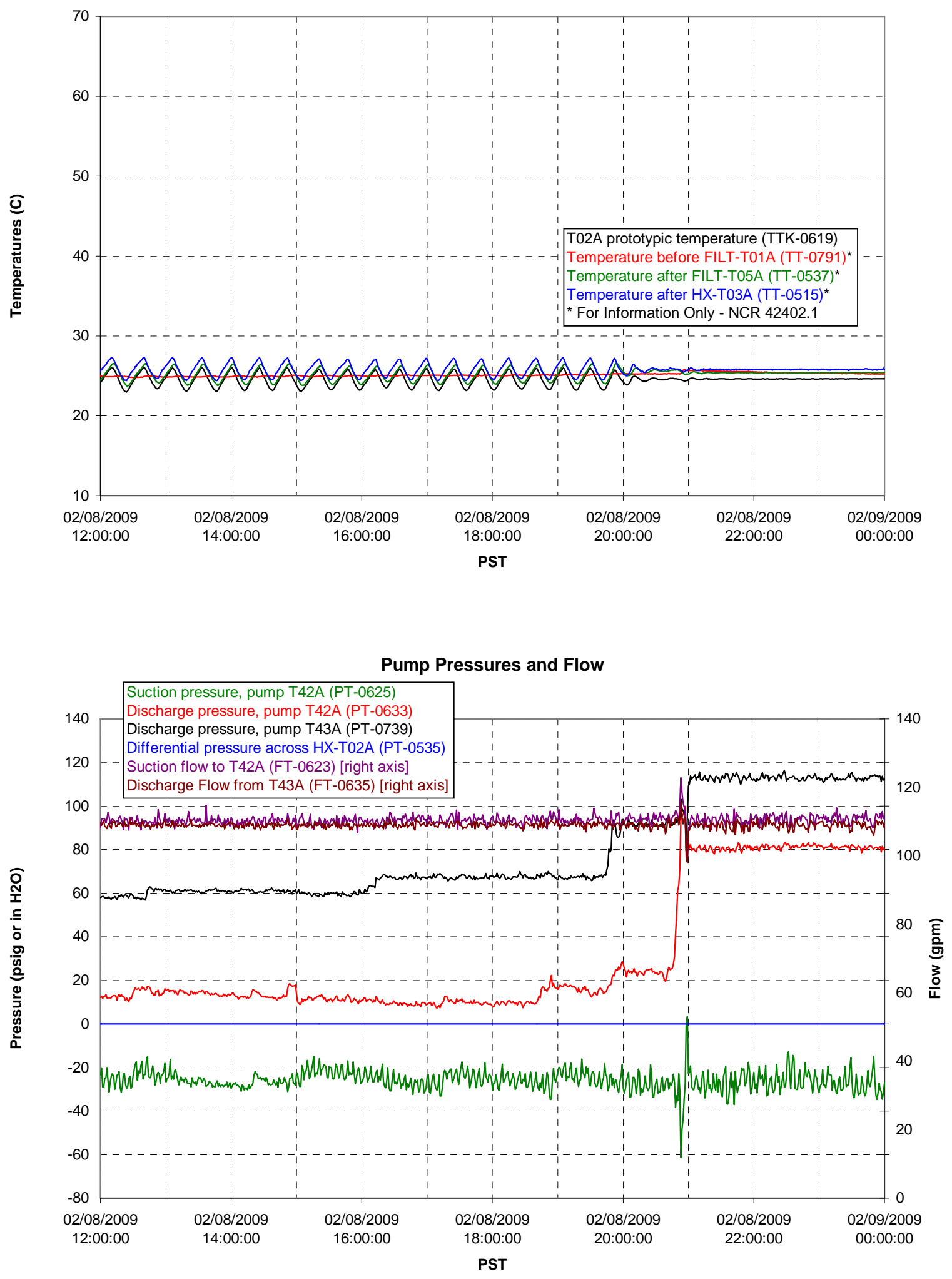
Axial pressure drop

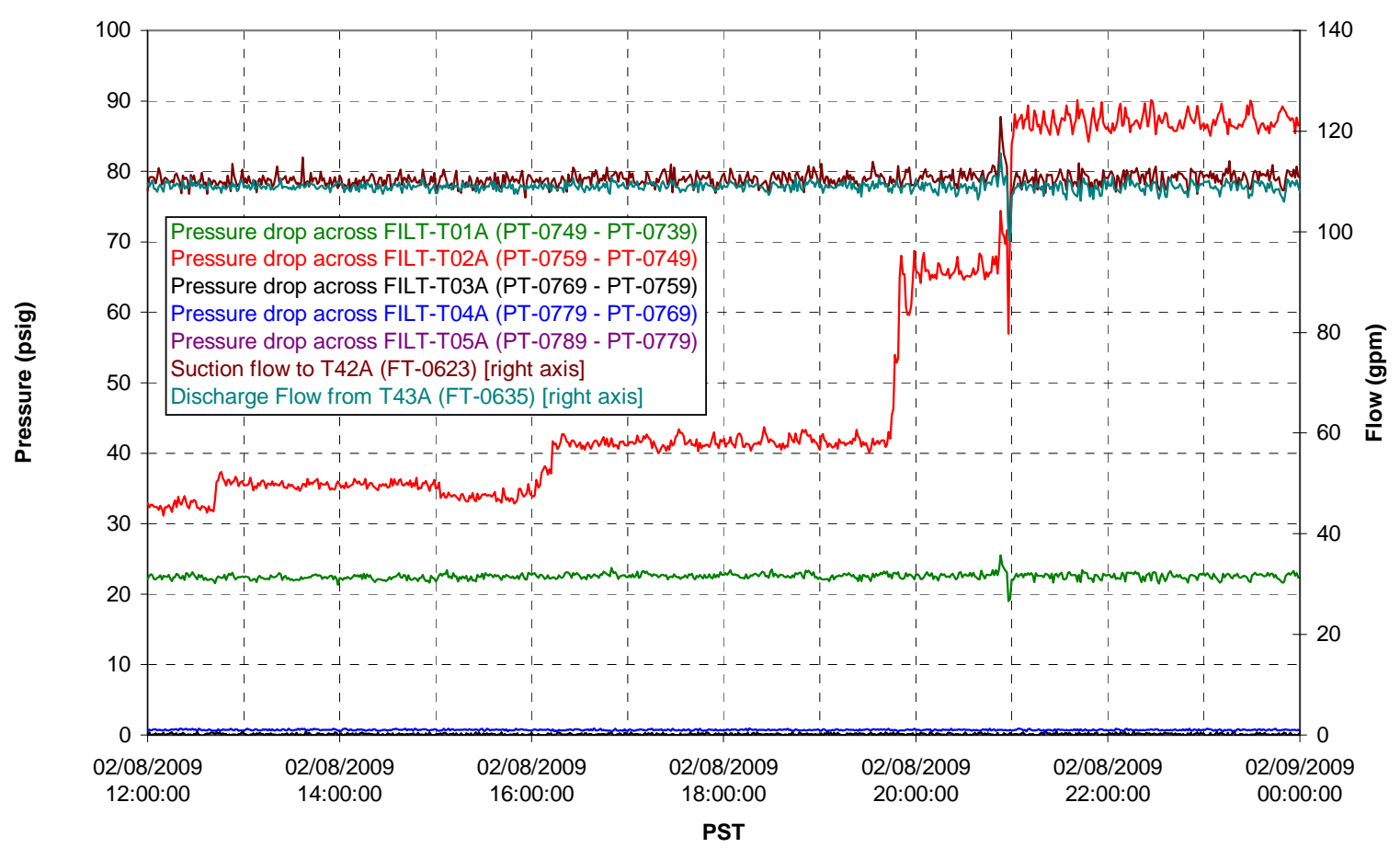

Permeate flow rates

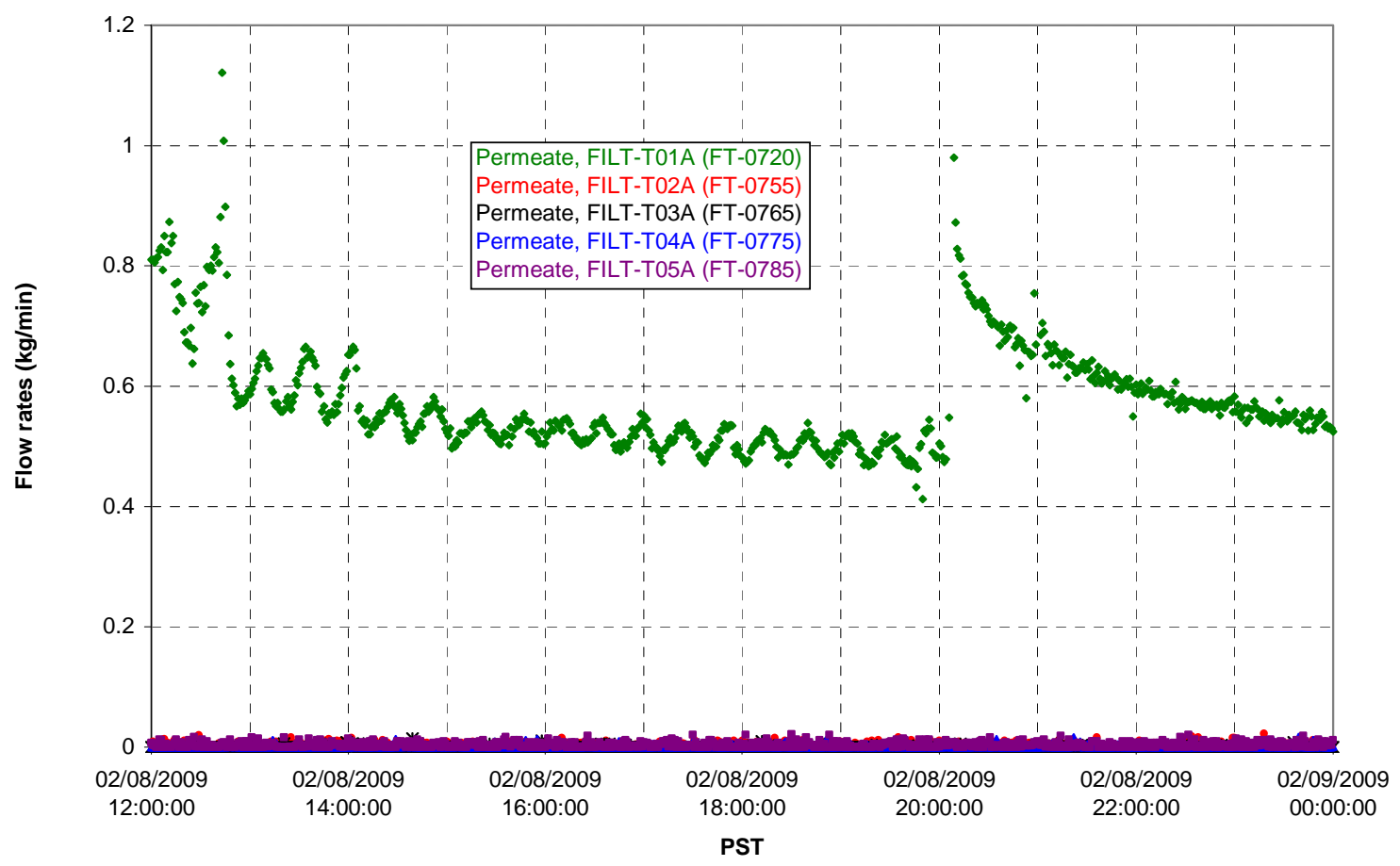

G.312 
T02A Inner Temperature Tree

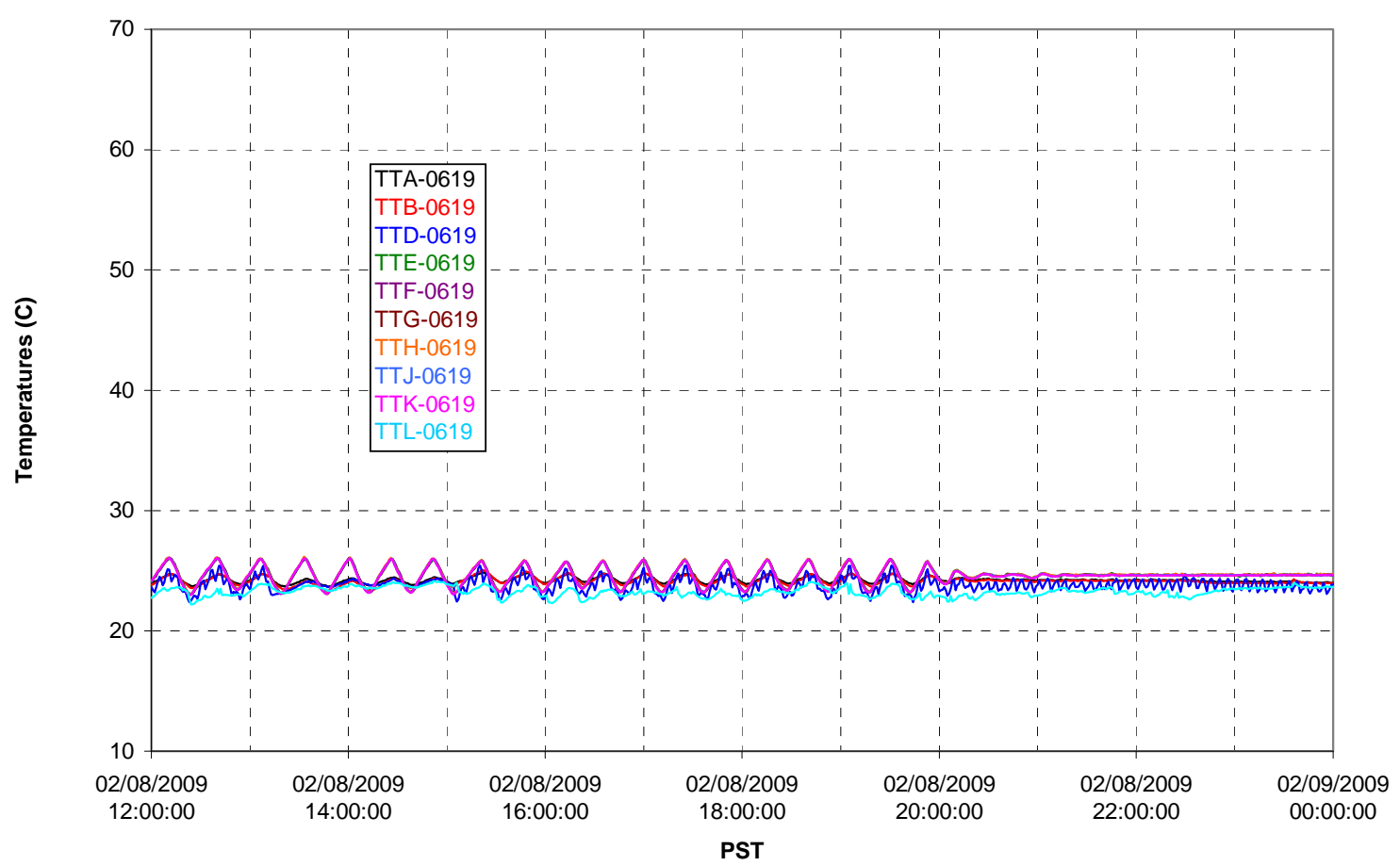

T02A Outer Temperature Tree

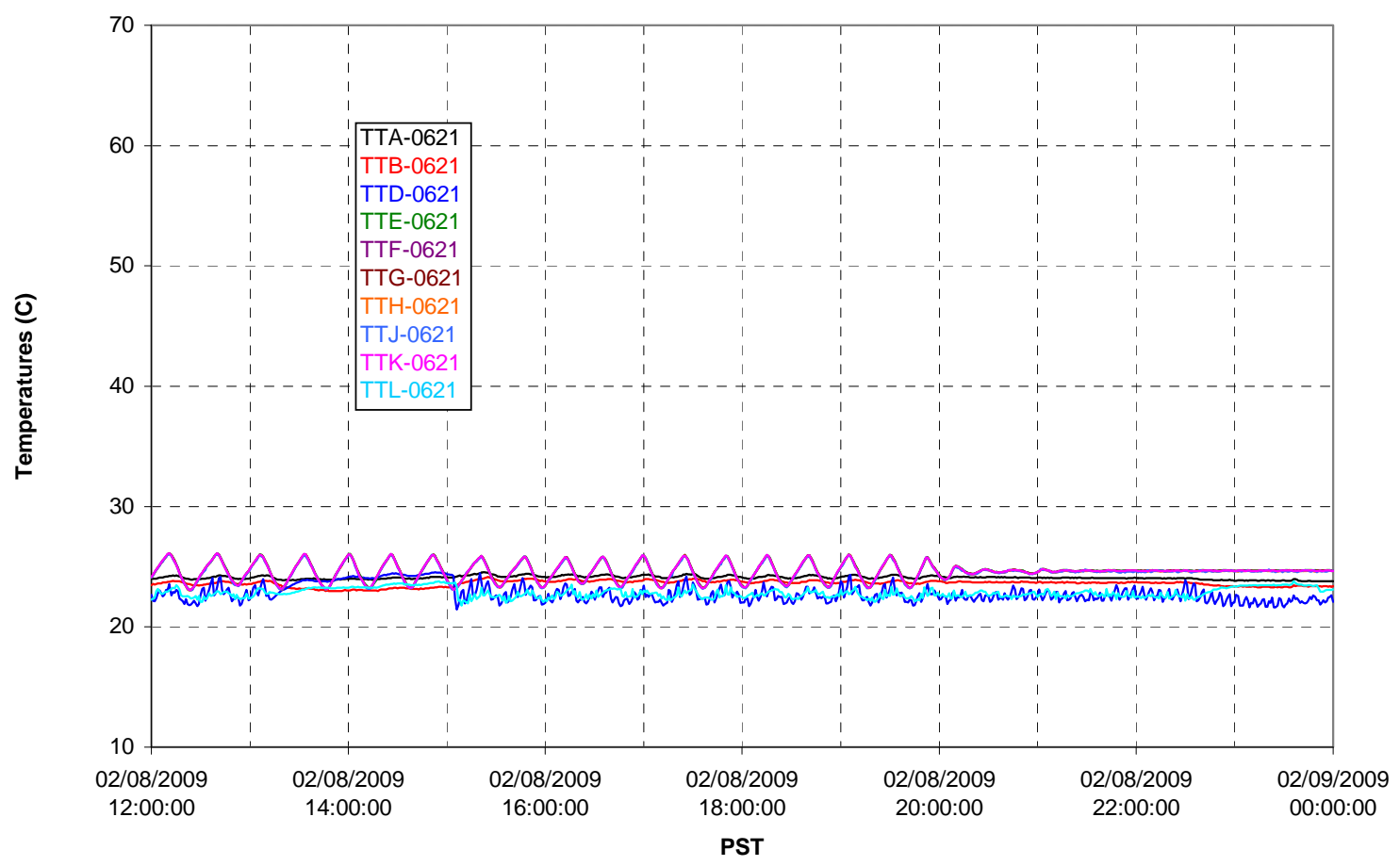


T02A temperatures

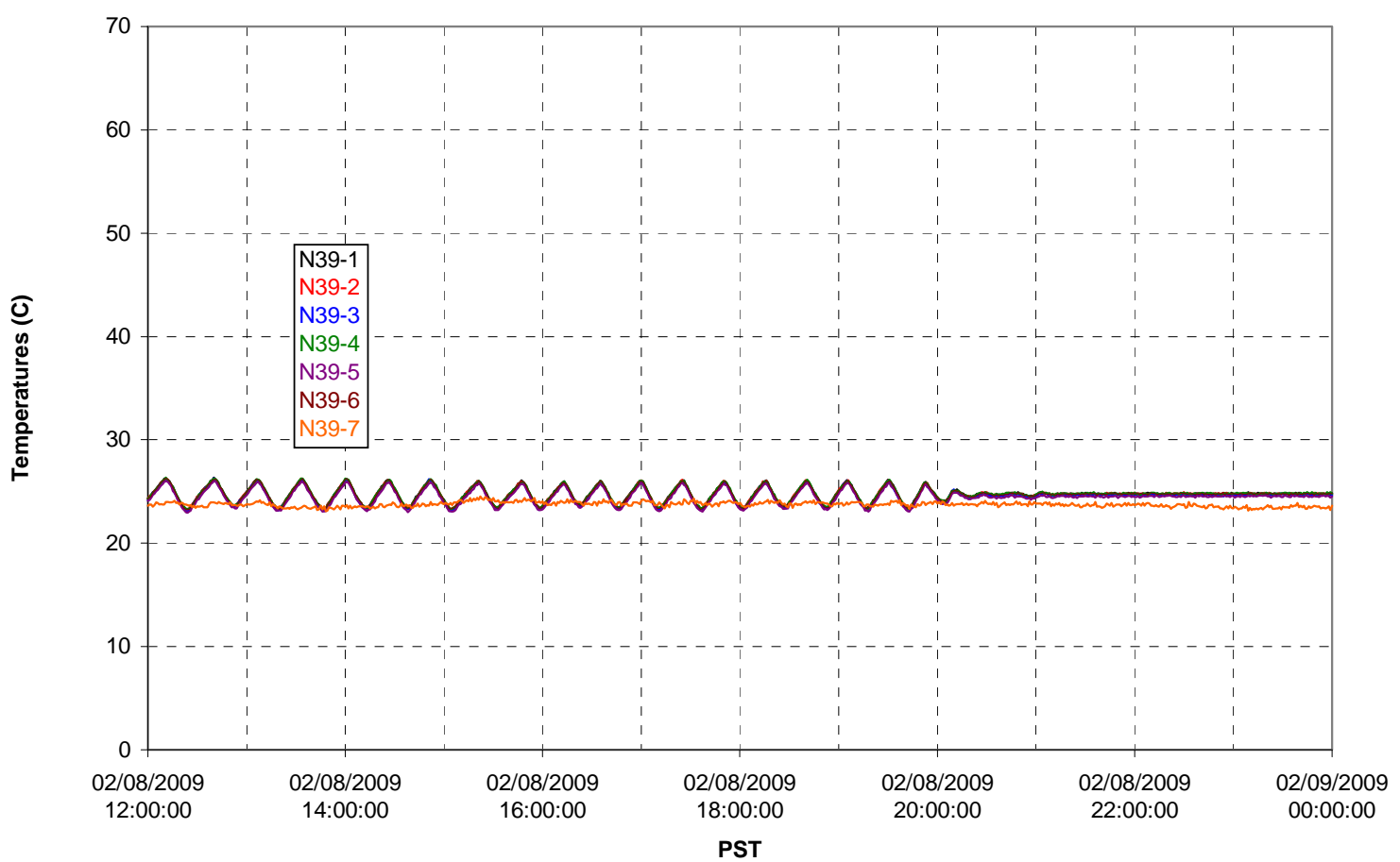

T02A temperatures

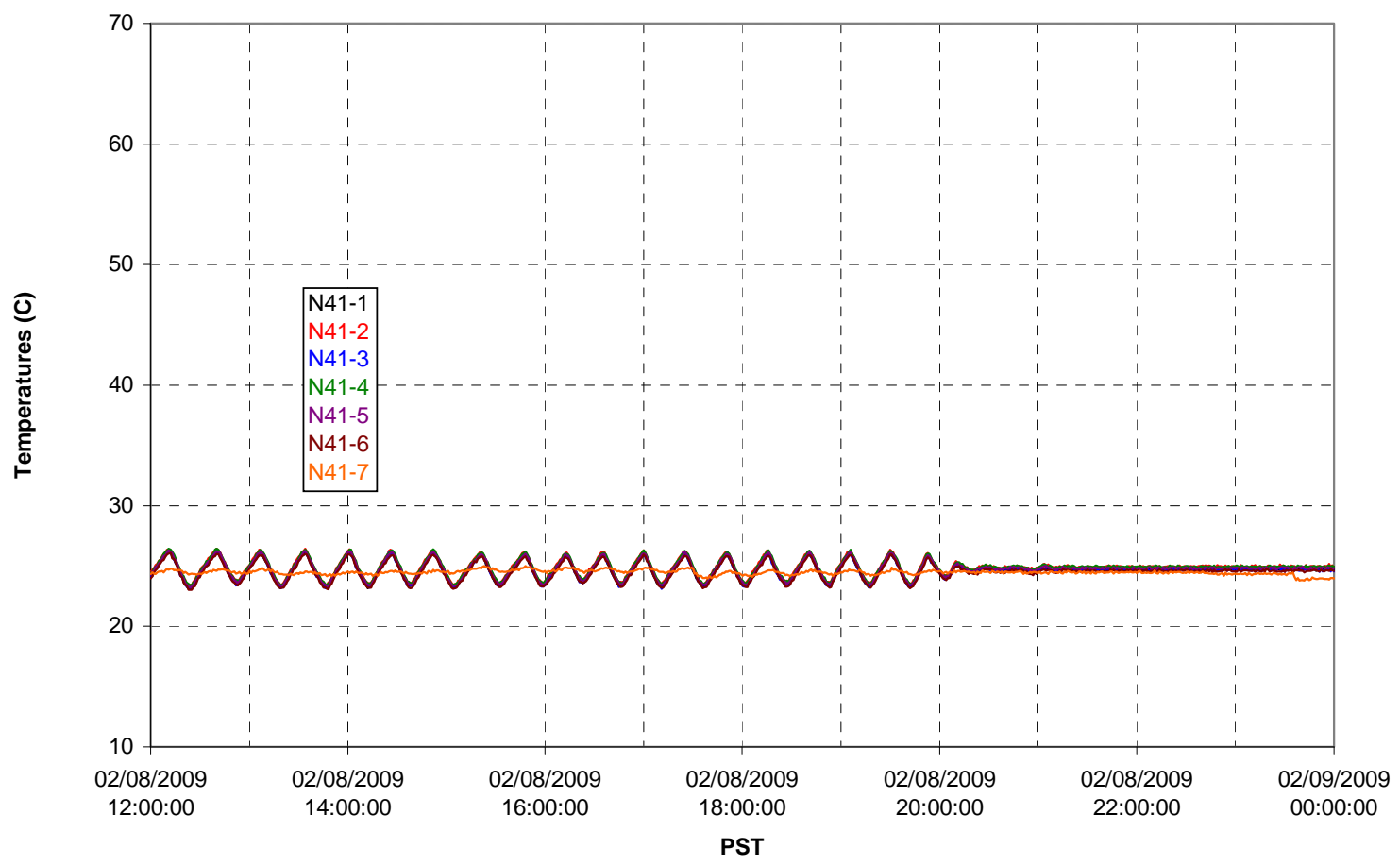


T02A temperatures

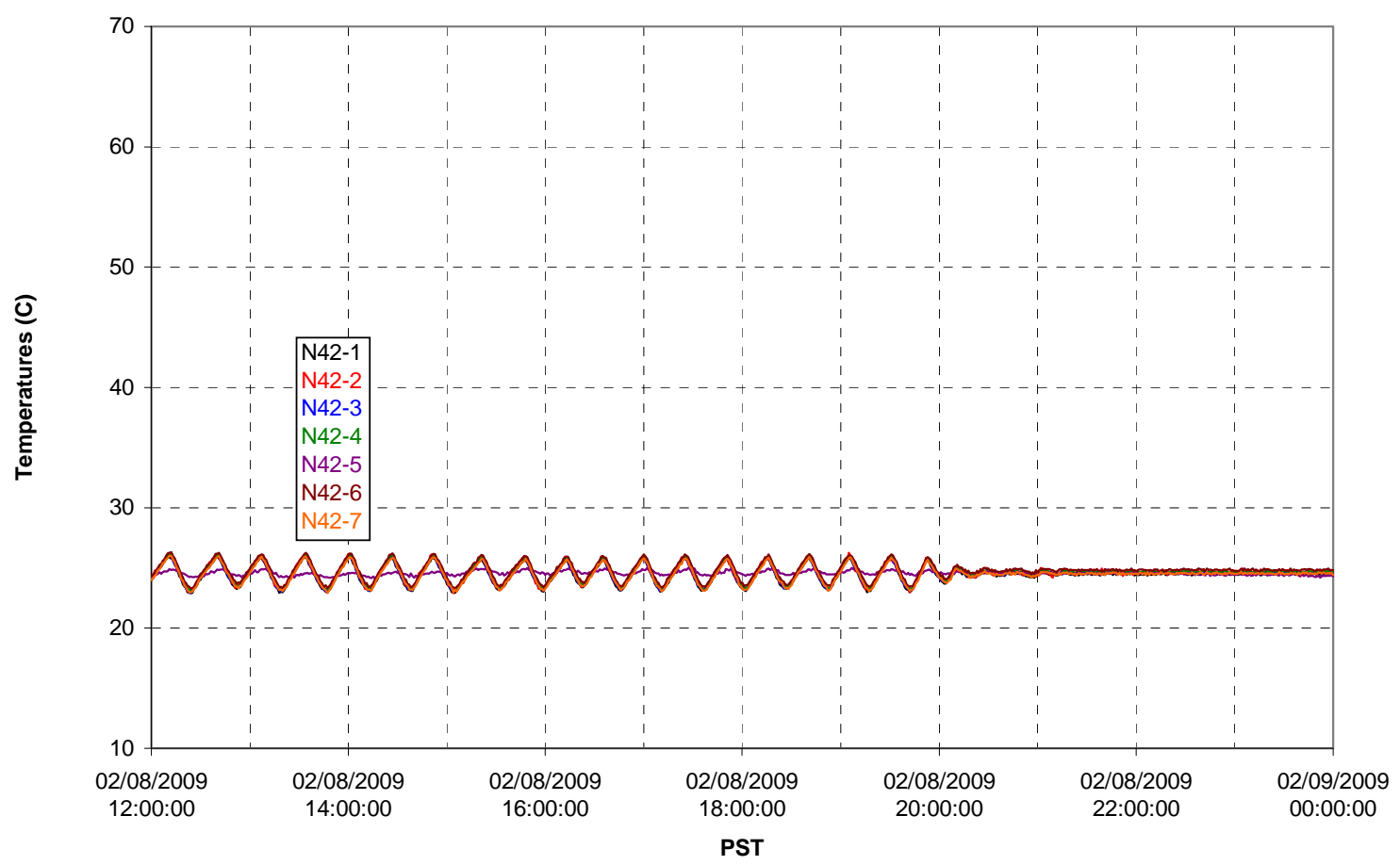

T02A temperatures

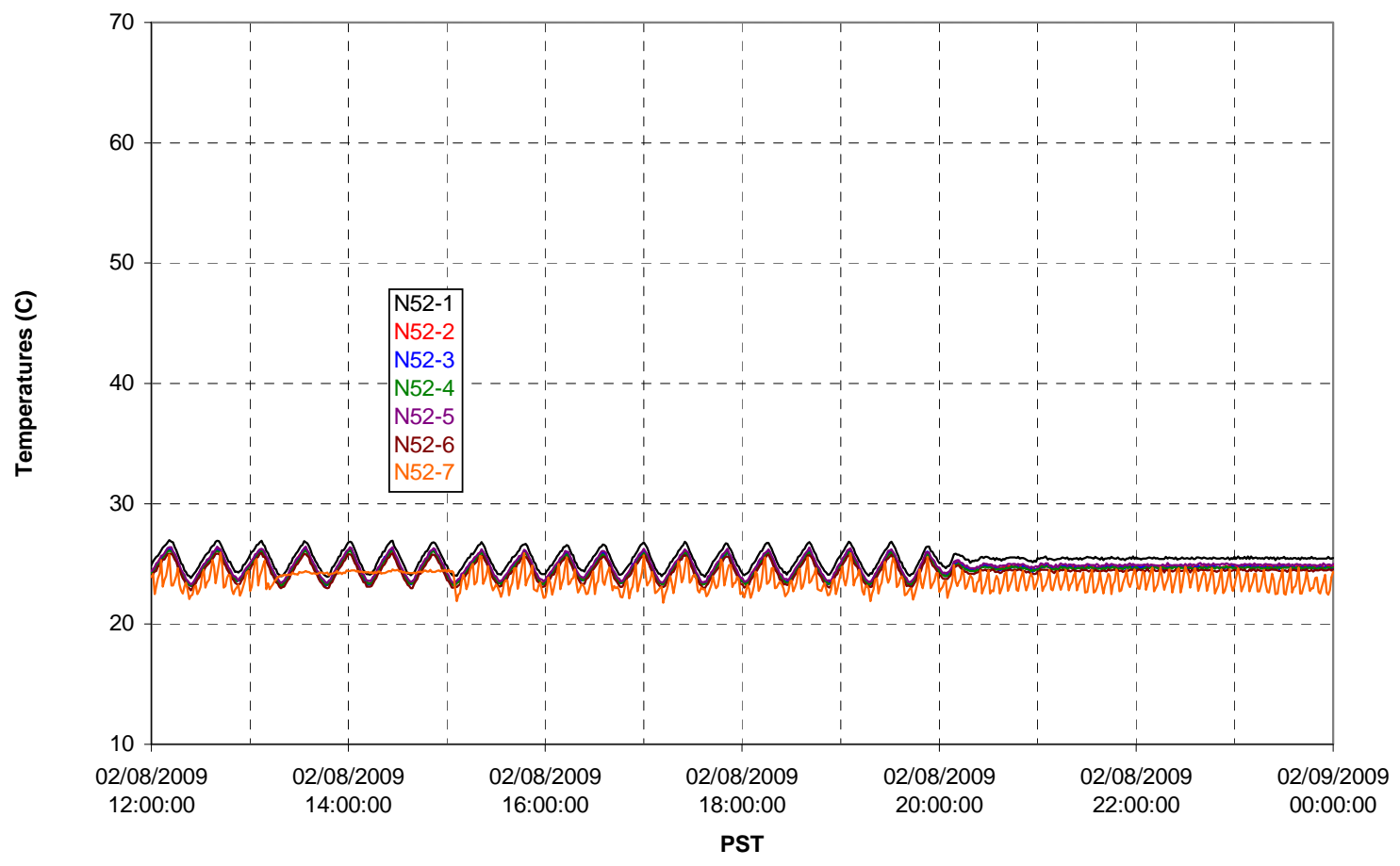


T02A Heating and Cooling

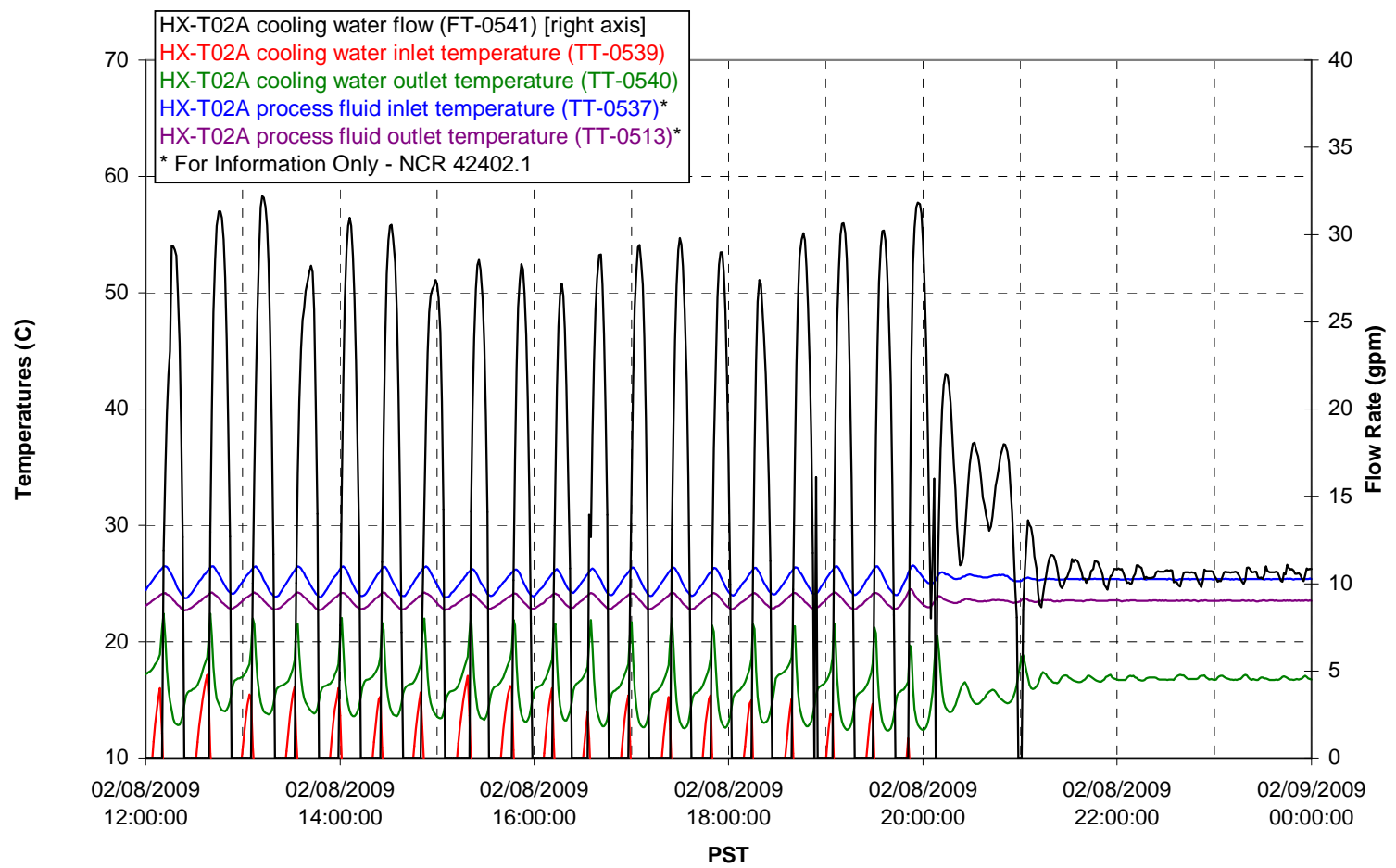

Pump Operation

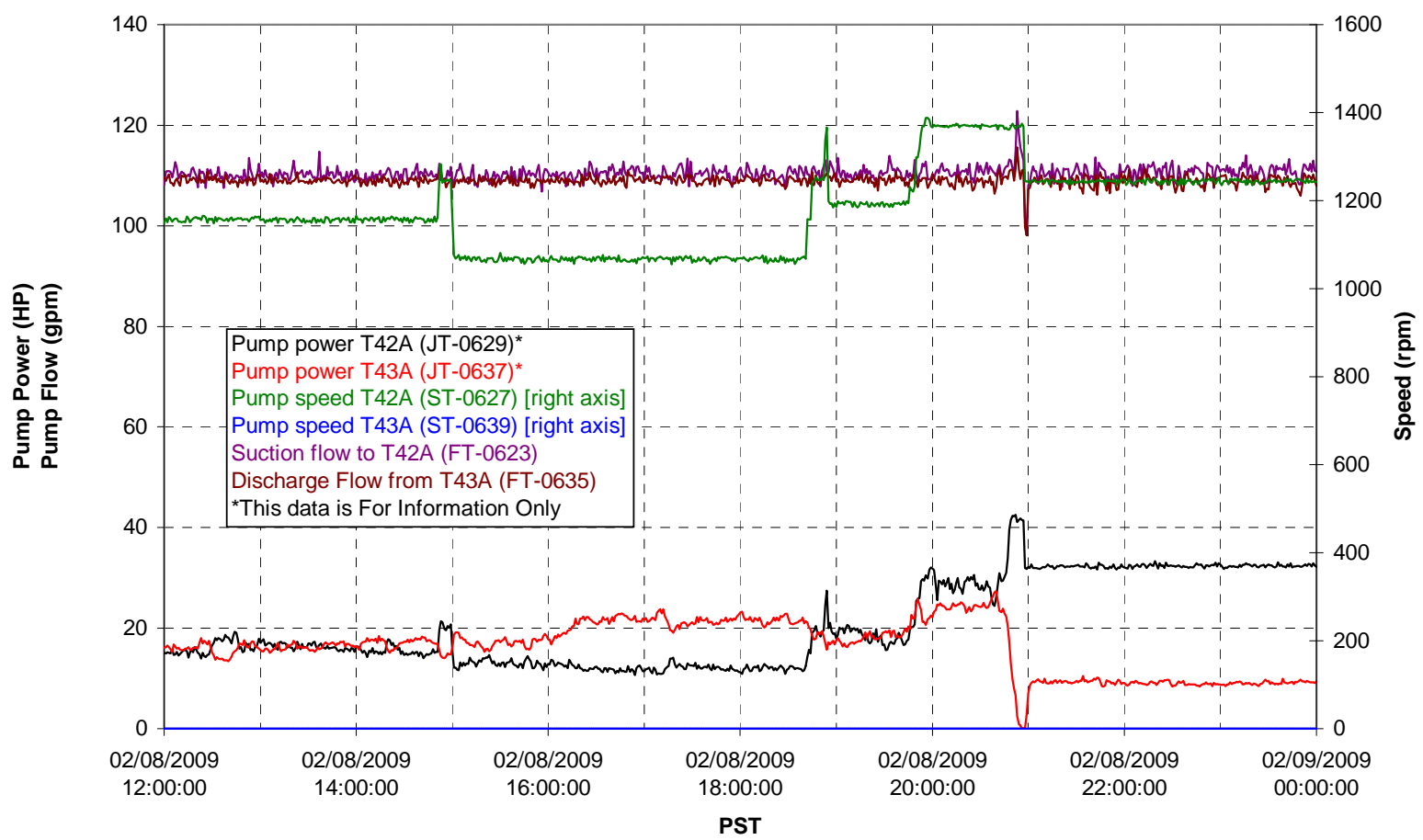


Pulsepot UFP-PP-T01A

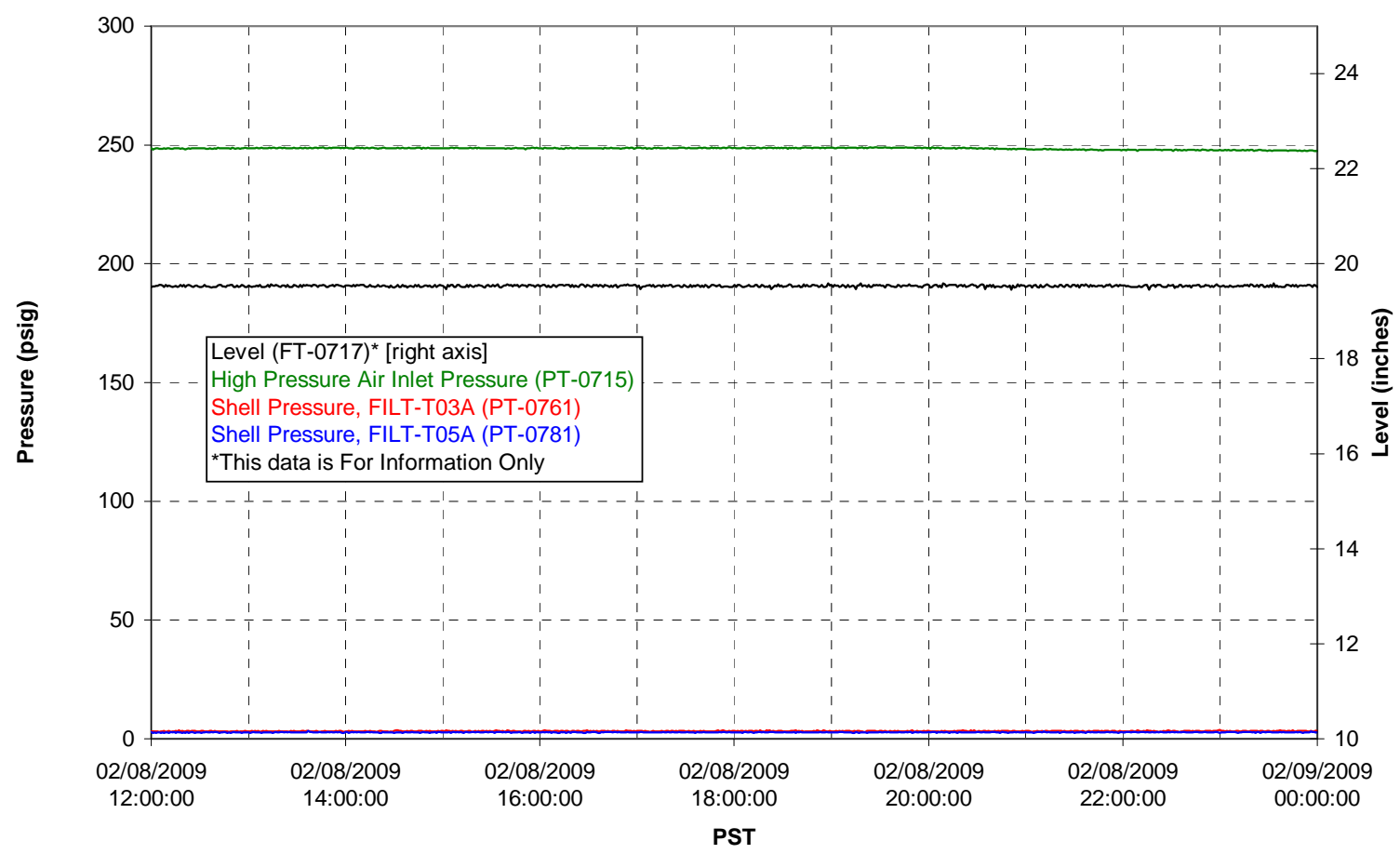

Pulsepot UFP-PP-T02A

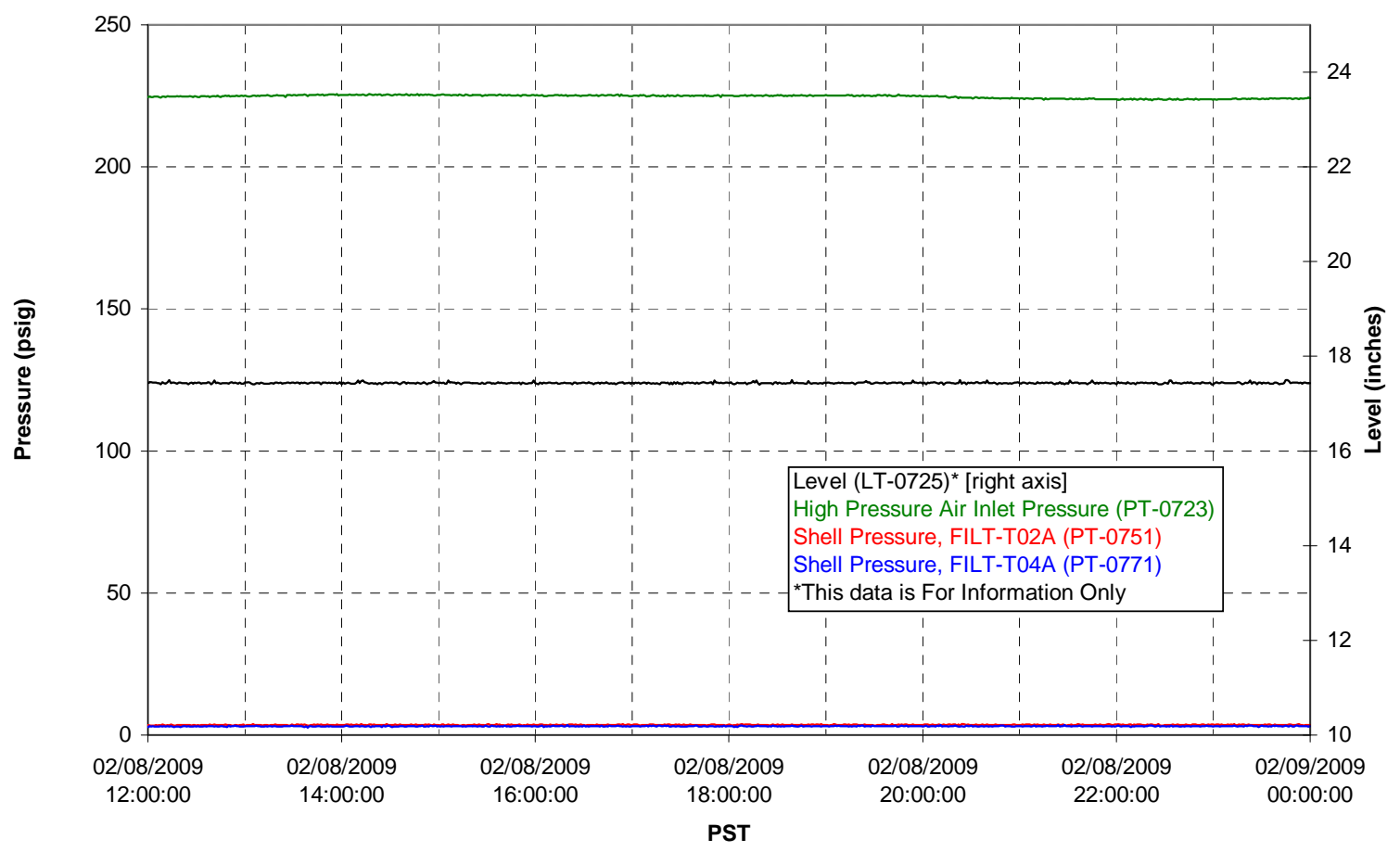


Pulsepot UFP-PP-T03A

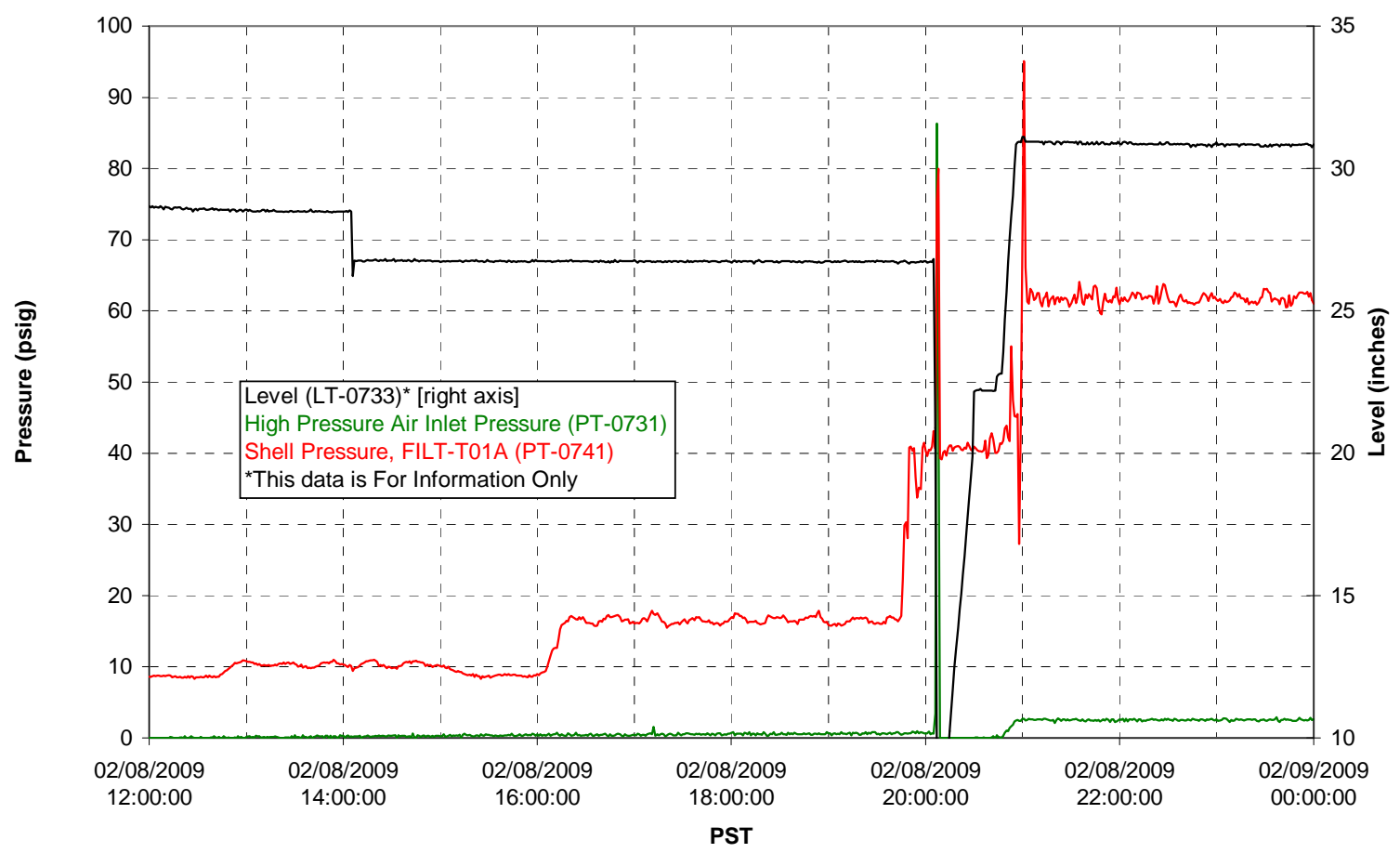

Pulsepot Levels

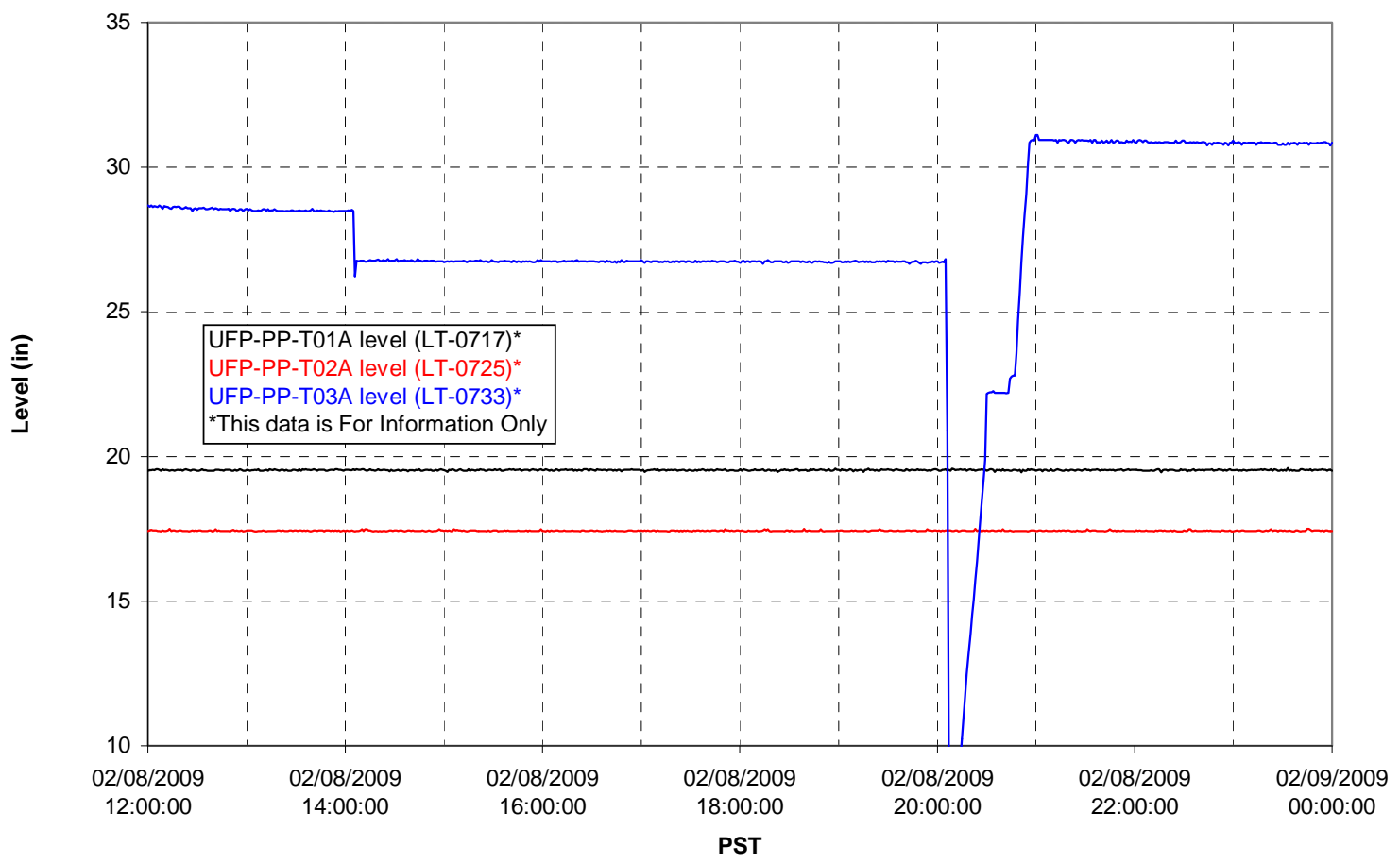


Filter UFP-FILT-T01A

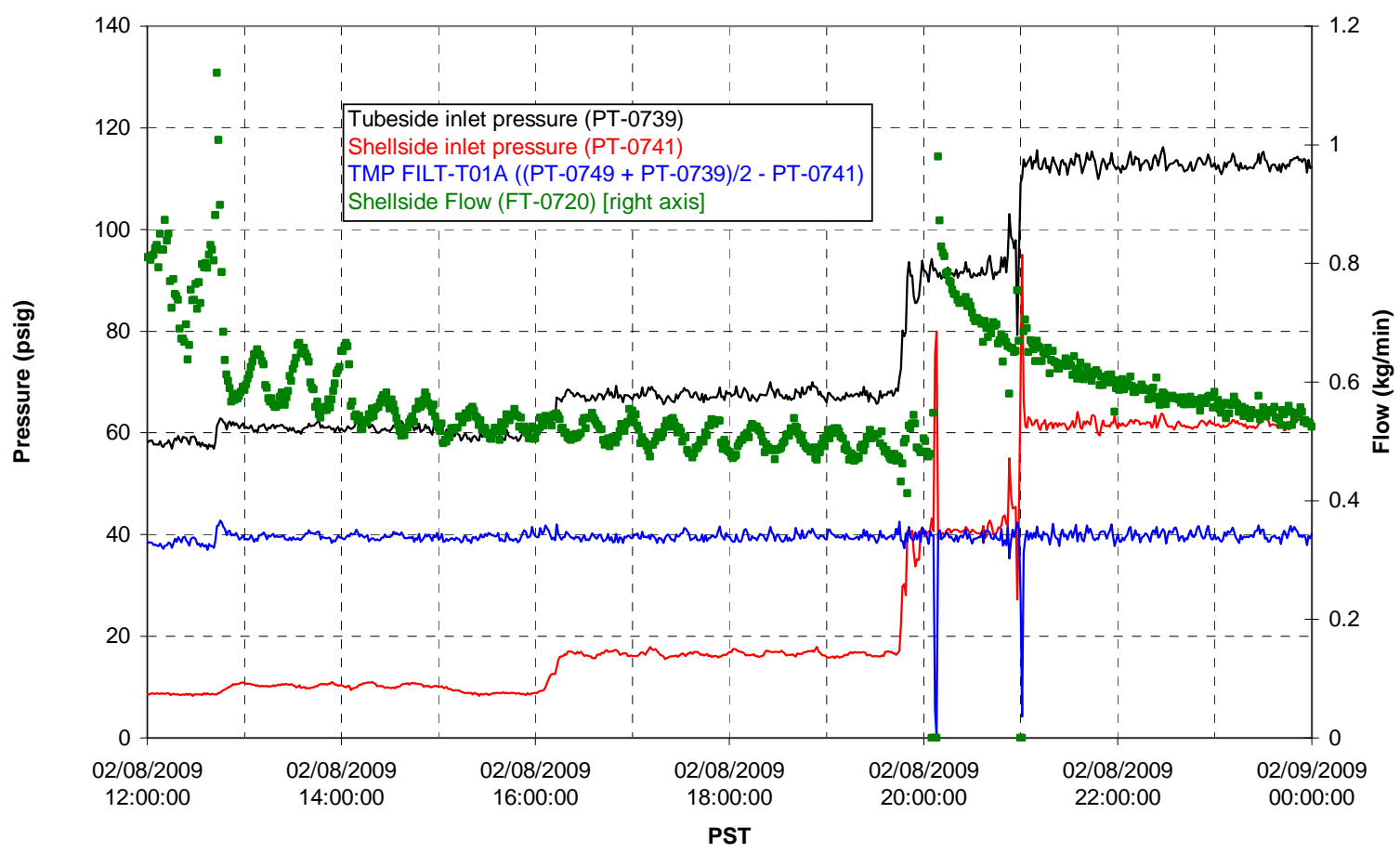

Filter UFP-FILT-T02A

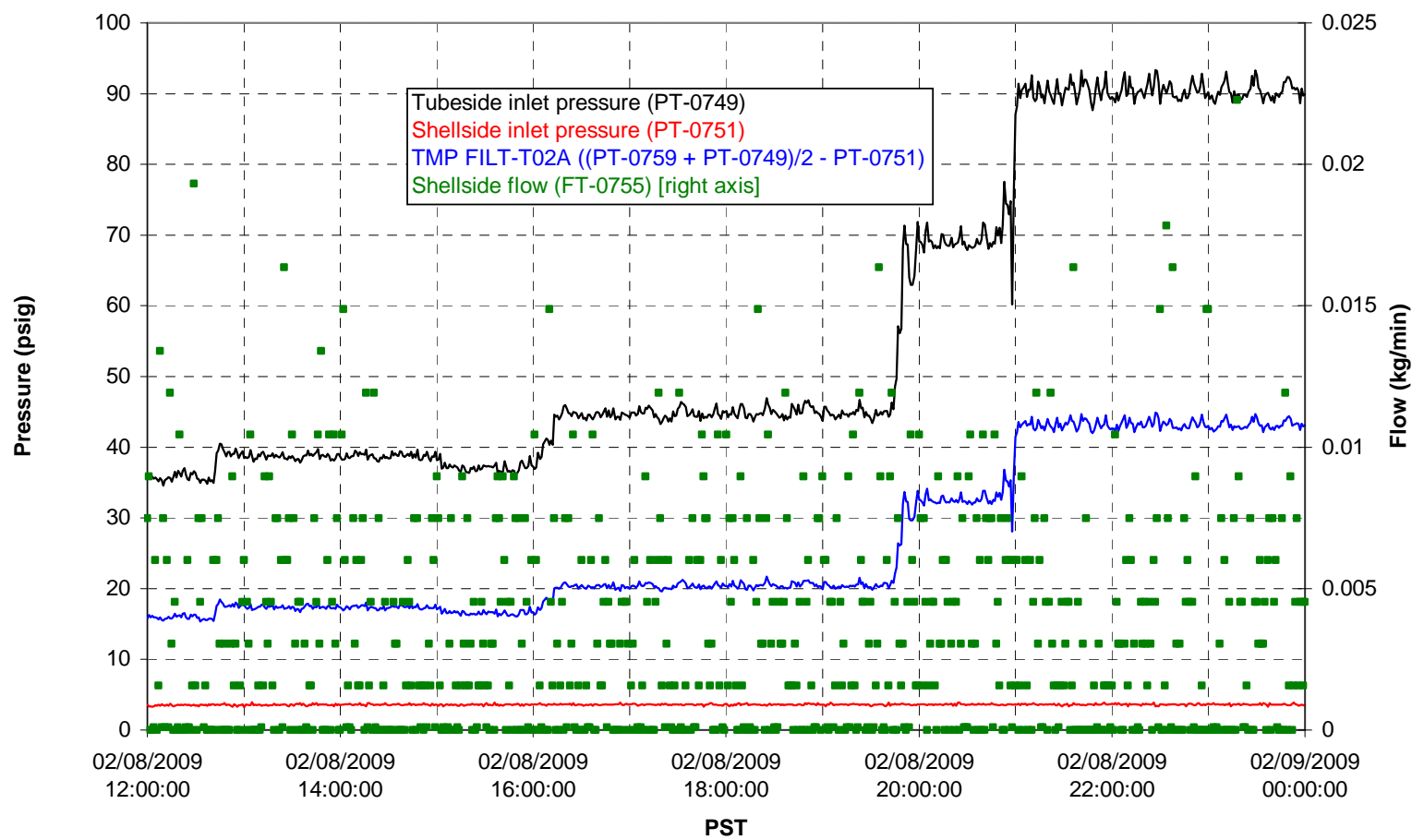


Filter UFP-FILT-T03A

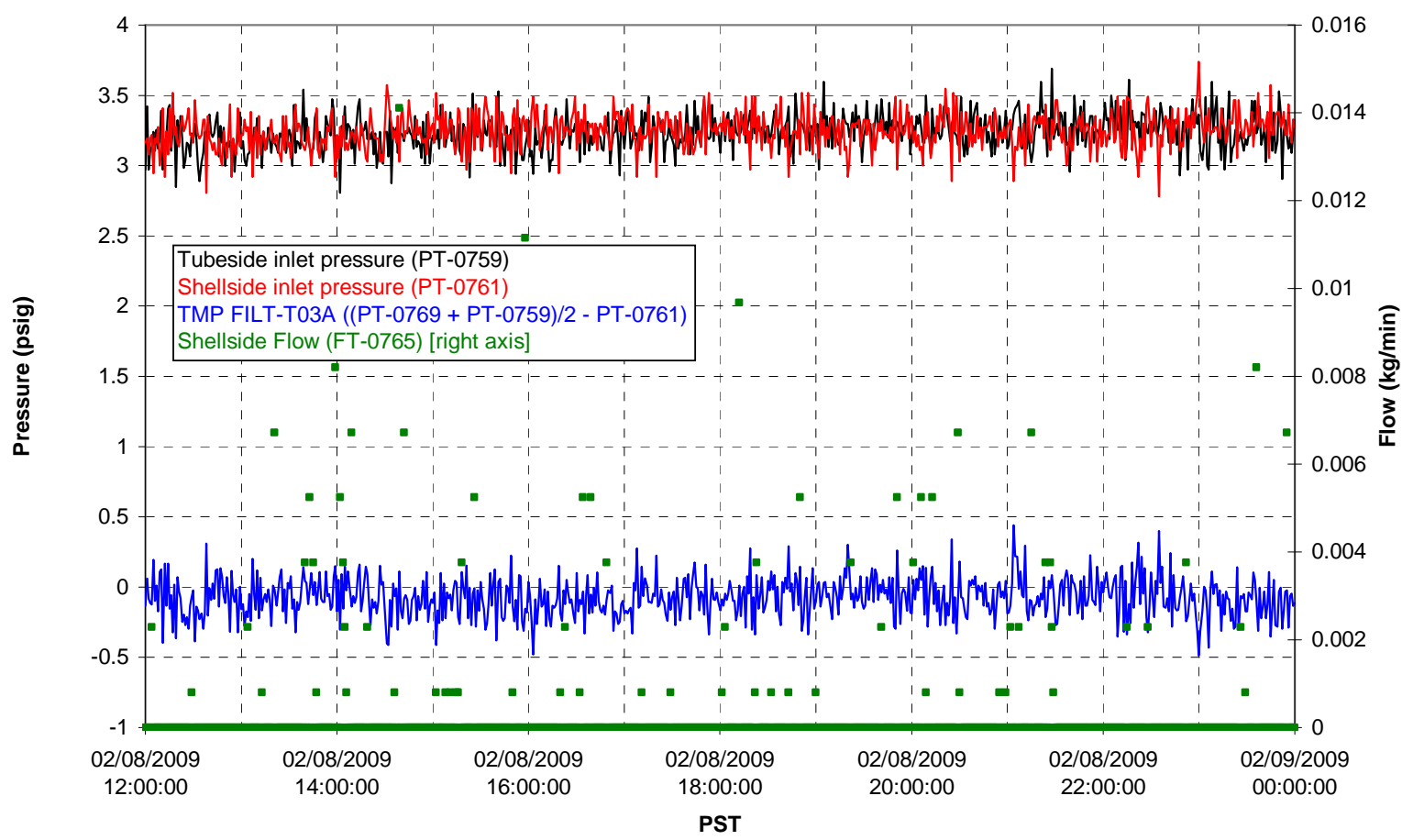

Filter UFP-FILT-T04A

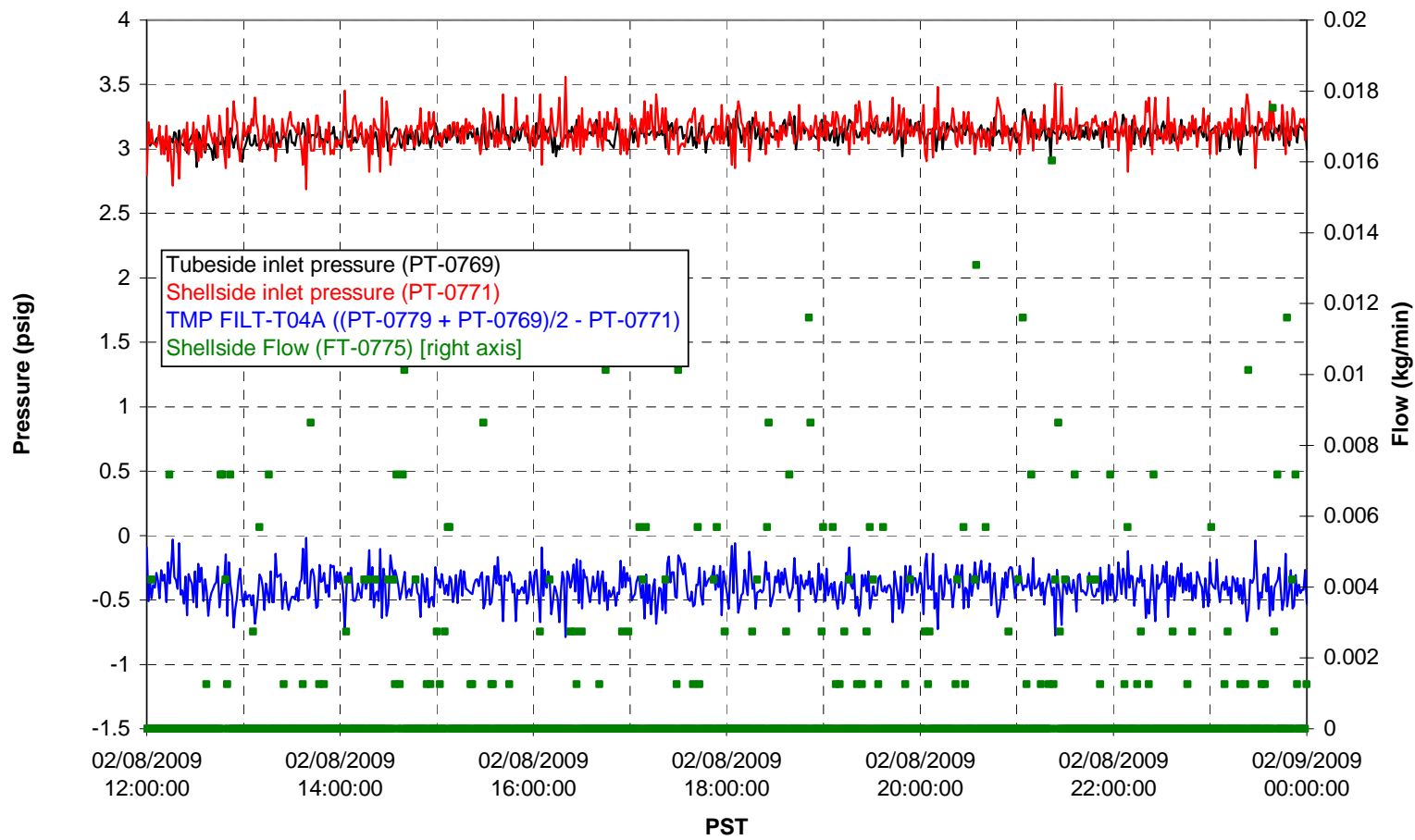


Filter UFP-FILT-T05A

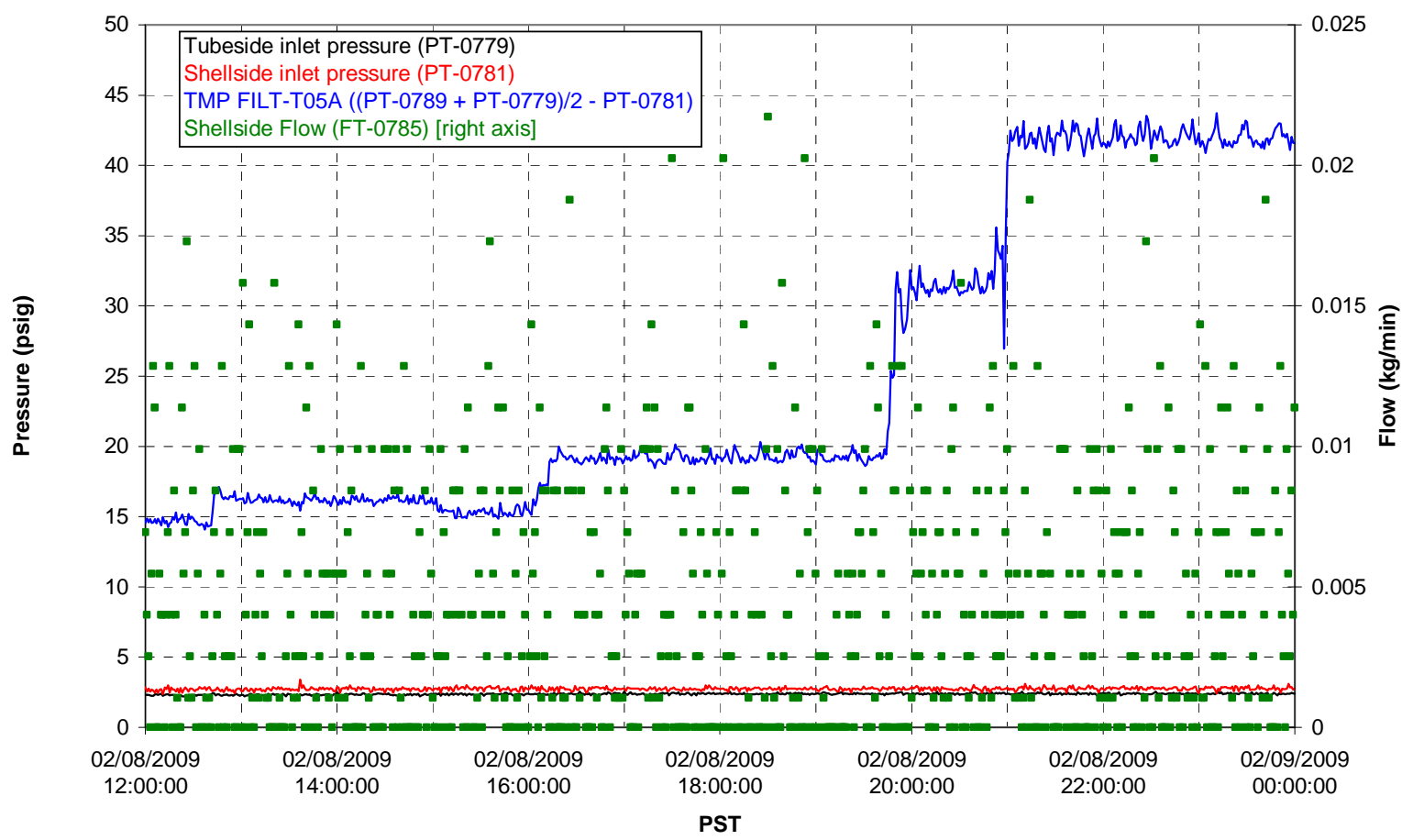

Chemical Flow

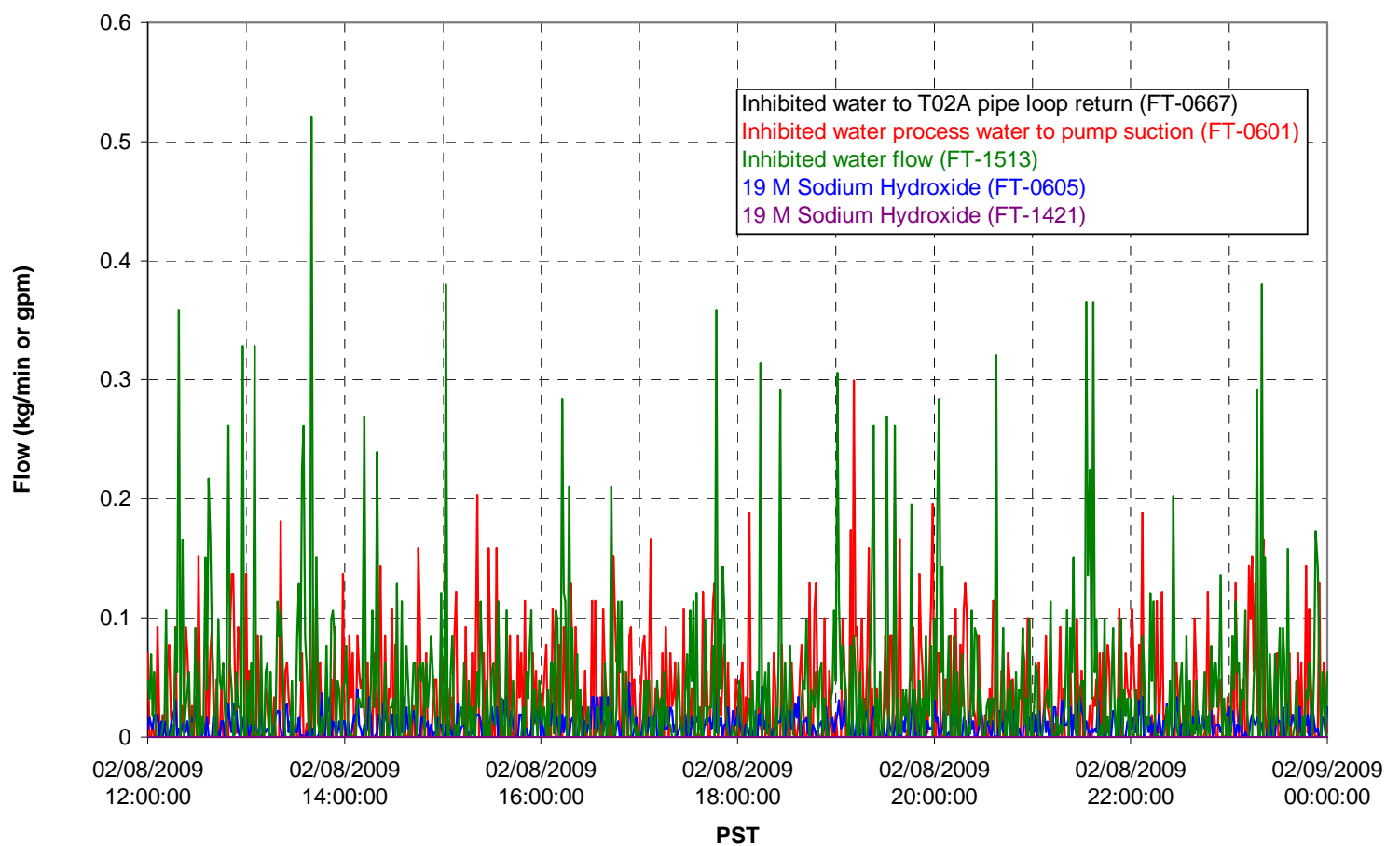




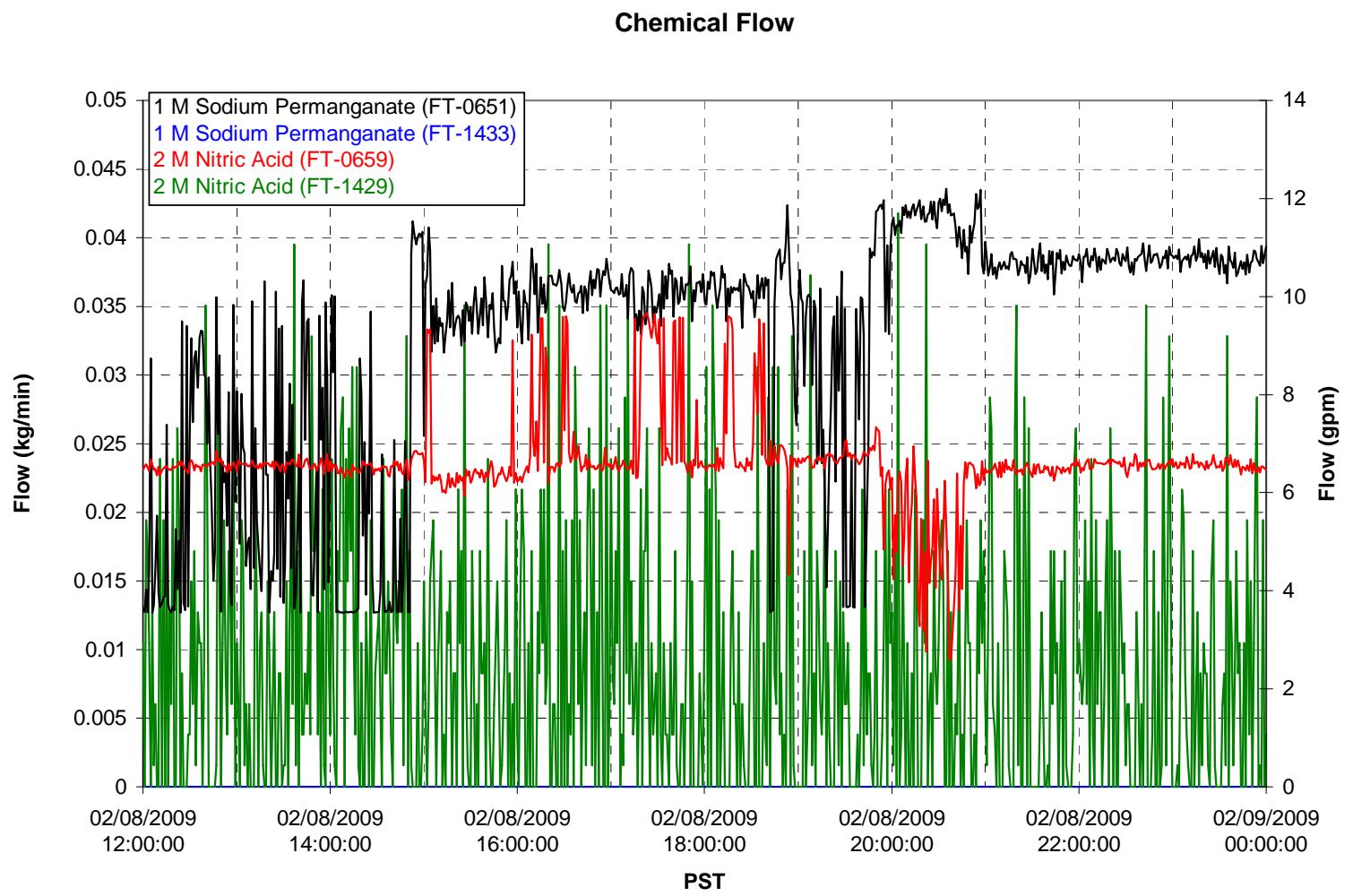

Air Flows

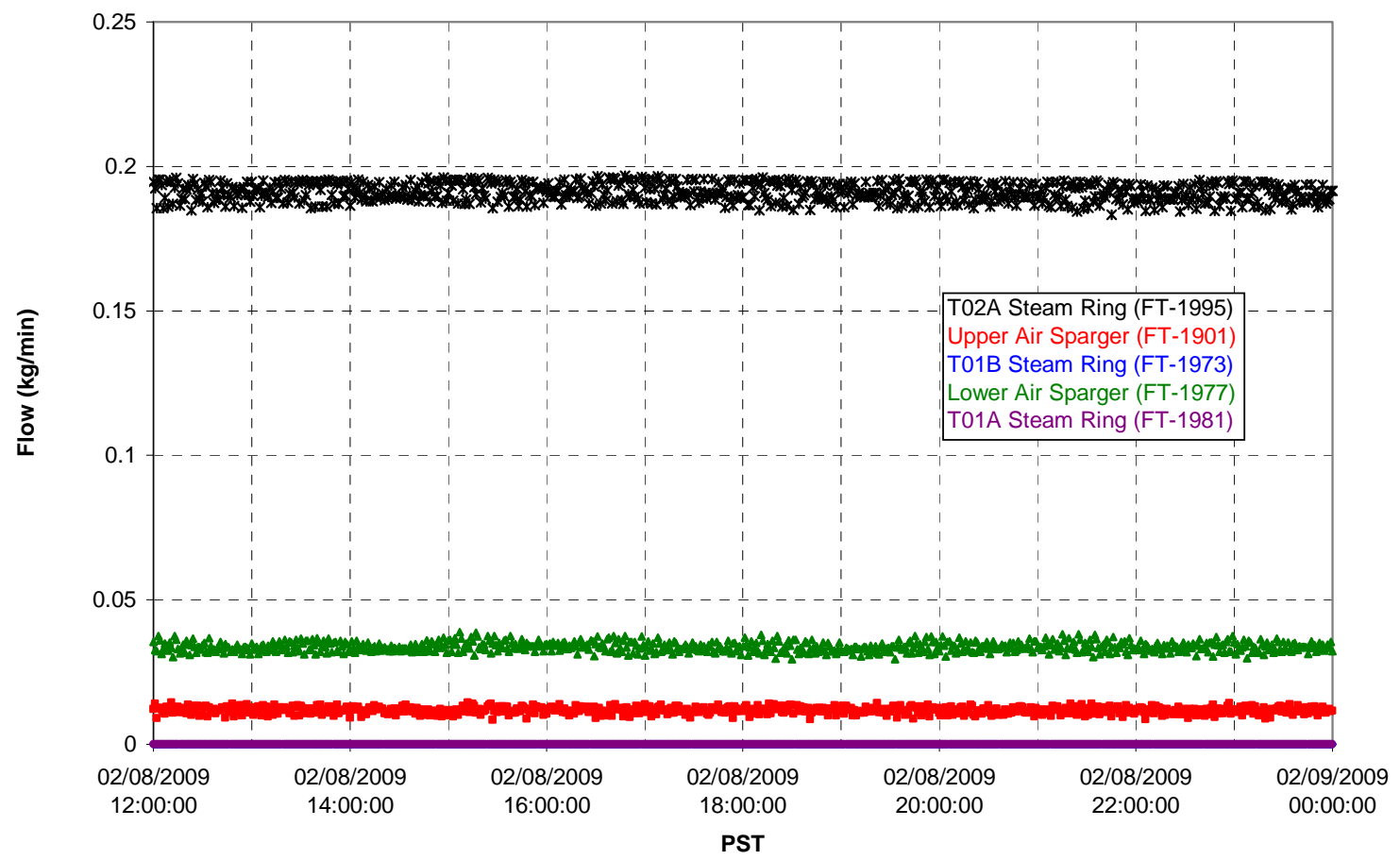


T02A Steam

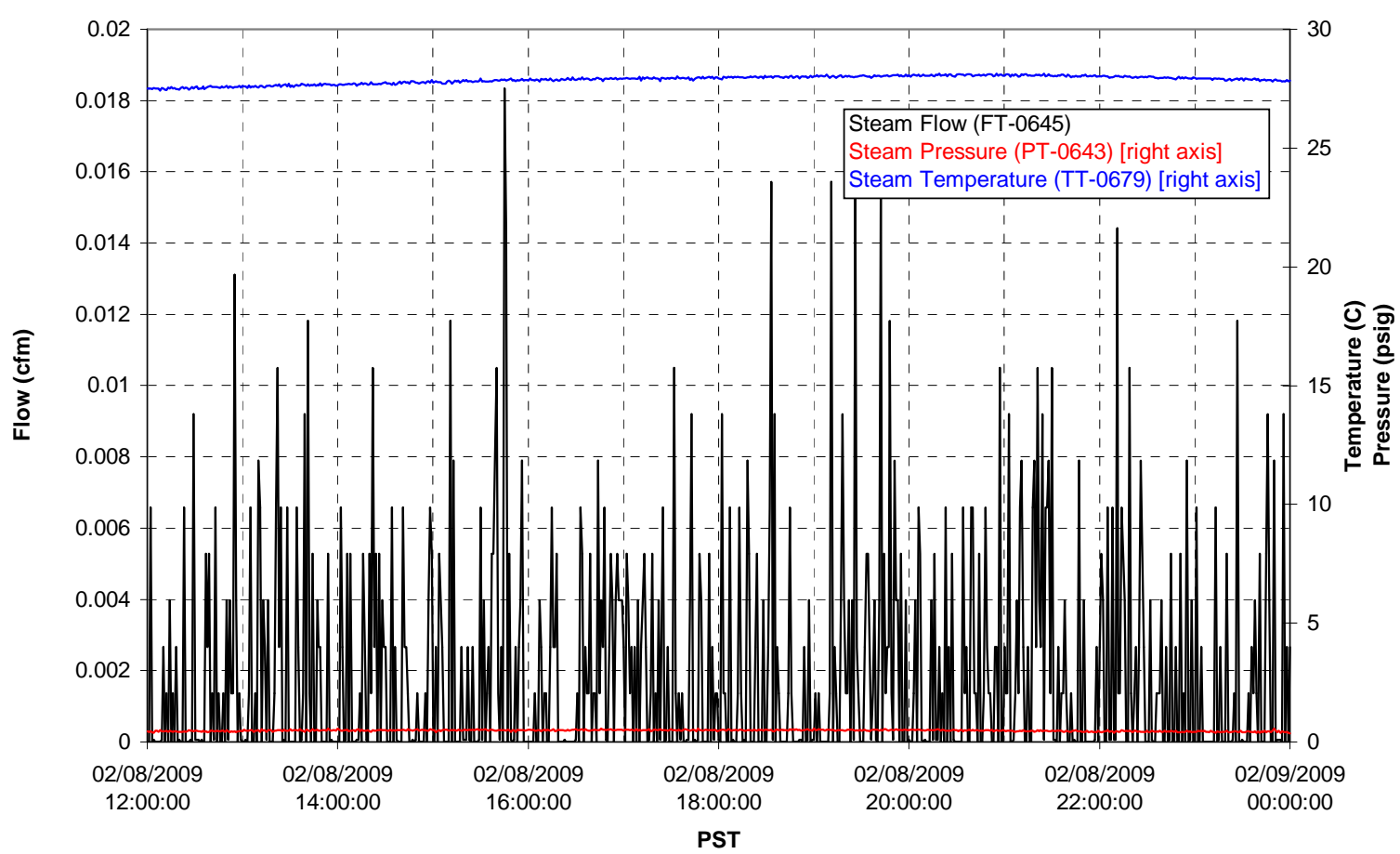

T01A Steam

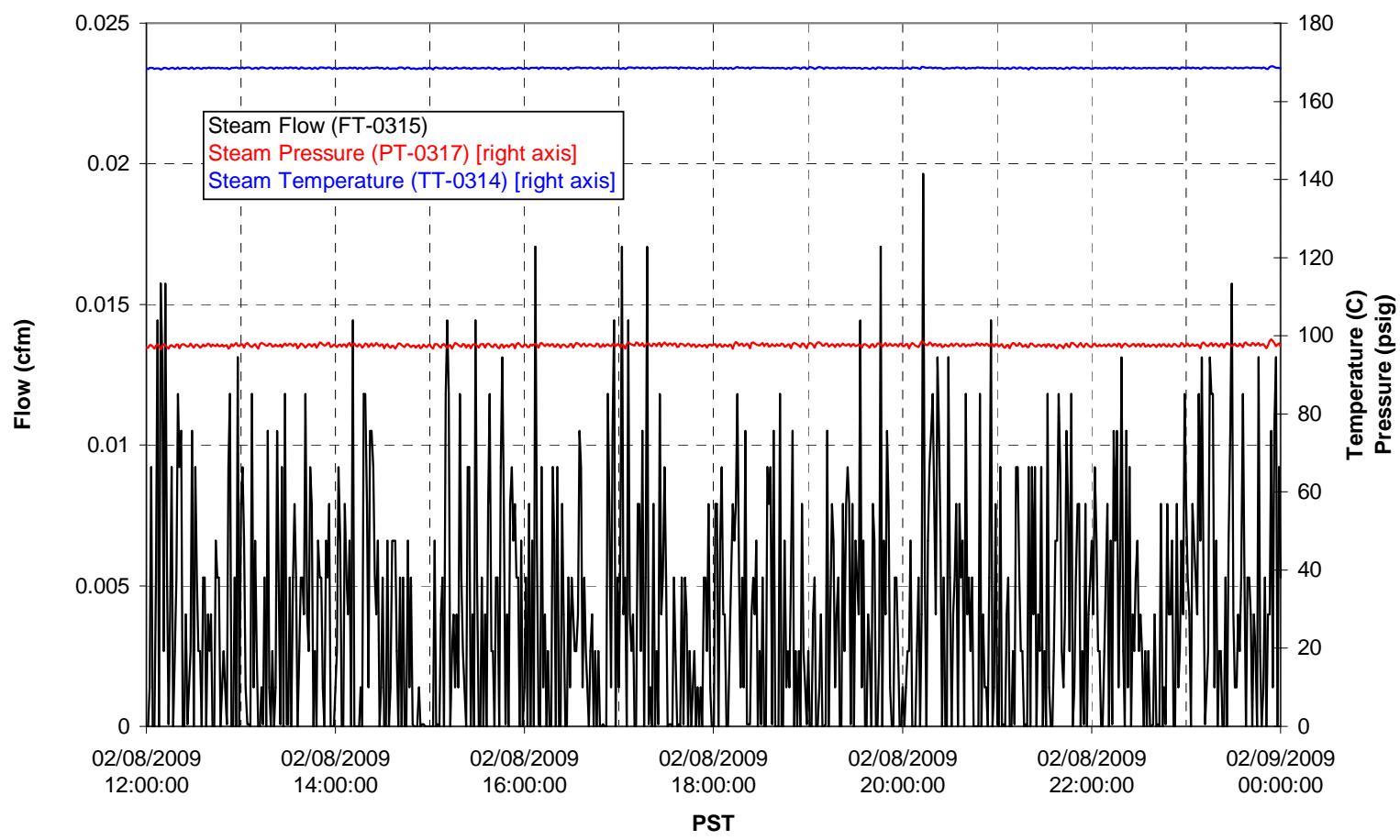


T01B Steam

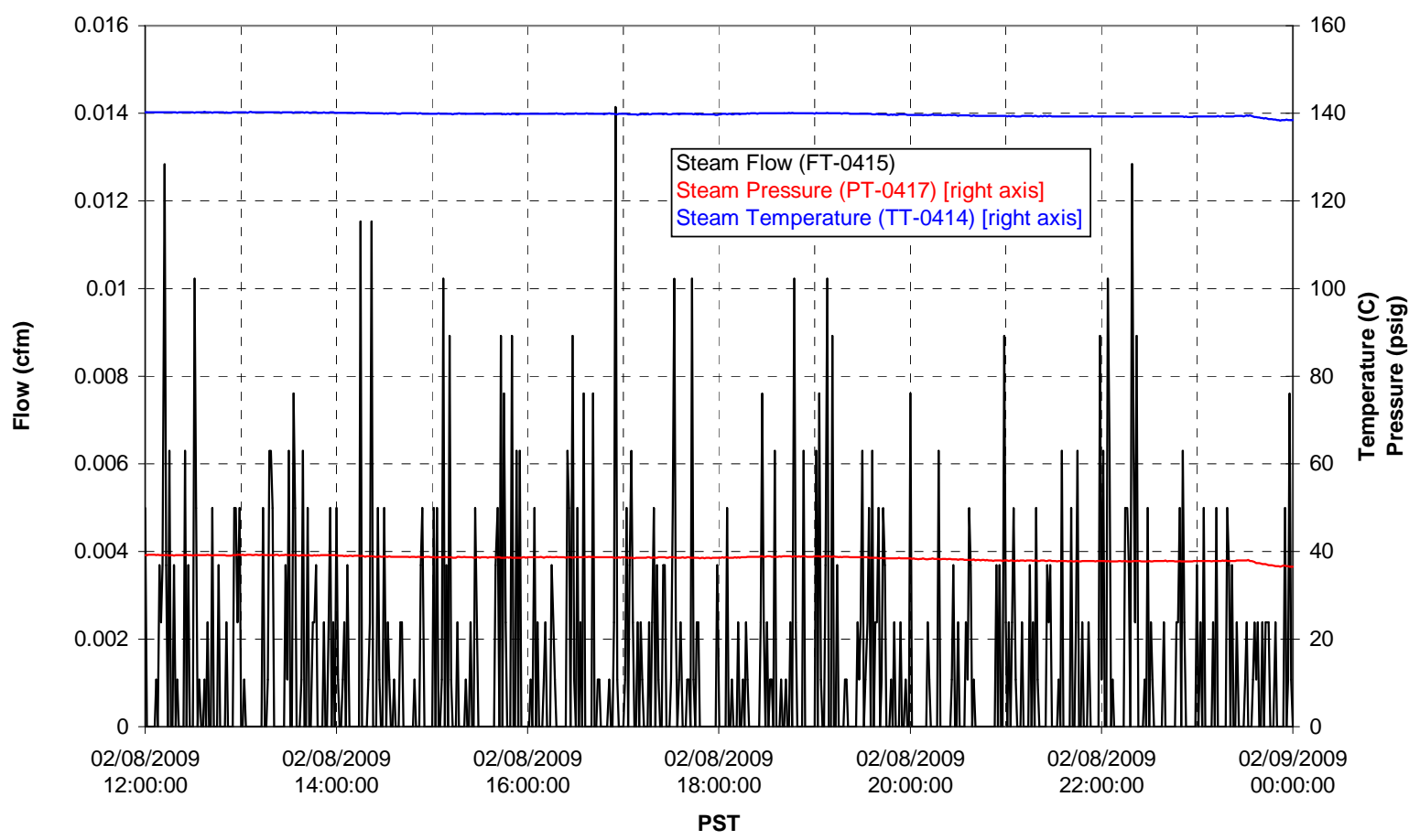




\section{Integrated Test A Data Plots 02/09/09 00:00 - 02/09/09 12:00}


T01A level

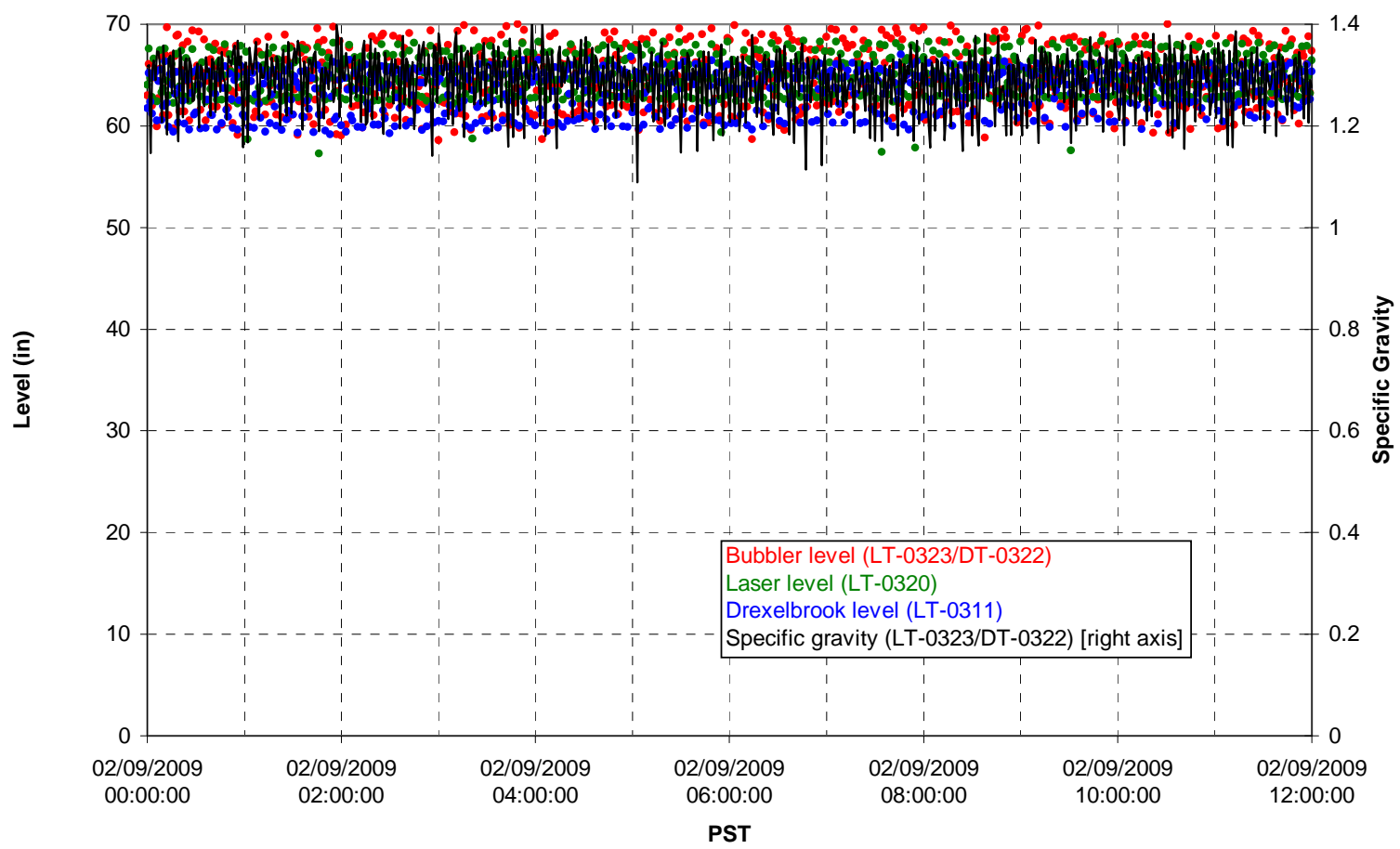

T01A temperatures

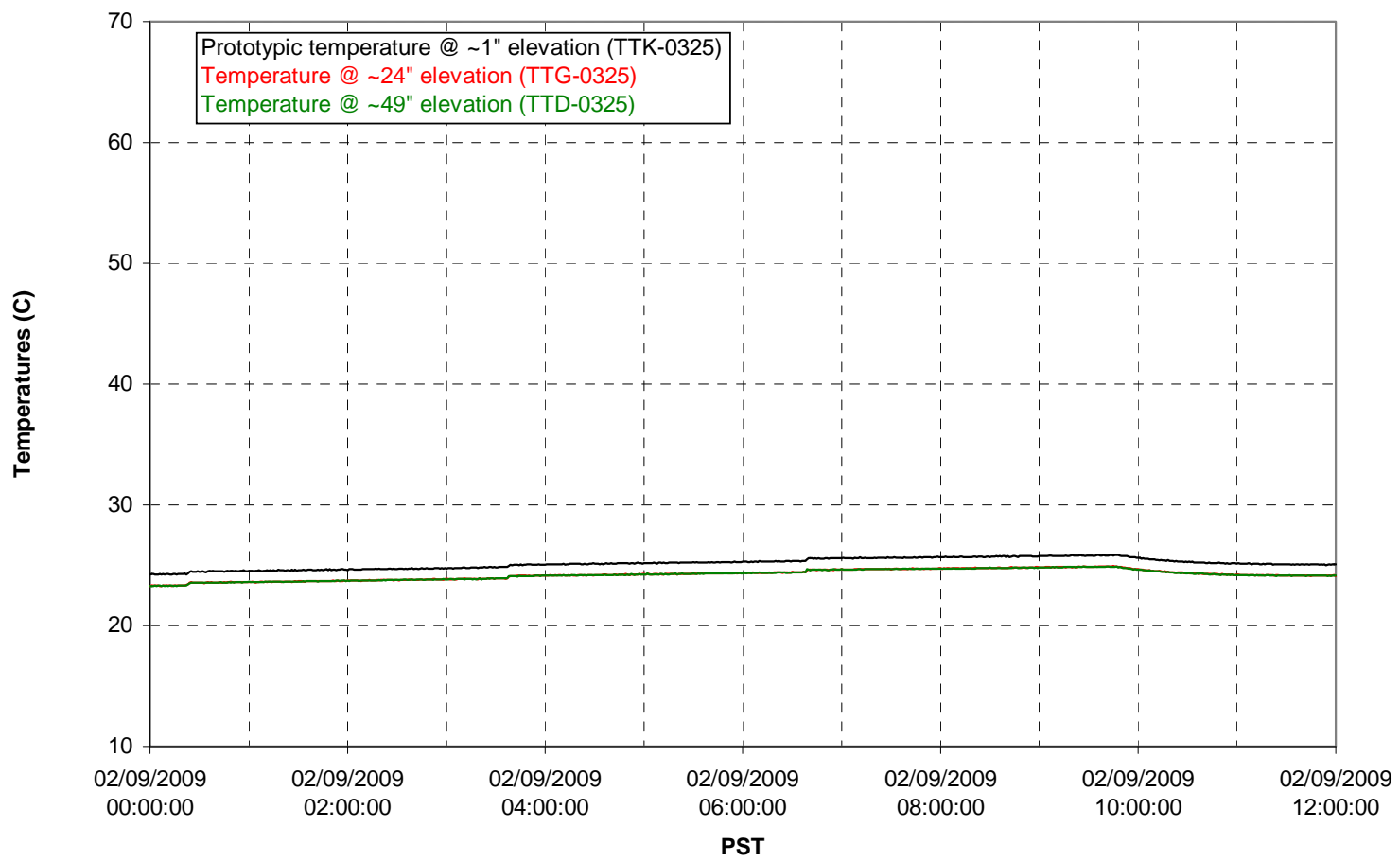


T01B level

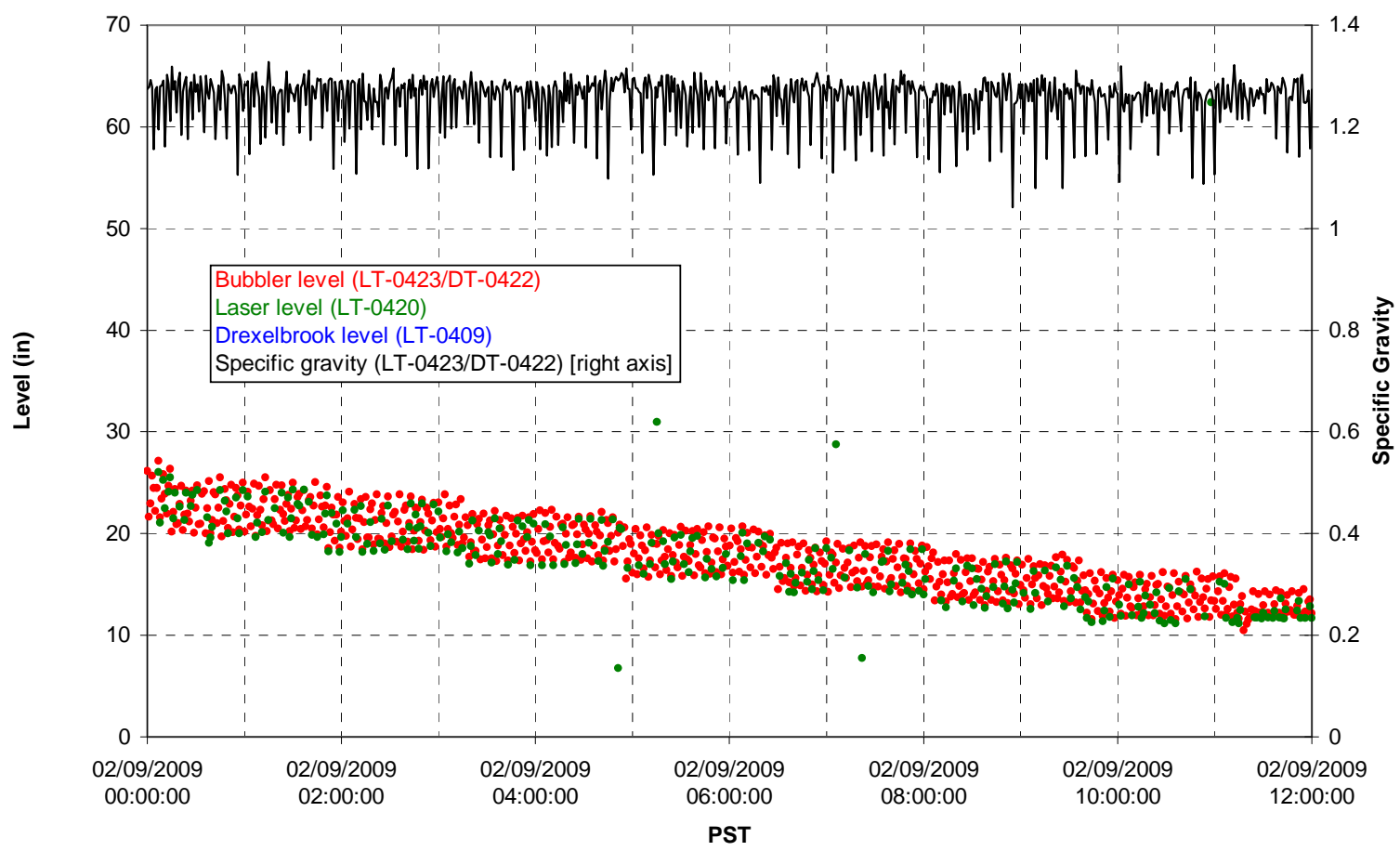

T01B temperatures

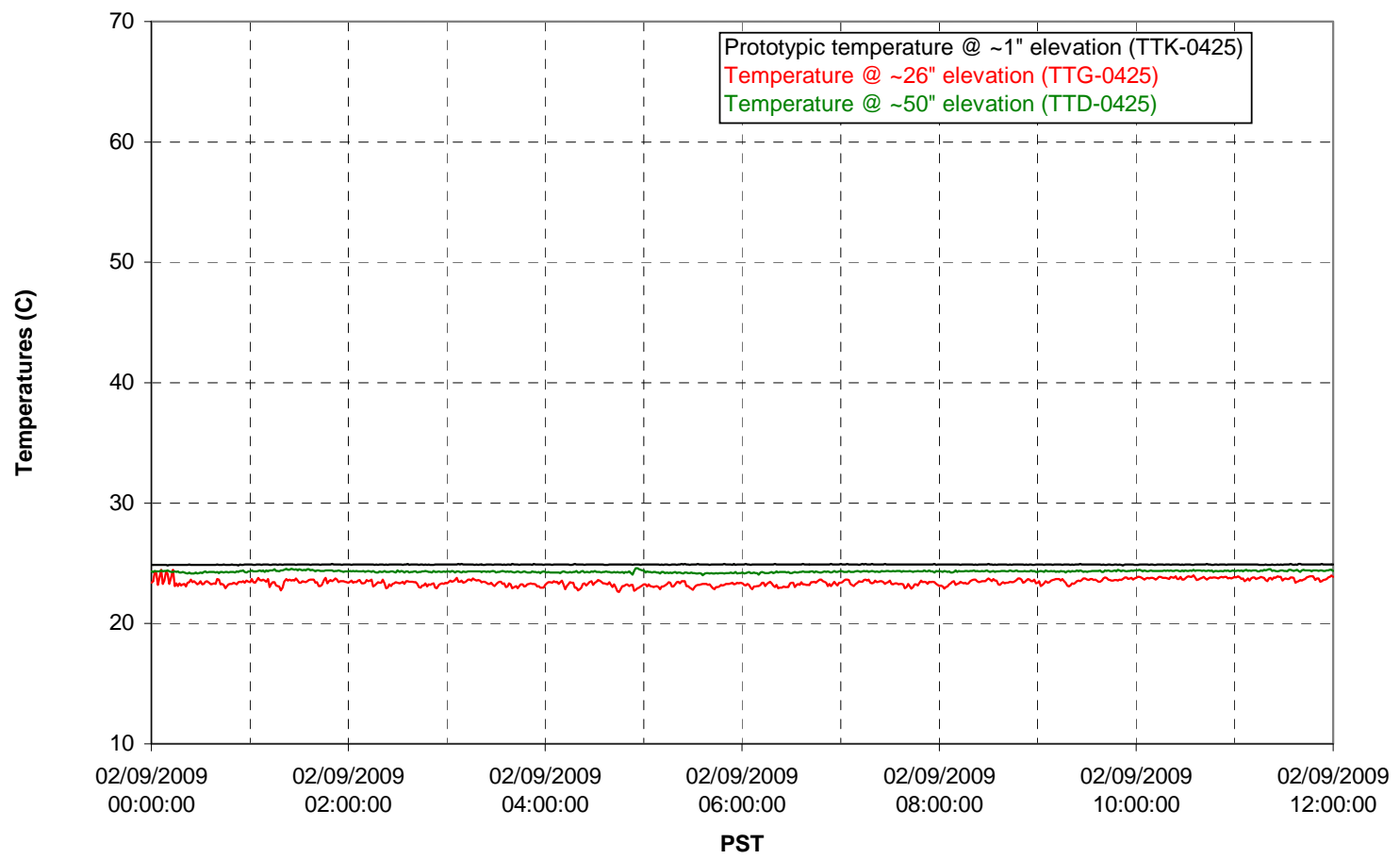


T02A level

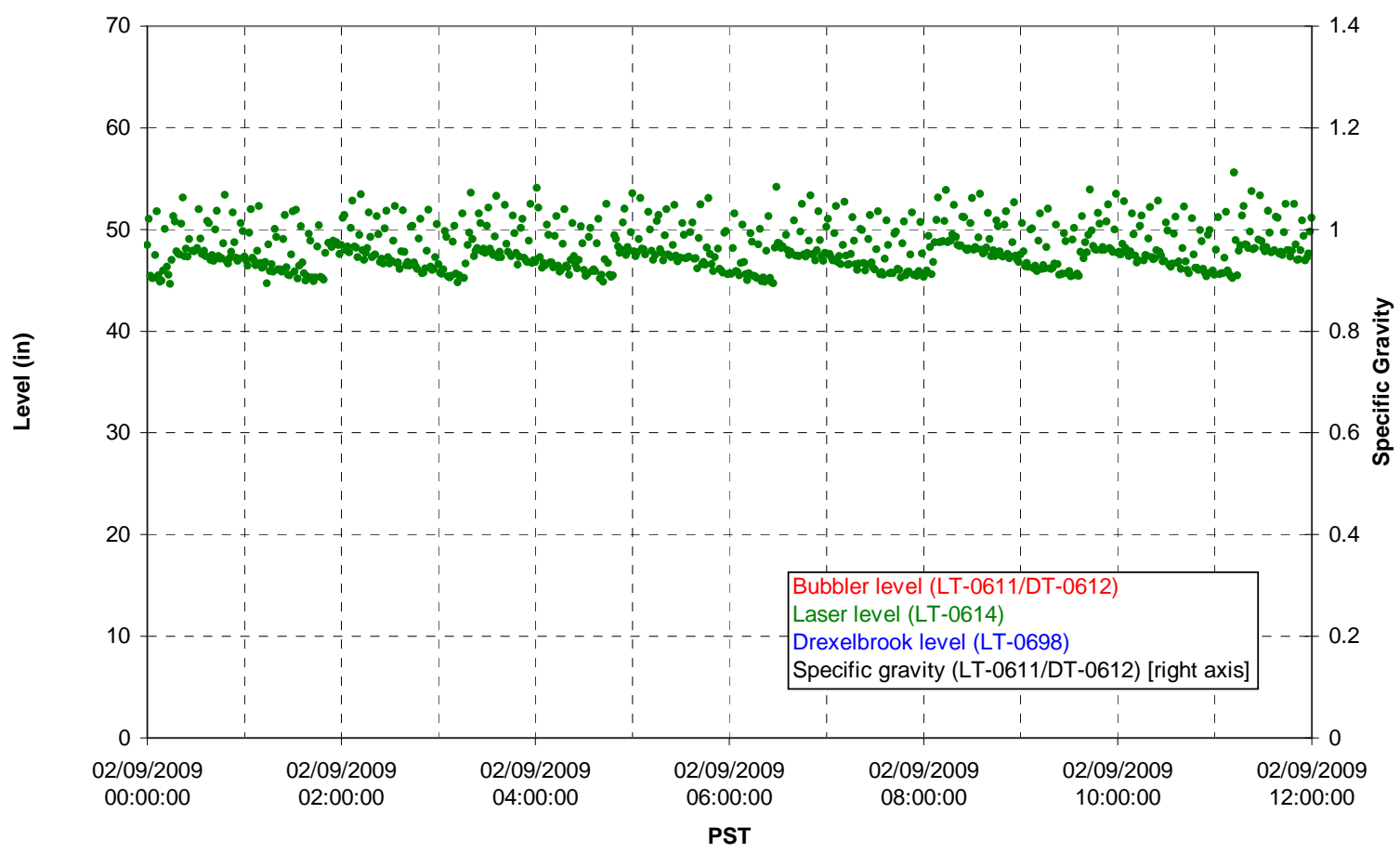

T02A temperatures

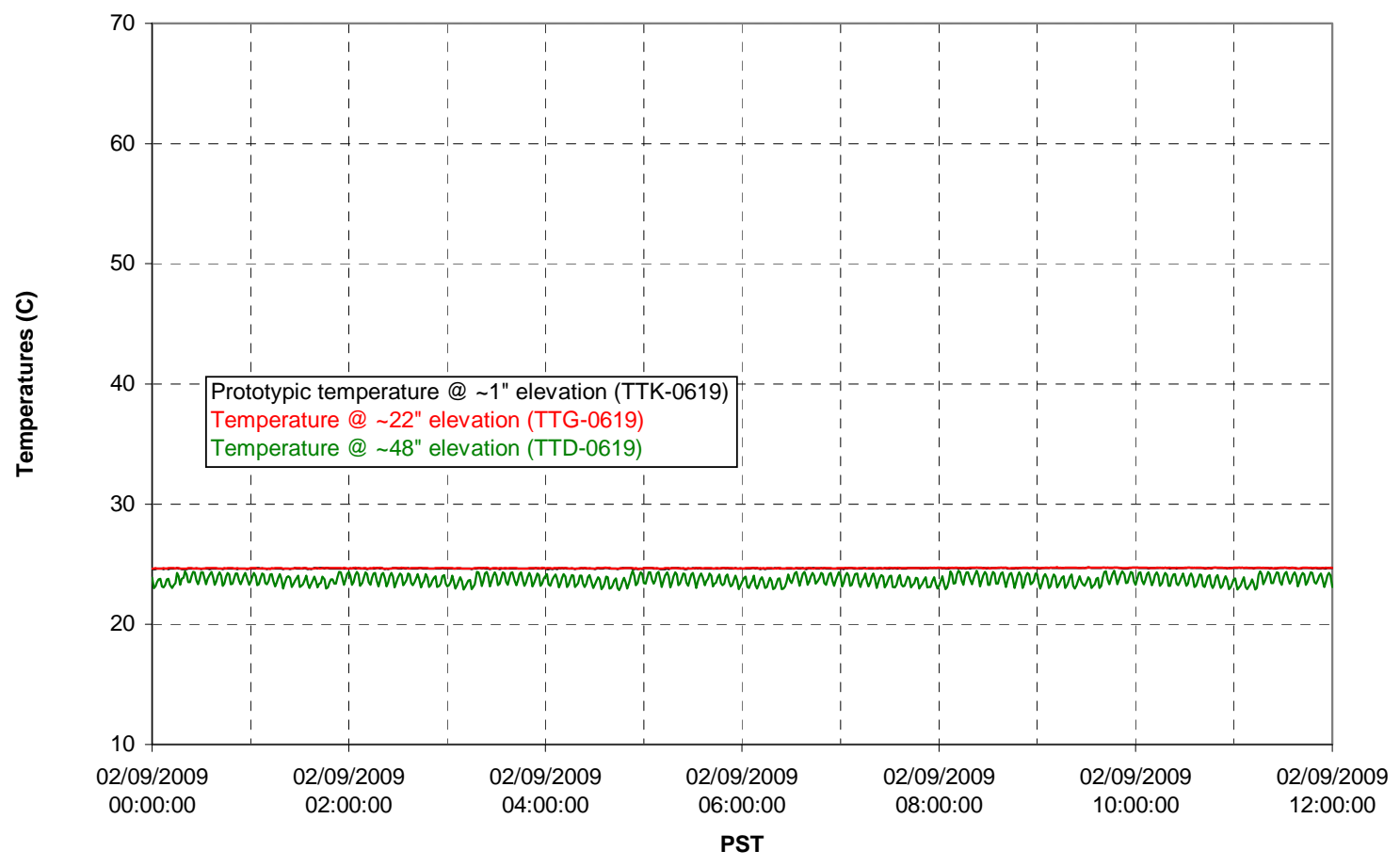


T02A and filter loop temperatures
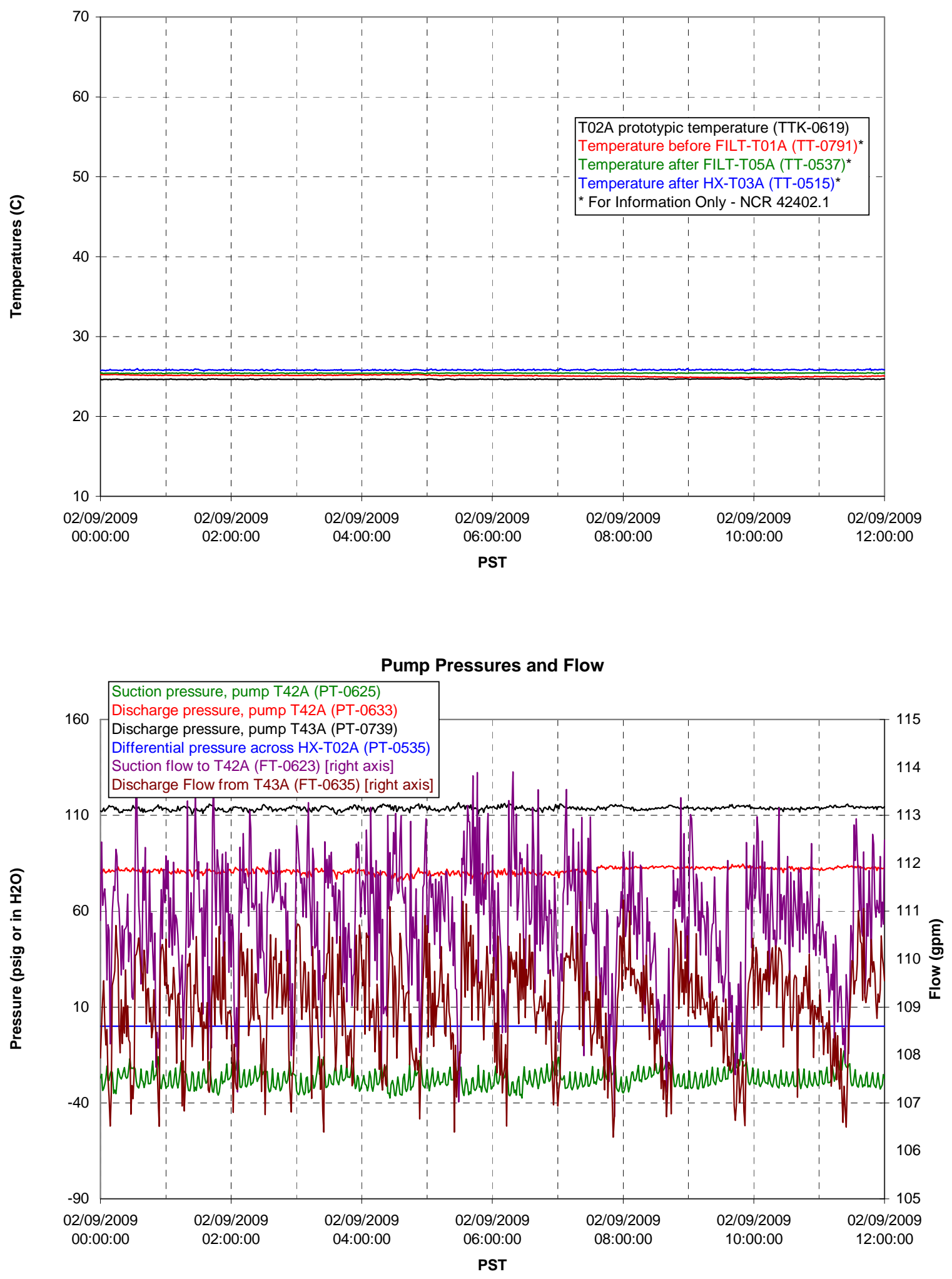


\section{Axial pressure drop}

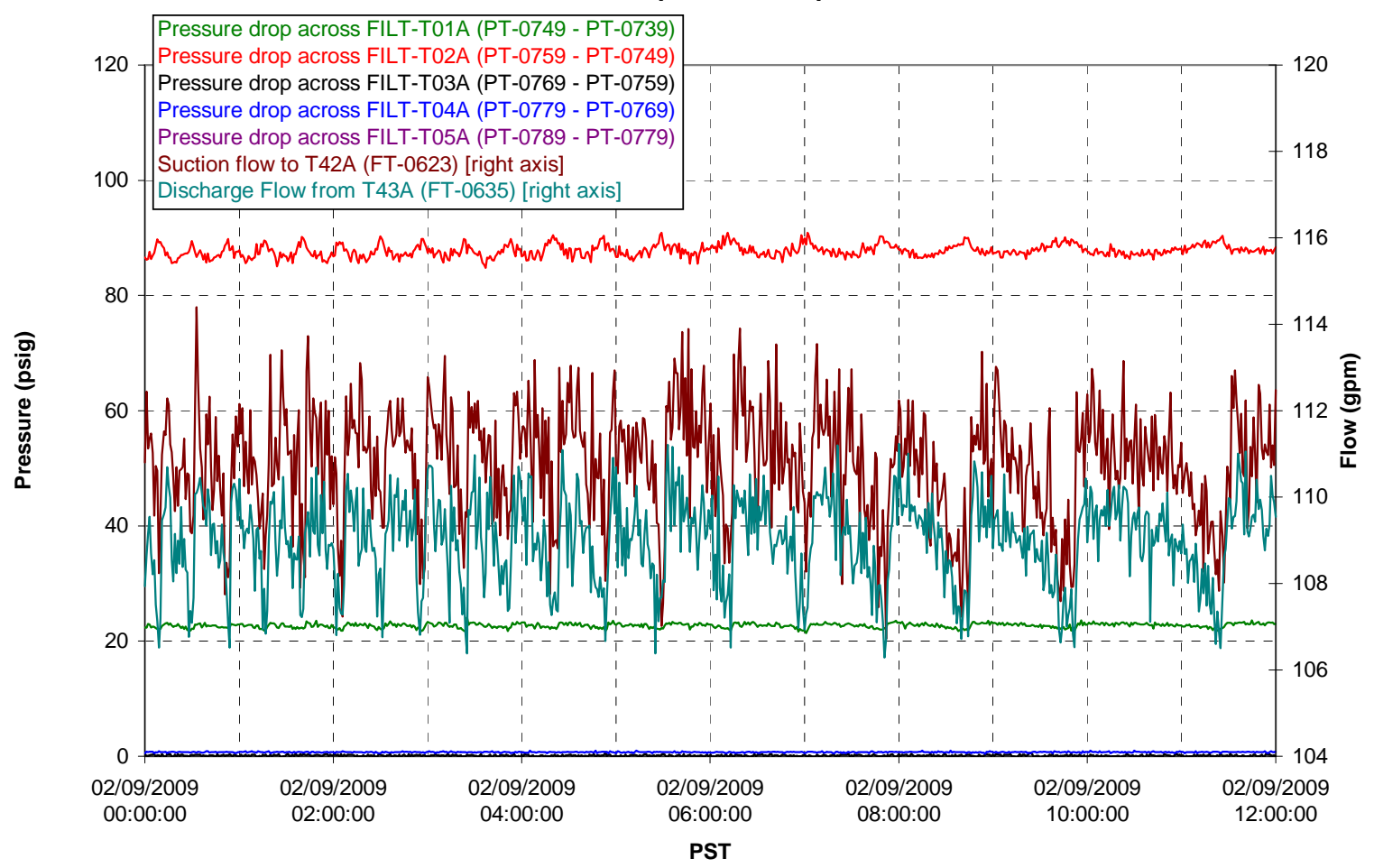

Permeate flow rates

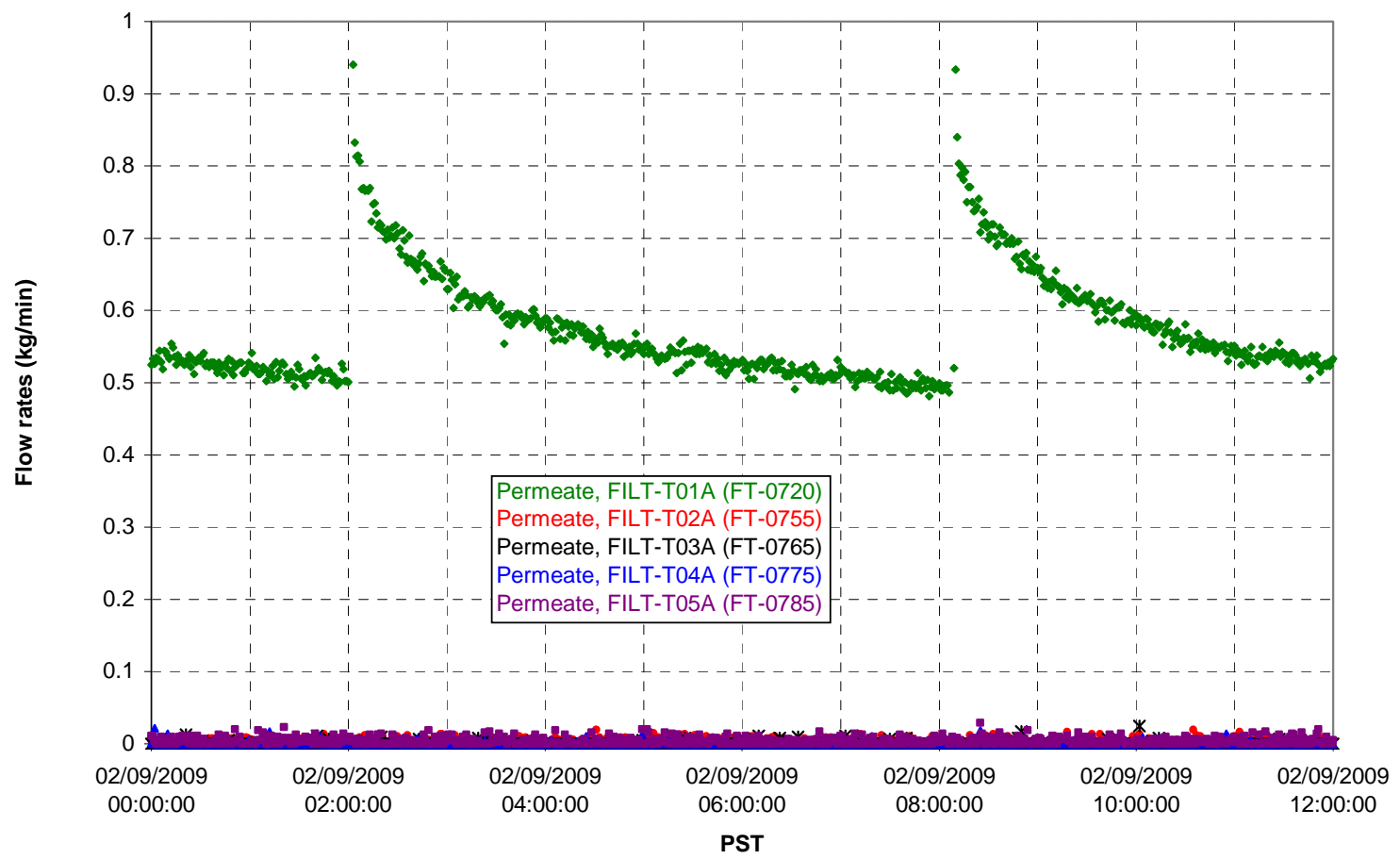


T02A Inner Temperature Tree

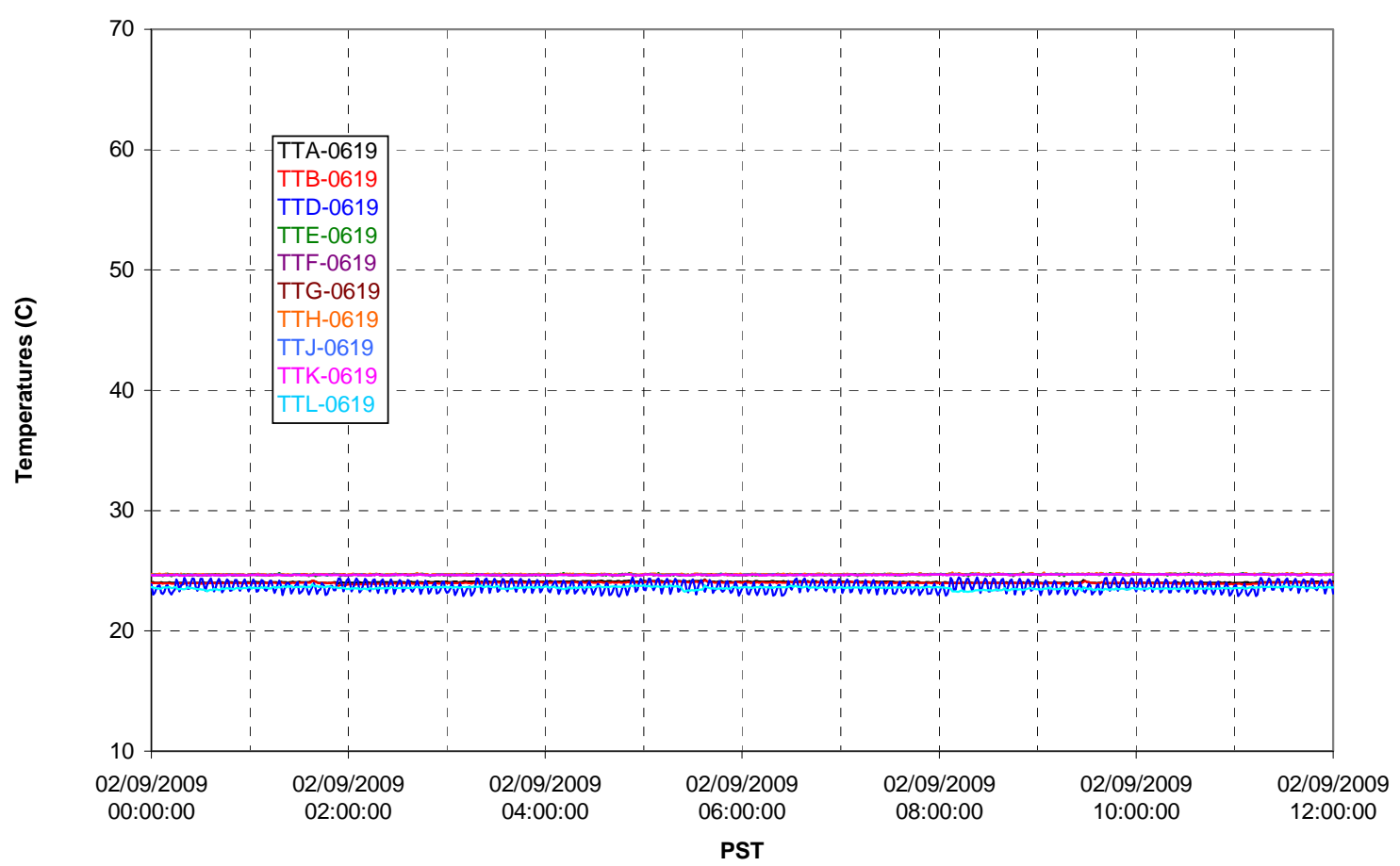

T02A Outer Temperature Tree

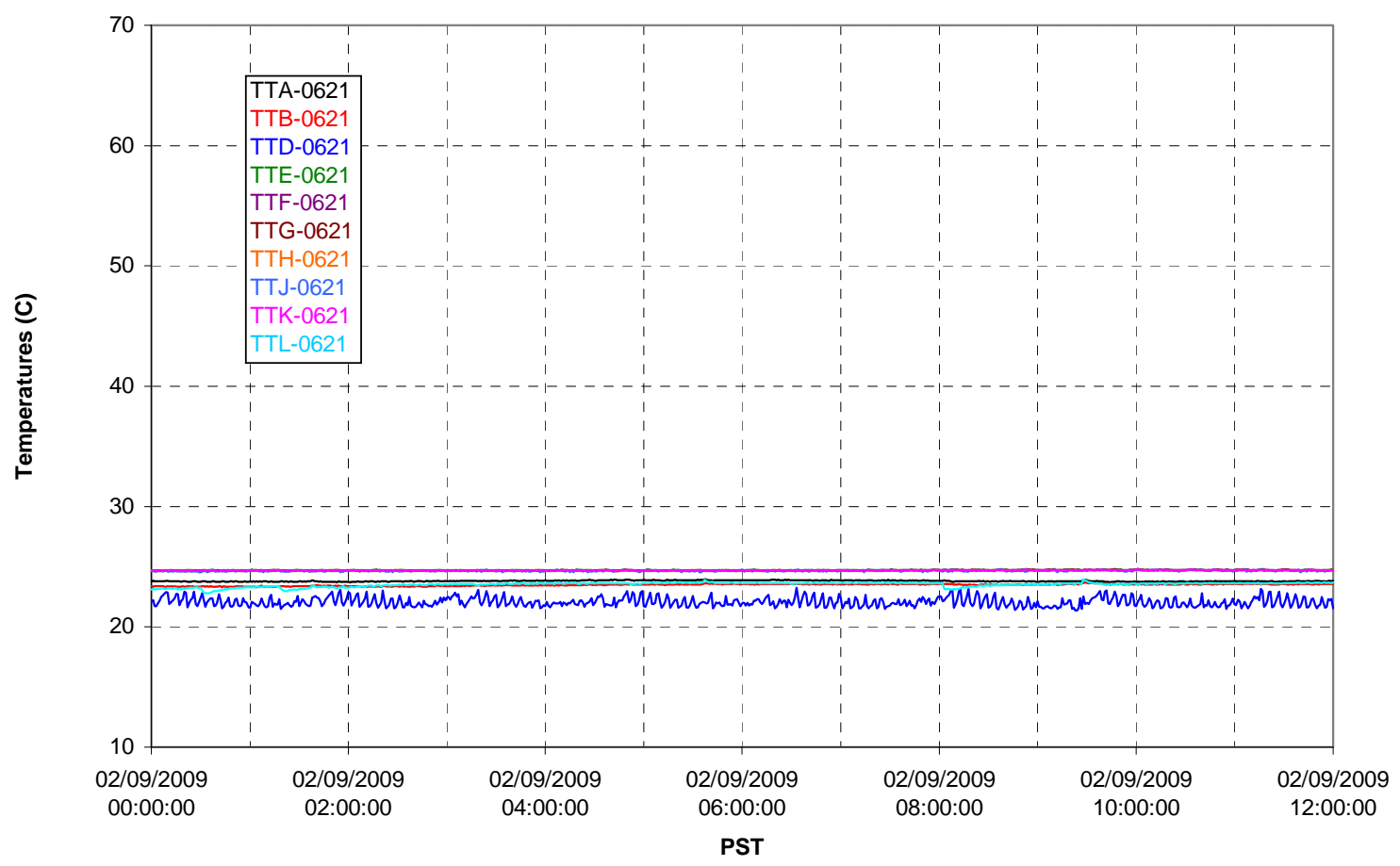


T02A temperatures

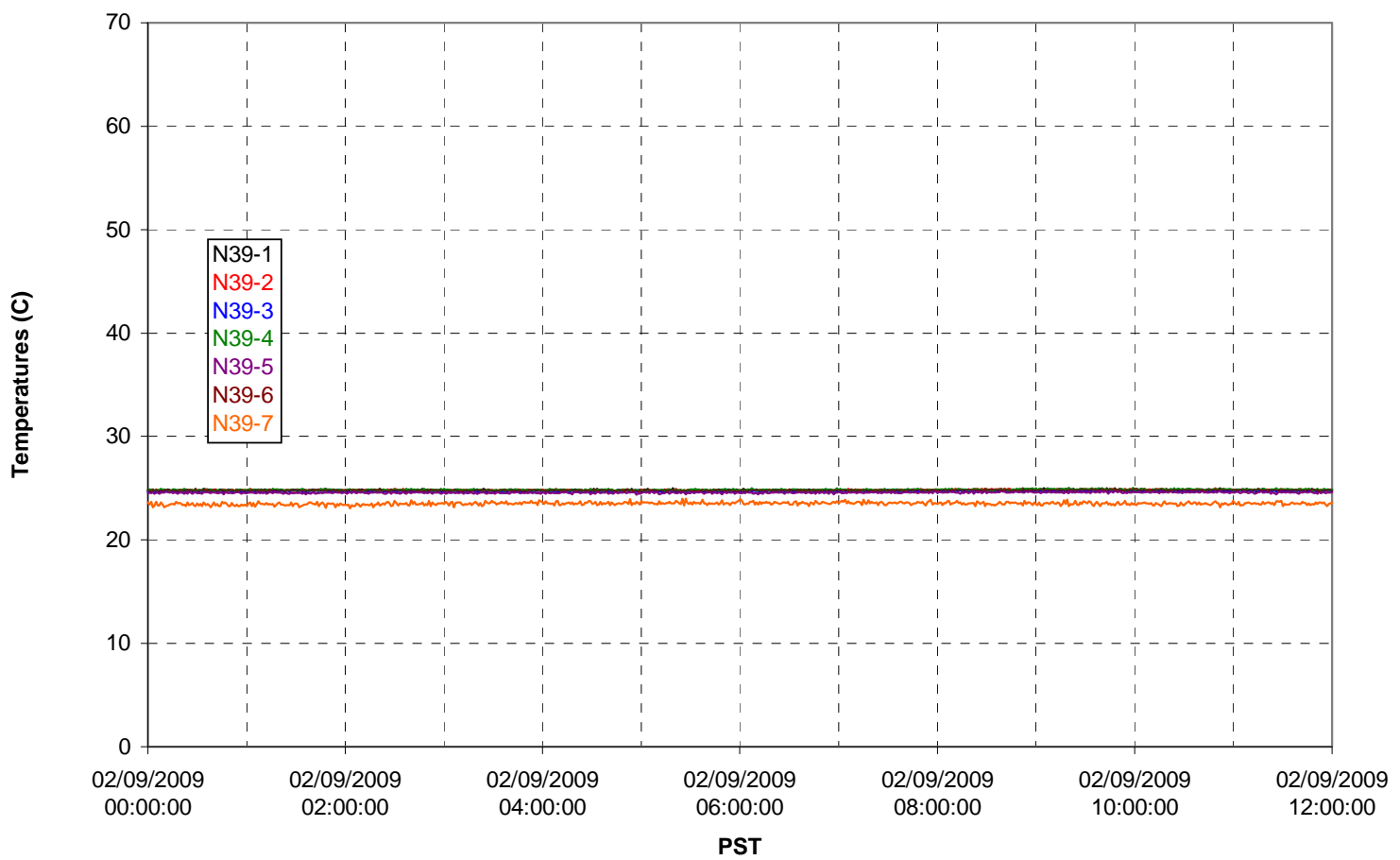

T02A temperatures

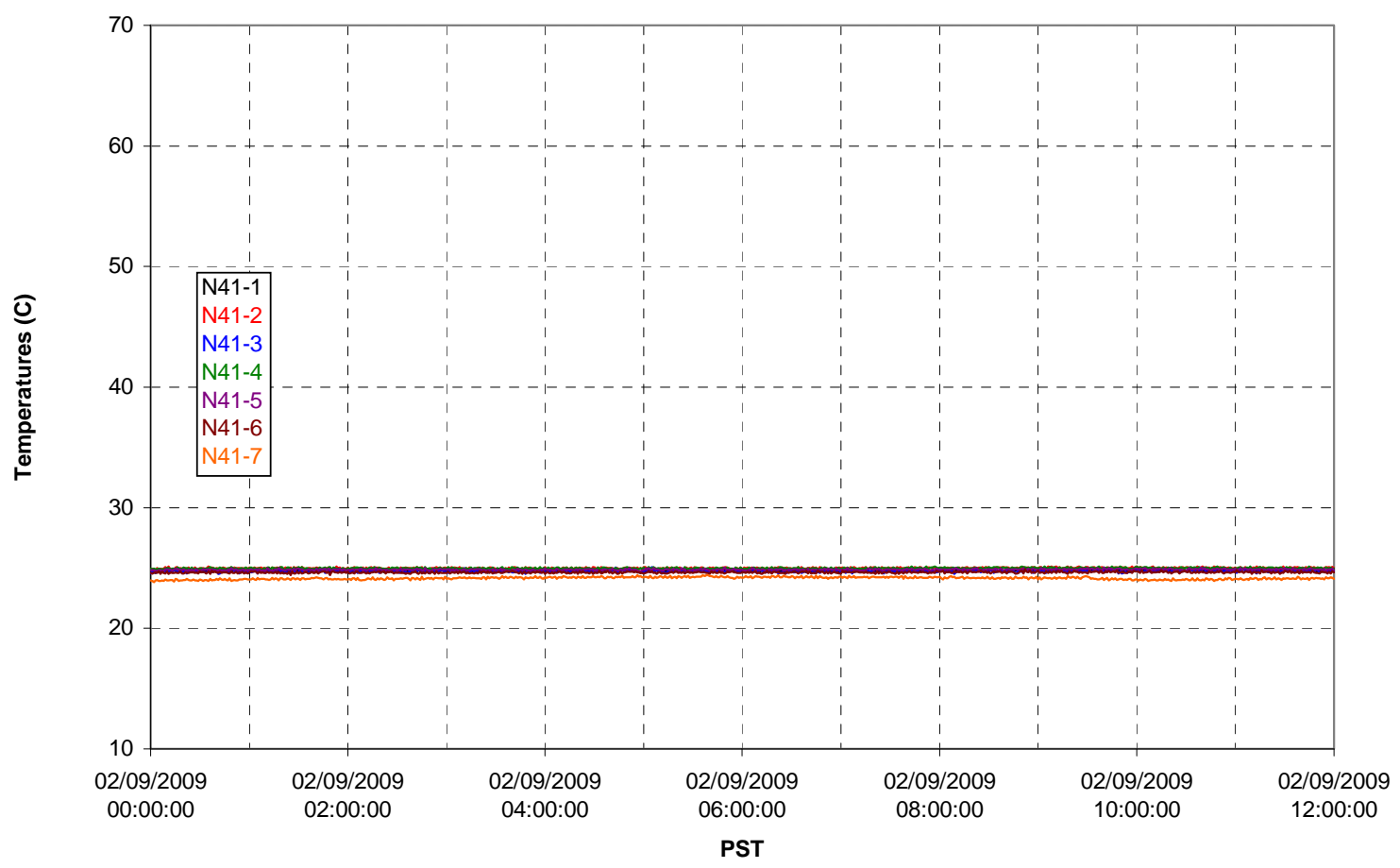


T02A temperatures

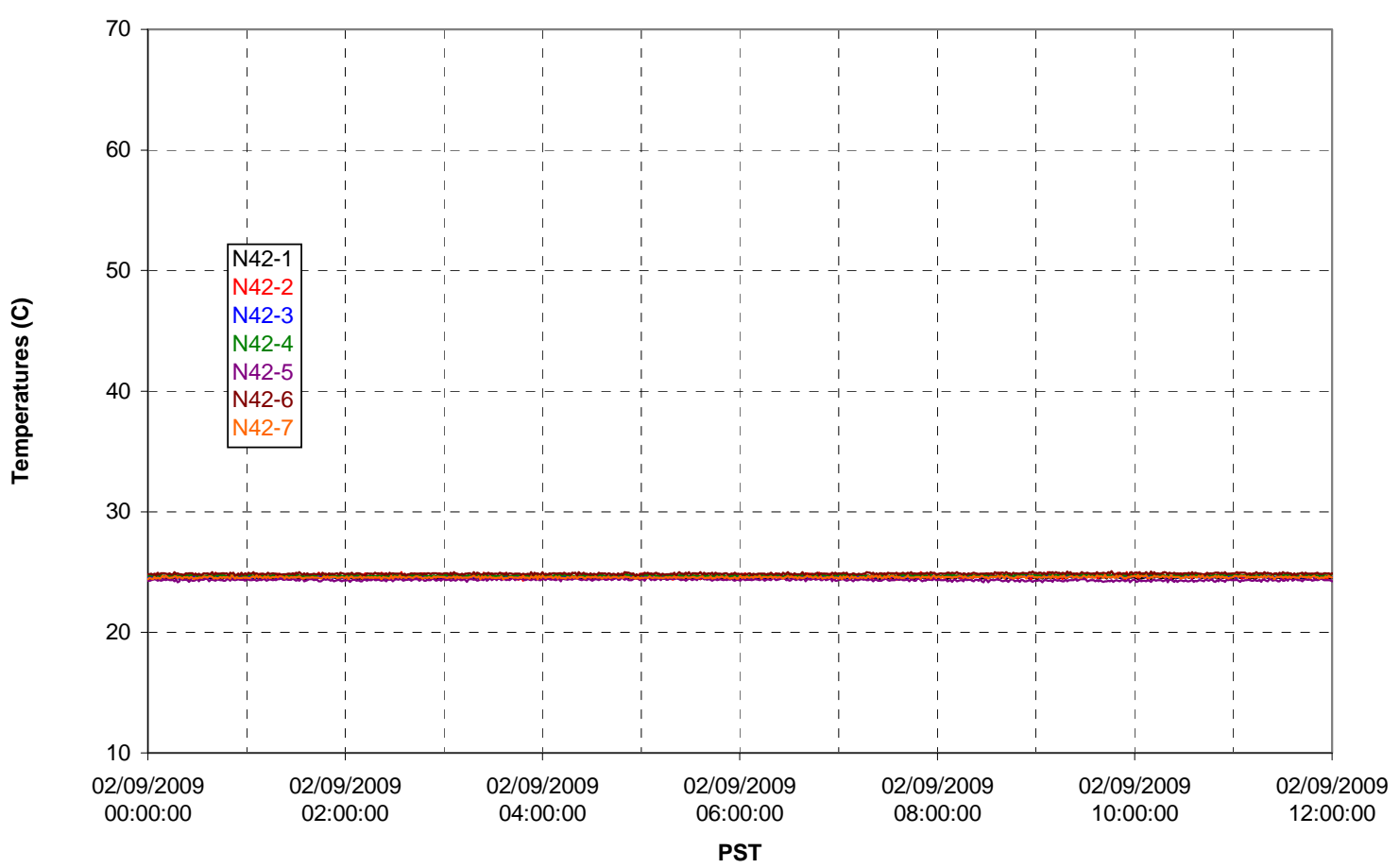

T02A temperatures

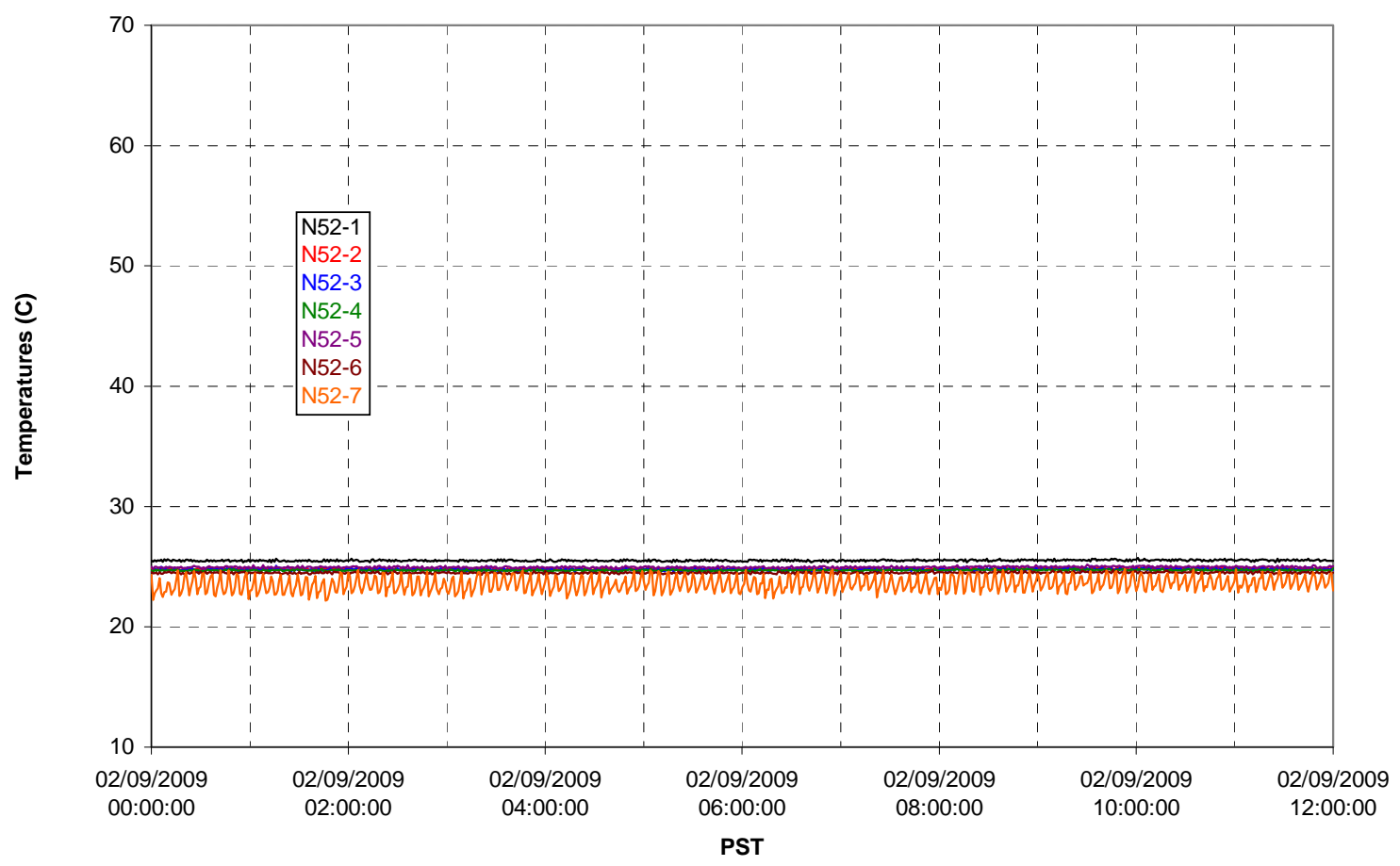


T02A Heating and Cooling

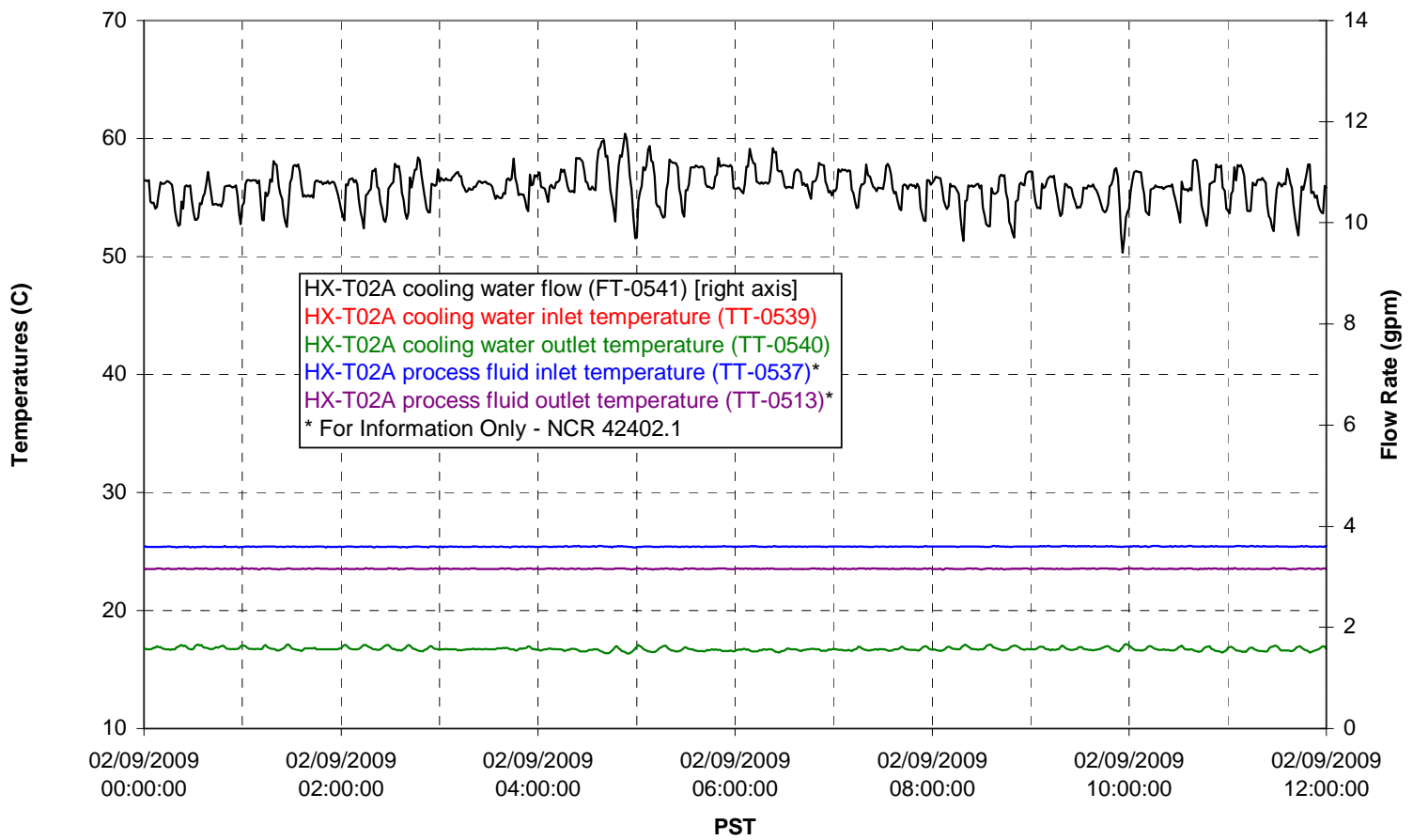

Pump Operation

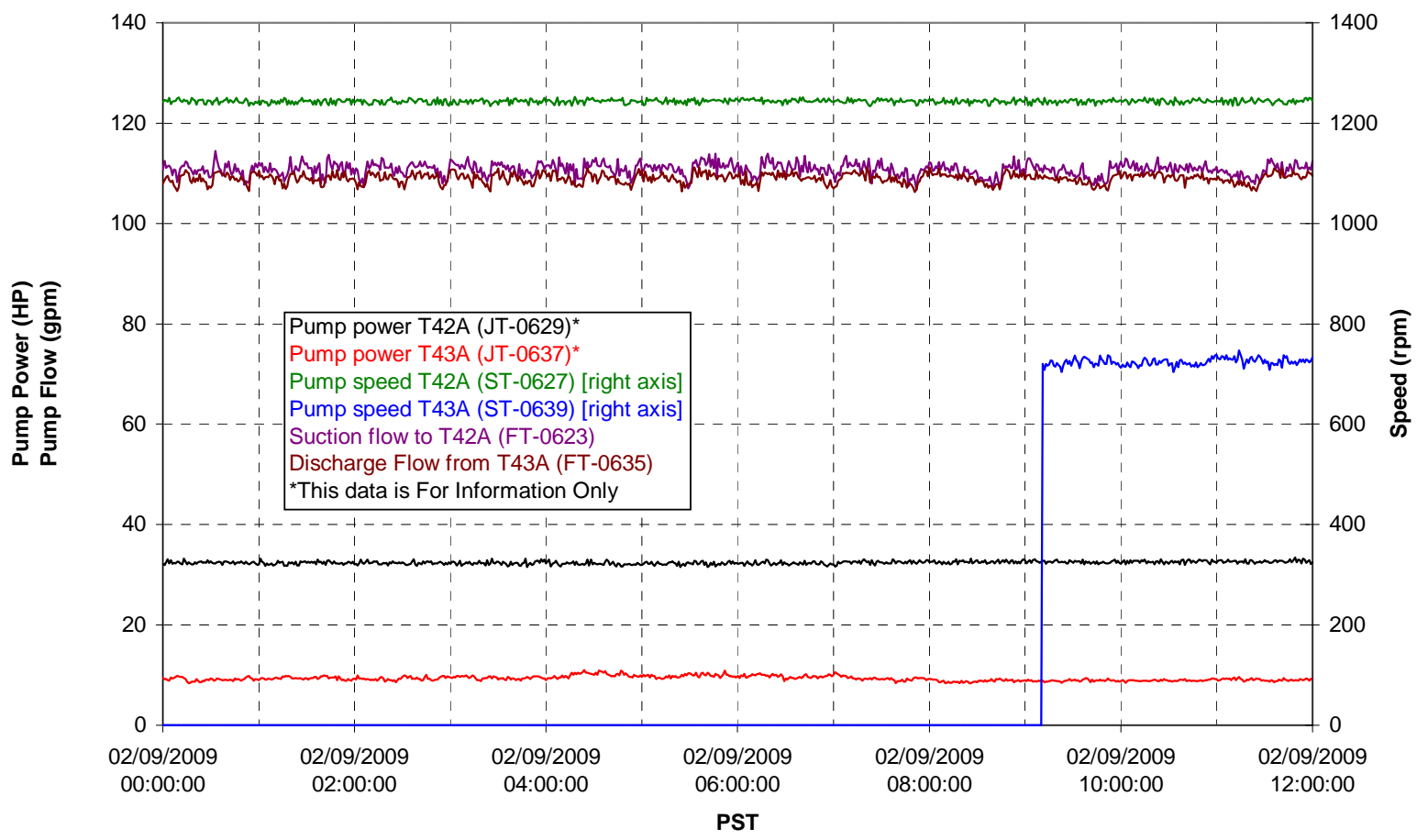


Pulsepot UFP-PP-T01A

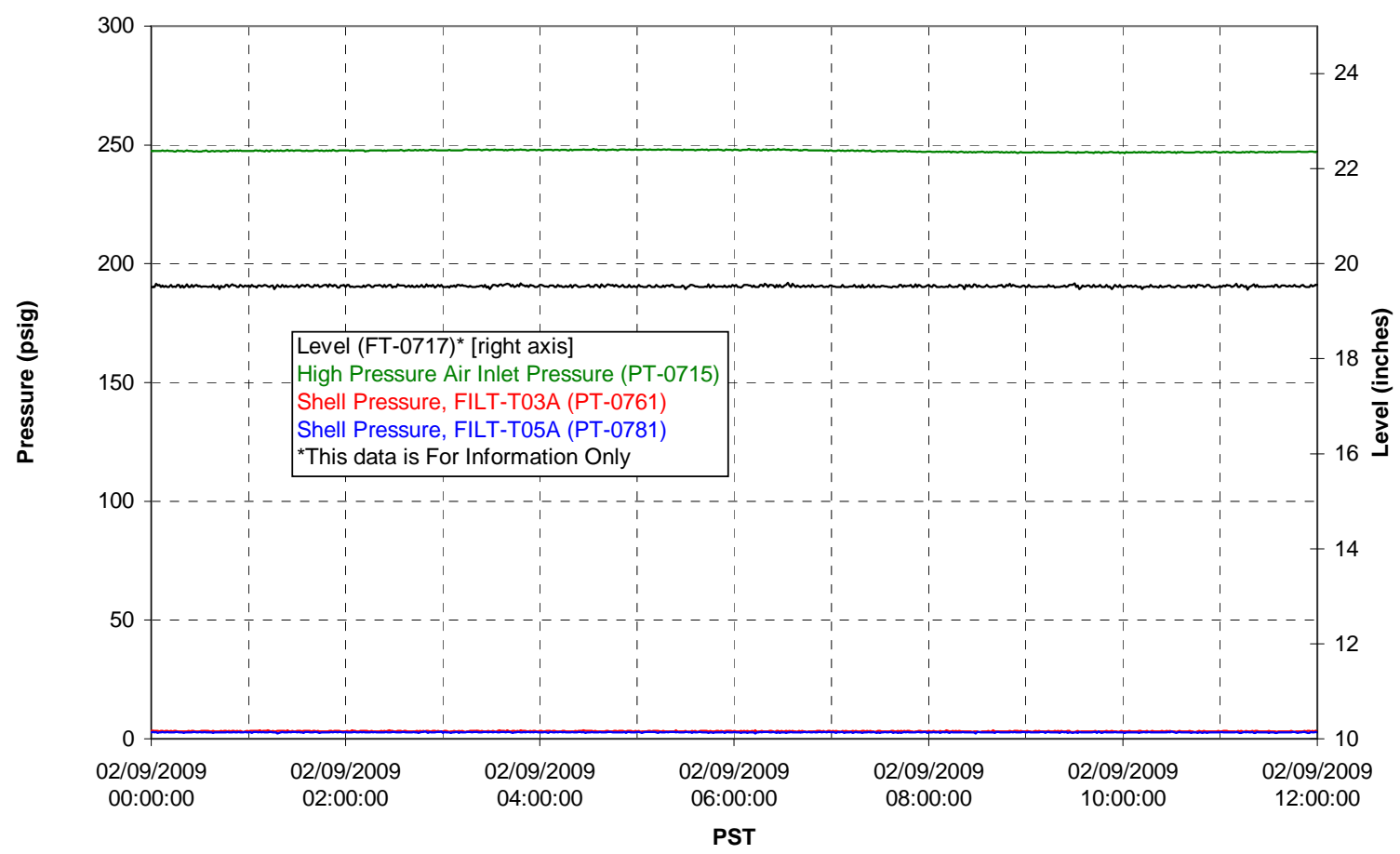

Pulsepot UFP-PP-T02A

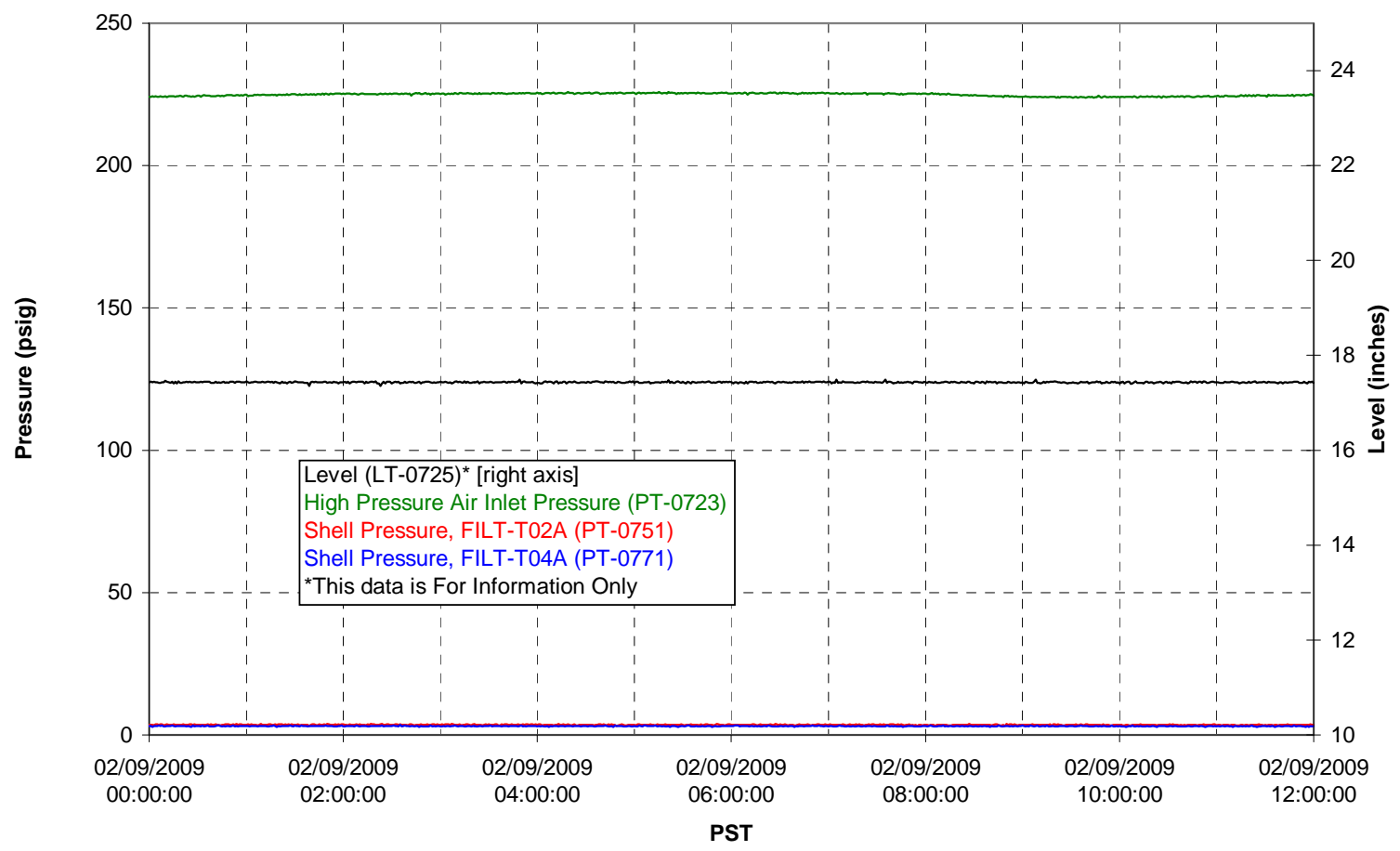


Pulsepot UFP-PP-T03A

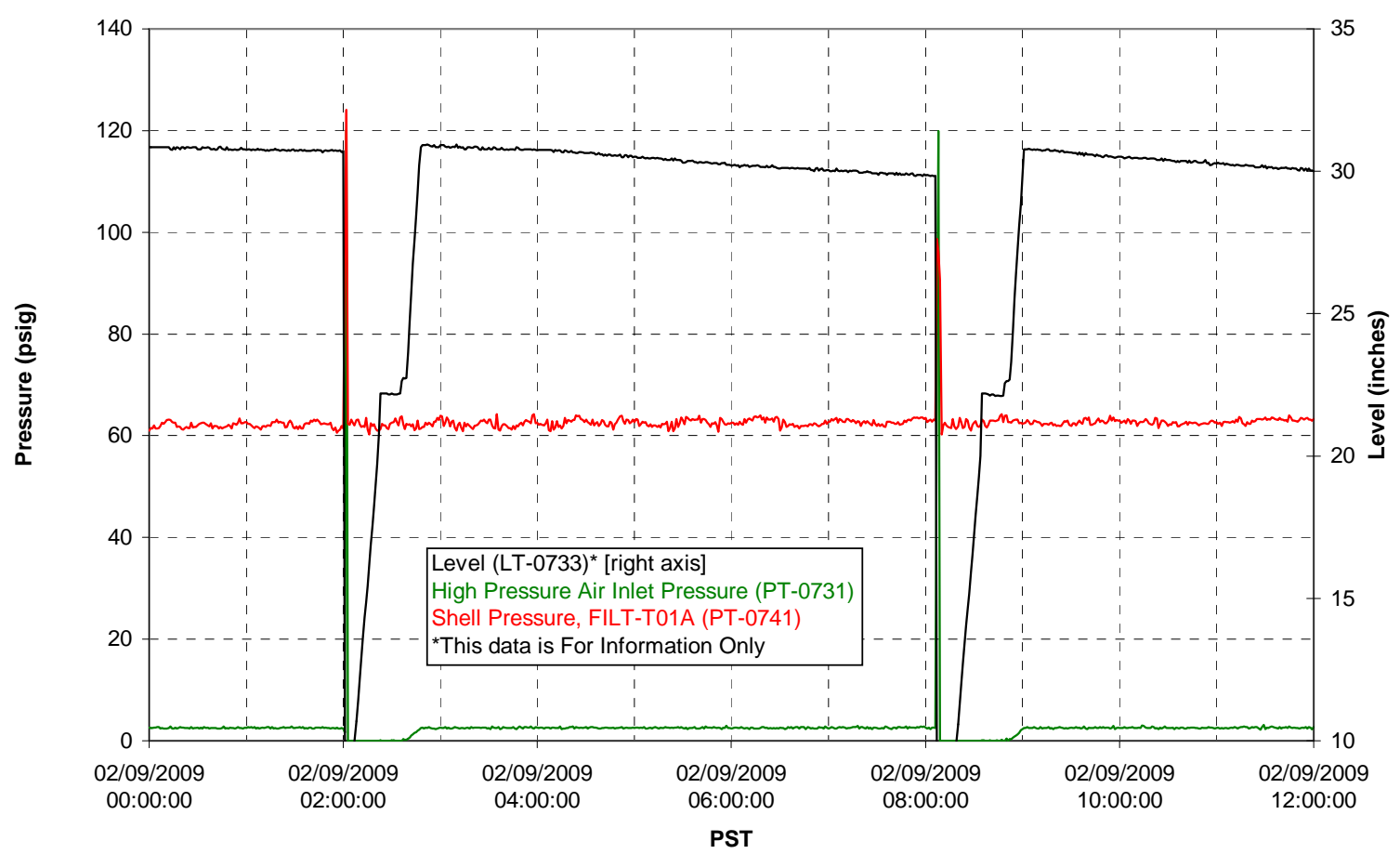

Pulsepot Levels

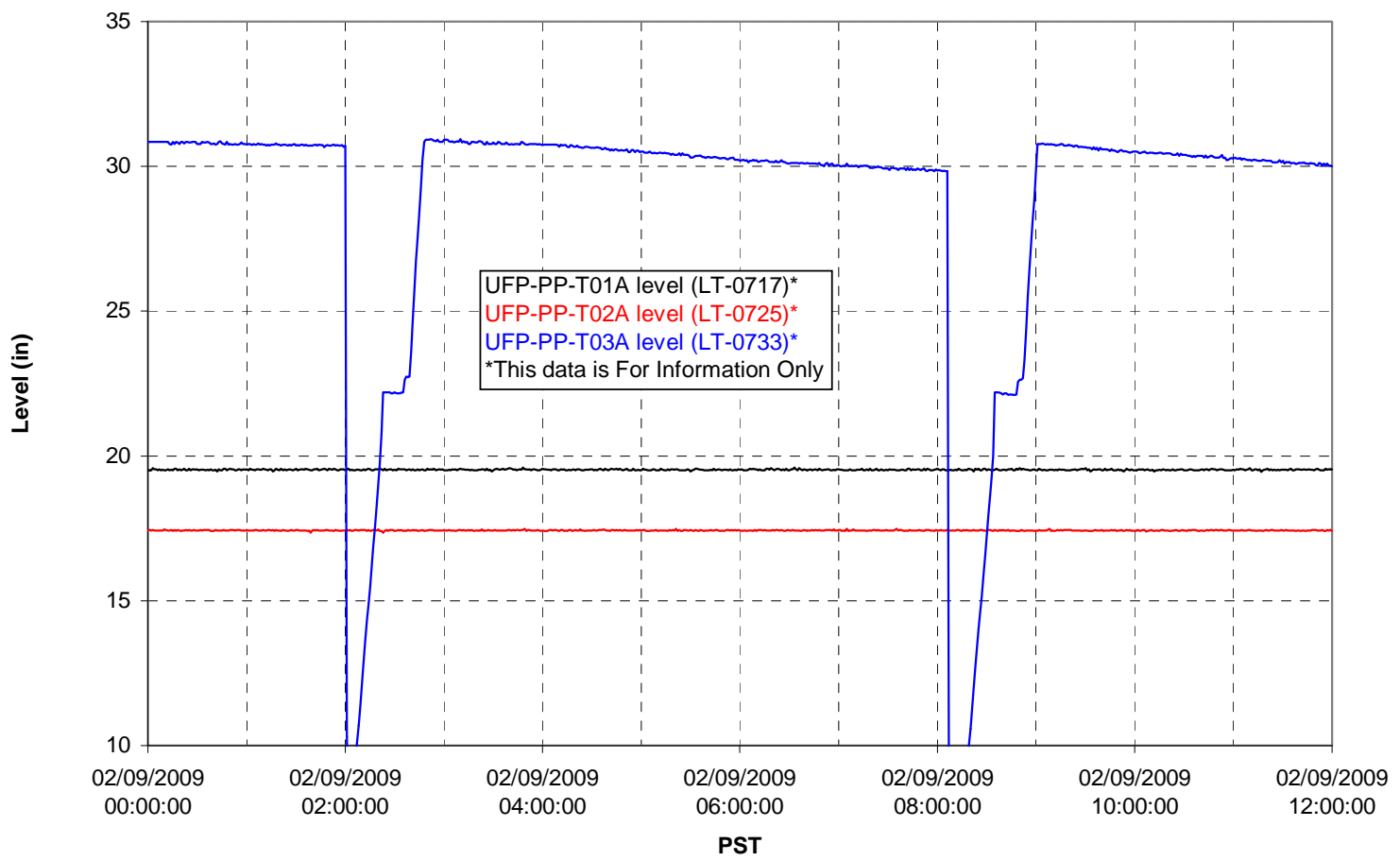


Filter UFP-FILT-T01A

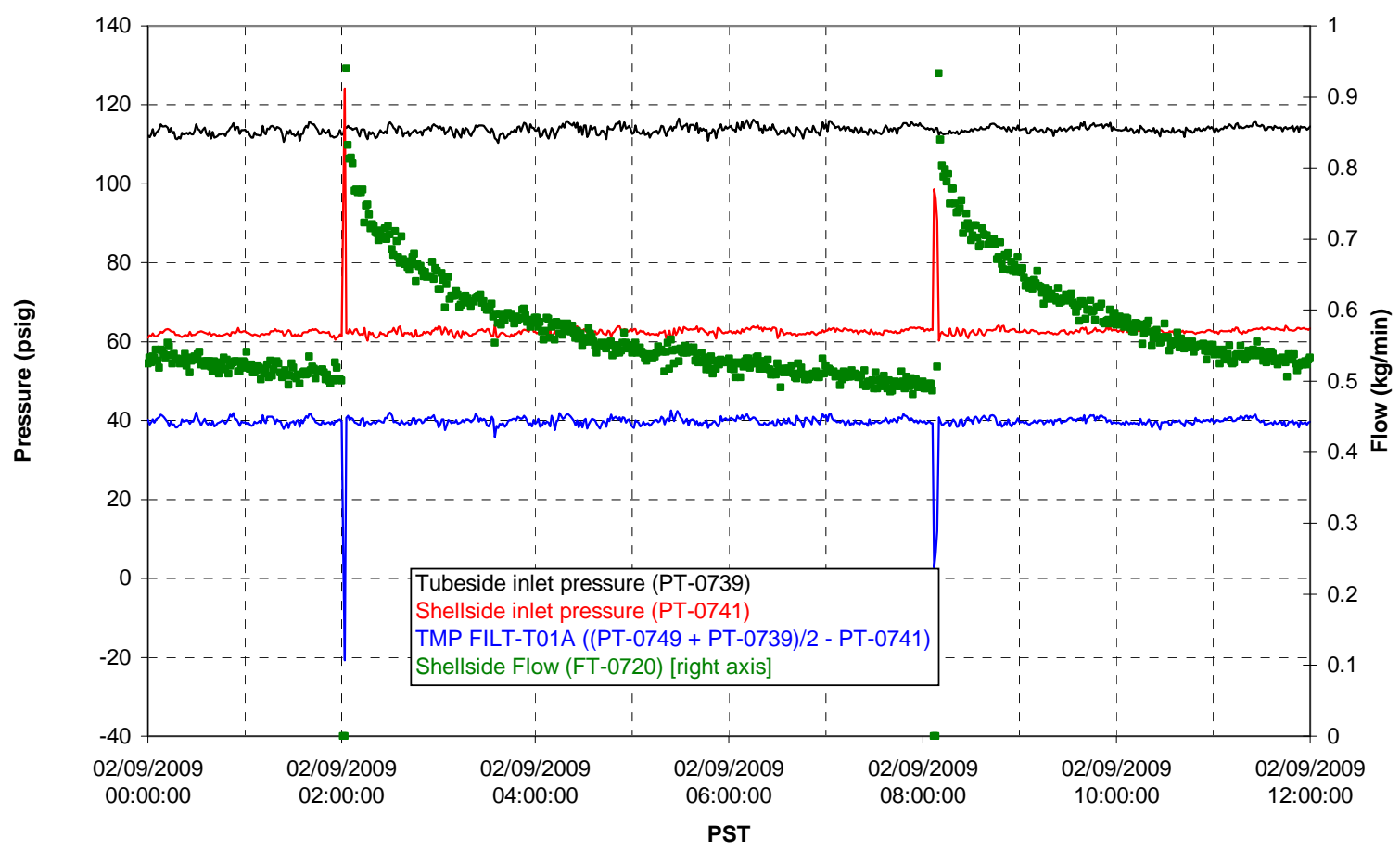

Filter UFP-FILT-T02A

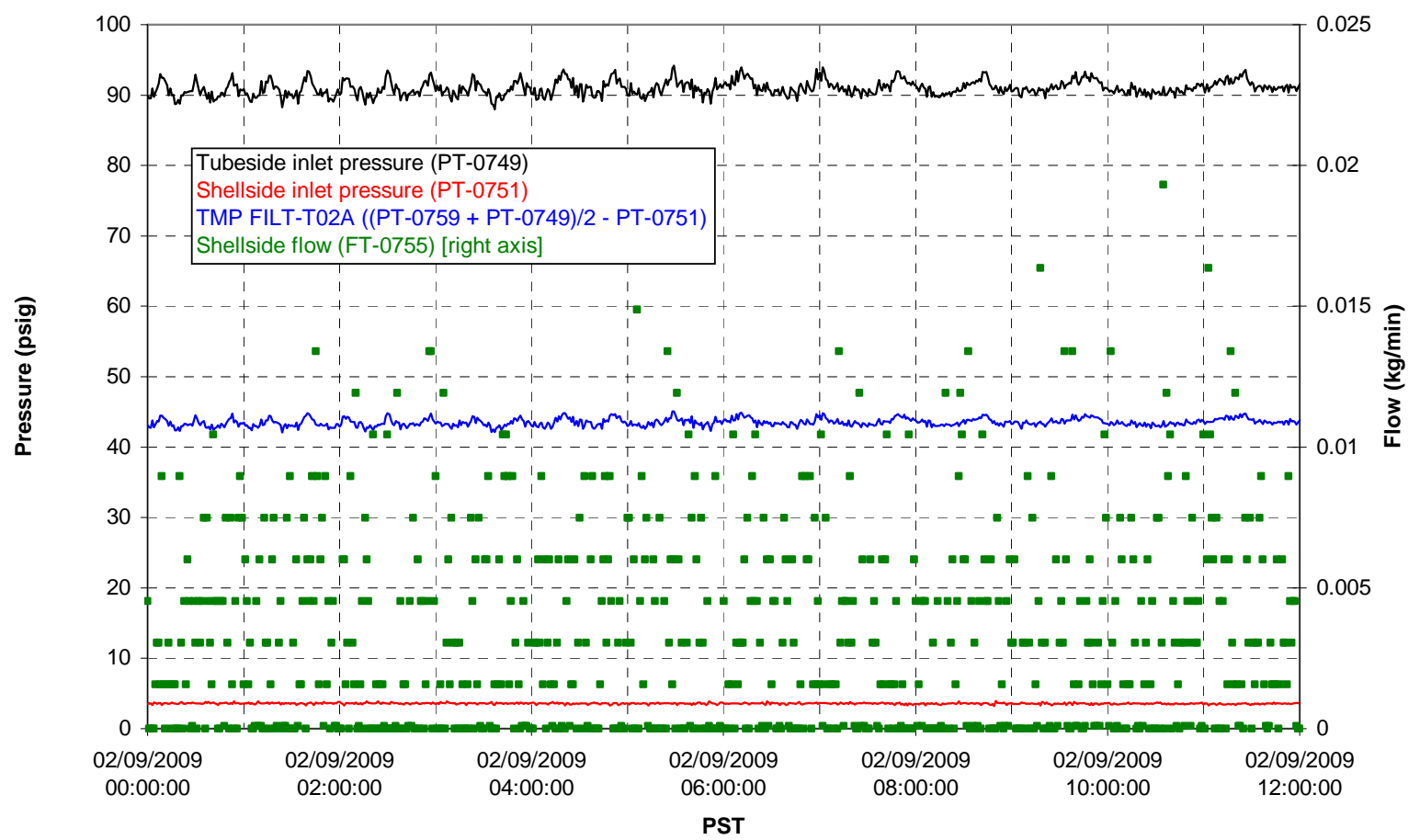


Filter UFP-FILT-T03A

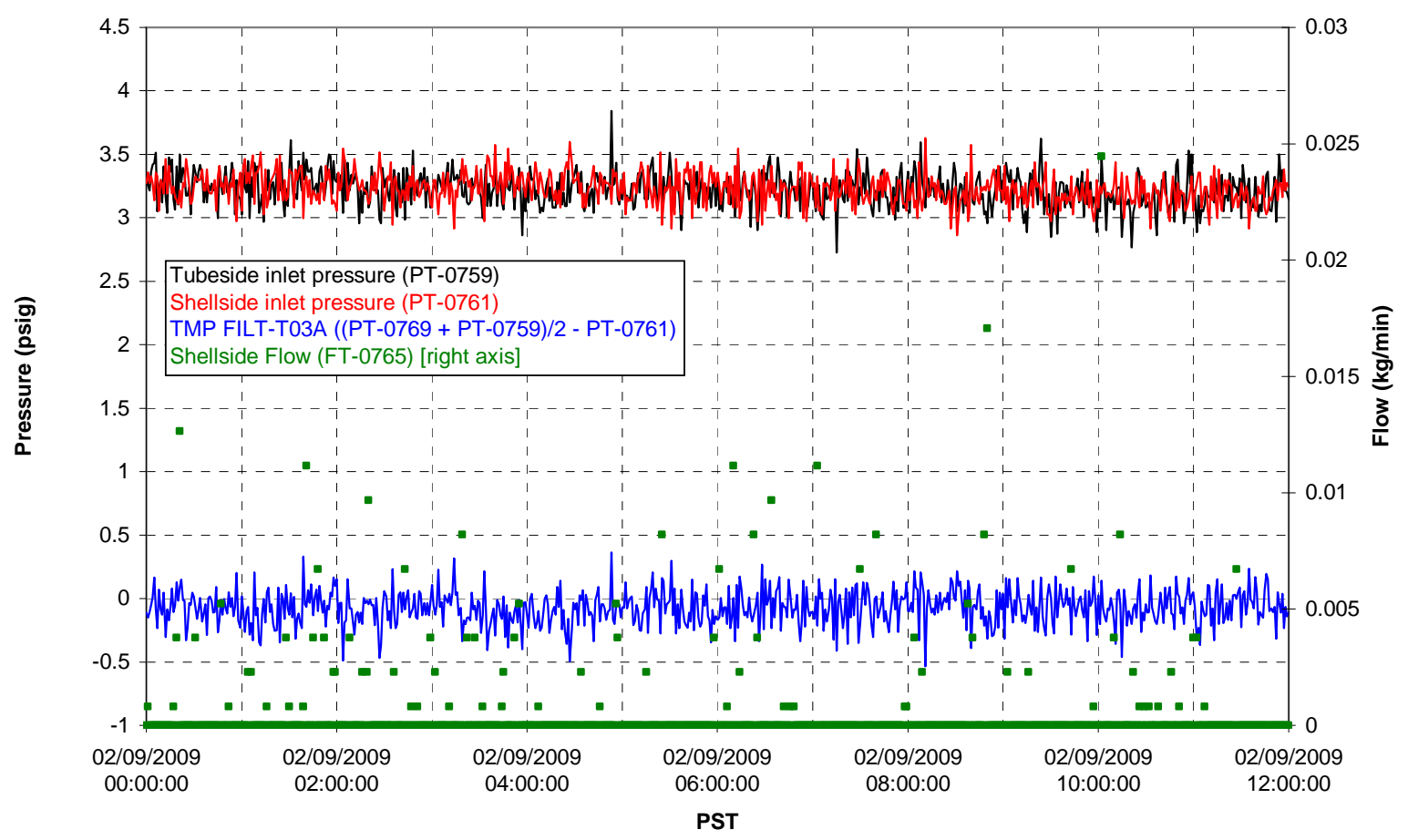

Filter UFP-FILT-T04A

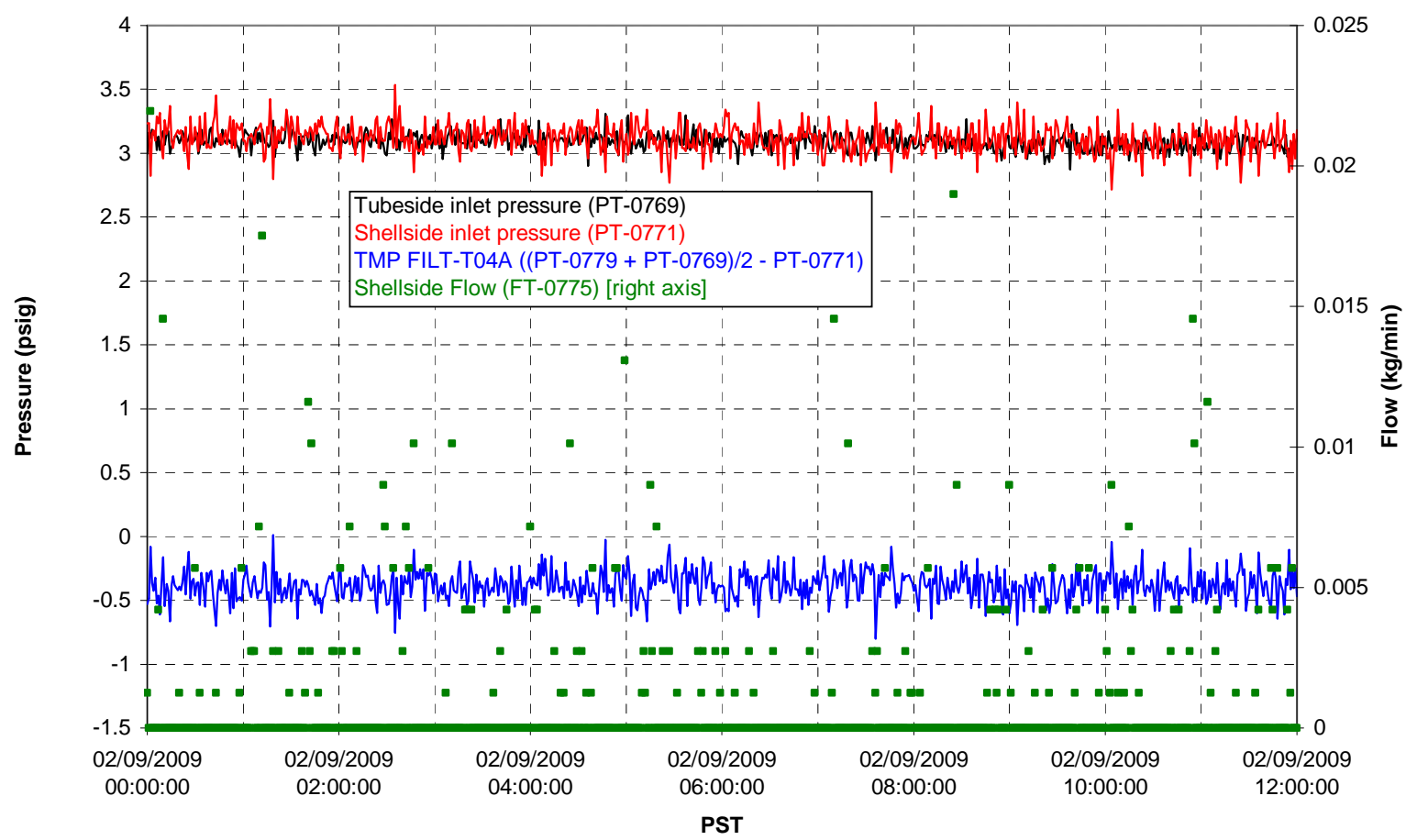


Filter UFP-FILT-T05A

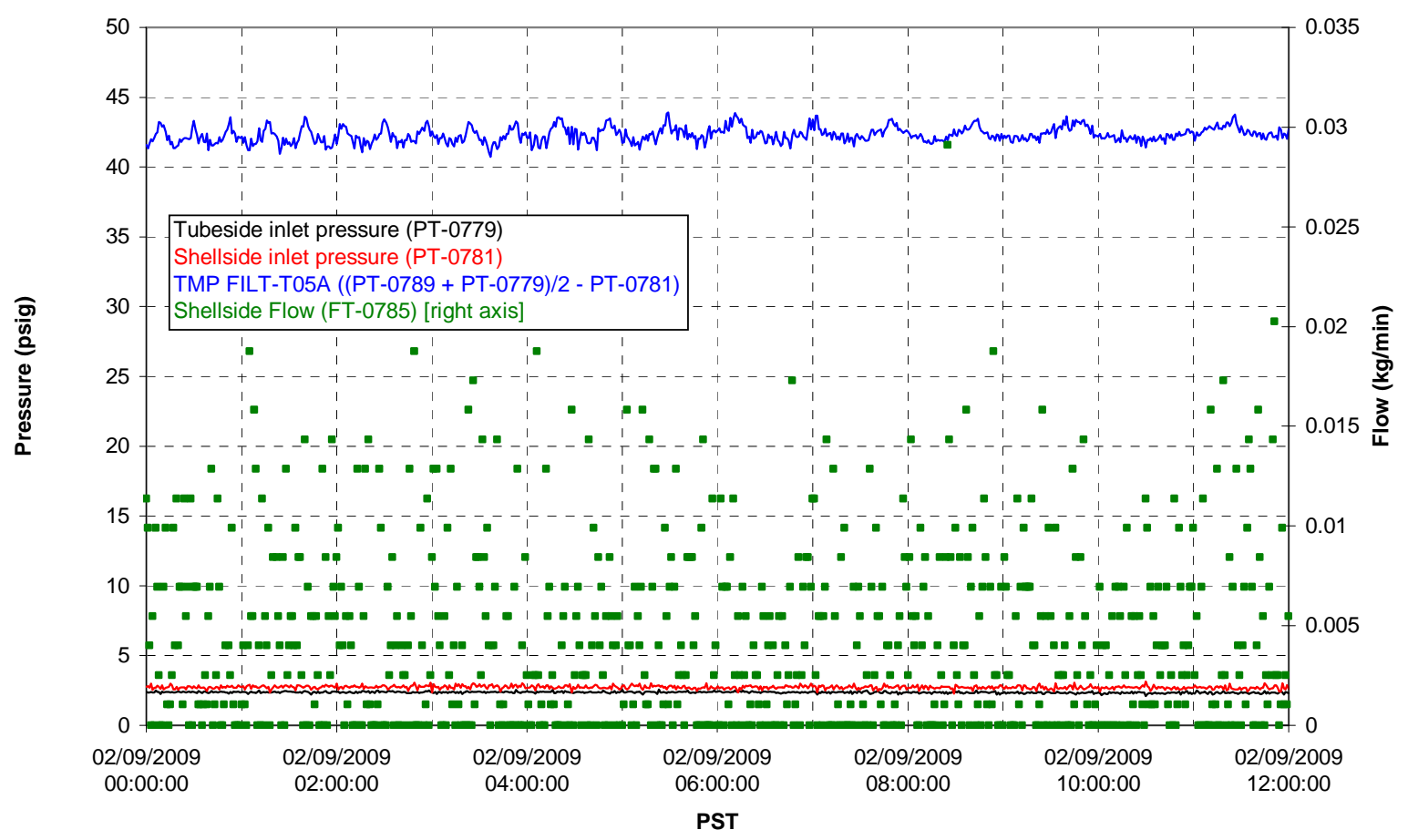

Chemical Flow

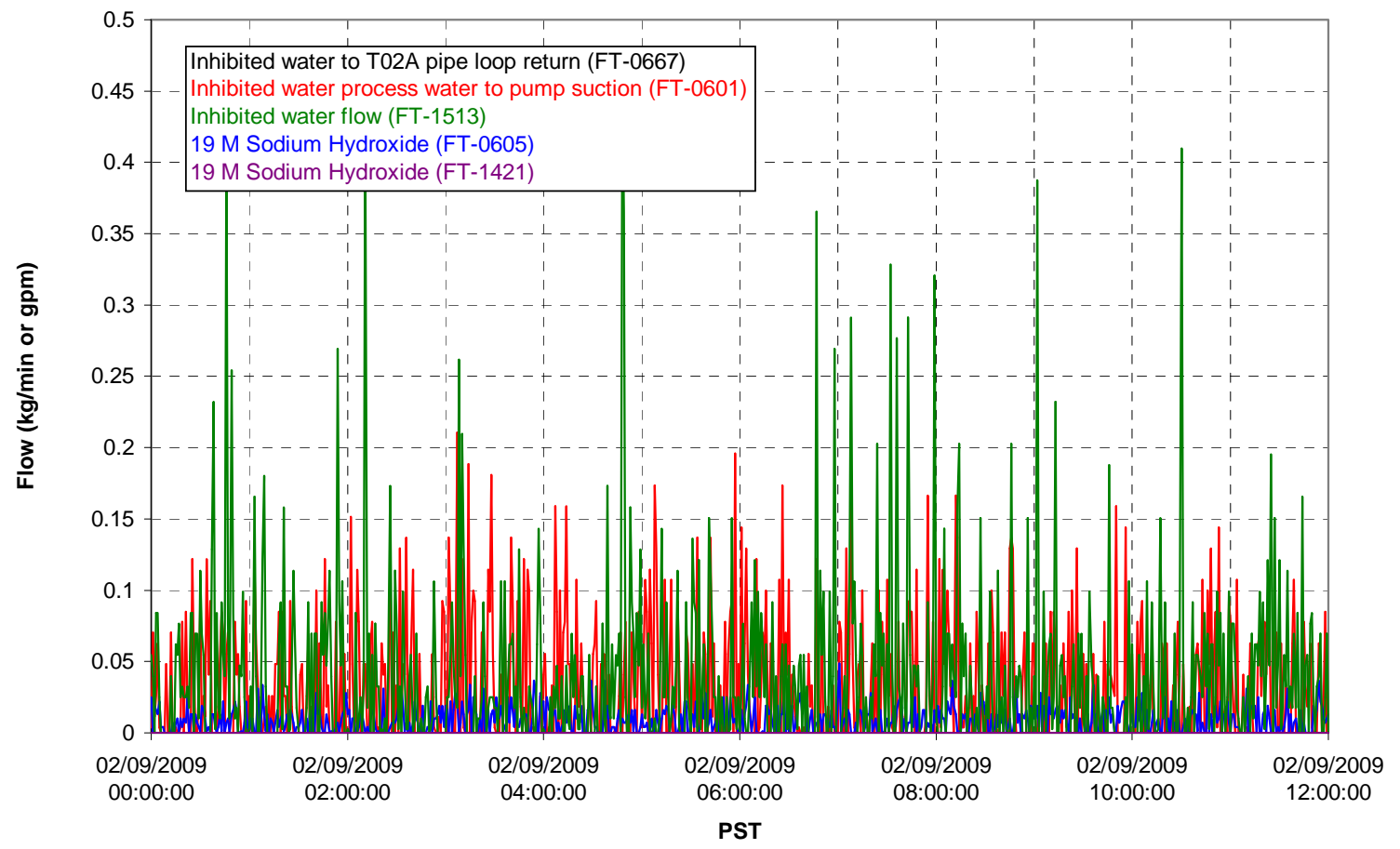


Chemical Flow

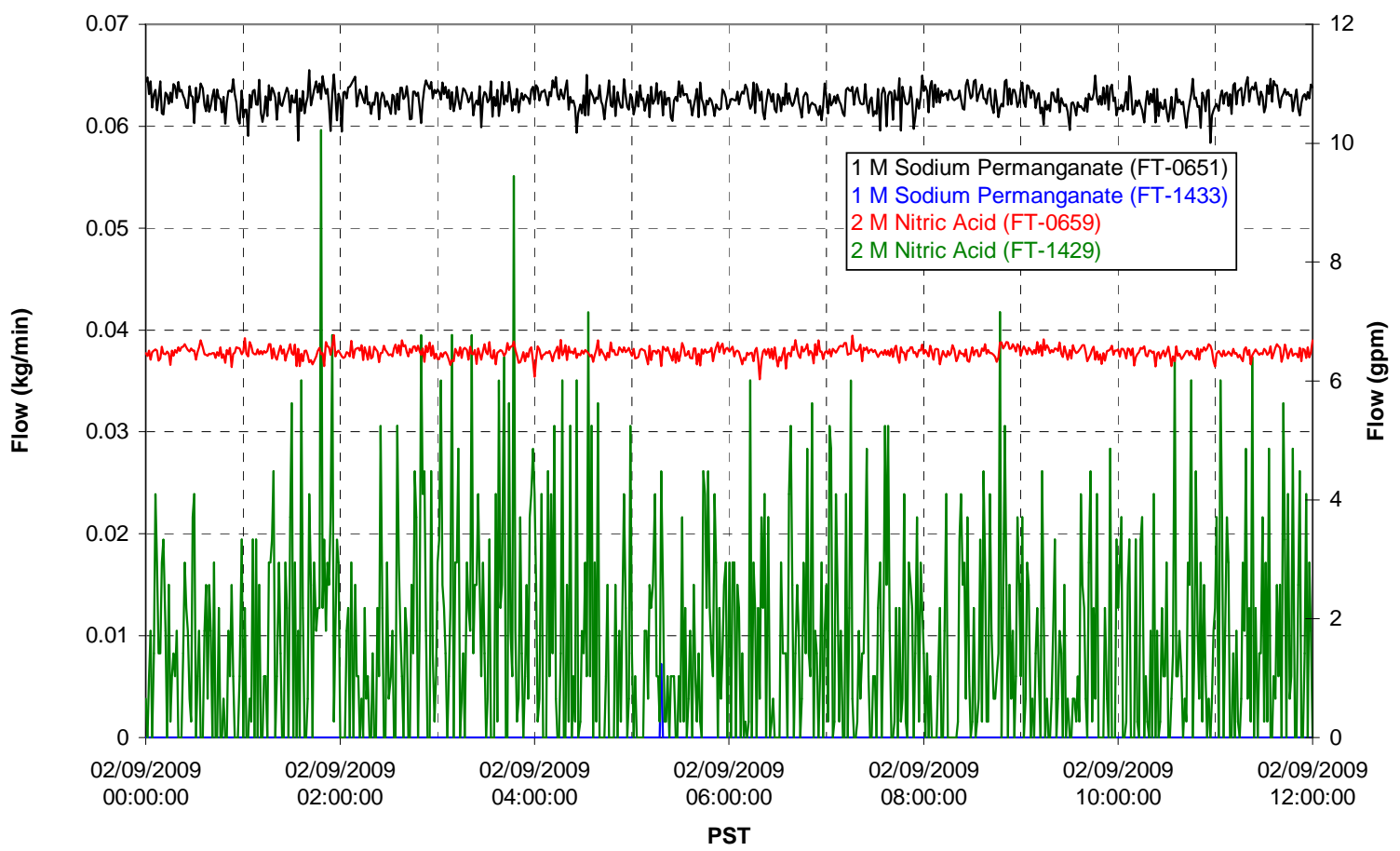

Air Flows

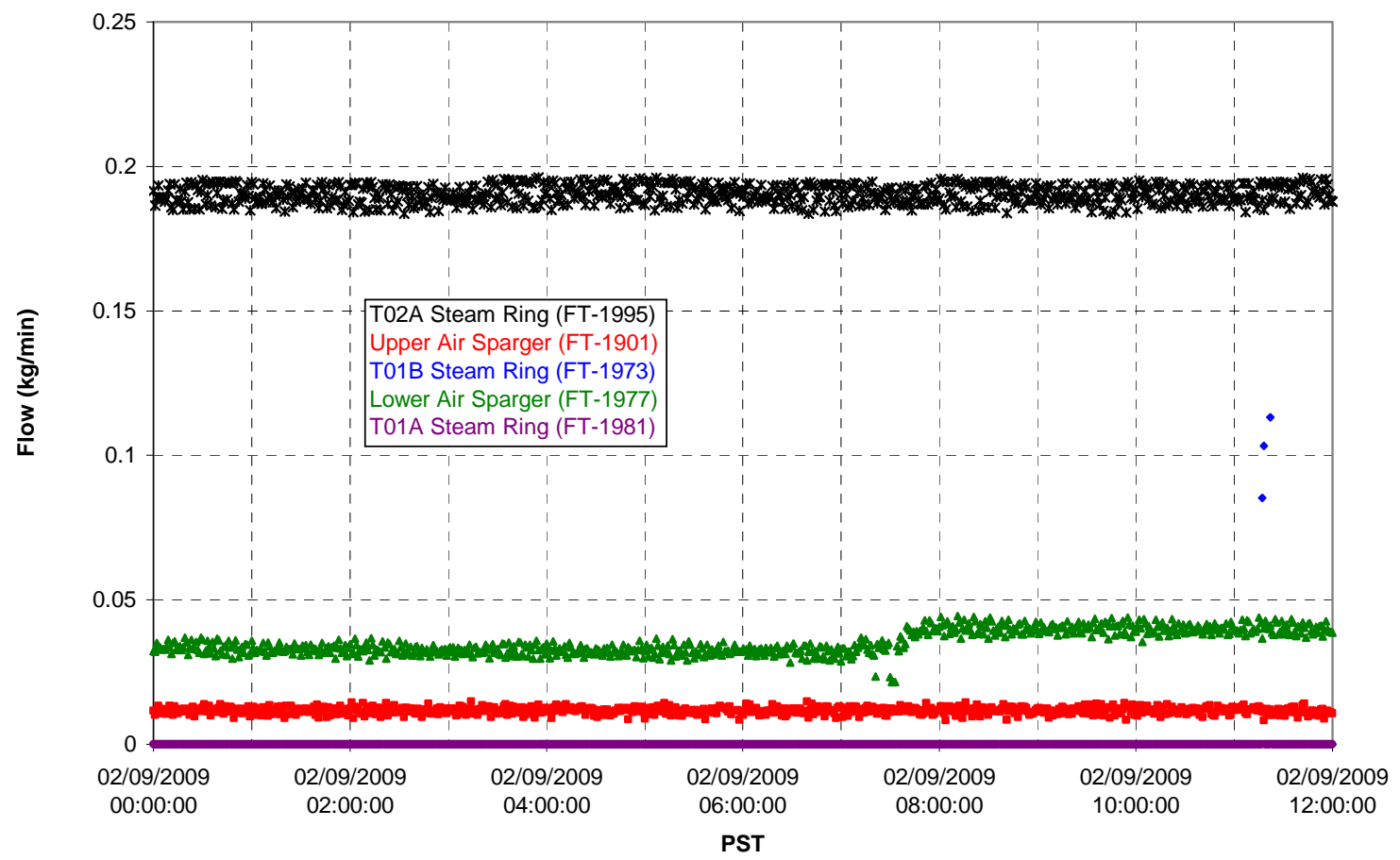

G.340 
T02A Steam

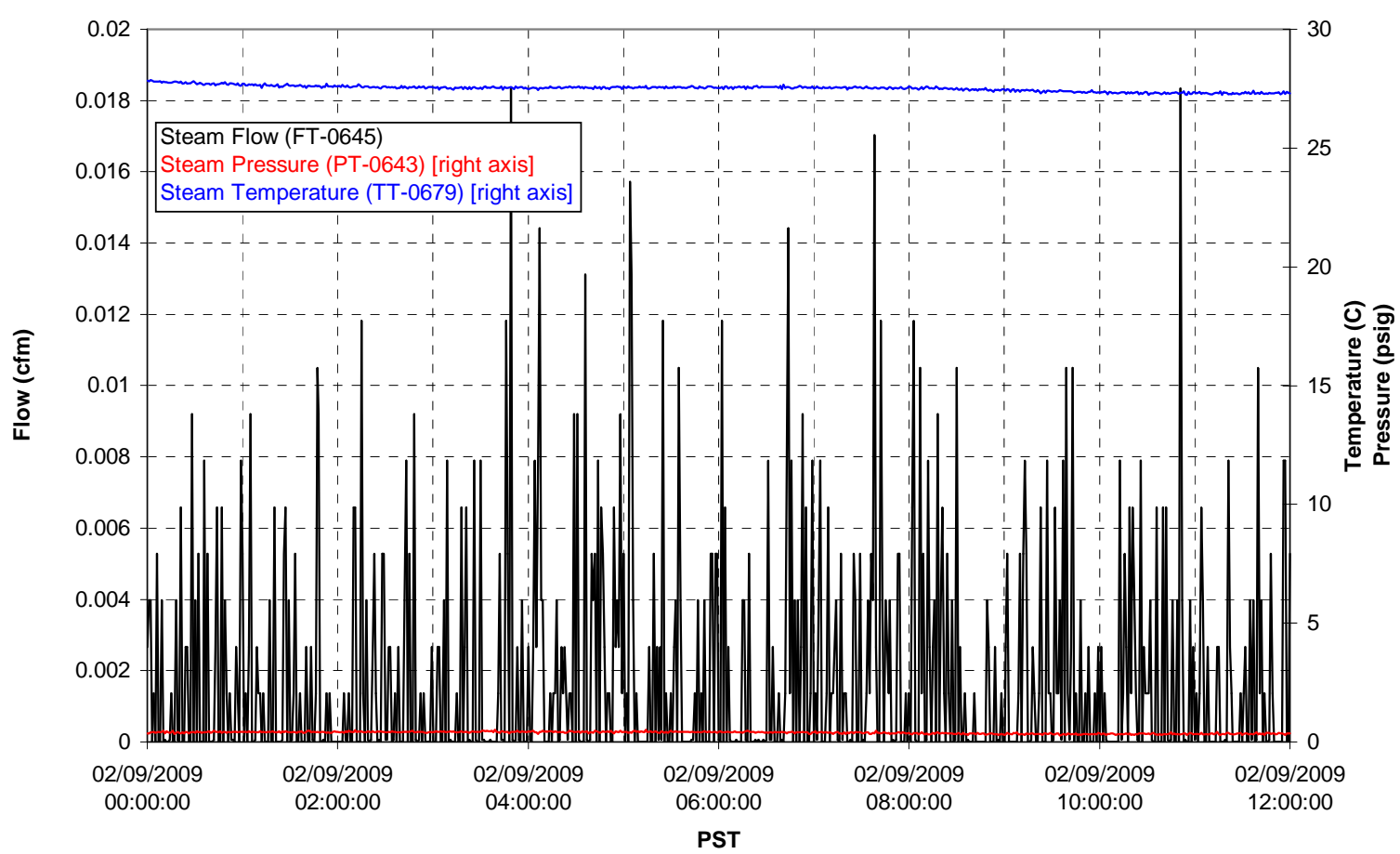

T01A Steam

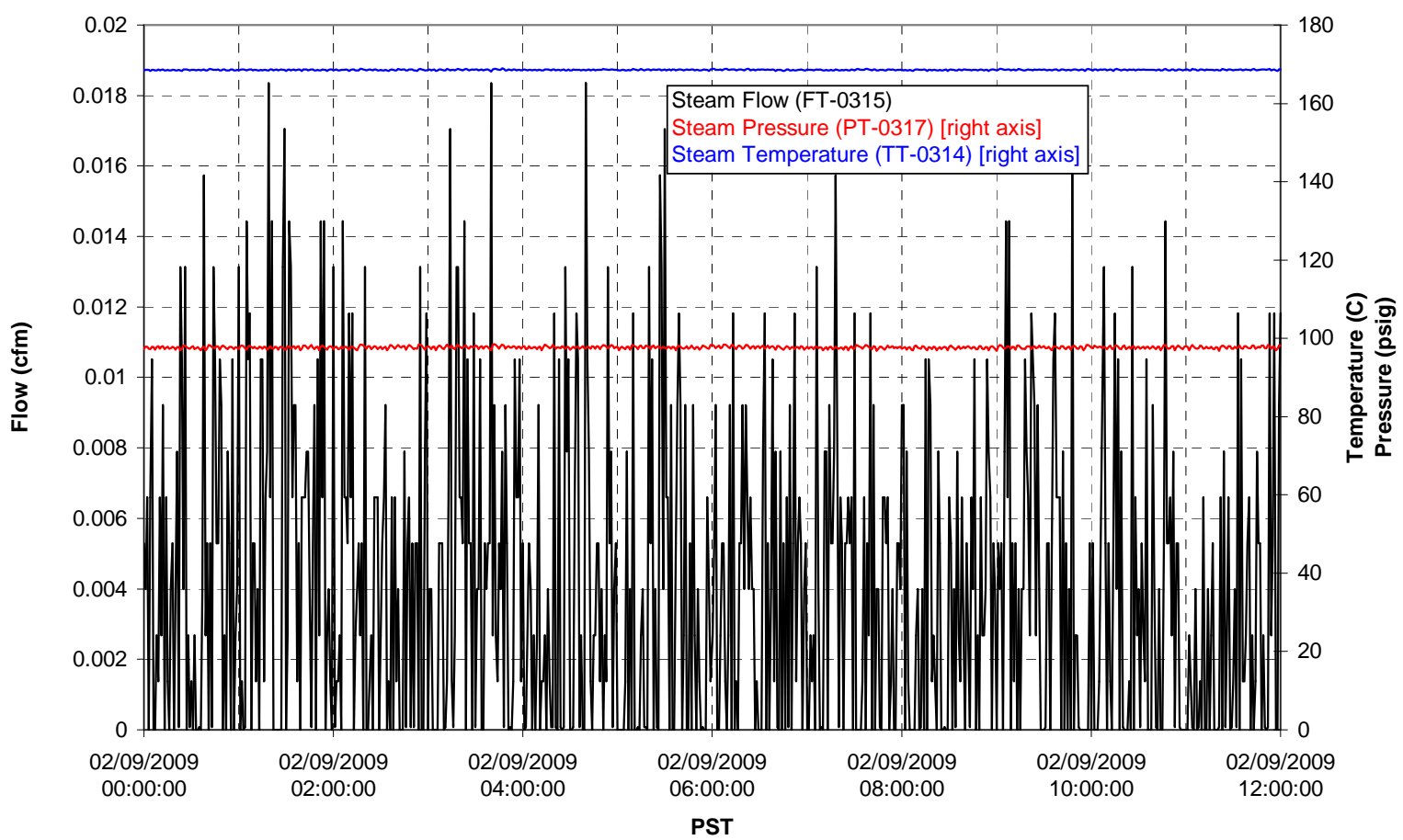


T01B Steam

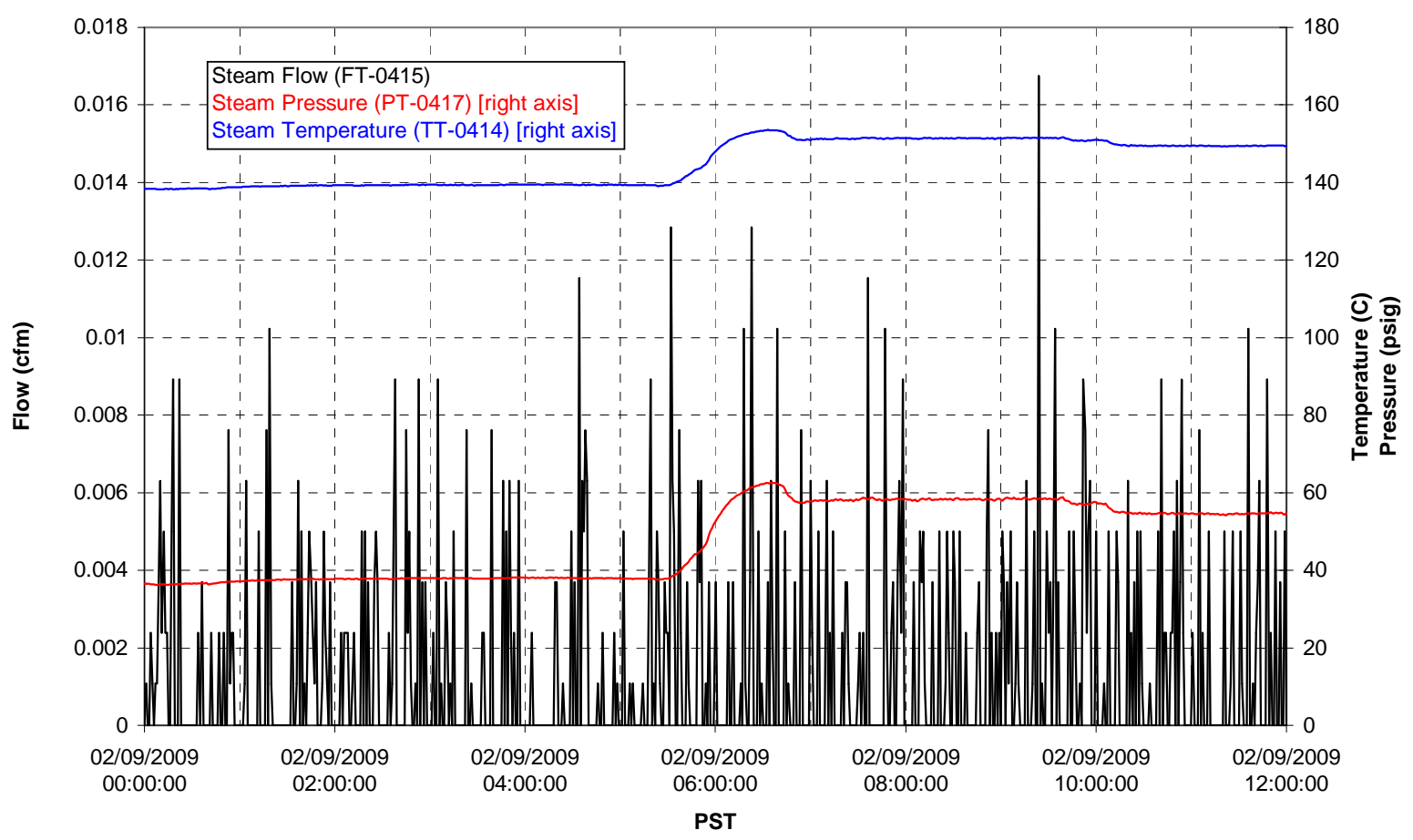




\section{Integrated Test A Data Plots 02/09/09 12:00 - 02/10/09 00:00}


T01A level

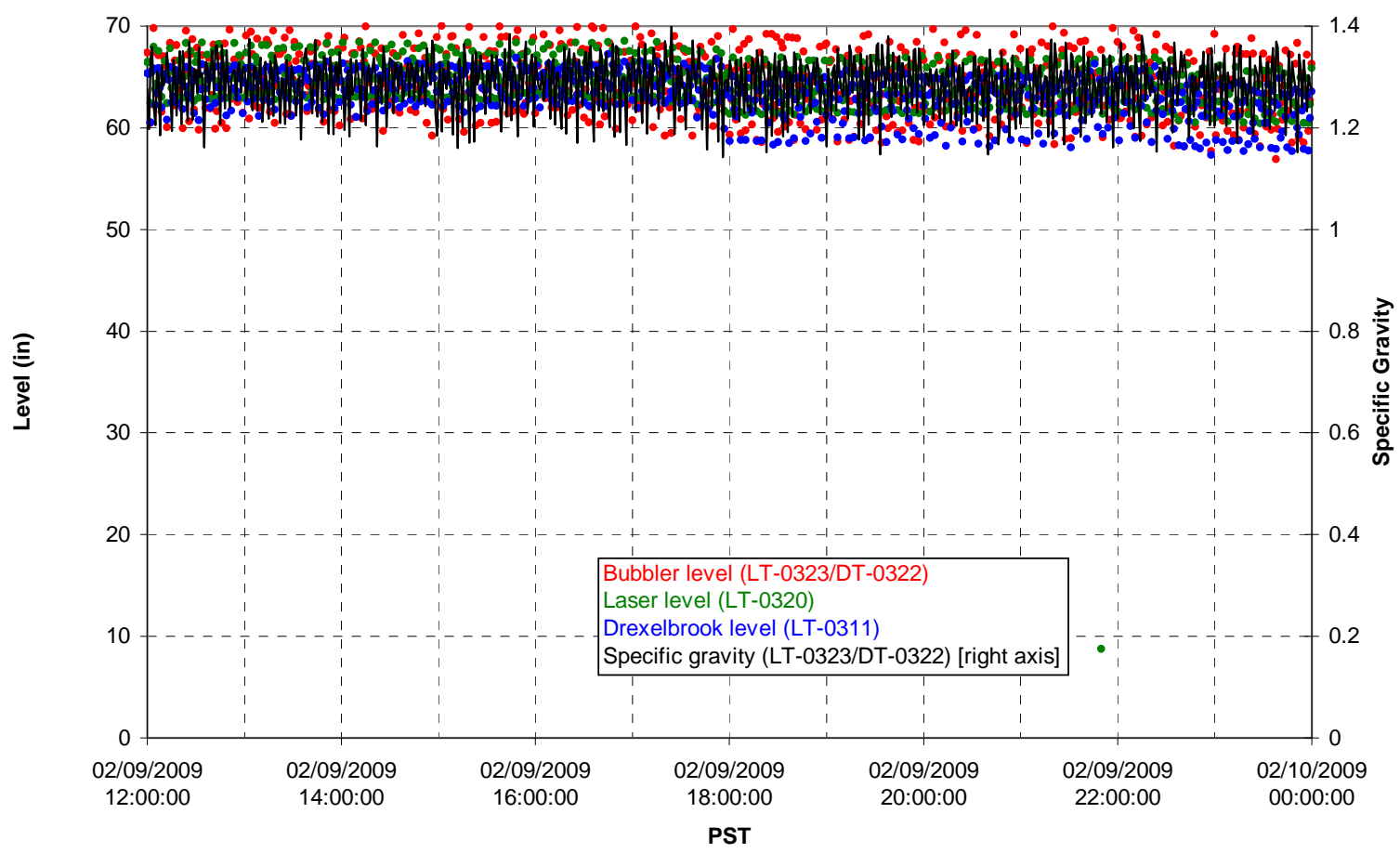

T01A temperatures

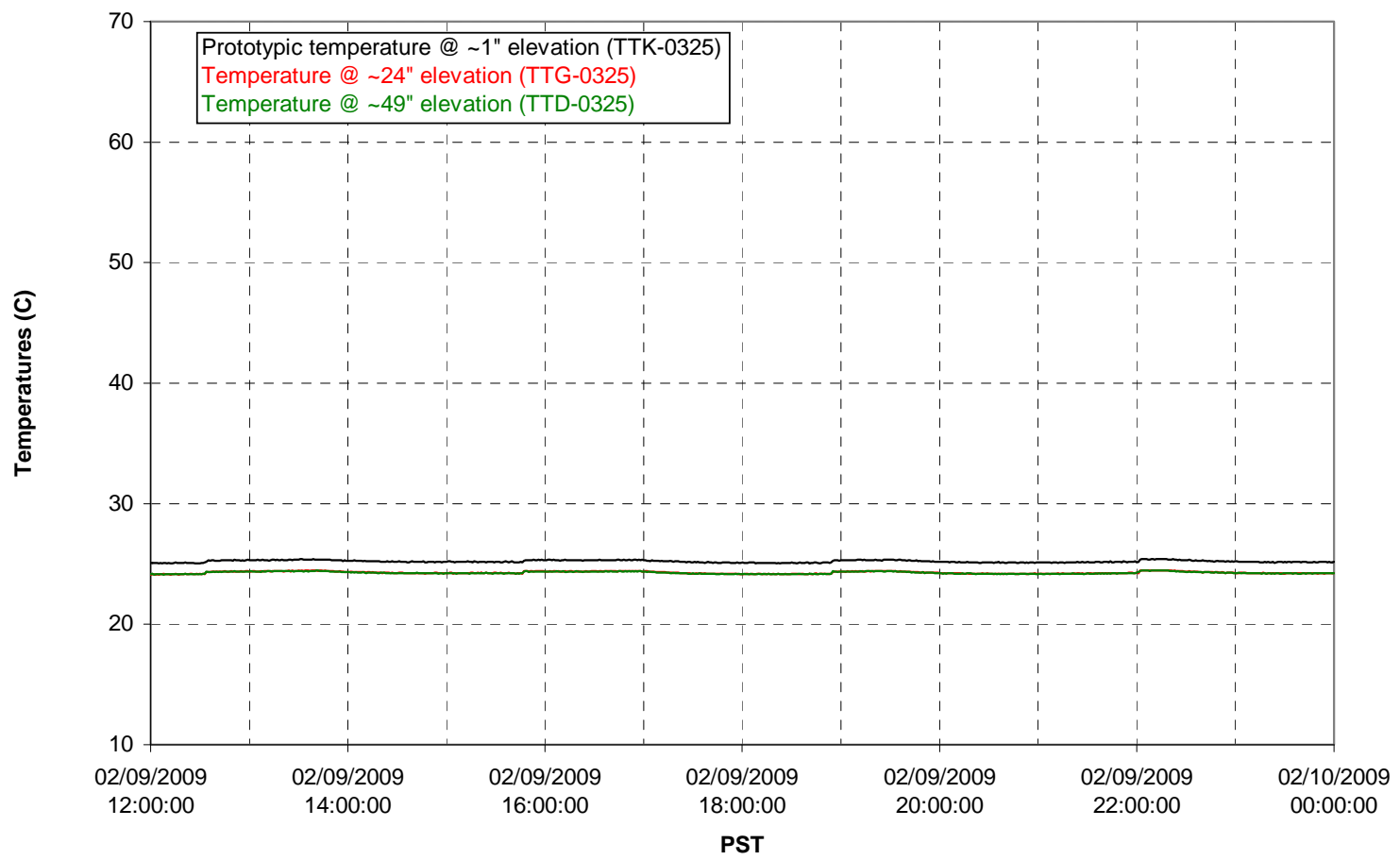


T01B level

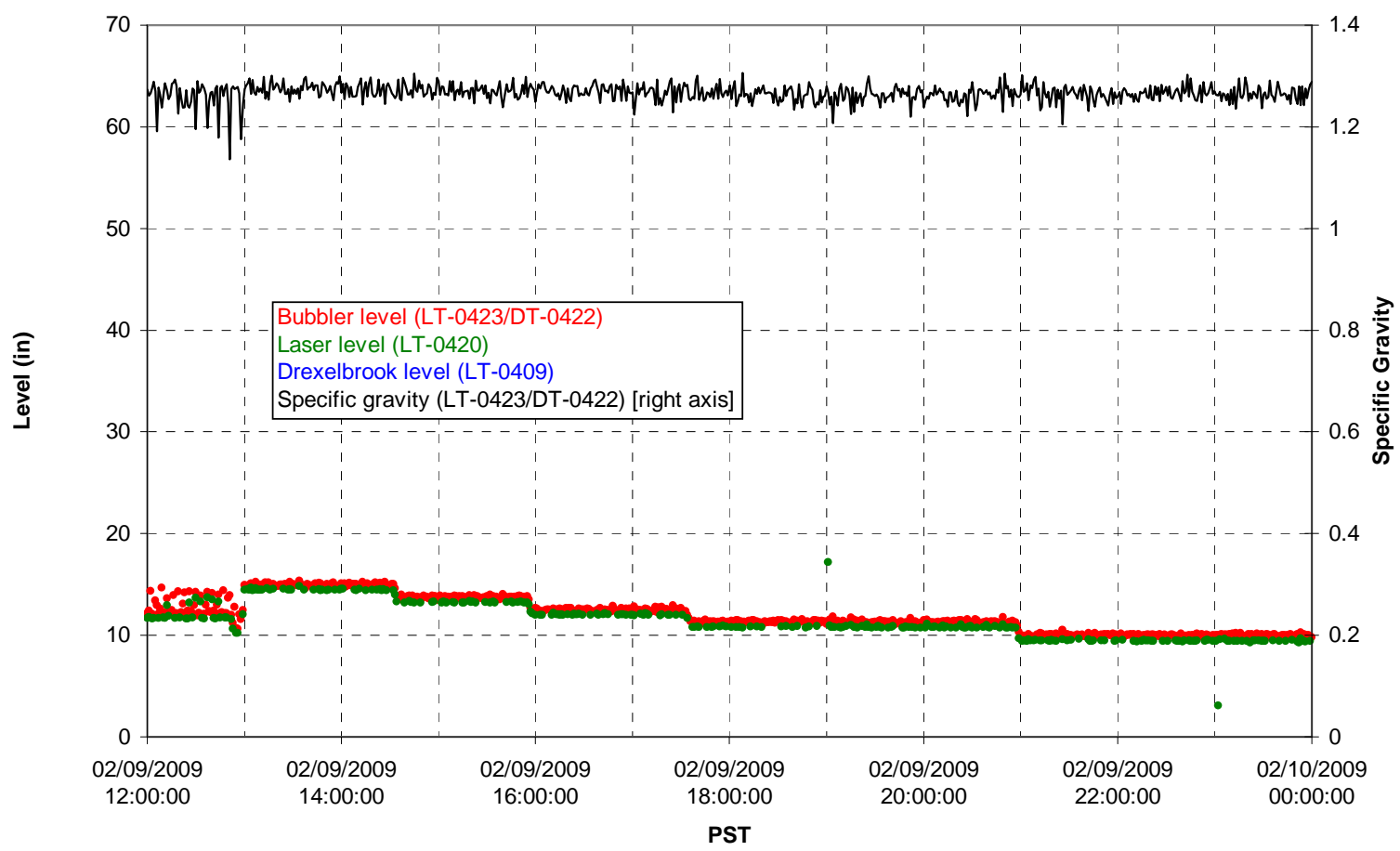

T01B temperatures

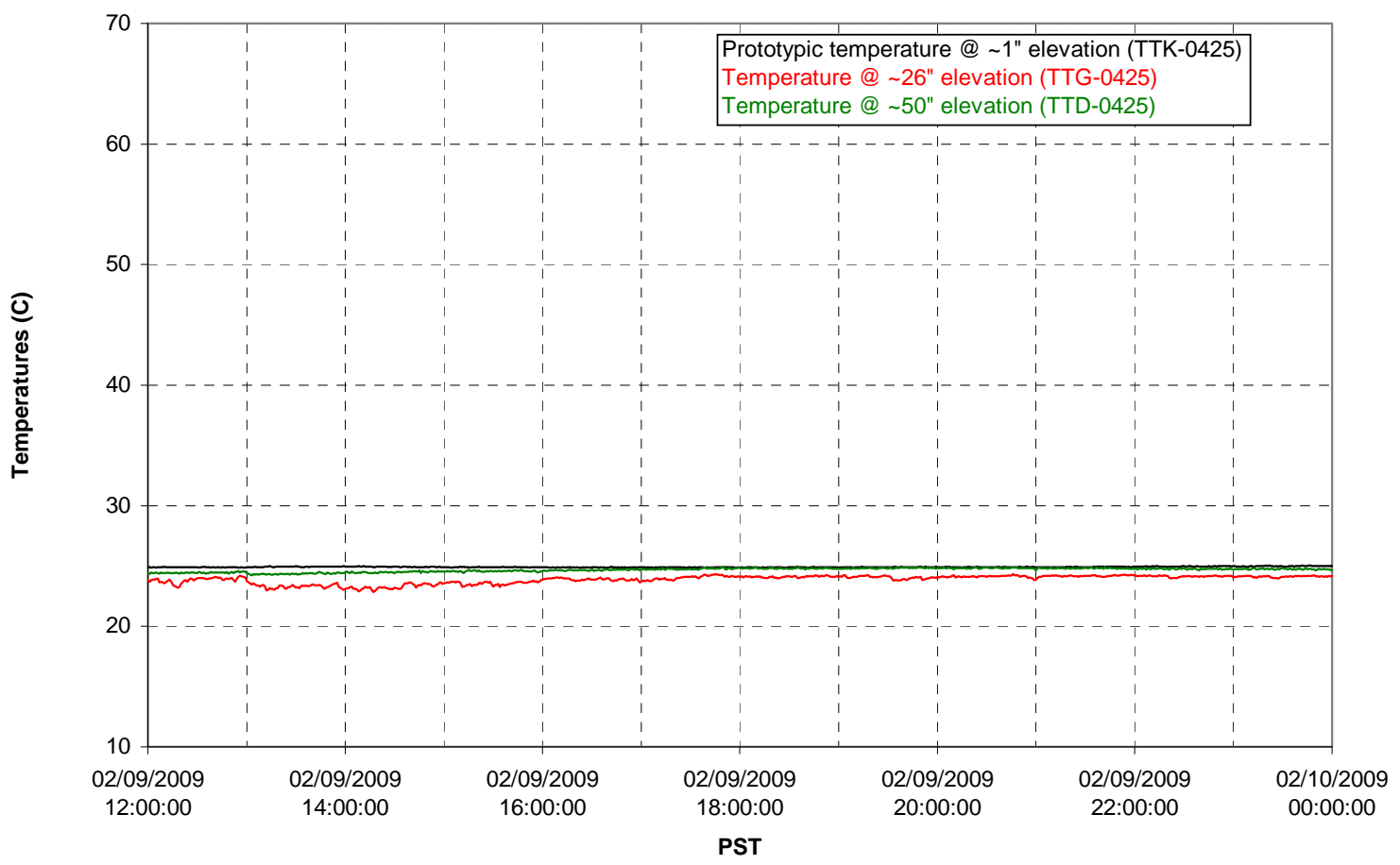


T02A level

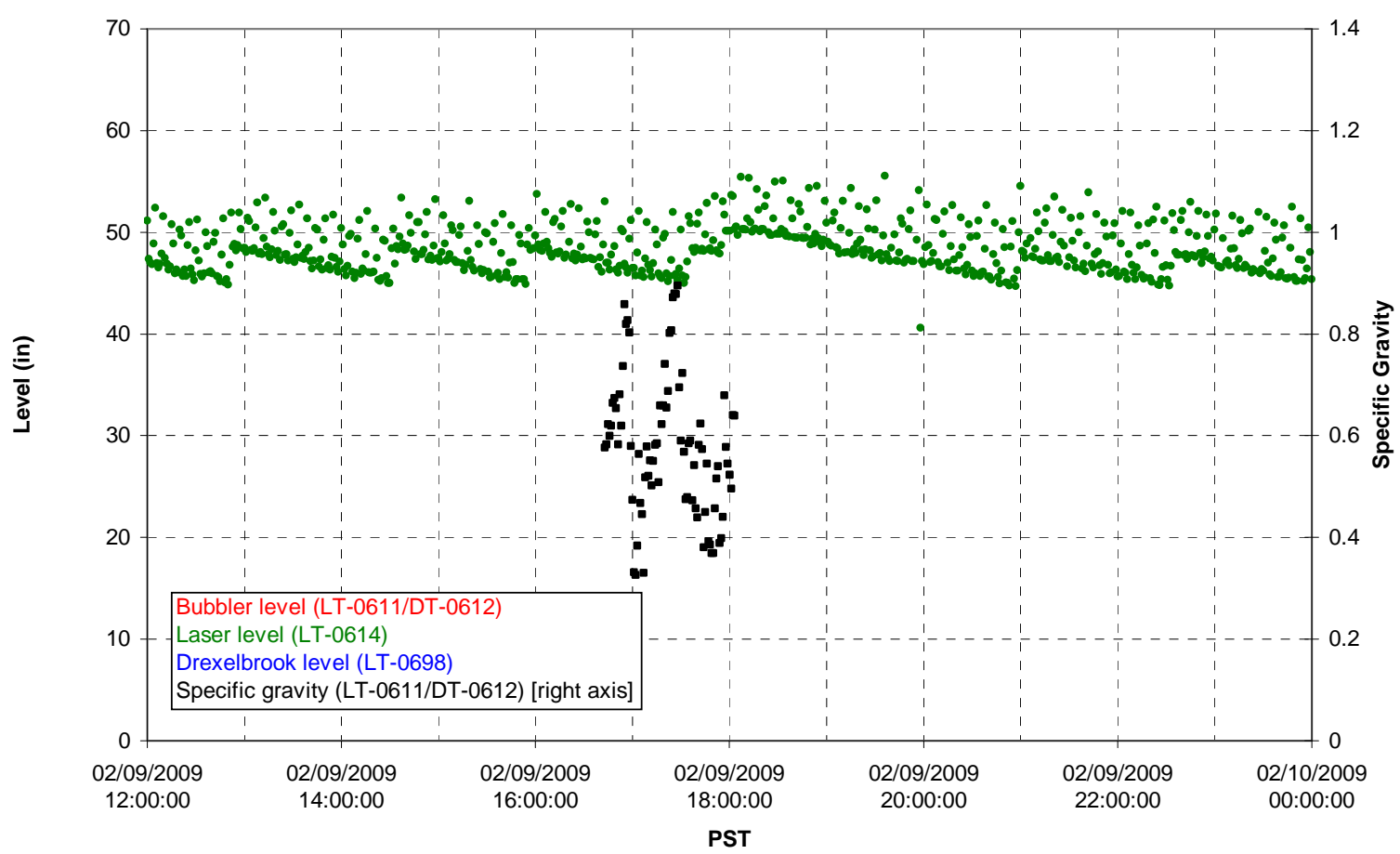

T02A temperatures

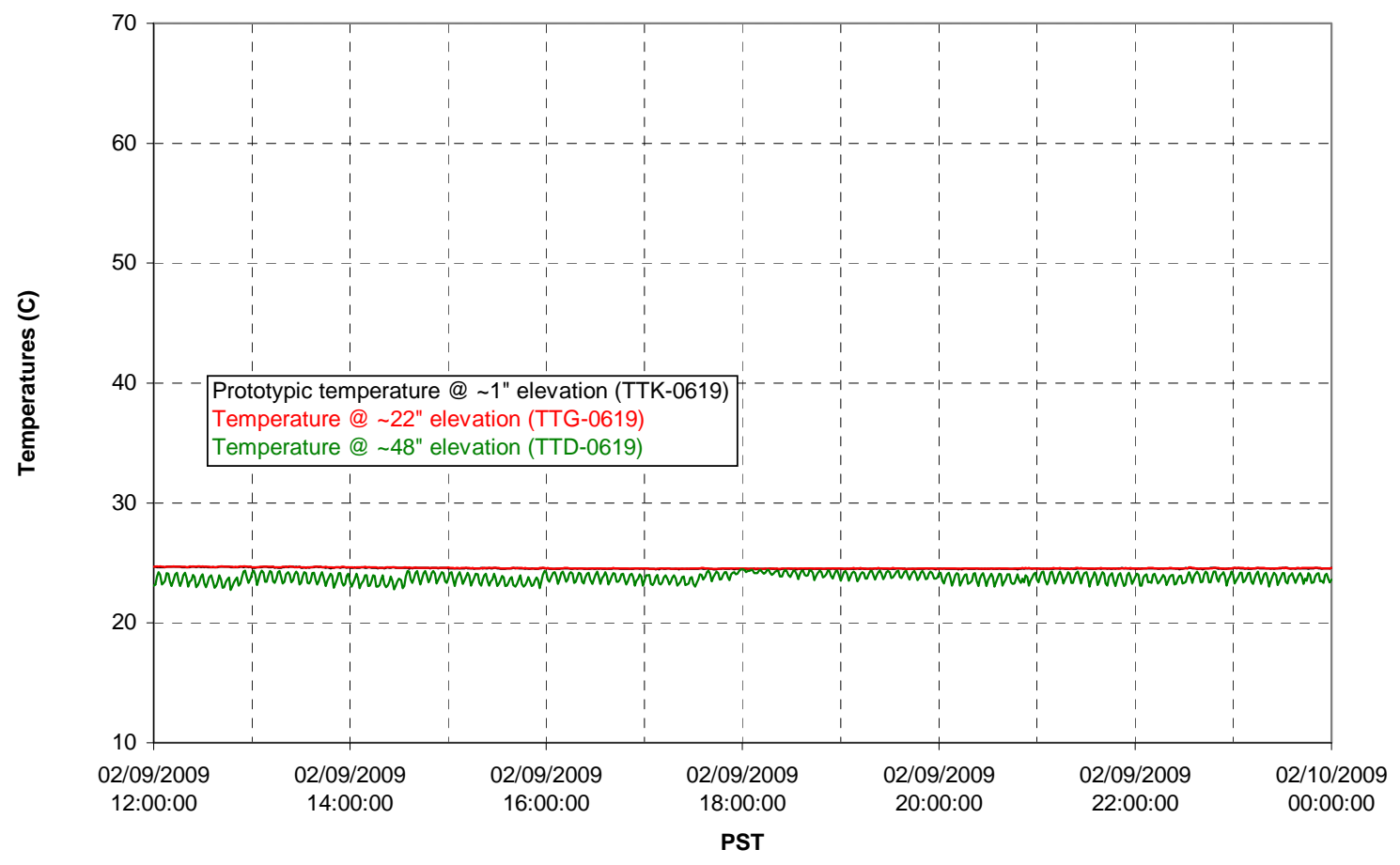


T02A and filter loop temperatures

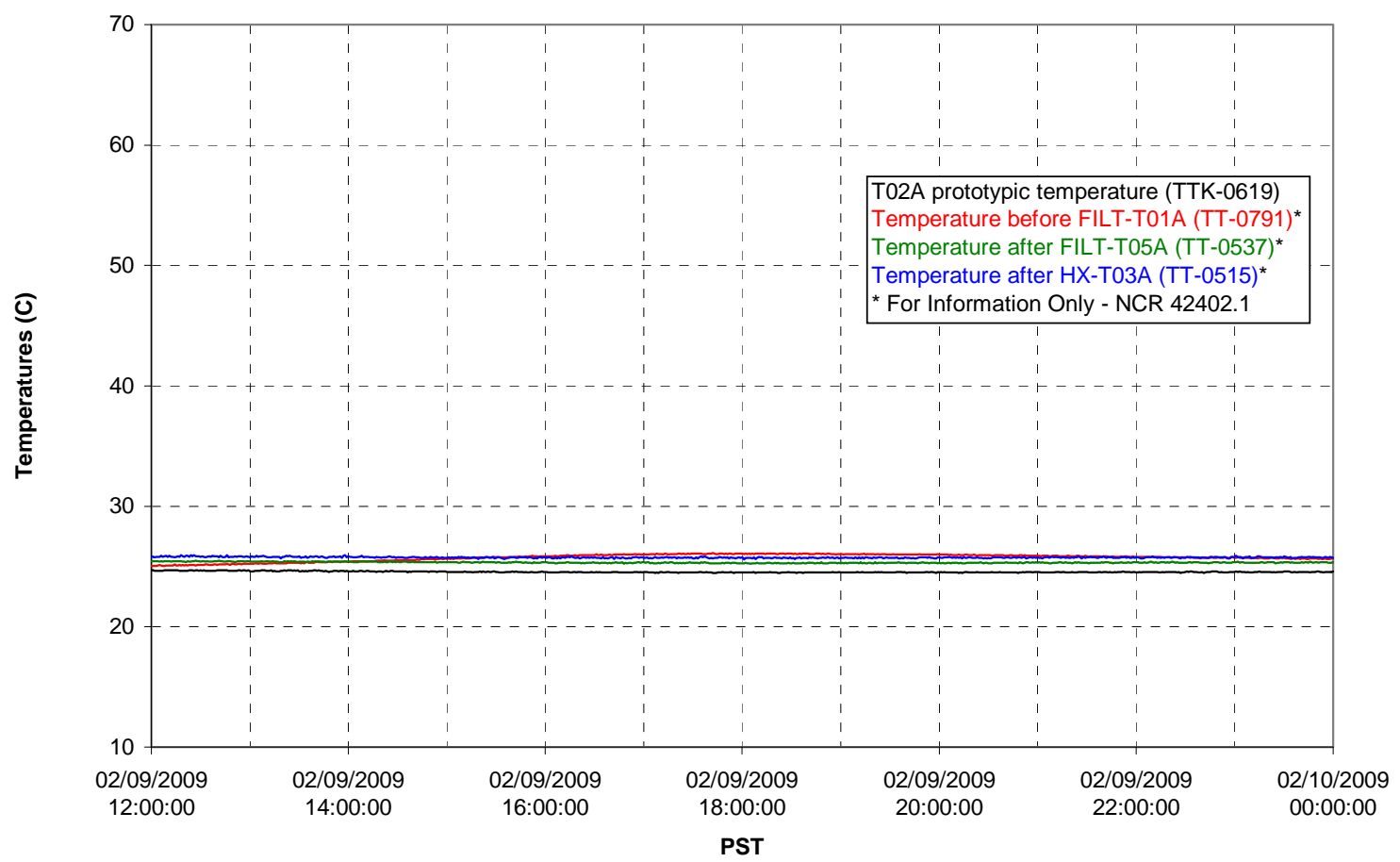

Pump Pressures and Flow

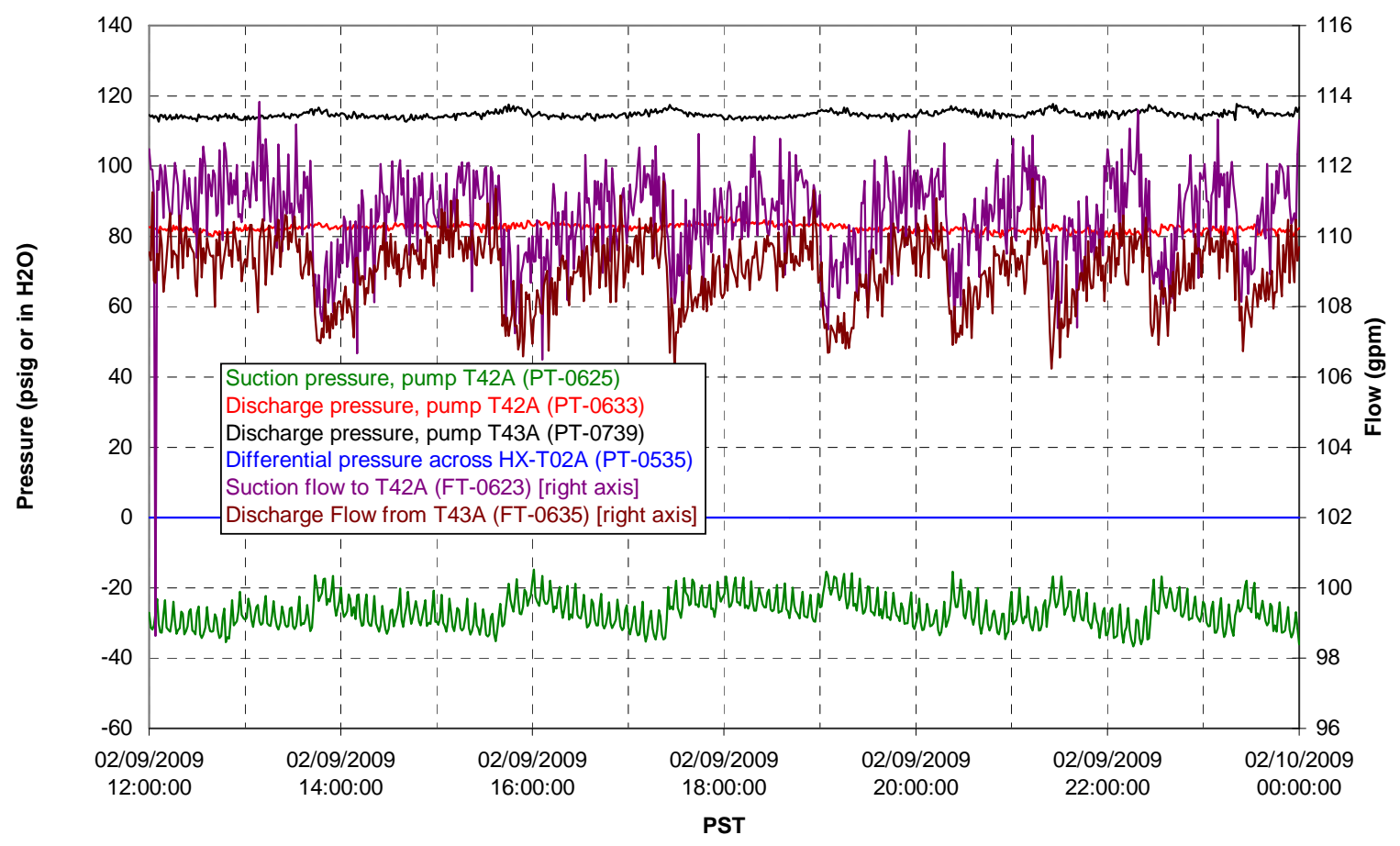


Axial pressure drop

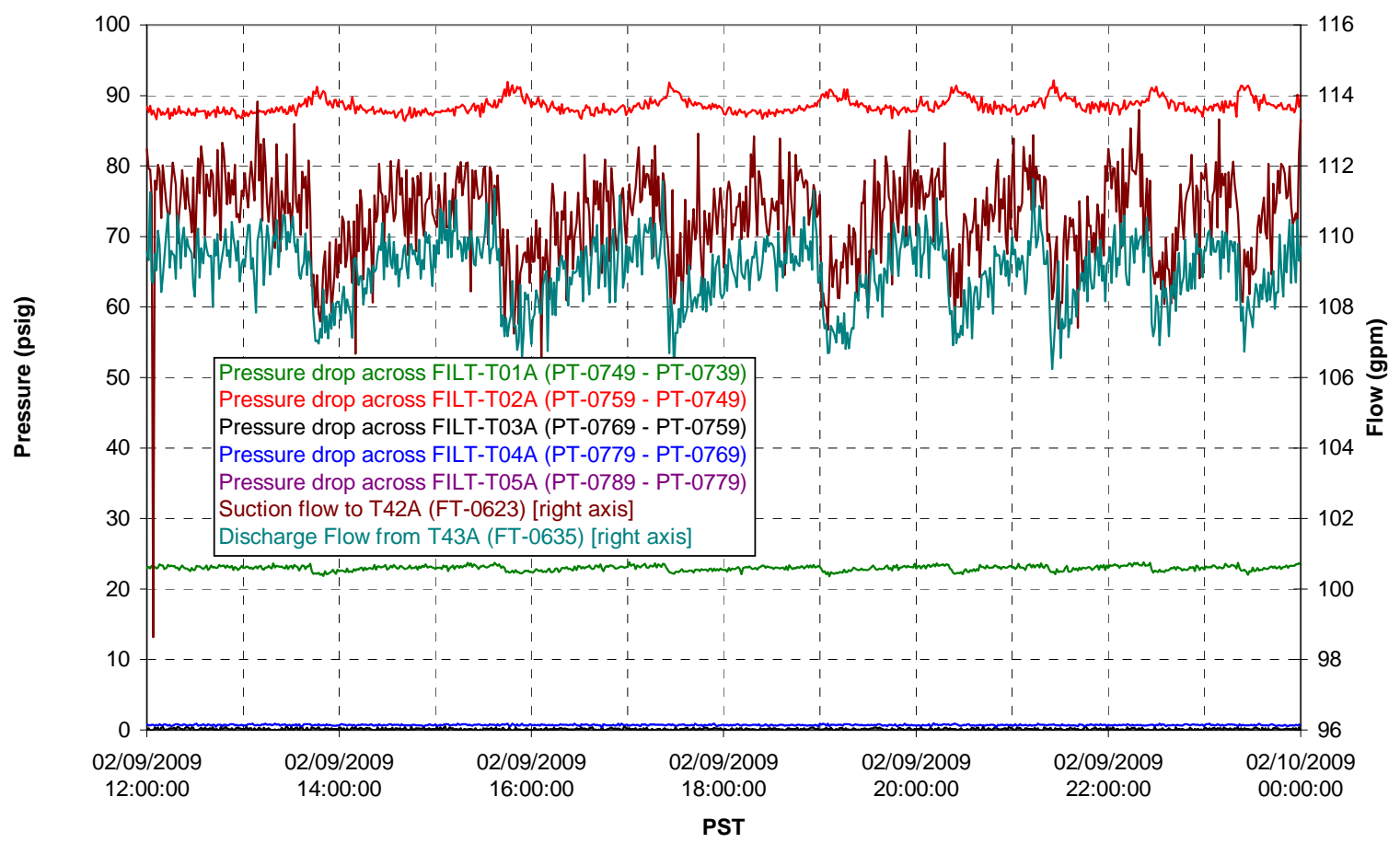

Permeate flow rates

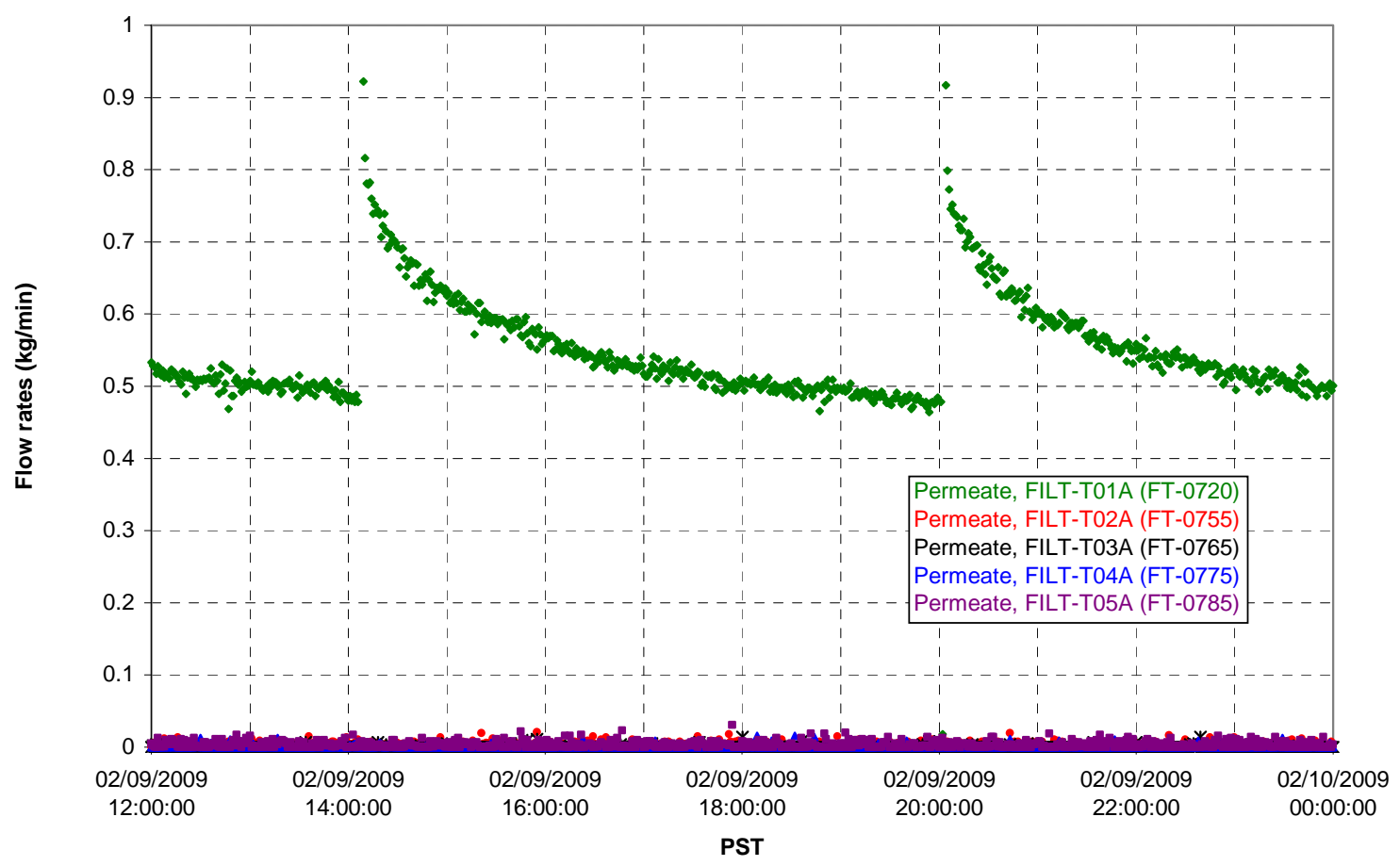


T02A Inner Temperature Tree

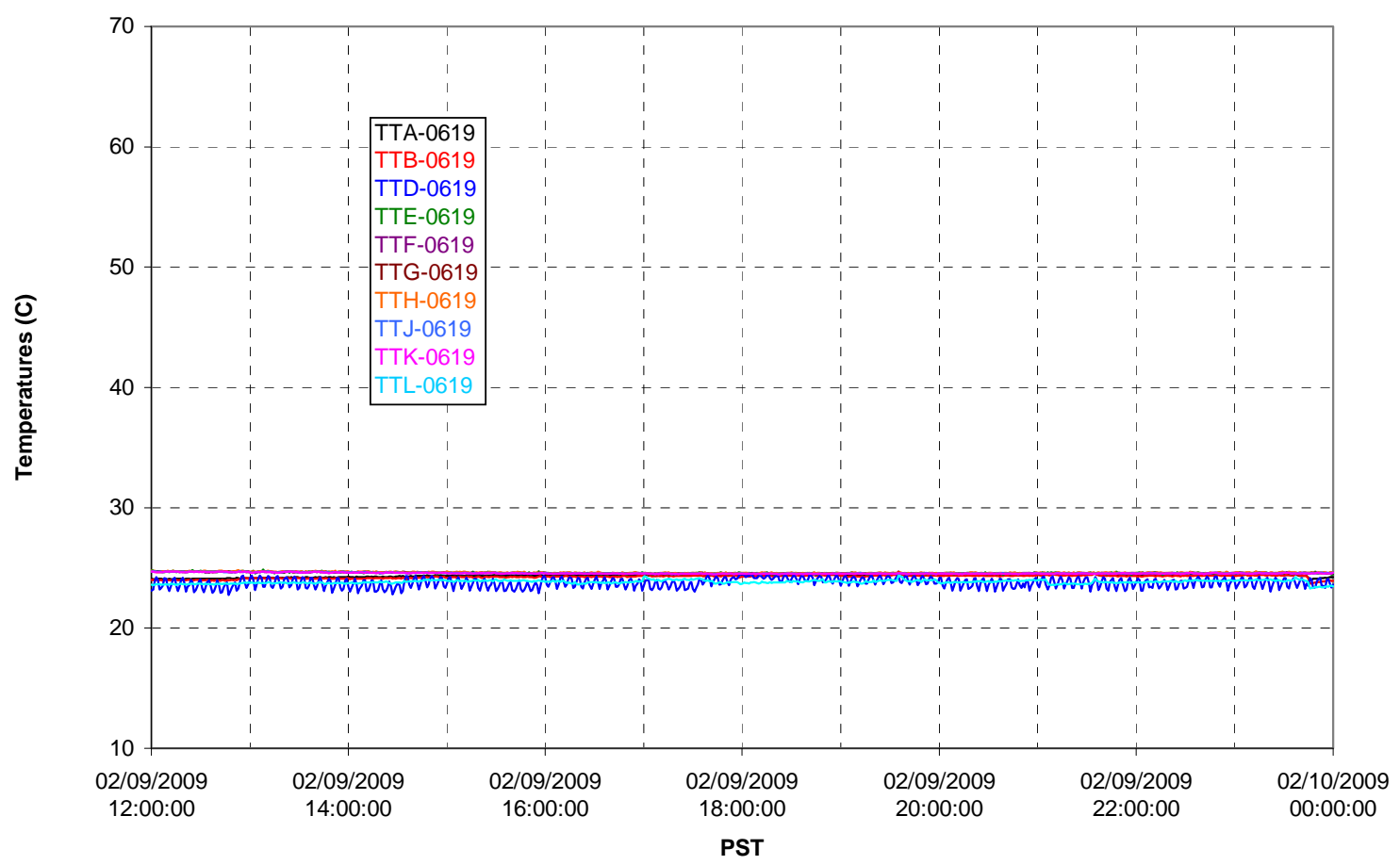

T02A Outer Temperature Tree

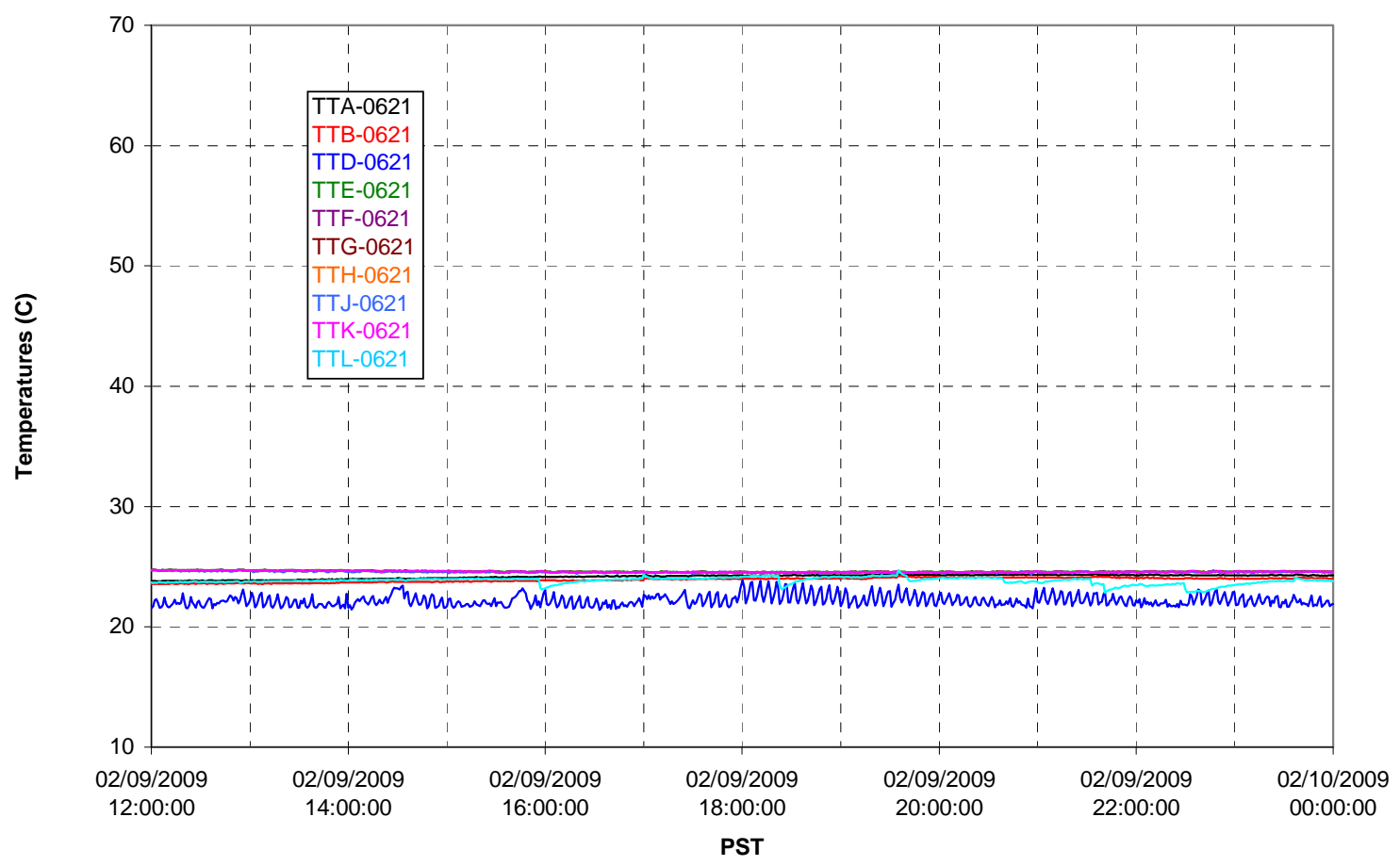


T02A temperatures

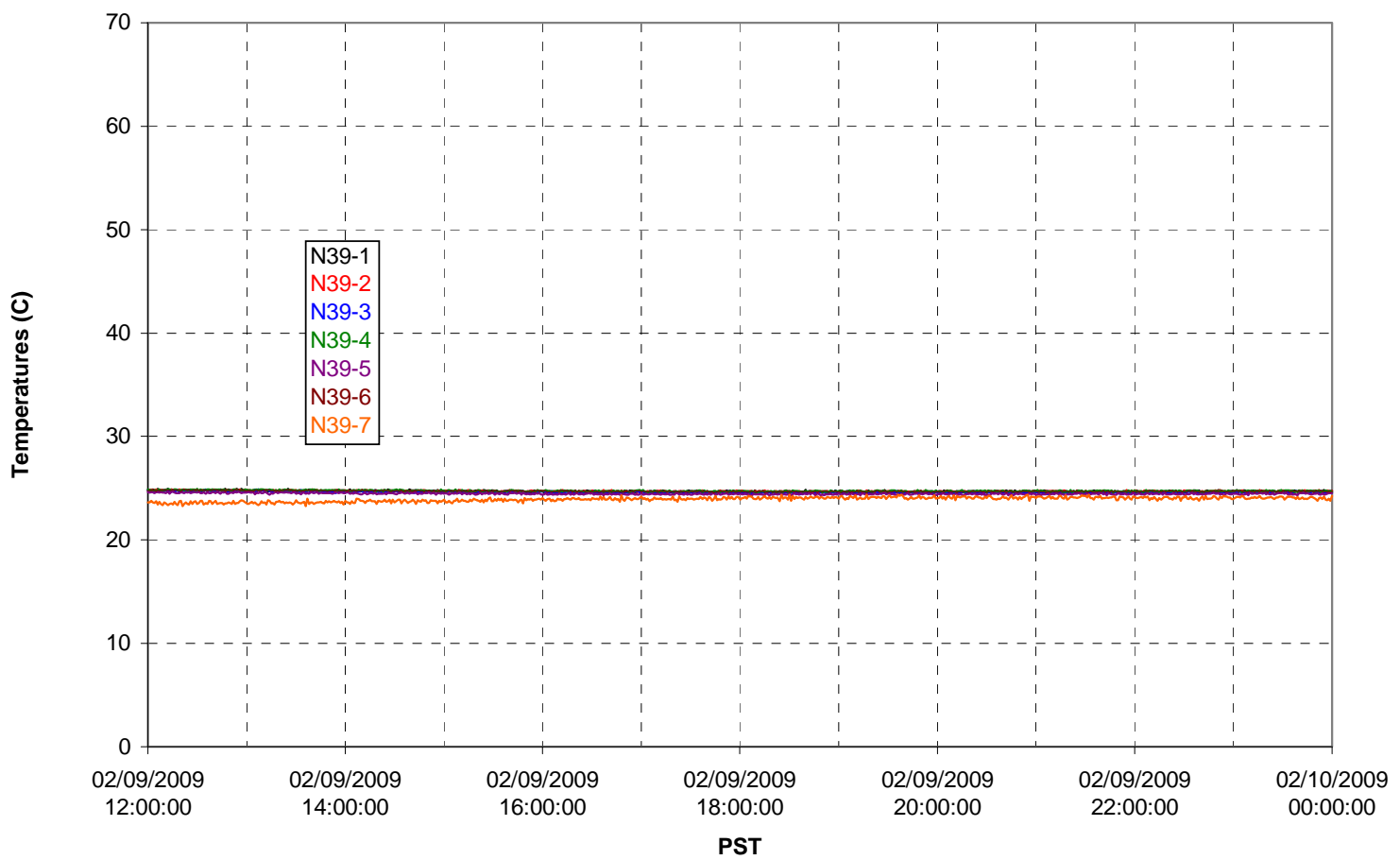

T02A temperatures

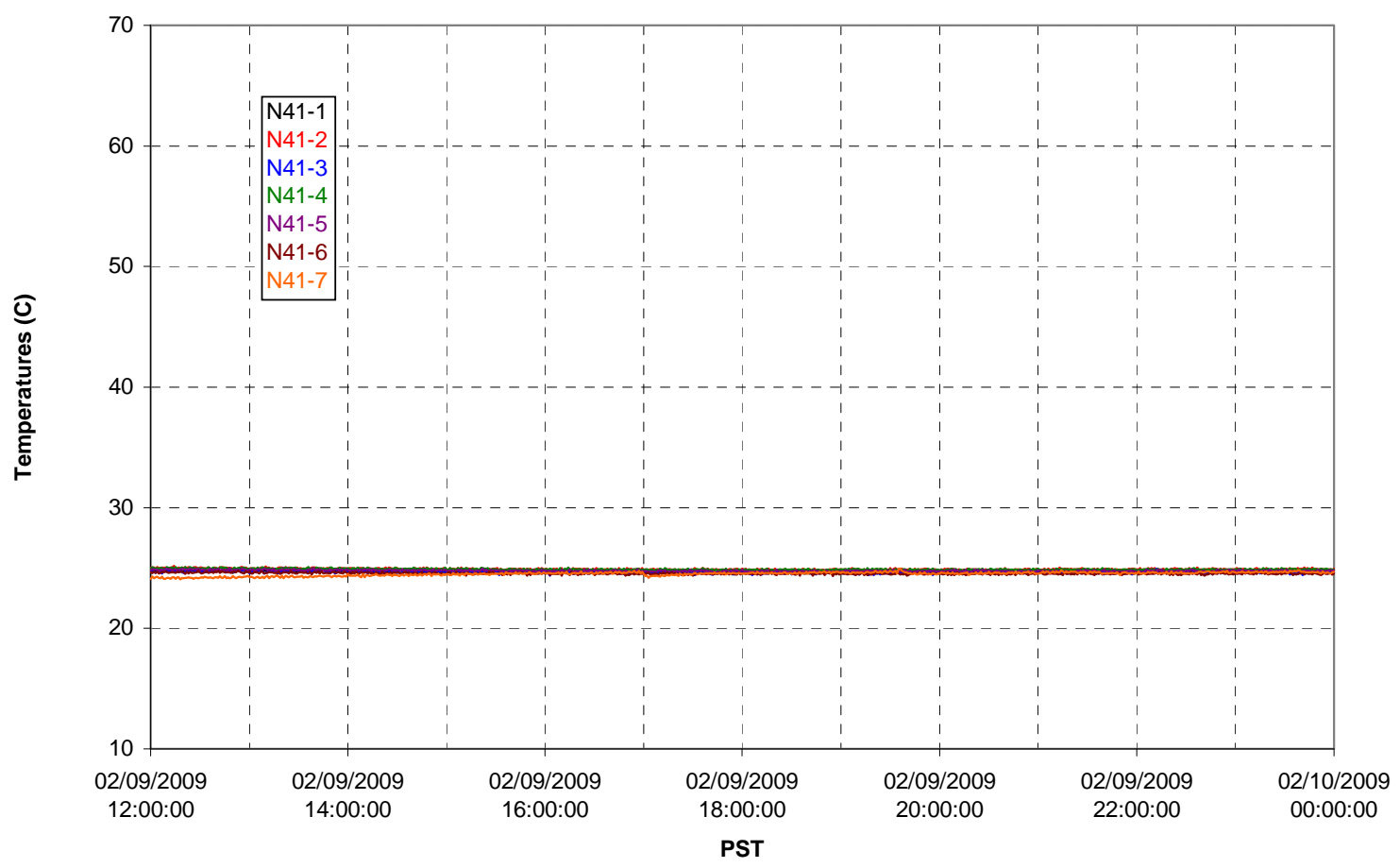


T02A temperatures

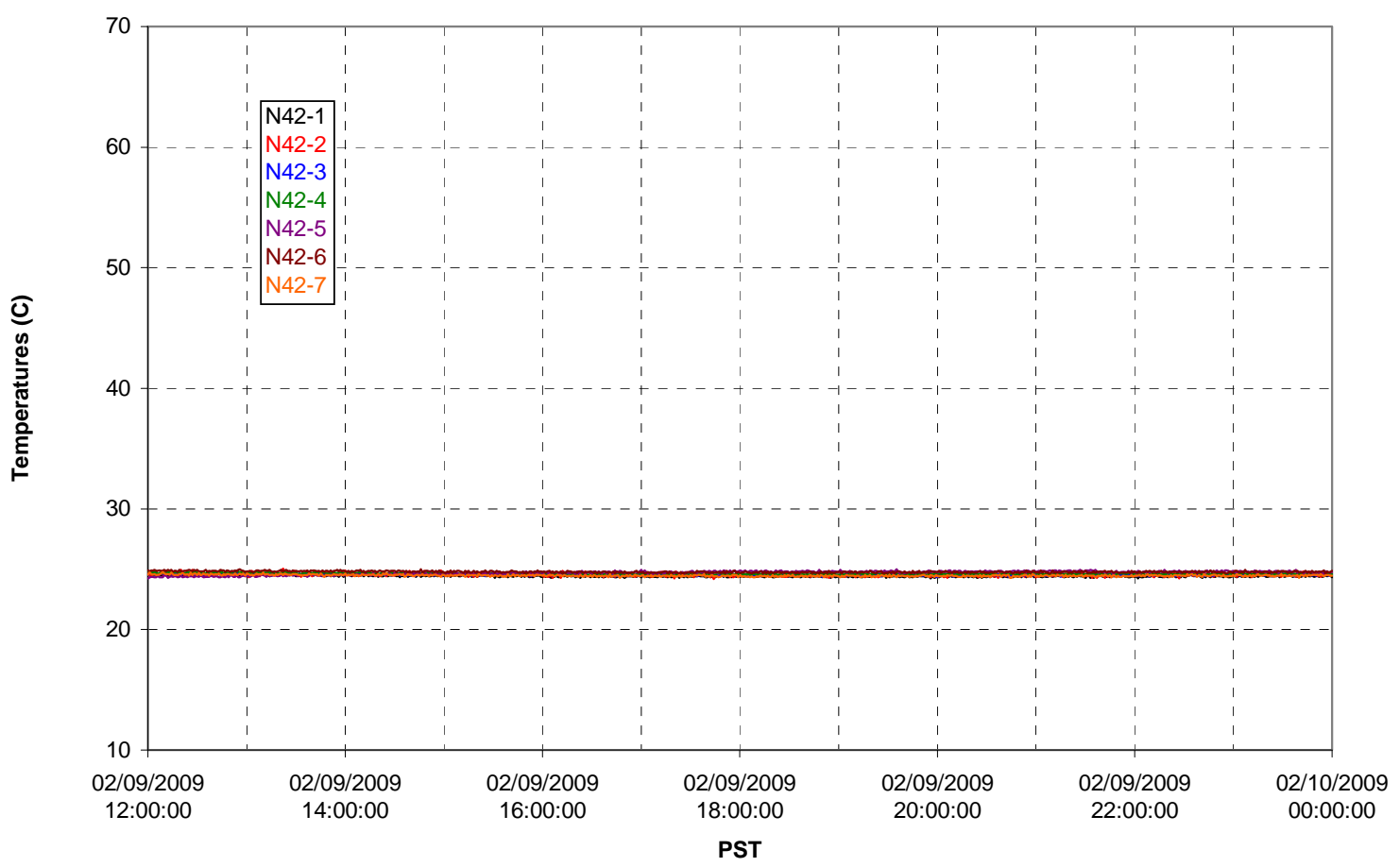

T02A temperatures

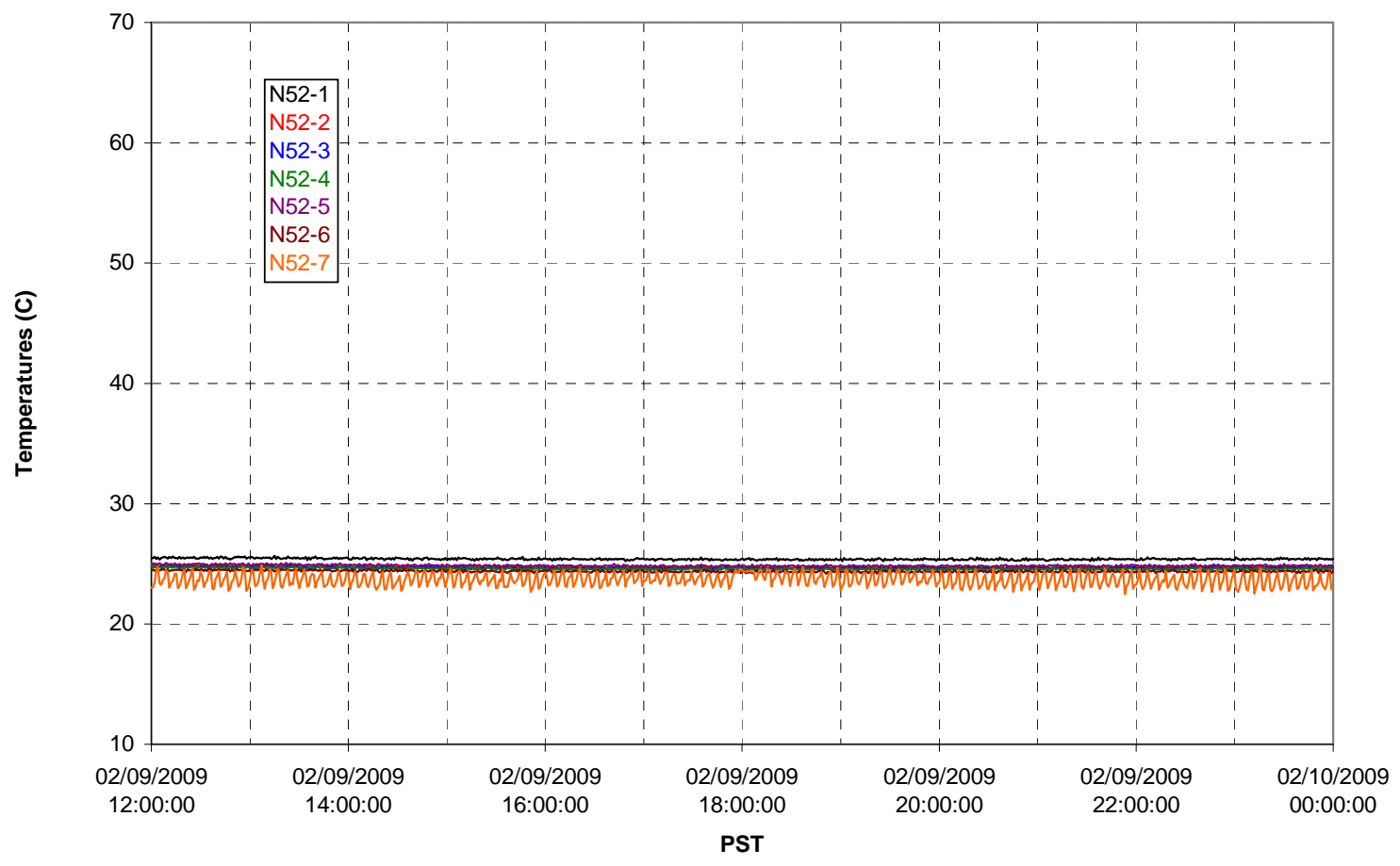


T02A Heating and Cooling
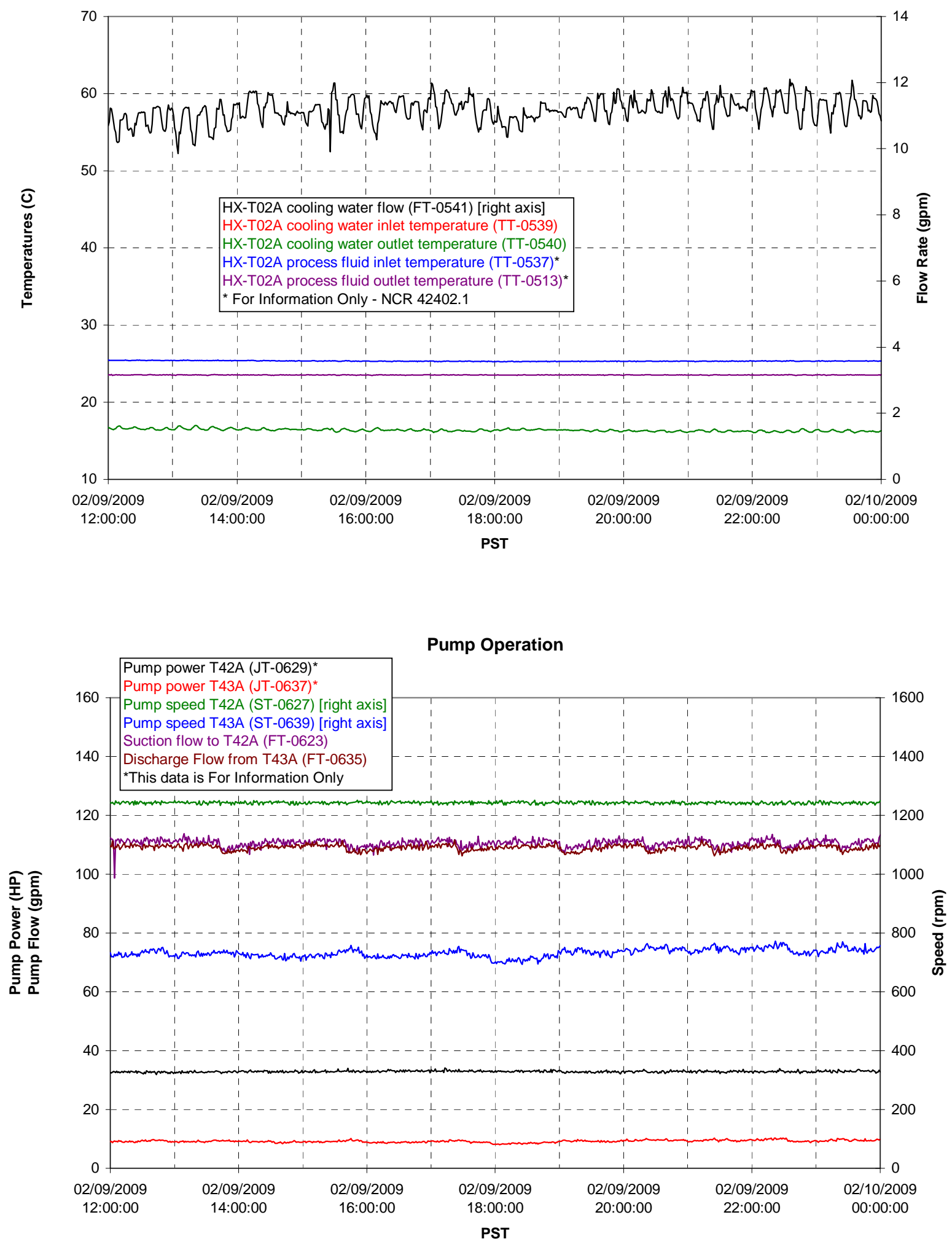
Pulsepot UFP-PP-T01A

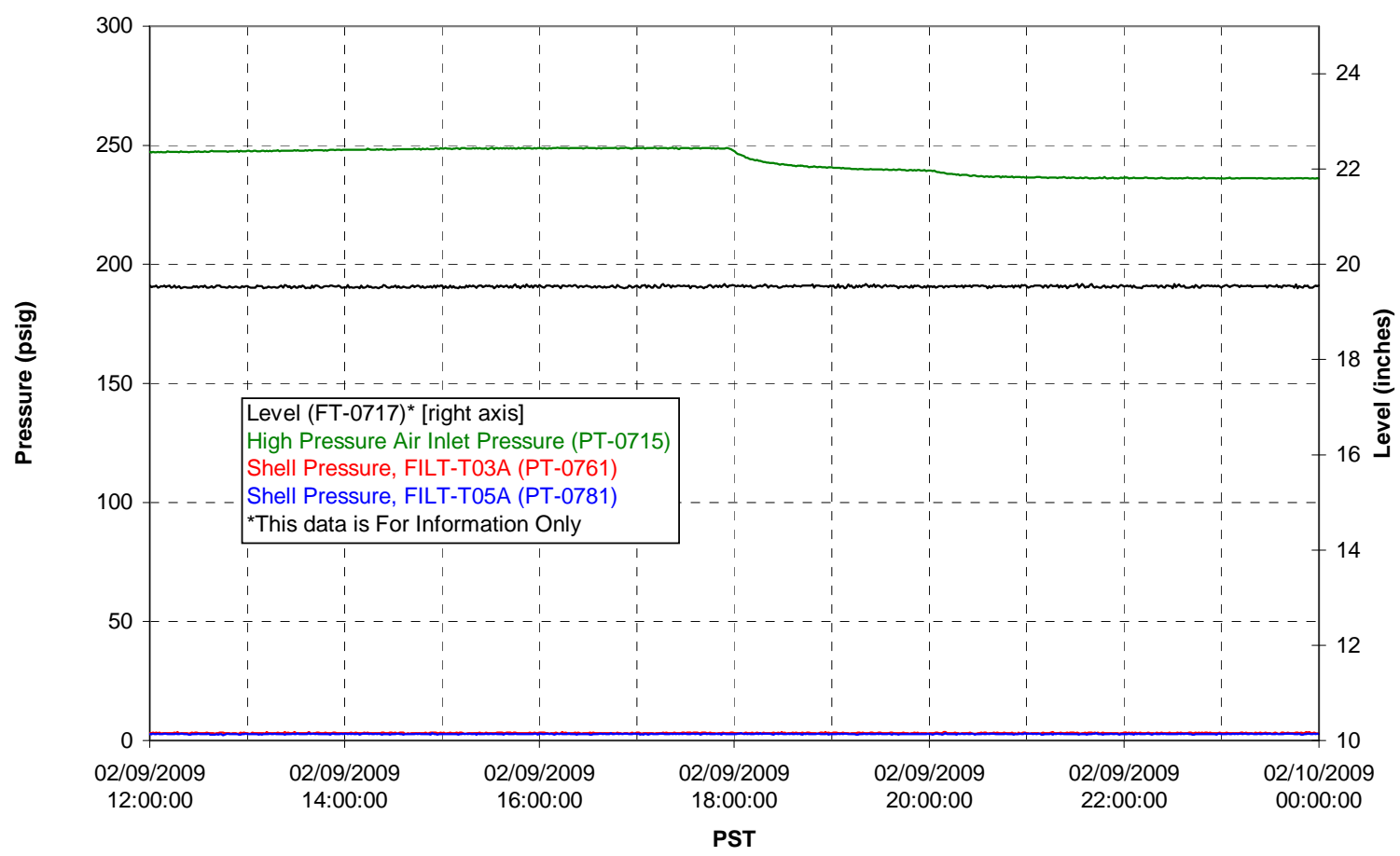

Pulsepot UFP-PP-T02A

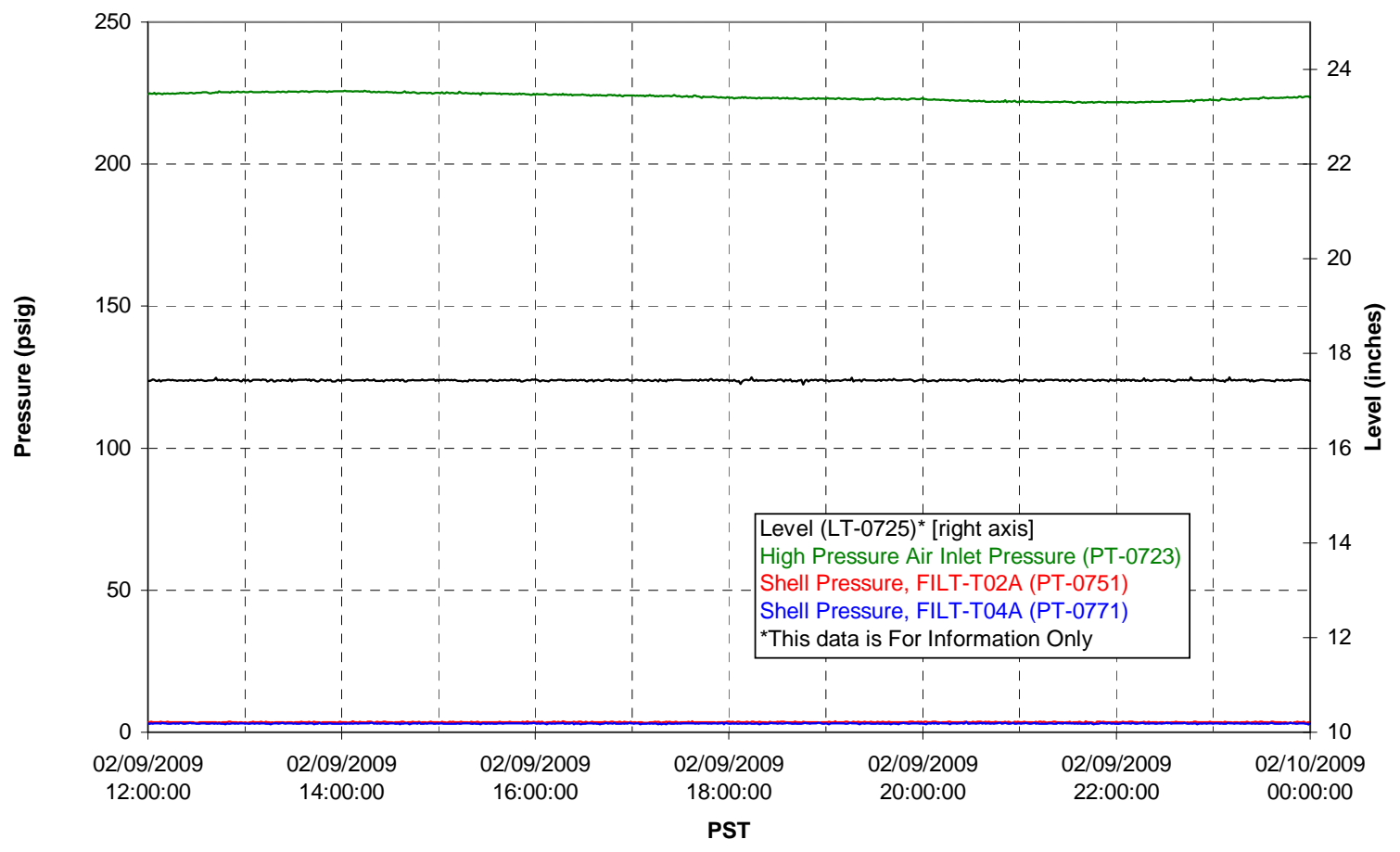


Pulsepot UFP-PP-T03A

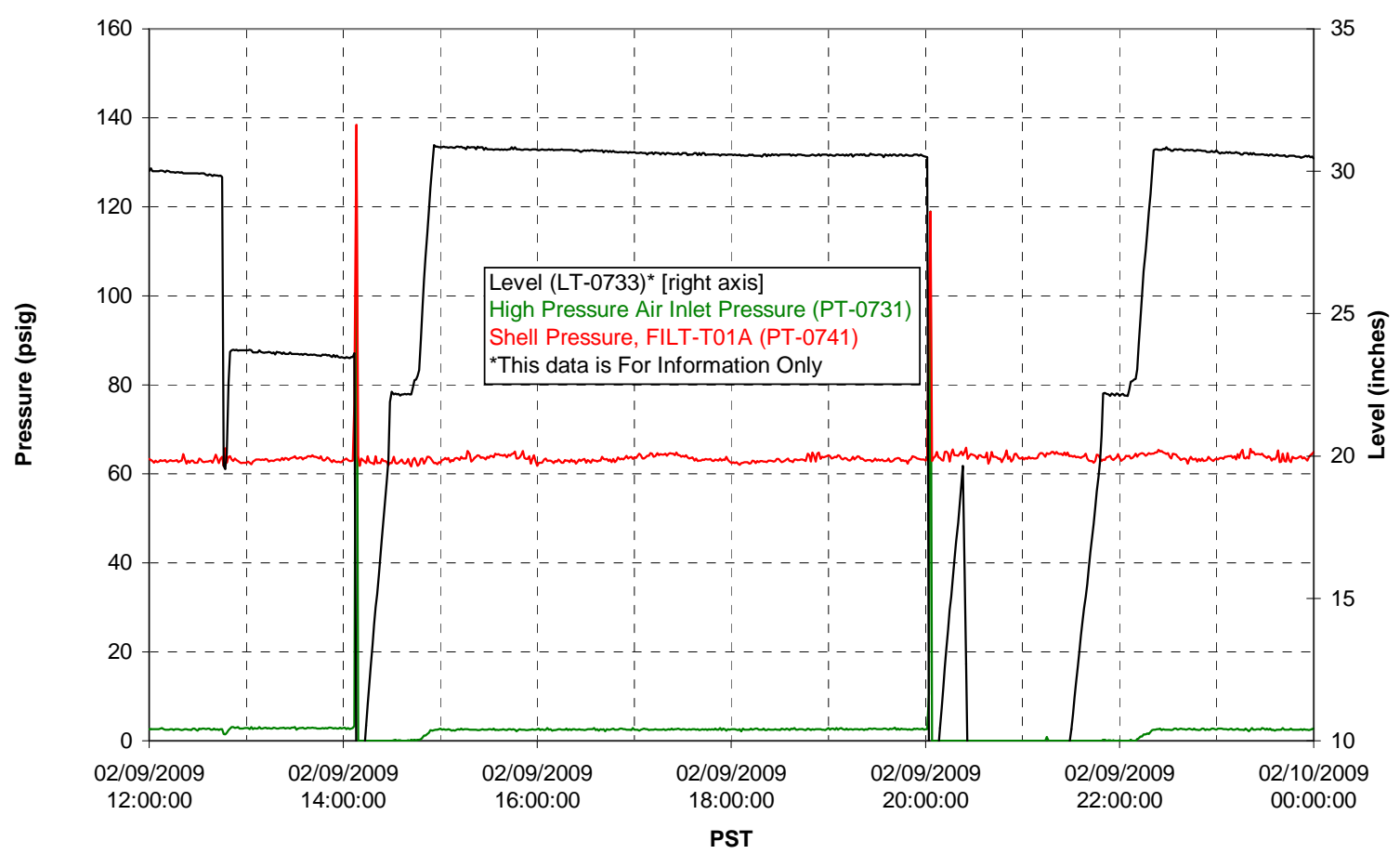

Pulsepot Levels

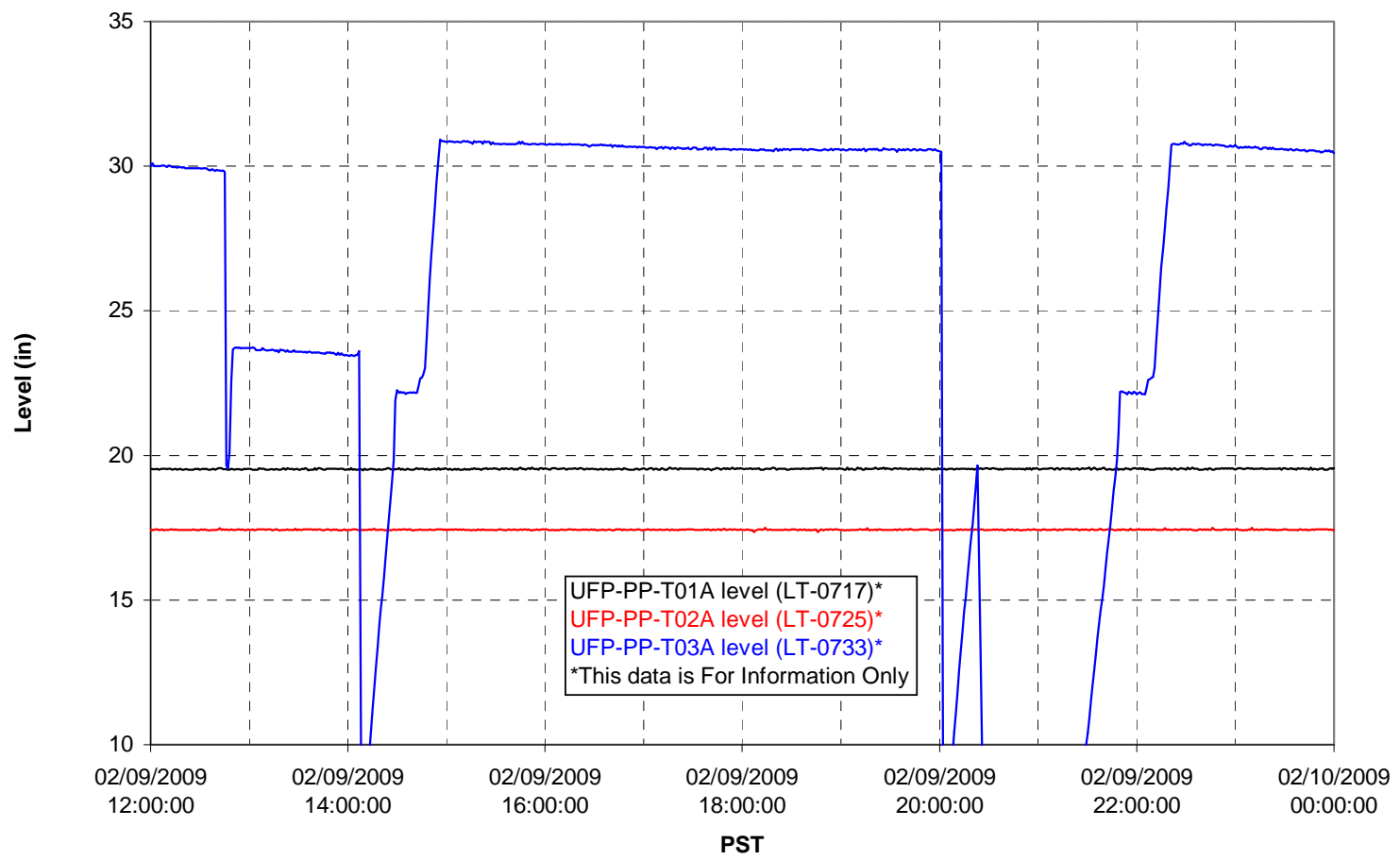


Filter UFP-FILT-T01A

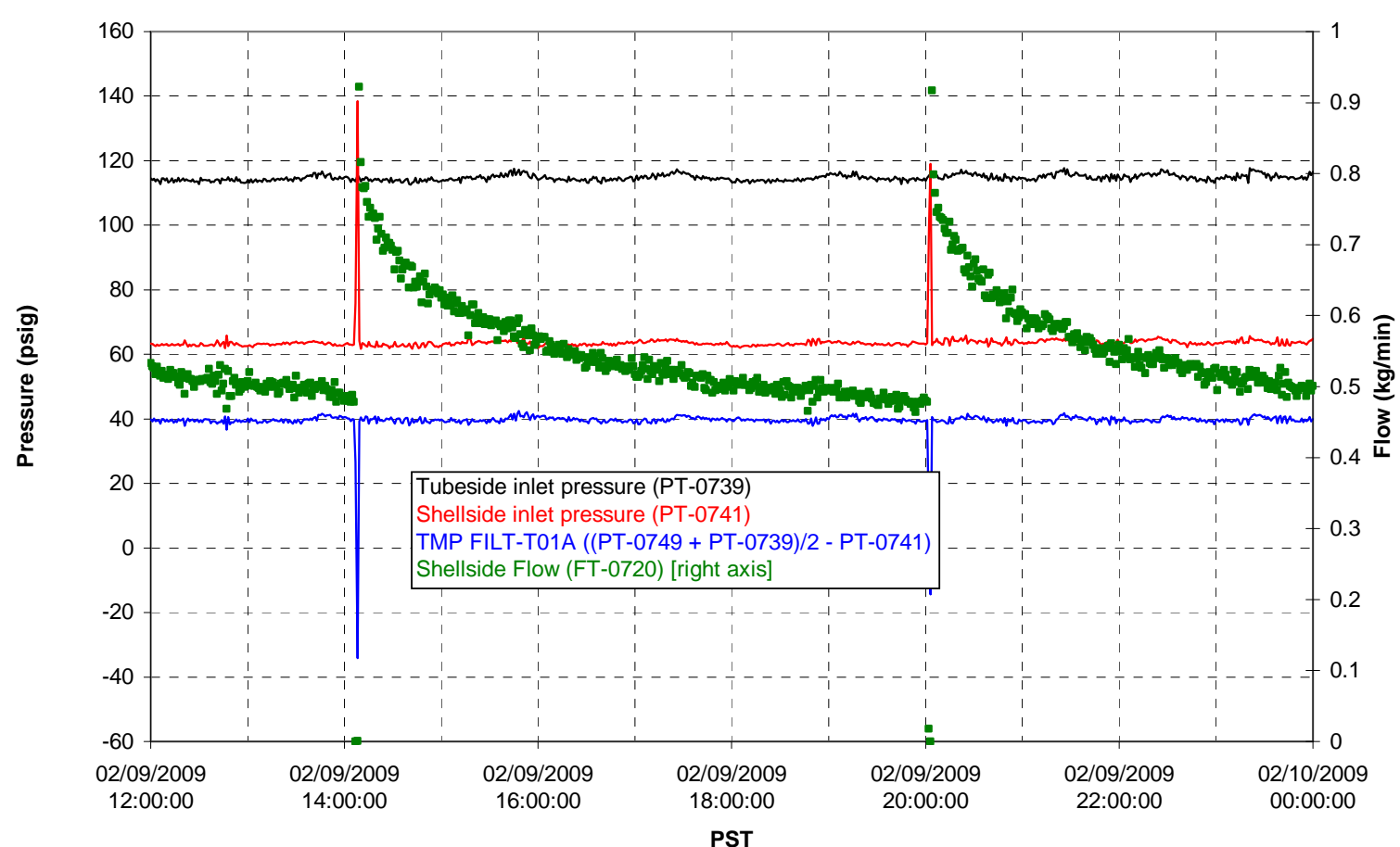

Filter UFP-FILT-T02A

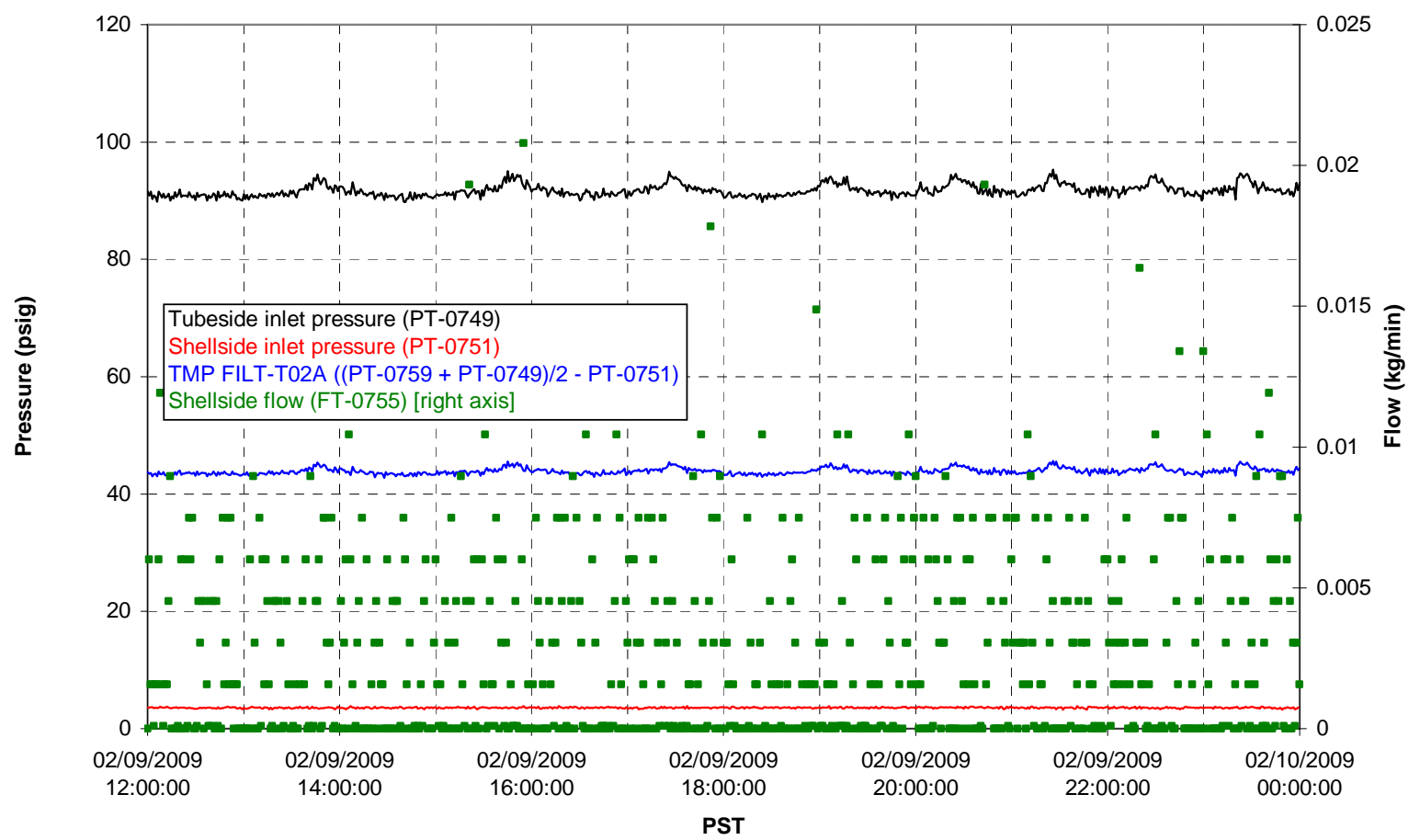


Filter UFP-FILT-T03A

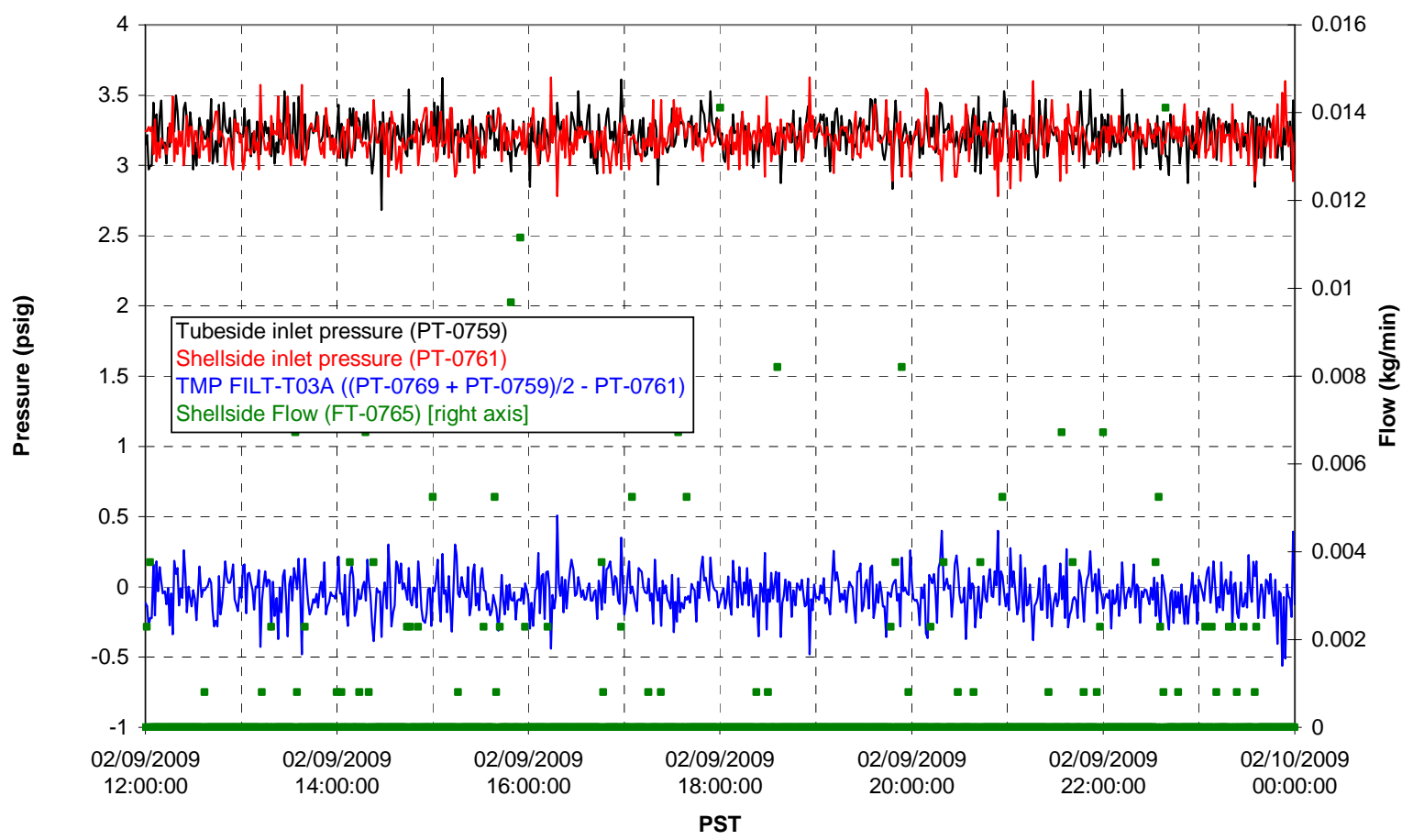

Filter UFP-FILT-T04A

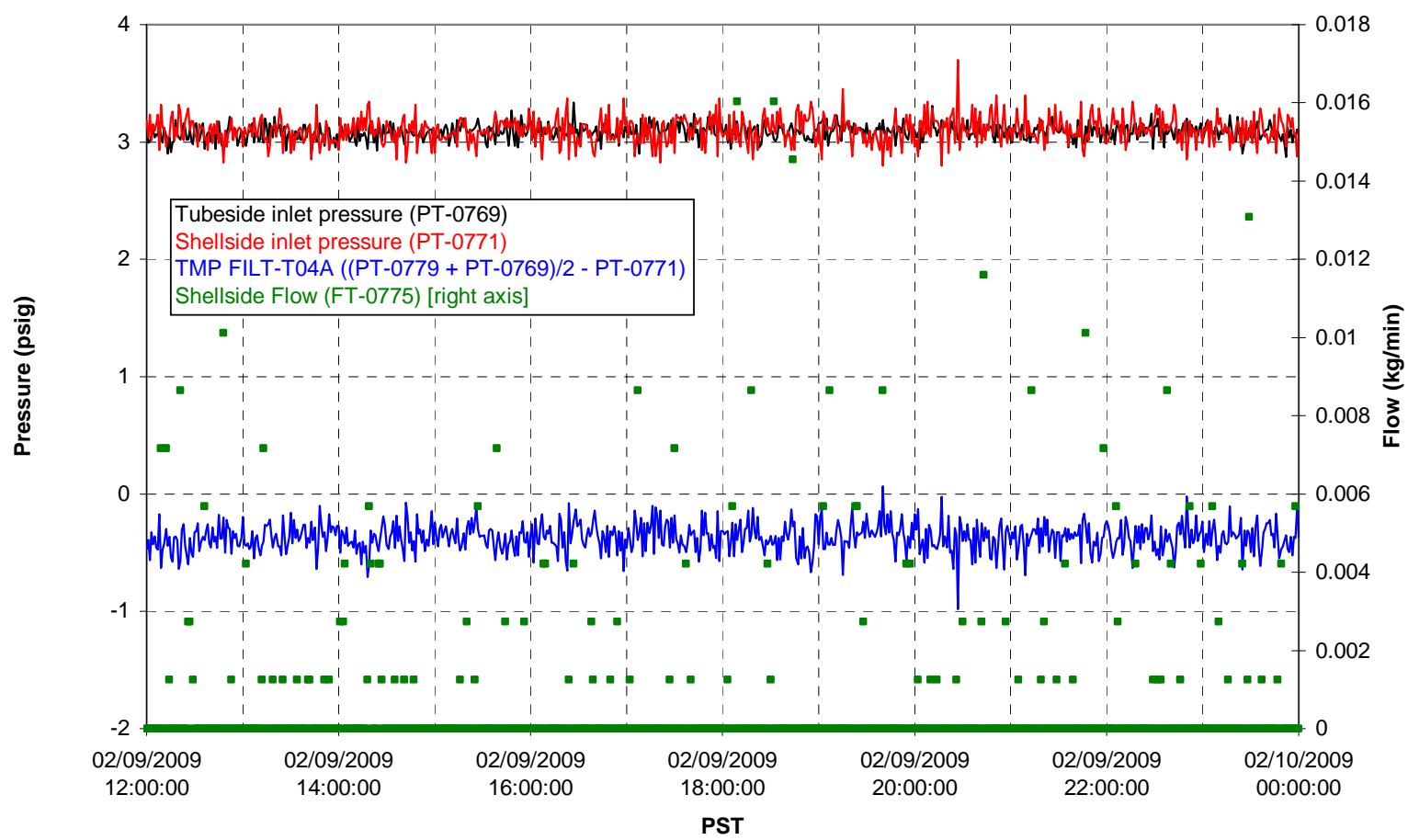


Filter UFP-FILT-T05A

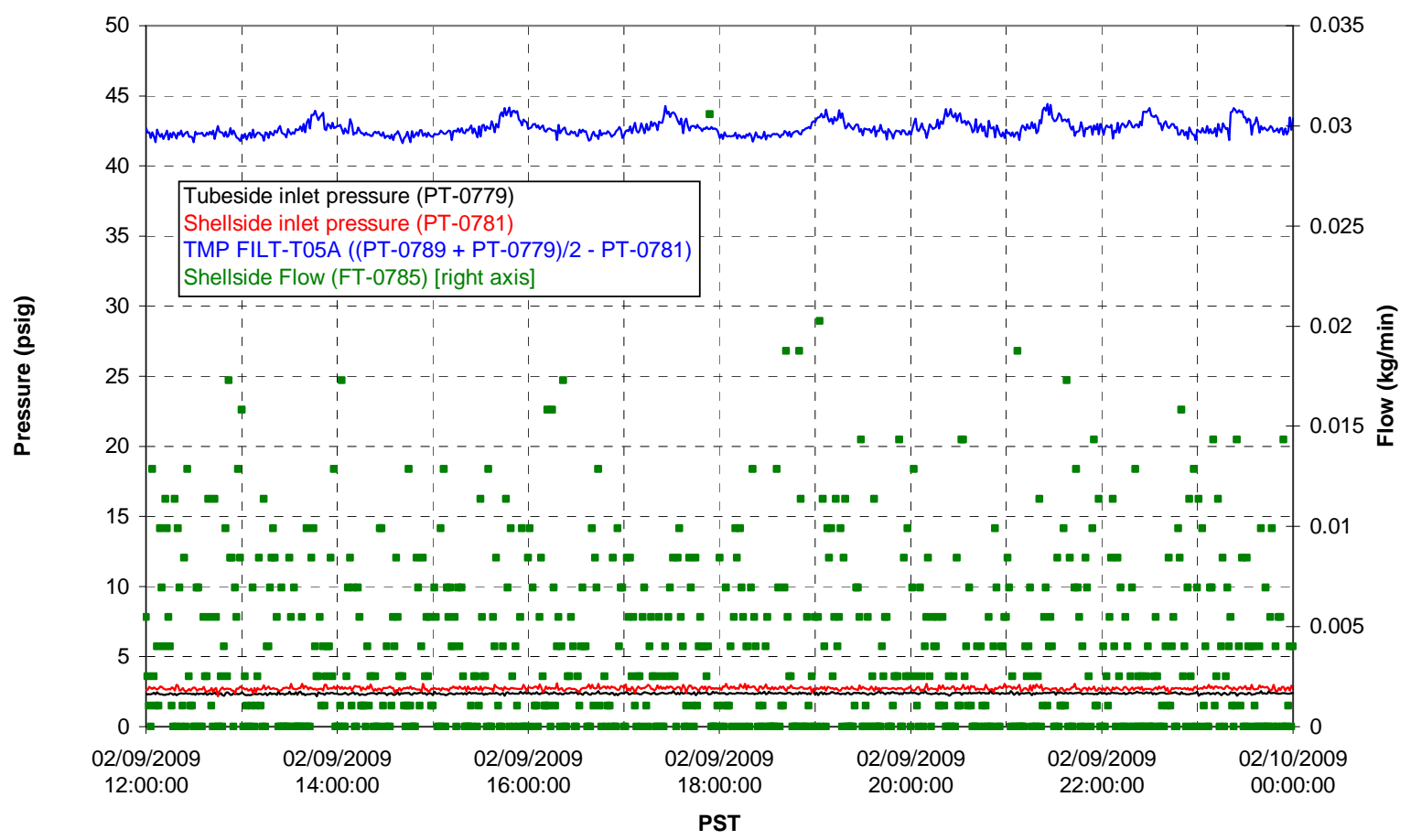

Chemical Flow

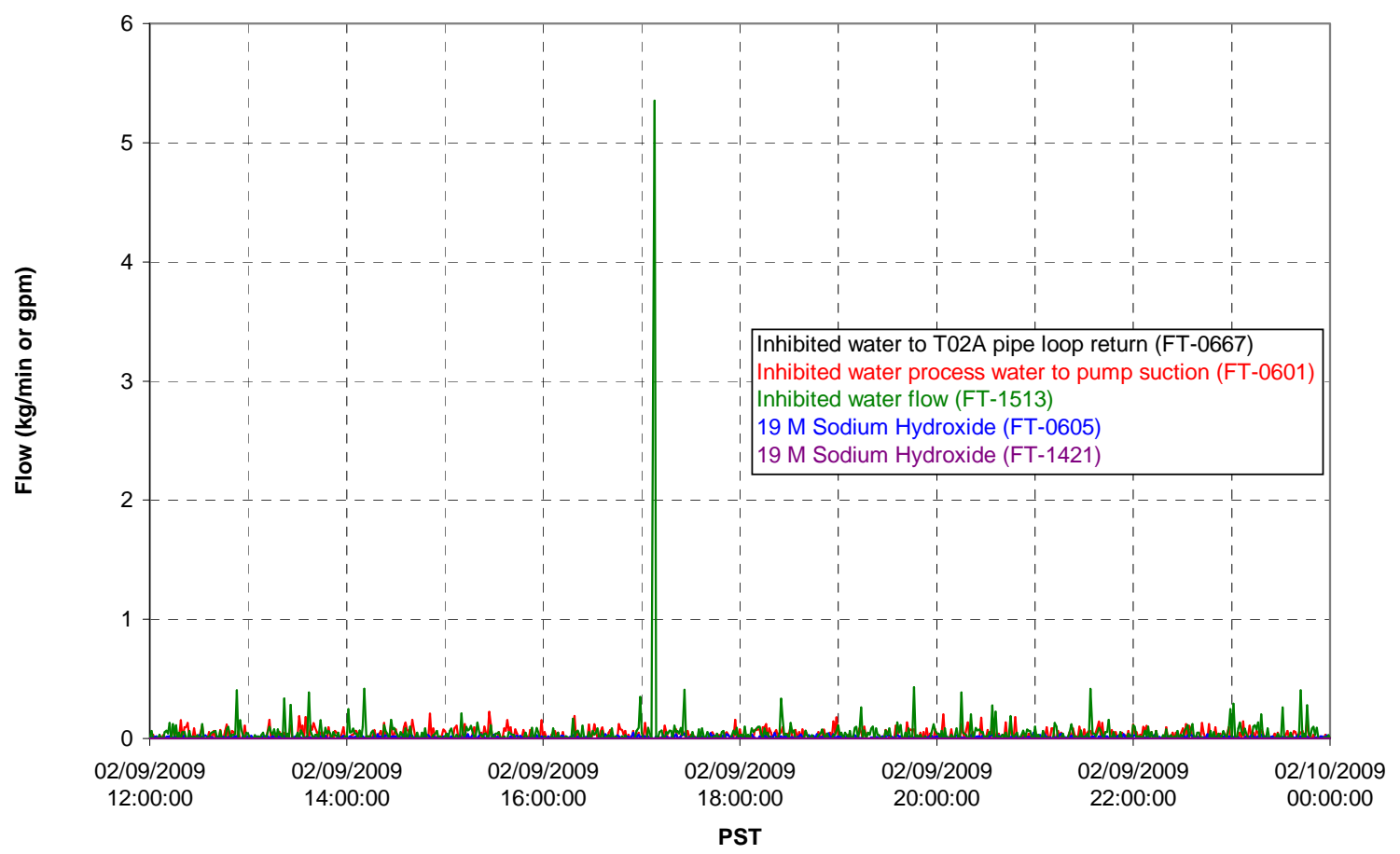


Chemical Flow

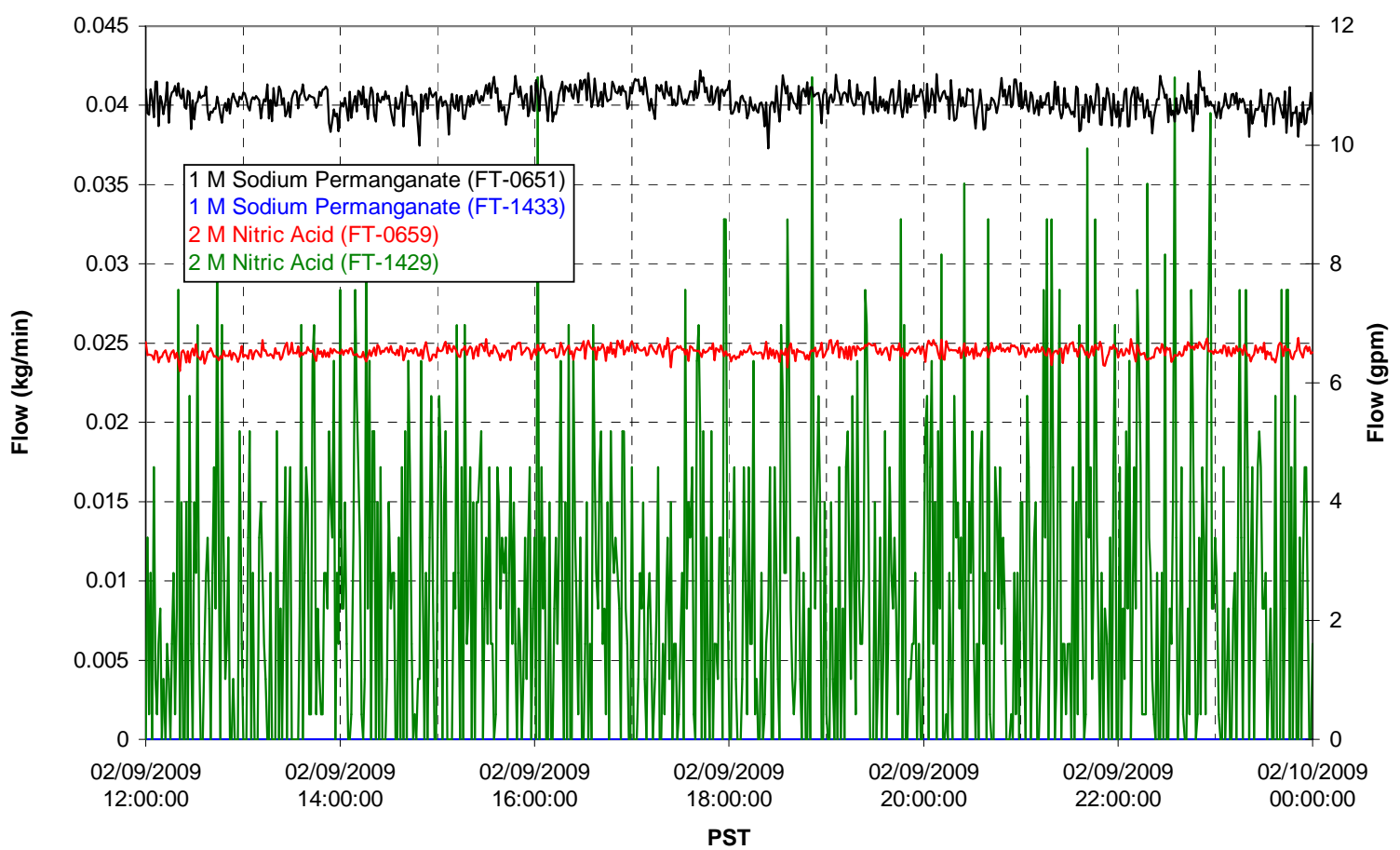

Air Flows

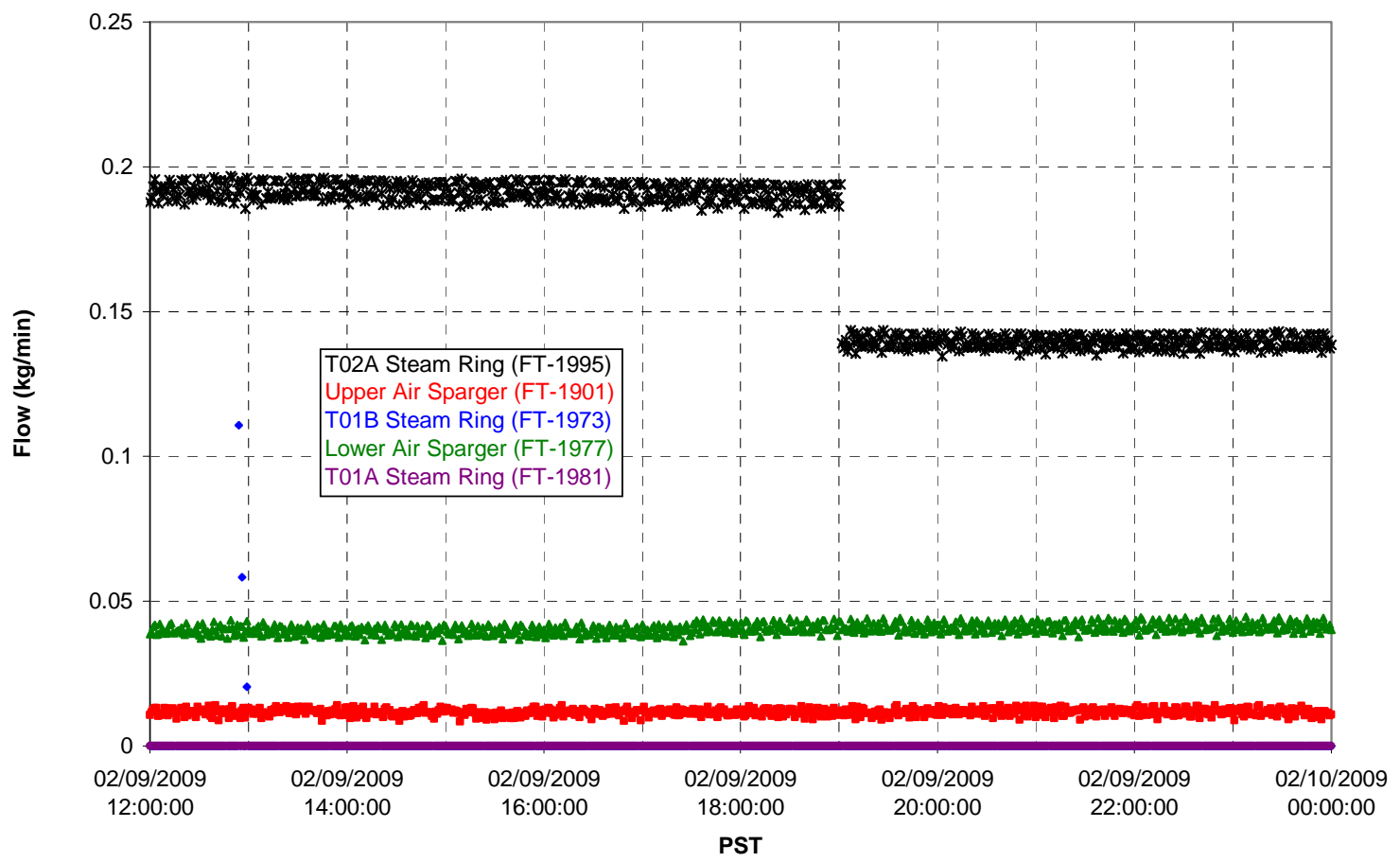


T02A Steam

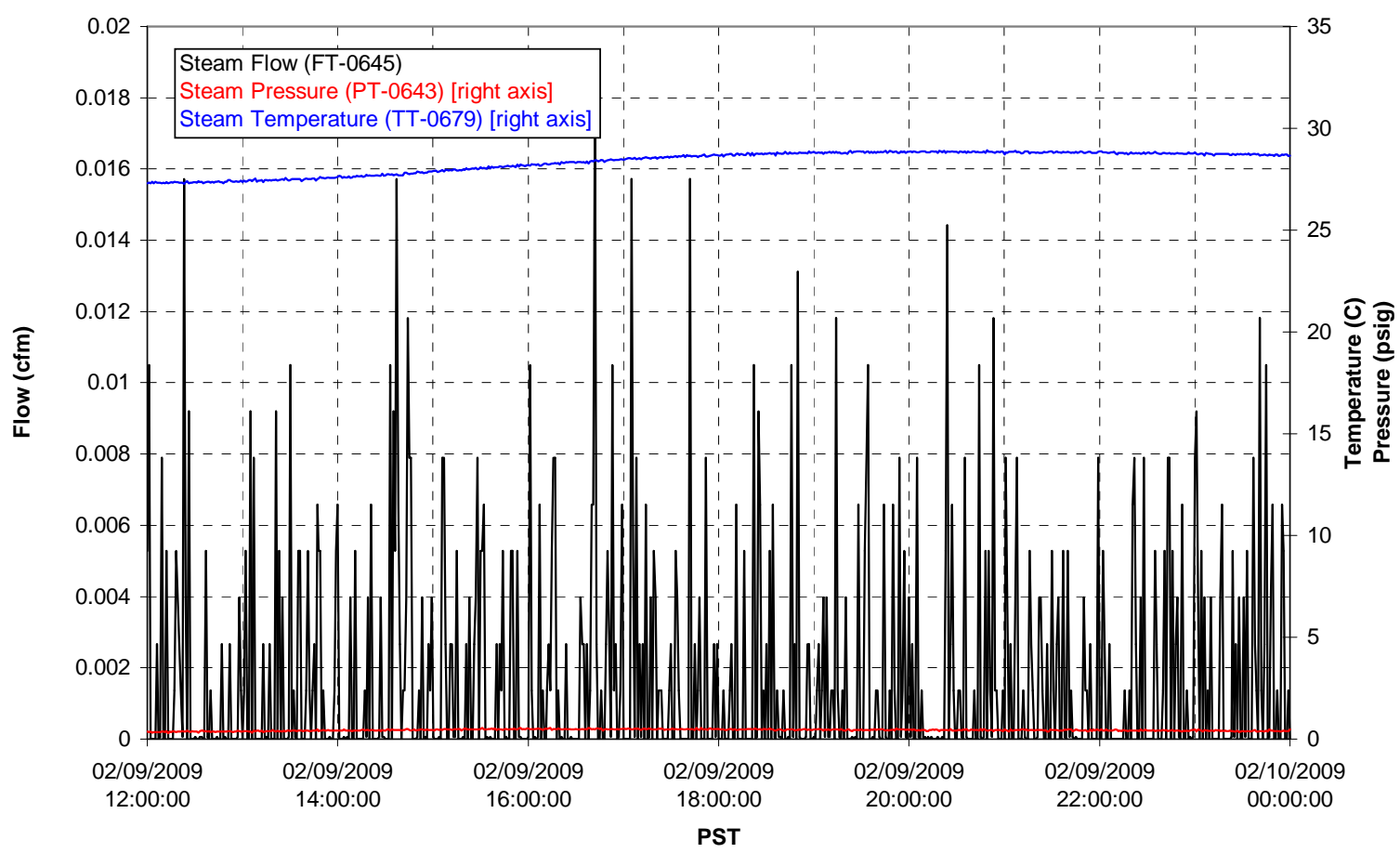

T01A Steam

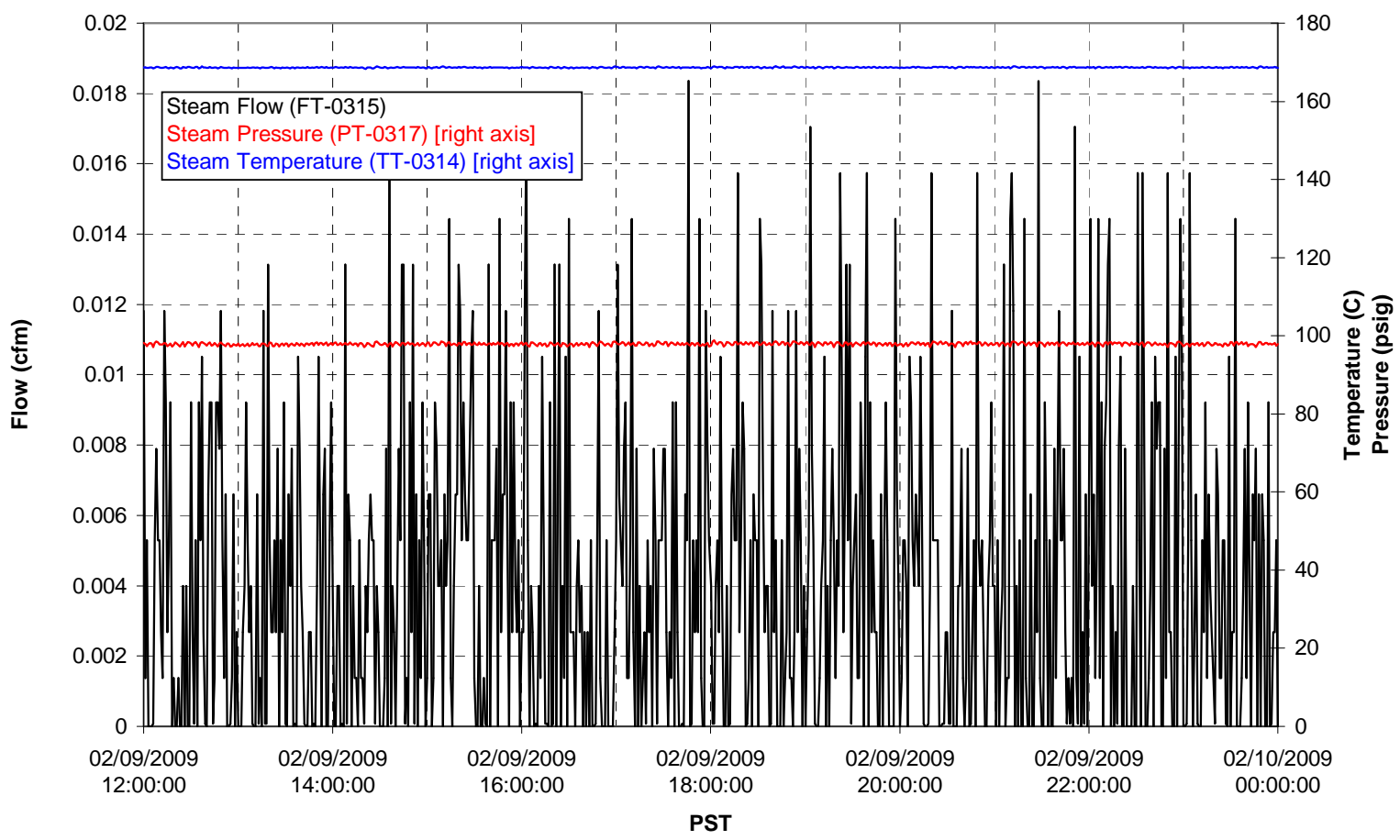


T01B Steam

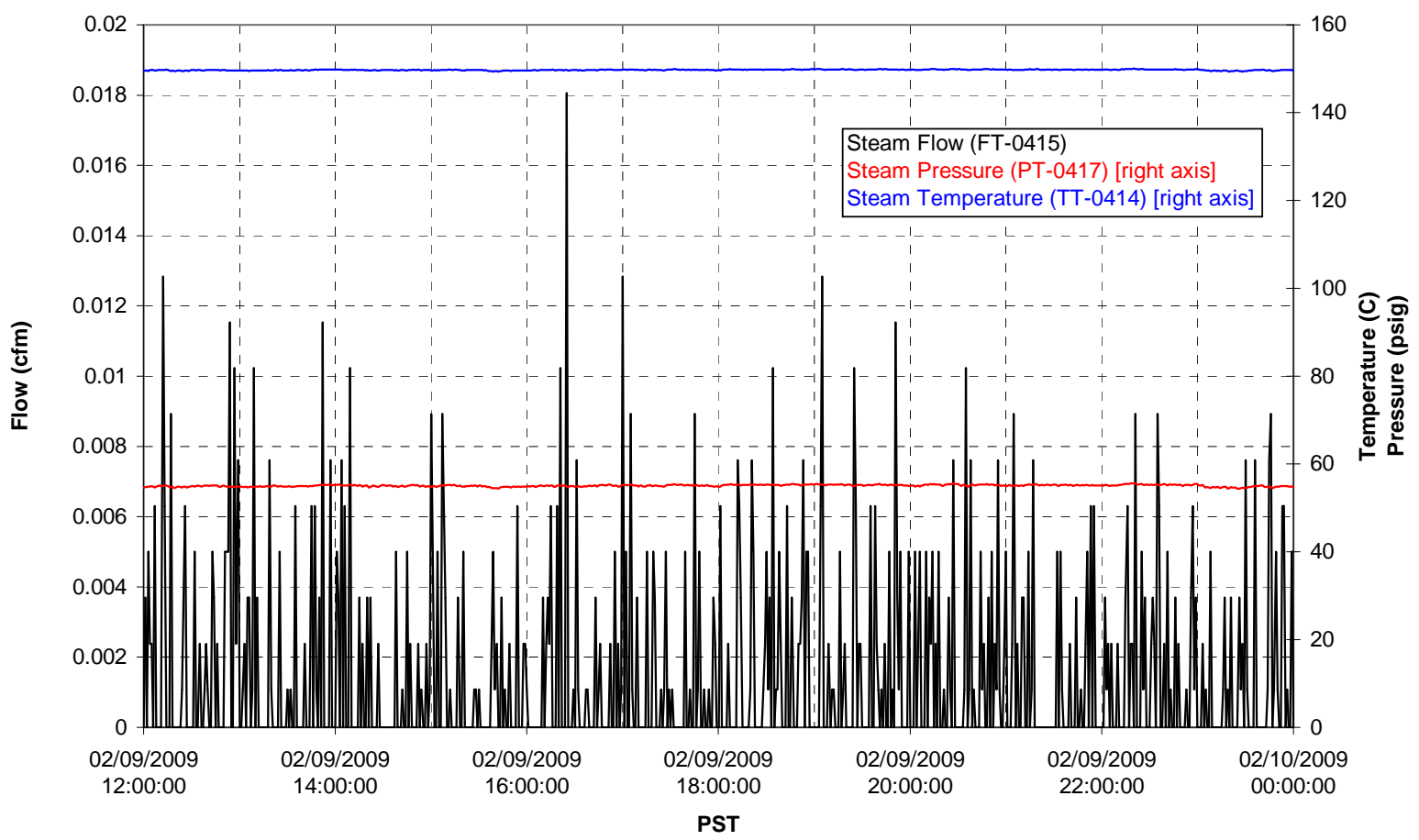




\section{Integrated Test A Data Plots 02/10/09 00:00 - 02/10/09 12:00}


T01A level

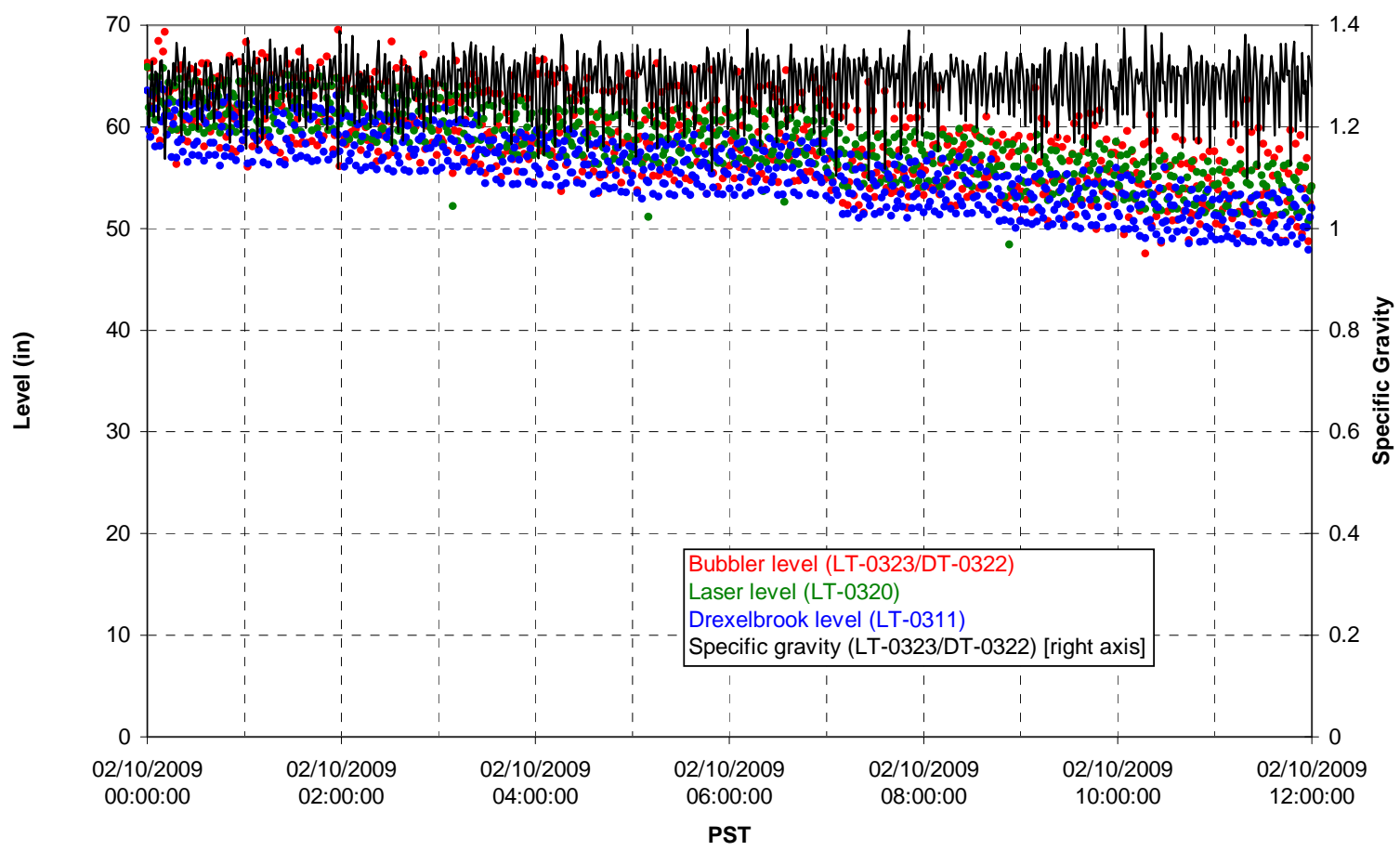

T01A temperatures

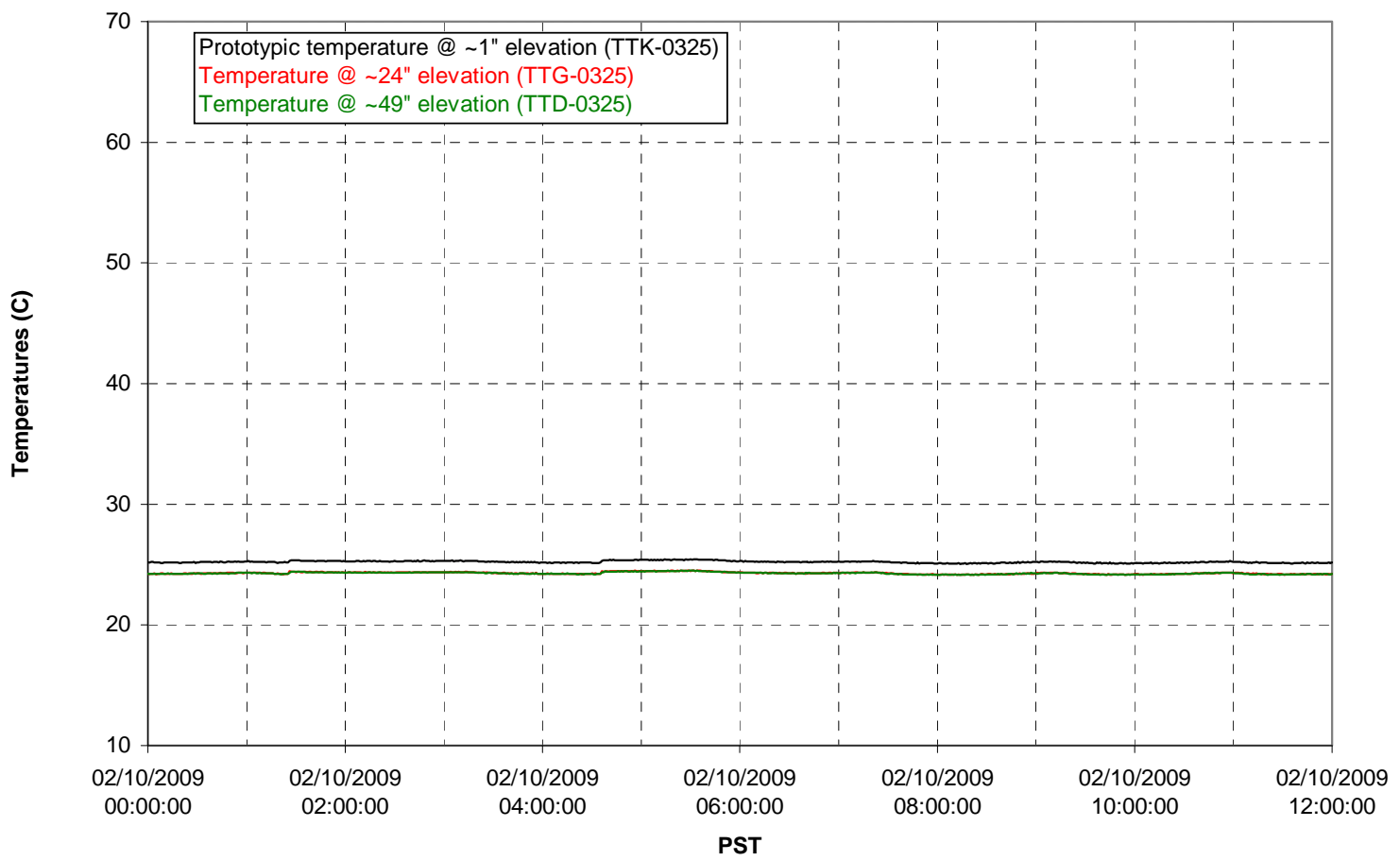


T01B level

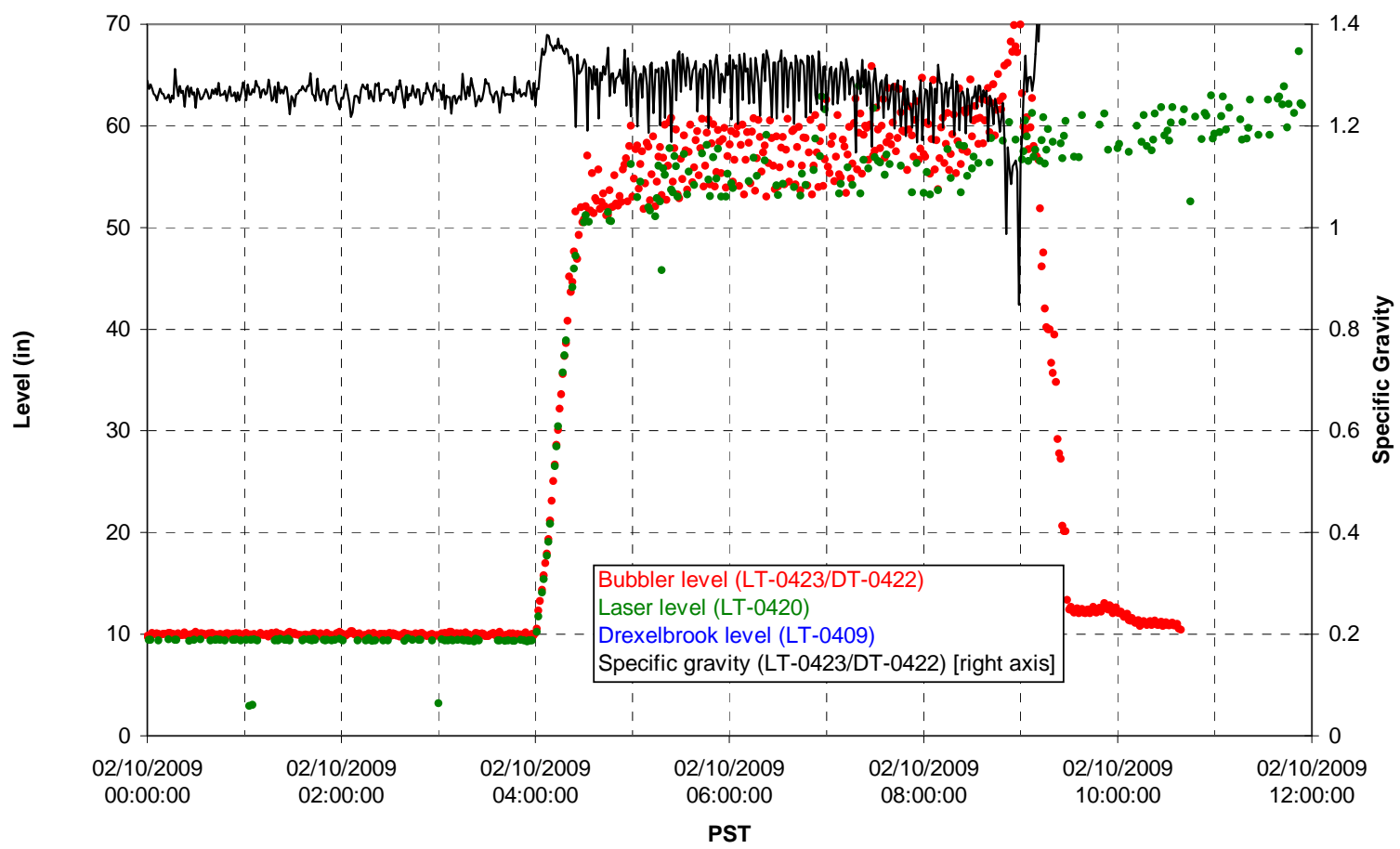

T01B temperatures

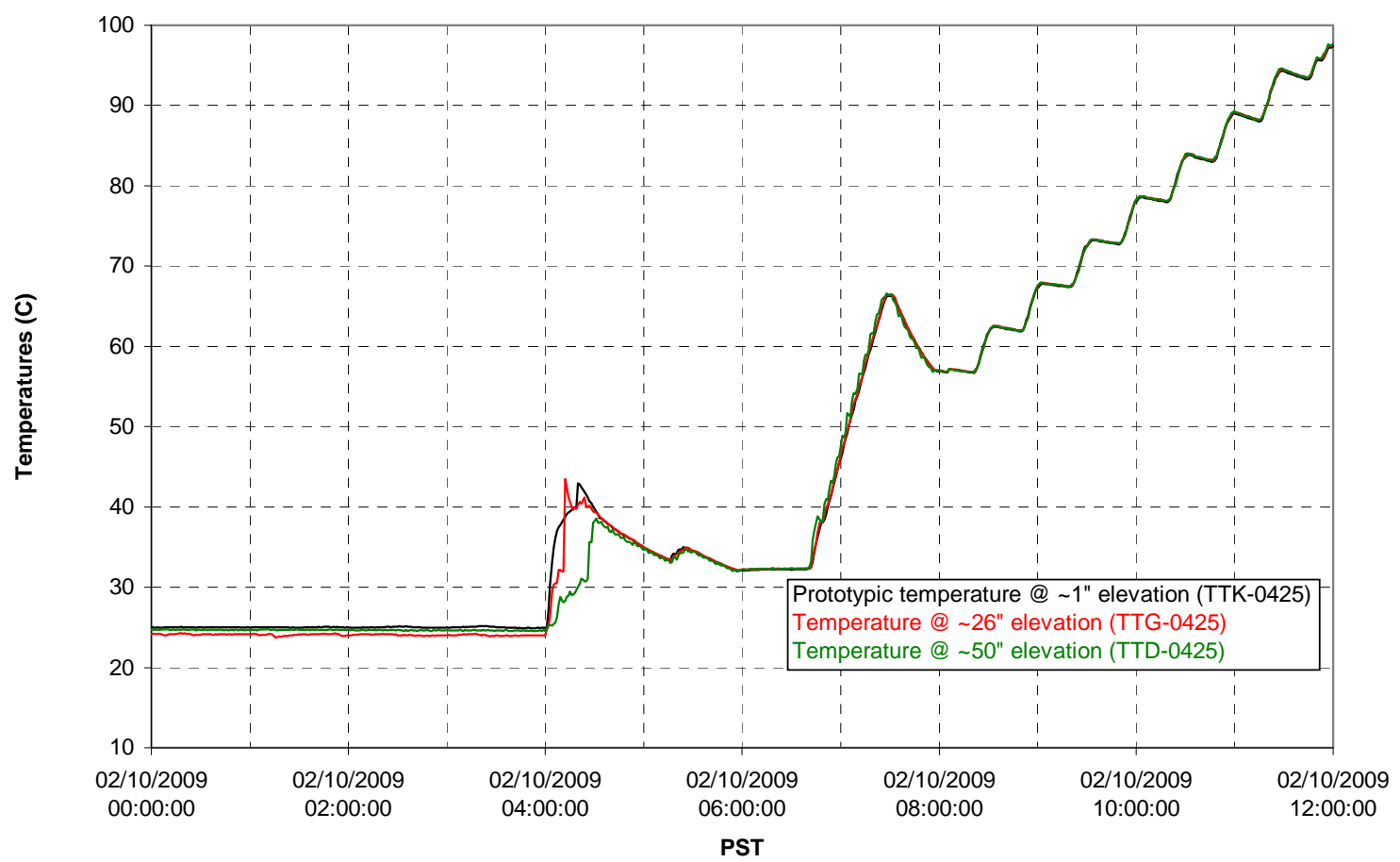


T02A level

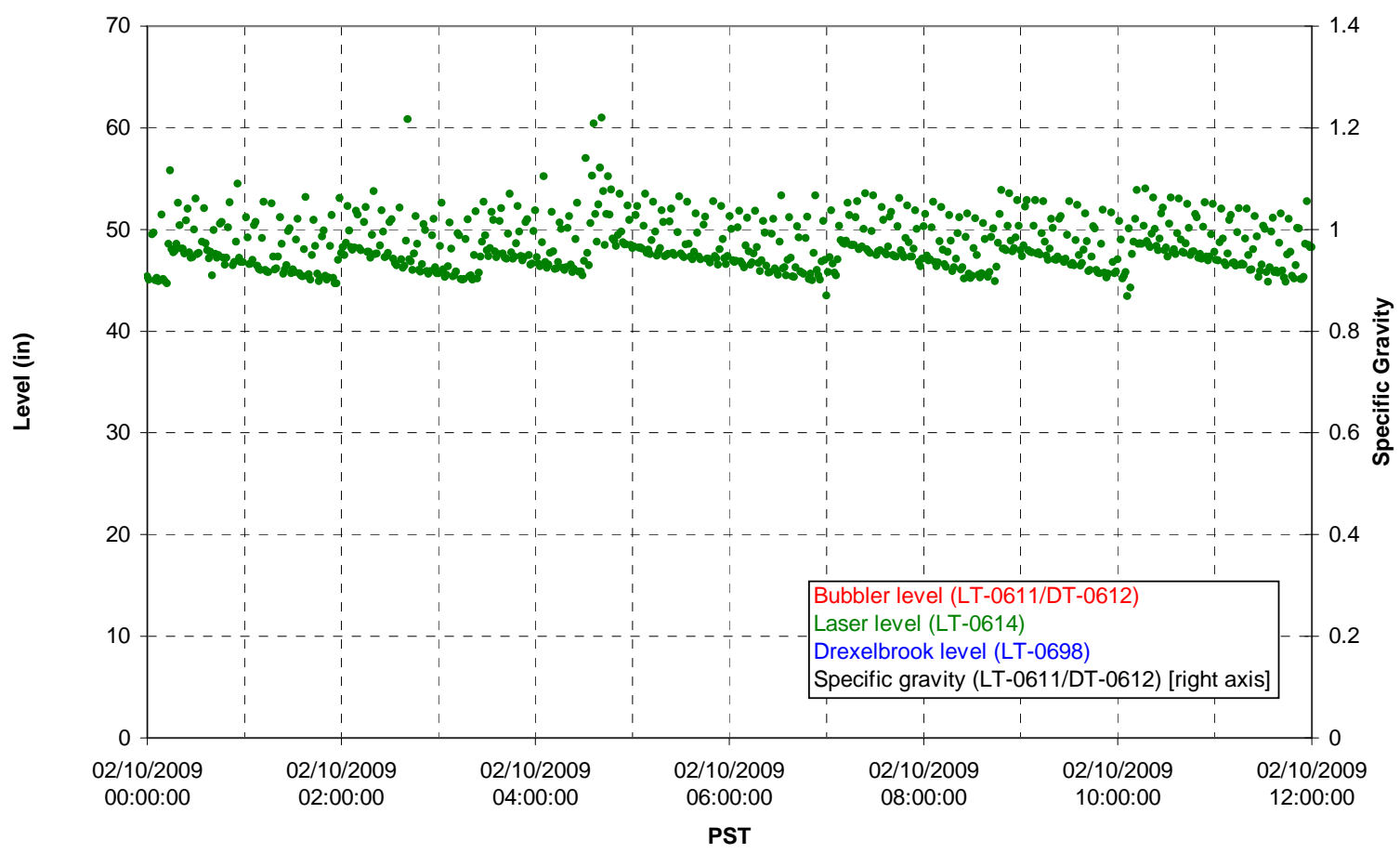

T02A temperatures

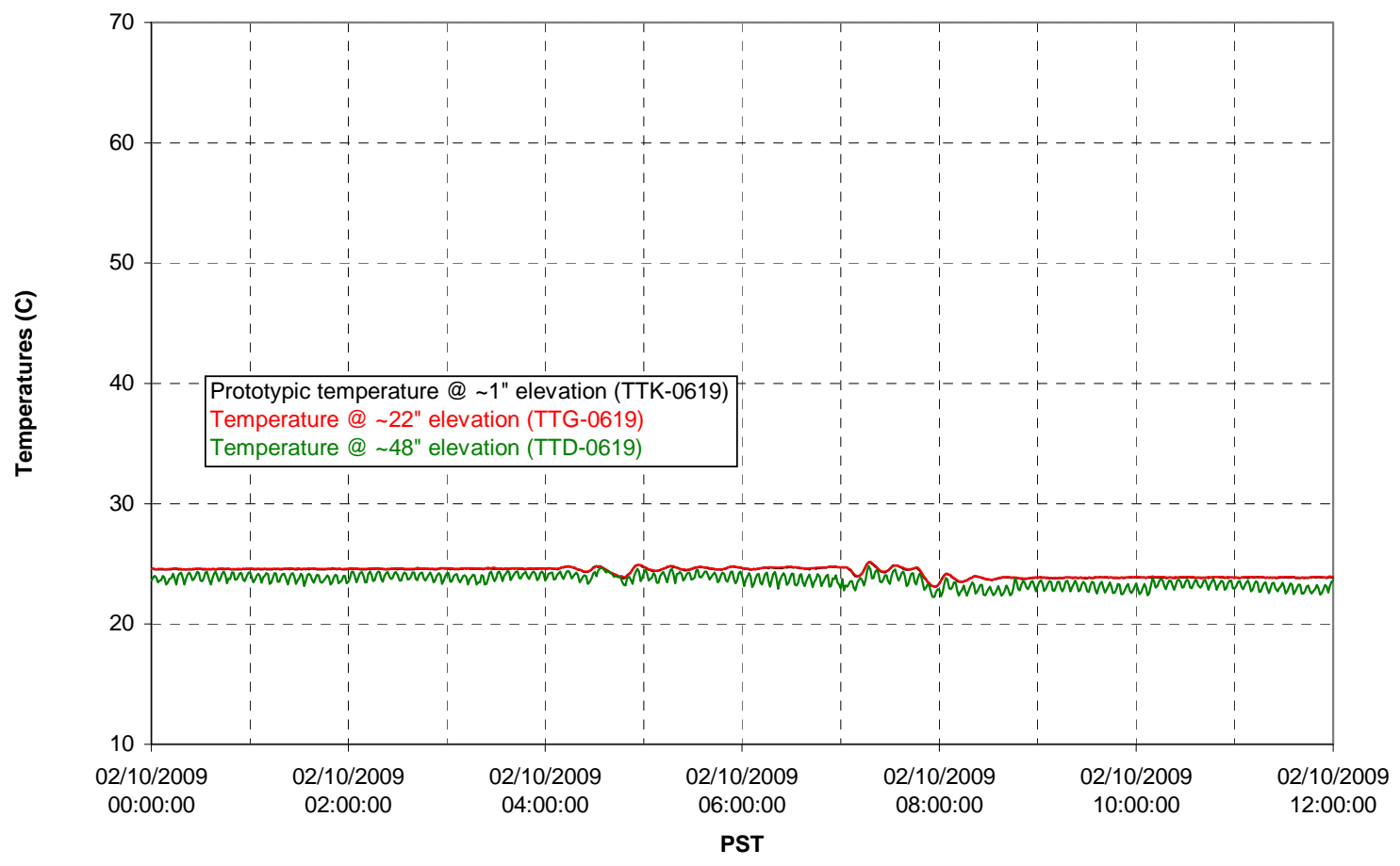


T02A and filter loop temperatures

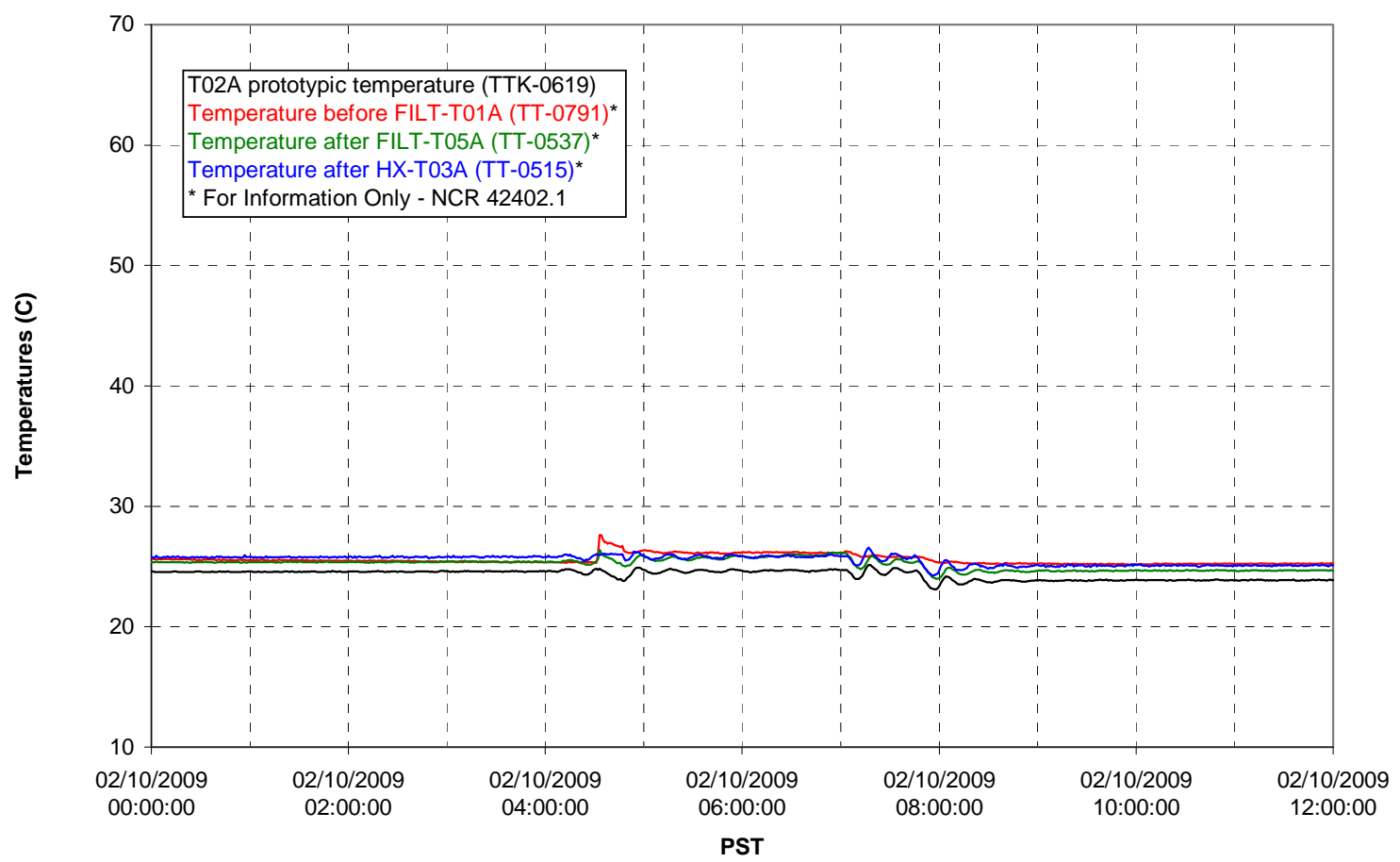

Pump Pressures and Flow

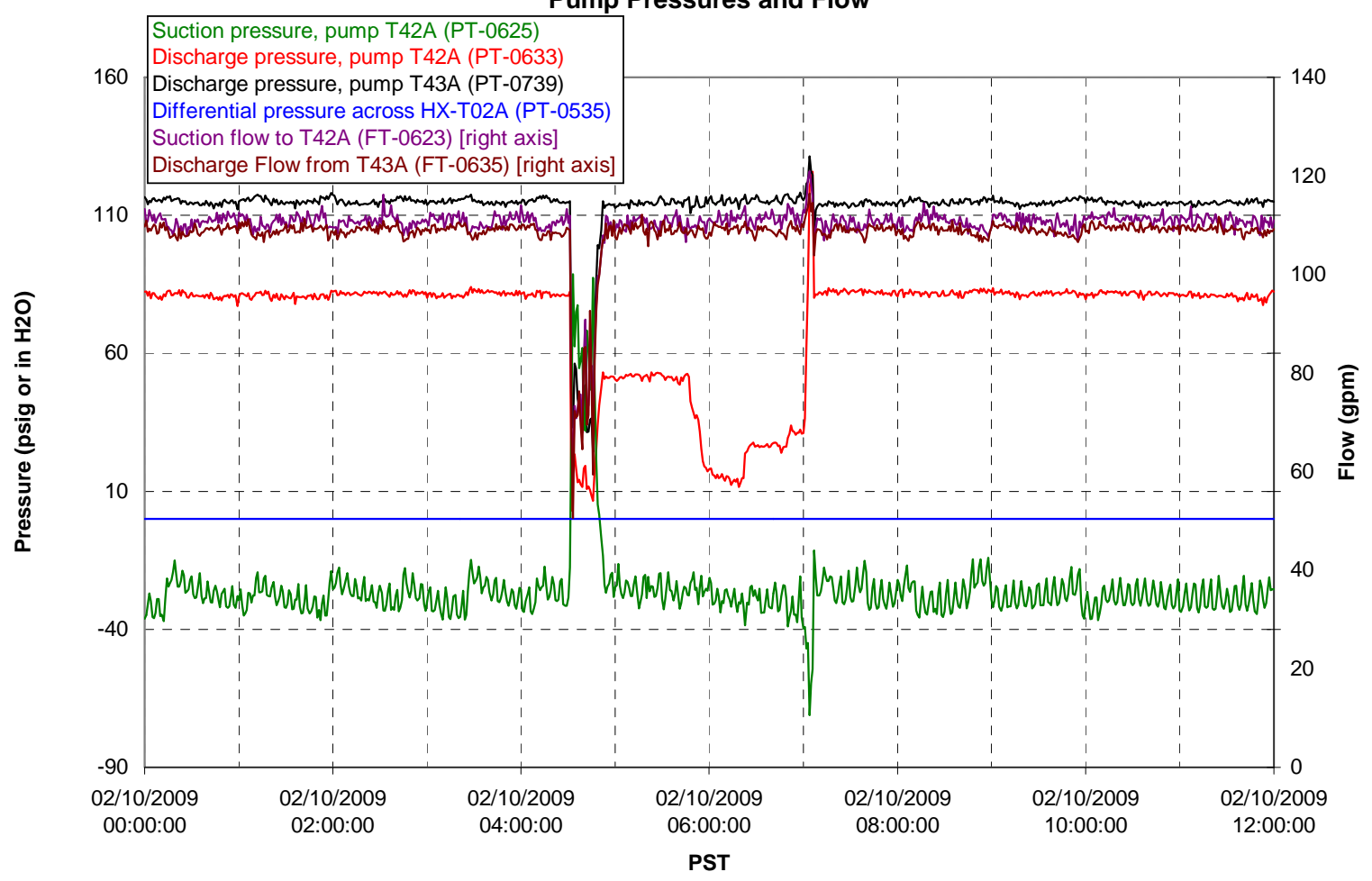




\section{Axial pressure drop}

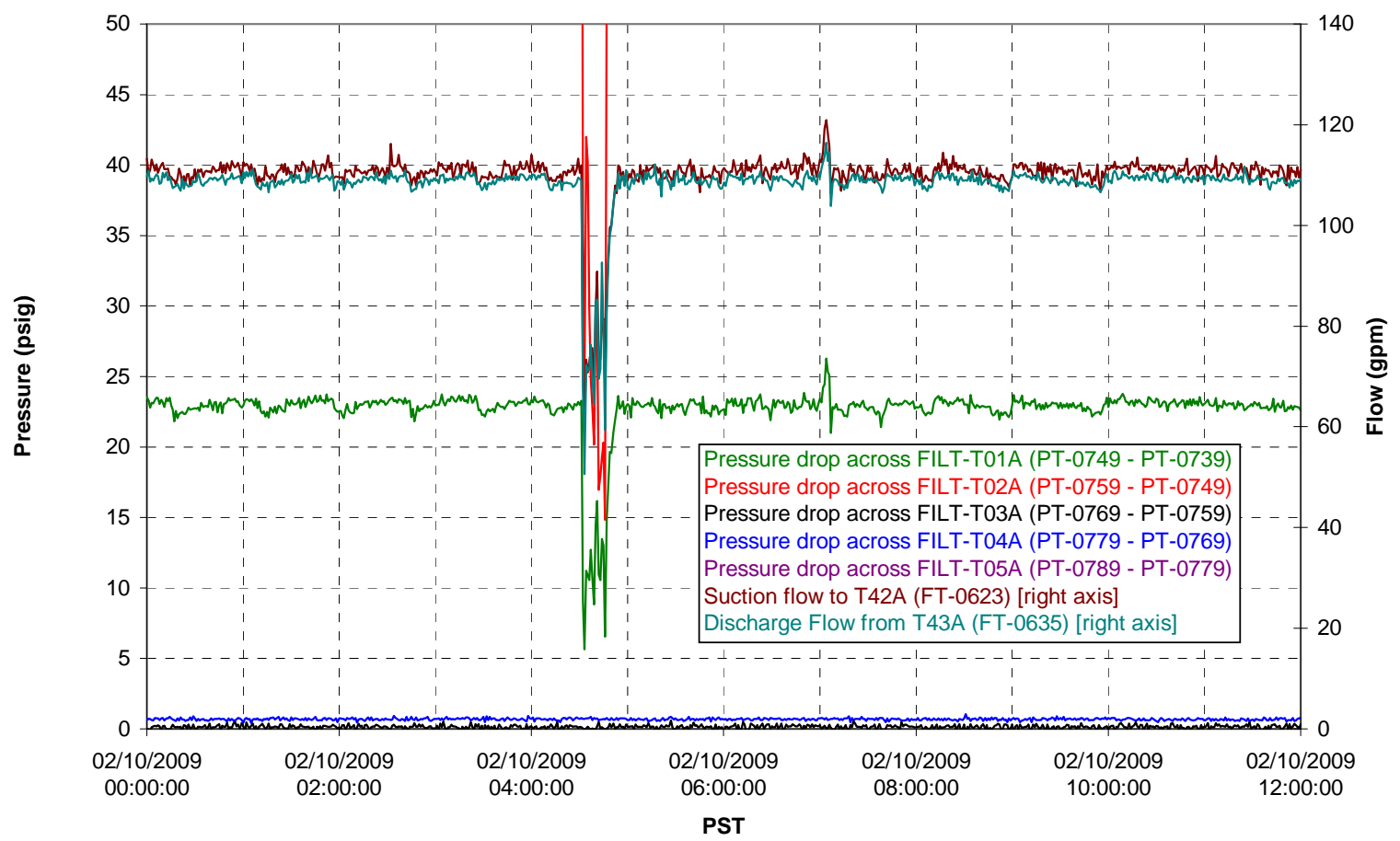

Permeate flow rates

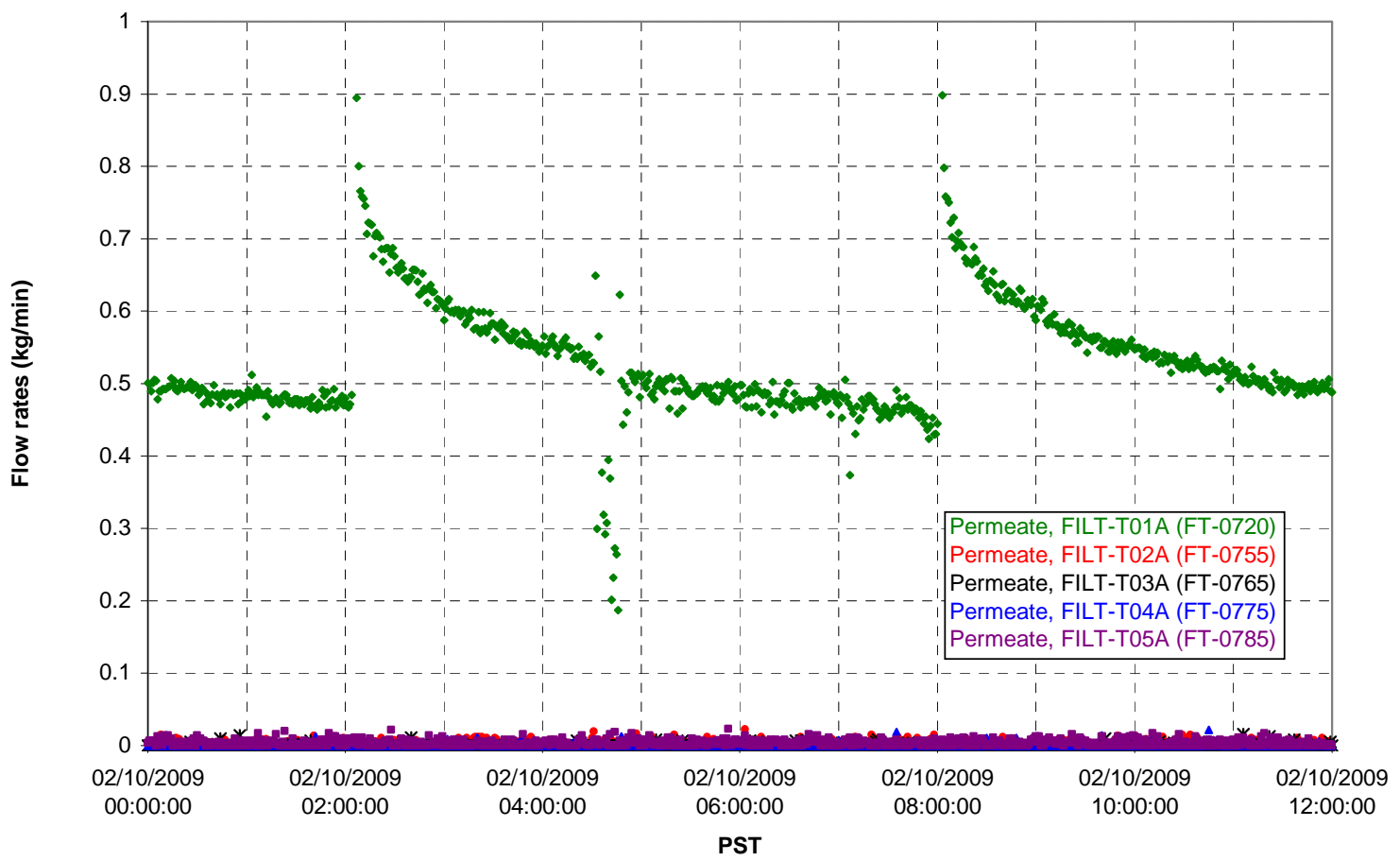


T02A Inner Temperature Tree

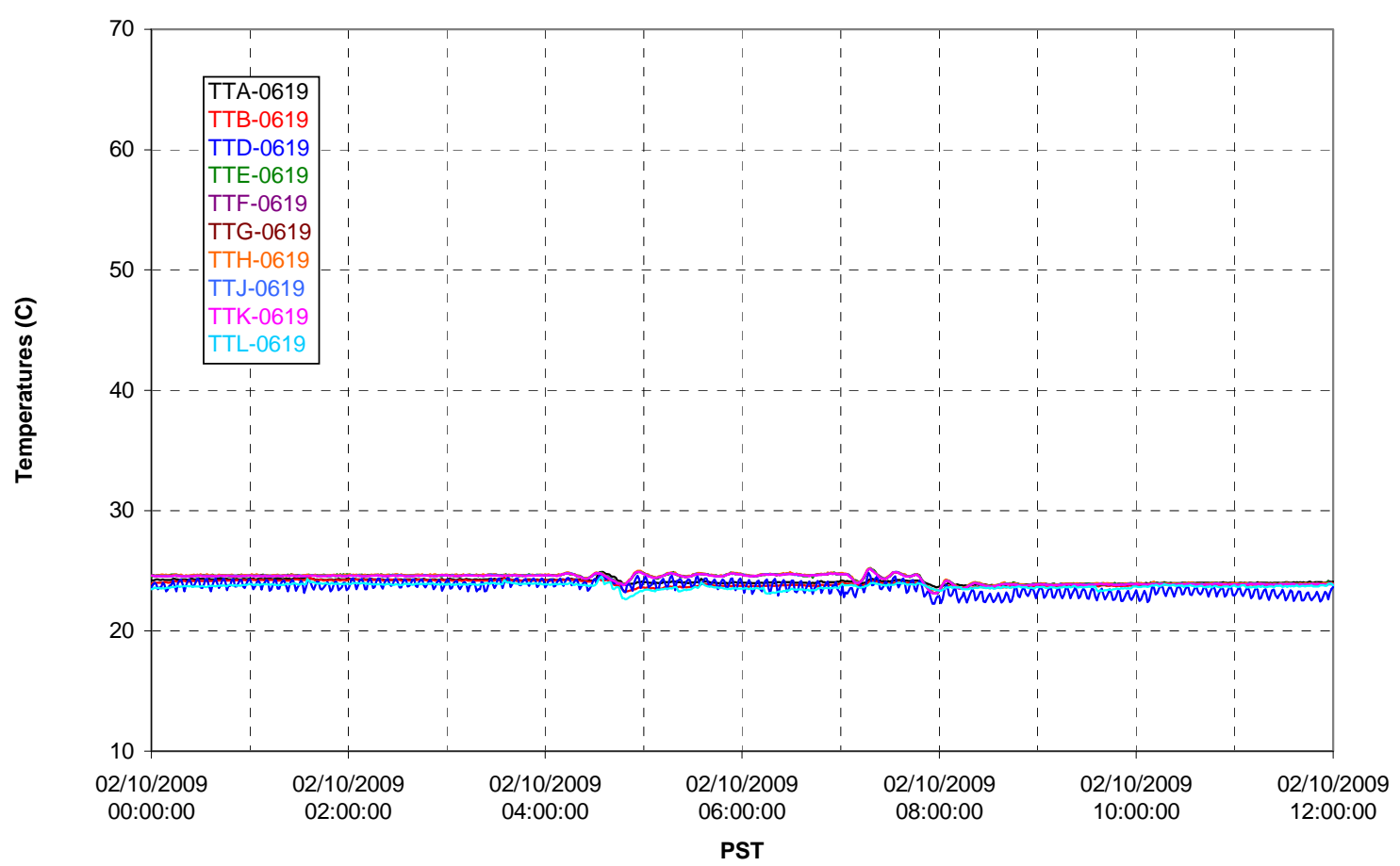

T02A Outer Temperature Tree

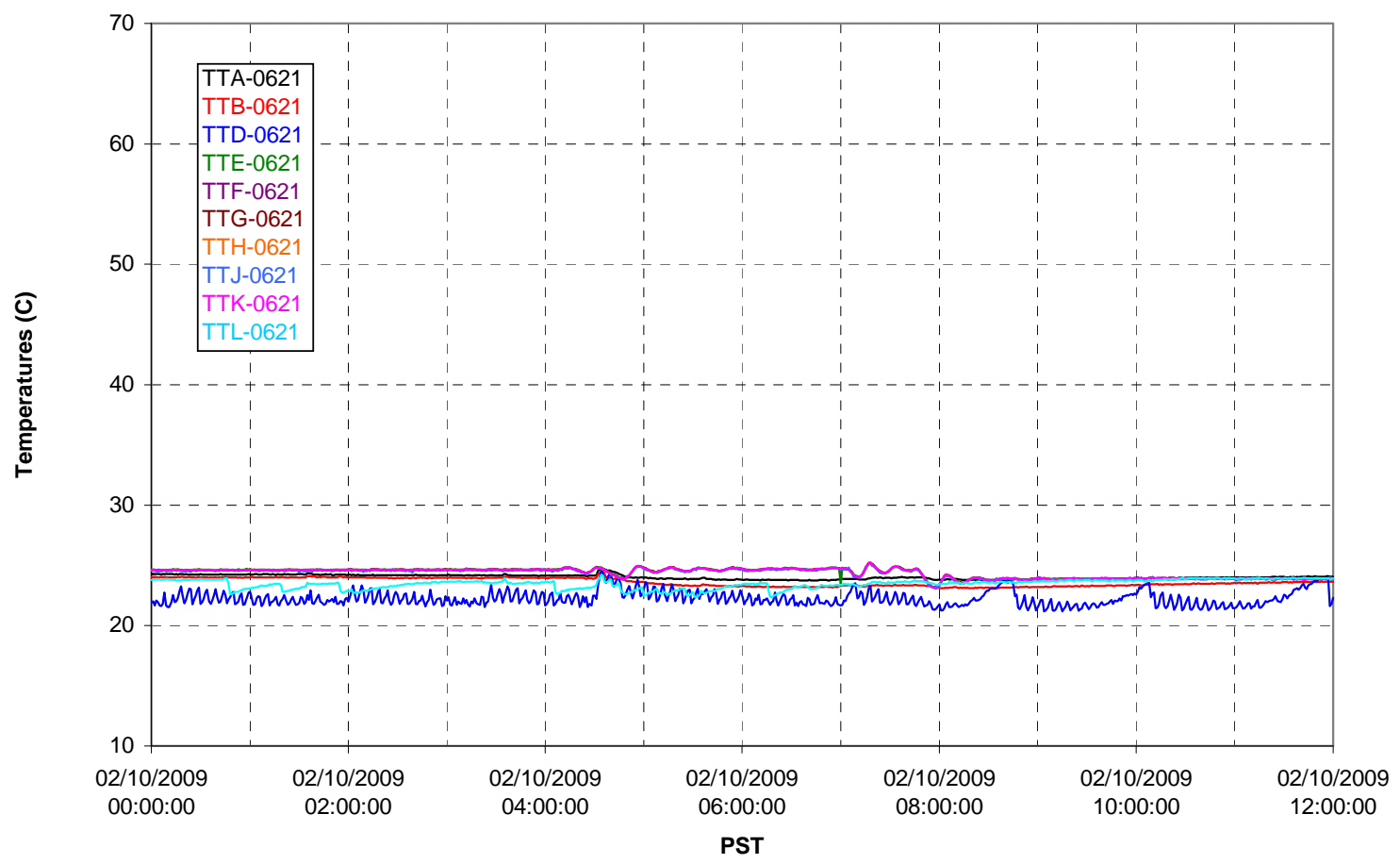


T02A temperatures

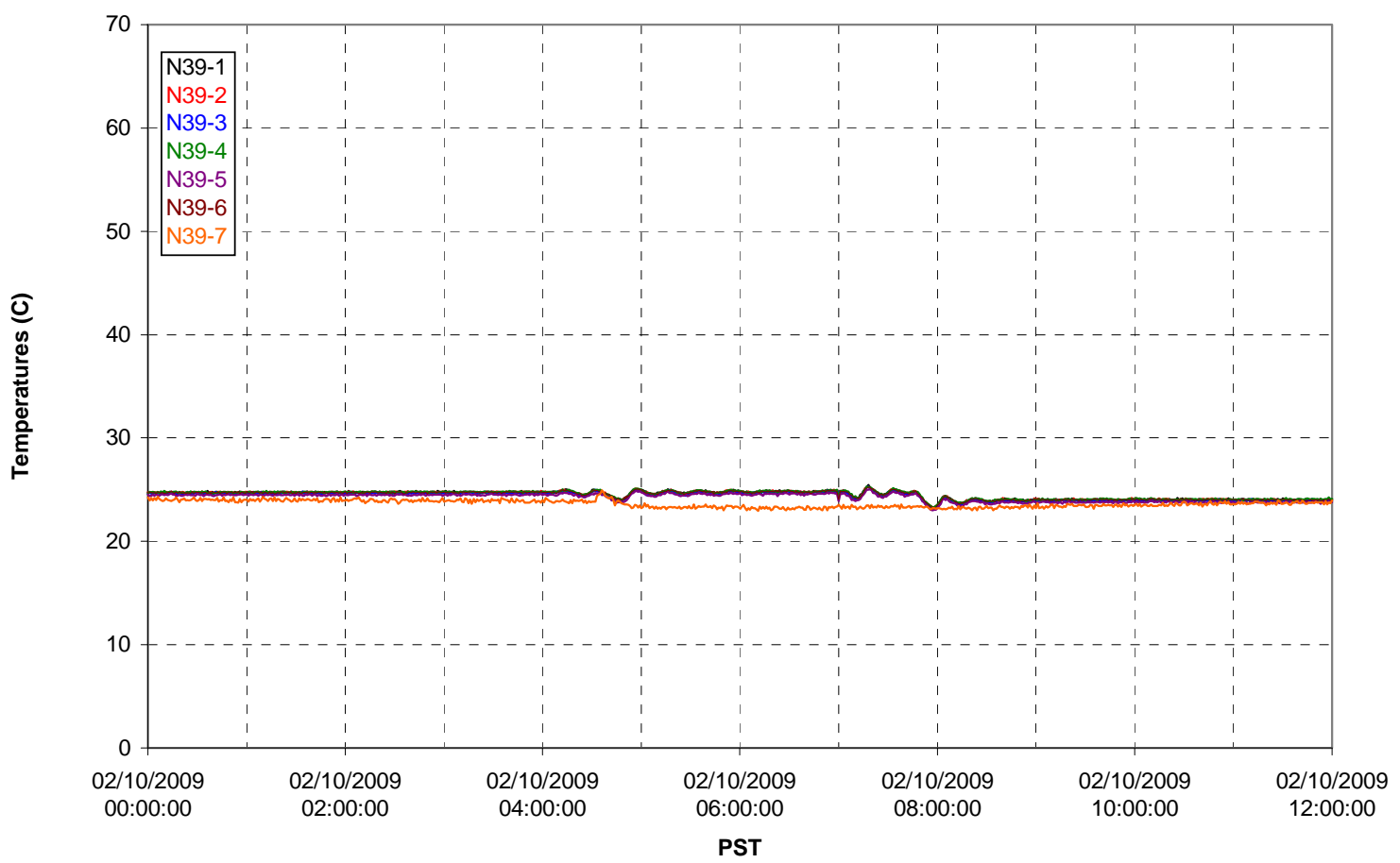

T02A temperatures

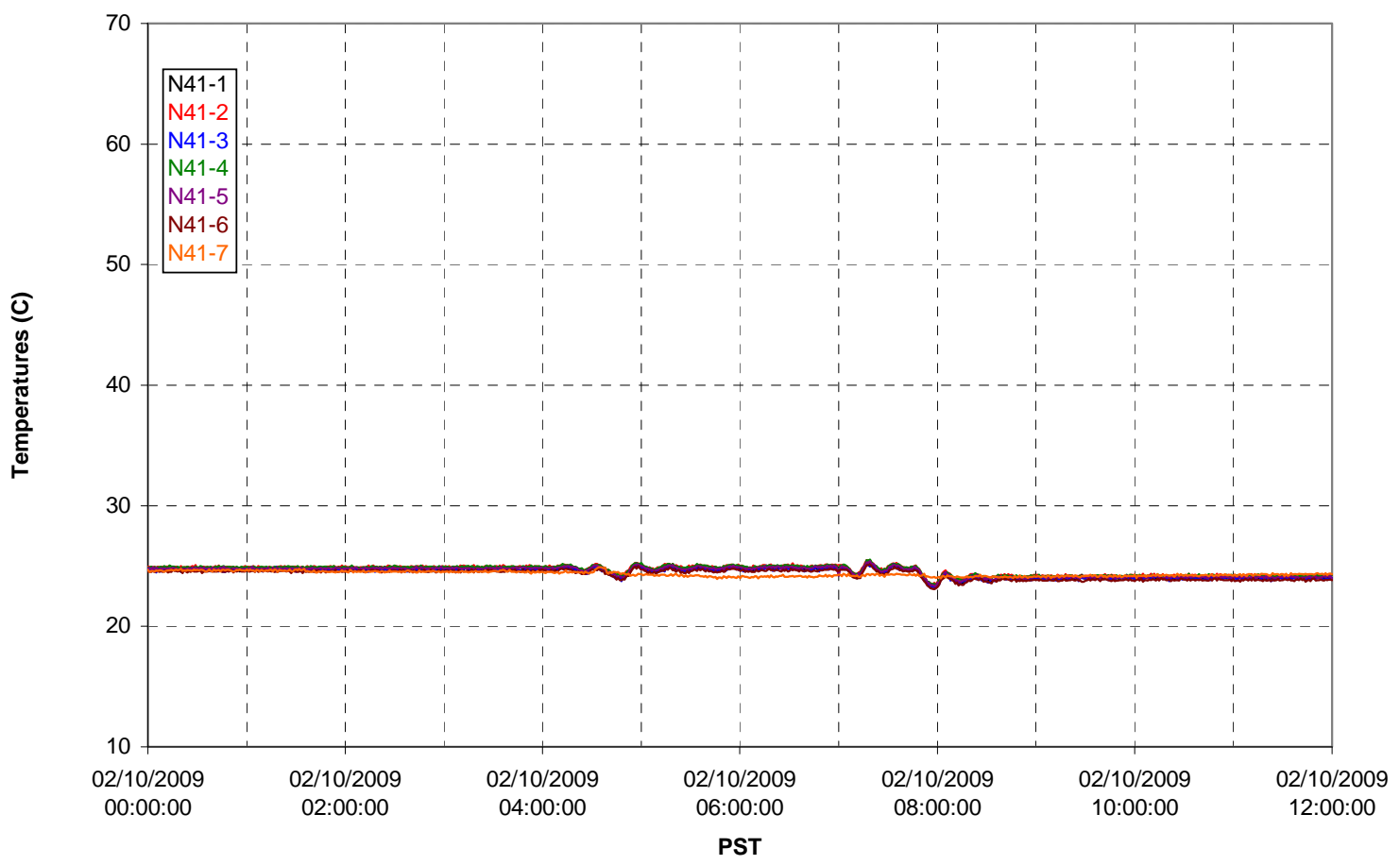


T02A temperatures

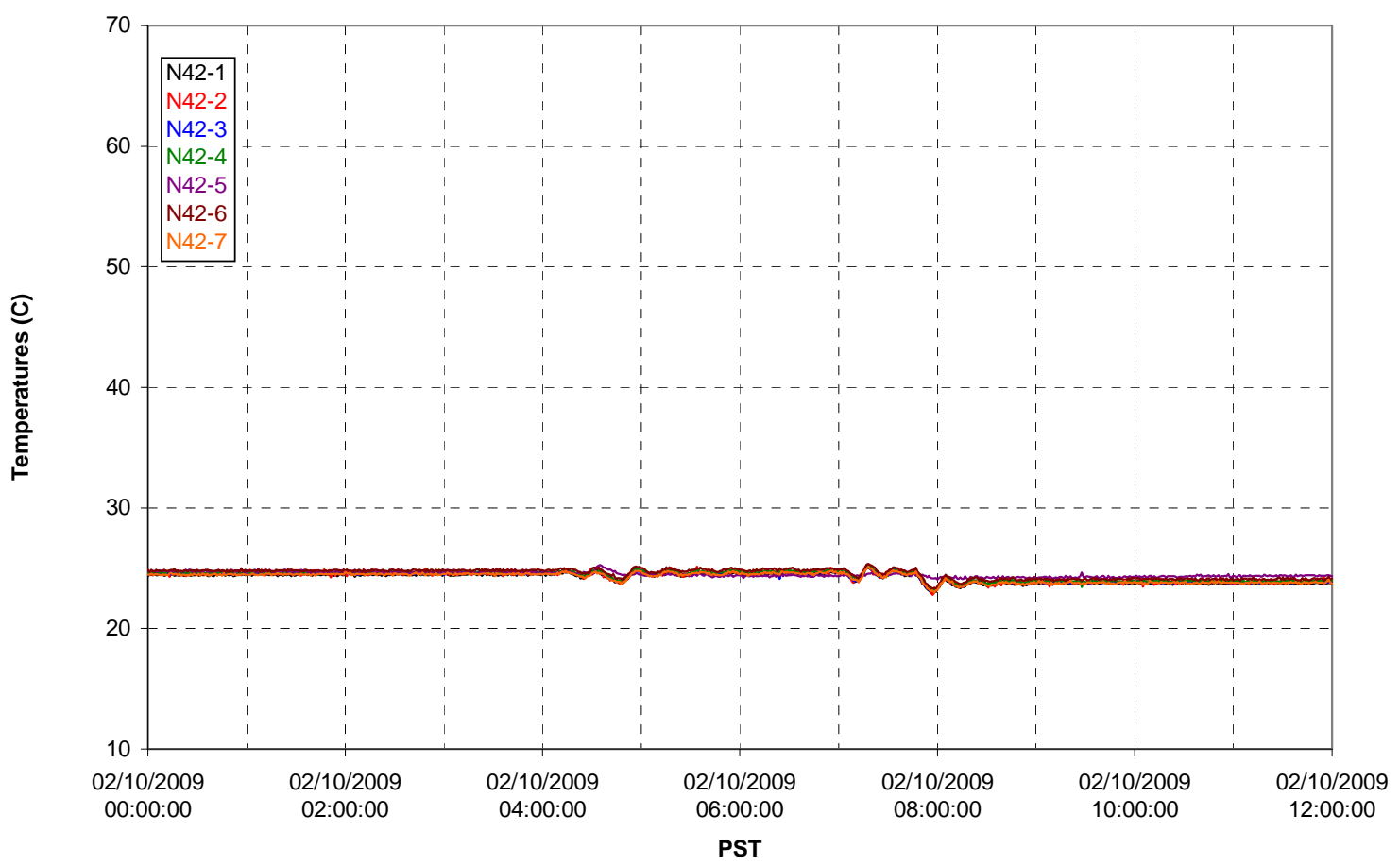

T02A temperatures

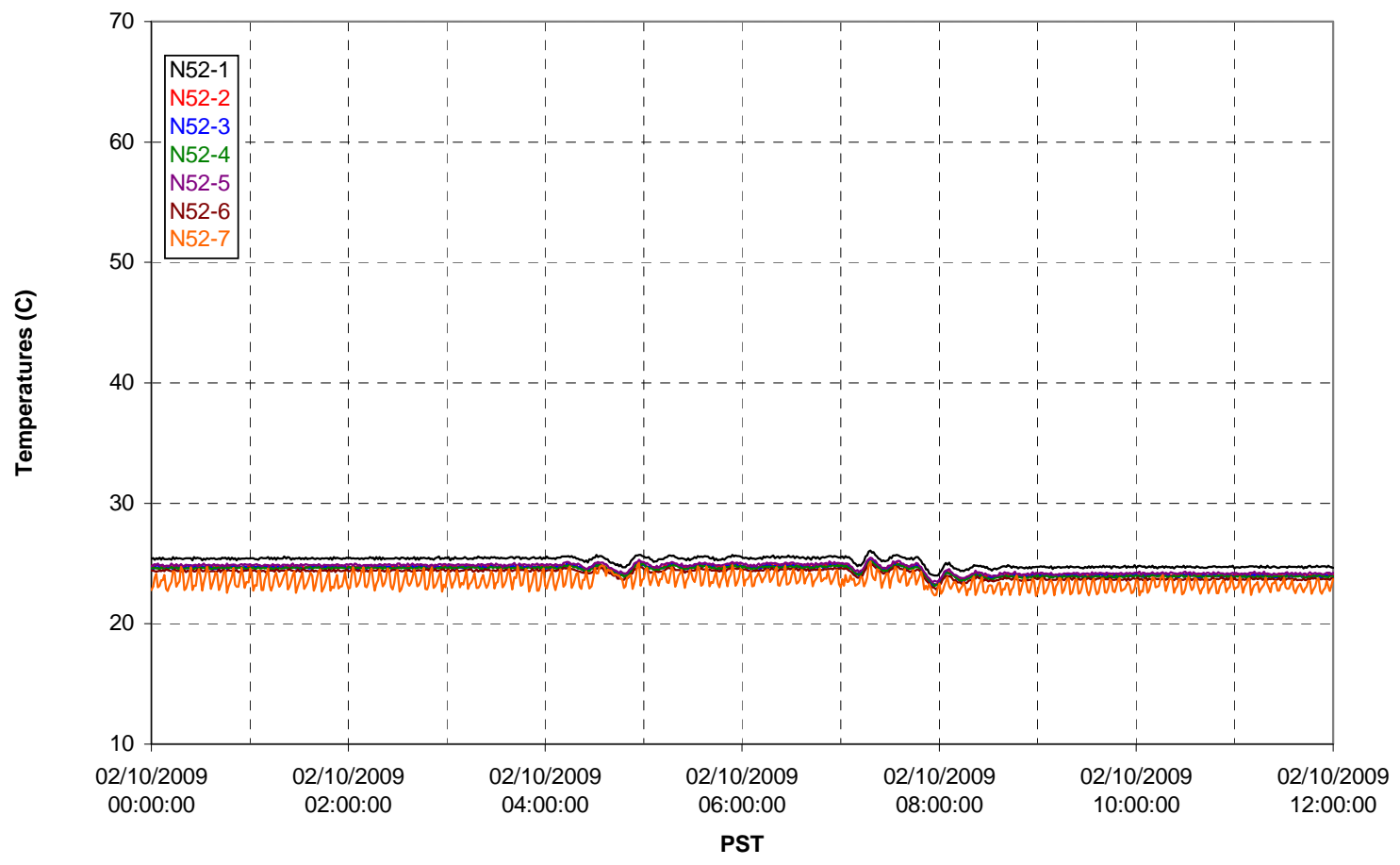


T02A Heating and Cooling
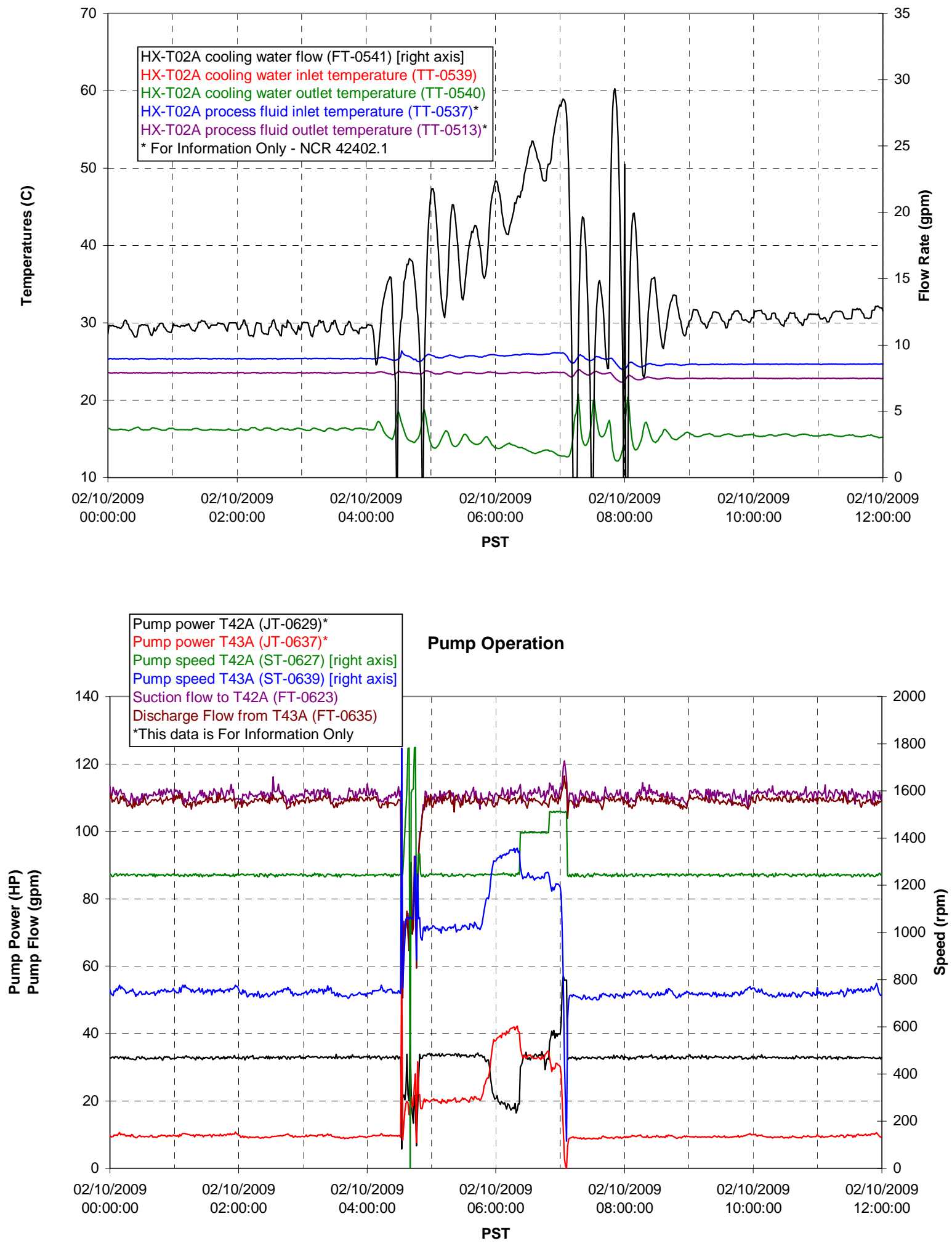
Pulsepot UFP-PP-T01A

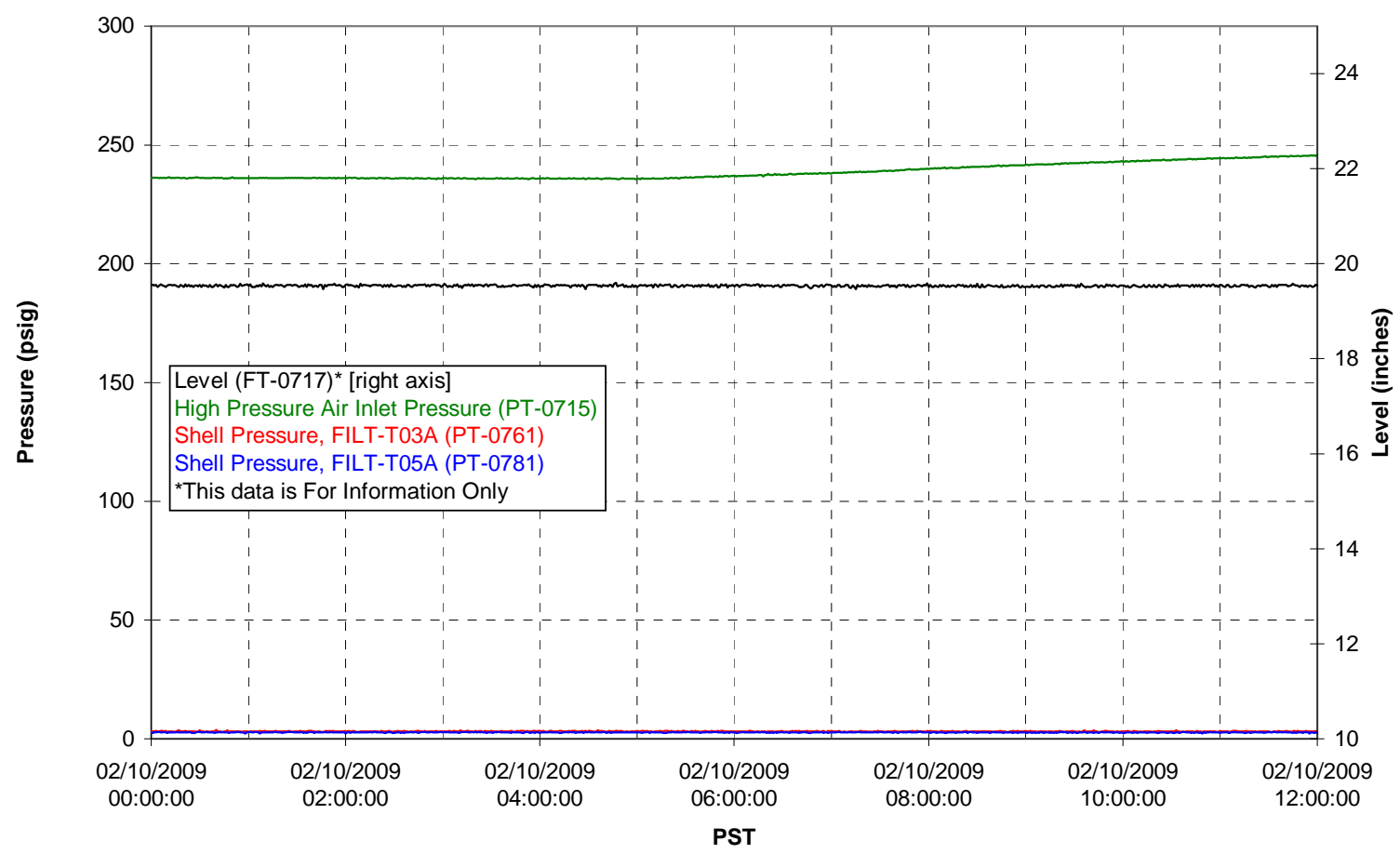

Pulsepot UFP-PP-T02A

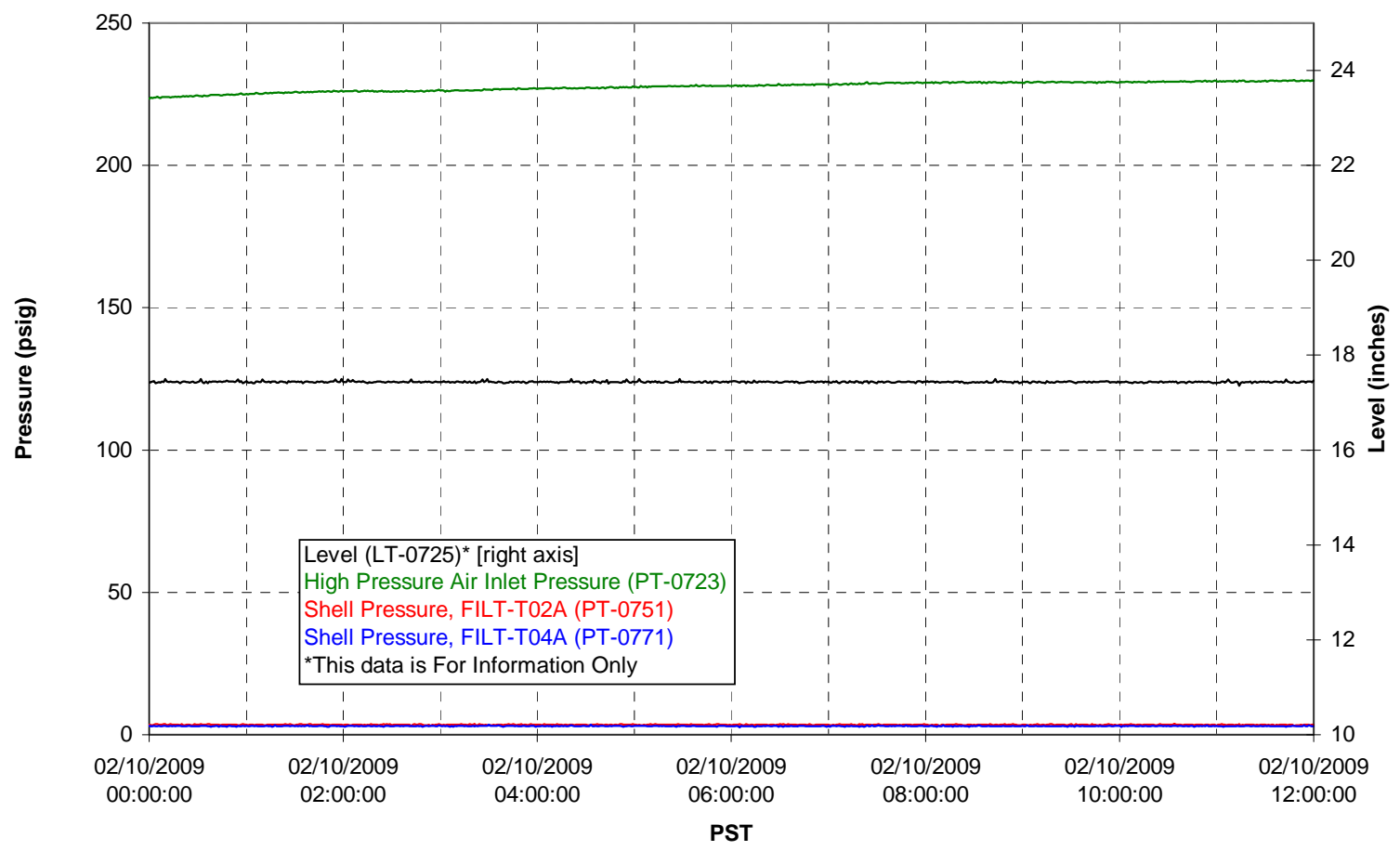


Pulsepot UFP-PP-T03A

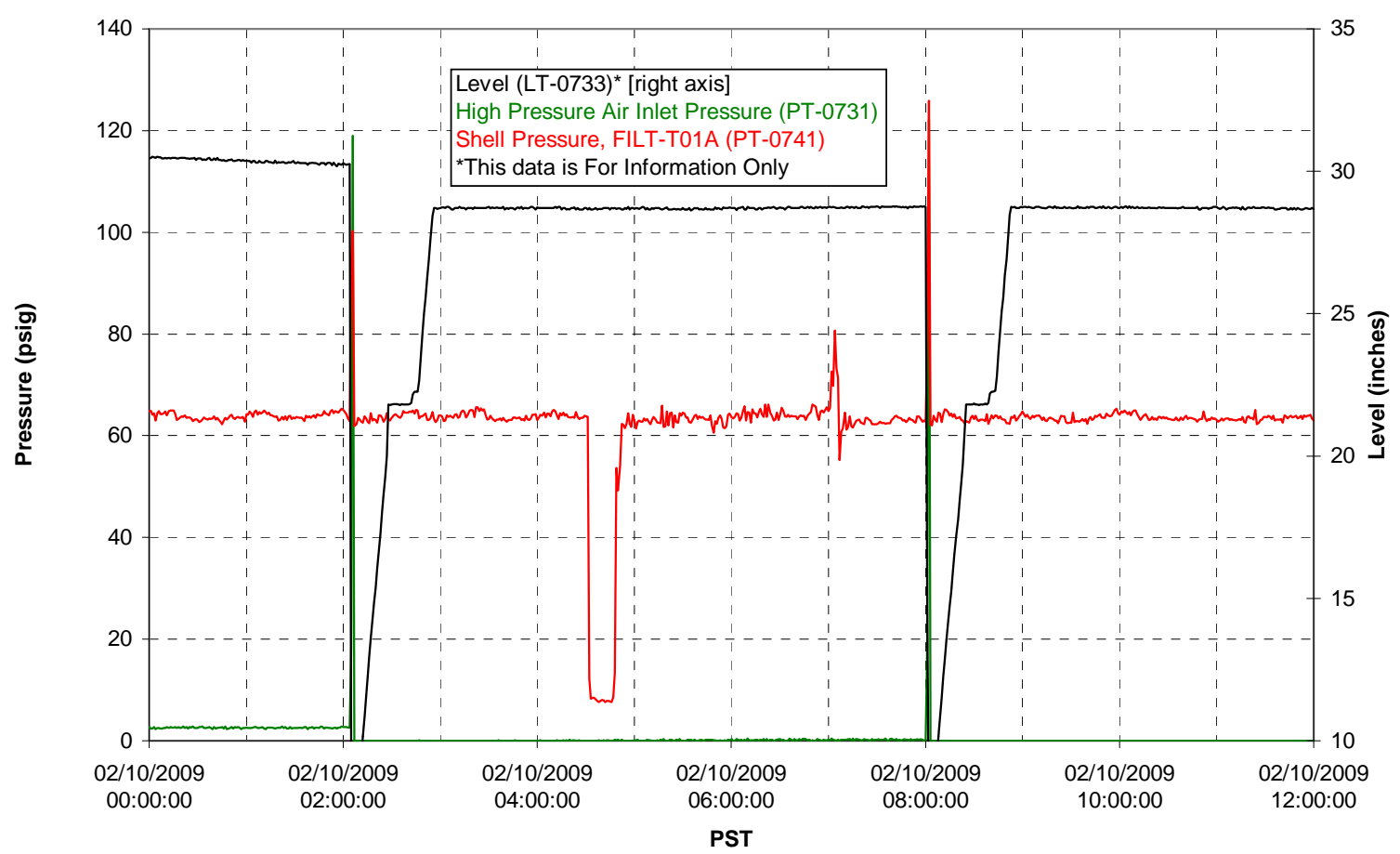

Pulsepot Levels

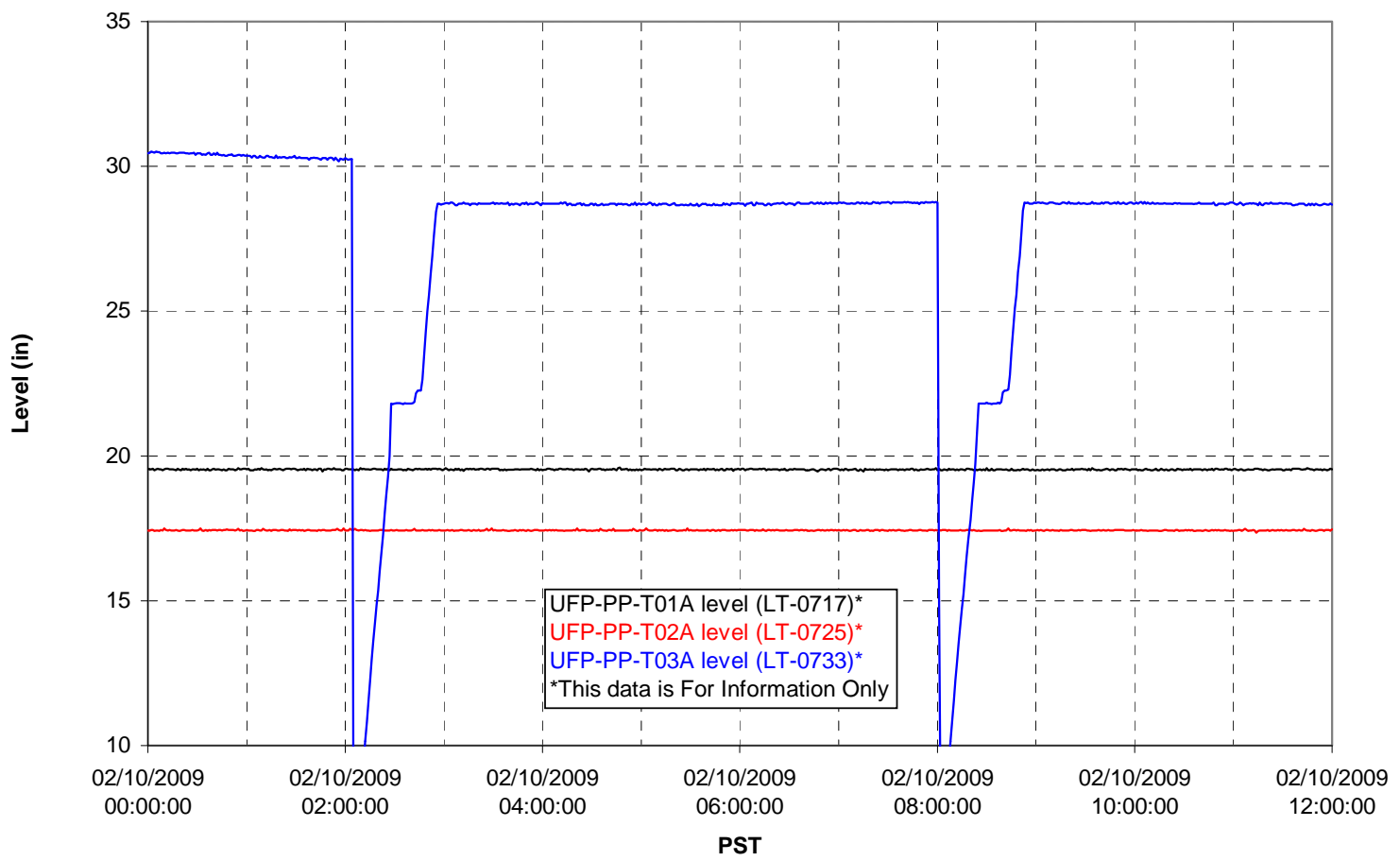


Filter UFP-FILT-T01A

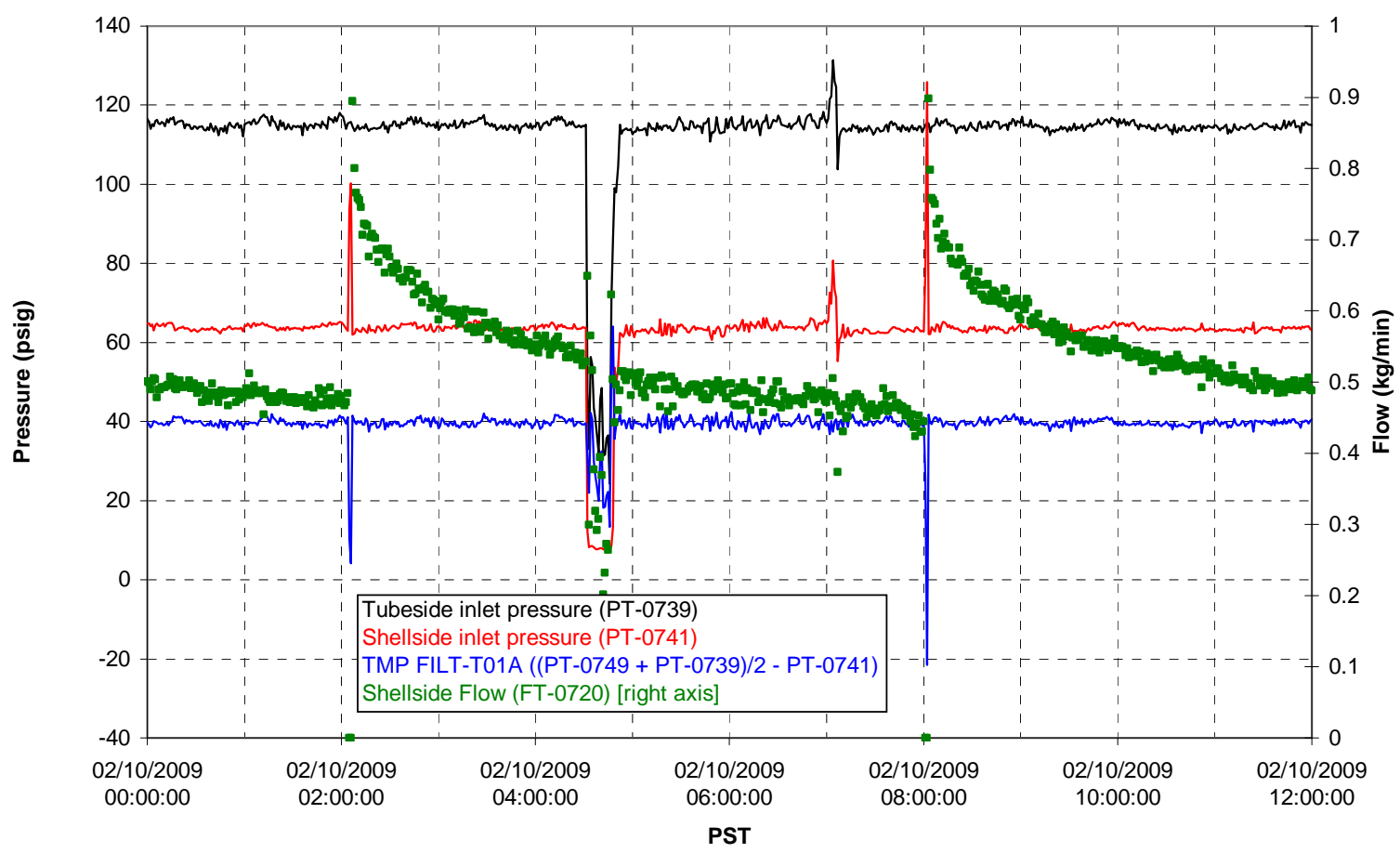

Filter UFP-FILT-T02A

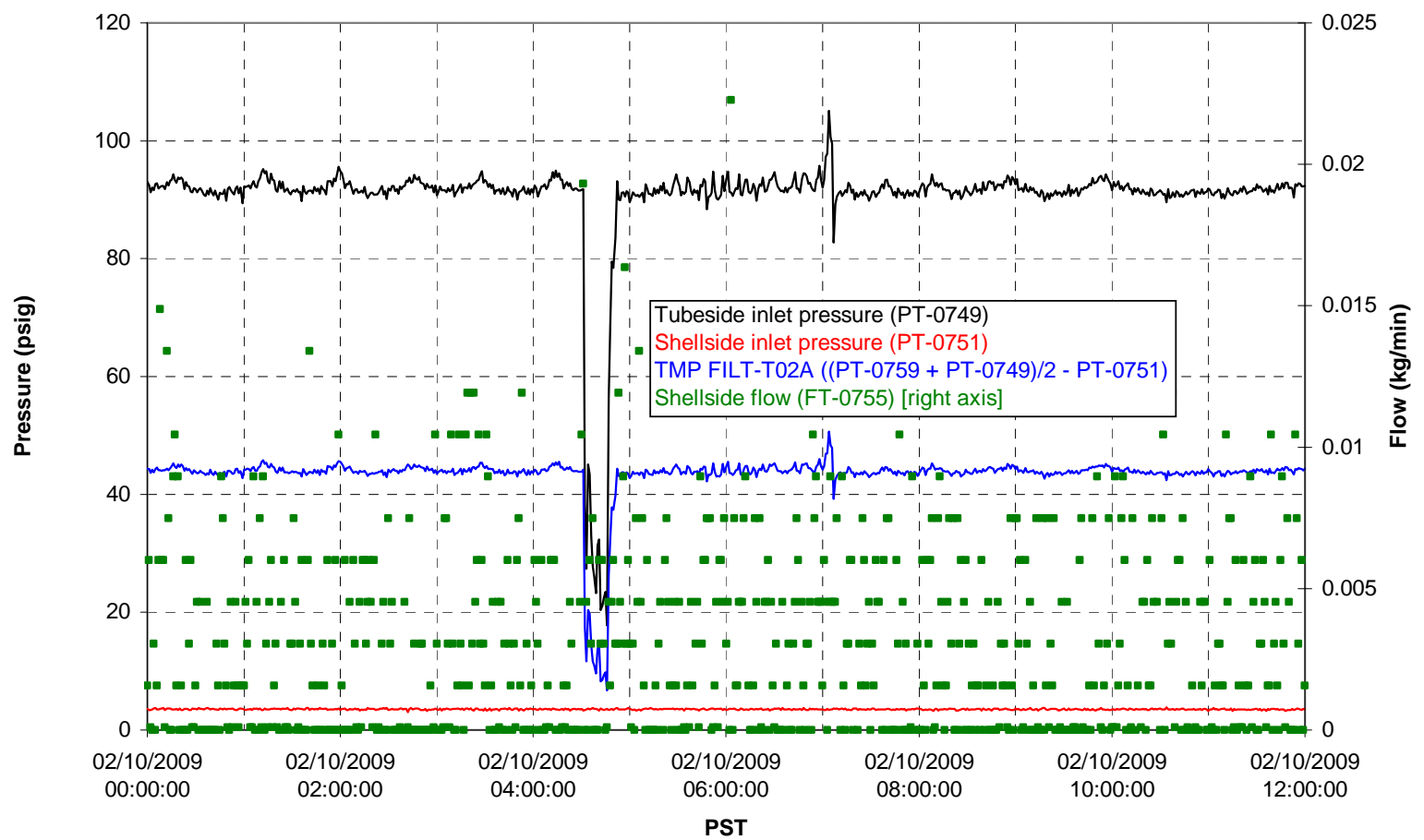


Filter UFP-FILT-T03A

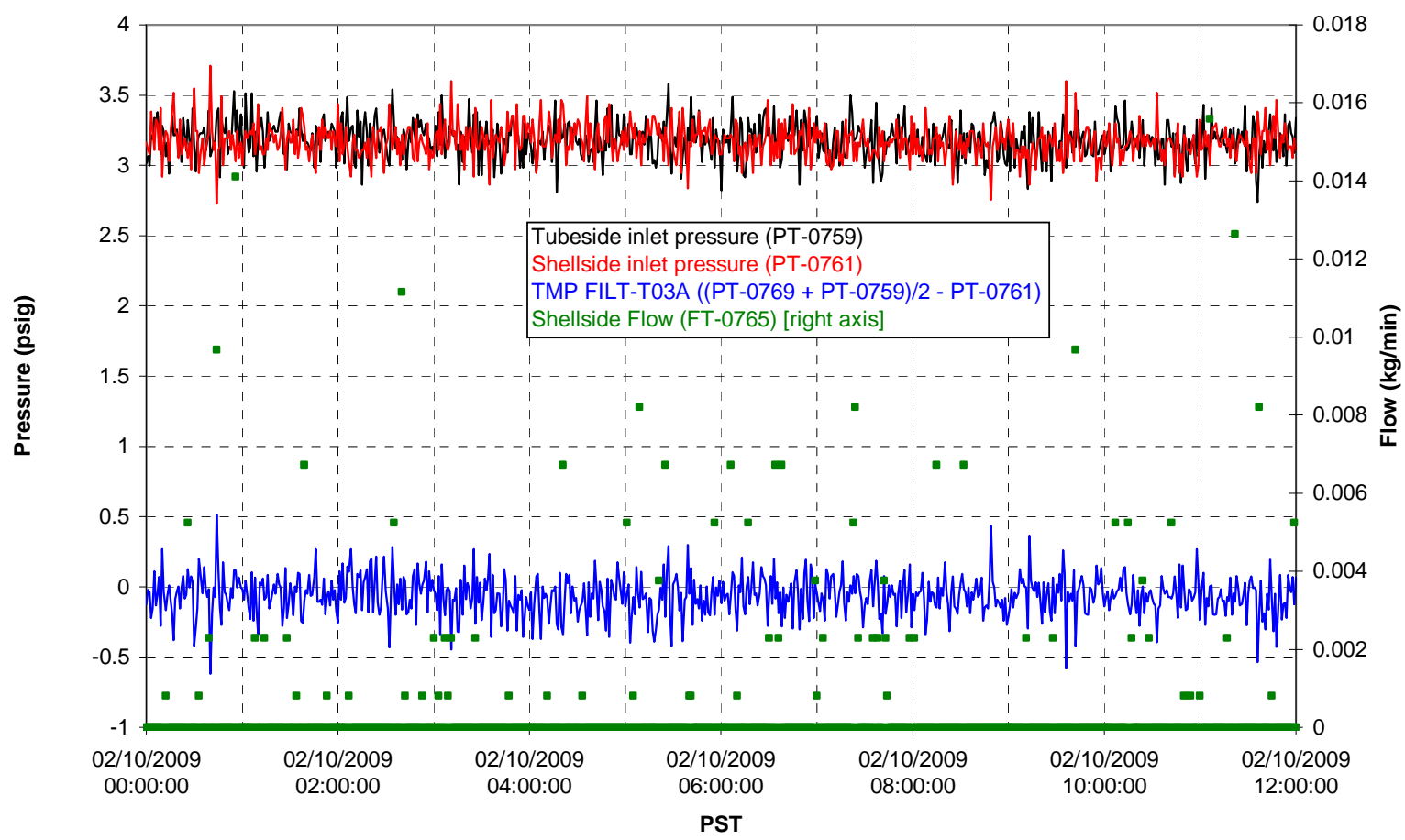

Filter UFP-FILT-T04A

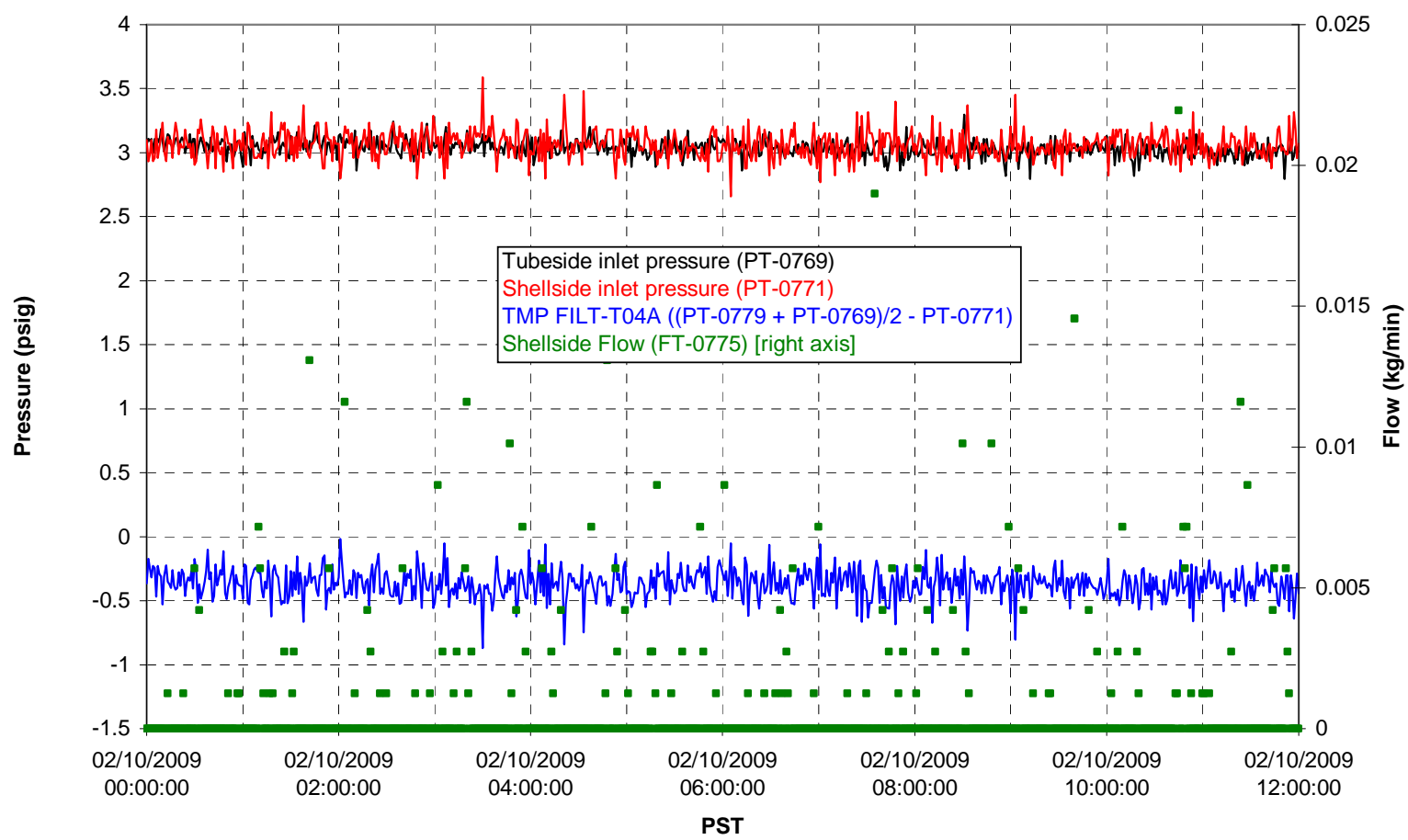


Filter UFP-FILT-T05A

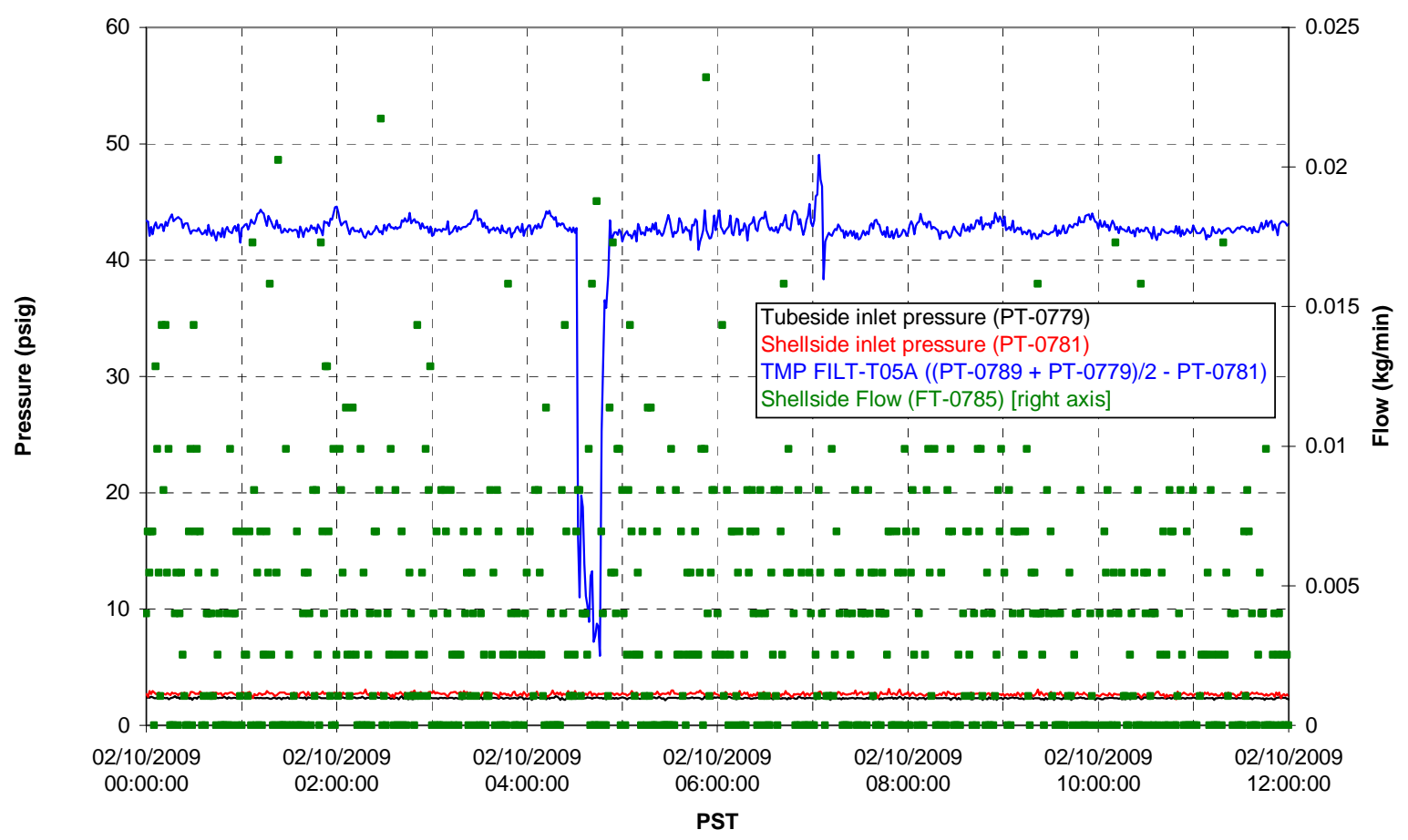

Chemical Flow

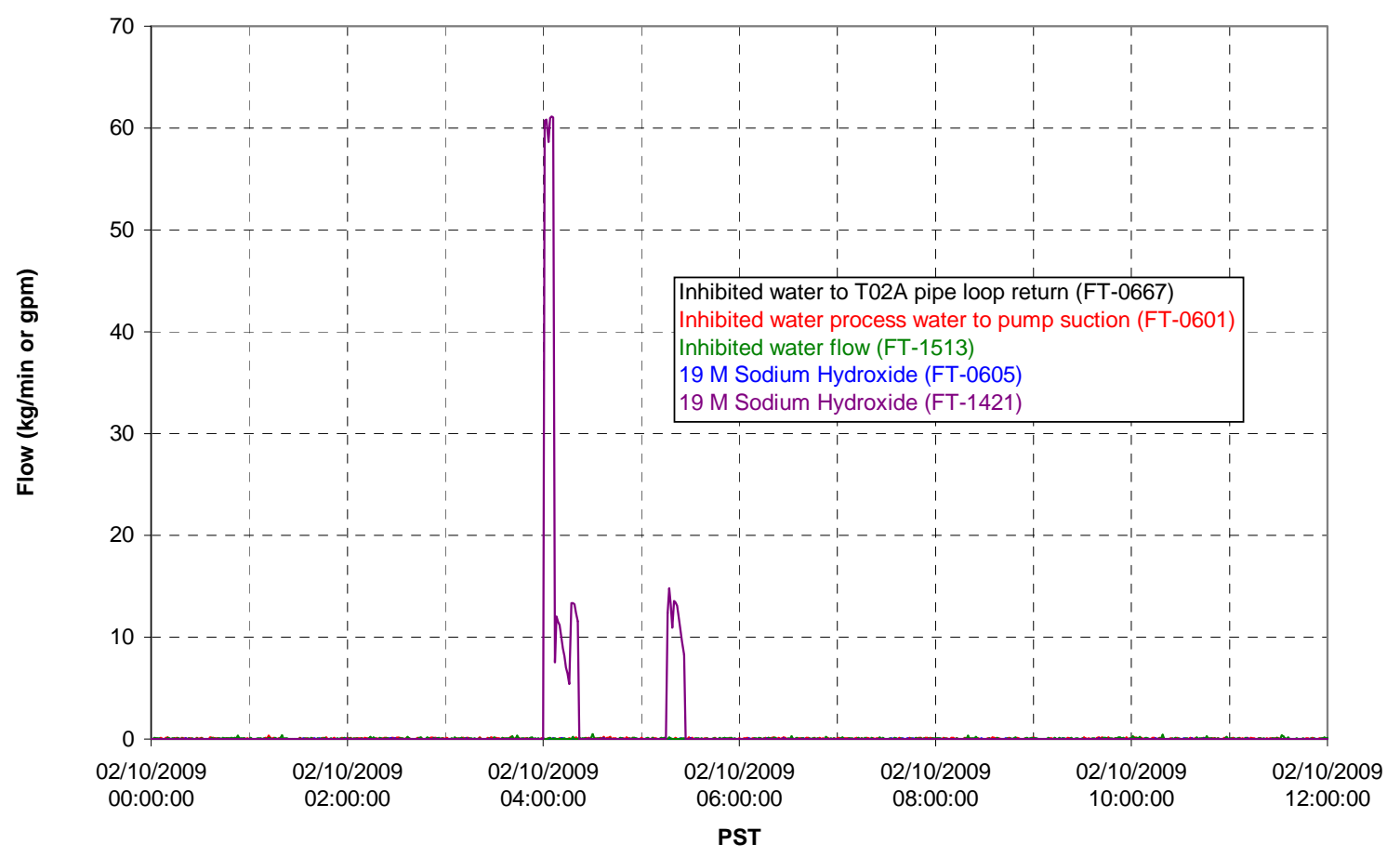


Chemical Flow

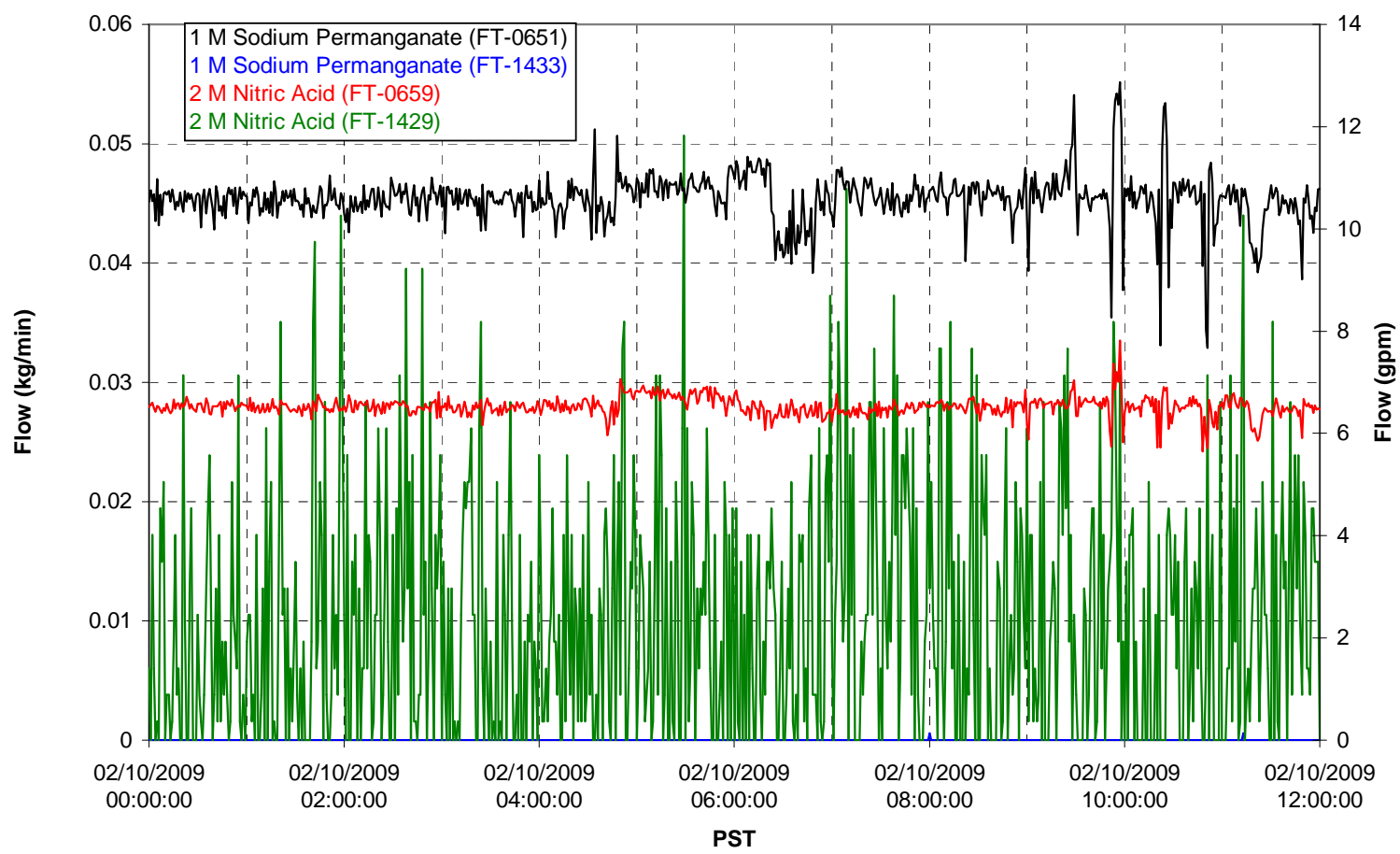

Air Flows

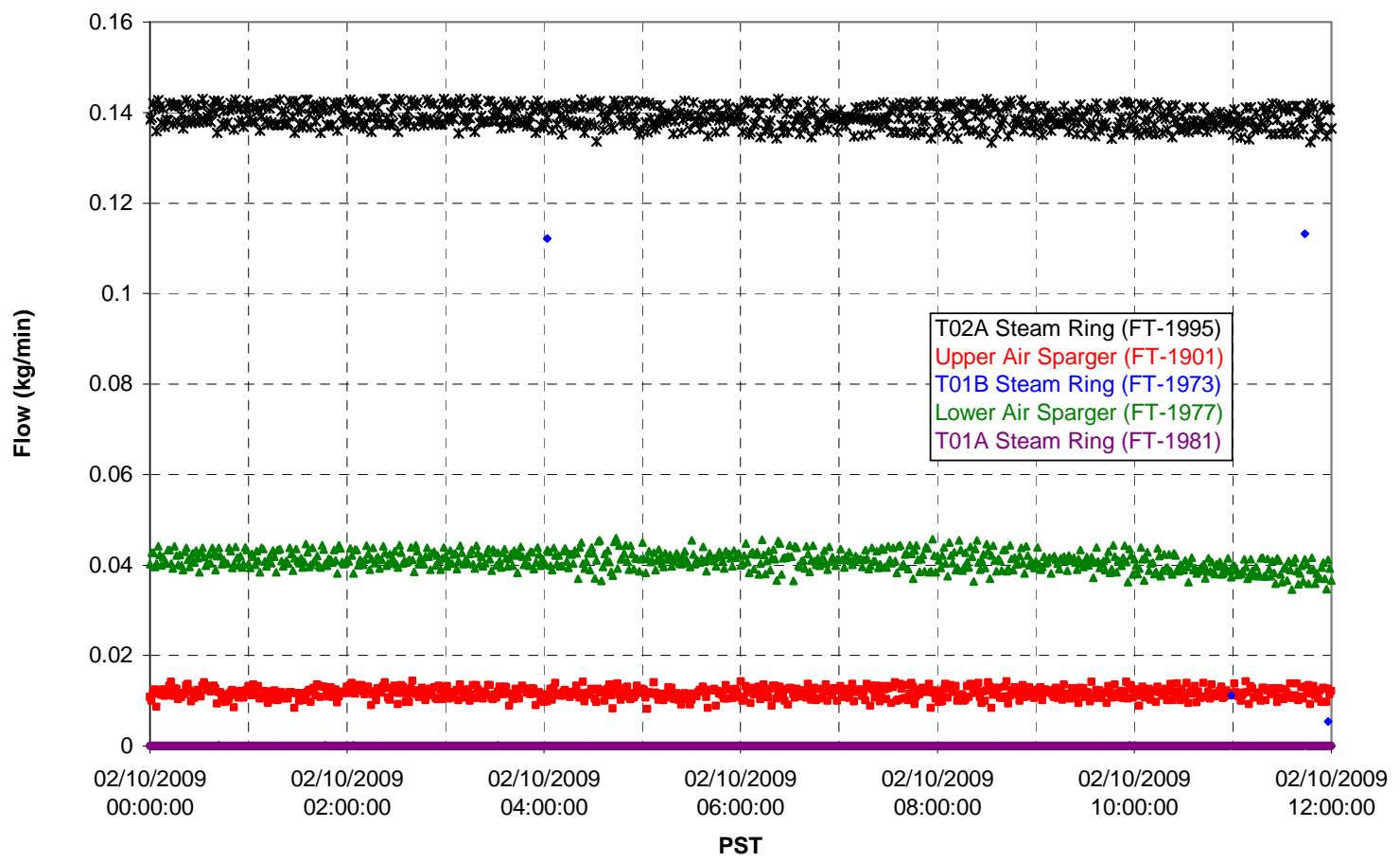


T02A Steam

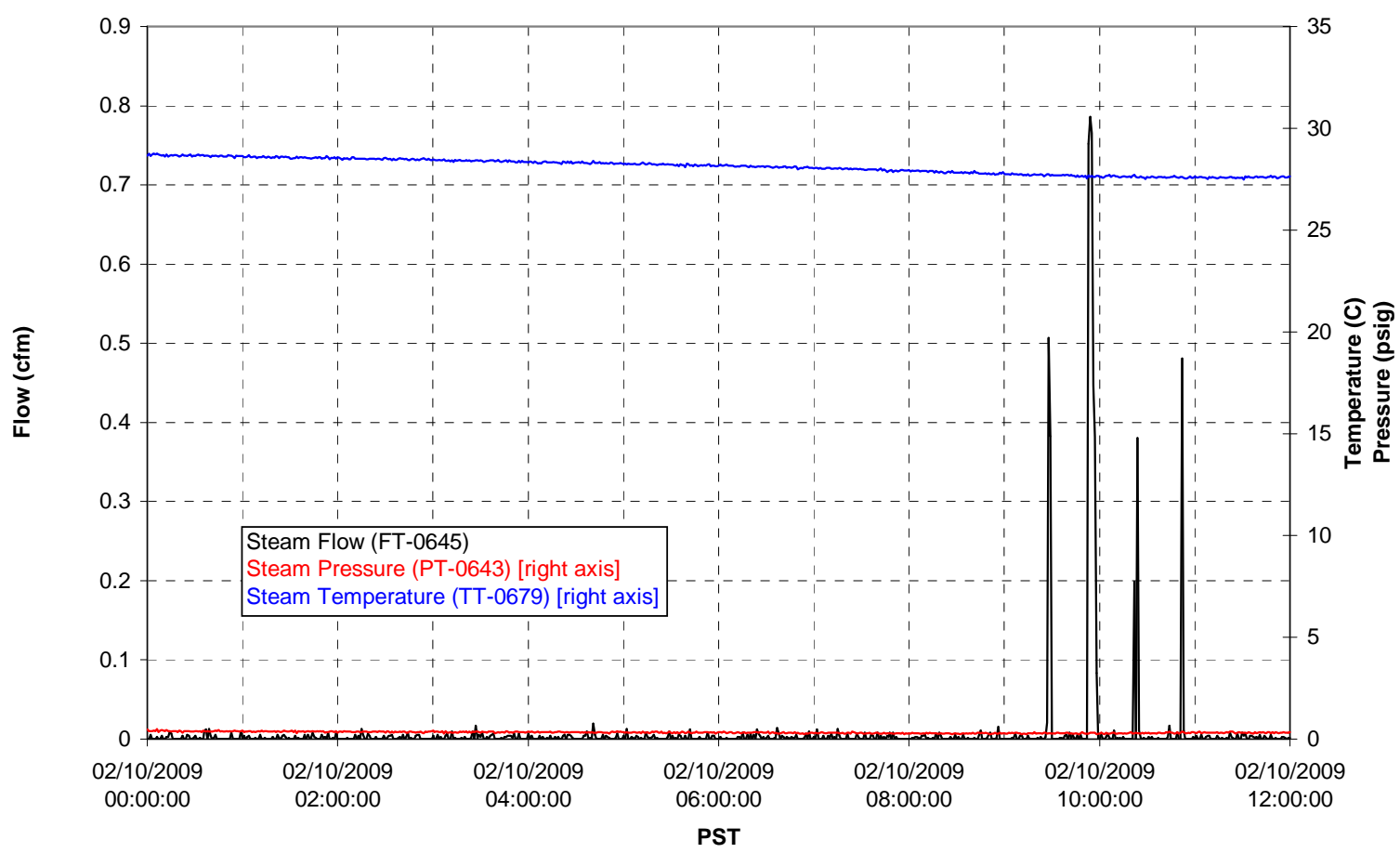

T01A Steam

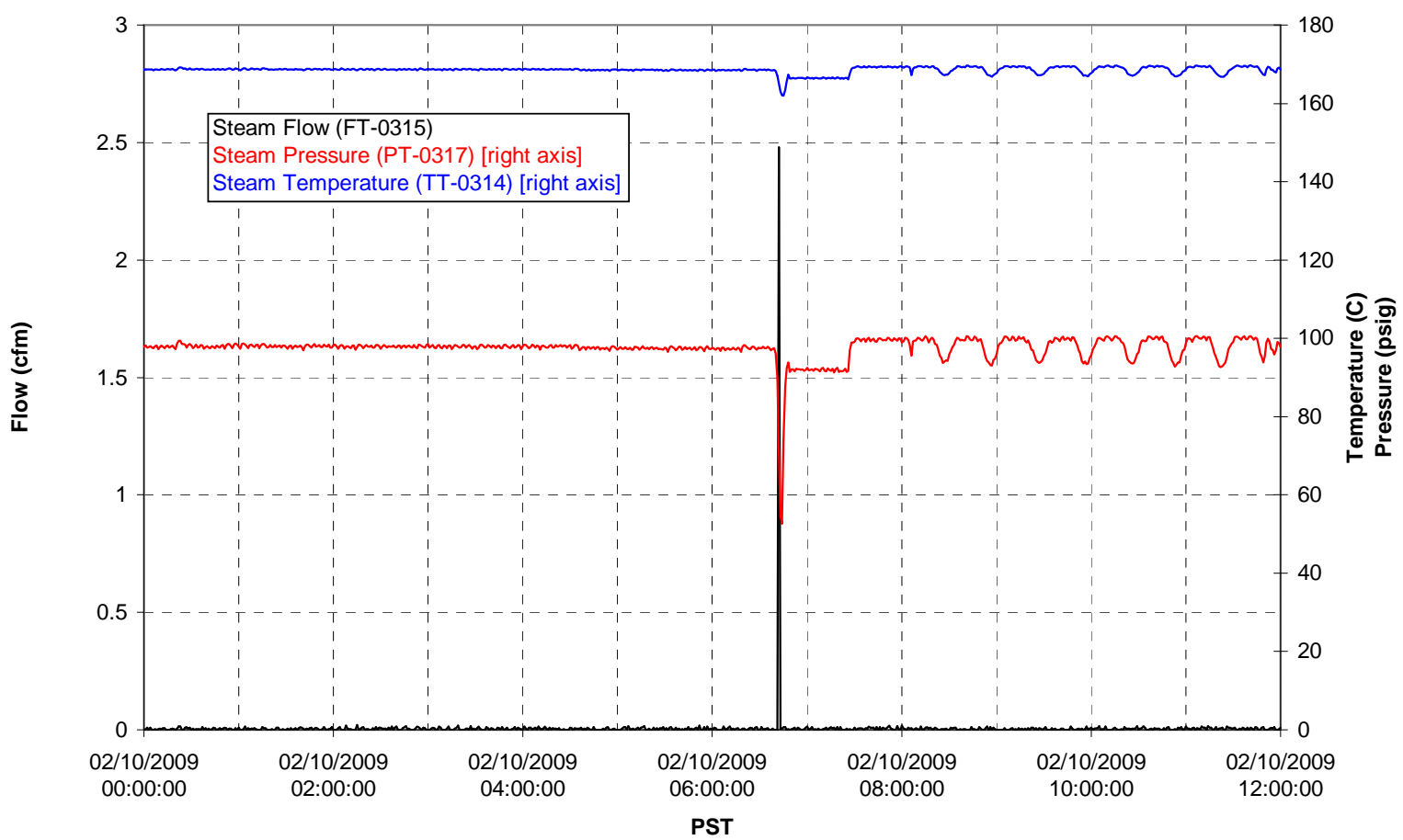


T01B Steam

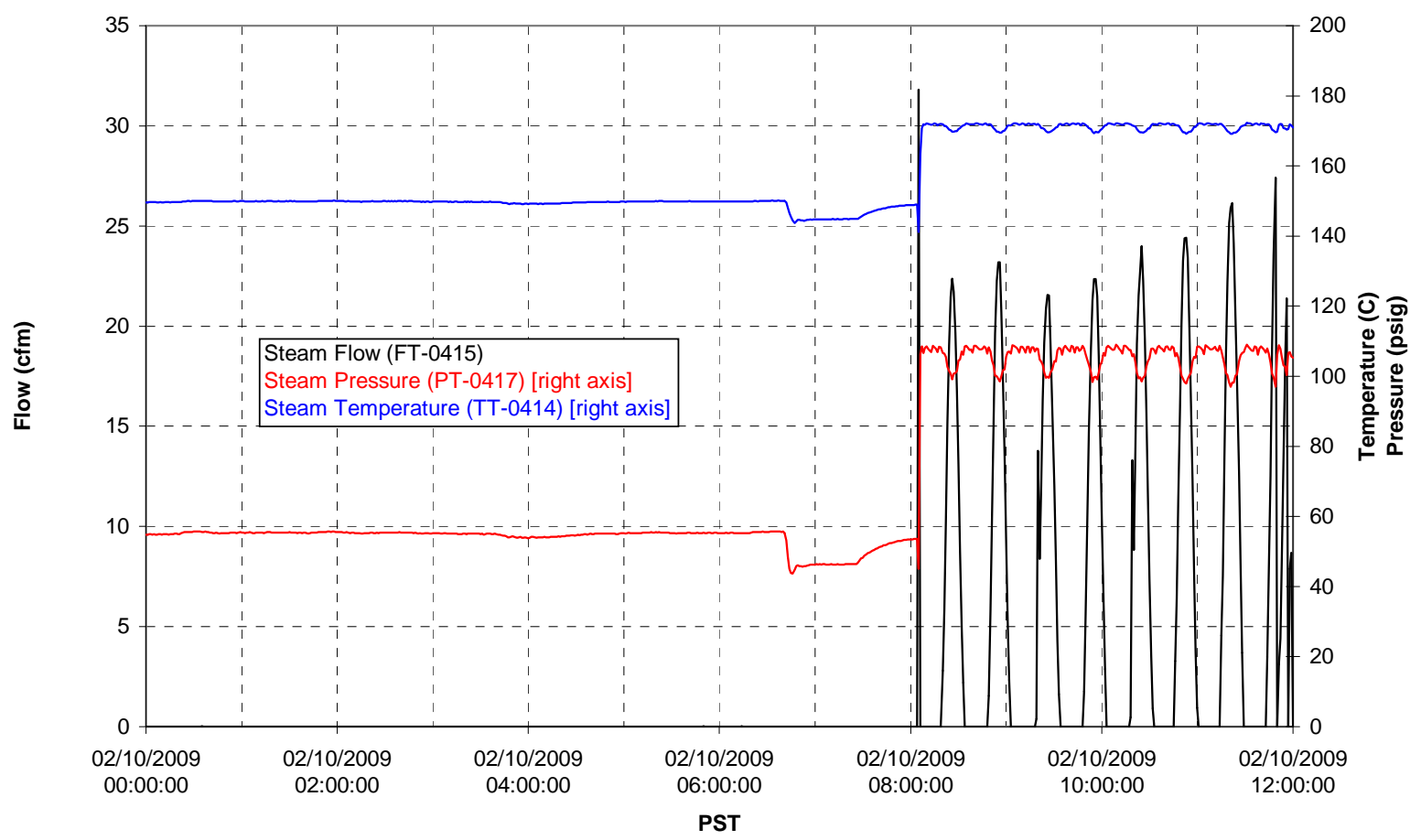




\section{Integrated Test A Data Plots 02/10/09 12:00 - 02/11/09 00:00}


T01A level

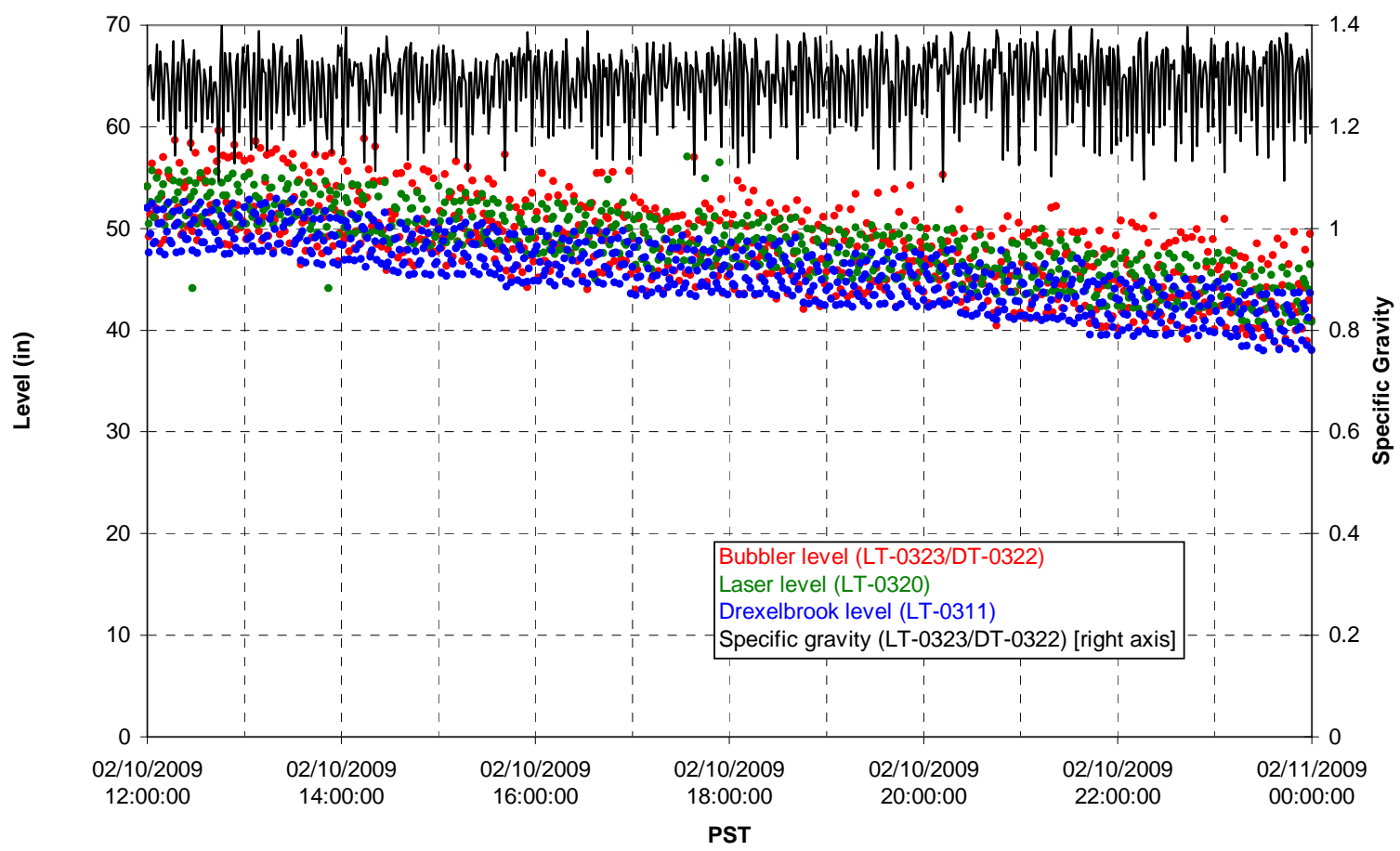

T01A temperatures

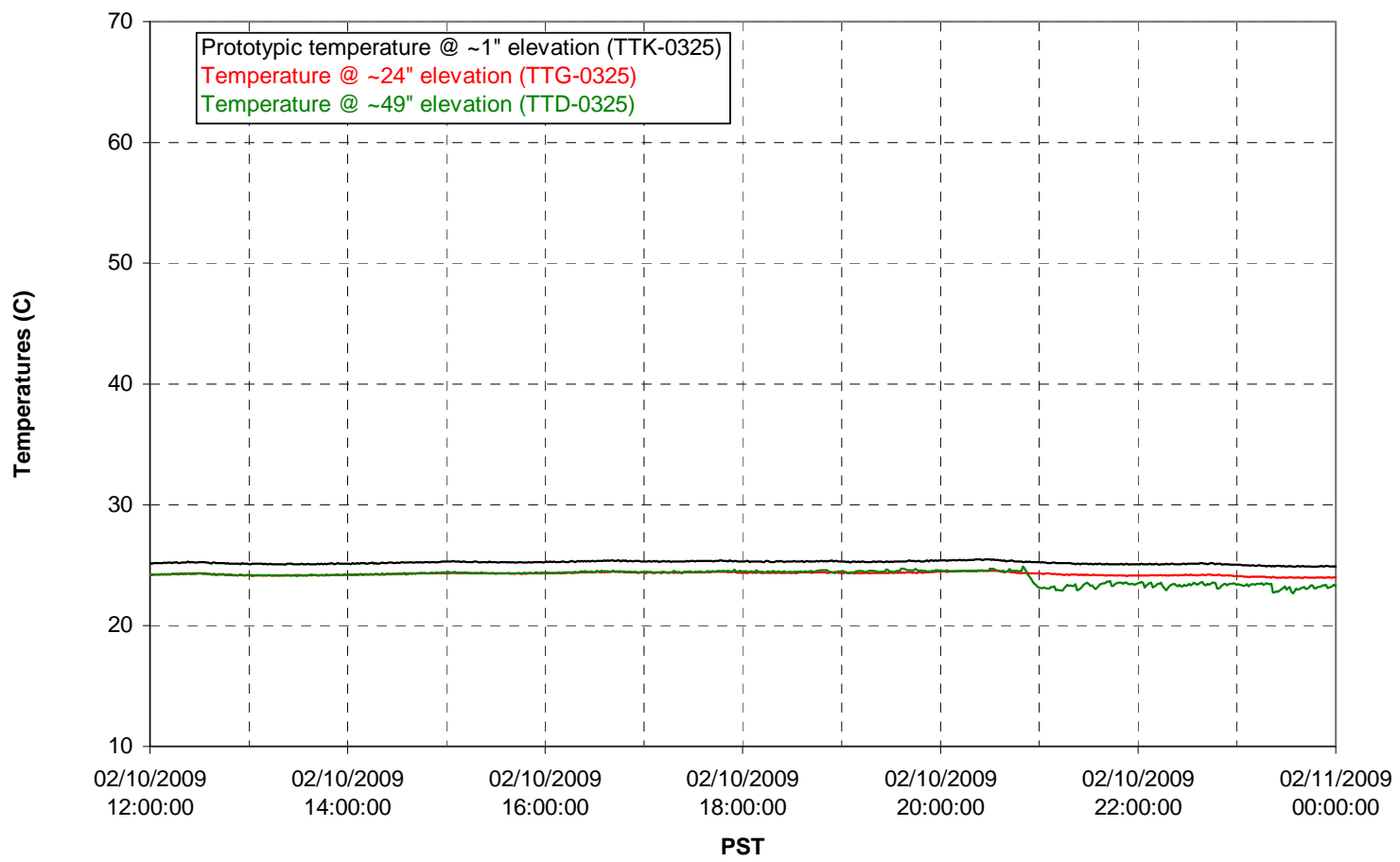


T01B level

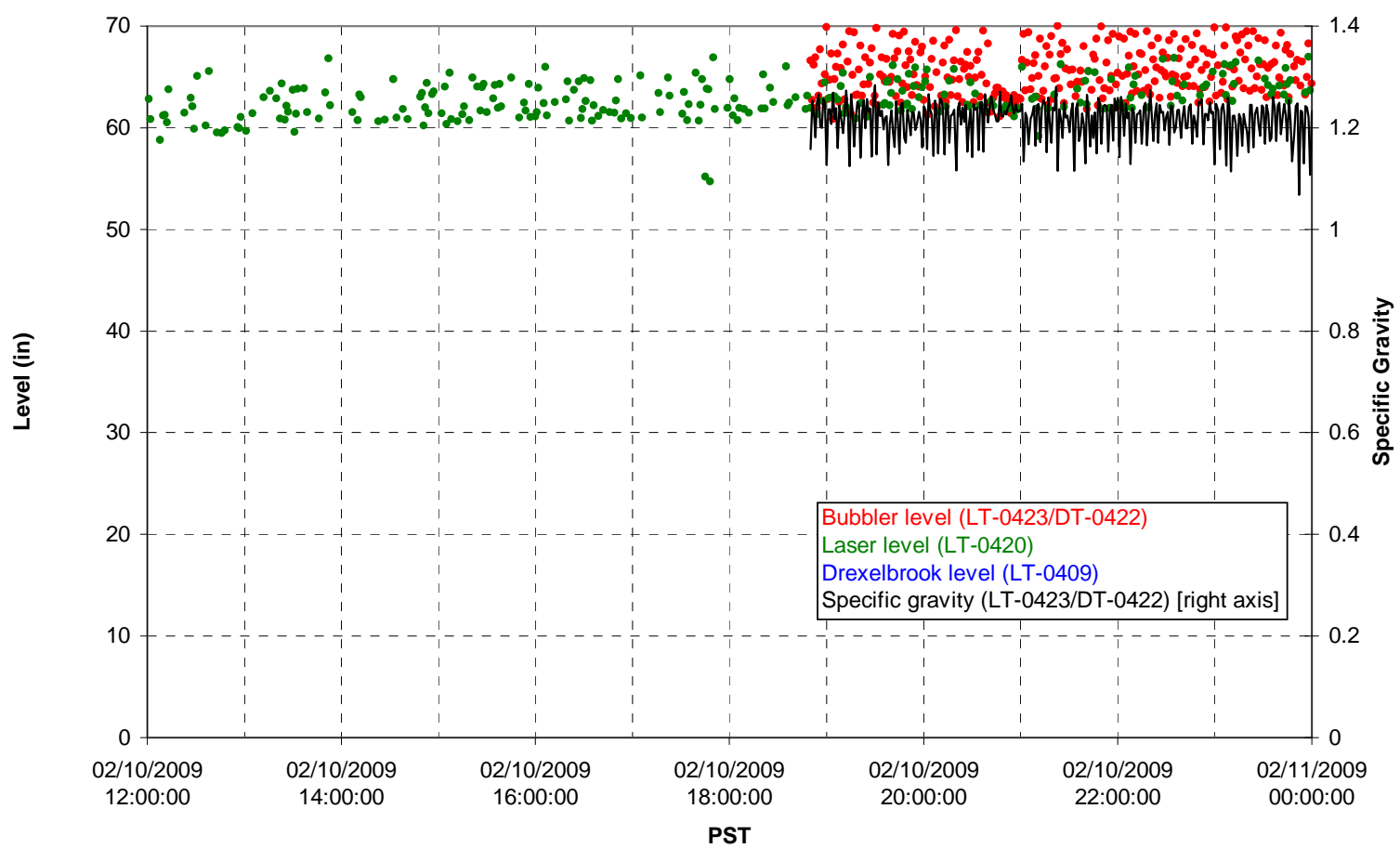

T01B temperatures

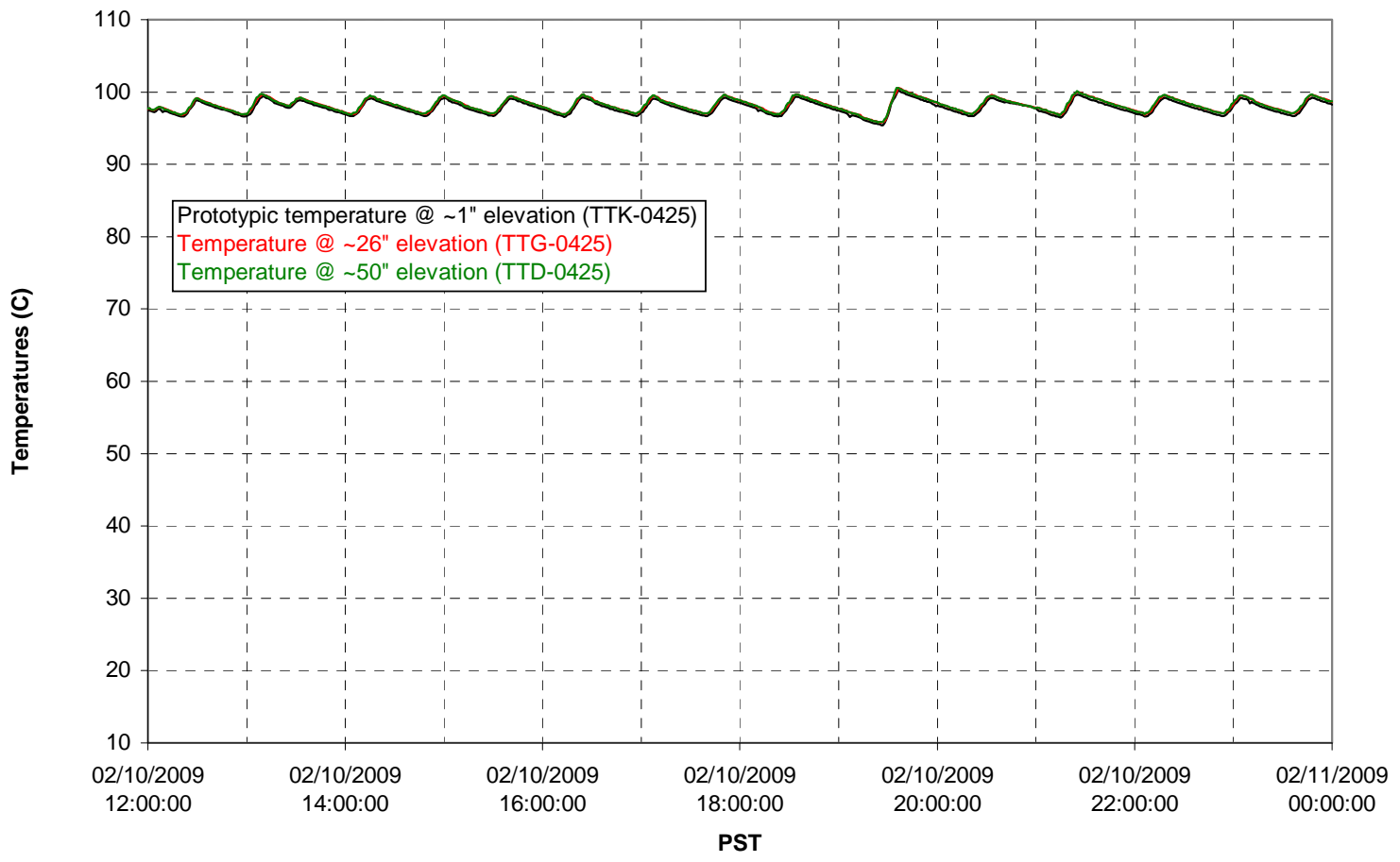


T02A level

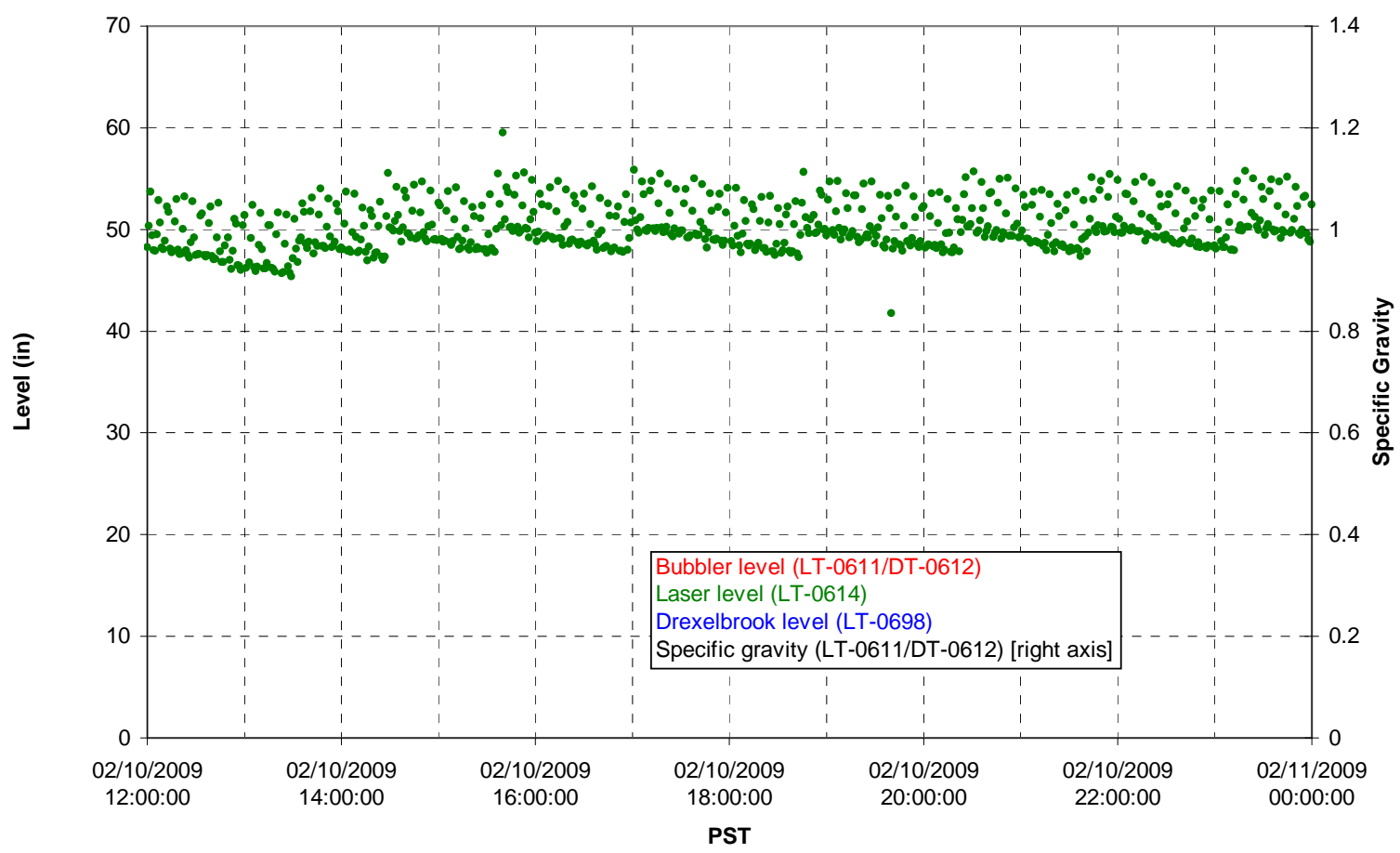

T02A temperatures

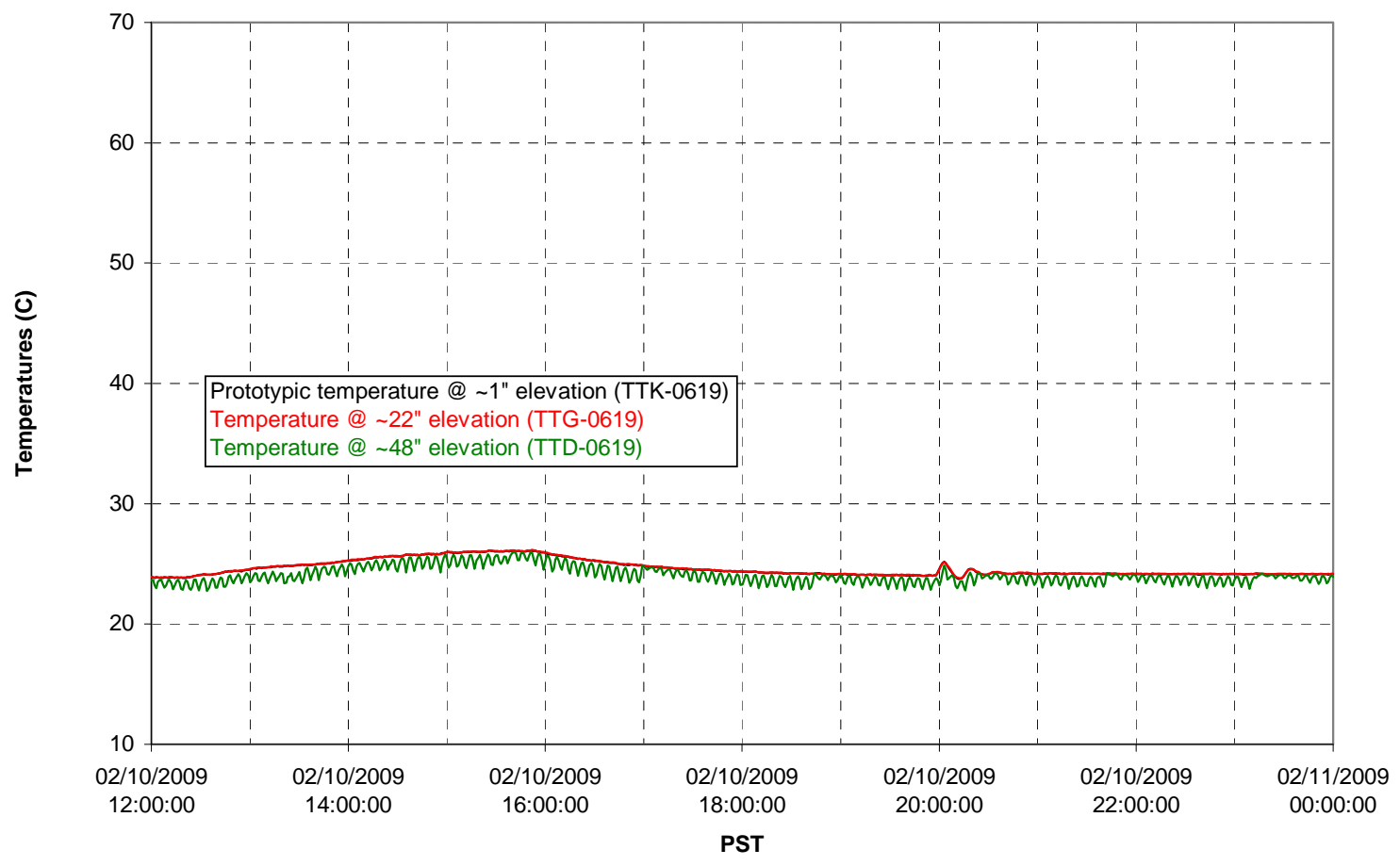


T02A and filter loop temperatures

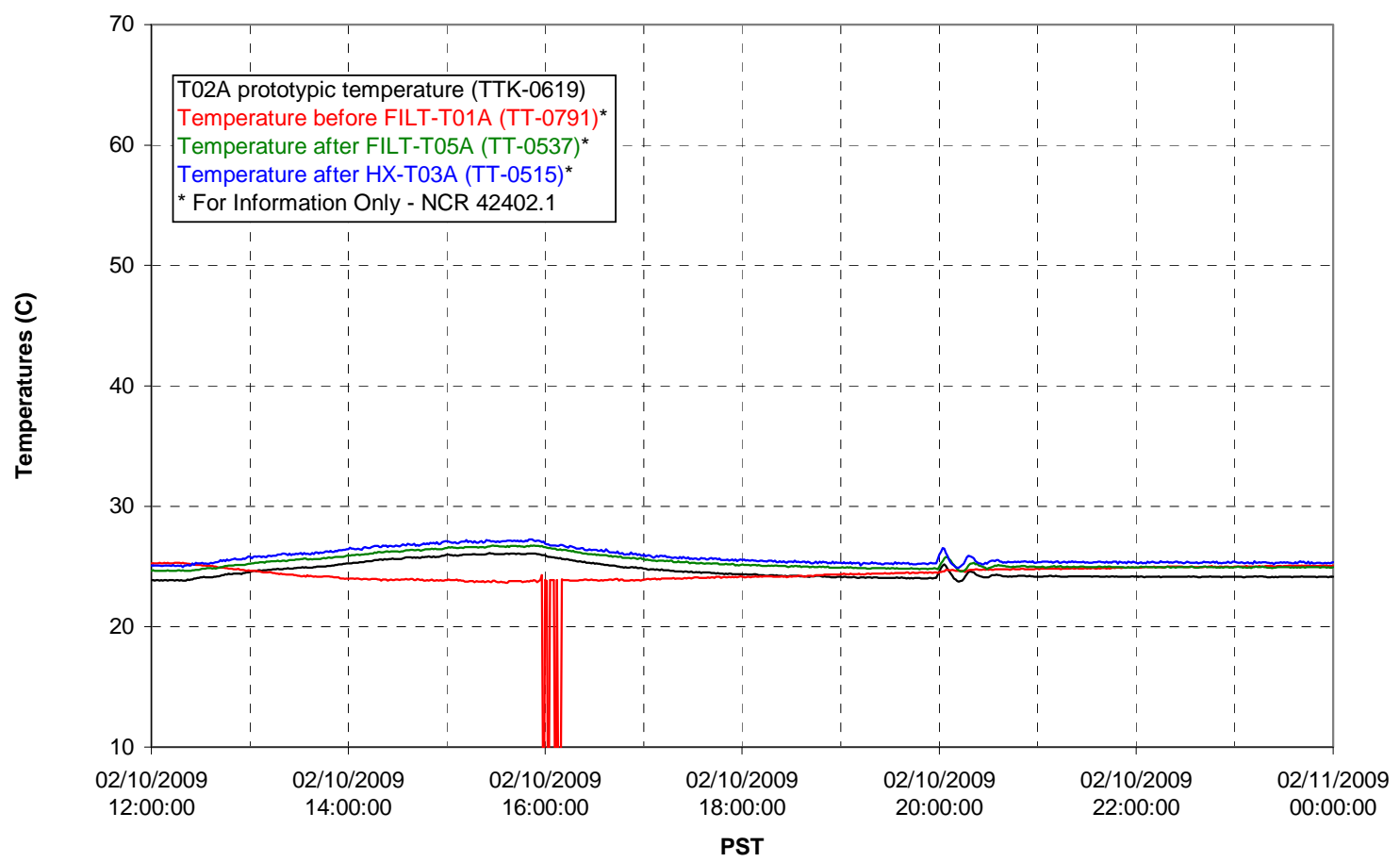

Pump Pressures and Flow

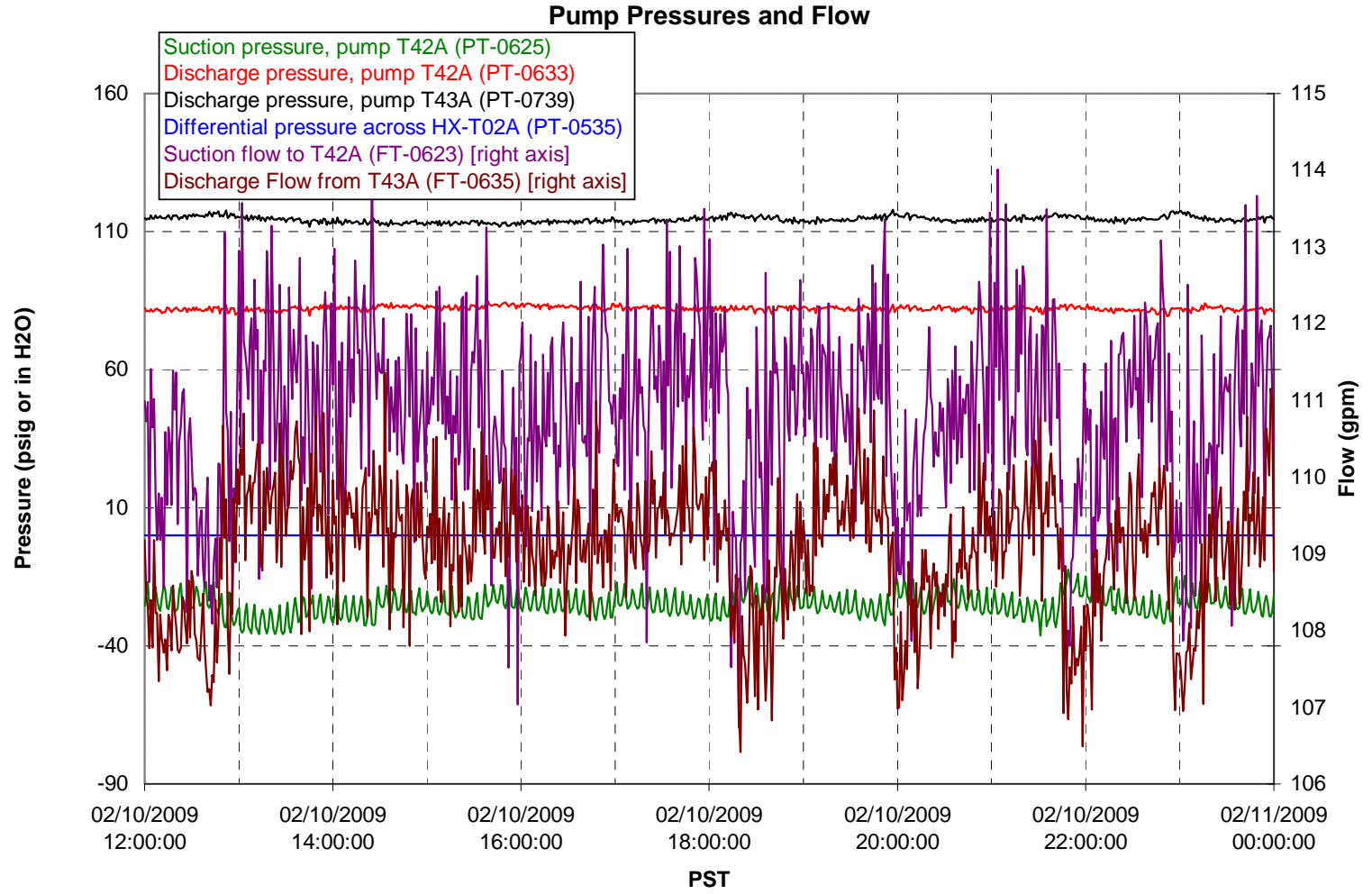


Axial pressure drop

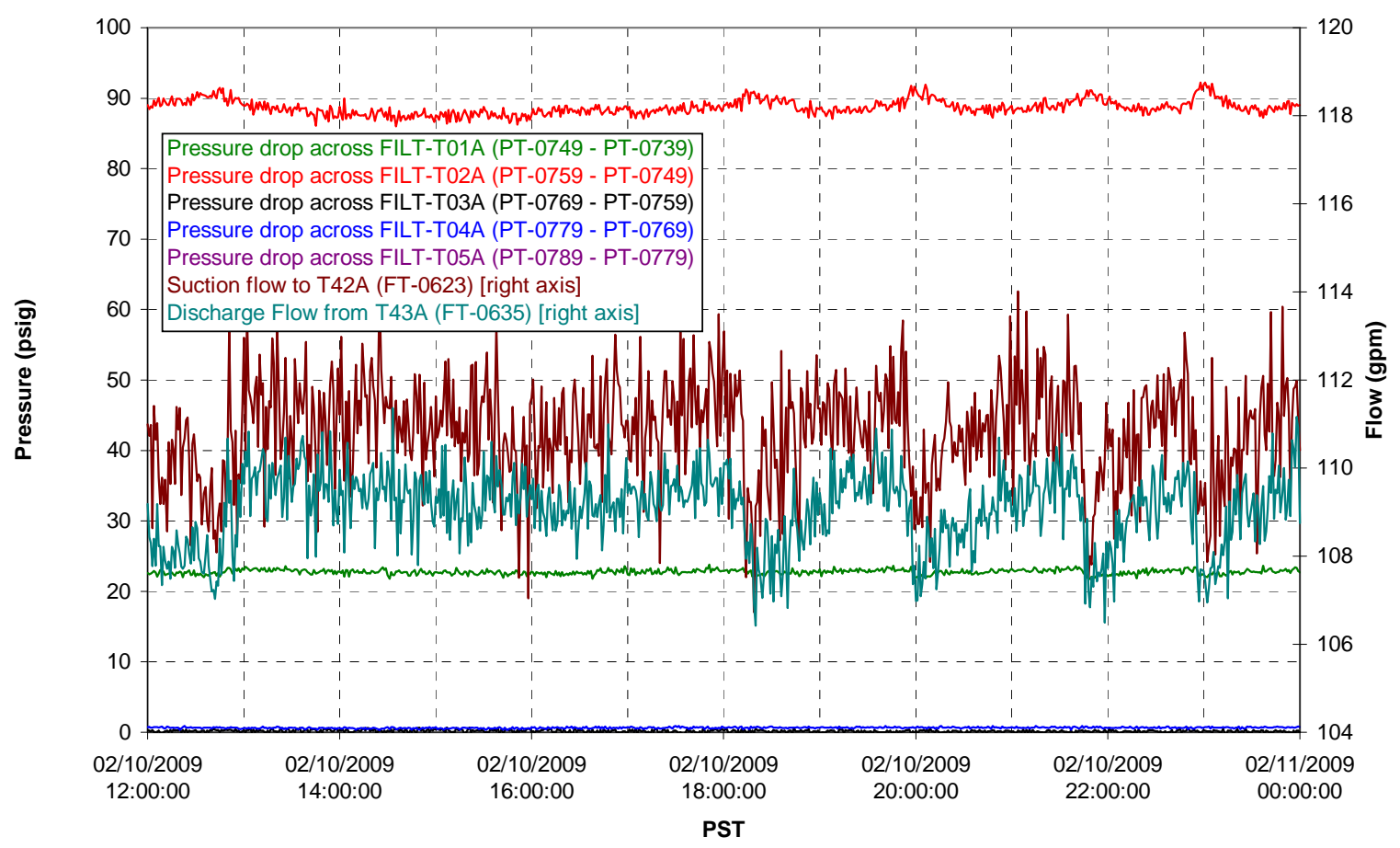

Permeate flow rates

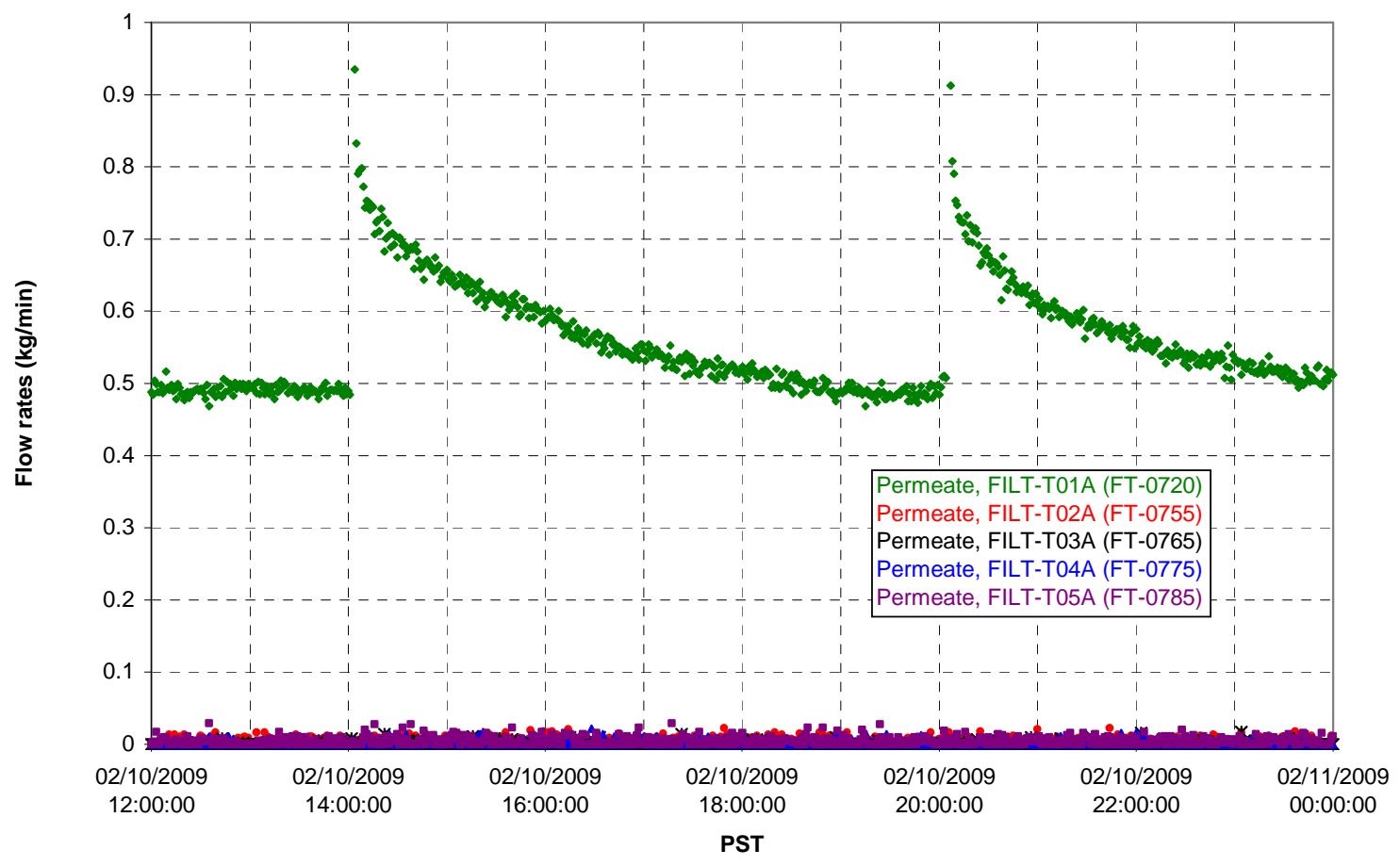


T02A Inner Temperature Tree

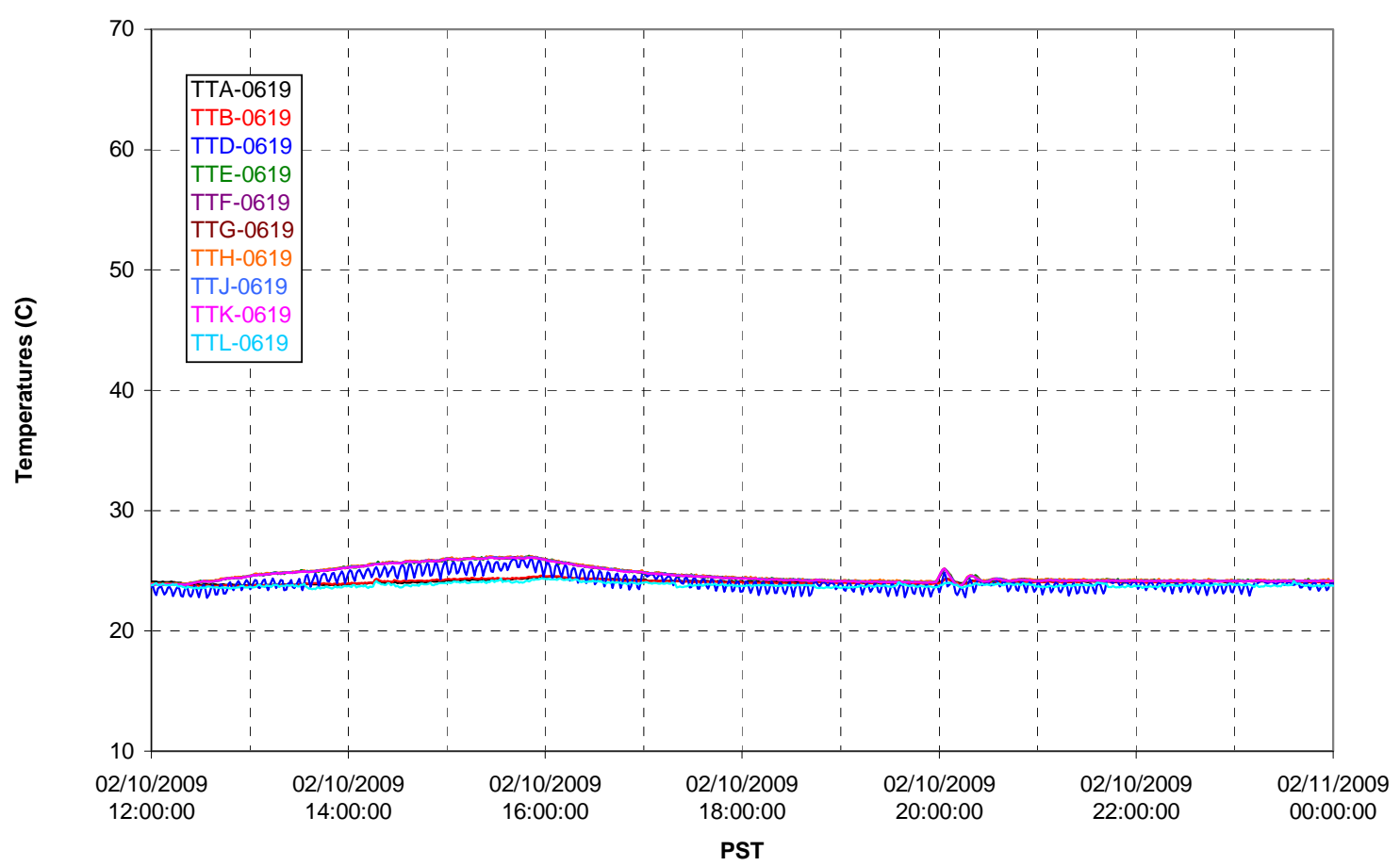

T02A Outer Temperature Tree

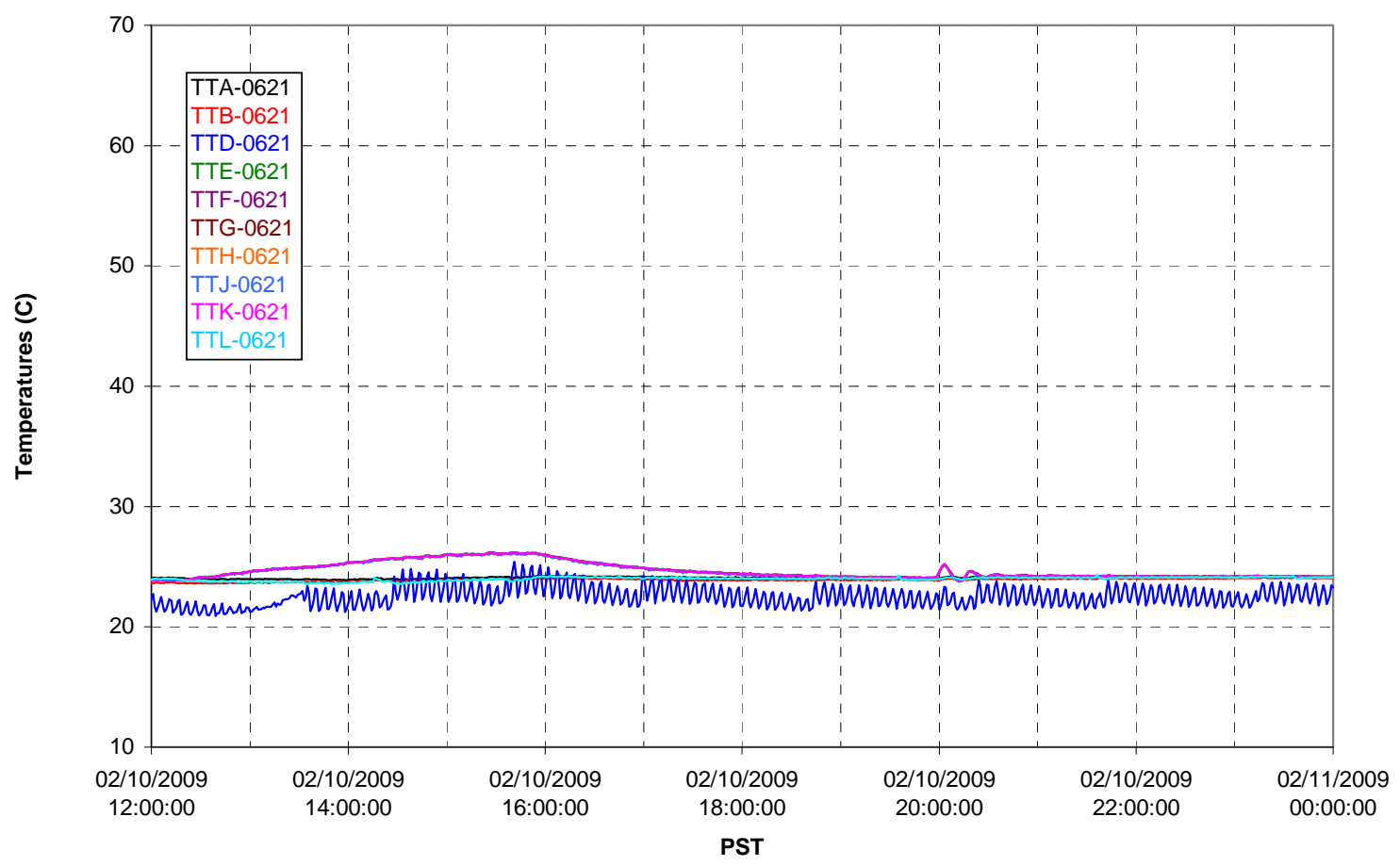


T02A temperatures

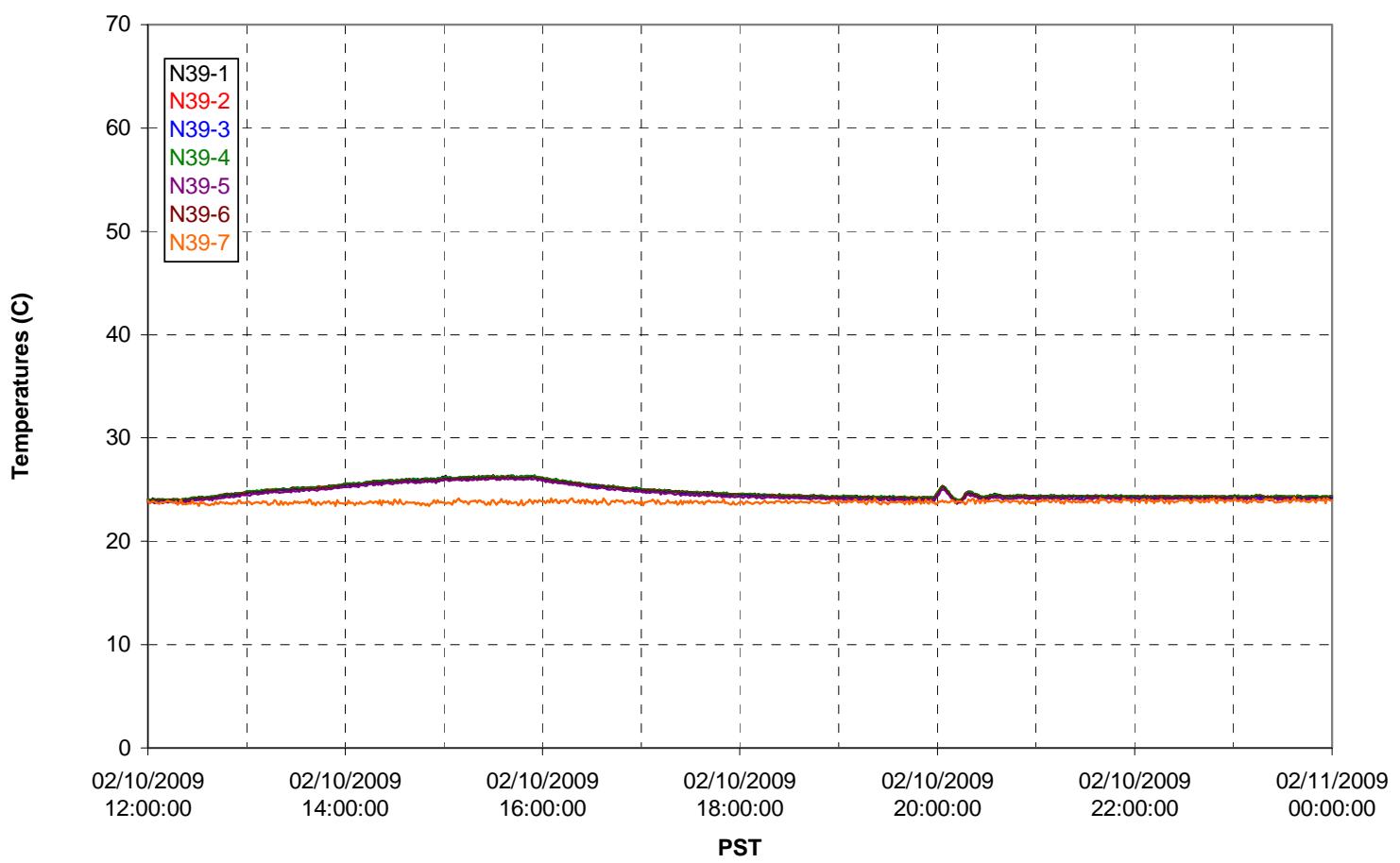

T02A temperatures

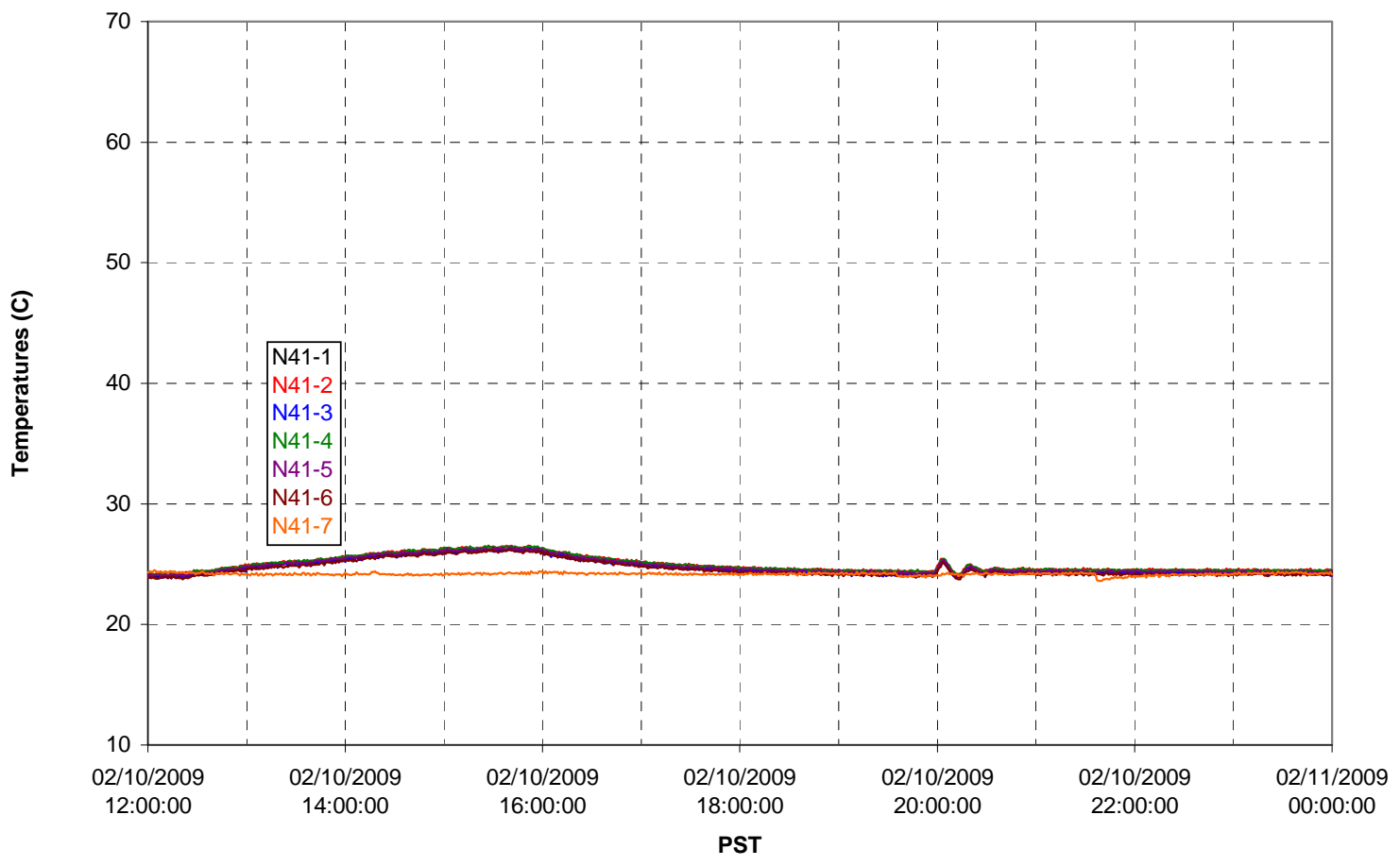


T02A temperatures

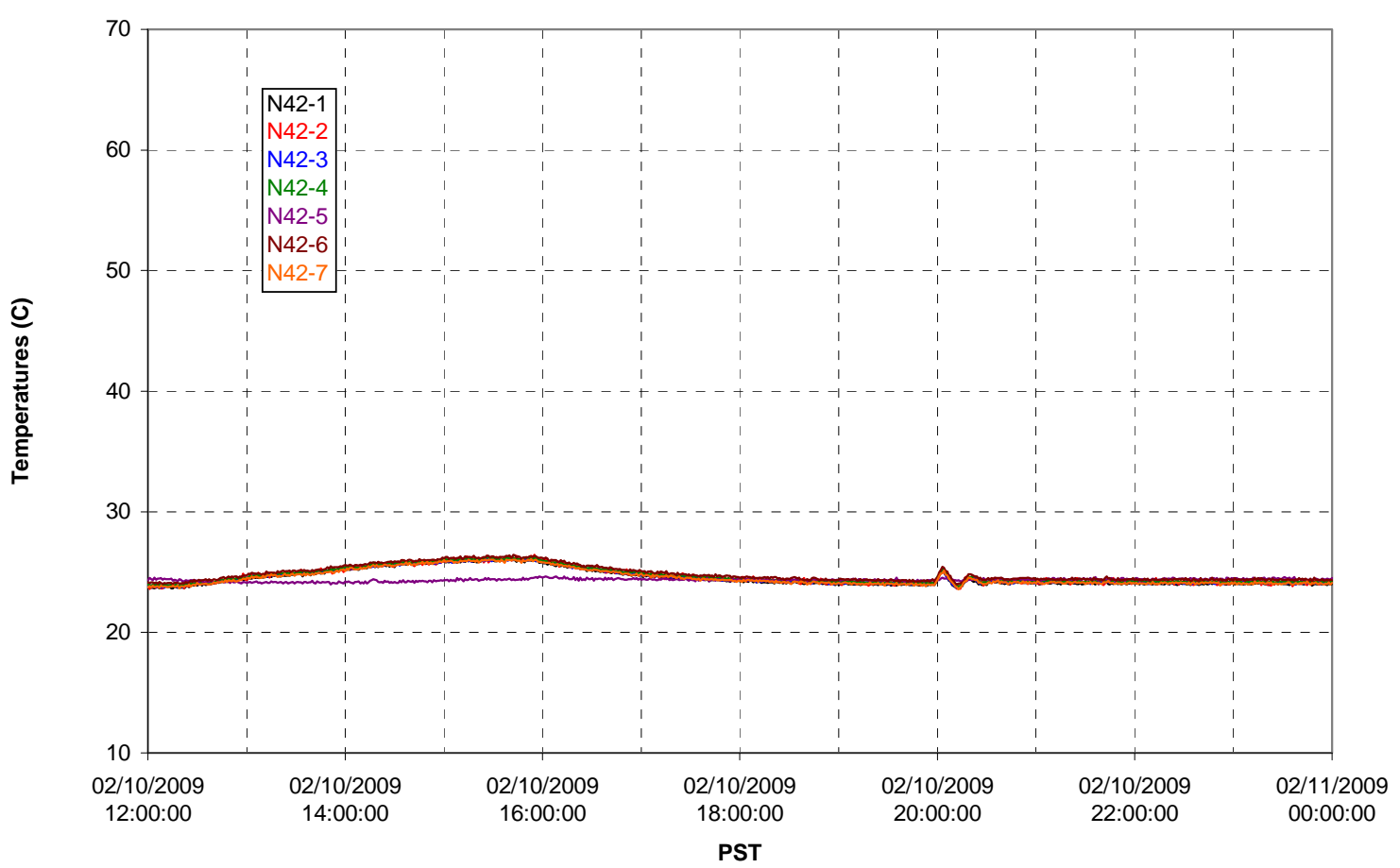

T02A temperatures

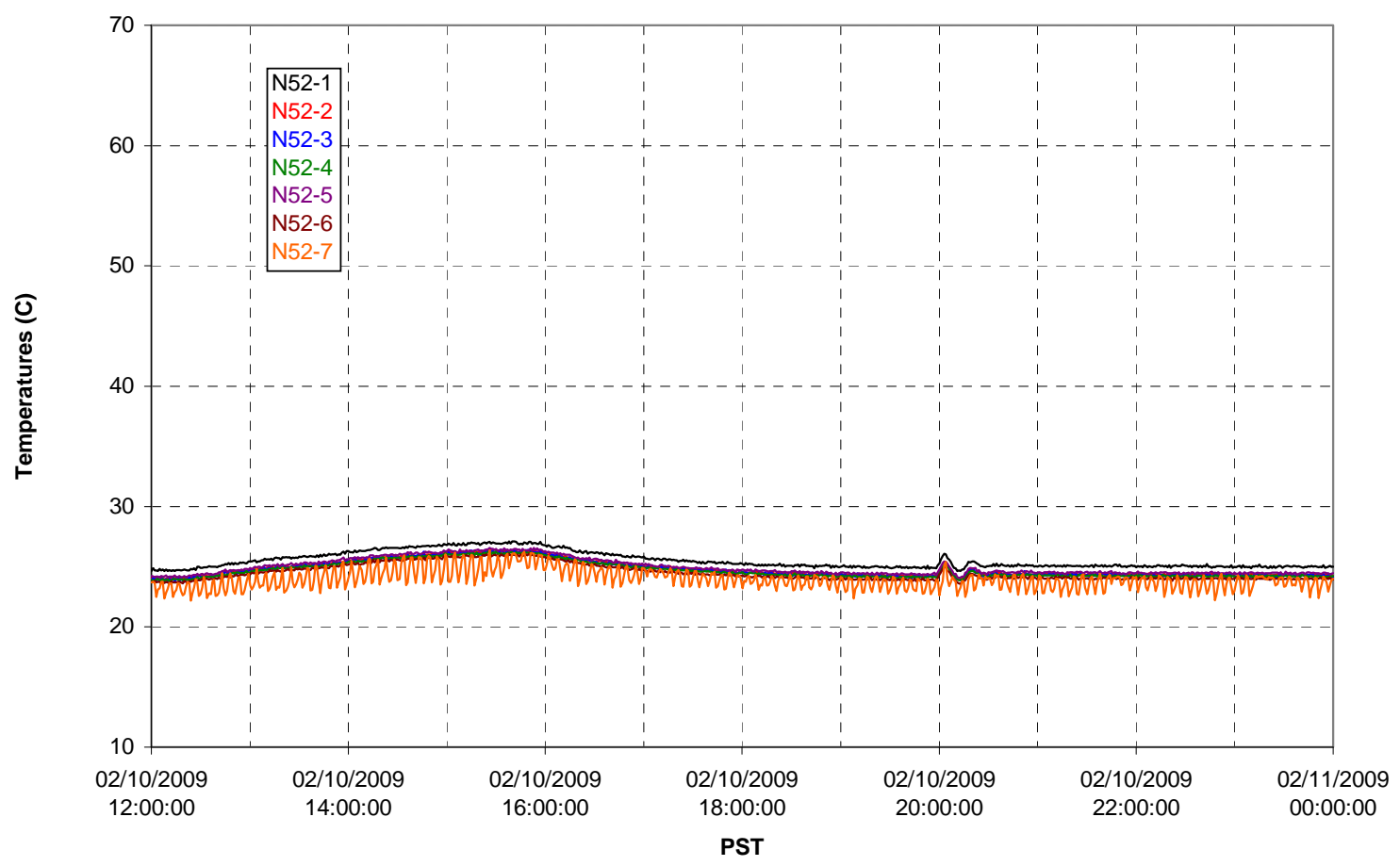


T02A Heating and Cooling
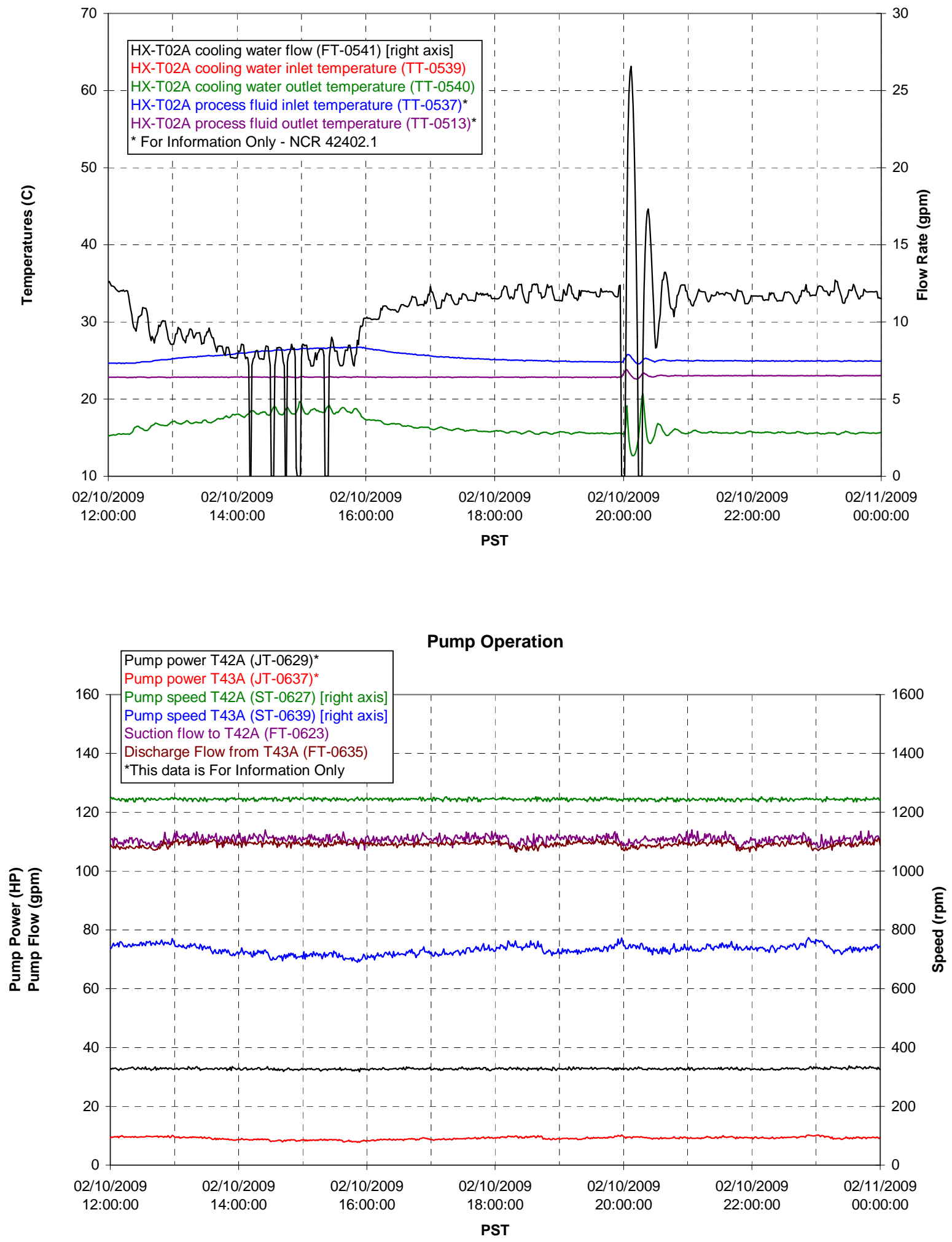
Pulsepot UFP-PP-T01A

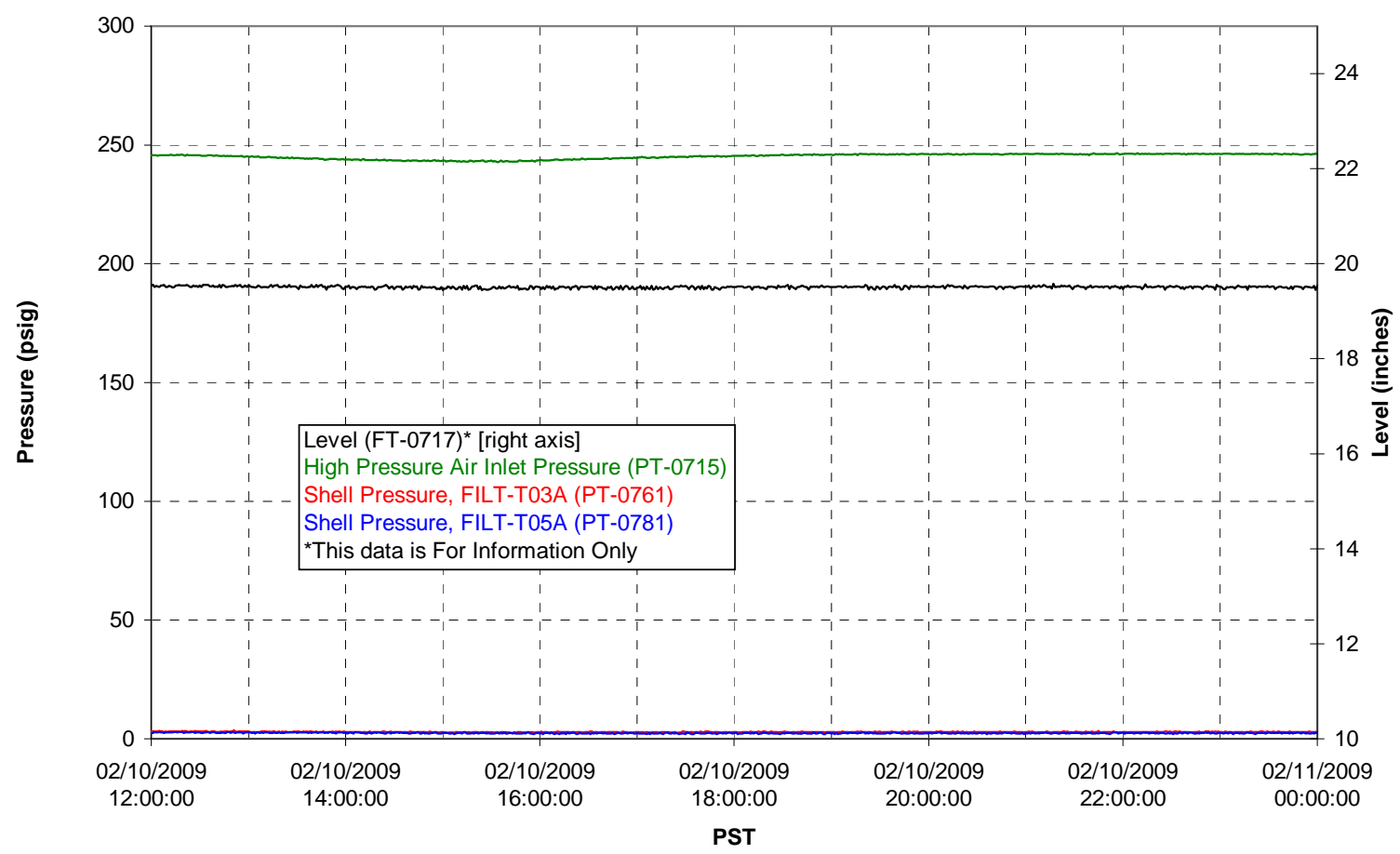

Pulsepot UFP-PP-T02A

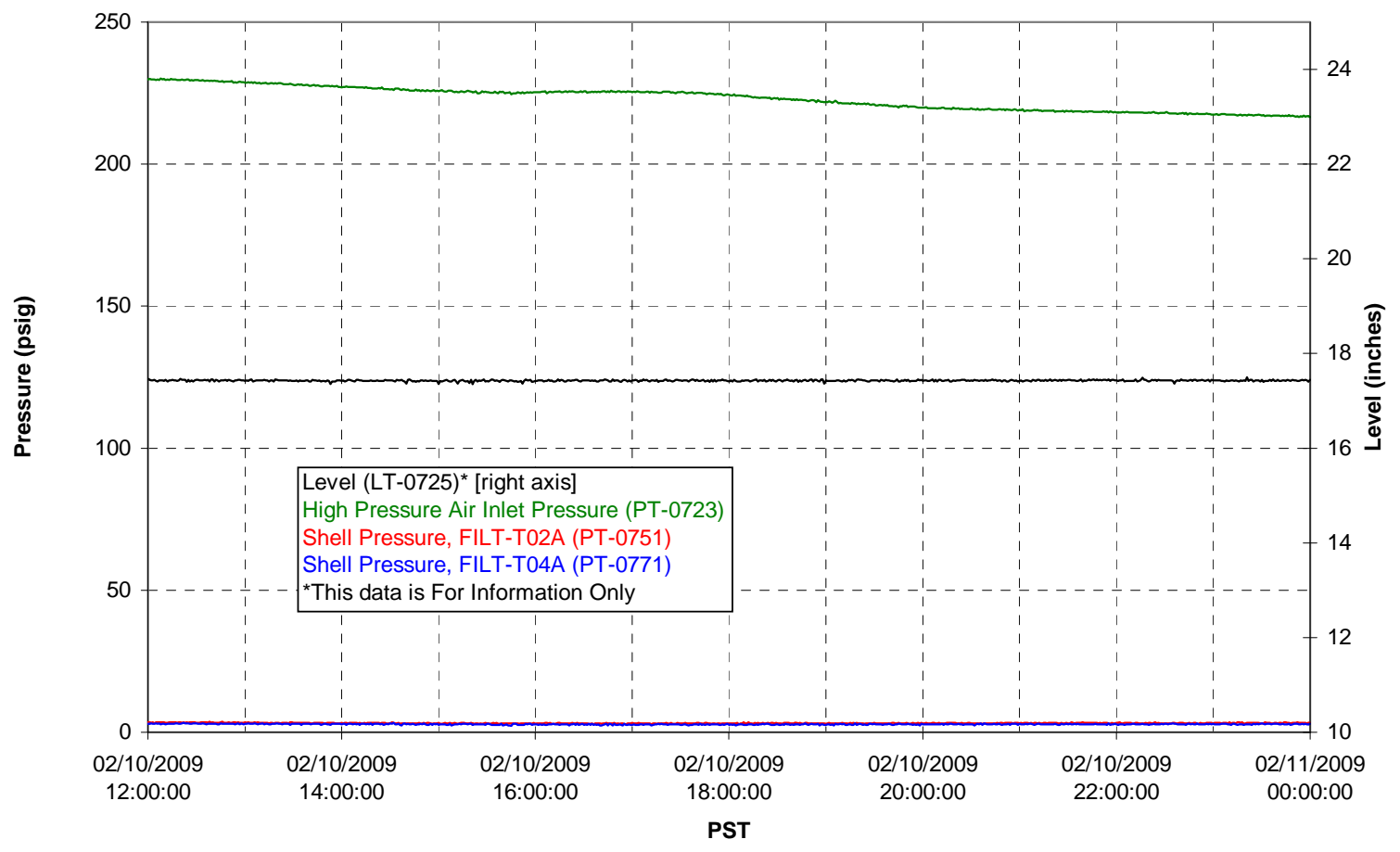


Pulsepot UFP-PP-T03A

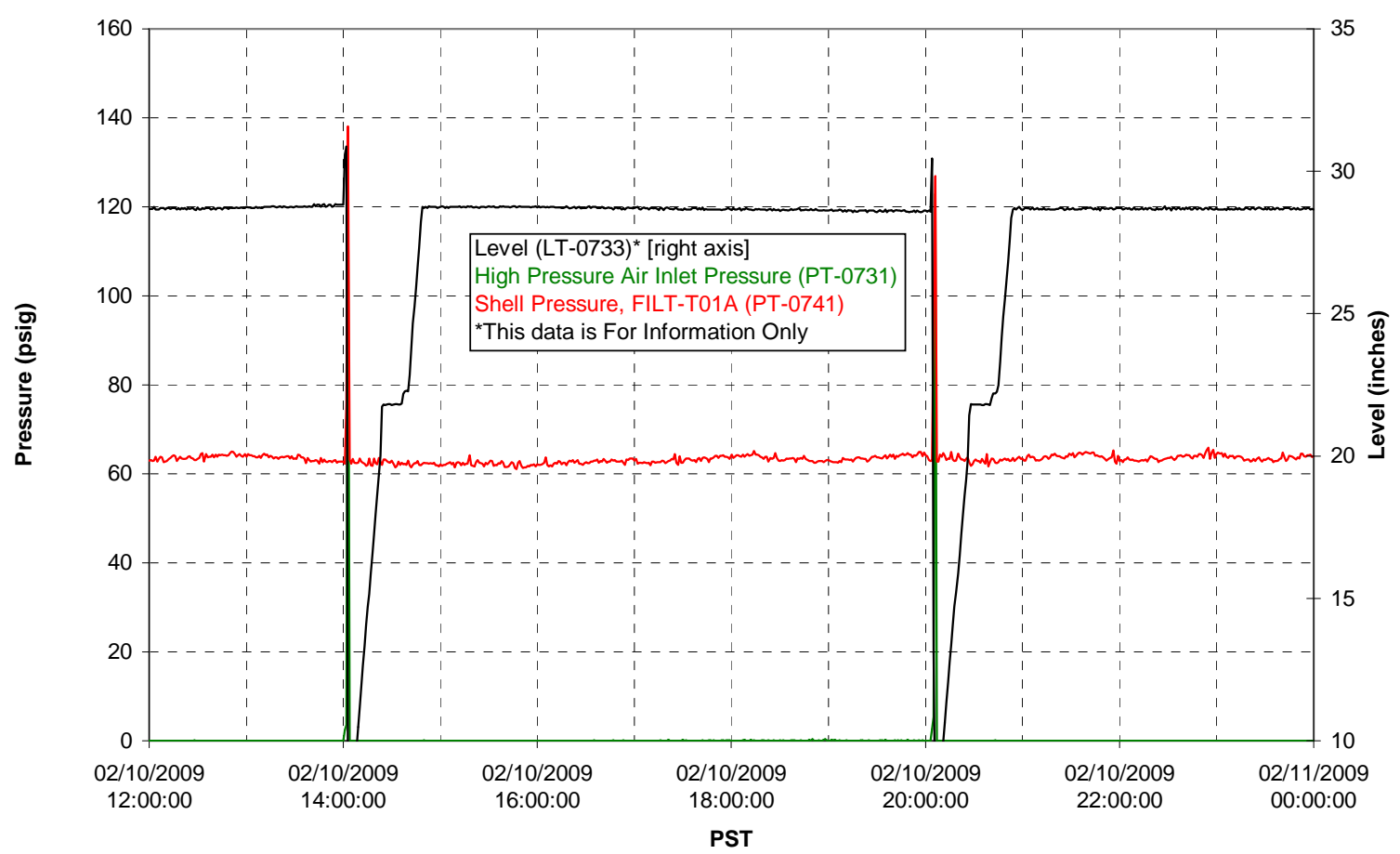

Pulsepot Levels

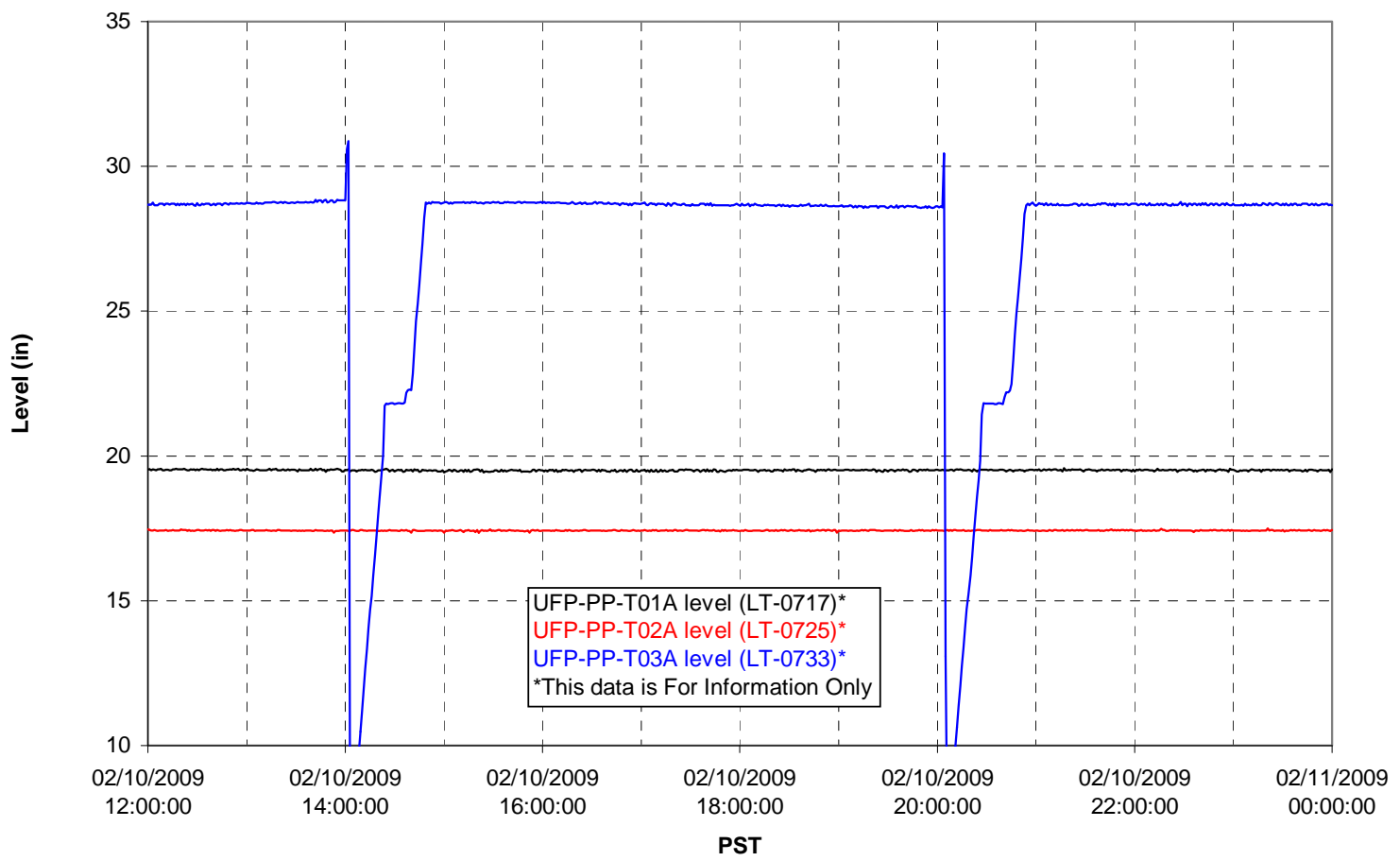


Filter UFP-FILT-T01A

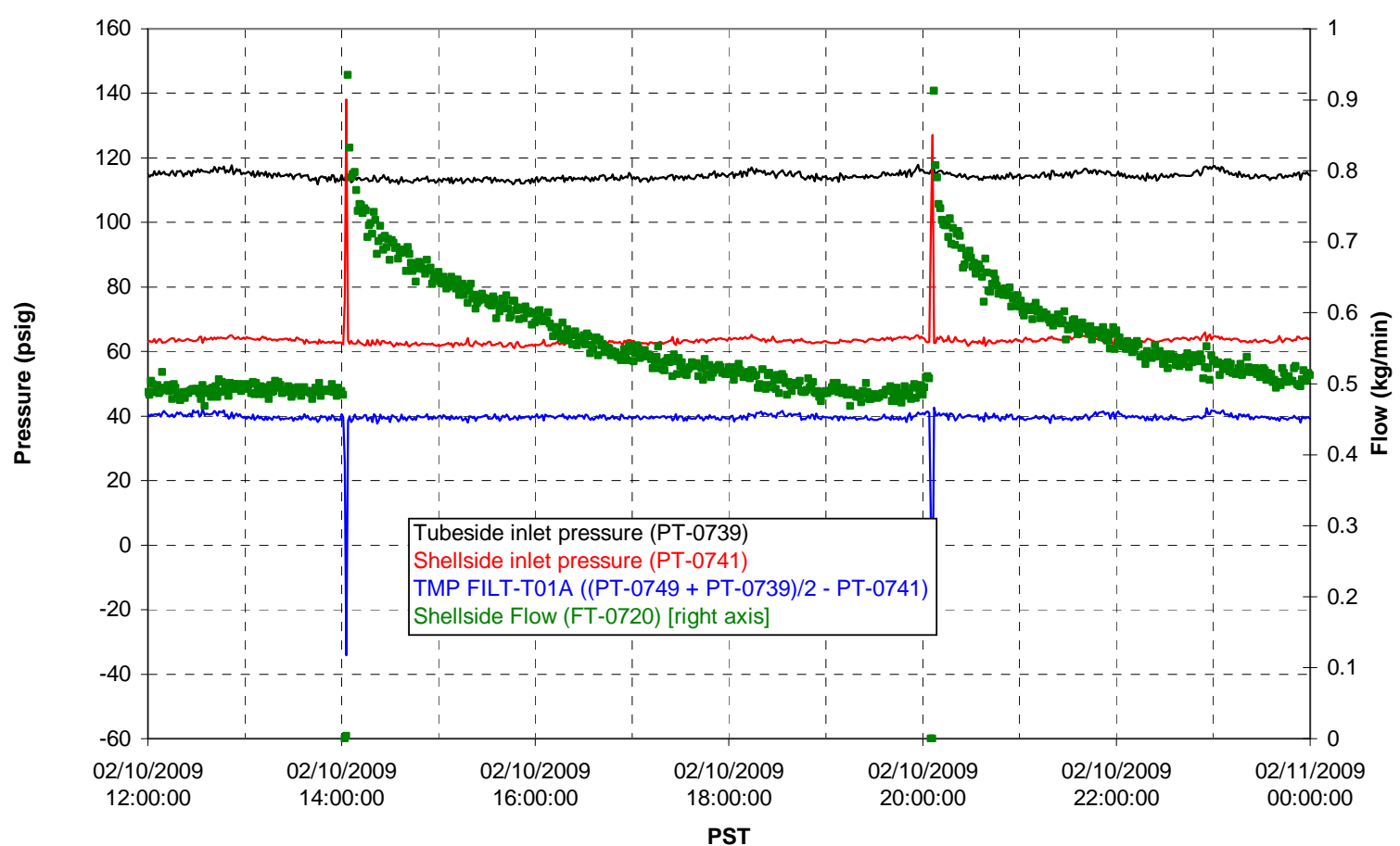

Filter UFP-FILT-T02A

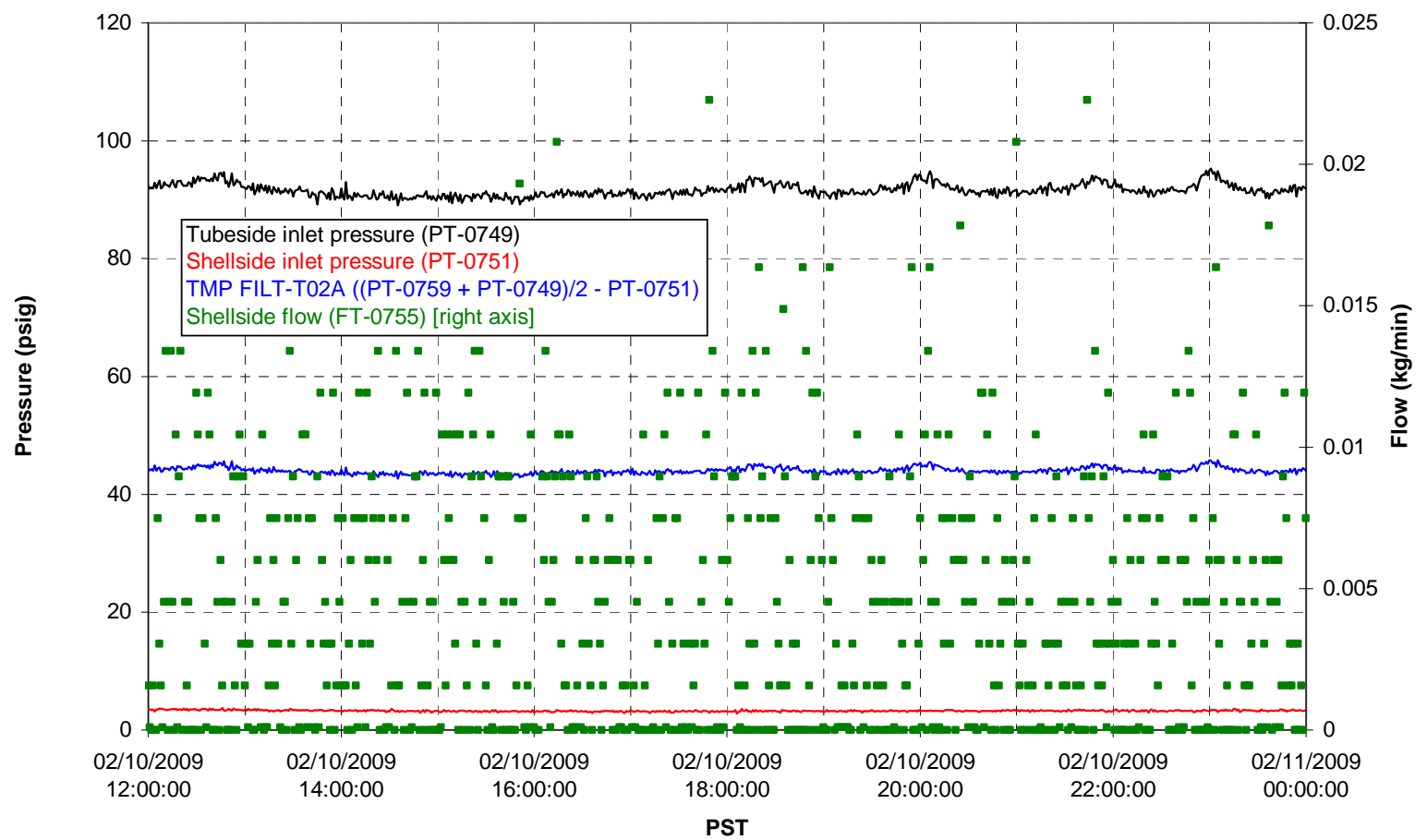


Filter UFP-FILT-T03A

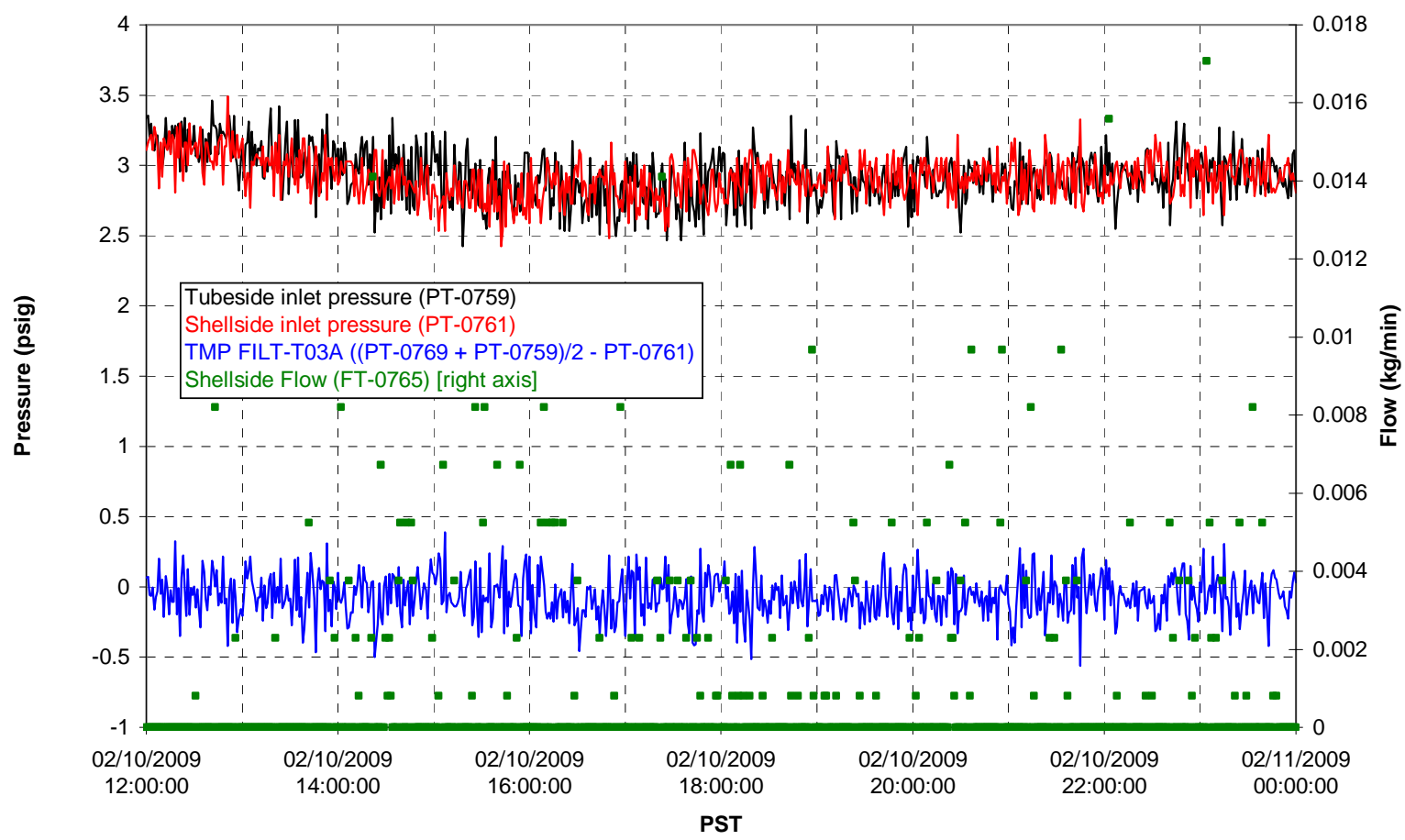

Filter UFP-FILT-T04A

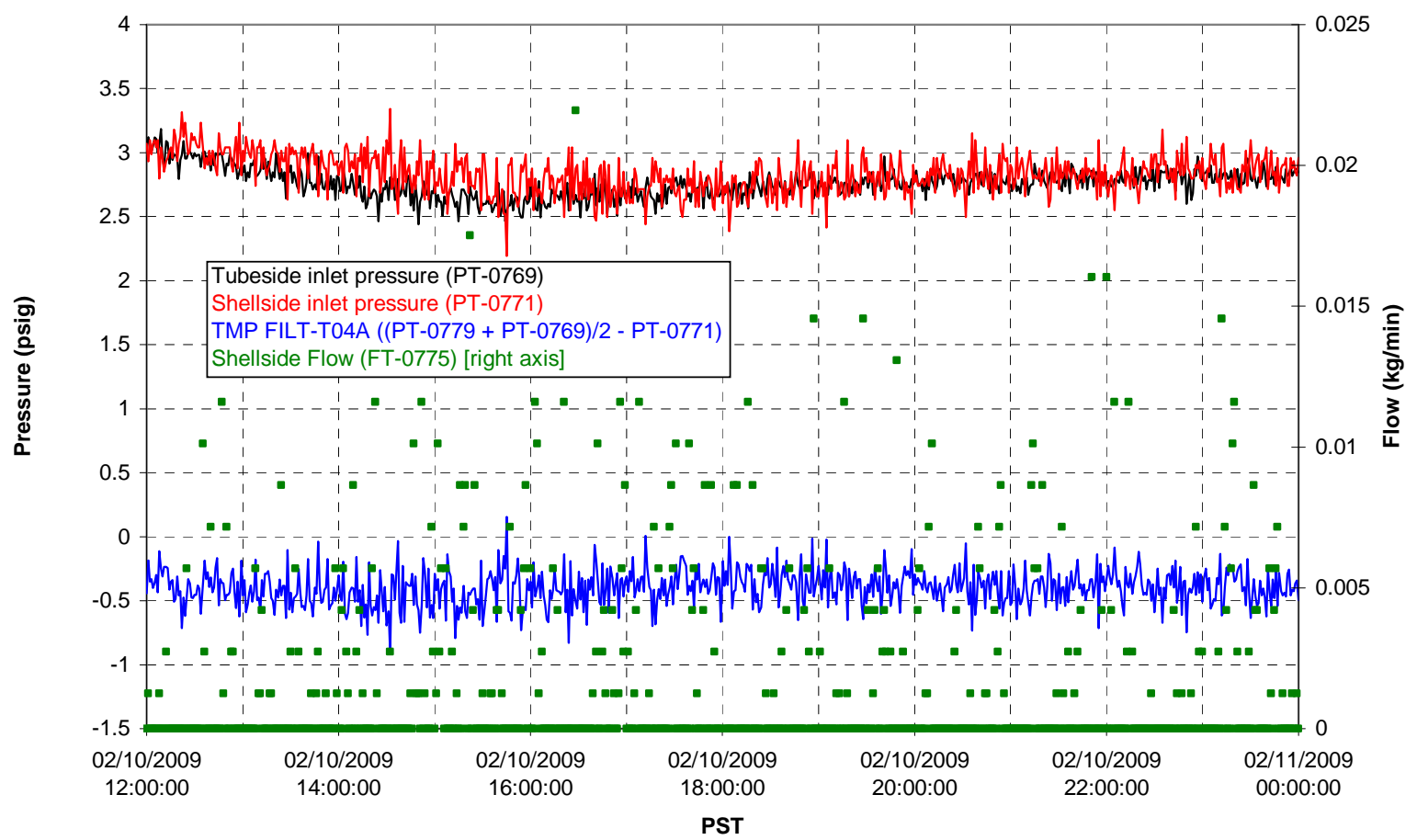


Filter UFP-FILT-T05A

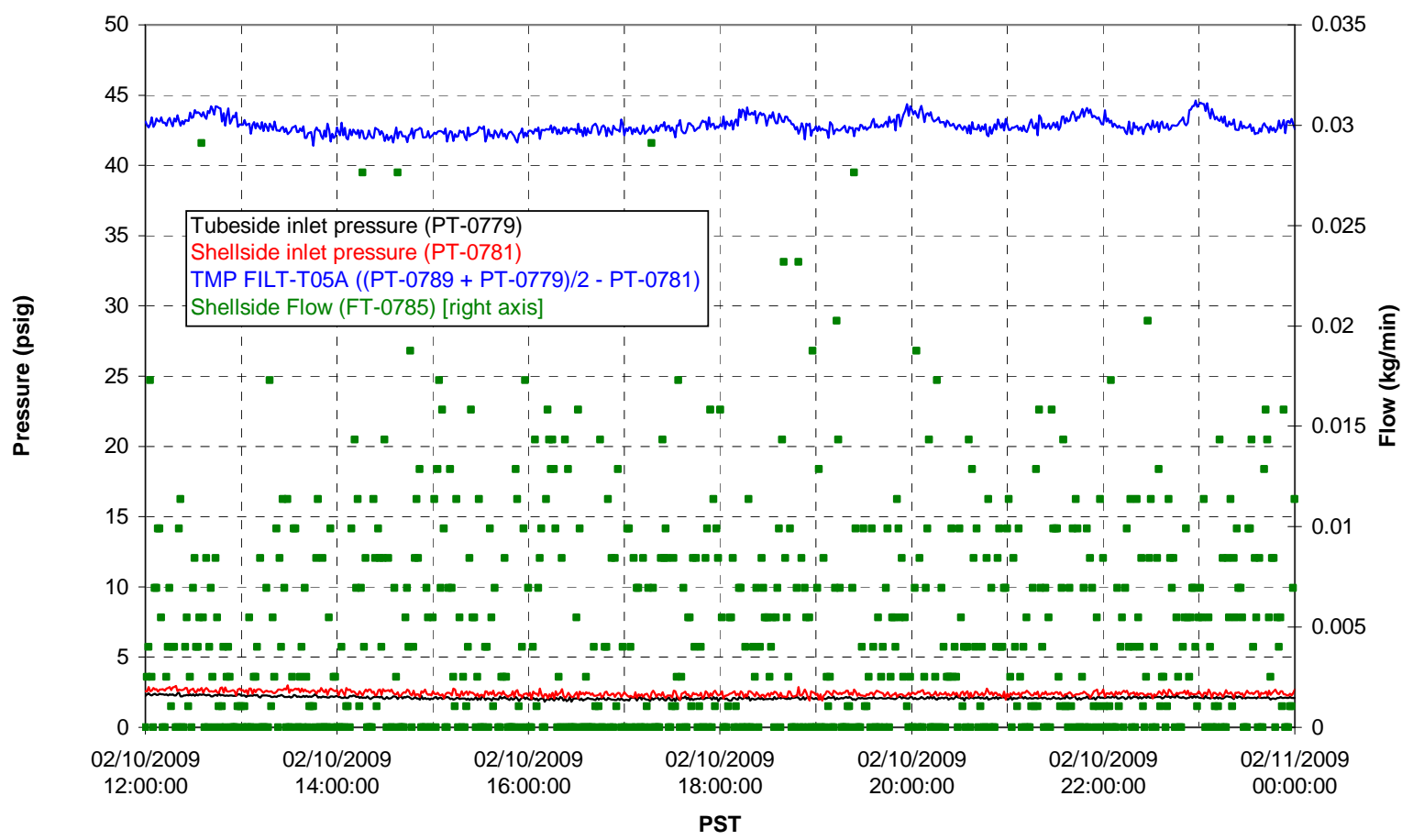

Chemical Flow

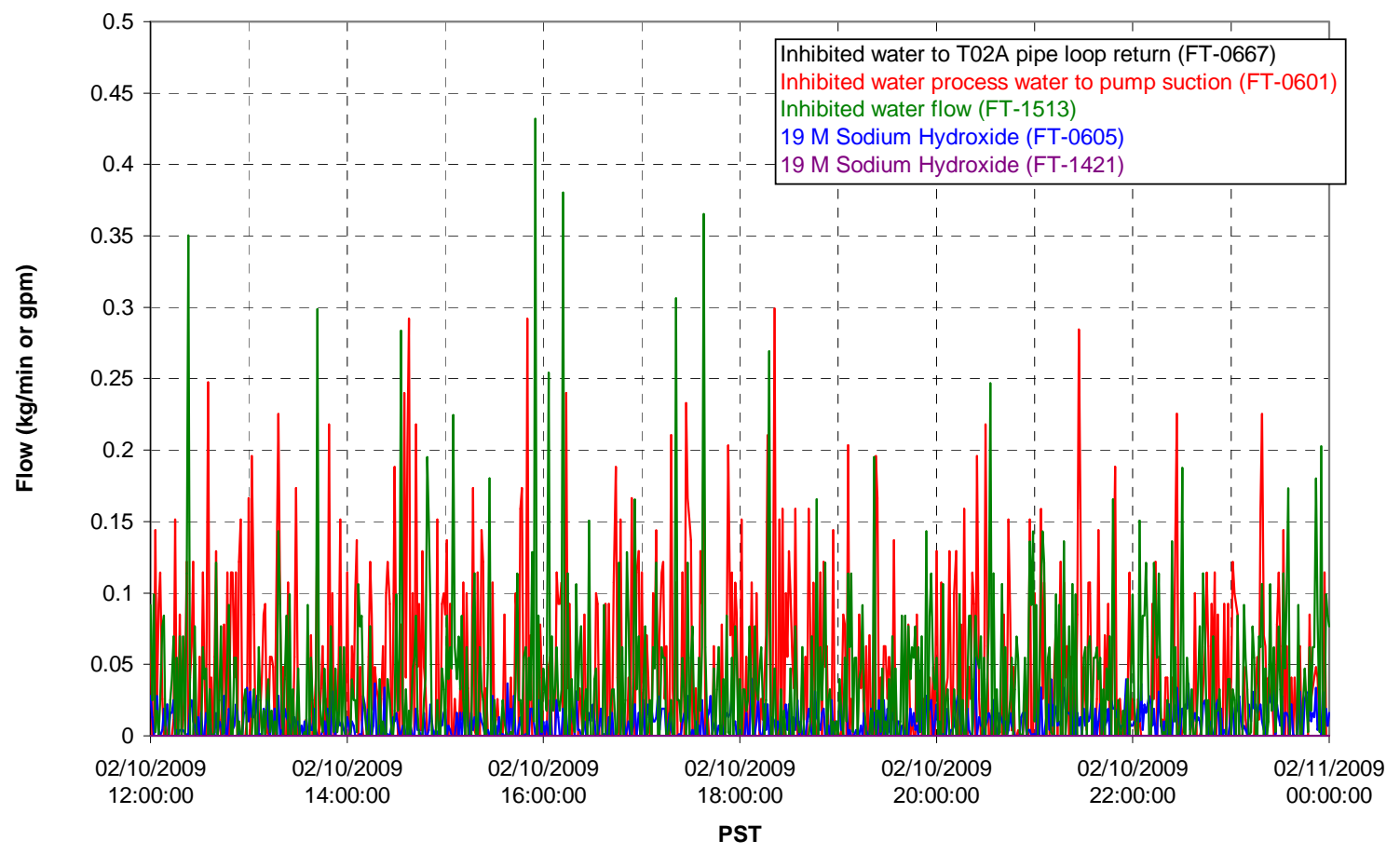


Chemical Flow

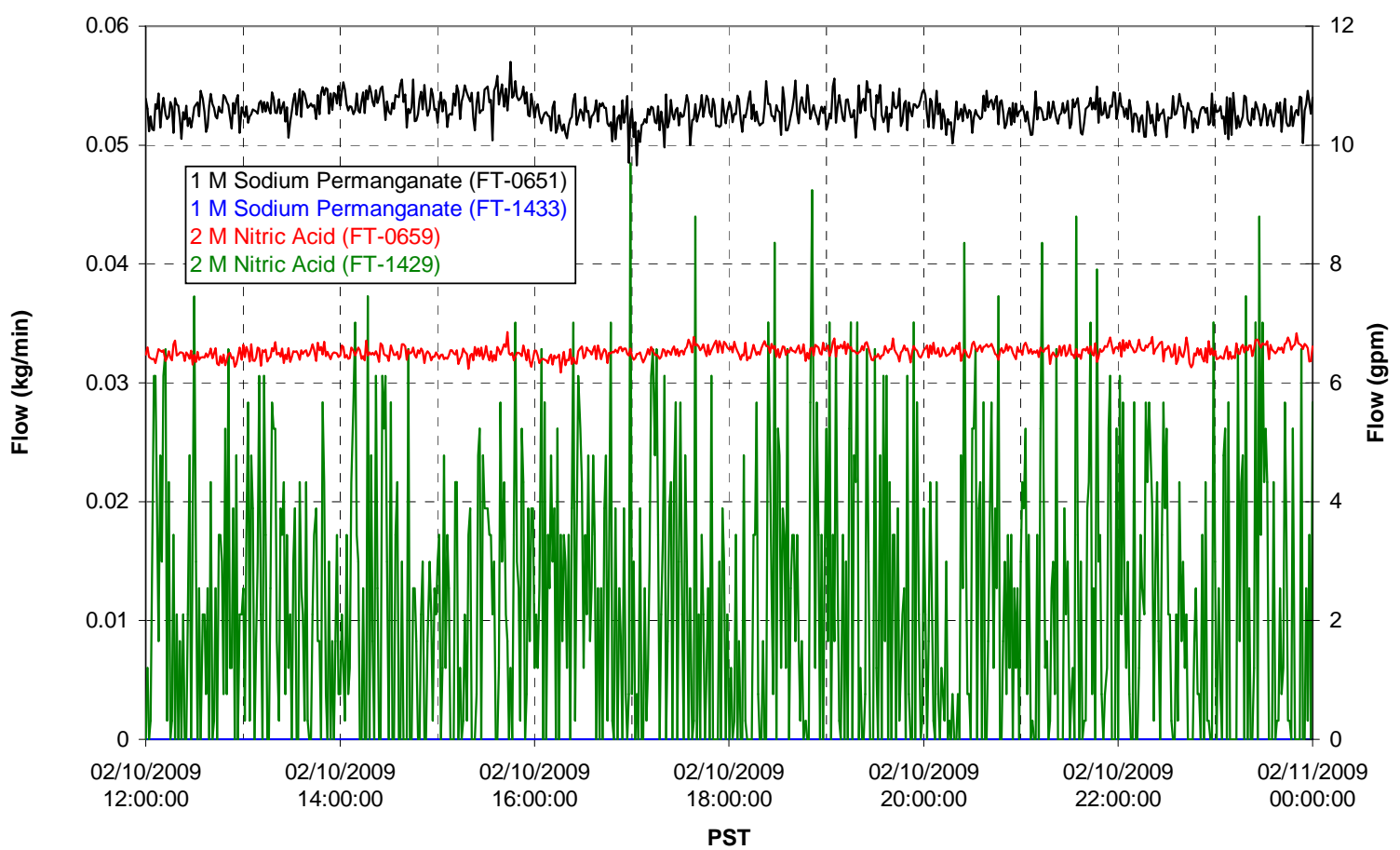

Air Flows

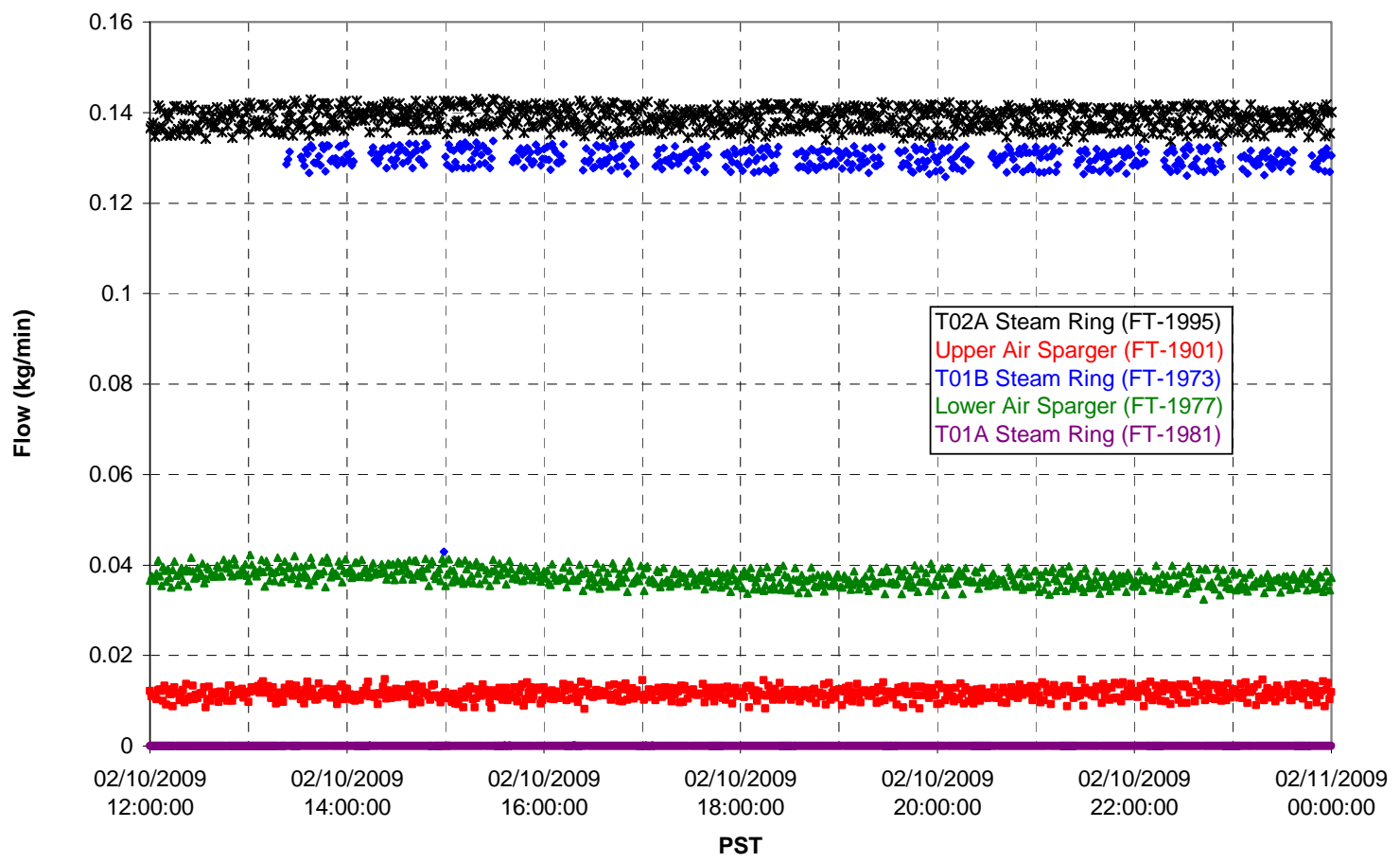


T02A Steam

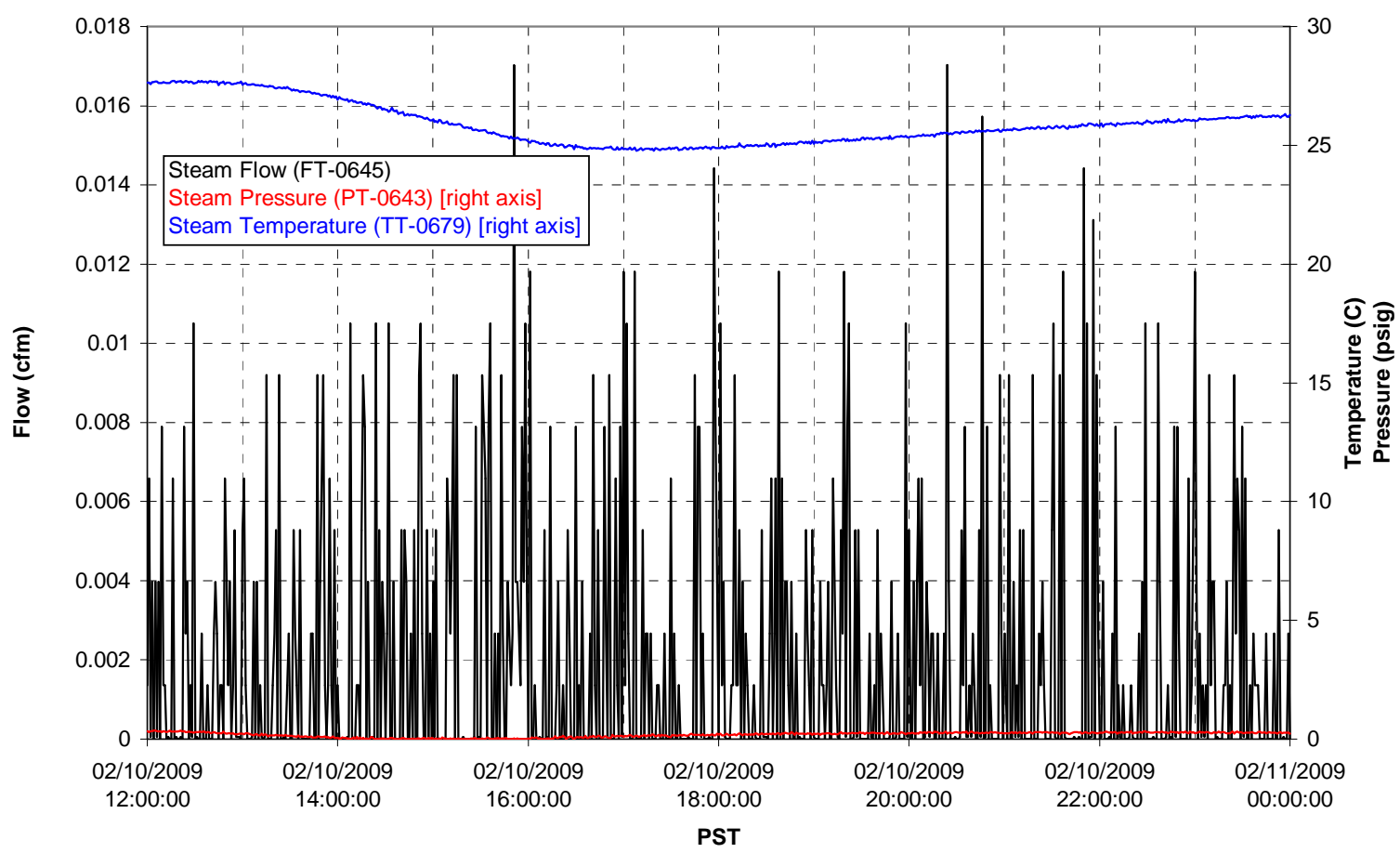

T01A Steam

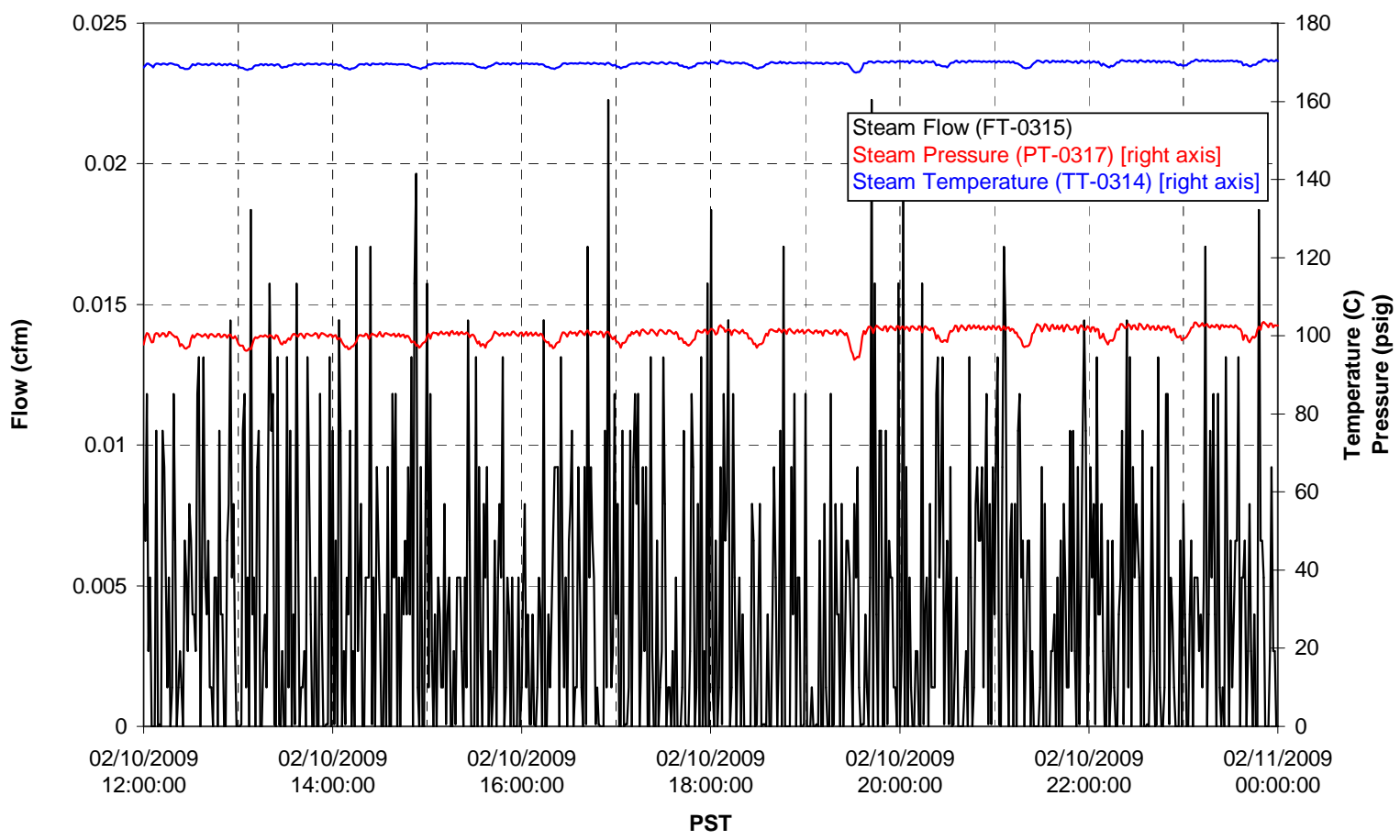


T01B Steam

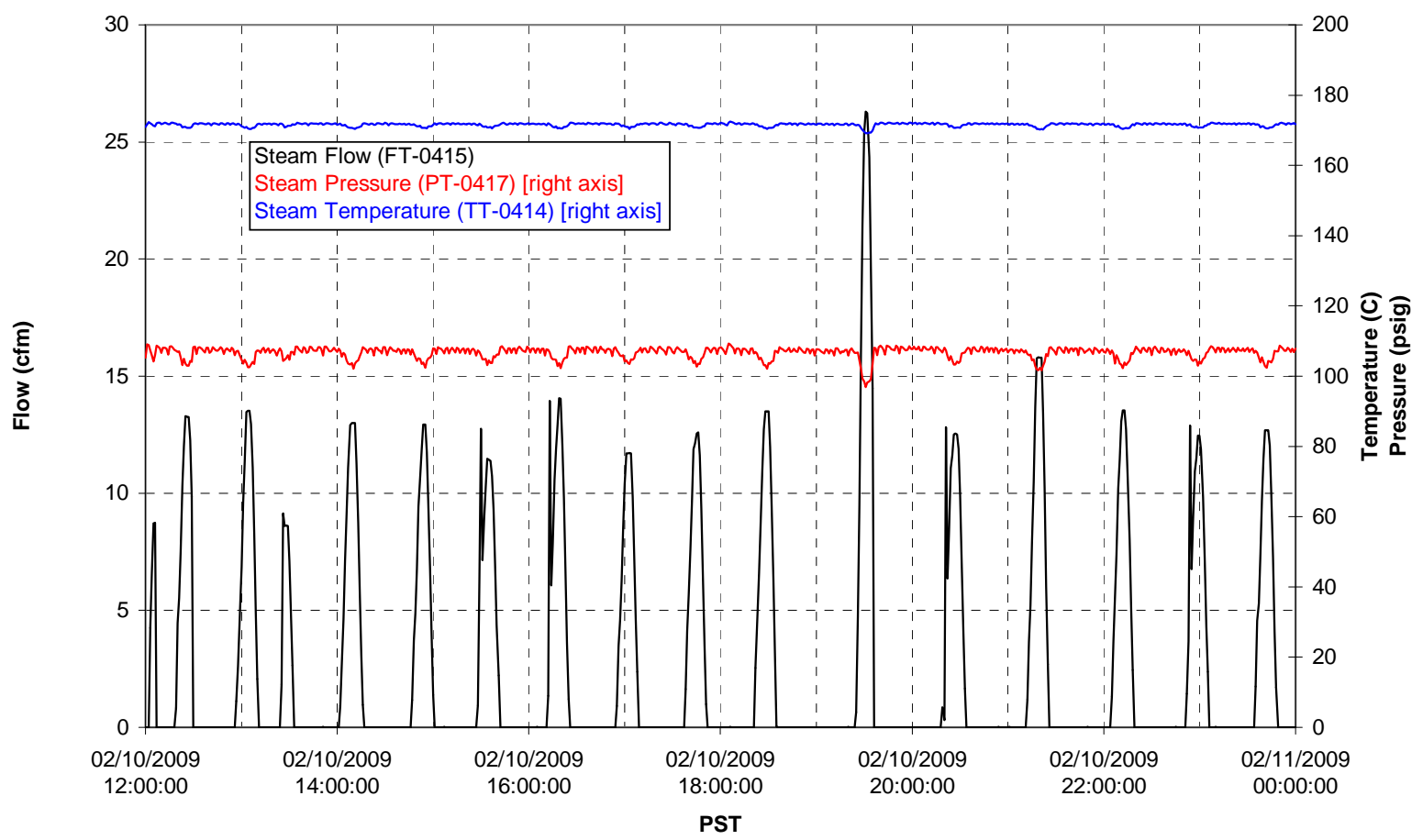




\section{Integrated Test A Data Plots 02/11/09 00:00 - 02/11/09 12:00}


T01A level

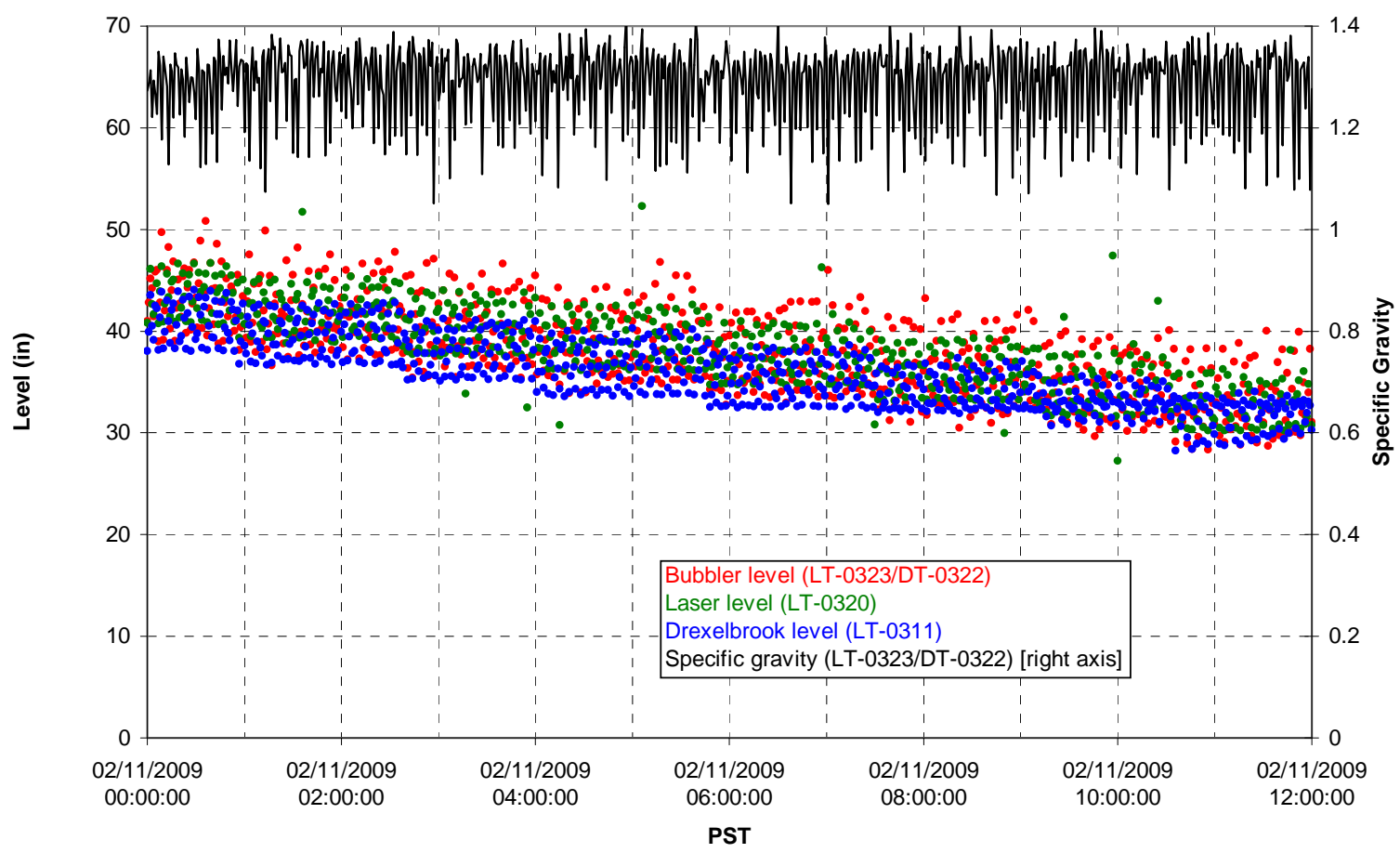

T01A temperatures

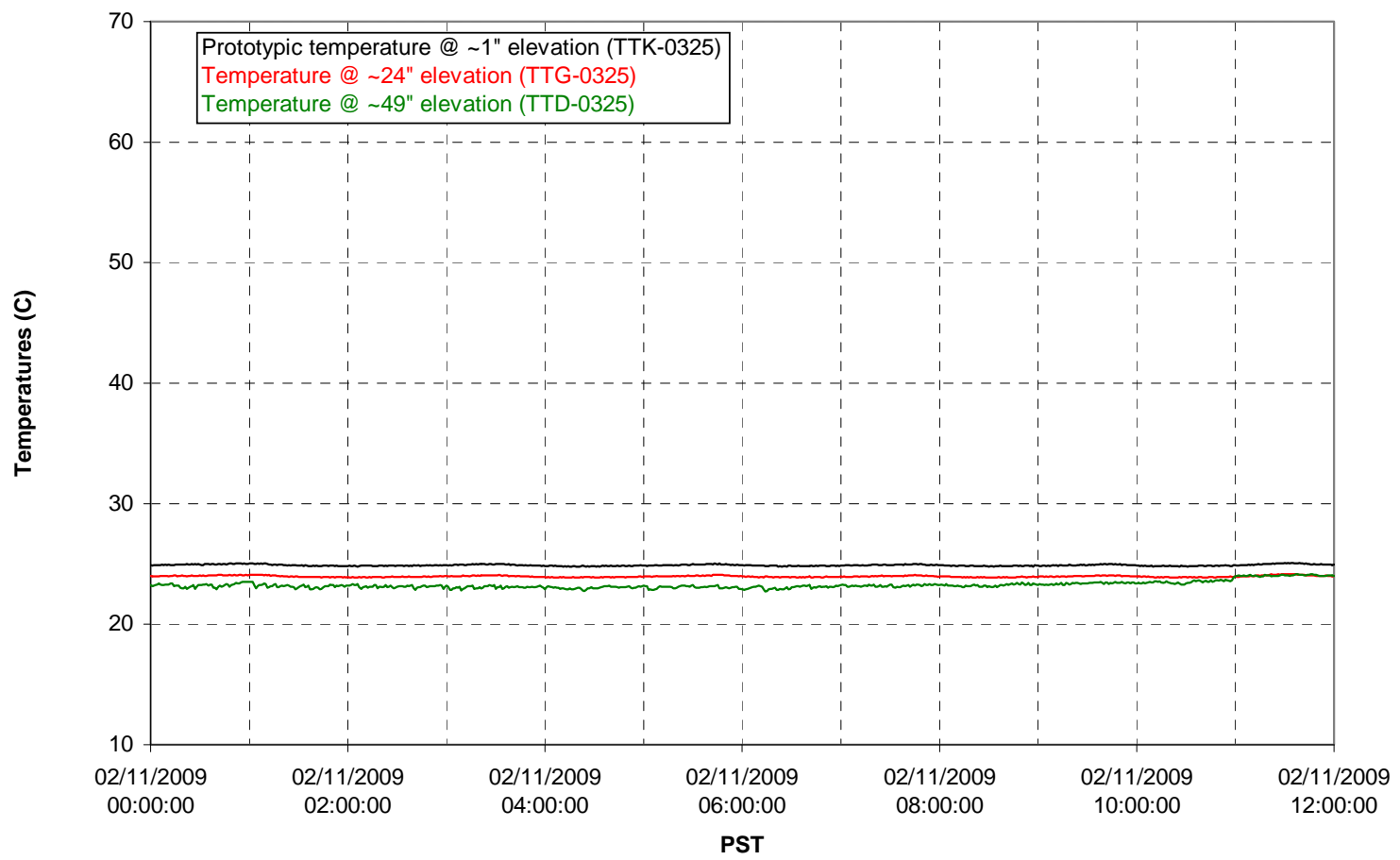


T01B level

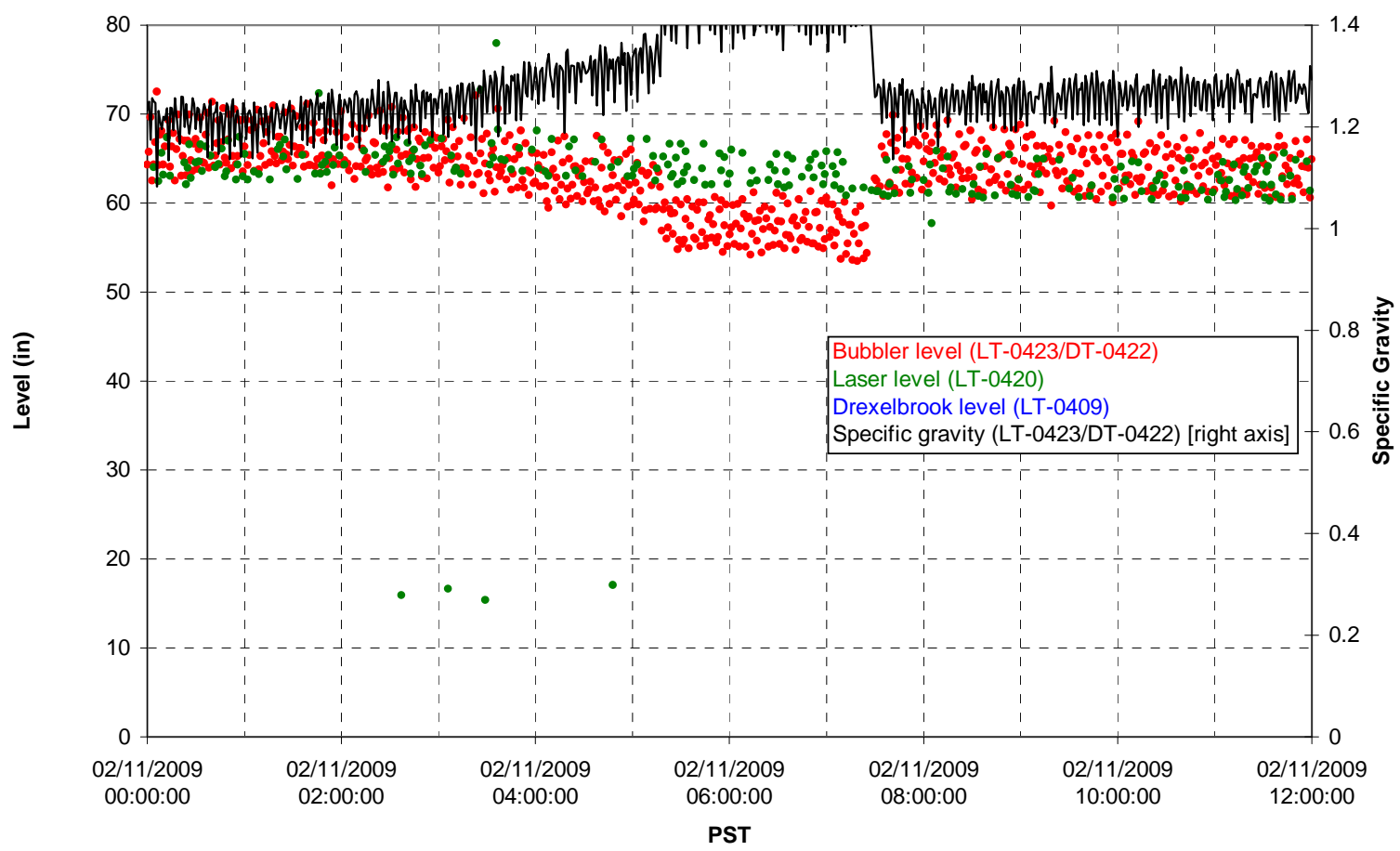

T01B temperatures

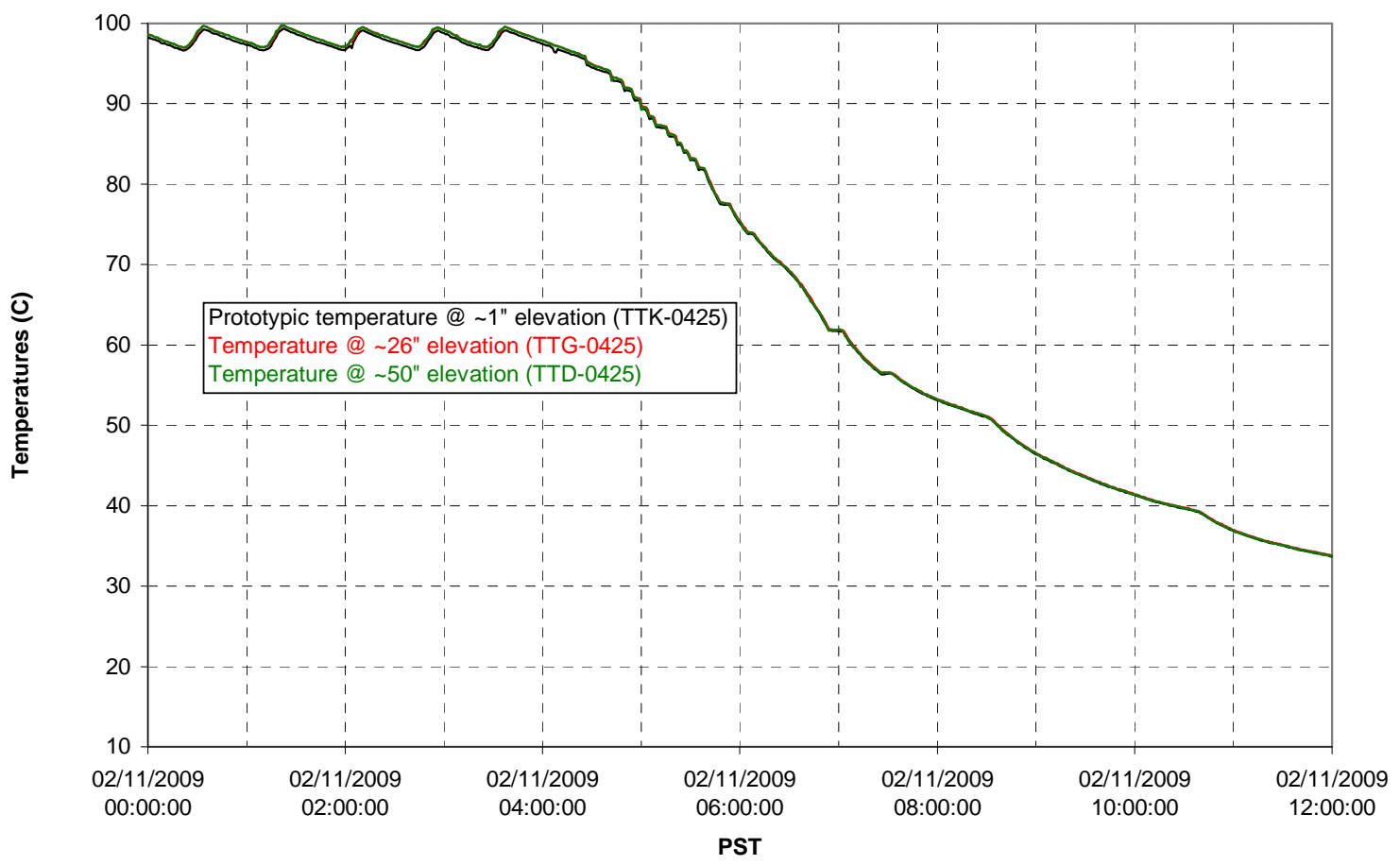


T02A level

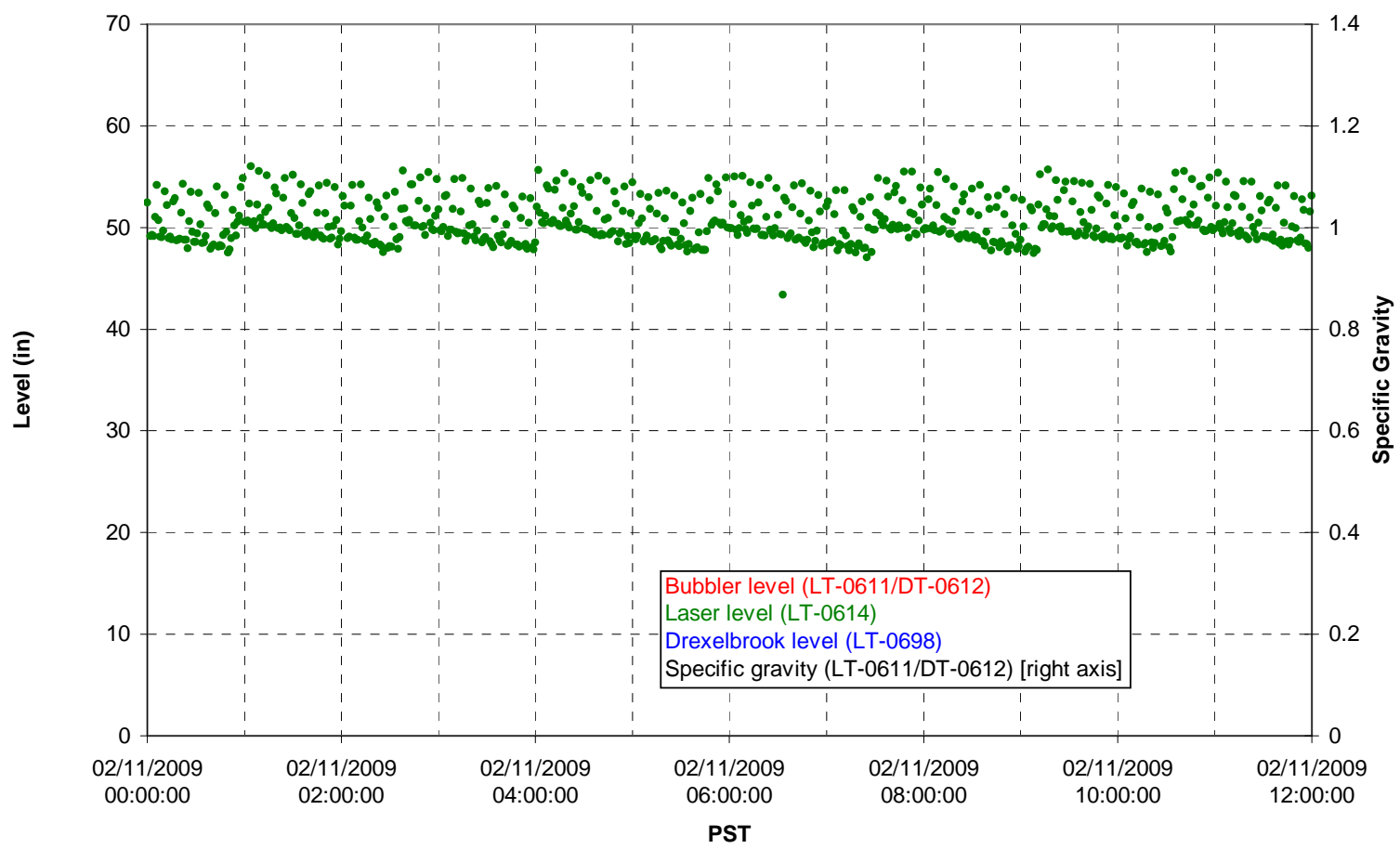

T02A temperatures

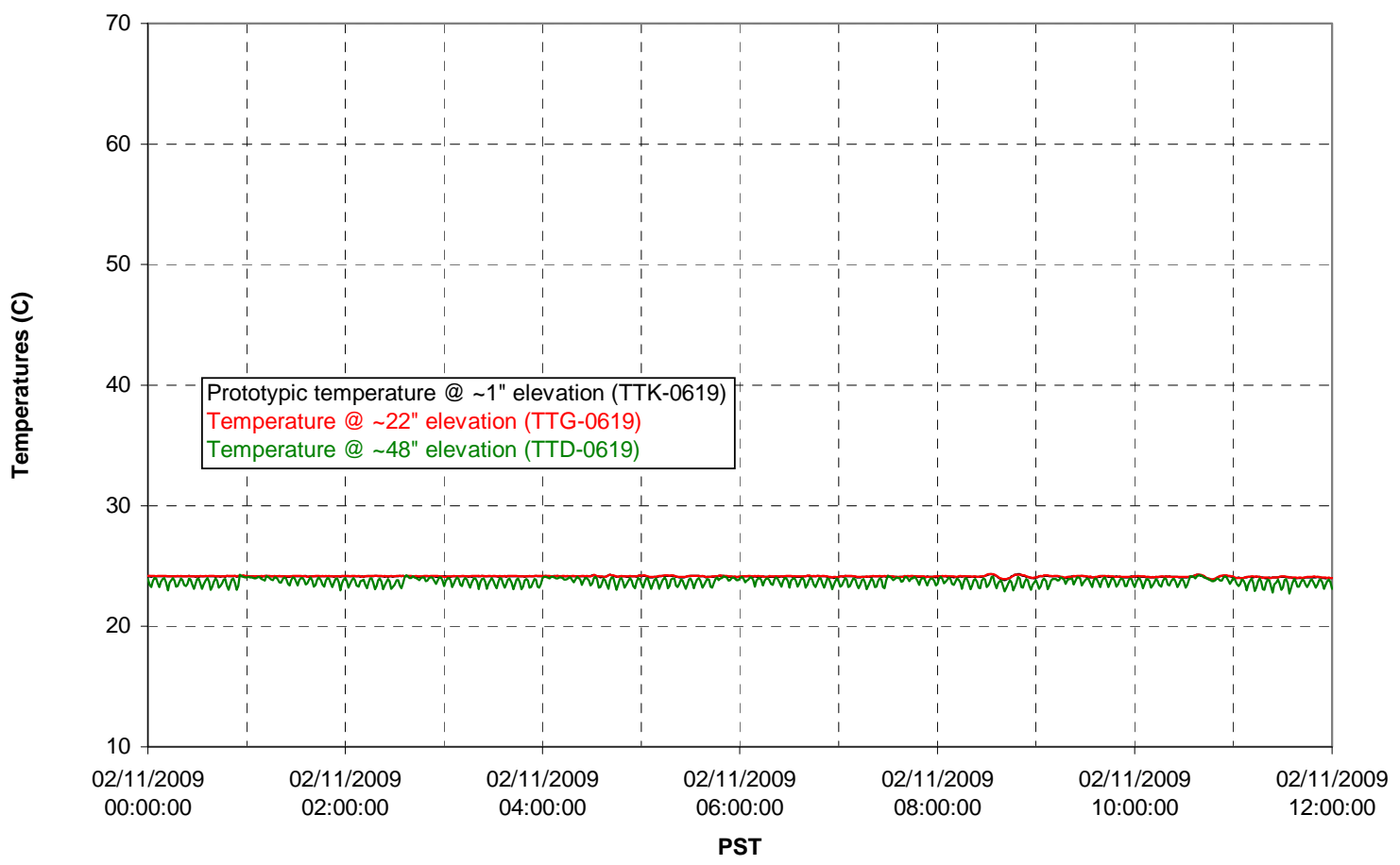


T02A and filter loop temperatures

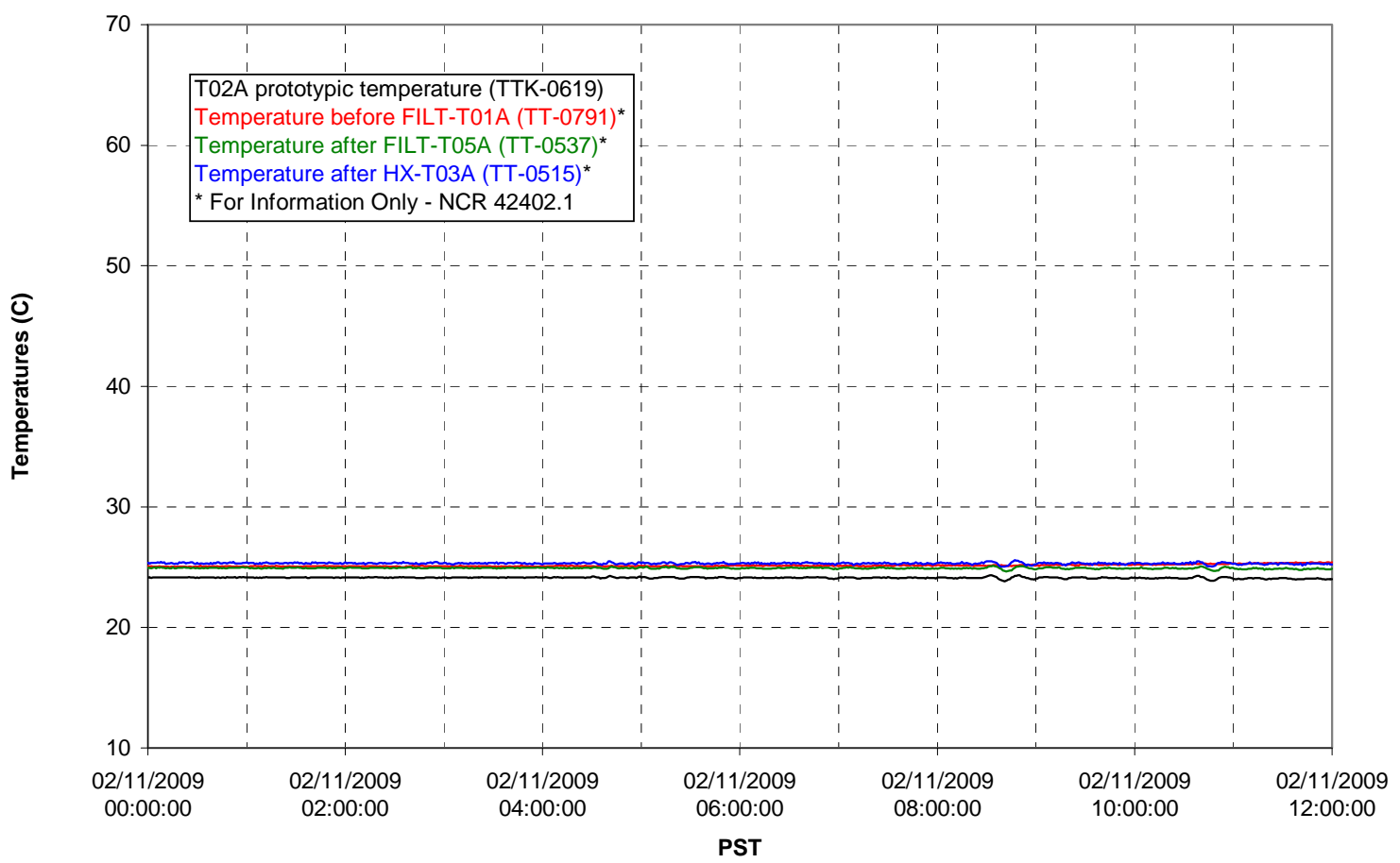

Pump Pressures and Flow

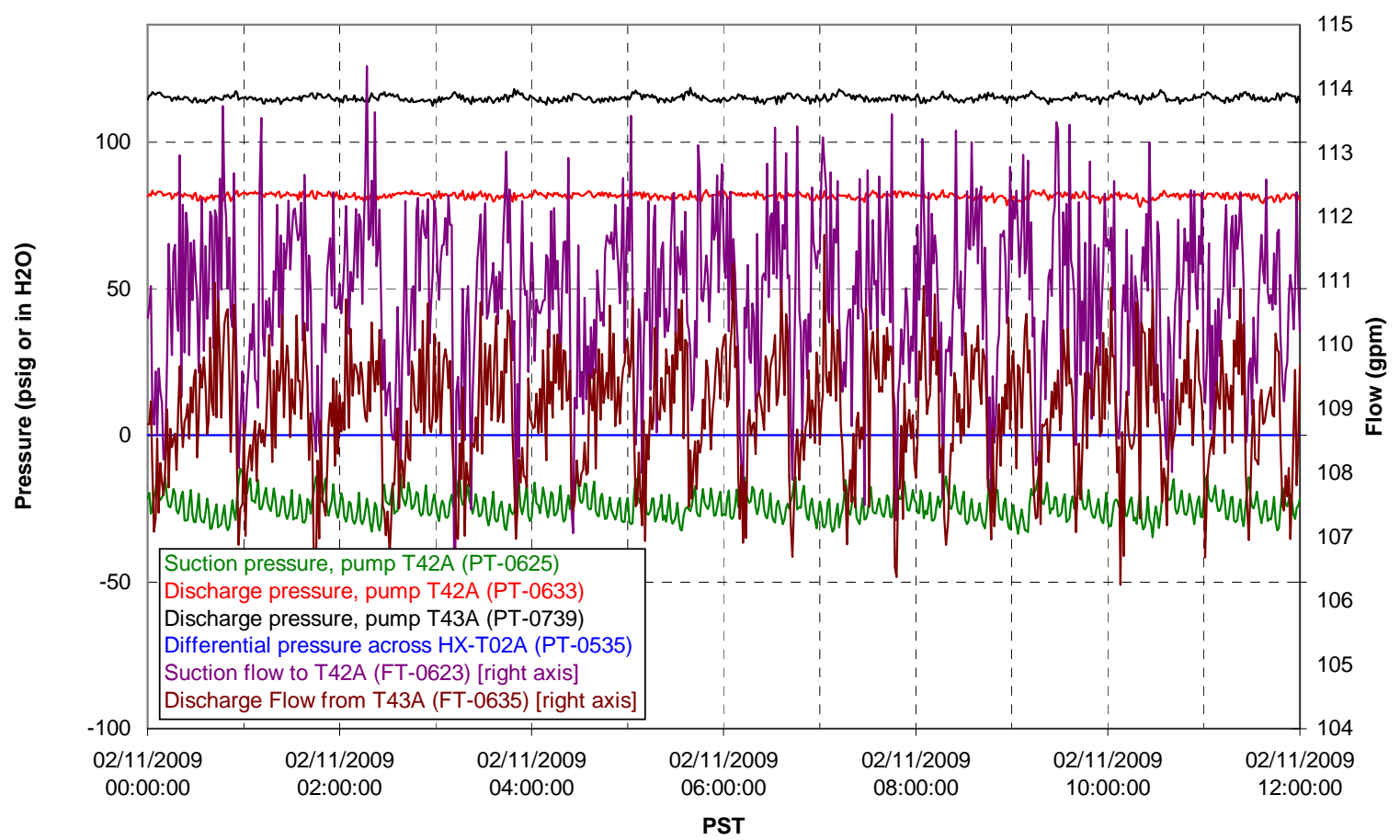


Axial pressure drop

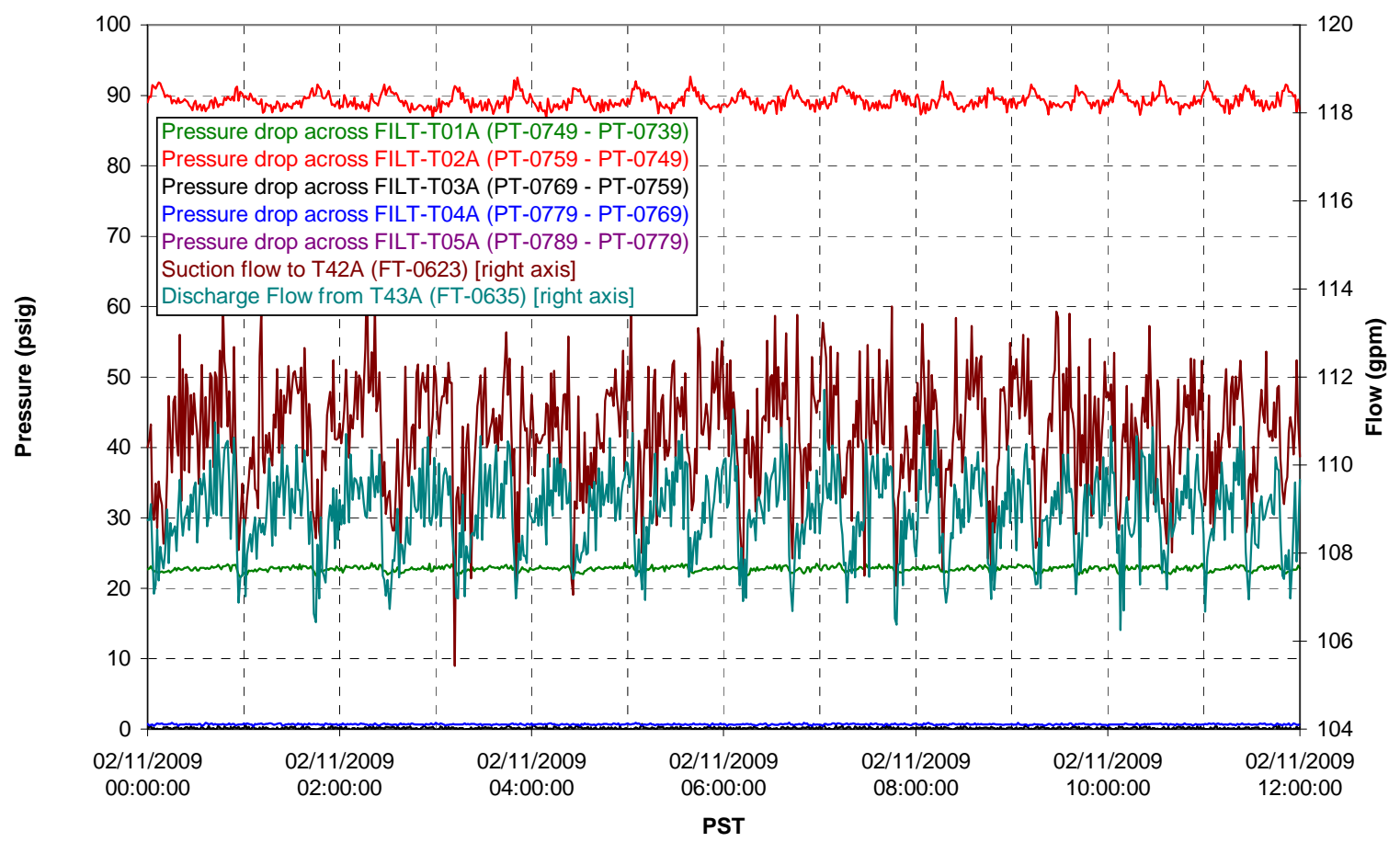

Permeate flow rates

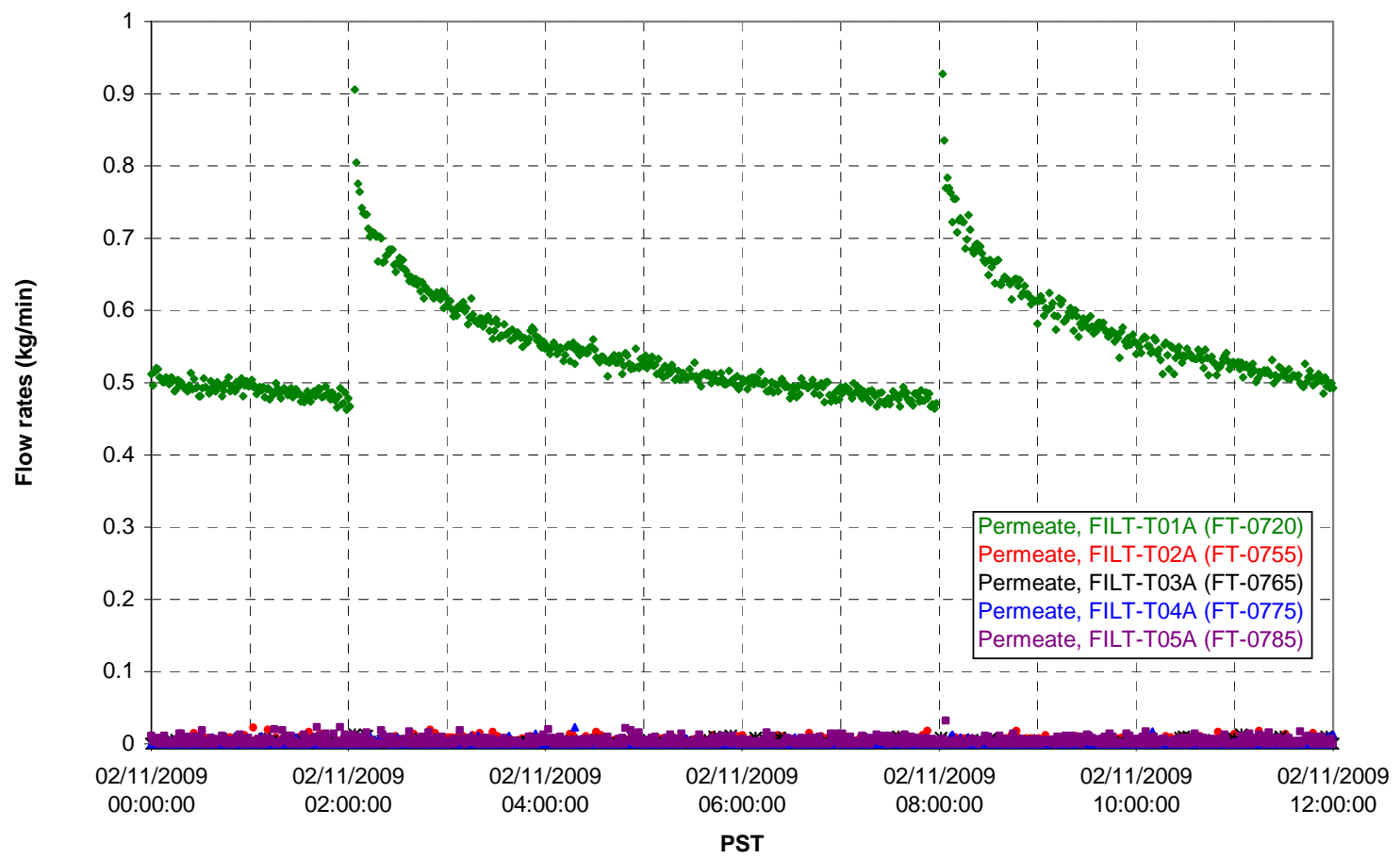


T02A Inner Temperature Tree

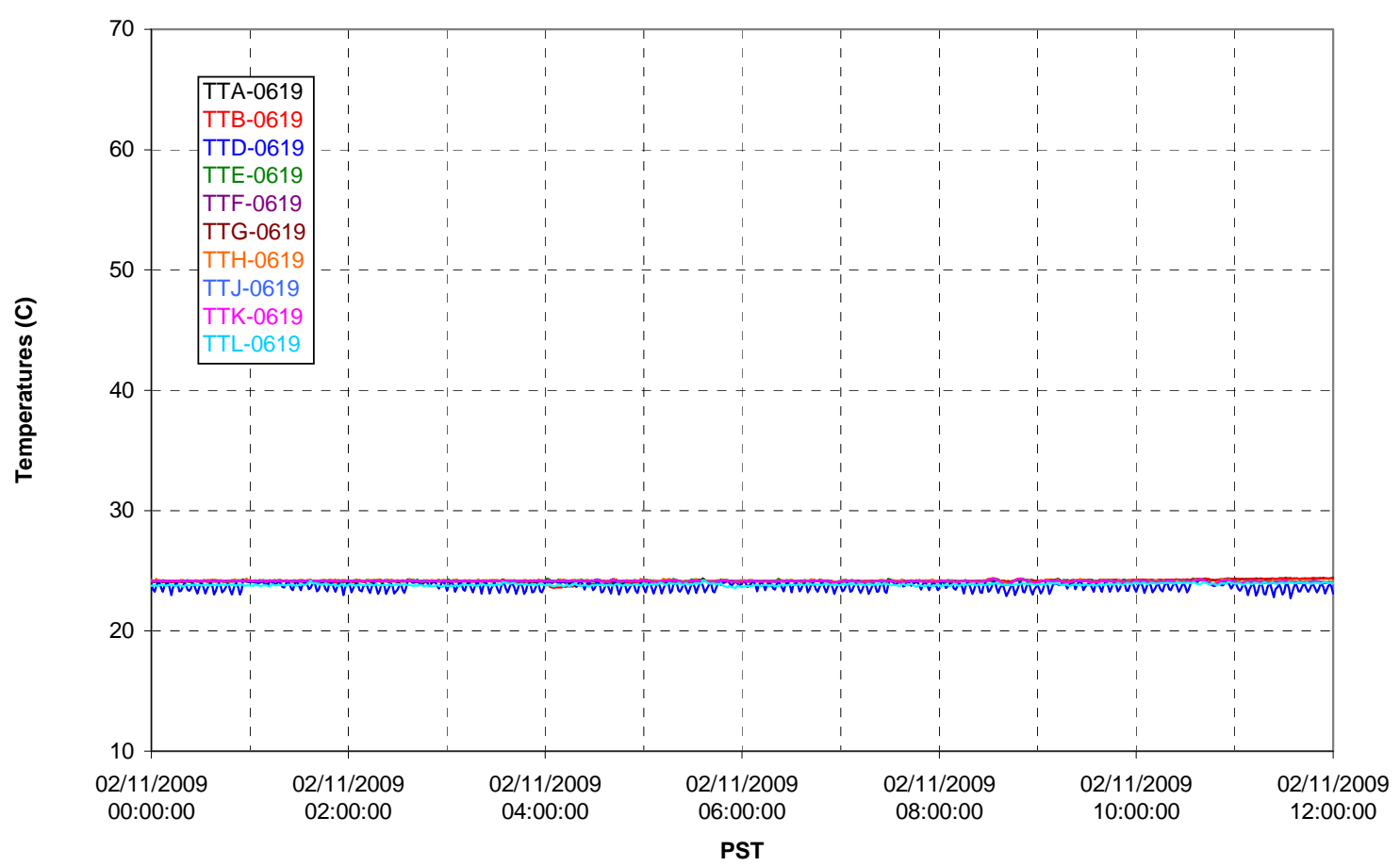

T02A Outer Temperature Tree

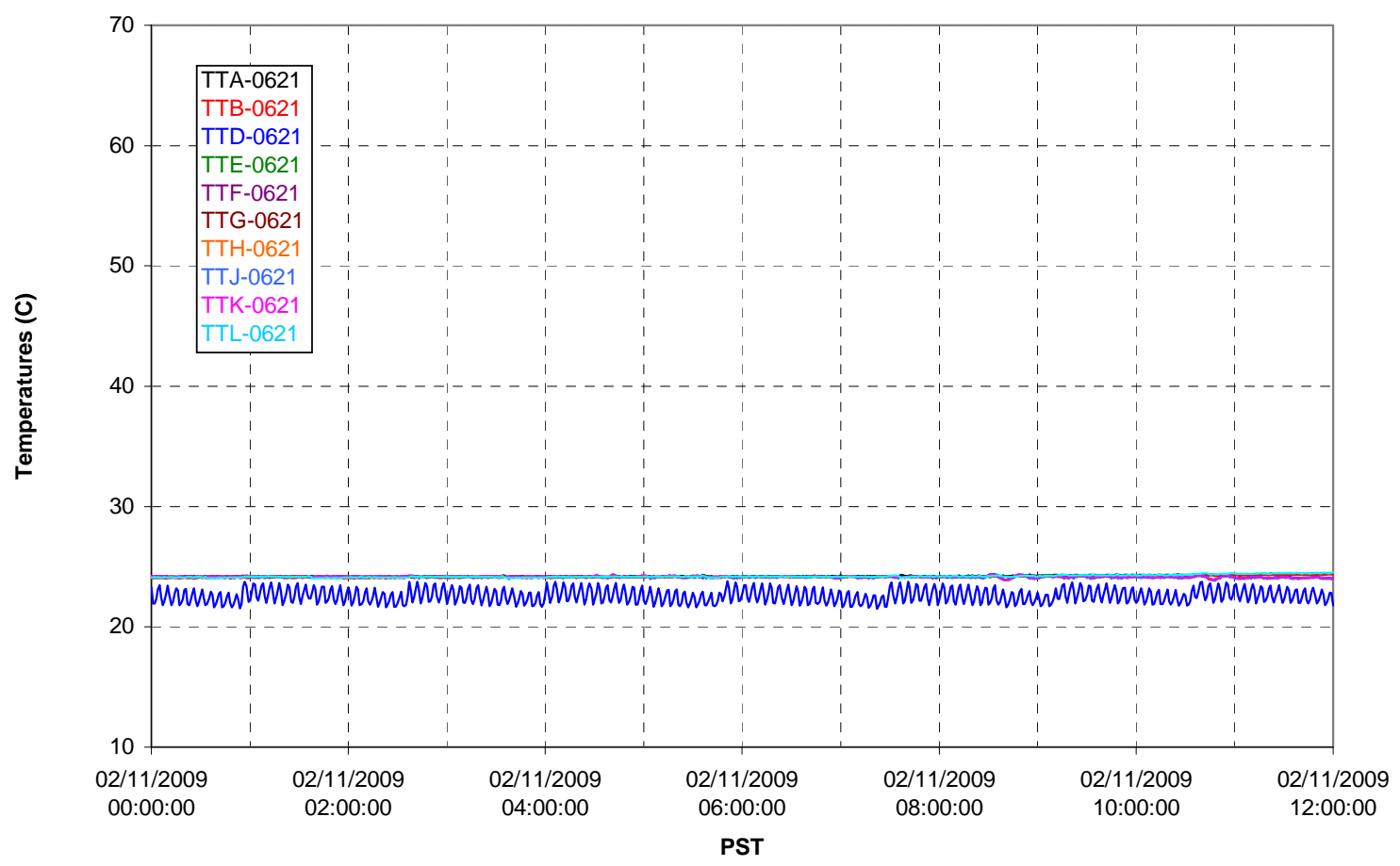


T02A temperatures

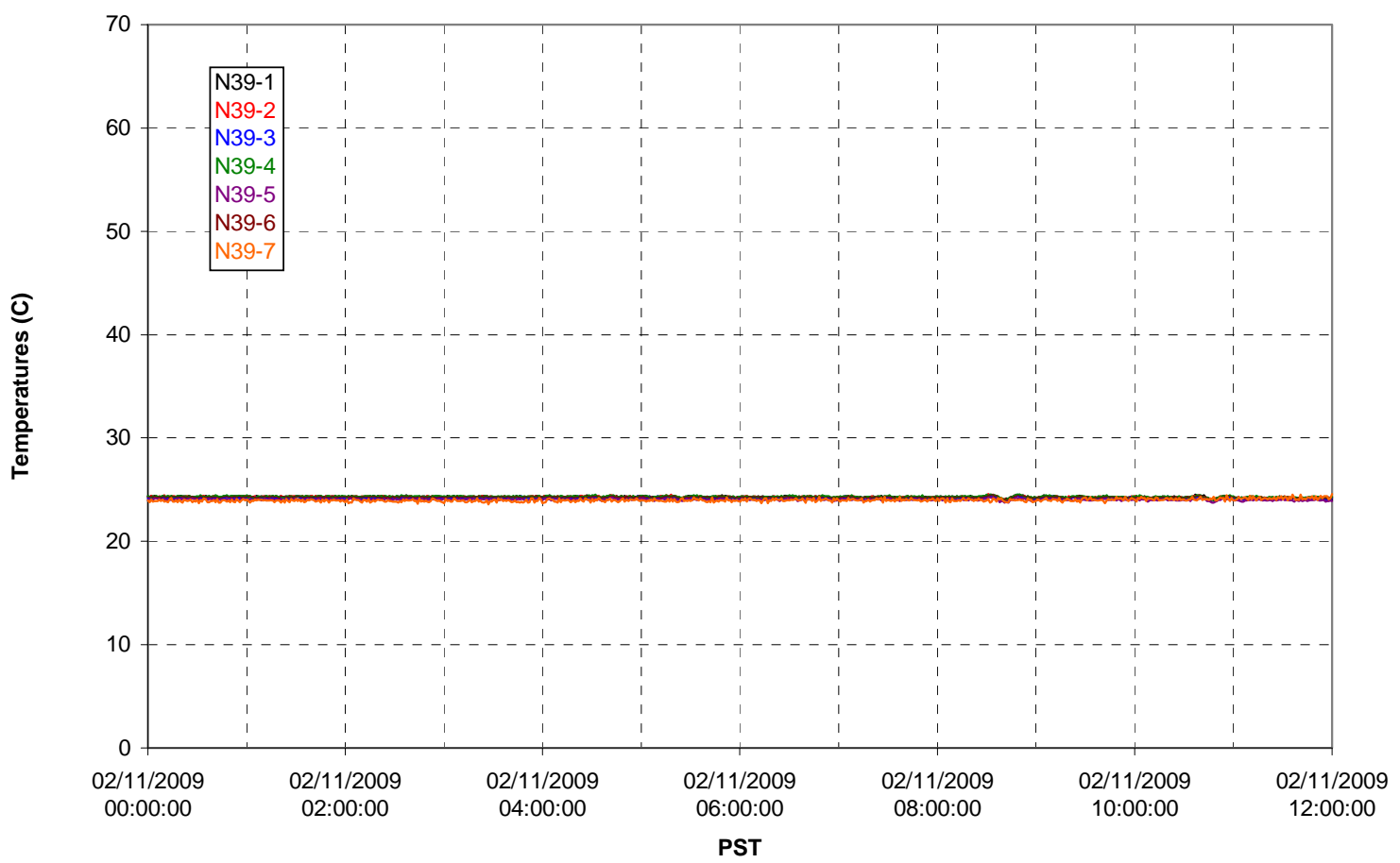

T02A temperatures

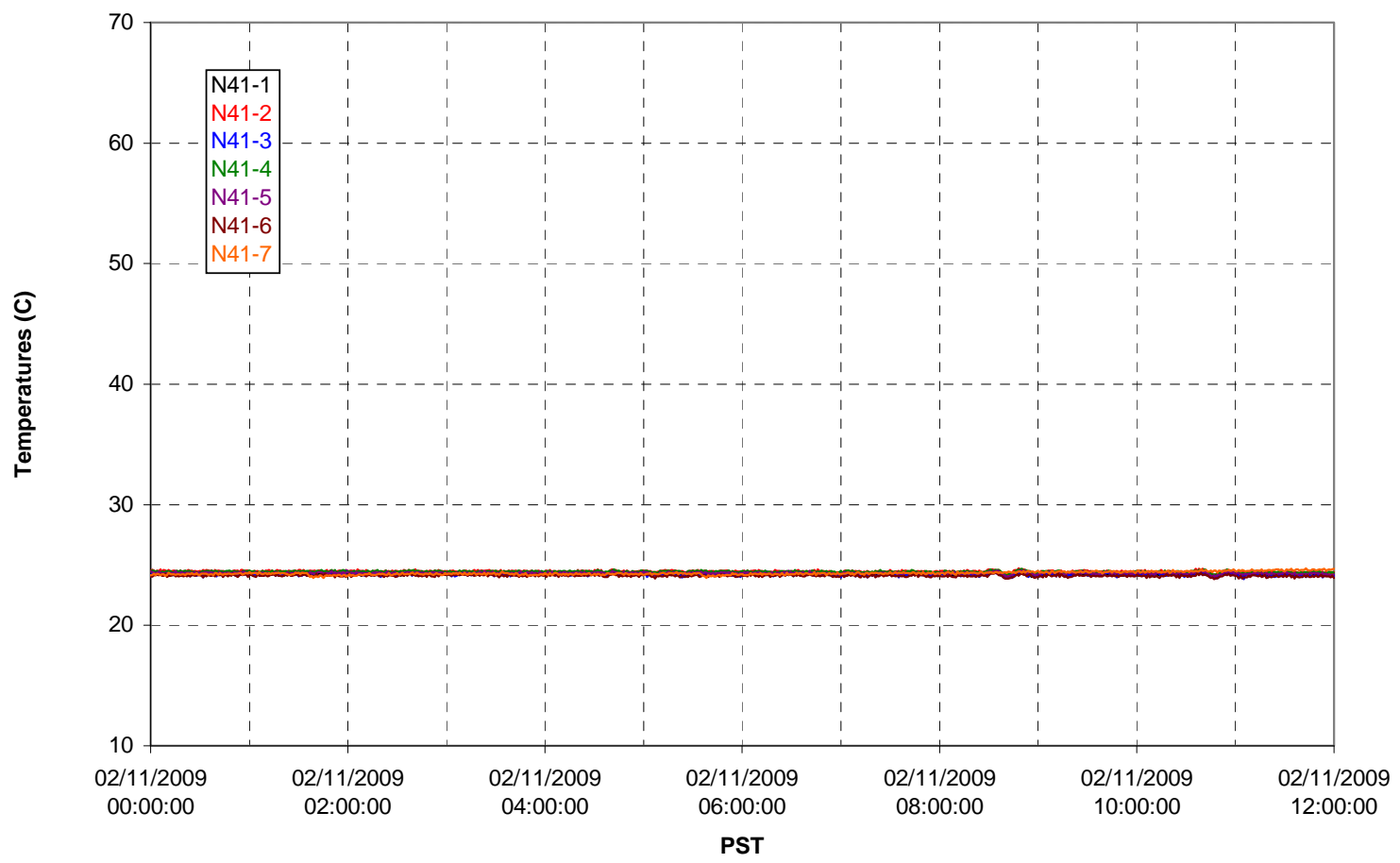


T02A temperatures

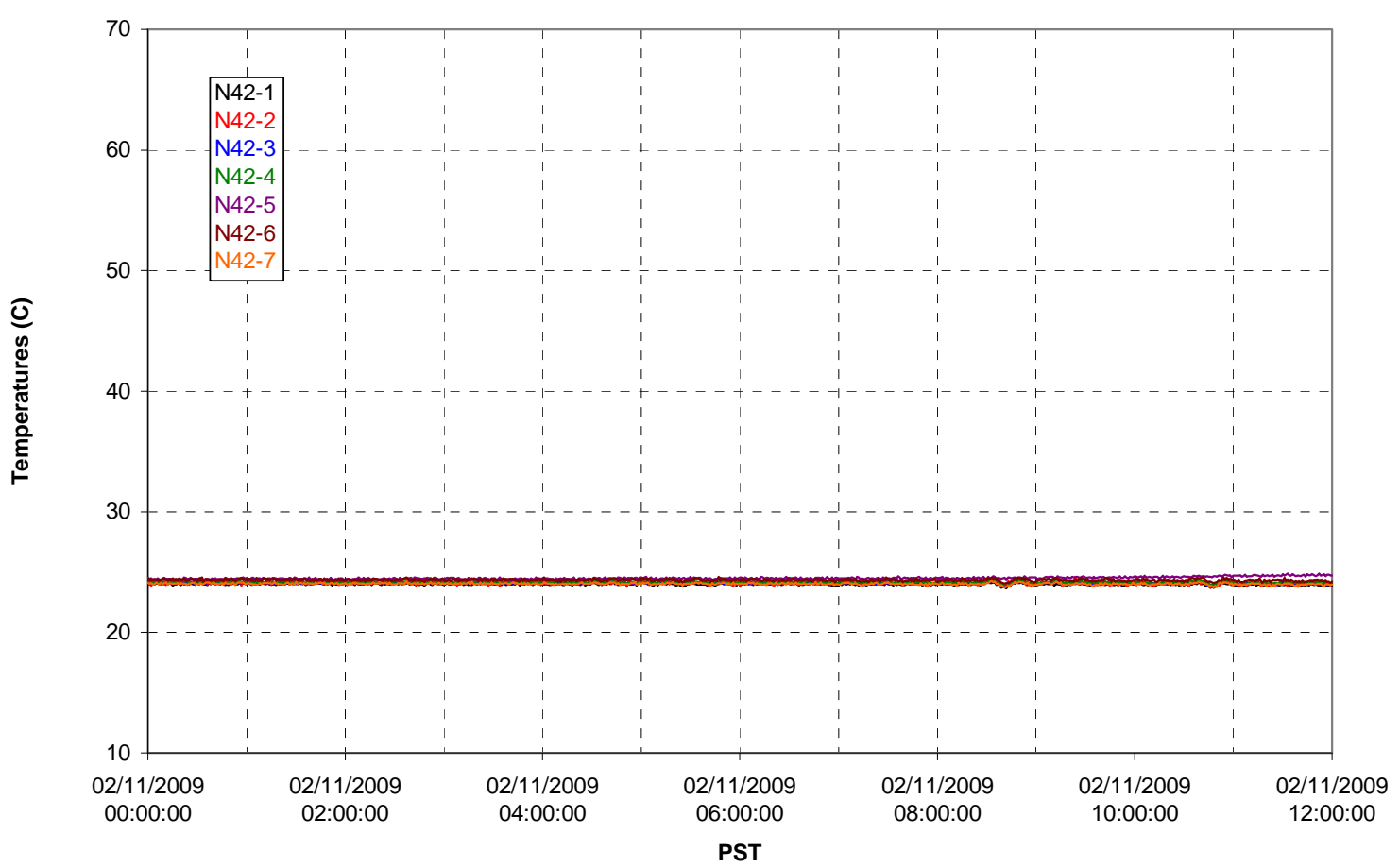

T02A temperatures

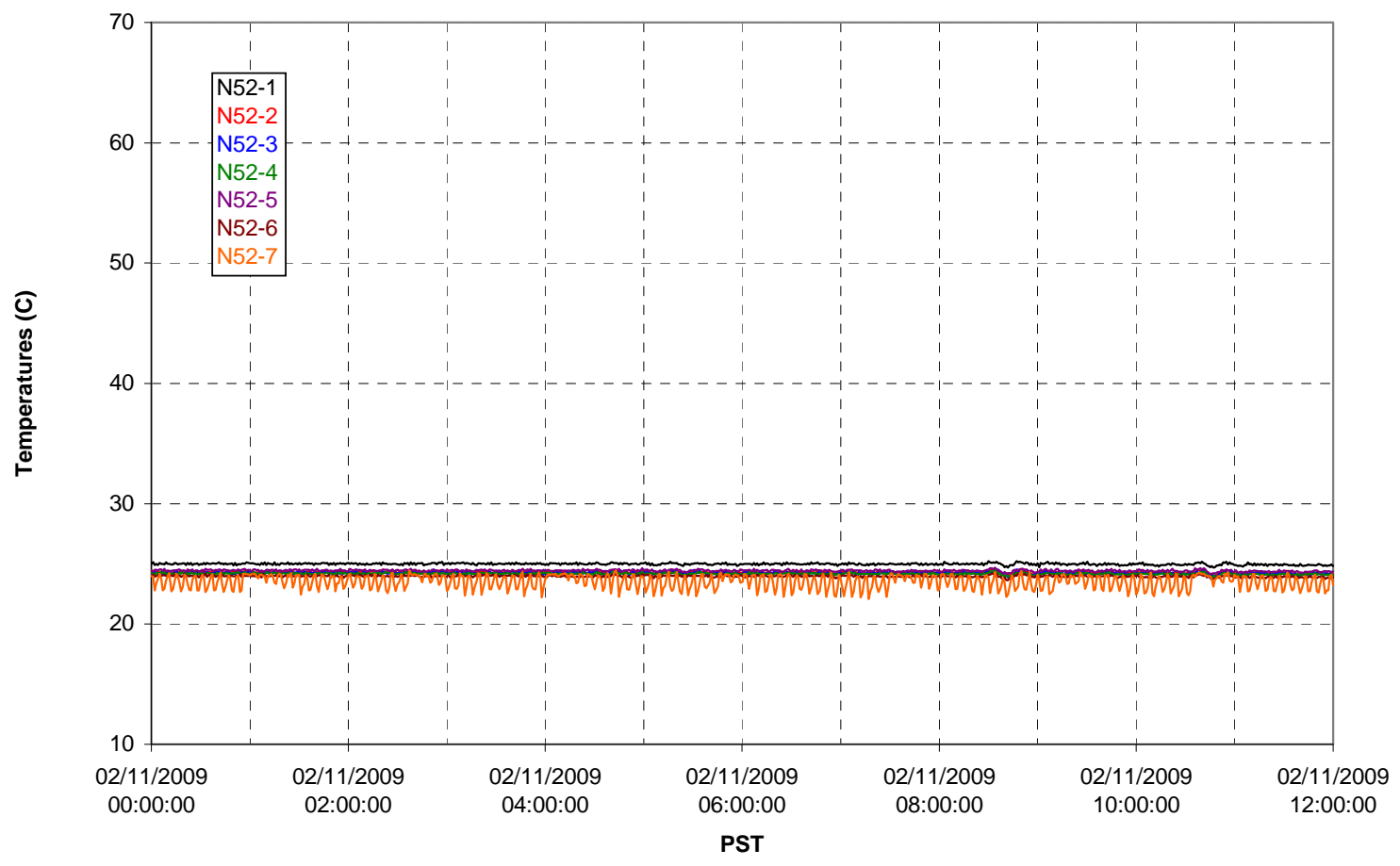


T02A Heating and Cooling
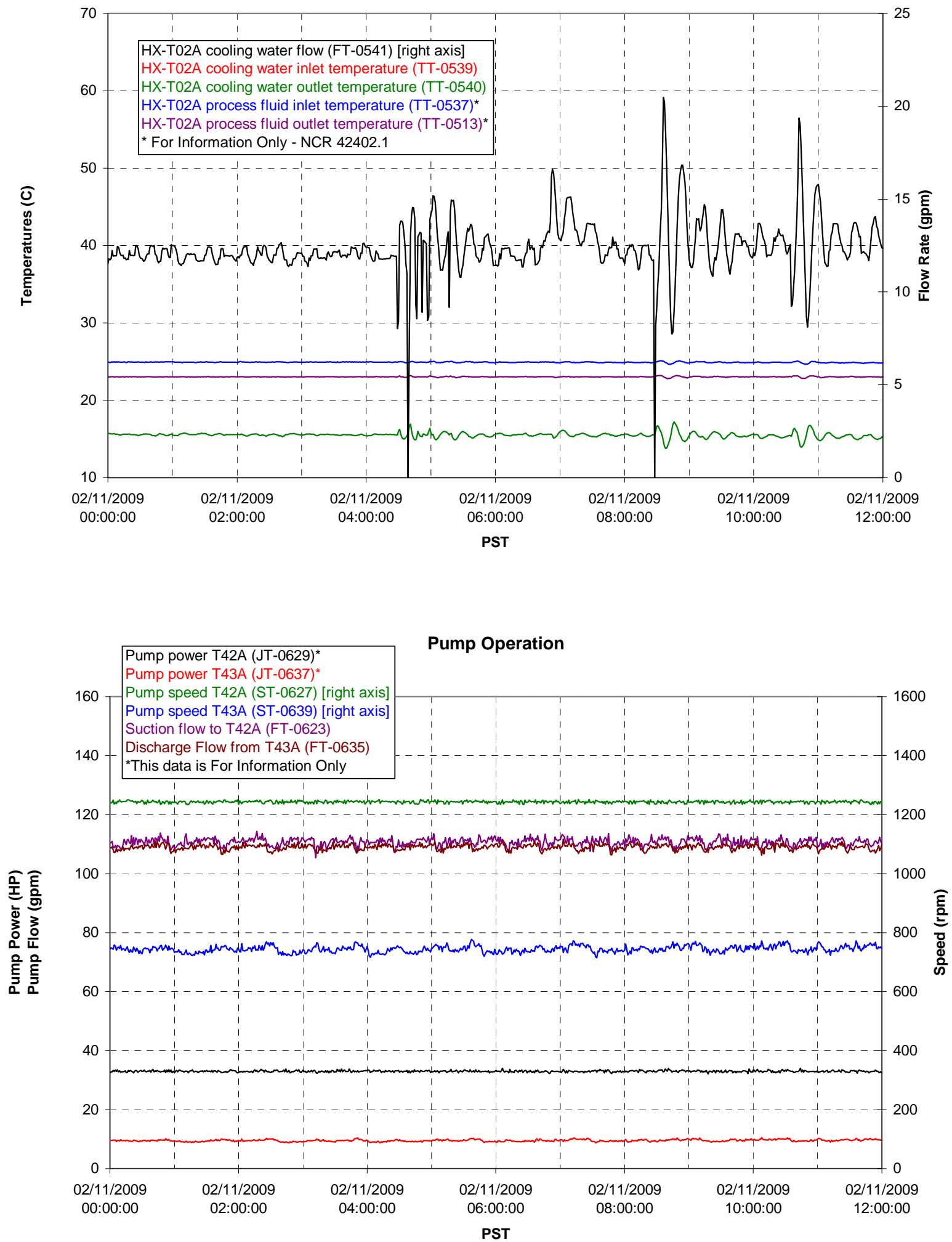
Pulsepot UFP-PP-T01A

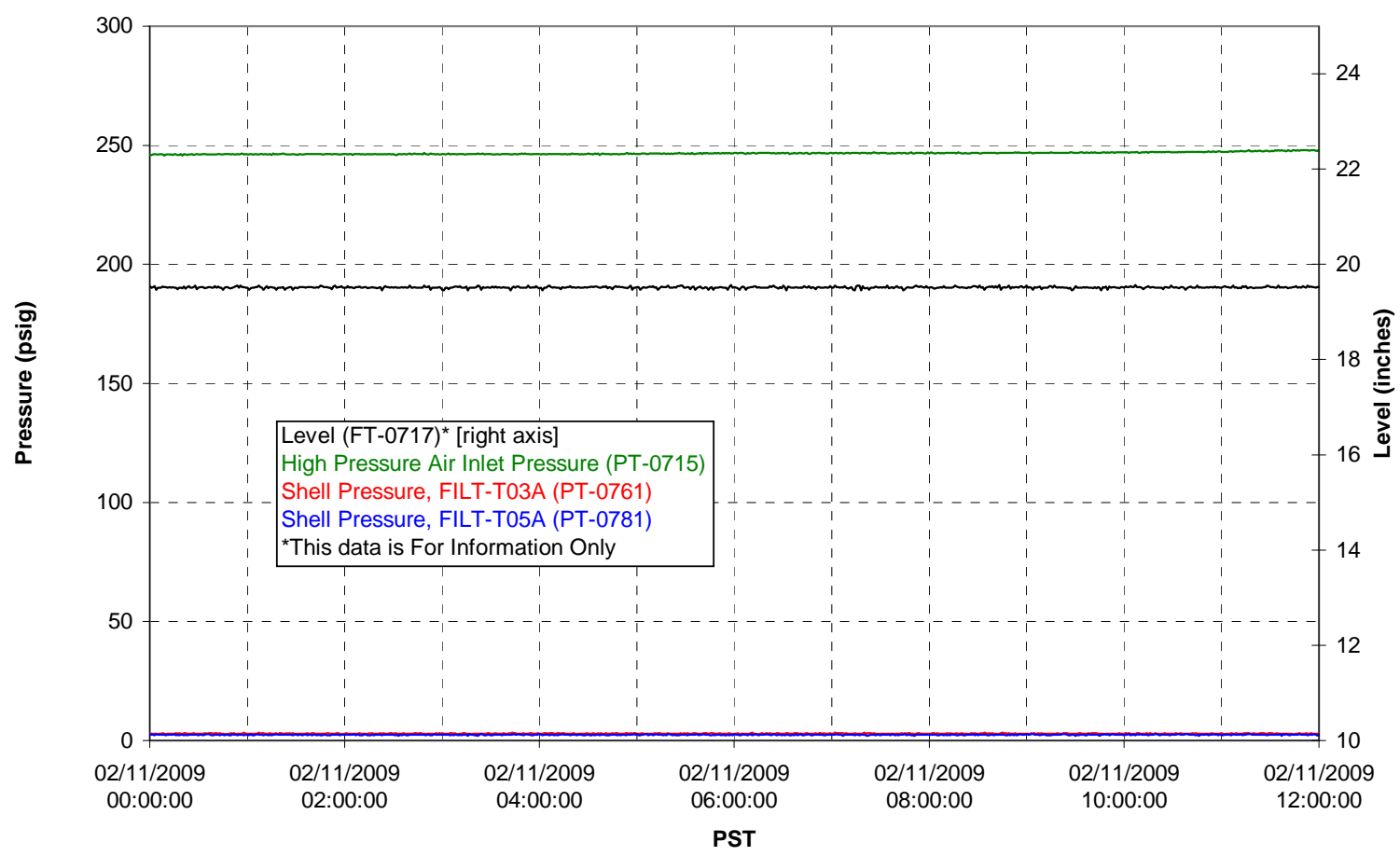

Pulsepot UFP-PP-T02A

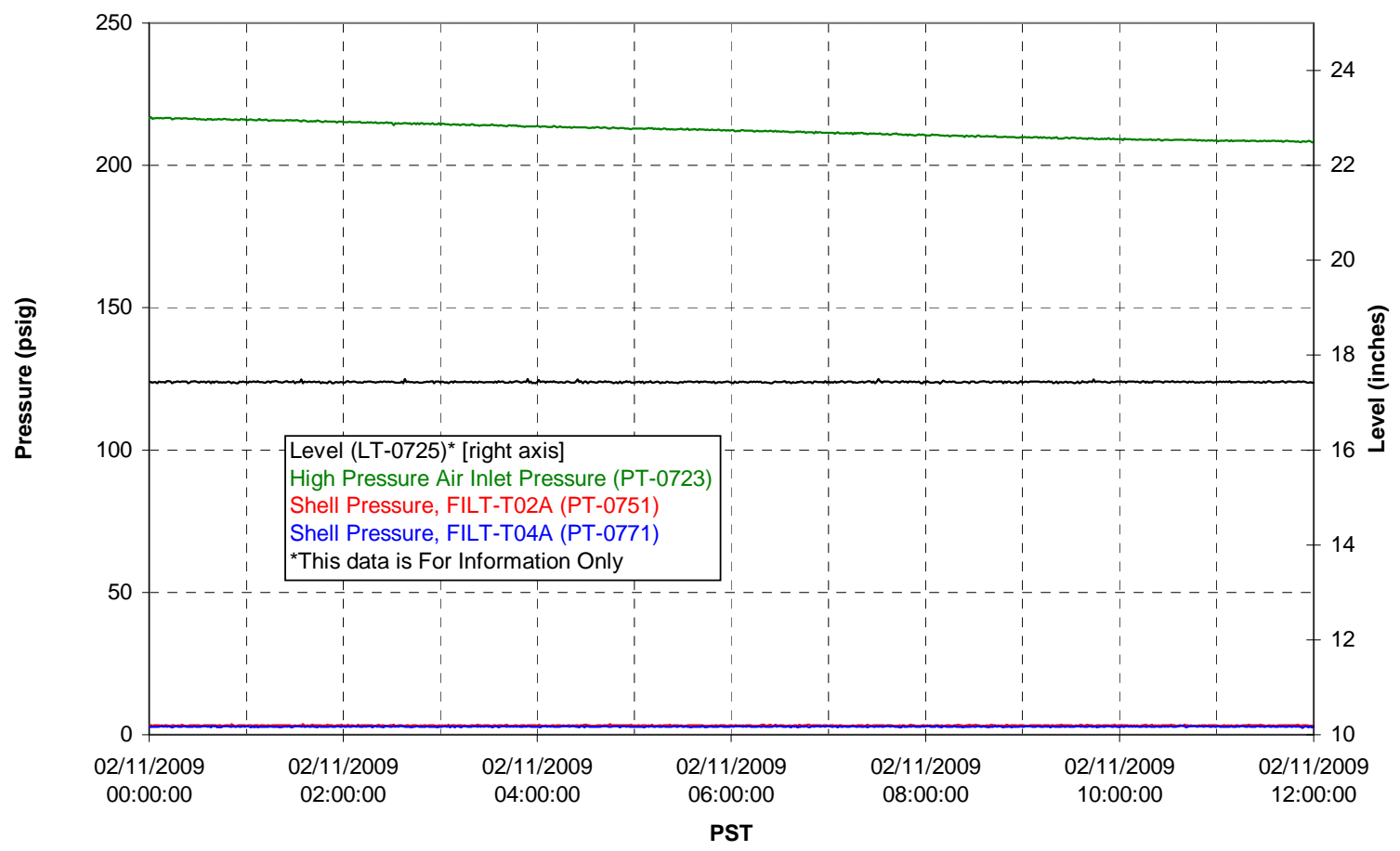


Pulsepot UFP-PP-T03A

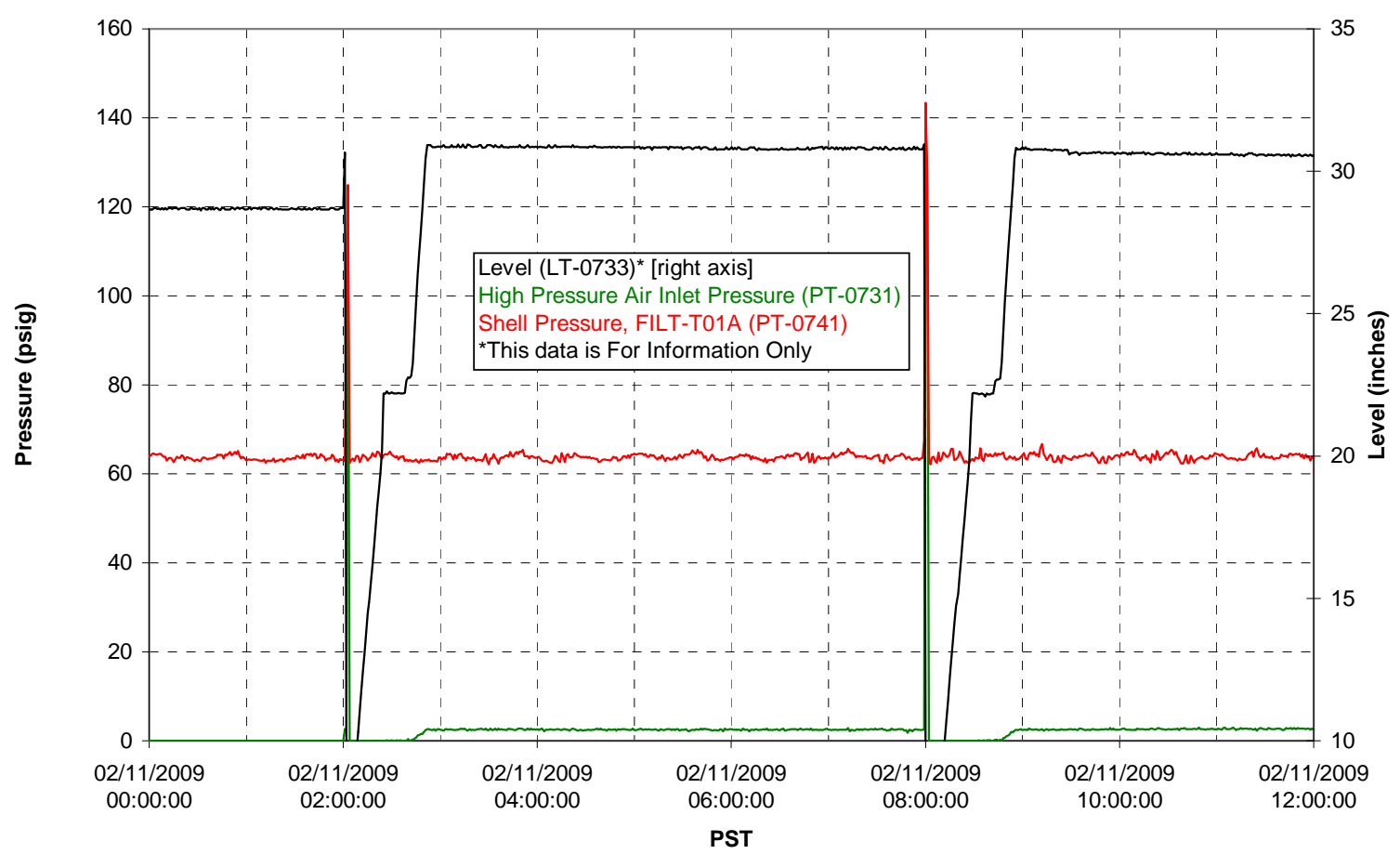

Pulsepot Levels

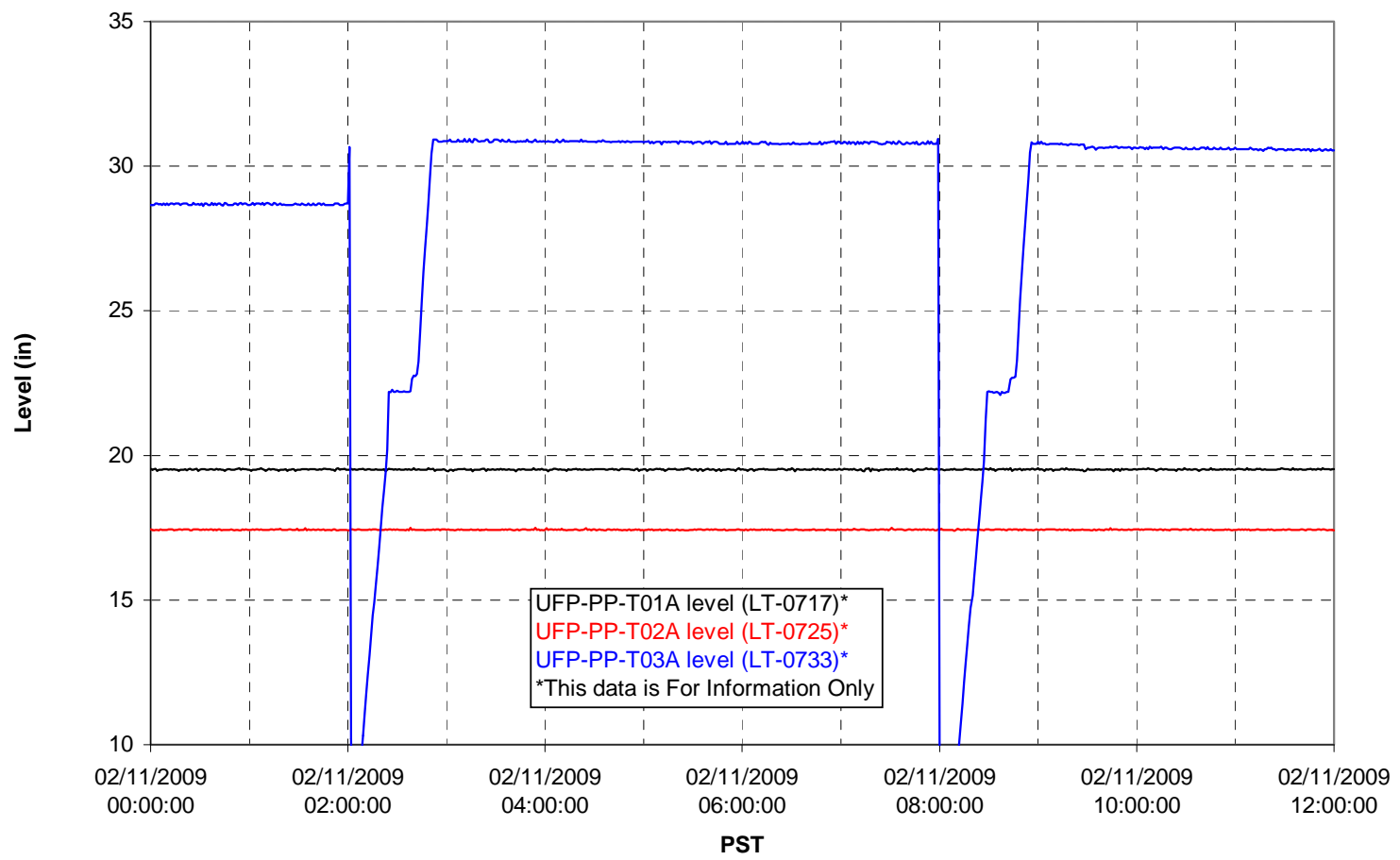


Filter UFP-FILT-T01A

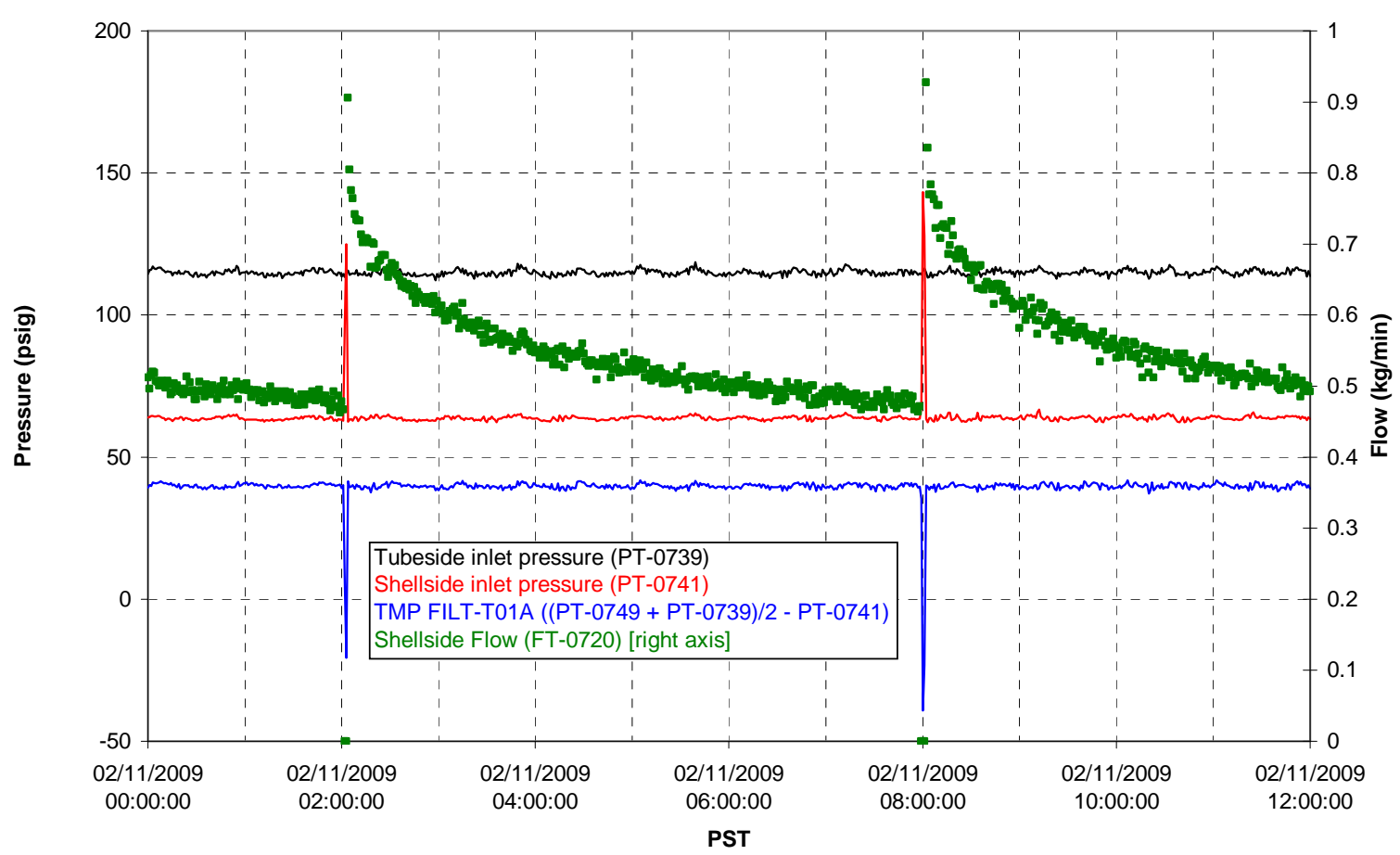

Filter UFP-FILT-T02A

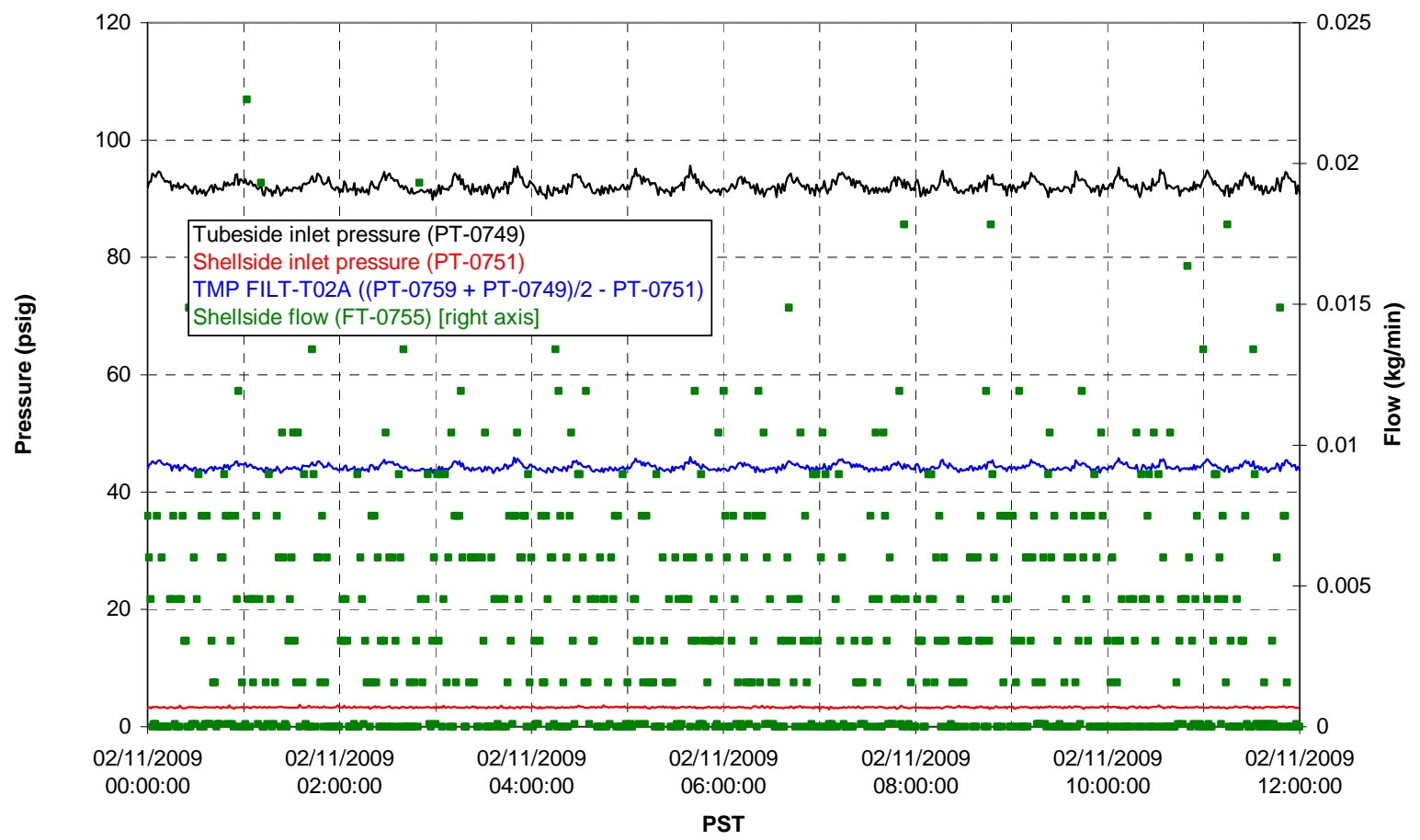


Filter UFP-FILT-T03A

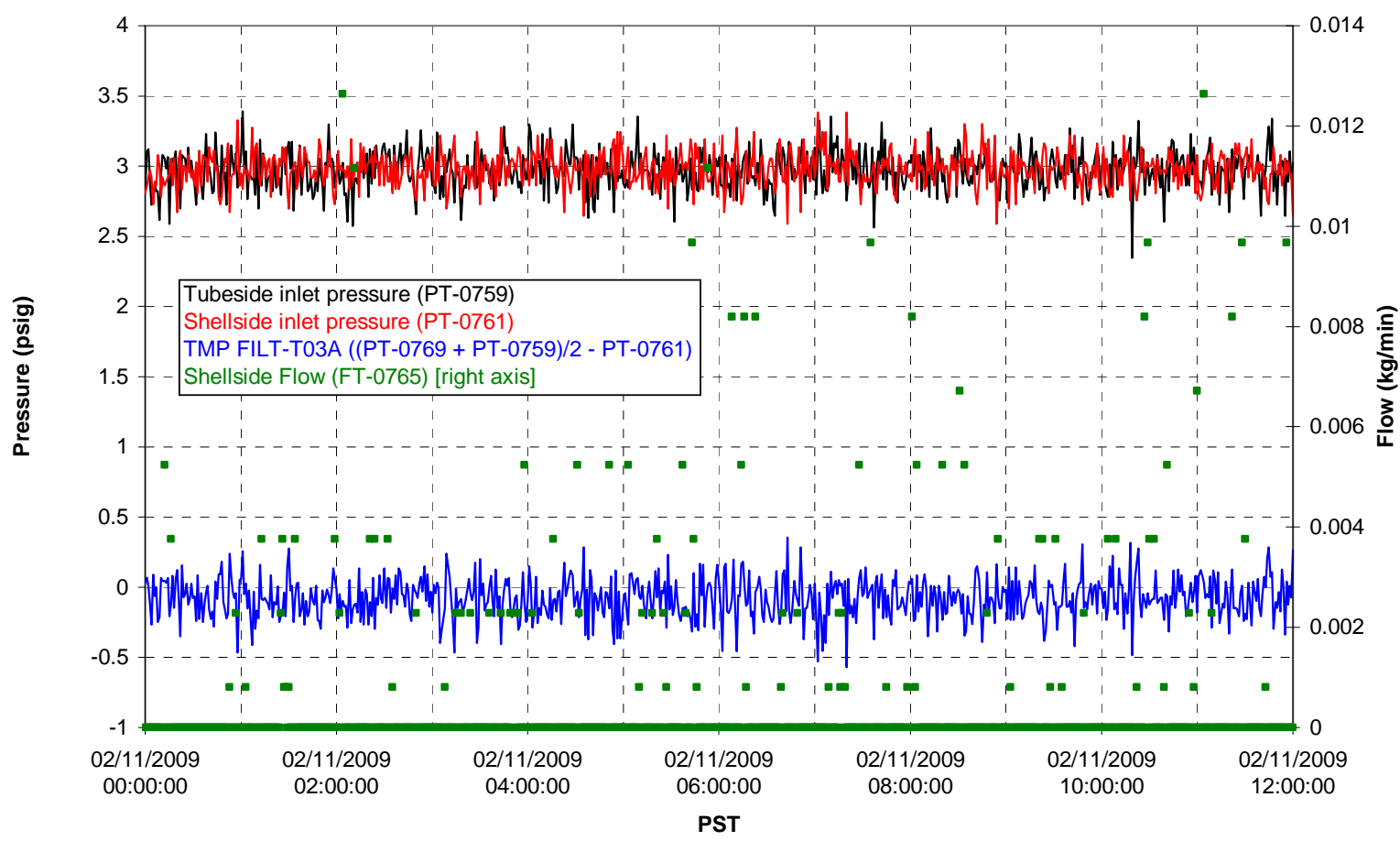

Filter UFP-FILT-T04A

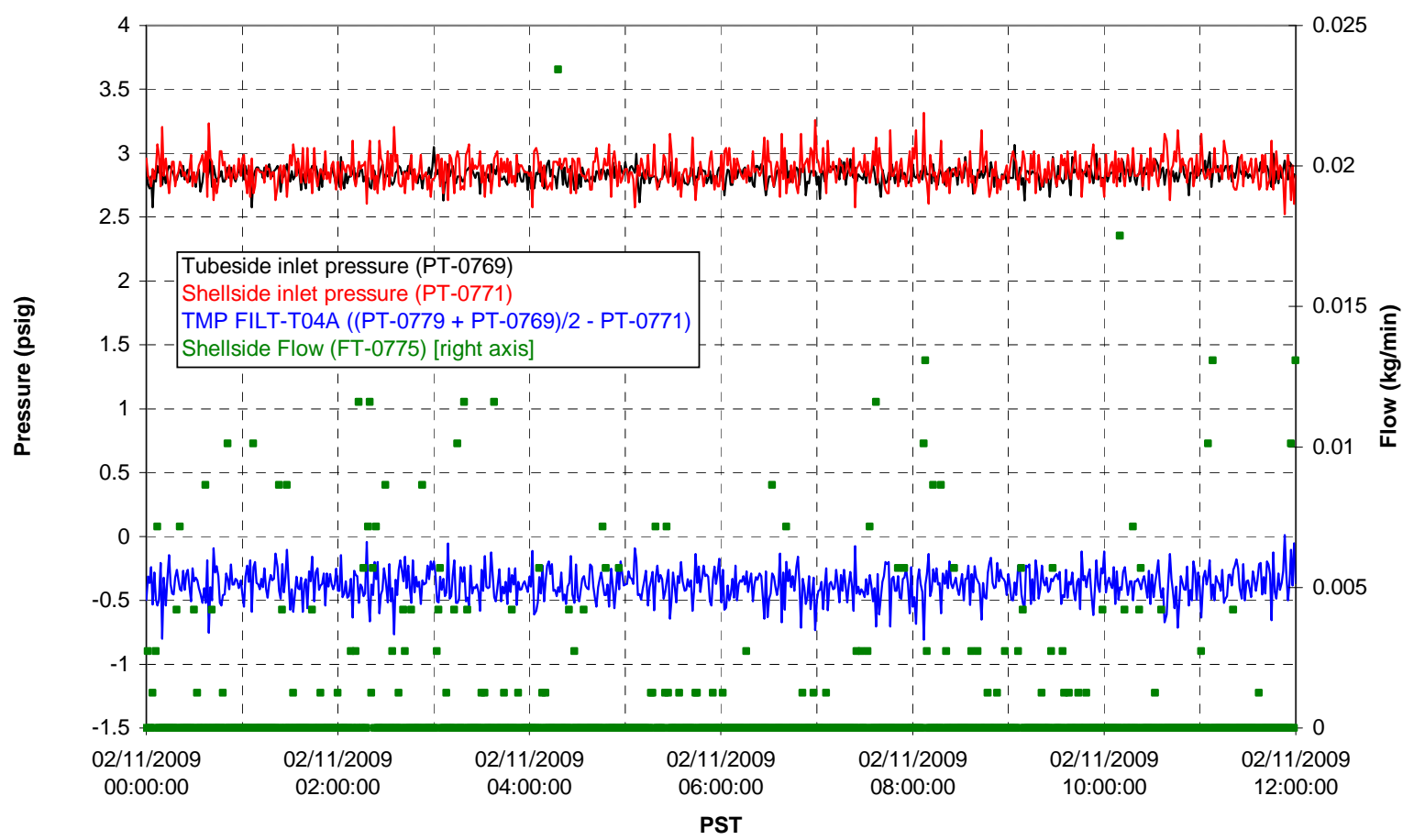


Filter UFP-FILT-T05A

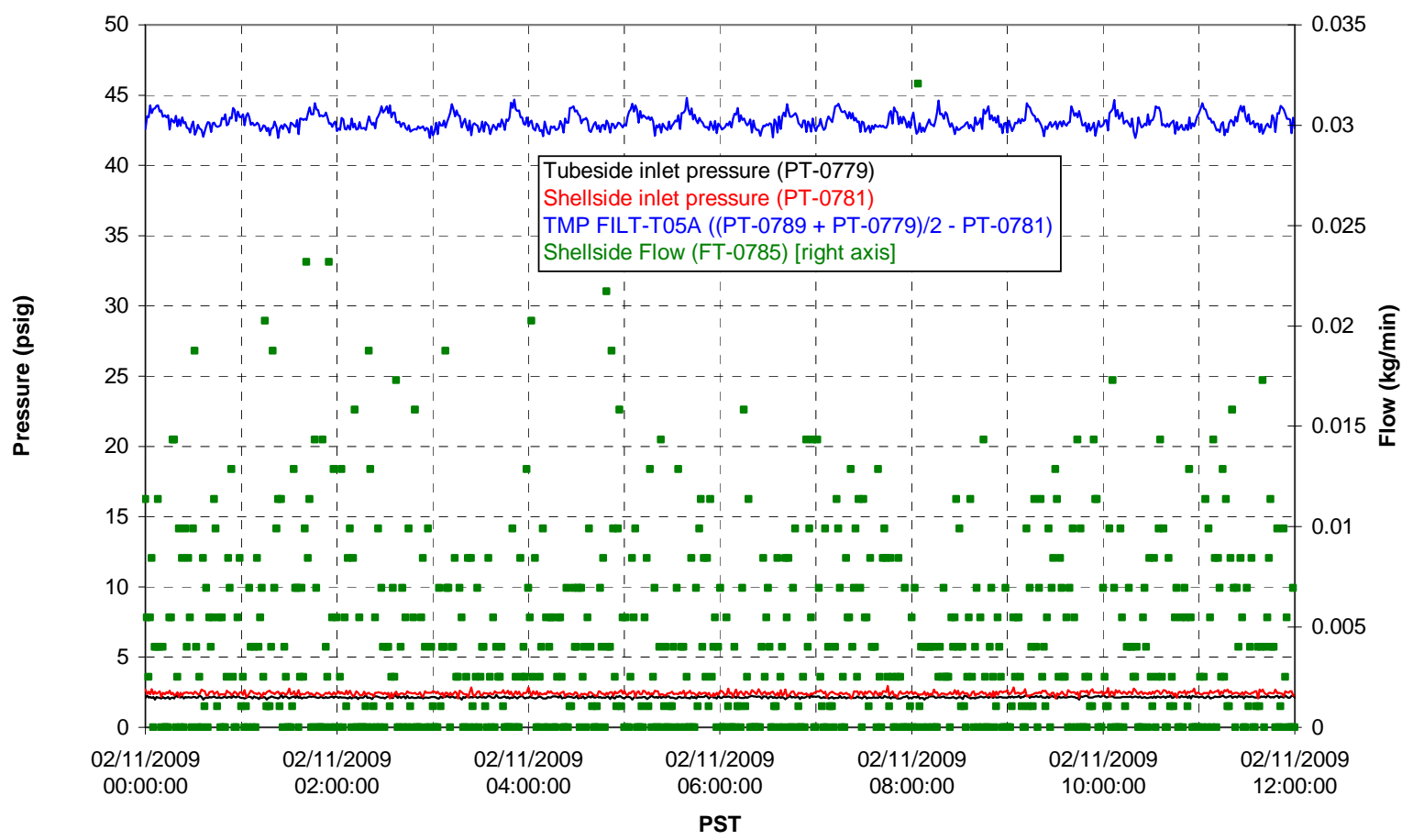

Chemical Flow

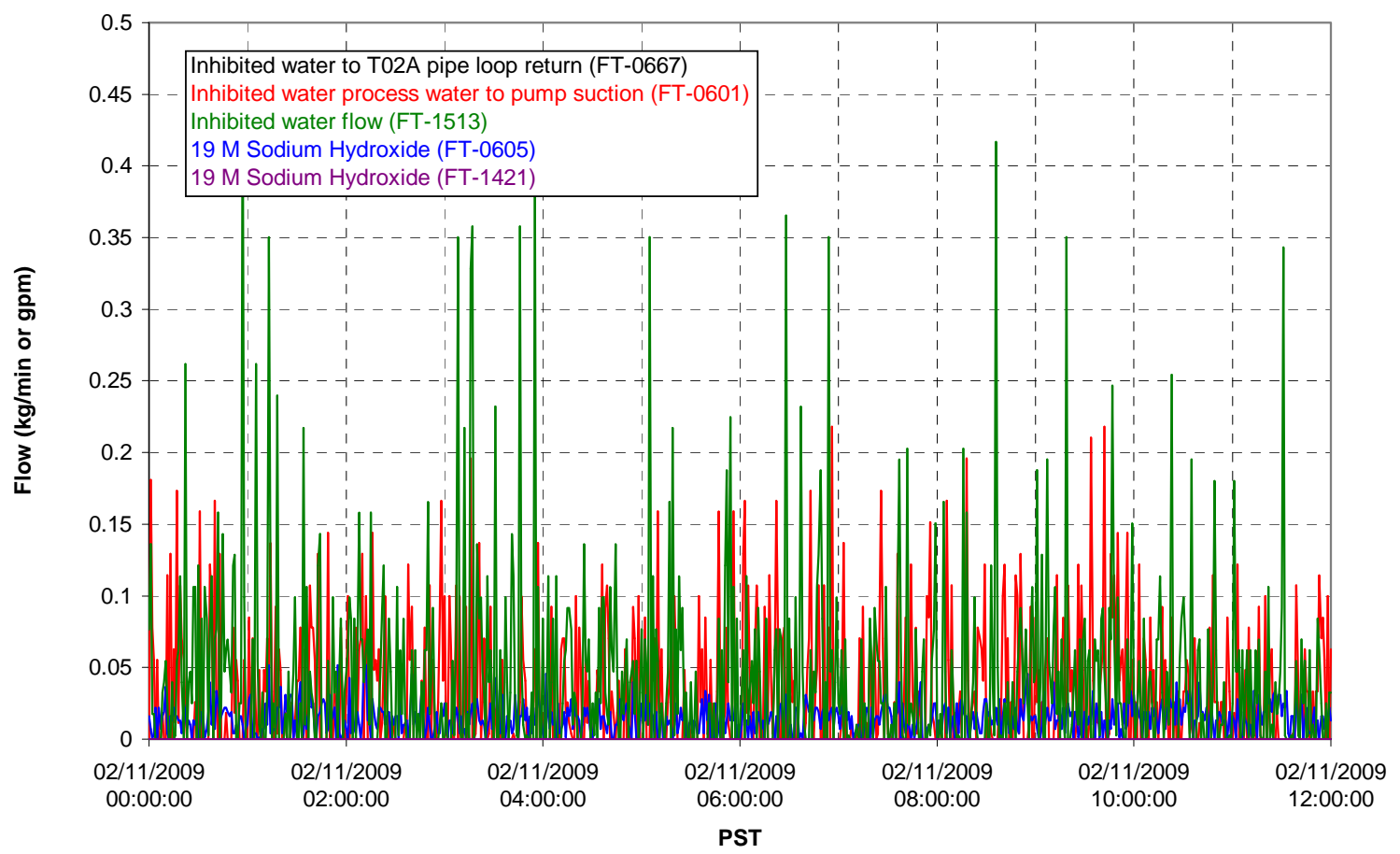


Chemical Flow

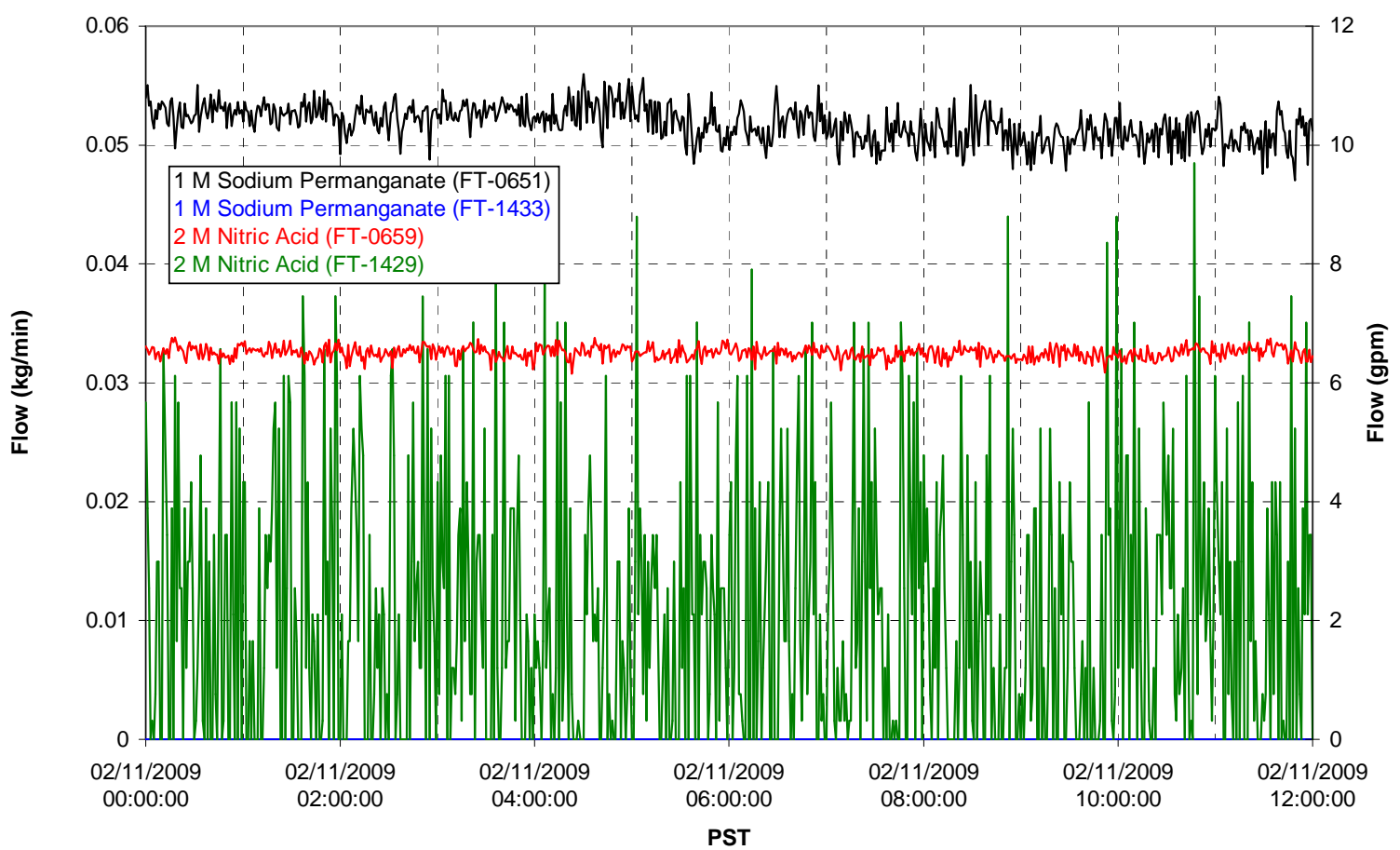

Air Flows

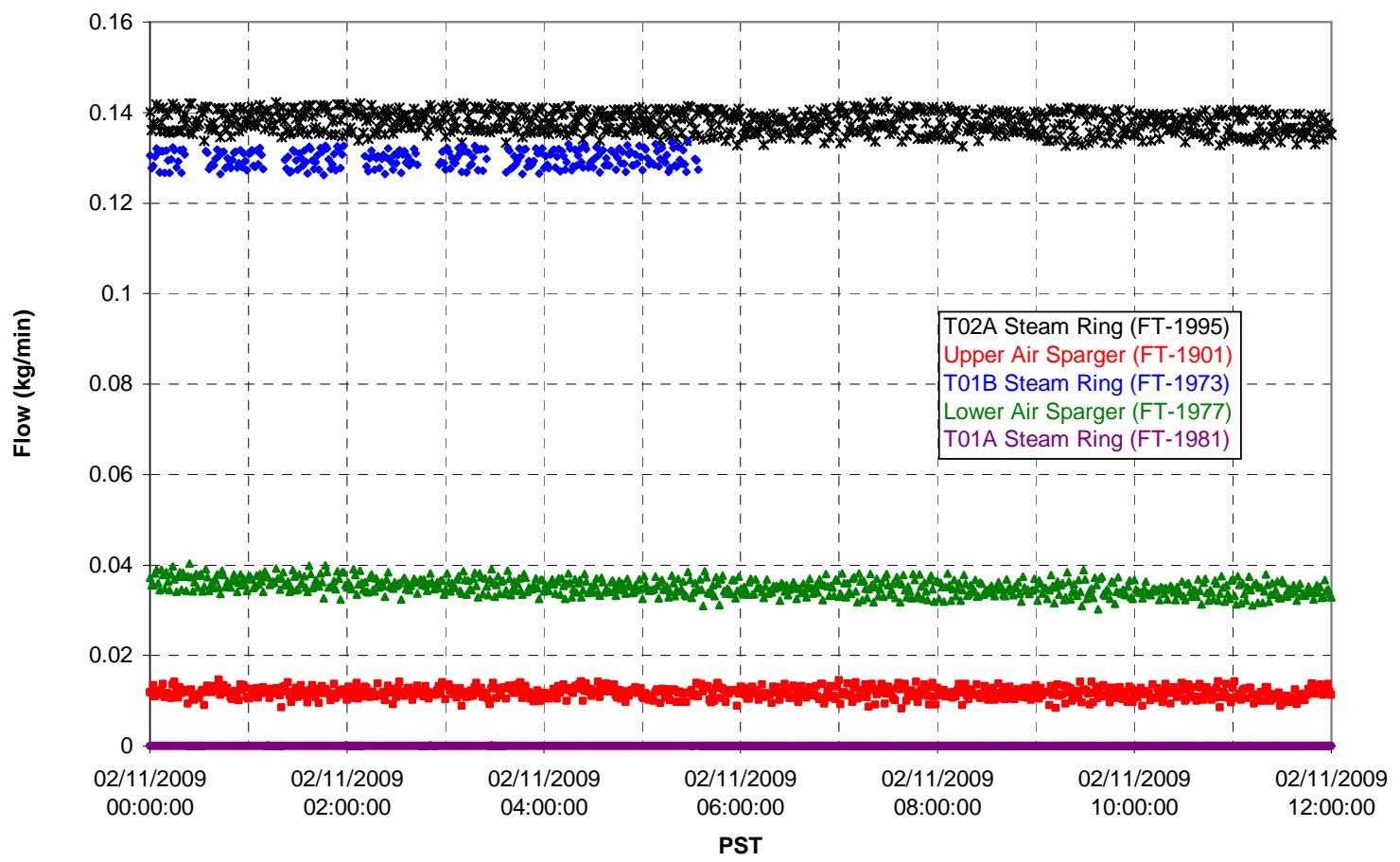


T02A Steam

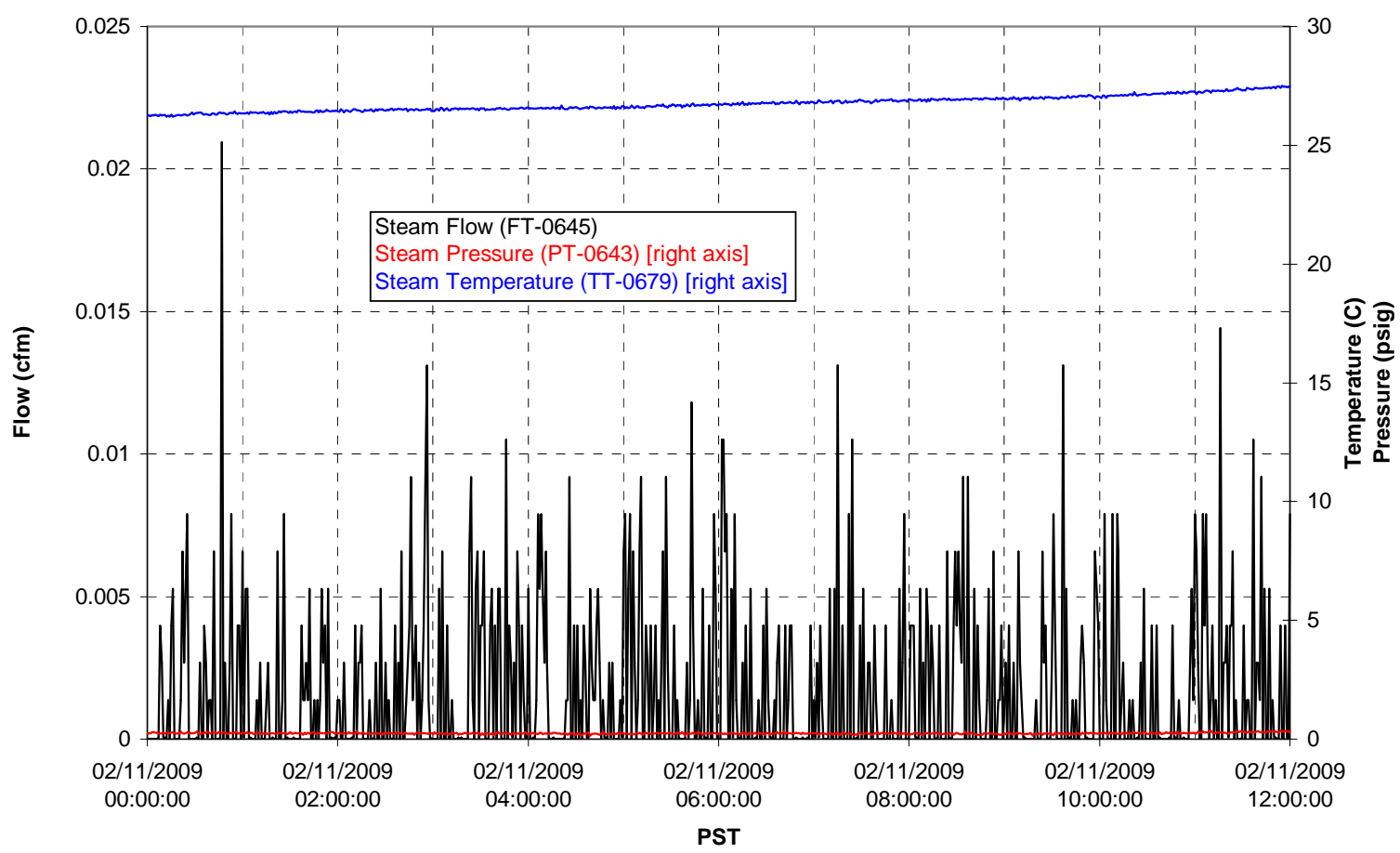

T01A Steam

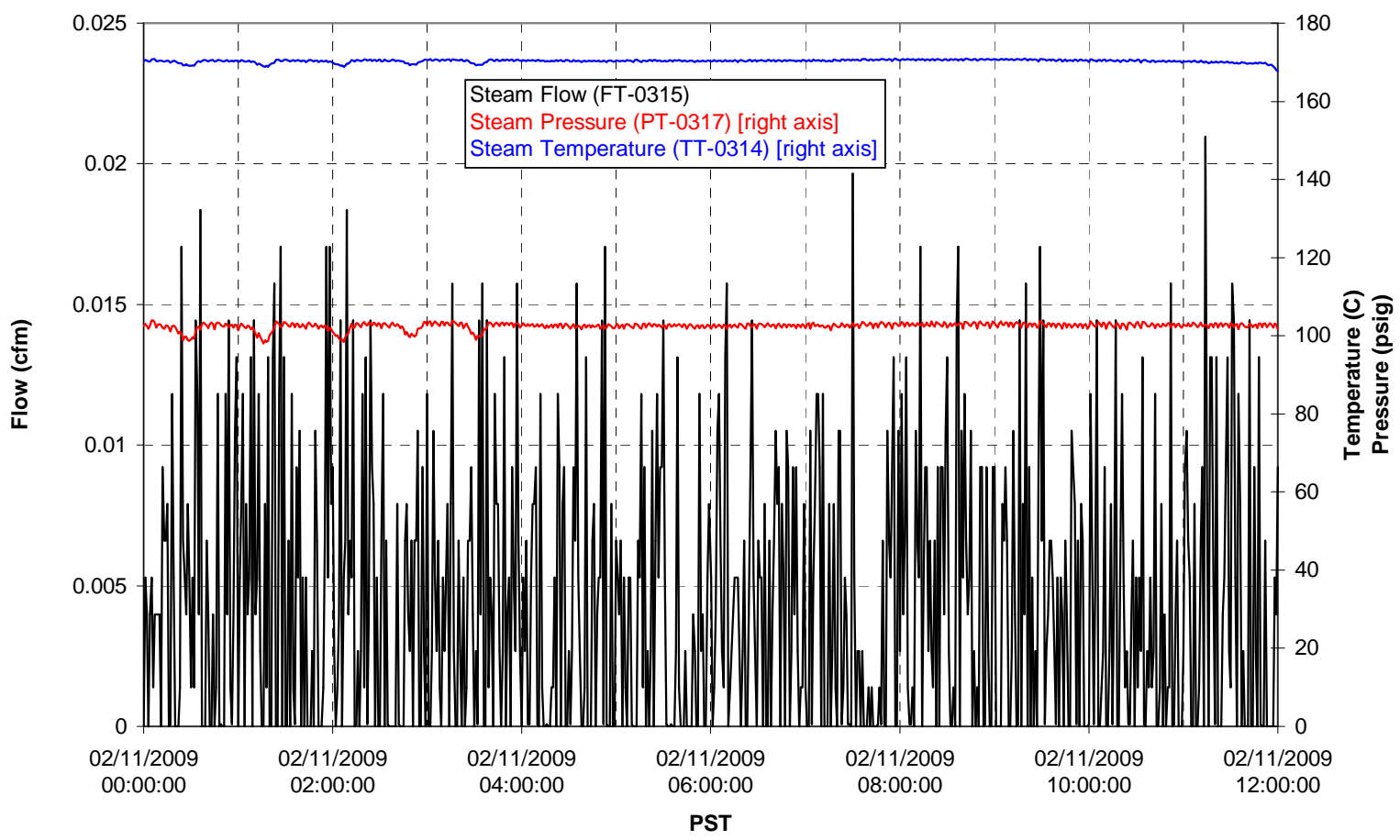


T01B Steam

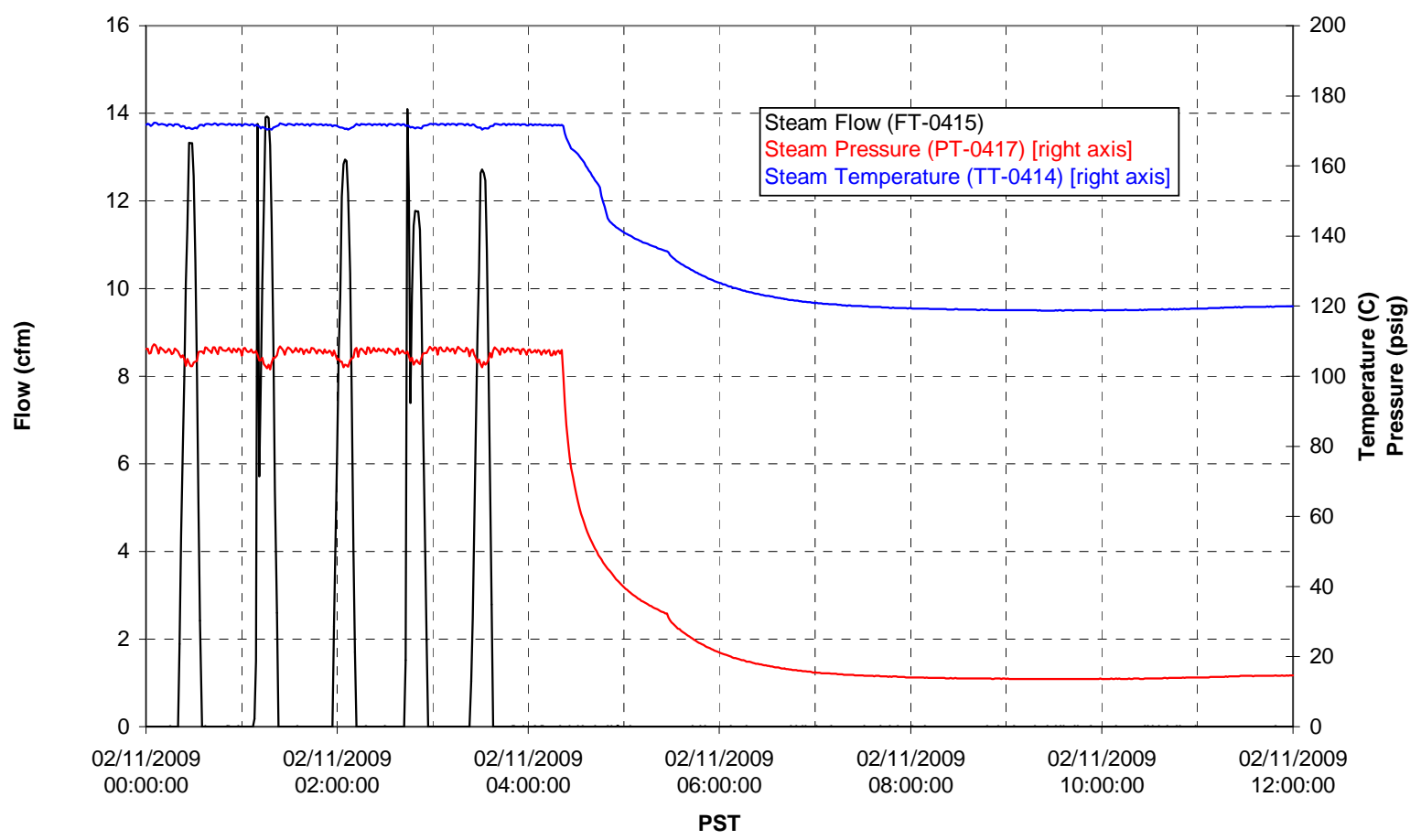




\section{Integrated Test A Data Plots 02/11/09 12:00 - 02/12/09 00:00}


T01A level

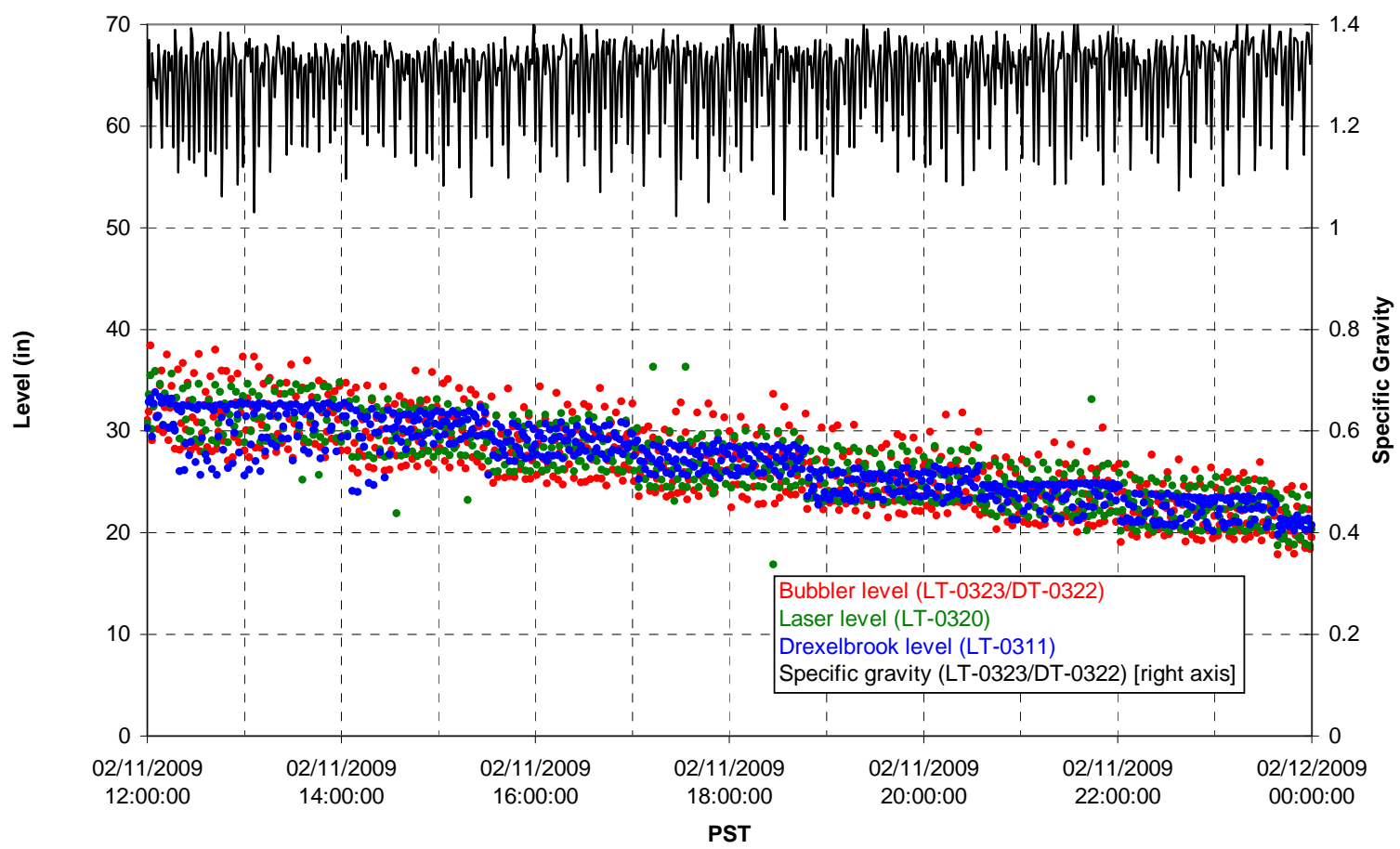

T01A temperatures

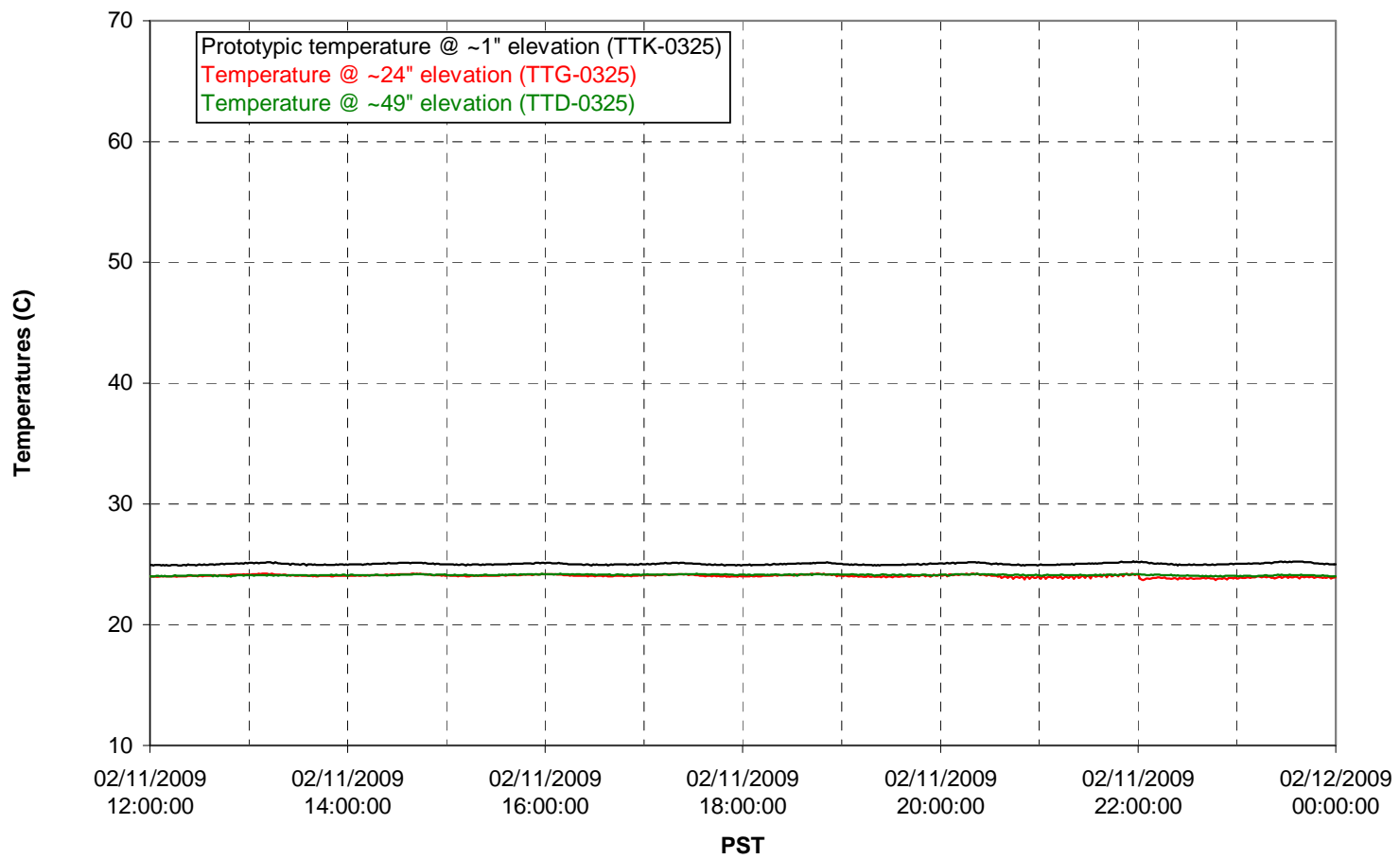


T01B level

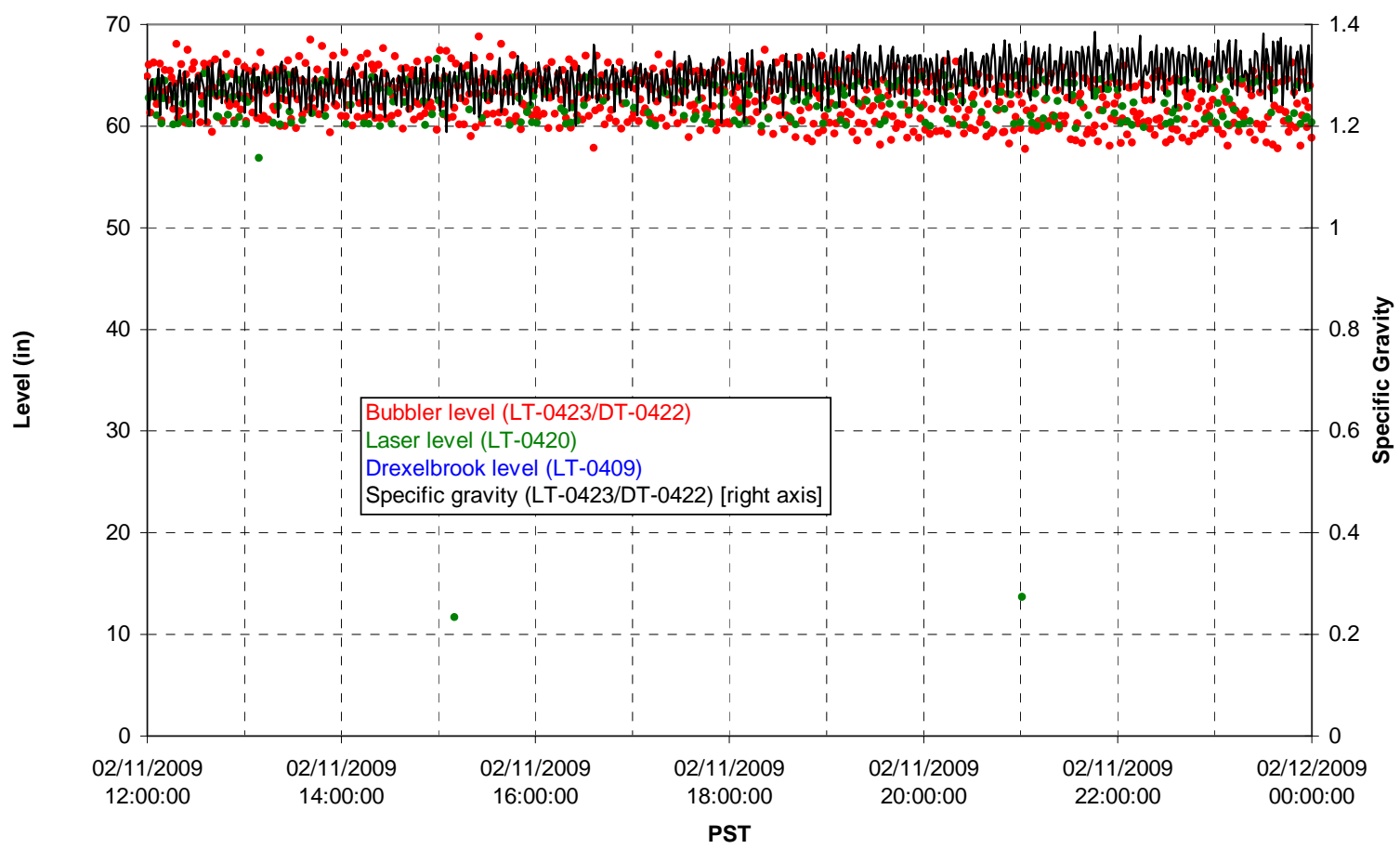

T01B temperatures

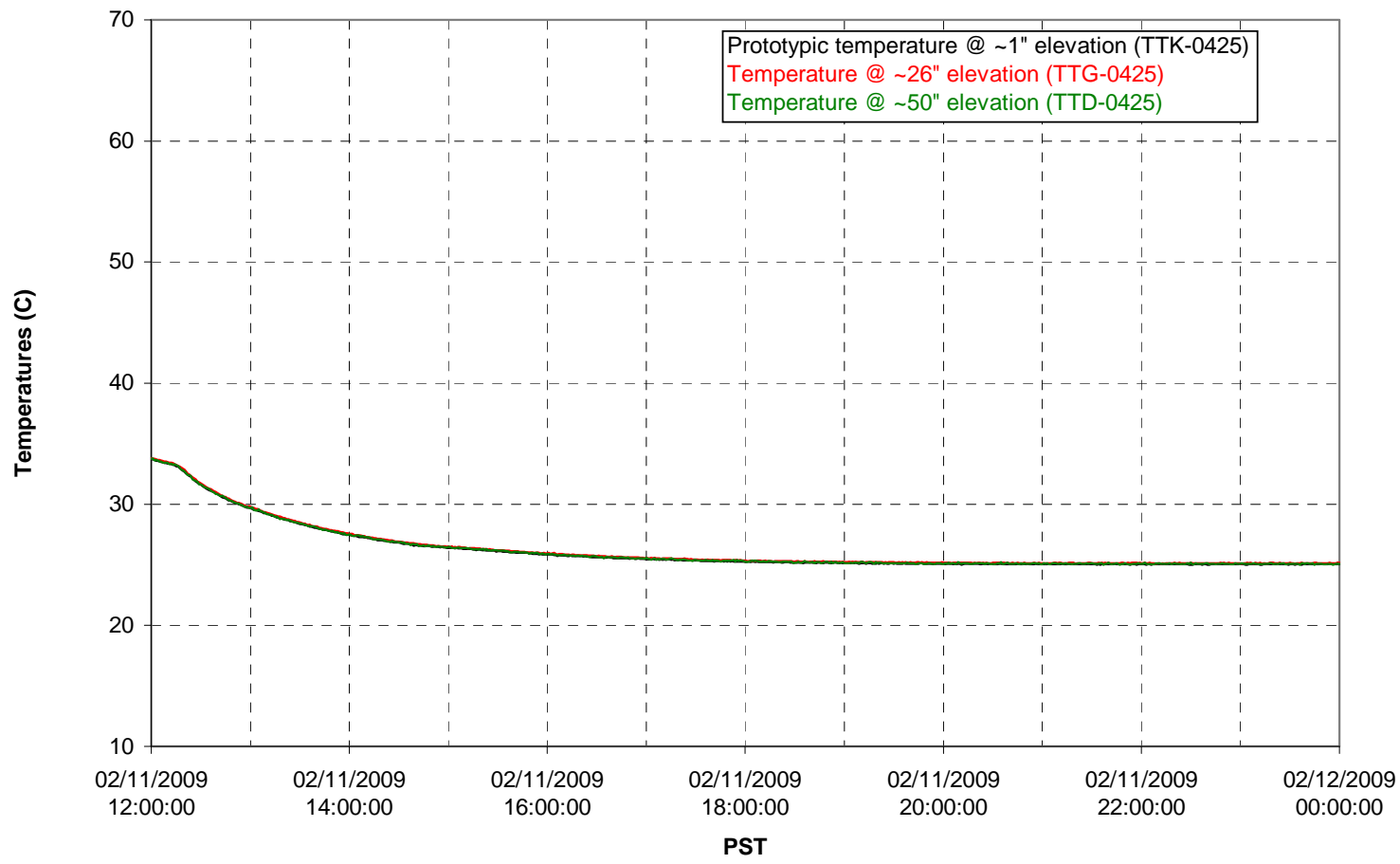


T02A level

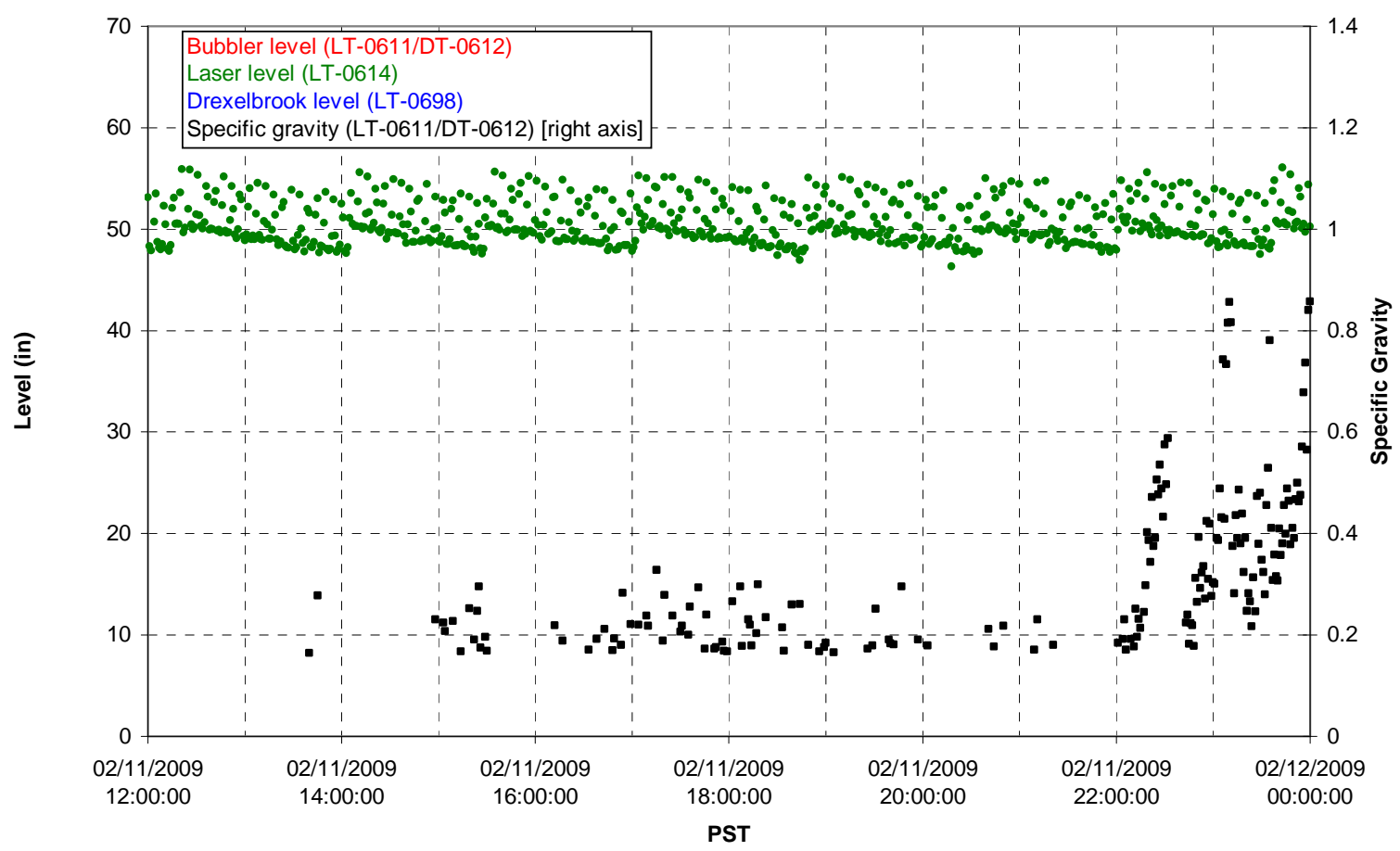

T02A temperatures

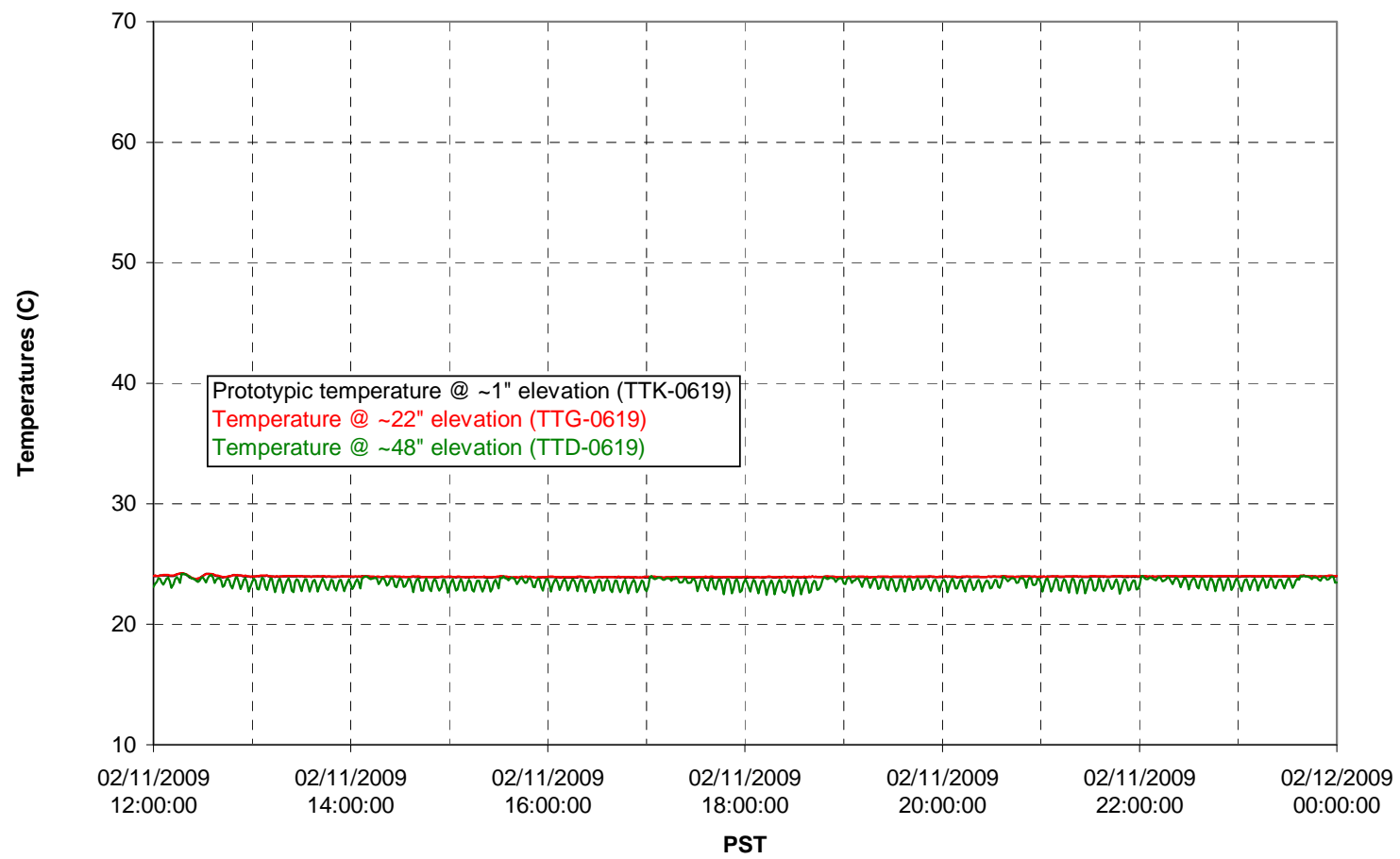


T02A and filter loop temperatures

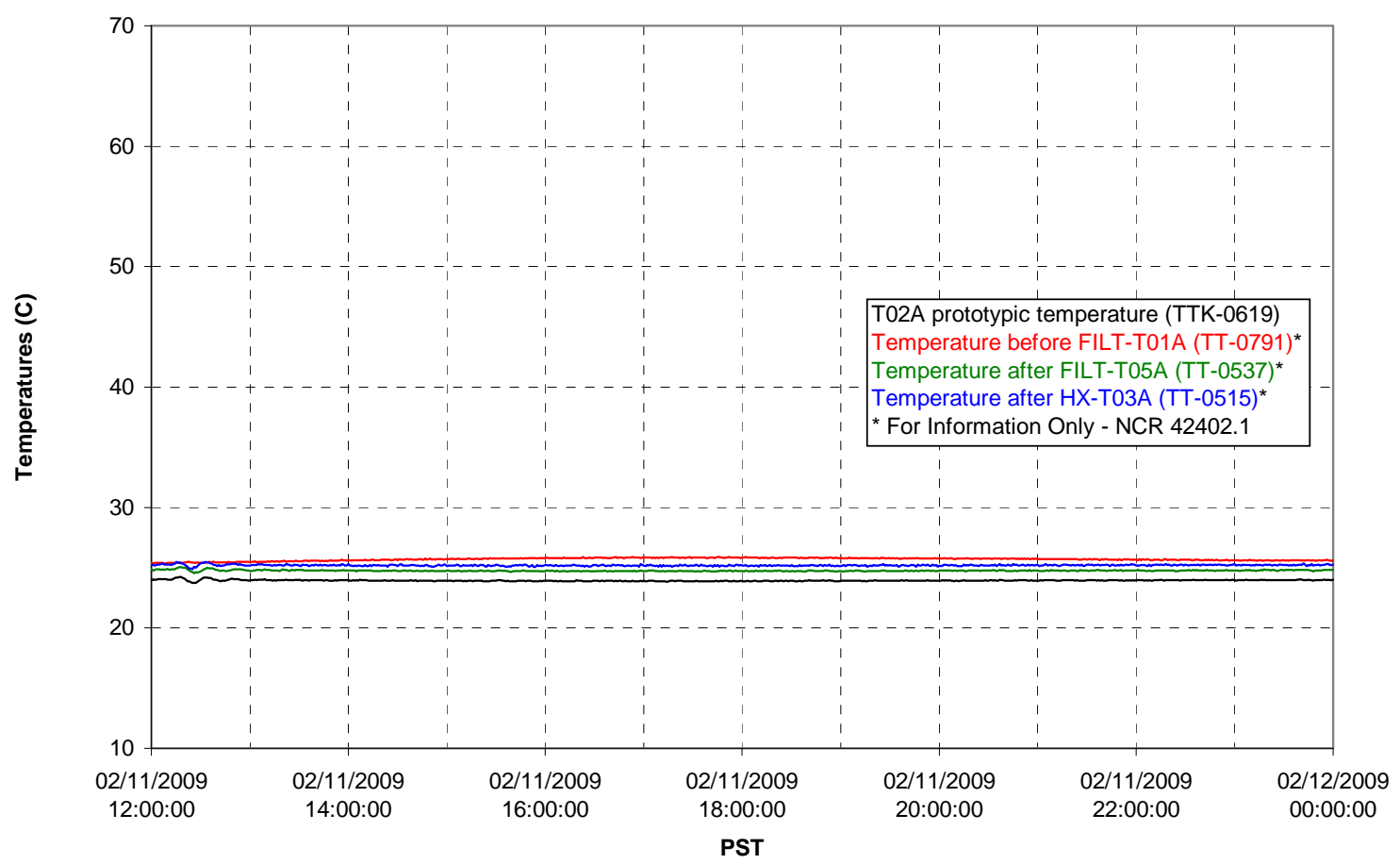

Pump Pressures and Flow

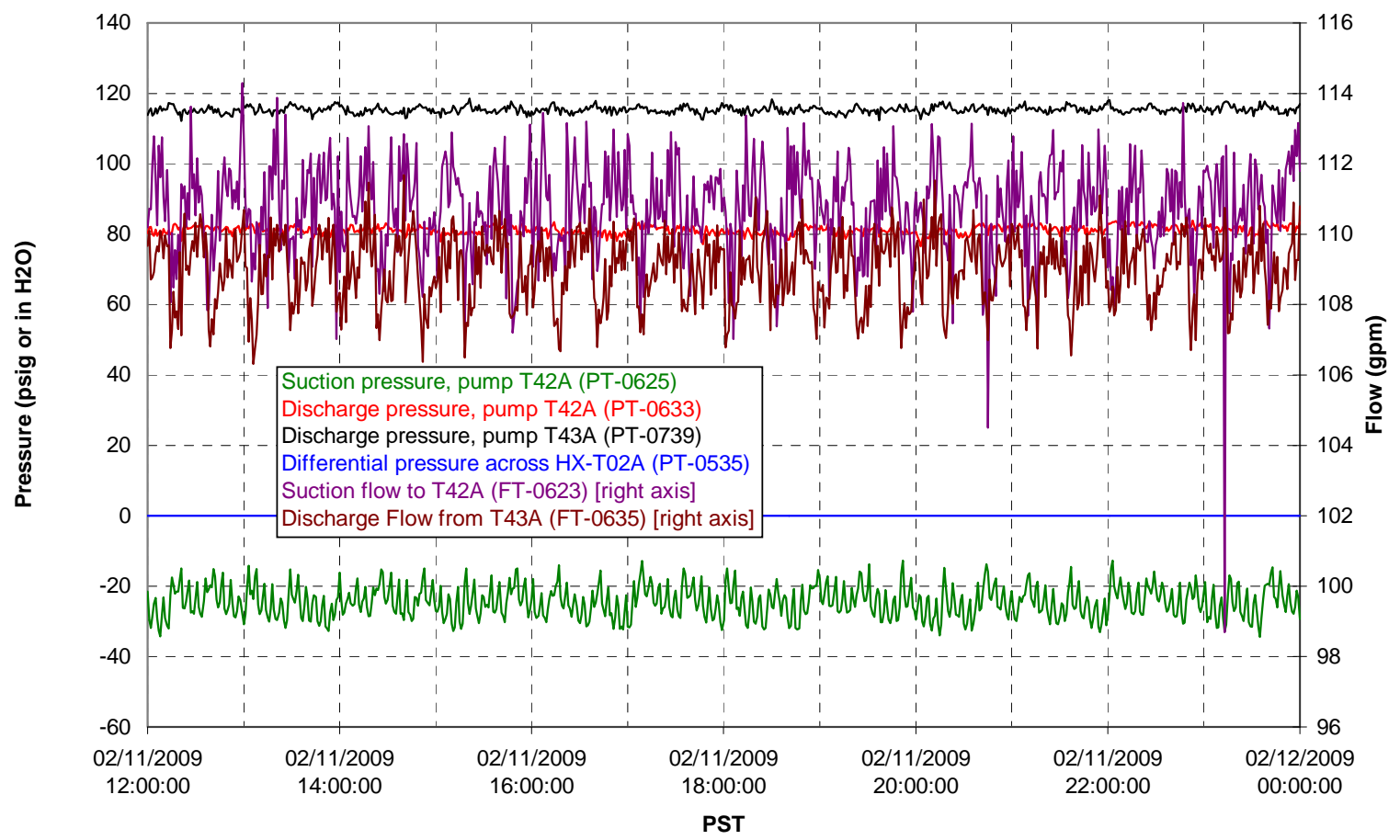


Axial pressure drop

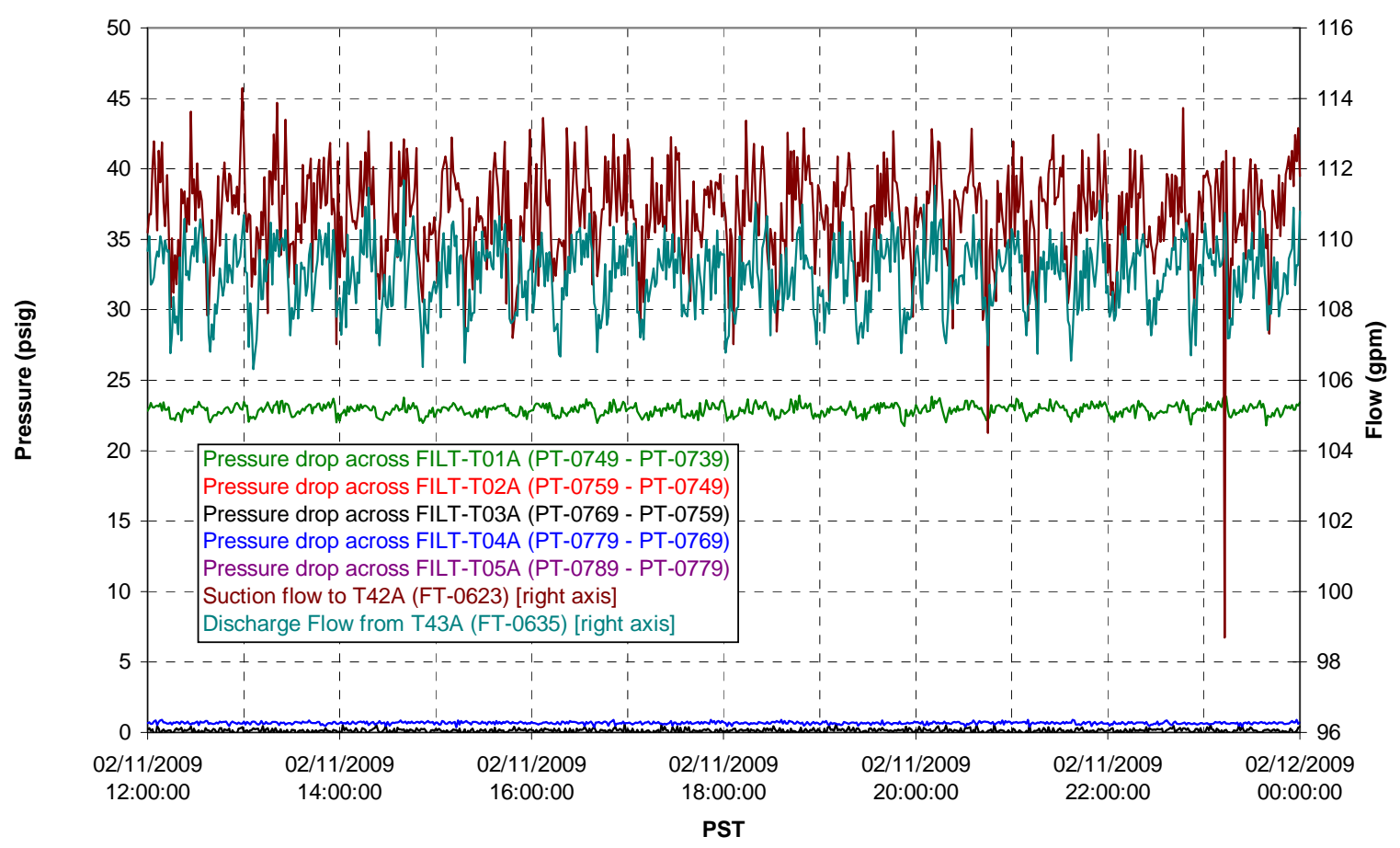

Permeate flow rates

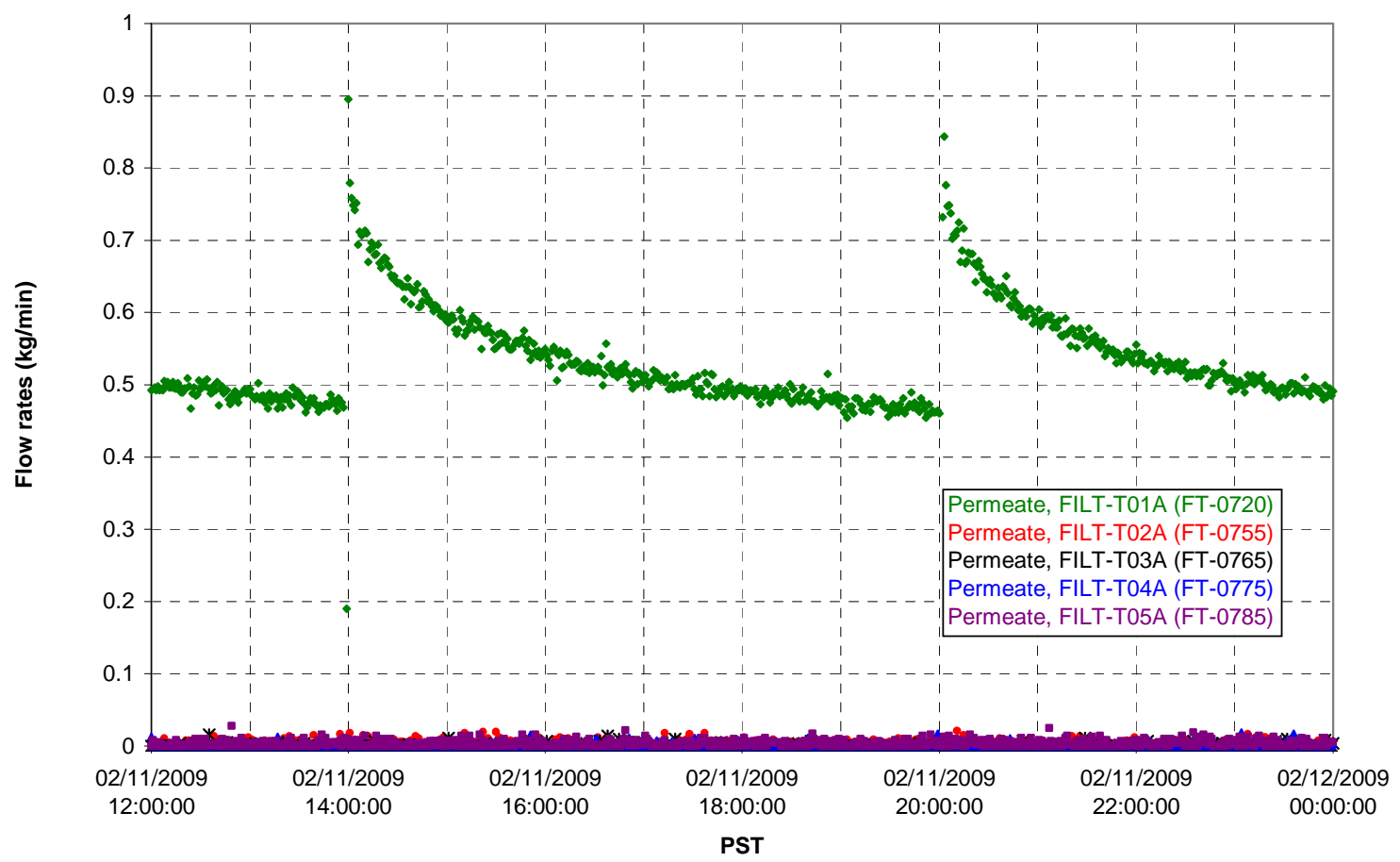


T02A Inner Temperature Tree

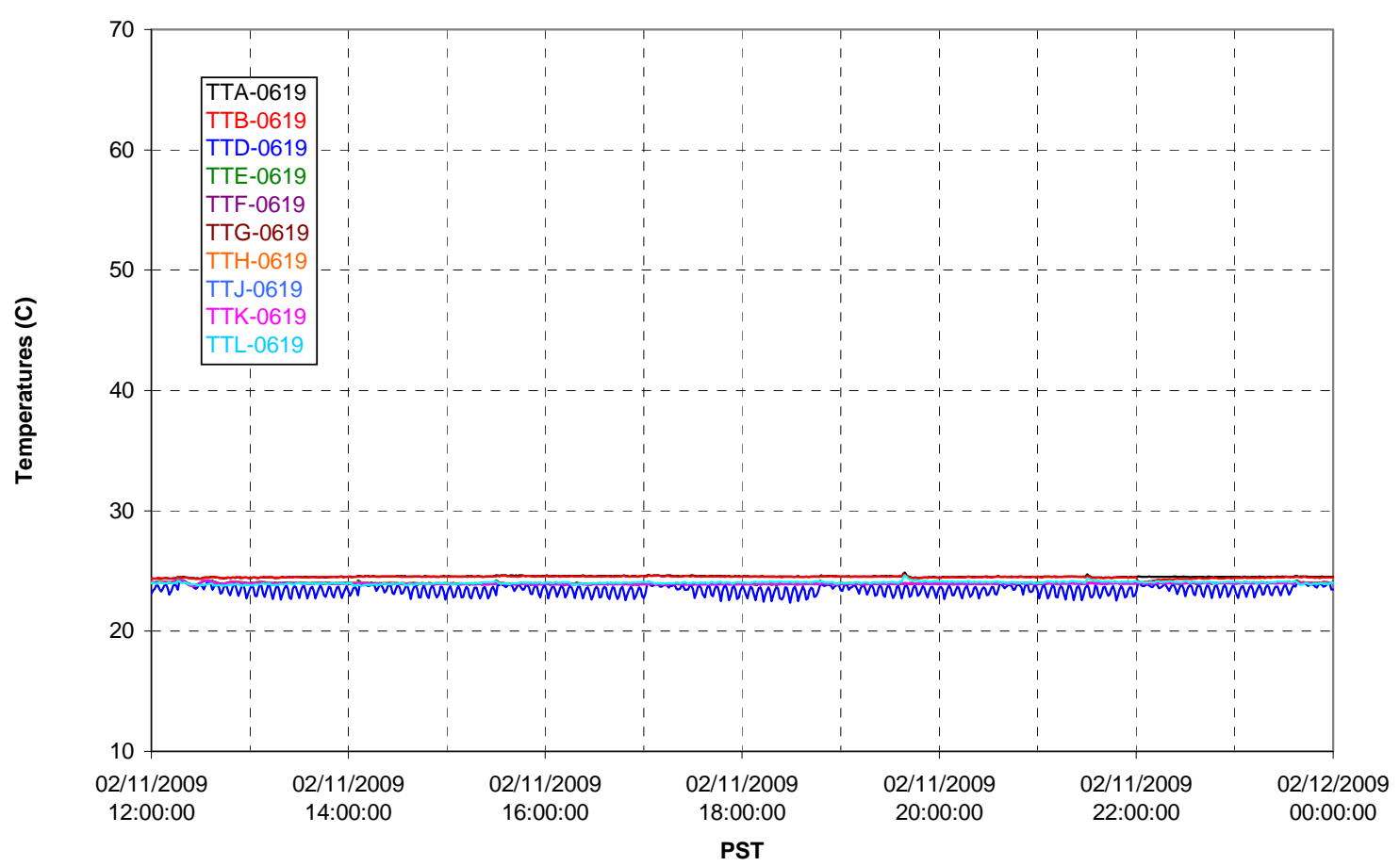

T02A Outer Temperature Tree

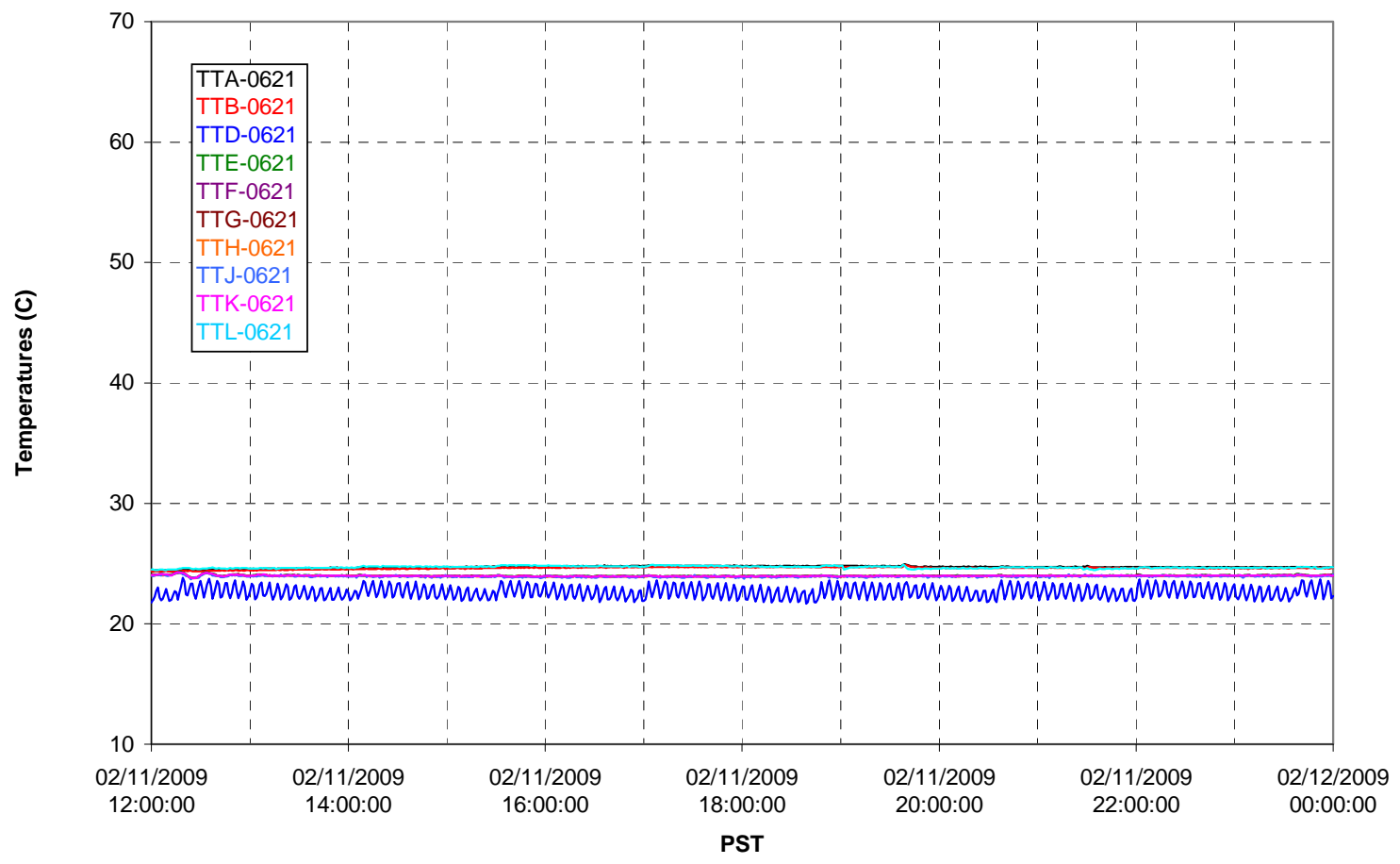


T02A temperatures

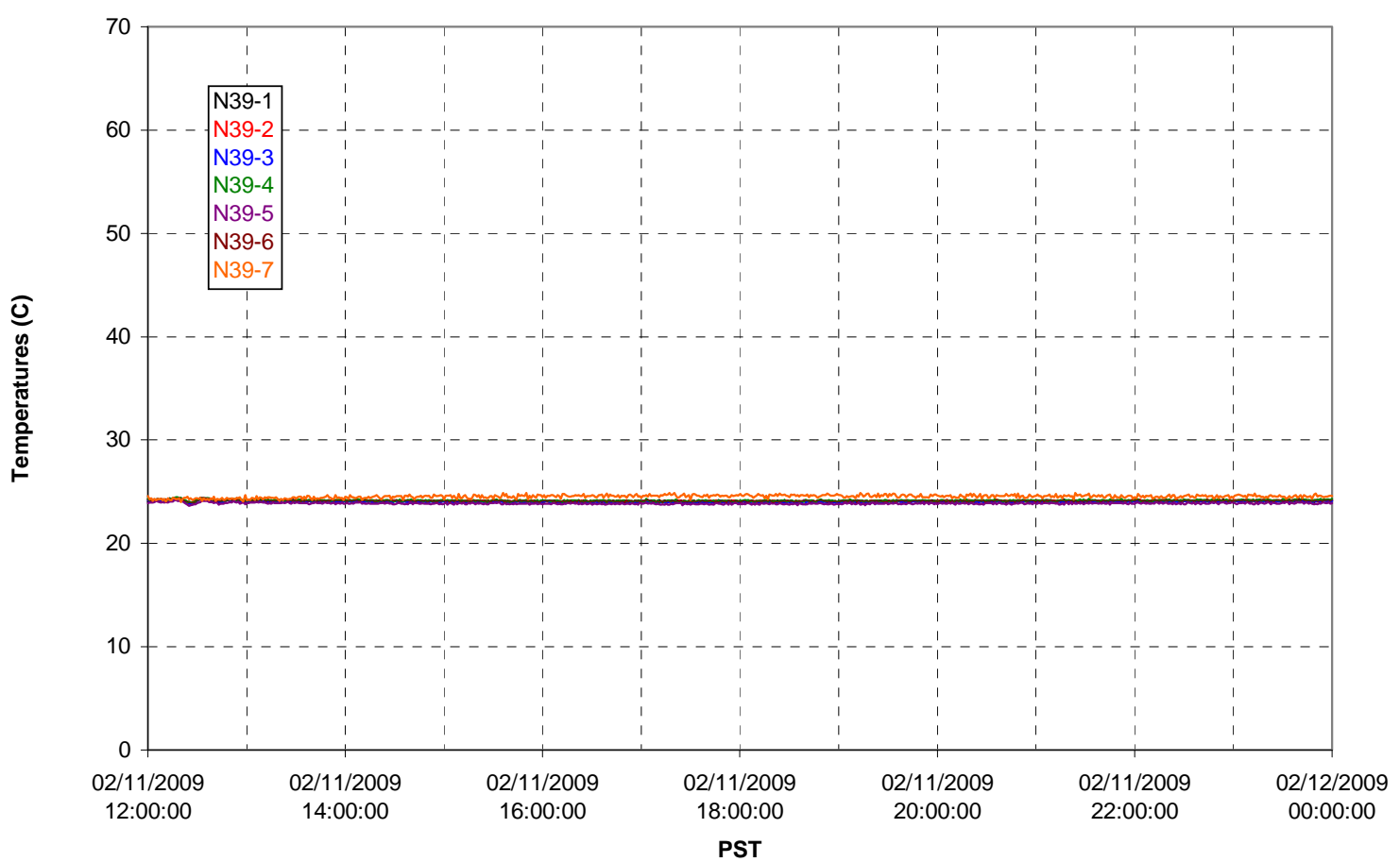

T02A temperatures

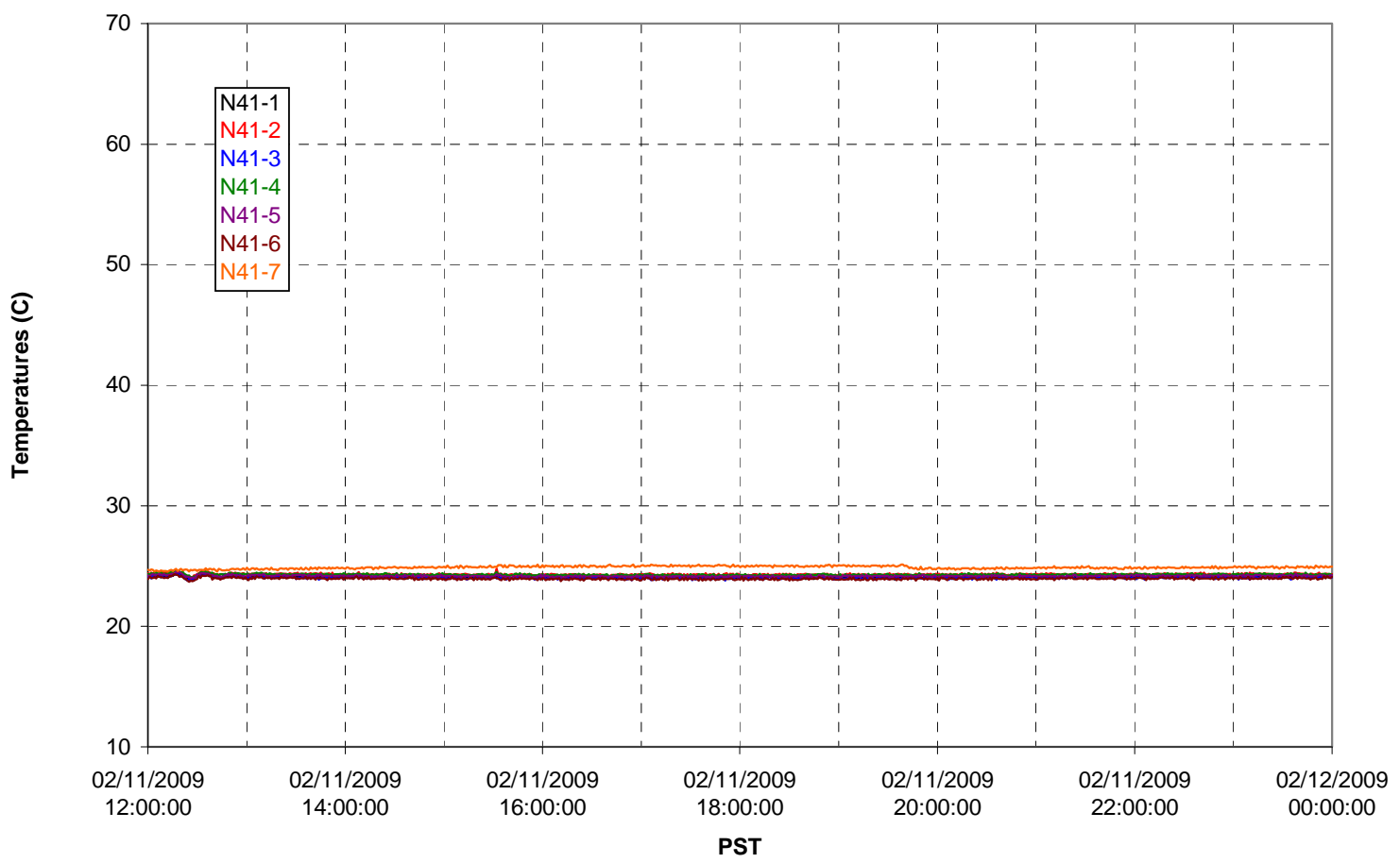


T02A temperatures

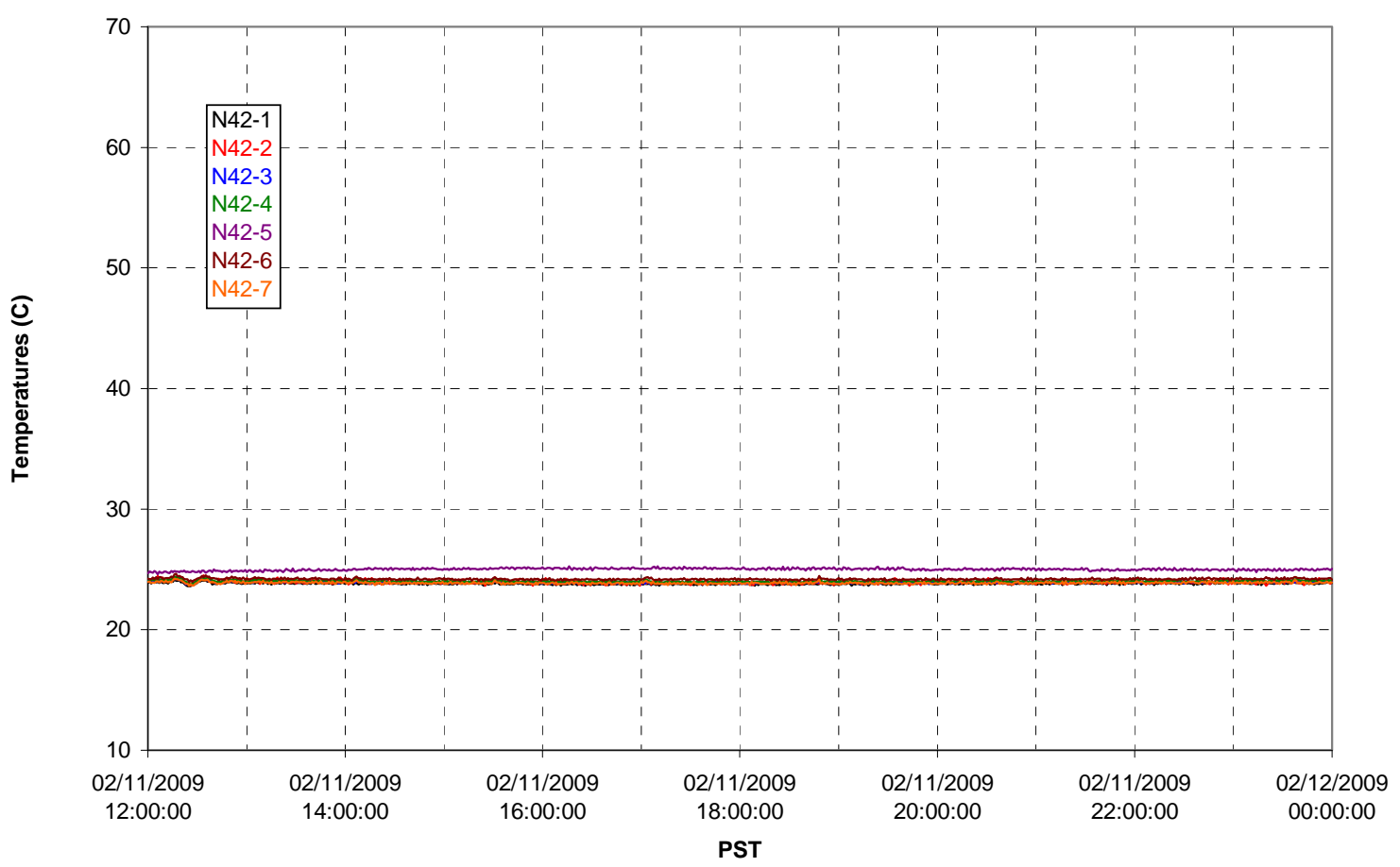

T02A temperatures

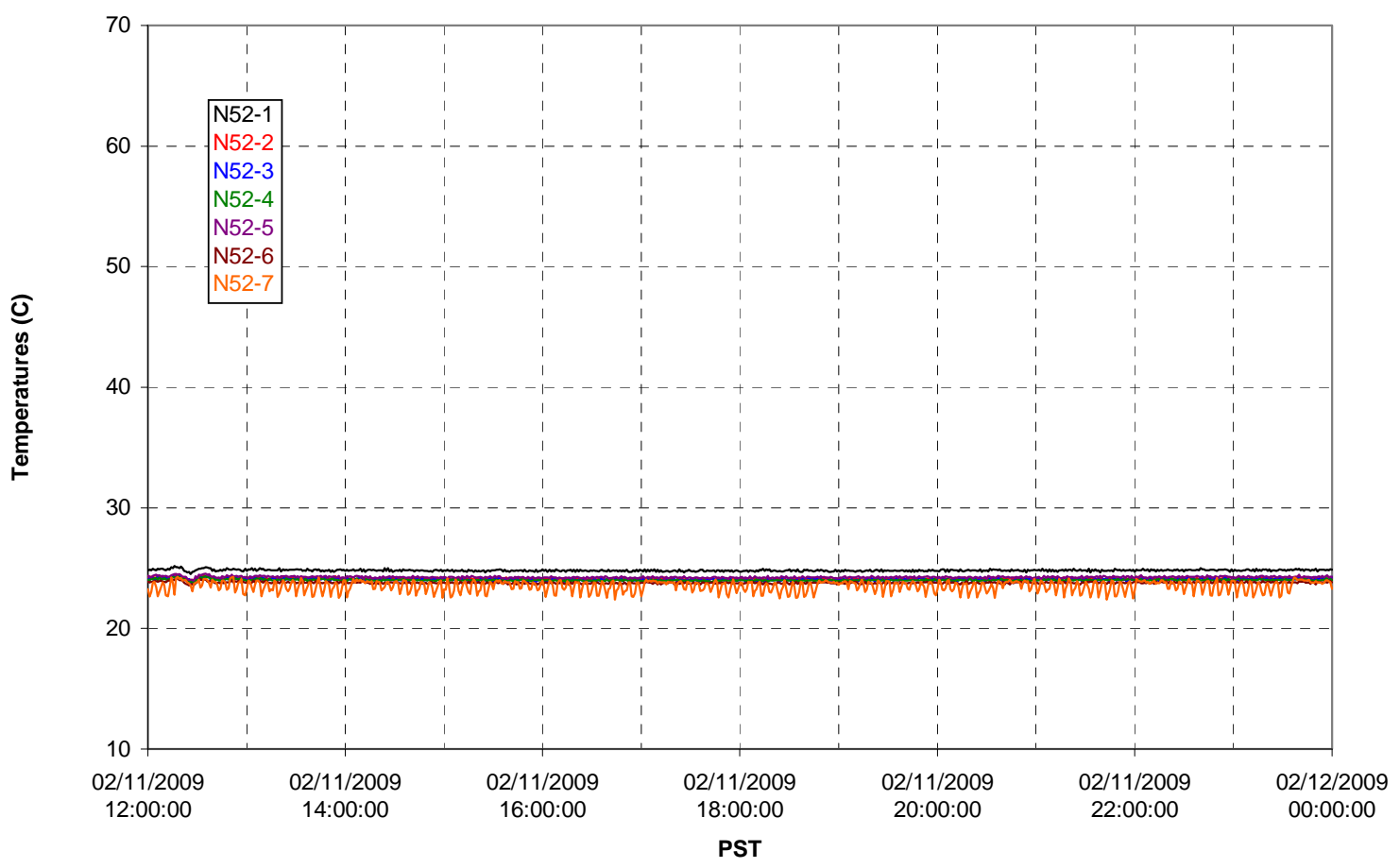


T02A Heating and Cooling

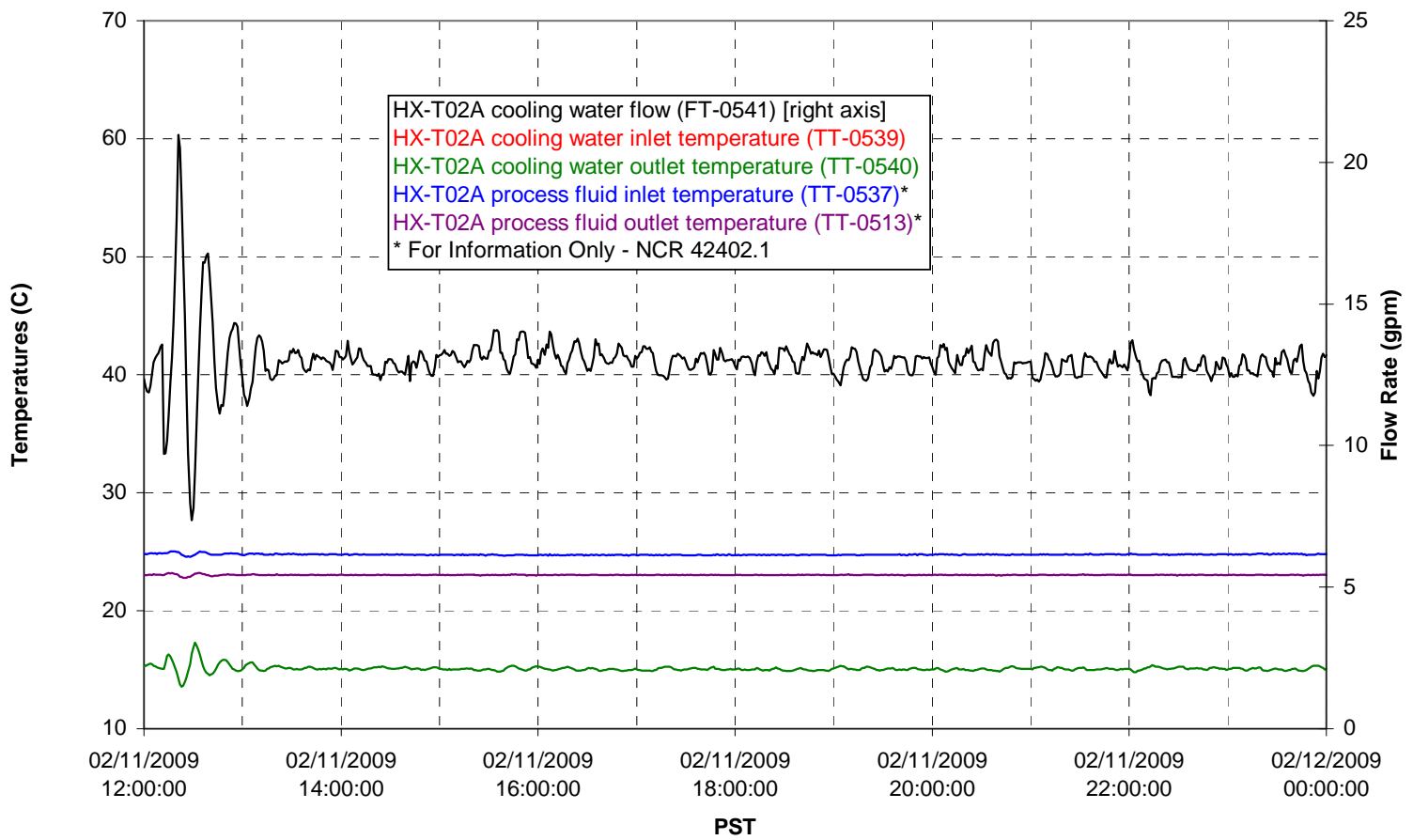

Pump Operation

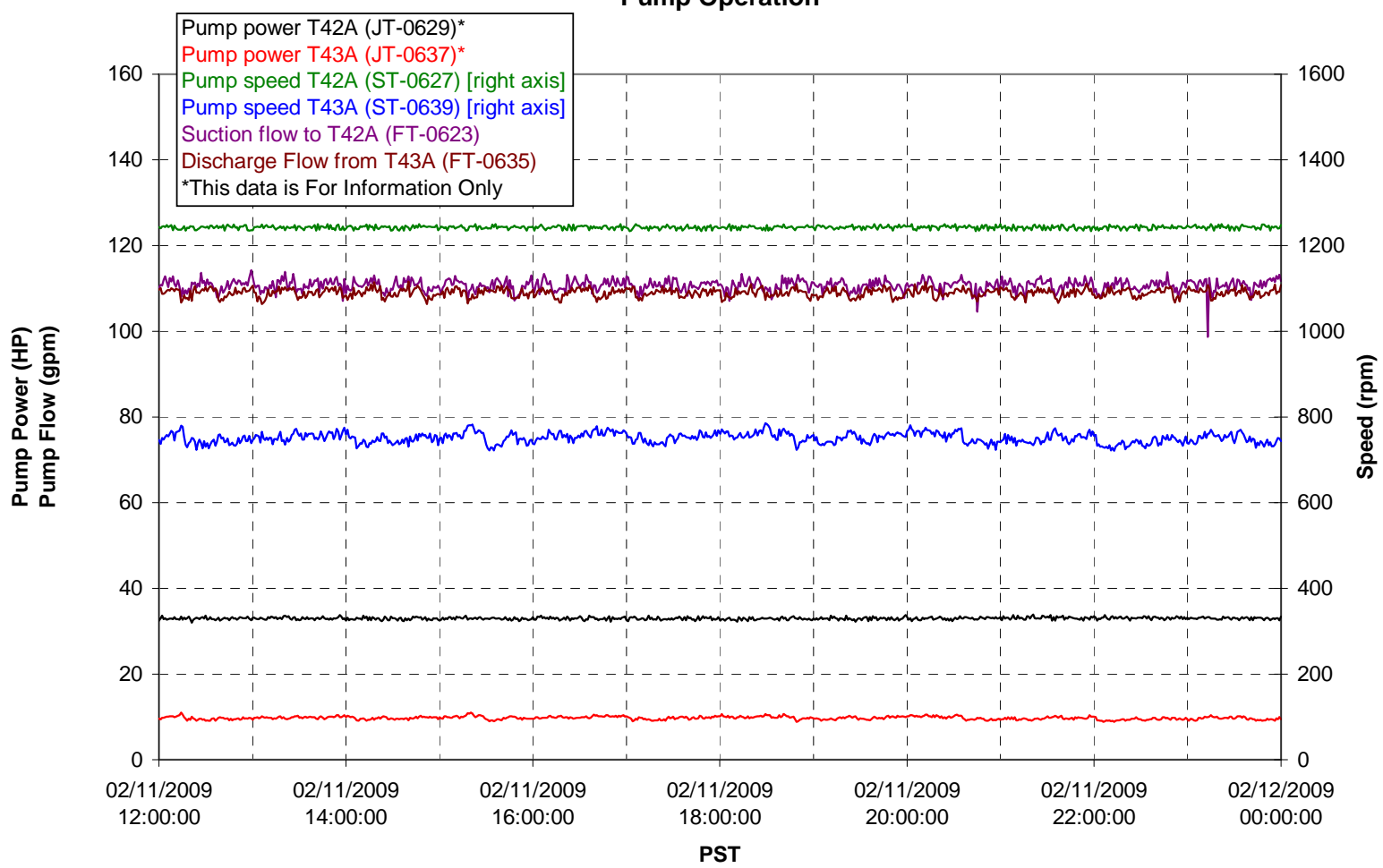


Pulsepot UFP-PP-T01A

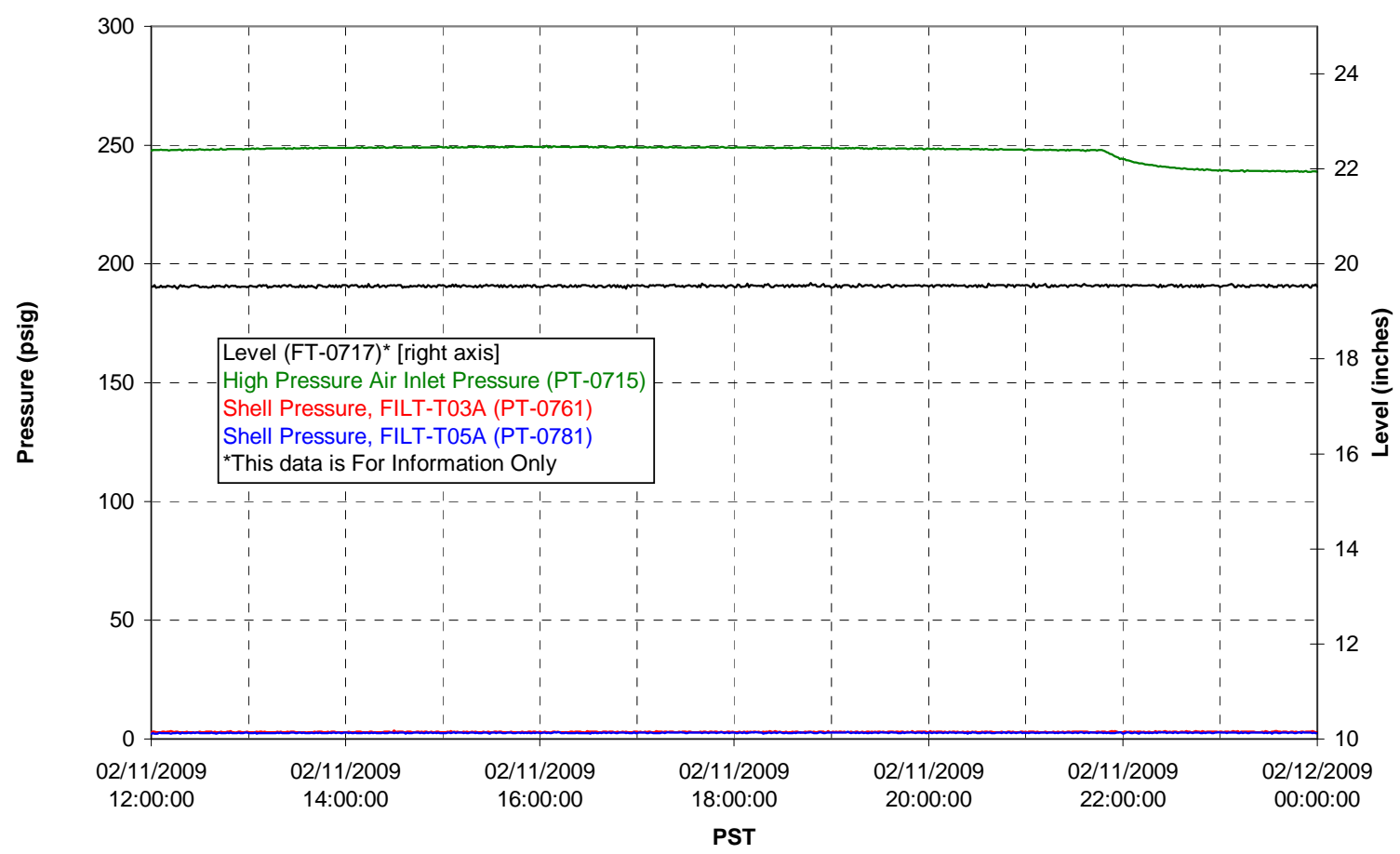

Pulsepot UFP-PP-T02A

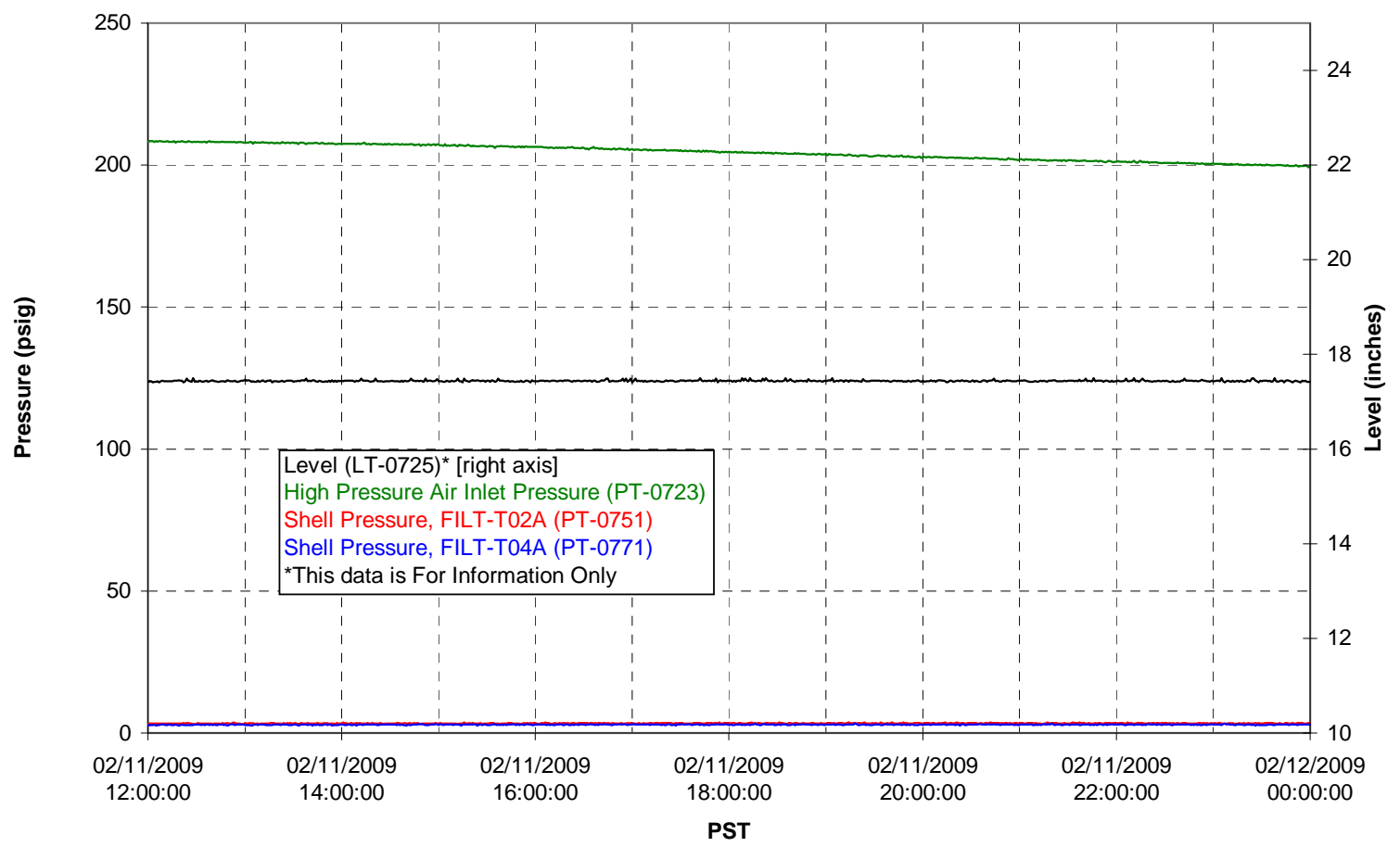


Pulsepot UFP-PP-T03A

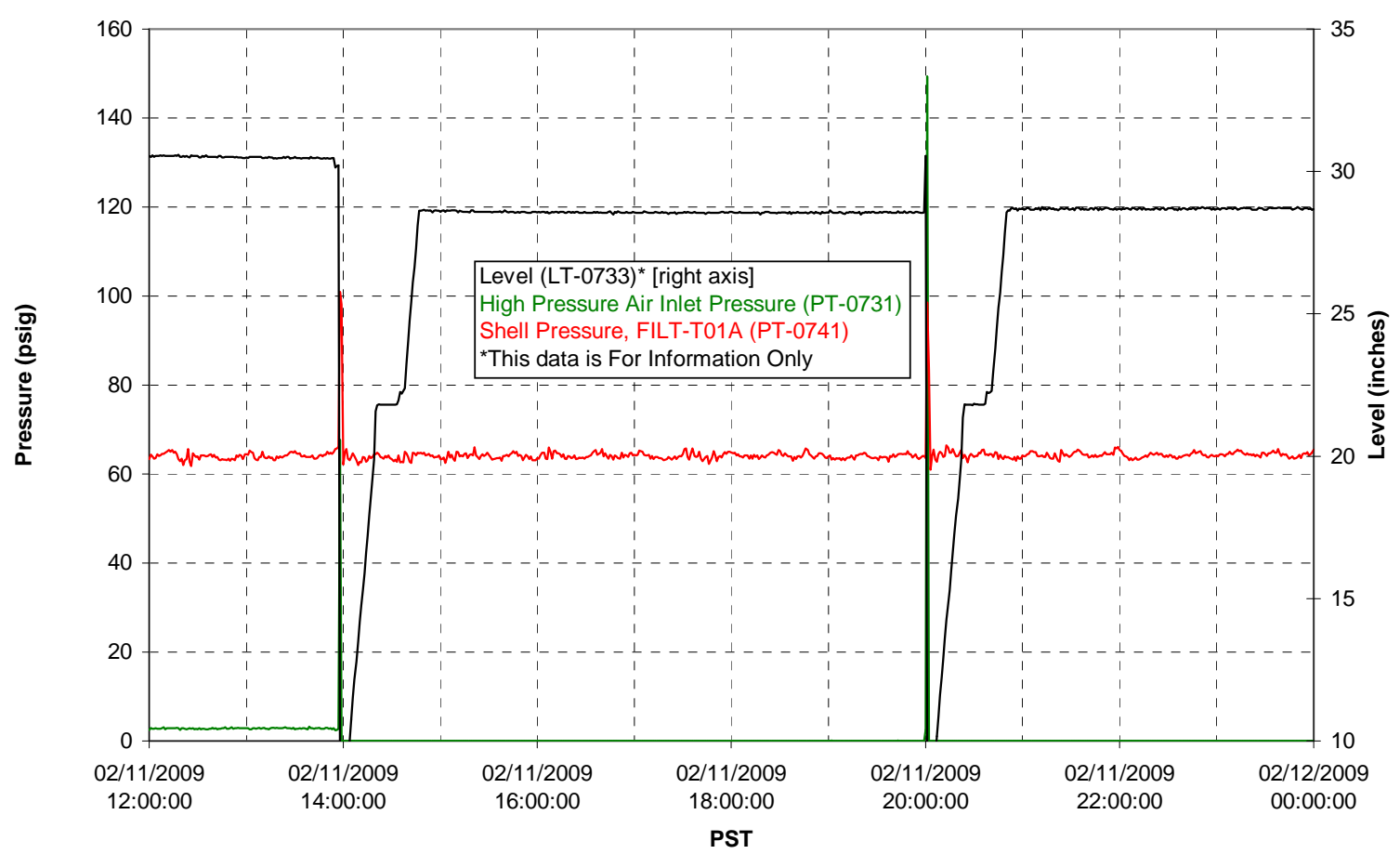

Pulsepot Levels

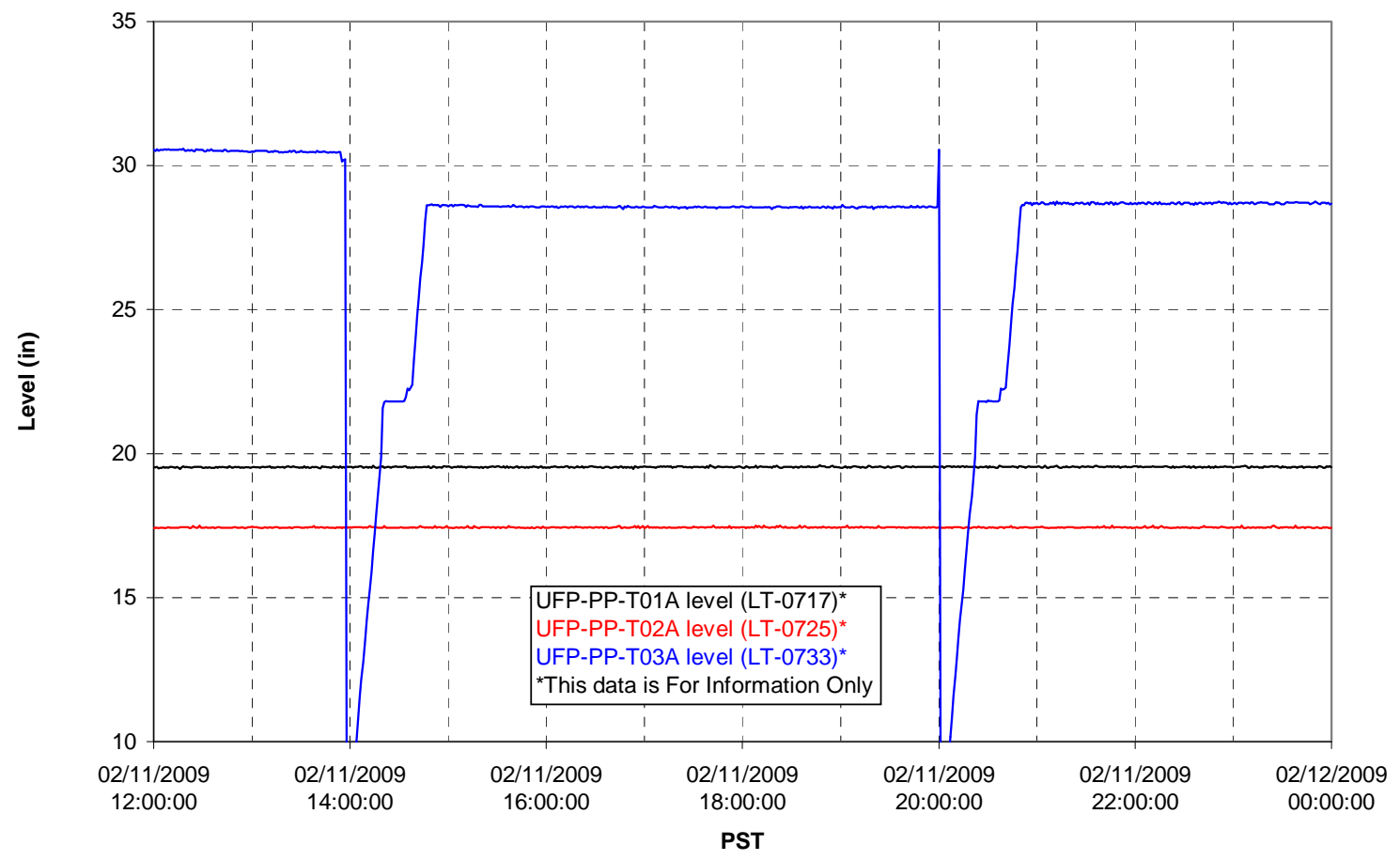


Filter UFP-FILT-T01A

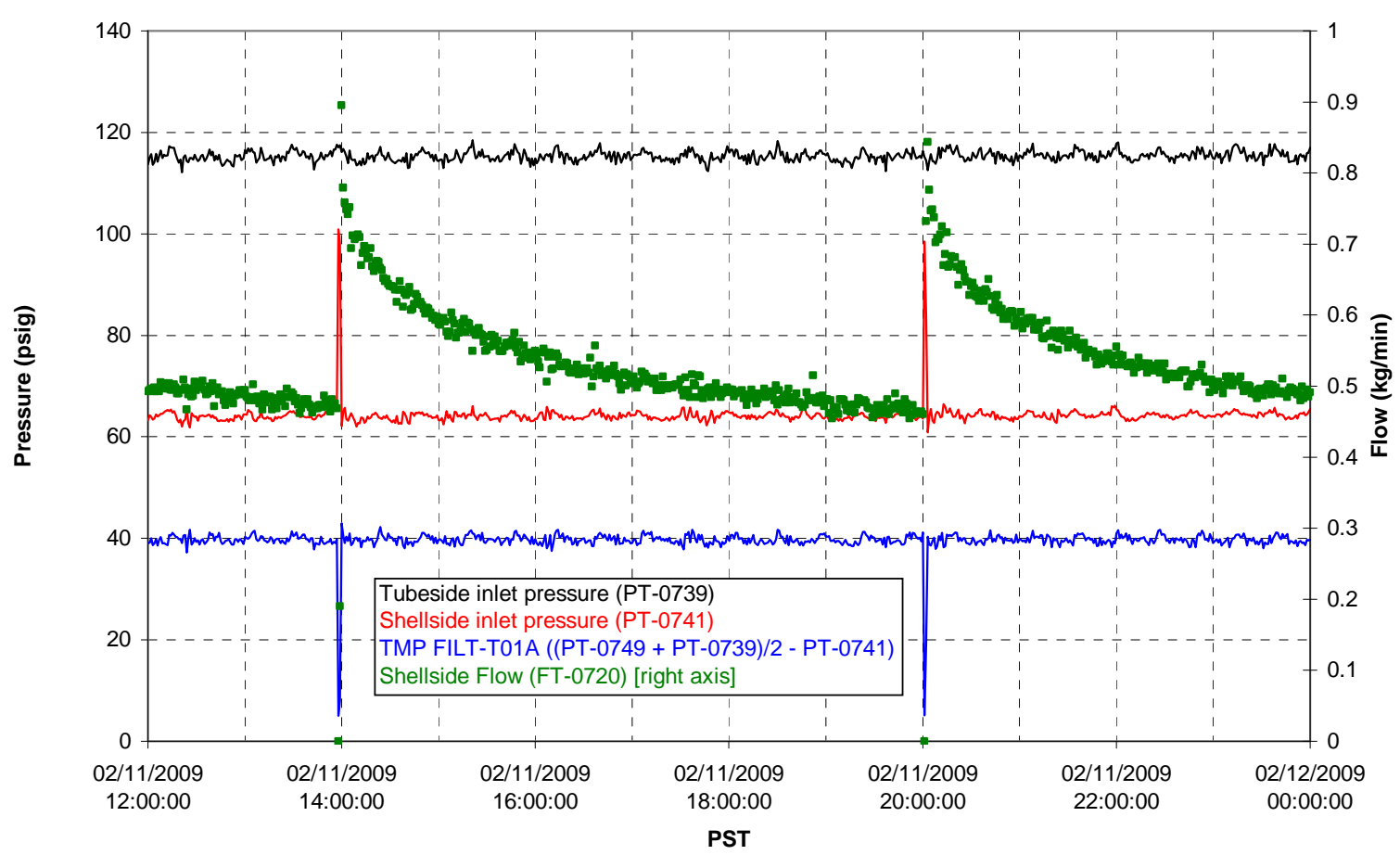

Filter UFP-FILT-T02A

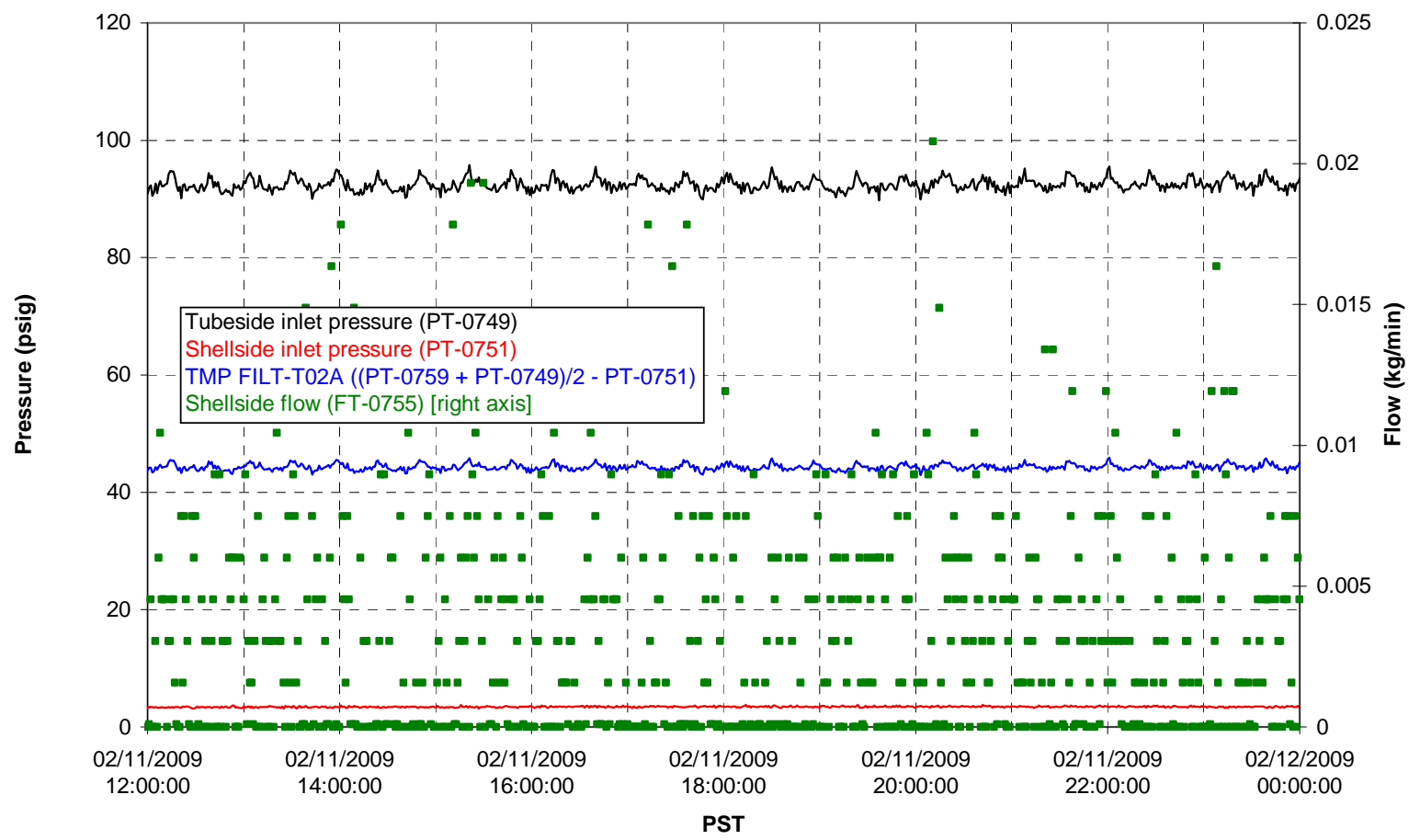


Filter UFP-FILT-T03A

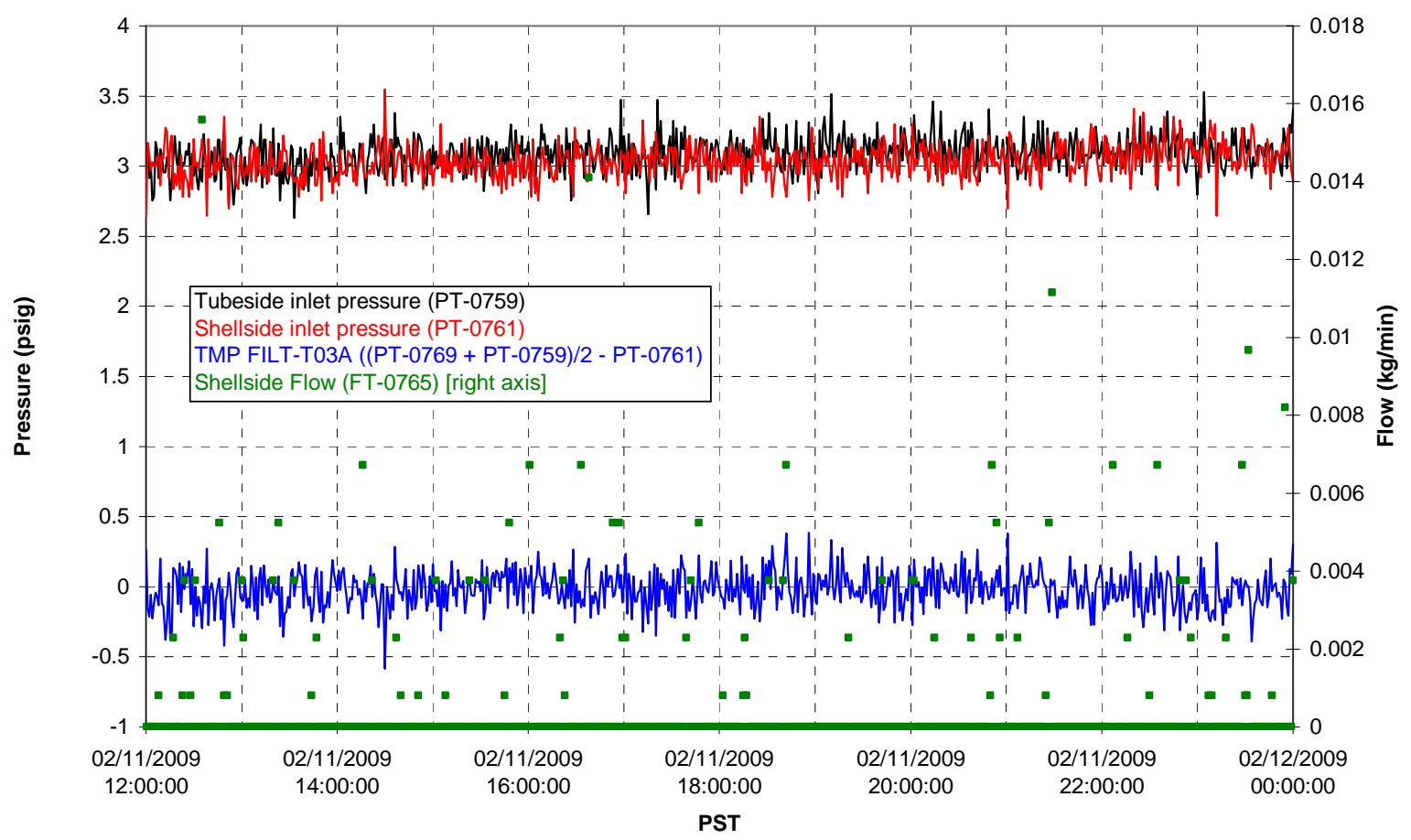

Filter UFP-FILT-T04A

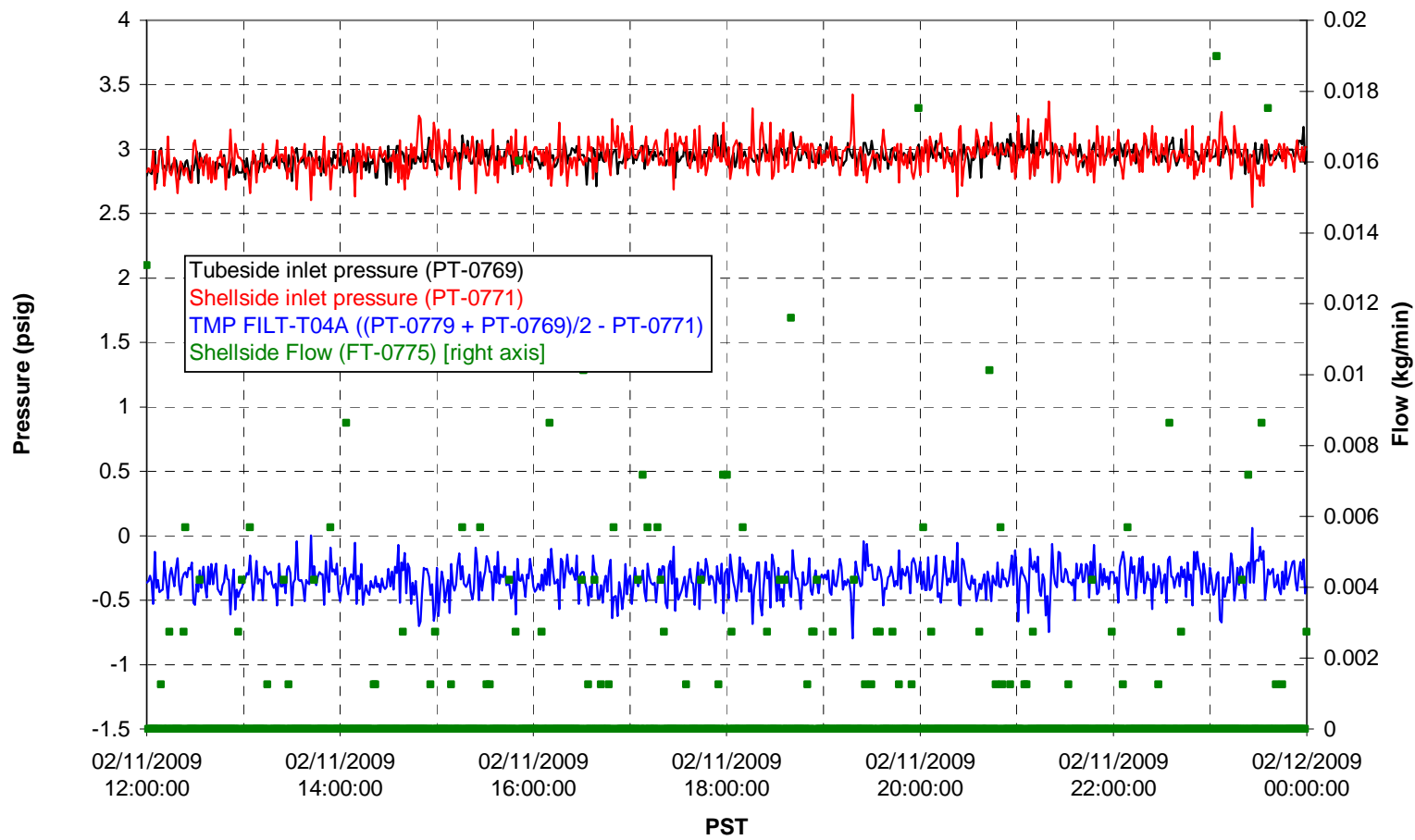


Filter UFP-FILT-T05A

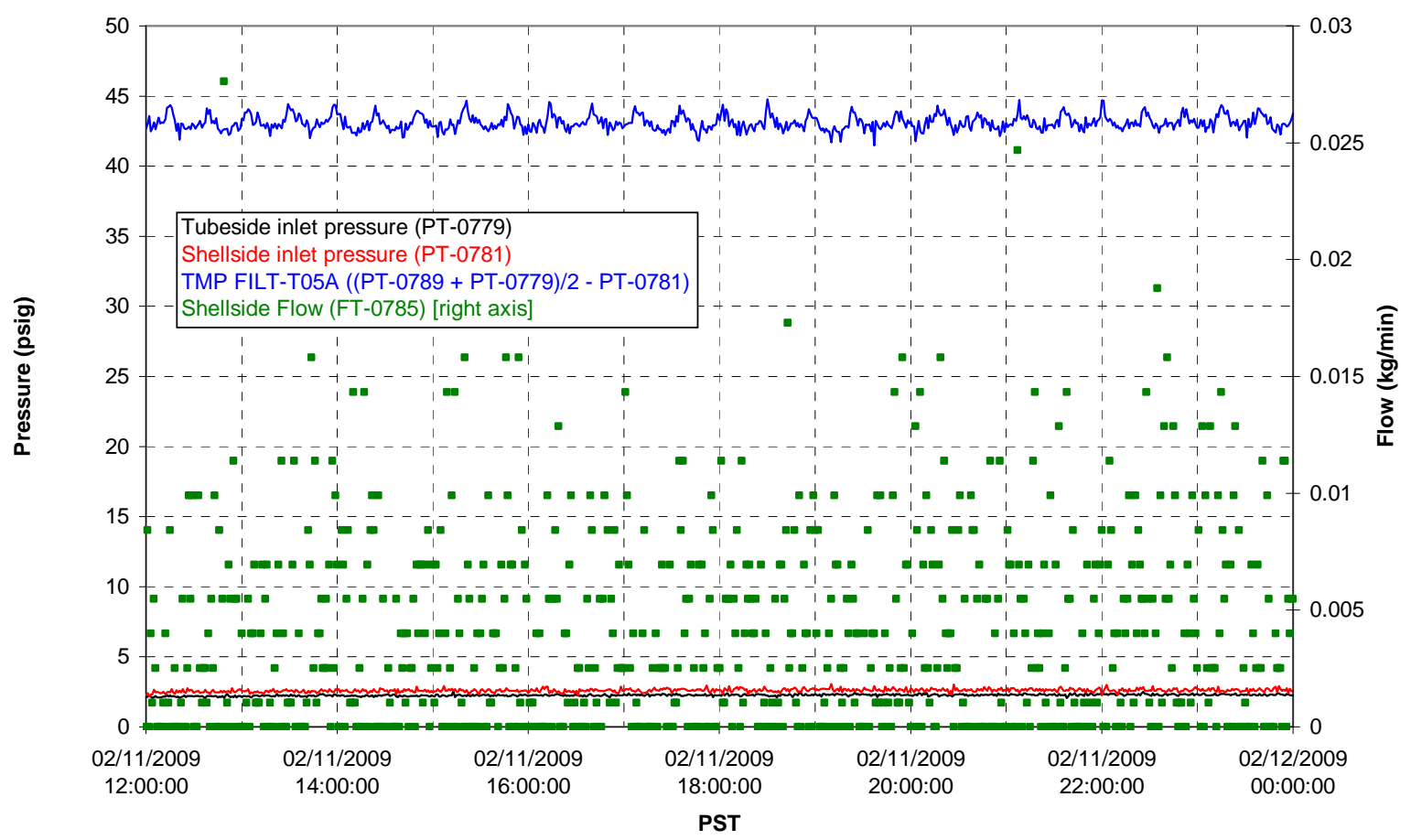

Chemical Flow

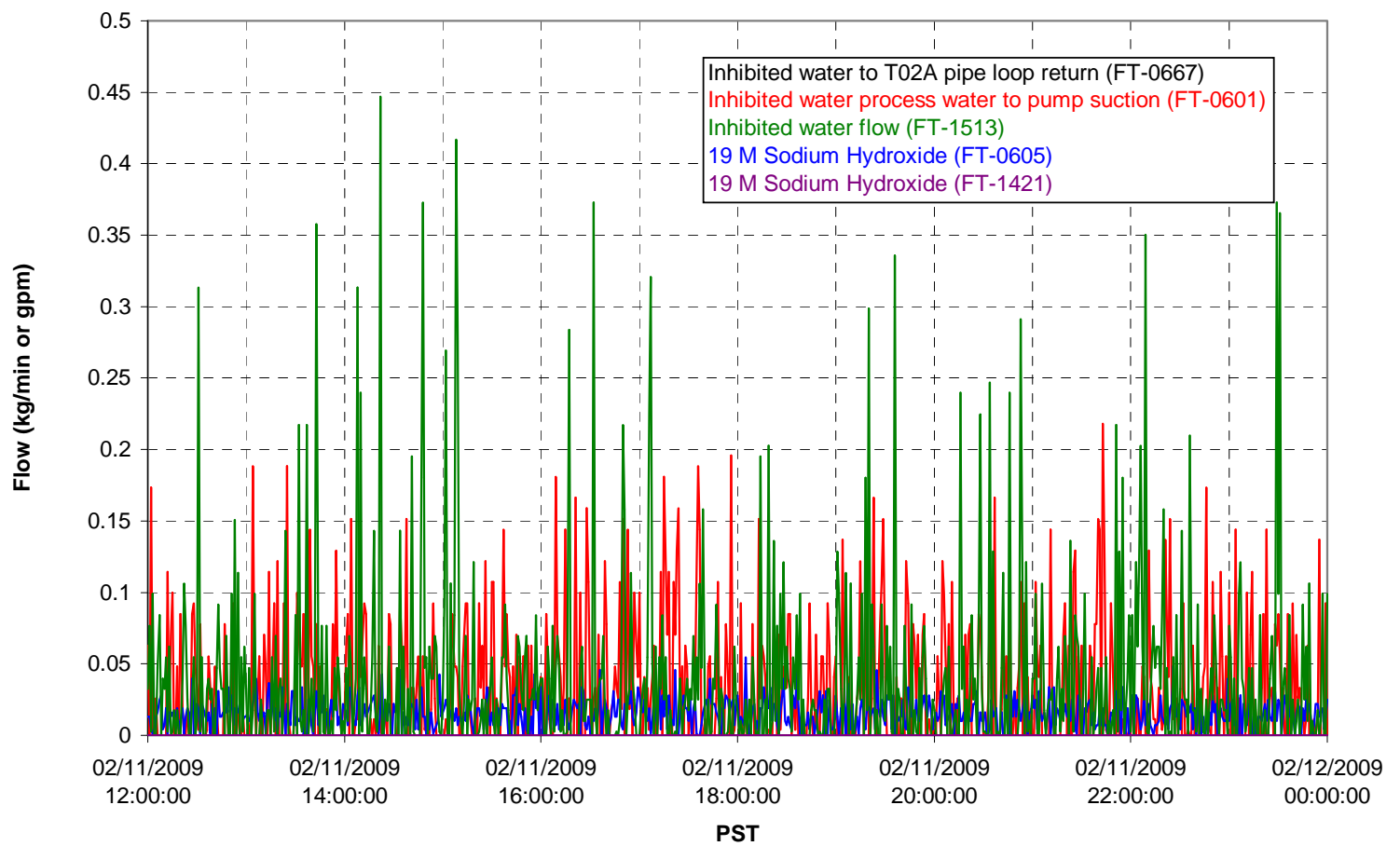


Chemical Flow

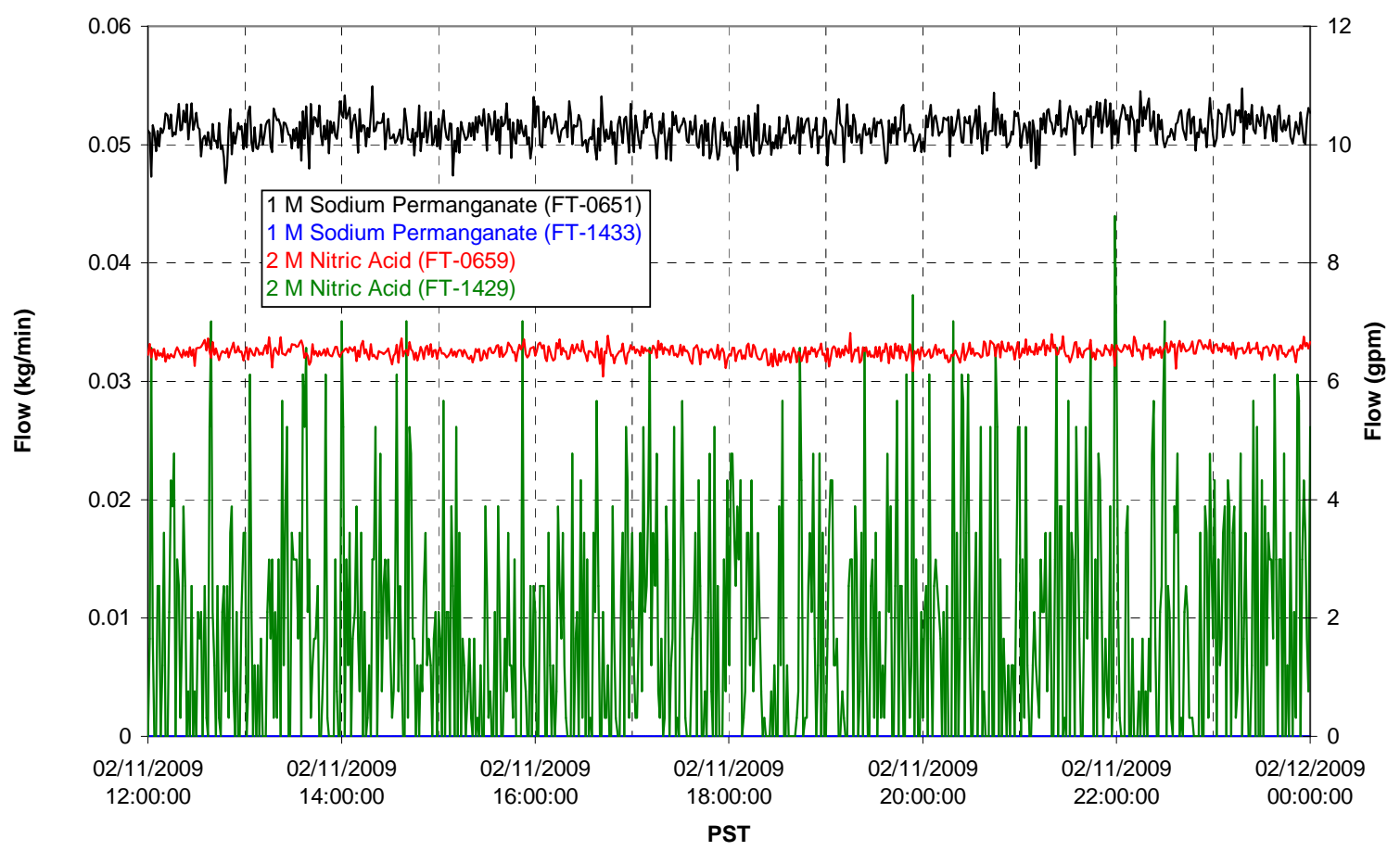

Air Flows

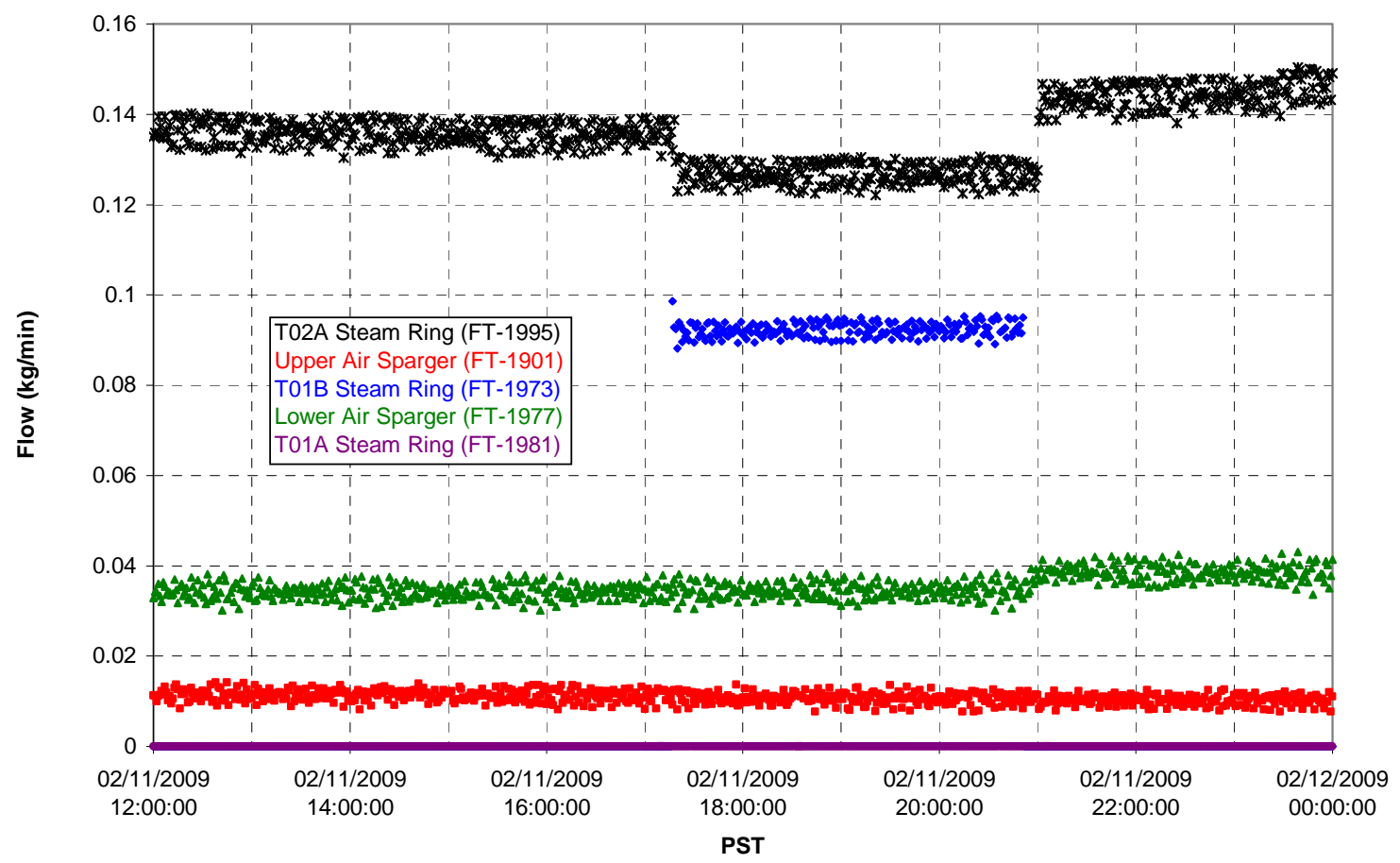


T02A Steam

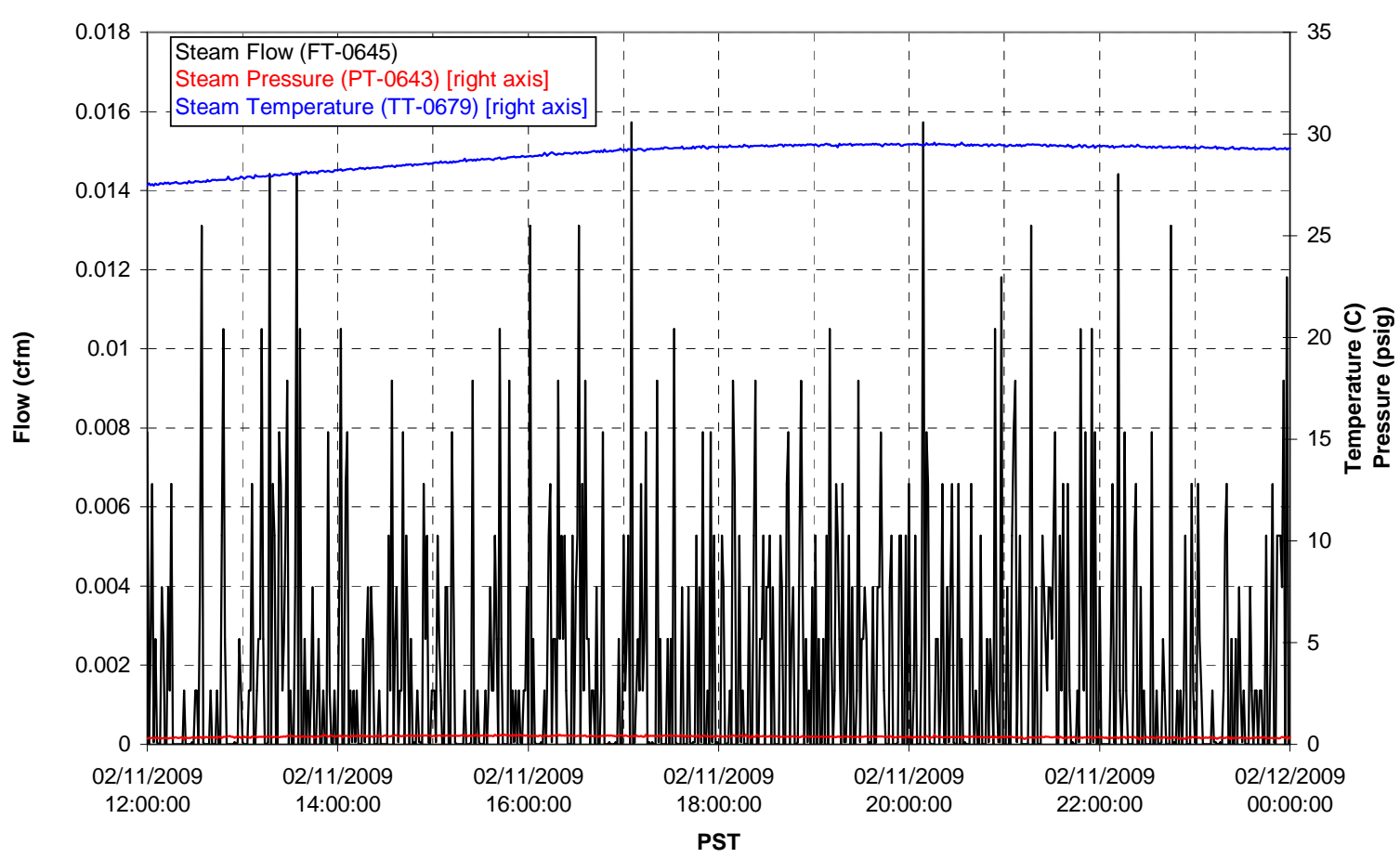

T01A Steam

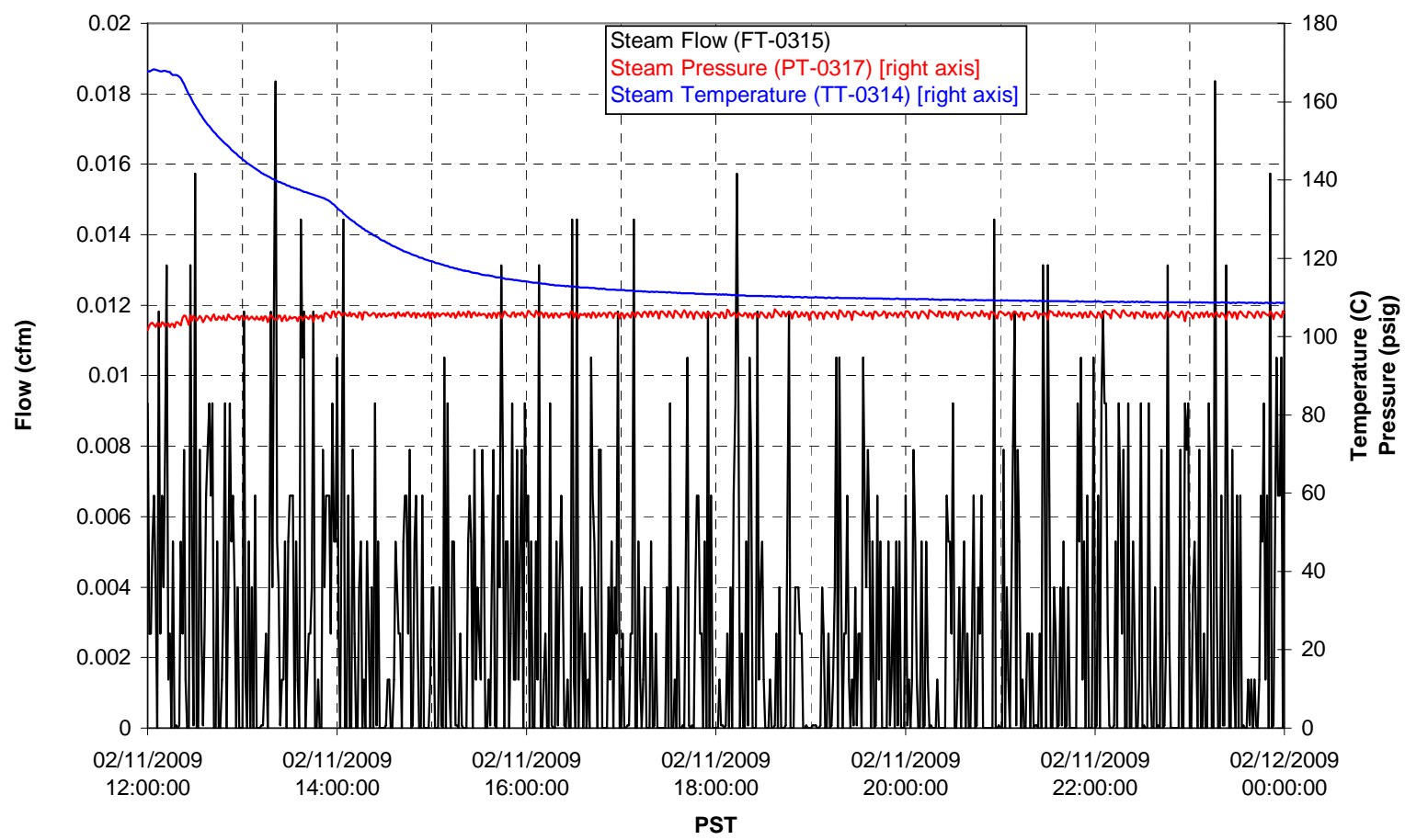


T01B Steam

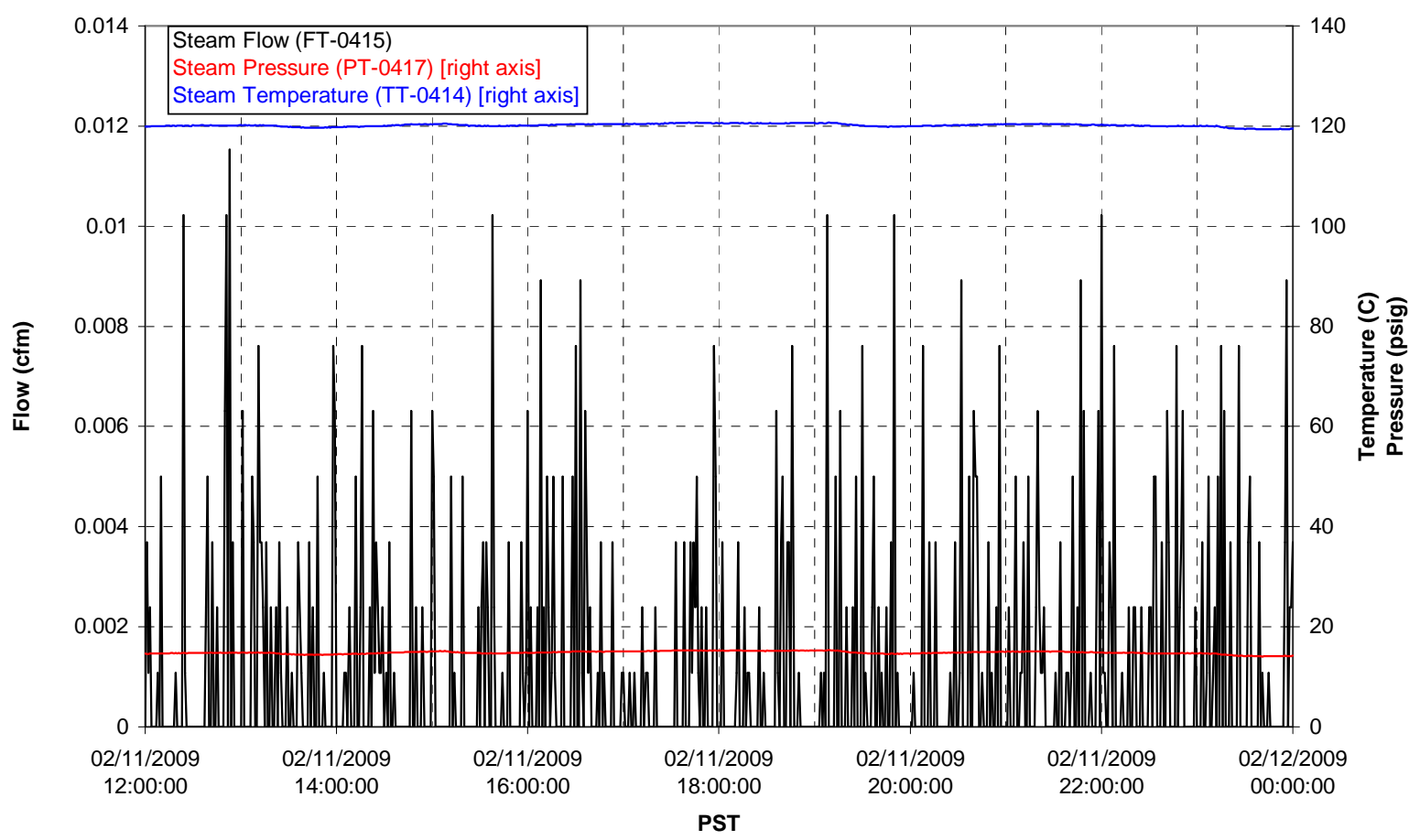




\section{Integrated Test A Data Plots 02/12/09 00:00 - 02/12/09 12:00}


T01A level

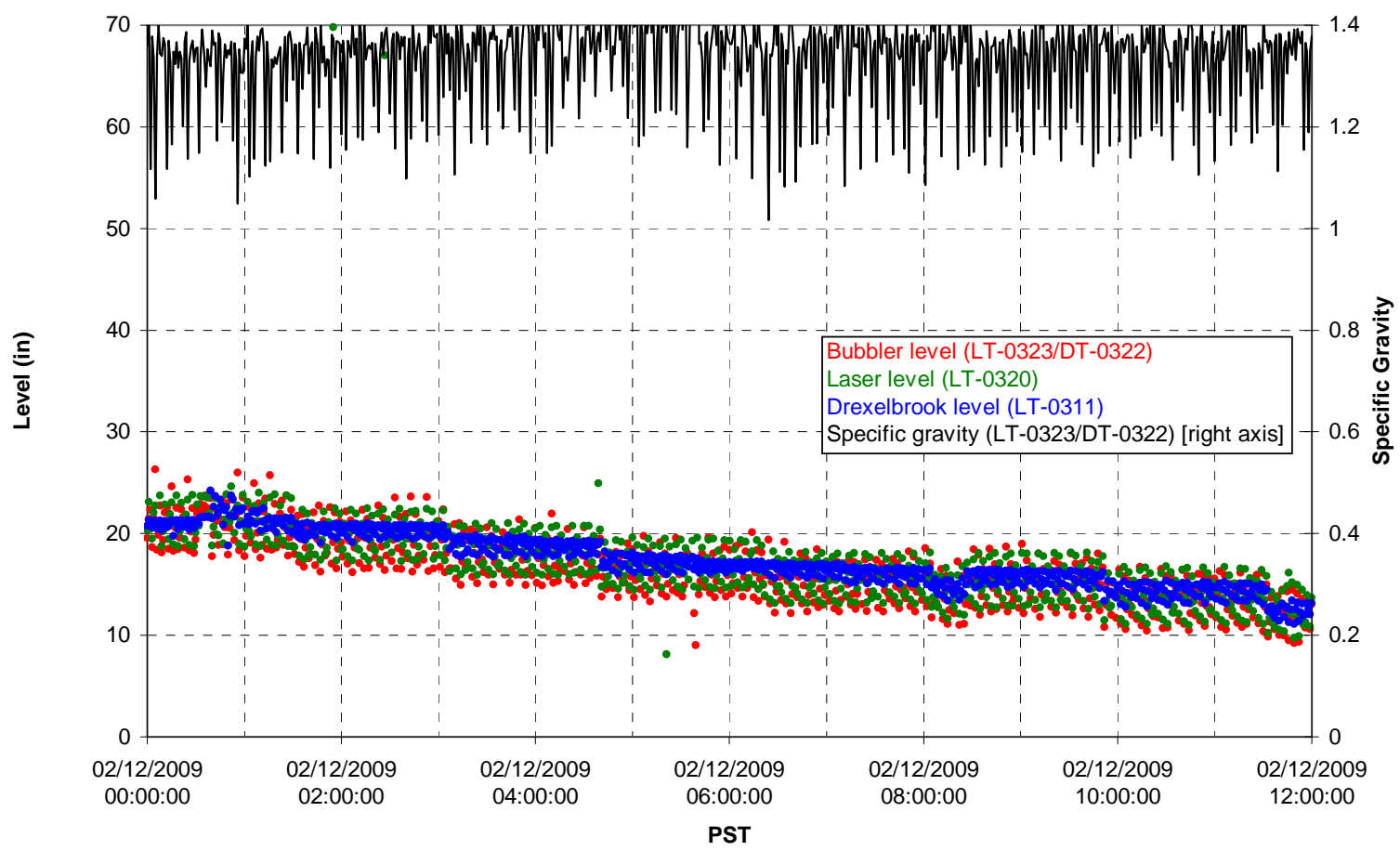

T01A temperatures

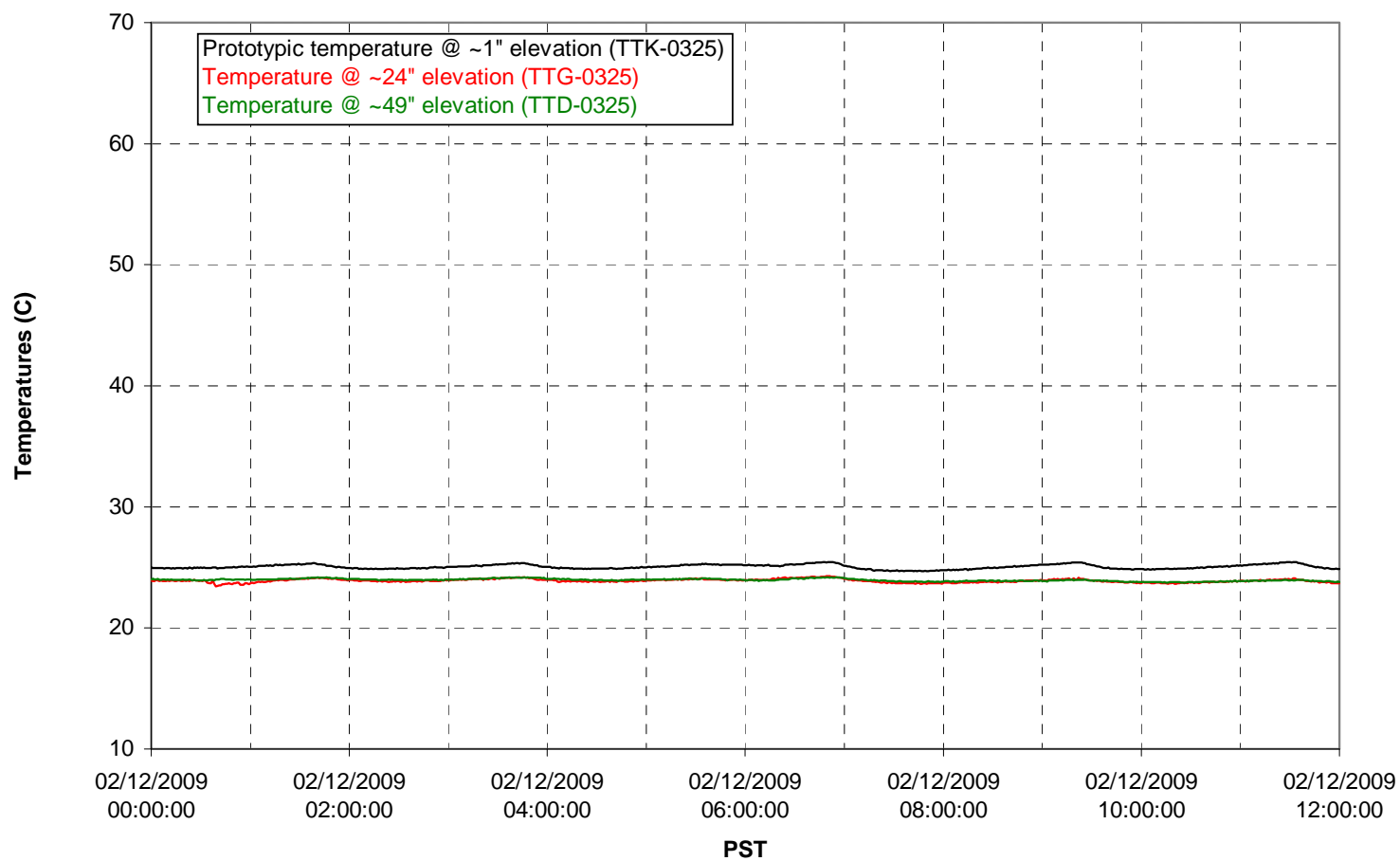


T01B level

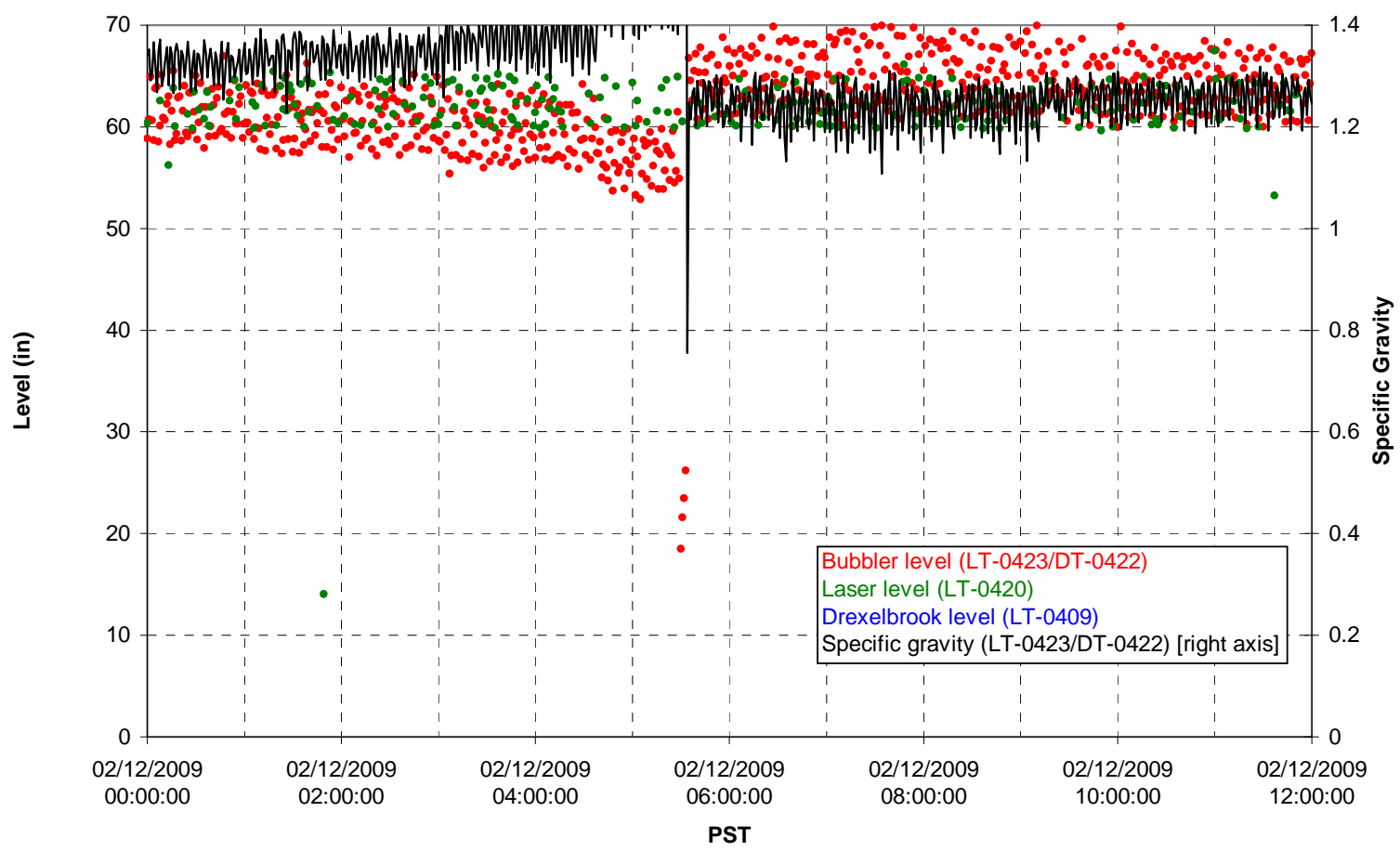

T01B temperatures

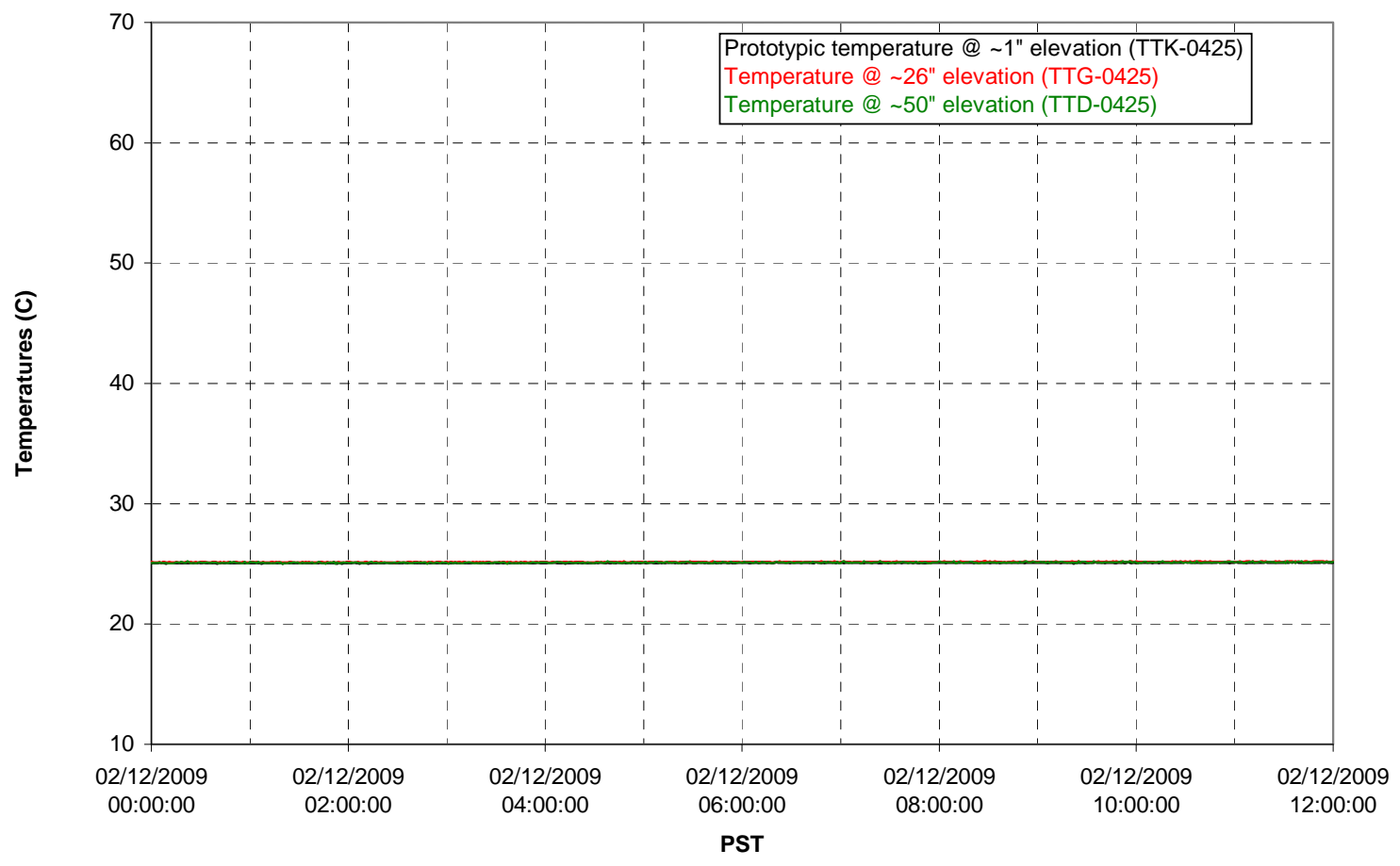


T02A level

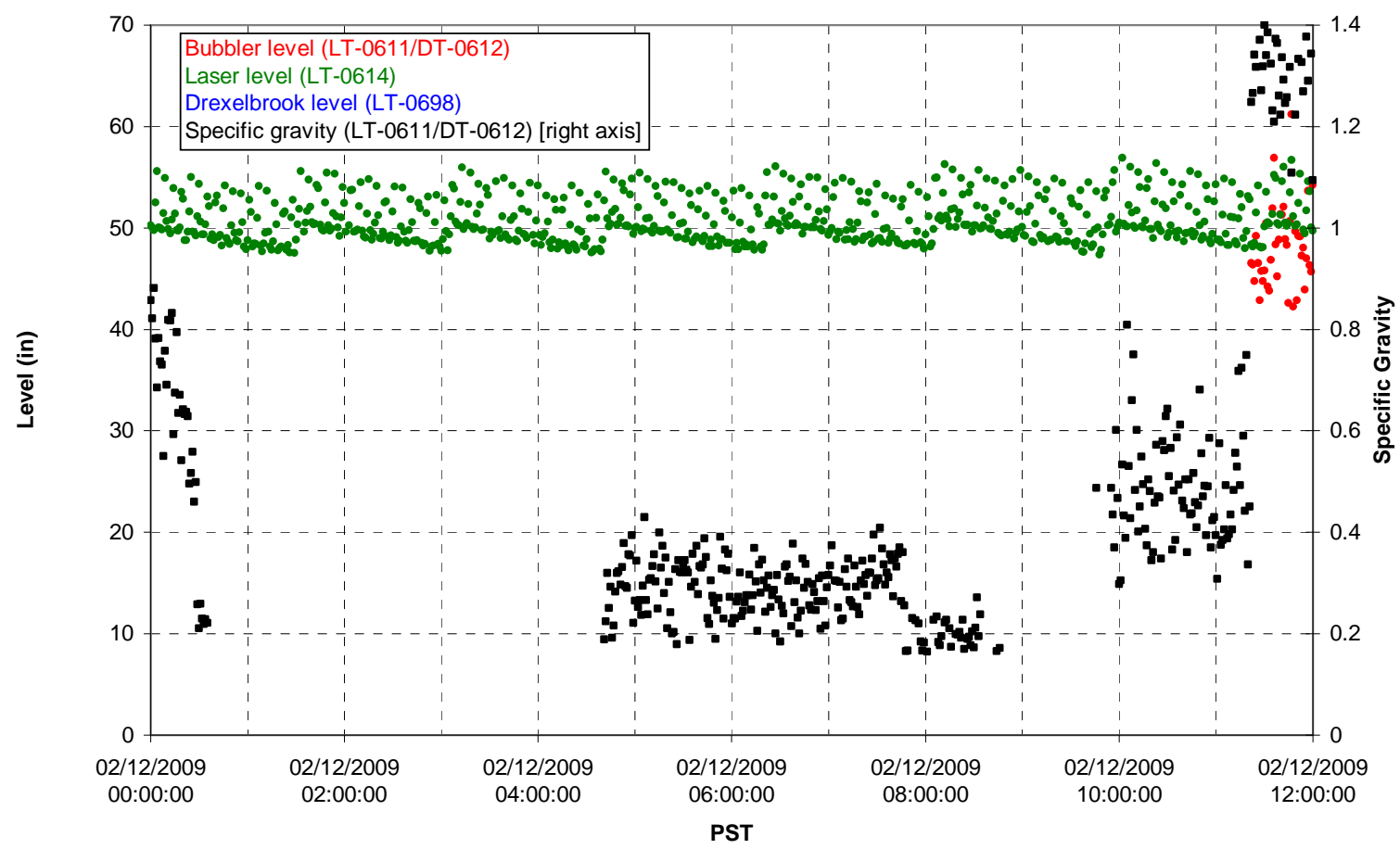

T02A temperatures

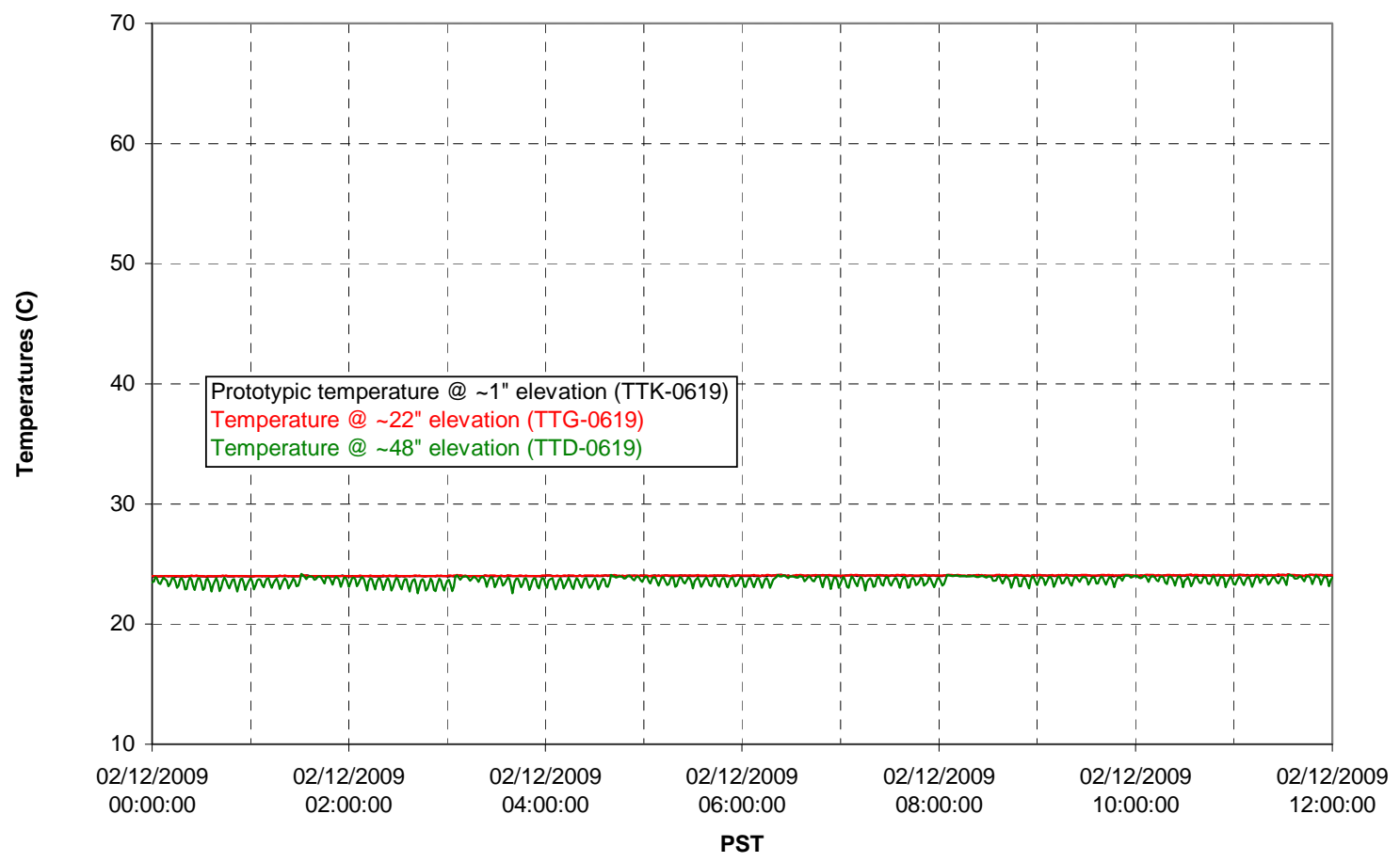


T02A and filter loop temperatures

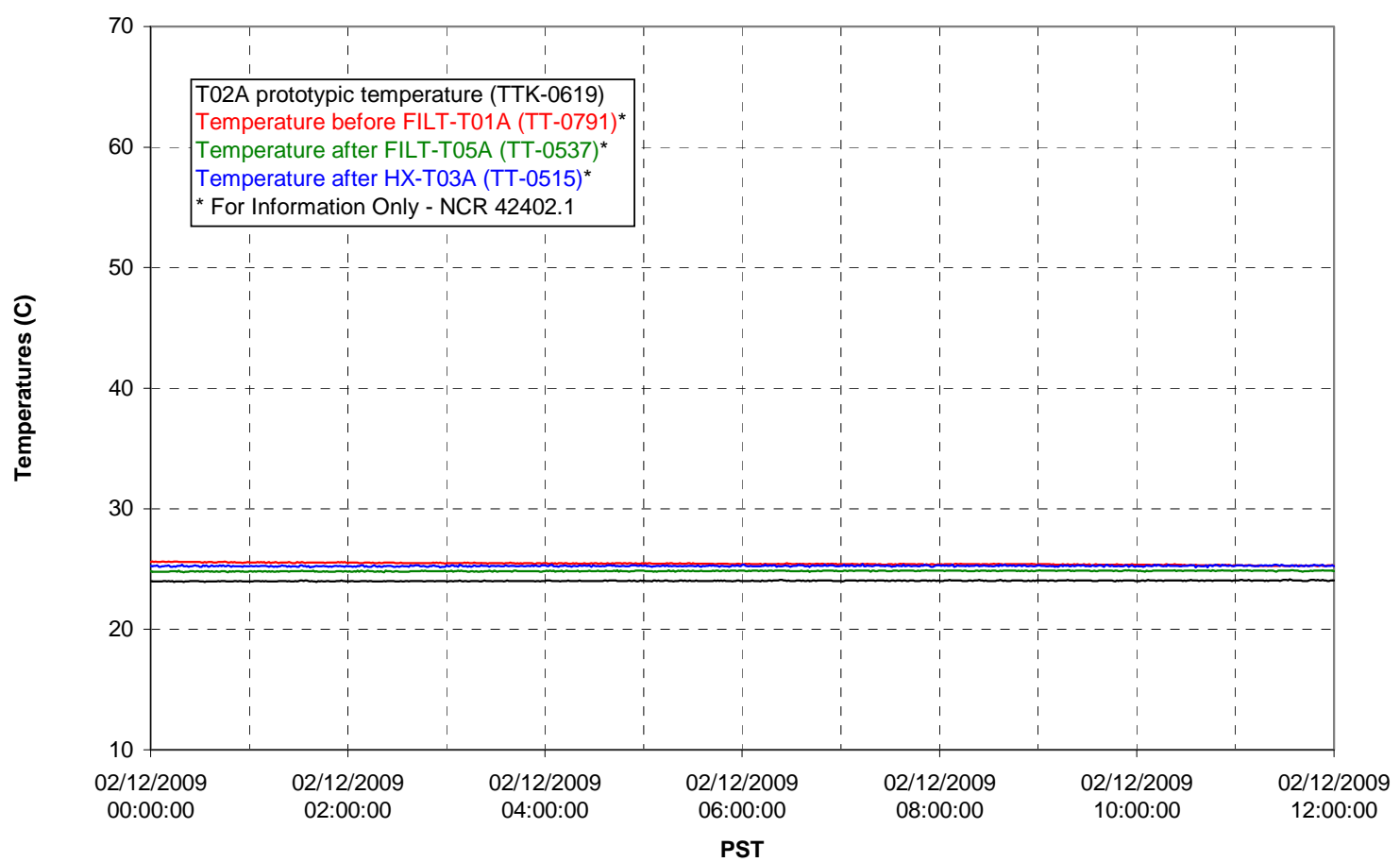

Pump Pressures and Flow

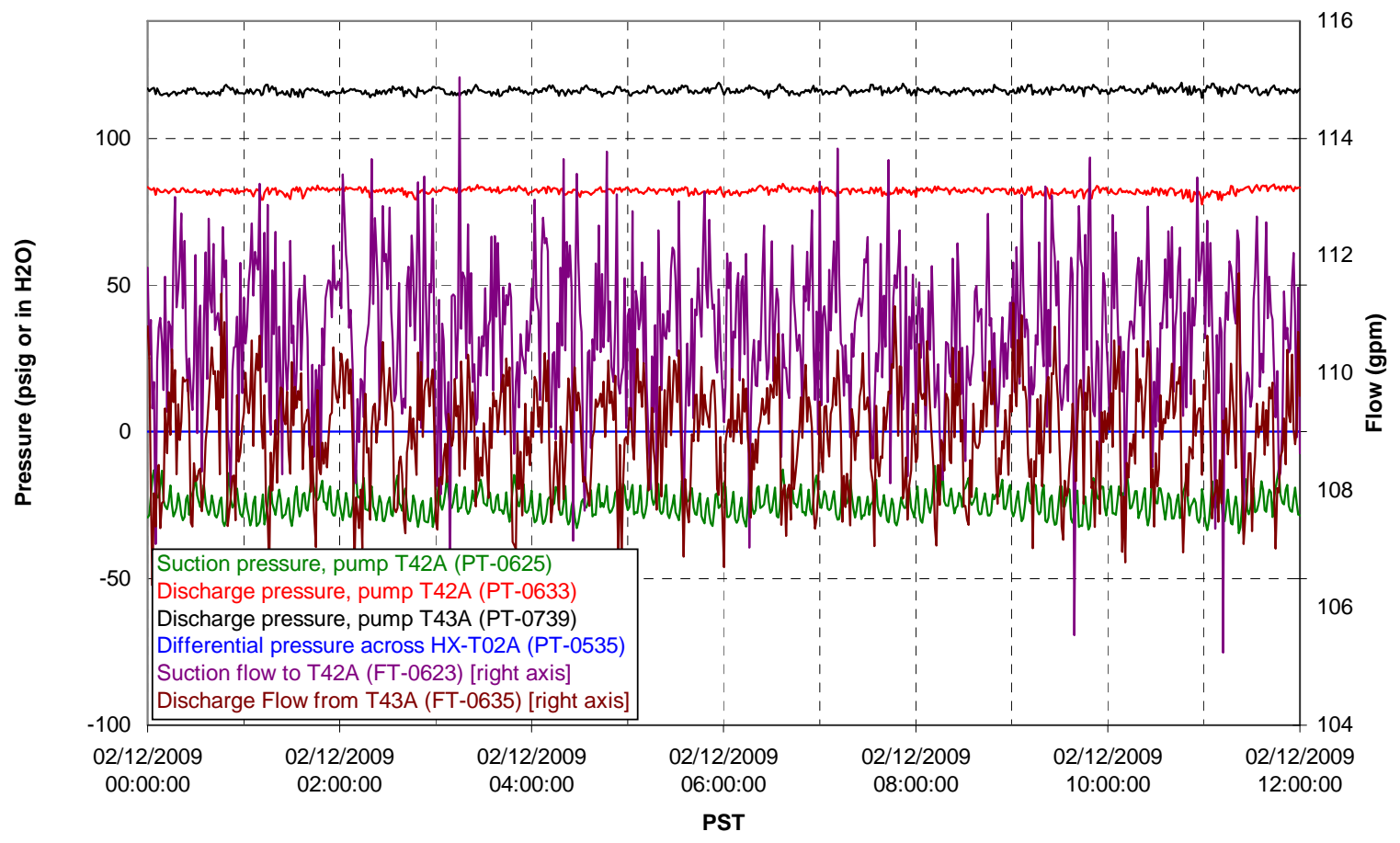




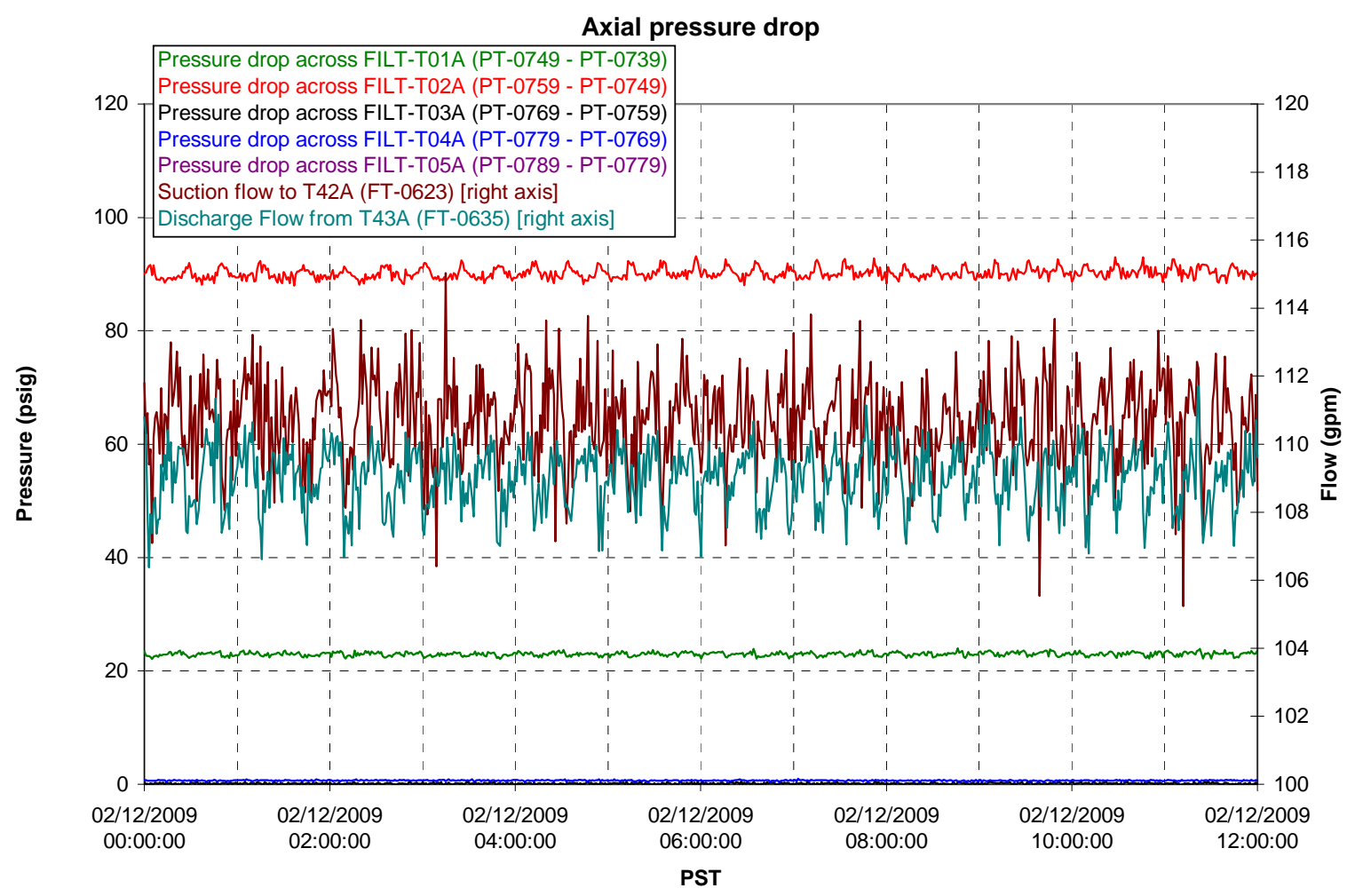

Permeate flow rates

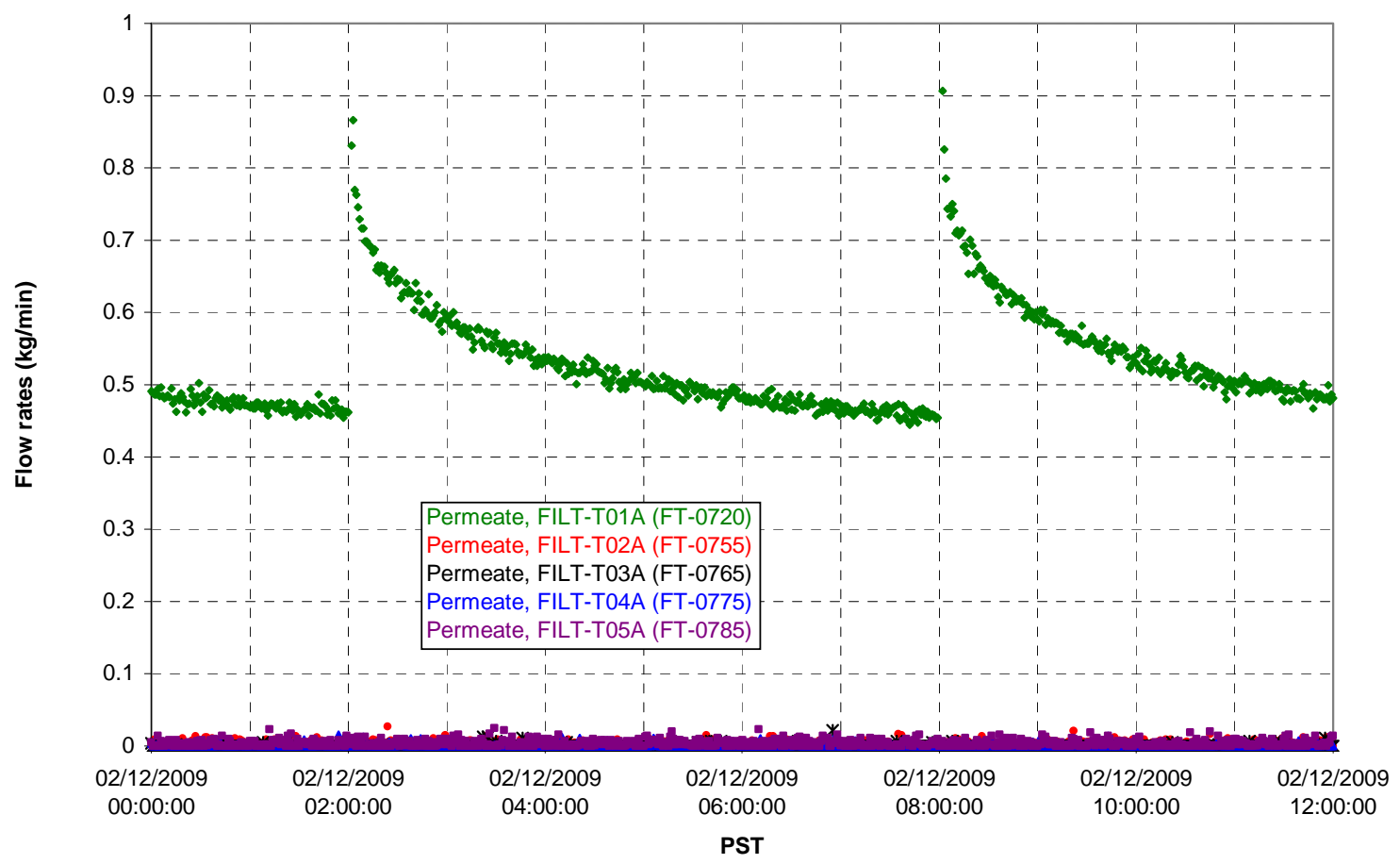


T02A Inner Temperature Tree

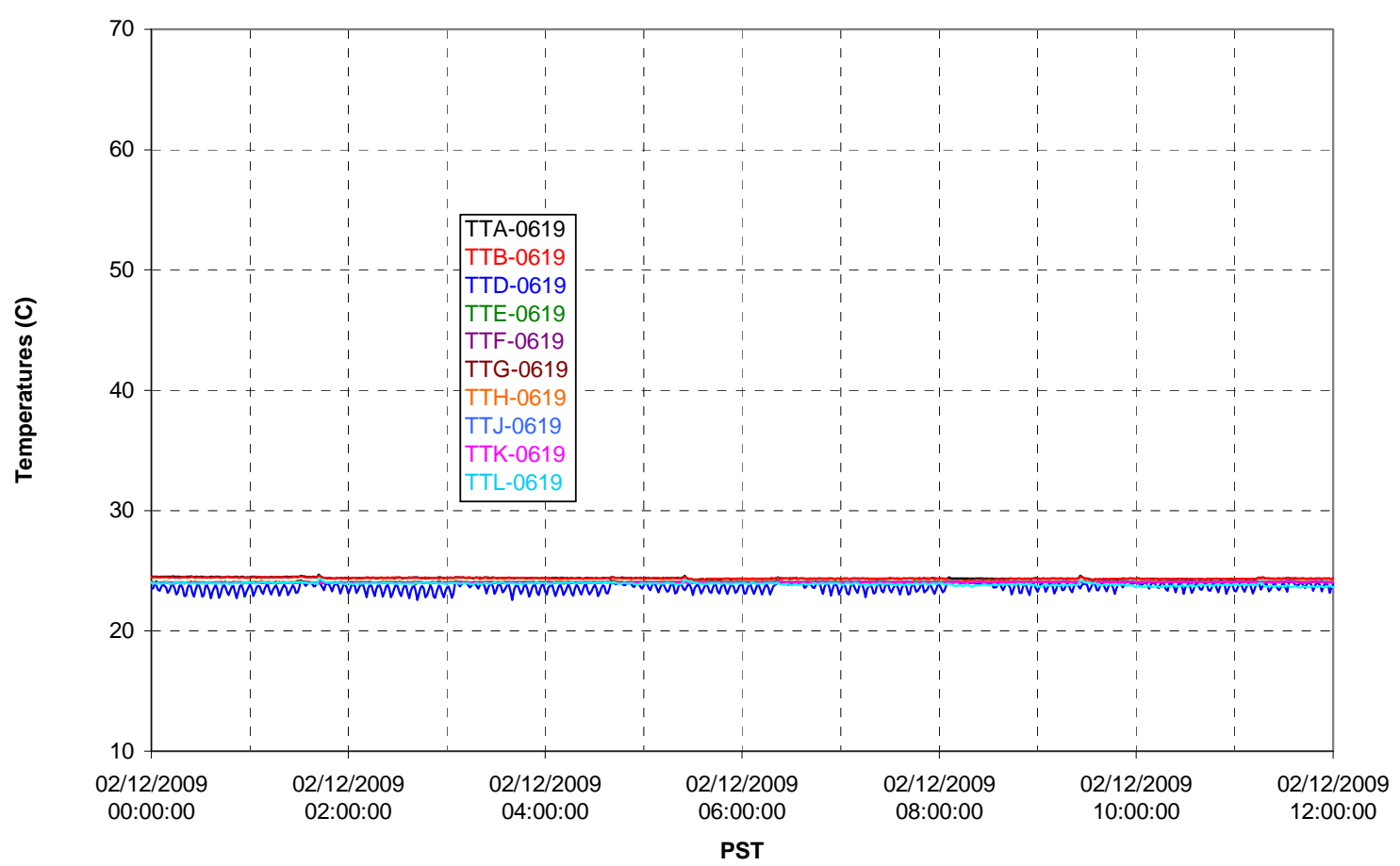

T02A Outer Temperature Tree

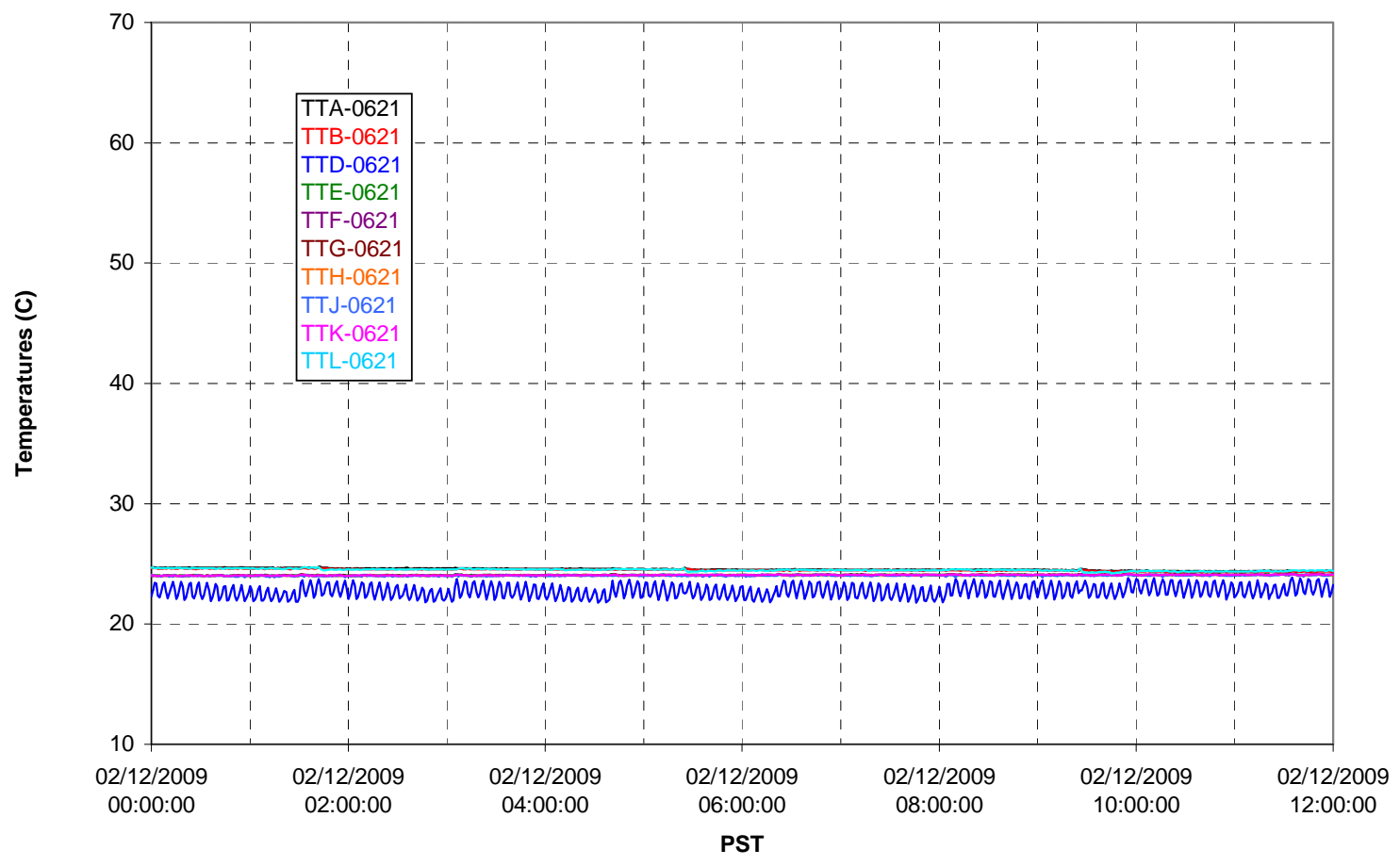


T02A temperatures

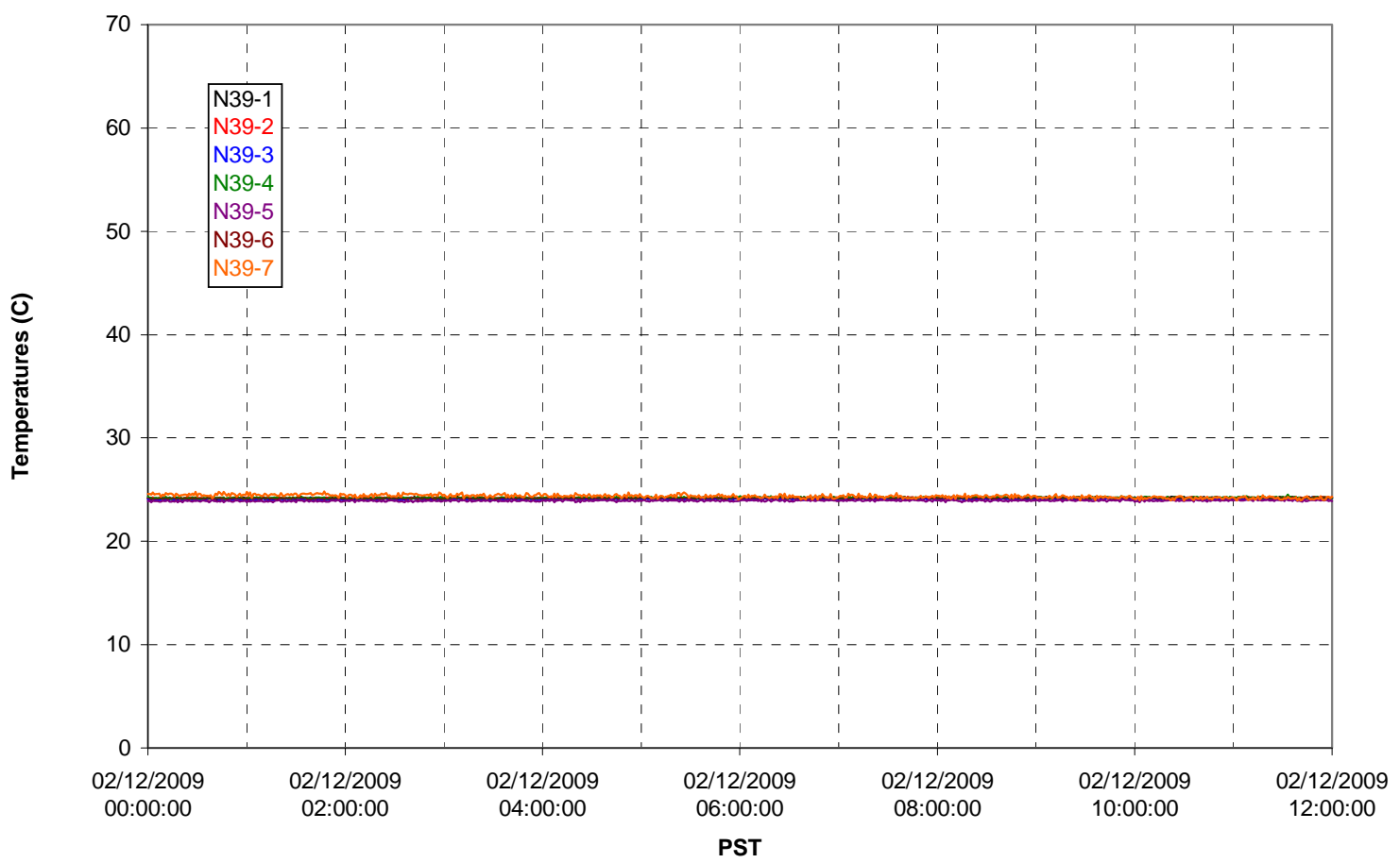

T02A temperatures

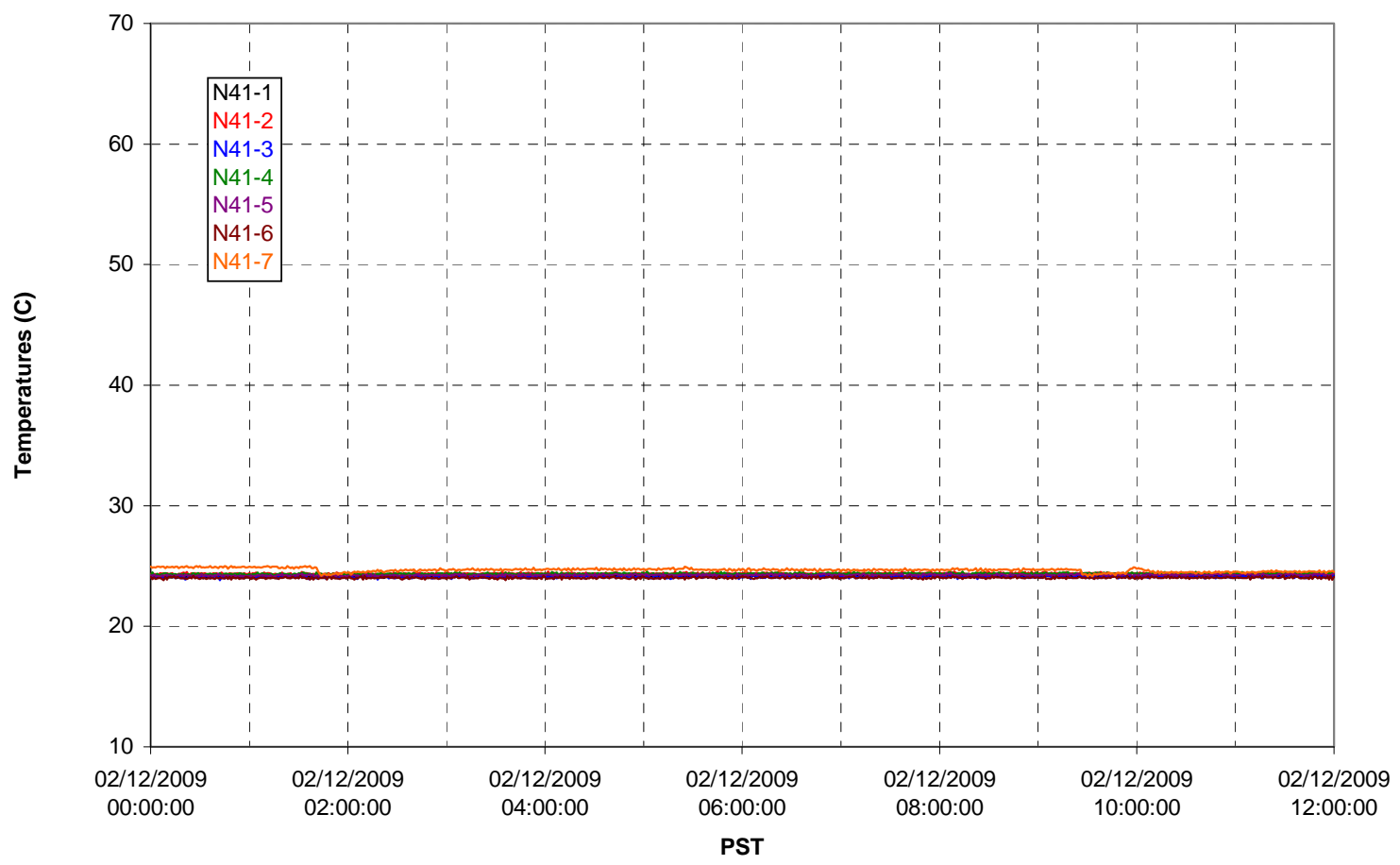


T02A temperatures

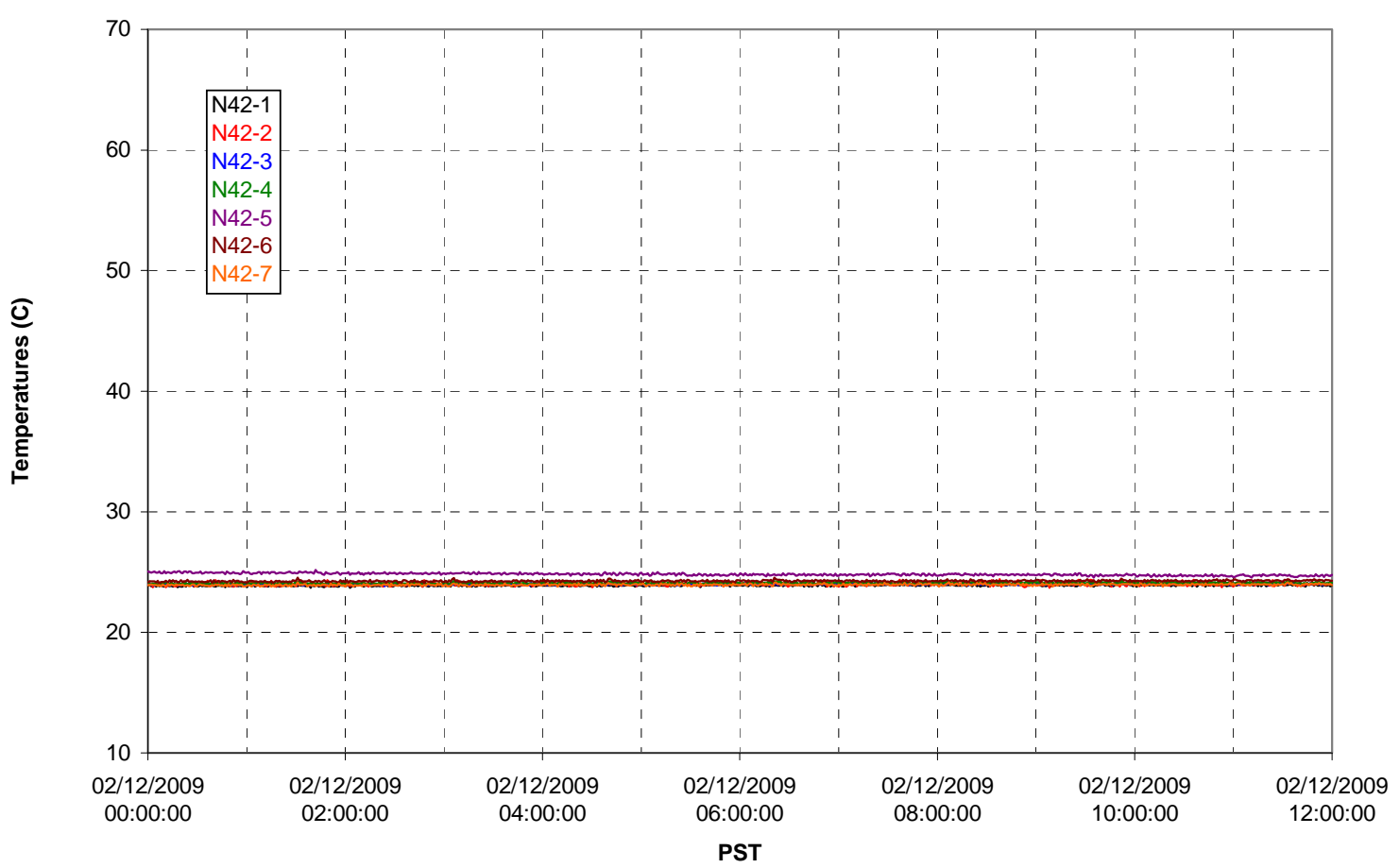

T02A temperatures

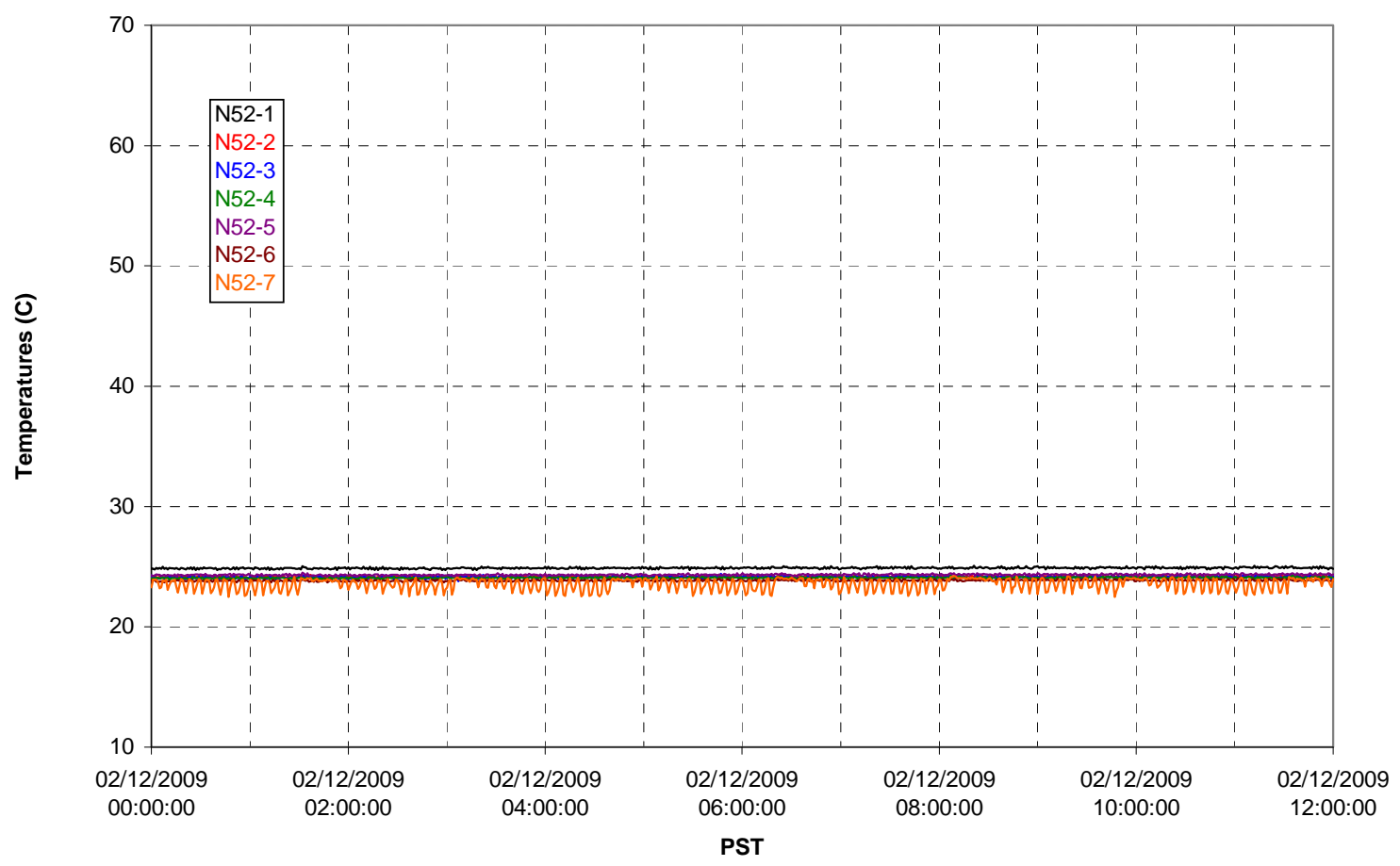


T02A Heating and Cooling
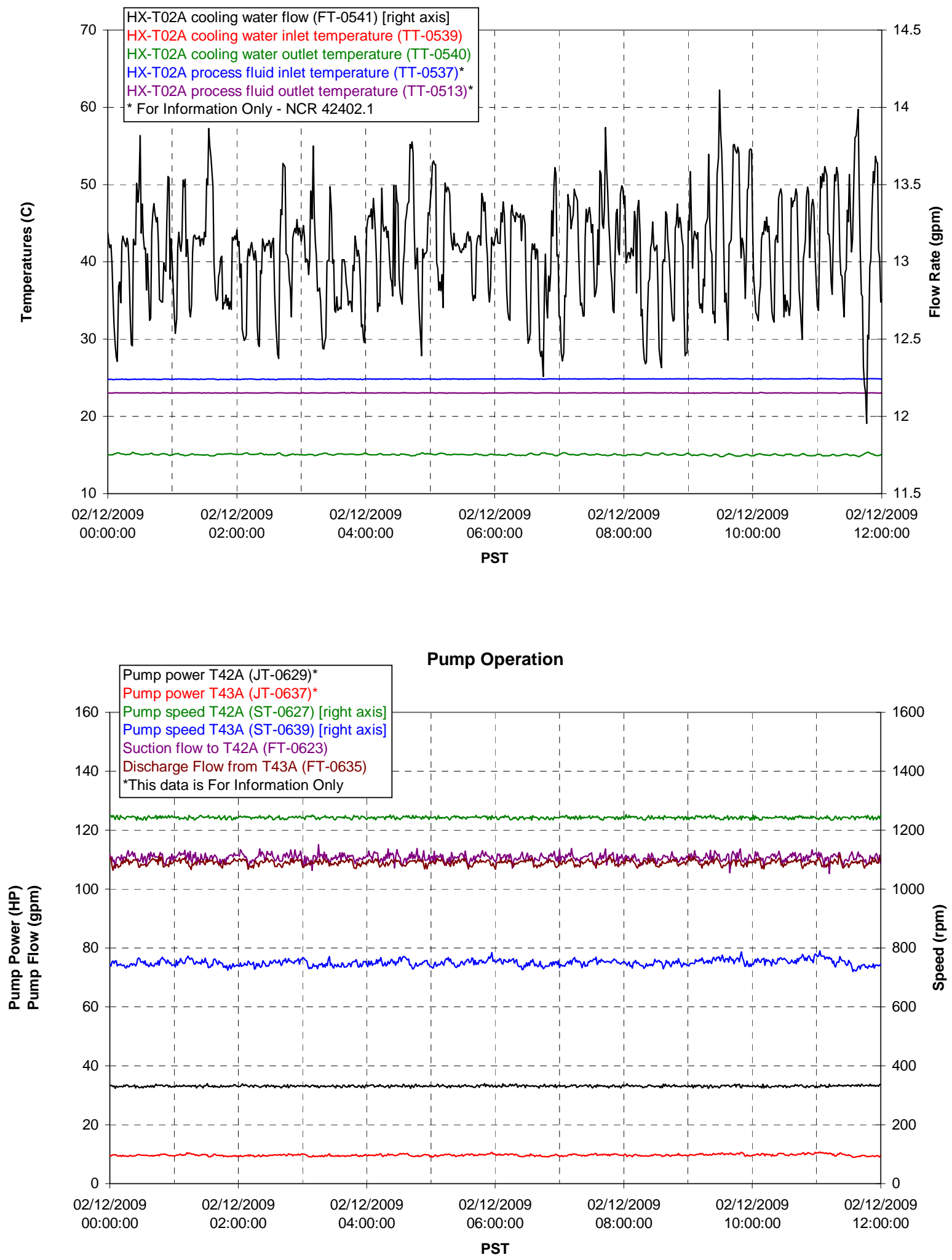
Pulsepot UFP-PP-T01A

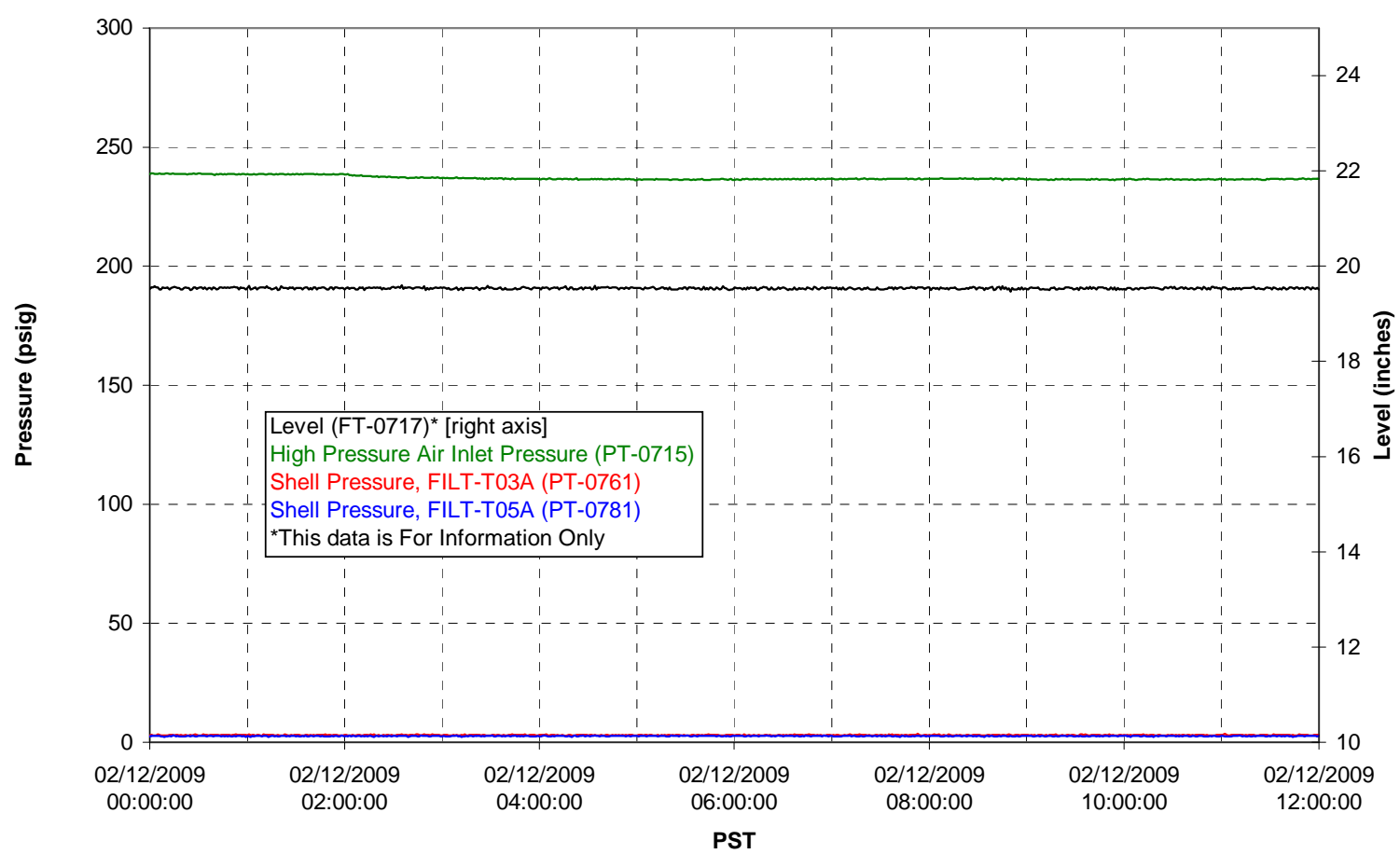

Pulsepot UFP-PP-T02A

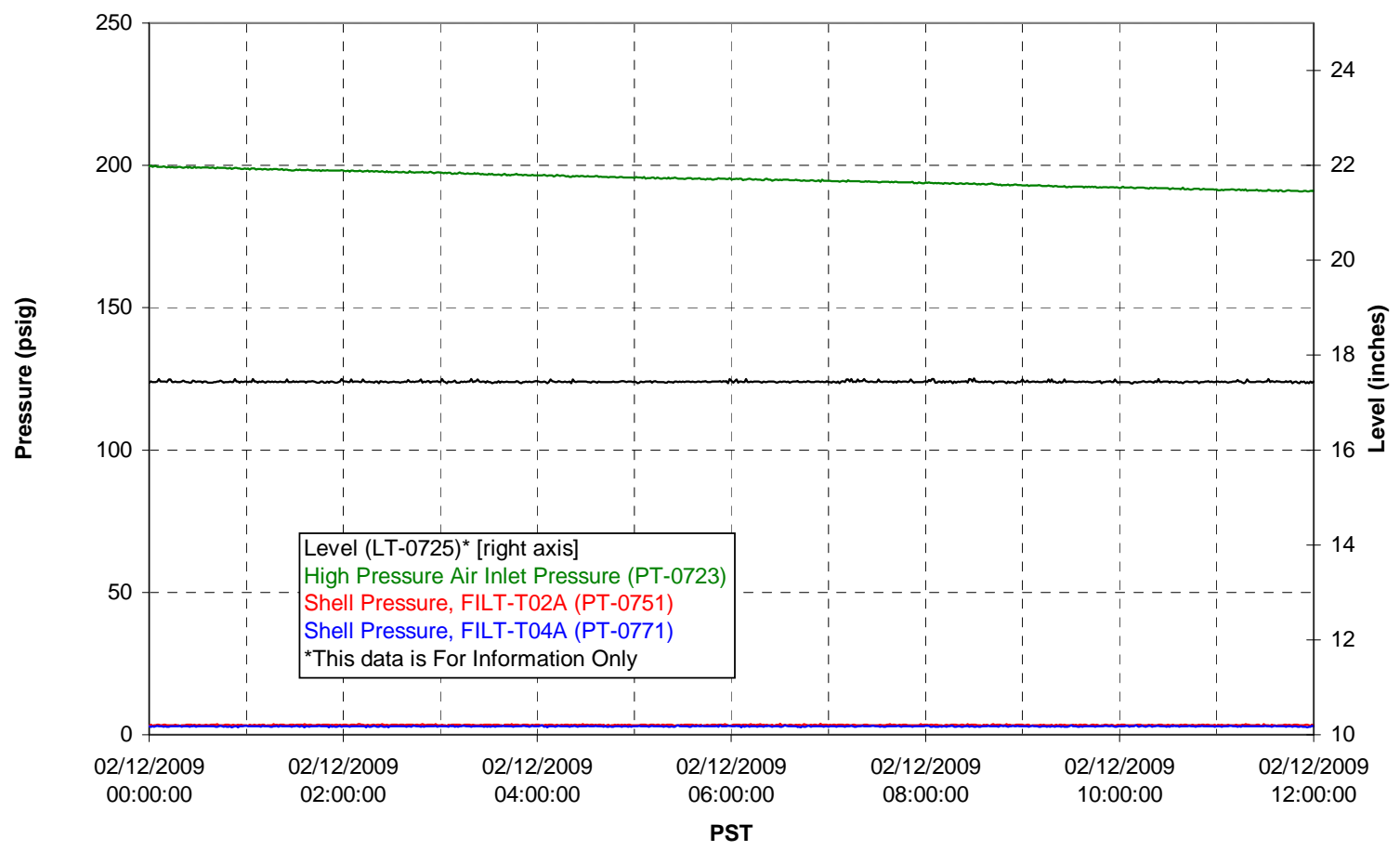


Pulsepot UFP-PP-T03A

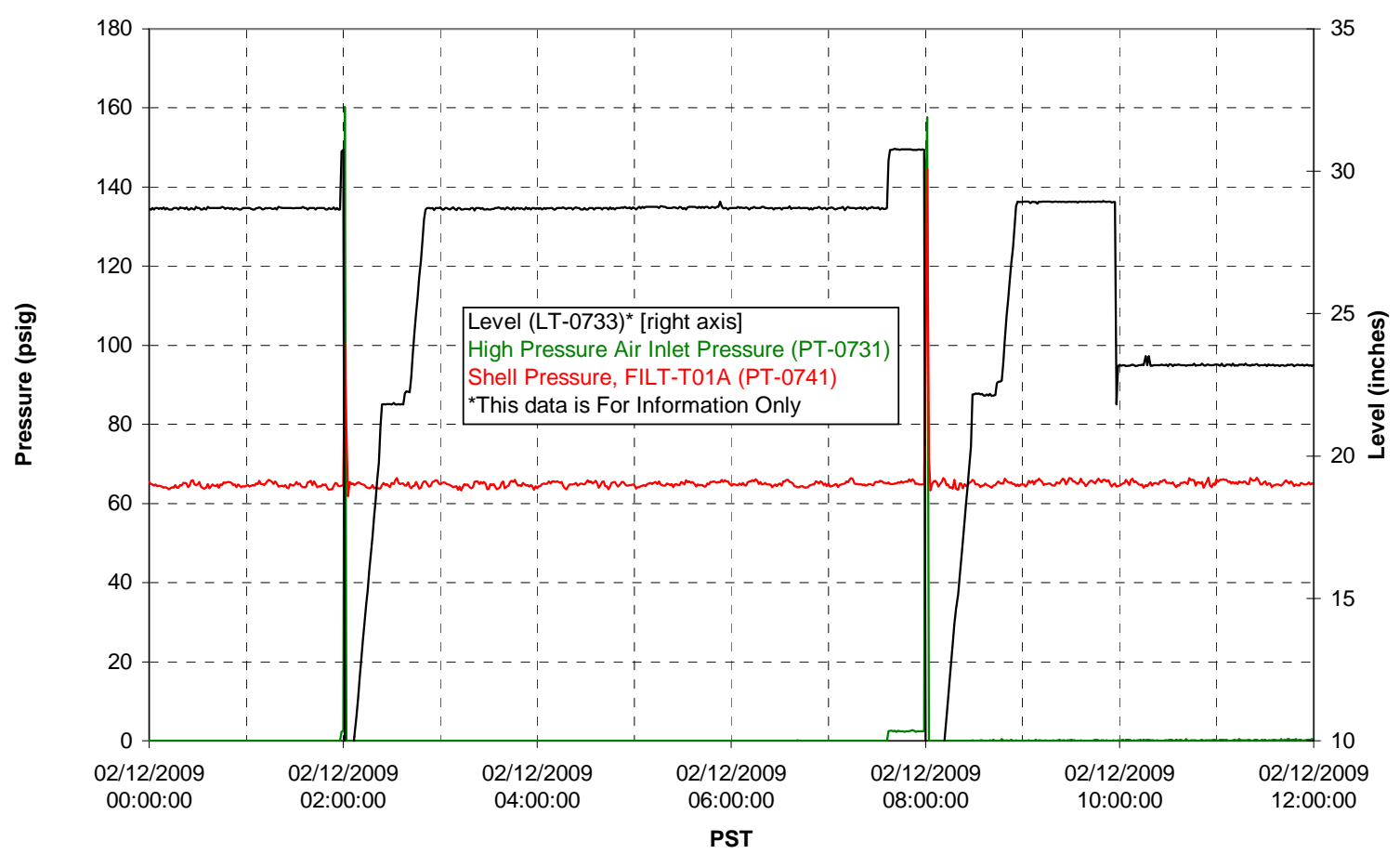

Pulsepot Levels

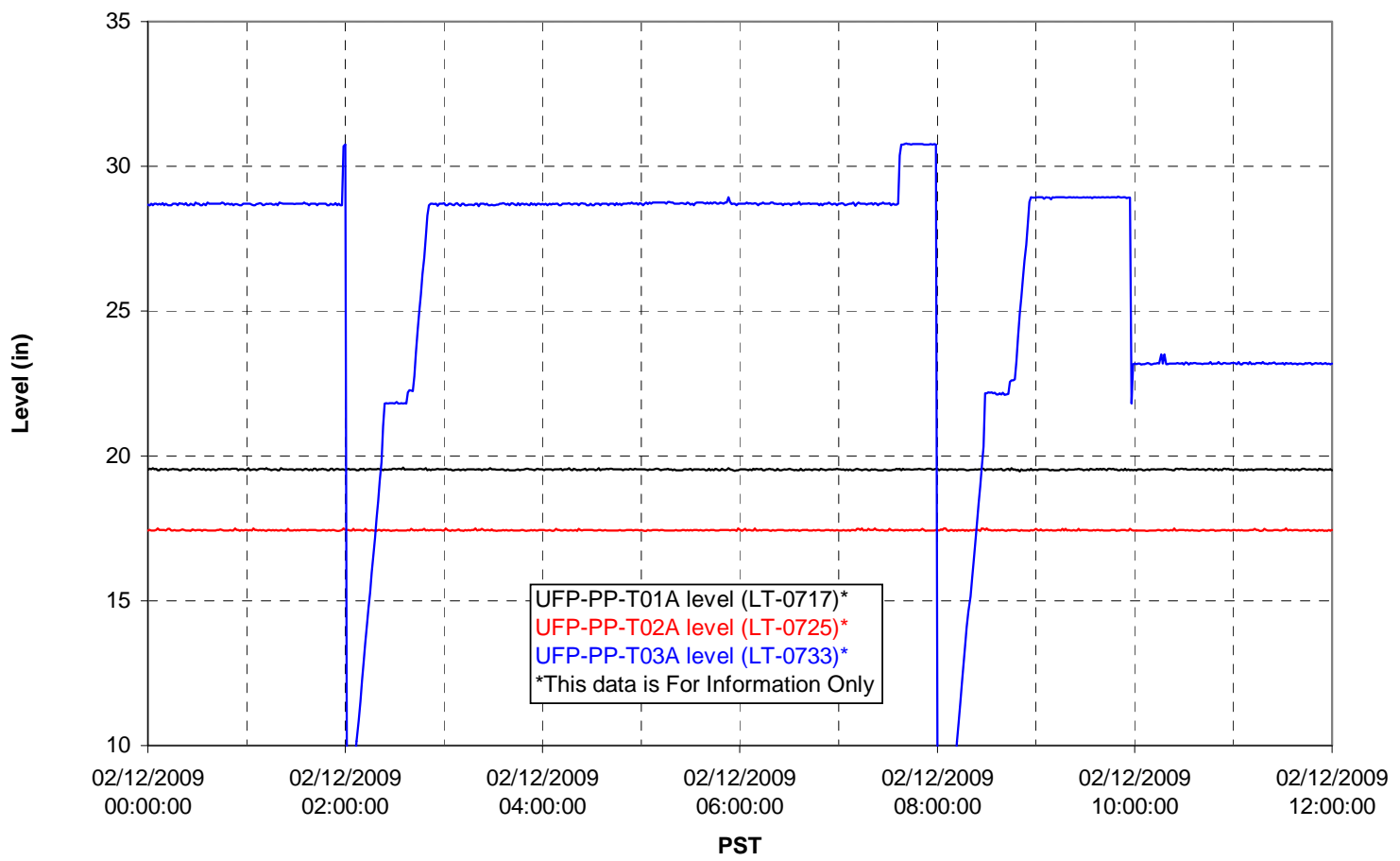


Filter UFP-FILT-T01A

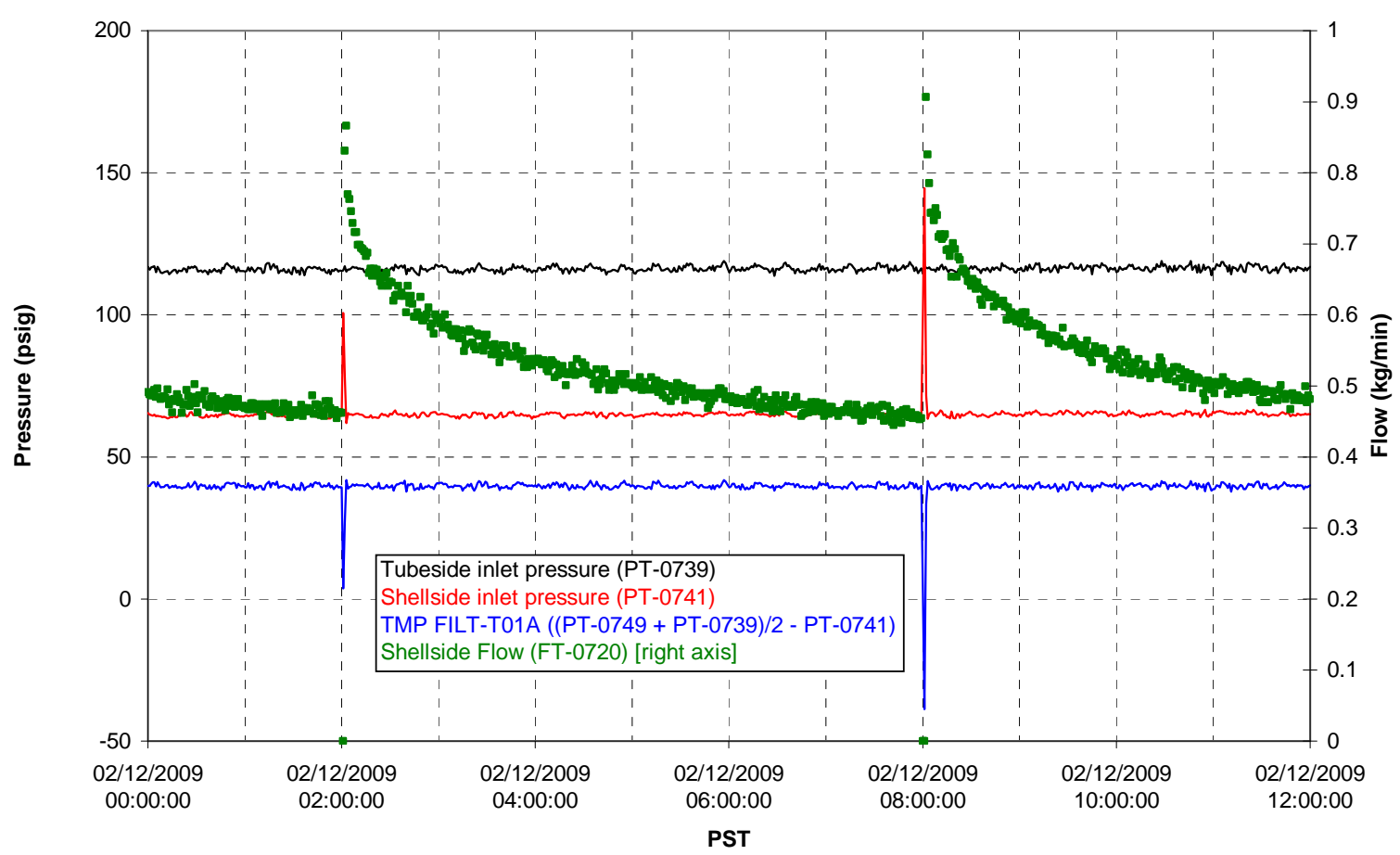

Filter UFP-FILT-T02A

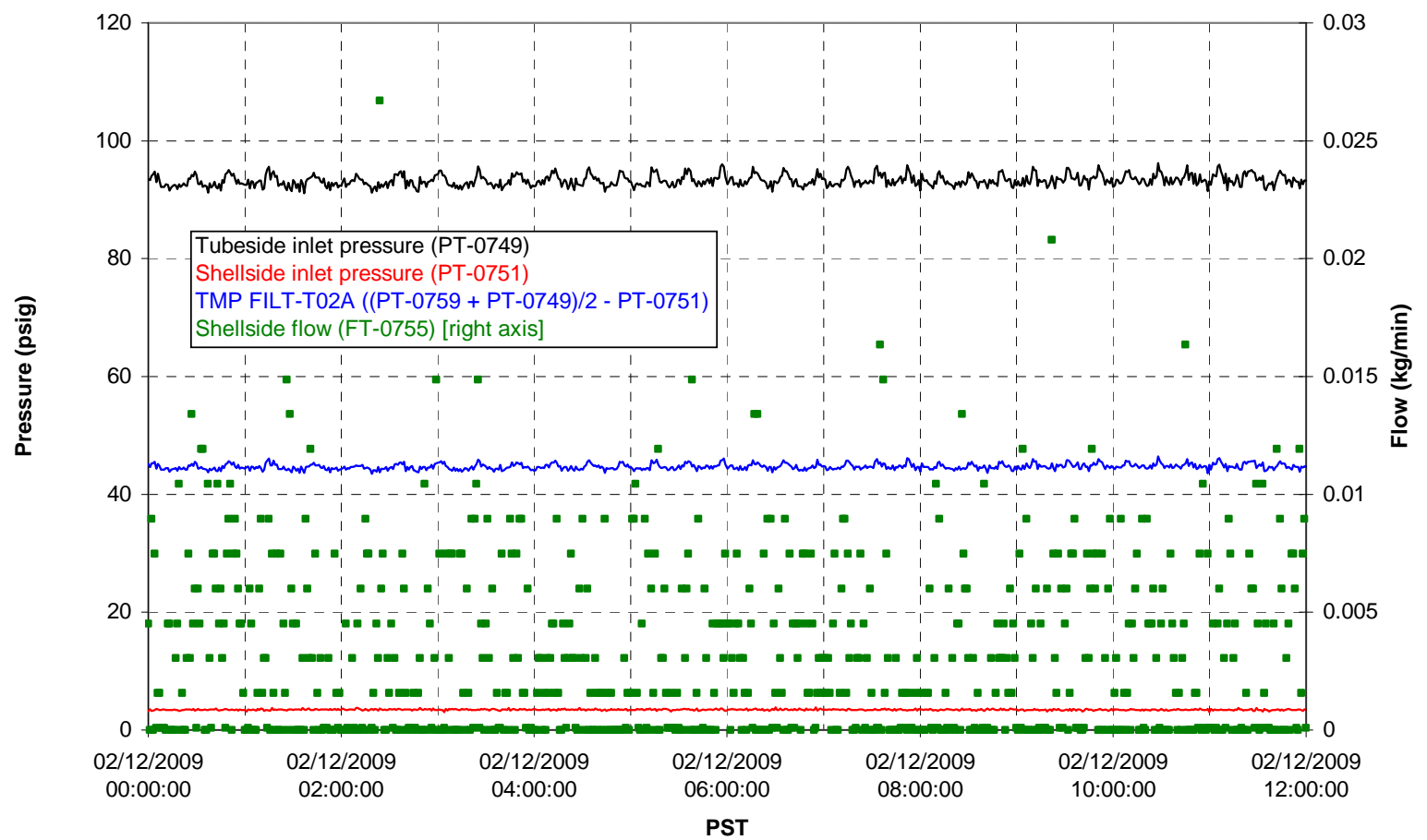


Filter UFP-FILT-T03A

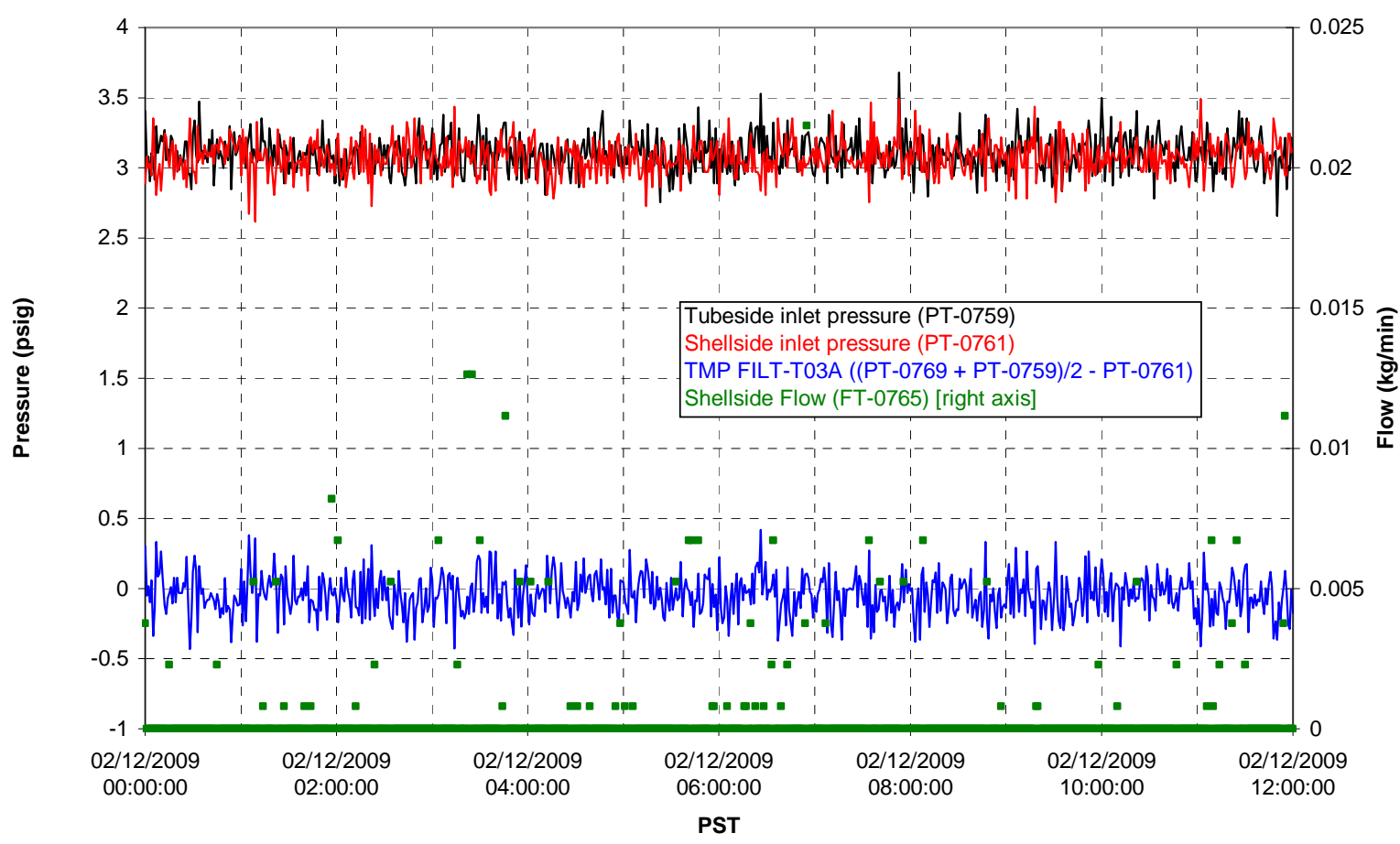

Filter UFP-FILT-T04A

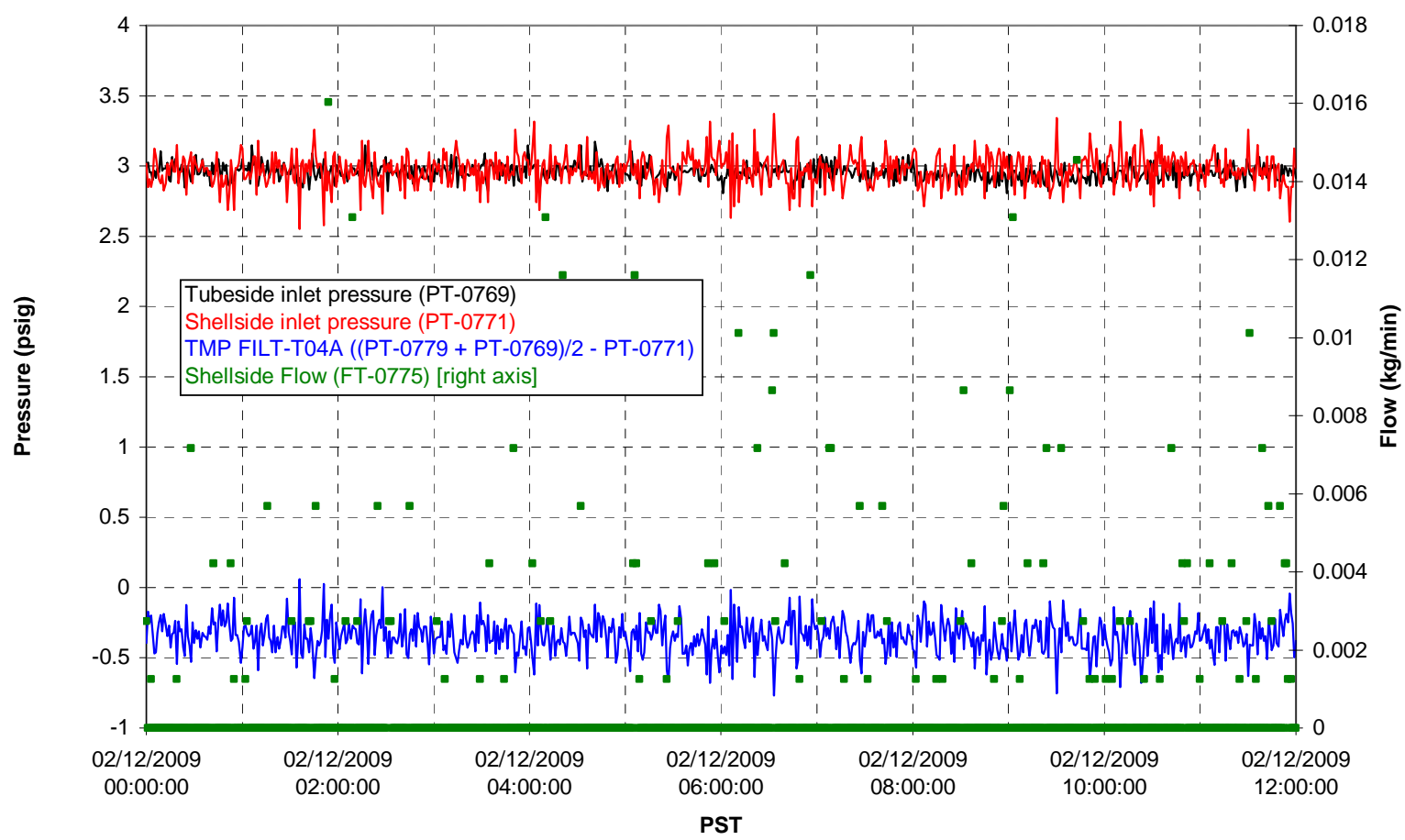


Filter UFP-FILT-T05A

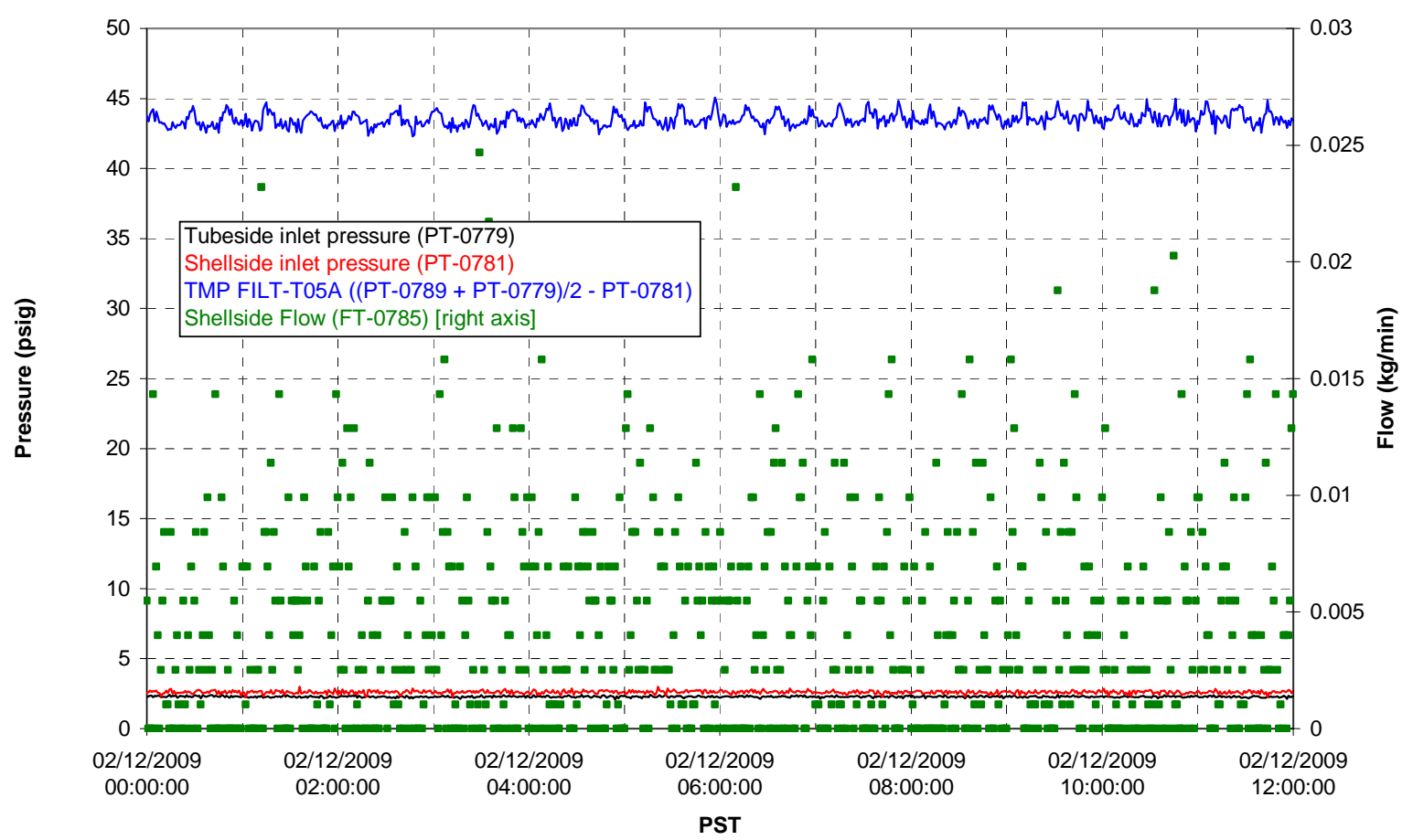

Chemical Flow

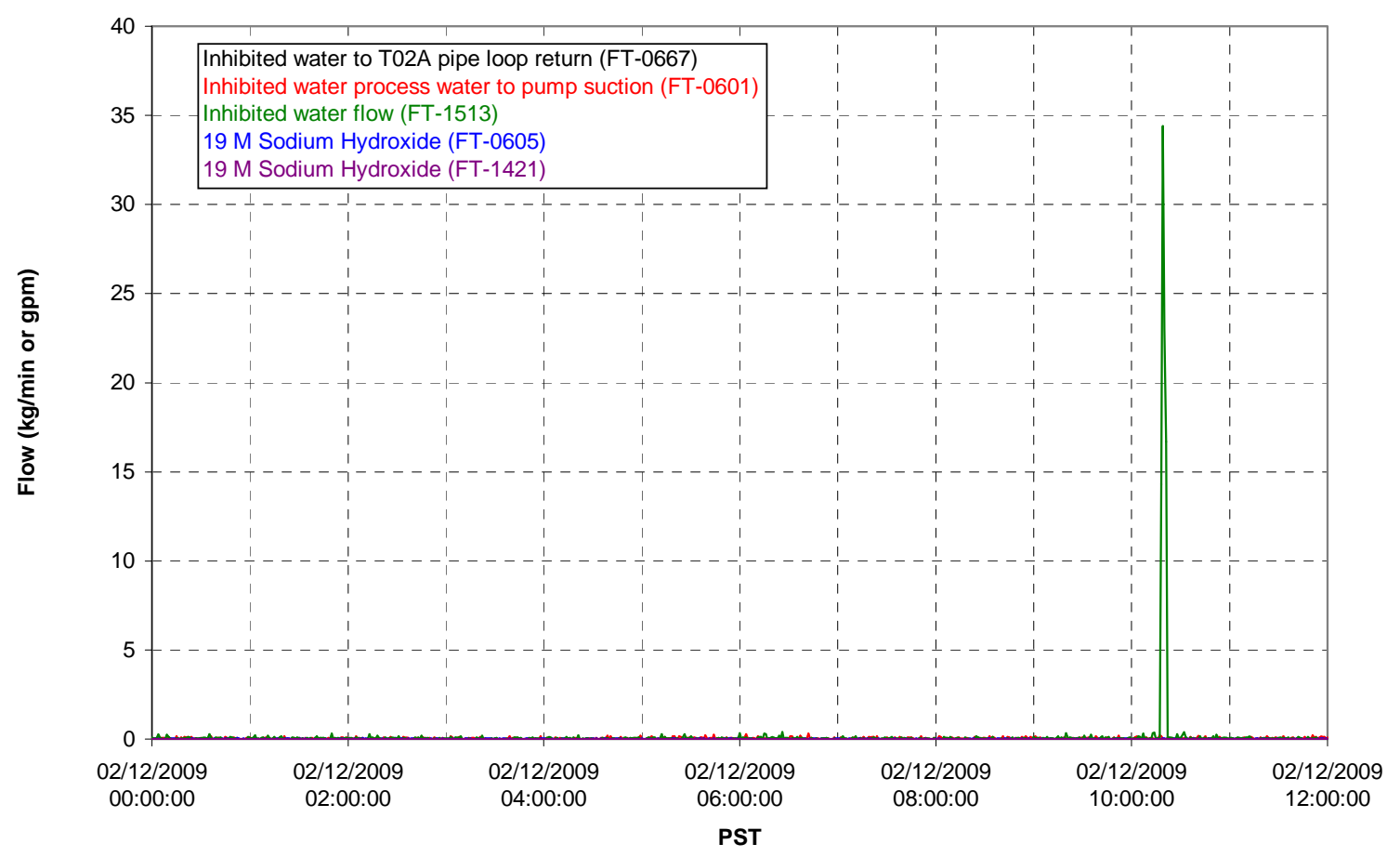


Chemical Flow

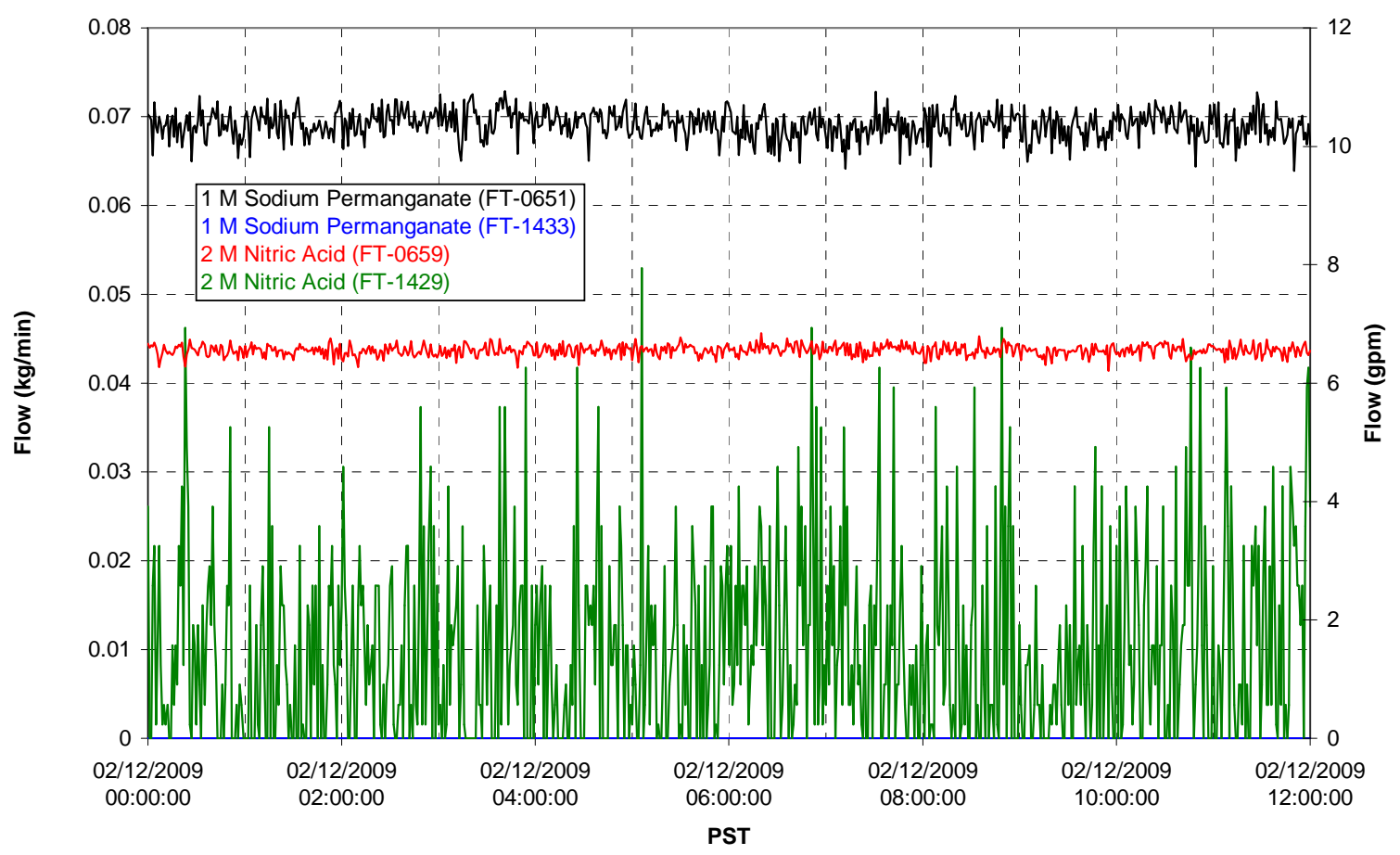

Air Flows

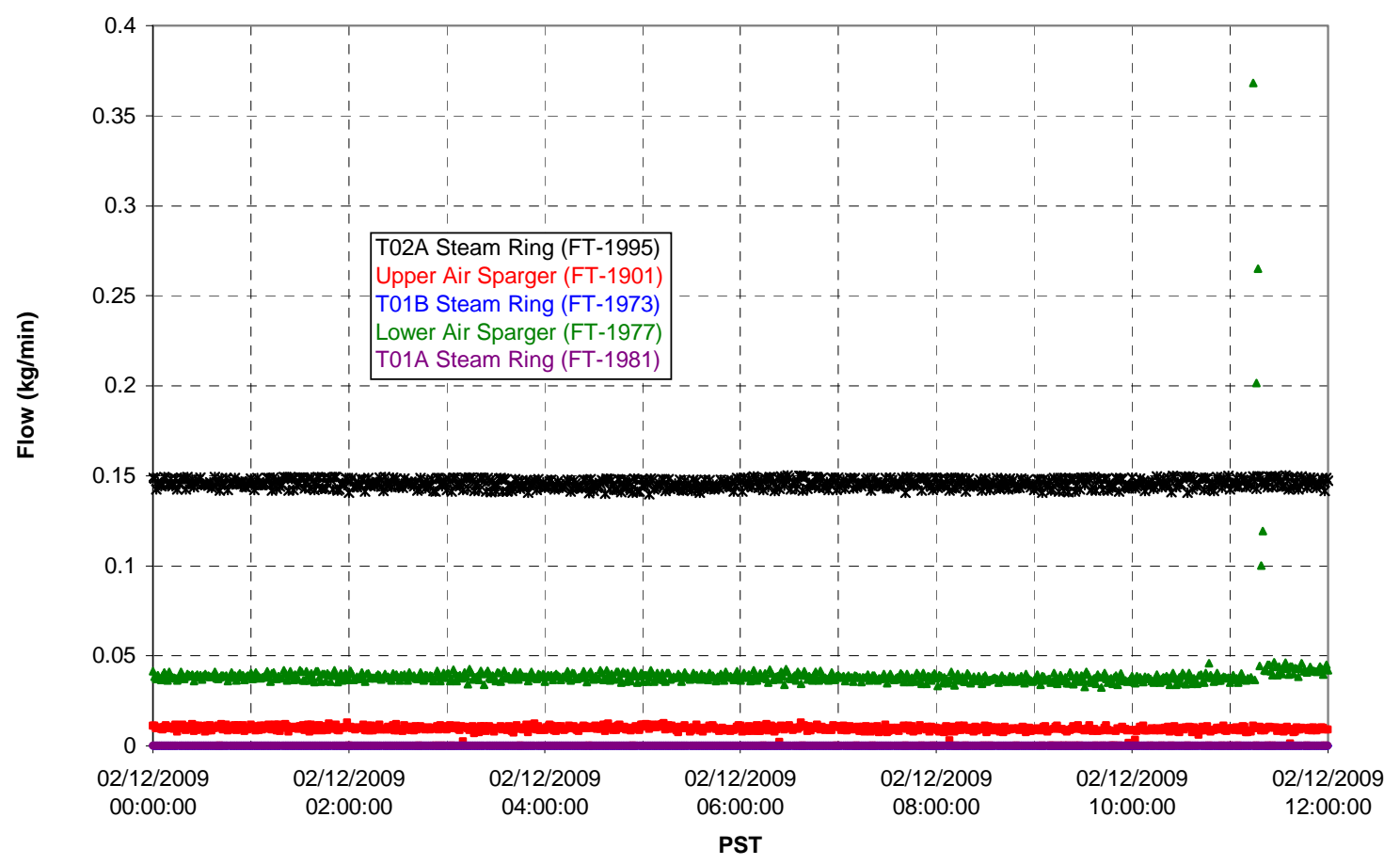


T02A Steam

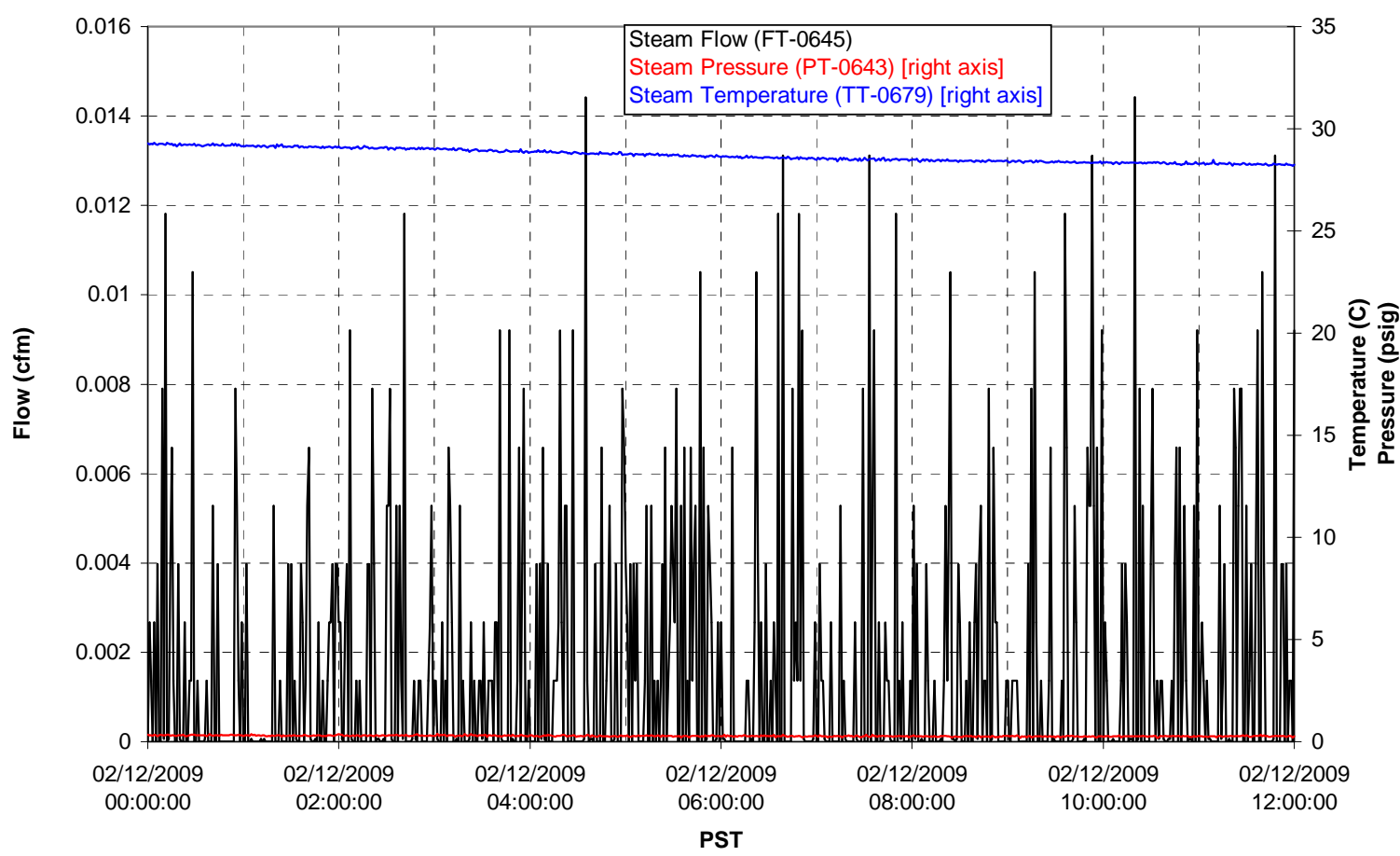

T01A Steam

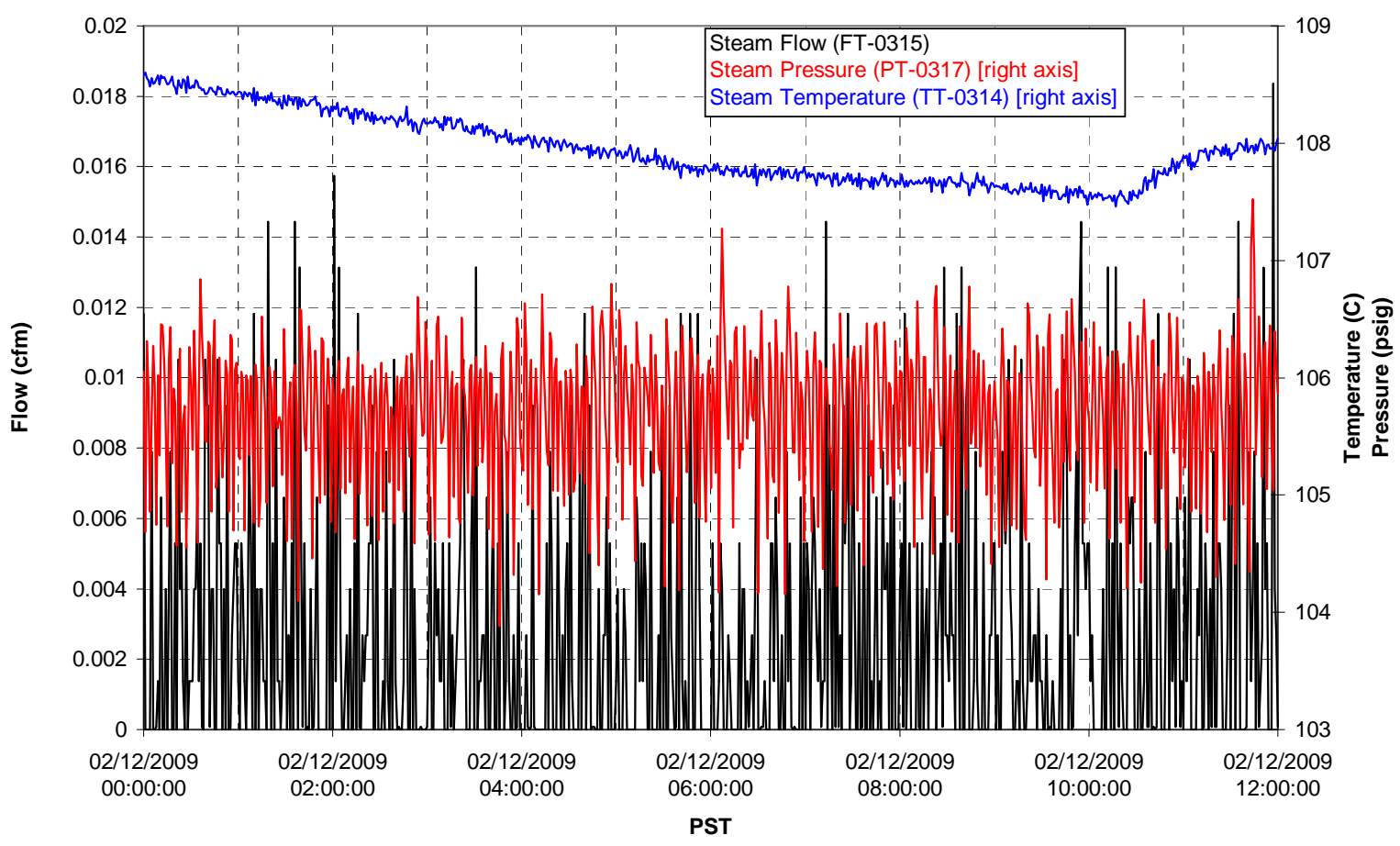


T01B Steam

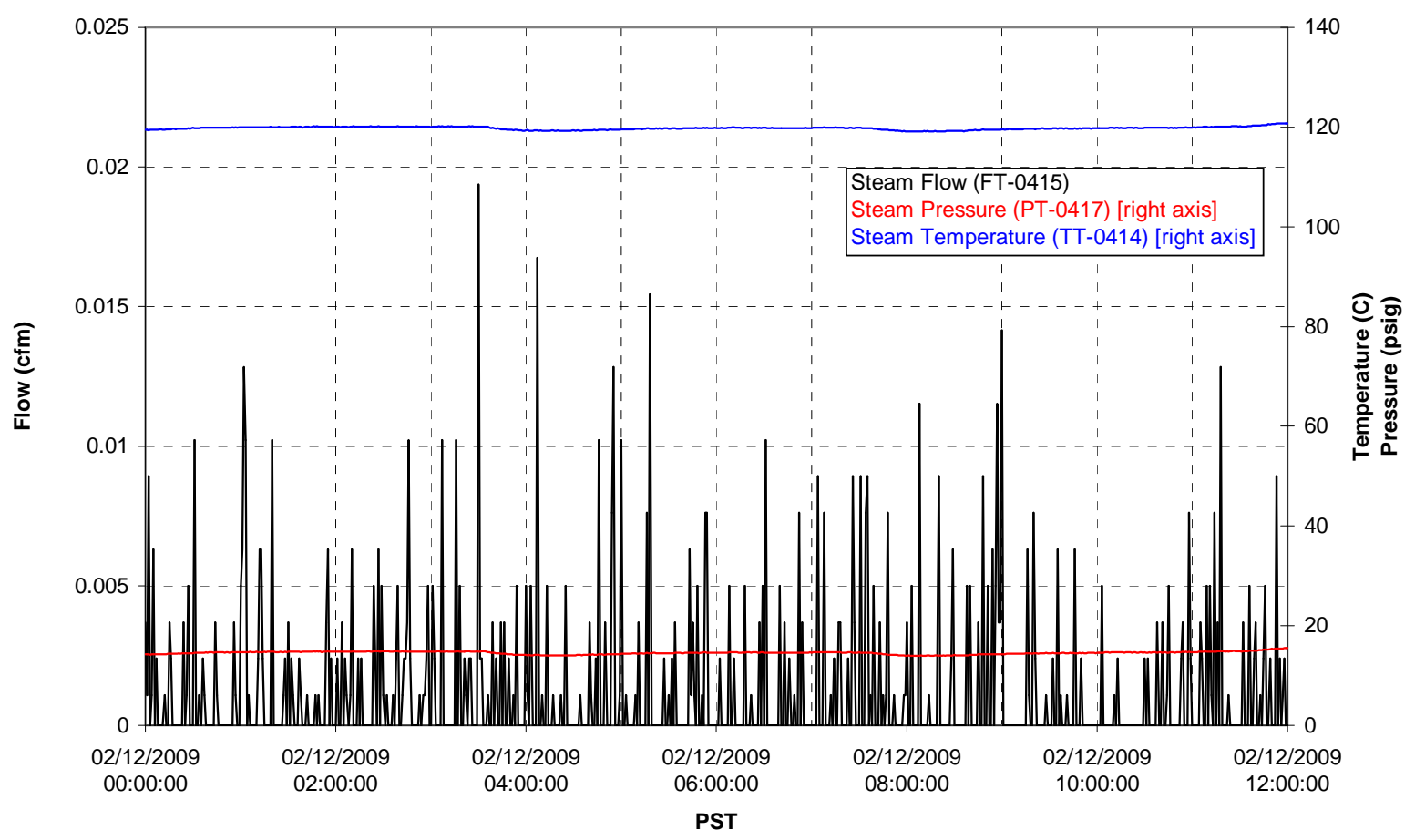




\section{Integrated Test A Data Plots 02/12/09 12:00 - 02/13/09 00:00}


T01A level

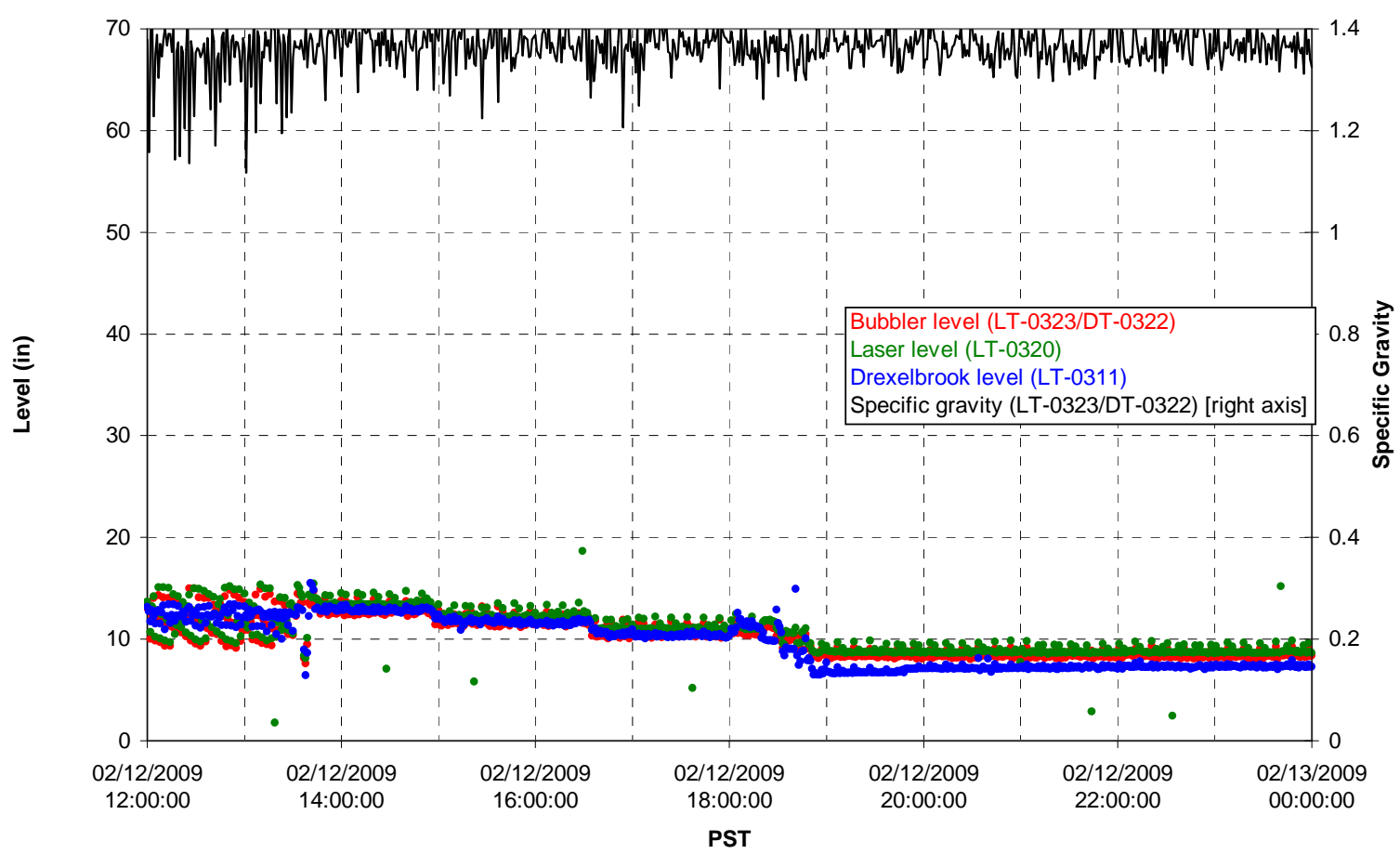

T01A temperatures

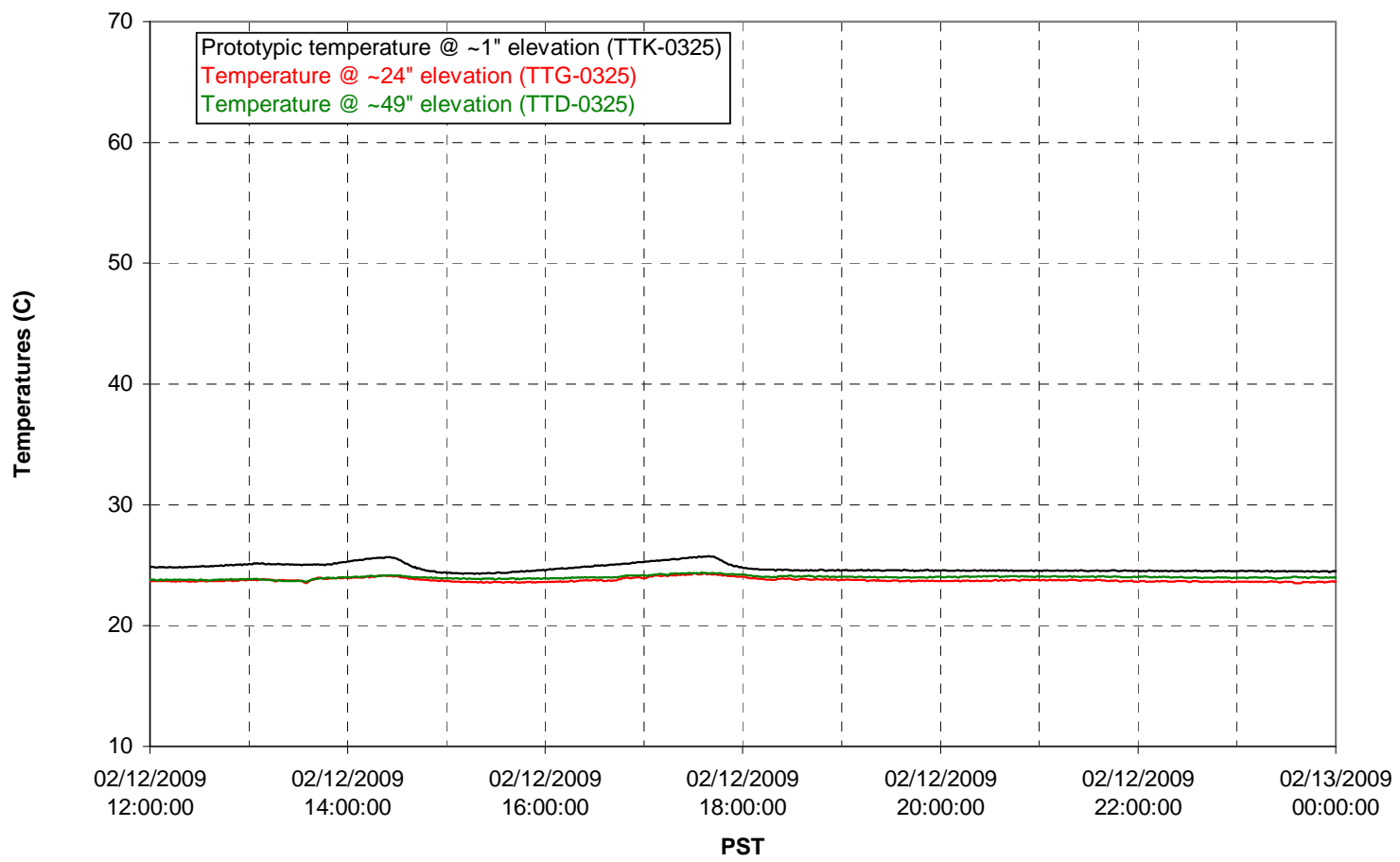


T01B level

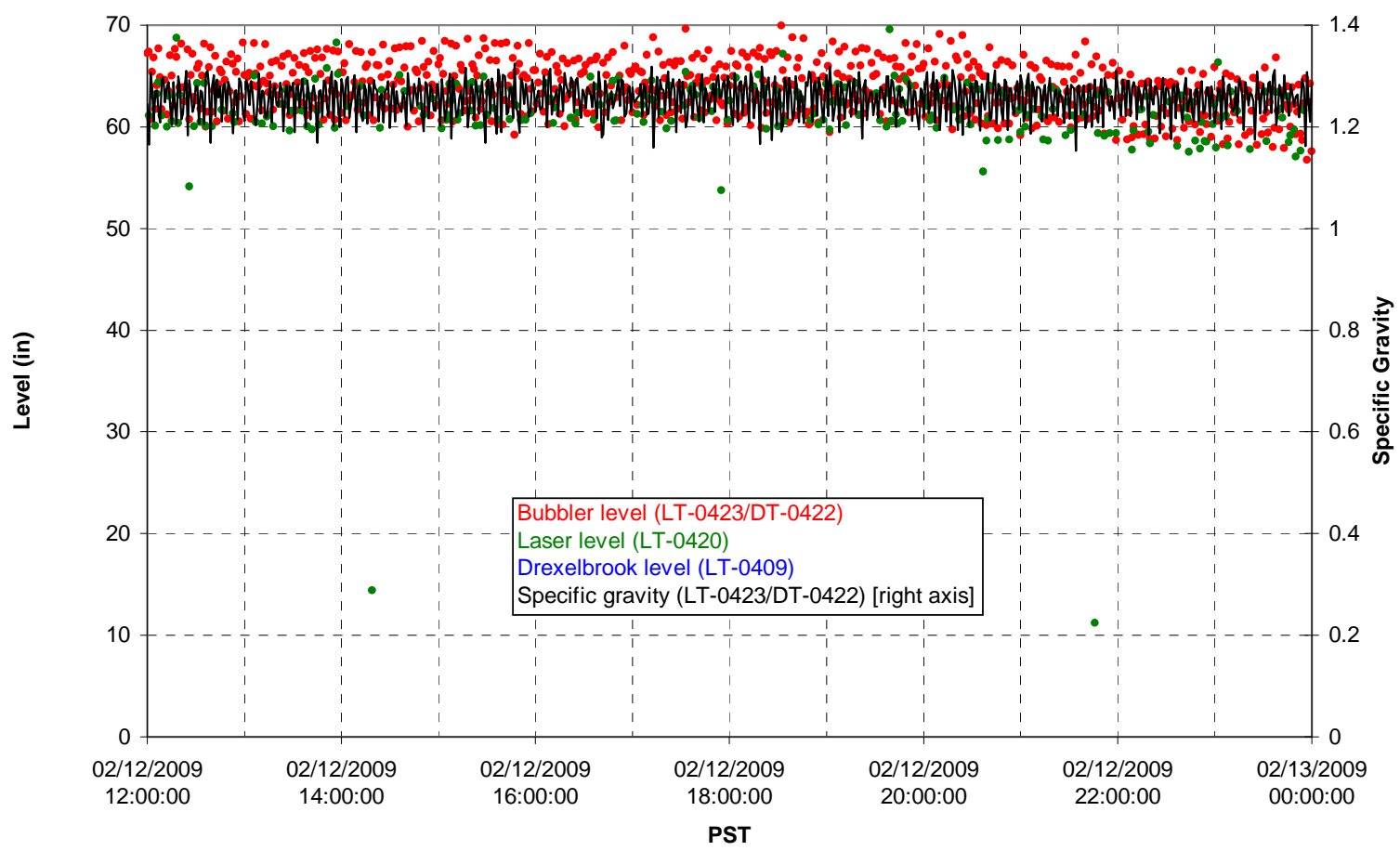

T01B temperatures

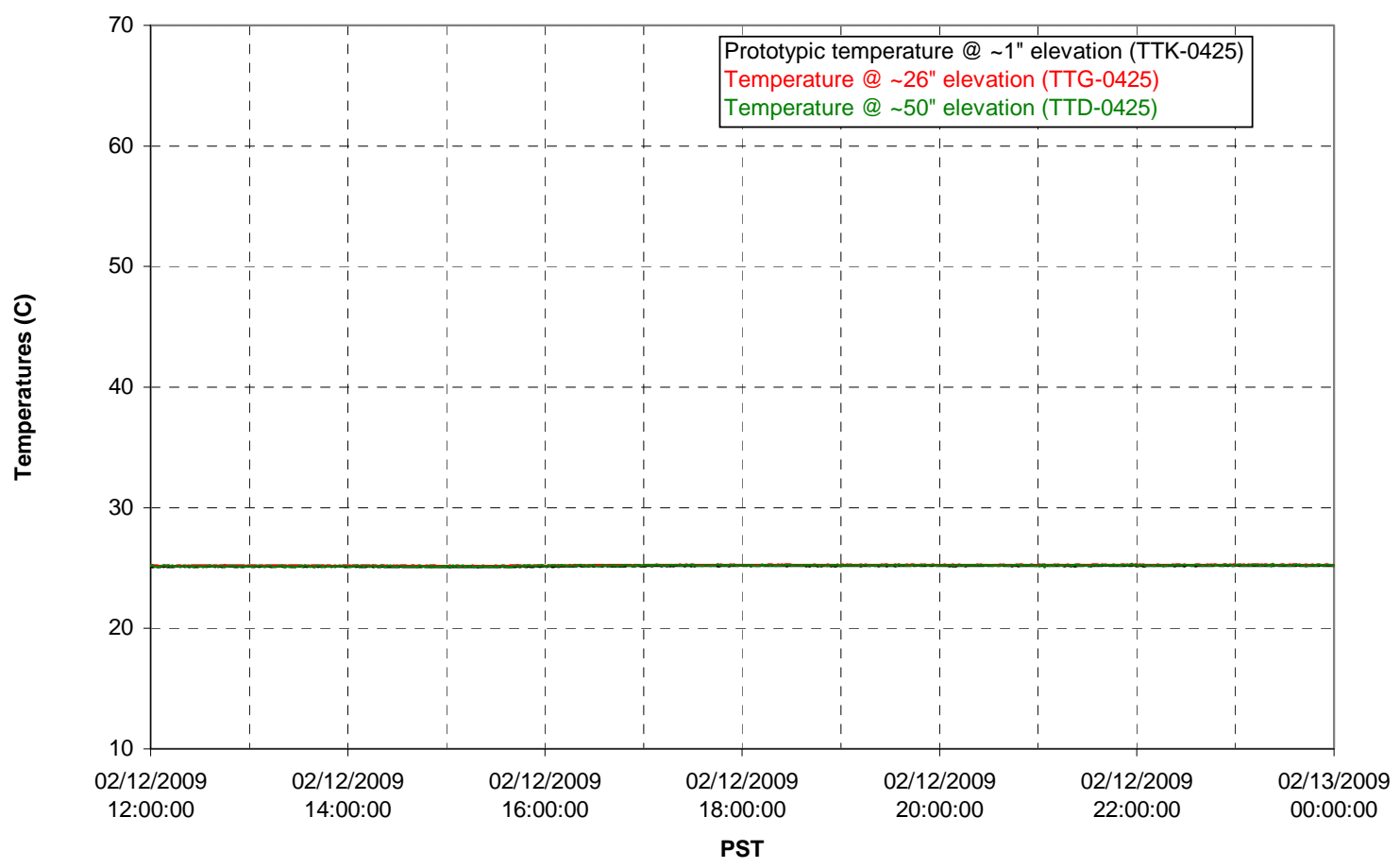


T02A level

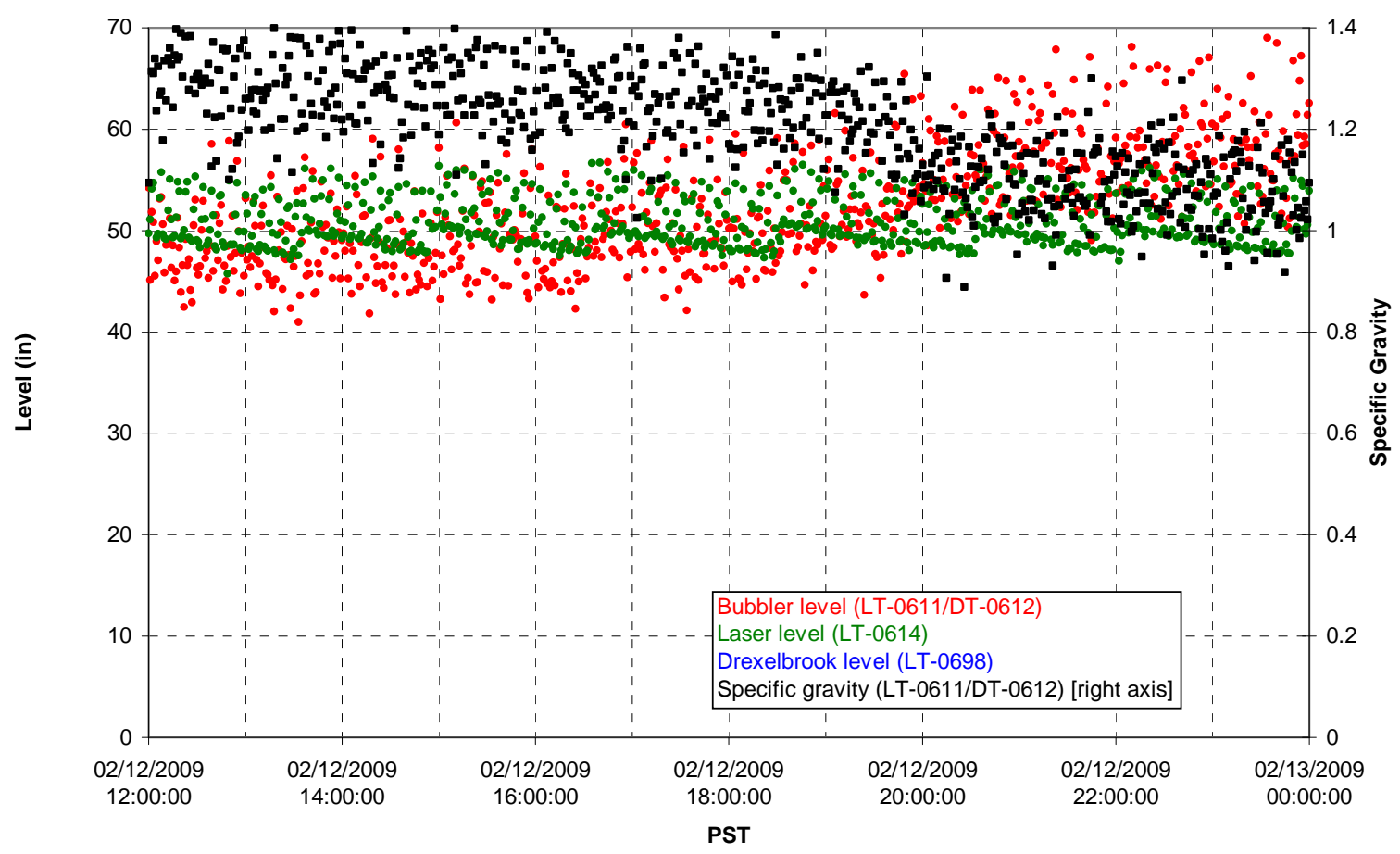

T02A temperatures

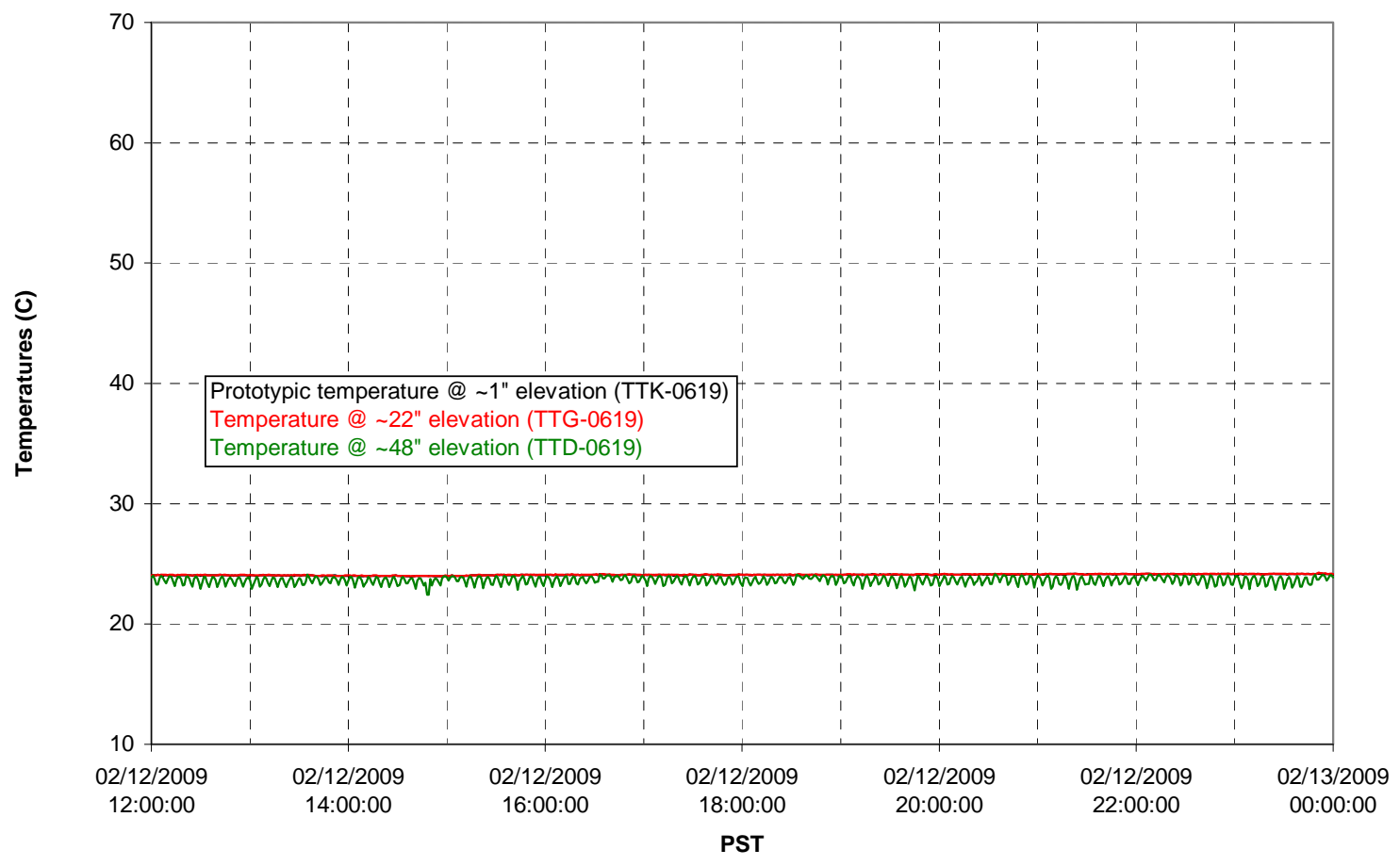


T02A and filter loop temperatures
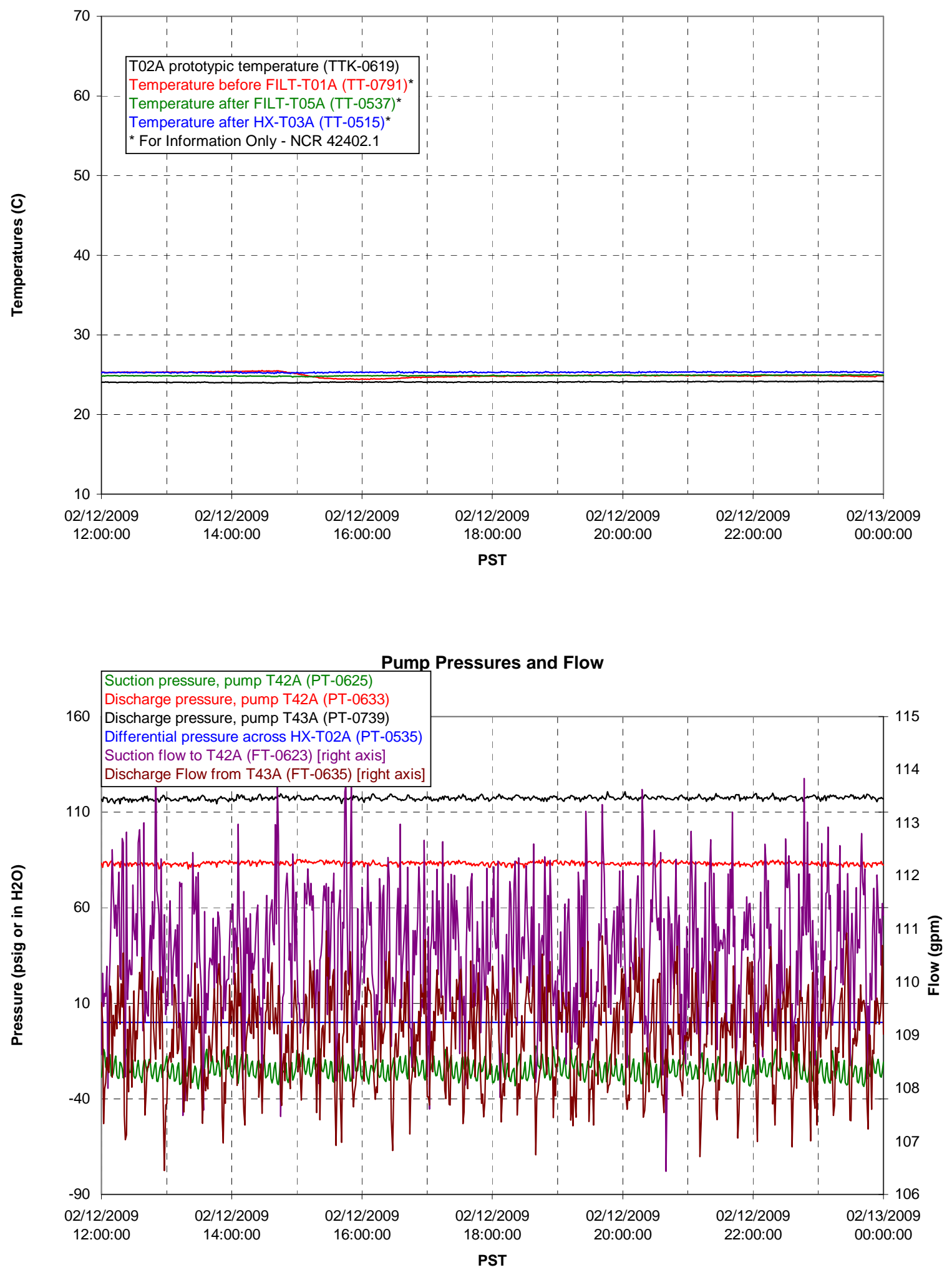


\section{Axial pressure drop}

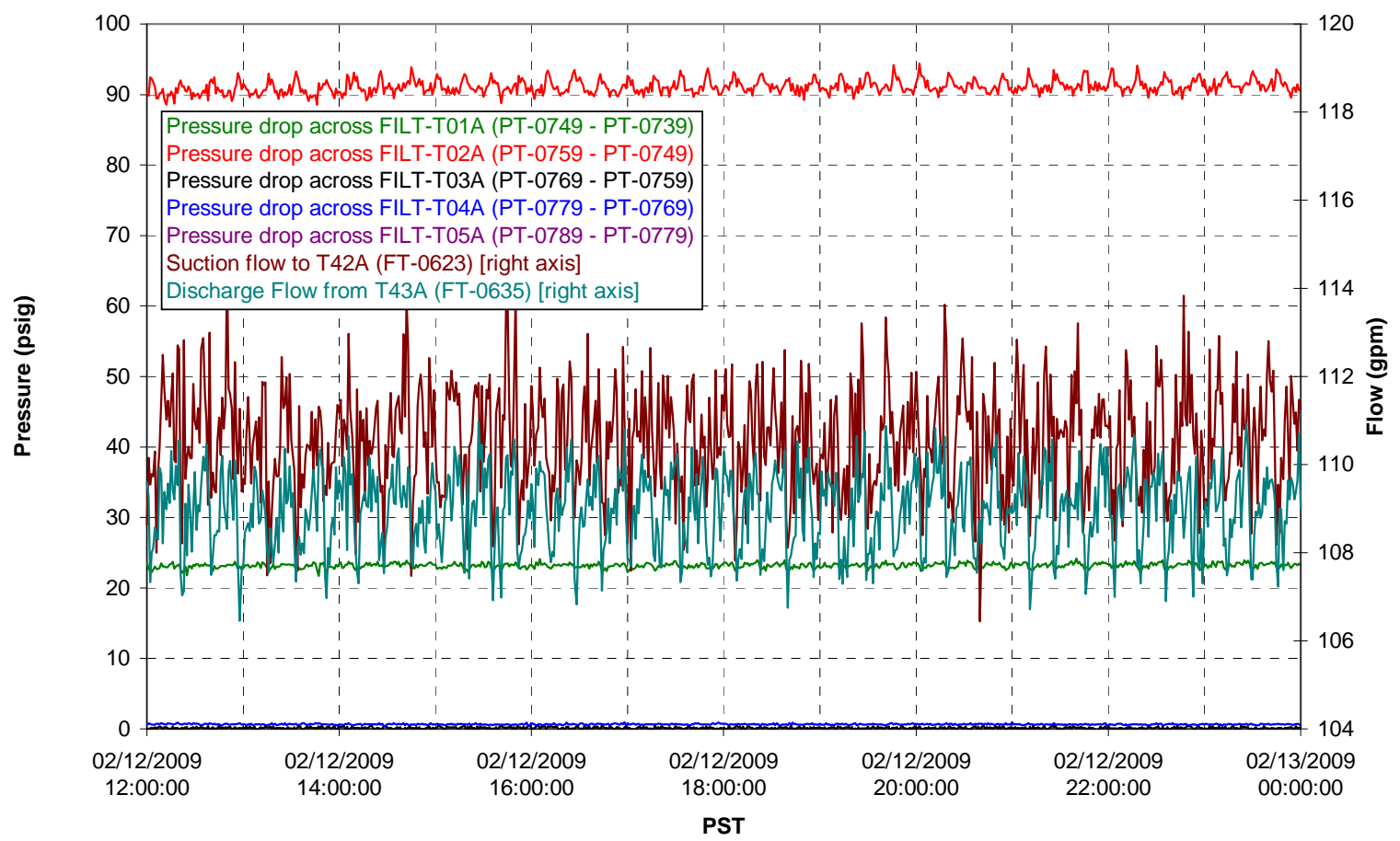

Permeate flow rates

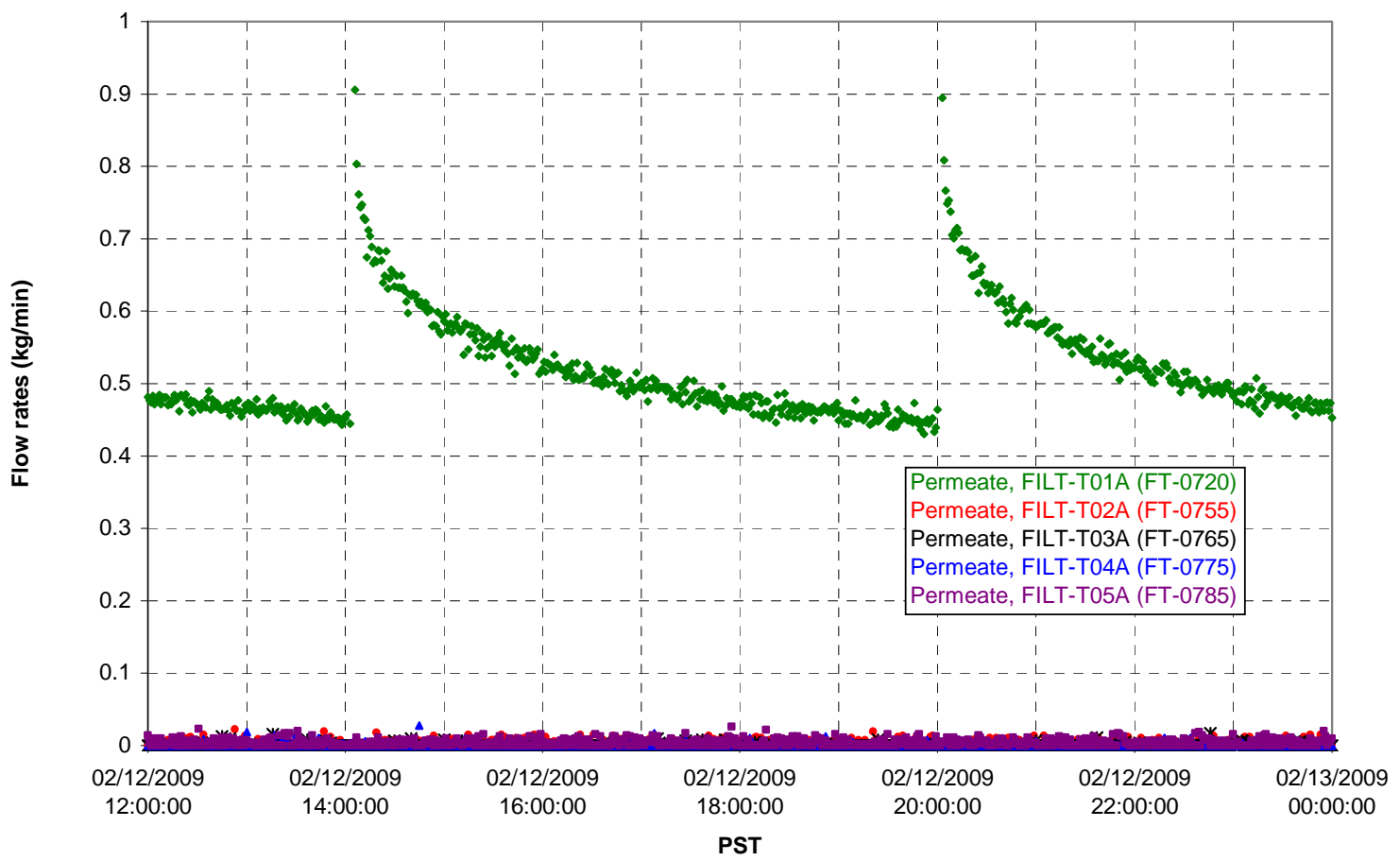


T02A Inner Temperature Tree

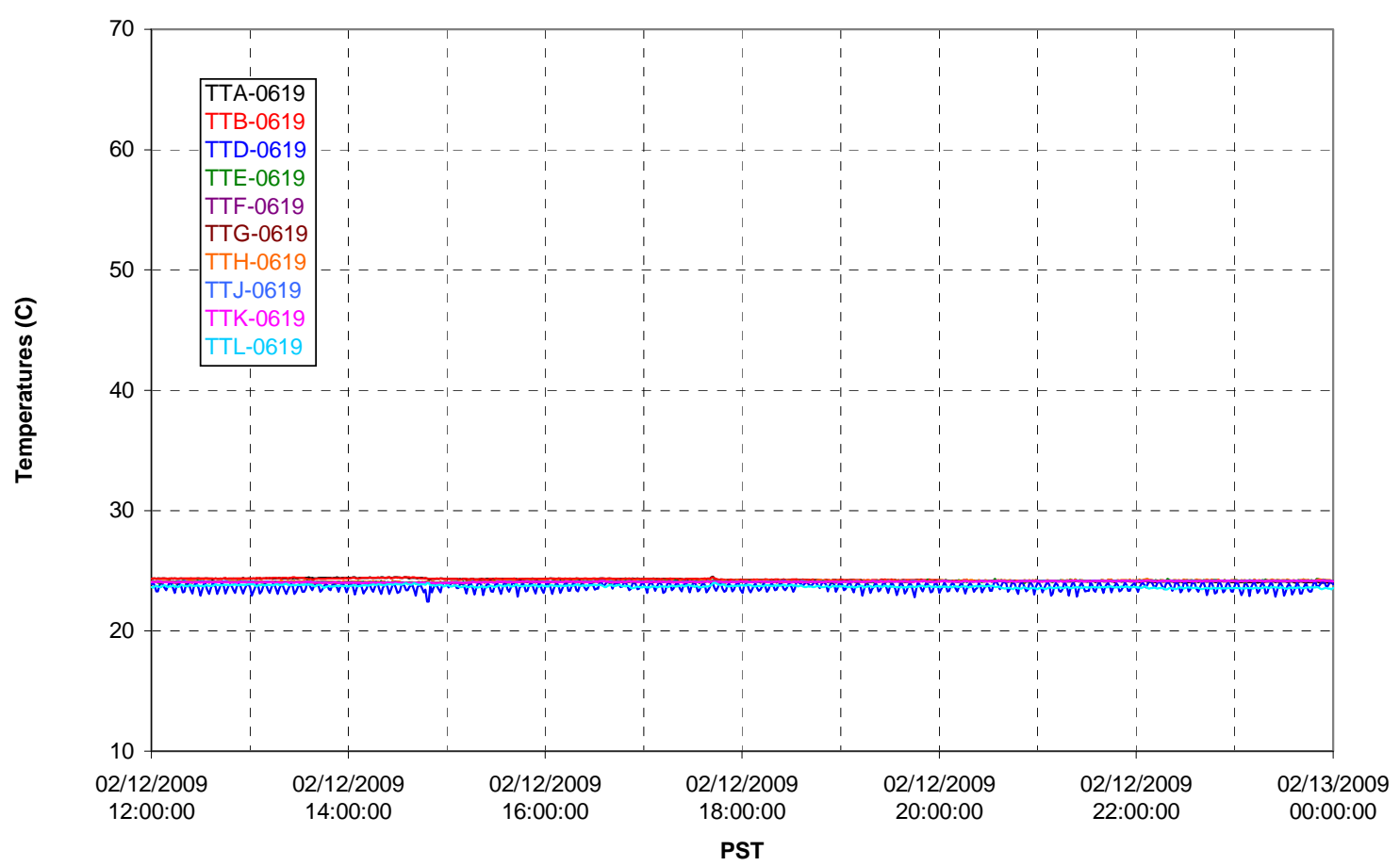

T02A Outer Temperature Tree

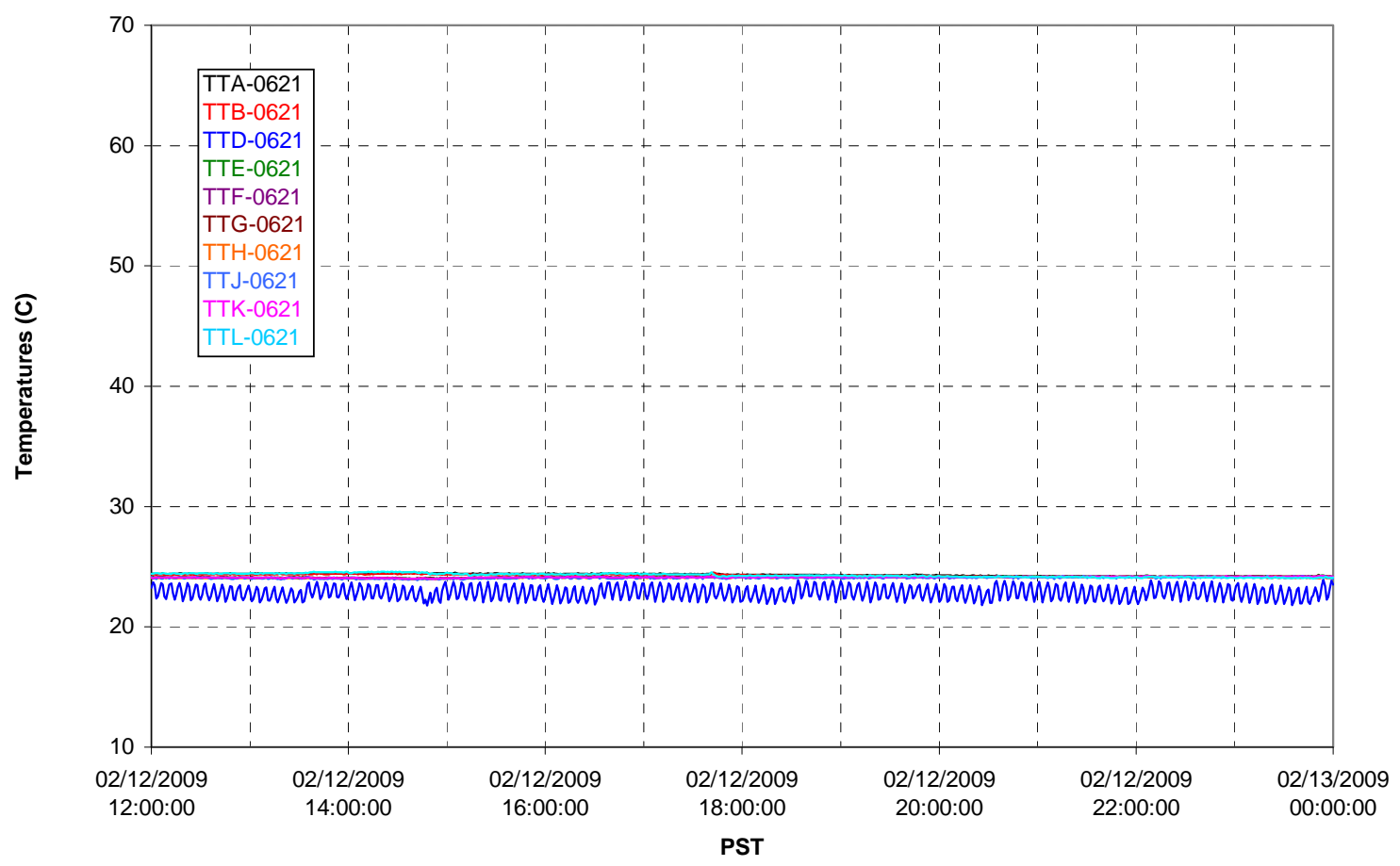


T02A temperatures

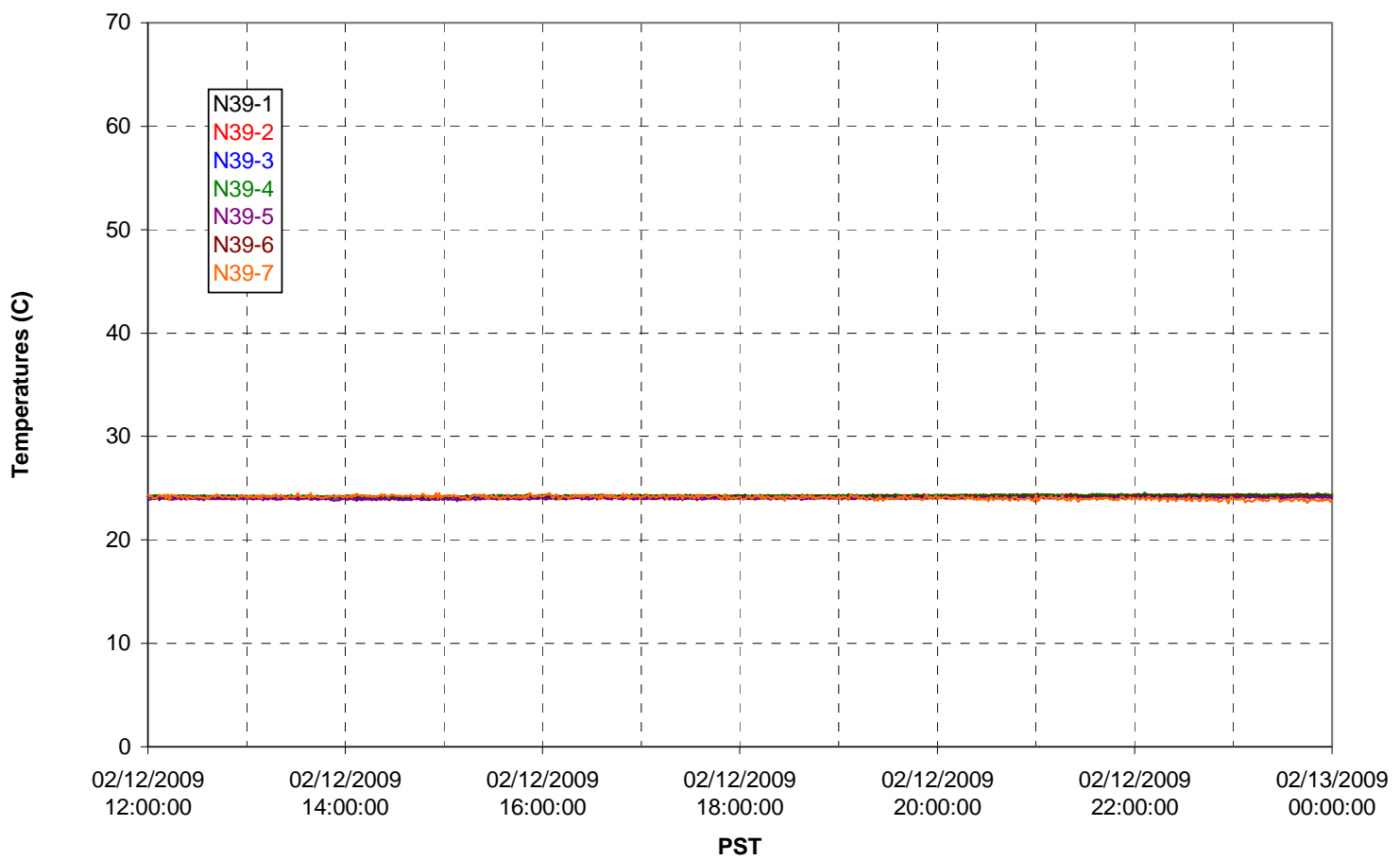

T02A temperatures

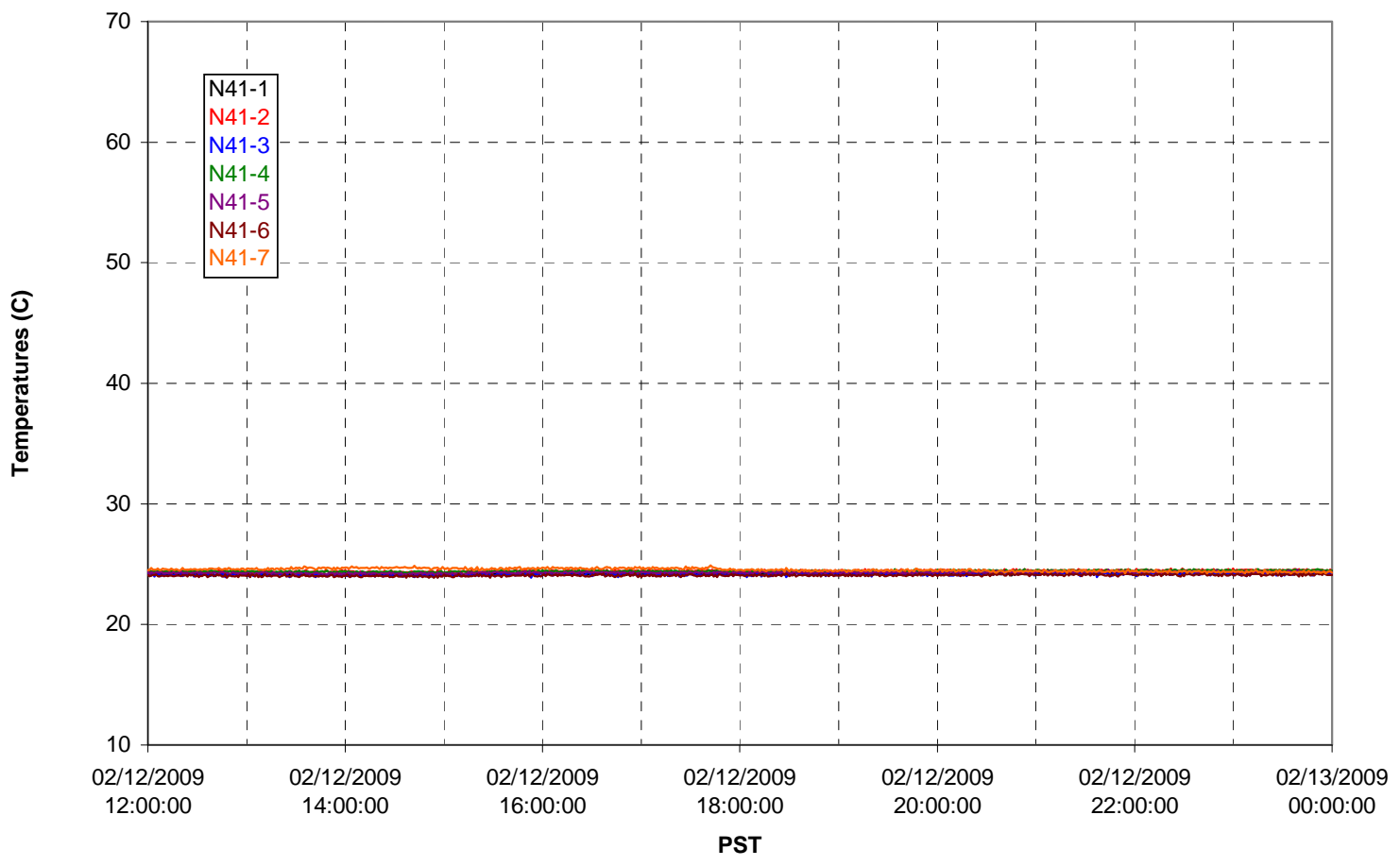


T02A temperatures

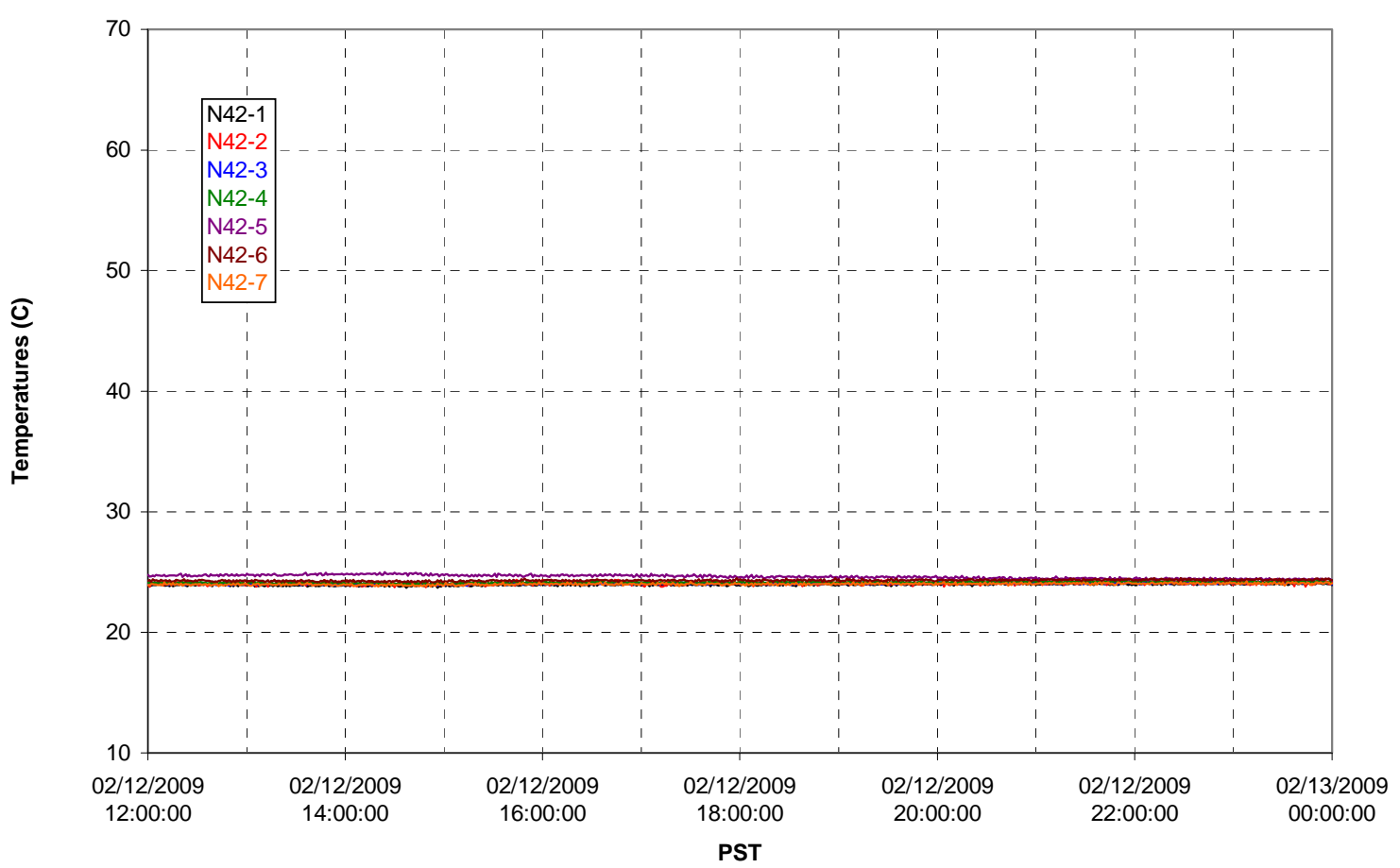

T02A temperatures

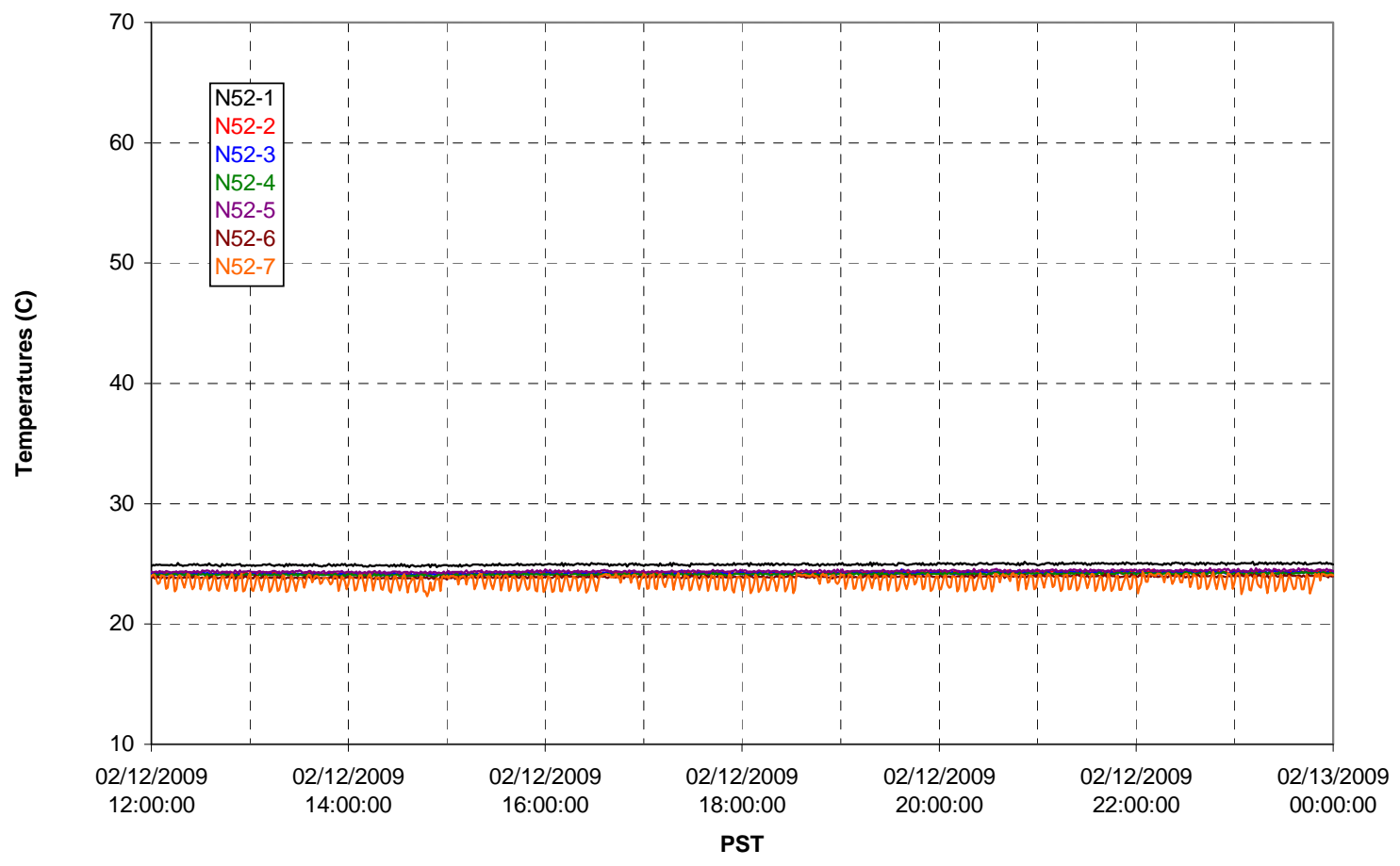


T02A Heating and Cooling
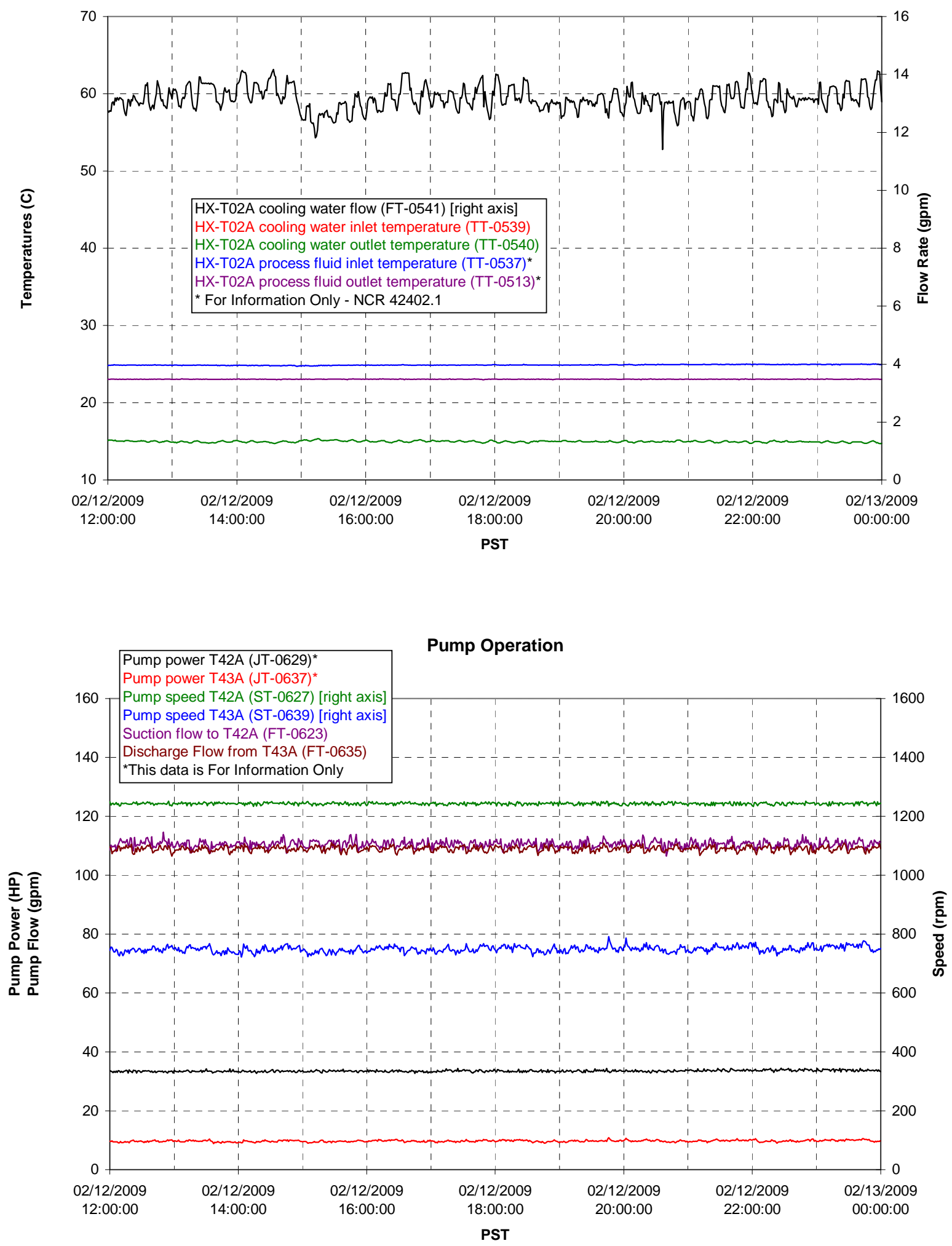
Pulsepot UFP-PP-T01A

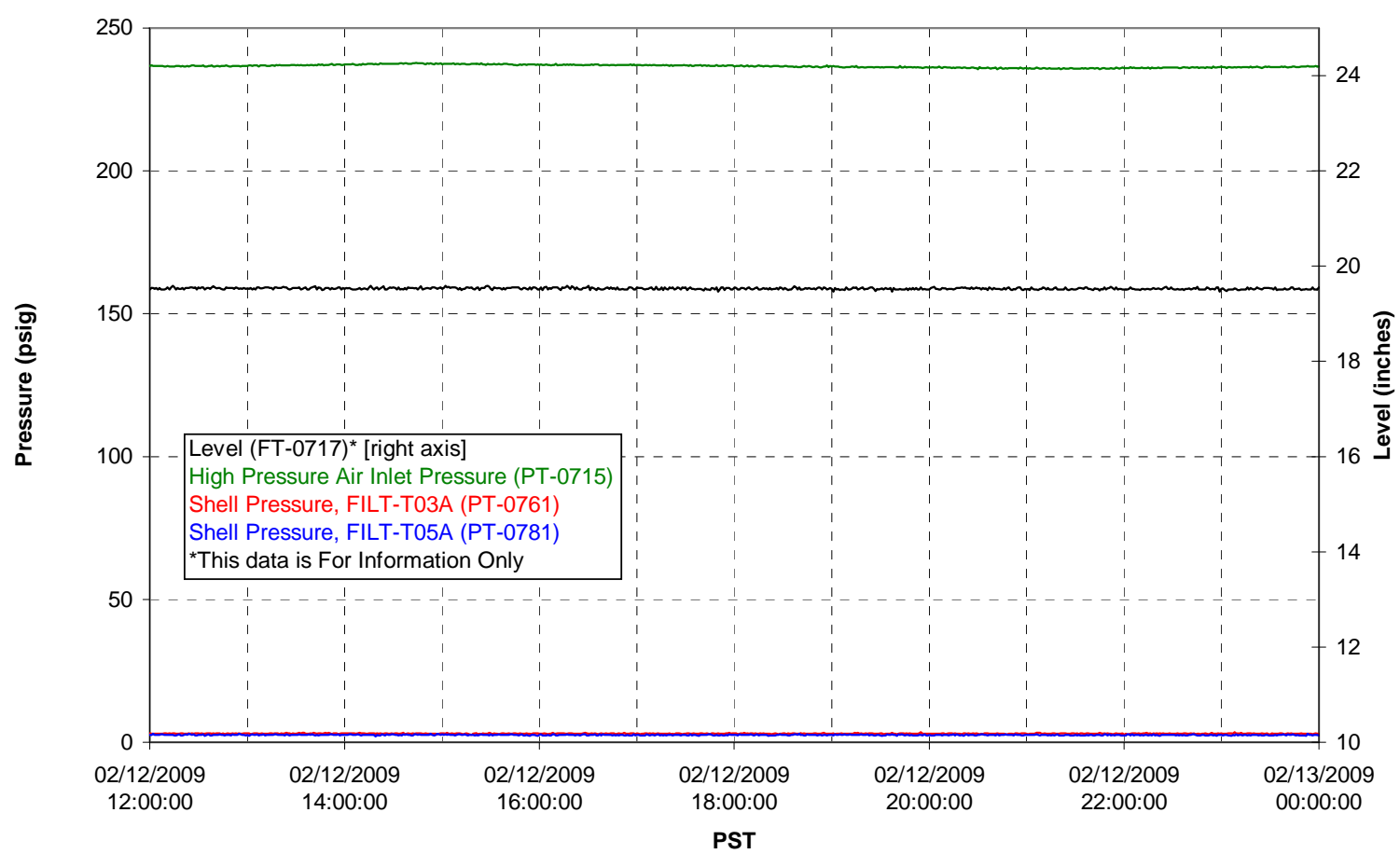

Pulsepot UFP-PP-T02A

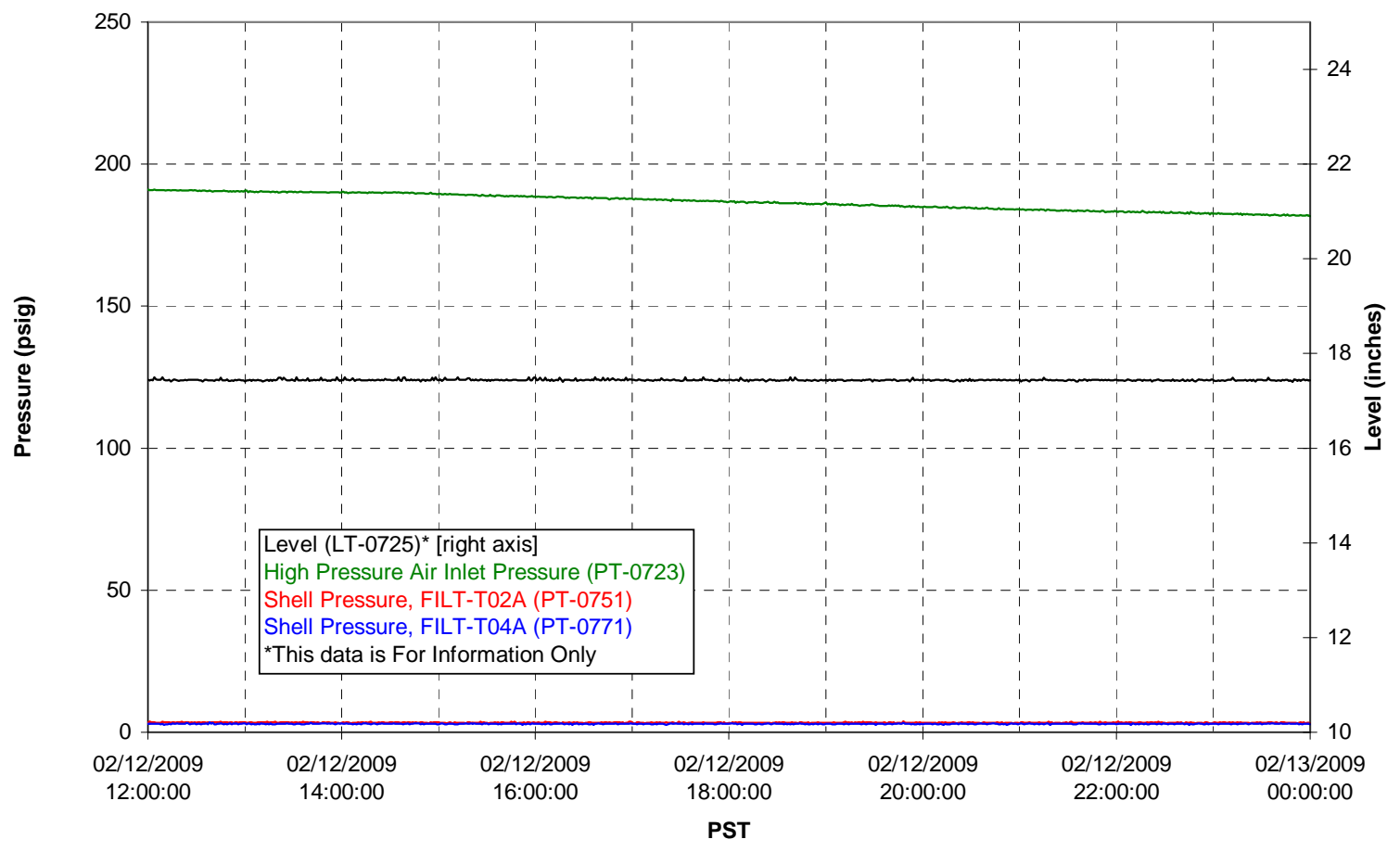


Pulsepot UFP-PP-T03A

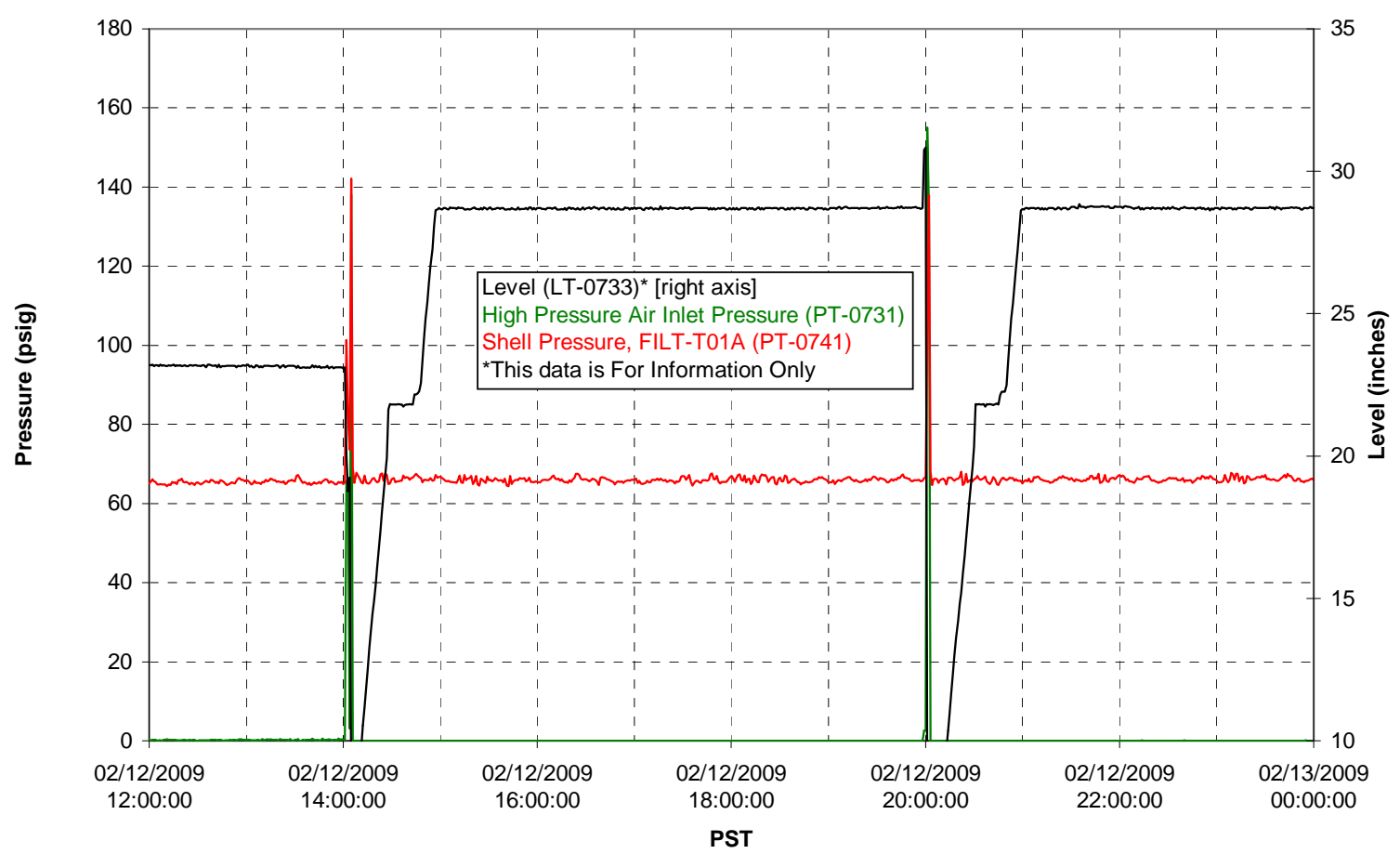

Pulsepot Levels

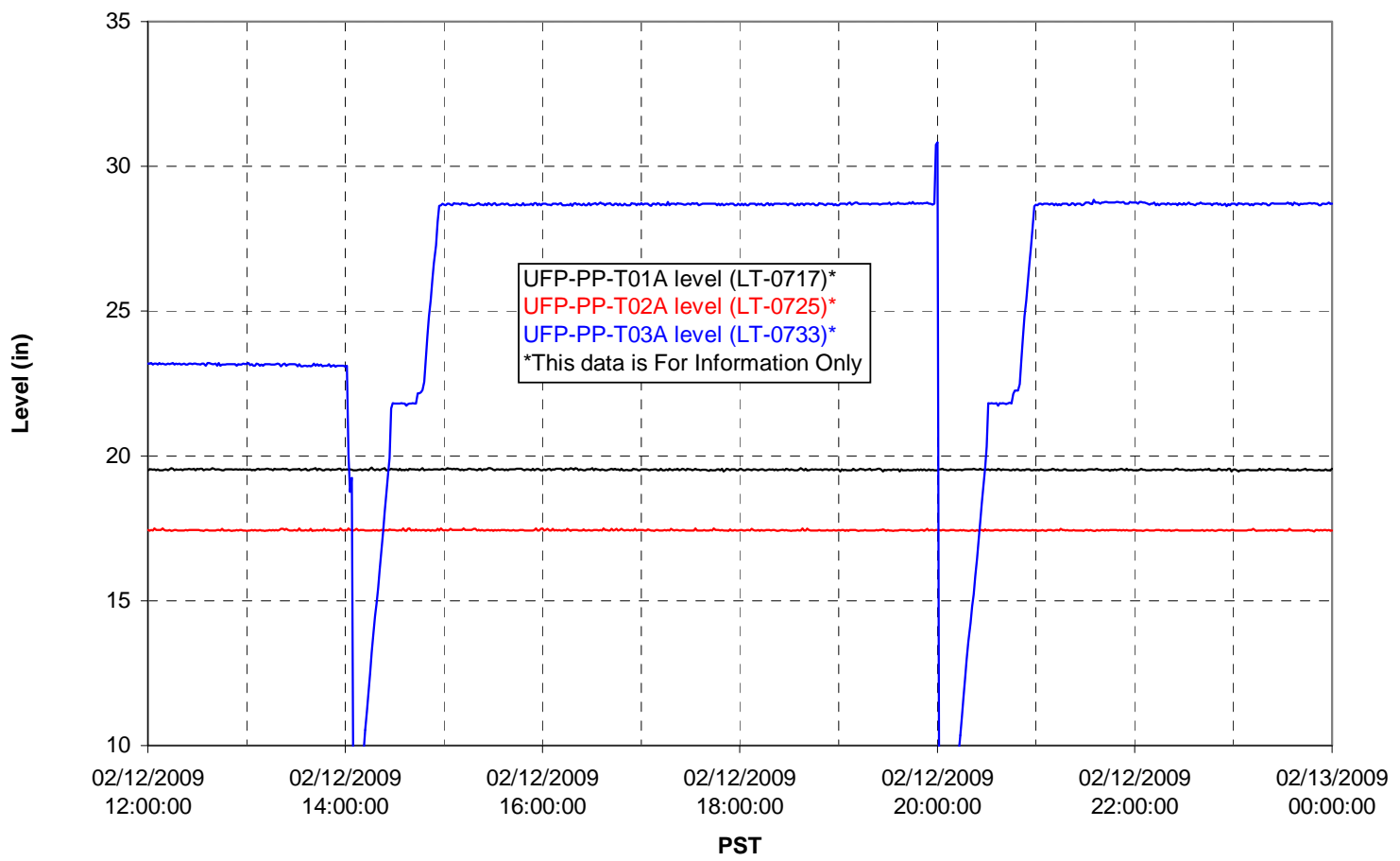


Filter UFP-FILT-T01A

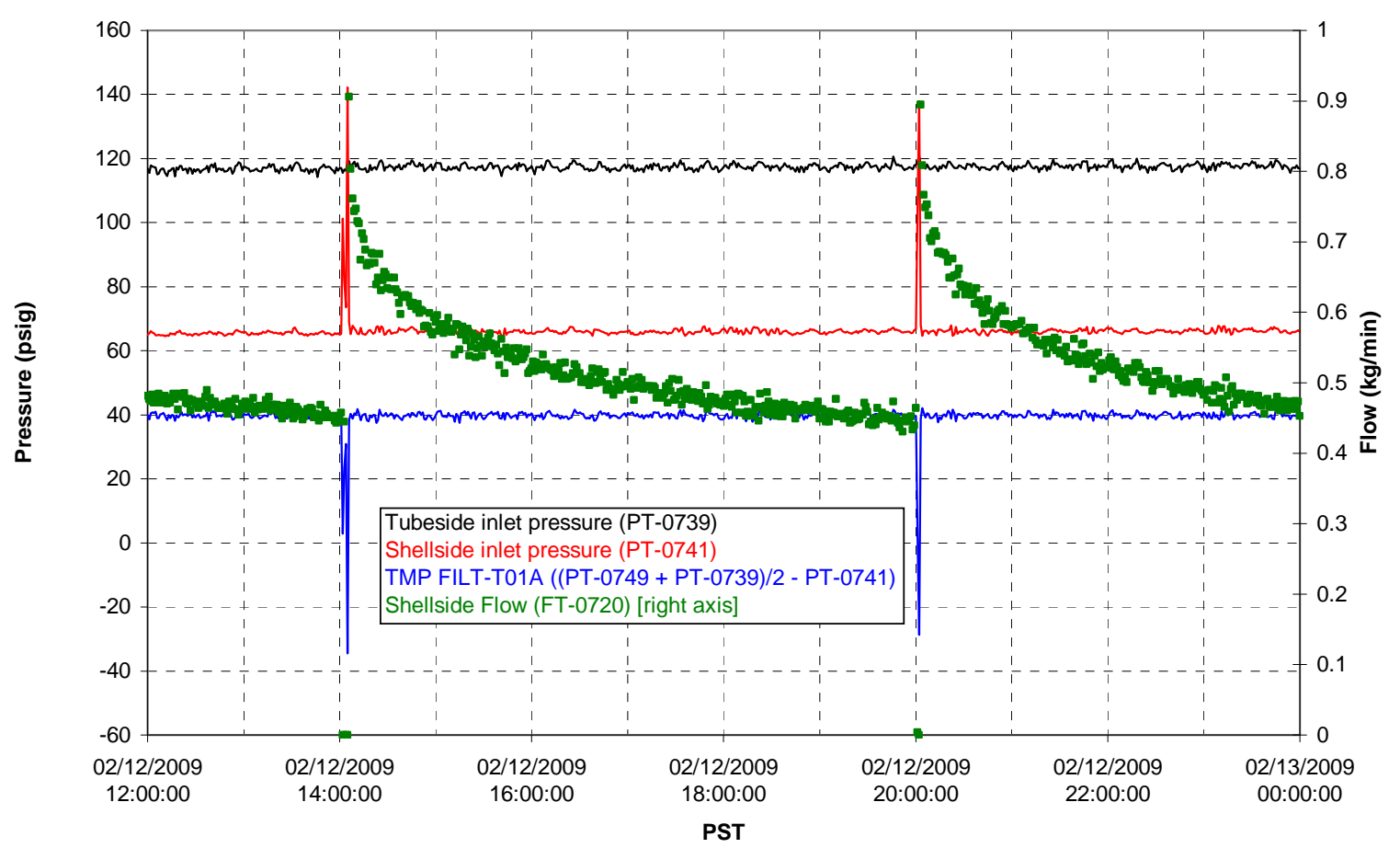

Filter UFP-FILT-T02A

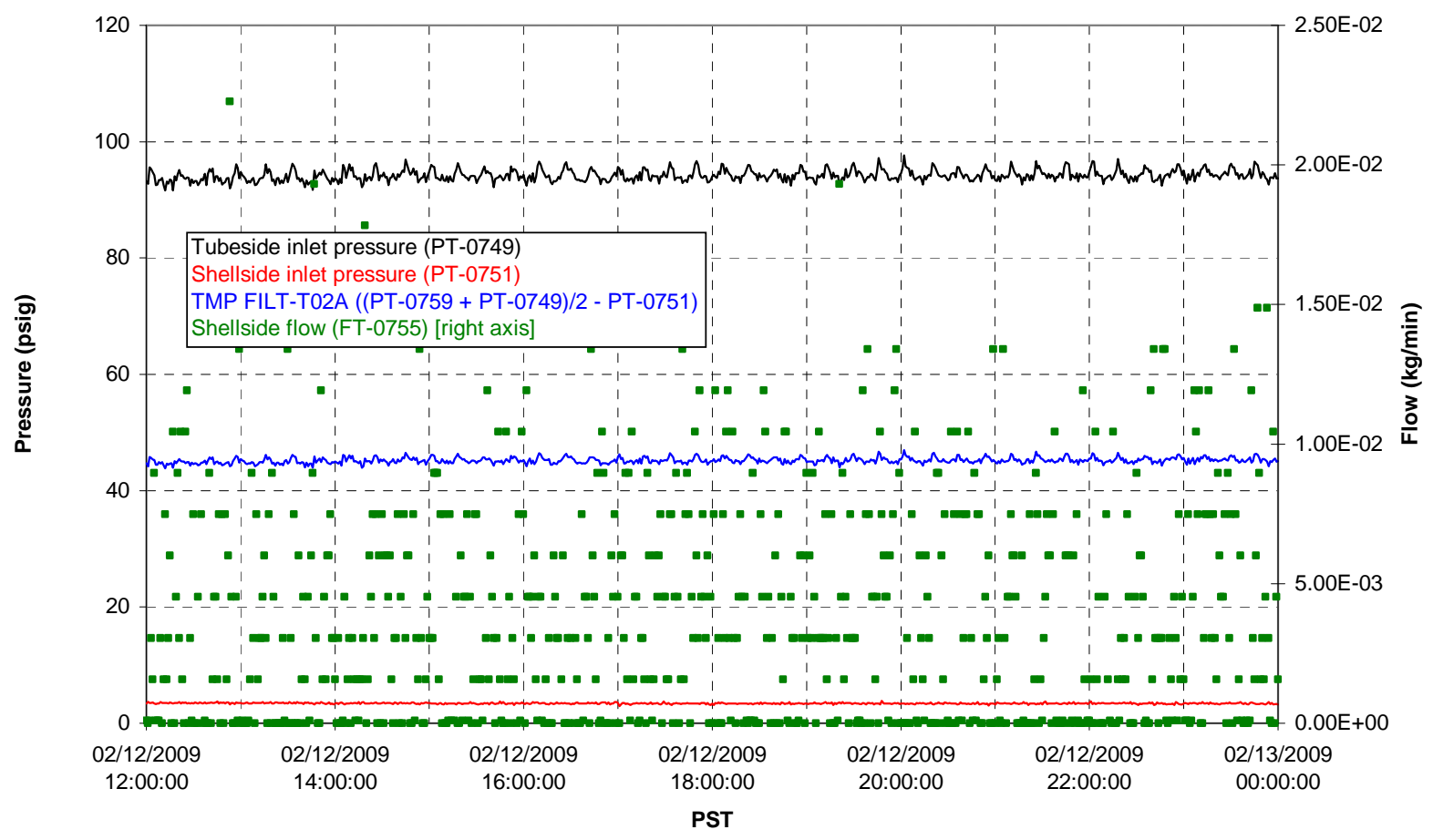


Filter UFP-FILT-T03A

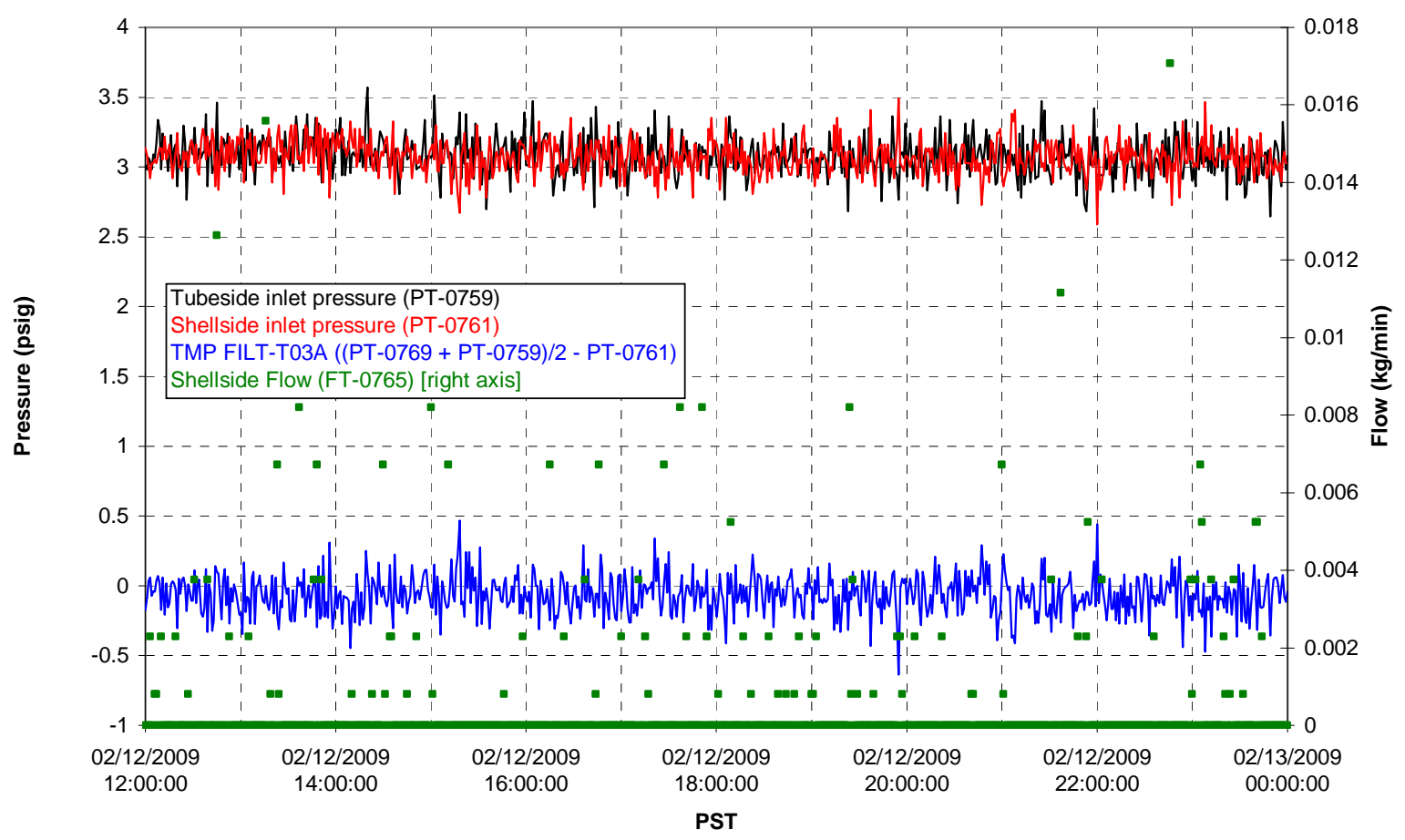

Filter UFP-FILT-T04A

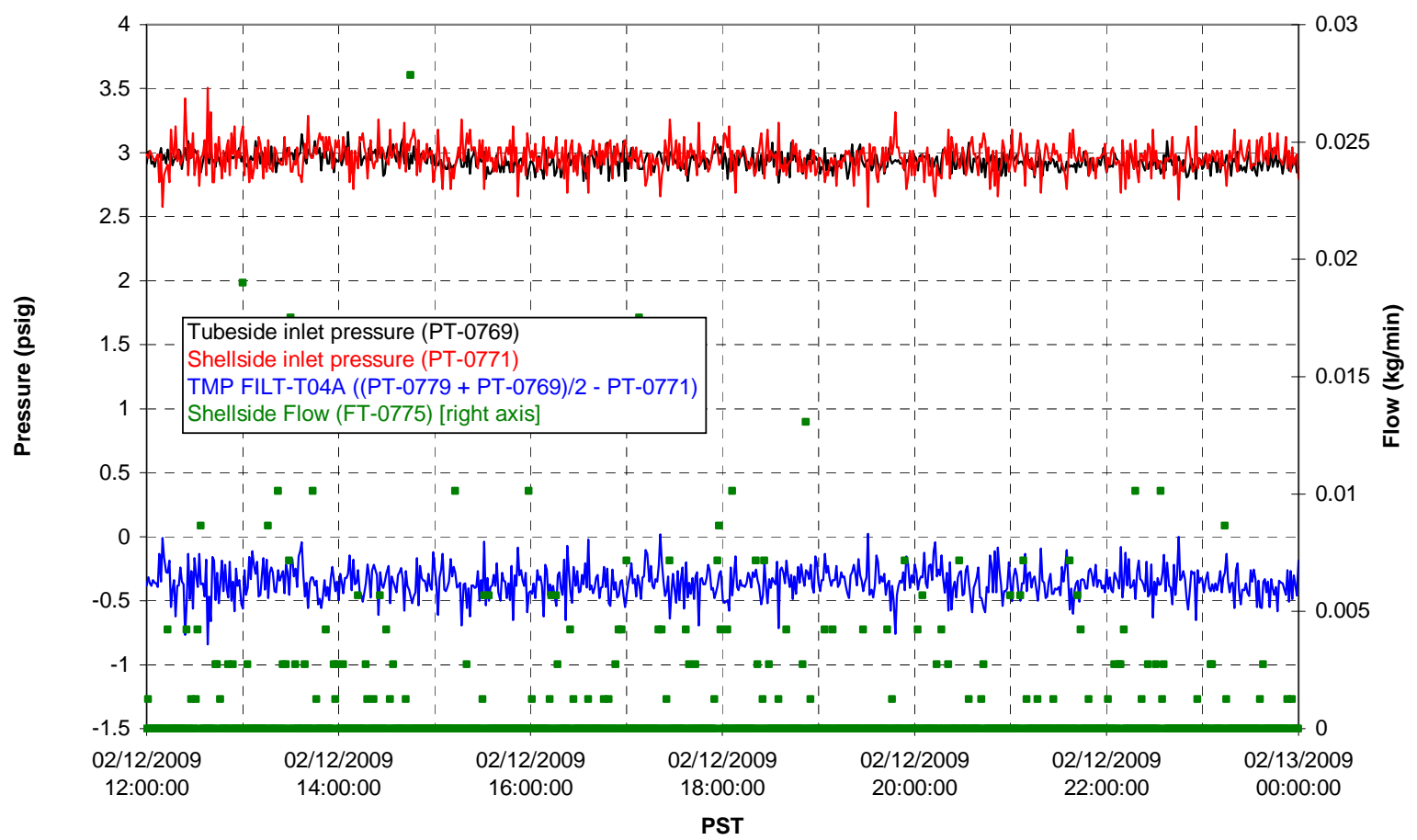


Filter UFP-FILT-T05A

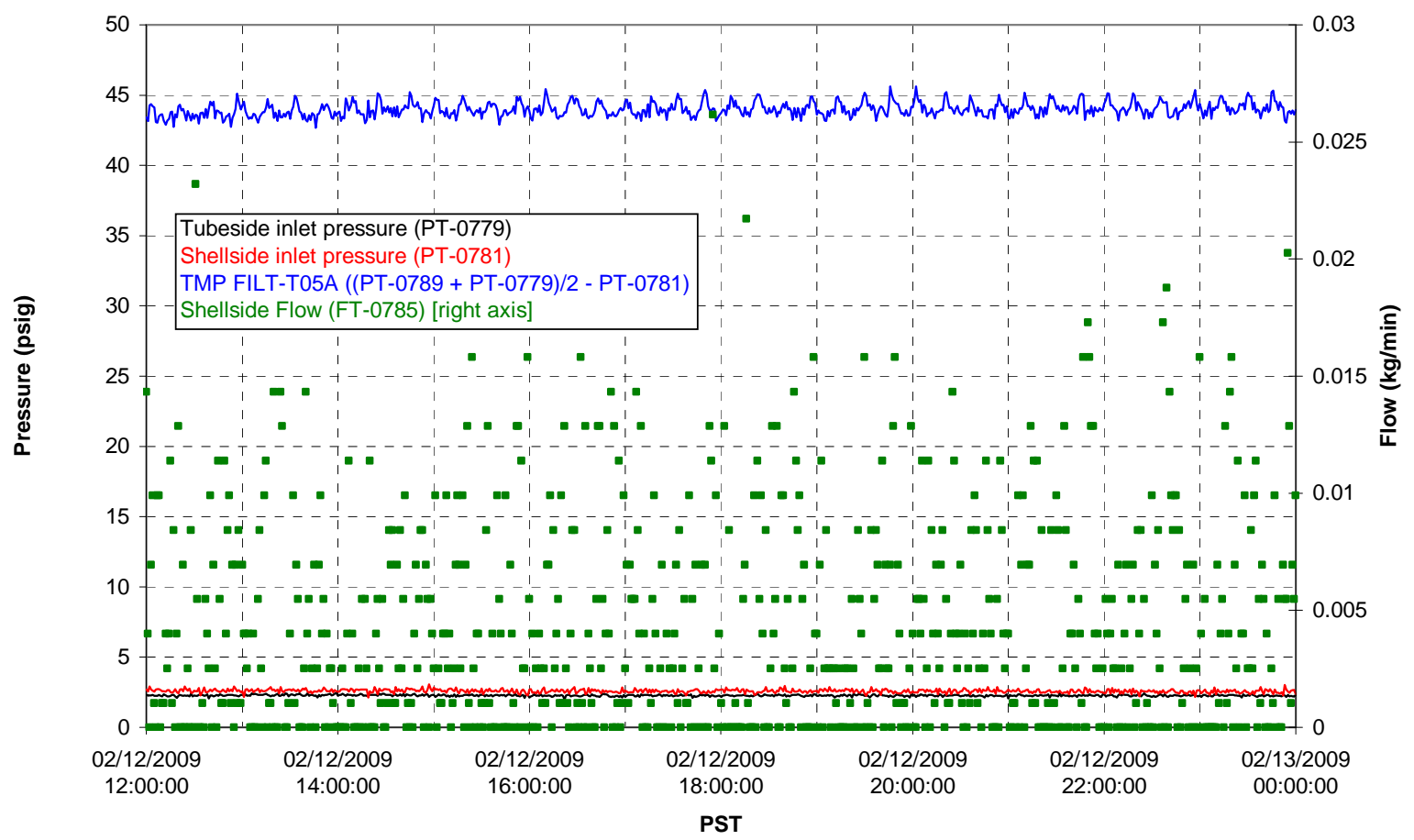

Chemical Flow

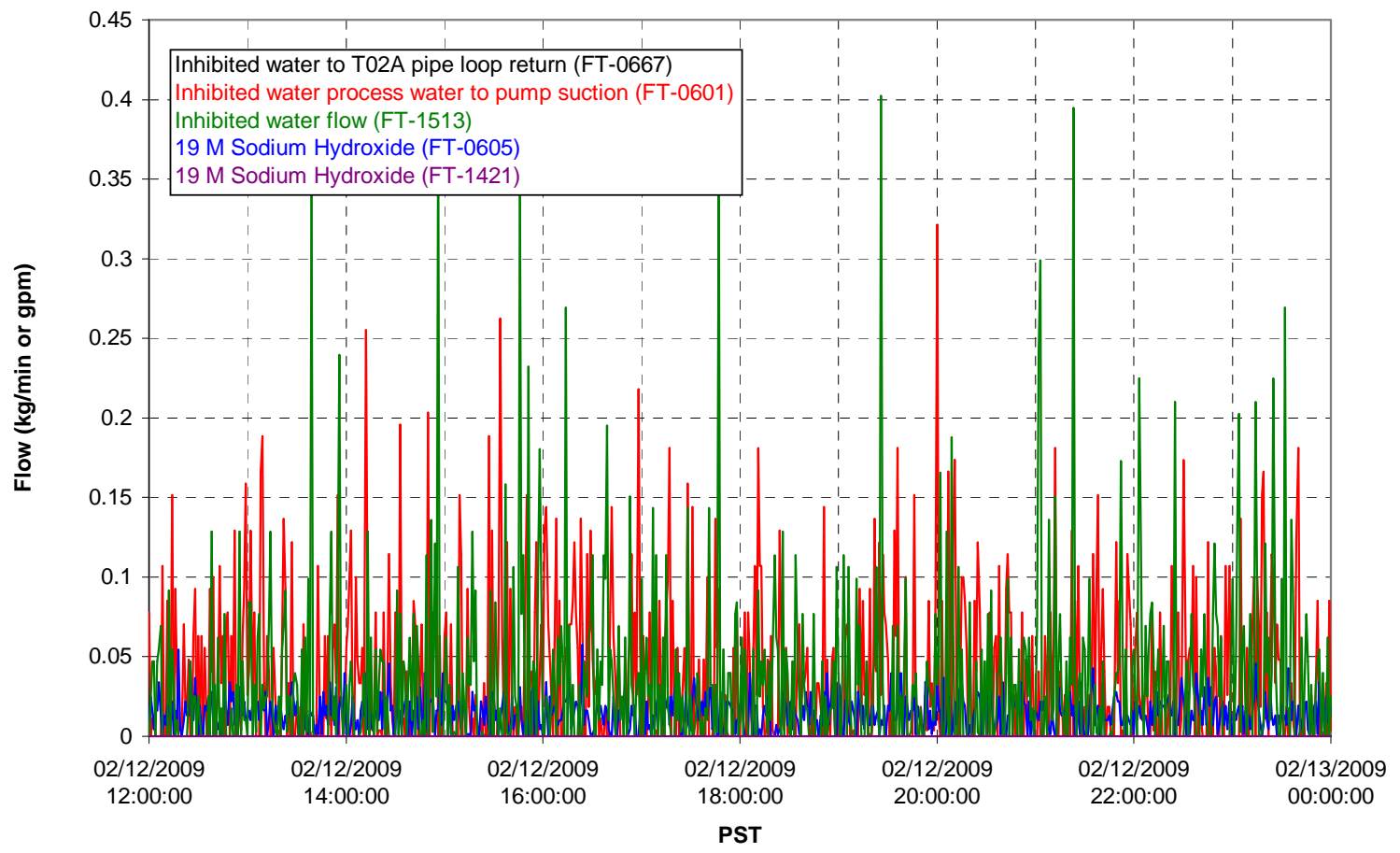


Chemical Flow

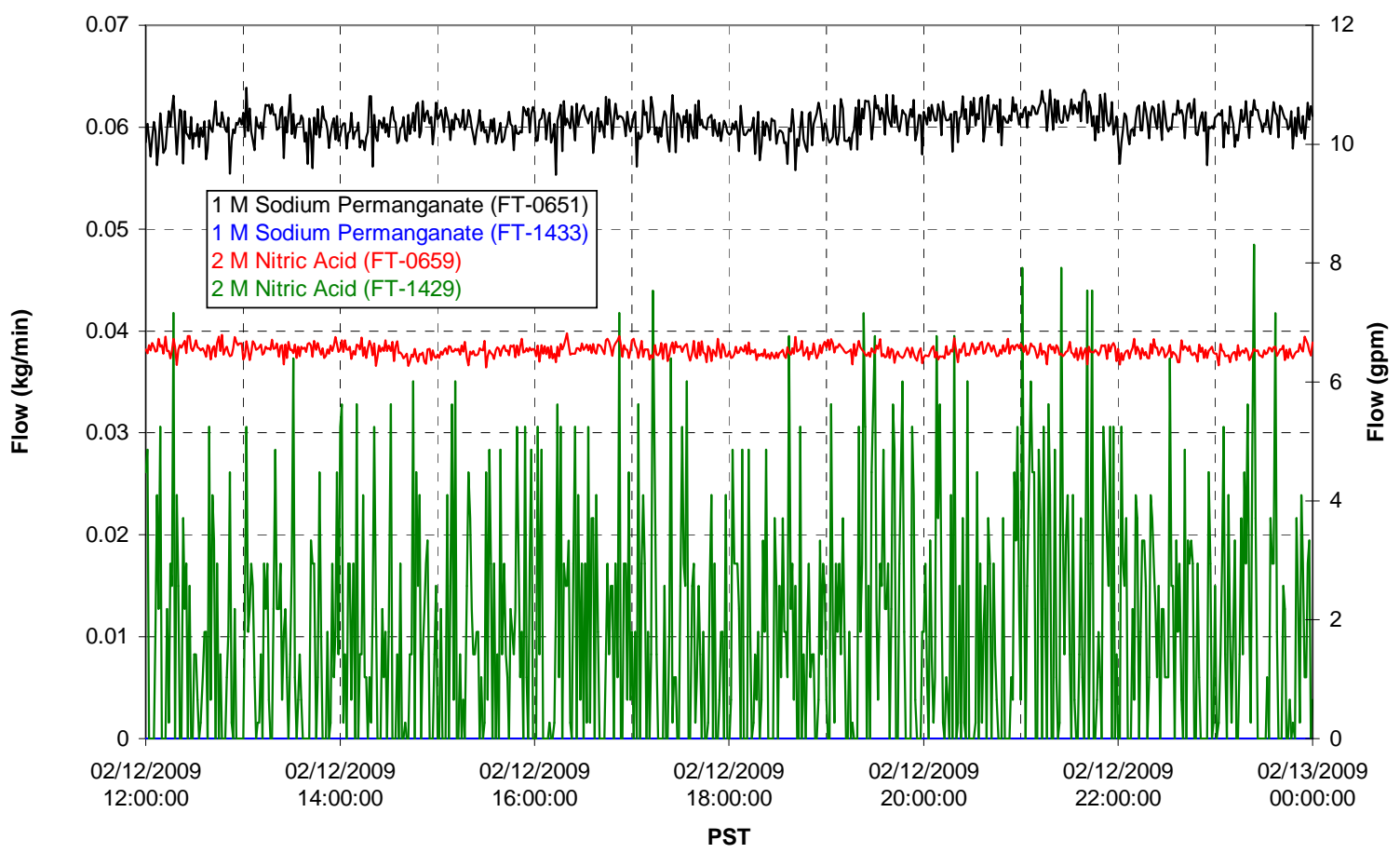

Air Flows

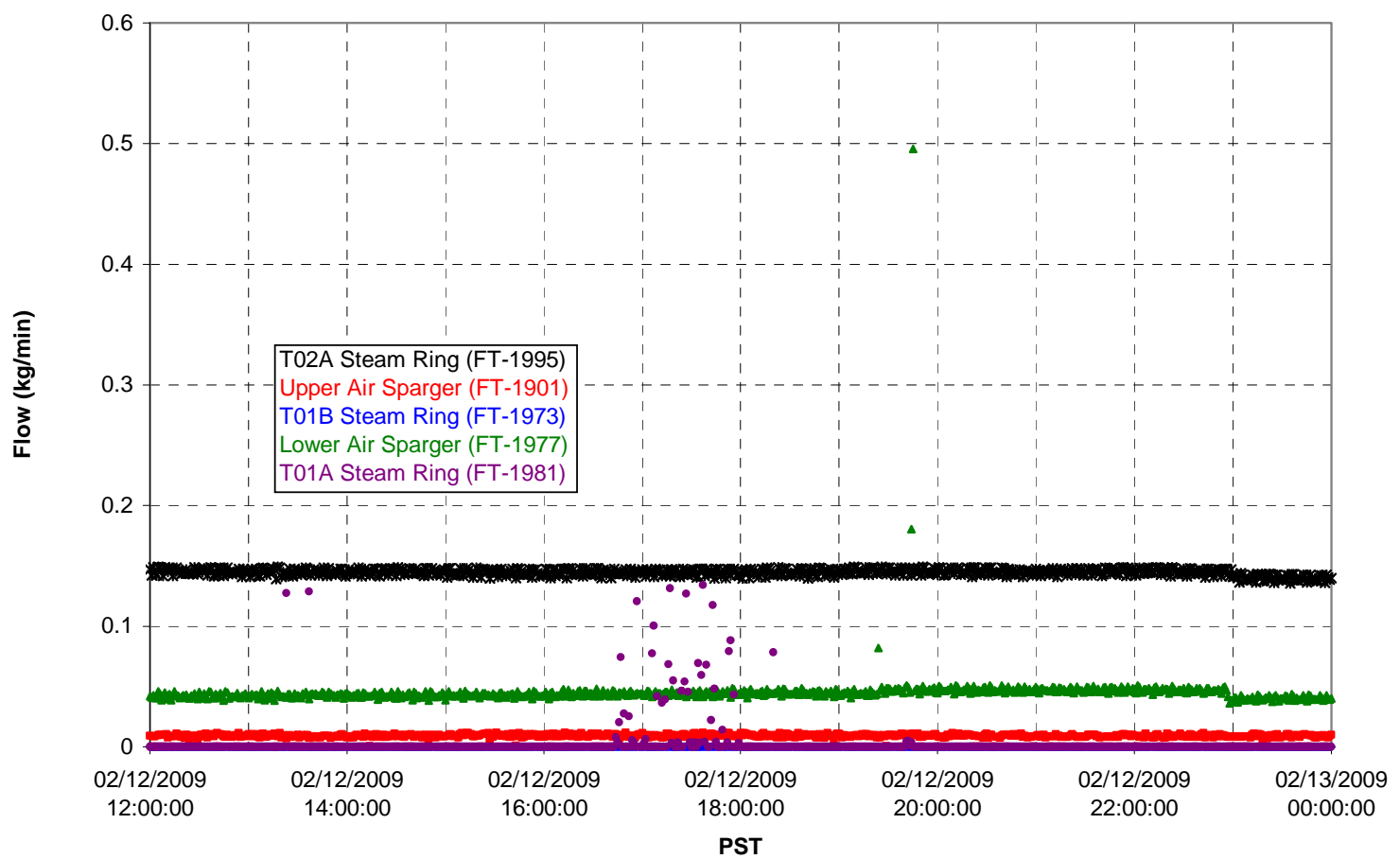


T02A Steam

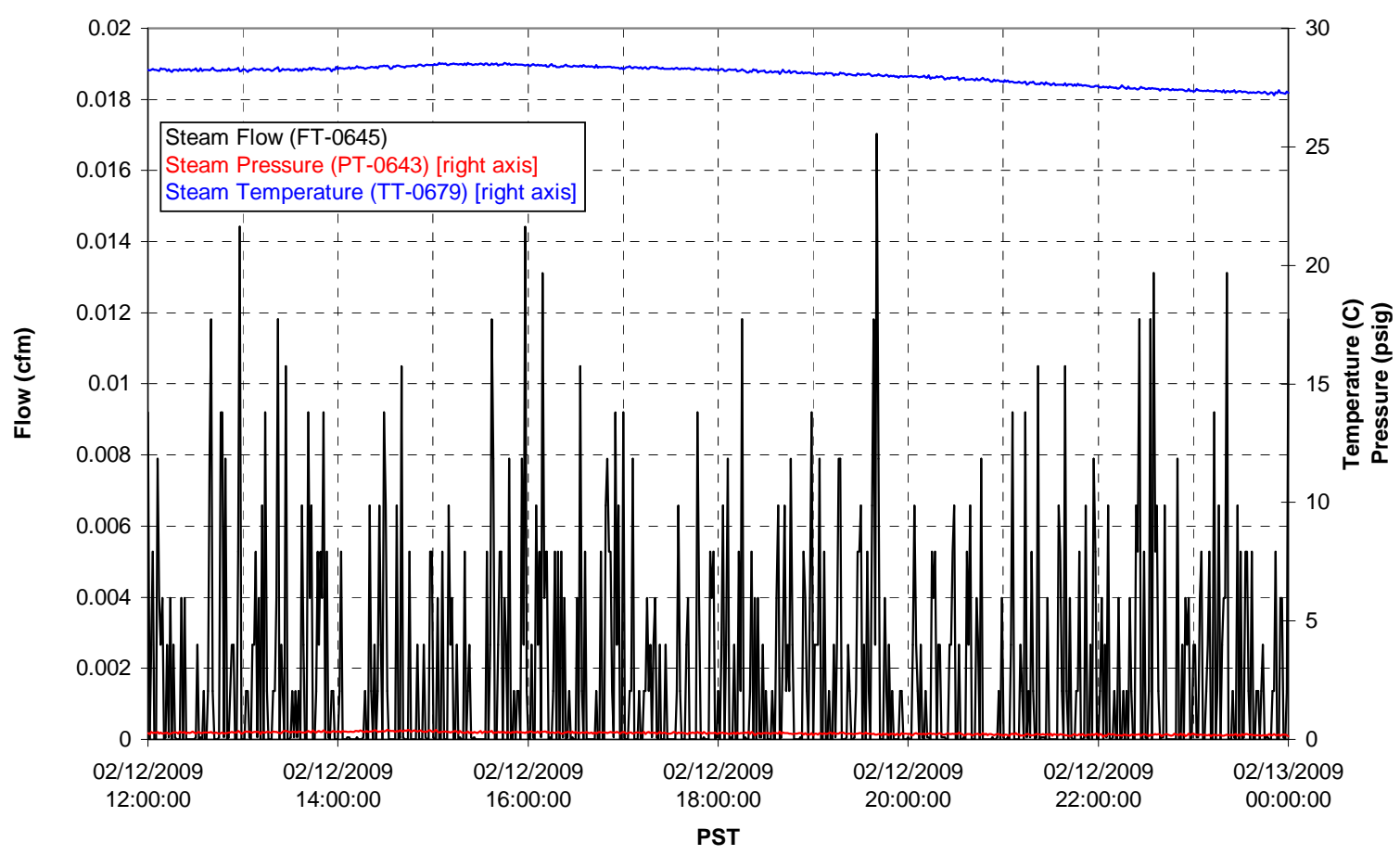

T01A Steam

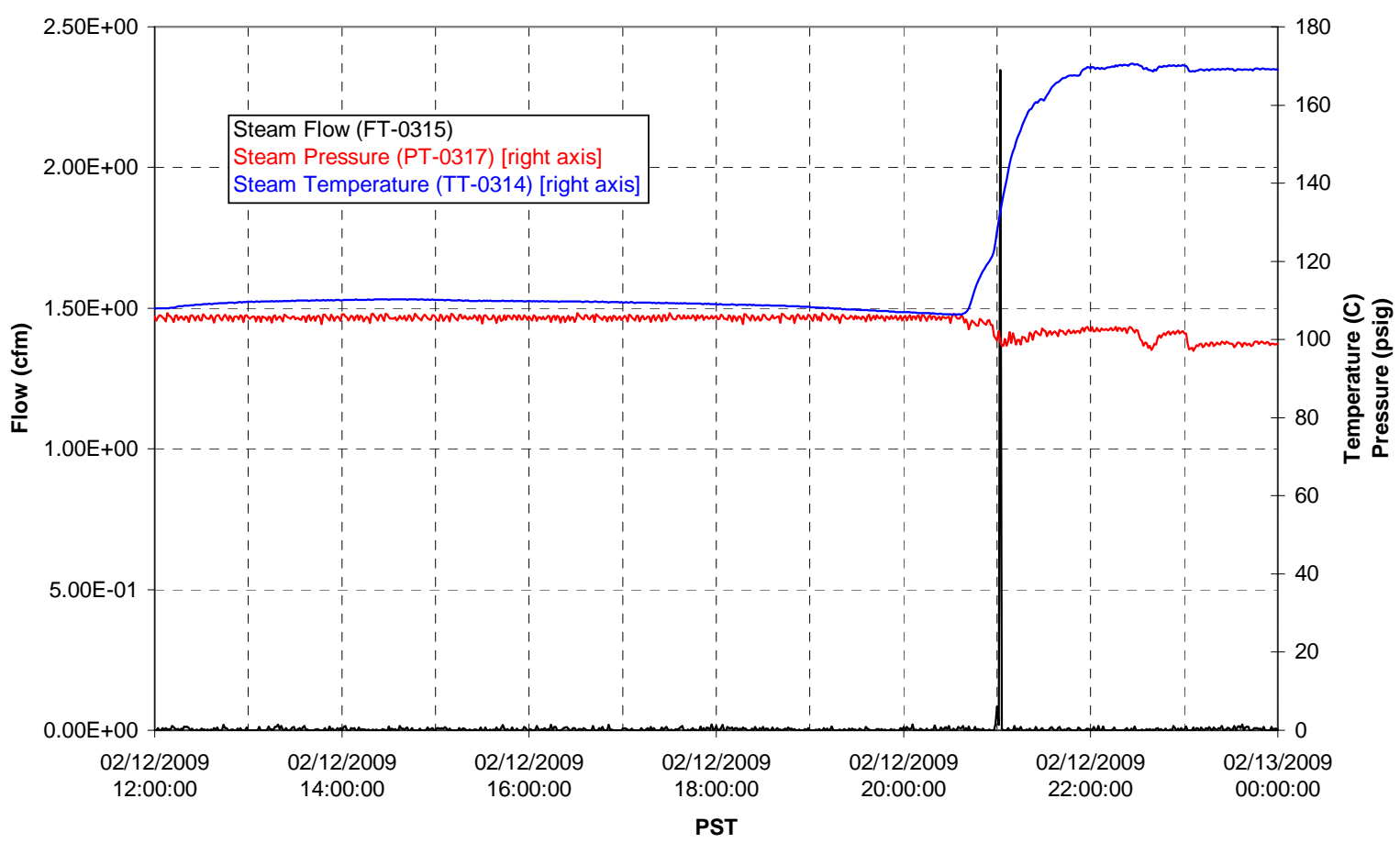


T01B Steam

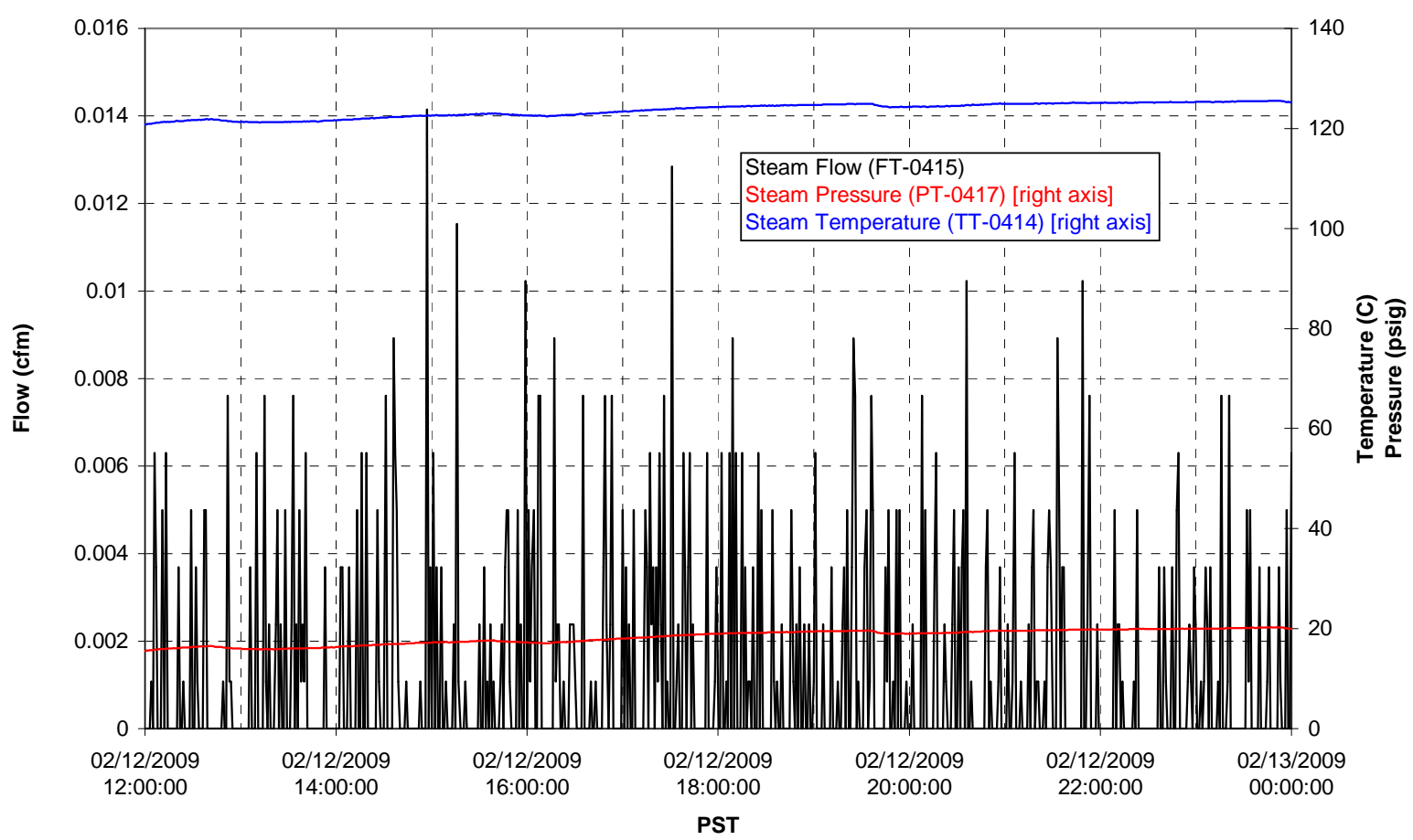




\section{Integrated Test A Data Plots 02/13/09 00:00 - 02/13/09 12:00}


T01A level

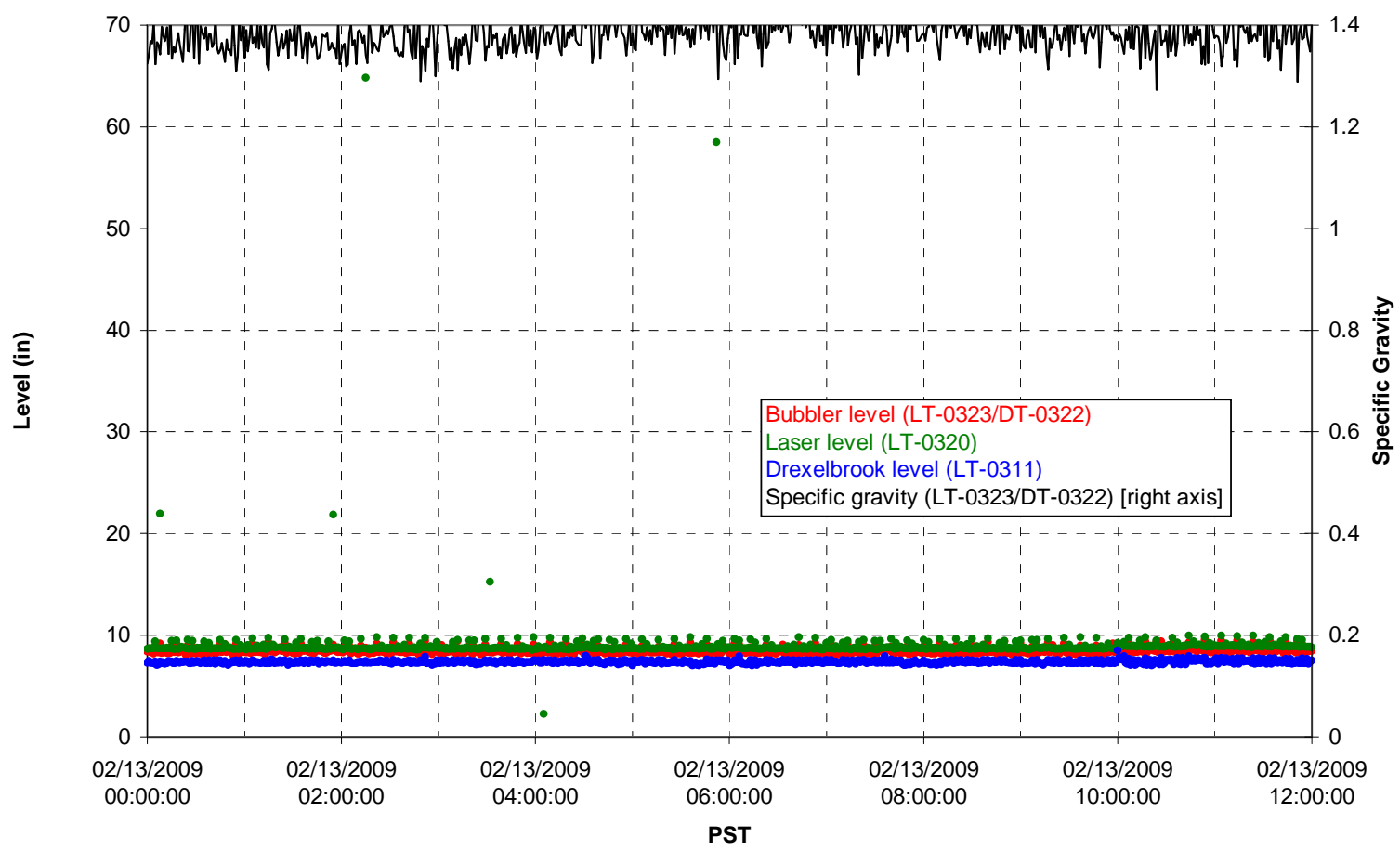

T01A temperatures

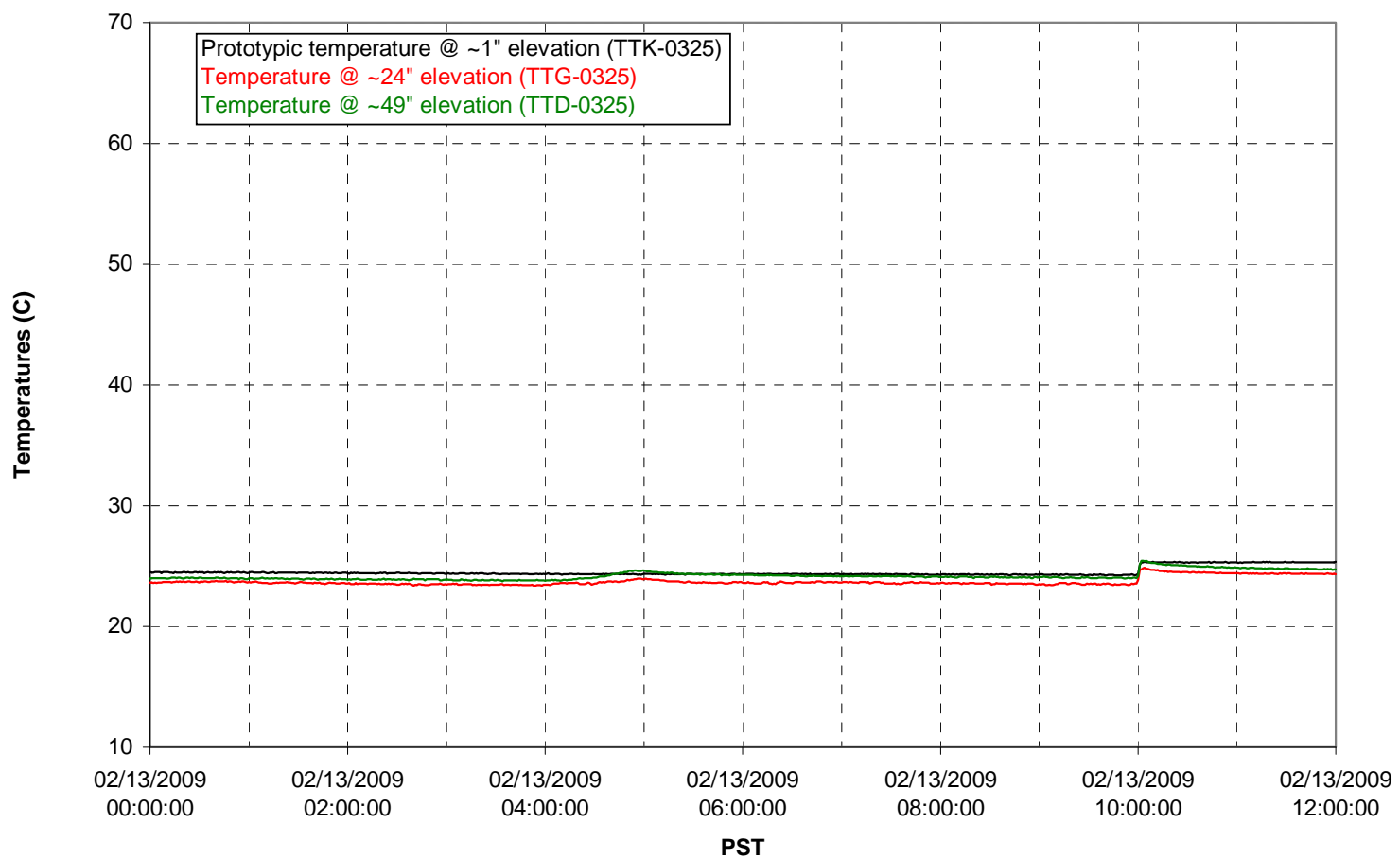


T01B level

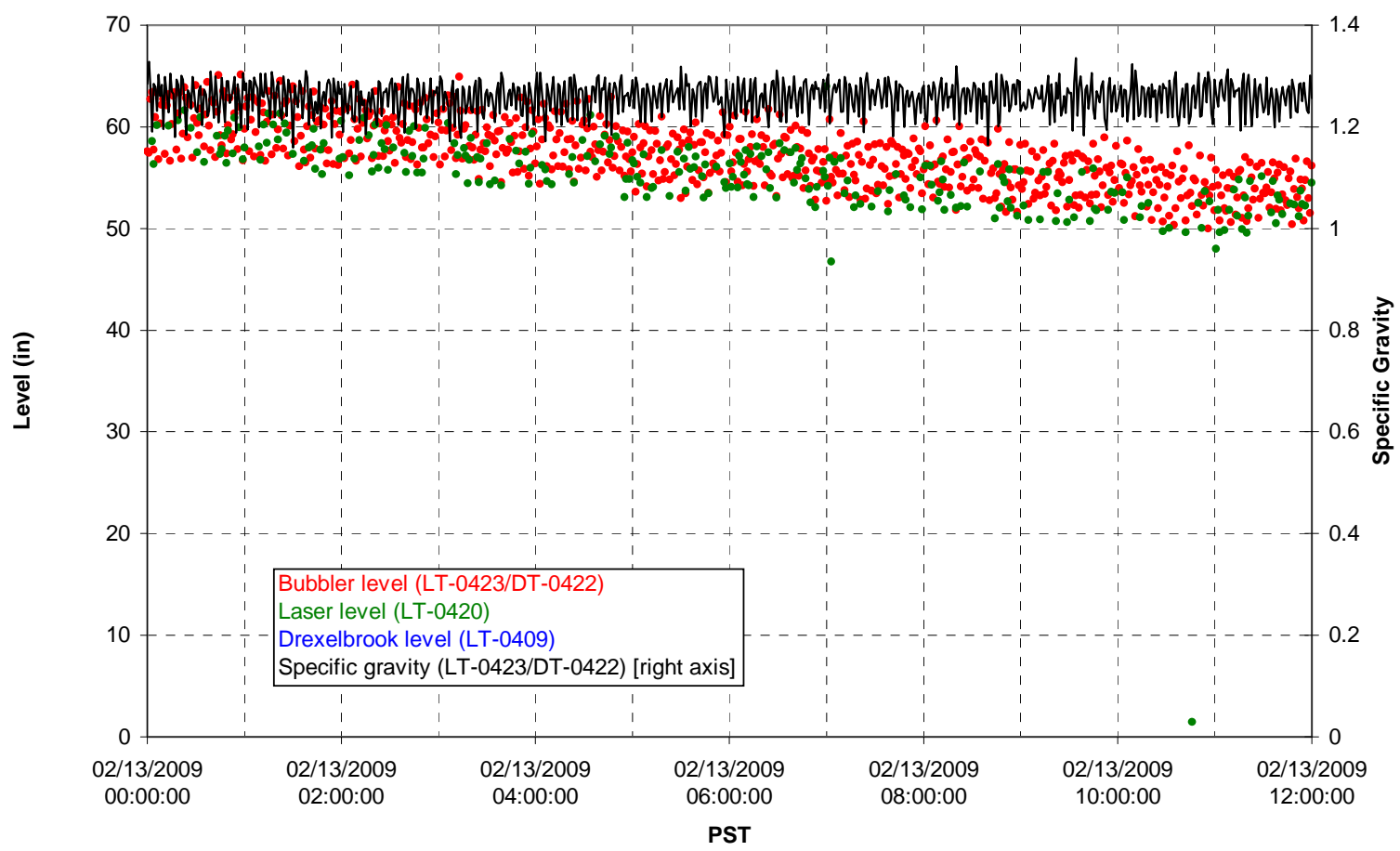

T01B temperatures

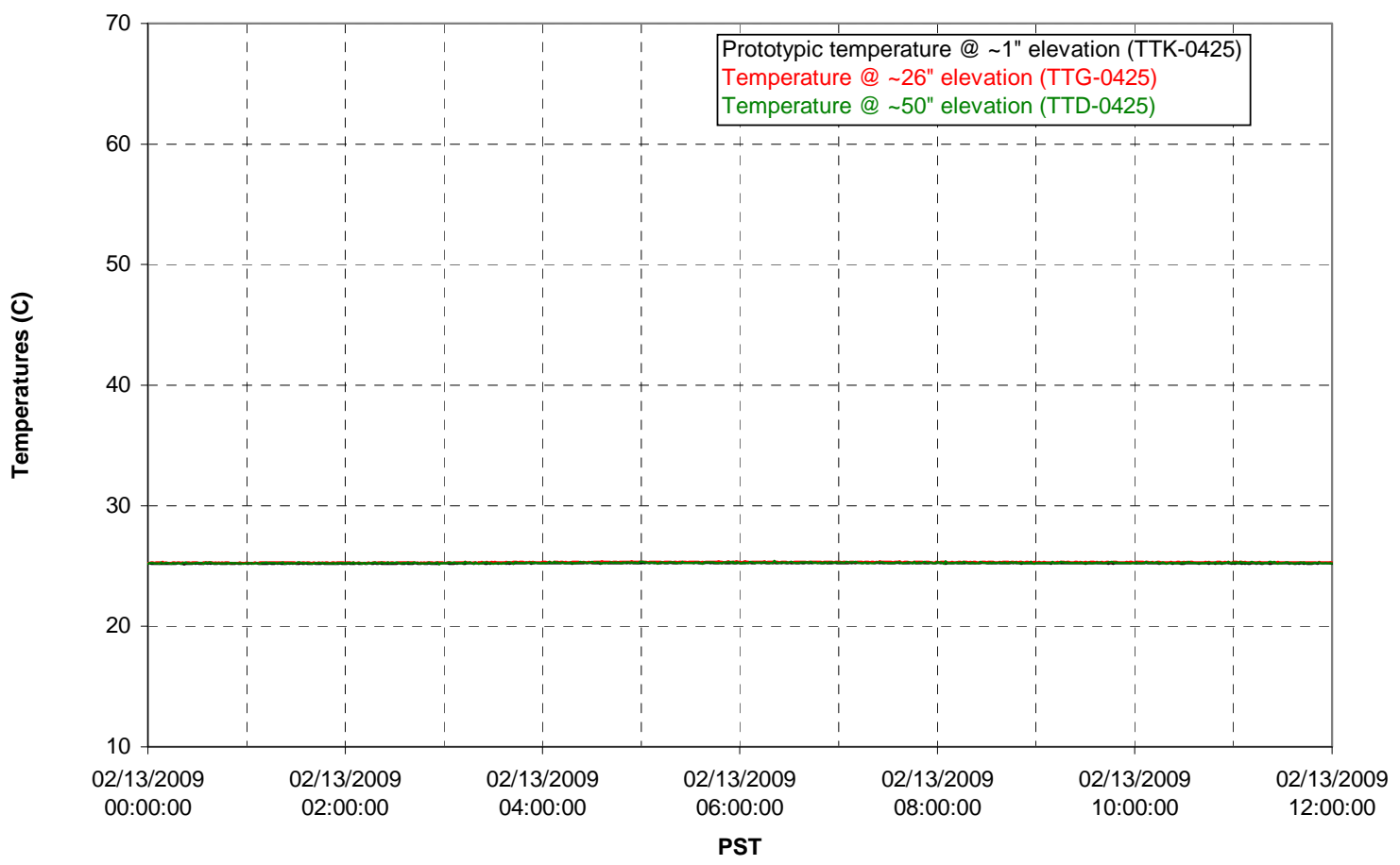


T02A level

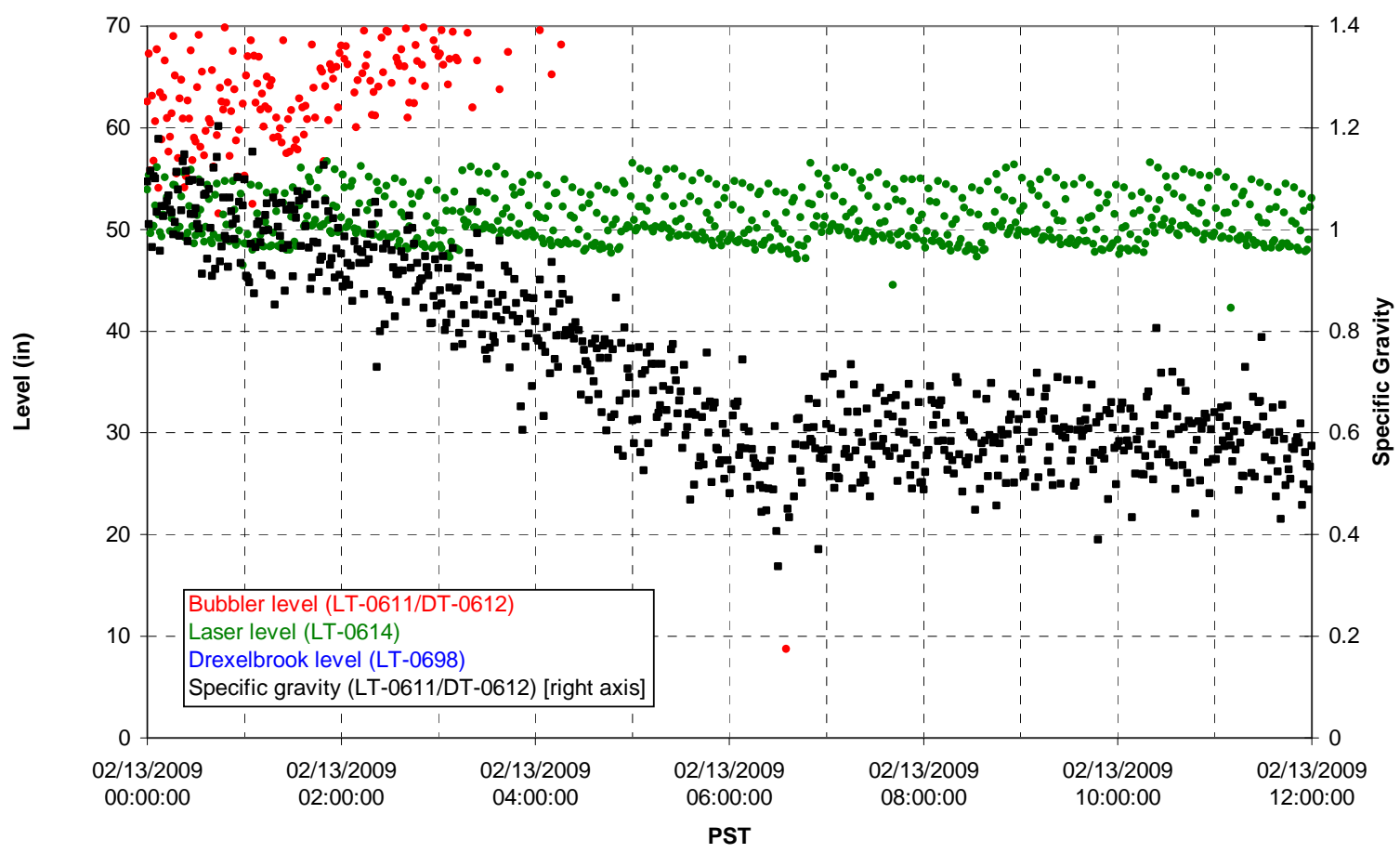

T02A temperatures

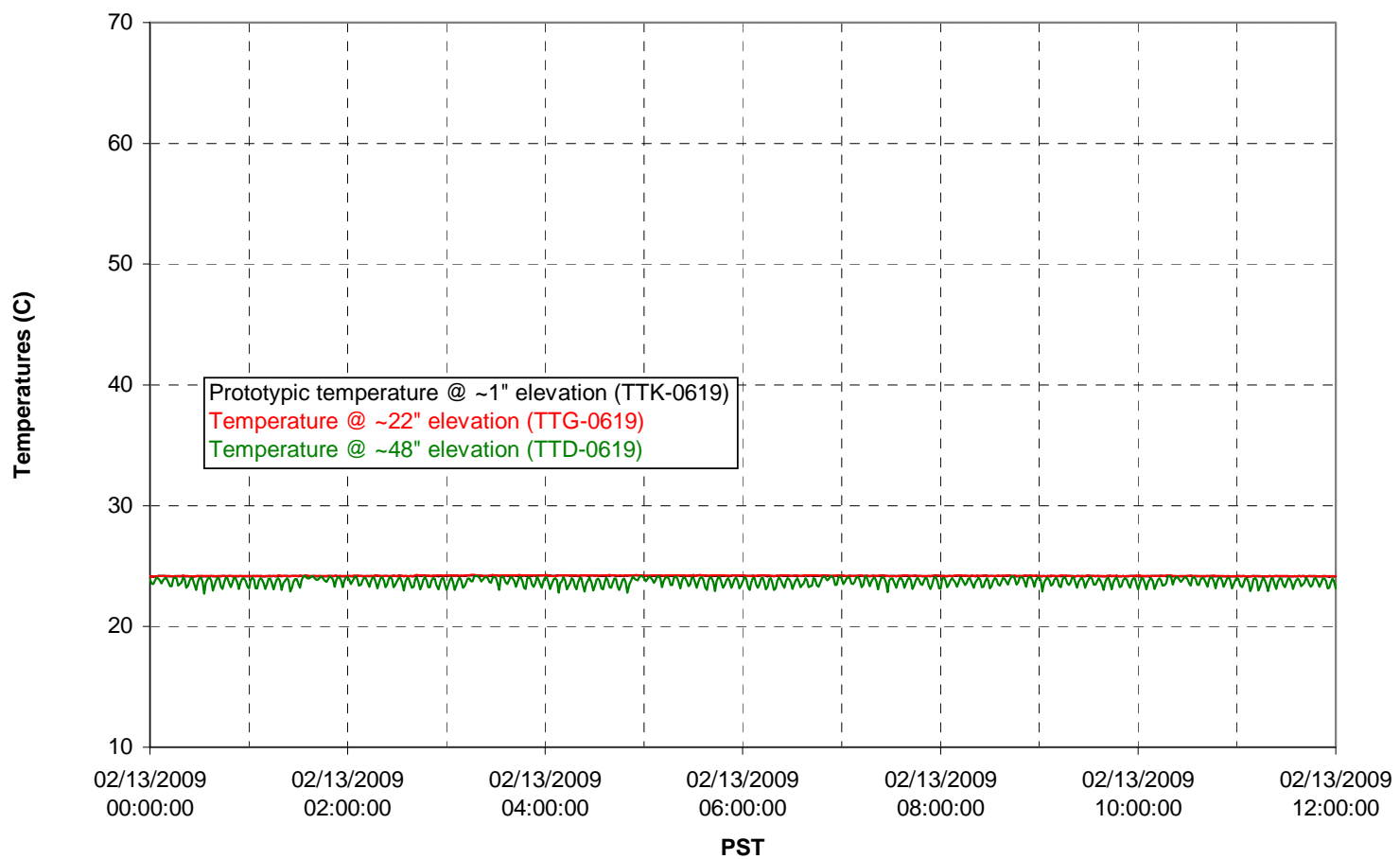


T02A and filter loop temperatures

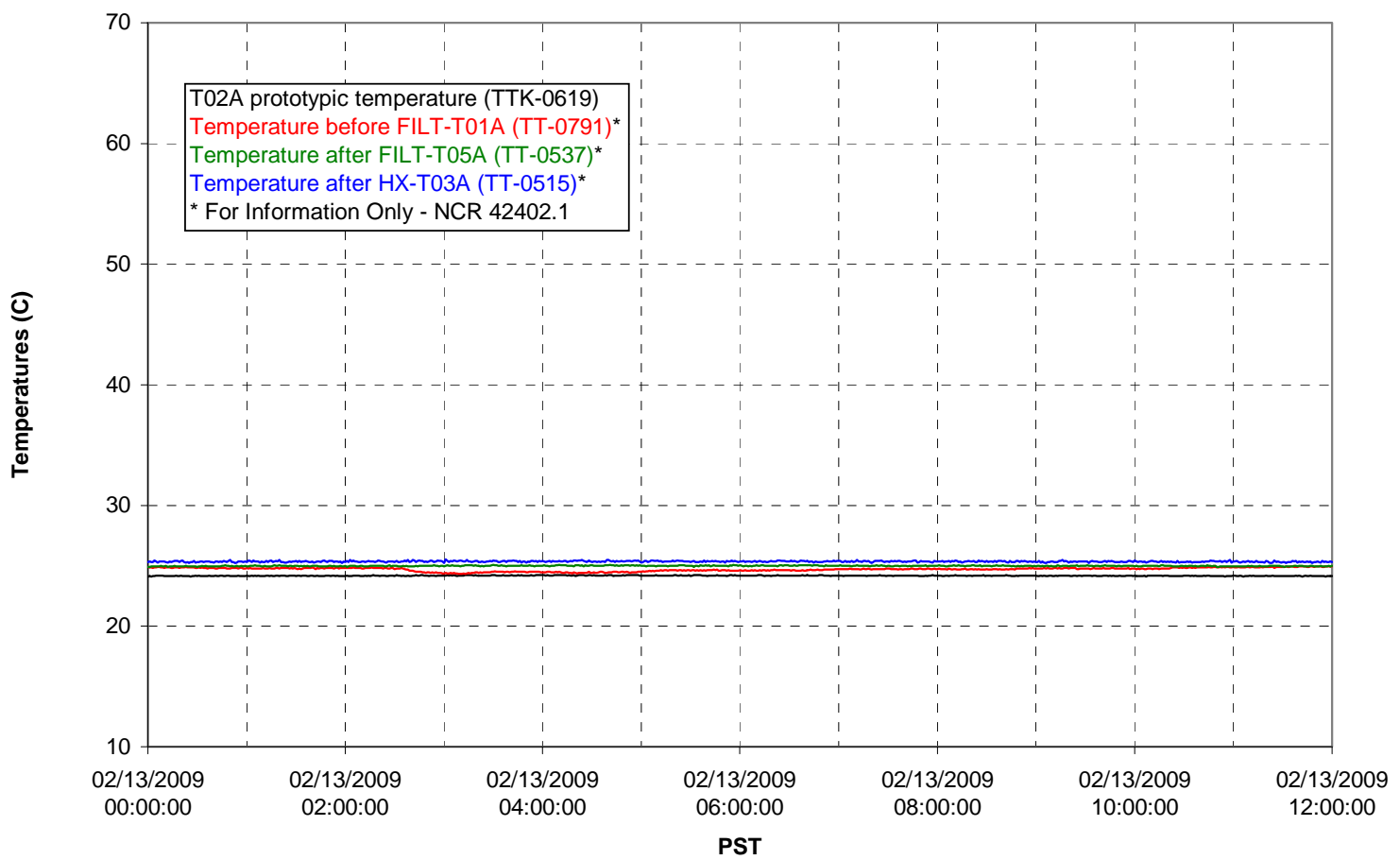

Pump Pressures and Flow

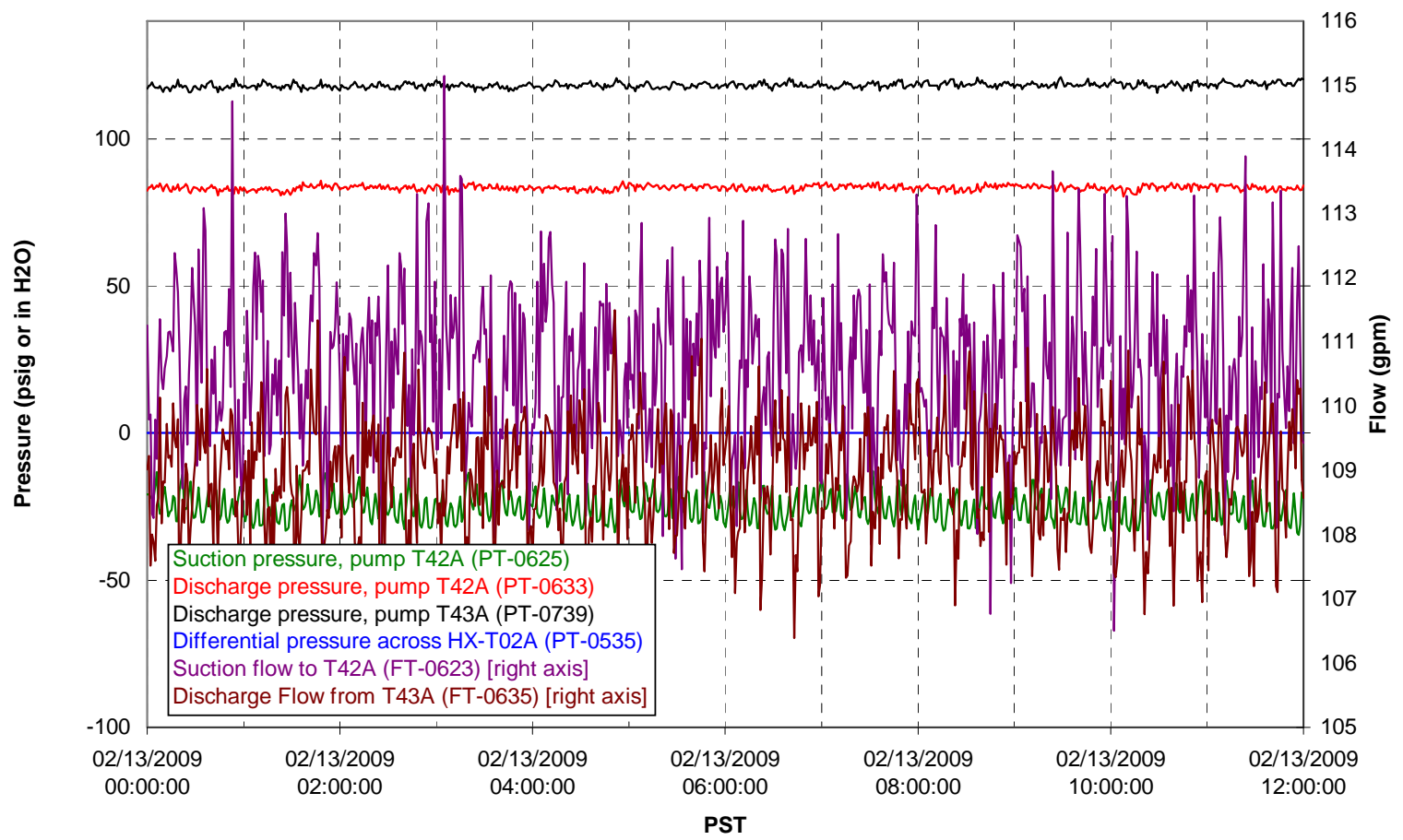


Axial pressure drop

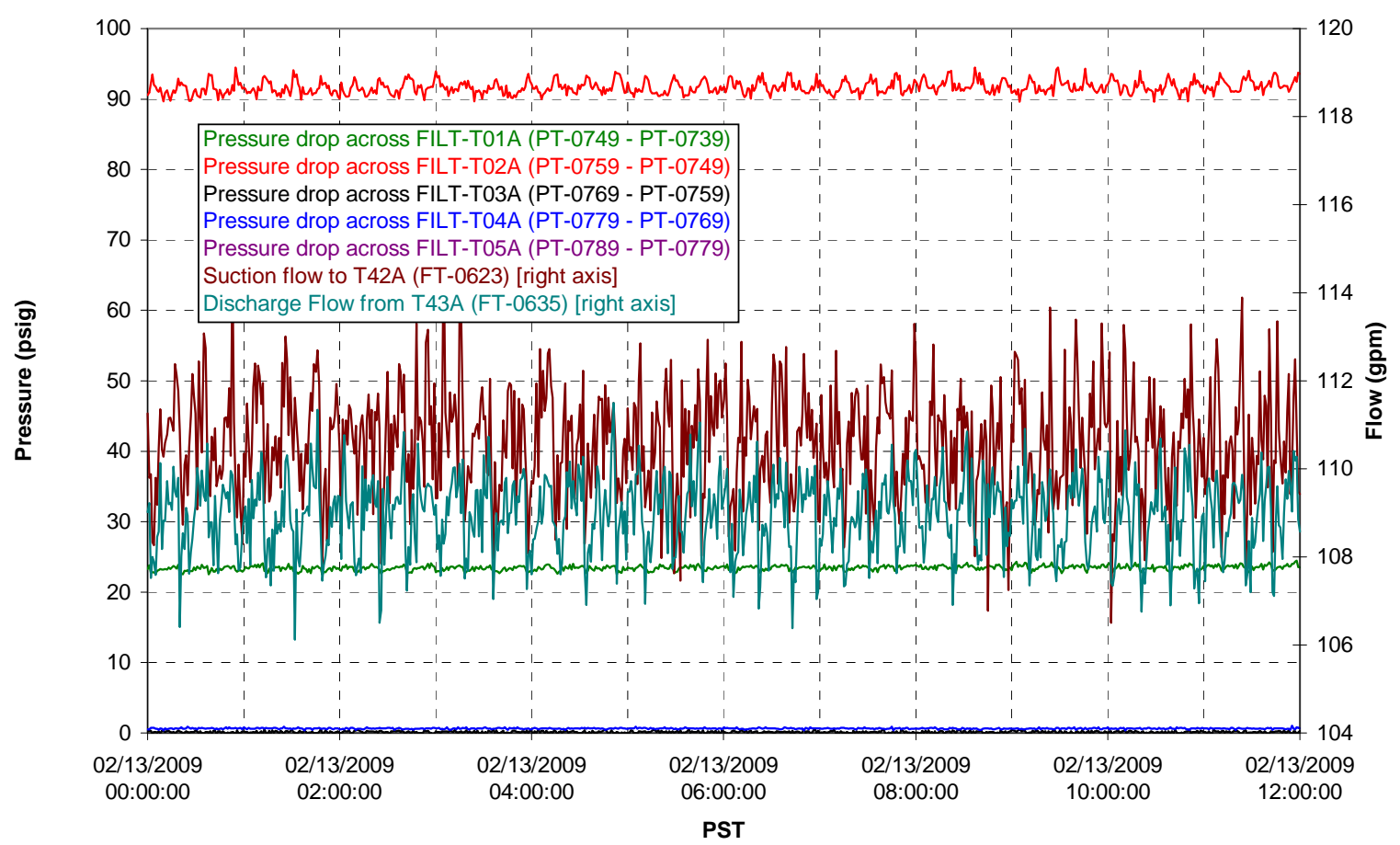

Permeate flow rates

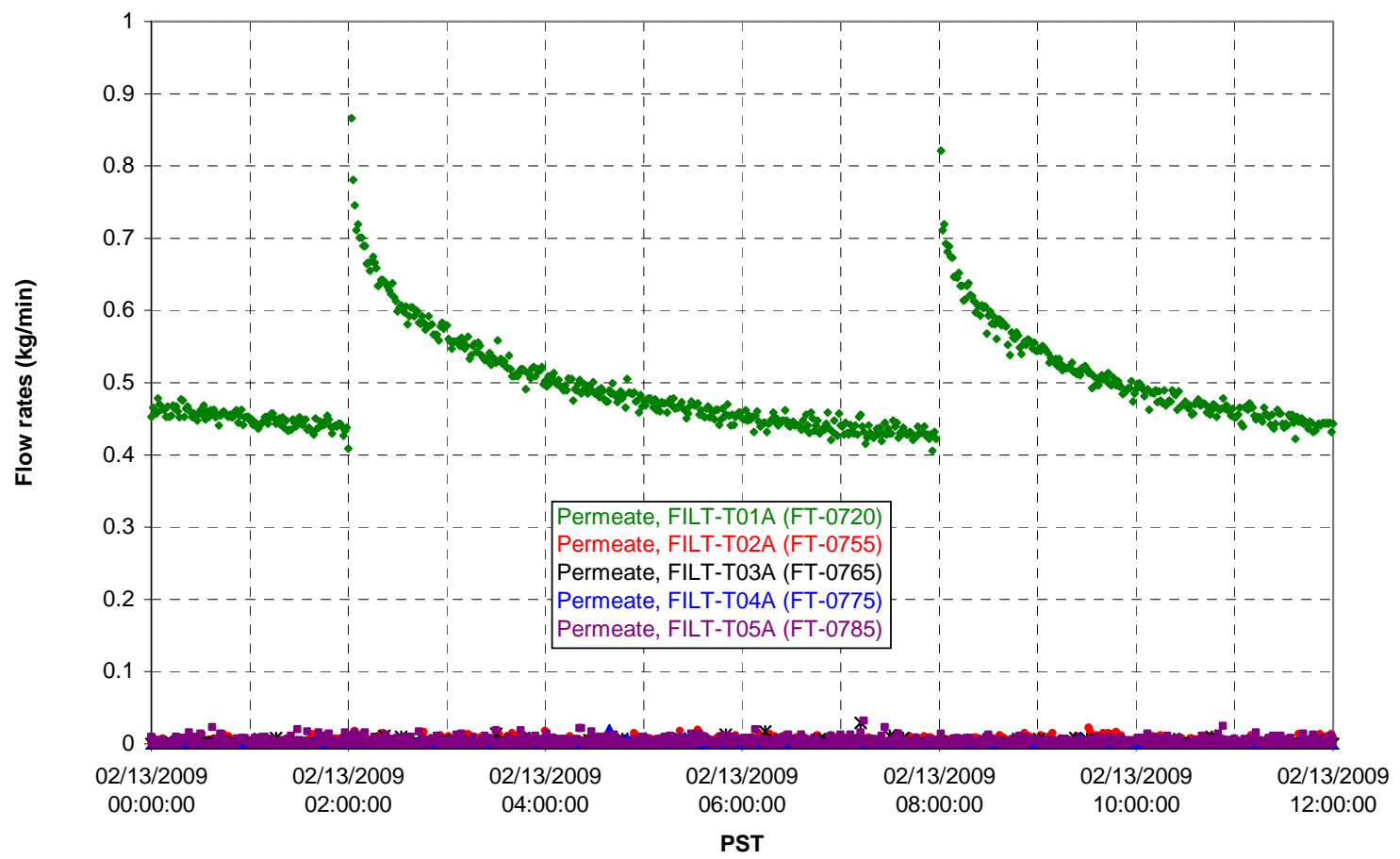


T02A Inner Temperature Tree

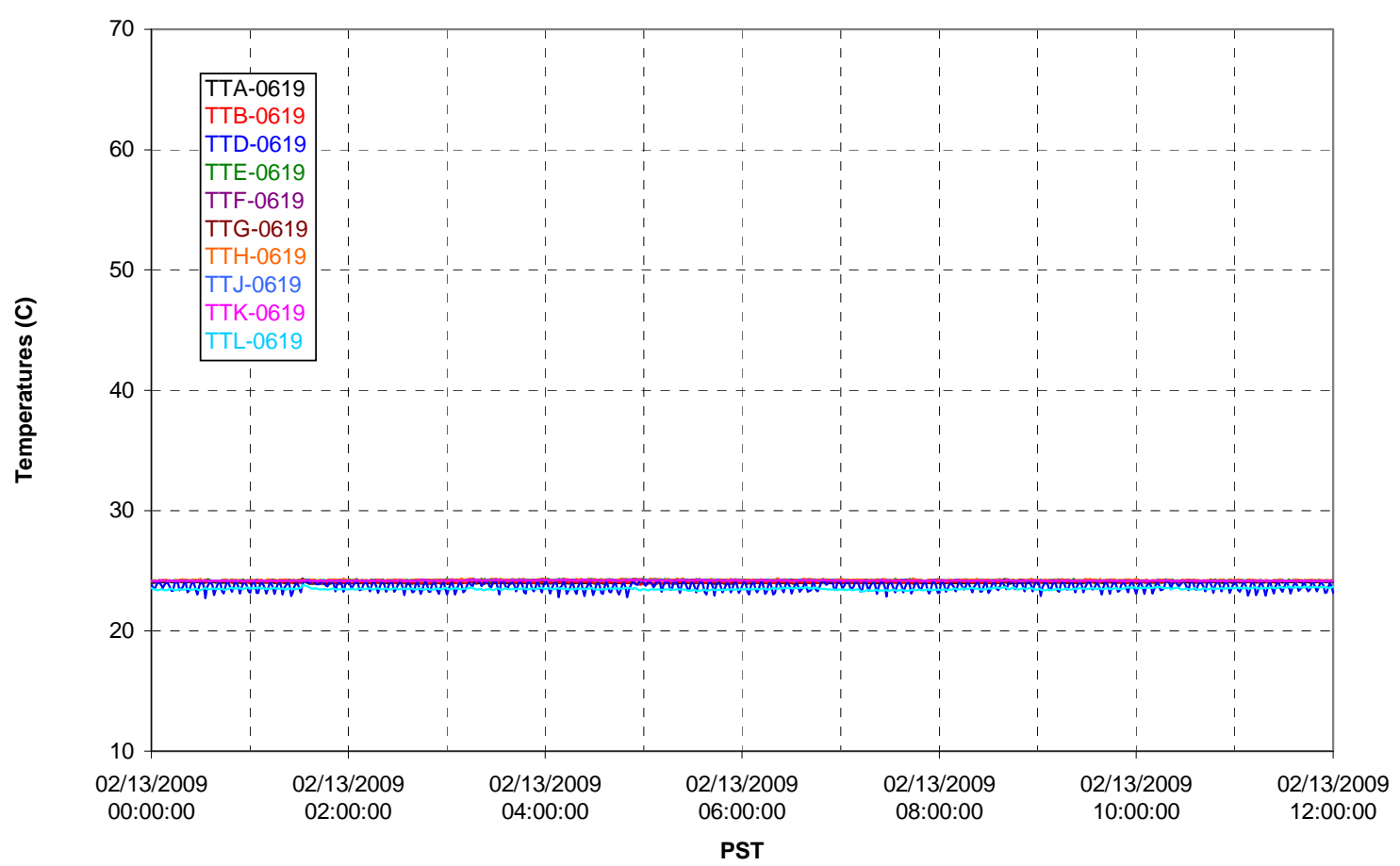

T02A Outer Temperature Tree

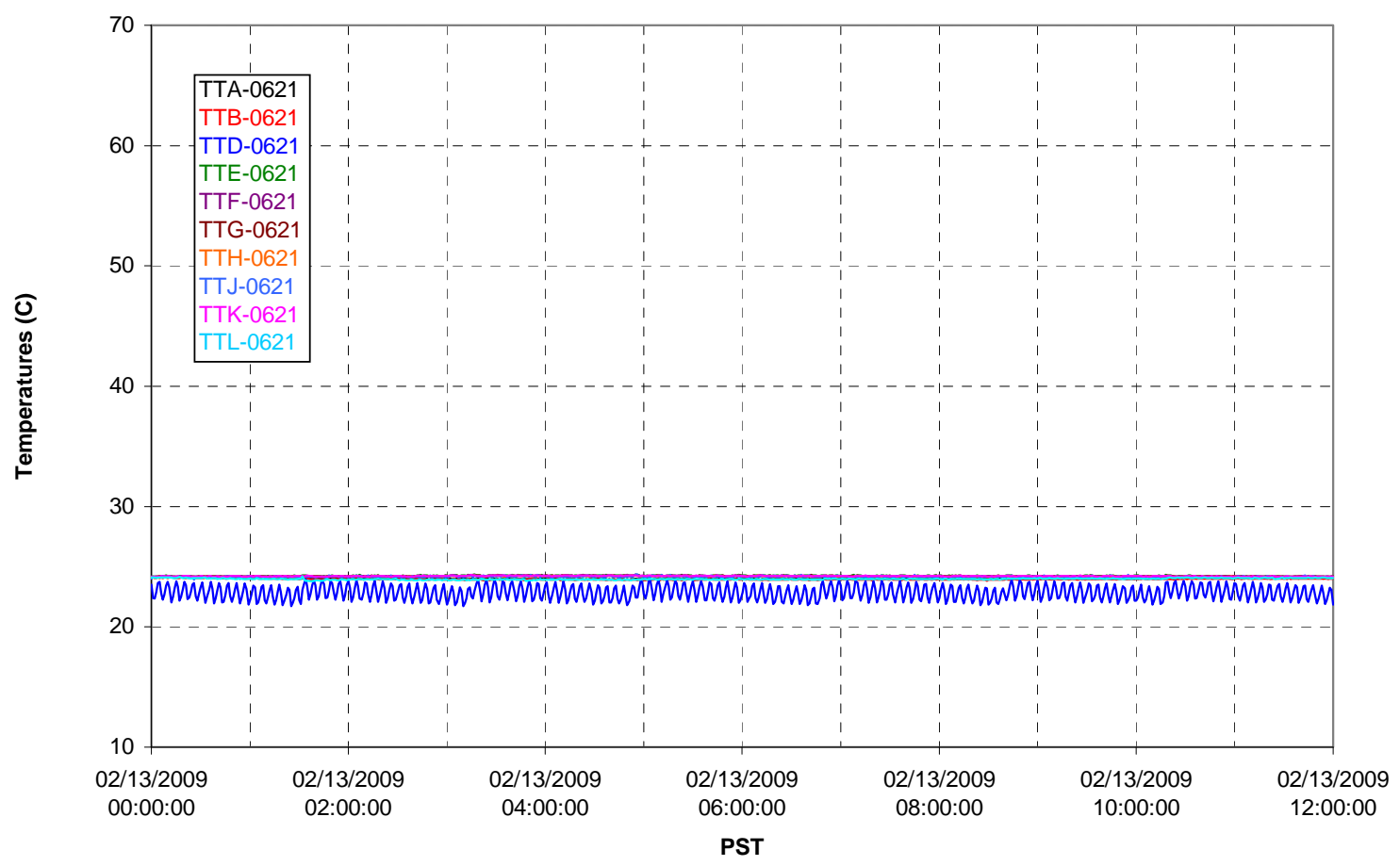


T02A temperatures

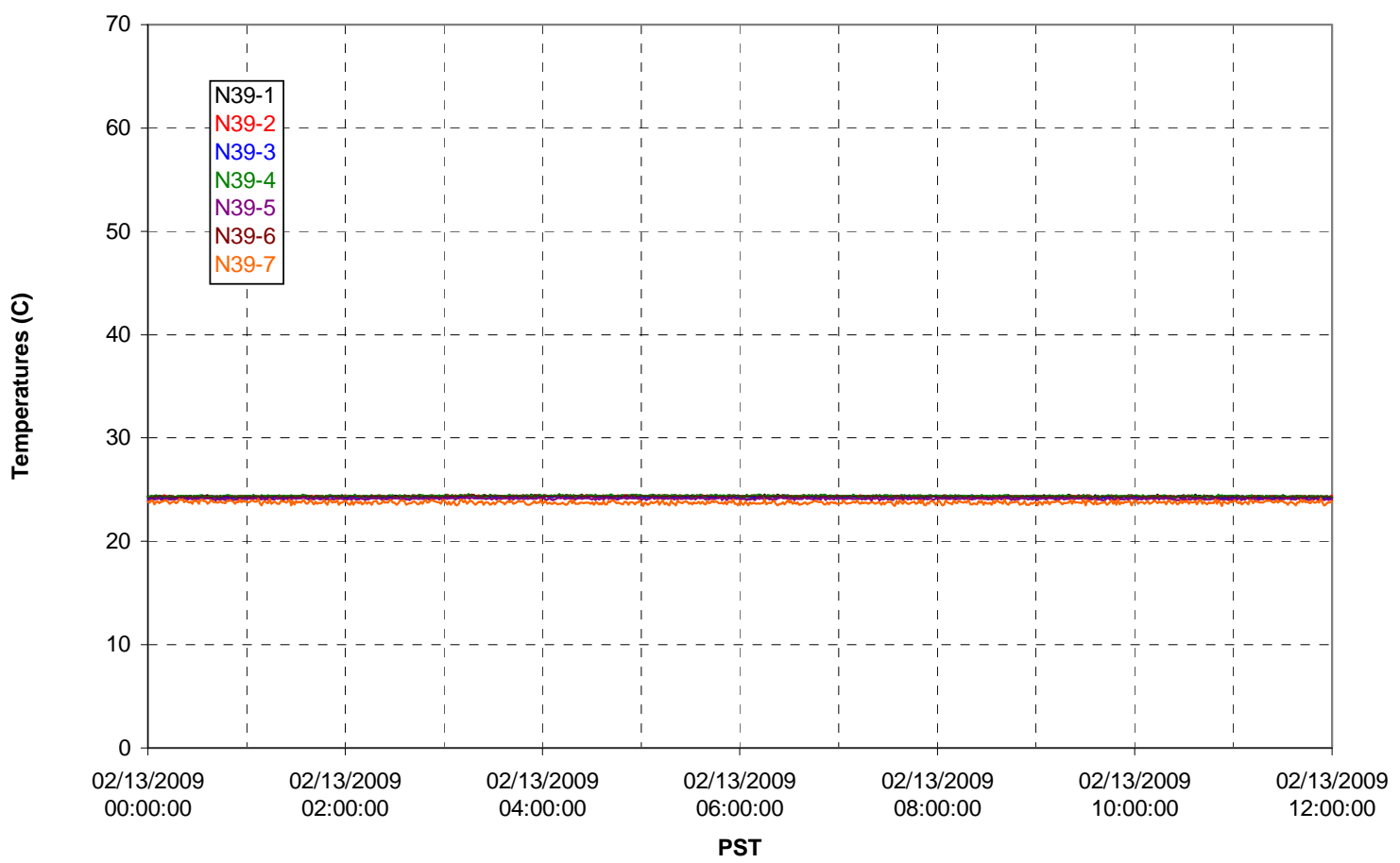

T02A temperatures

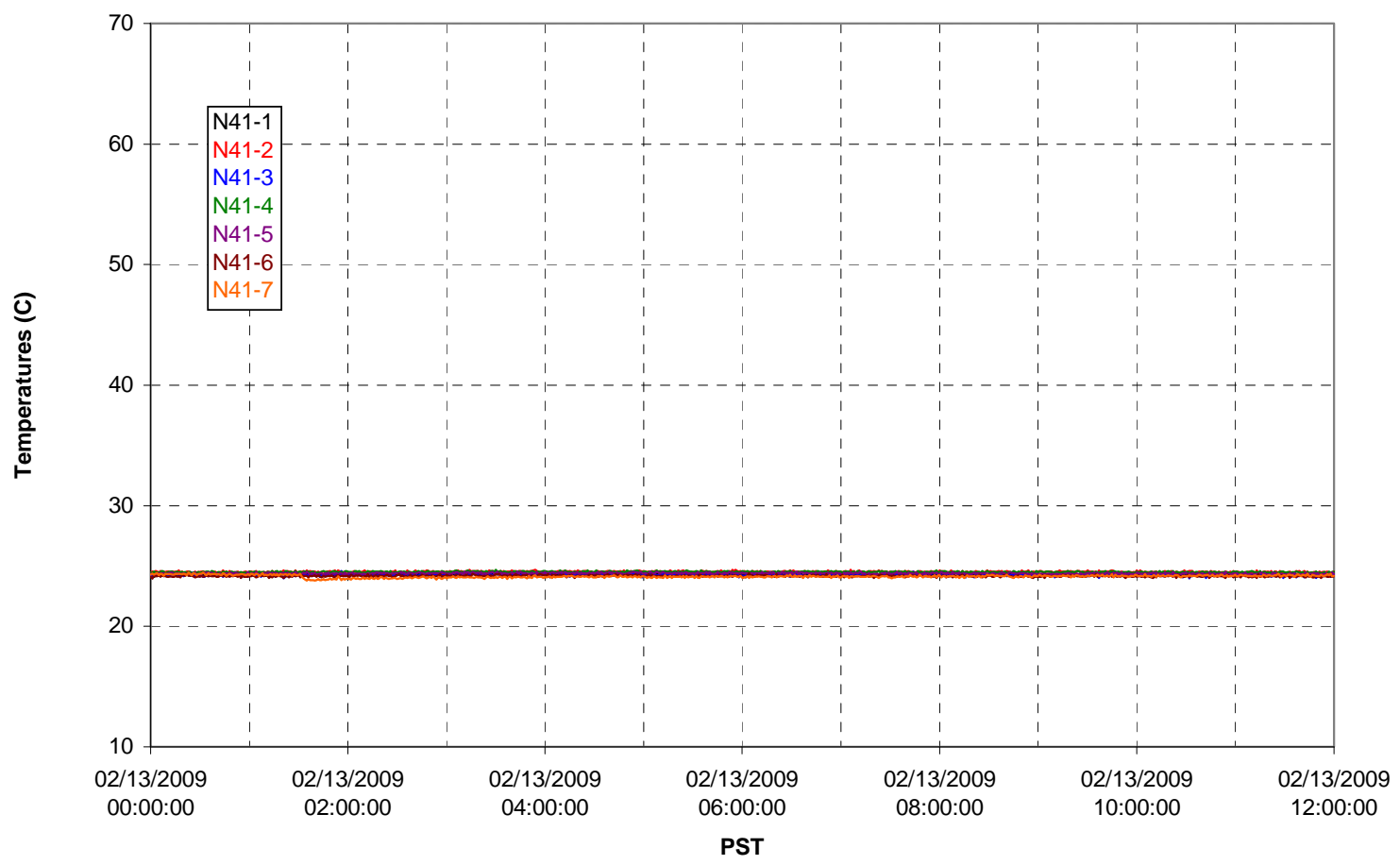


T02A temperatures

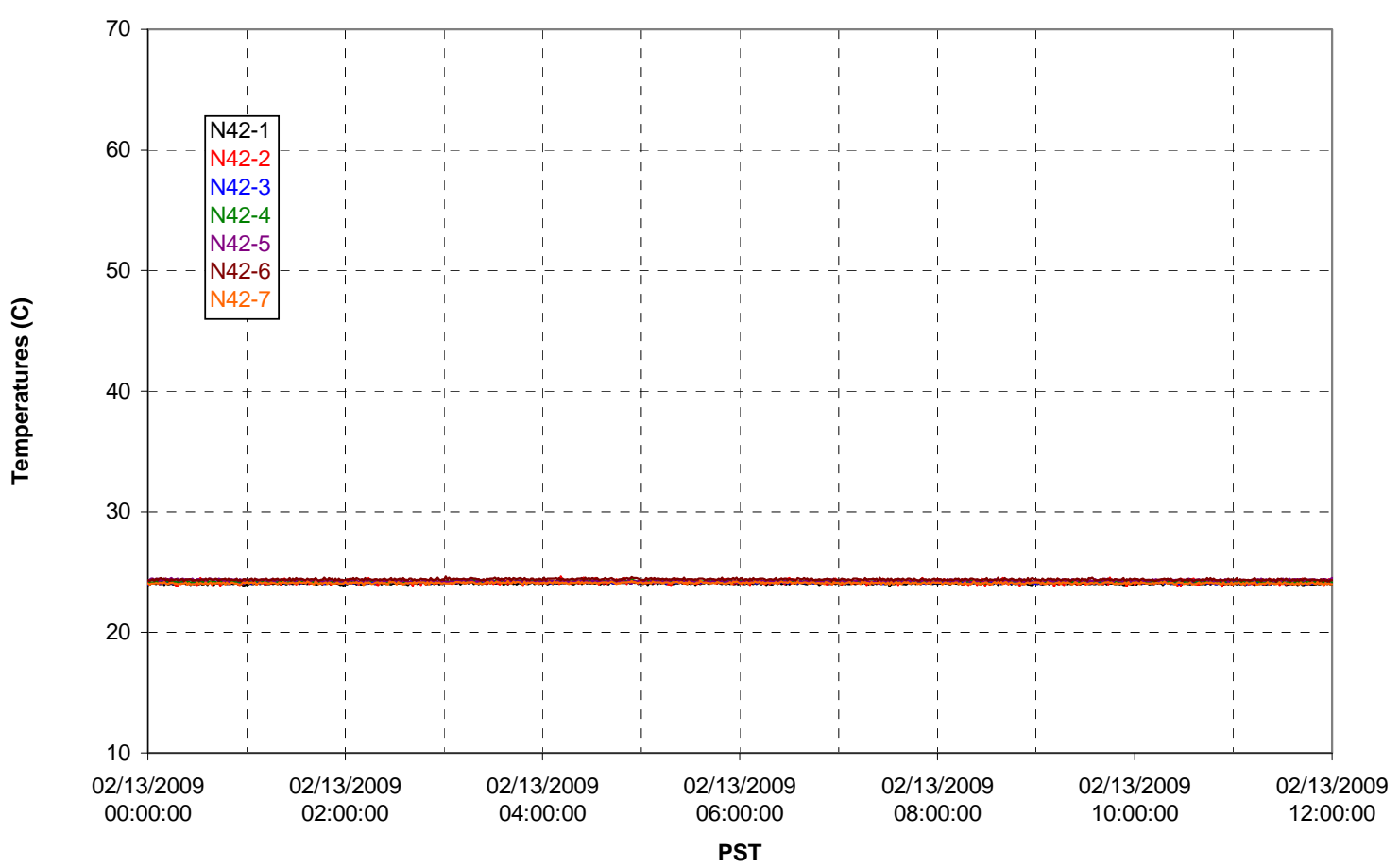

T02A temperatures

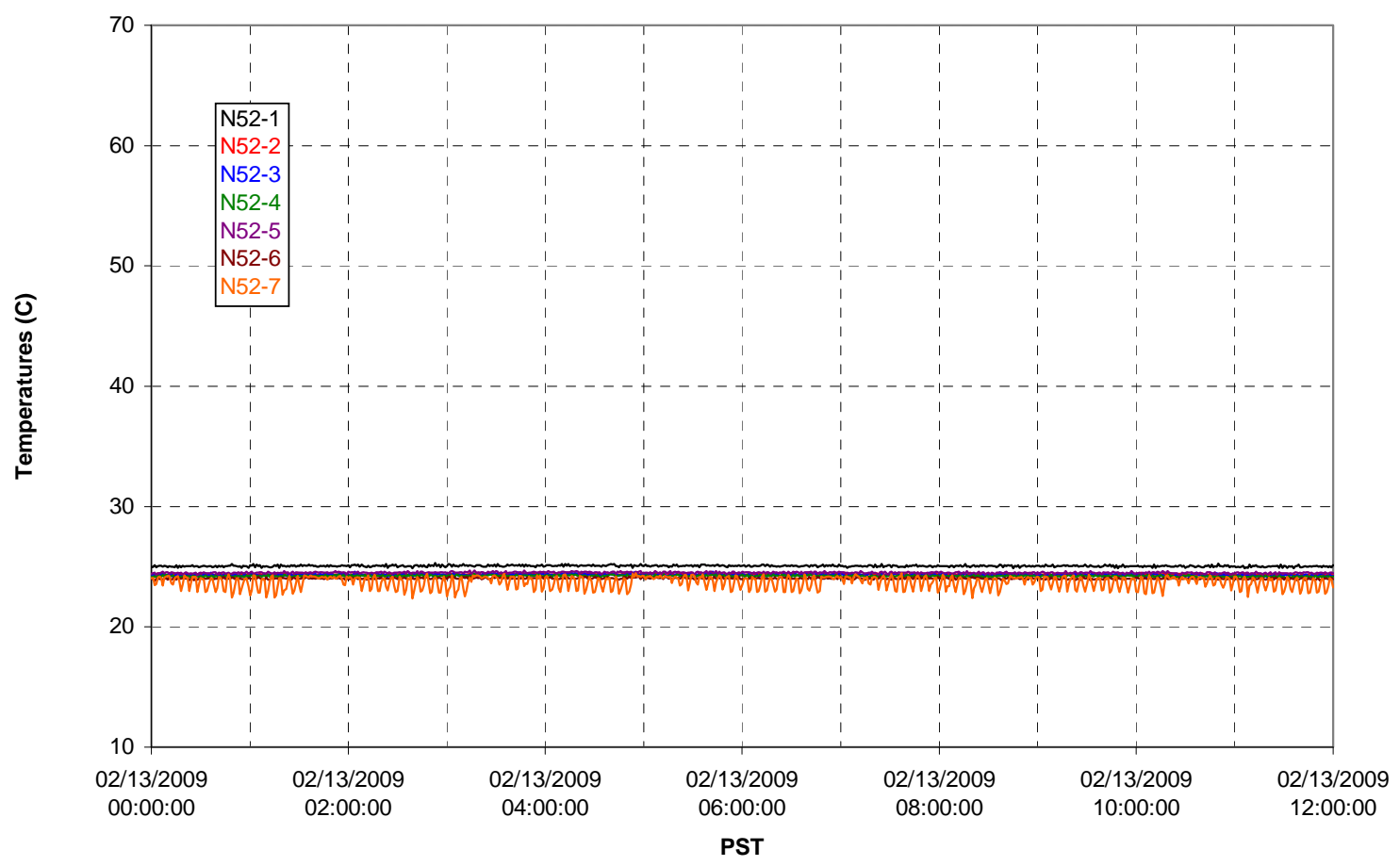


T02A Heating and Cooling
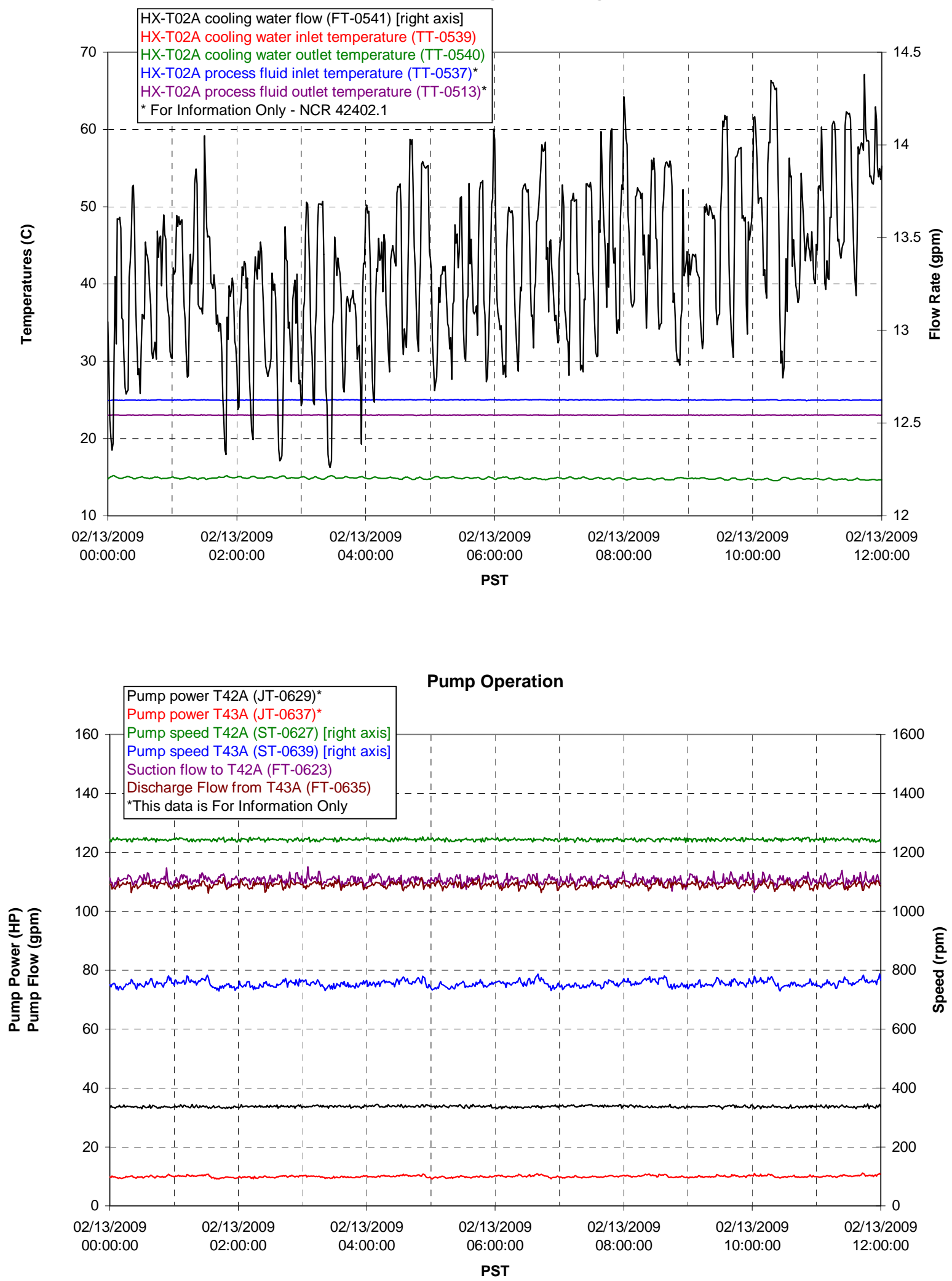
Pulsepot UFP-PP-T01A

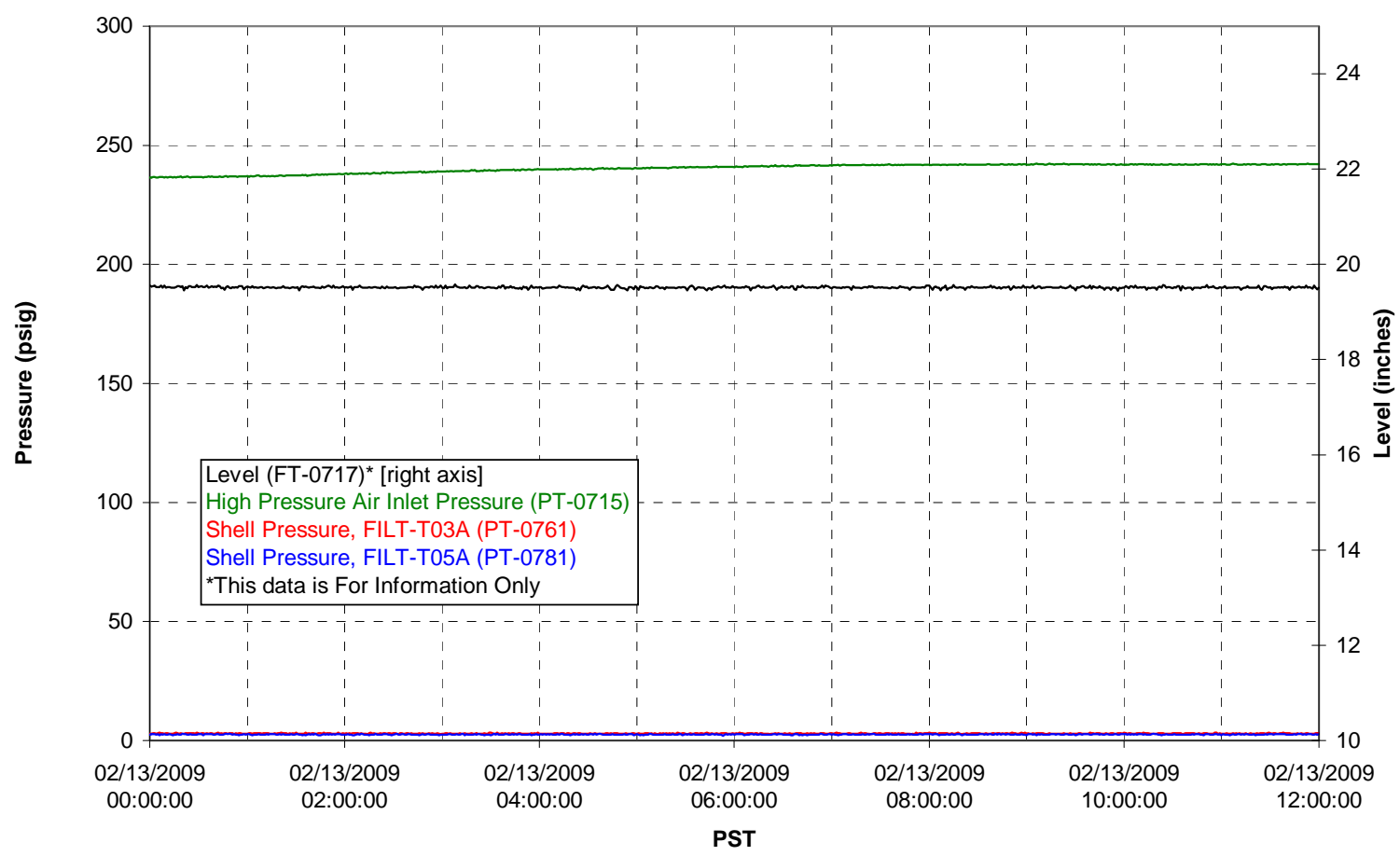

Pulsepot UFP-PP-T02A

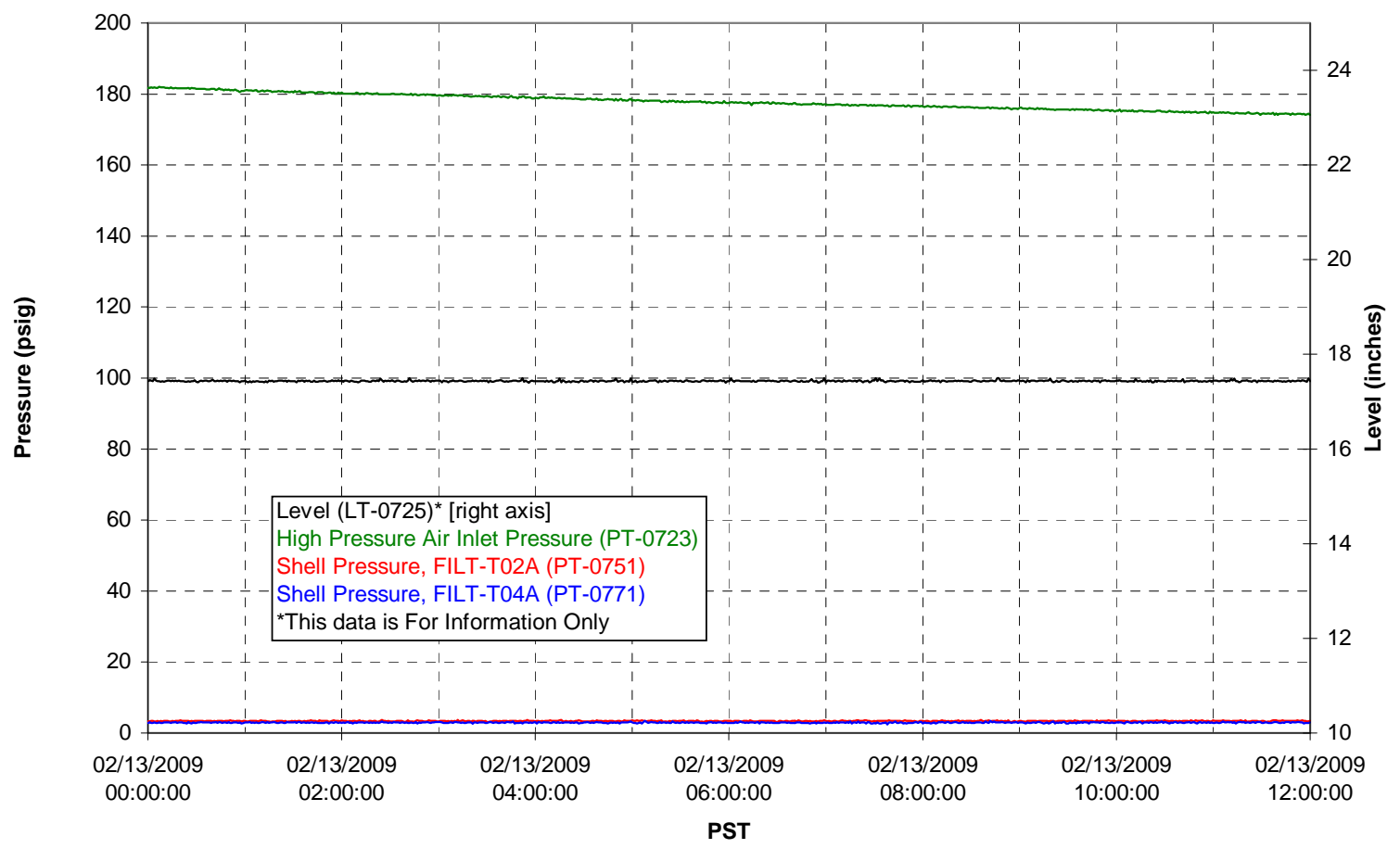


Pulsepot UFP-PP-T03A

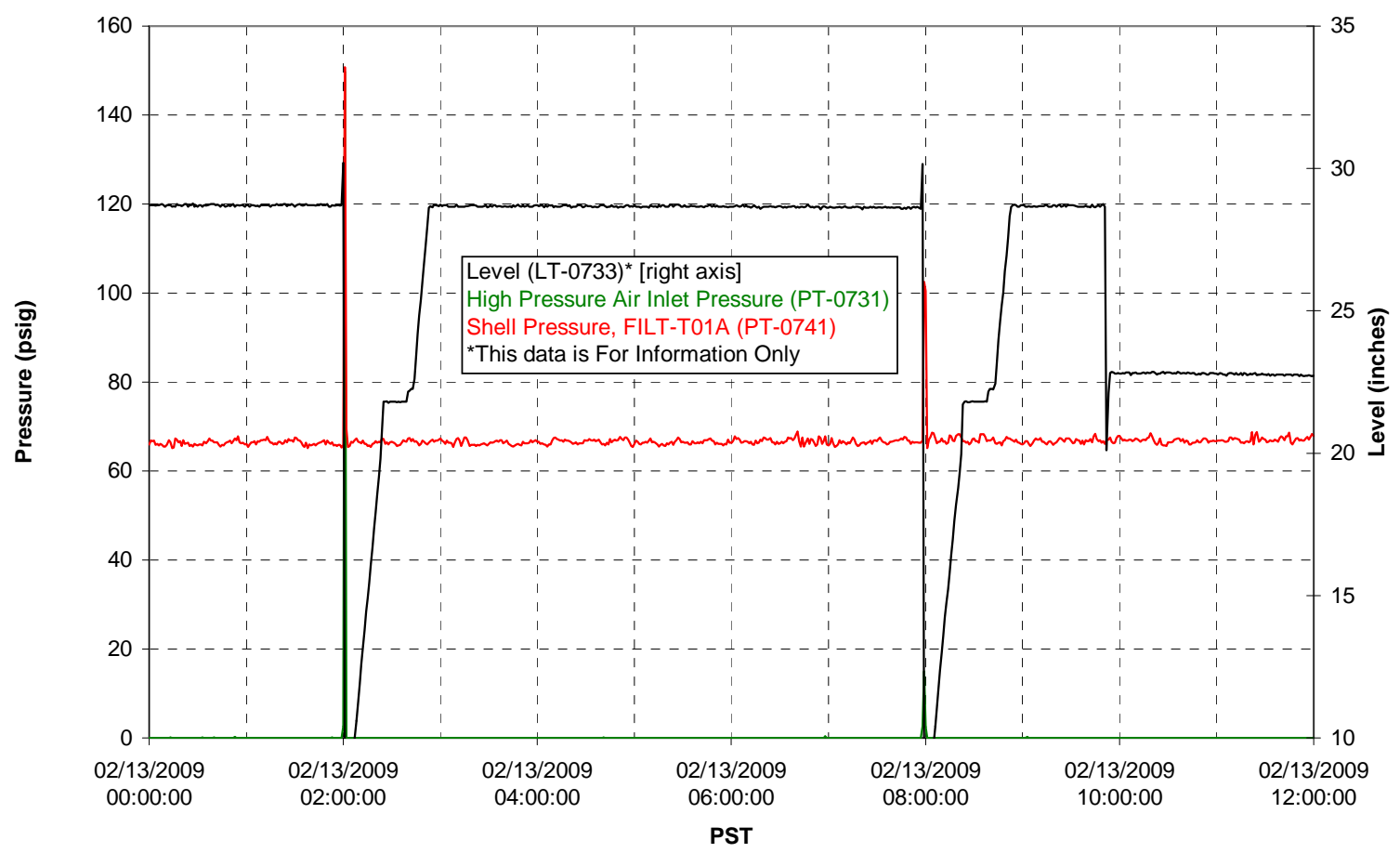

Pulsepot Levels

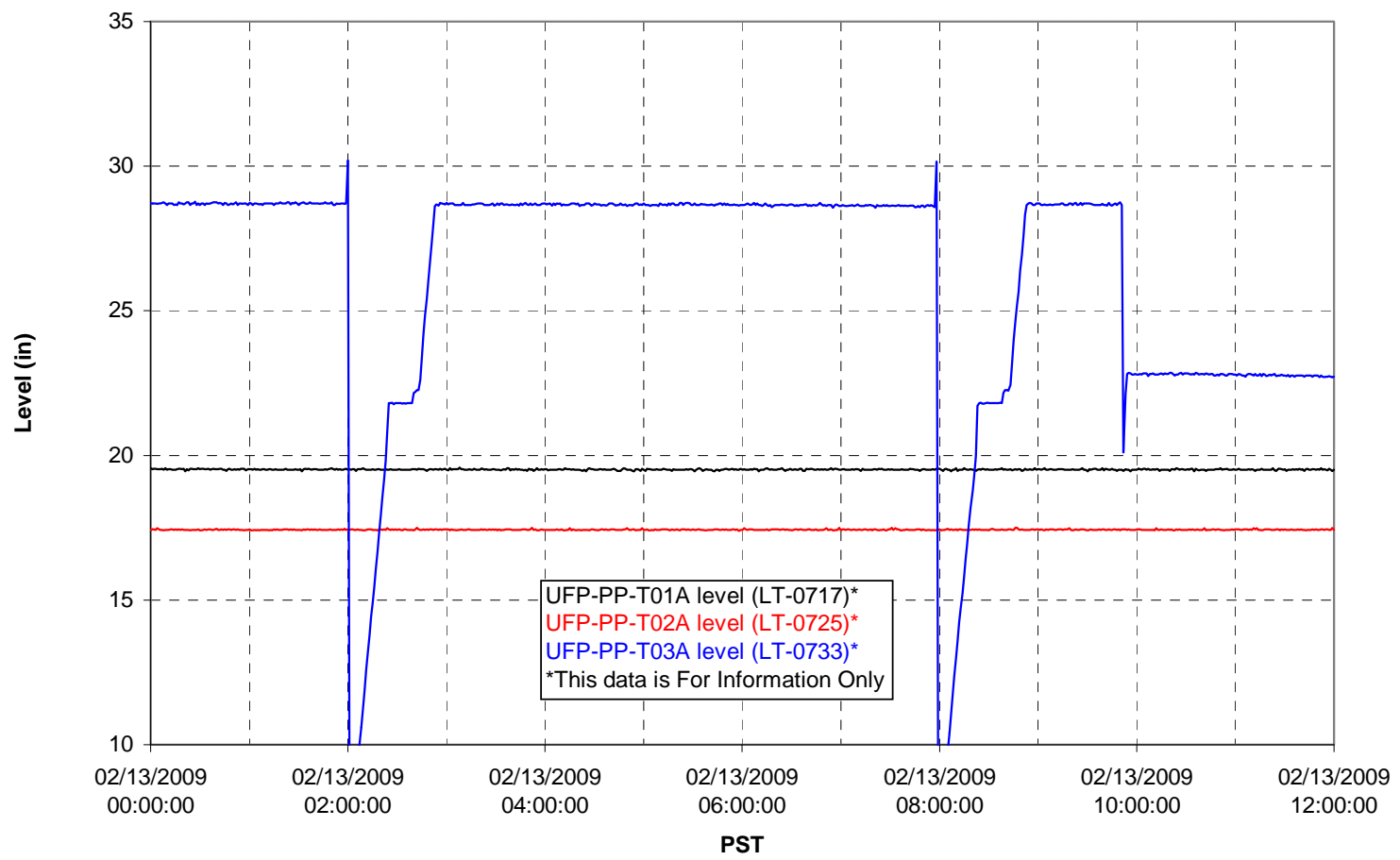


Filter UFP-FILT-T01A

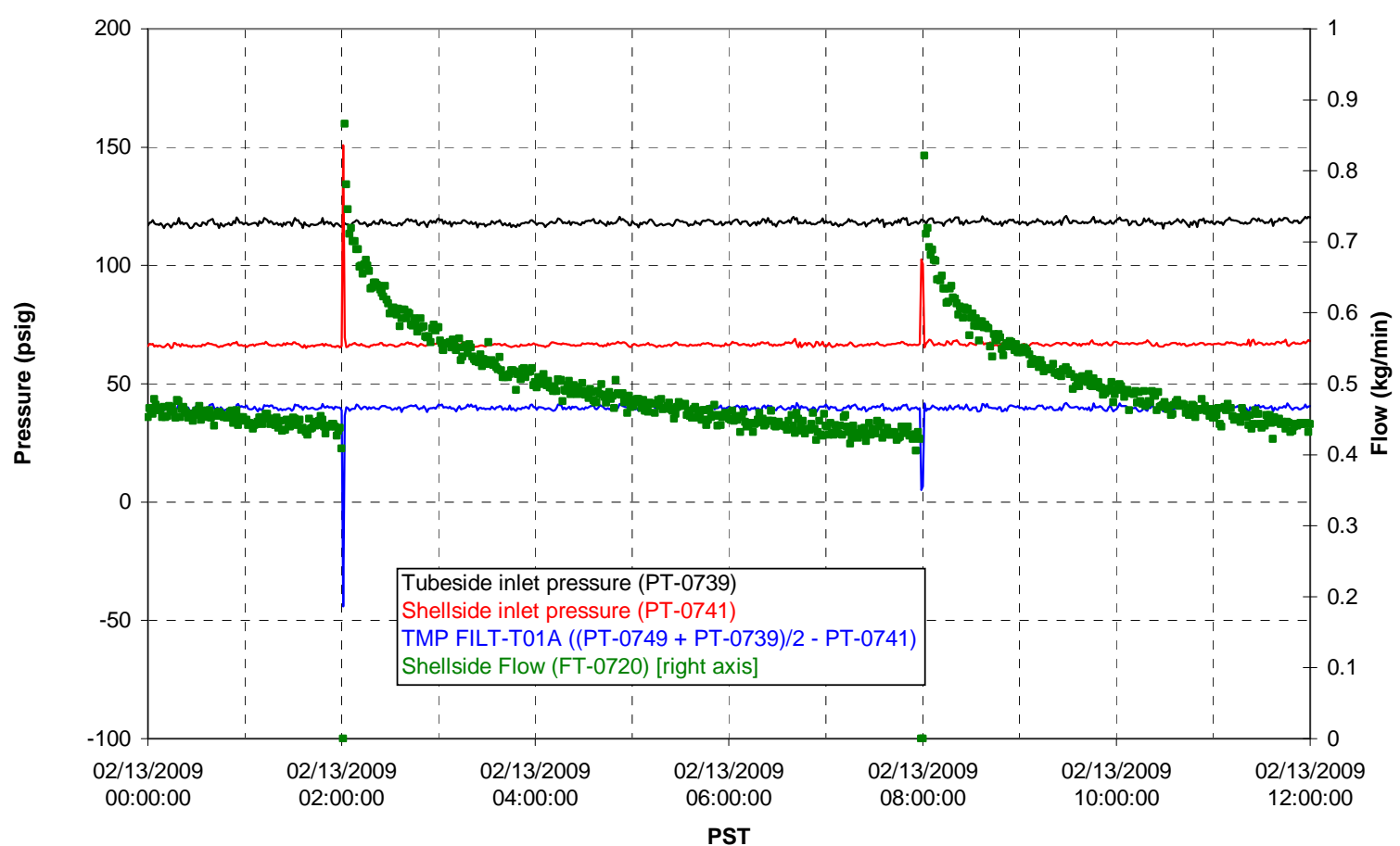

Filter UFP-FILT-T02A

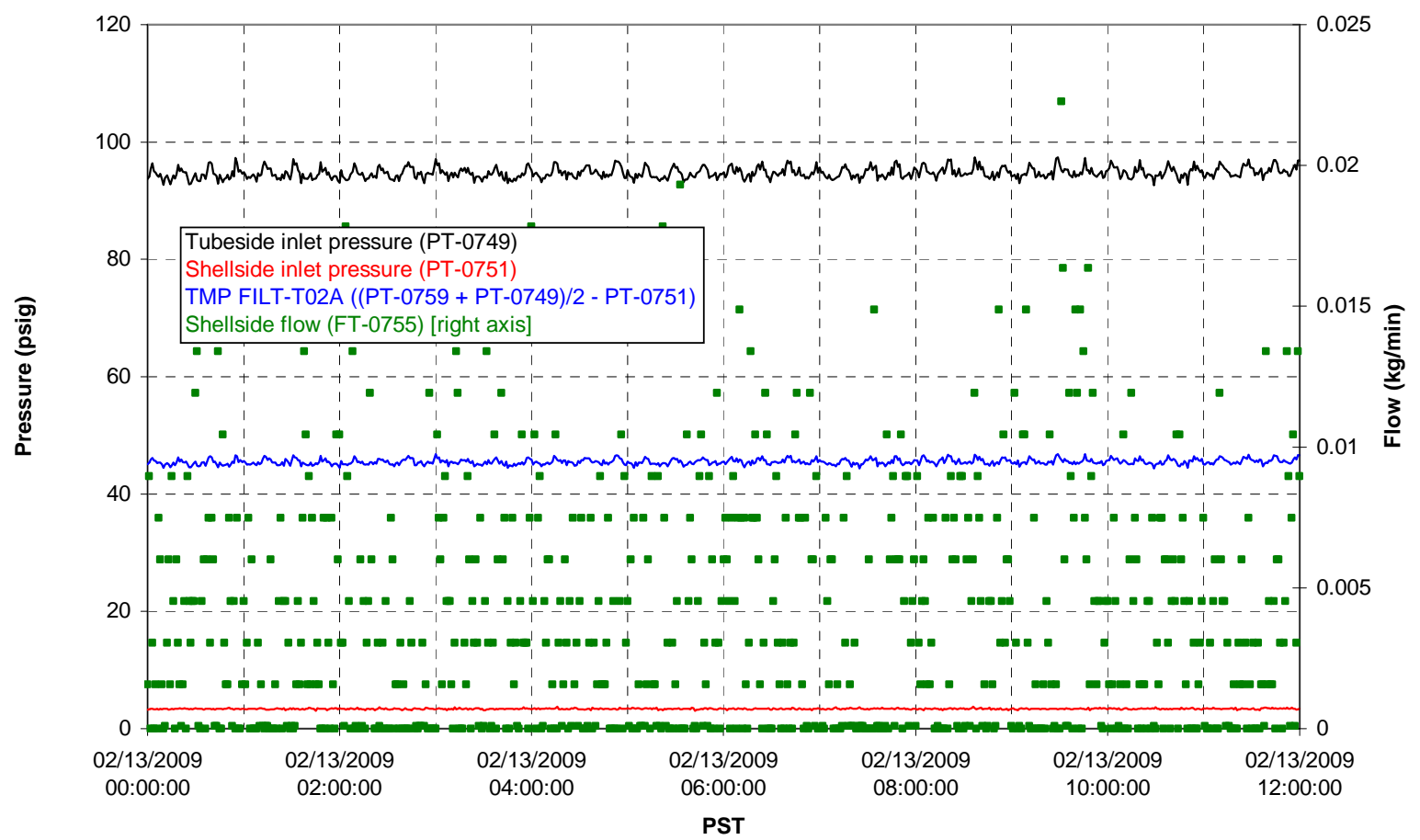


Filter UFP-FILT-T03A

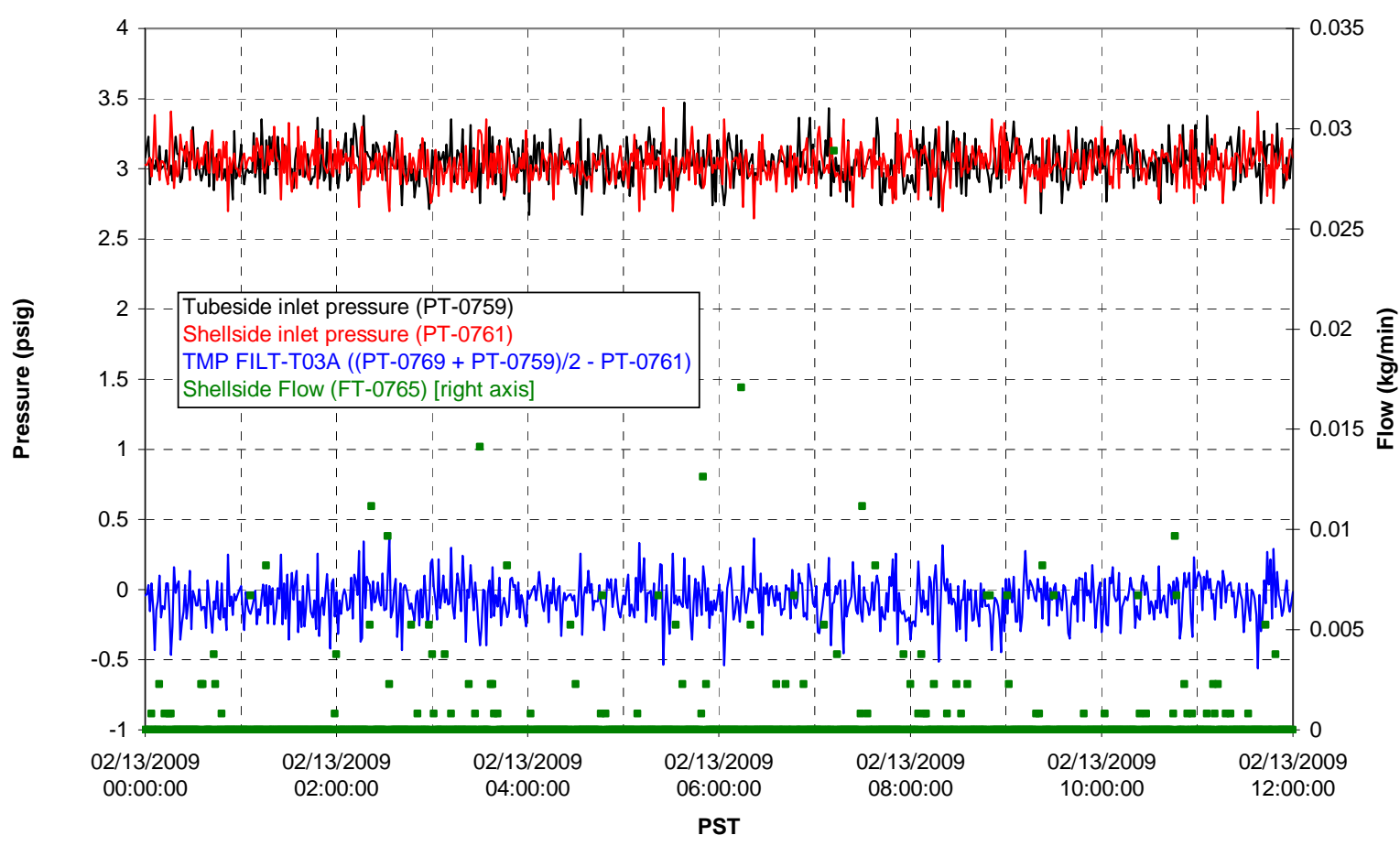

Filter UFP-FILT-T04A

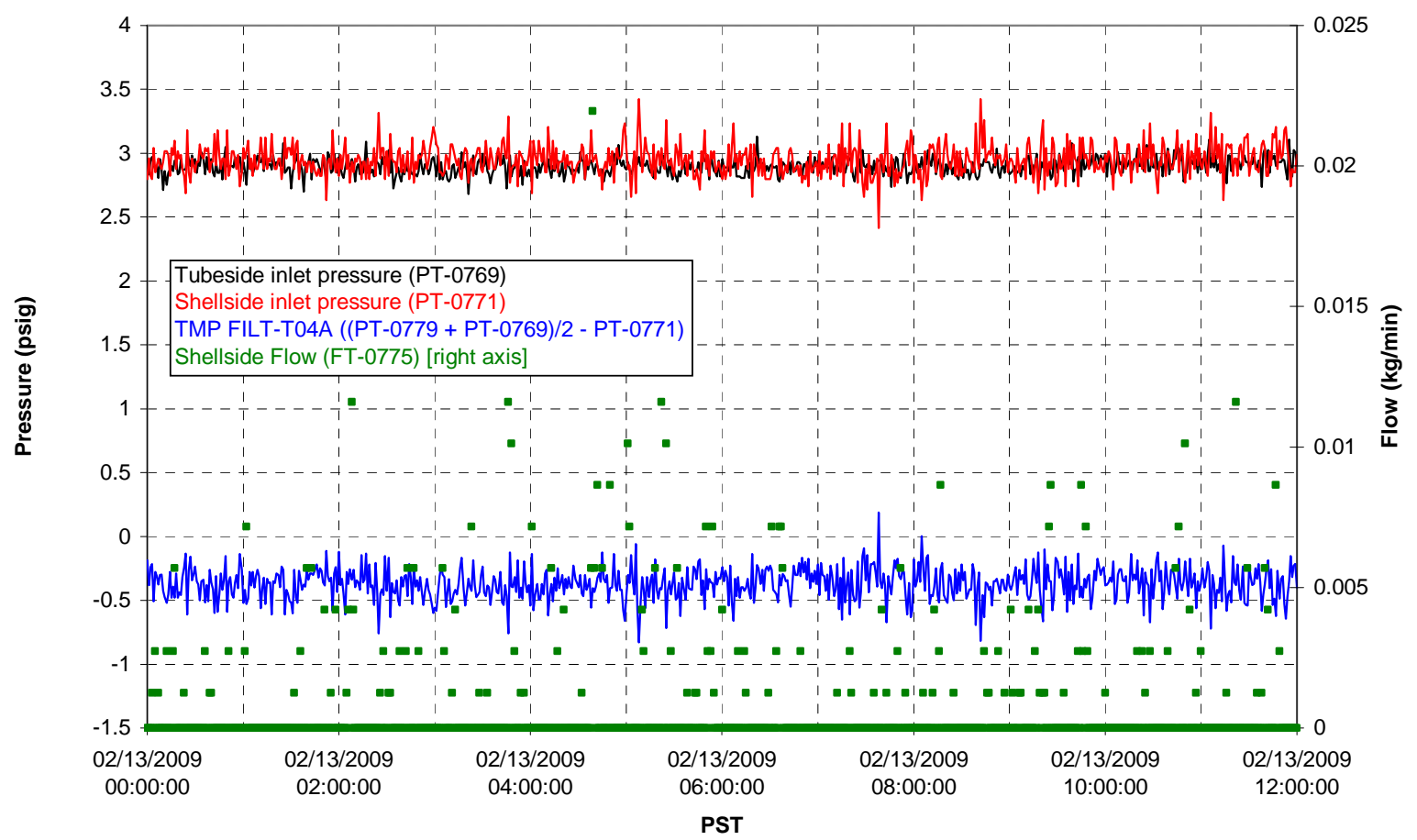


Filter UFP-FILT-T05A

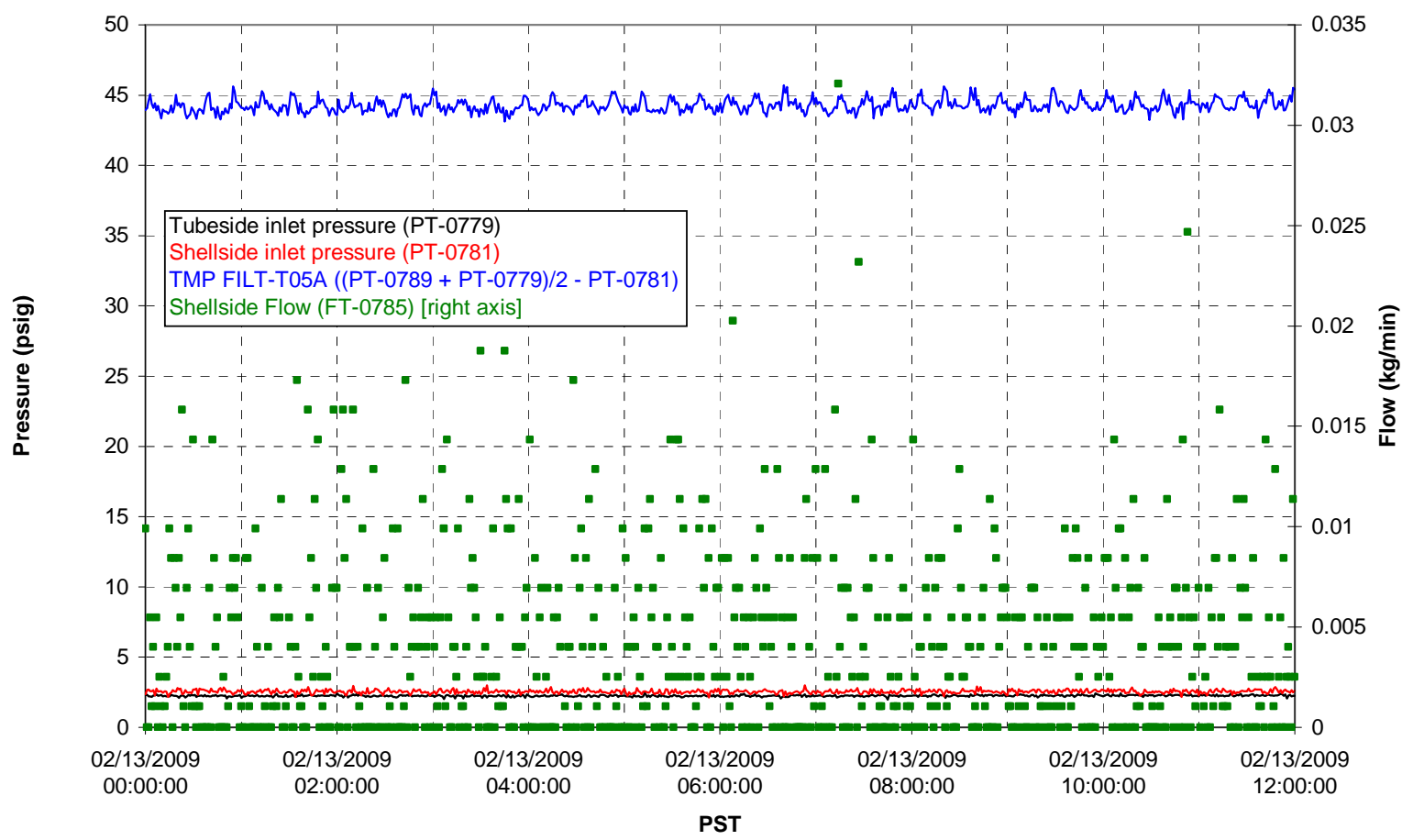

Chemical Flow

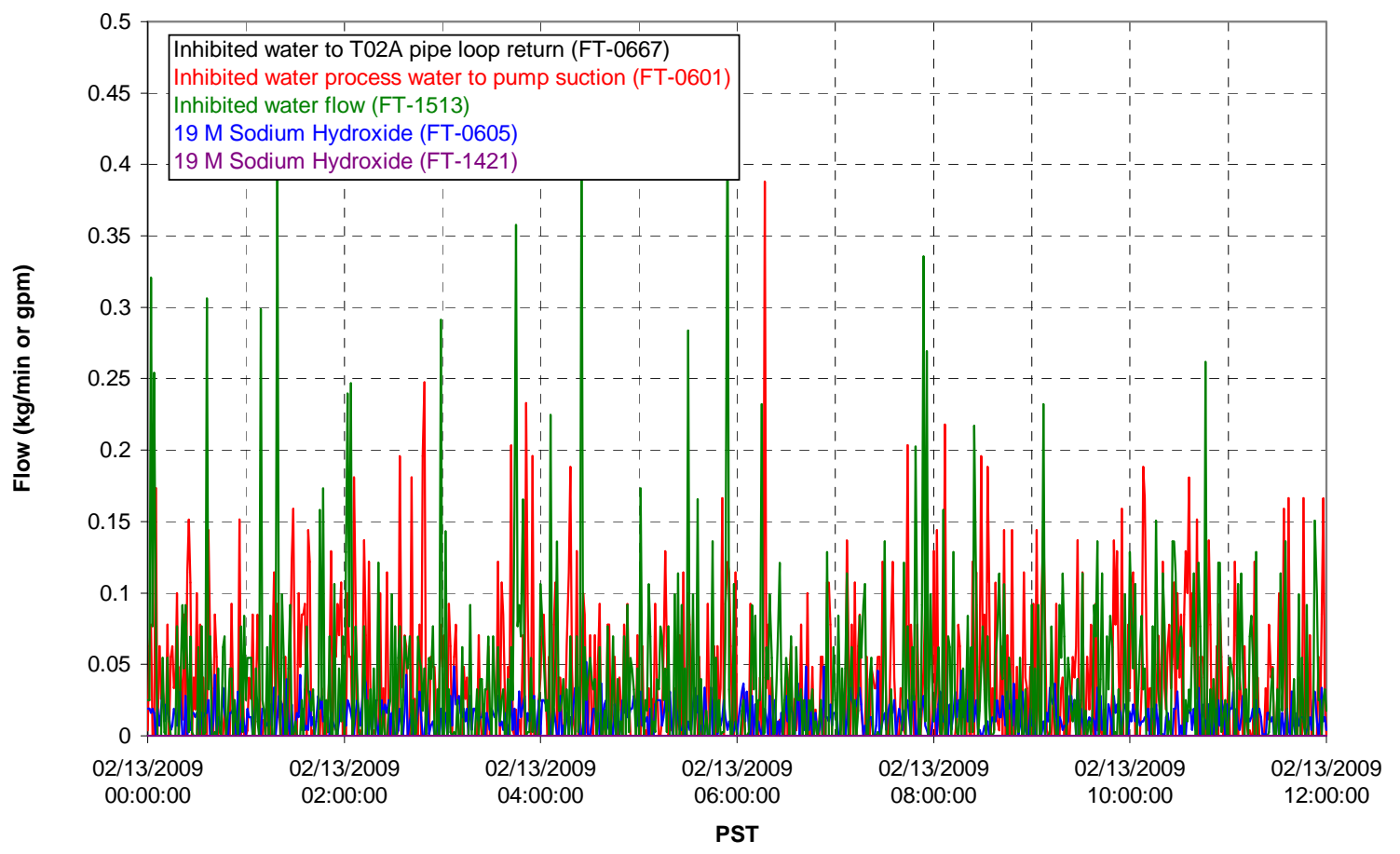


Chemical Flow

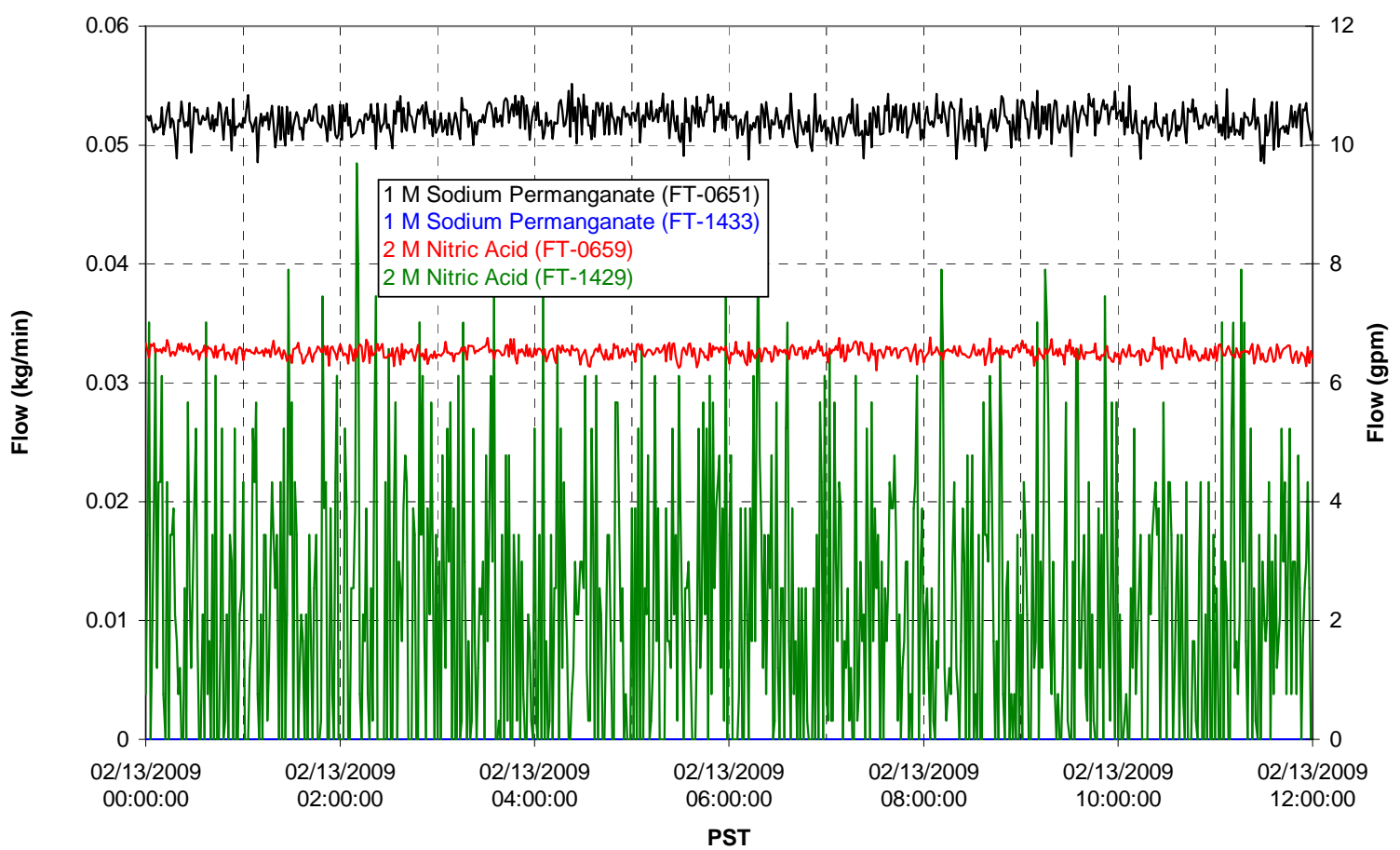

Air Flows

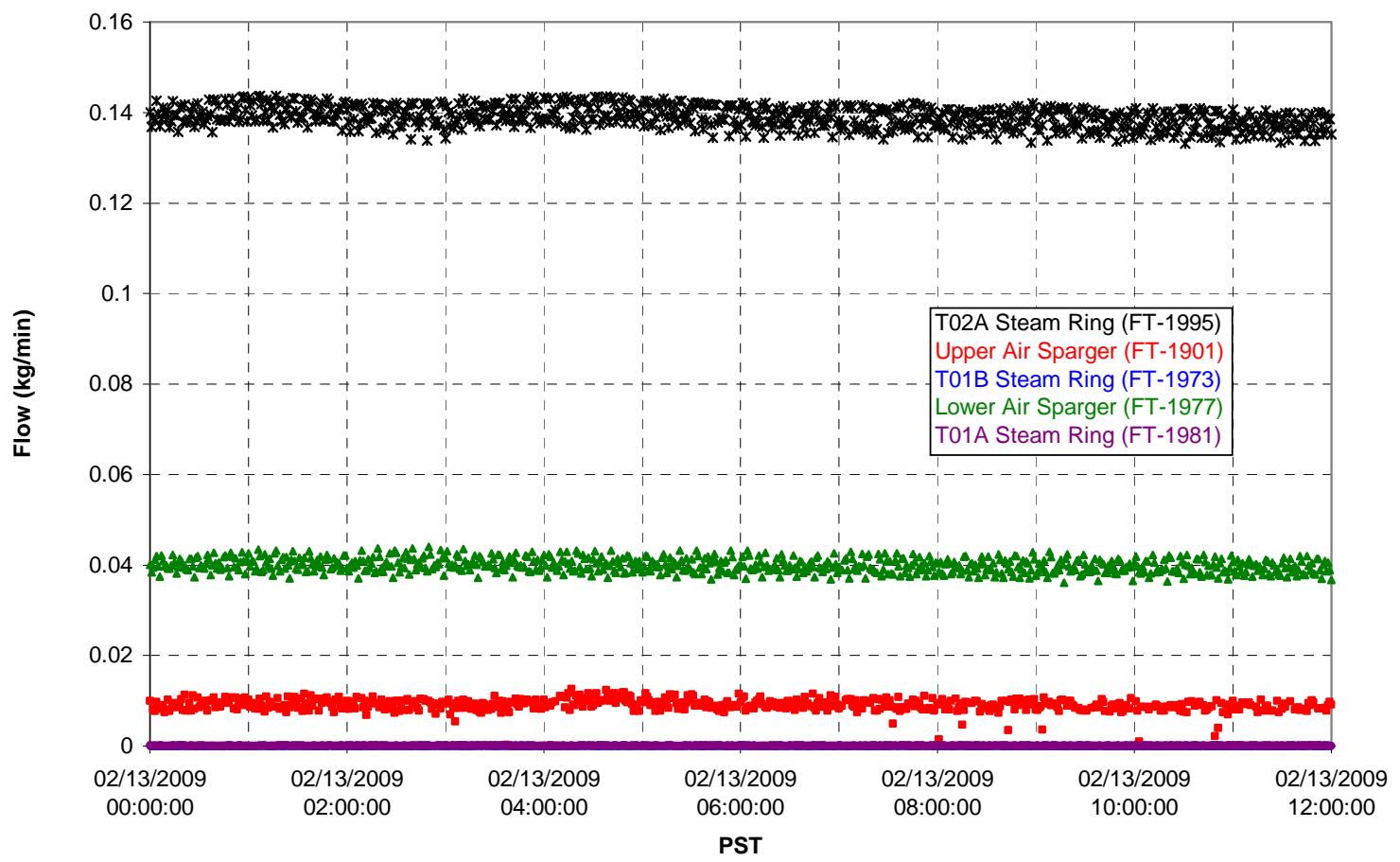


T02A Steam

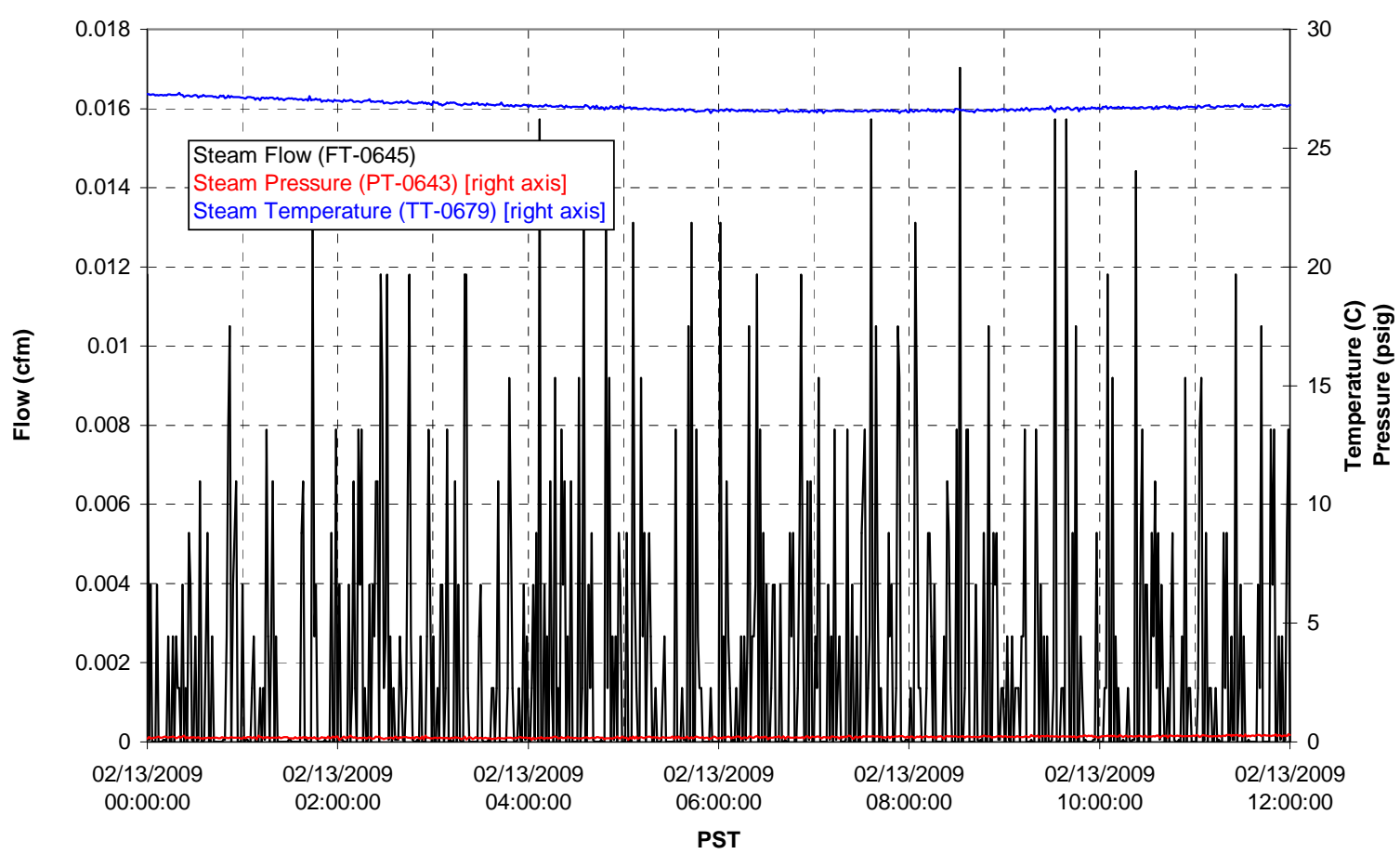

T01A Steam

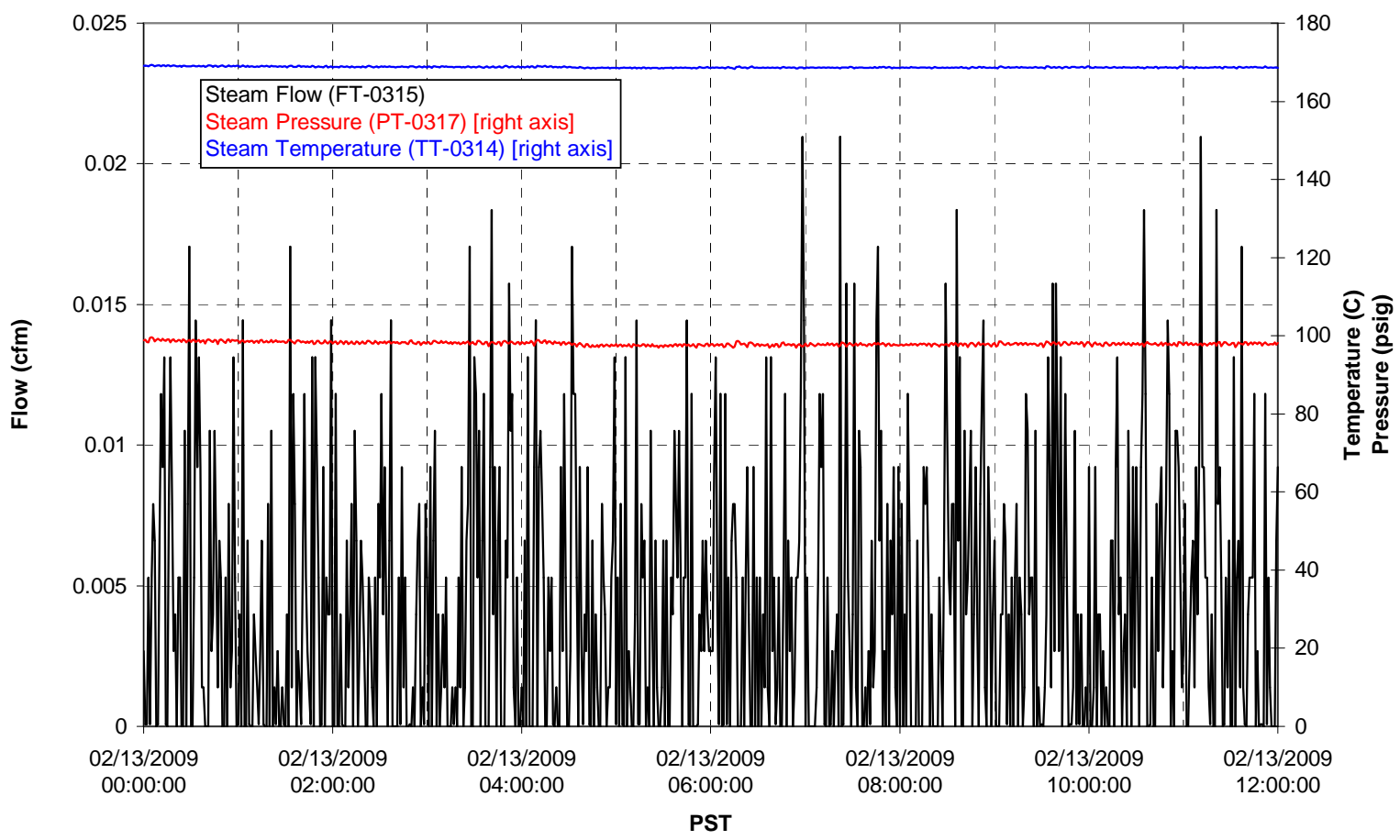


T01B Steam

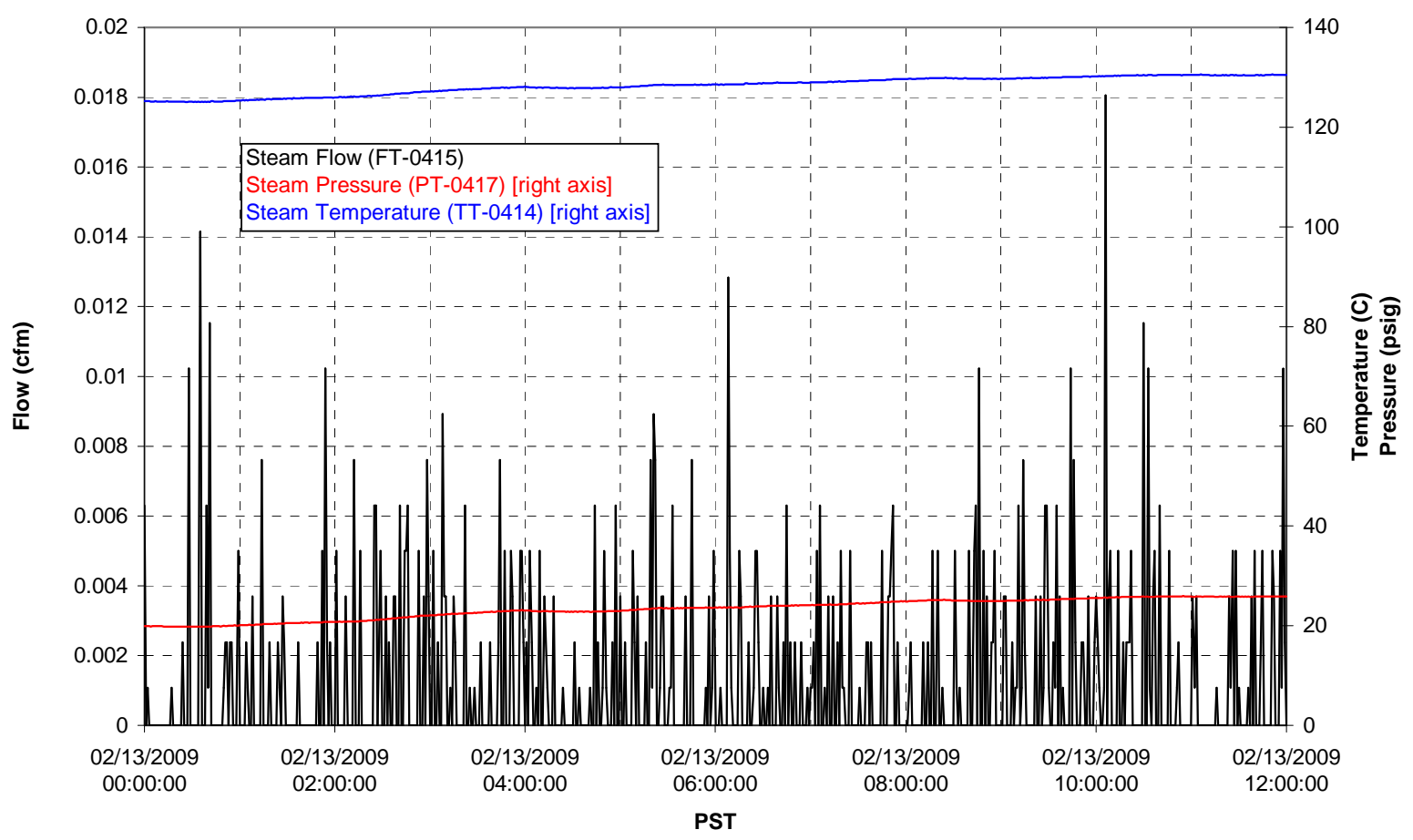




\section{Integrated Test A Data Plots 02/13/09 12:00 - 02/14/09 00:00}


T01A level

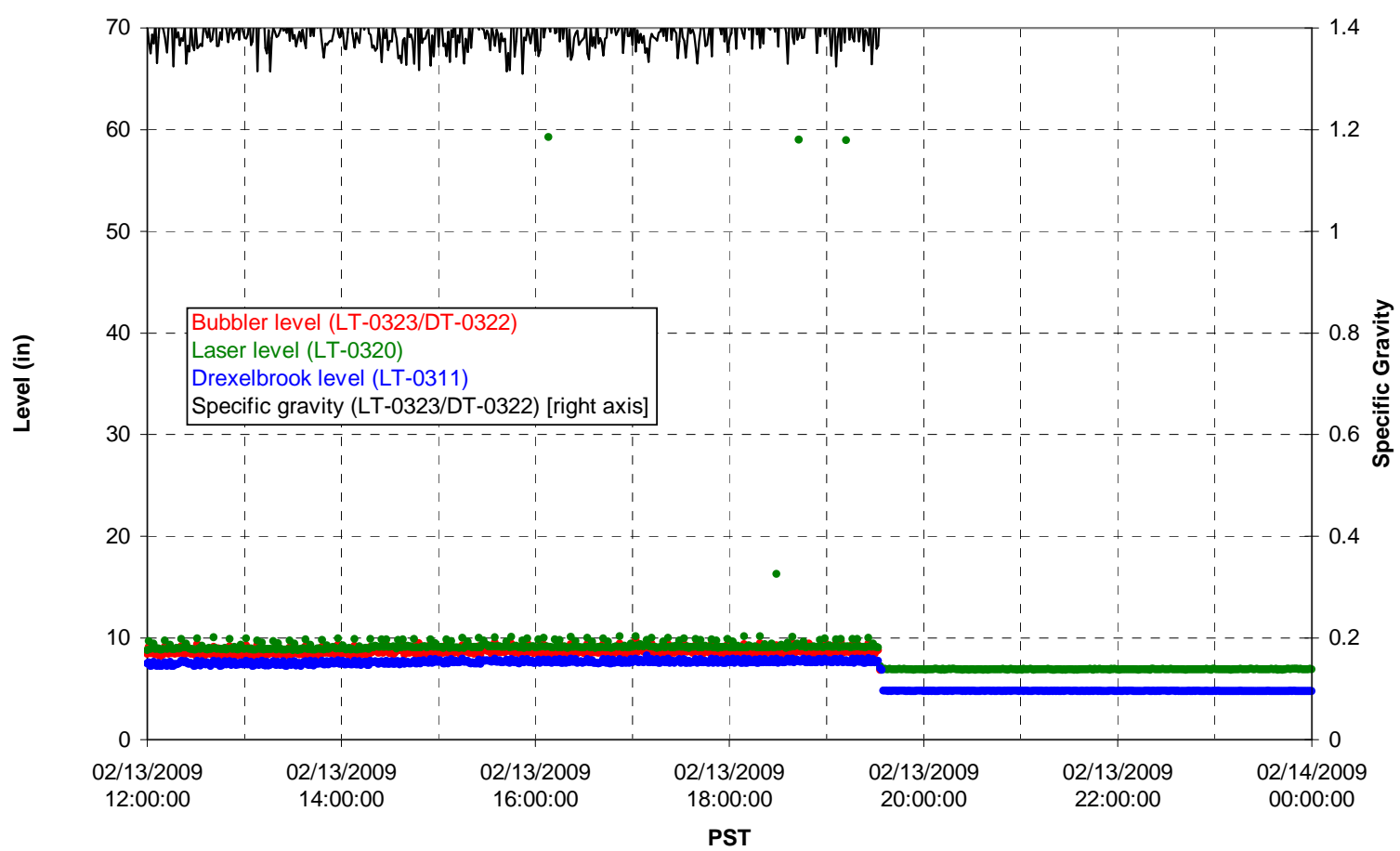

T01A temperatures

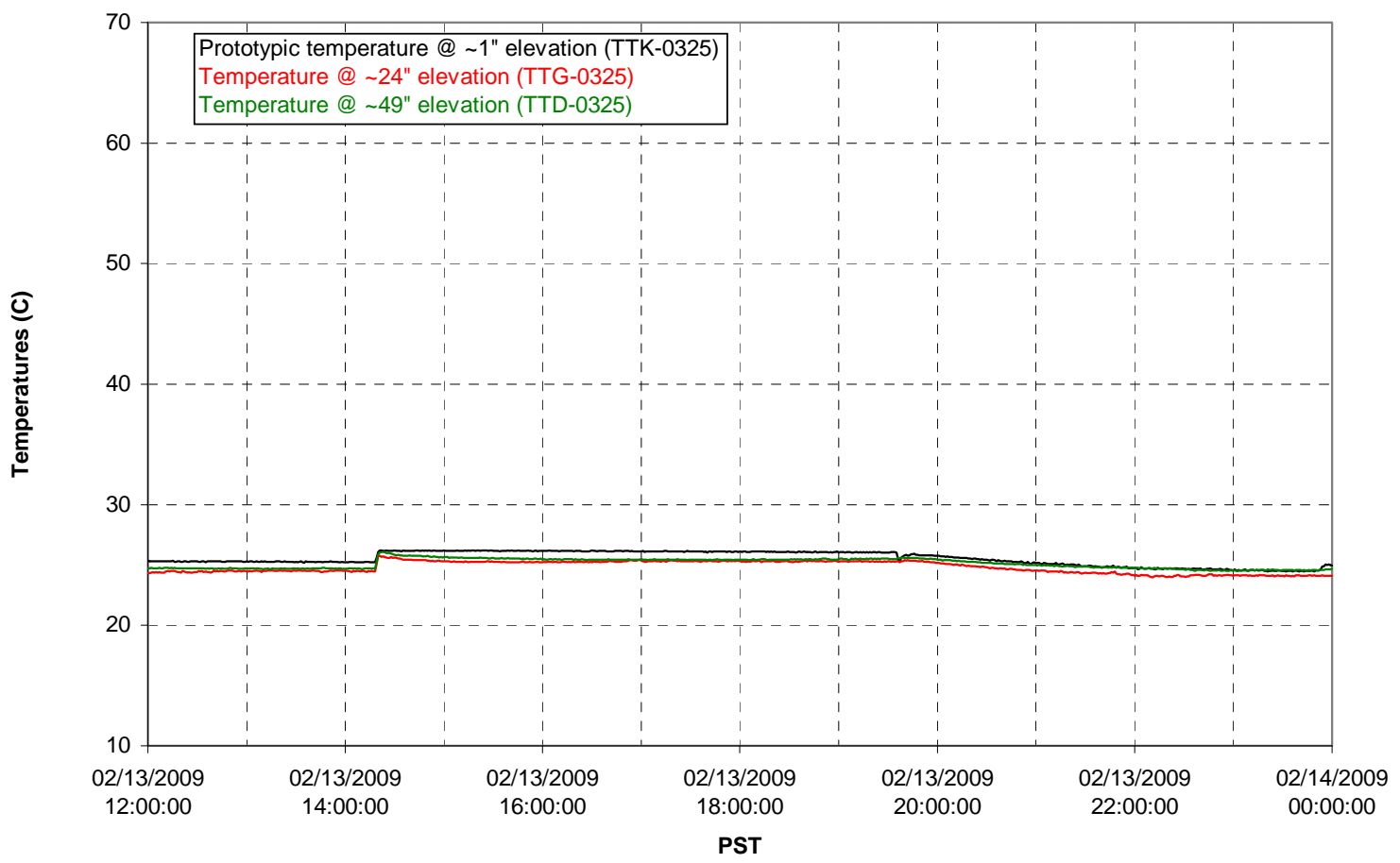


T01B level

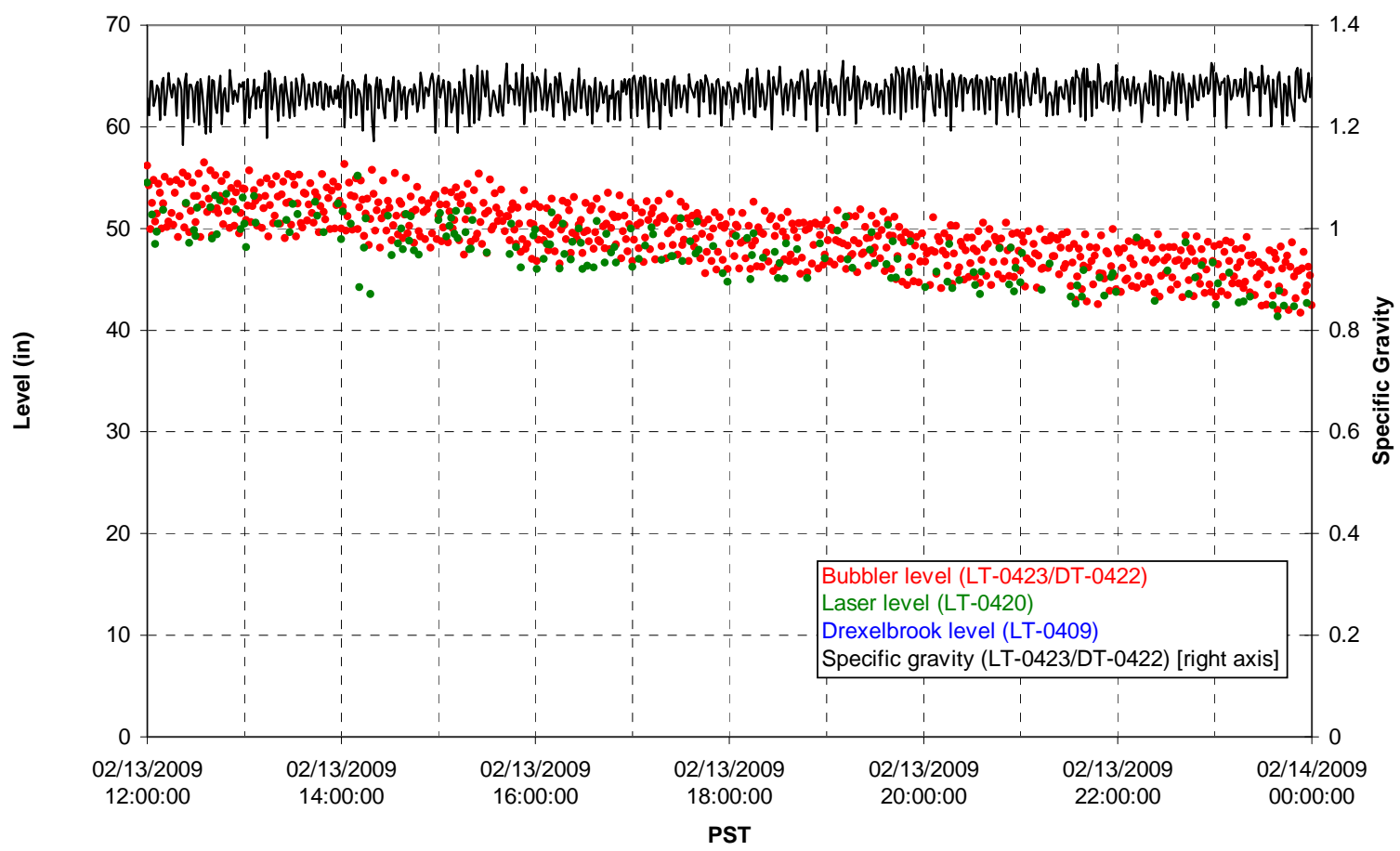

T01B temperatures

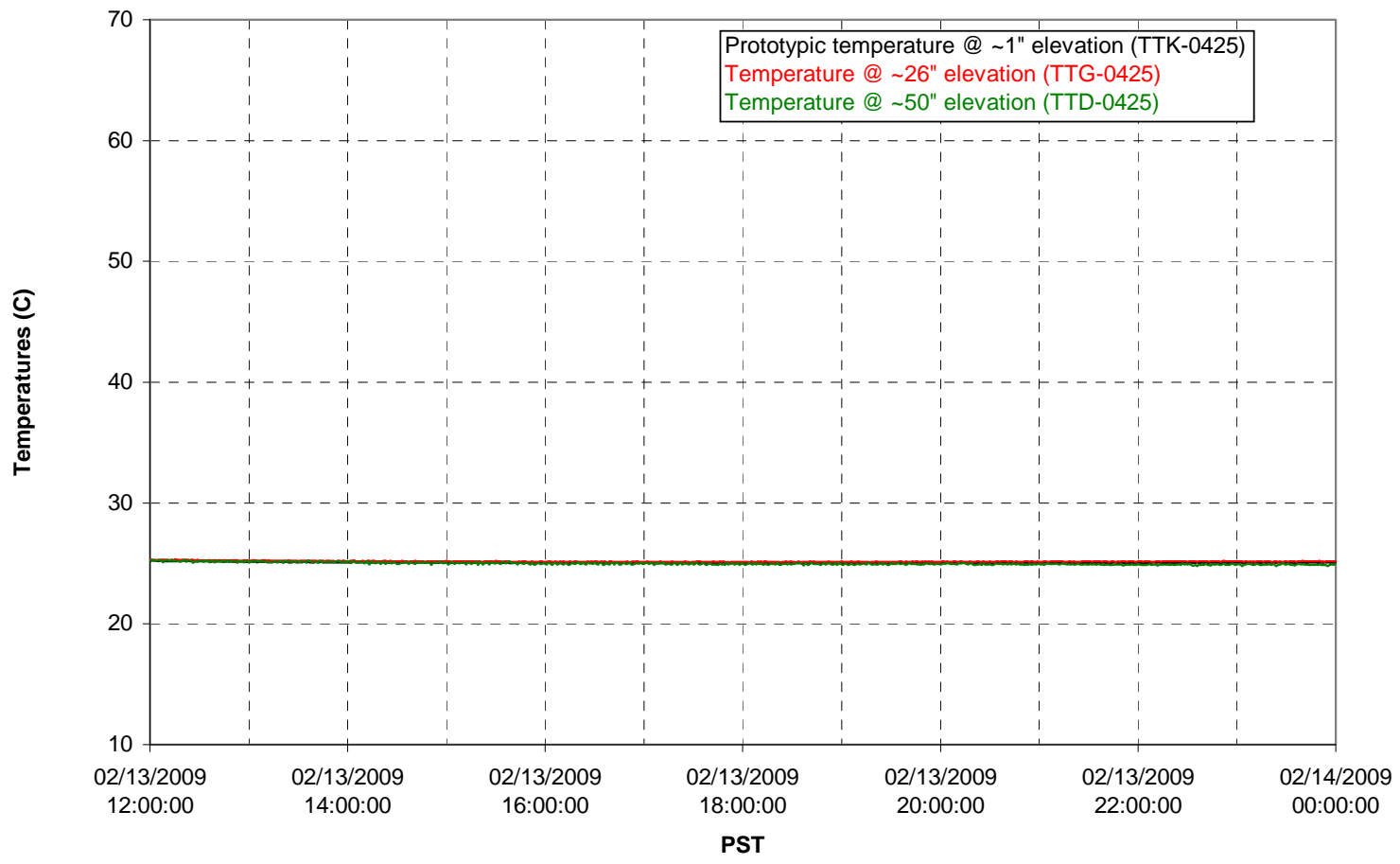


T02A level

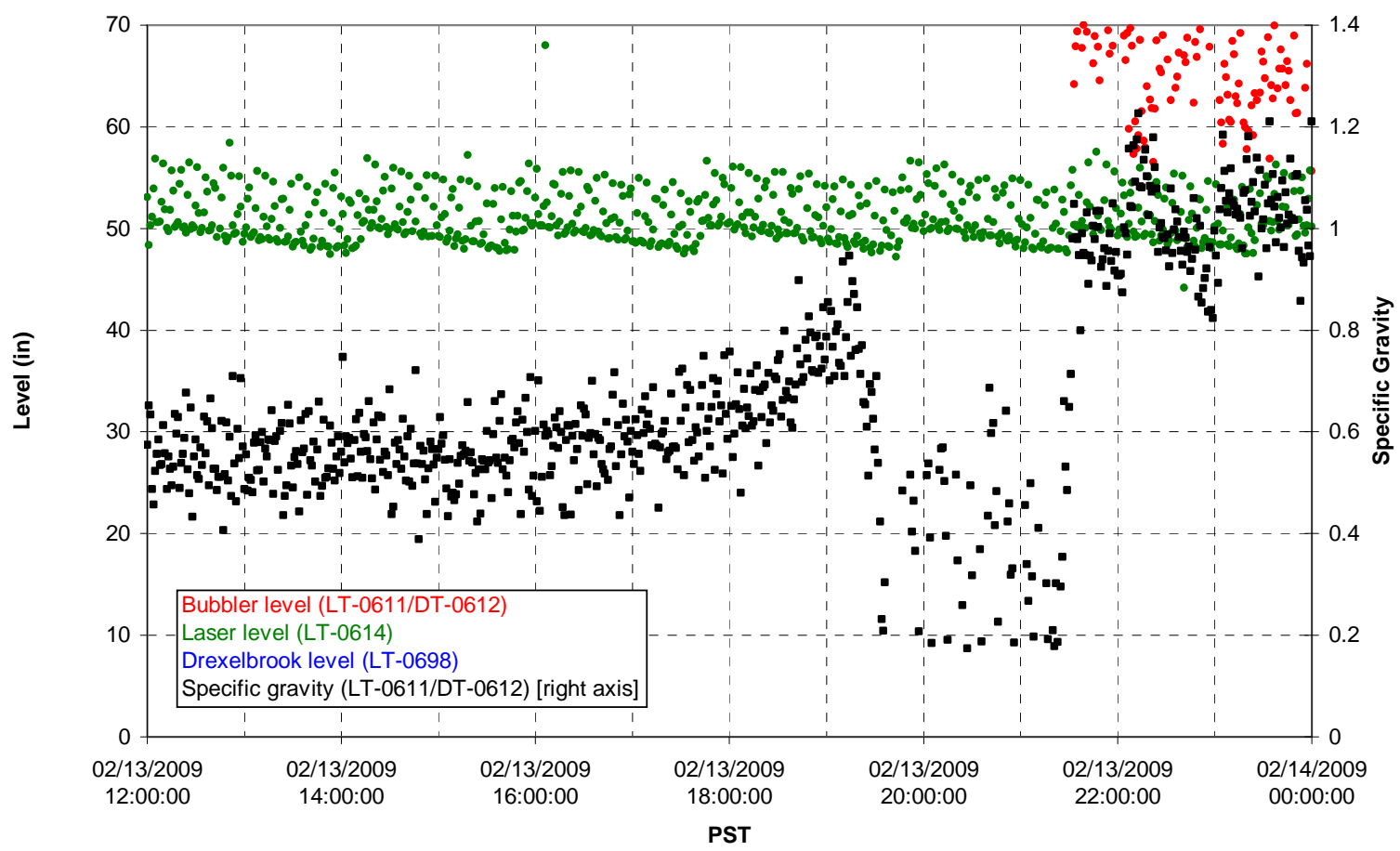

T02A temperatures

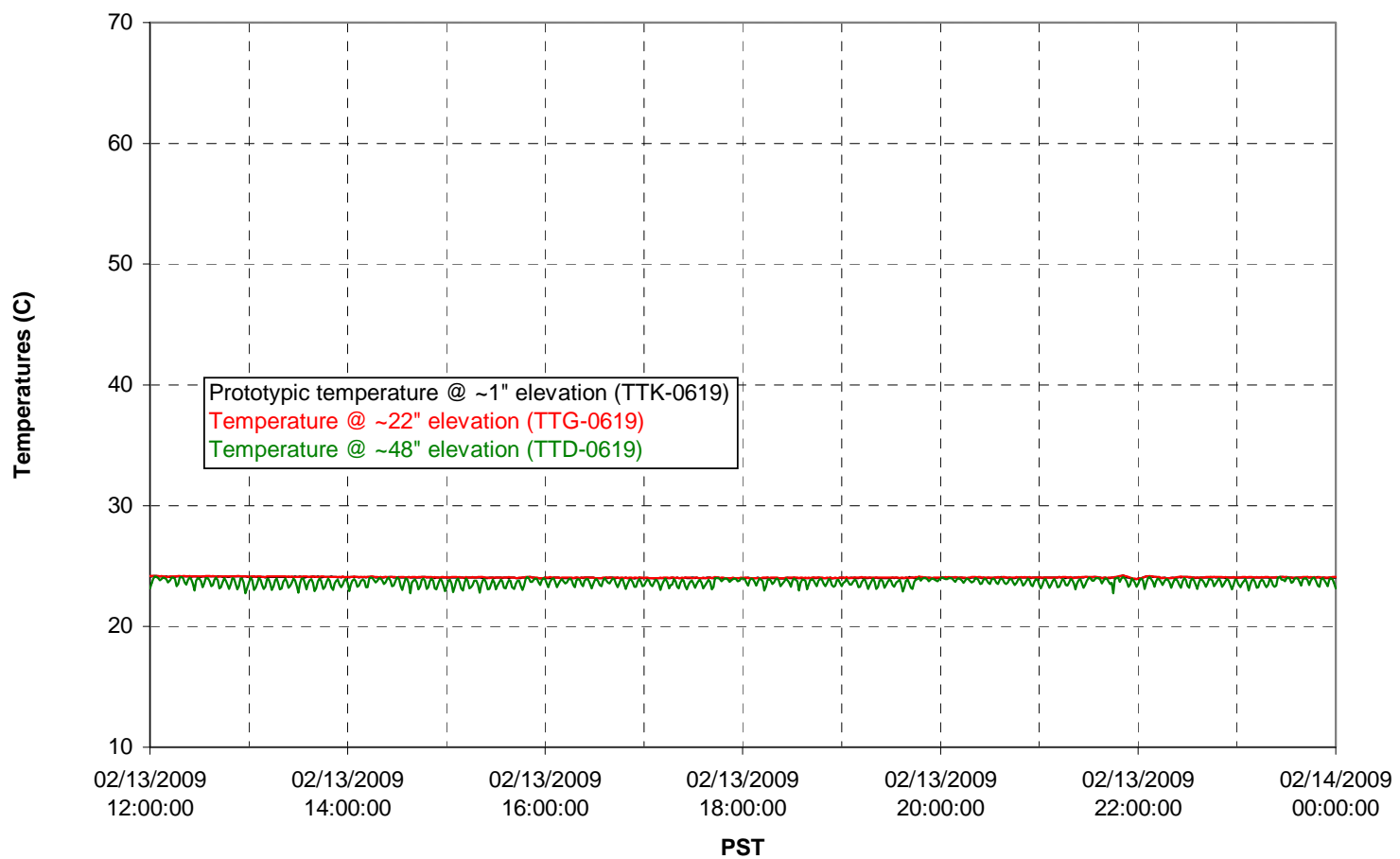


T02A and filter loop temperatures

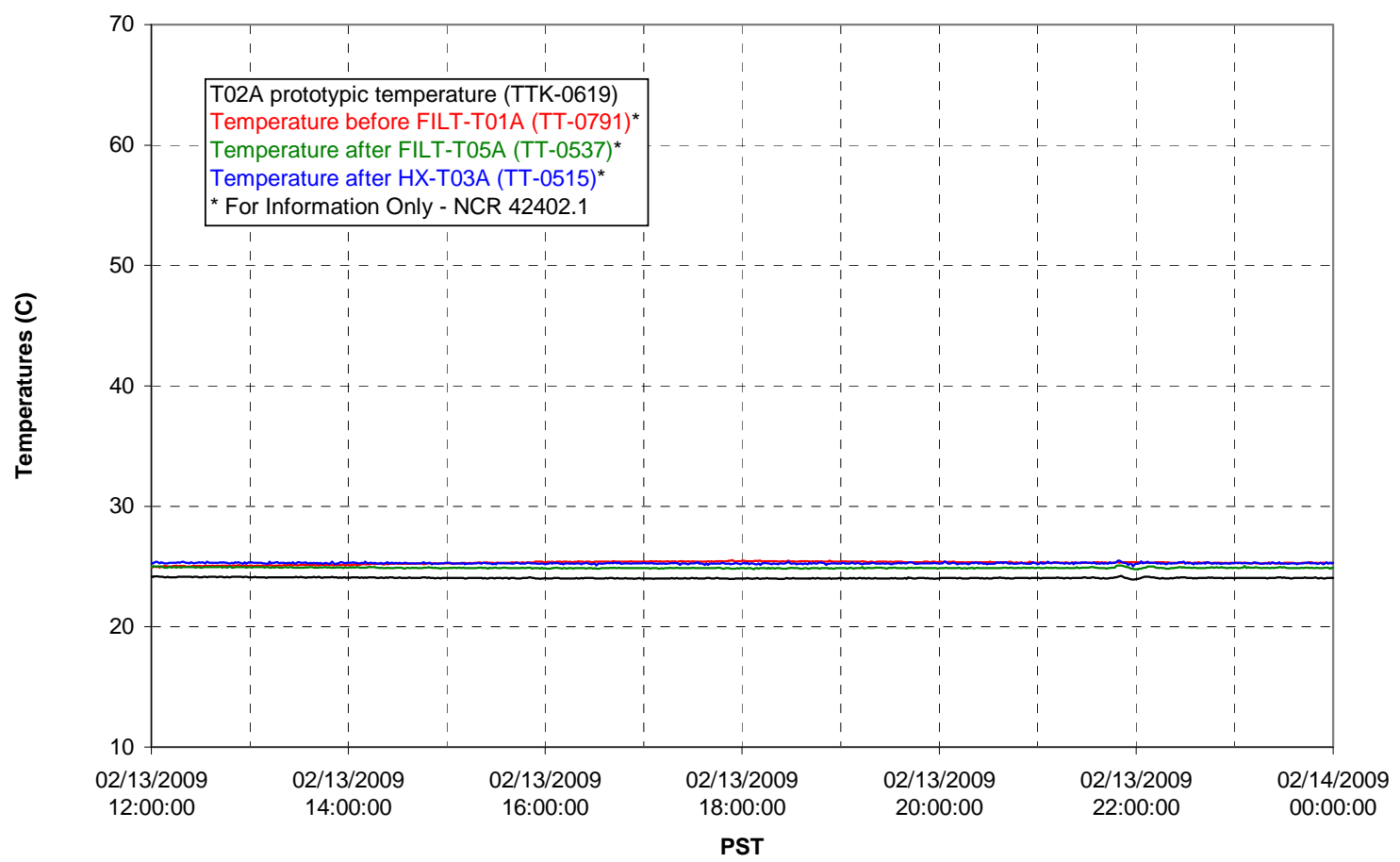

Pump Pressures and Flow

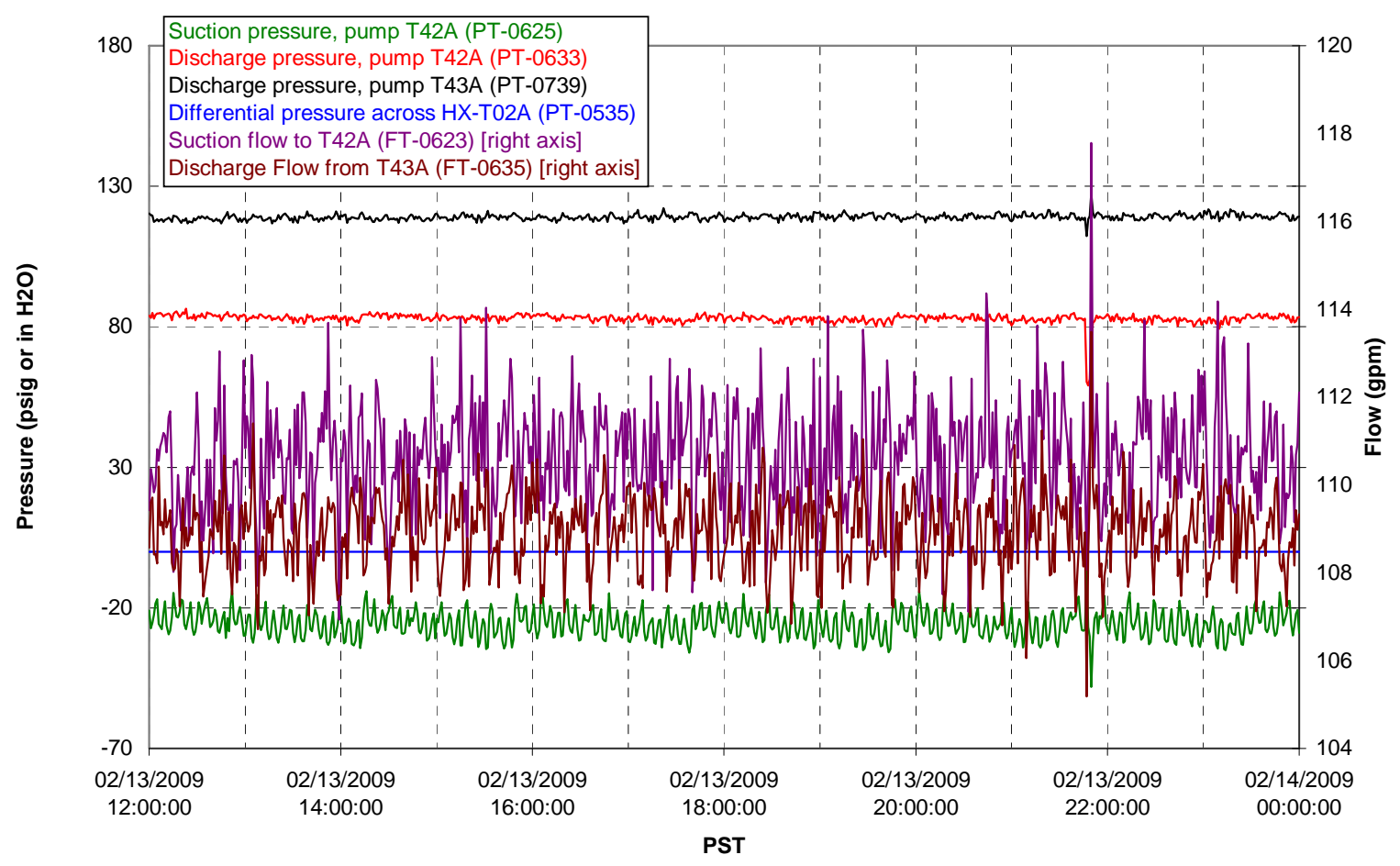


Axial pressure drop

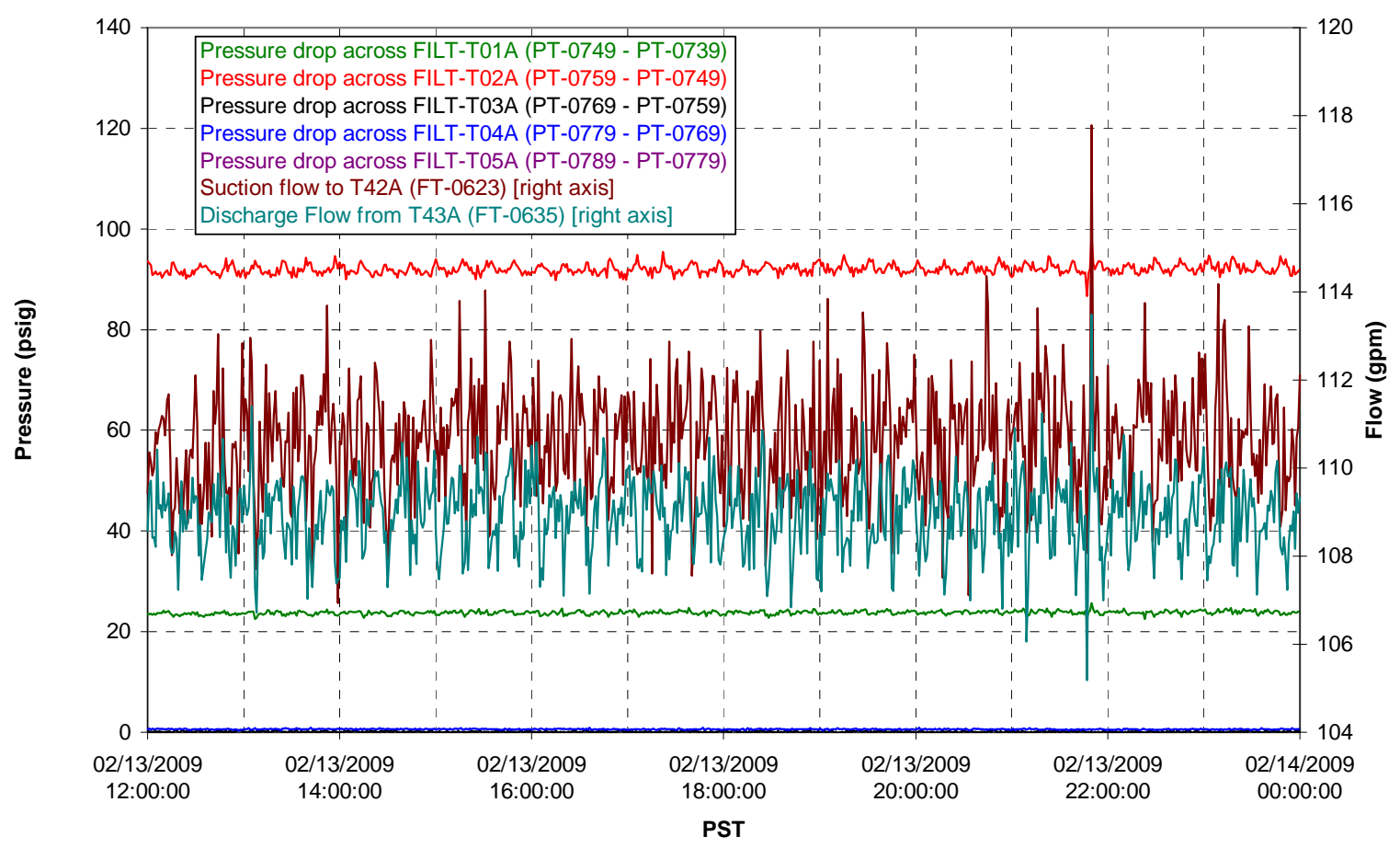

Permeate flow rates

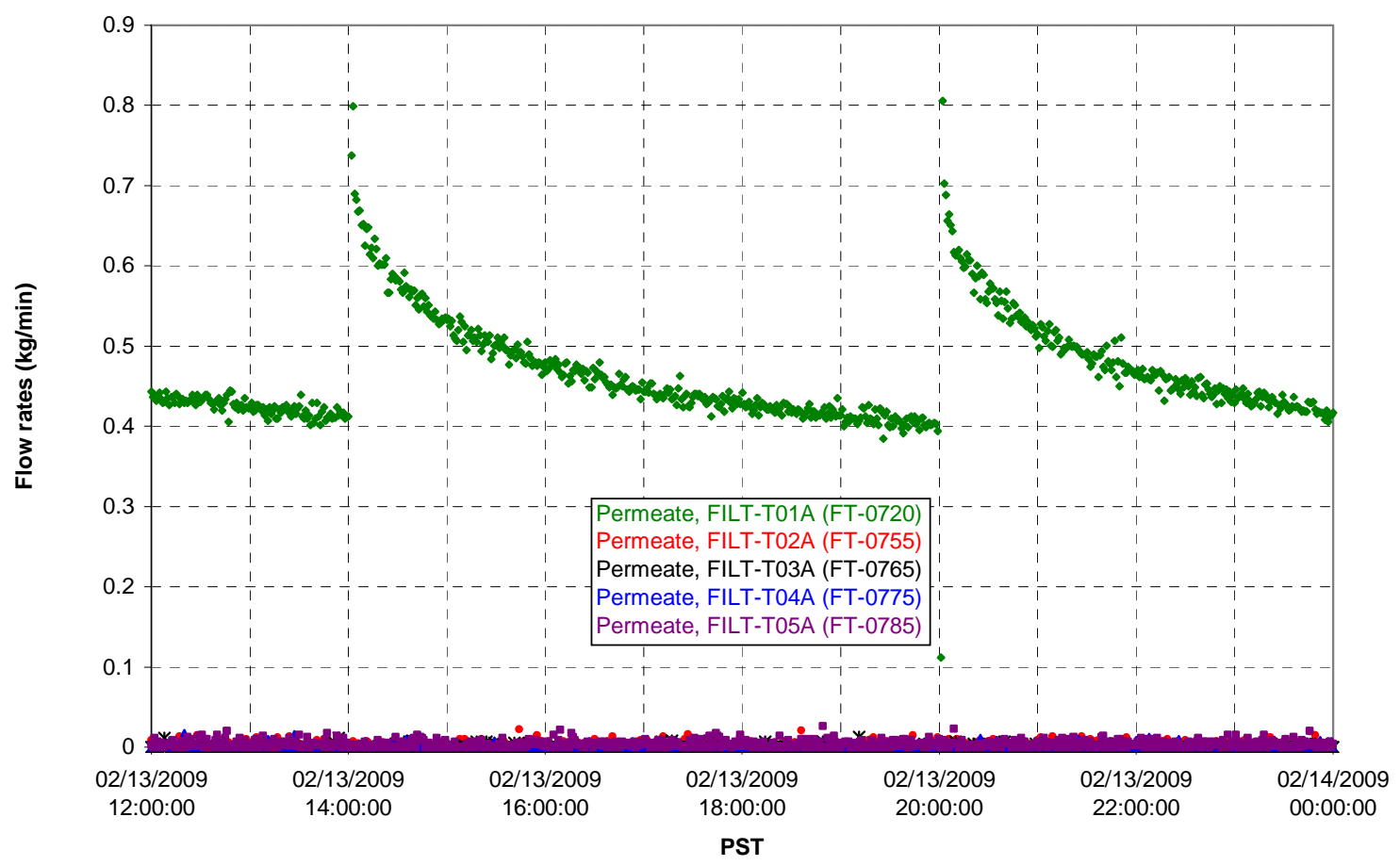


T02A Inner Temperature Tree

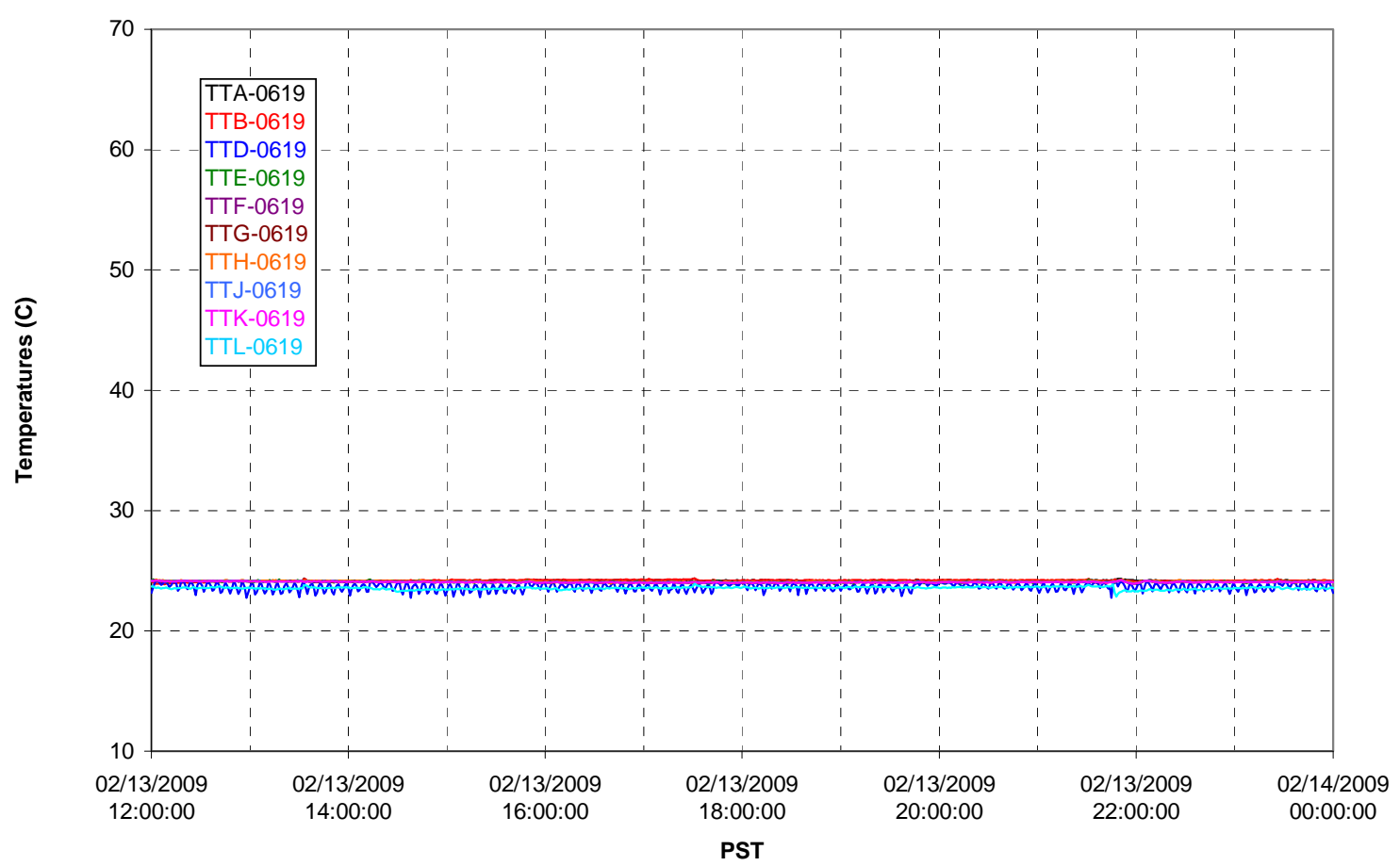

T02A Outer Temperature Tree

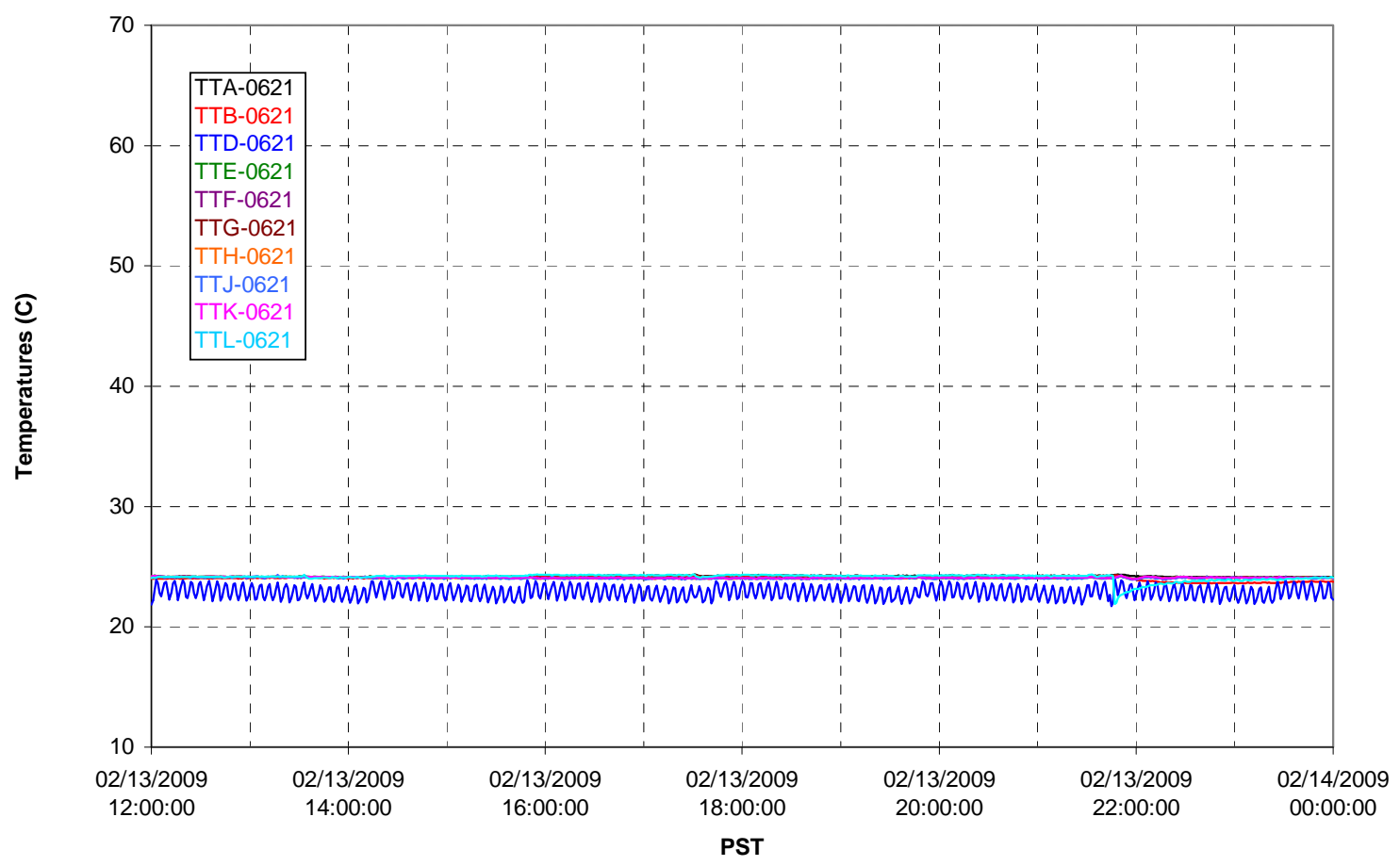


T02A temperatures

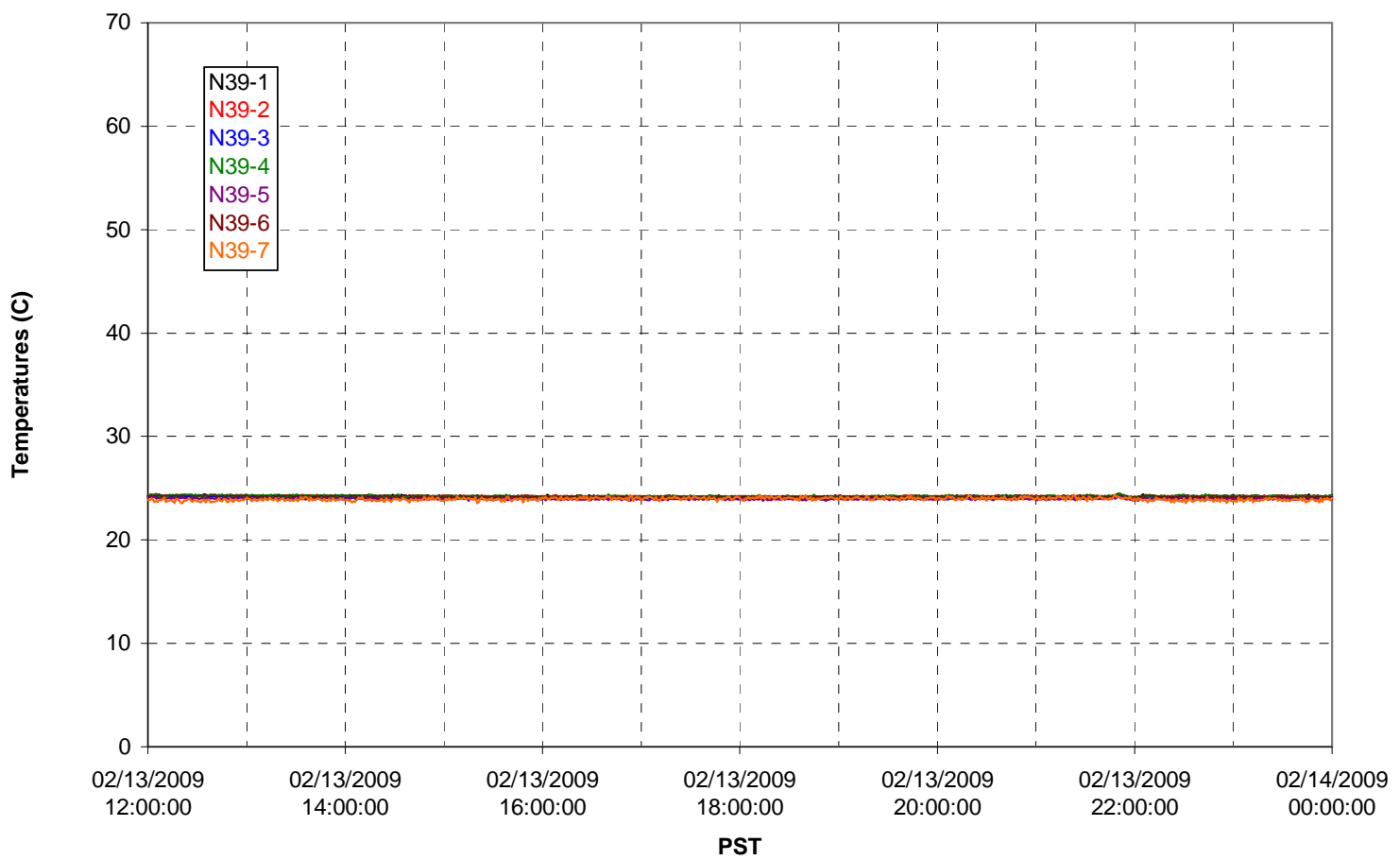

T02A temperatures

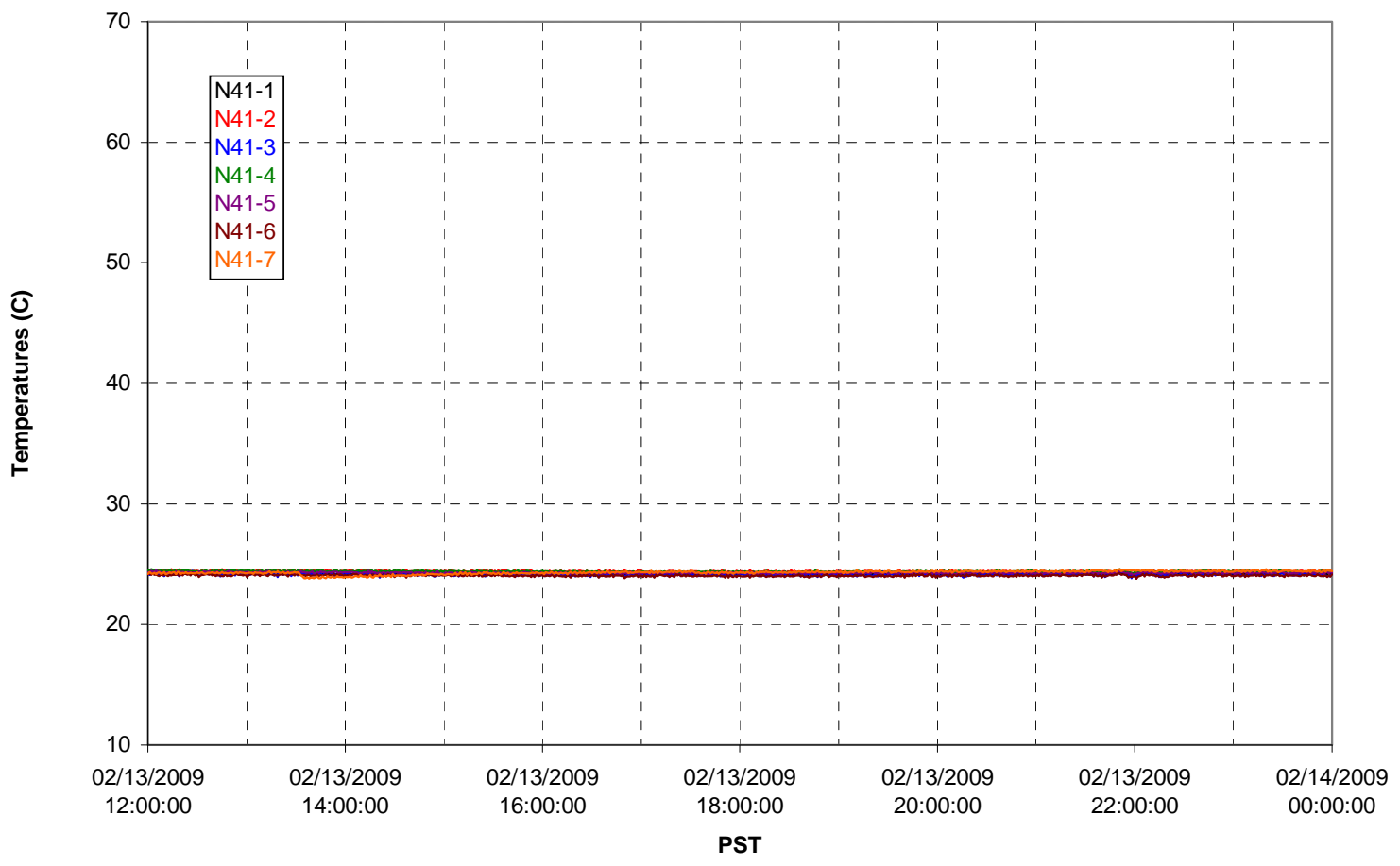


T02A temperatures

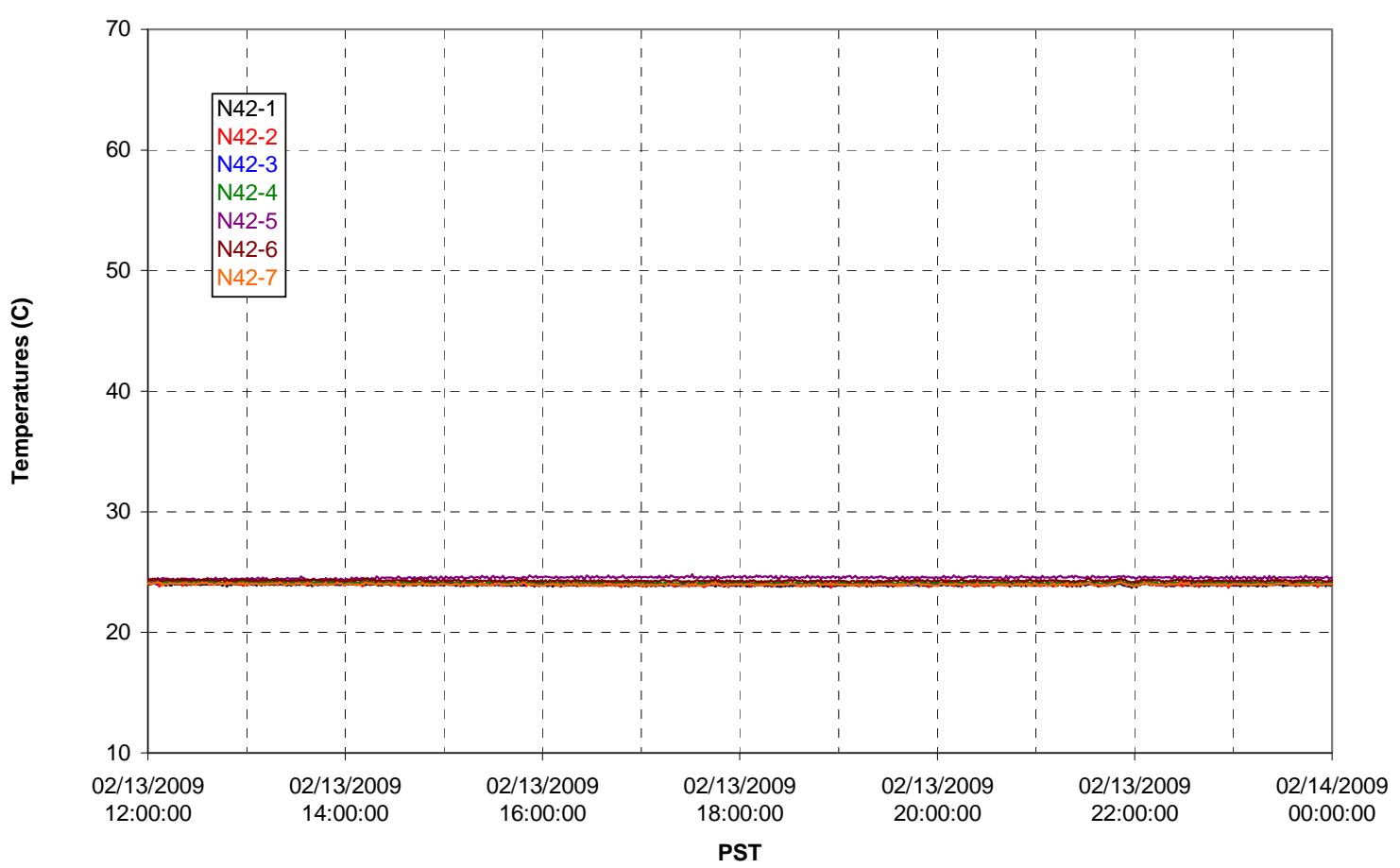

T02A temperatures

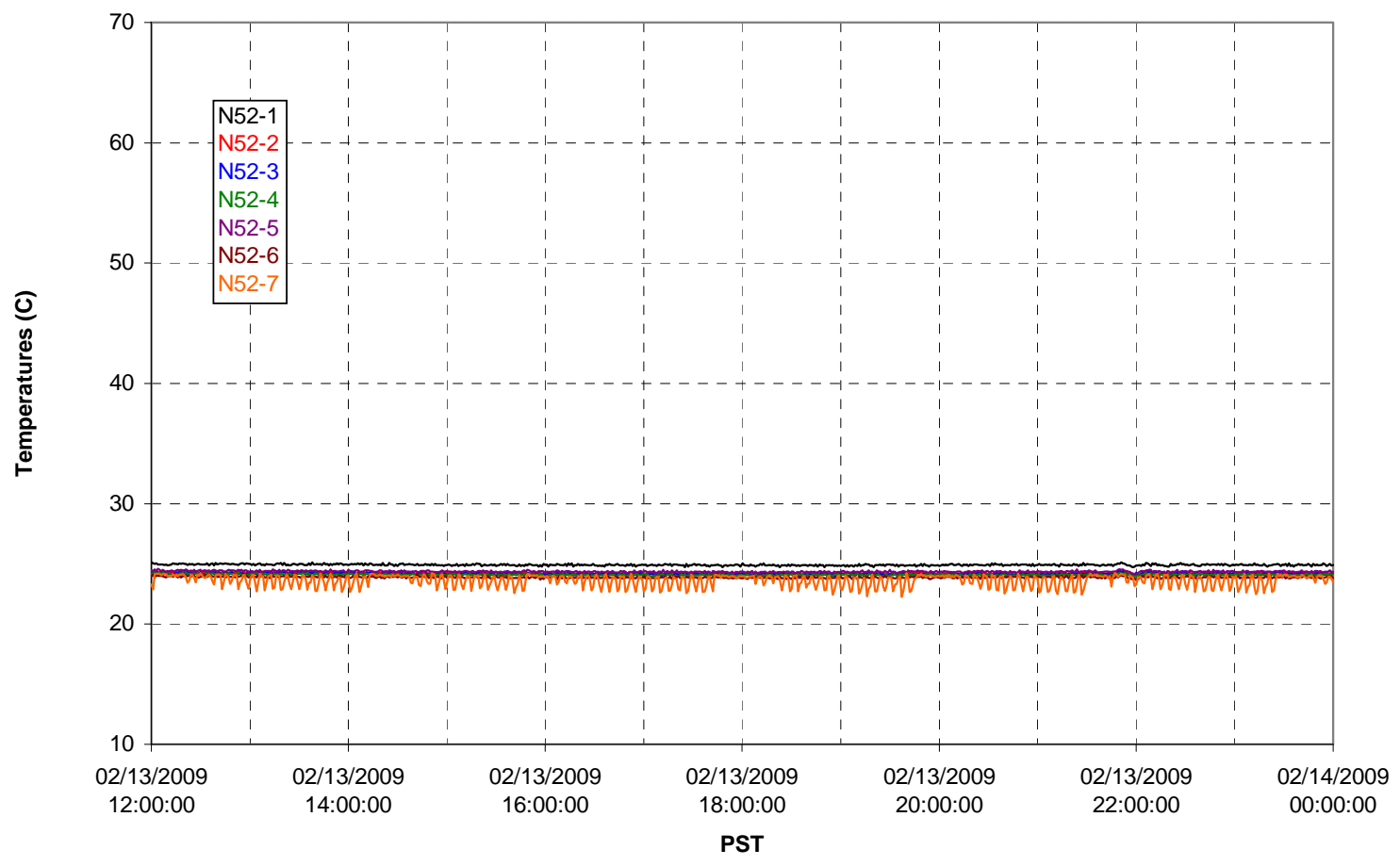


T02A Heating and Cooling
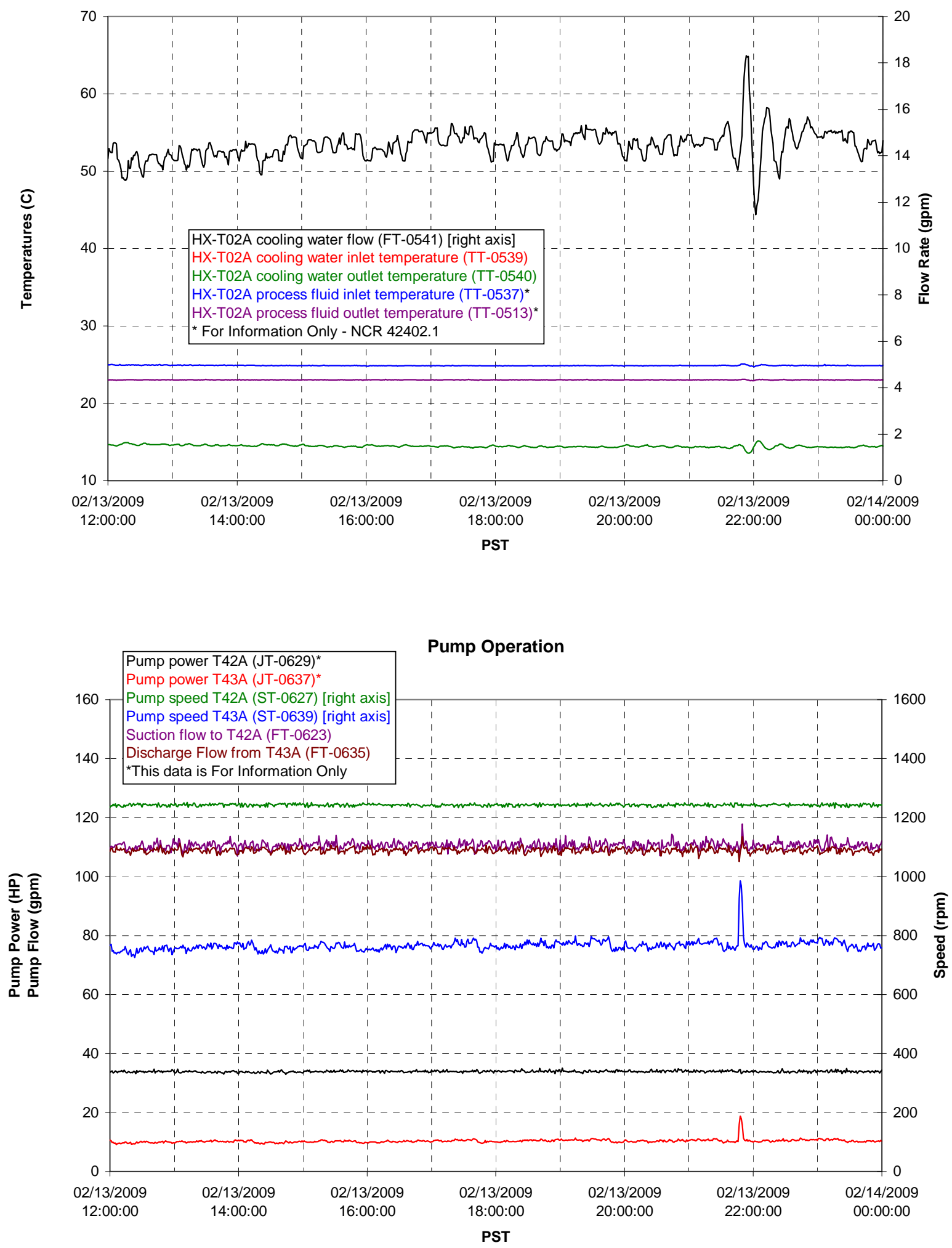
Pulsepot UFP-PP-T01A

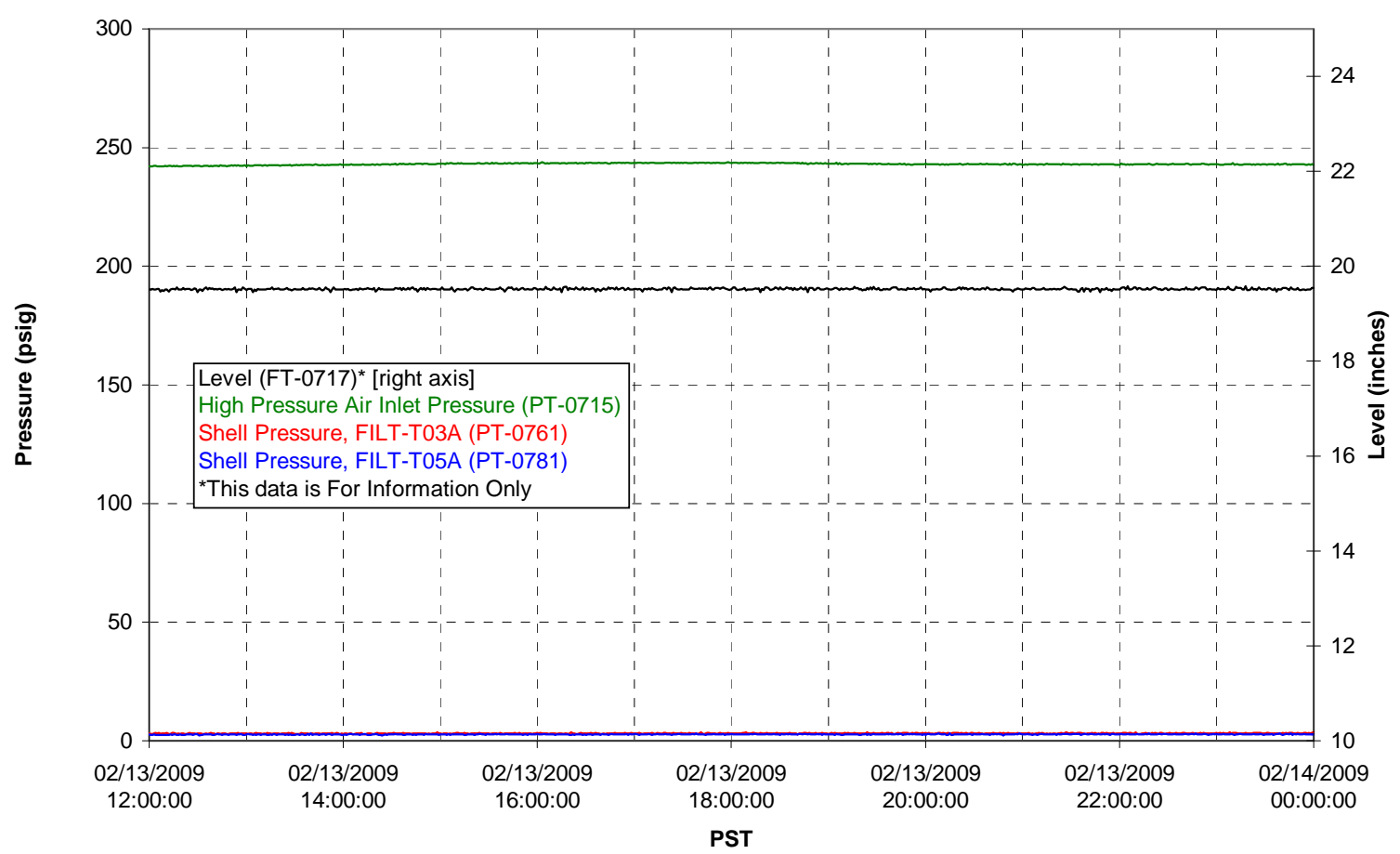

Pulsepot UFP-PP-T02A

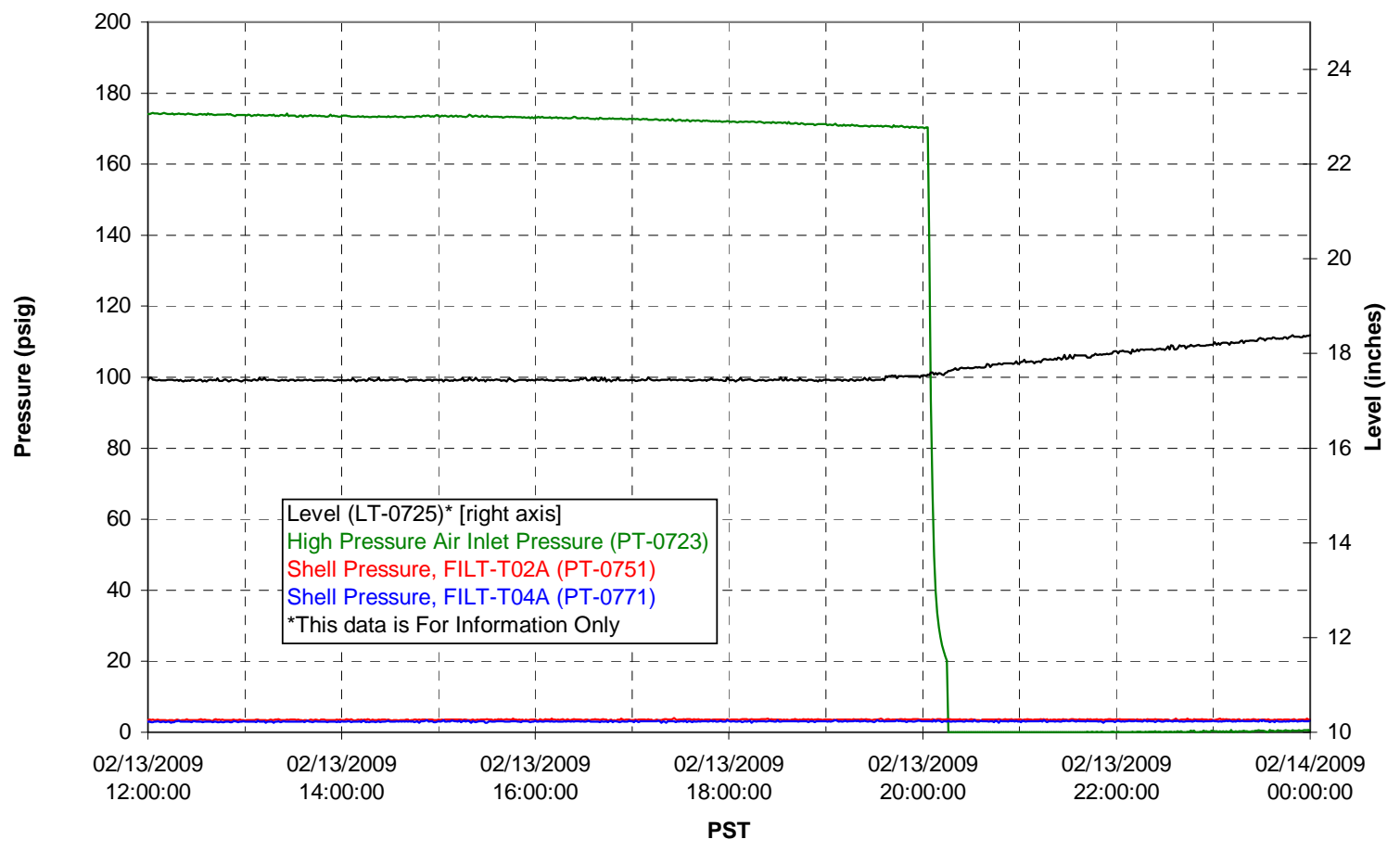


Pulsepot UFP-PP-T03A

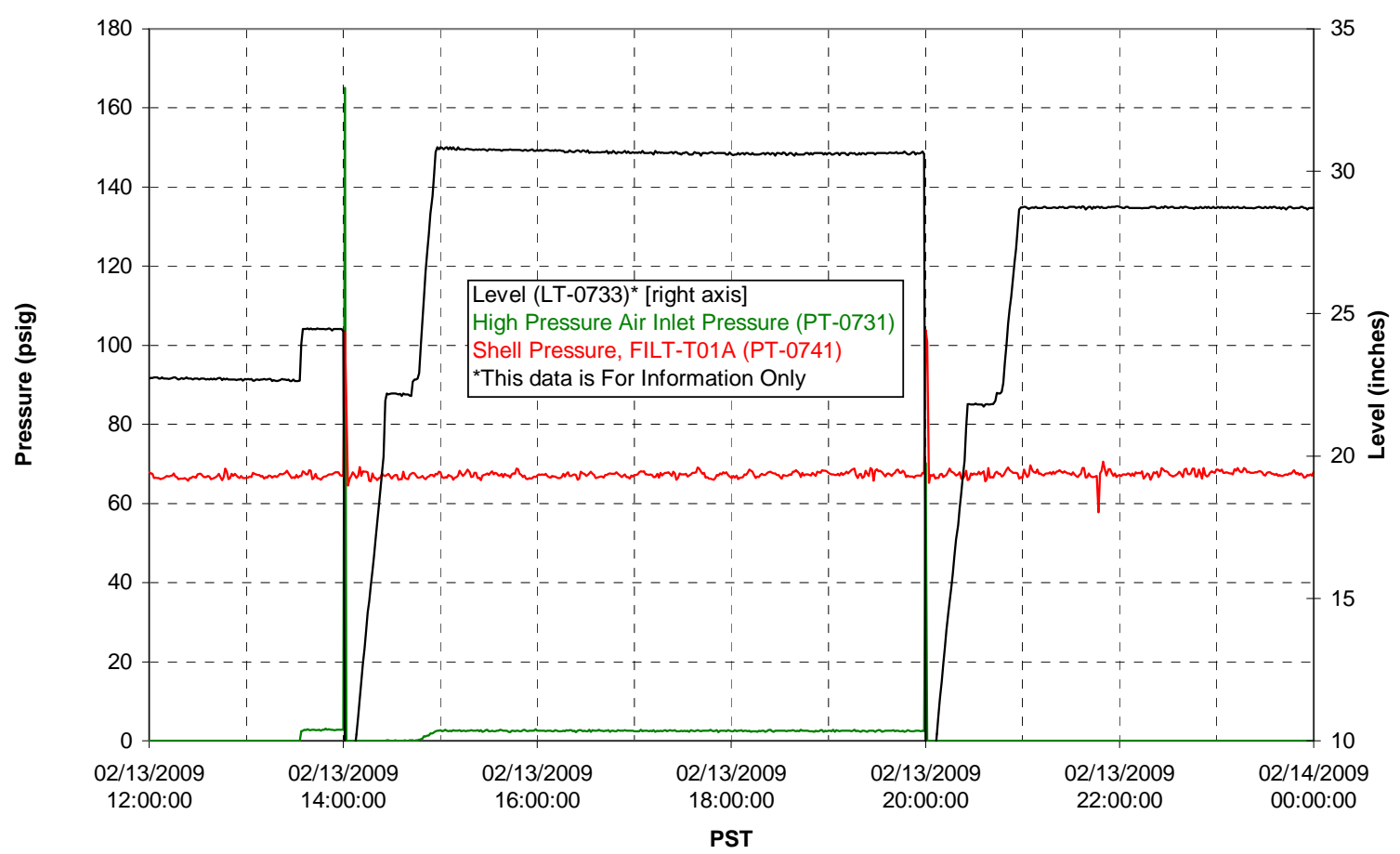

Pulsepot Levels

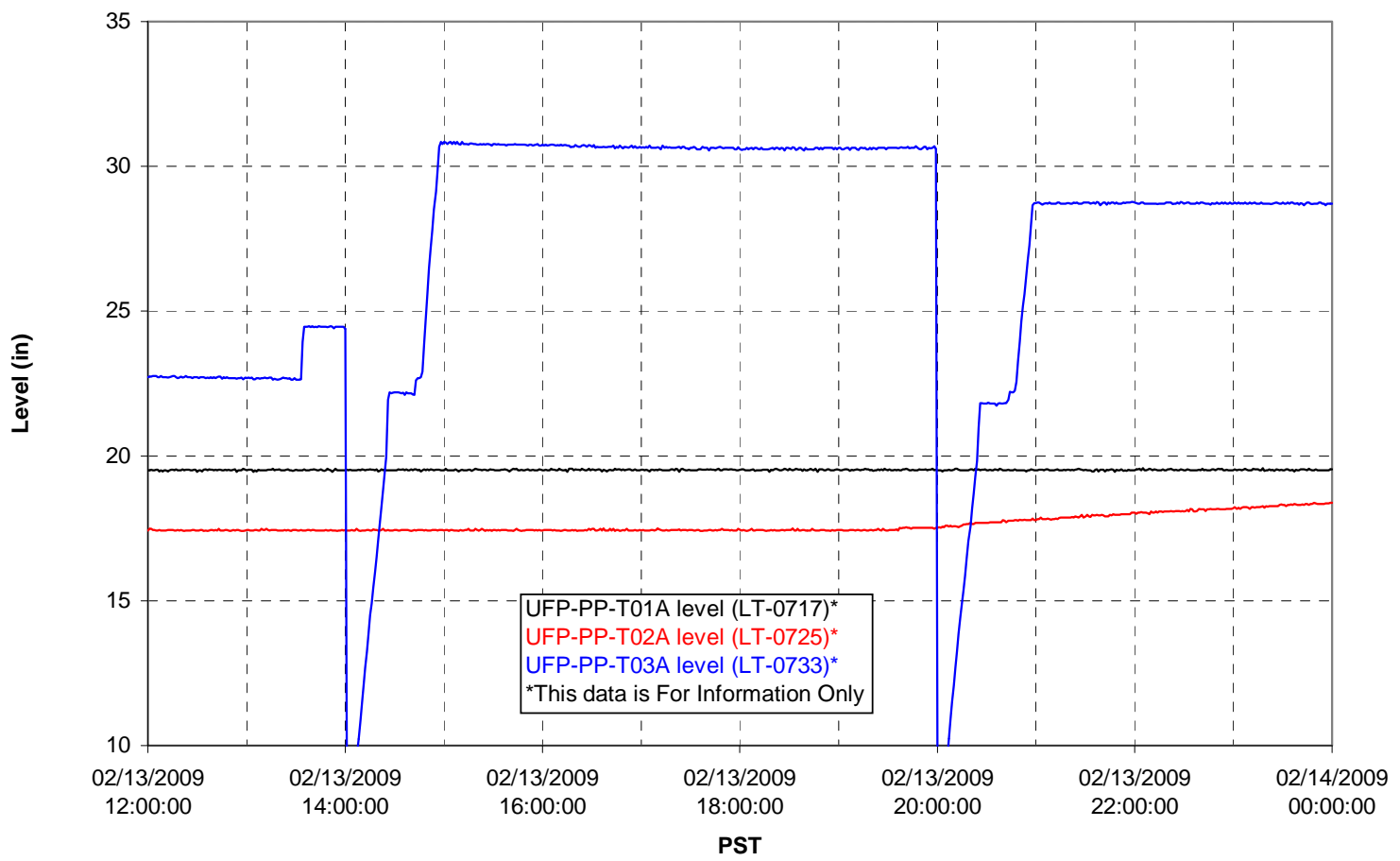


Filter UFP-FILT-T01A

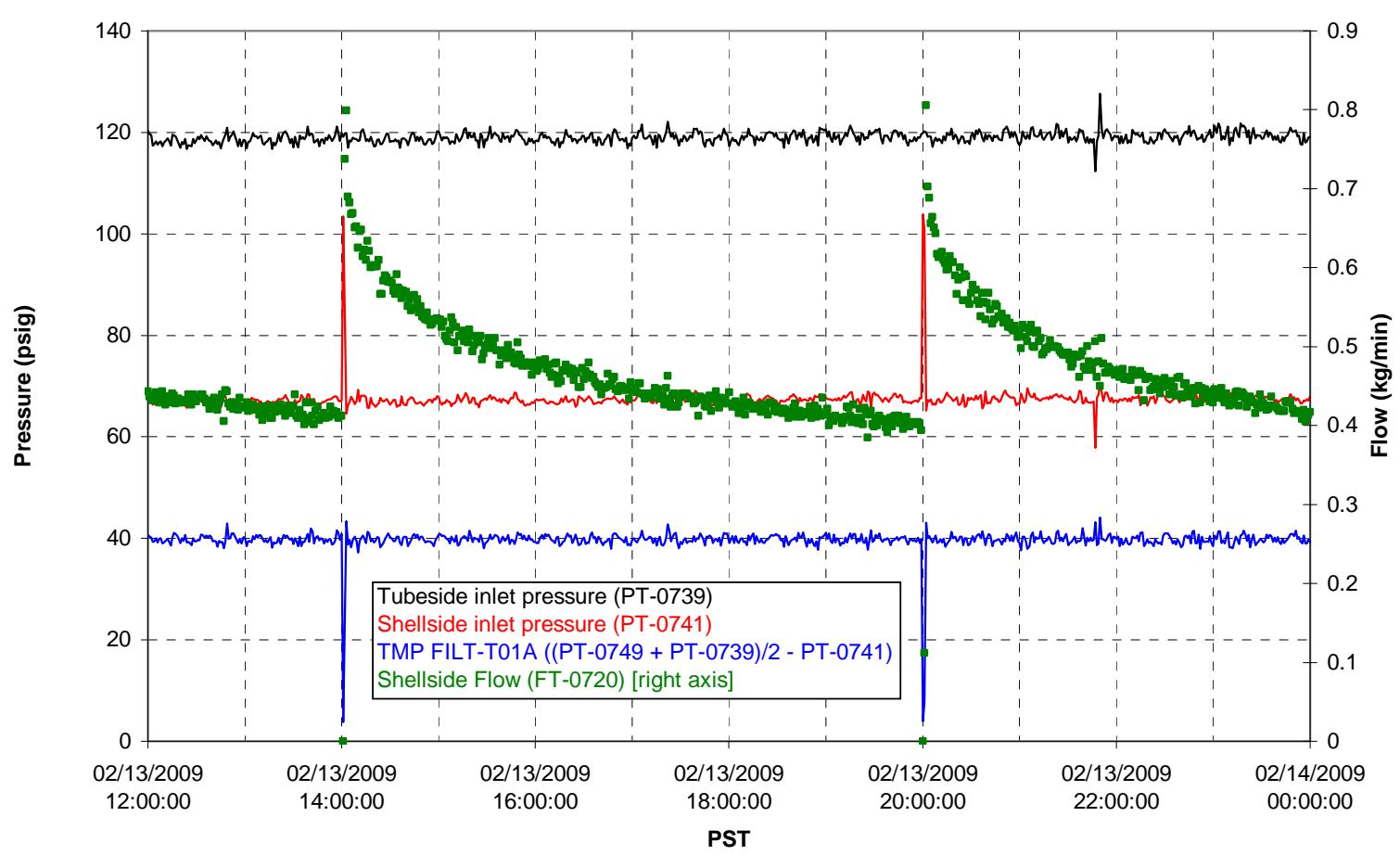

Filter UFP-FILT-T02A

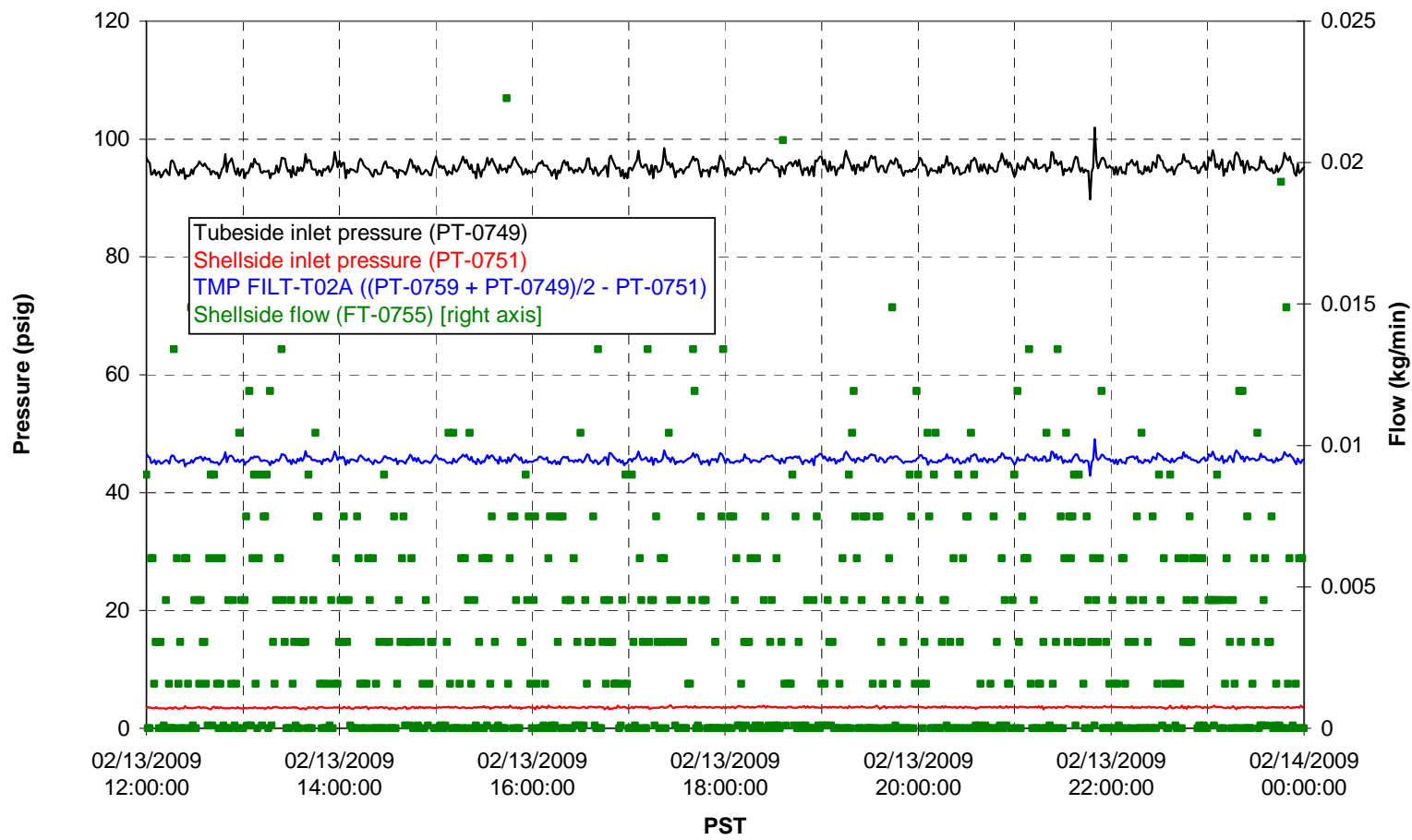


Filter UFP-FILT-T03A

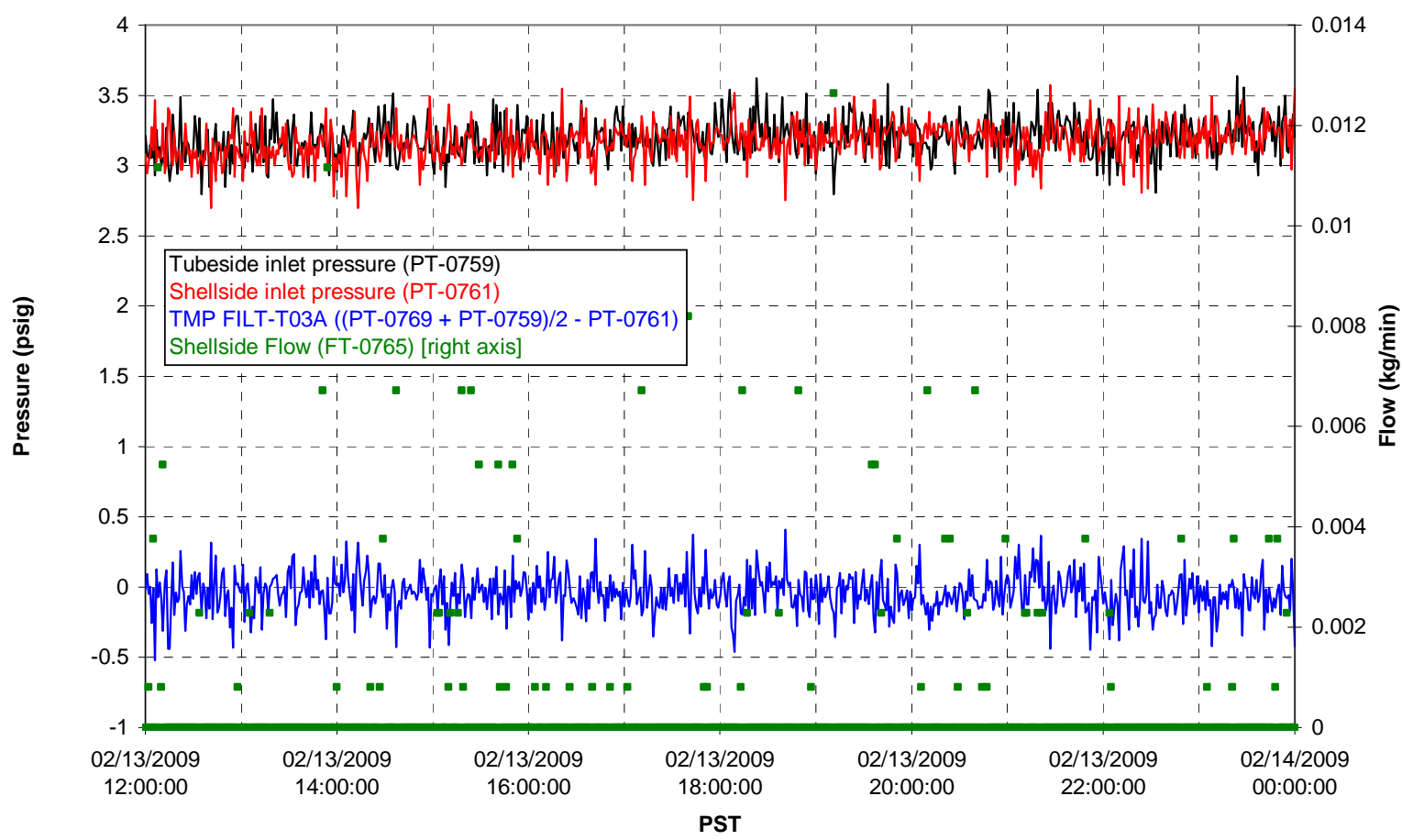

Filter UFP-FILT-T03A

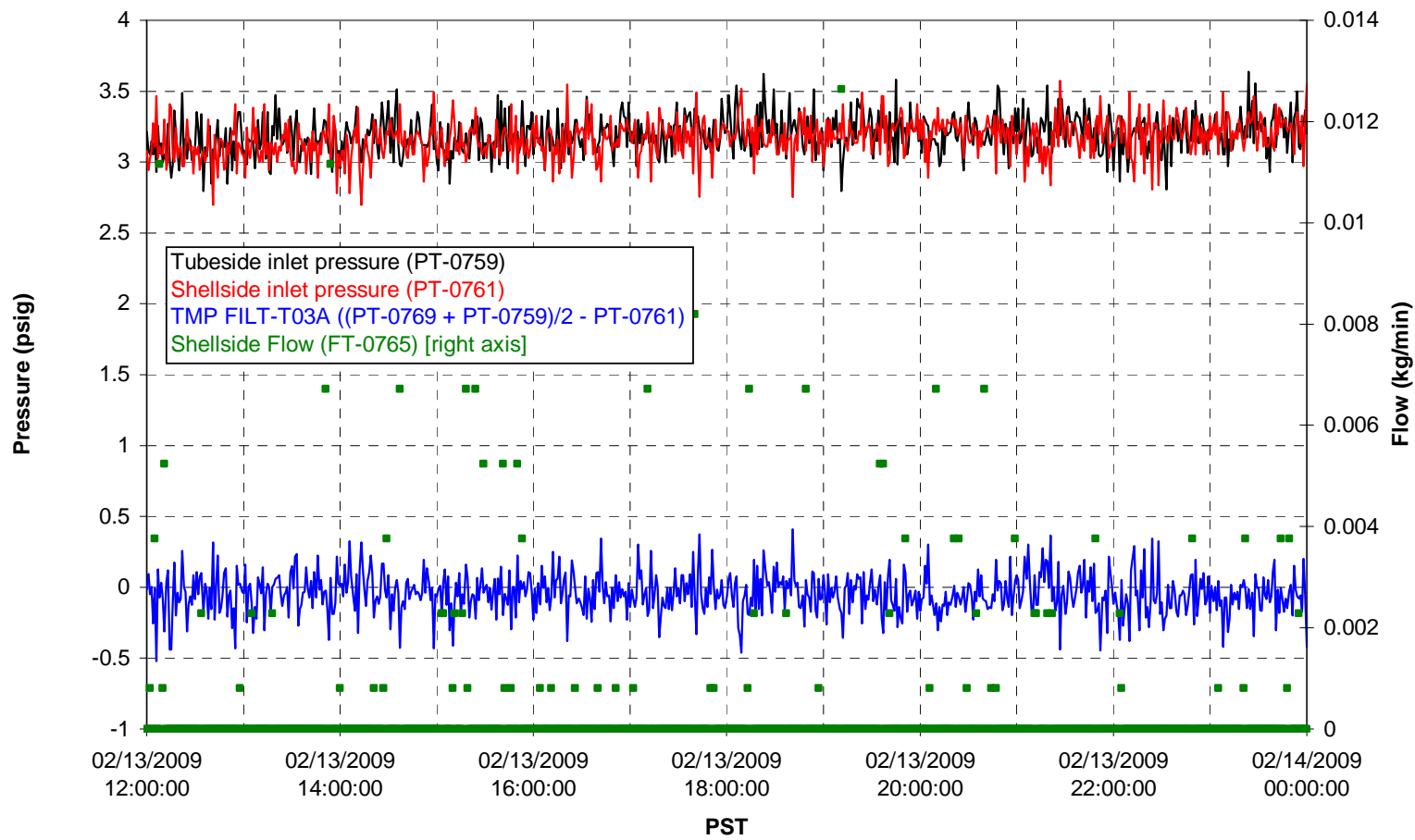


Filter UFP-FILT-T05A

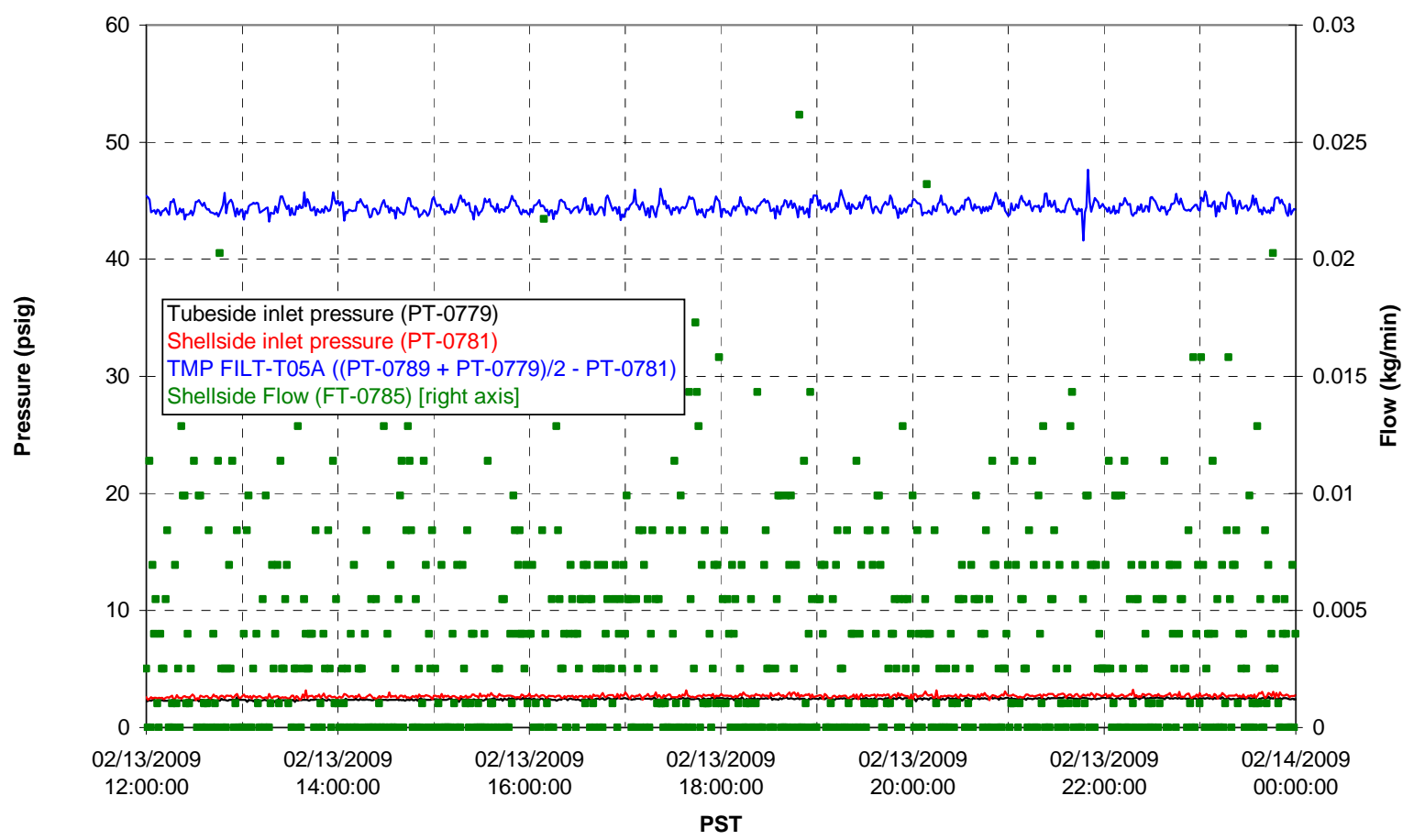

Chemical Flow

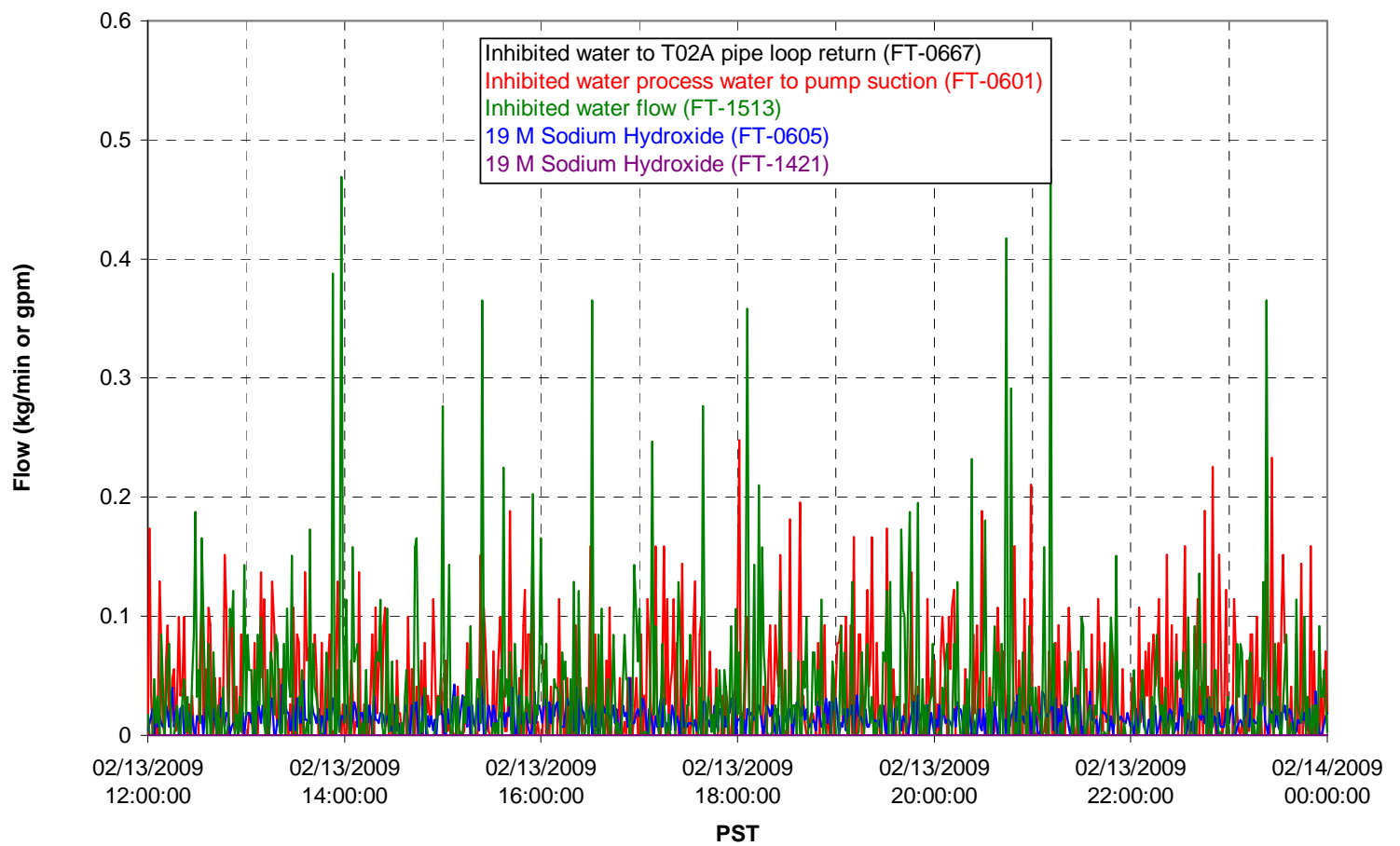


Chemical Flow

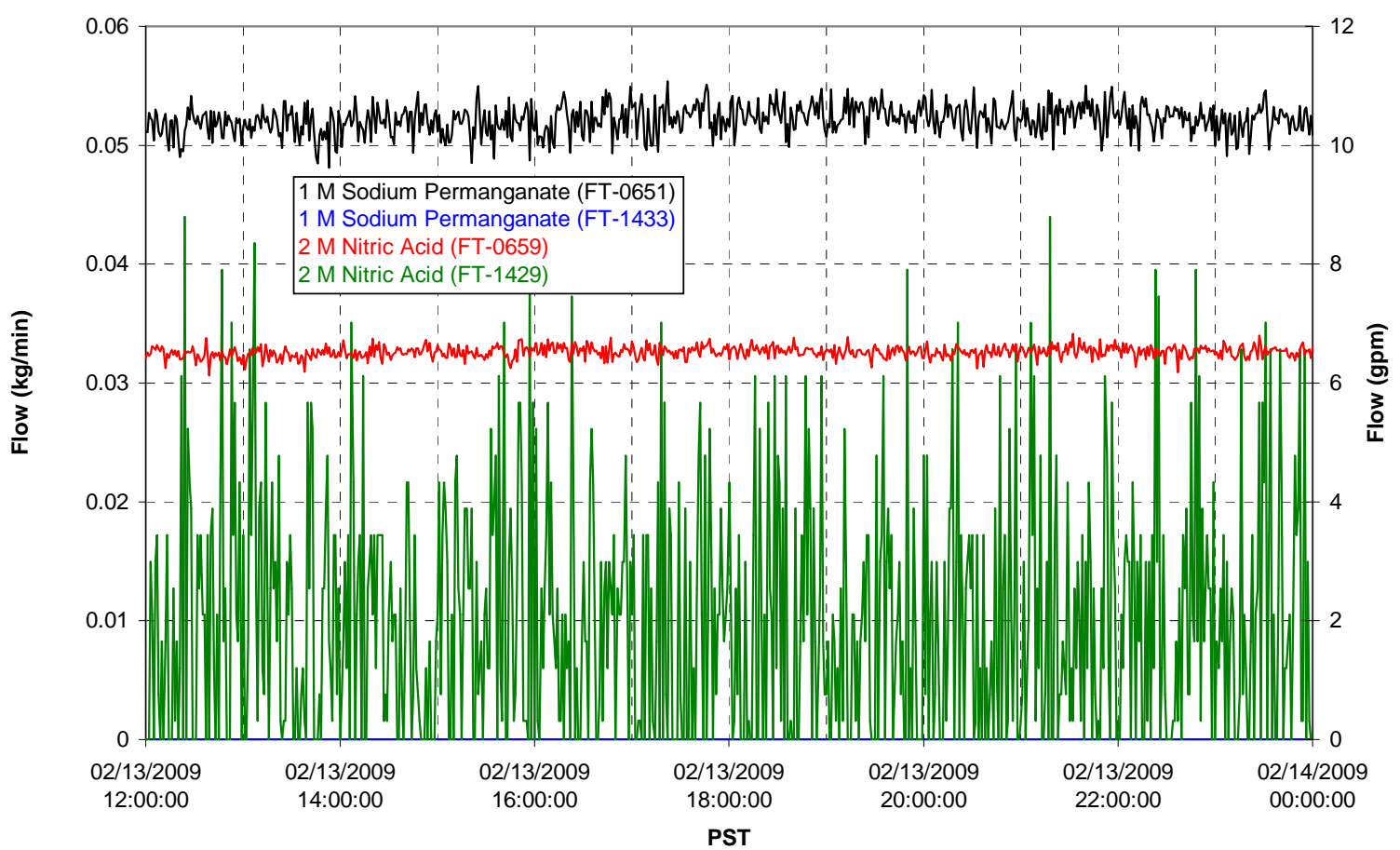

Air Flows

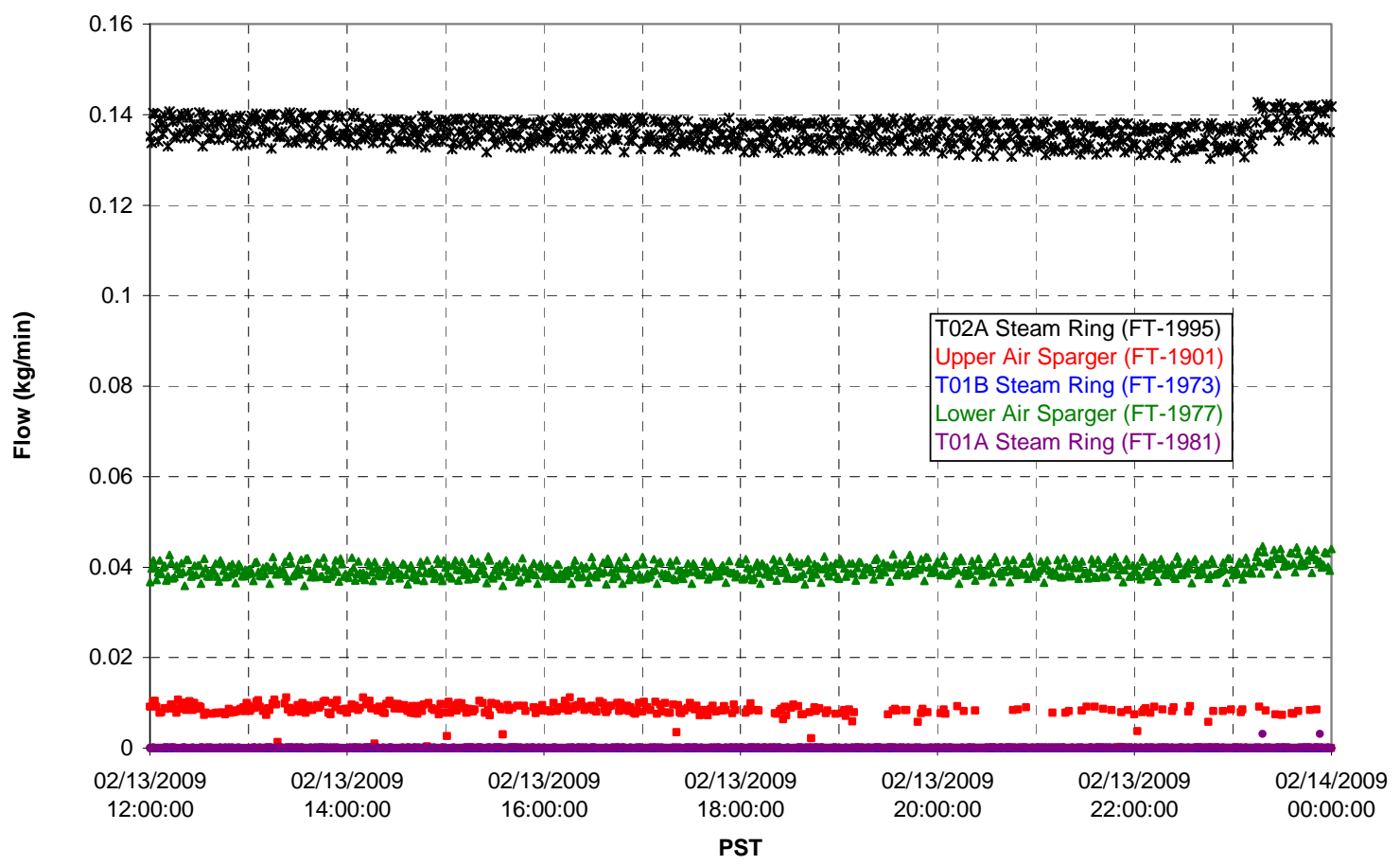


T02A Steam

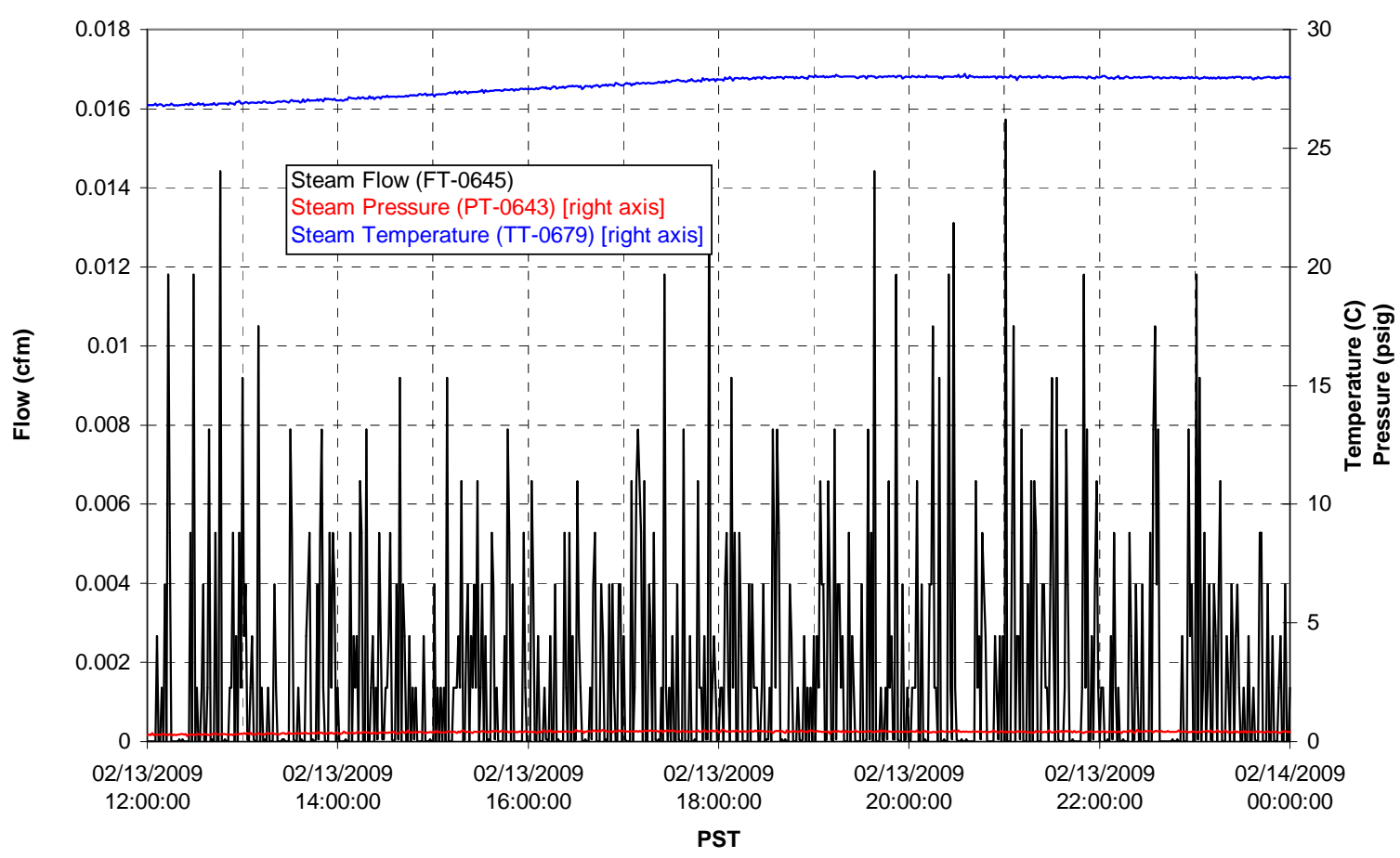

T01A Steam

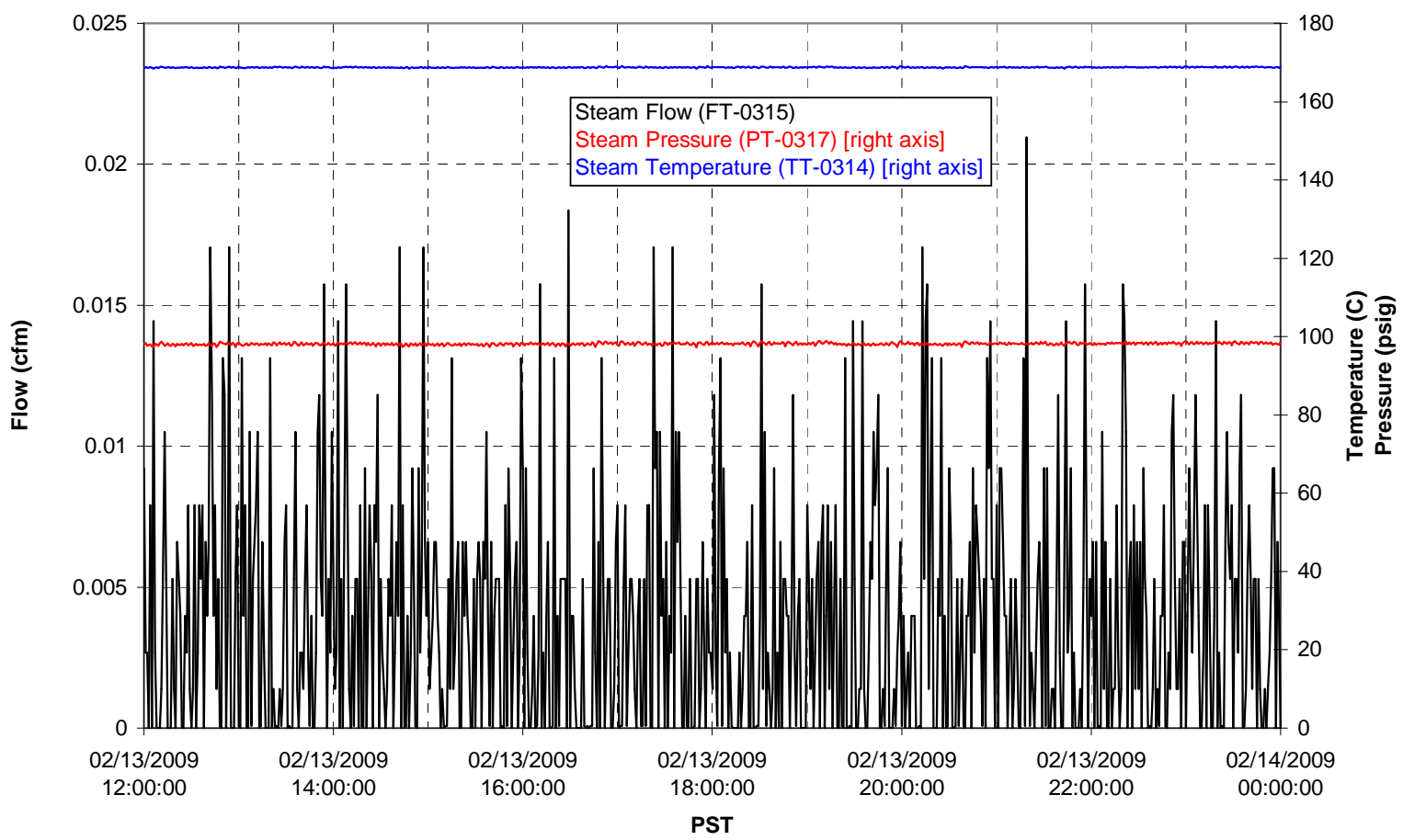


T01B Steam

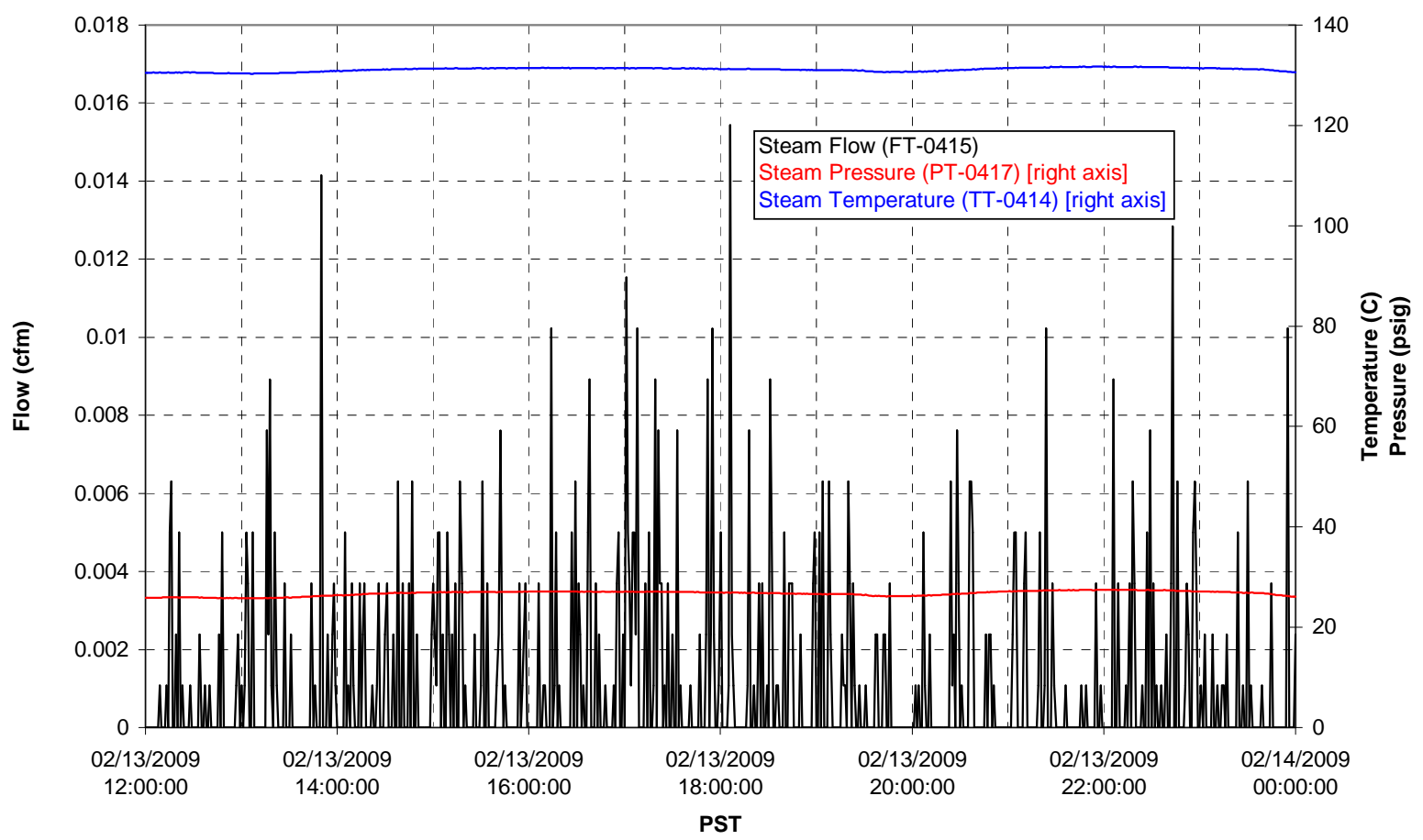




\section{Integrated Test A Data Plots 02/14/09 00:00 - 02/14/09 12:00}


T01A level

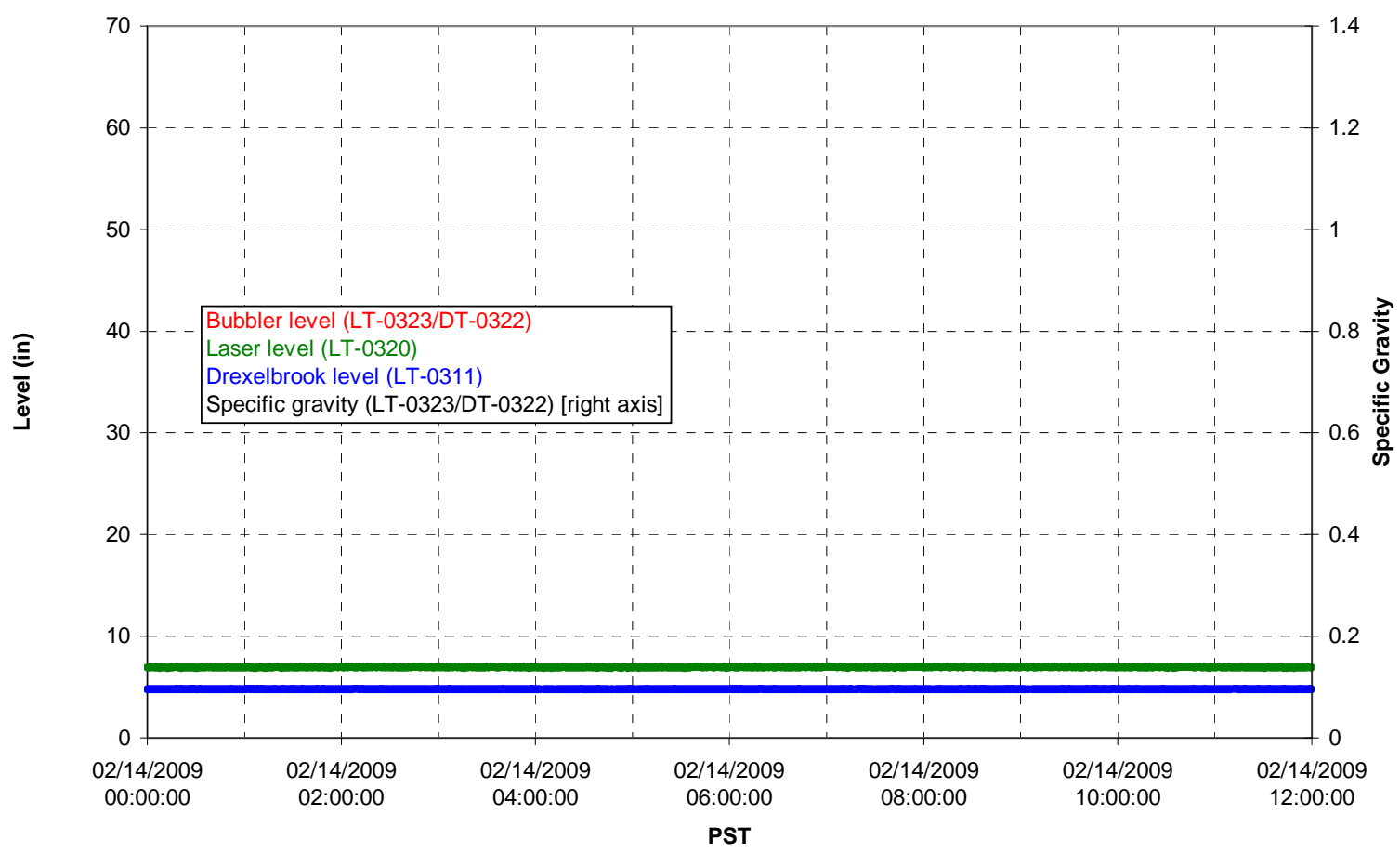

T01A temperatures

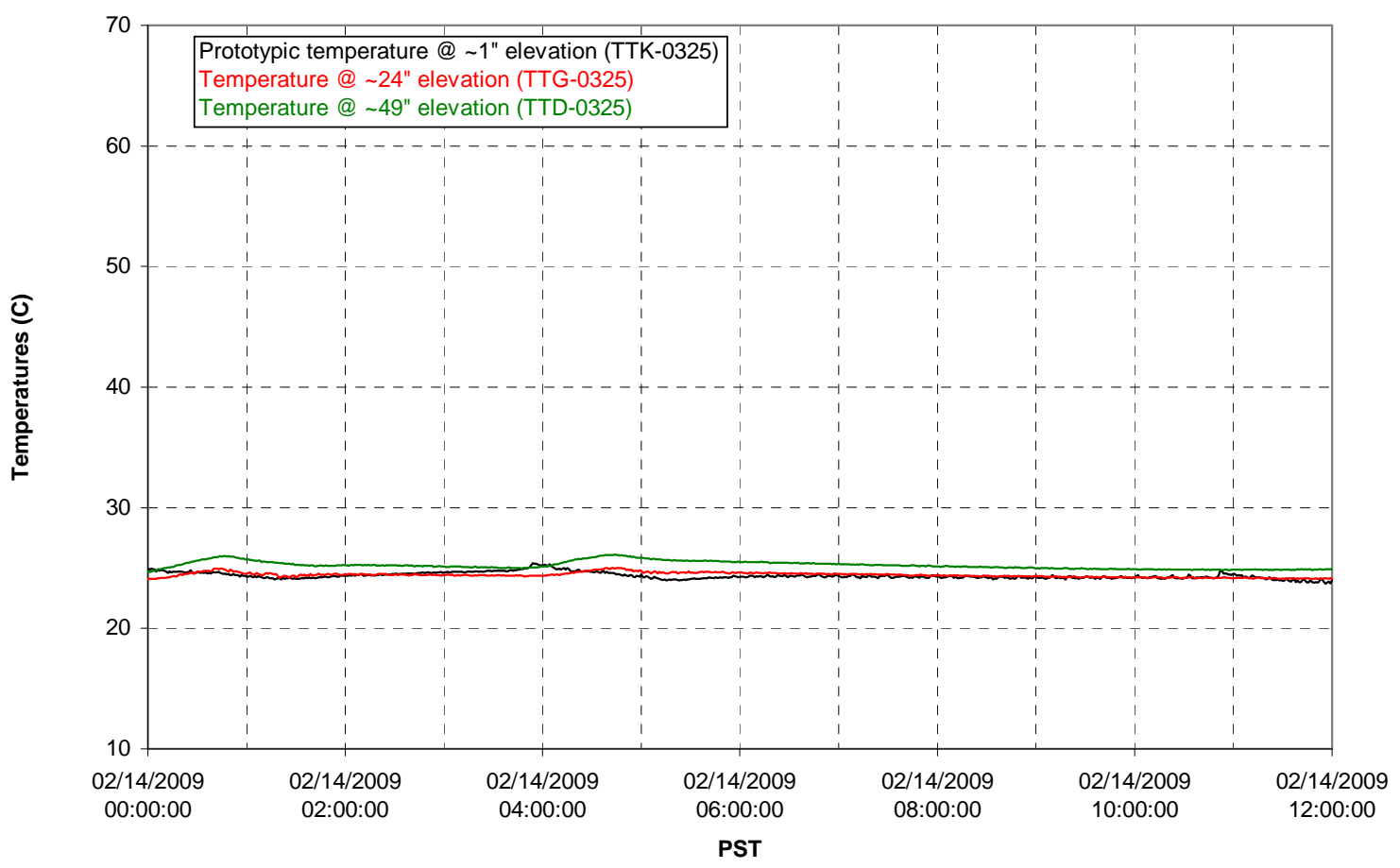


T01B level

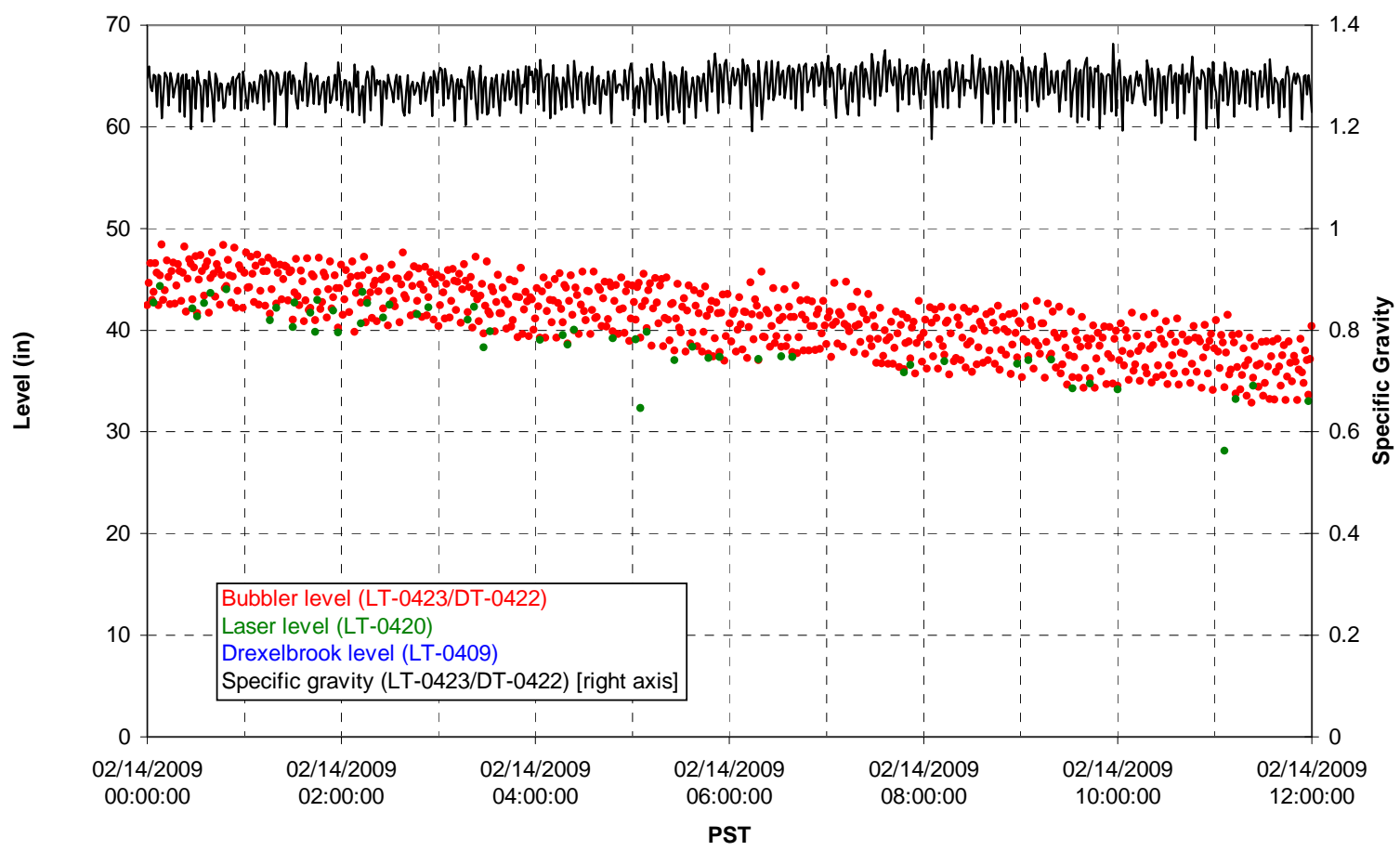

T01B temperatures

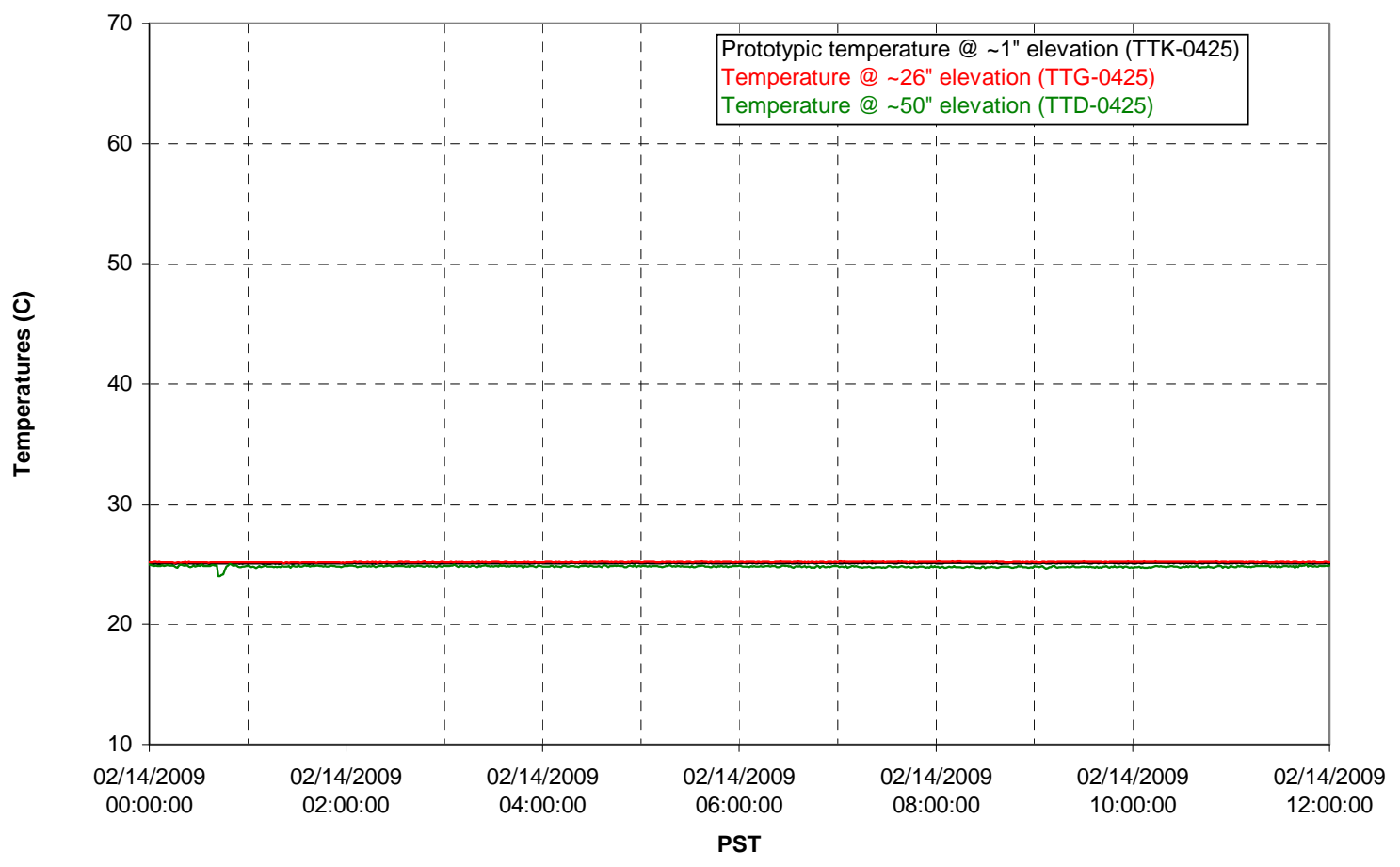


T02A level

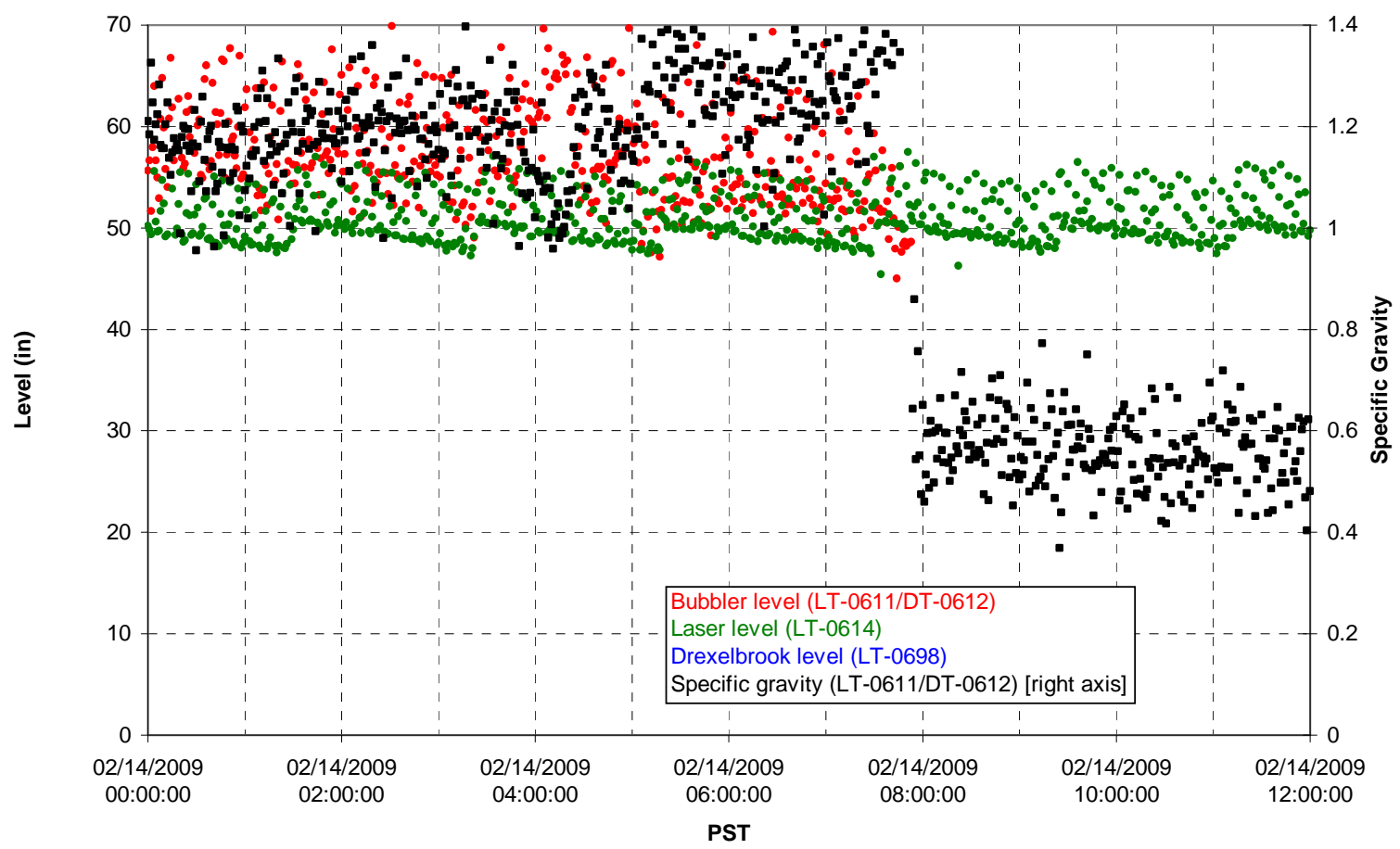

T02A temperatures

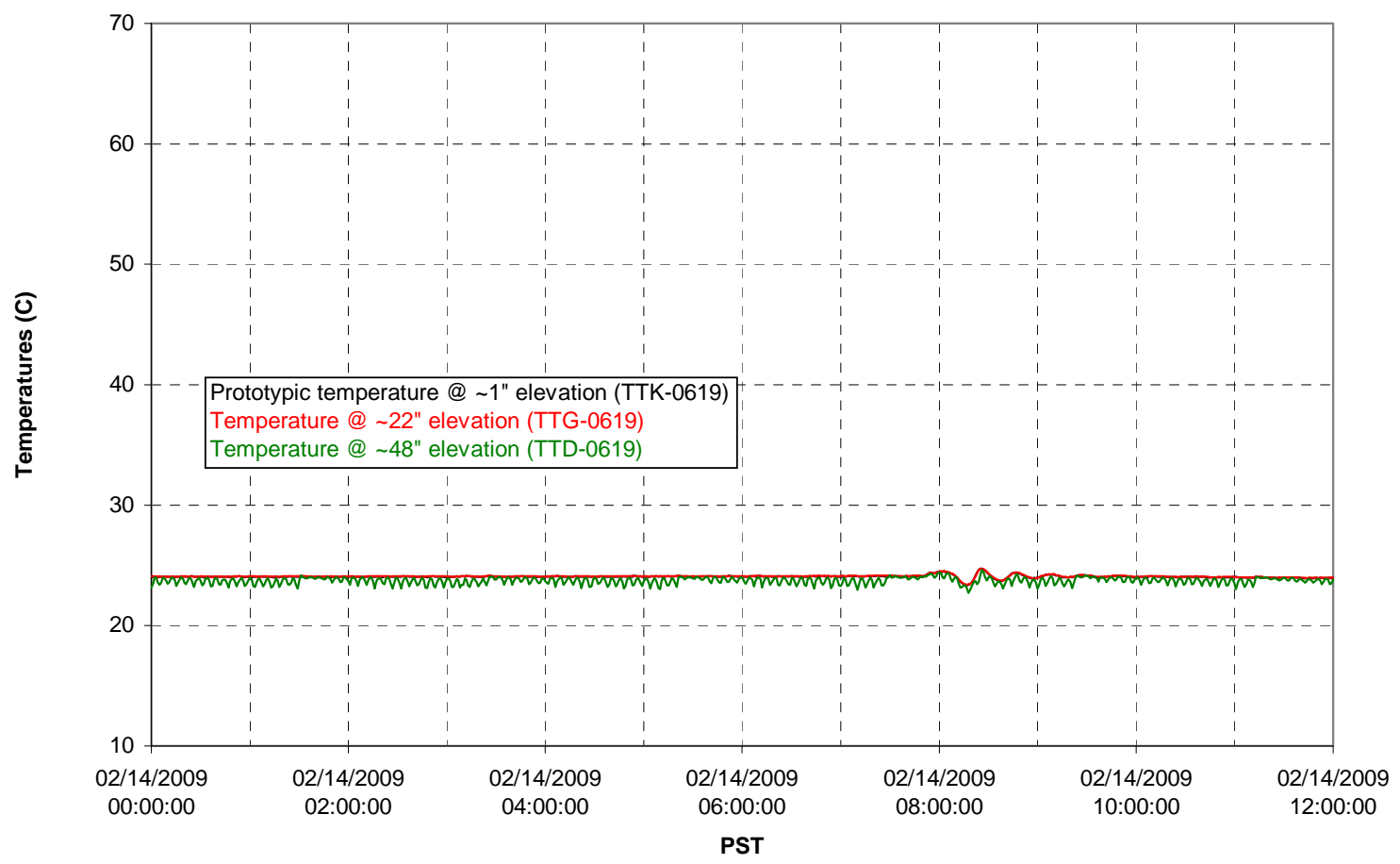


T02A and filter loop temperatures

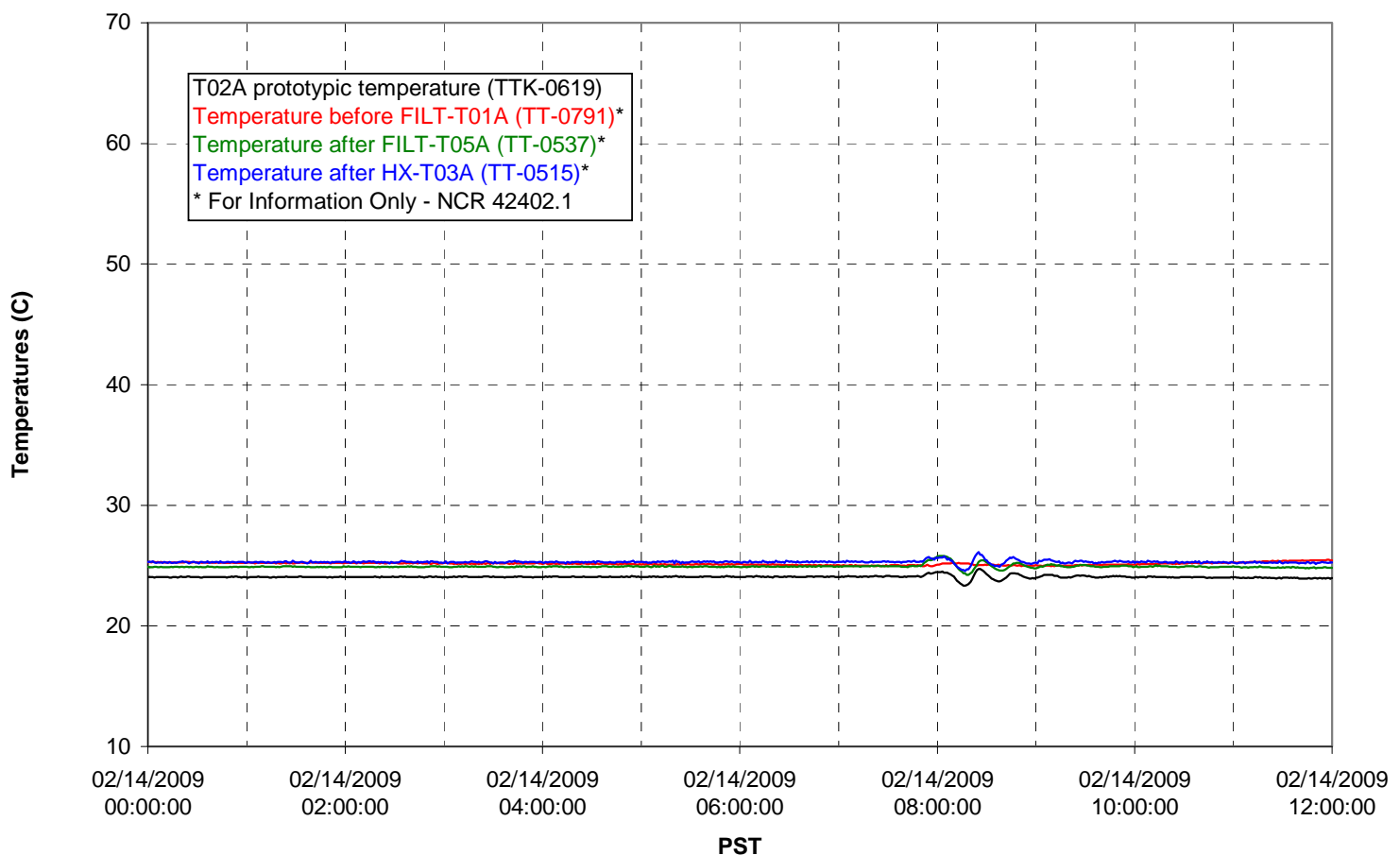

Pump Pressures and Flow

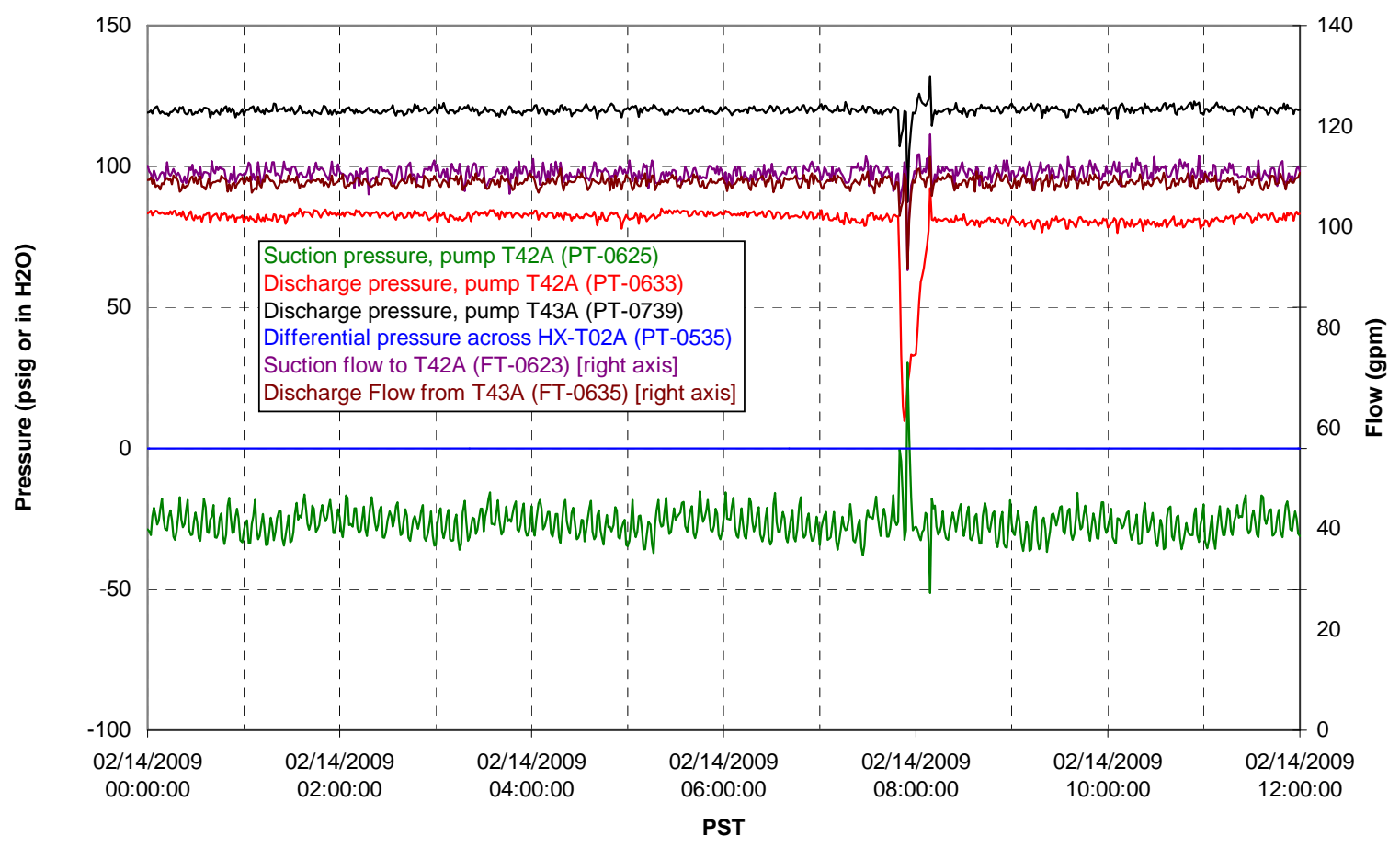


Axial pressure drop

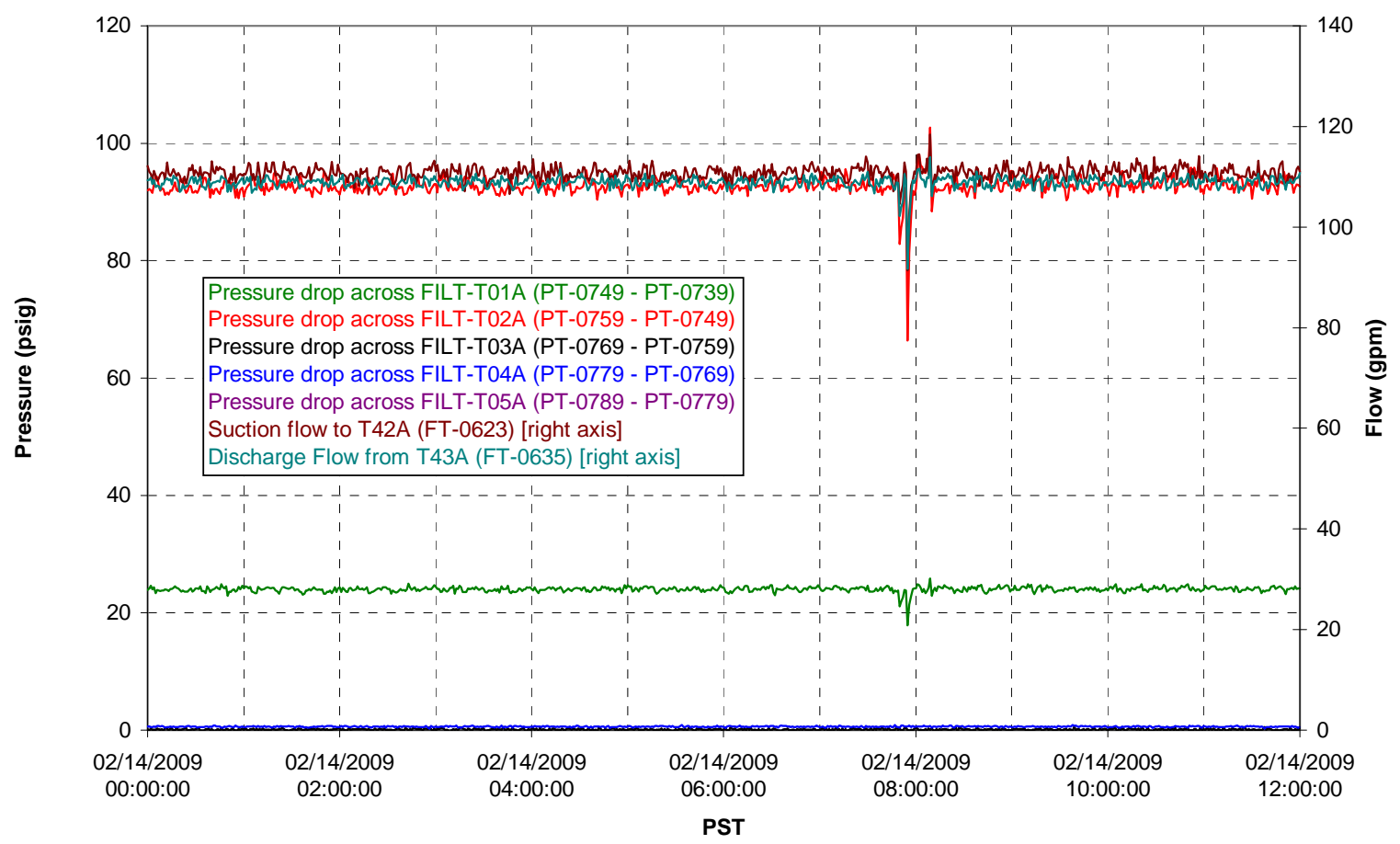

Permeate flow rates

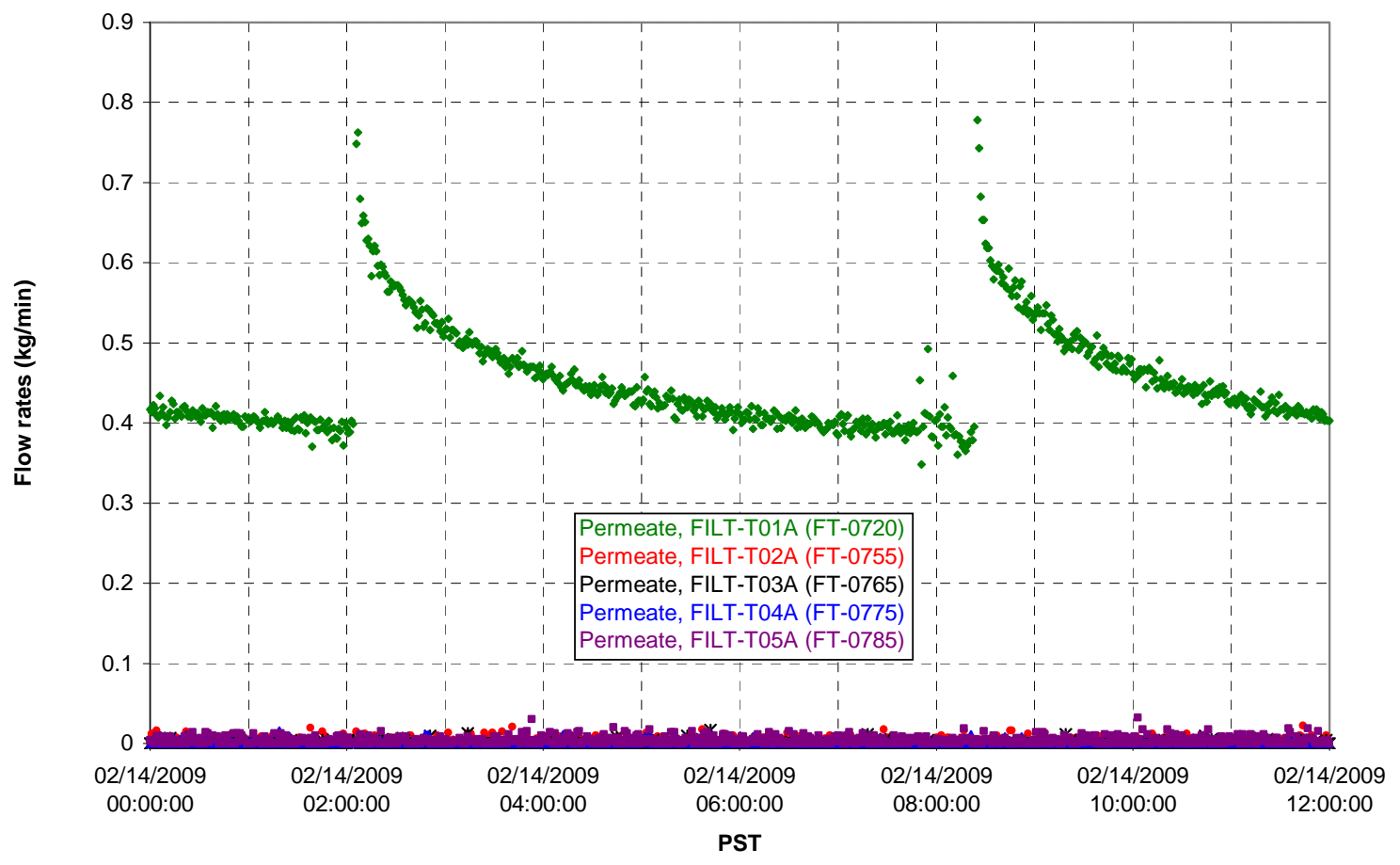


T02A Inner Temperature Tree

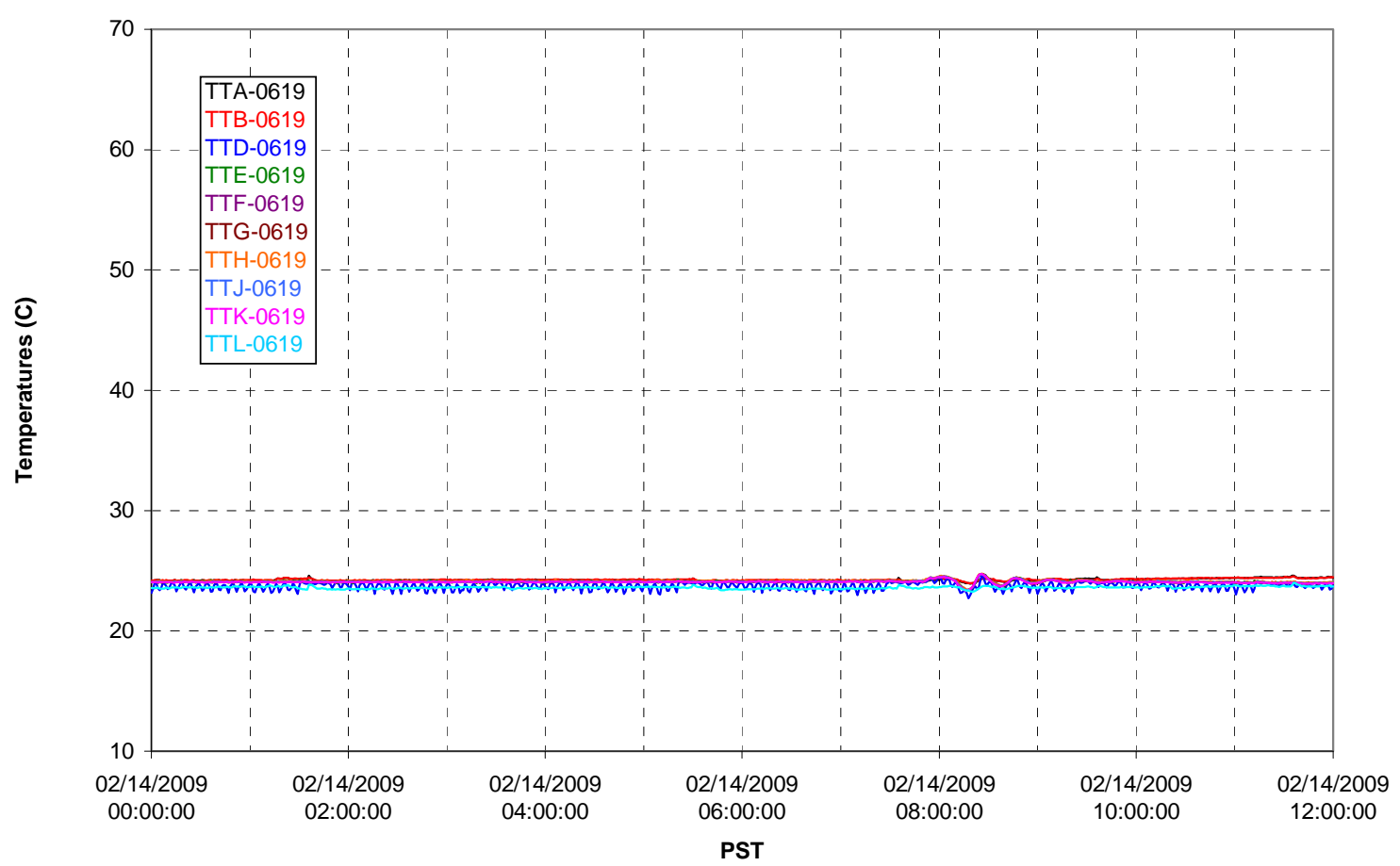

T02A Outer Temperature Tree

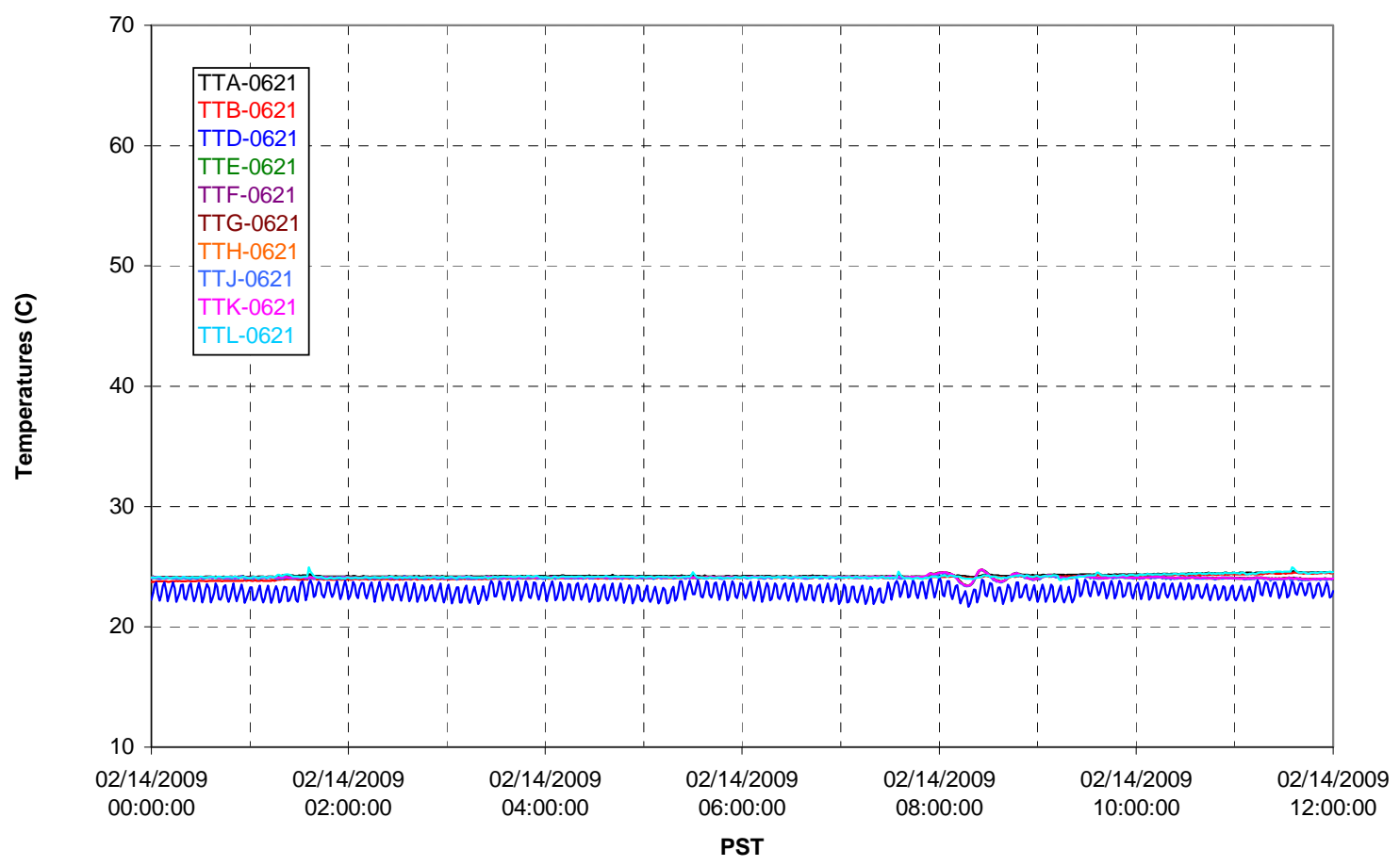


T02A temperatures

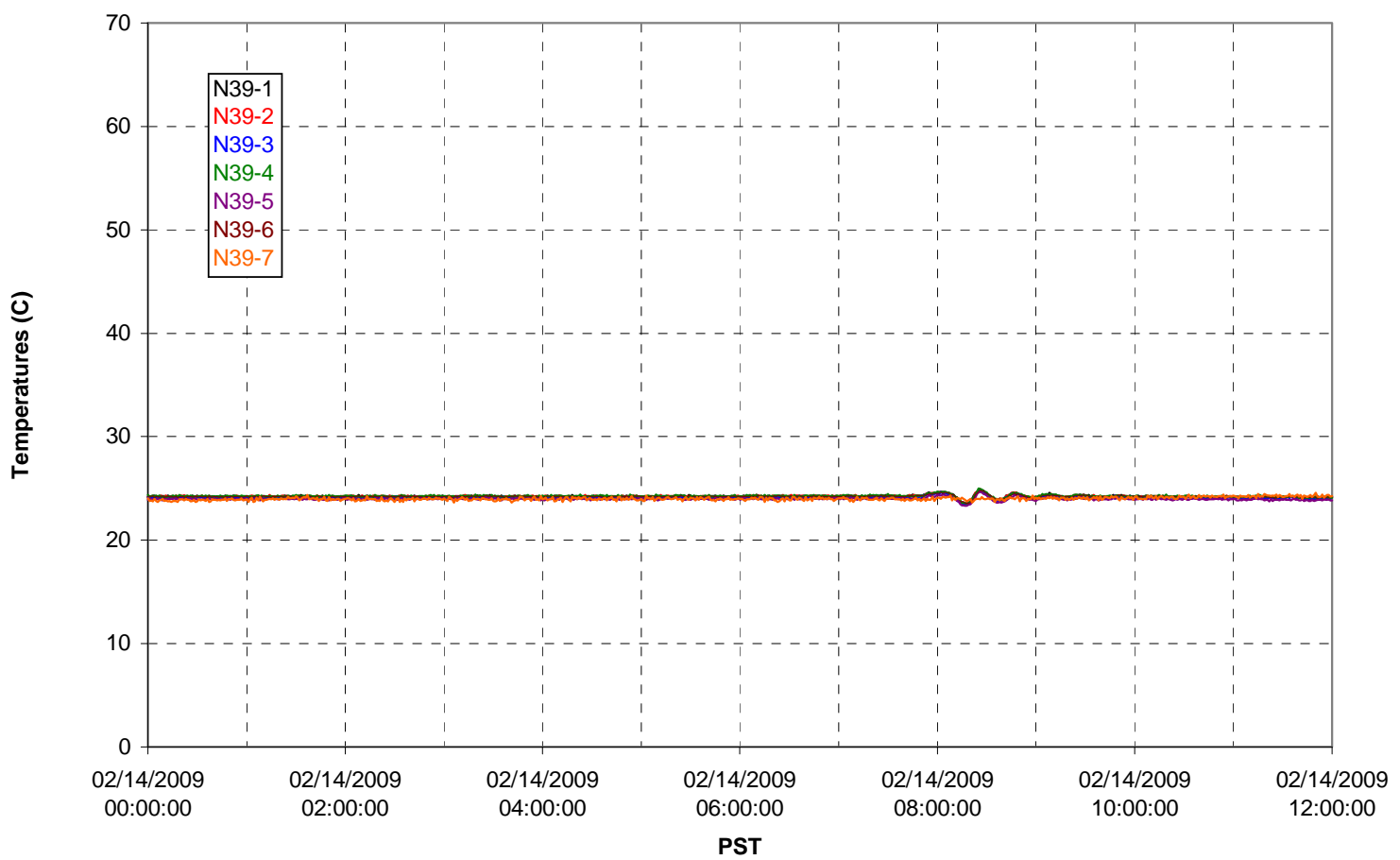

T02A temperatures

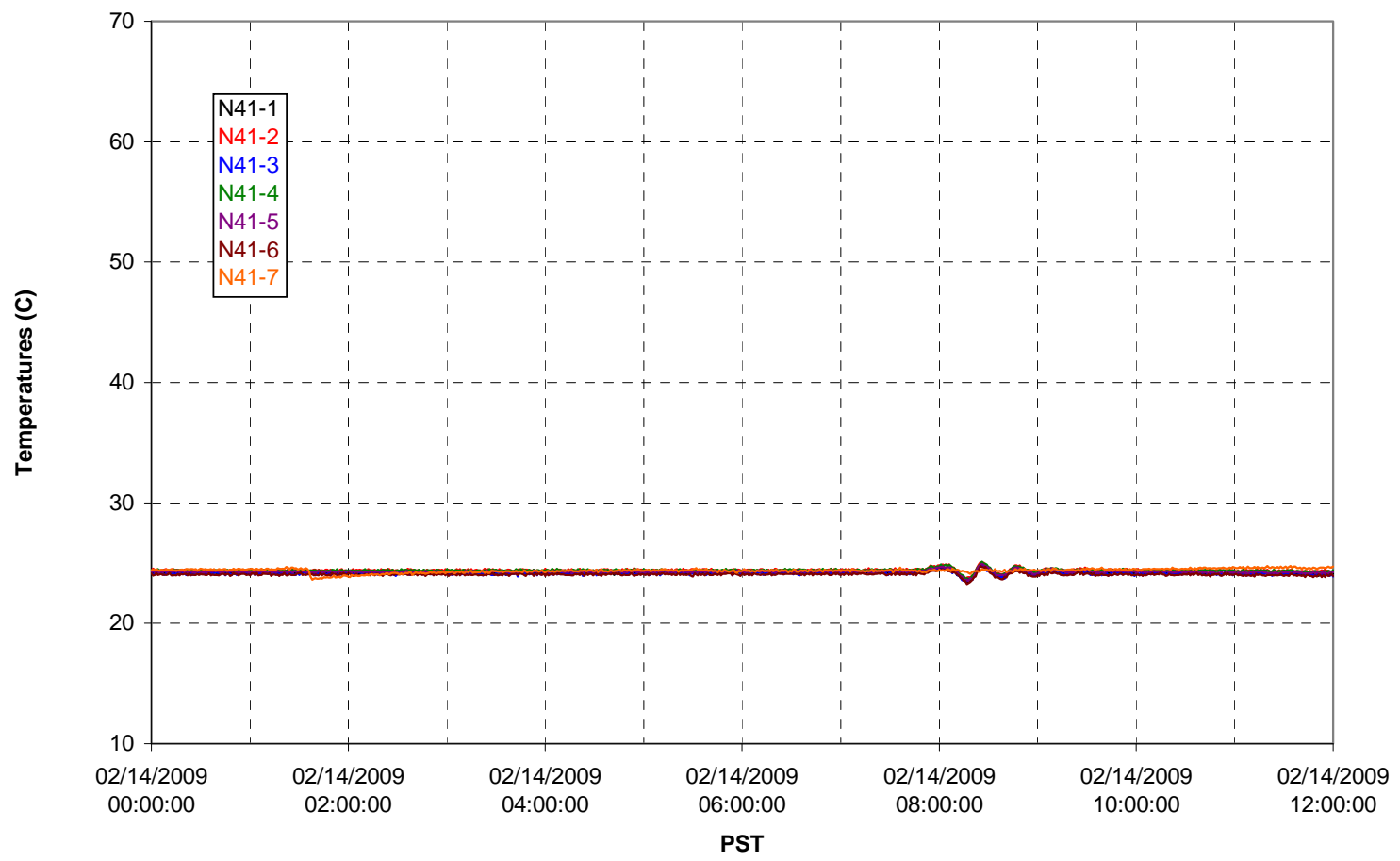


T02A temperatures

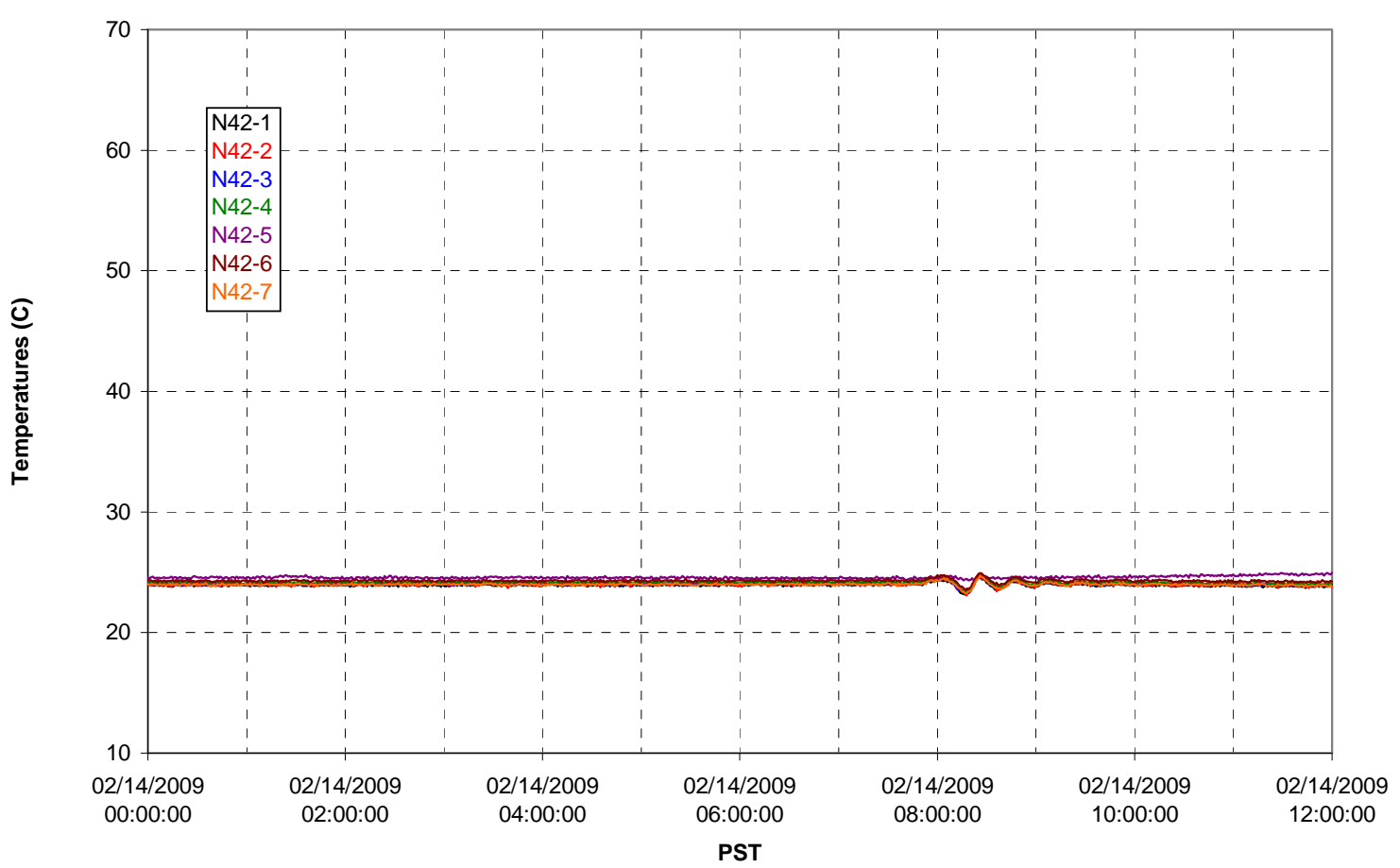

T02A temperatures

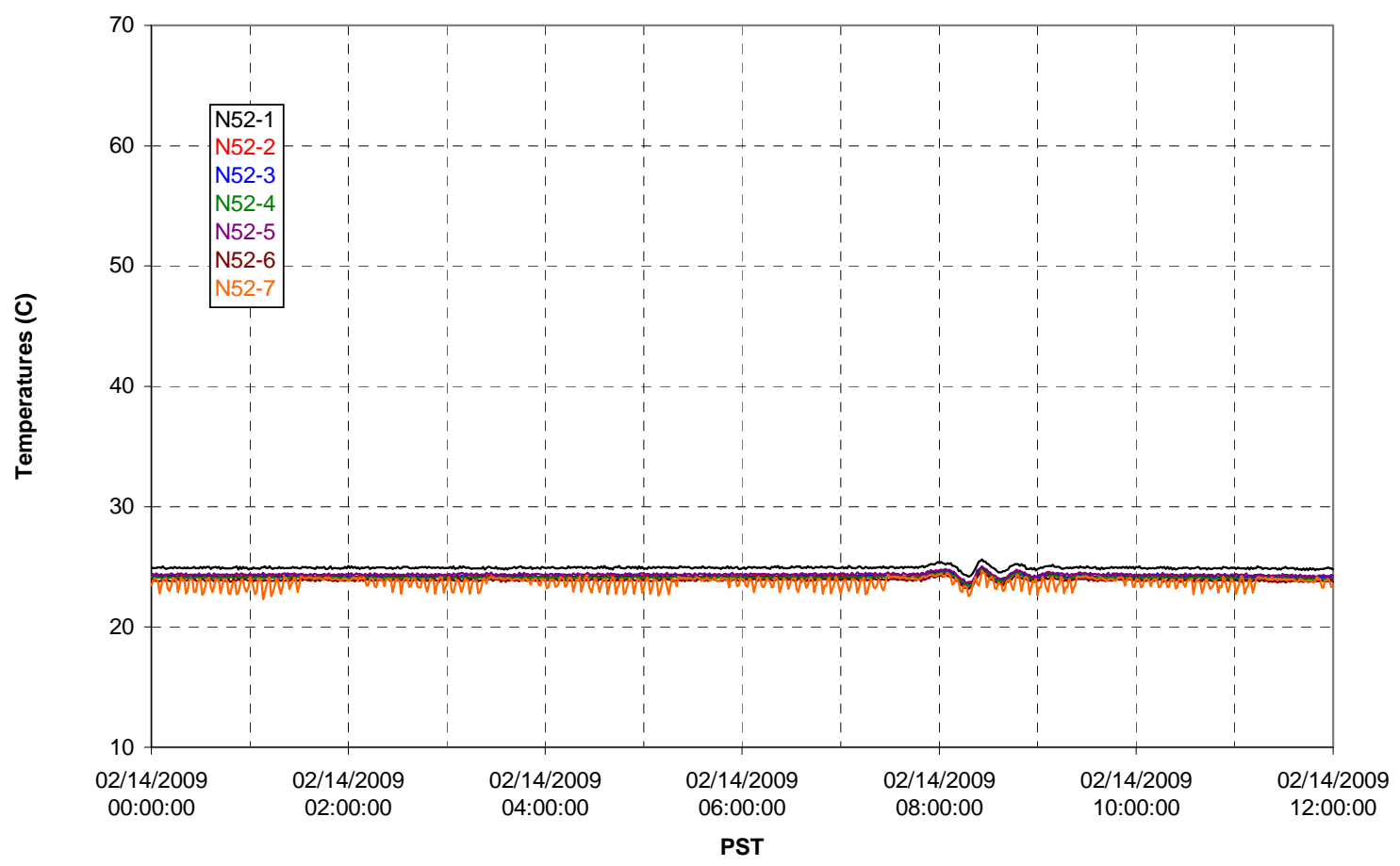


T02A Heating and Cooling

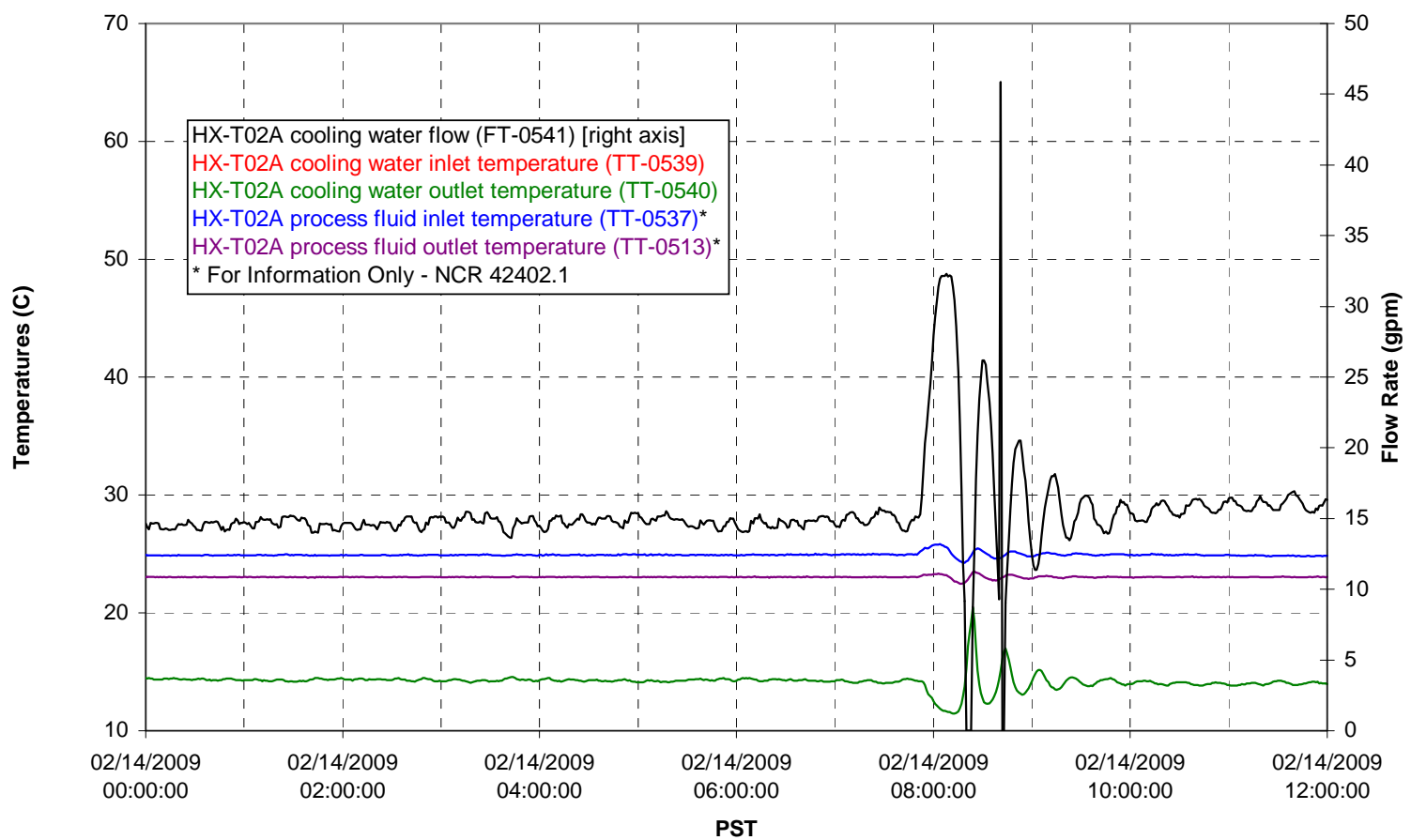

Pump Operation

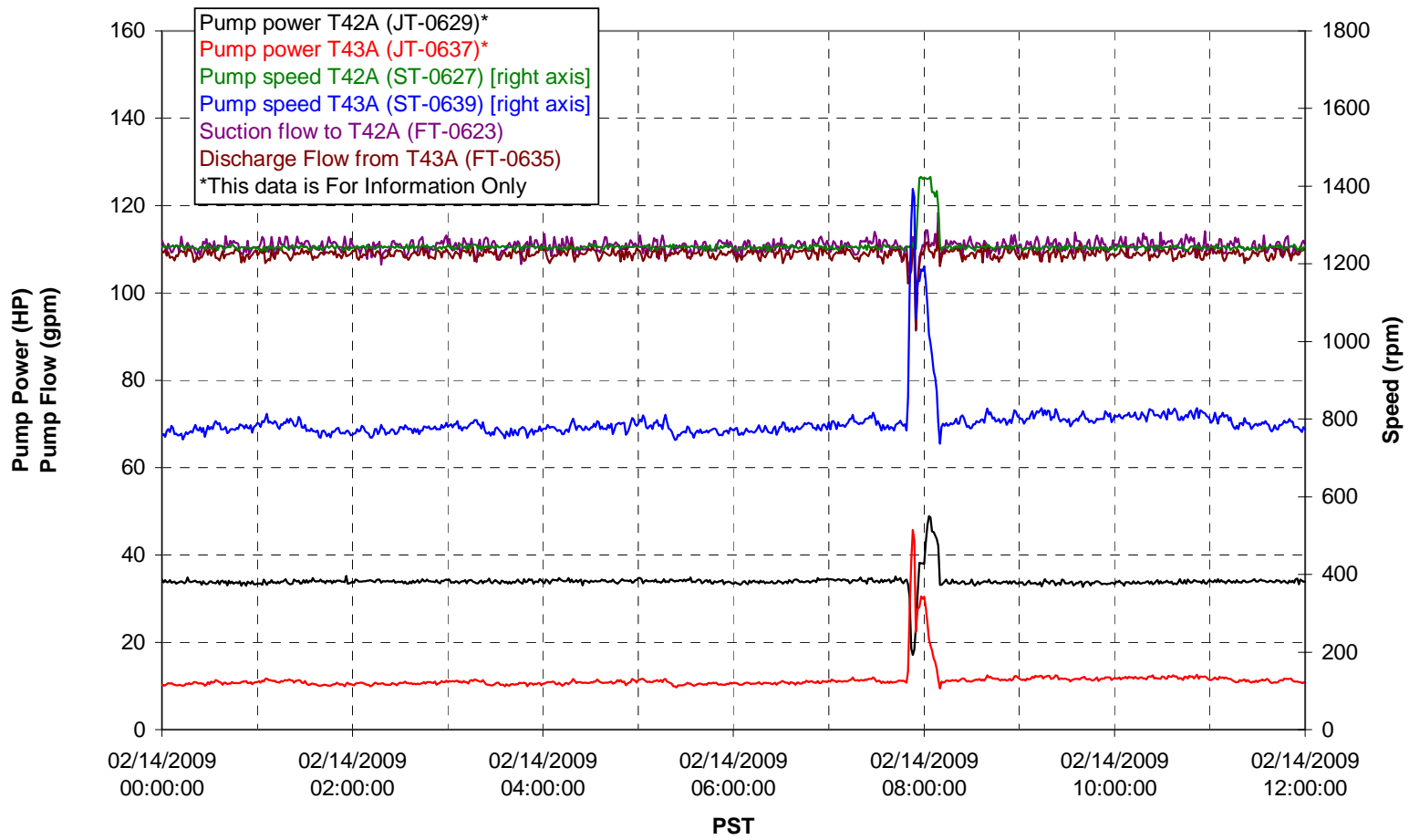


Pulsepot UFP-PP-T01A

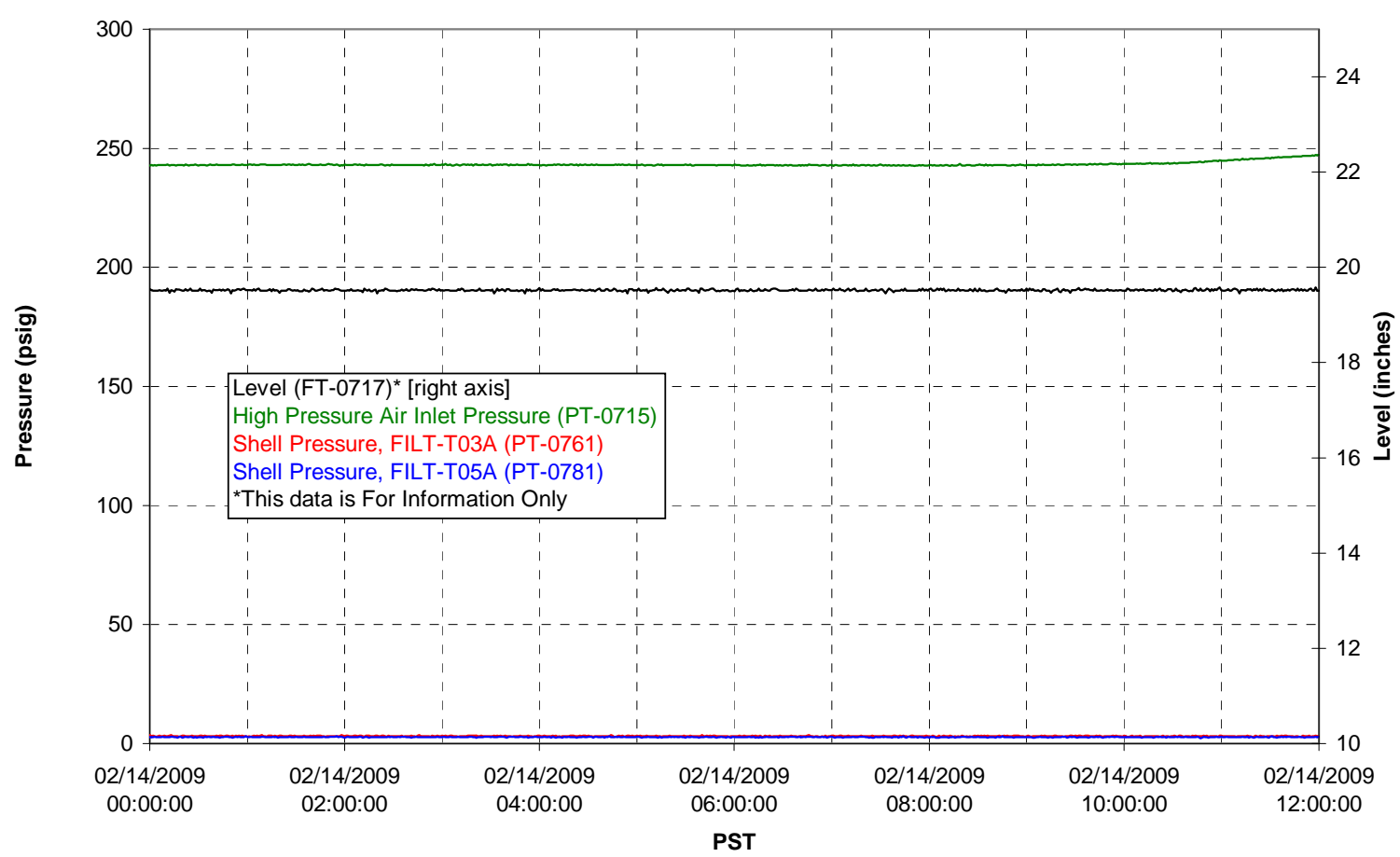

Pulsepot UFP-PP-T02A

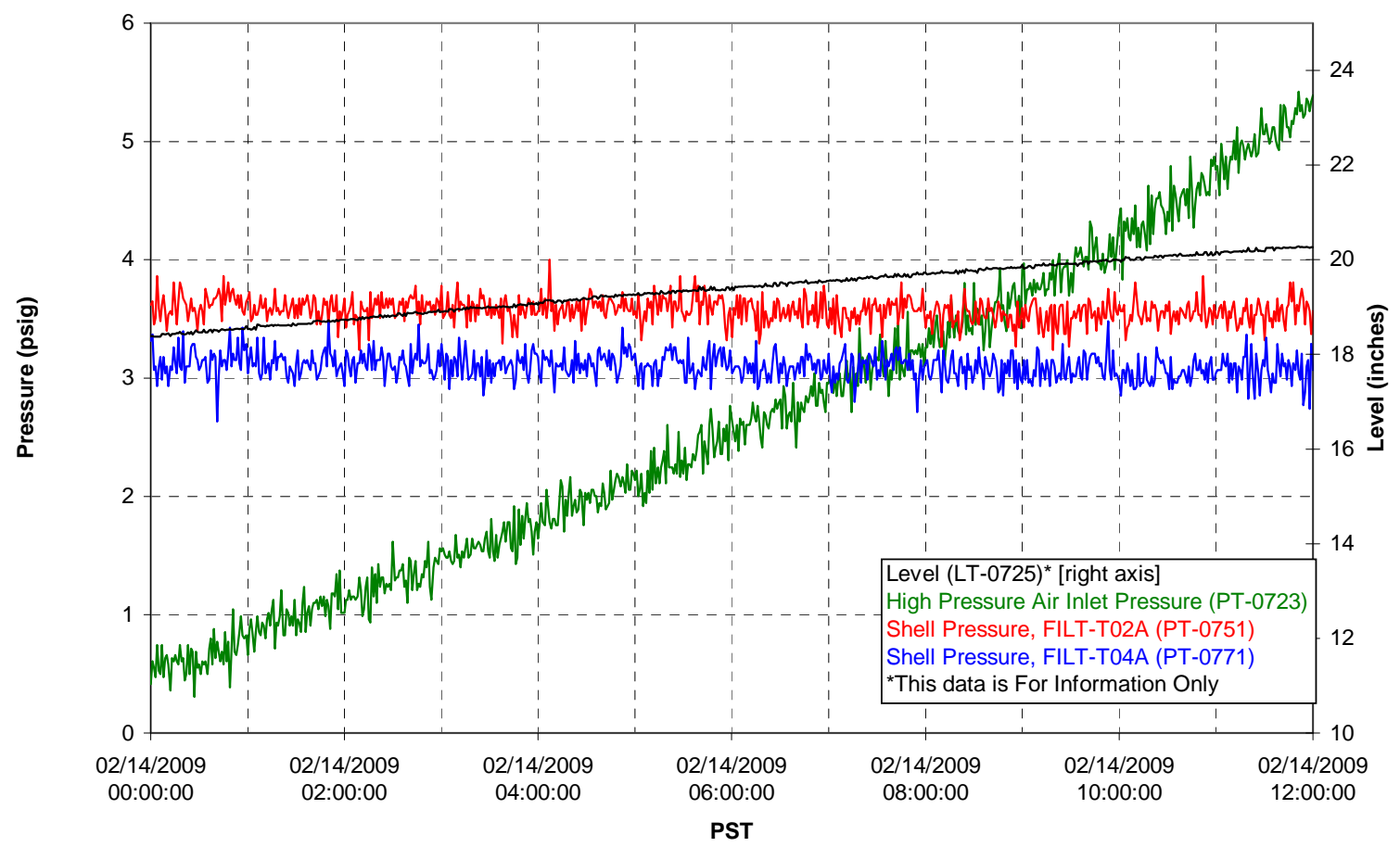


Pulsepot UFP-PP-T03A

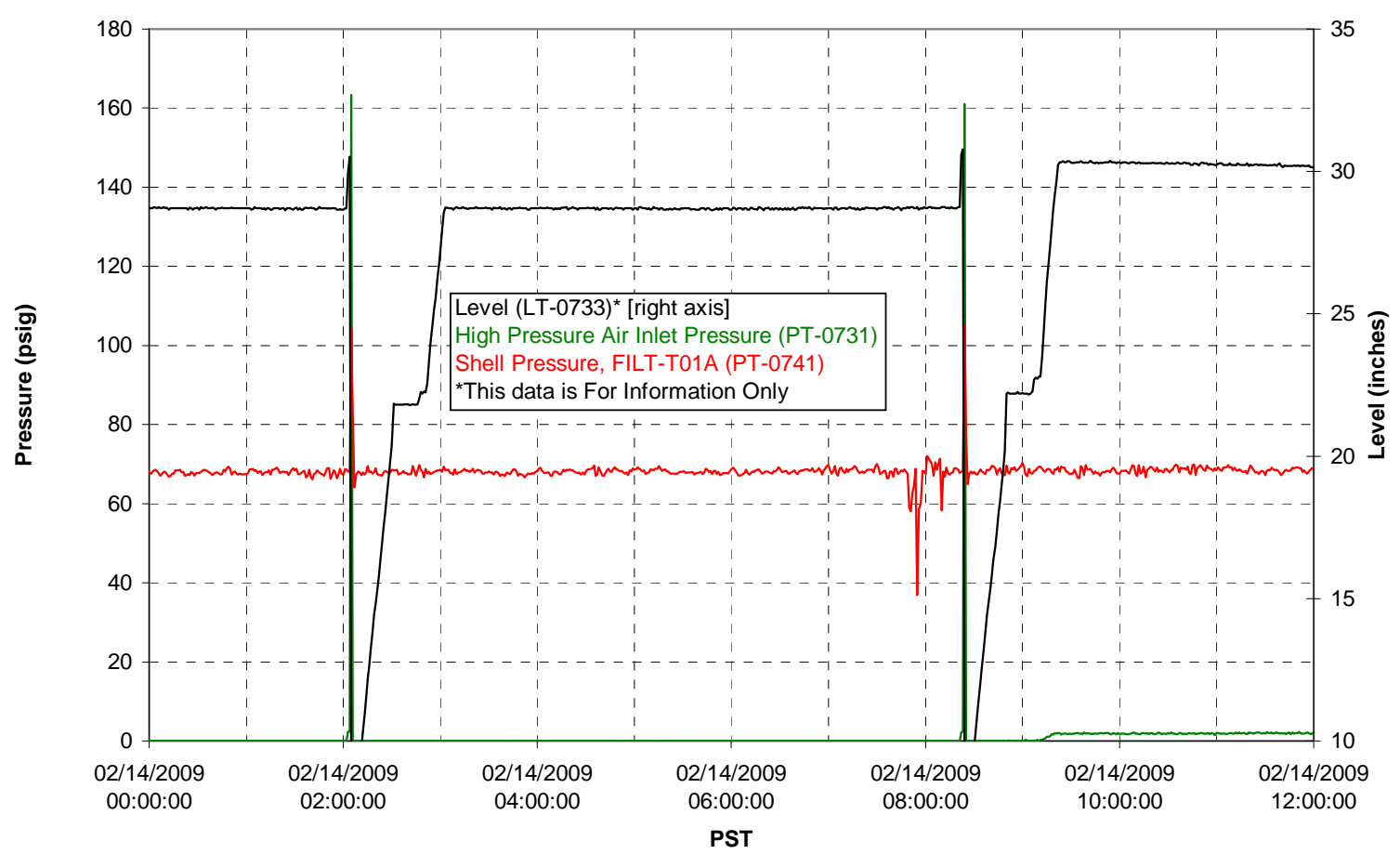

Pulsepot Levels

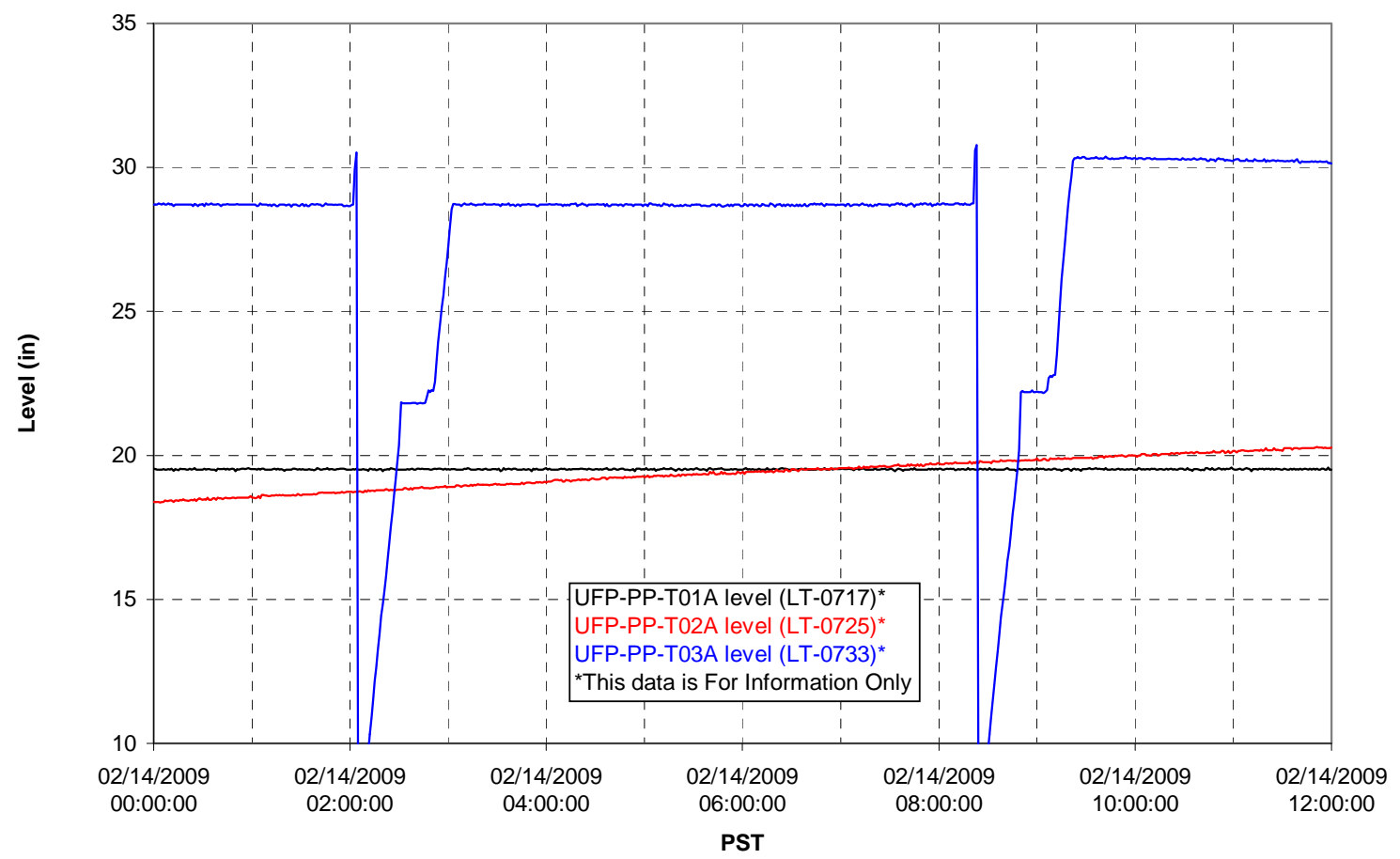


Filter UFP-FILT-T01A

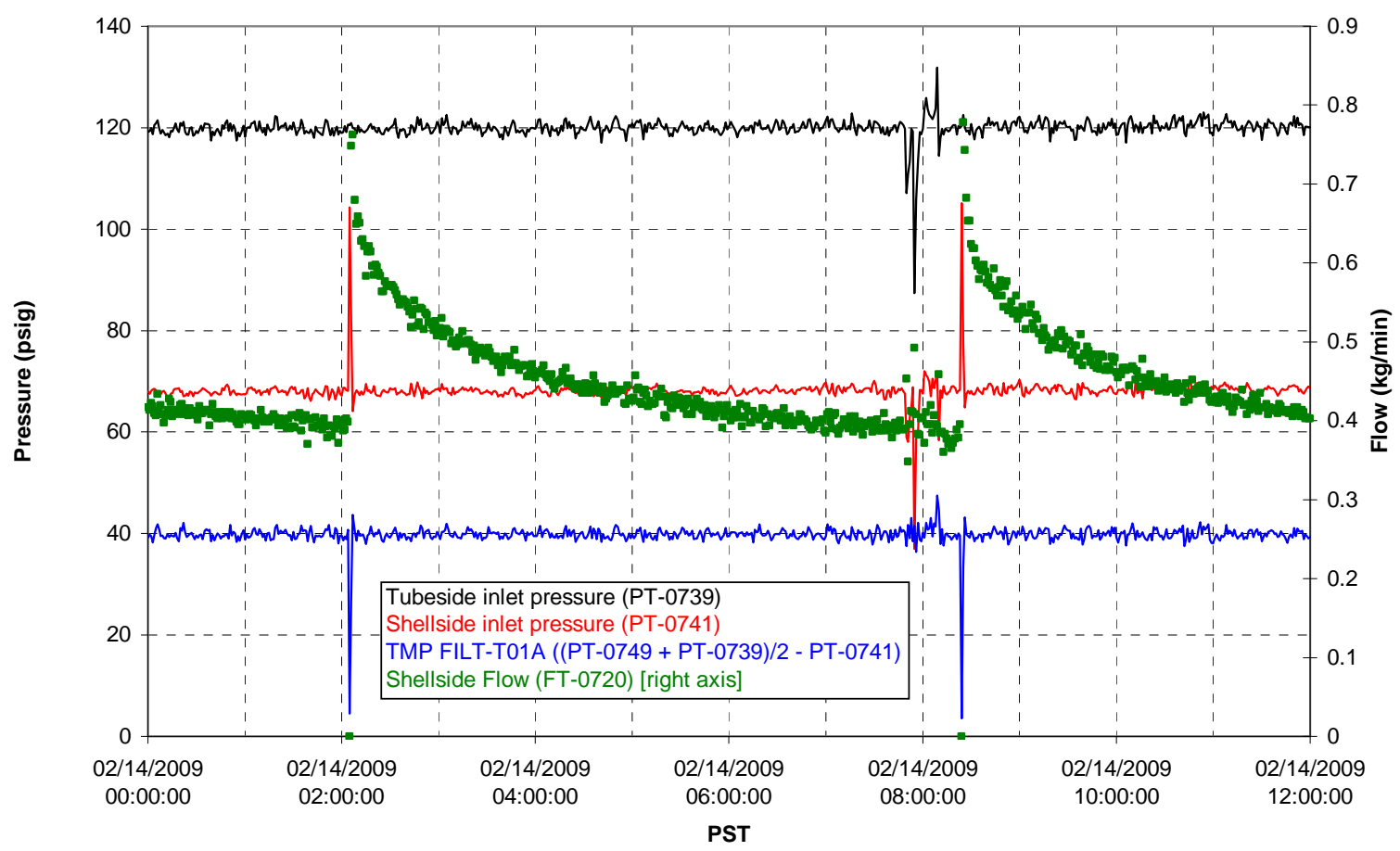

Filter UFP-FILT-T02A

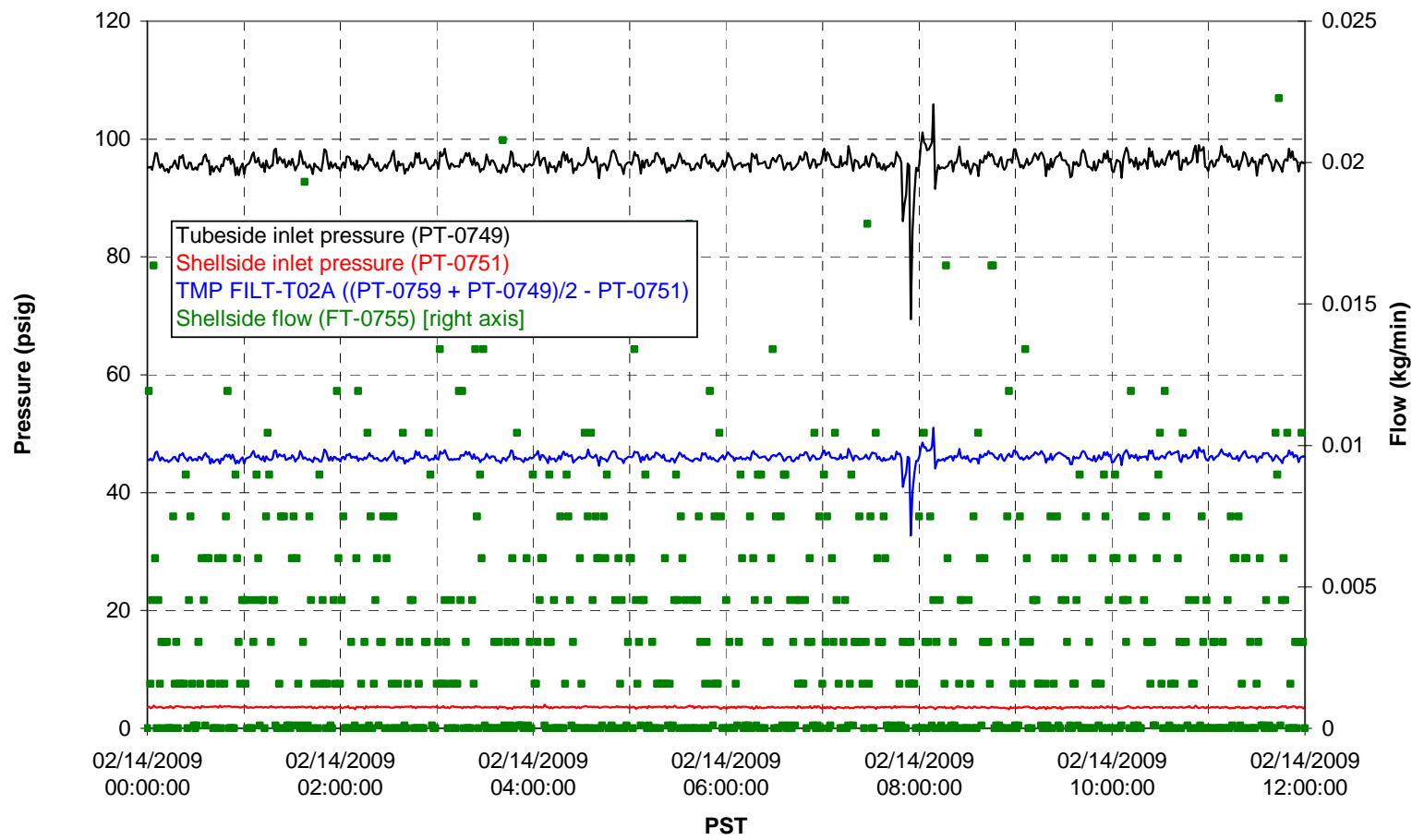


Filter UFP-FILT-T03A

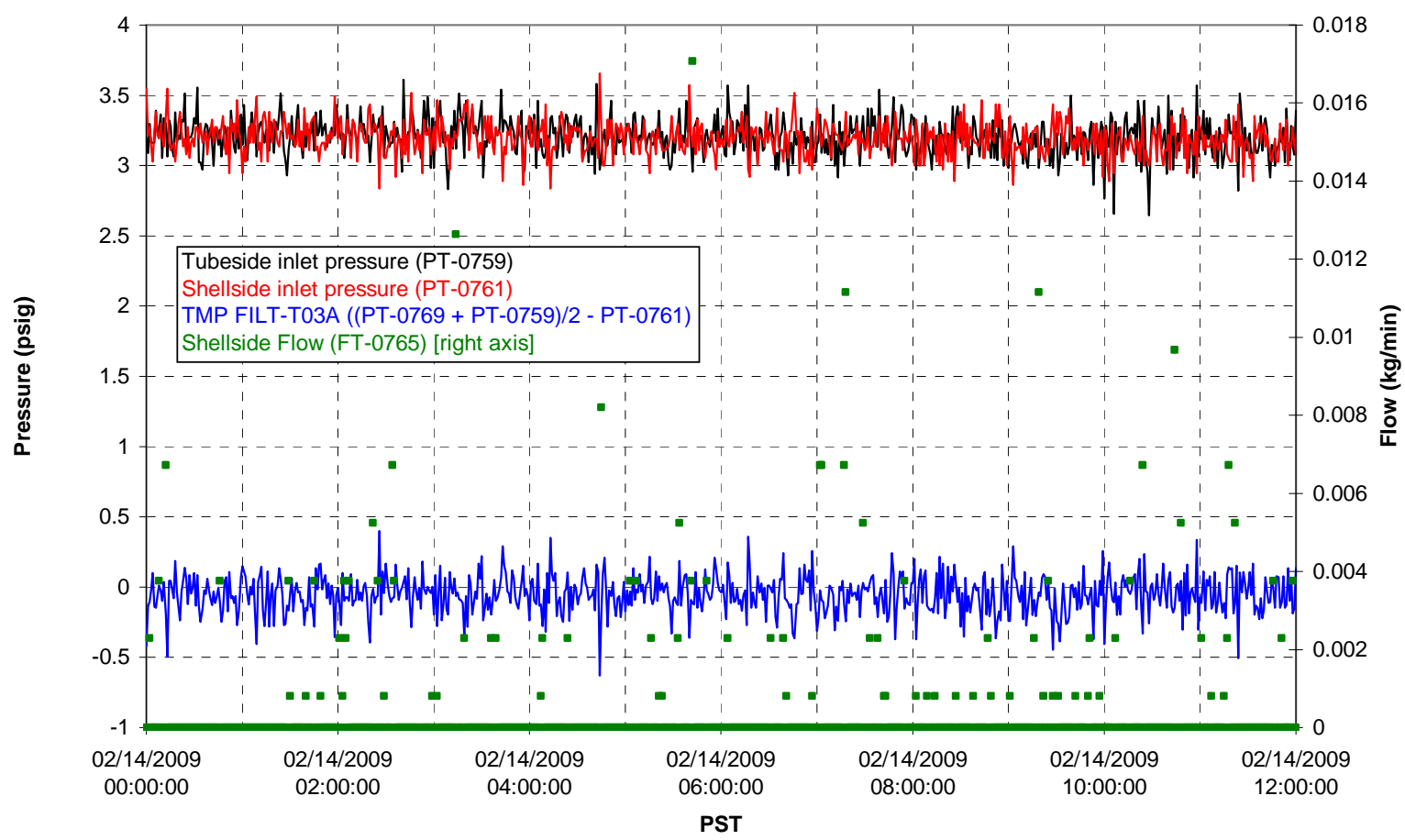

Filter UFP-FILT-T04A

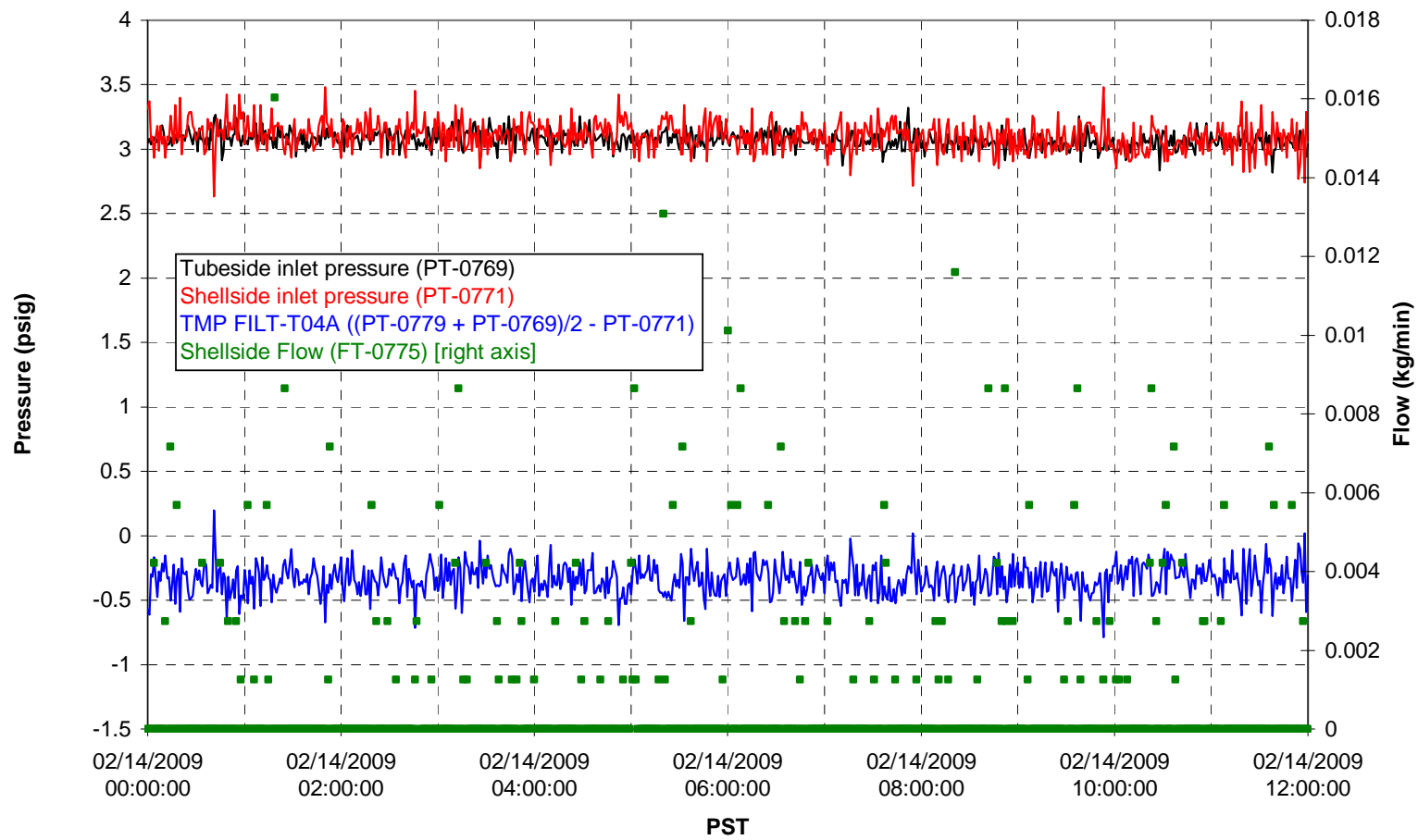


Filter UFP-FILT-T05A

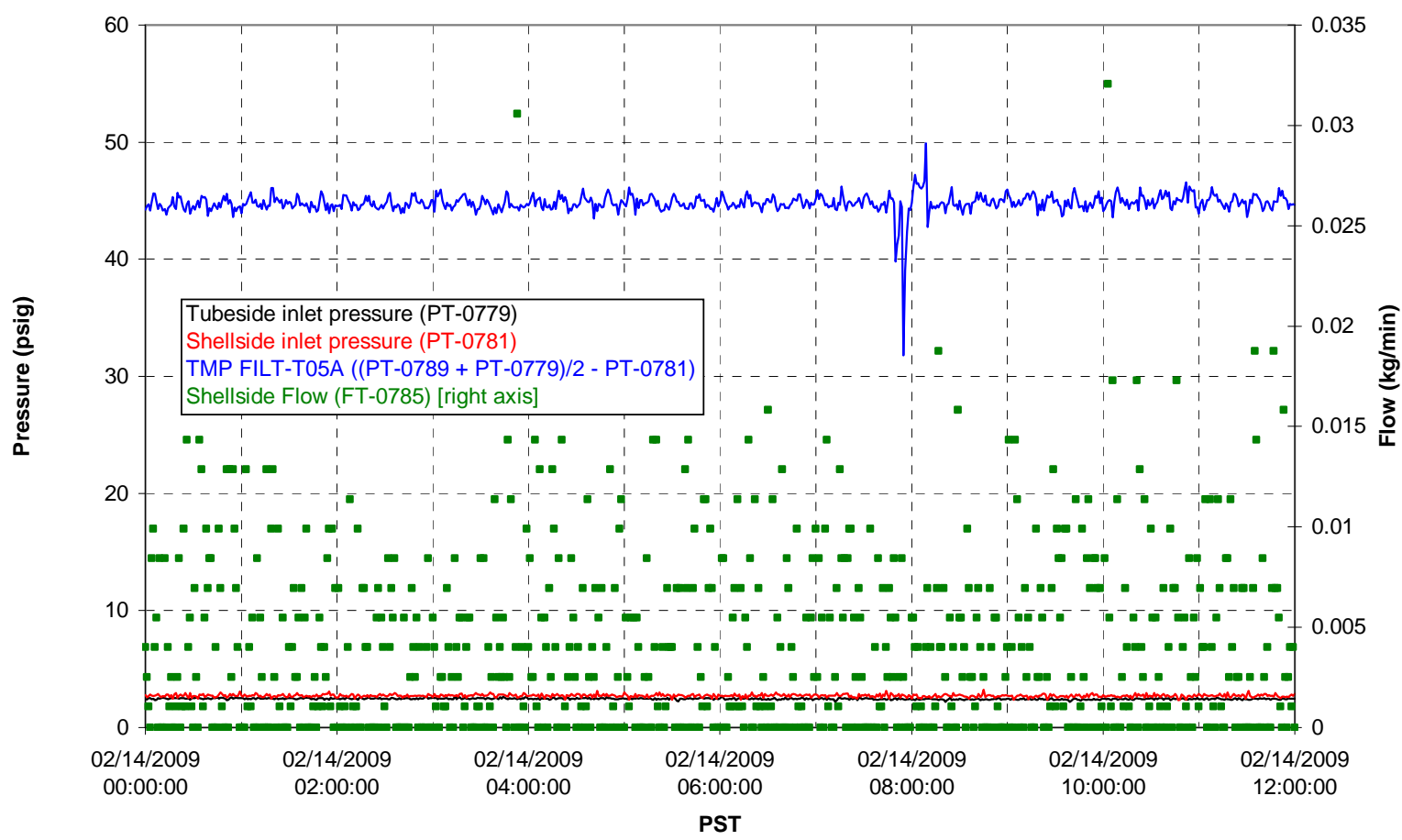

Chemical Flow

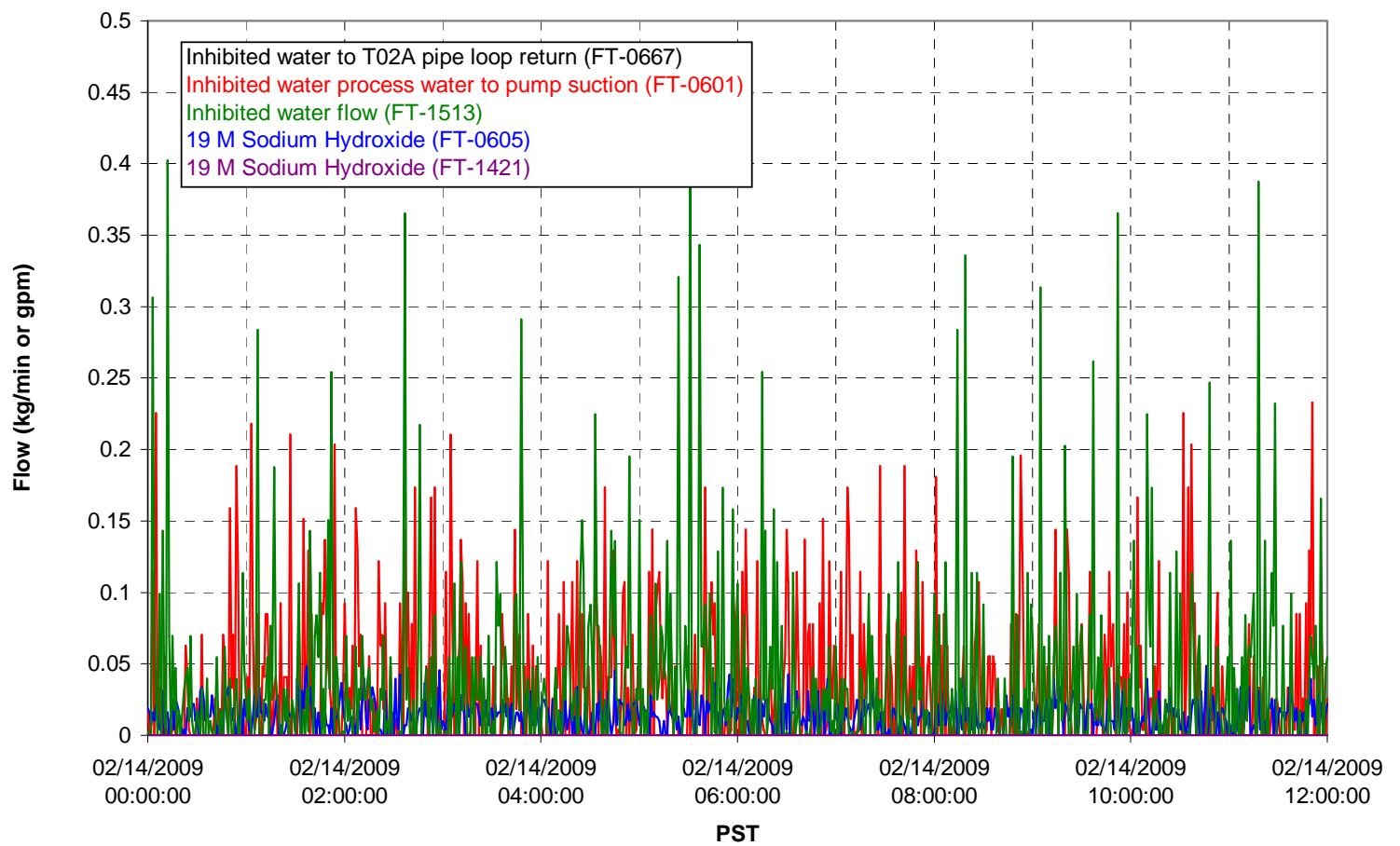


Chemical Flow

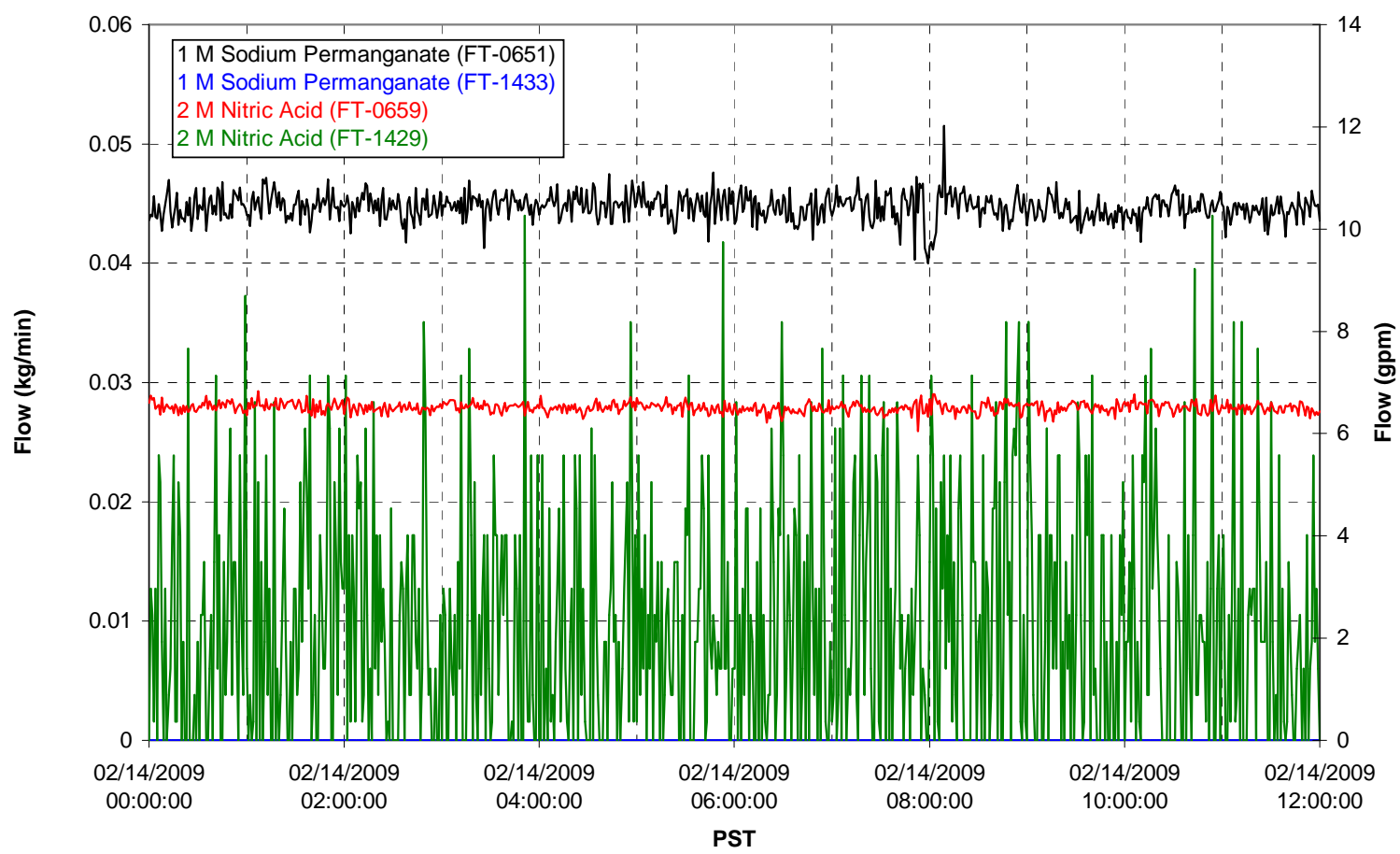

Air Flows

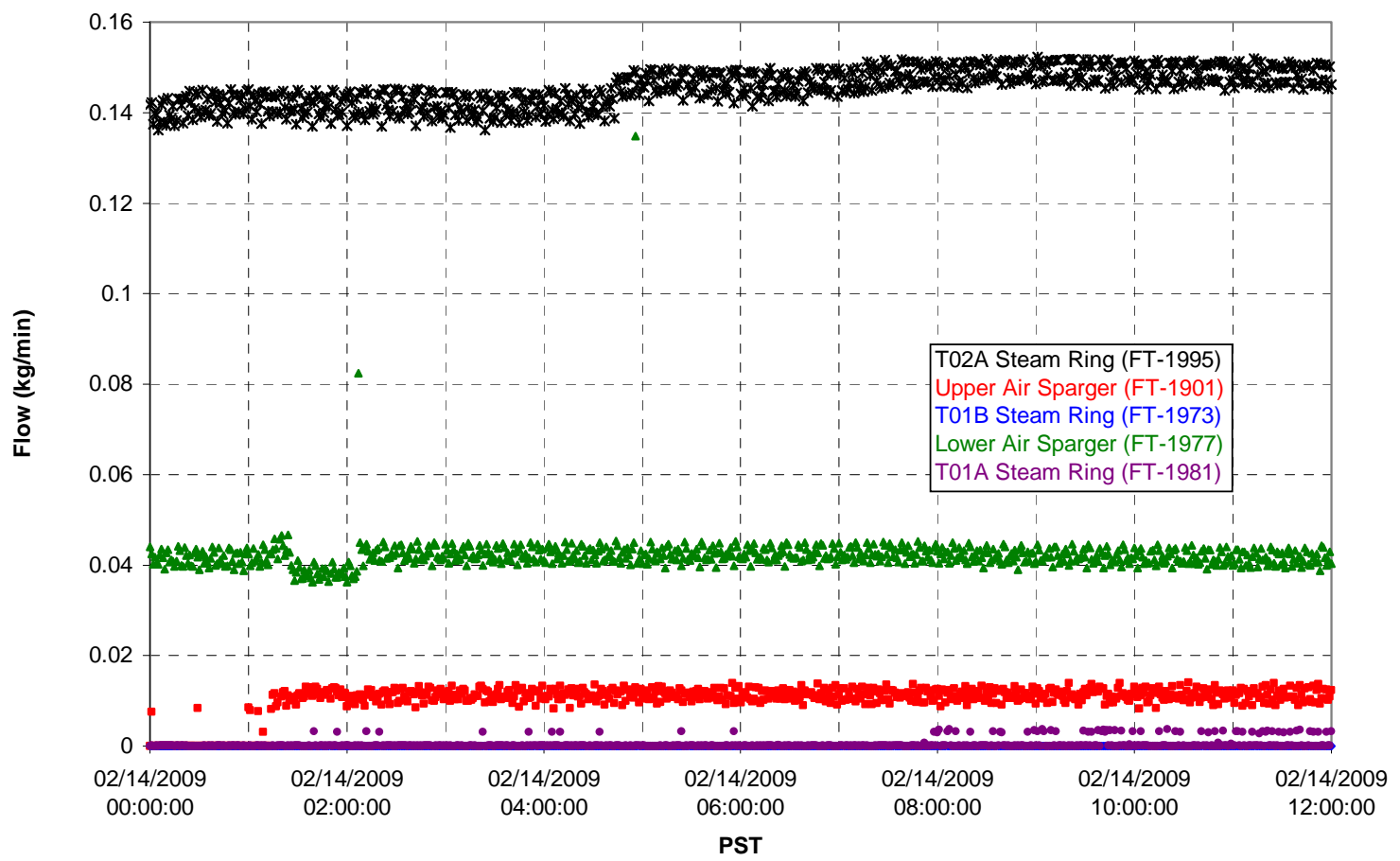


T02A Steam

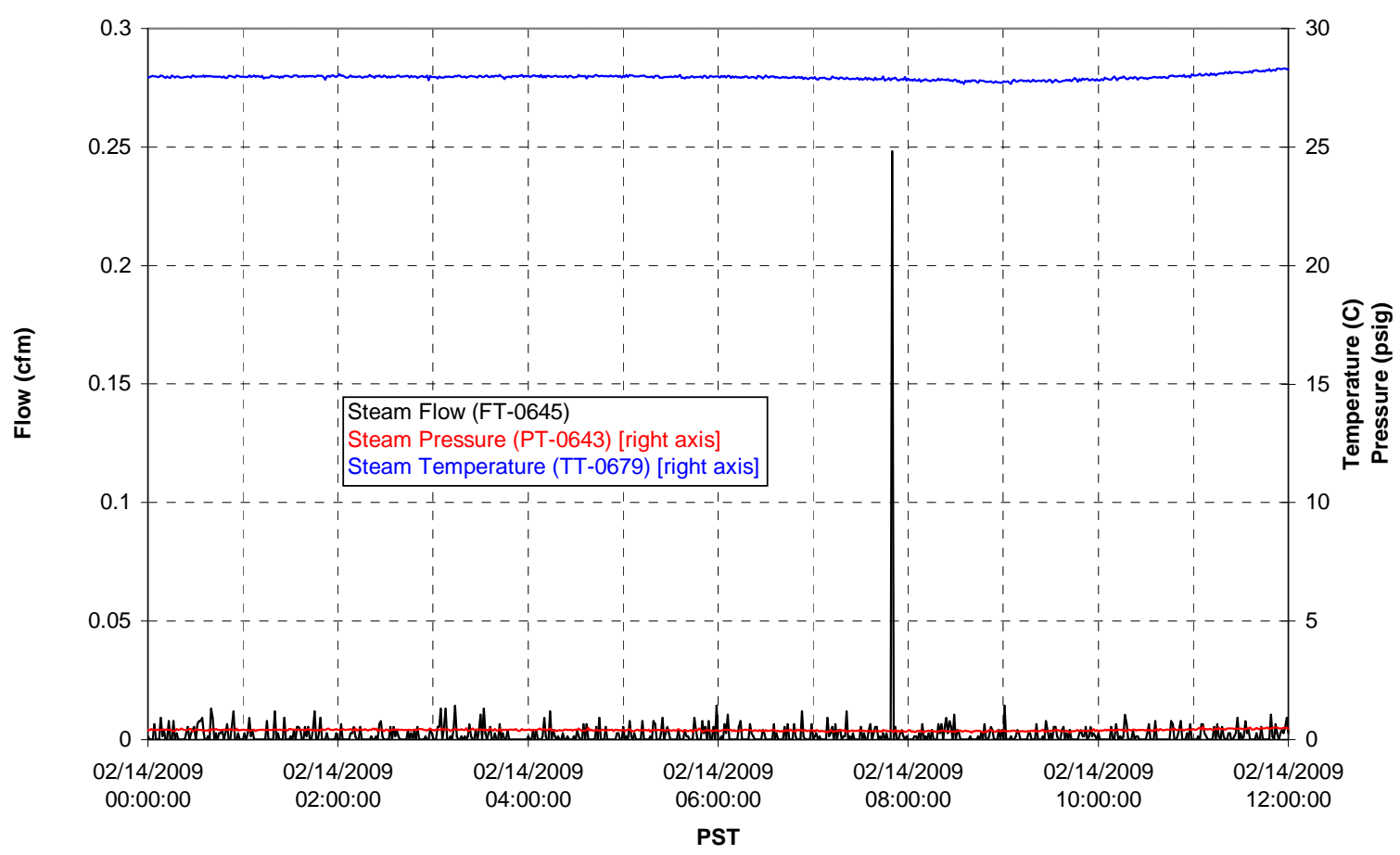

T01A Steam

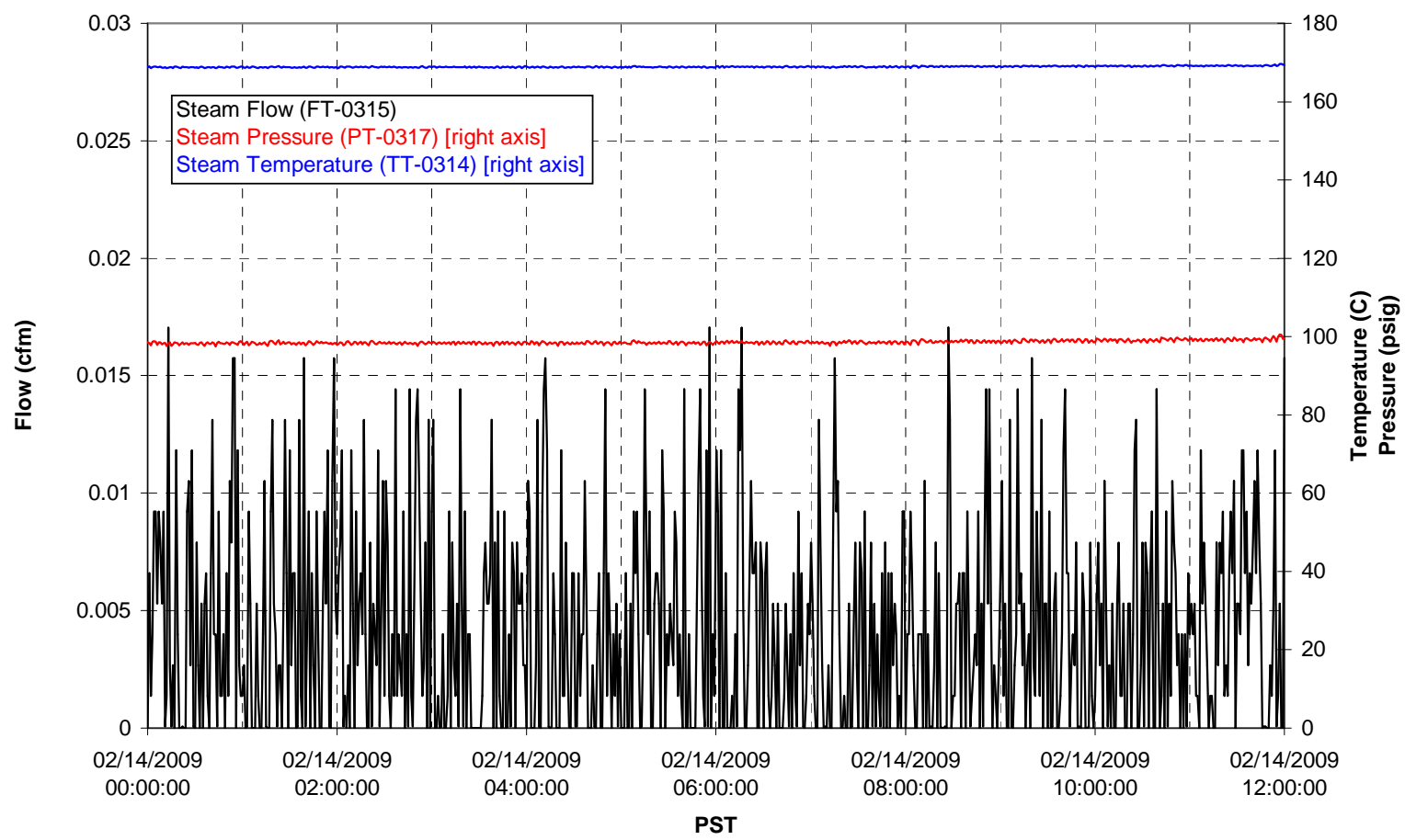


T01B Steam

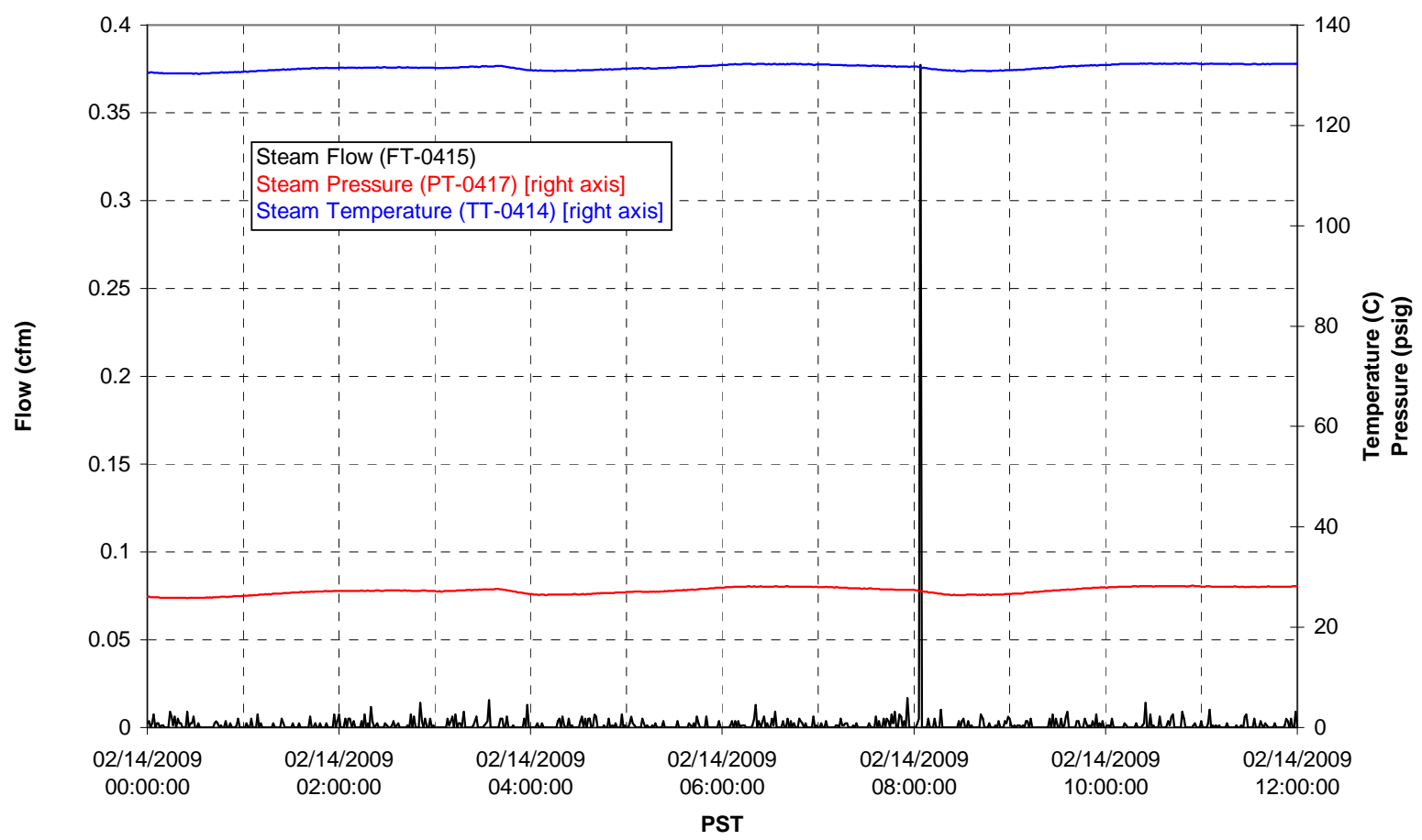




\section{Integrated Test A Data Plots 02/14/09 12:00 - 02/15/09 00:00}


T01A level

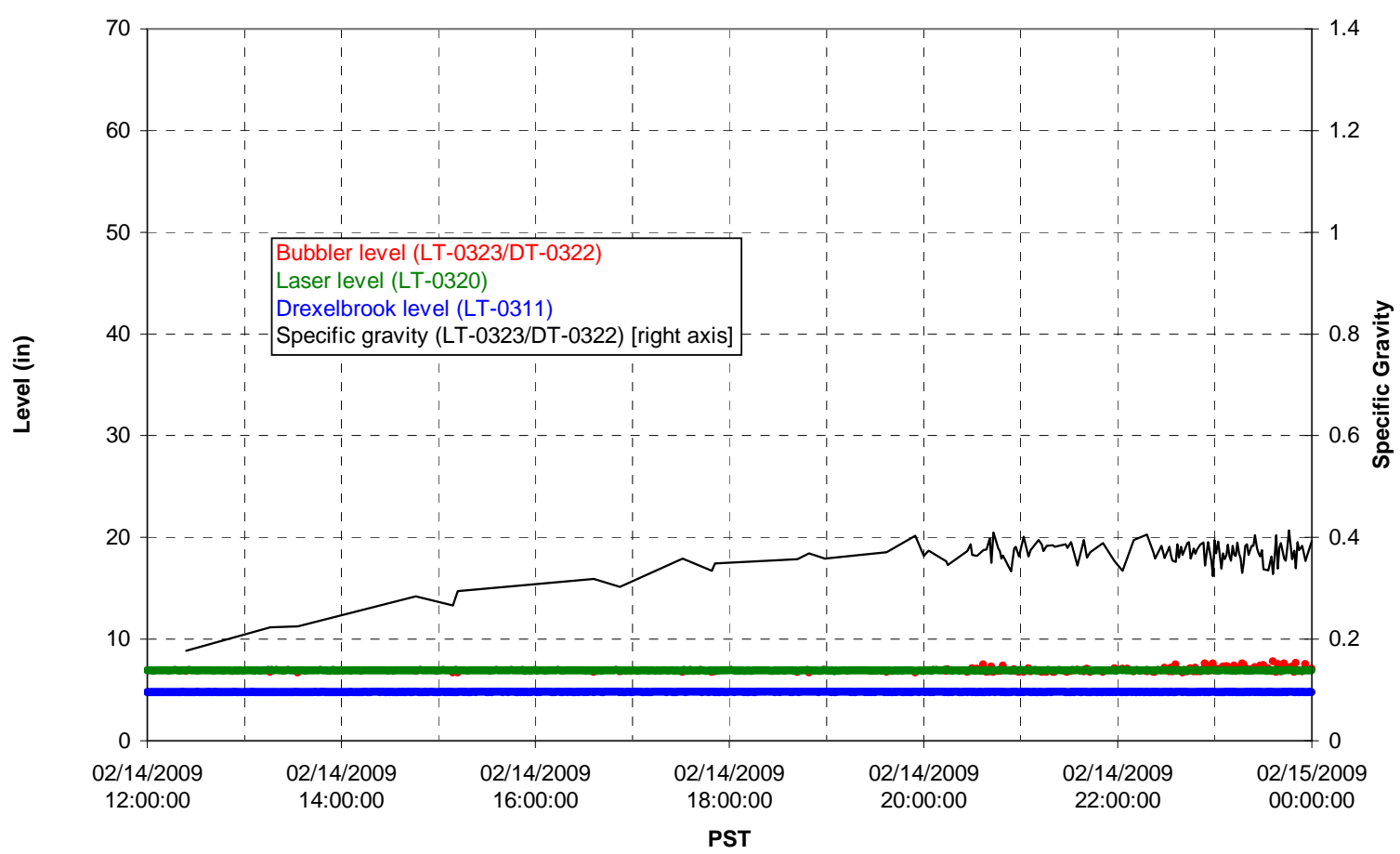

T01A temperatures

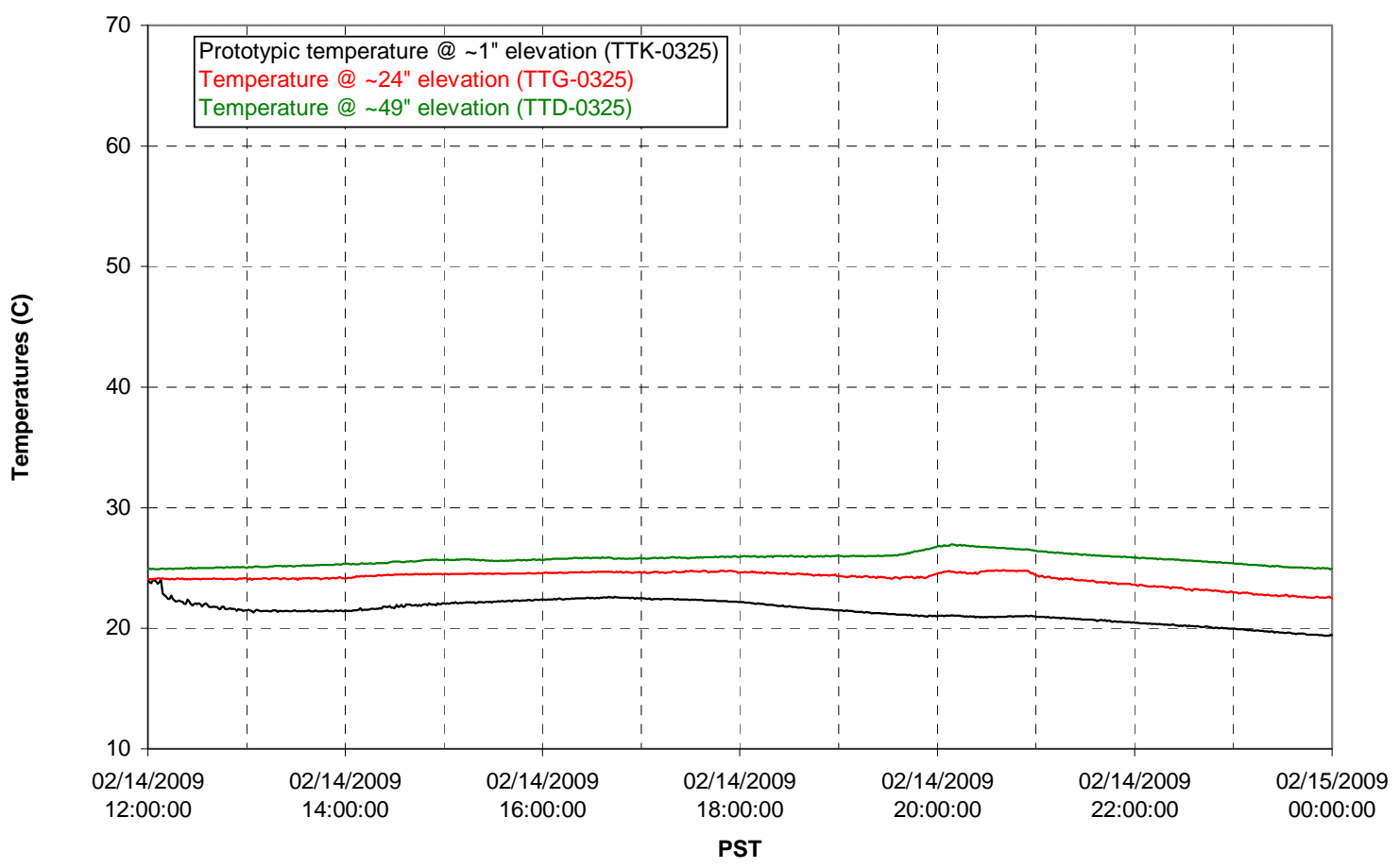


T01B level

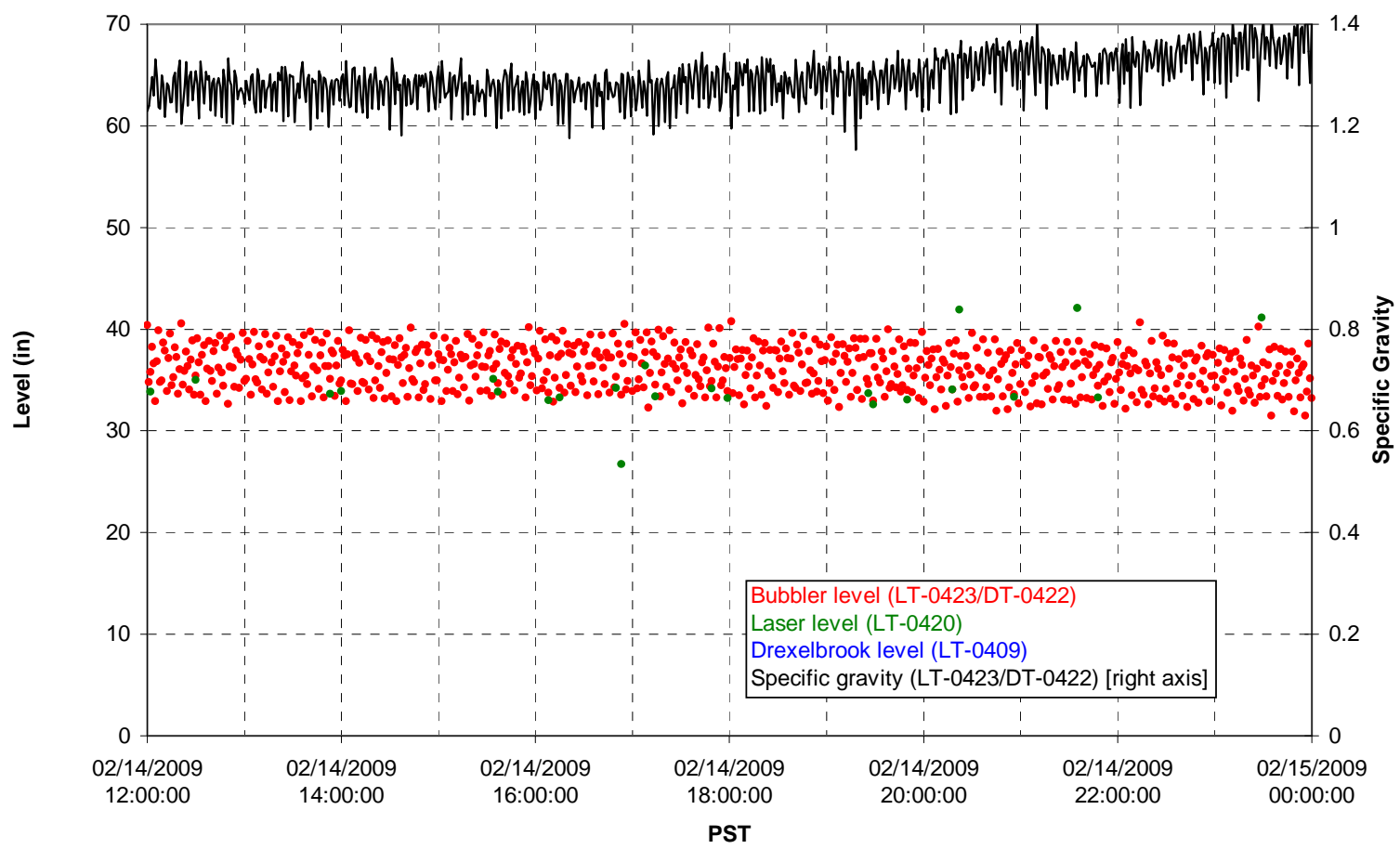

T01B temperatures

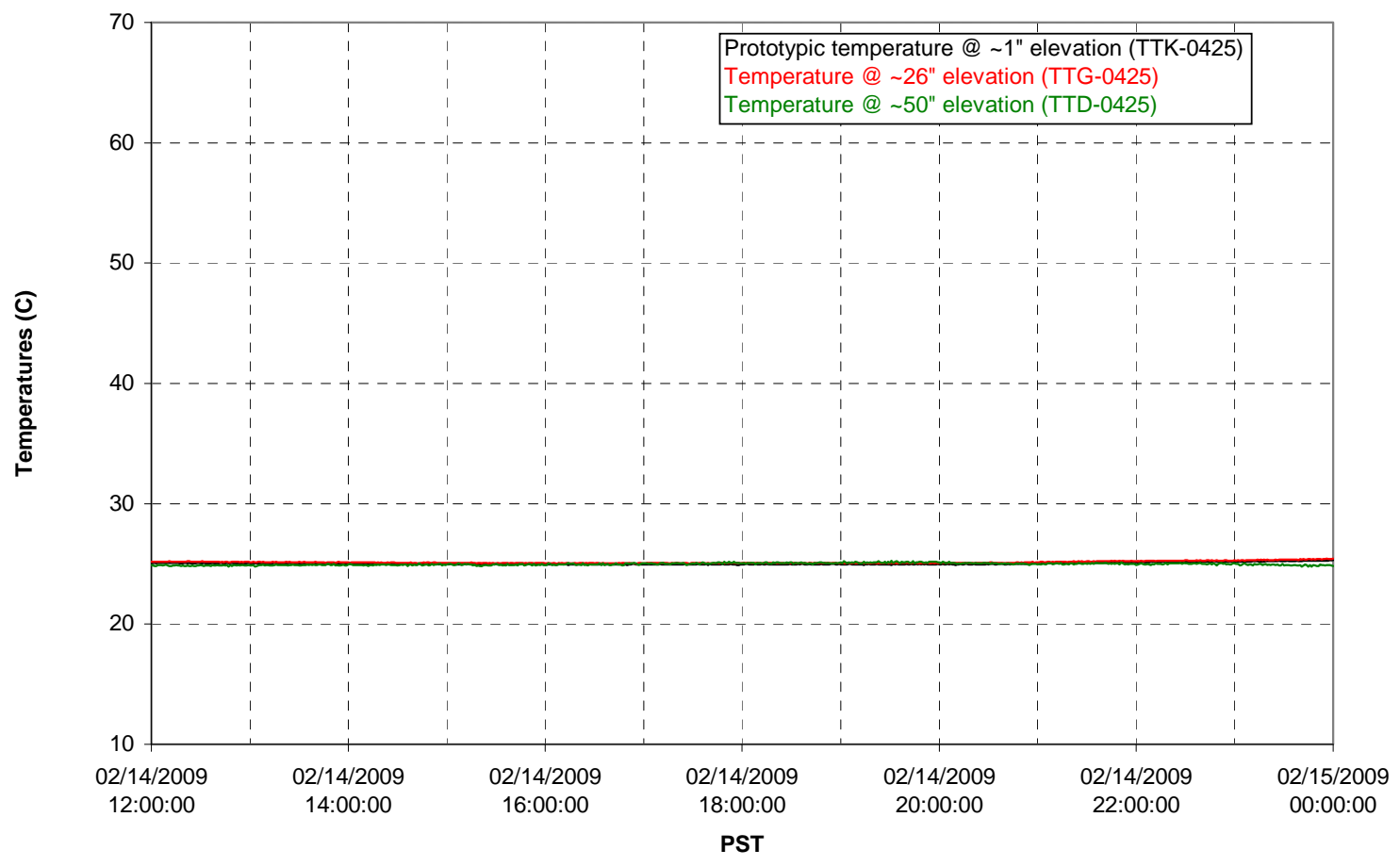


T02A level

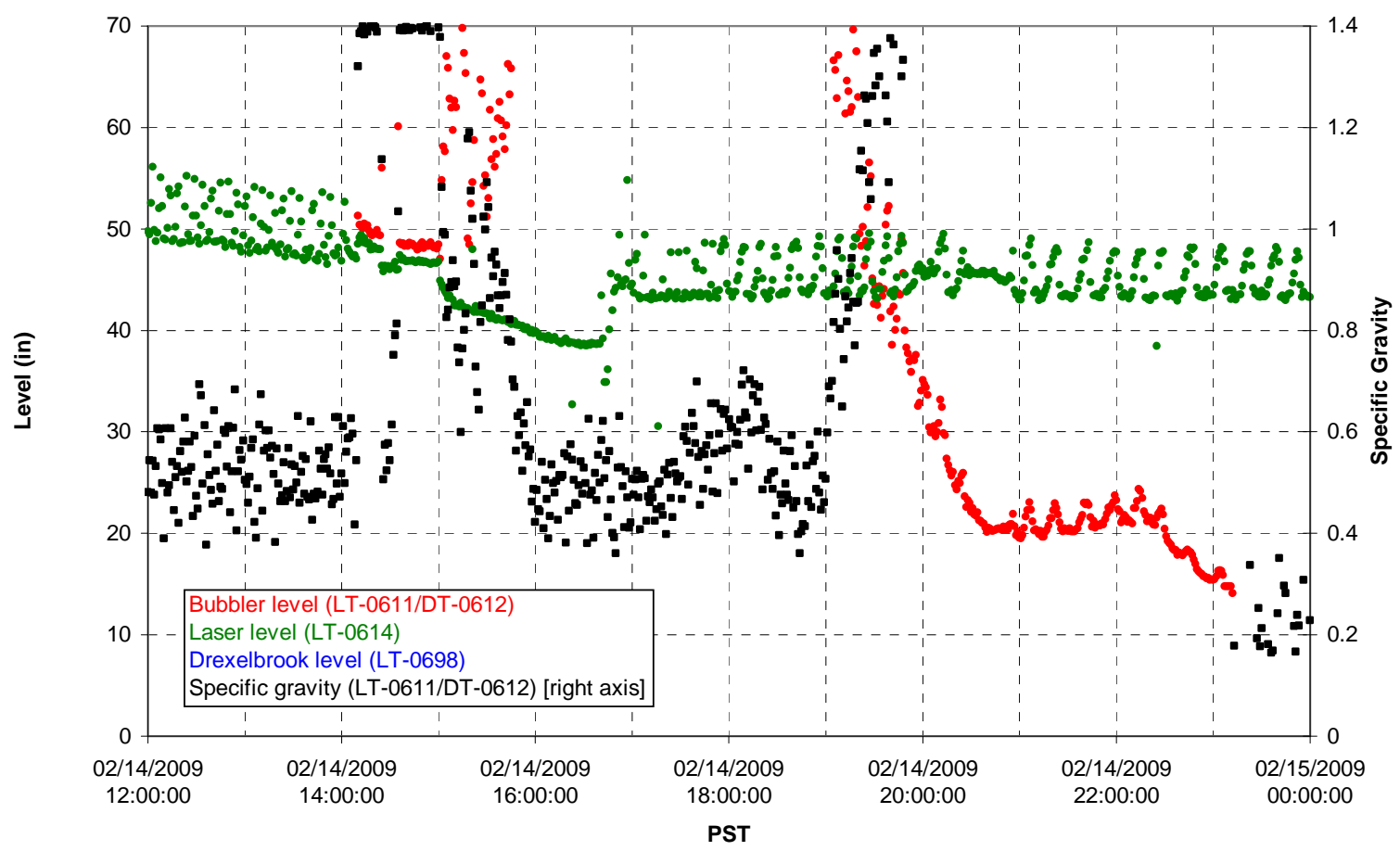

T02A temperatures

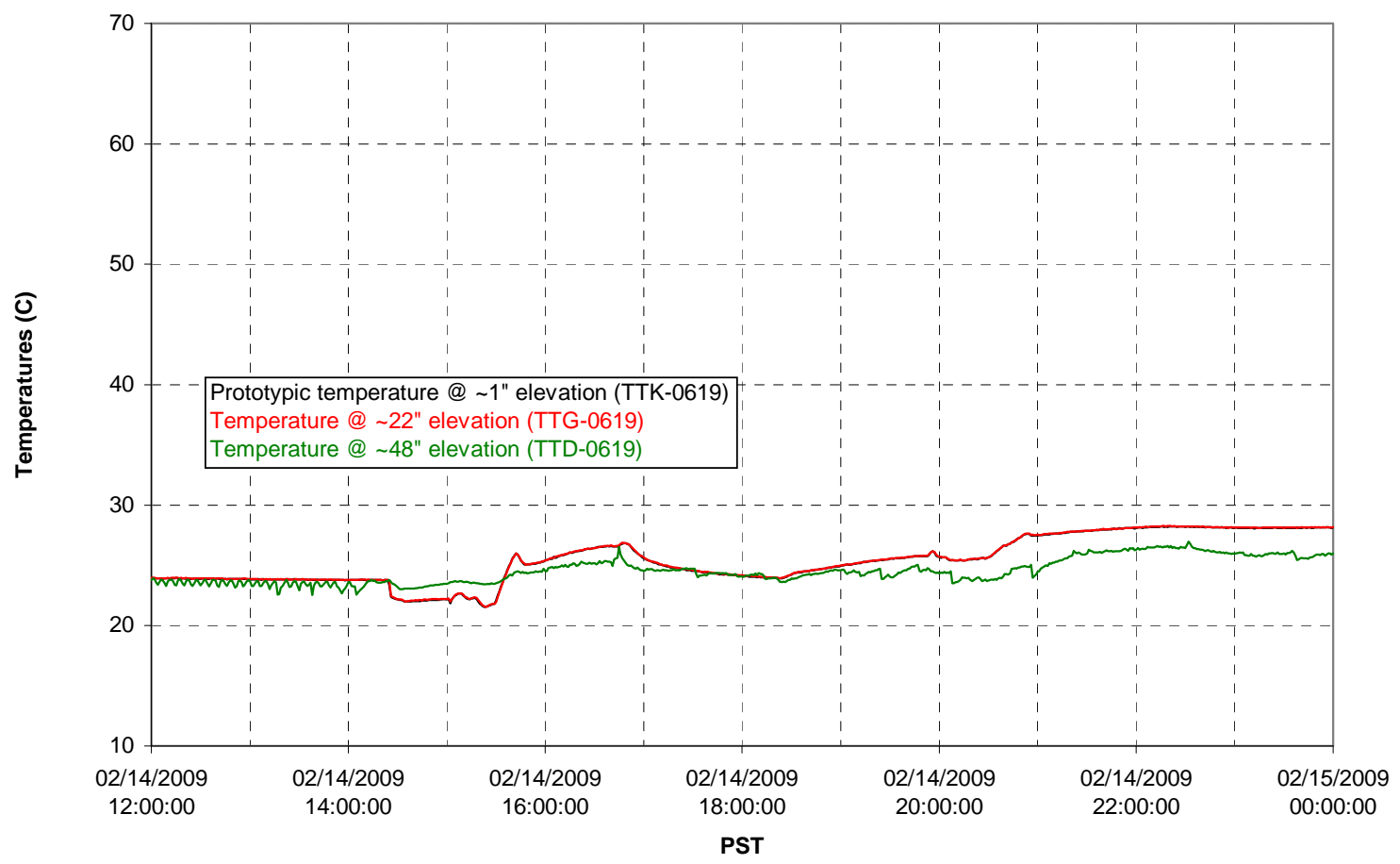

G.526 
T02A and filter loop temperatures

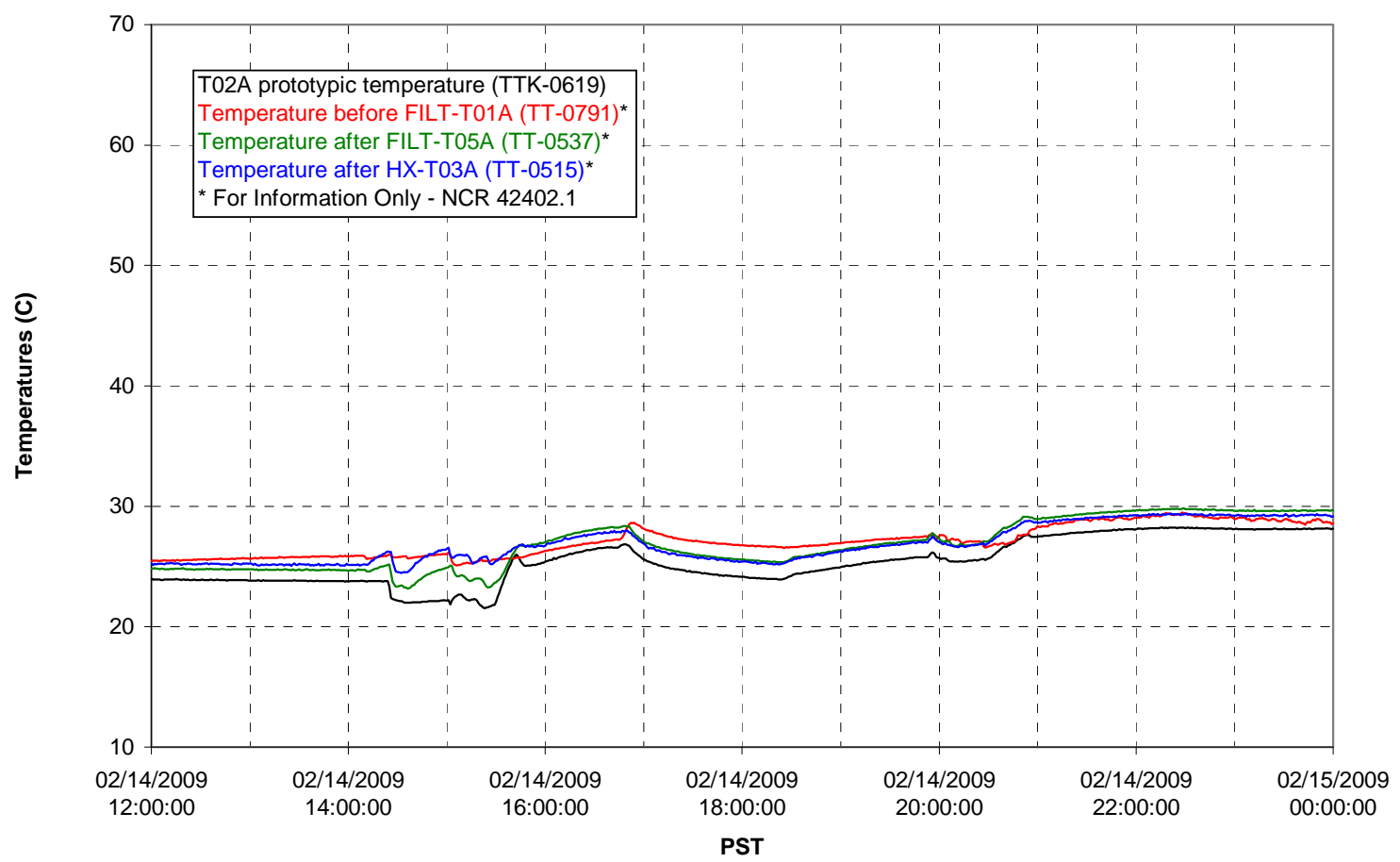

Pump Pressures and Flow

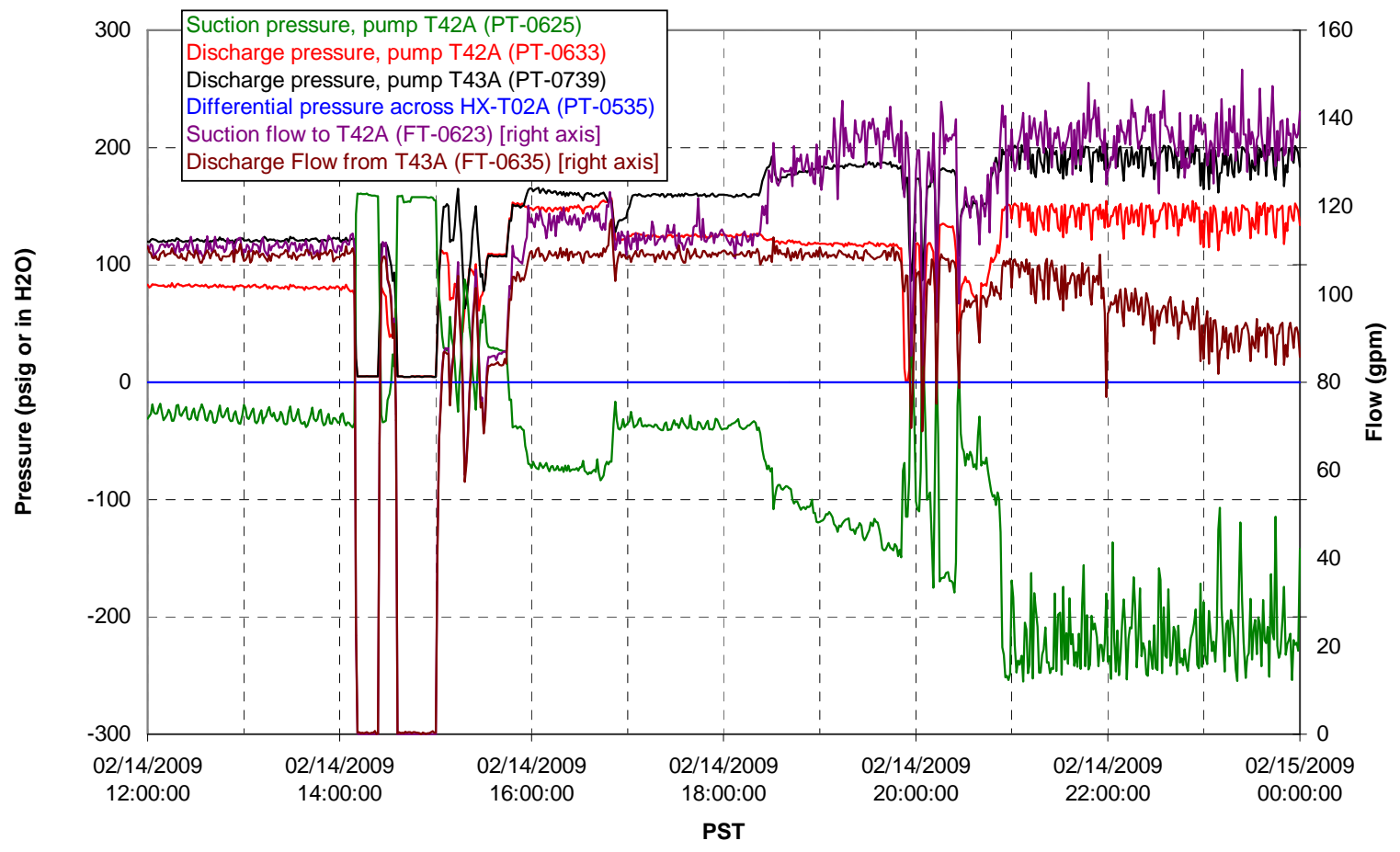




\section{Axial pressure drop}

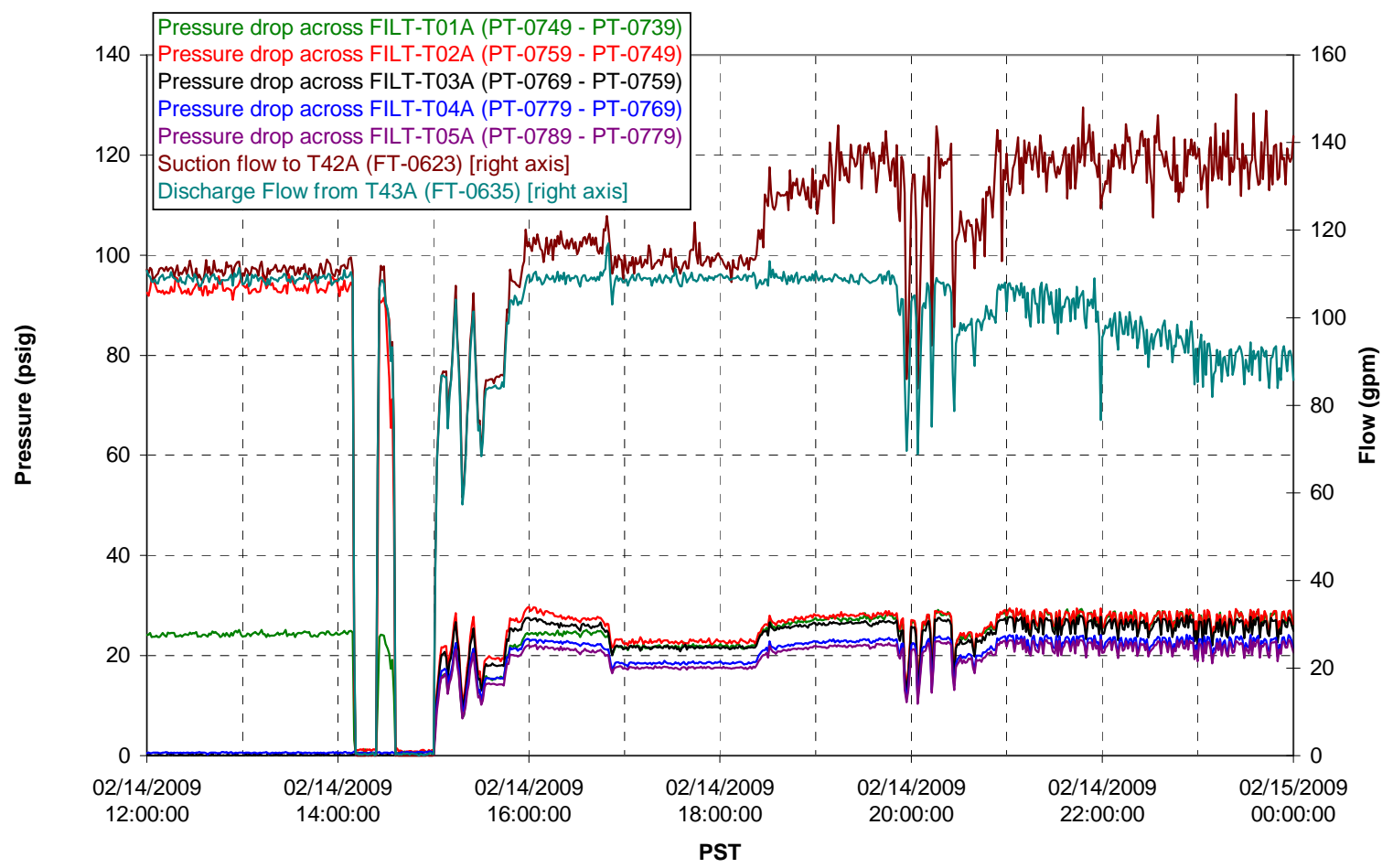

Permeate flow rates

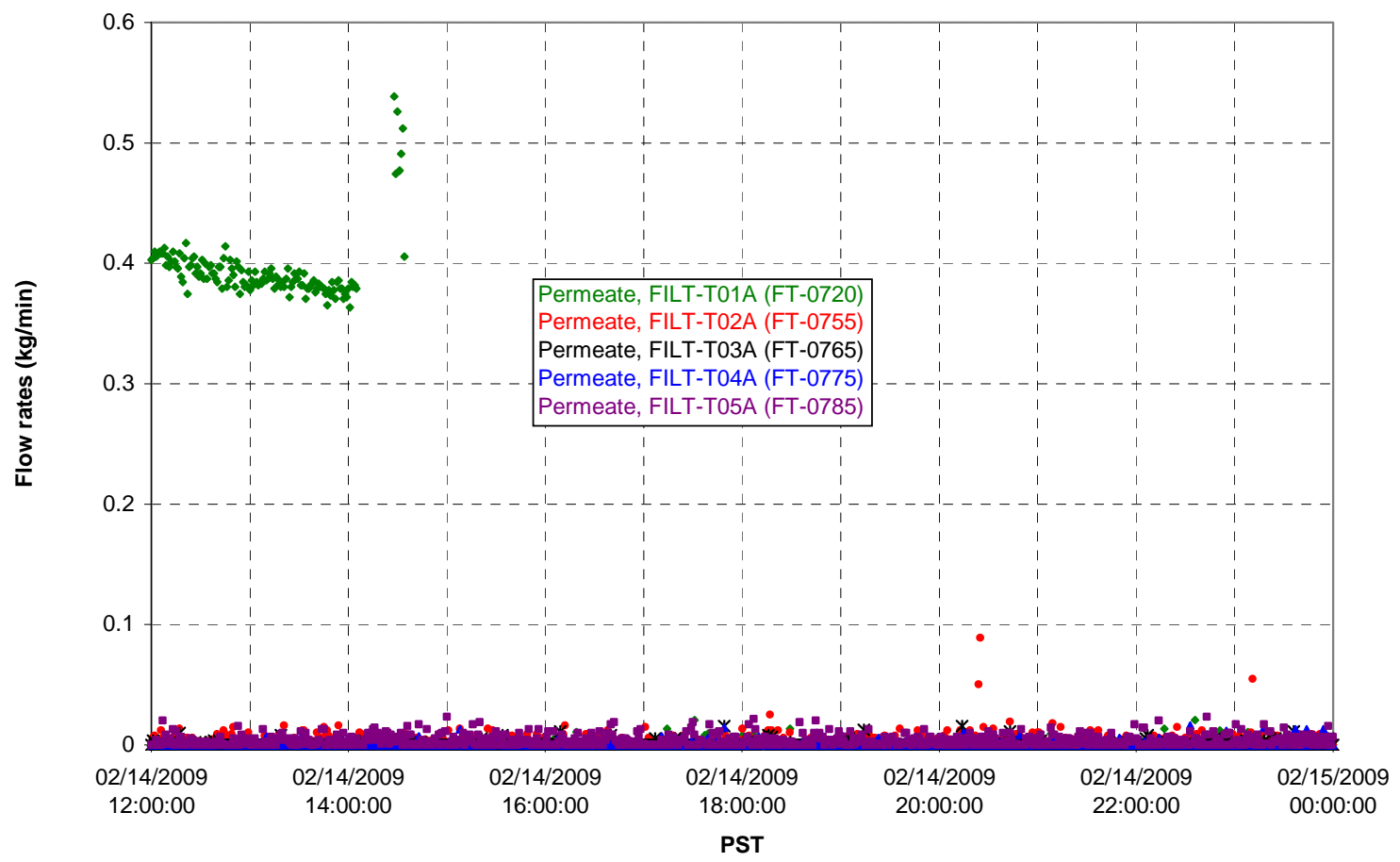


T02A Inner Temperature Tree

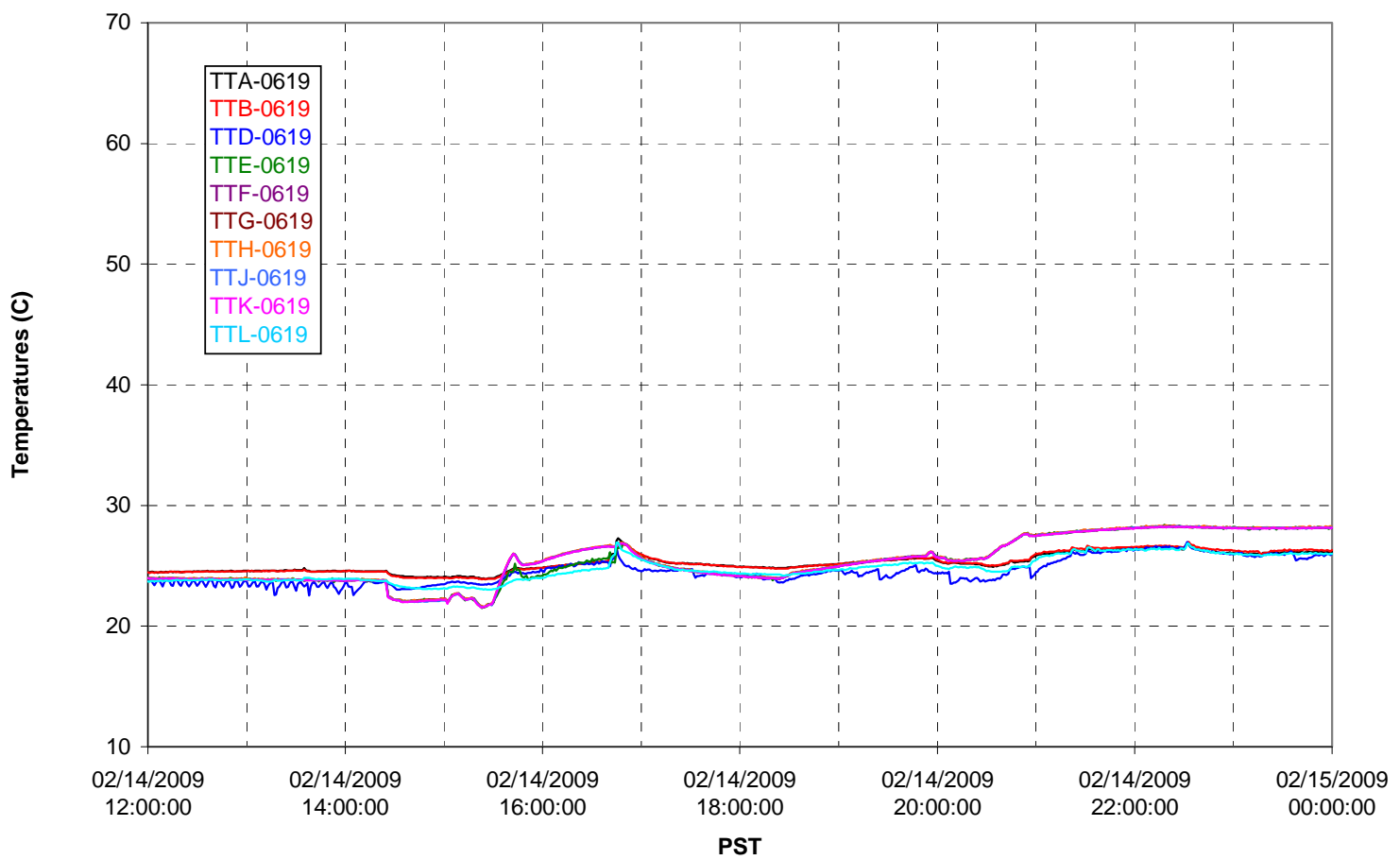

T02A Outer Temperature Tree

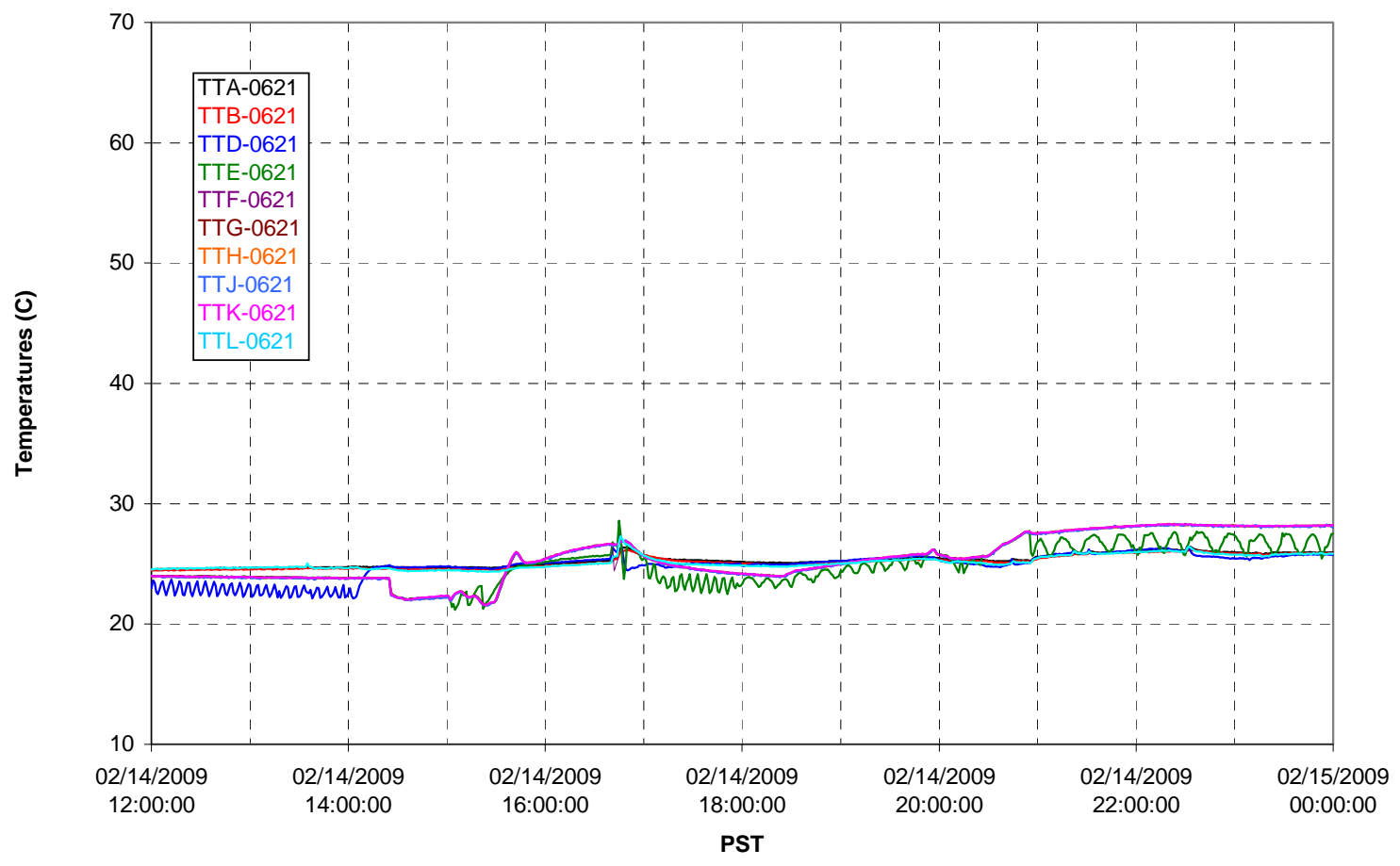


T02A temperatures

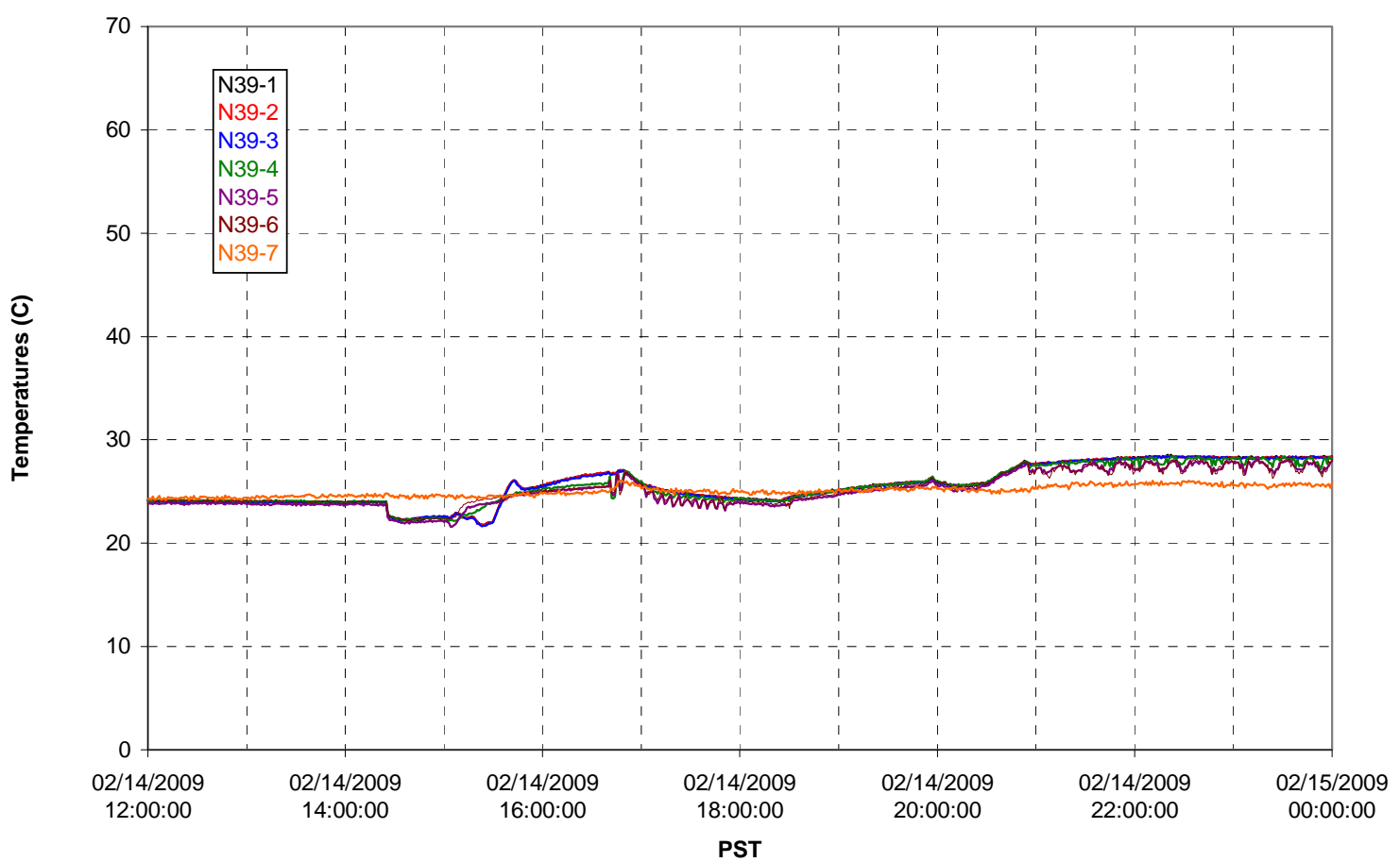

T02A temperatures

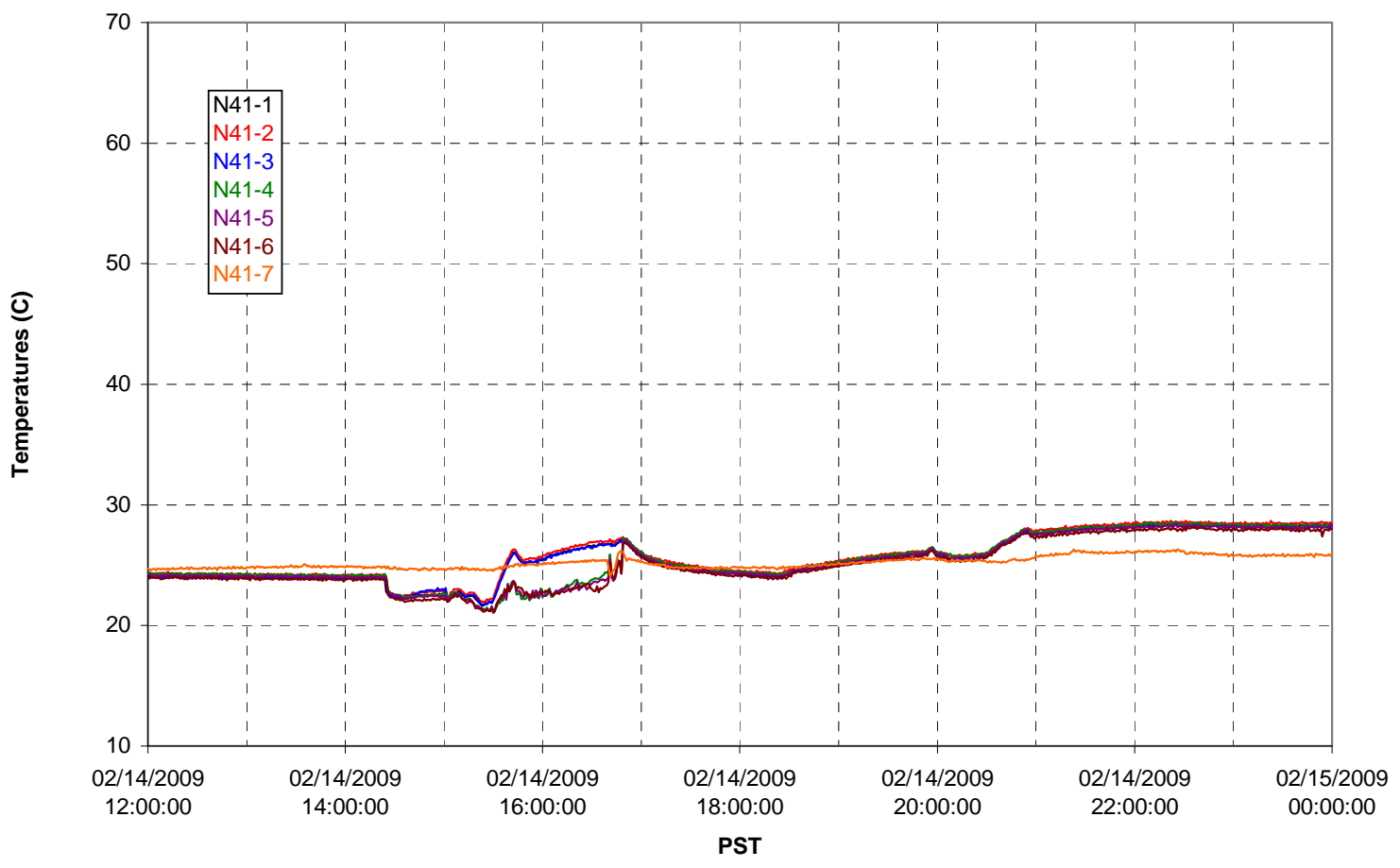


T02A temperatures

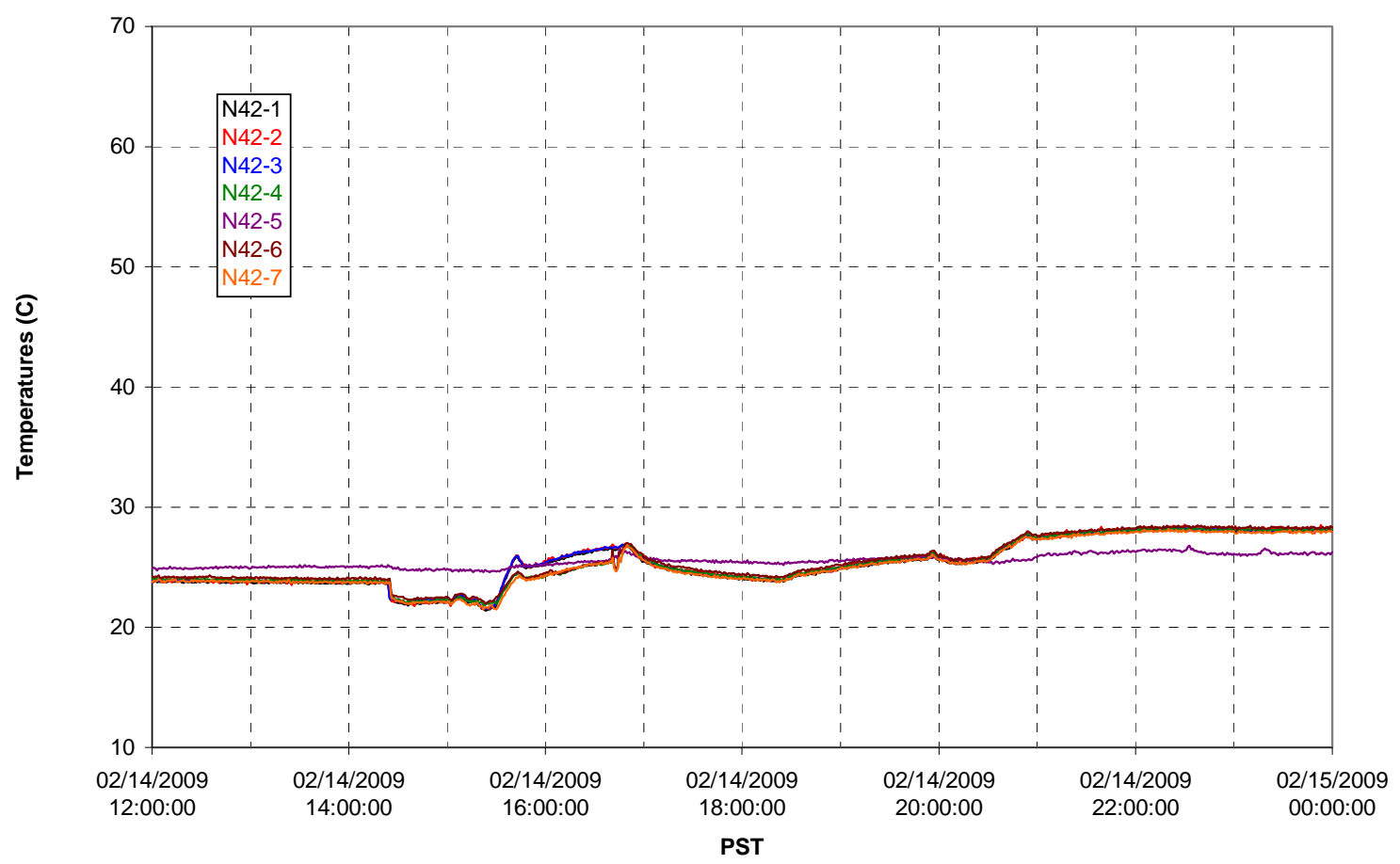

T02A temperatures

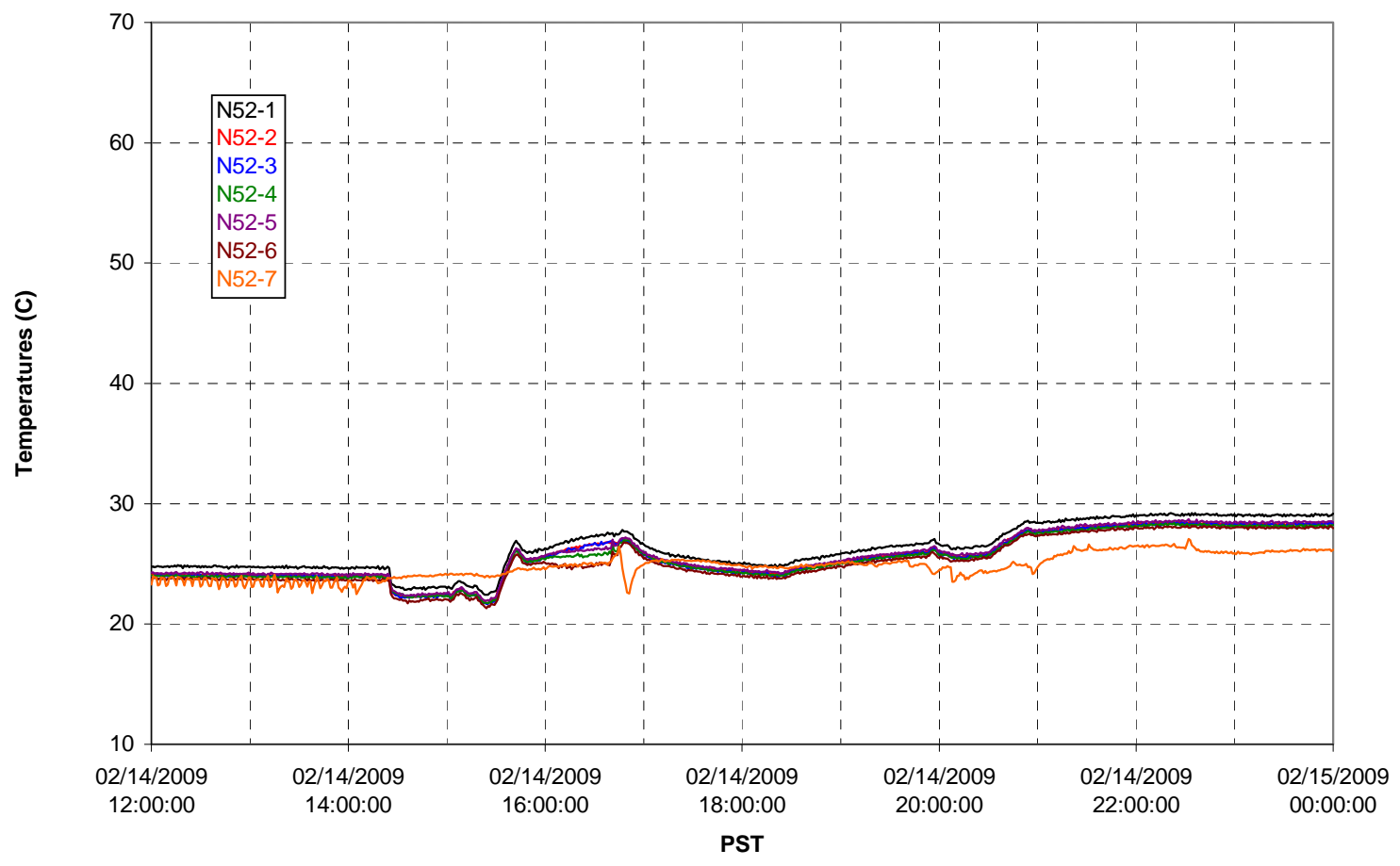


T02A Heating and Cooling
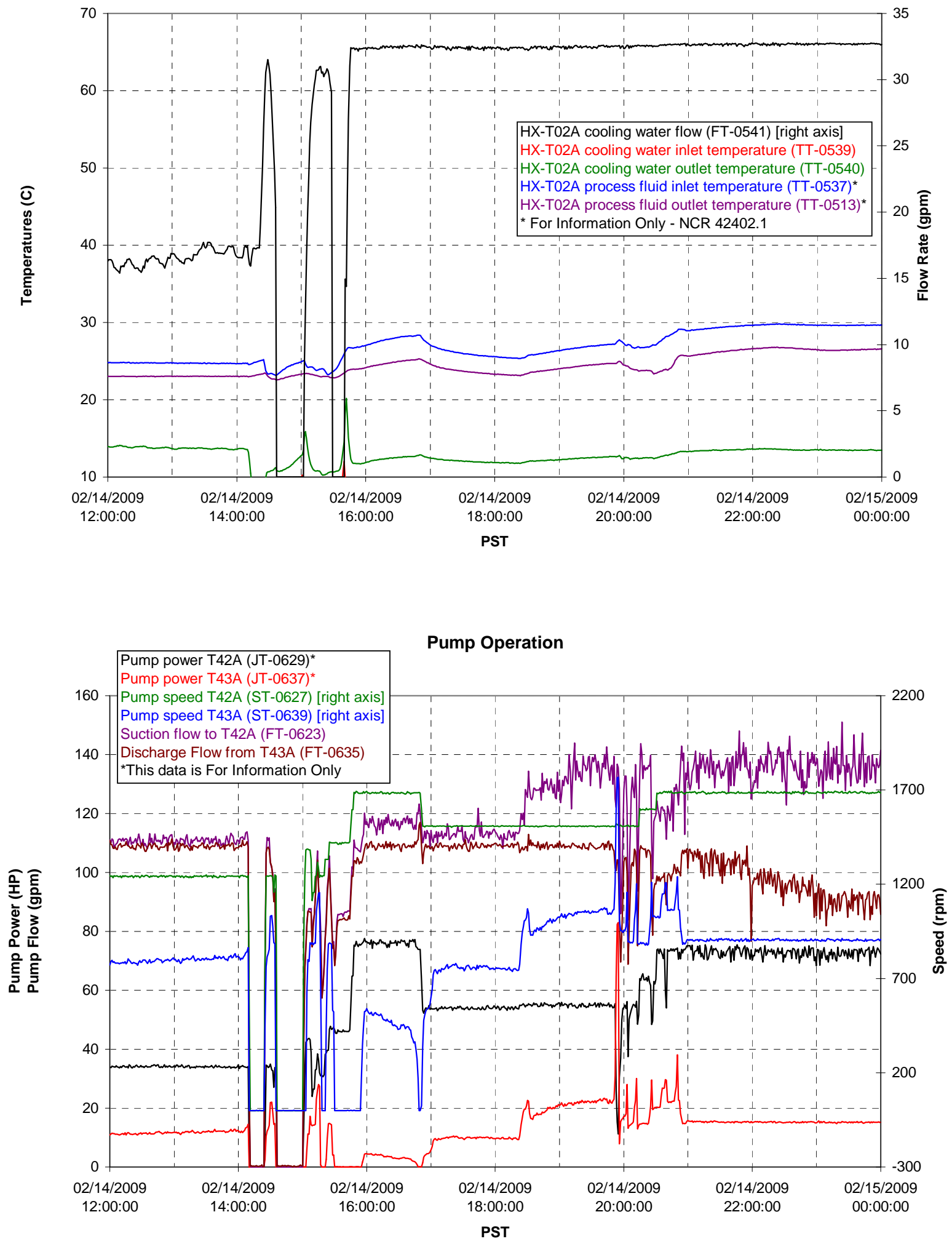
Pulsepot UFP-PP-T01A

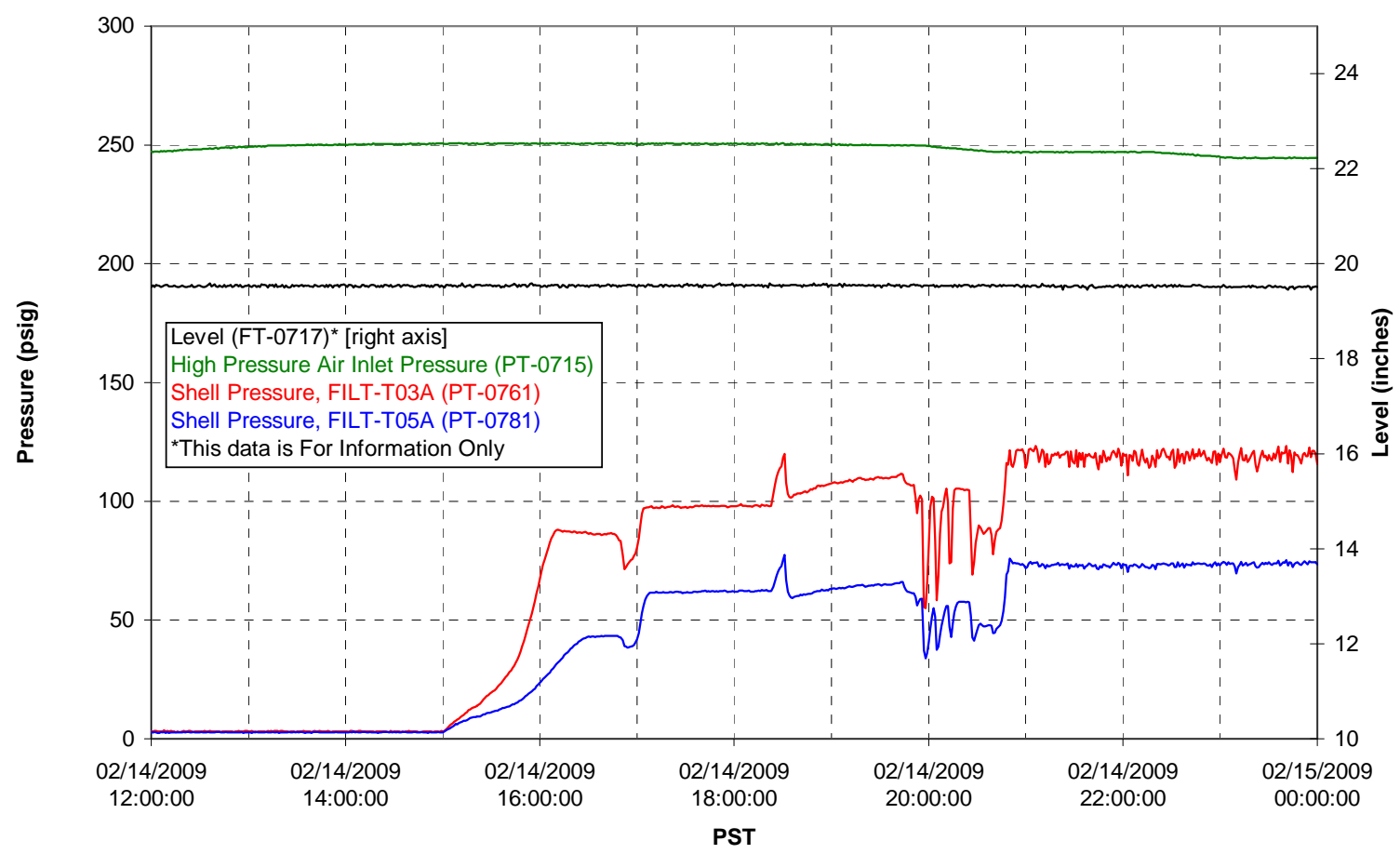

Pulsepot UFP-PP-T02A

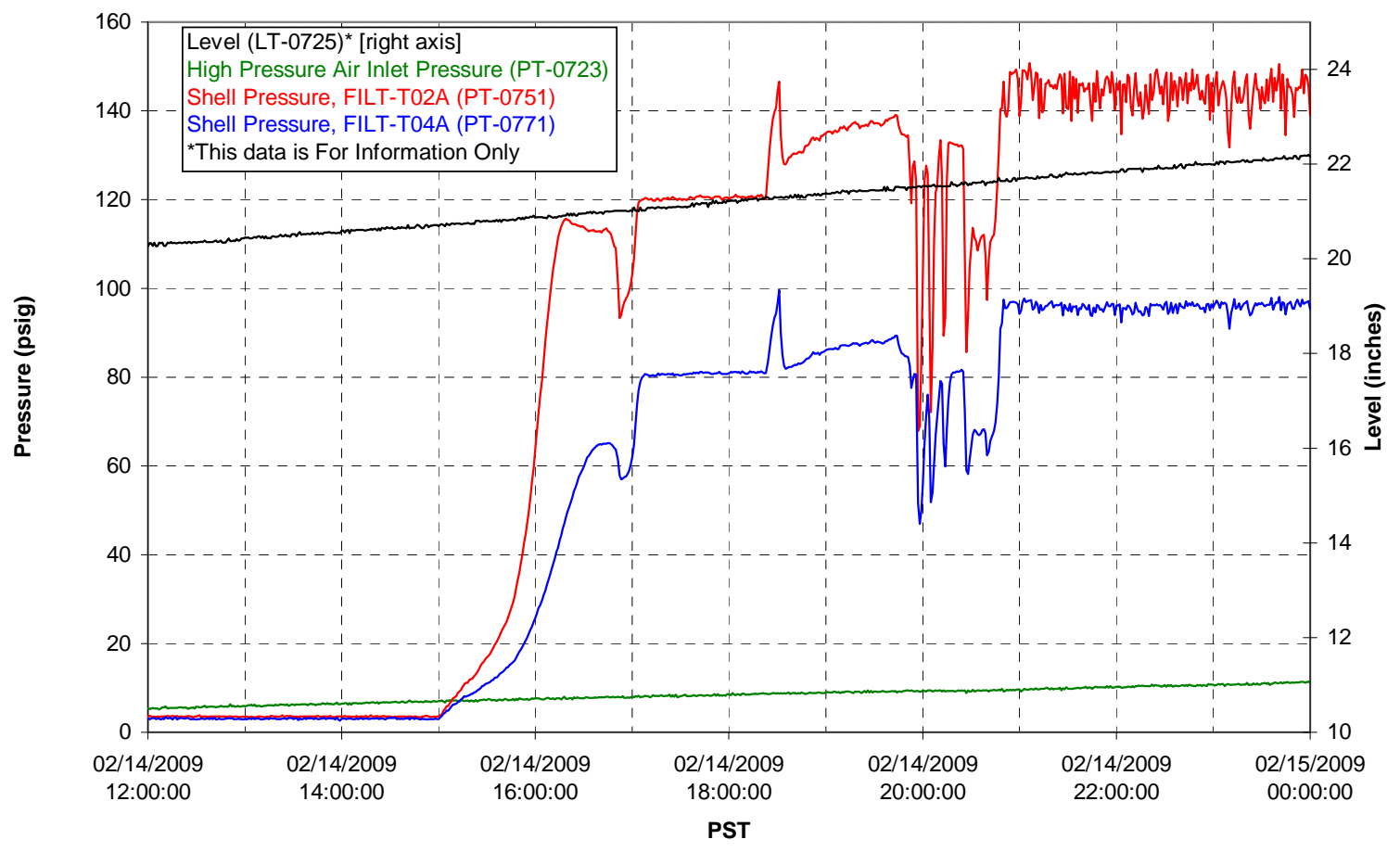


Pulsepot UFP-PP-T03A

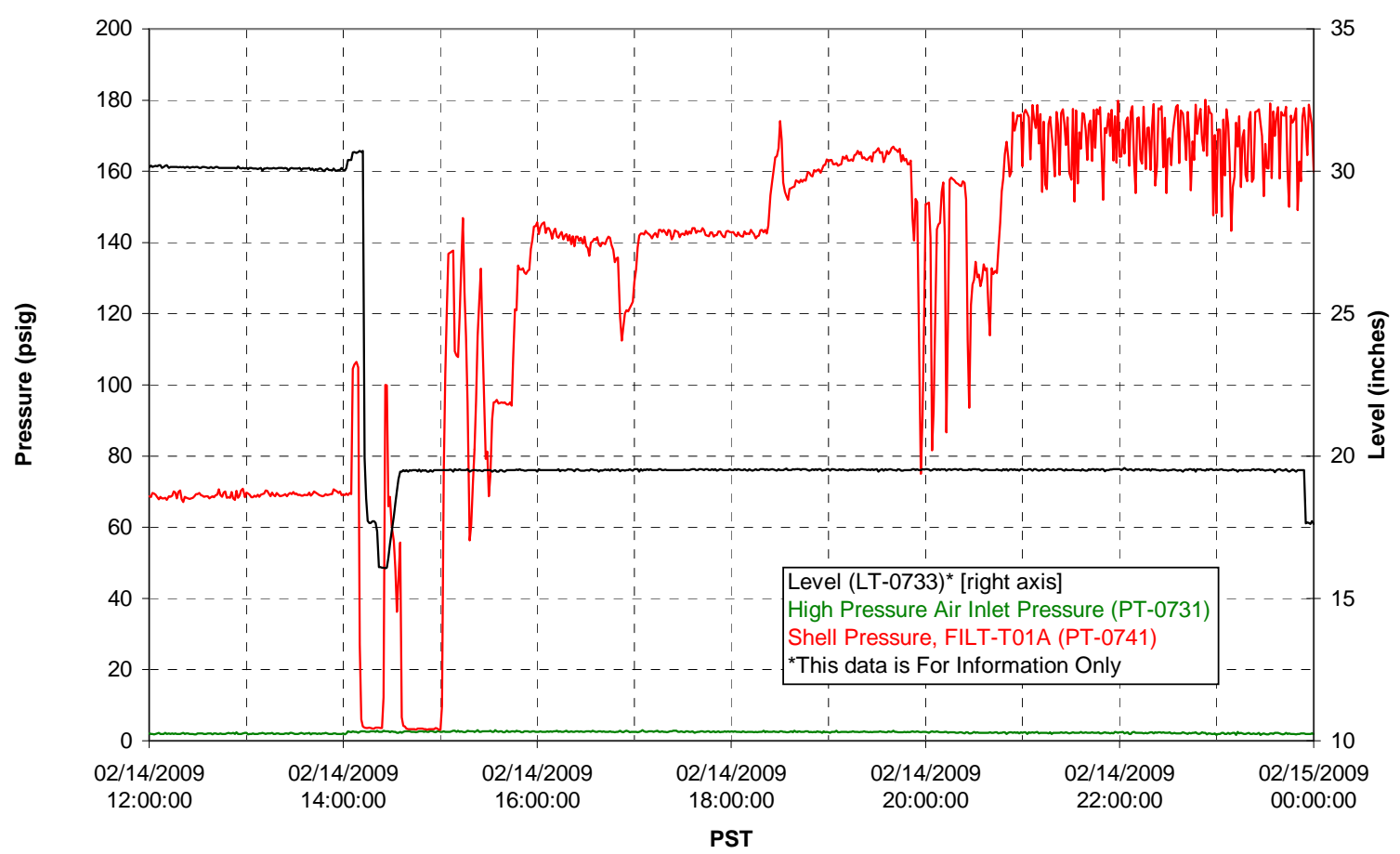

Pulsepot Levels

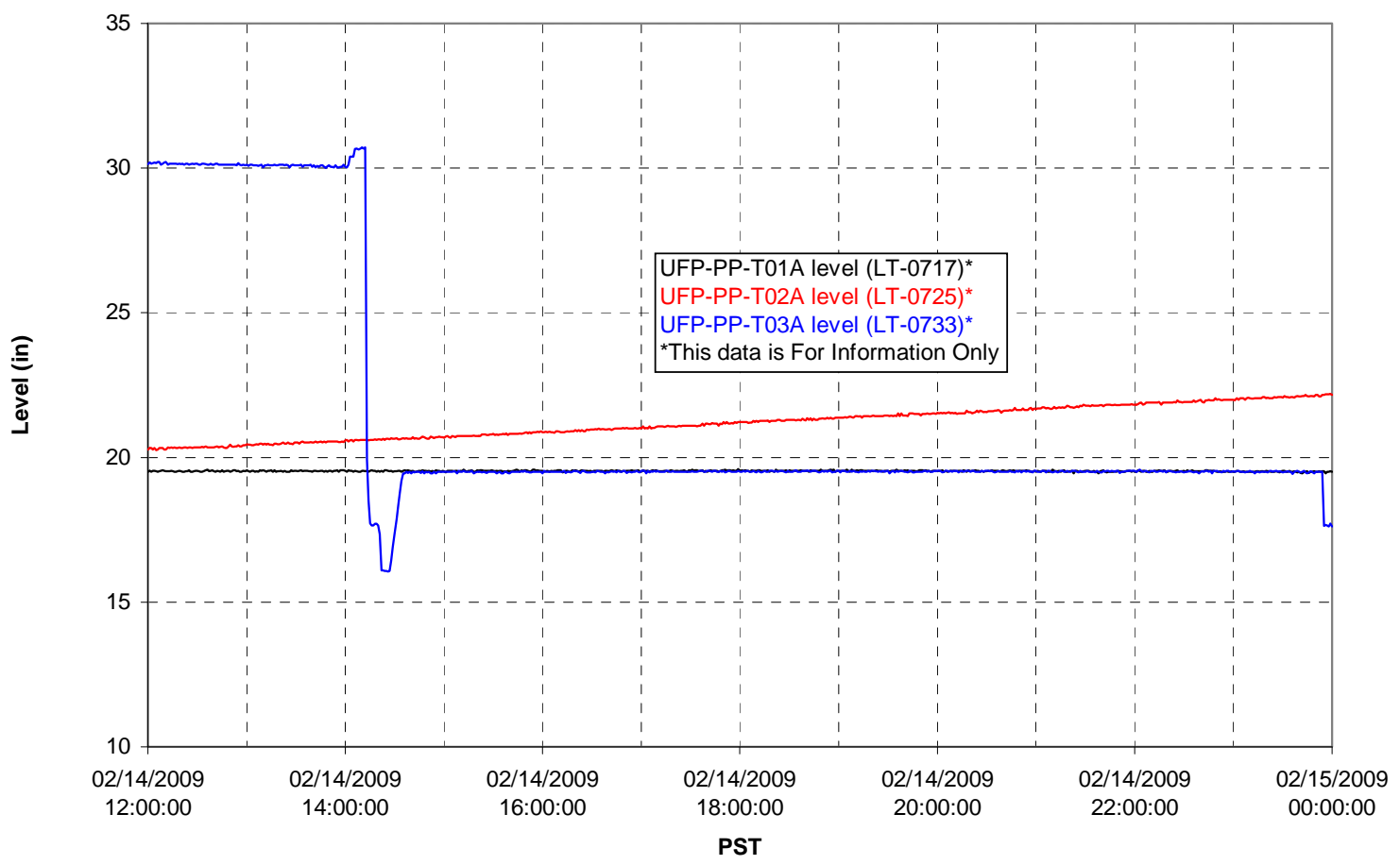


Filter UFP-FILT-T01A

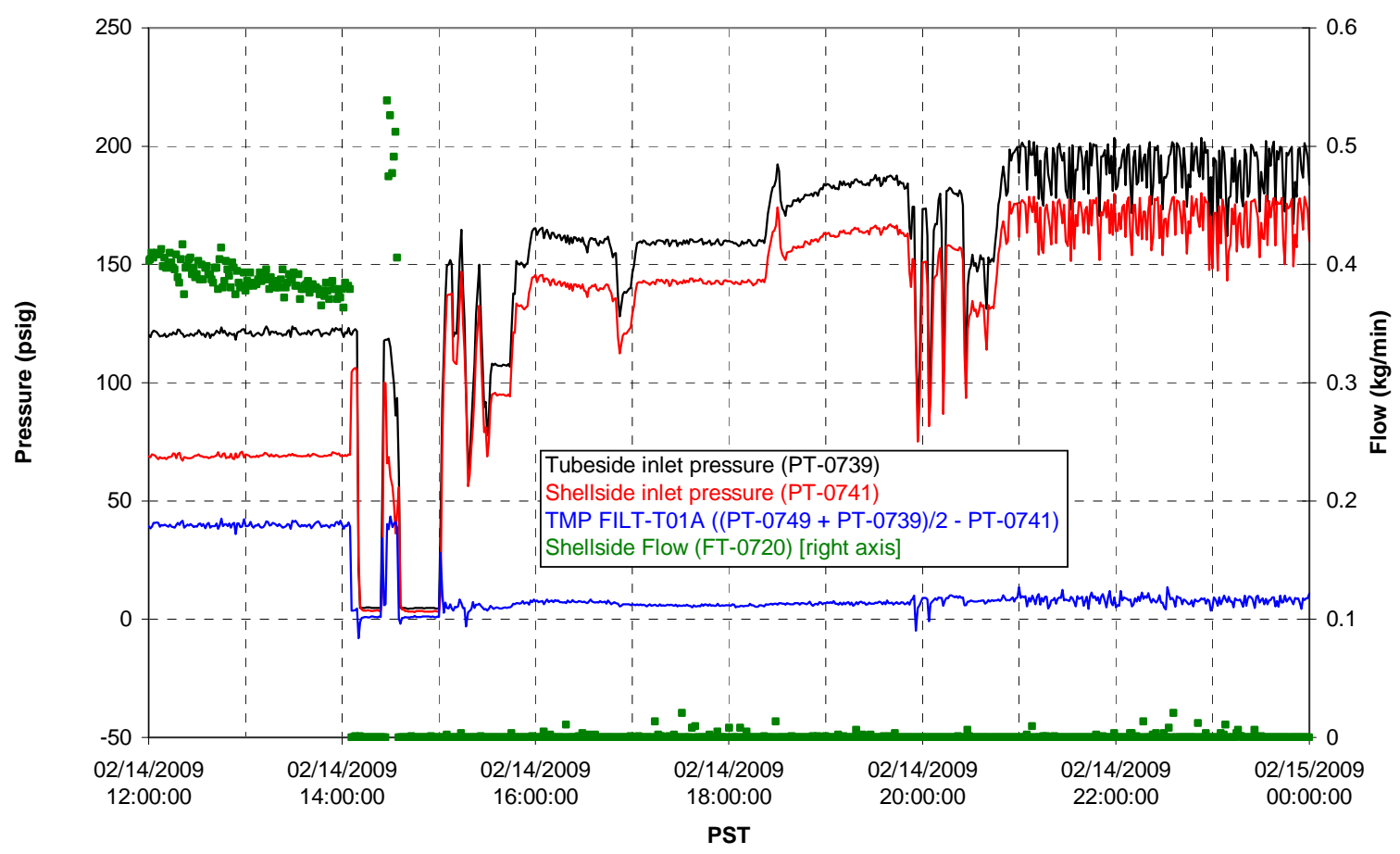

Filter UFP-FILT-T02A

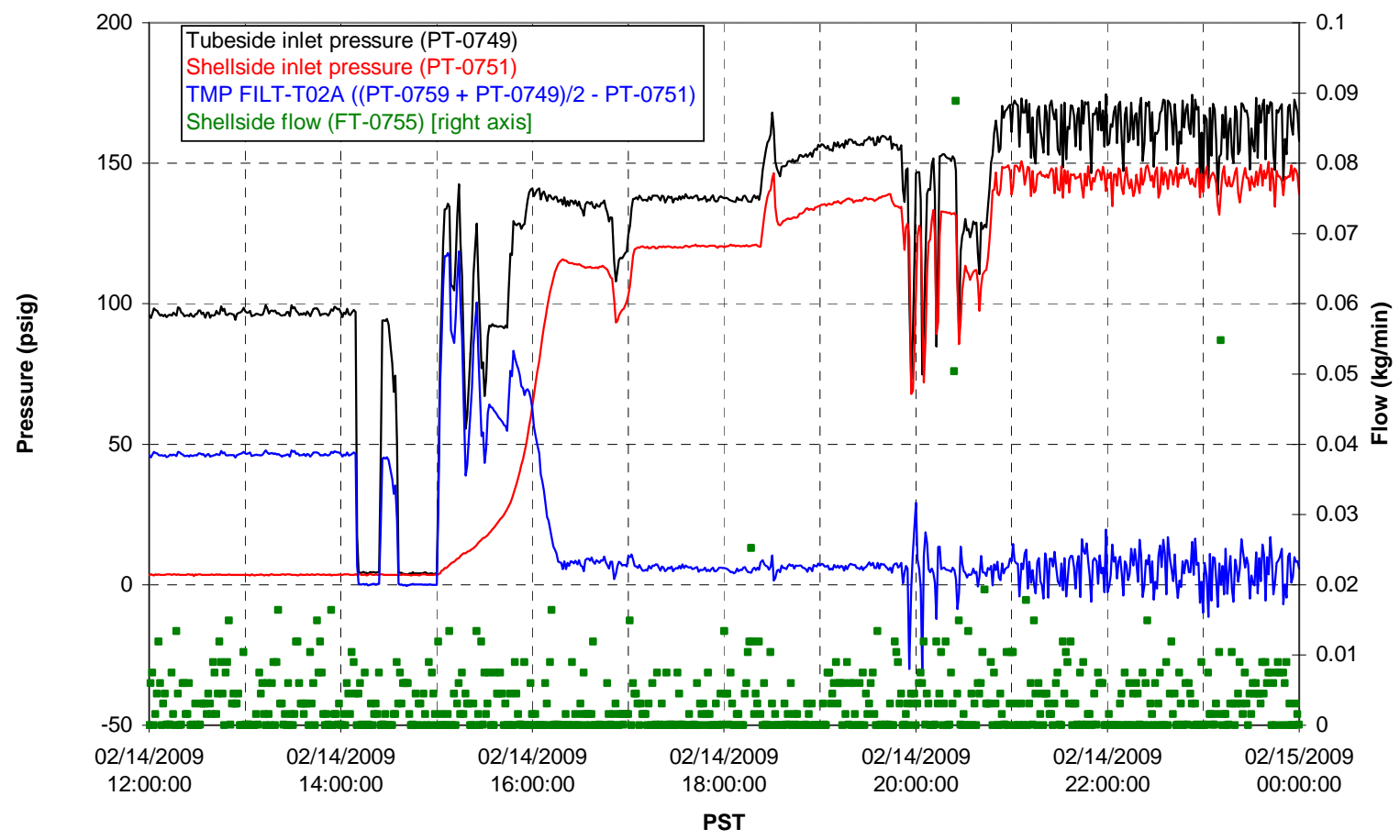


Filter UFP-FILT-T03A

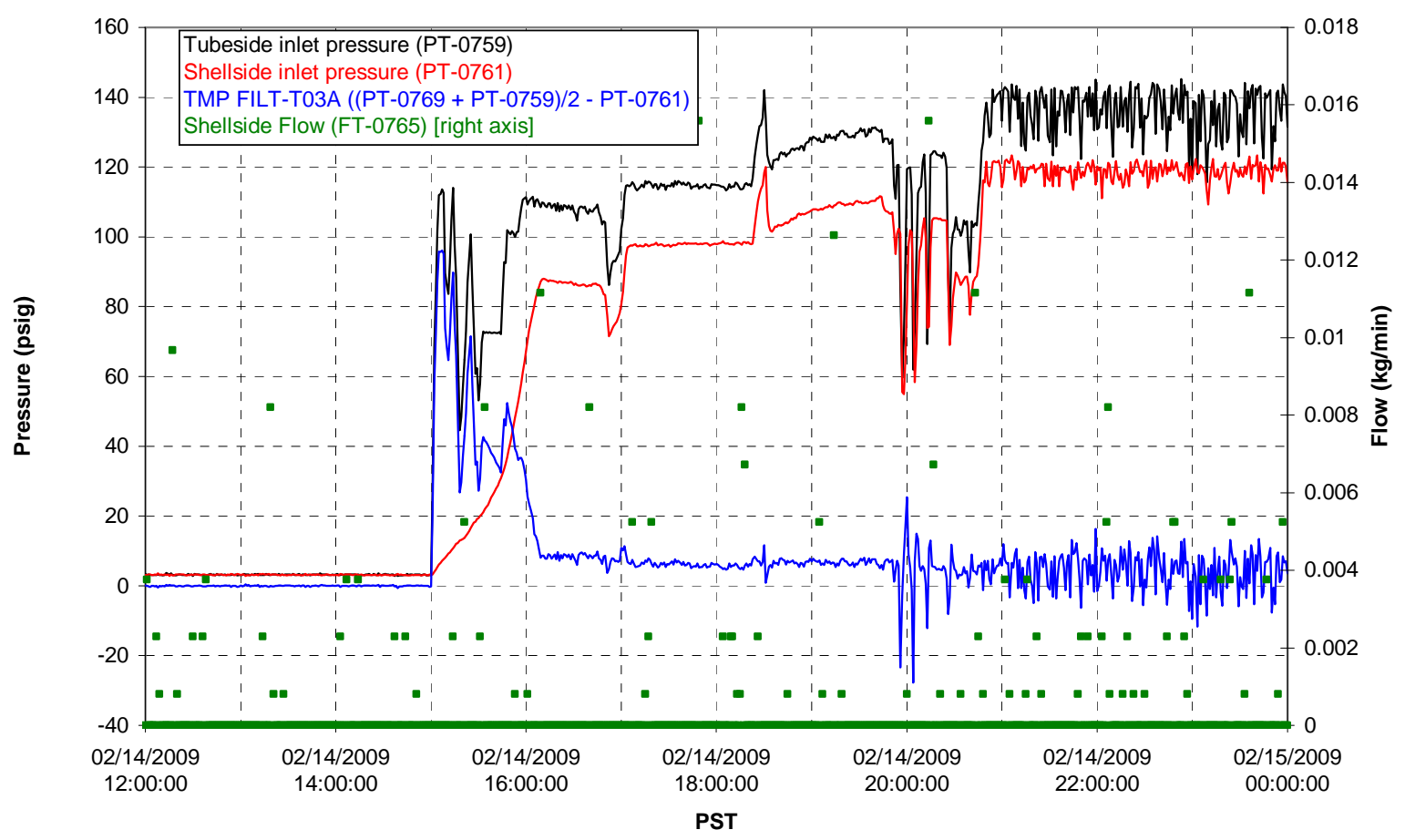

Filter UFP-FILT-T04A

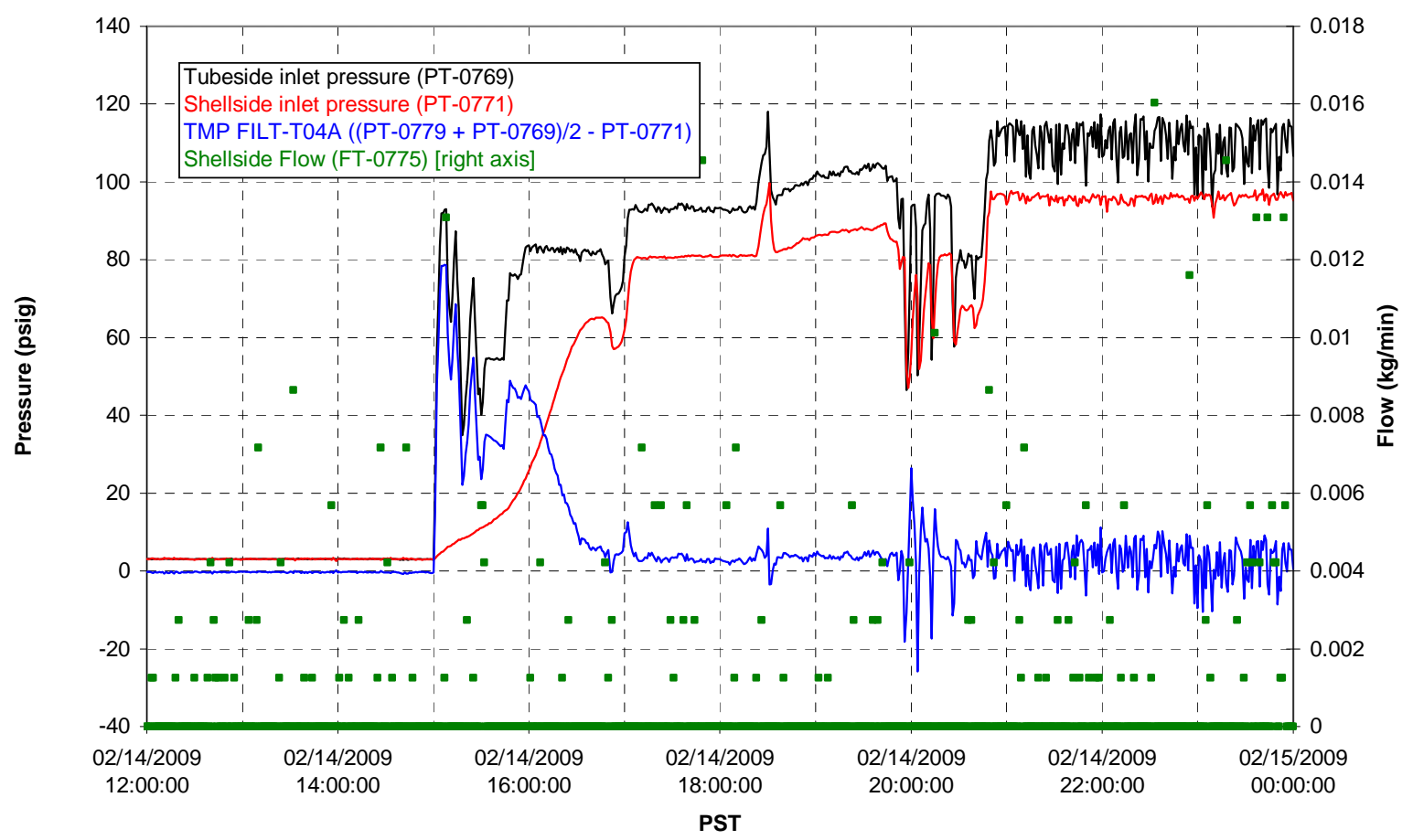


Filter UFP-FILT-T05A

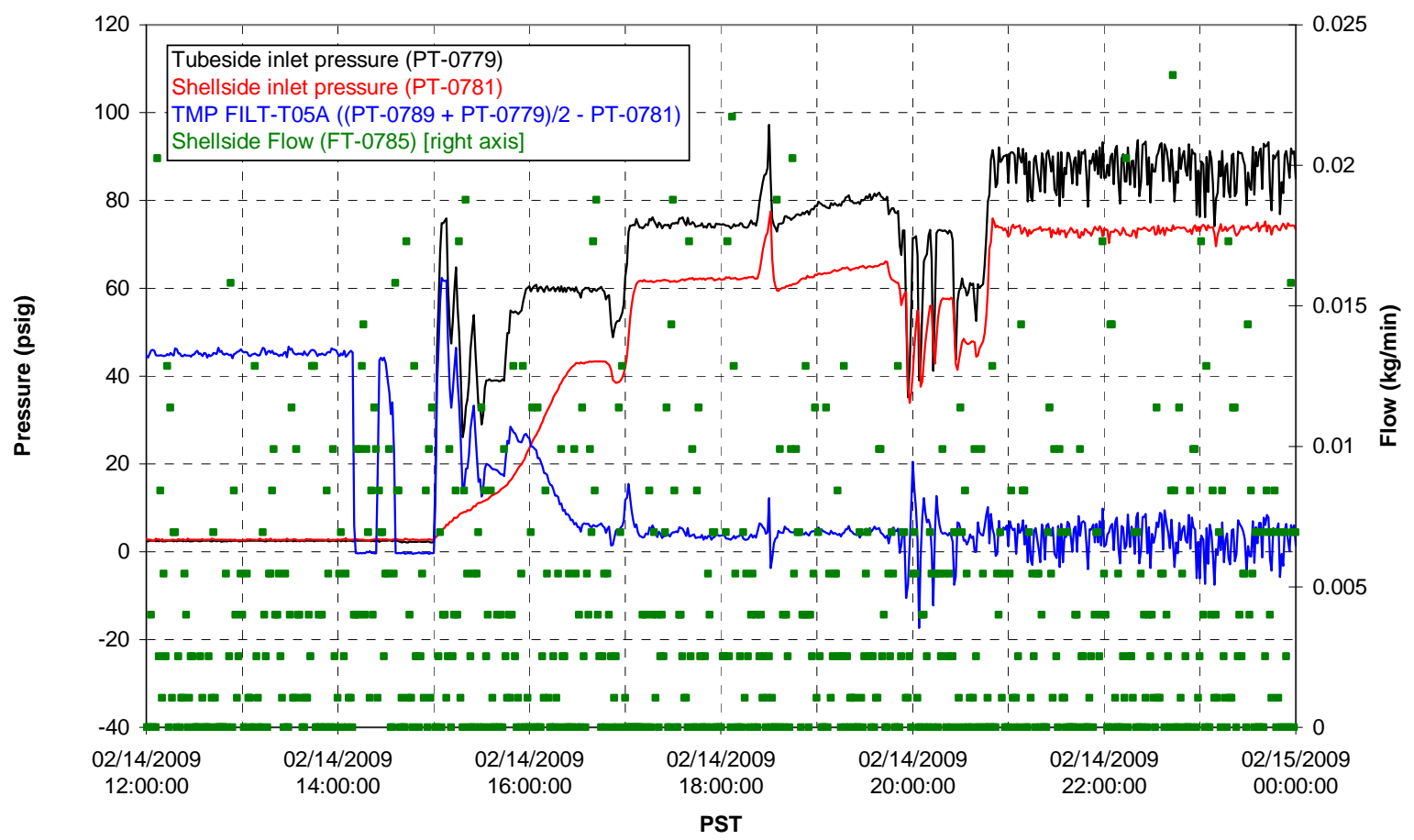

Chemical Flow

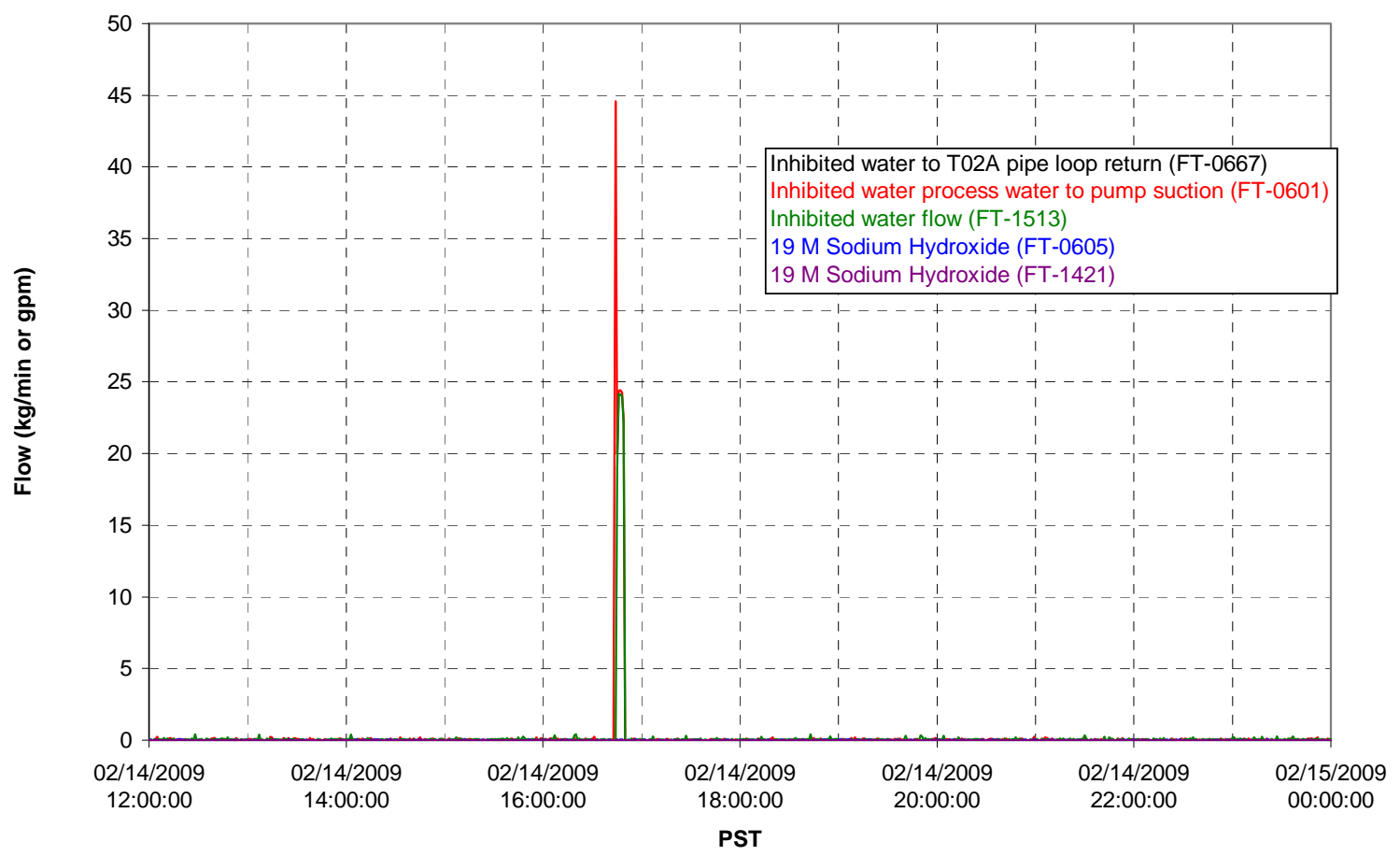


Chemical Flow

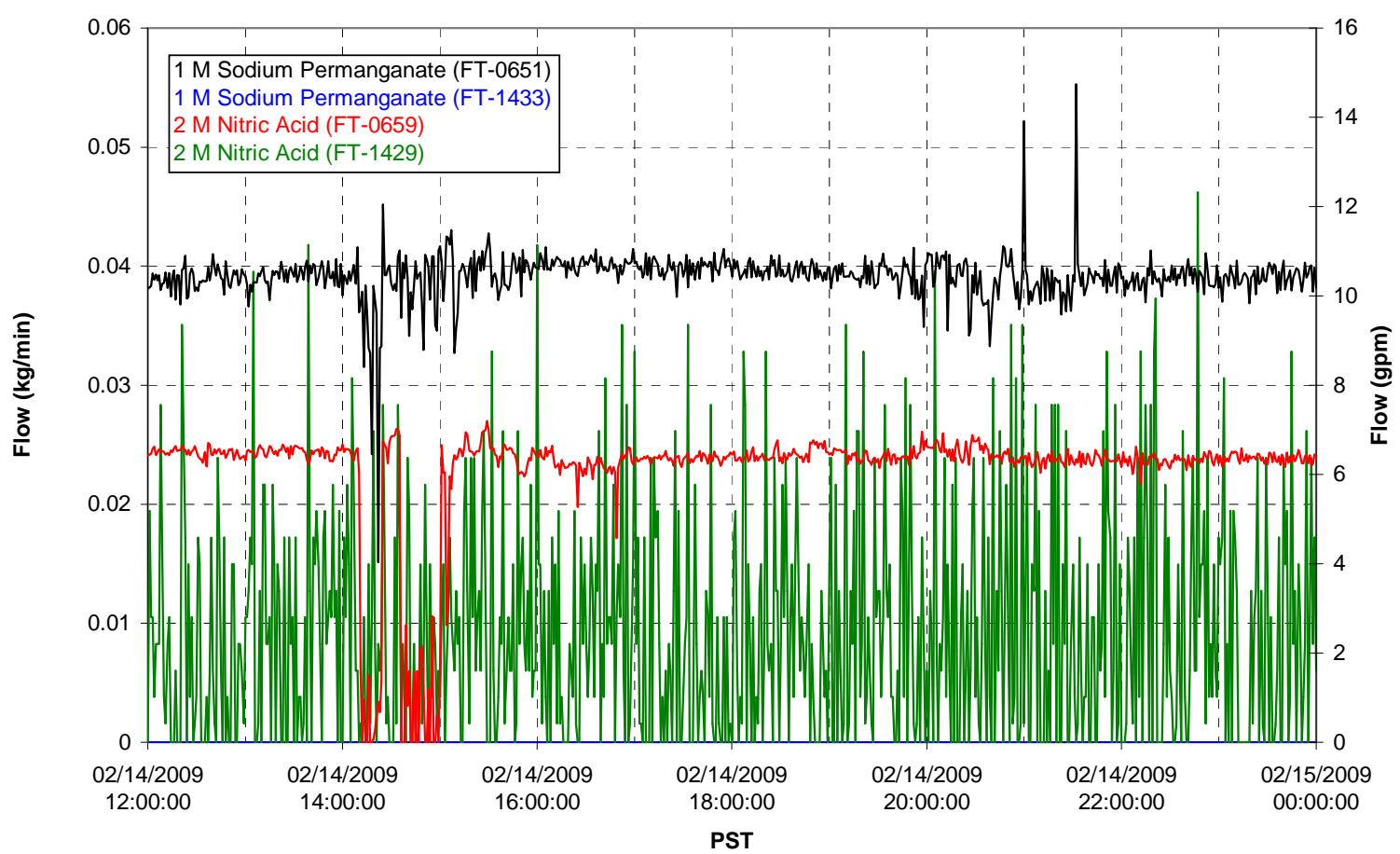

Air Flows

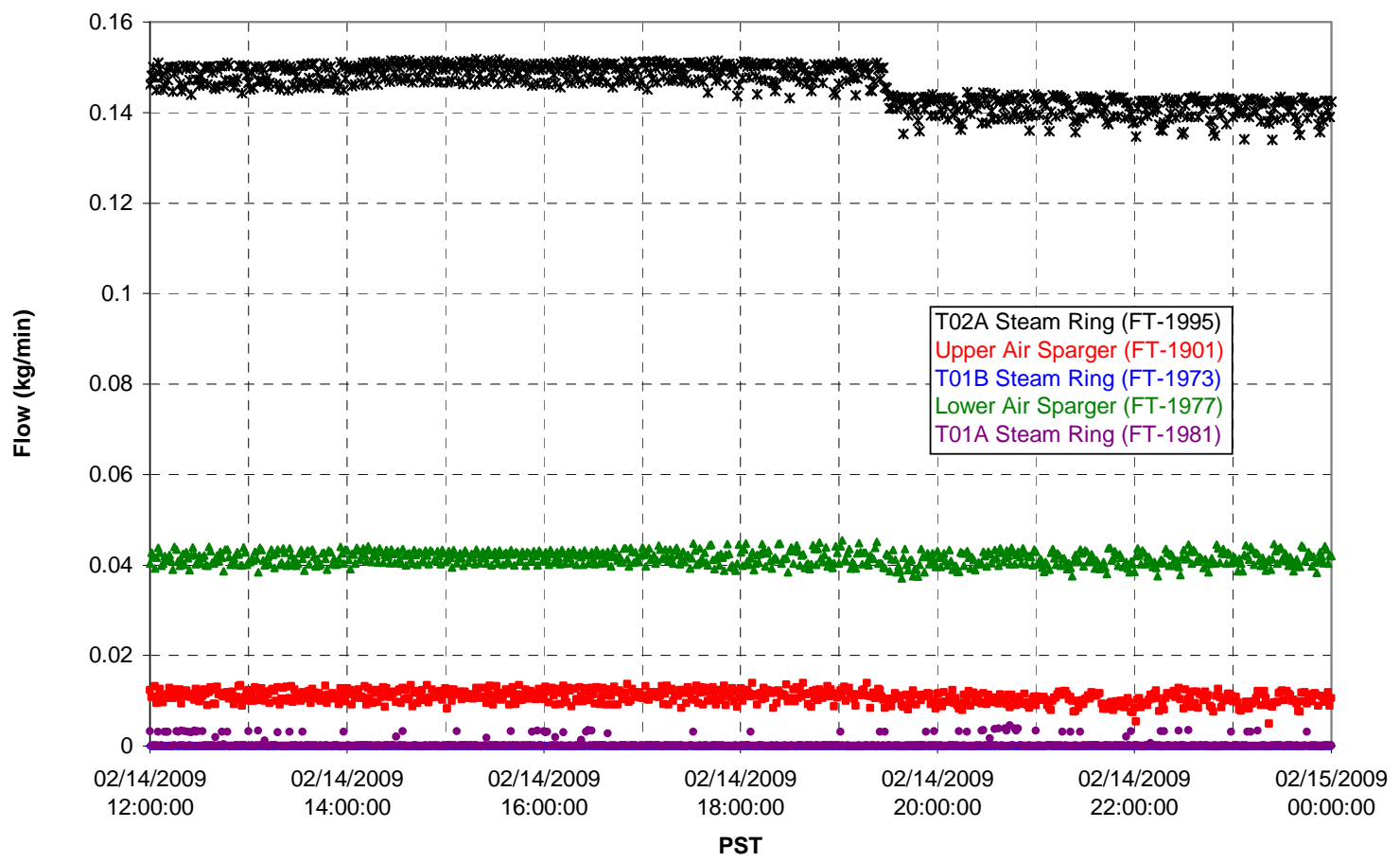


T02A Steam

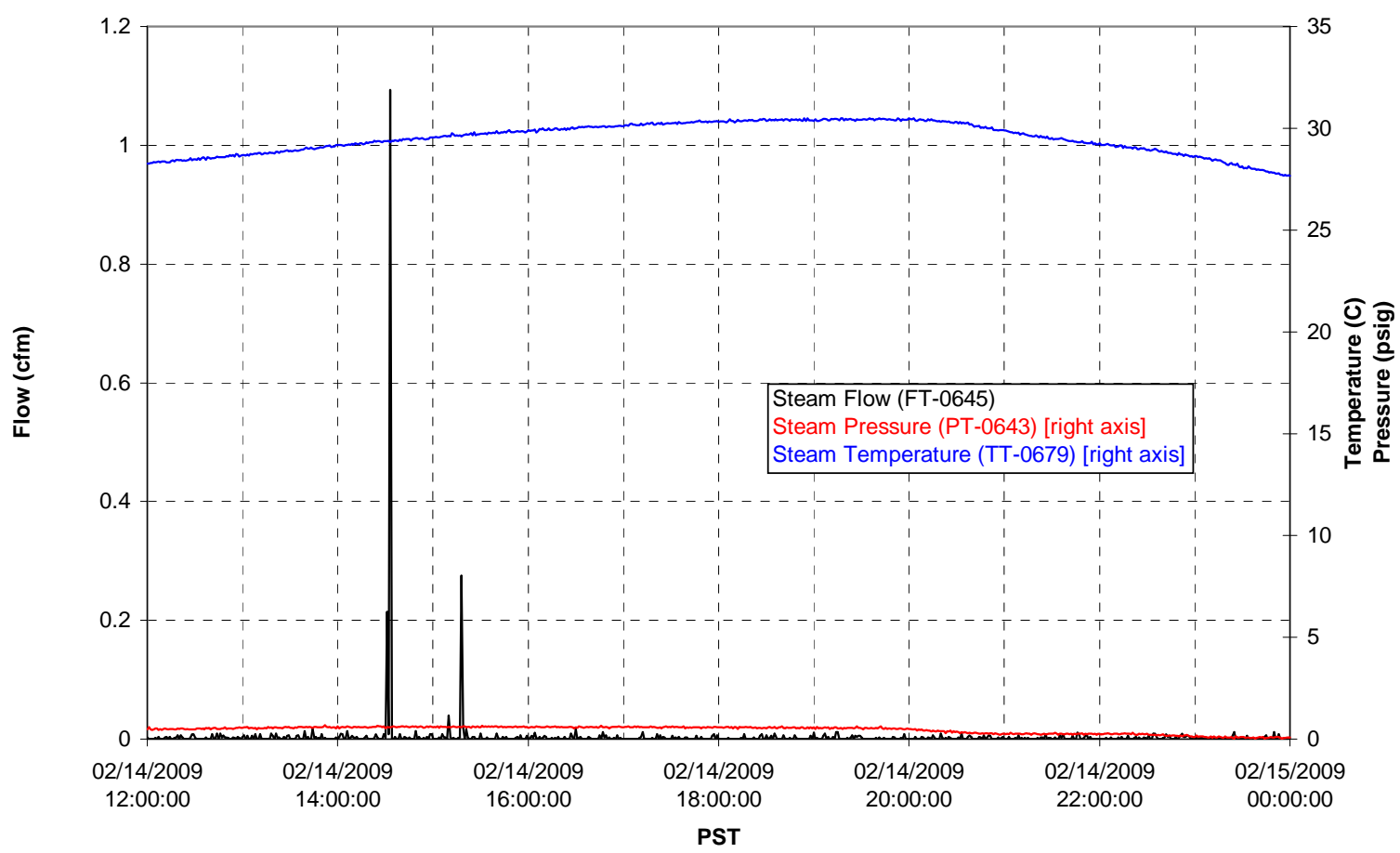

T01A Steam

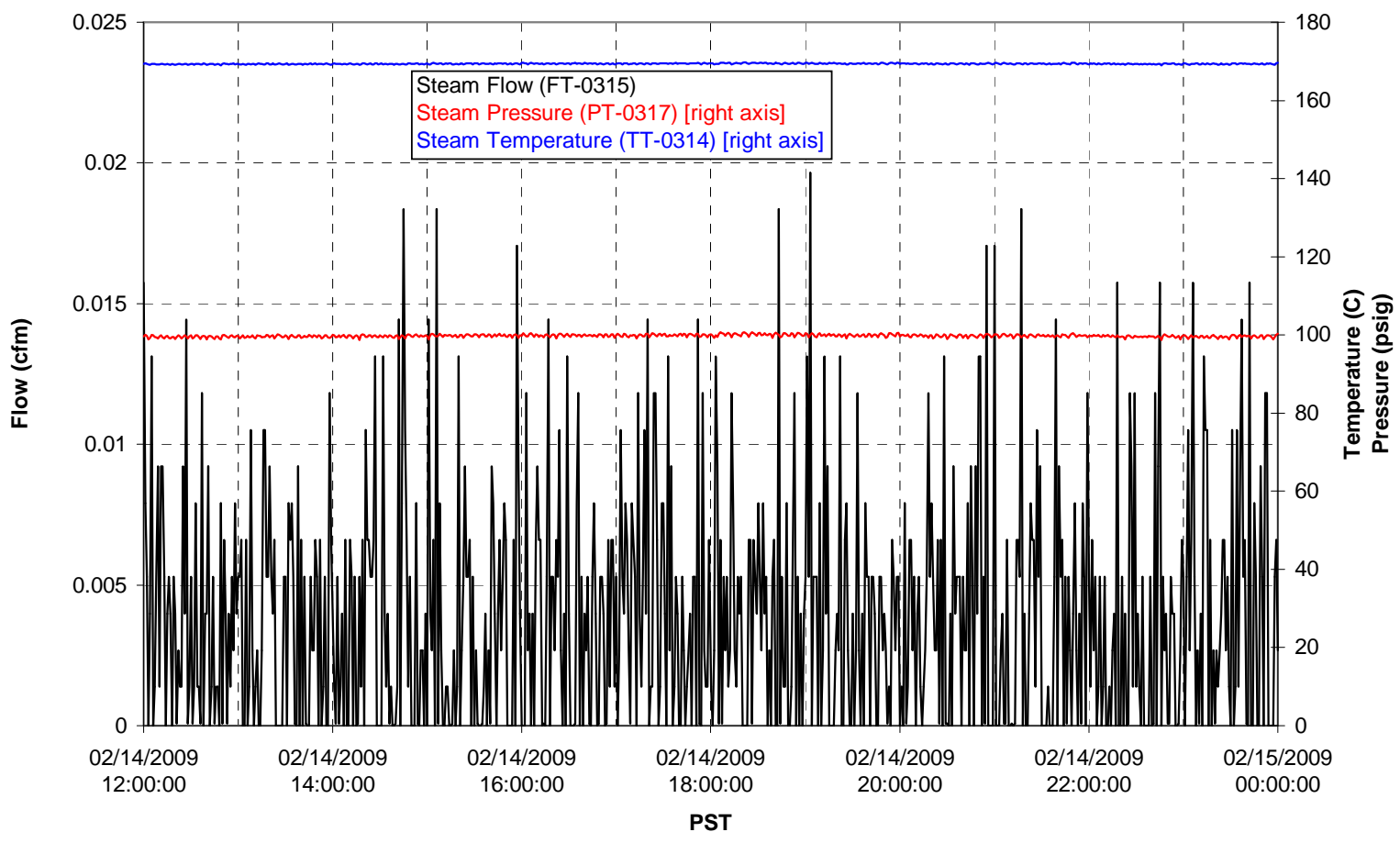


T01B Steam

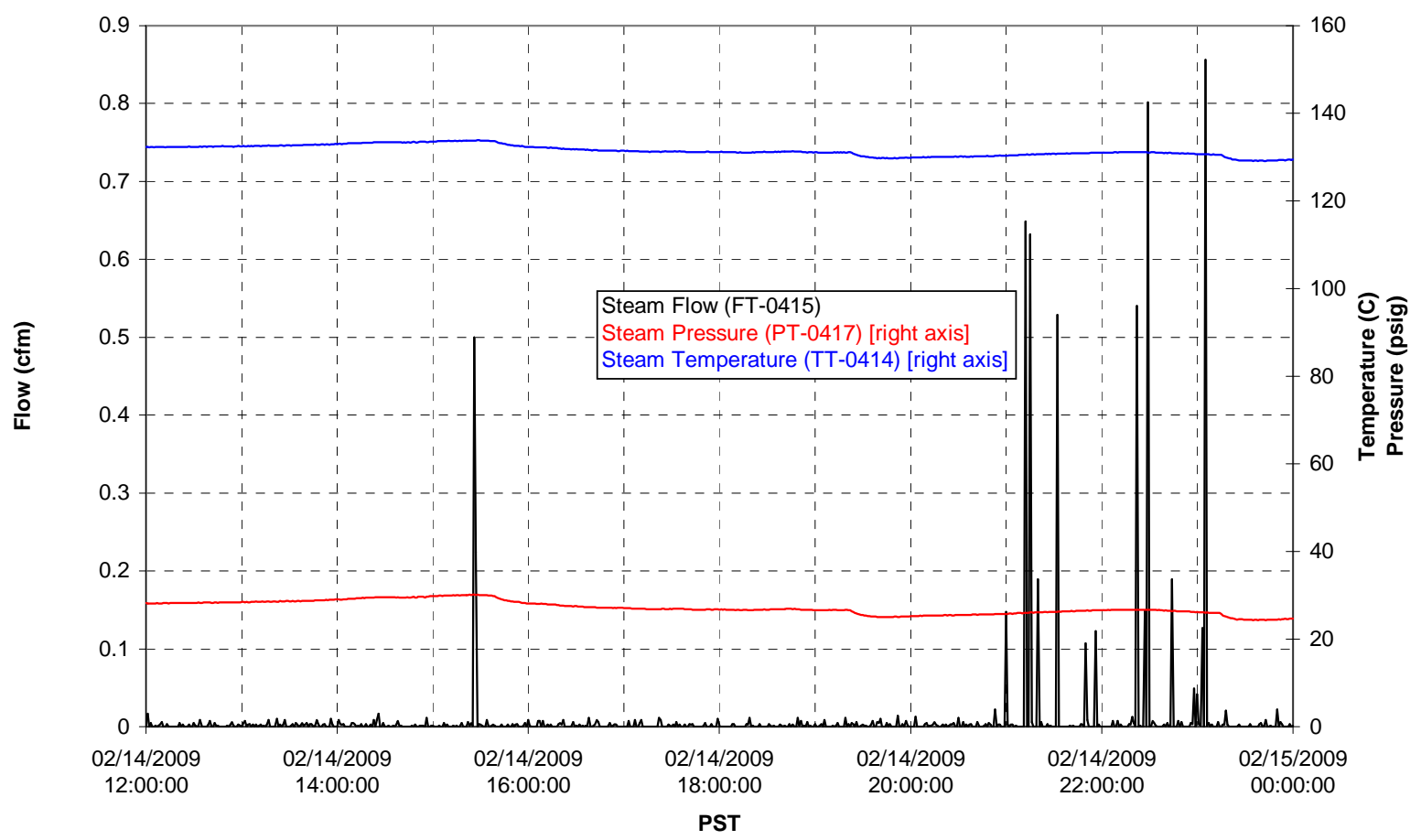




\section{Integrated Test A Data Plots 02/15/09 00:00 - 02/15/09 12:00}


T01A level

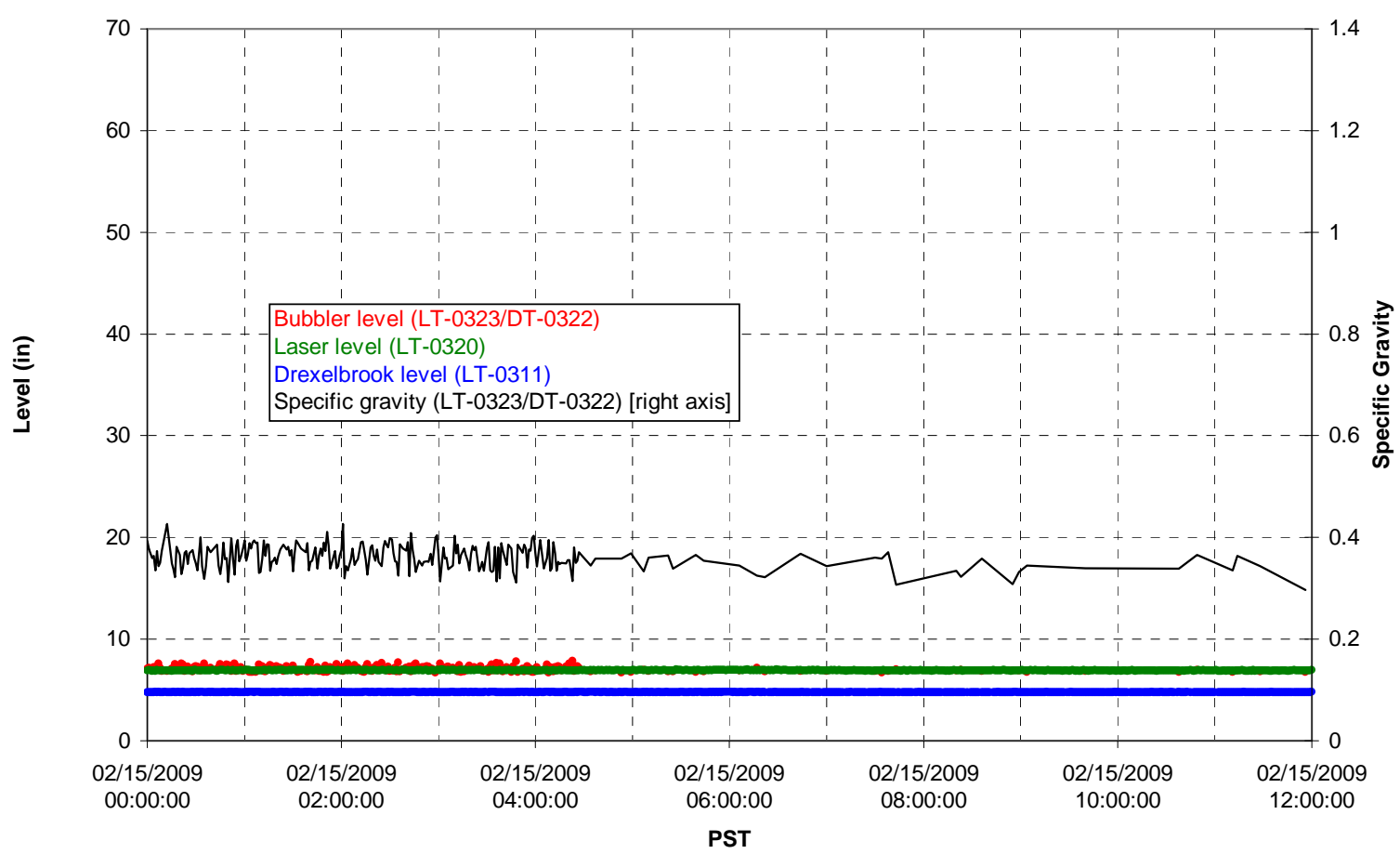

T01A temperatures

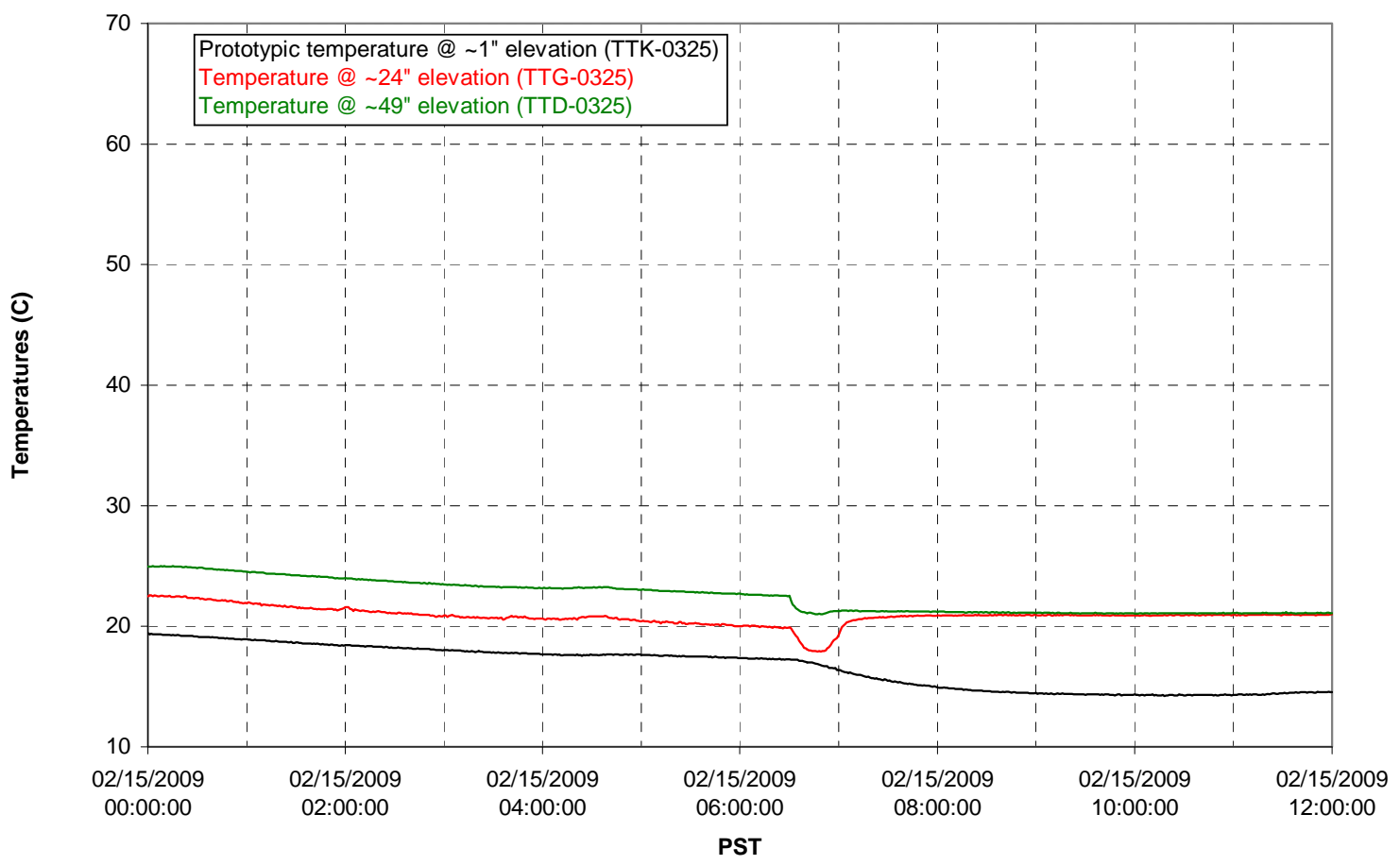


T01B level

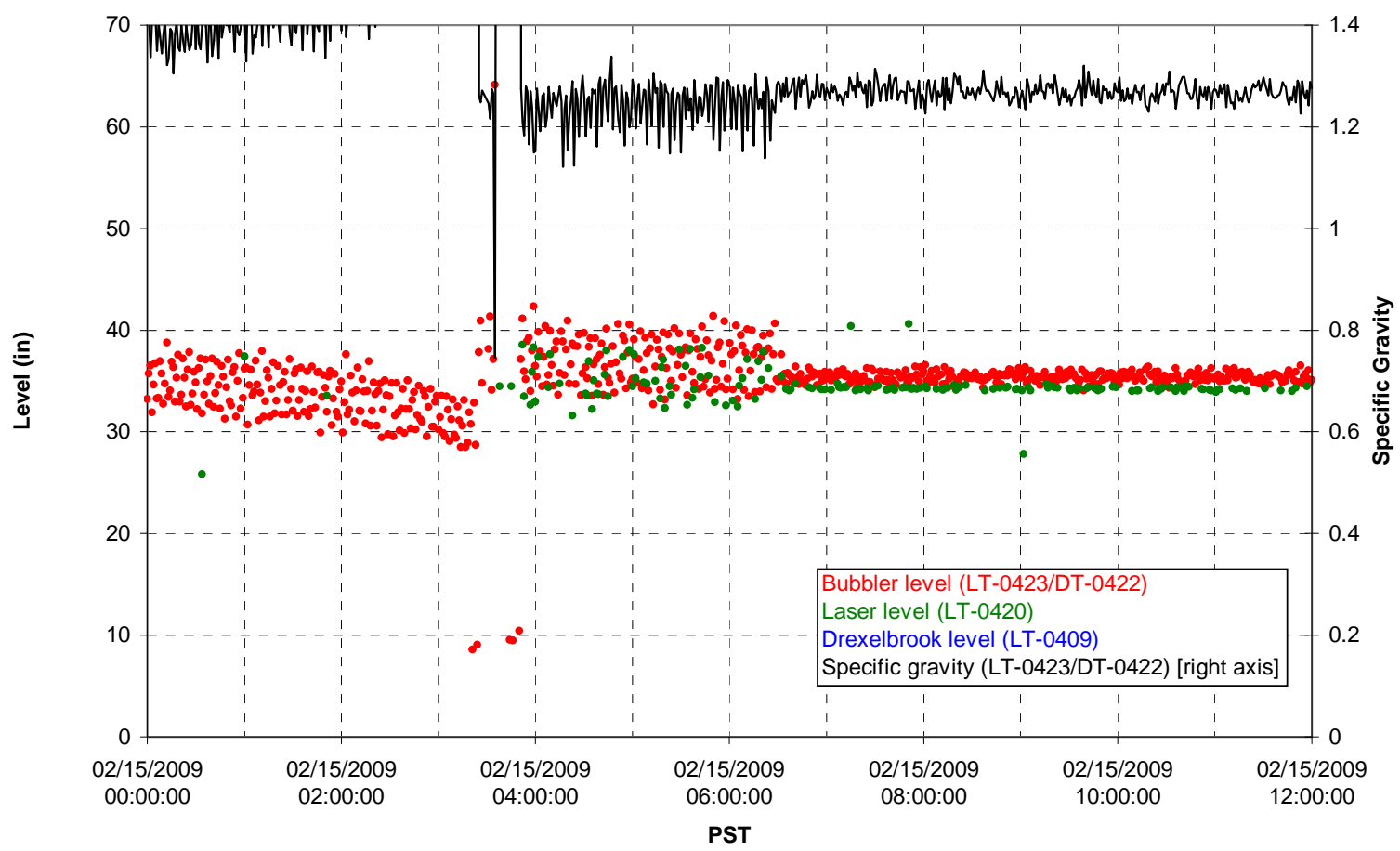

T01B temperatures

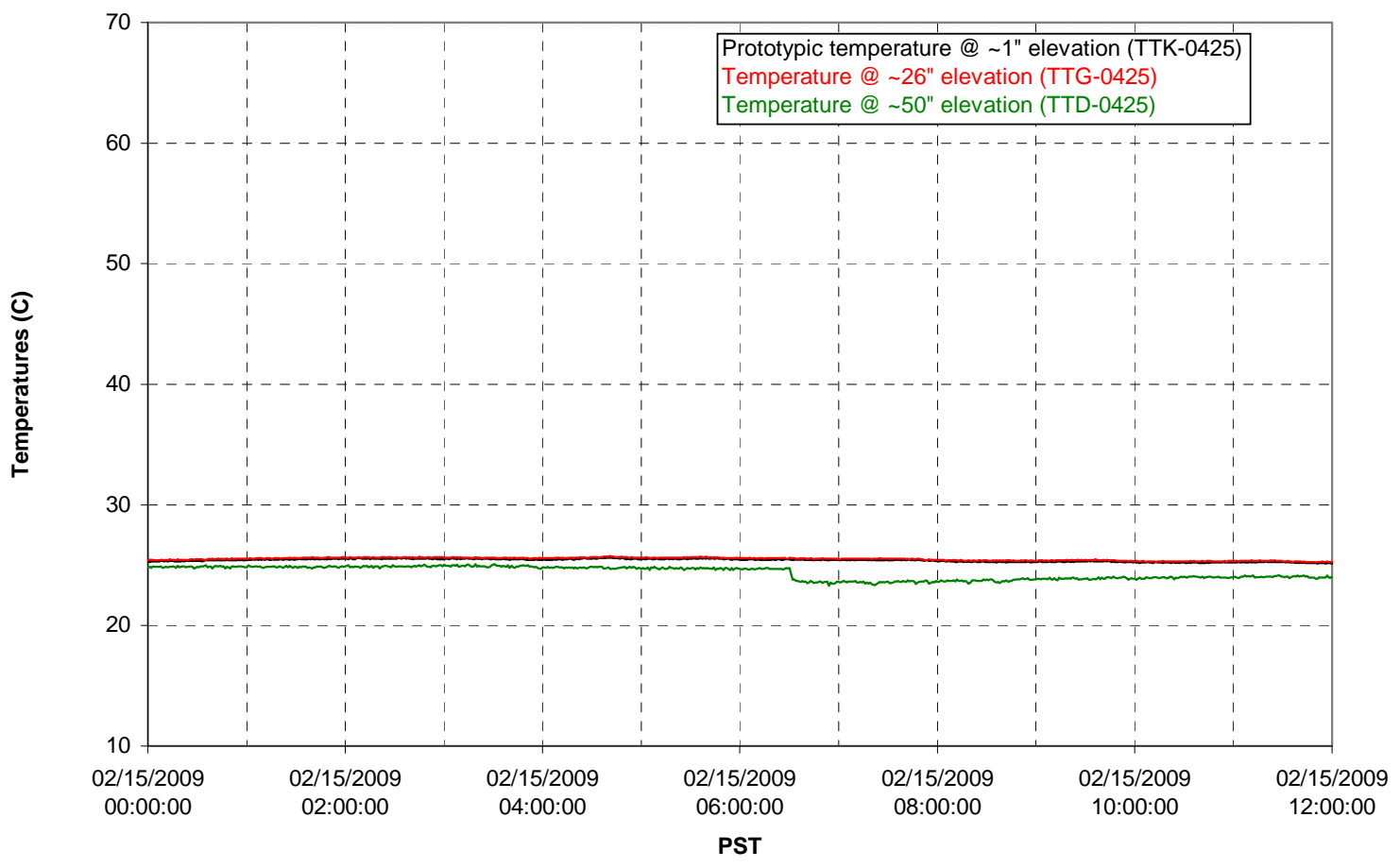


T02A level

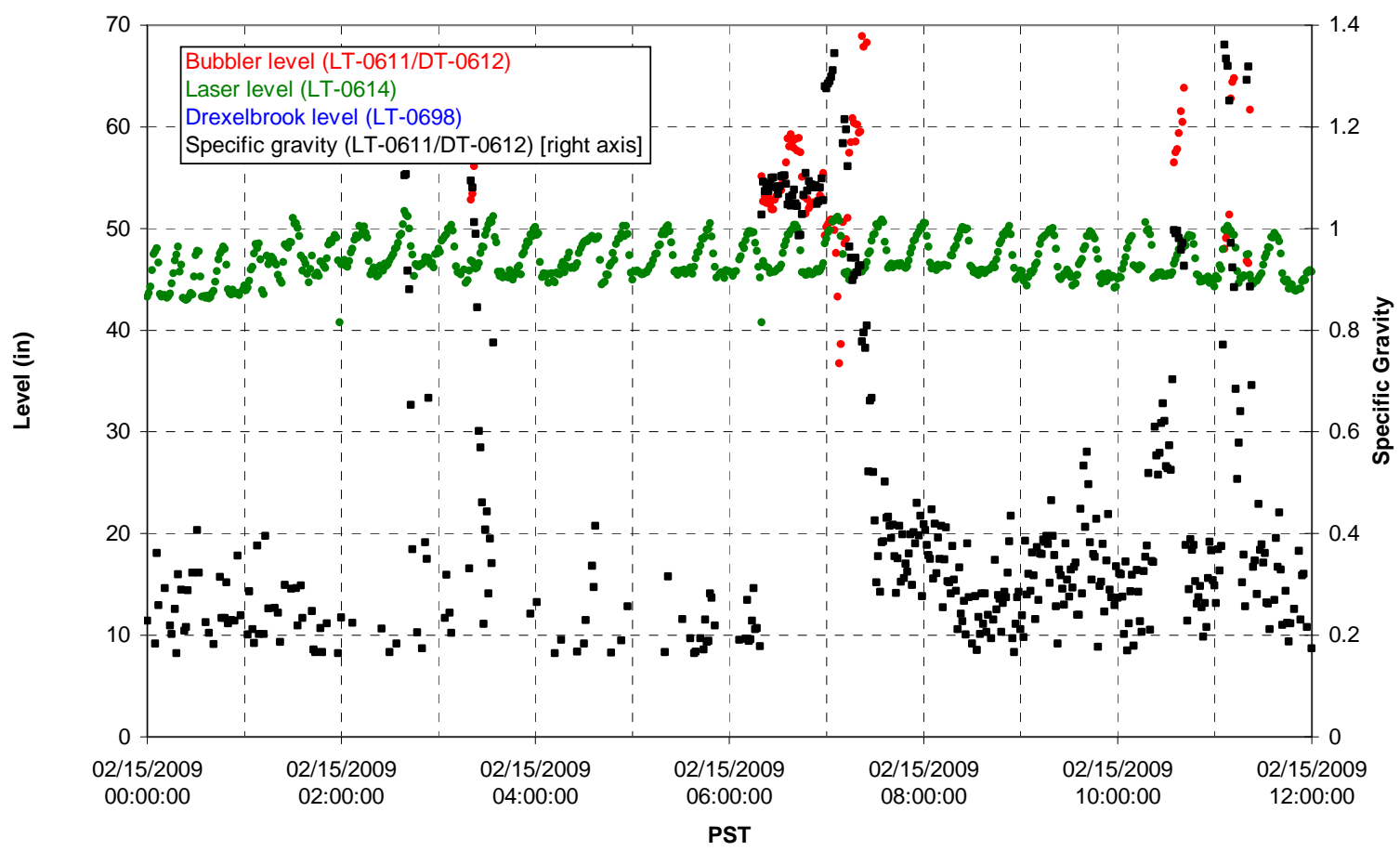

T02A temperatures

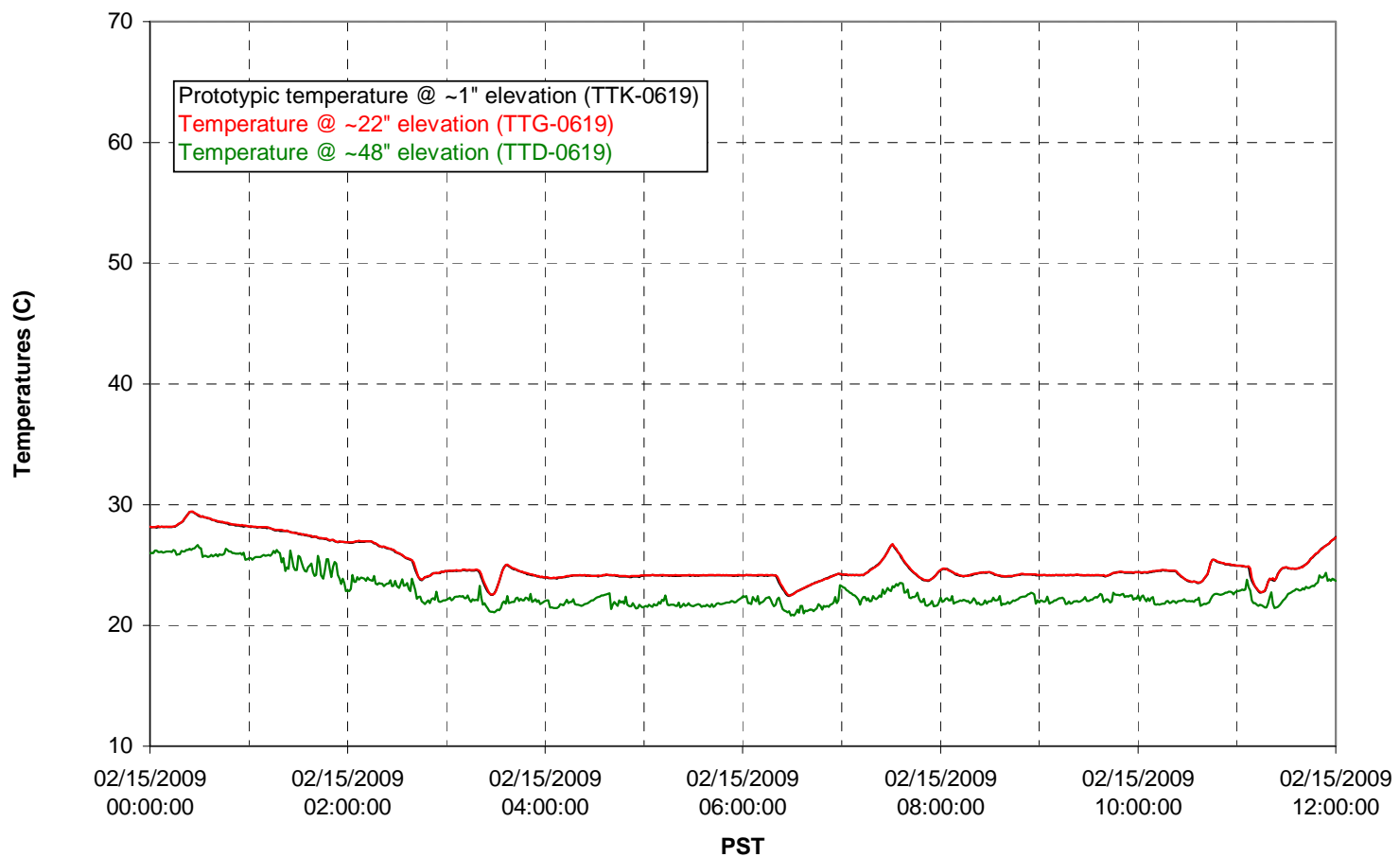


T02A and filter loop temperatures

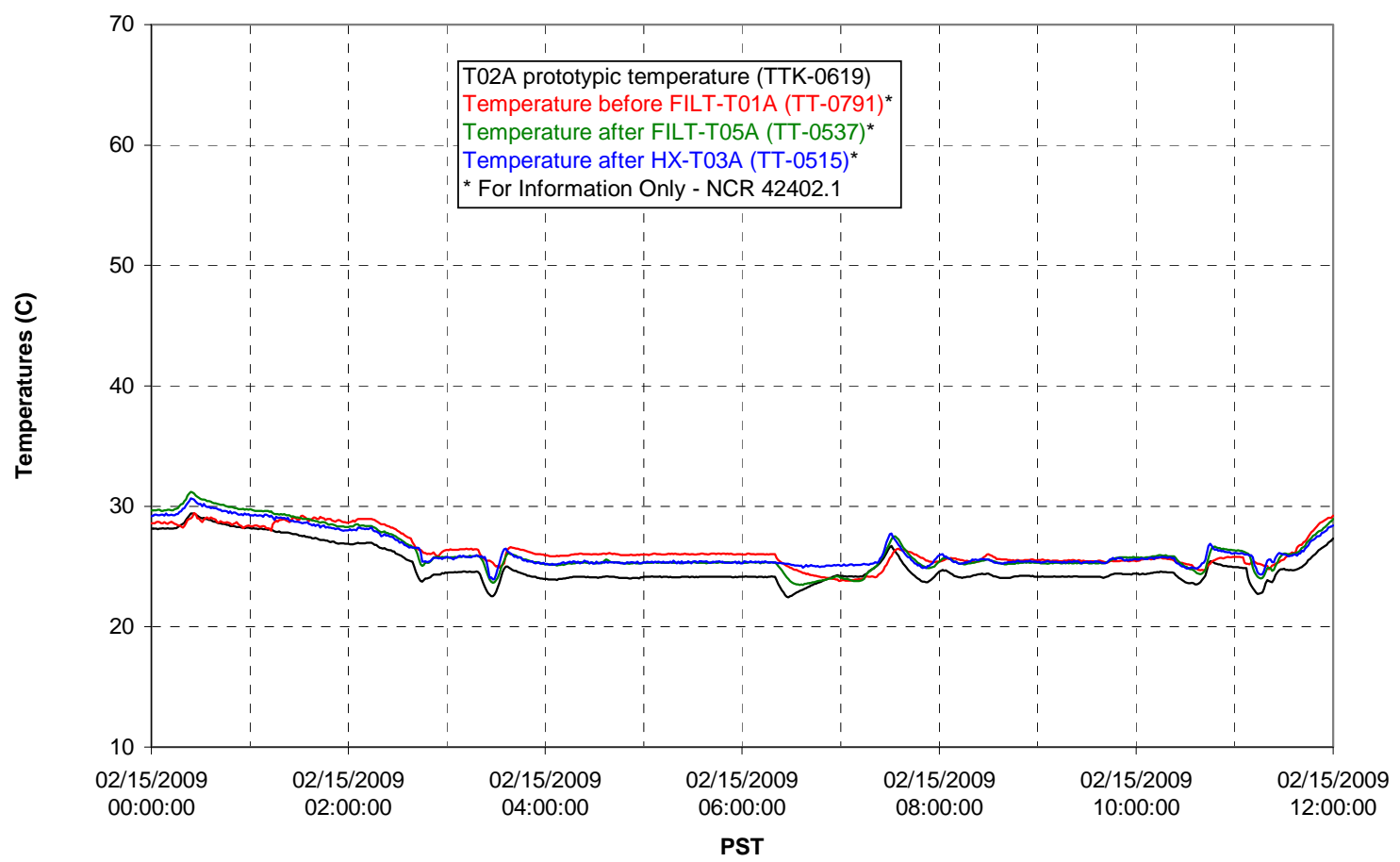

Pump Pressures and Flow

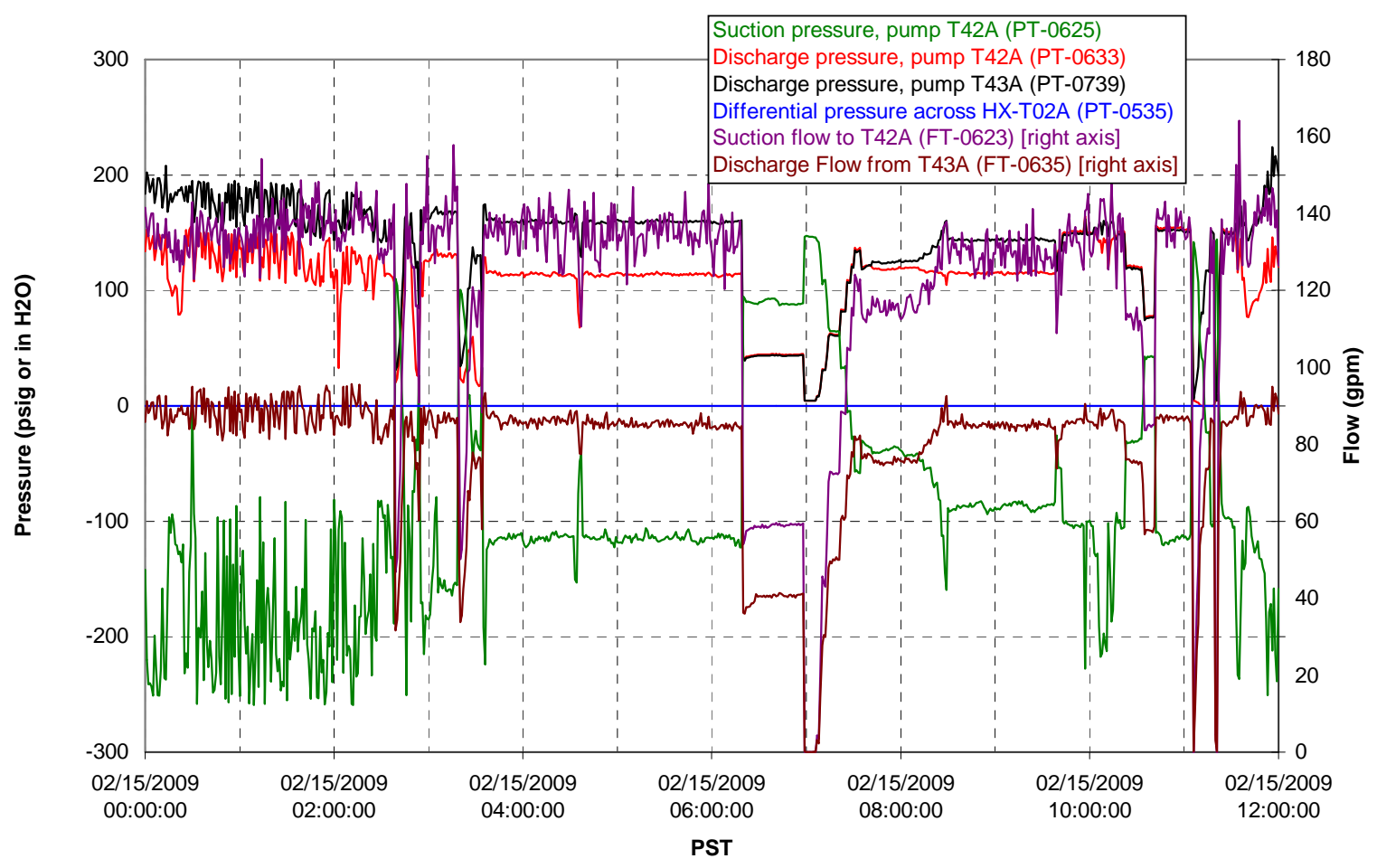




\section{Axial pressure drop}

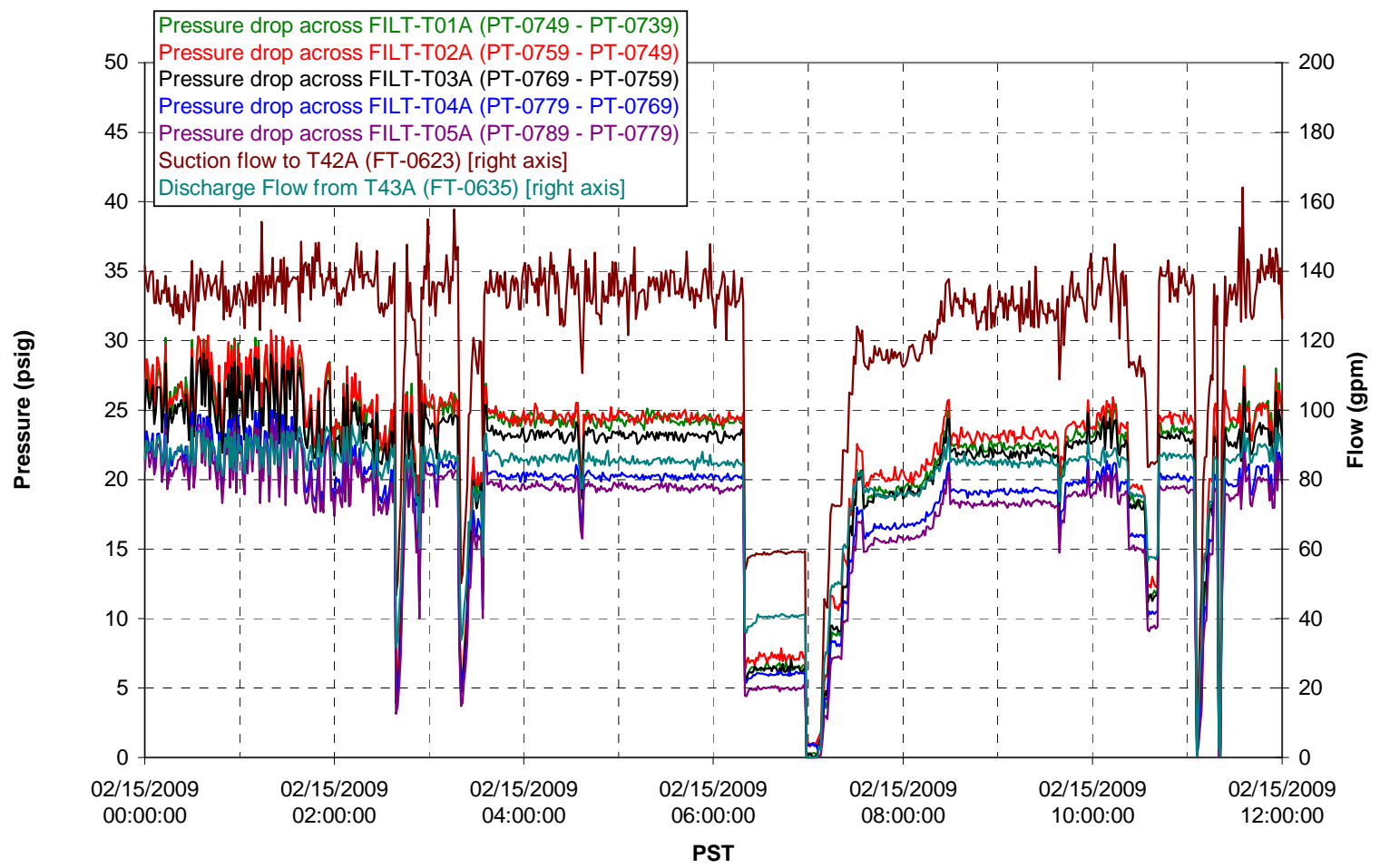

Permeate flow rates

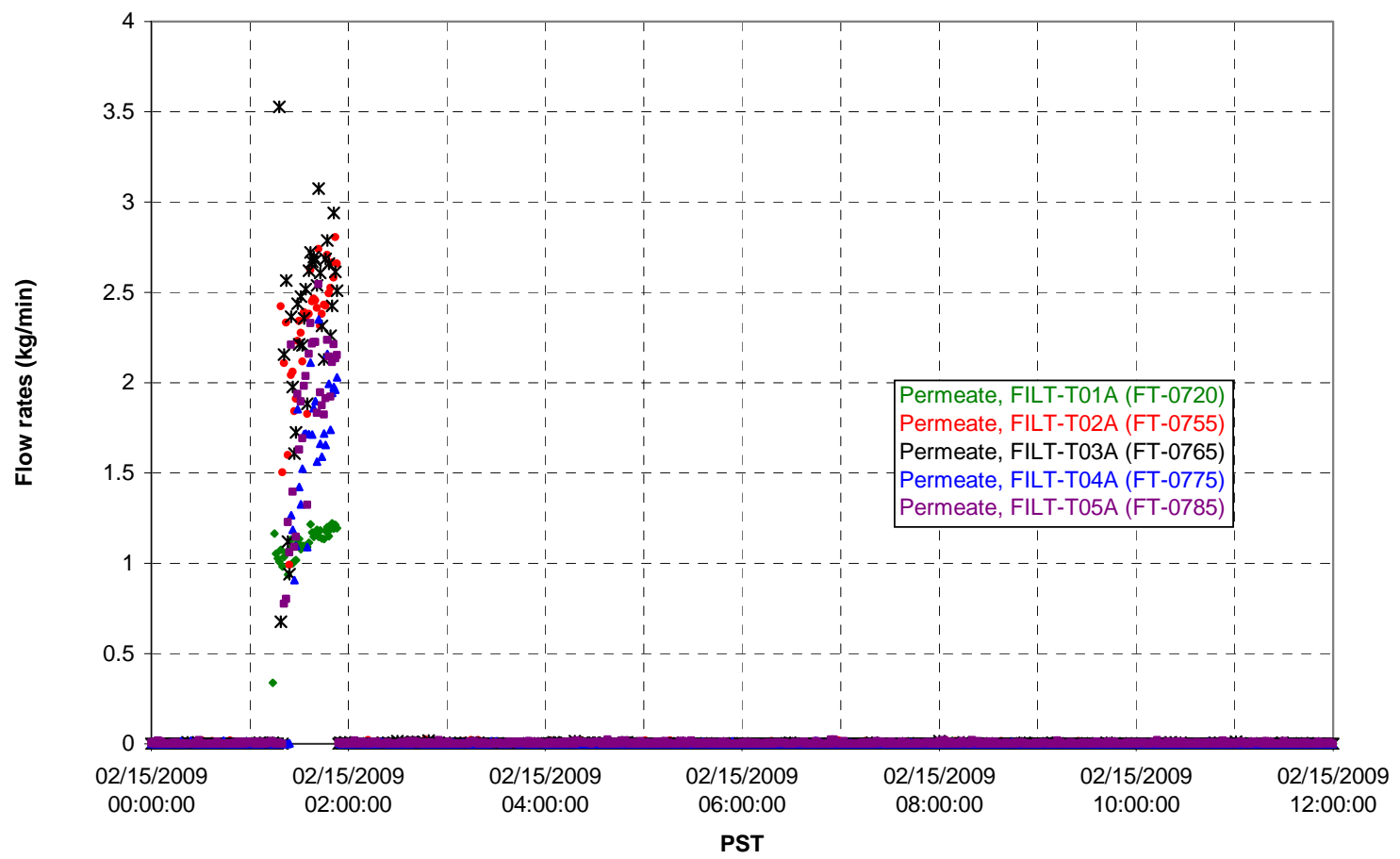


T02A Inner Temperature Tree

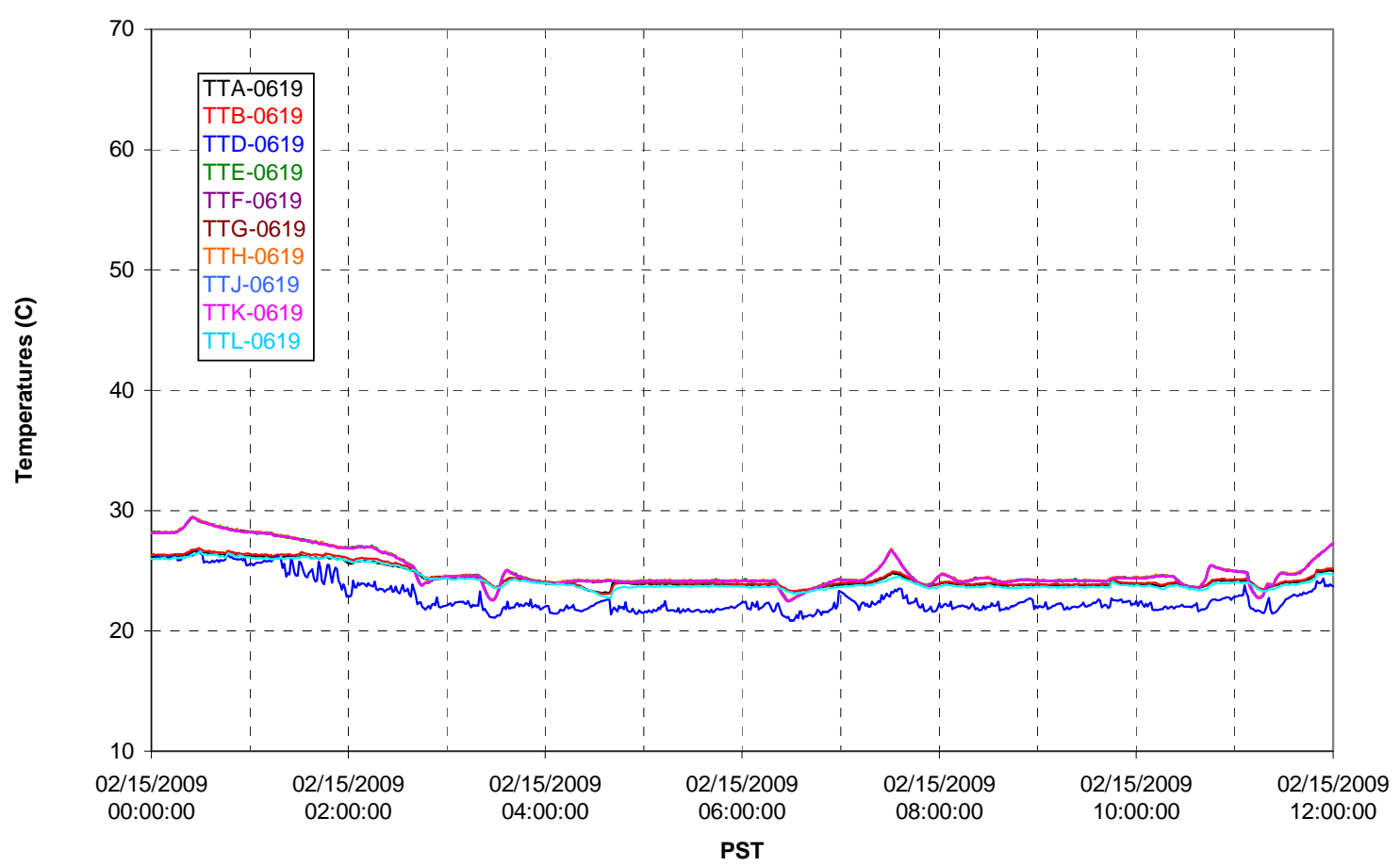

T02A Outer Temperature Tree

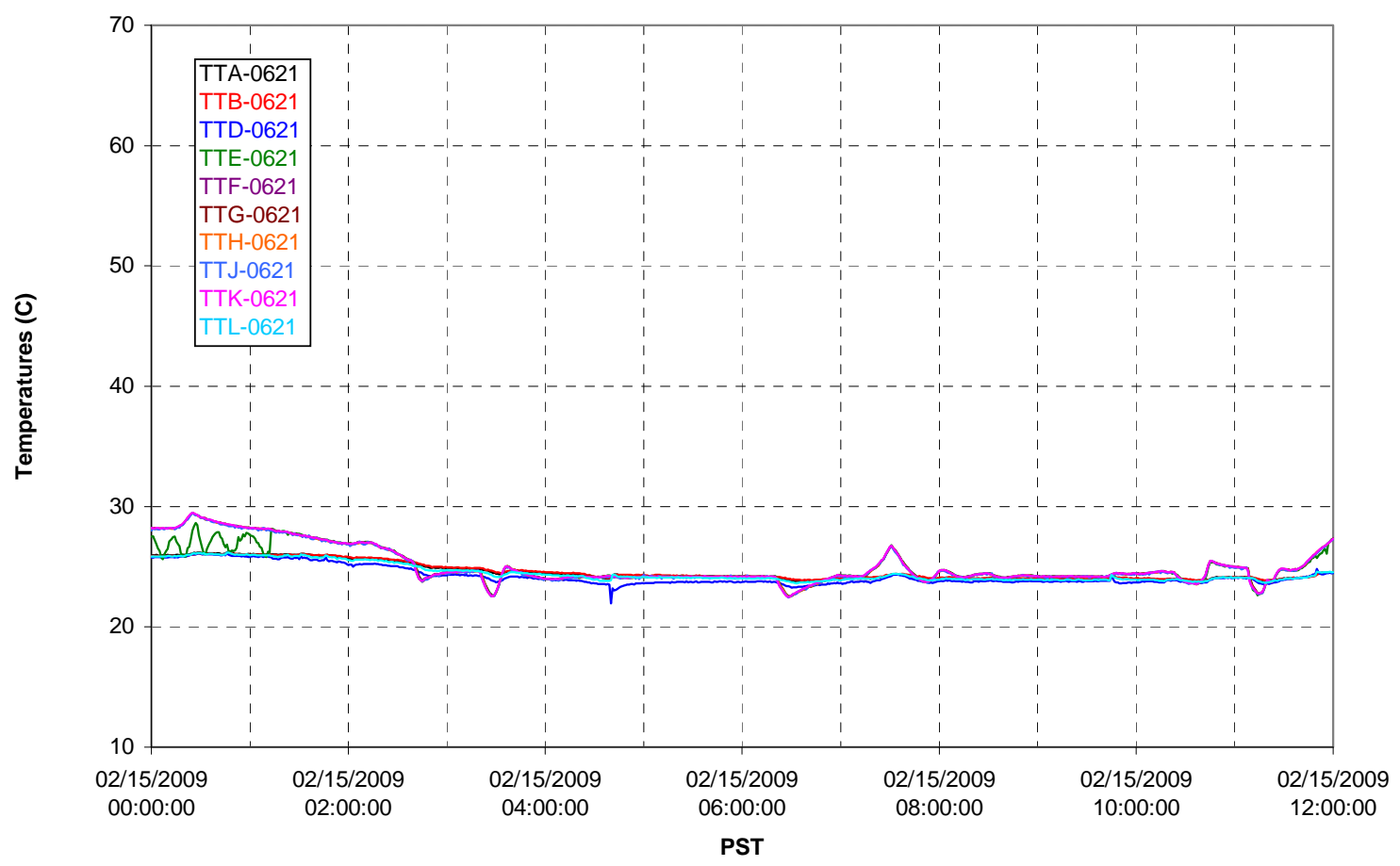


T02A temperatures

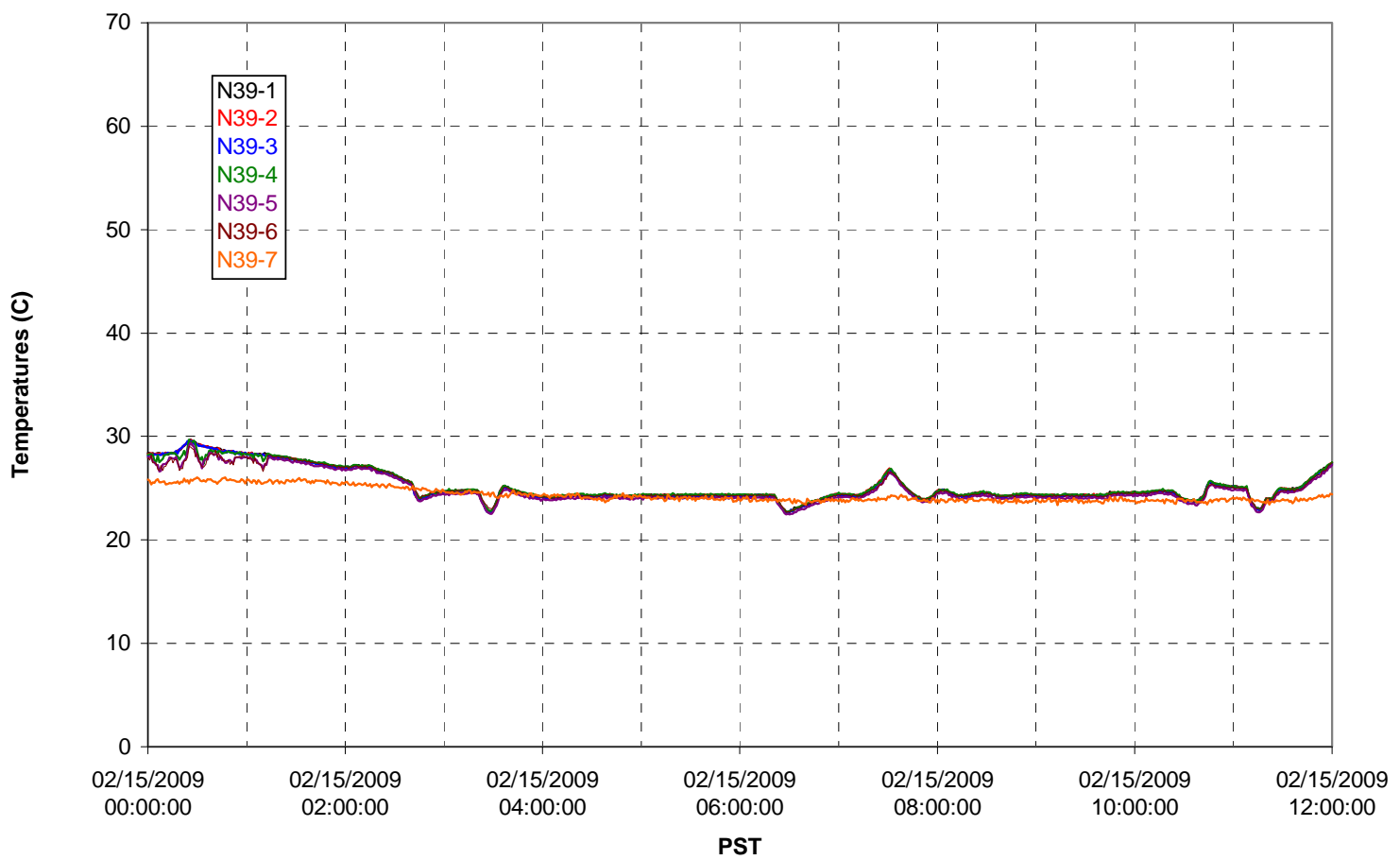

T02A temperatures

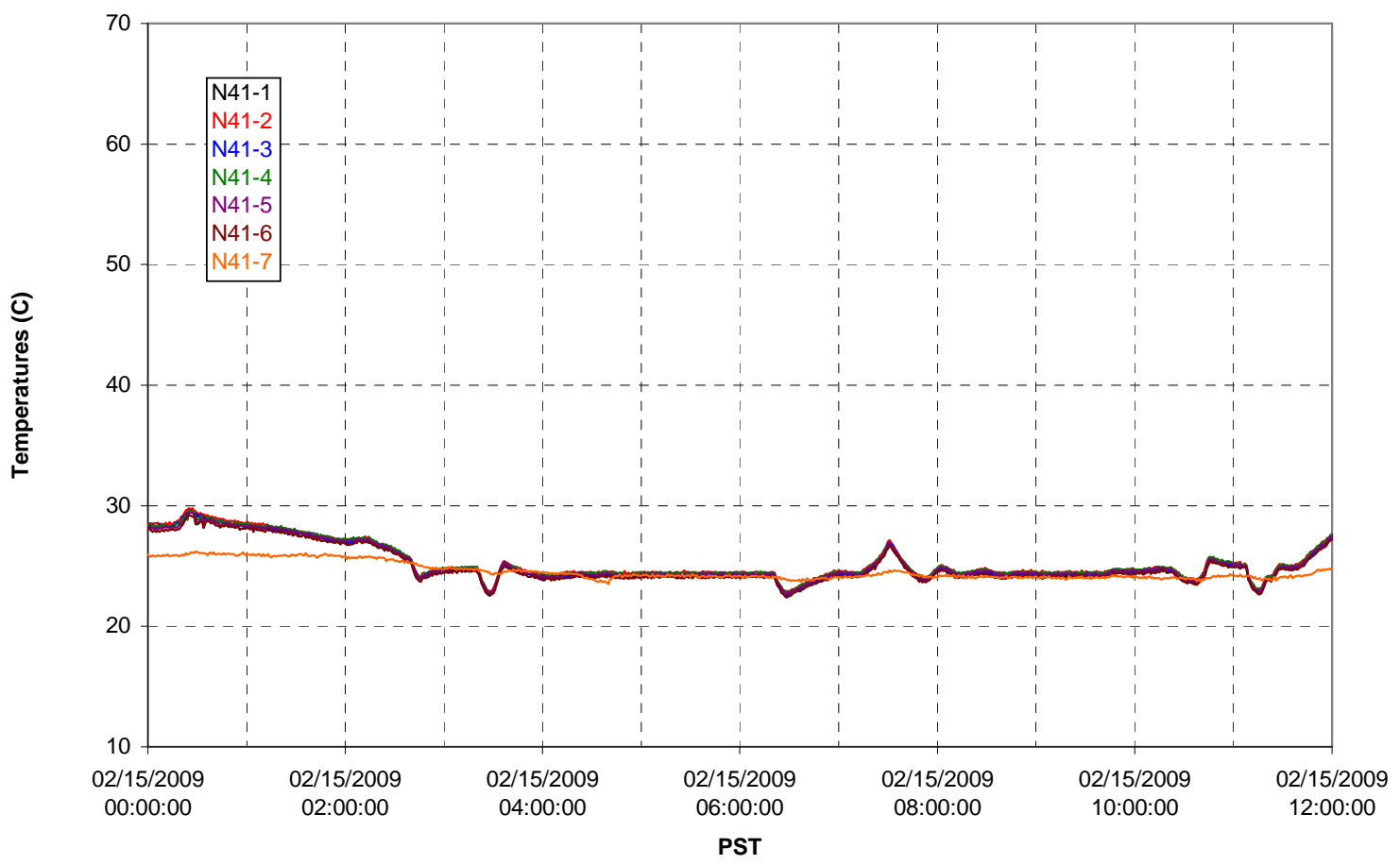


T02A temperatures

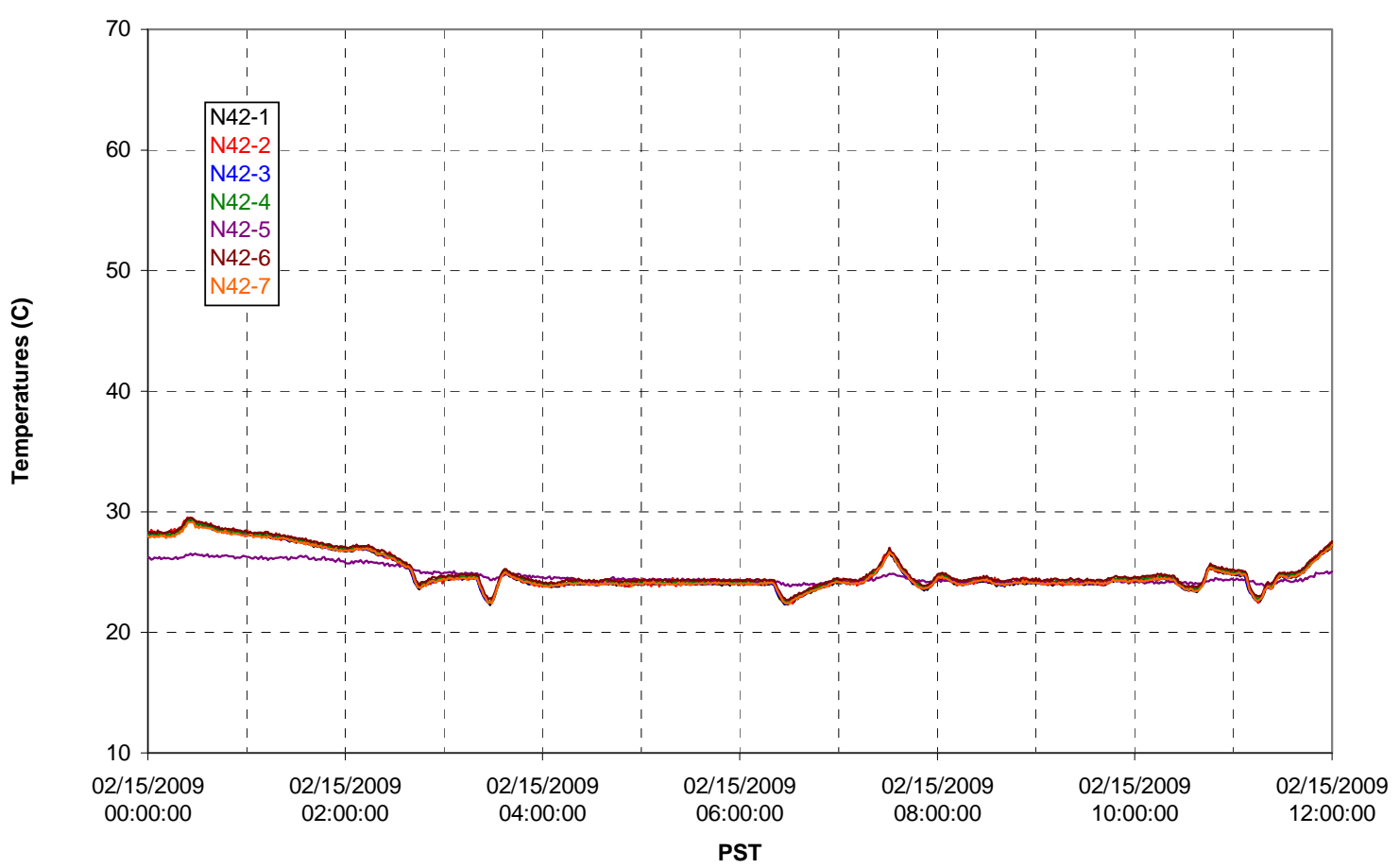

T02A temperatures

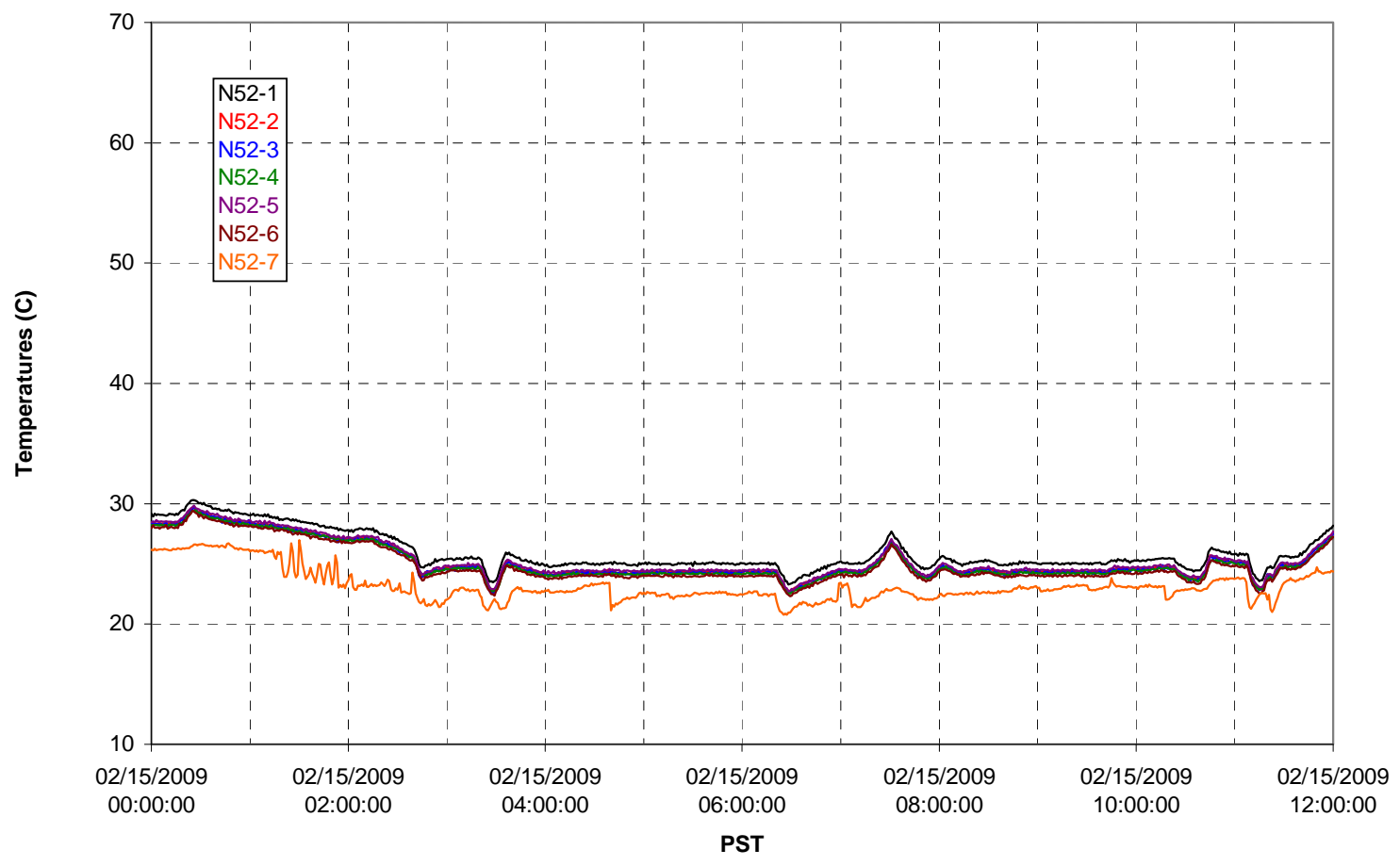


T02A Heating and Cooling
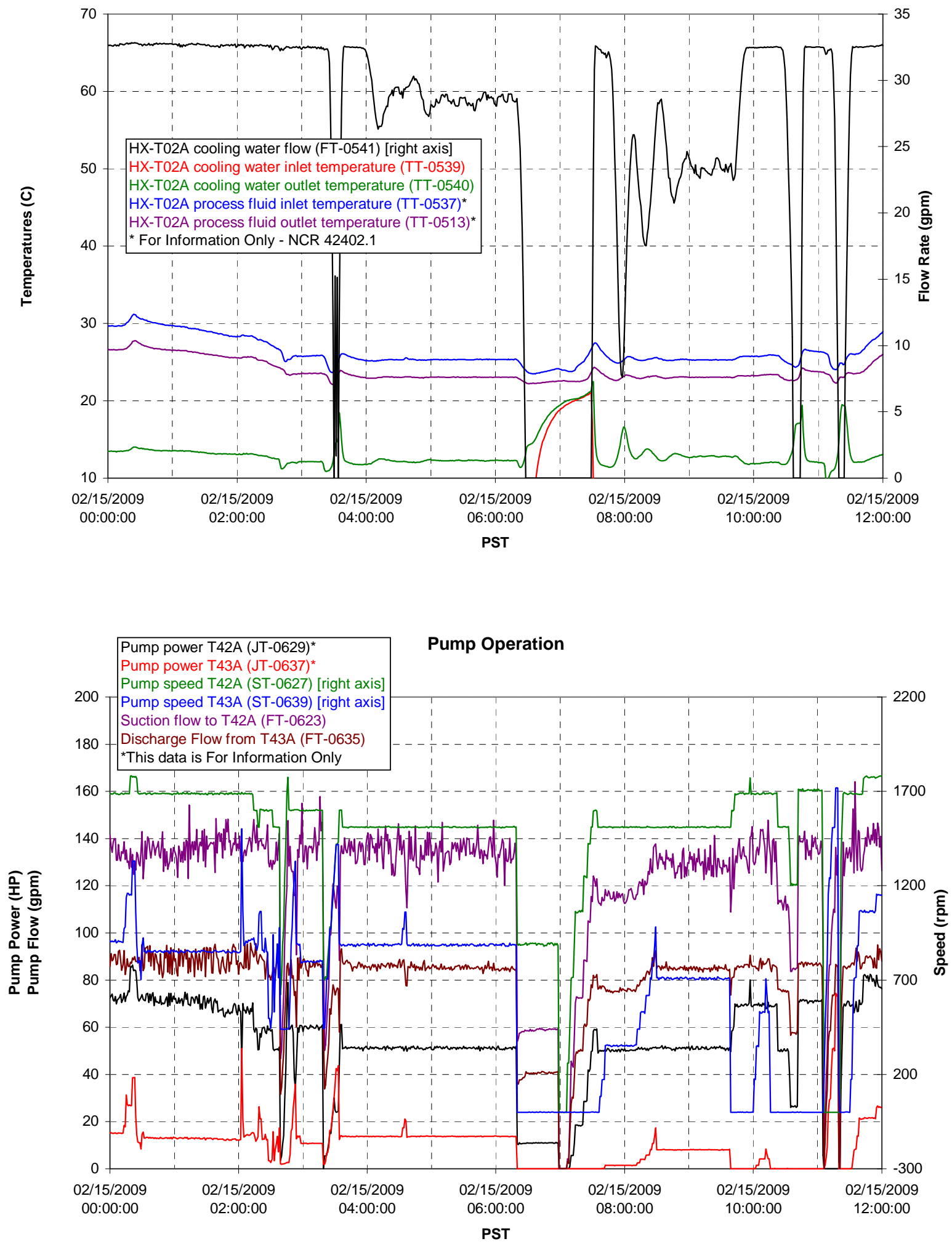
Pulsepot UFP-PP-T01A

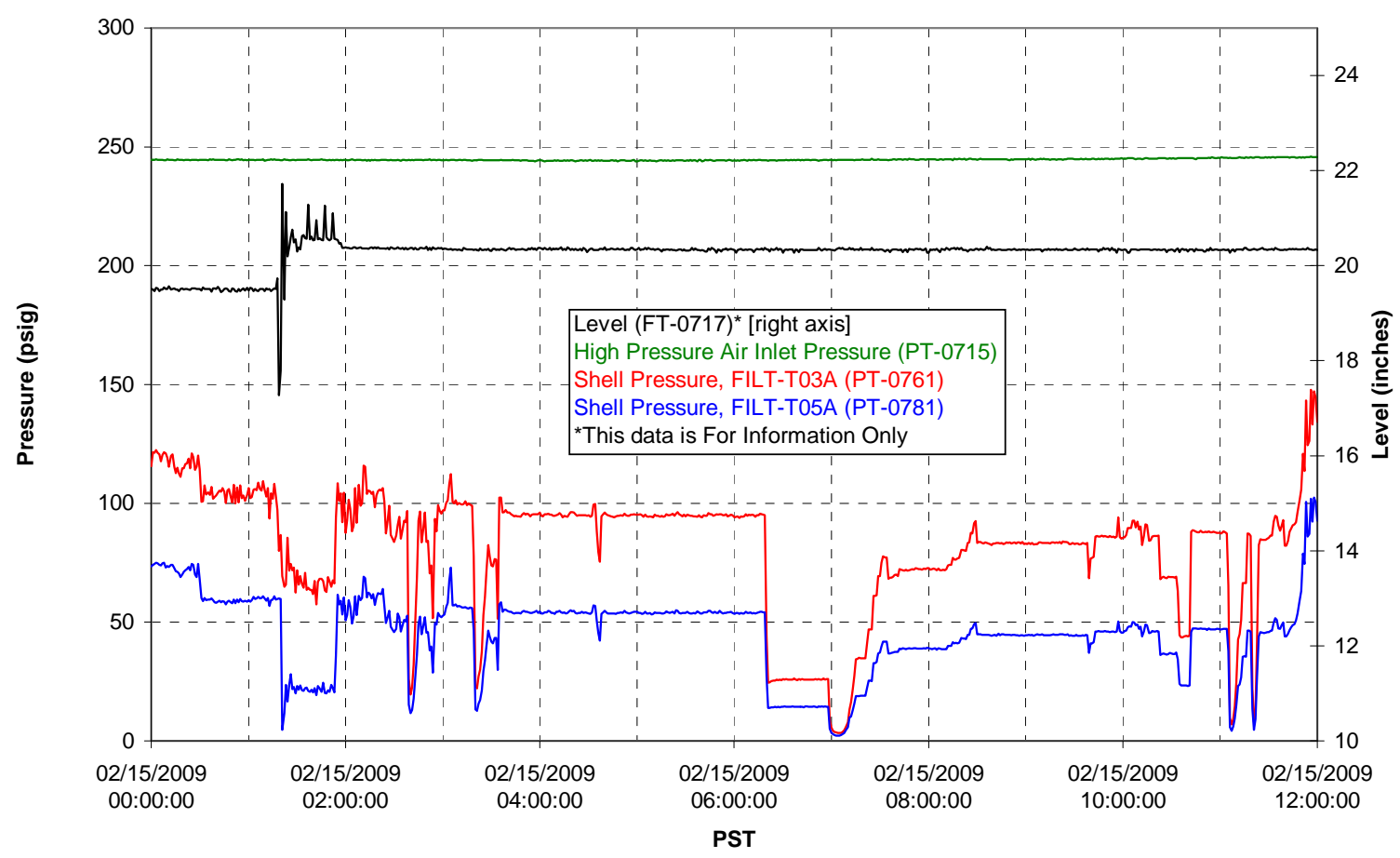

Pulsepot UFP-PP-T02A

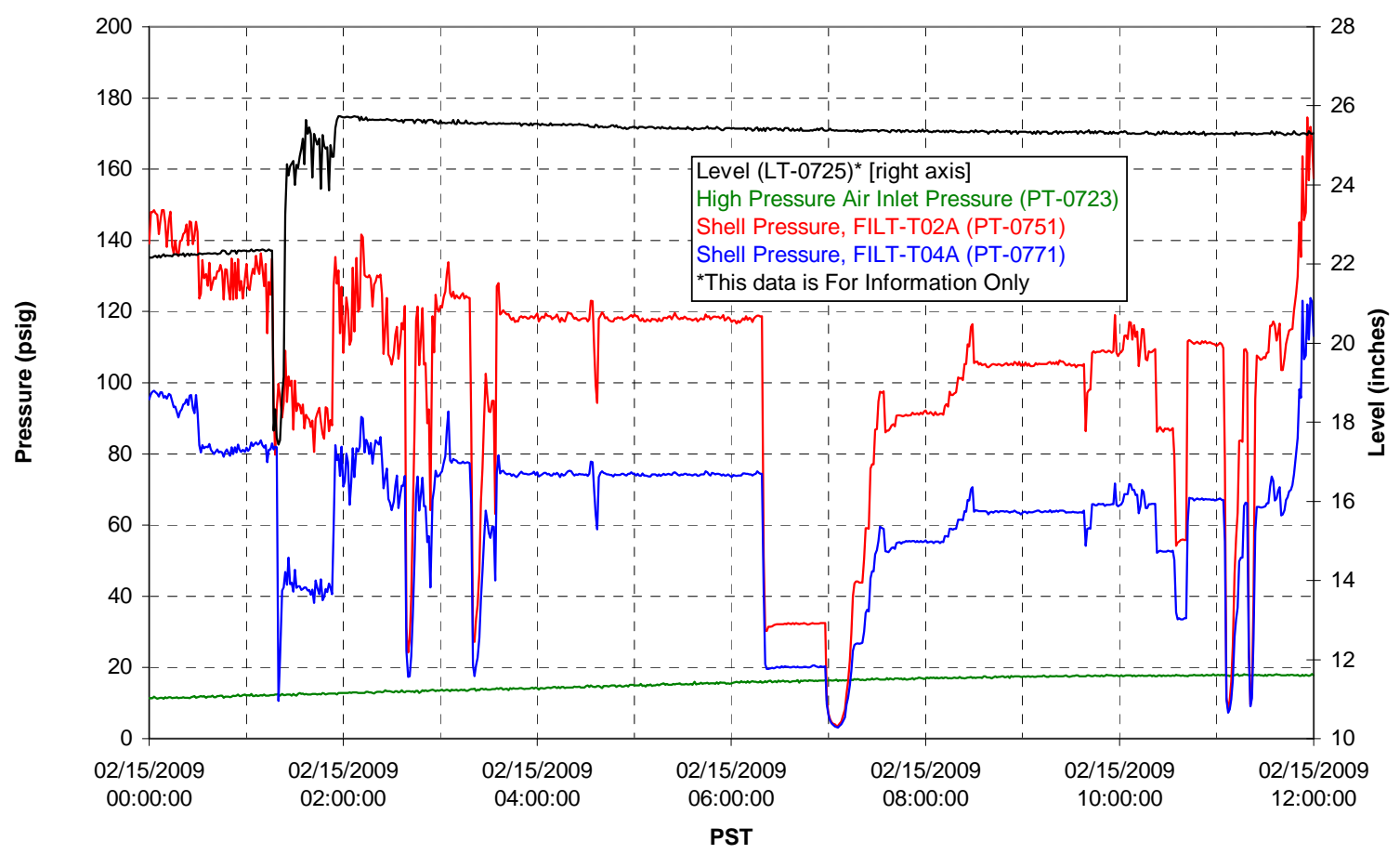


Pulsepot UFP-PP-T03A

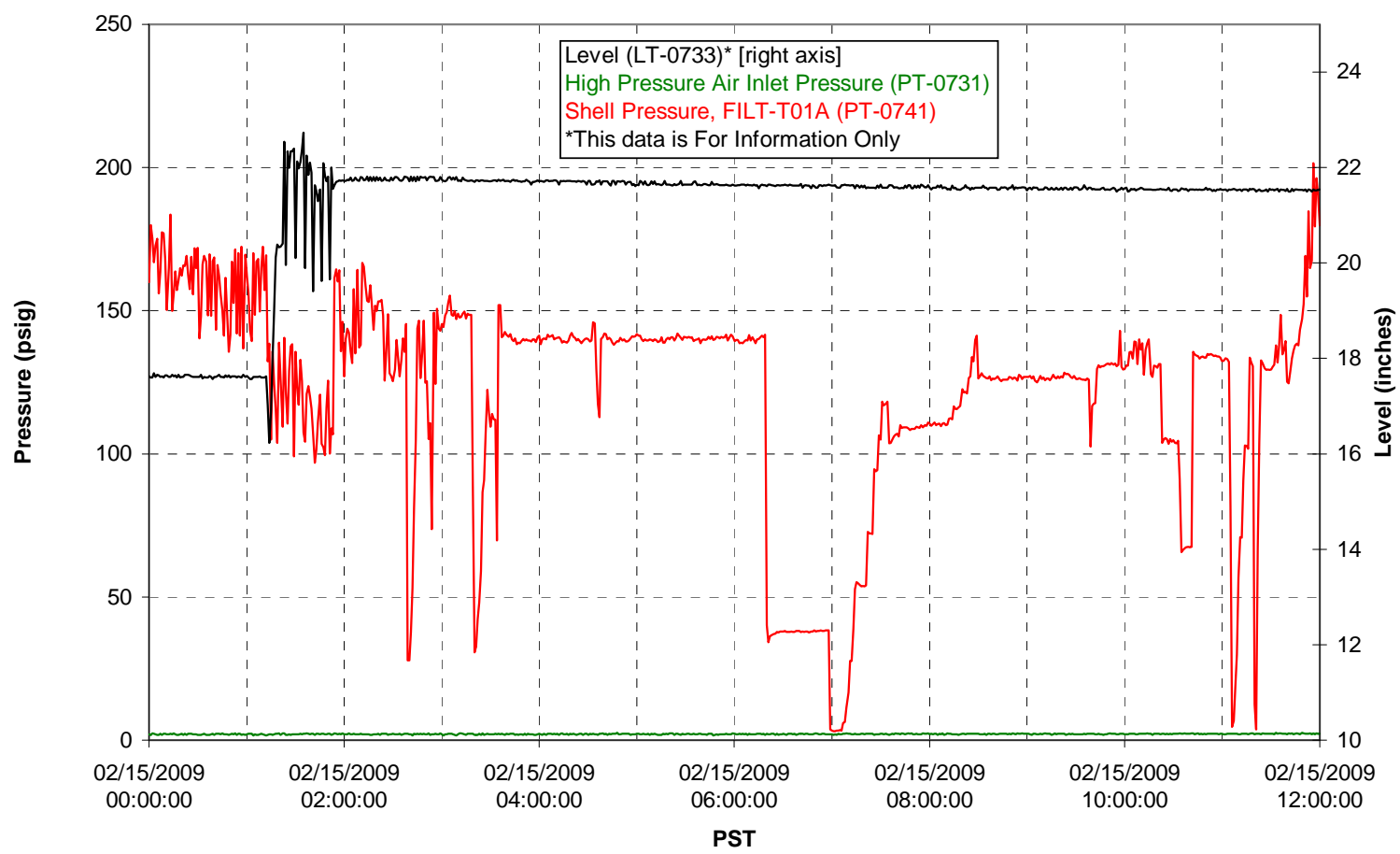

Pulsepot Levels

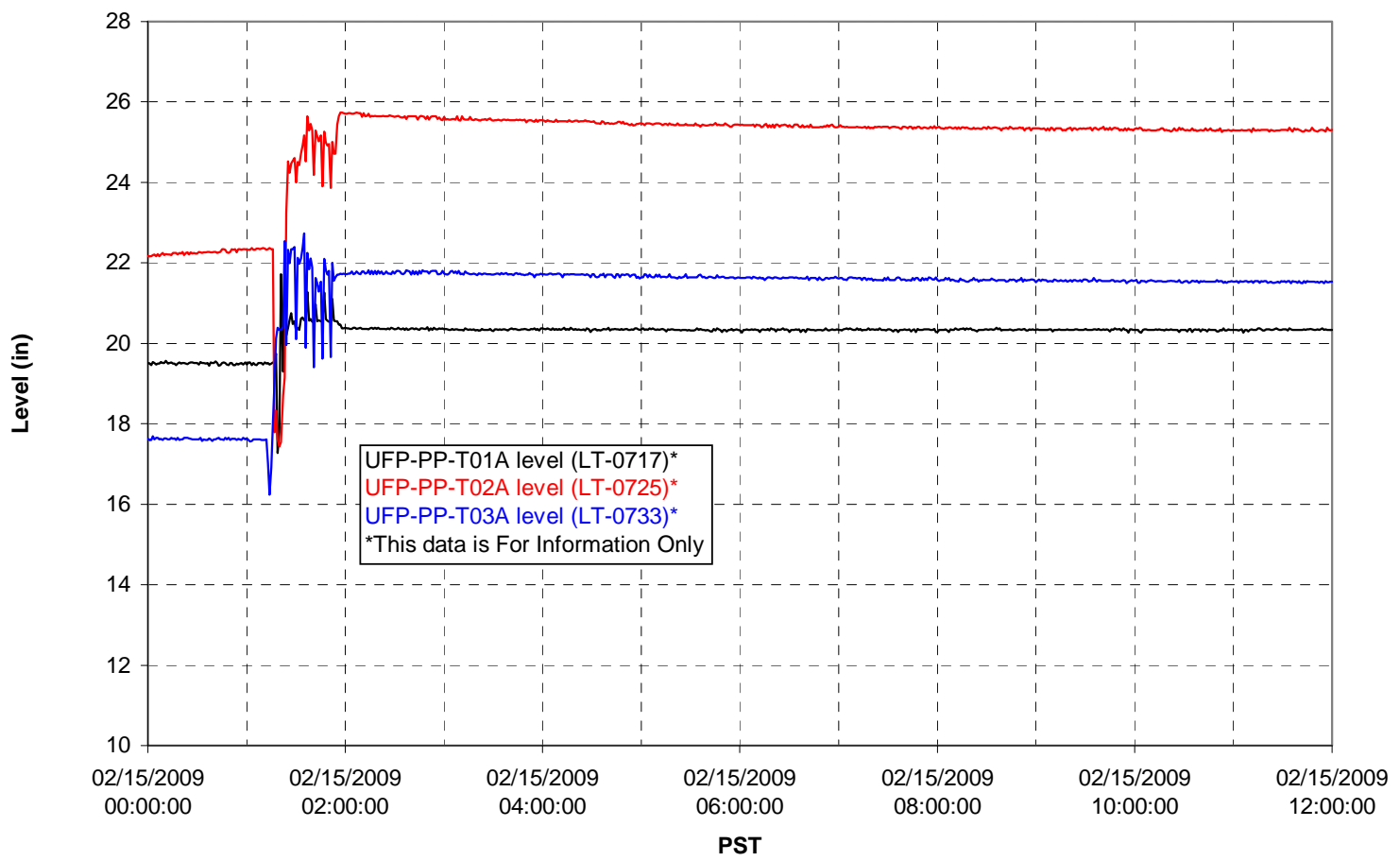


Filter UFP-FILT-T01A

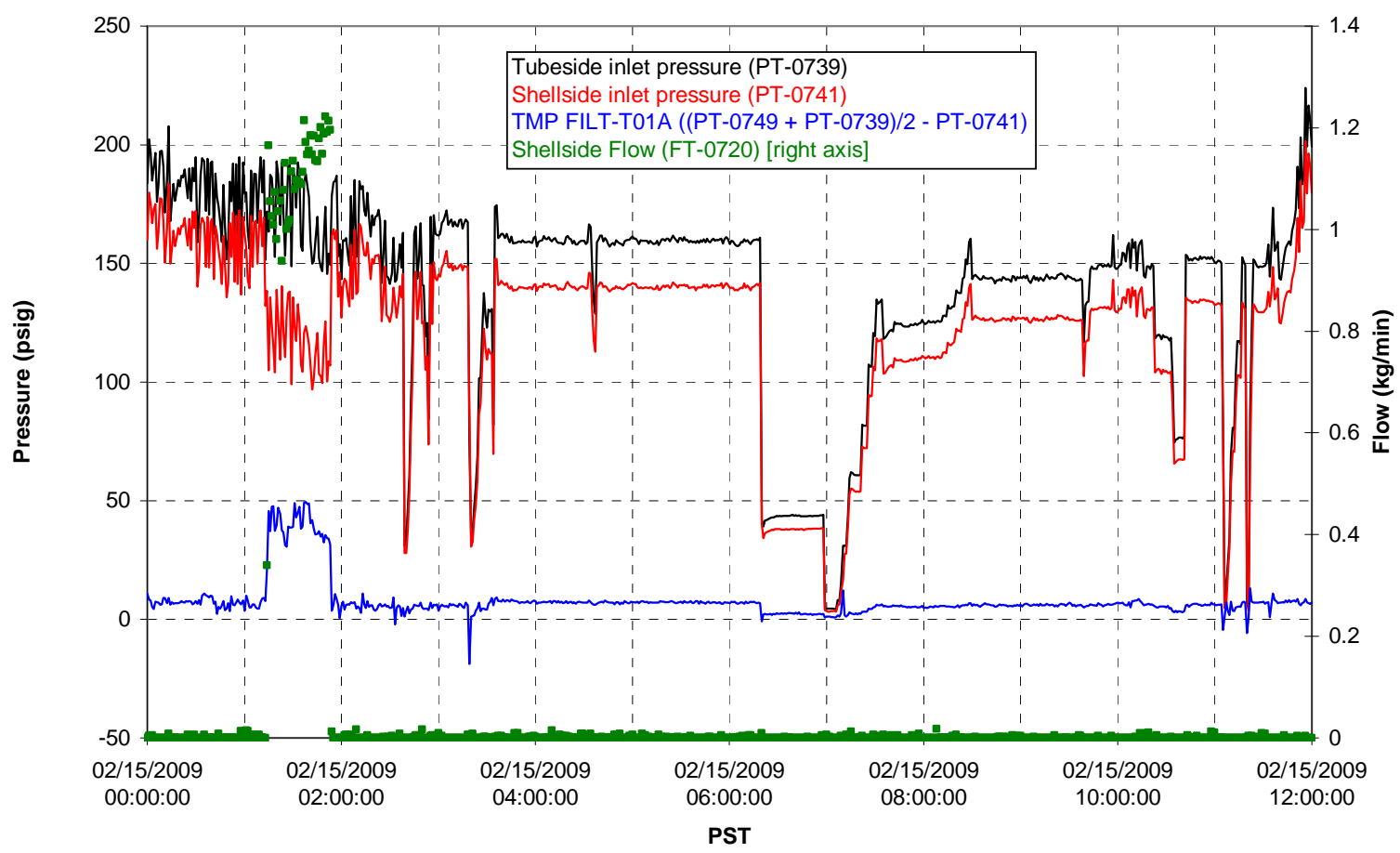

Filter UFP-FILT-T02A

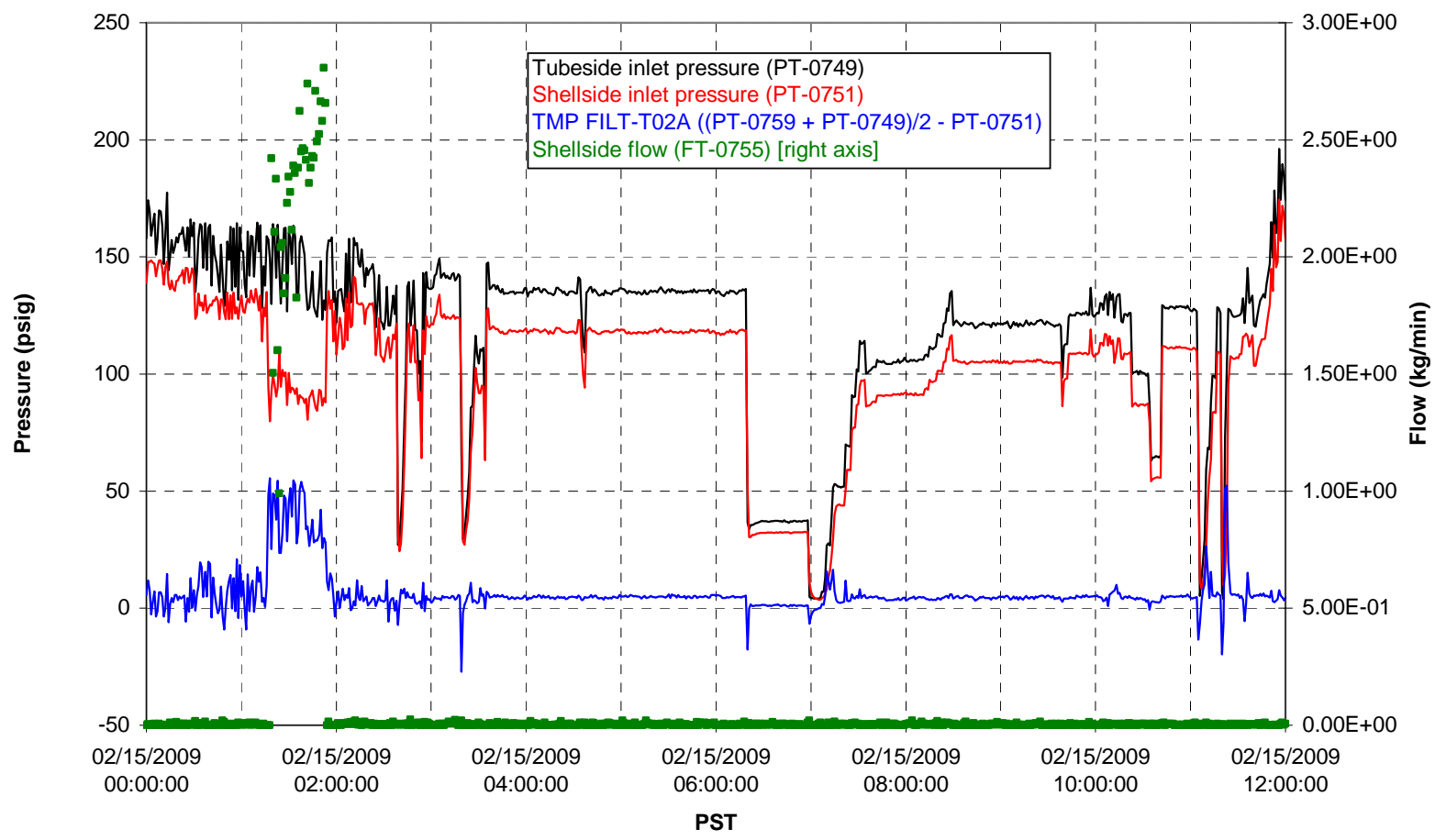


Filter UFP-FILT-T03A

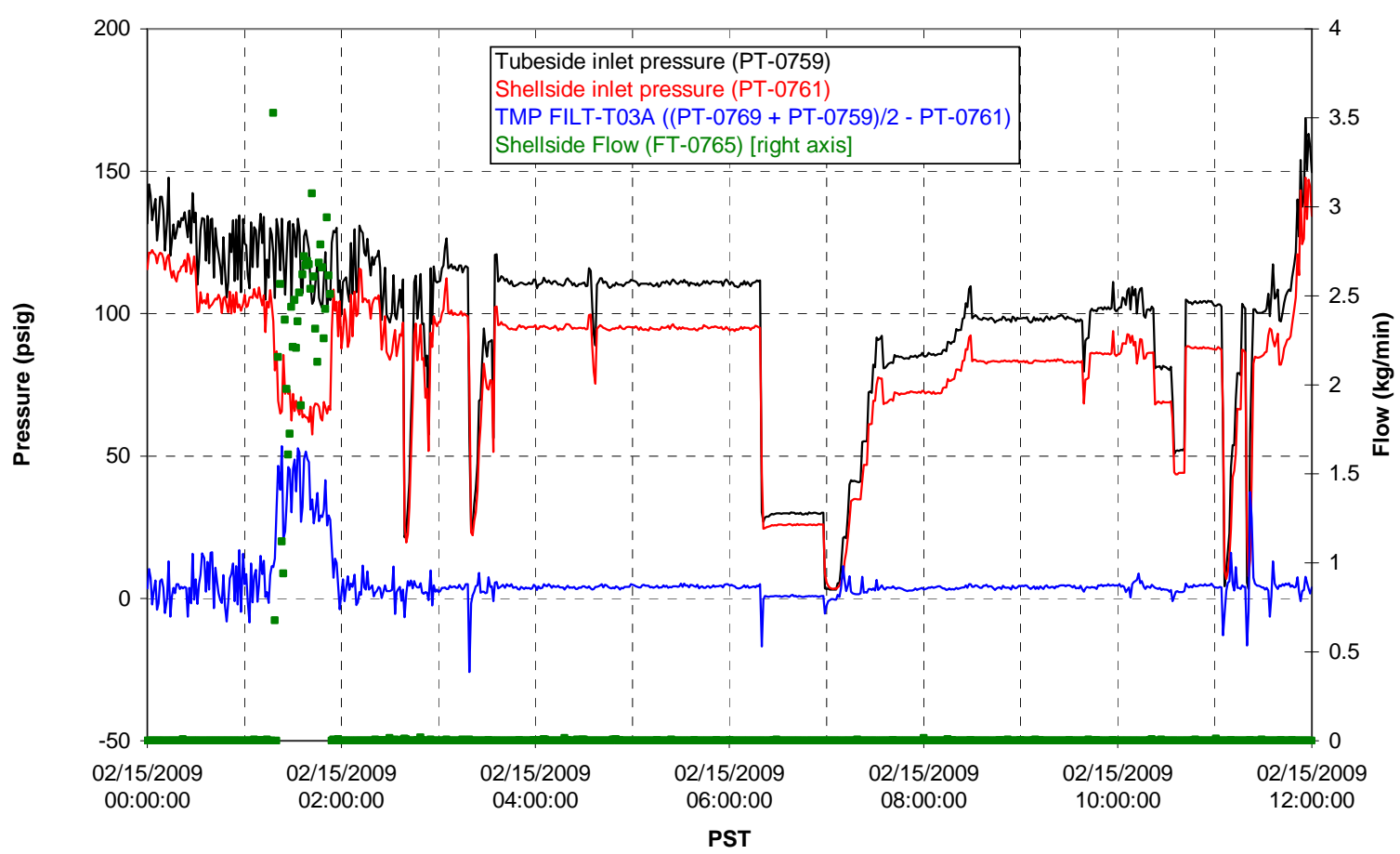

Filter UFP-FILT-T04A

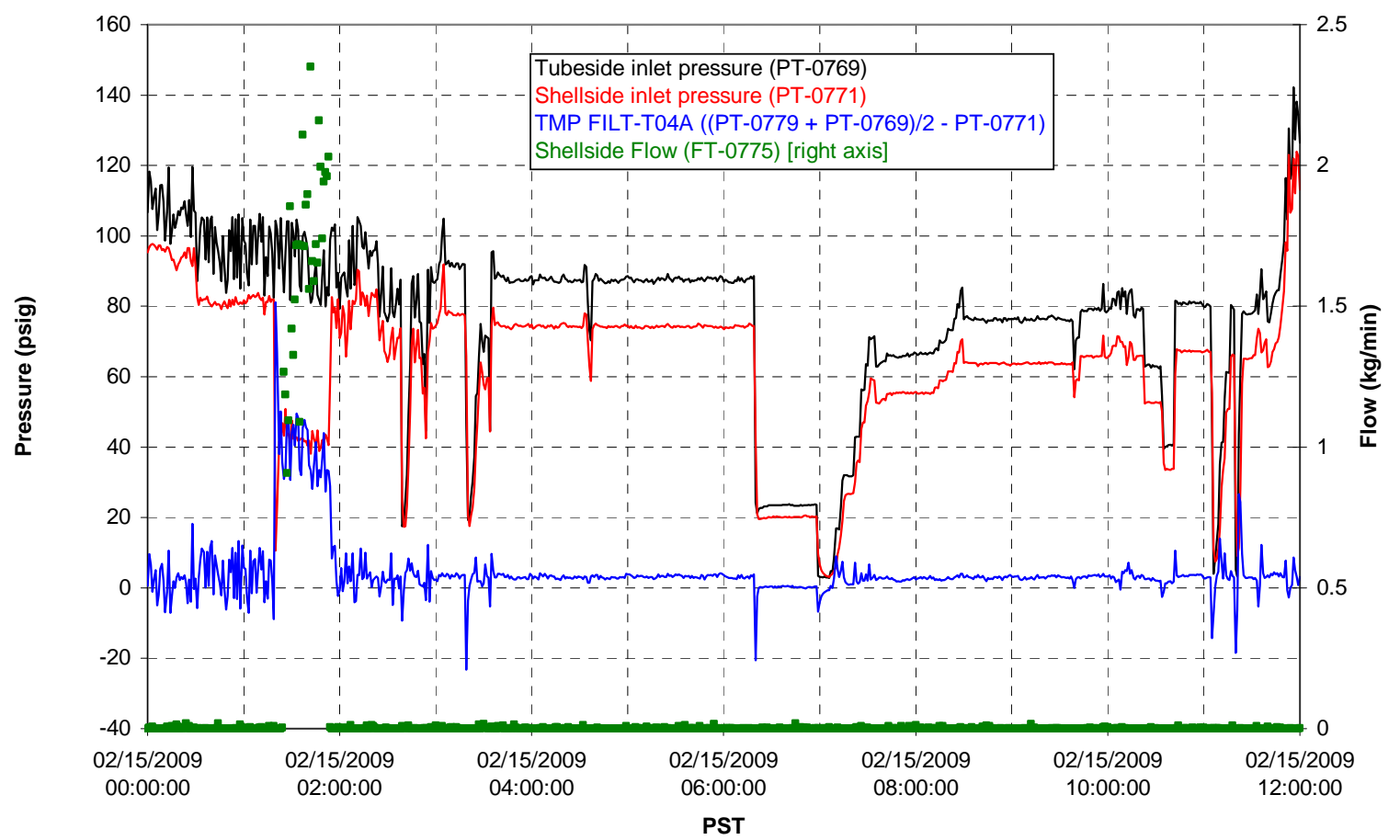


Filter UFP-FILT-T05A

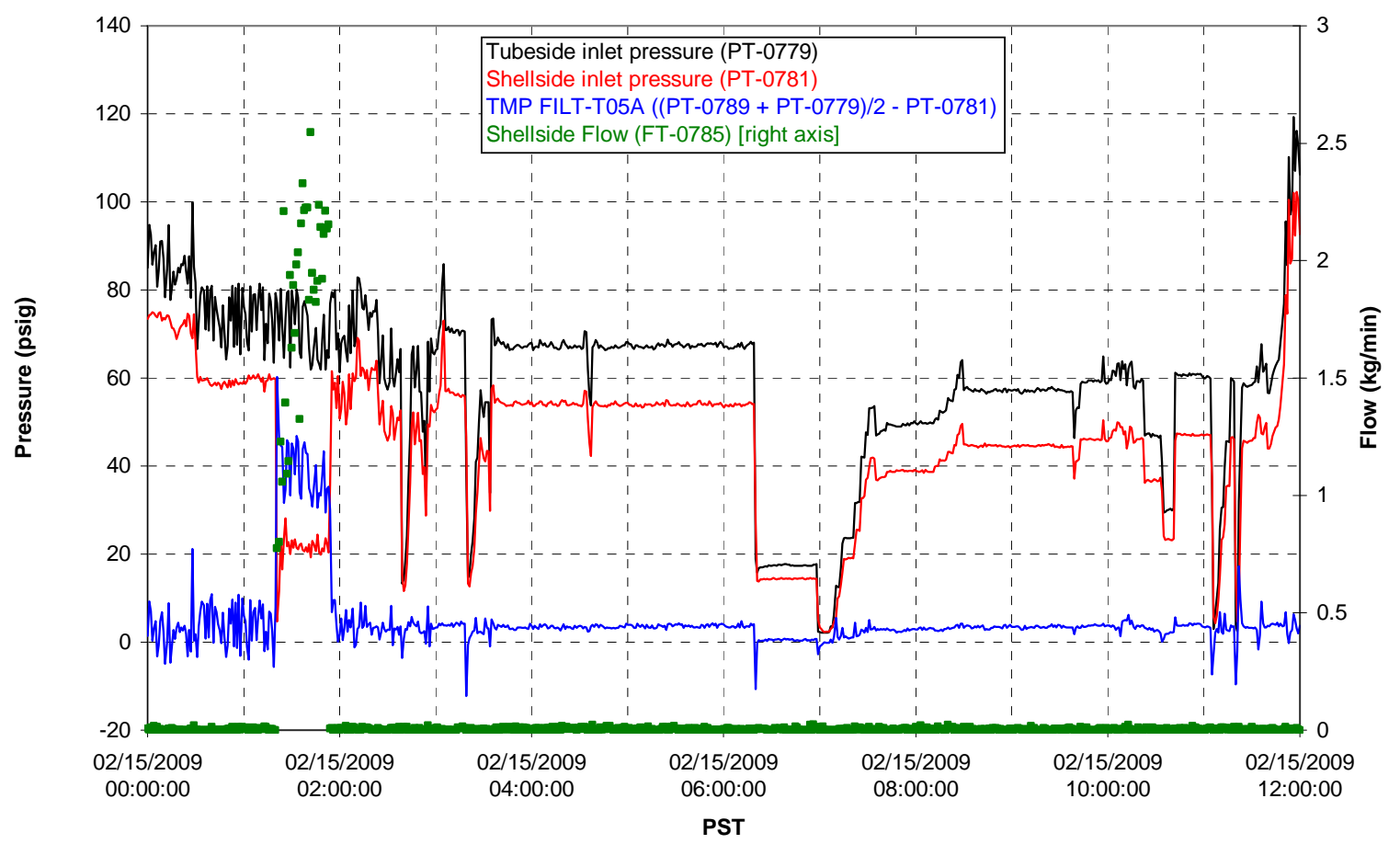

Chemical Flow

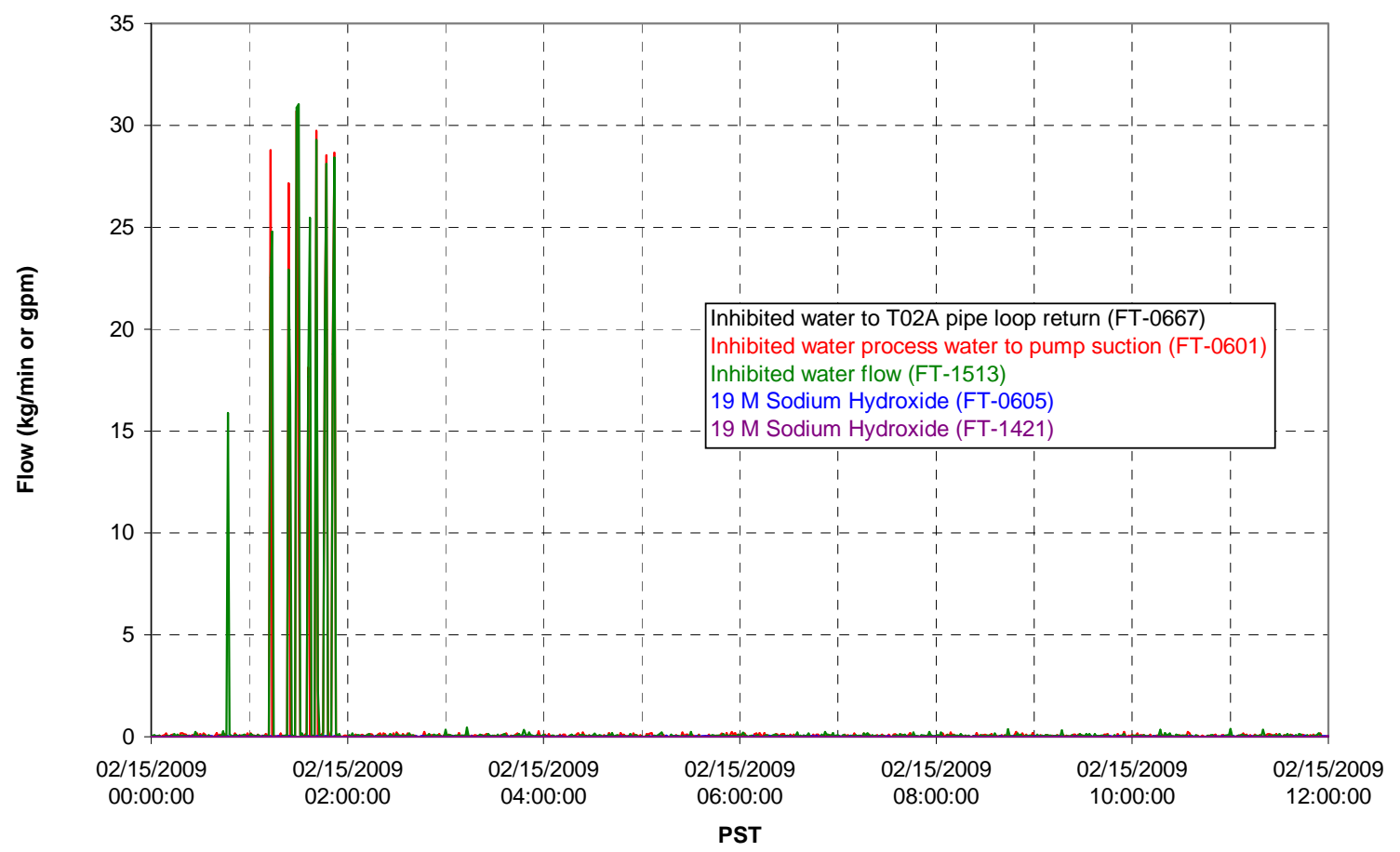


Chemical Flow

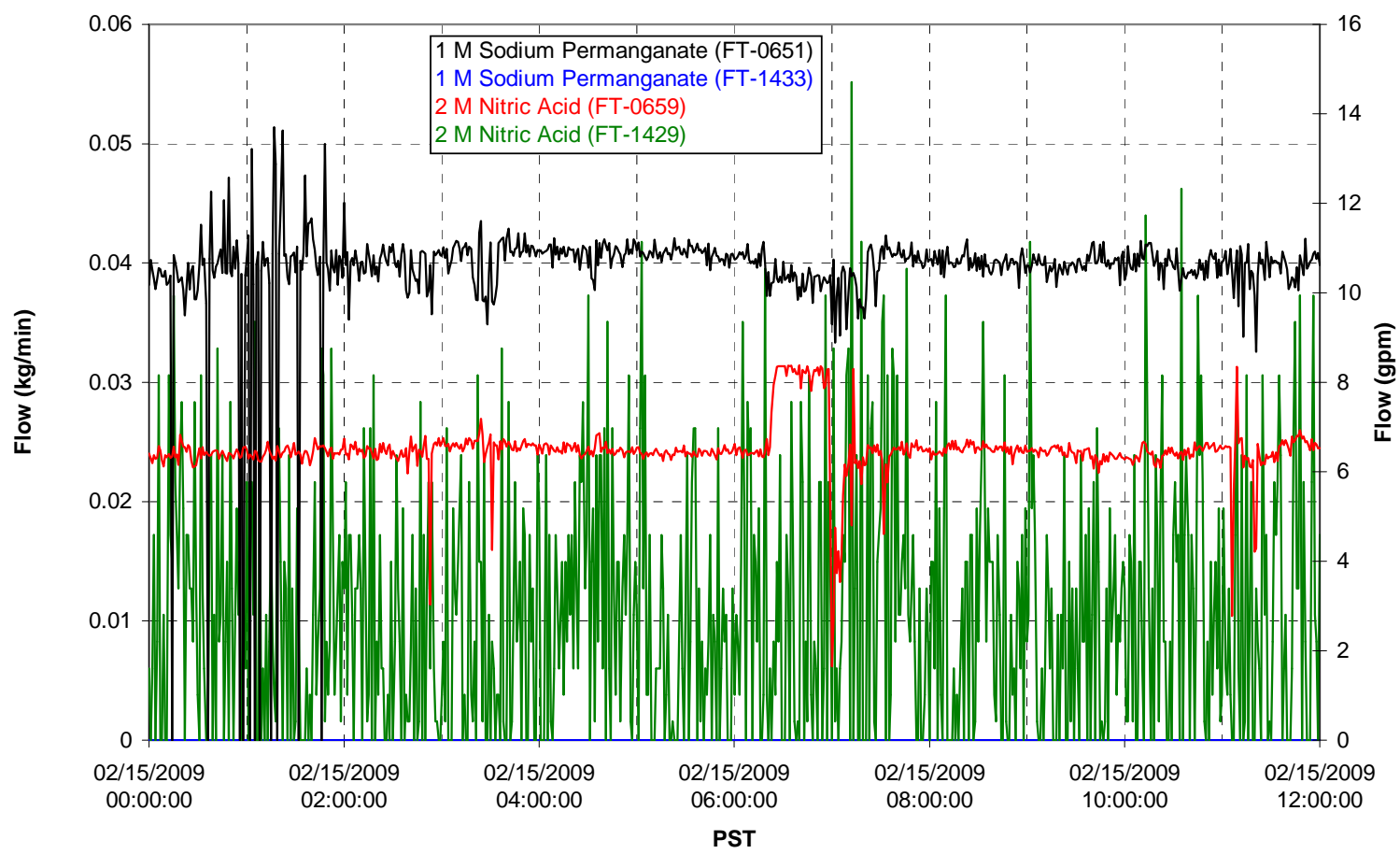

Air Flows

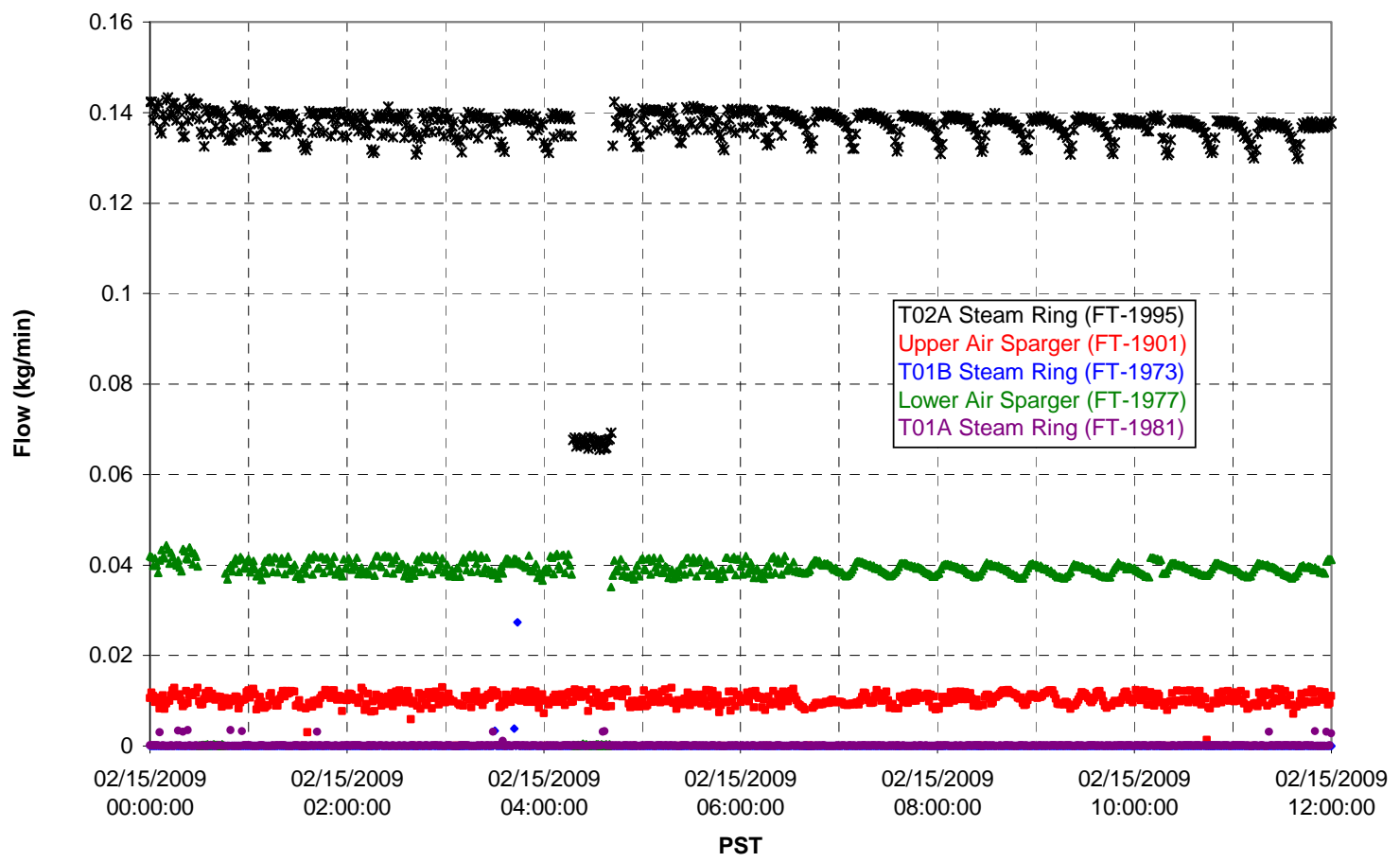


T02A Steam

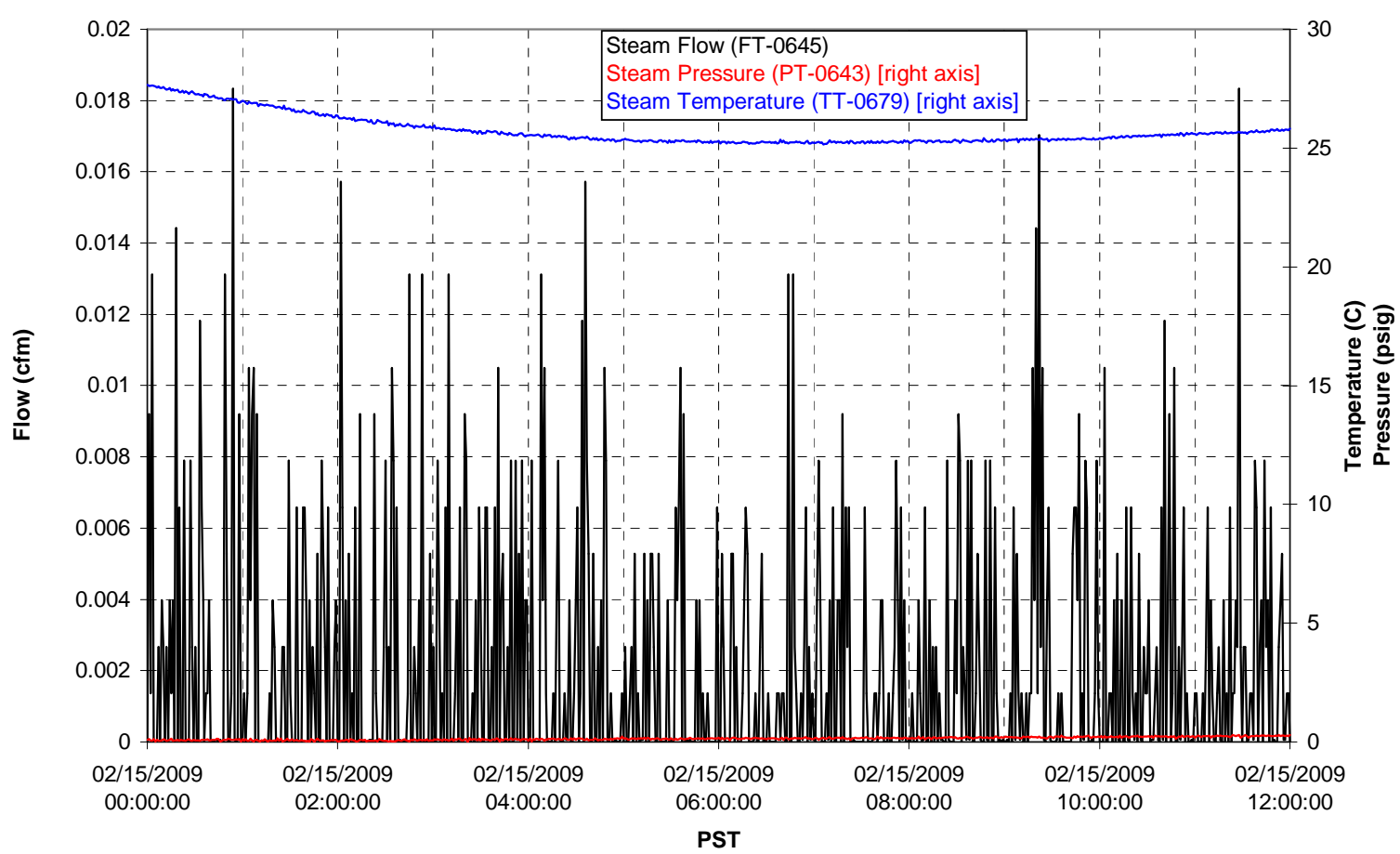

T01A Steam

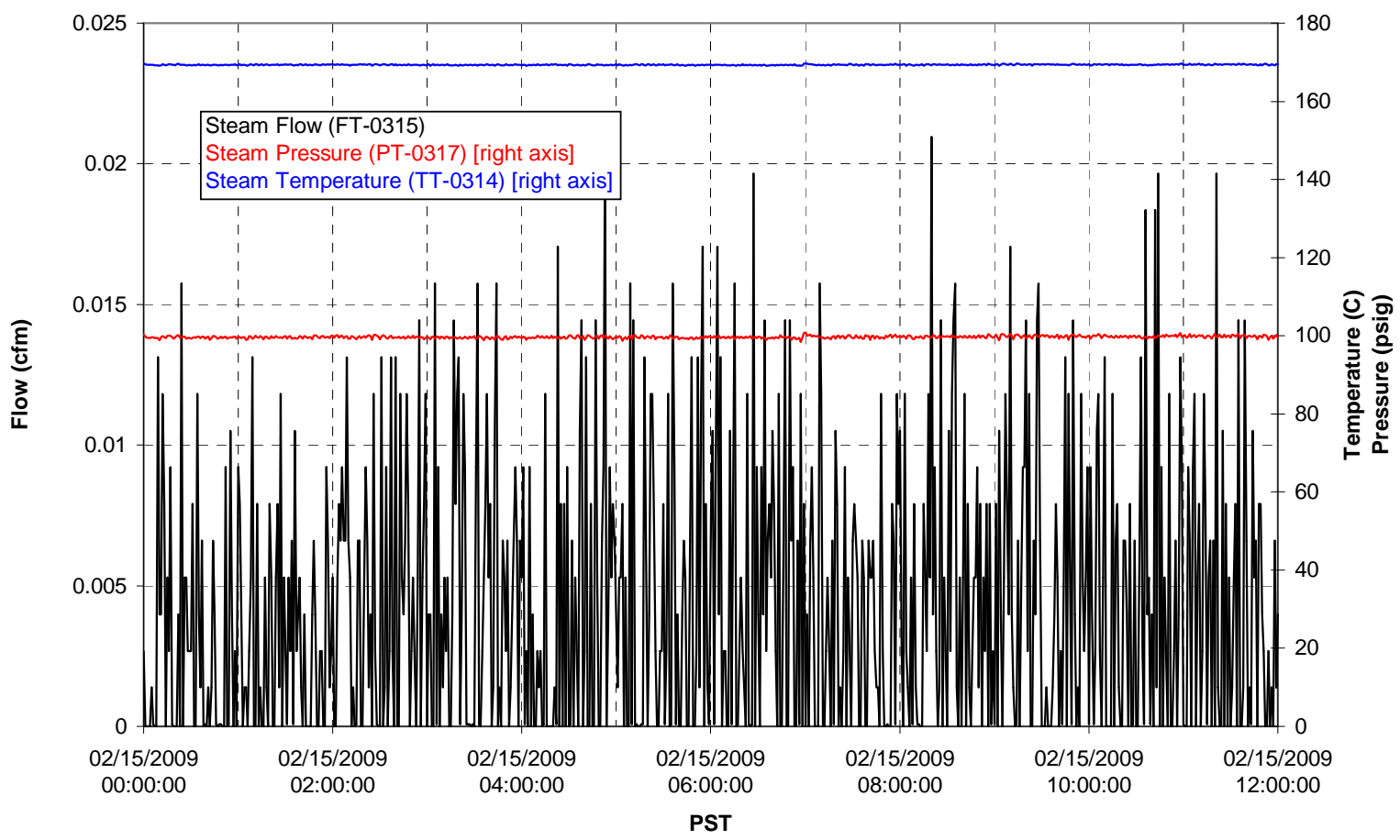


T01B Steam

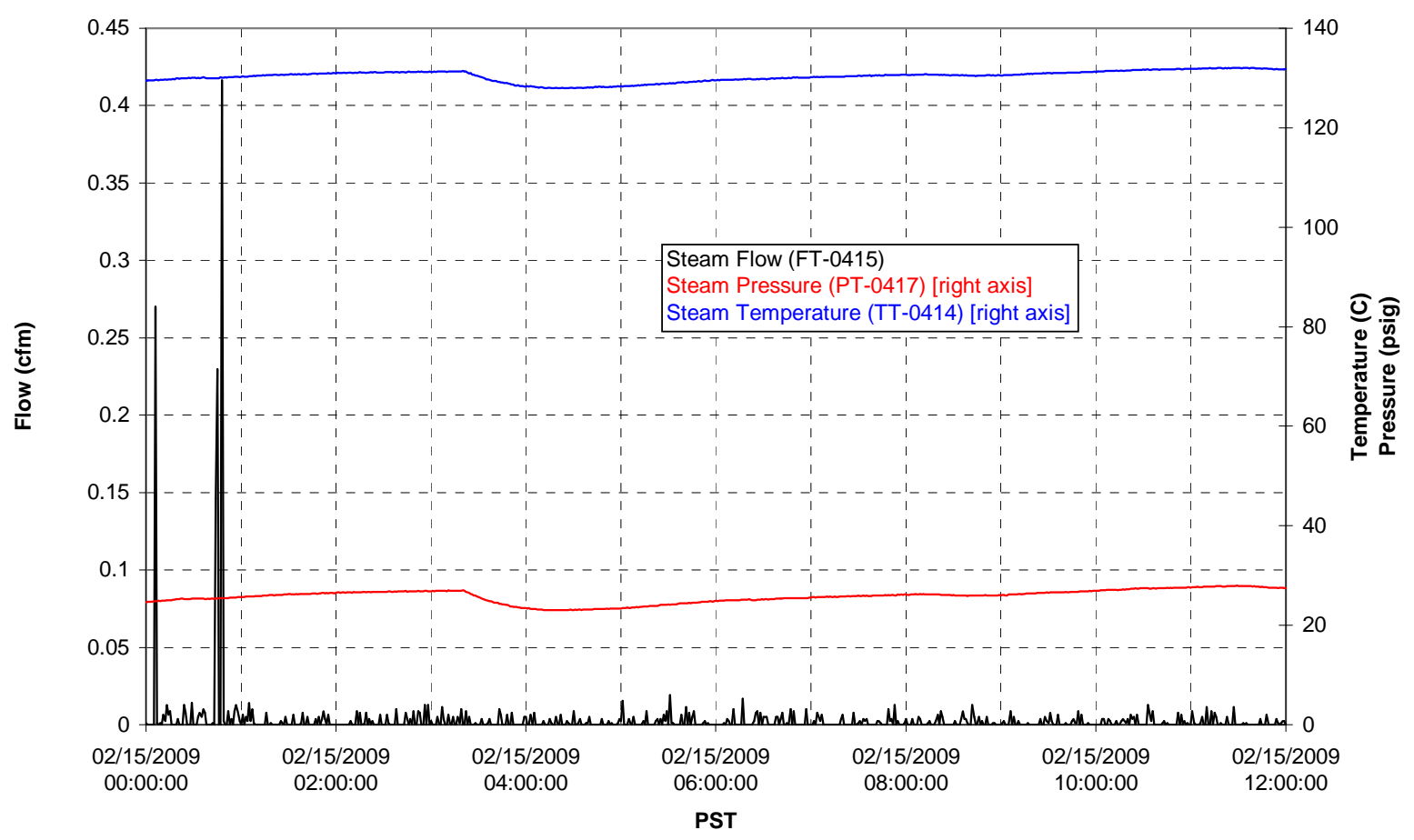




\section{Integrated Test A Data Plots 02/15/09 12:00 - 02/16/09 00:00}


T01A level

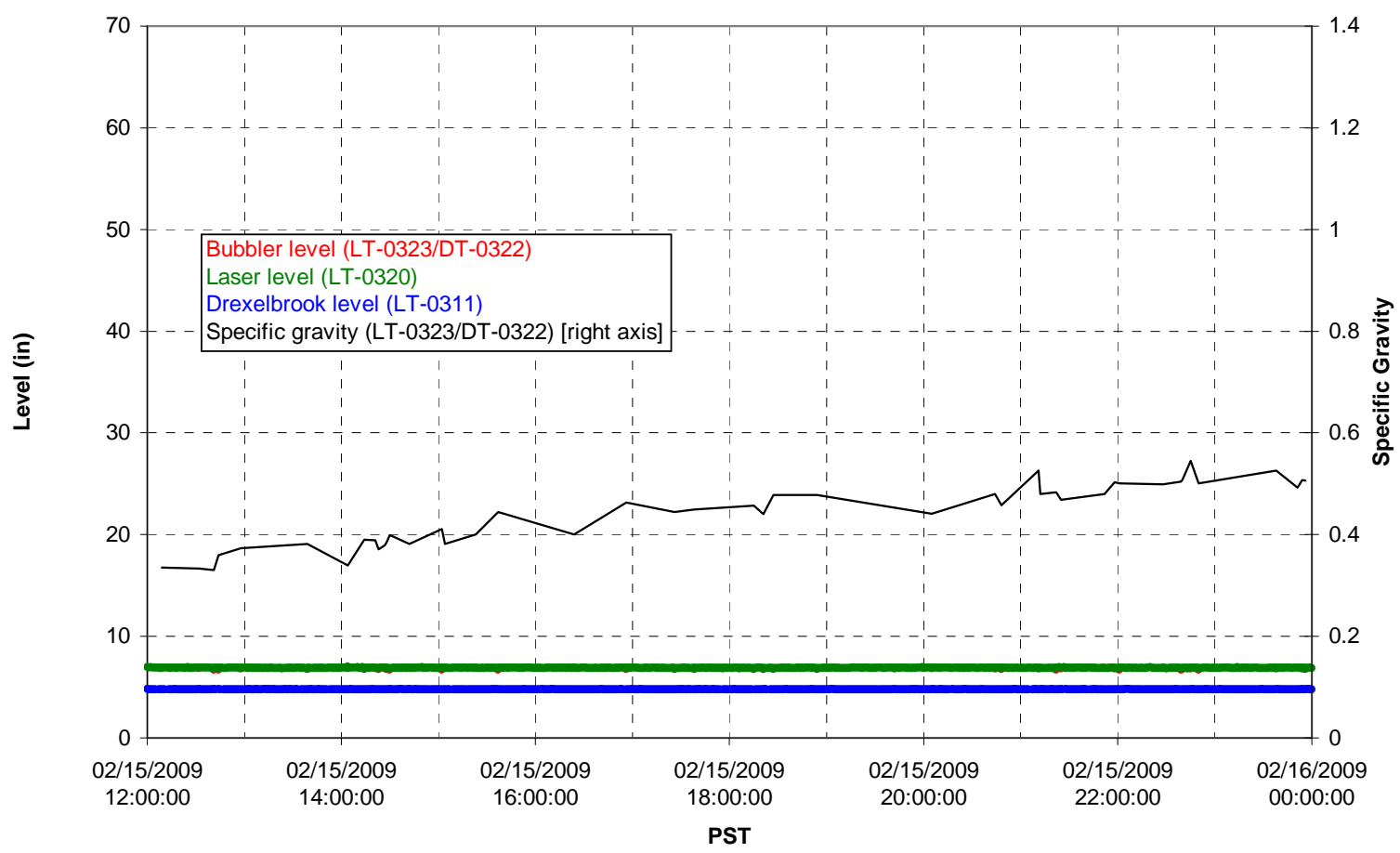

T01A temperatures

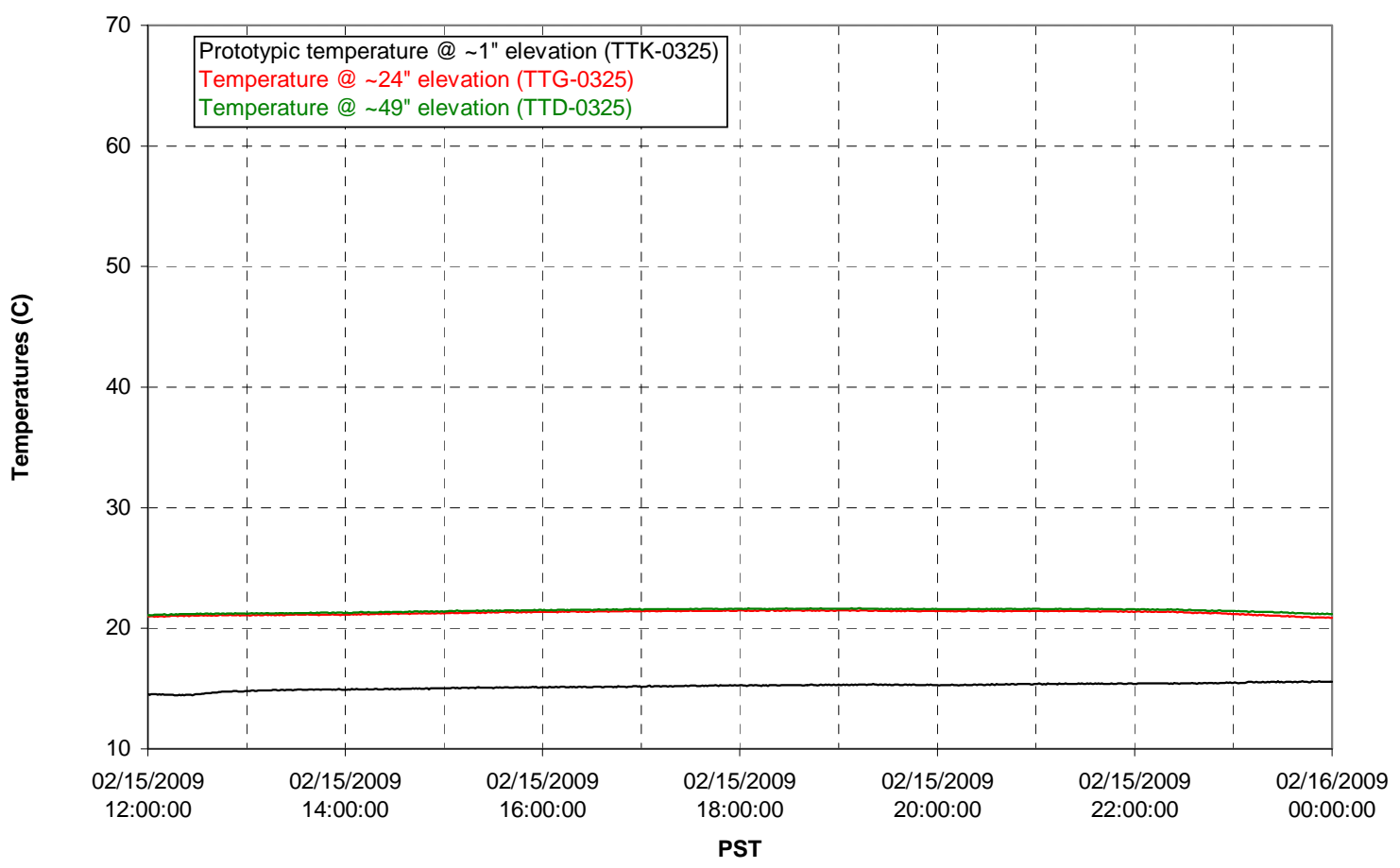


T01B level

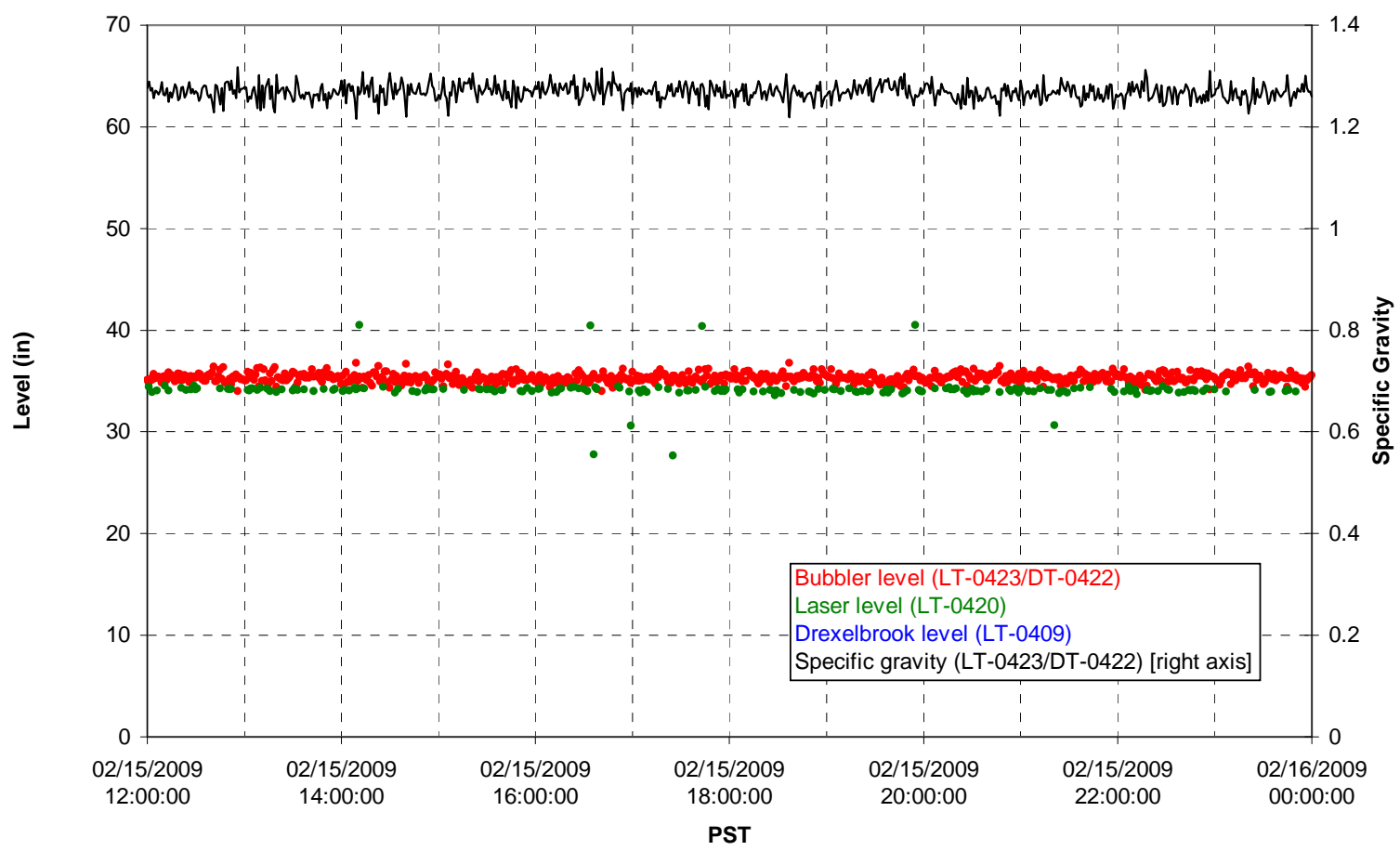

T01B temperatures

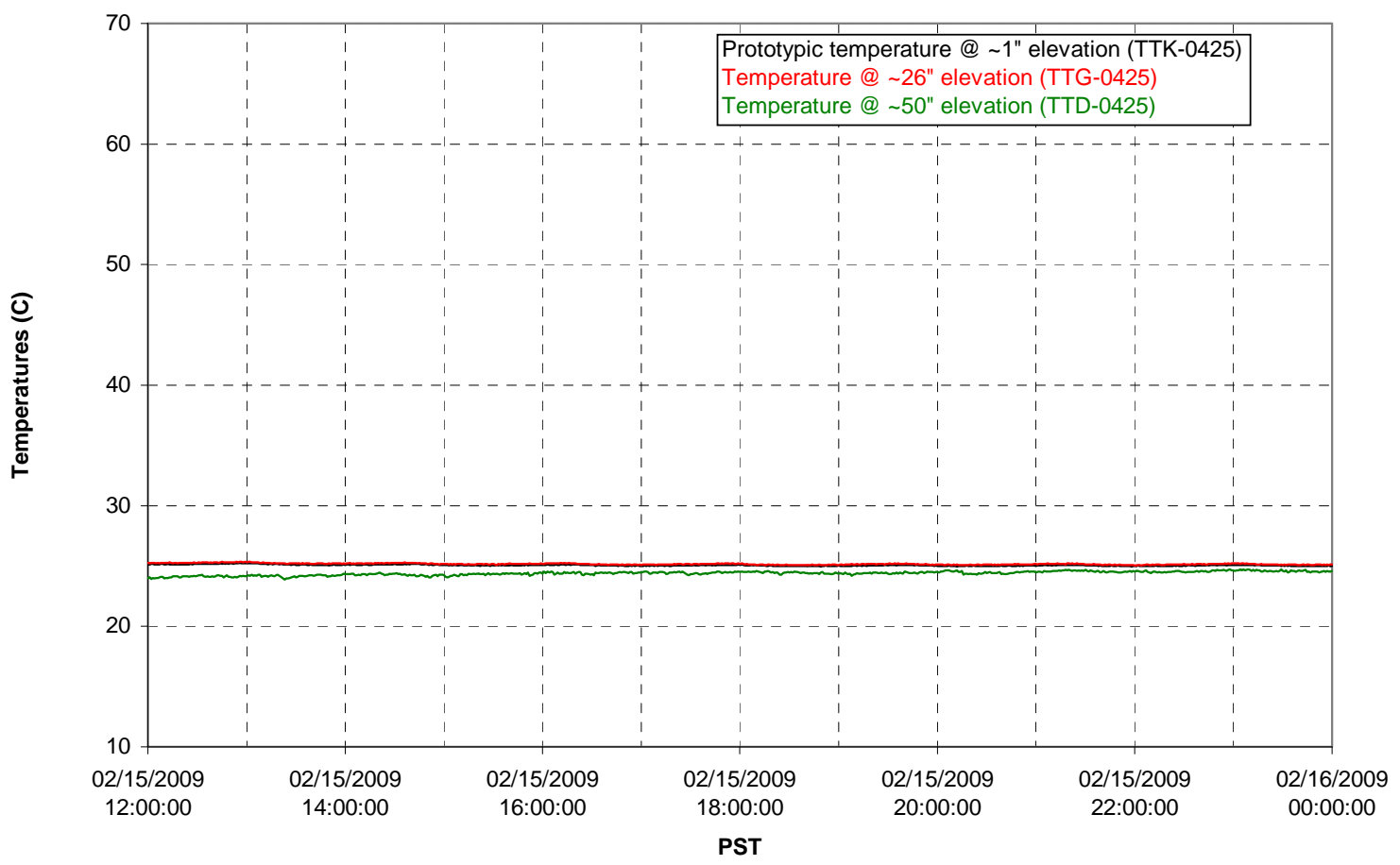


T02A level

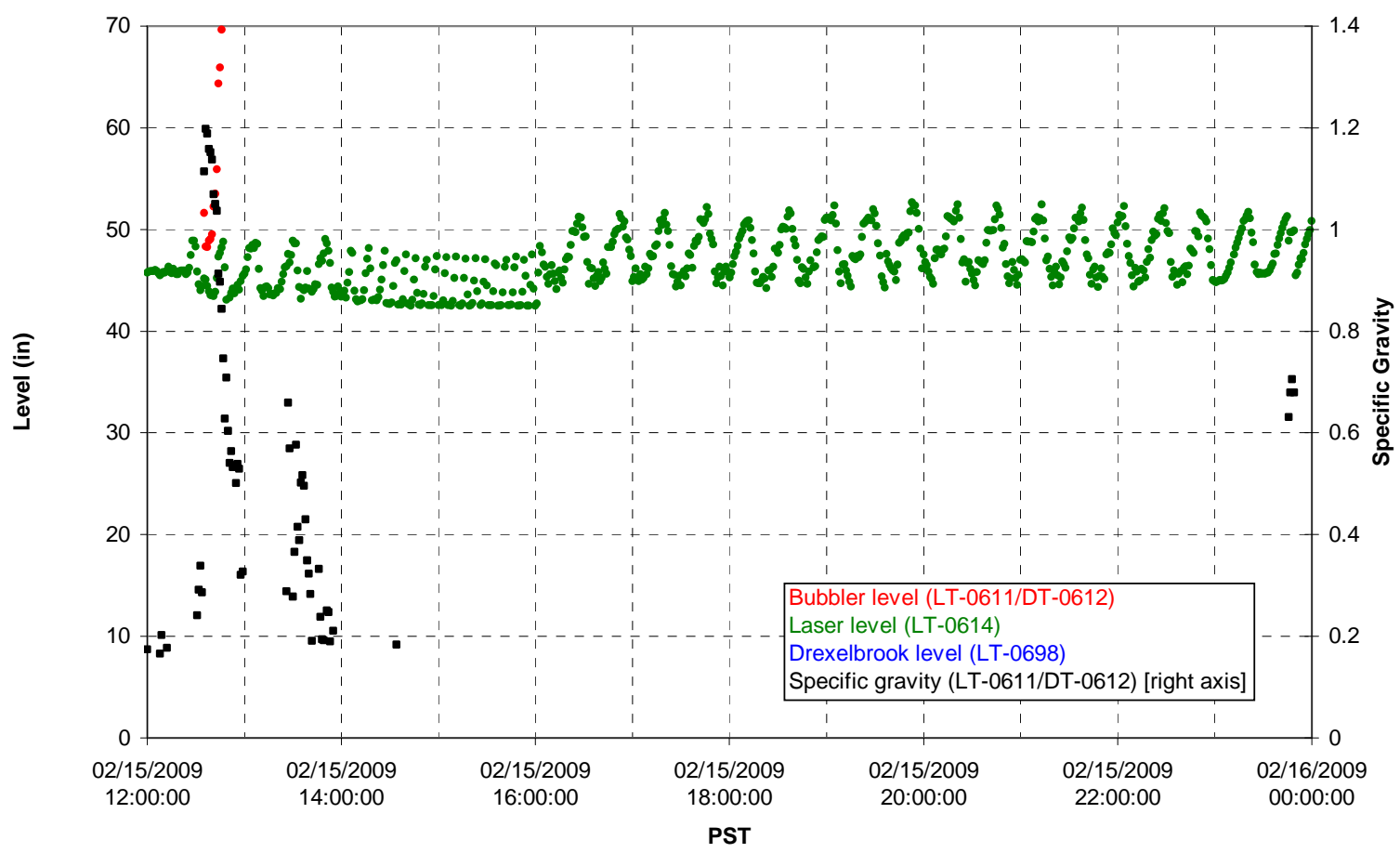

T02A temperatures

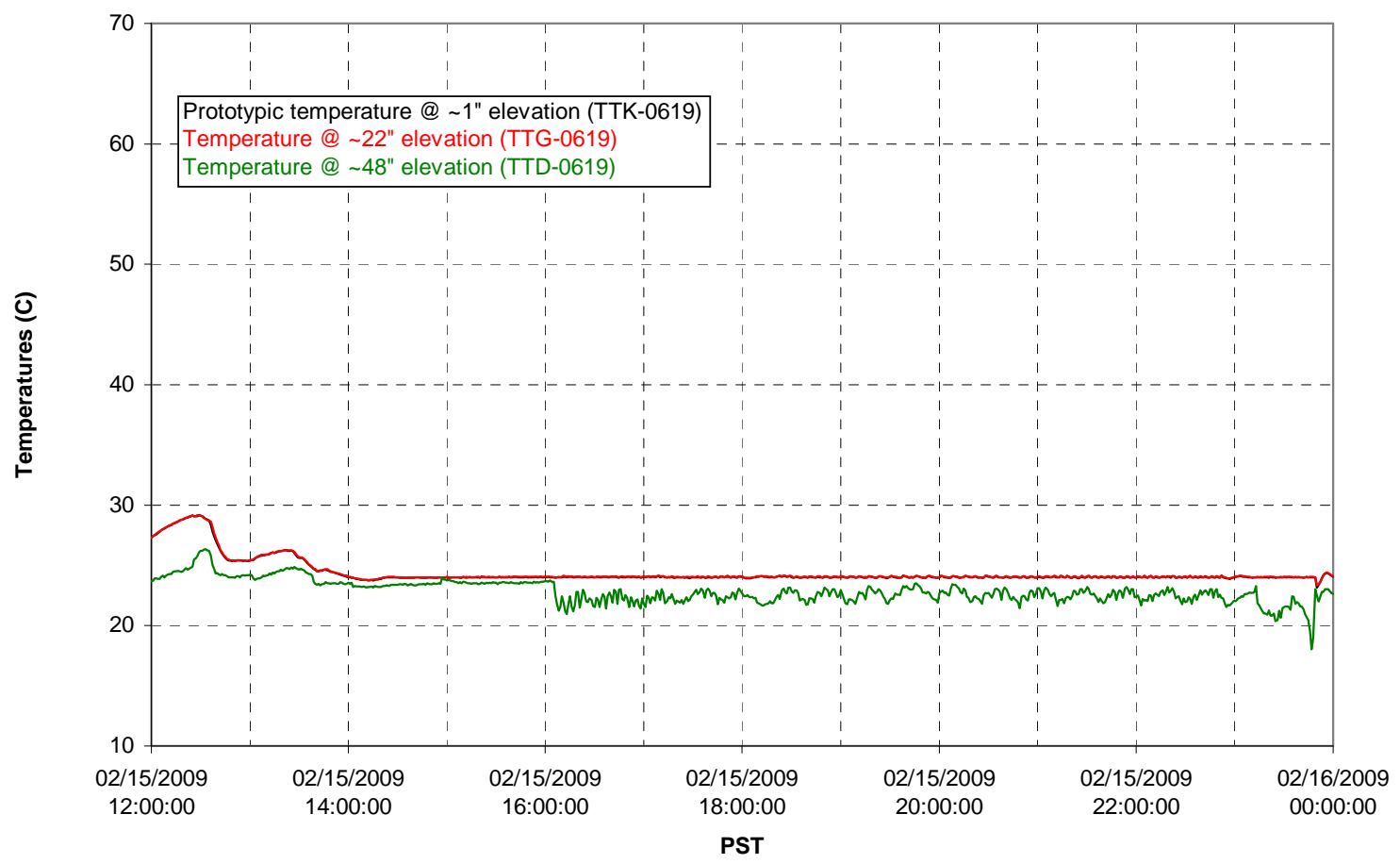


T02A and filter loop temperatures

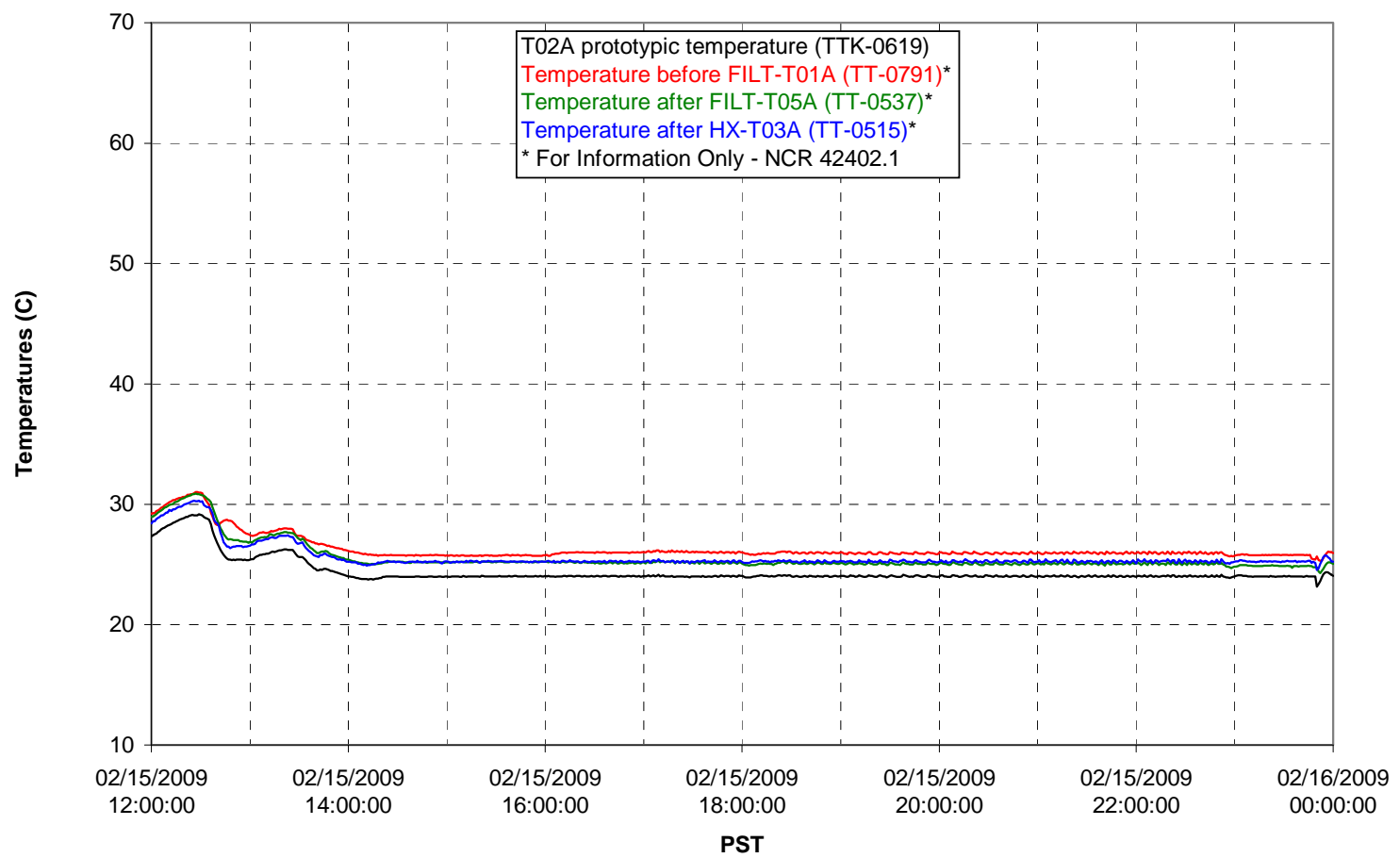

Pump Pressures and Flow

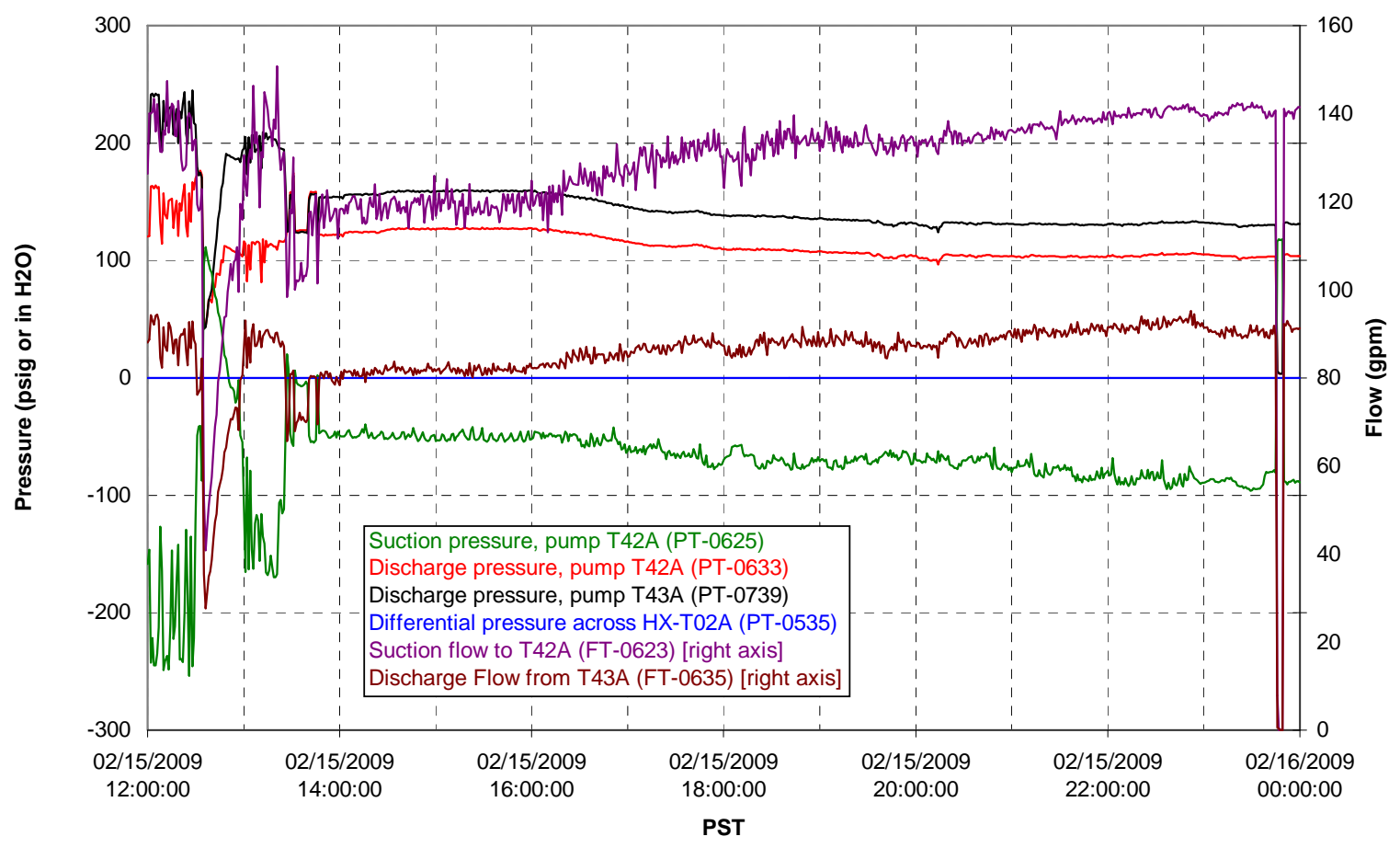


Axial pressure drop

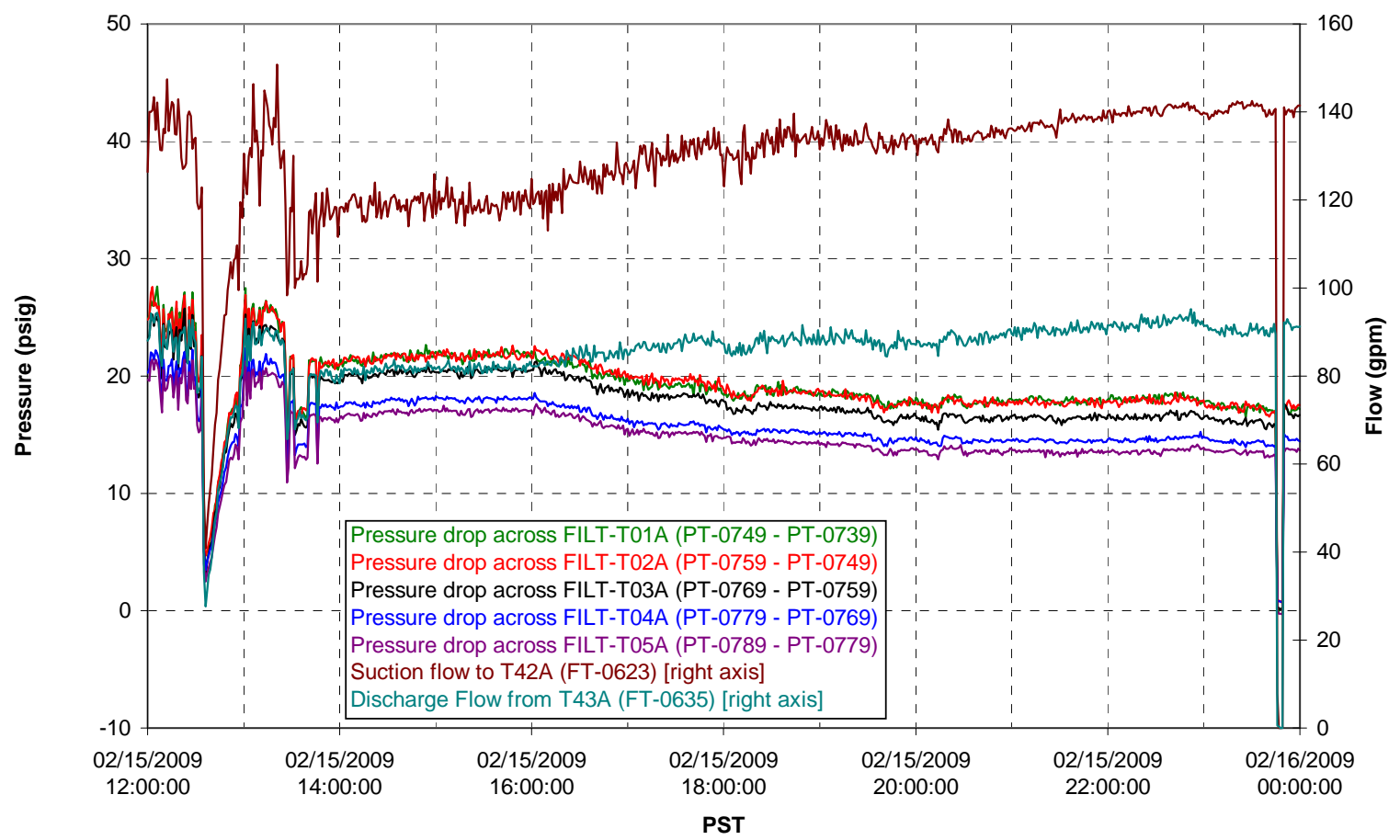

Permeate flow rates

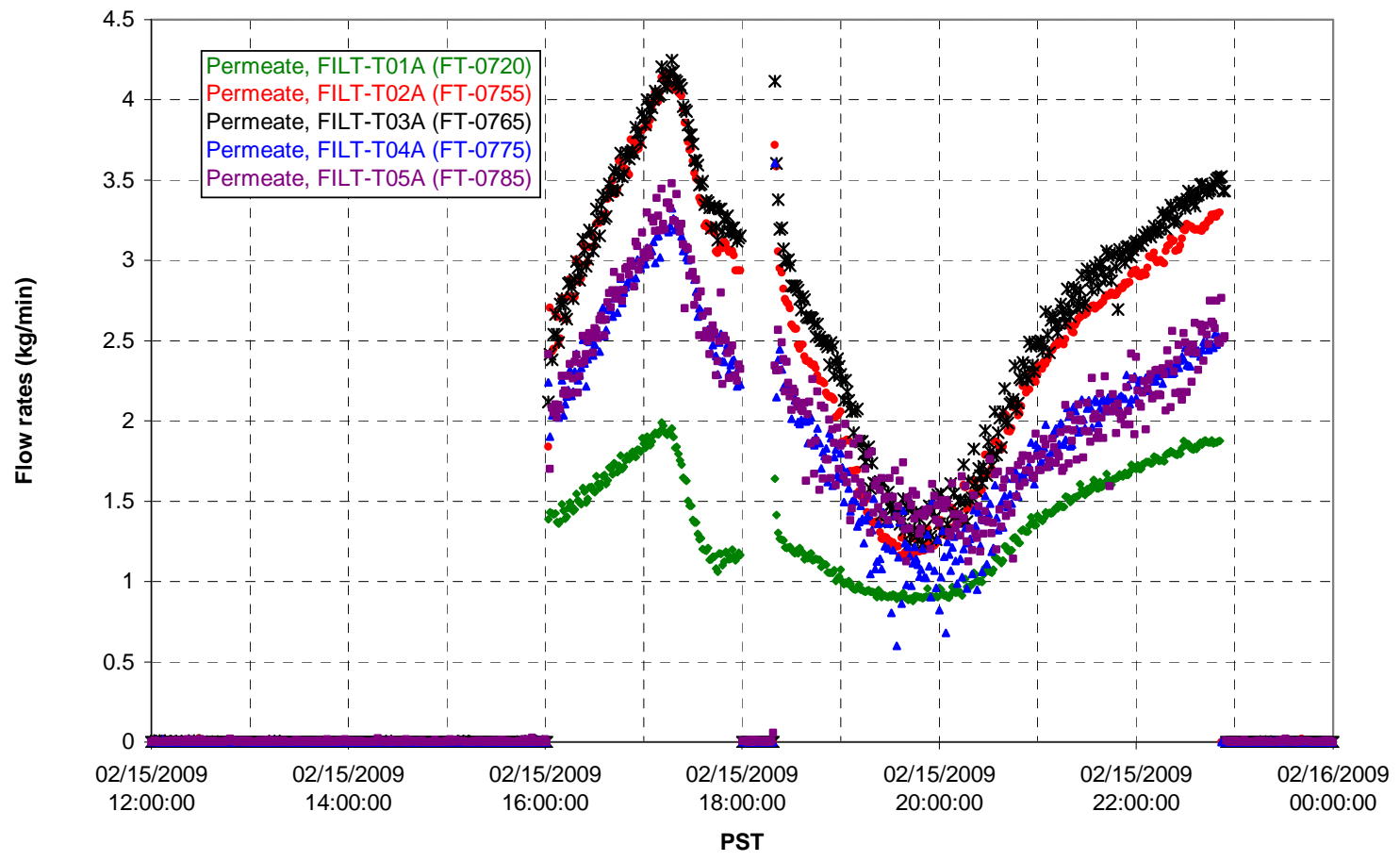


T02A Inner Temperature Tree

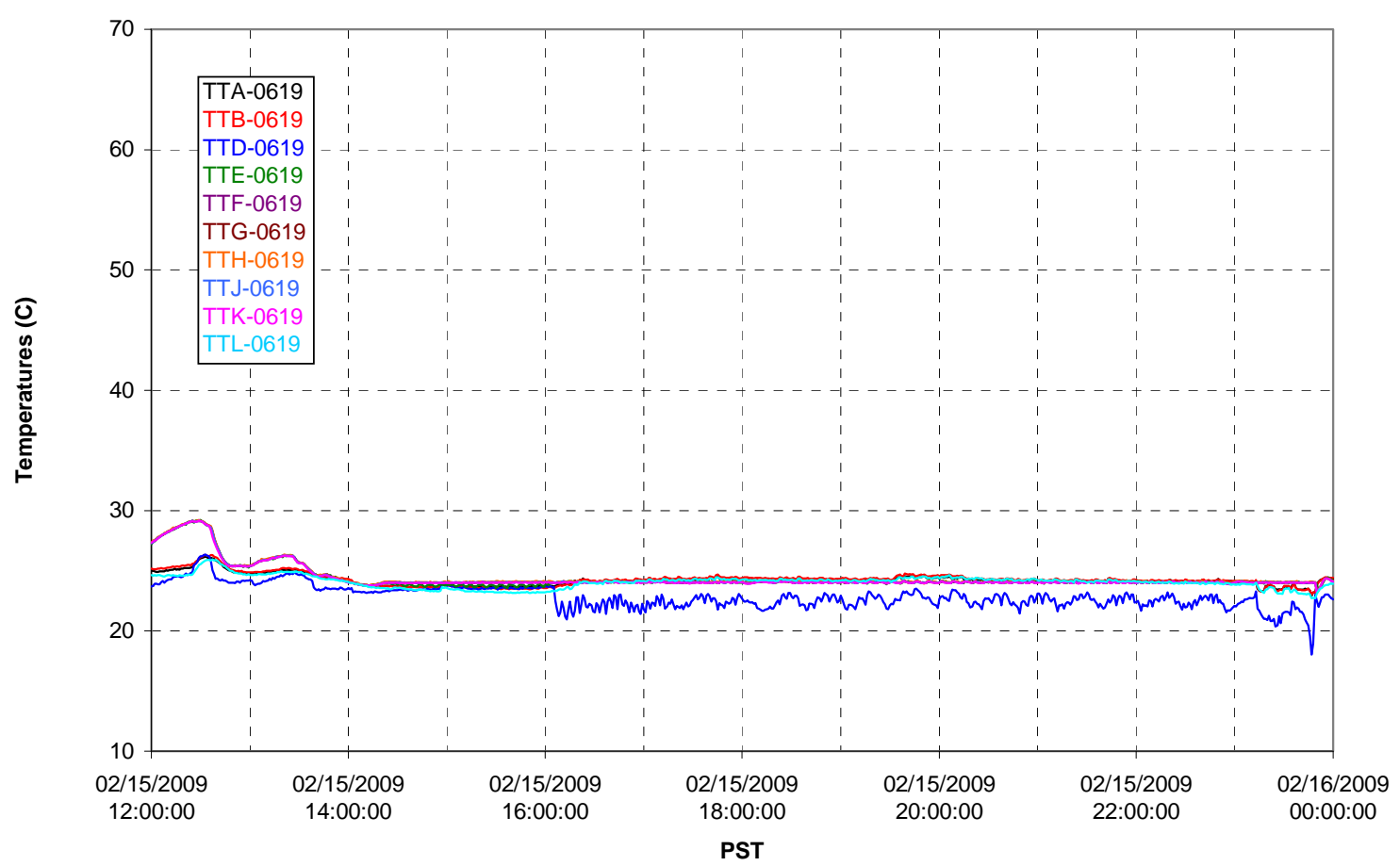

T02A Outer Temperature Tree

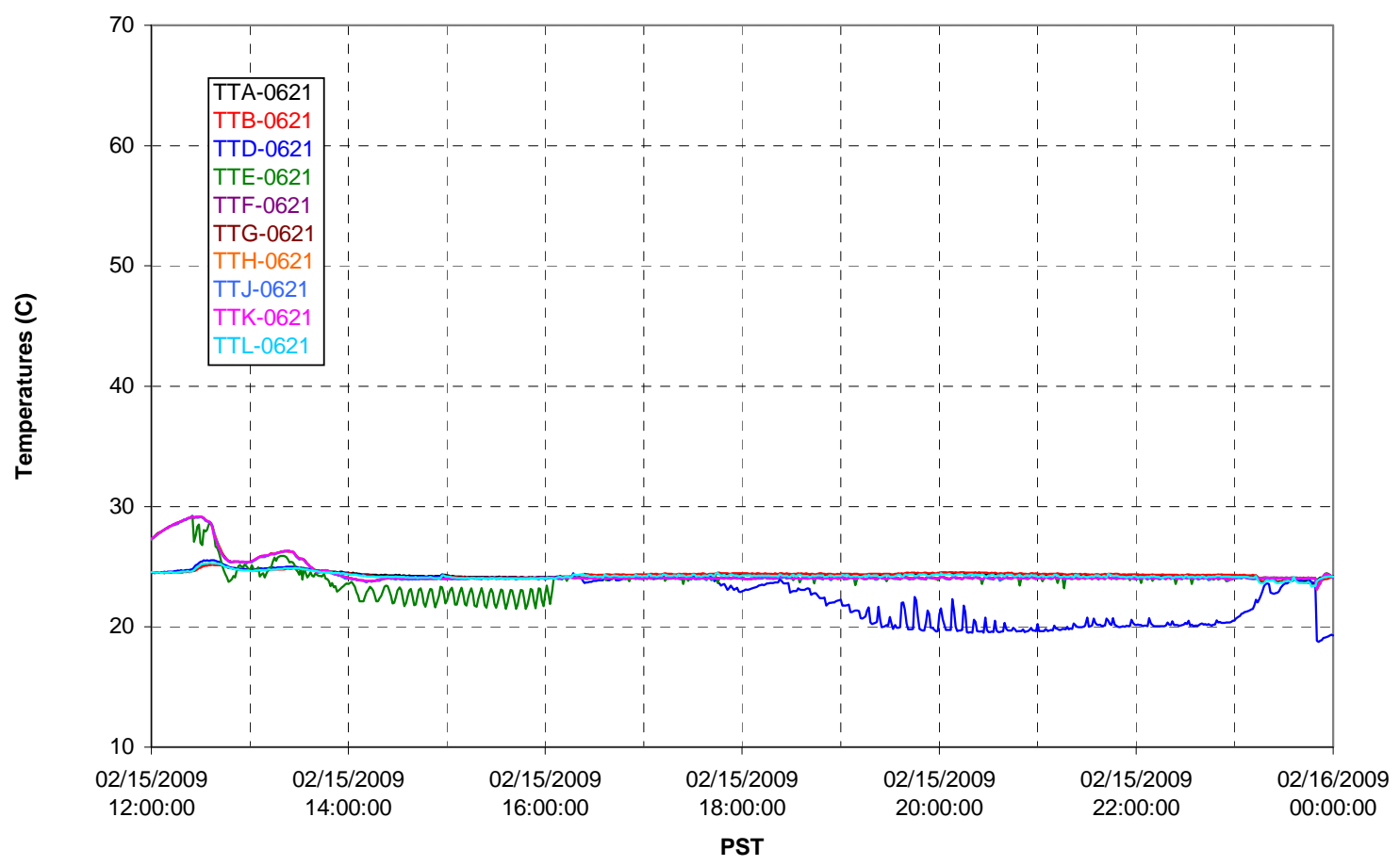


T02A temperatures

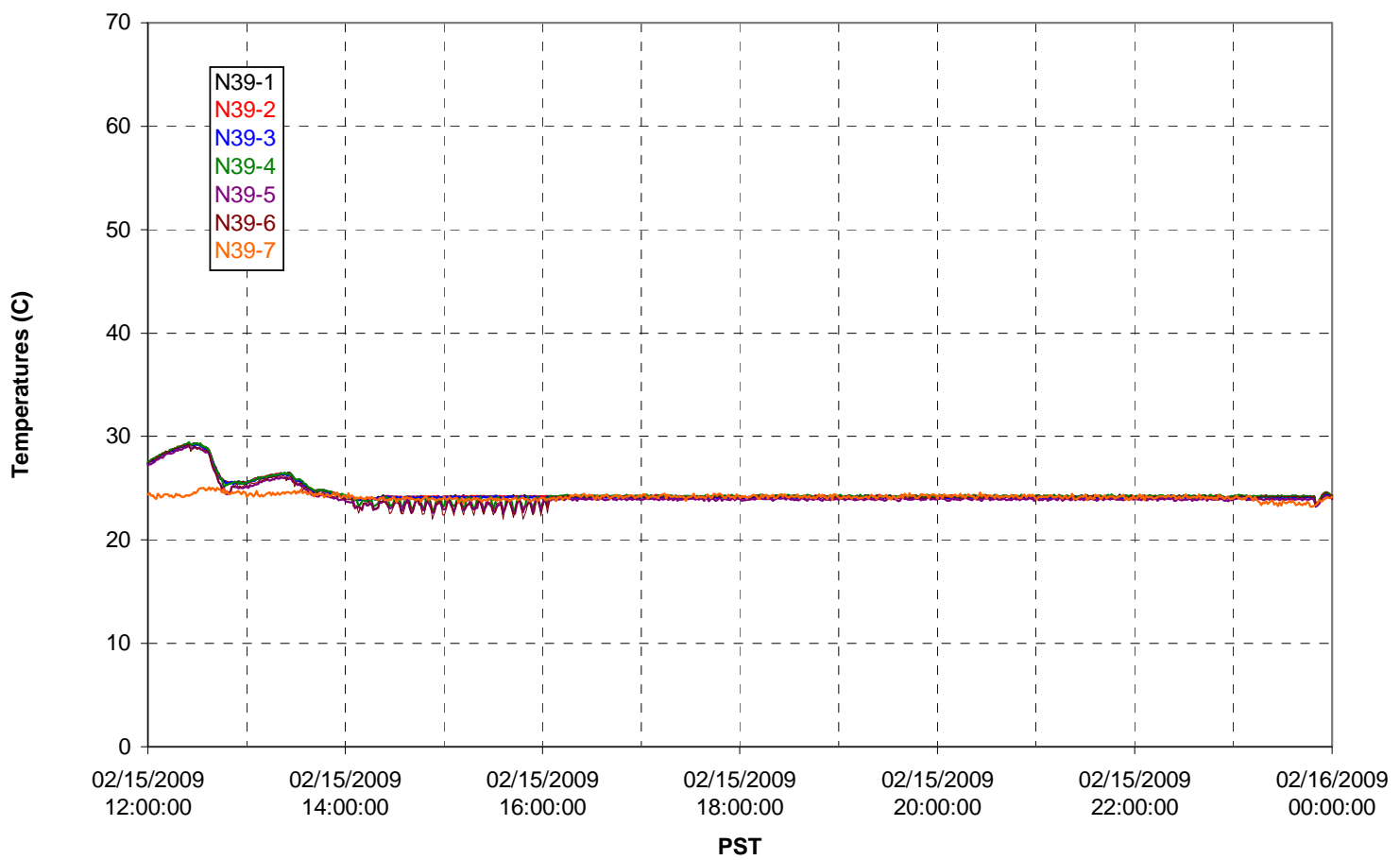

T02A temperatures

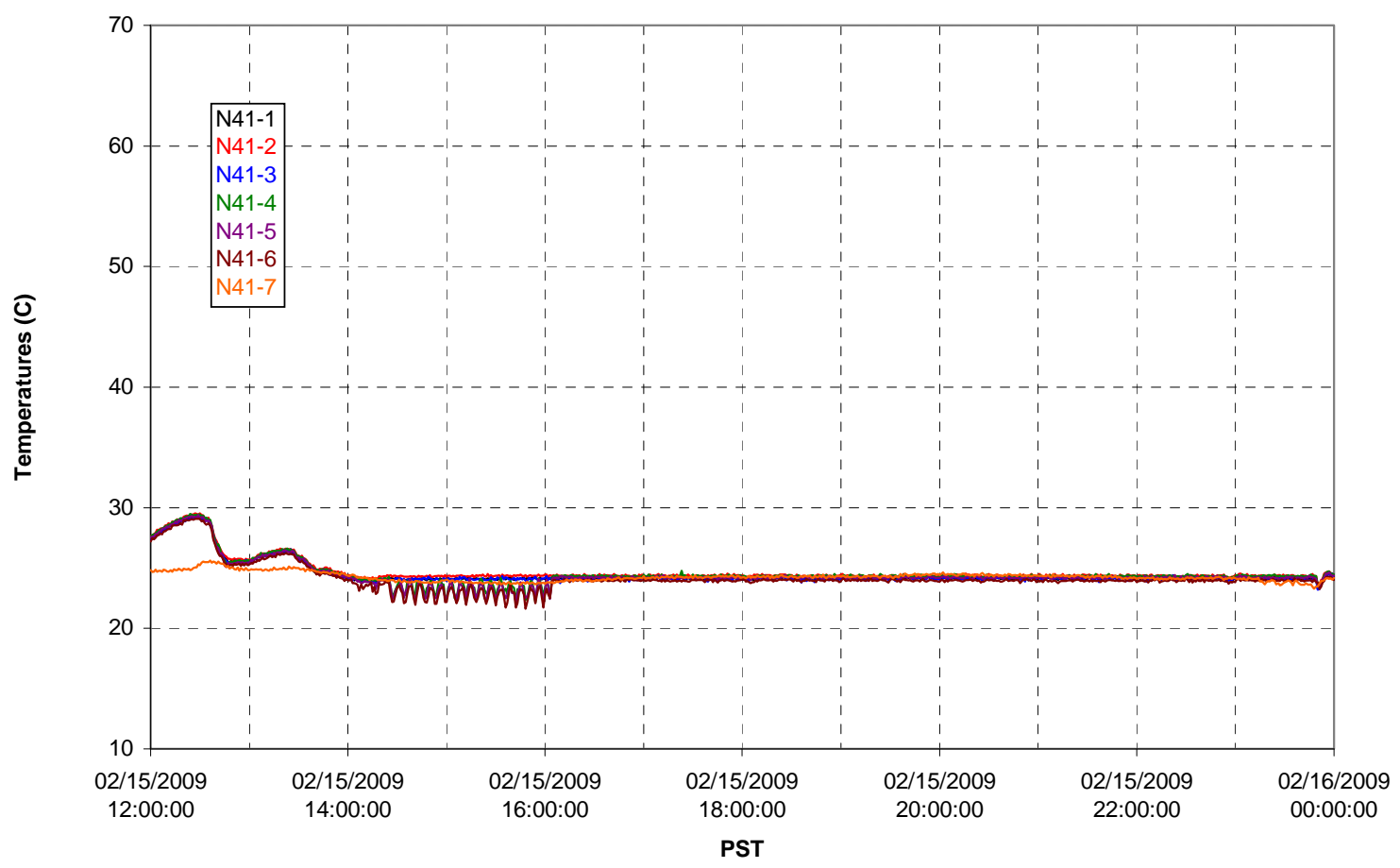


T02A temperatures

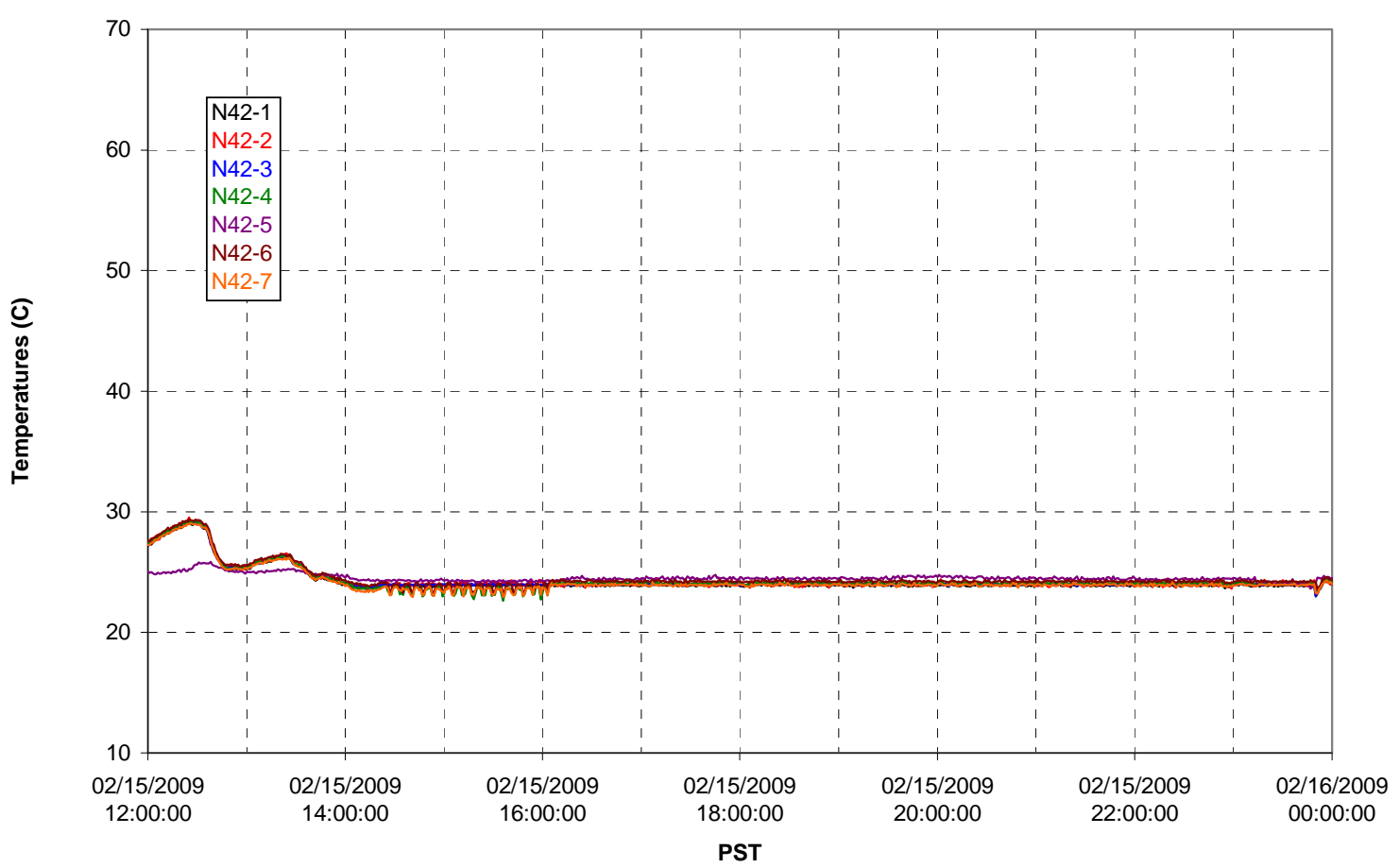

T02A temperatures

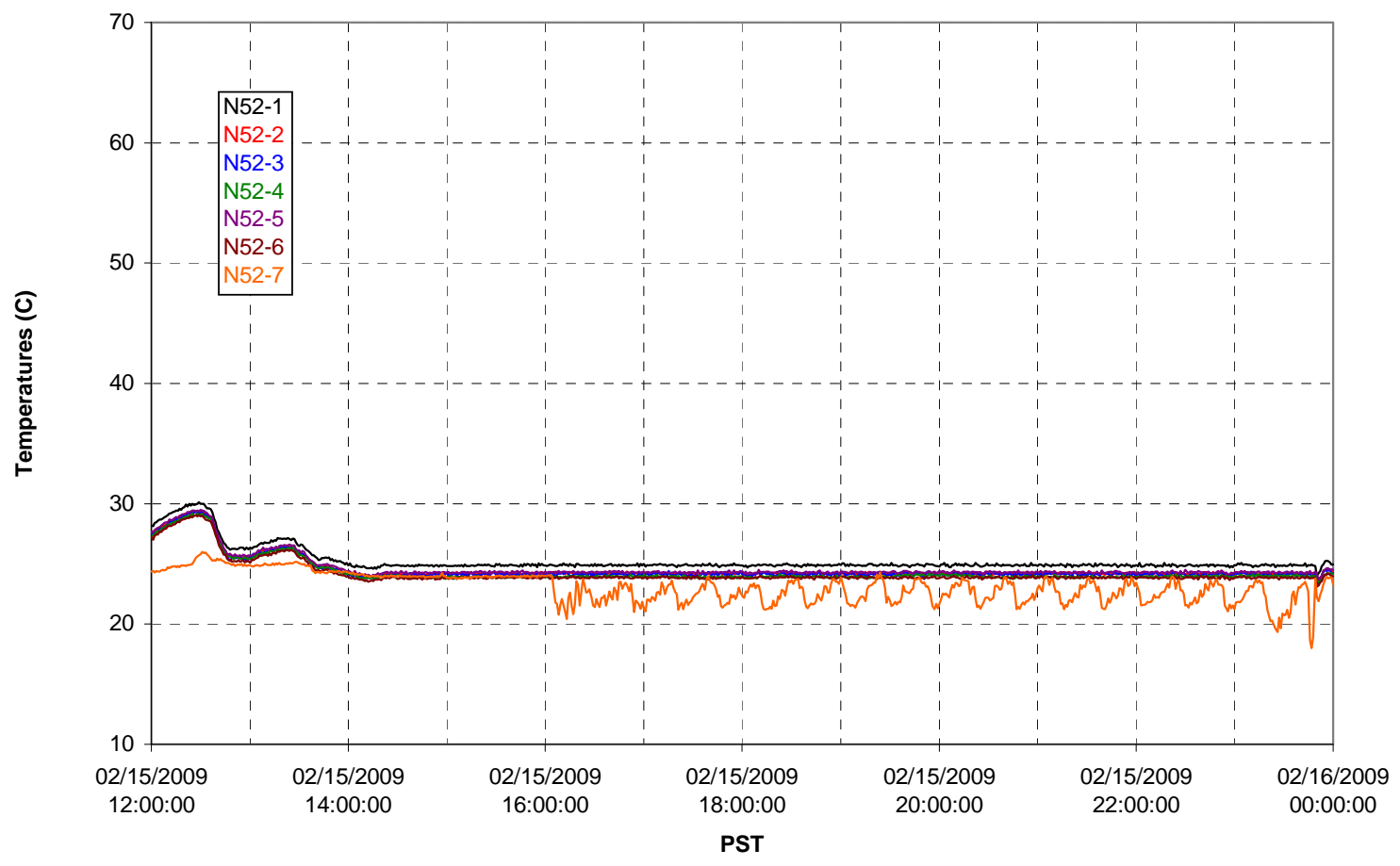


T02A Heating and Cooling
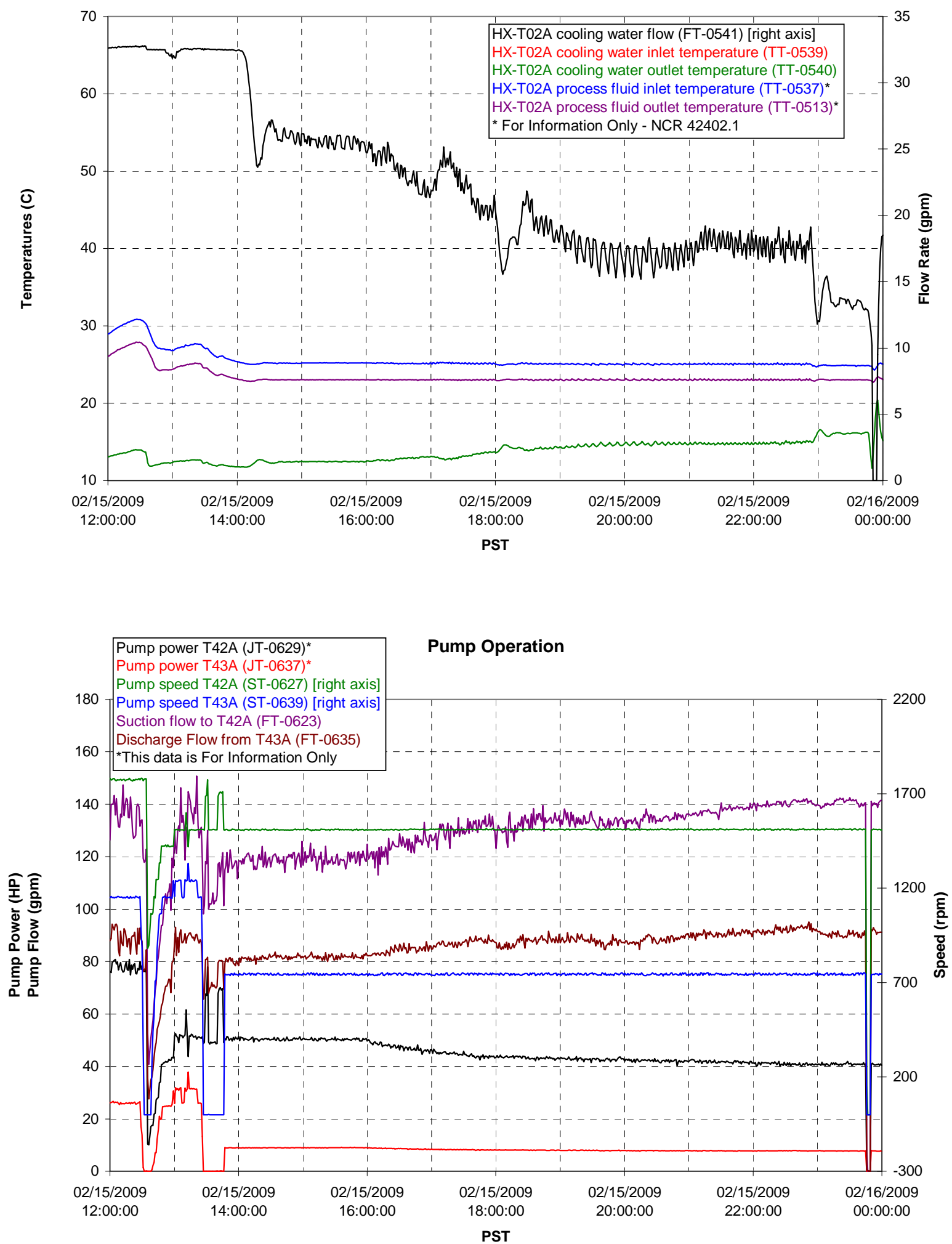
Pulsepot UFP-PP-T01A

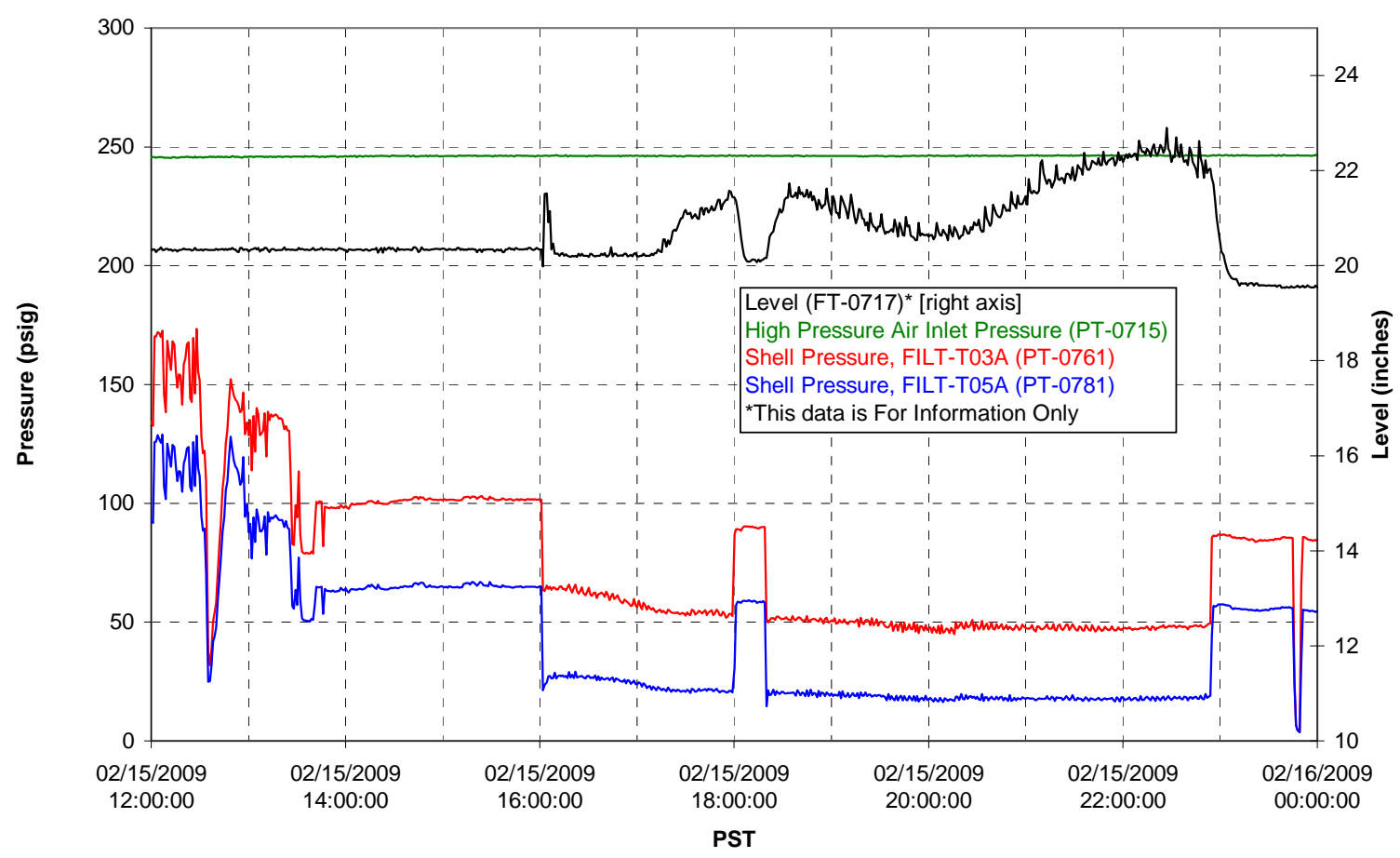

Pulsepot UFP-PP-T02A

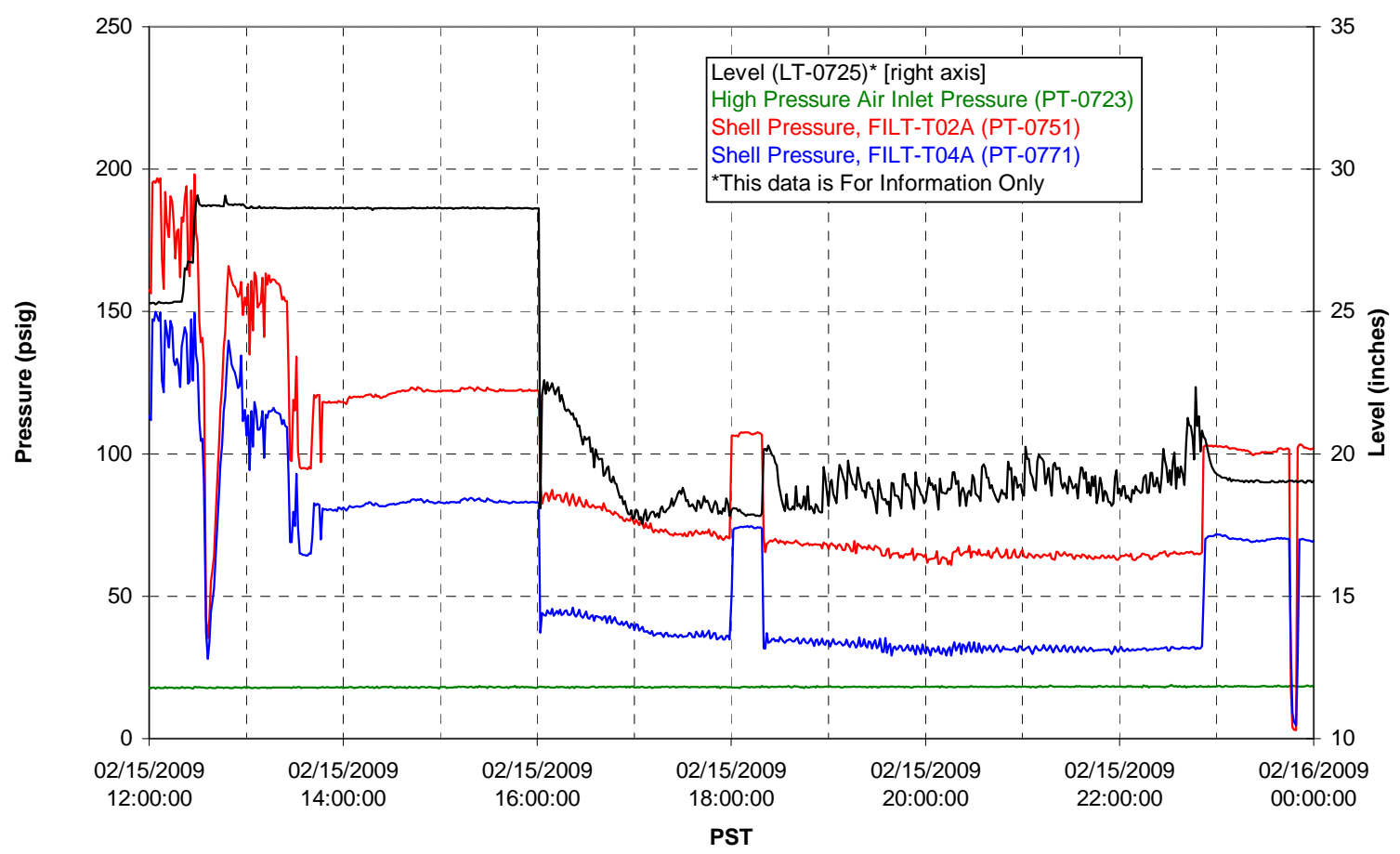


Pulsepot UFP-PP-T03A

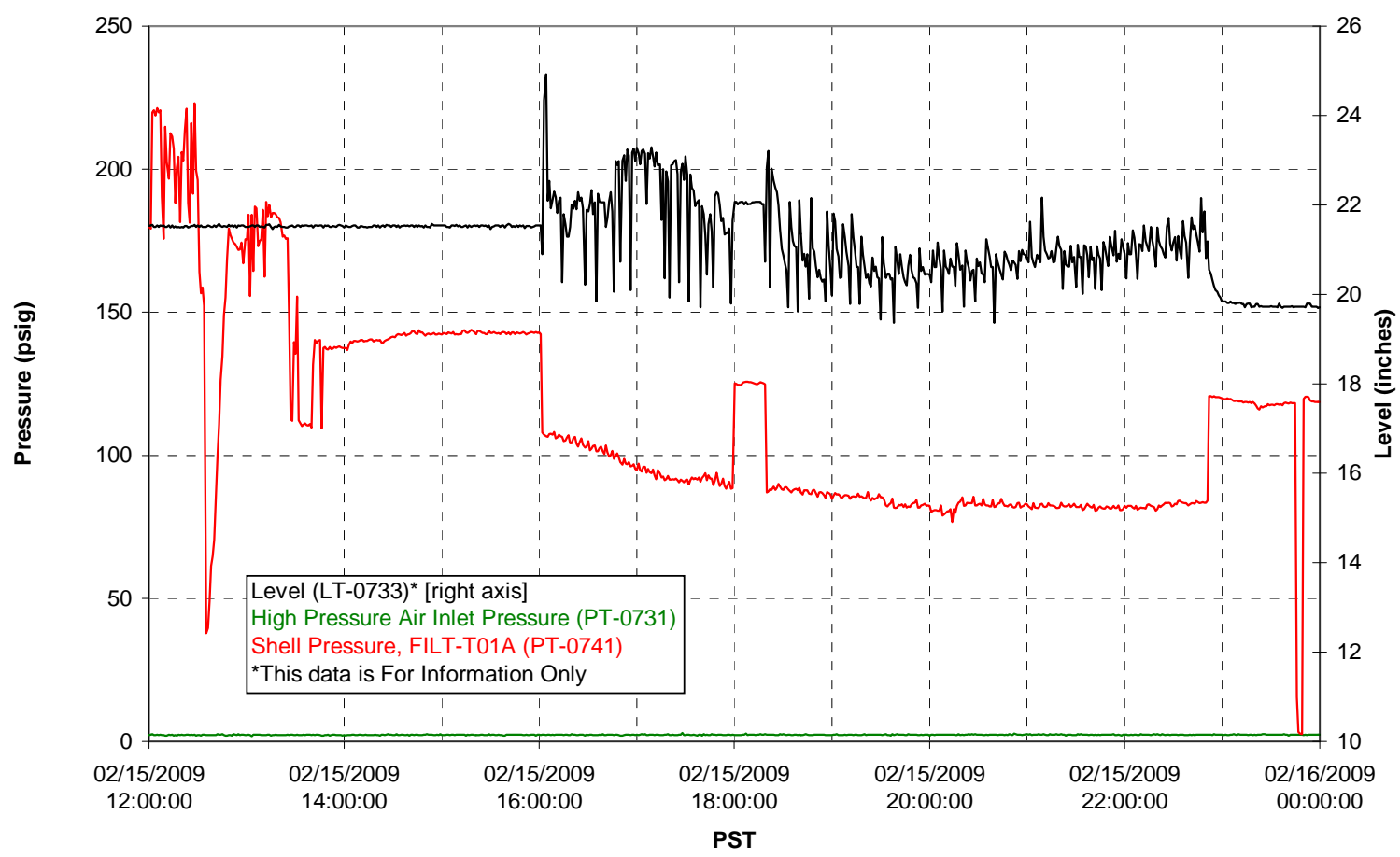

Pulsepot Levels

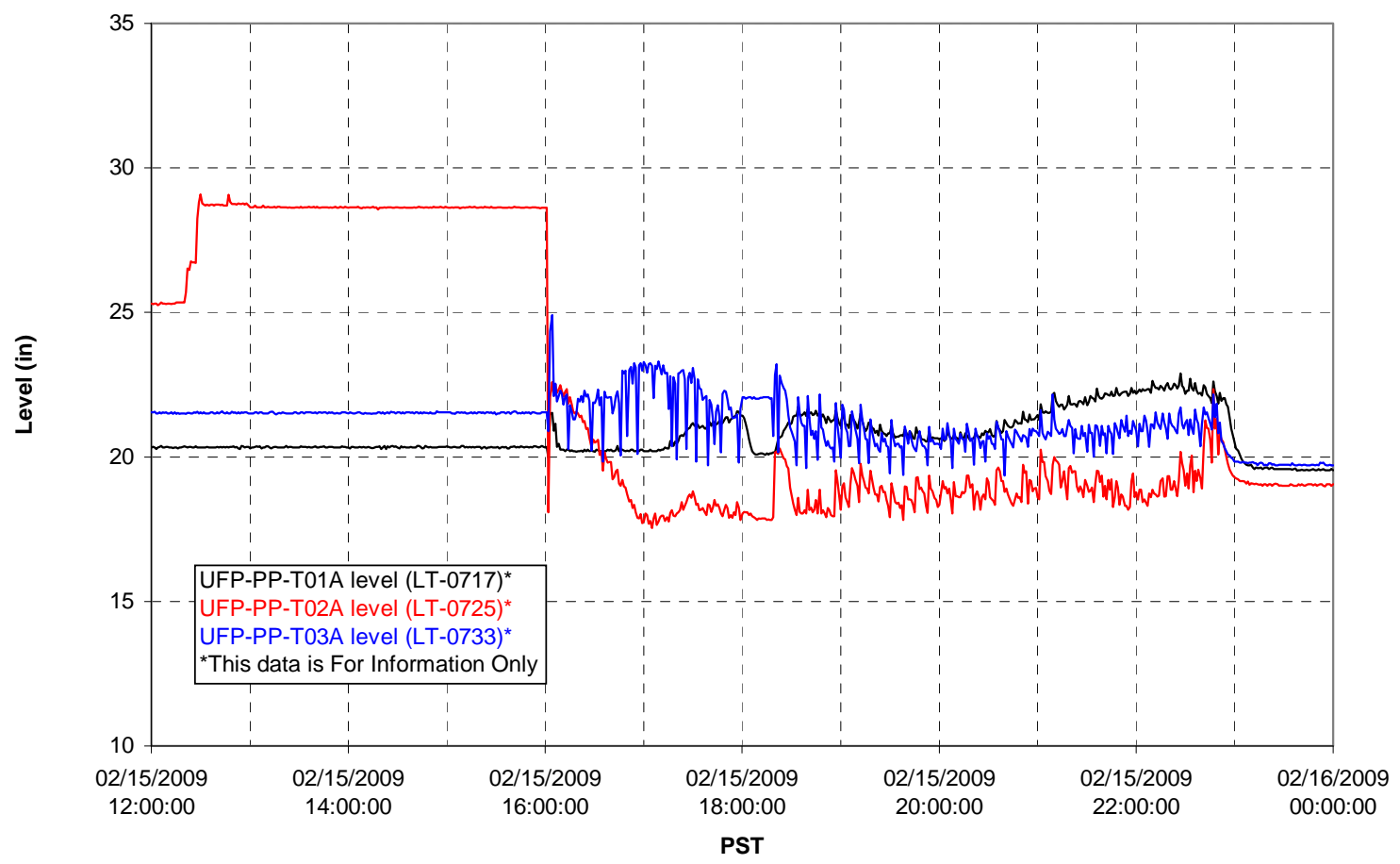


Filter UFP-FILT-T01A

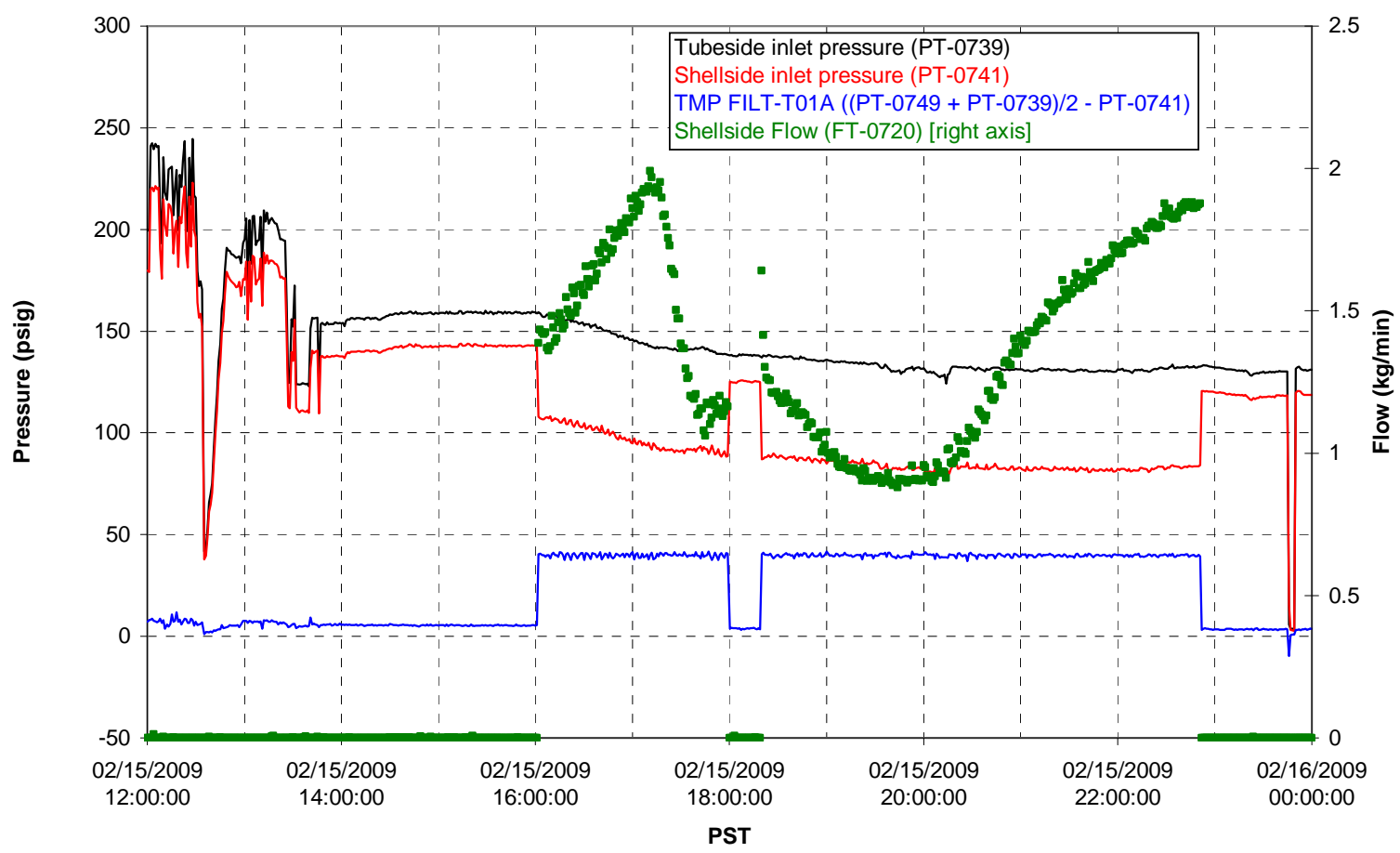

Filter UFP-FILT-T02A

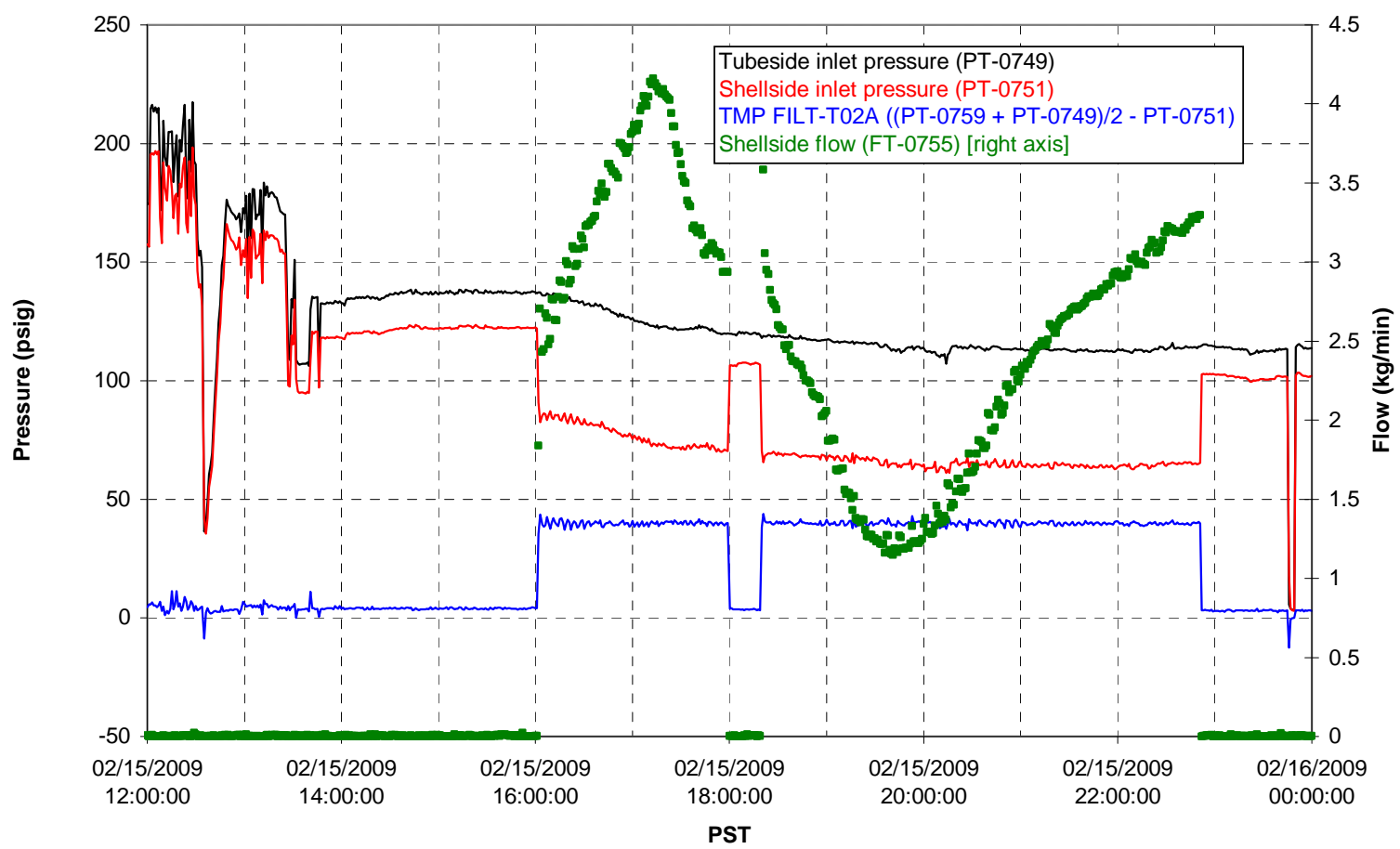


Filter UFP-FILT-T03A

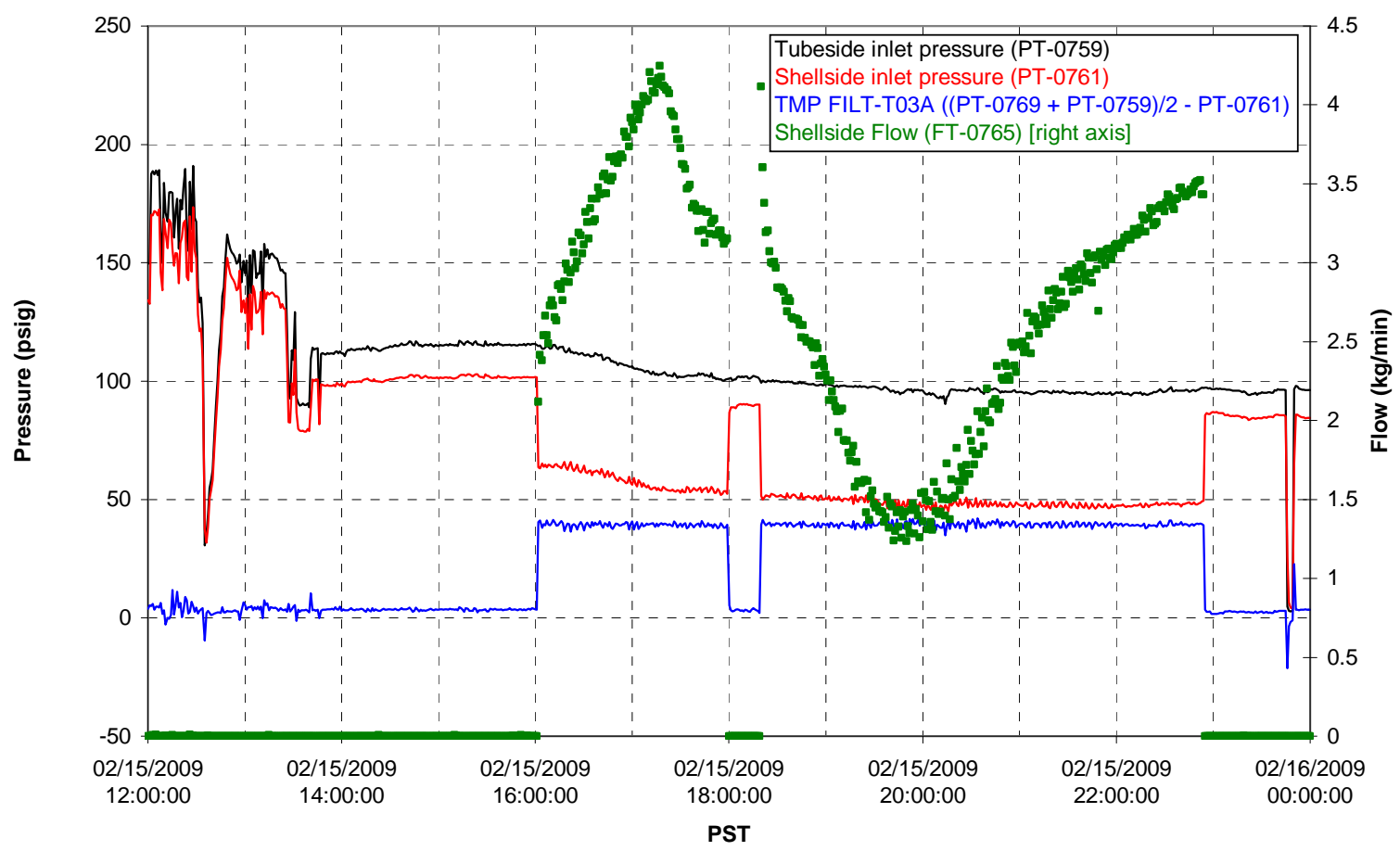

Filter UFP-FILT-T04A

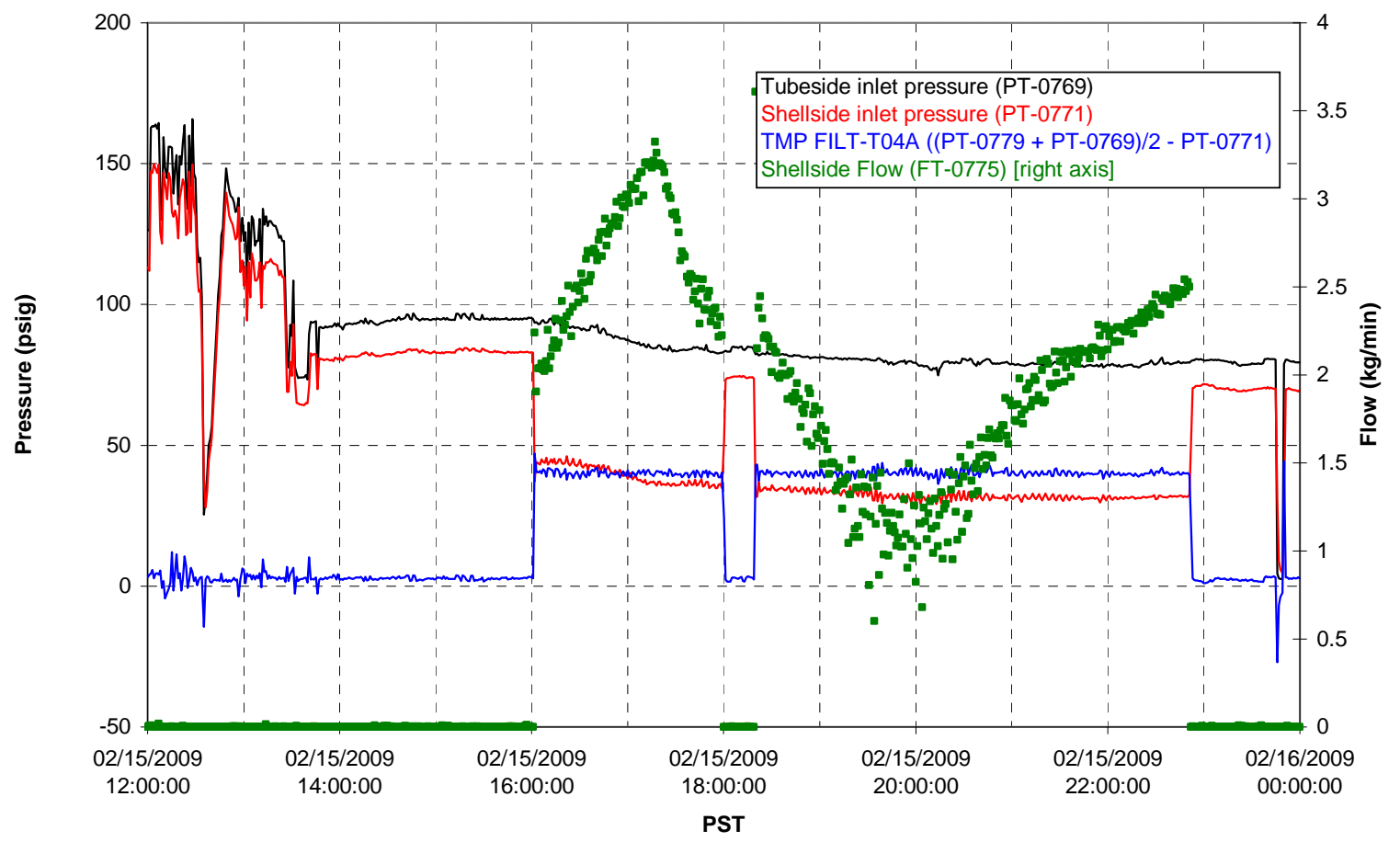


Filter UFP-FILT-T05A

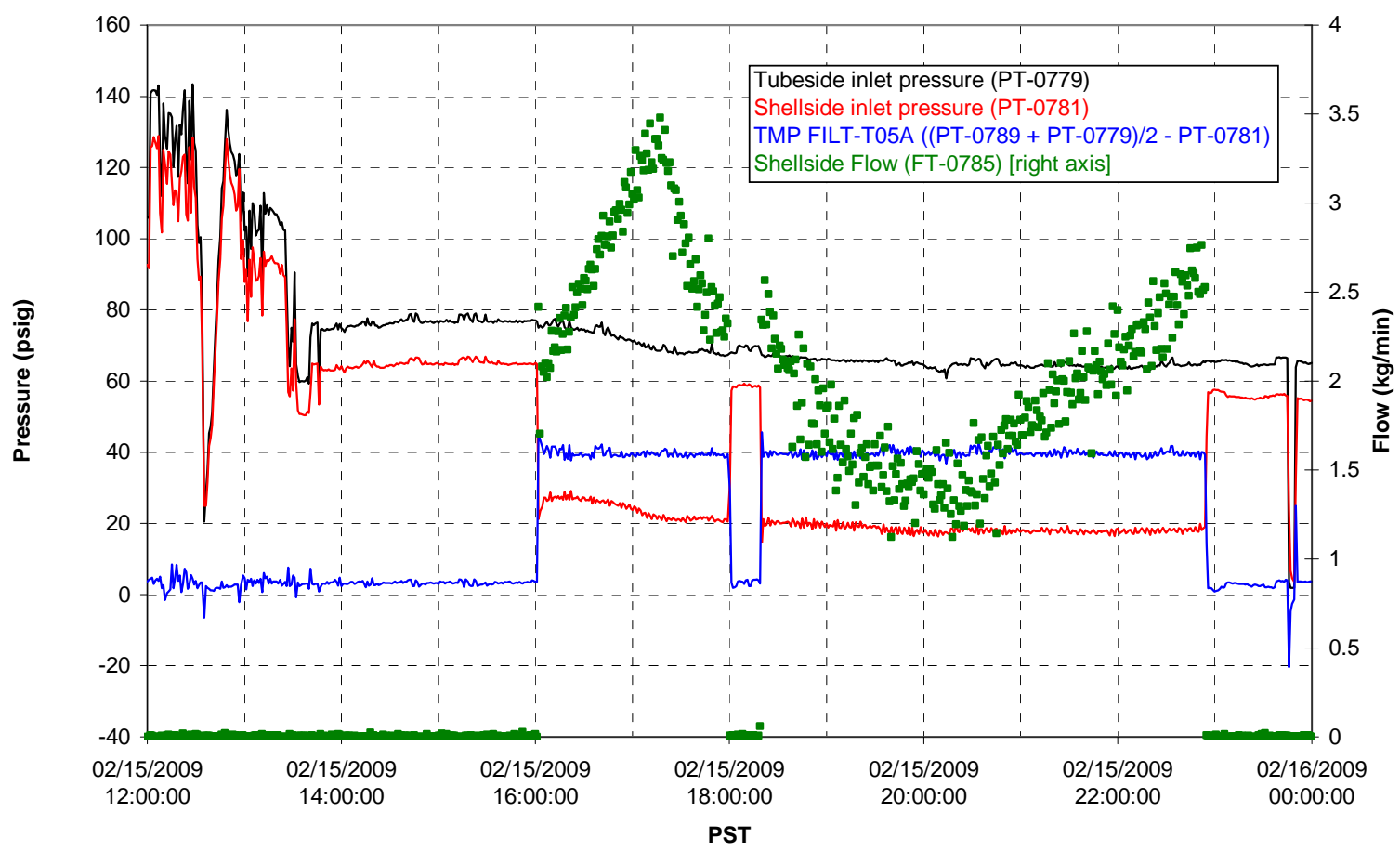

Chemical Flow

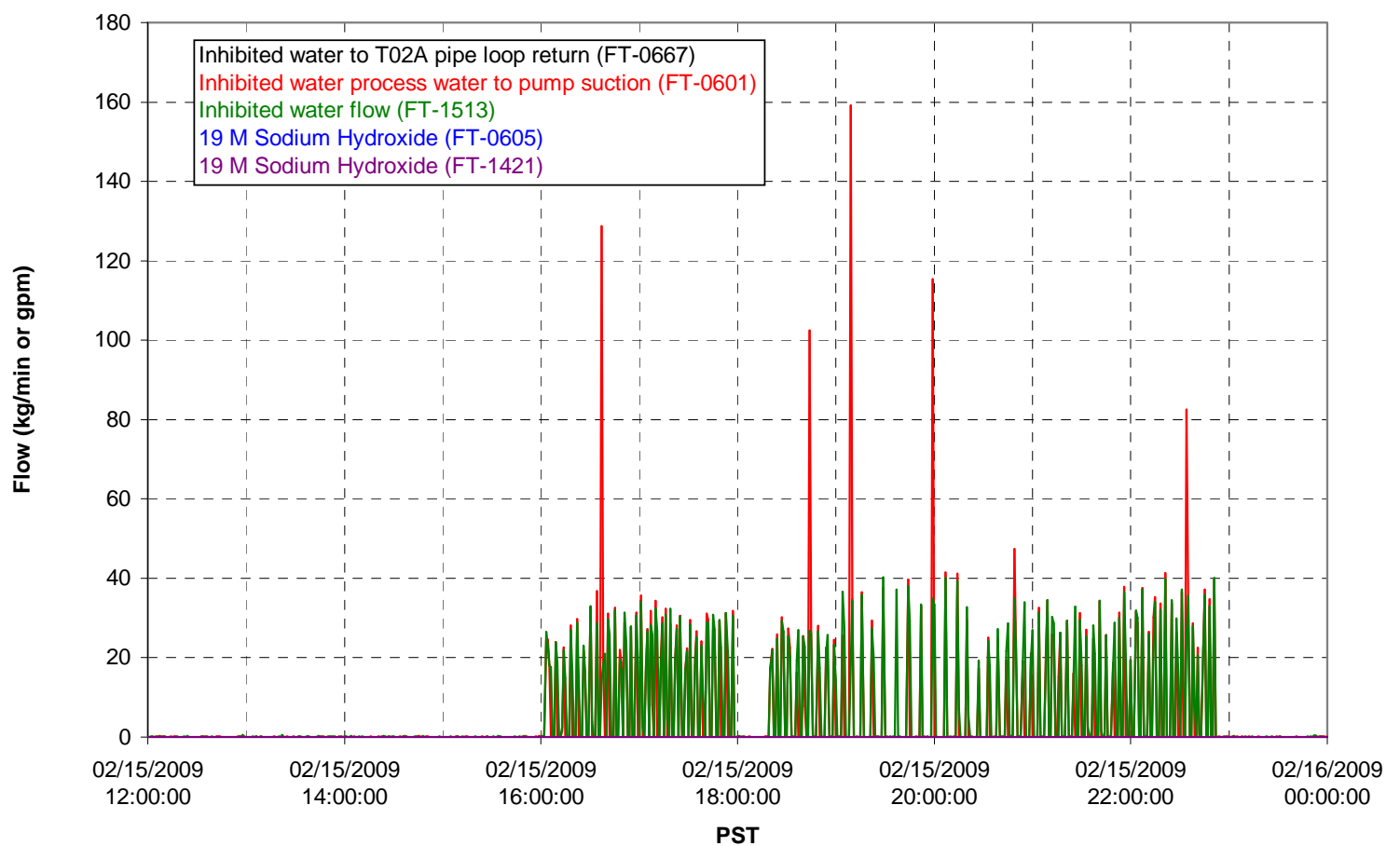


Chemical Flow

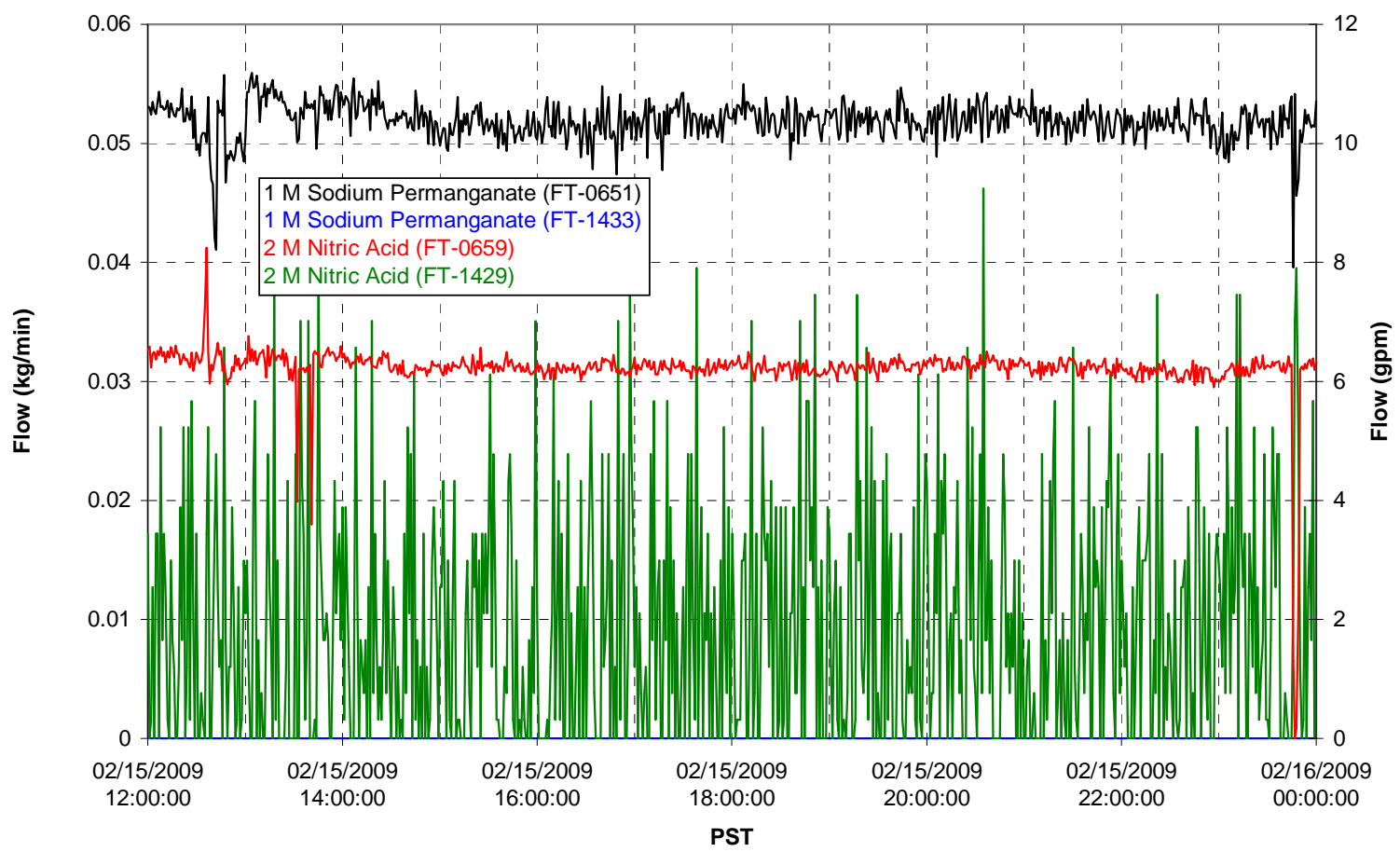

Air Flows

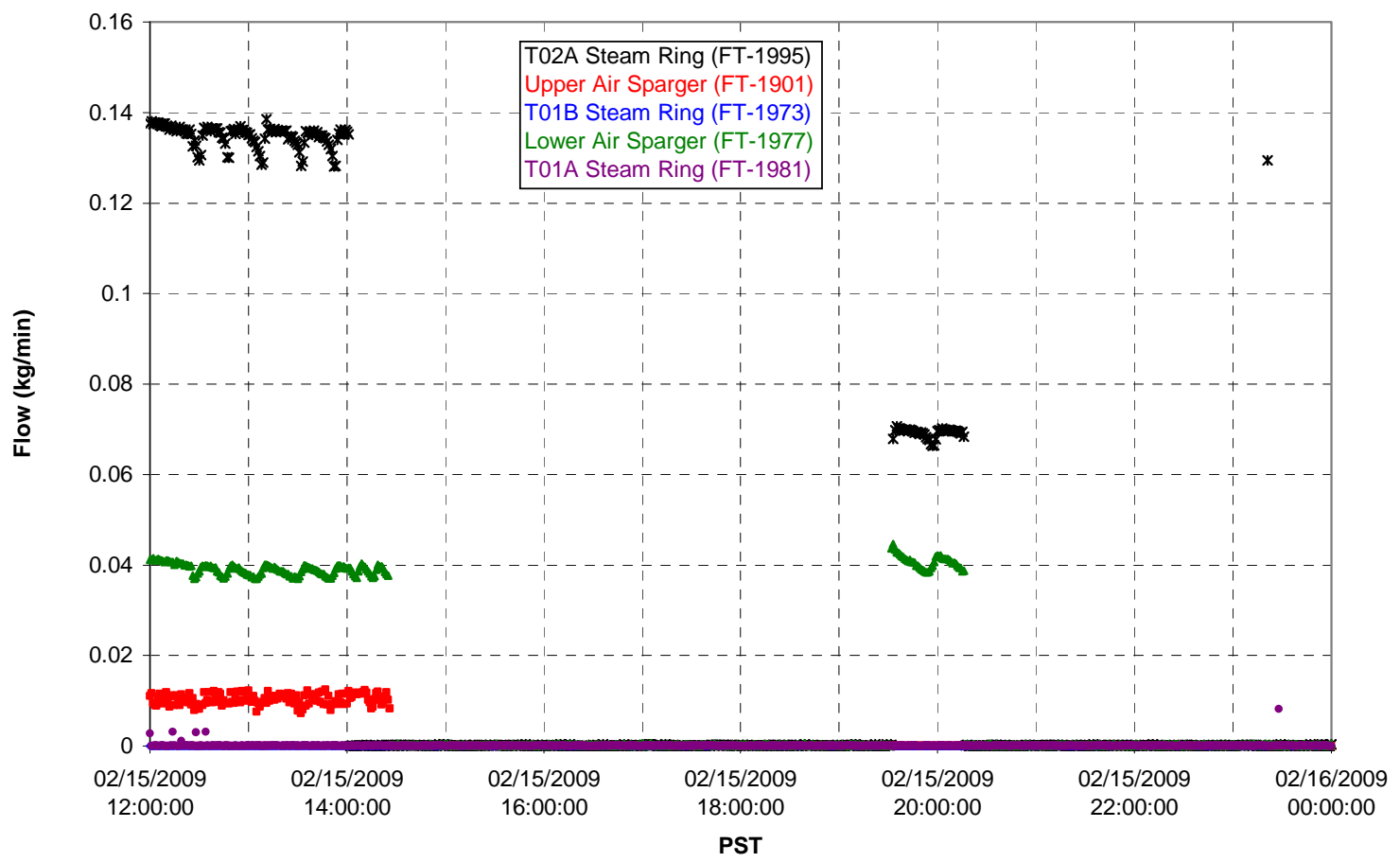


T02A Steam

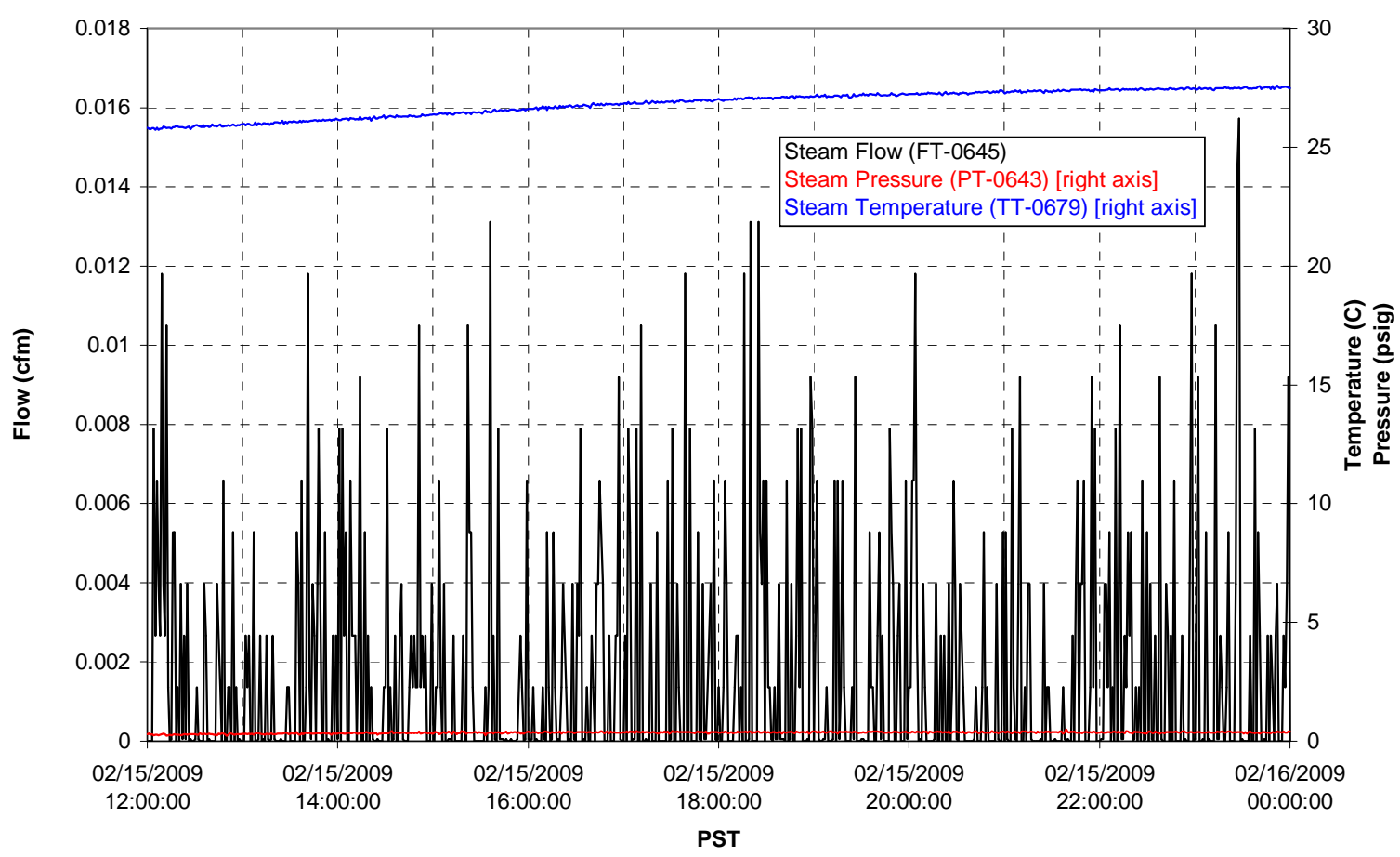

T01A Steam

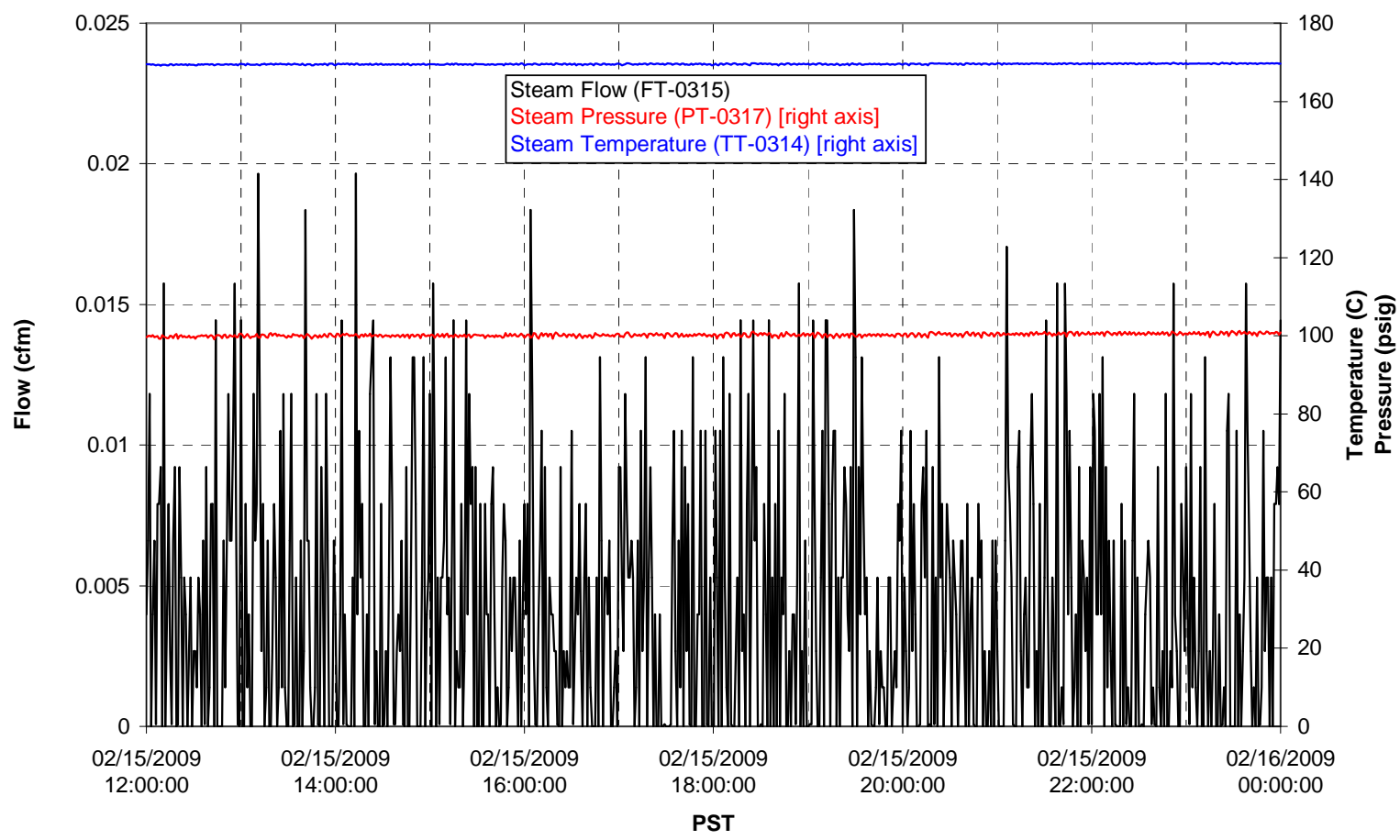


T01B Steam

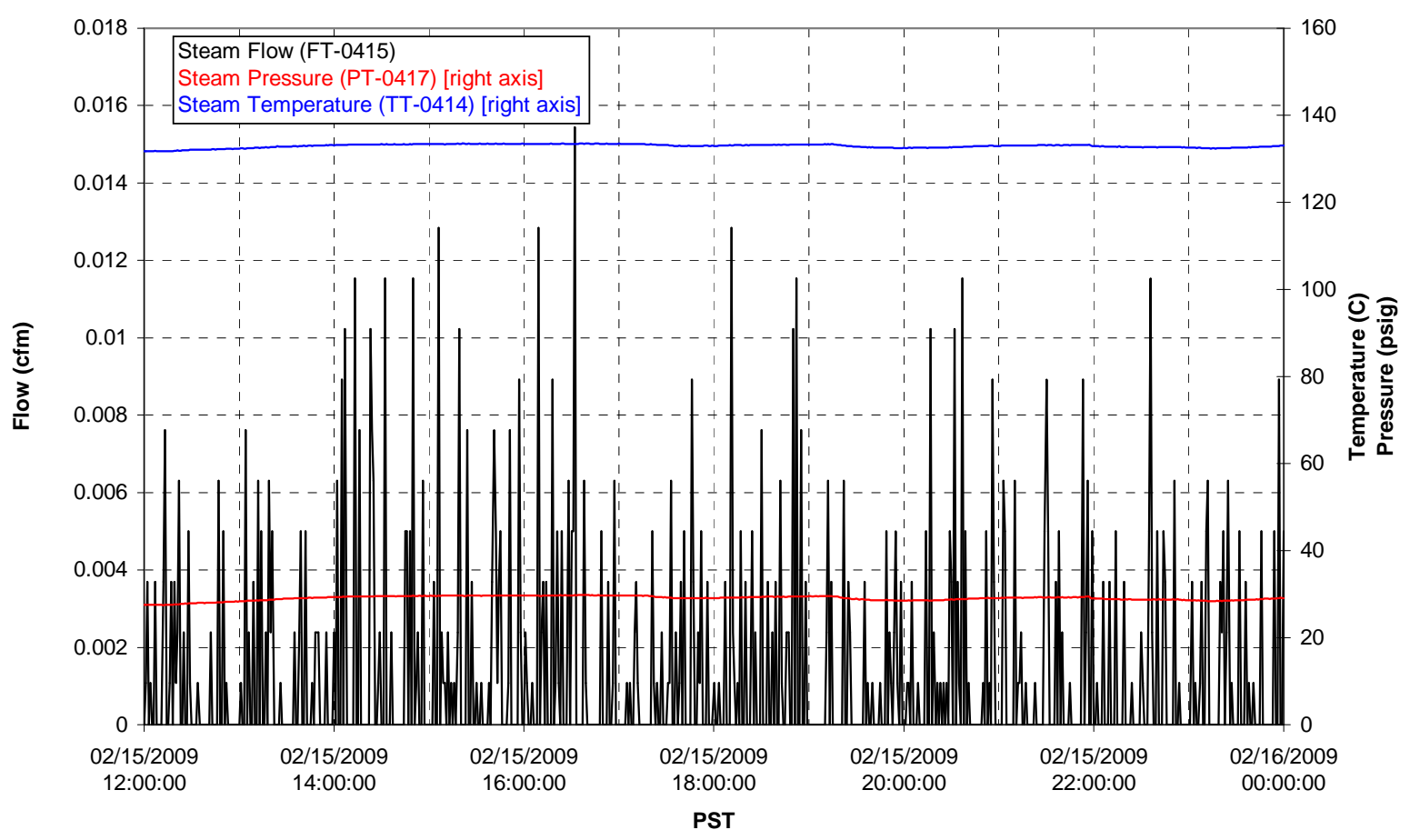




\section{Integrated Test A Data Plots 02/16/09 00:00 - 02/16/09 12:00}


T01A level

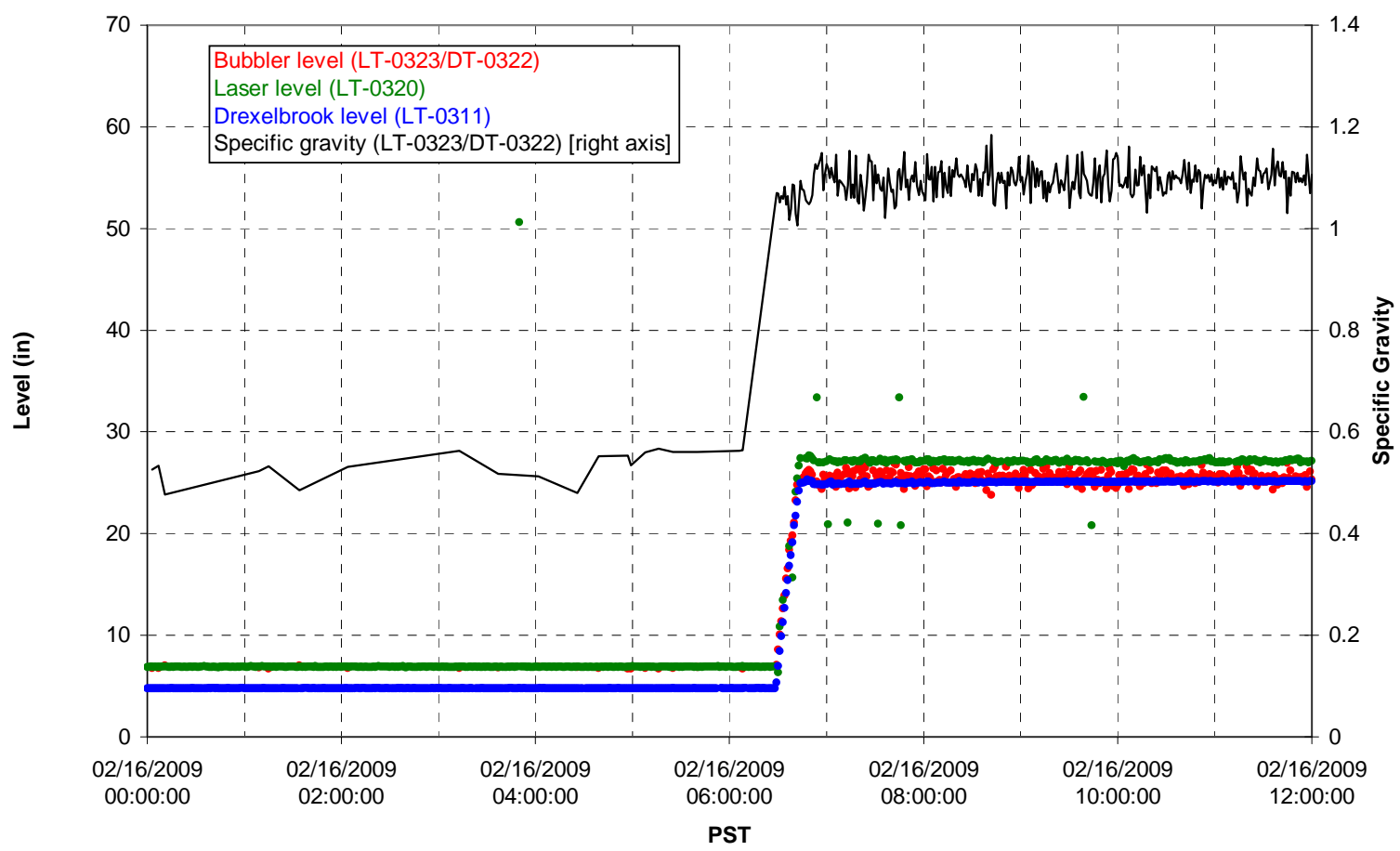

T01A temperatures

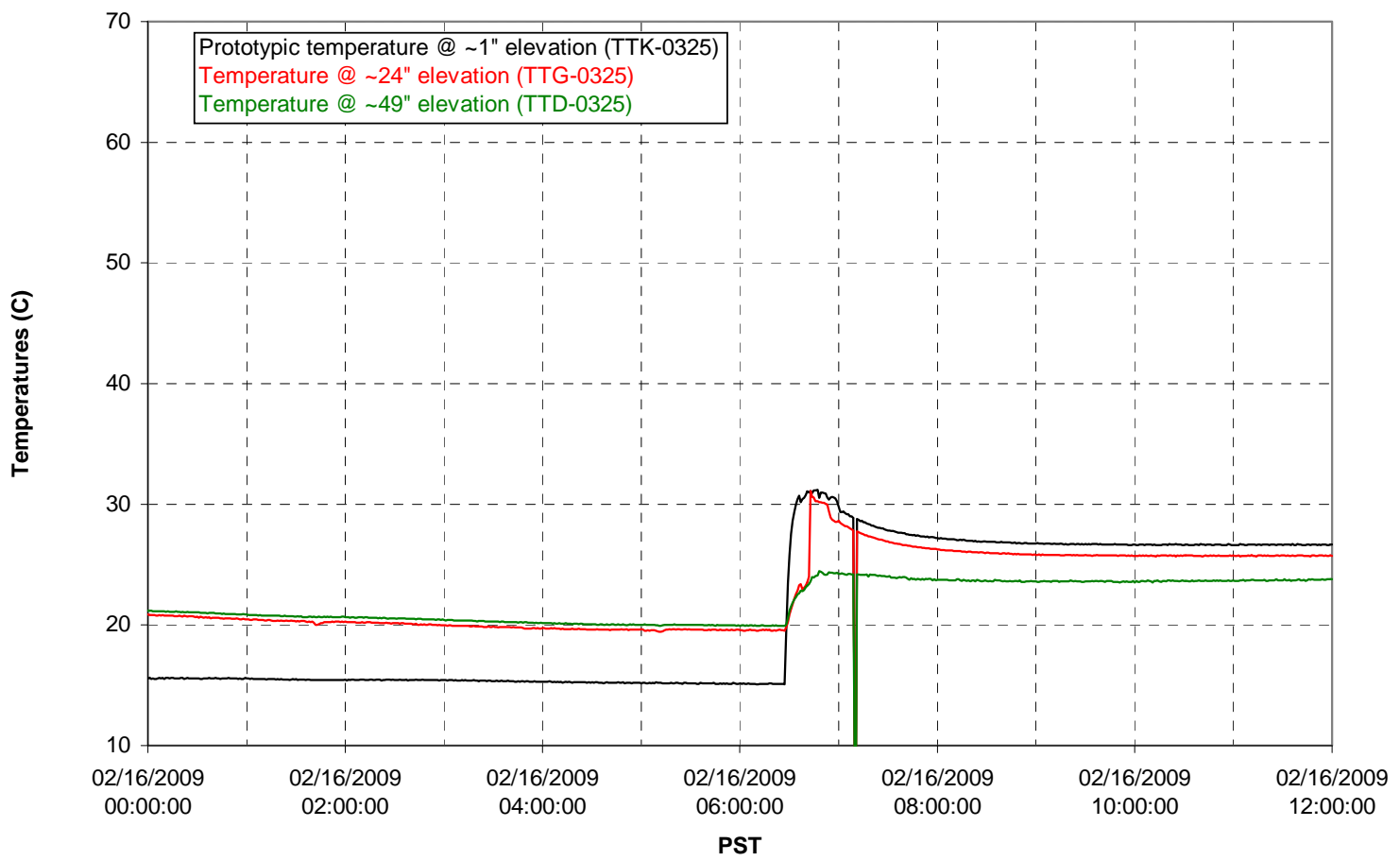


T01B level

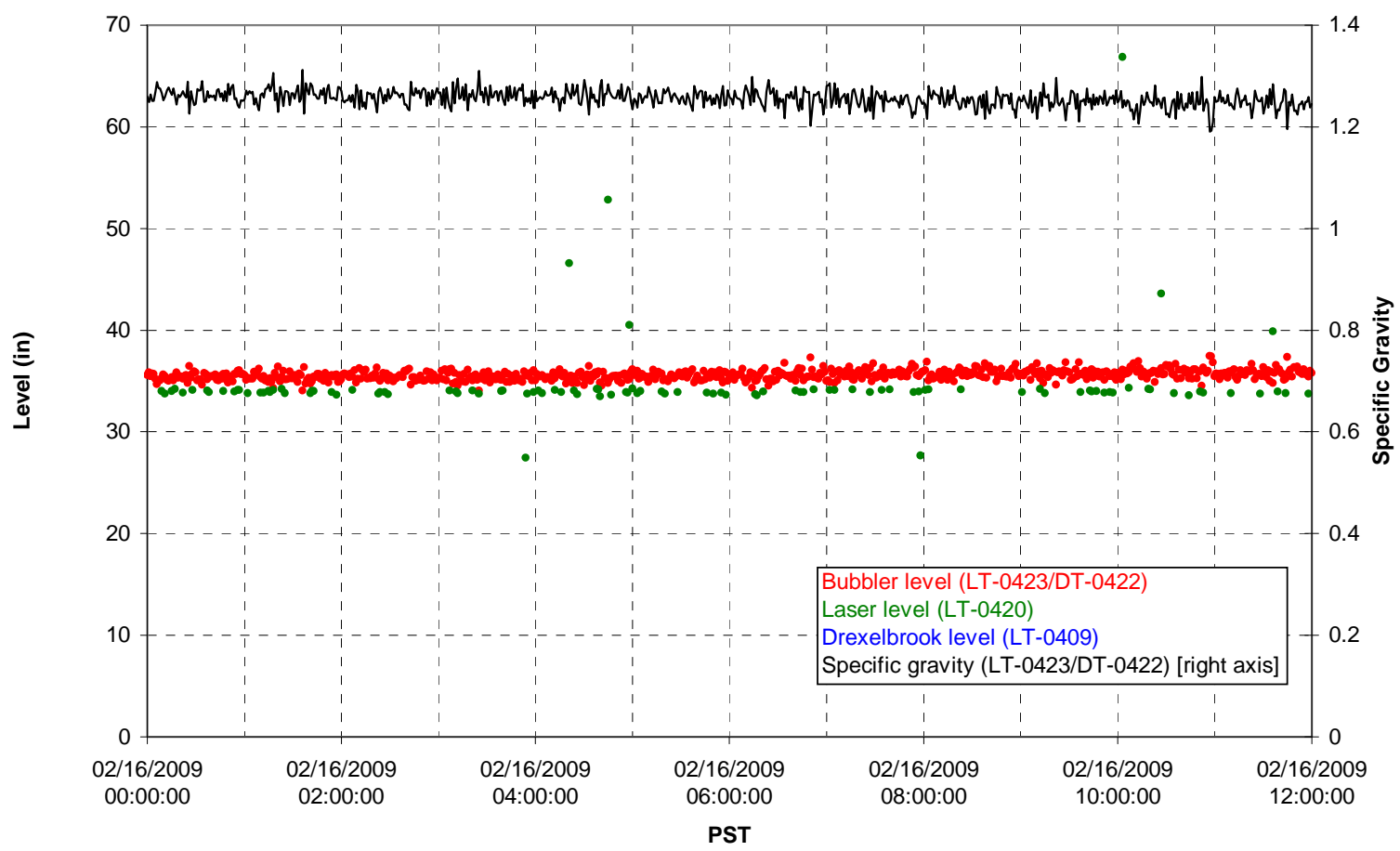

T01B temperatures

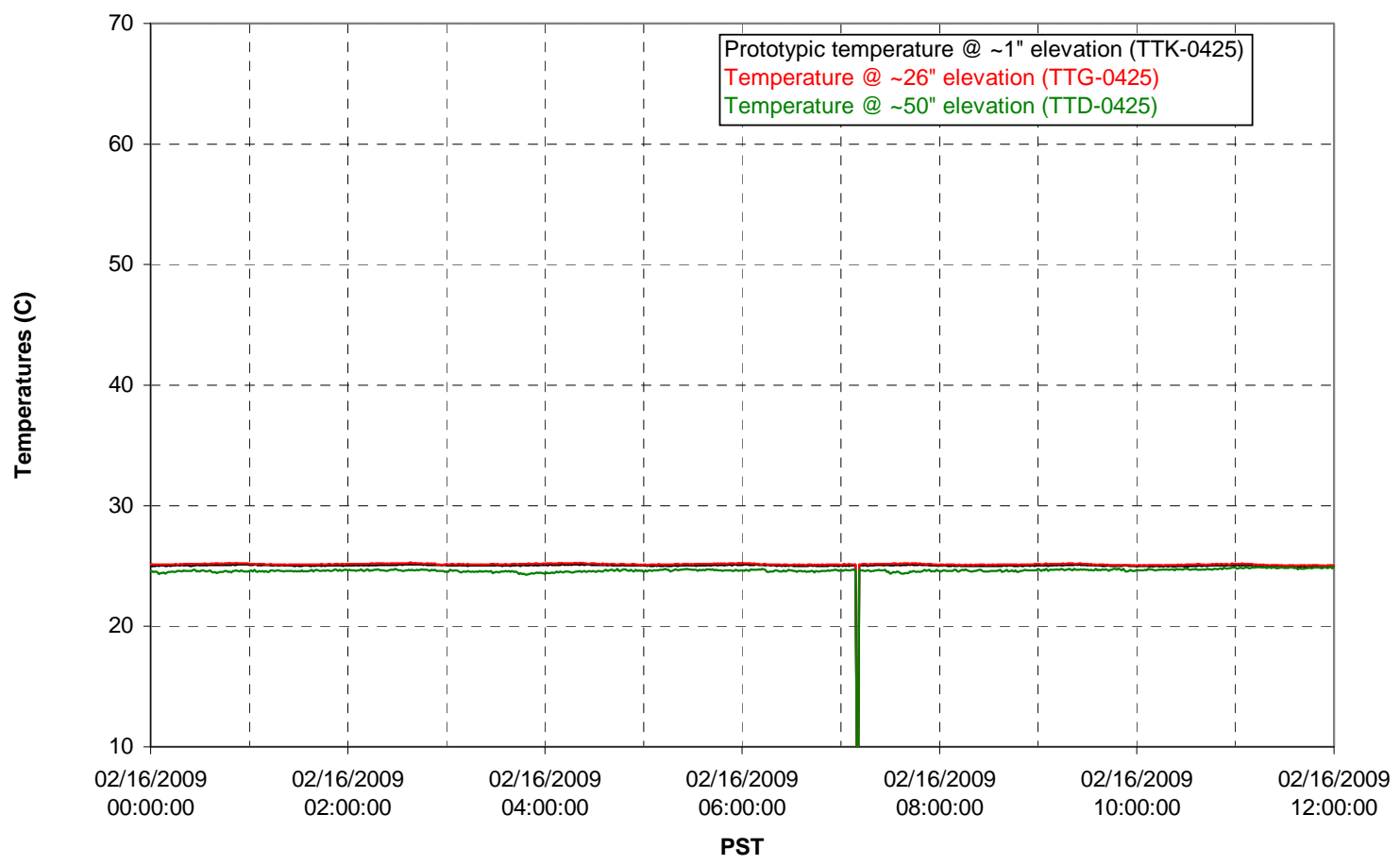


T02A level

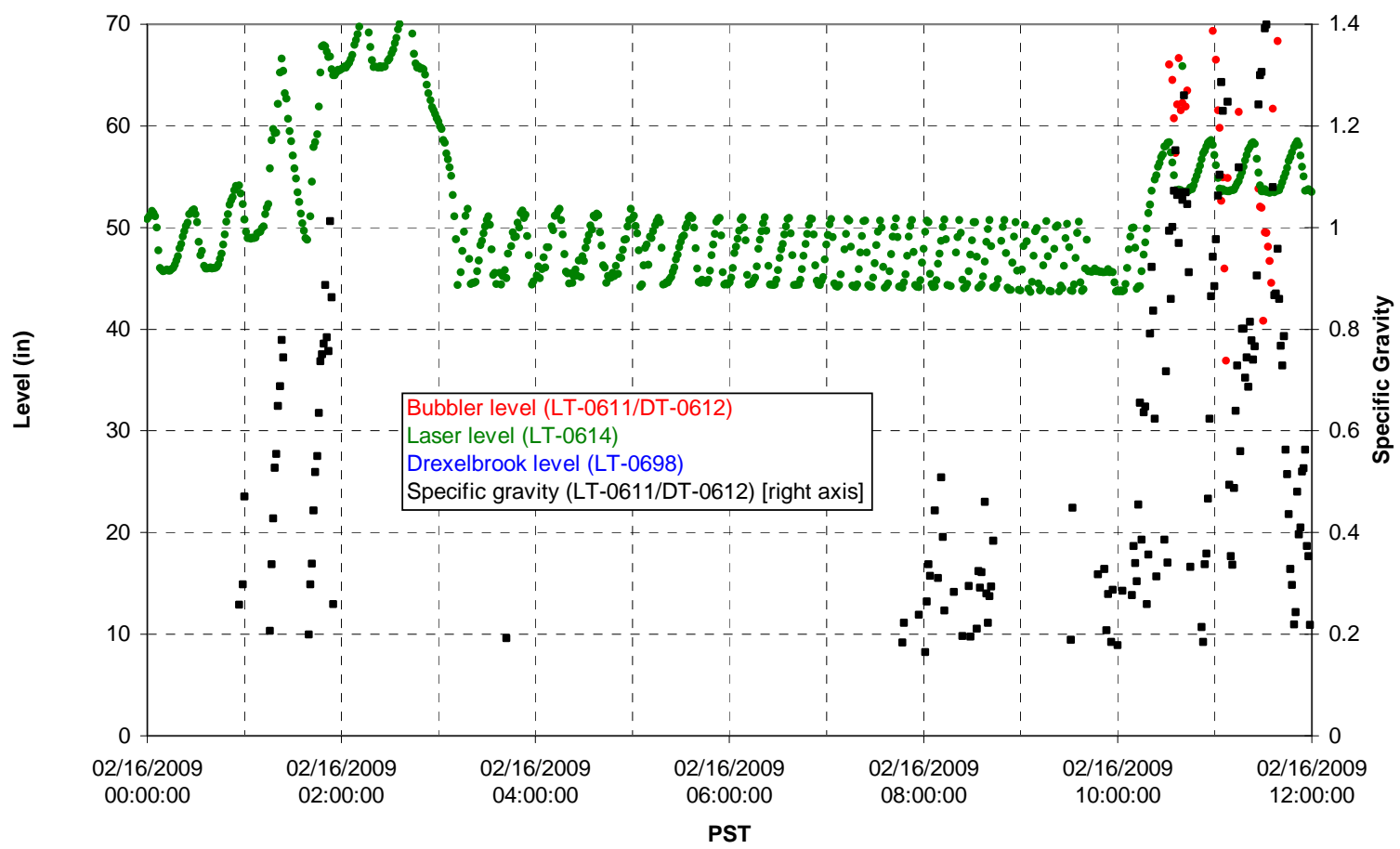

T02A temperatures

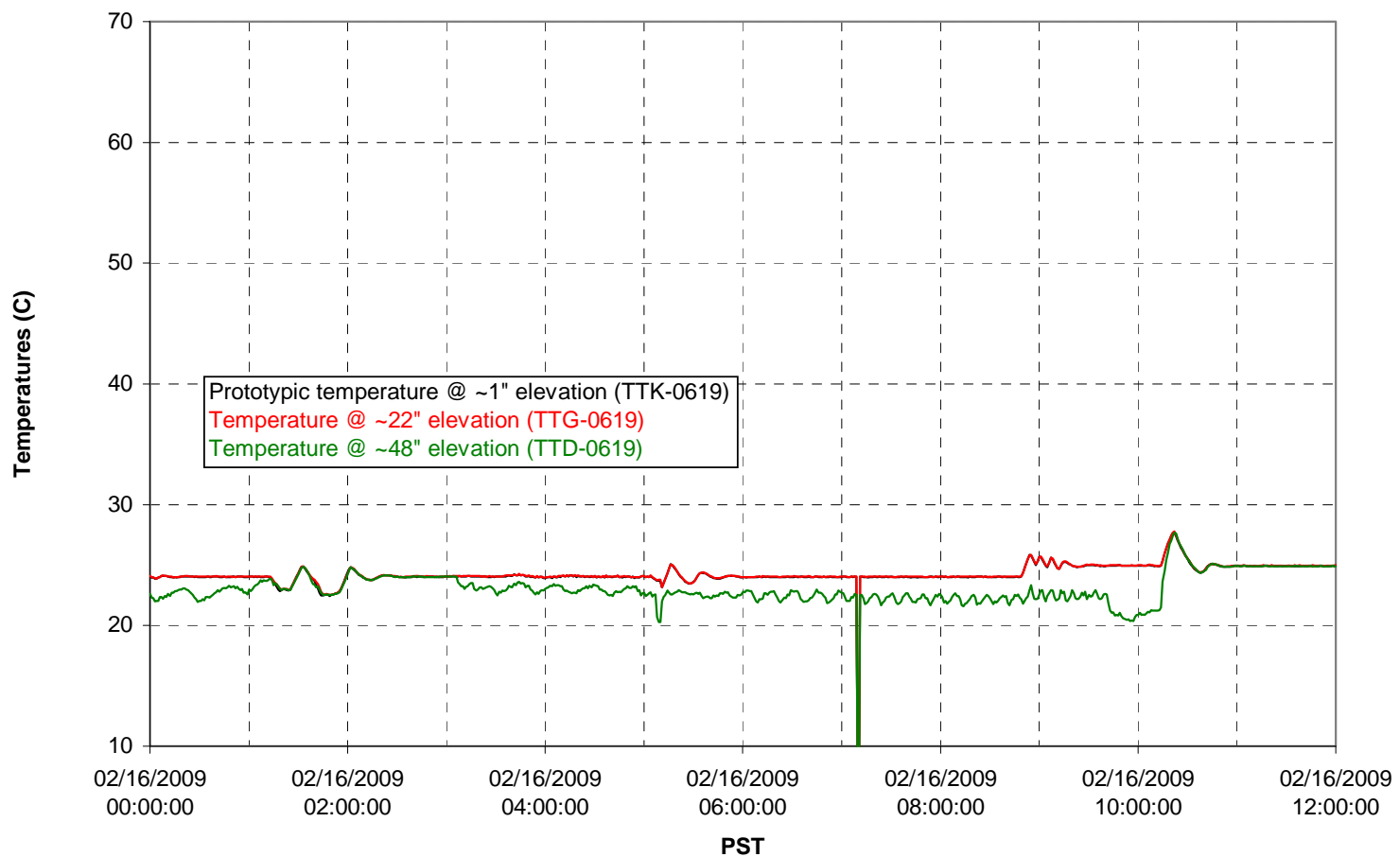


T02A and filter loop temperatures

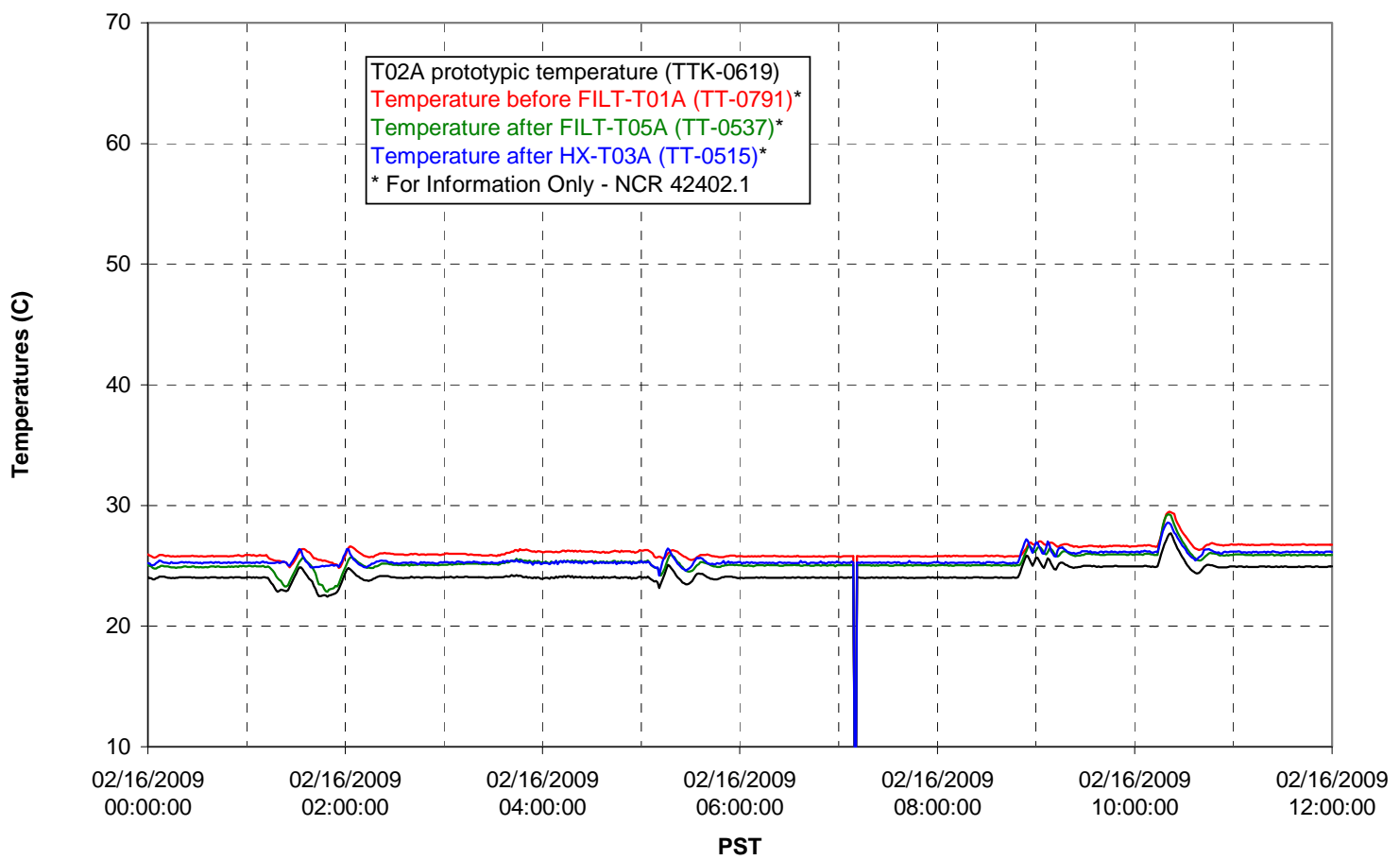

Pump Pressures and Flow

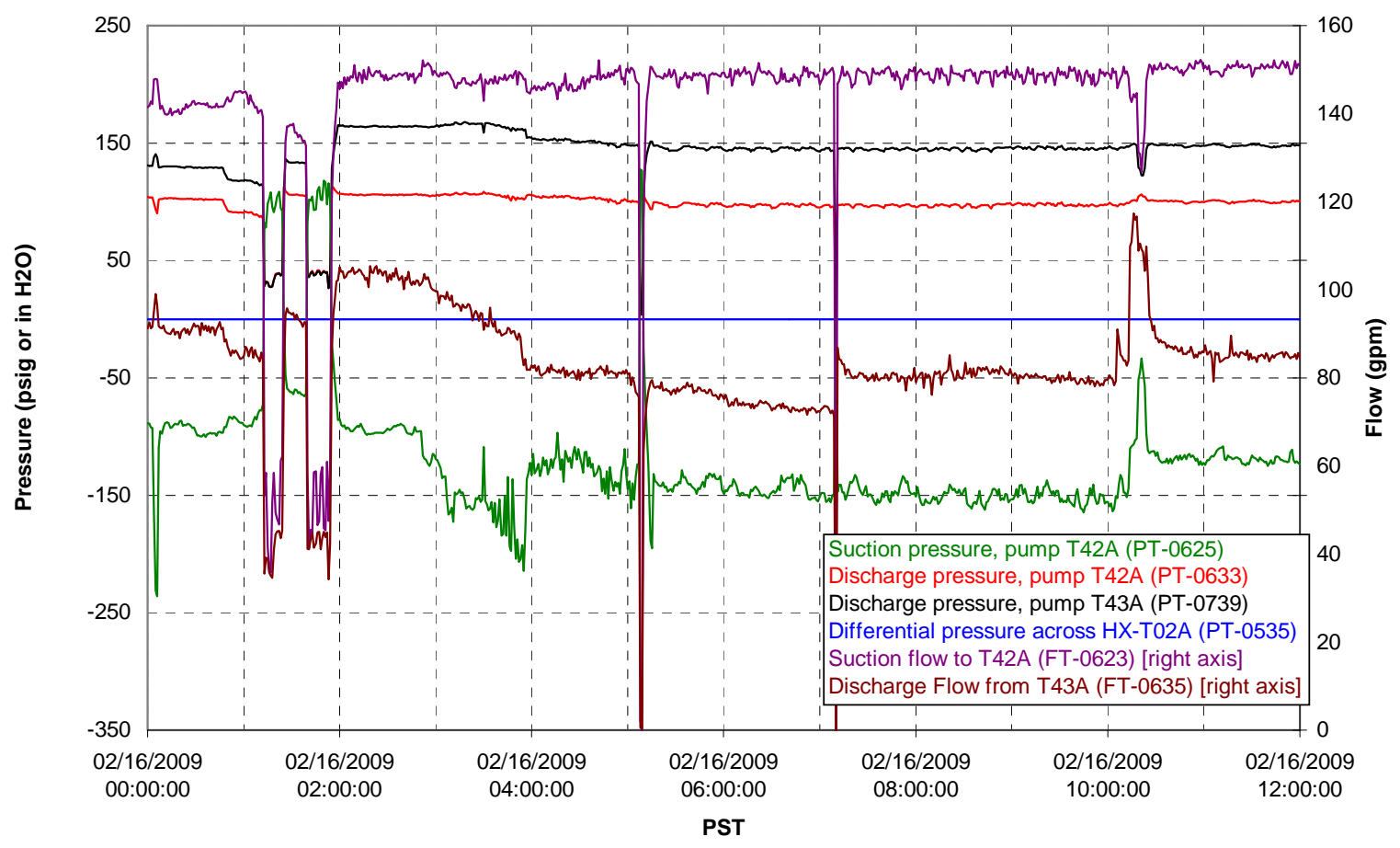




\section{Axial pressure drop}

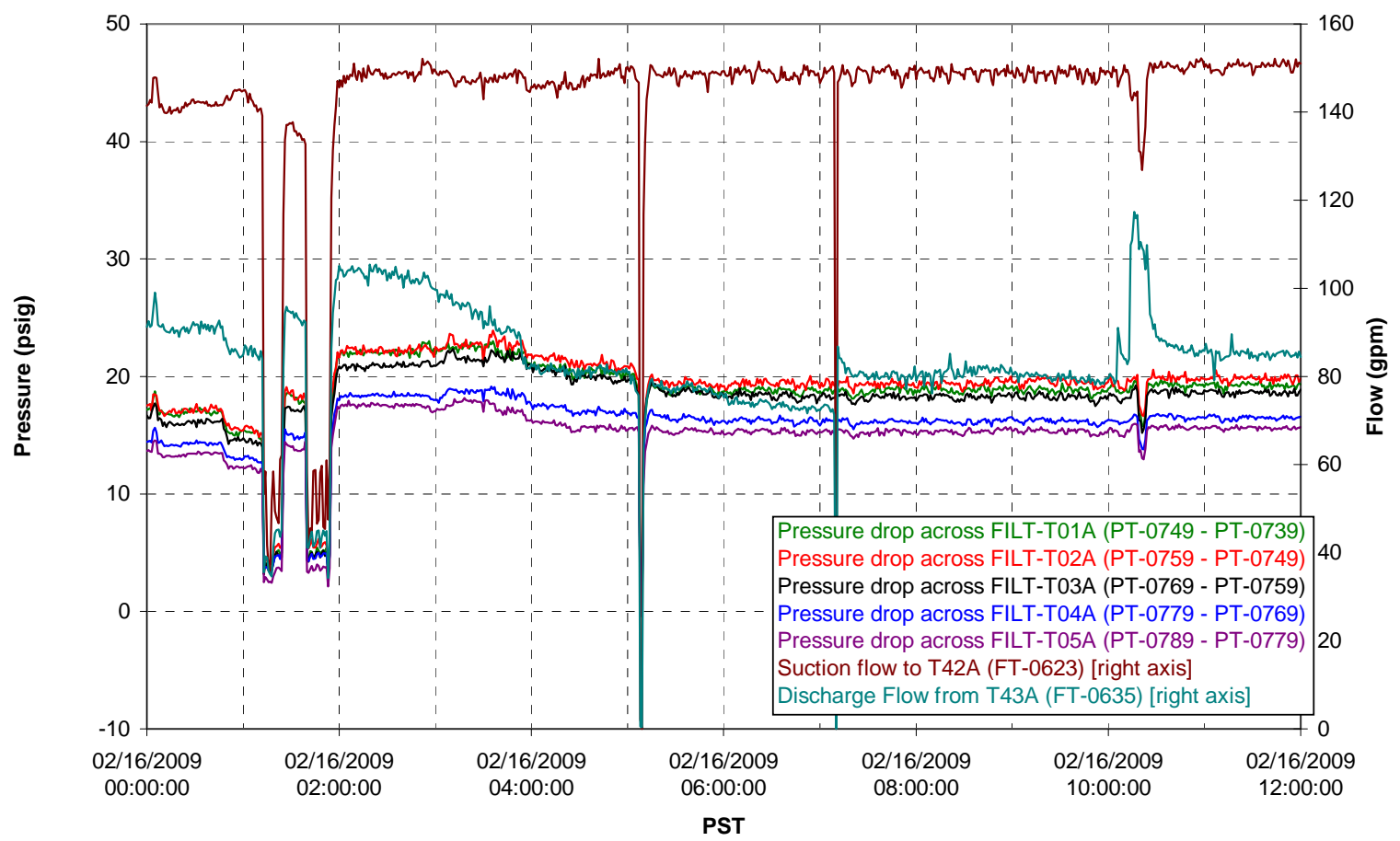

Permeate flow rates

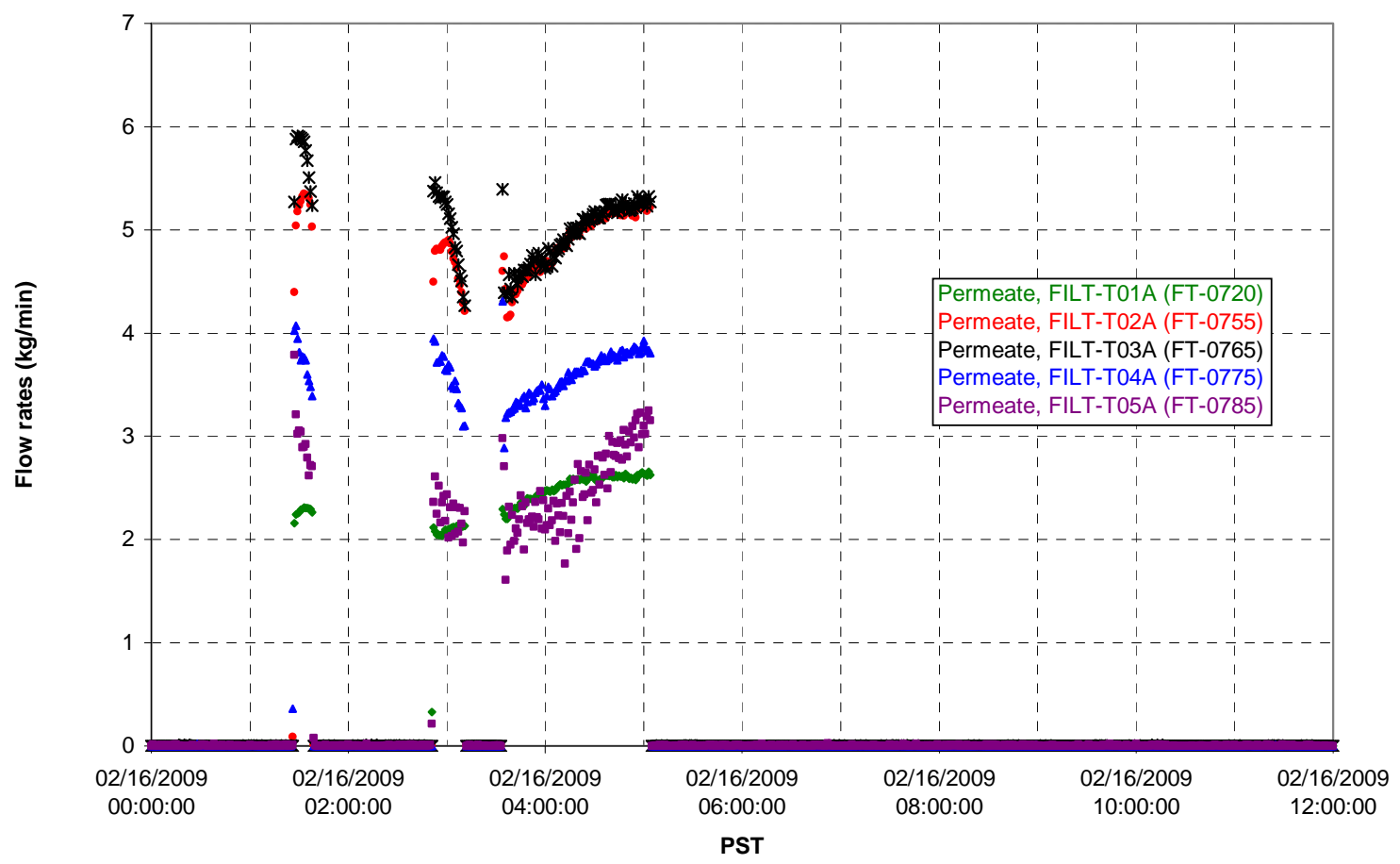


T02A Inner Temperature Tree

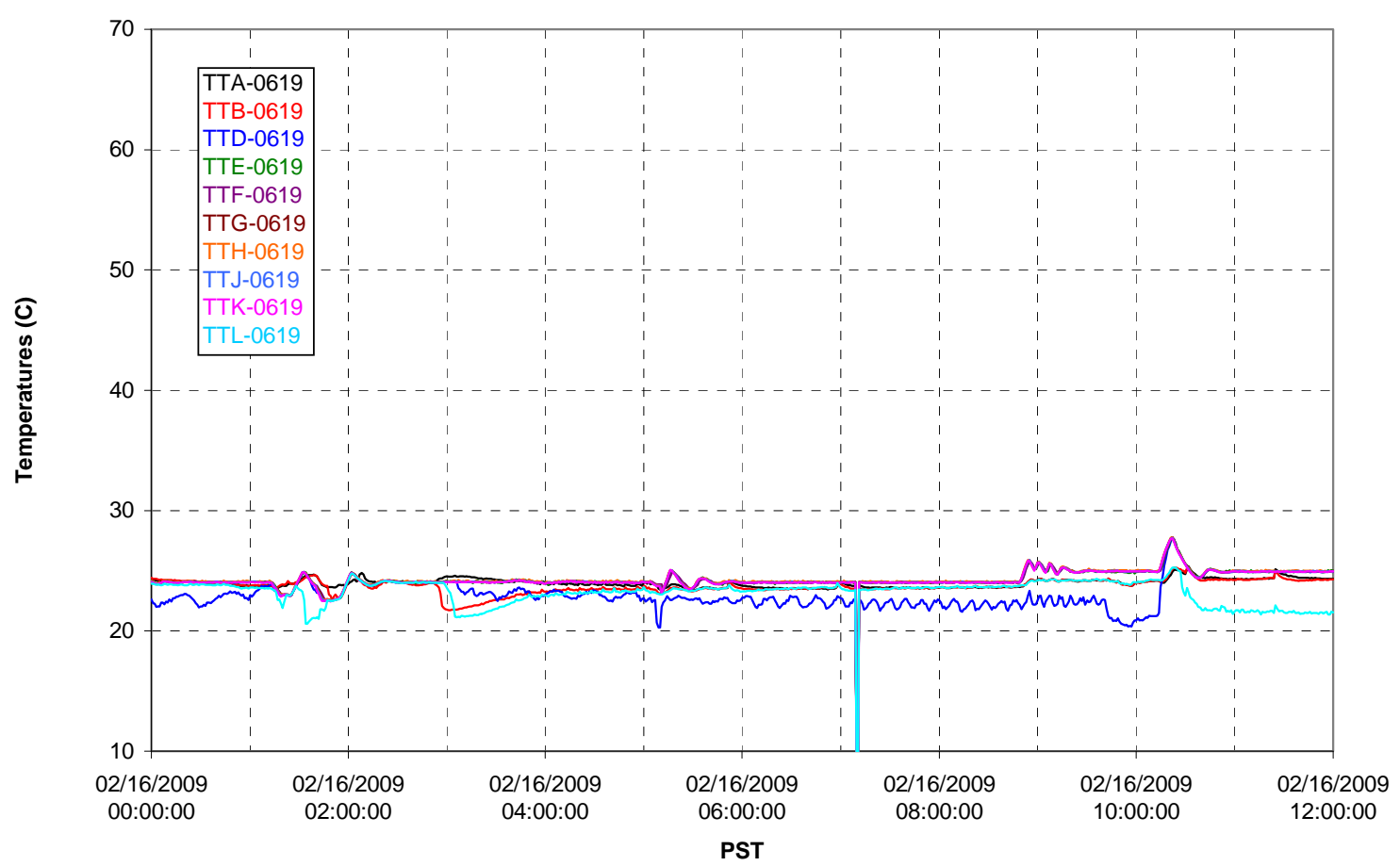

T02A Outer Temperature Tree

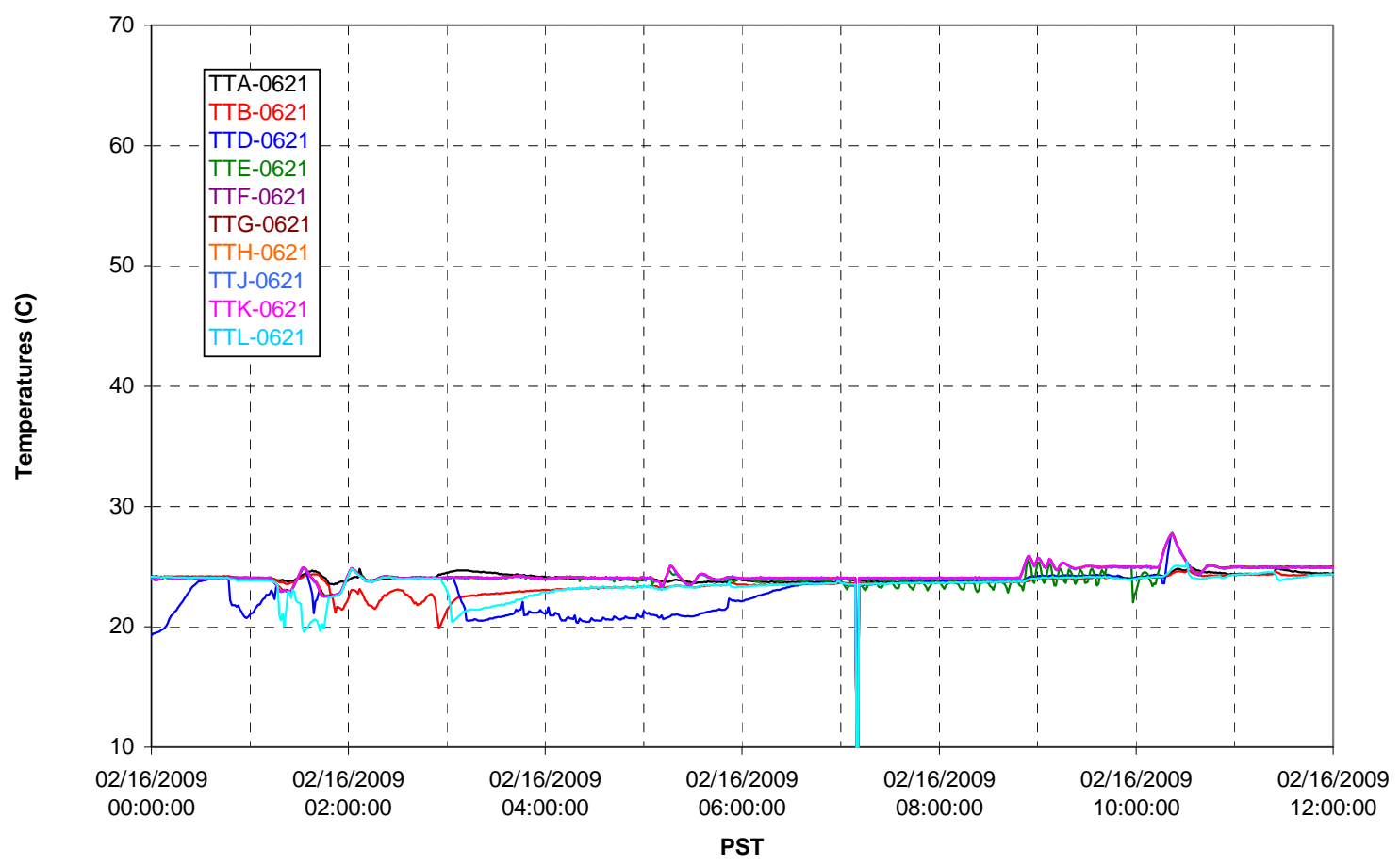


T02A temperatures

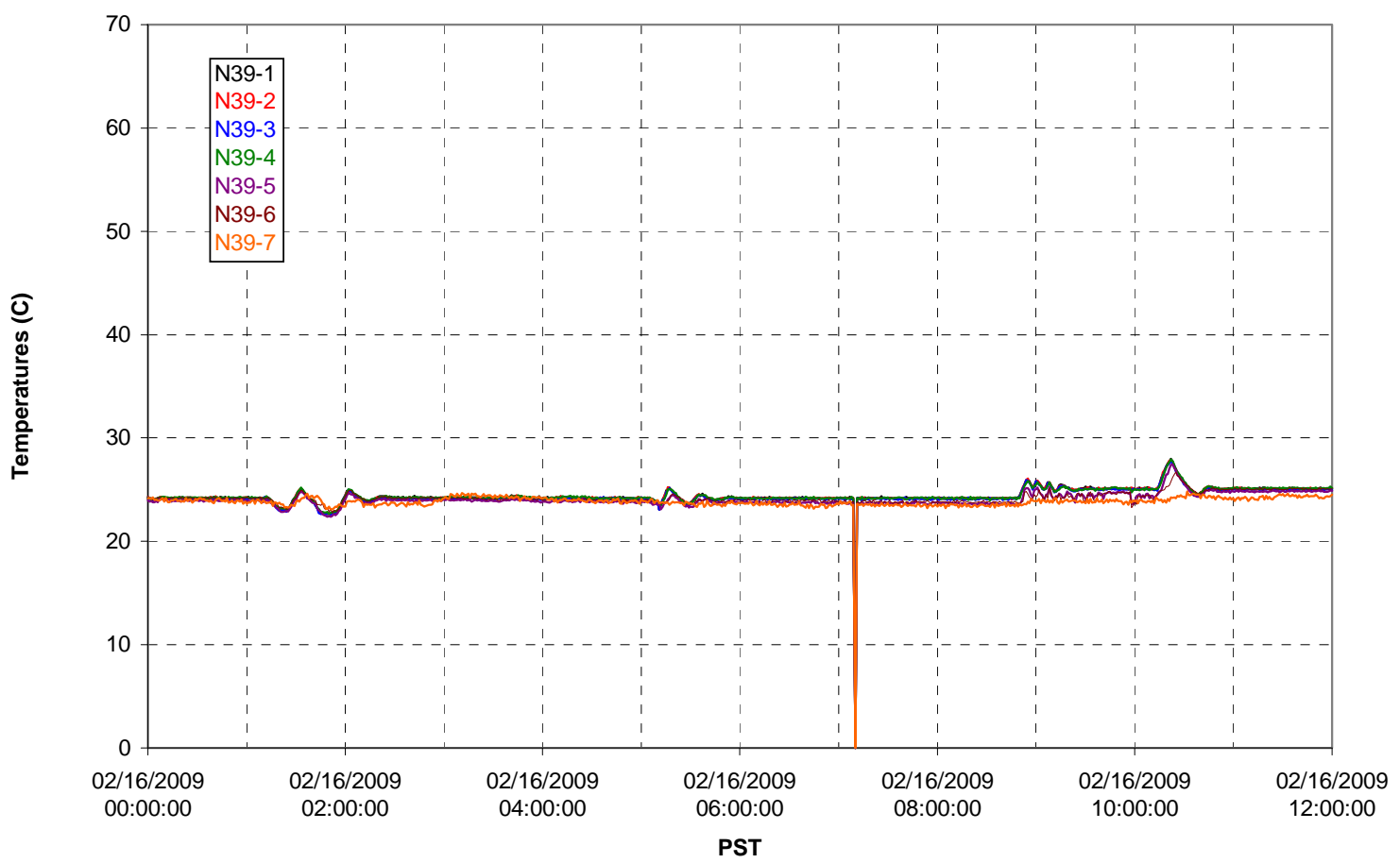

T02A temperatures

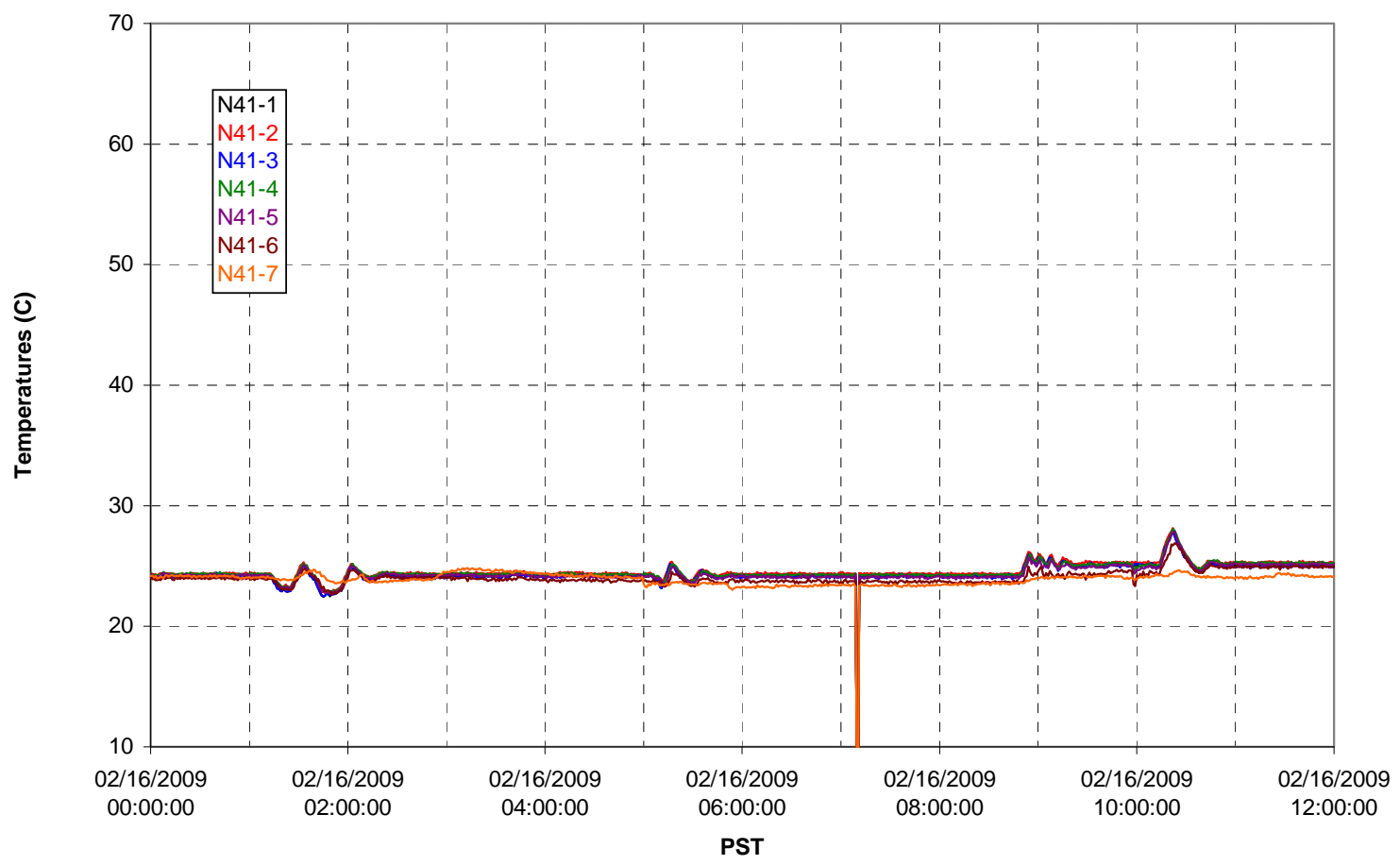


T02A temperatures

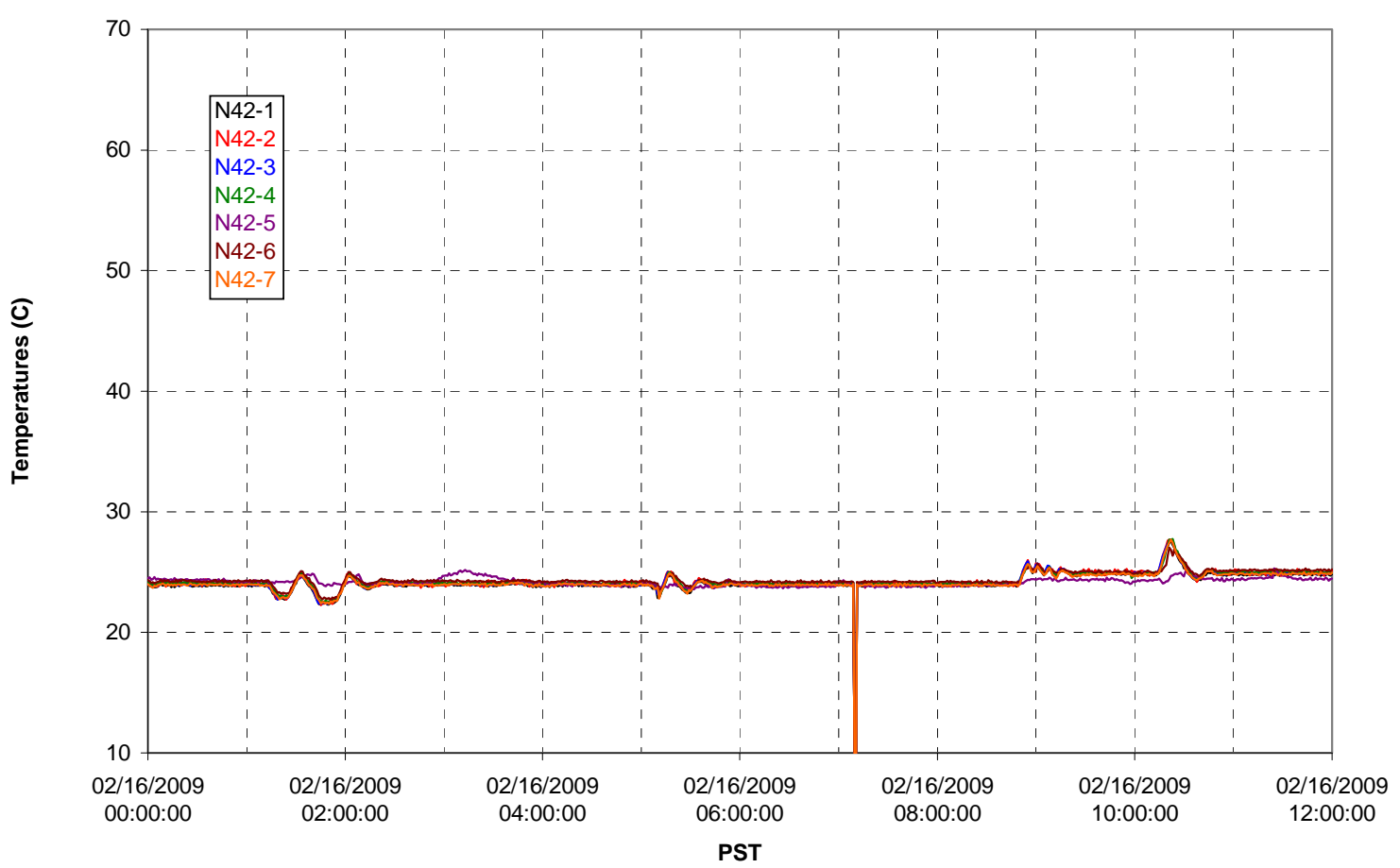

T02A temperatures

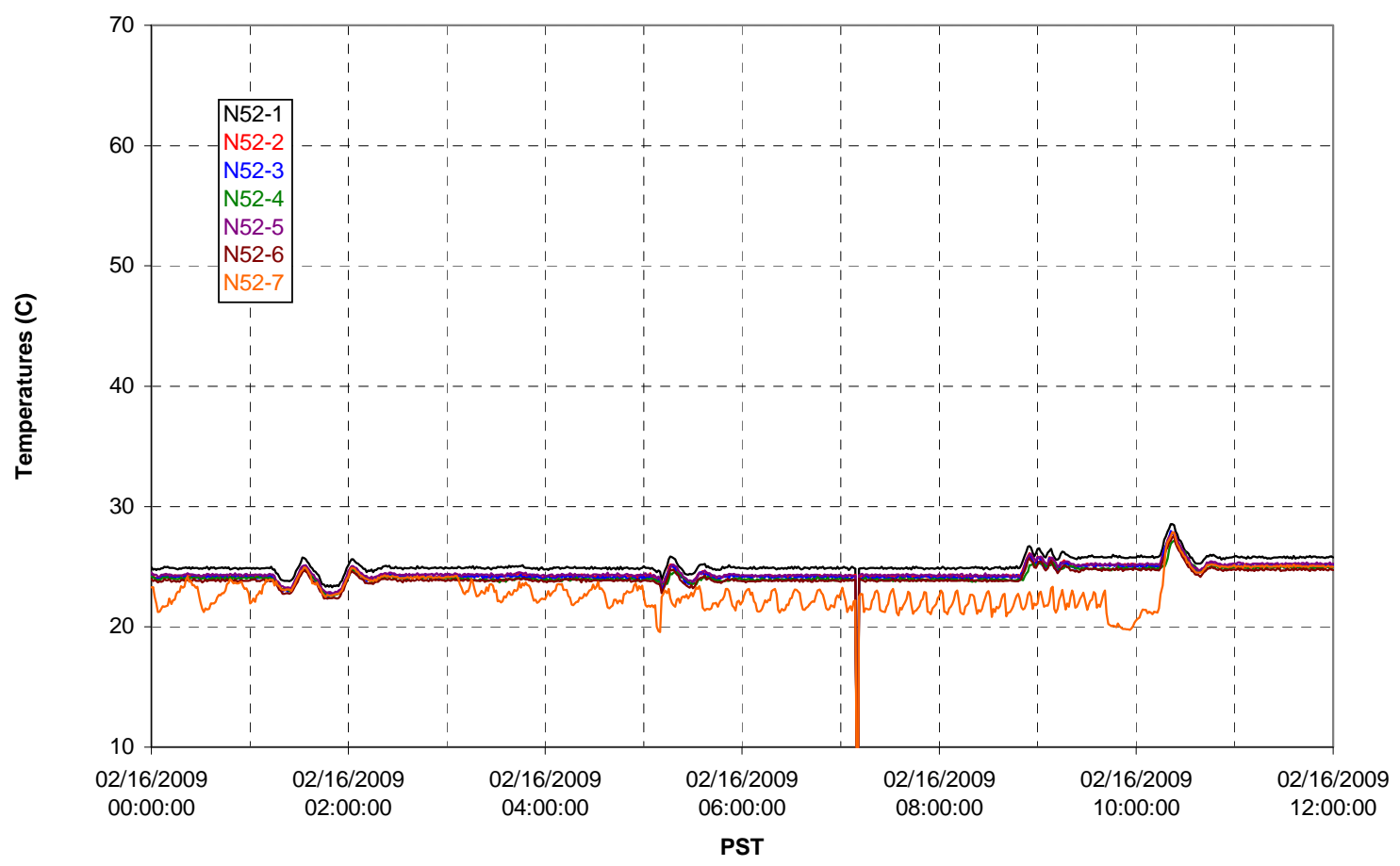


T02A Heating and Cooling
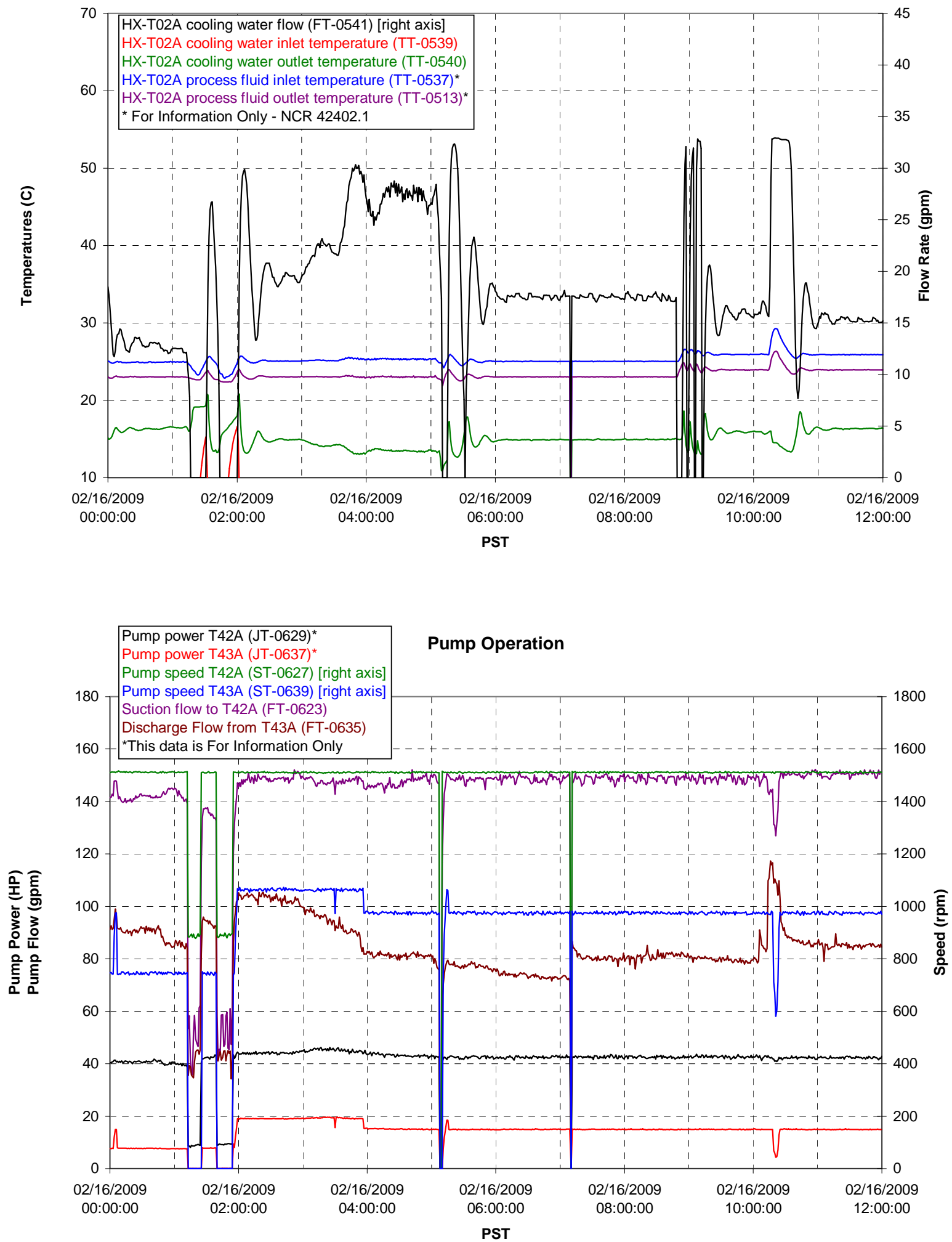
Pulsepot UFP-PP-T01A

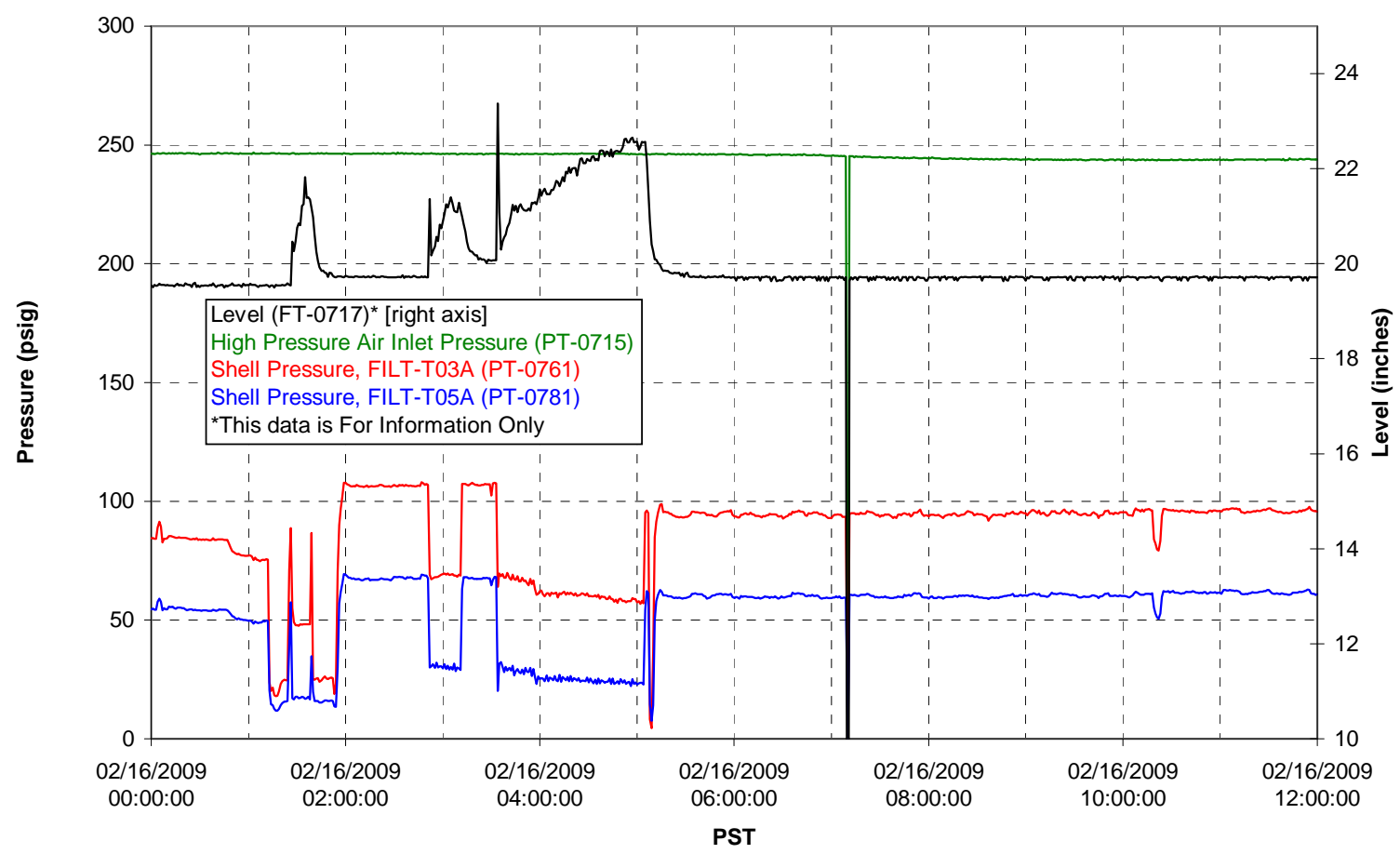

Pulsepot UFP-PP-T02A

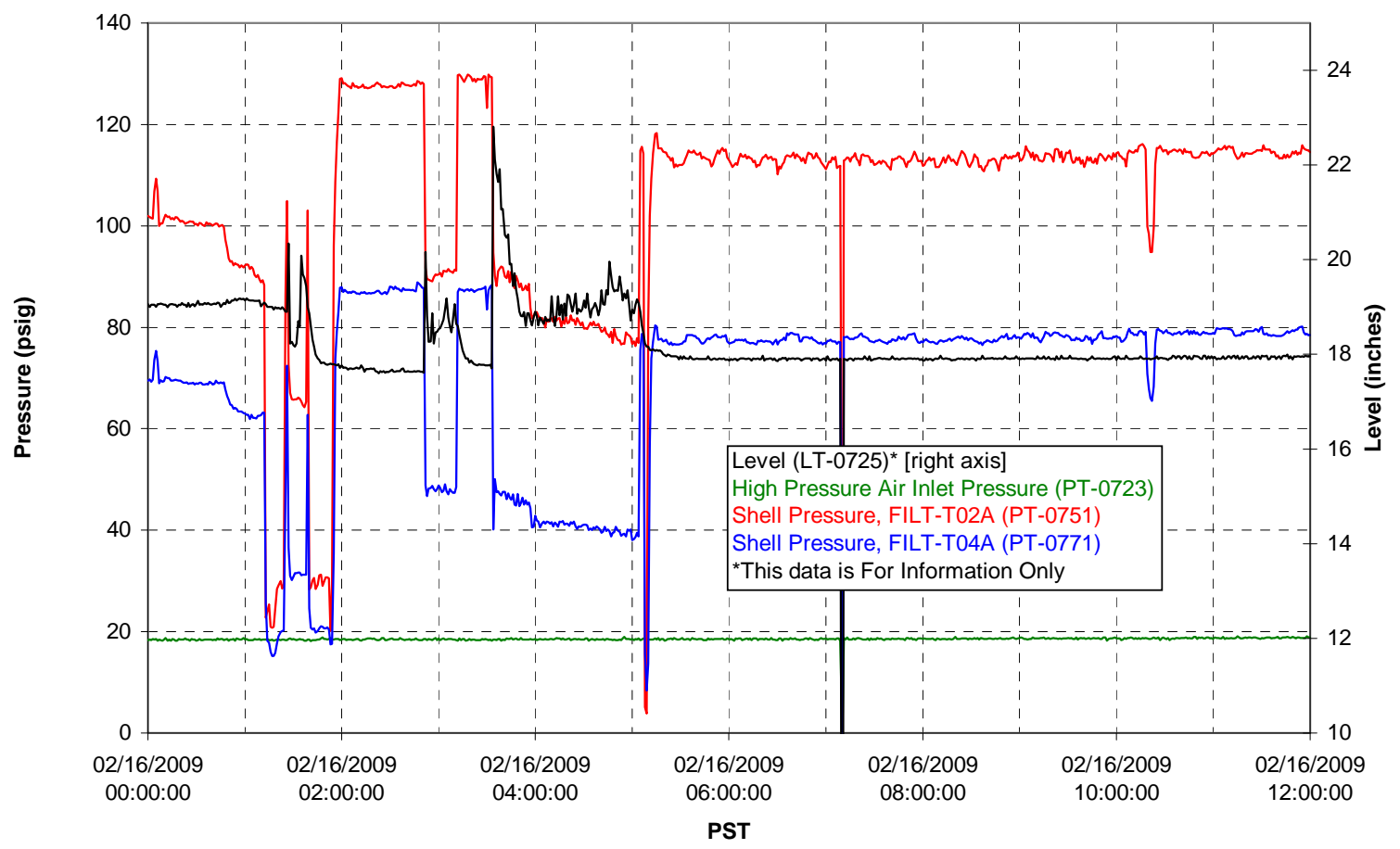


Pulsepot UFP-PP-T03A

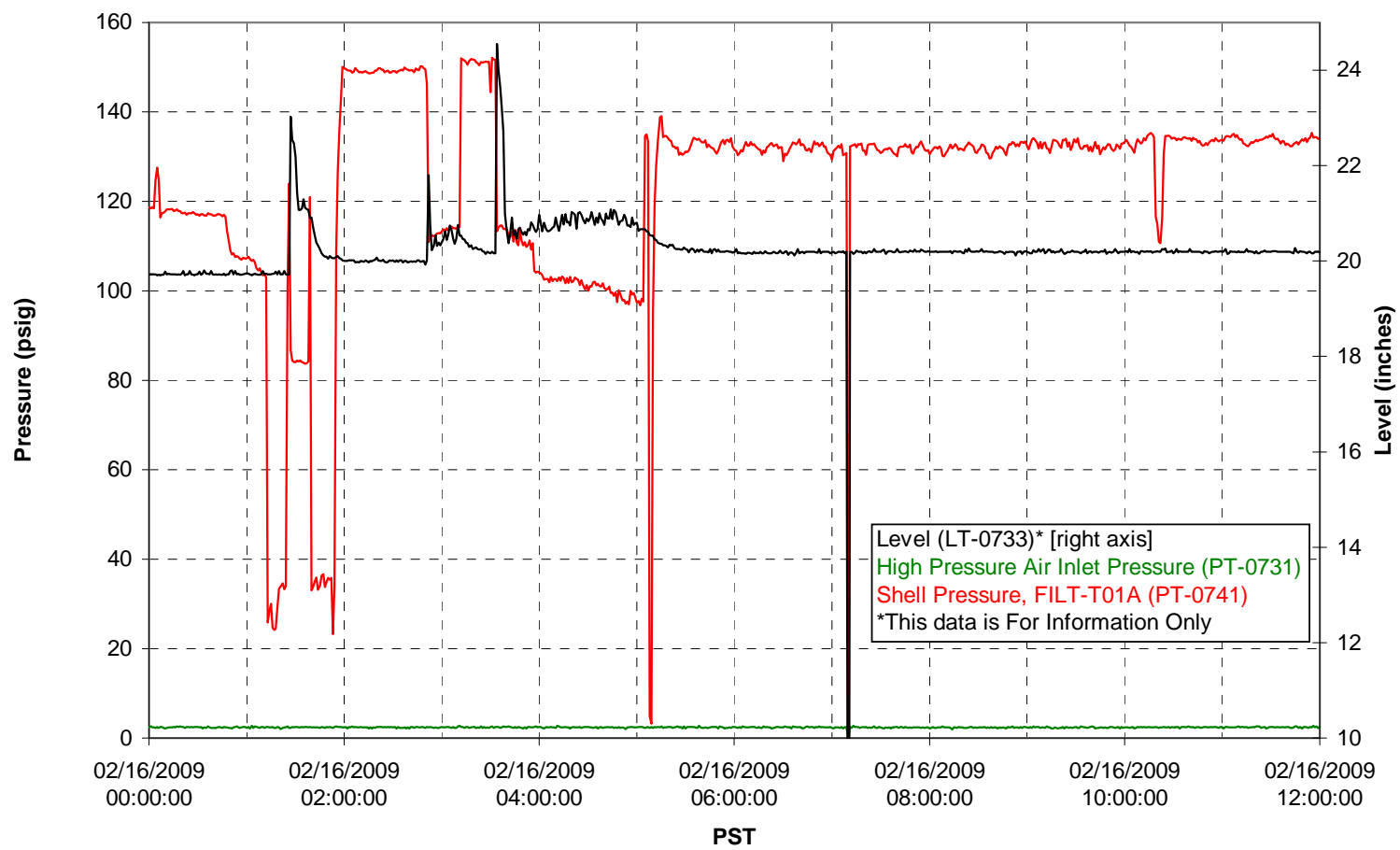

Pulsepot Levels

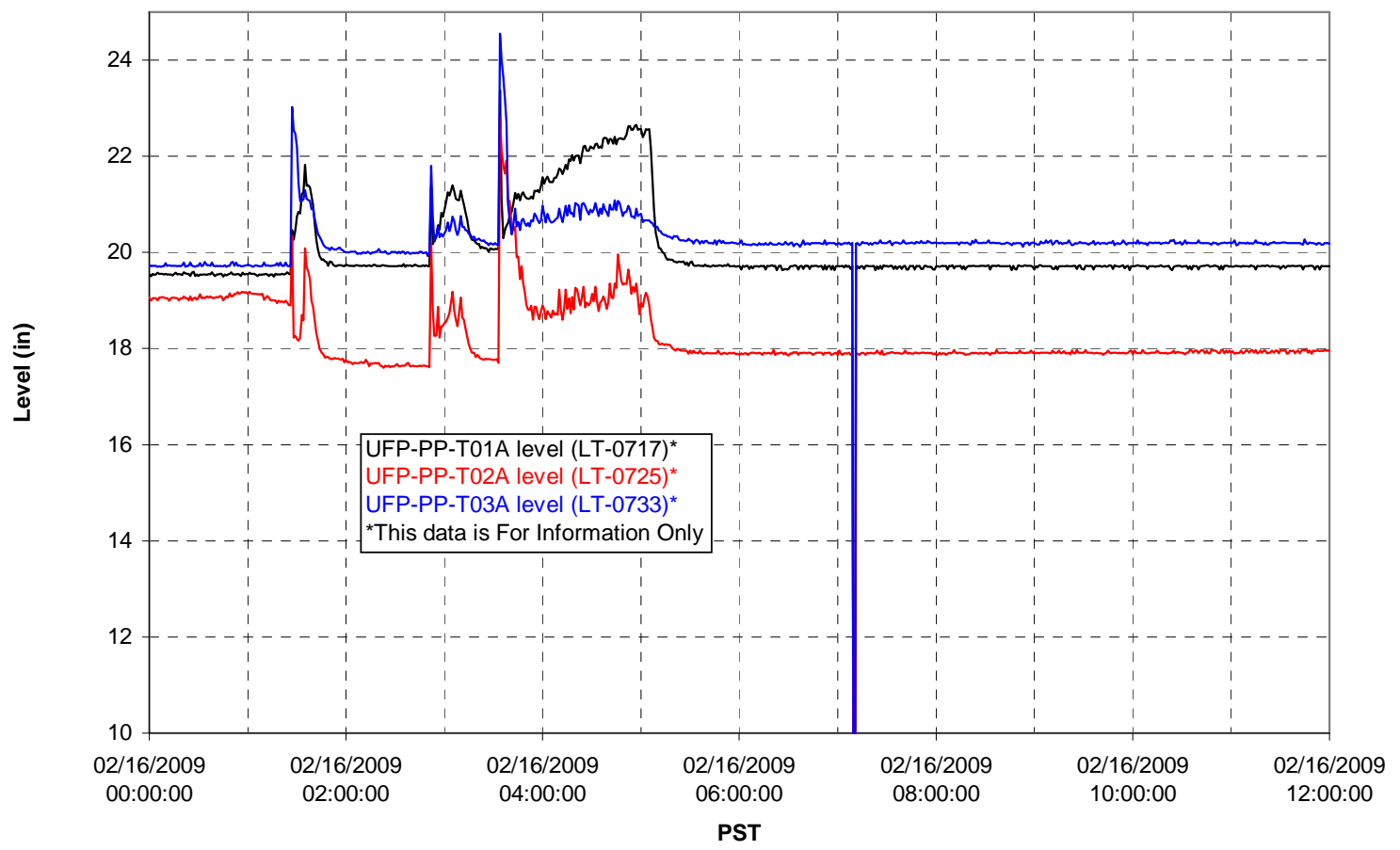


Filter UFP-FILT-T01A

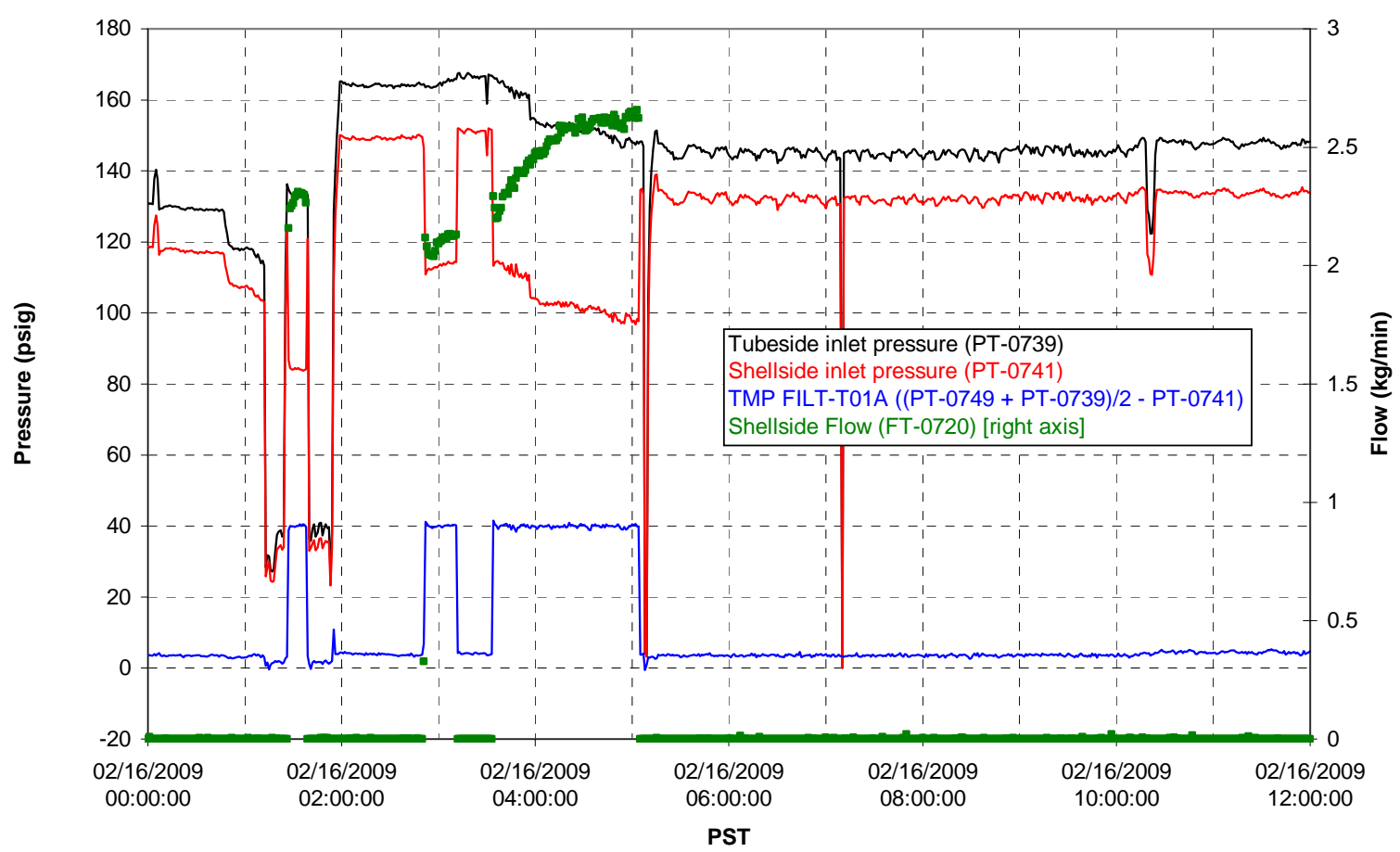

Filter UFP-FILT-T02A

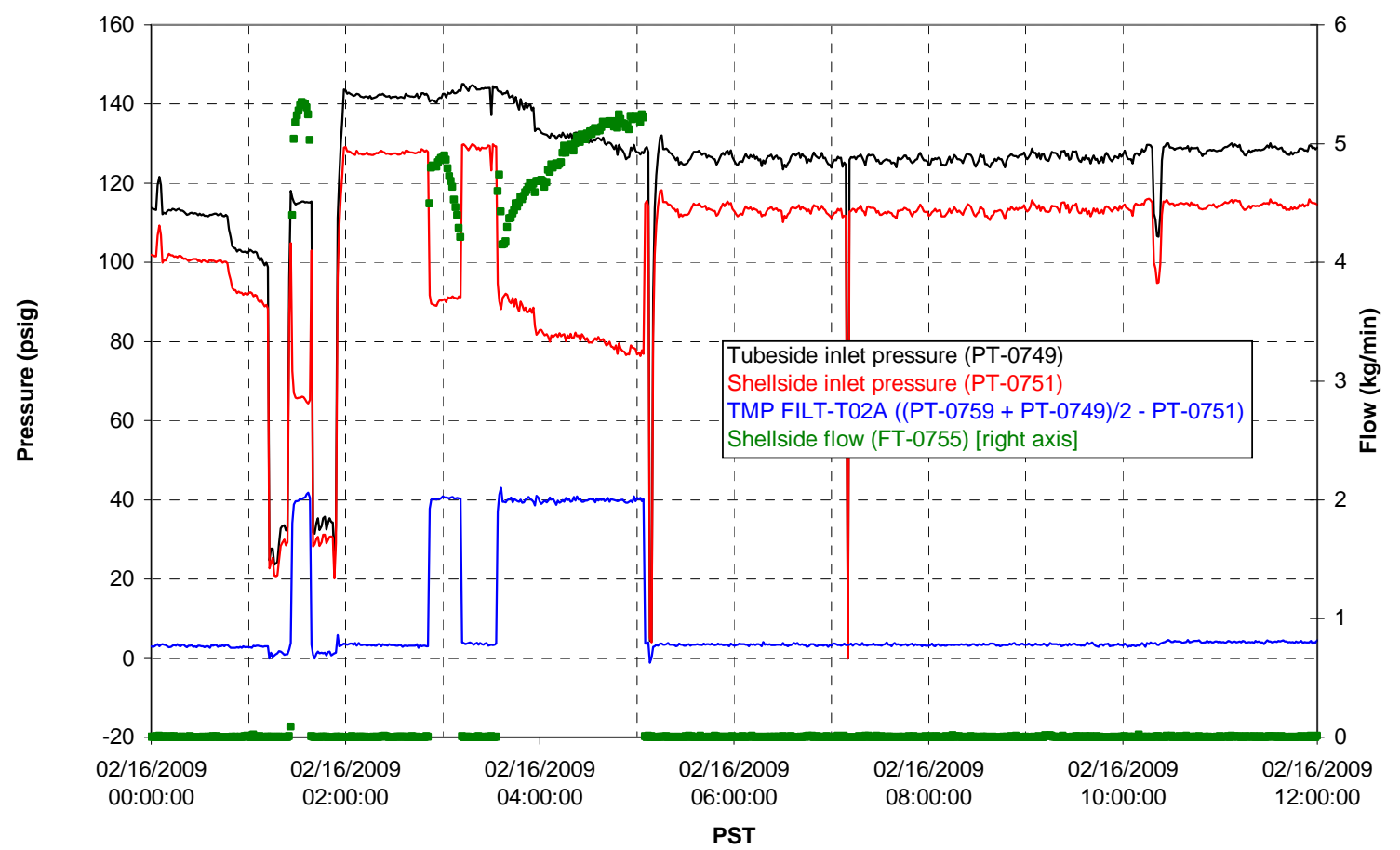


Filter UFP-FILT-T03A

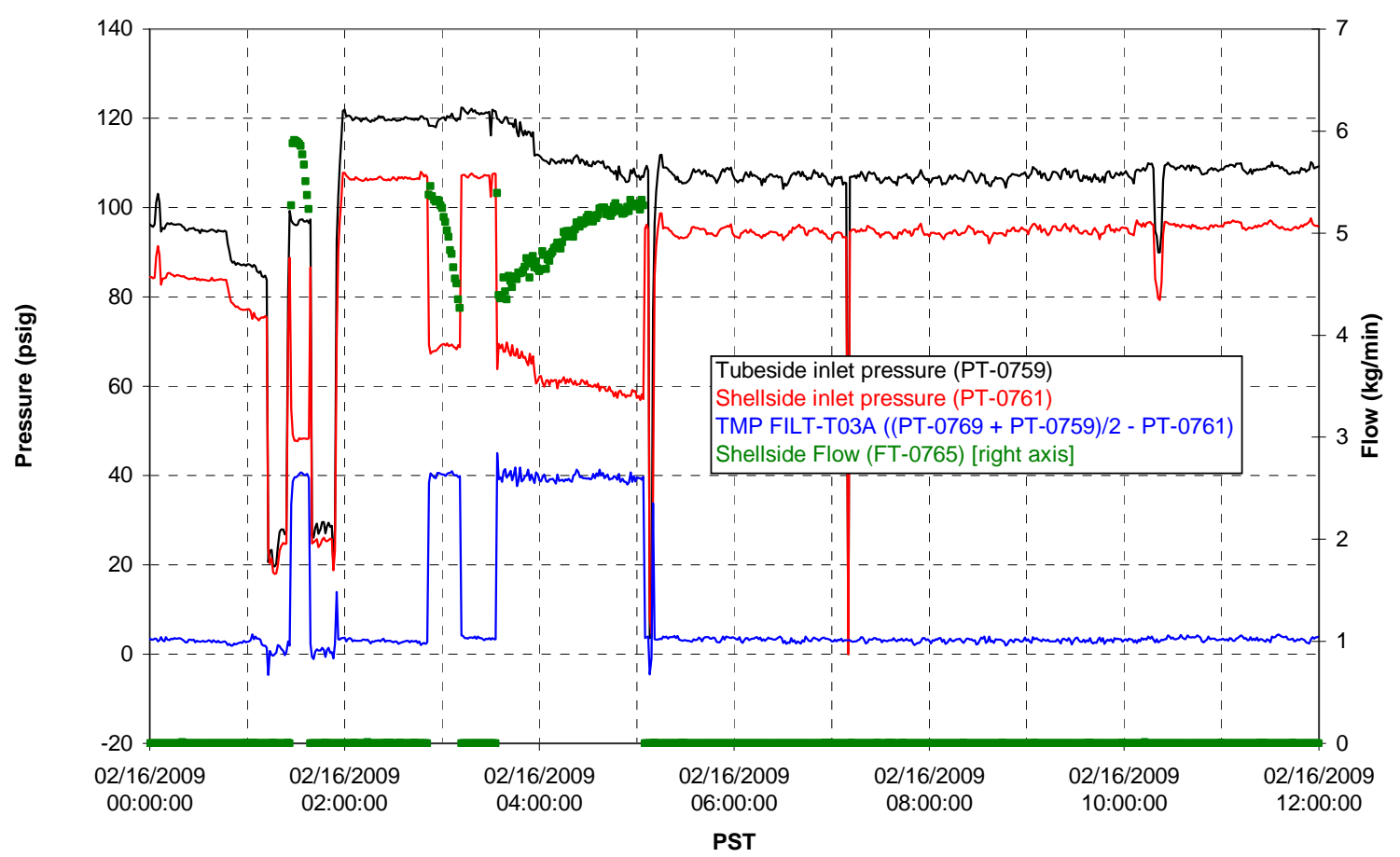

Filter UFP-FILT-T04A

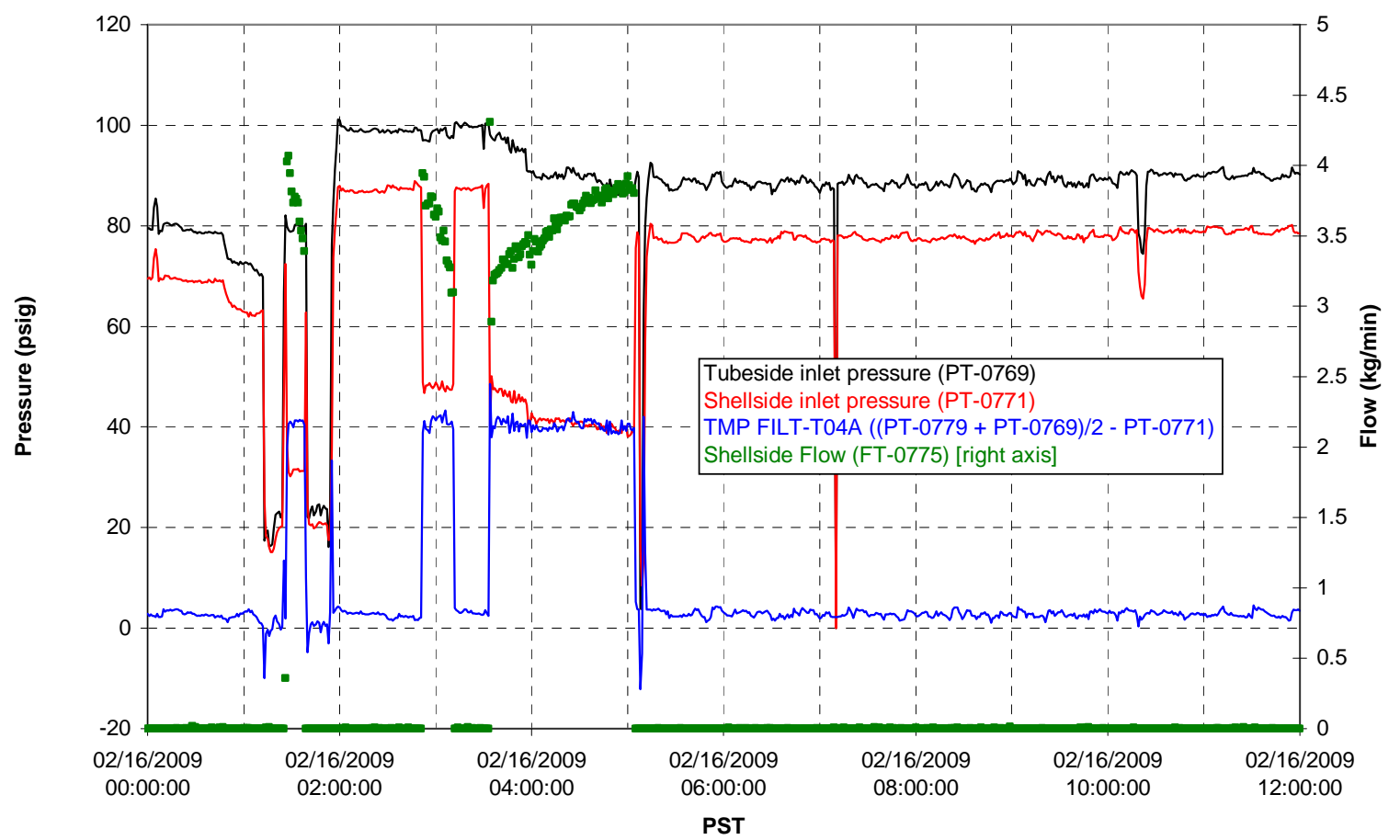


Filter UFP-FILT-T05A

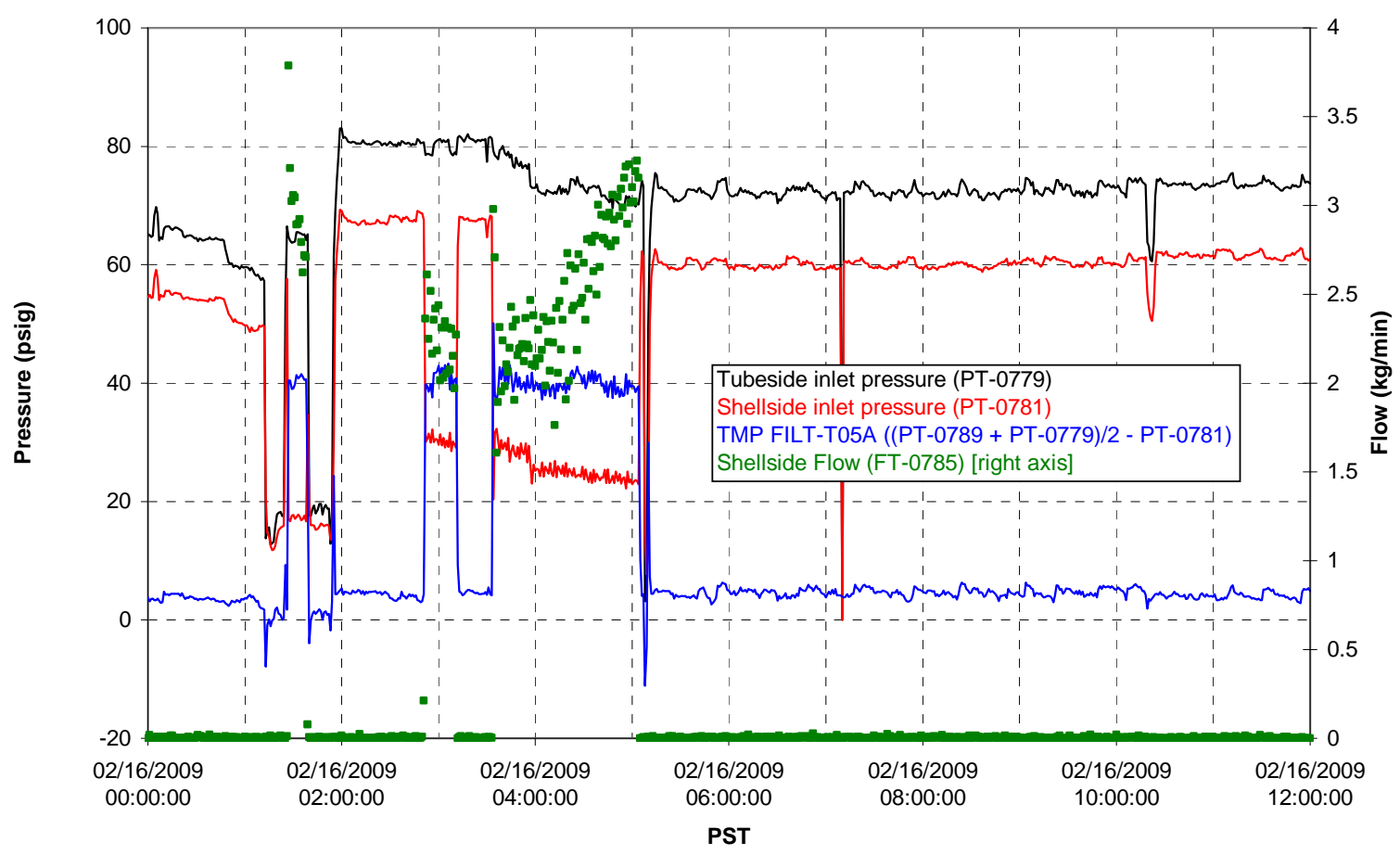

Chemical Flow

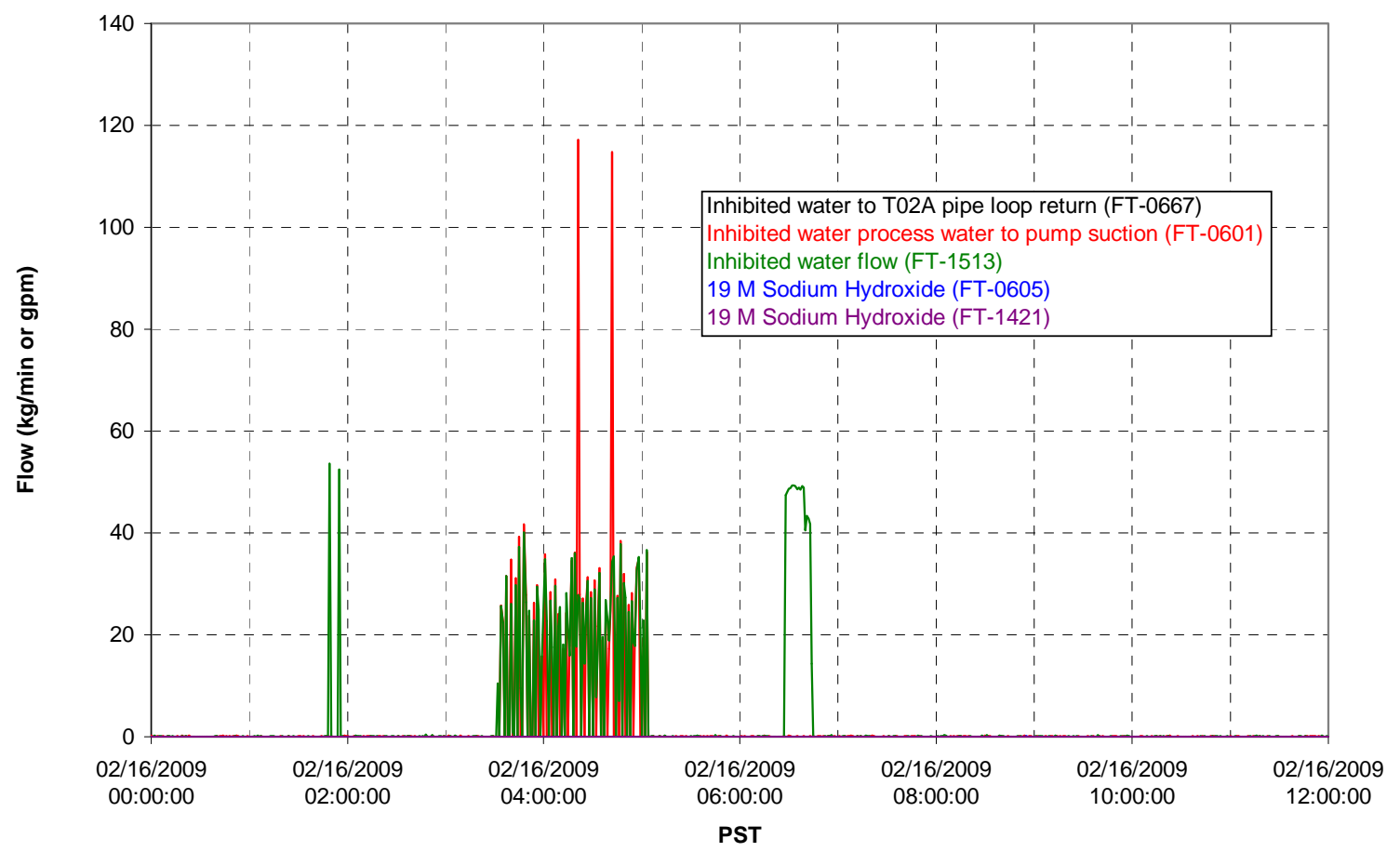




\section{Chemical Flow}

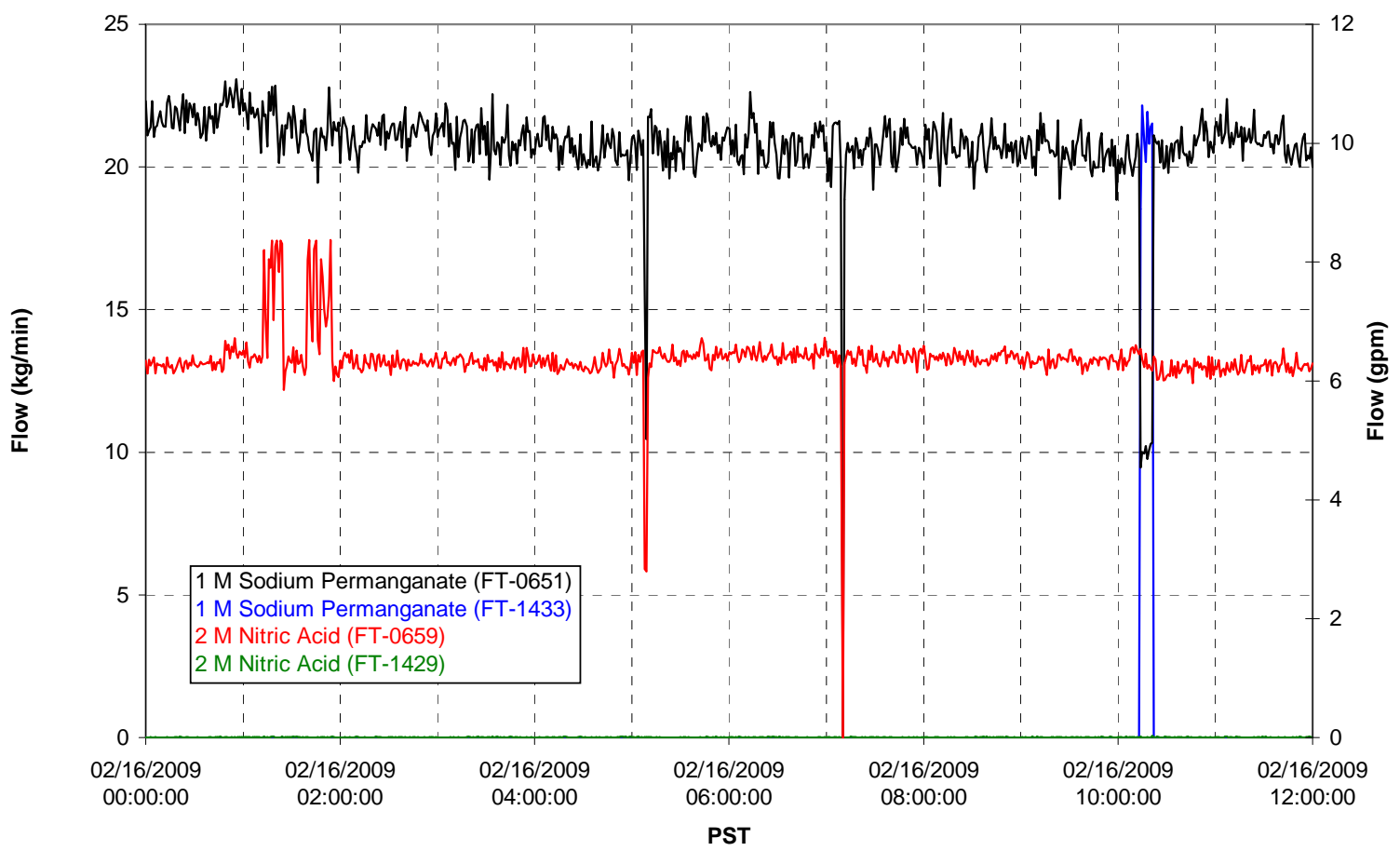

Air Flows

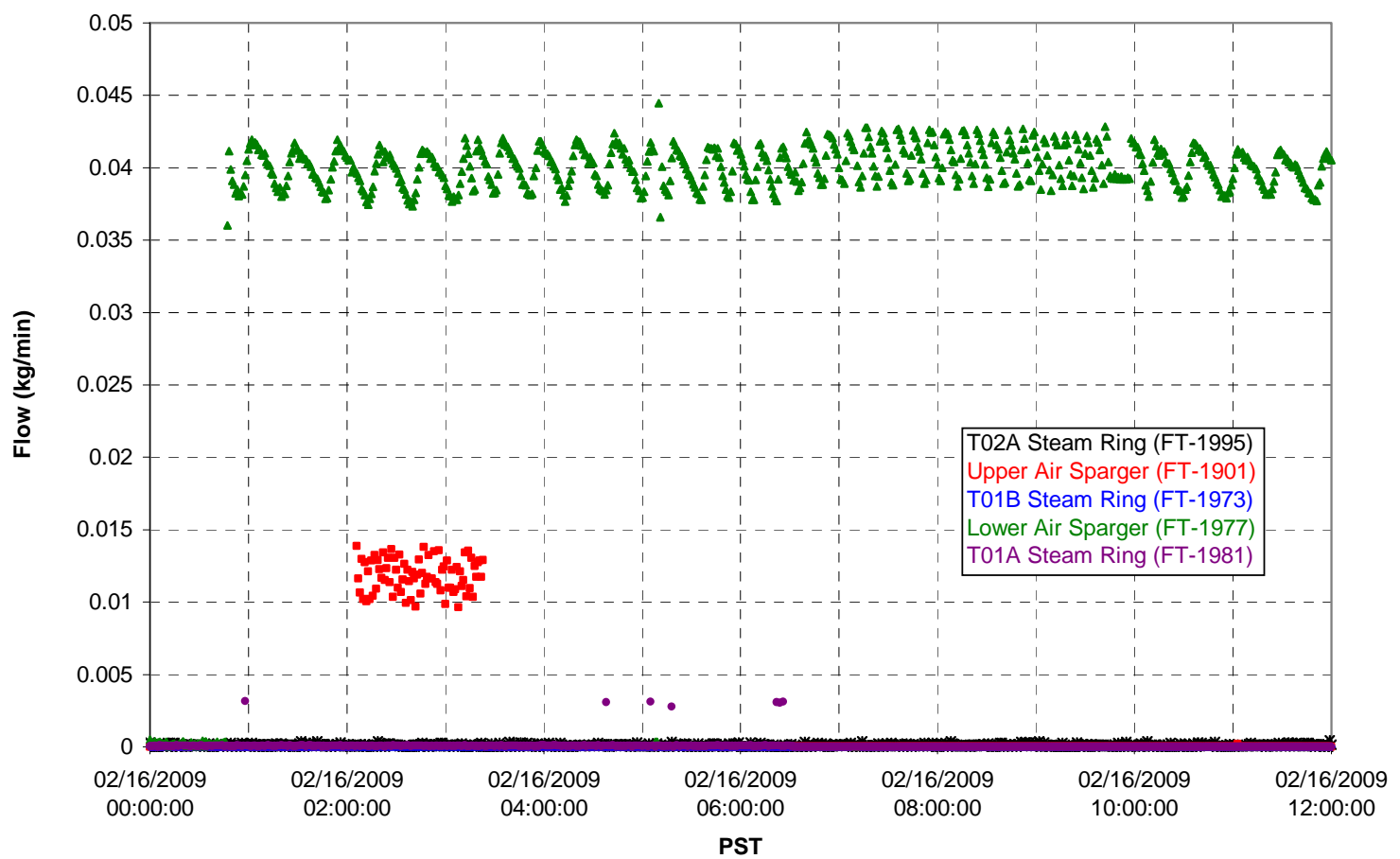

G.592 
T02A Steam

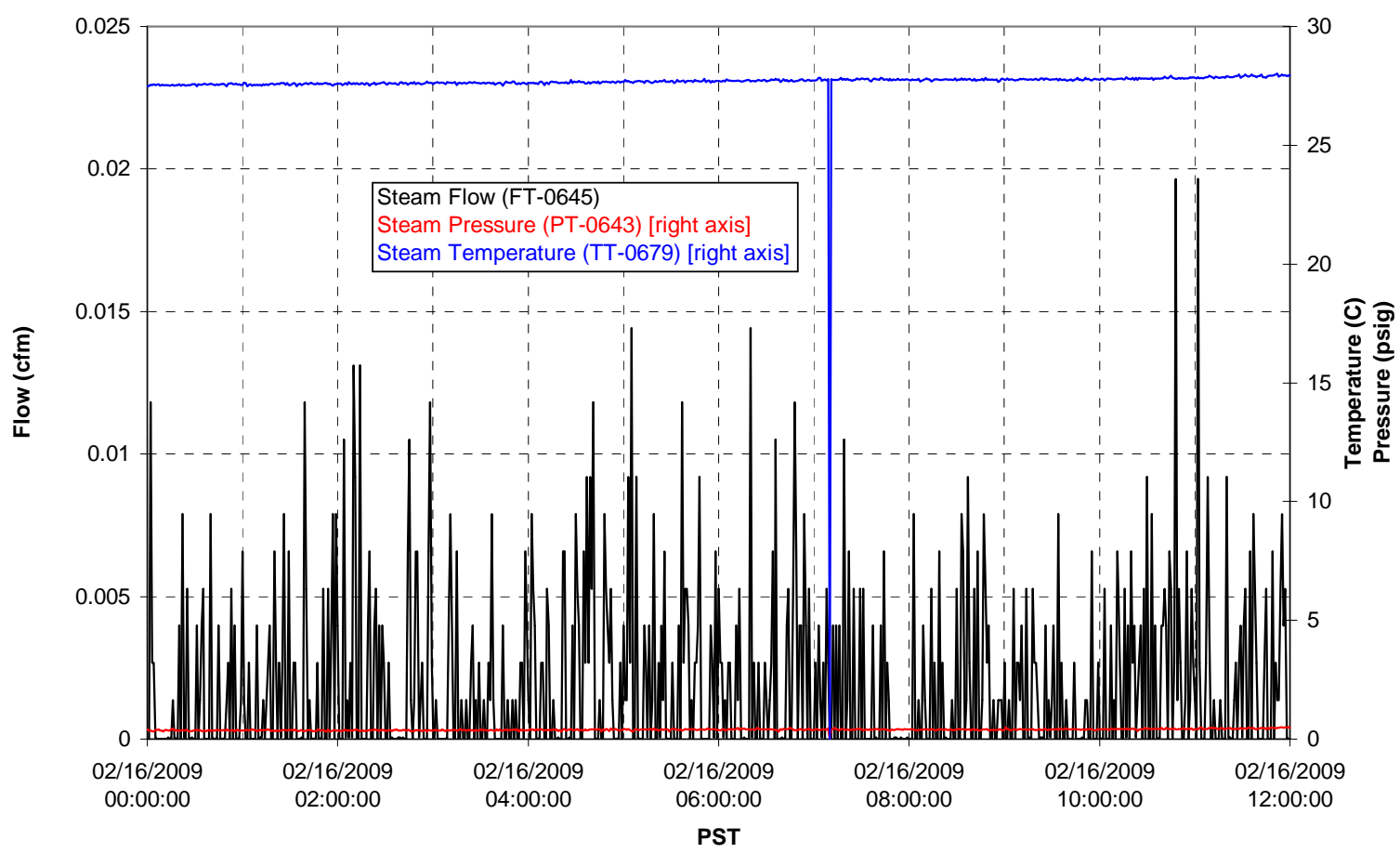

T01A Steam

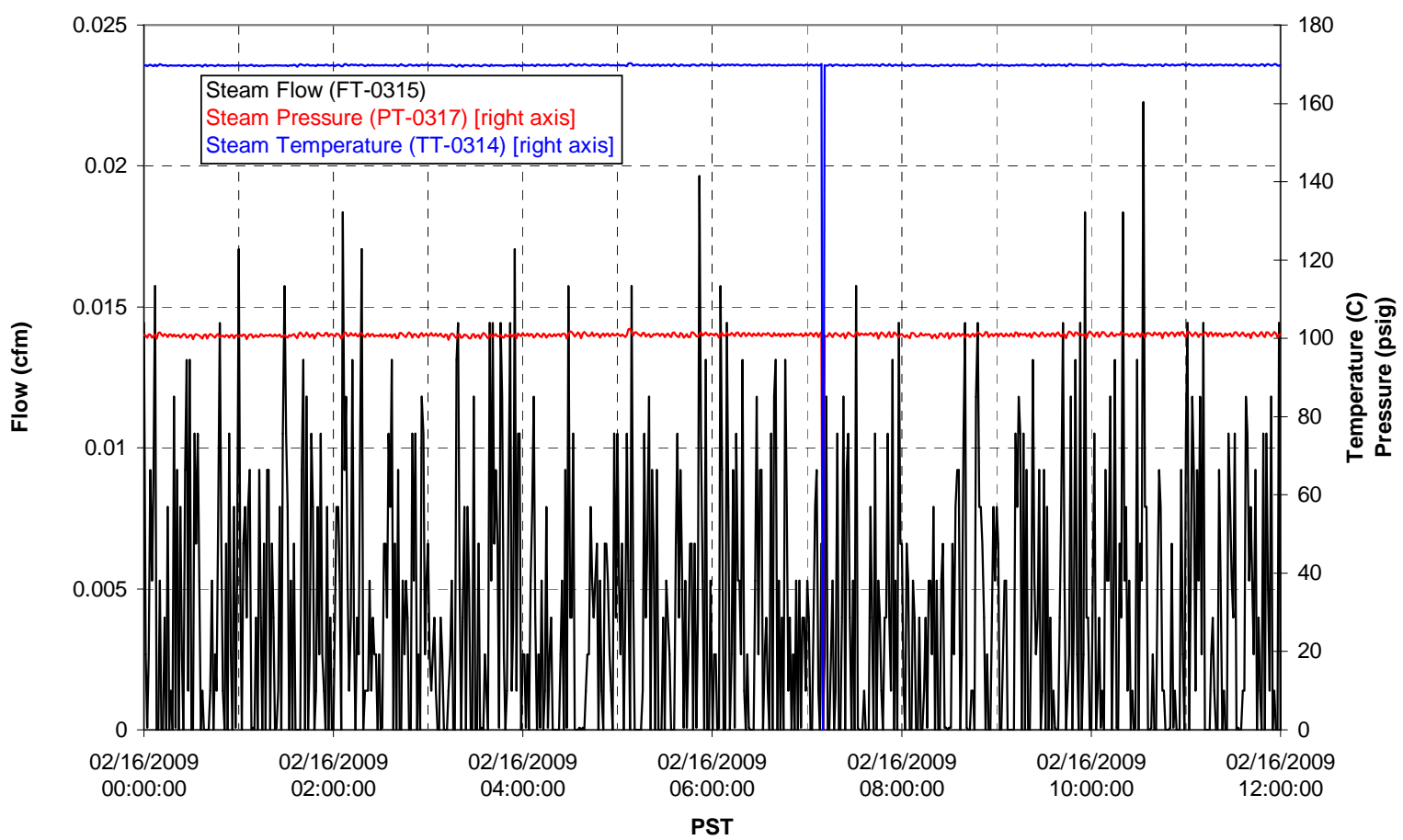


T01B Steam

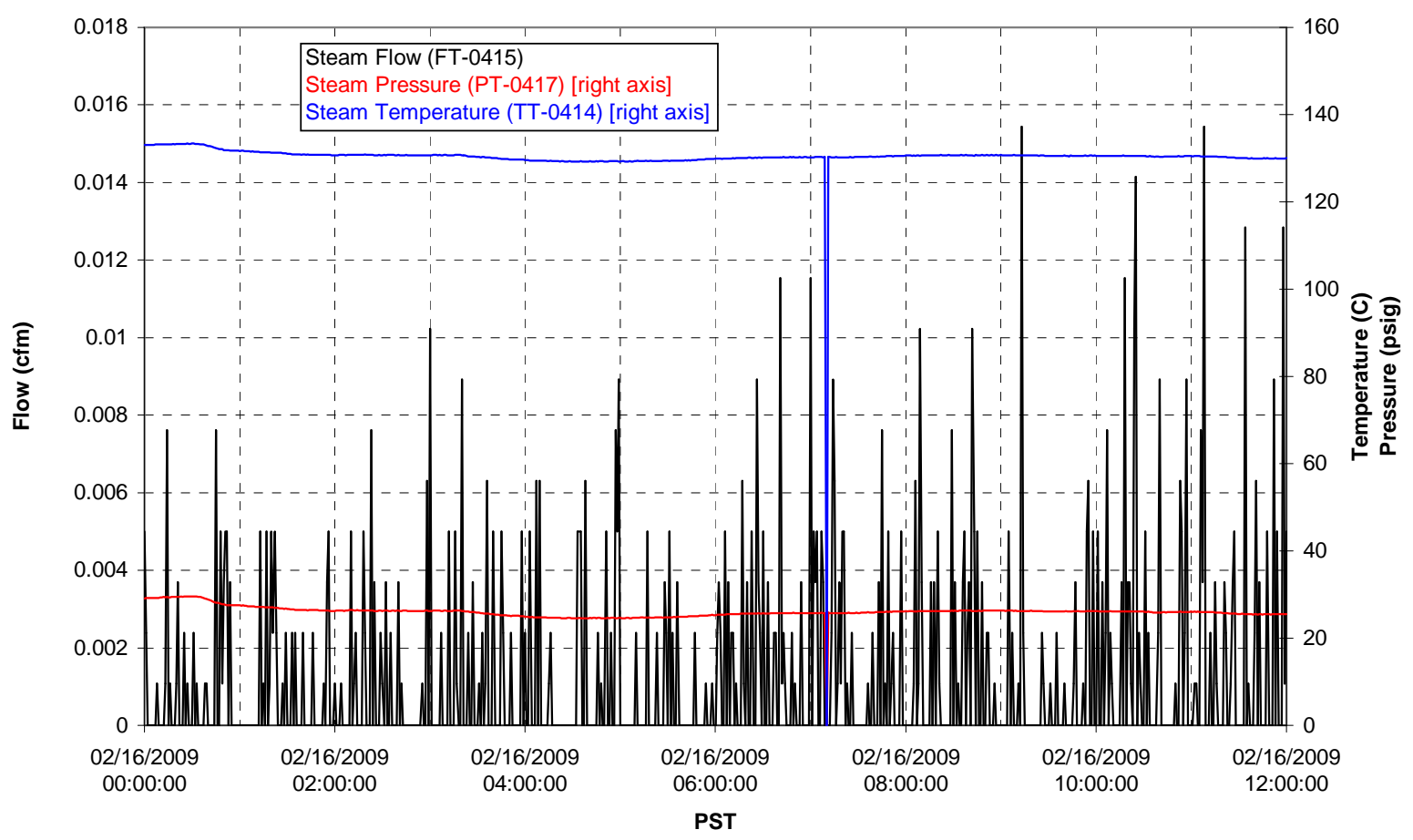




\section{Integrated Test A Data Plots 02/16/09 12:00 - 02/17/09 00:00}


T01A level

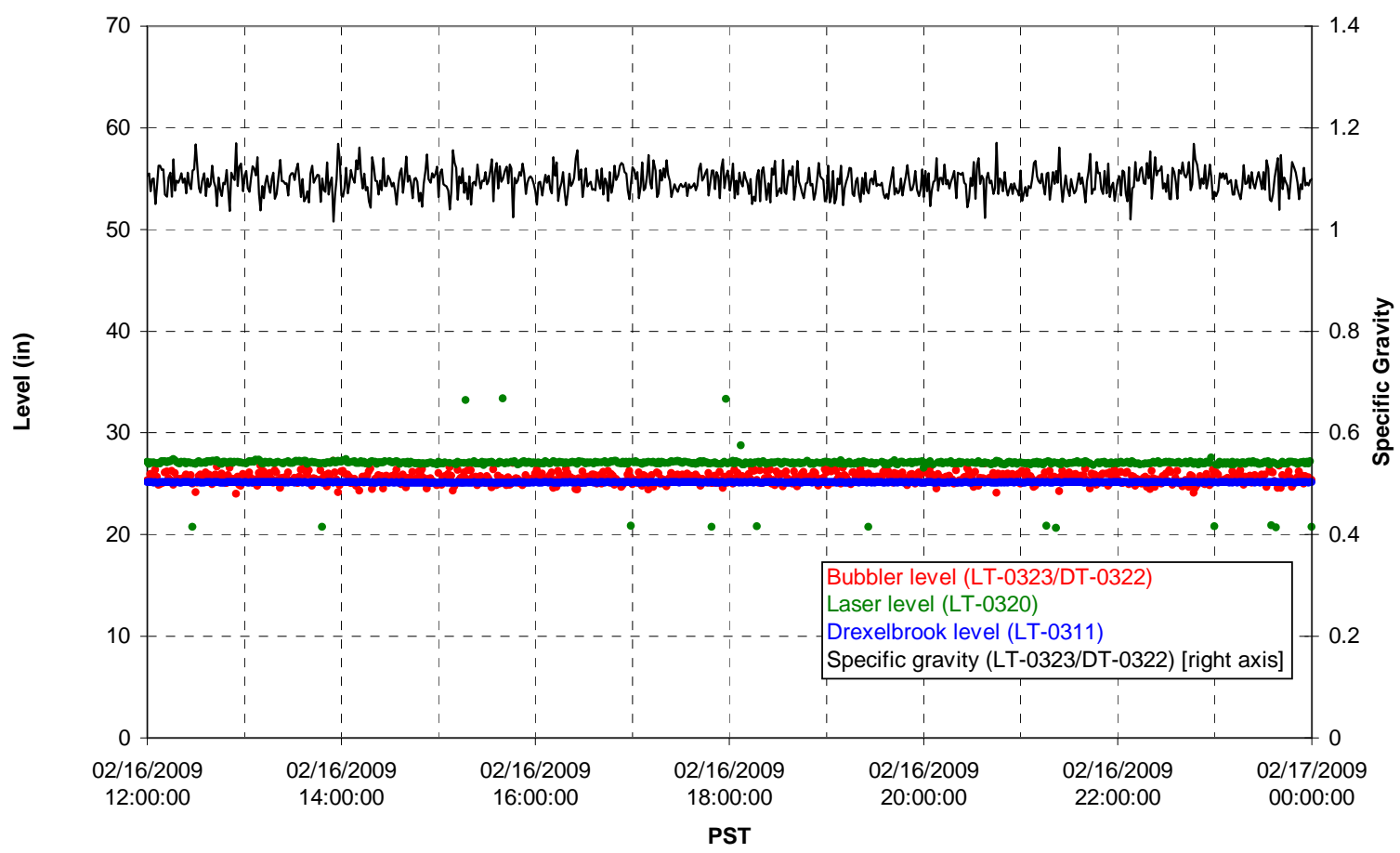

T01A temperatures

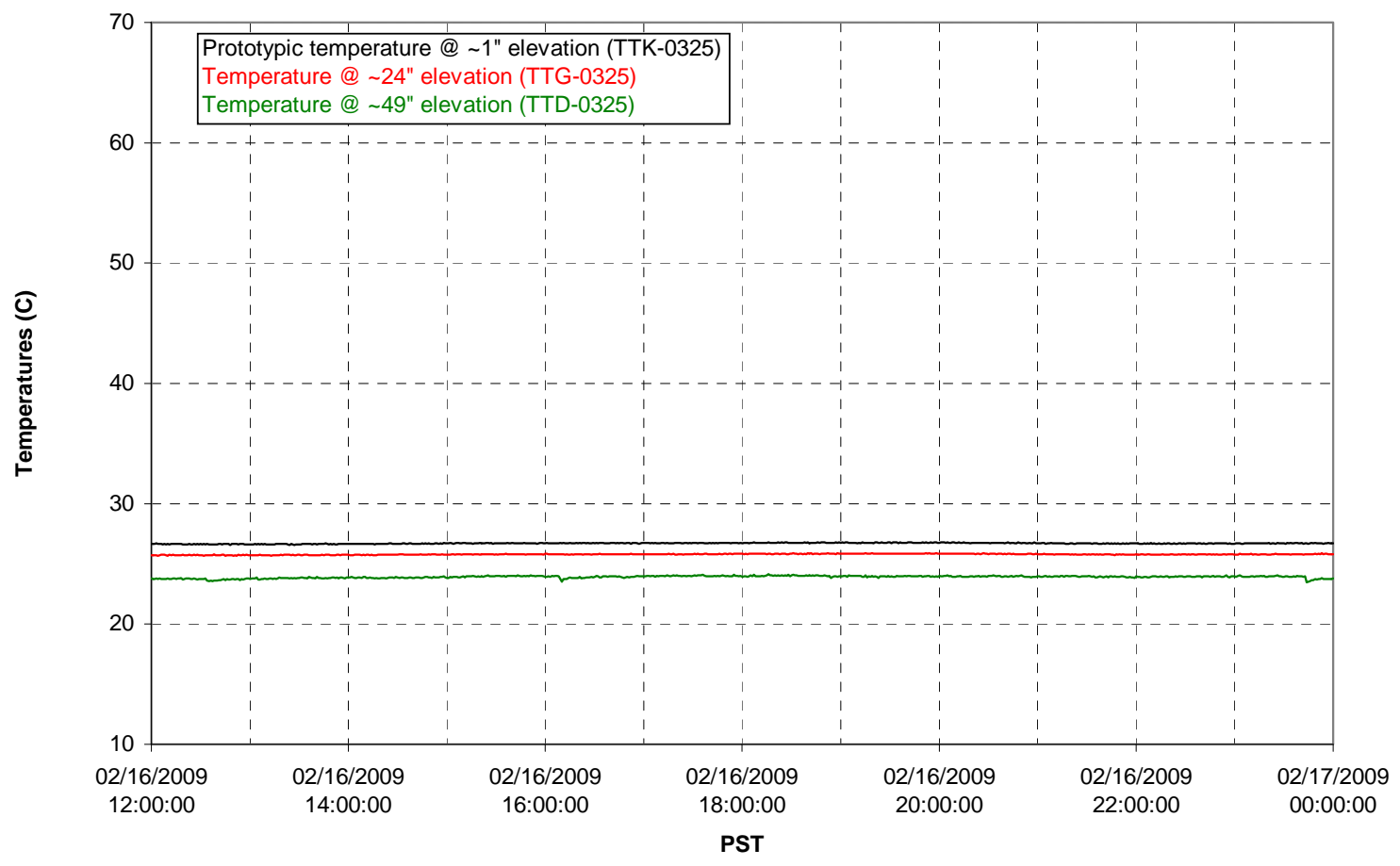


T01B level

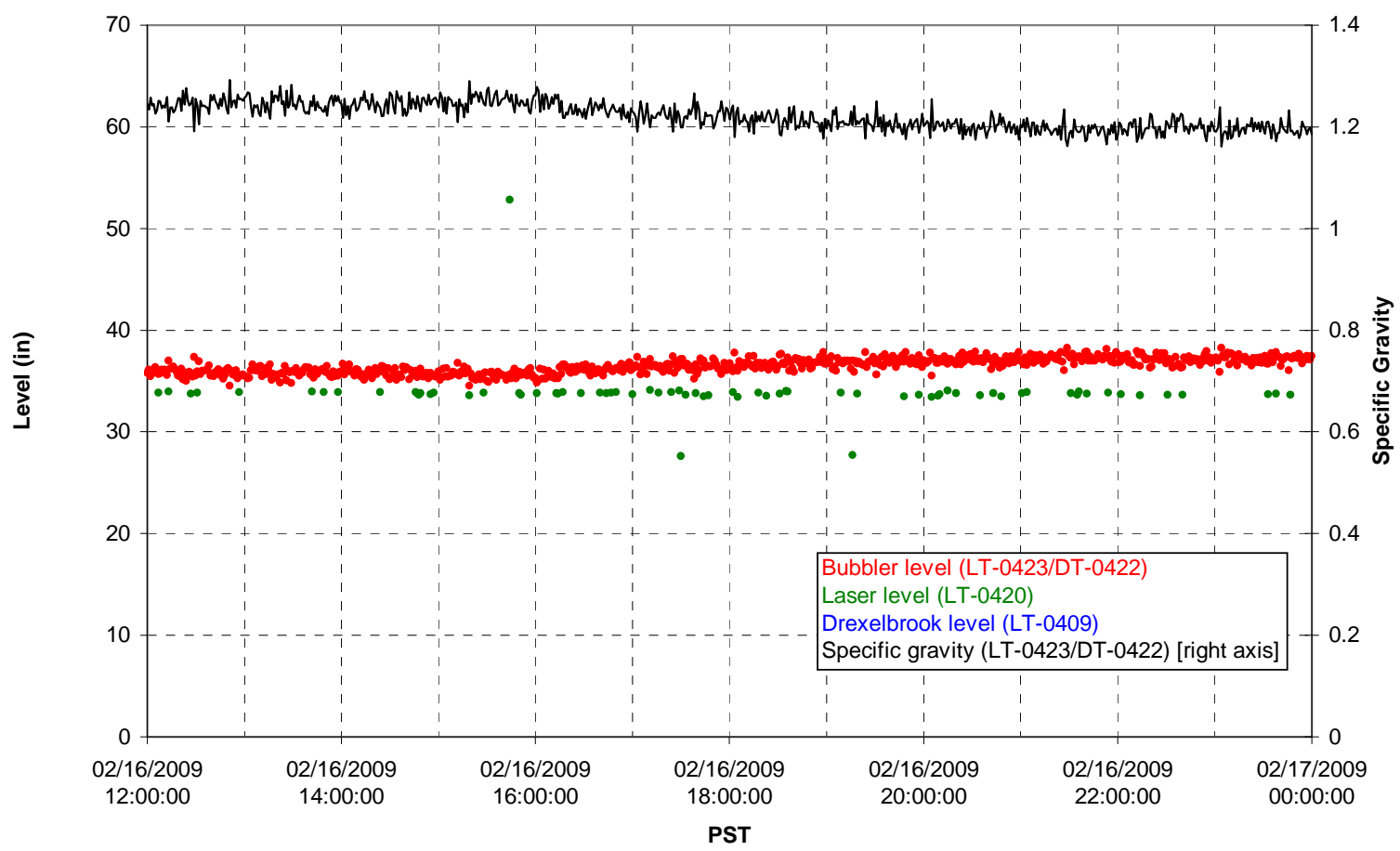

T01B temperatures

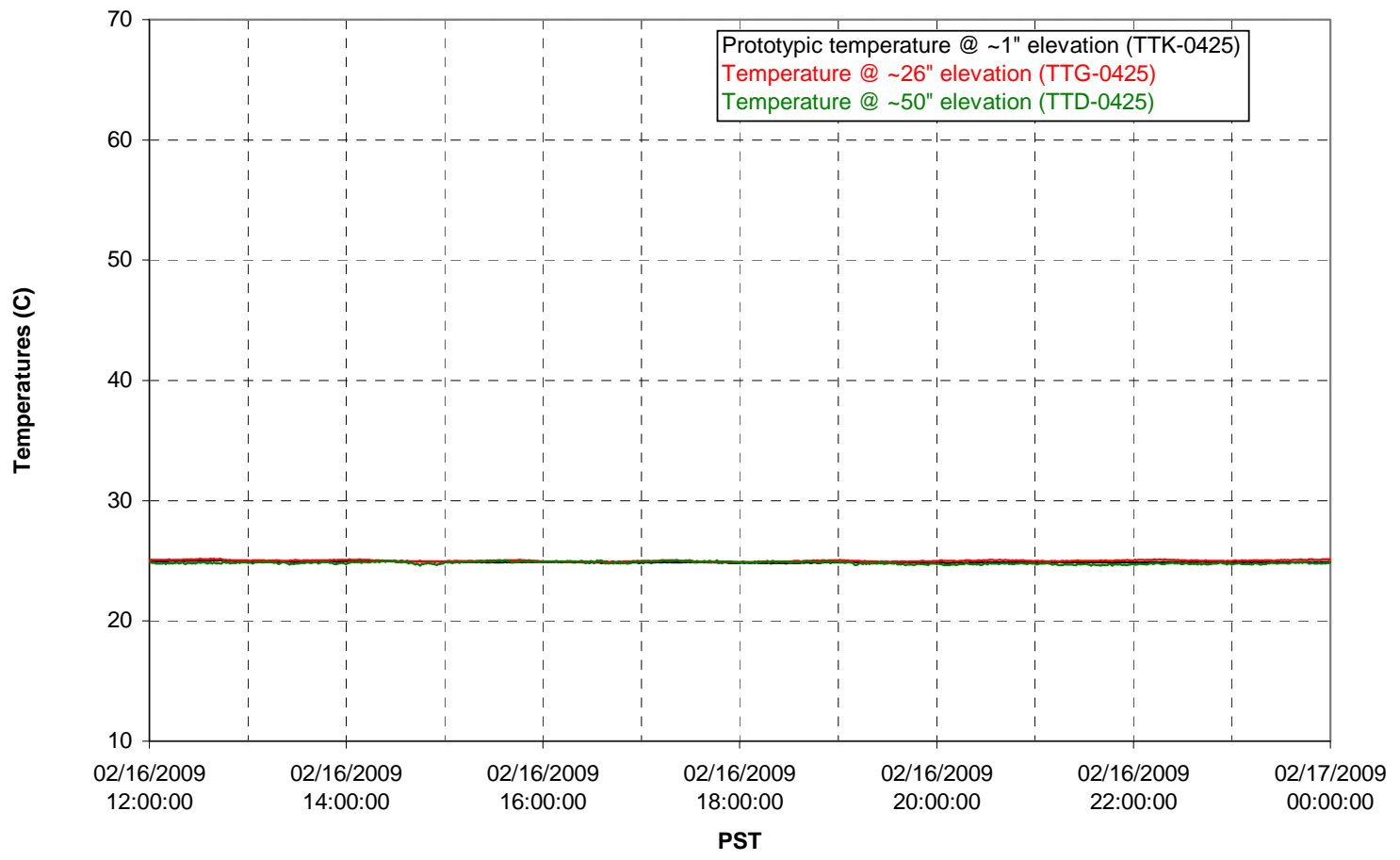


T02A level

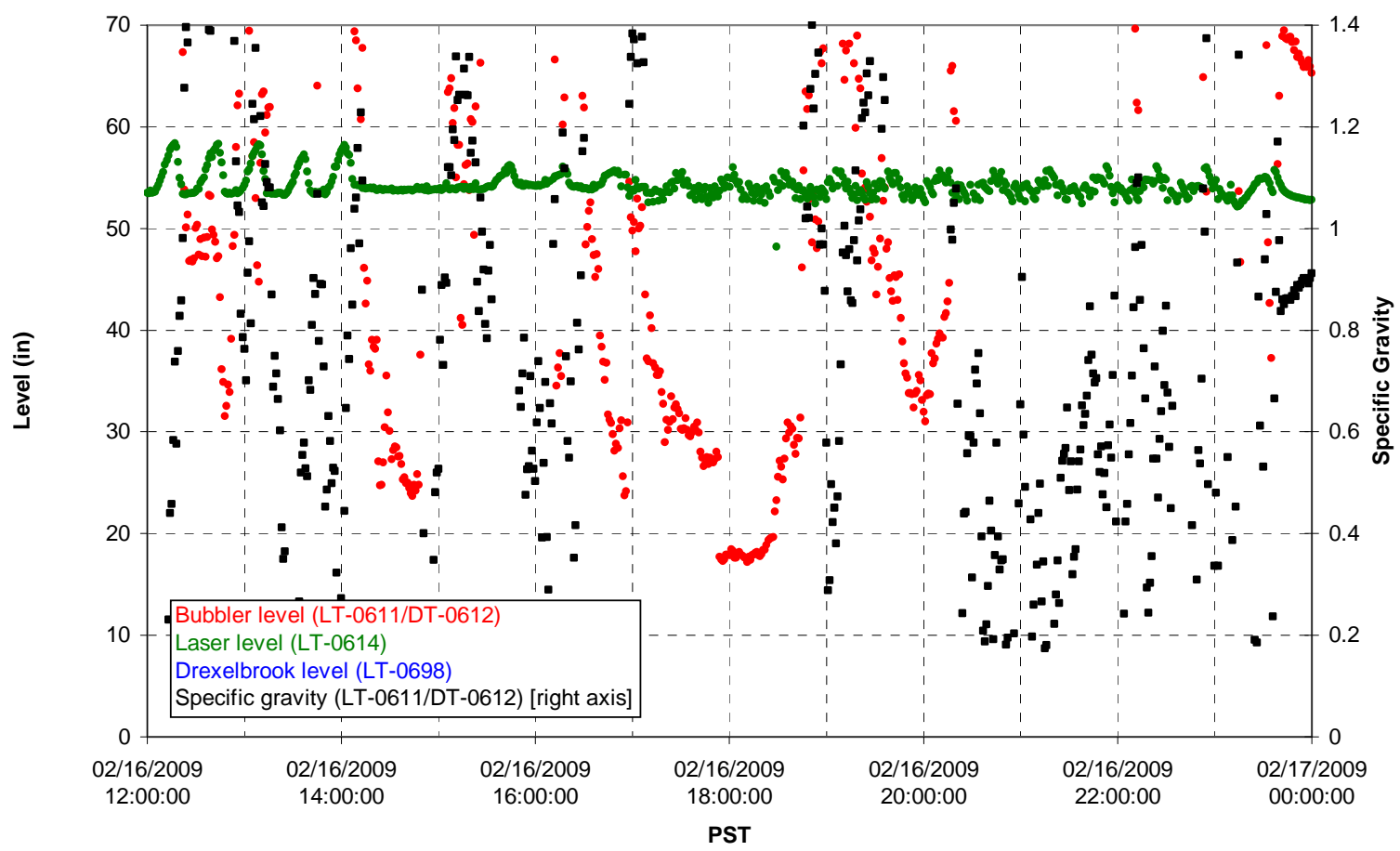

T02A temperatures

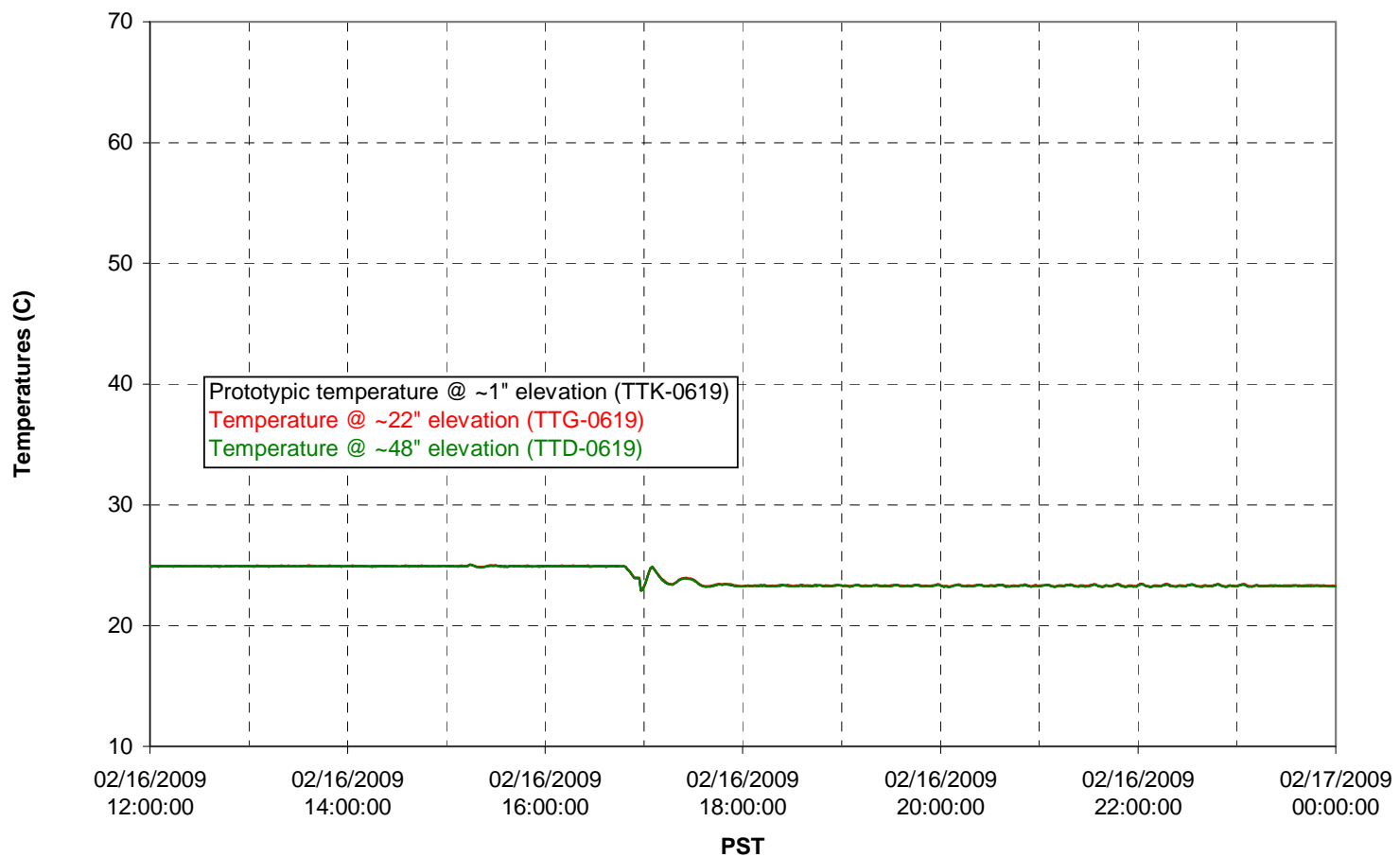


T02A and filter loop temperatures

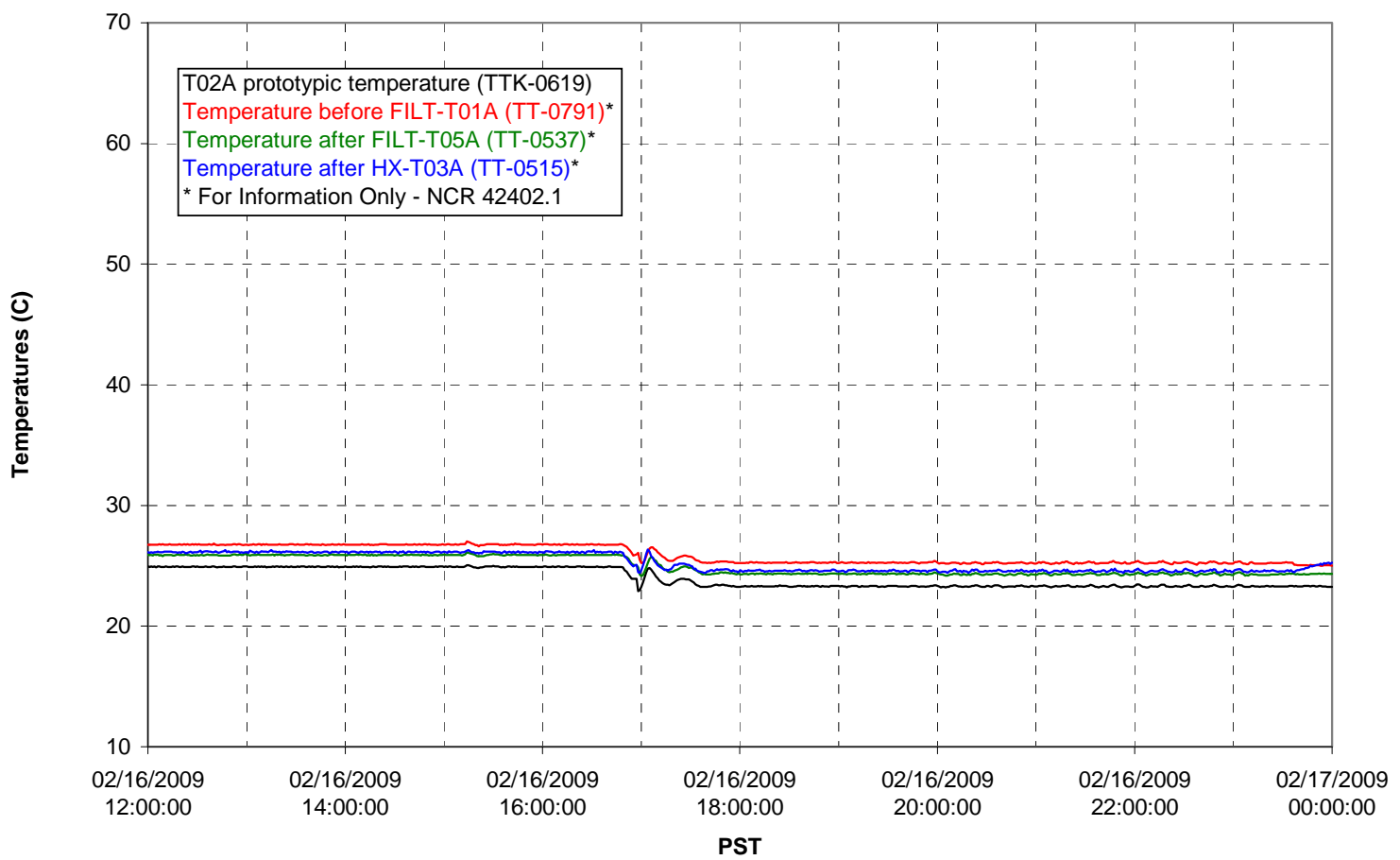

Pump Pressures and Flow

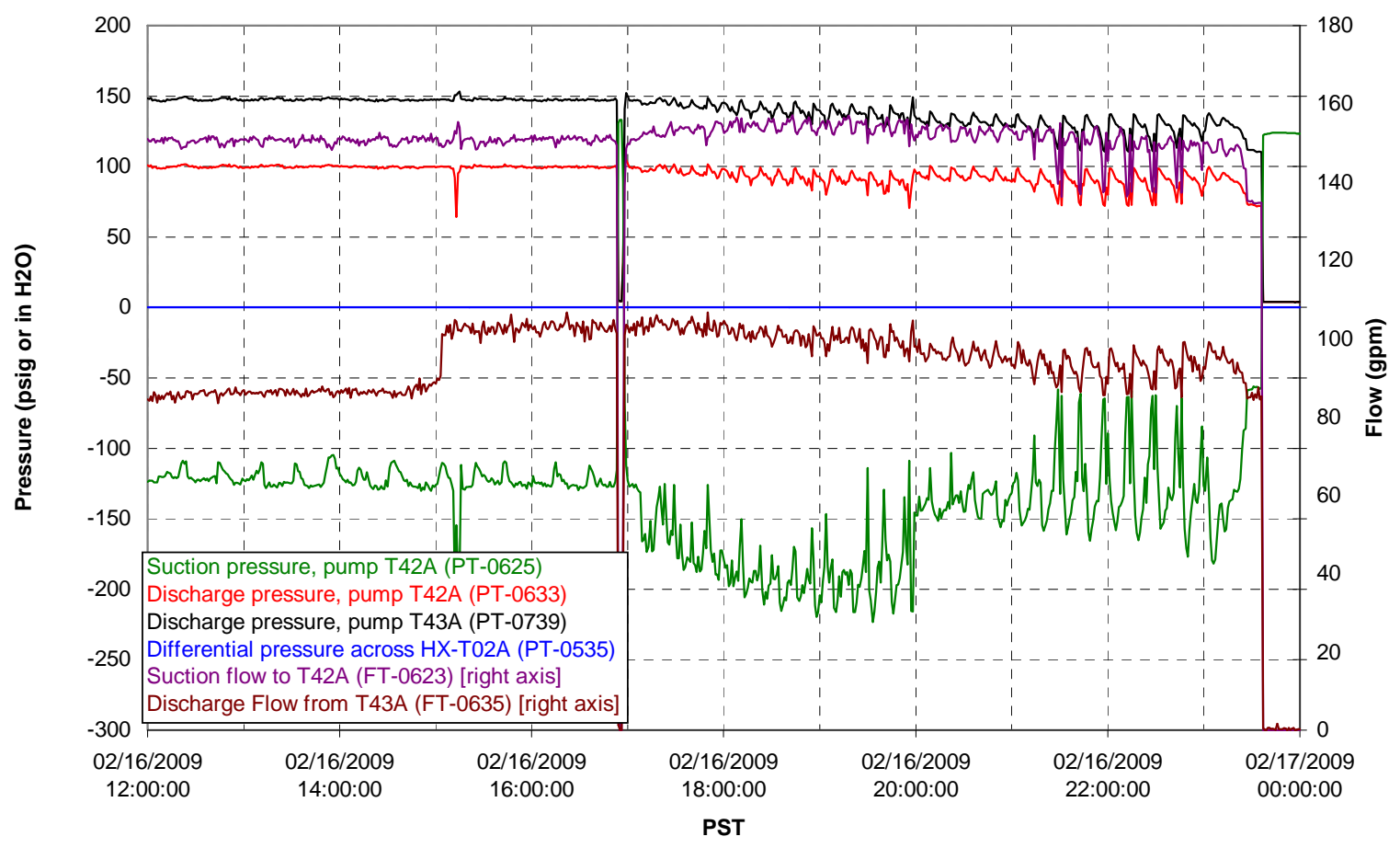




\section{Axial pressure drop}

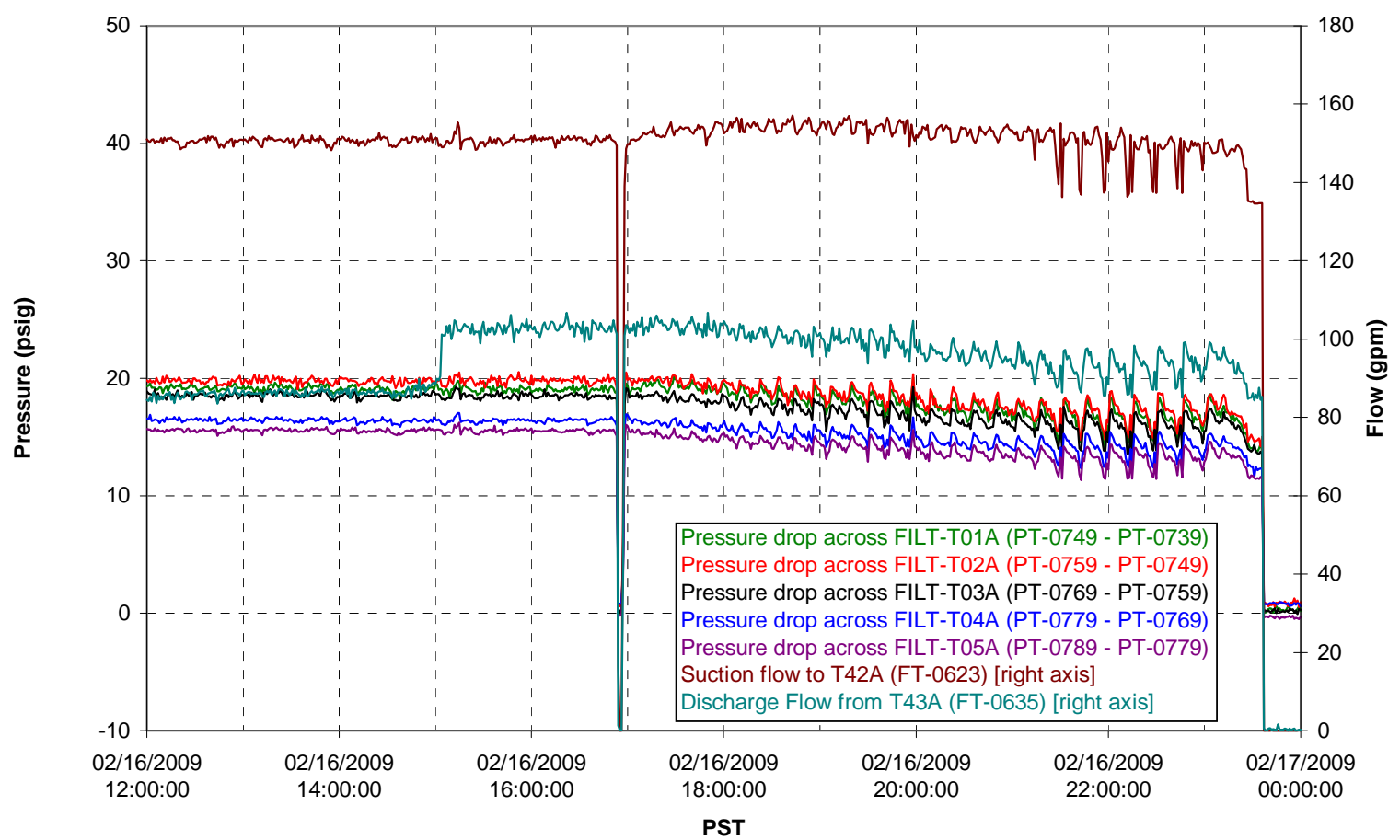

Permeate flow rates

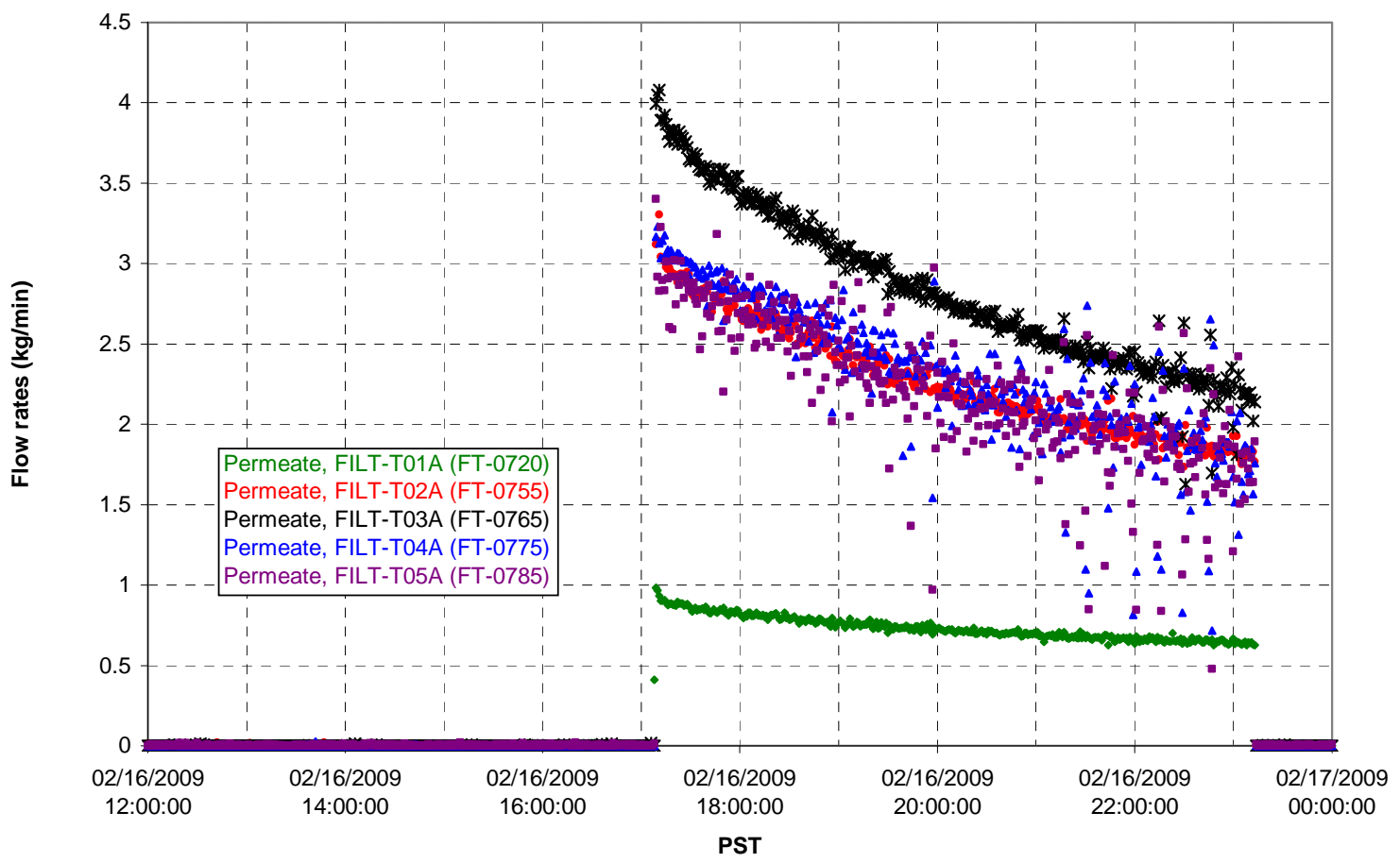


T02A Inner Temperature Tree

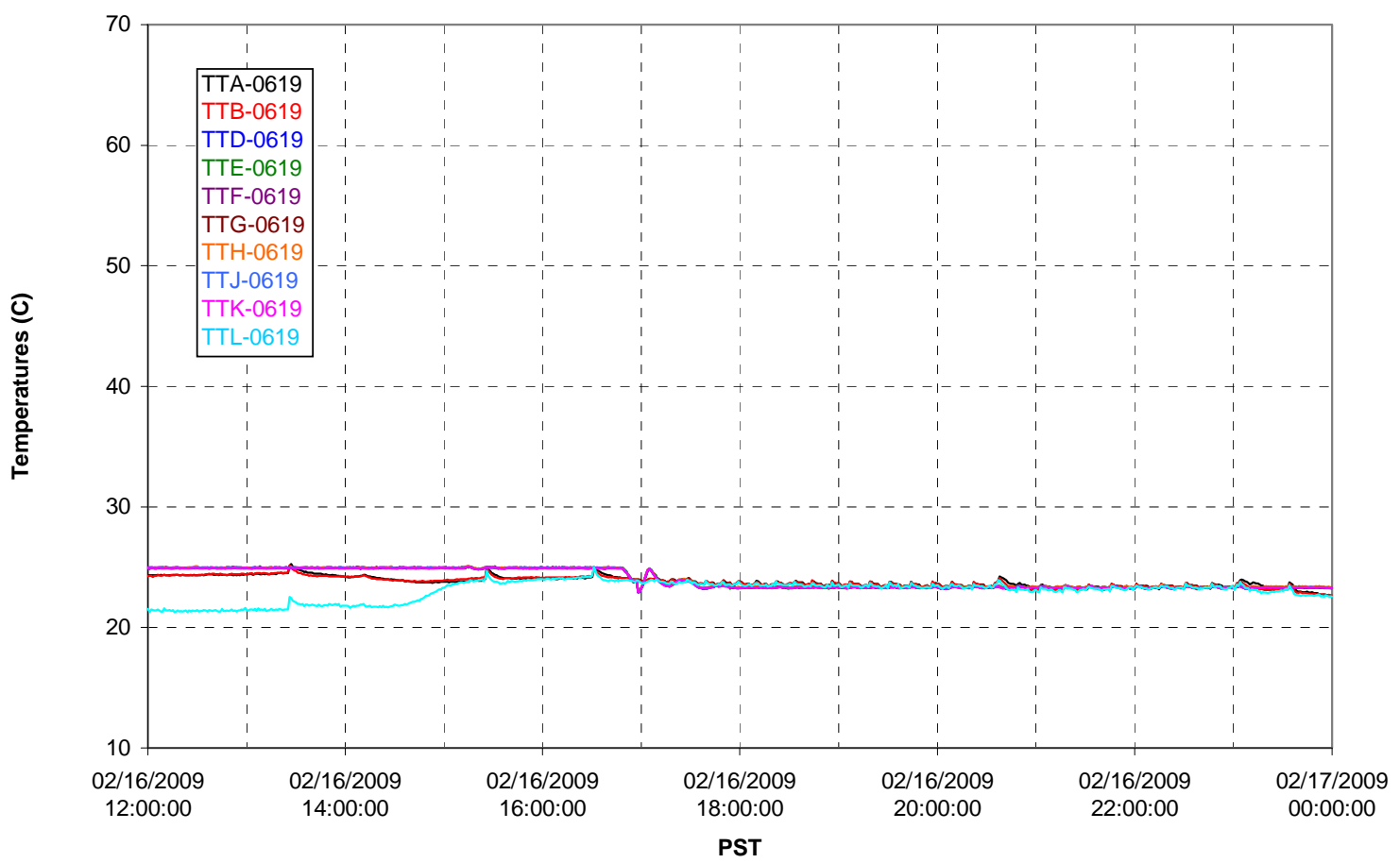

T02A Outer Temperature Tree

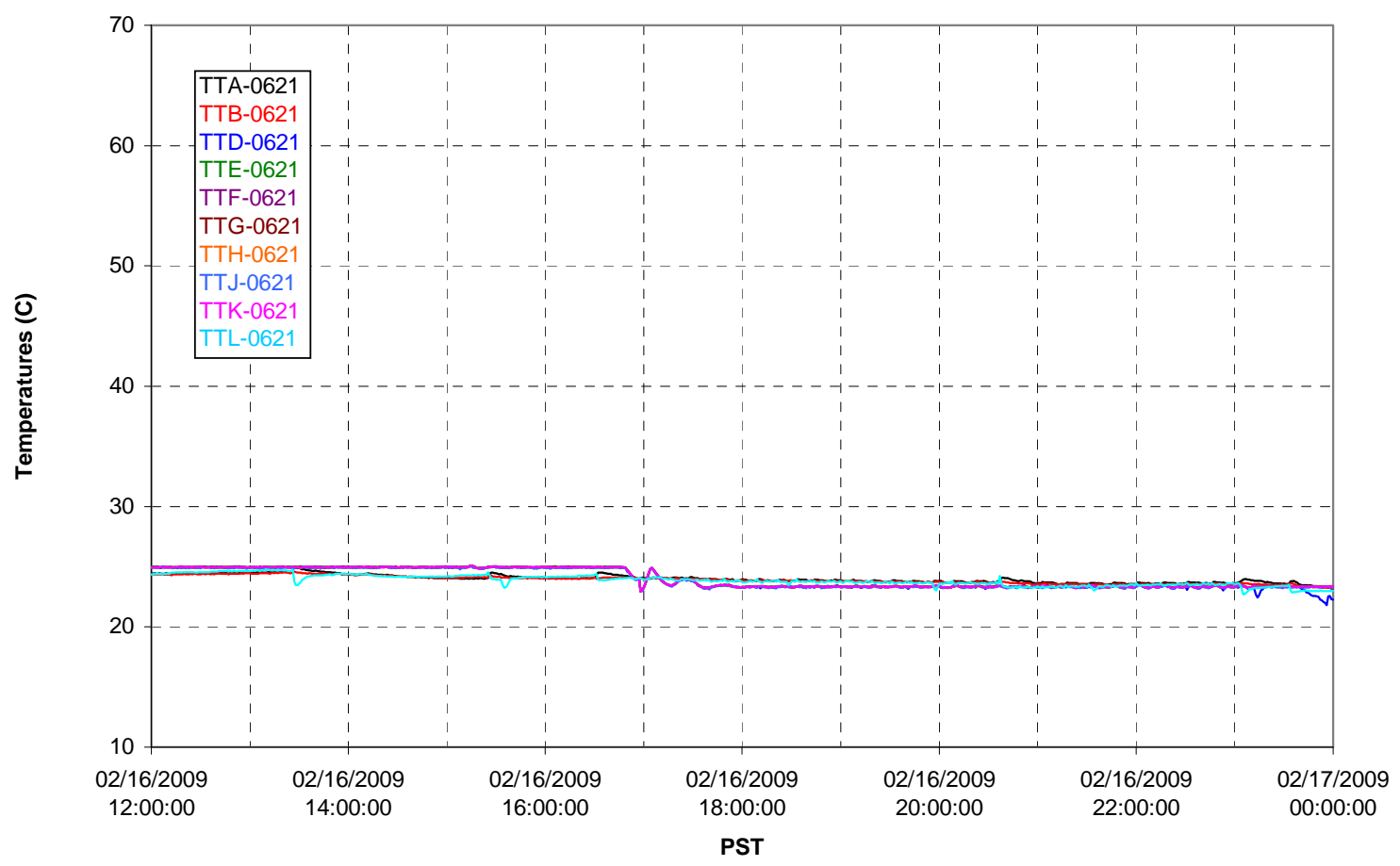


T02A temperatures

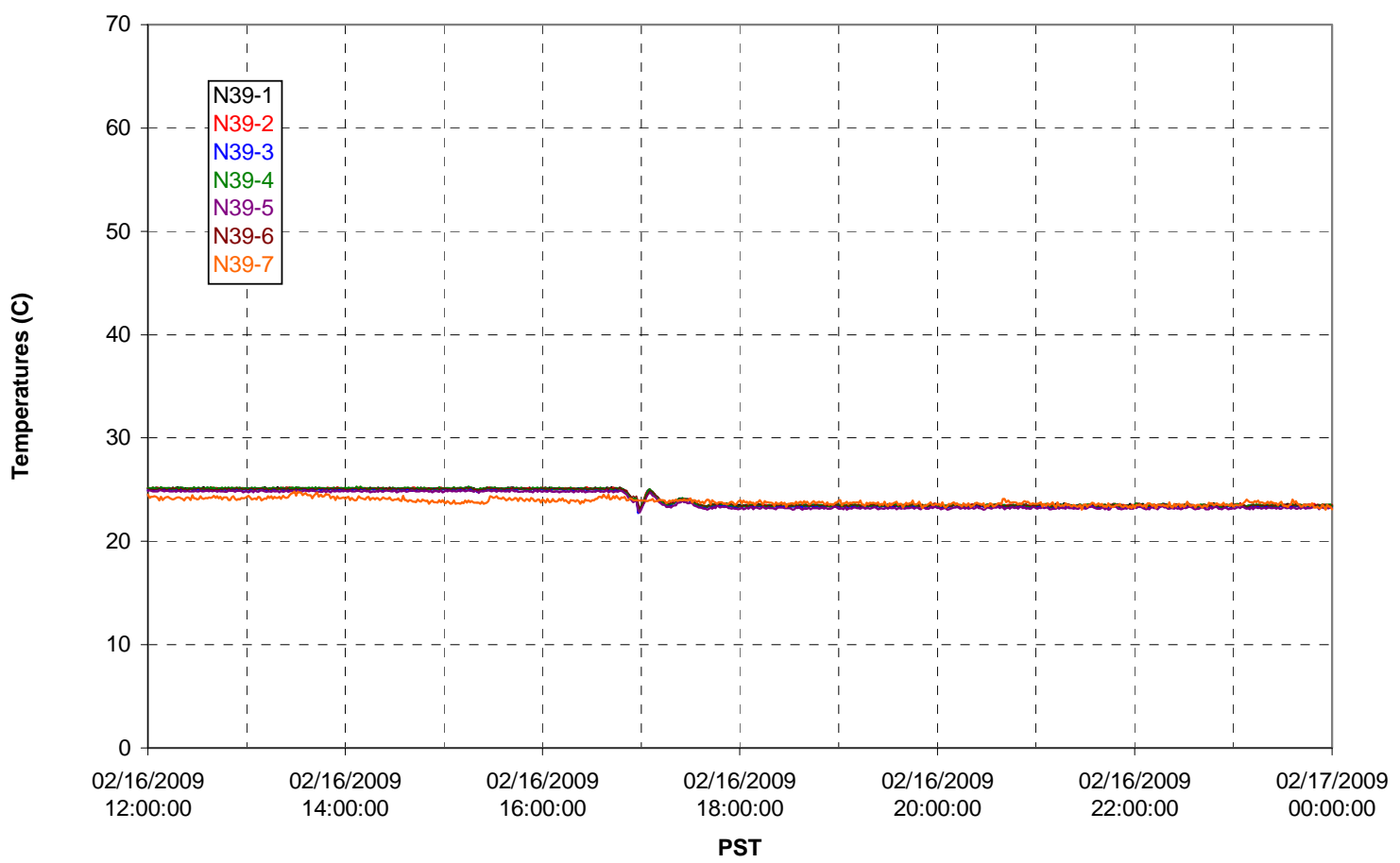

T02A temperatures

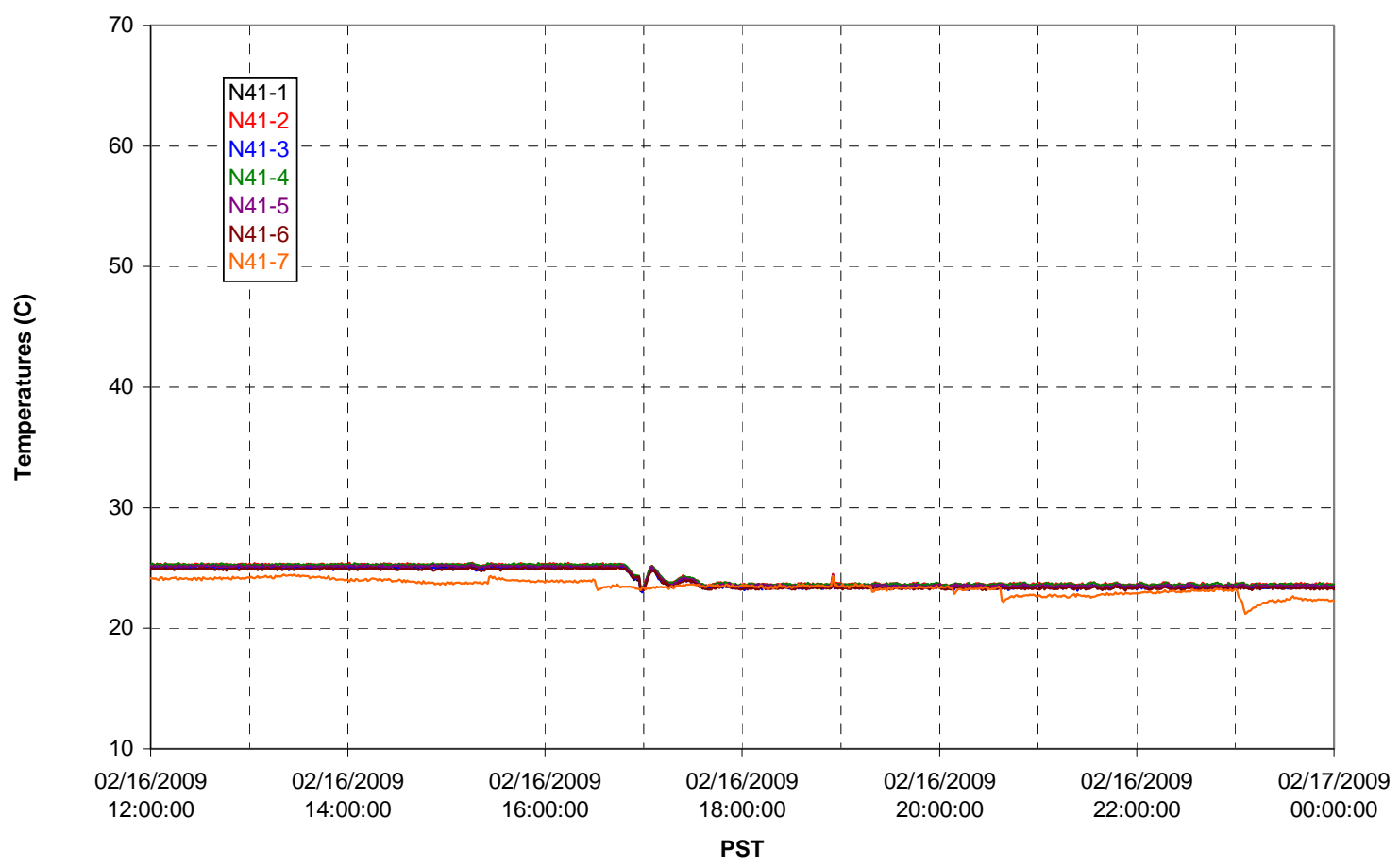


T02A temperatures

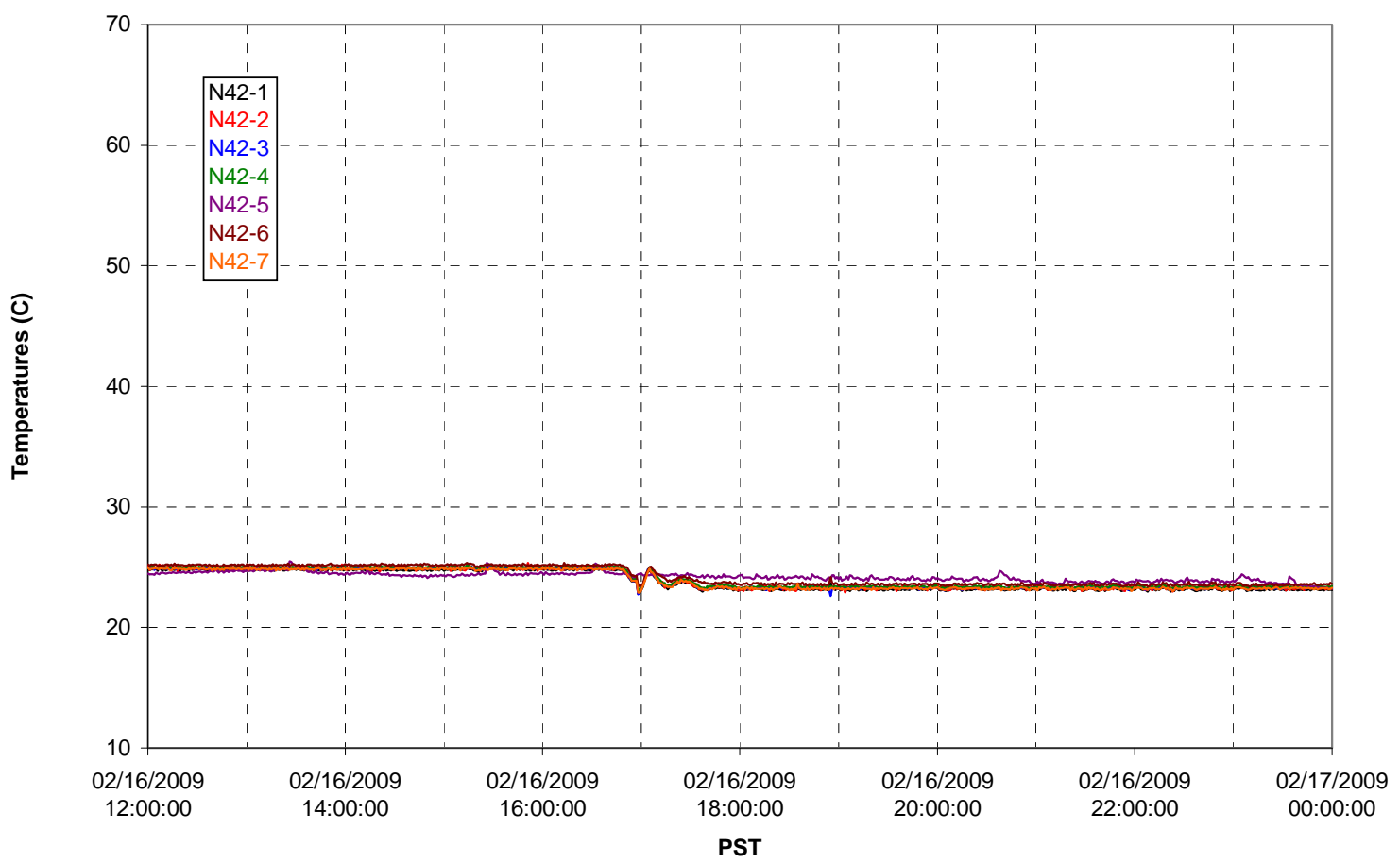

T02A temperatures

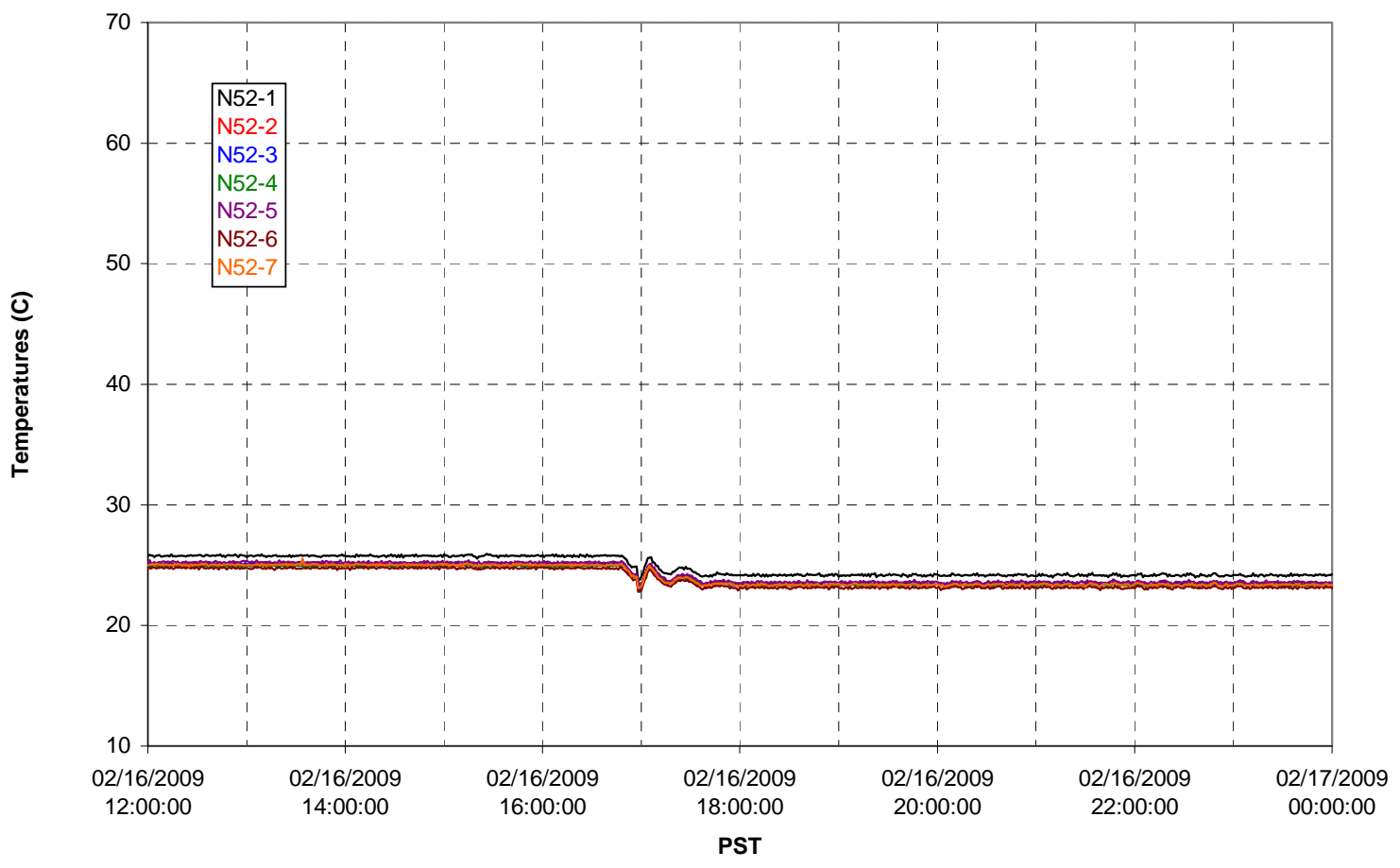


T02A Heating and Cooling
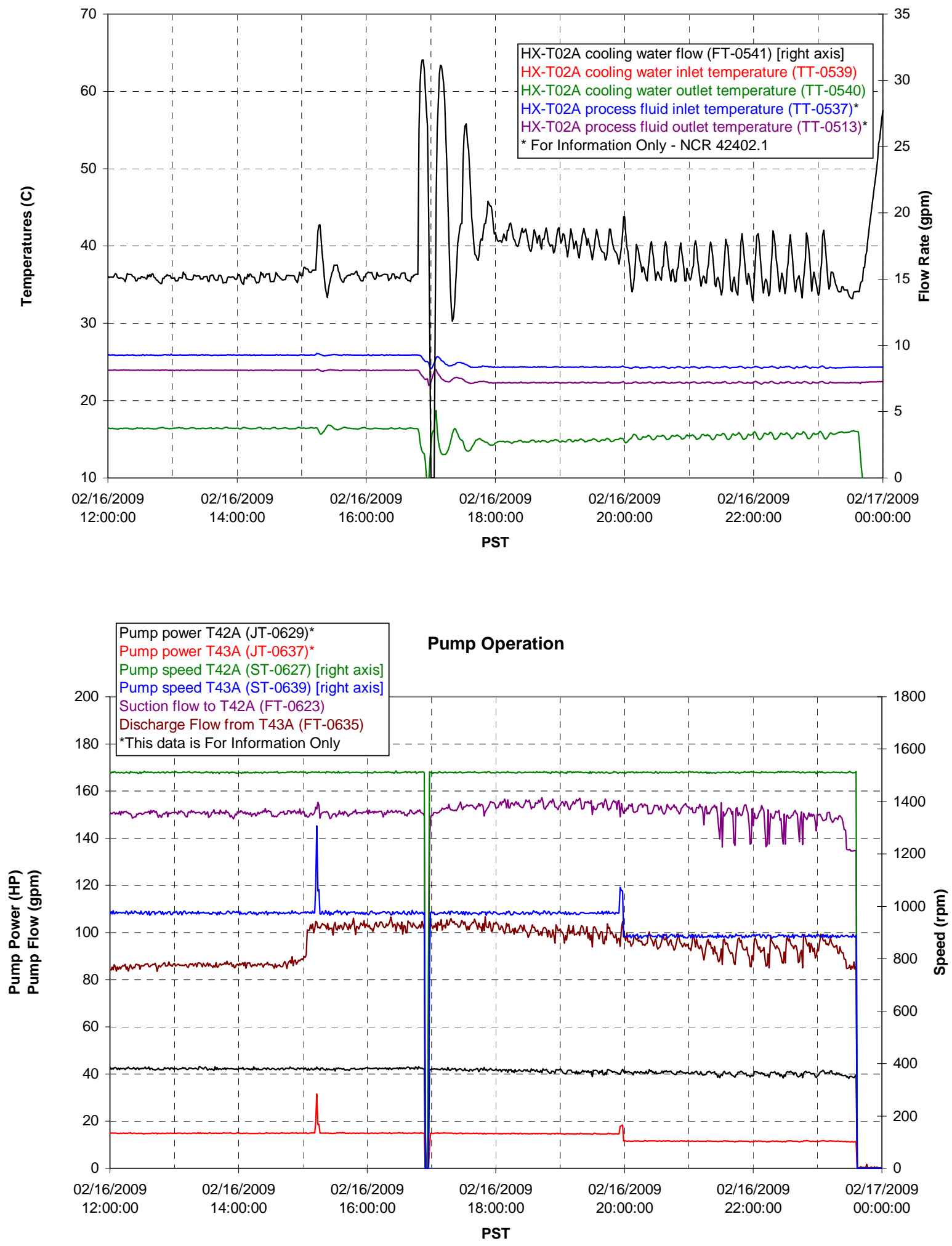
Pulsepot UFP-PP-T01A

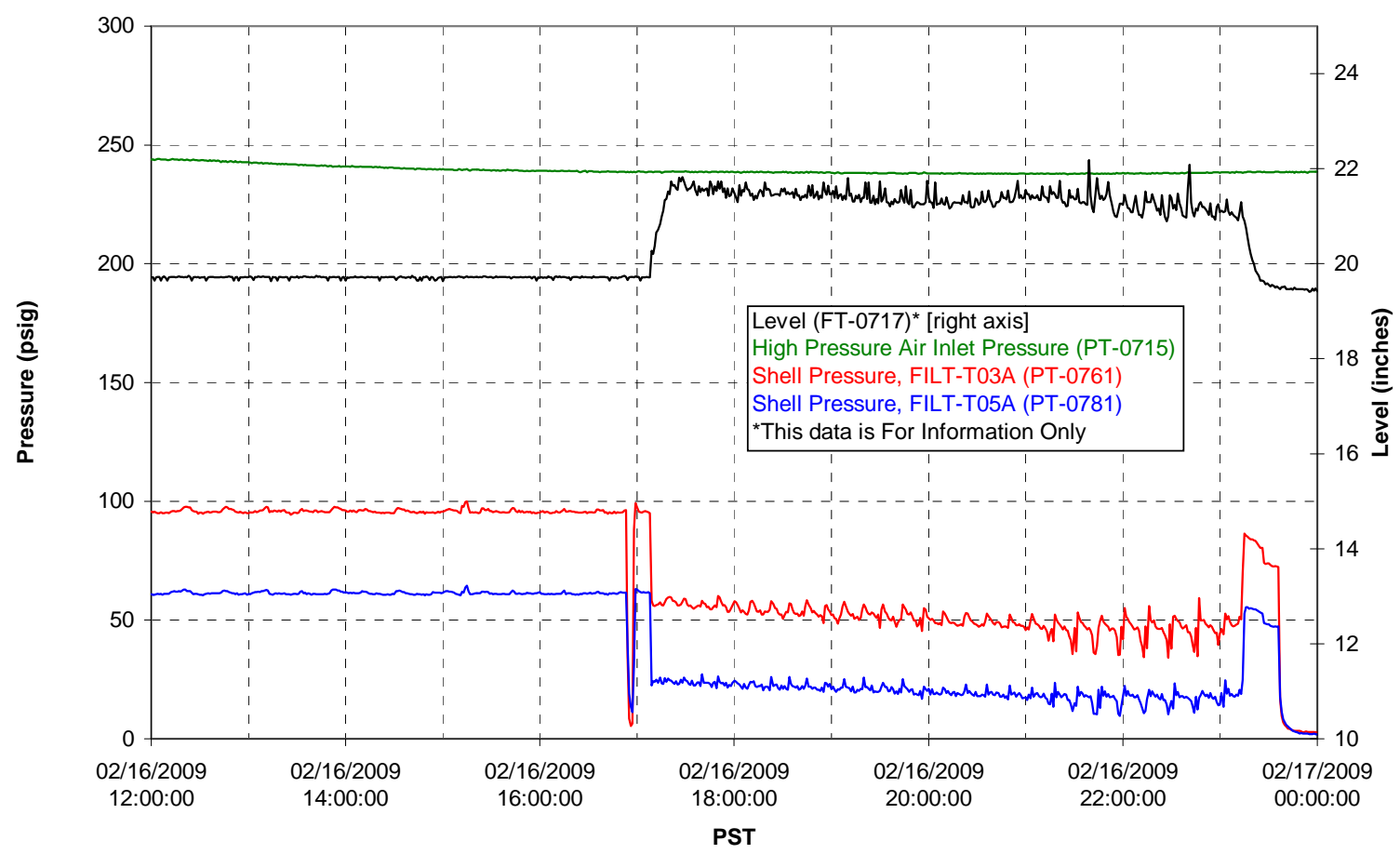

Pulsepot UFP-PP-T02A

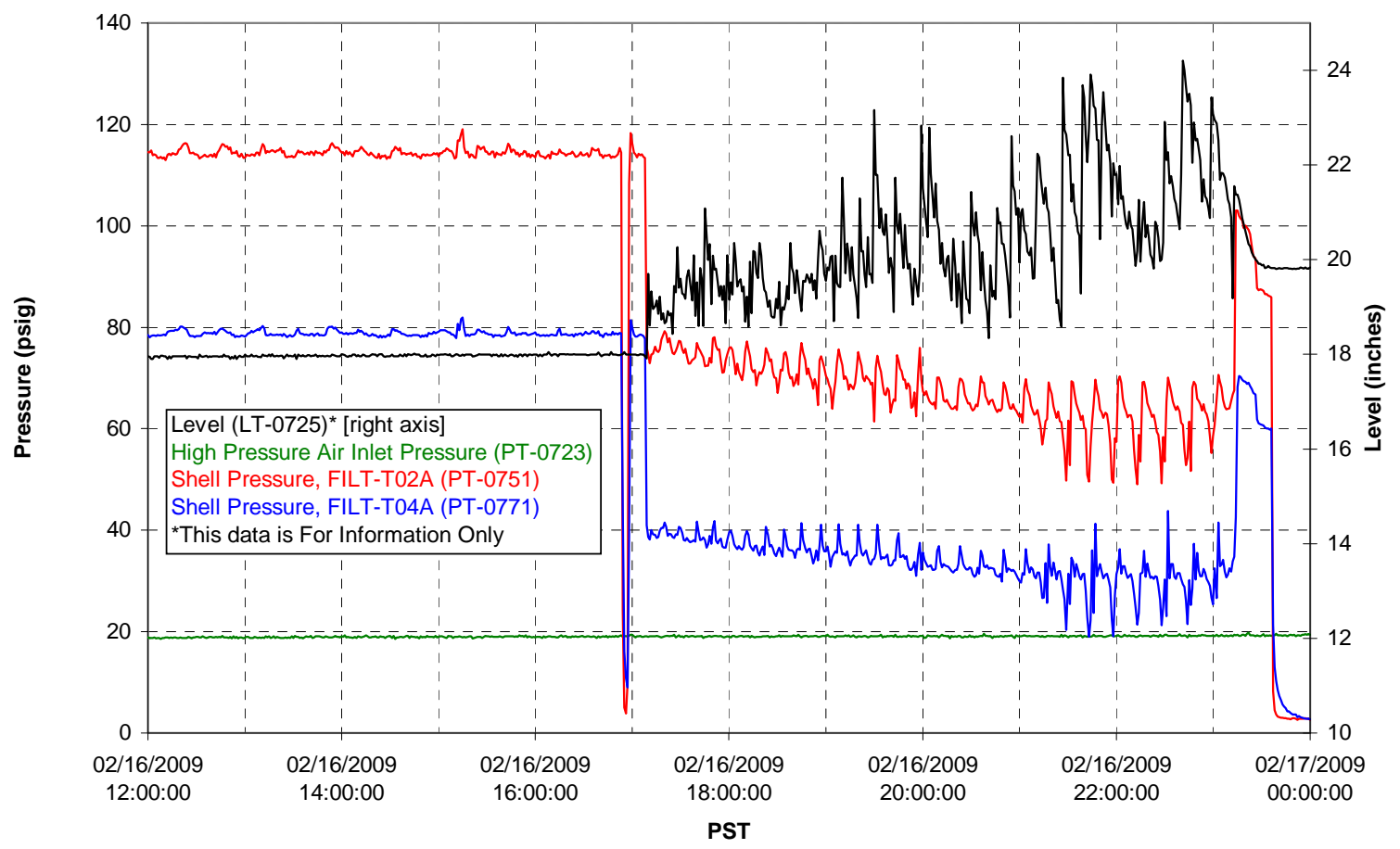


Pulsepot UFP-PP-T03A

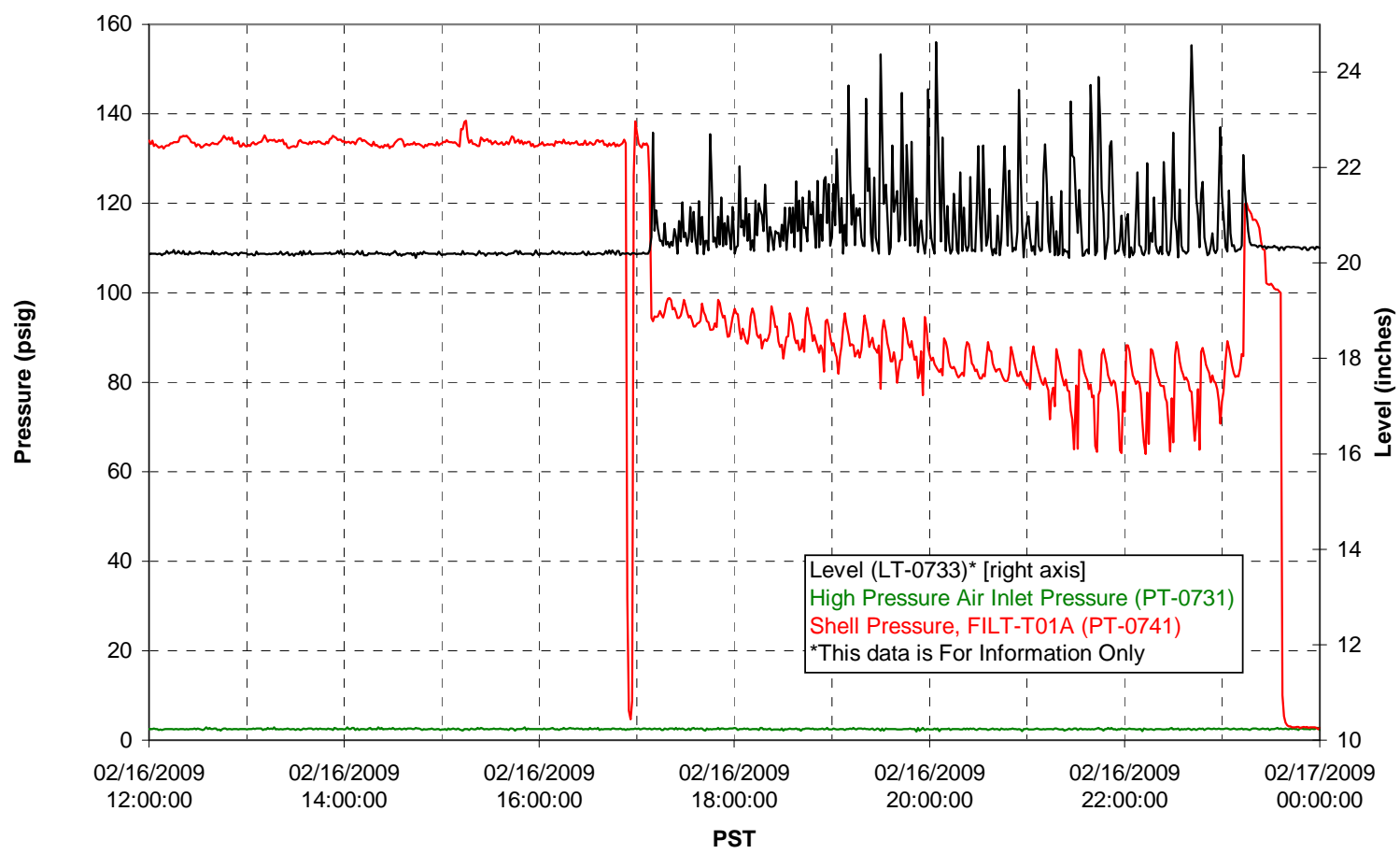

Pulsepot Levels

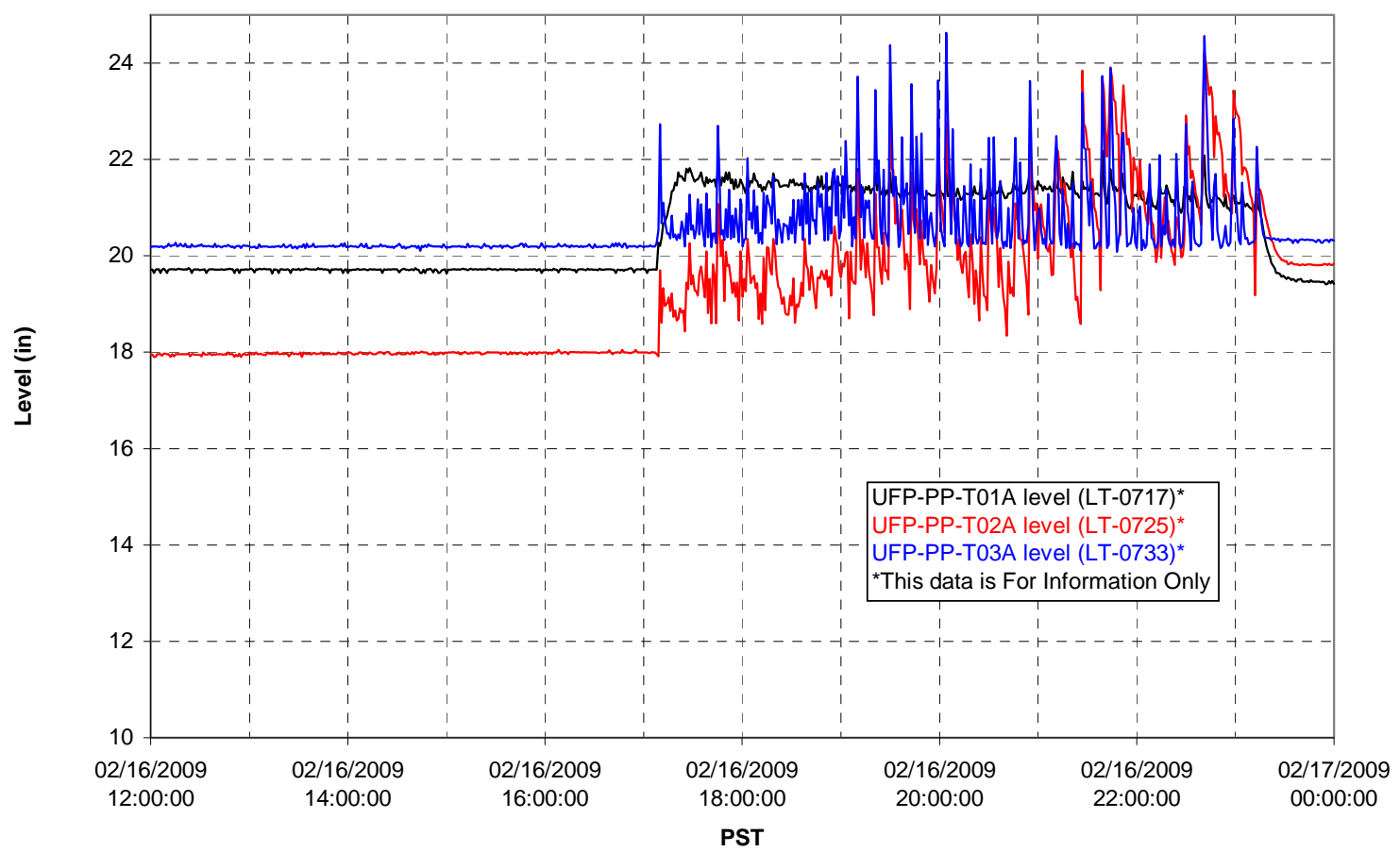


Filter UFP-FILT-T01A

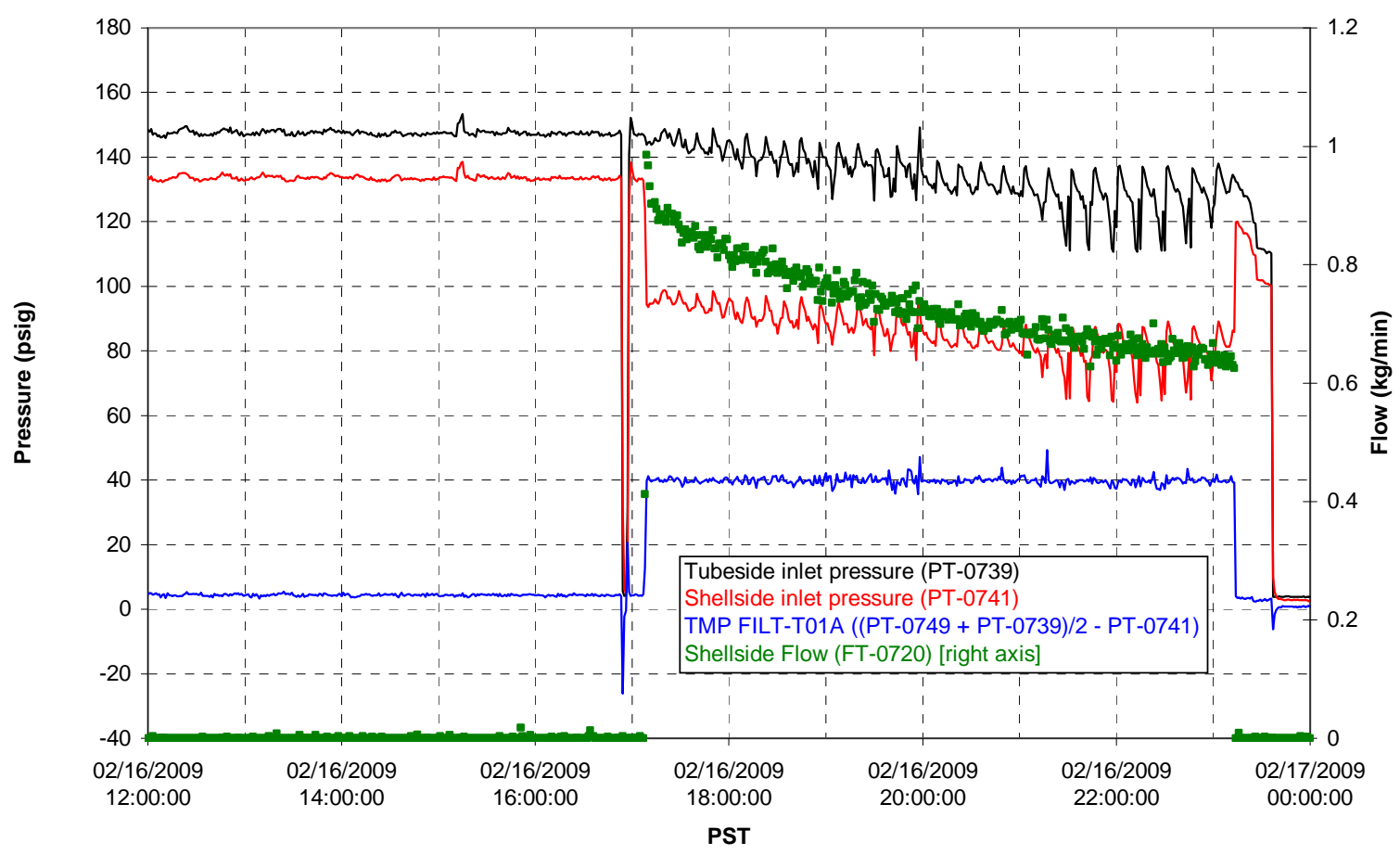

Filter UFP-FILT-T02A

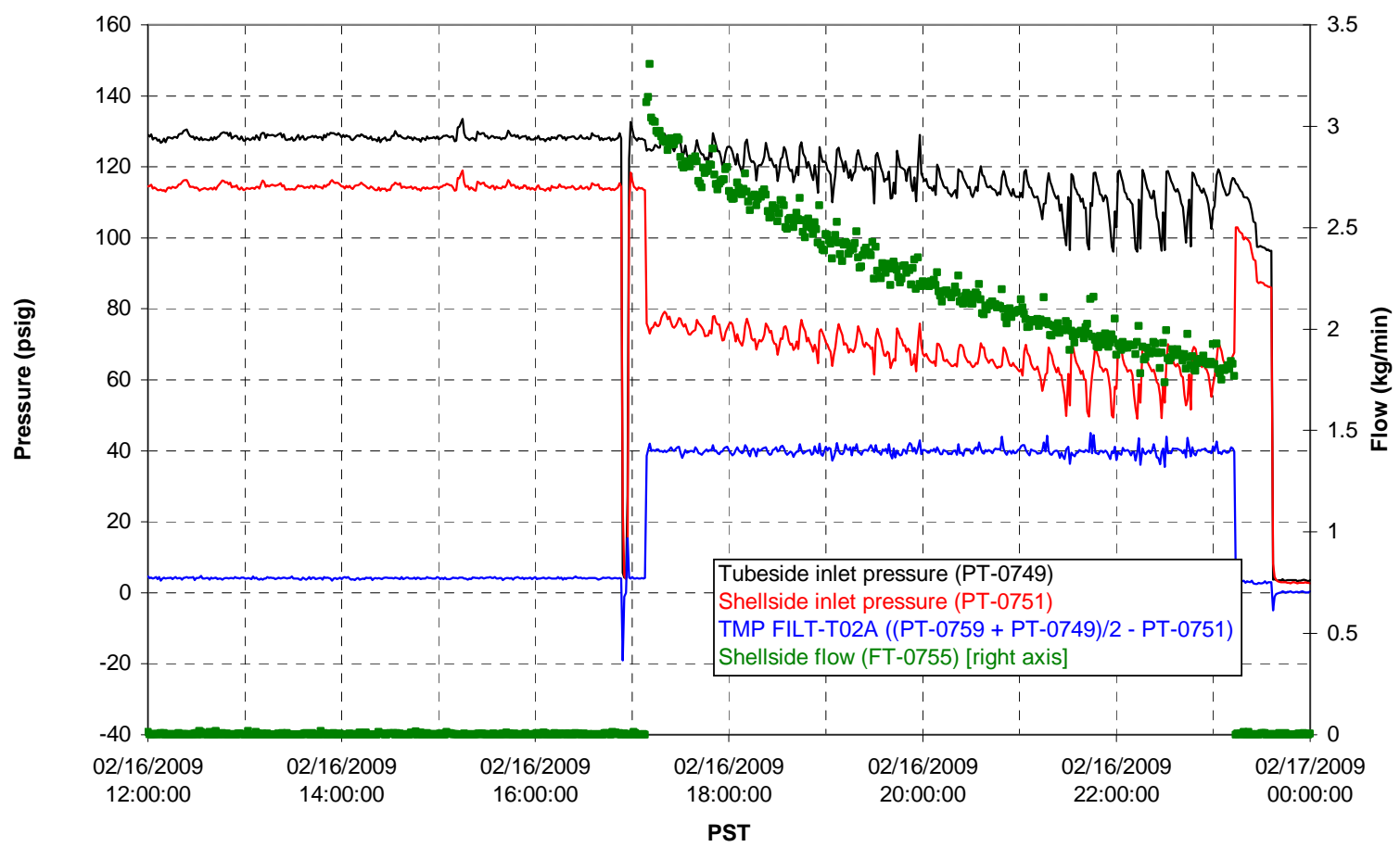


Filter UFP-FILT-T03A

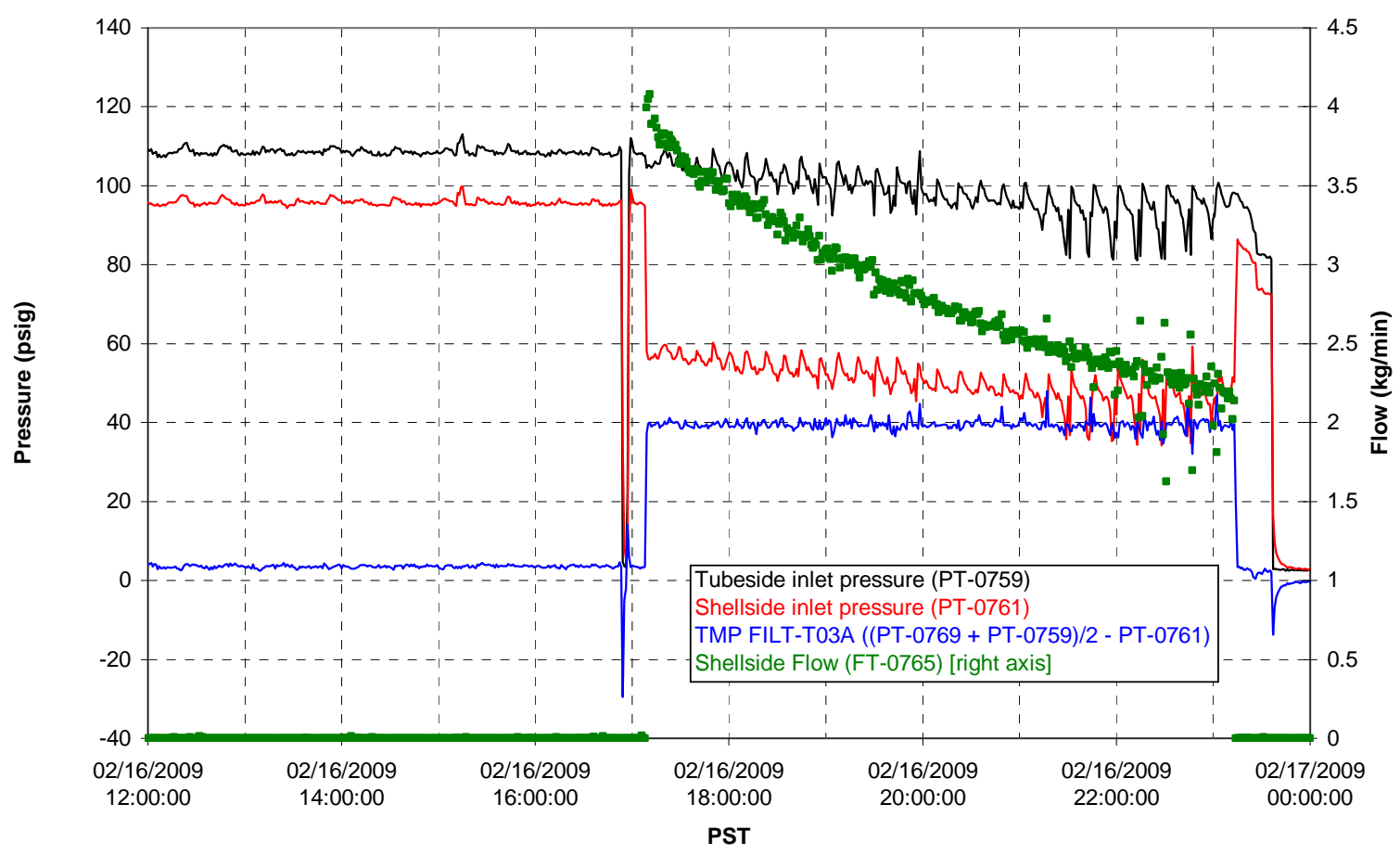

Filter UFP-FILT-T04A

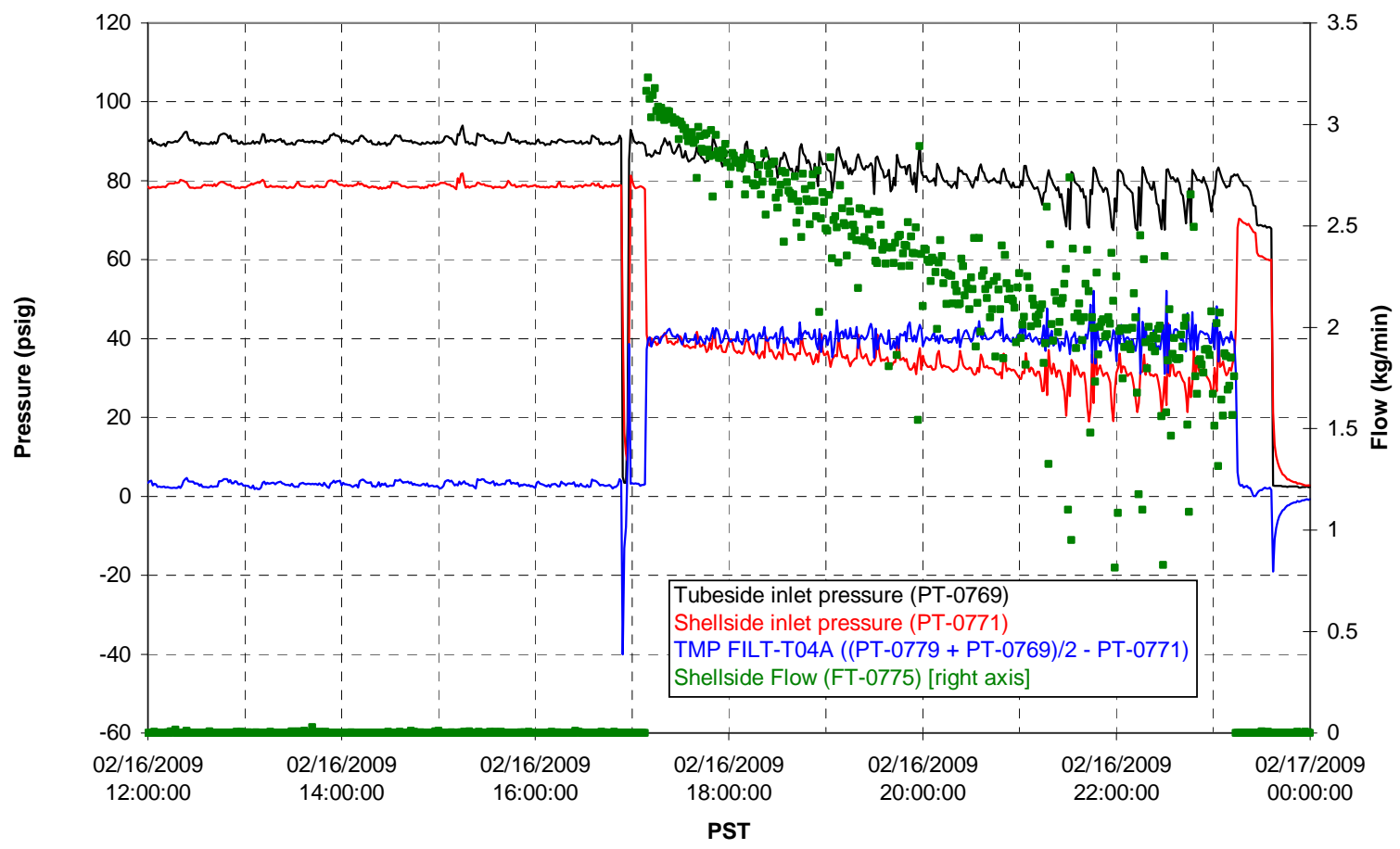


Filter UFP-FILT-T05A

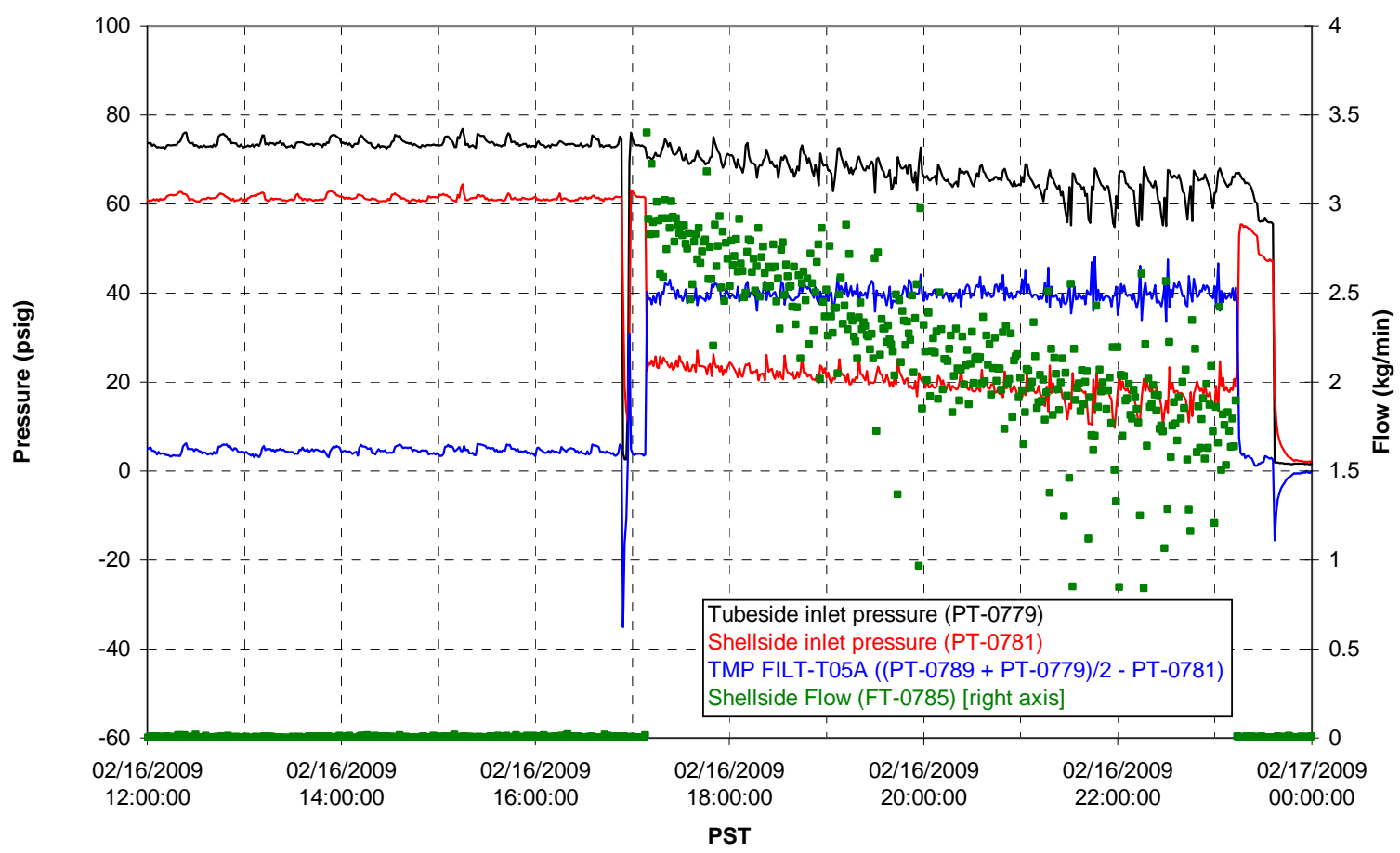

Chemical Flow

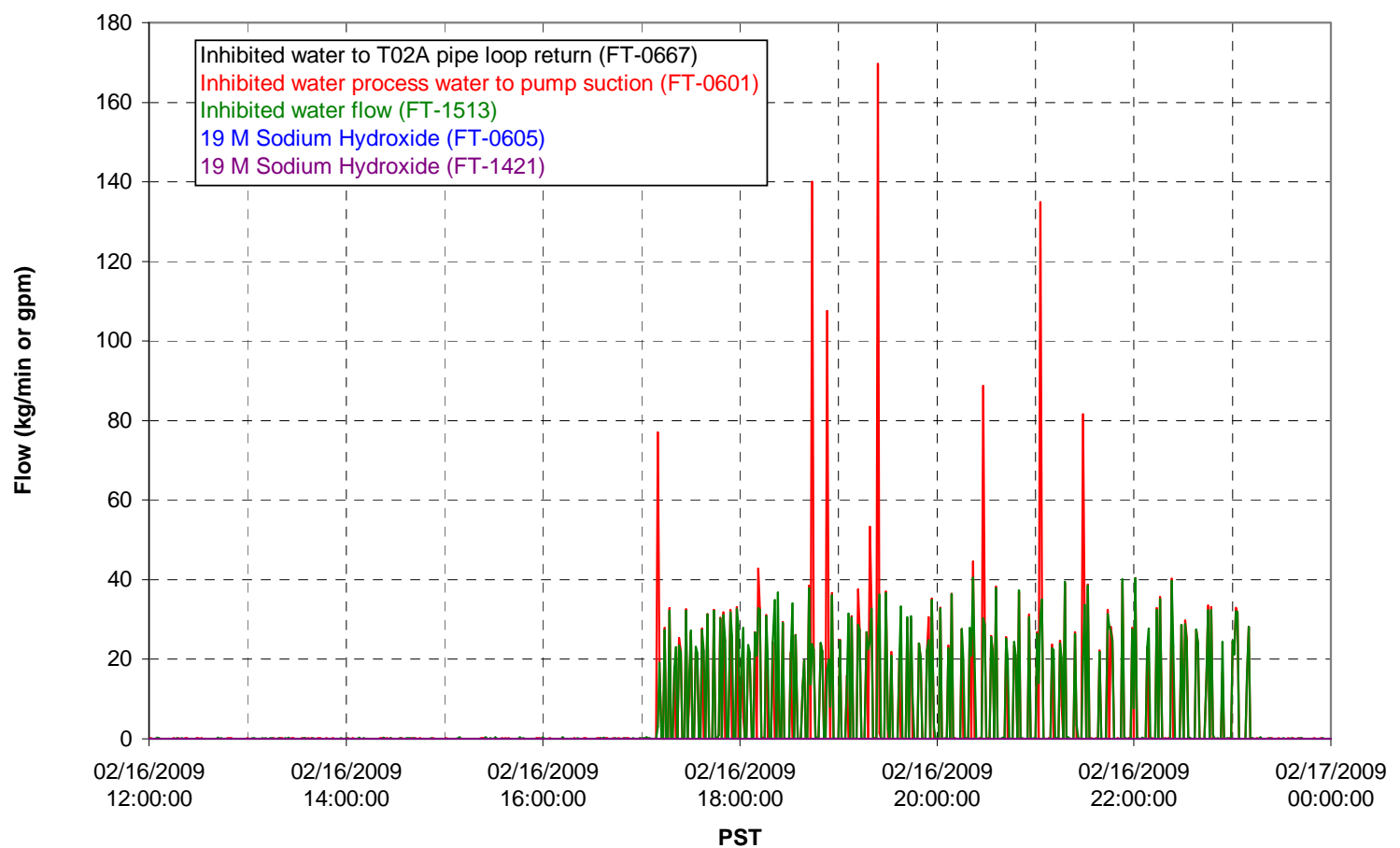




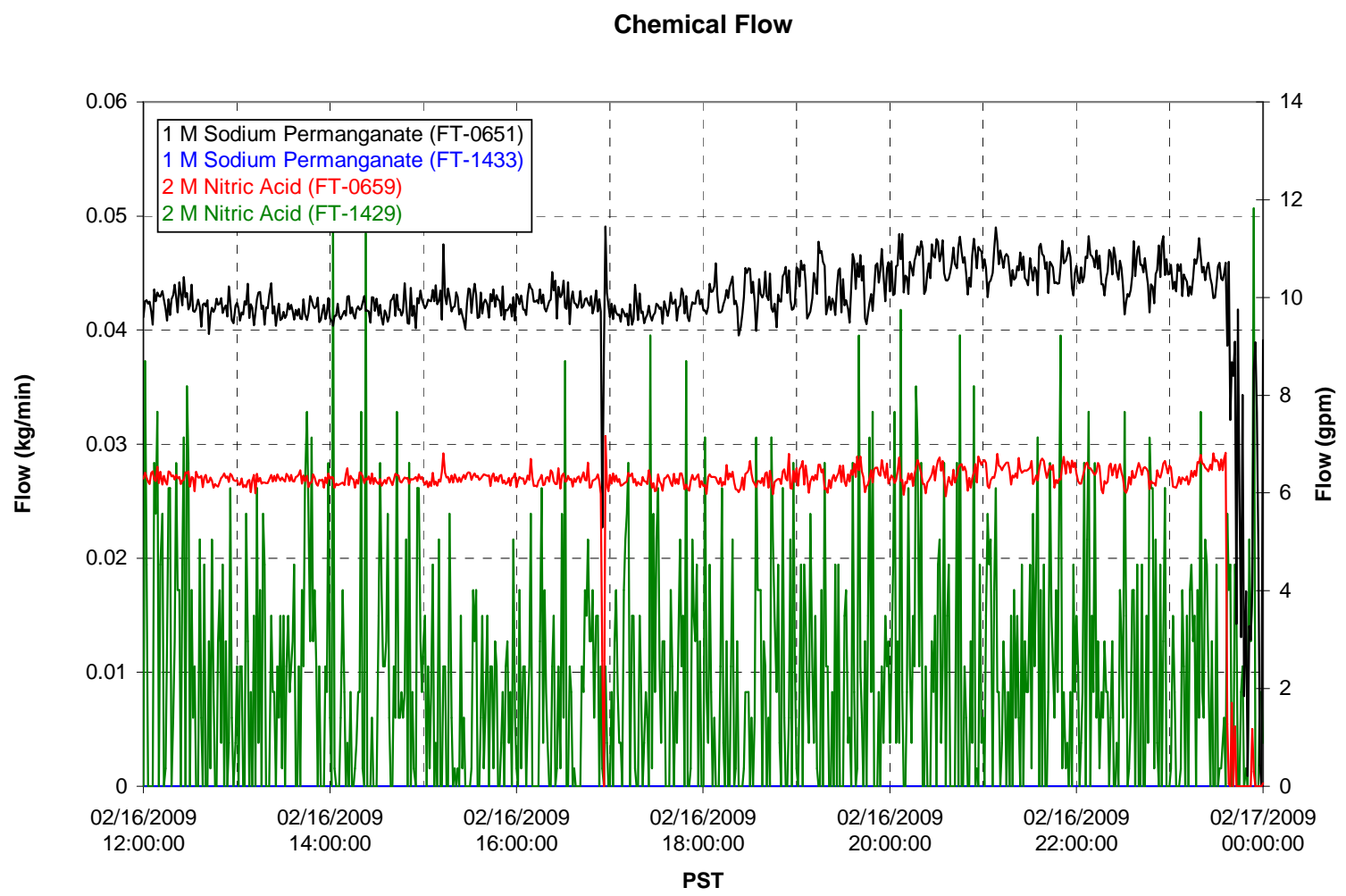

Air Flows

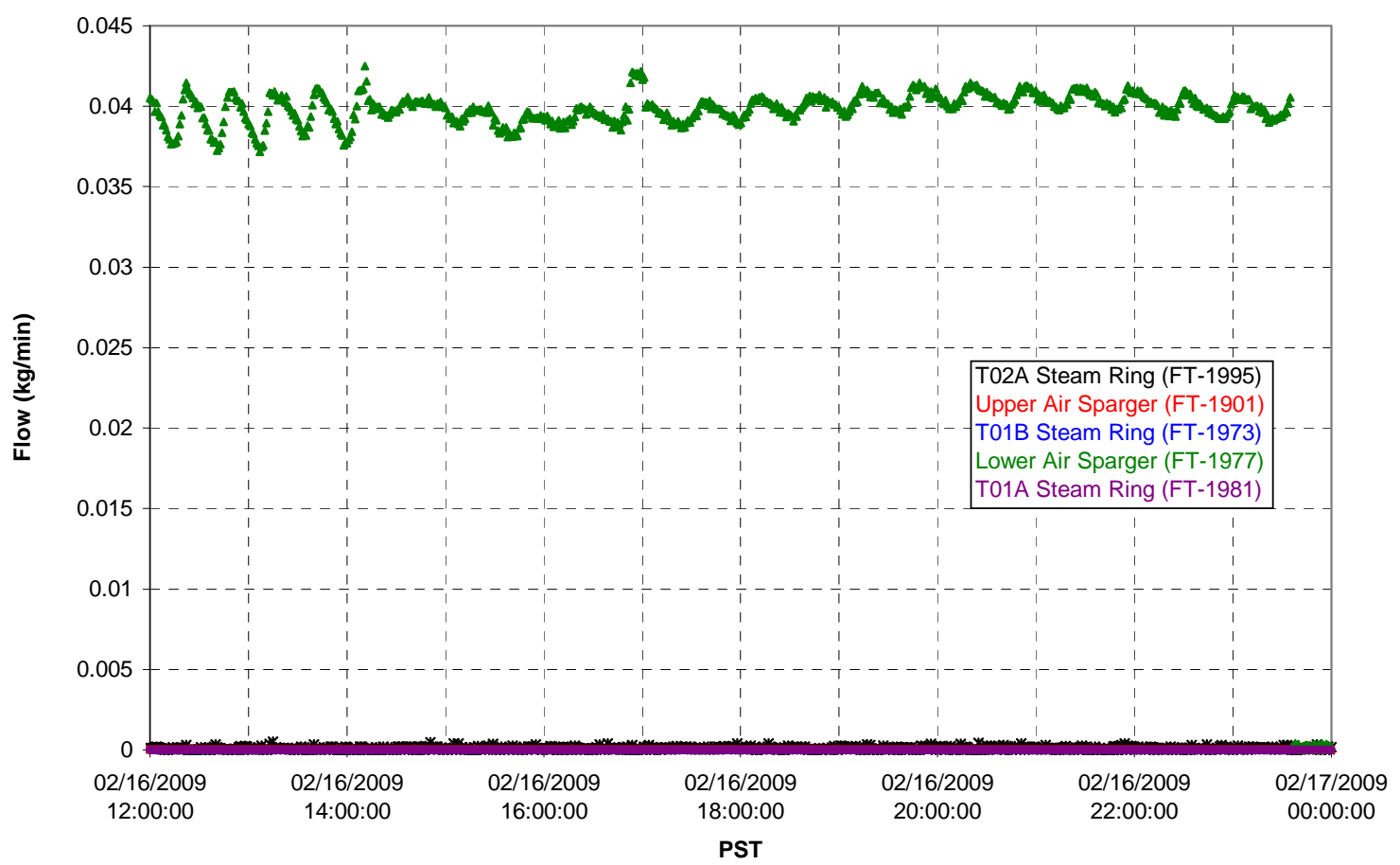


T02A Steam

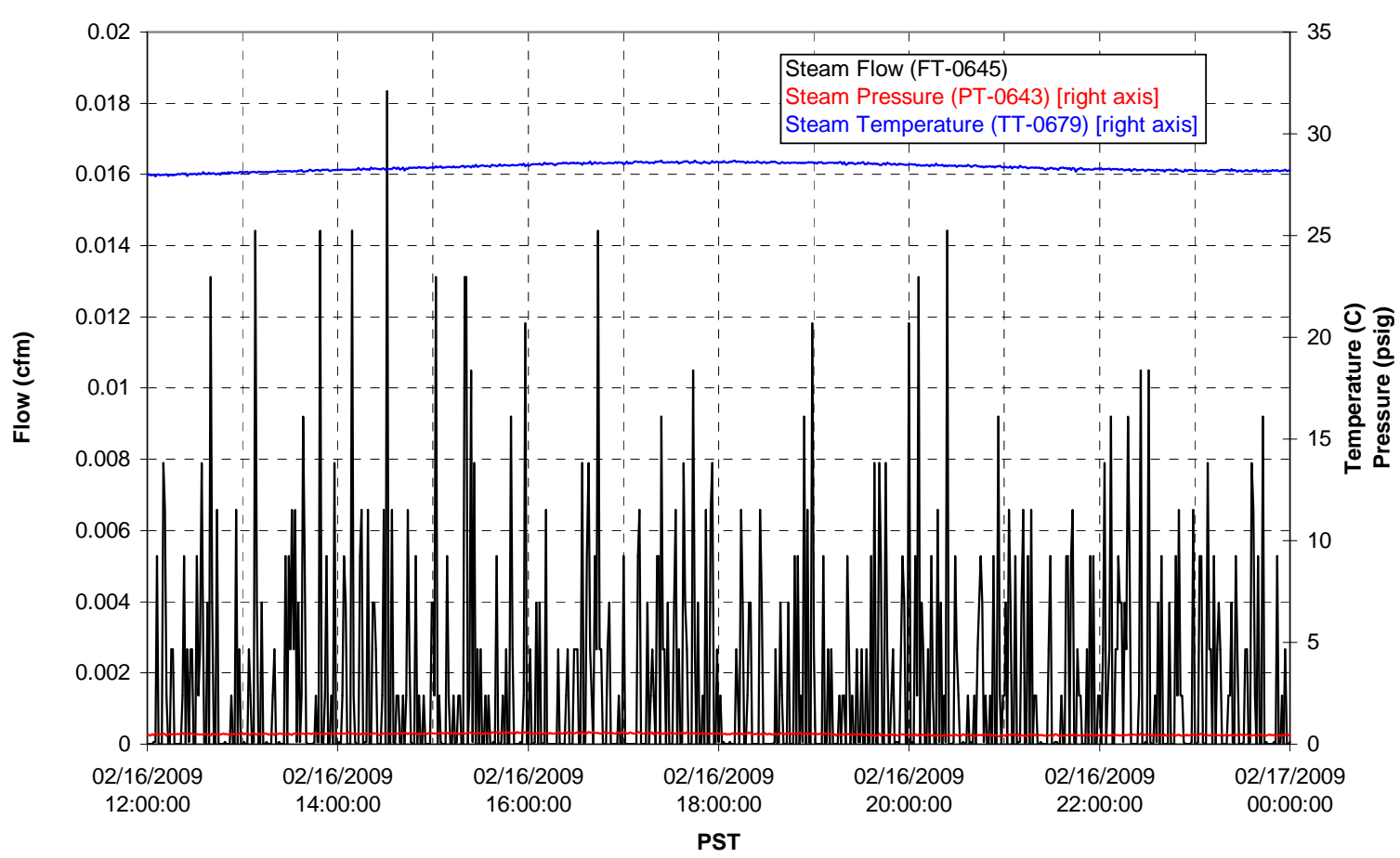

T01A Steam

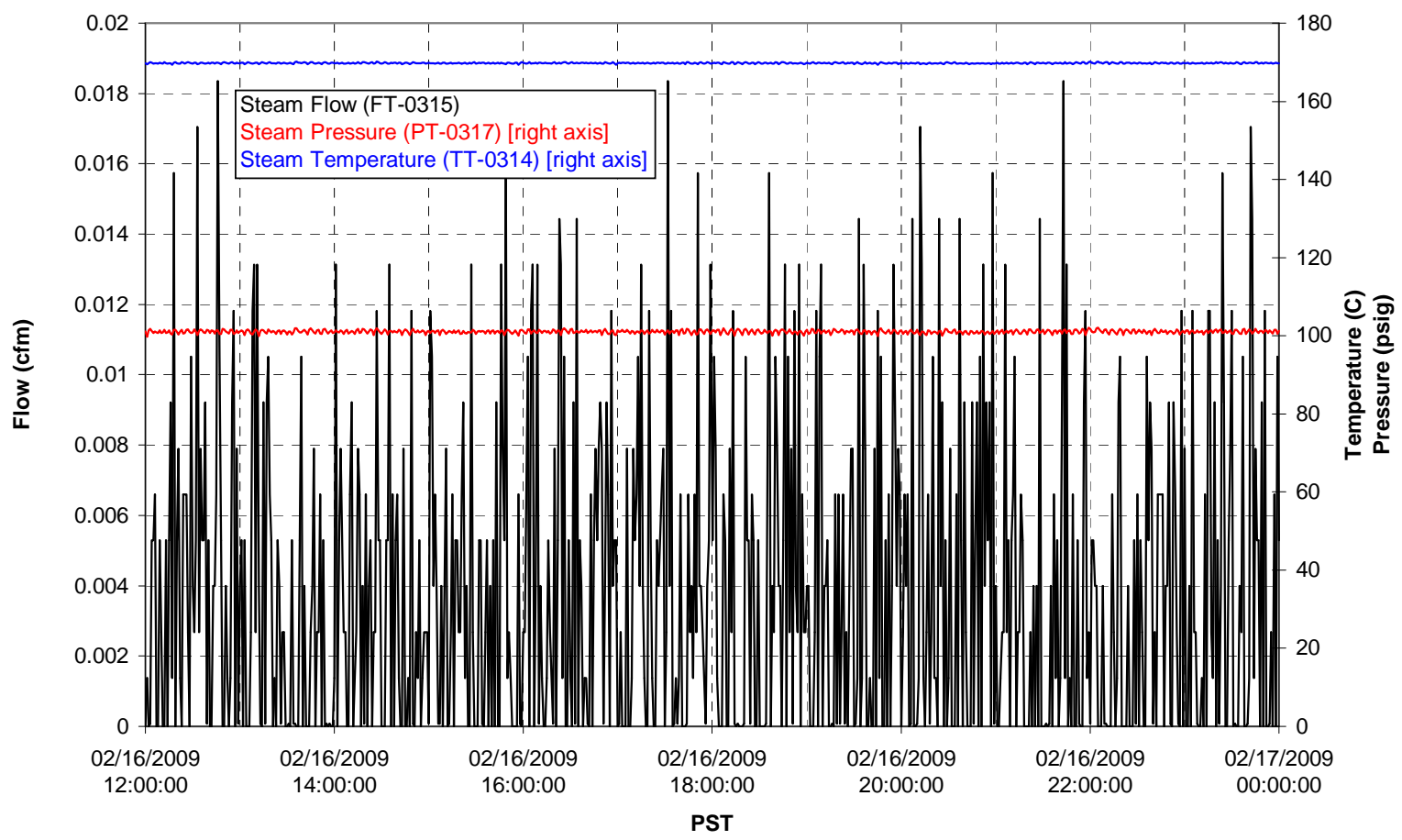


T01B Steam

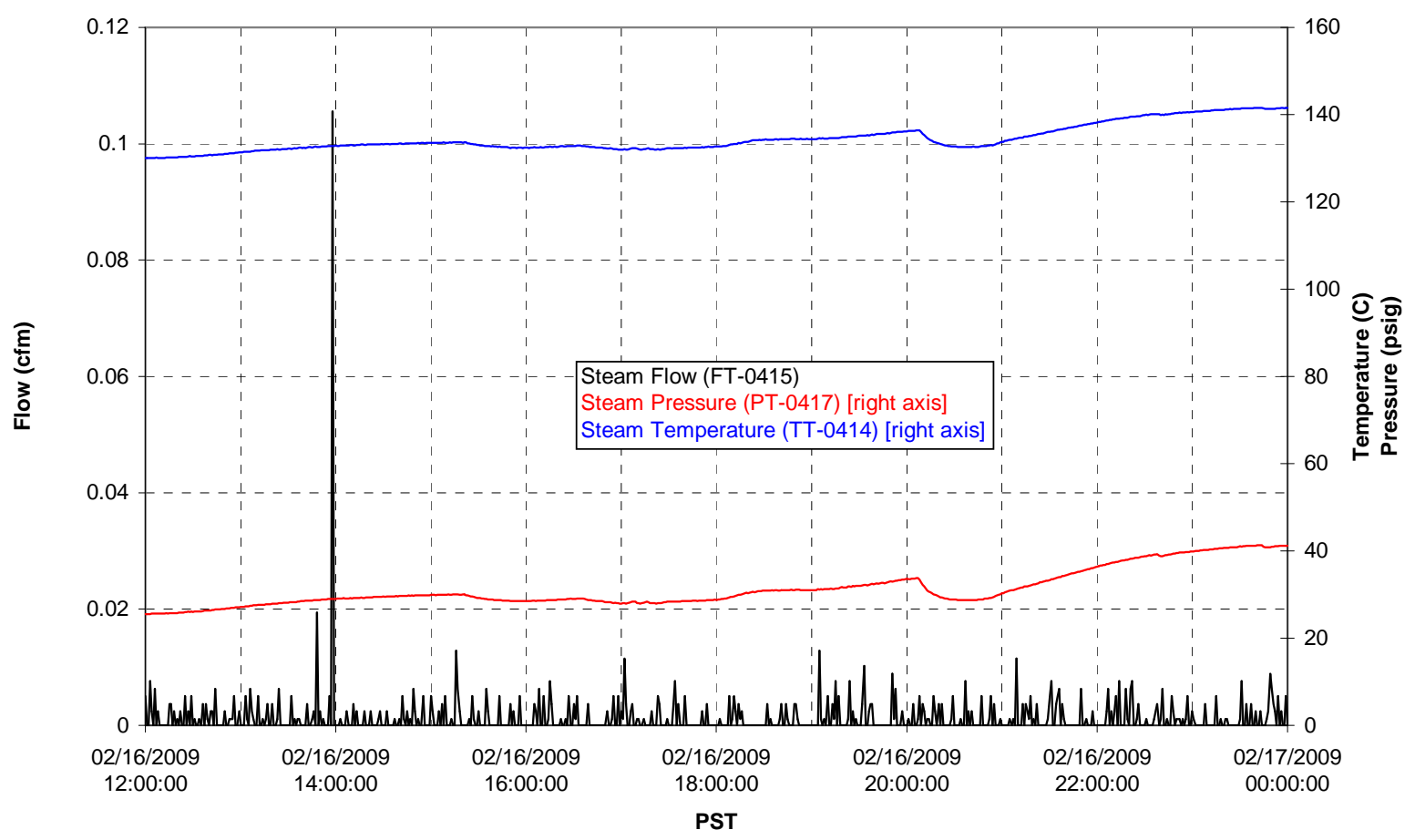




\section{Integrated Test A Data Plots 02/17/09 00:00 - 02/17/09 12:00}


T01A level

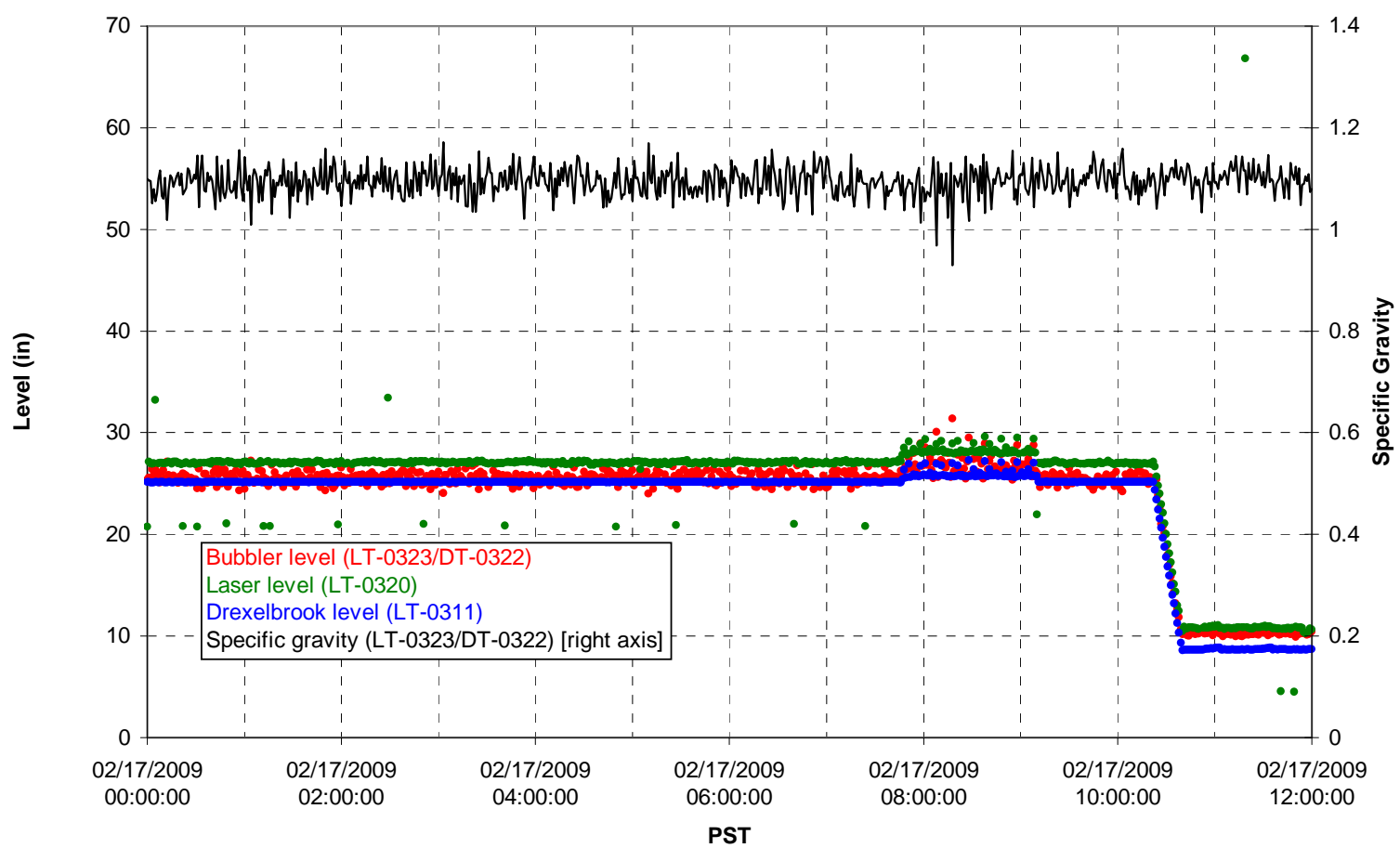

T01A temperatures

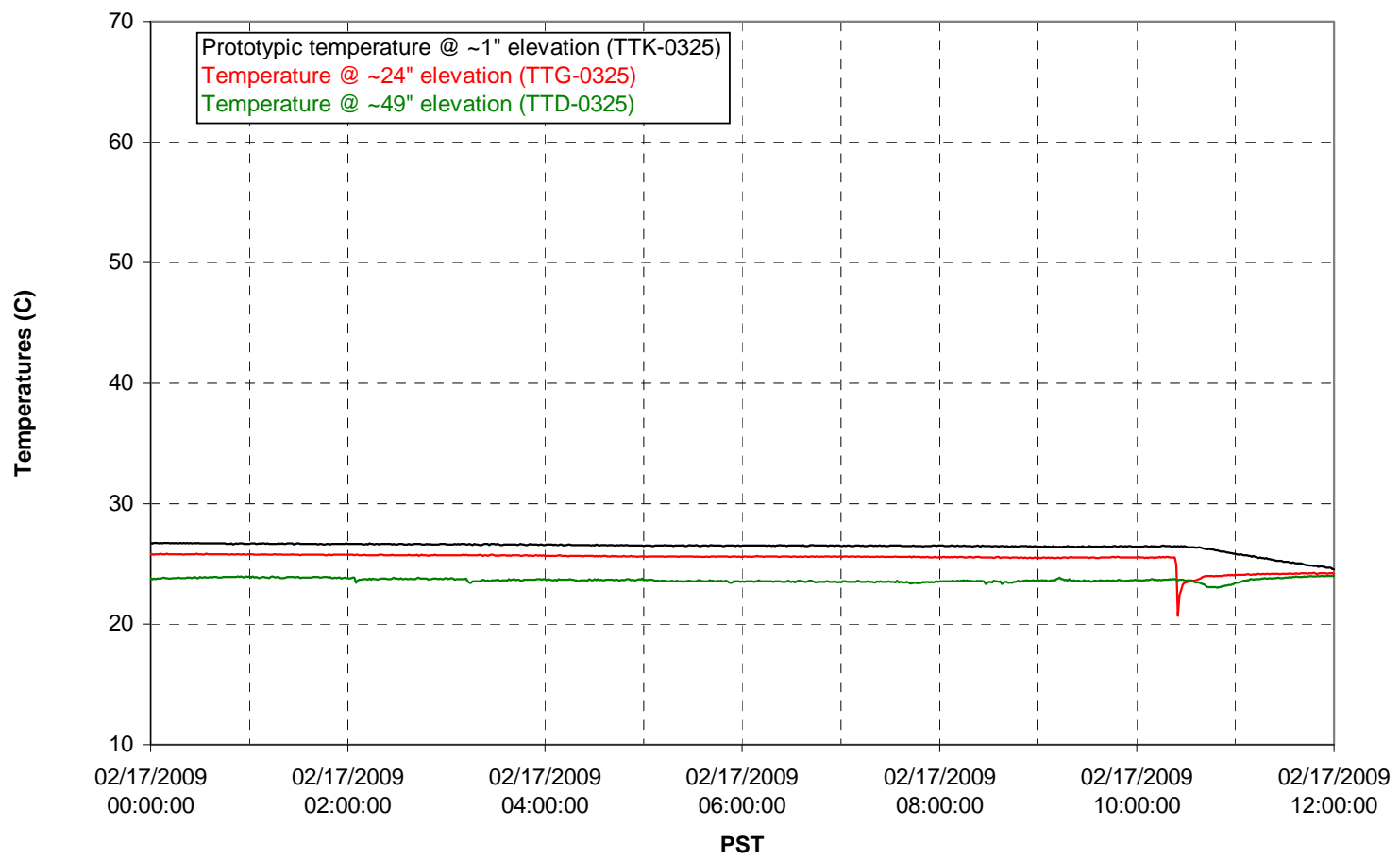


T01B level

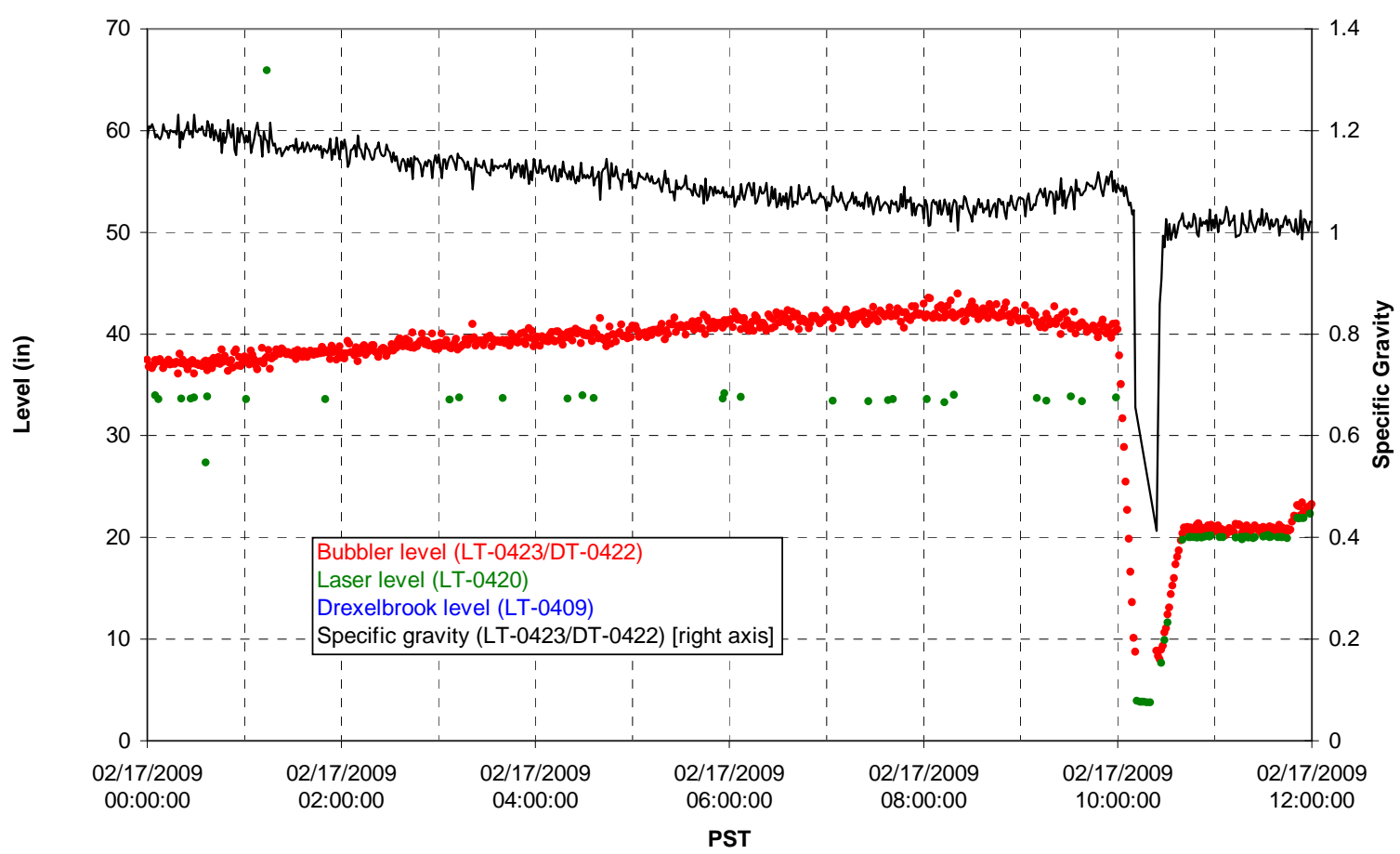

T01B temperatures

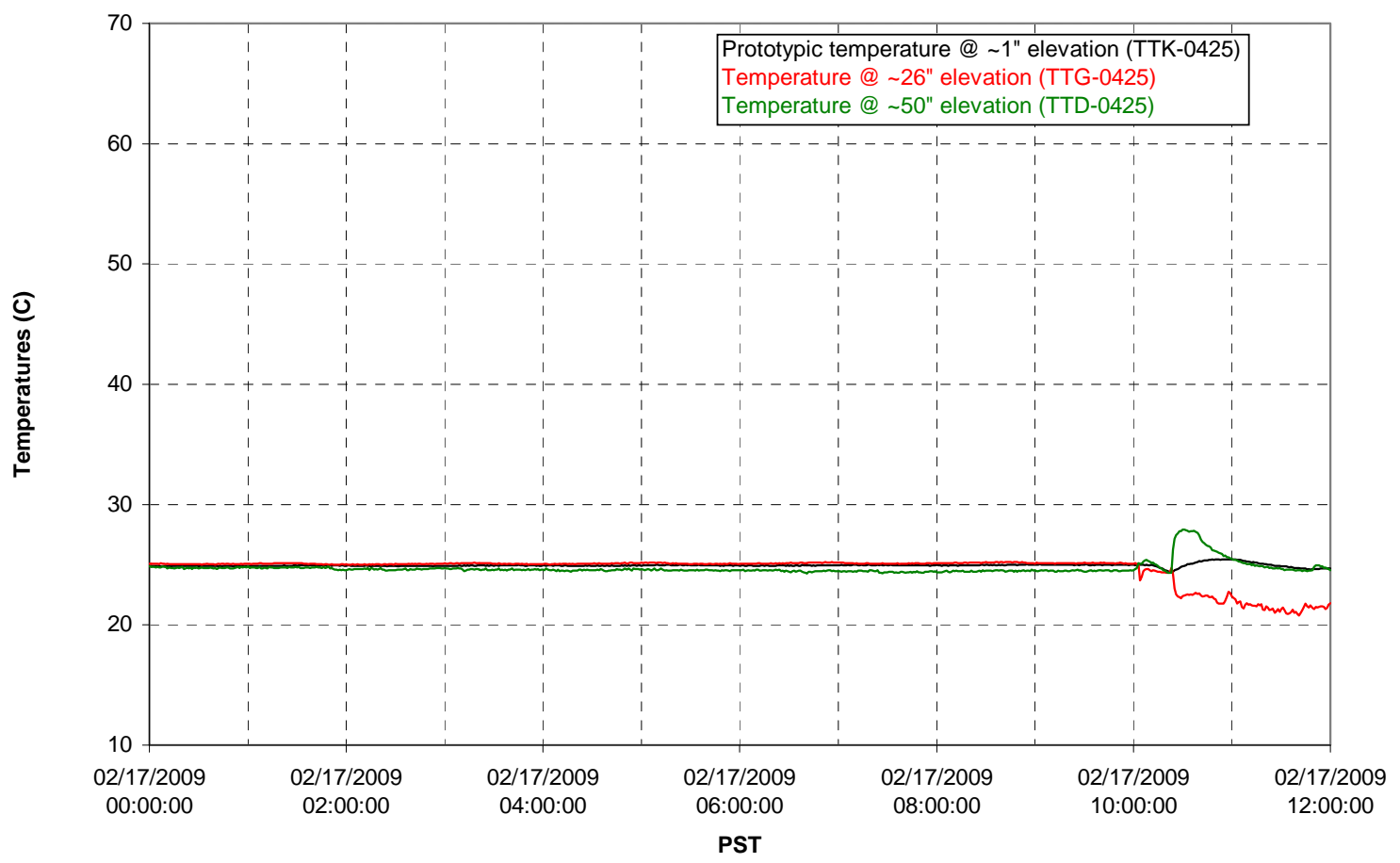


T02A level

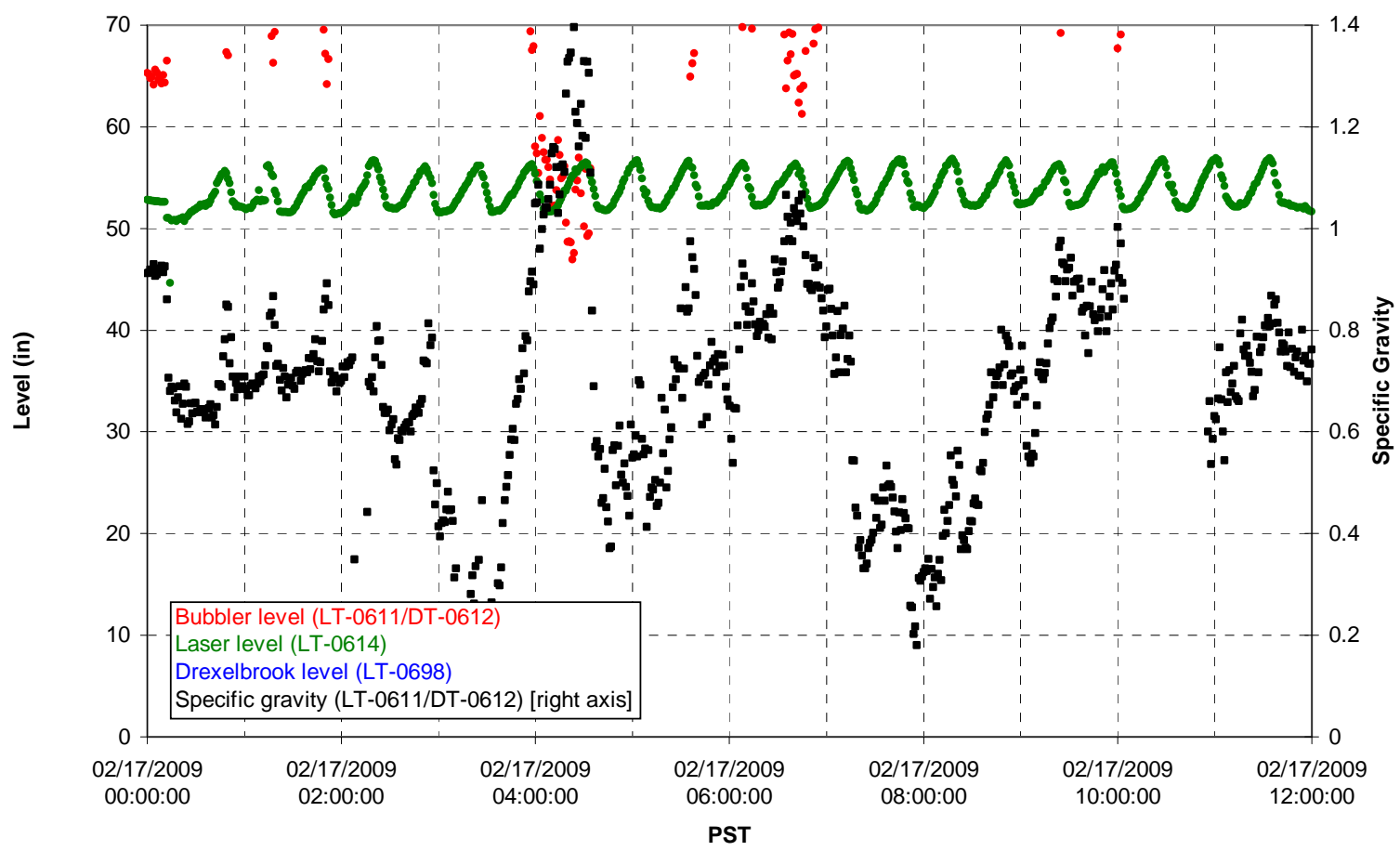

T02A temperatures

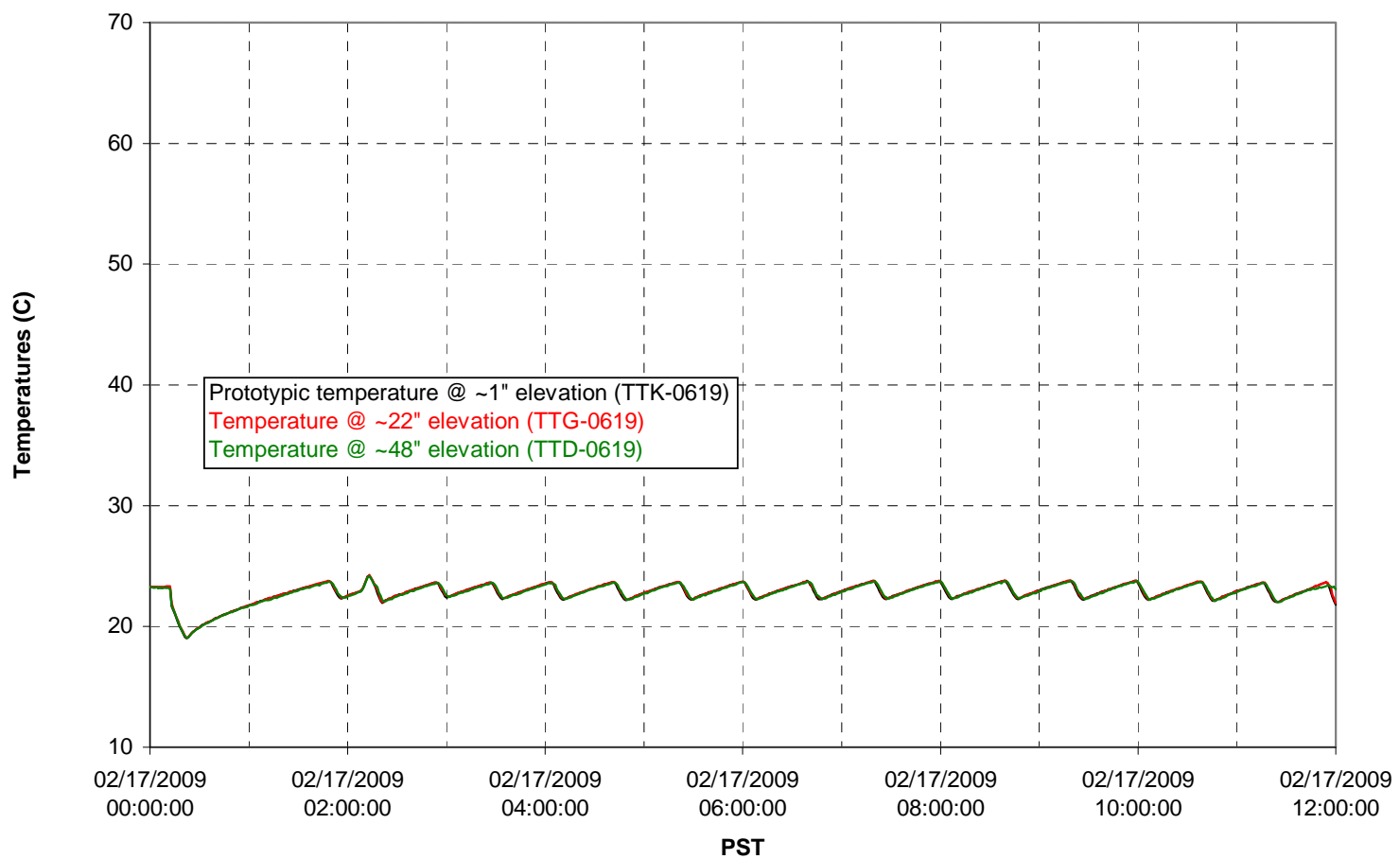


T02A and filter loop temperatures

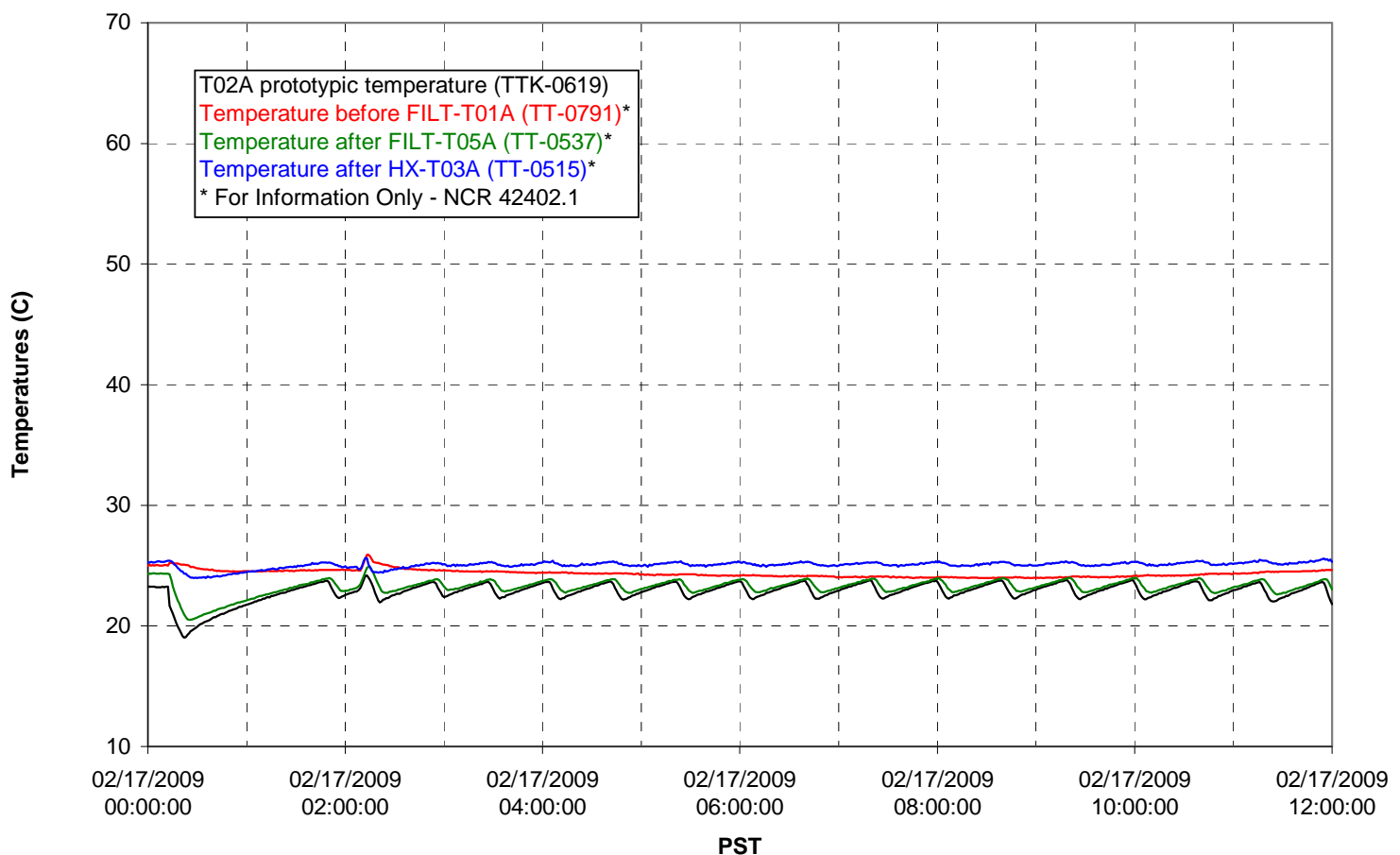

Pump Pressures and Flow

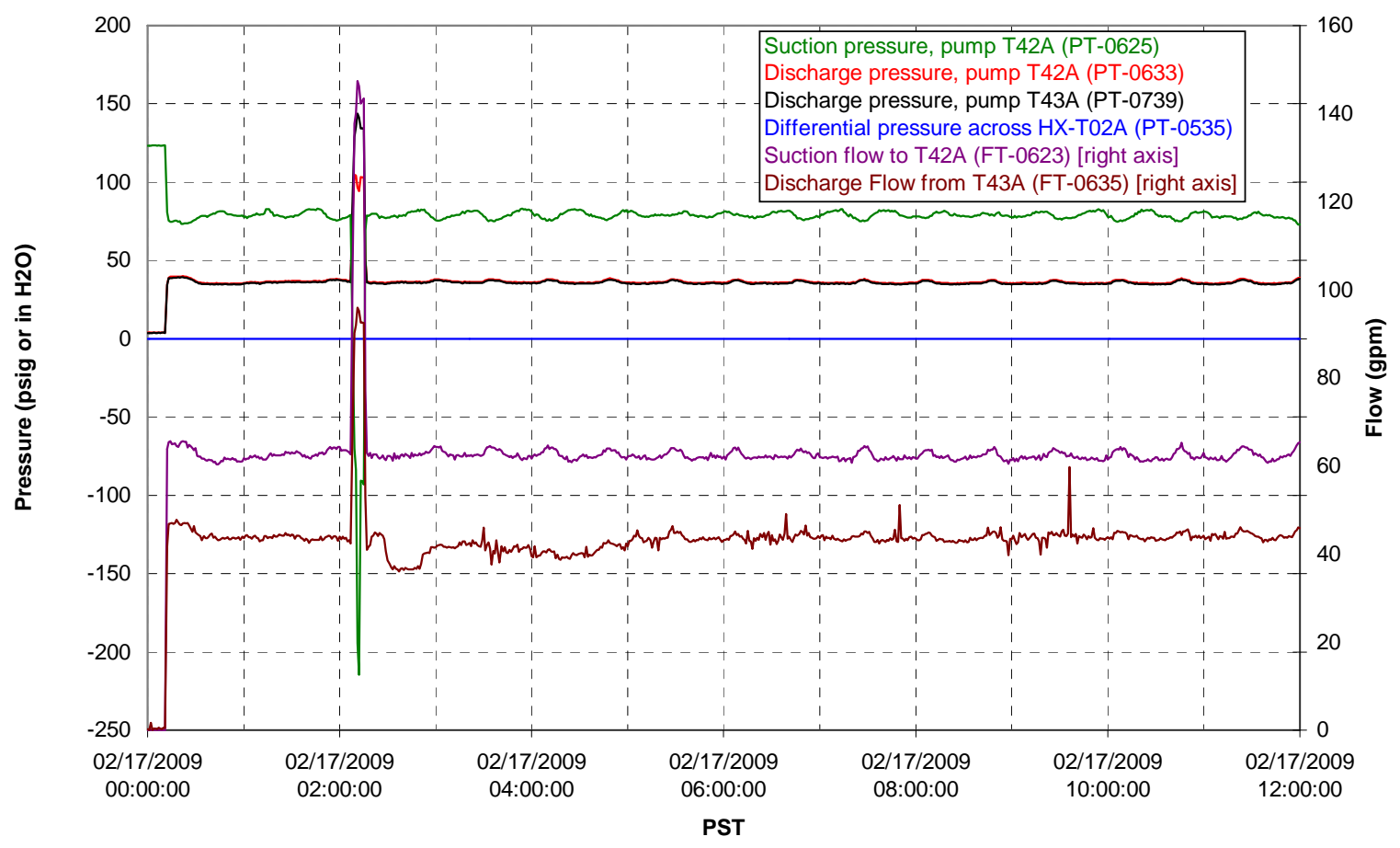


Axial pressure drop

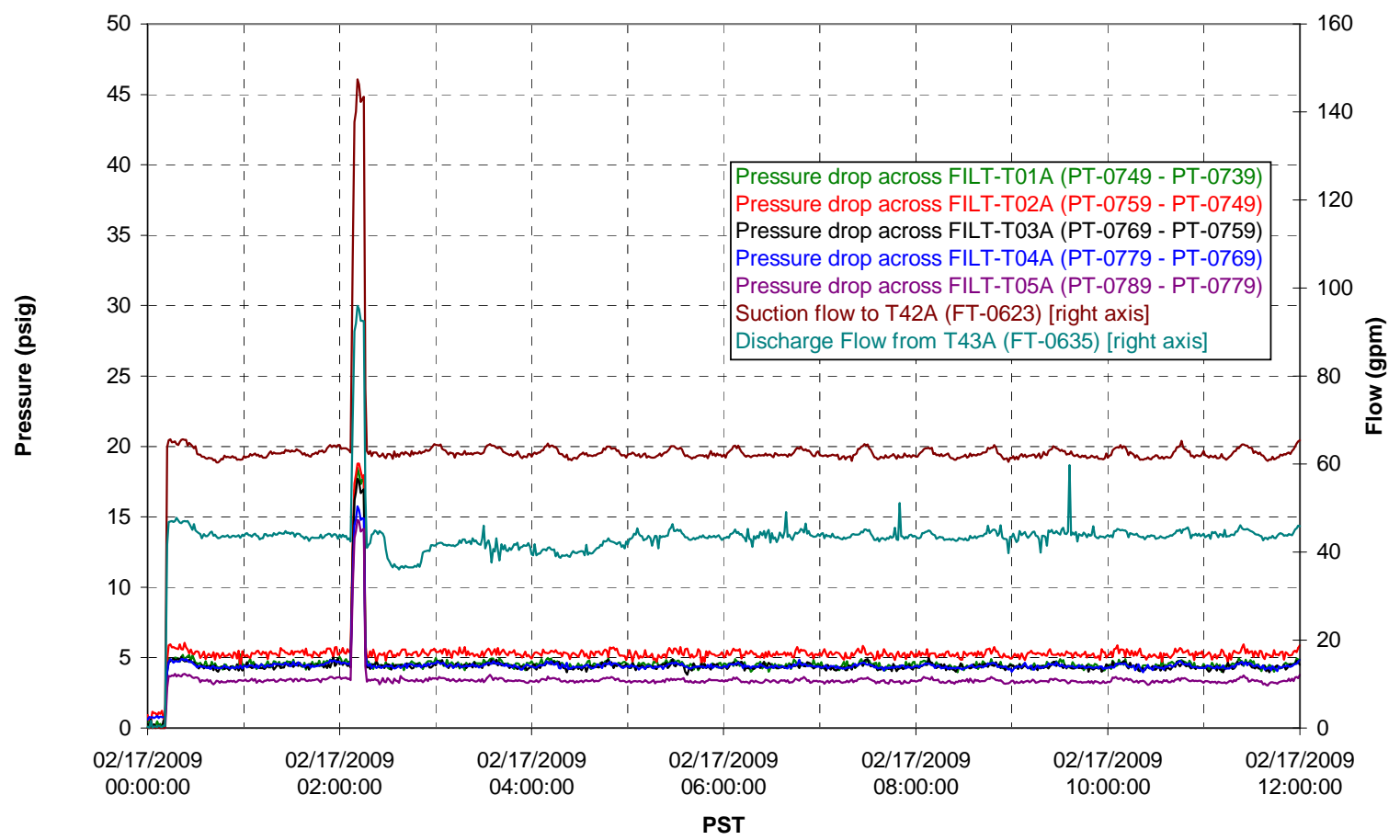

Permeate flow rates

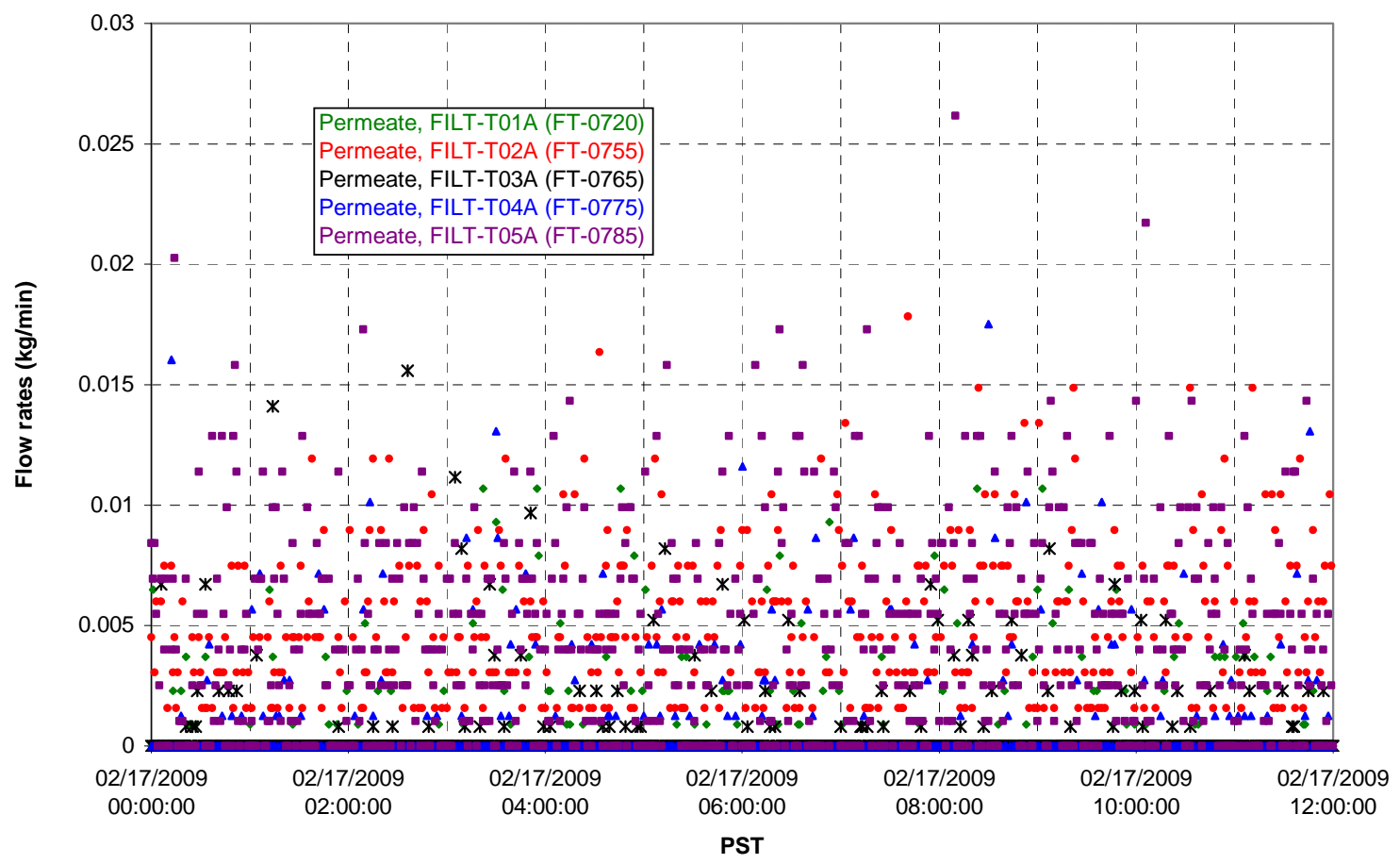


T02A Inner Temperature Tree

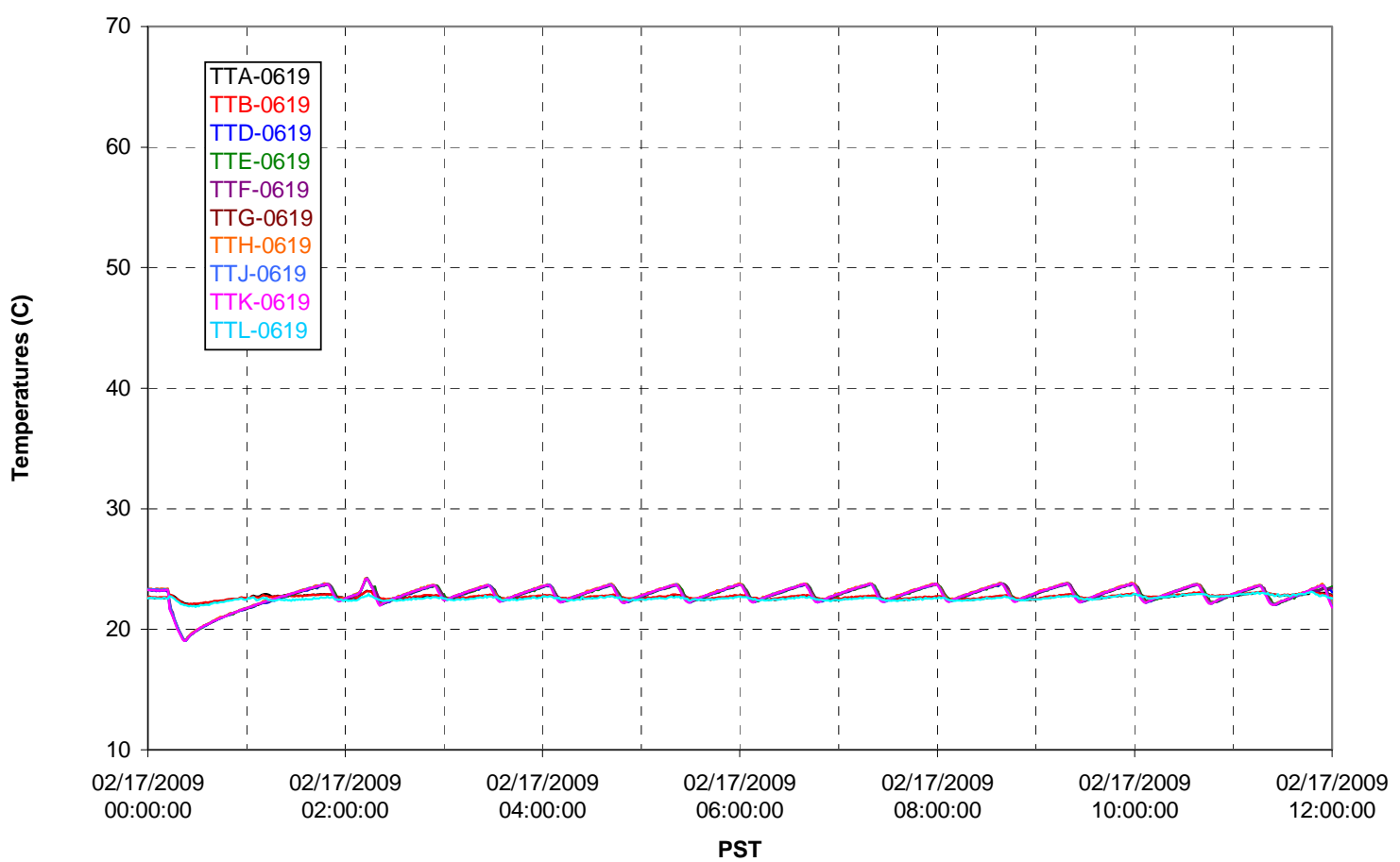

T02A Outer Temperature Tree

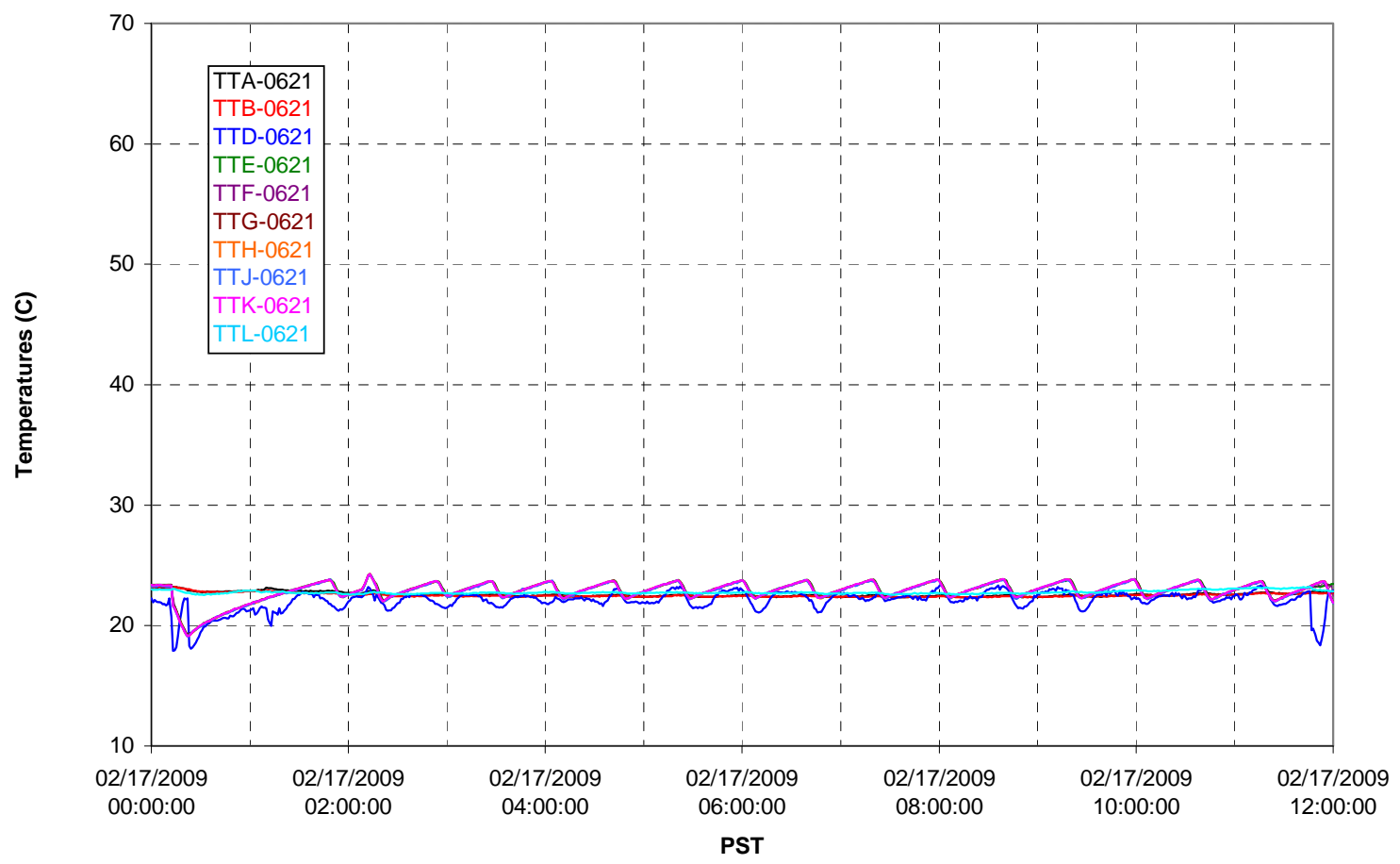


T02A temperatures

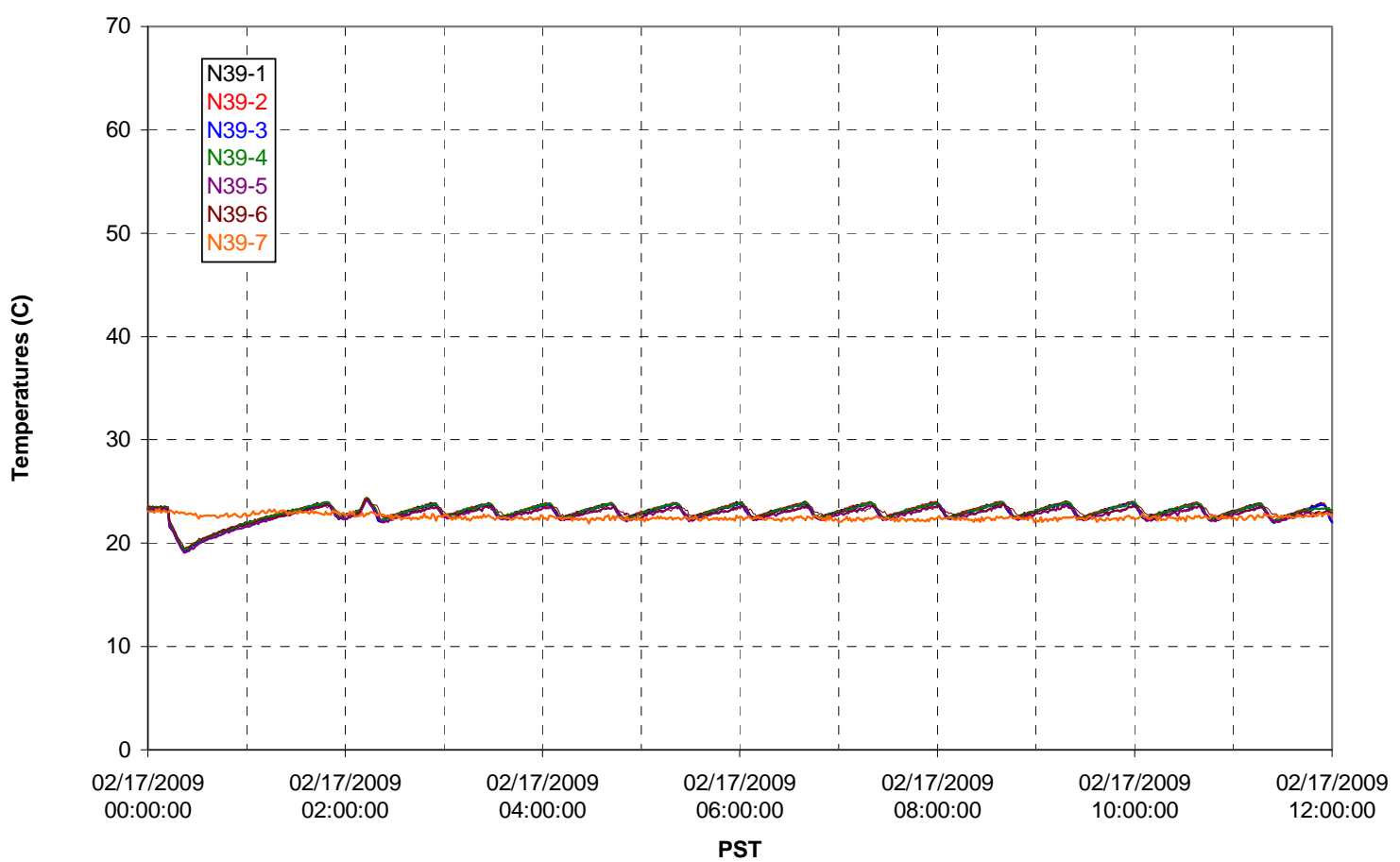

T02A temperatures

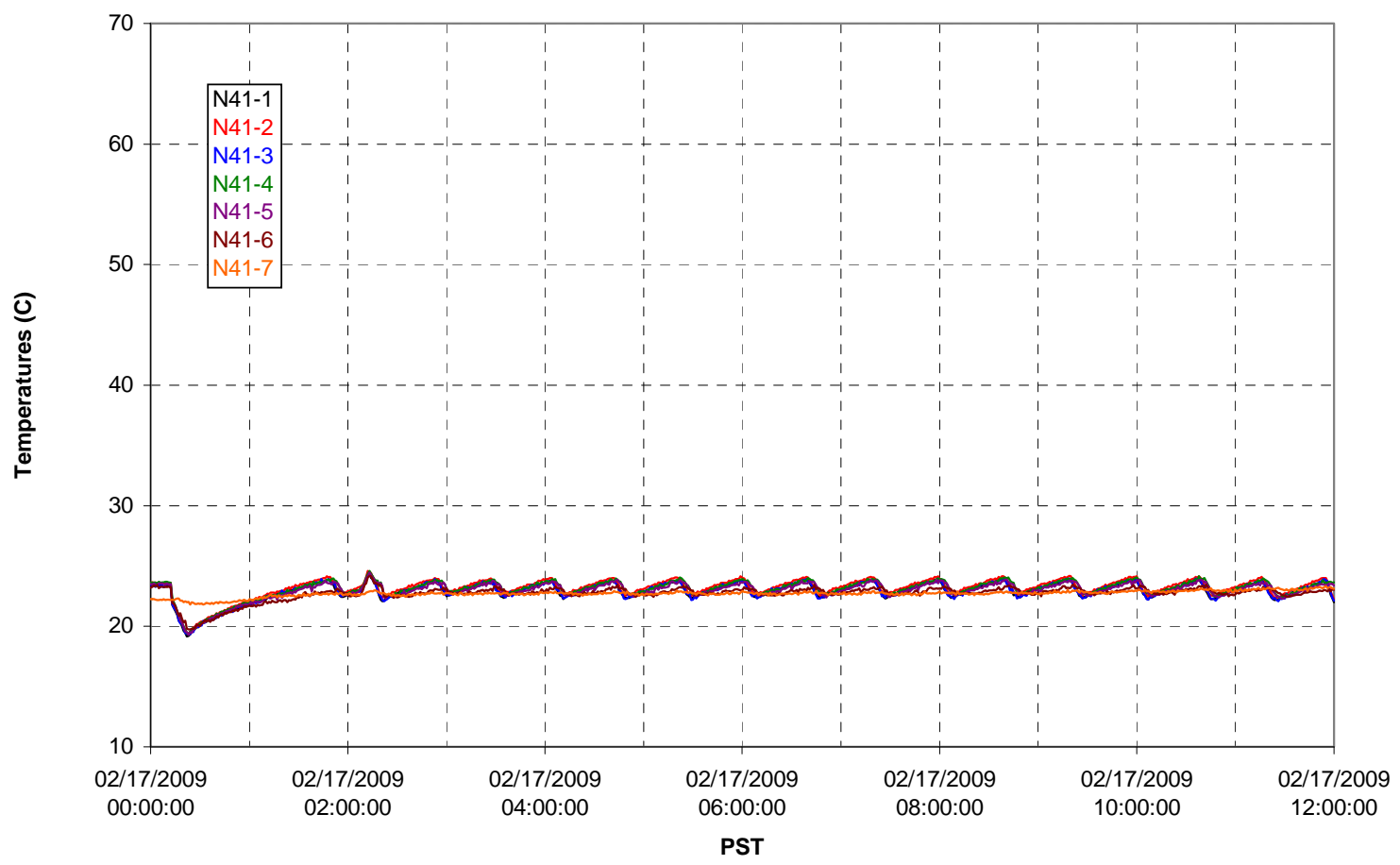


T02A temperatures

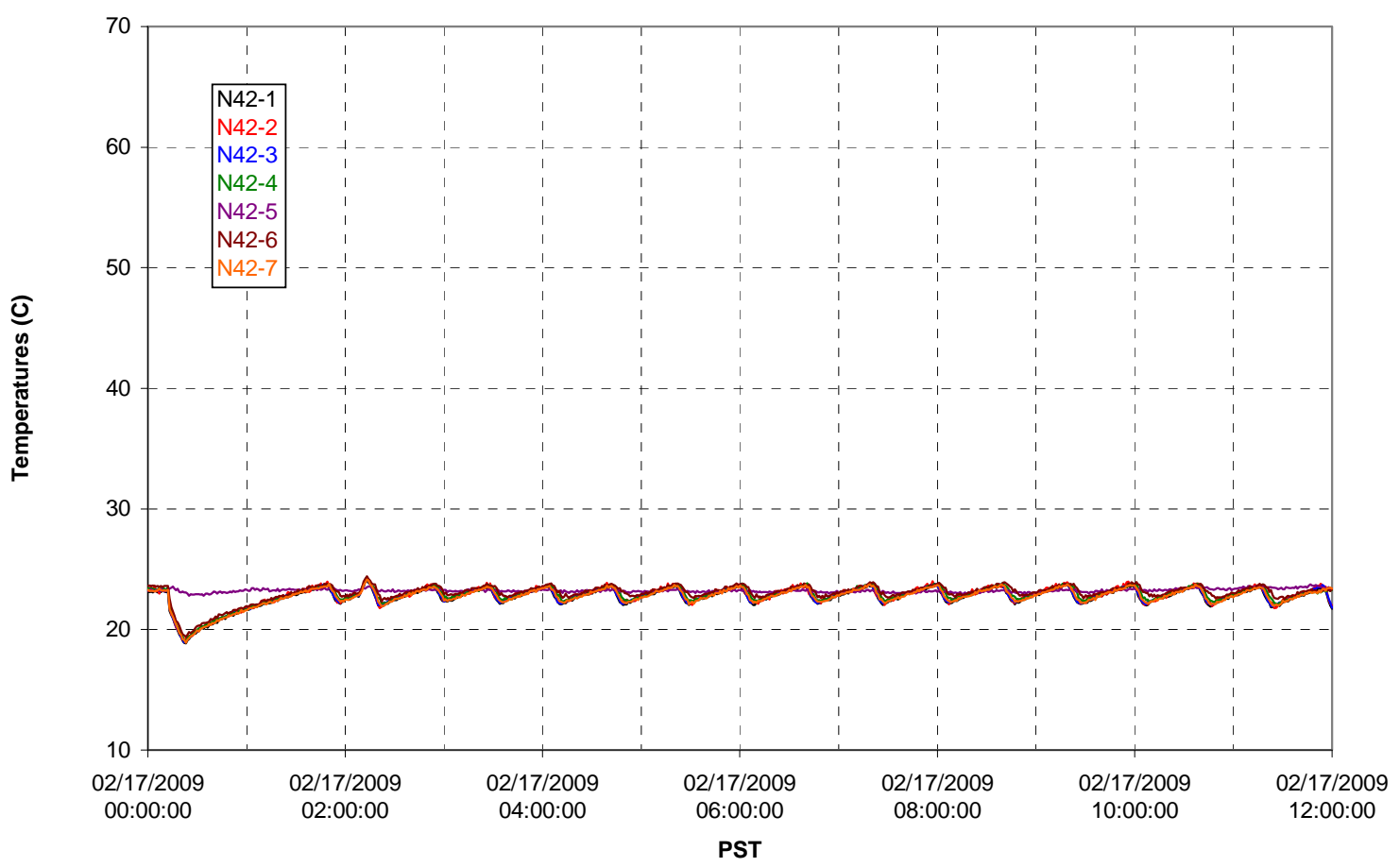

T02A temperatures

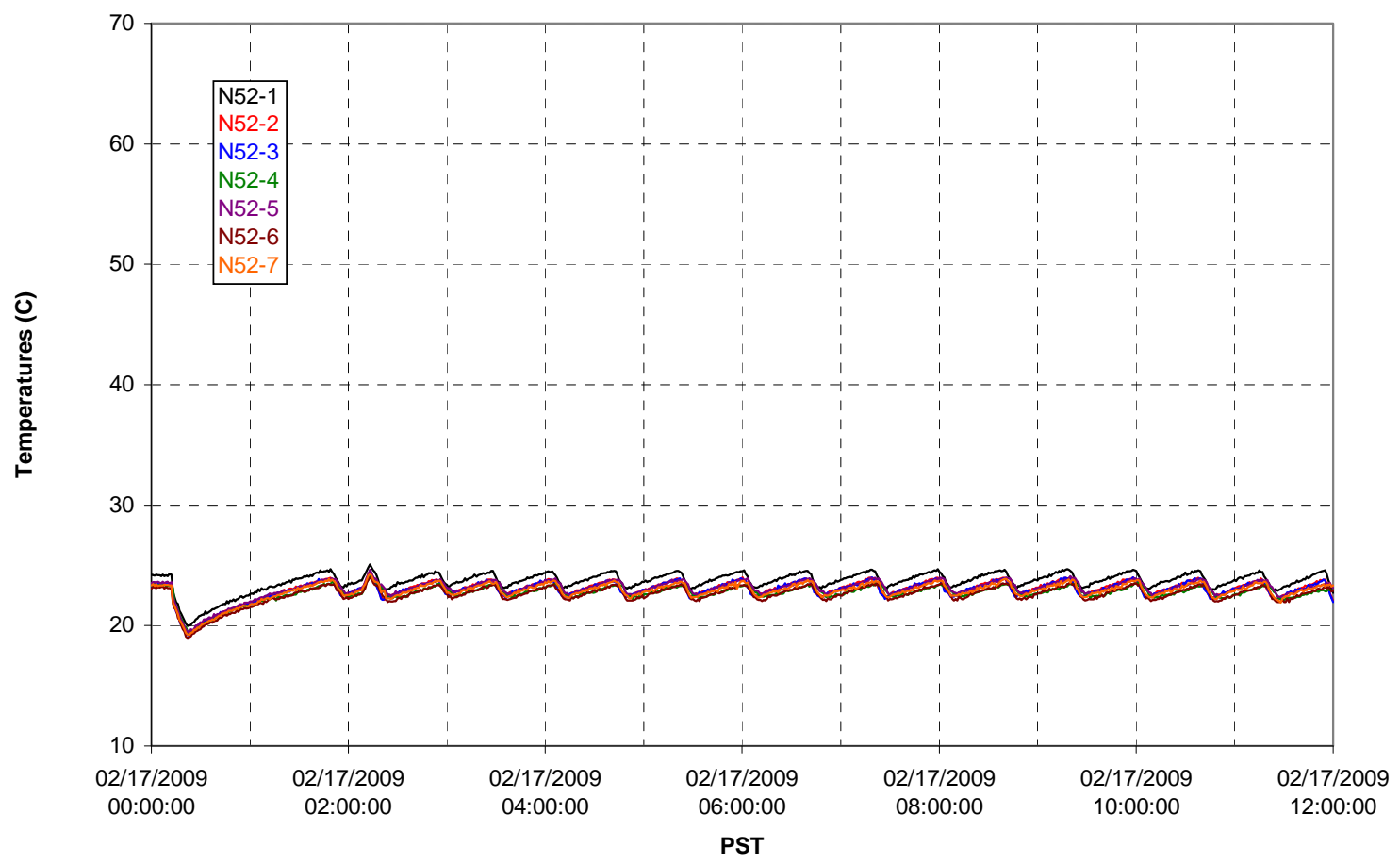


T02A Heating and Cooling

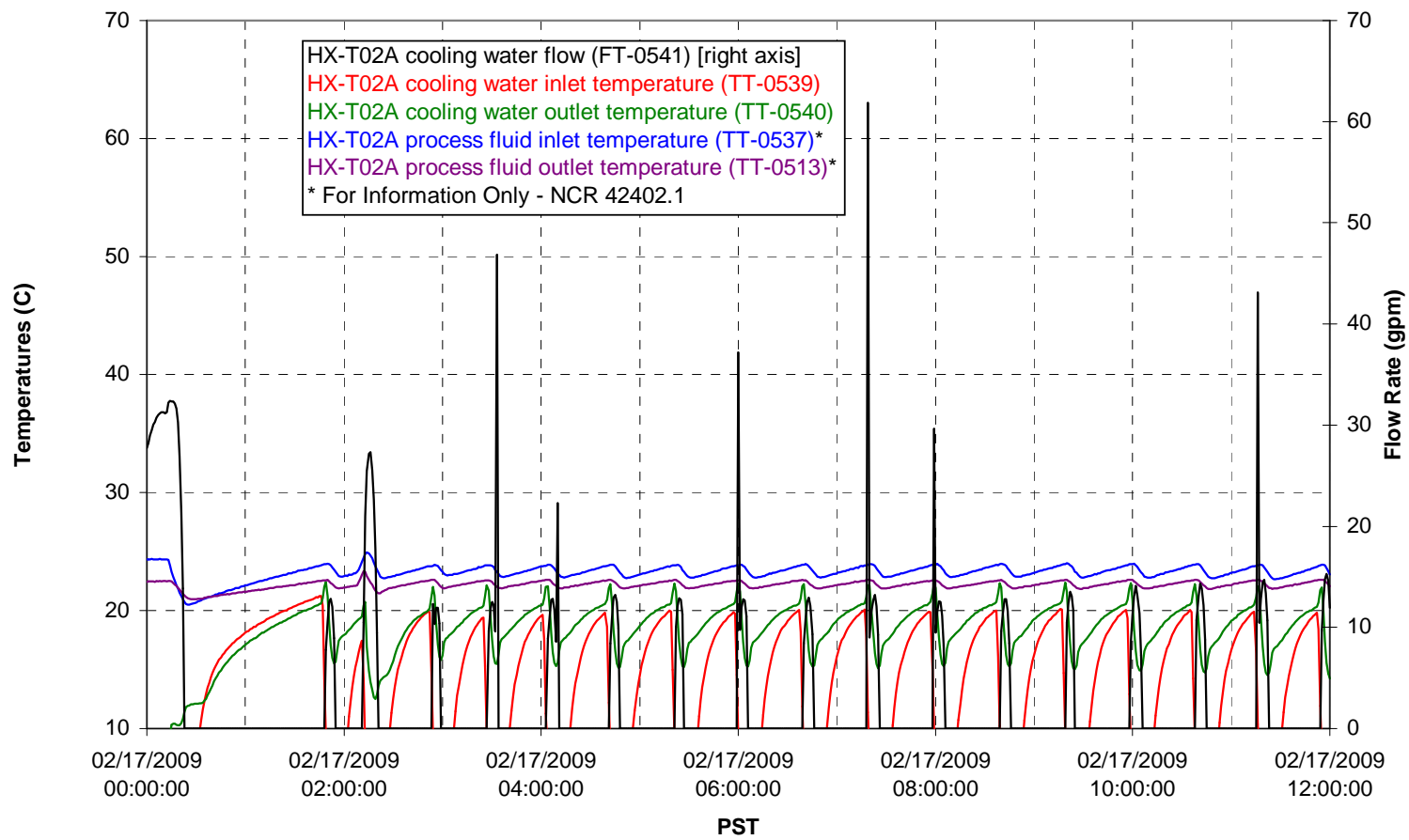

Pump Operation

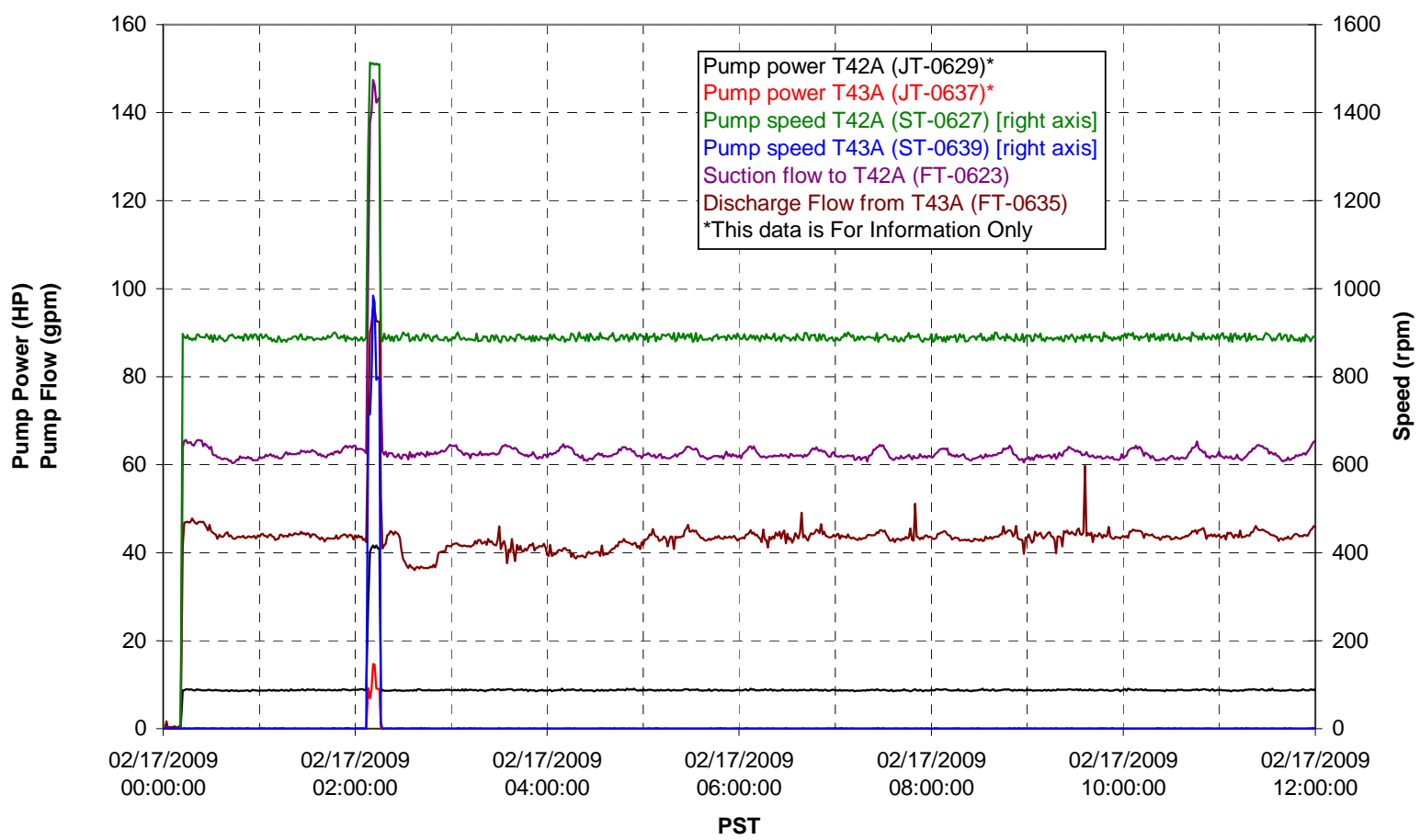


Pulsepot UFP-PP-T01A

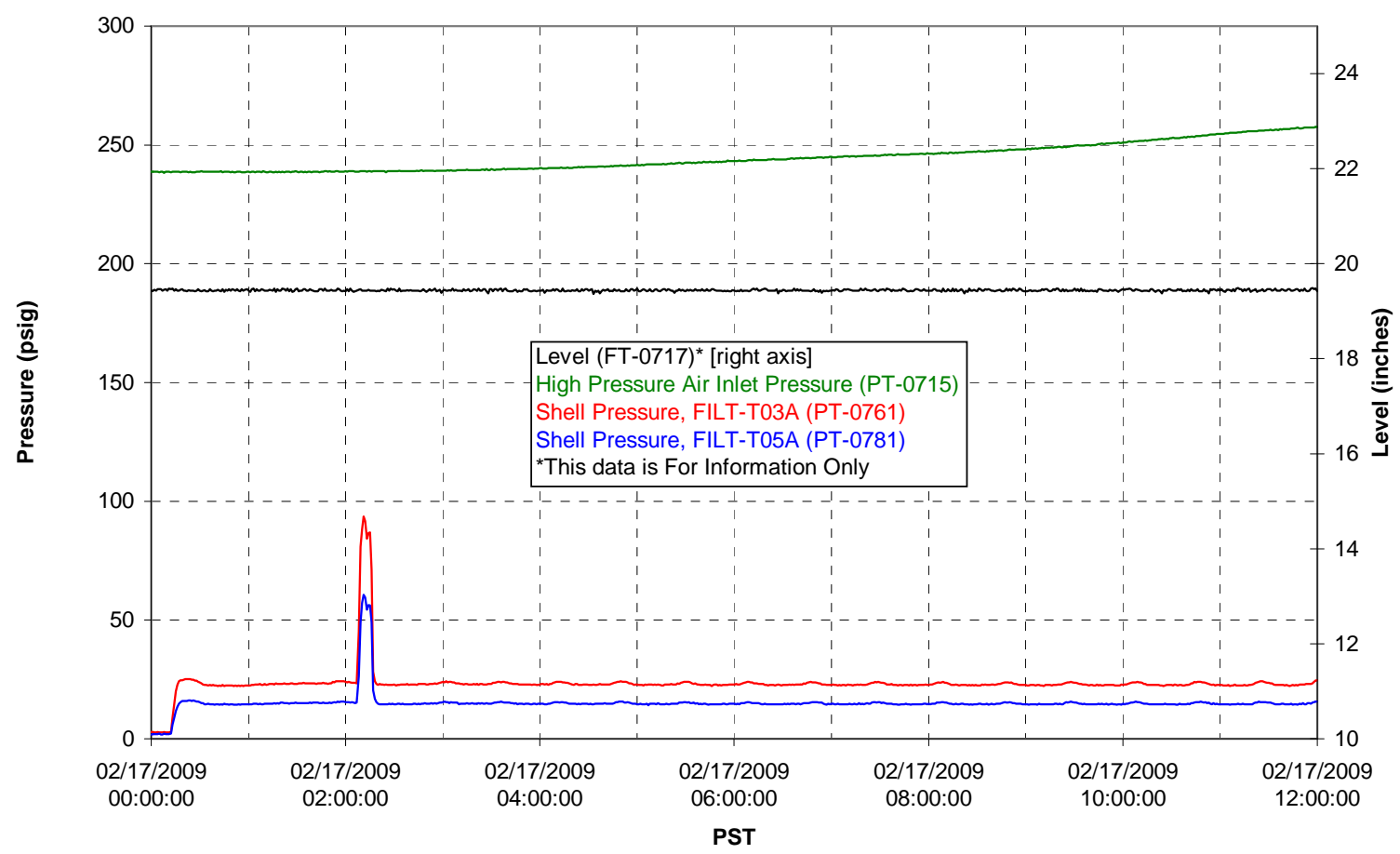

Pulsepot UFP-PP-T02A

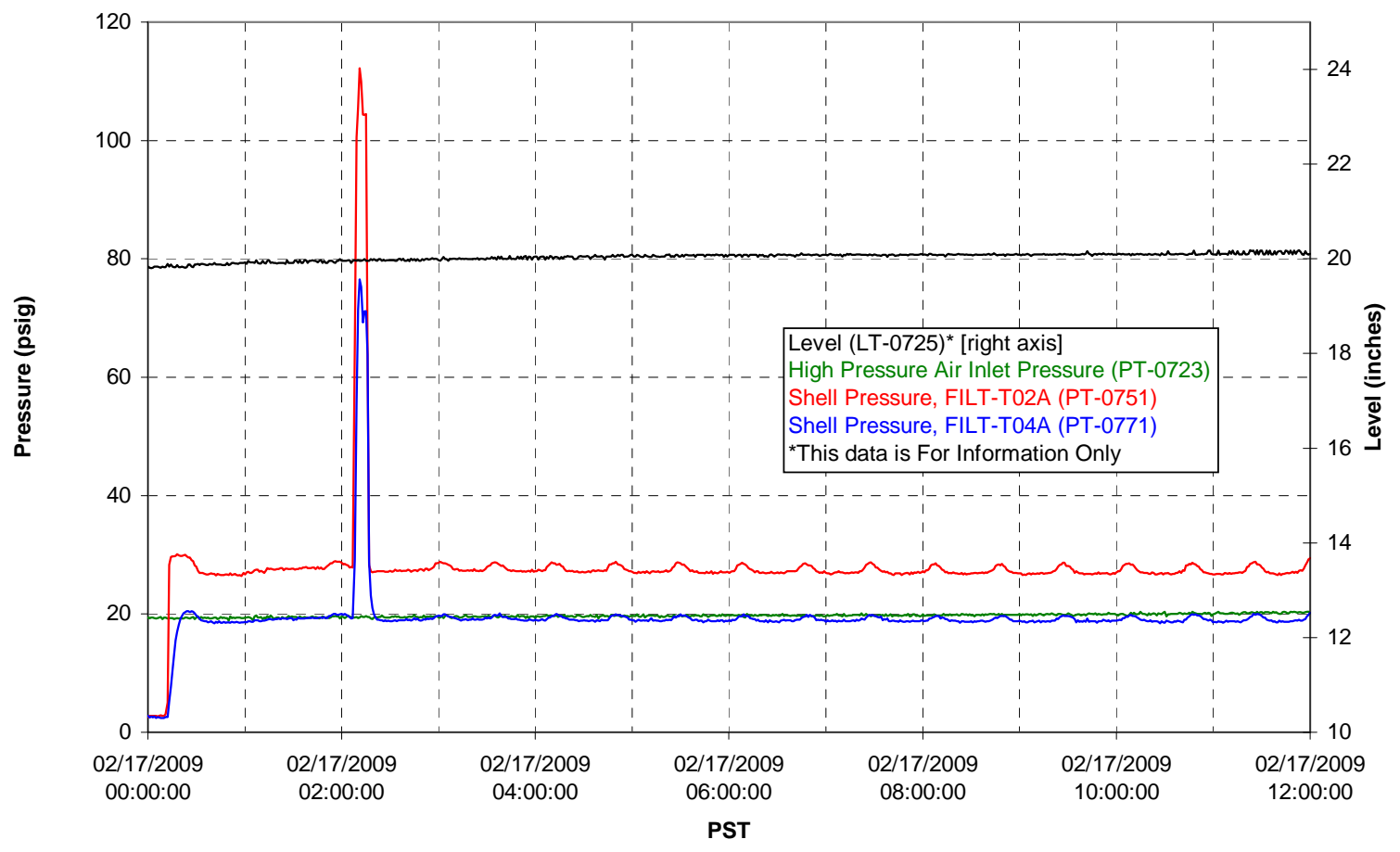


Pulsepot UFP-PP-T03A

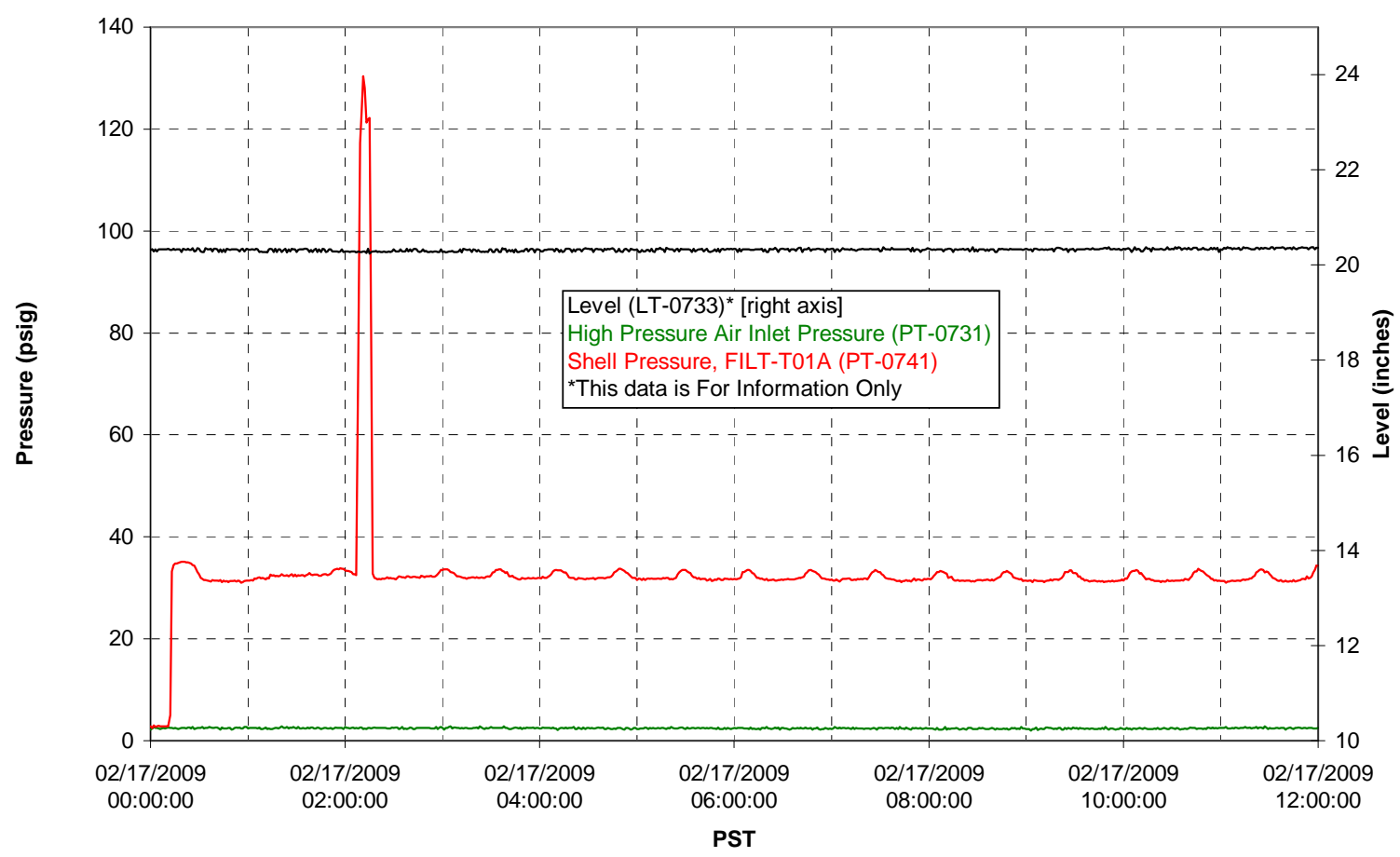

Pulsepot Levels

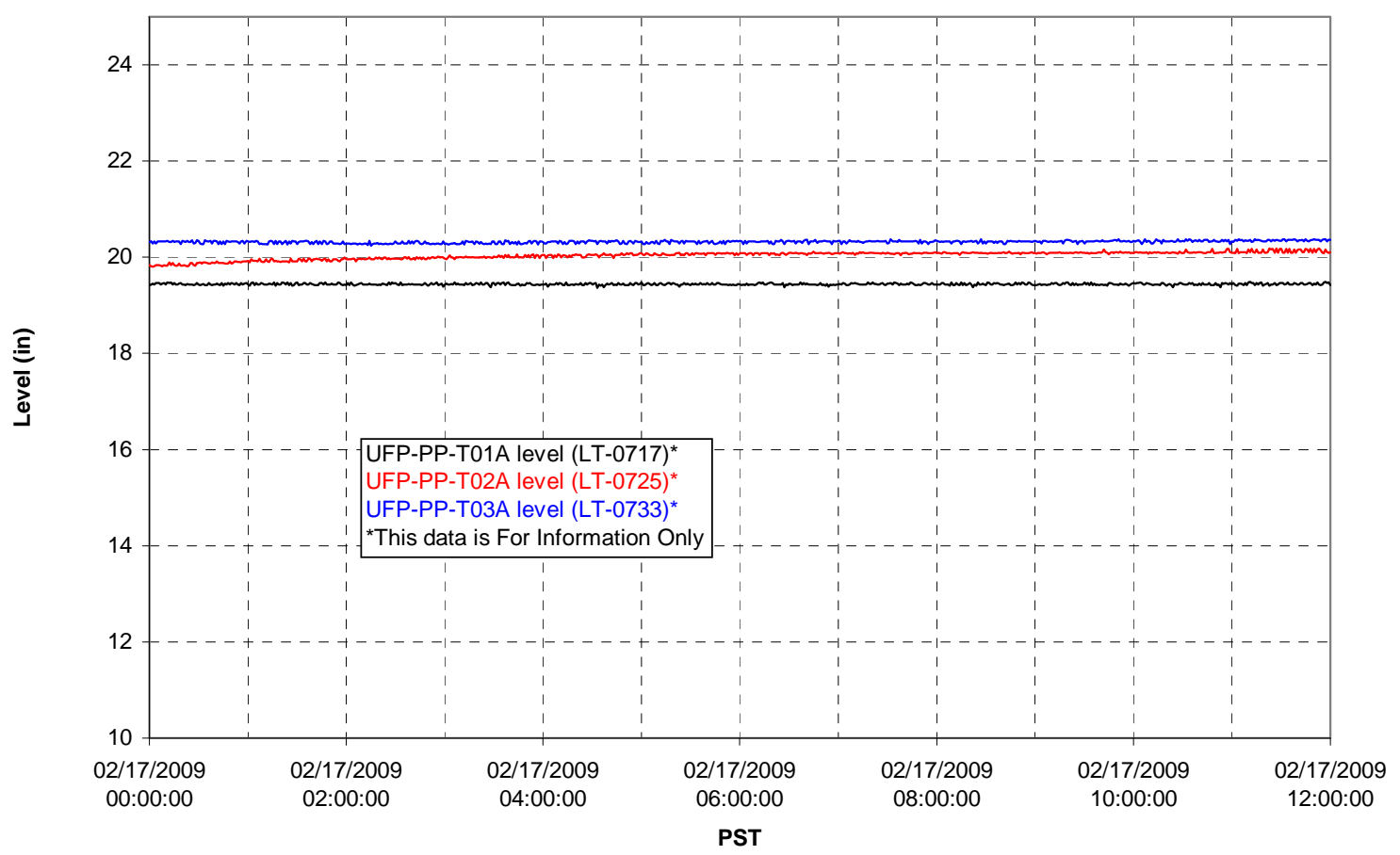


Filter UFP-FILT-T01A

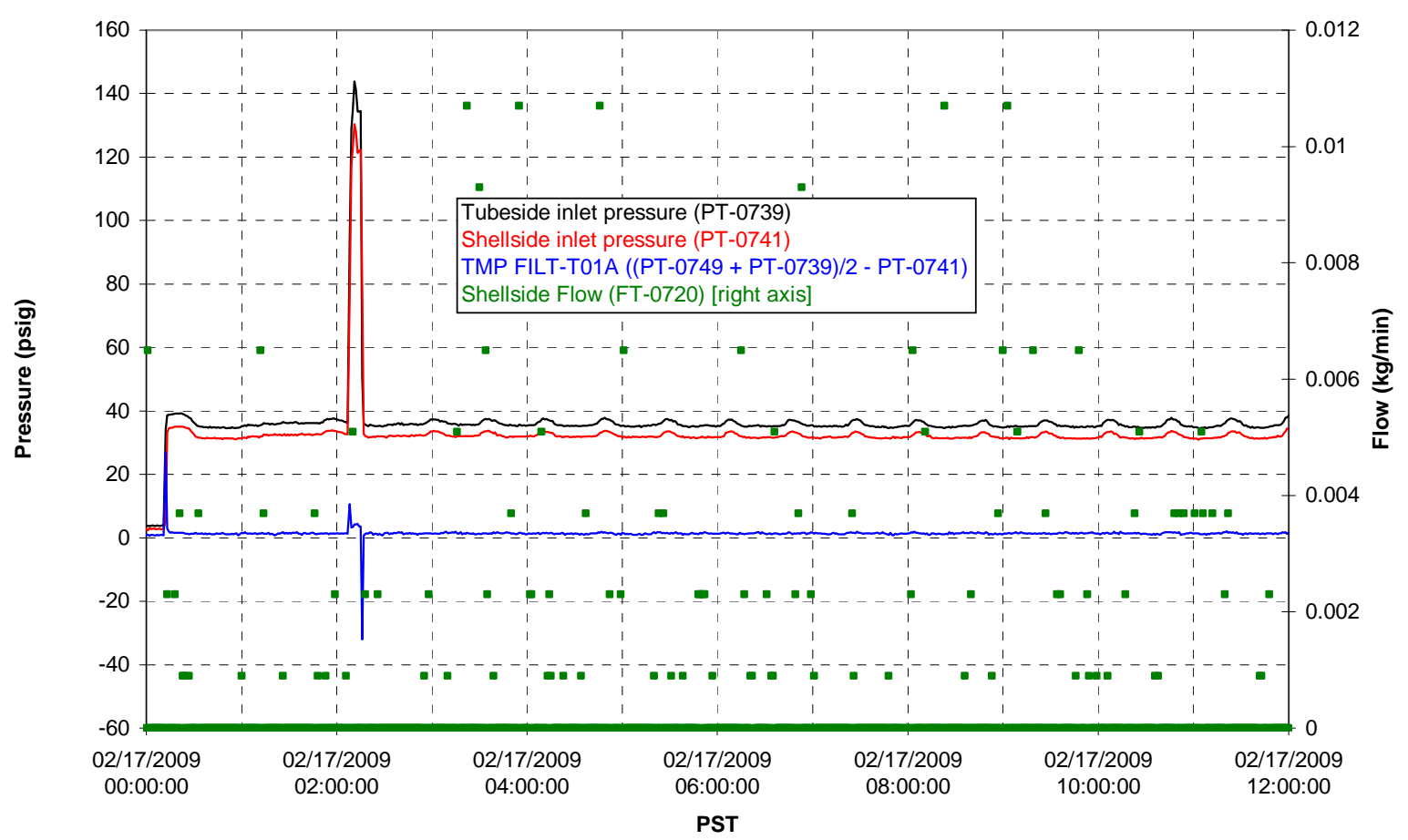

Filter UFP-FILT-T02A

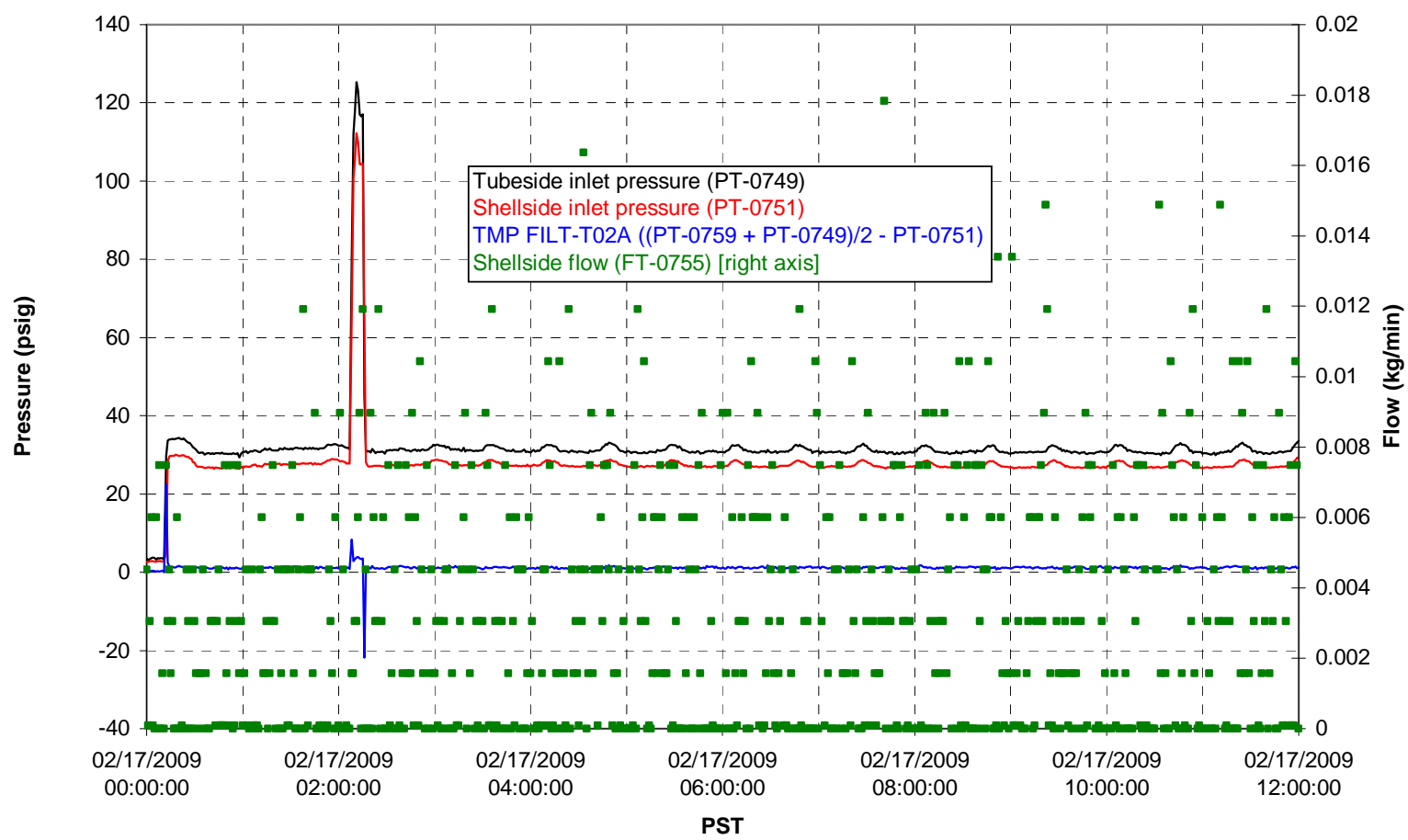


Filter UFP-FILT-T03A

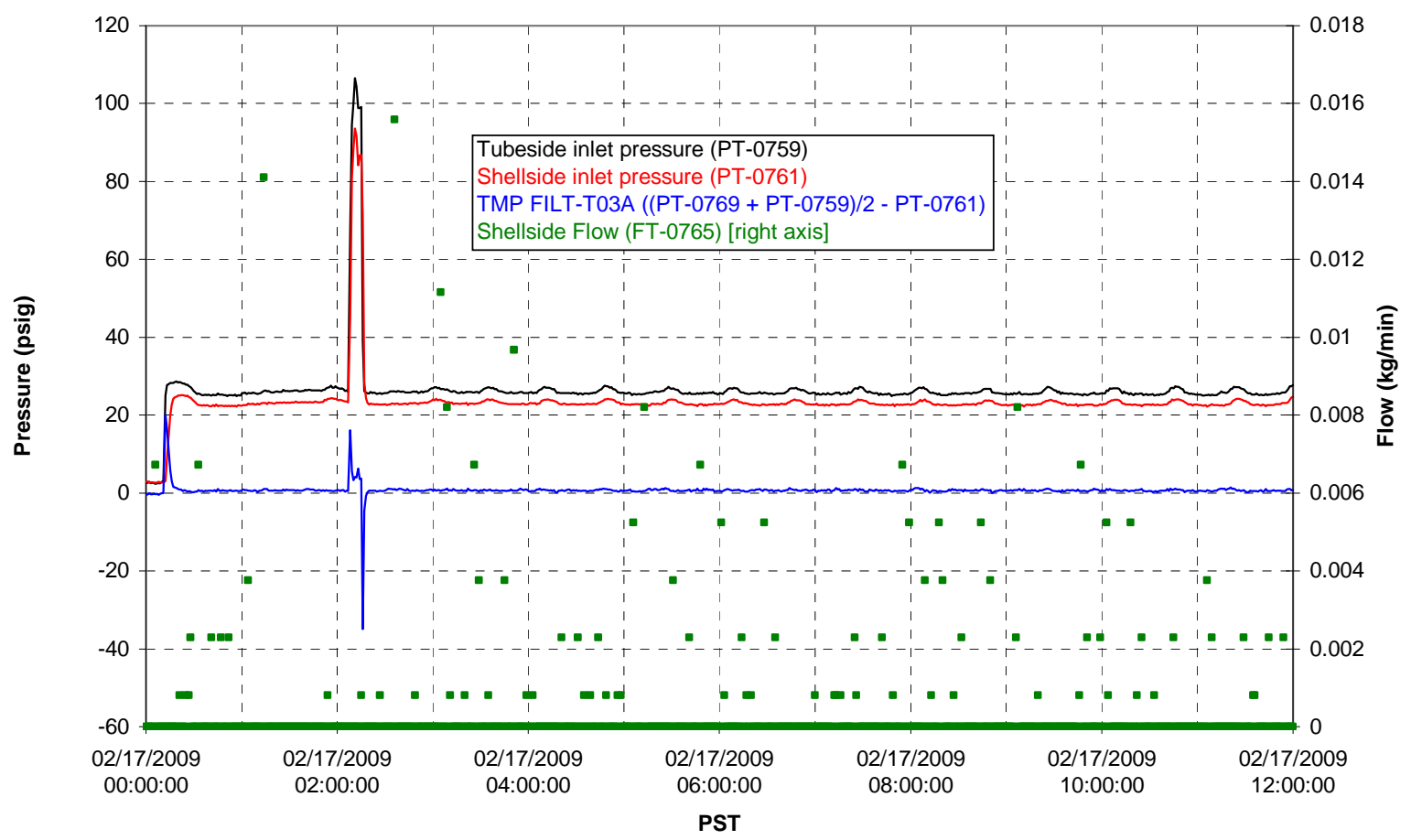

Filter UFP-FILT-T04A

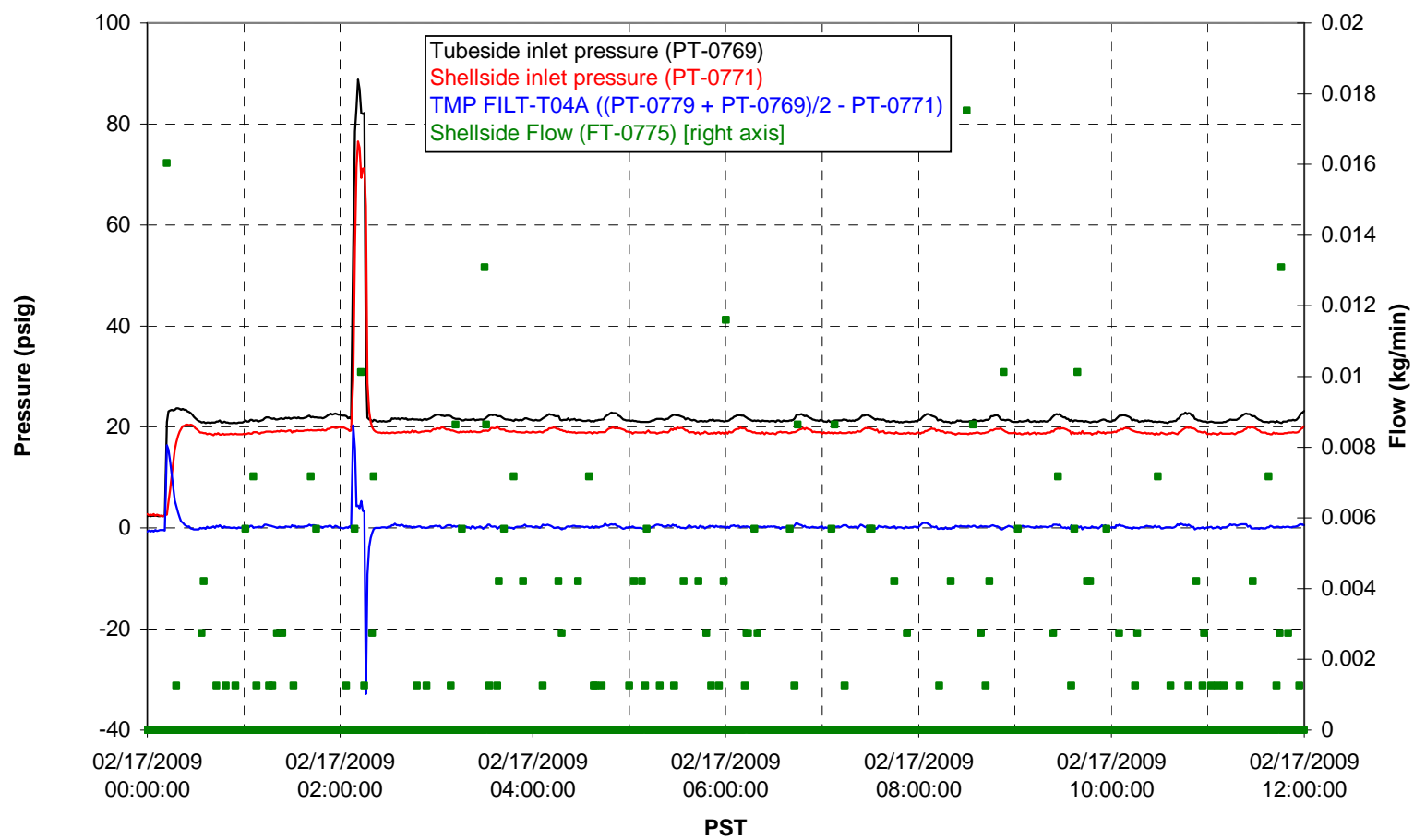


Filter UFP-FILT-T05A

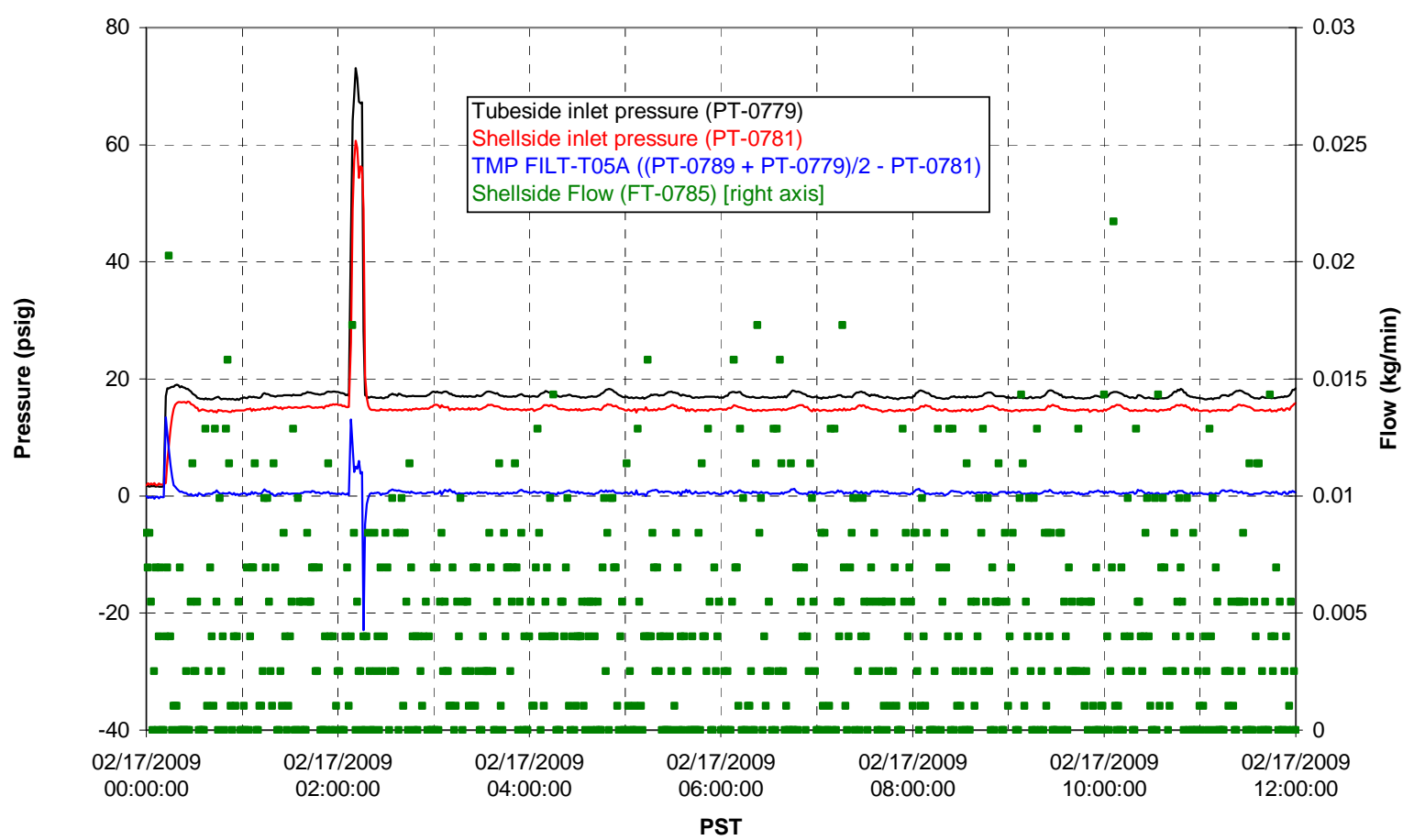

Chemical Flow

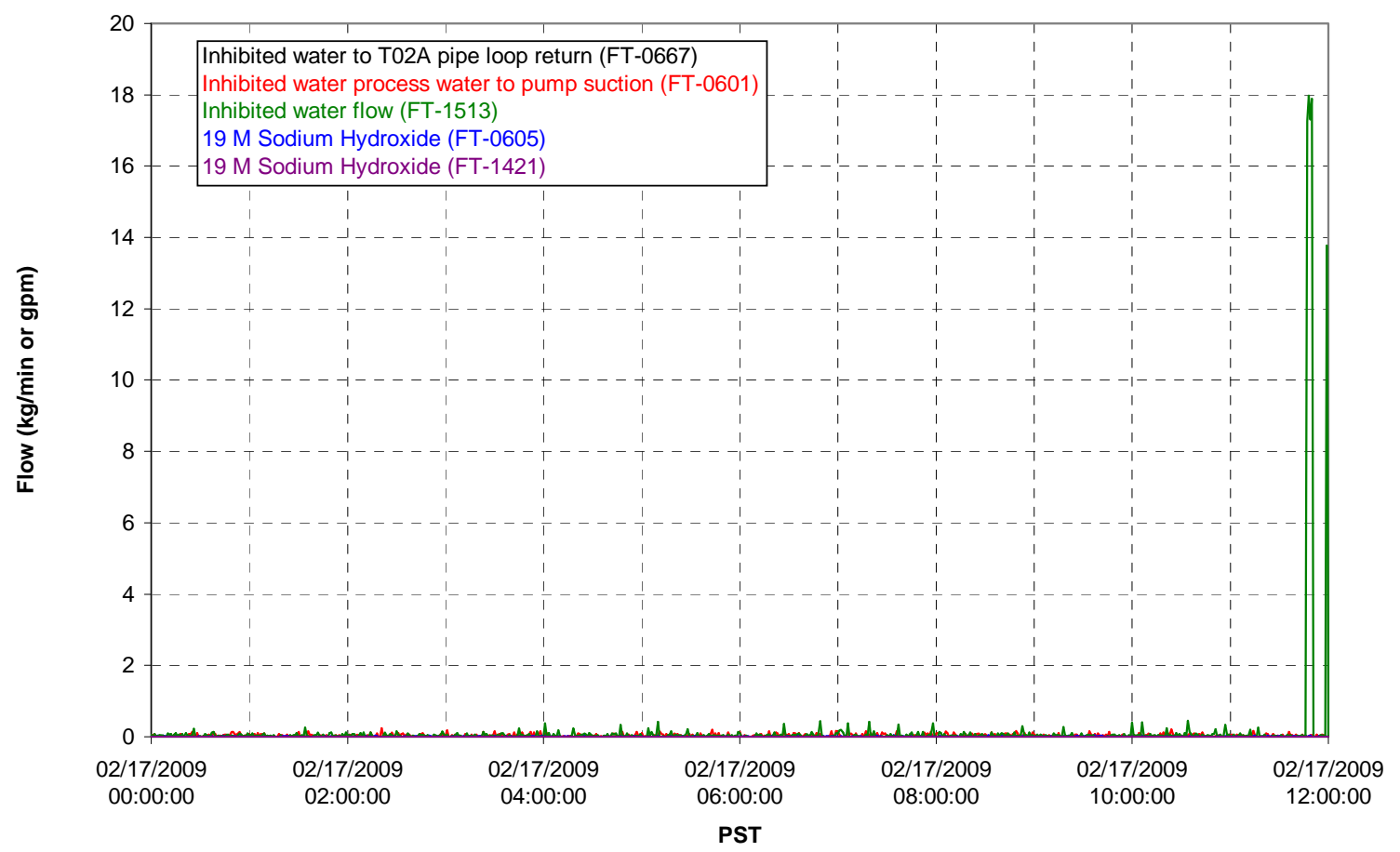




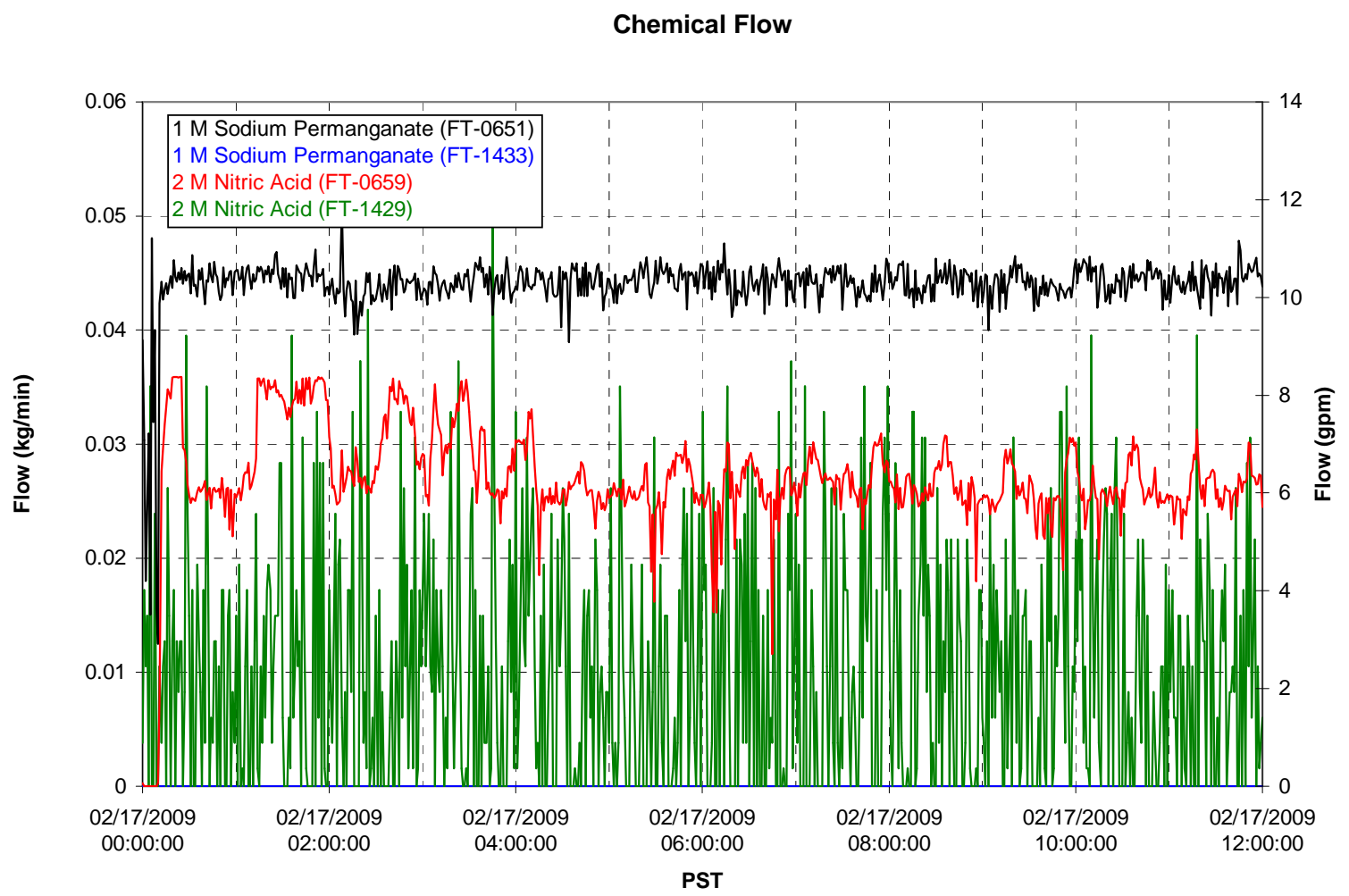

Air Flows

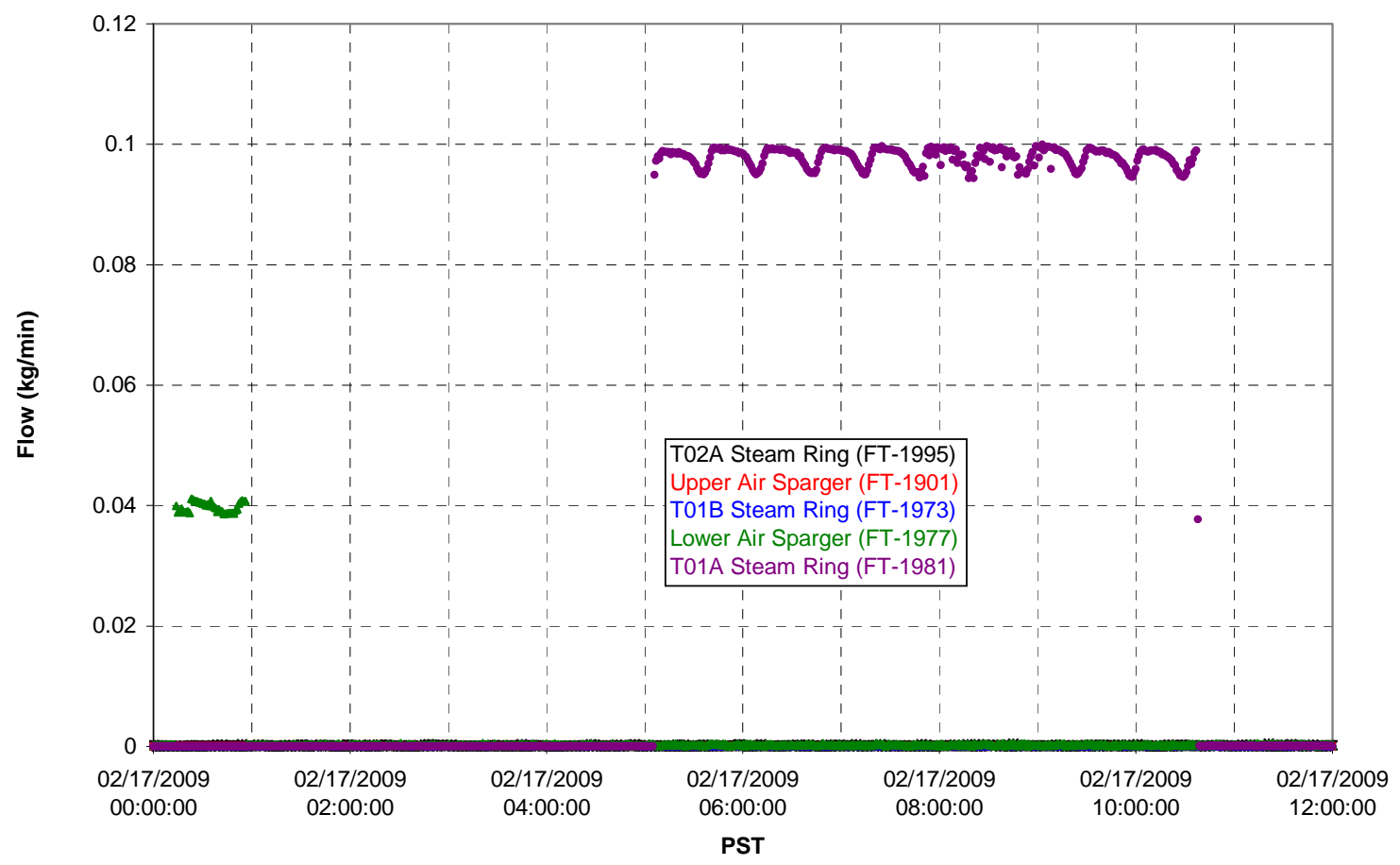


T02A Steam

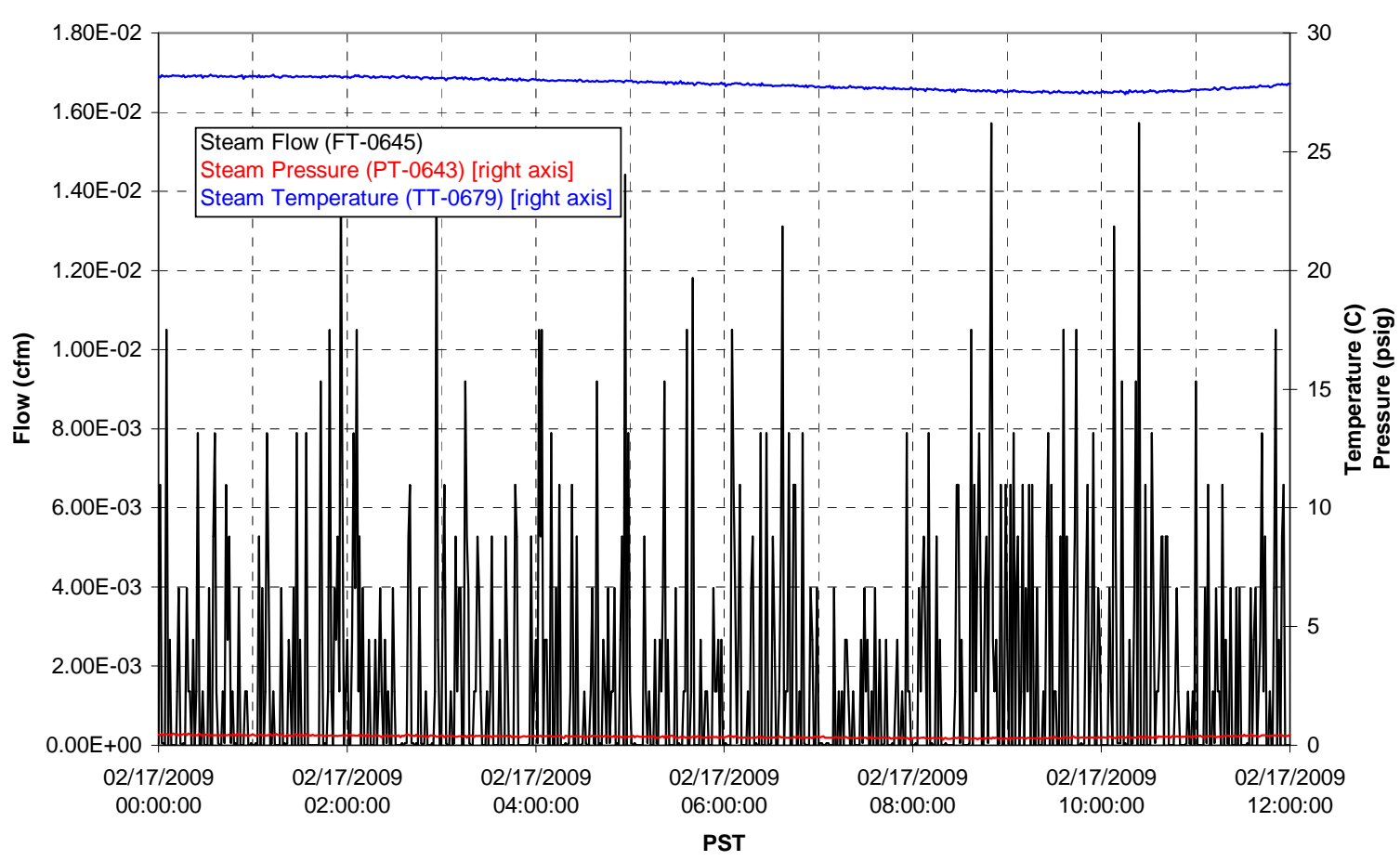

T01A Steam

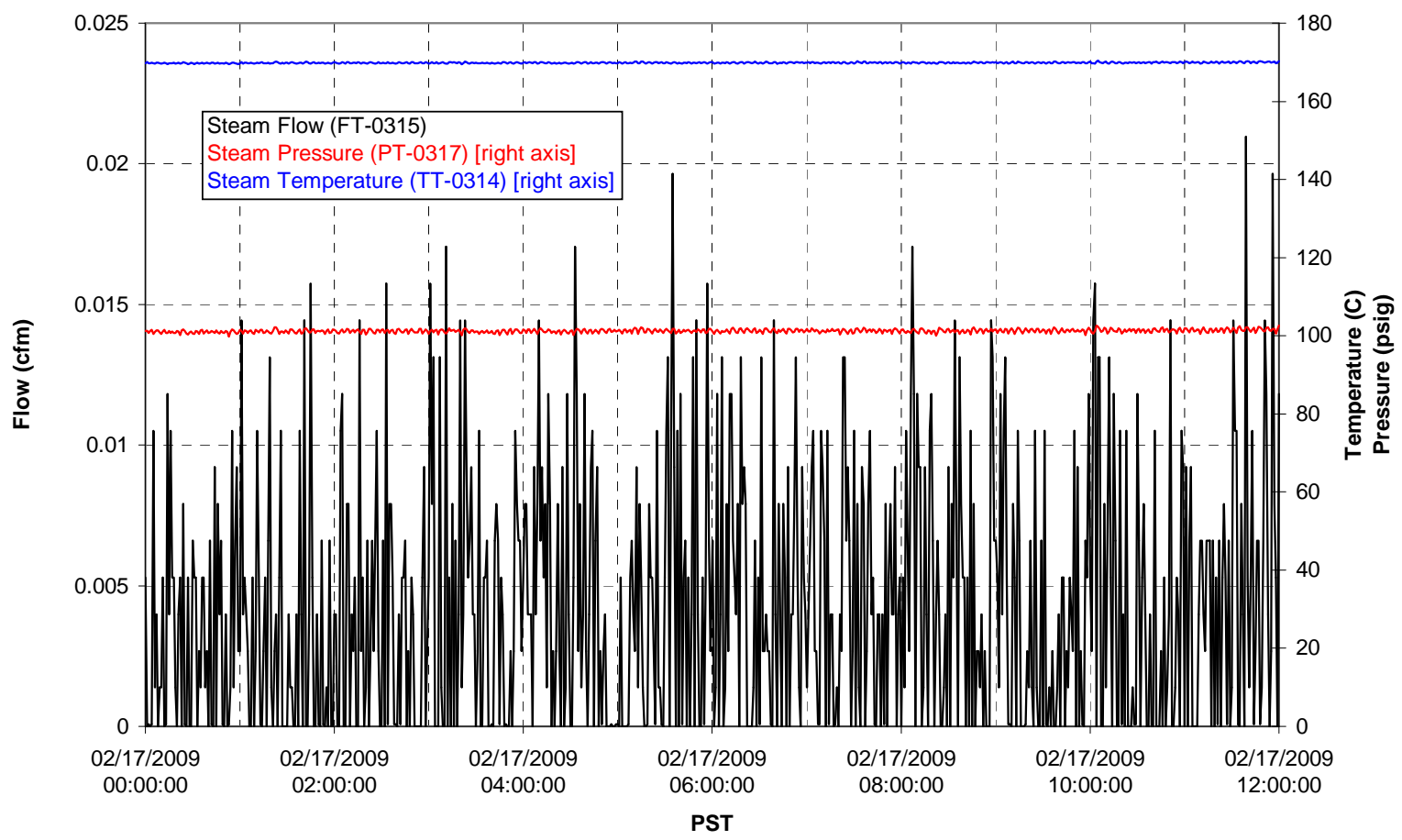


T01B Steam

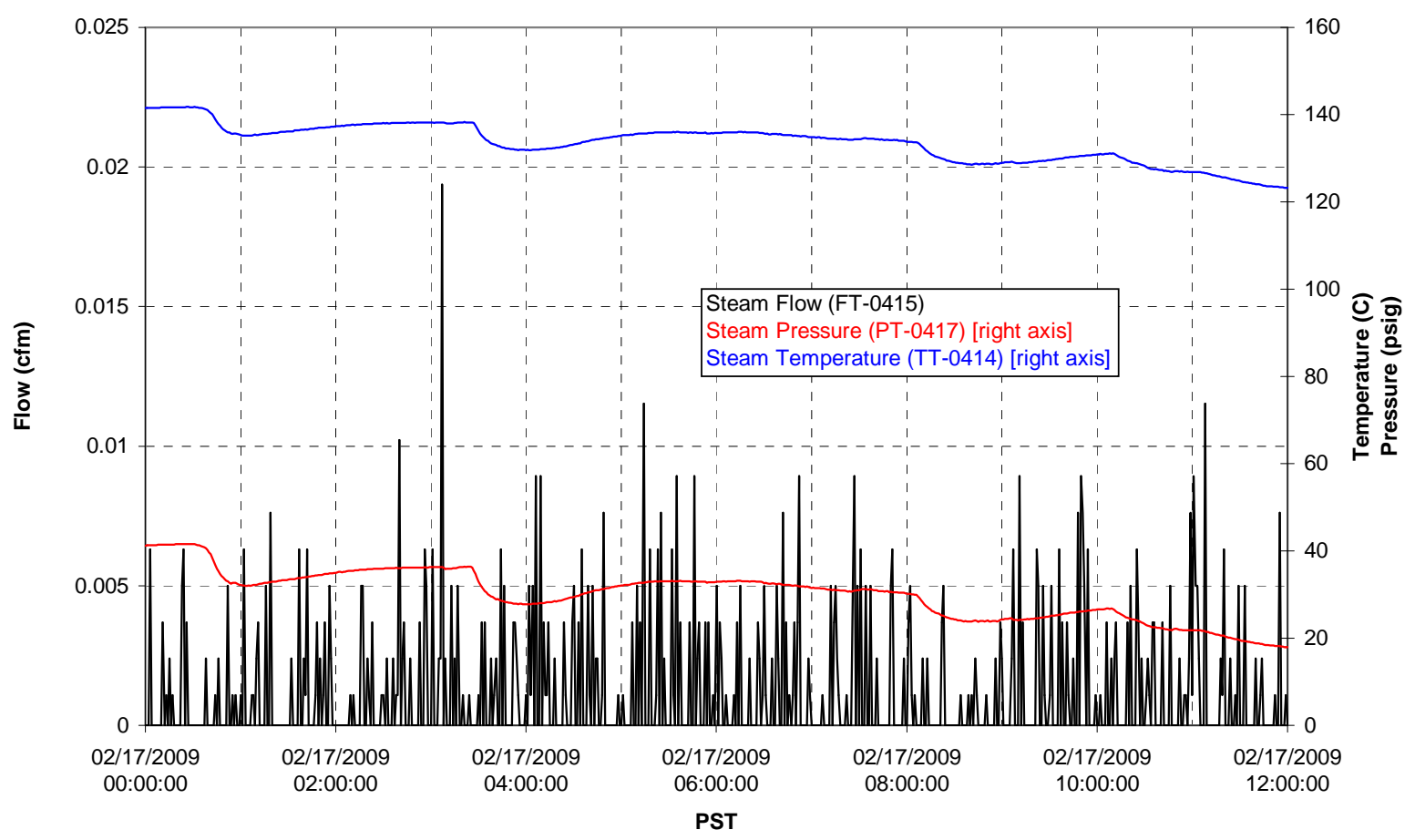




\section{Integrated Test A Data Plots 02/17/09 12:00 - 02/18/09 00:00}


T01A level

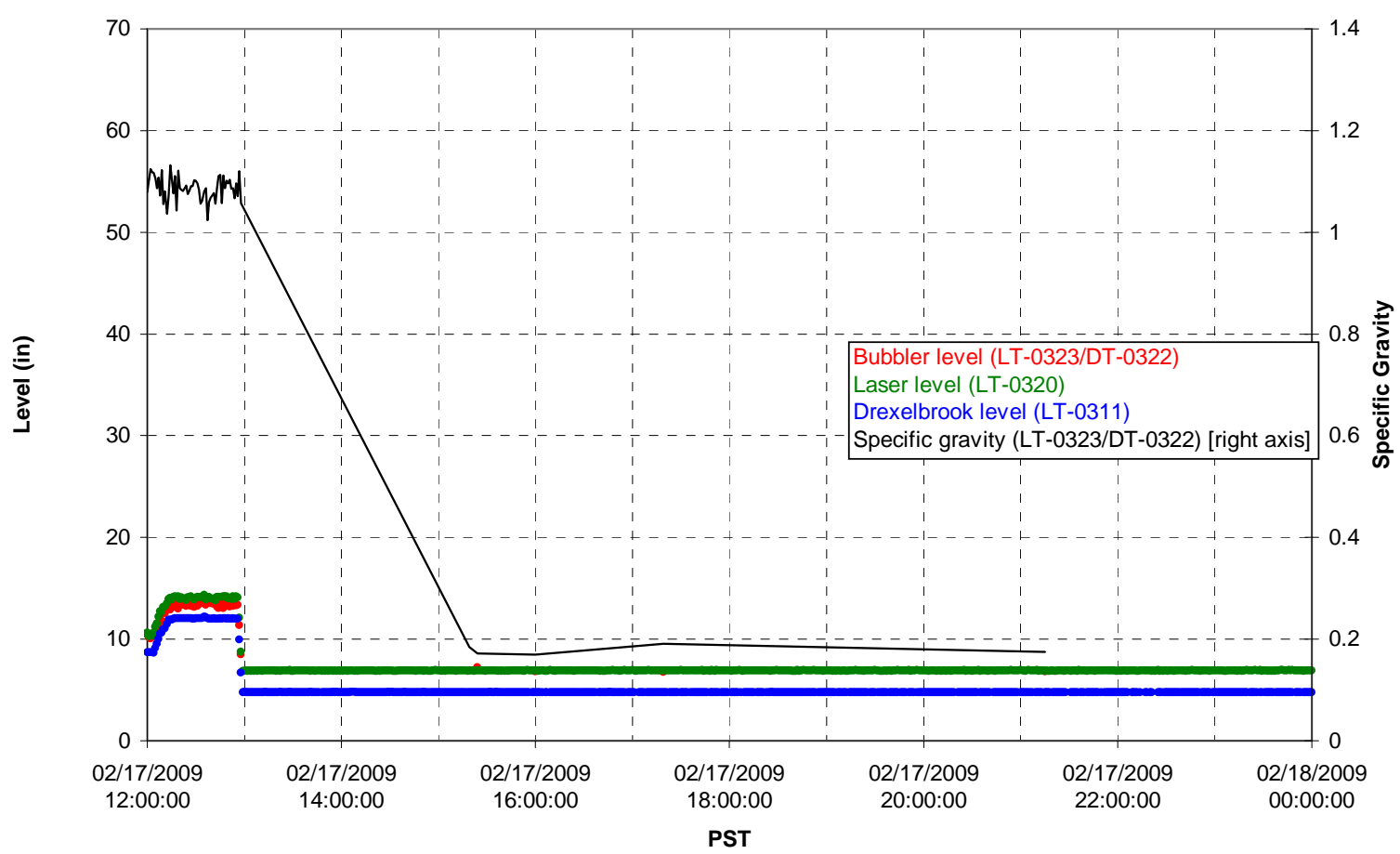

T01A temperatures

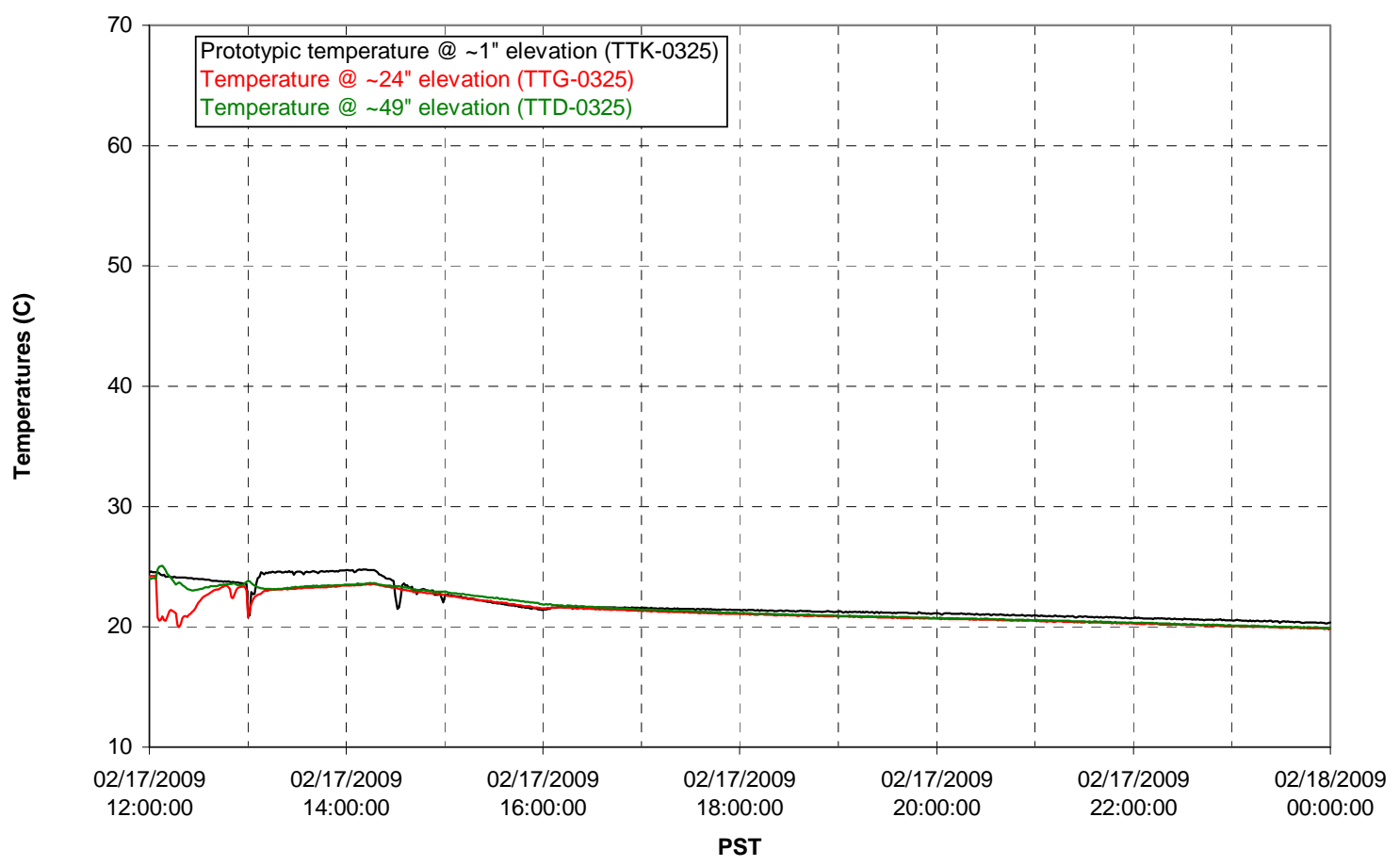


T01B level

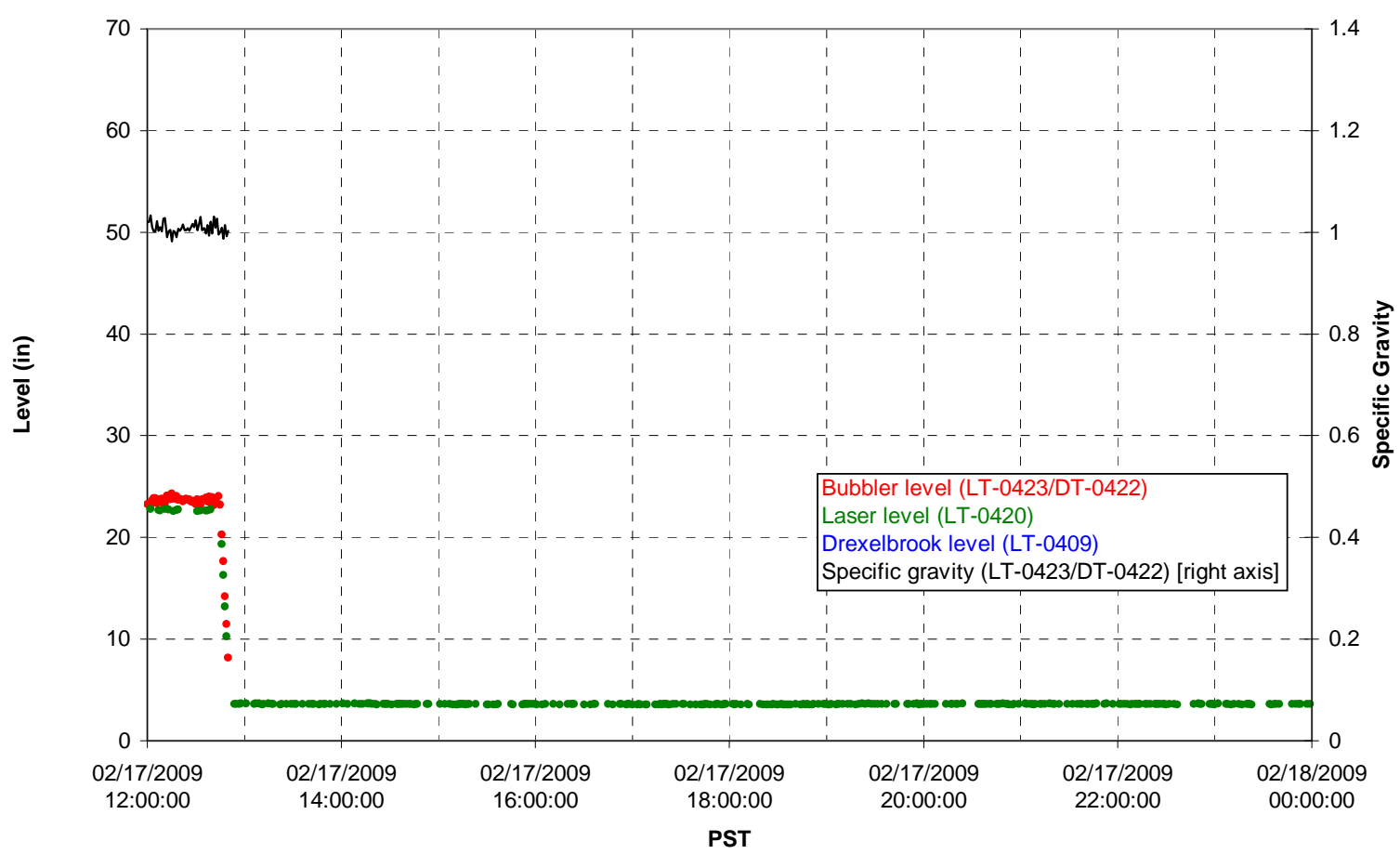

T01B temperatures

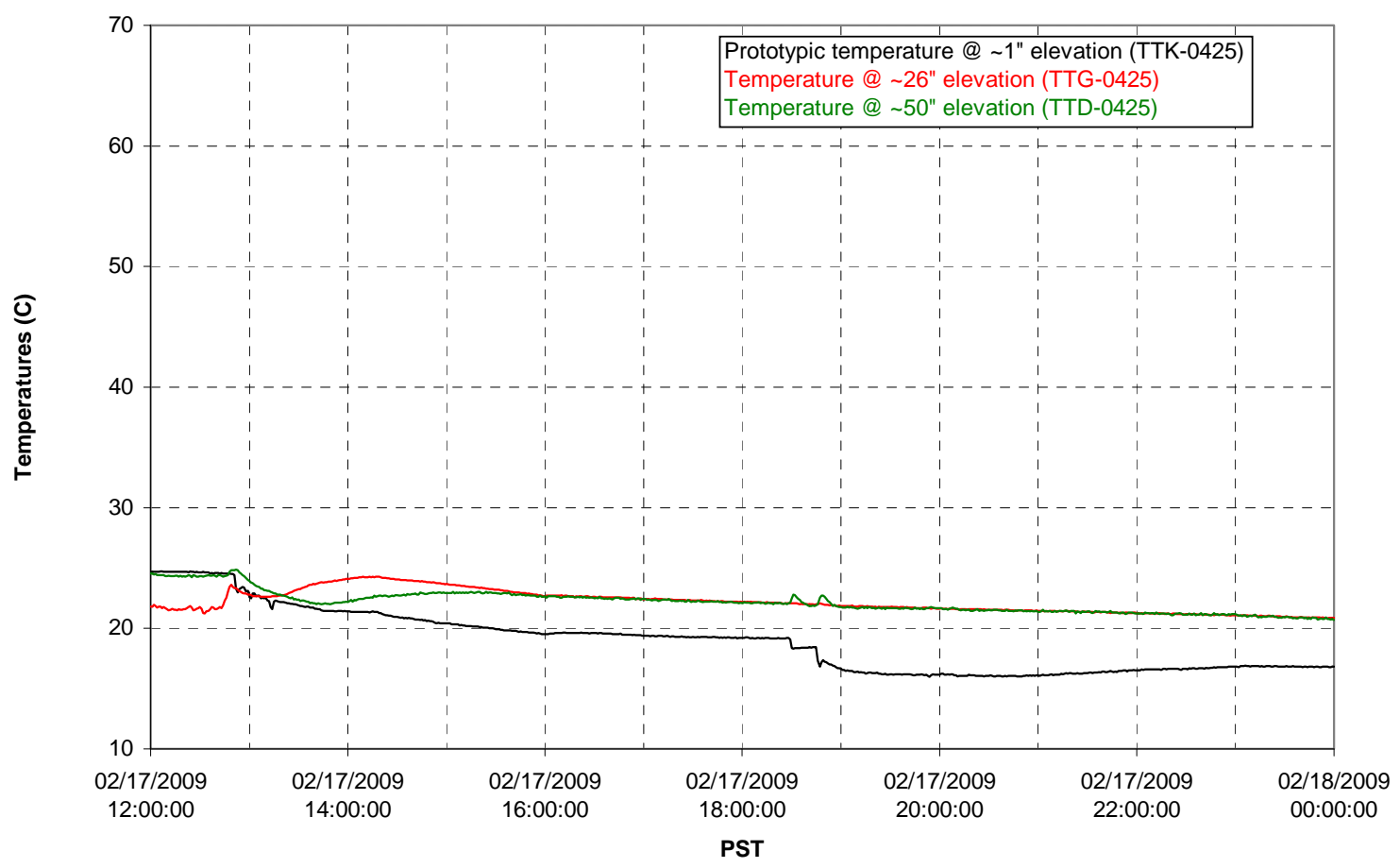


T02A level

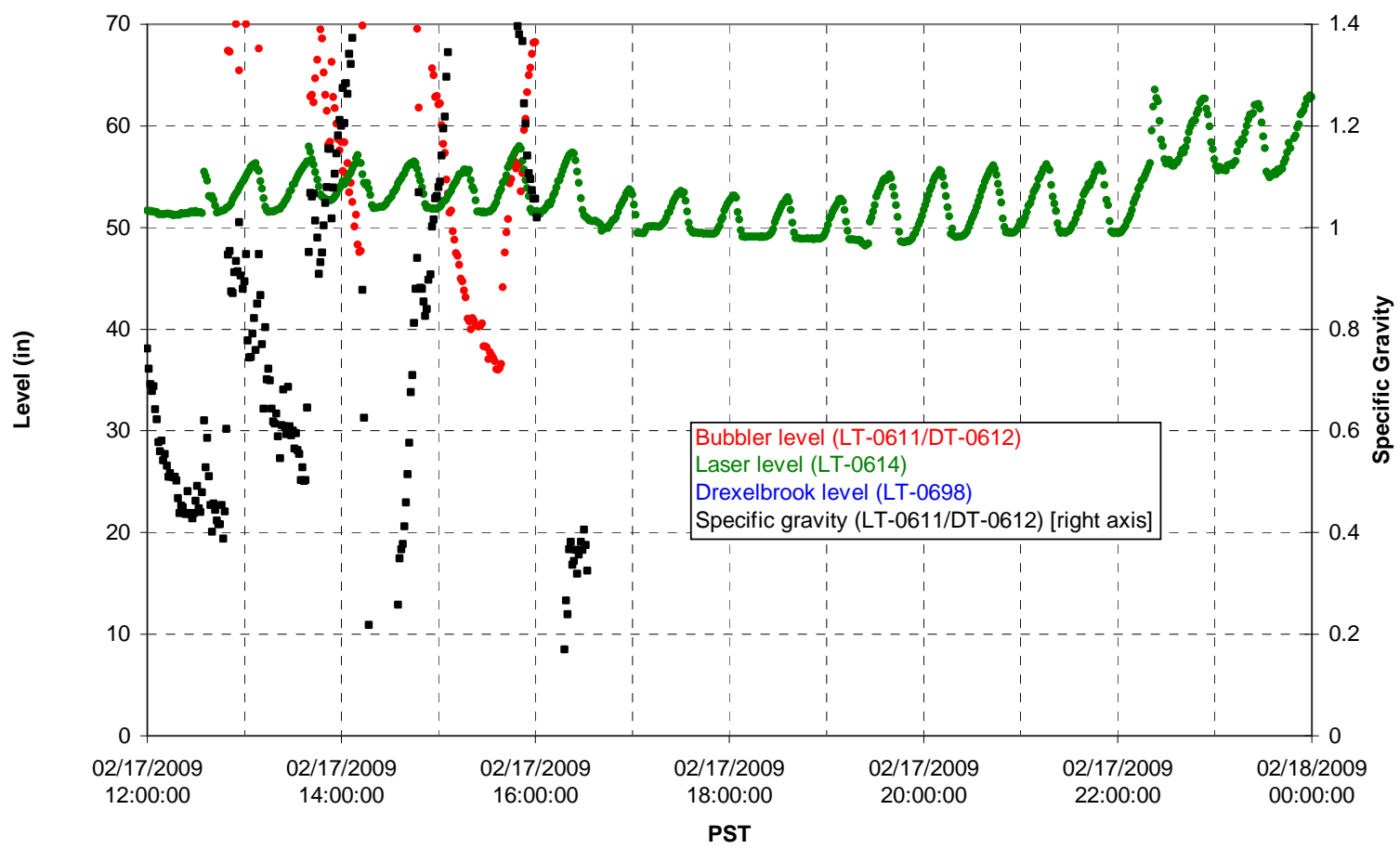

T02A temperatures

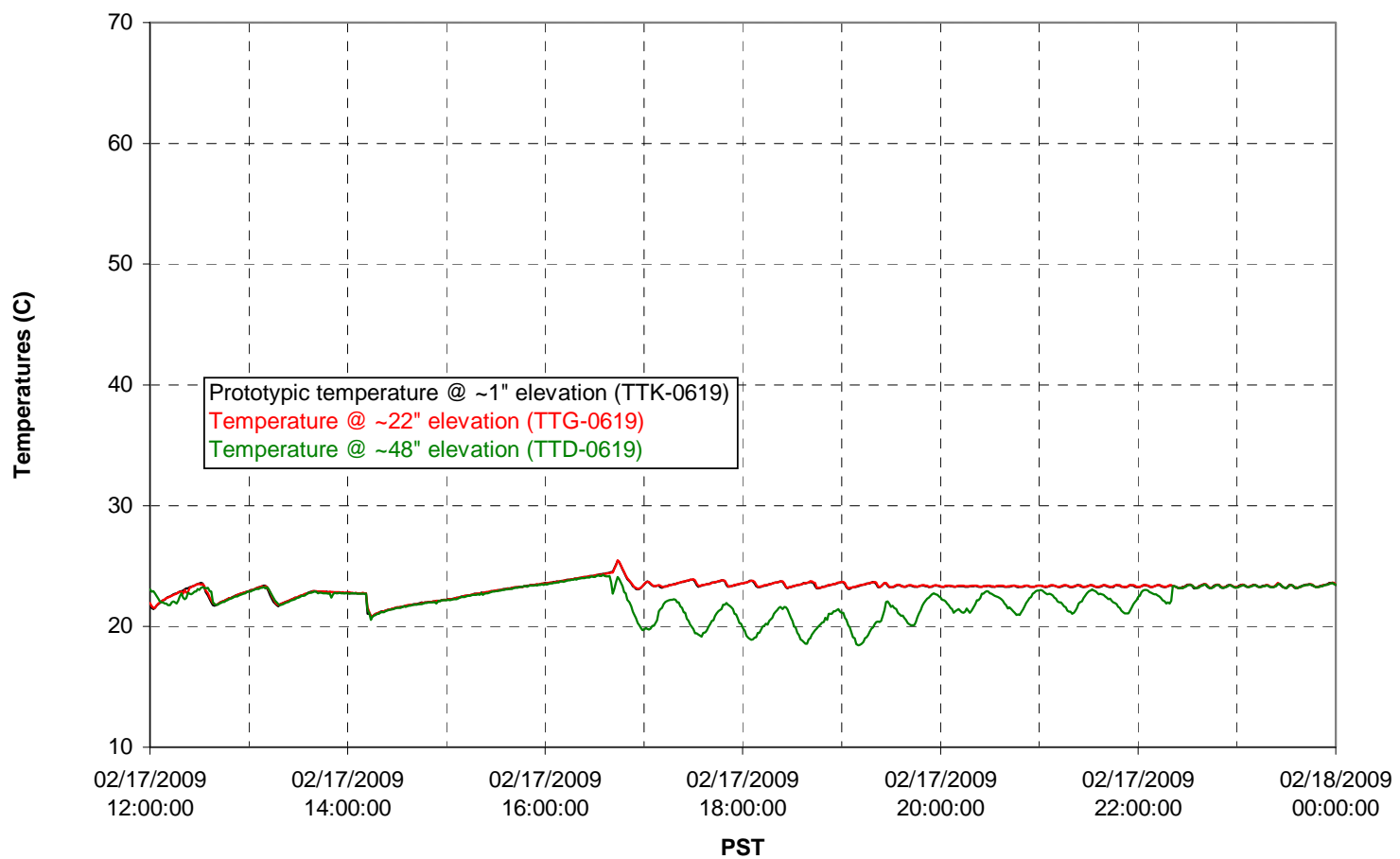


T02A and filter loop temperatures

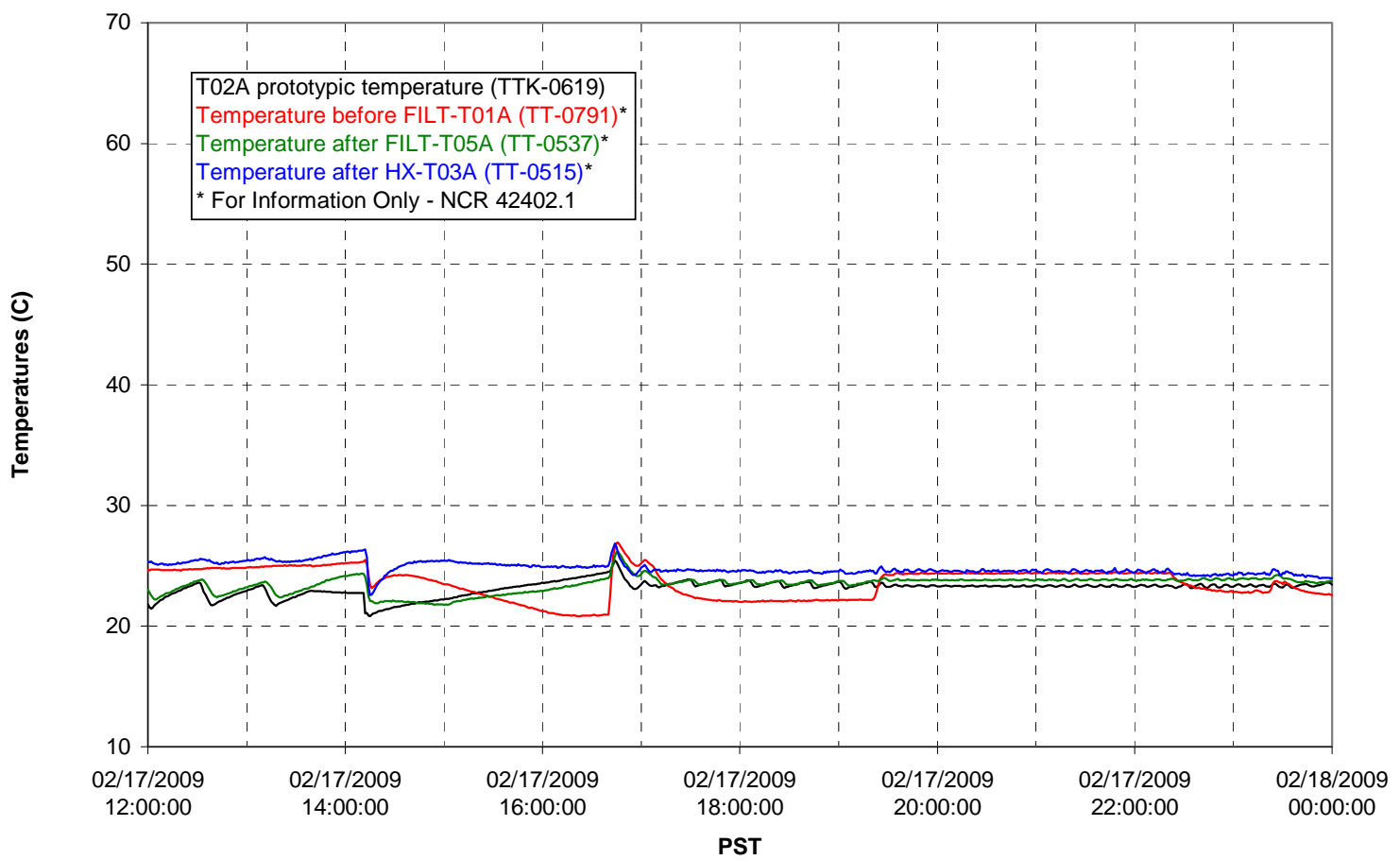

Pump Pressures and Flow

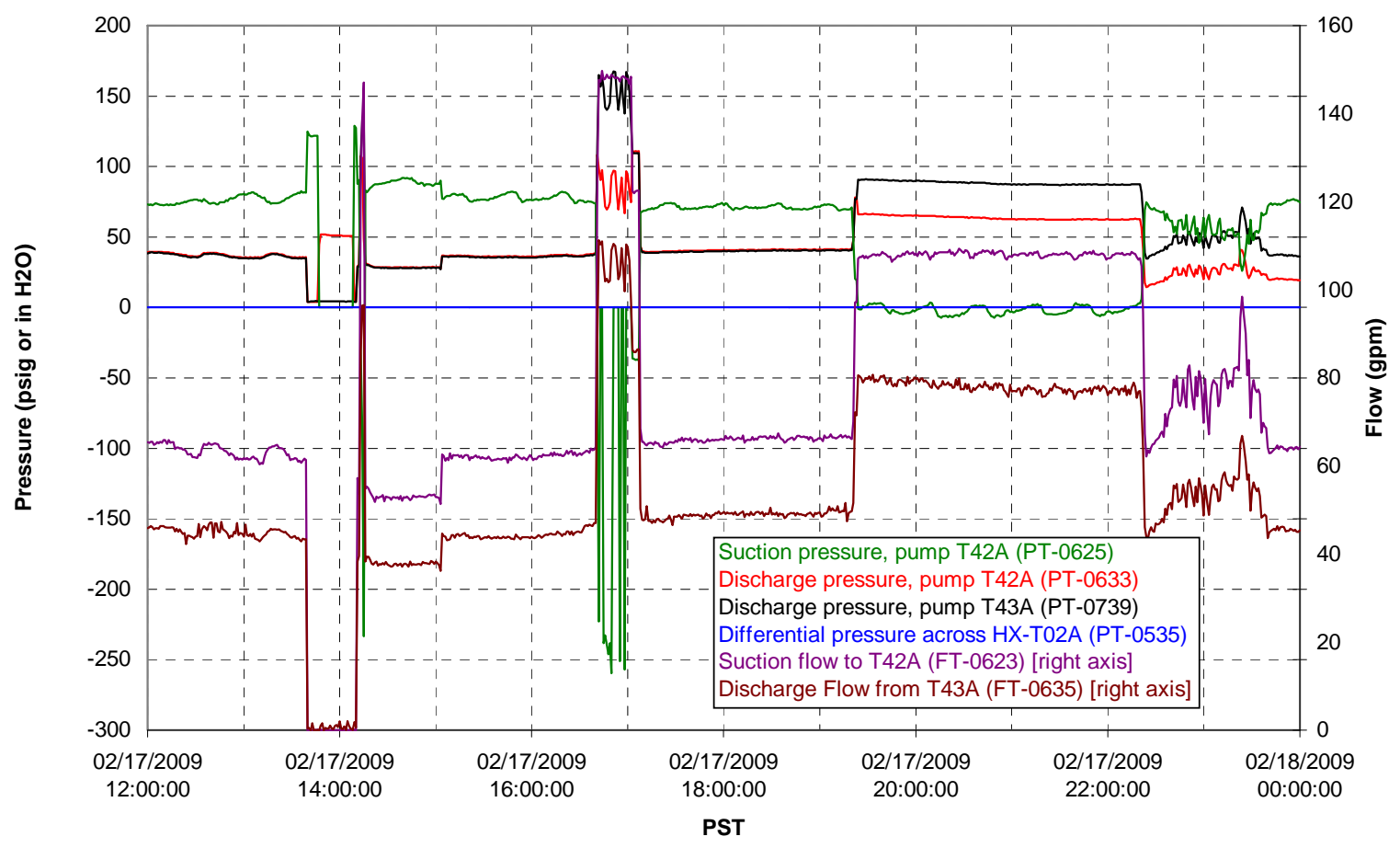




\section{Axial pressure drop}

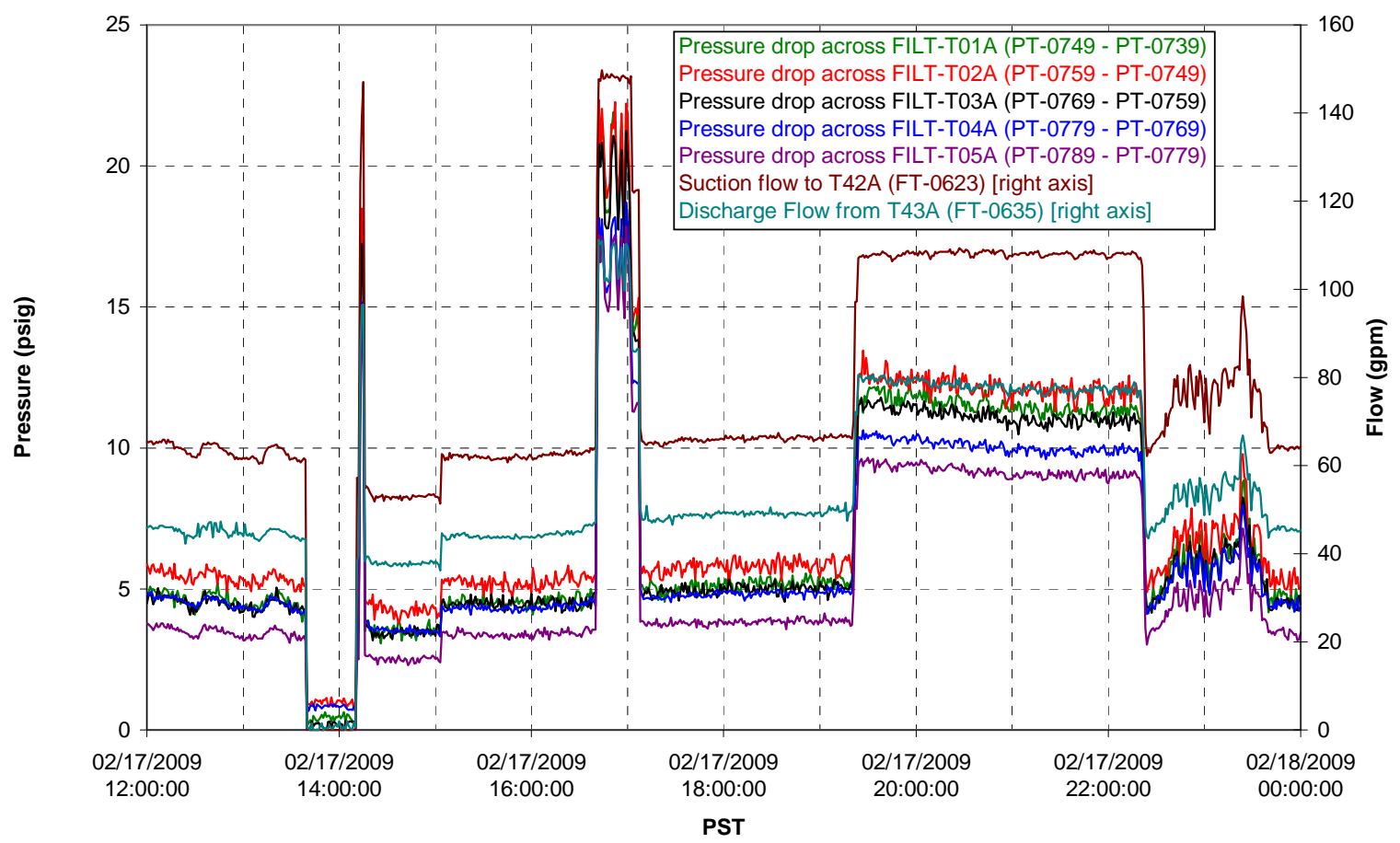

Permeate flow rates

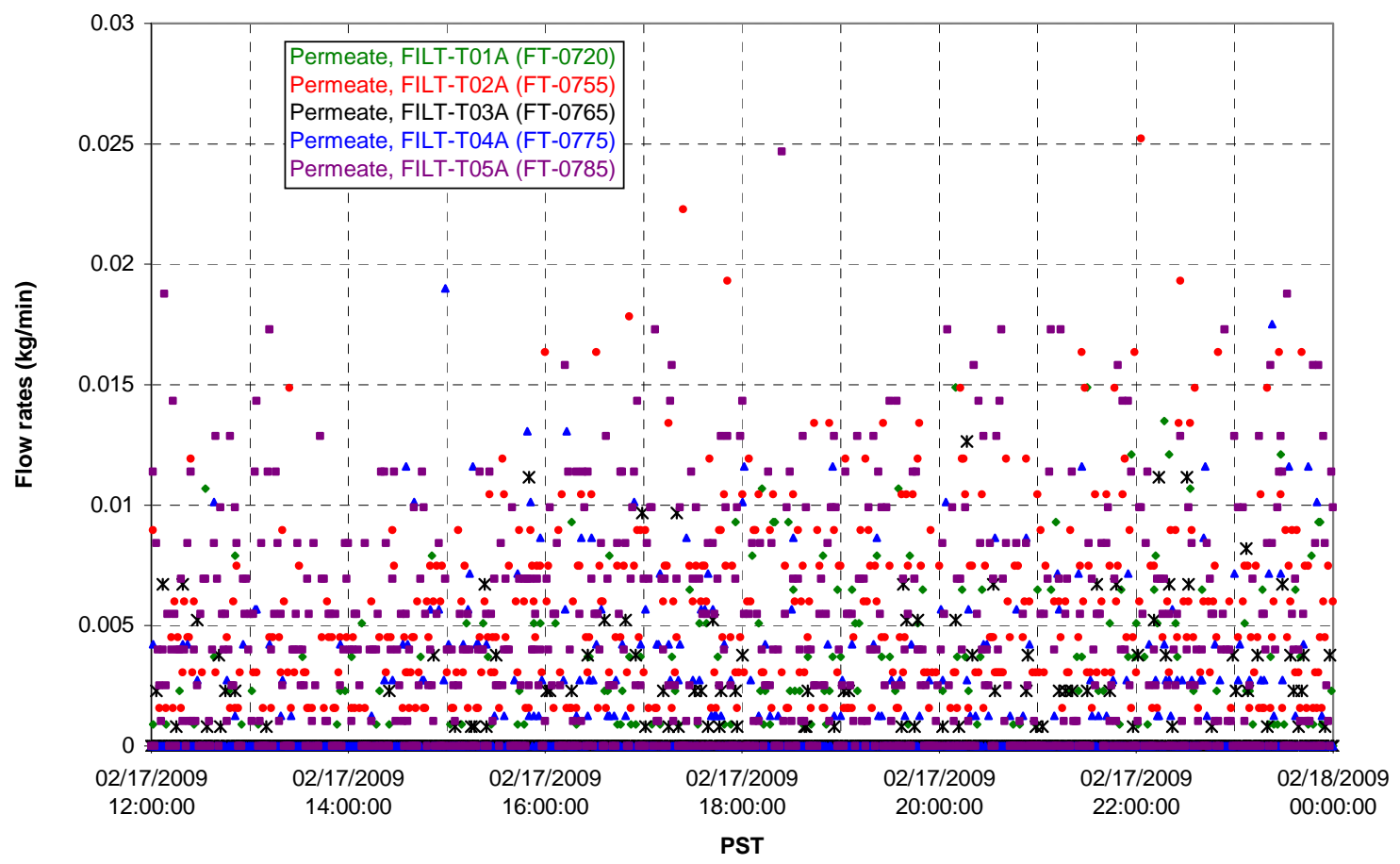


T02A Inner Temperature Tree

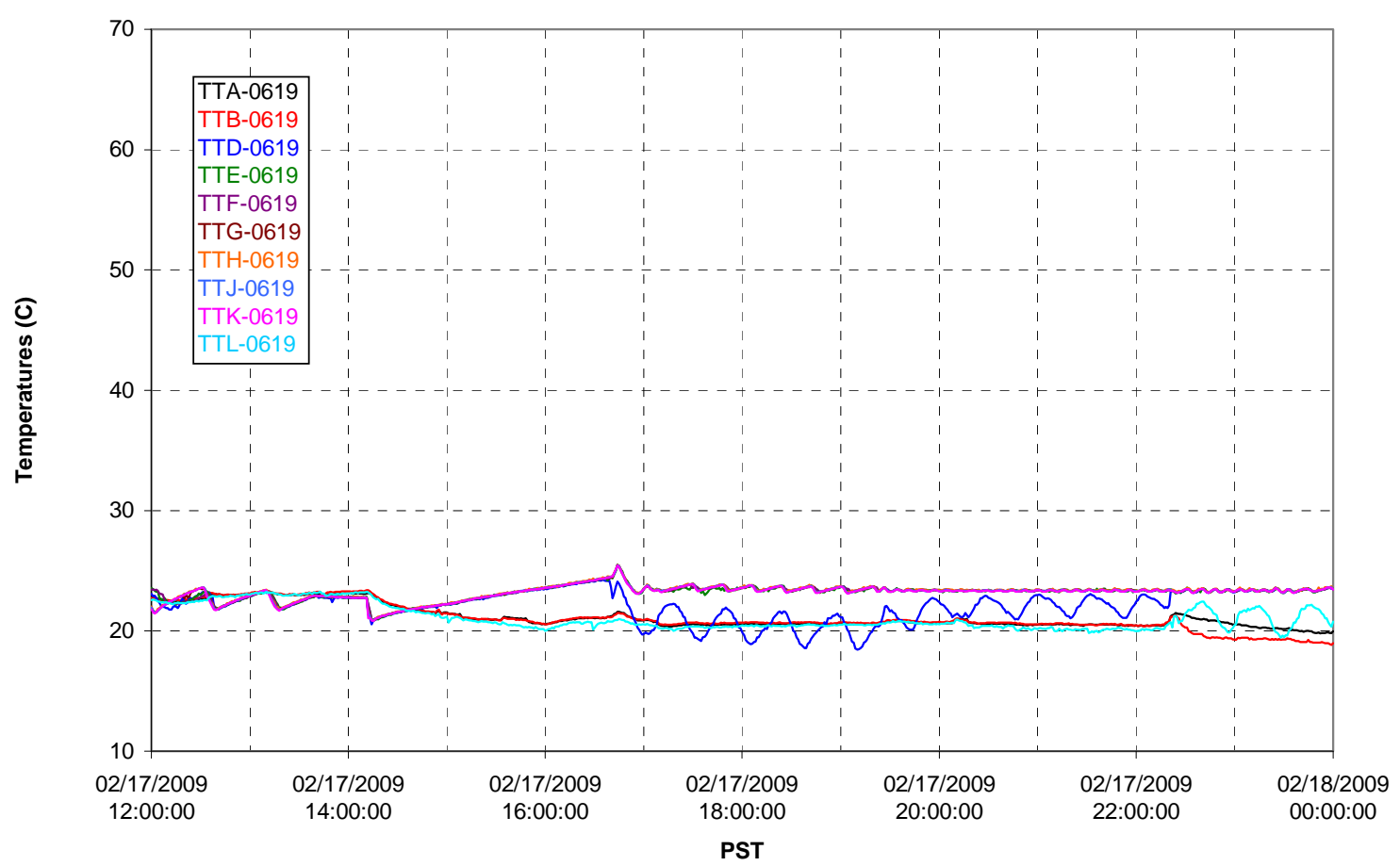

T02A Outer Temperature Tree

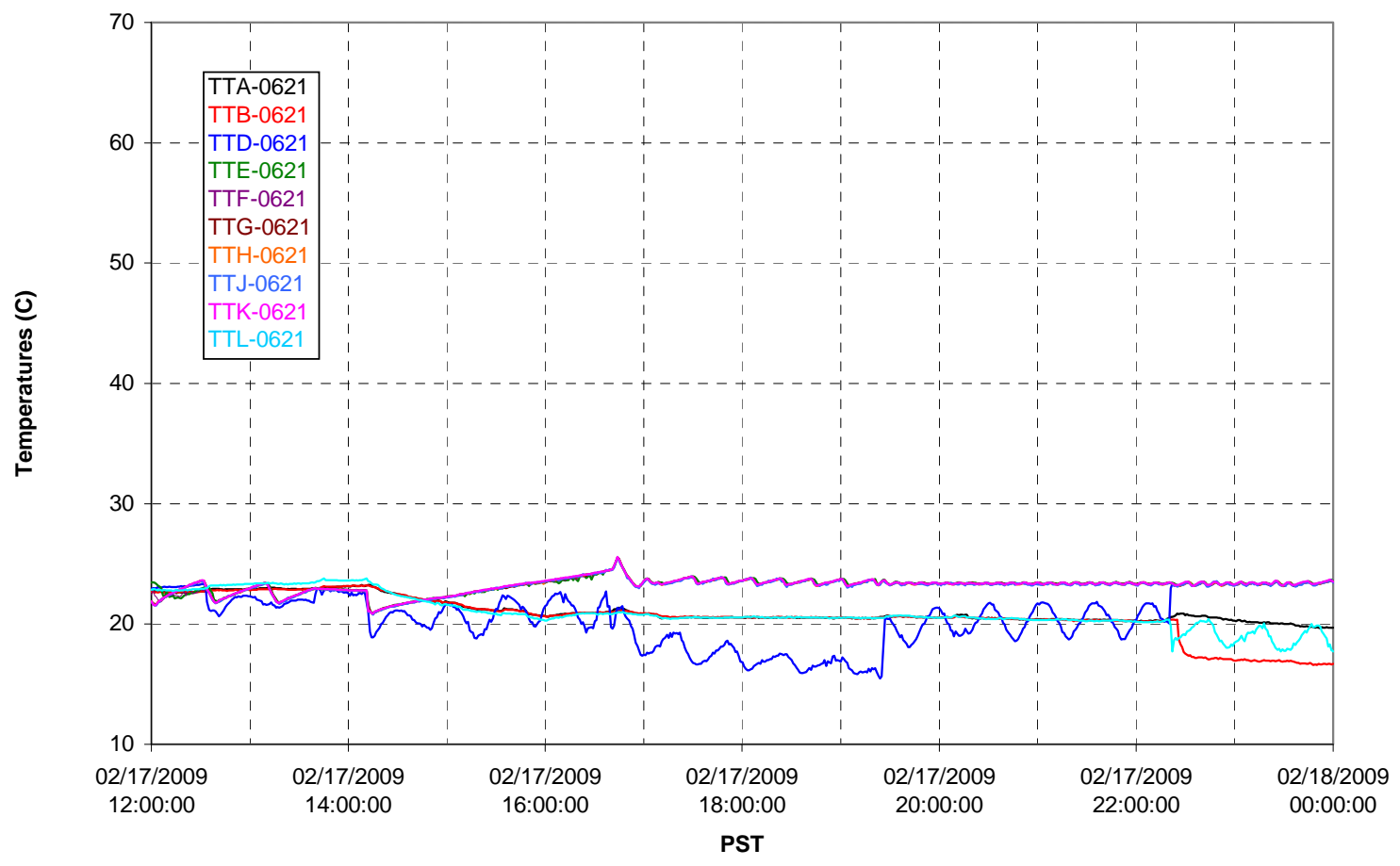


T02A temperatures

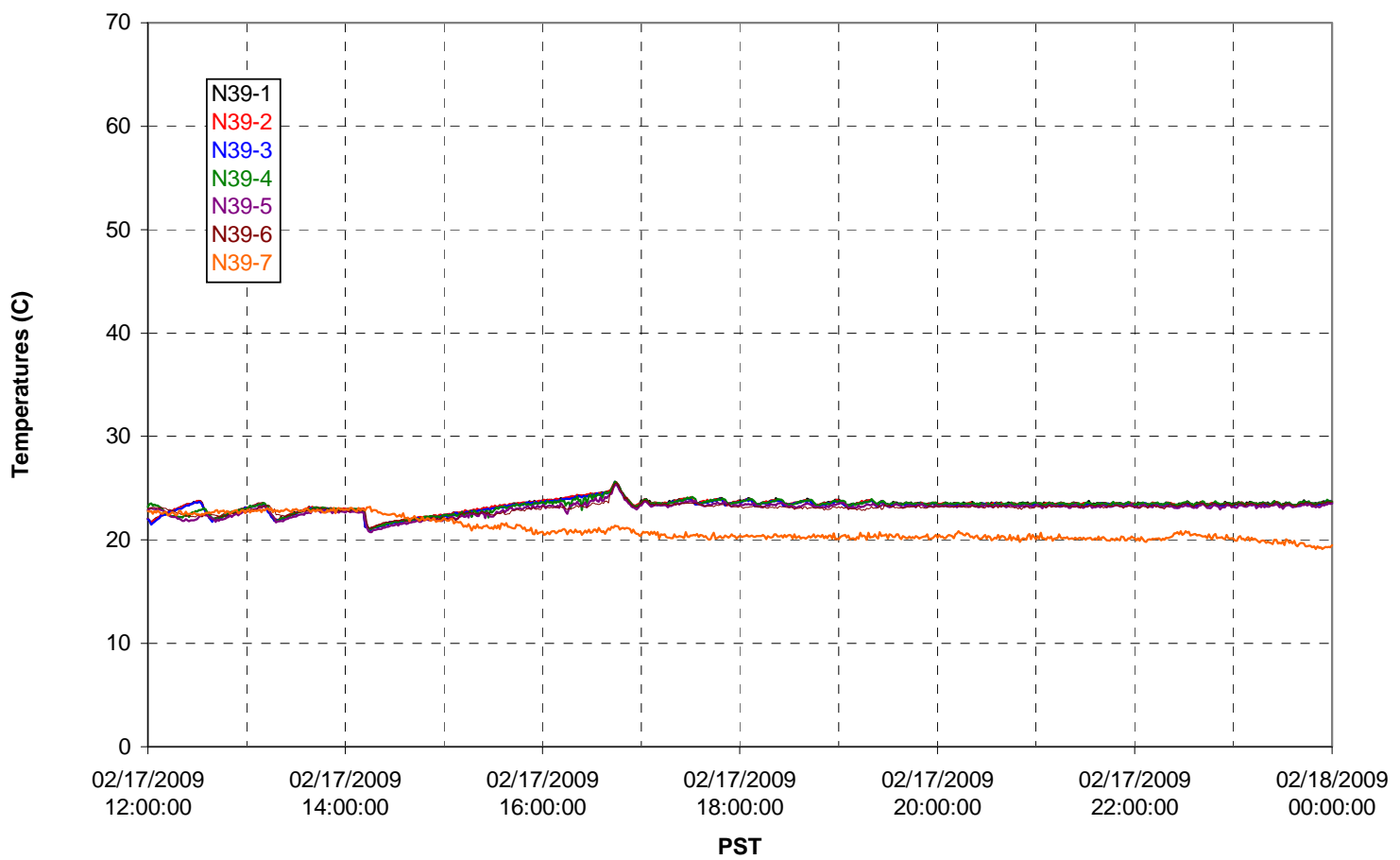

T02A temperatures

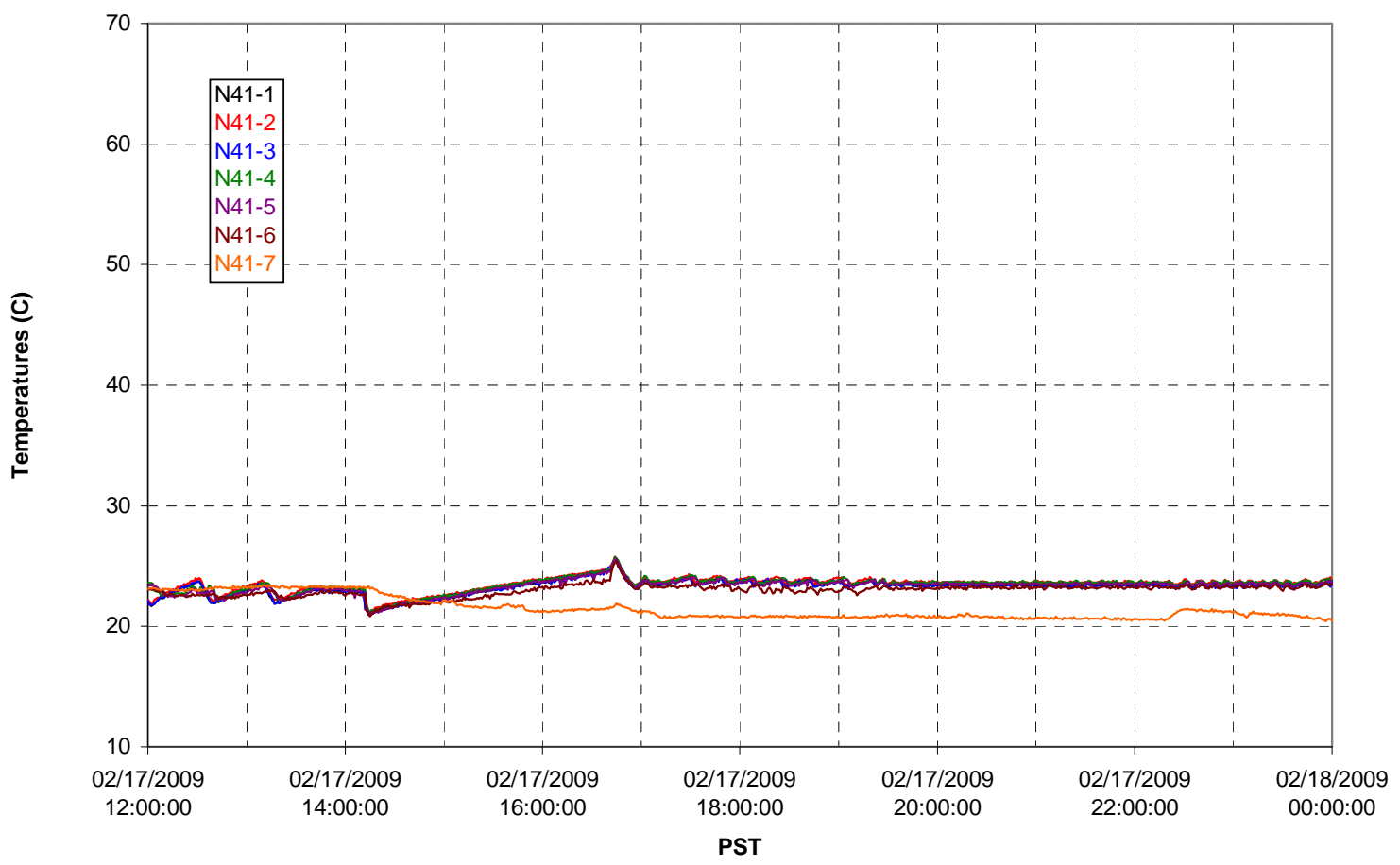


T02A temperatures

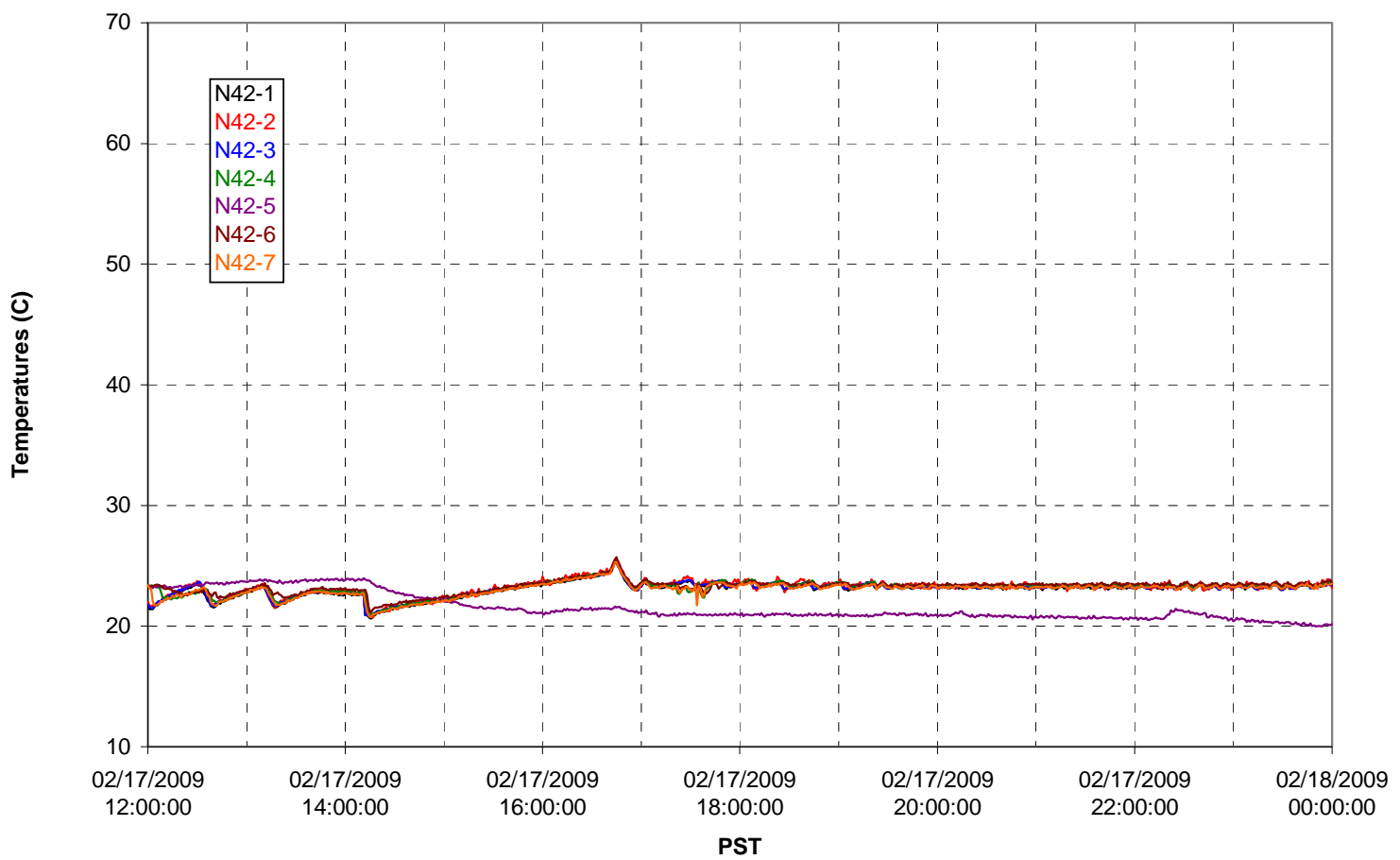

T02A temperatures

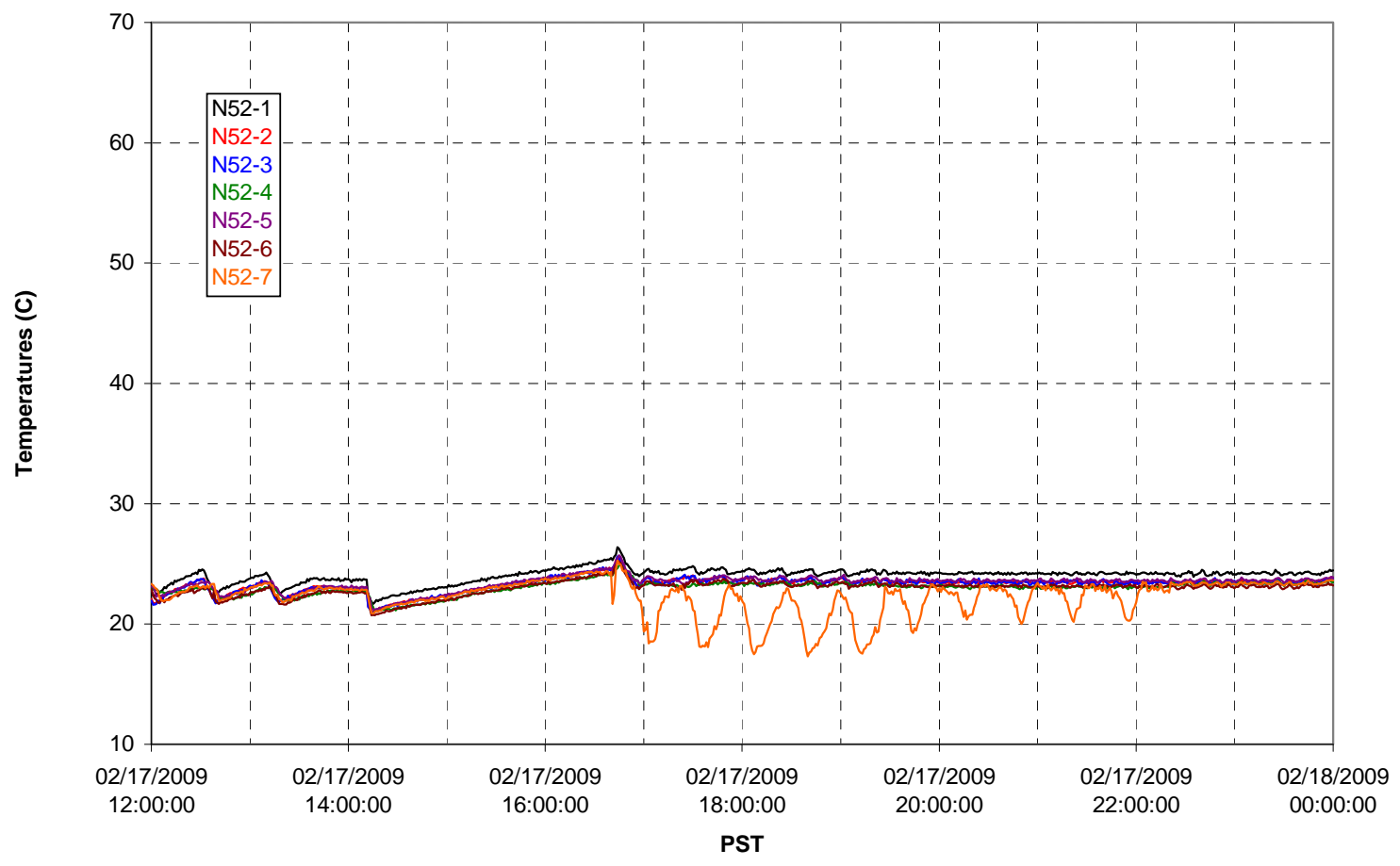


T02A Heating and Cooling

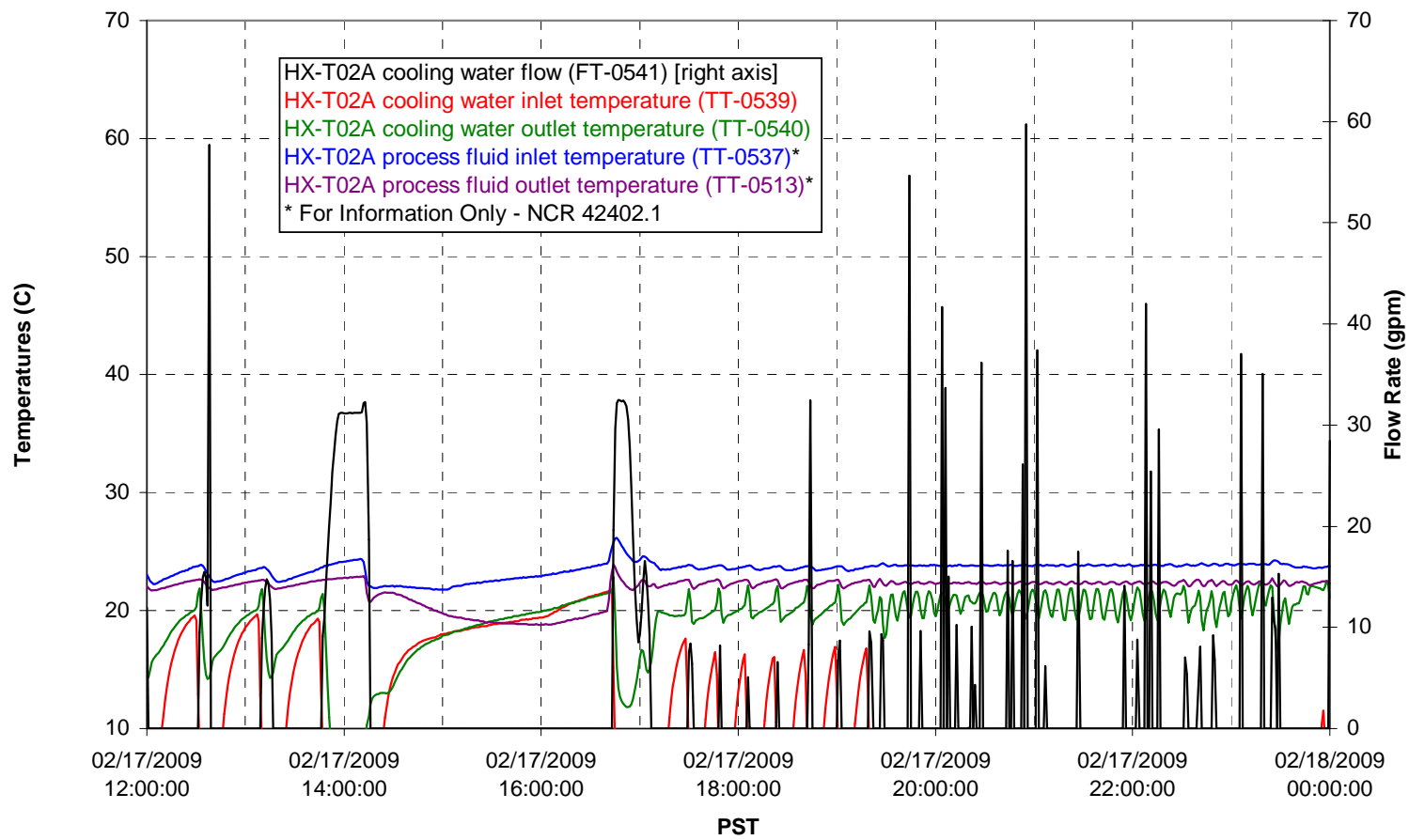

Pump Operation

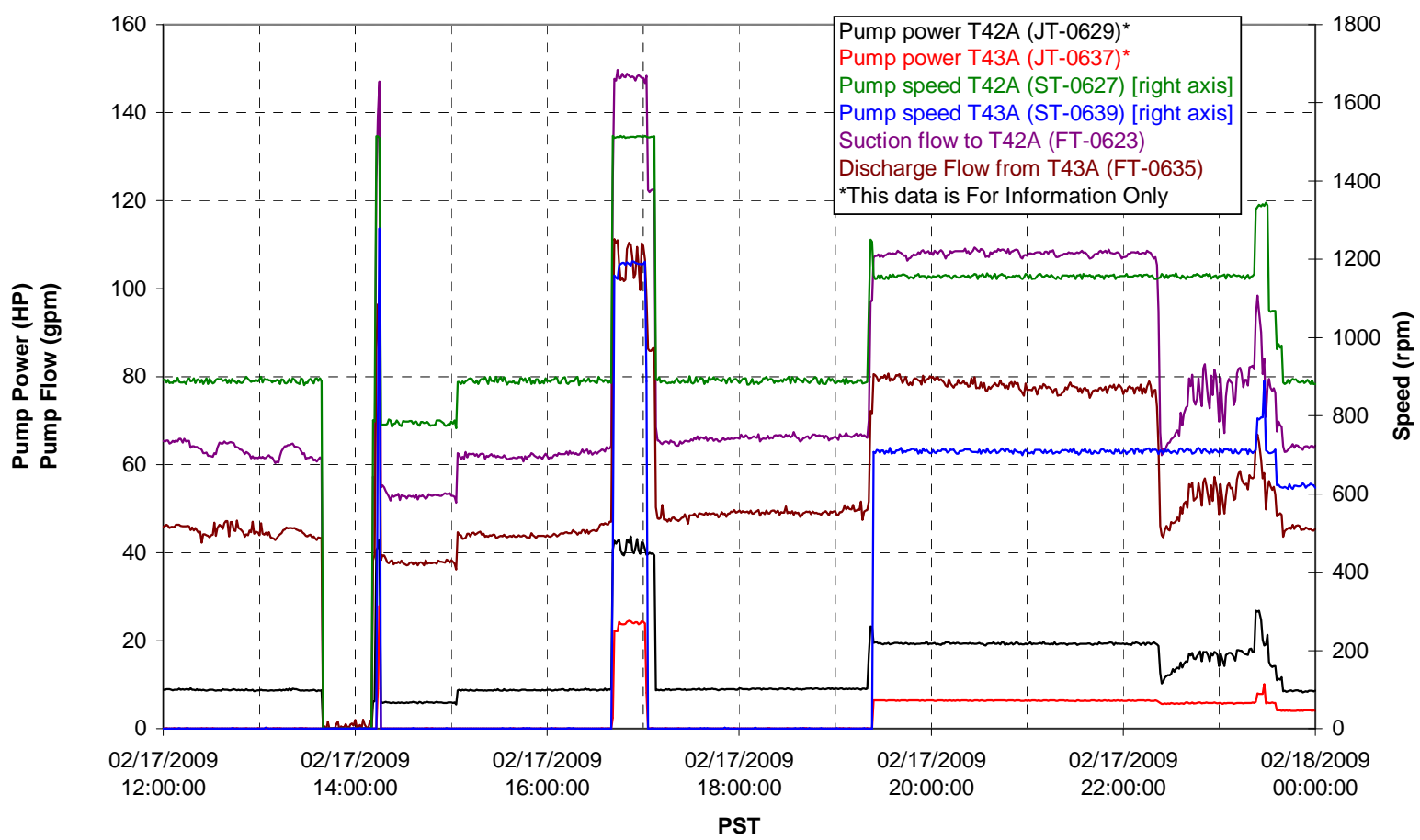


Pulsepot UFP-PP-T01A

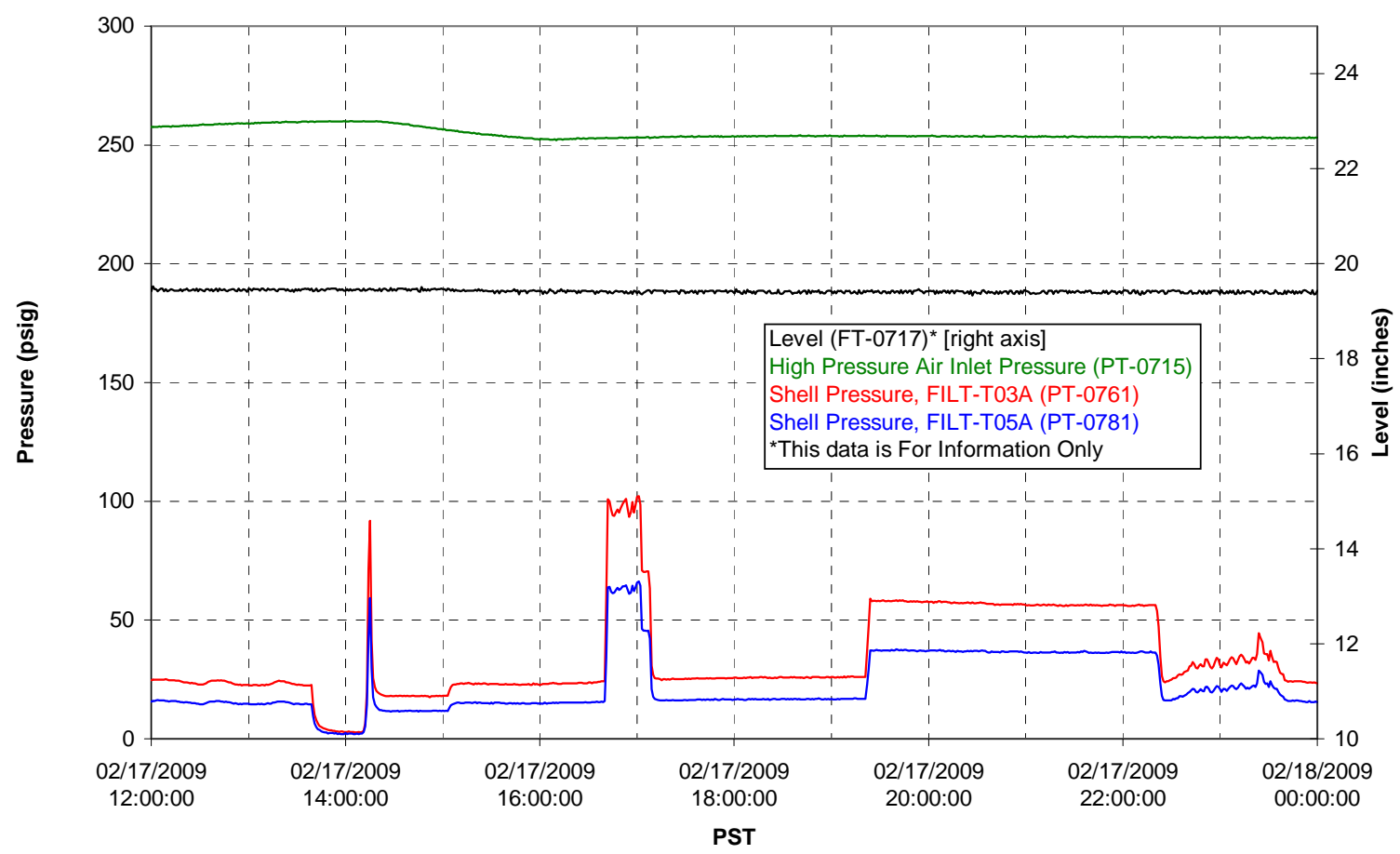

Pulsepot UFP-PP-T02A

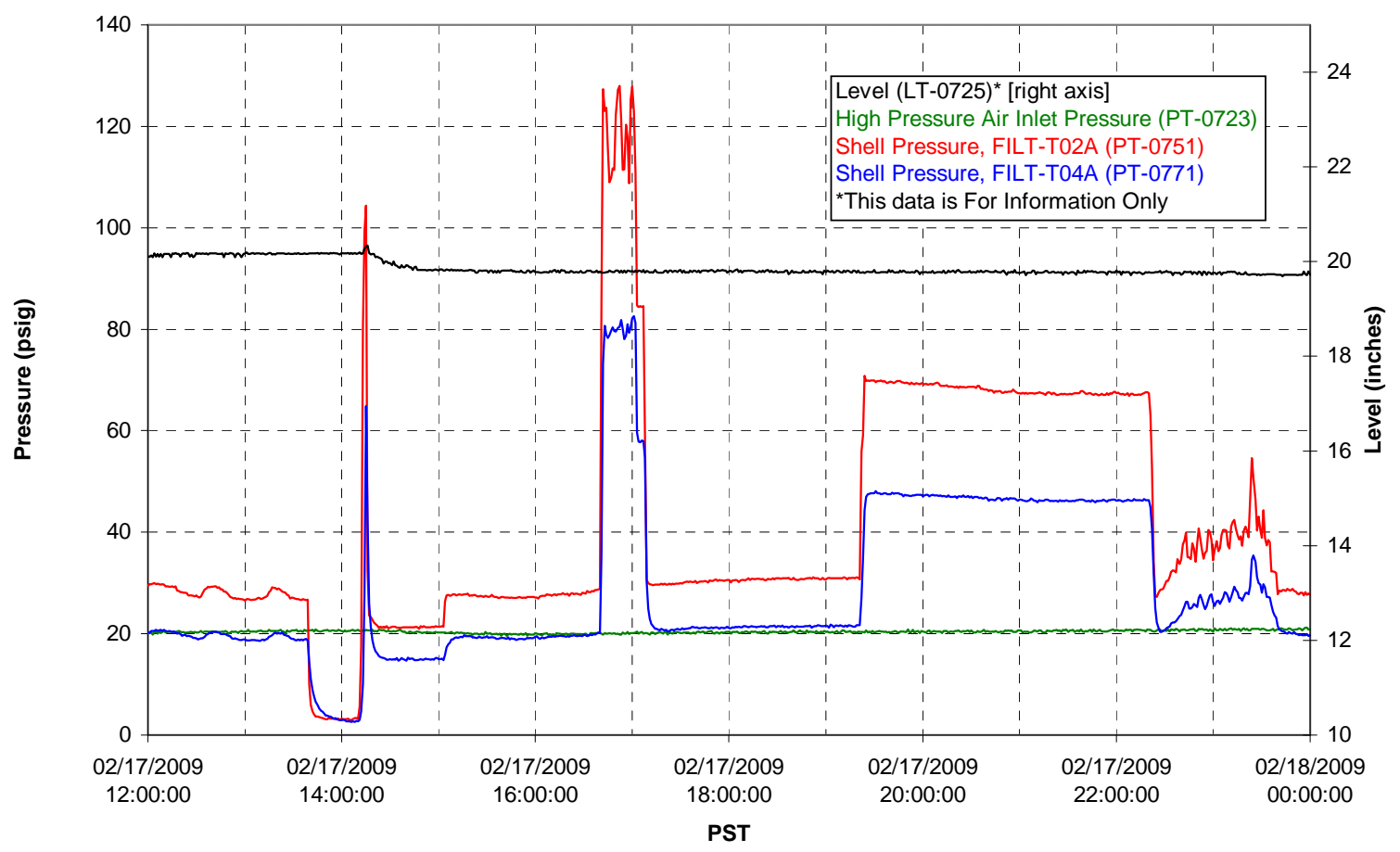


Pulsepot UFP-PP-T03A

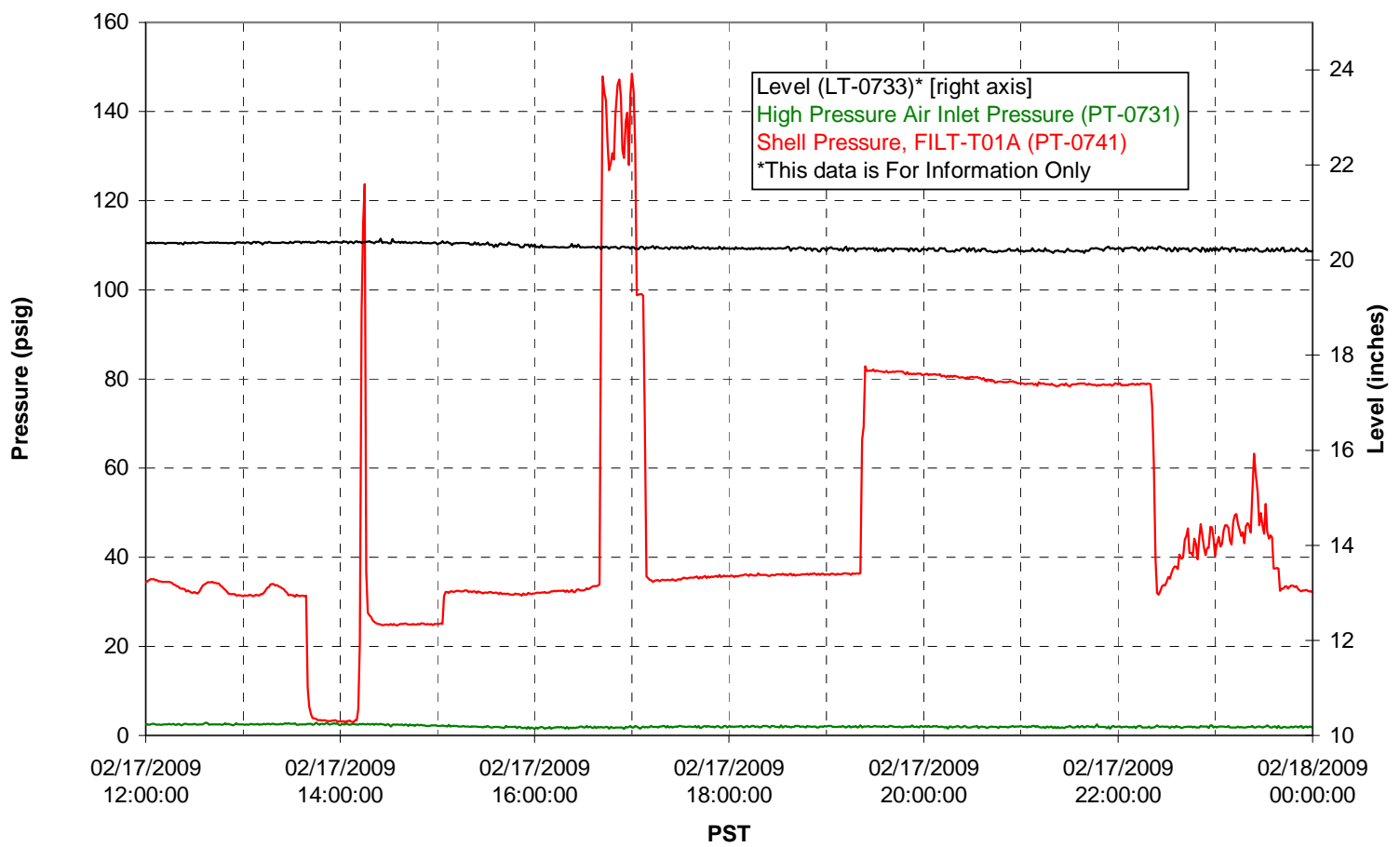

Pulsepot Levels

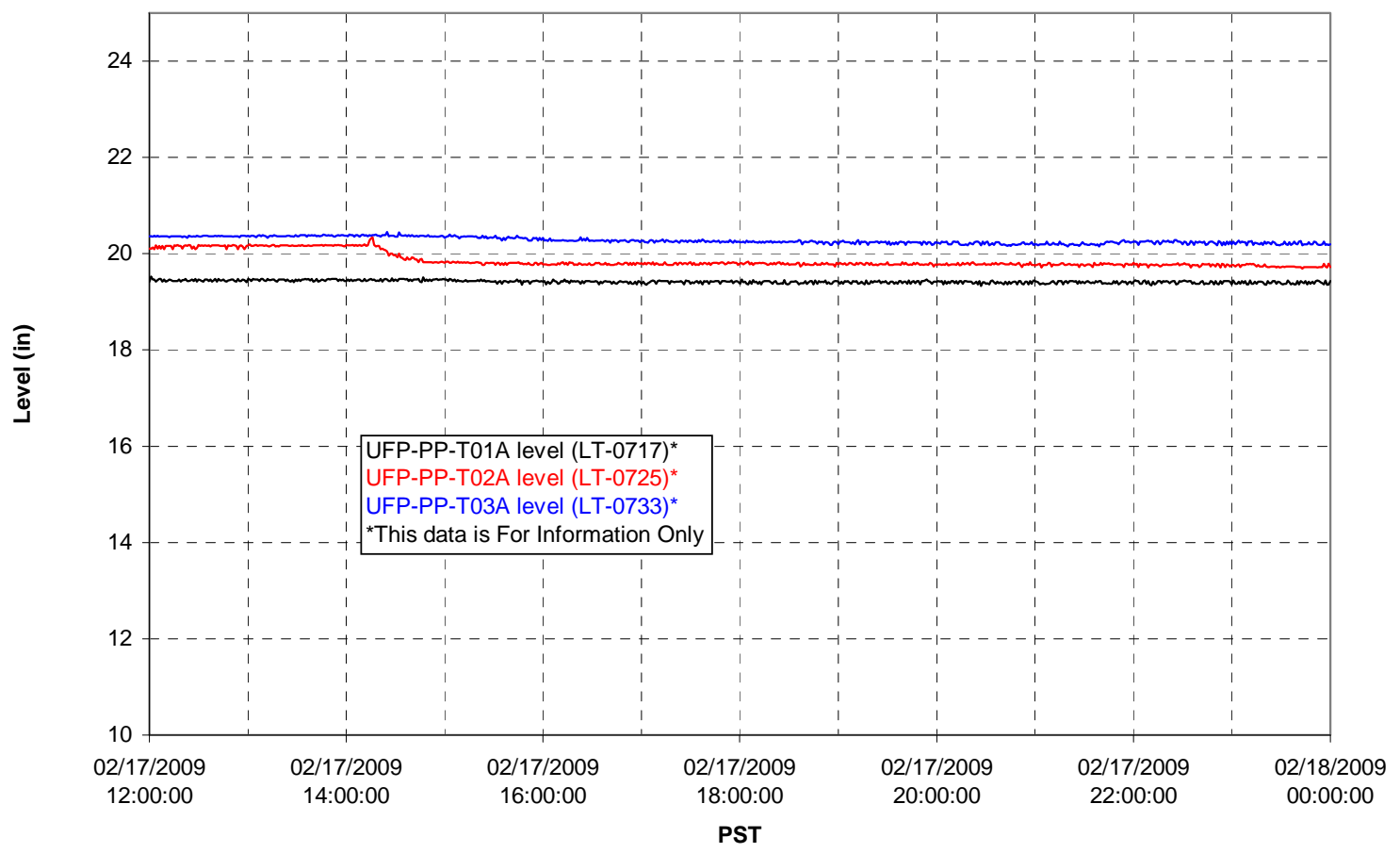


Filter UFP-FILT-T01A

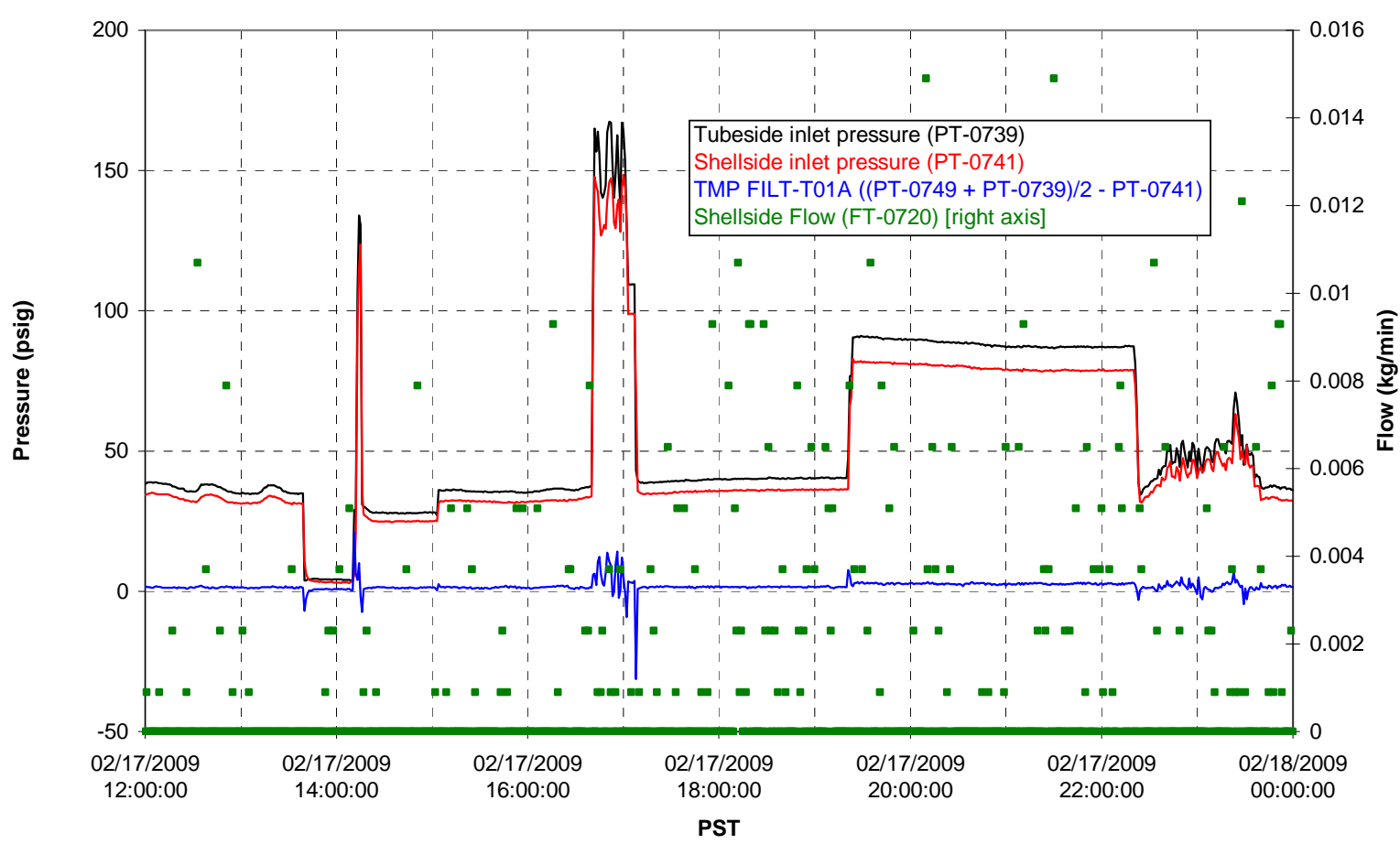

Filter UFP-FILT-T02A

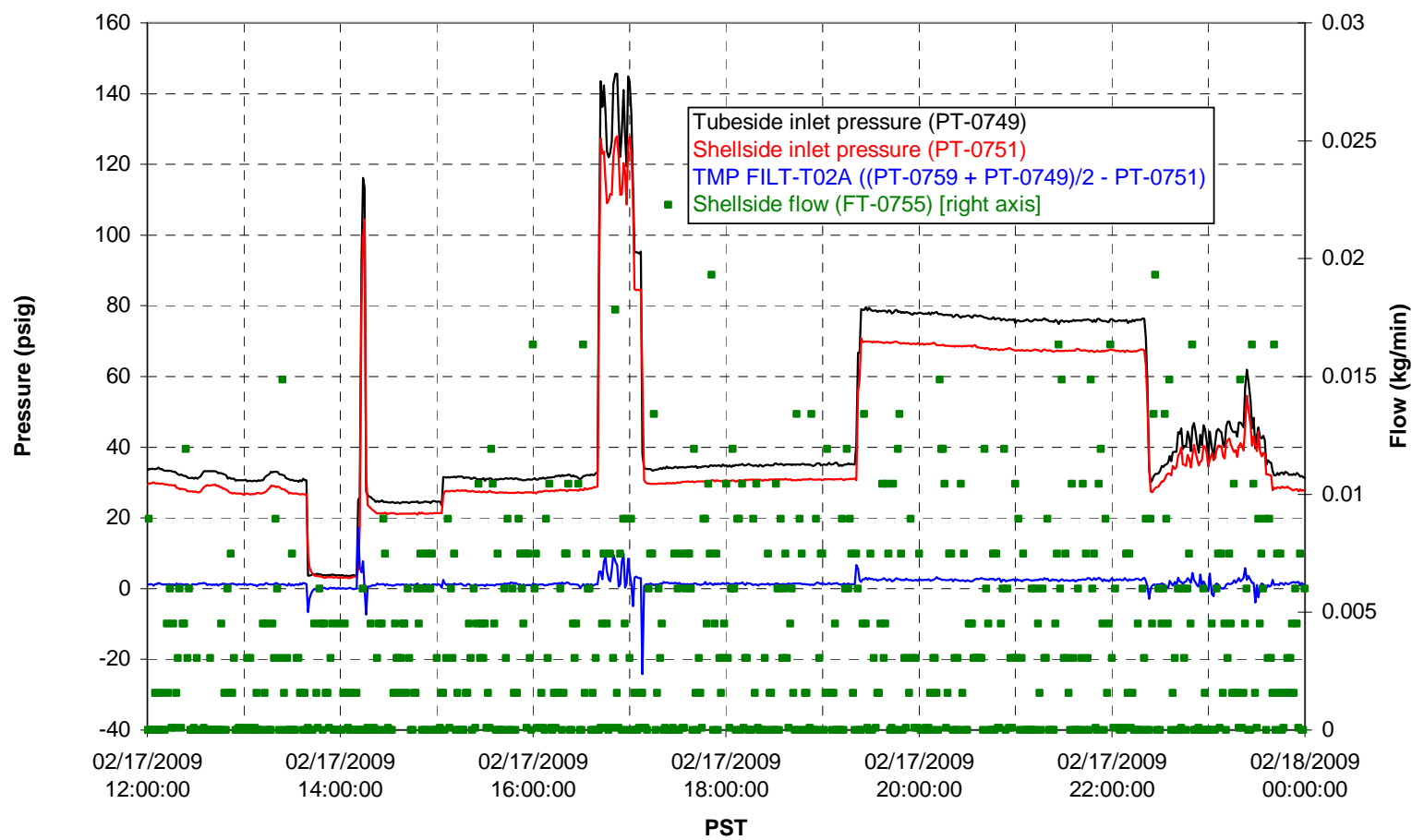


Filter UFP-FILT-T03A

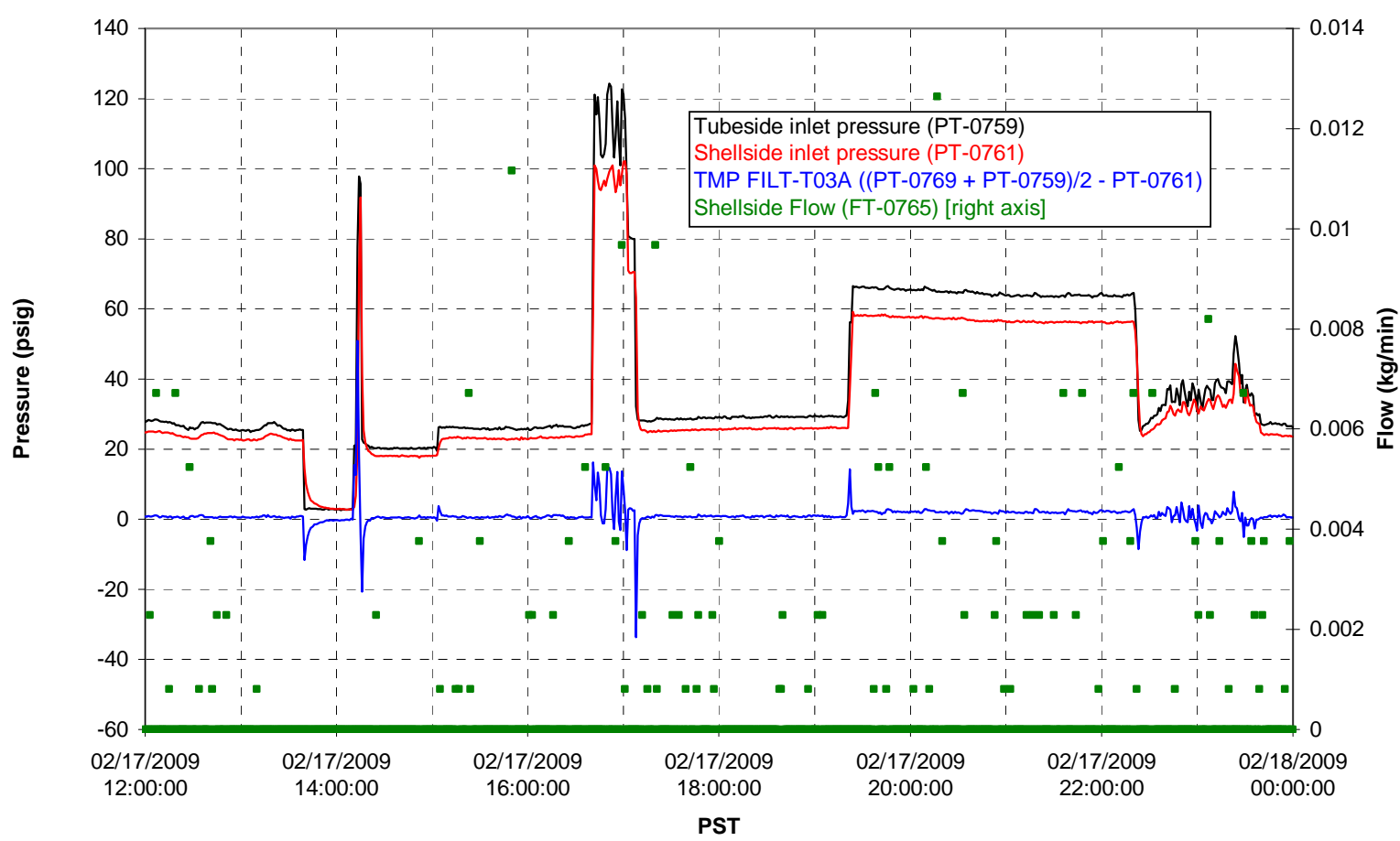

Filter UFP-FILT-T04A

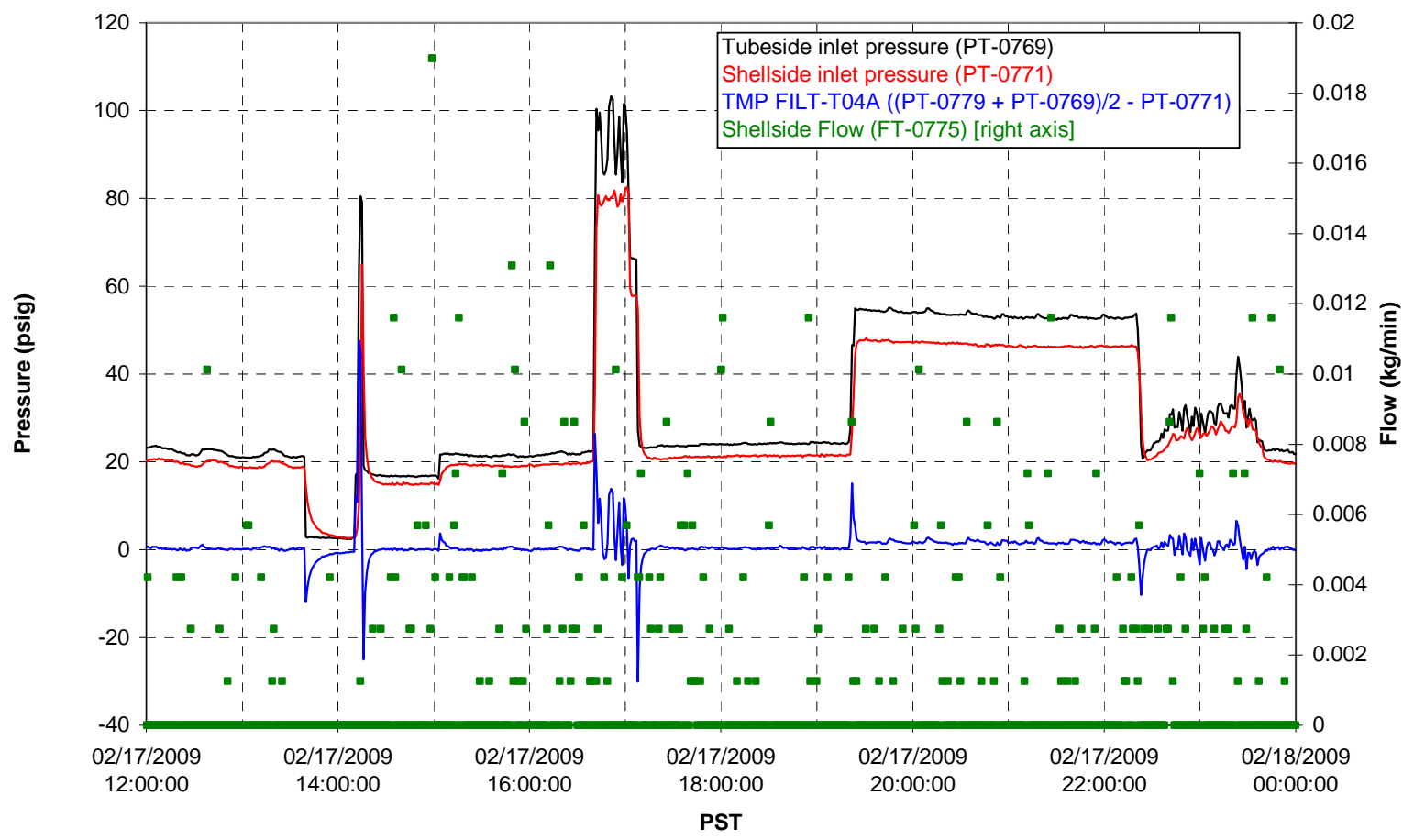


Filter UFP-FILT-T05A

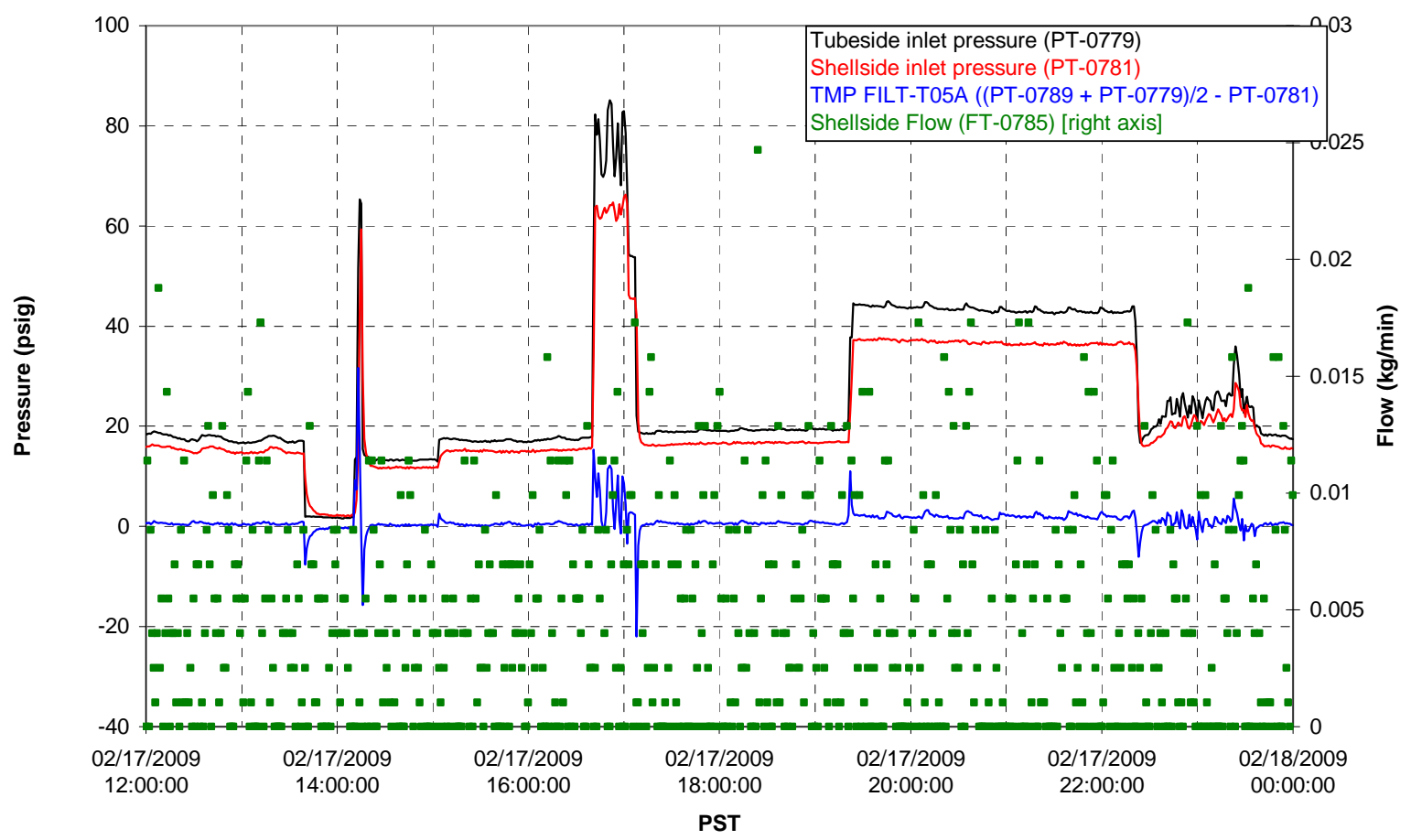

Chemical Flow

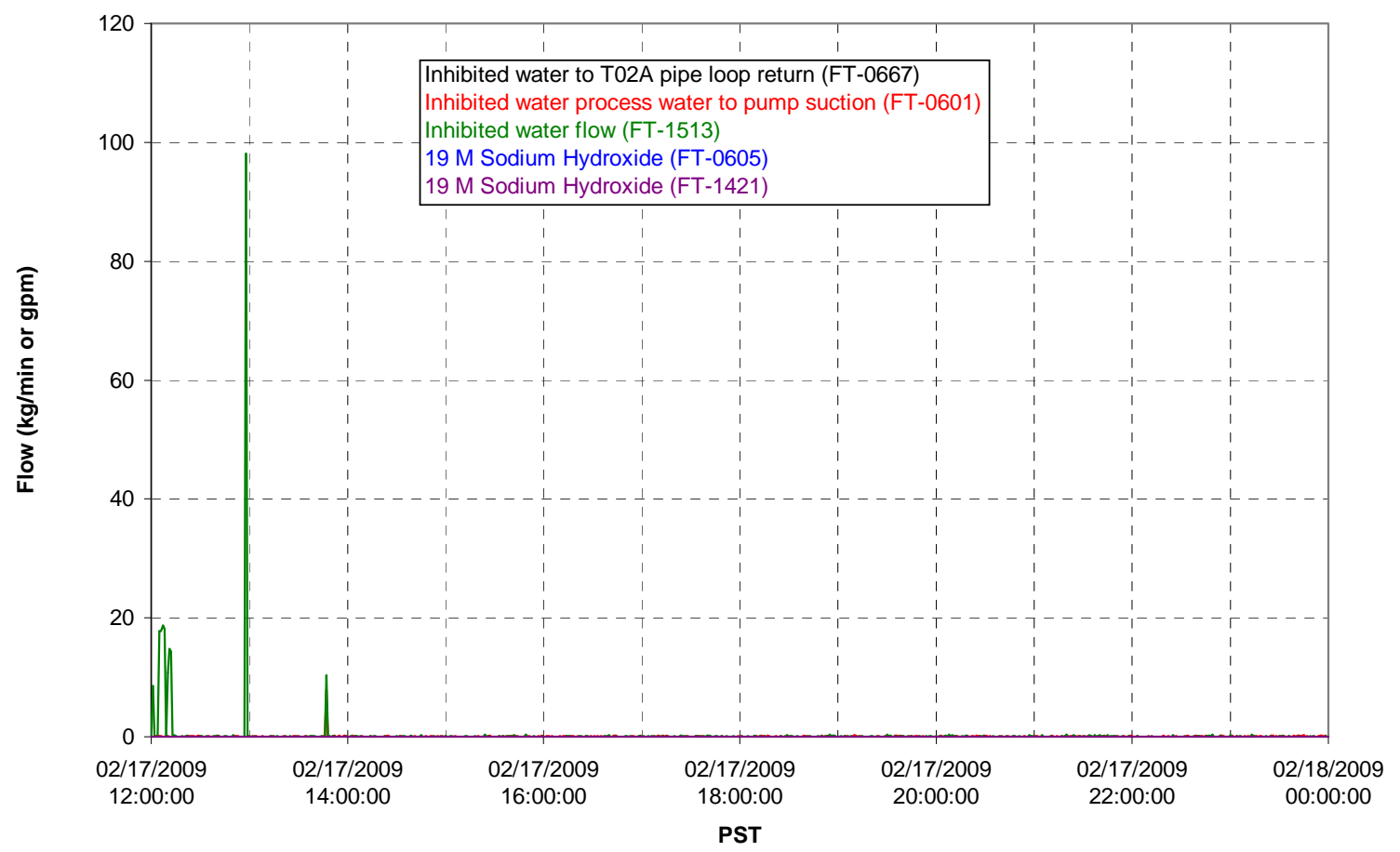




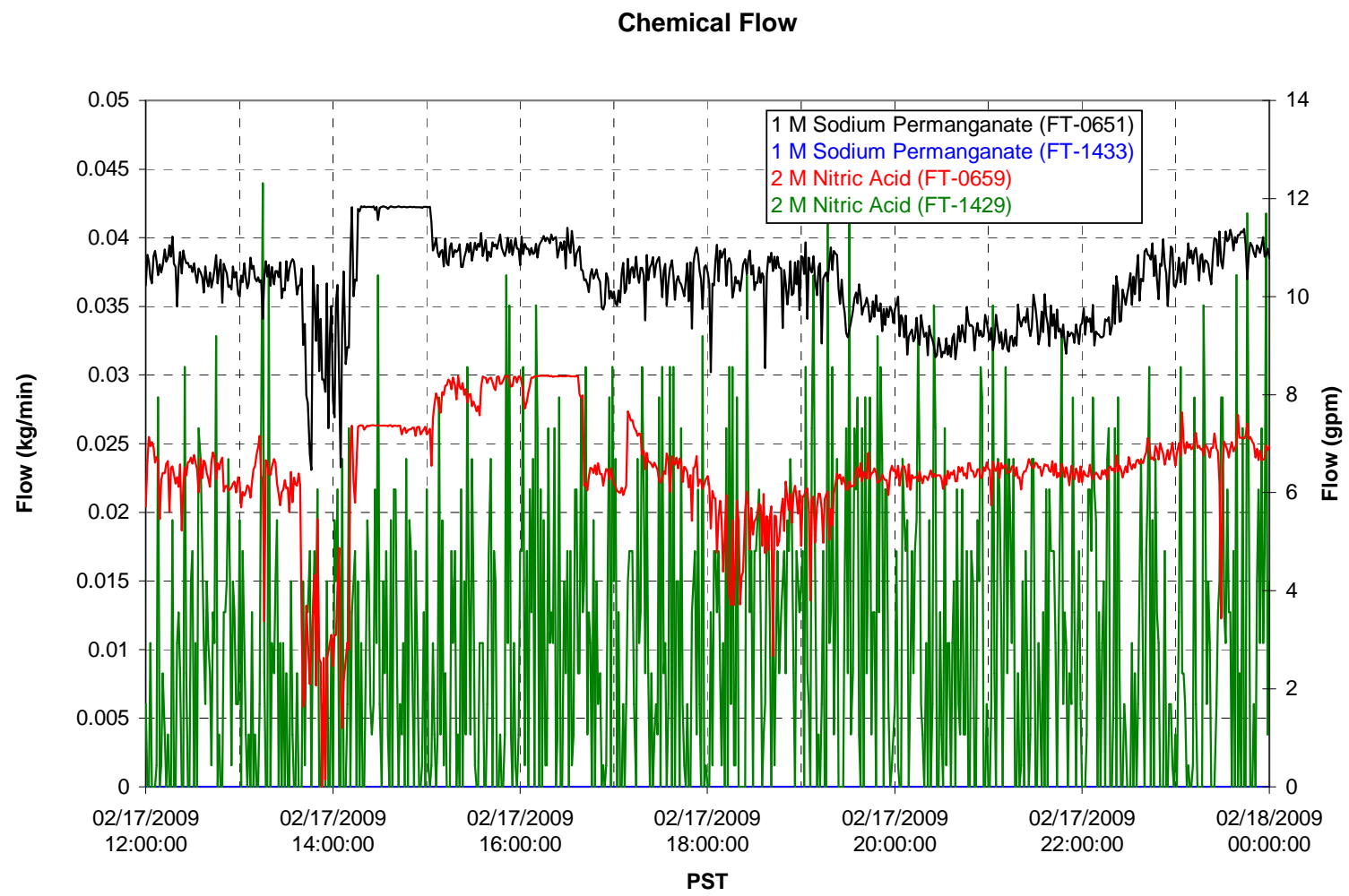

Air Flows

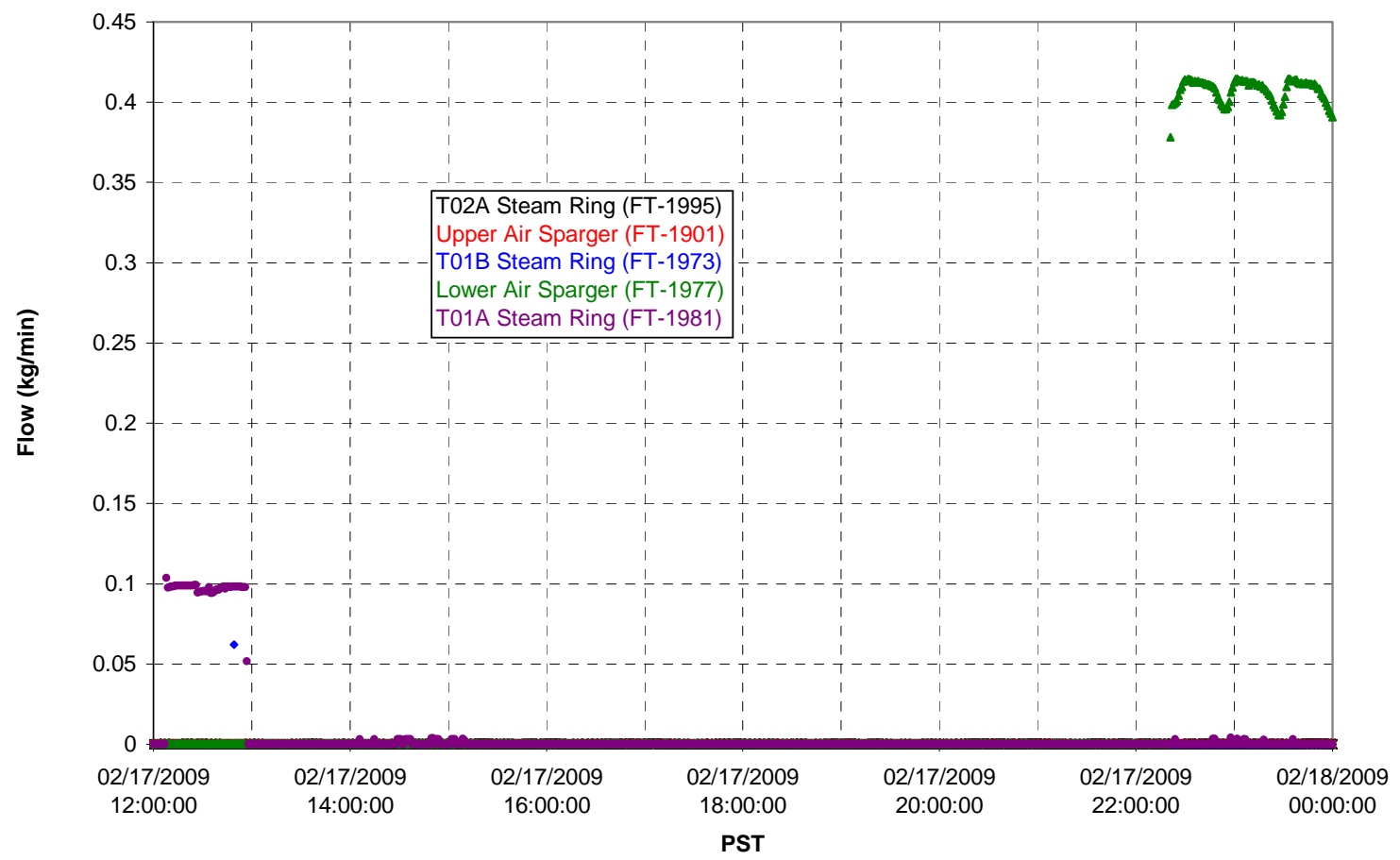


T02A Steam

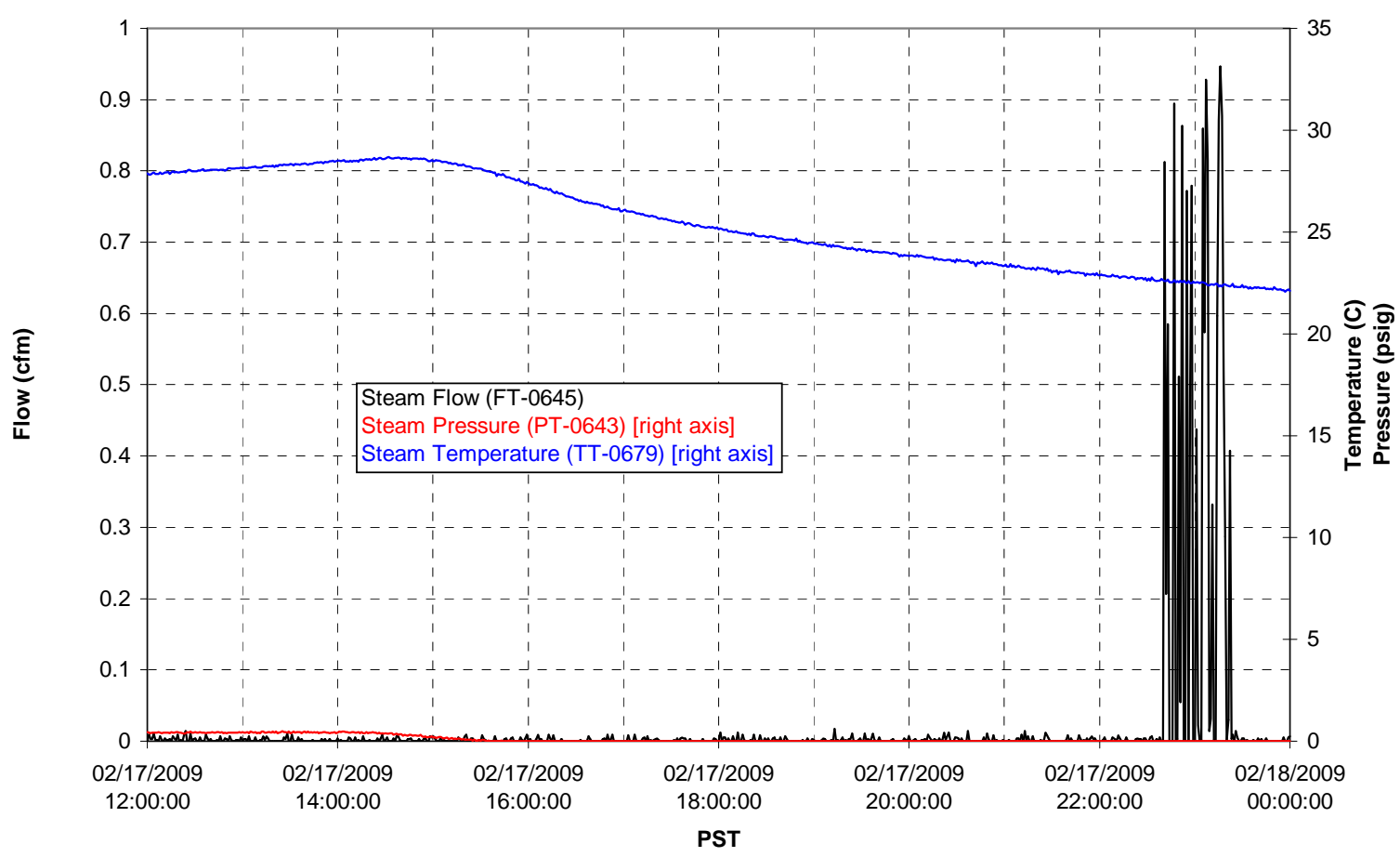

T01A Steam

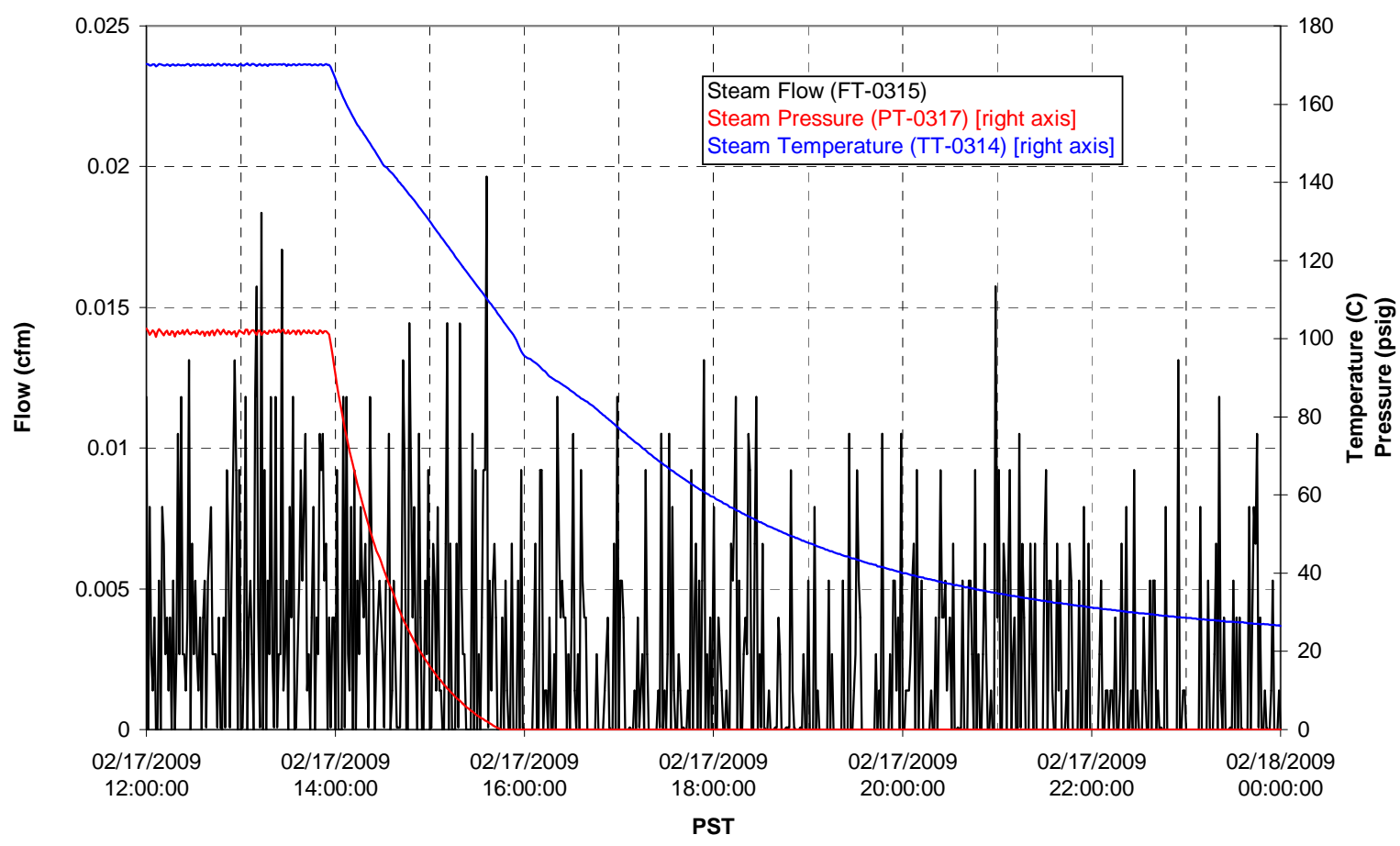


T01B Steam

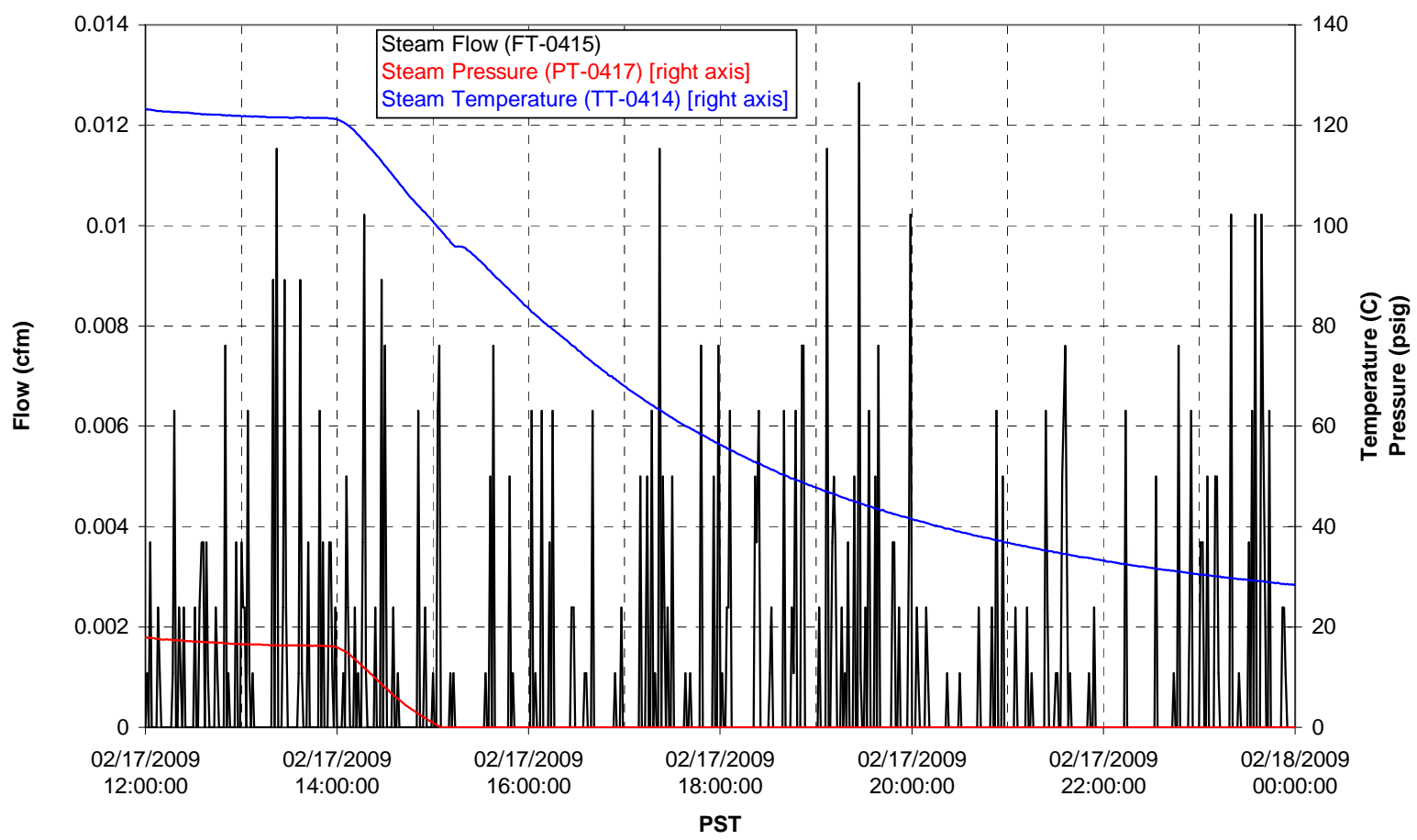




\section{Integrated Test A Data Plots 02/18/09 00:00 - 02/18/09 12:00}


T01A level

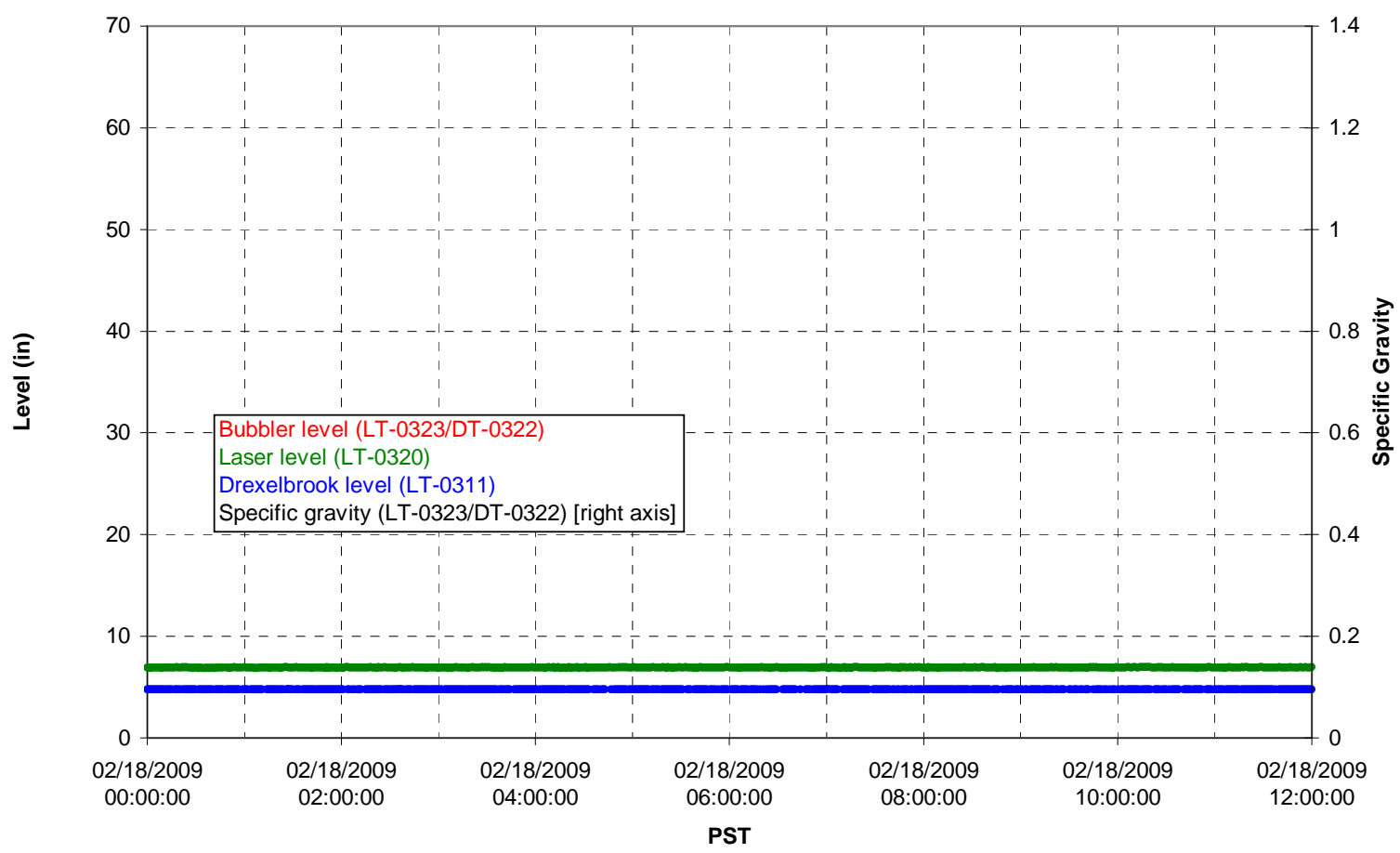

T01A temperatures

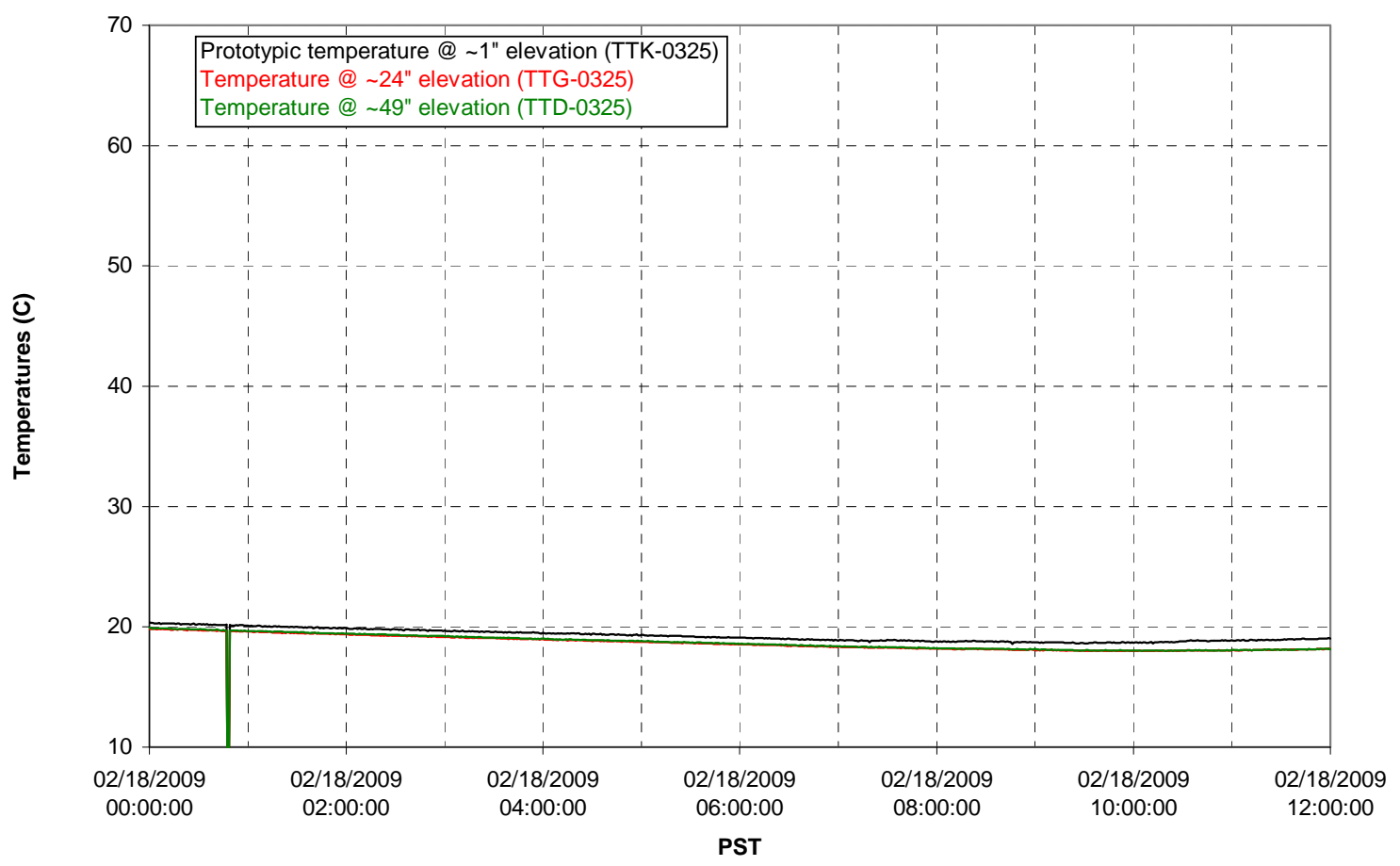


T01B level

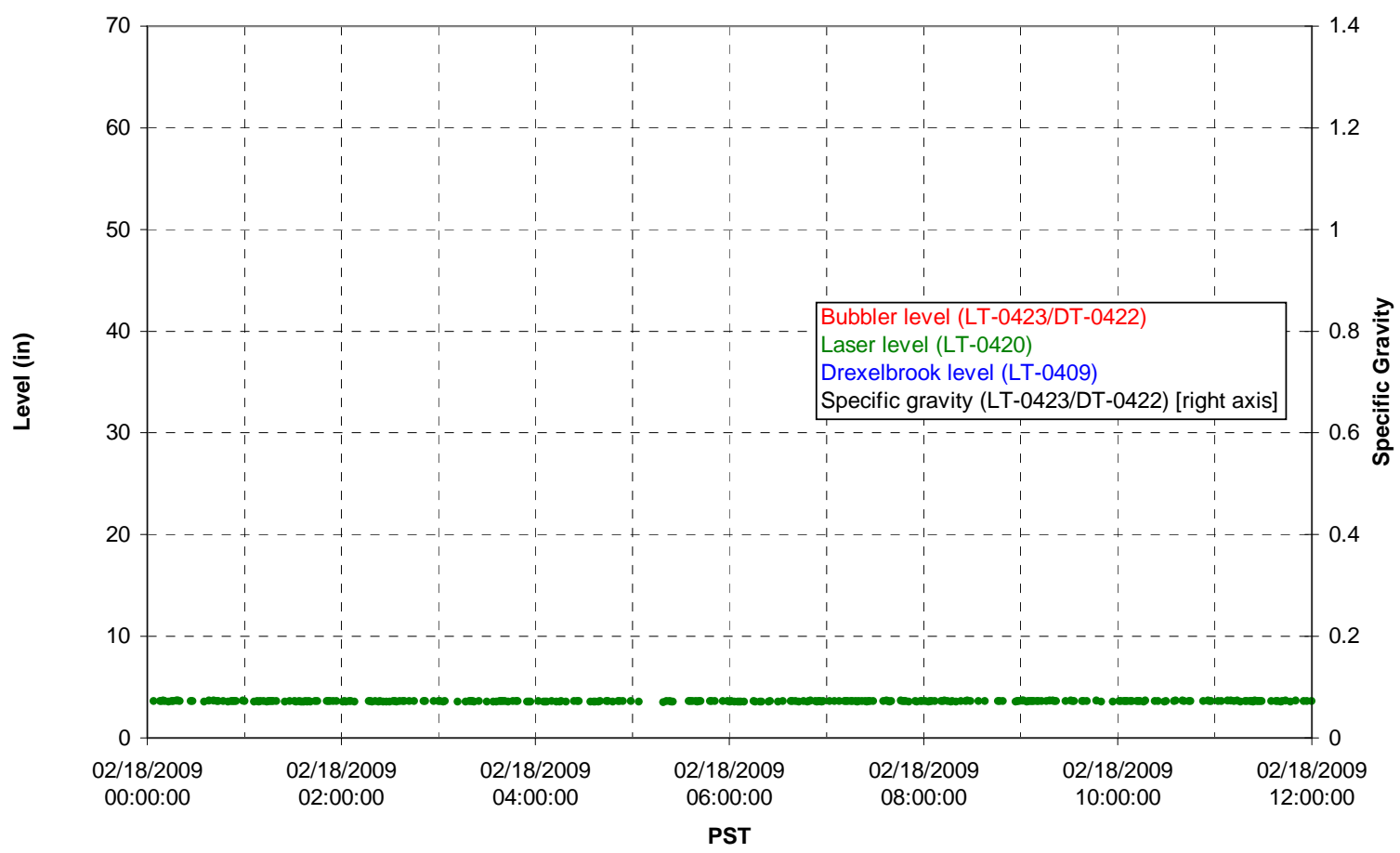

T01B temperatures

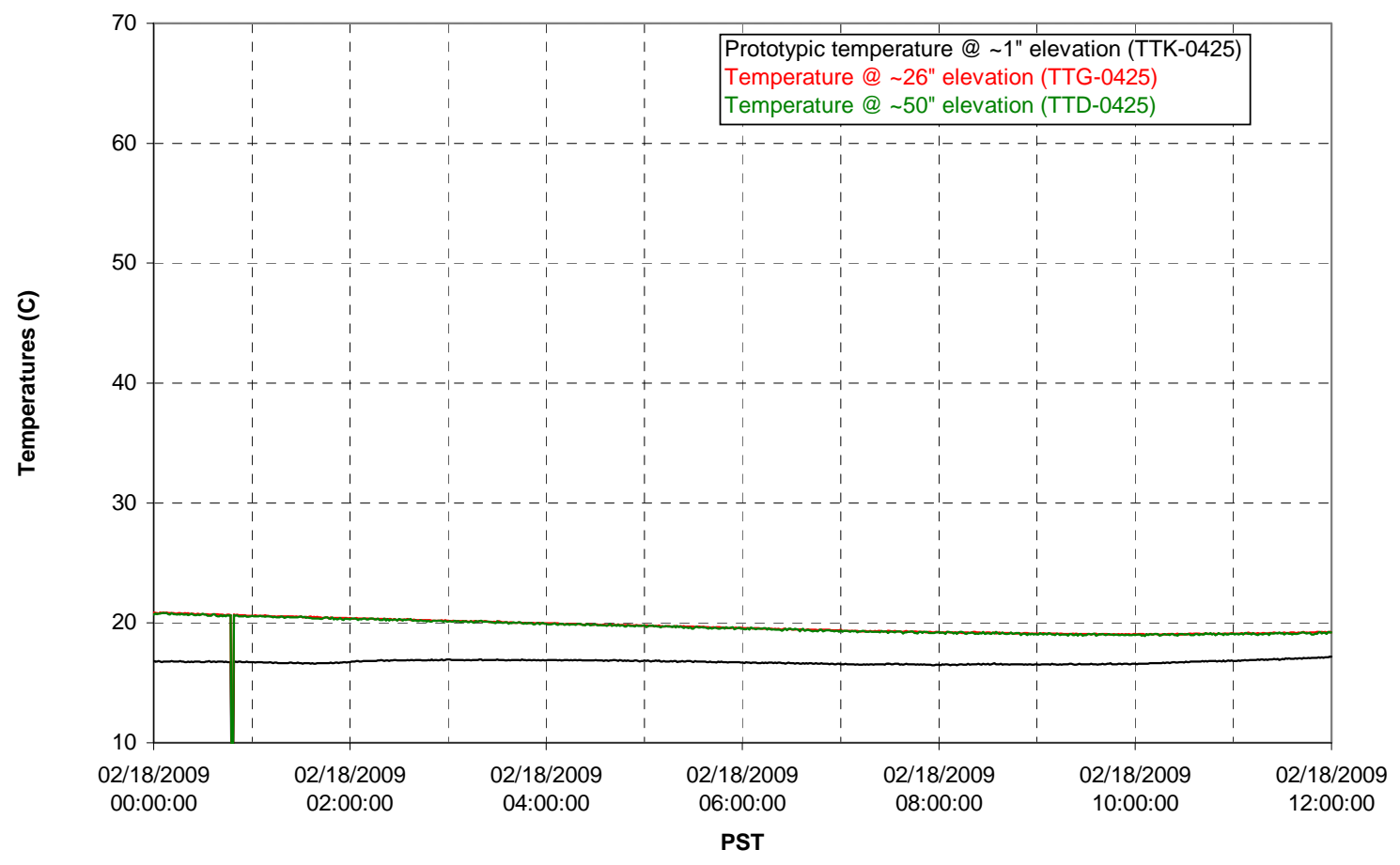


T02A level

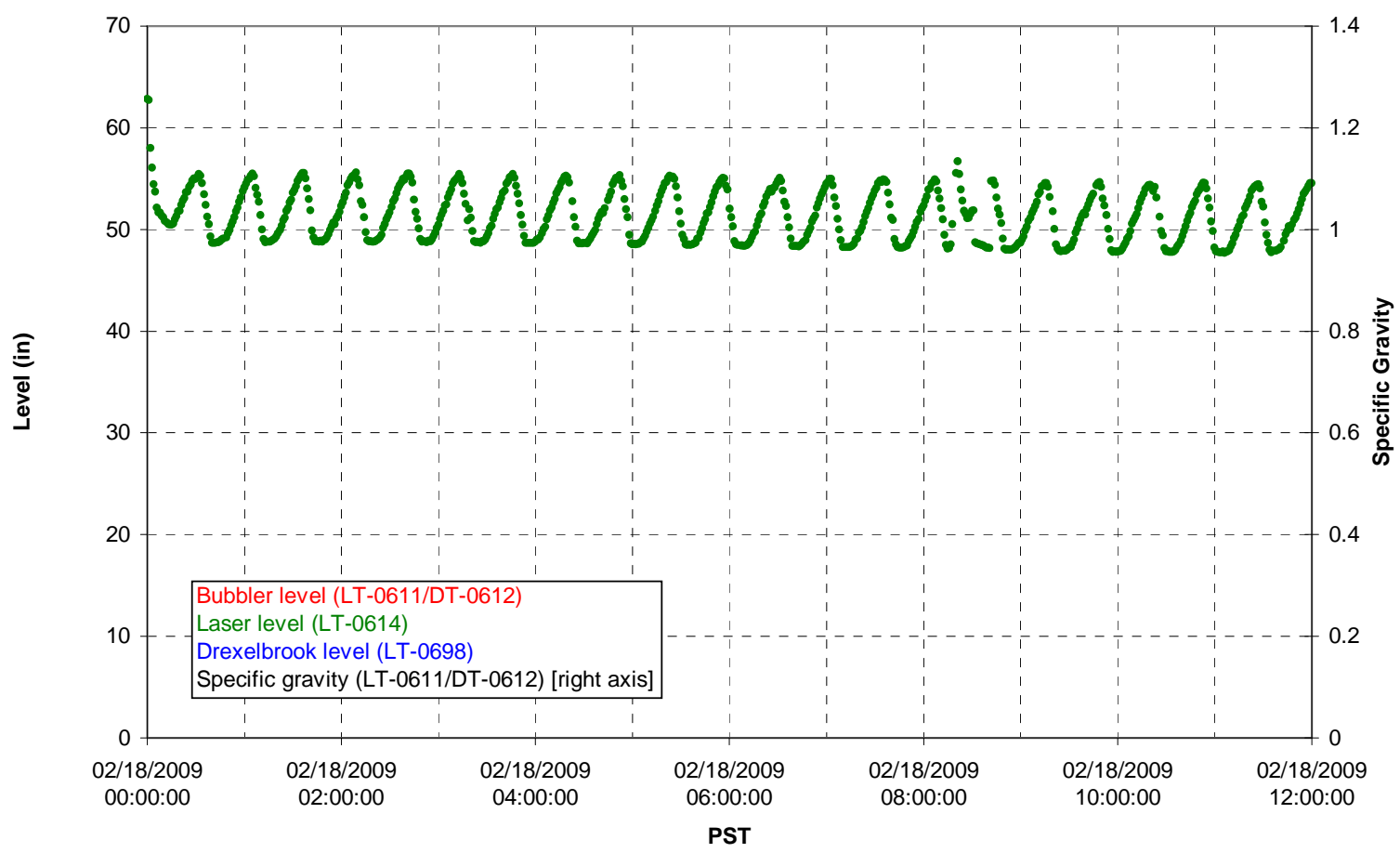

T02A temperatures

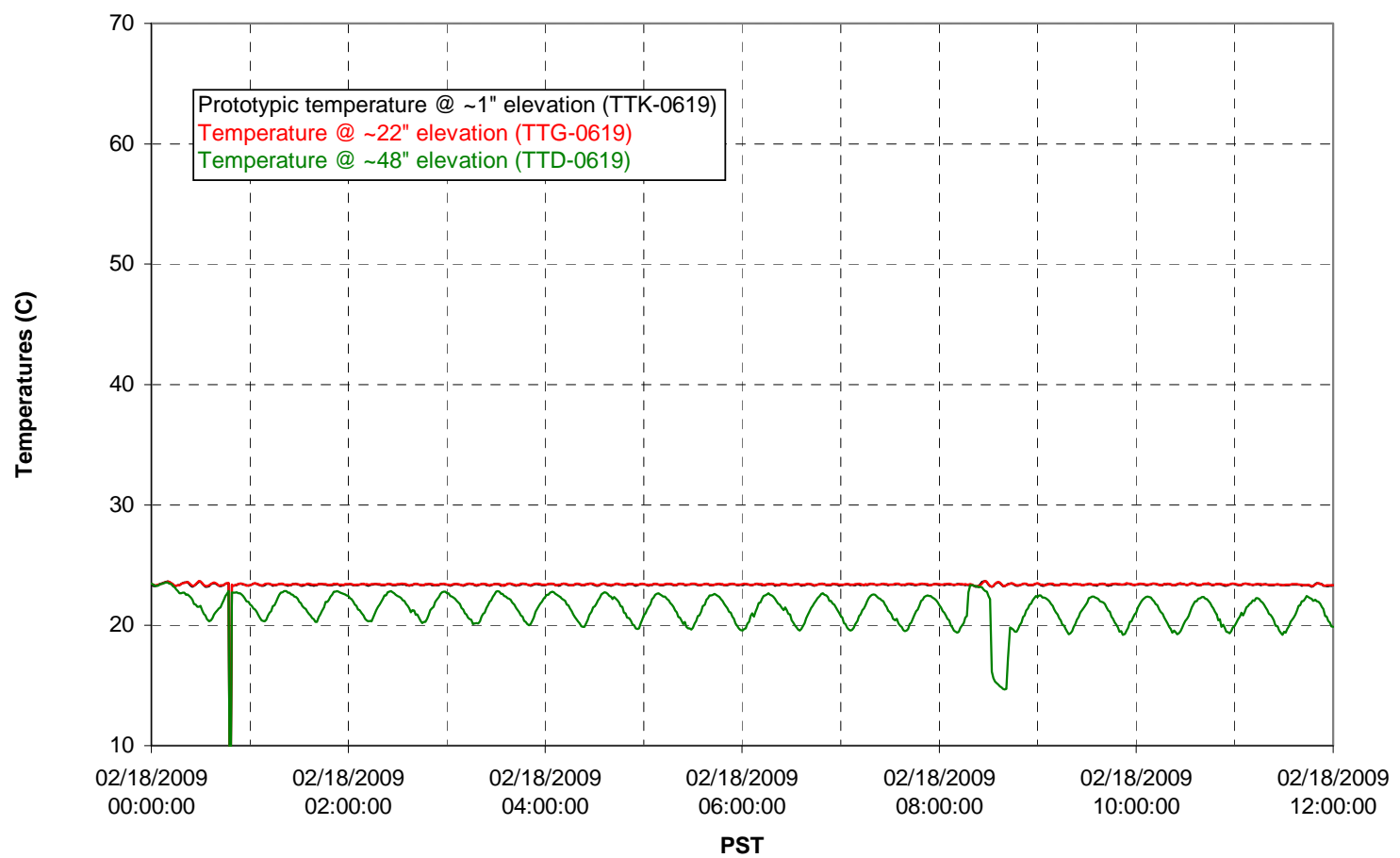


T02A and filter loop temperatures

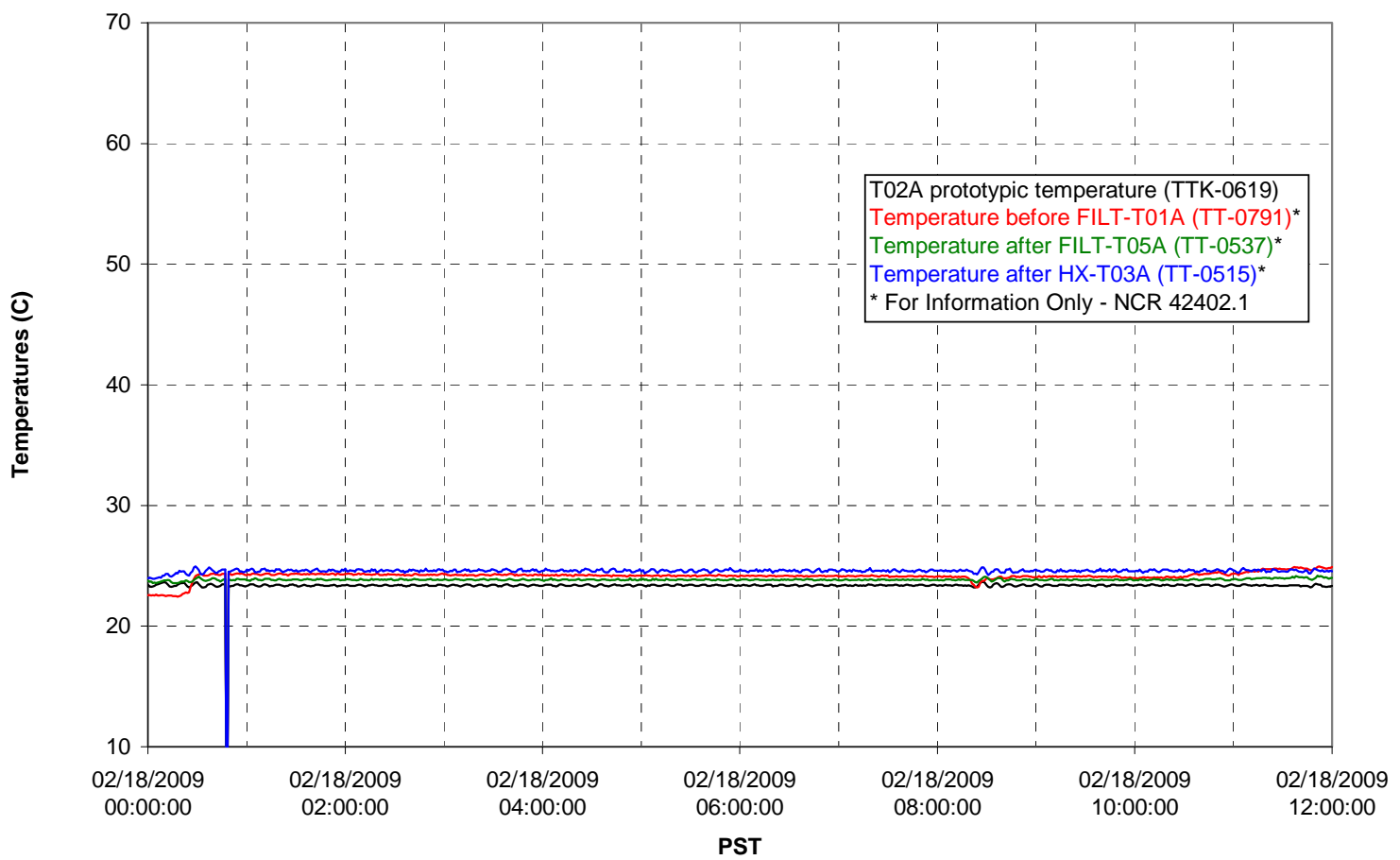

Pump Pressures and Flow

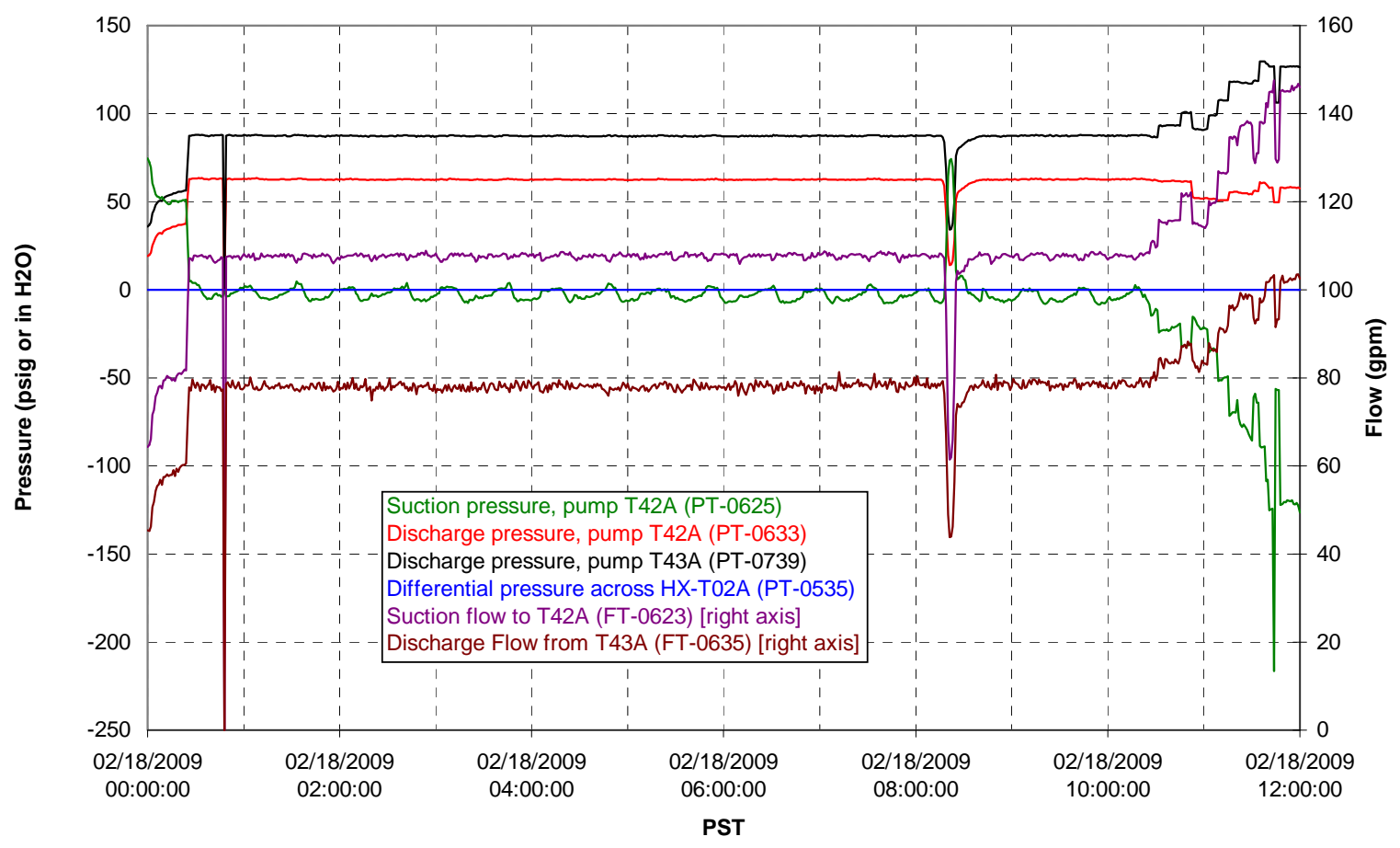


Axial pressure drop

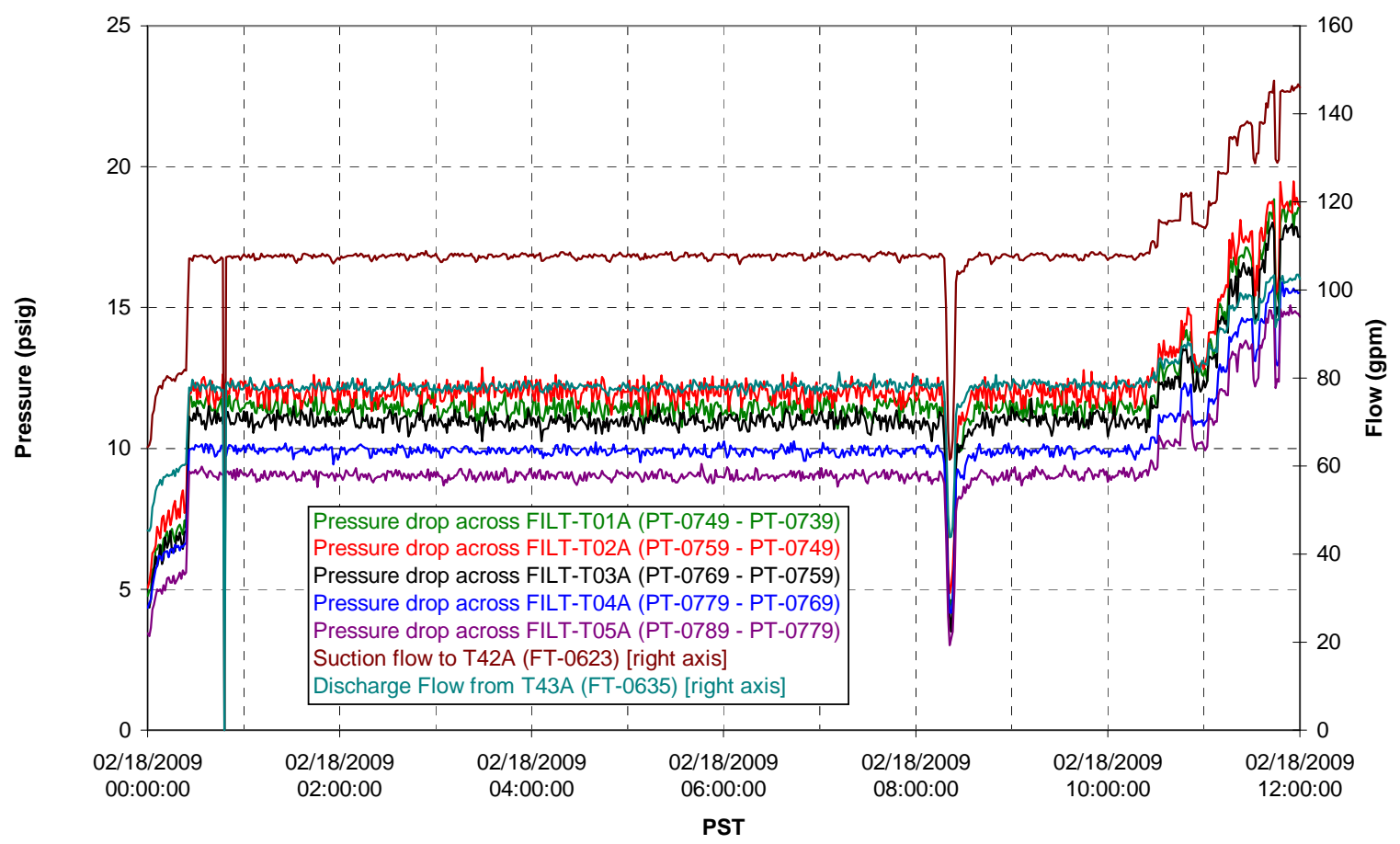

Permeate flow rates

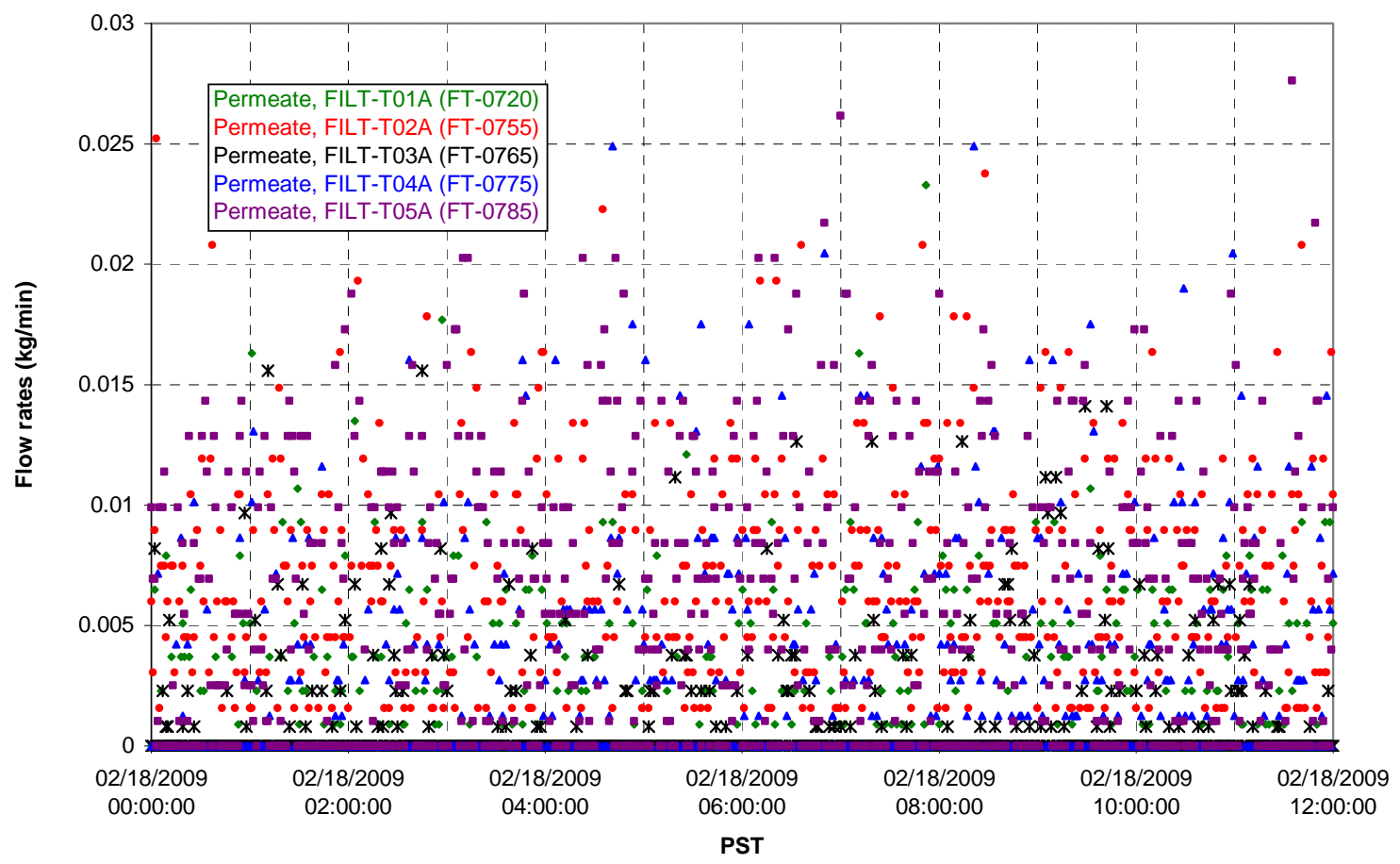


T02A Inner Temperature Tree

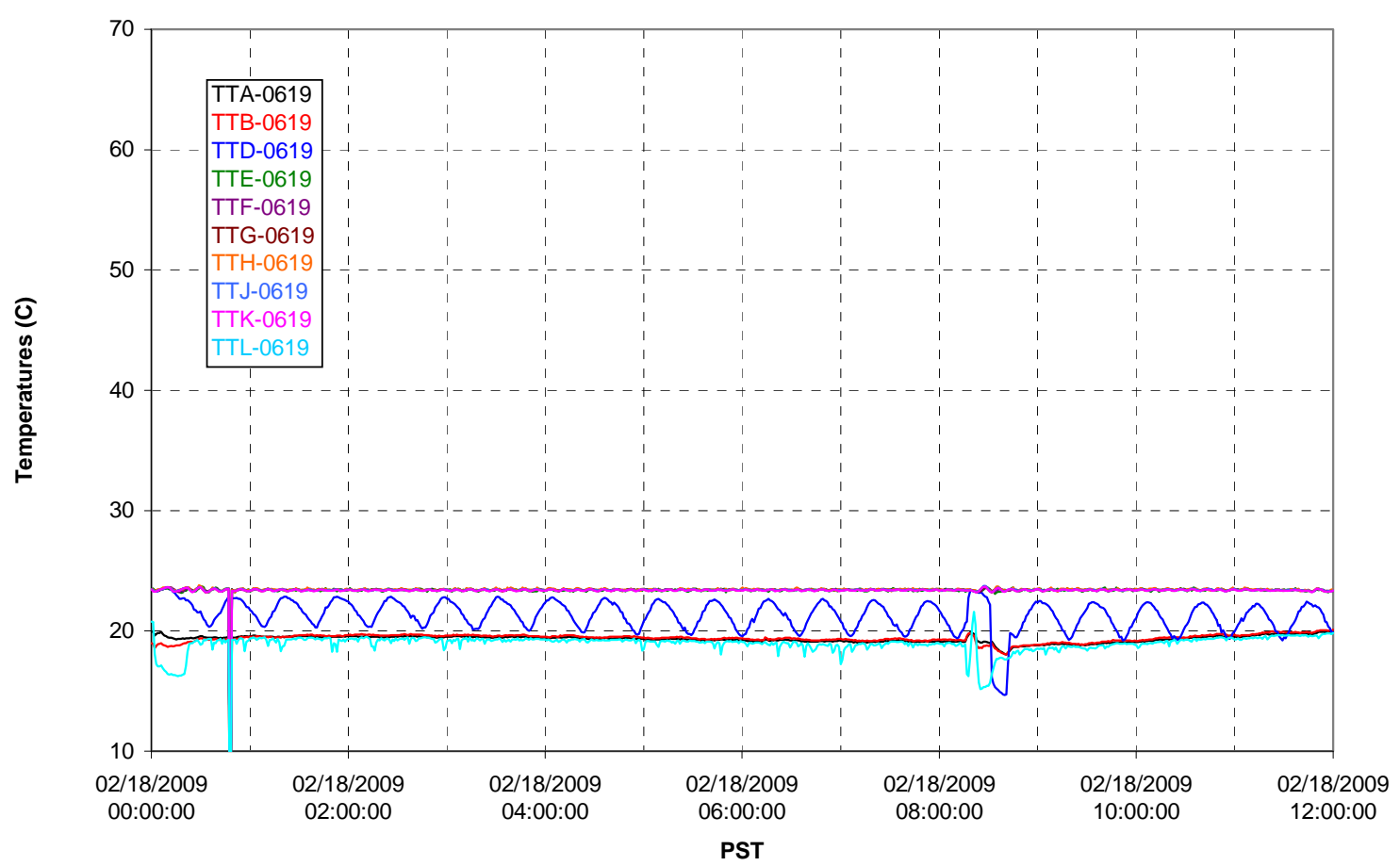

T02A Outer Temperature Tree

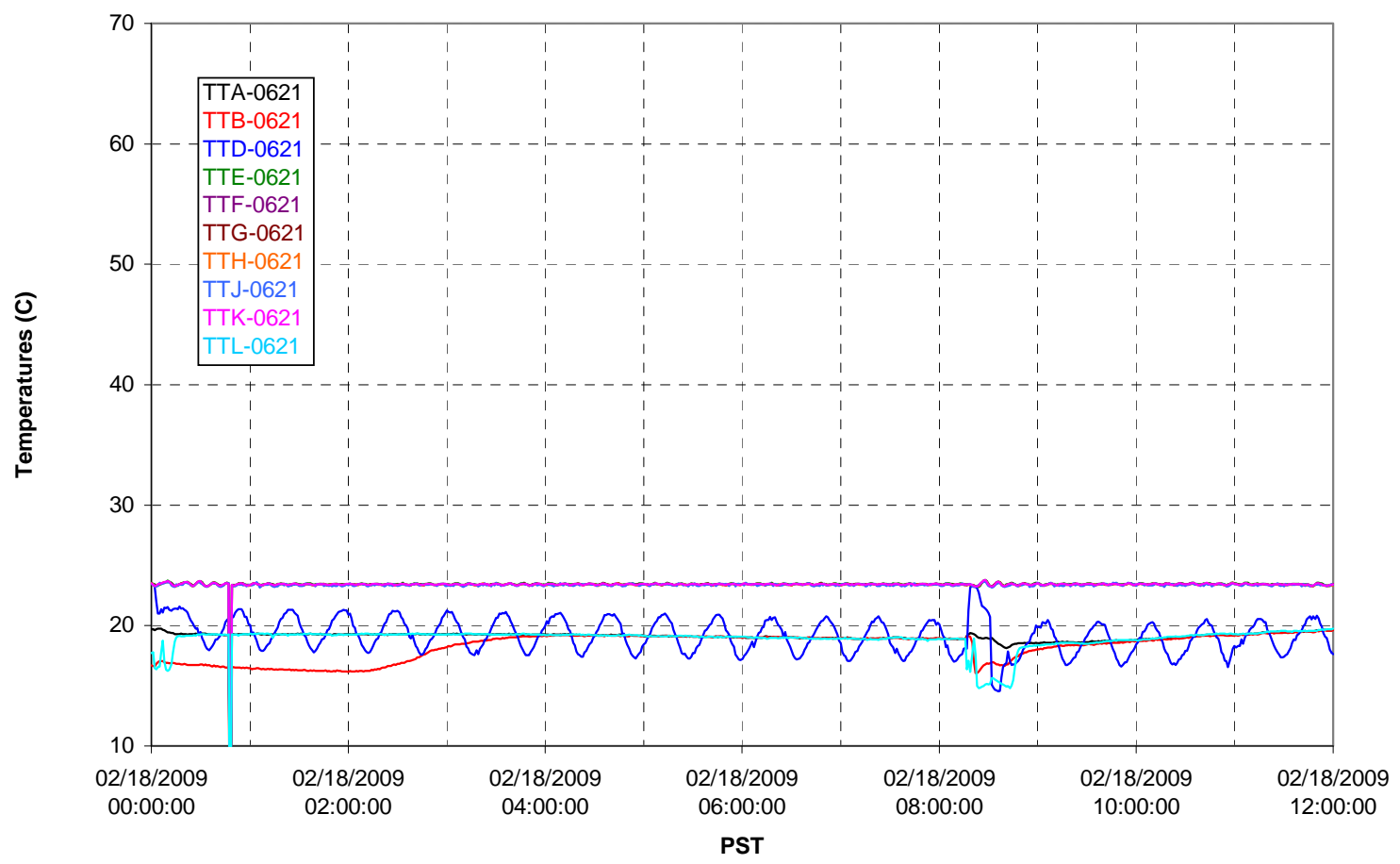


T02A temperatures

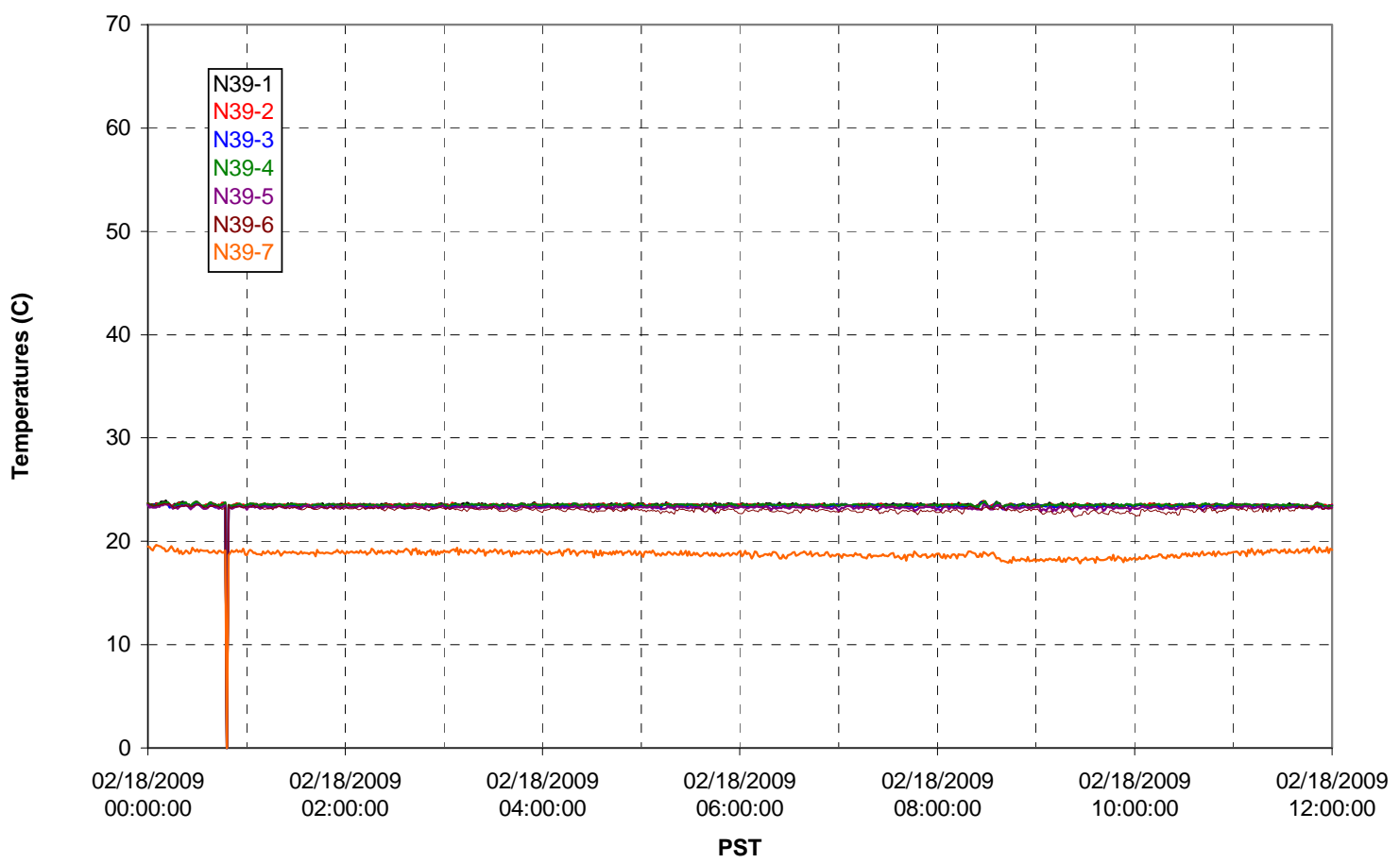

T02A temperatures

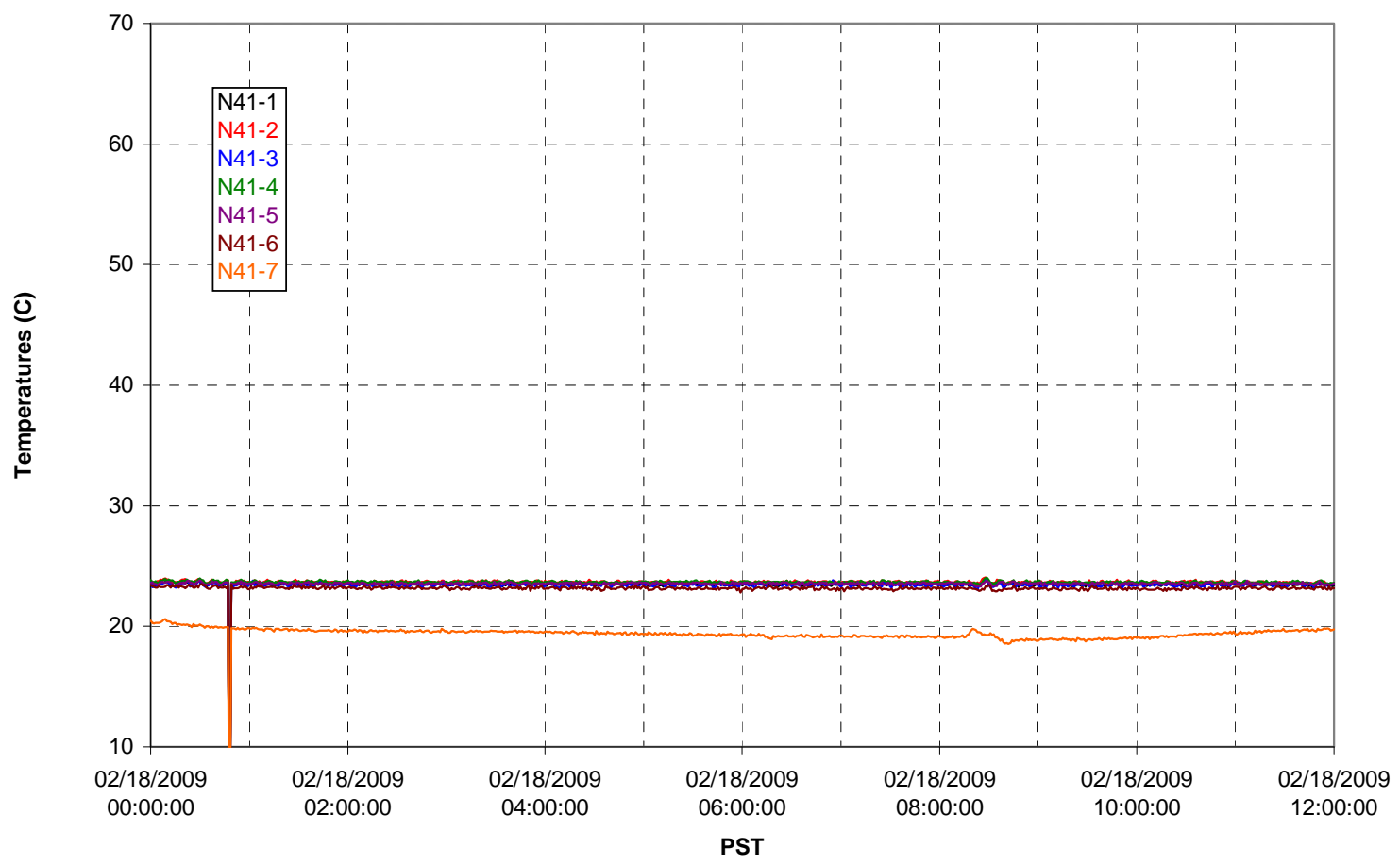


T02A temperatures

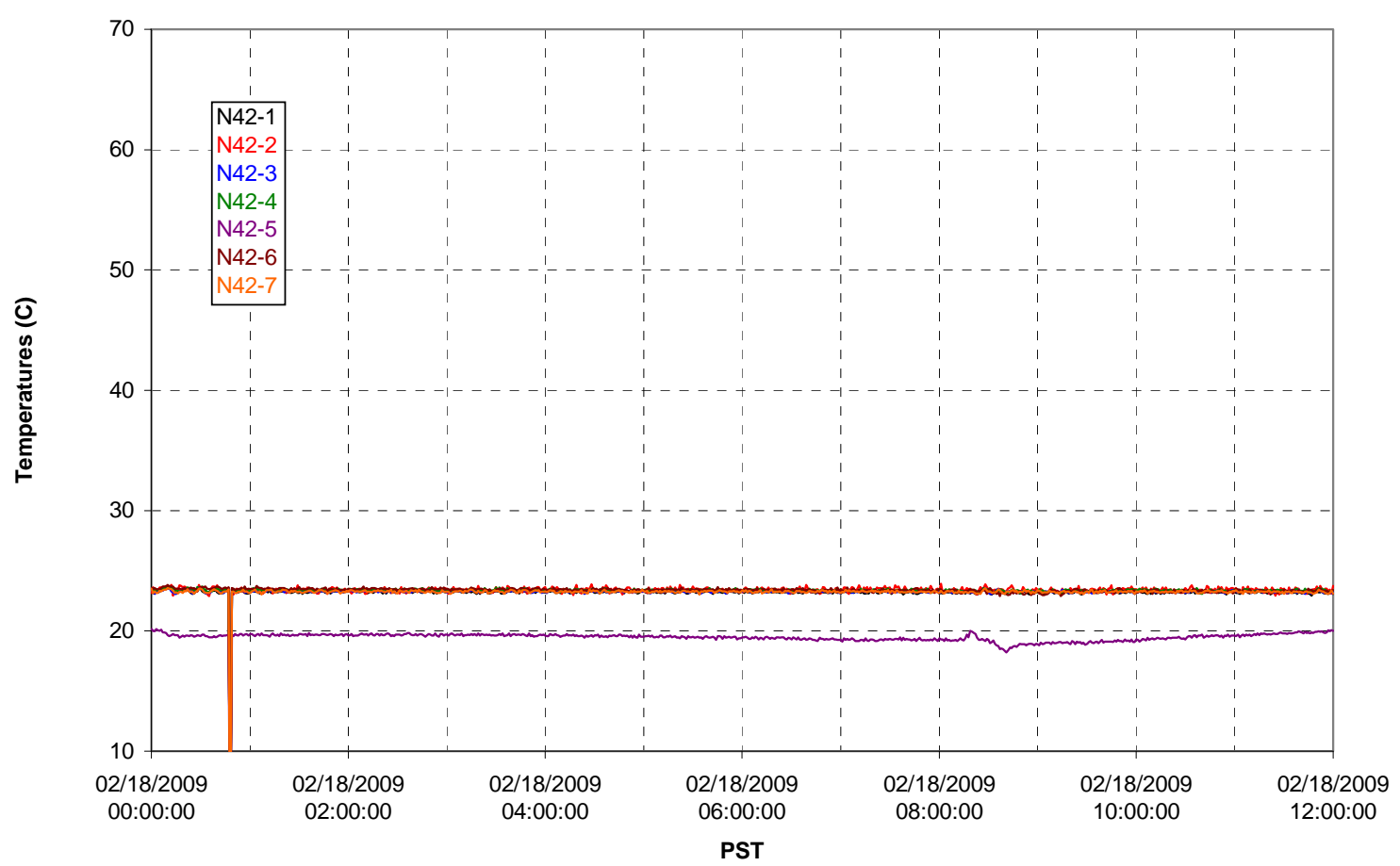

T02A temperatures

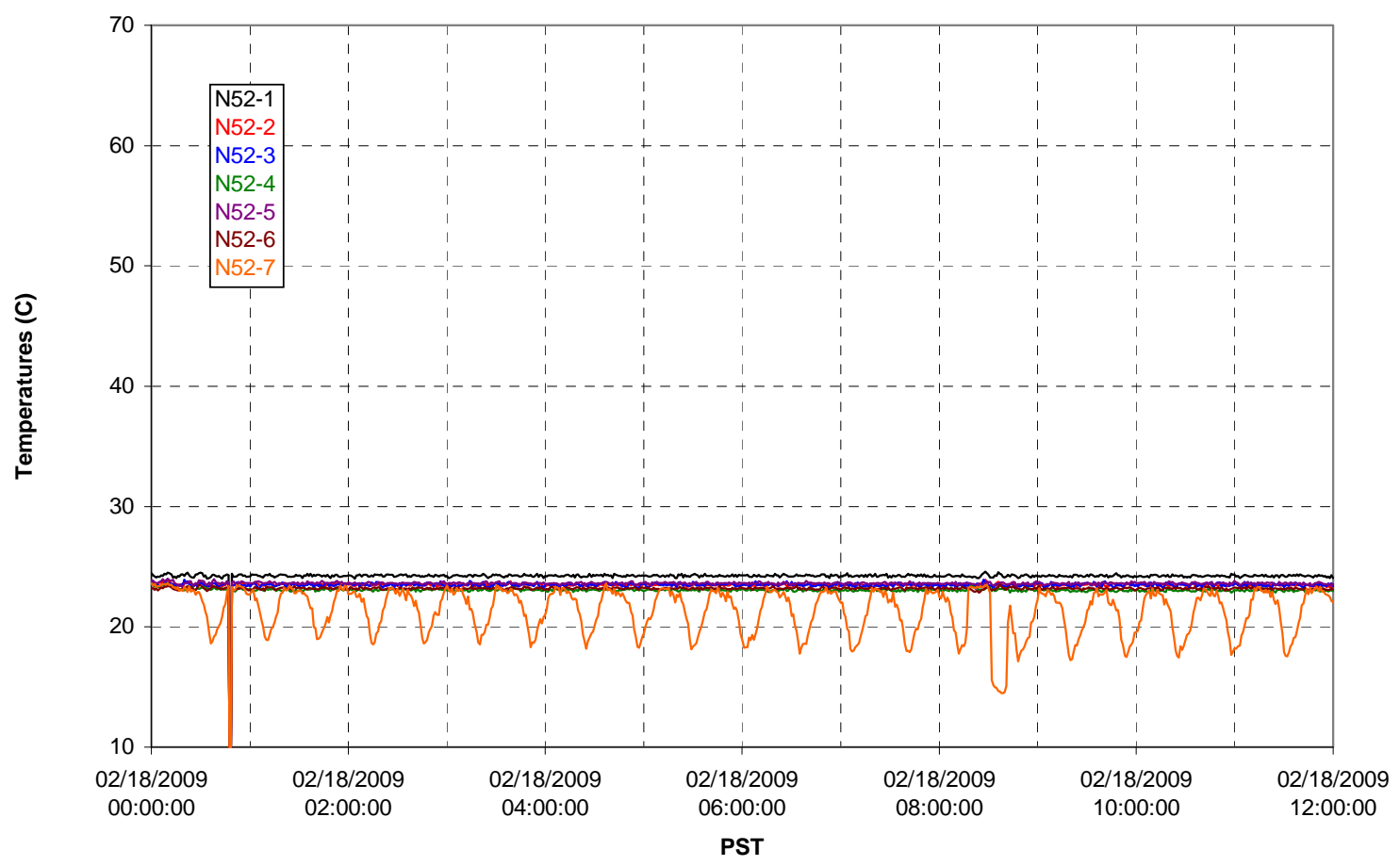


T02A Heating and Cooling

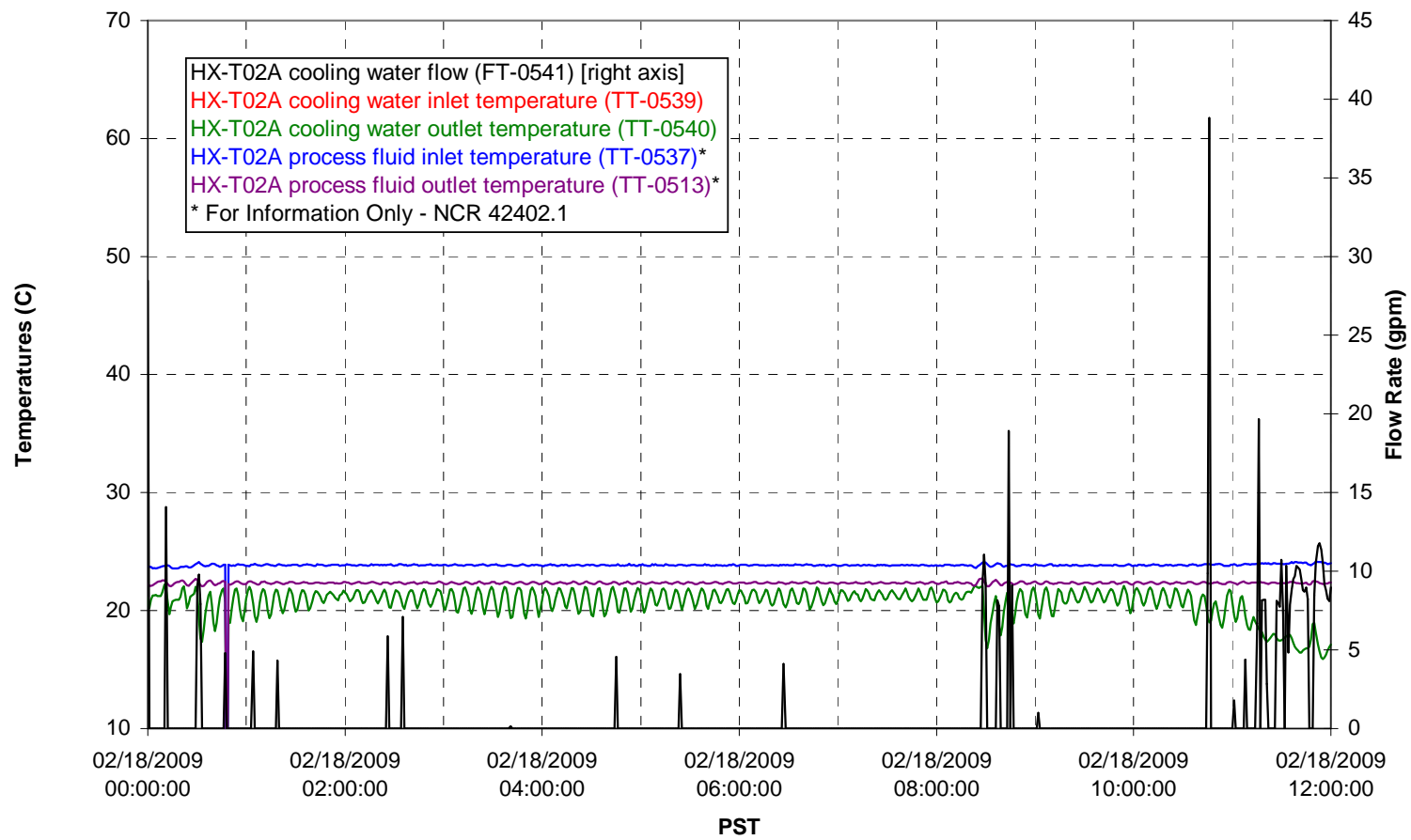

Pump Operation

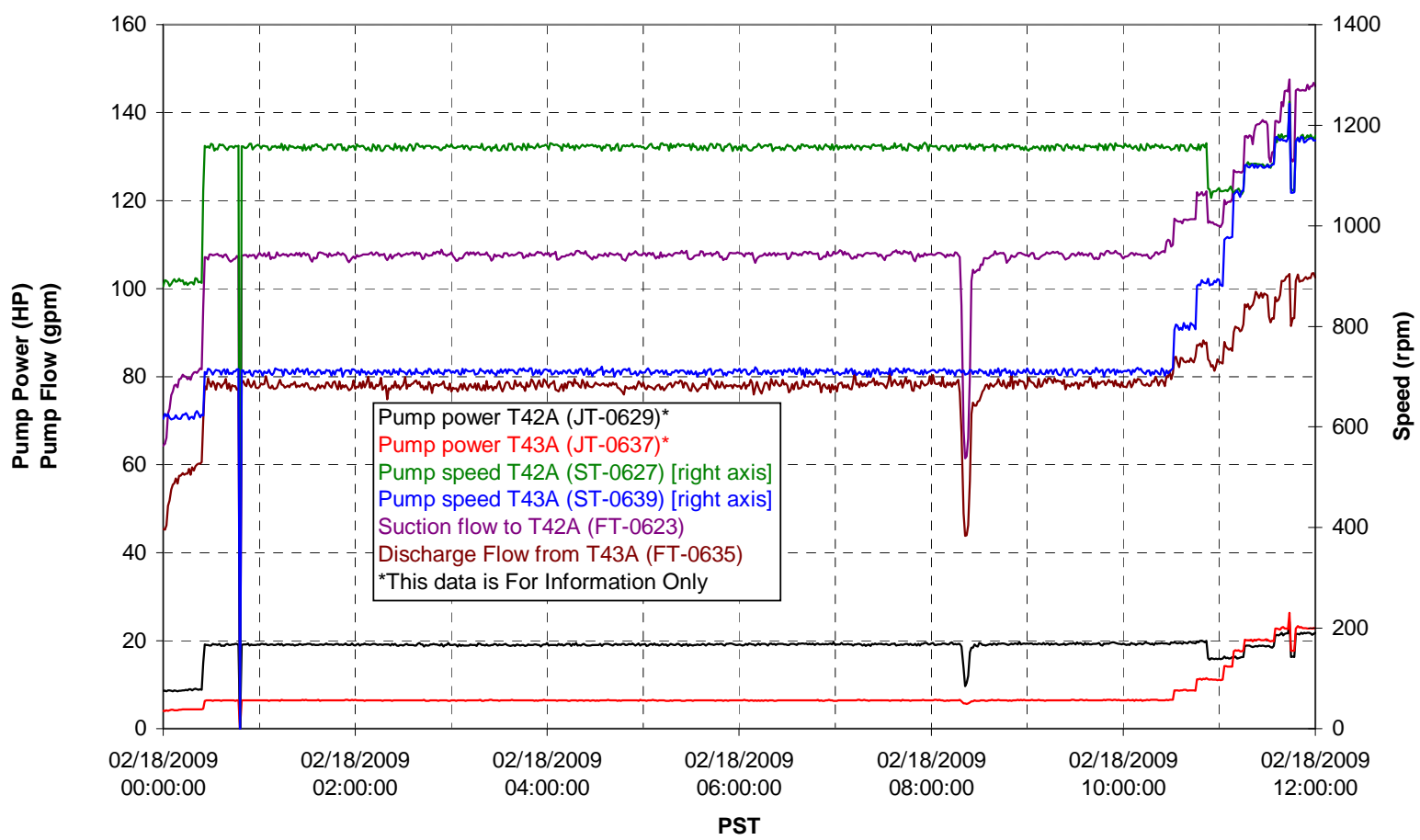


Pulsepot UFP-PP-T01A

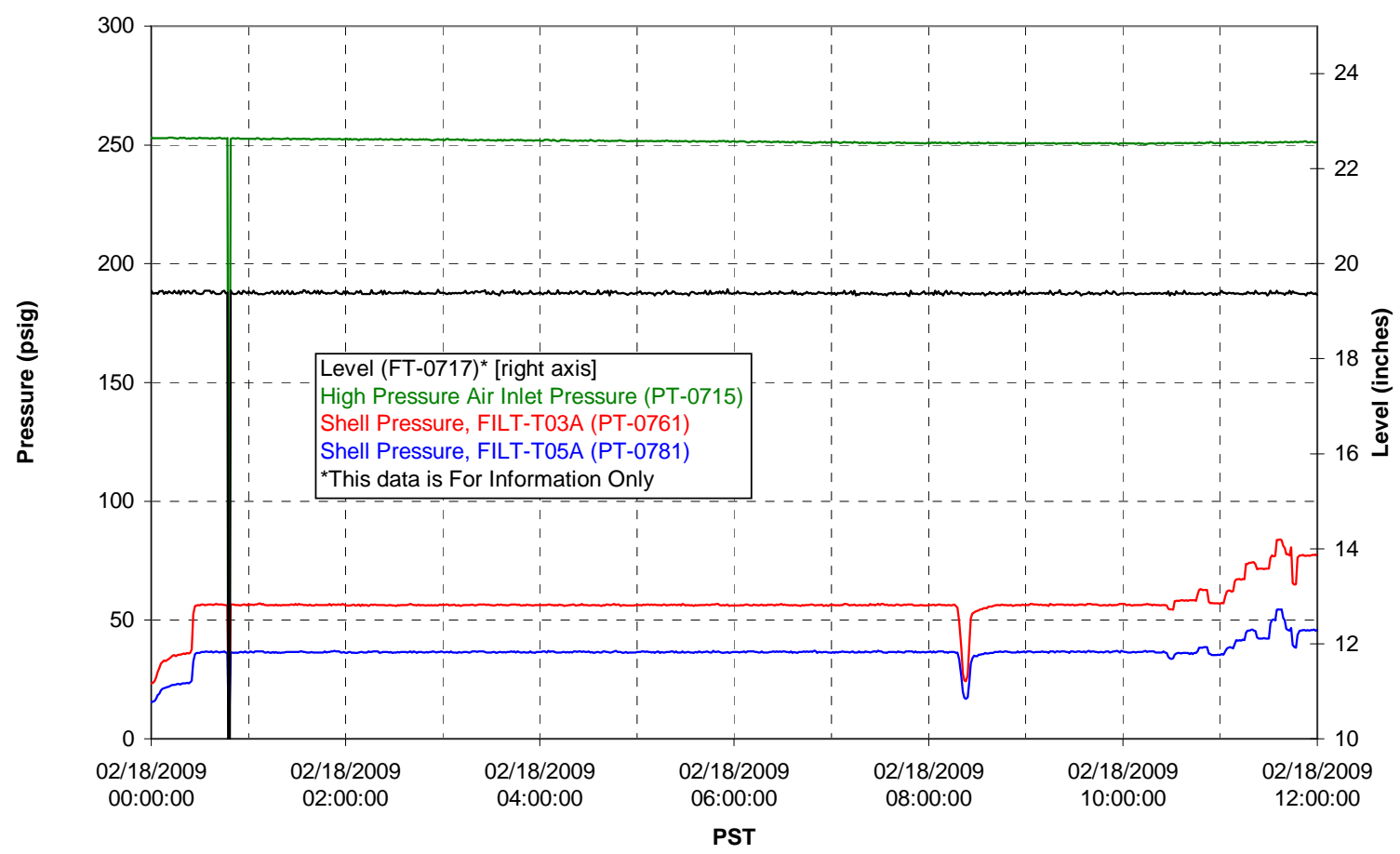

Pulsepot UFP-PP-T02A

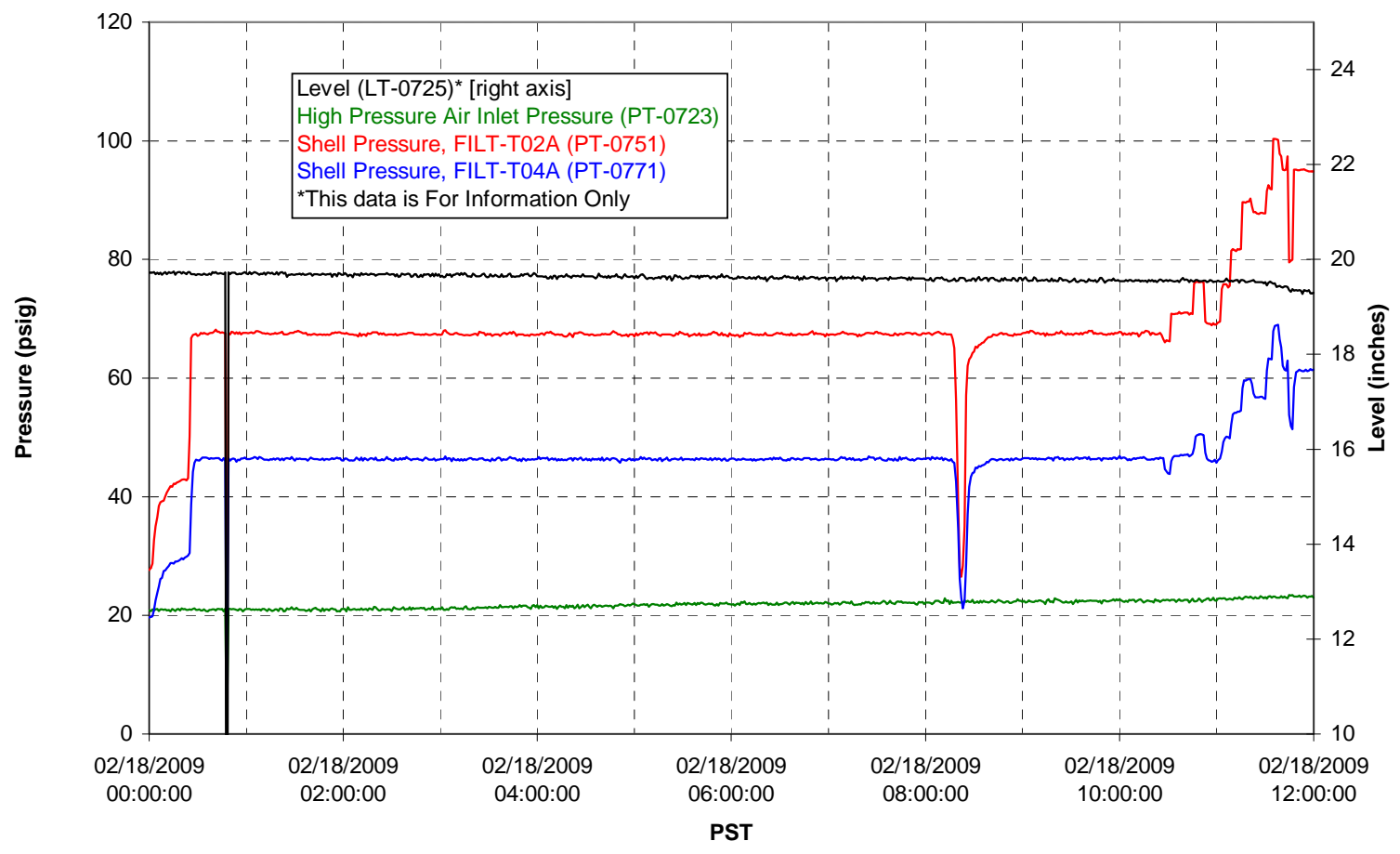


Pulsepot UFP-PP-T03A

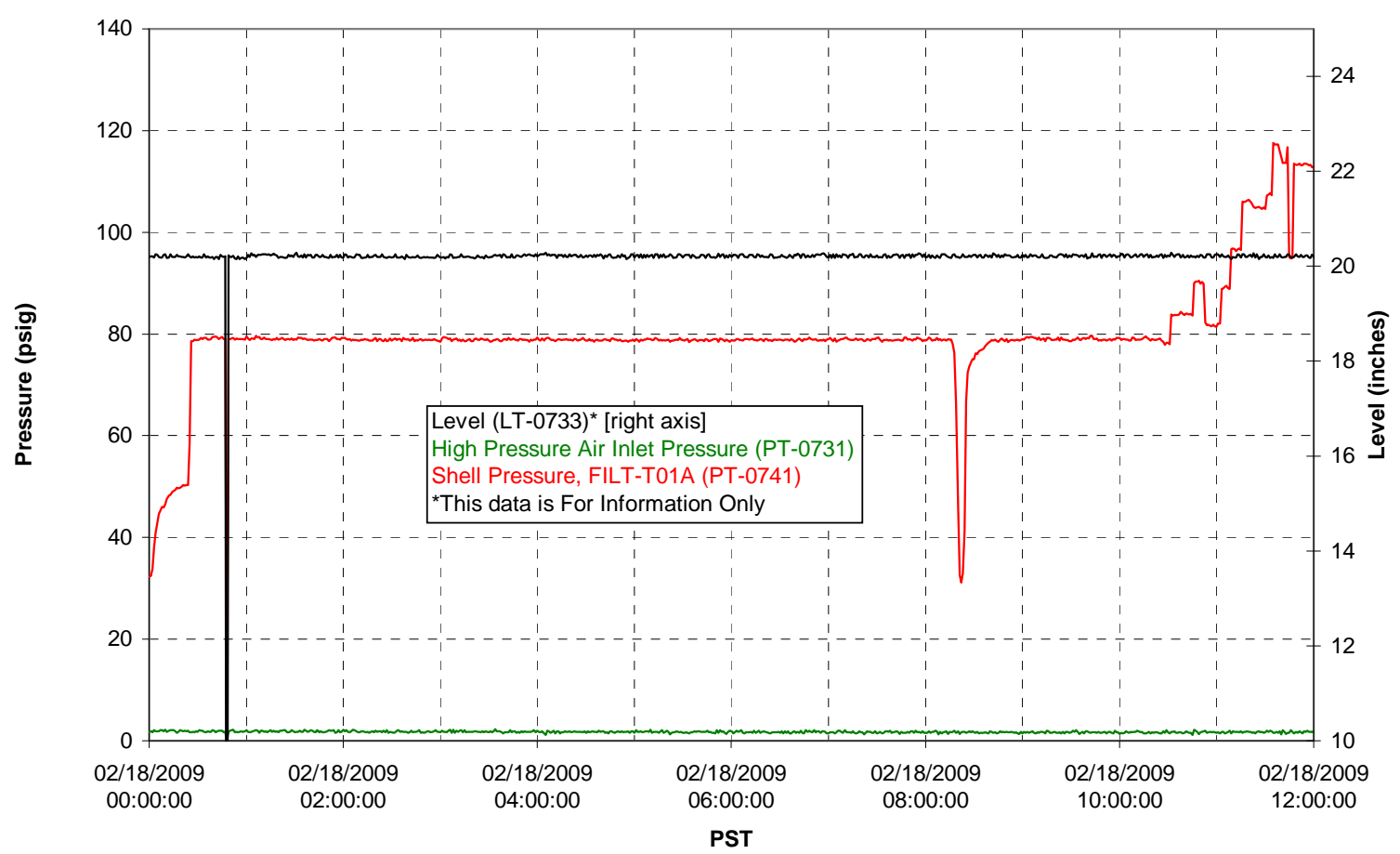

Pulsepot Levels

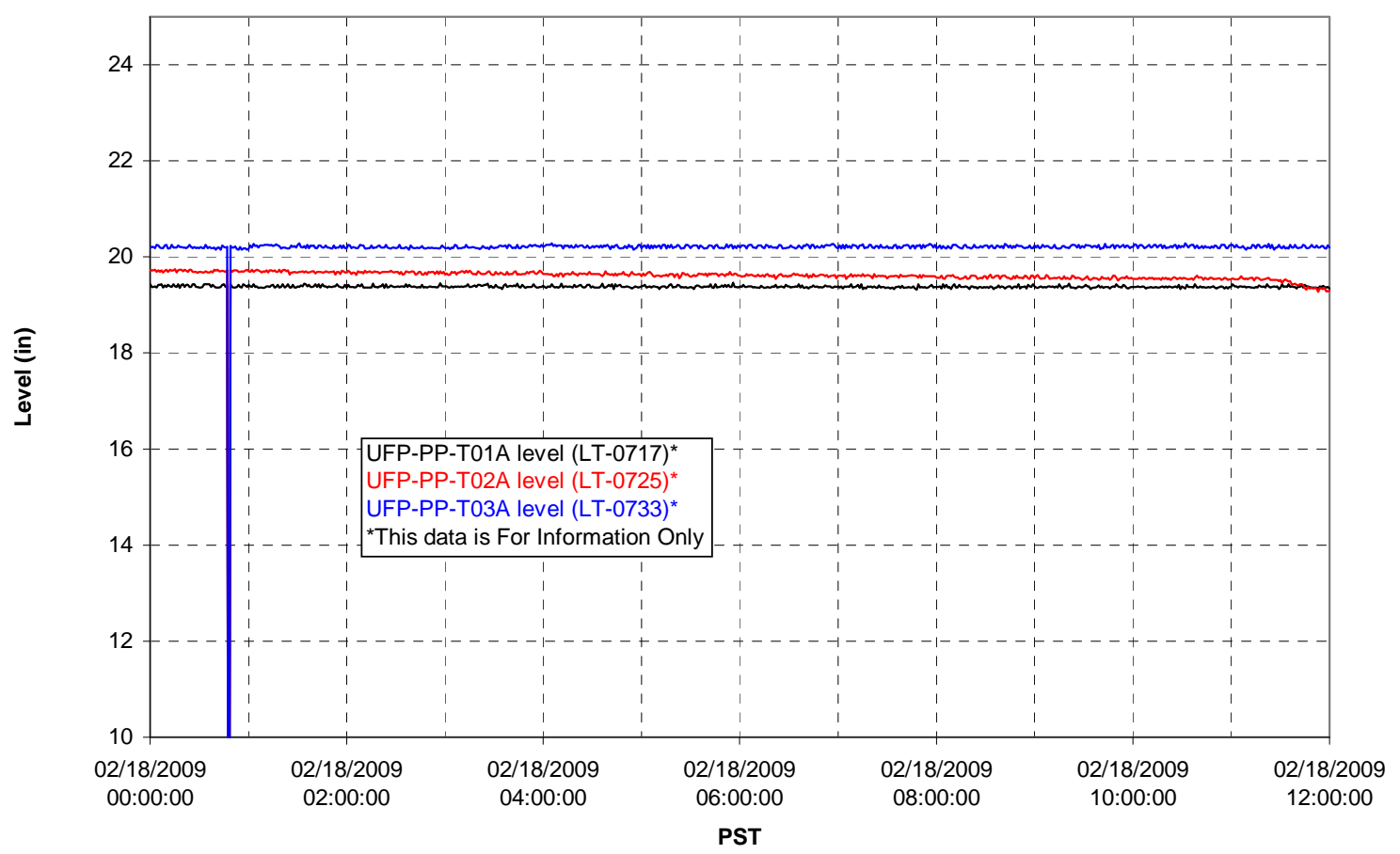


Filter UFP-FILT-T01A

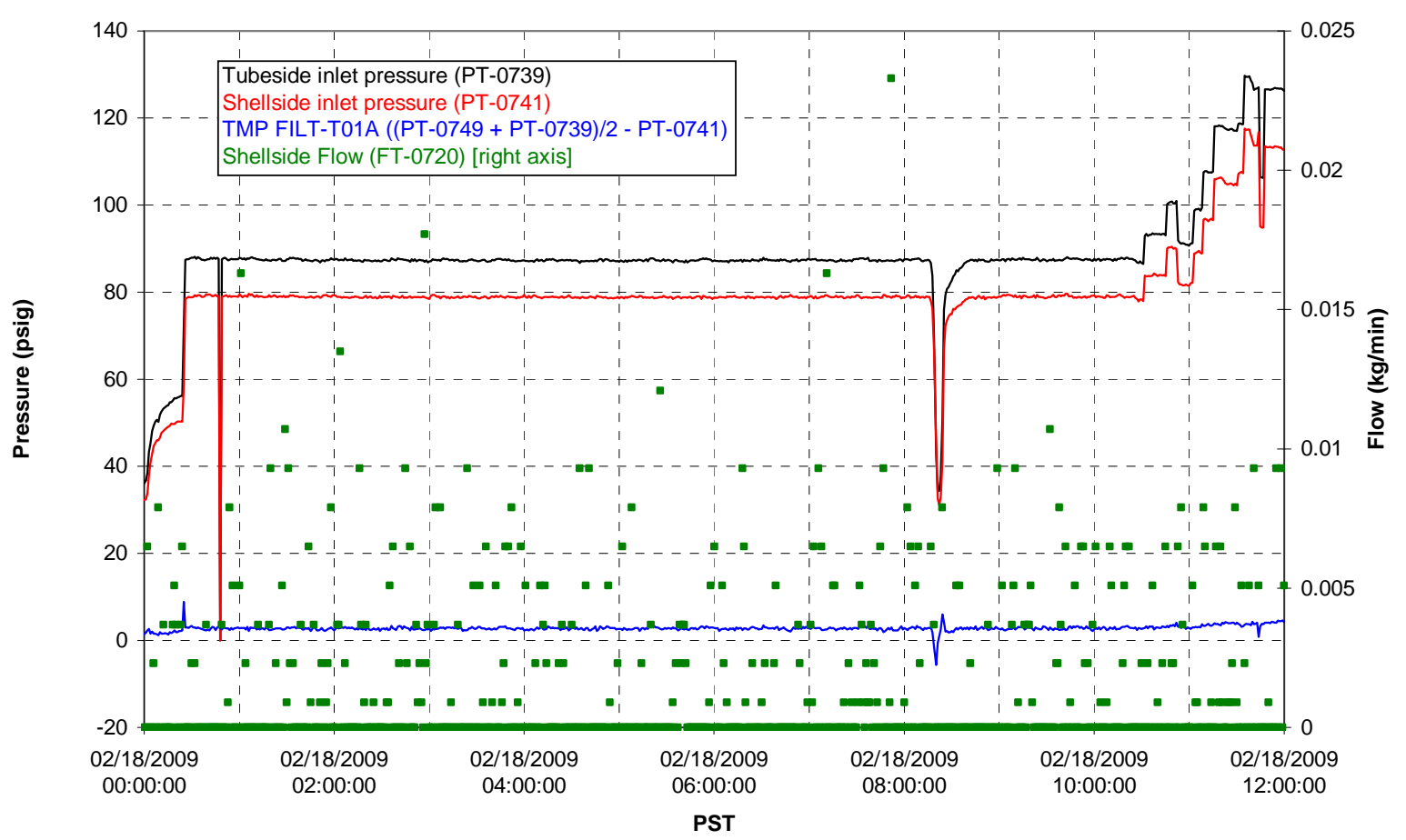

Filter UFP-FILT-T02A

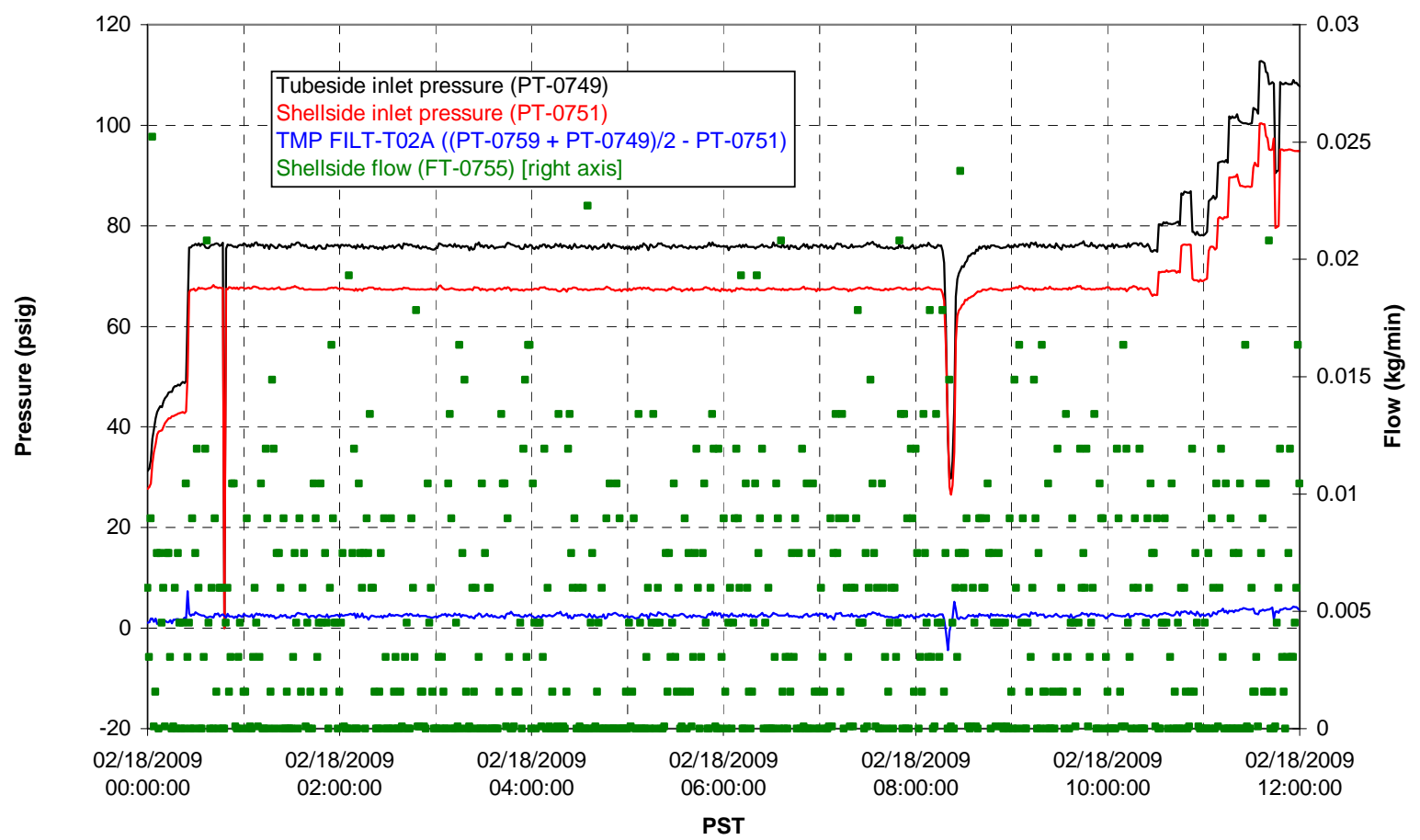


Filter UFP-FILT-T03A

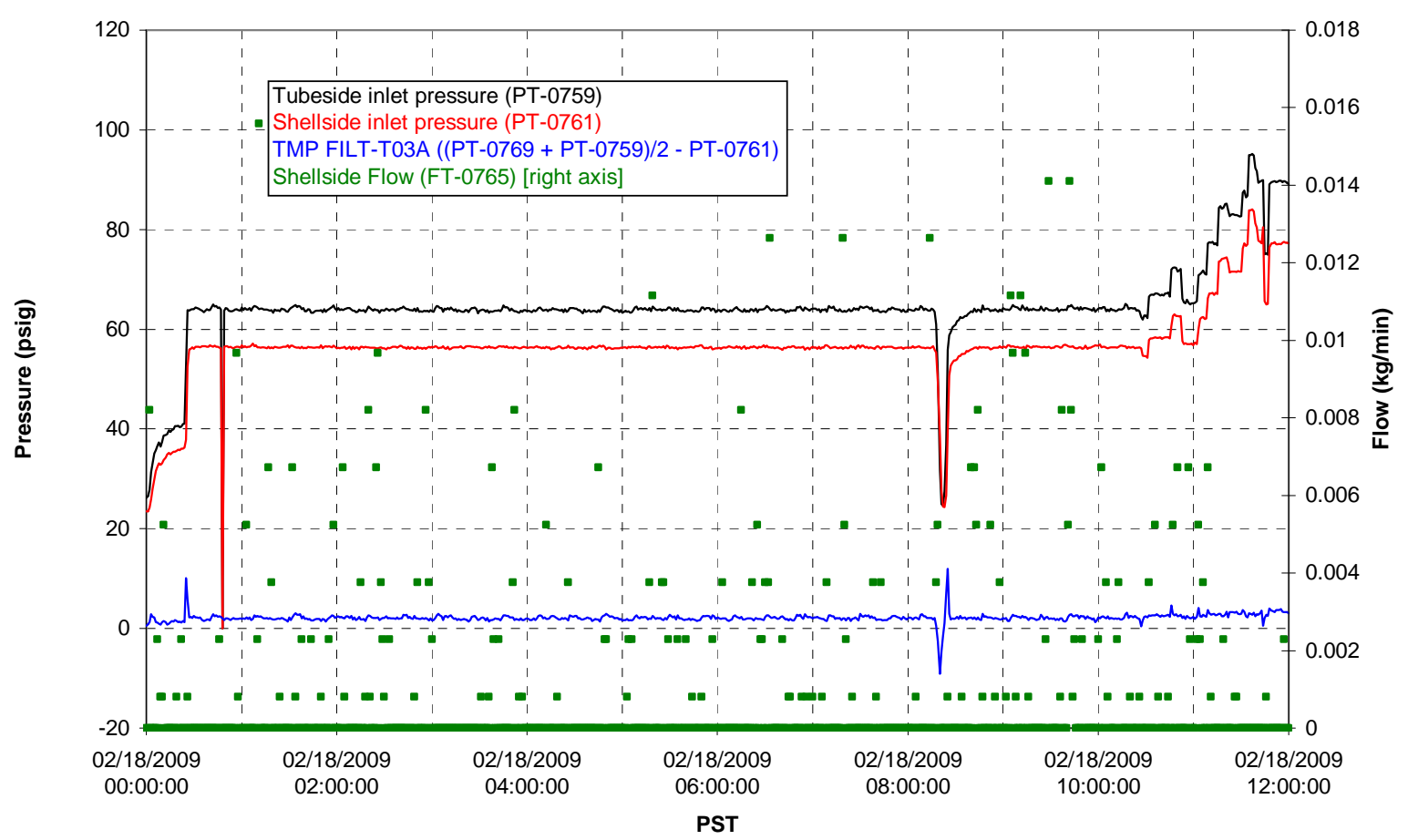

Filter UFP-FILT-T04A

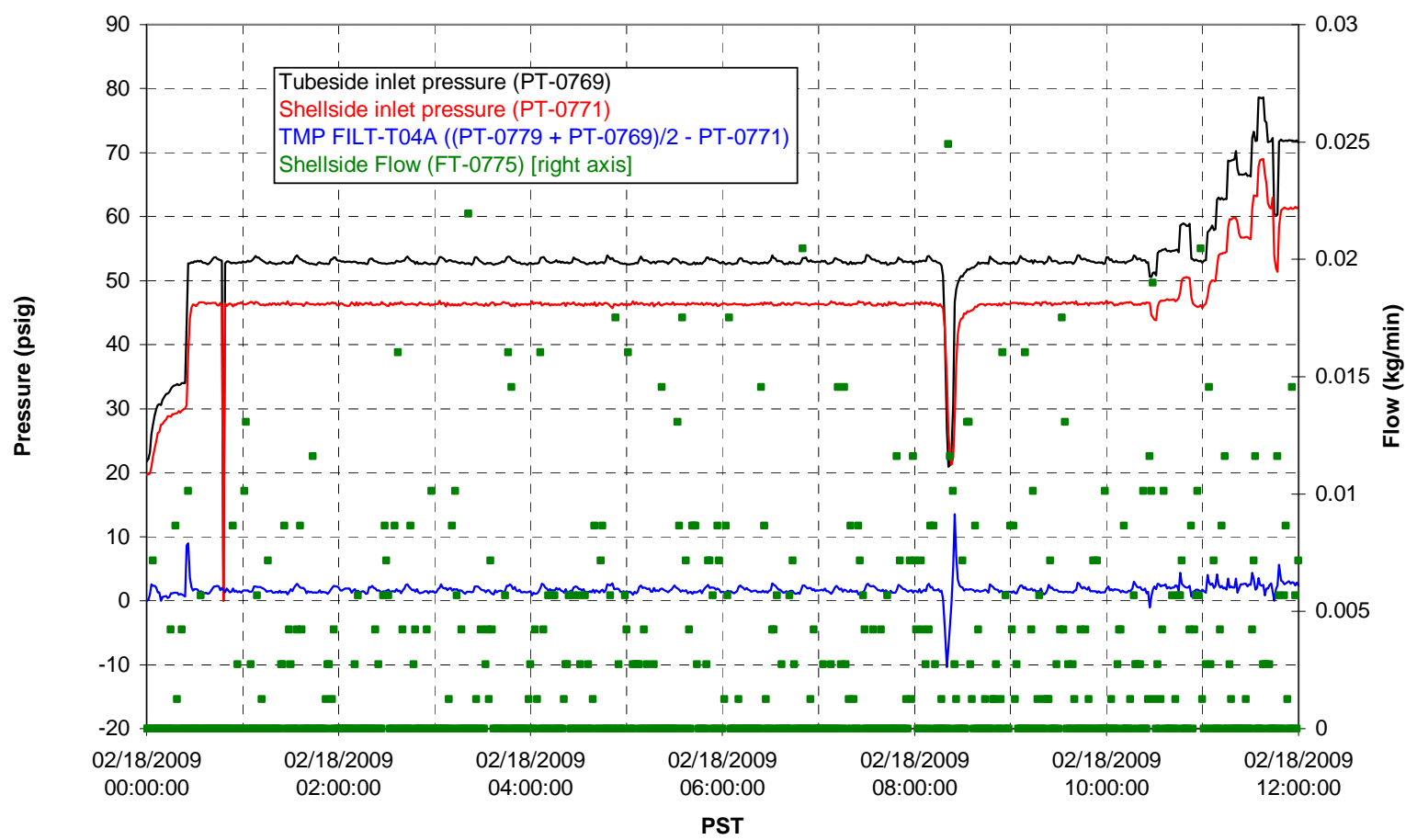


Filter UFP-FILT-T05A

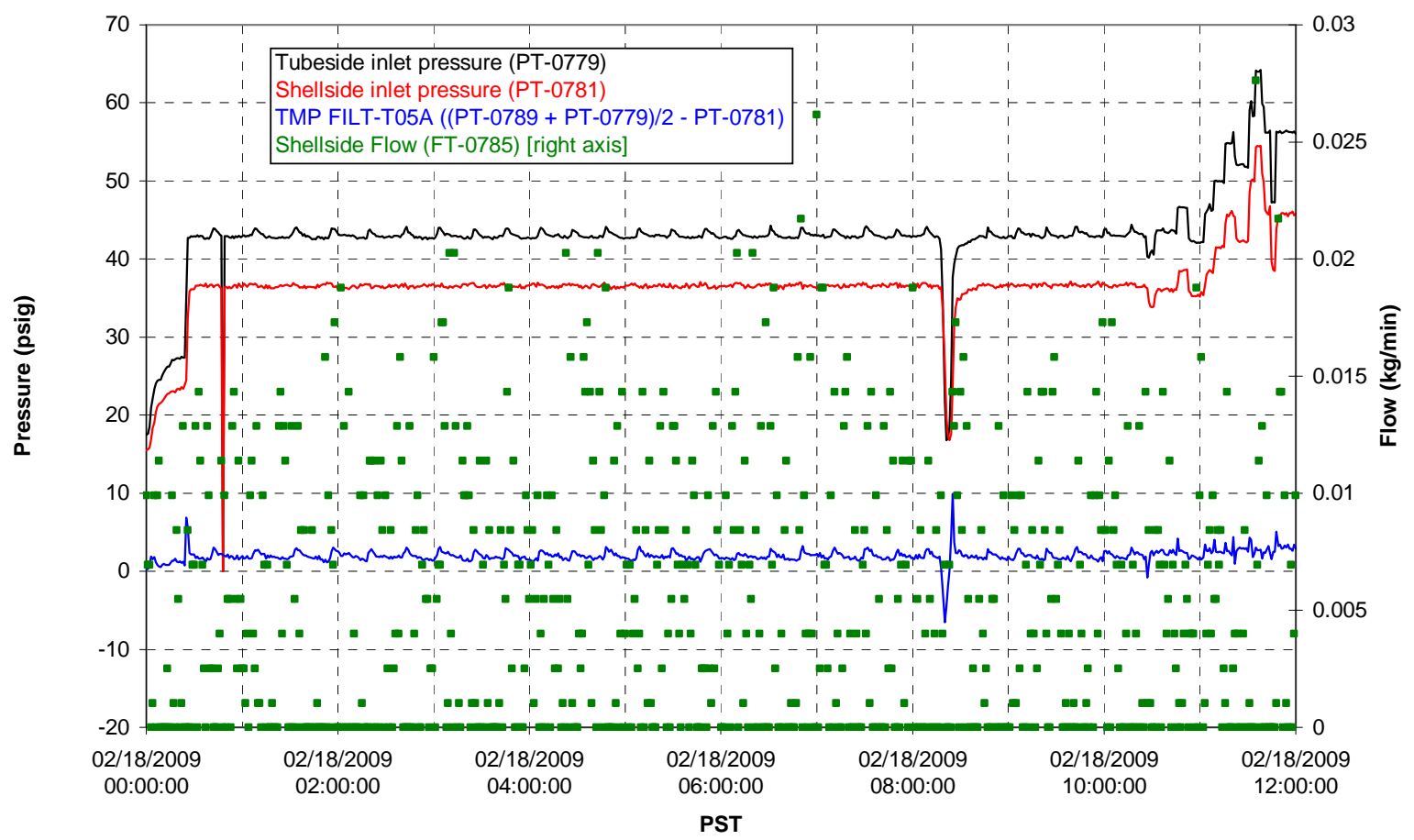

Chemical Flow

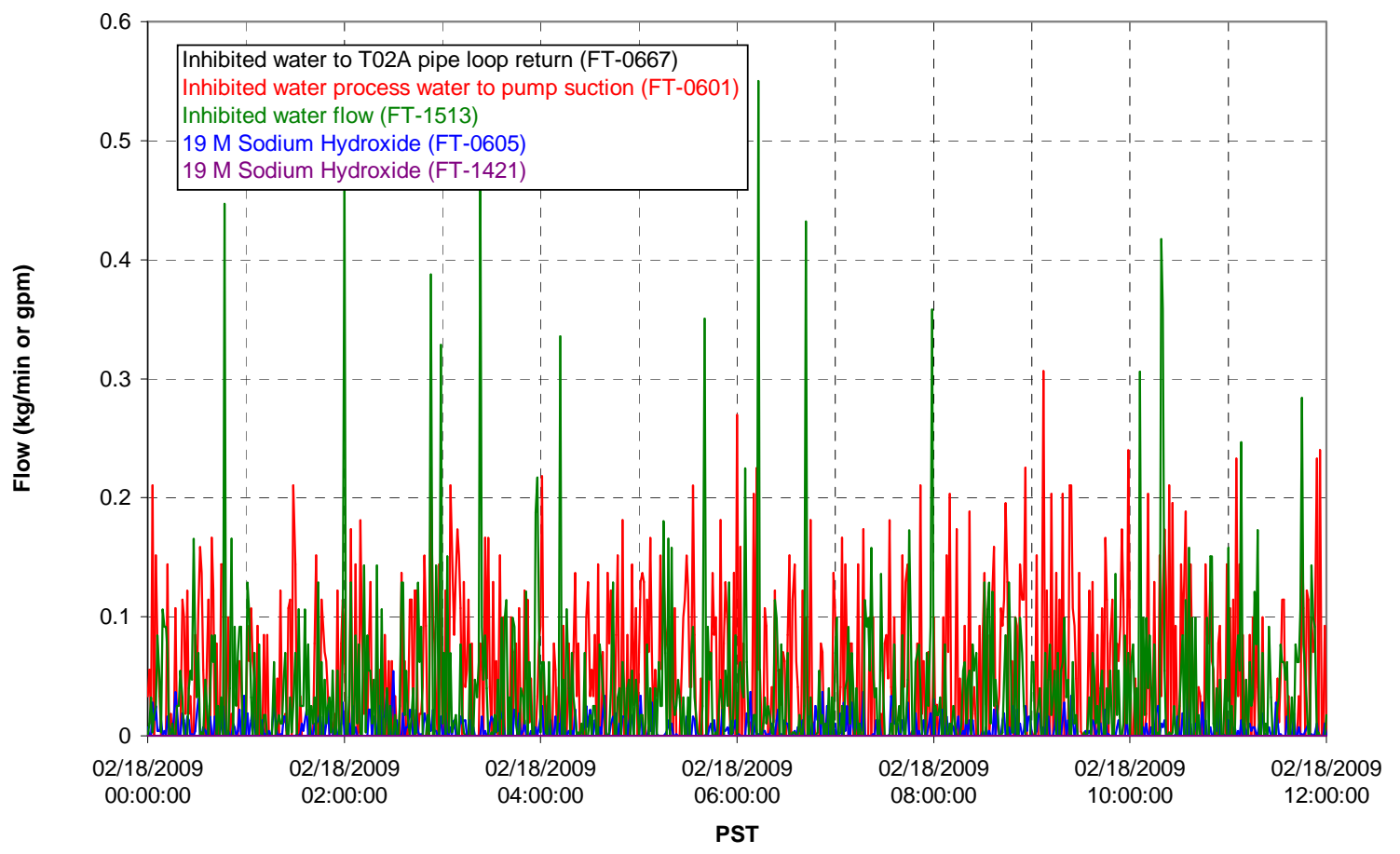




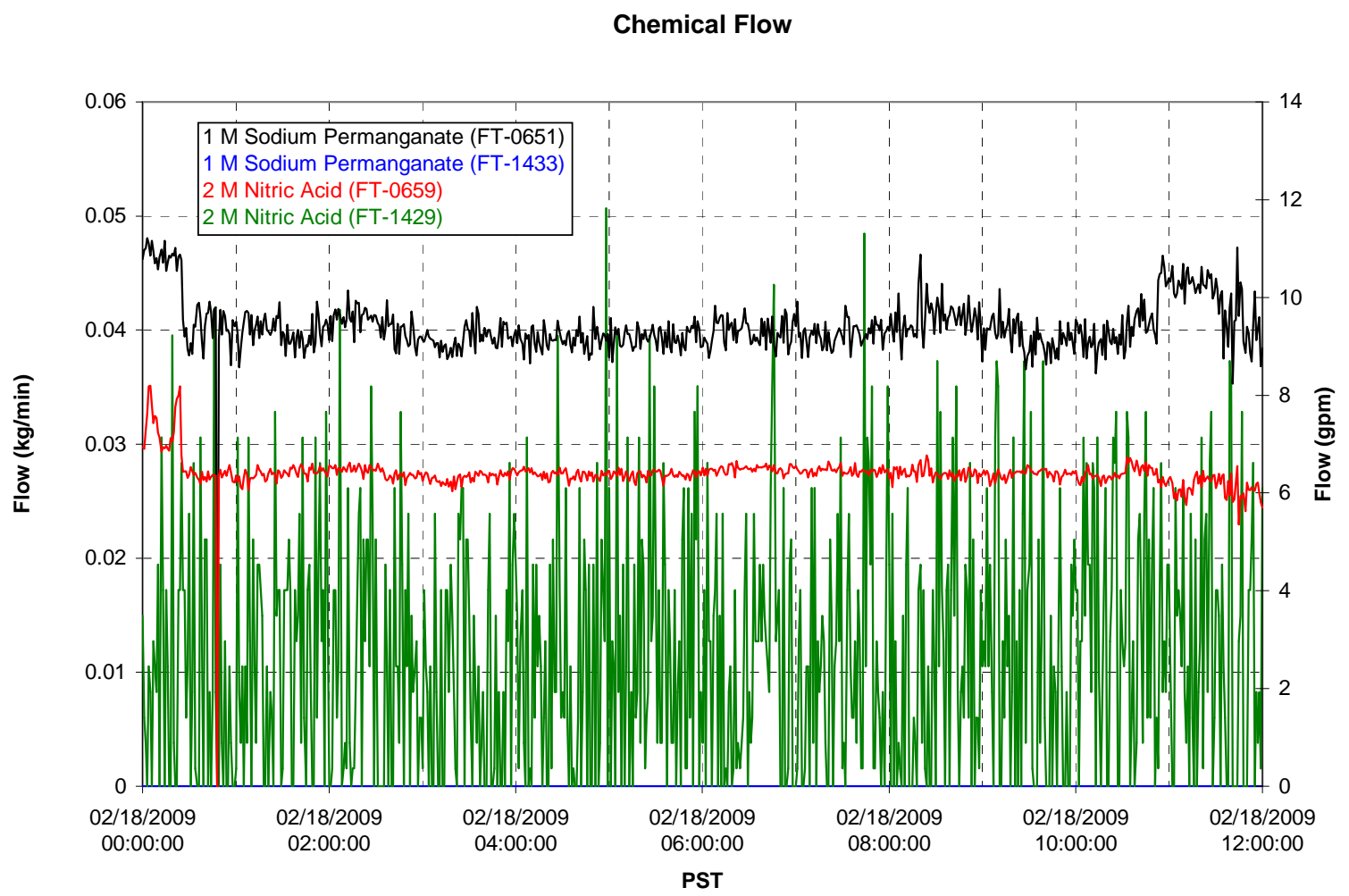

Air Flows

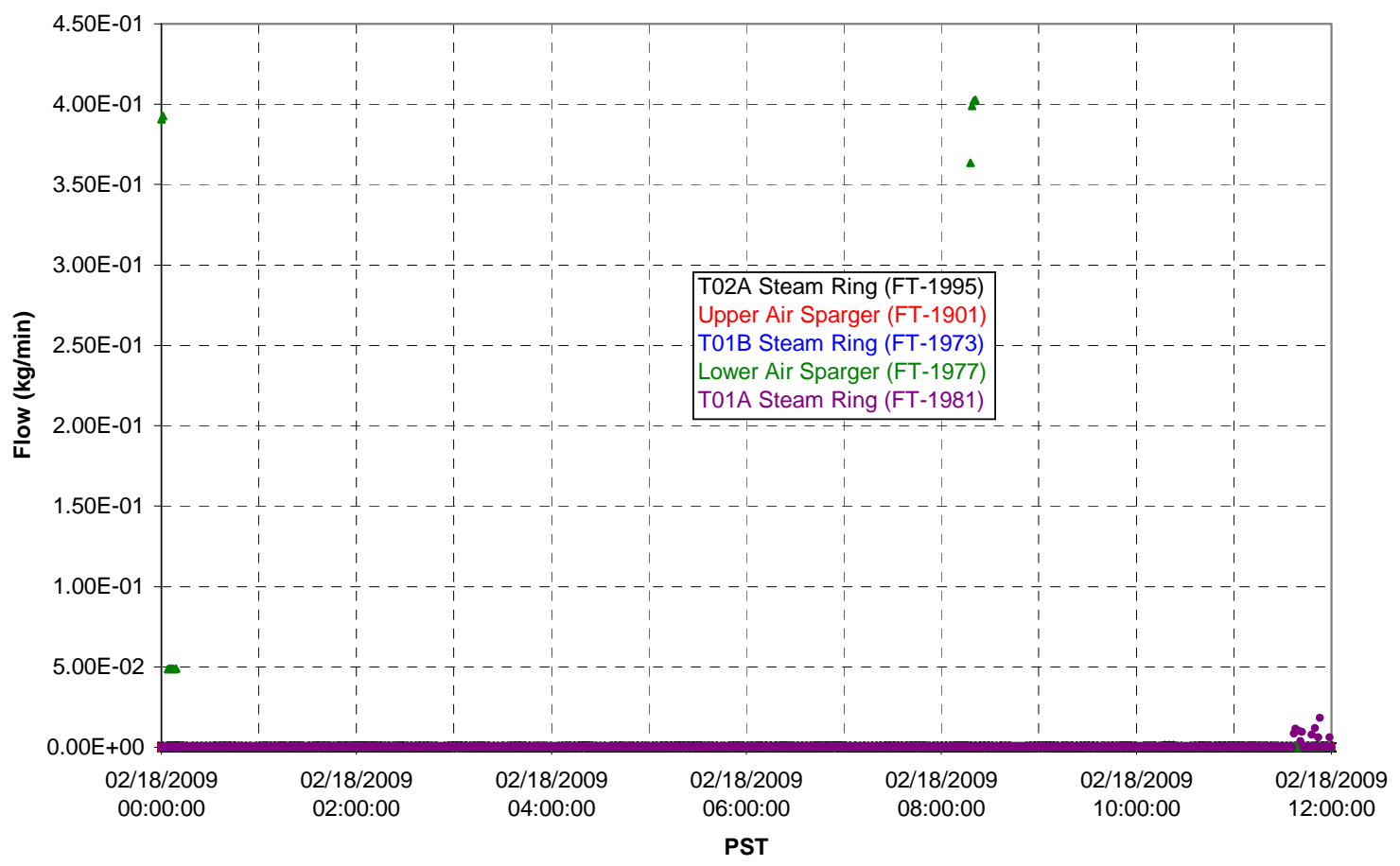


T02A Steam

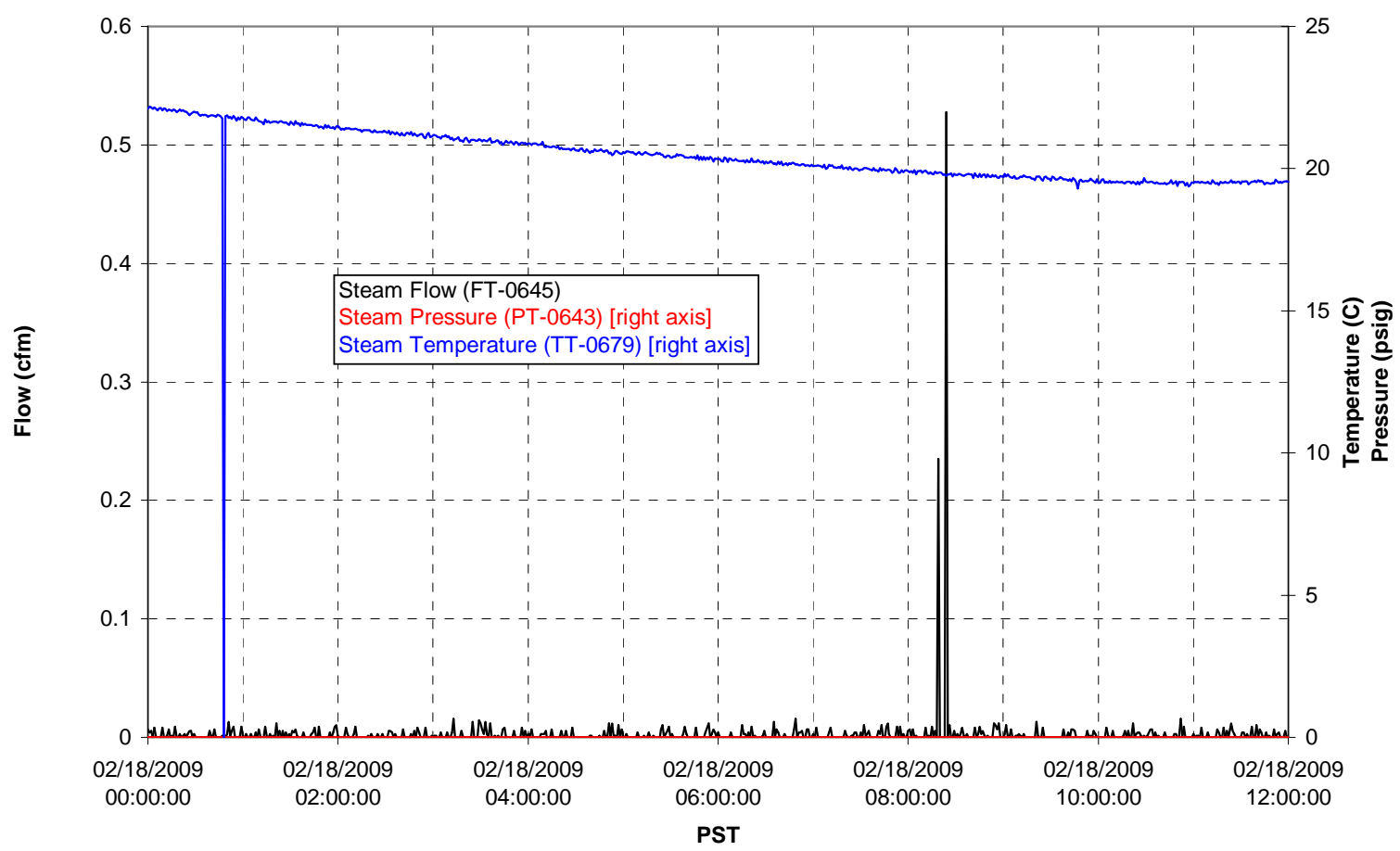

T01A Steam

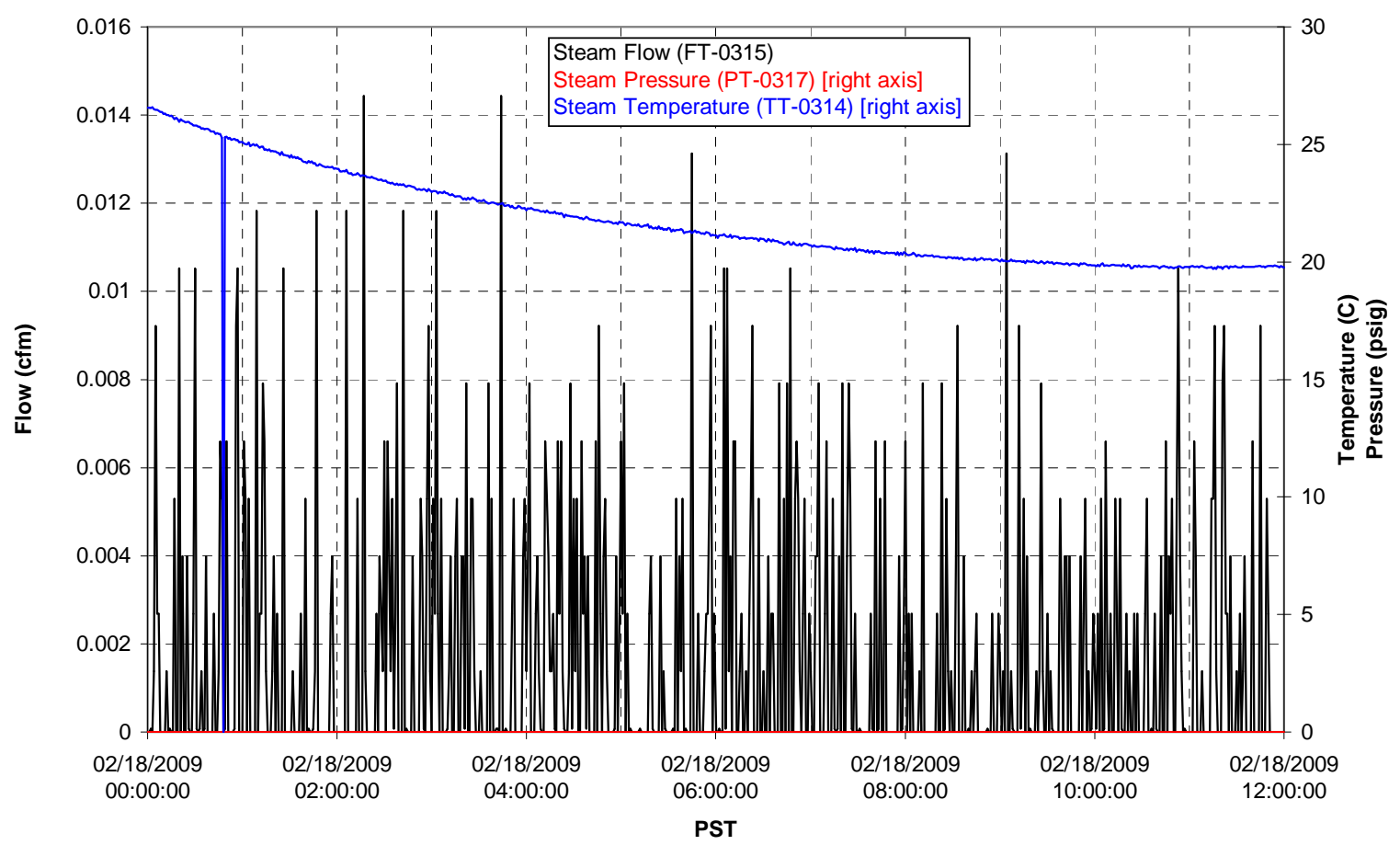


T01B Steam

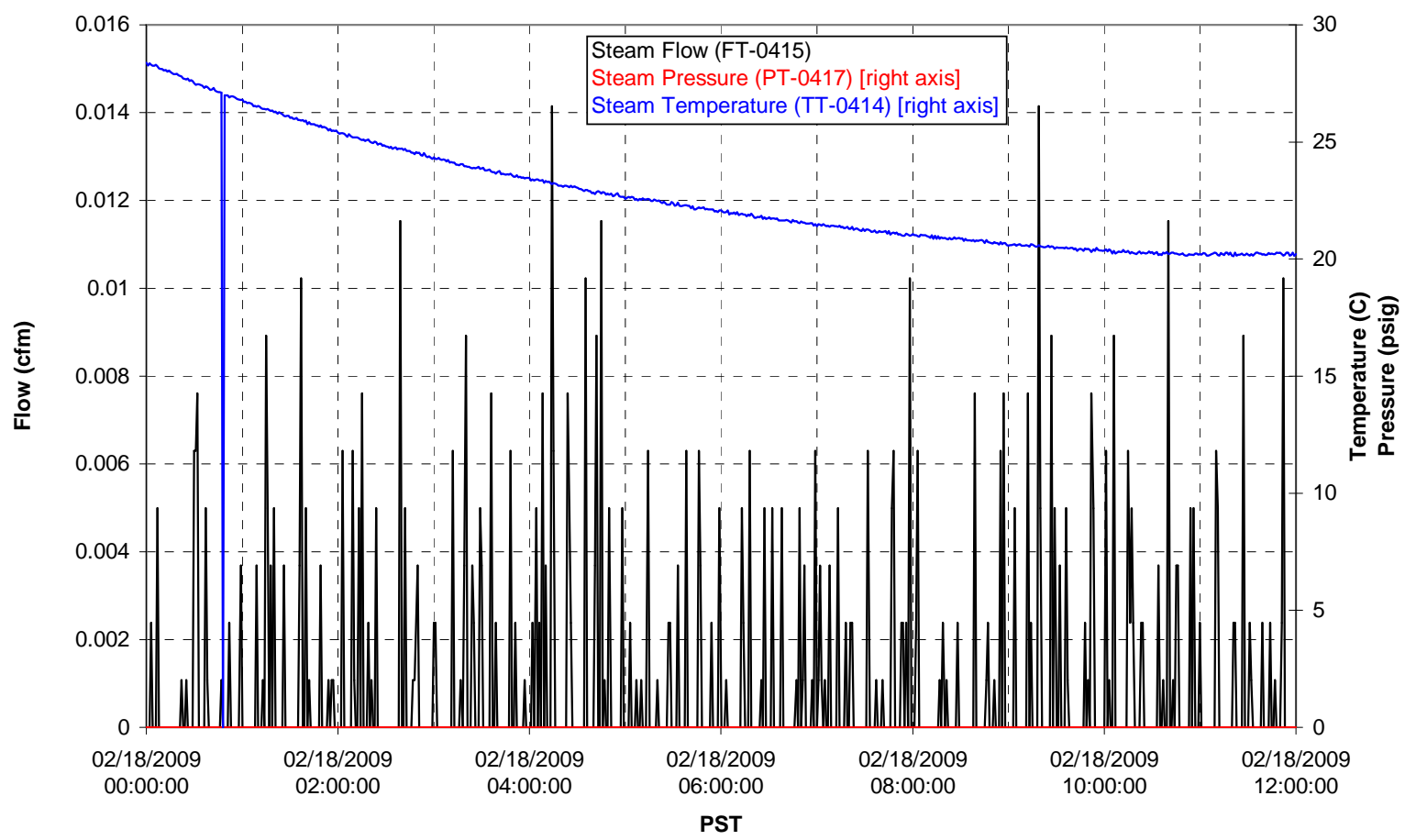




\section{Integrated Test A Data Plots 02/18/09 12:00 - 02/19/09 00:00}


T01A level

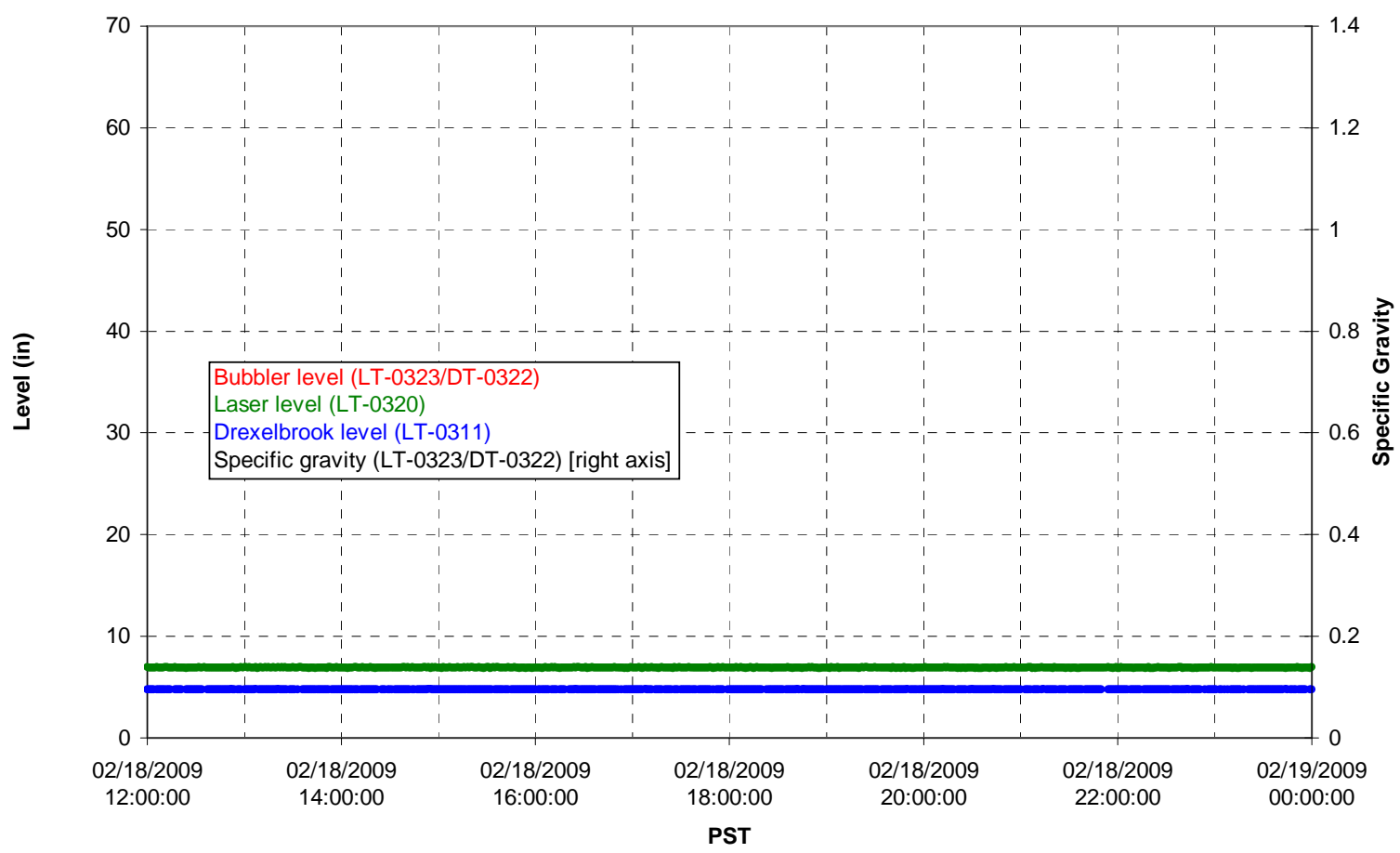

T01A temperatures

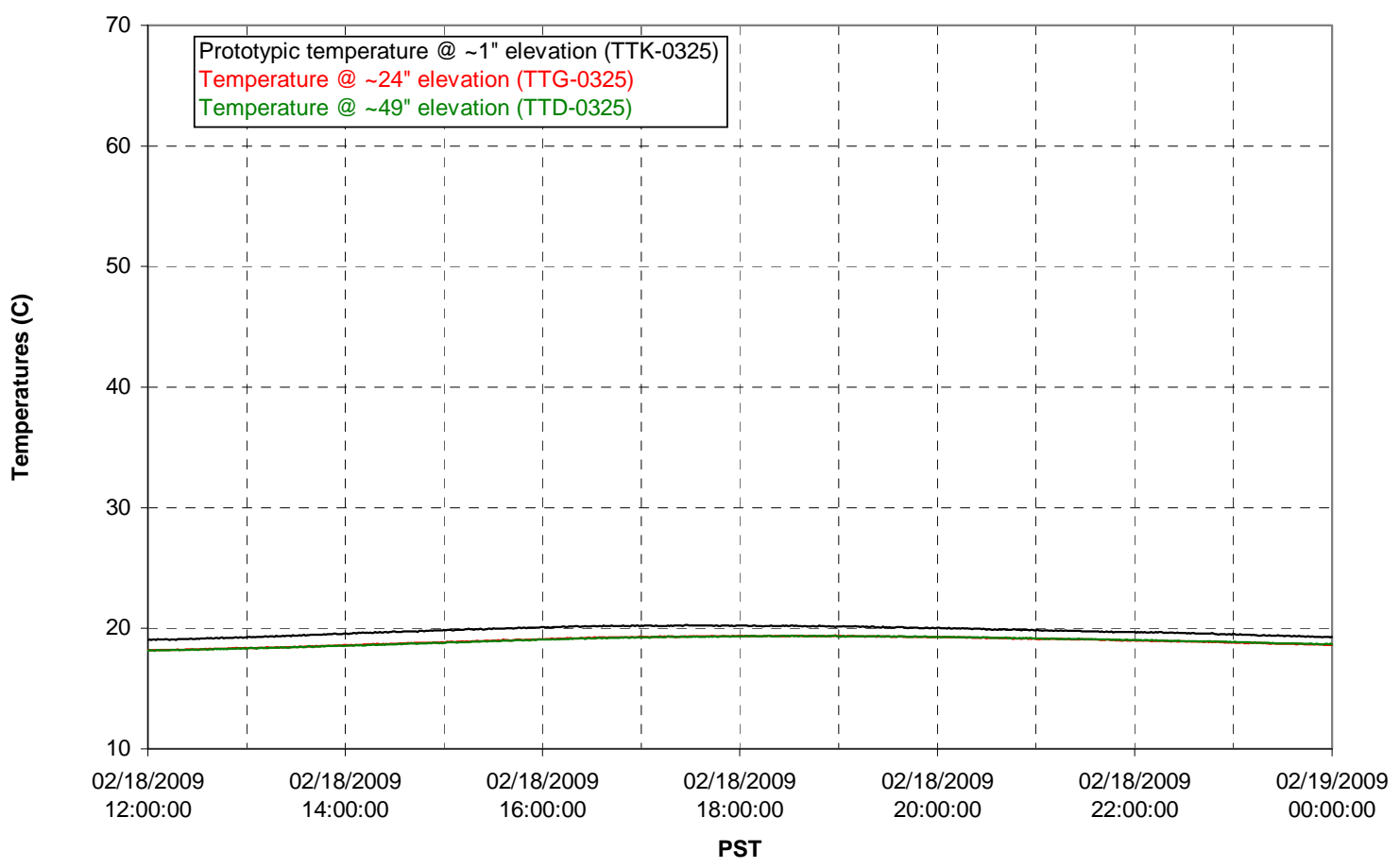


T01B level

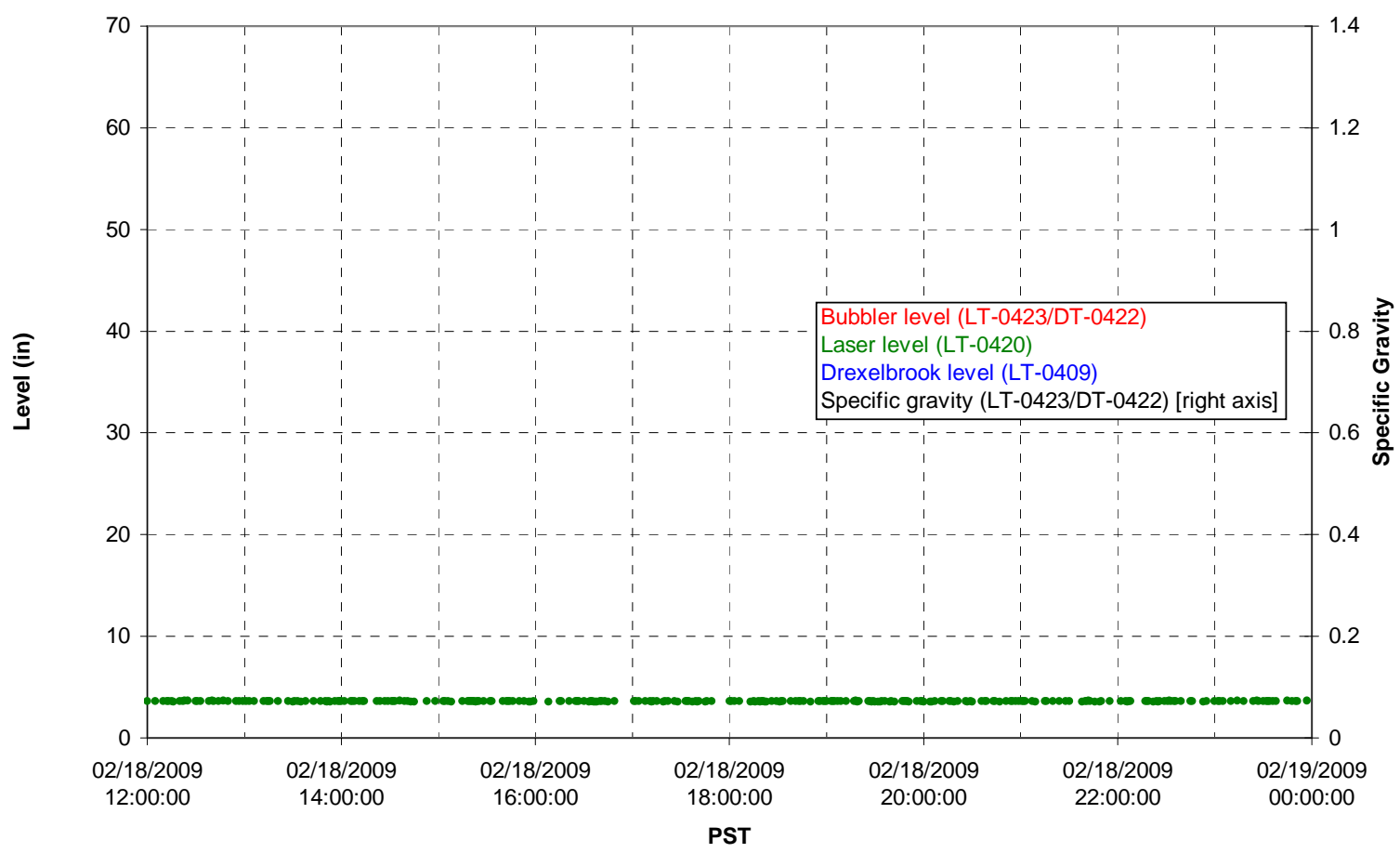

T01B temperatures

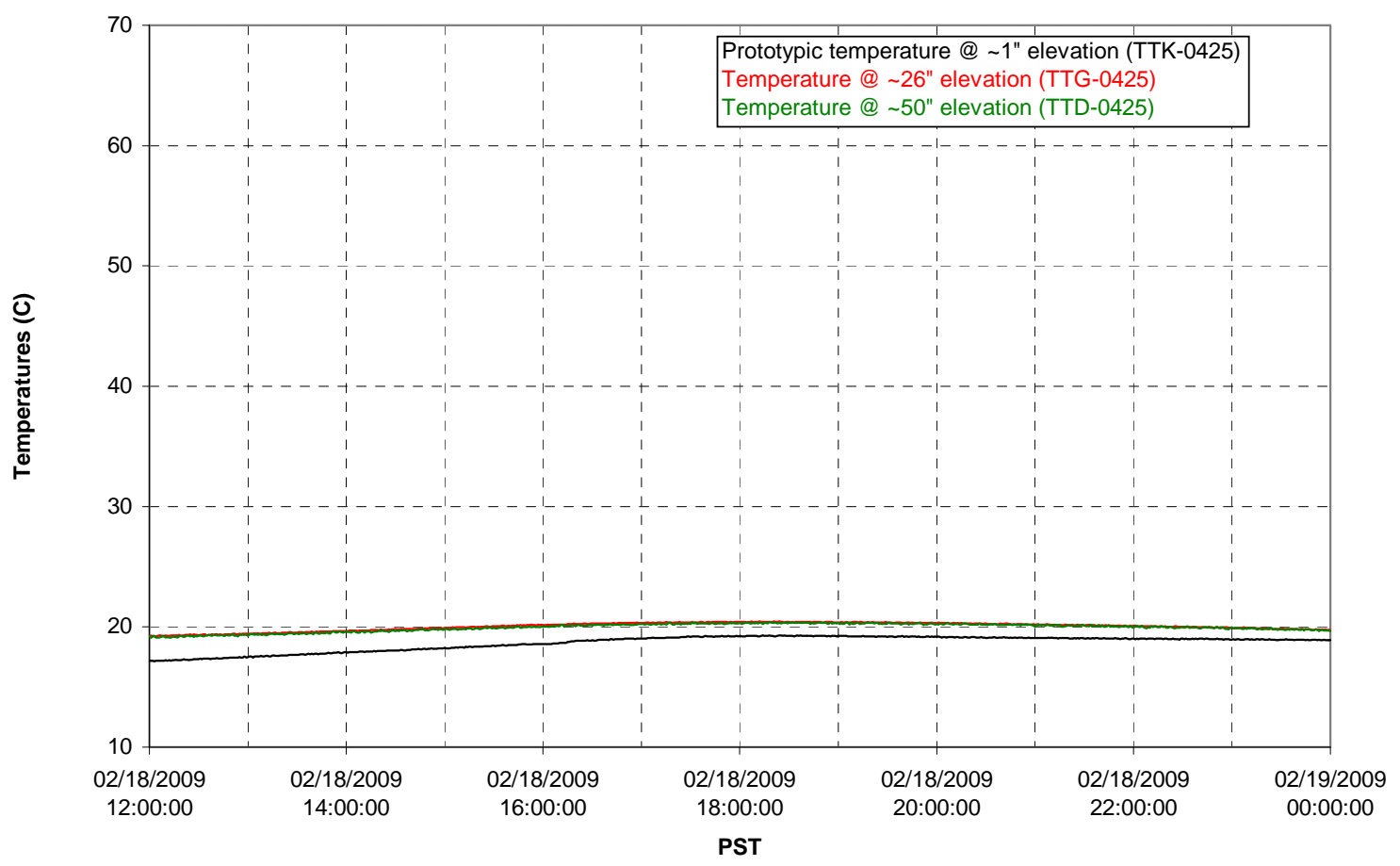


T02A level

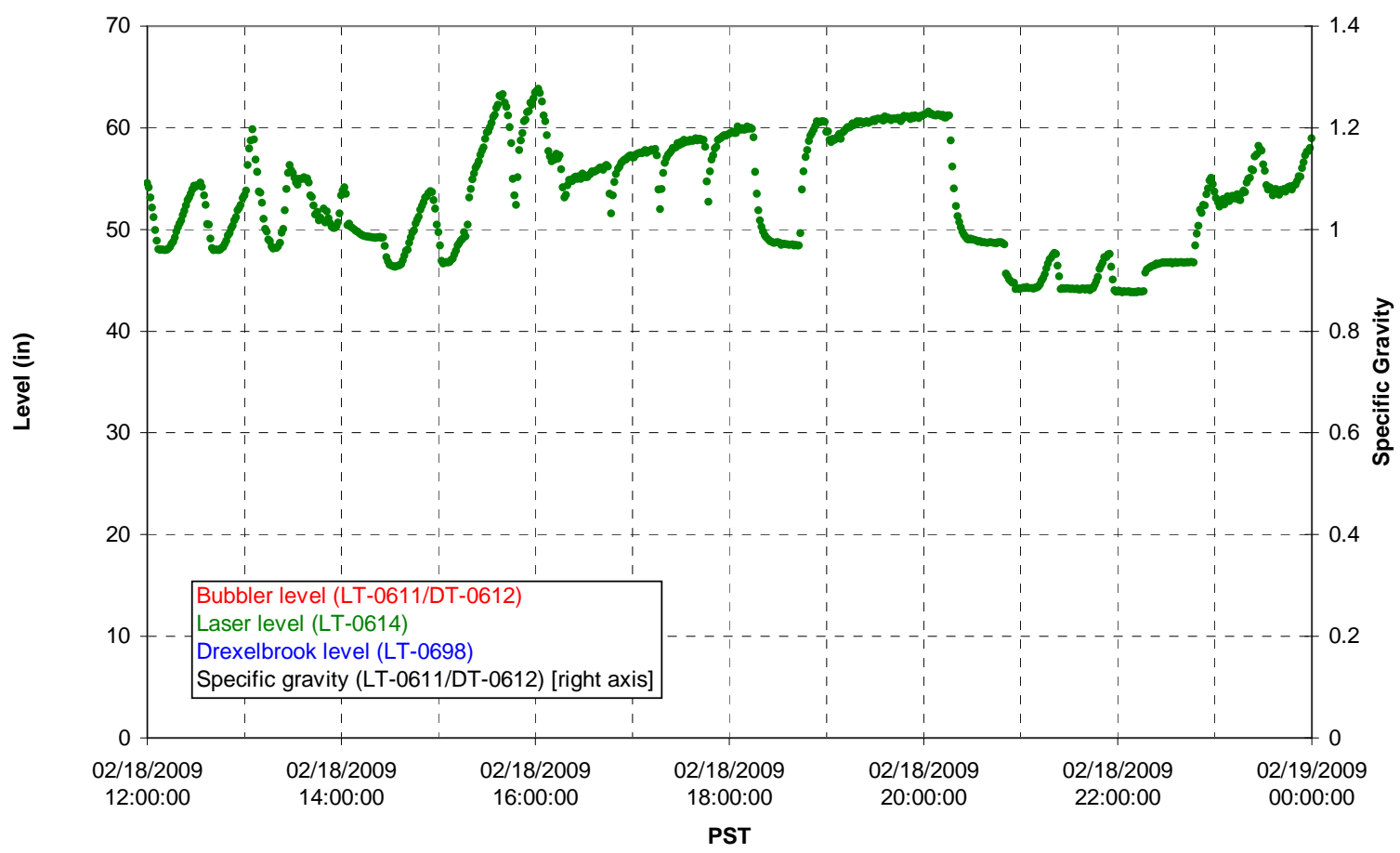

T02A temperatures

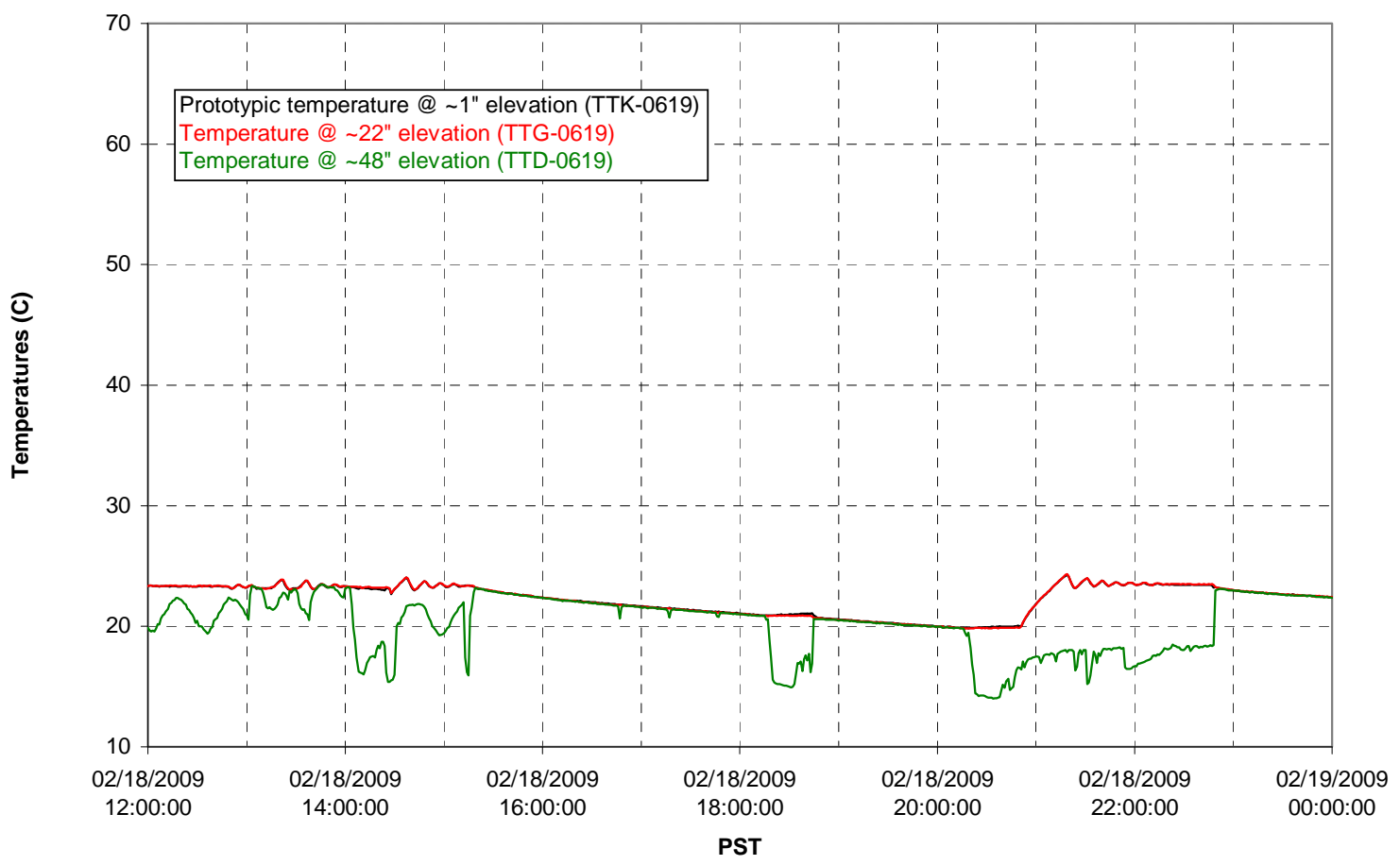


T02A and filter loop temperatures

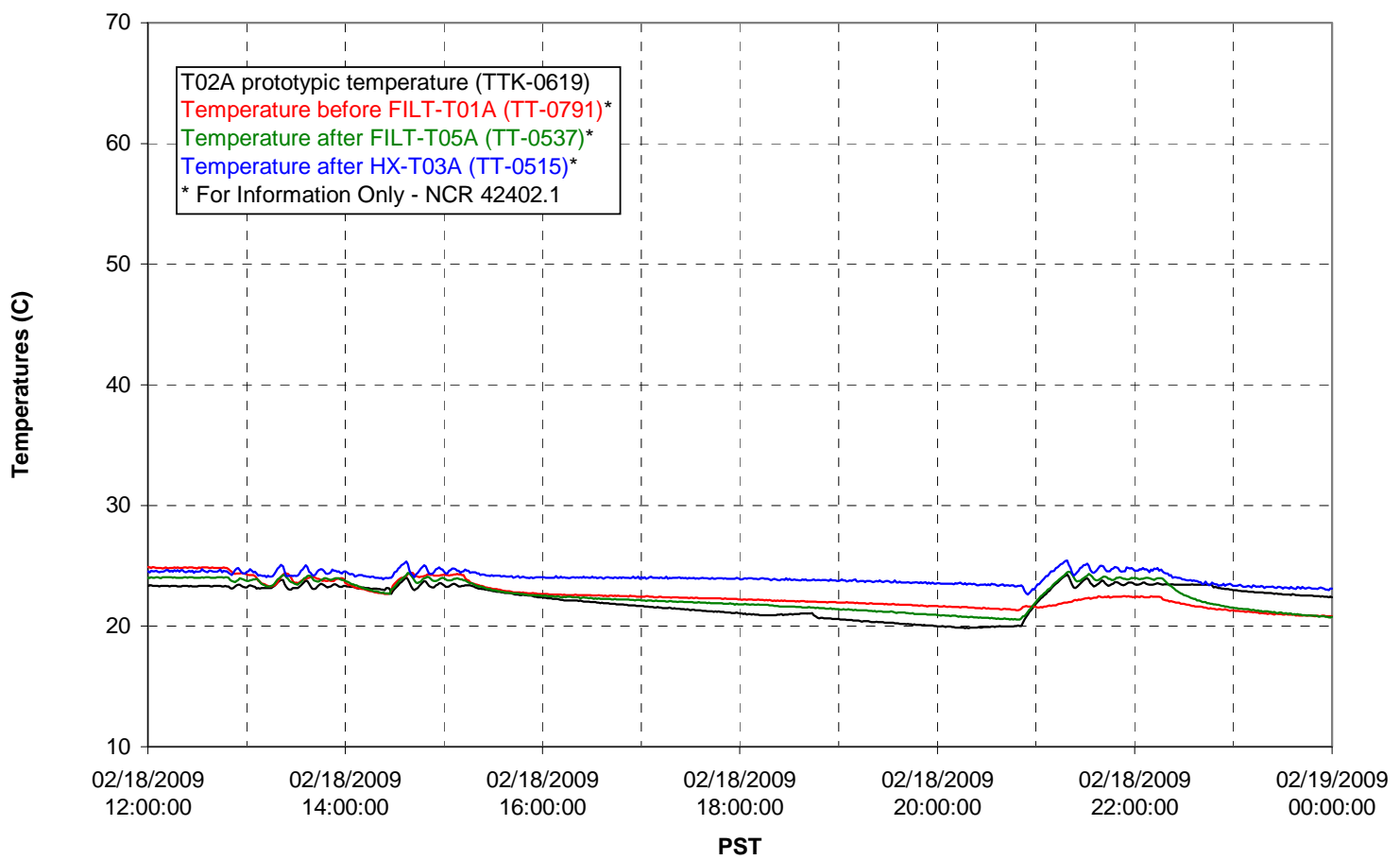

Pump Pressures and Flow

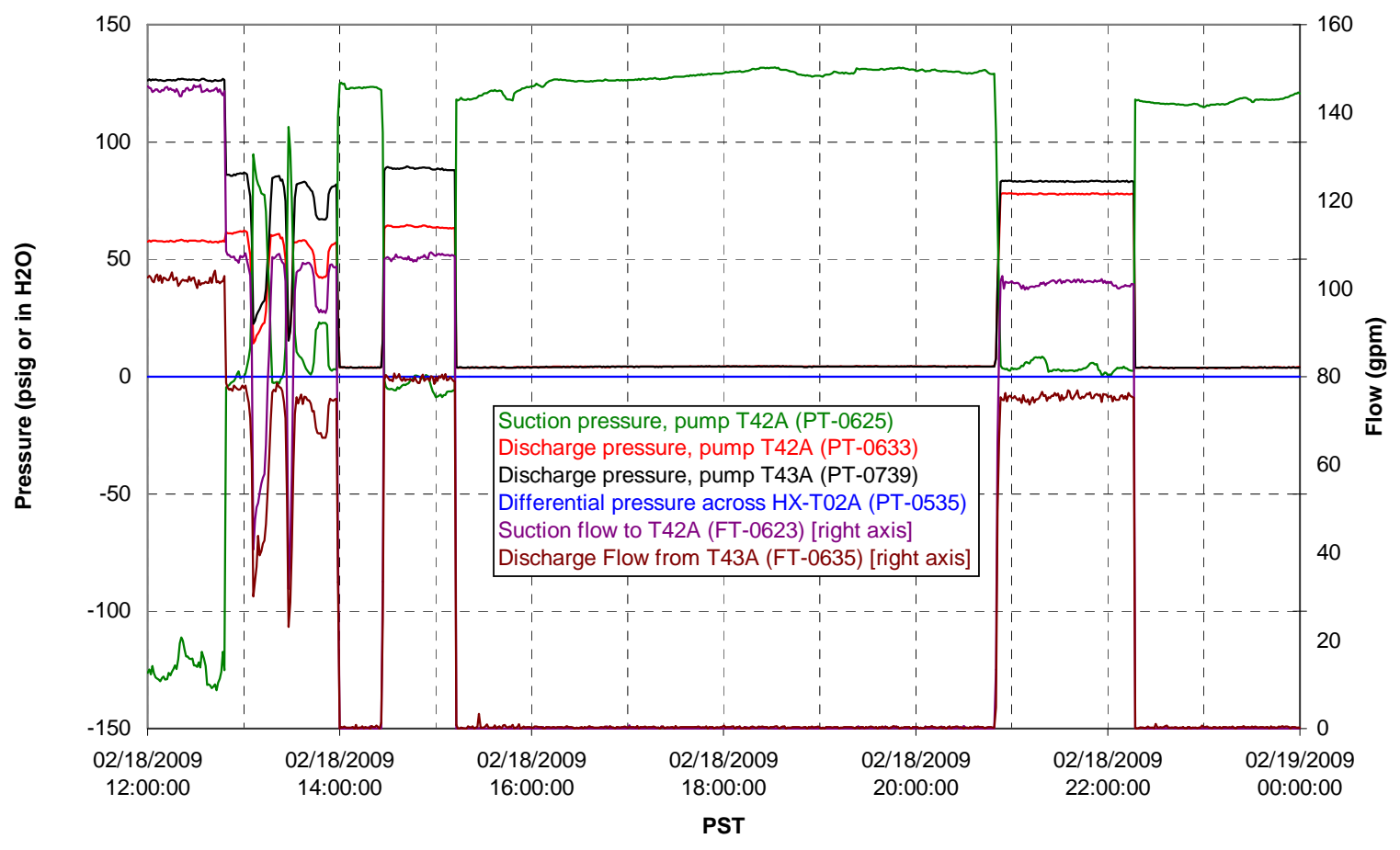


Axial pressure drop

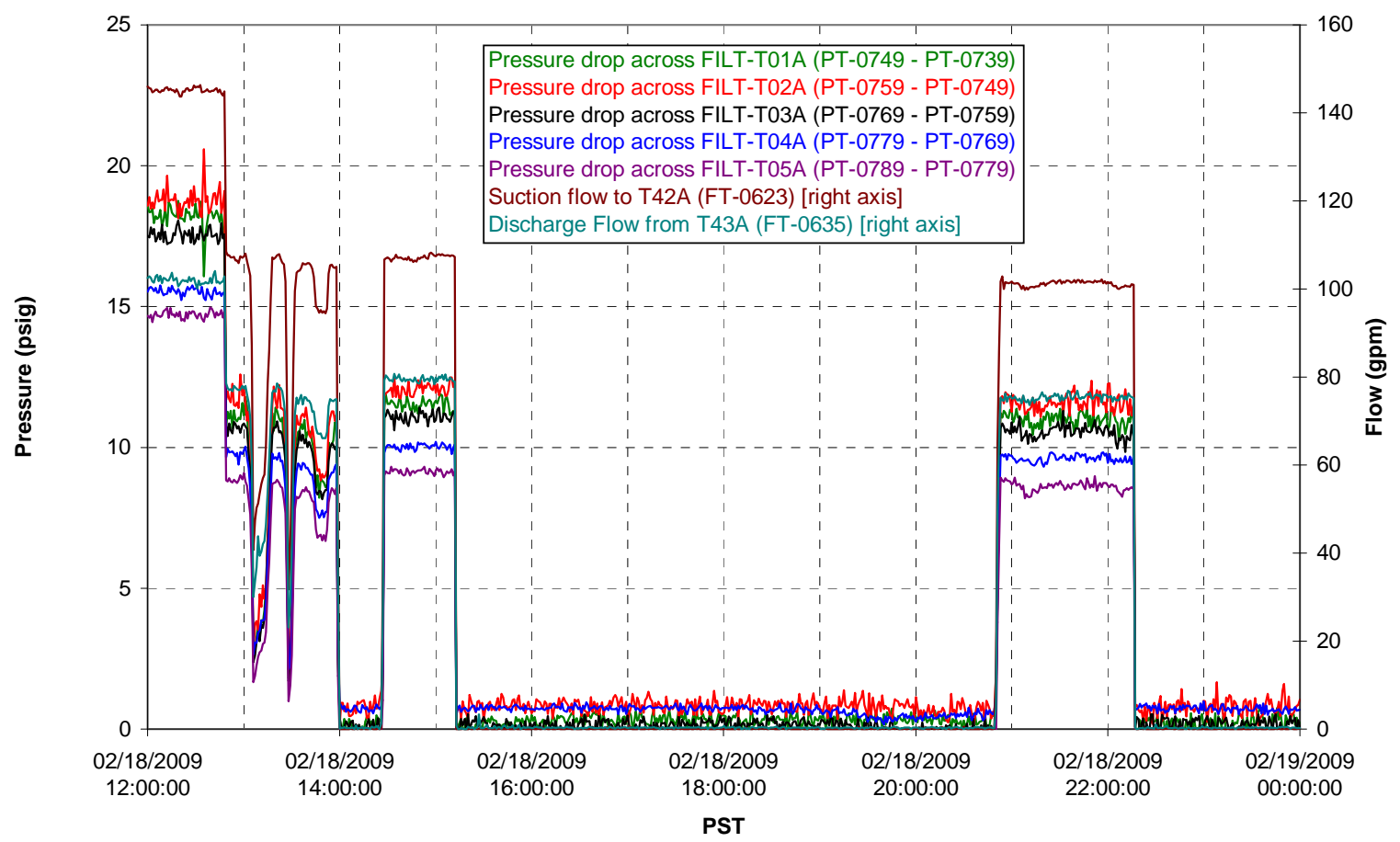

Permeate flow rates

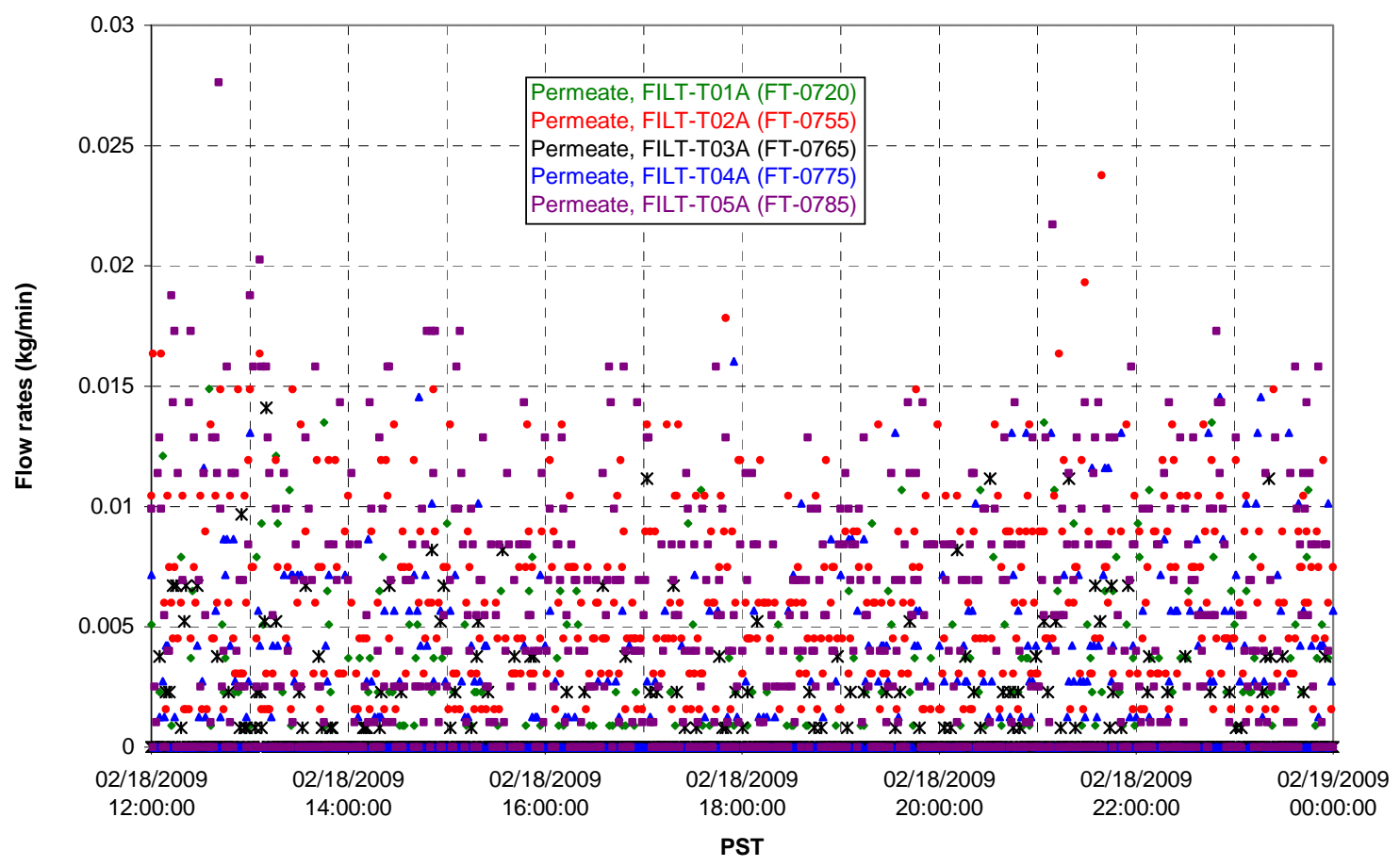


T02A Inner Temperature Tree

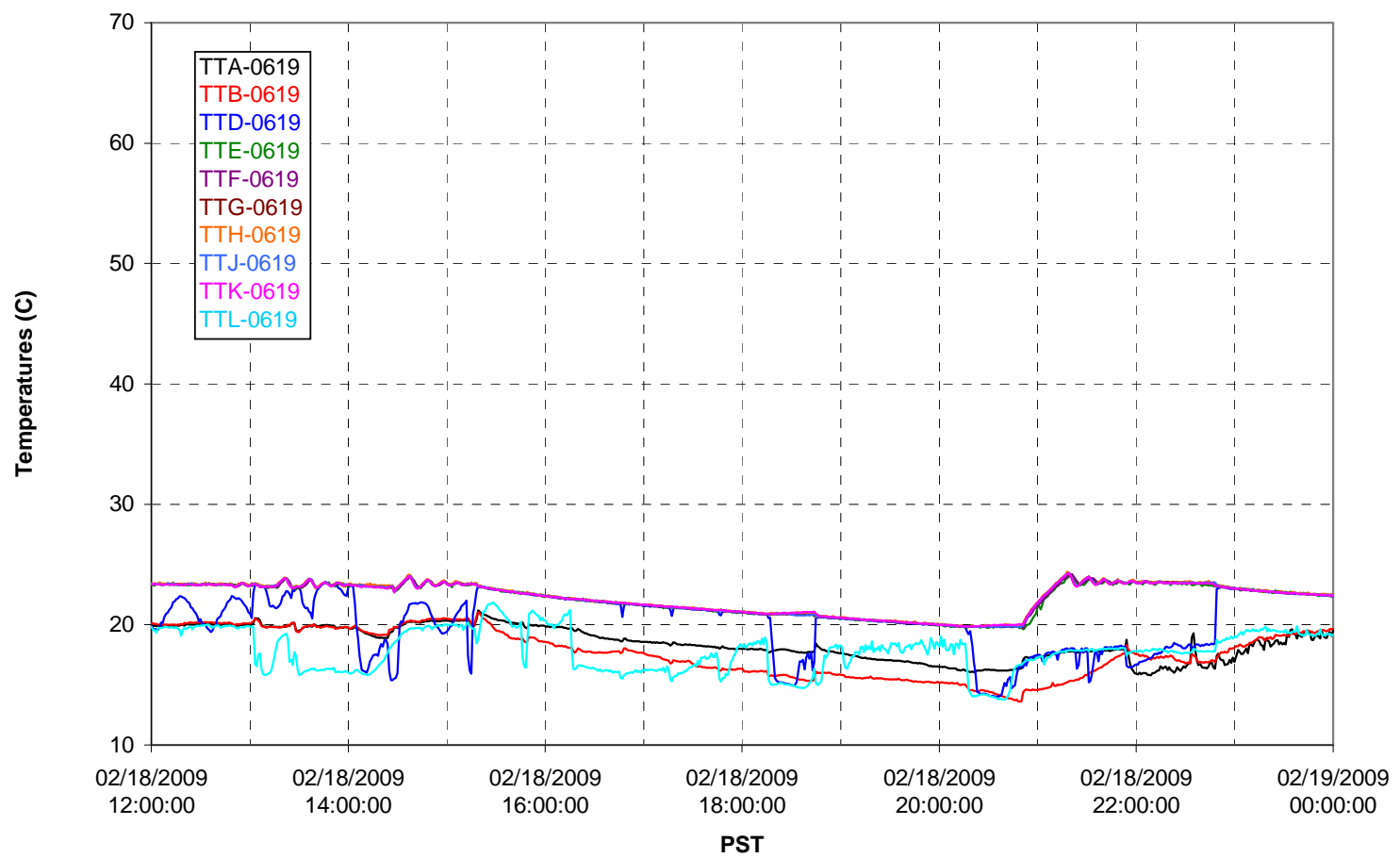

T02A Outer Temperature Tree

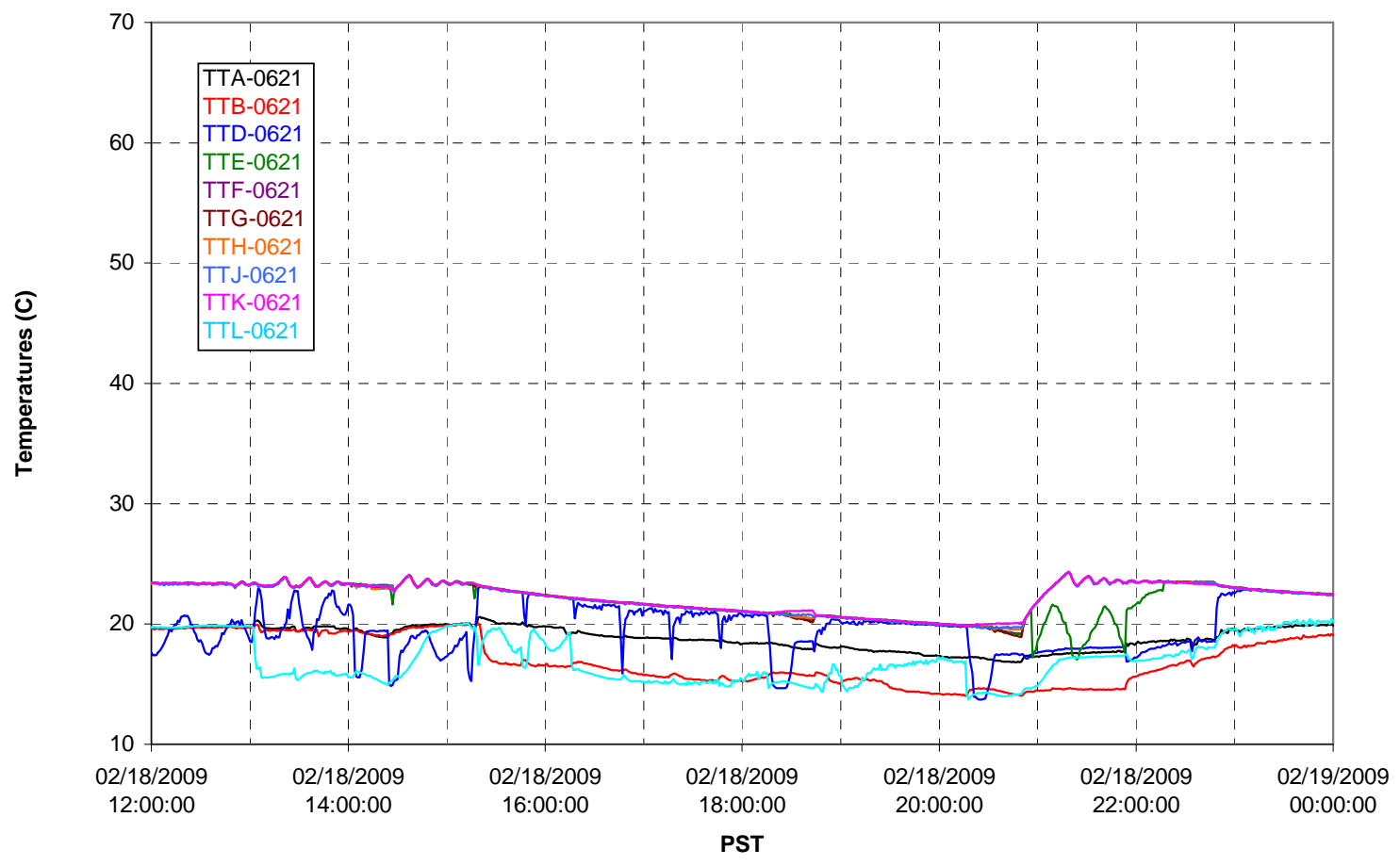


T02A temperatures

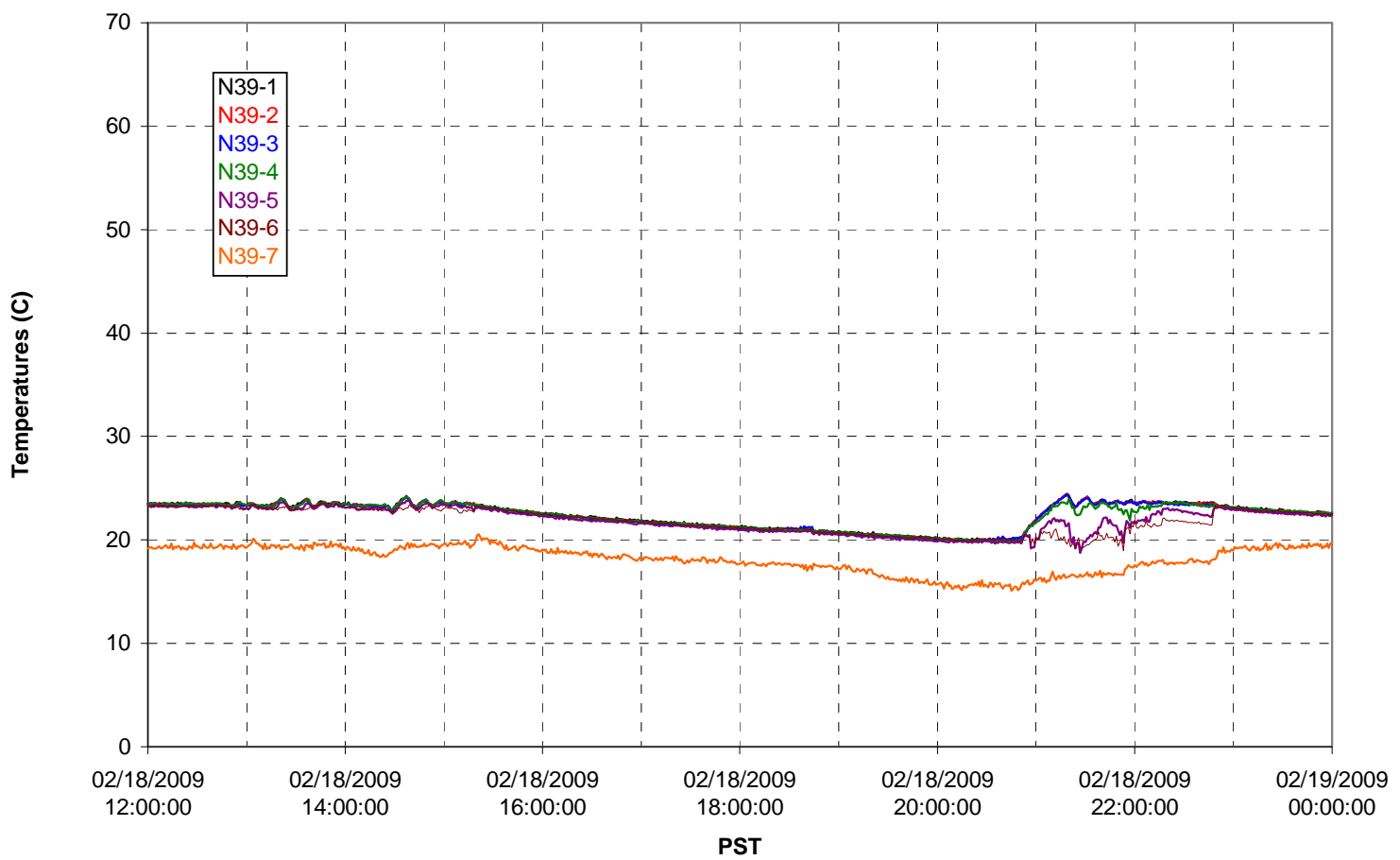

T02A temperatures

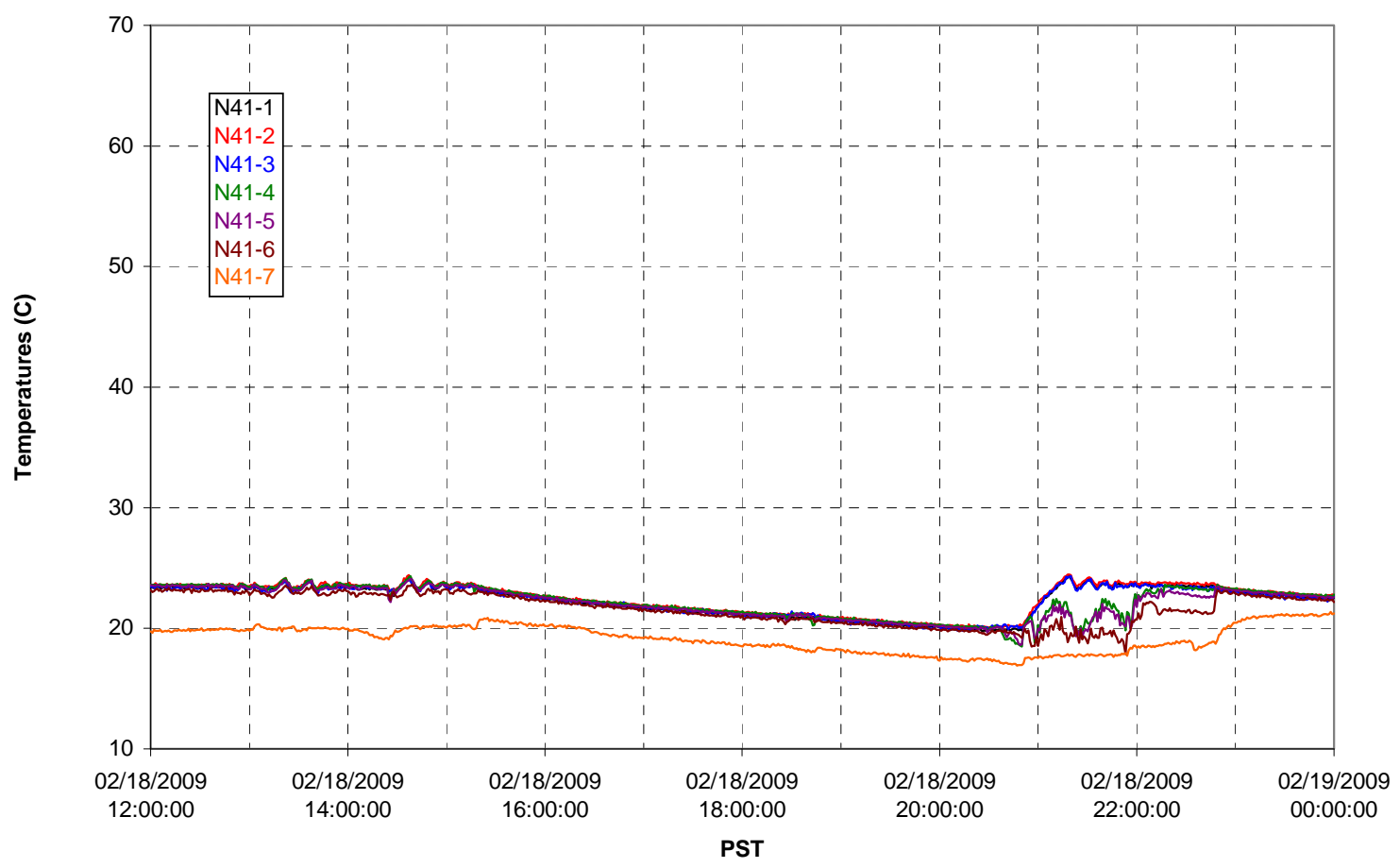


T02A temperatures

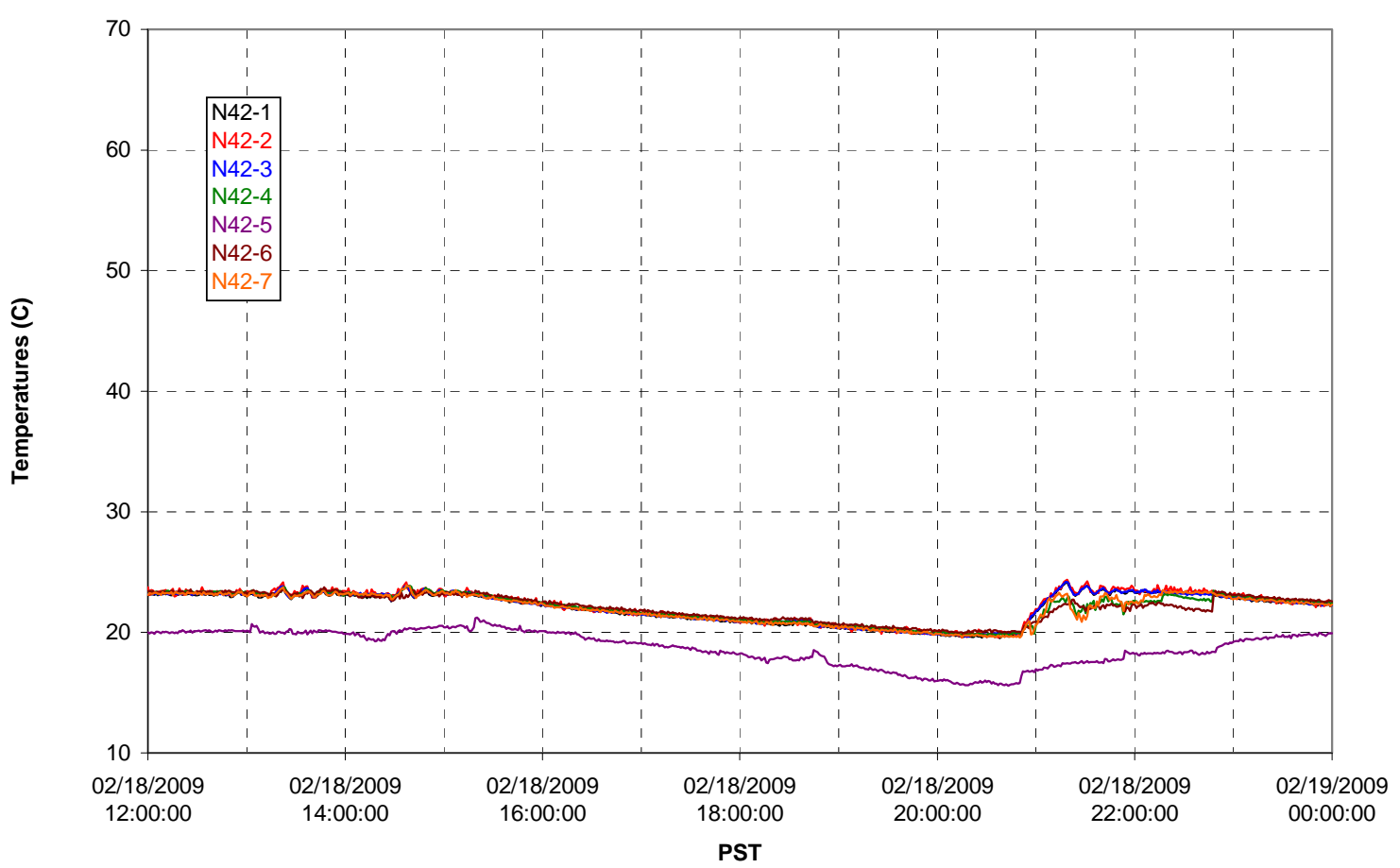

T02A temperatures

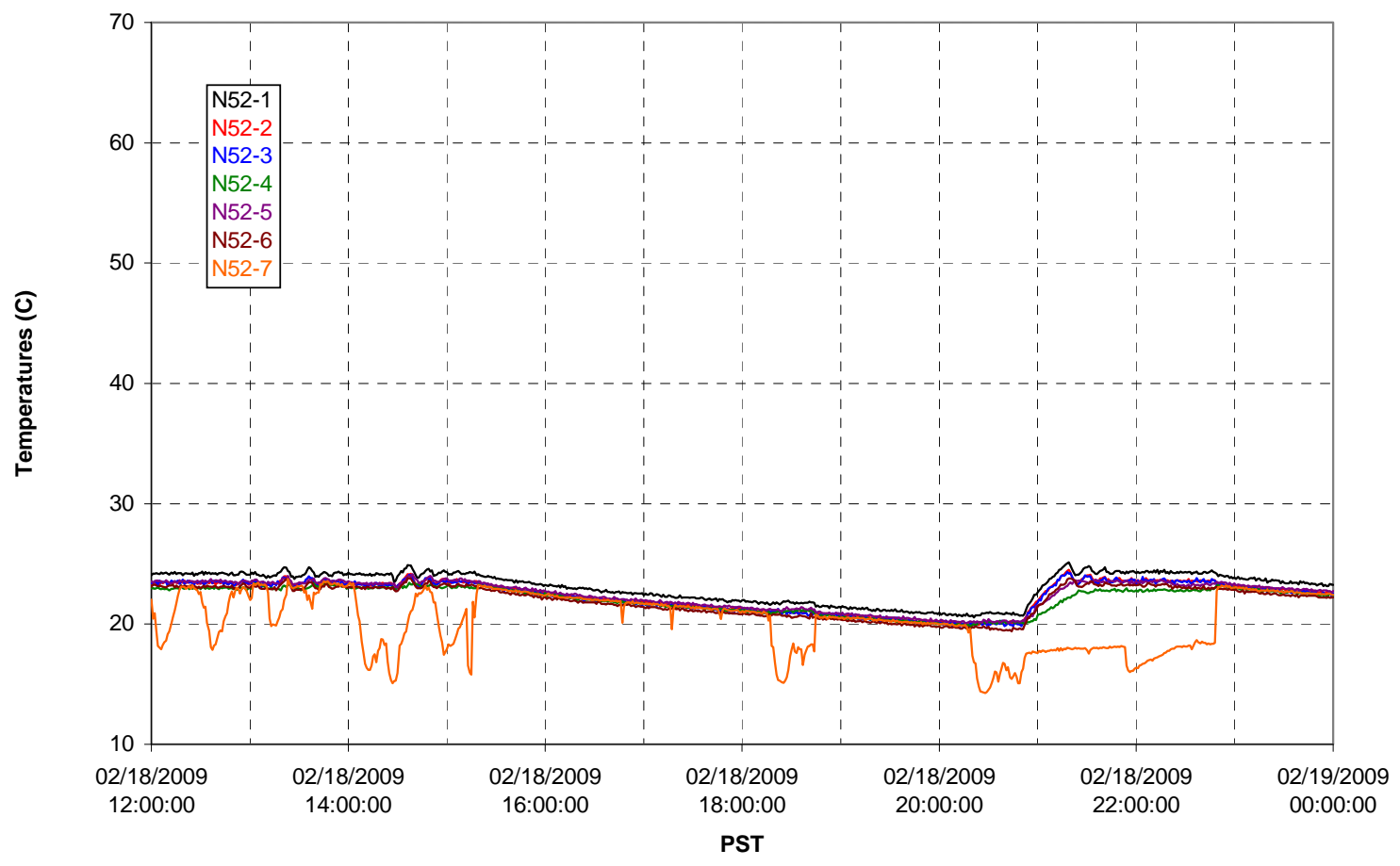


T02A Heating and Cooling

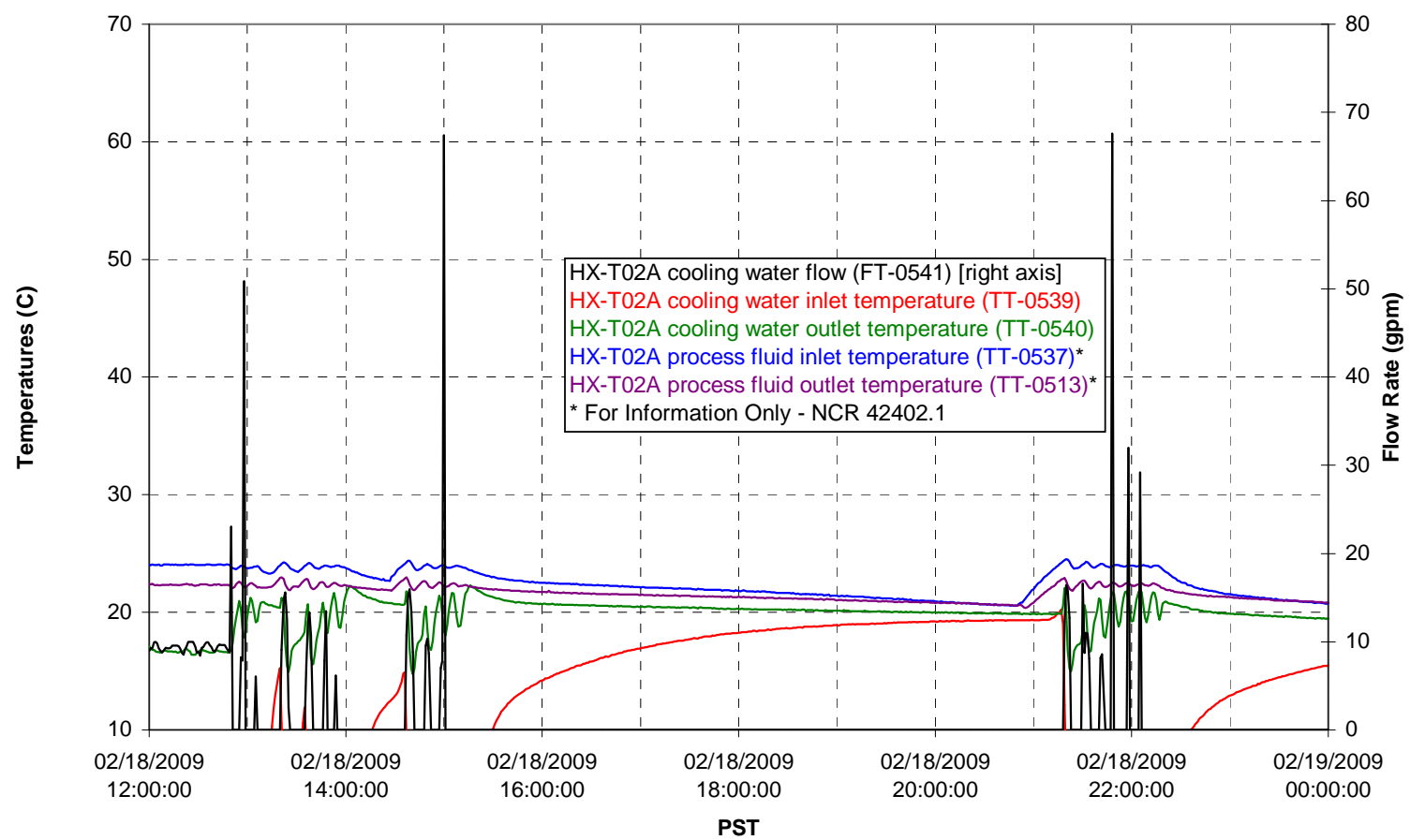

Pump Operation

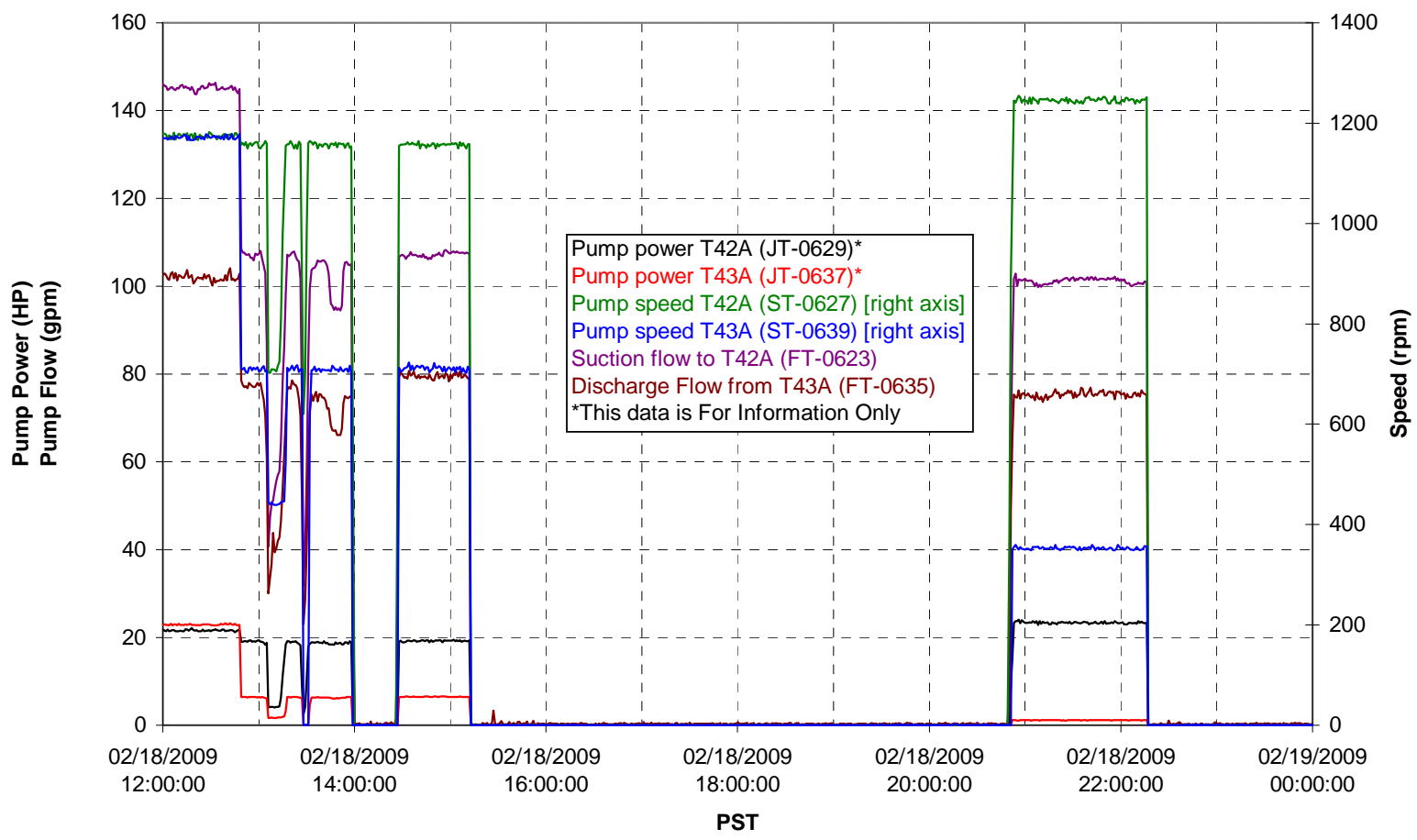


Pulsepot UFP-PP-T01A

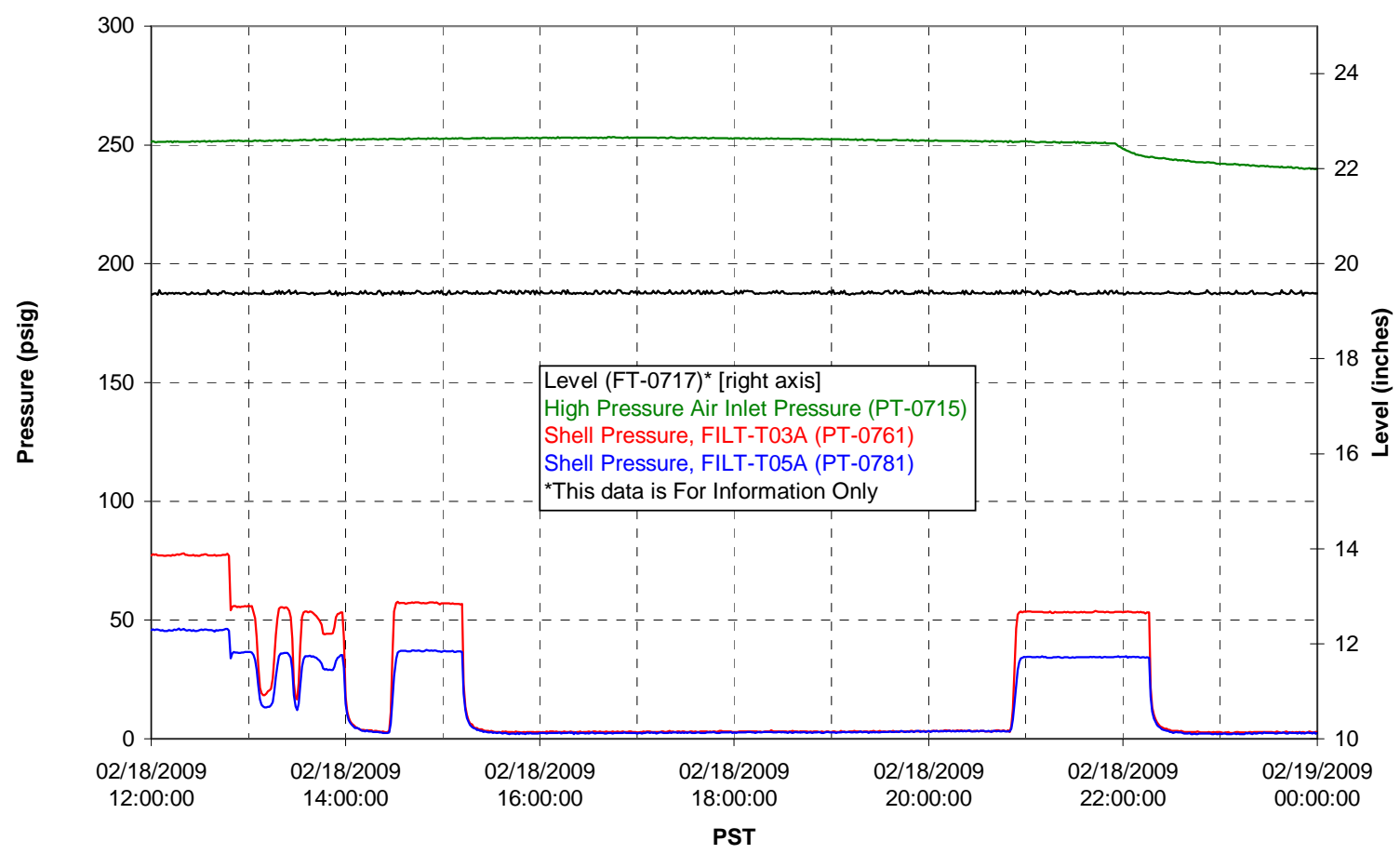

Pulsepot UFP-PP-T02A

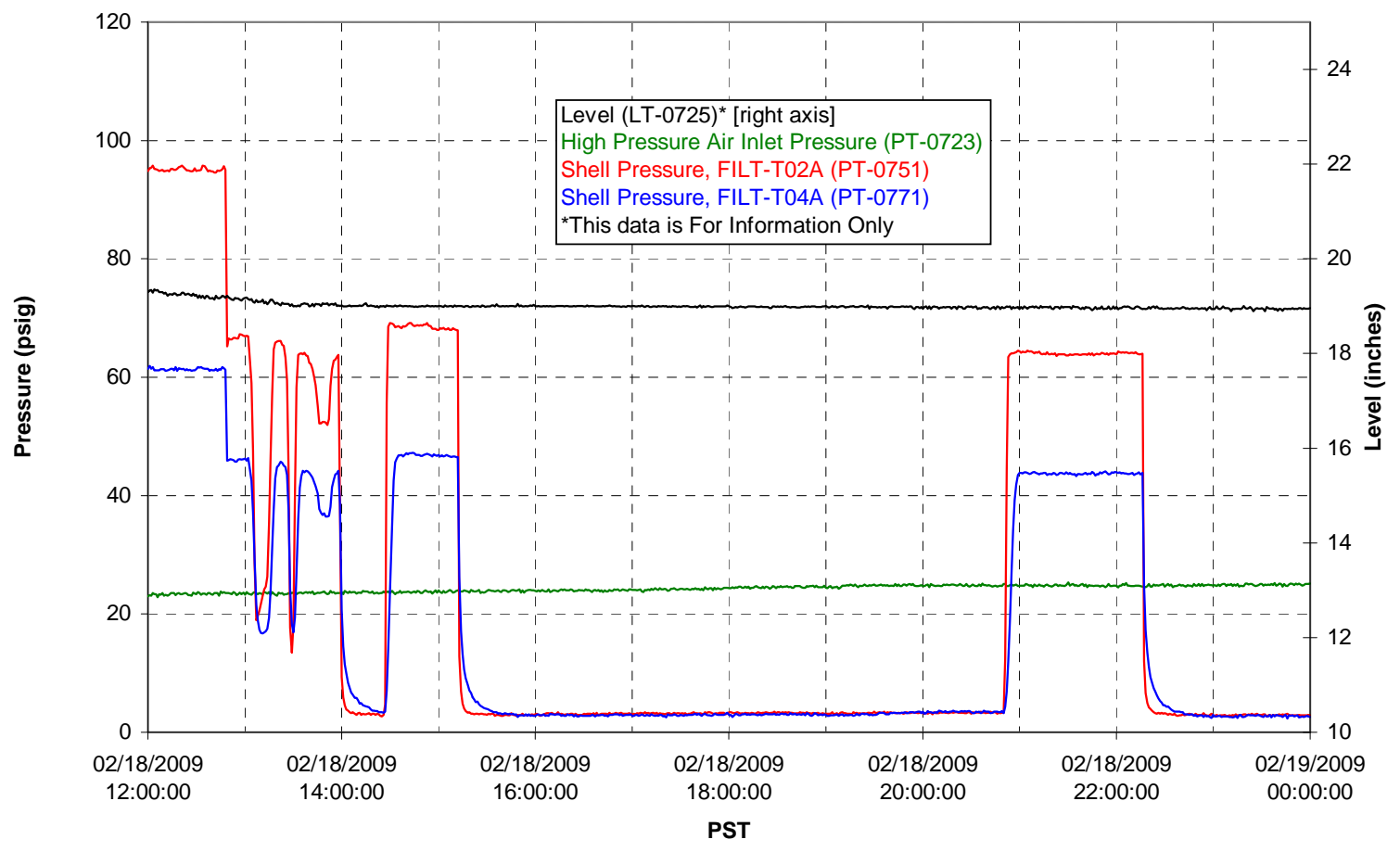


Pulsepot UFP-PP-T03A

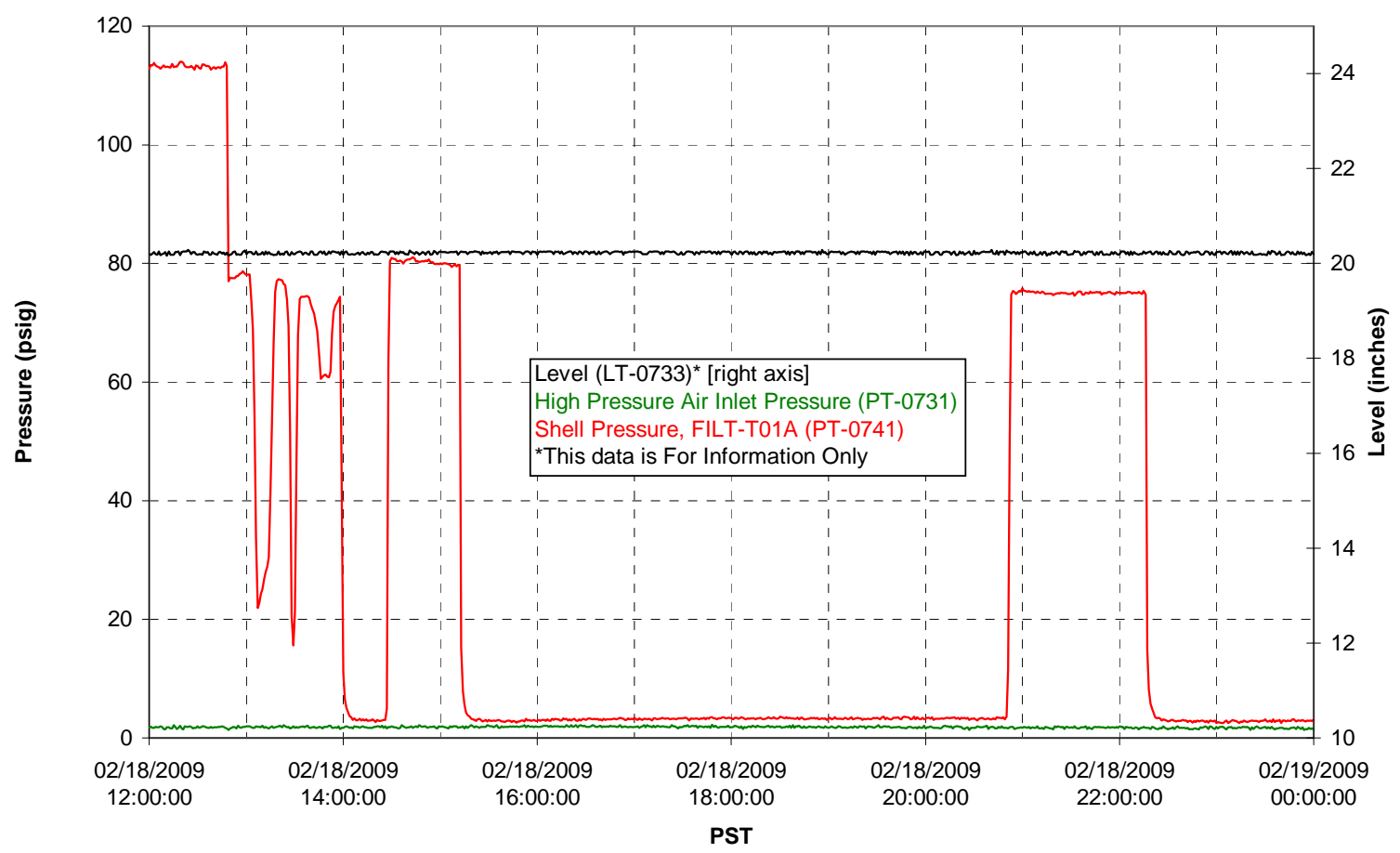

Pulsepot Levels

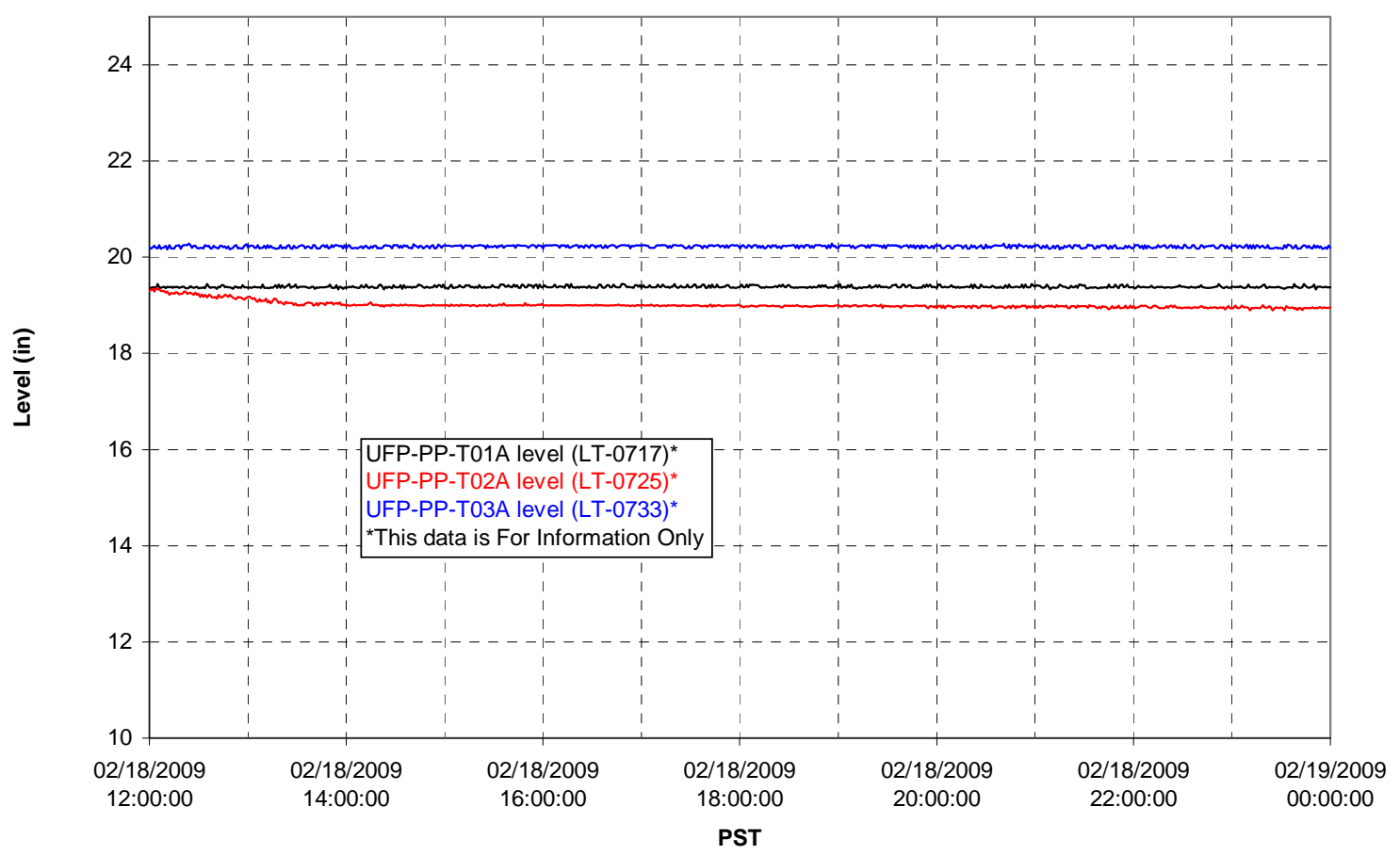


Filter UFP-FILT-T01A

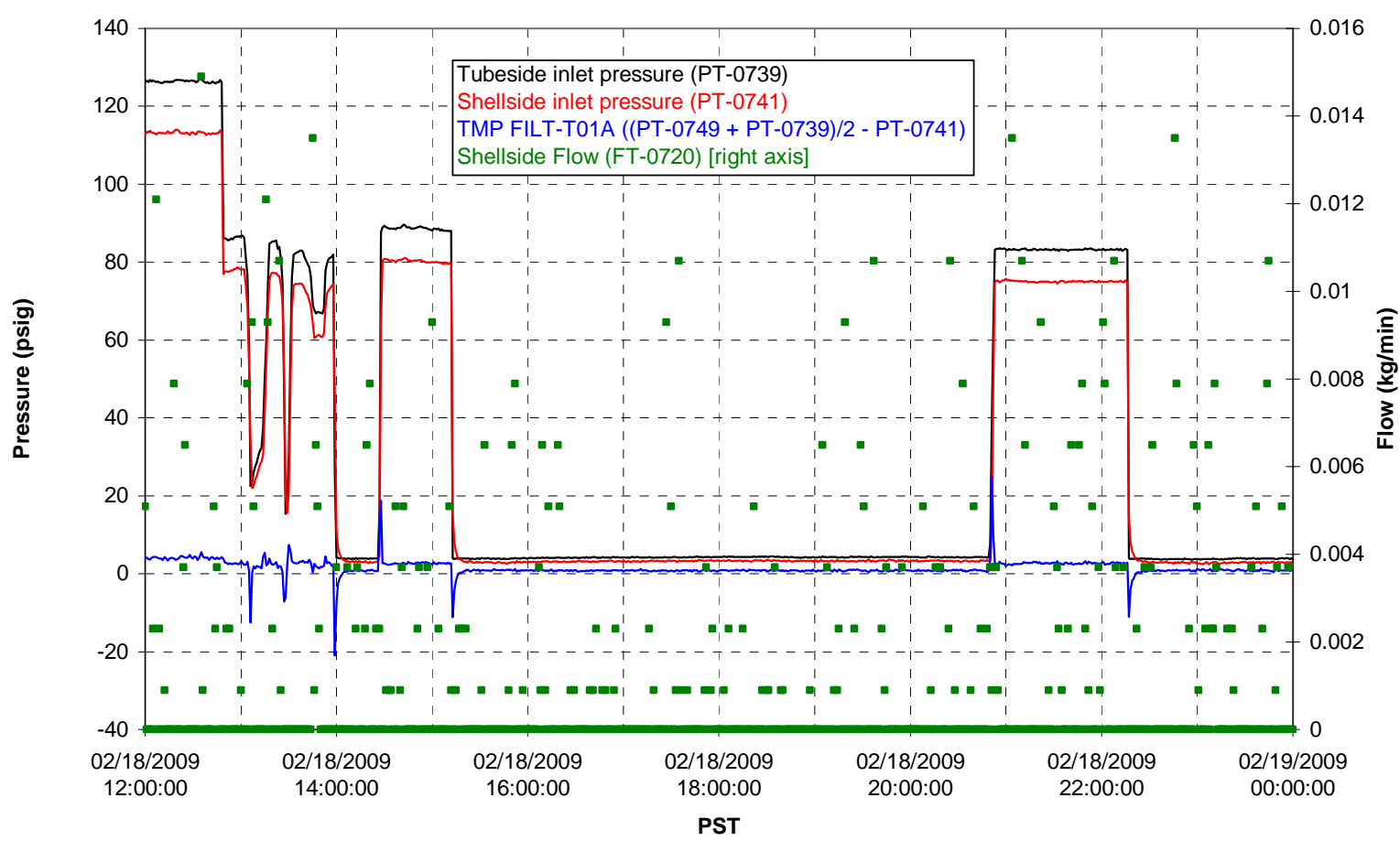

Filter UFP-FILT-T02A

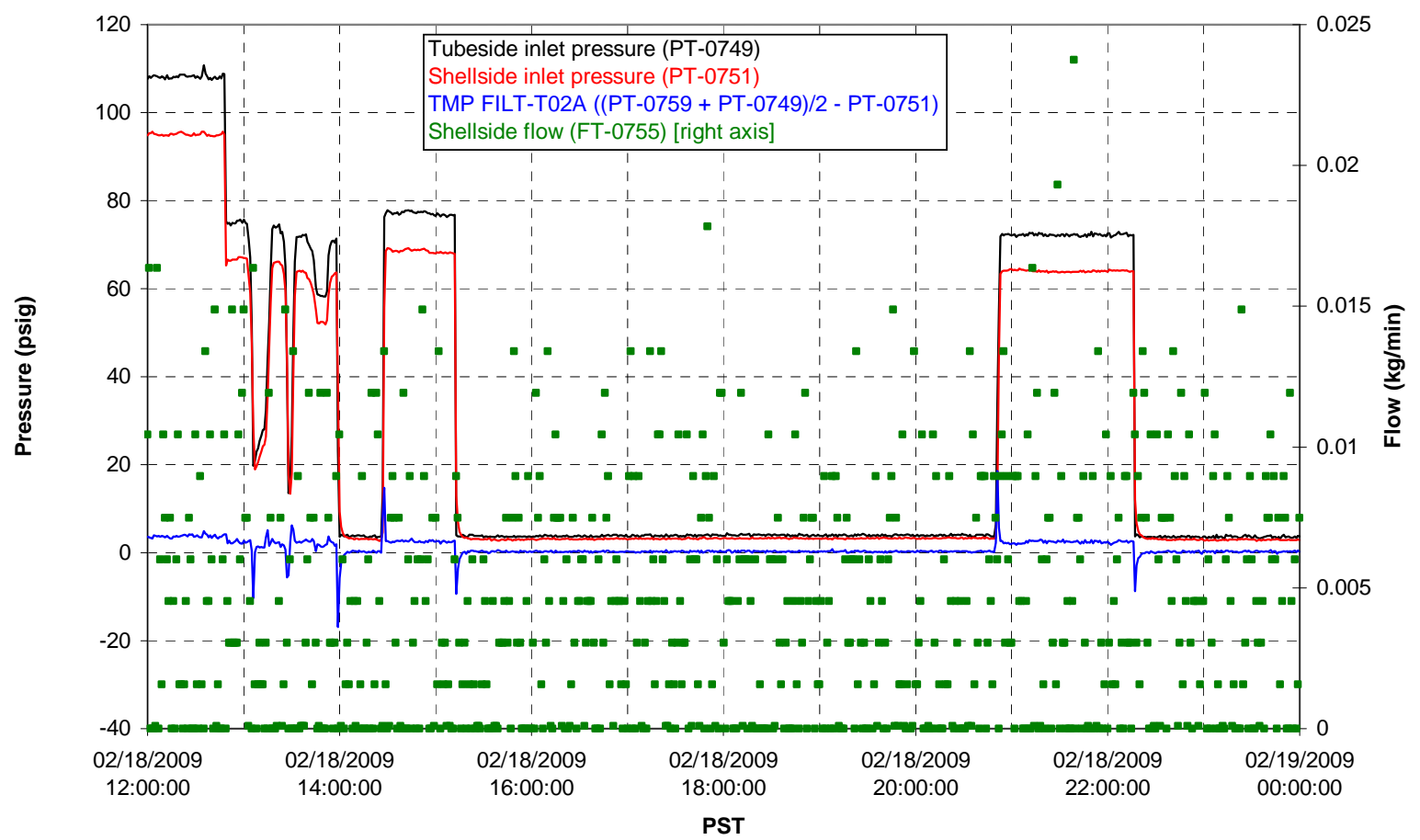


Filter UFP-FILT-T03A

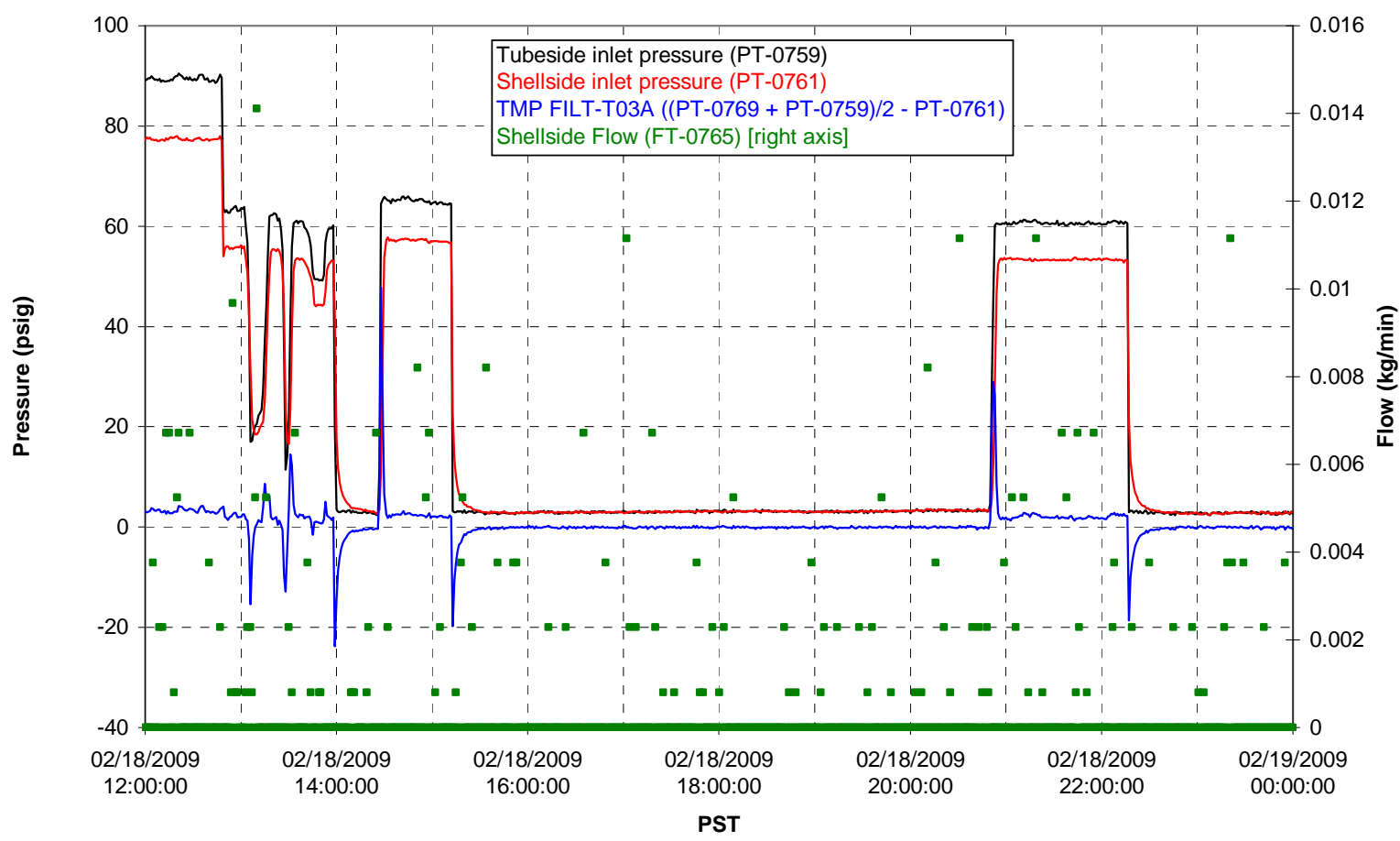

Filter UFP-FILT-T04A

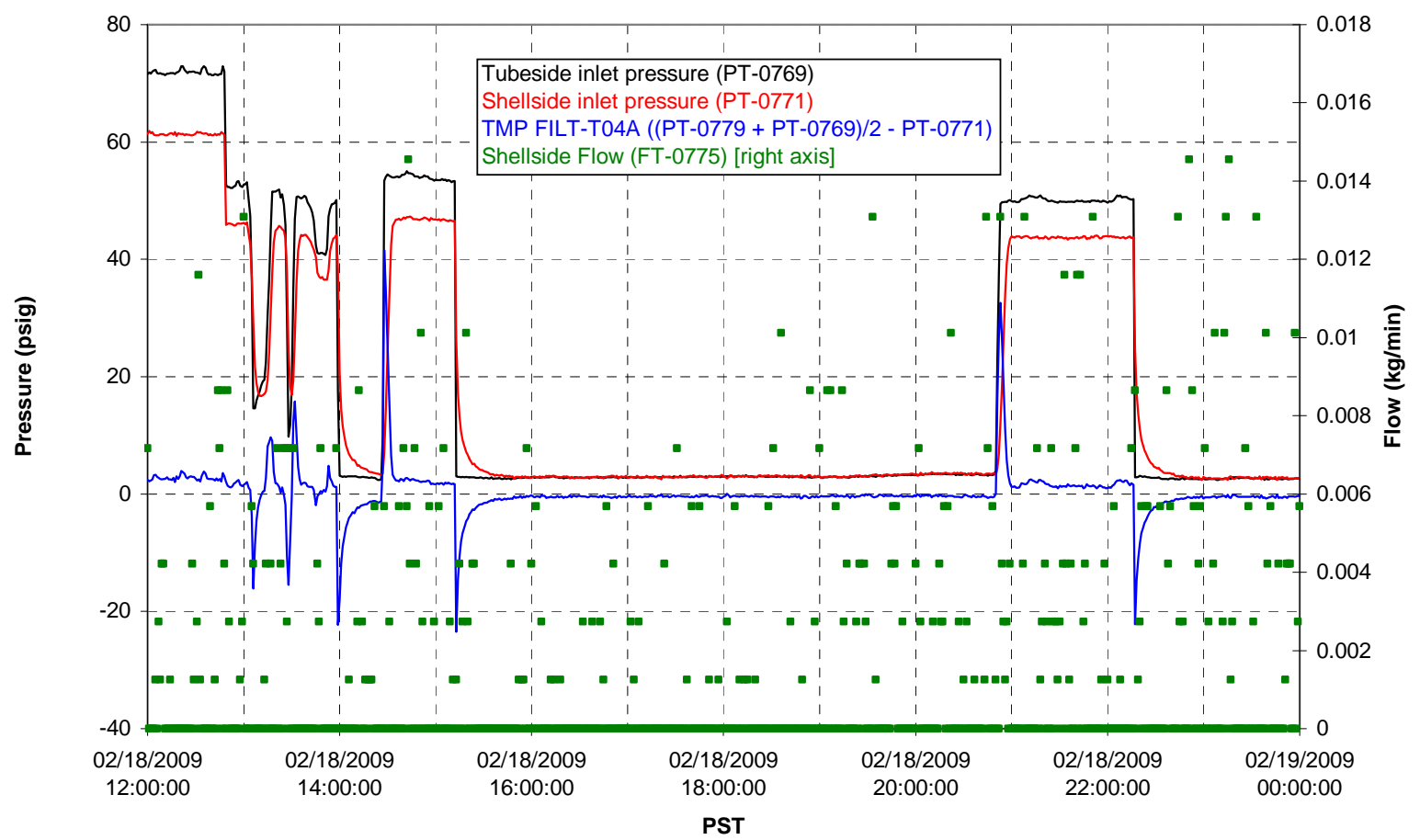


Filter UFP-FILT-T05A

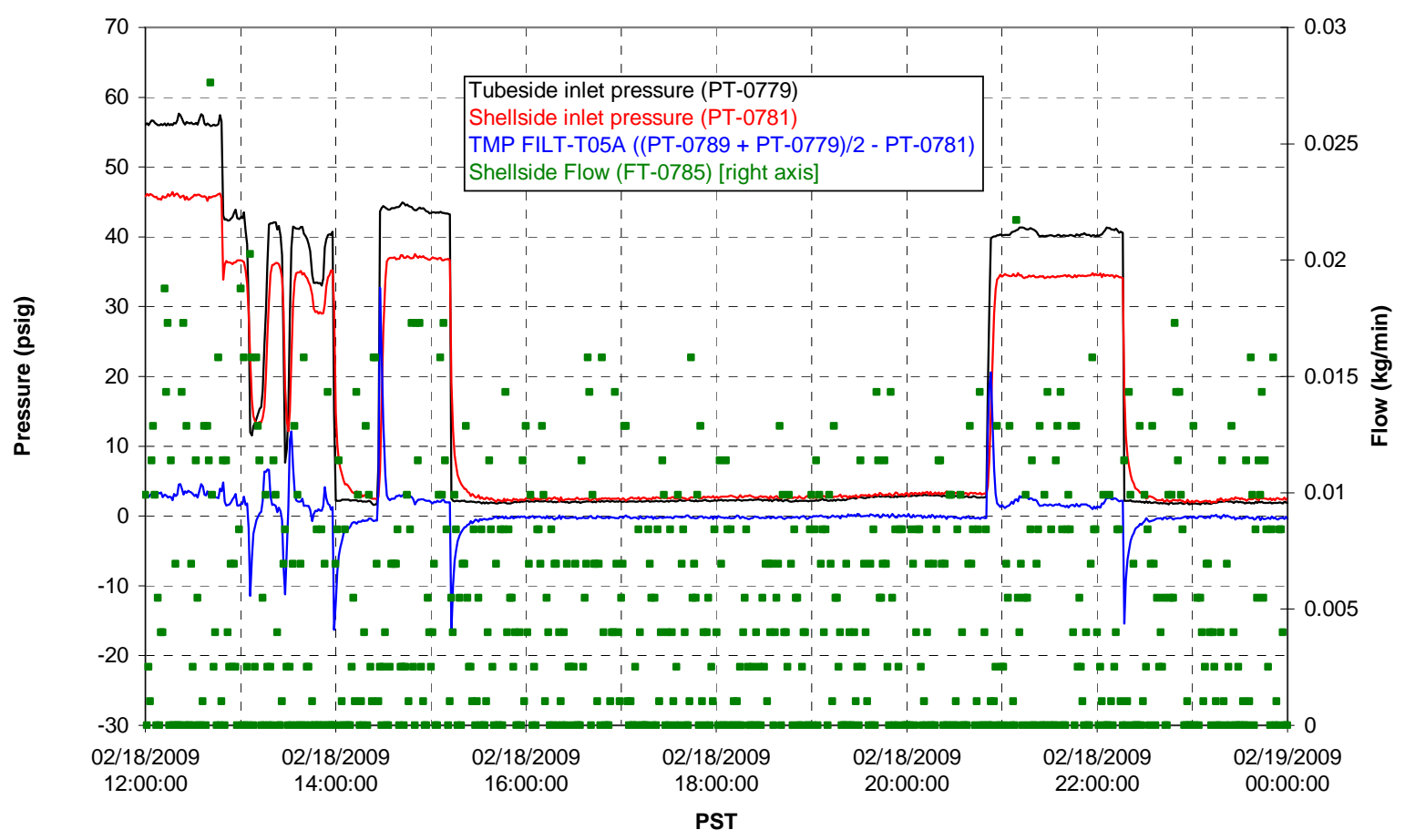

Chemical Flow

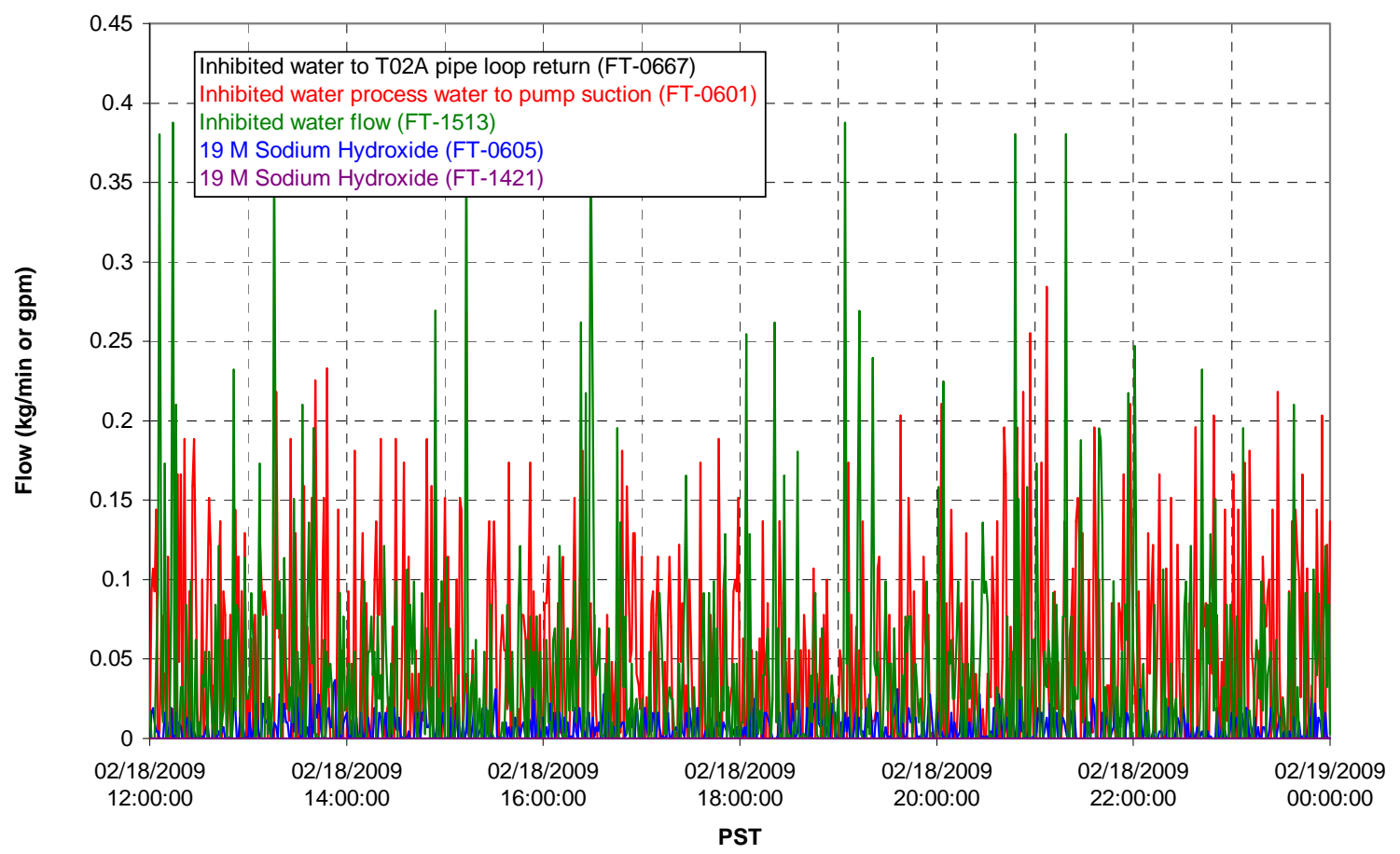




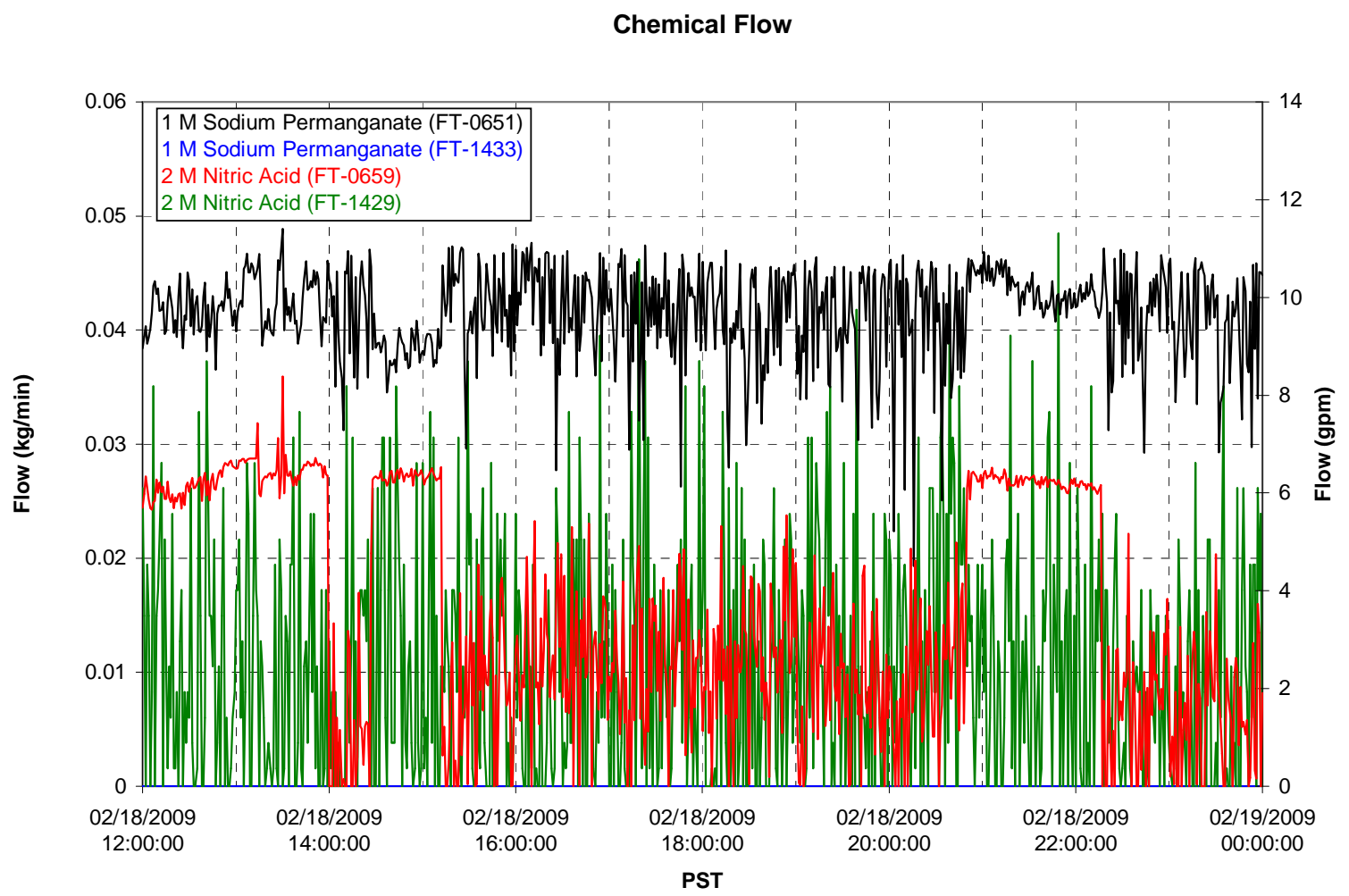

Air Flows

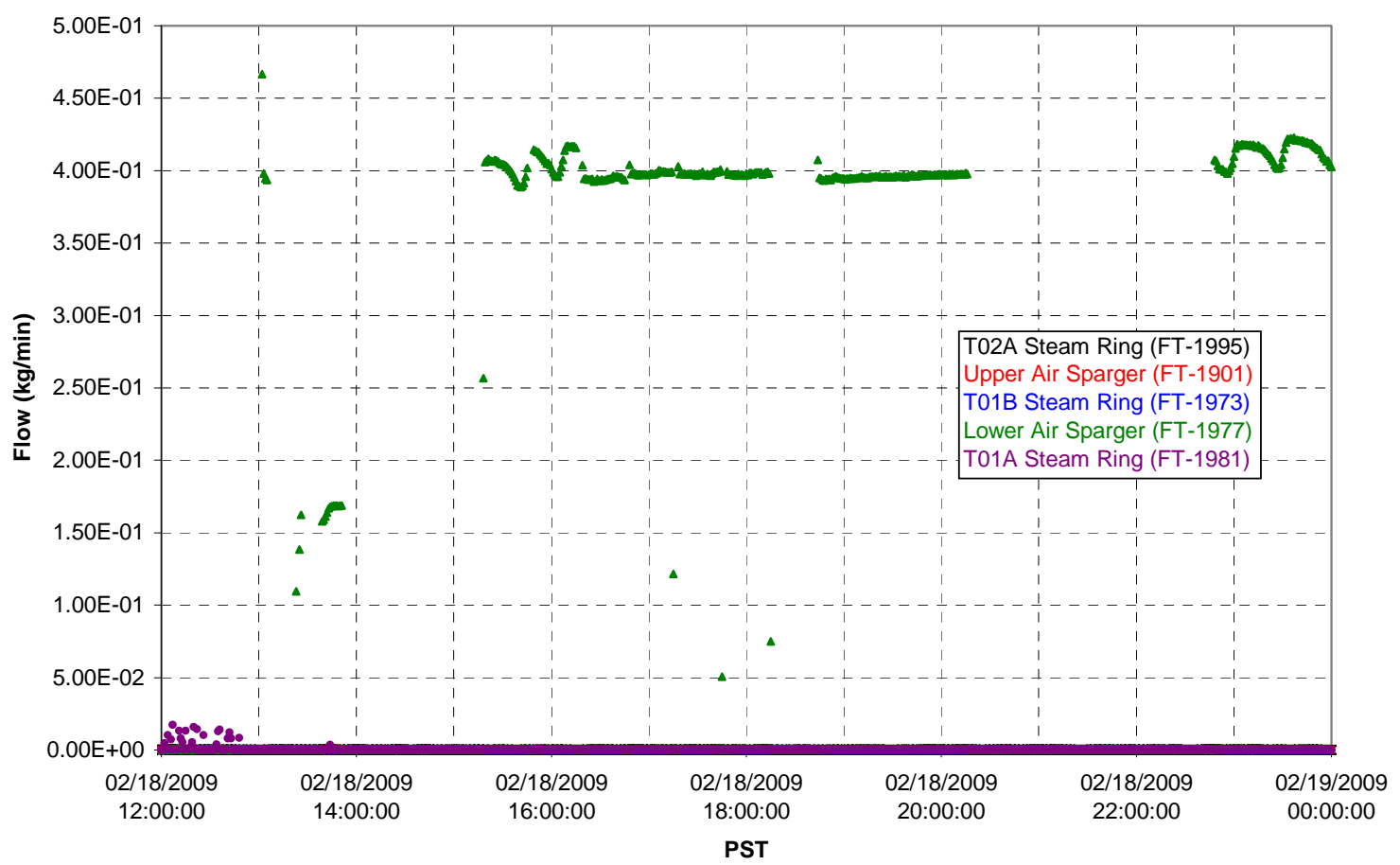


T02A Steam

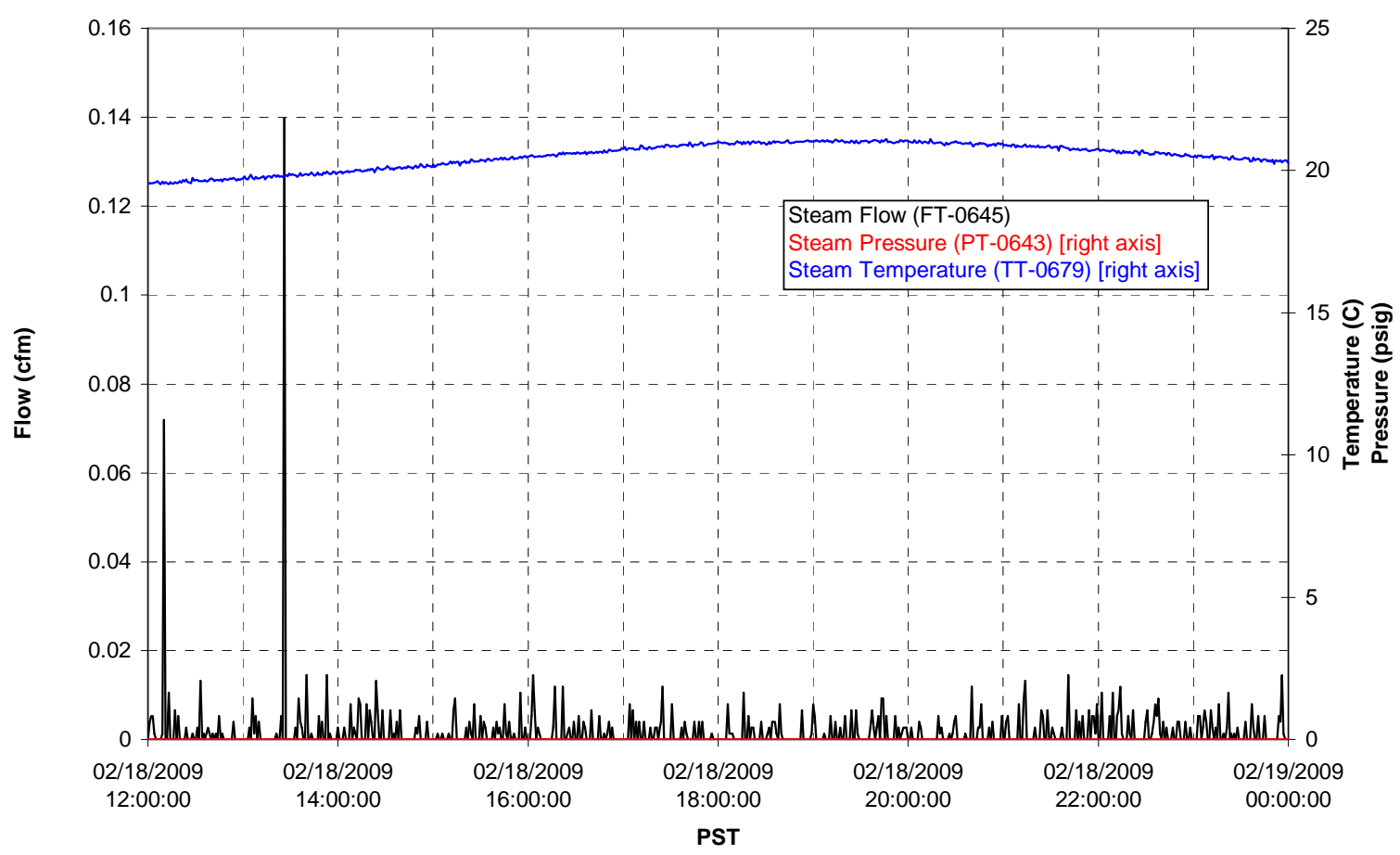

T01A Steam

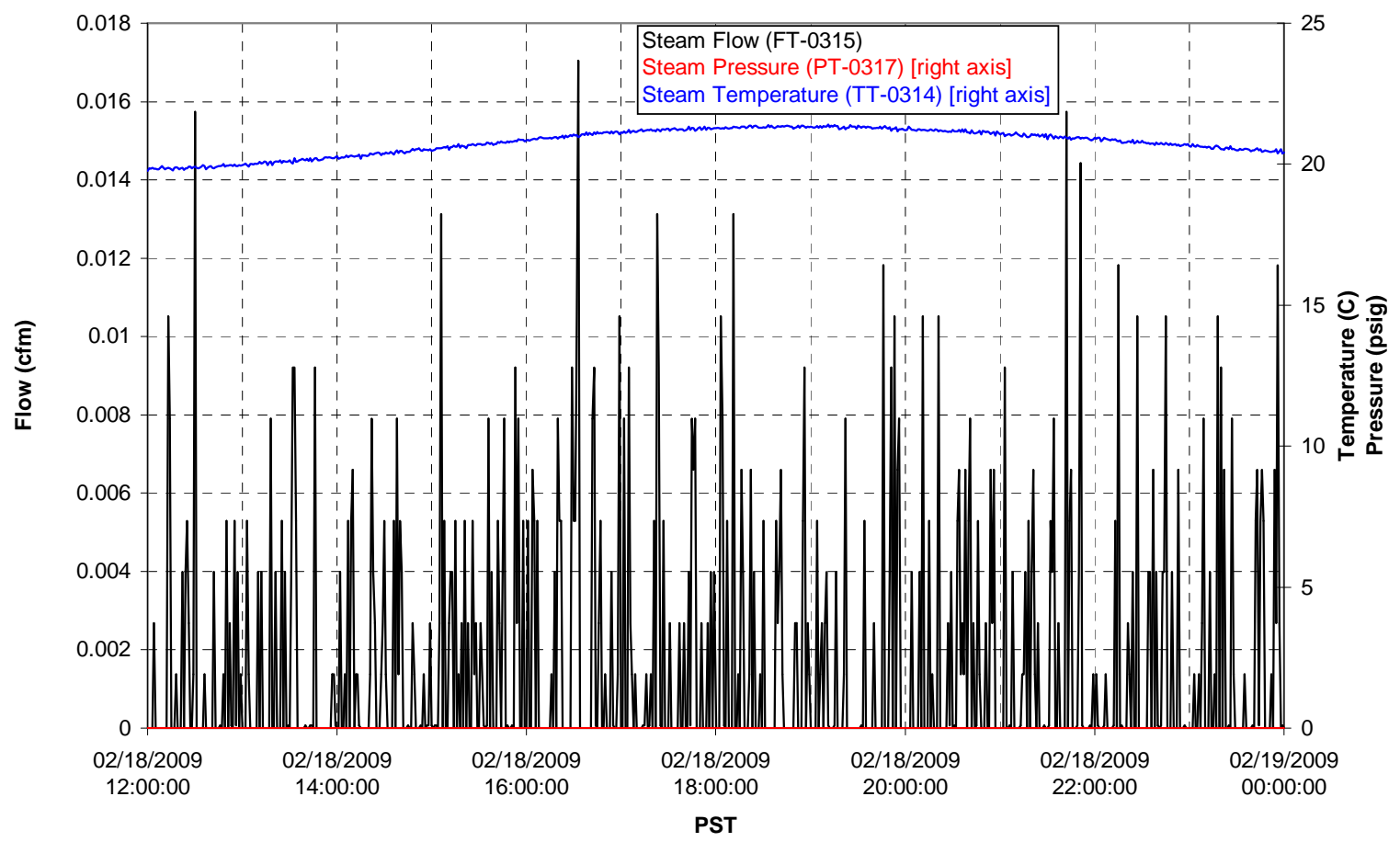


T01B Steam

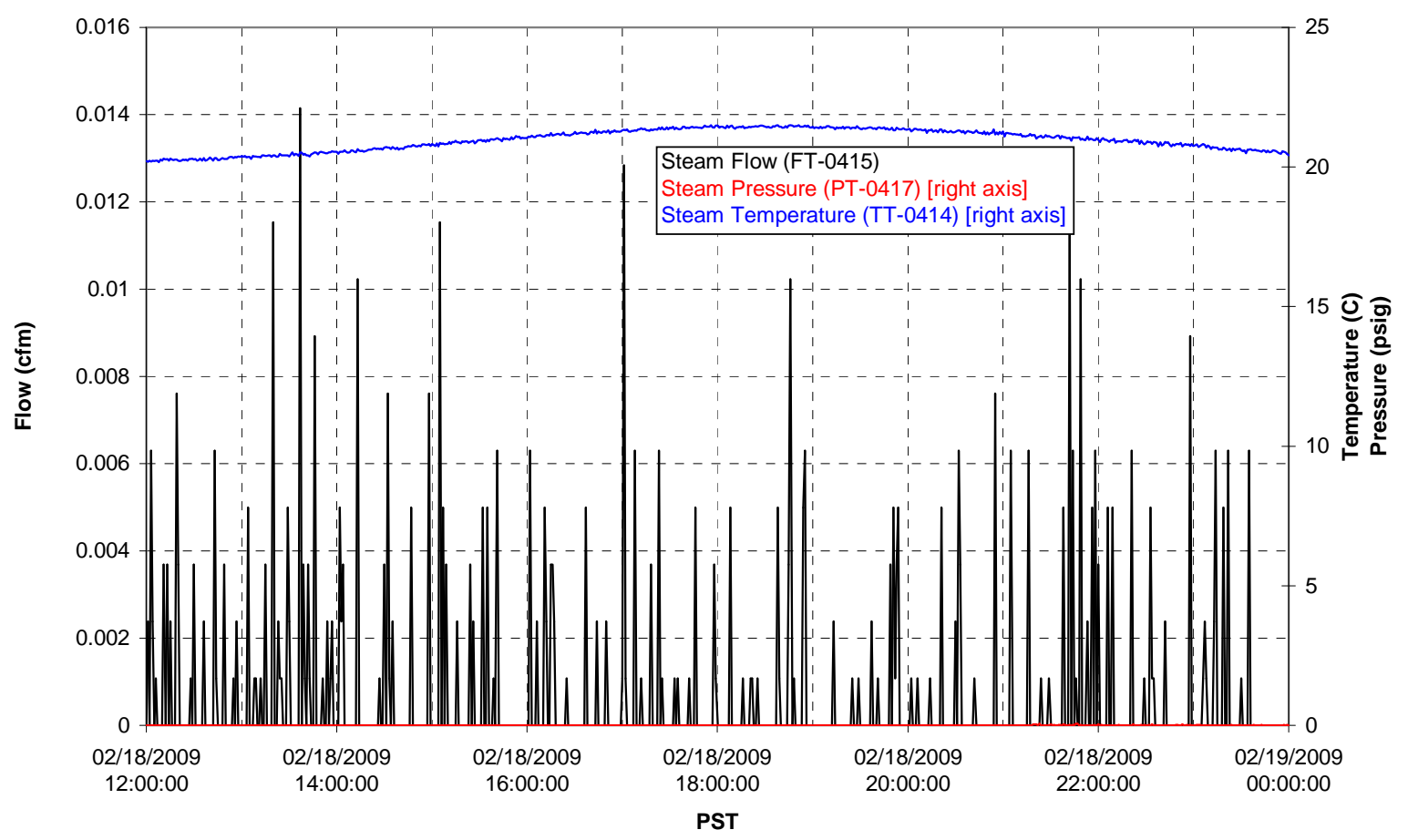




\section{Integrated Test A Data Plots 02/19/09 00:00 - 02/19/09 12:00}


T01A level

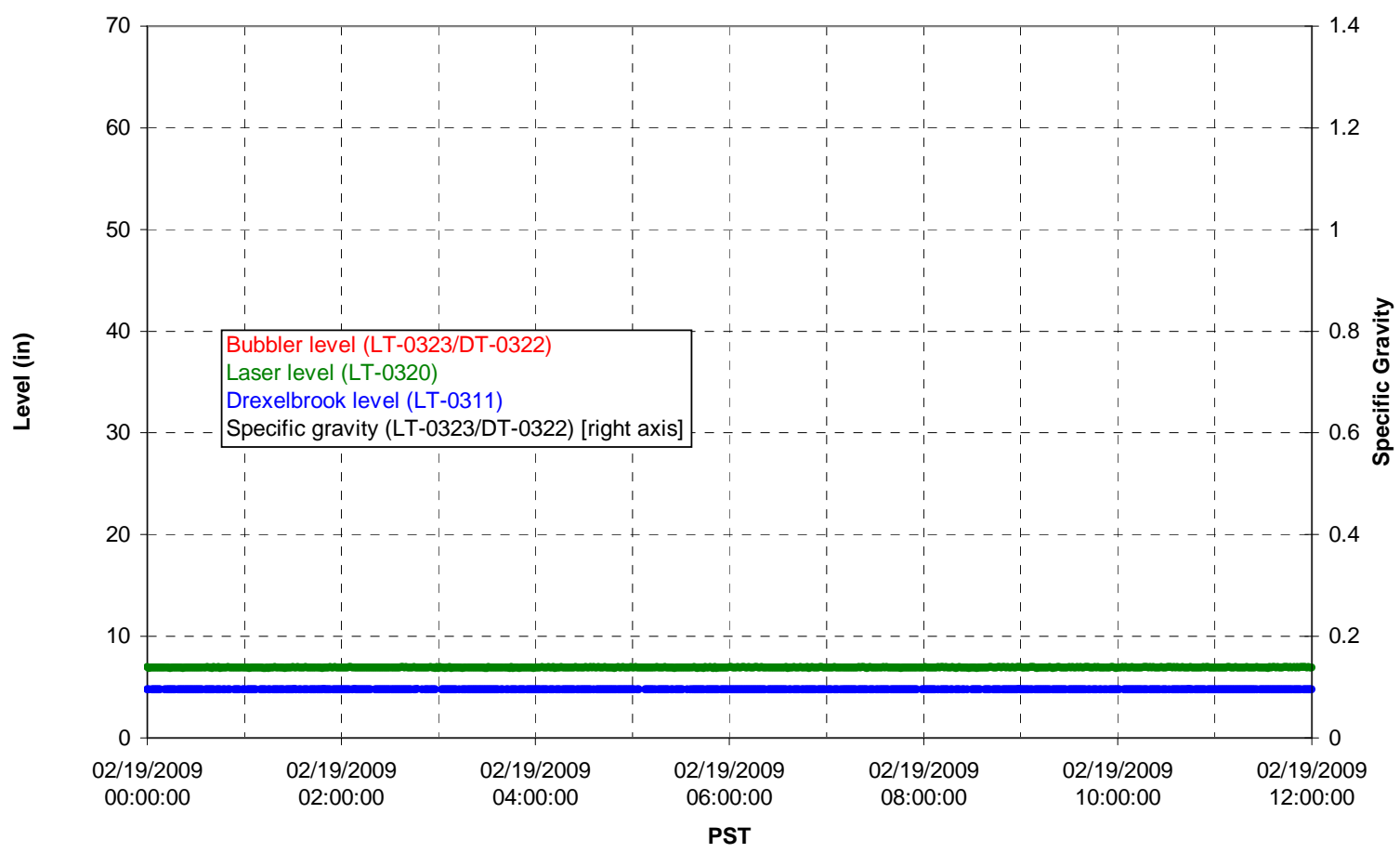

T01A temperatures

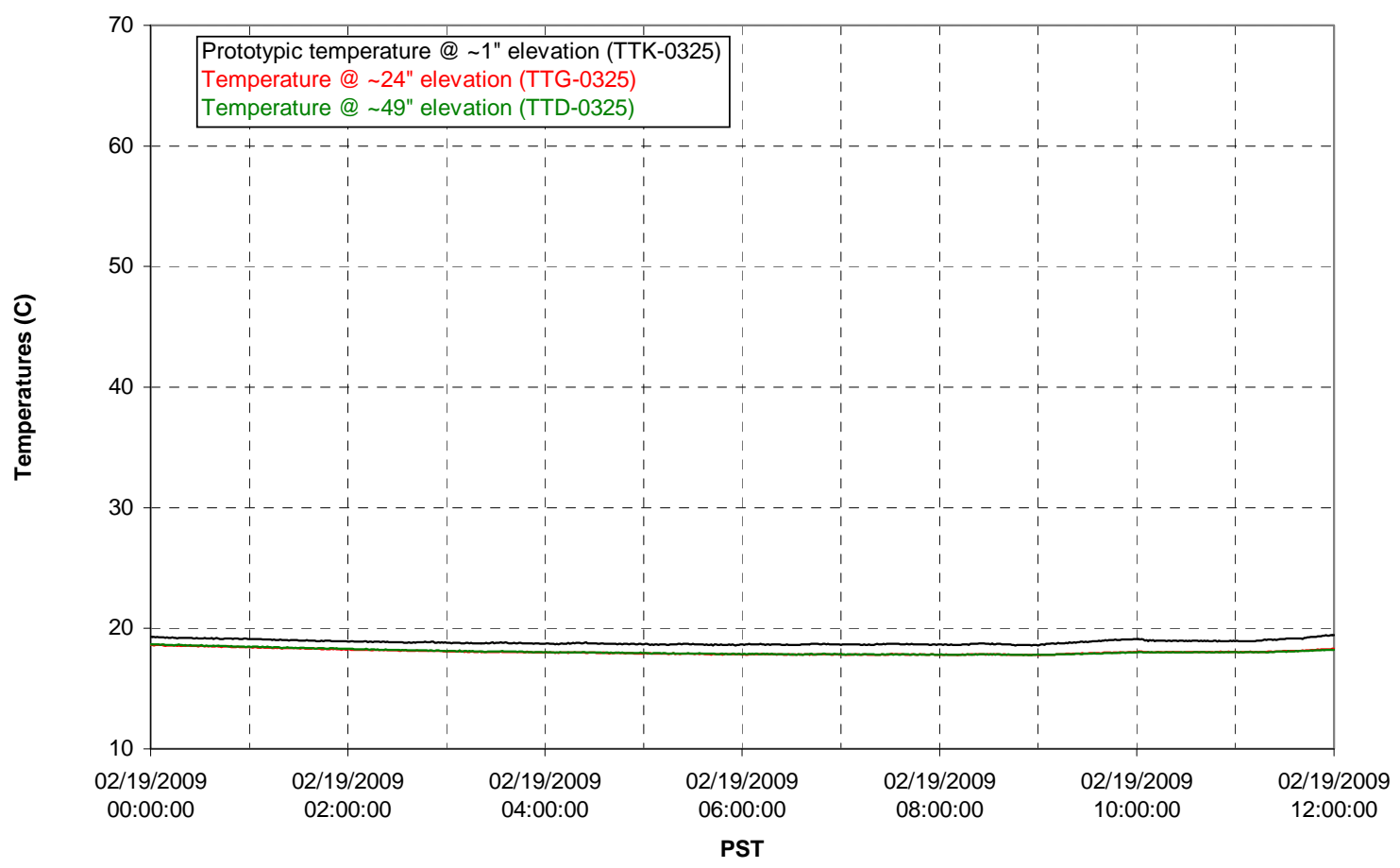


T01B level

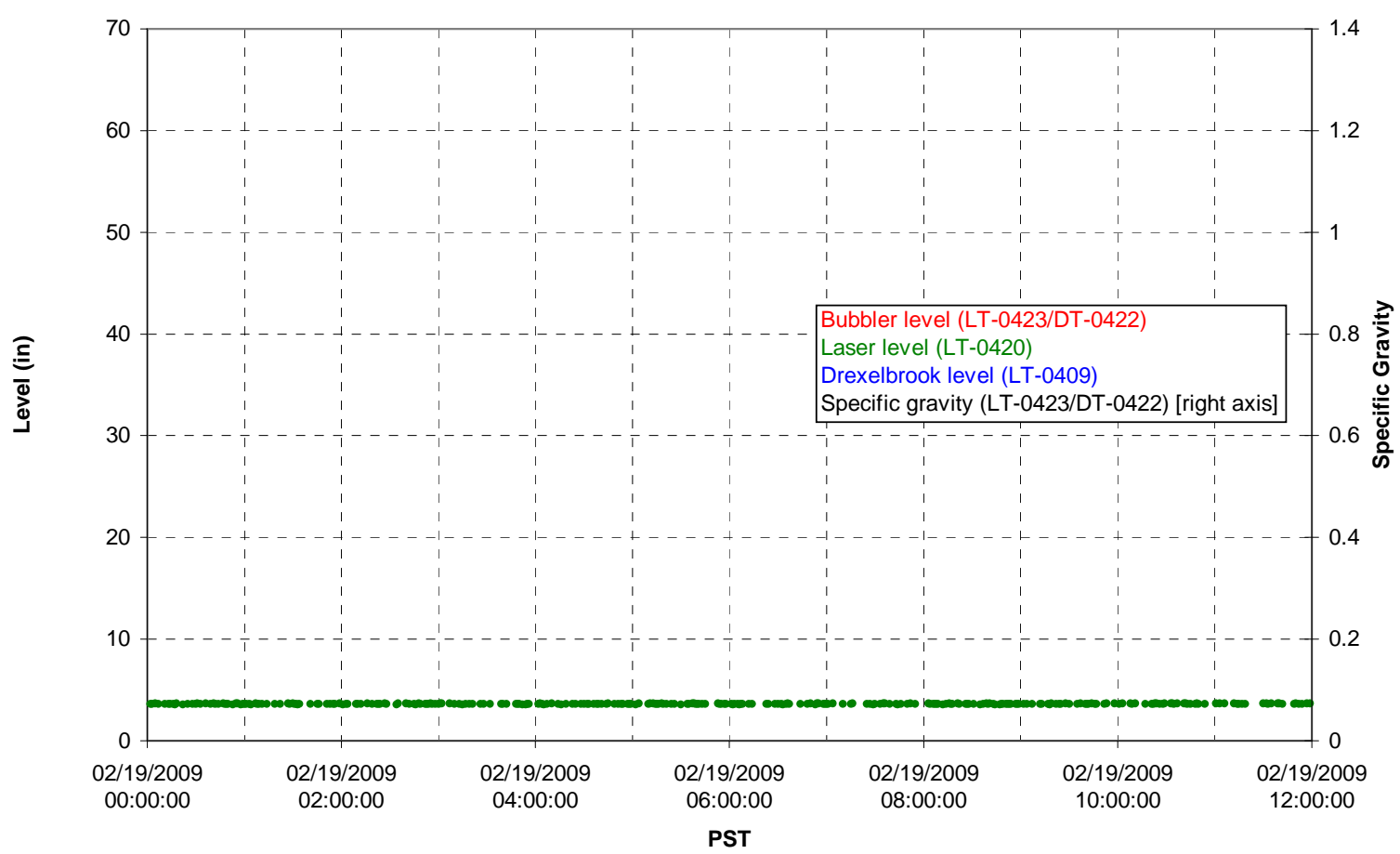

T01B temperatures

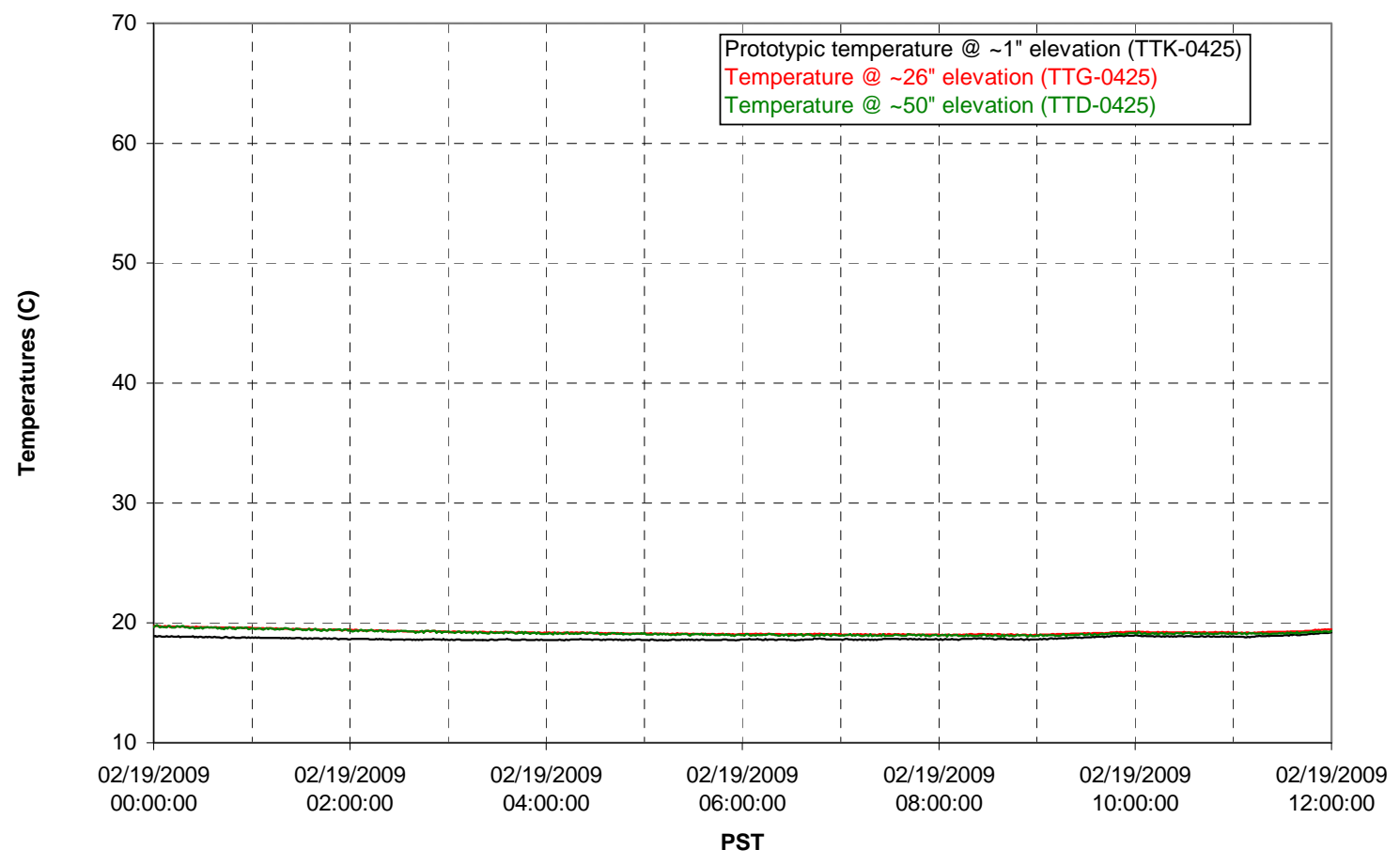


T02A level

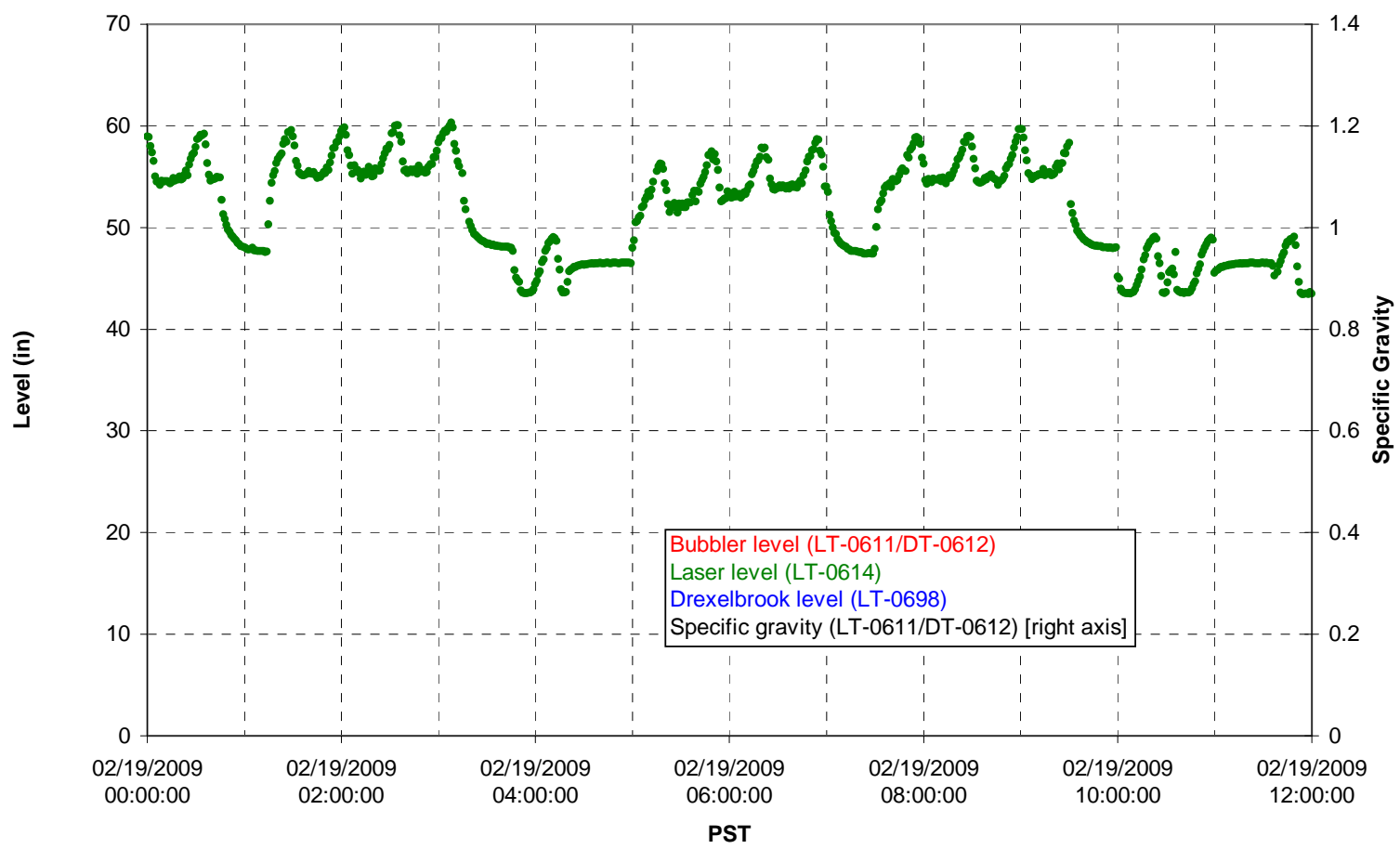

T02A temperatures

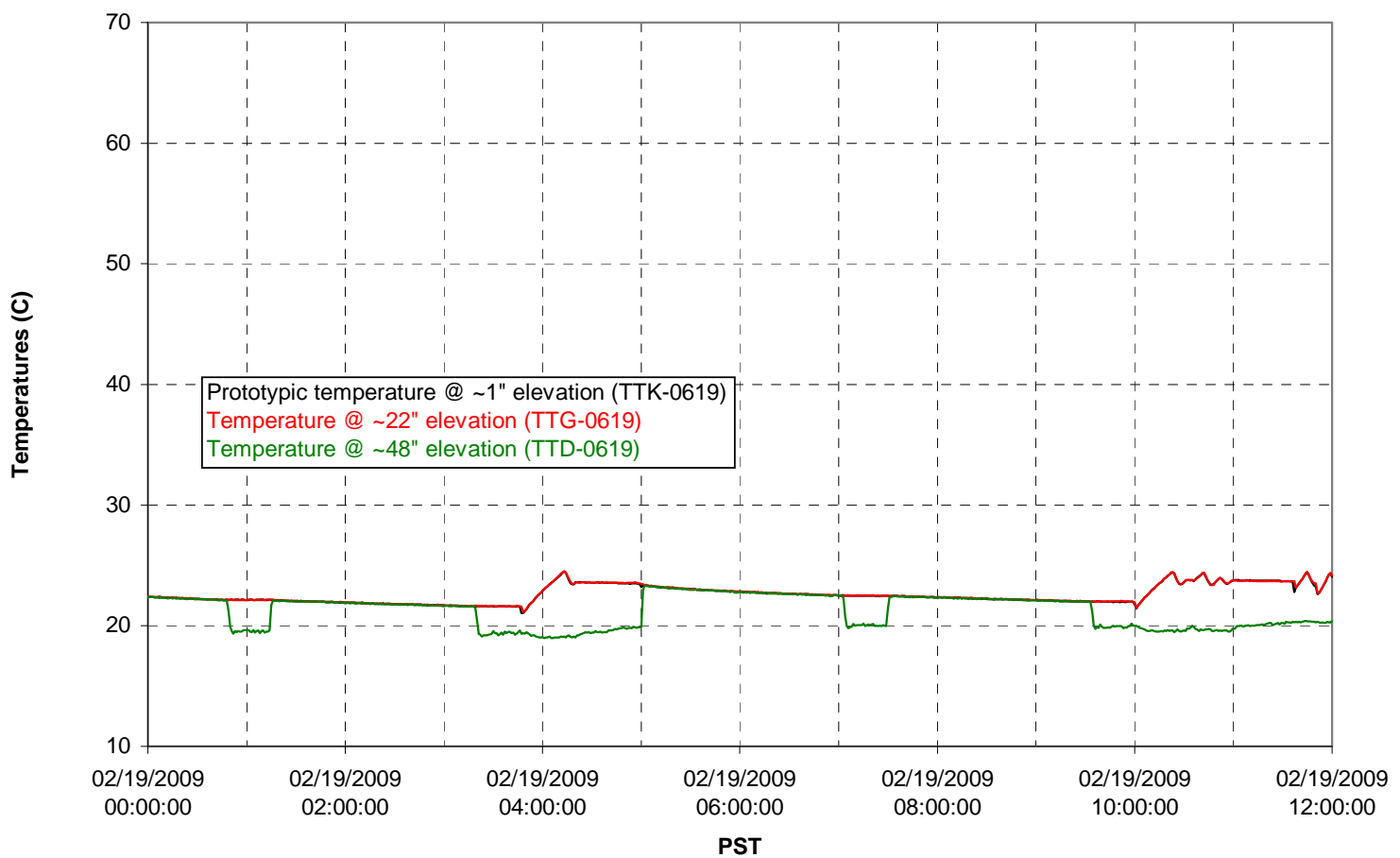


T02A and filter loop temperatures

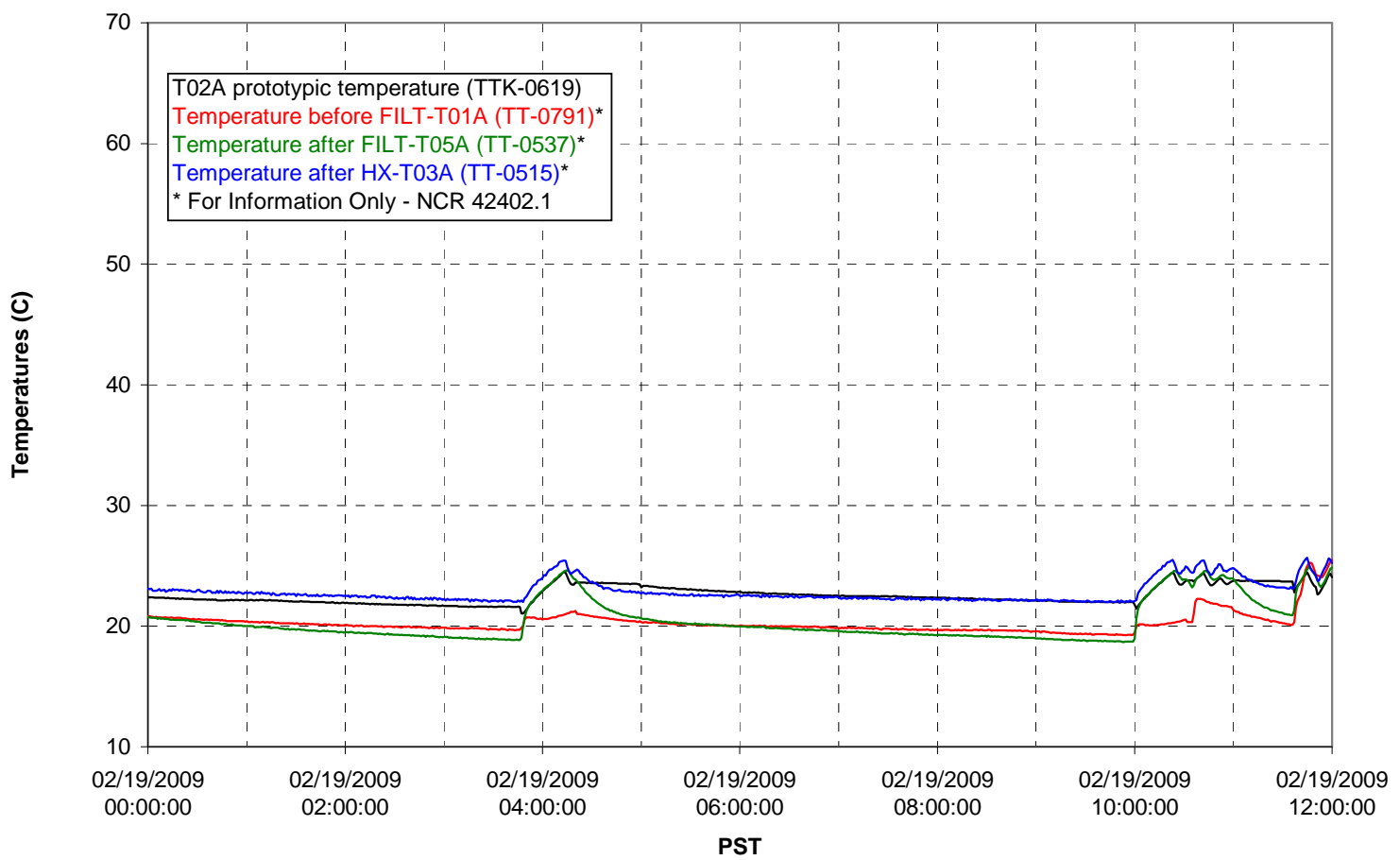

Pump Pressures and Flow

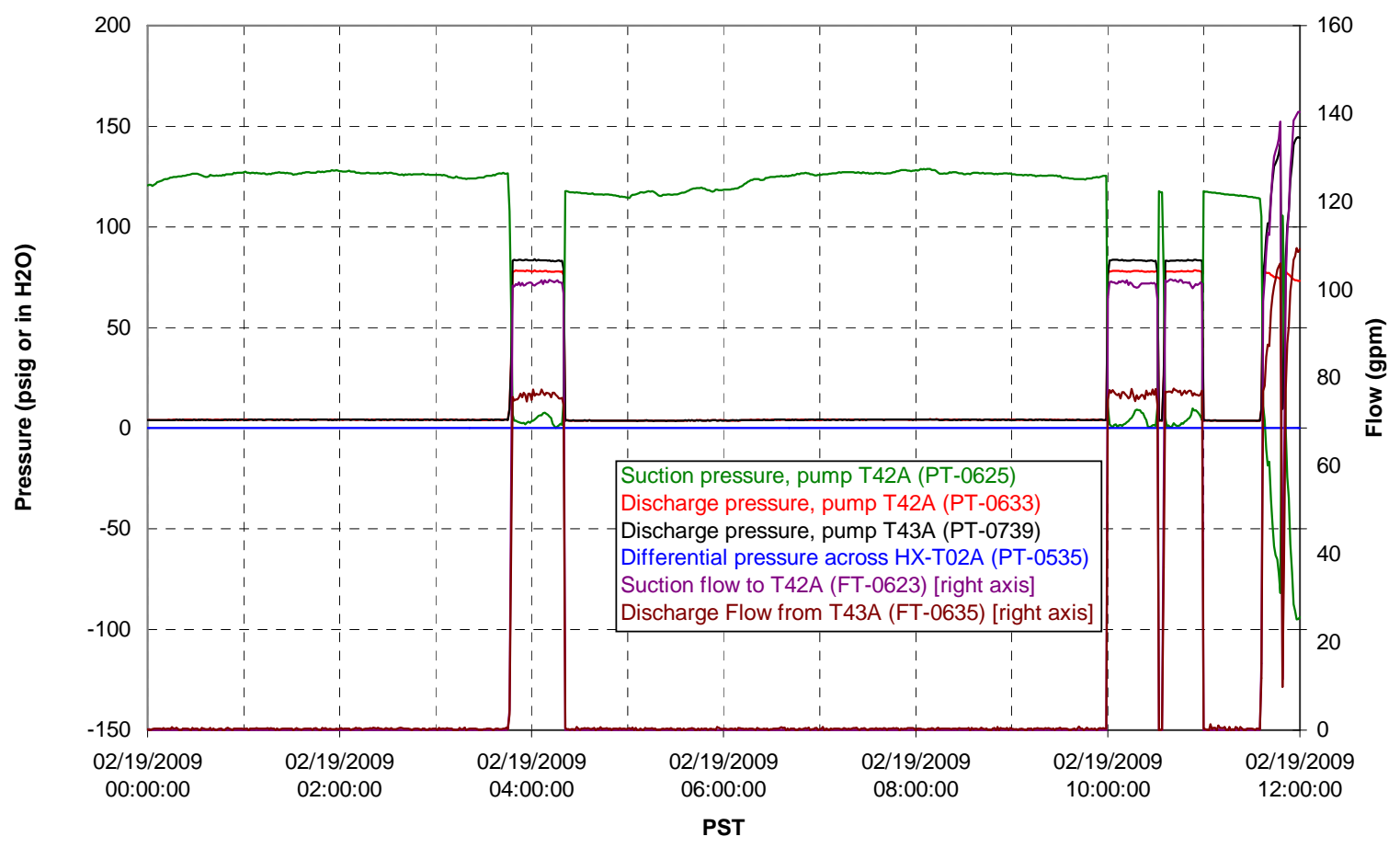


Axial pressure drop

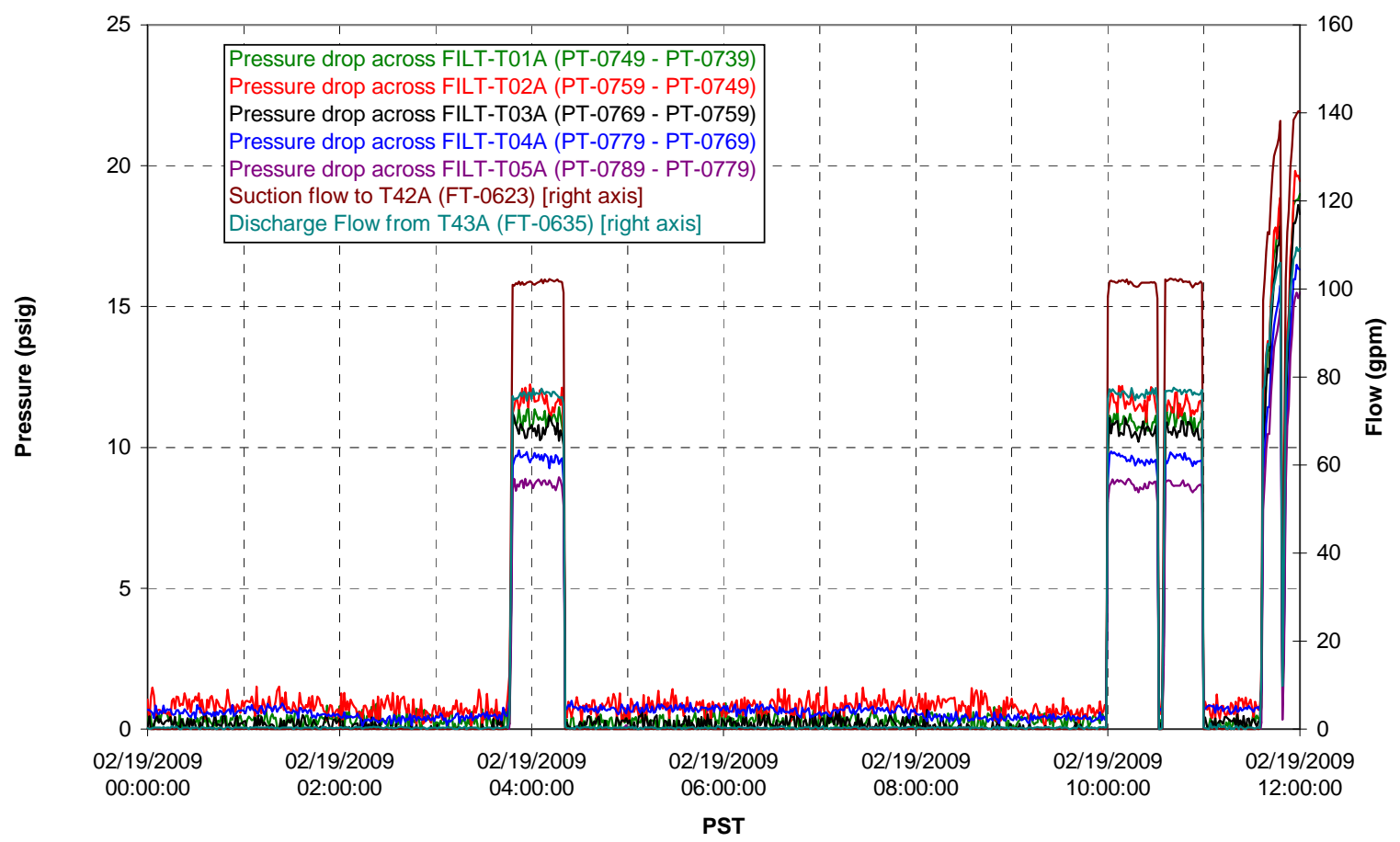

Permeate flow rates

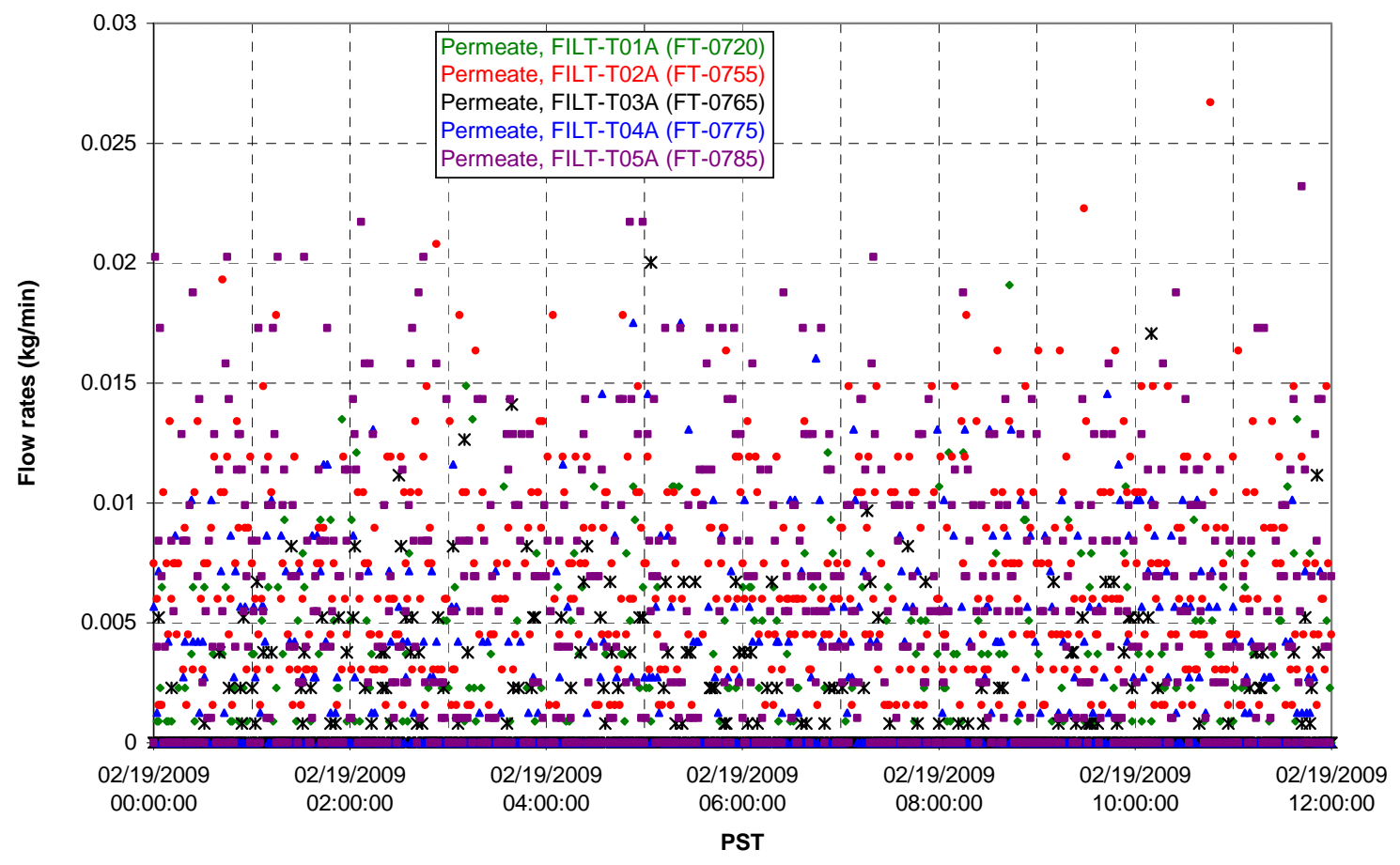


T02A Inner Temperature Tree

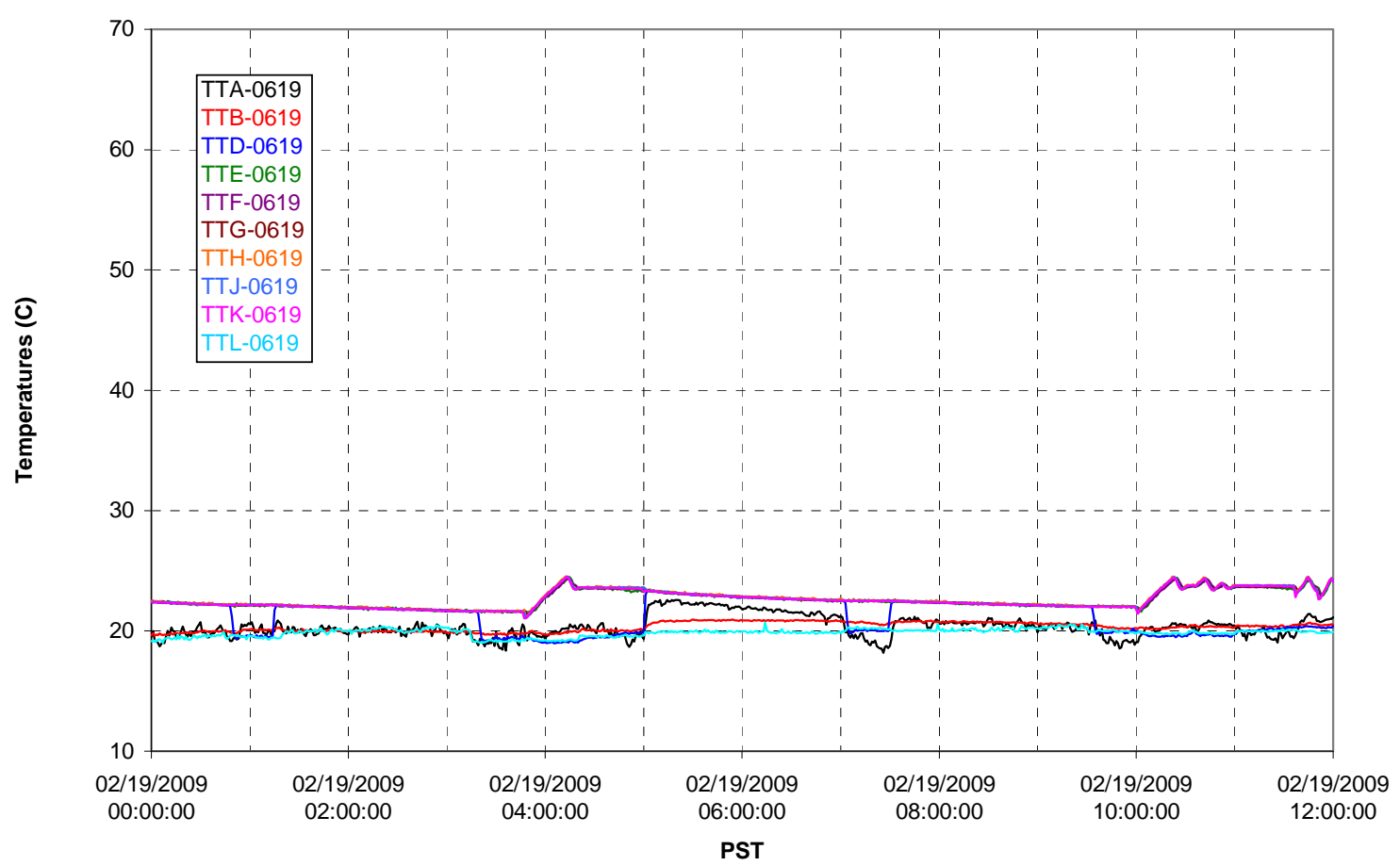

T02A Outer Temperature Tree

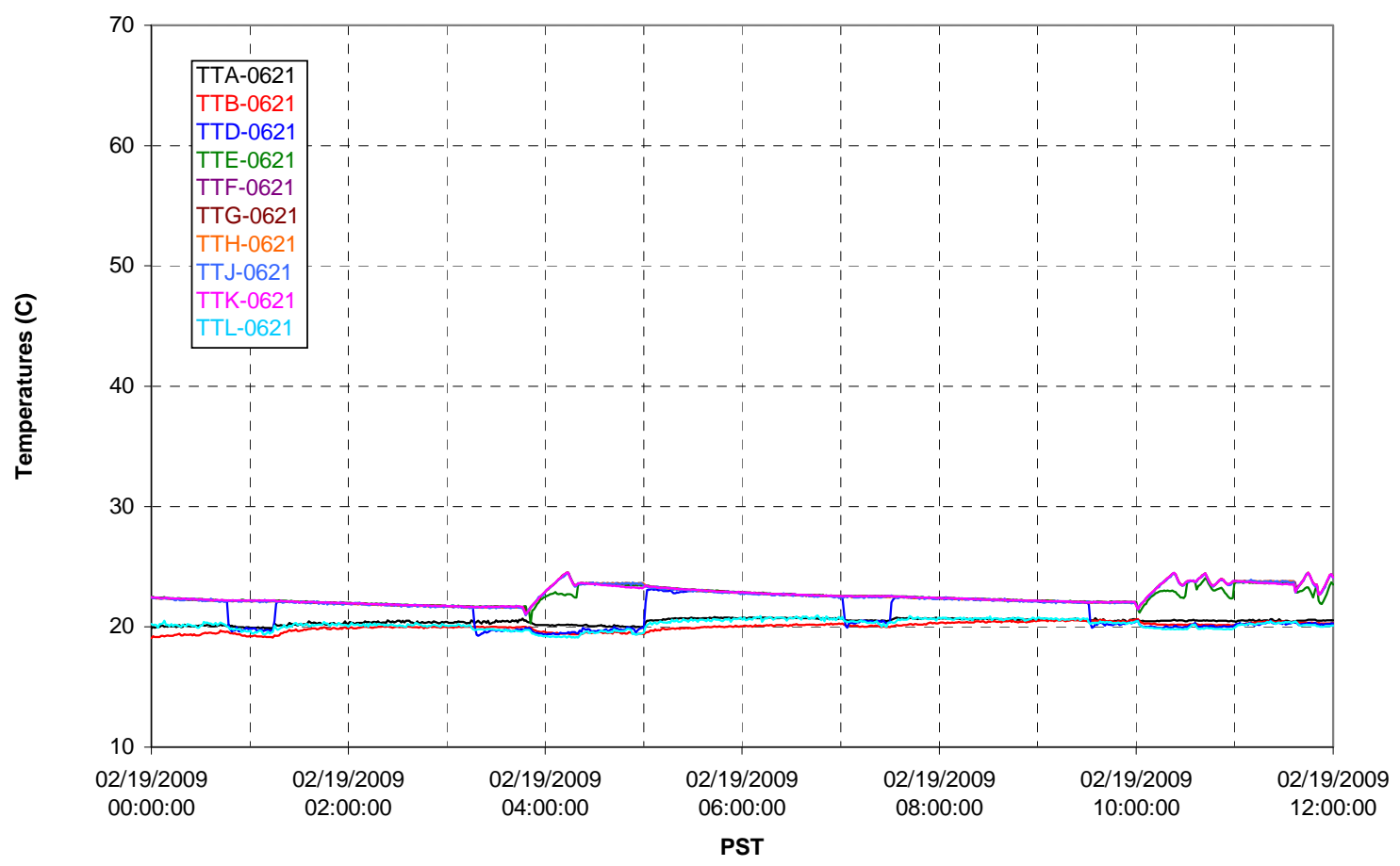


T02A temperatures

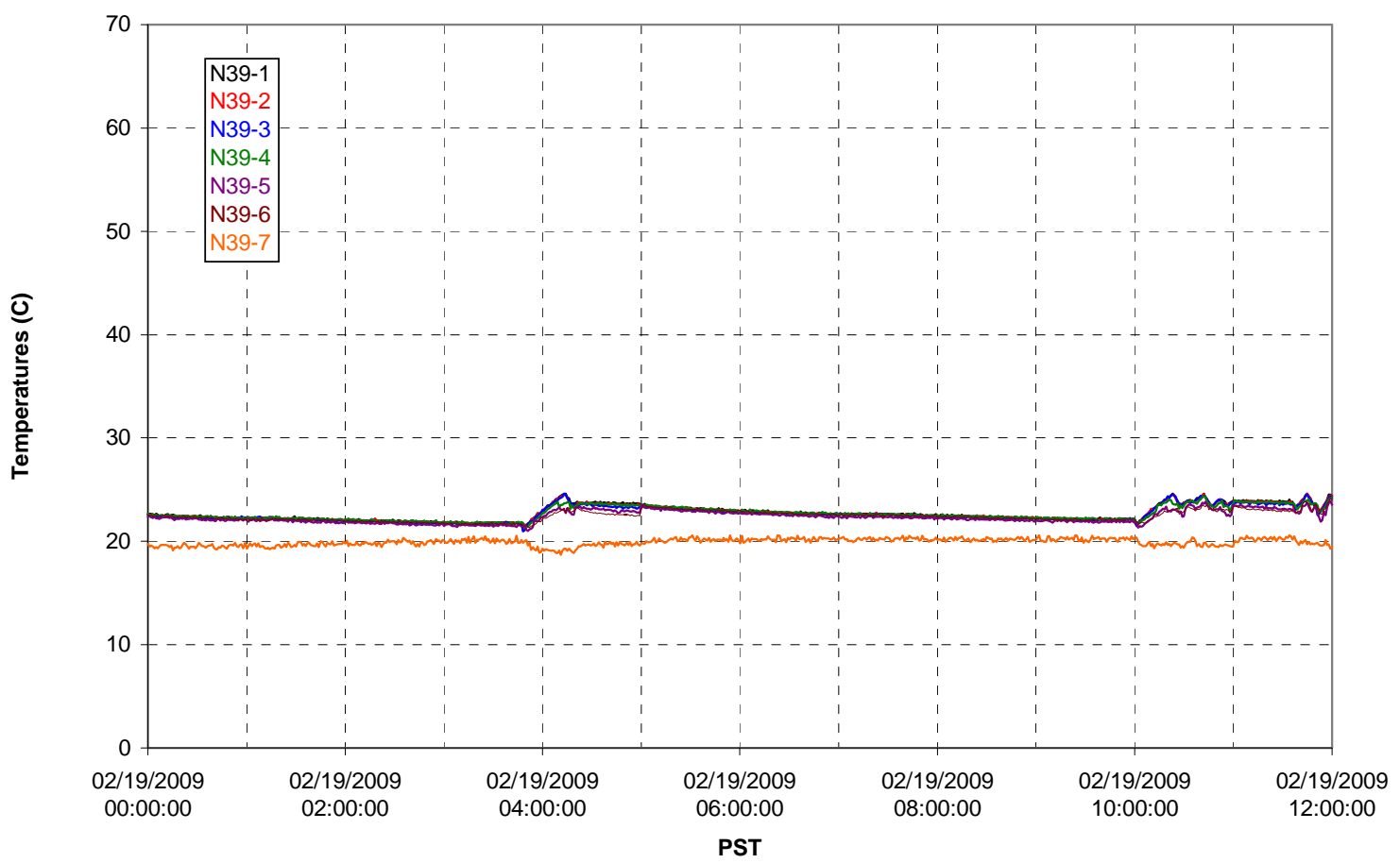

T02A temperatures

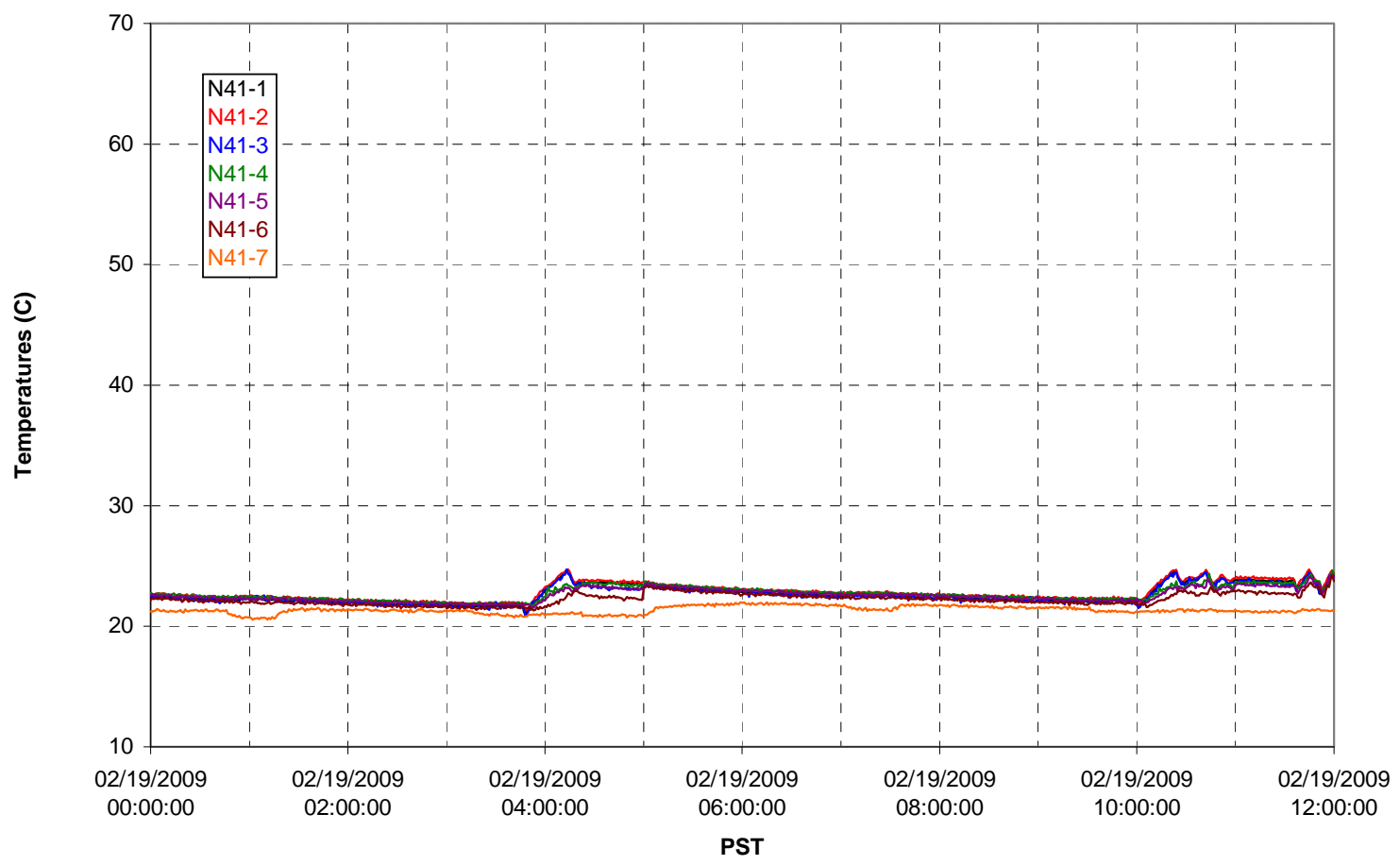


T02A temperatures

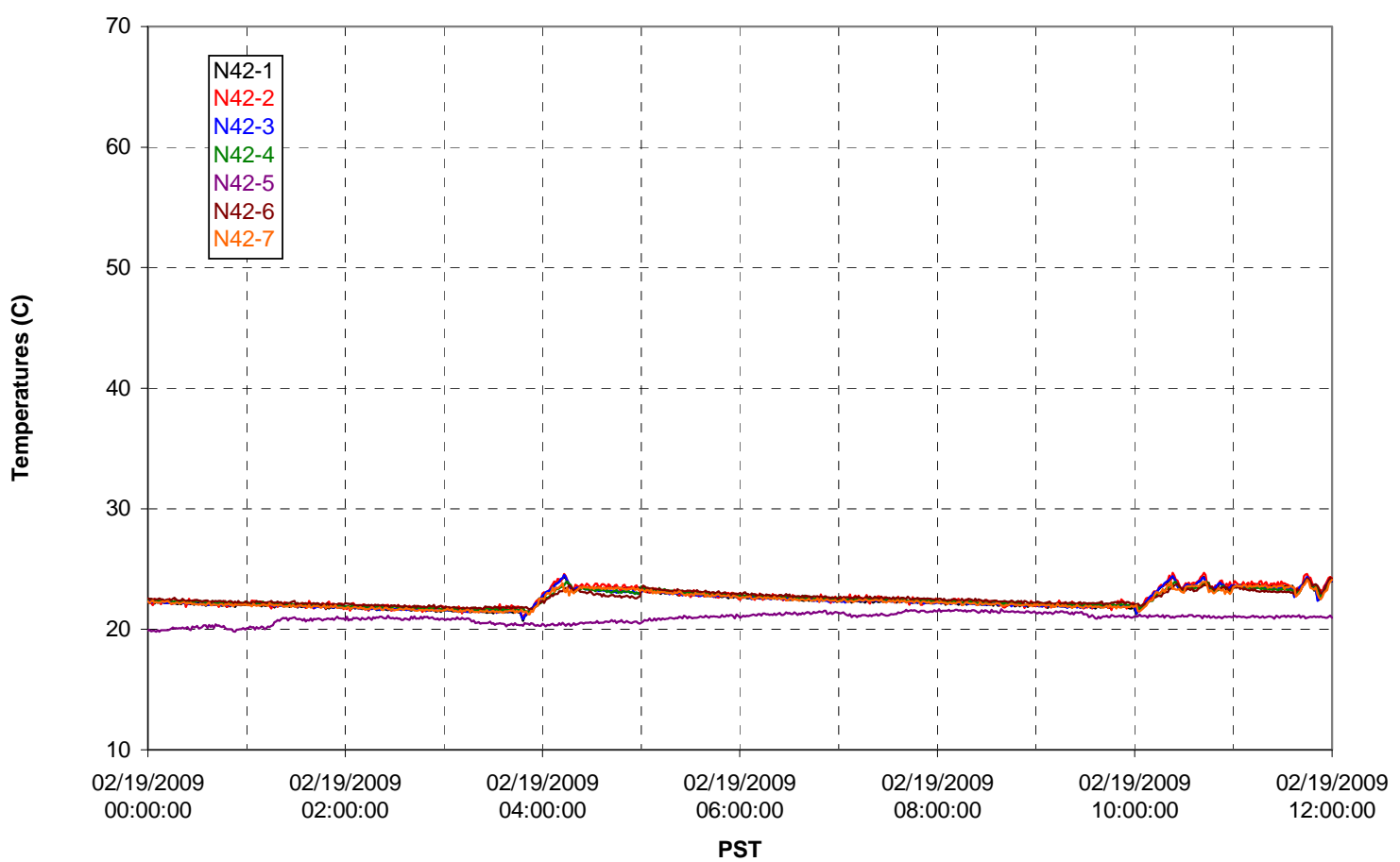

T02A temperatures

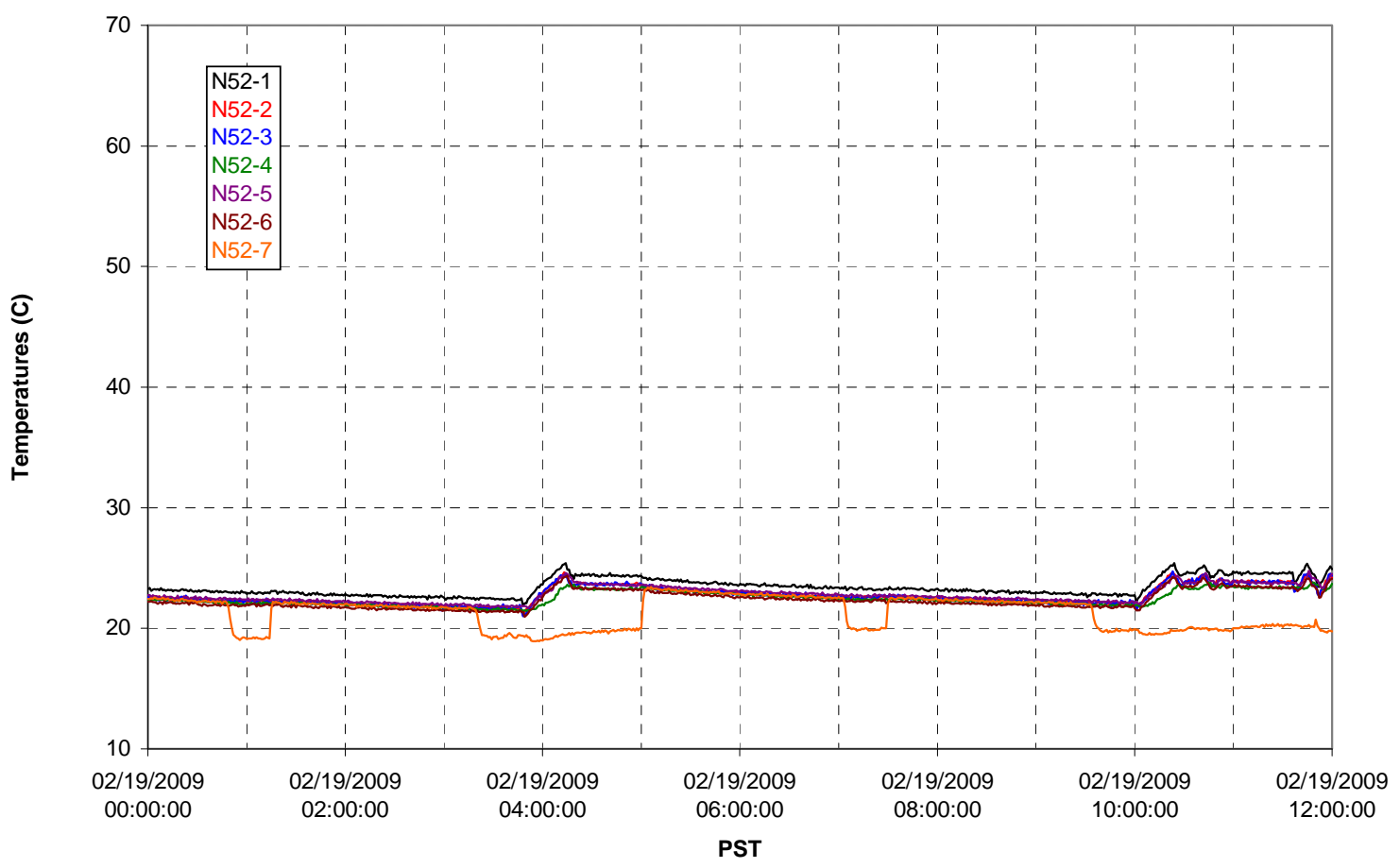


T02A Heating and Cooling

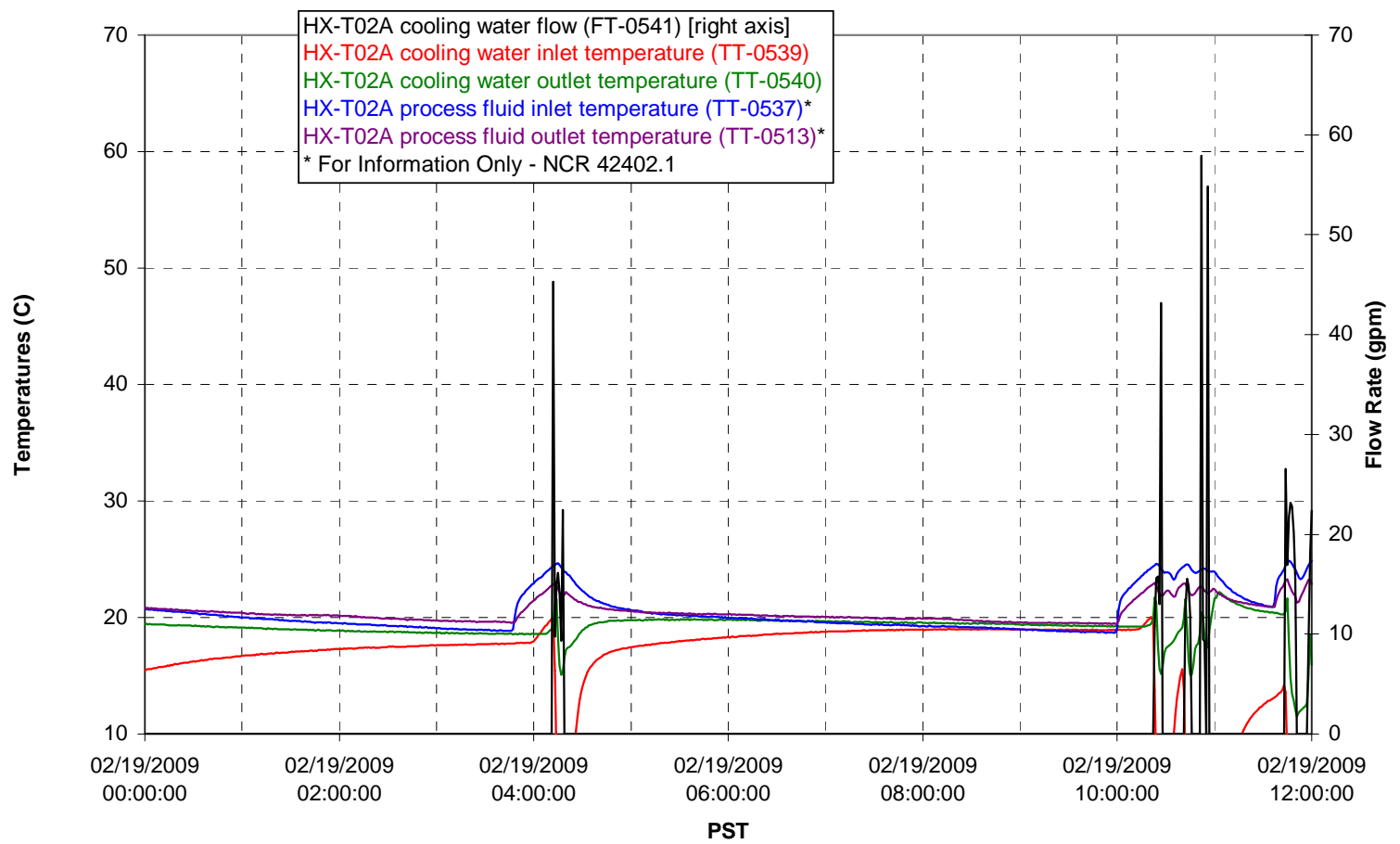

Pump Operation

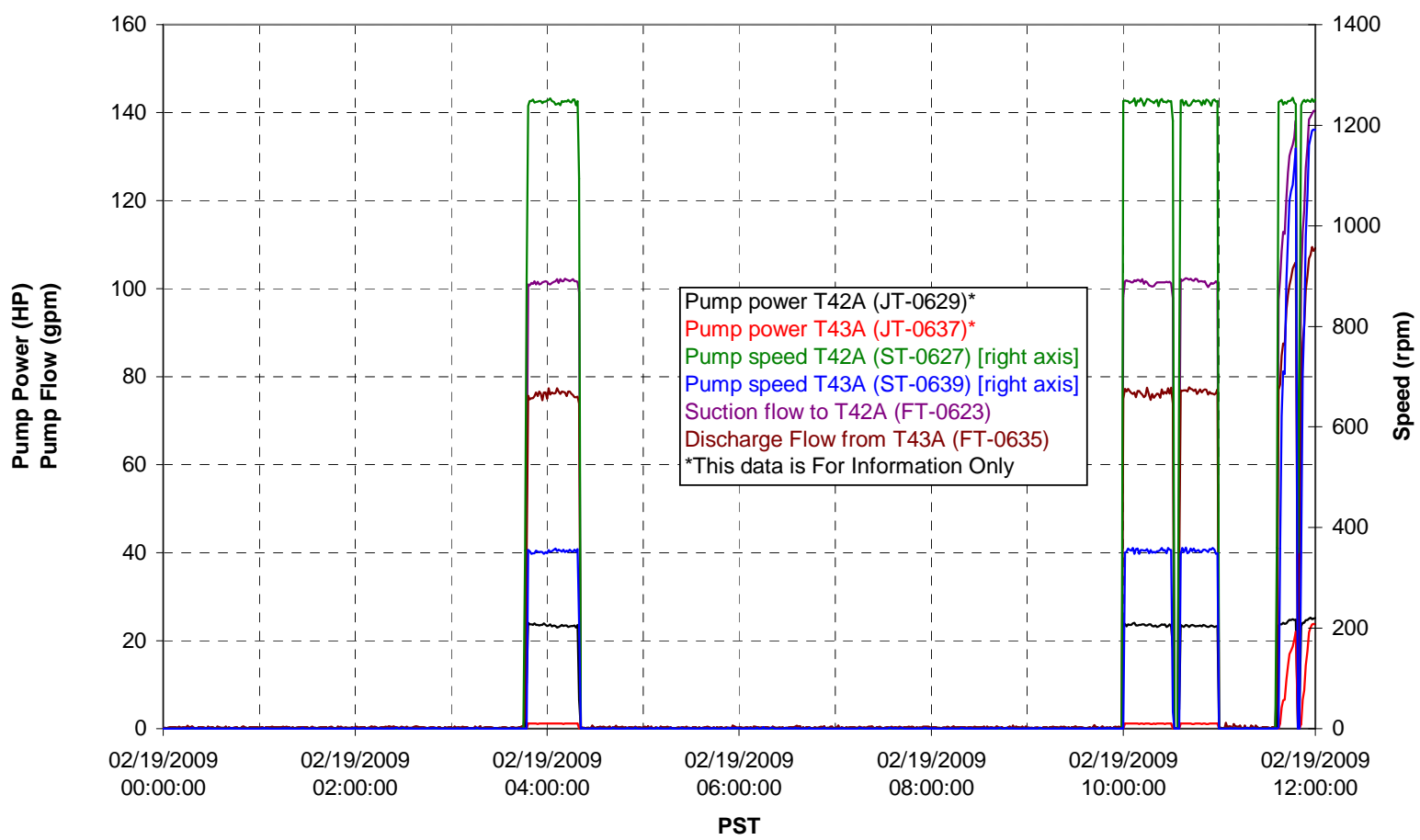


Pulsepot UFP-PP-T01A

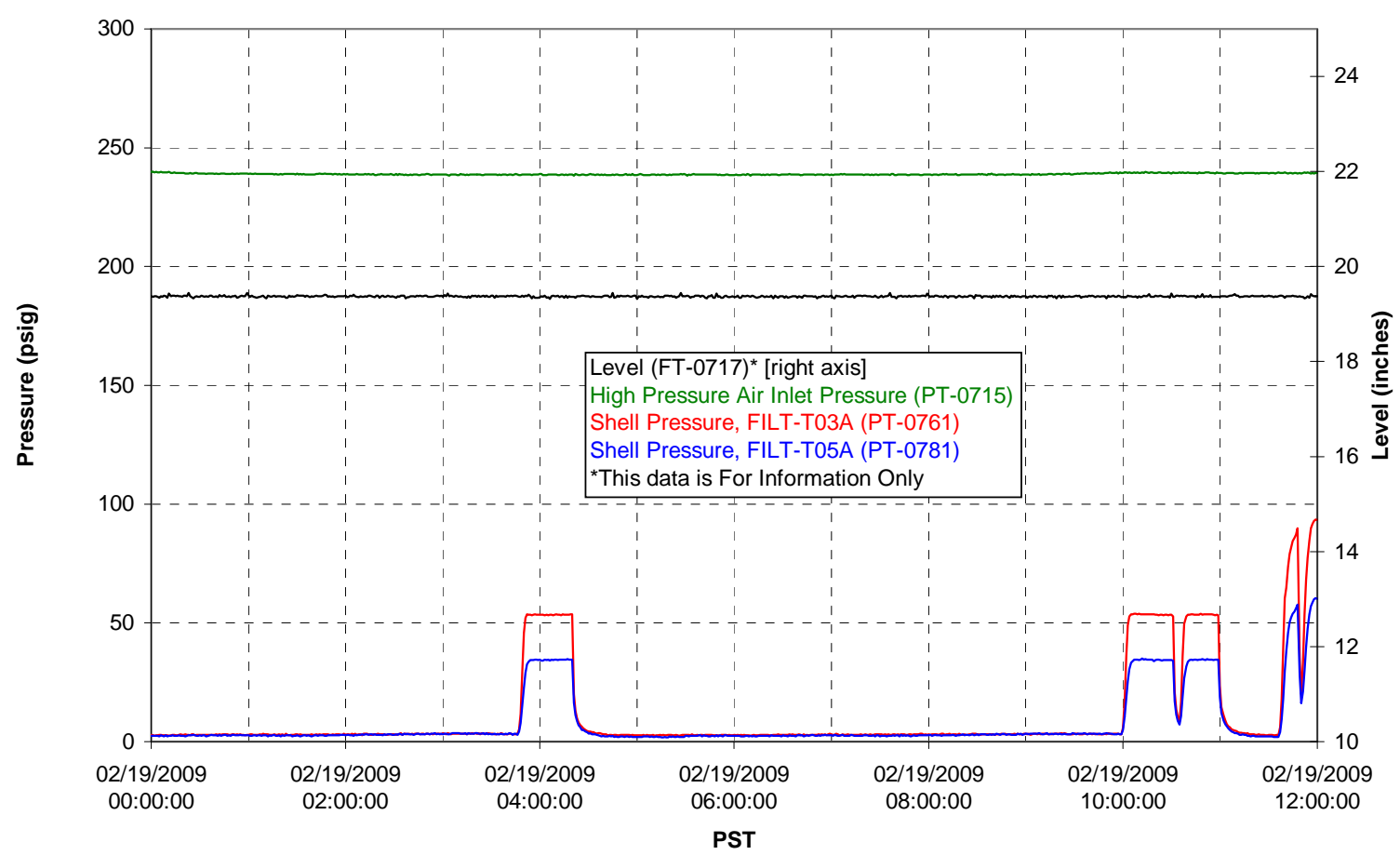

Pulsepot UFP-PP-T02A

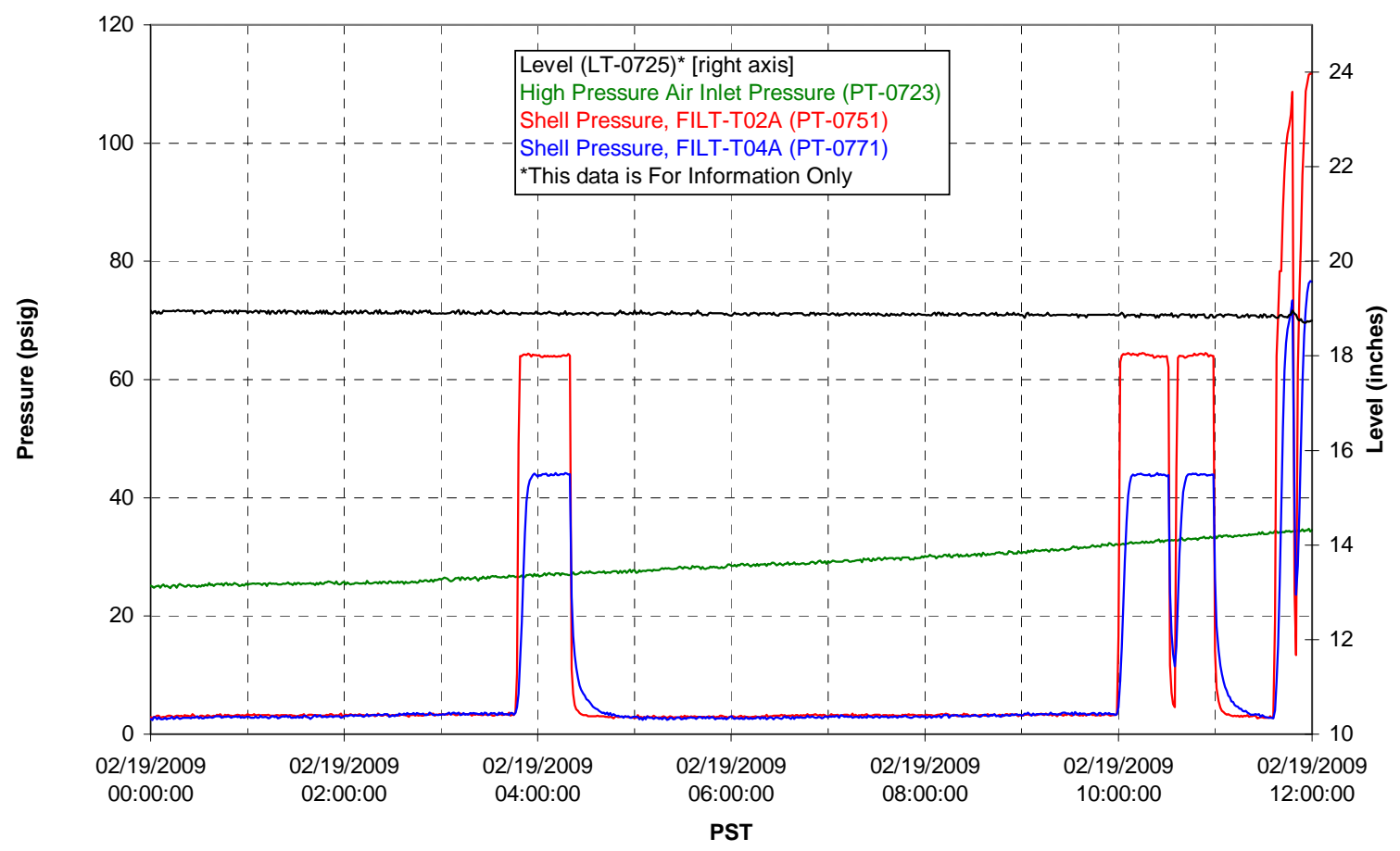


Pulsepot UFP-PP-T03A

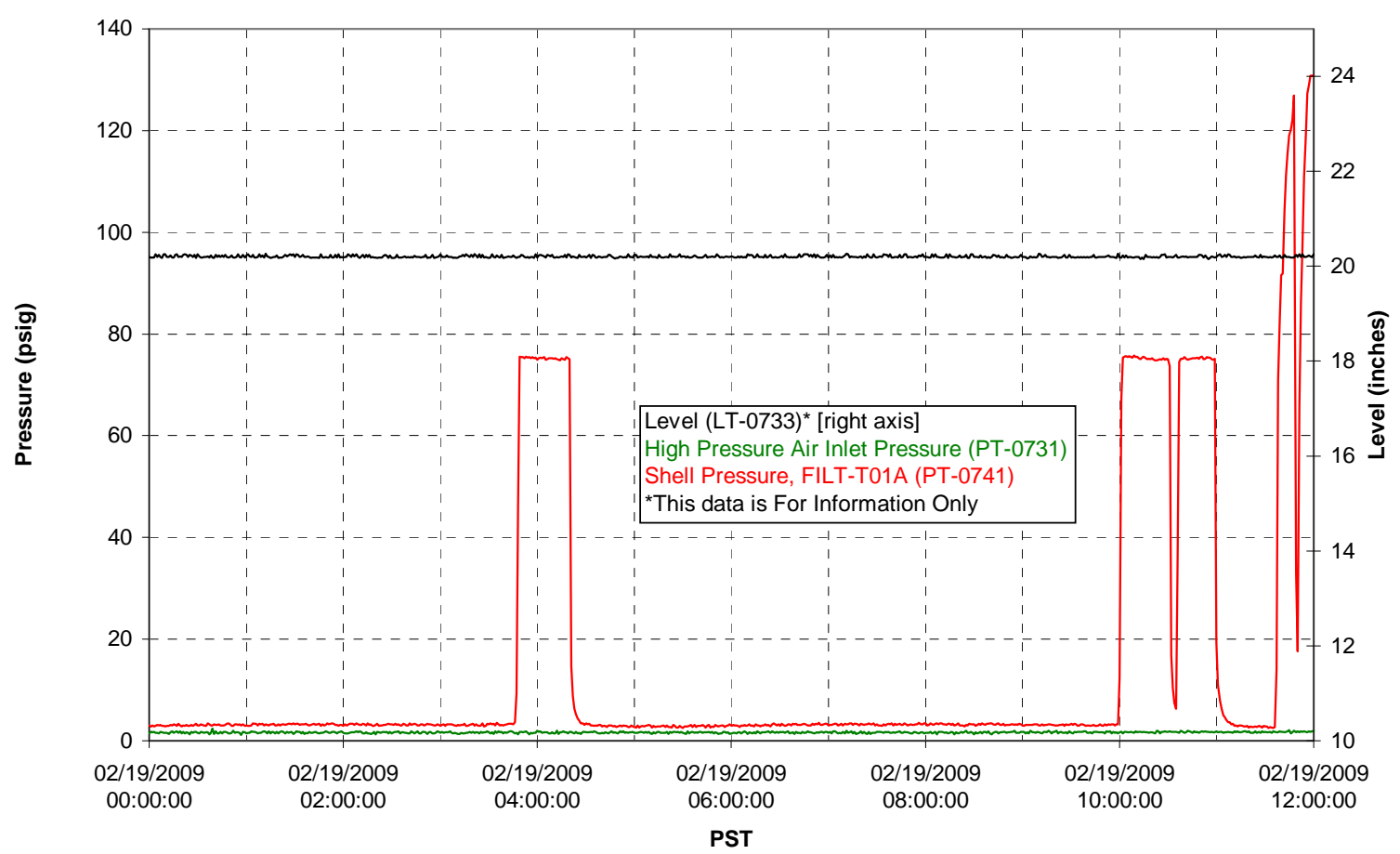

Pulsepot Levels

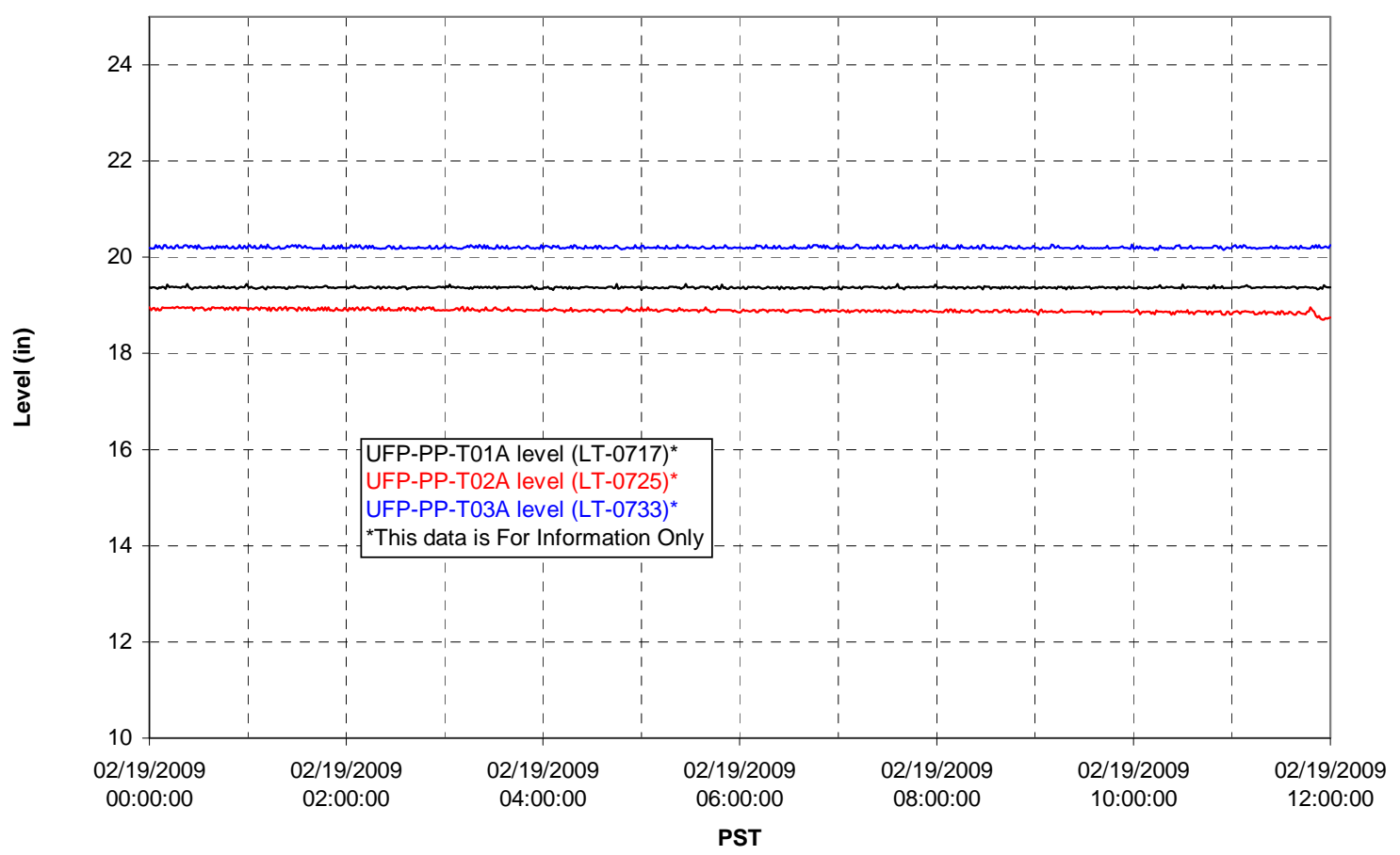


Filter UFP-FILT-T01A

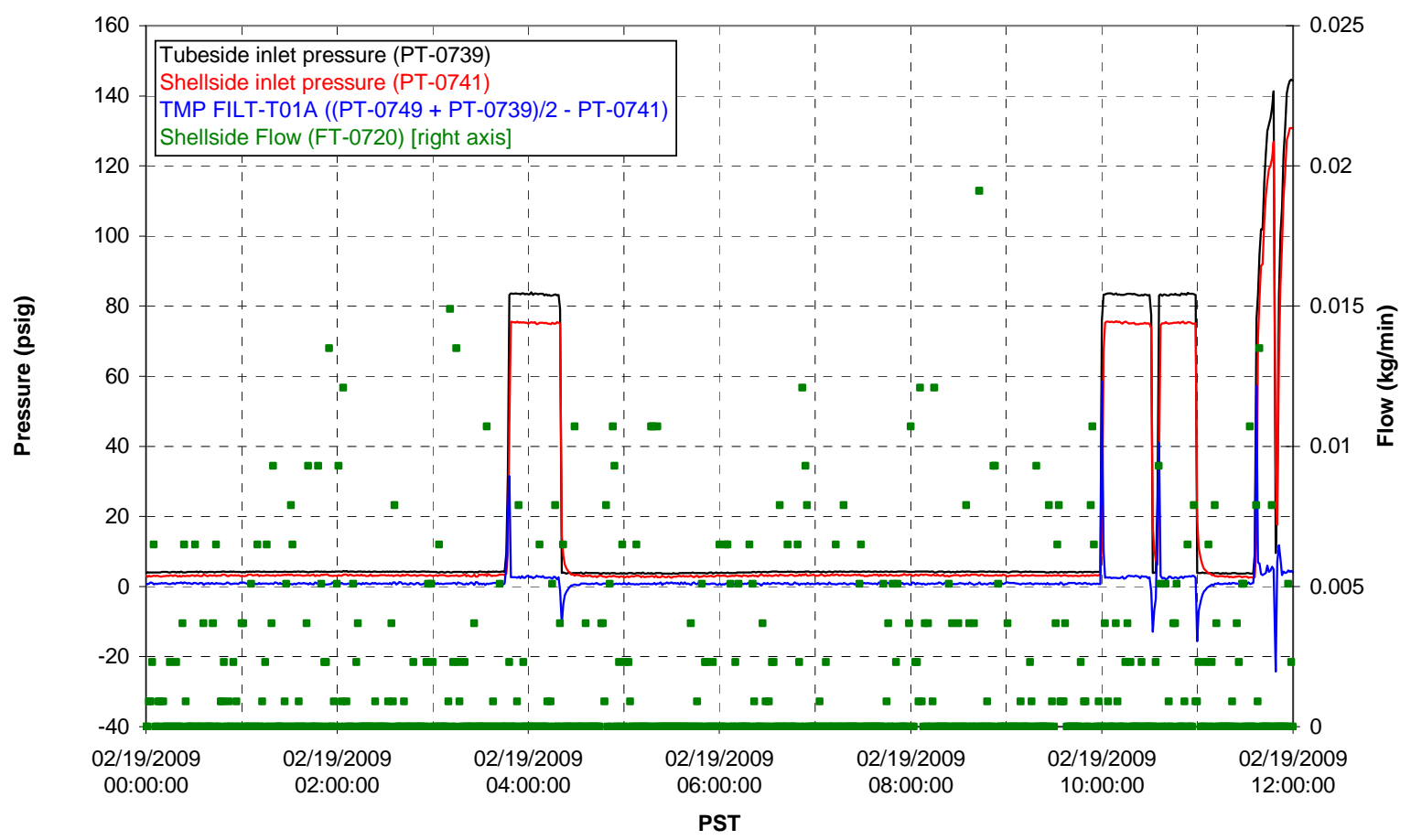

Filter UFP-FILT-T02A

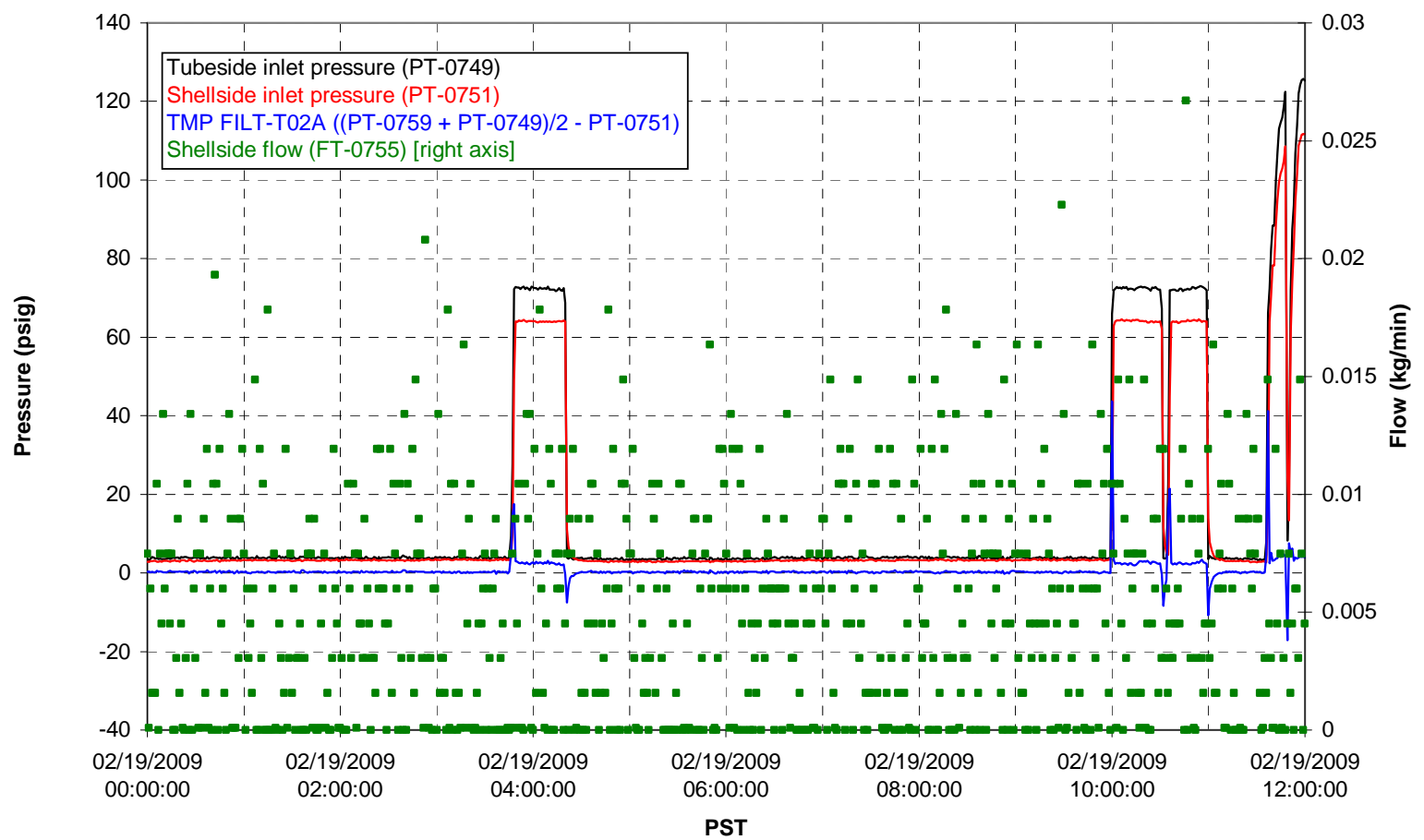


Filter UFP-FILT-T03A

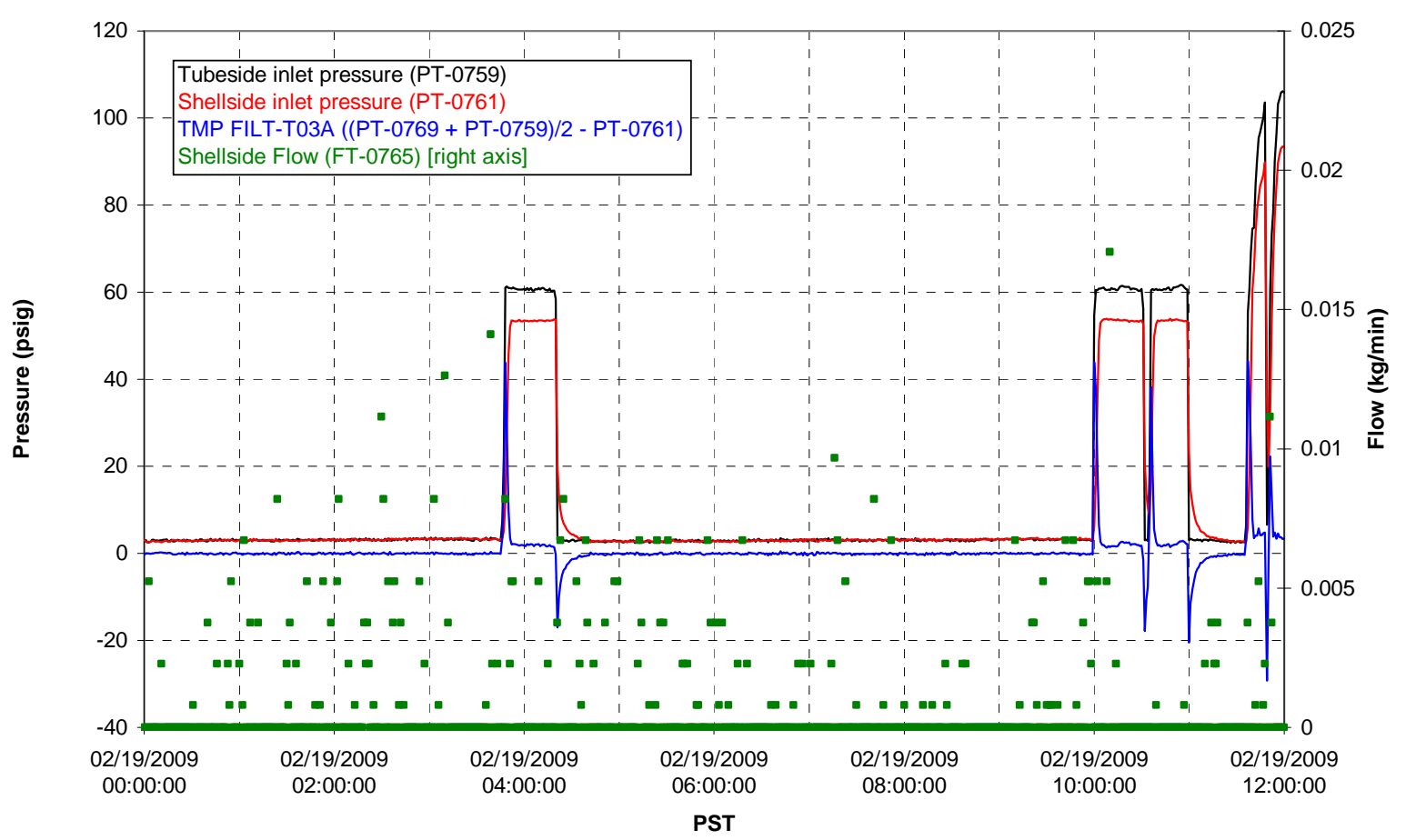

Filter UFP-FILT-T04A

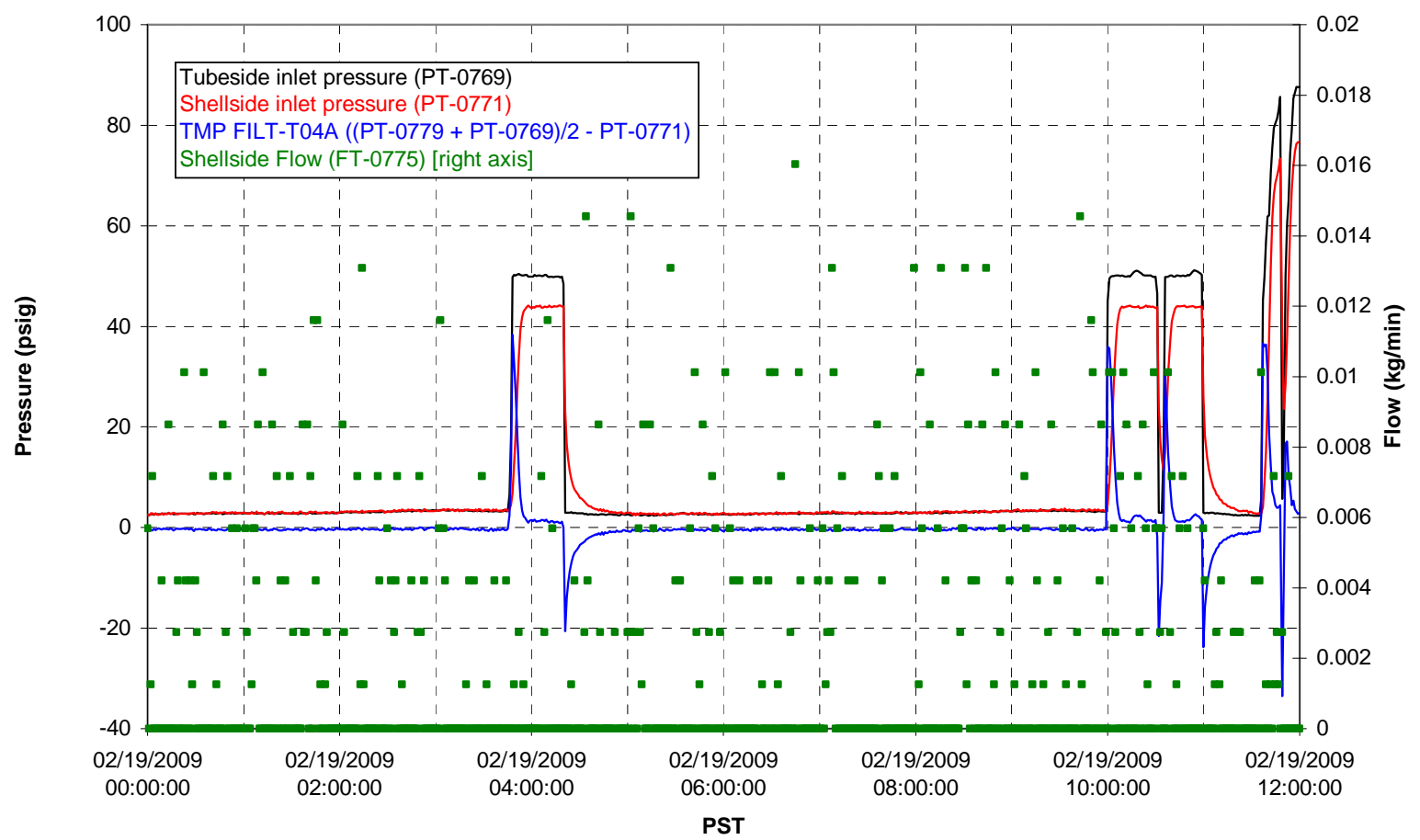


Filter UFP-FILT-T05A

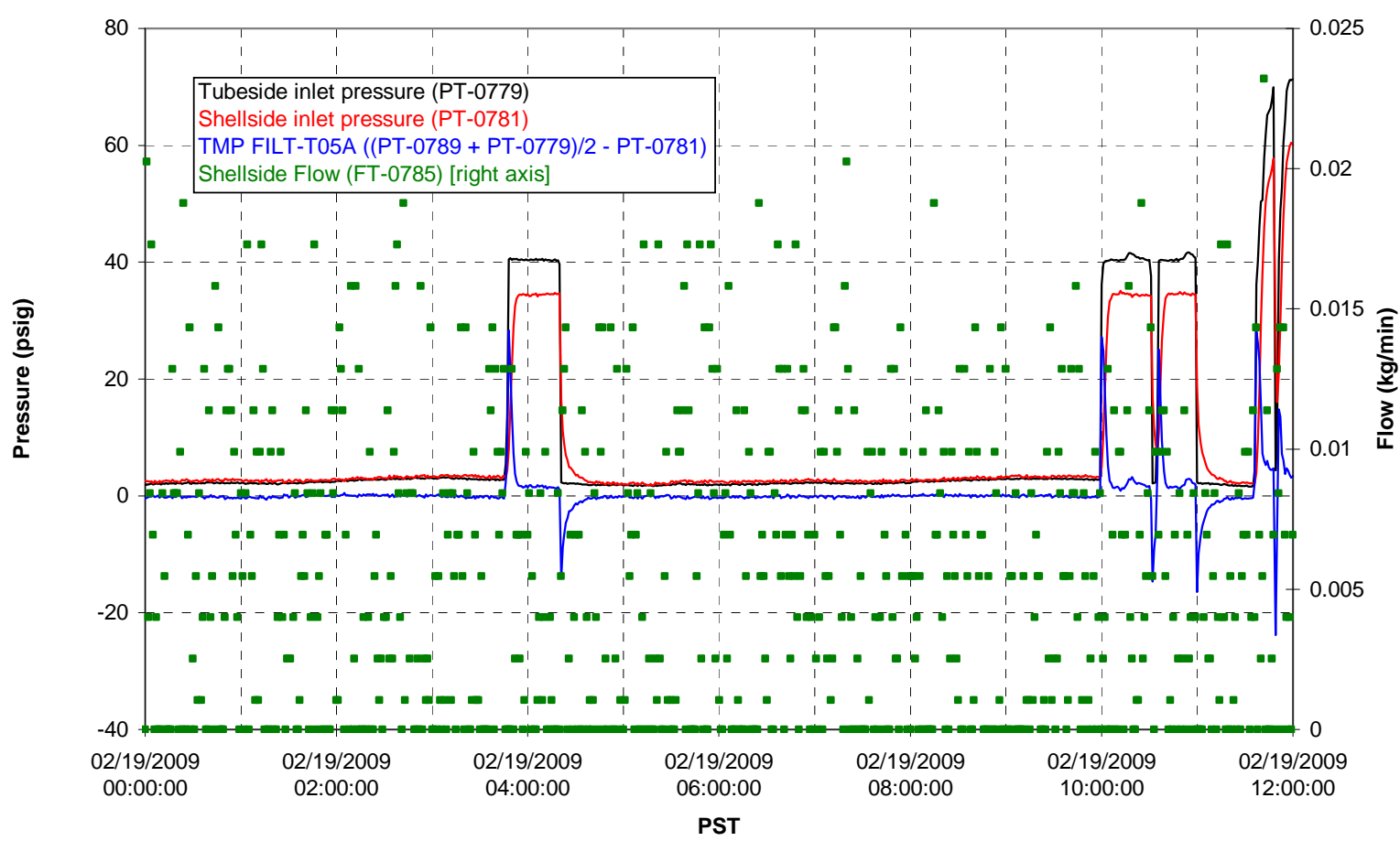

Chemical Flow

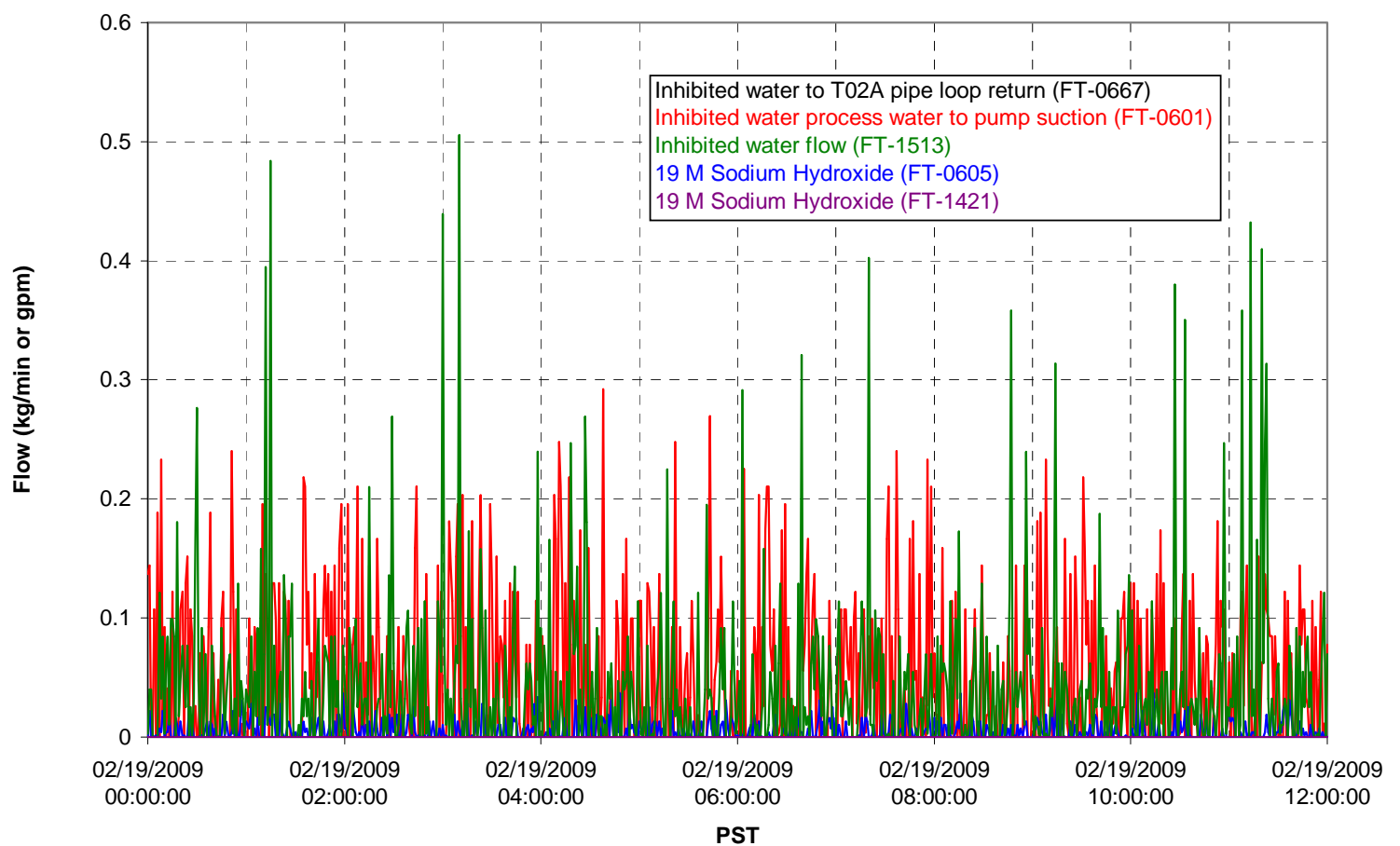




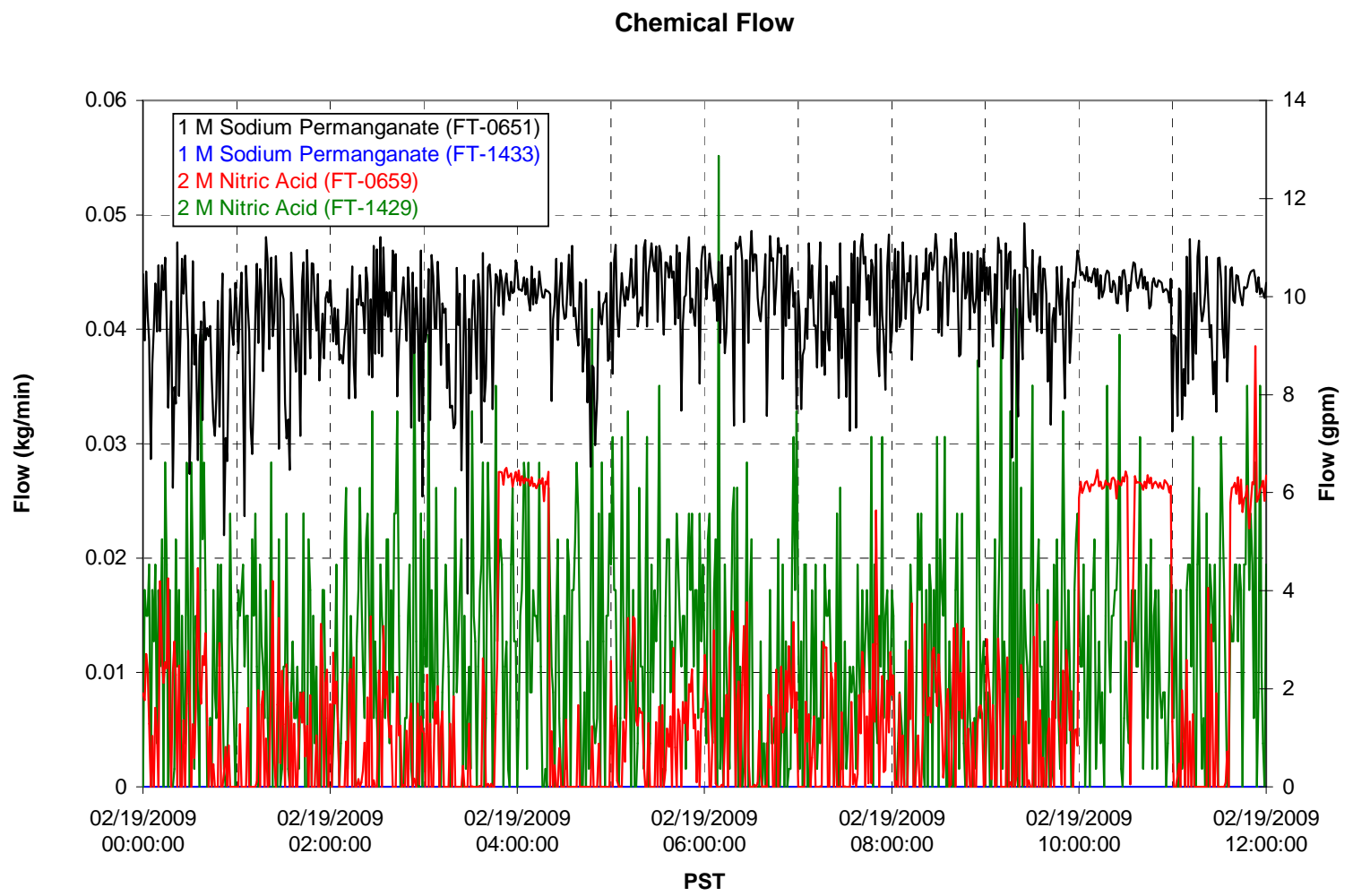

Air Flows

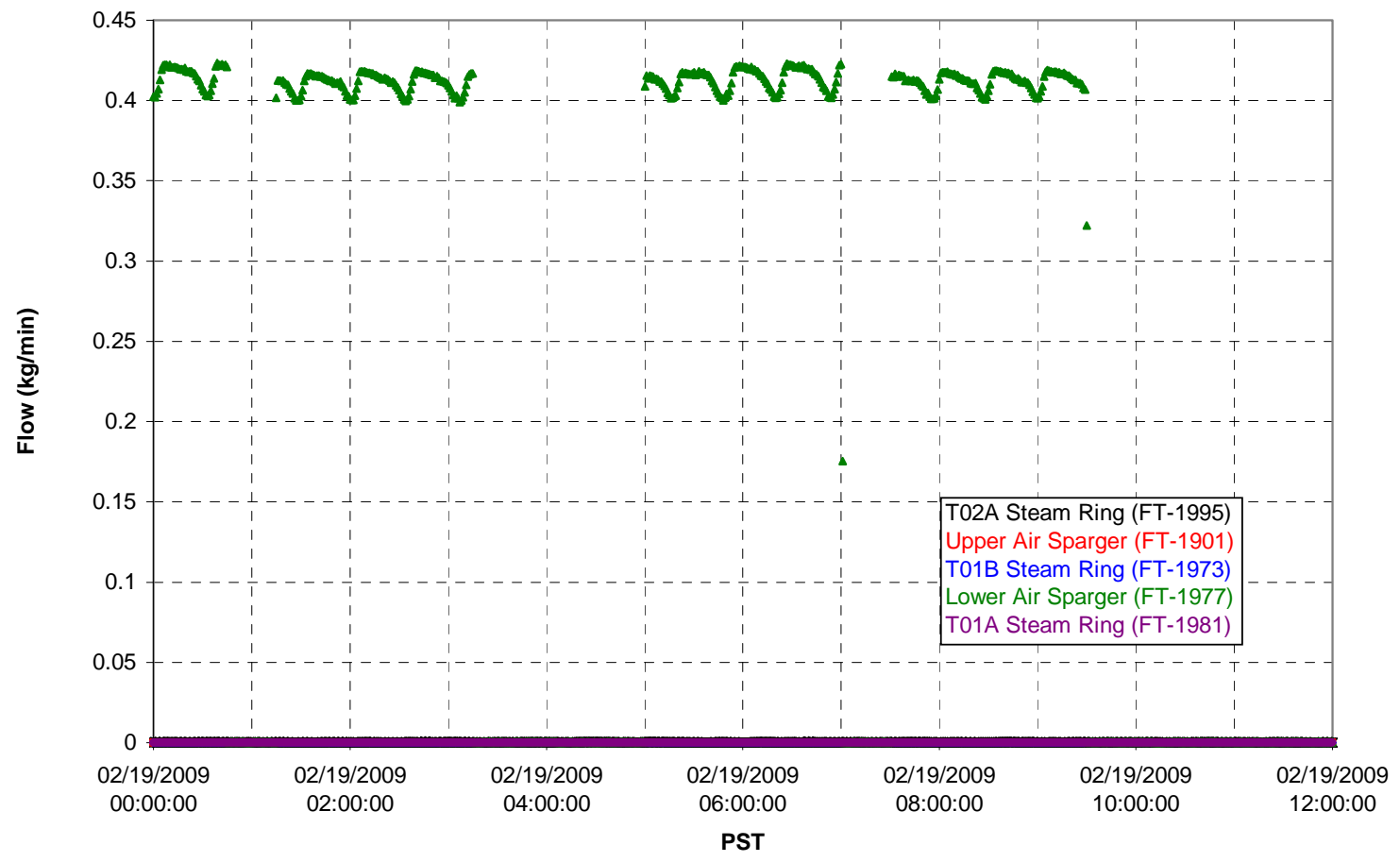


T02A Steam

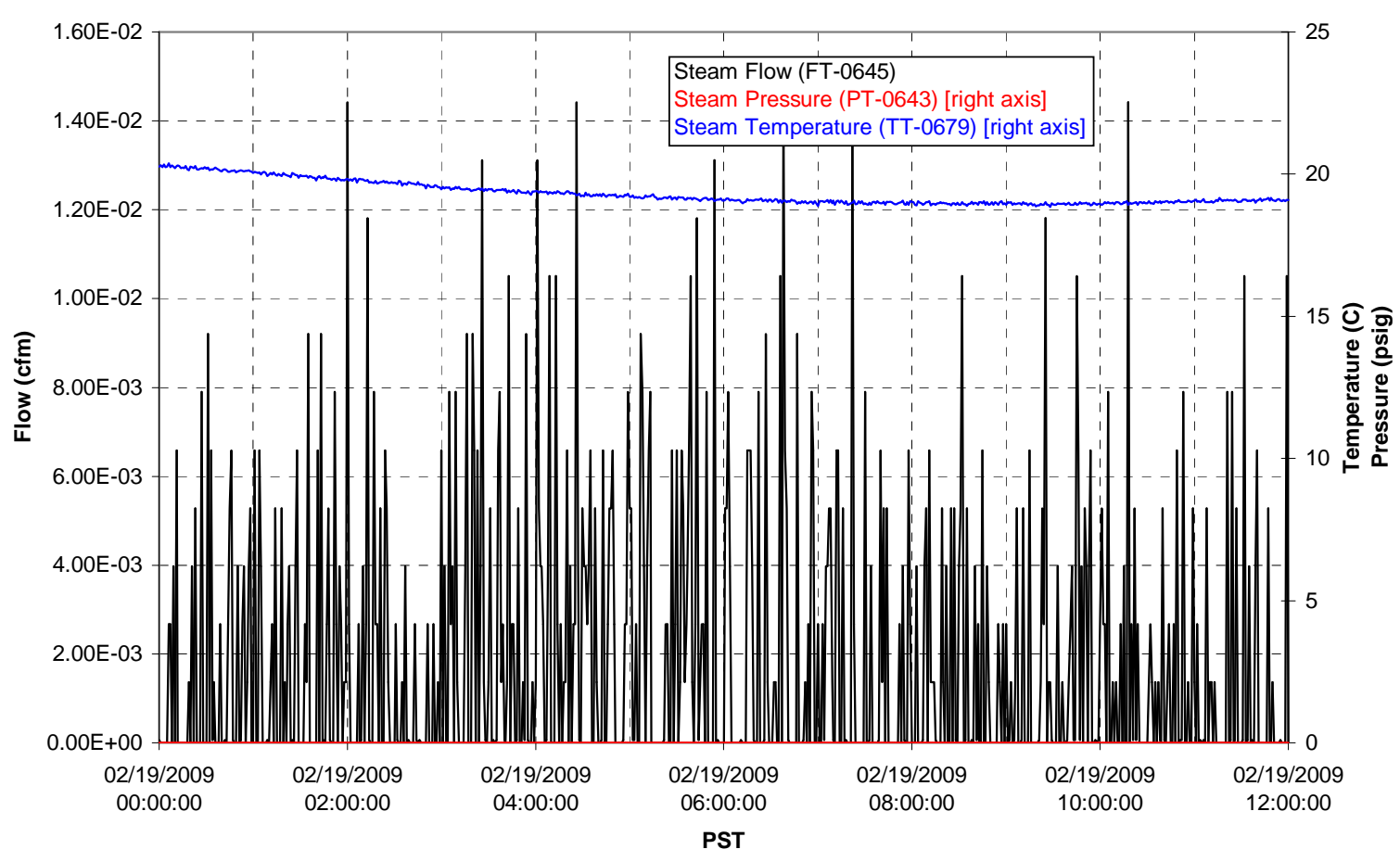

T01A Steam

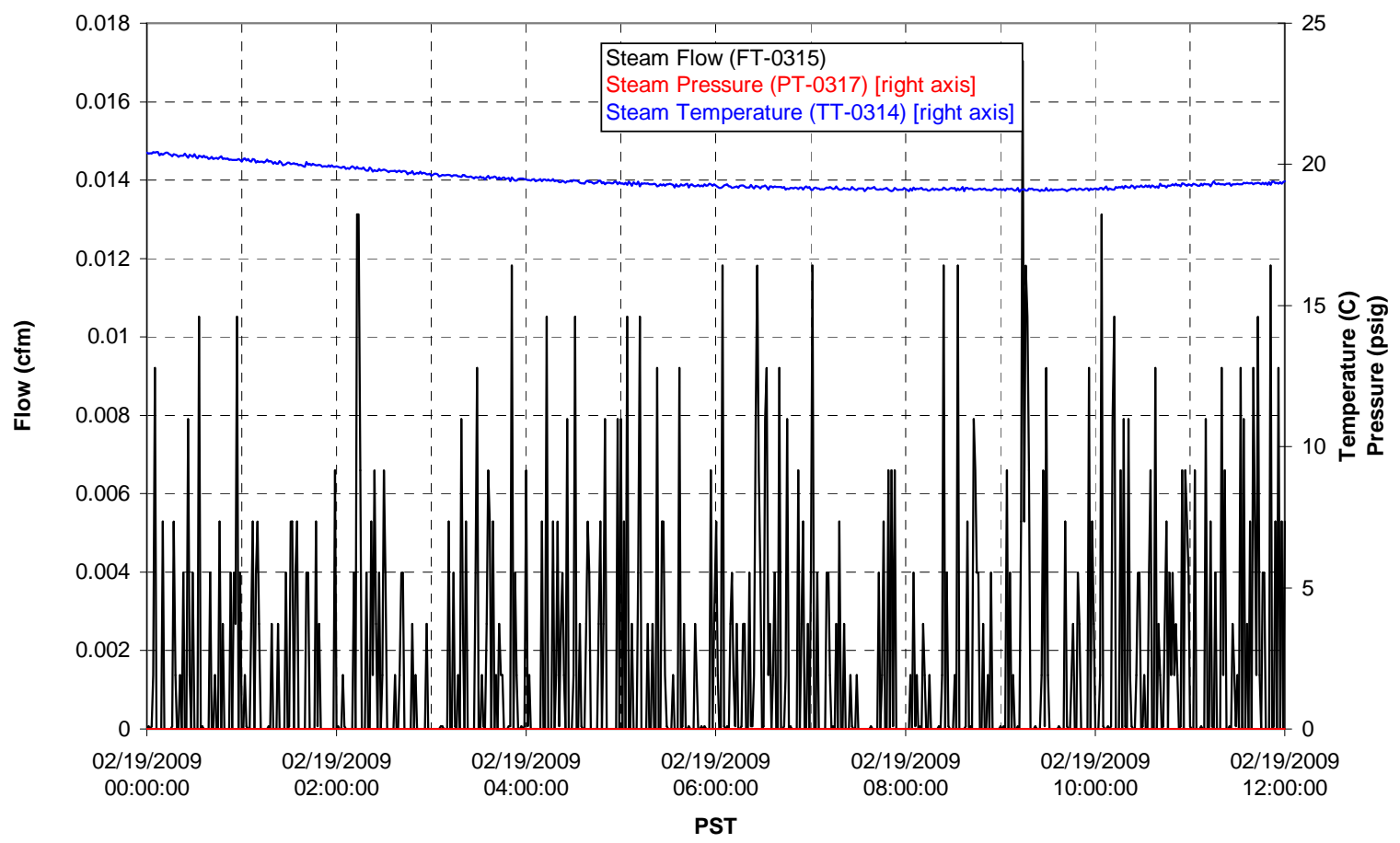


T01B Steam

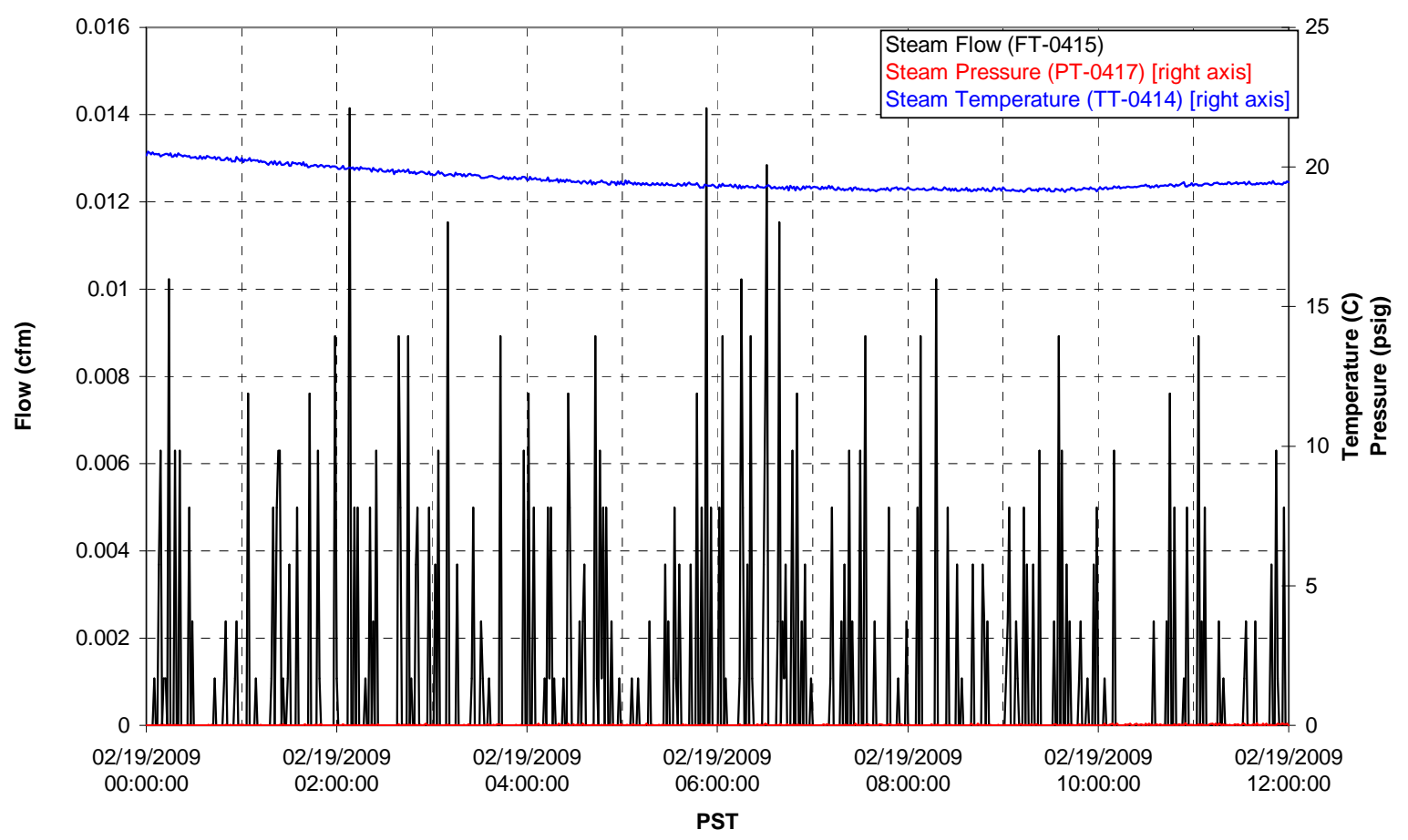




\section{Integrated Test A Data Plots 02/19/09 12:00 - 02/20/09 00:00}


T01A level

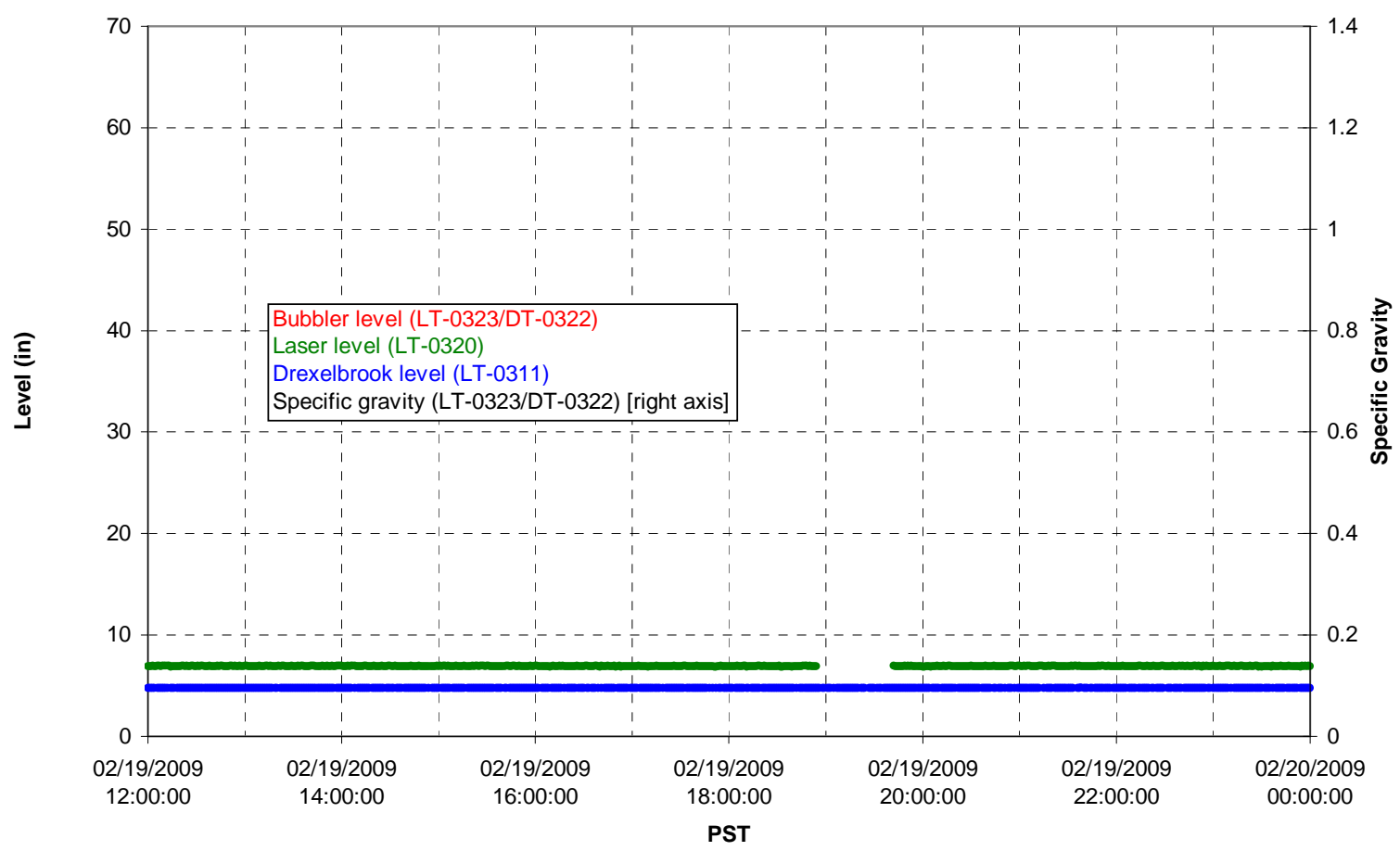

T01A temperatures

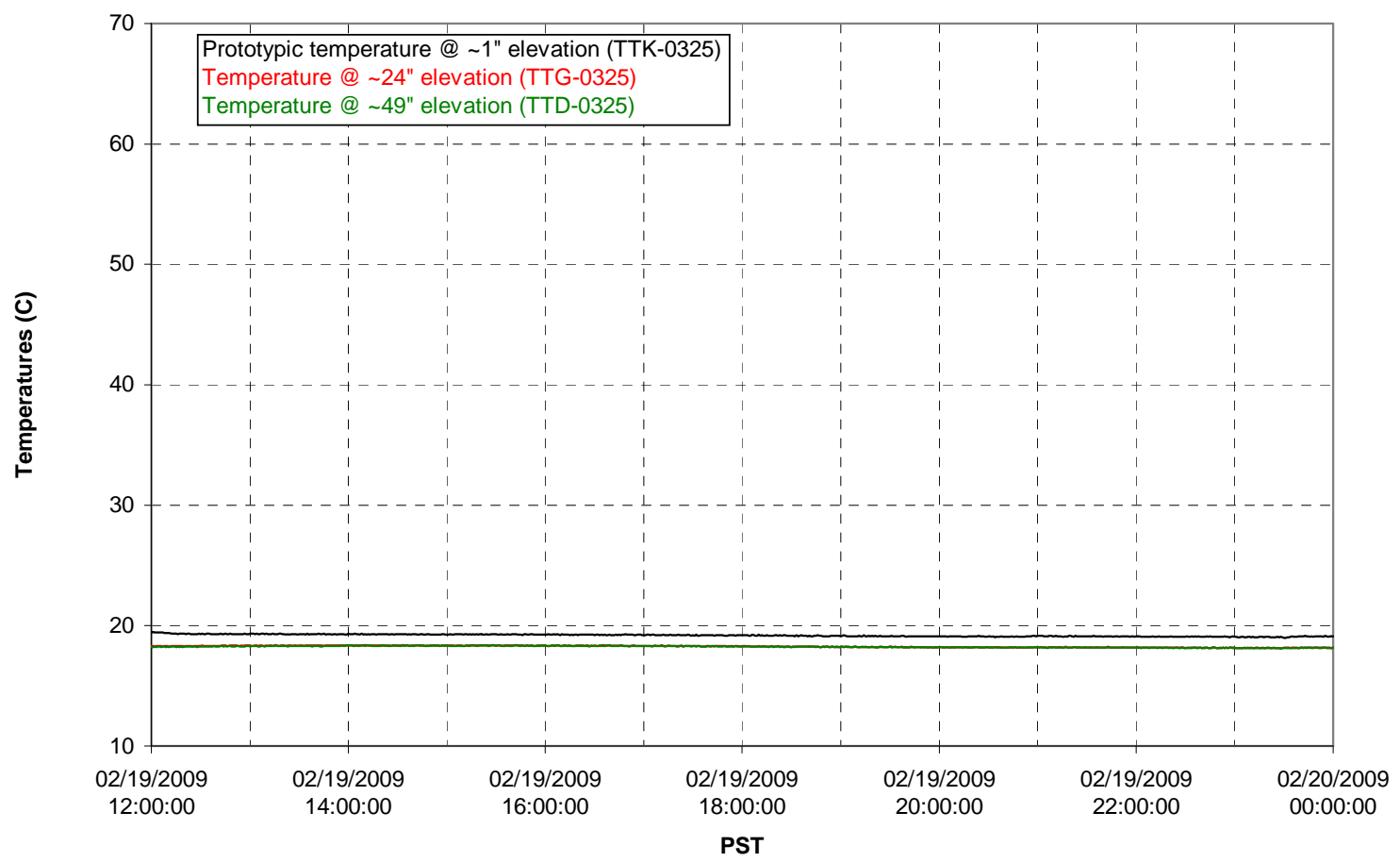


T01B level

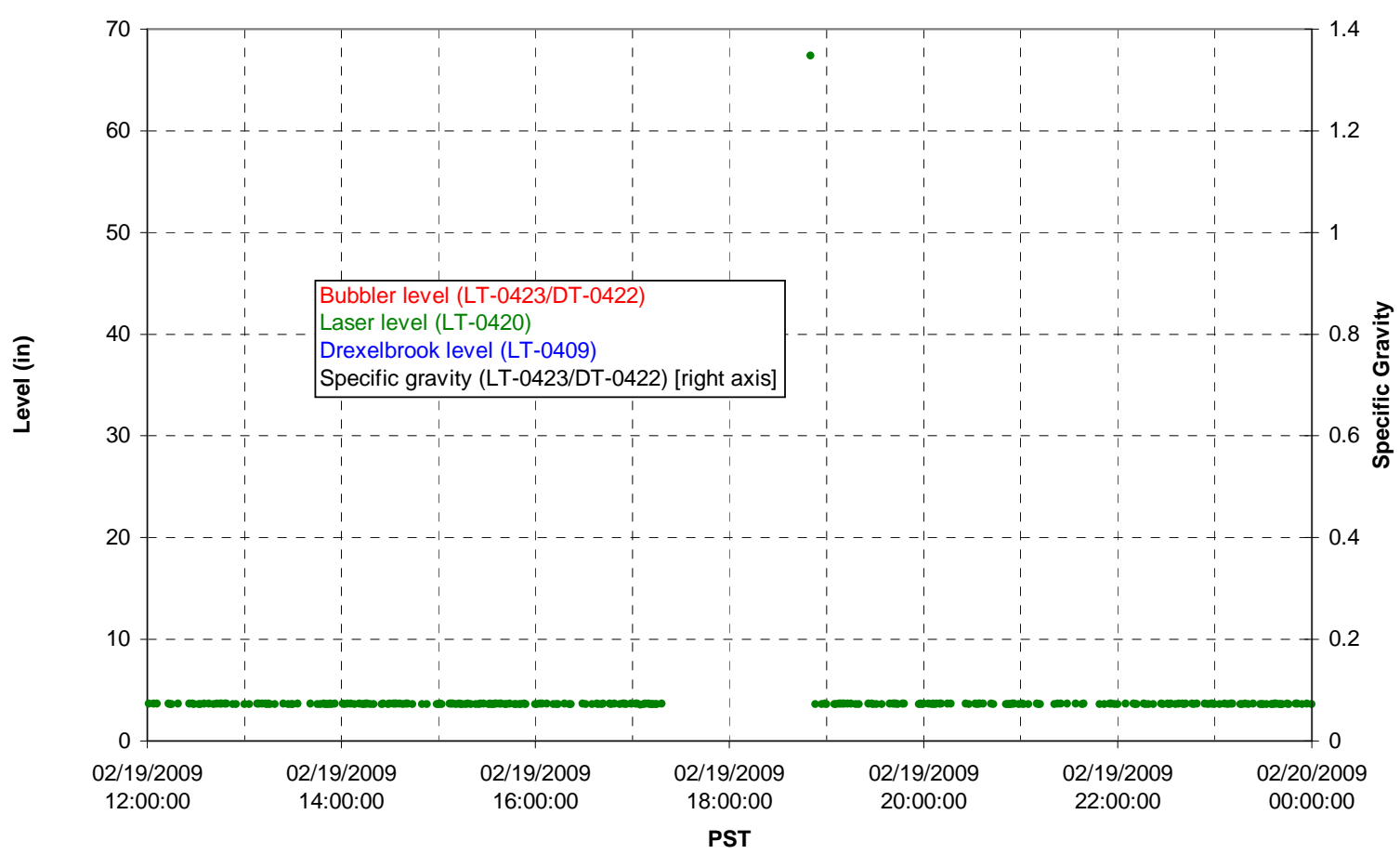

T01B temperatures

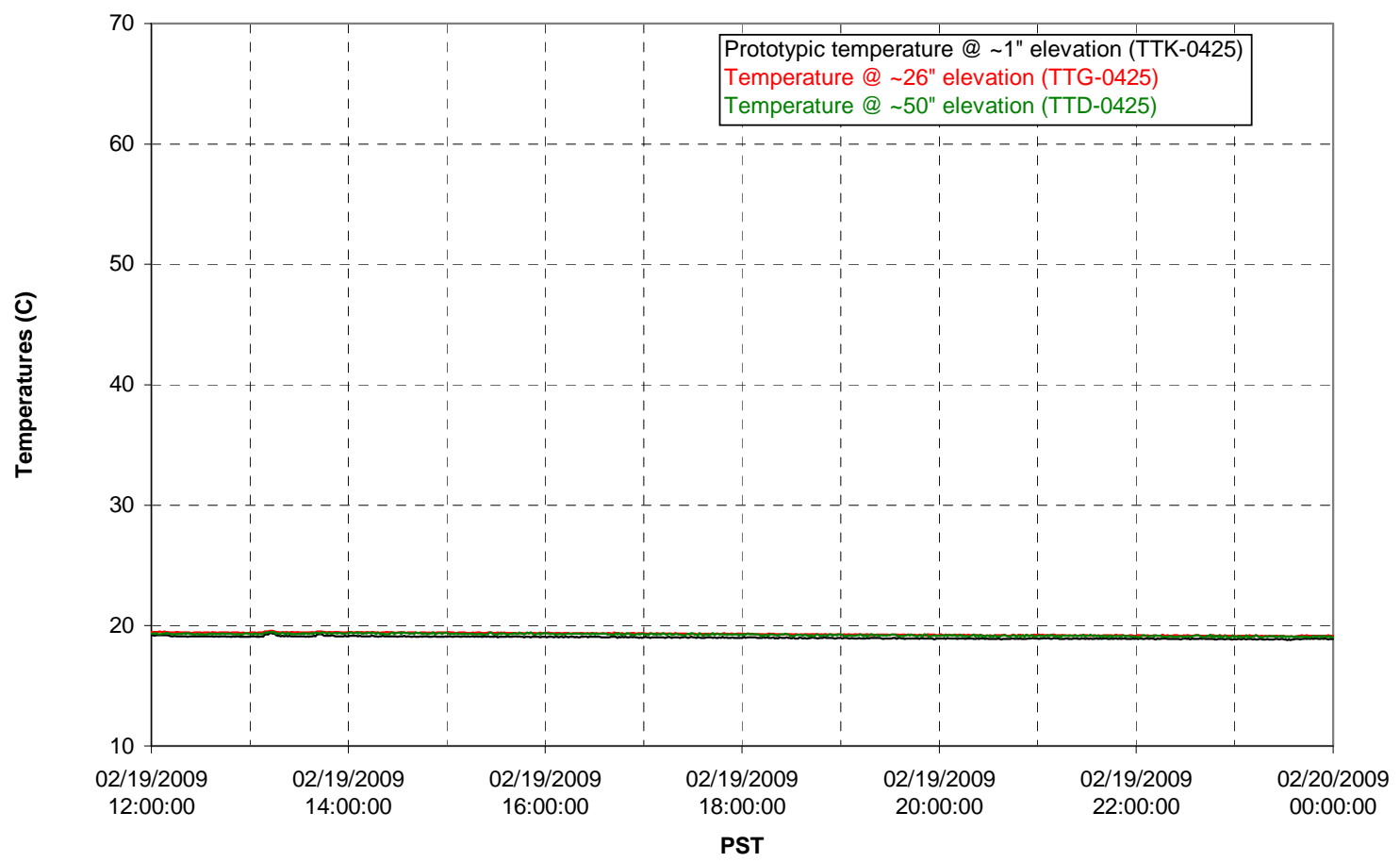


T02A level

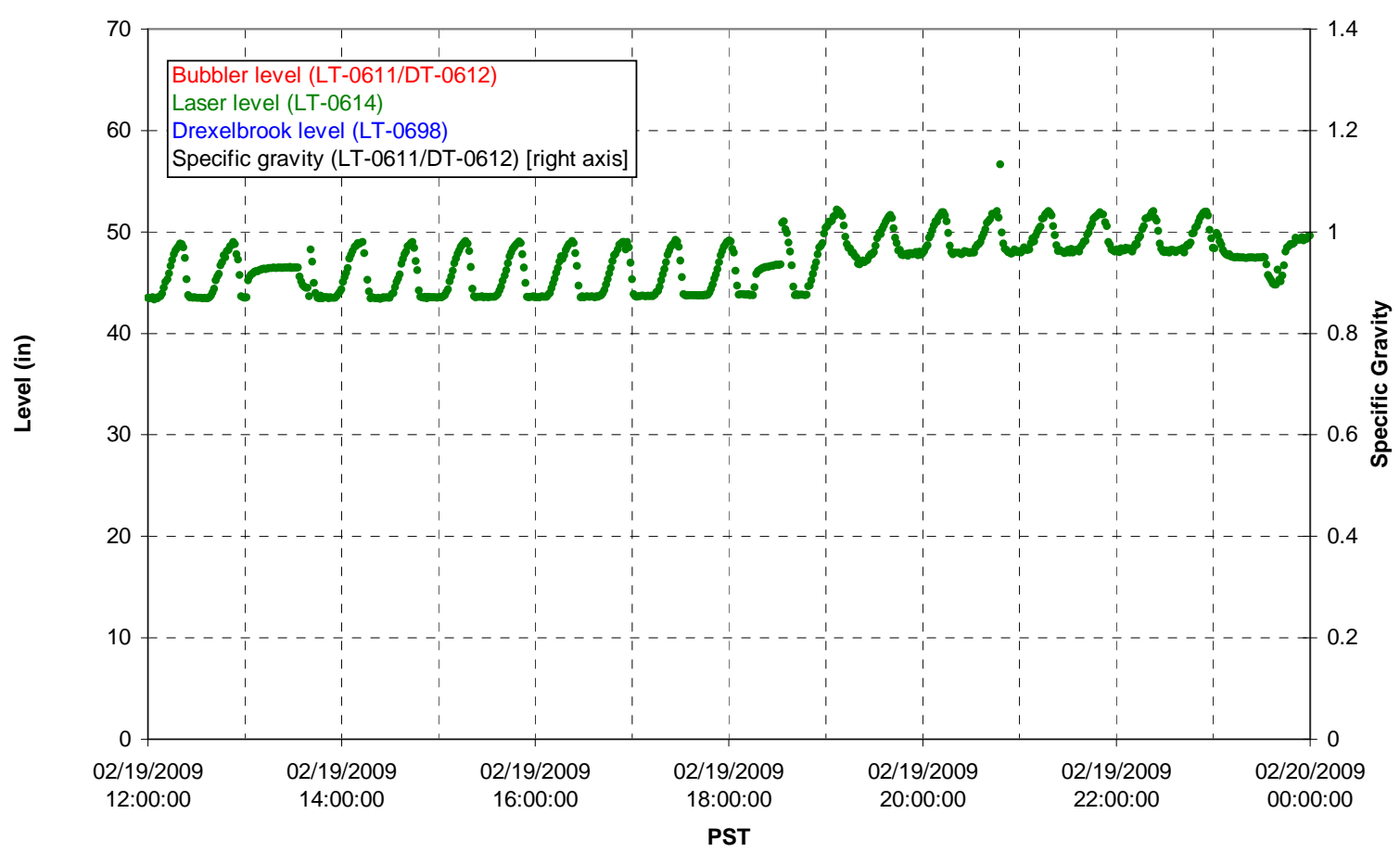

T02A temperatures

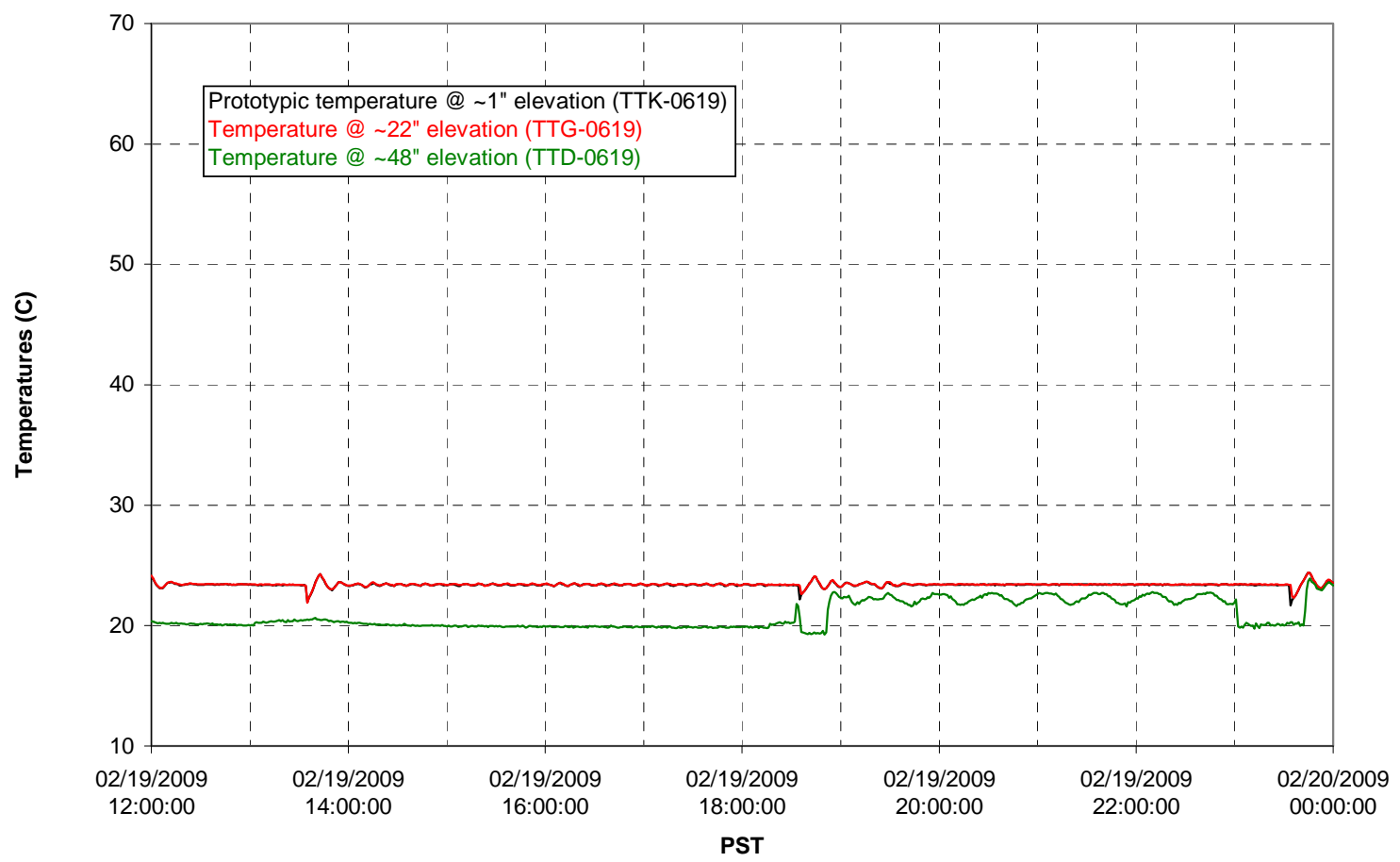


T02A and filter loop temperatures

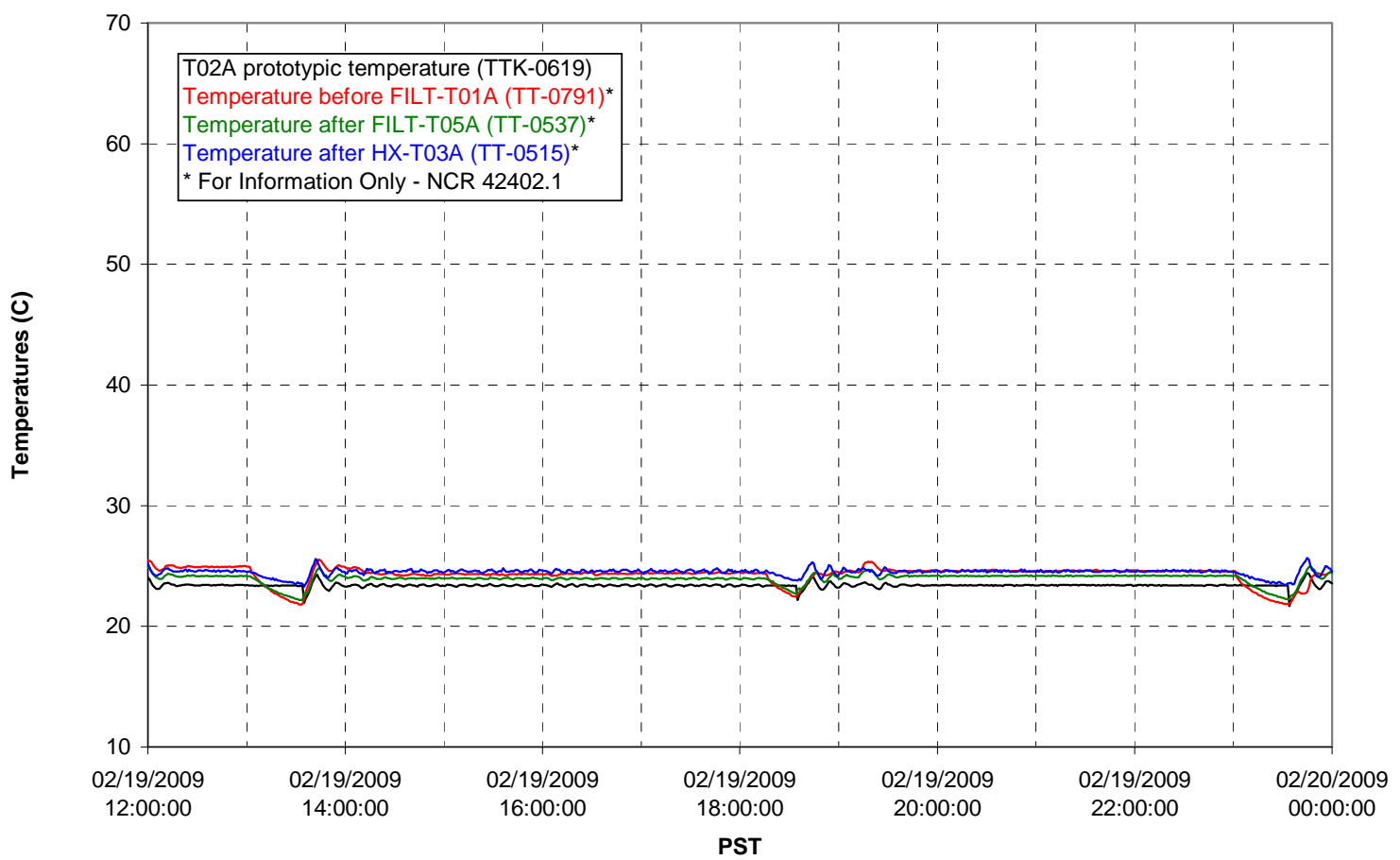

Pump Pressures and Flow

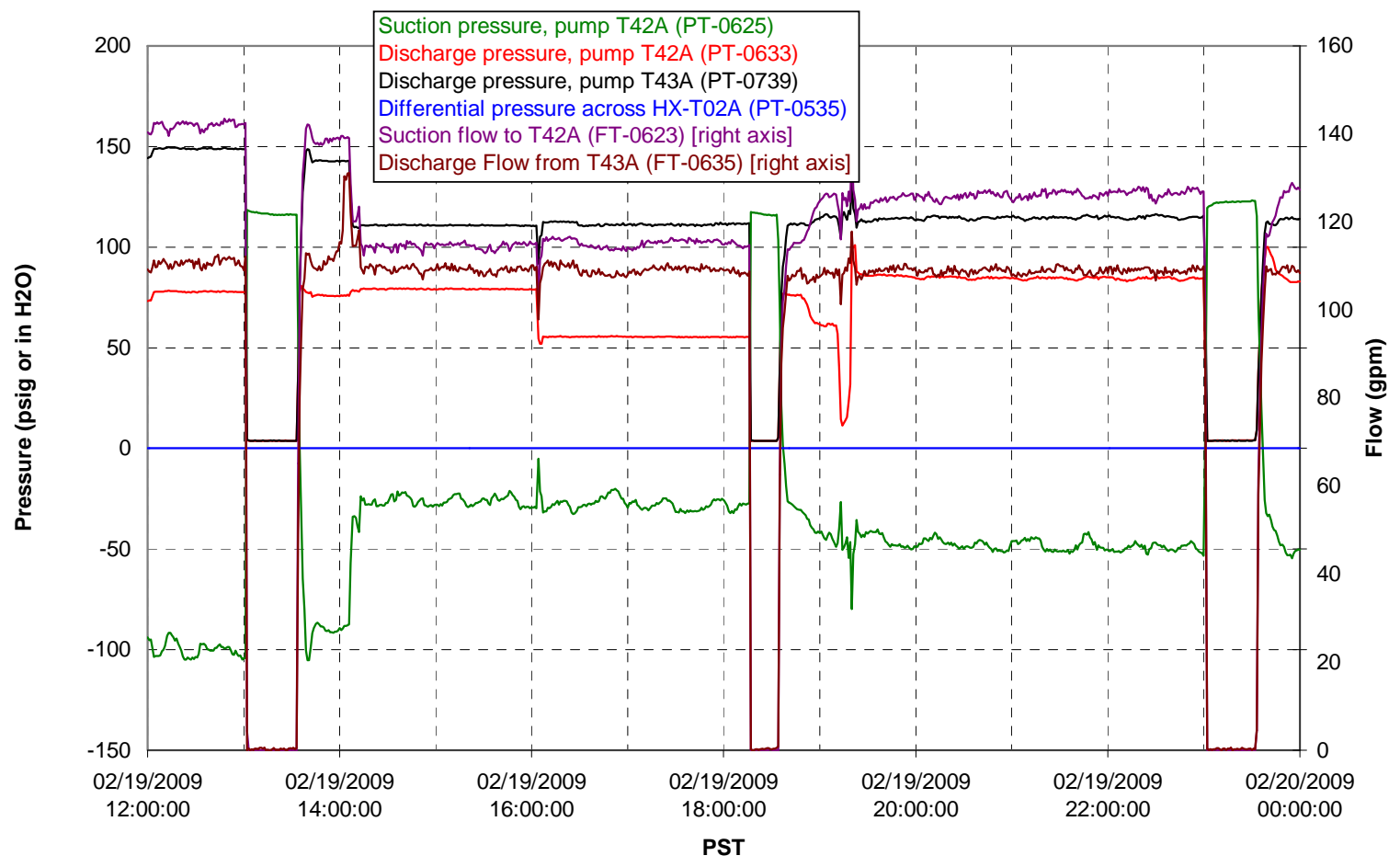


Axial pressure drop

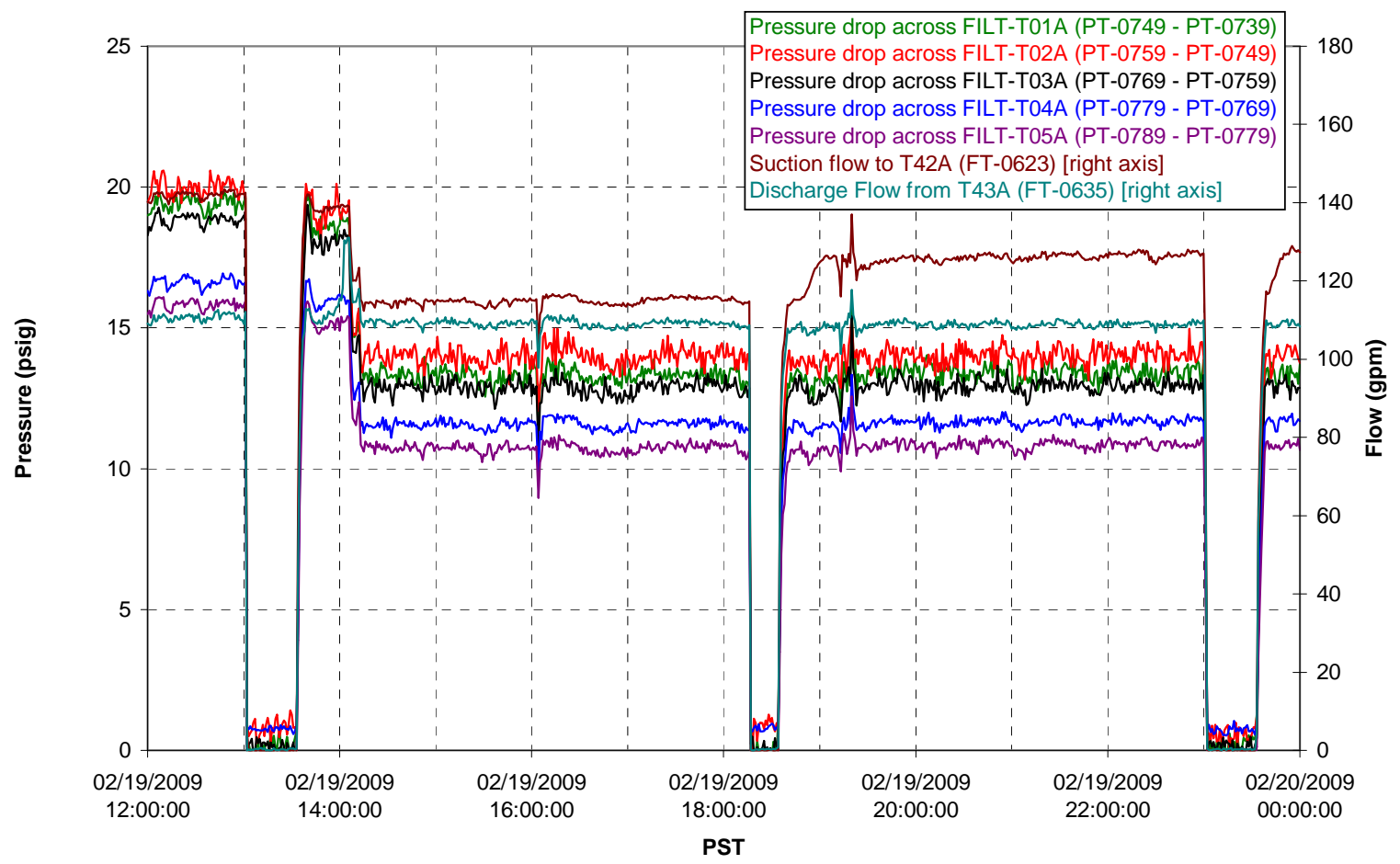

Permeate flow rates

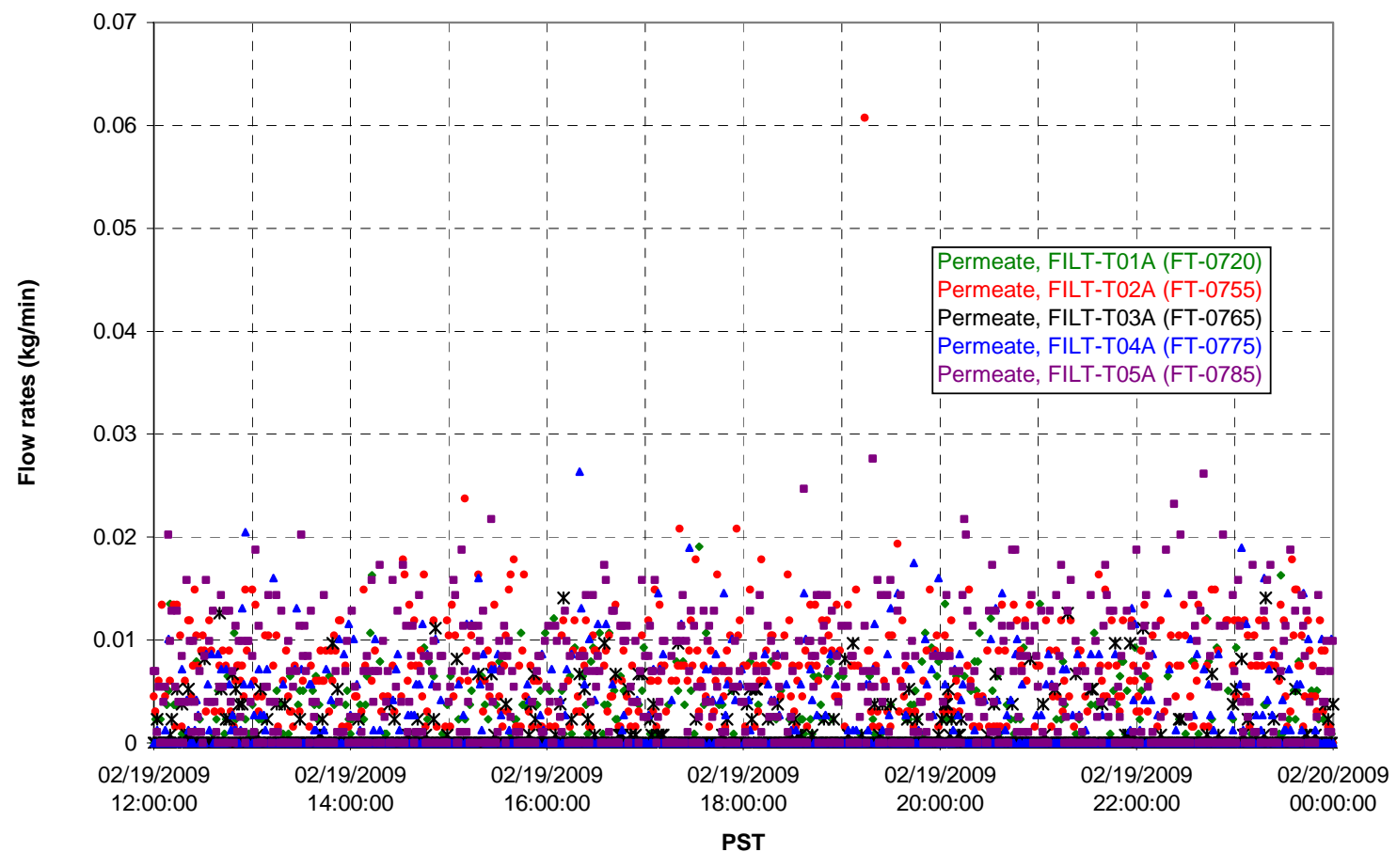


T02A Inner Temperature Tree

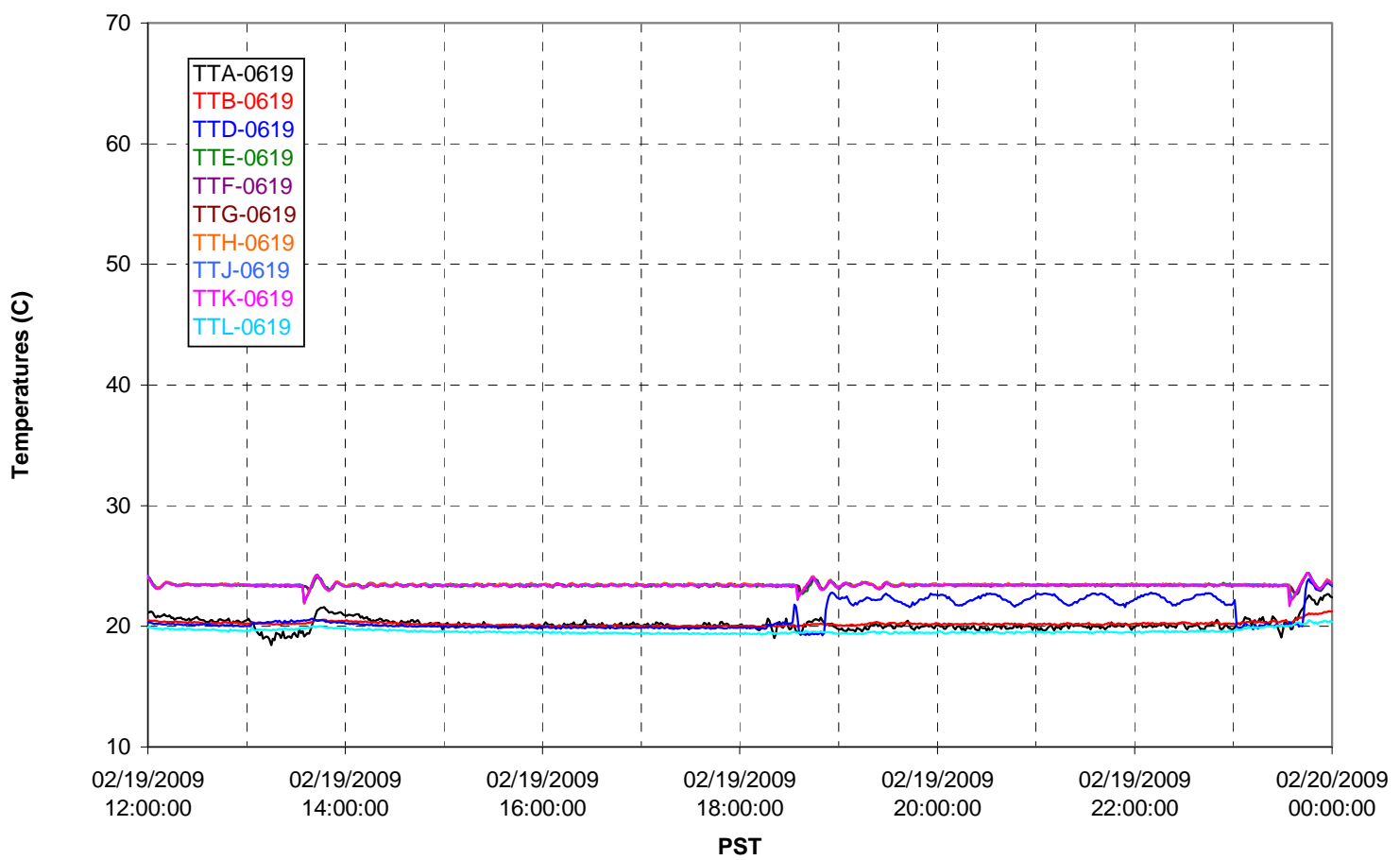

T02A Outer Temperature Tree

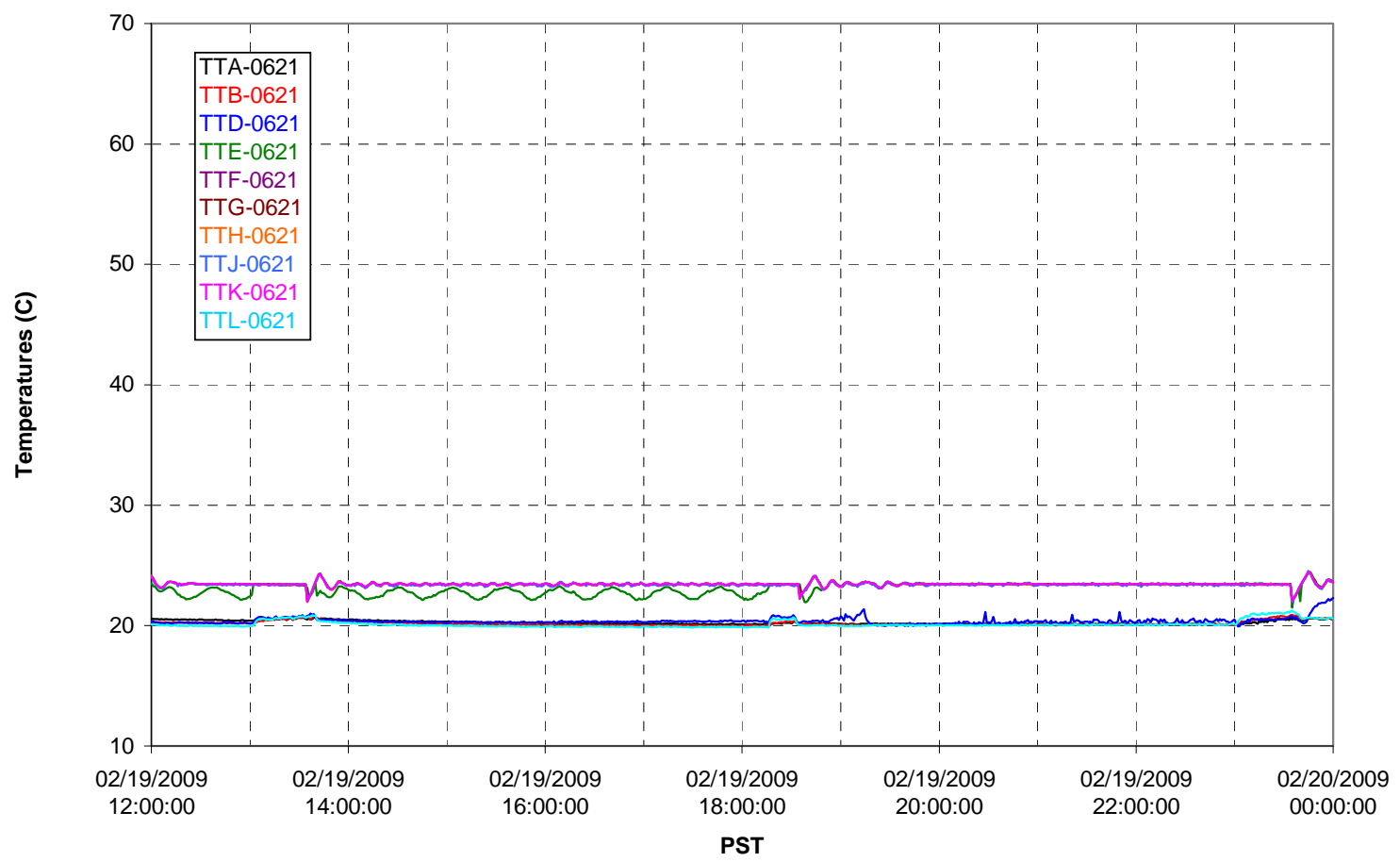


T02A temperatures

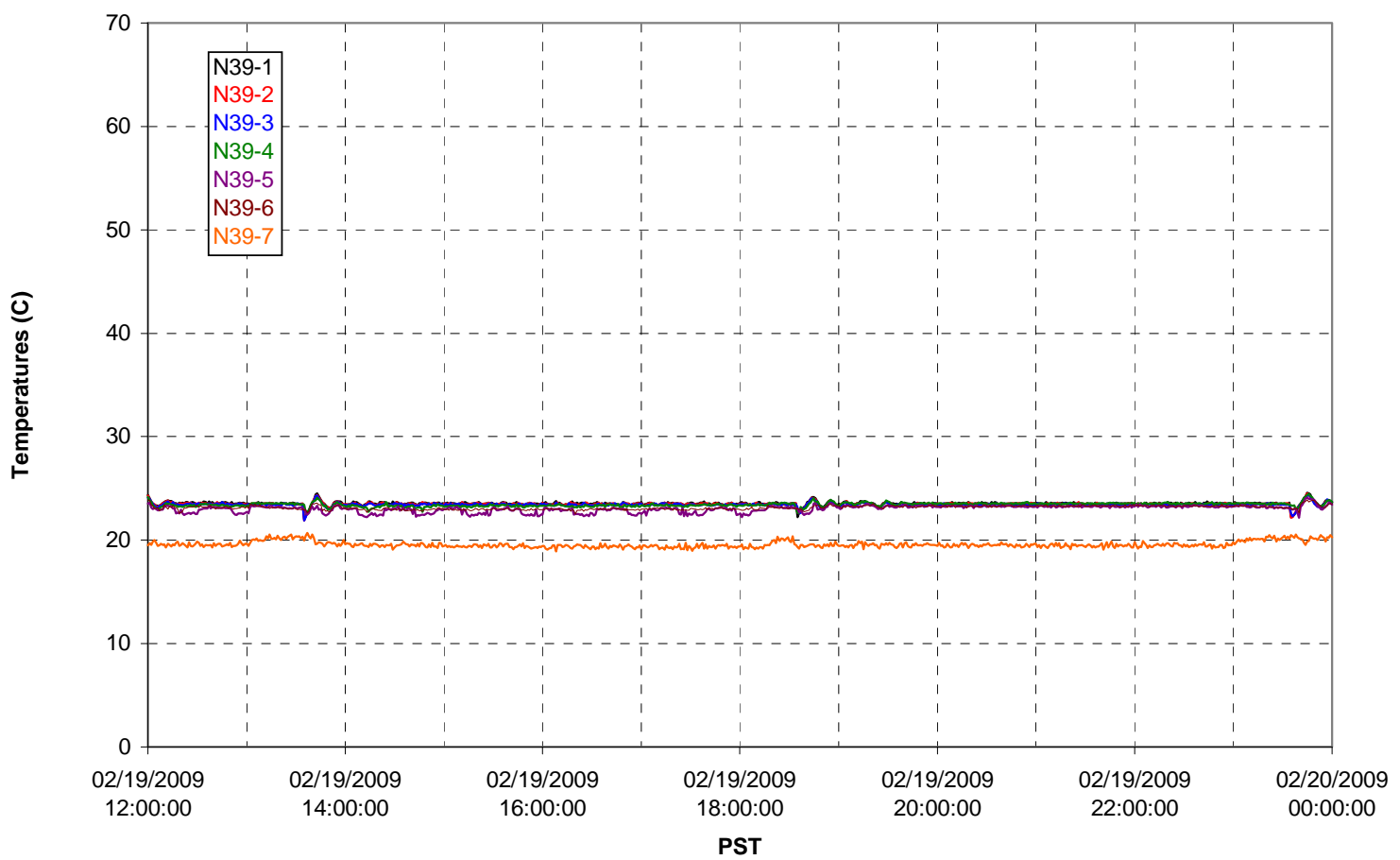

T02A temperatures

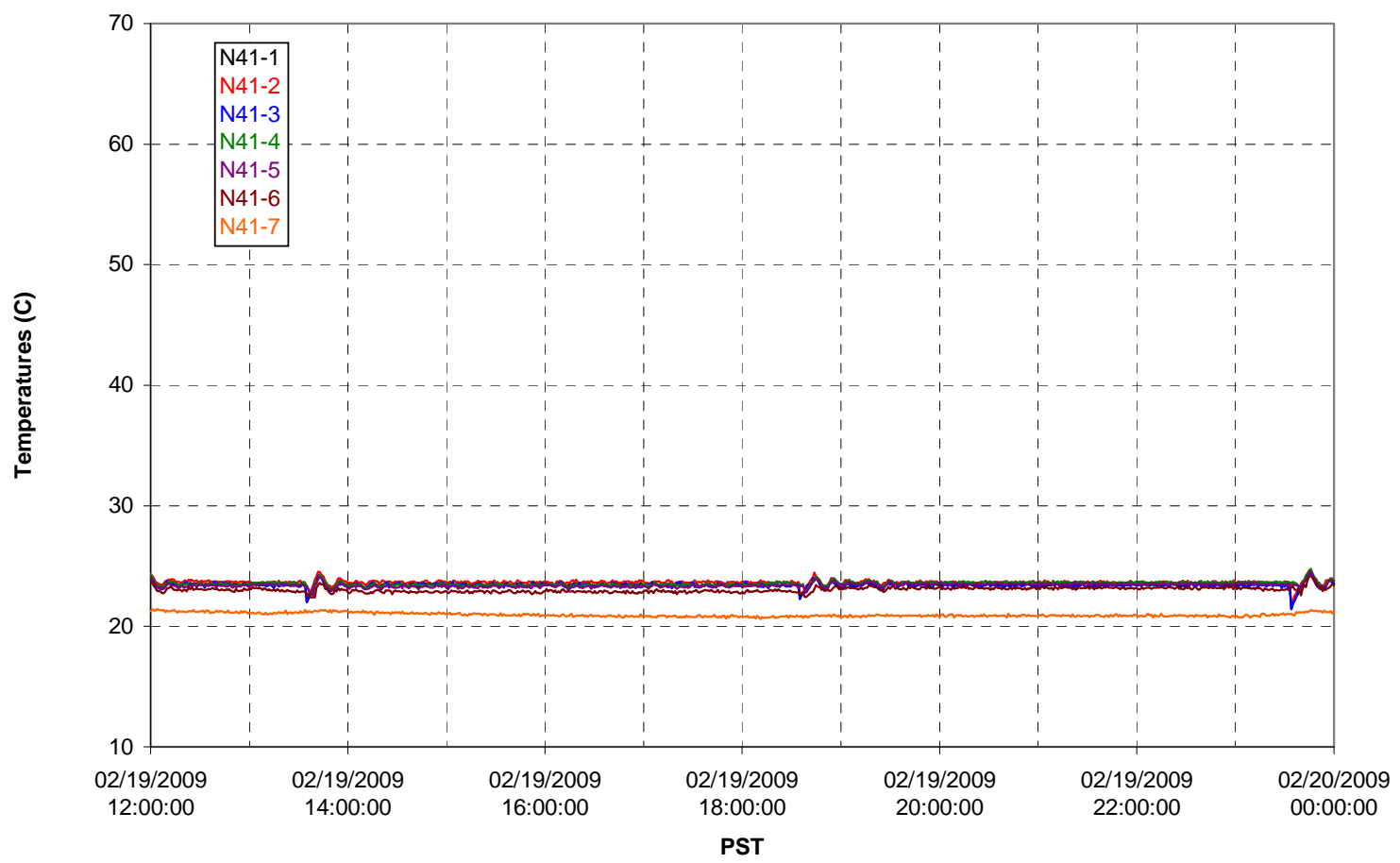


T02A temperatures

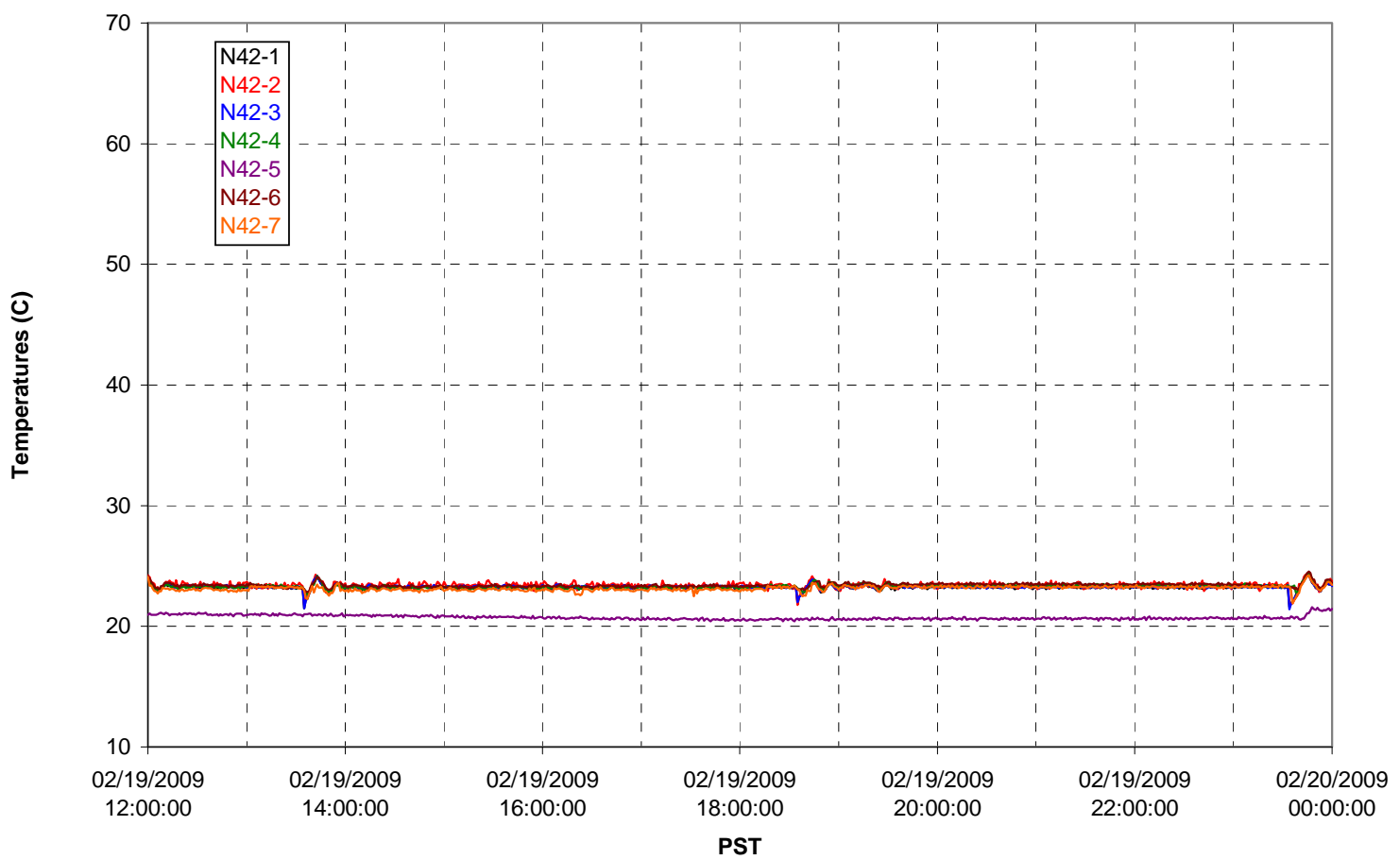

T02A temperatures

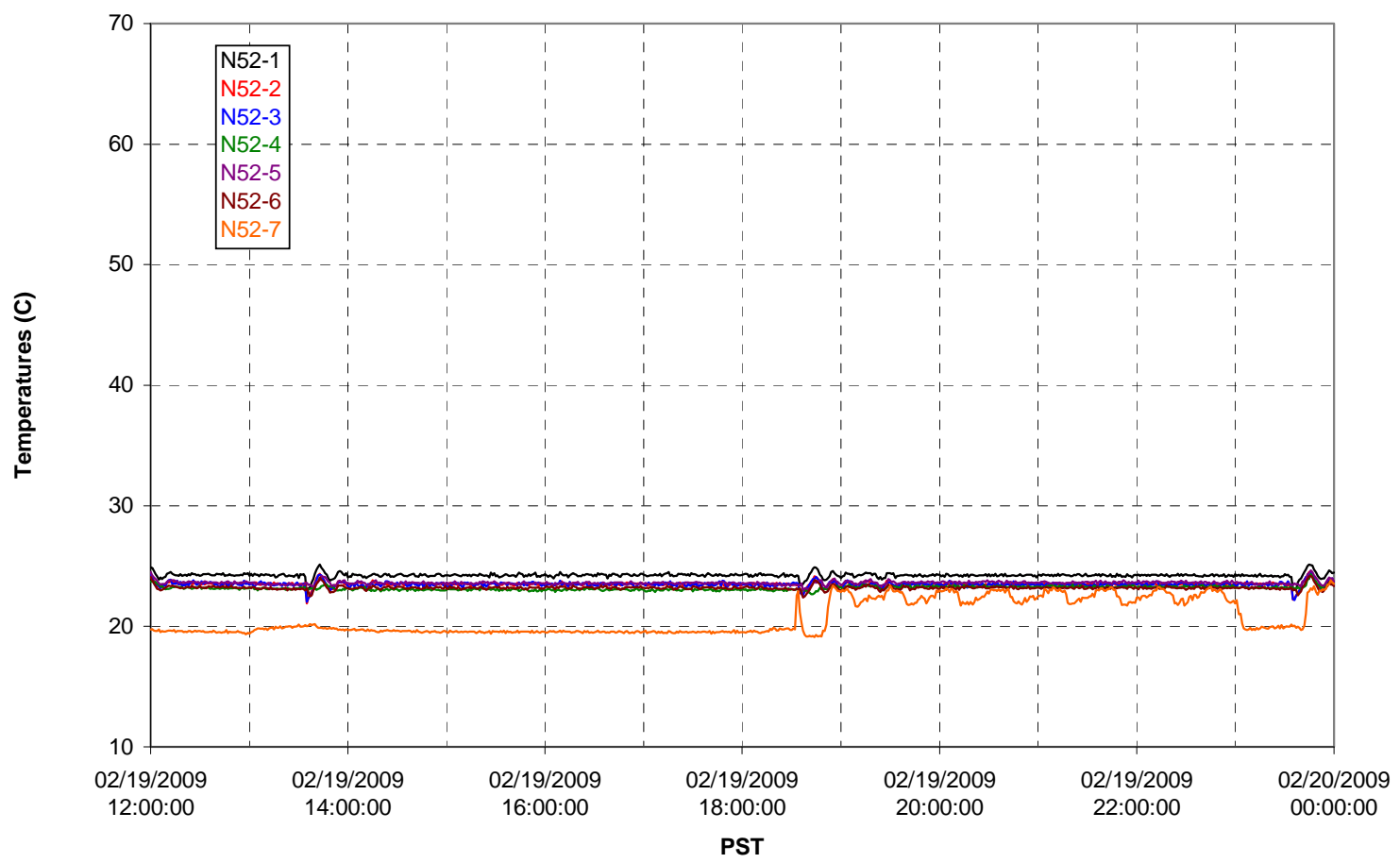


T02A Heating and Cooling
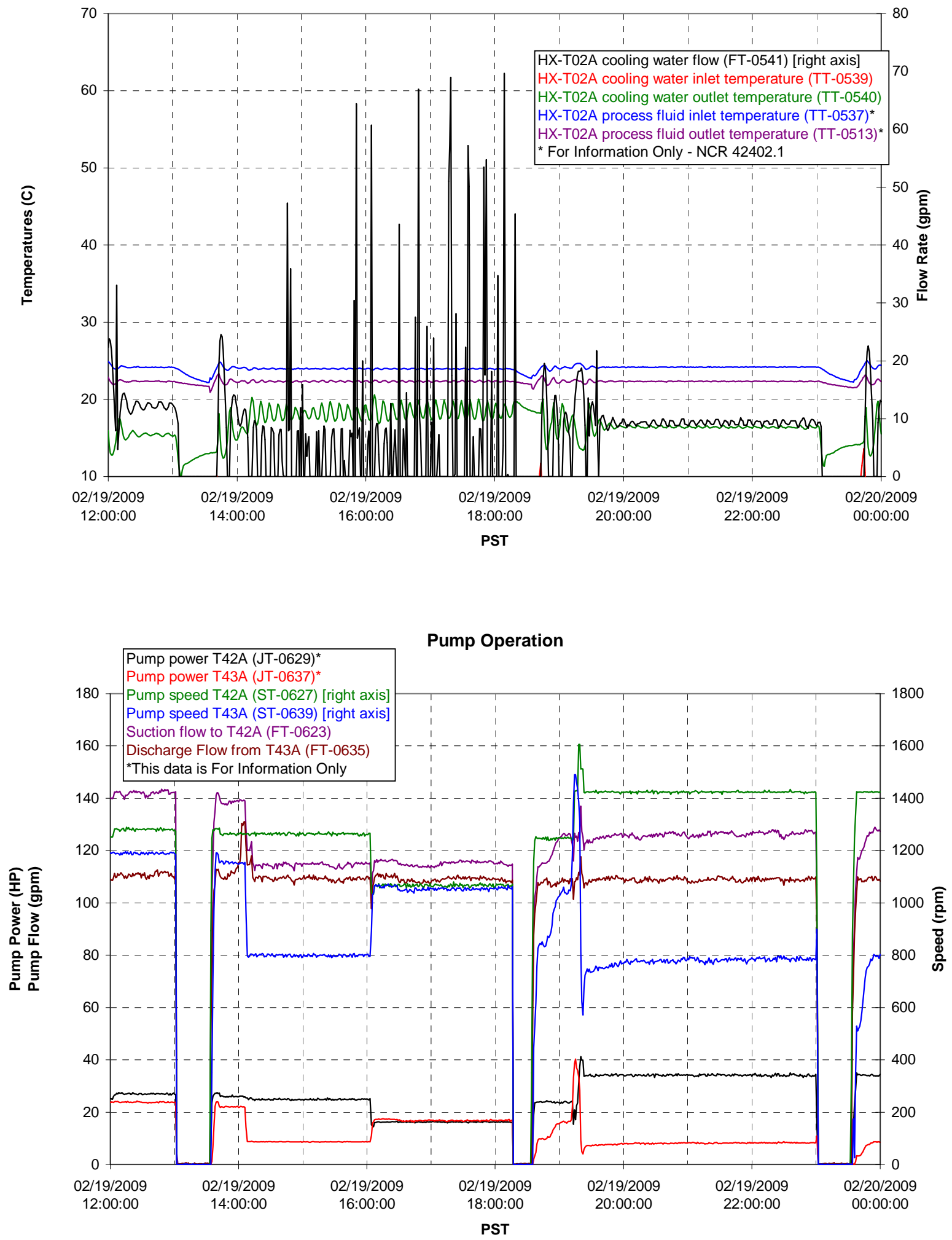
Pulsepot UFP-PP-T01A

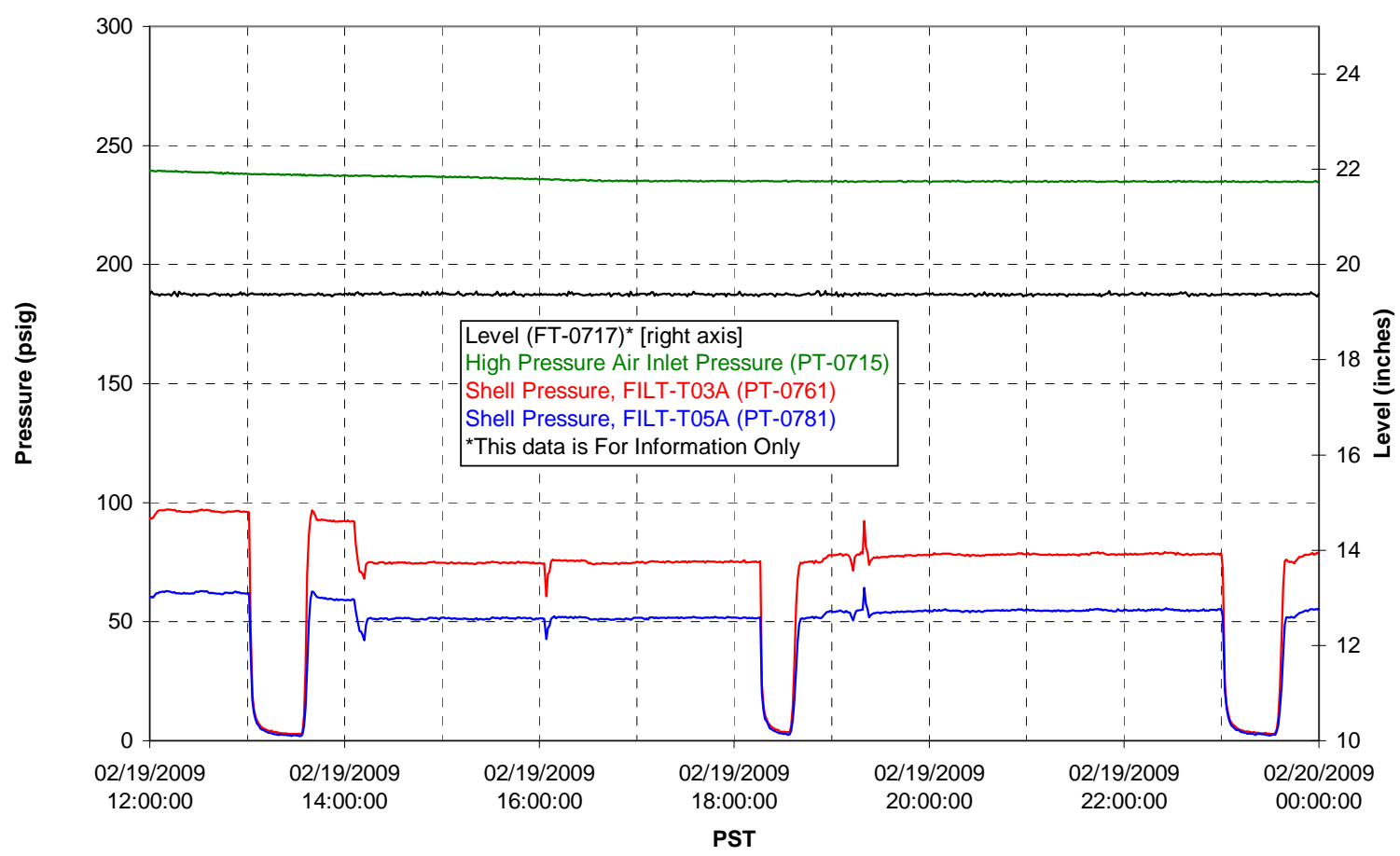

Pulsepot UFP-PP-T02A

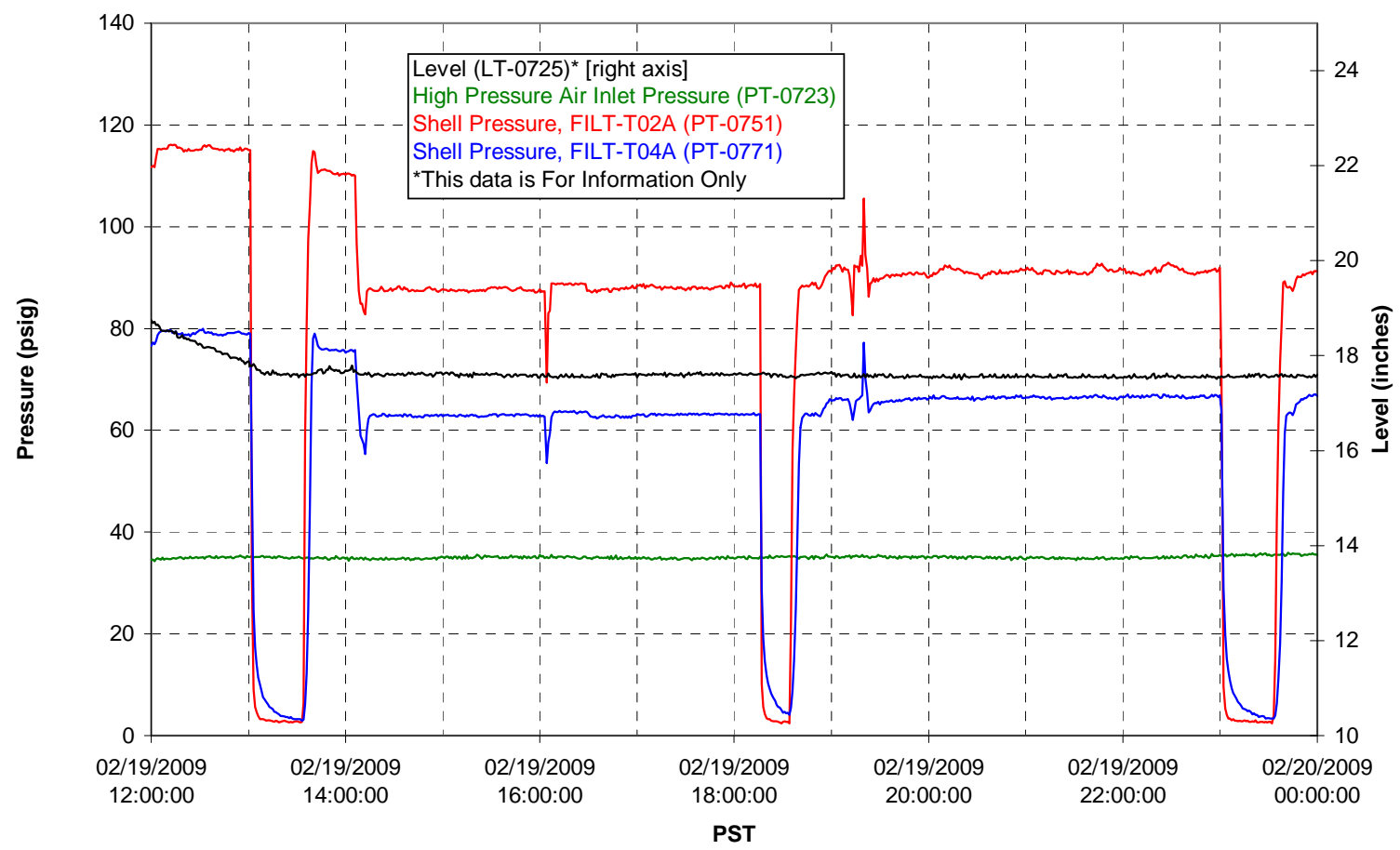




\section{Pulsepot UFP-PP-T03A}

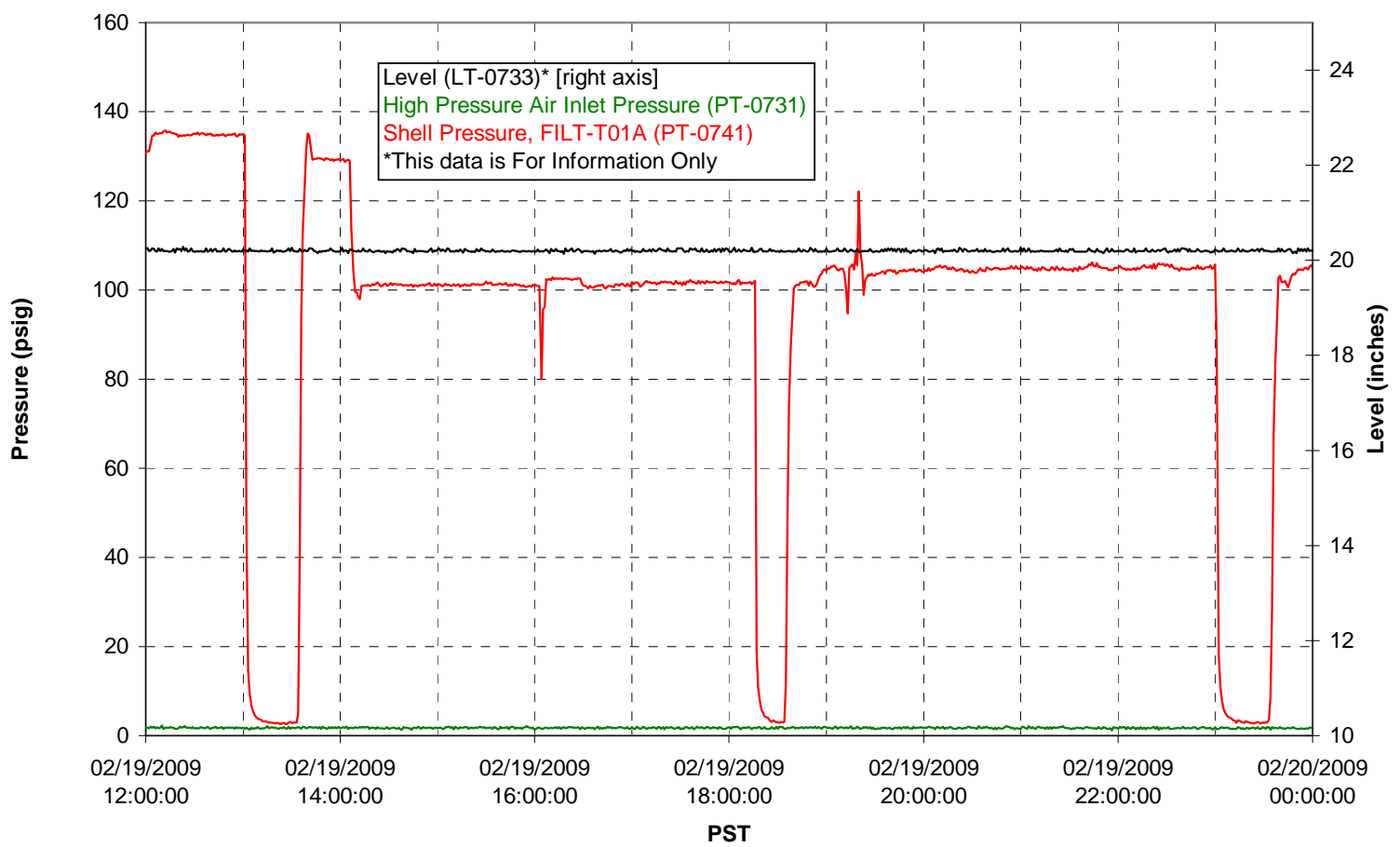

Pulsepot Levels

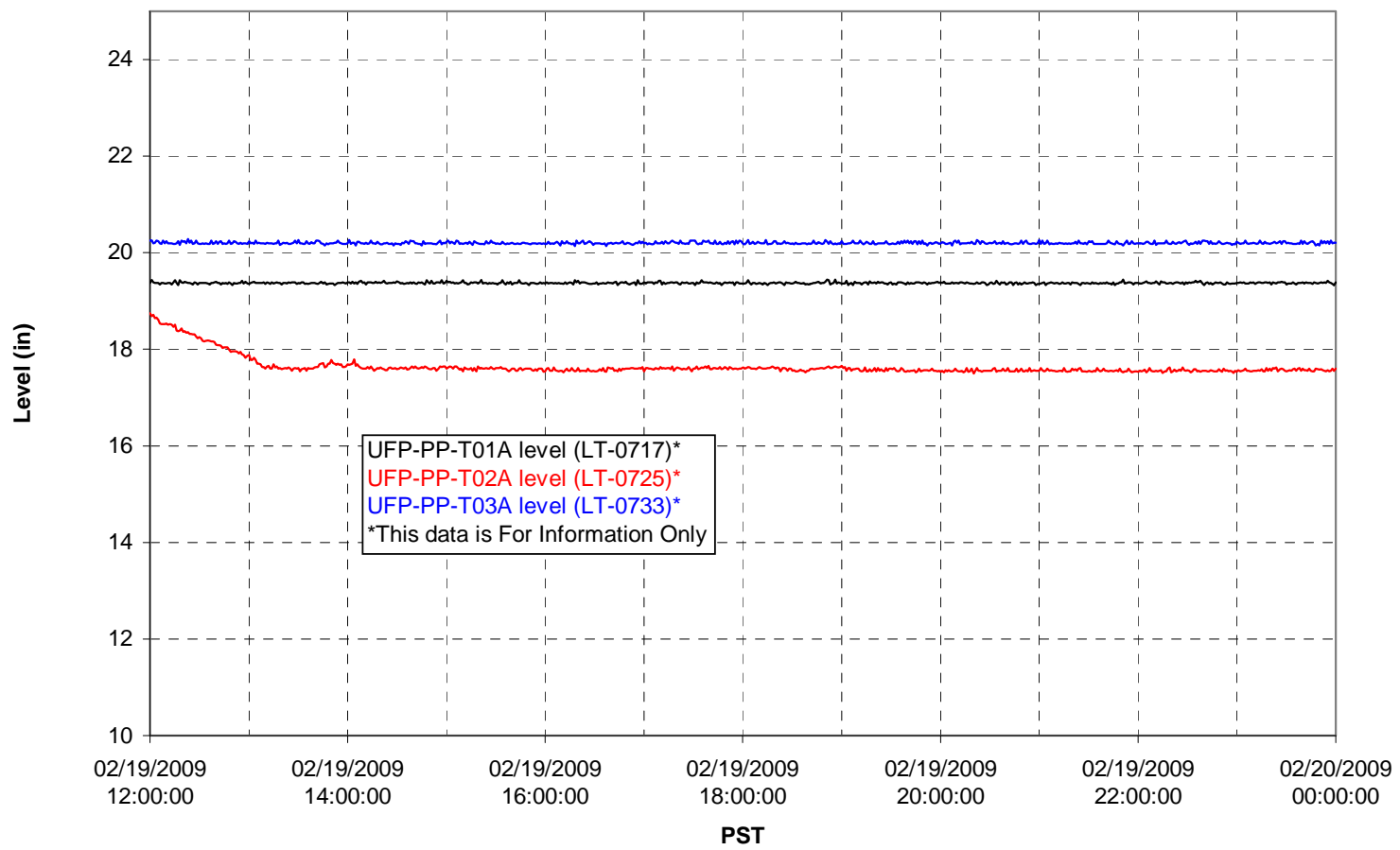


Filter UFP-FILT-T01A

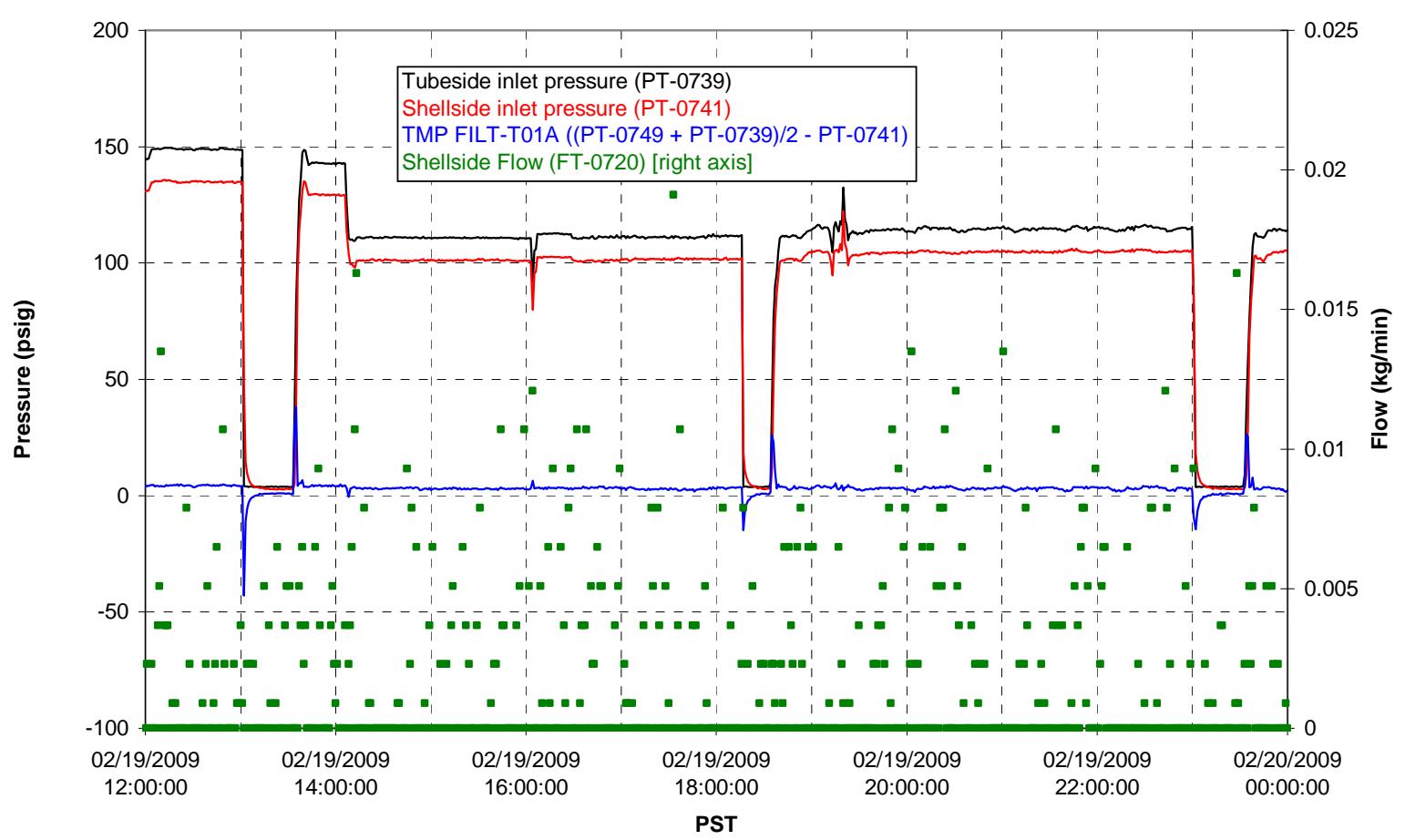

Filter UFP-FILT-T02A

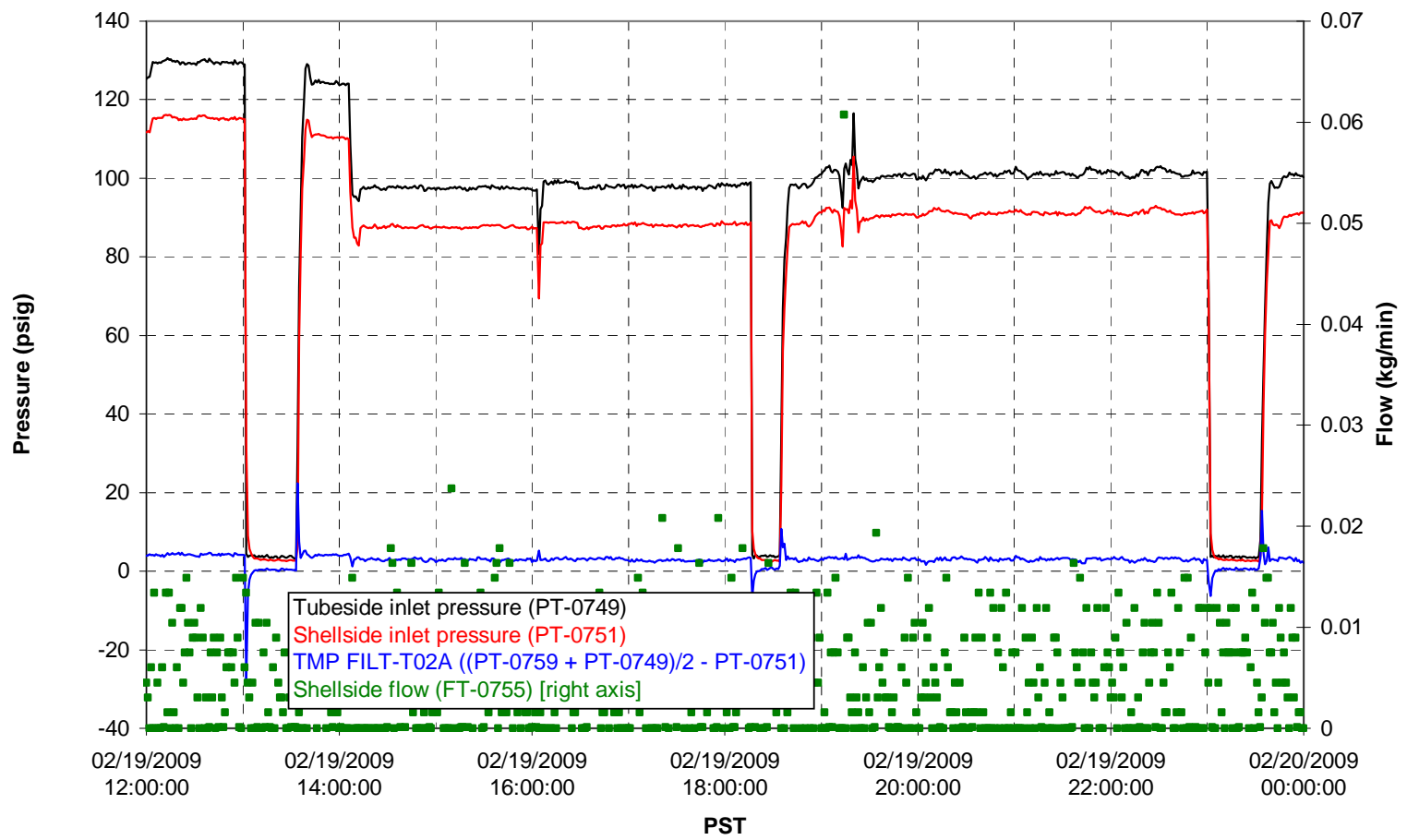


Filter UFP-FILT-T03A

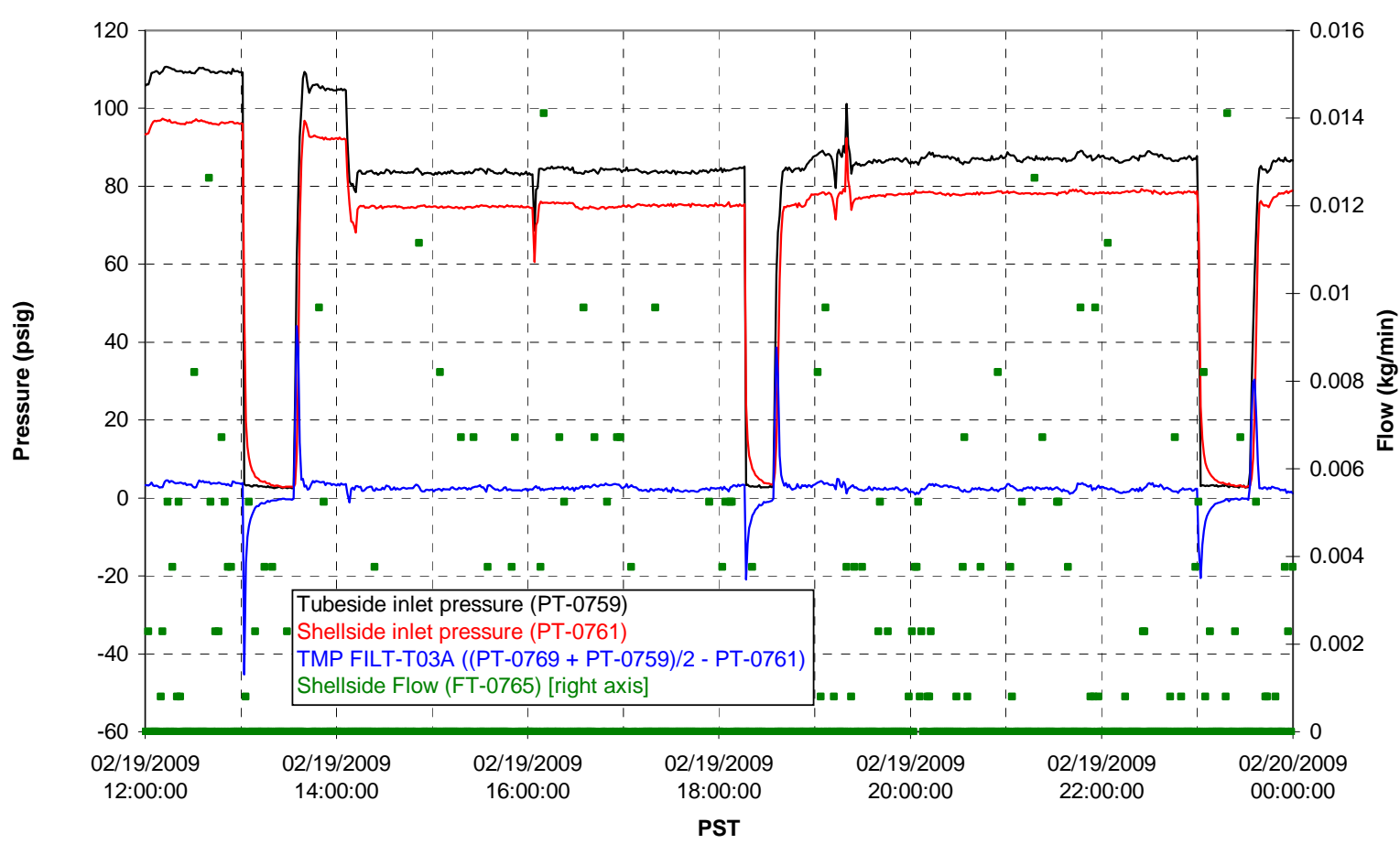

Filter UFP-FILT-T04A

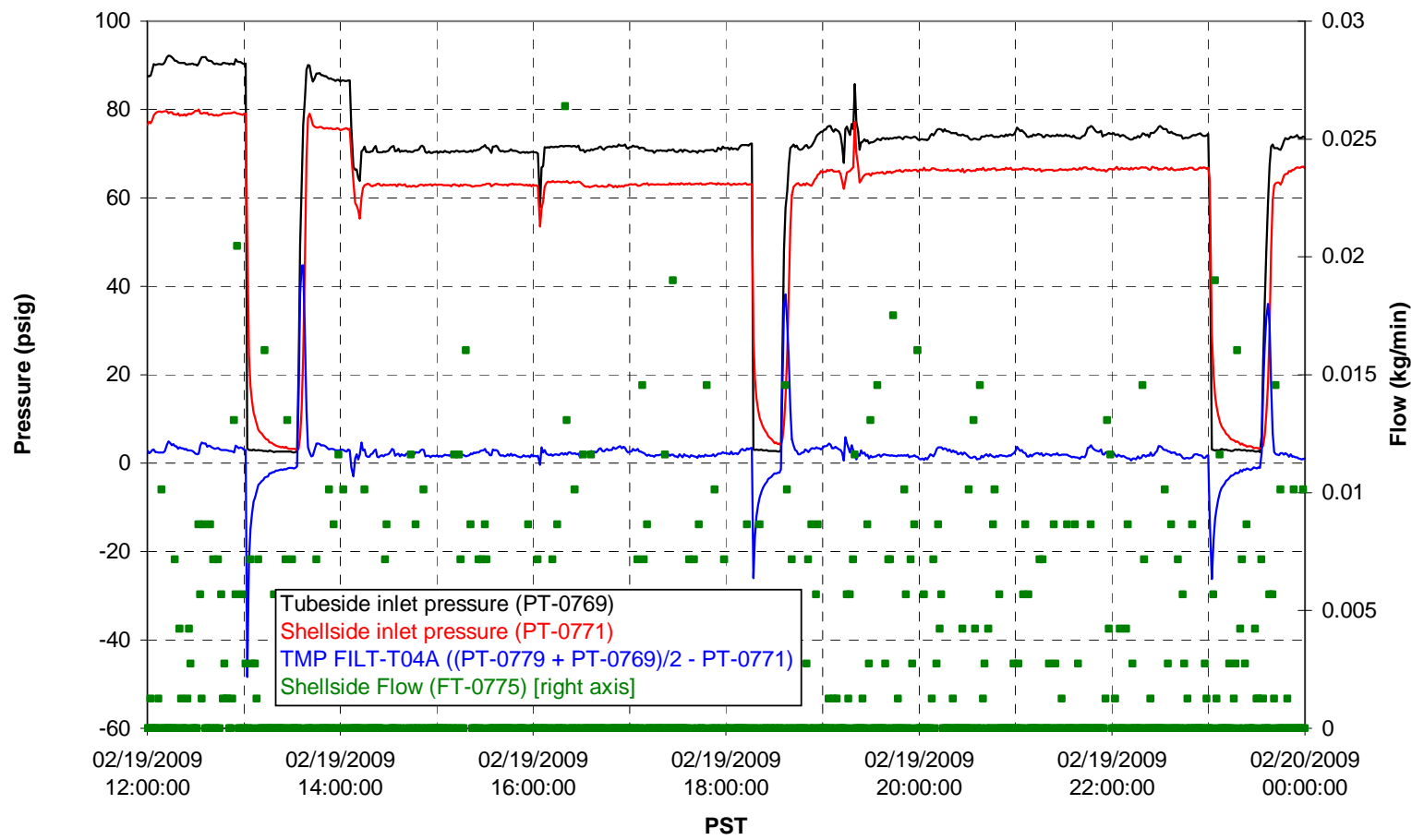

G.716 
Filter UFP-FILT-T05A

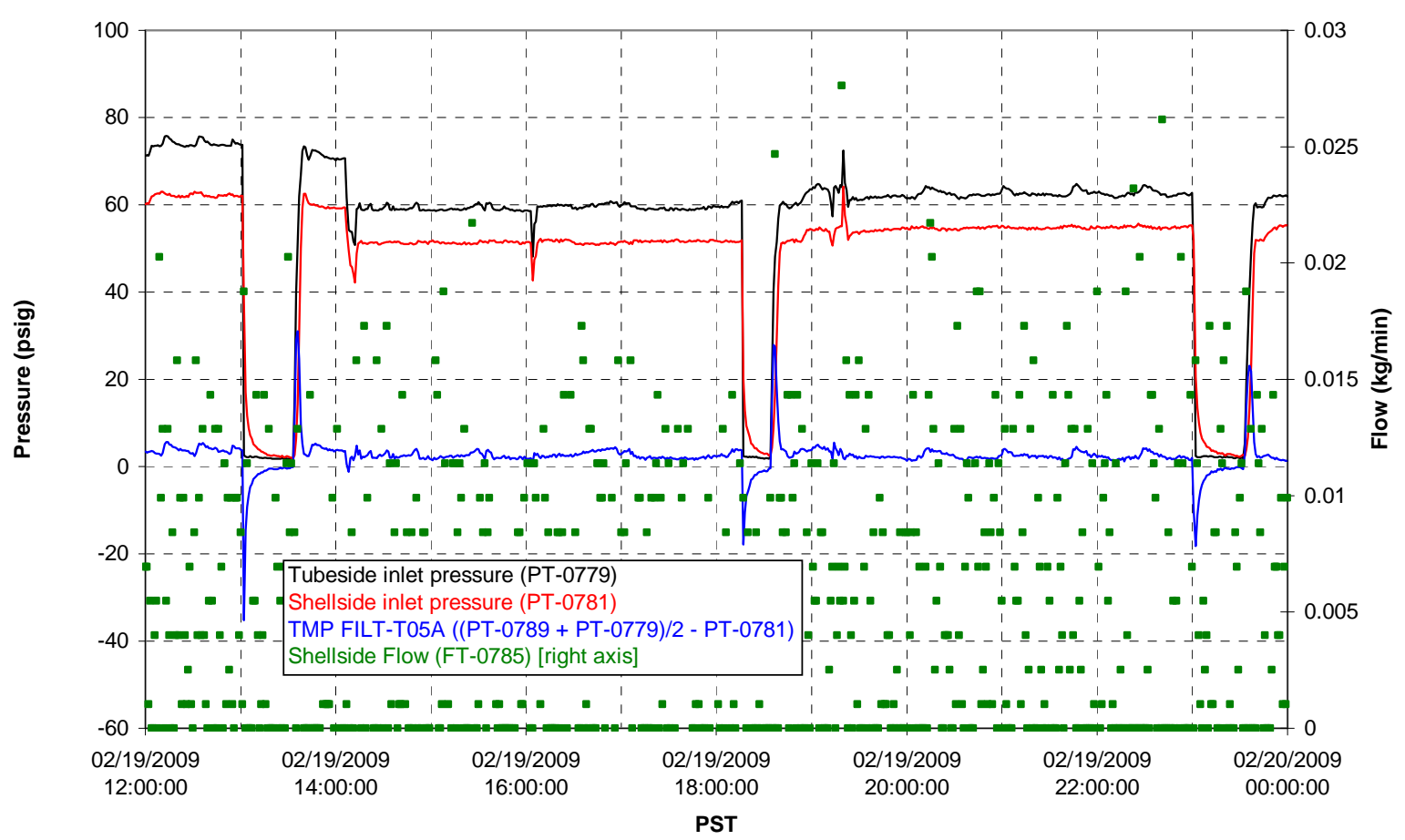

Chemical Flow

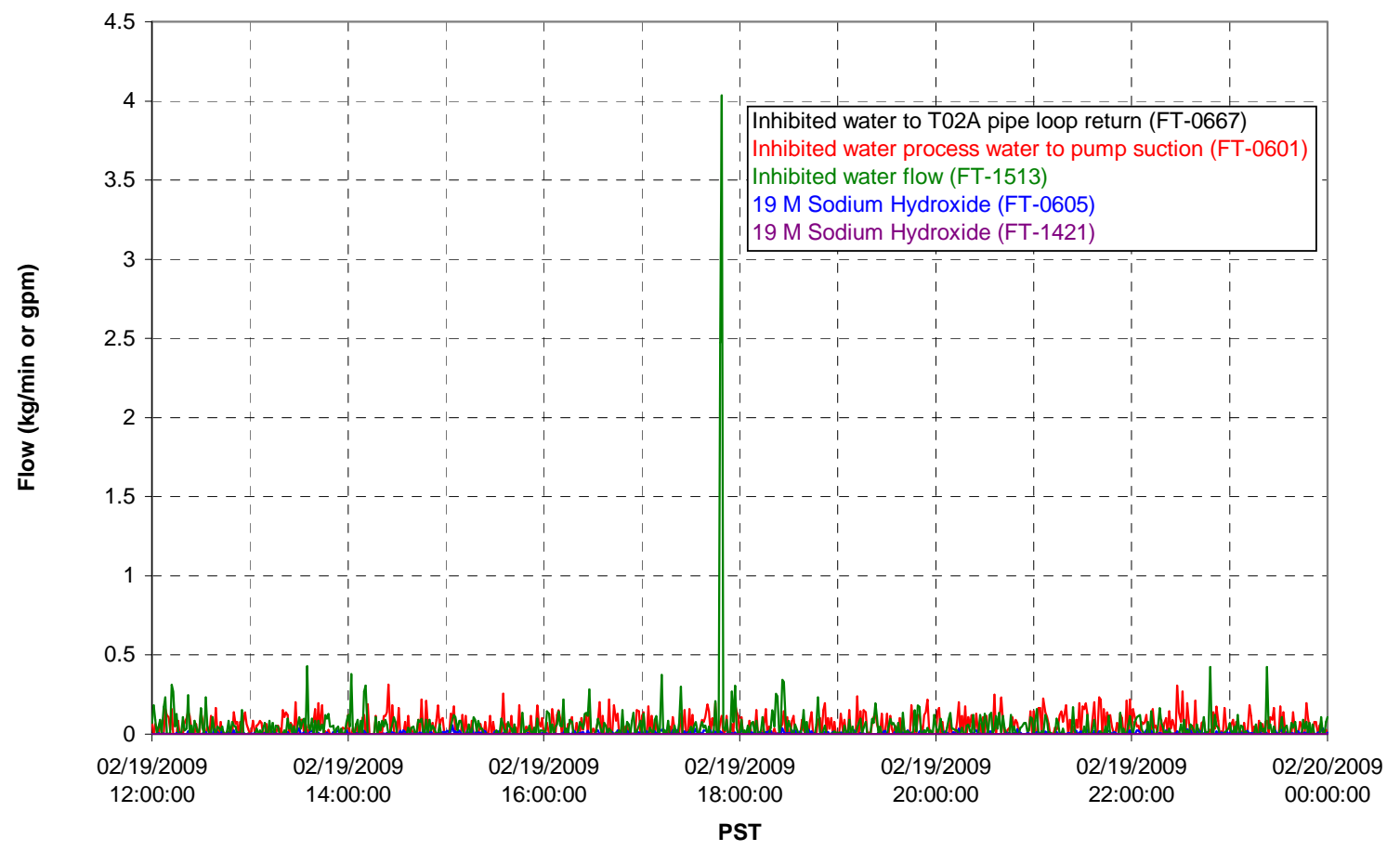

G.717 


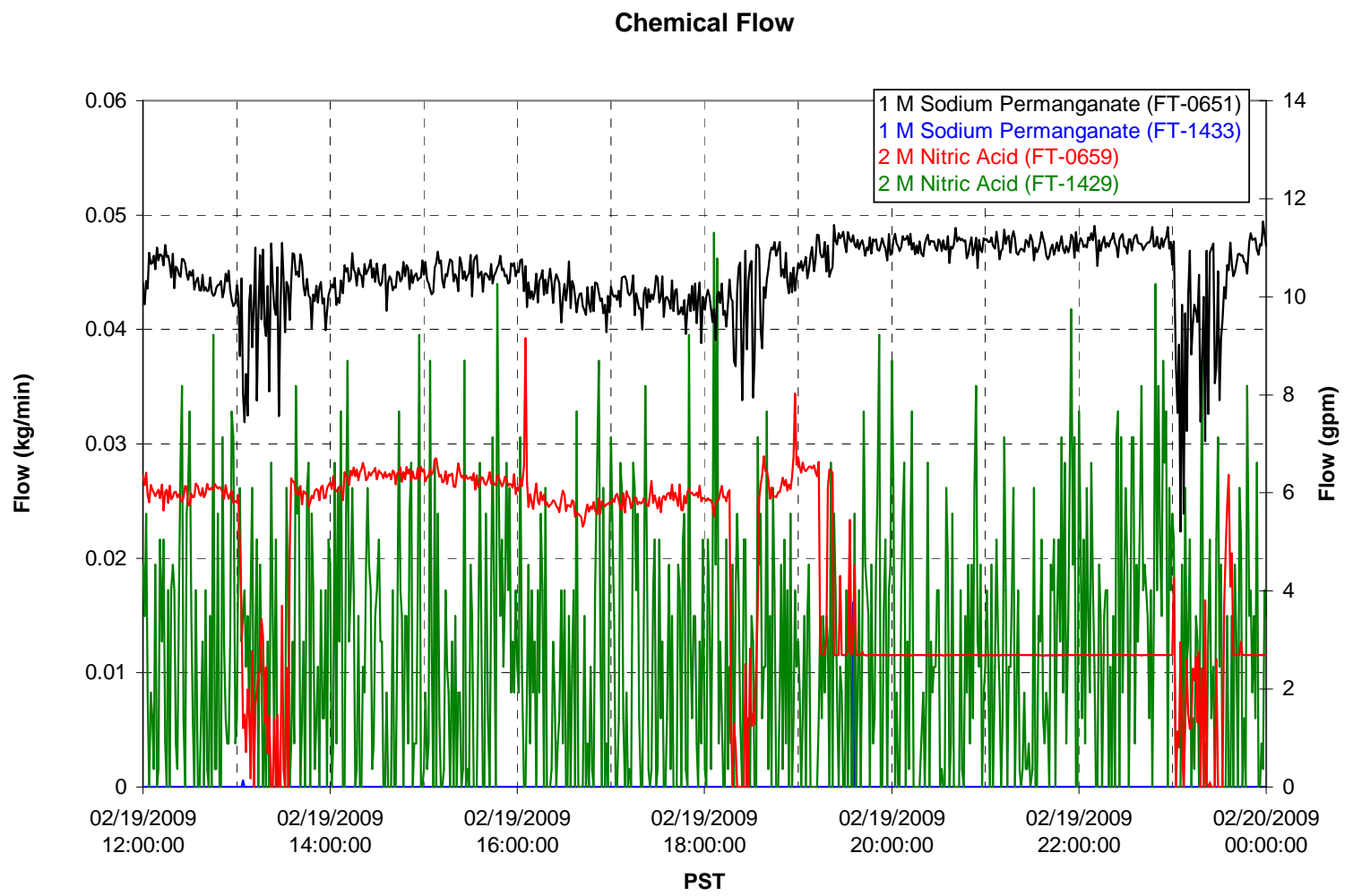

Air Flows

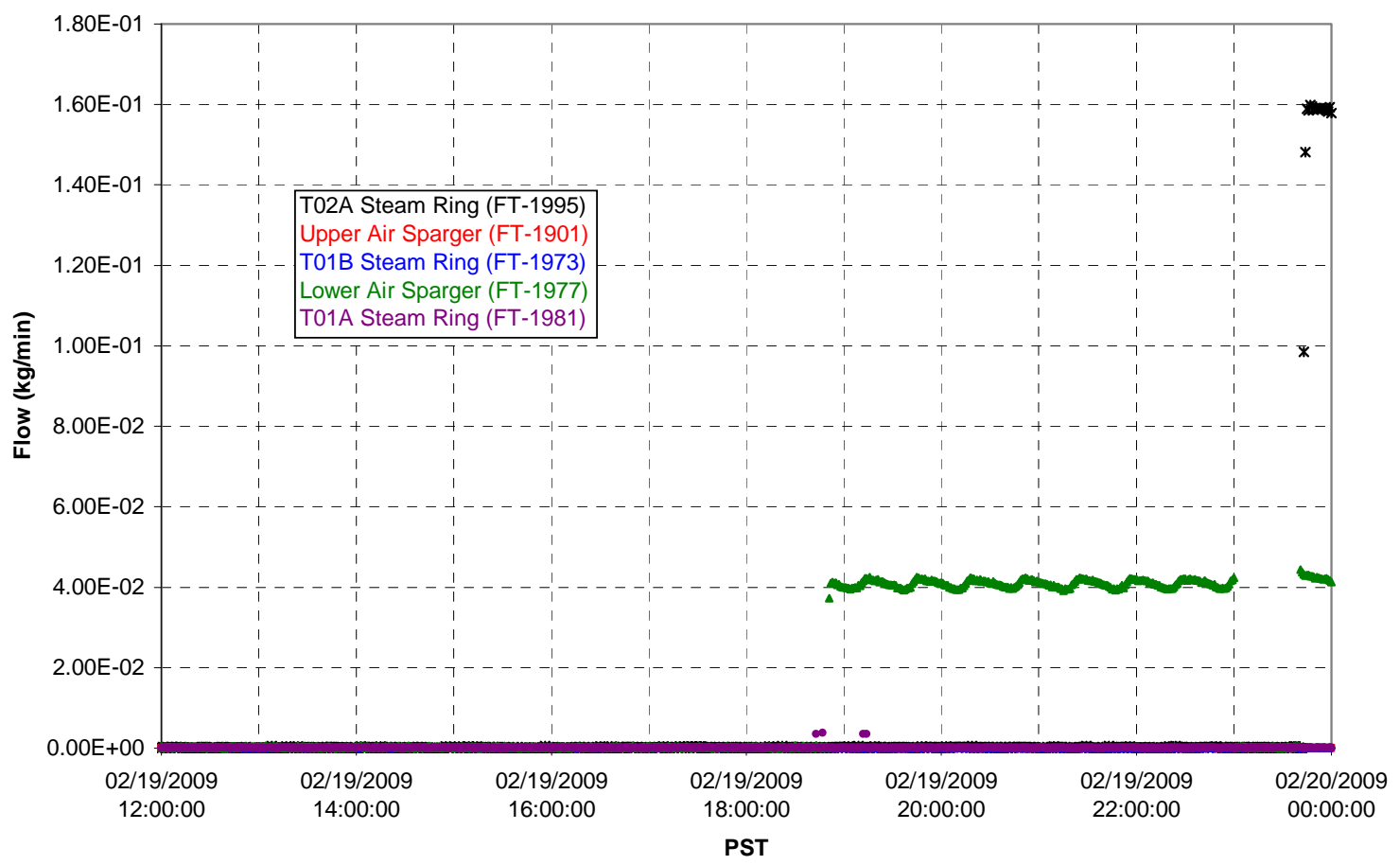


T02A Steam

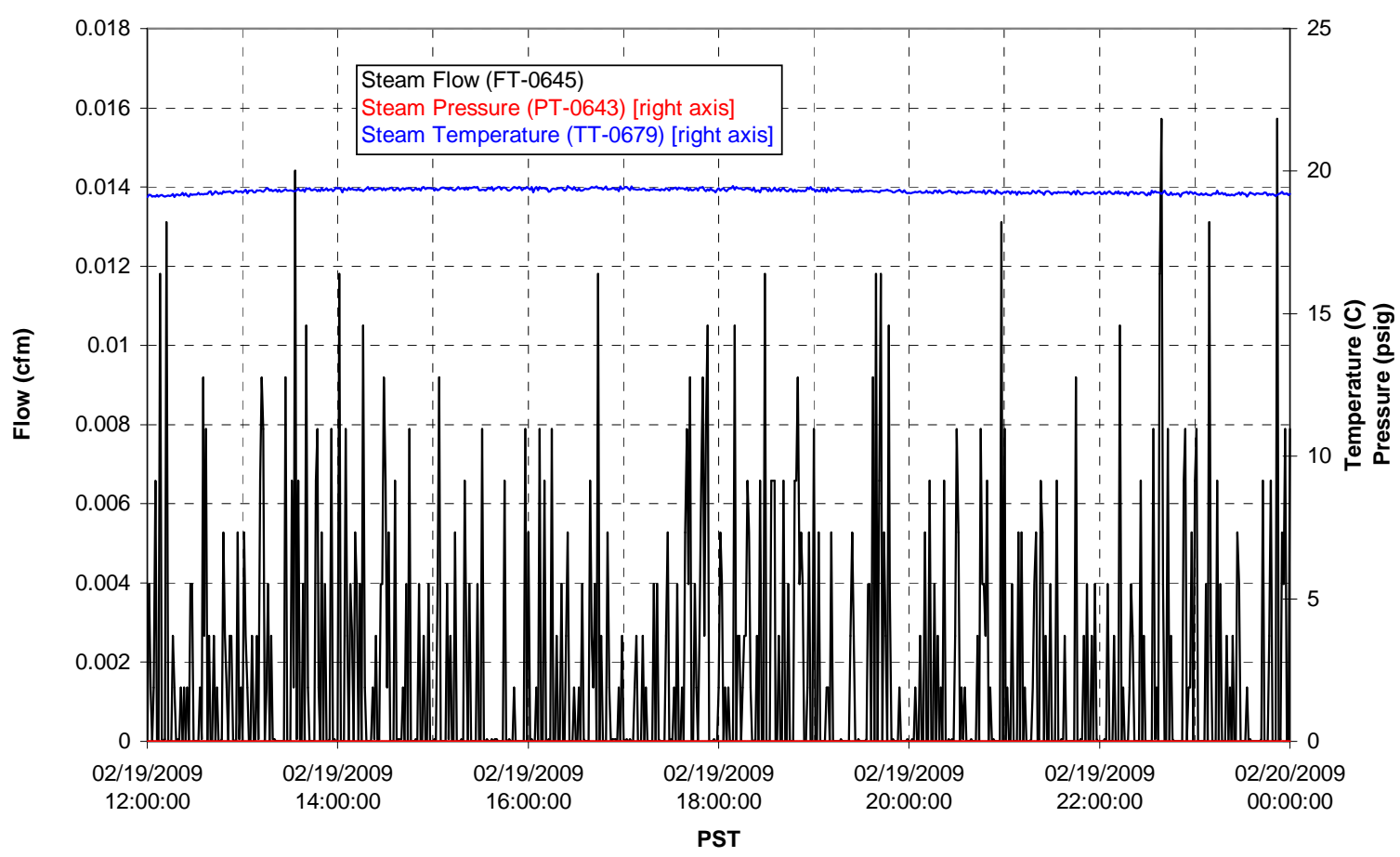

T01A Steam

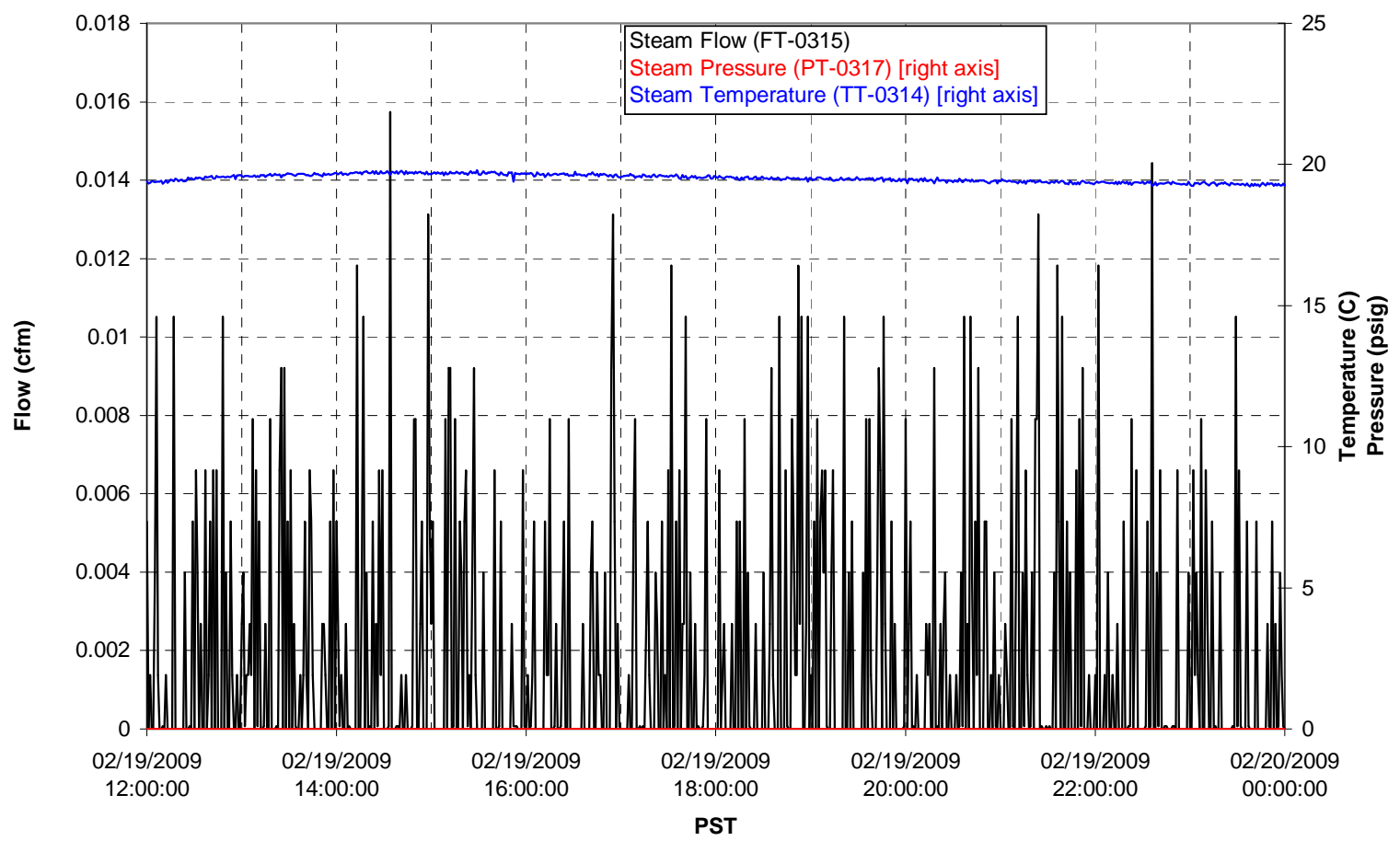


T01B Steam

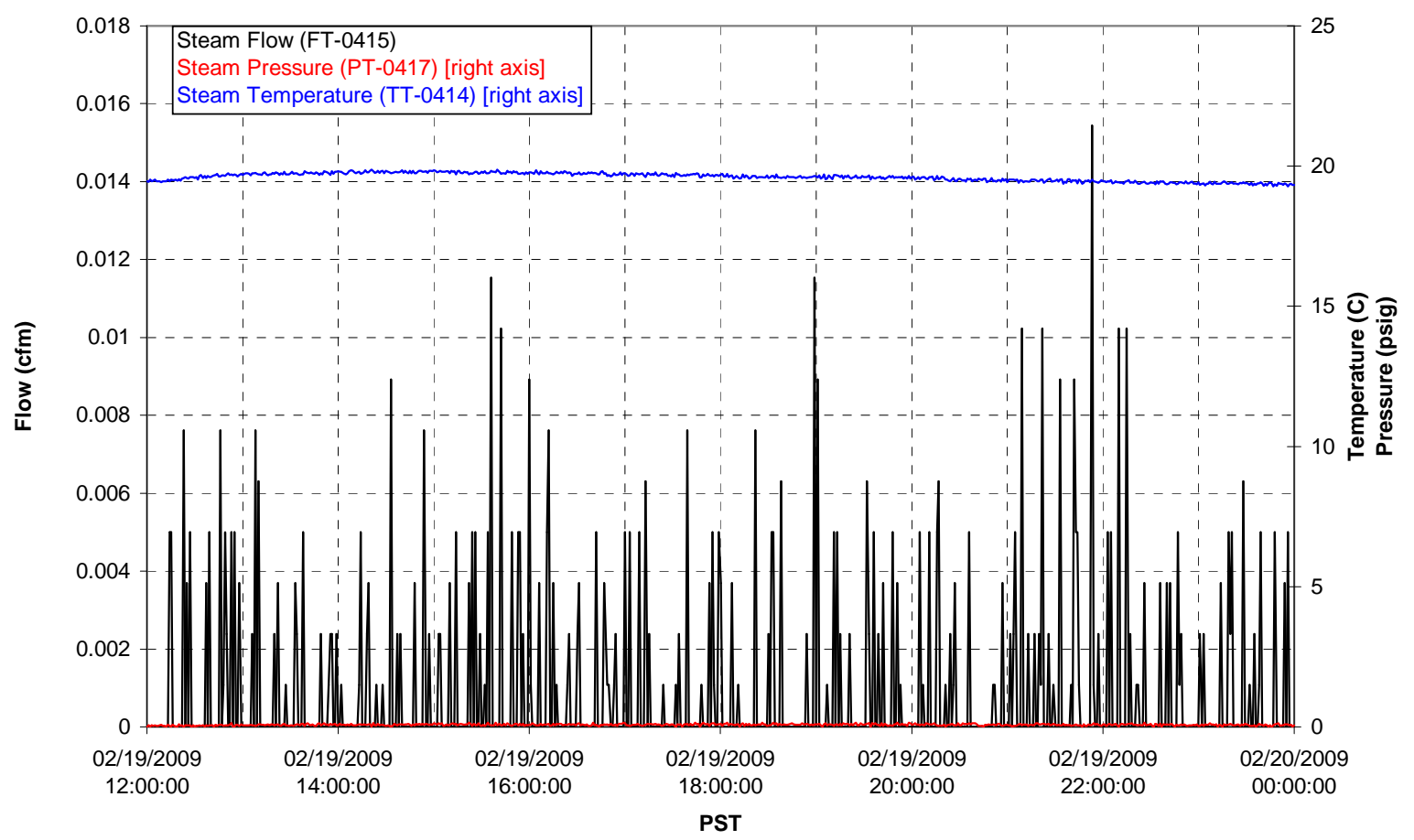




\section{Integrated Test A Data Plots 02/20/09 00:00 - 02/20/09 12:00}


T01A level

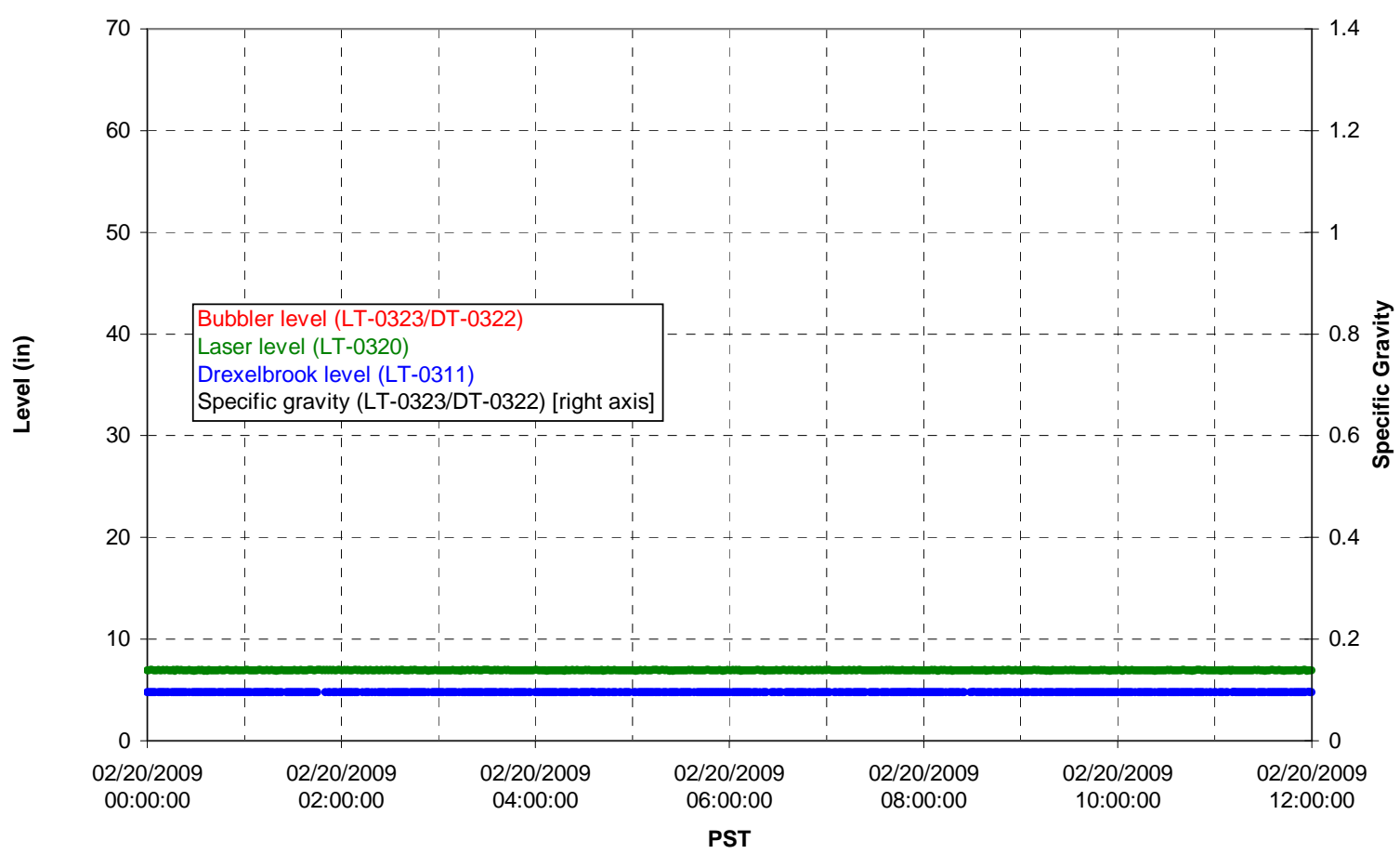

T01A temperatures

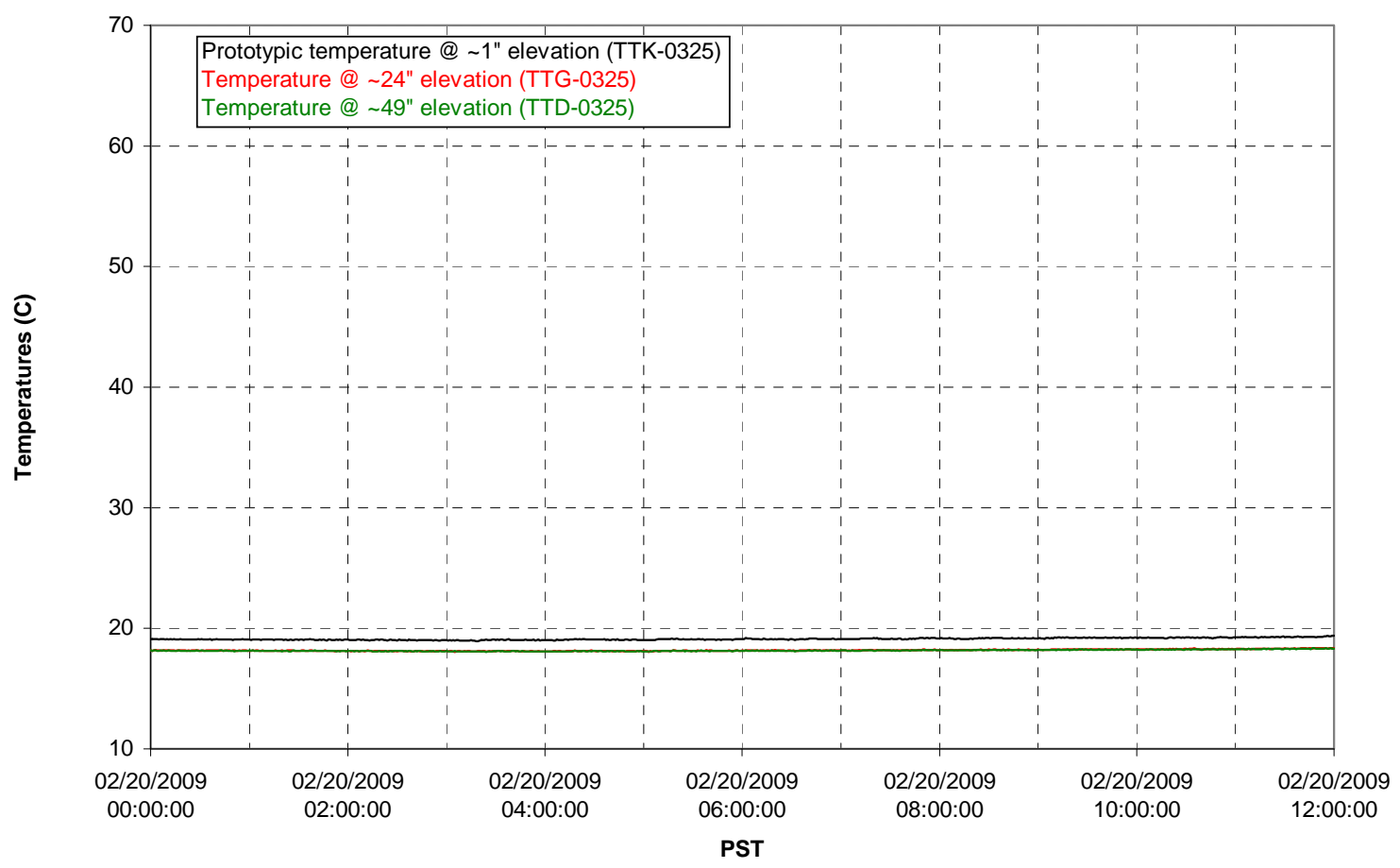

G.722 
T01B level

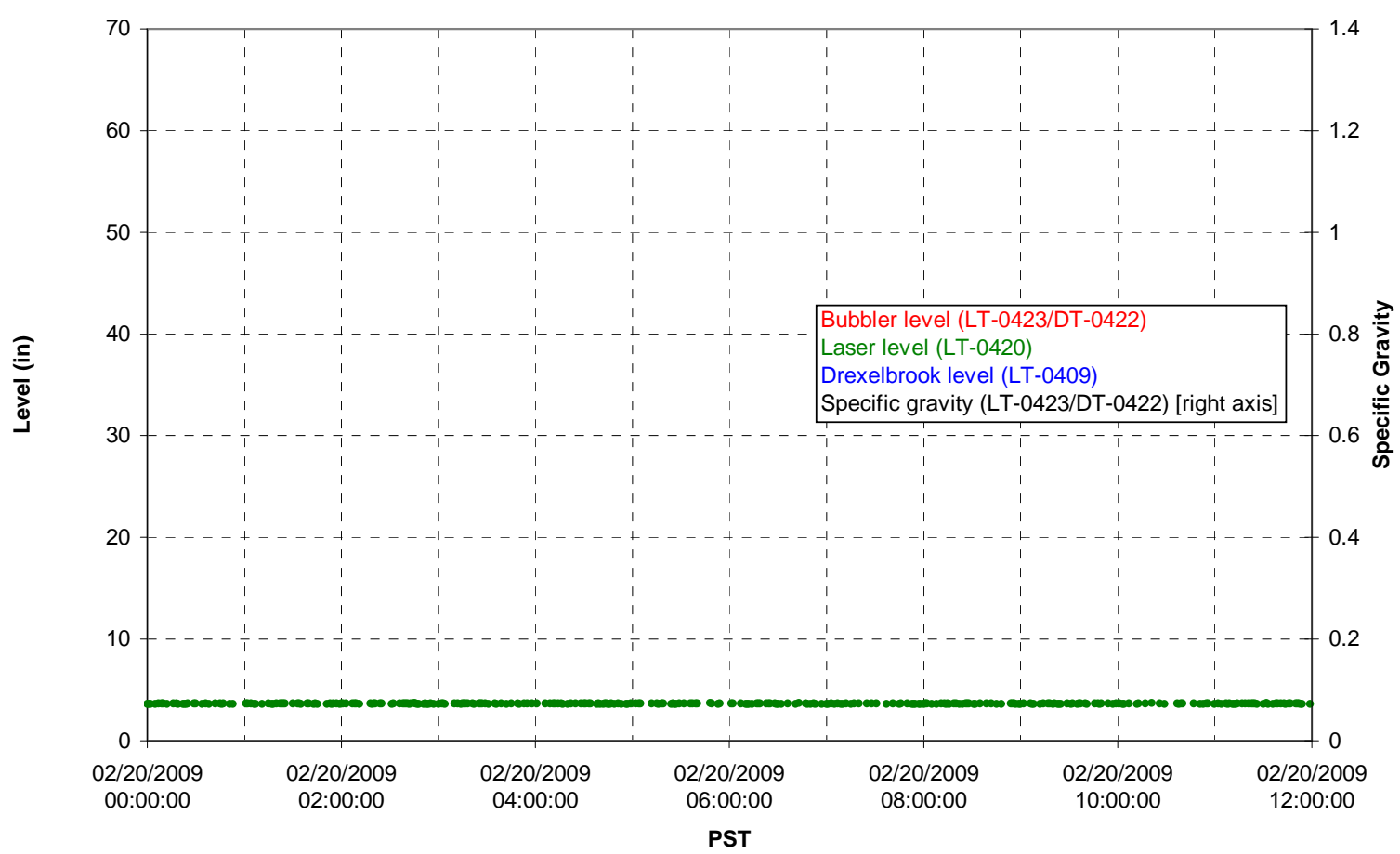

T01B temperatures

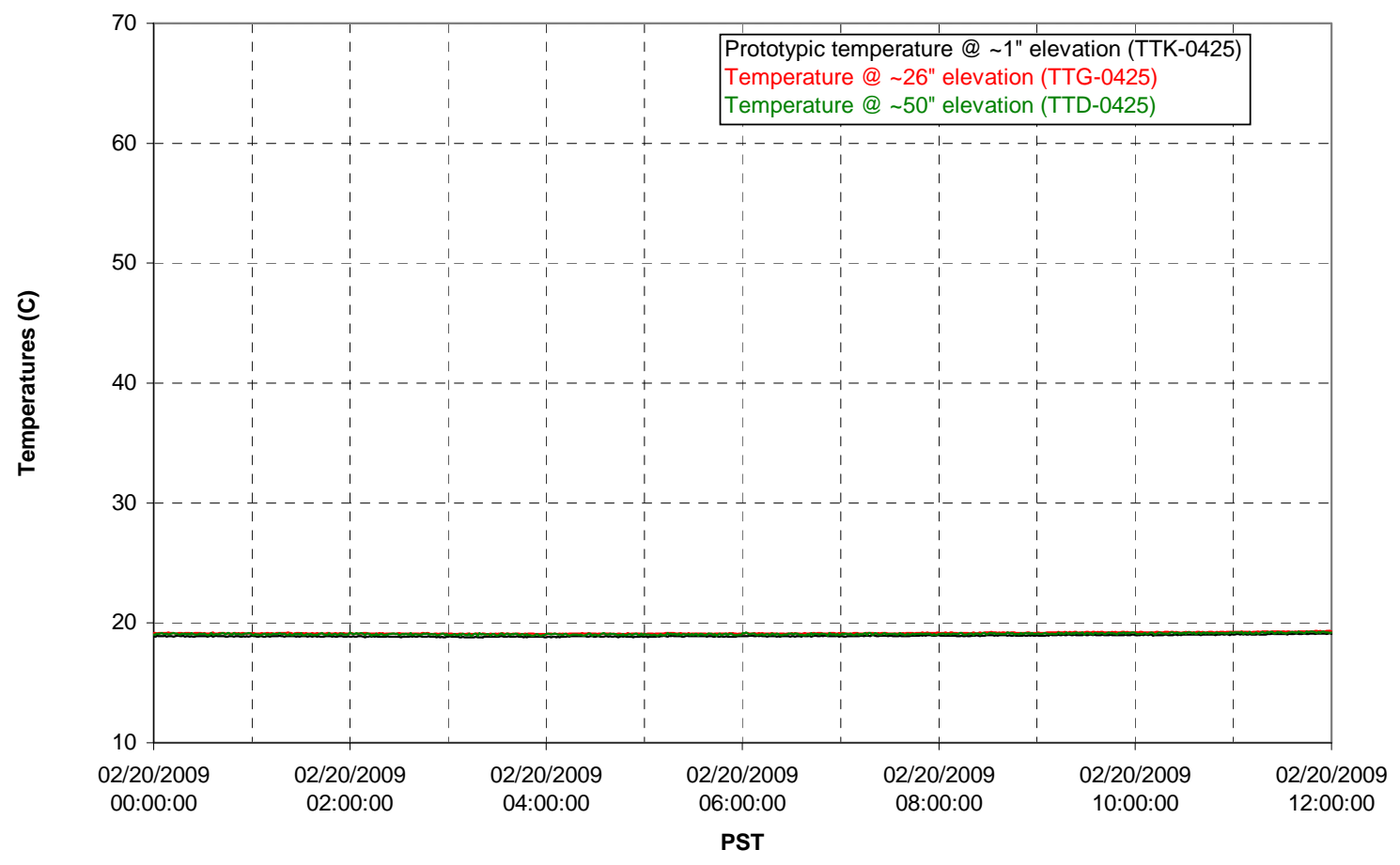

G.723 
T02A level

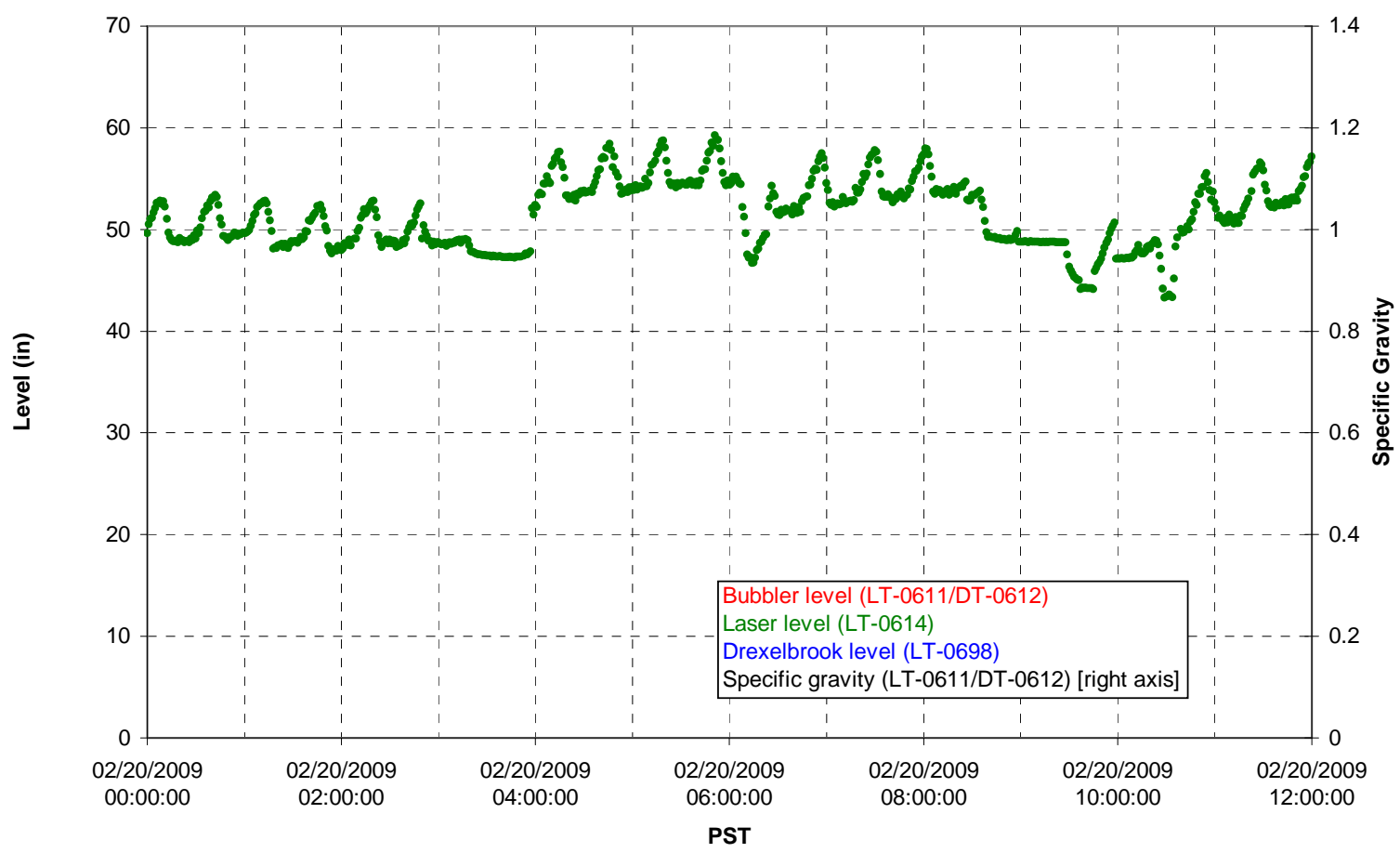

T02A temperatures

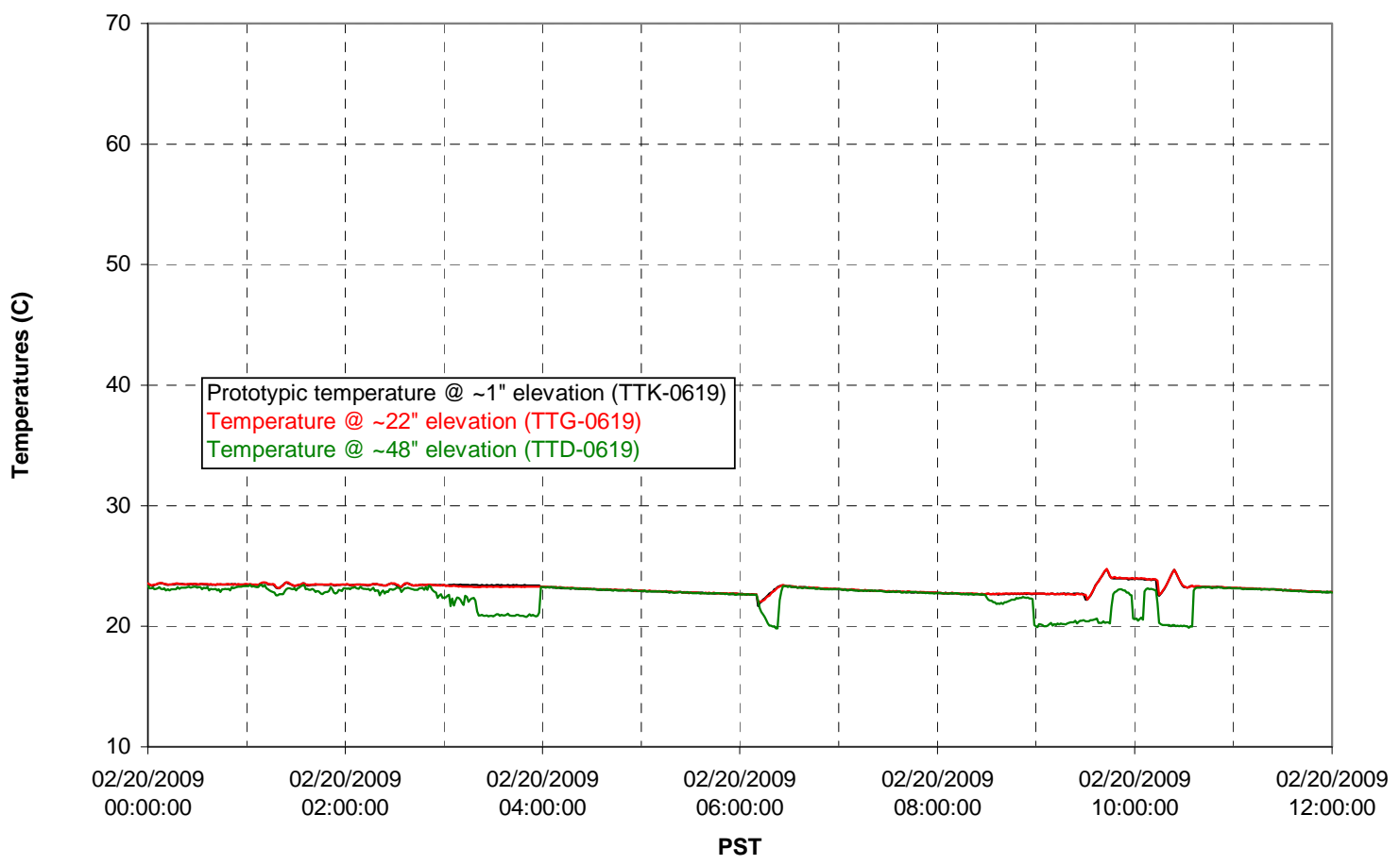

G.724 
T02A and filter loop temperatures

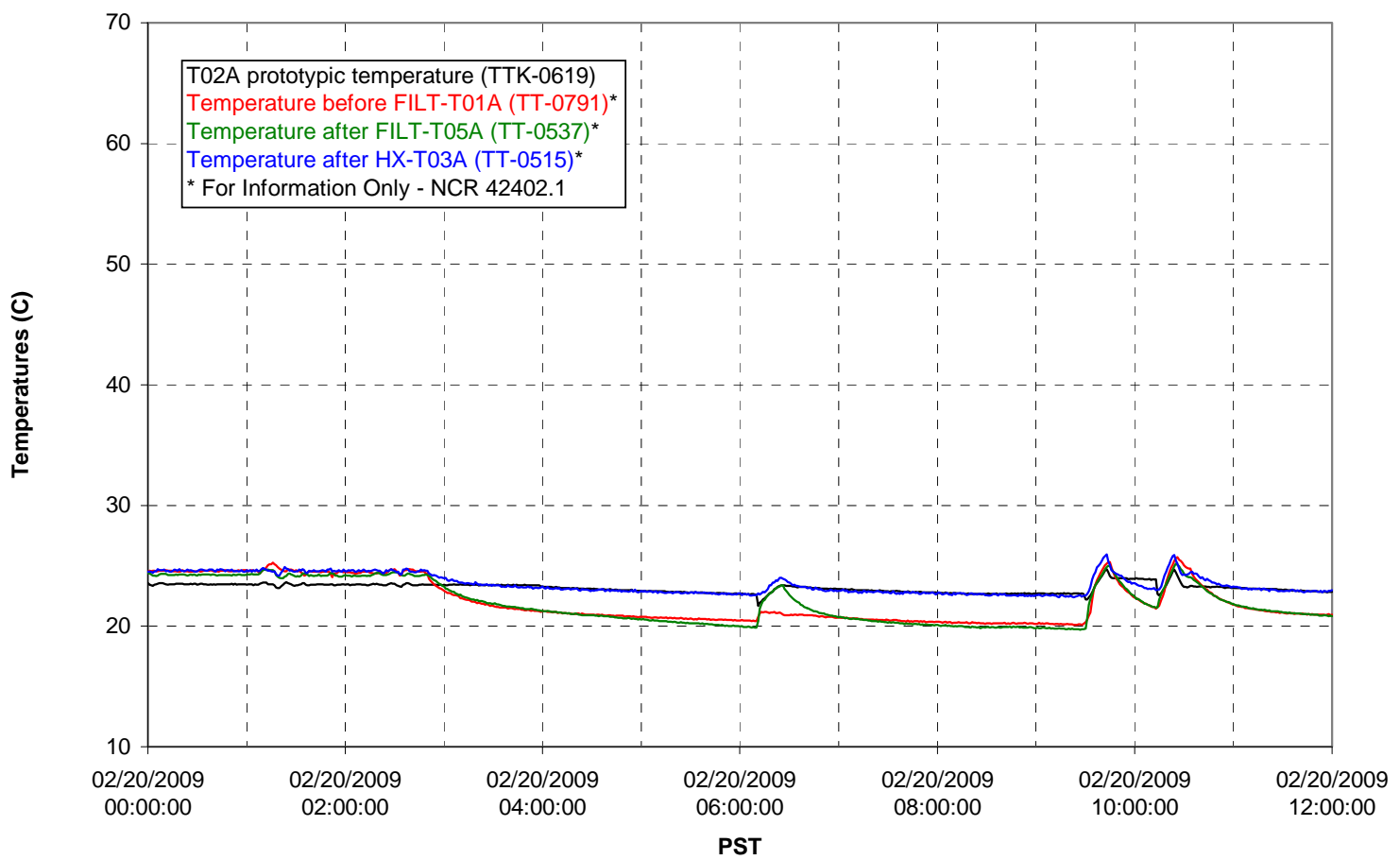

Pump Pressures and Flow

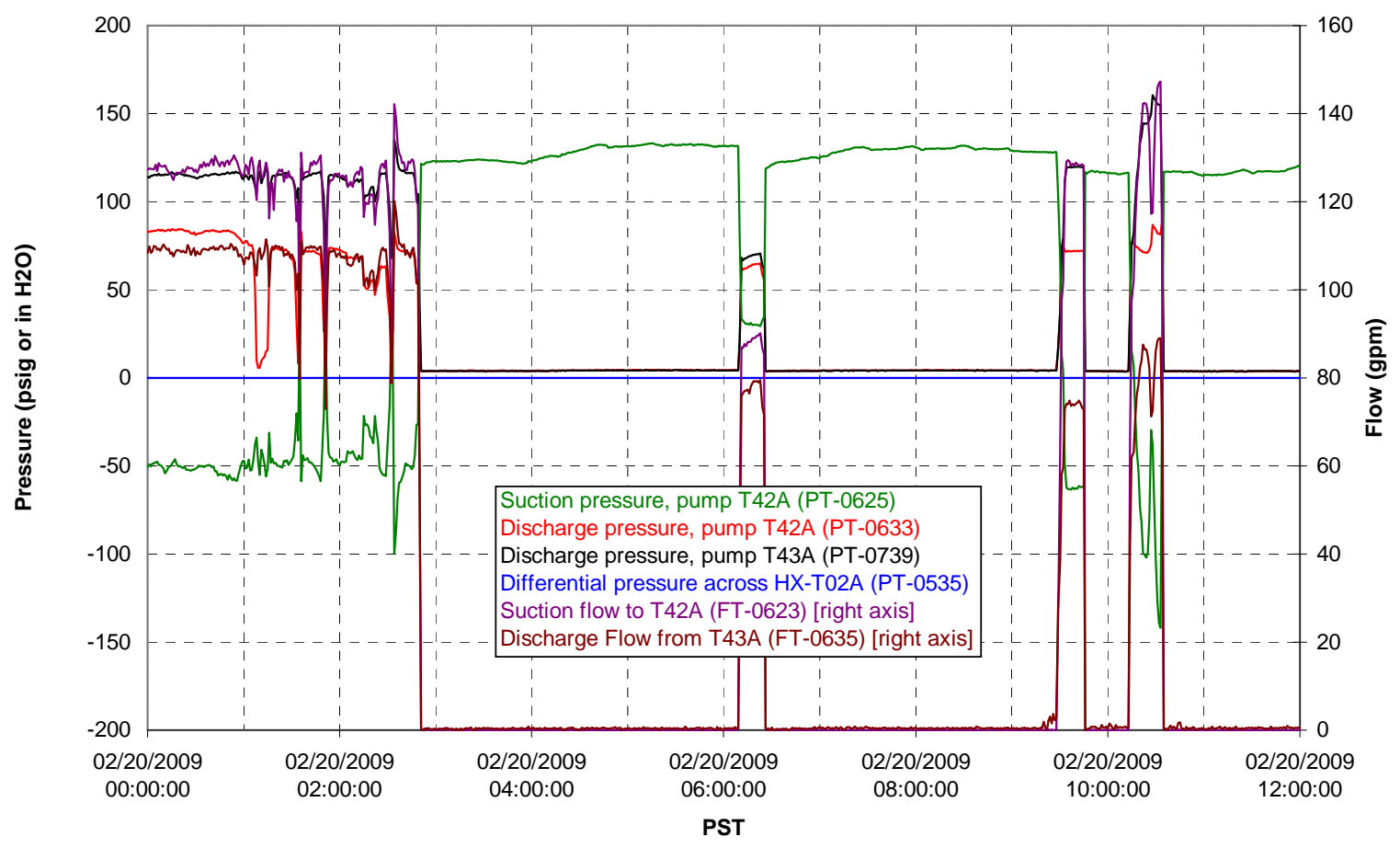

G.725 
Axial pressure drop

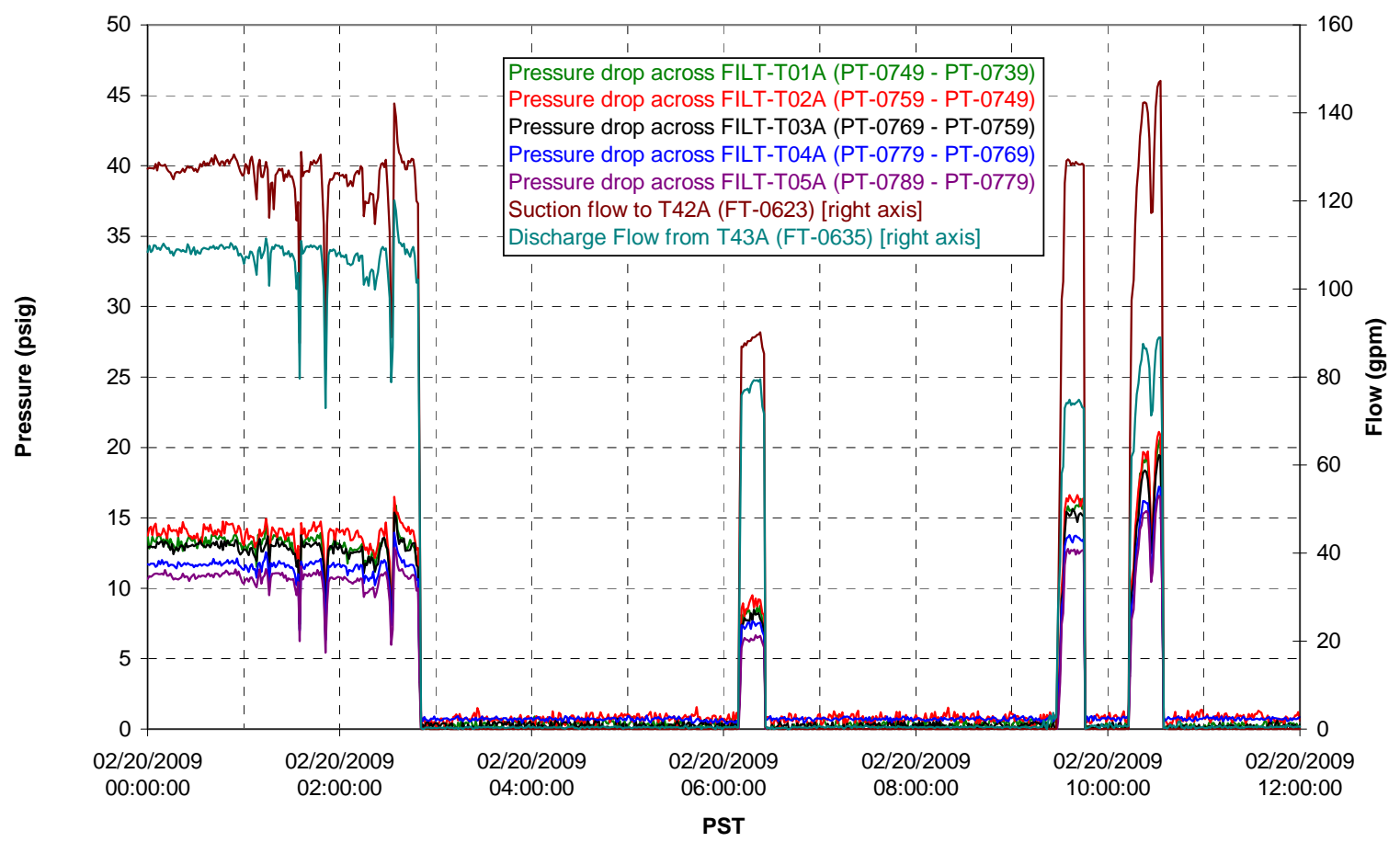

Permeate flow rates

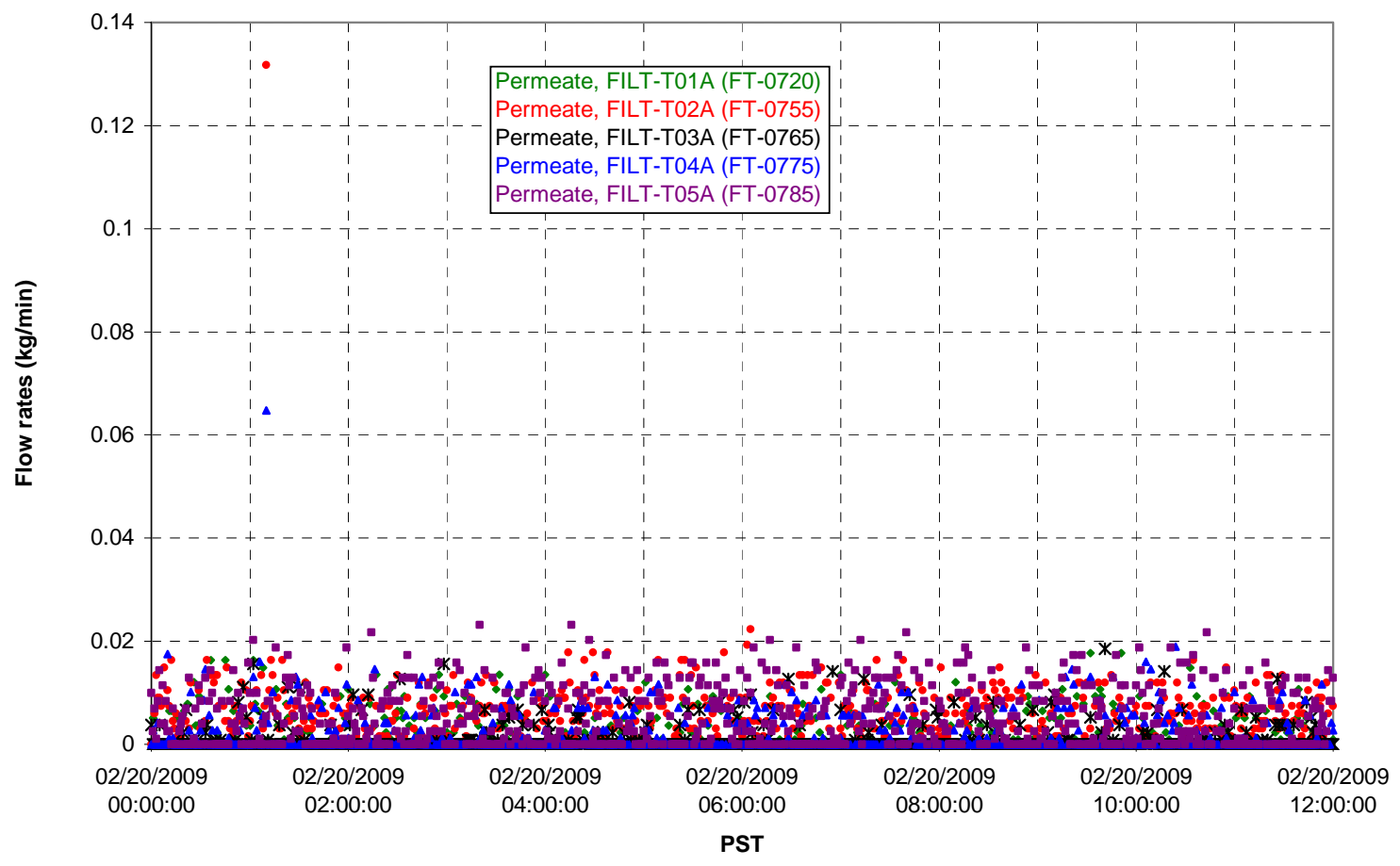

G.726 
T02A Inner Temperature Tree

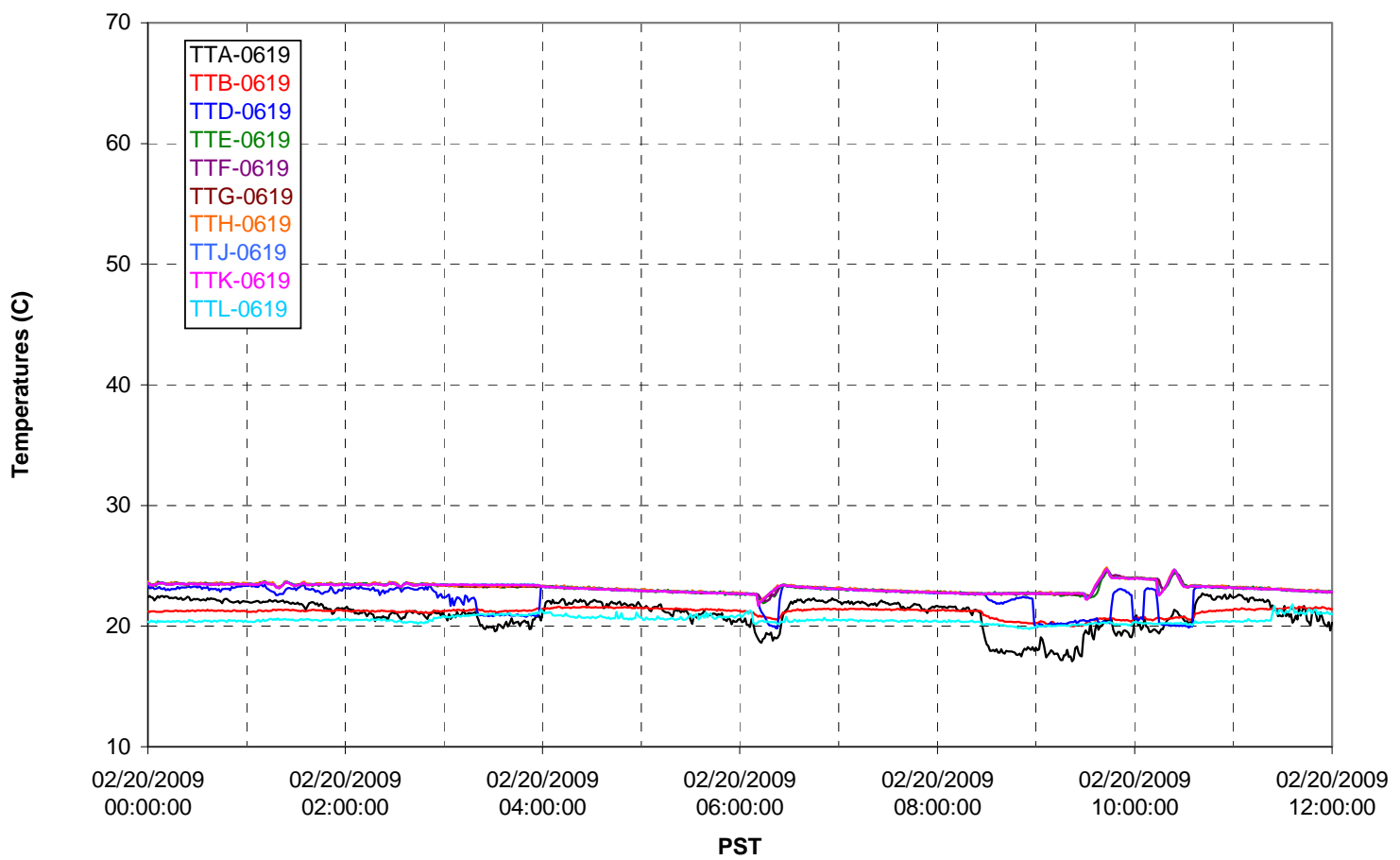

T02A Outer Temperature Tree

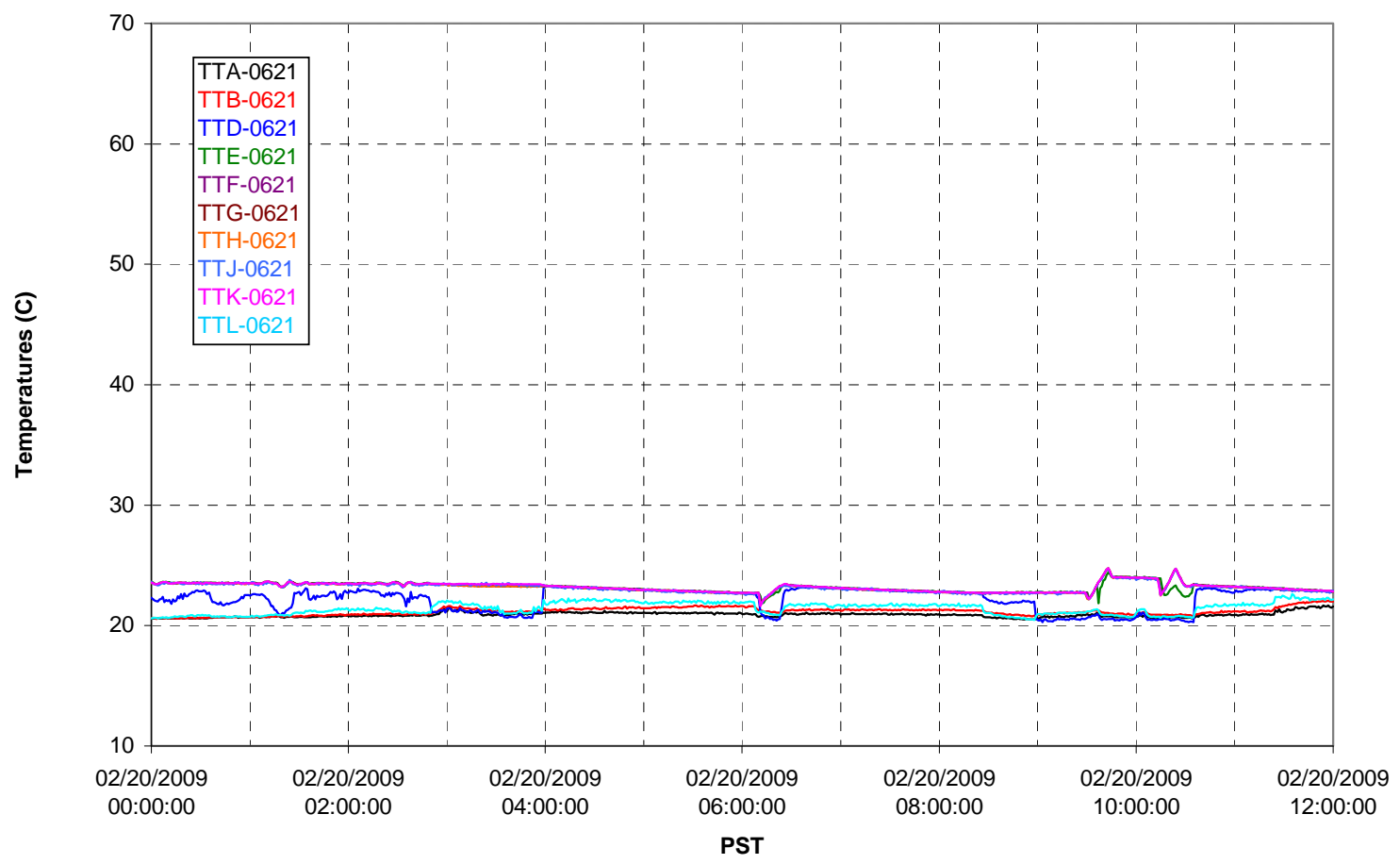


T02A temperatures

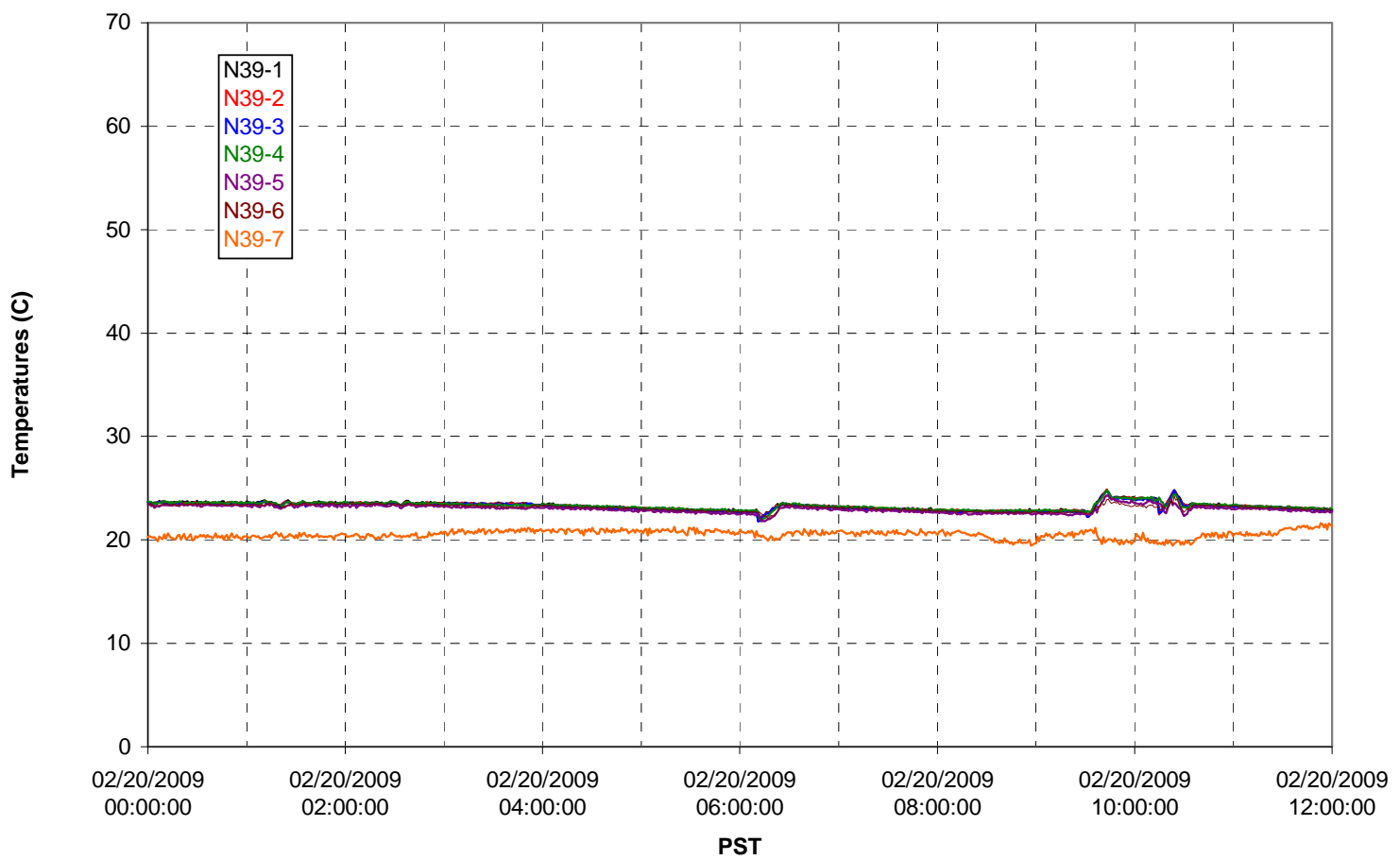

T02A temperatures

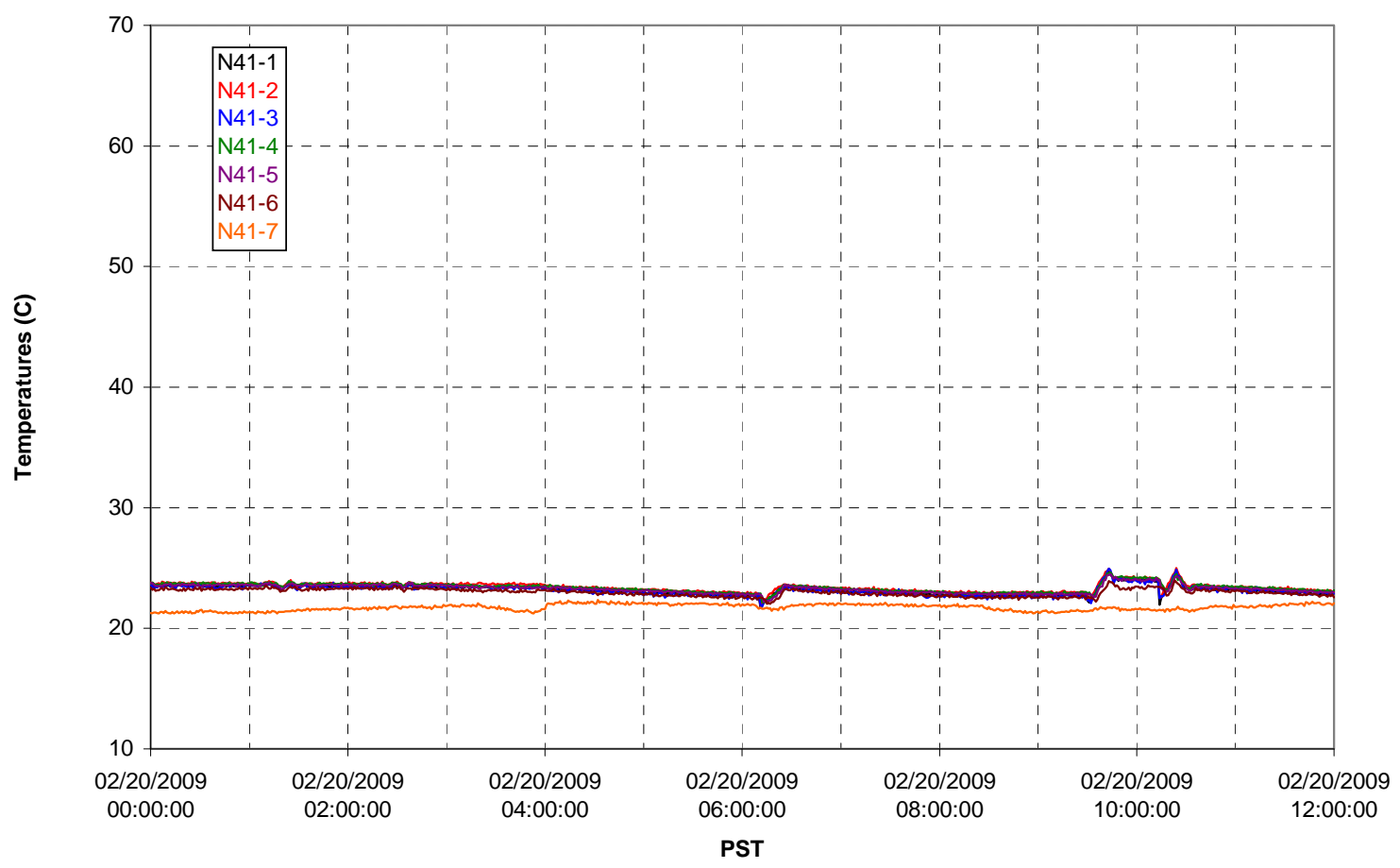


T02A temperatures

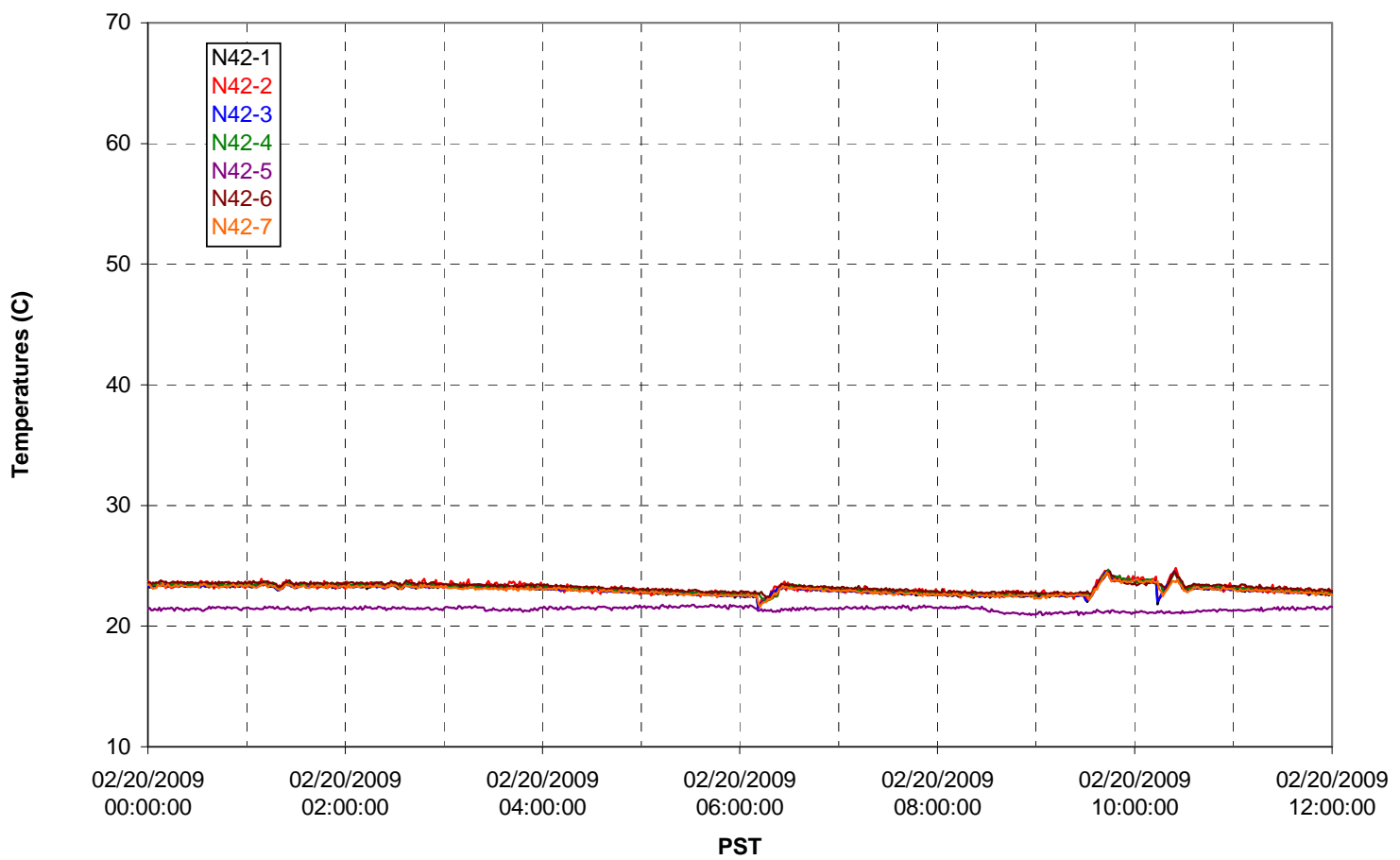

T02A temperatures

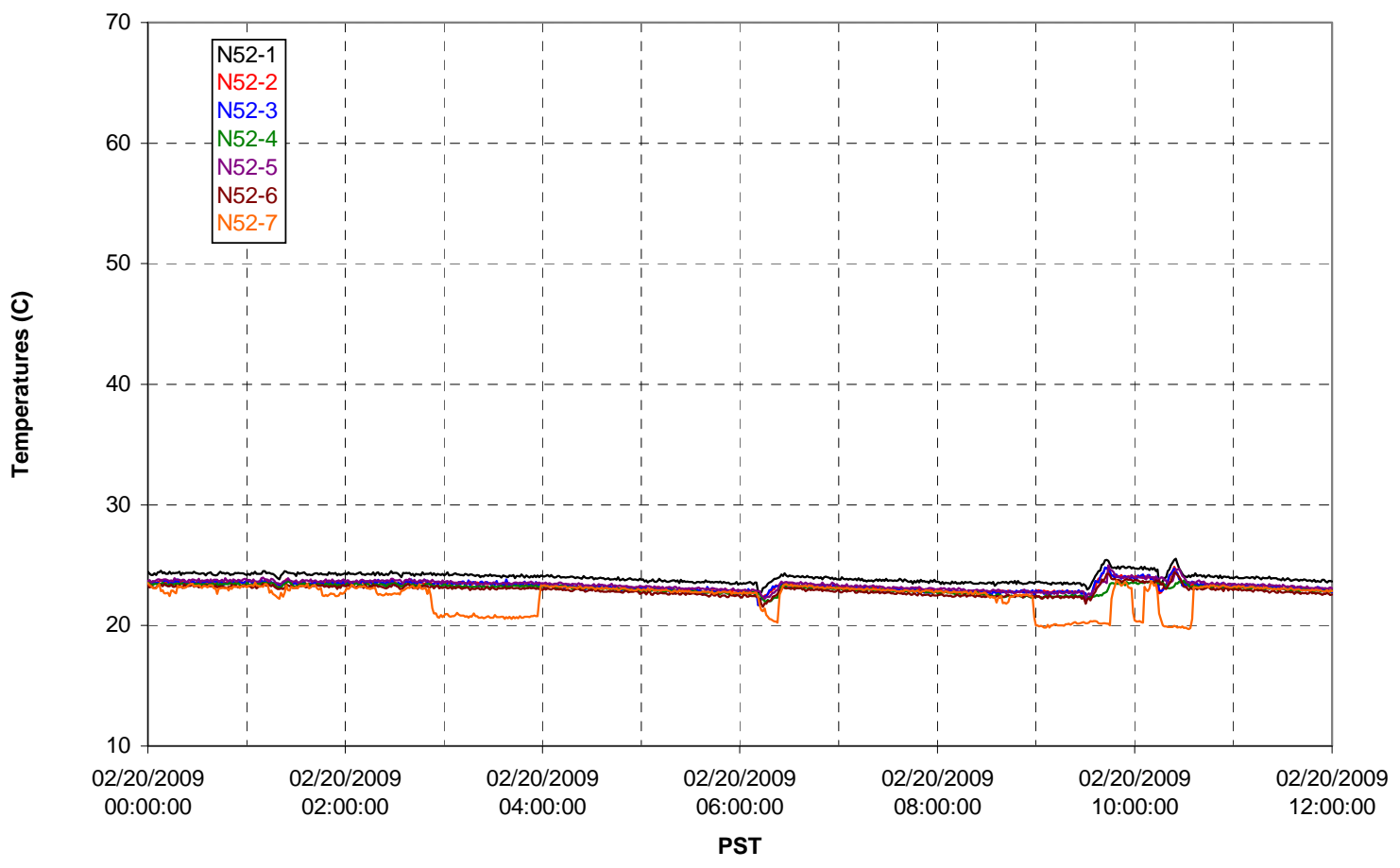

G.729 
T02A Heating and Cooling

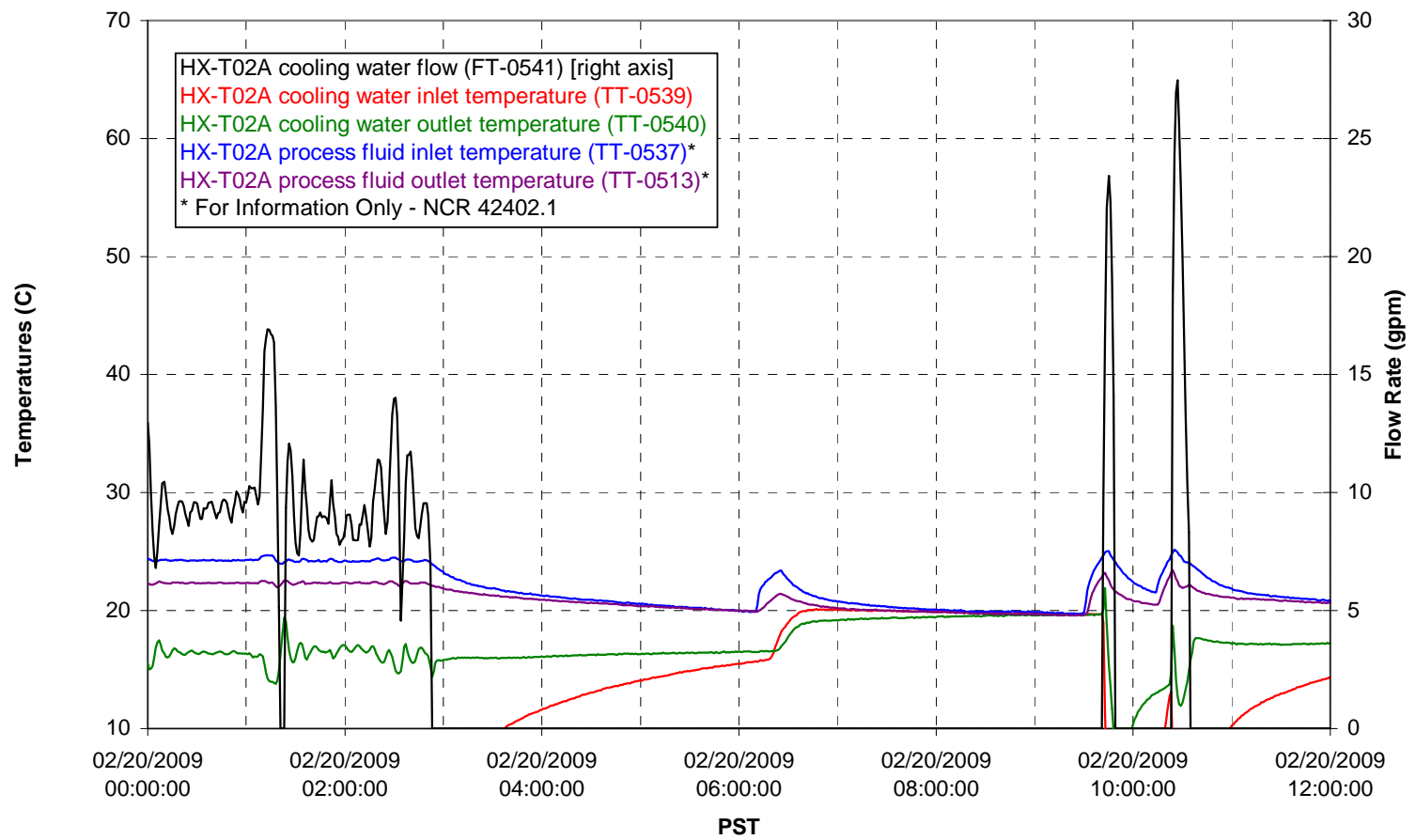

Pump Operation

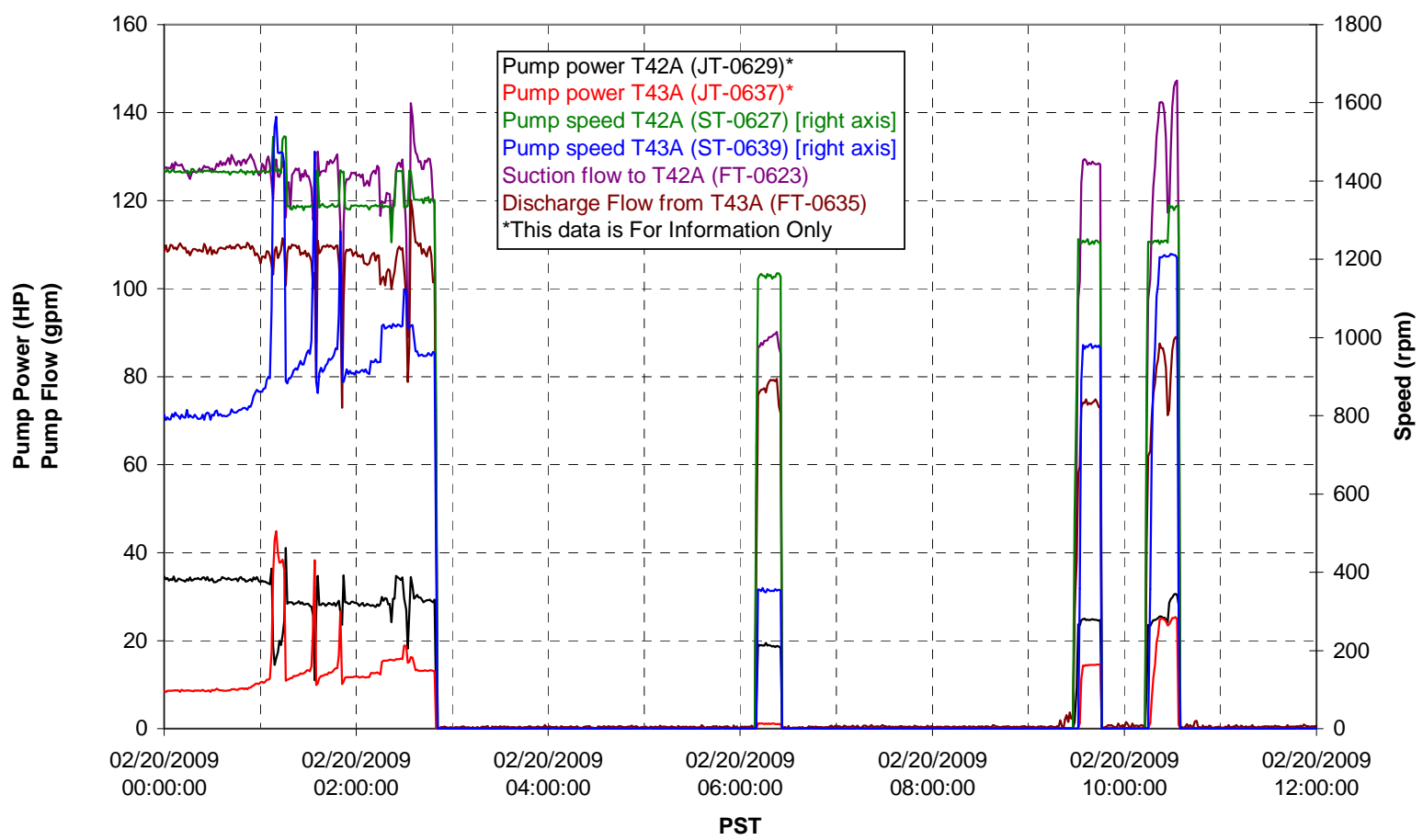

G.730 
Pulsepot UFP-PP-T01A

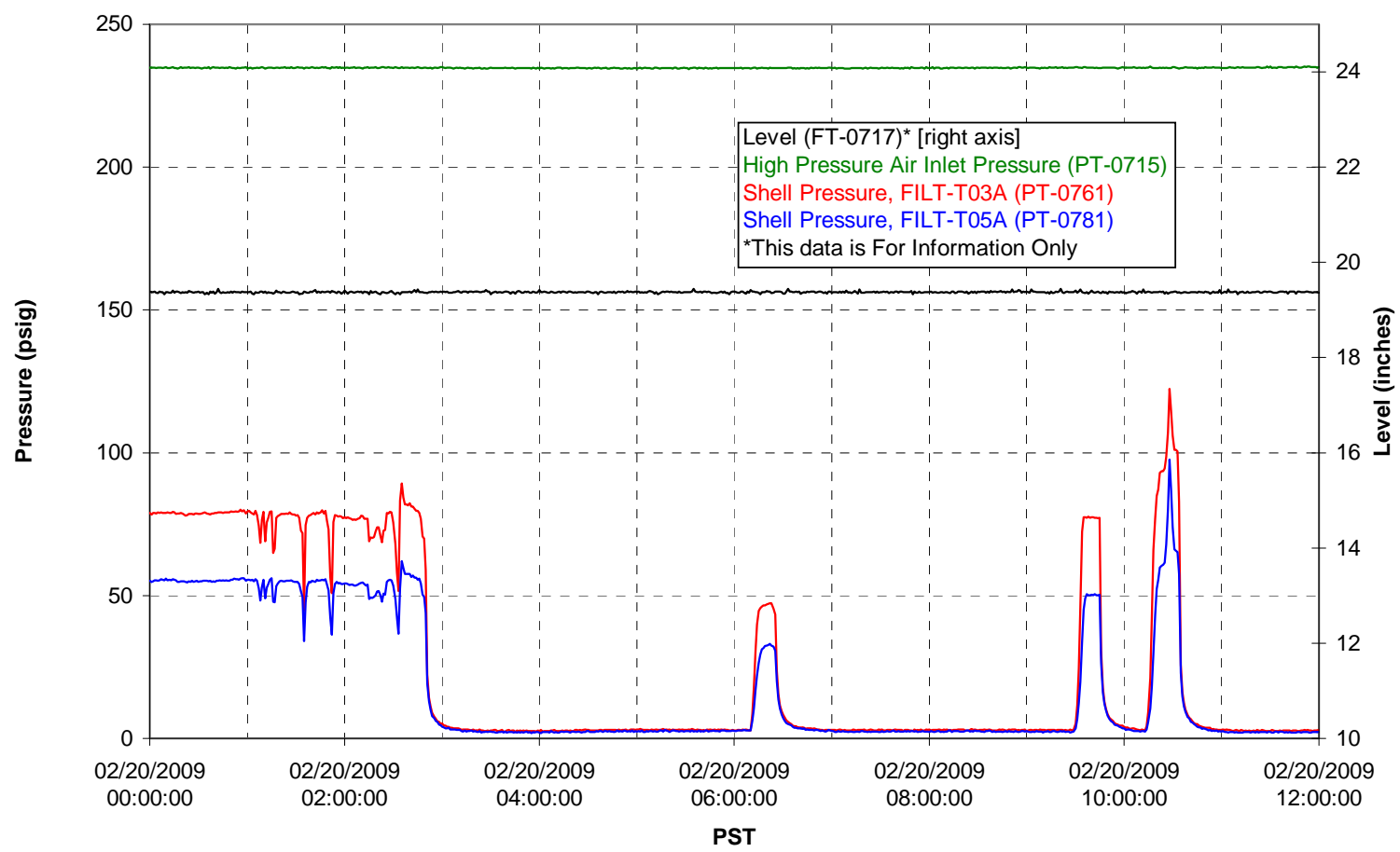

Pulsepot UFP-PP-T02A

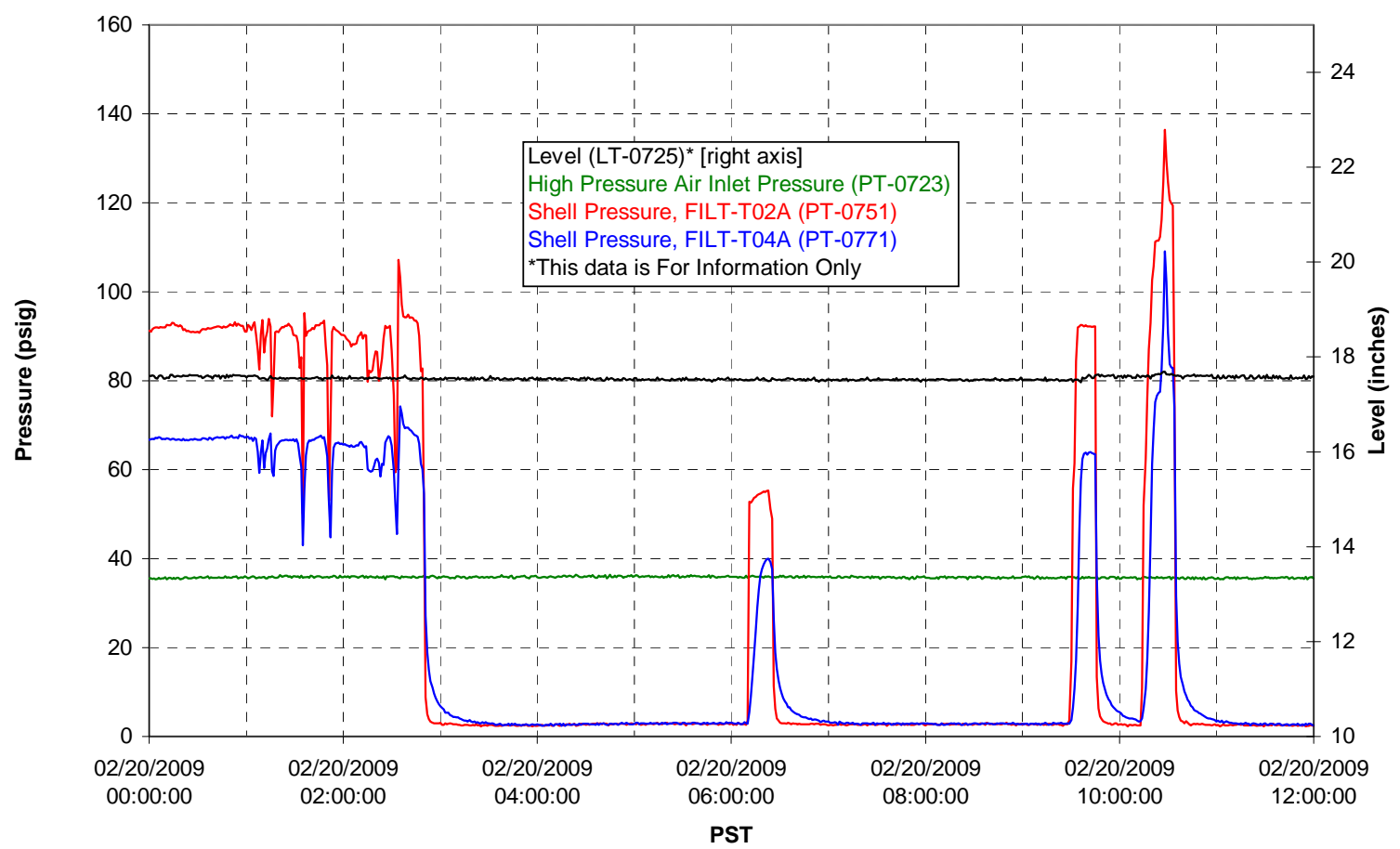


Pulsepot UFP-PP-T03A

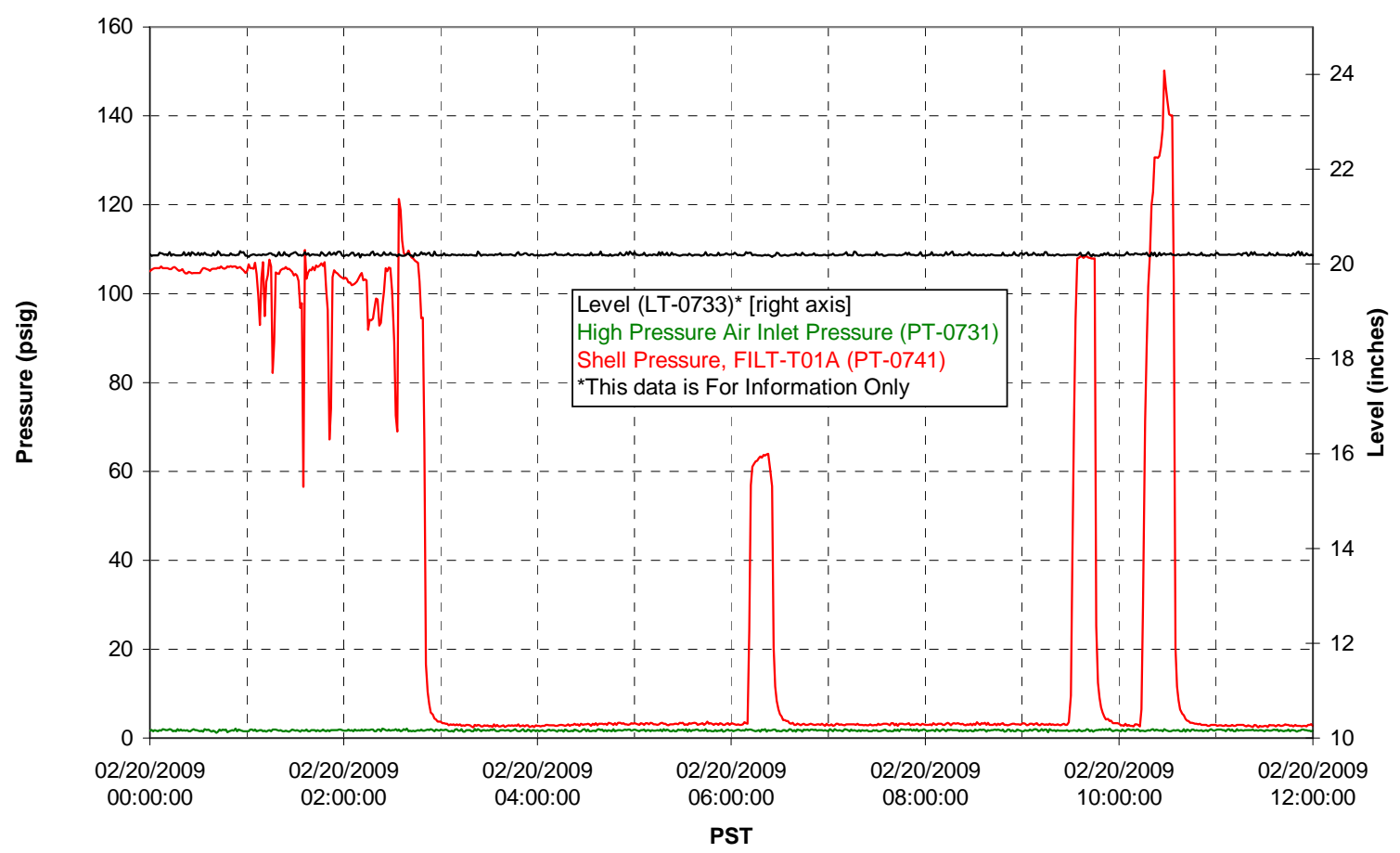

Pulsepot Levels

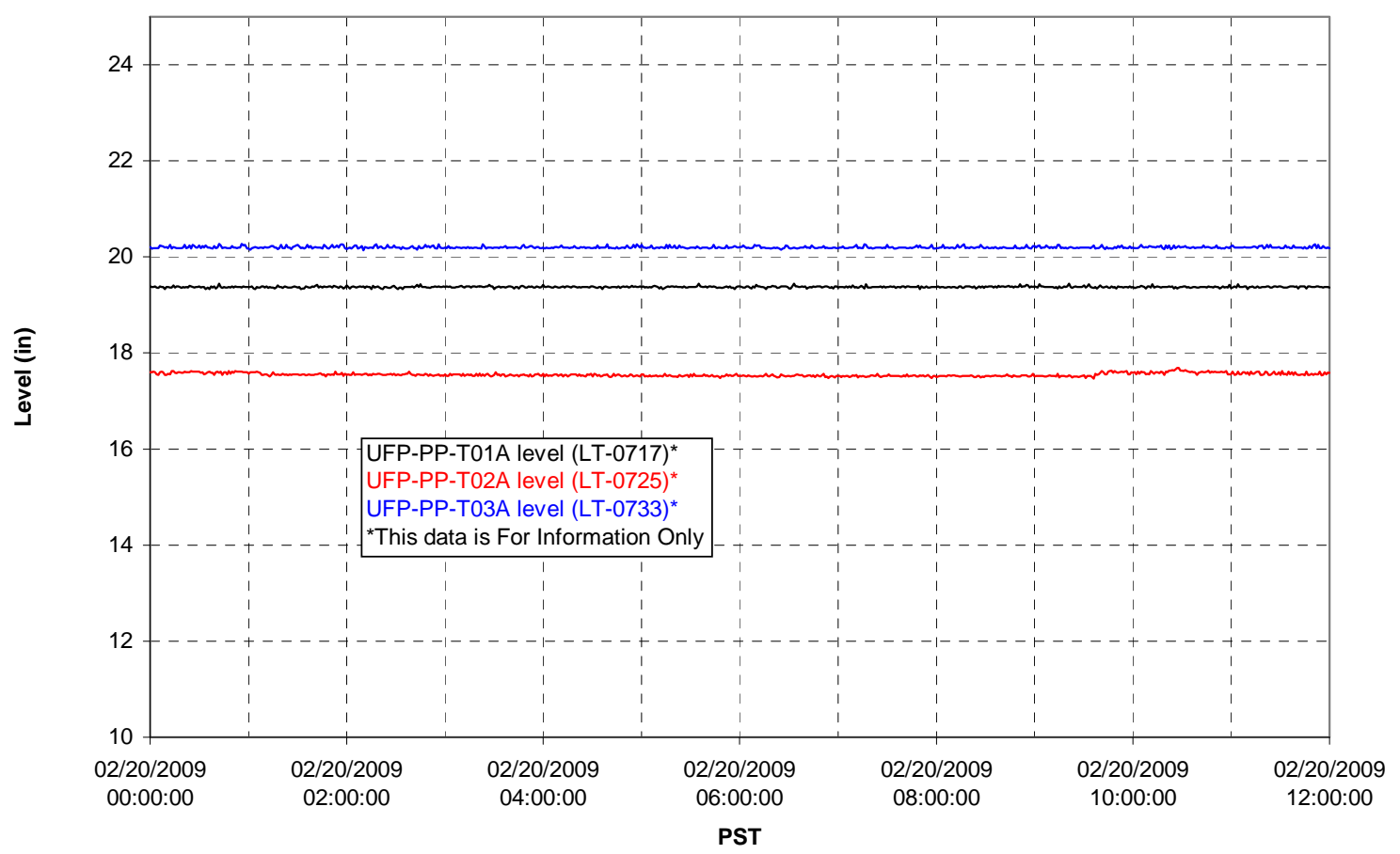

G.732 
Filter UFP-FILT-T01A

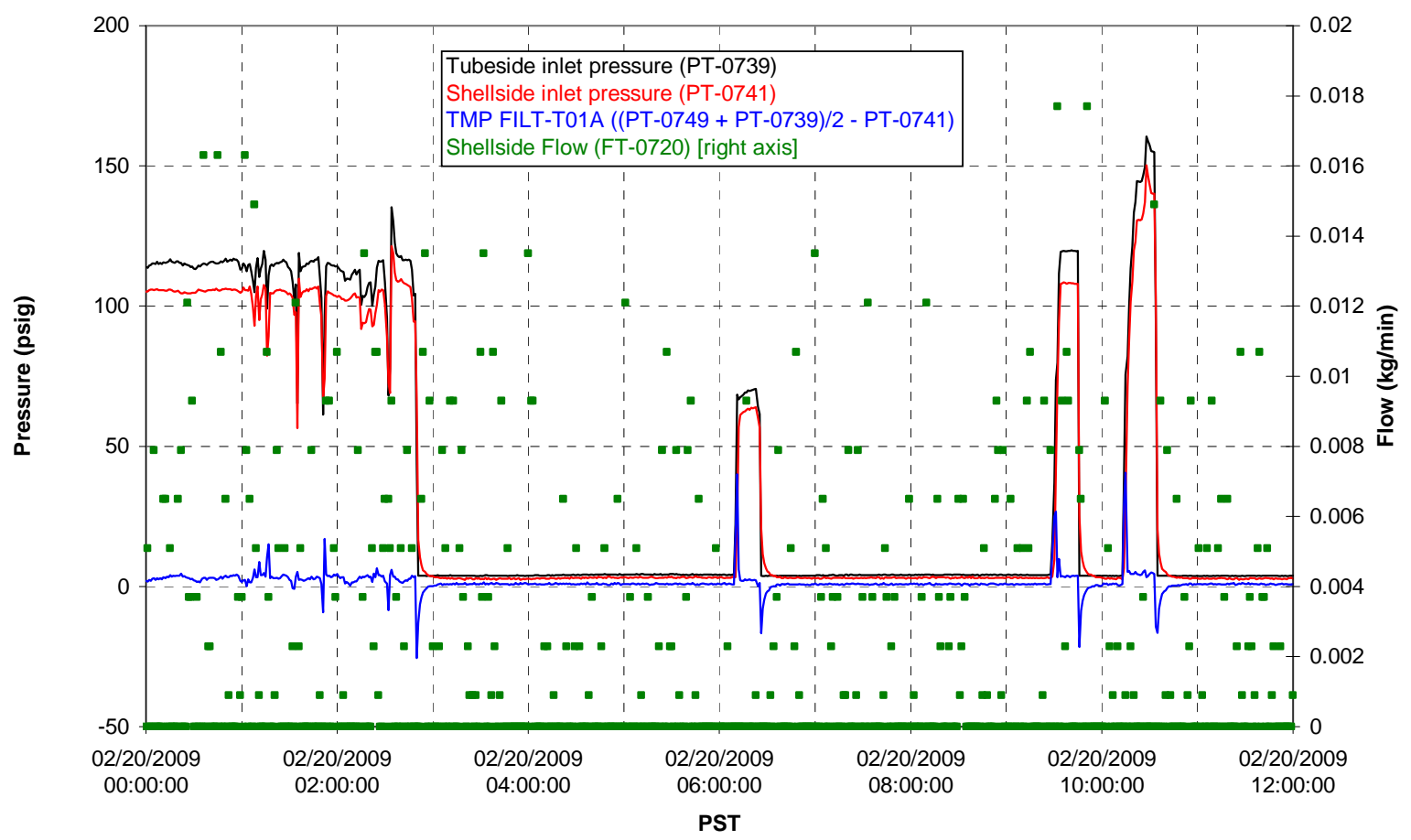

Filter UFP-FILT-T02A

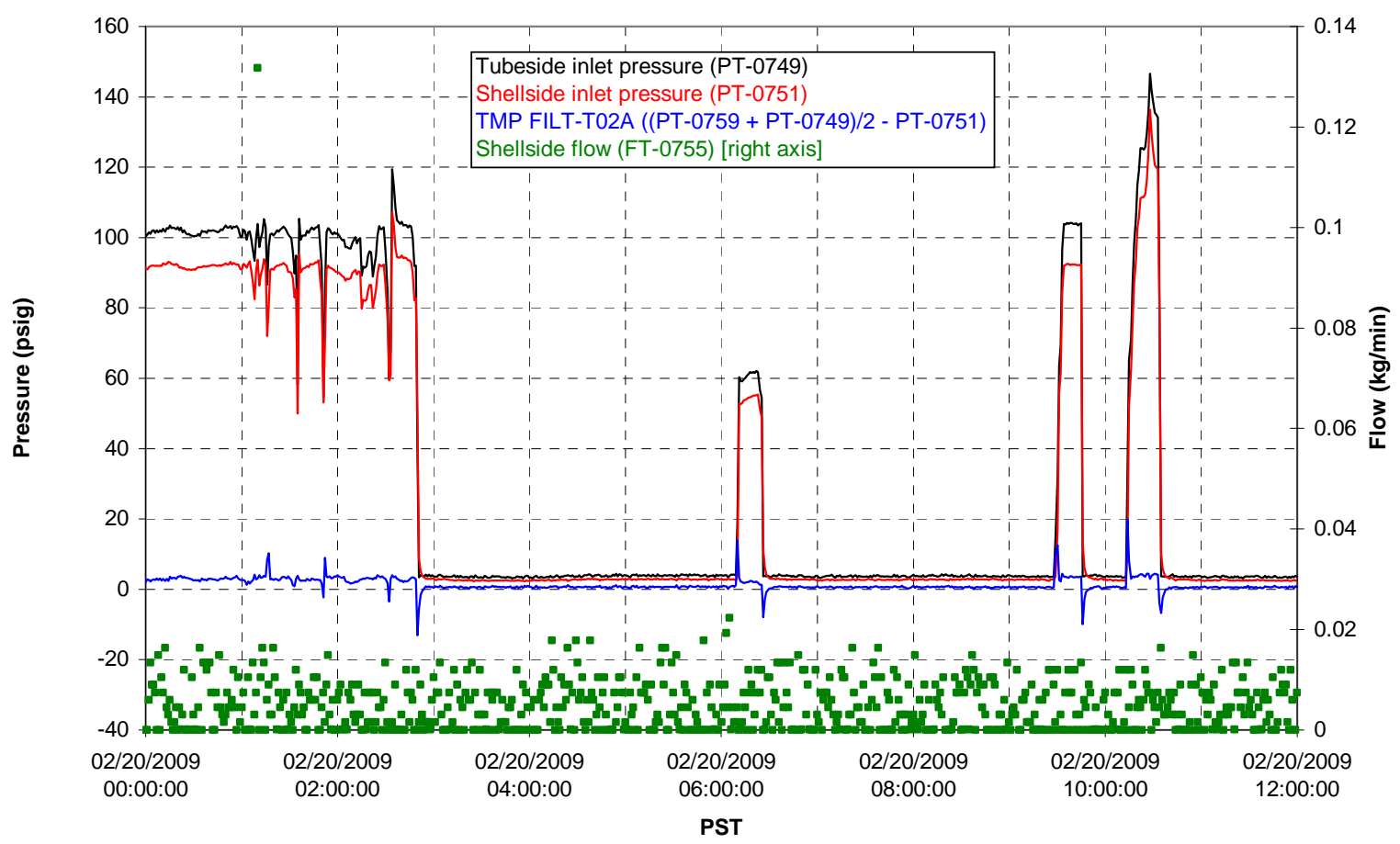


Filter UFP-FILT-T03A

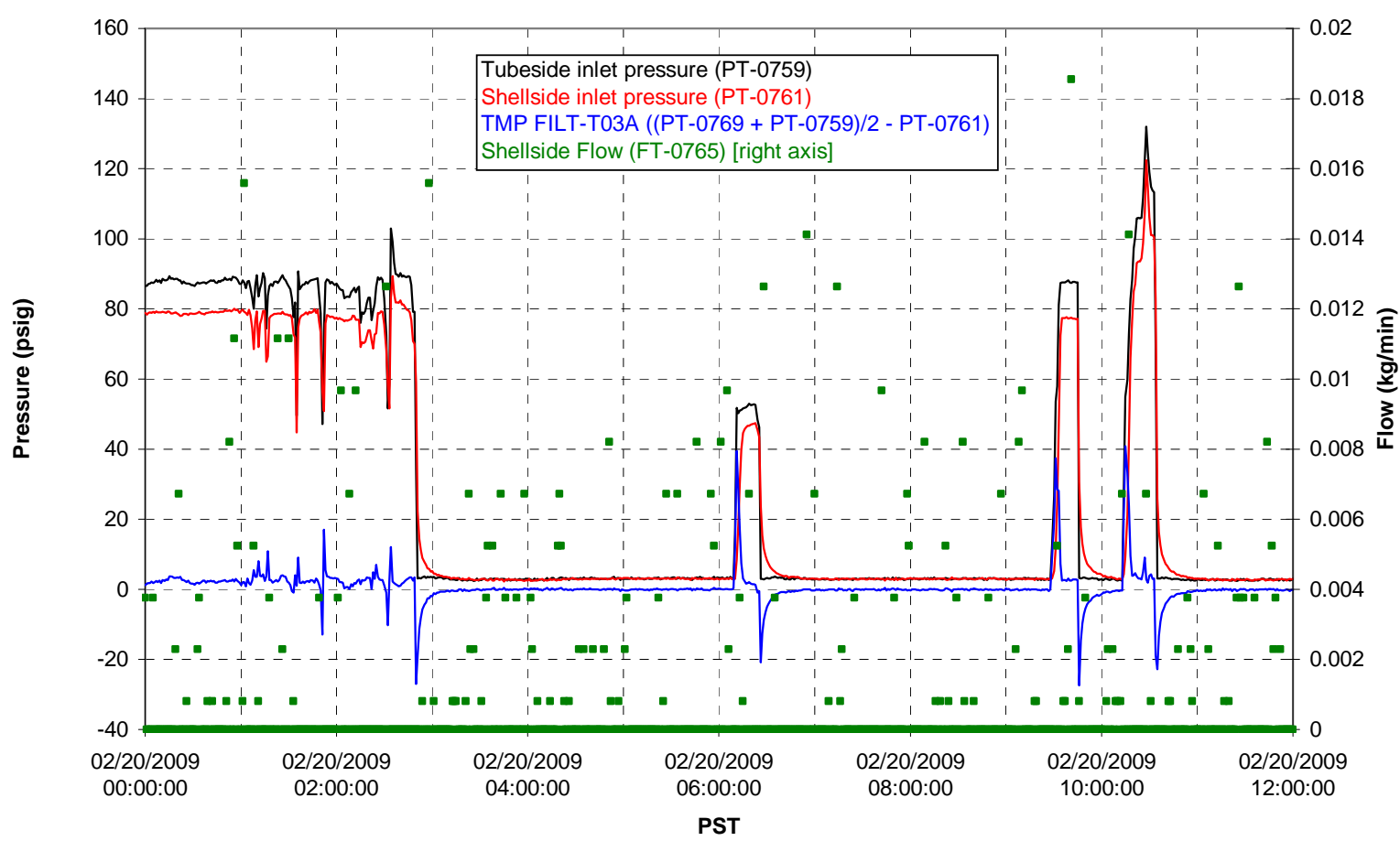

Filter UFP-FILT-T04A

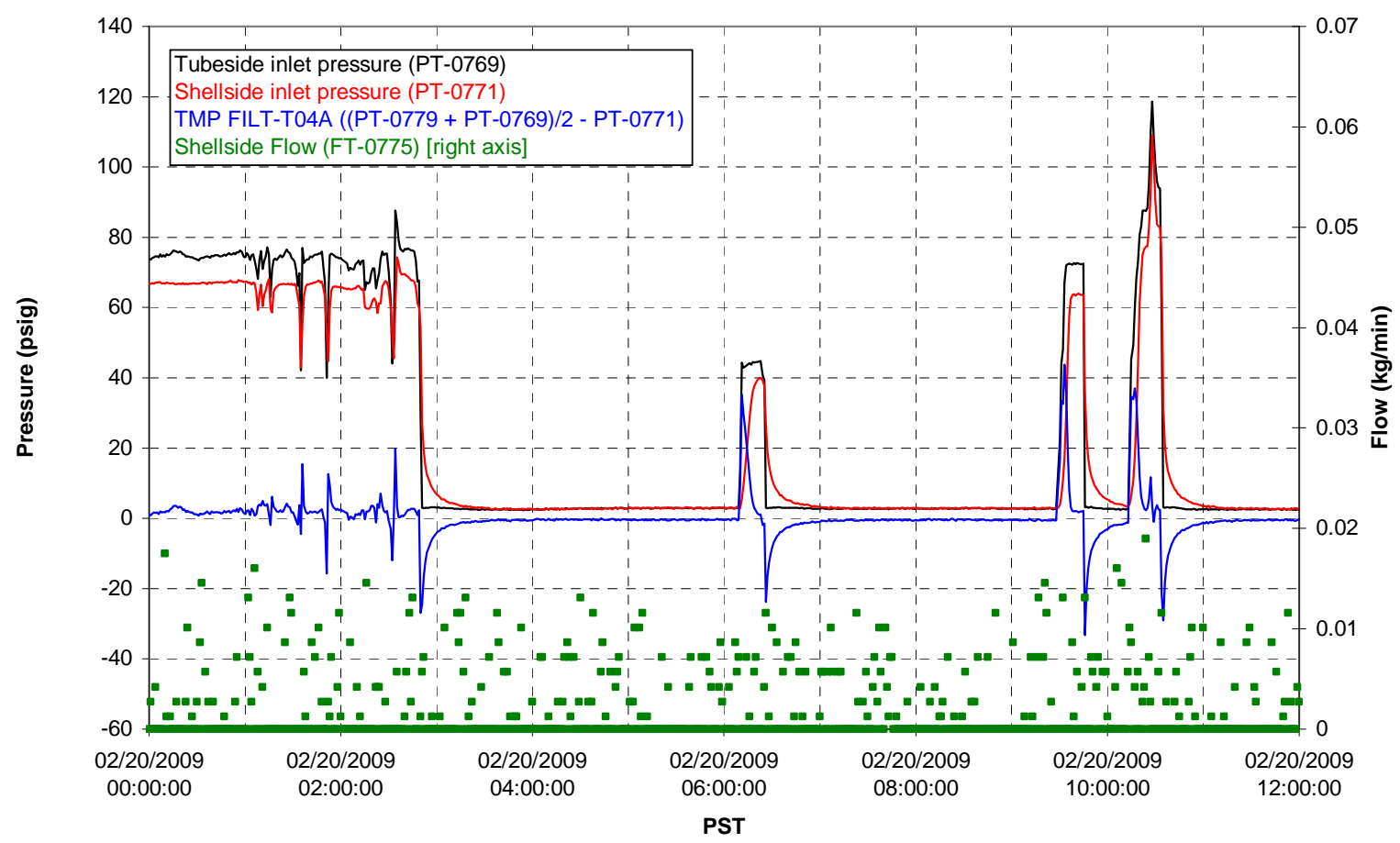


Filter UFP-FILT-T05A

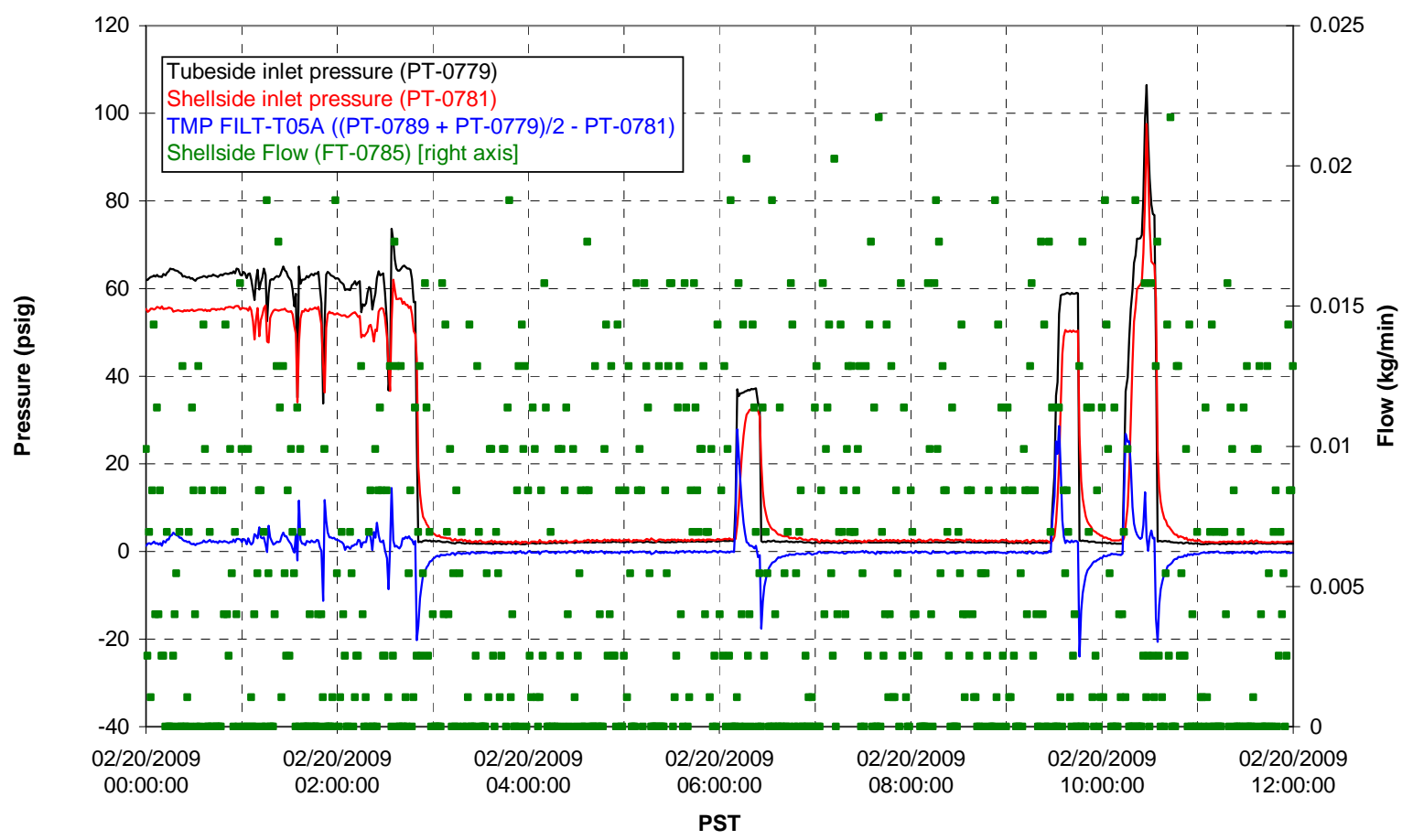

Chemical Flow

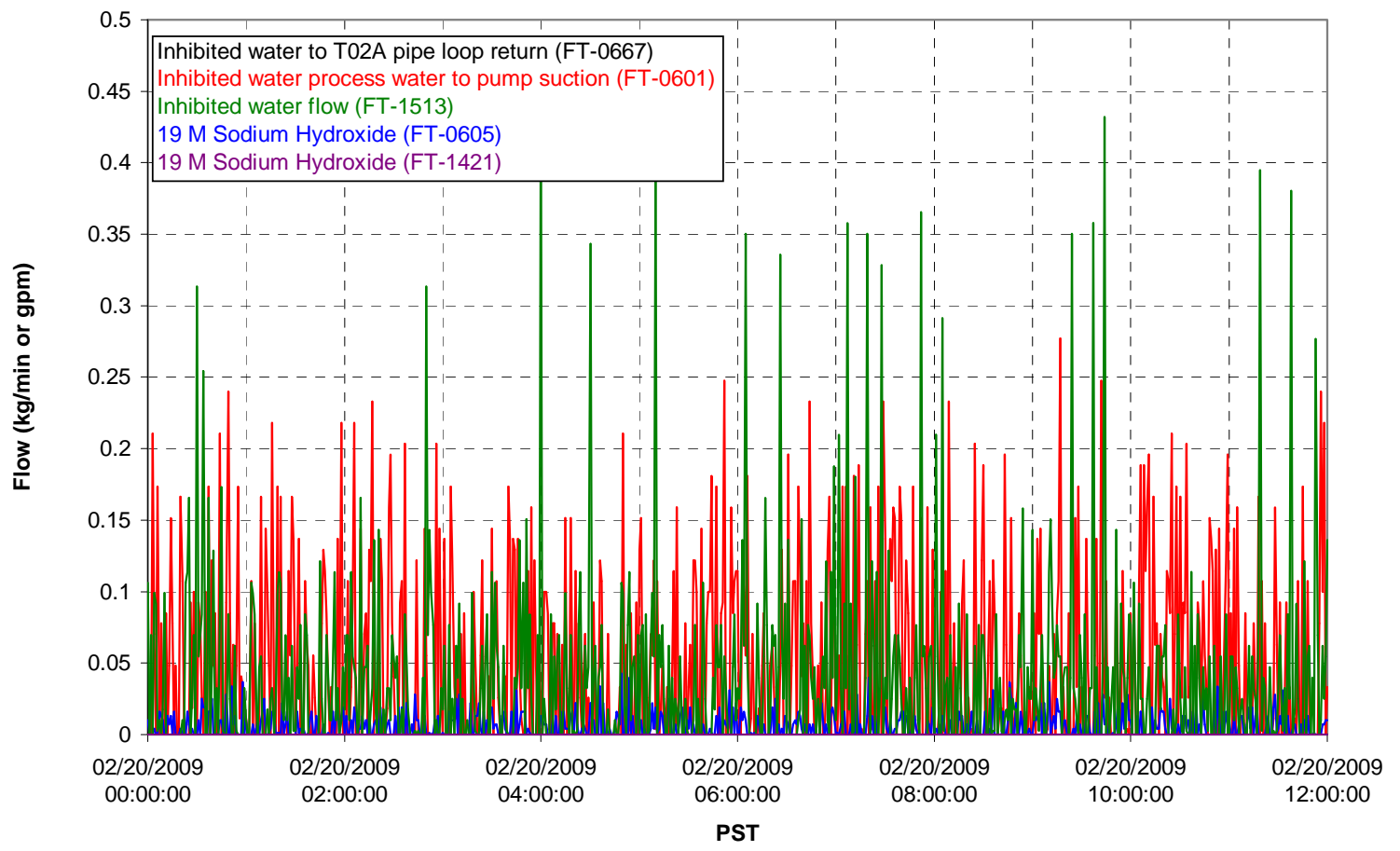




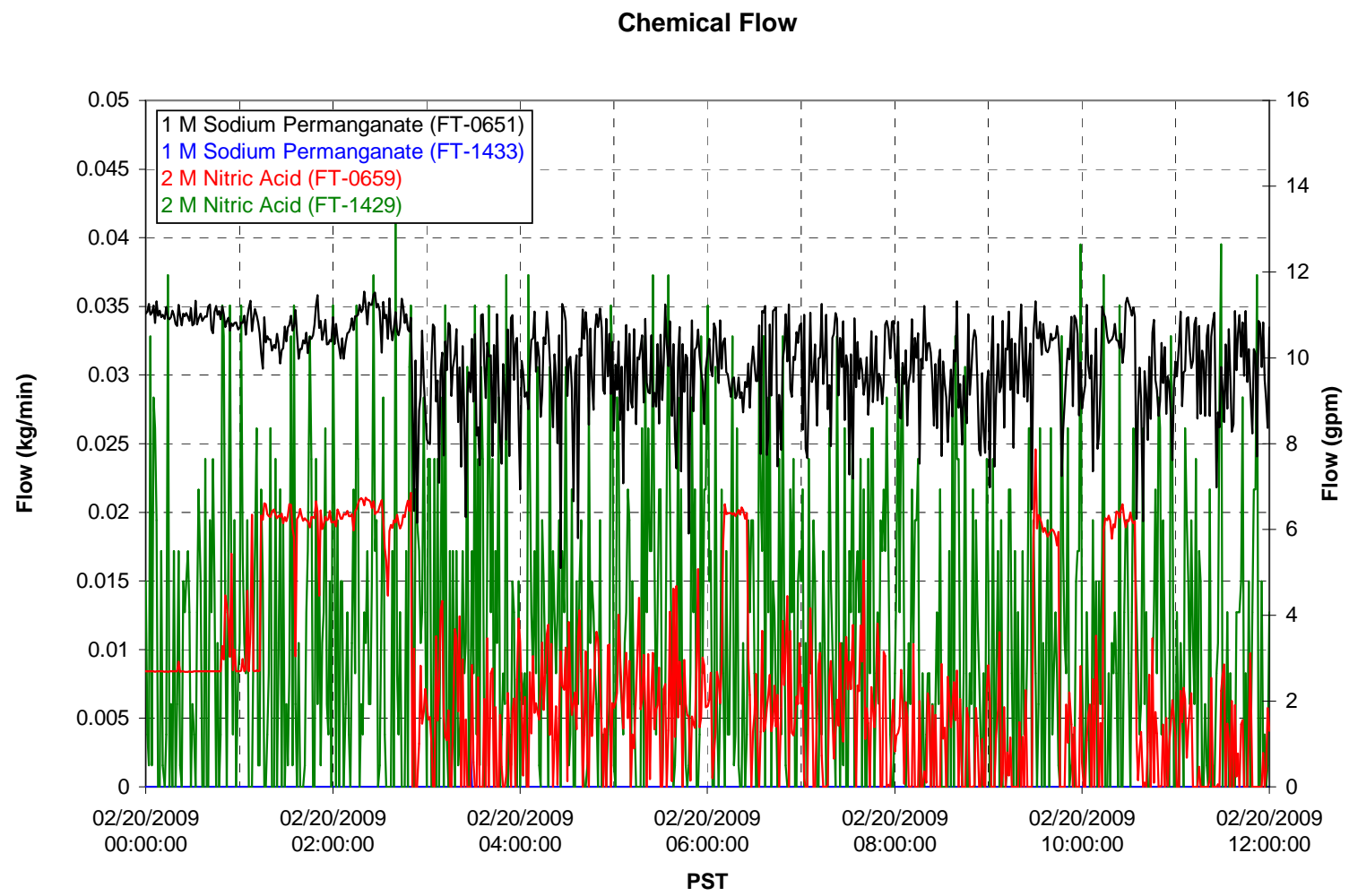

Air Flows

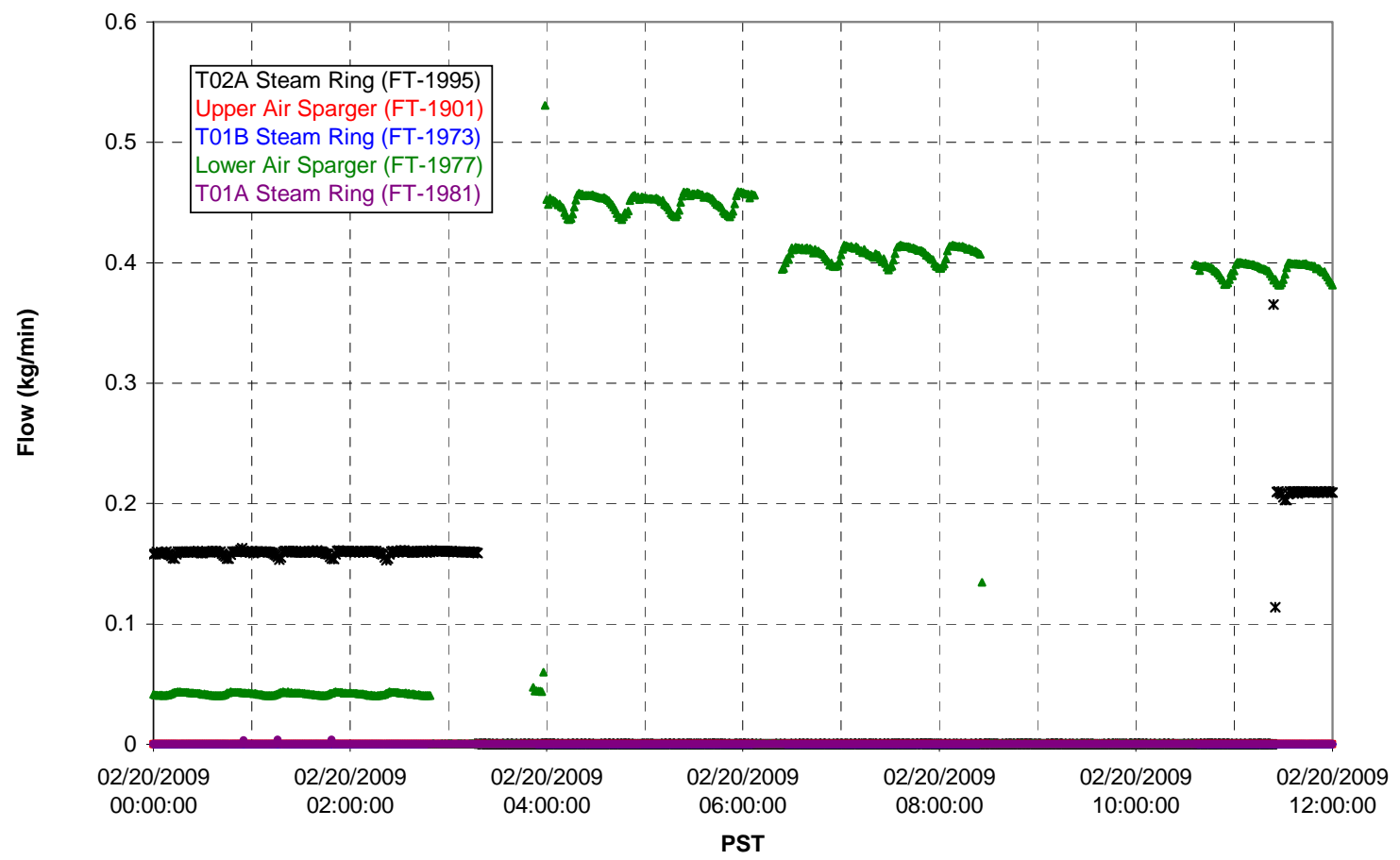


T02A Steam

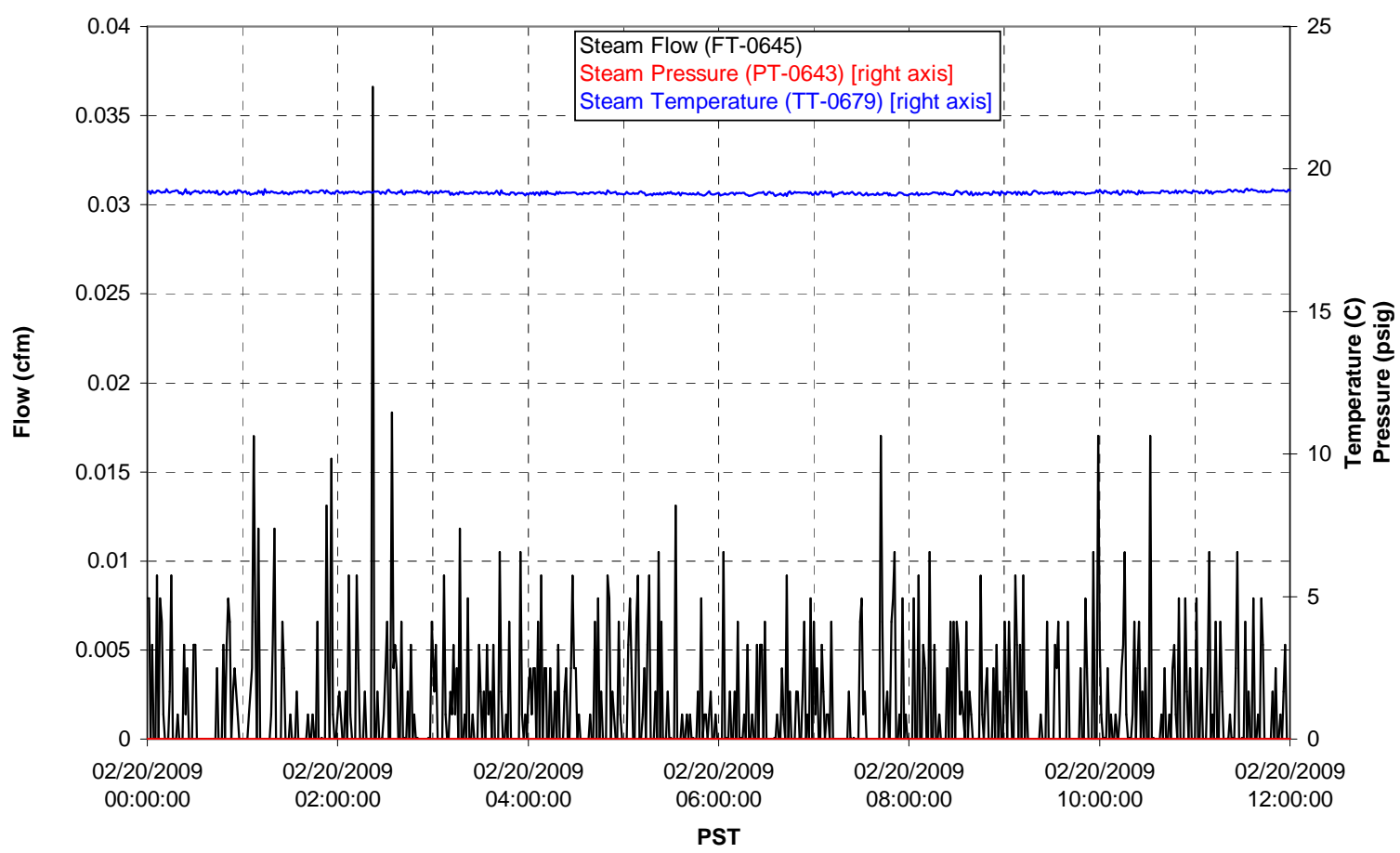

T01A Steam

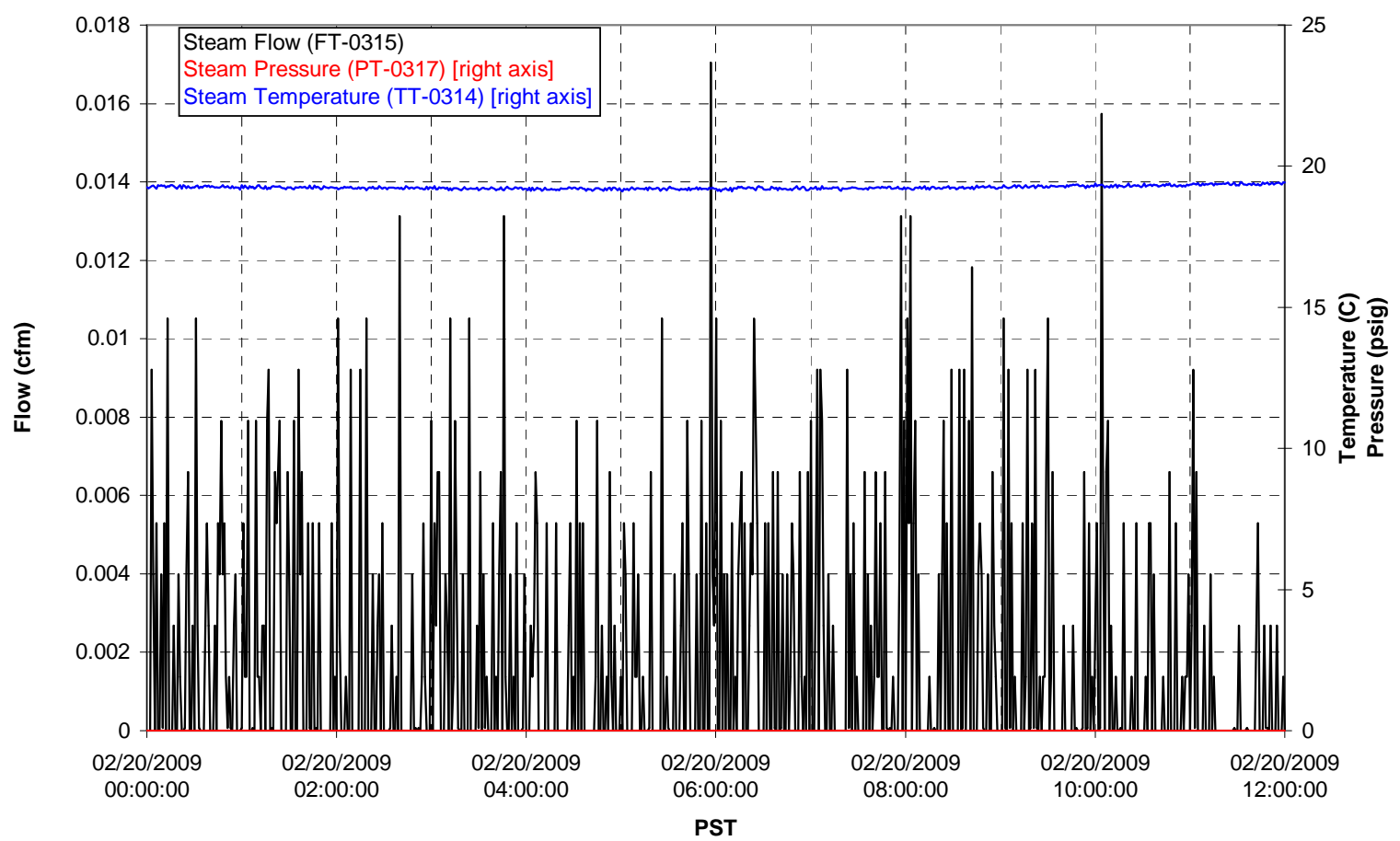


T01B Steam

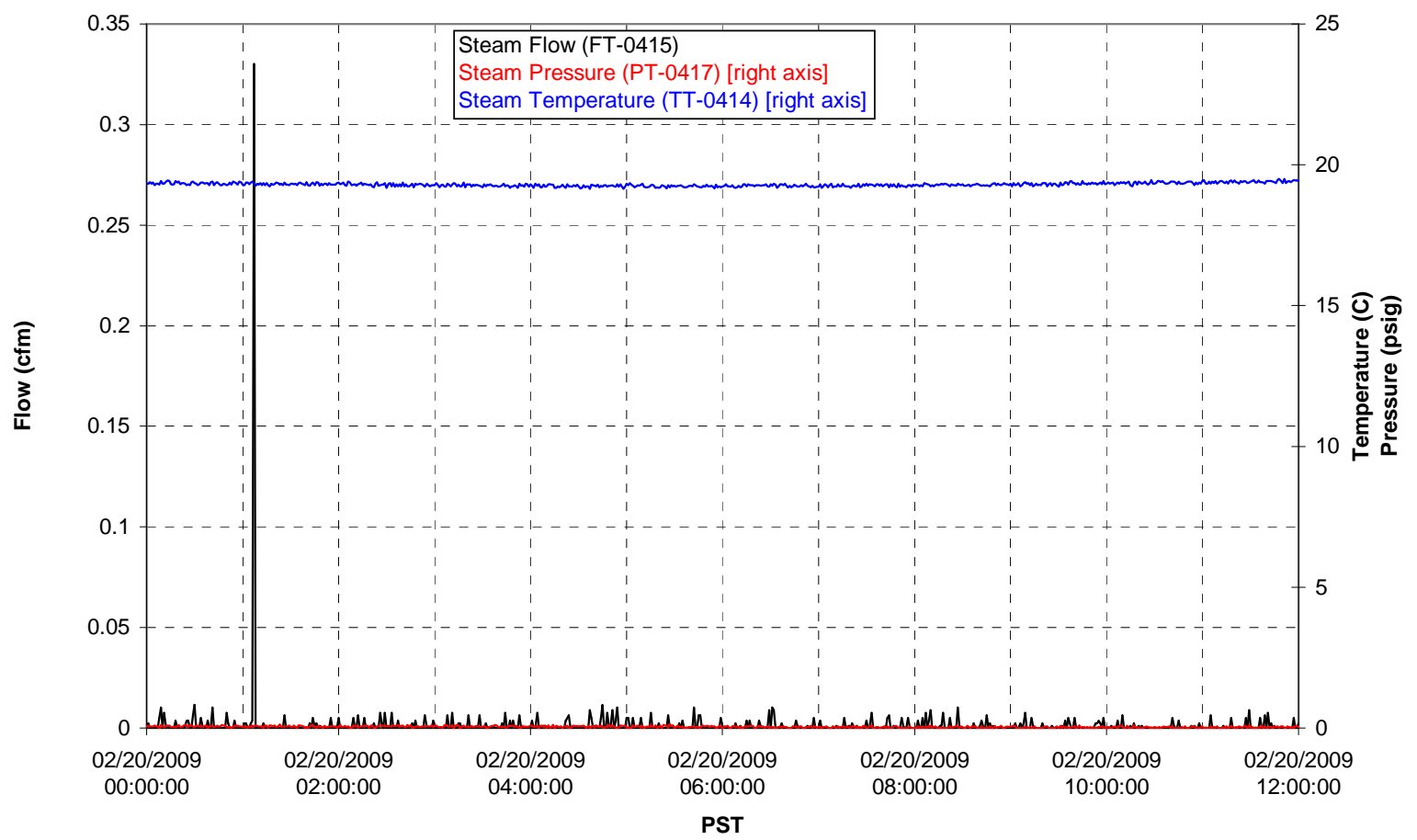




\section{Integrated Test A Data Plots 02/20/09 12:00 - 02/21/09 00:00}


T01A level

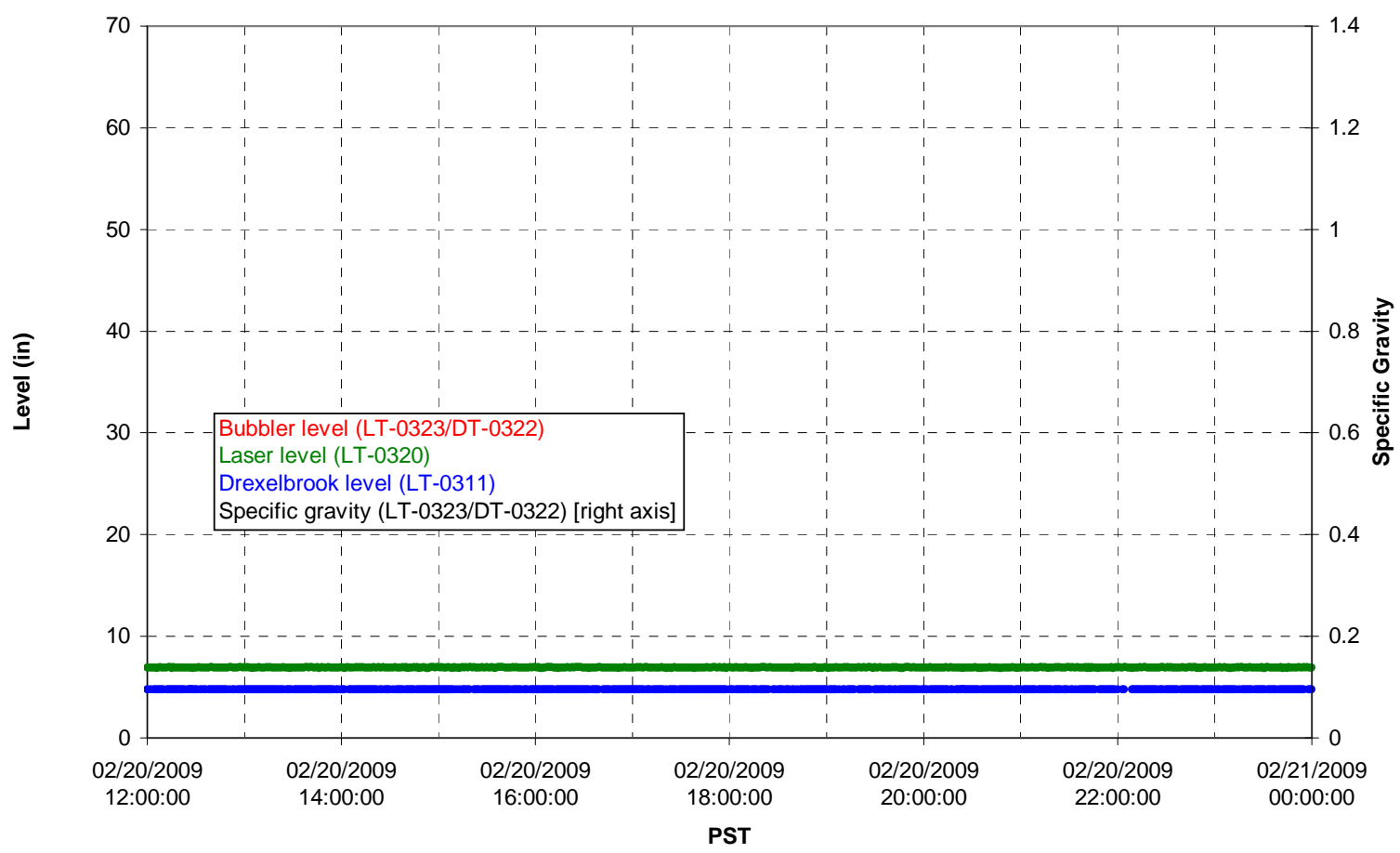

T01A temperatures

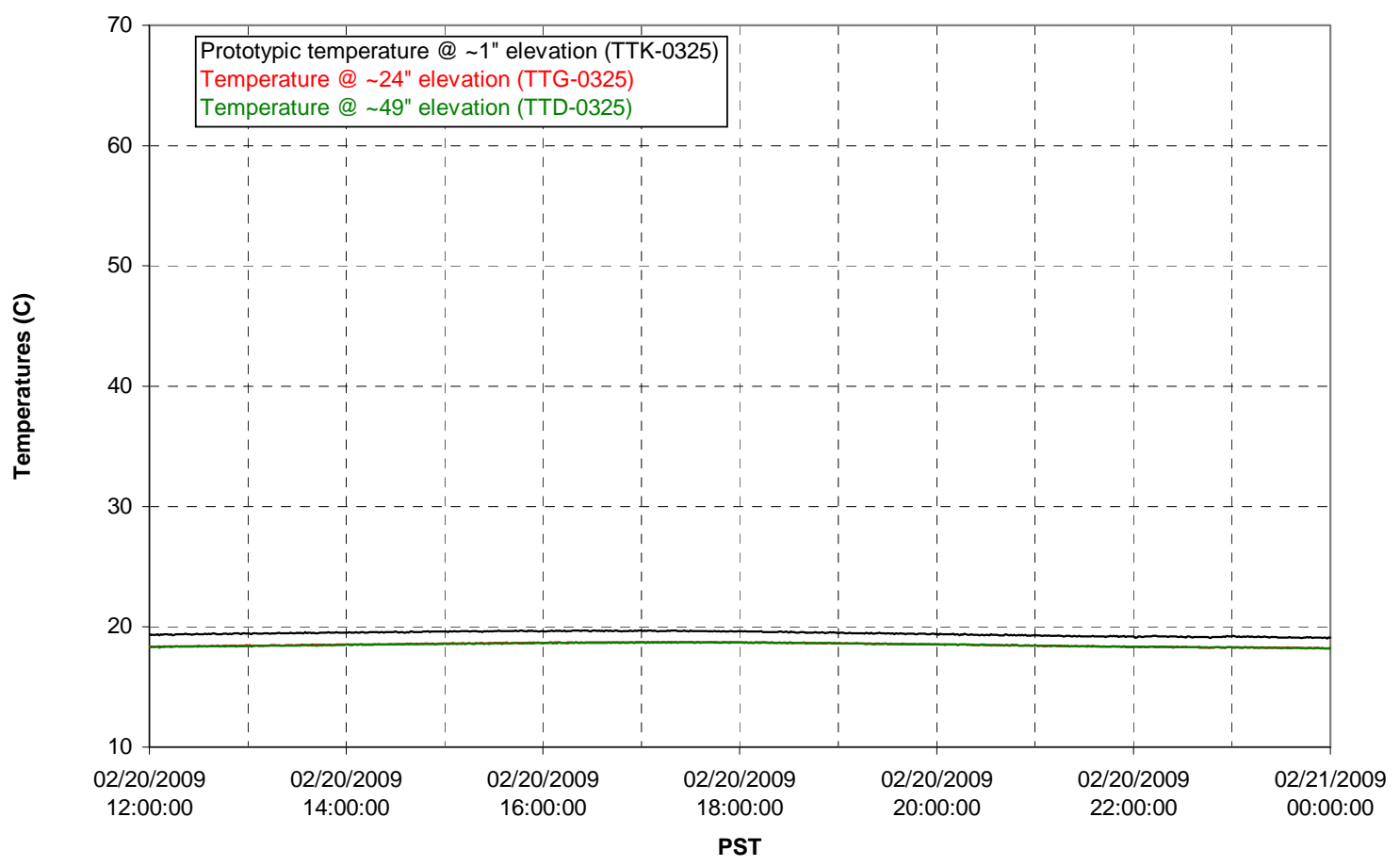


T01B level

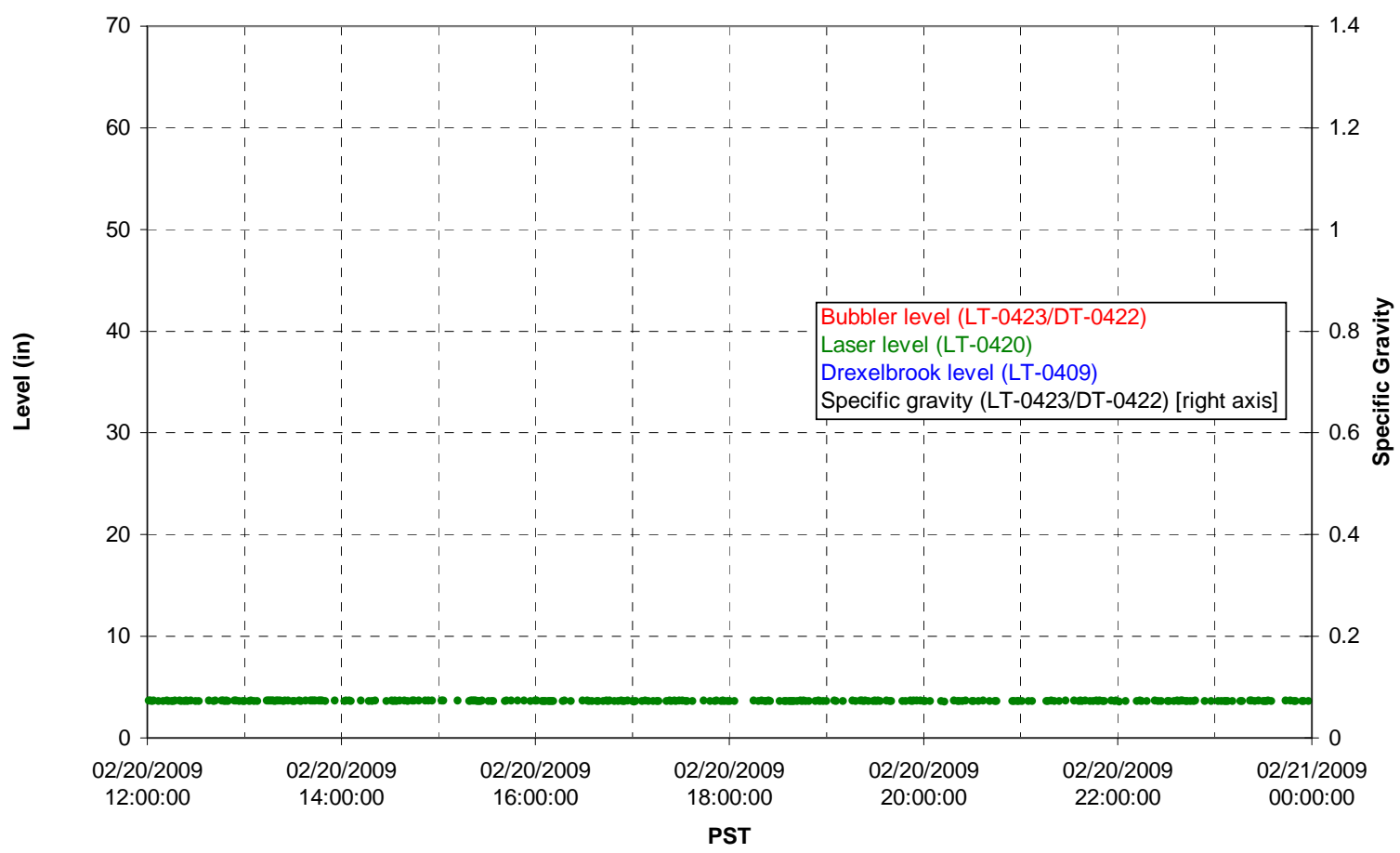

T01B temperatures

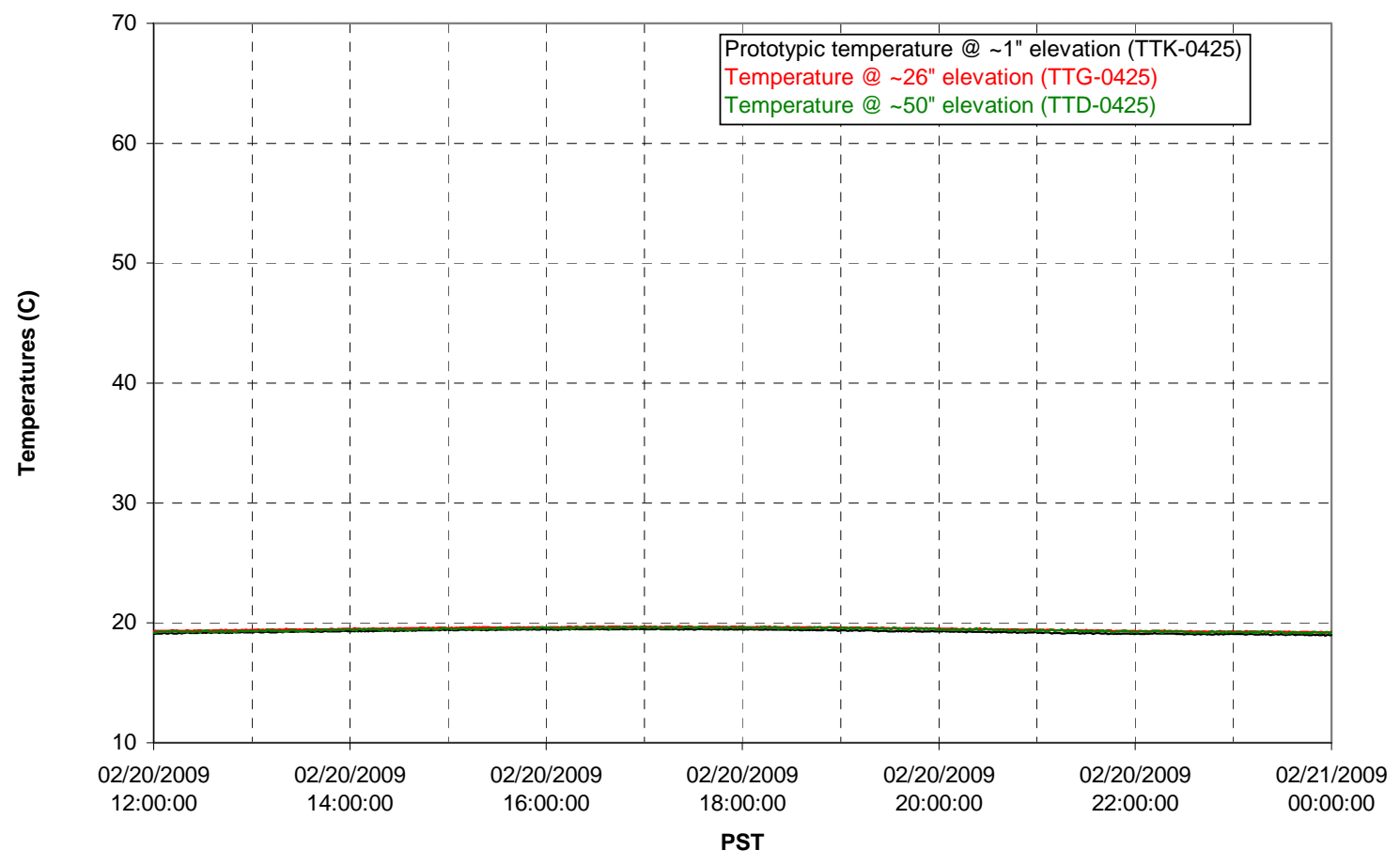


T02A level

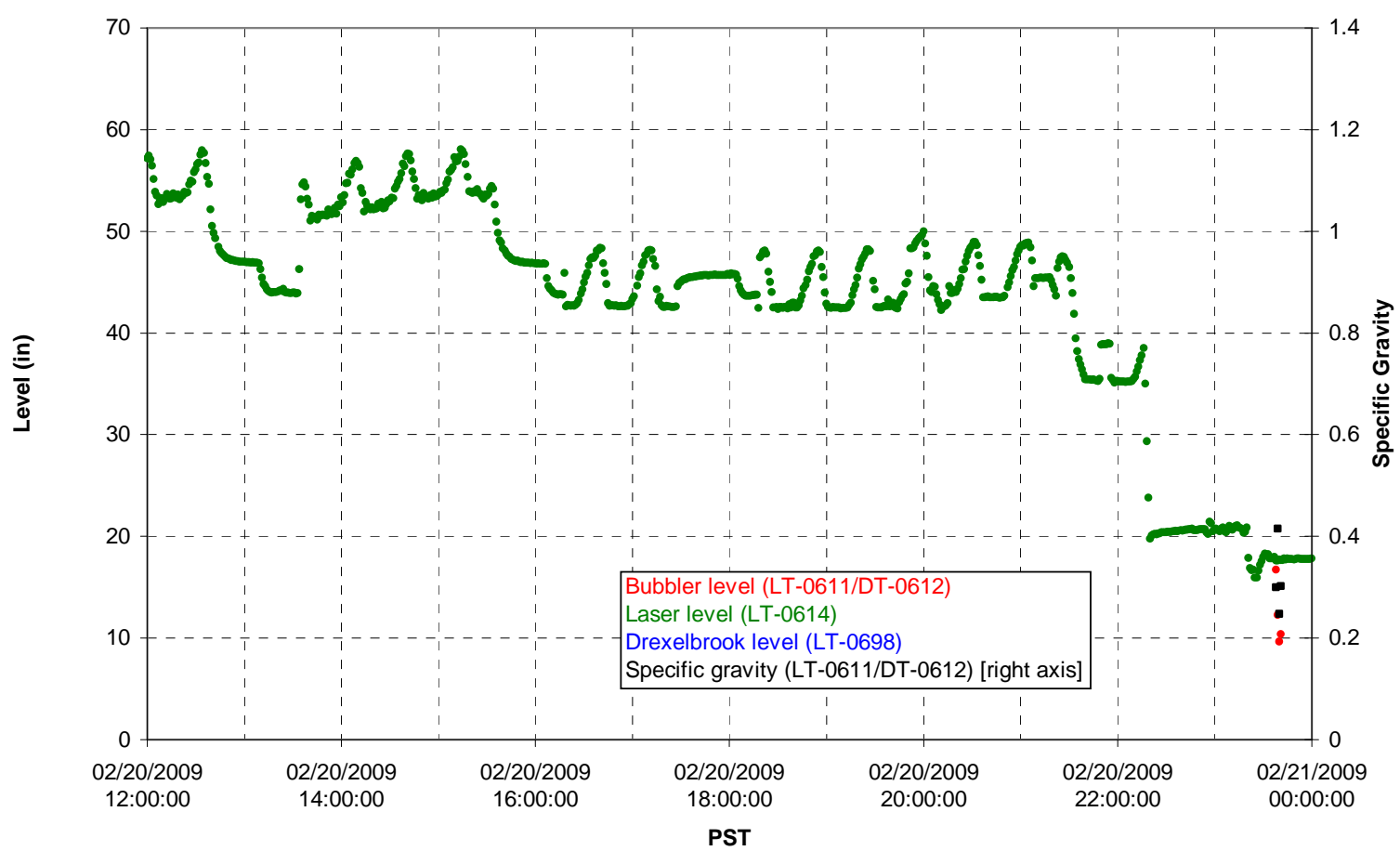

T02A temperatures

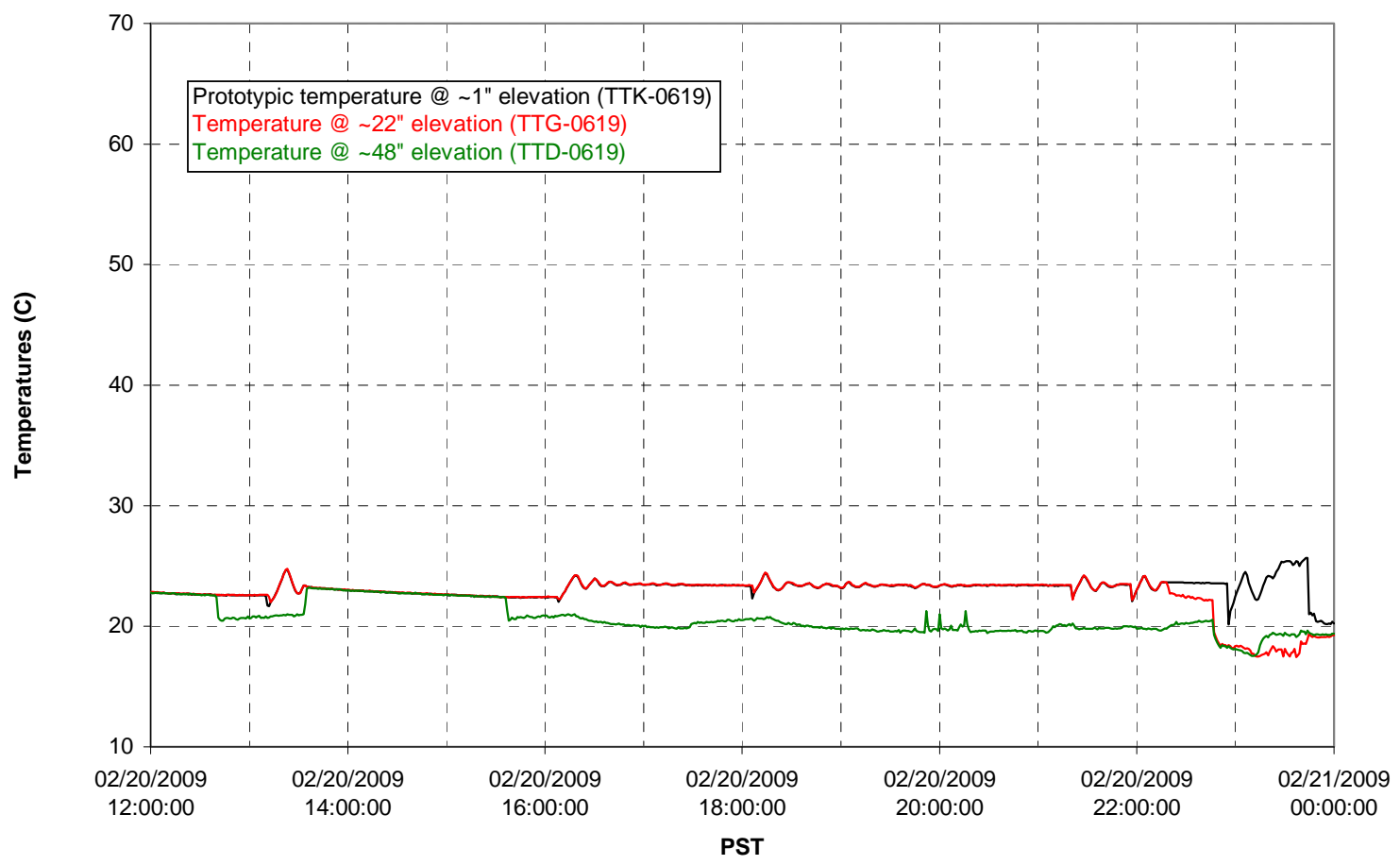

G.742 
T02A and filter loop temperatures
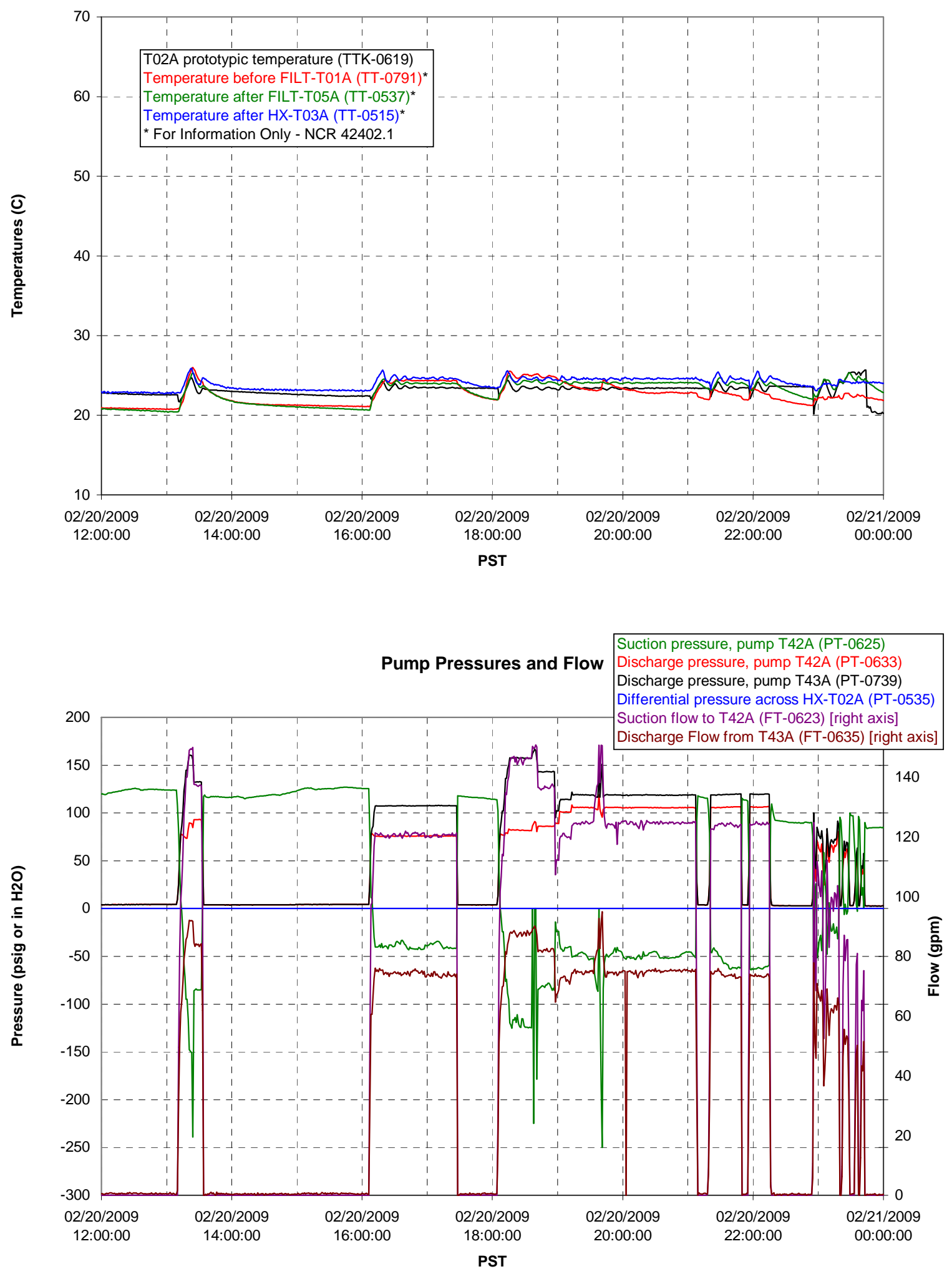


\section{Axial pressure drop}

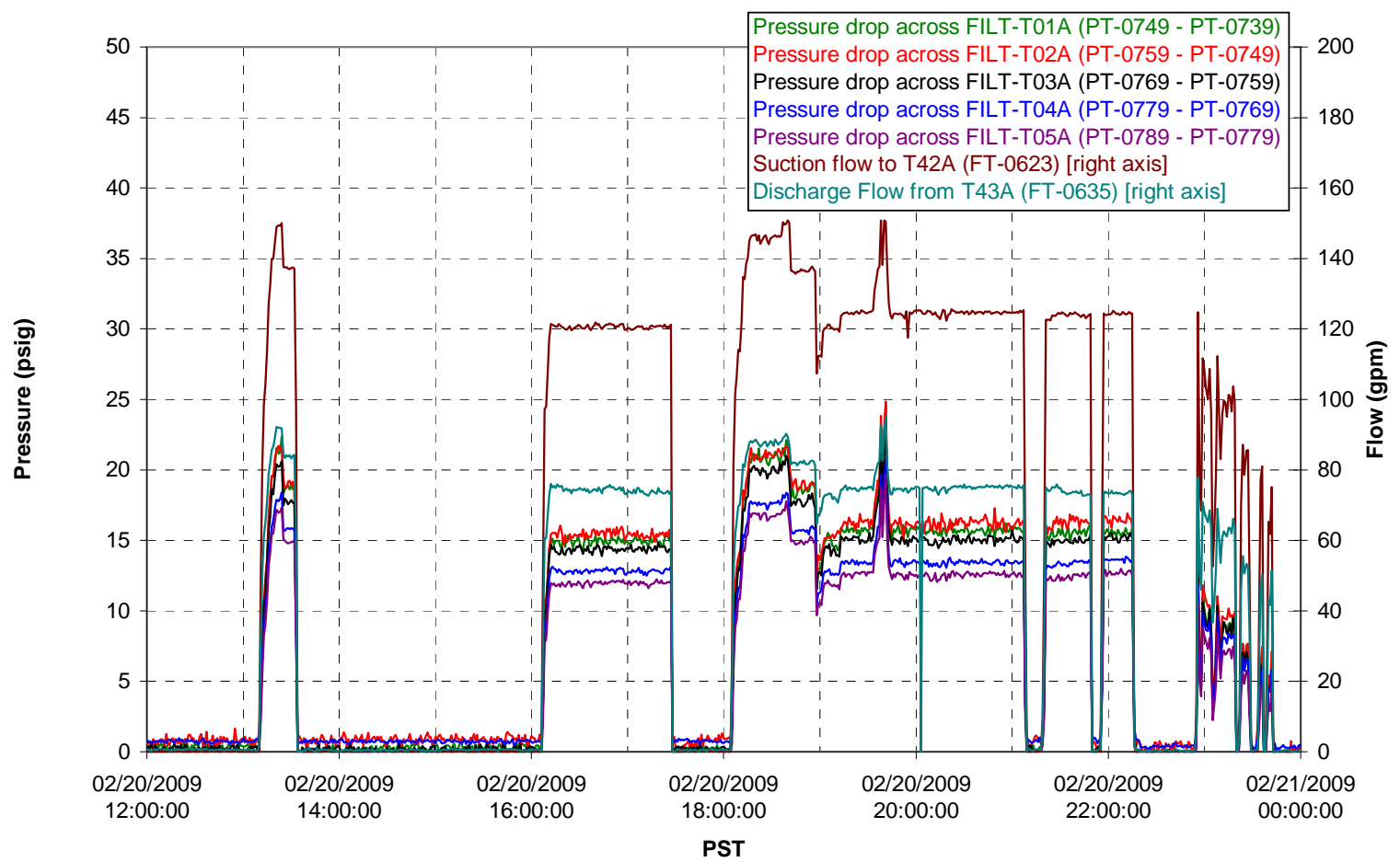

Permeate flow rates

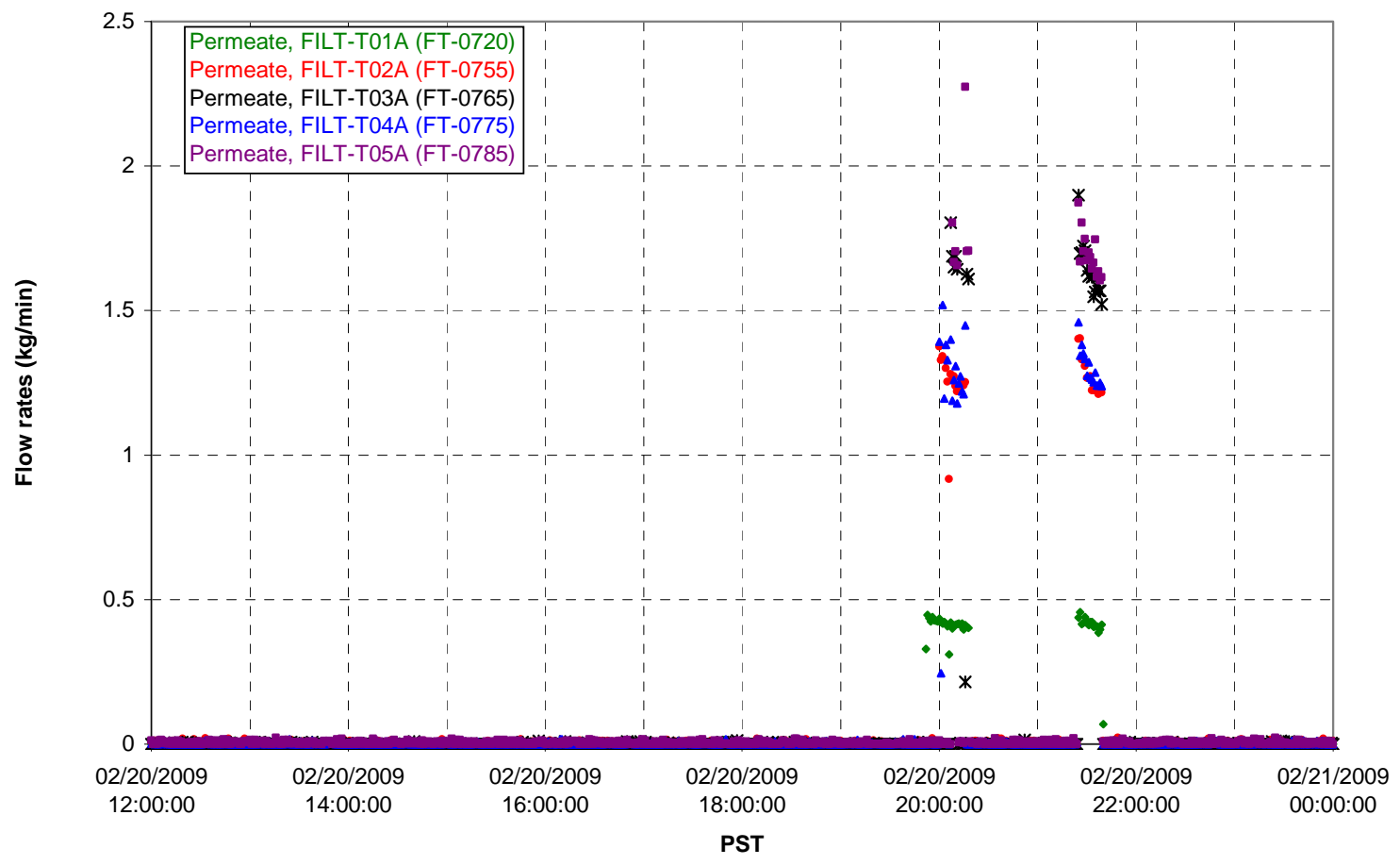


T02A Inner Temperature Tree

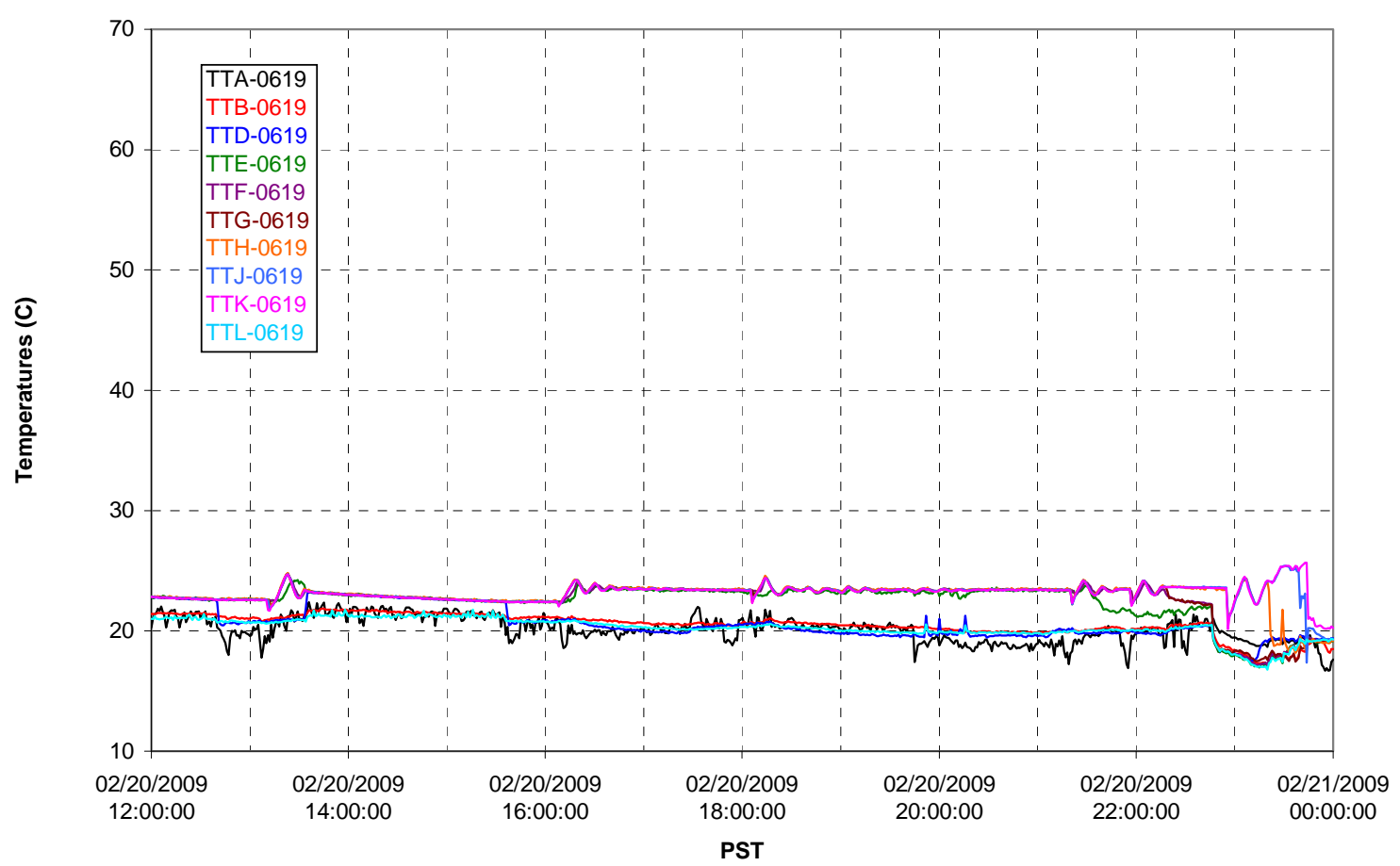

T02A Outer Temperature Tree

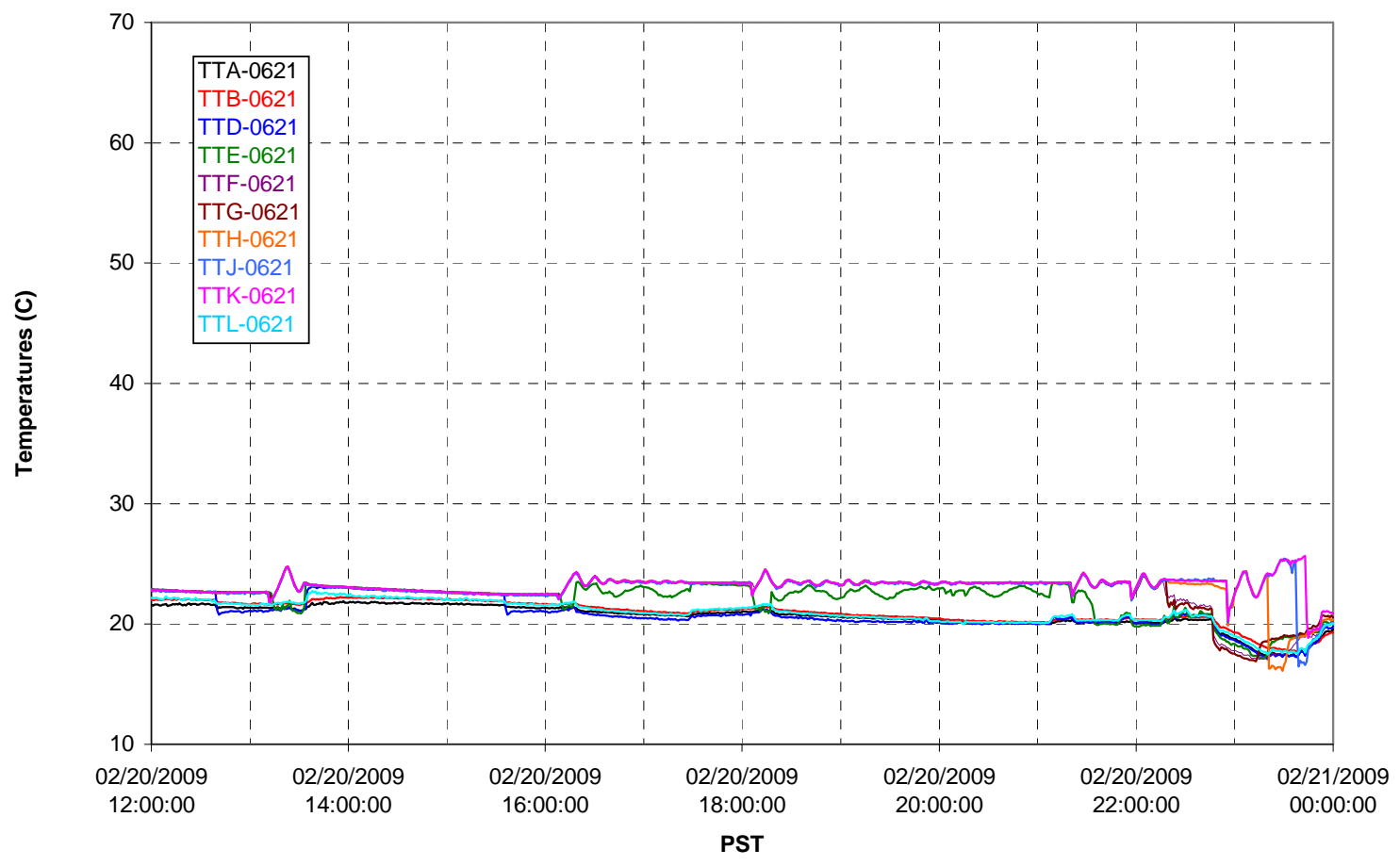


T02A temperatures

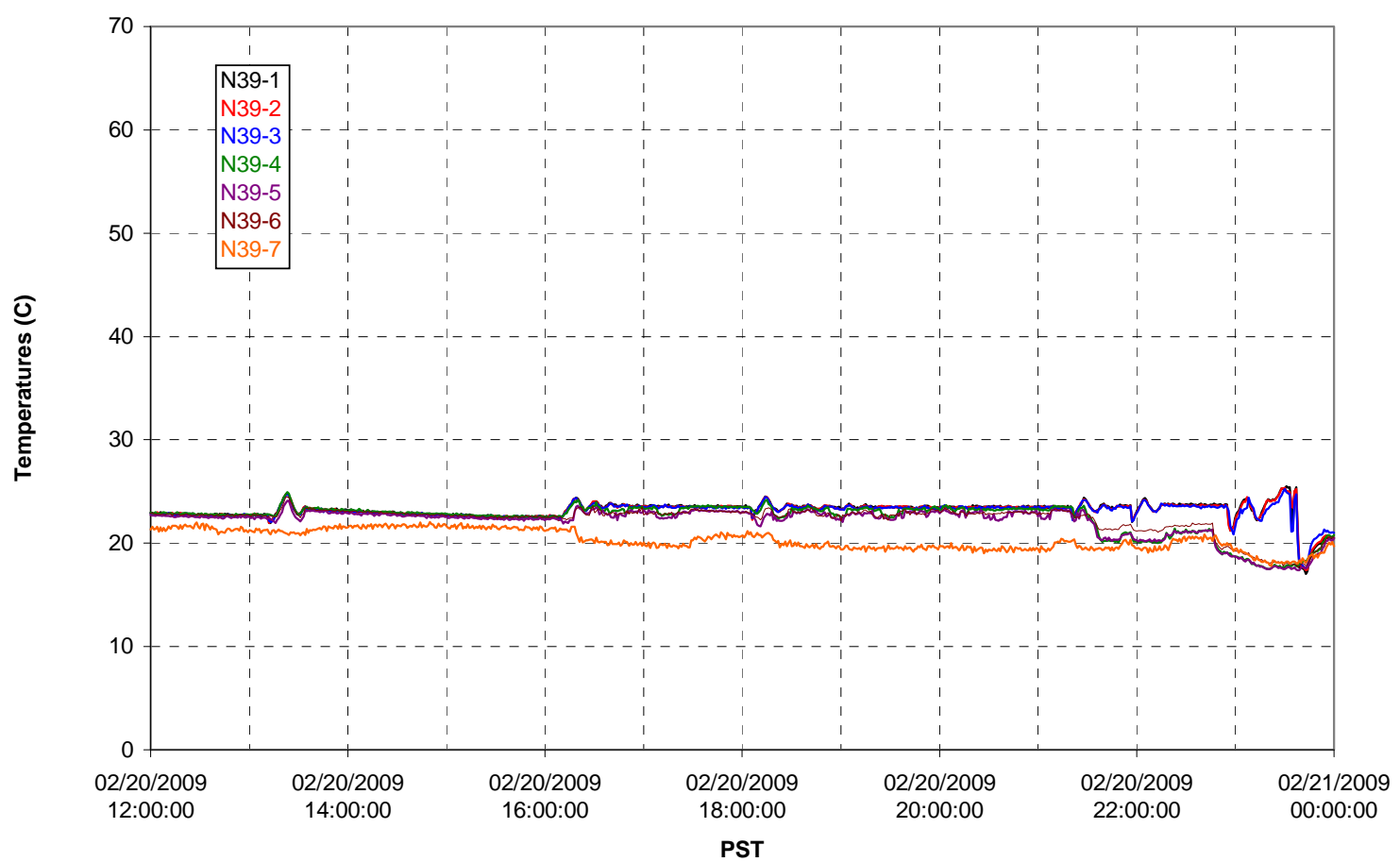

T02A temperatures

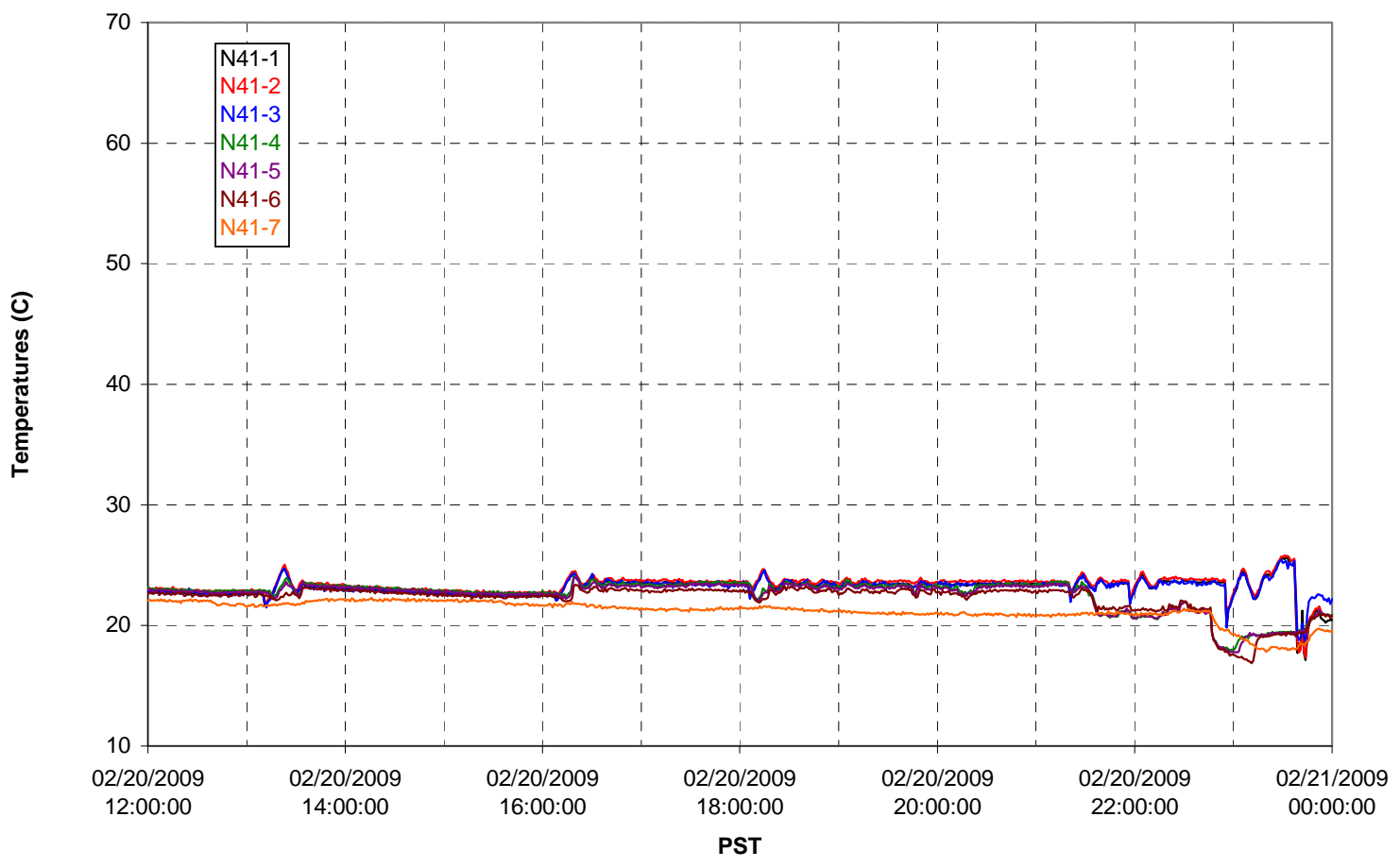


T02A temperatures

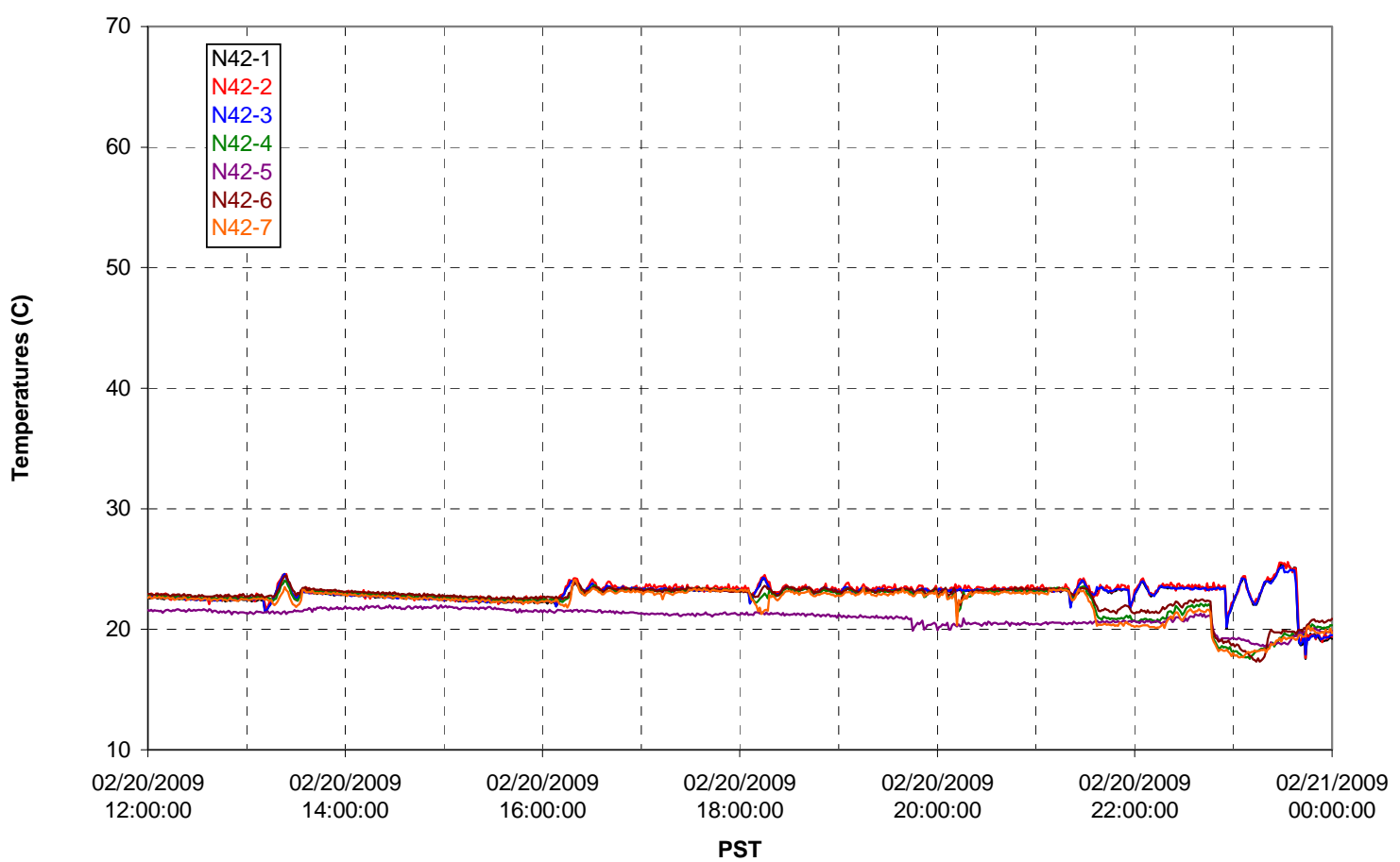

T02A temperatures

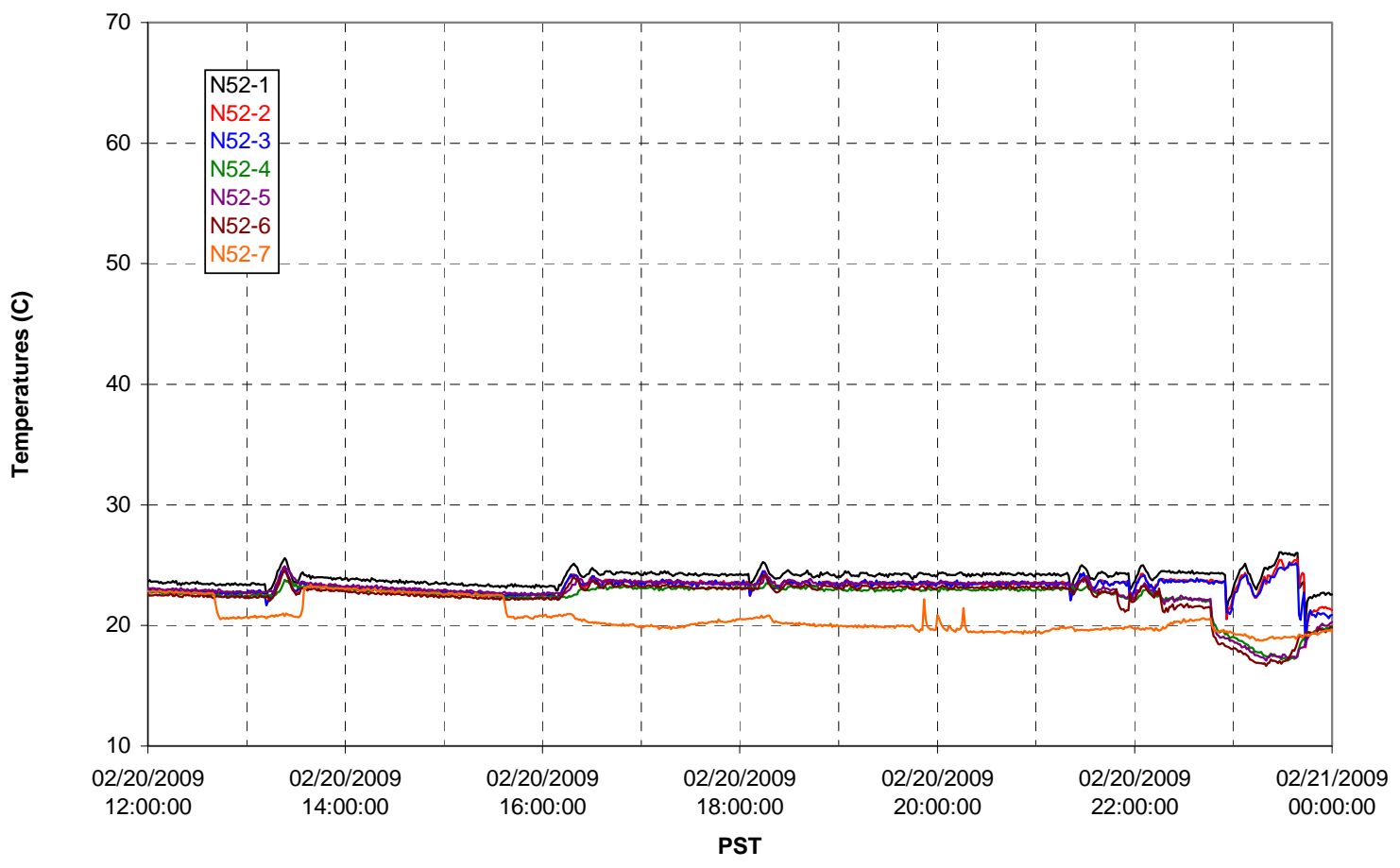


T02A Heating and Cooling
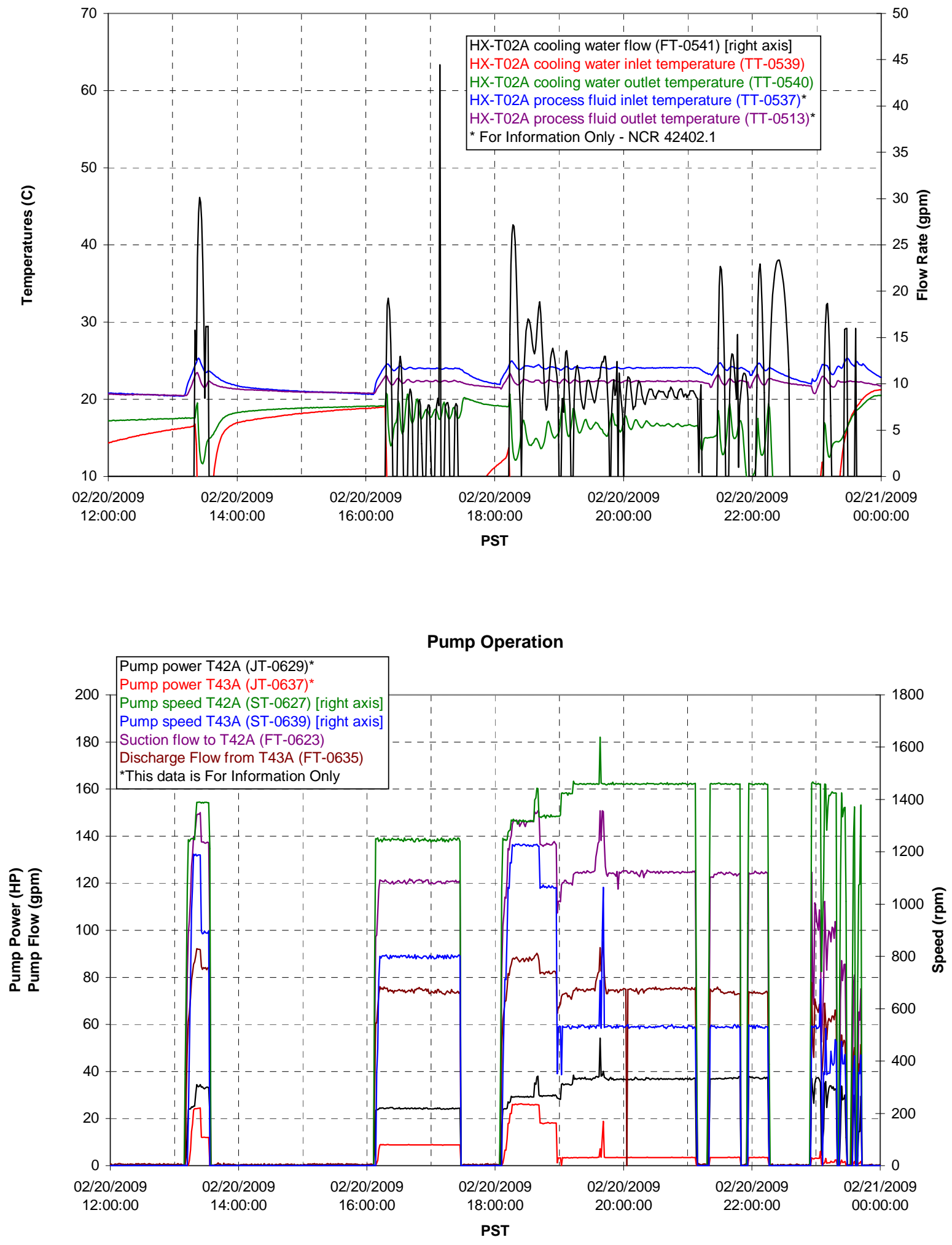
Pulsepot UFP-PP-T01A

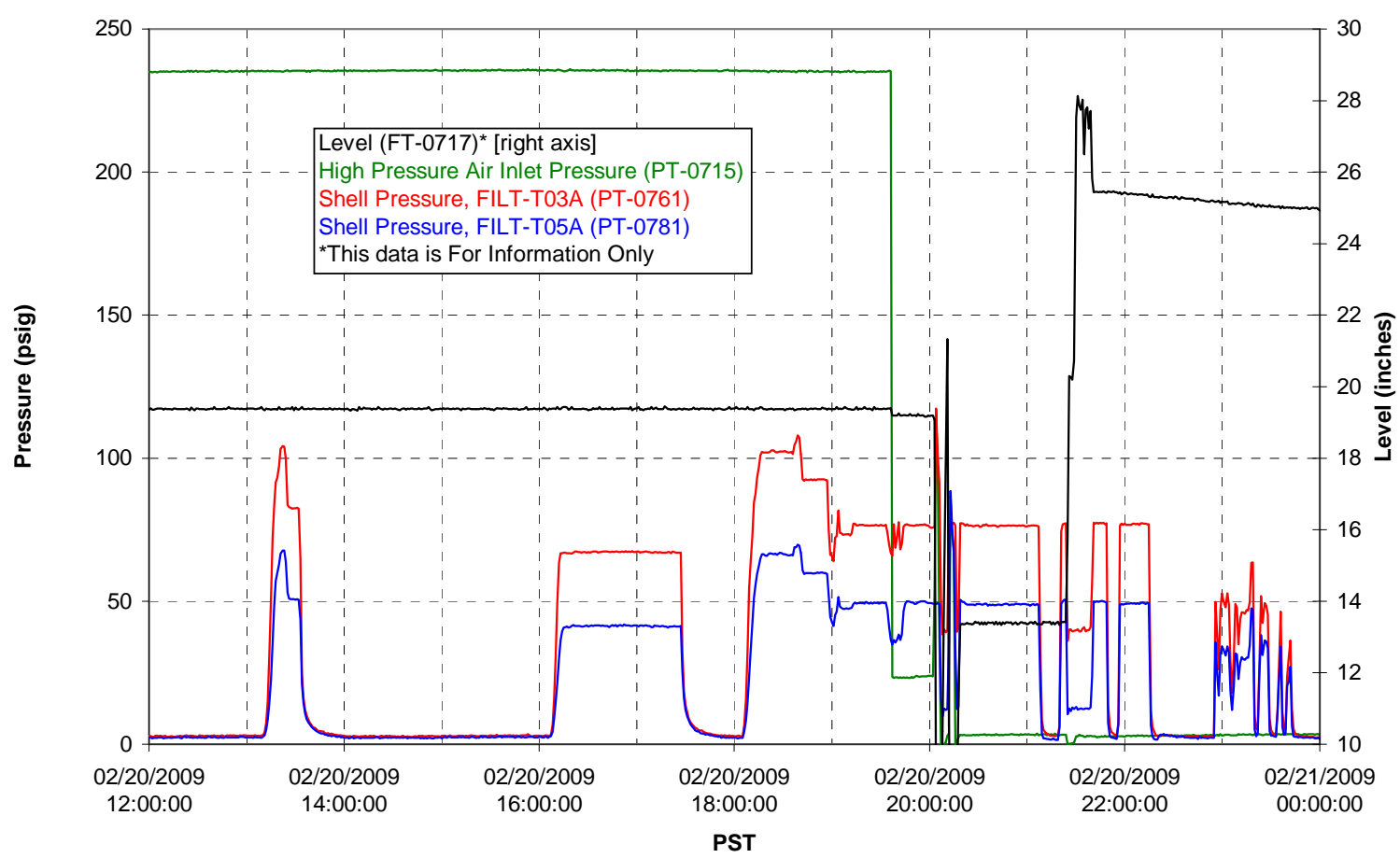

Pulsepot UFP-PP-T02A

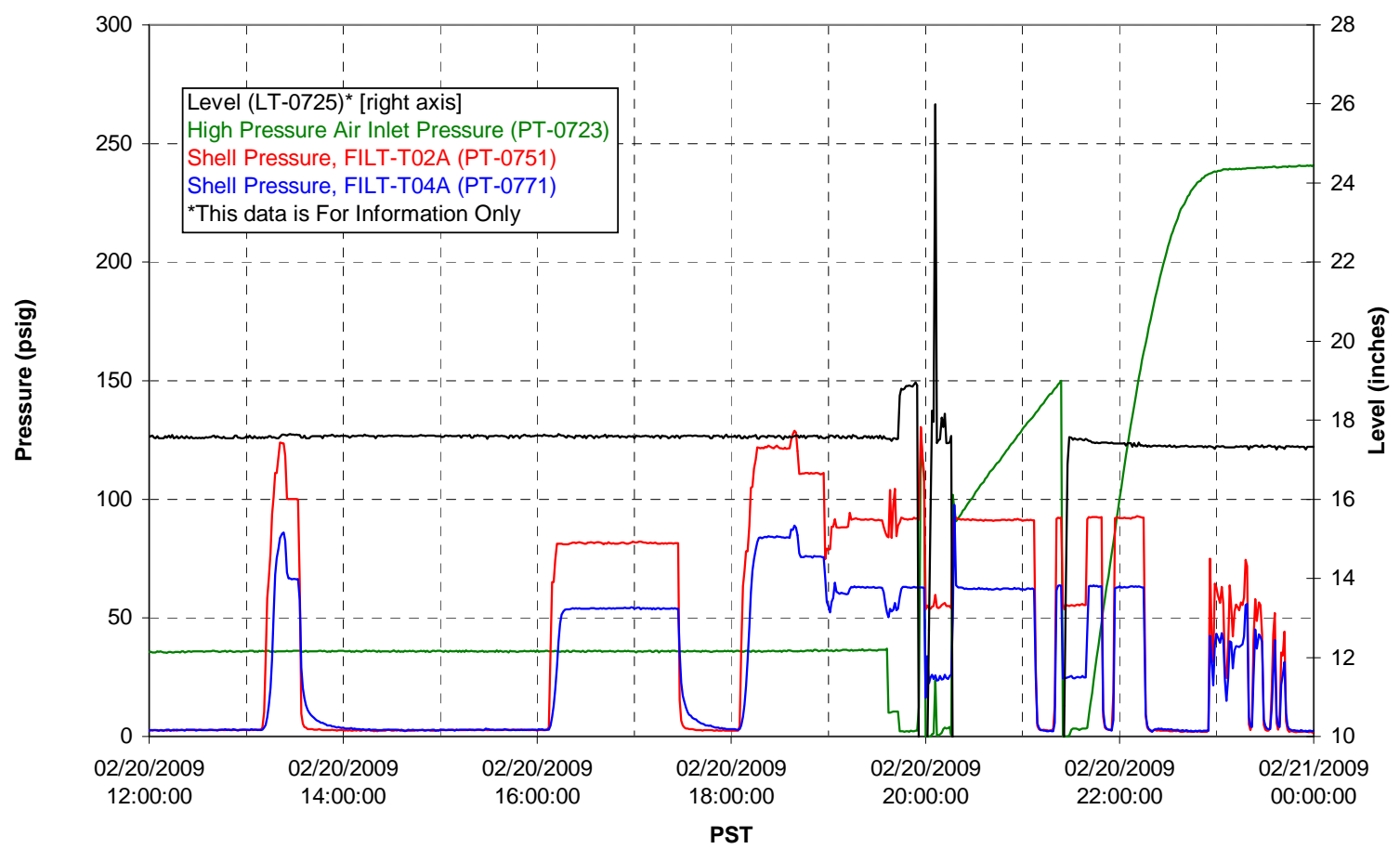


Pulsepot UFP-PP-T03A

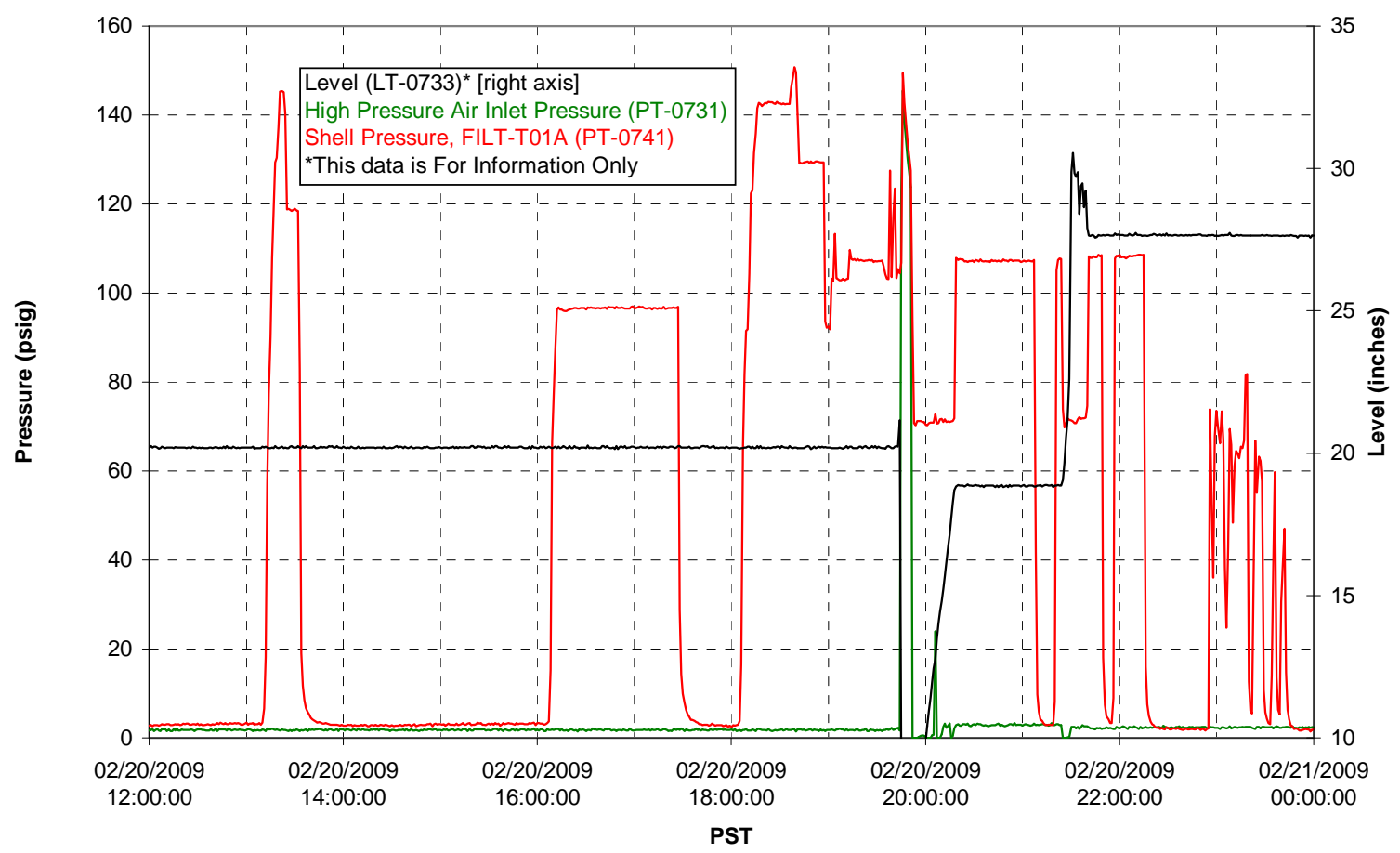

Pulsepot Levels

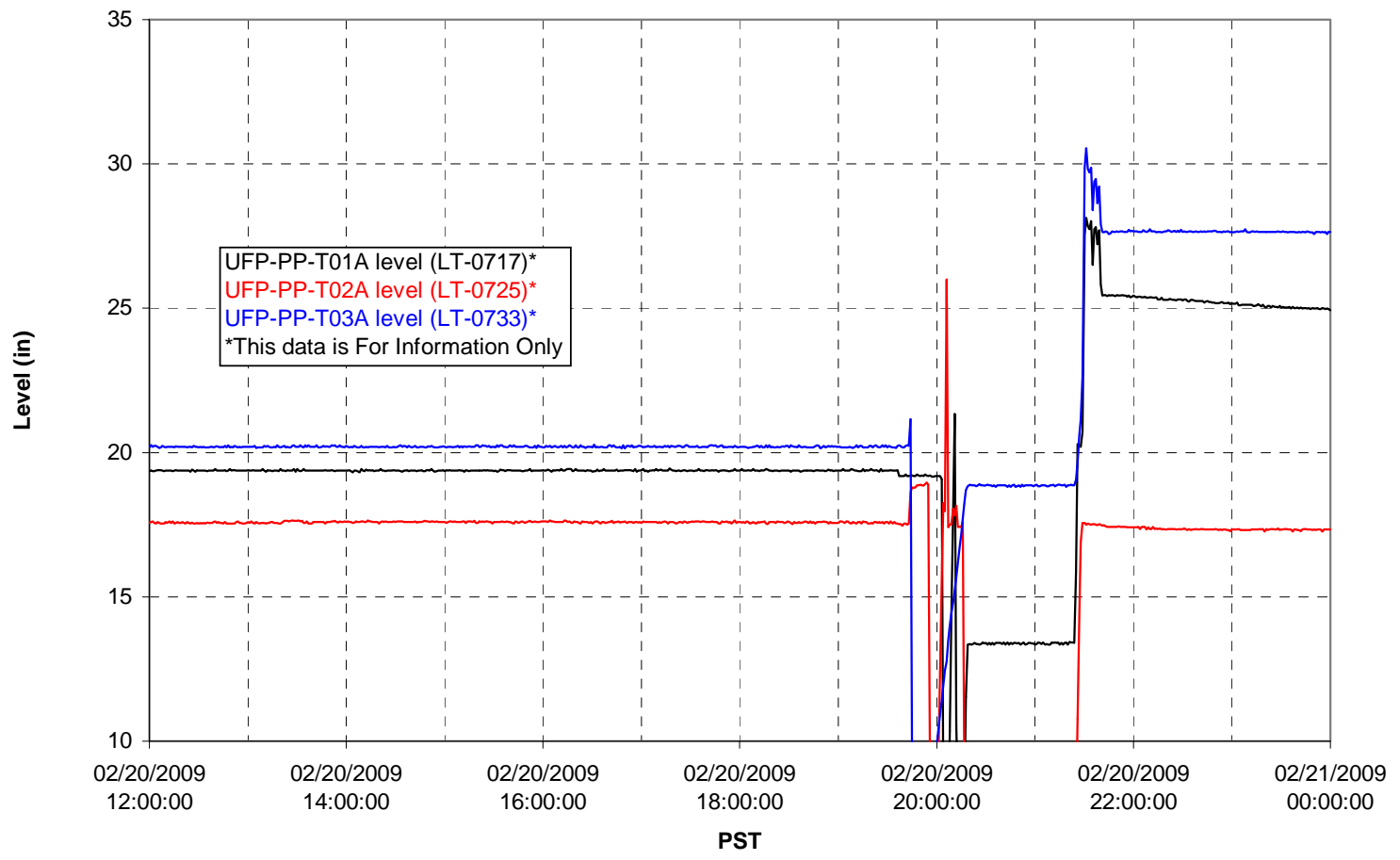

G.750 
Filter UFP-FILT-T01A

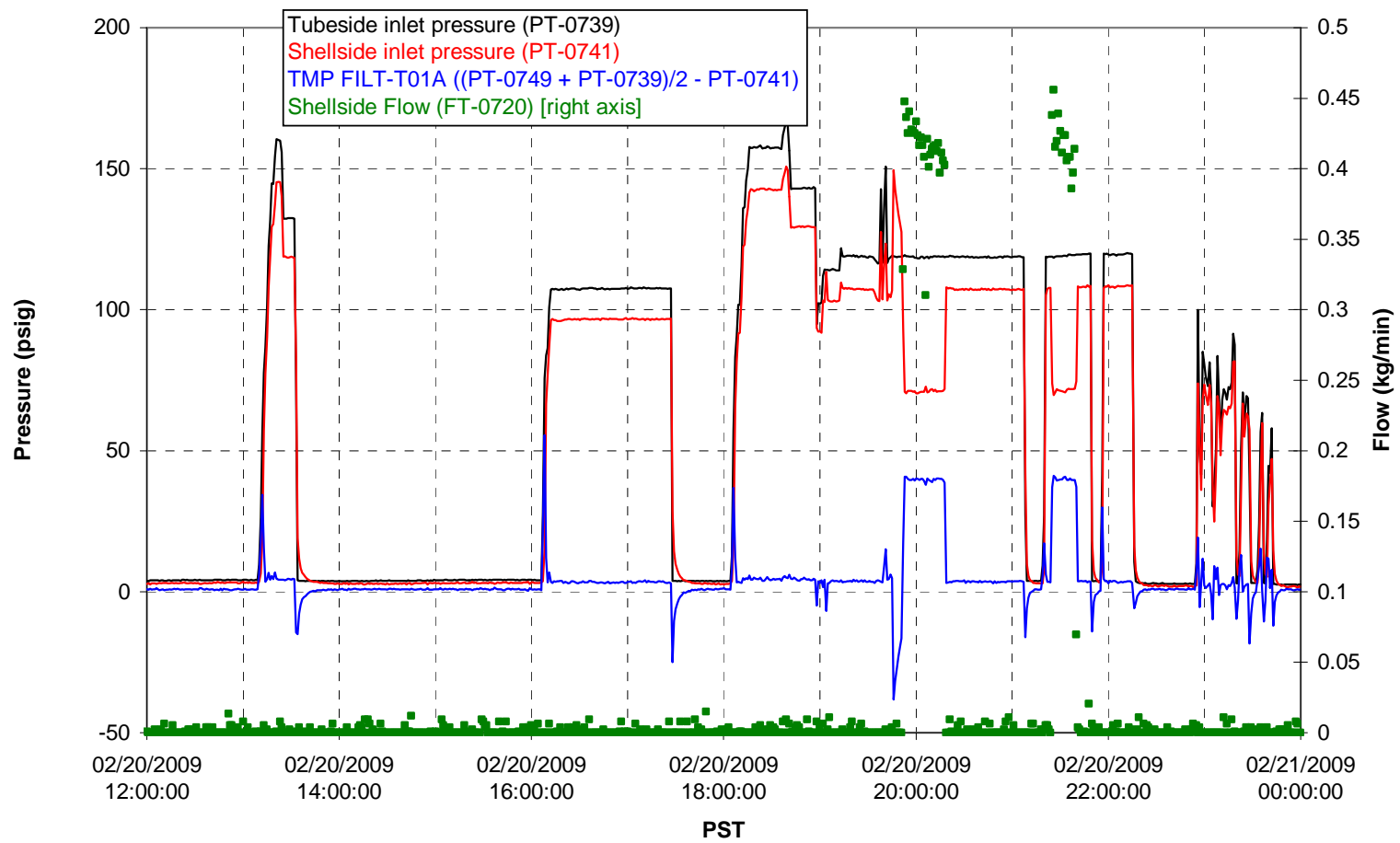

Filter UFP-FILT-T02A

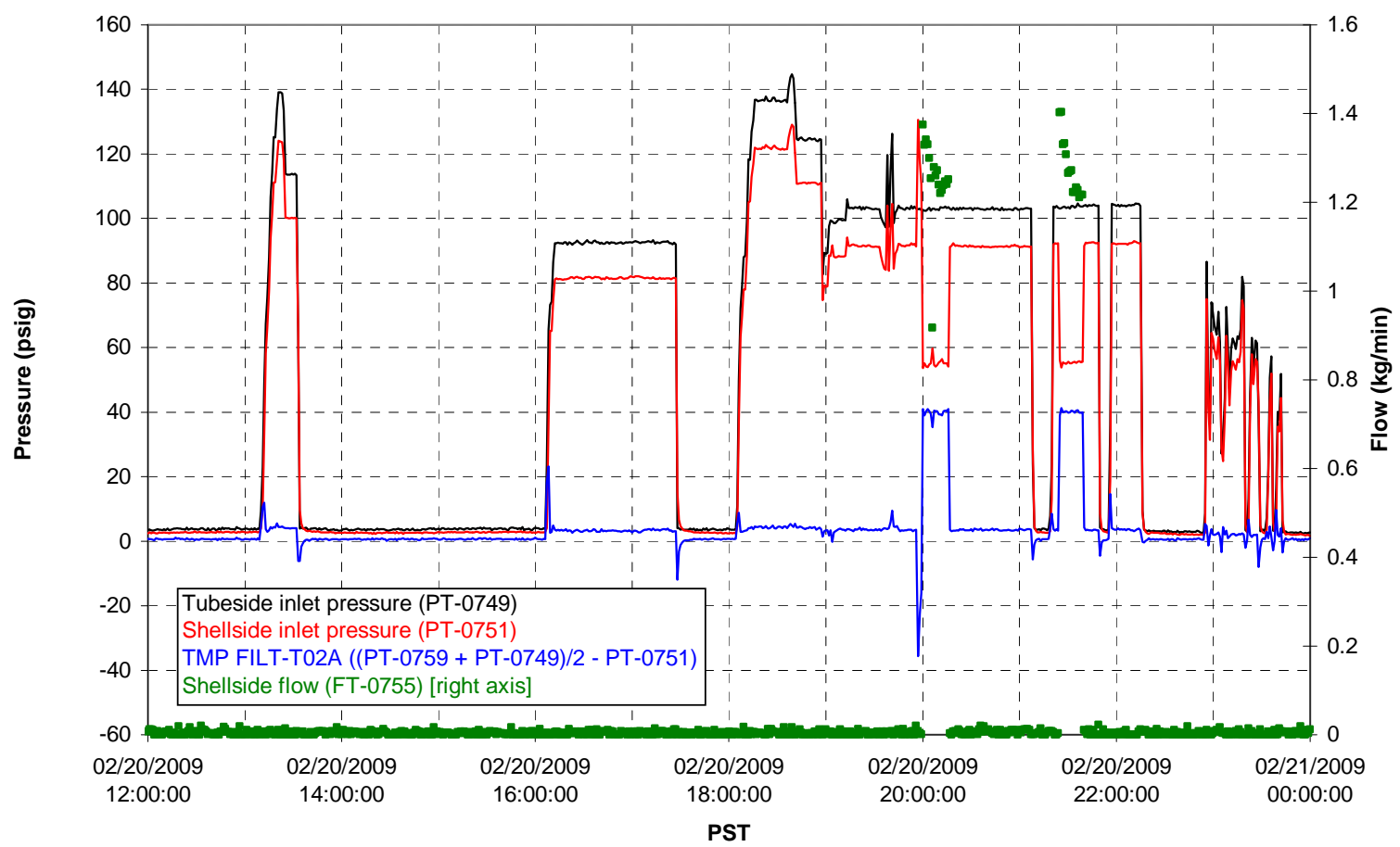


Filter UFP-FILT-T03A

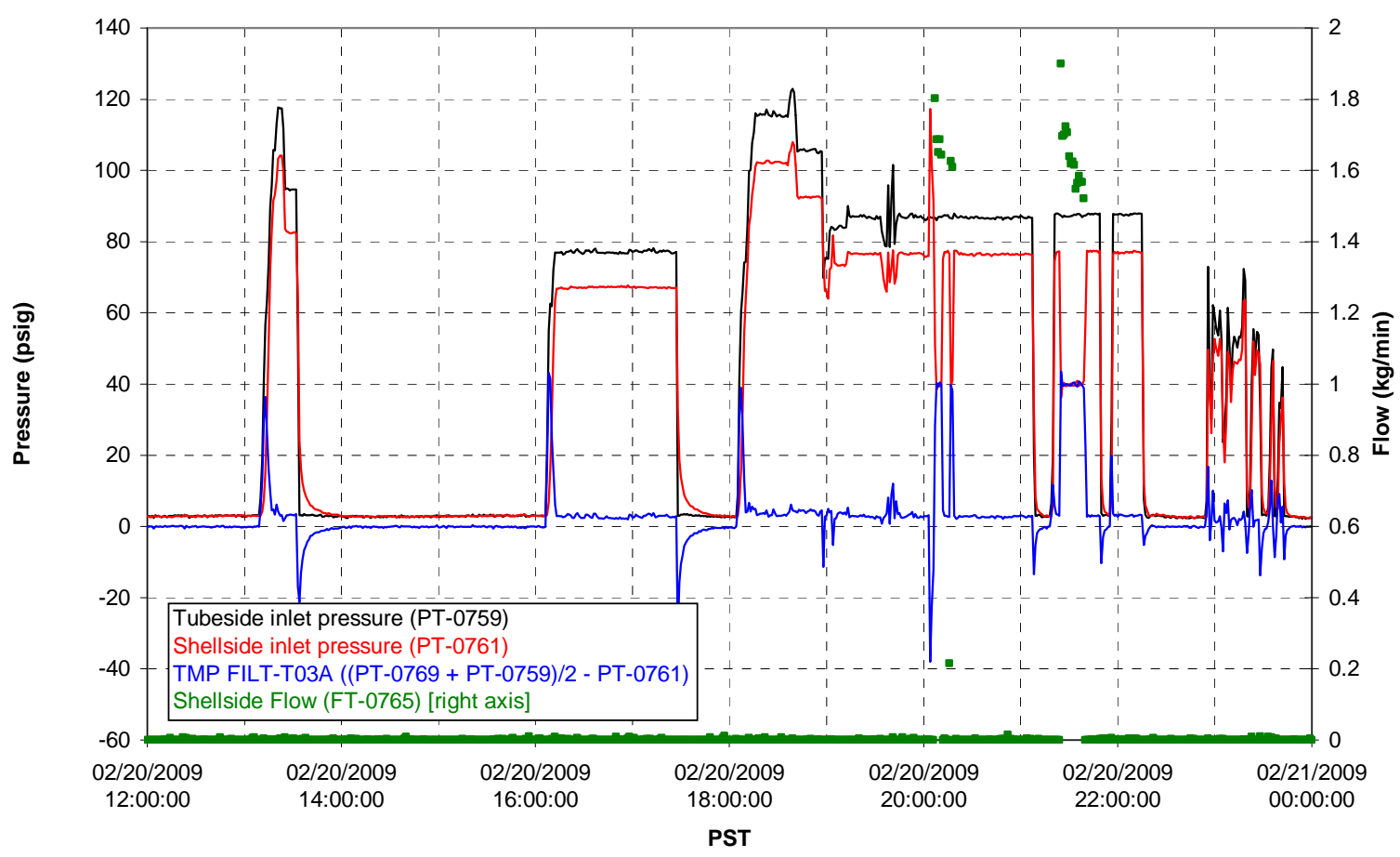

Filter UFP-FILT-T04A

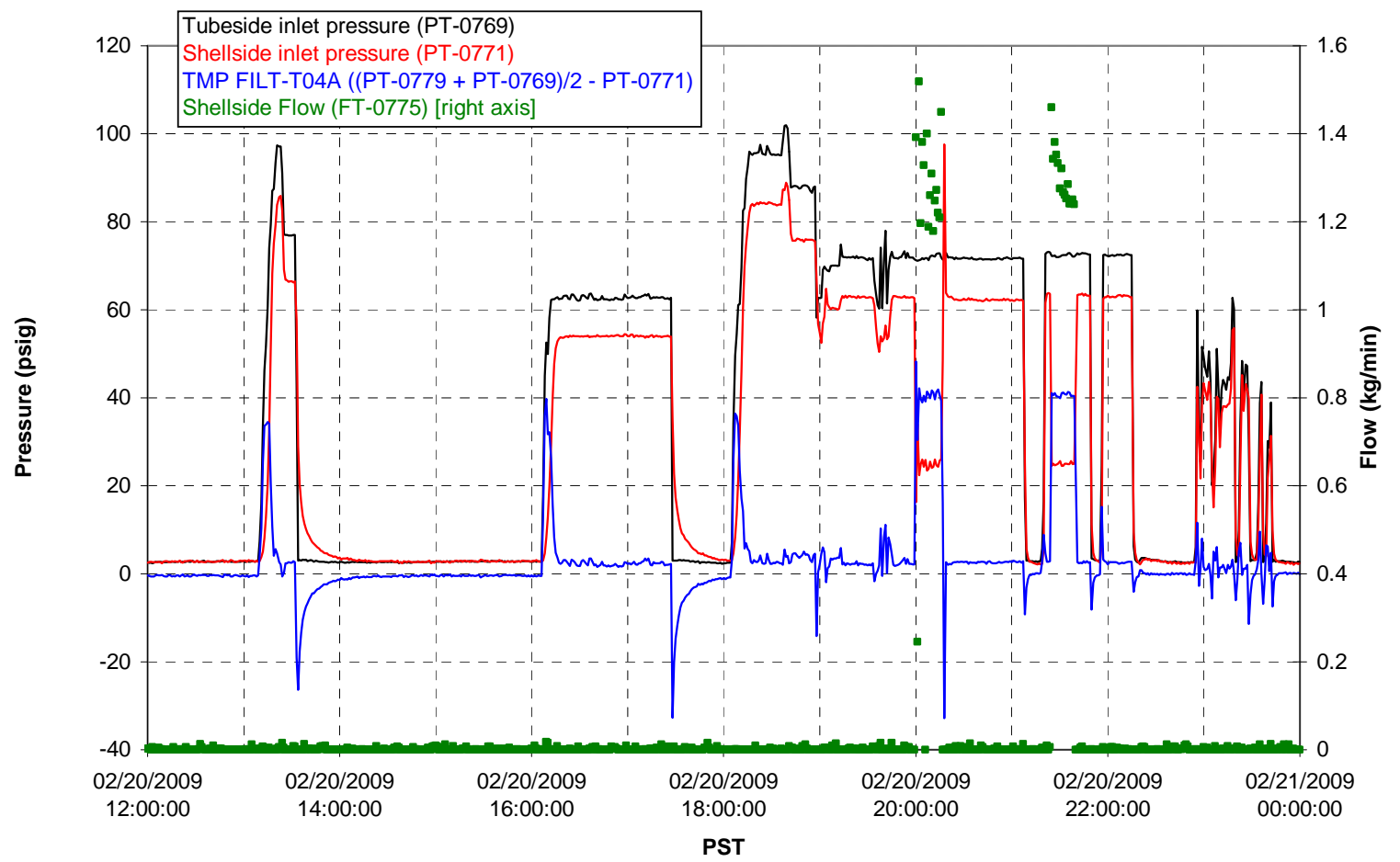


Filter UFP-FILT-T05A

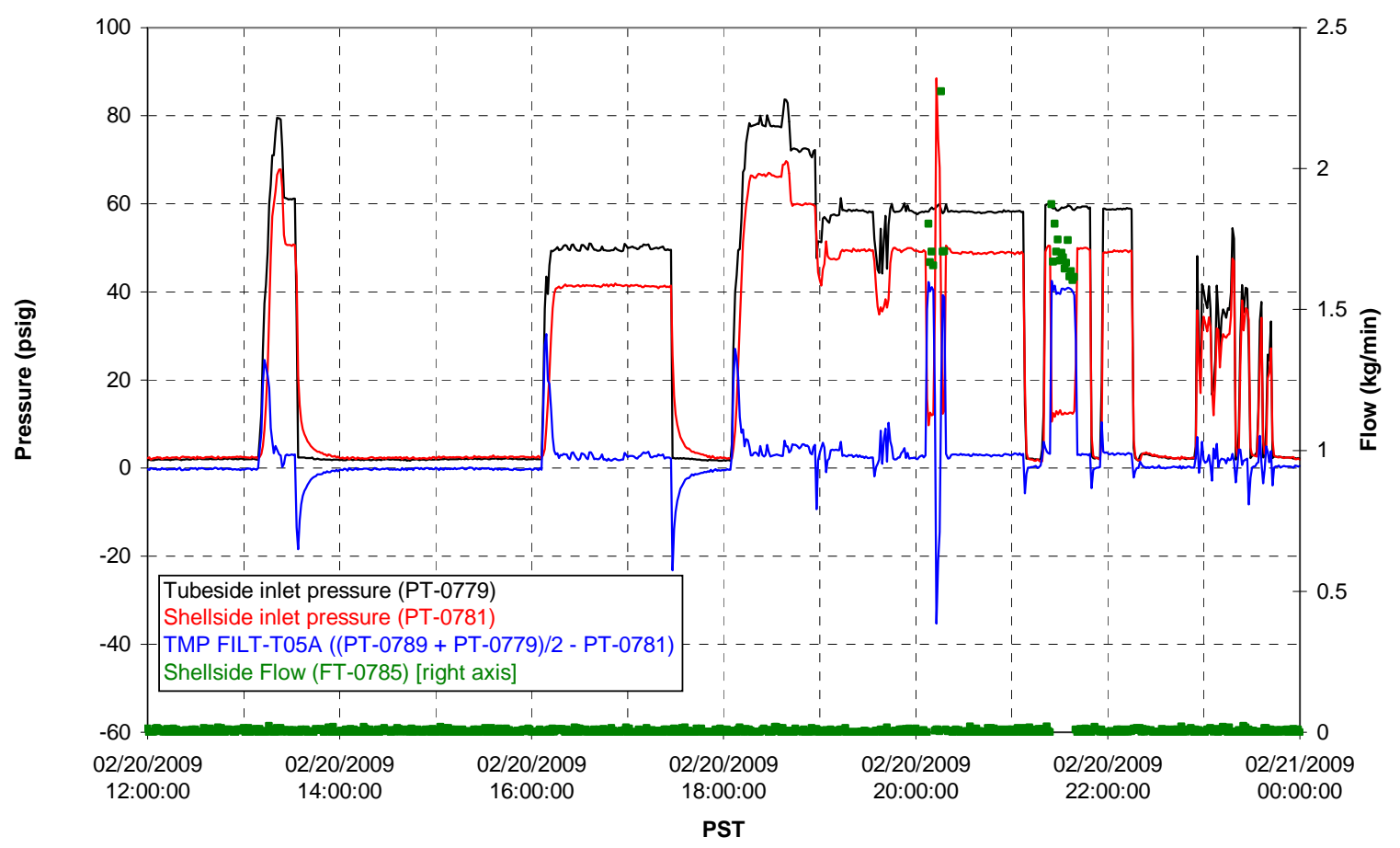

Chemical Flow

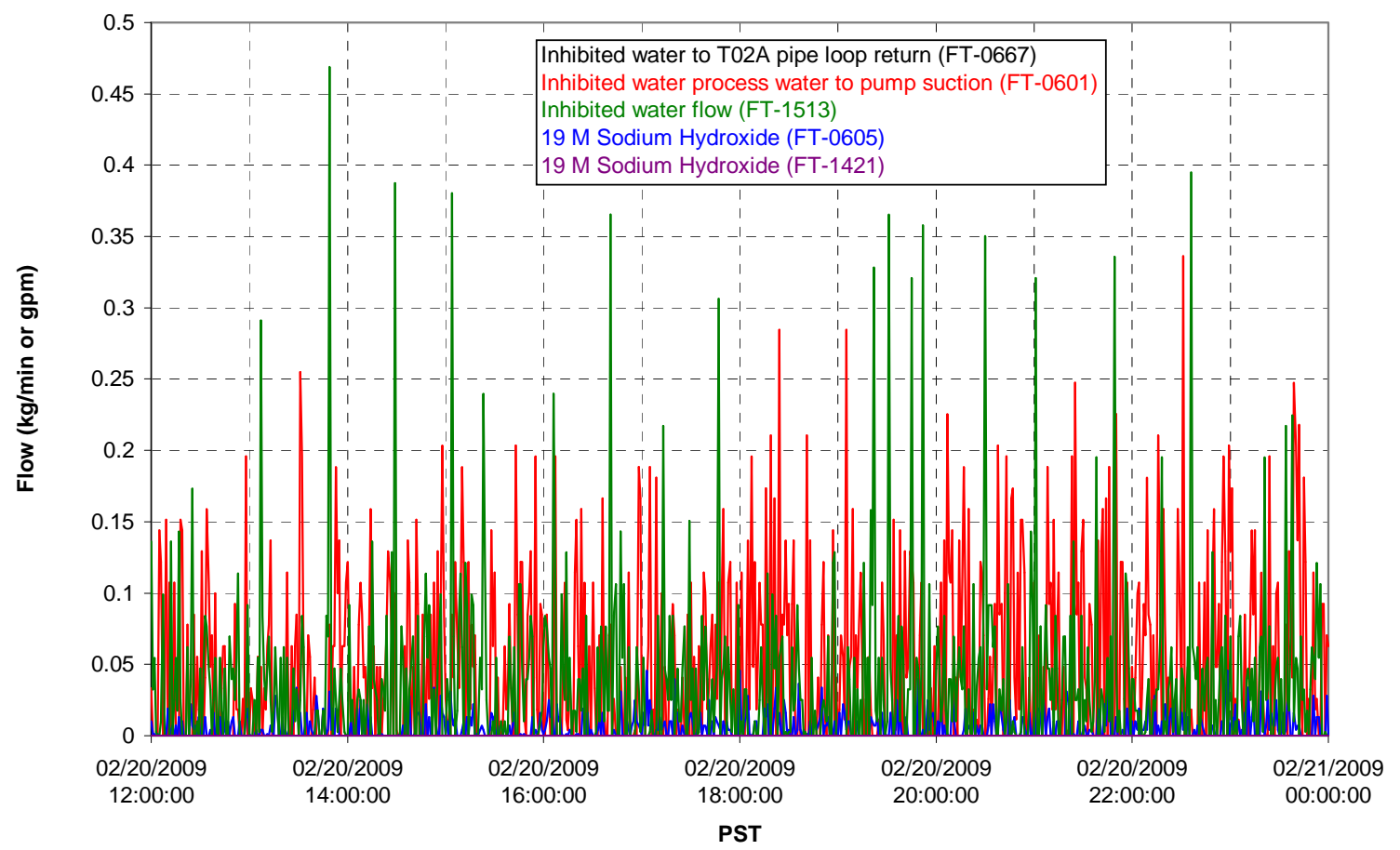




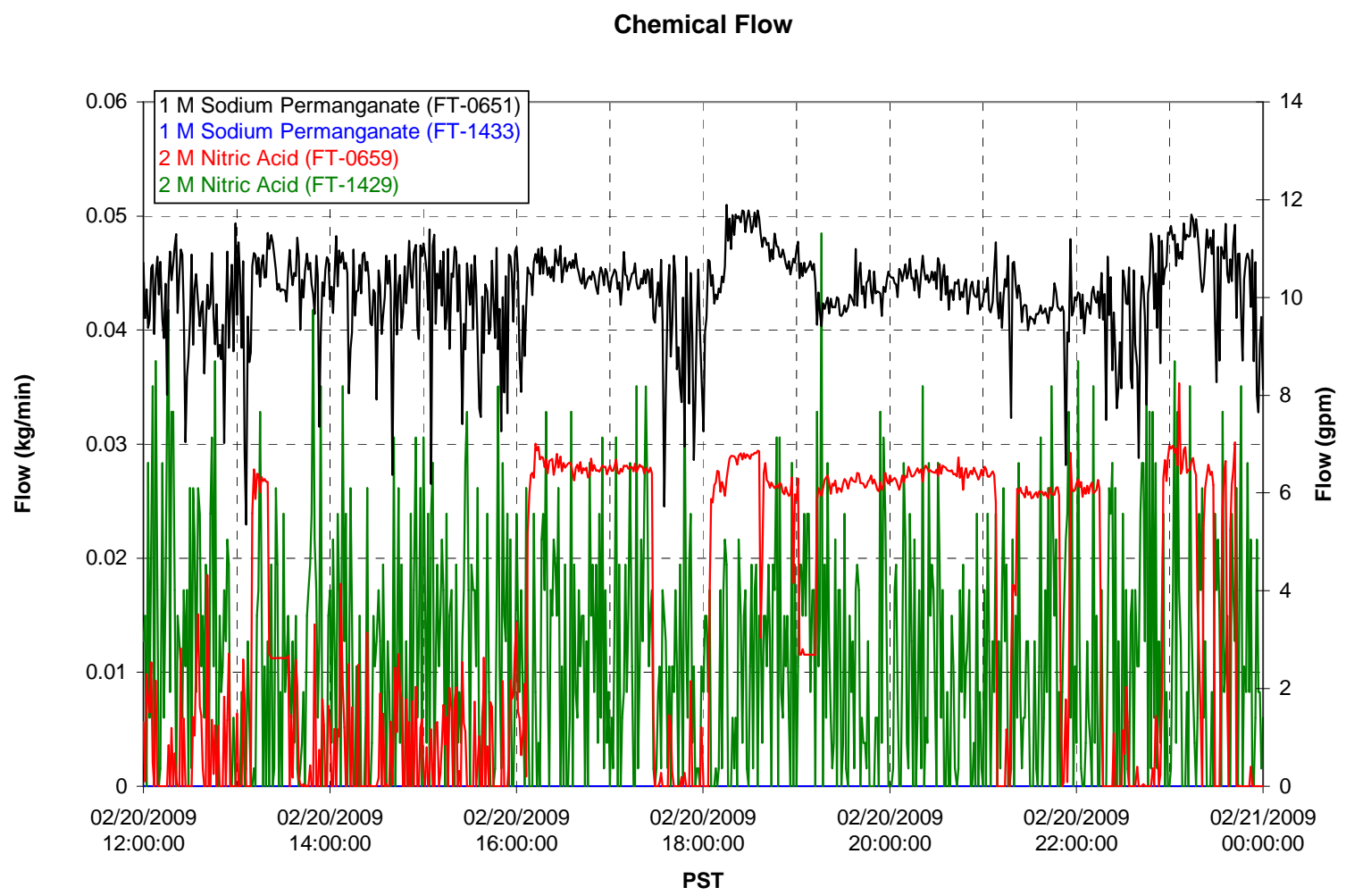

Air Flows

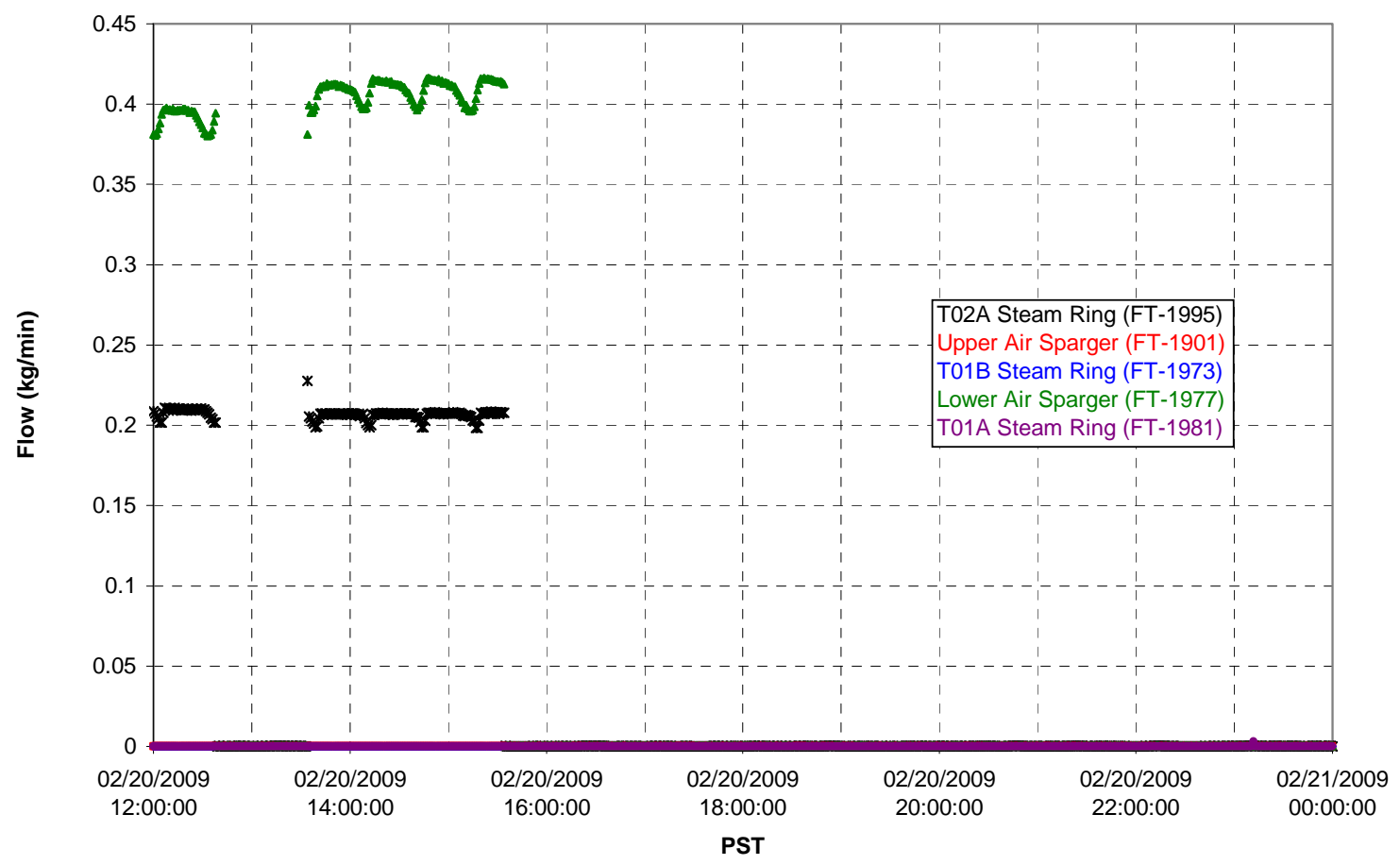


T02A Steam

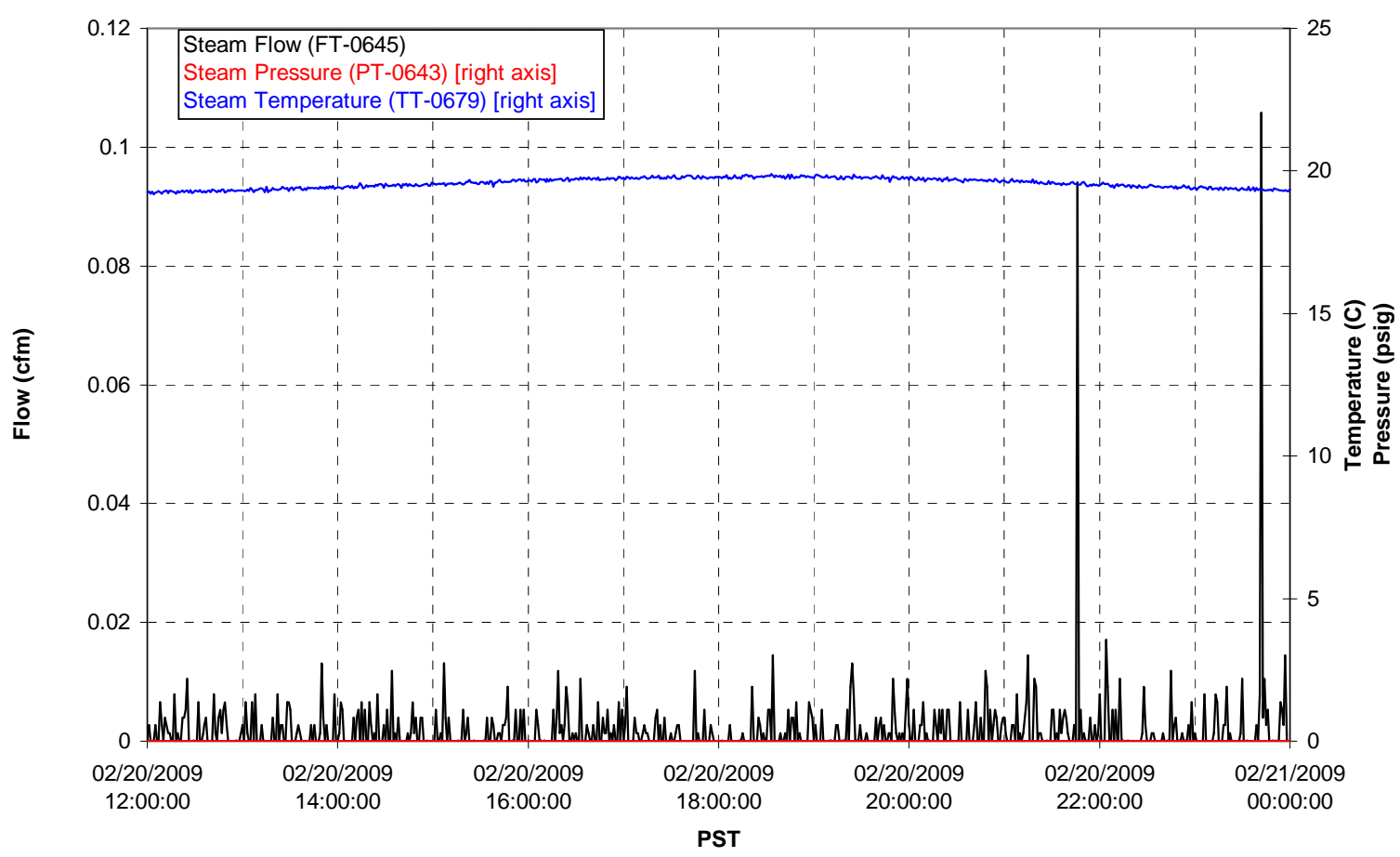

T01A Steam

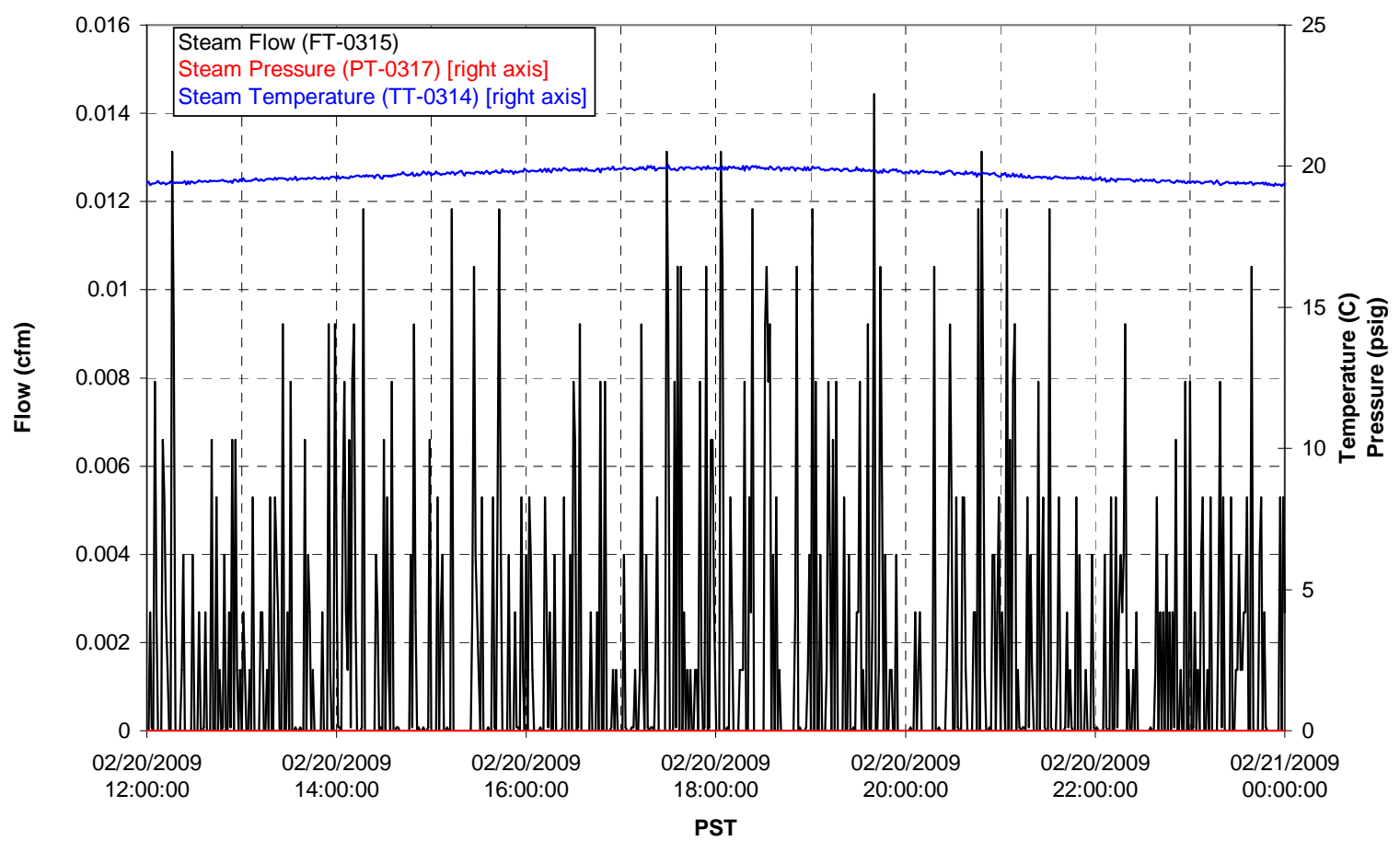


T01B Steam

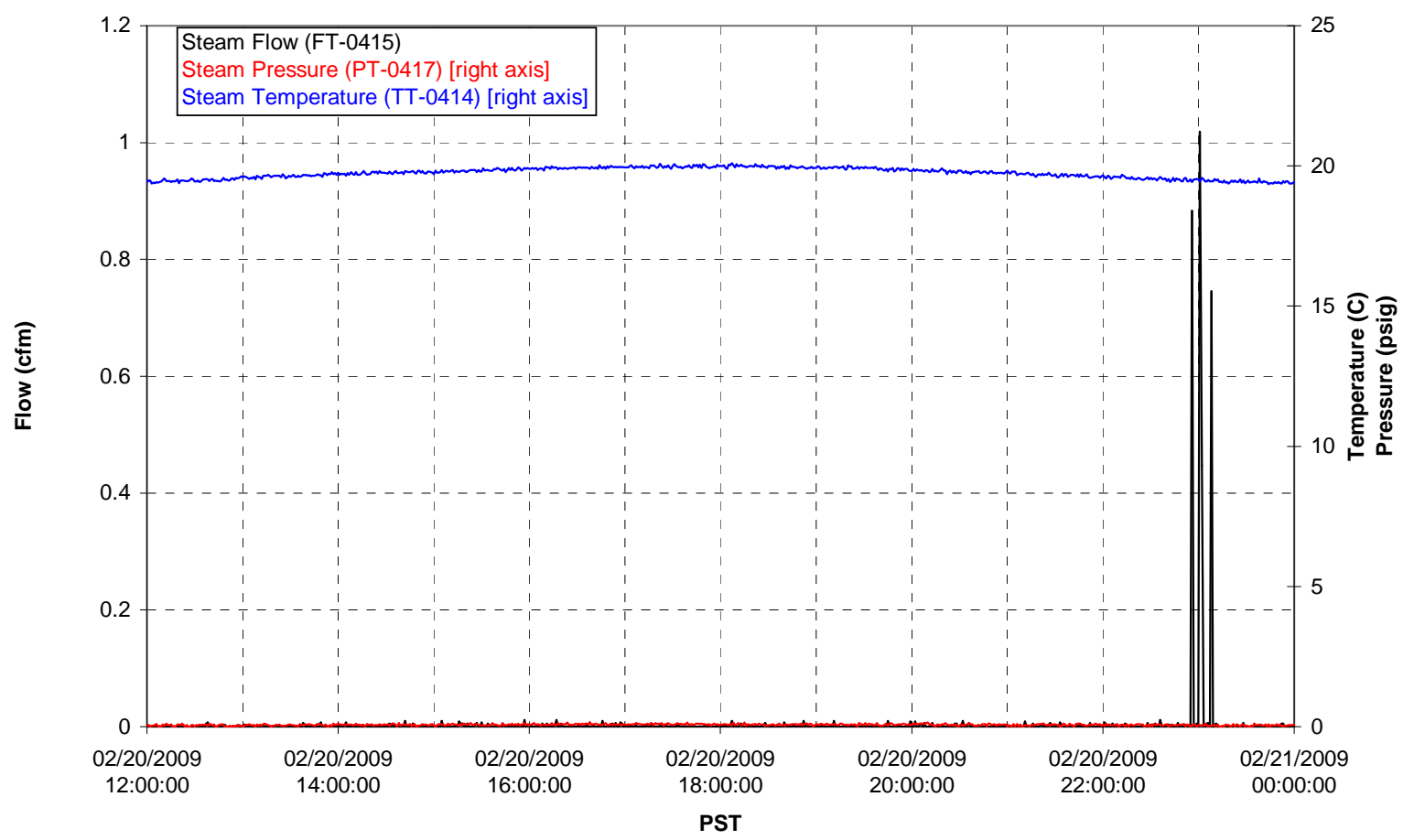




\section{Integrated Test A Data Plots 02/21/09 00:00 - 02/21/09 12:00}


T01A level

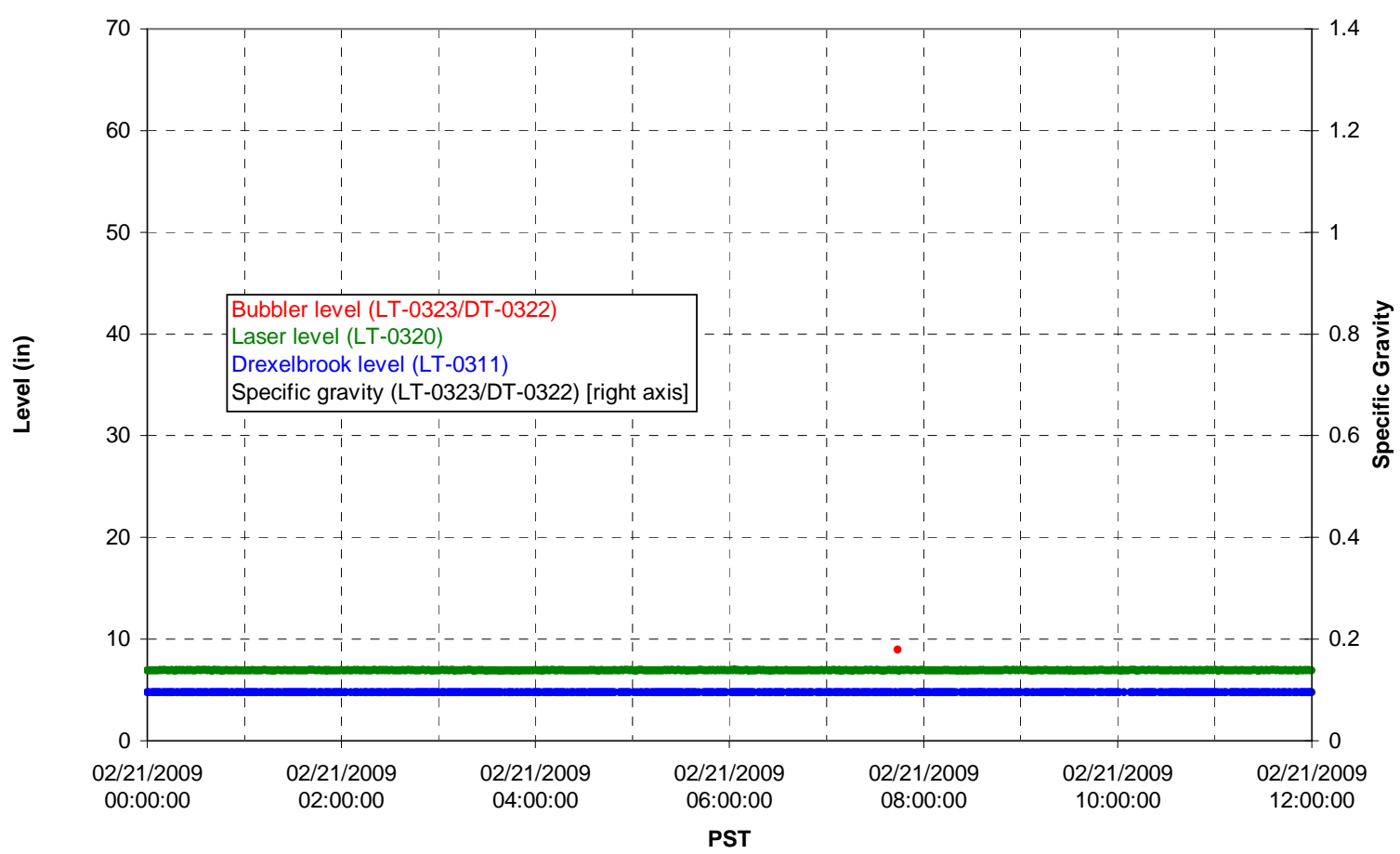

T01A temperatures

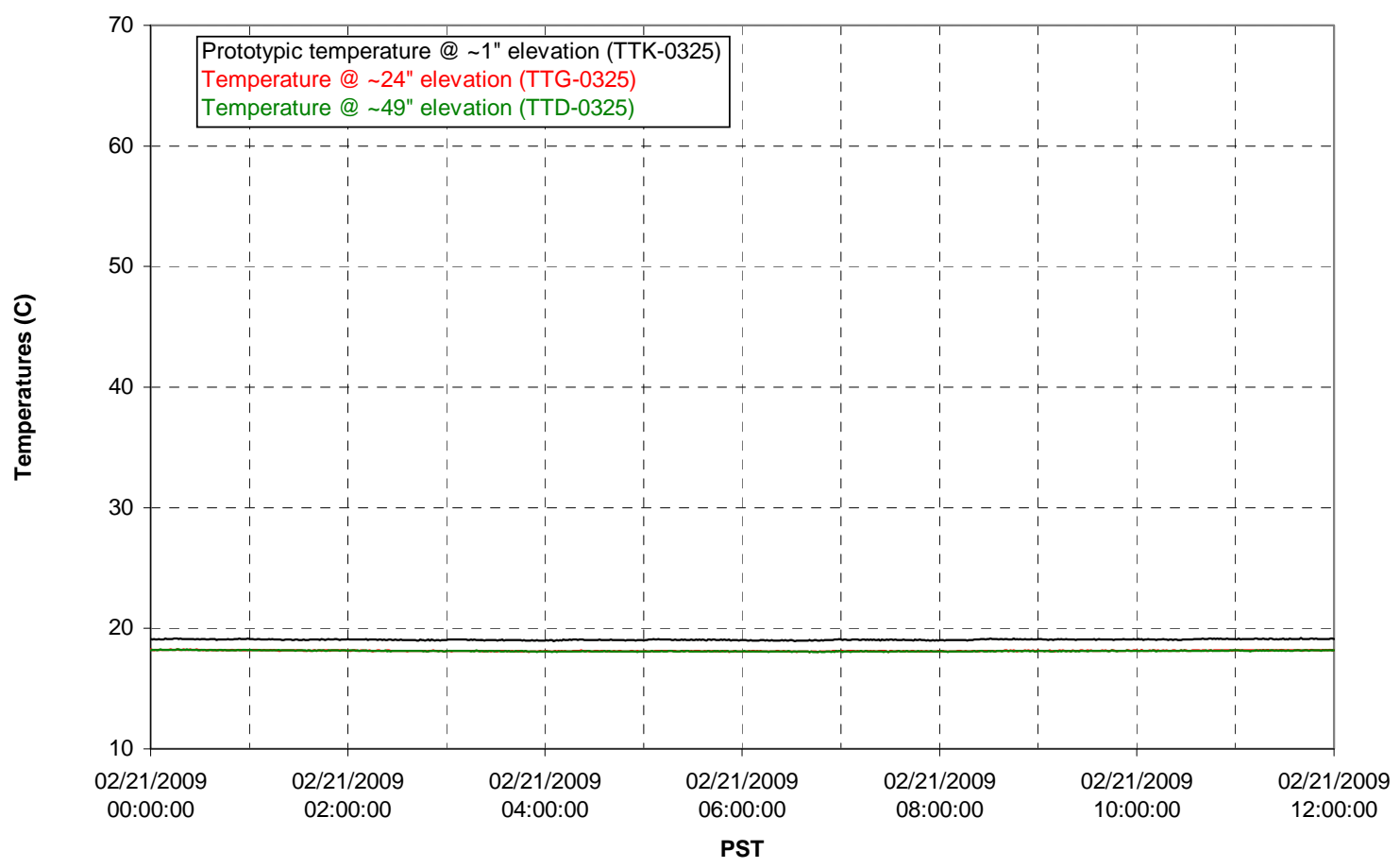


T01B level

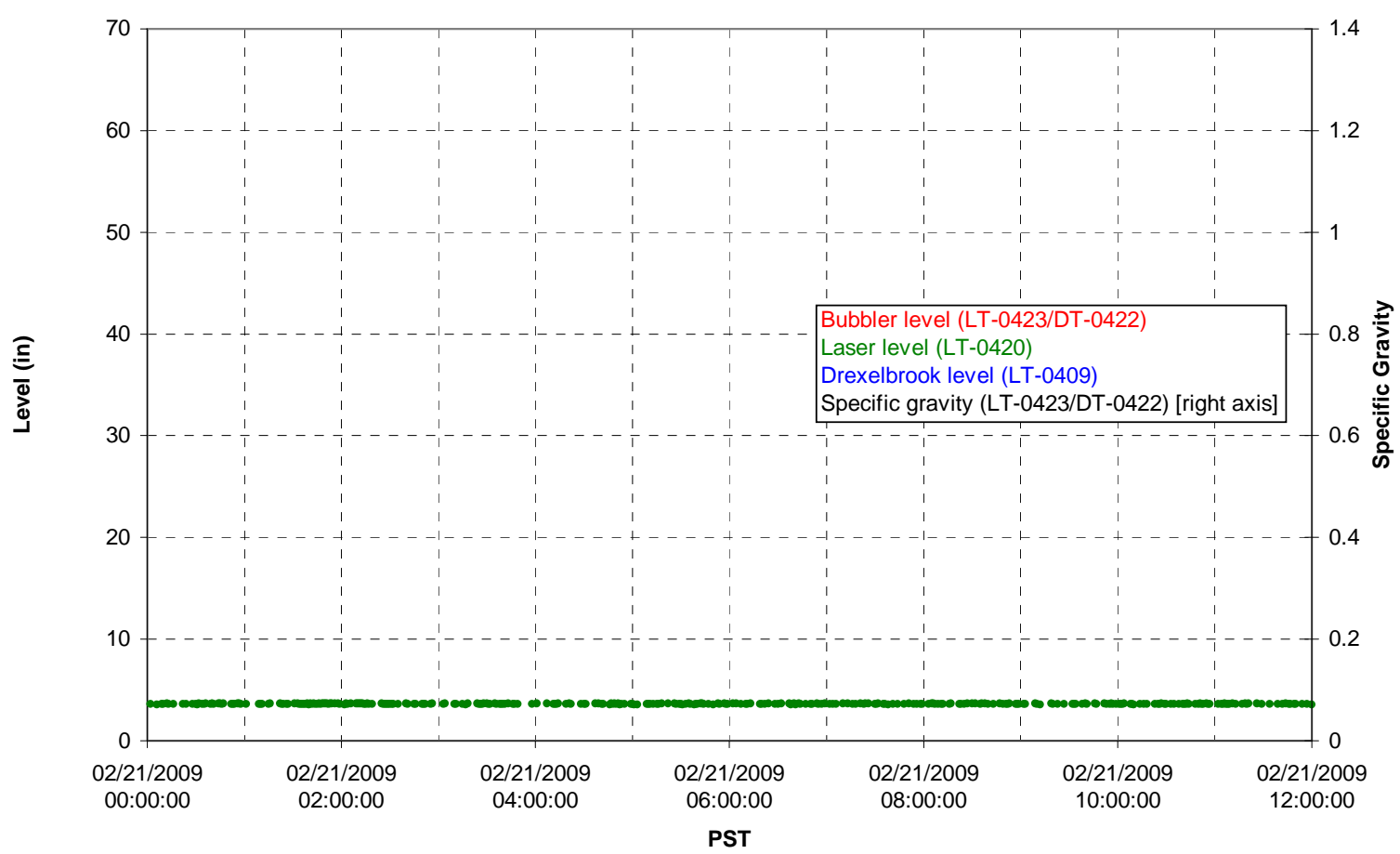

T01B temperatures

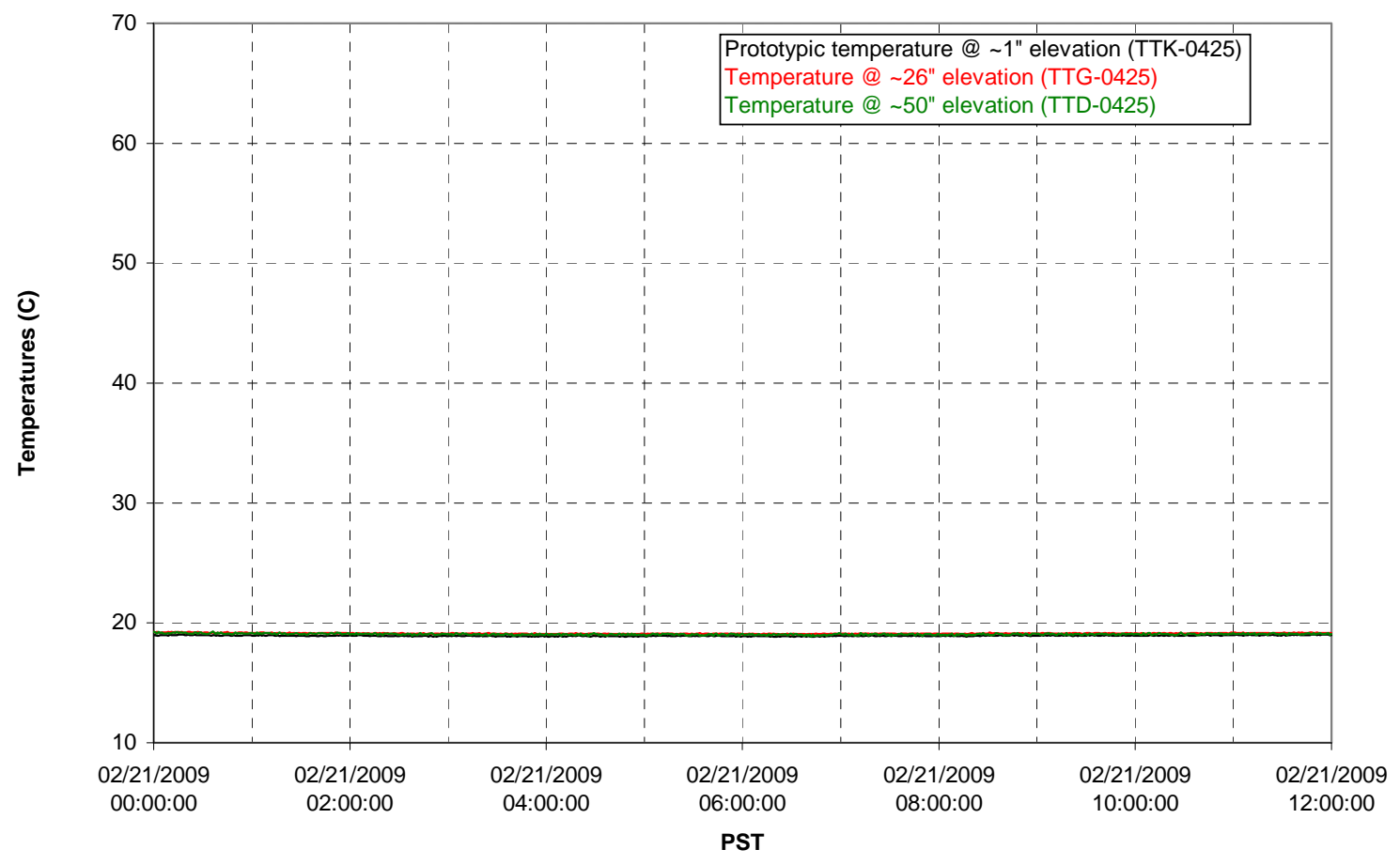


T02A level

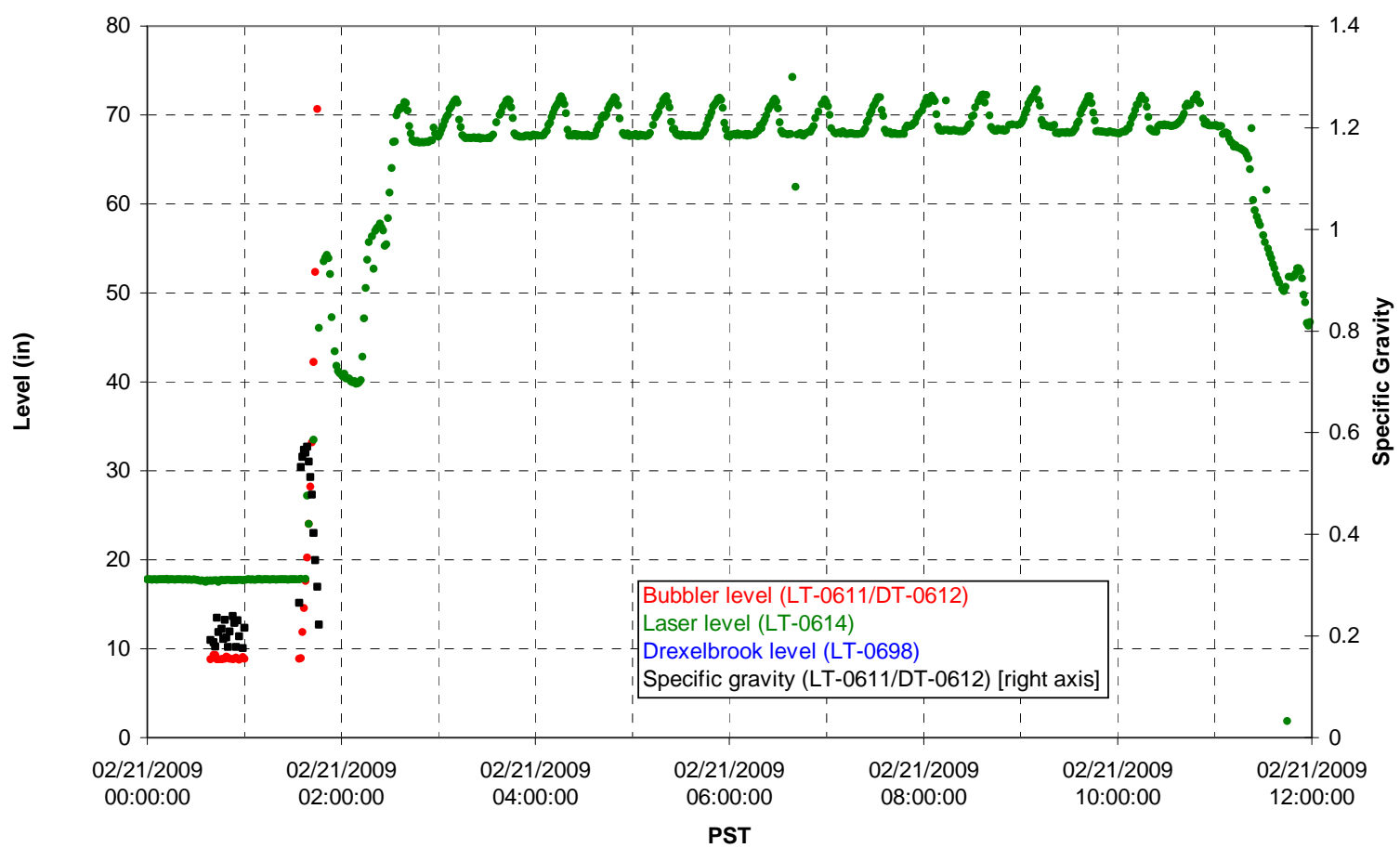

T02A temperatures

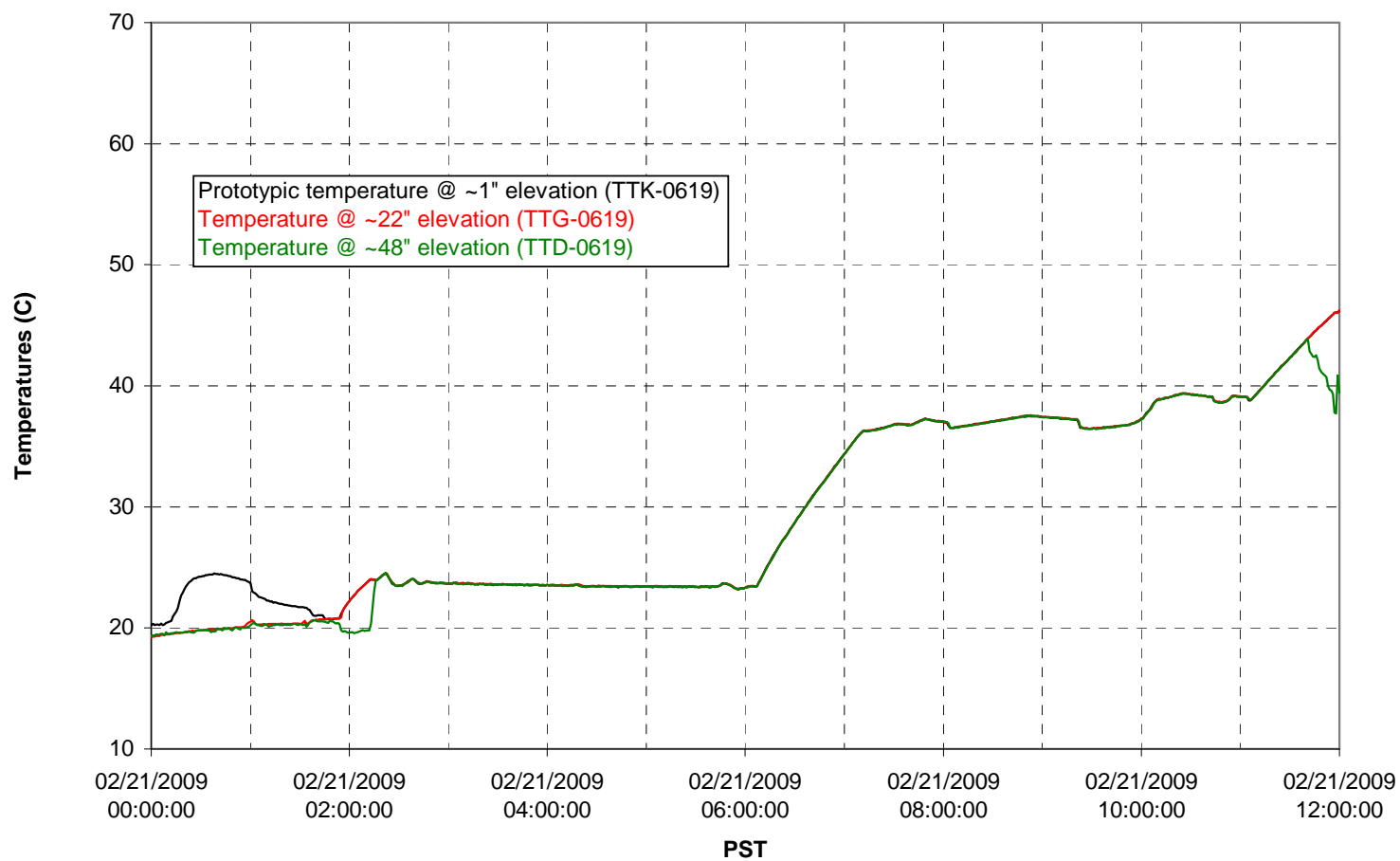


T02A and filter loop temperatures

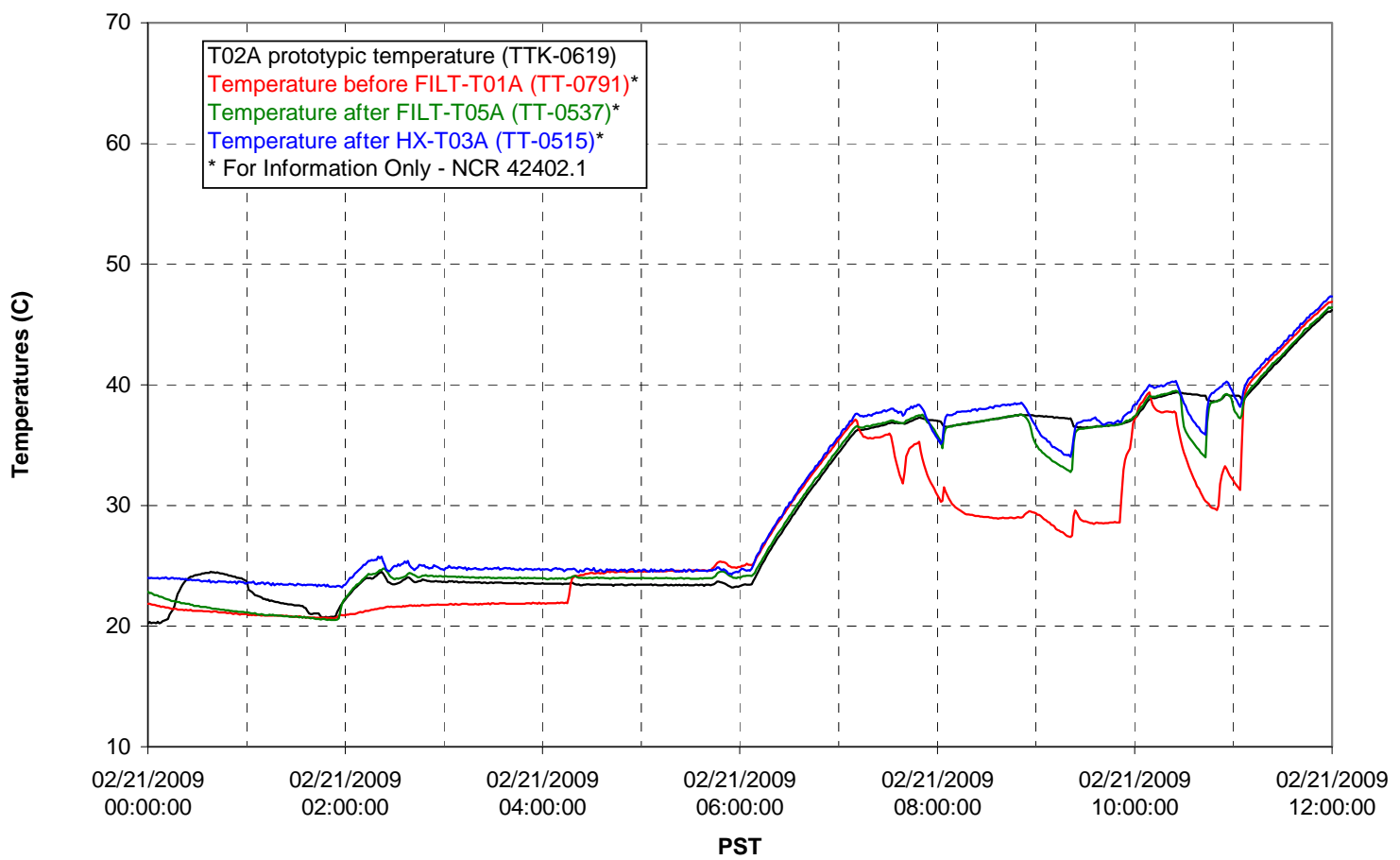

Pump Pressures and Flow

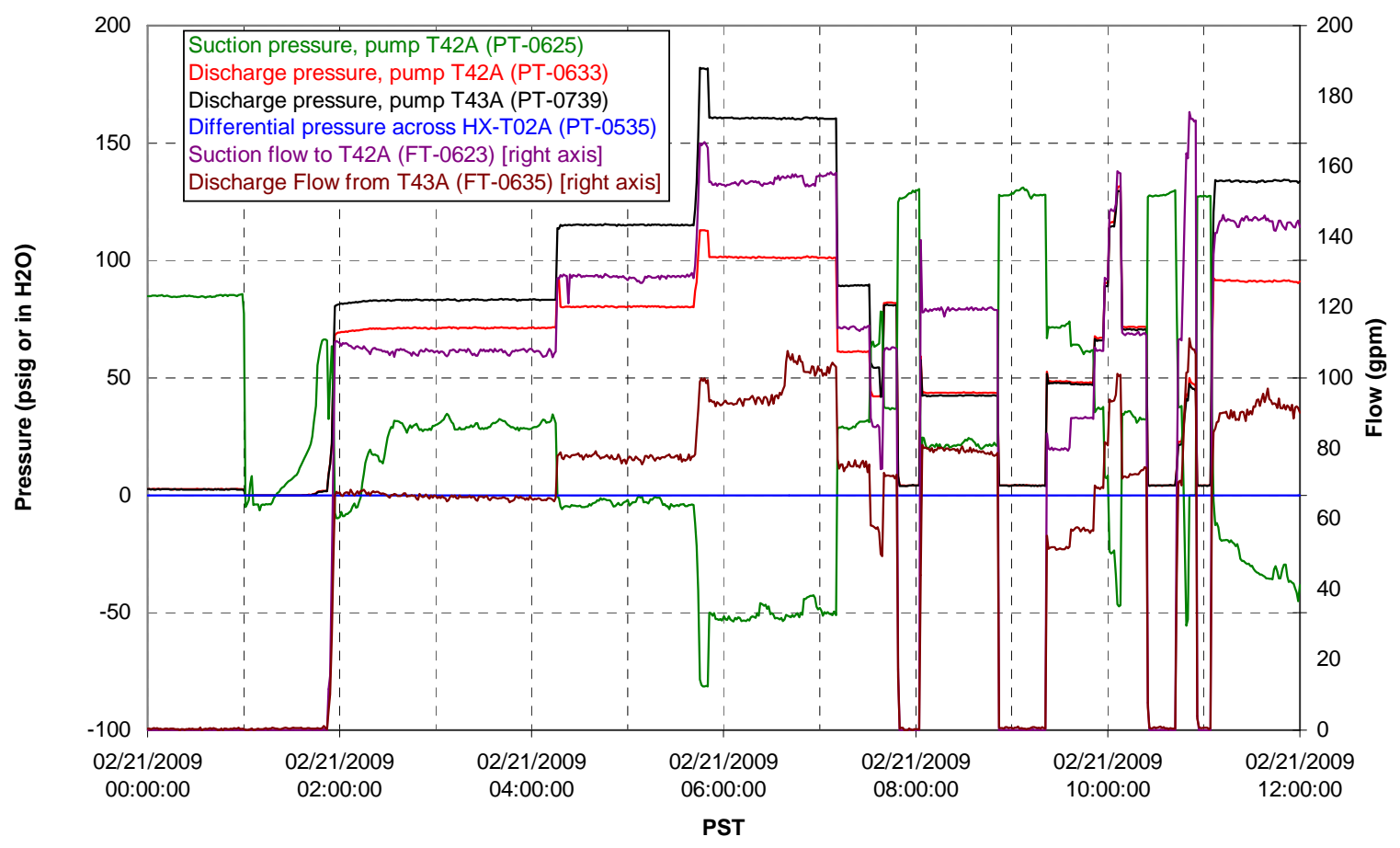




\section{Axial pressure drop}

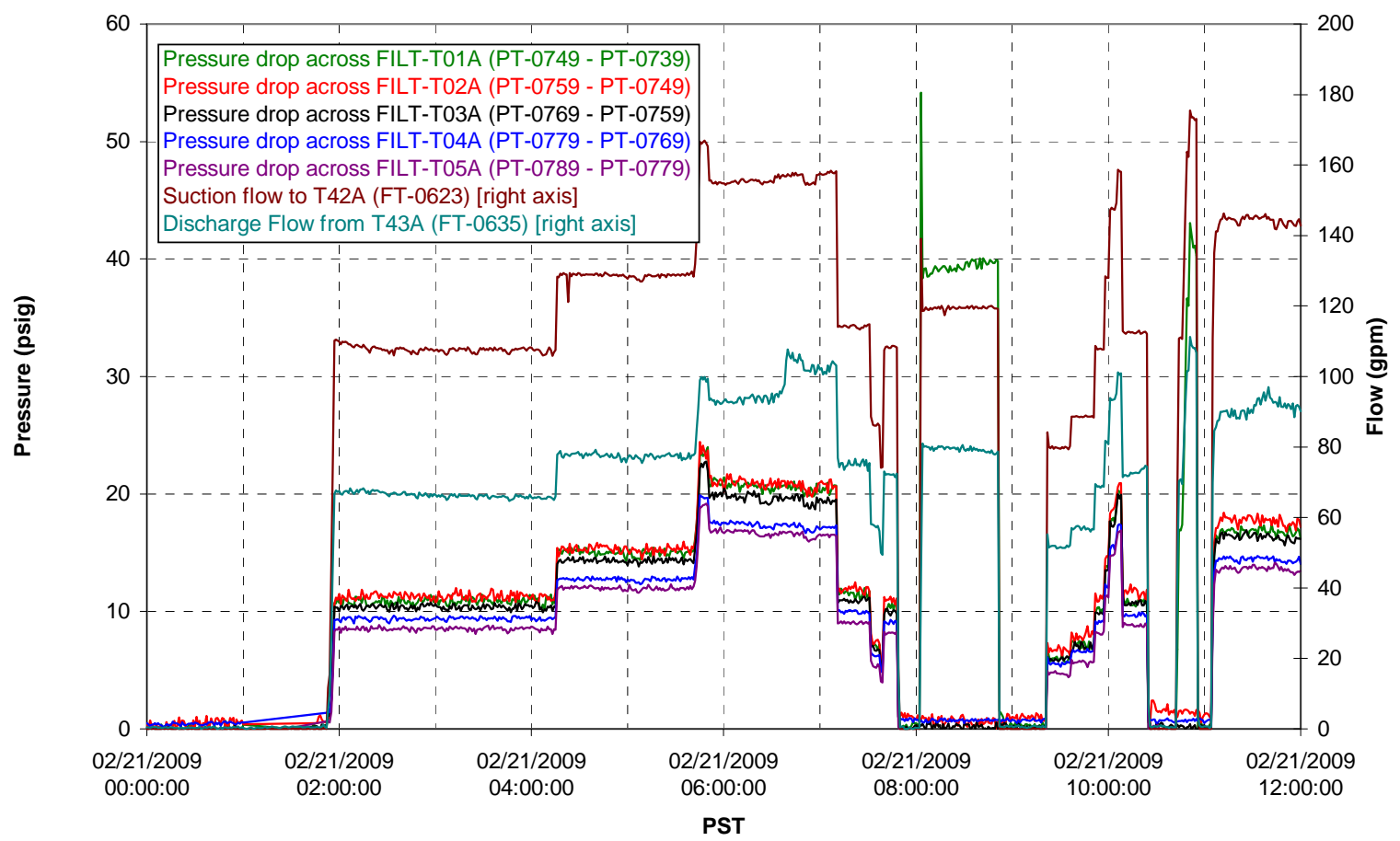

Permeate flow rates

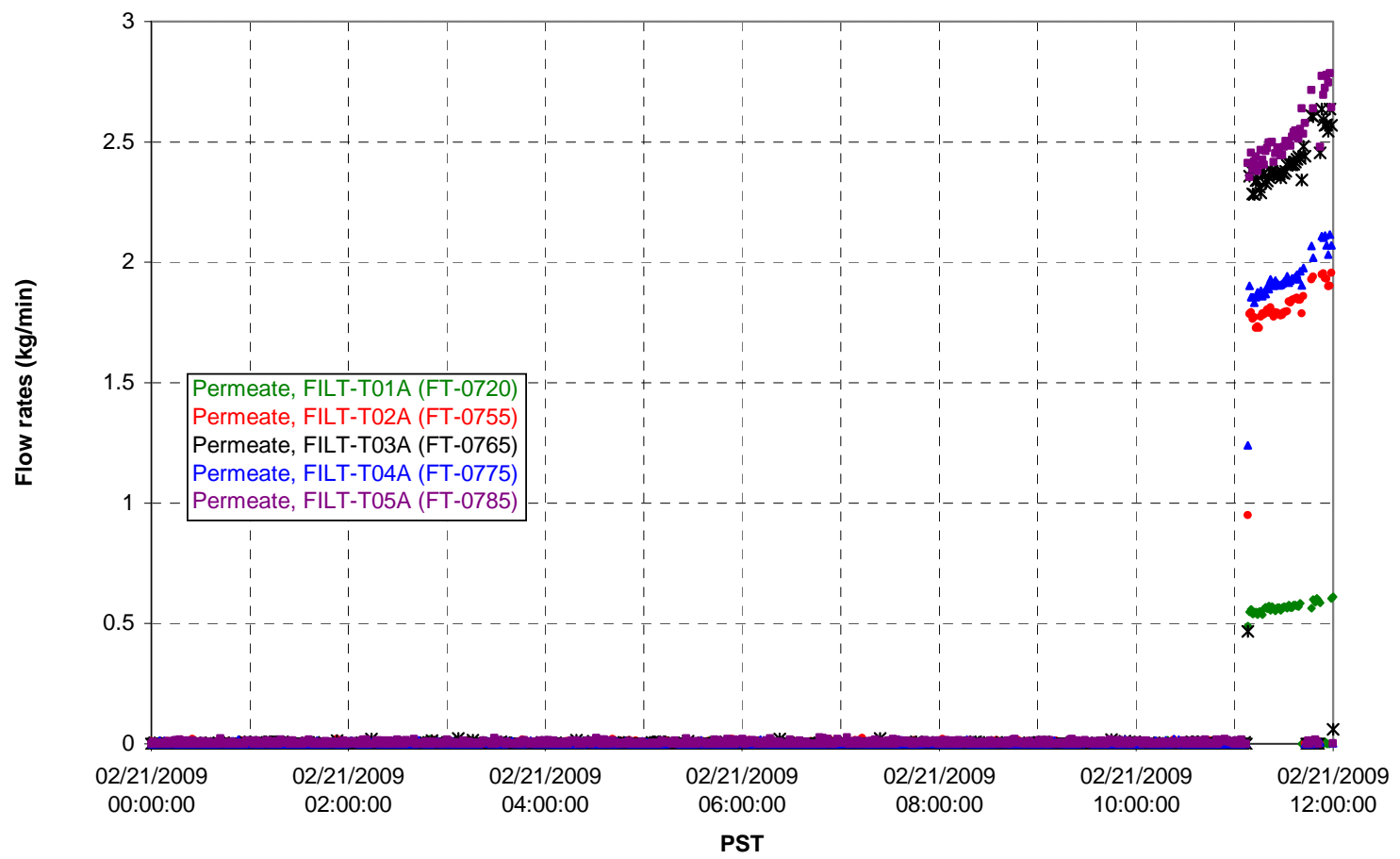


T02A Inner Temperature Tree

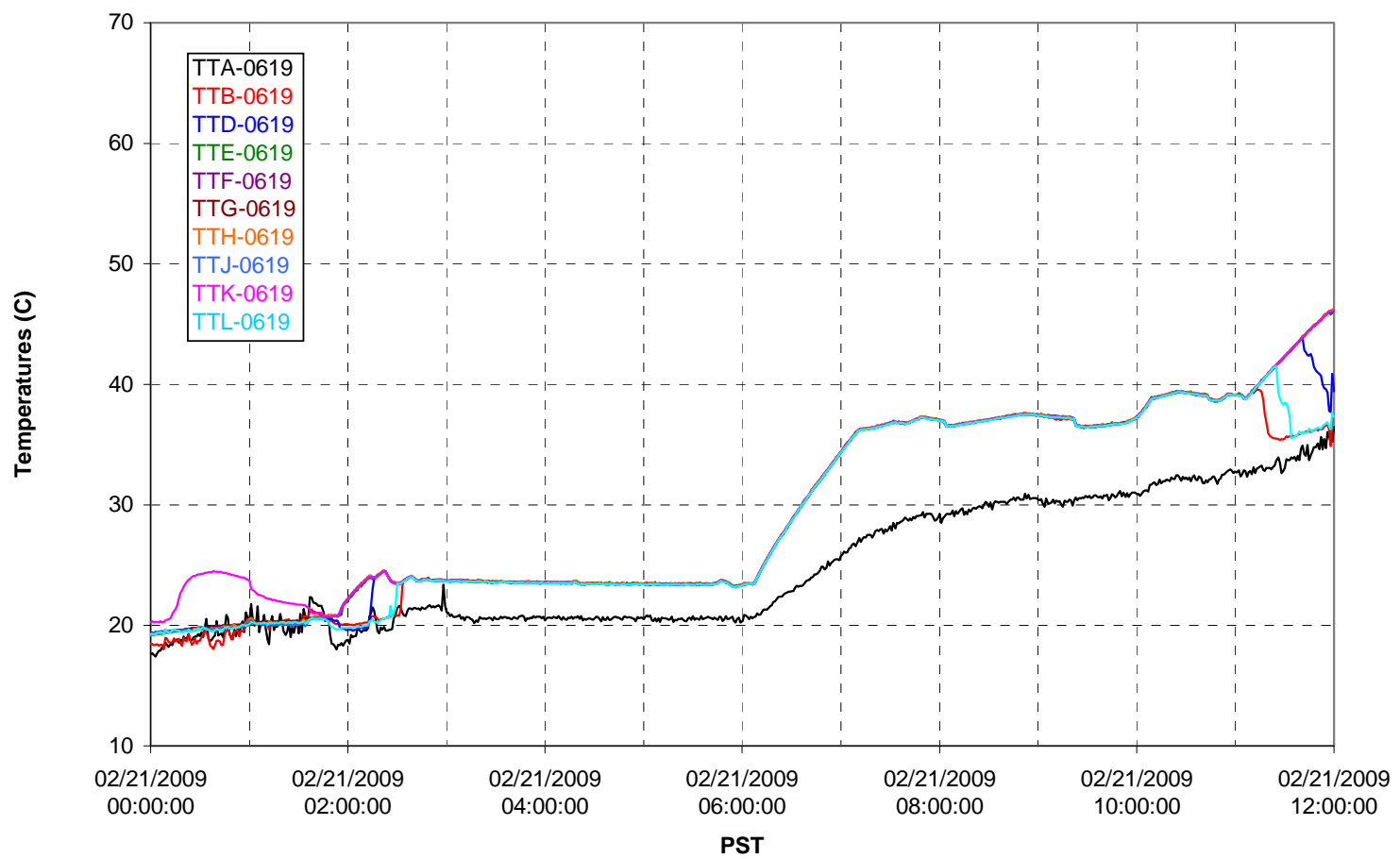

T02A Outer Temperature Tree

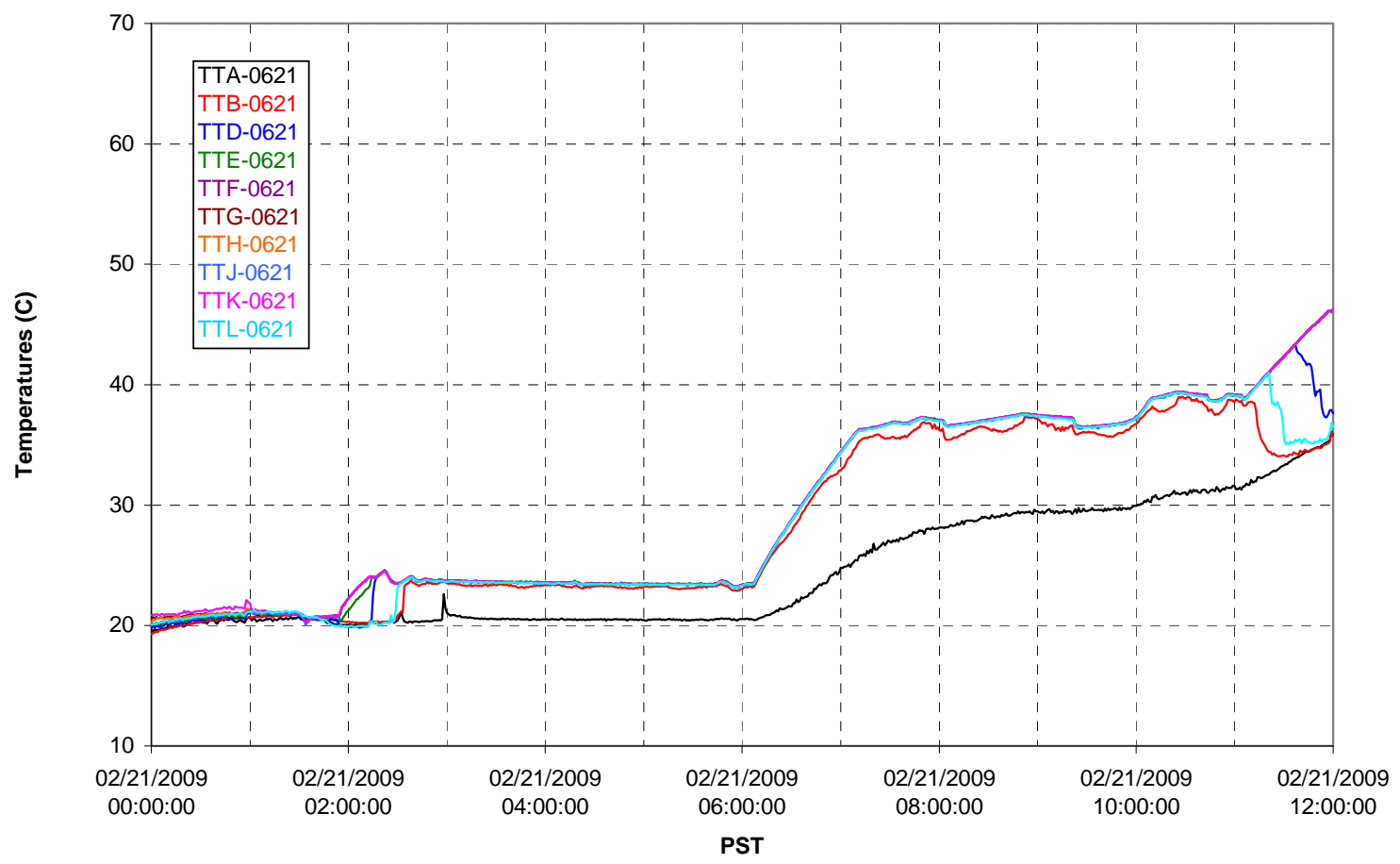


T02A temperatures

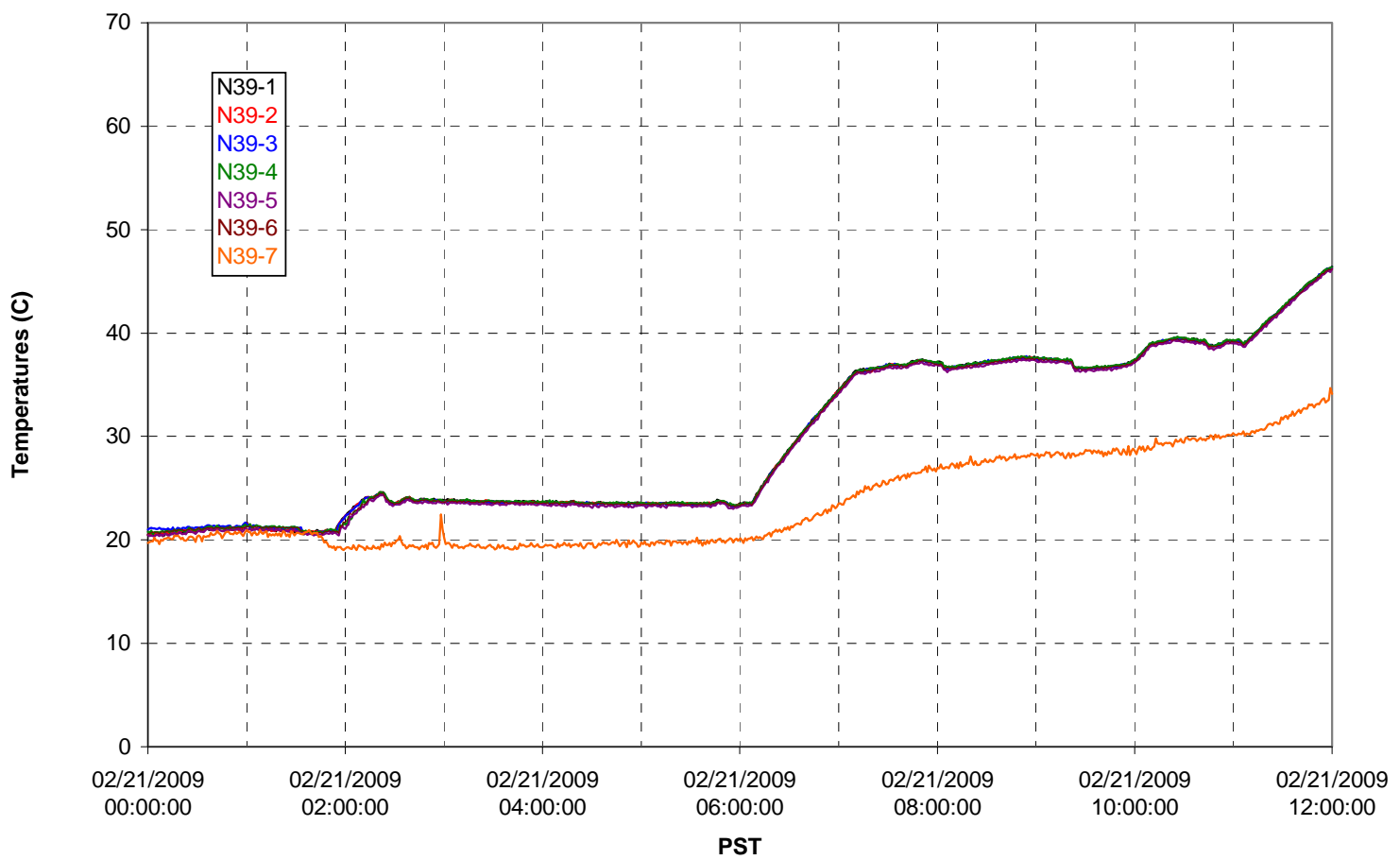

T02A temperatures

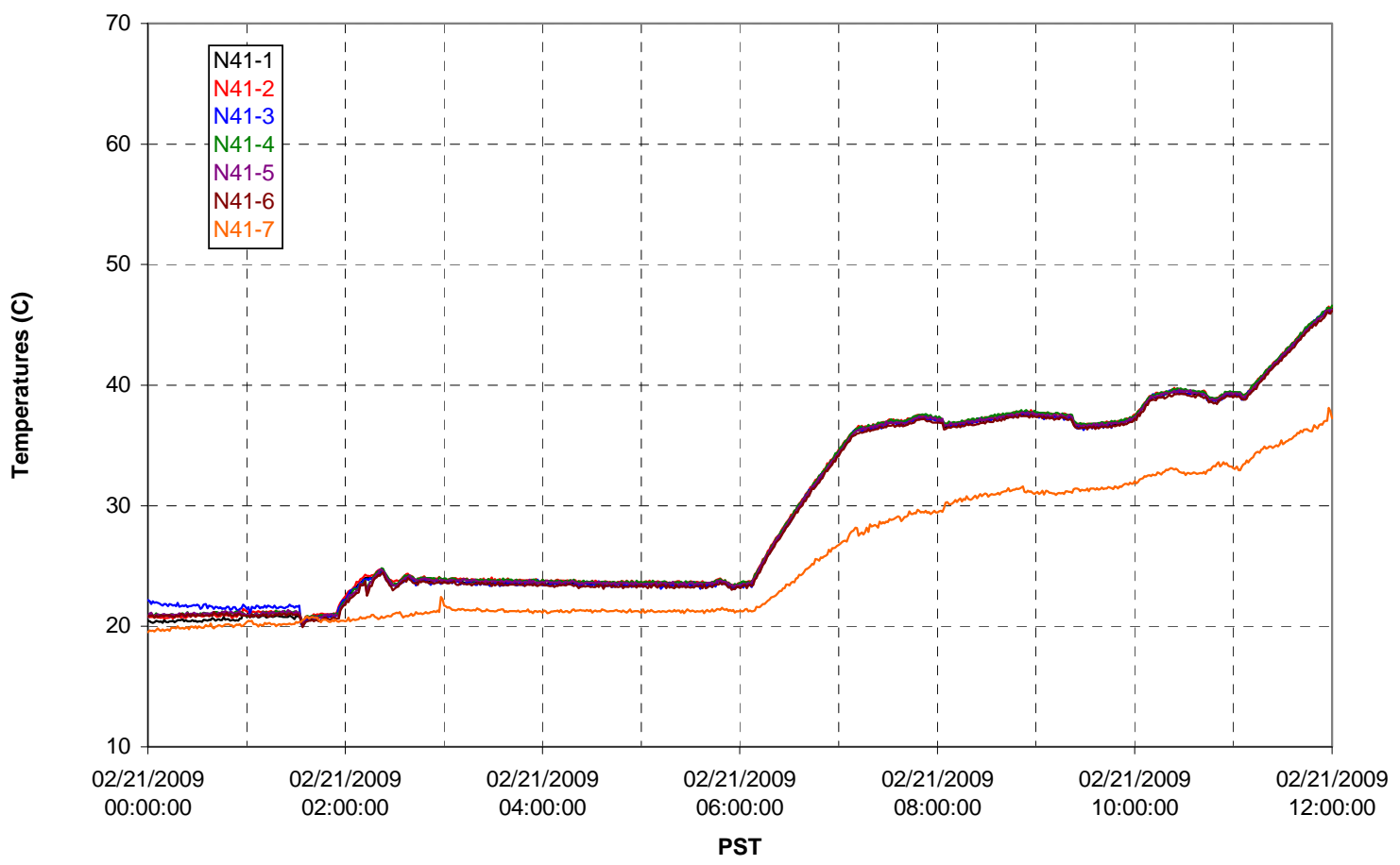


T02A temperatures

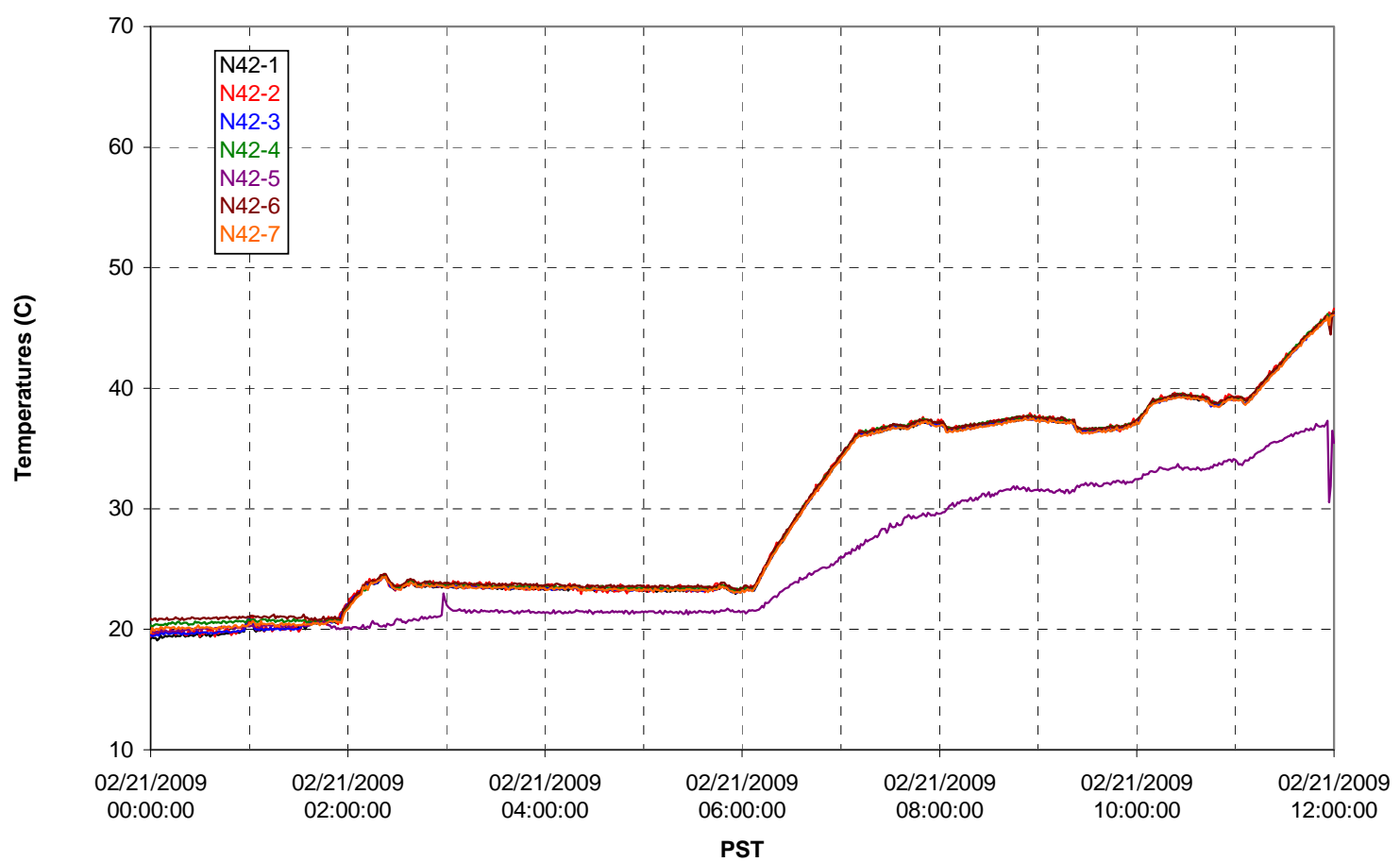

T02A temperatures

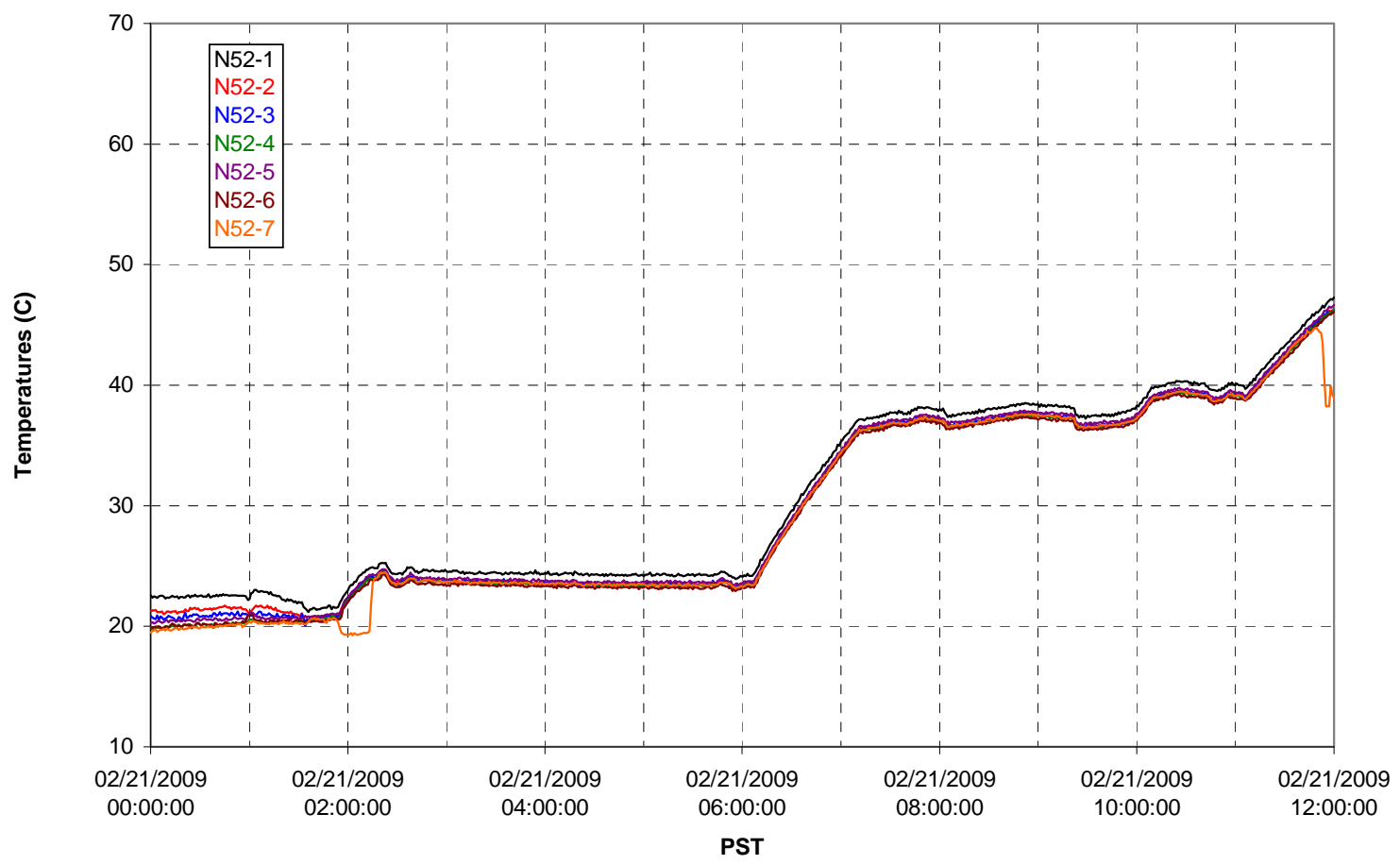


T02A Heating and Cooling

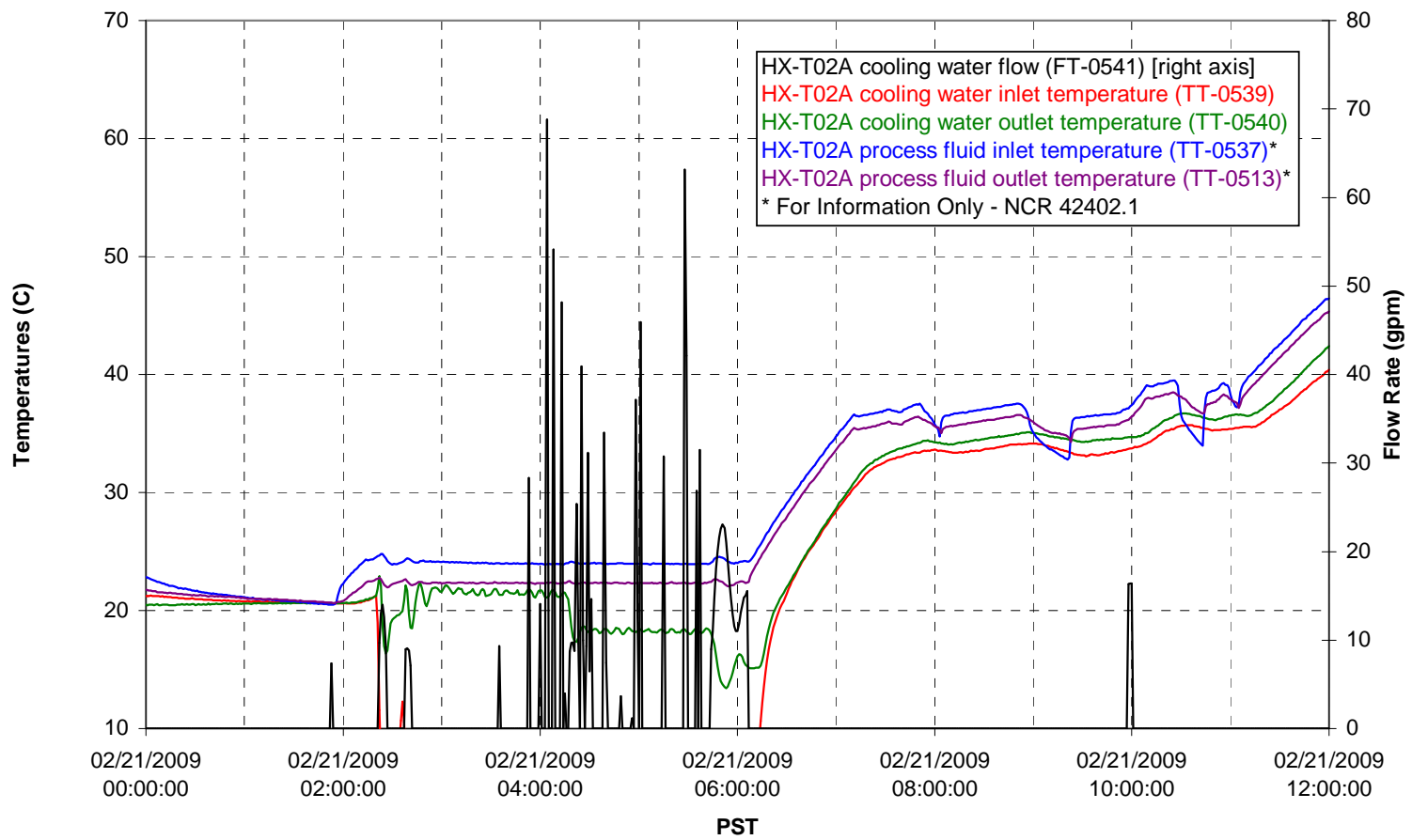

Pump Operation

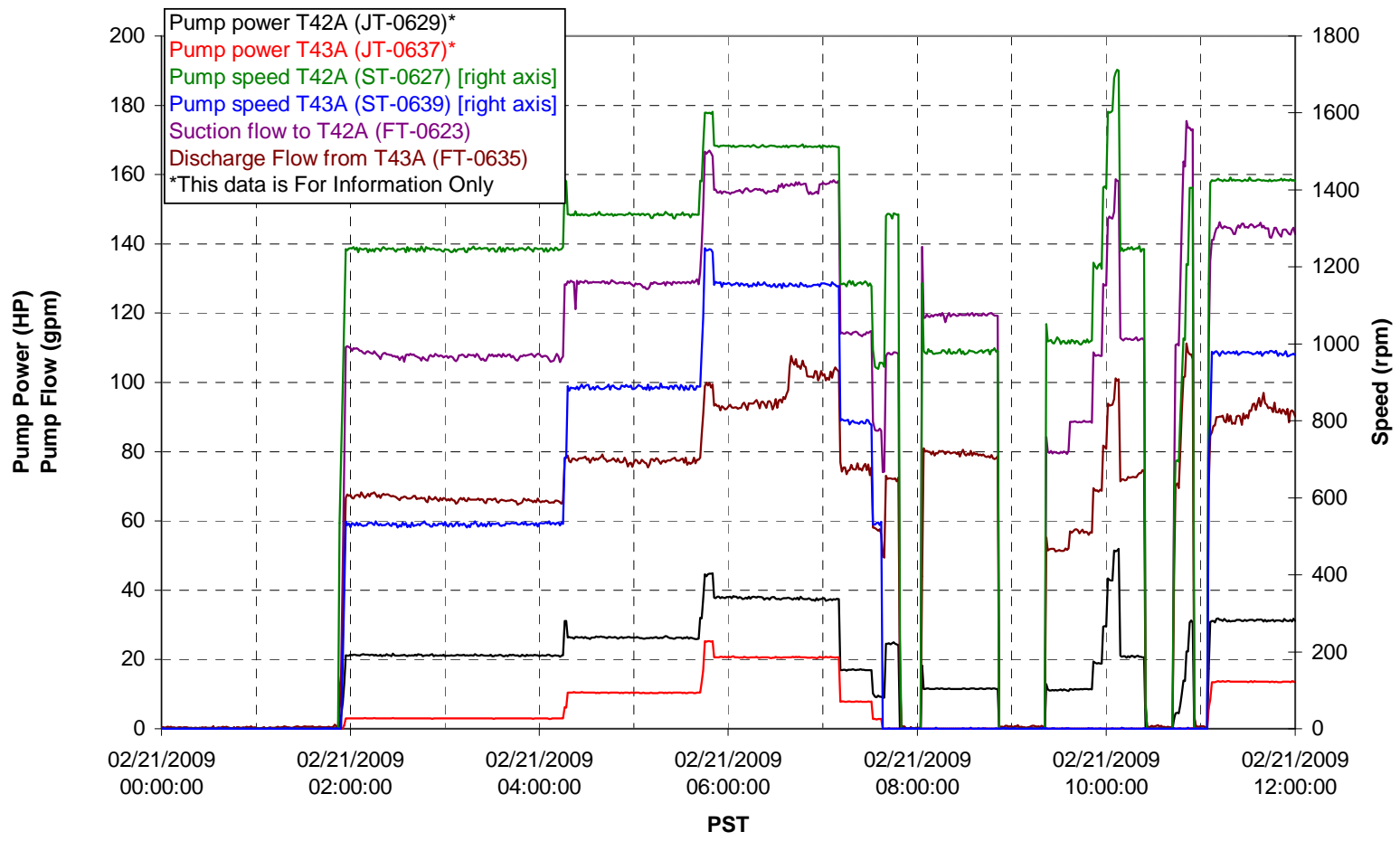


Pulsepot UFP-PP-T01A

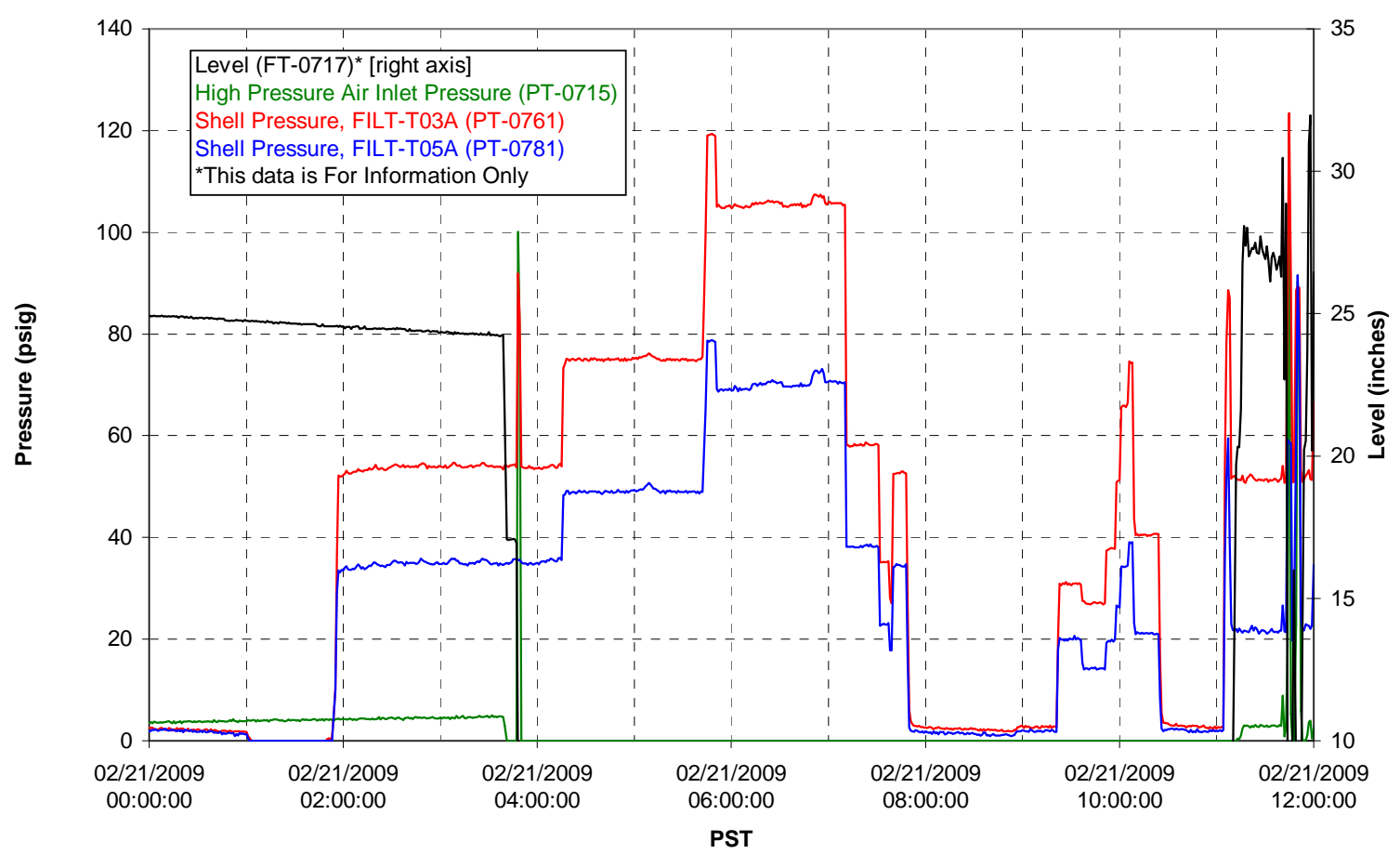

Pulsepot UFP-PP-T02A

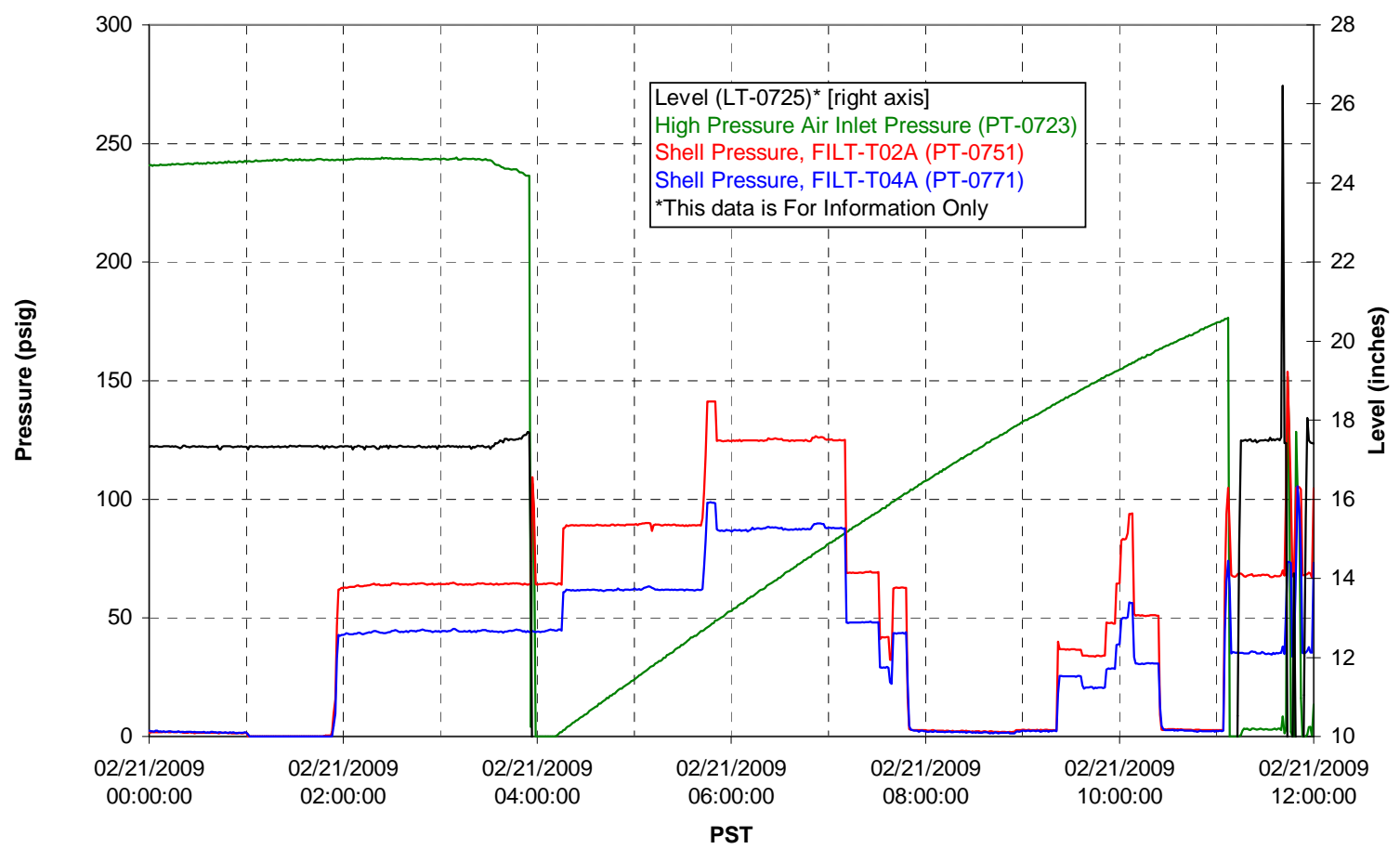


Pulsepot UFP-PP-T03A

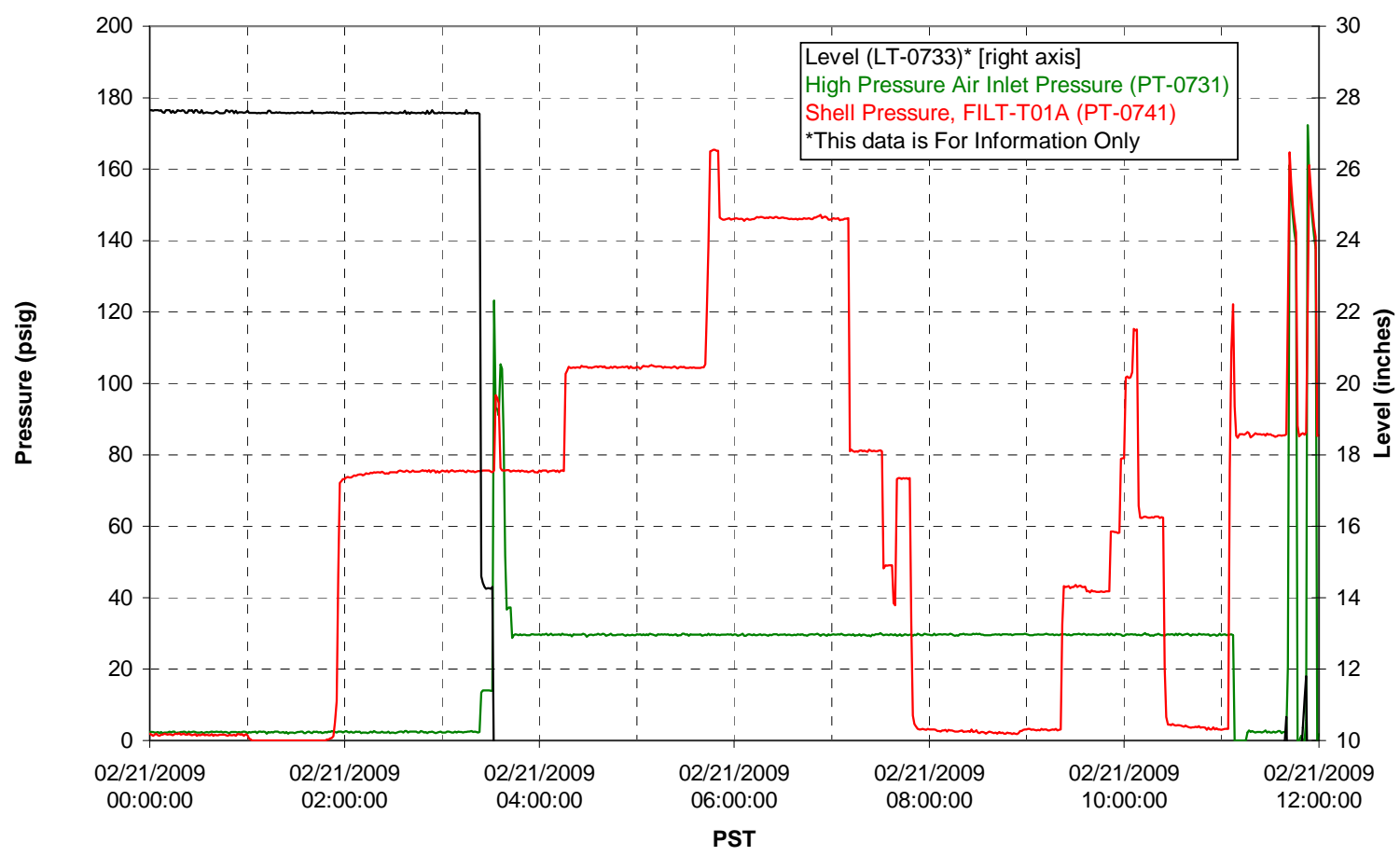

Pulsepot Levels

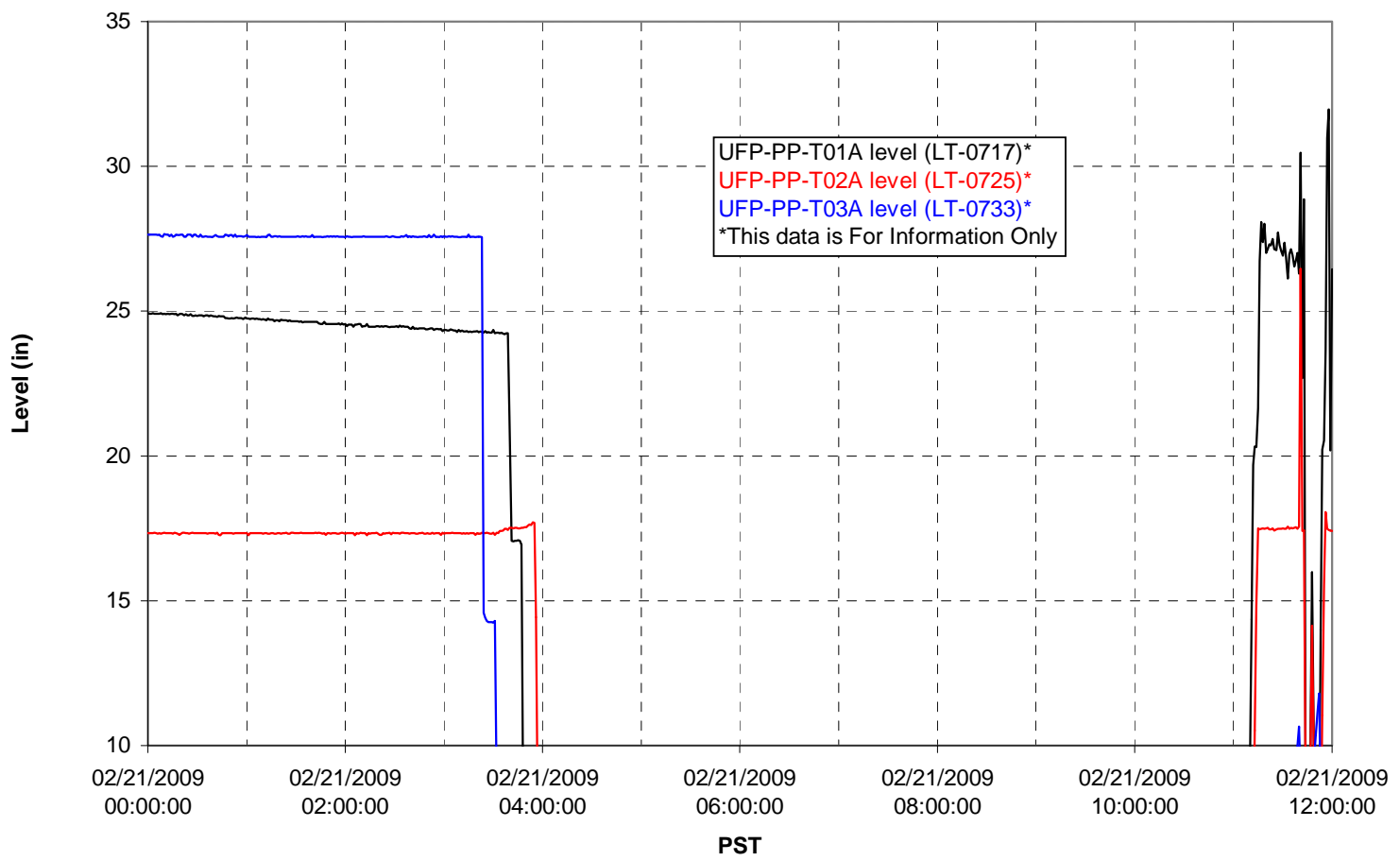


Filter UFP-FILT-T01A

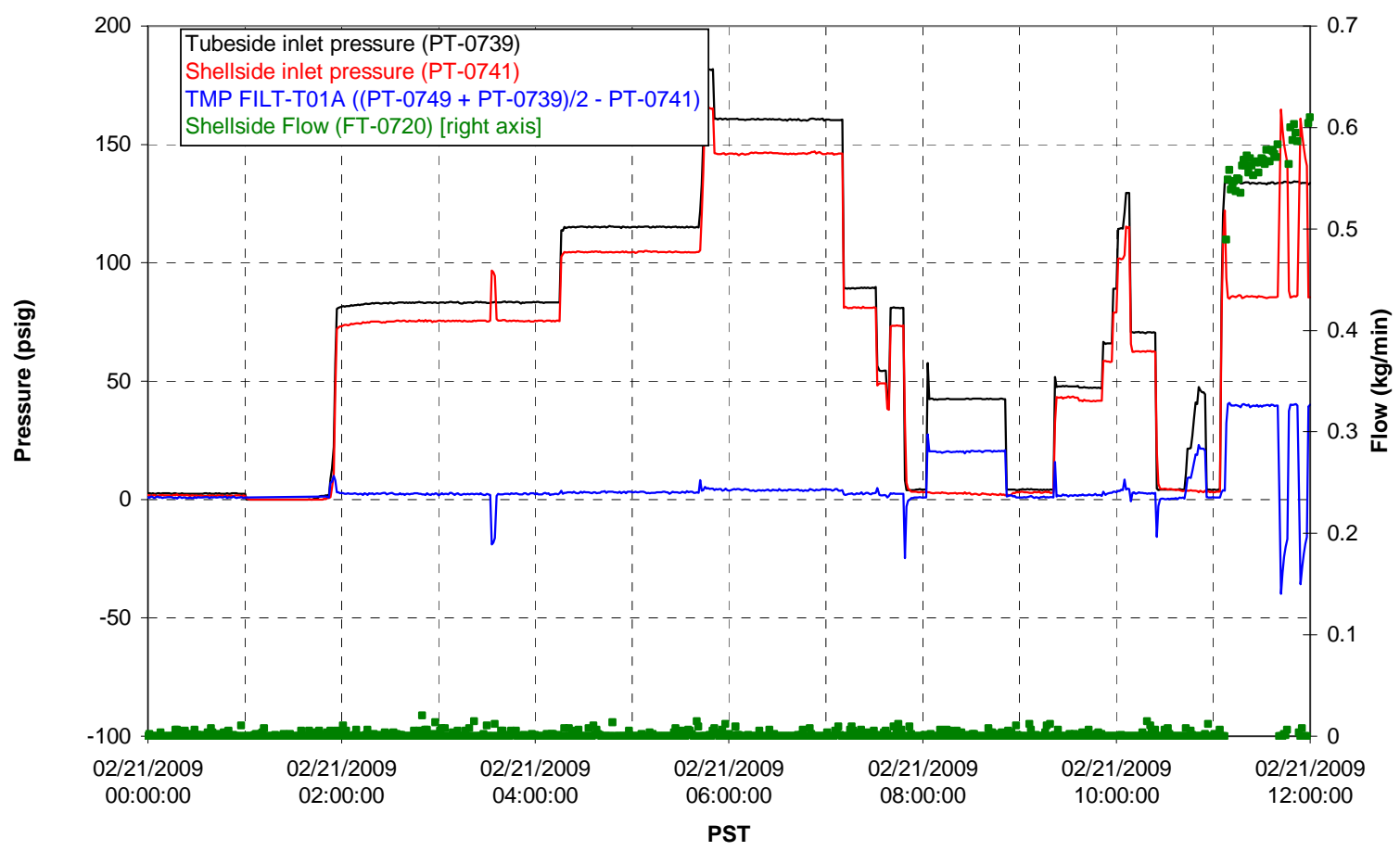

Filter UFP-FILT-T02A

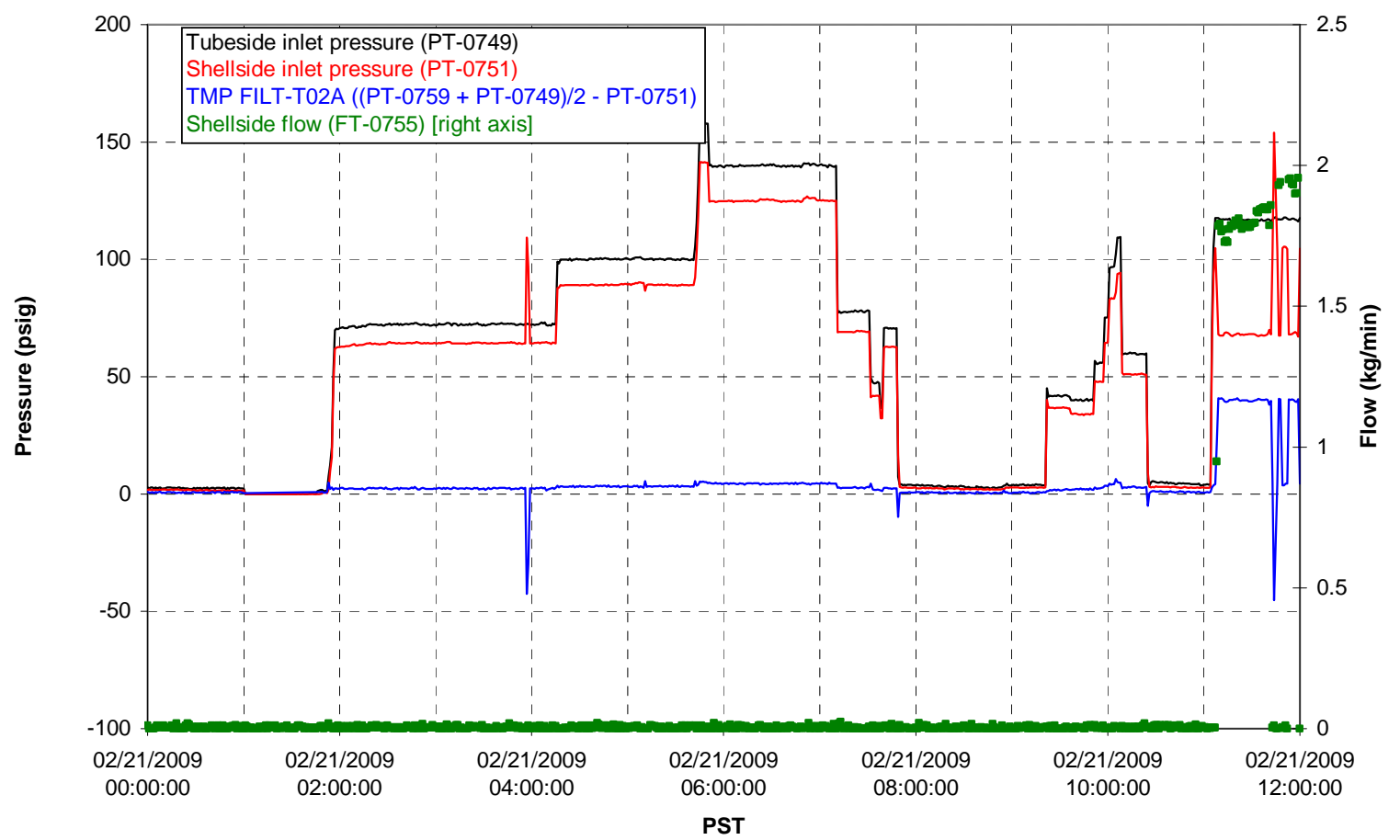


Filter UFP-FILT-T03A

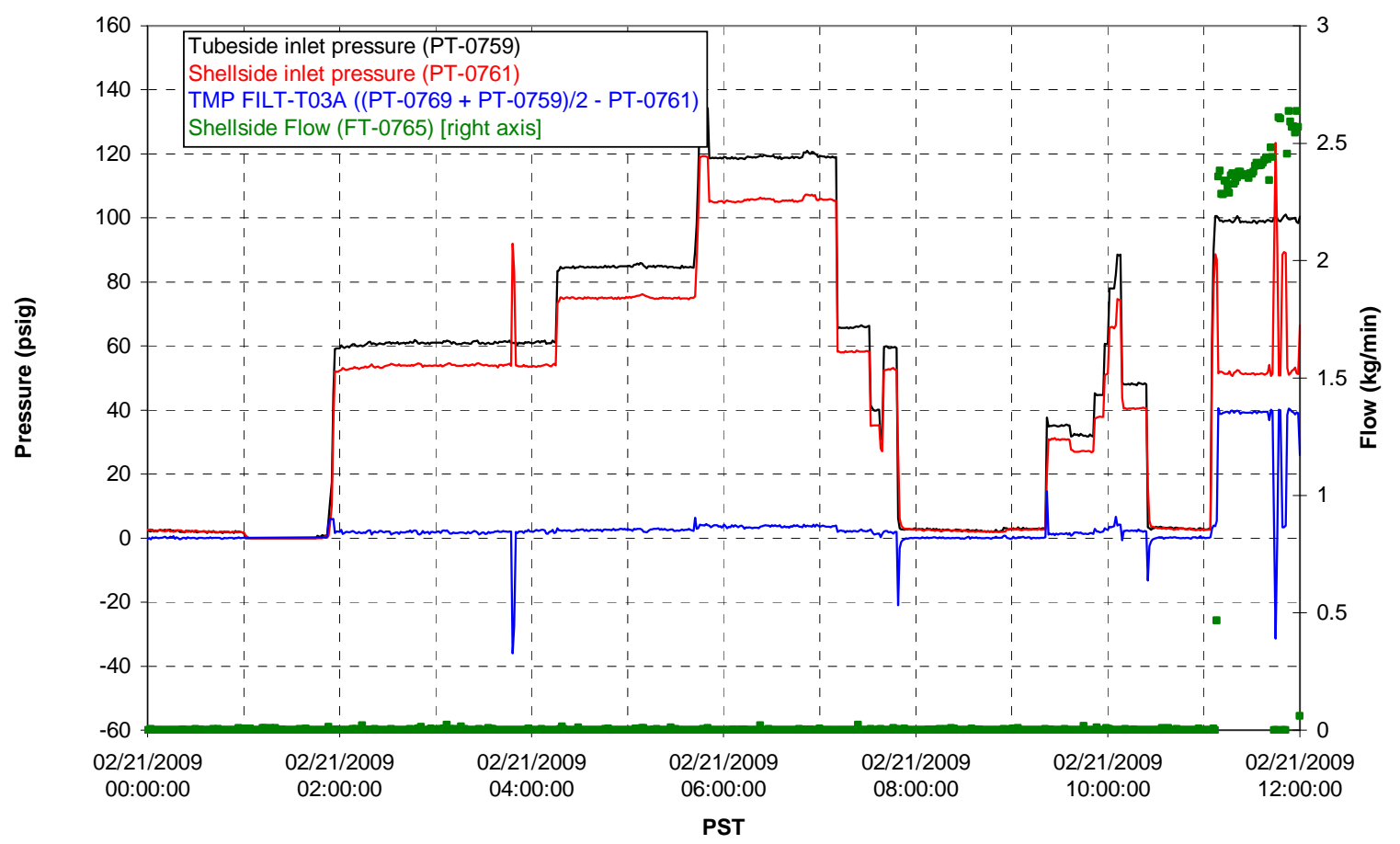

Filter UFP-FILT-T04A

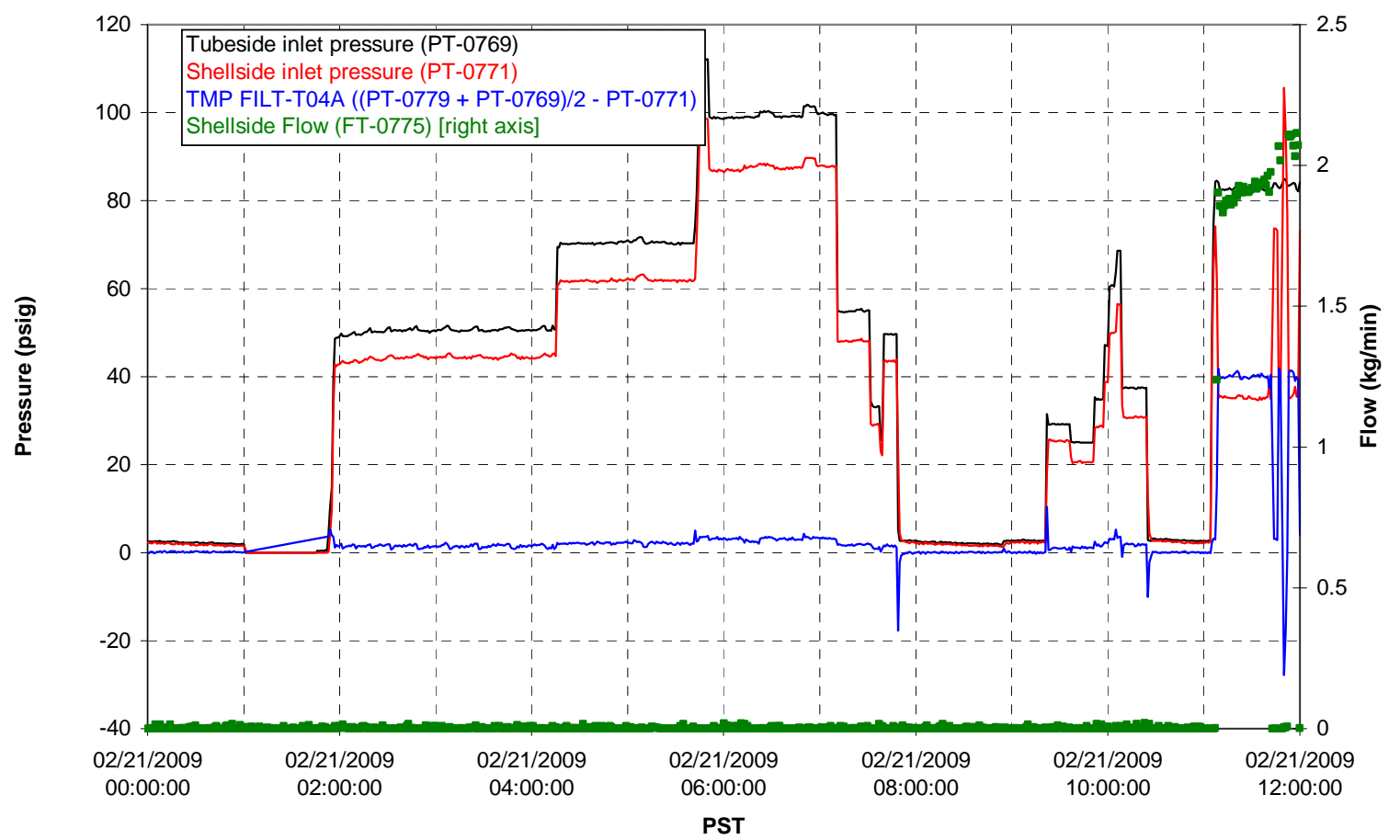

G.770 
Filter UFP-FILT-T05A

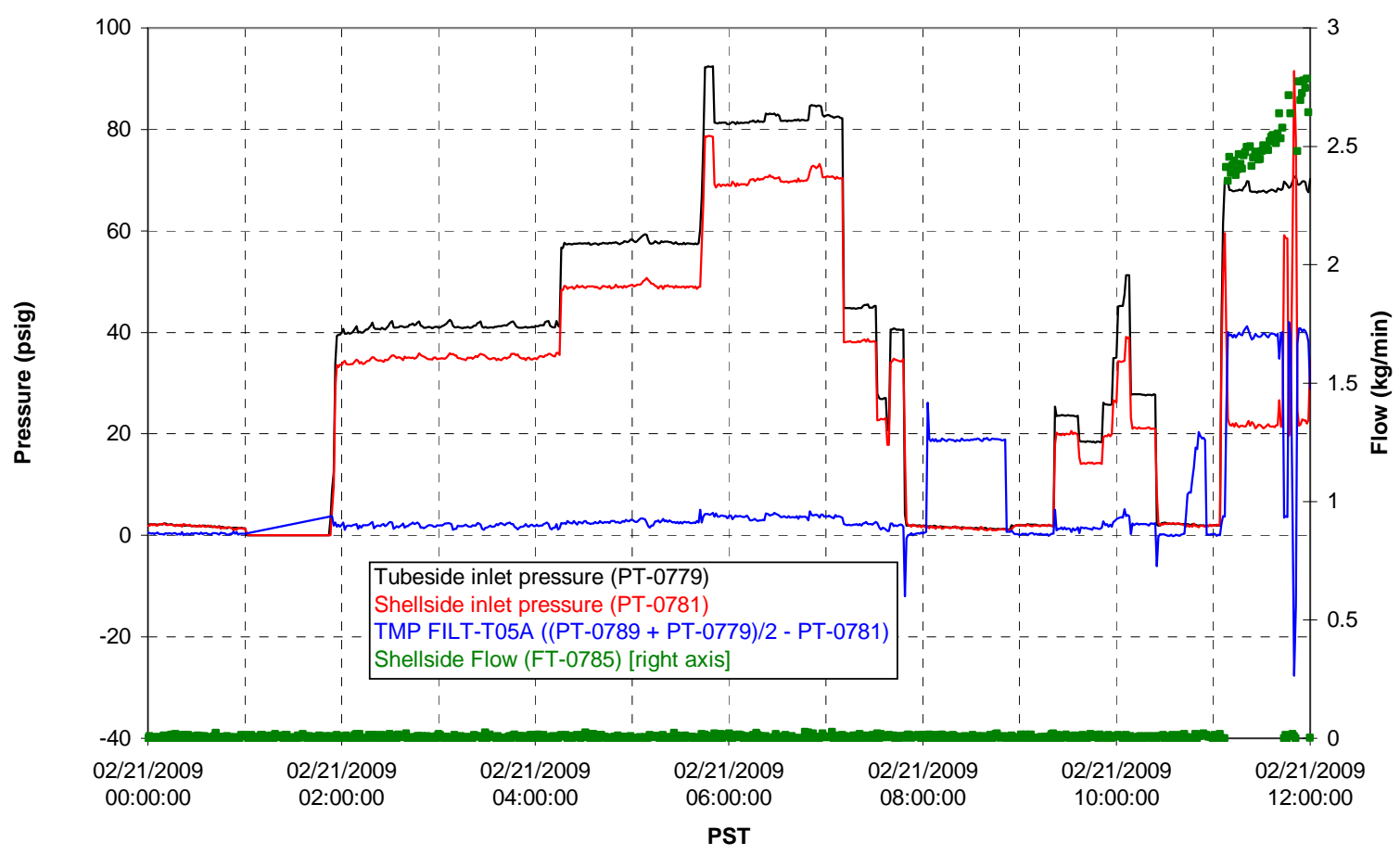

Chemical Flow

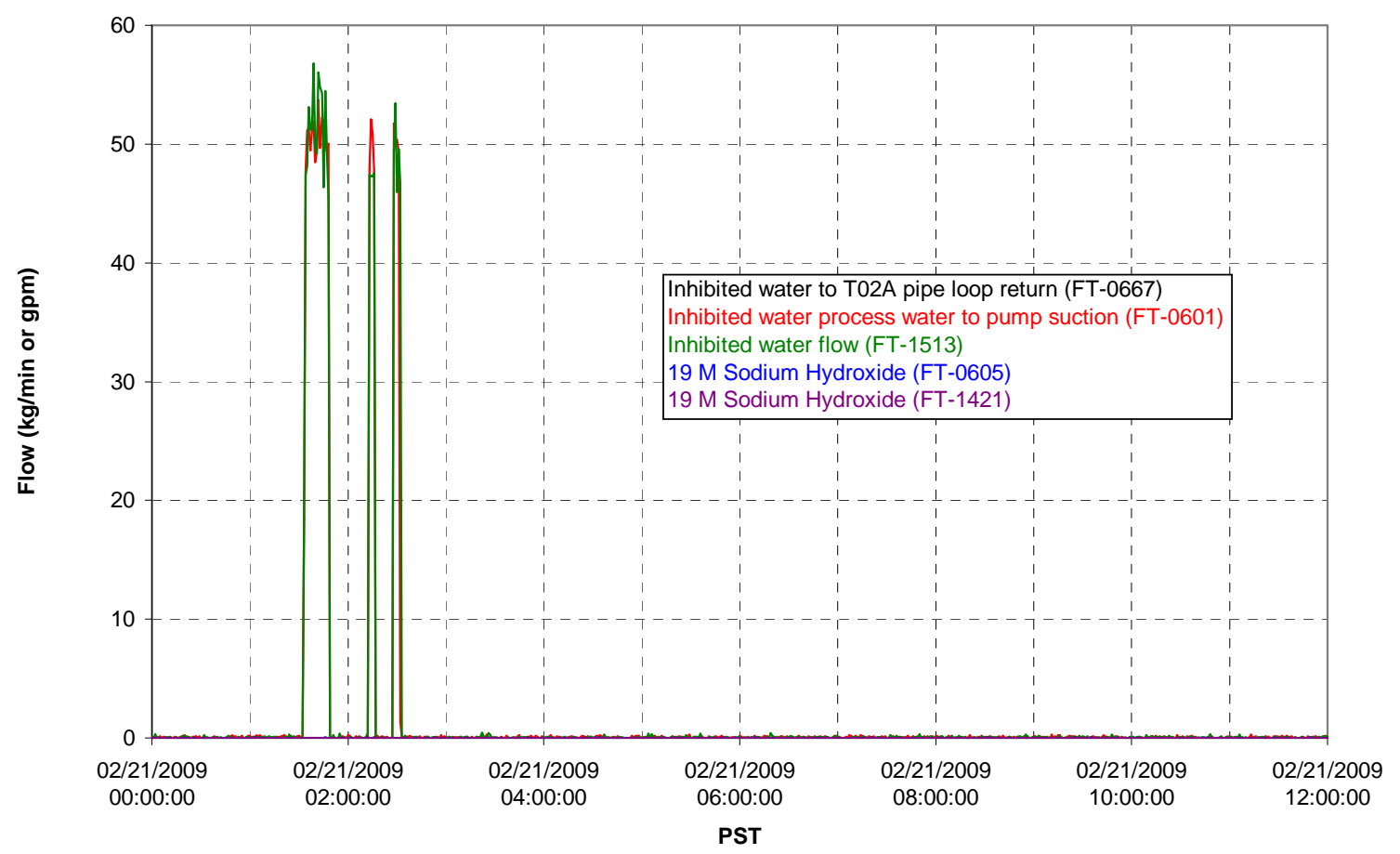


Chemical Flow

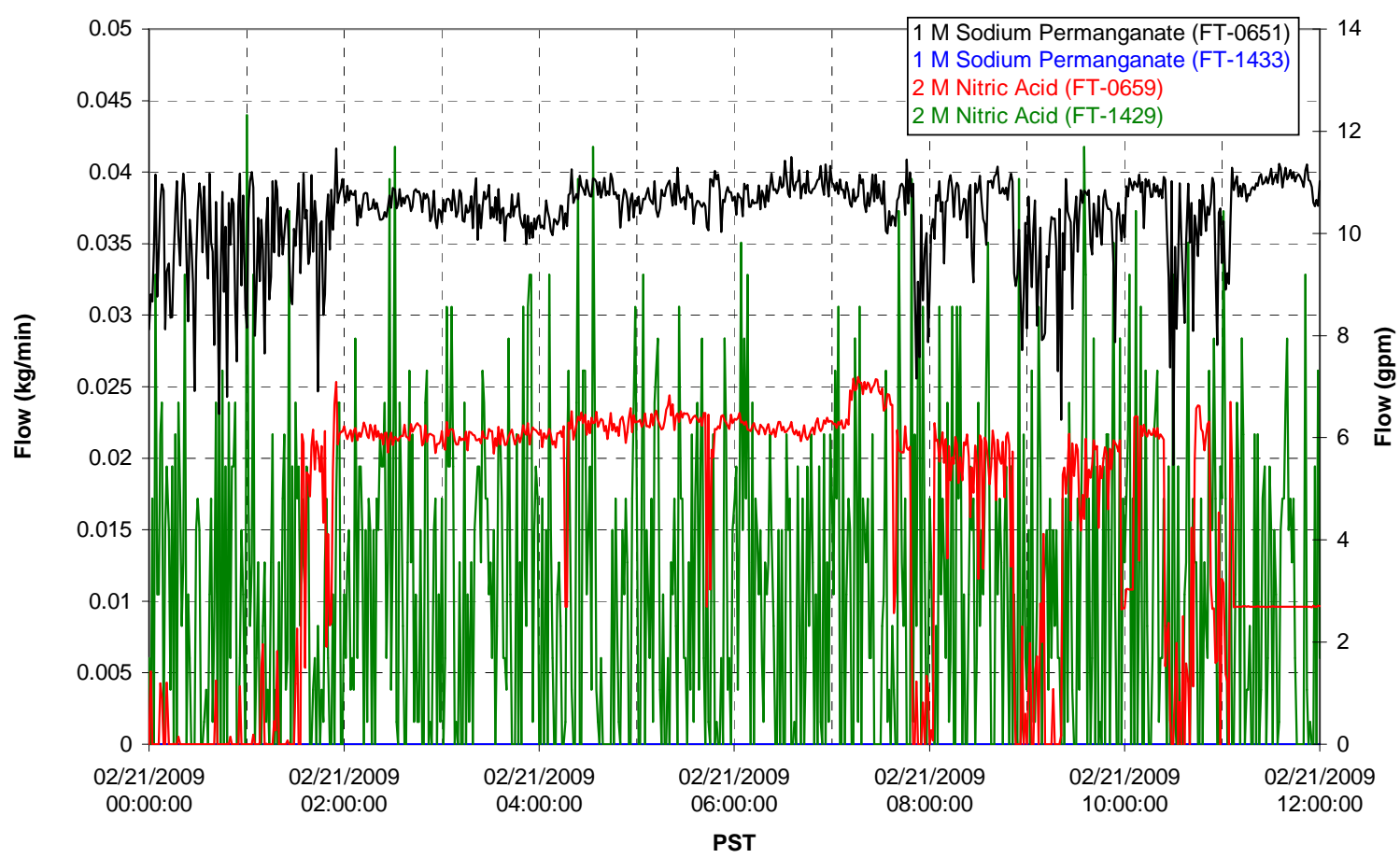

Air Flows

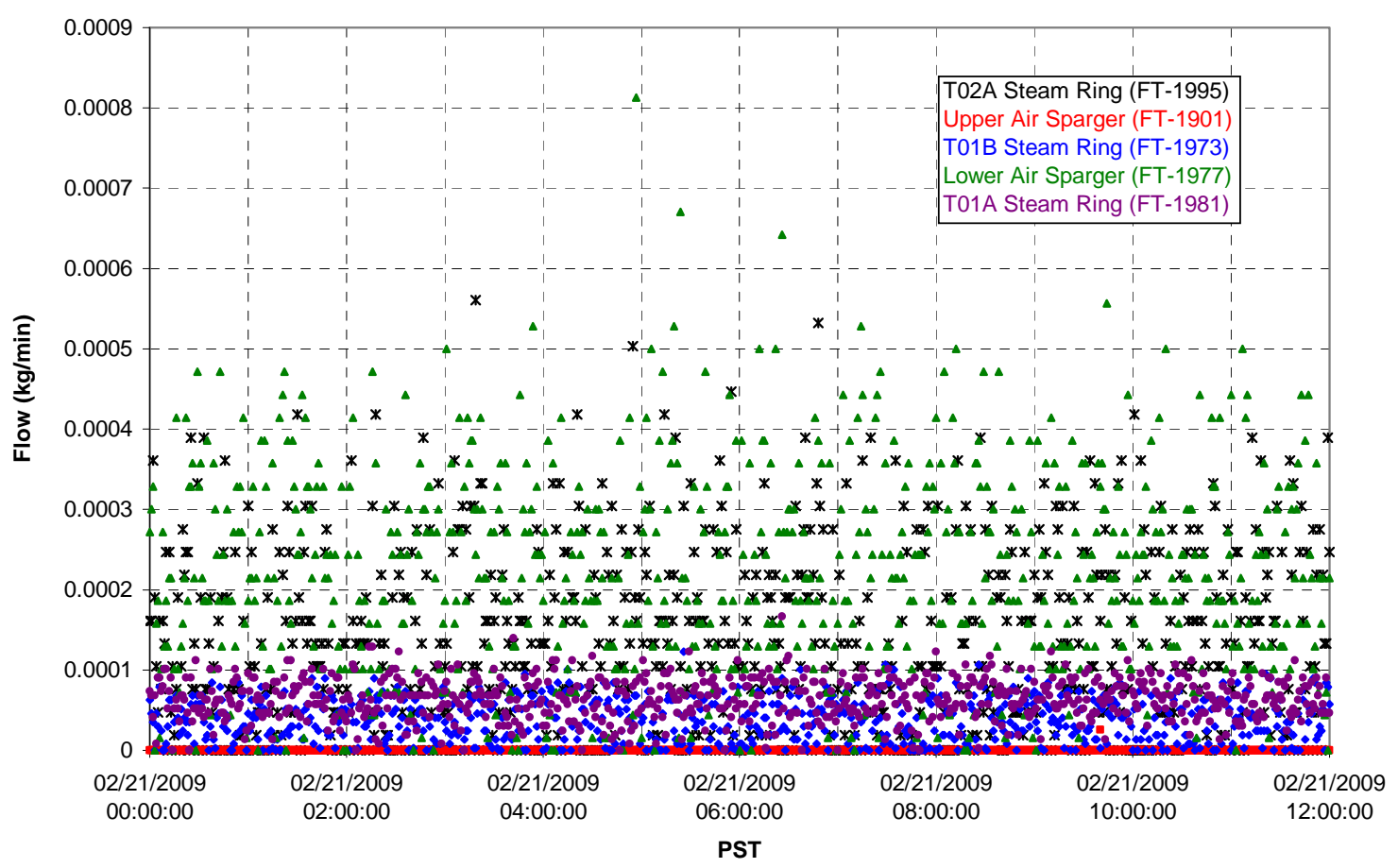


T02A Steam

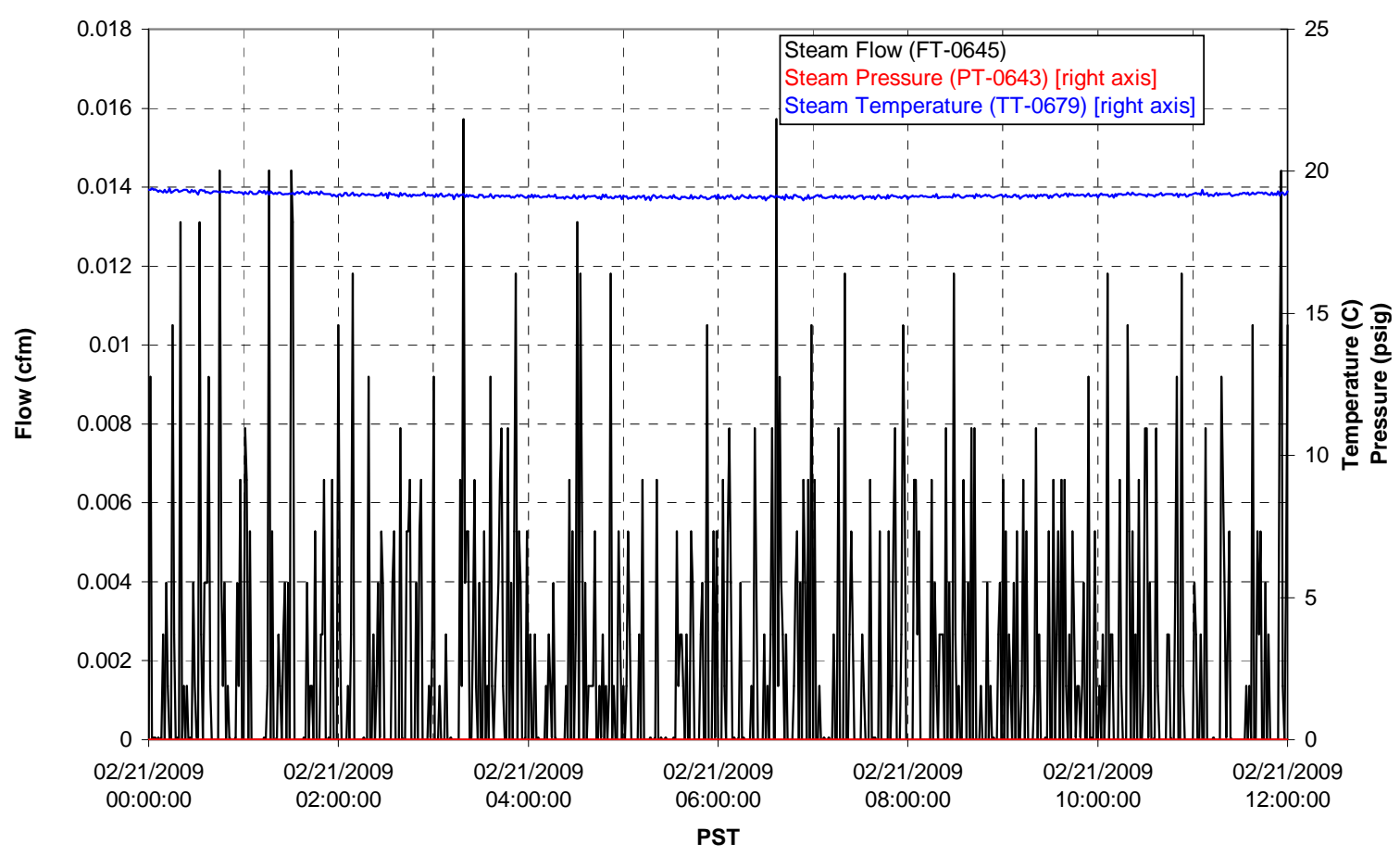

T01A Steam

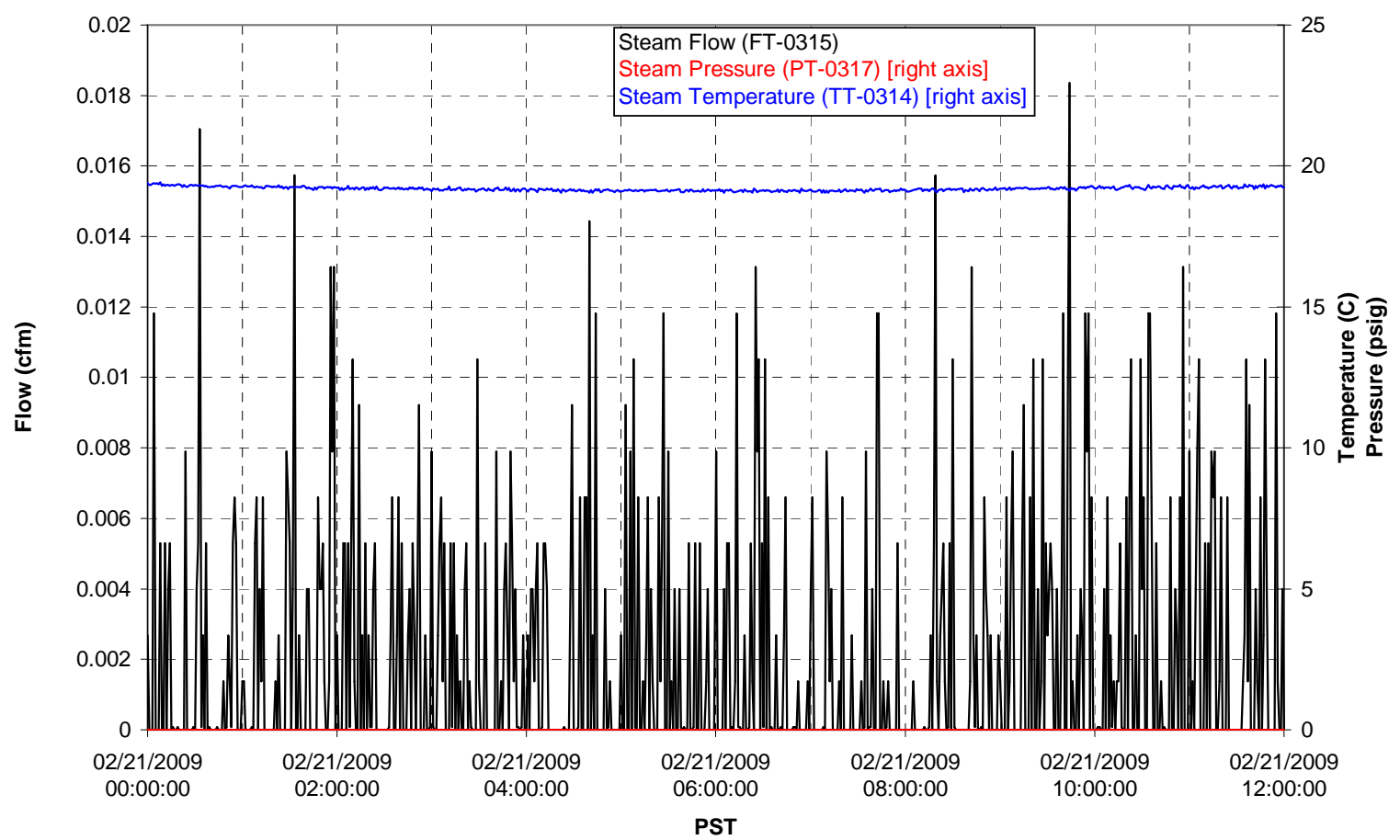


T01B Steam

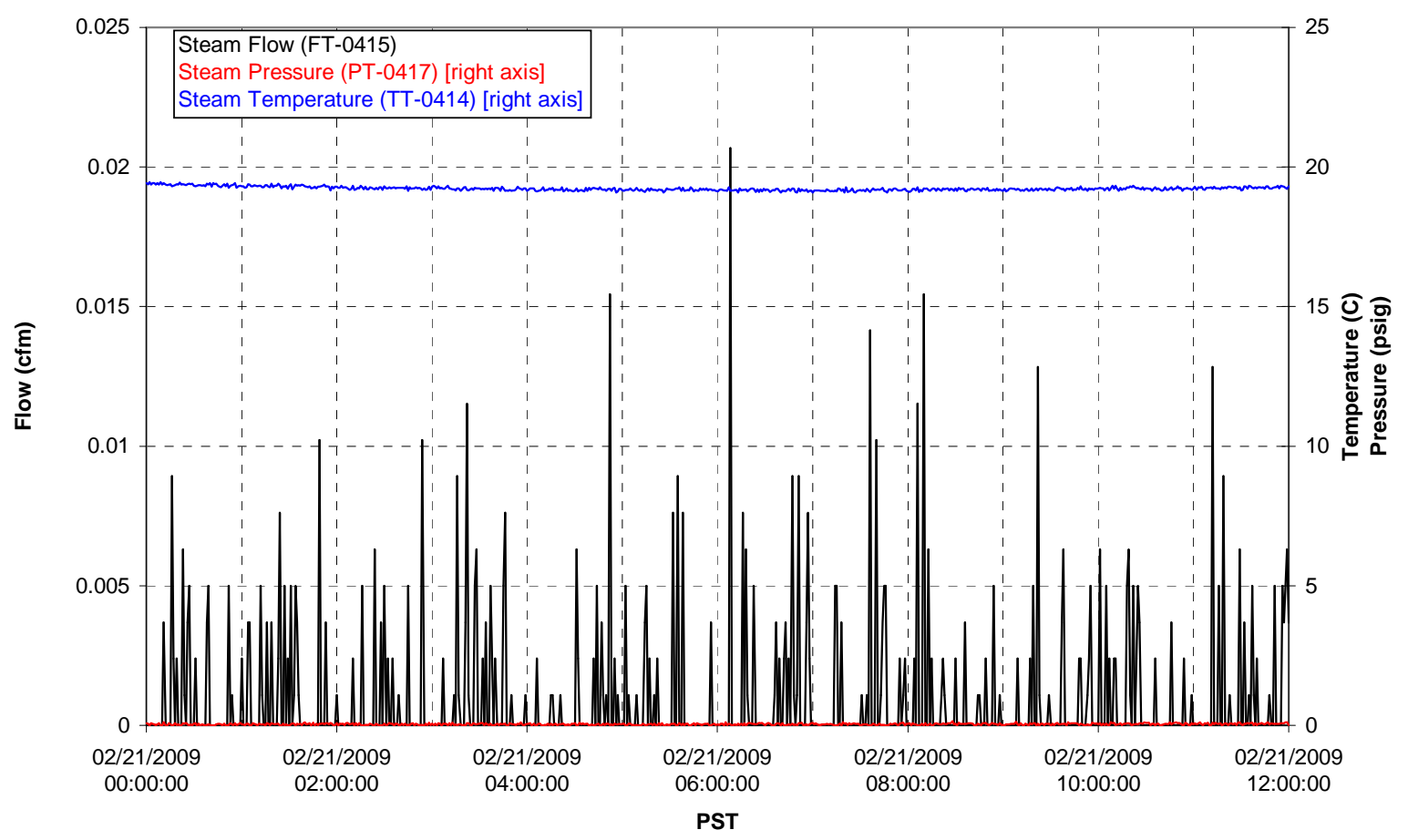




\section{Integrated Test A Data Plots 02/21/09 12:00 - 02/22/09 00:00}


T01A level

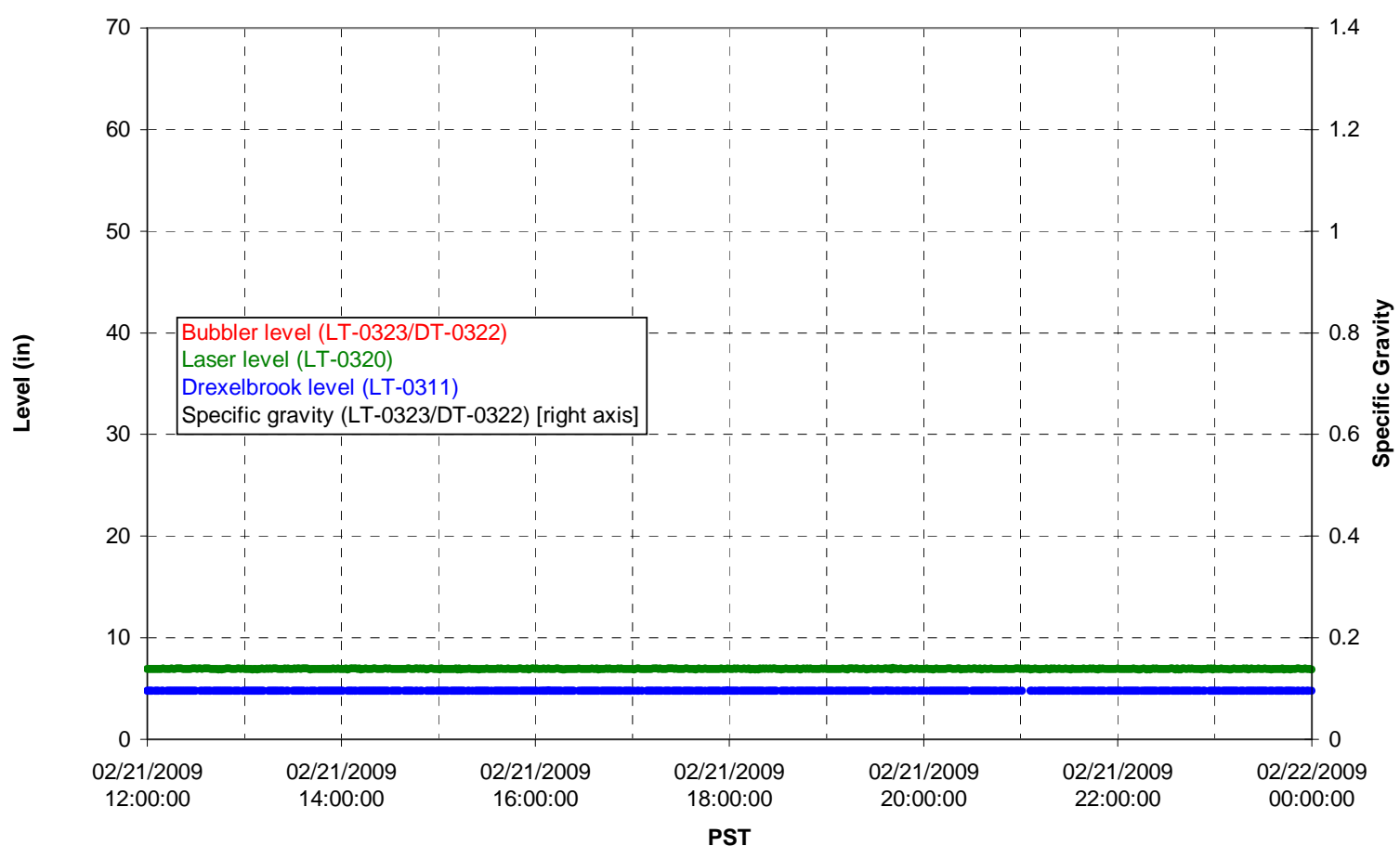

T01A level

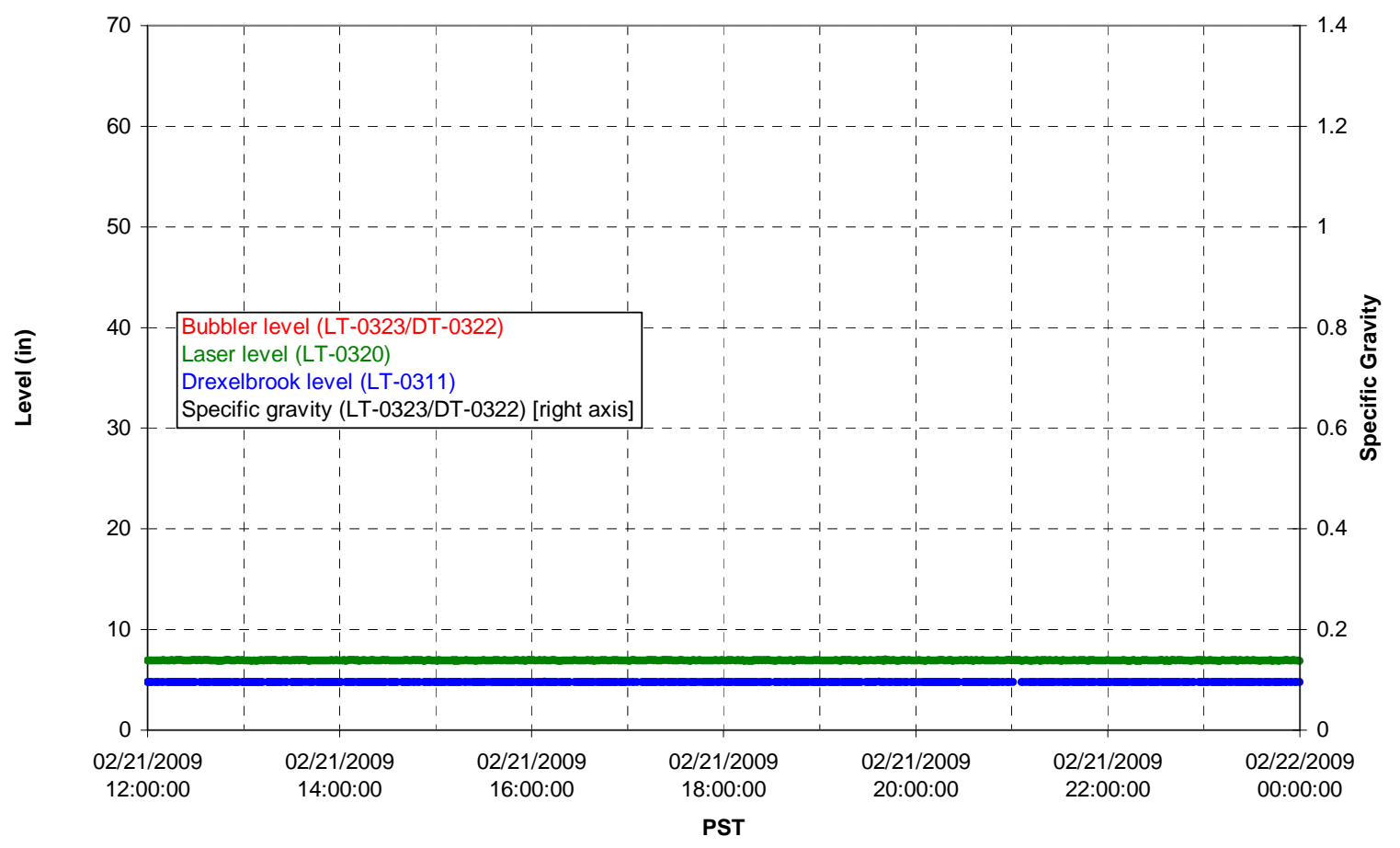

G.776 
T01B level

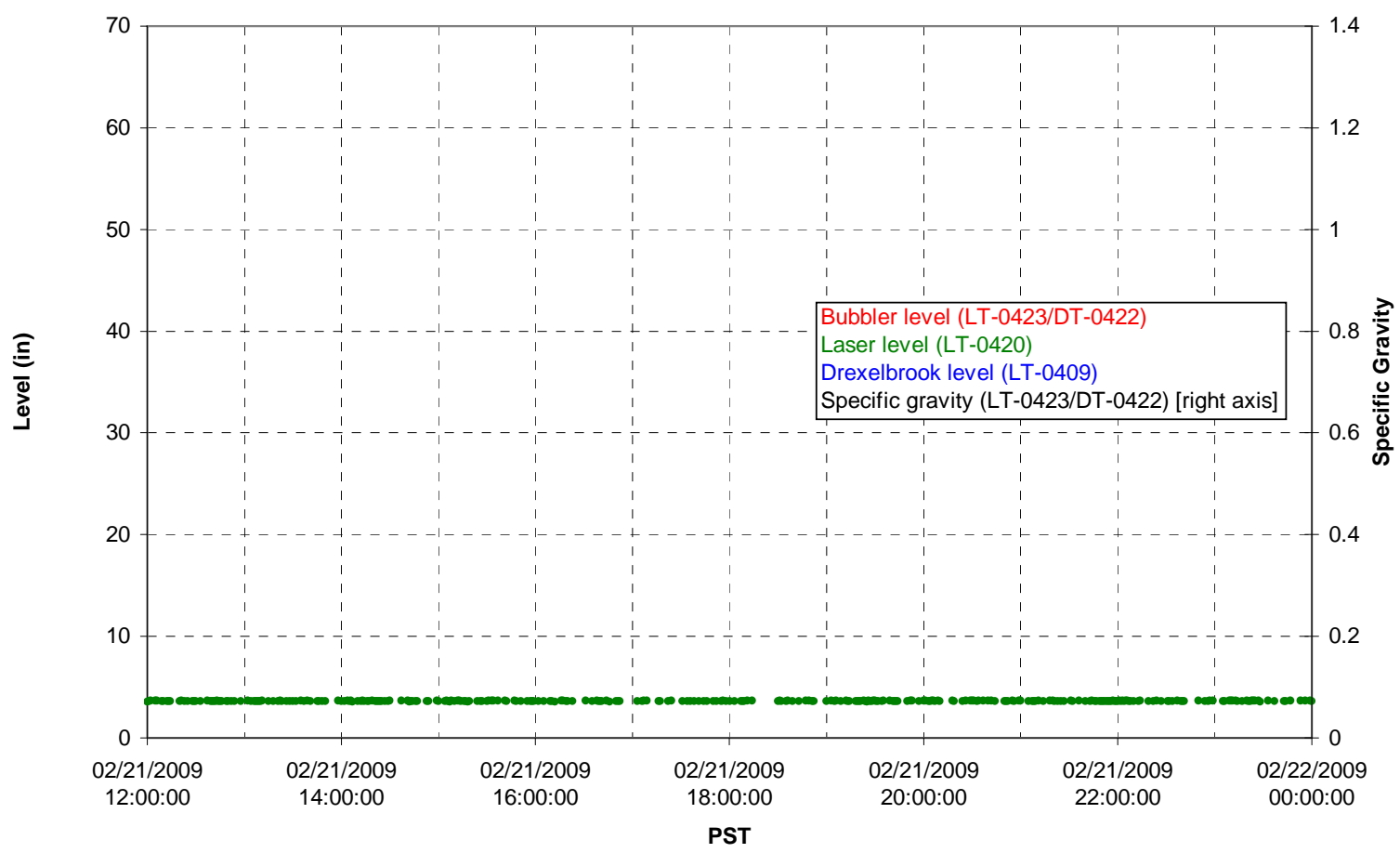

T01B temperatures

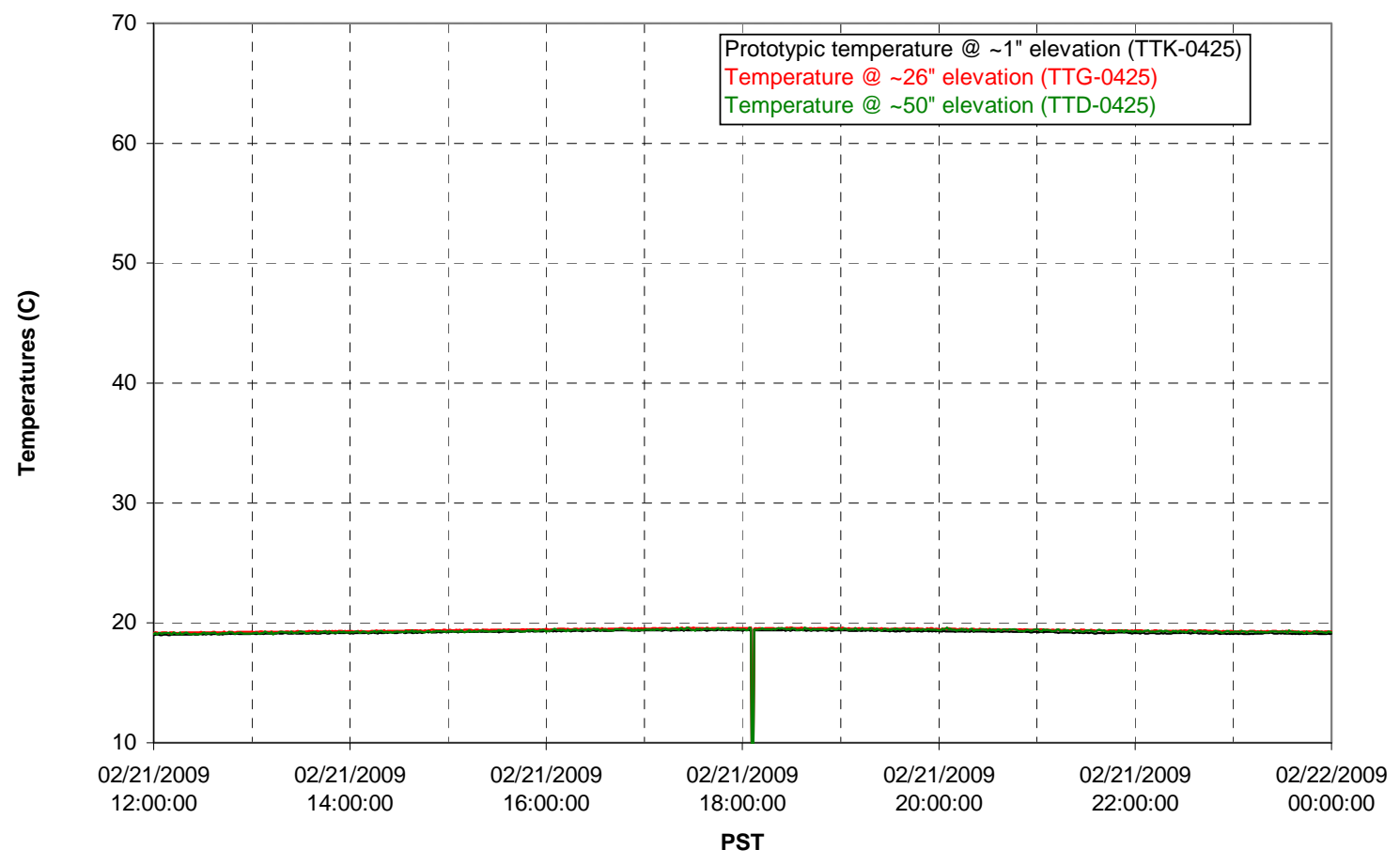

G.777 
T02A level

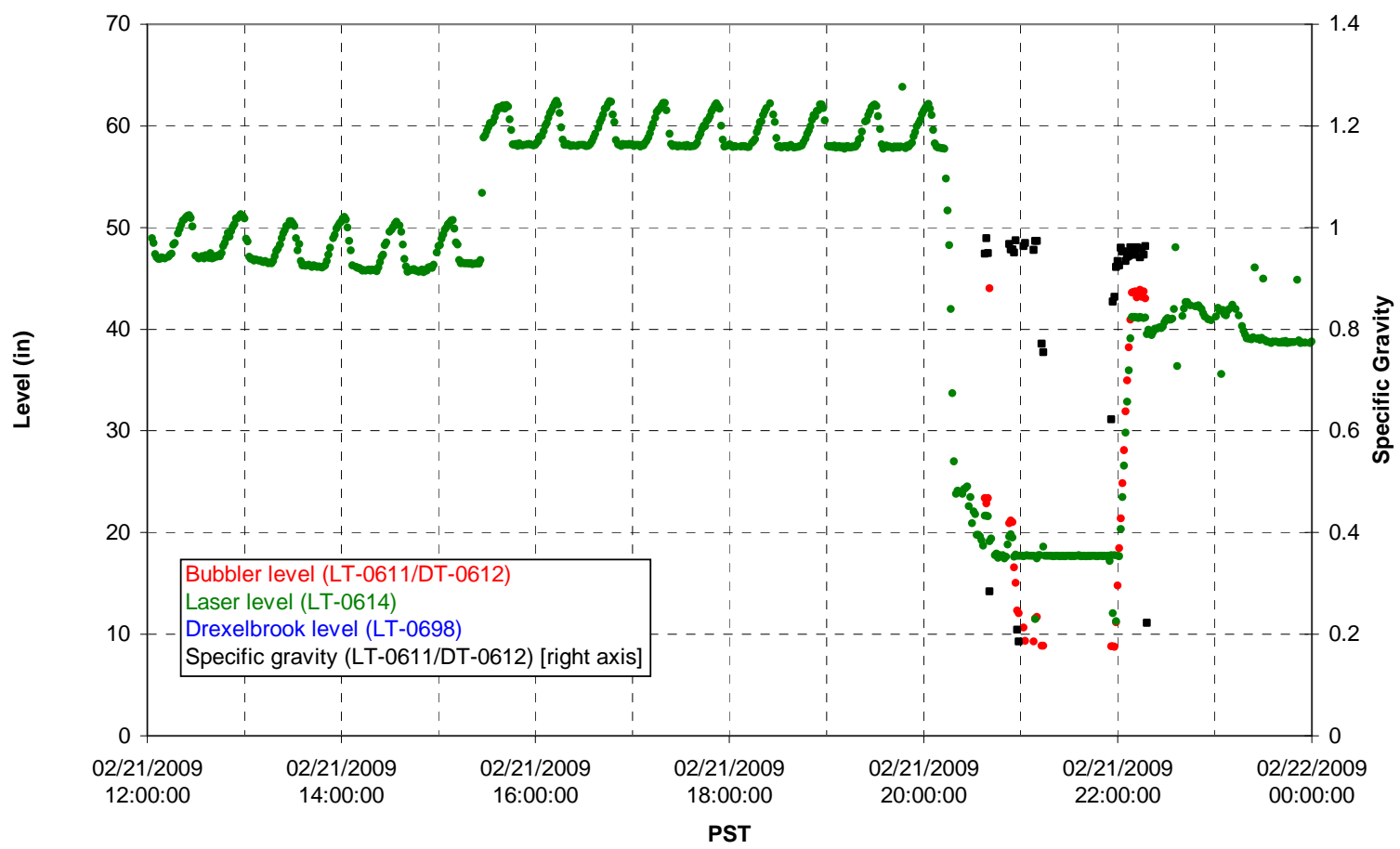

T02A temperatures

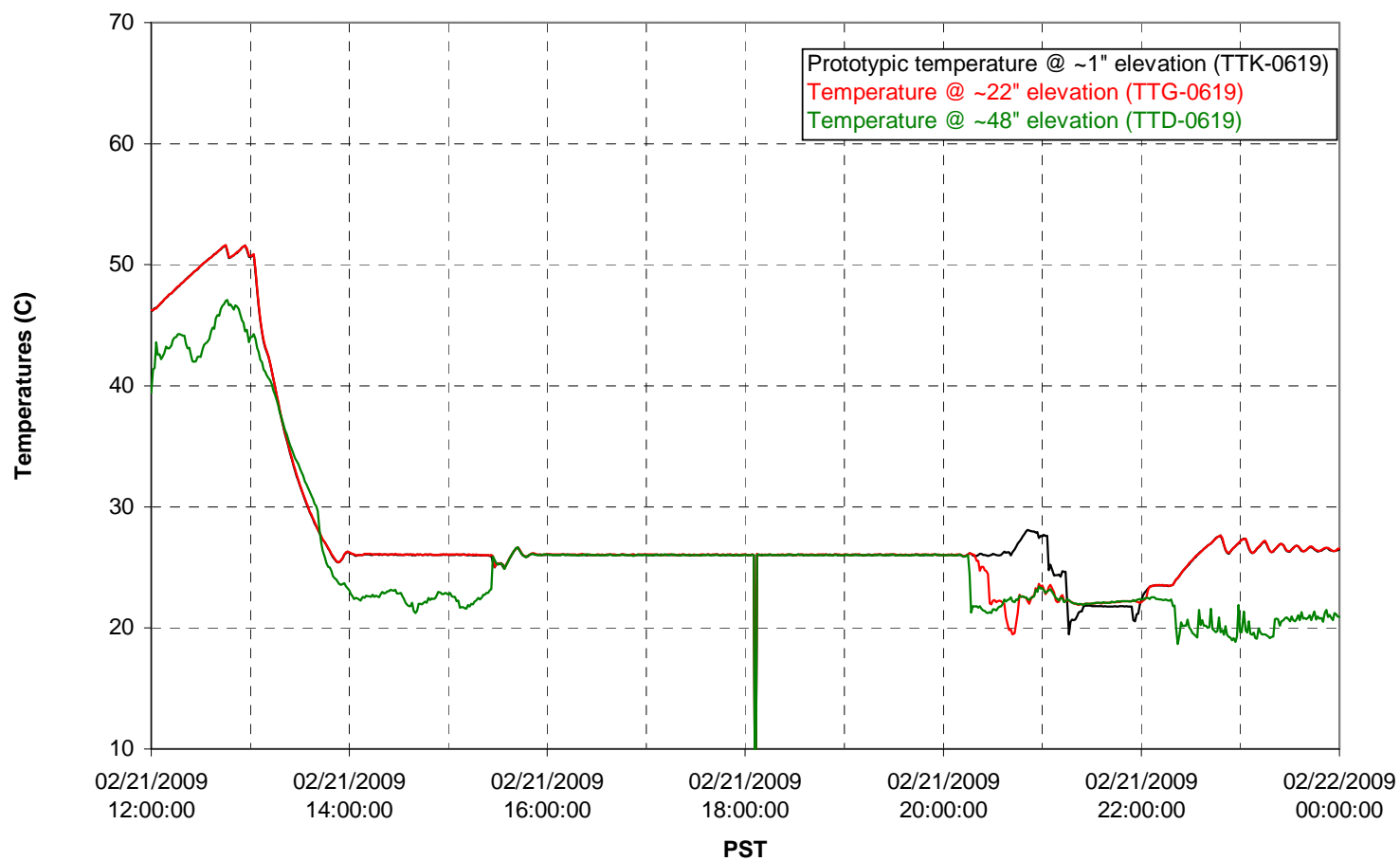


T02A and filter loop temperatures
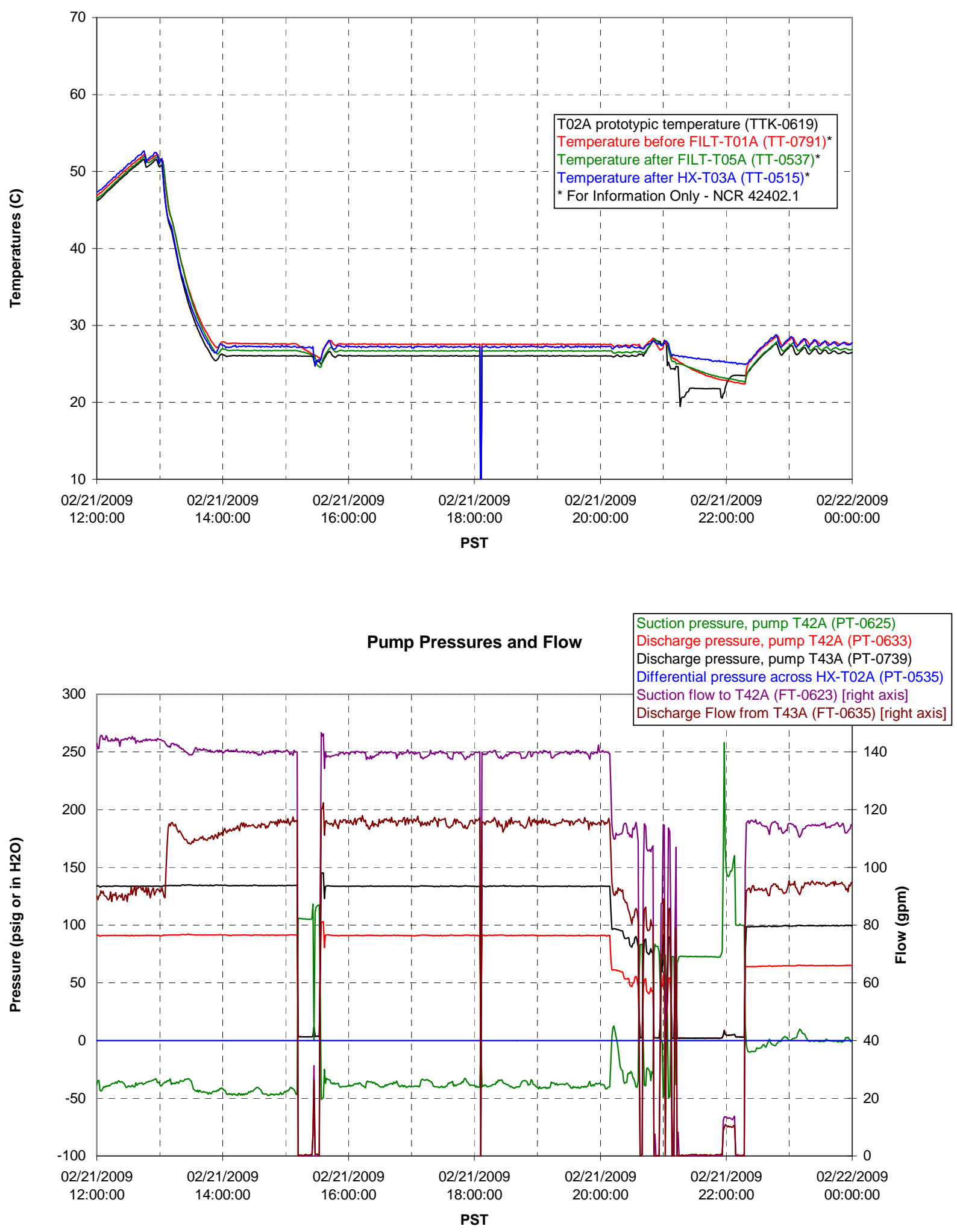


\section{Axial pressure drop}

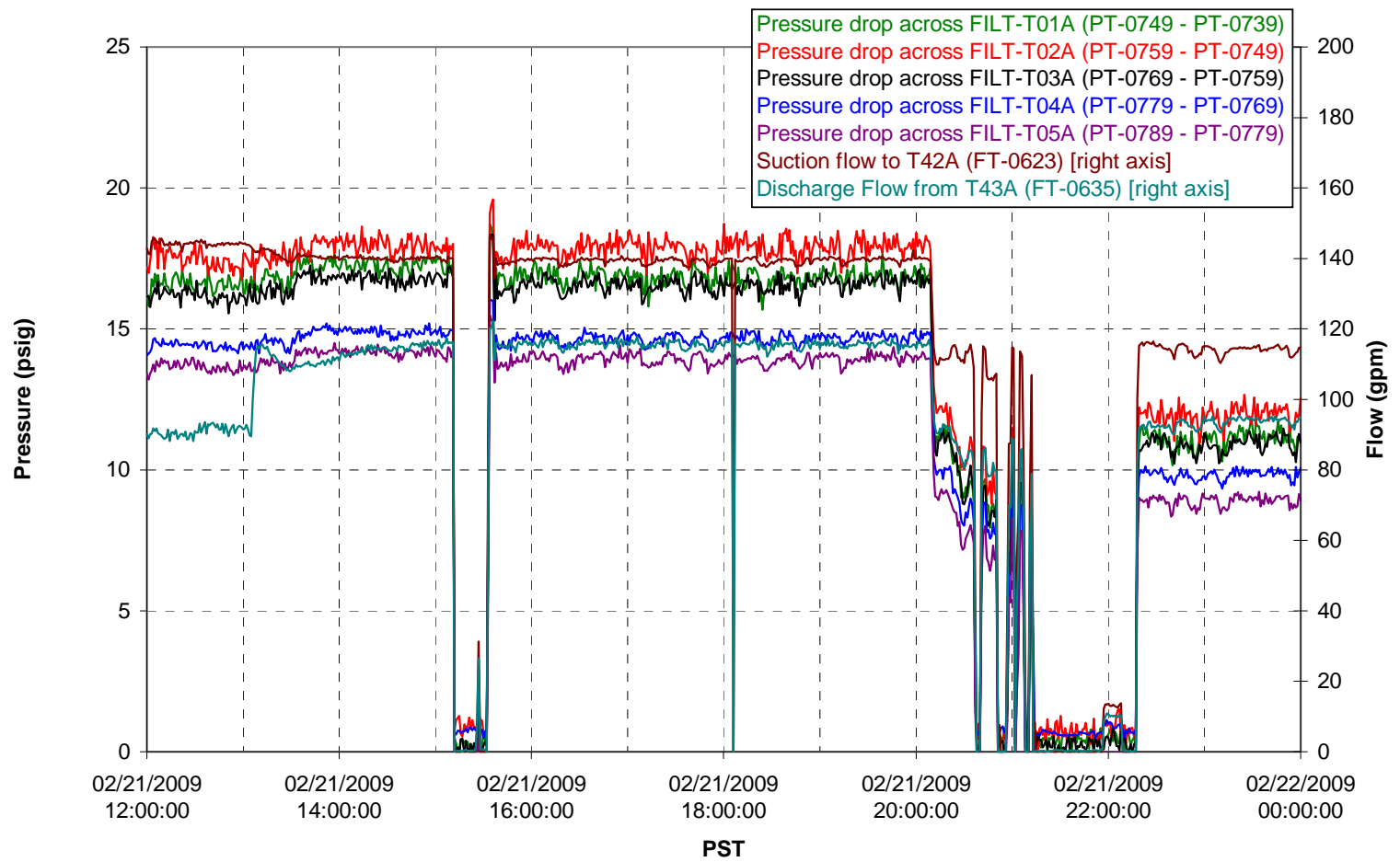

Permeate flow rates

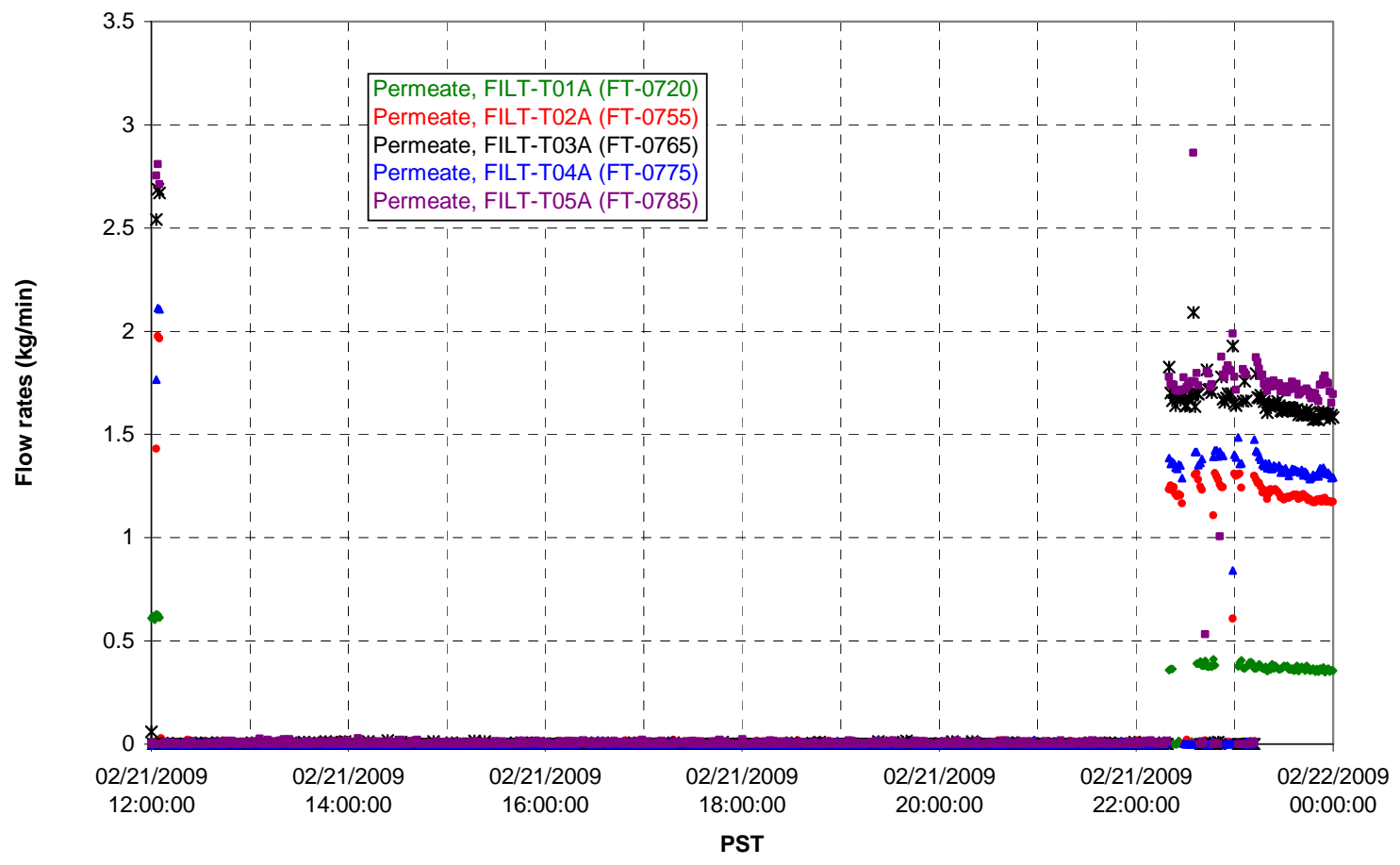

G.780 
T02A Inner Temperature Tree

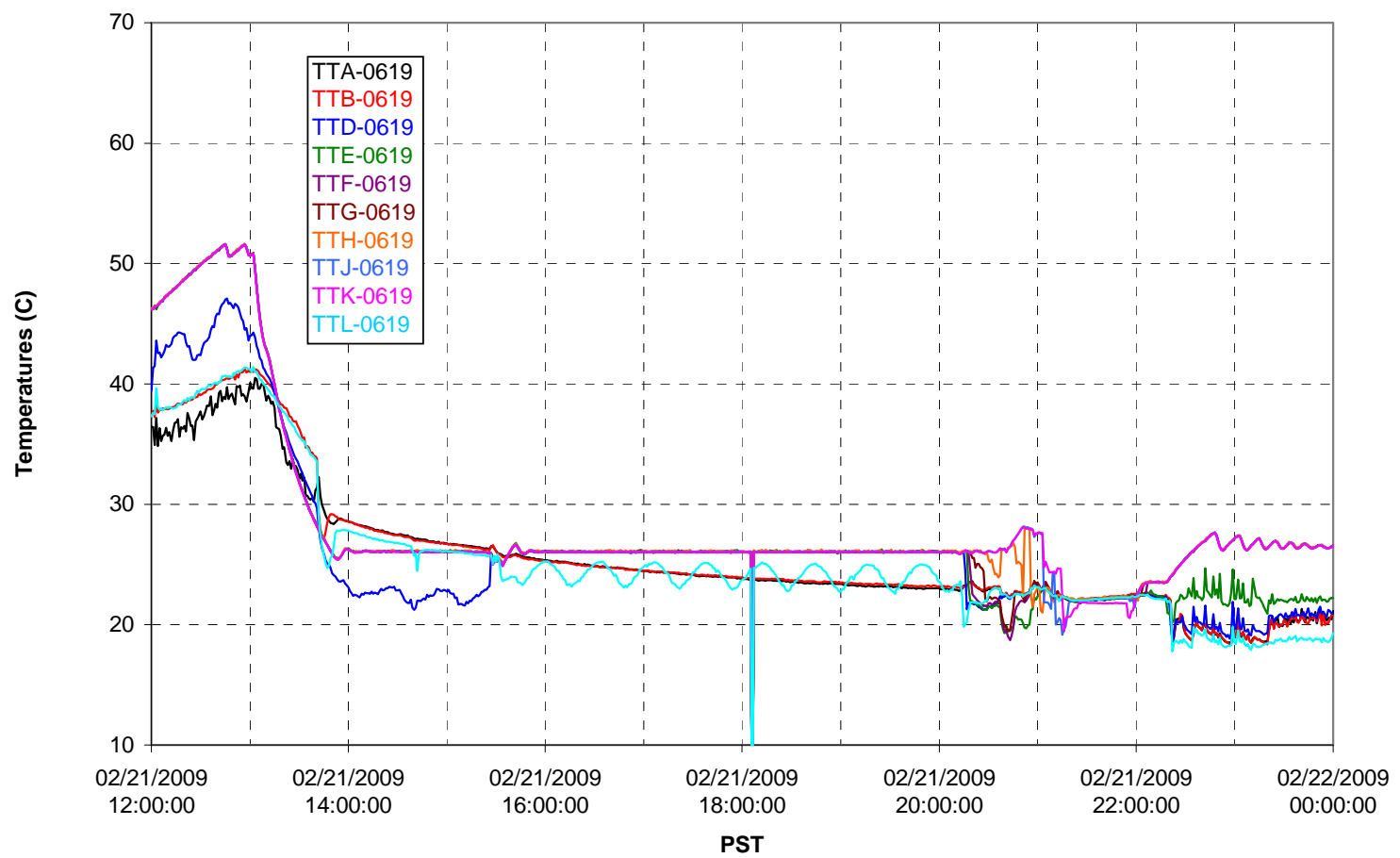

T02A Outer Temperature Tree

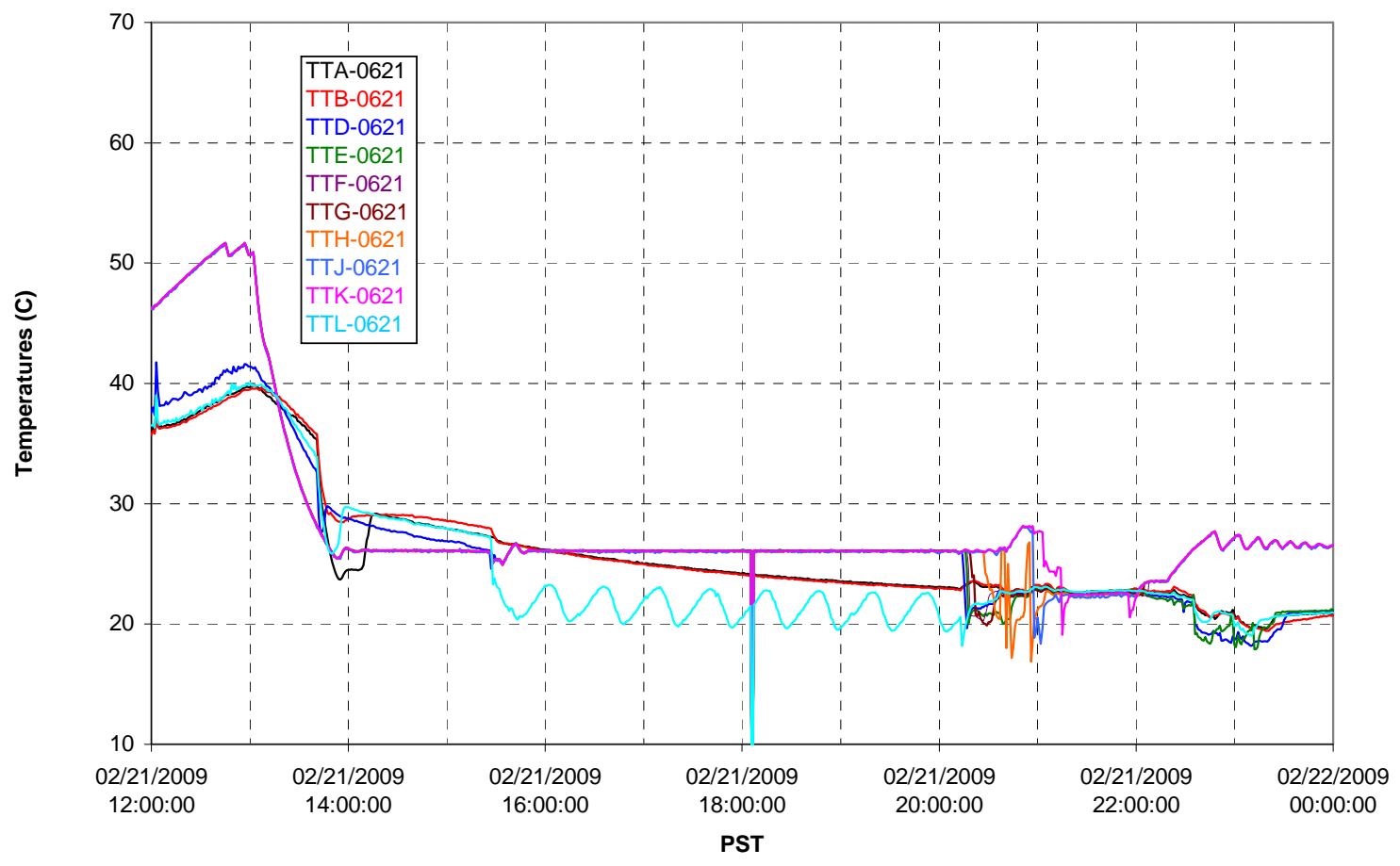


T02A temperatures

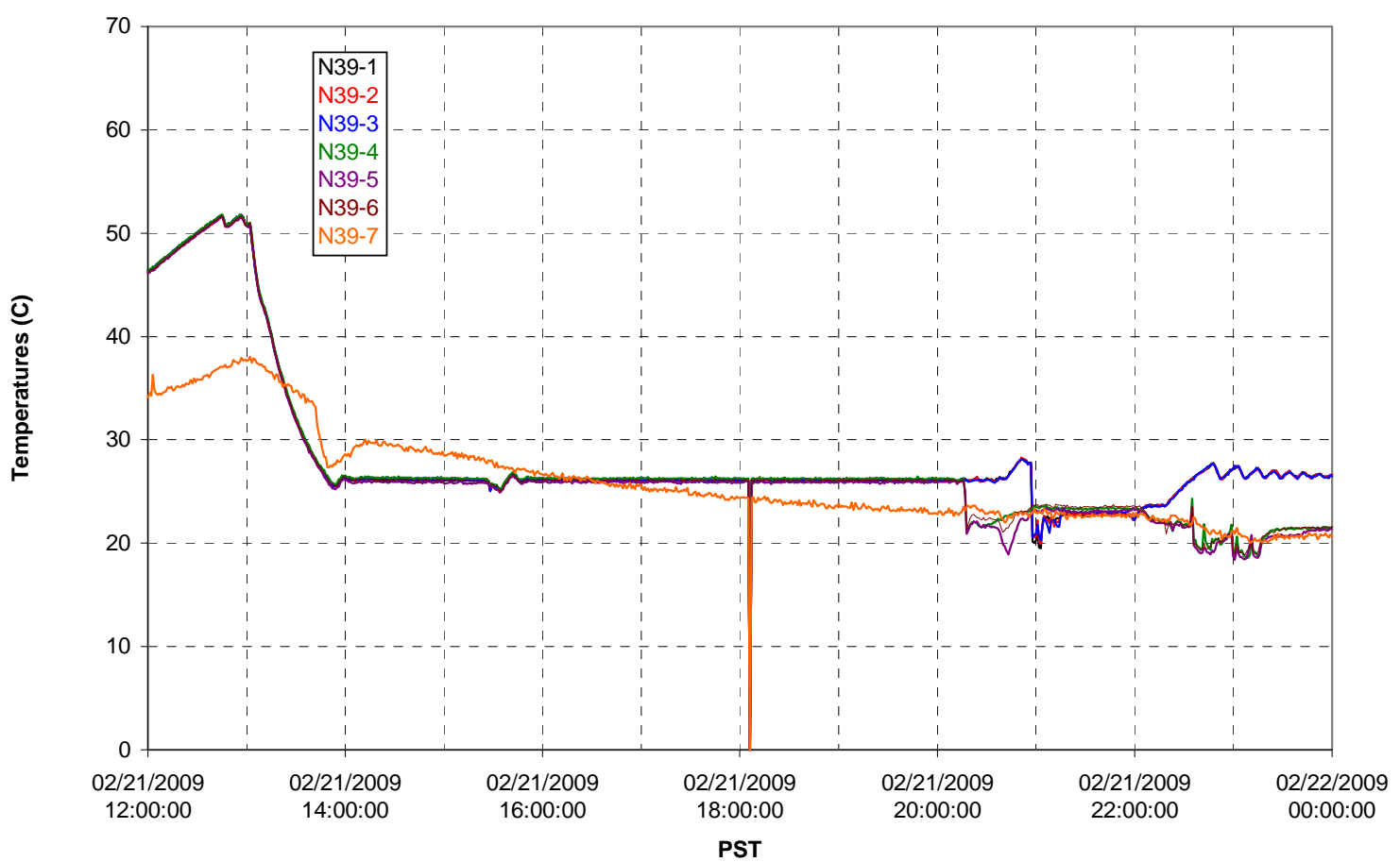

T02A temperatures

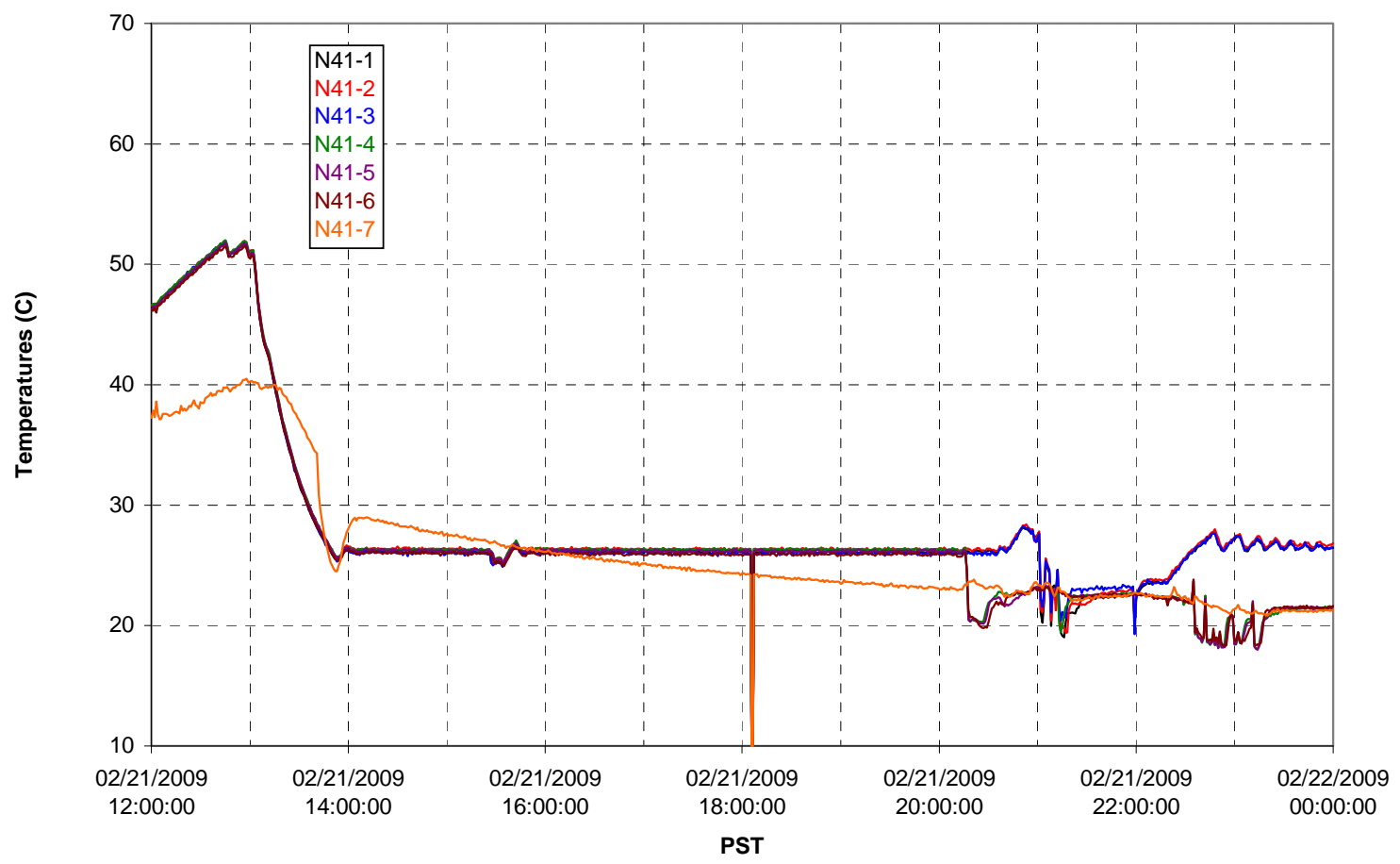

G.782 
T02A temperatures

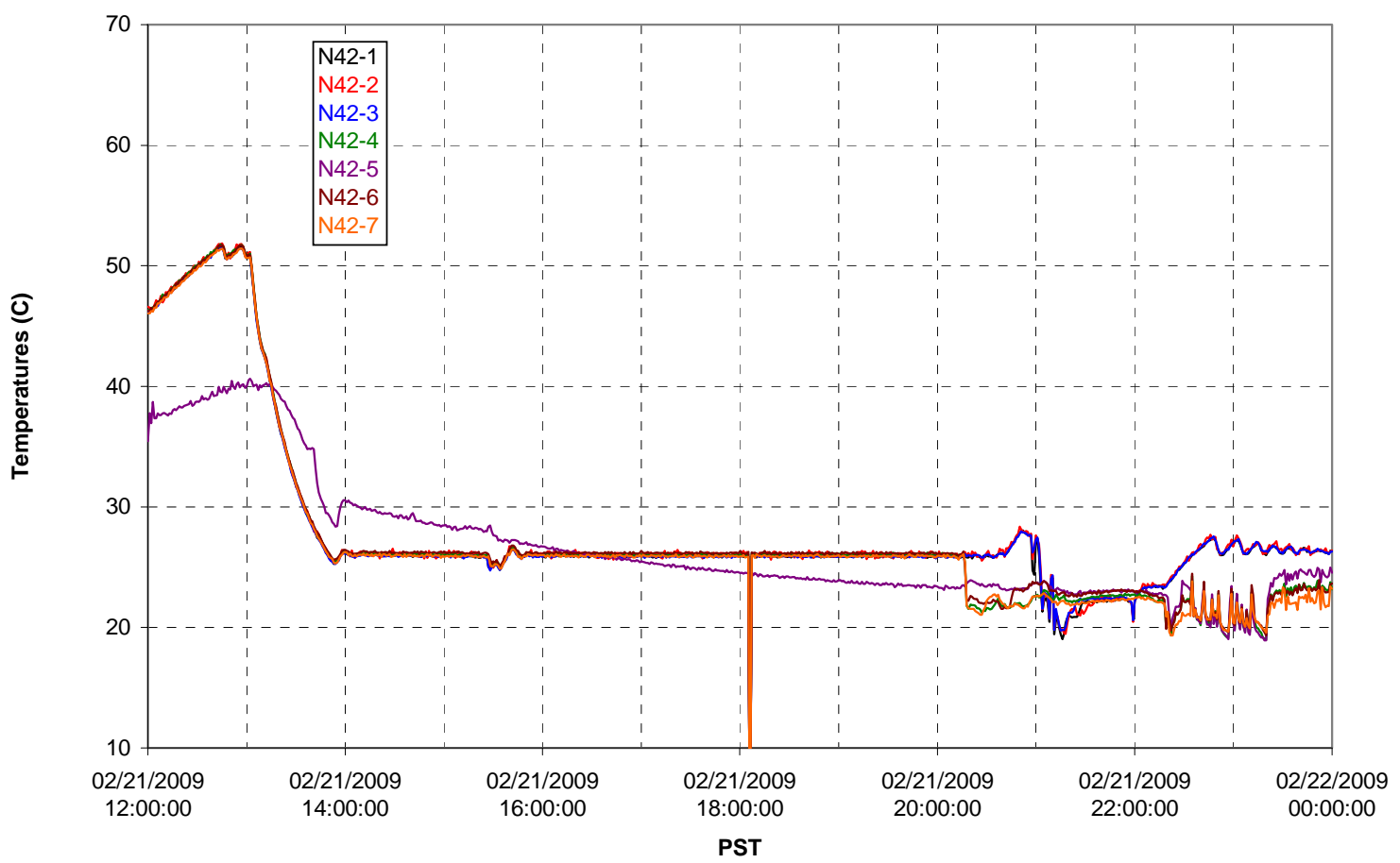

T02A temperatures

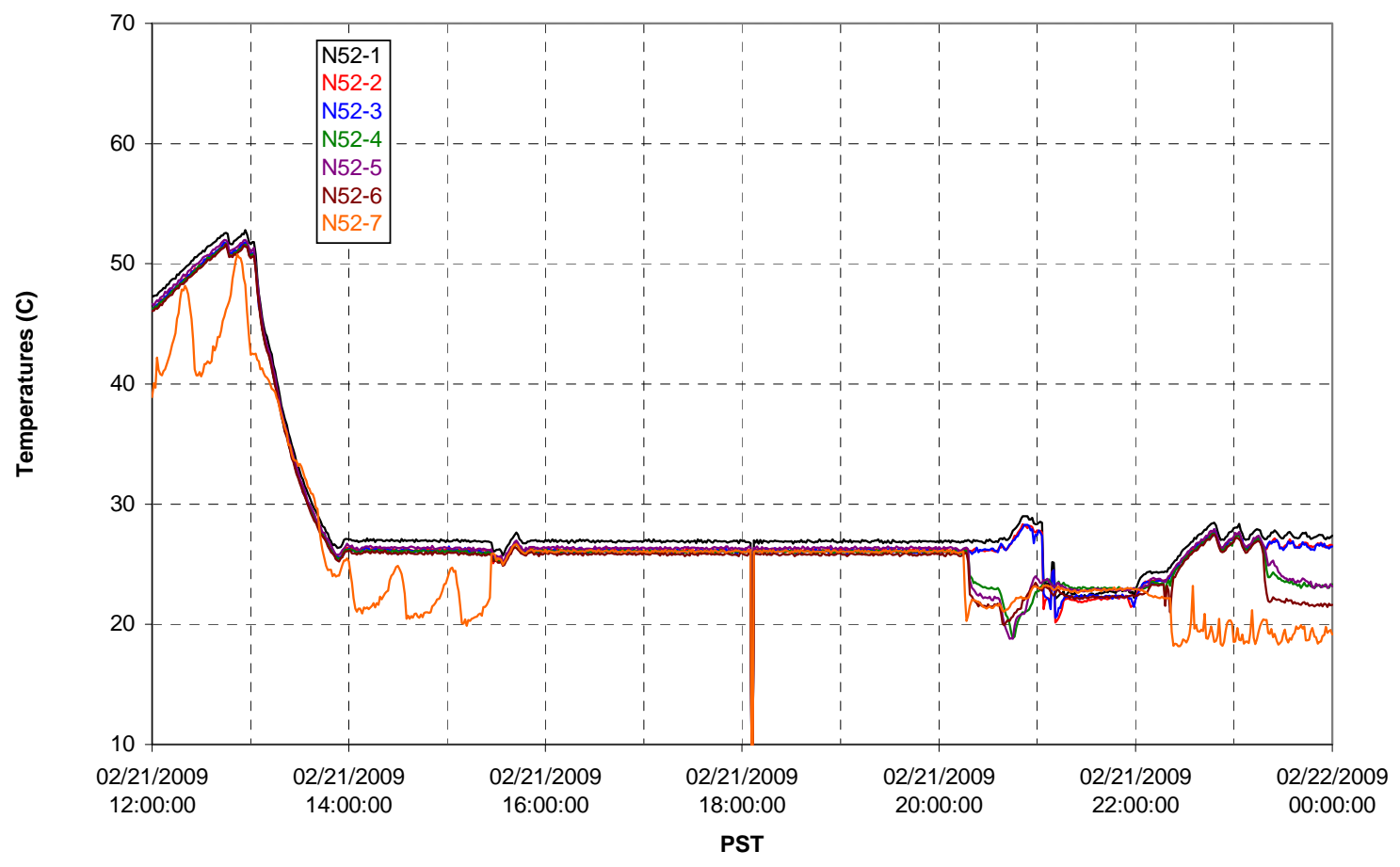


T02A Heating and Cooling
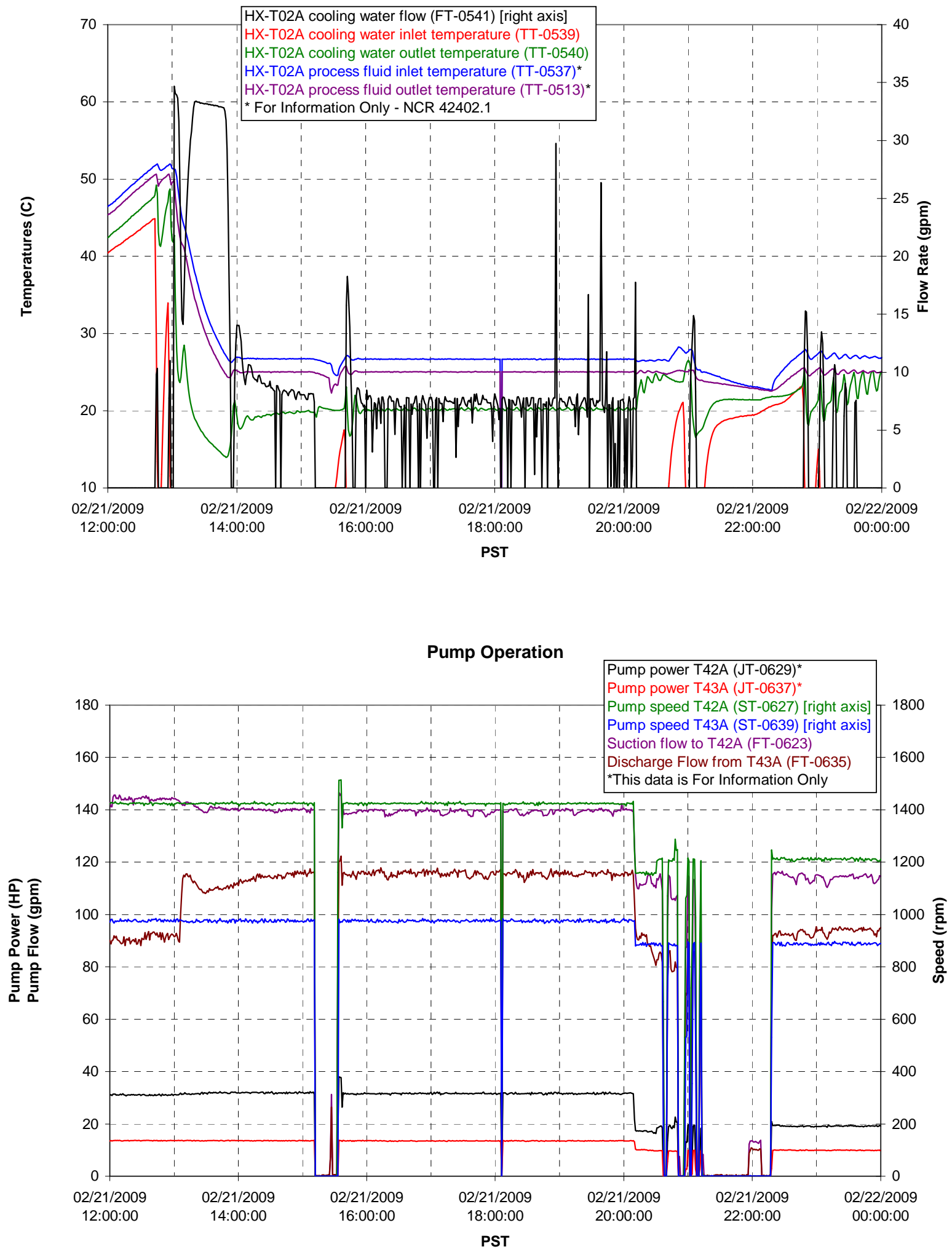
Pulsepot UFP-PP-T01A

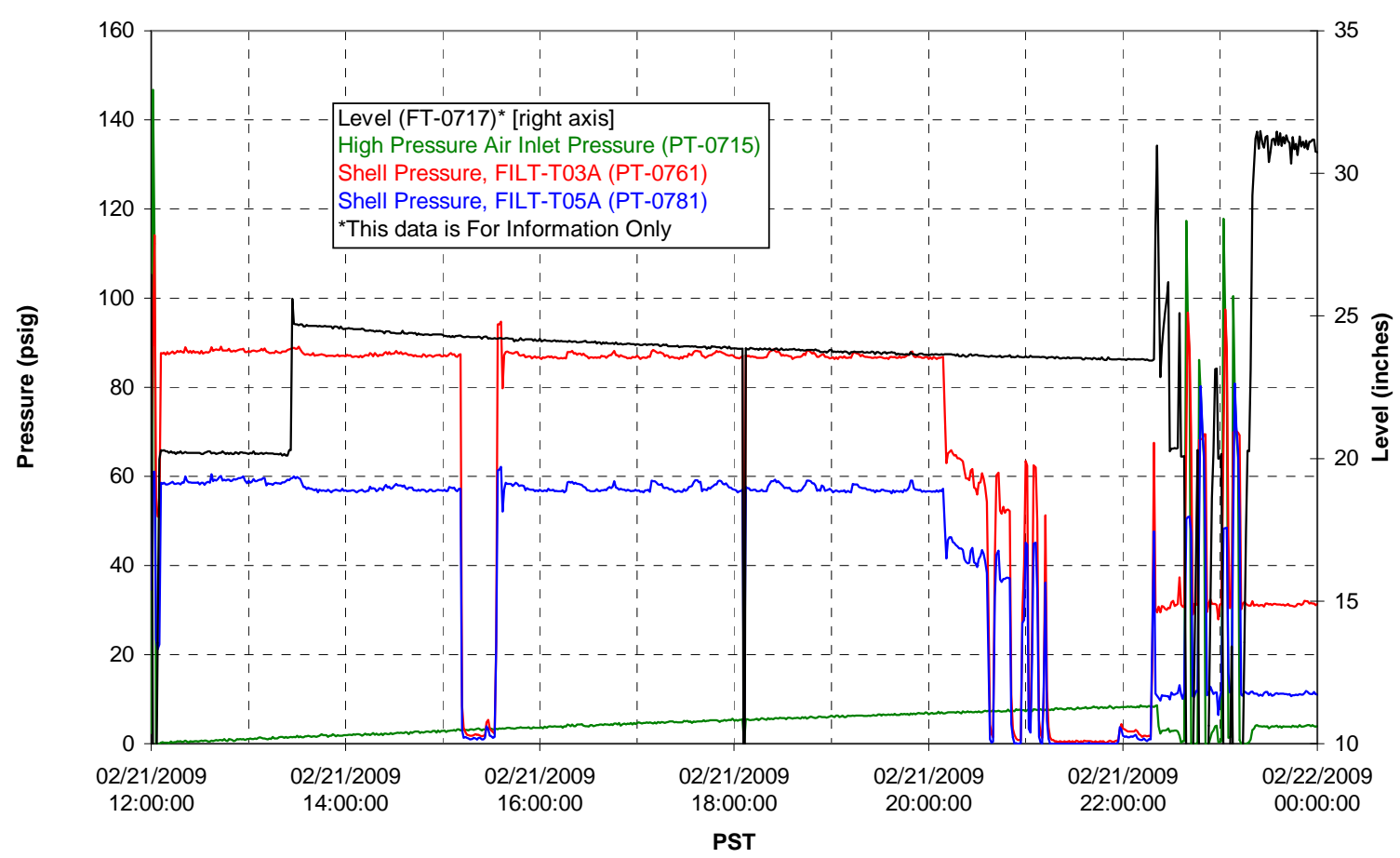

Pulsepot UFP-PP-T02A

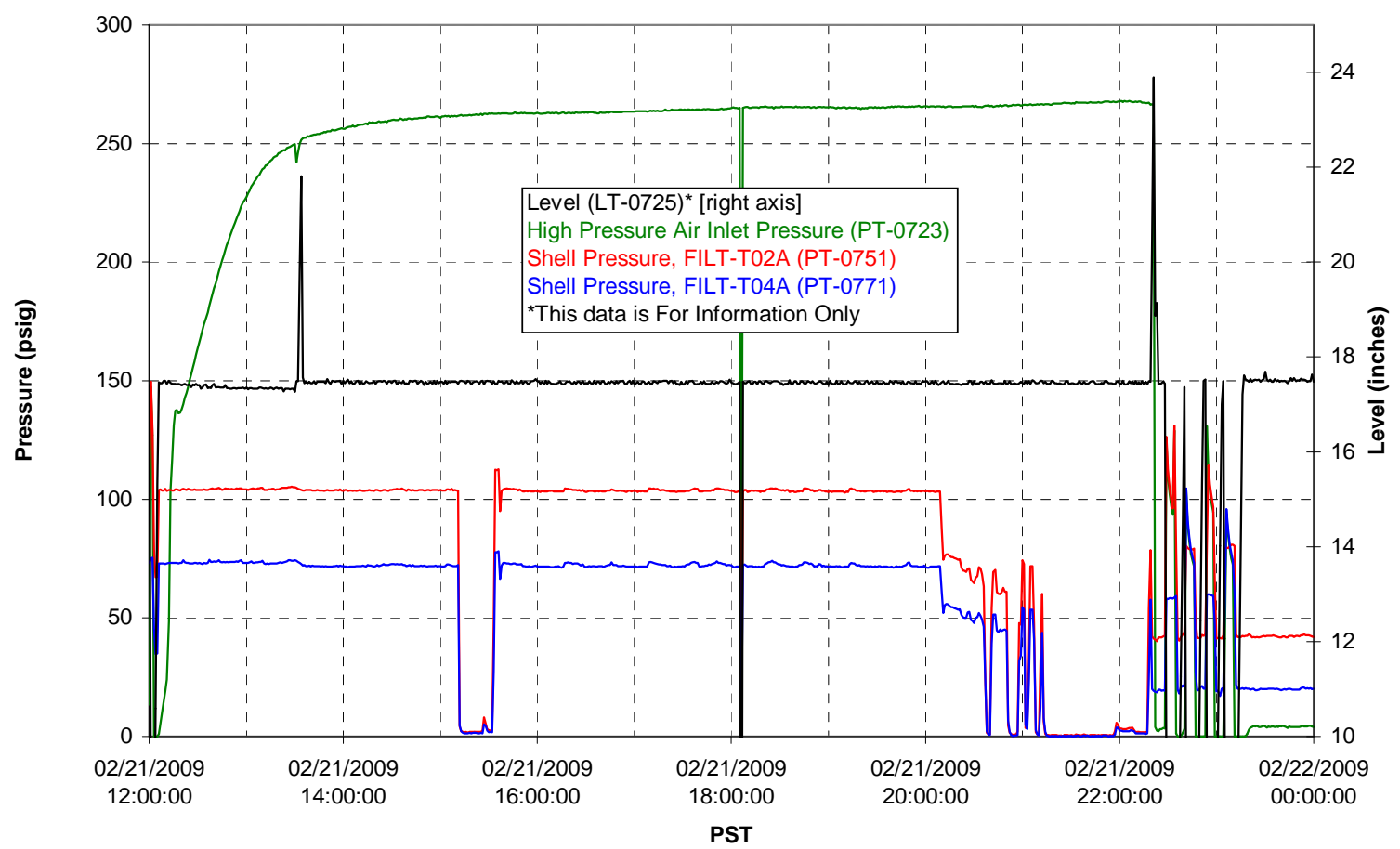


Pulsepot UFP-PP-T03A

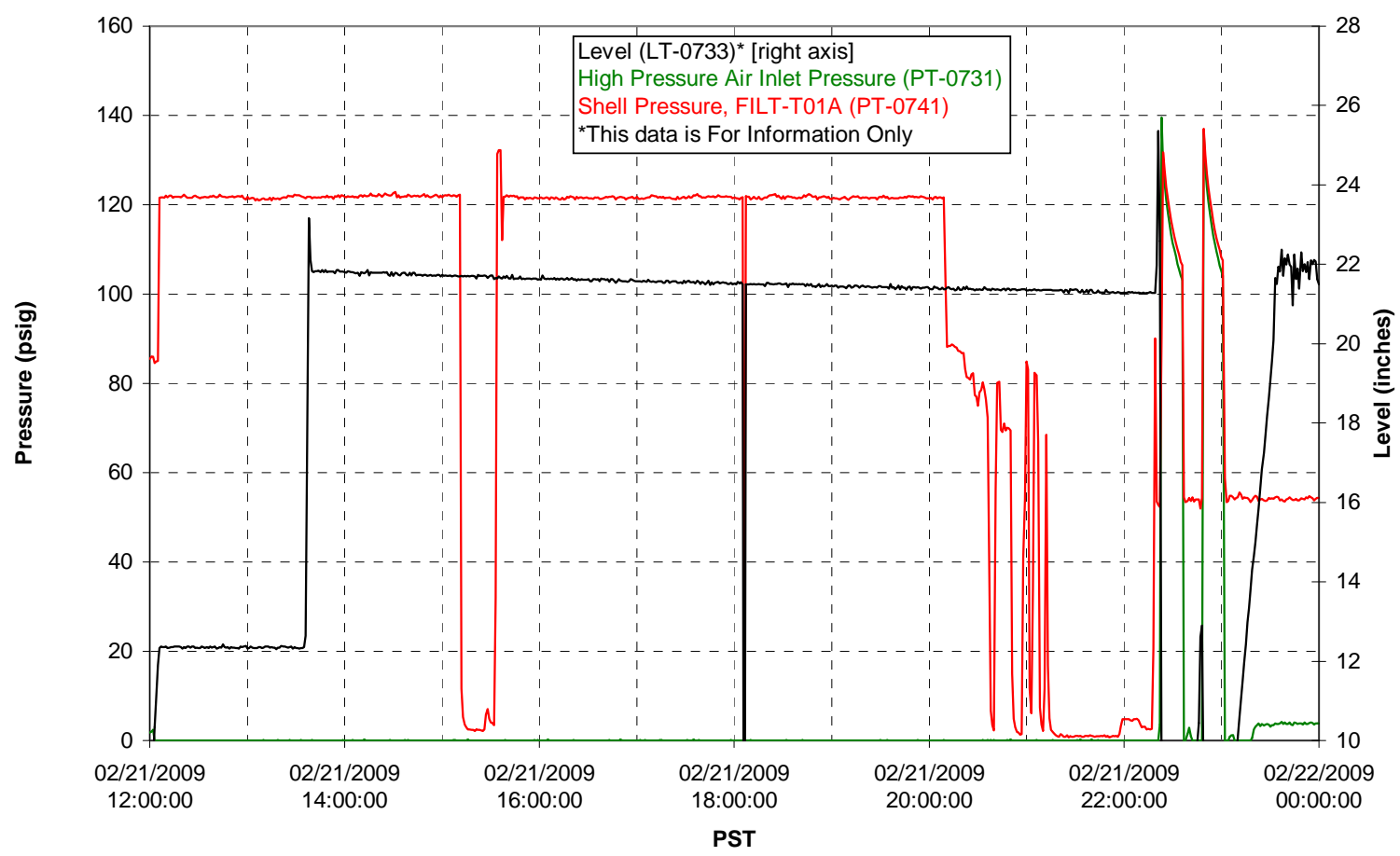

Pulsepot Levels

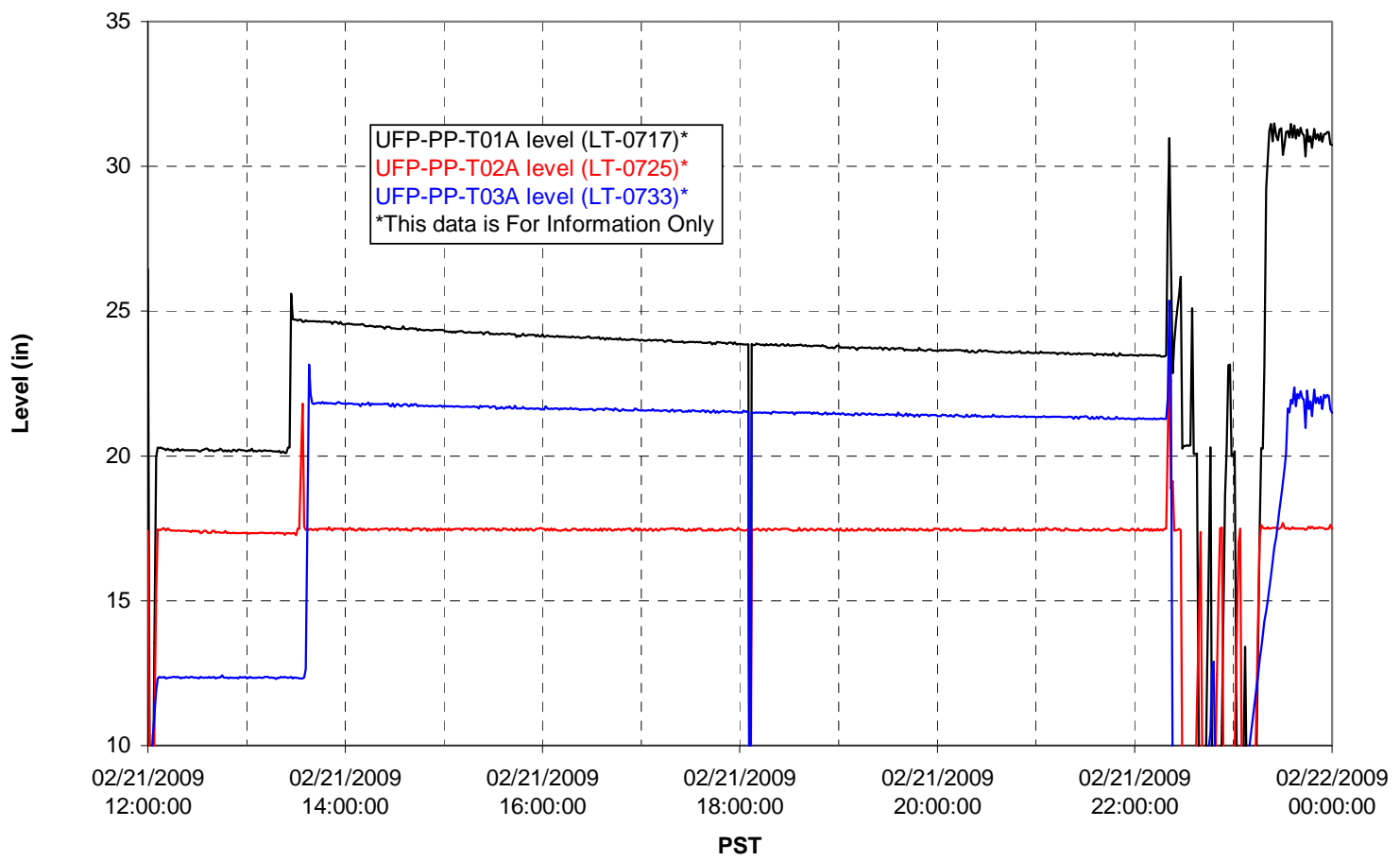


Filter UFP-FILT-T01A

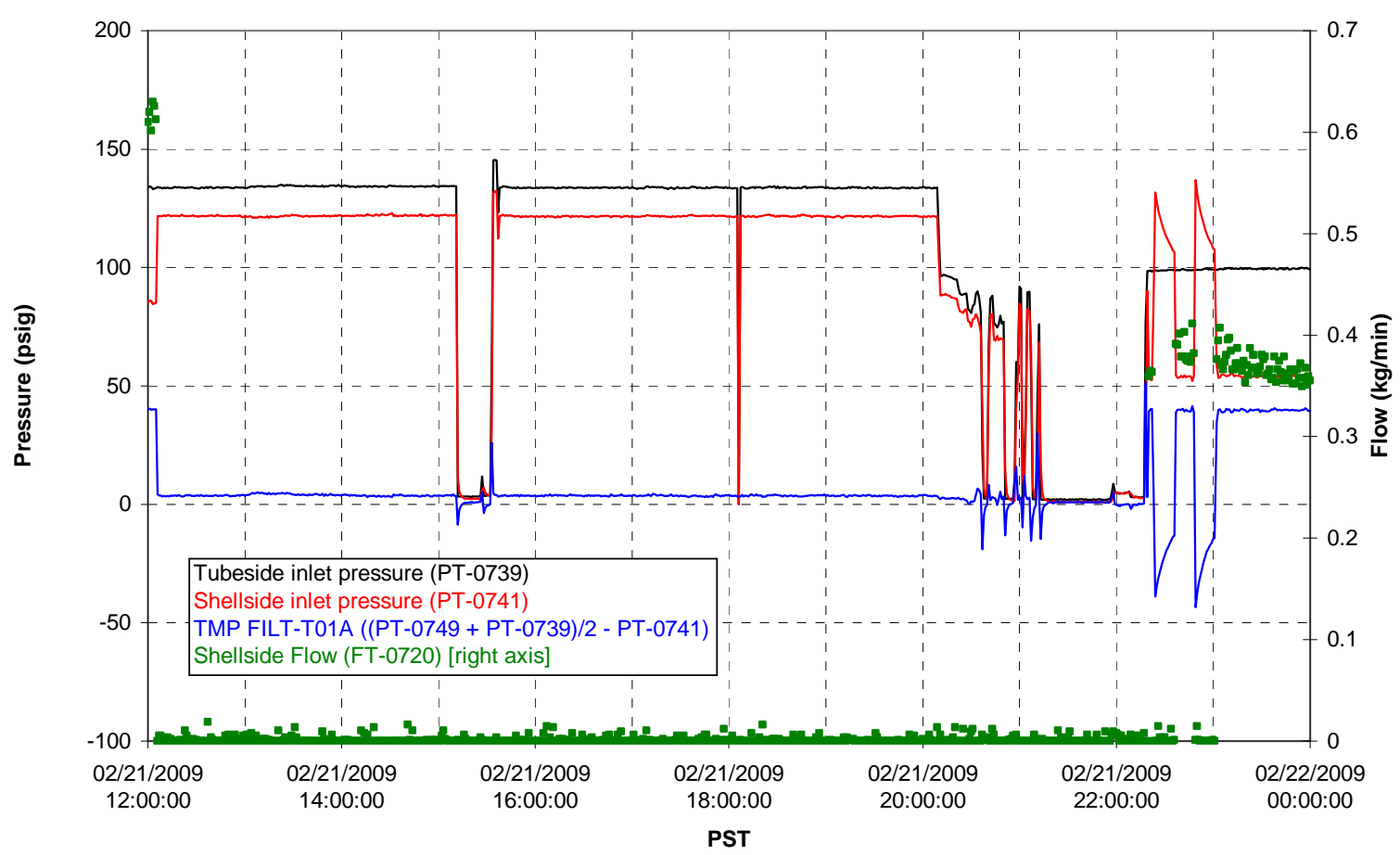

Filter UFP-FILT-T02A

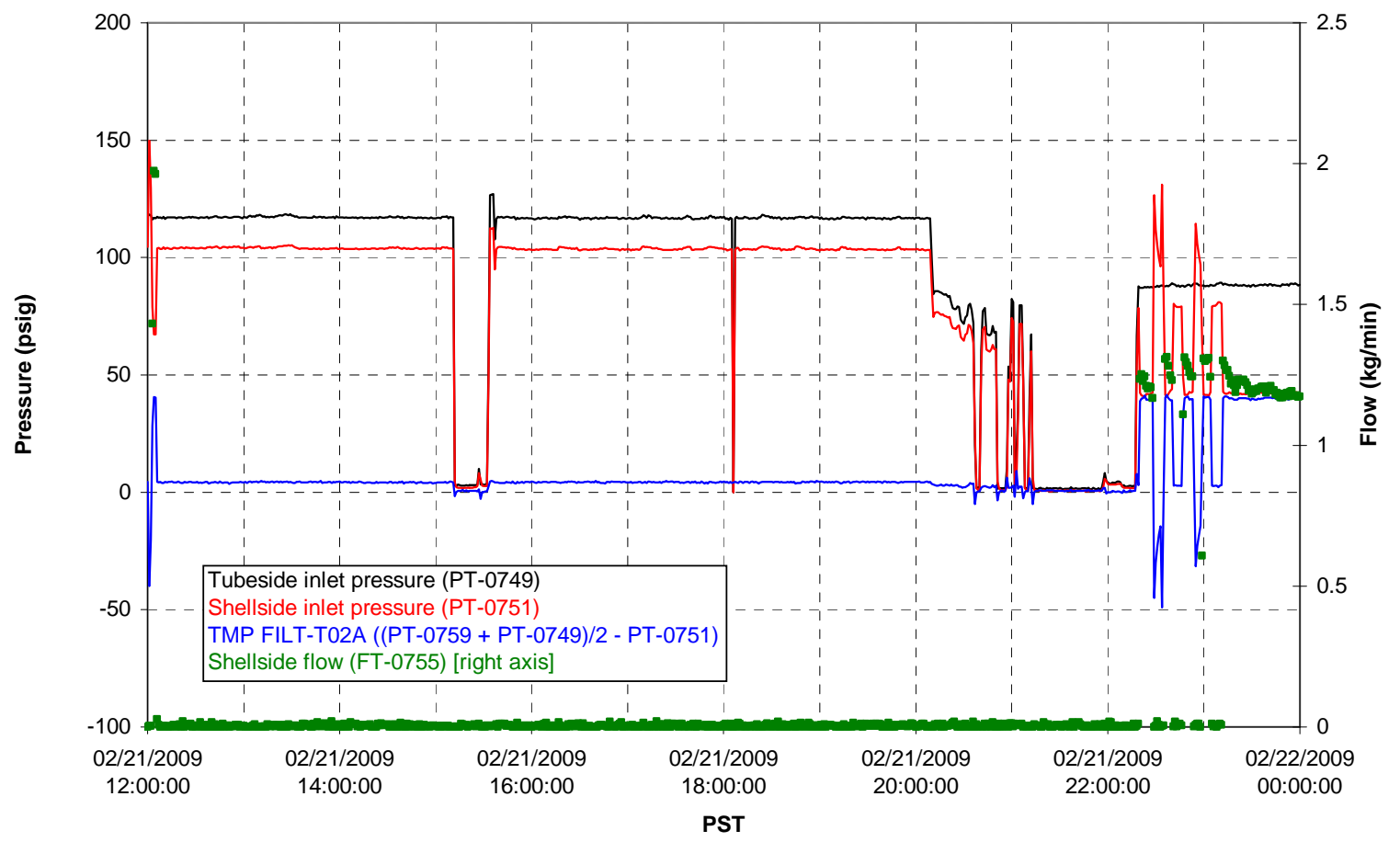

G.787 
Filter UFP-FILT-T03A

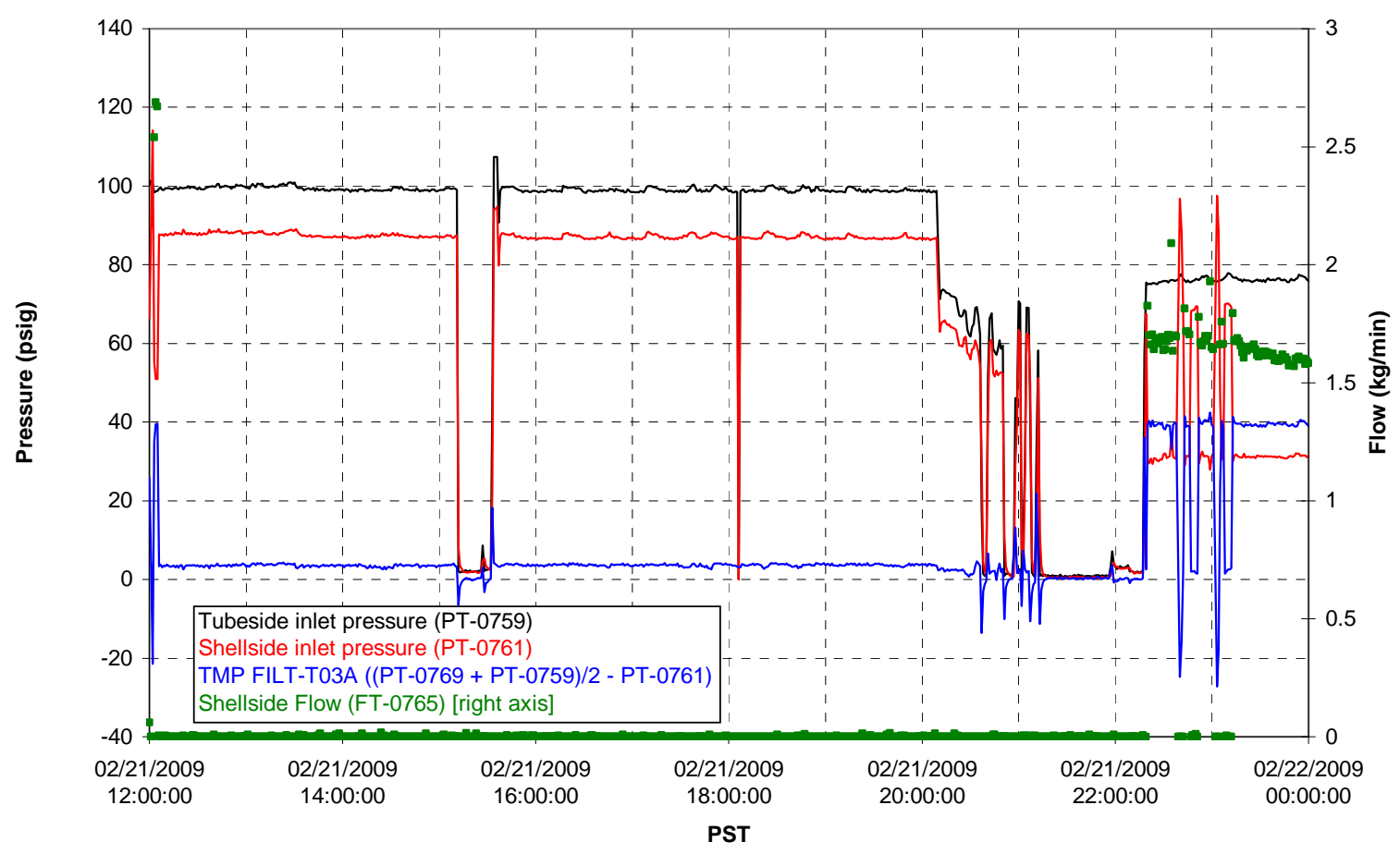

Filter UFP-FILT-T04A

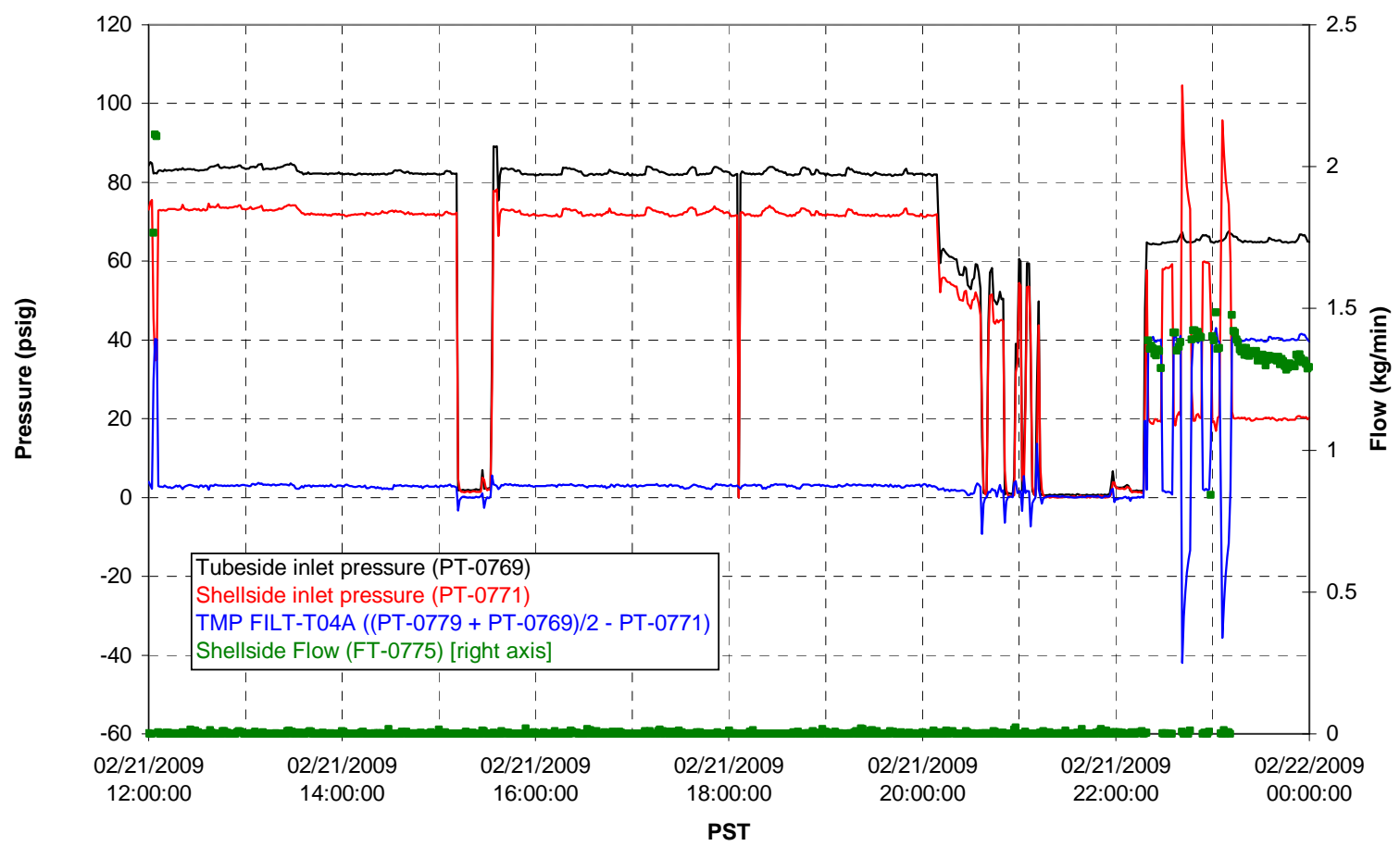


Filter UFP-FILT-T05A

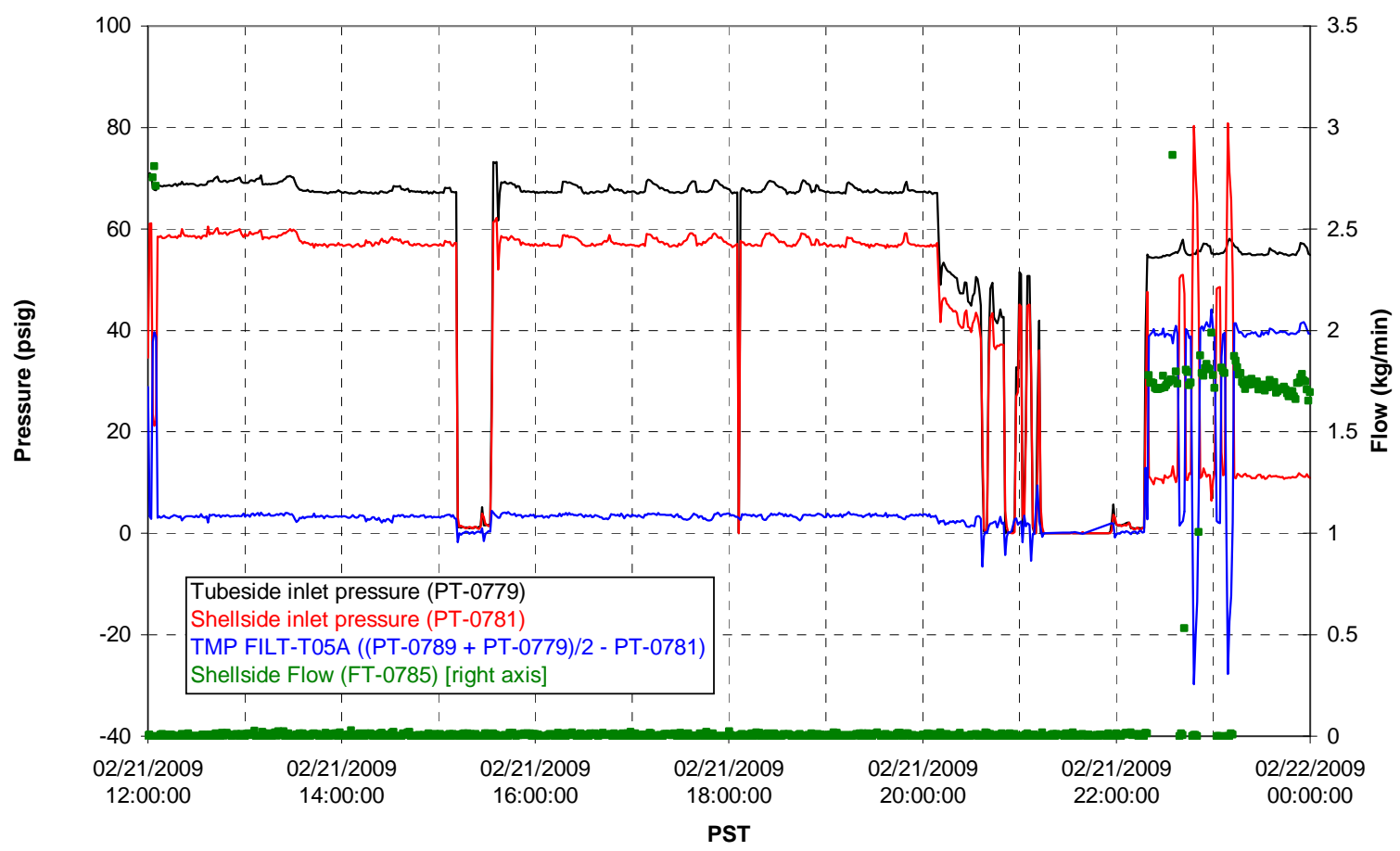

Chemical Flow

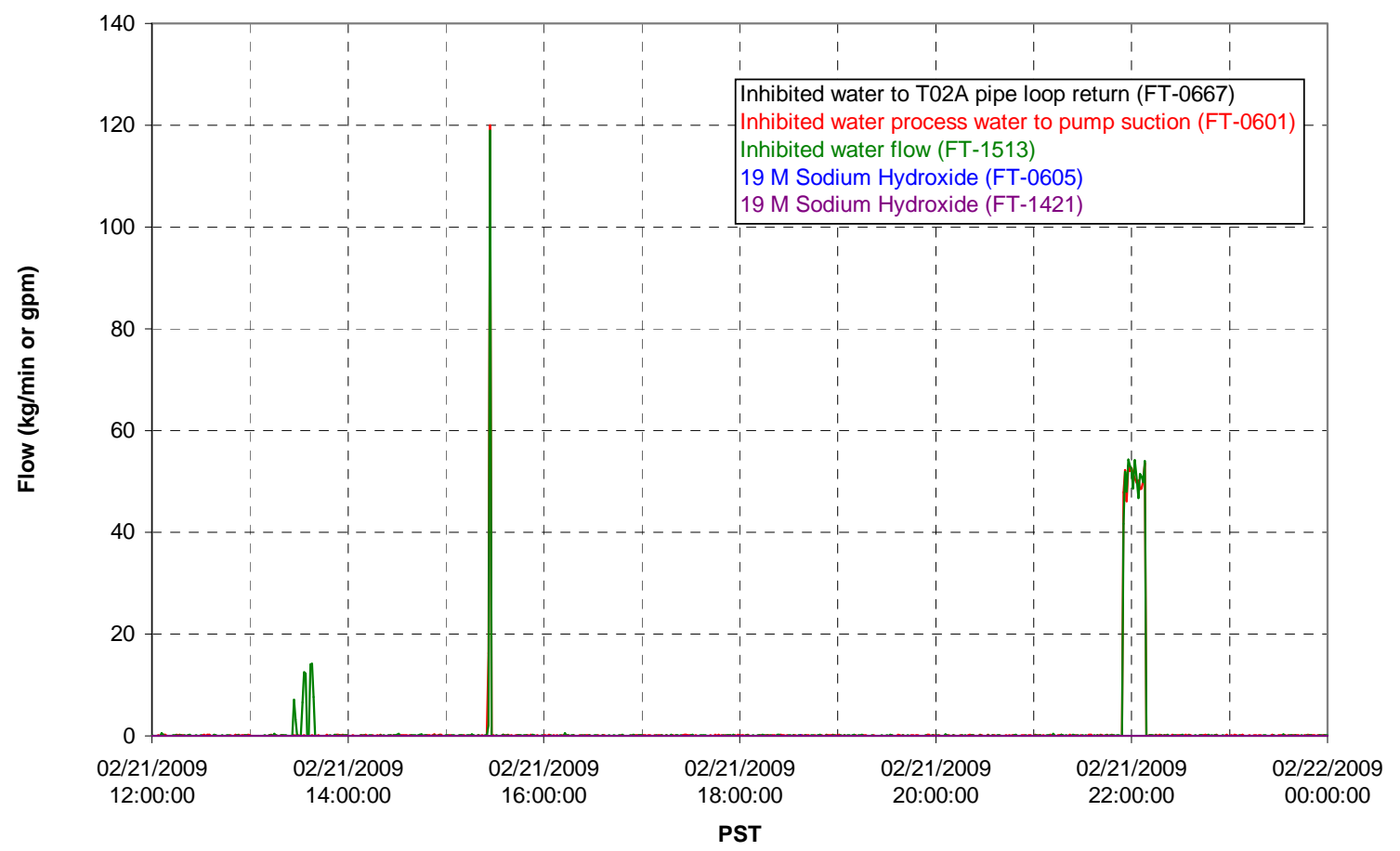




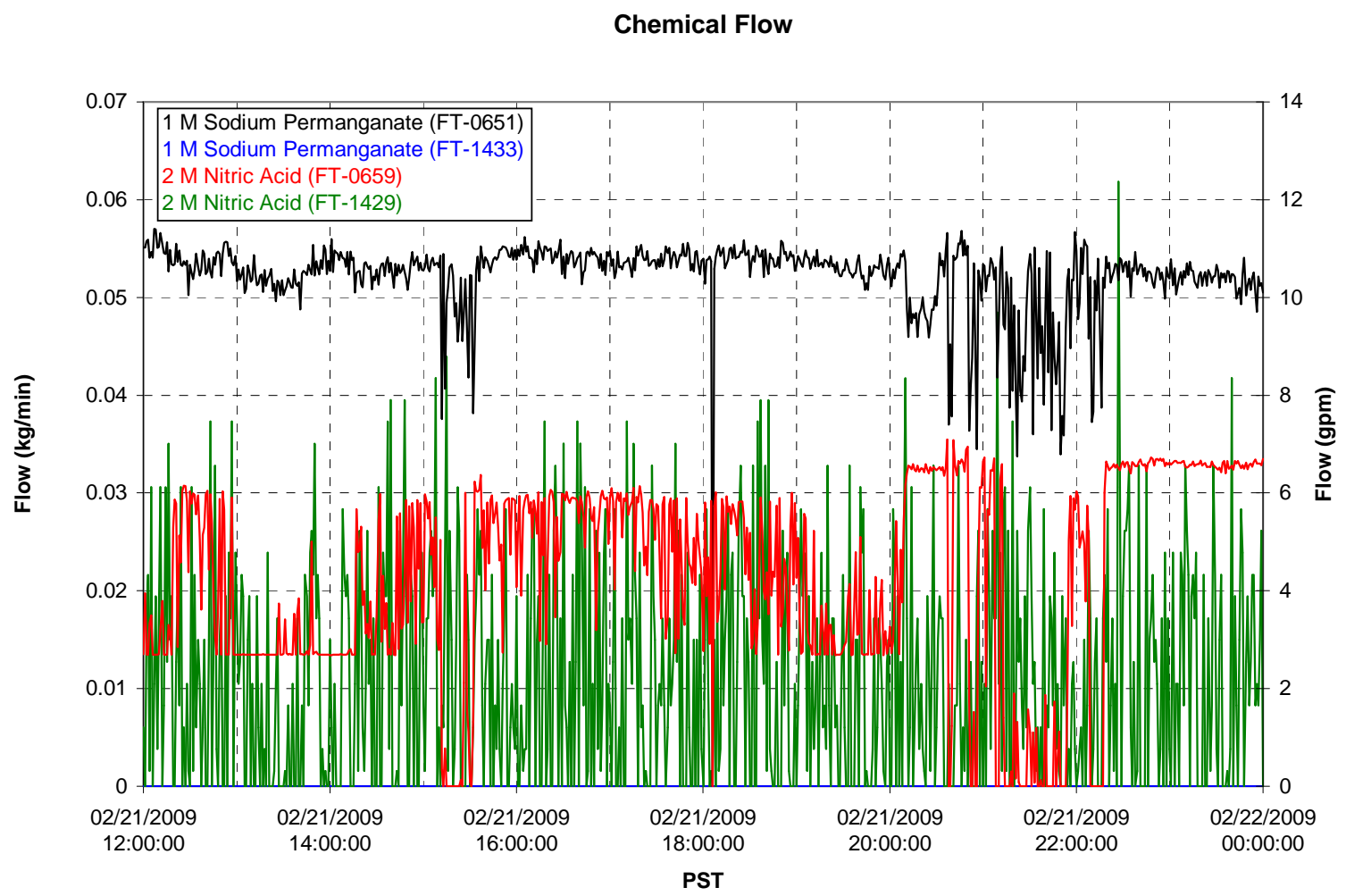

Air Flows

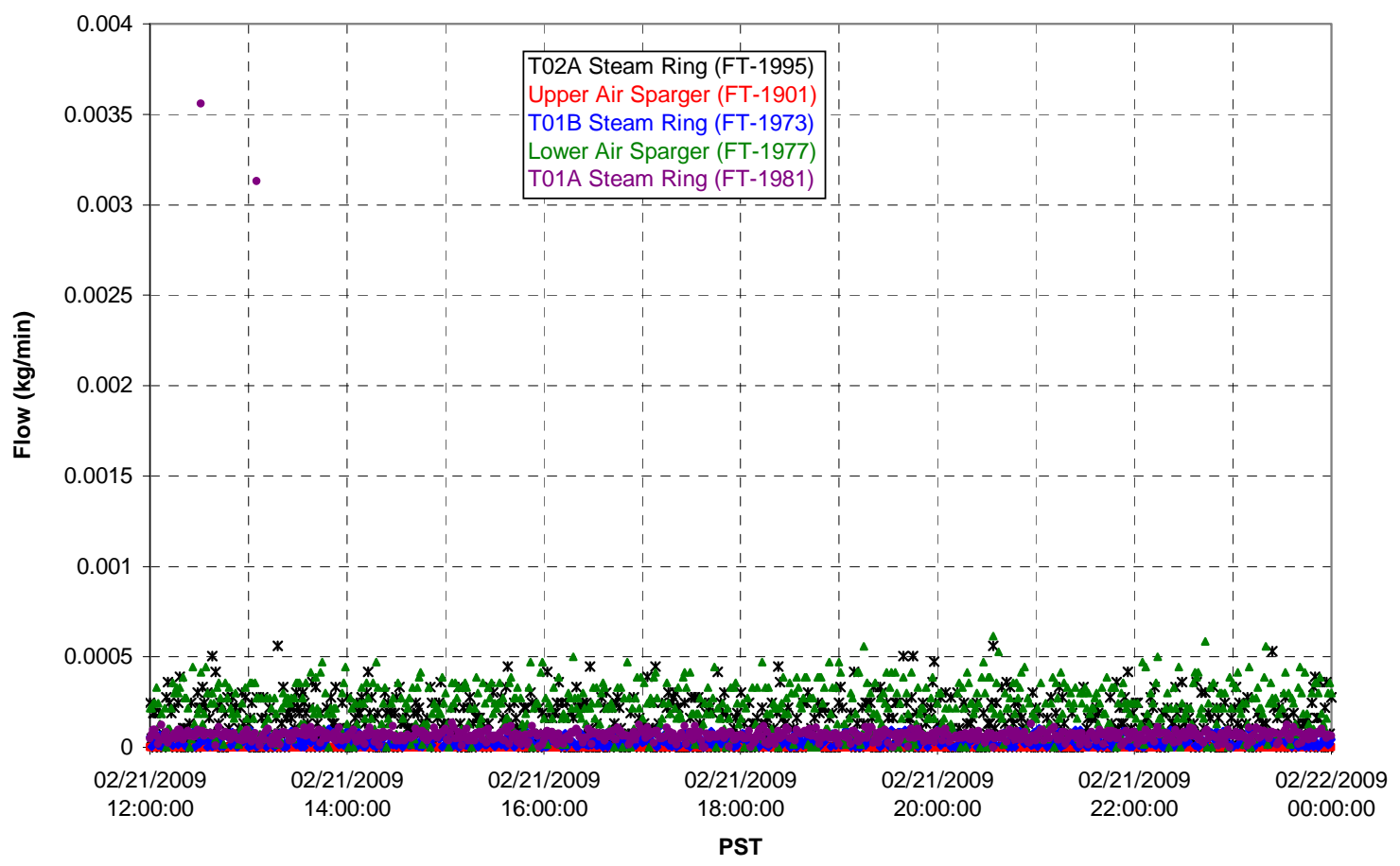


T02A Steam

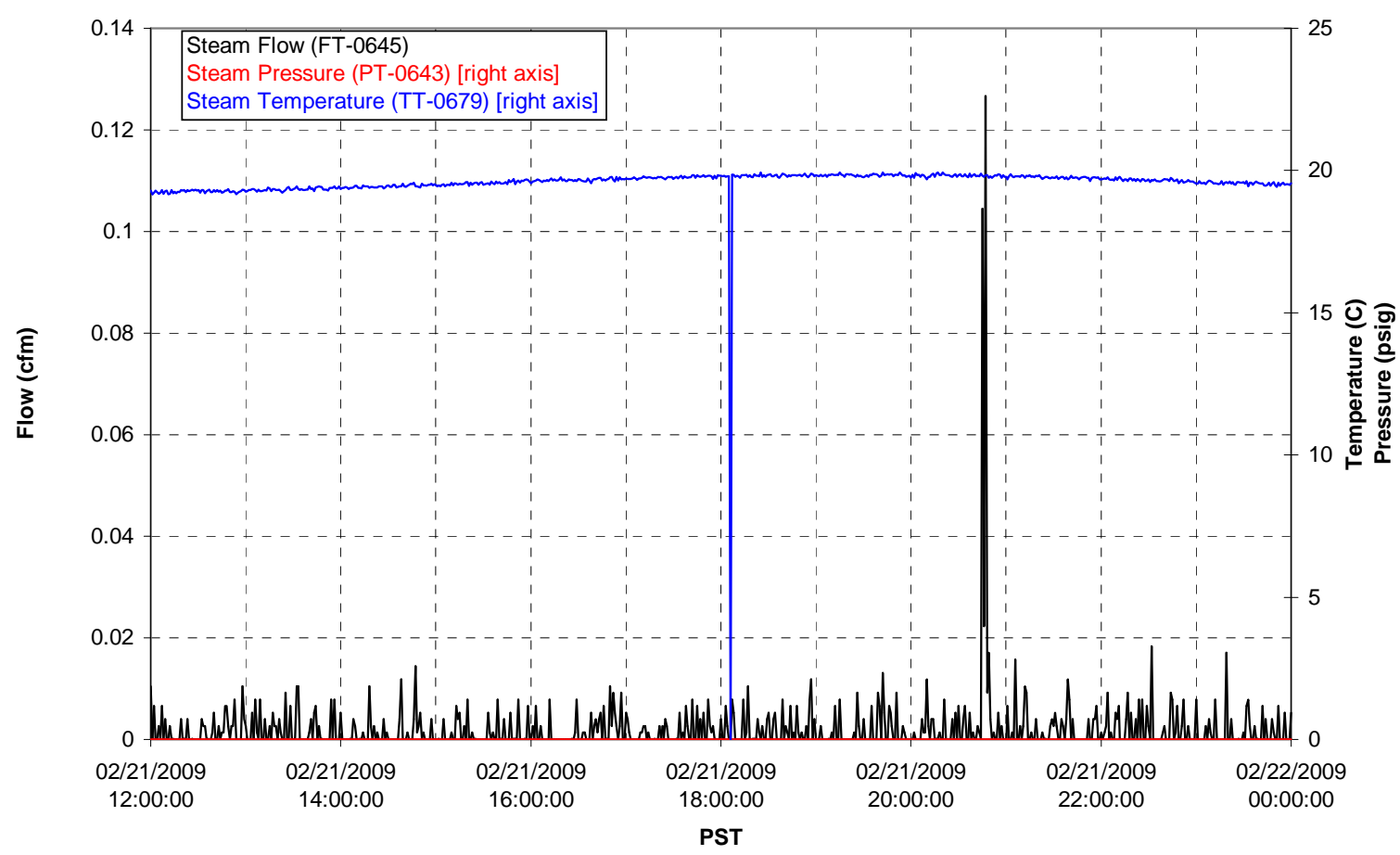

T01A Steam

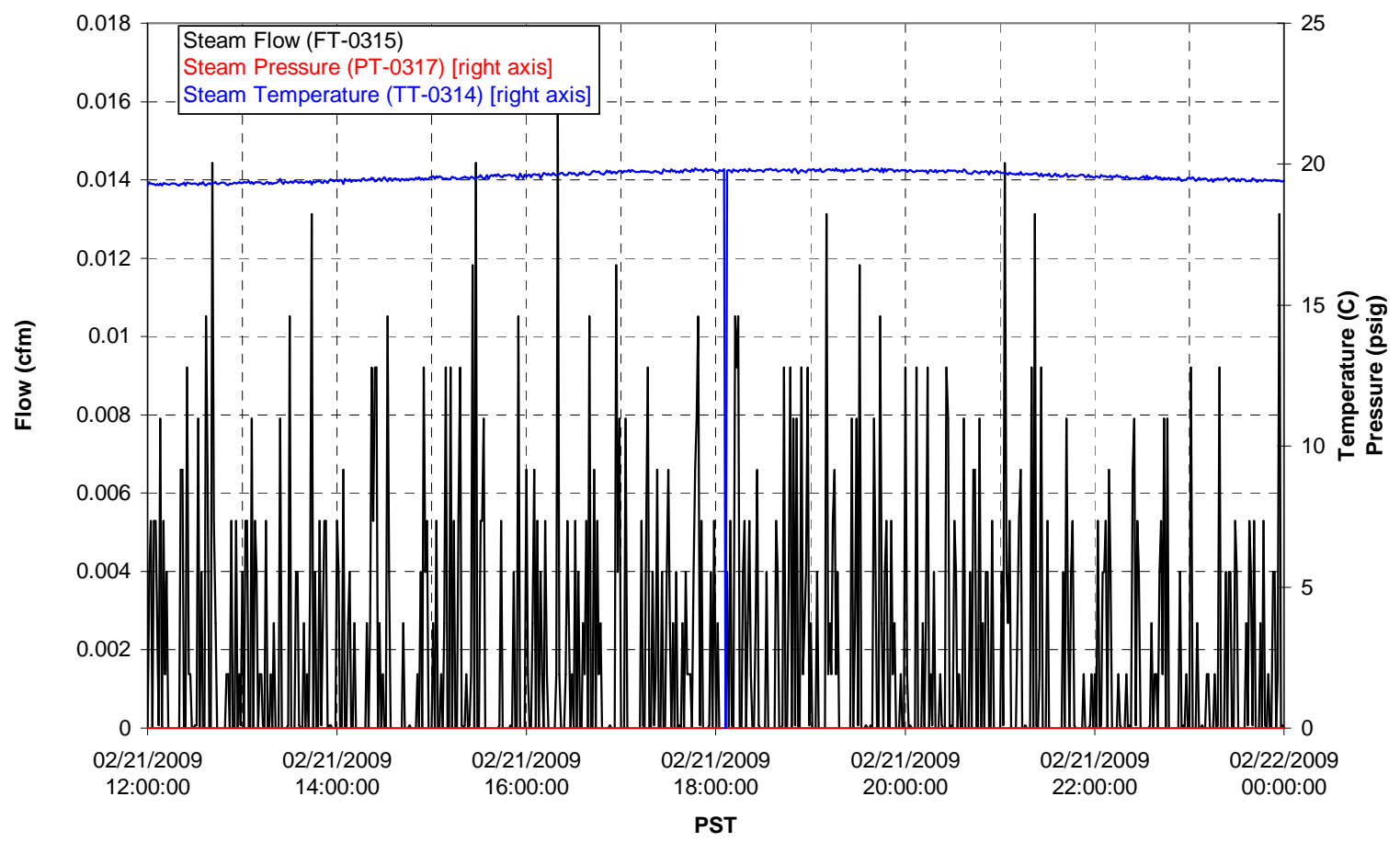


T01B Steam

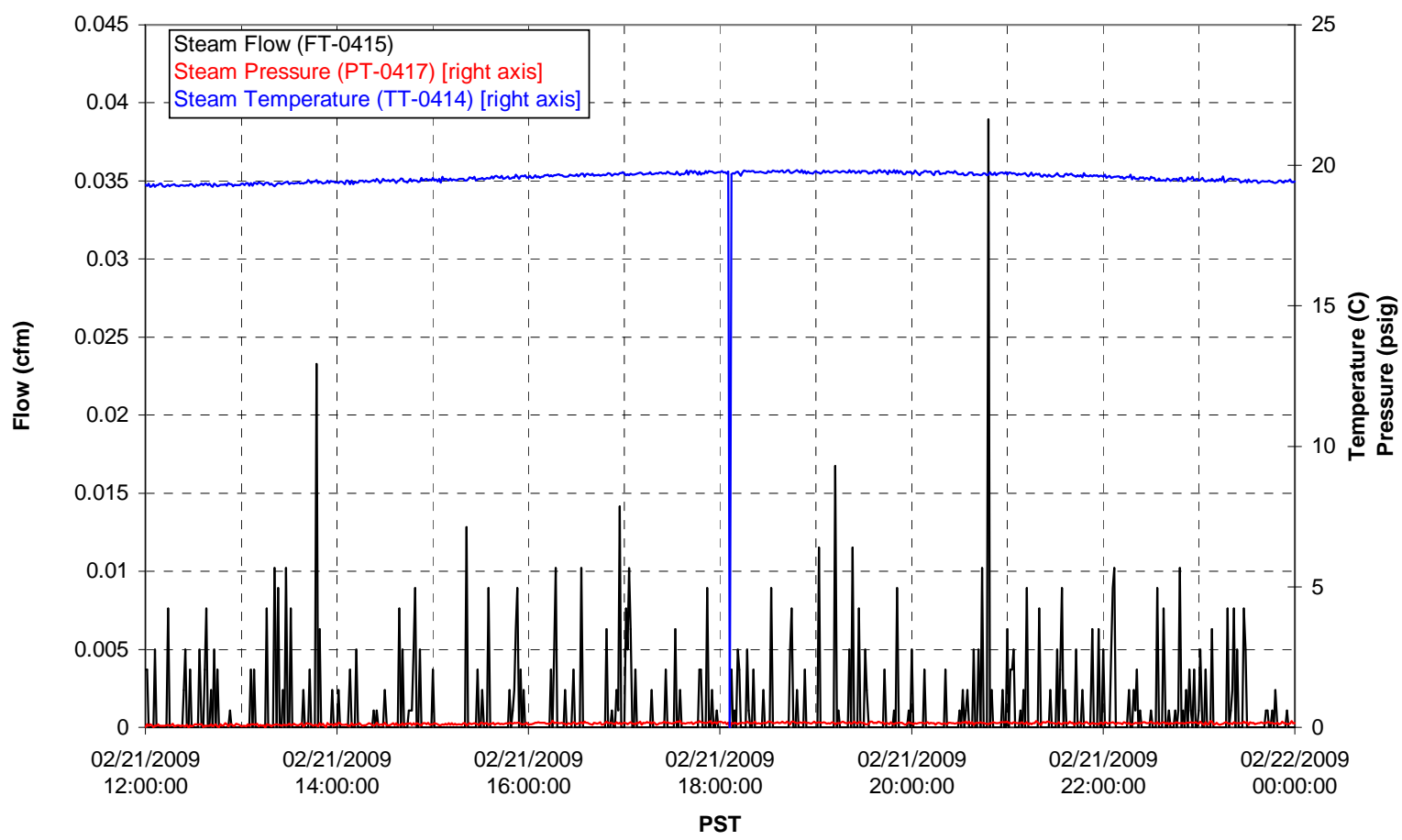




\section{Integrated Test A Data Plots 03/20/09 00:00 - 03/20/09 12:00}


T01A level

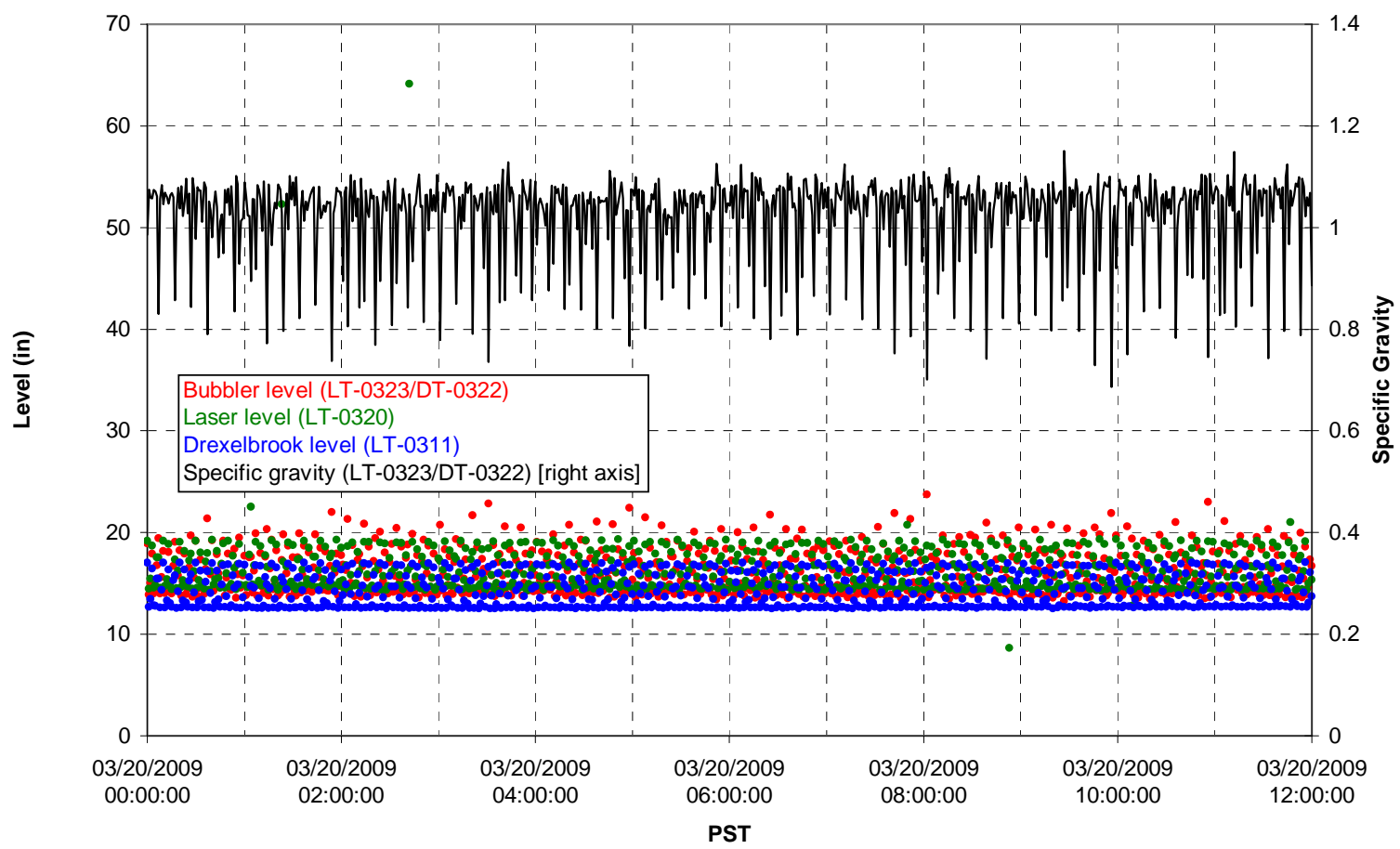

T01A temperatures

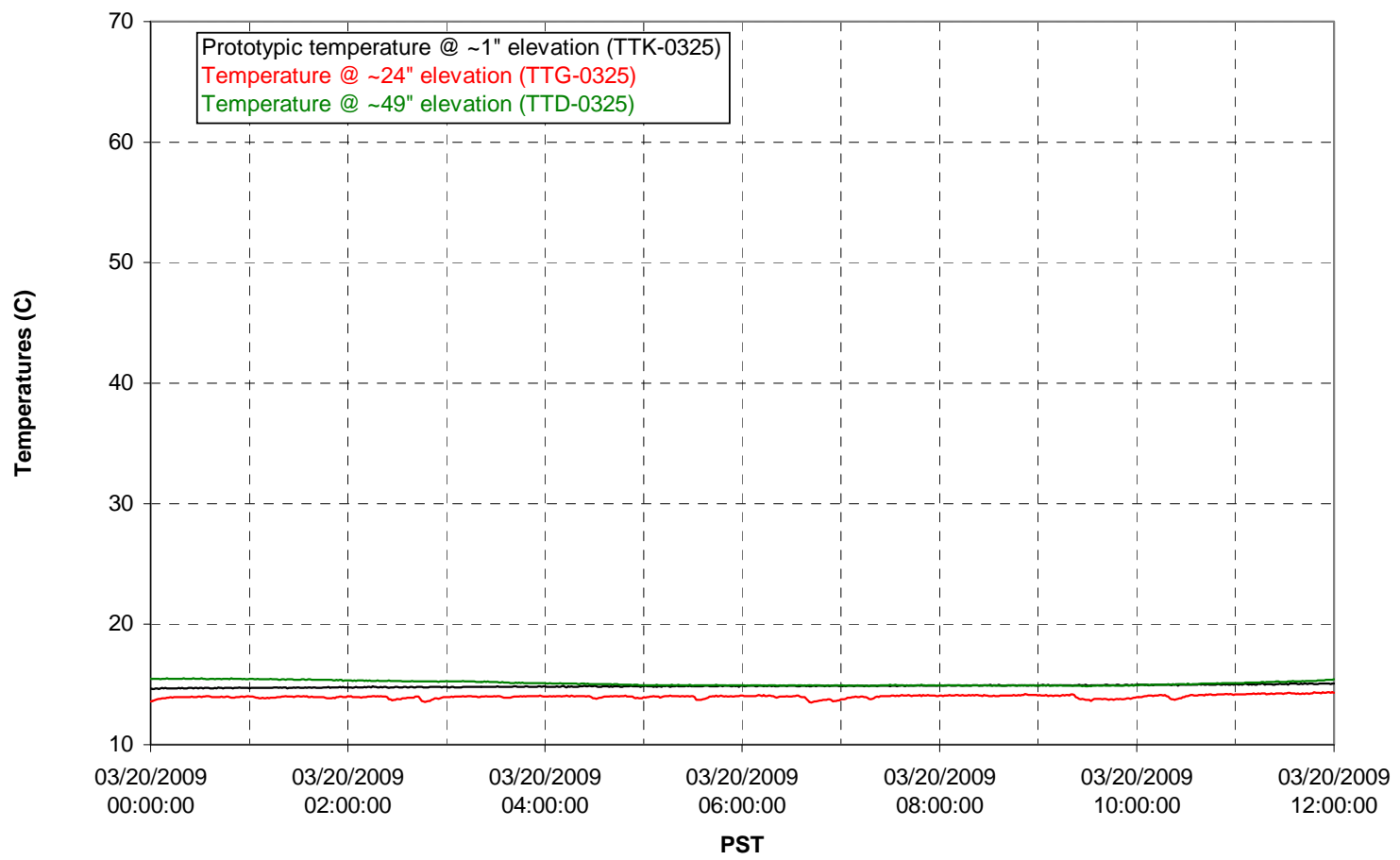


T01B level

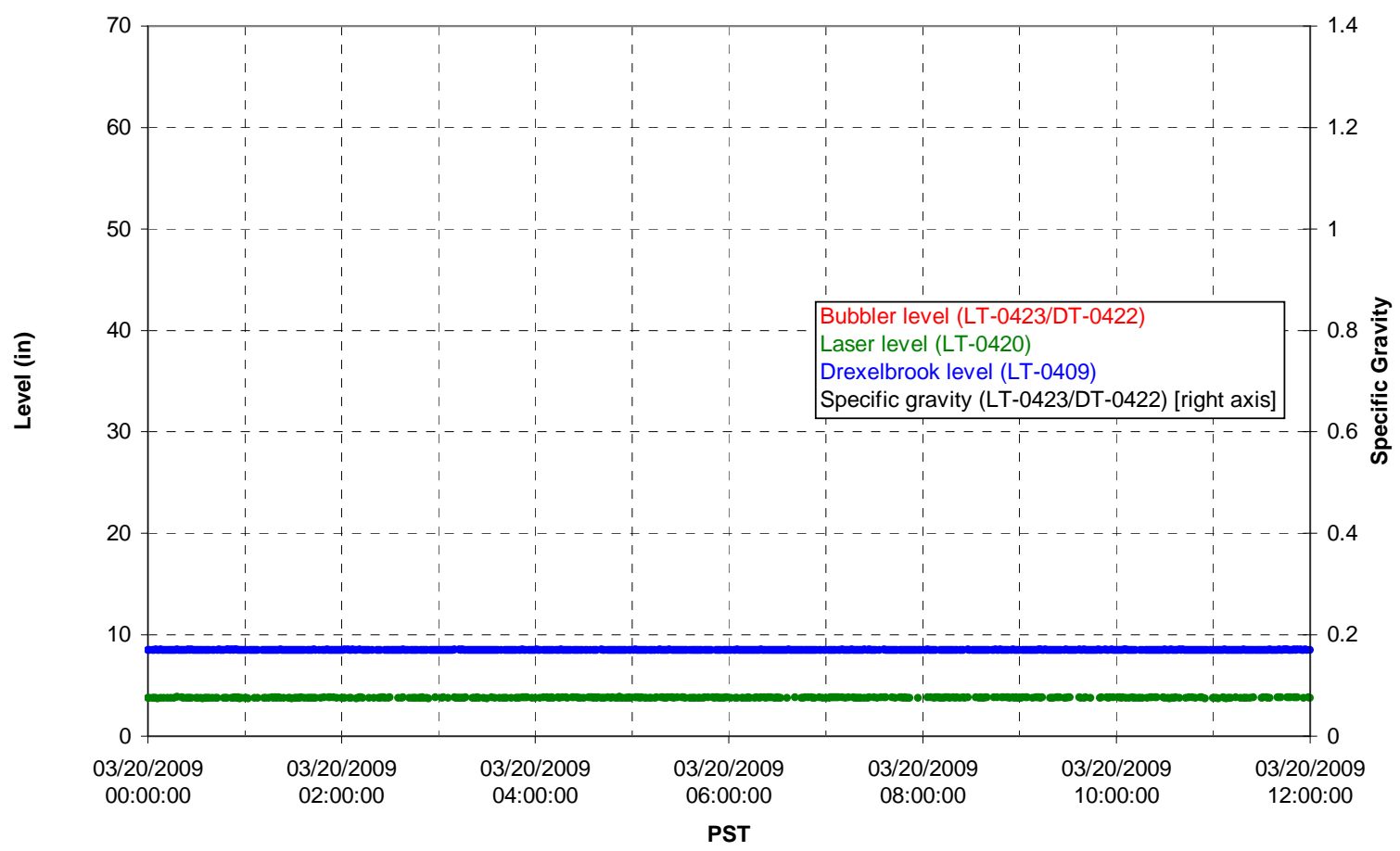

T01B temperatures

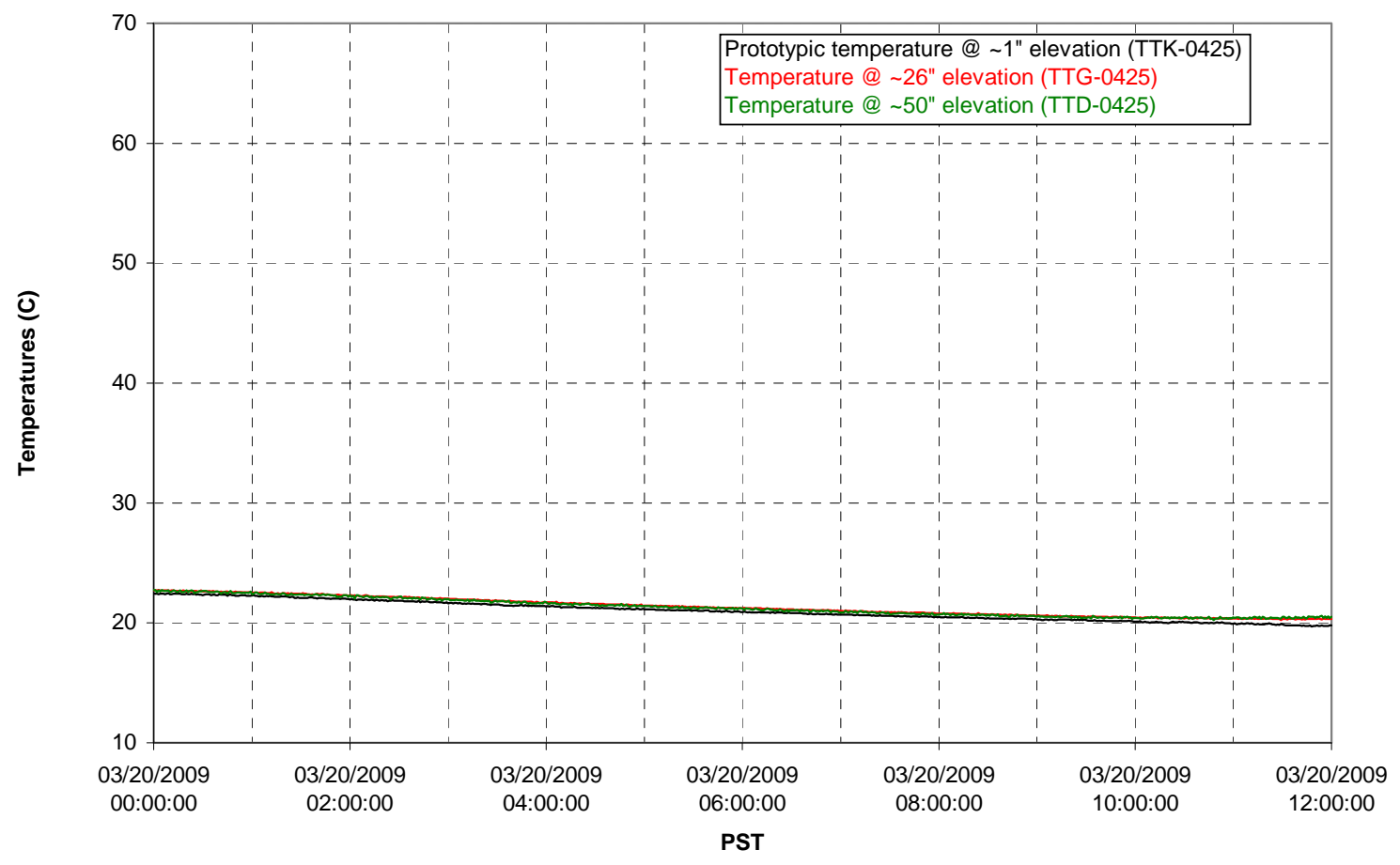

G.795 
T02A level

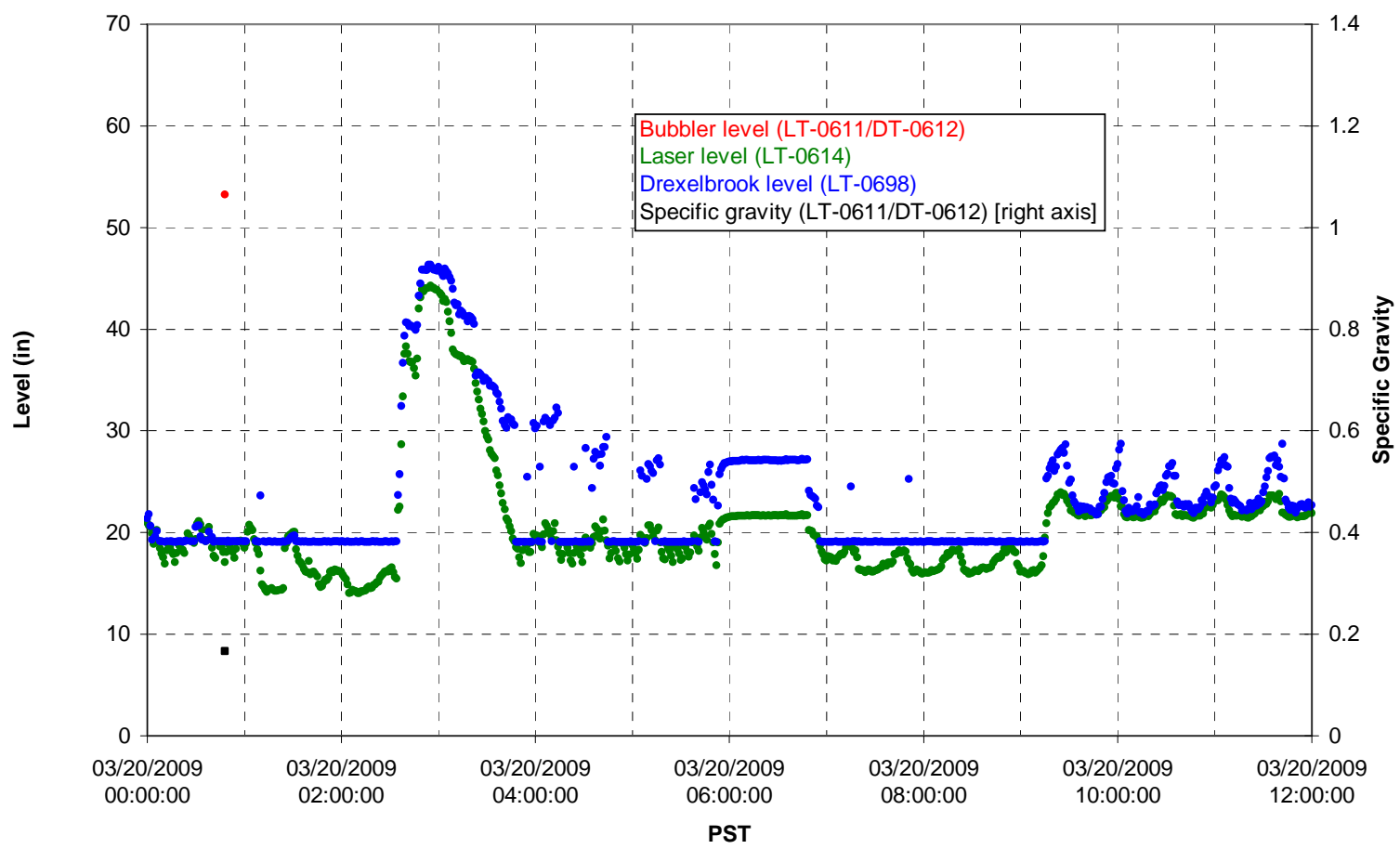

T02A temperatures

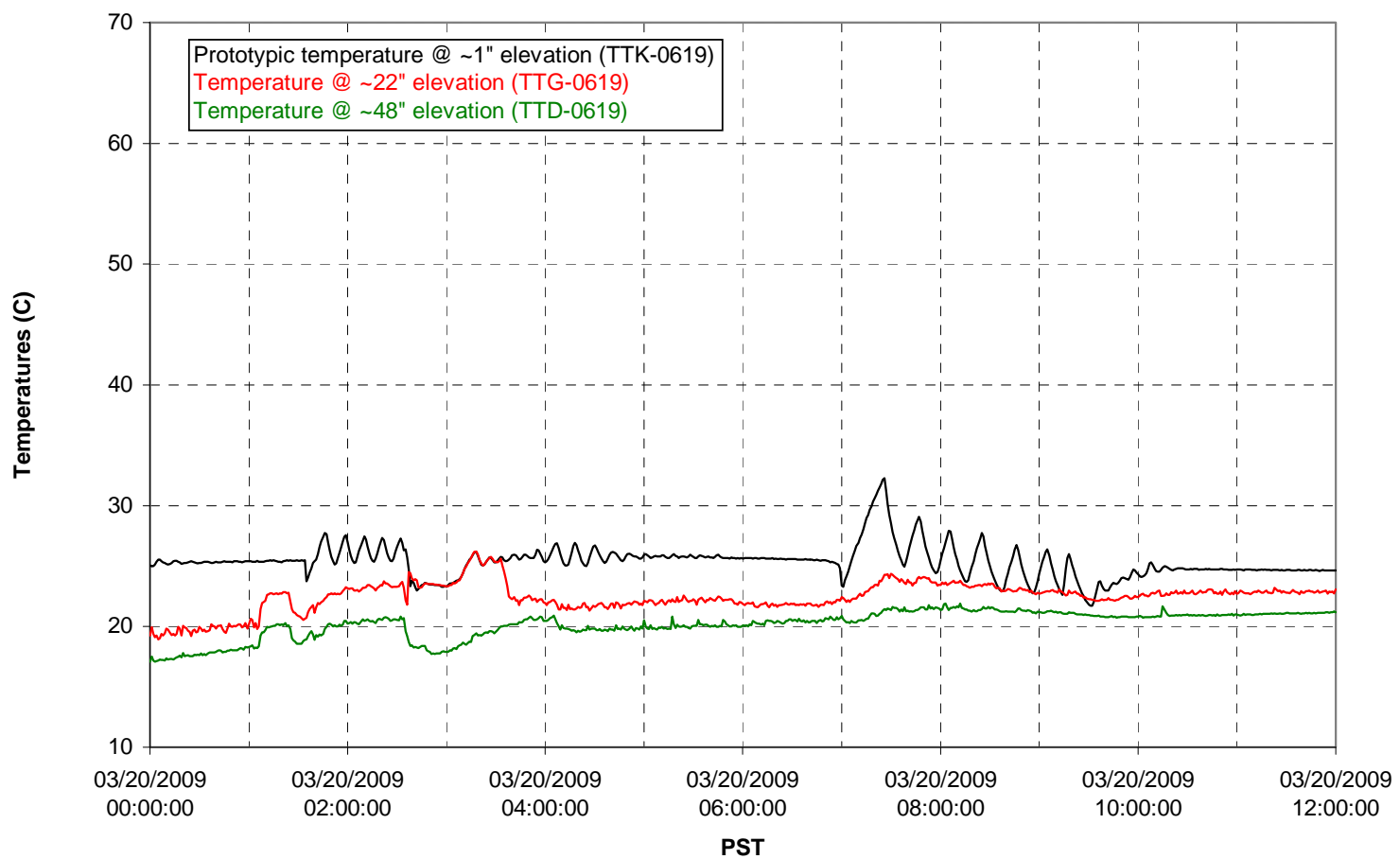


T02A and filter loop temperatures

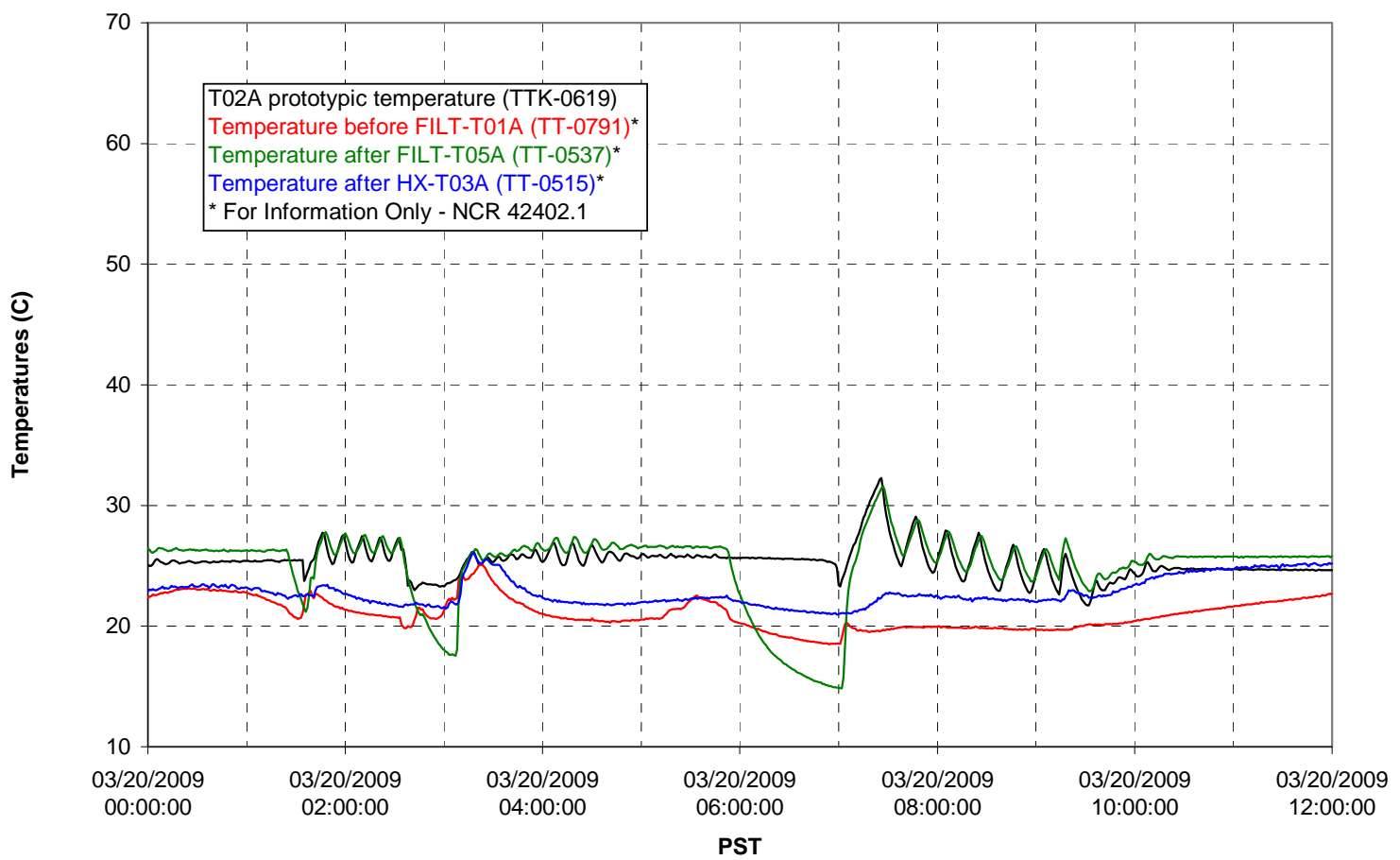

Pump Pressures and Flow

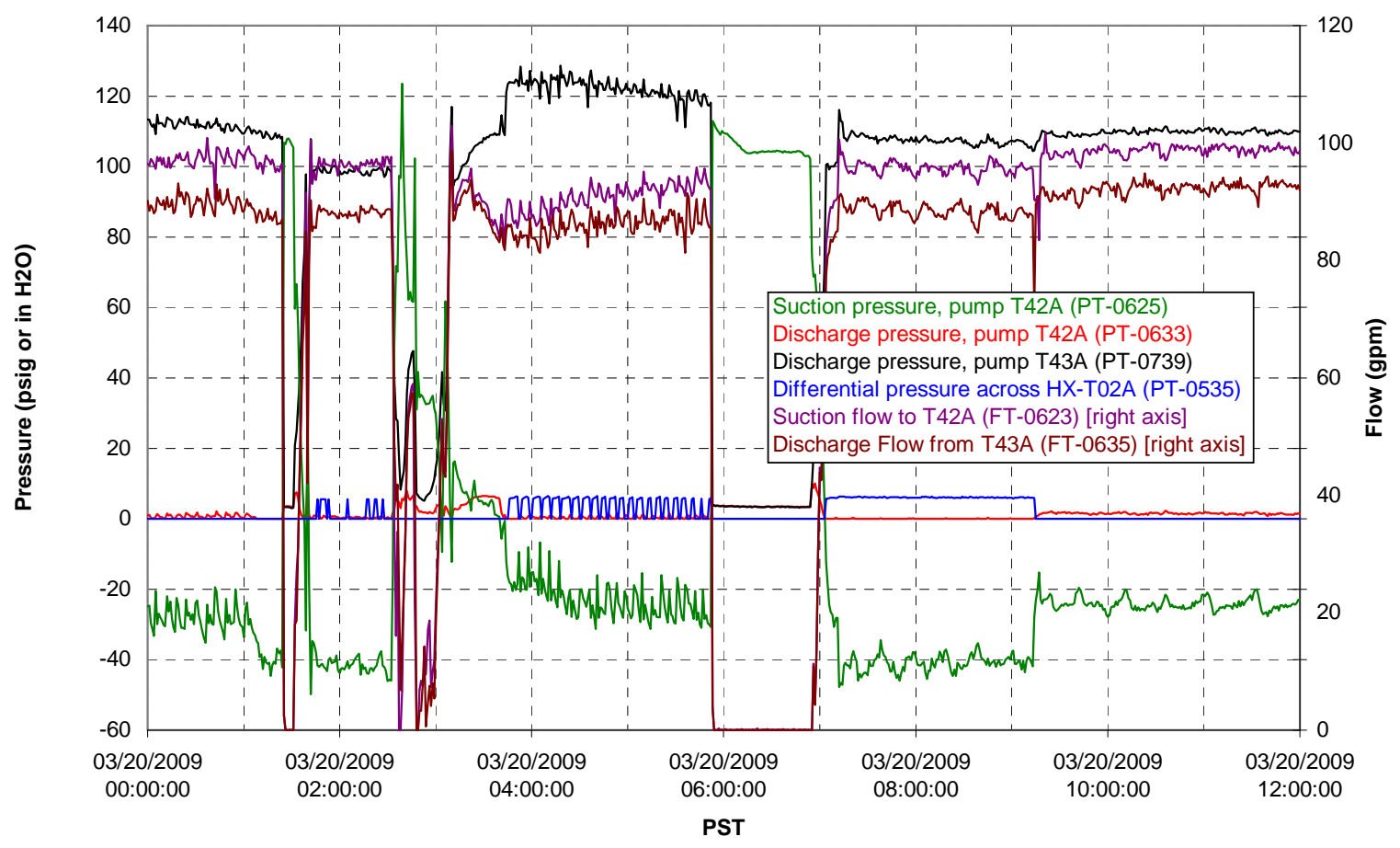

G.797 
Axial pressure drop

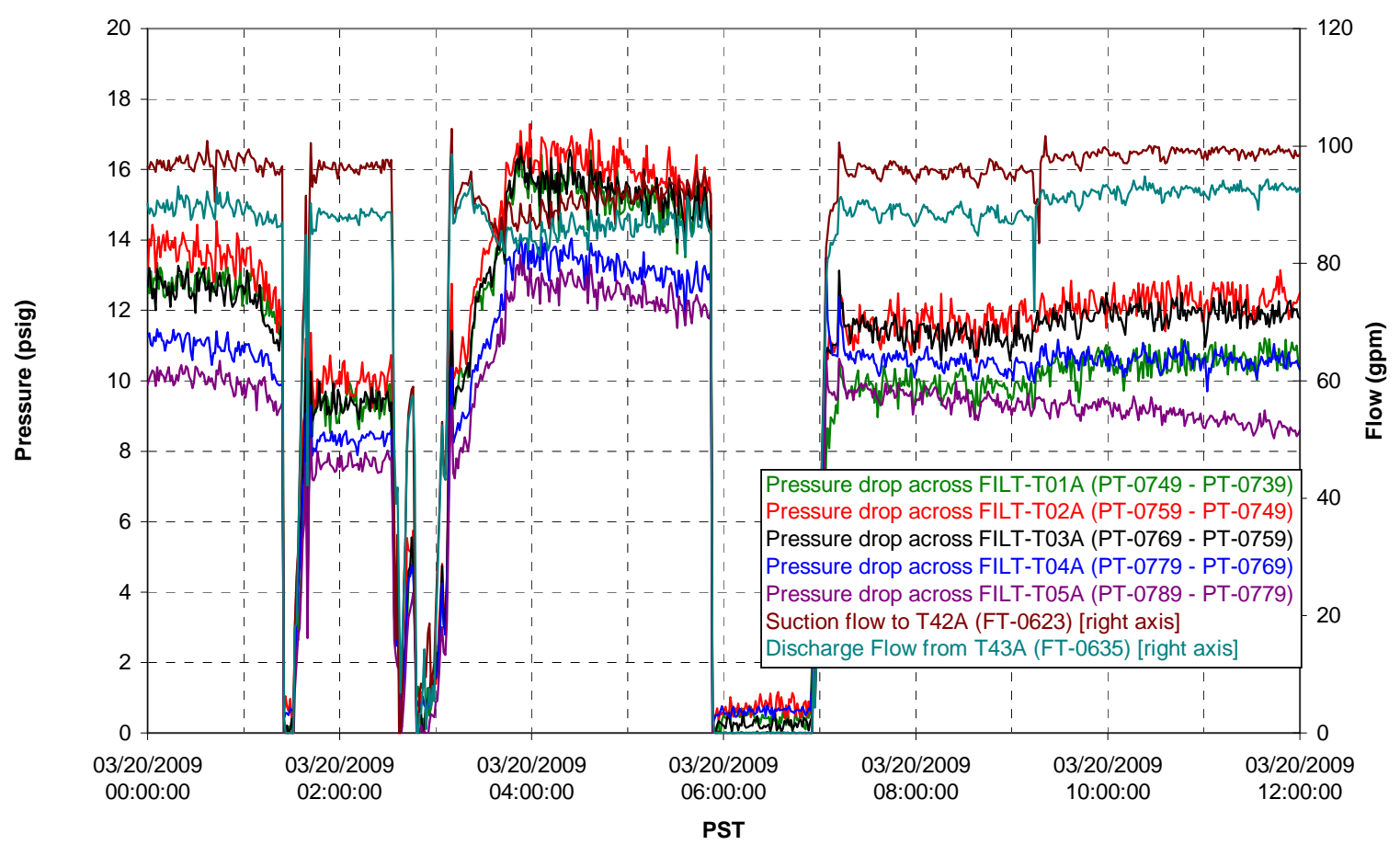

Permeate flow rates

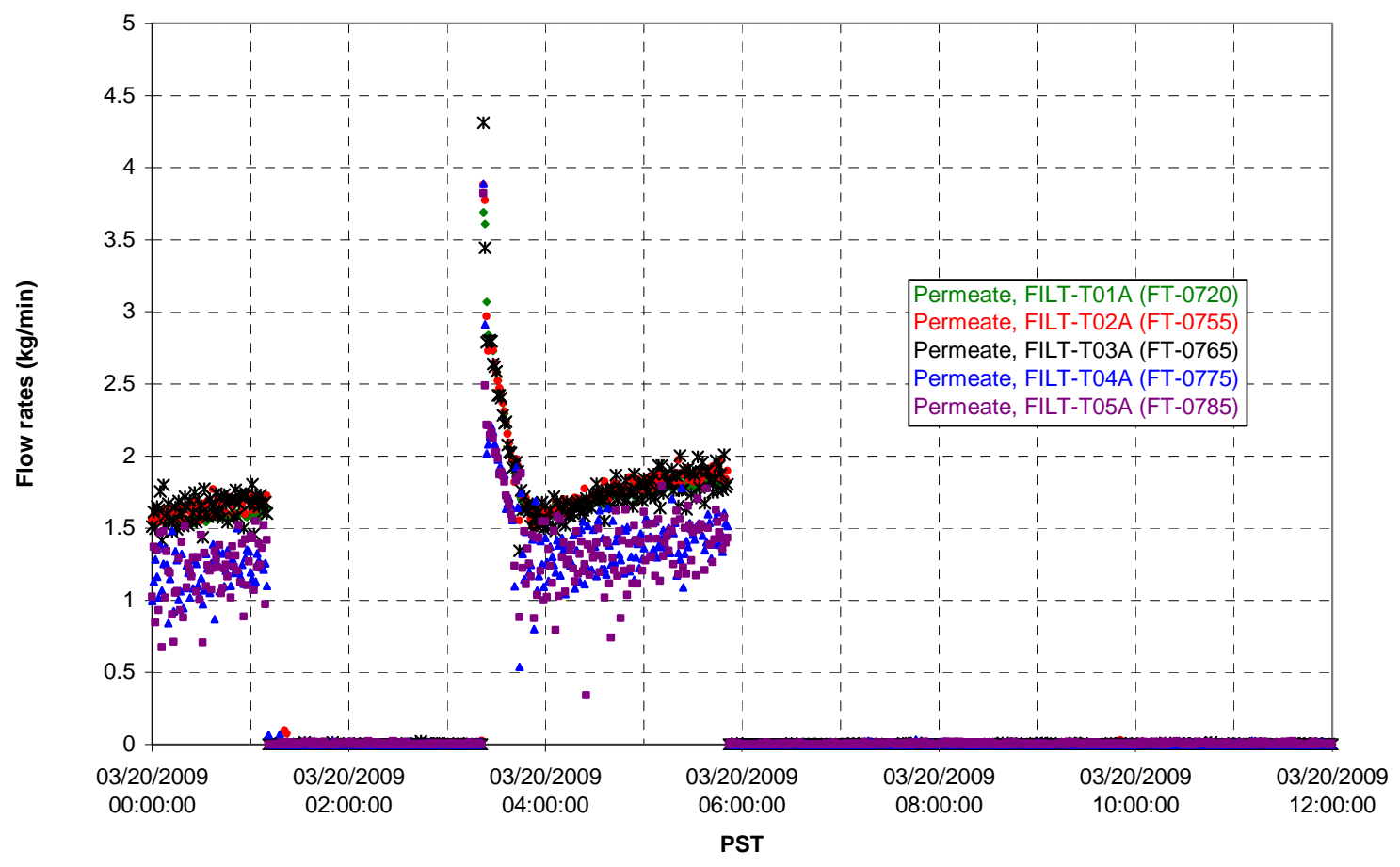


T02A Inner Temperature Tree

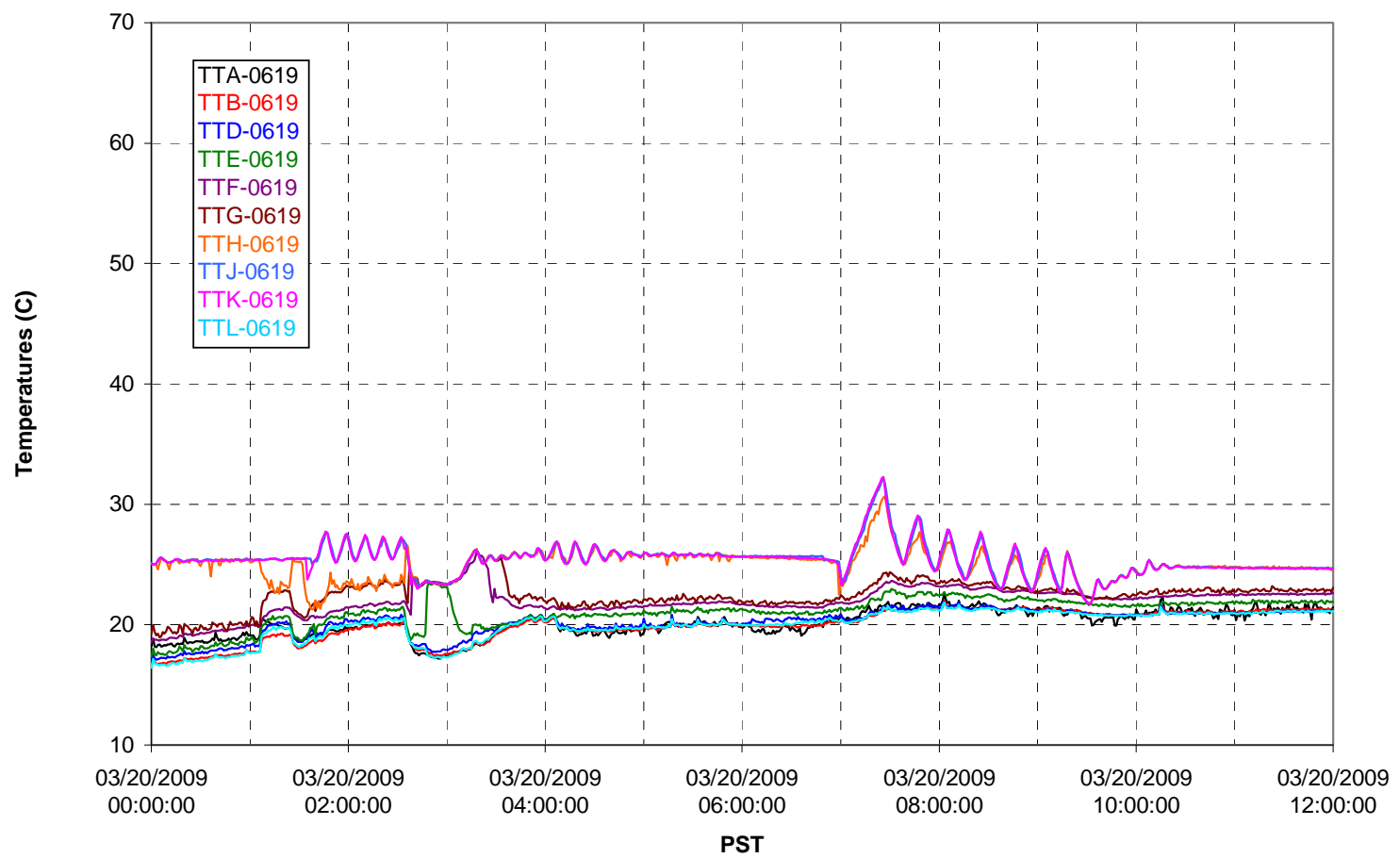

T02A Outer Temperature Tree

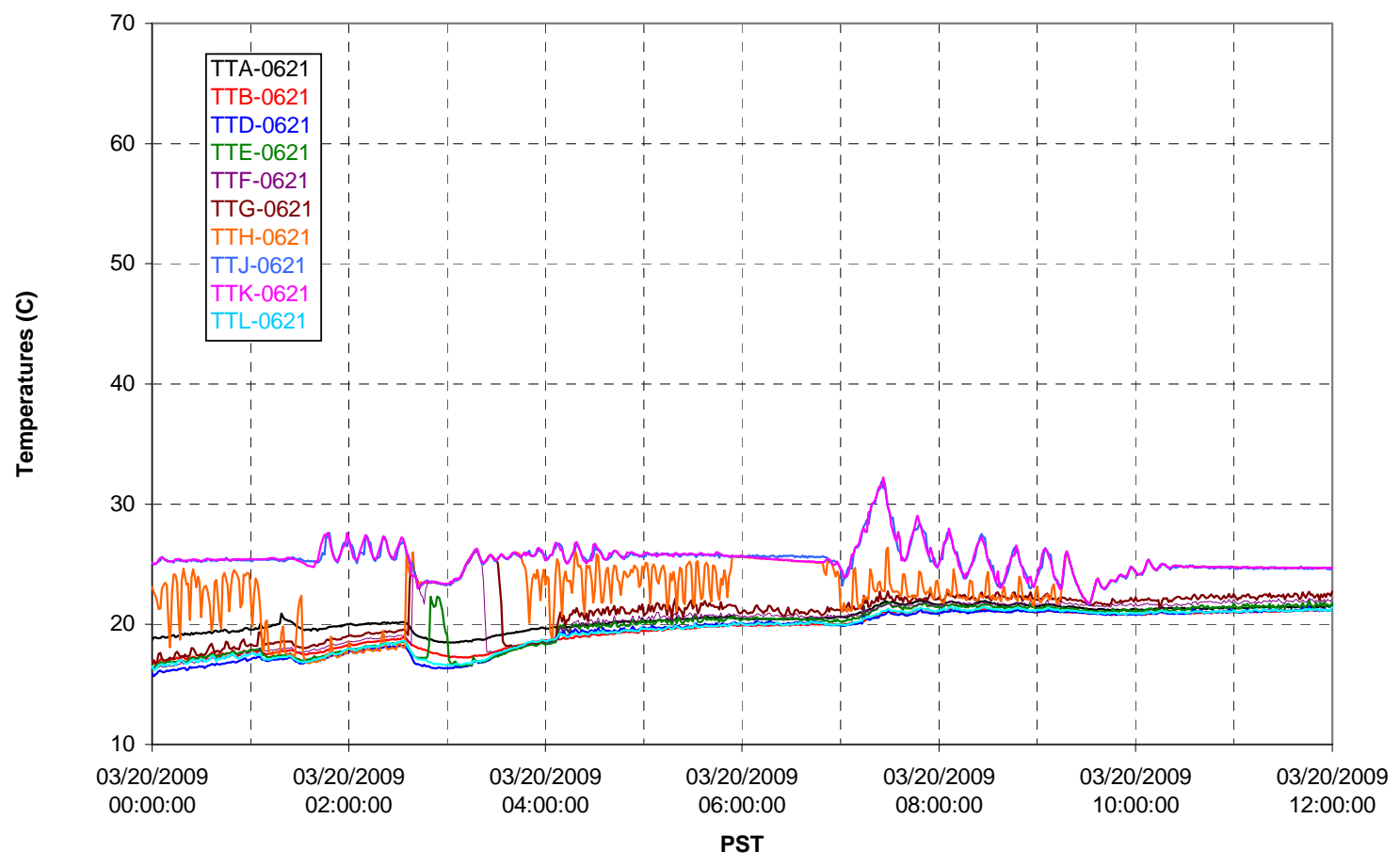


T02A temperatures

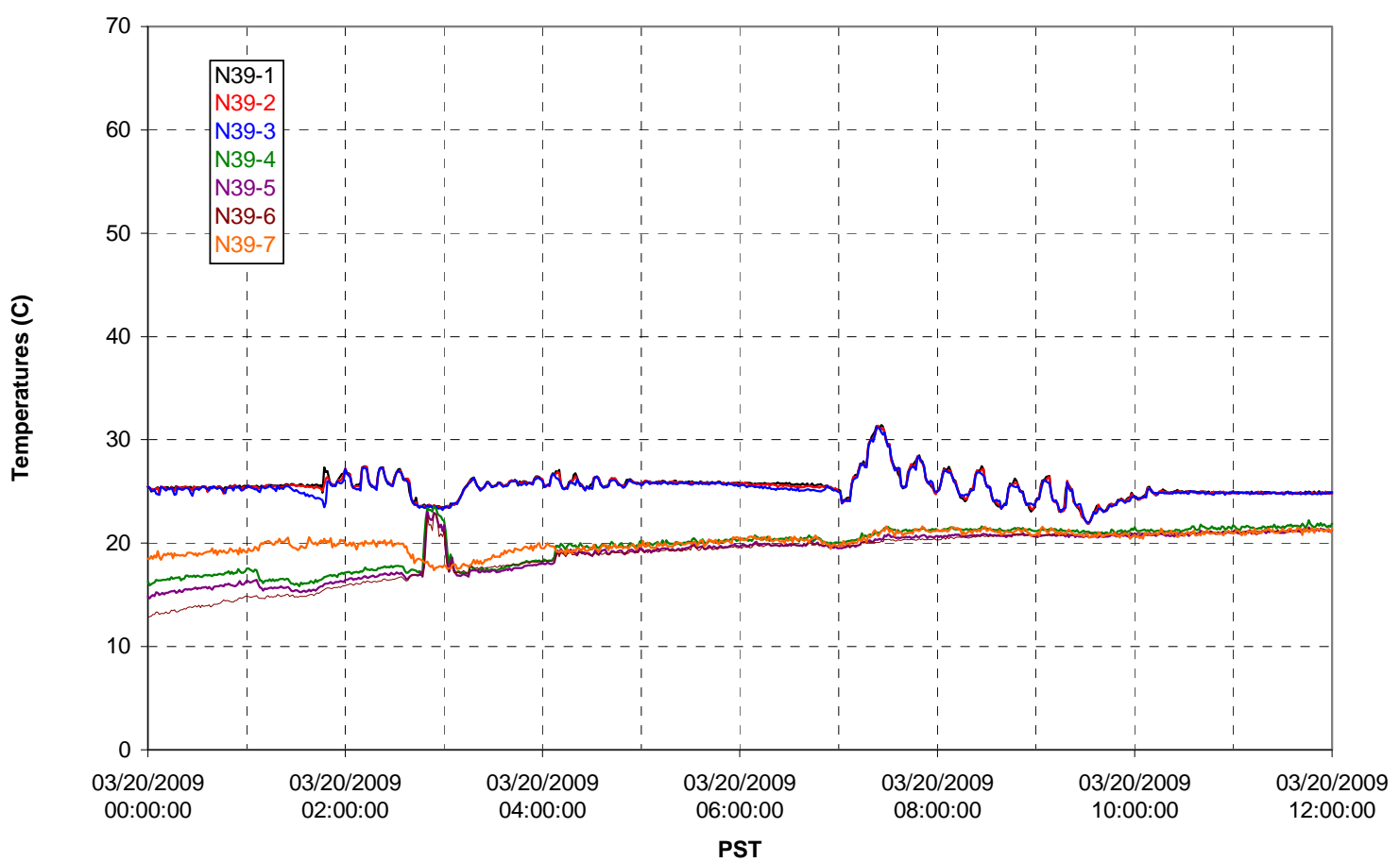

T02A temperatures

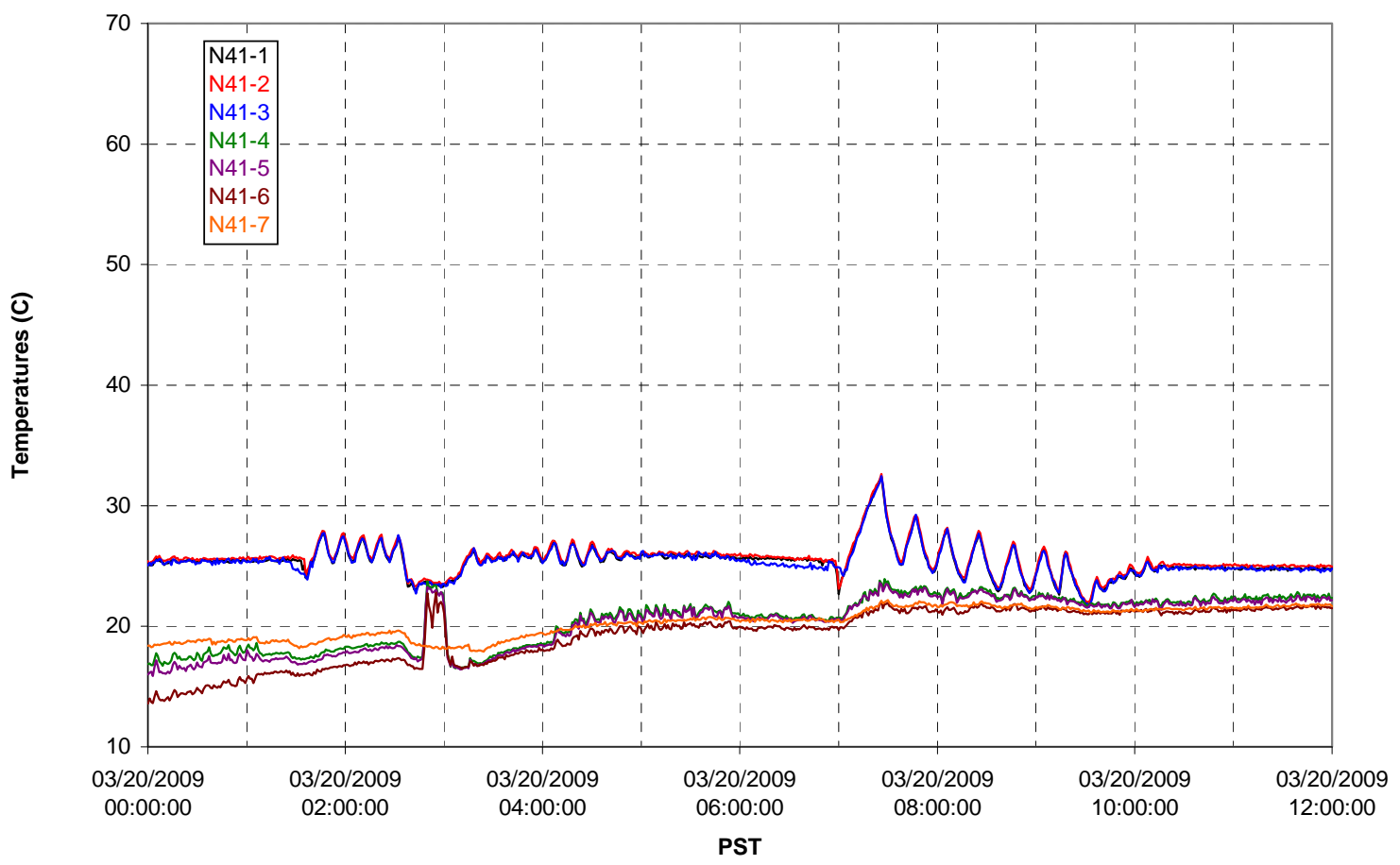


T02A temperatures

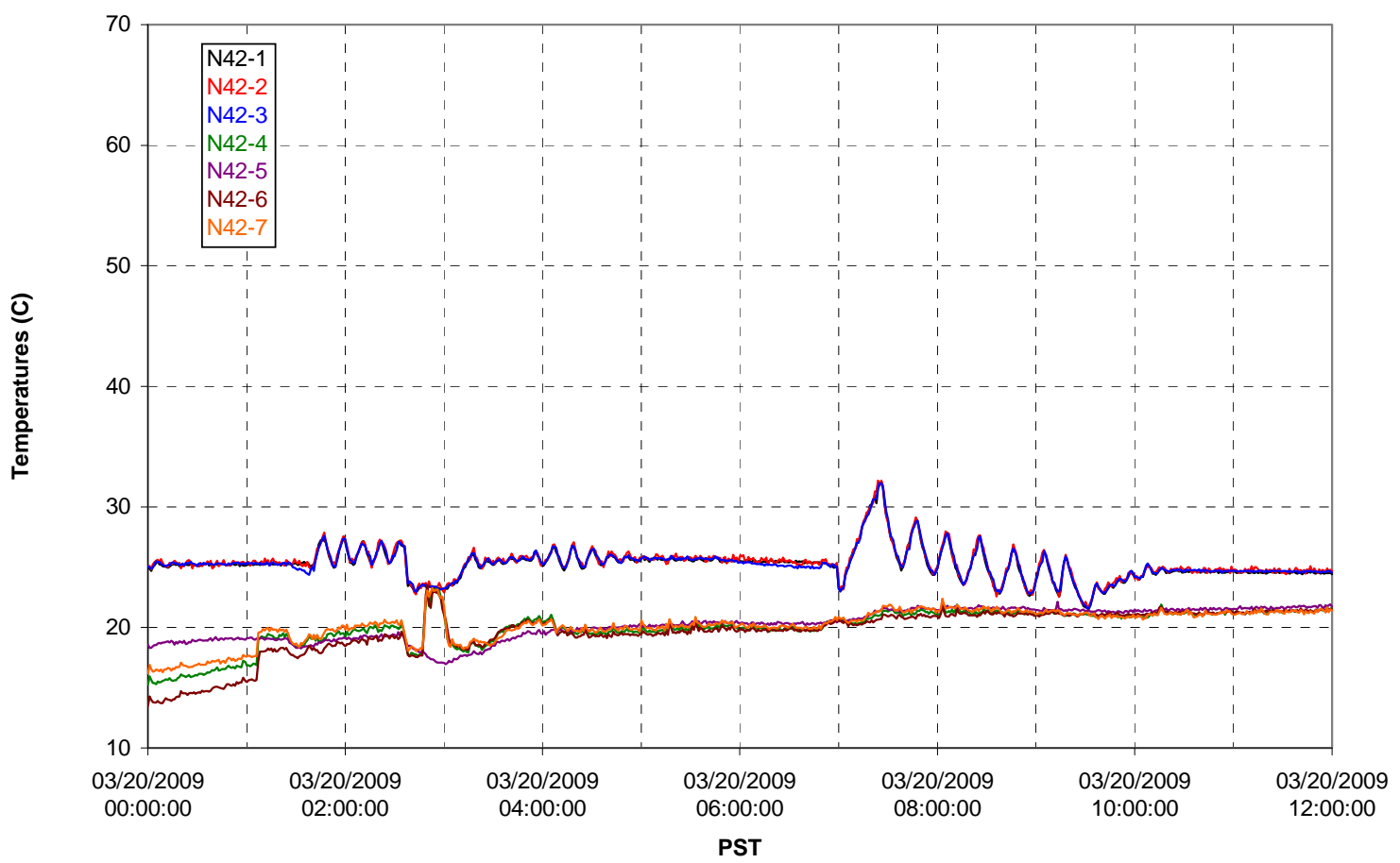

T02A temperatures

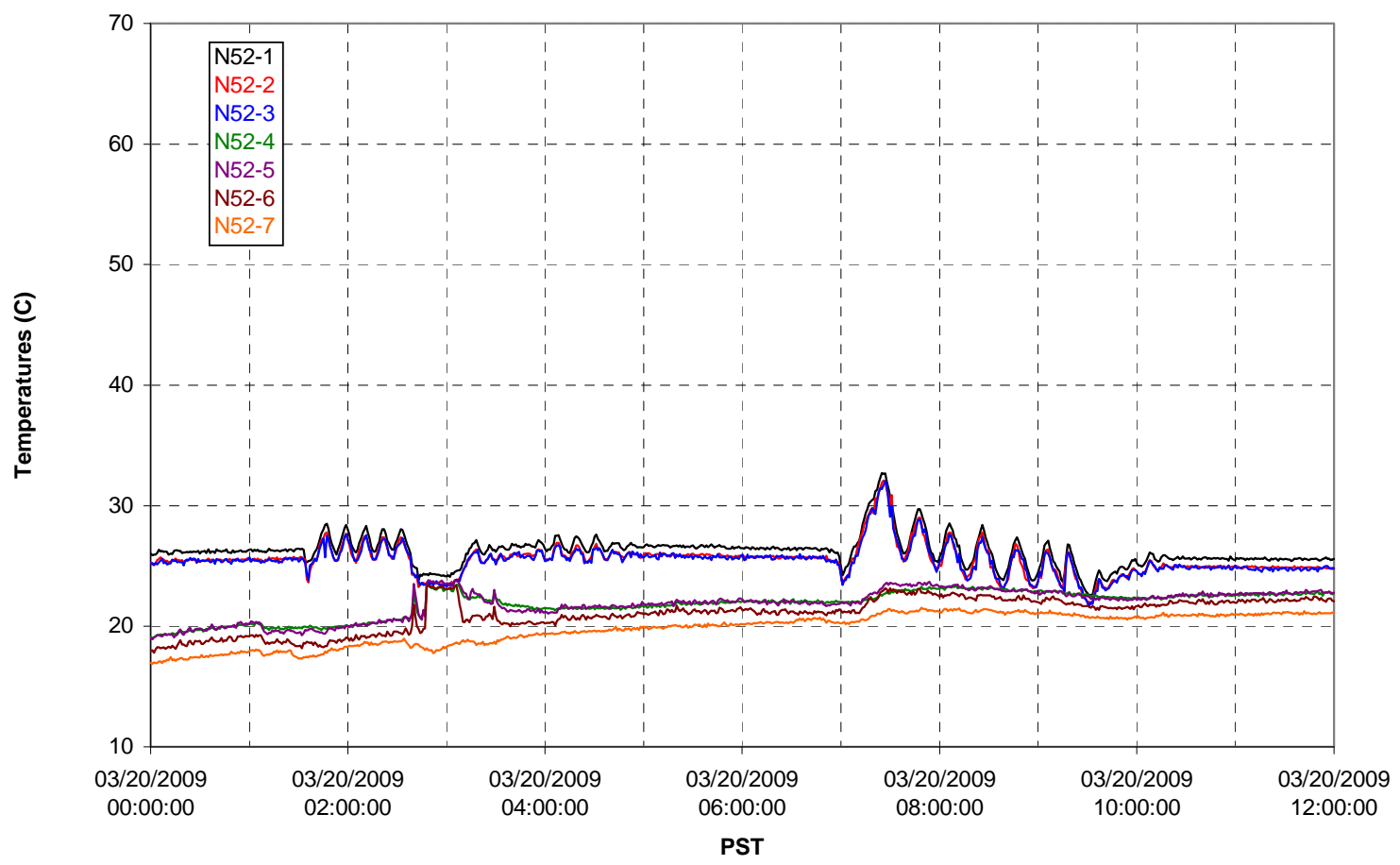


T02A Heating and Cooling

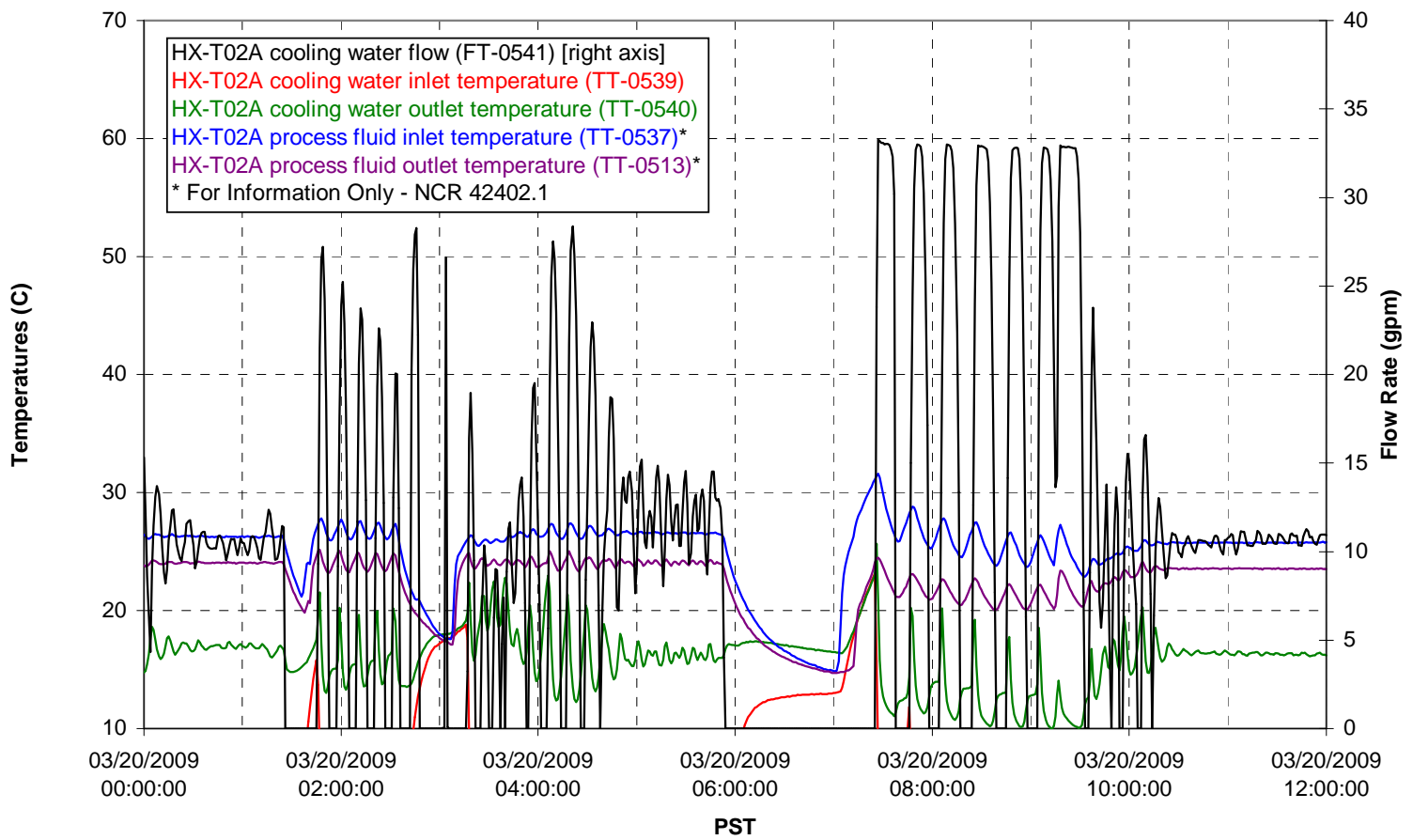

Pump Operation

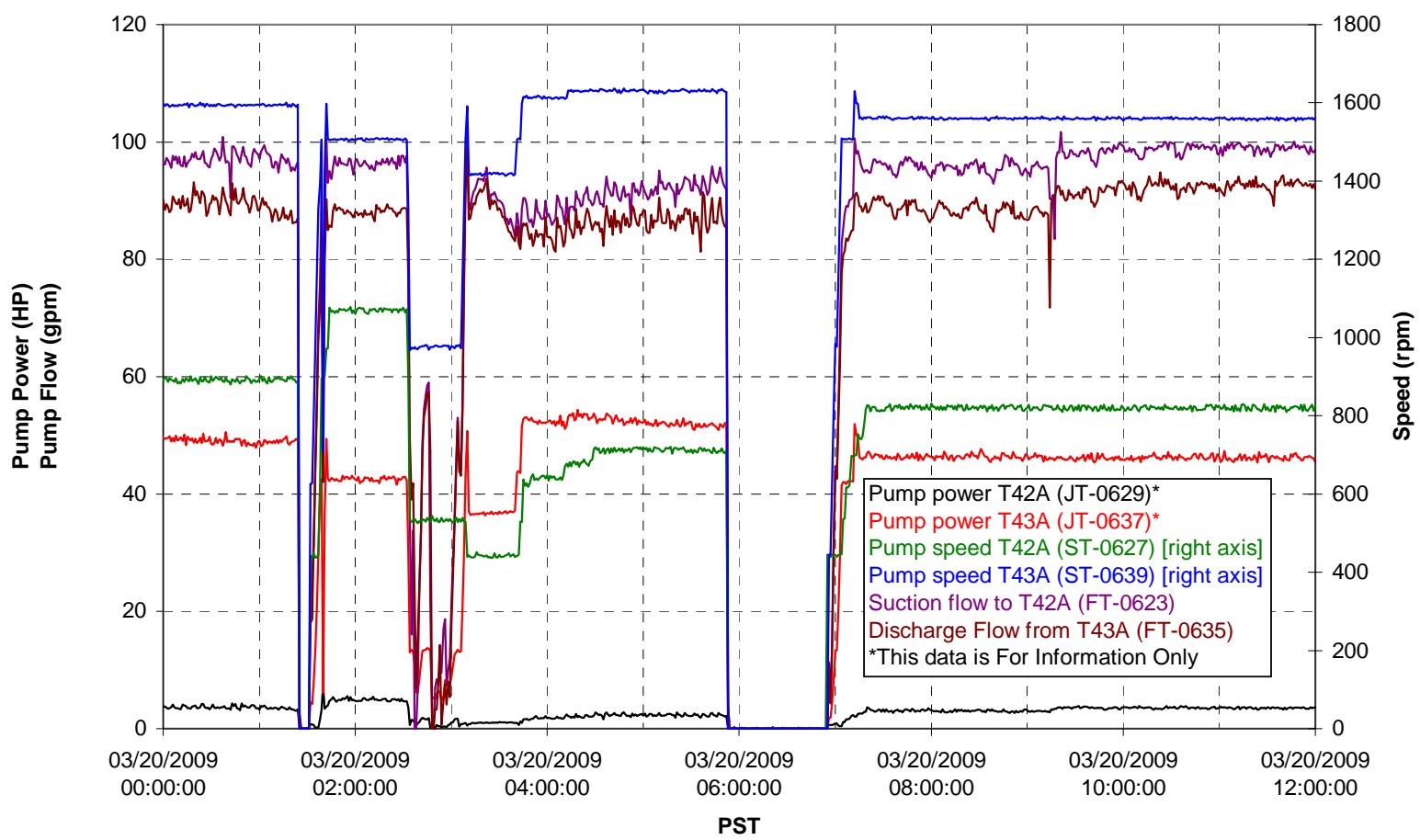


Pulsepot UFP-PP-T01A

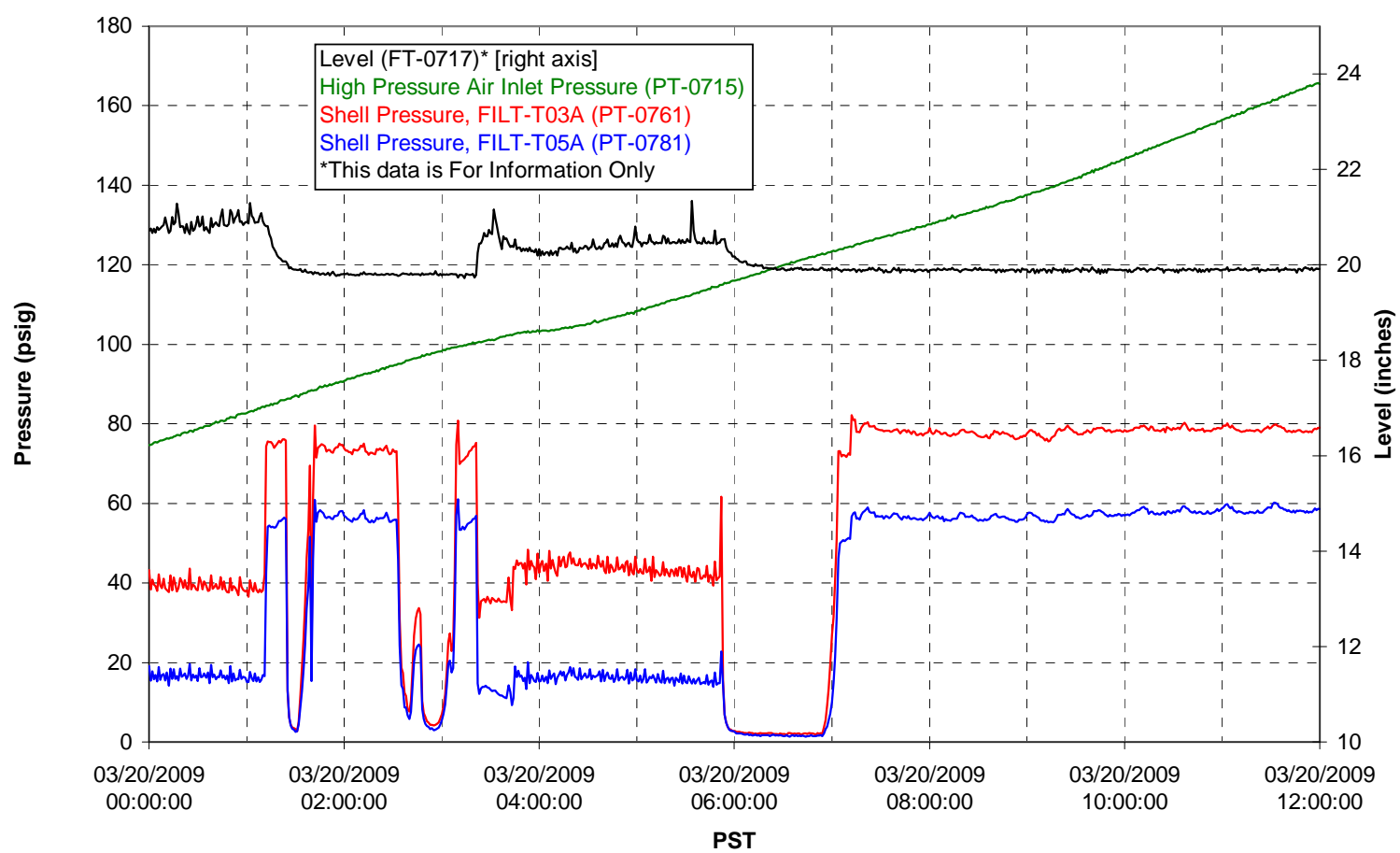

Pulsepot UFP-PP-T02A

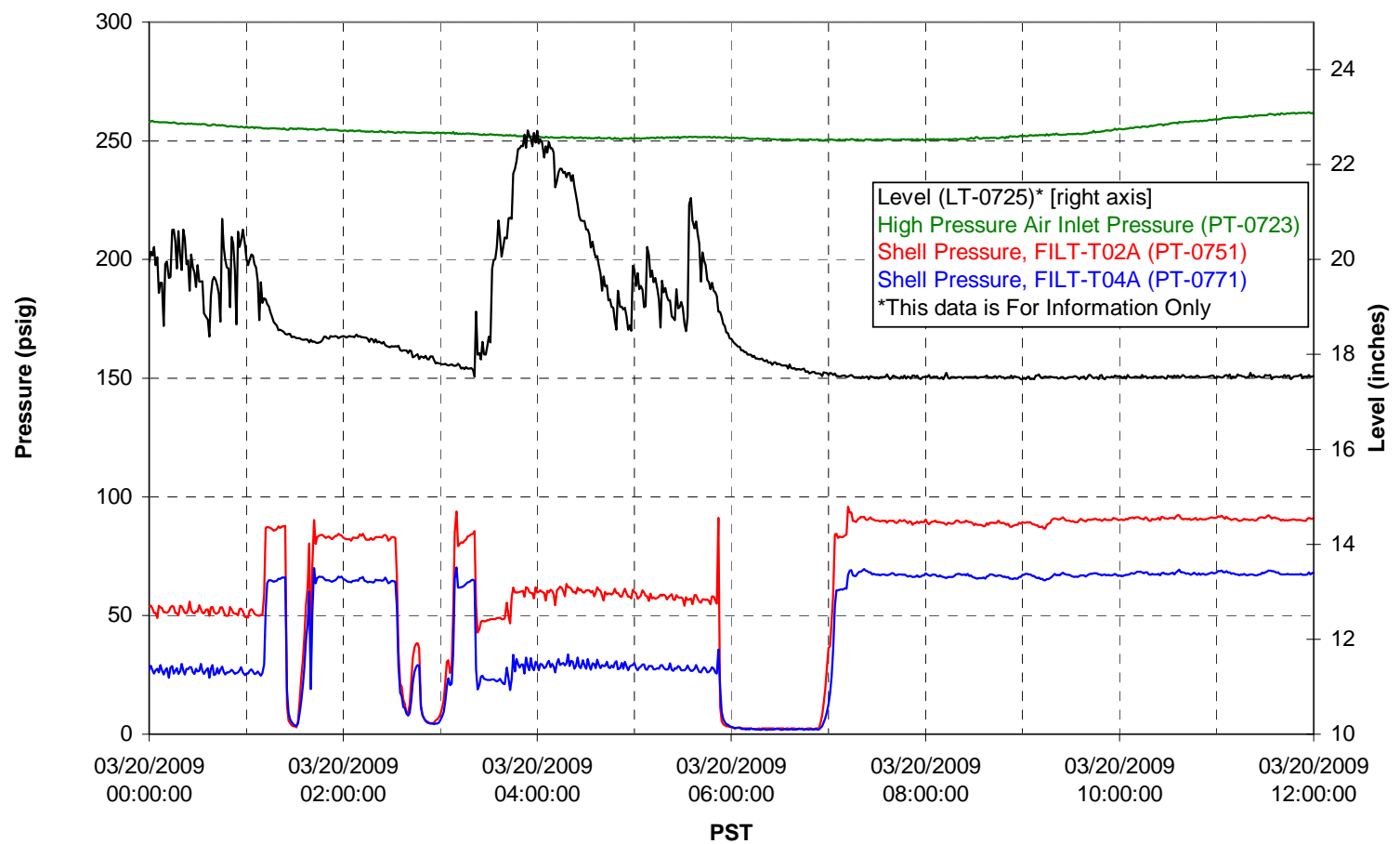


Pulsepot UFP-PP-T03A

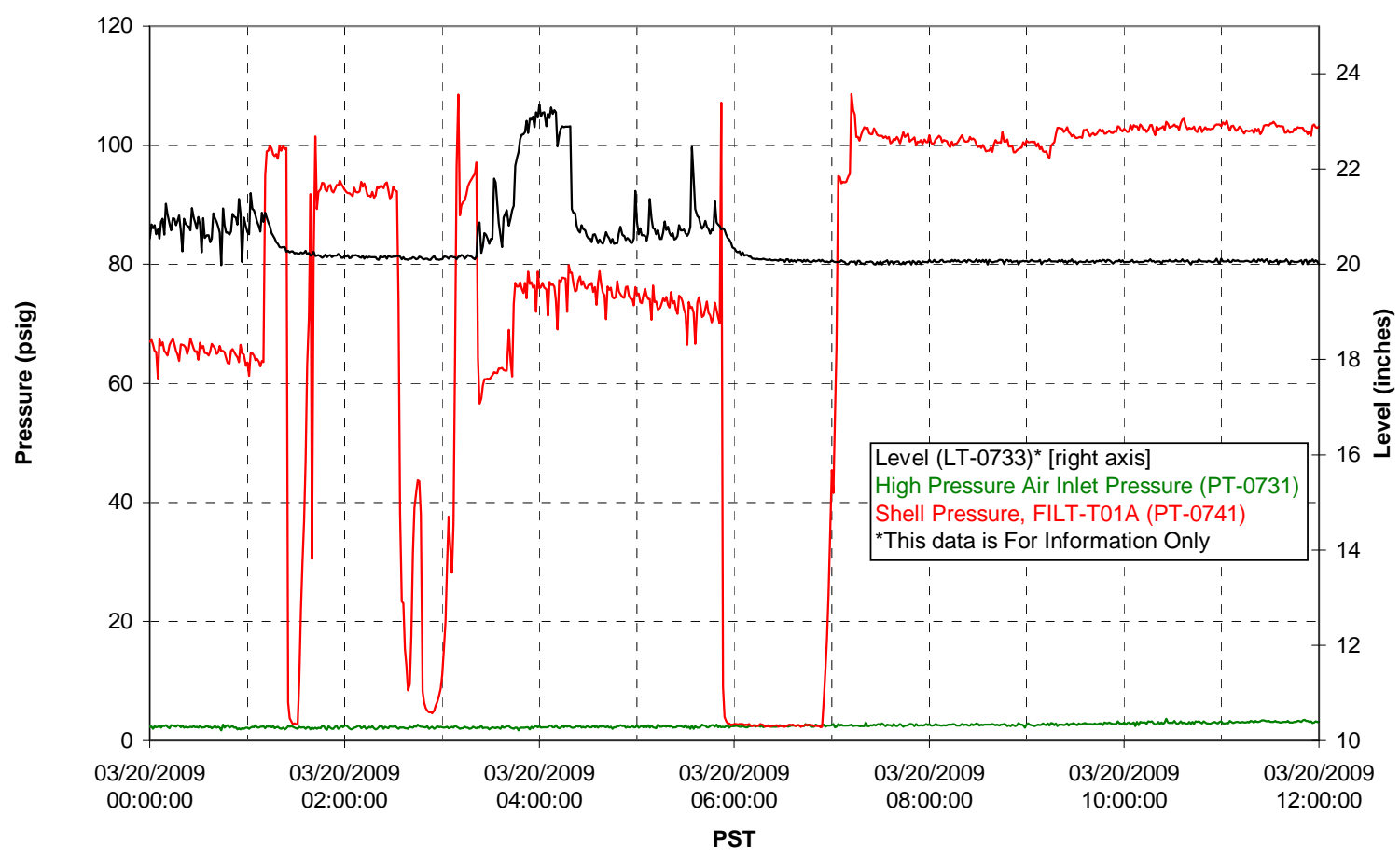

Pulsepot Levels

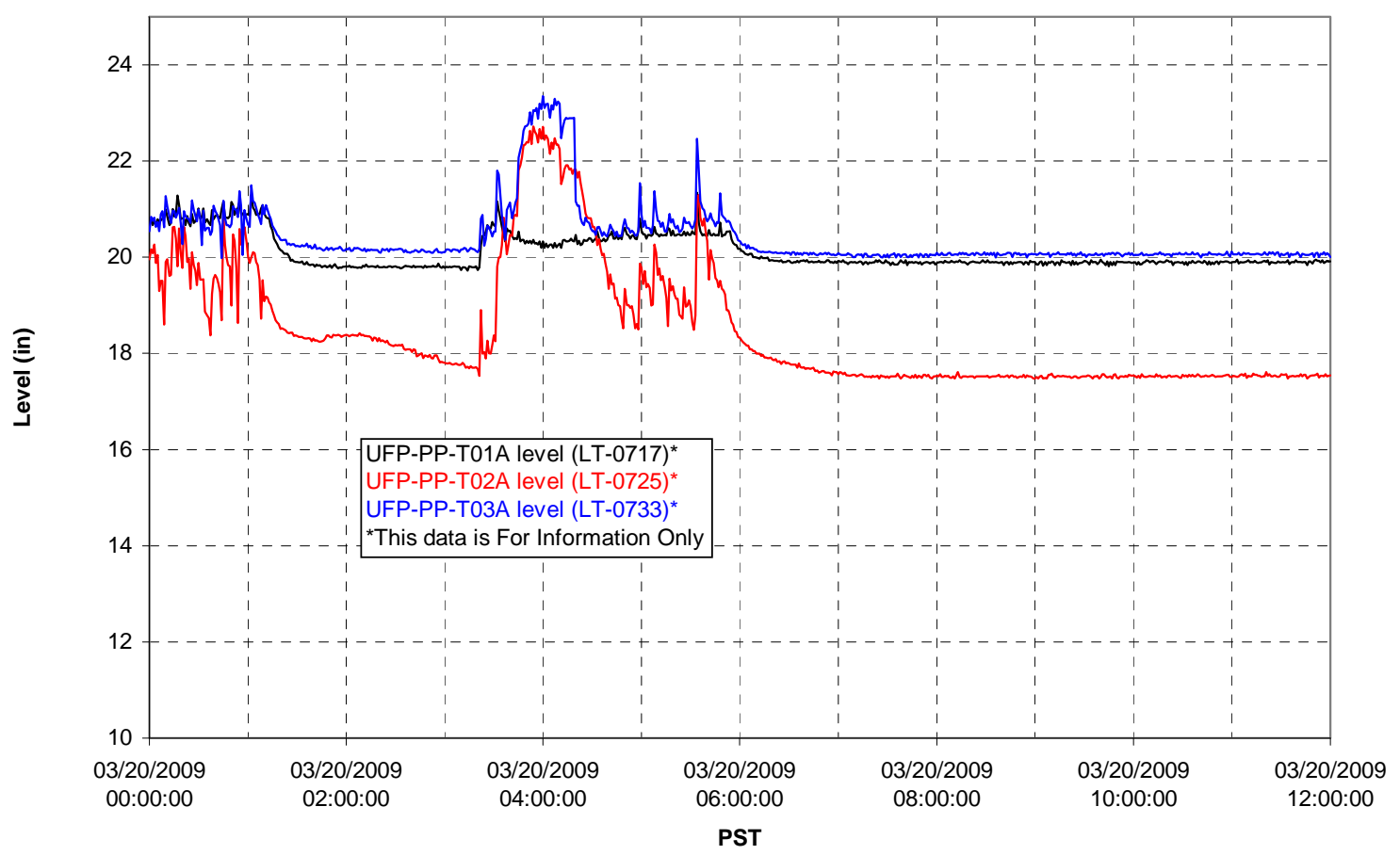


Filter UFP-FILT-T01A

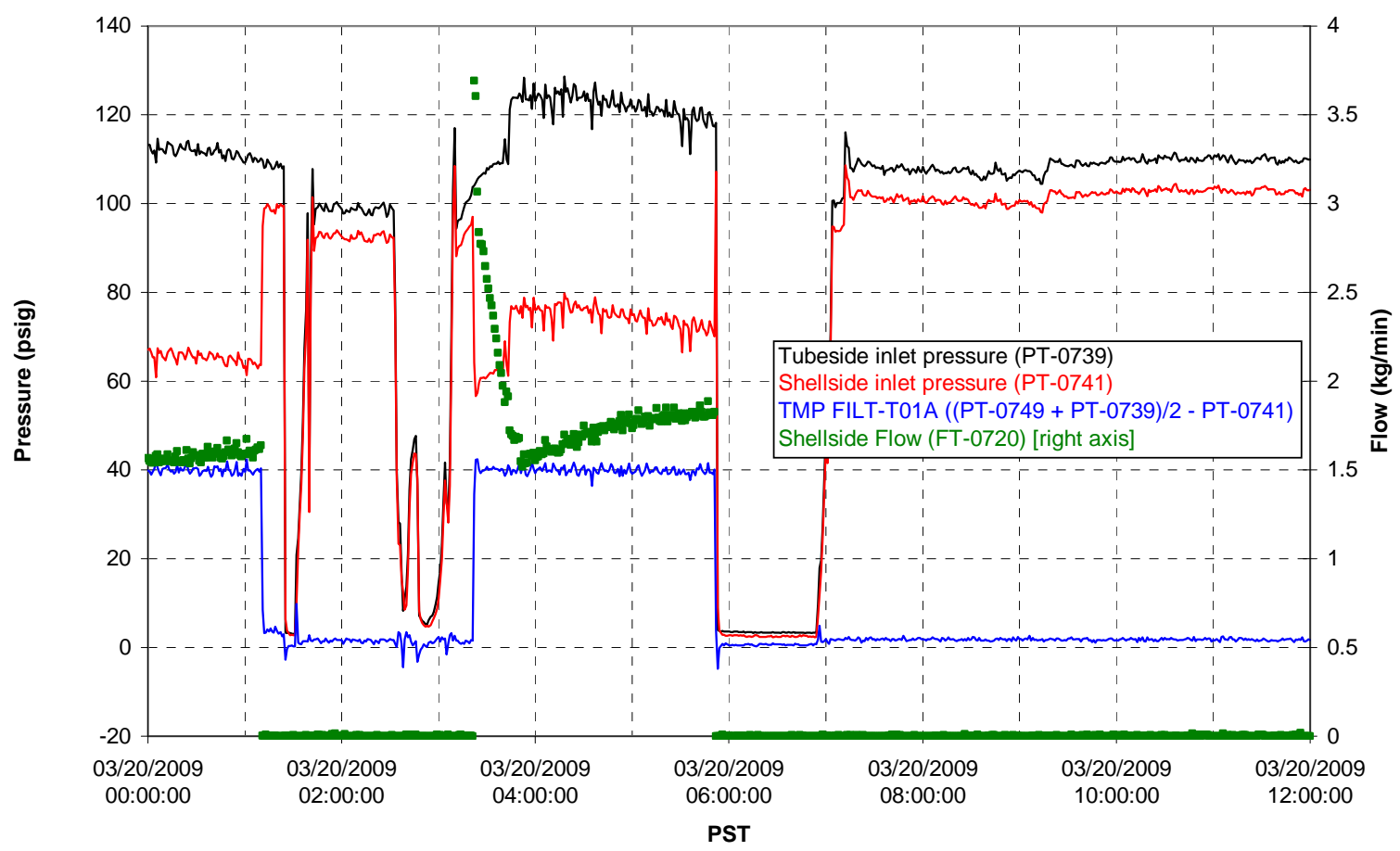

Filter UFP-FILT-T02A

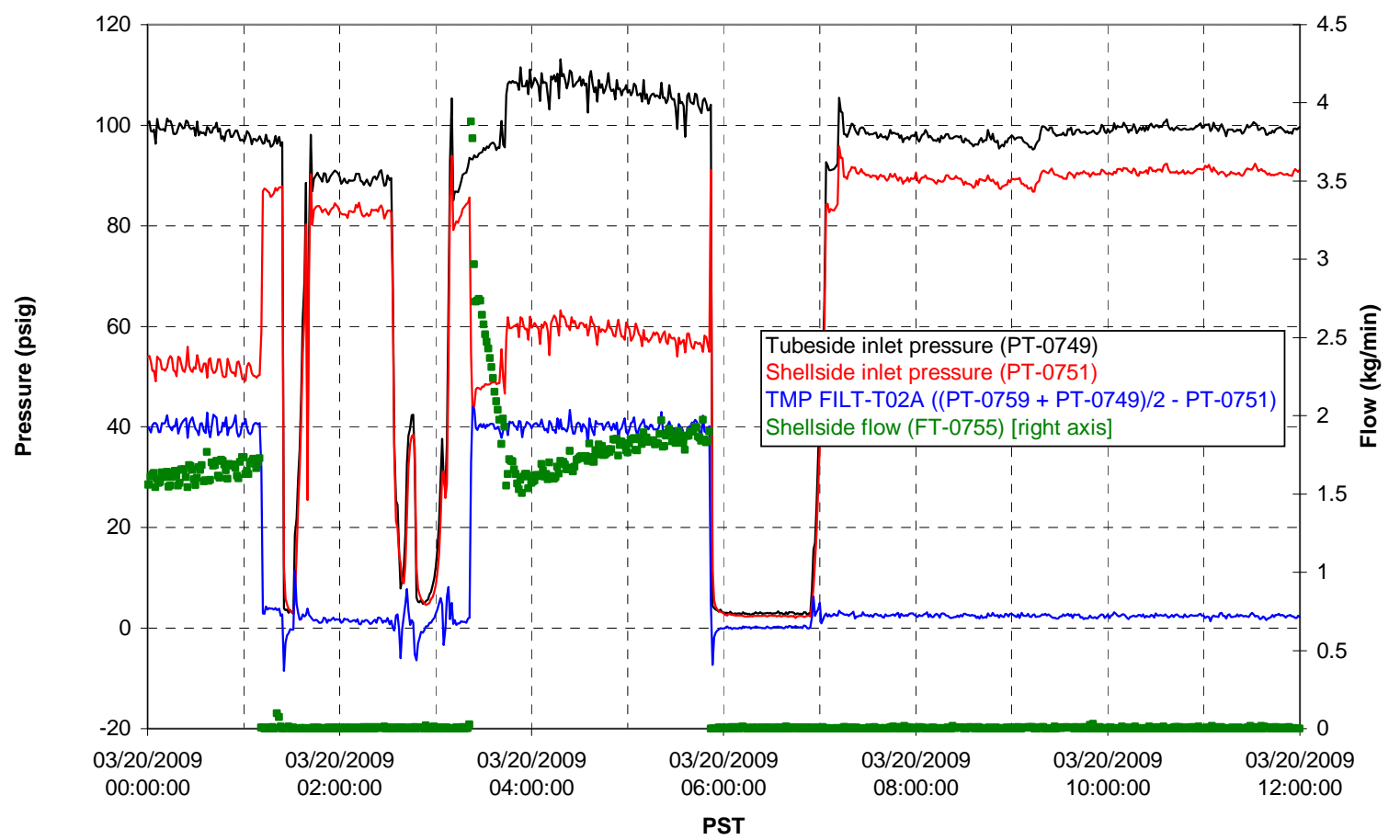


Filter UFP-FILT-T03A

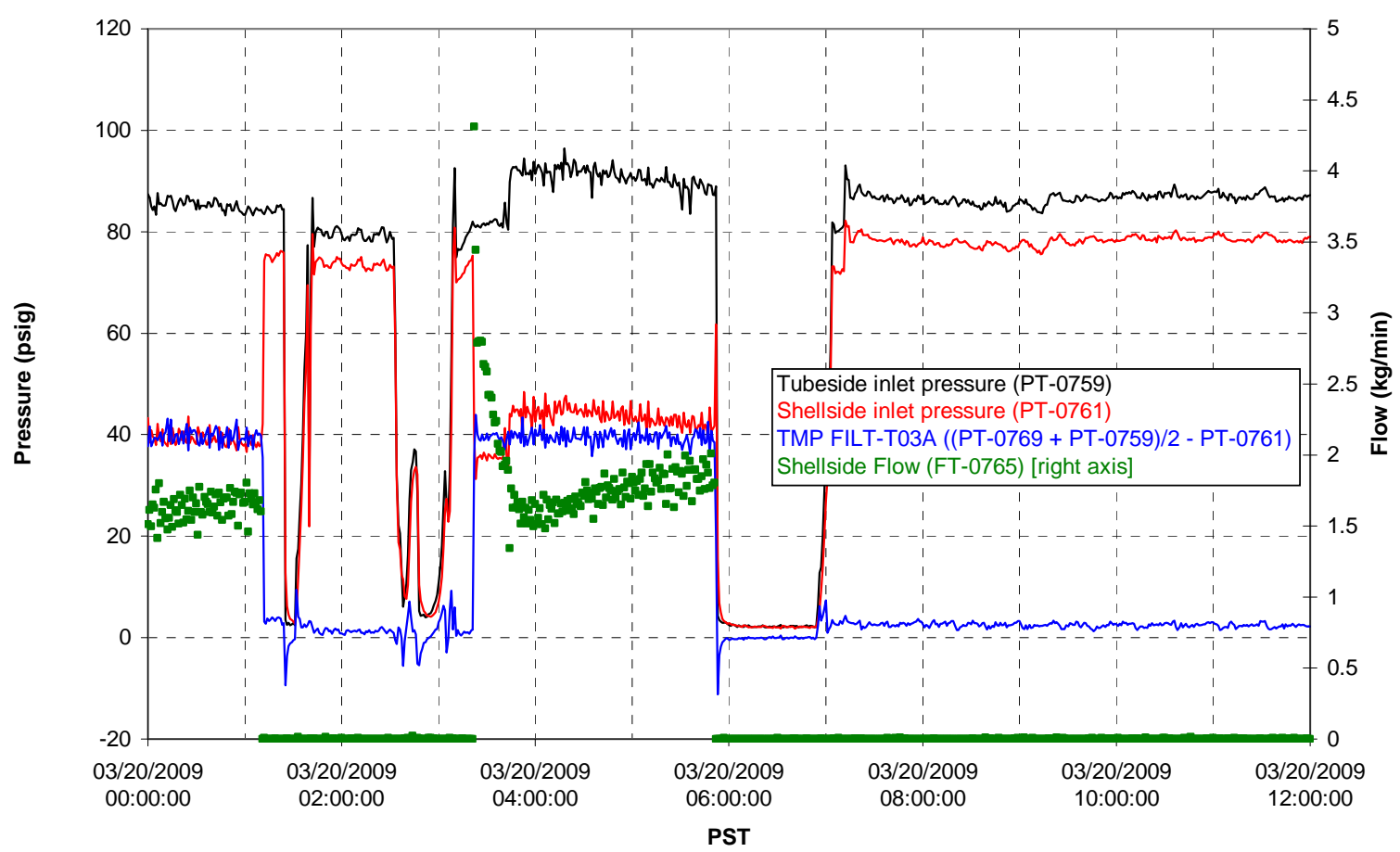

Filter UFP-FILT-T04A

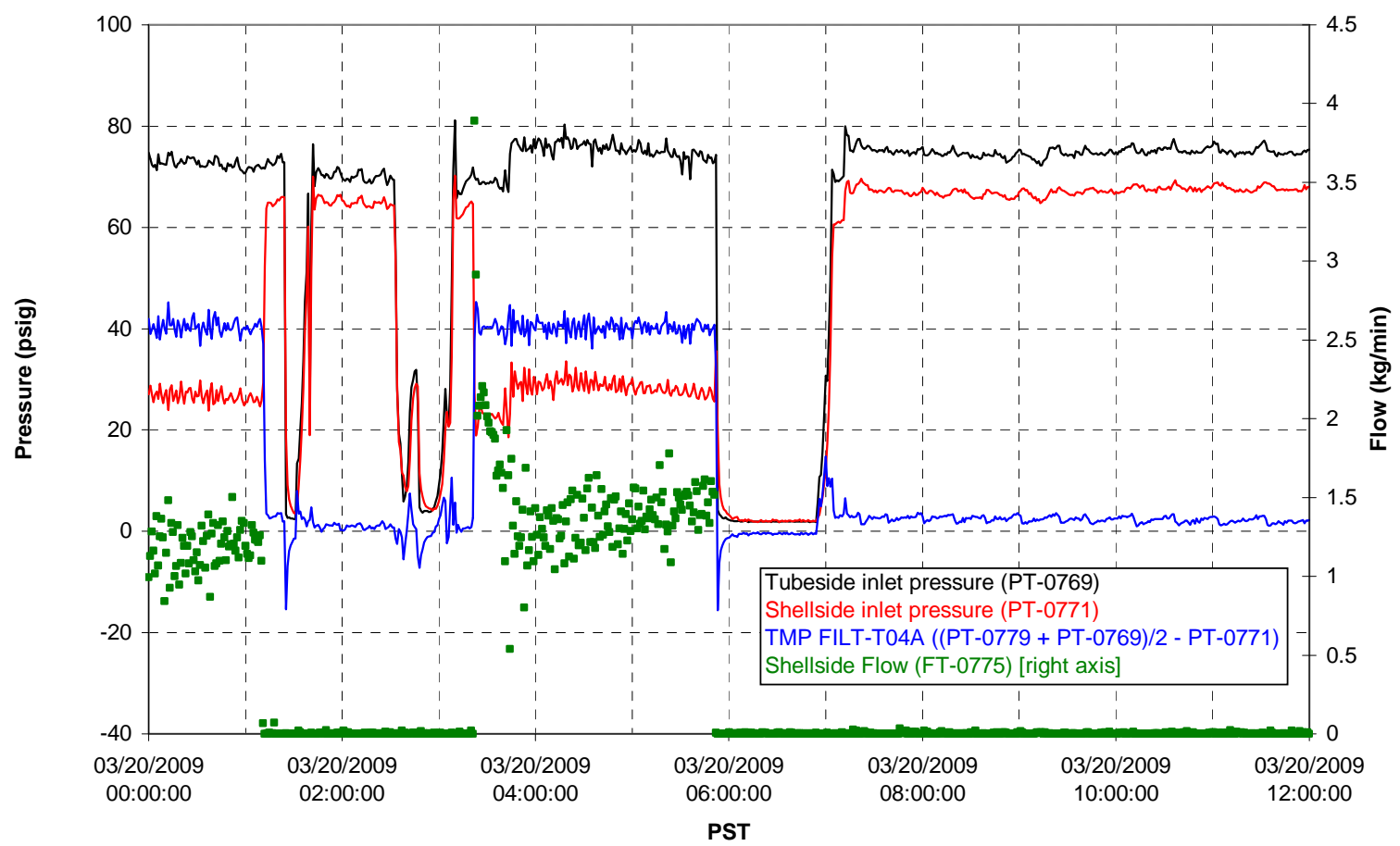


Filter UFP-FILT-T05A

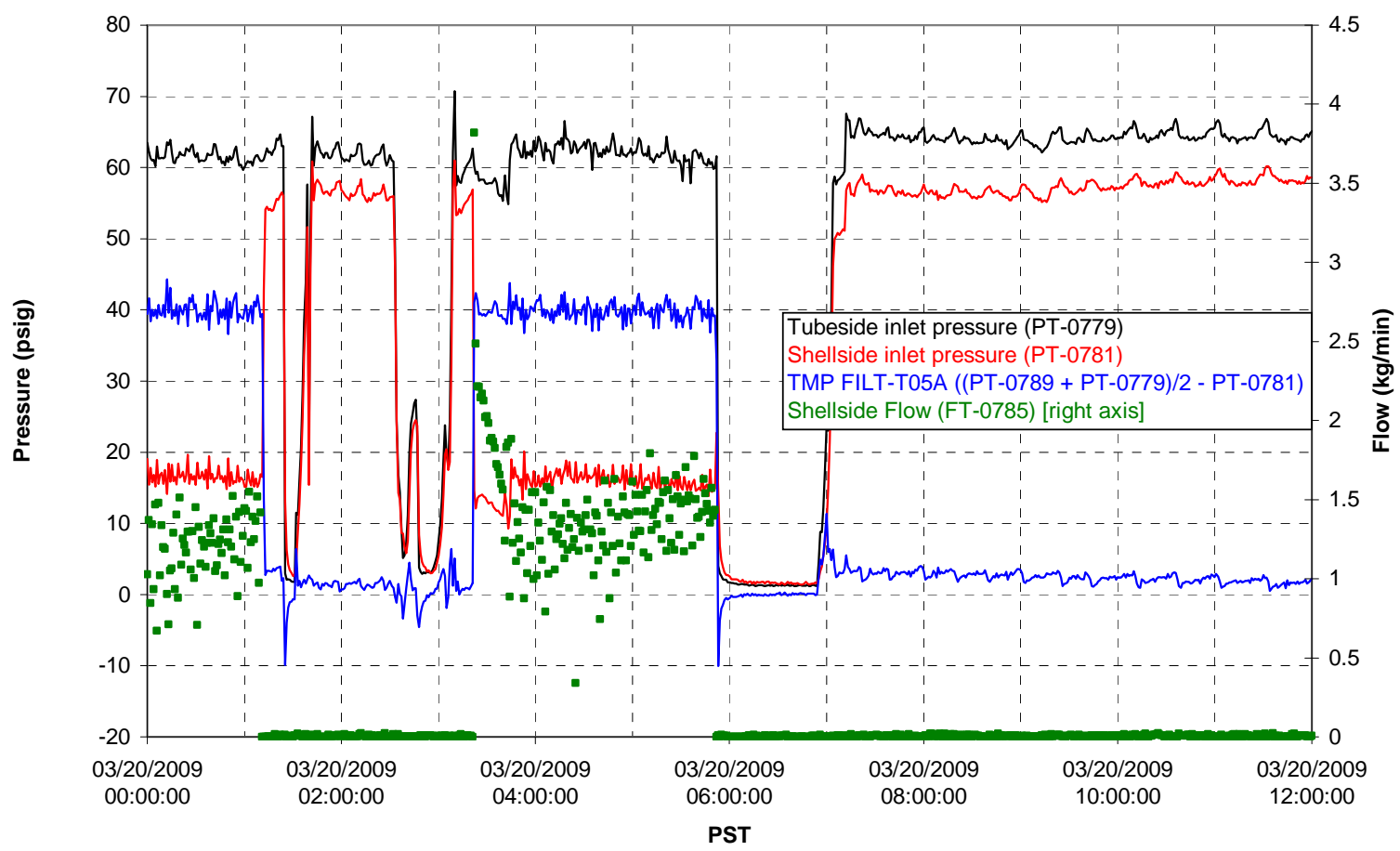

Chemical Flow

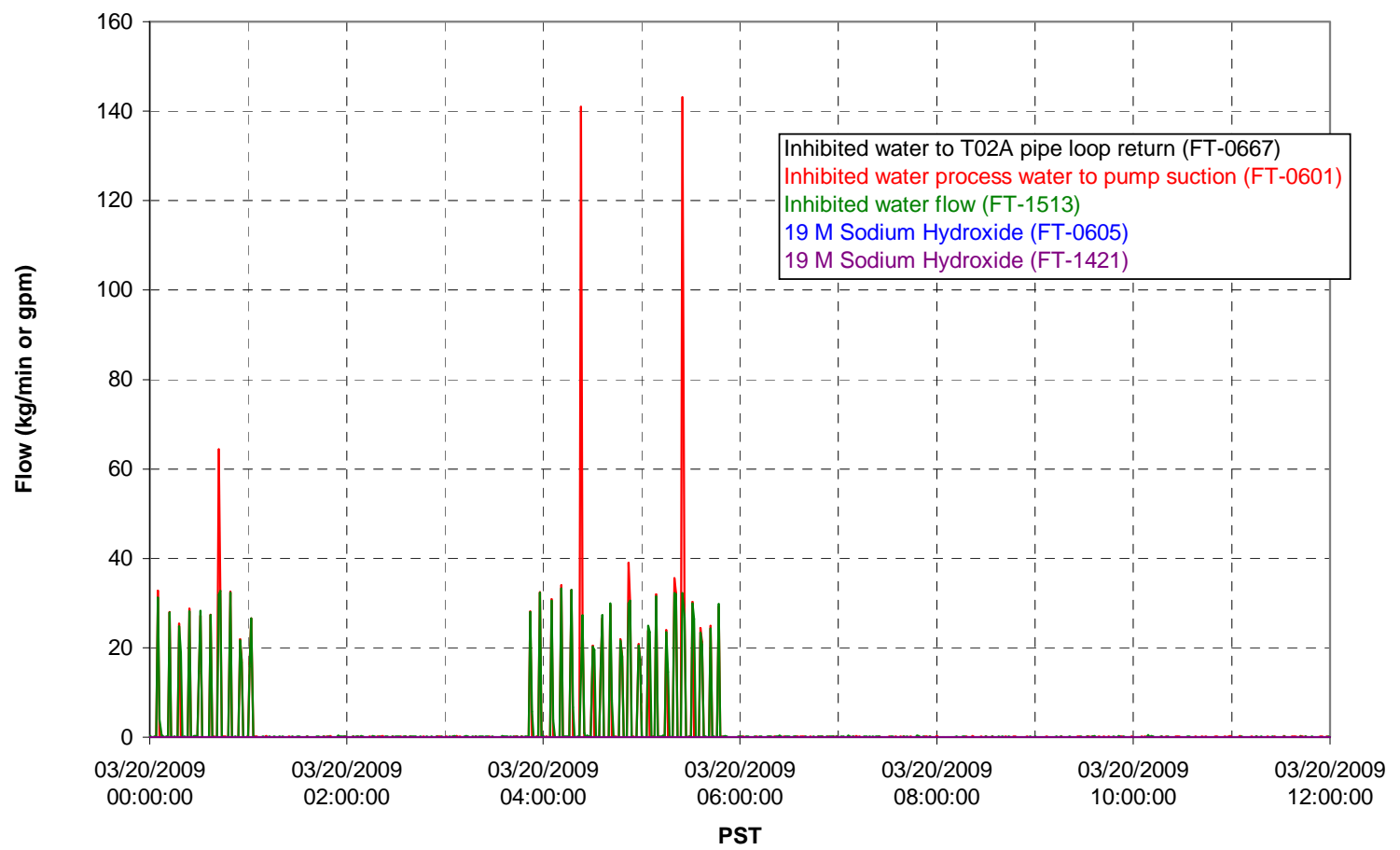


Chemical Flow

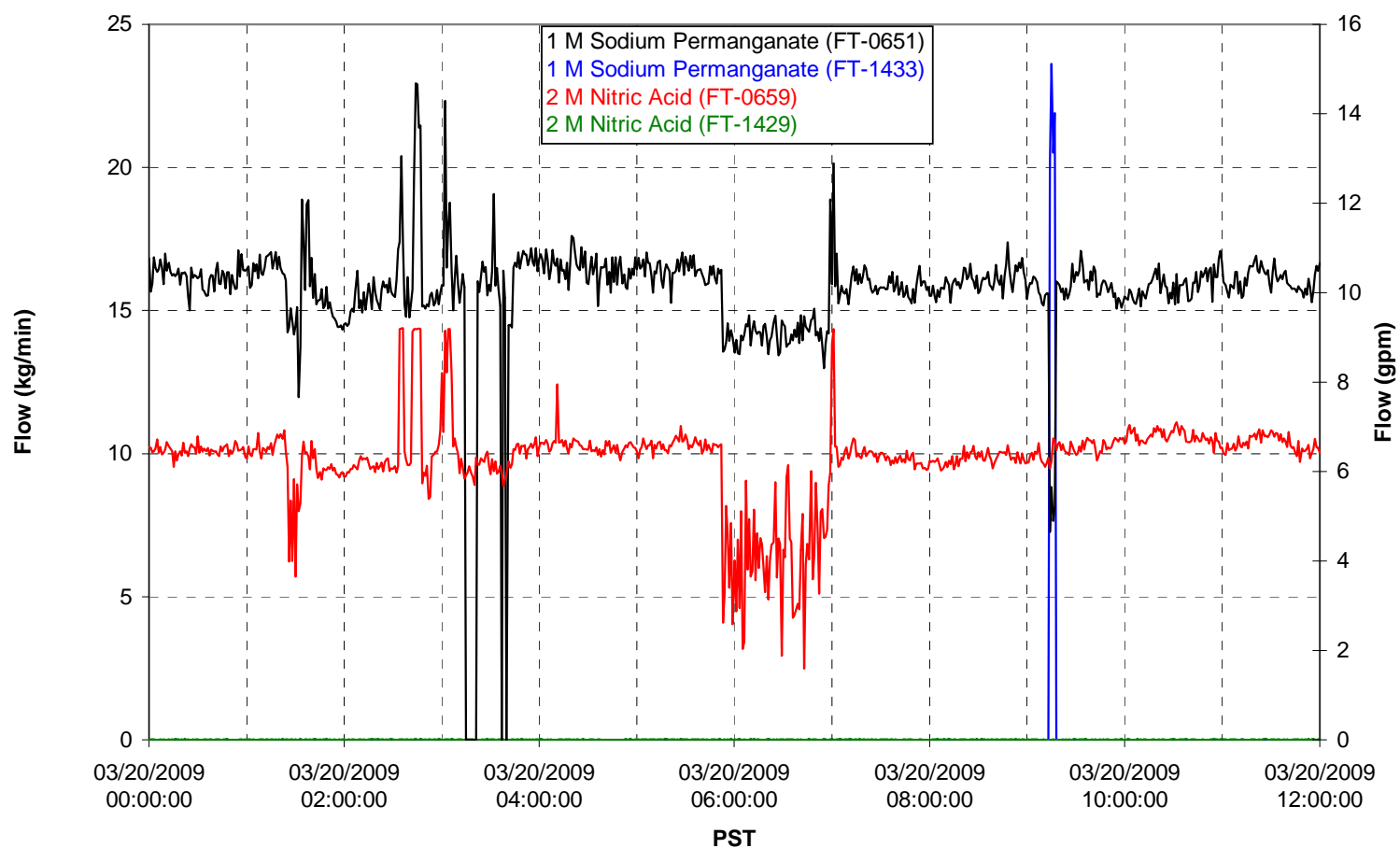

Air Flows

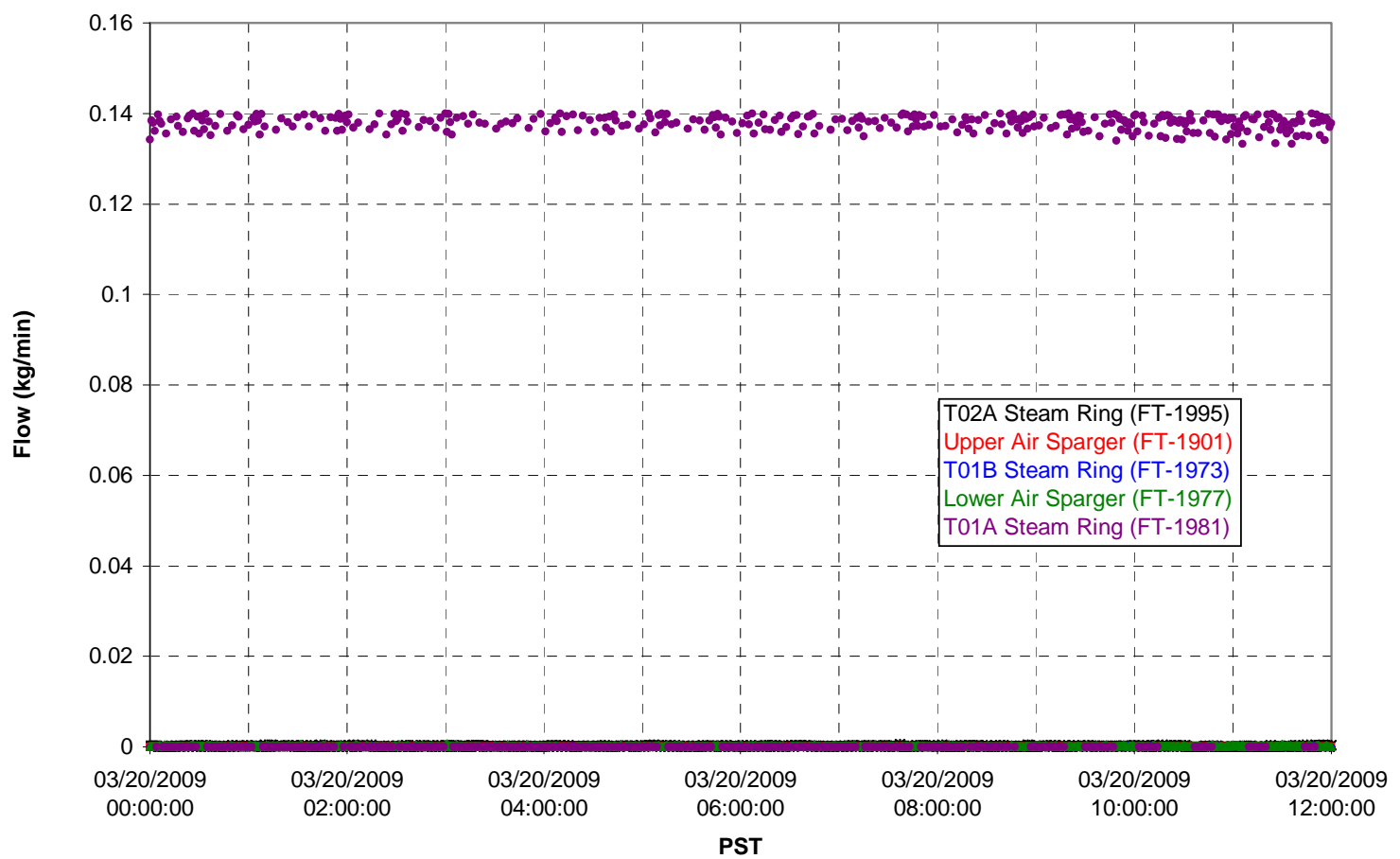


T02A Steam

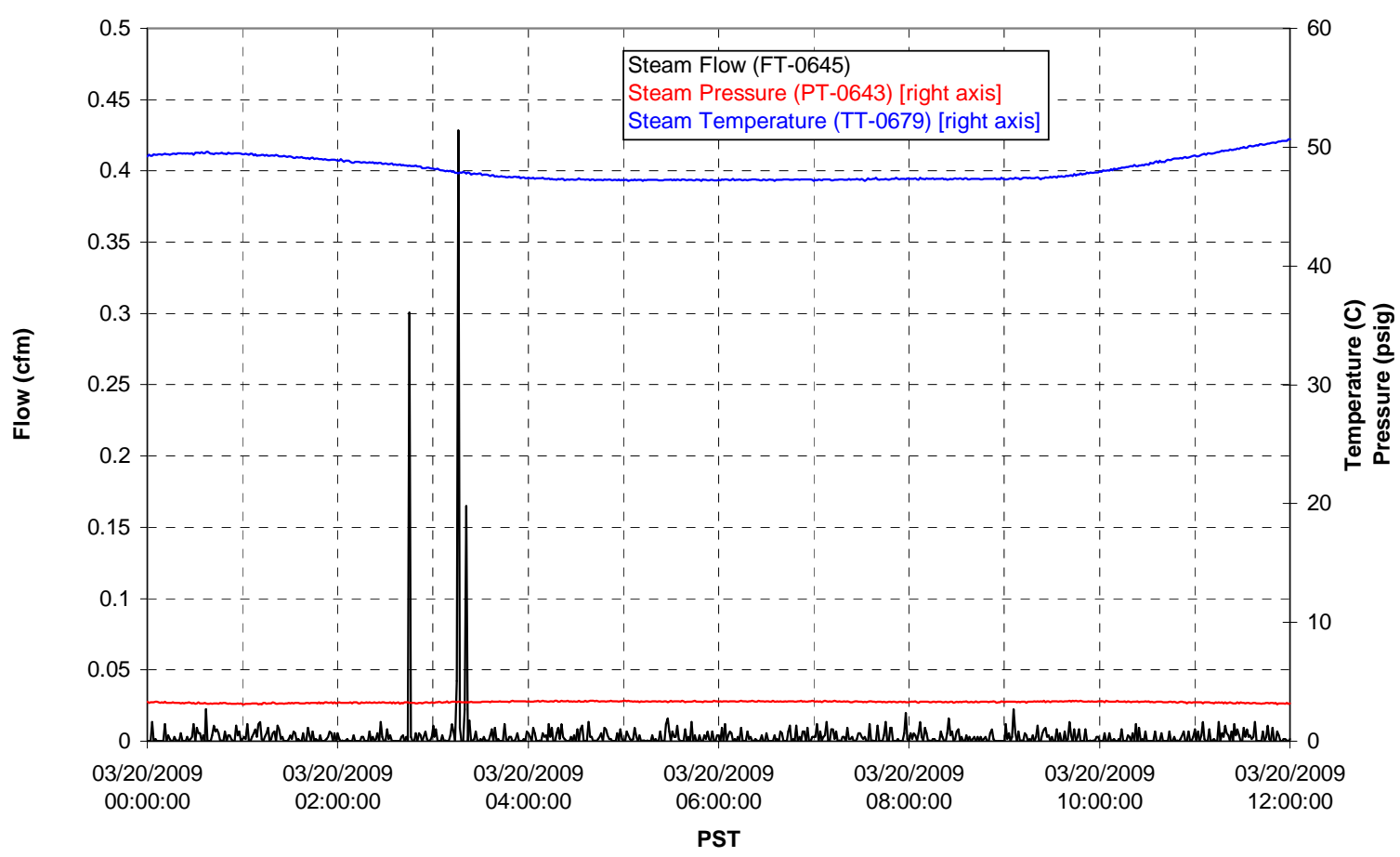

T01A Steam

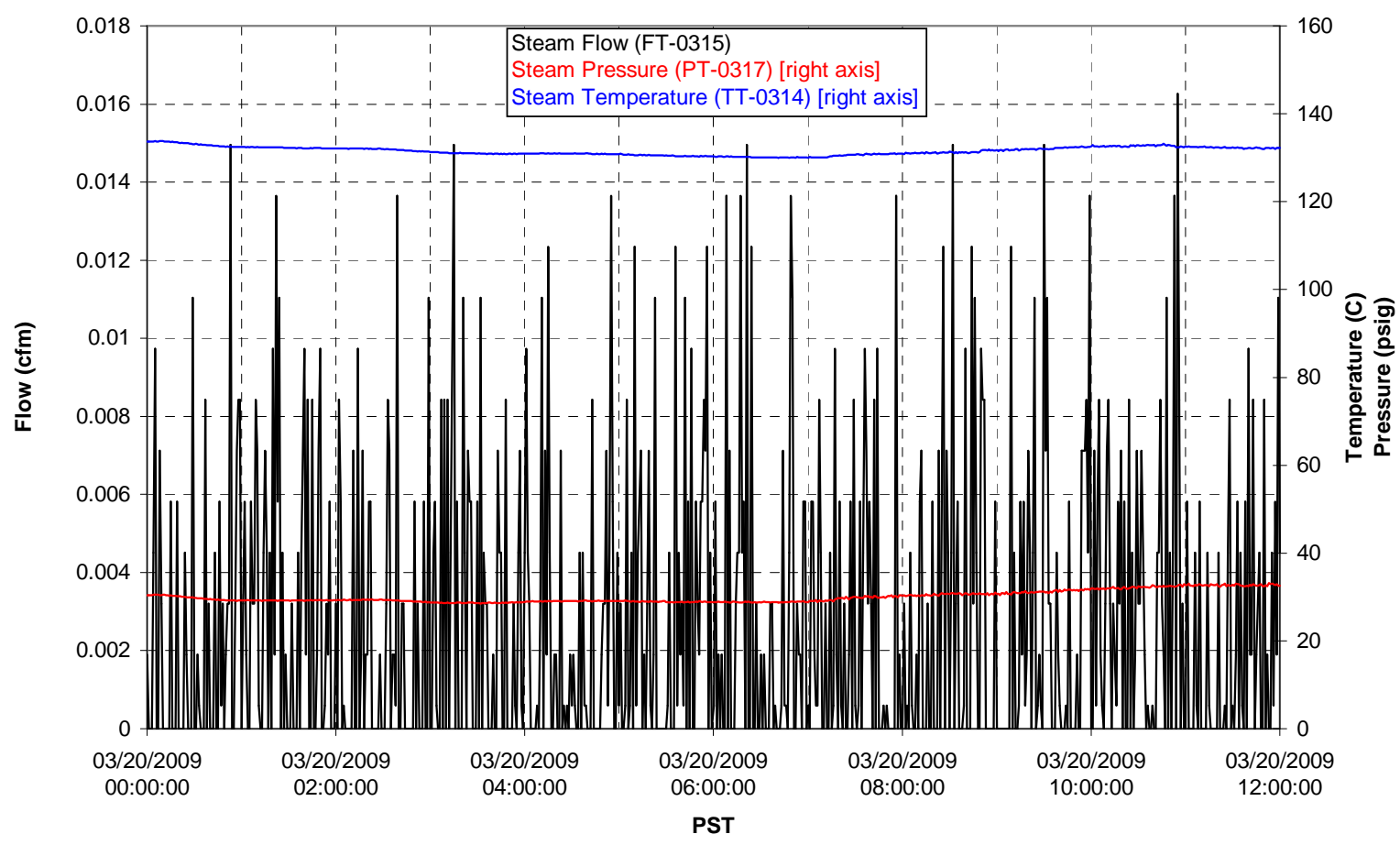


T01B Steam

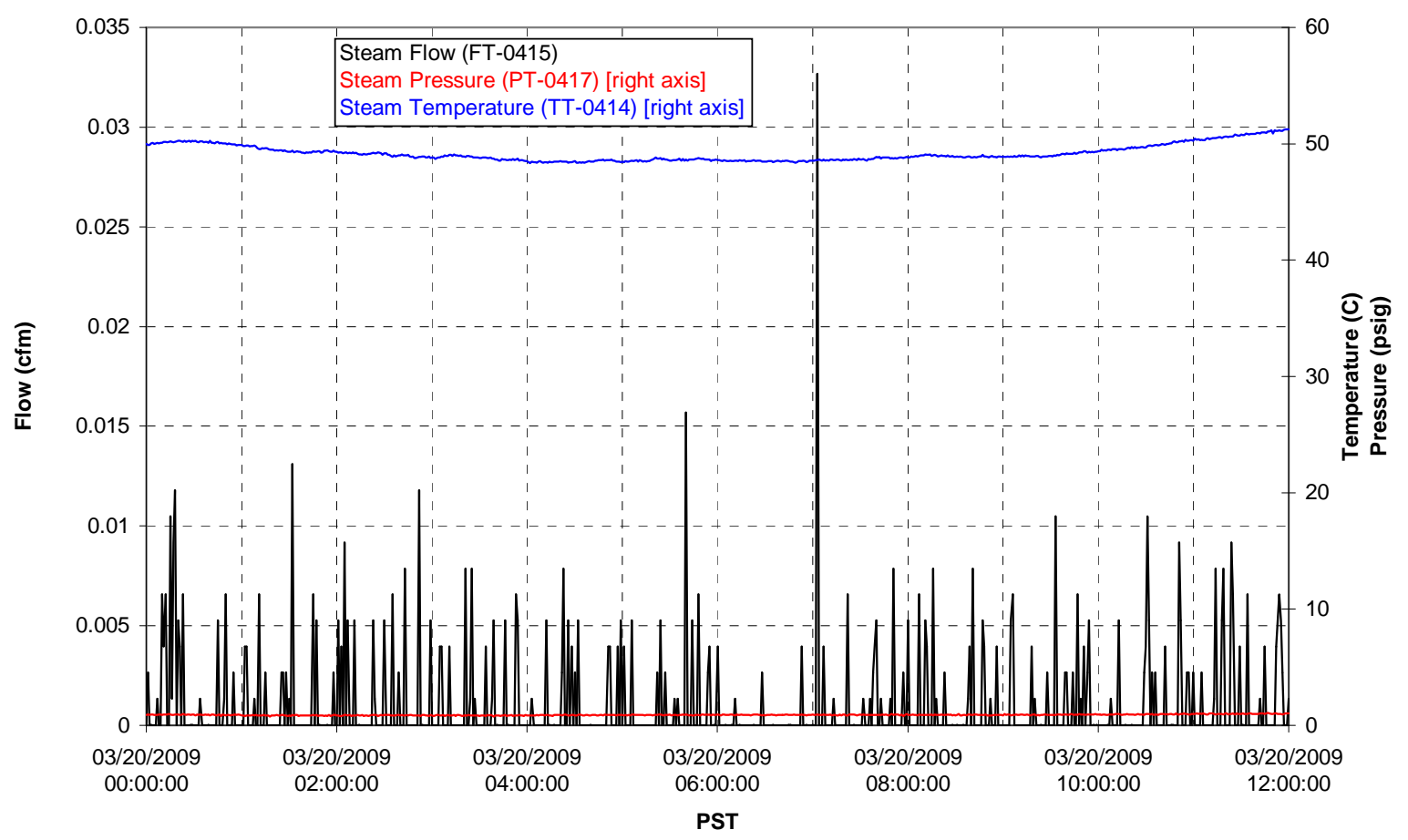




\section{Integrated Test A Data Plots 03/20/09 12:00 - 03/21/09 00:00}


T01A level

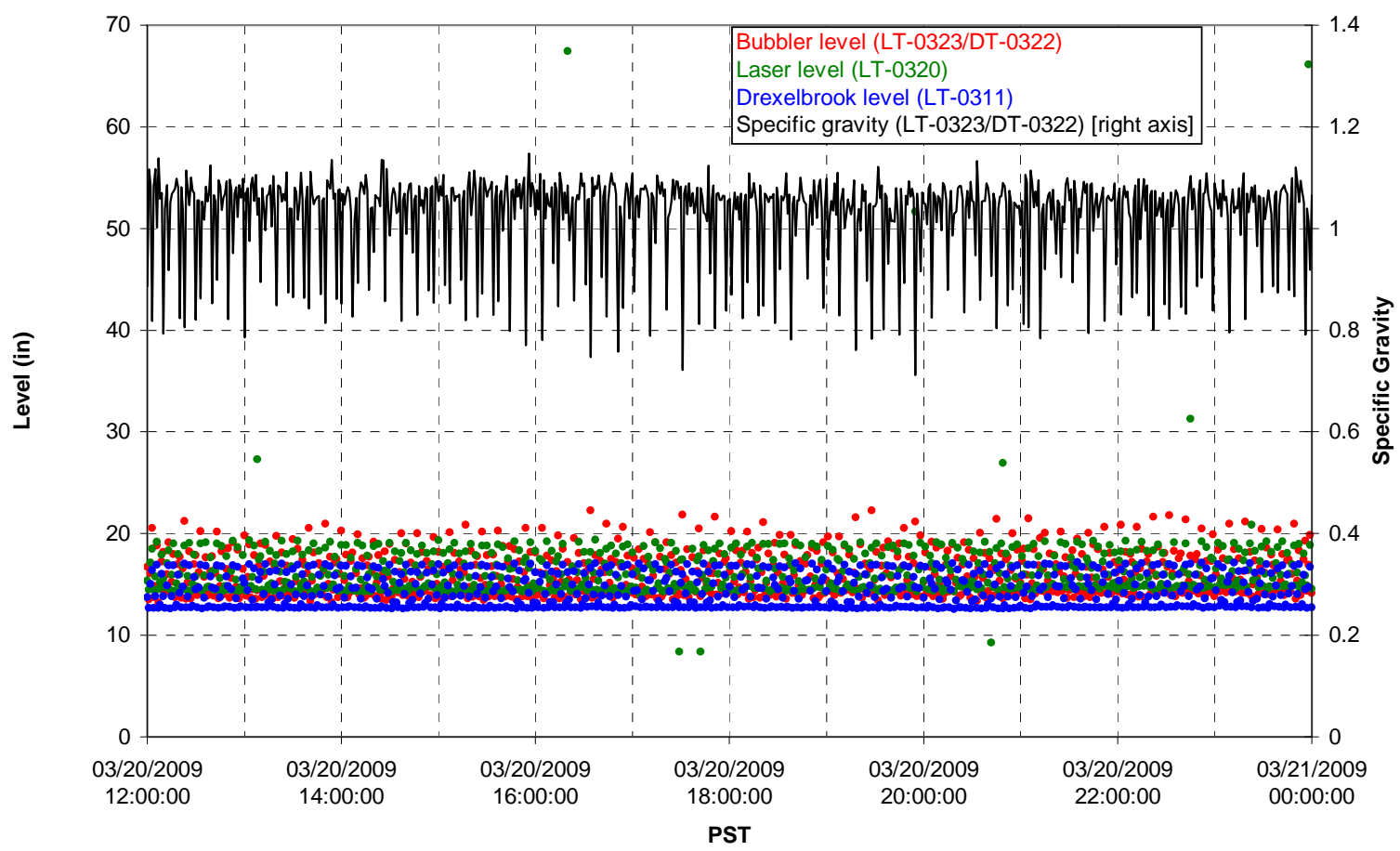

T01A temperatures

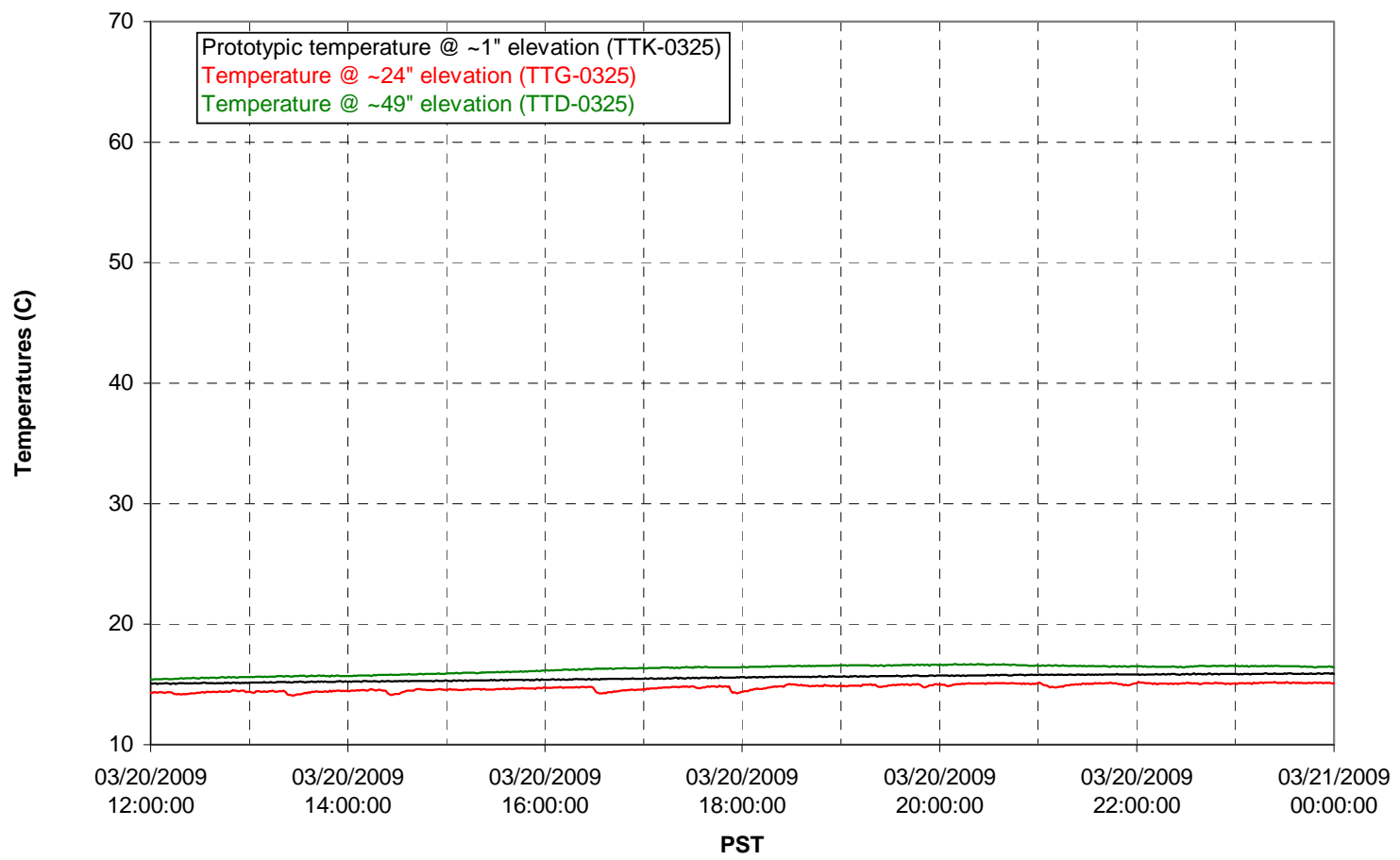


T01B level

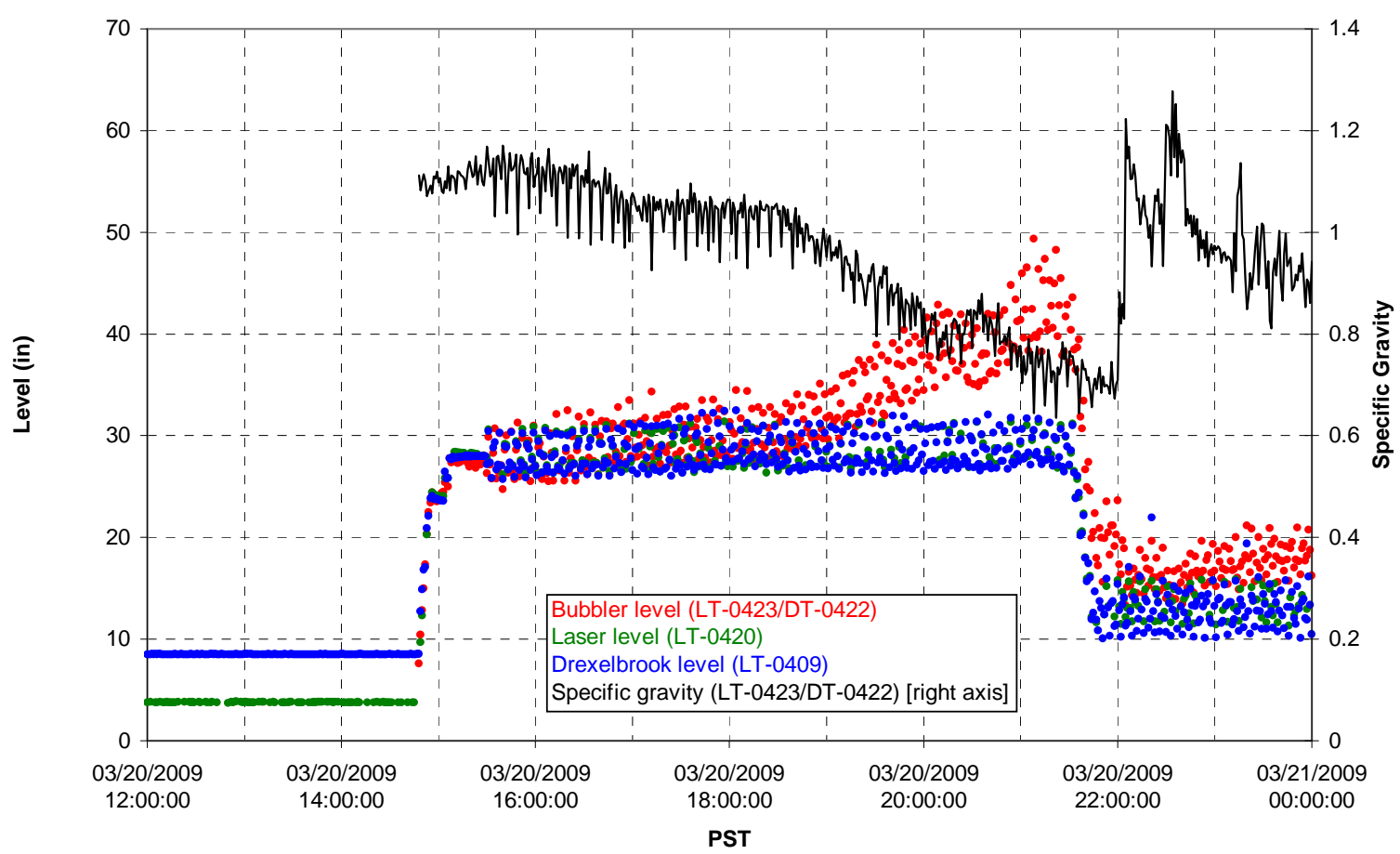

T01B temperatures

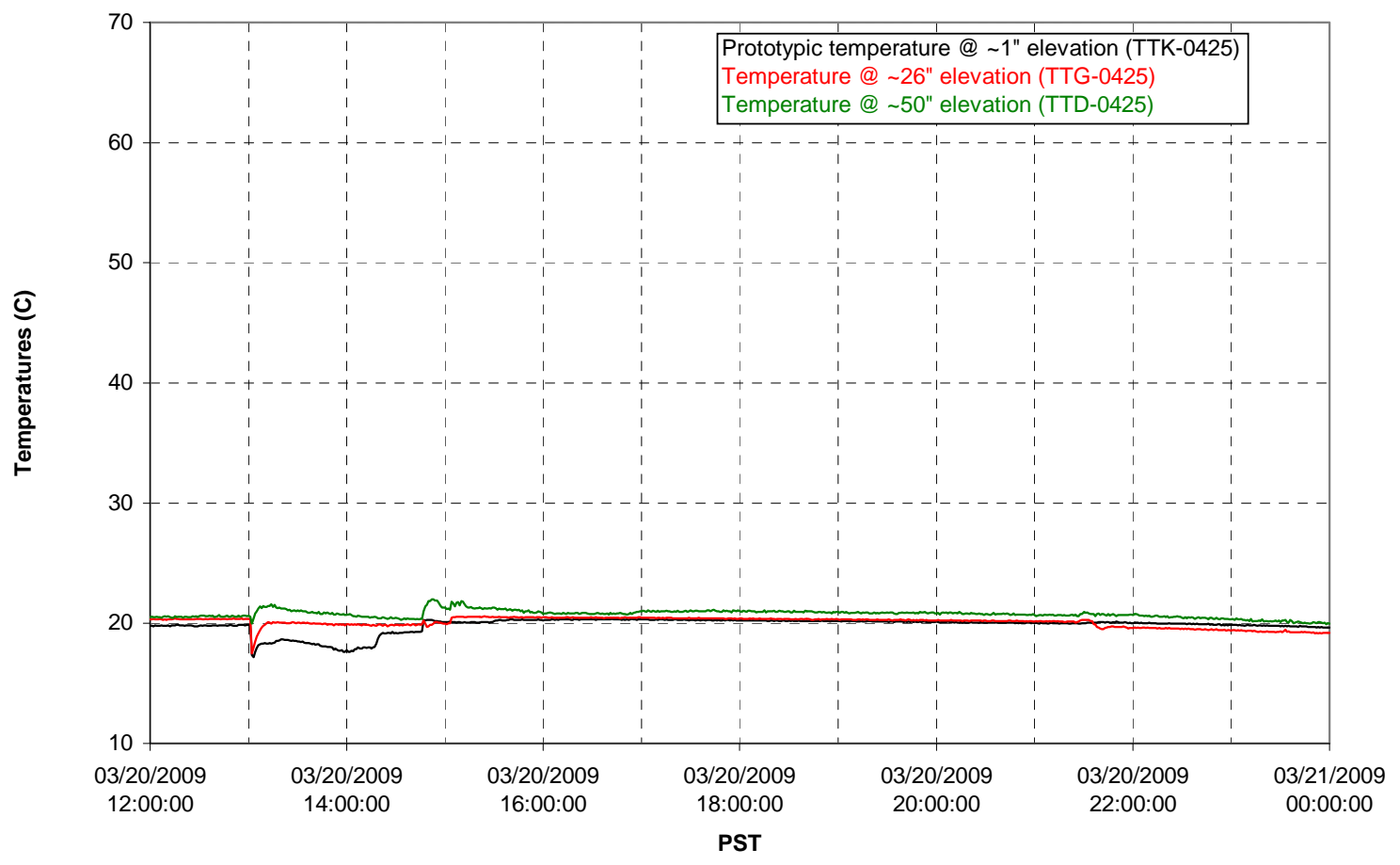


T02A level

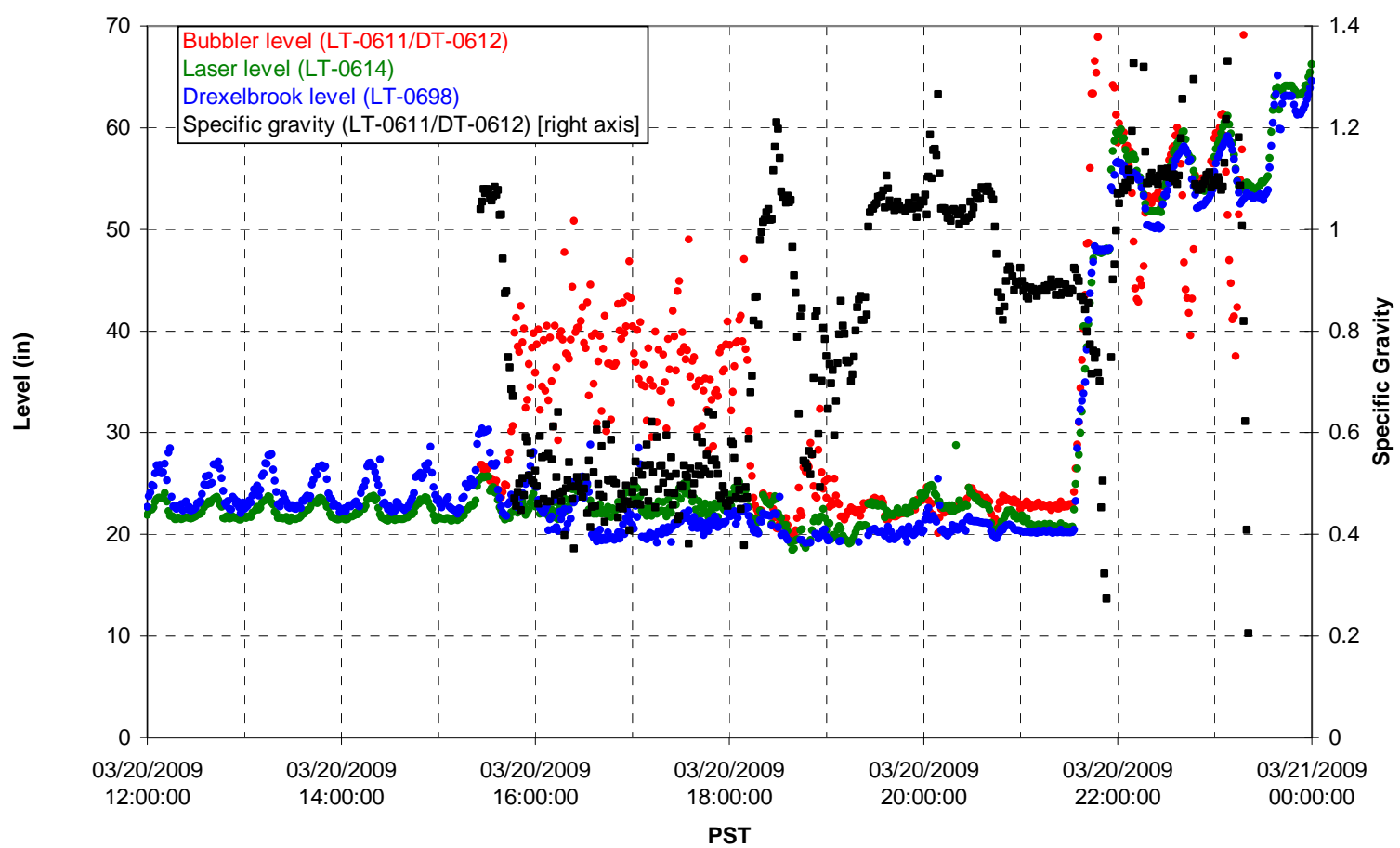

T02A temperatures

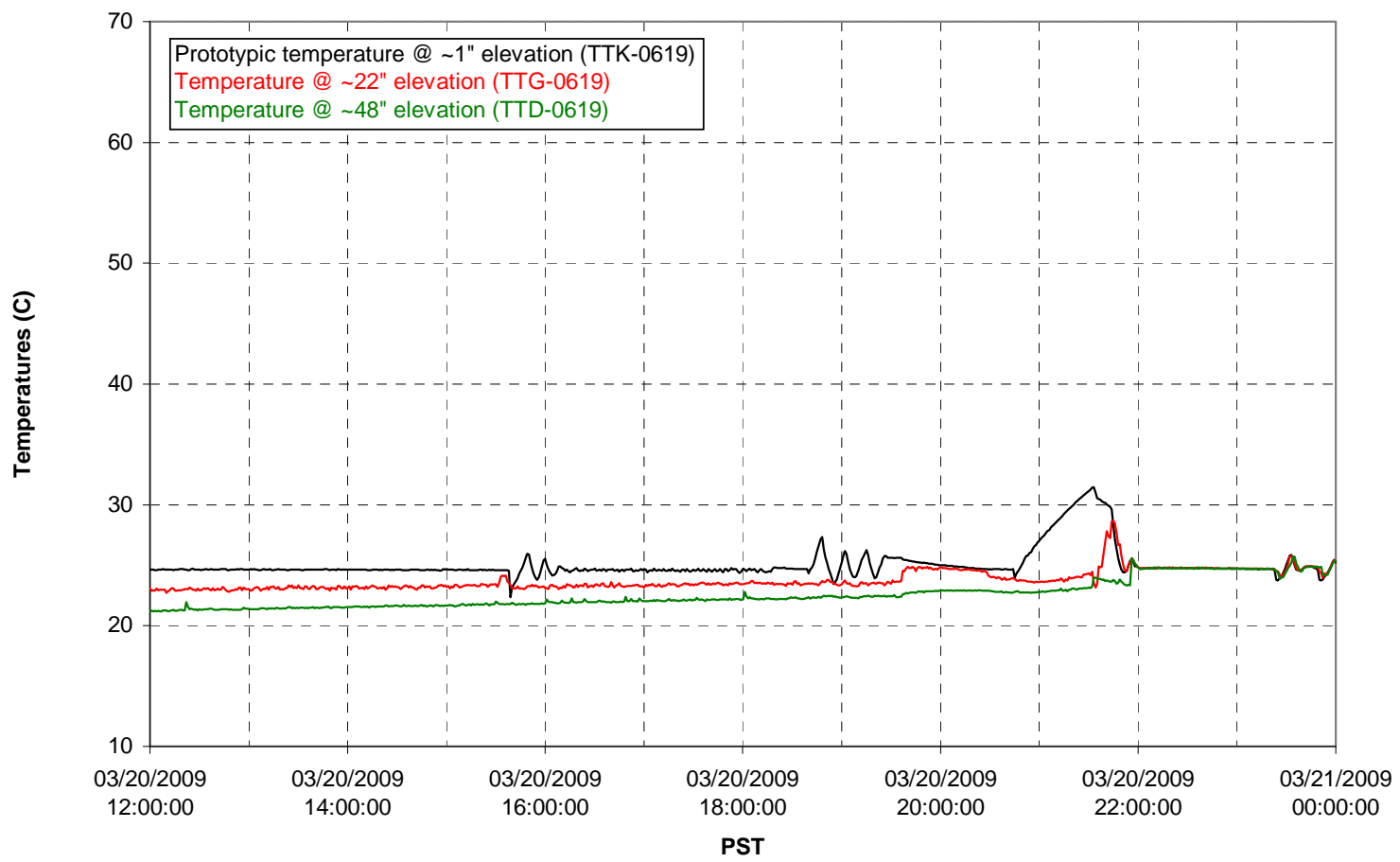


T02A and filter loop temperatures

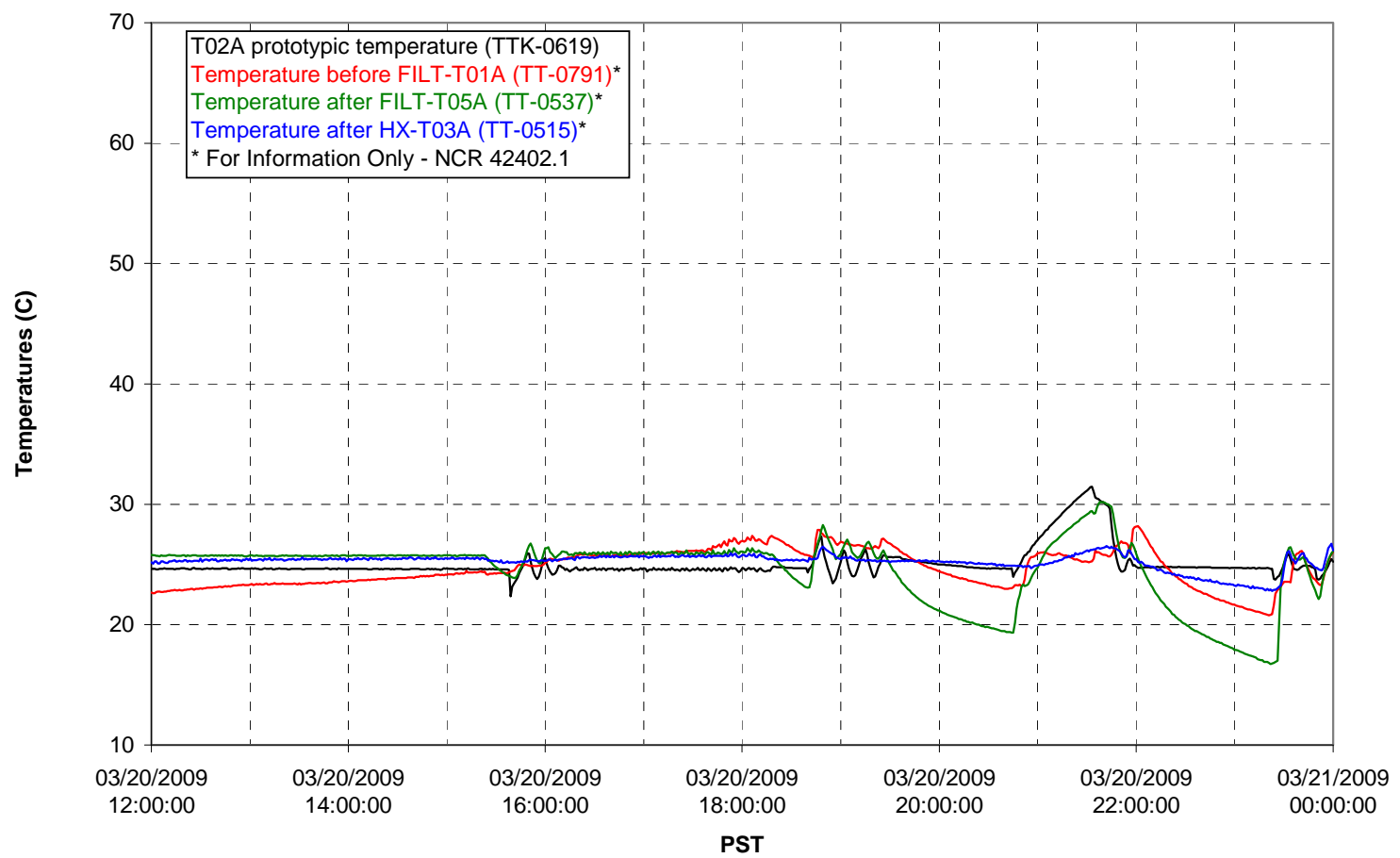

Pump Pressures and Flow

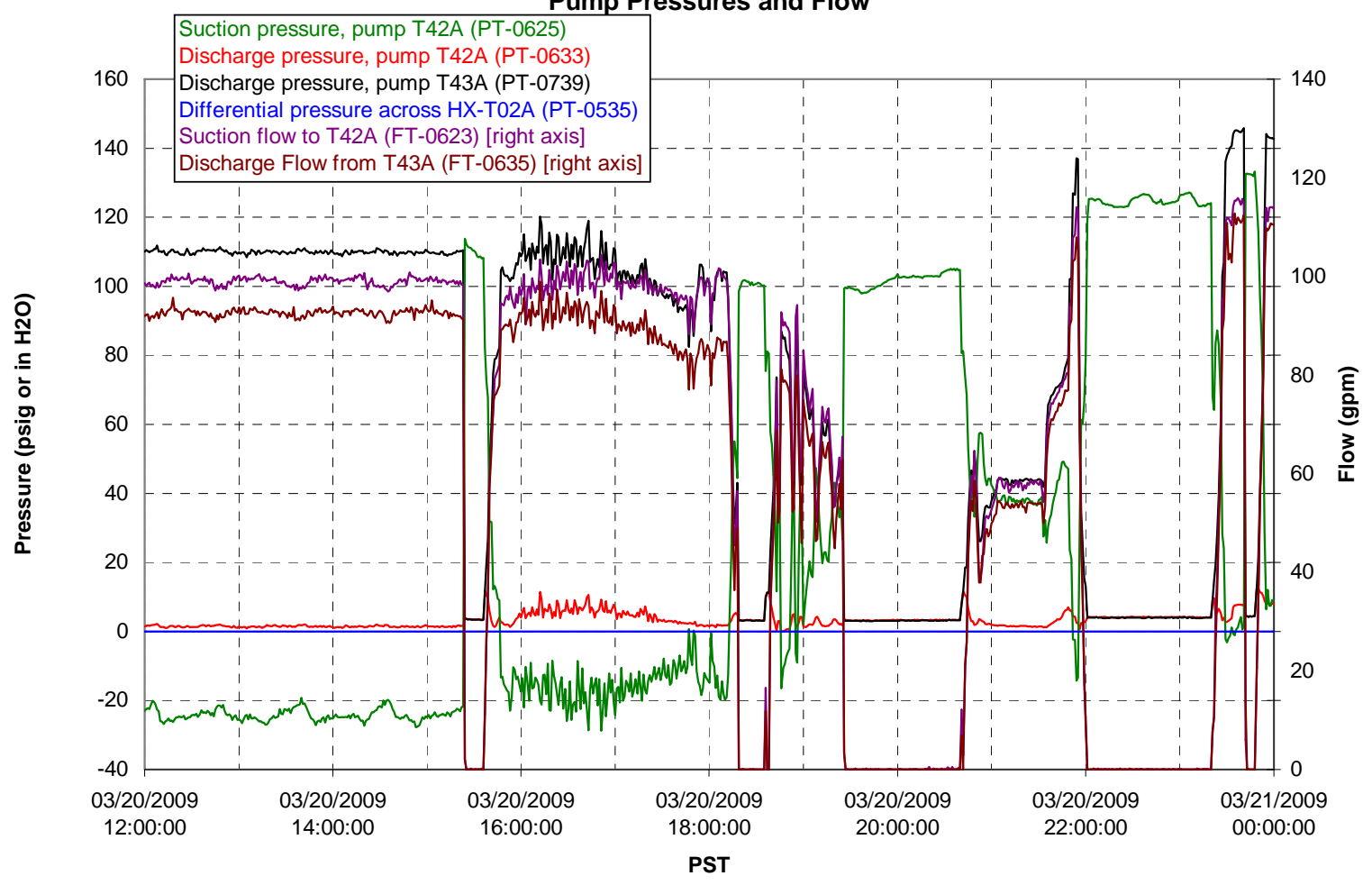




\section{Axial pressure drop}

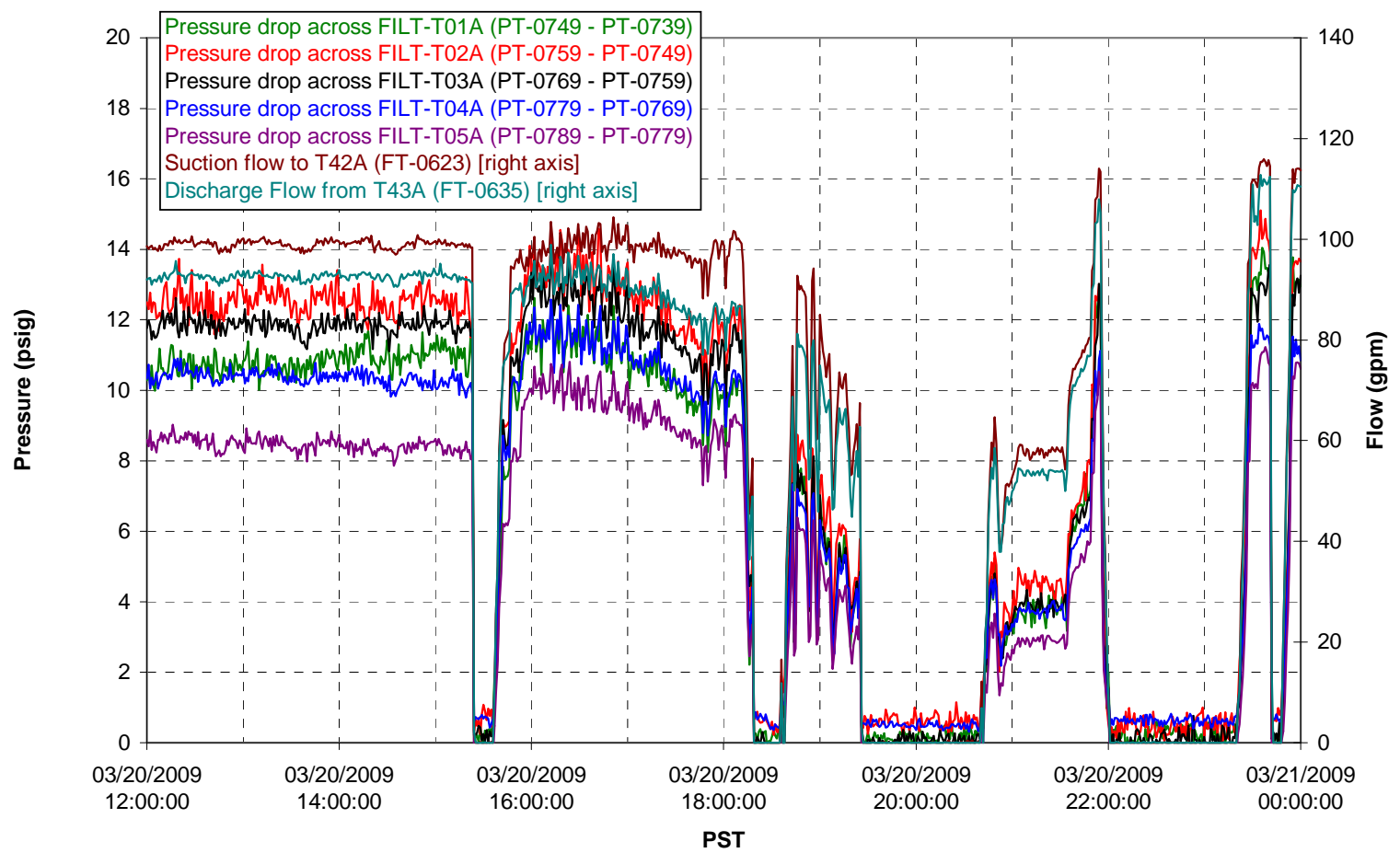

Permeate flow rates

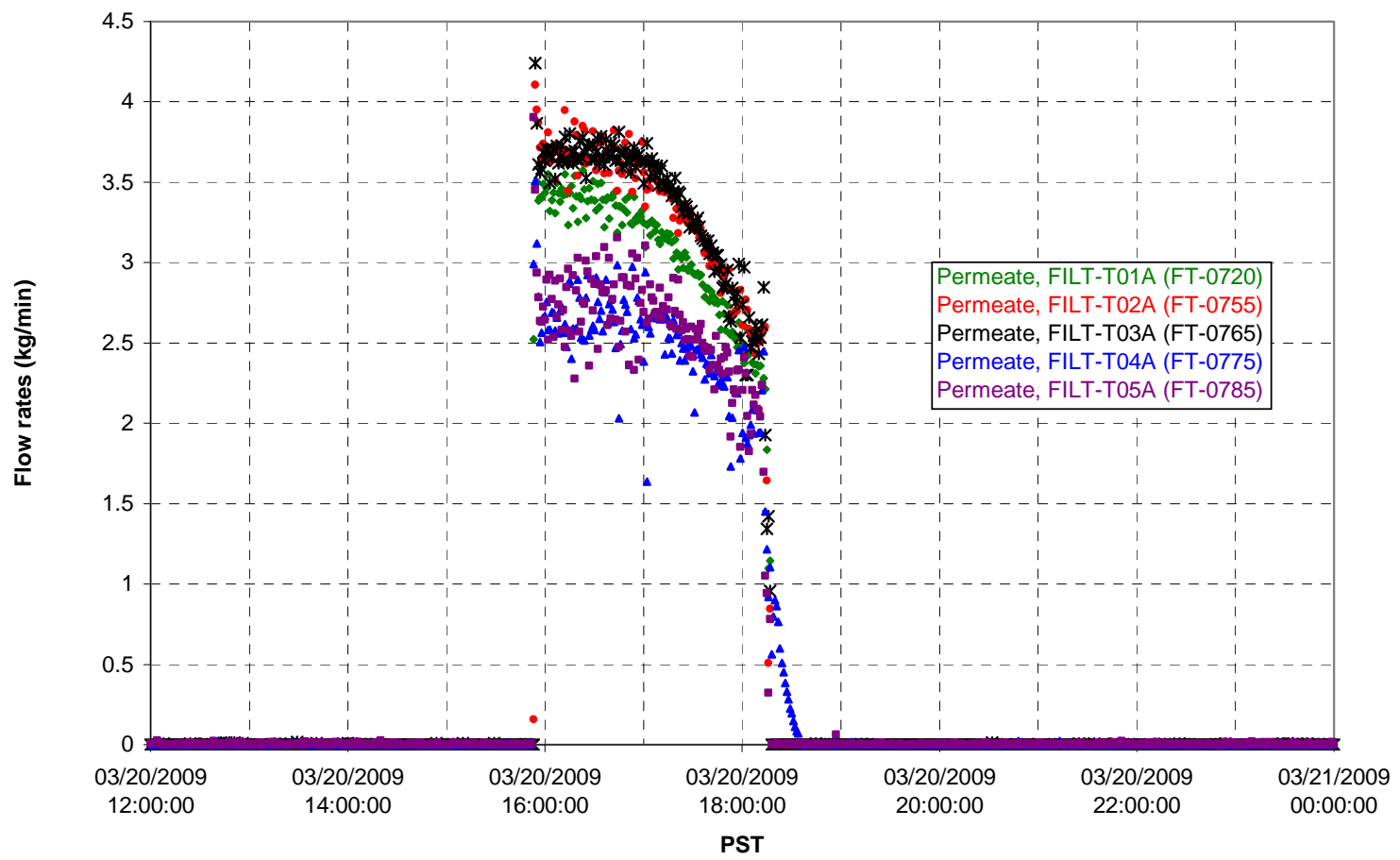


T02A Inner Temperature Tree

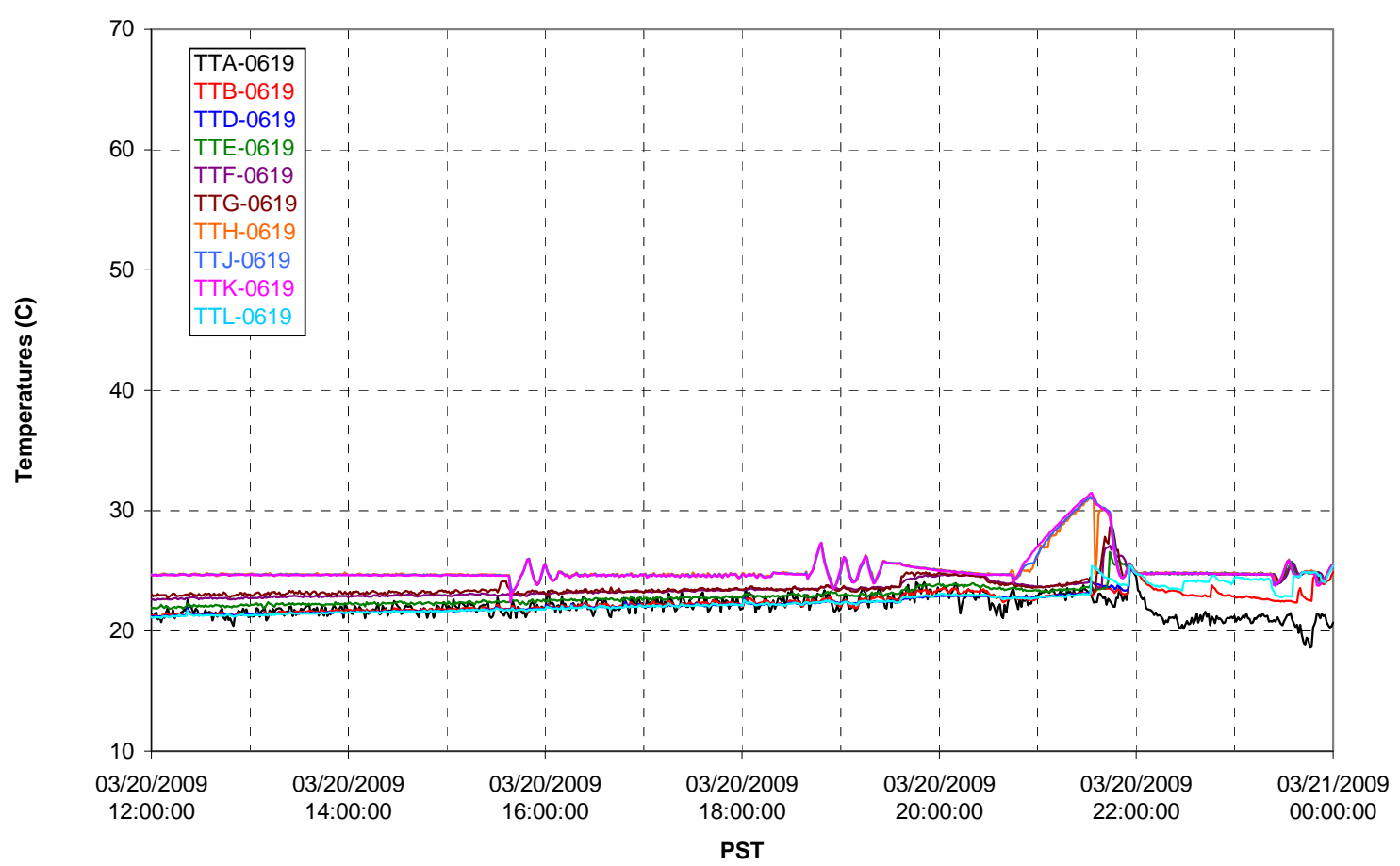

T02A Outer Temperature Tree

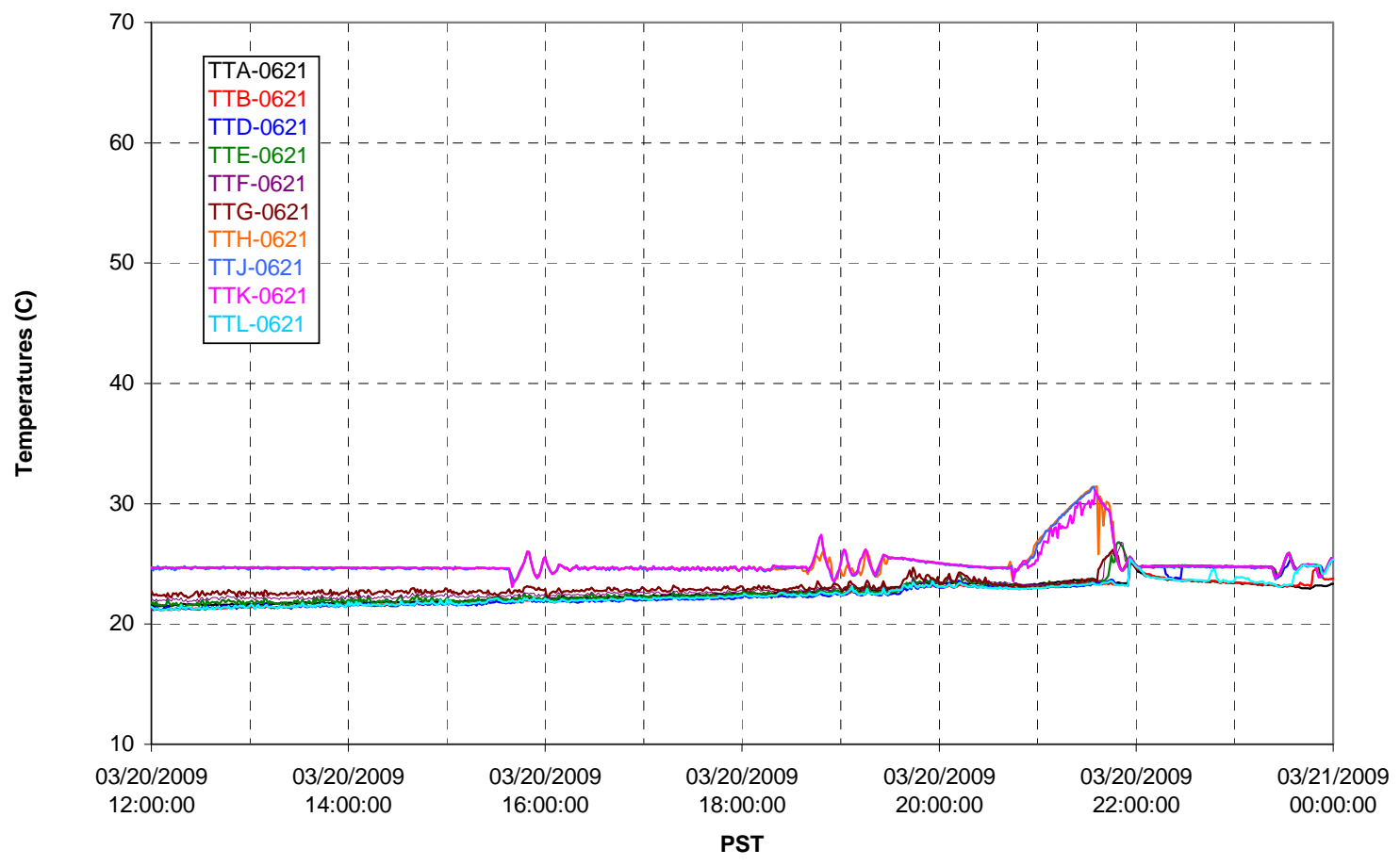


T02A temperatures

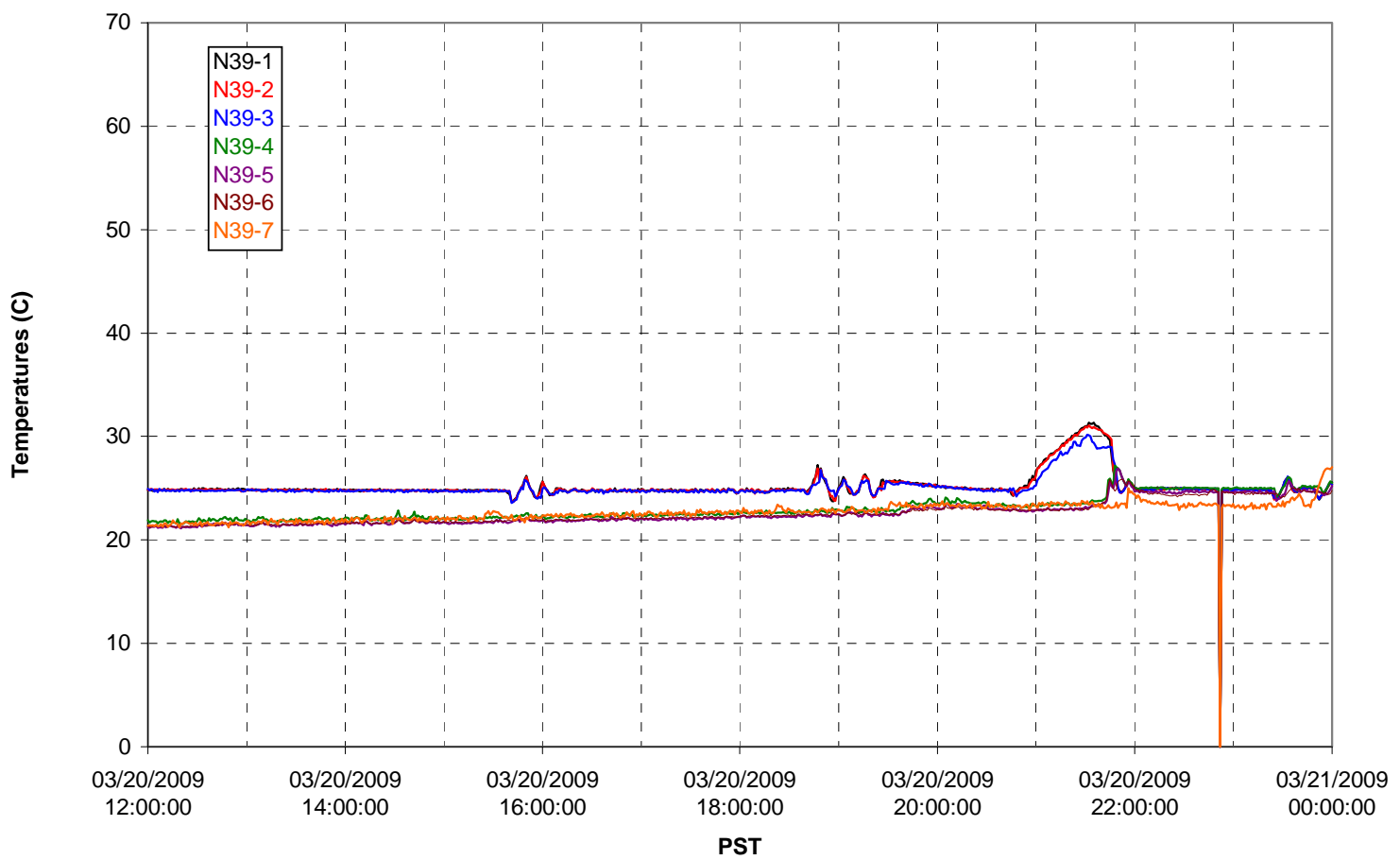

T02A temperatures

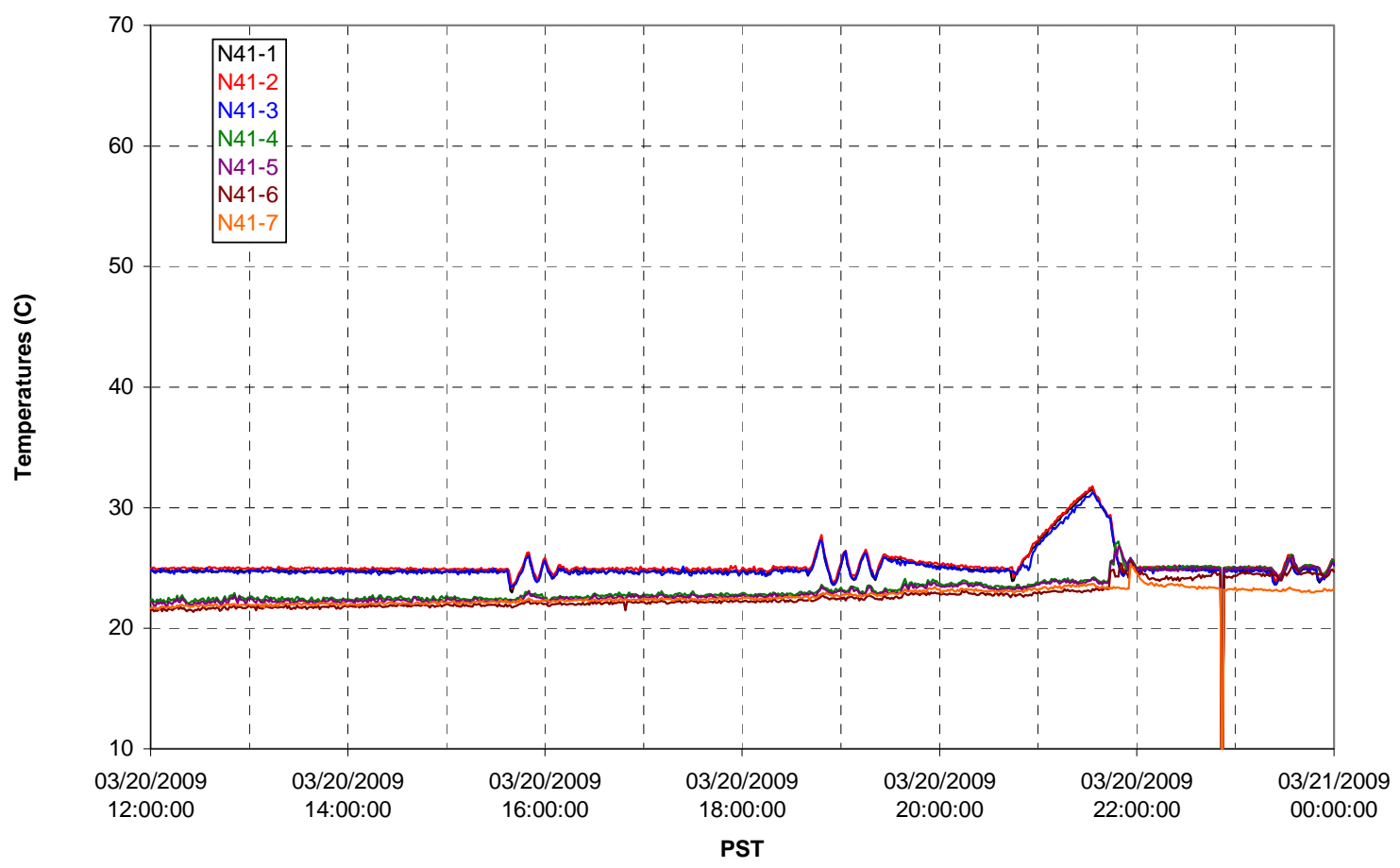


T02A temperatures

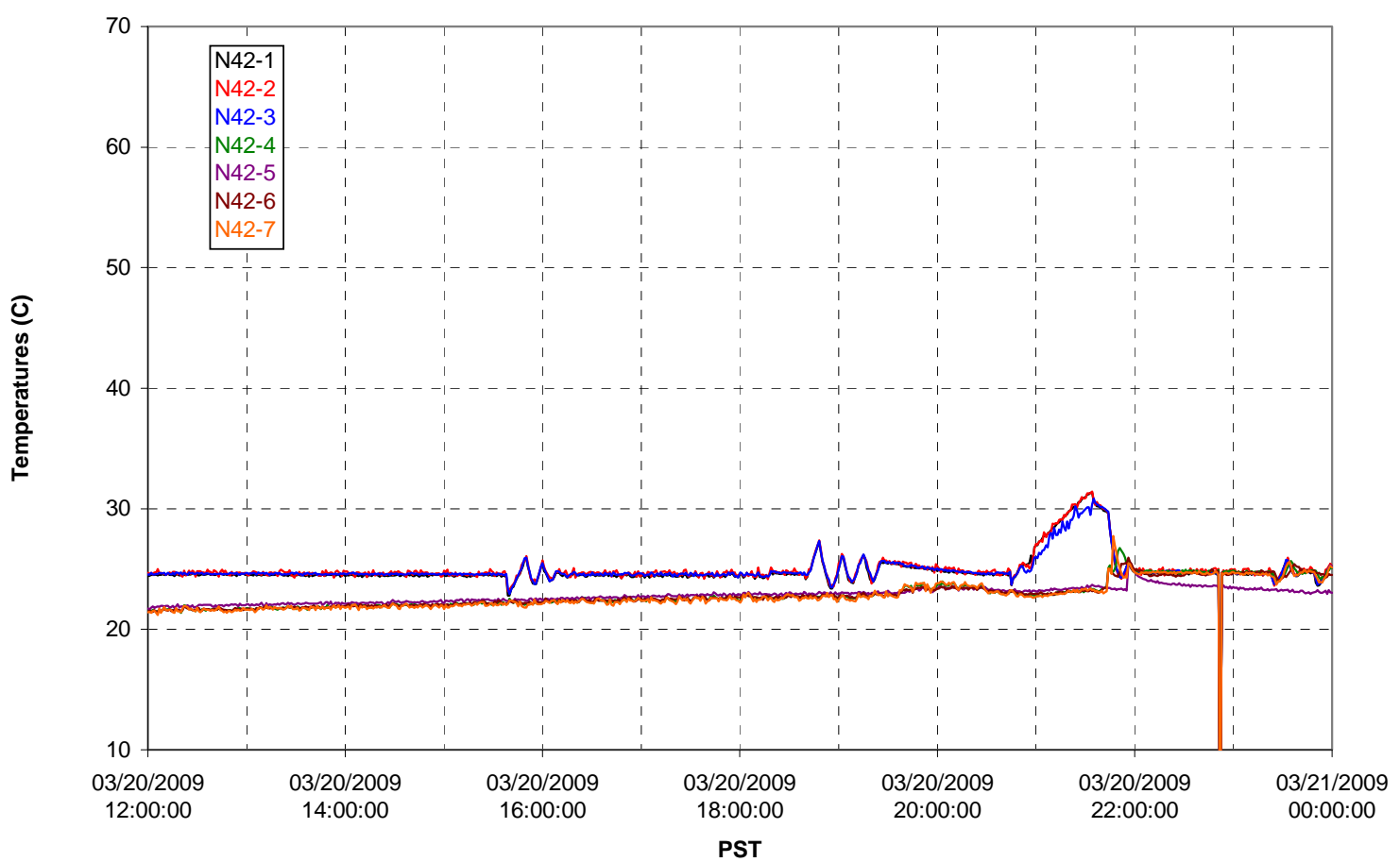

T02A temperatures

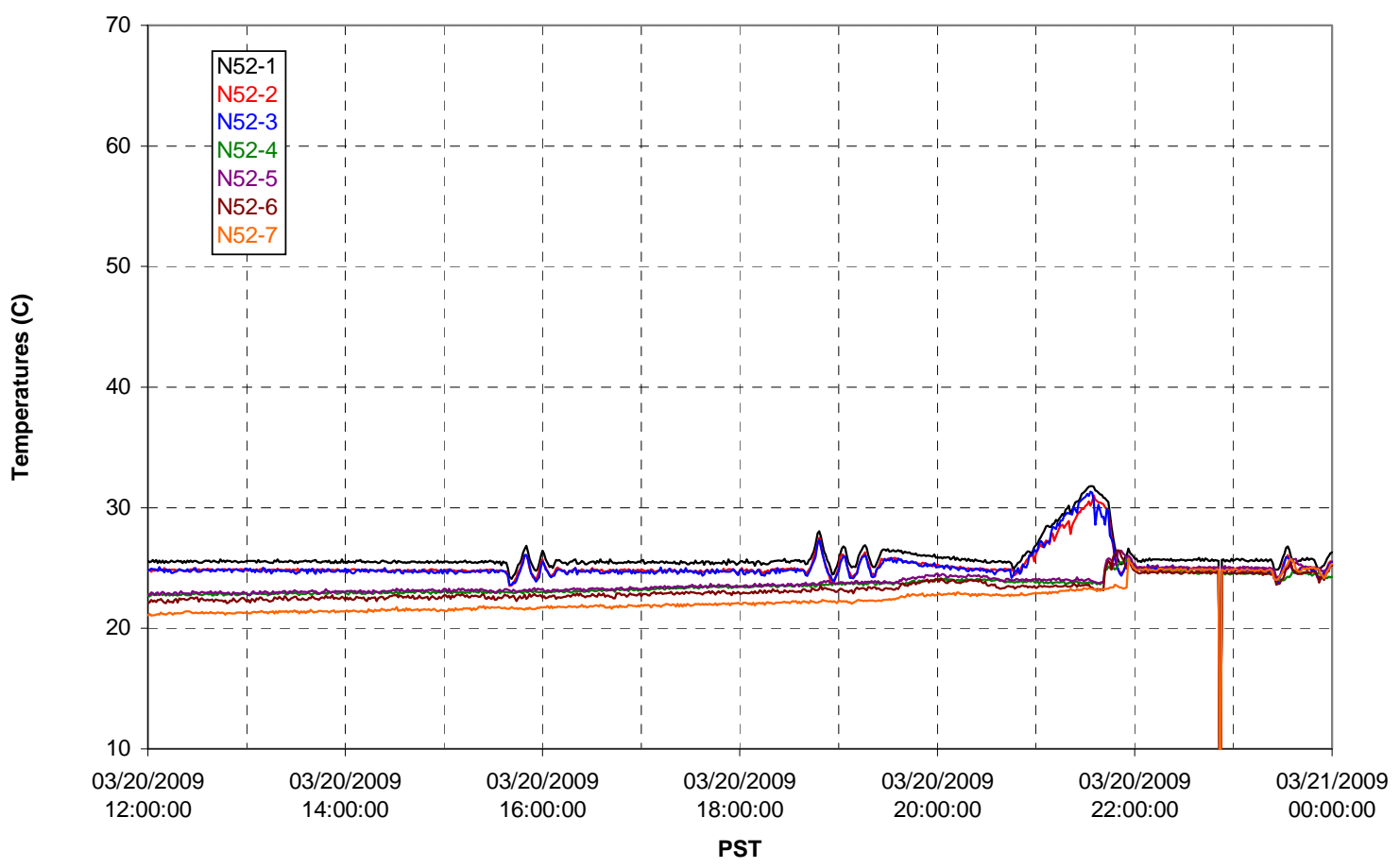


T02A Heating and Cooling
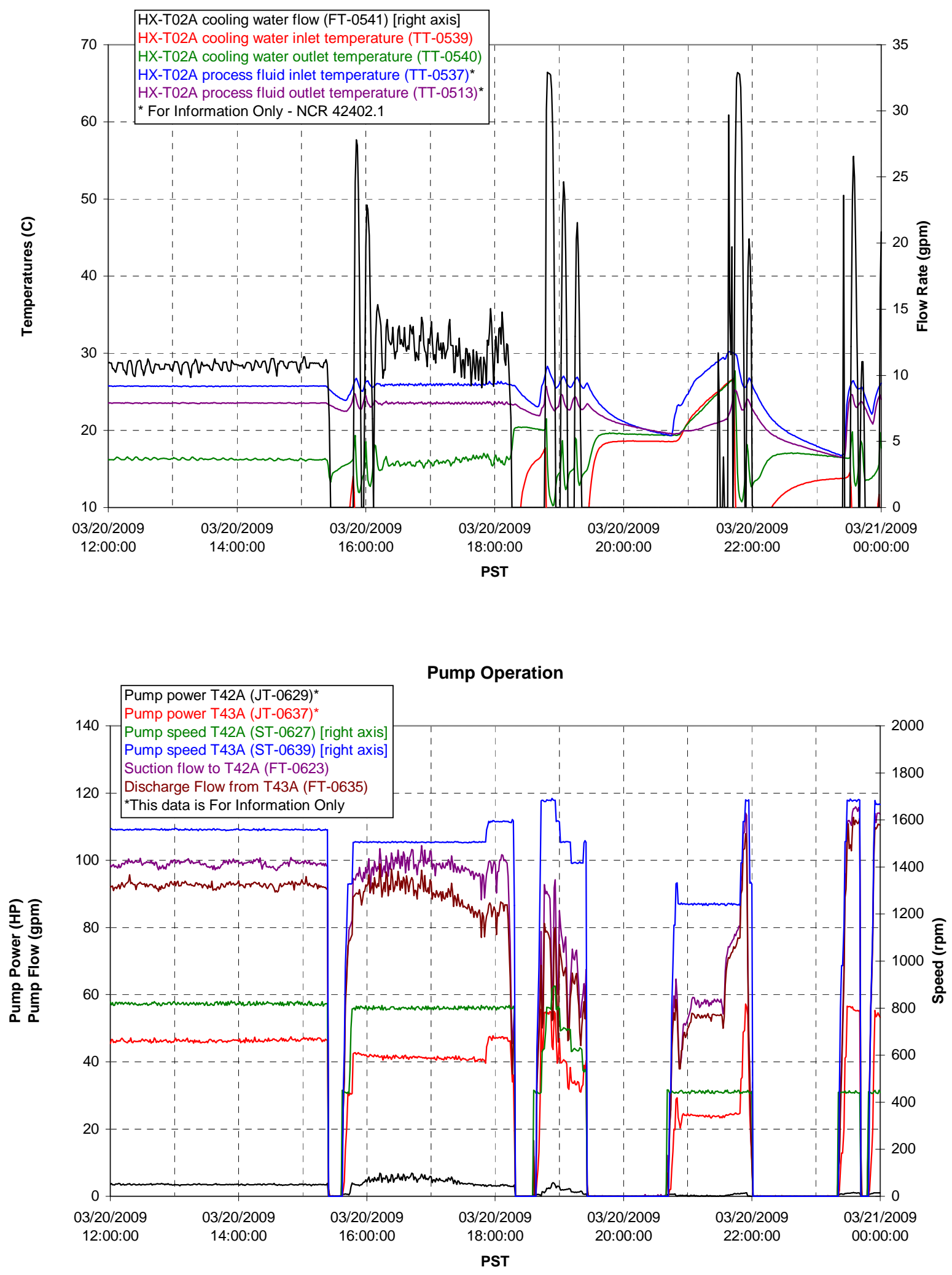
Pulsepot UFP-PP-T01A

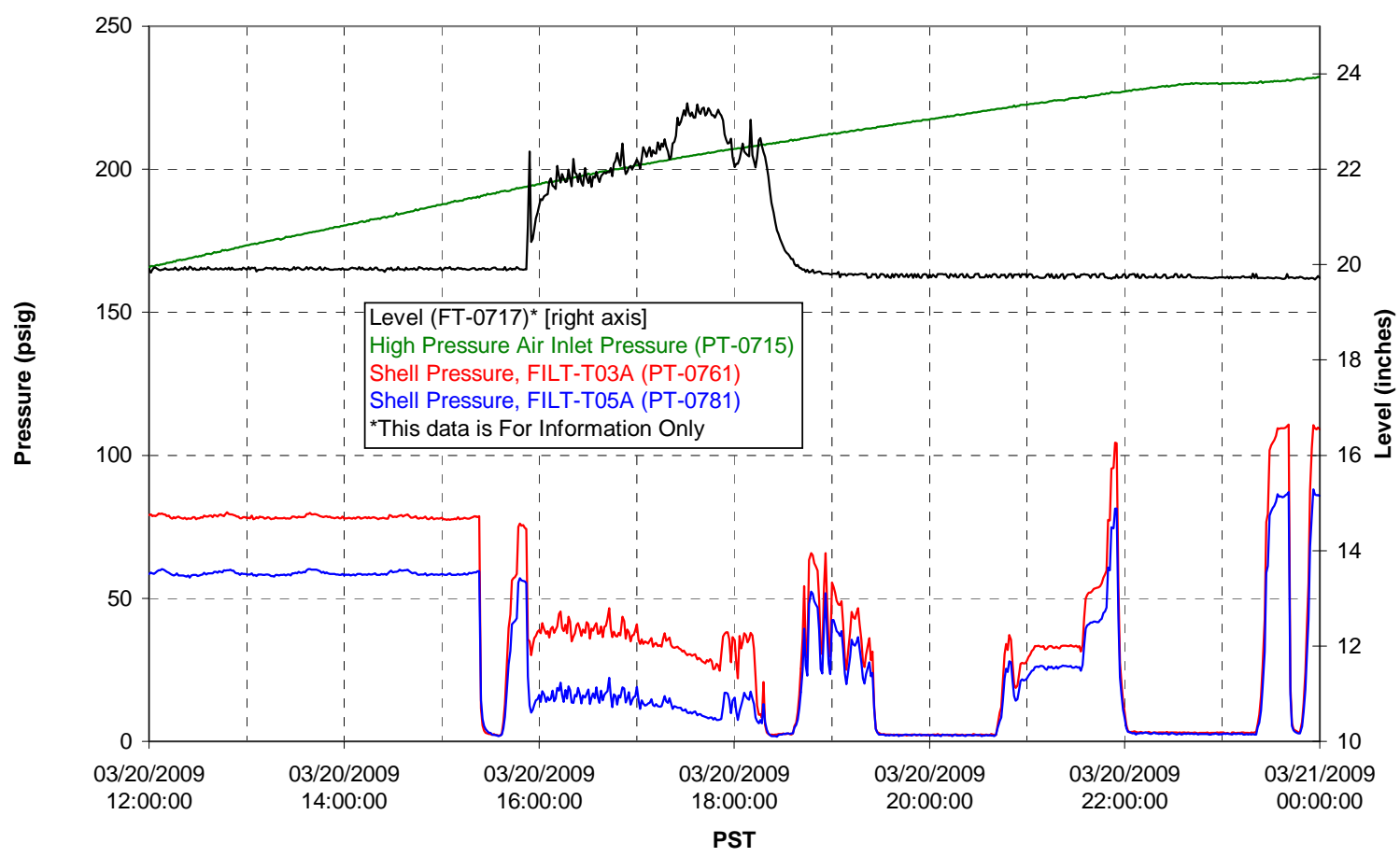

Pulsepot UFP-PP-T02A

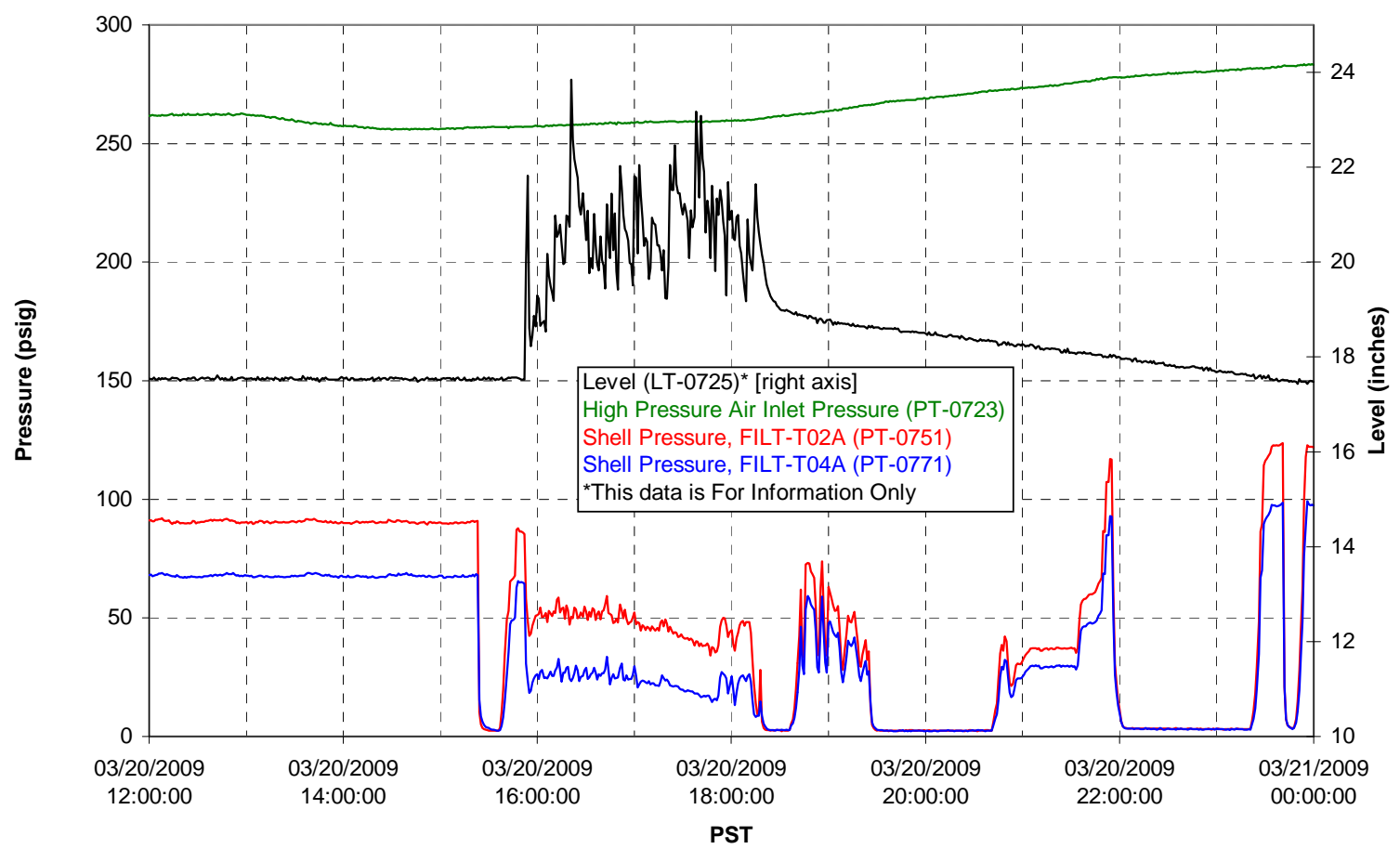


Pulsepot UFP-PP-T03A

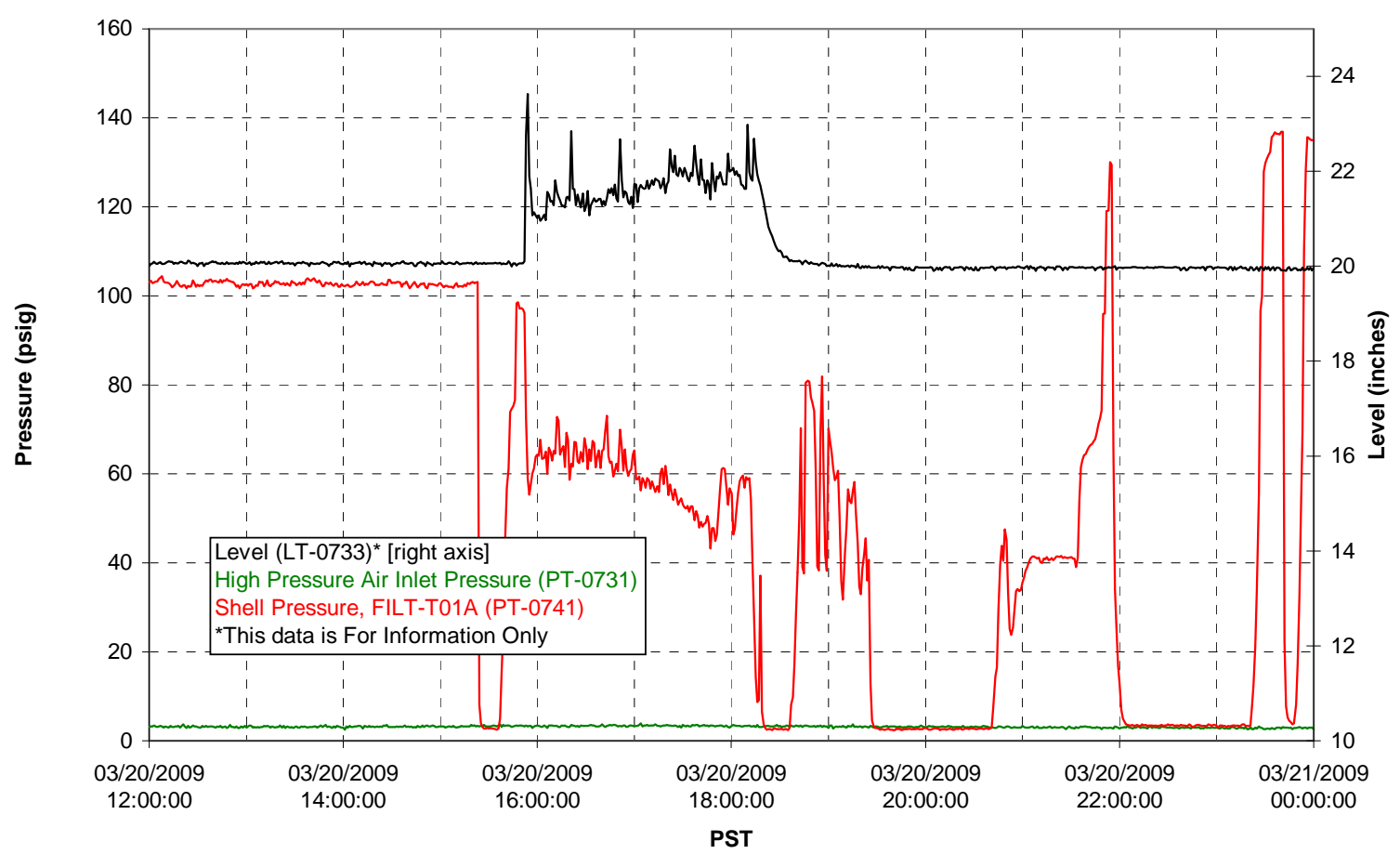

Pulsepot Levels

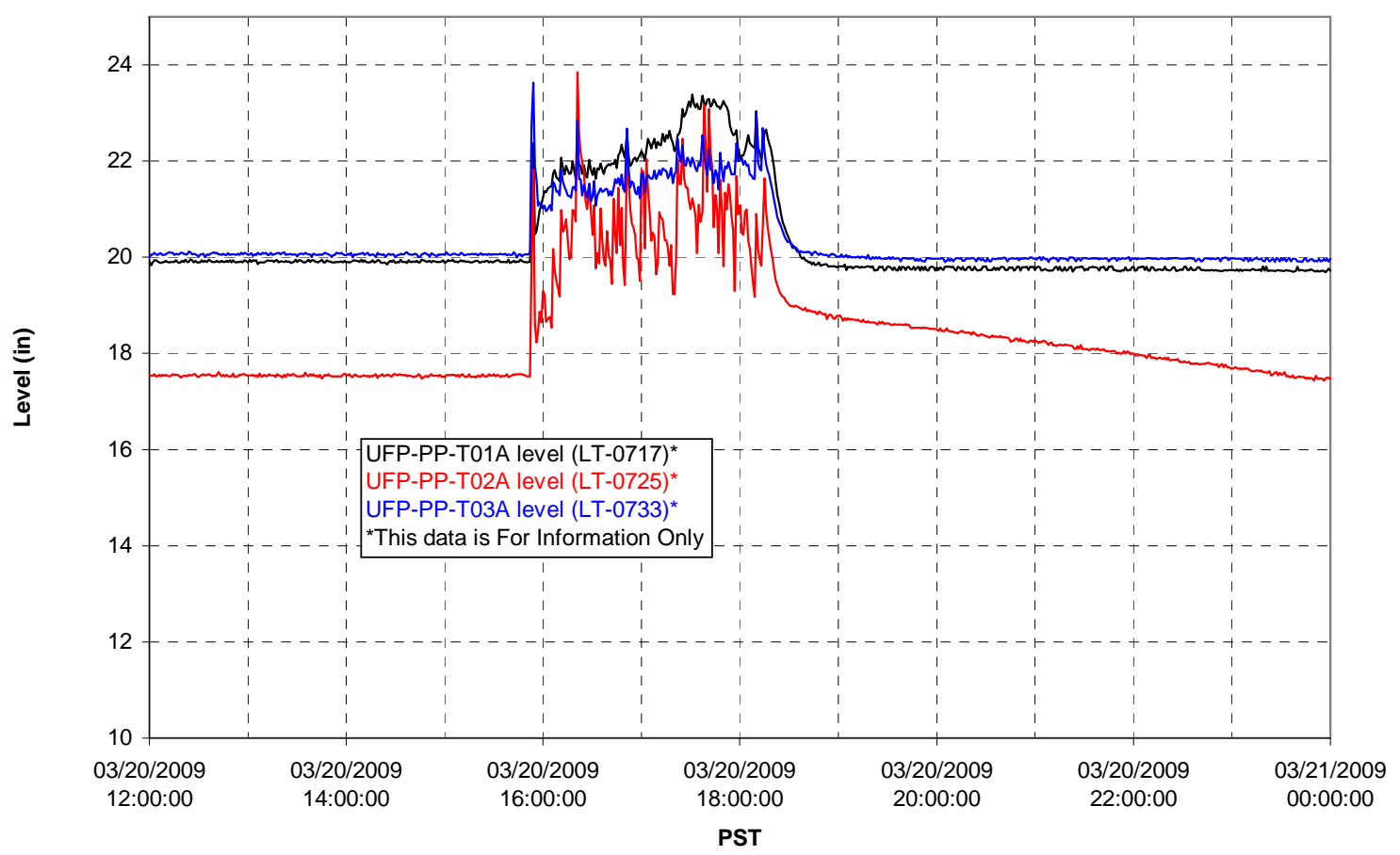


Filter UFP-FILT-T01A

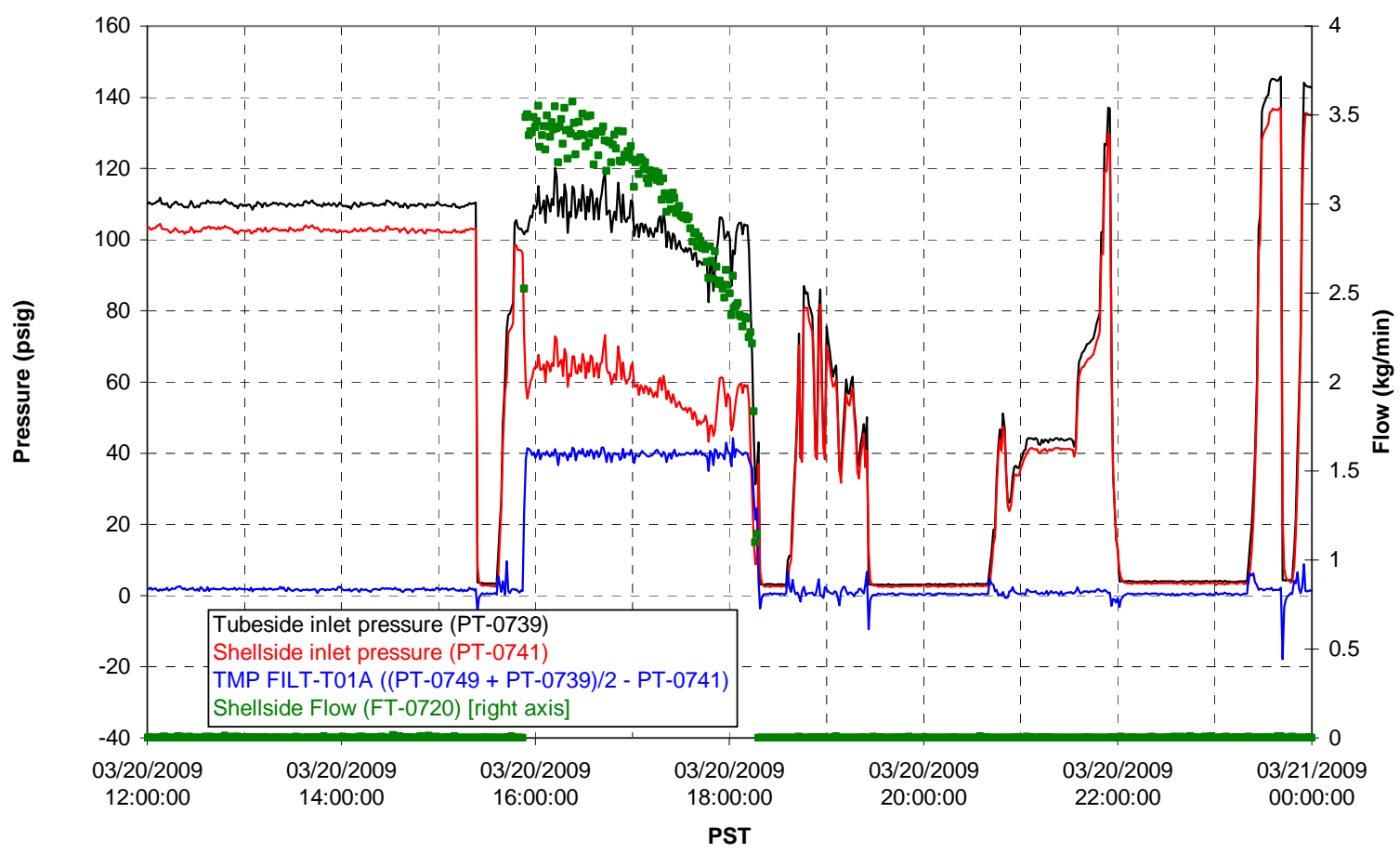

Filter UFP-FILT-T02A

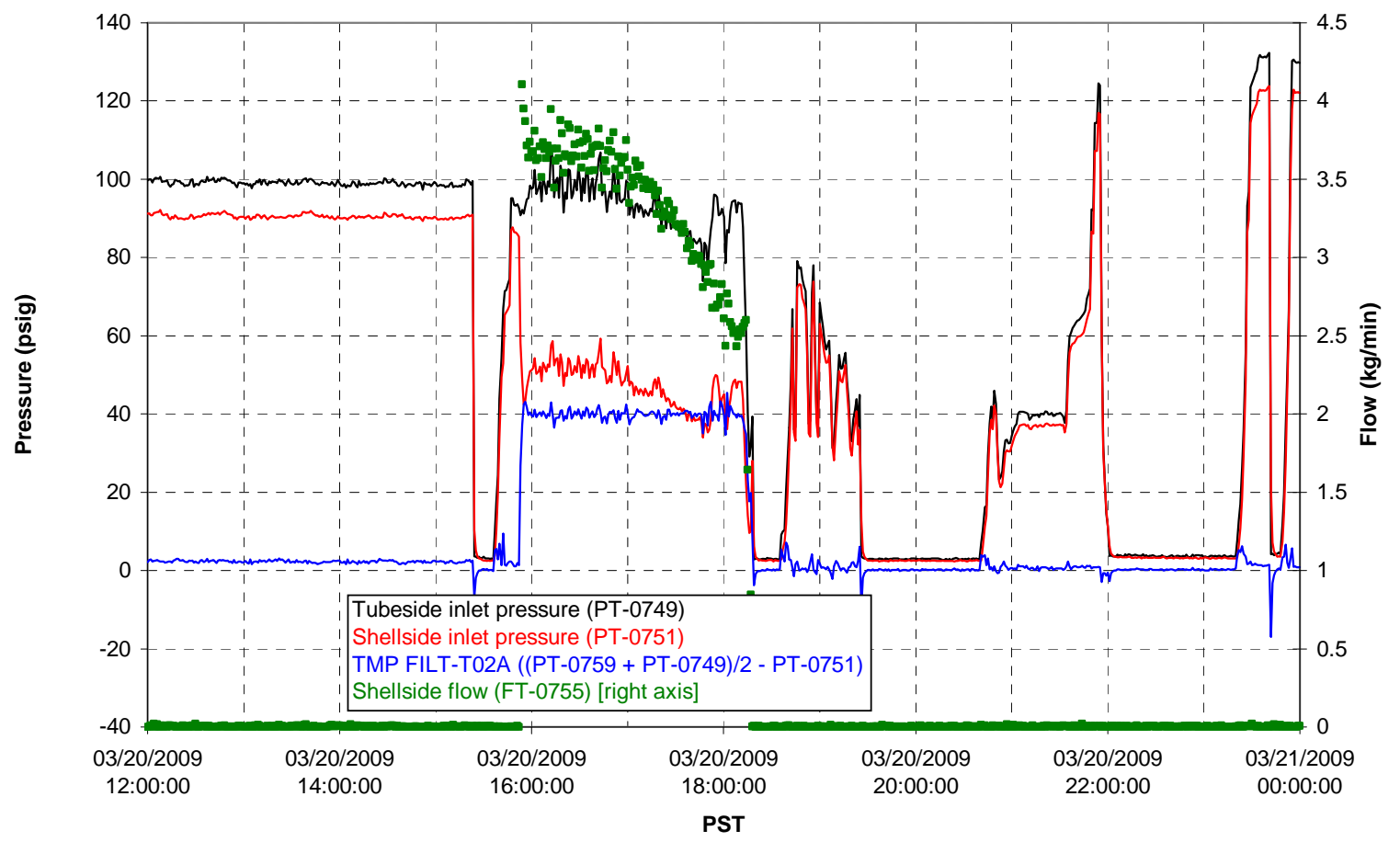


Filter UFP-FILT-T03A

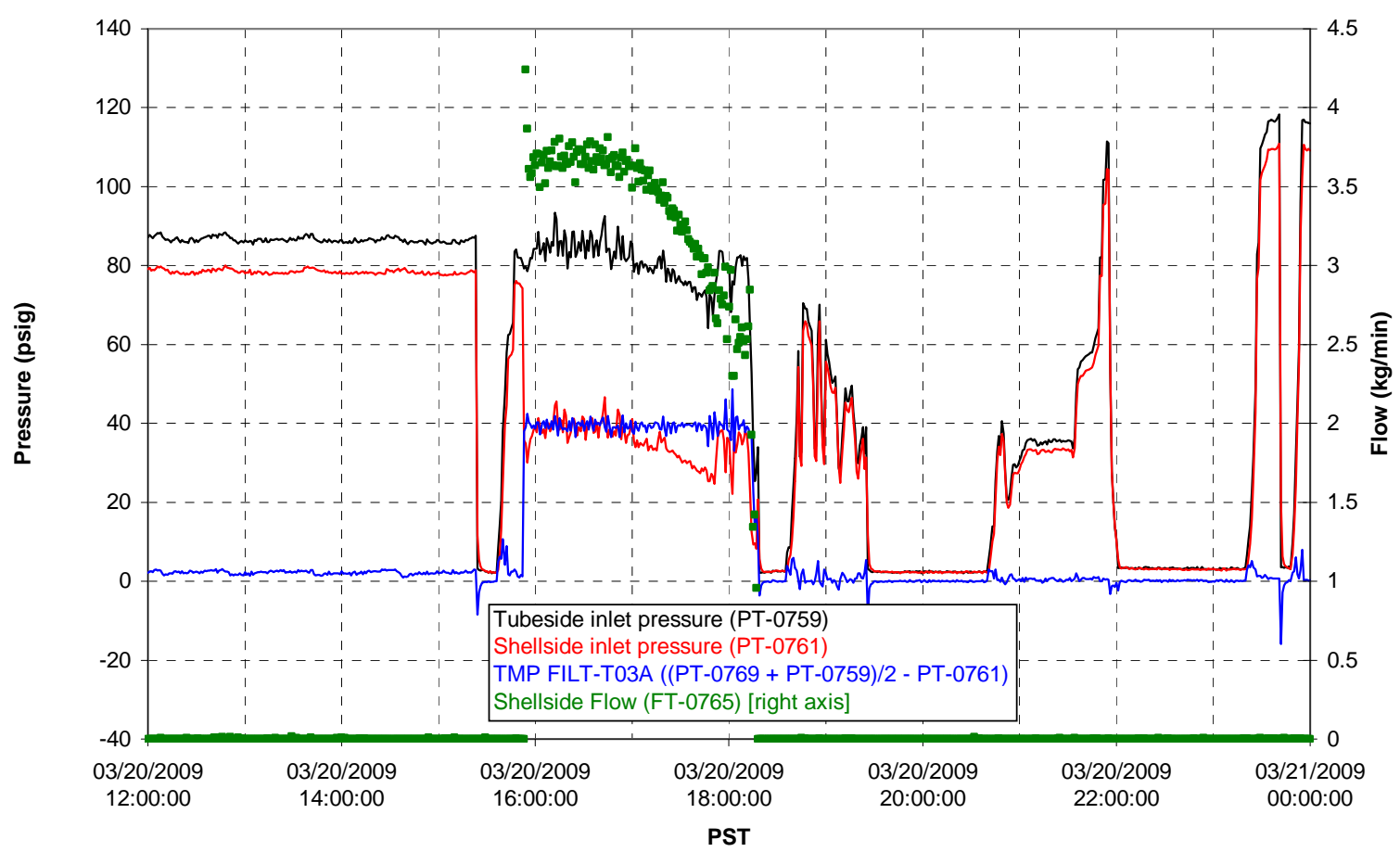

Filter UFP-FILT-T04A

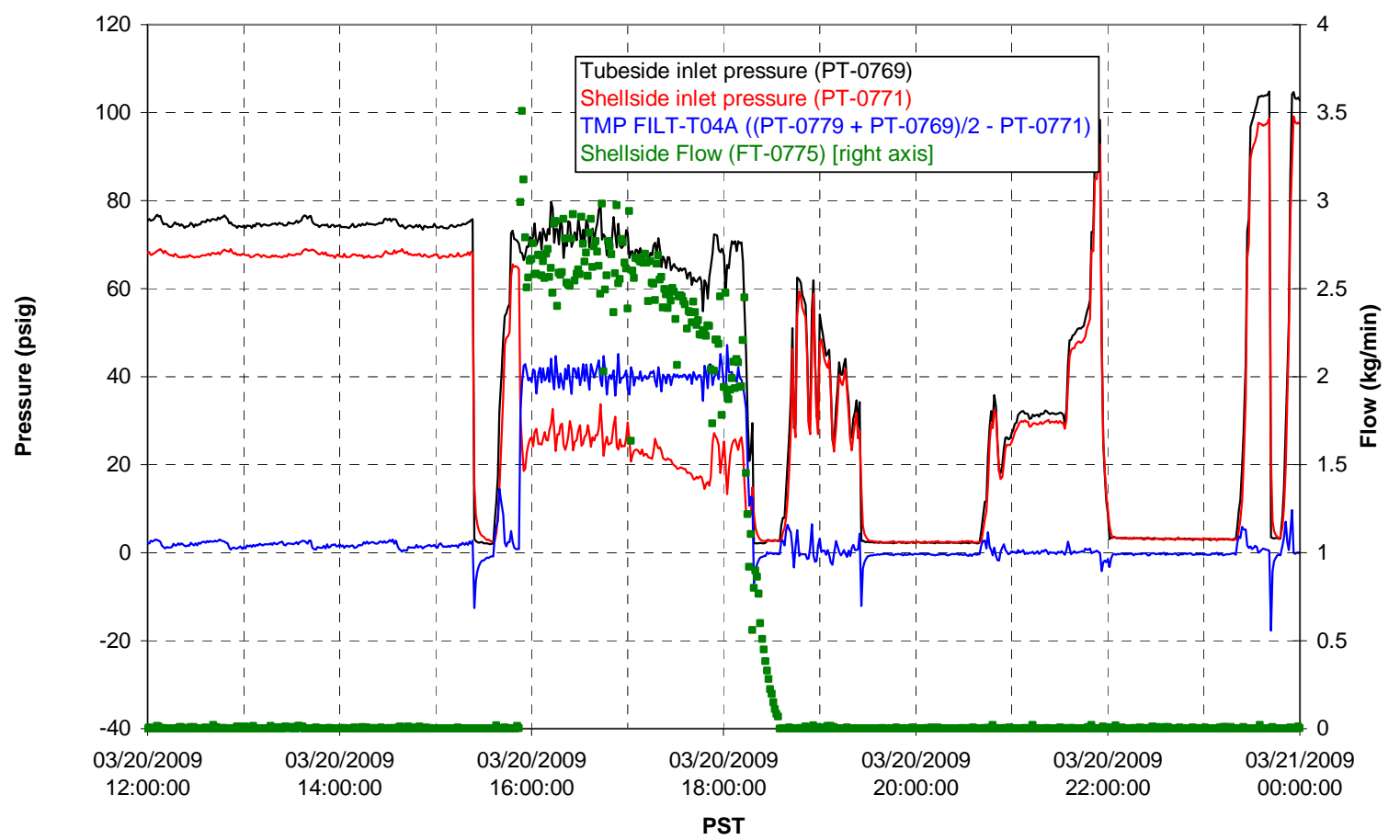


Filter UFP-FILT-T05A

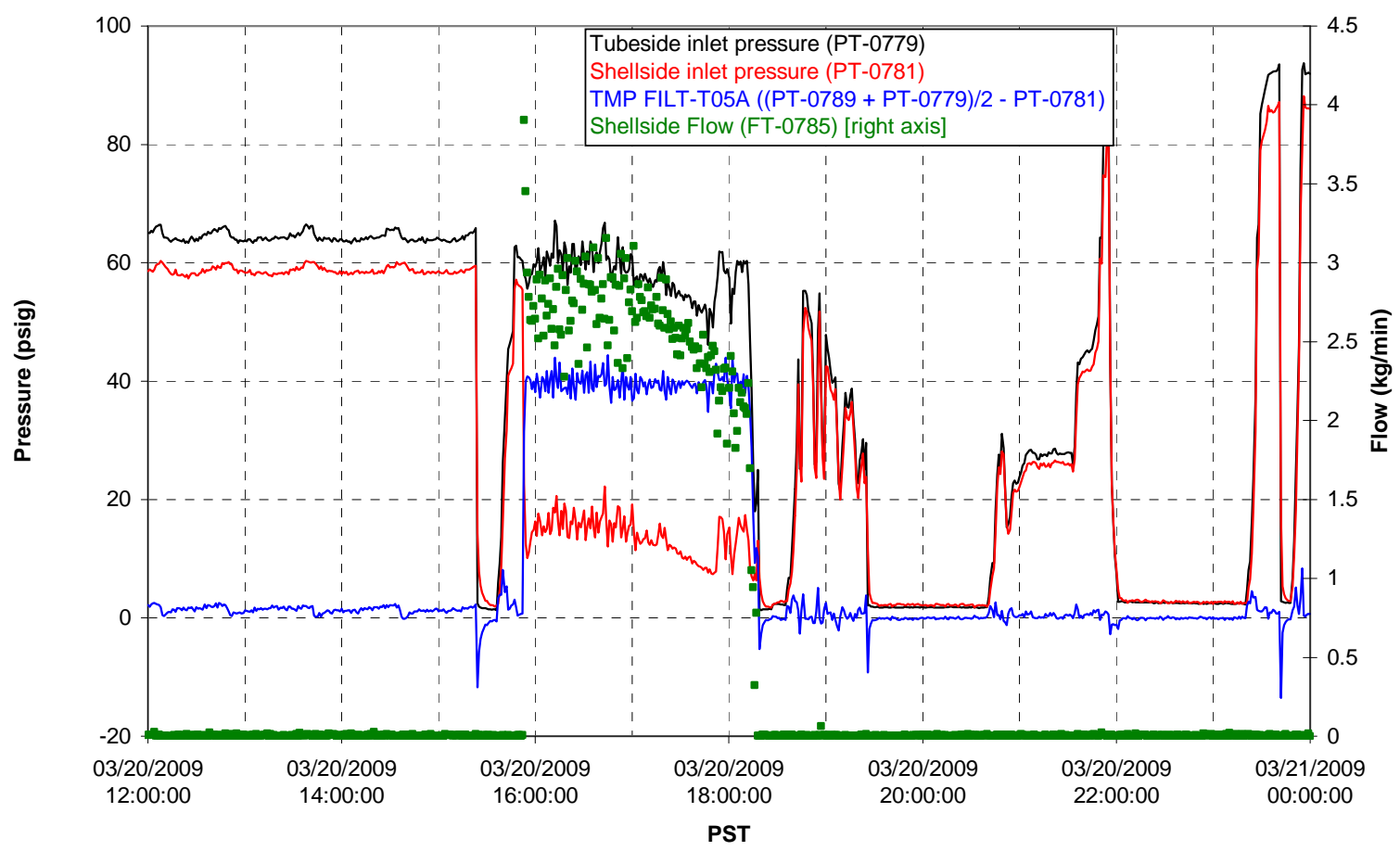

Chemical Flow

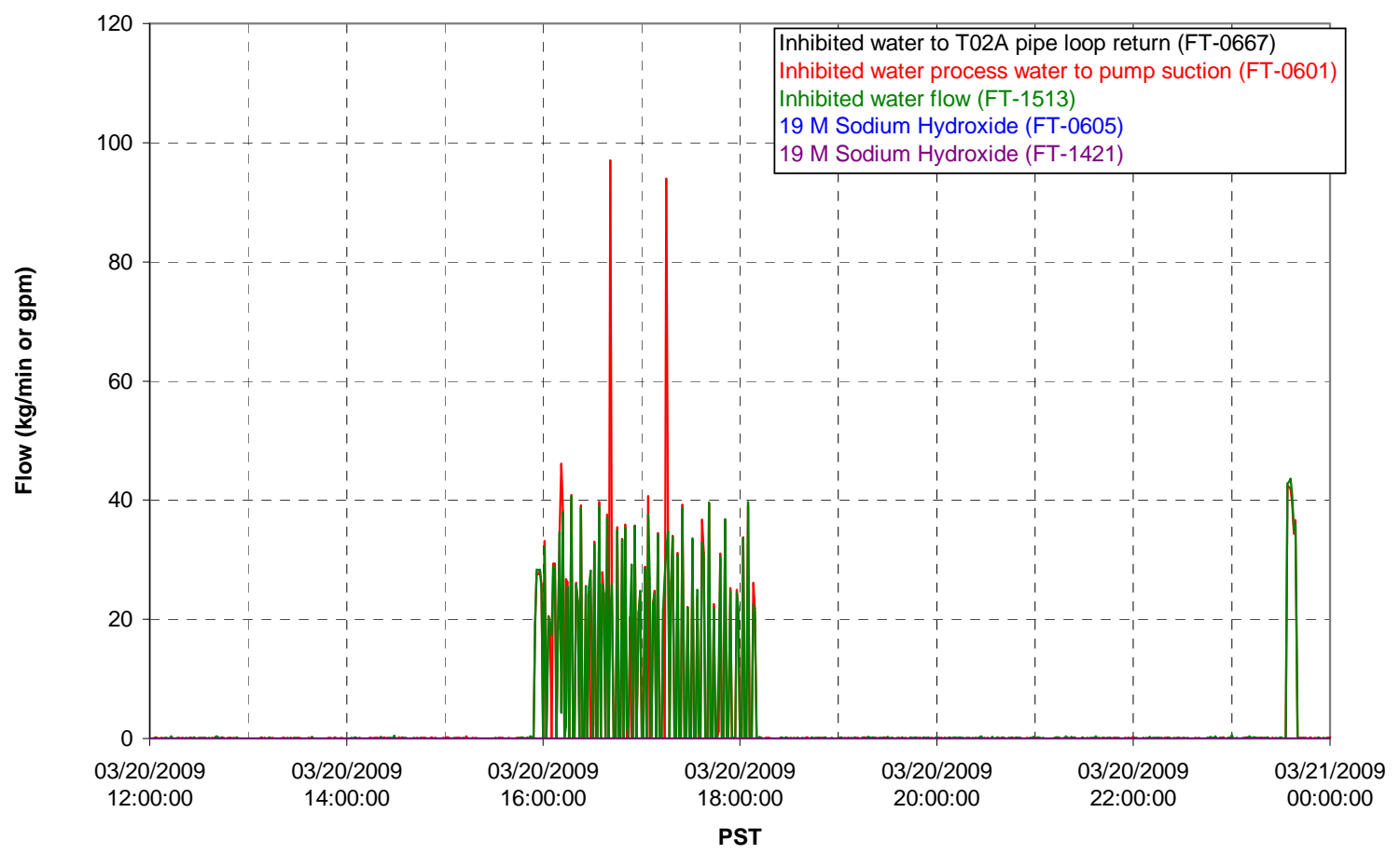




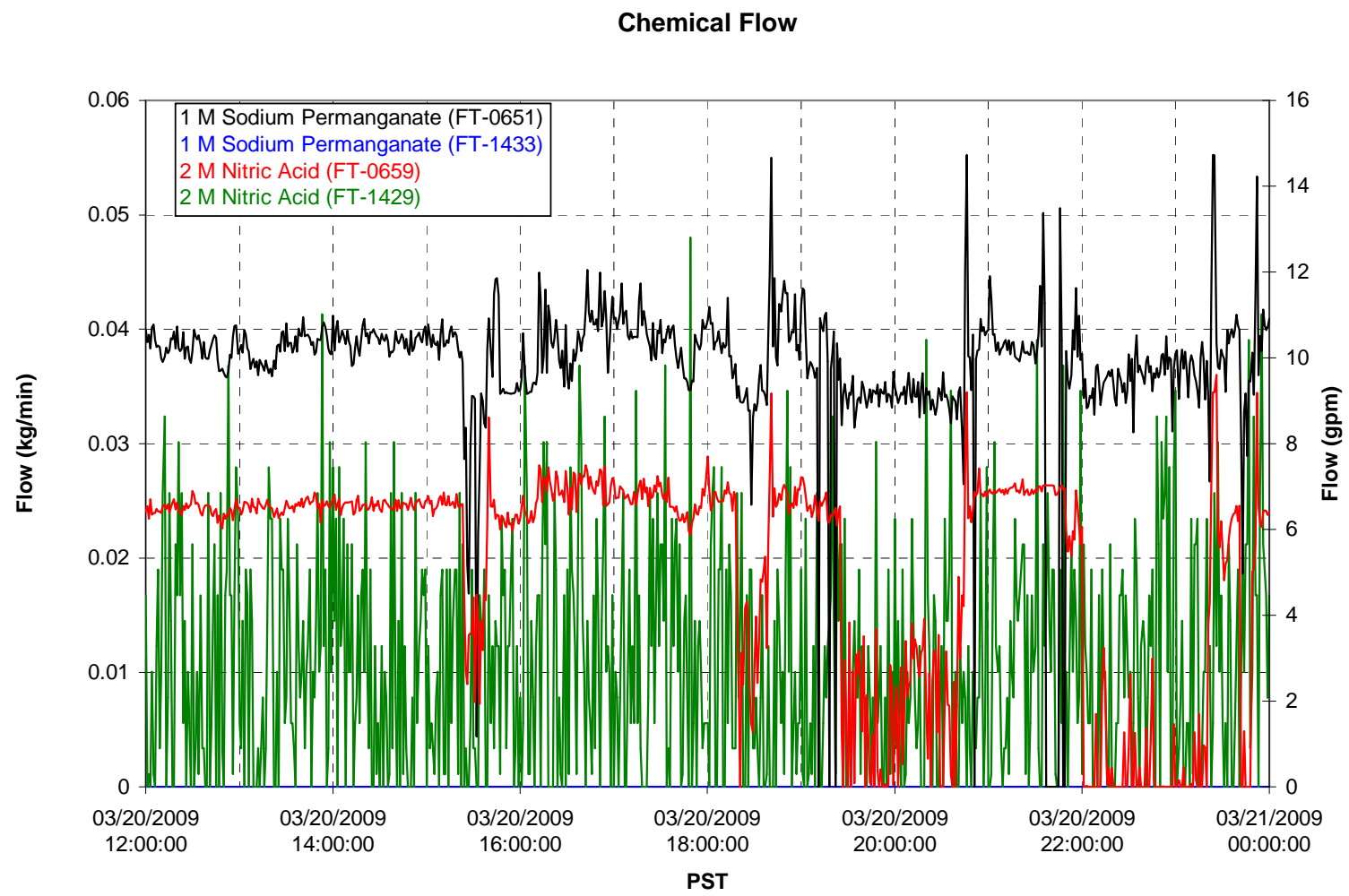

Air Flows

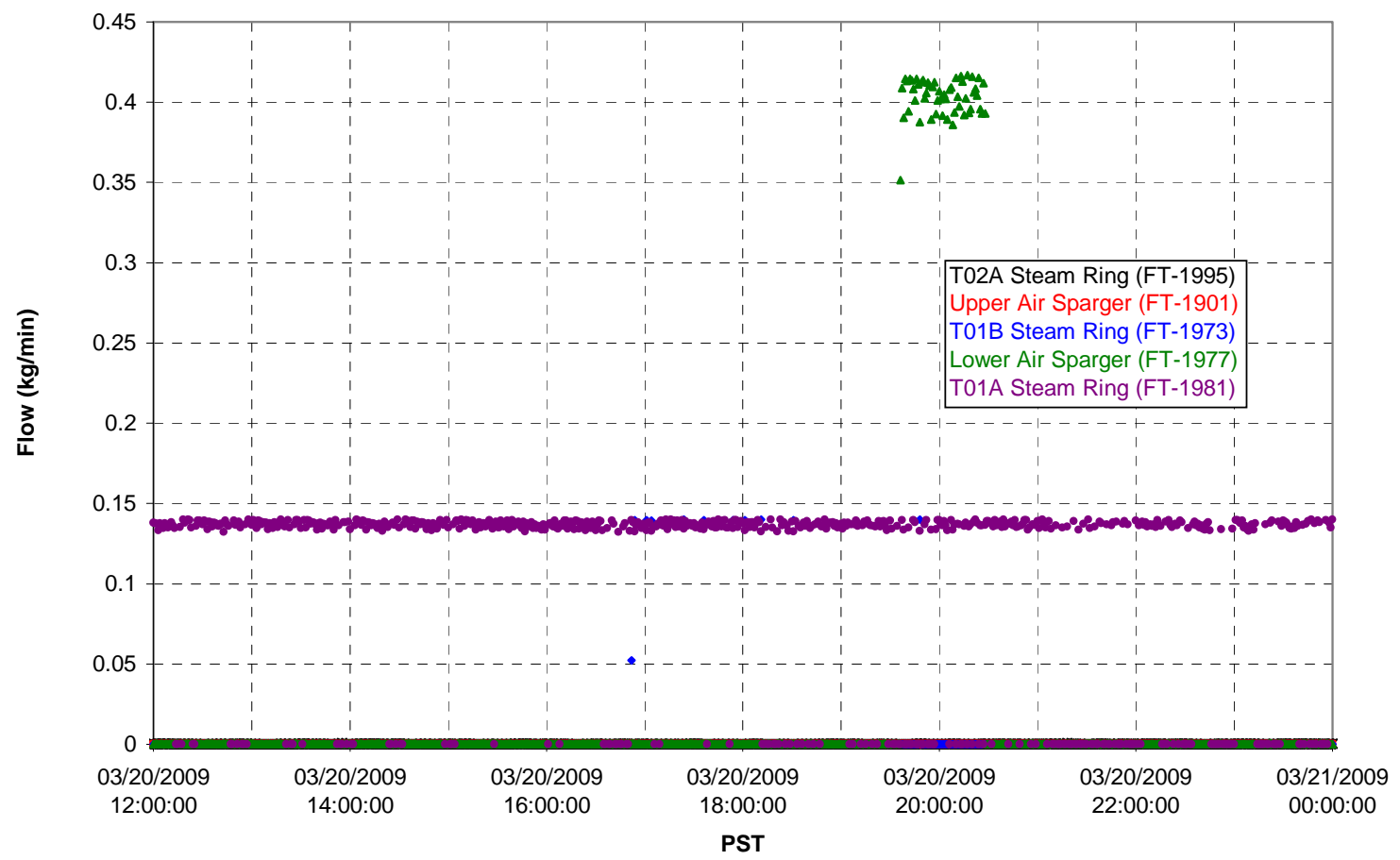


T02A Steam

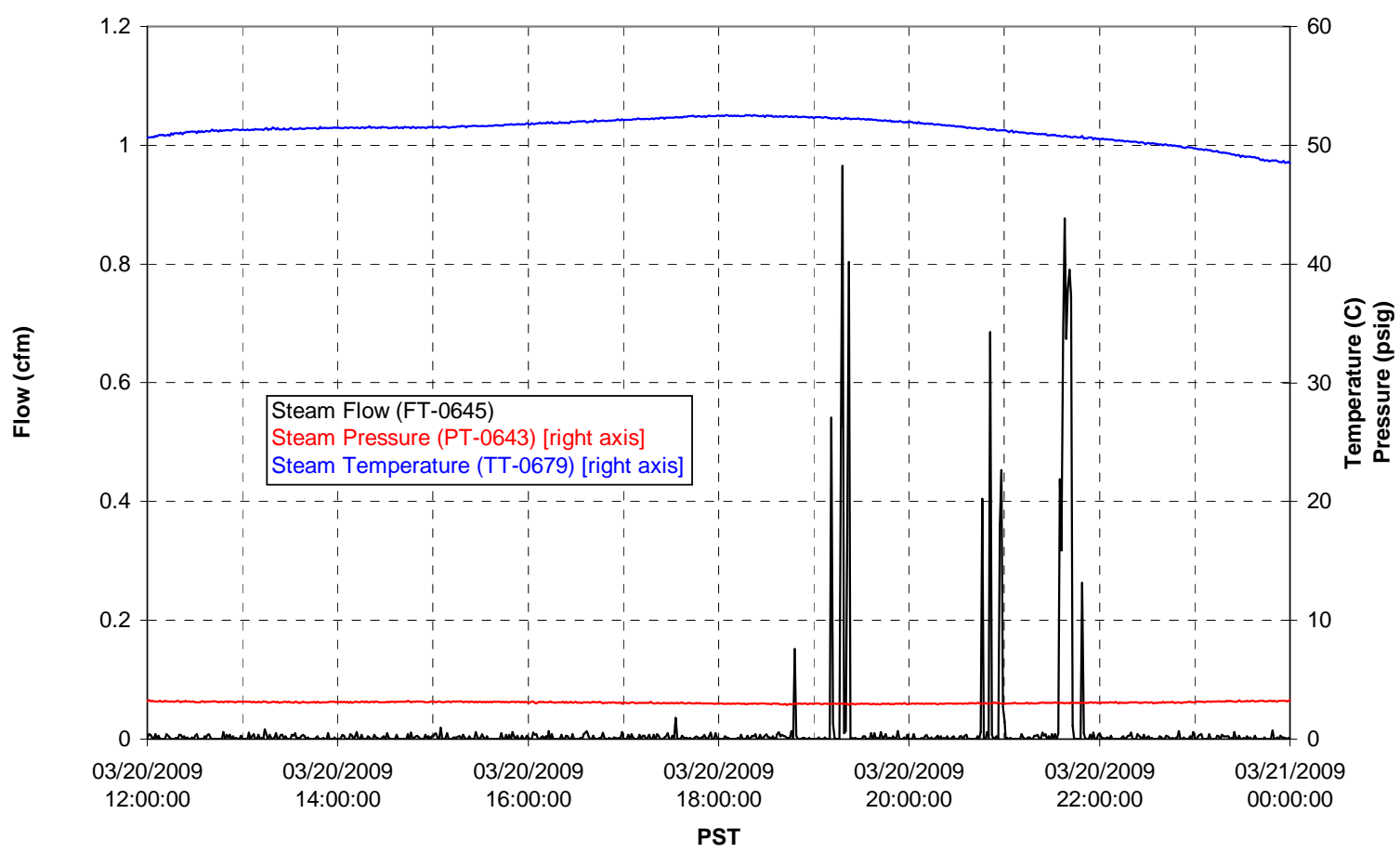

T01A Steam

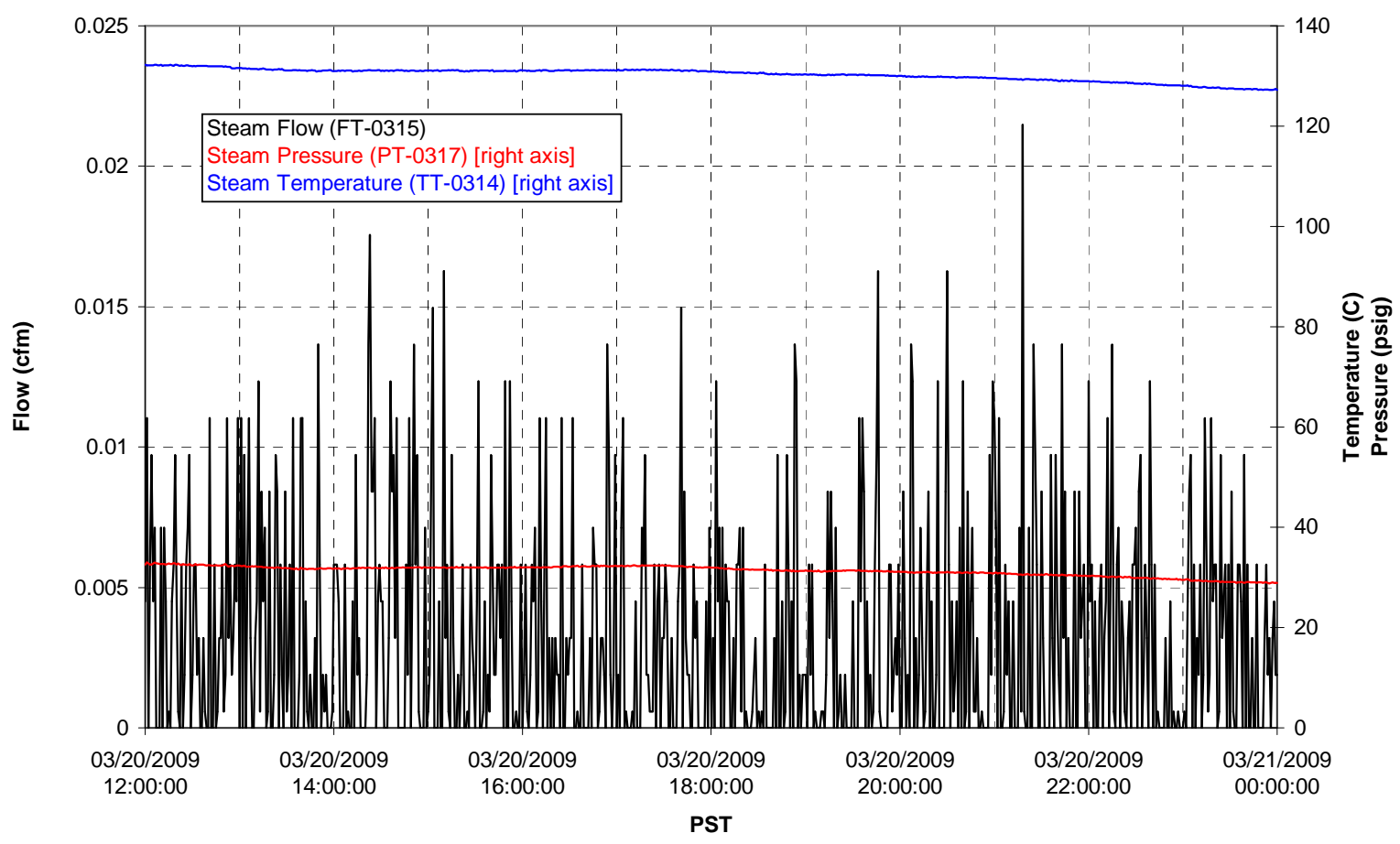


T01B Steam

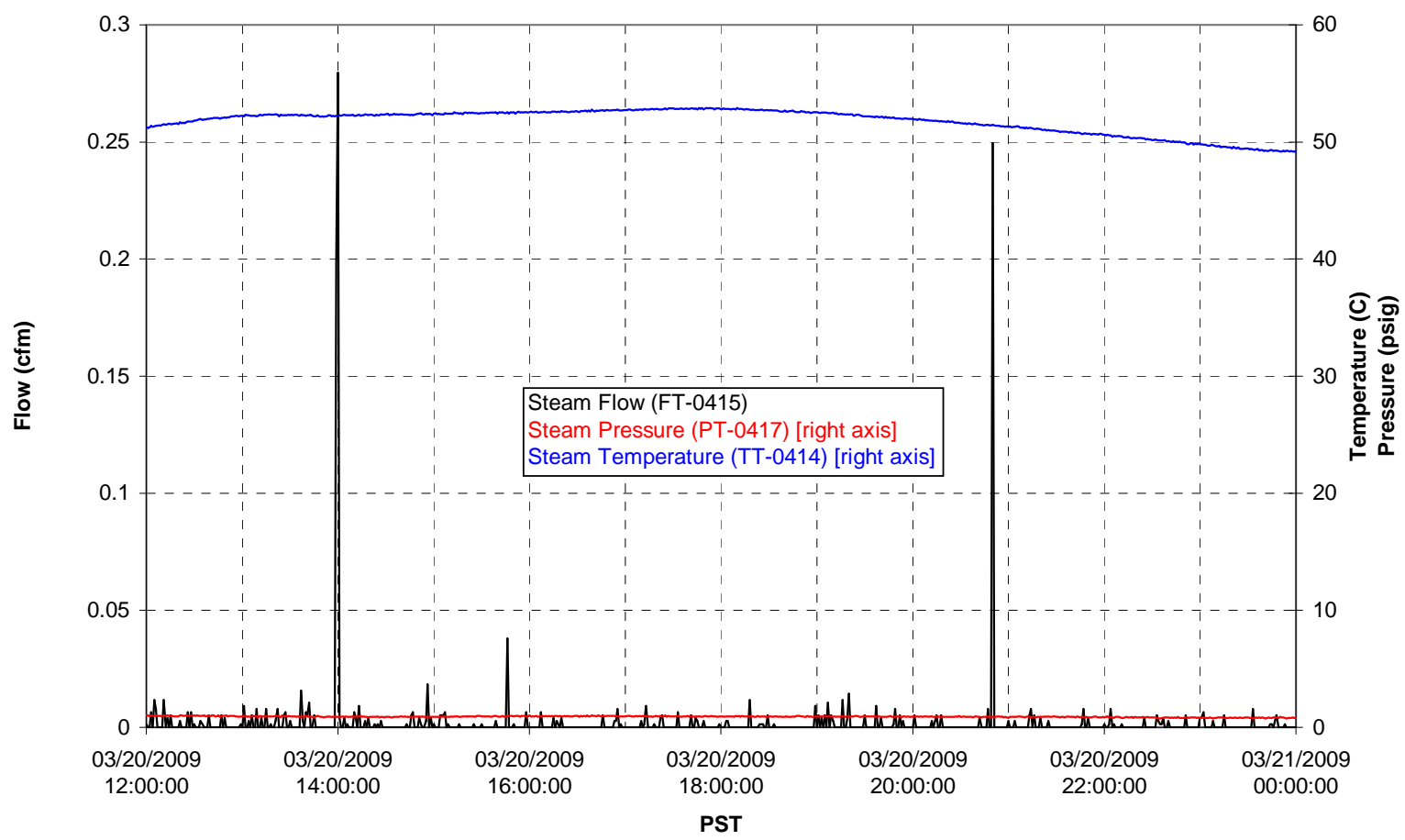




\section{Integrated Test A Data Plots 03/21/09 00:00 - 03/21/09 12:00}


T01A level

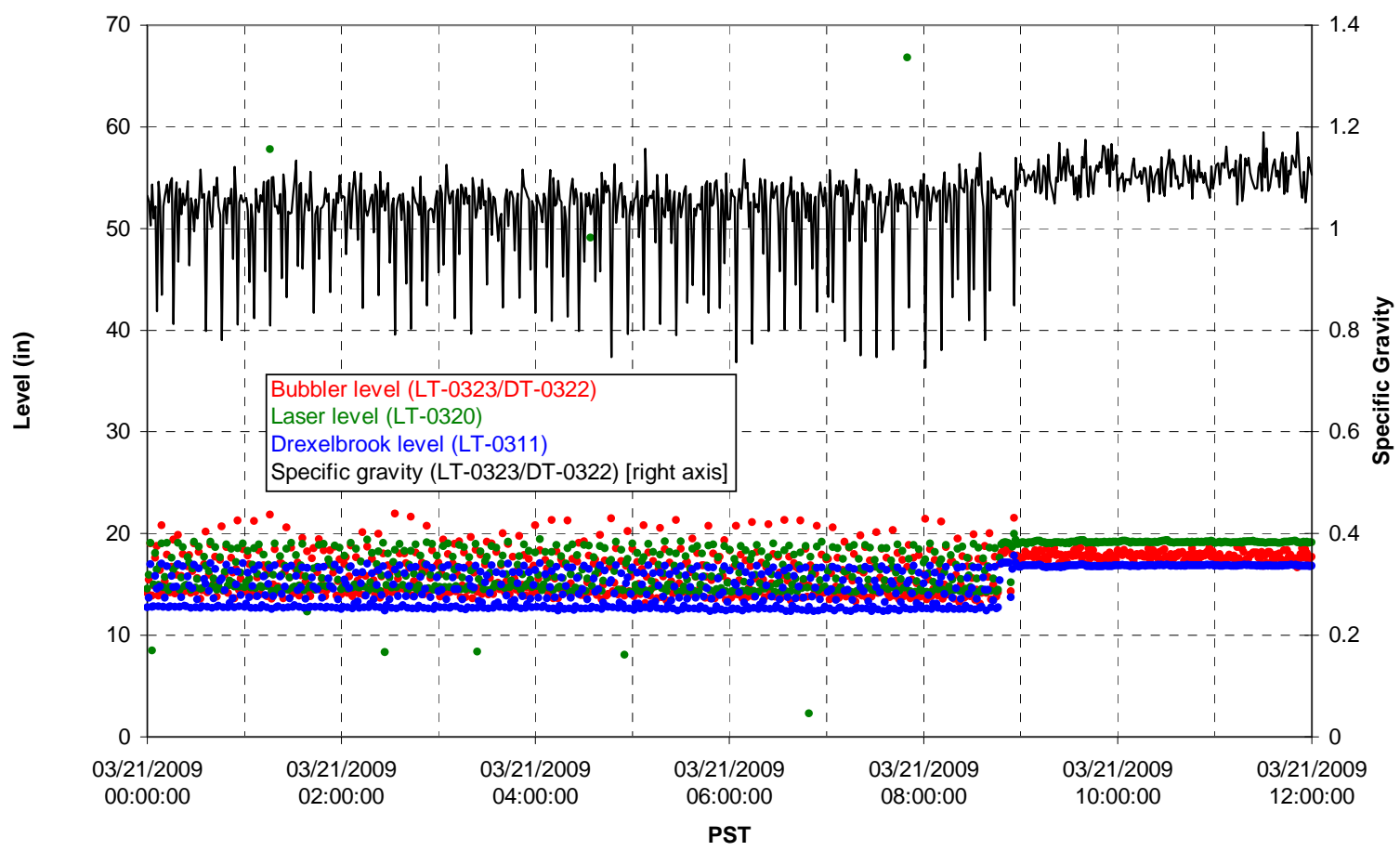

T01A temperatures

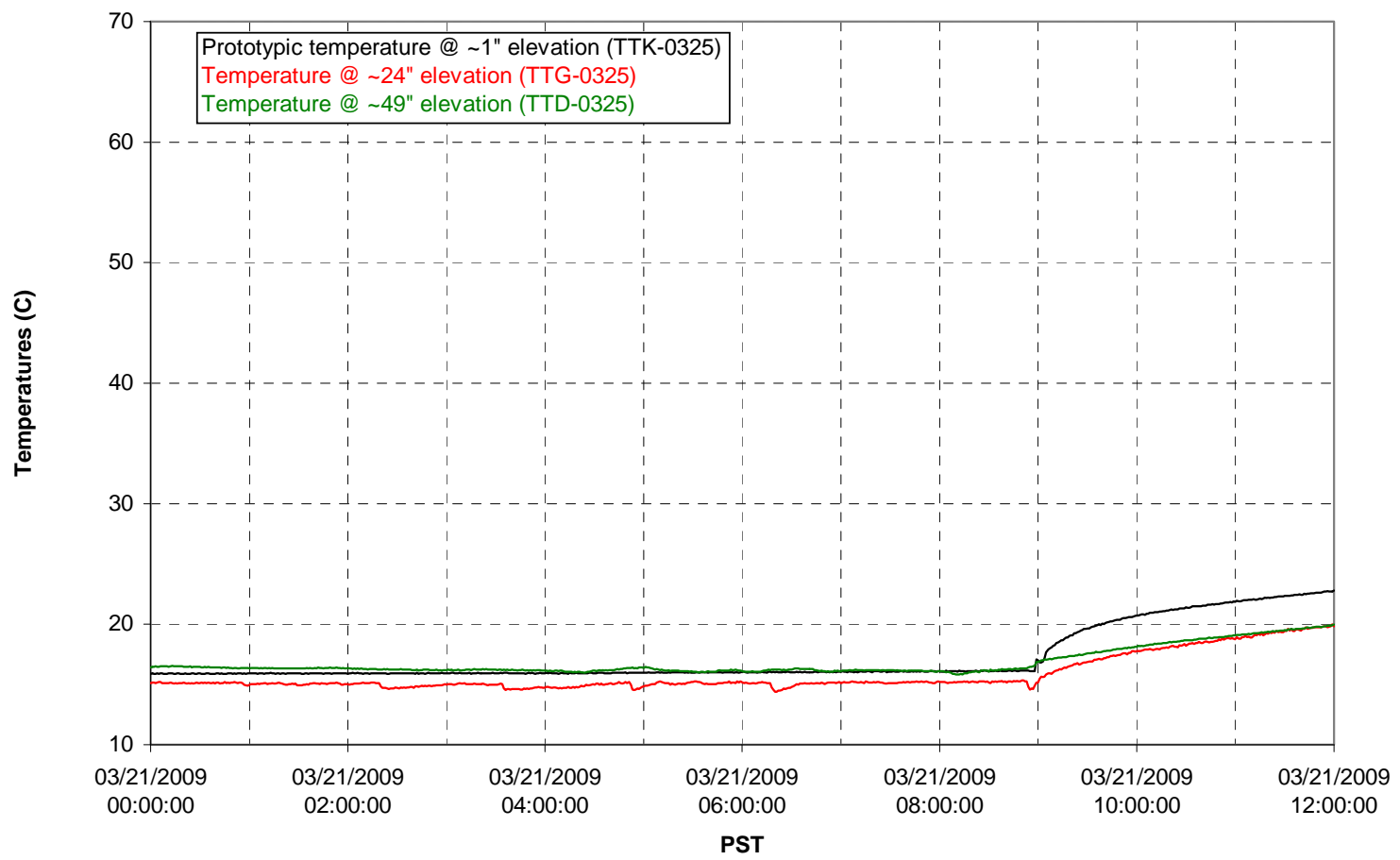


T01B level

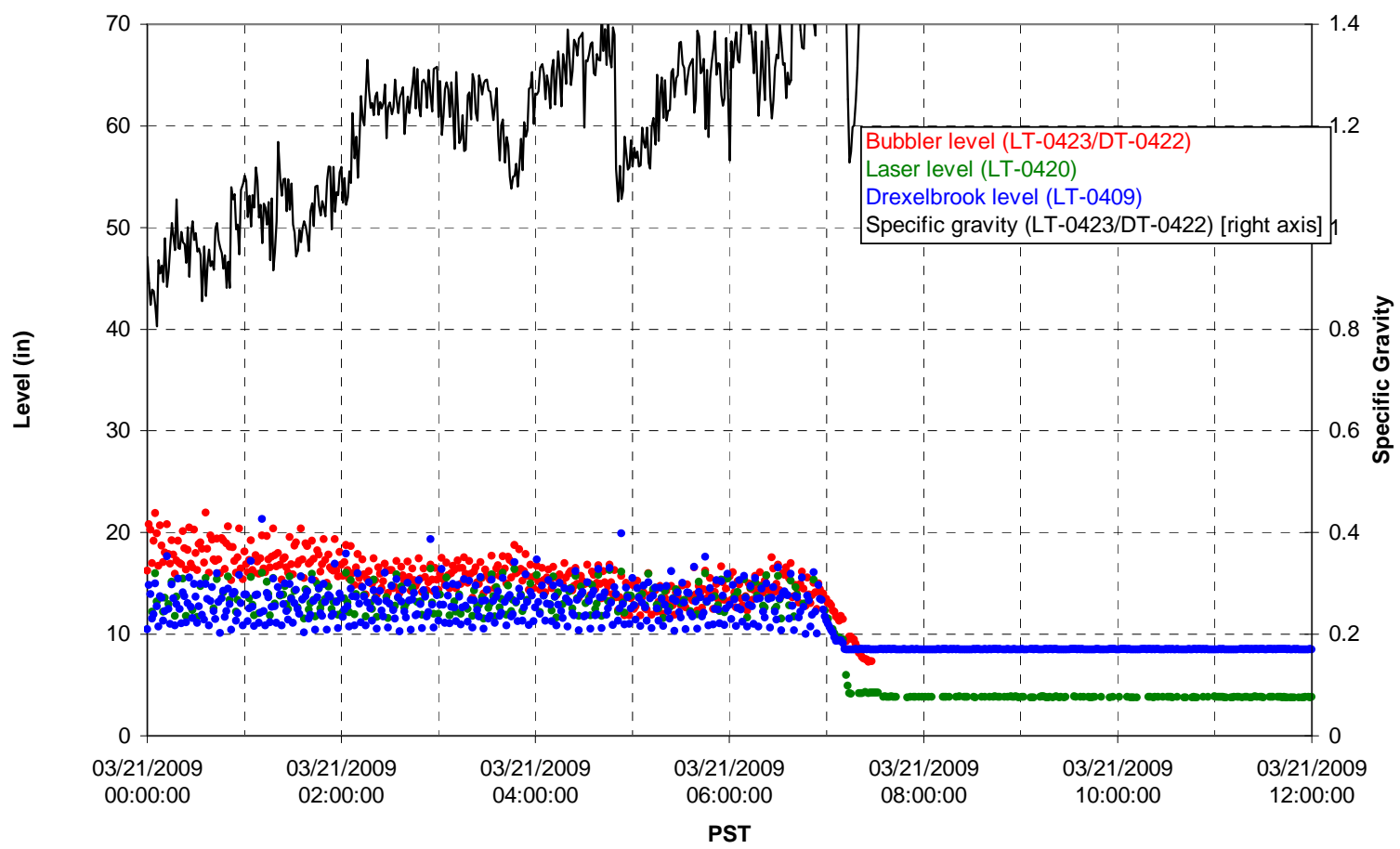

T01B temperatures

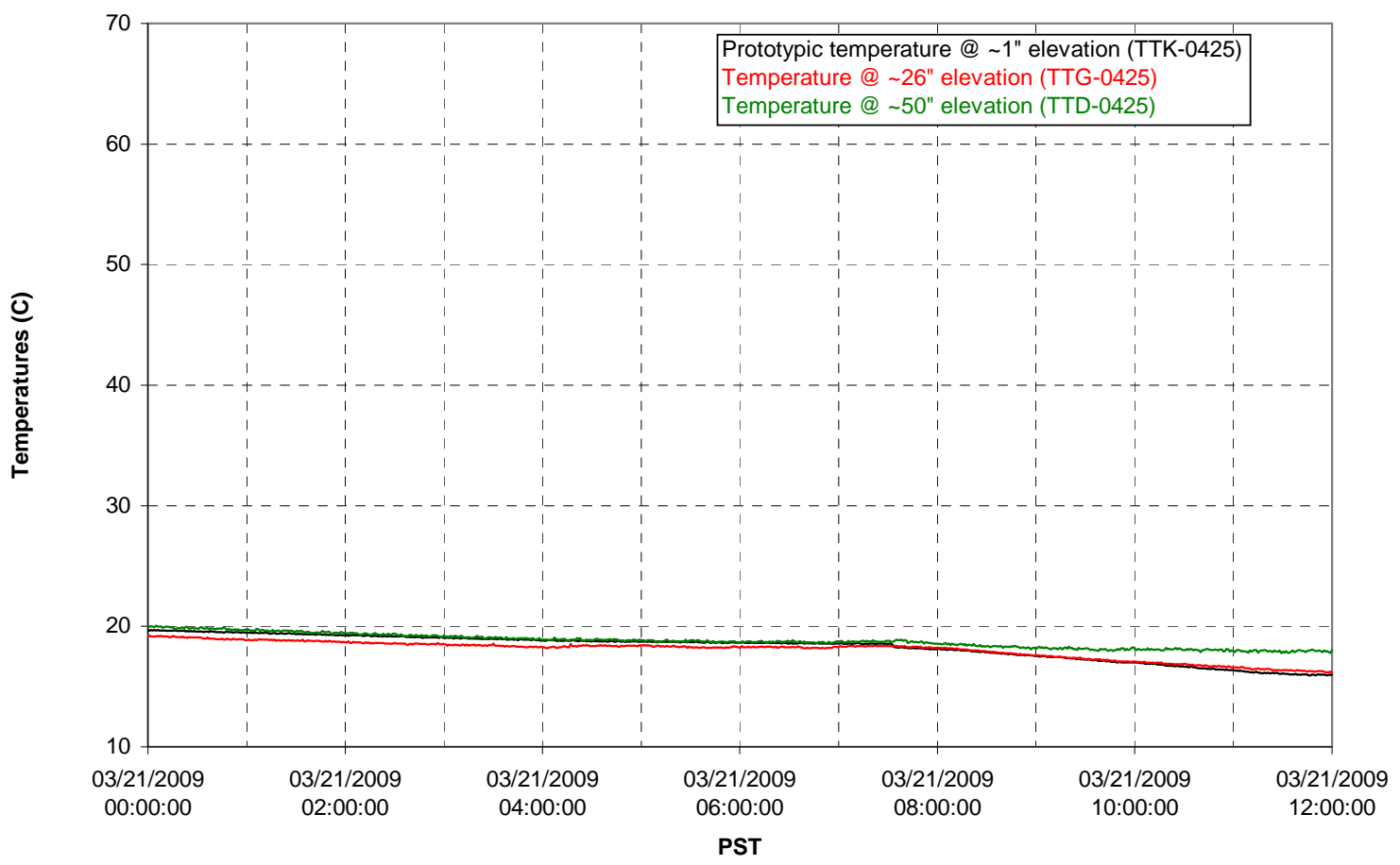


T02A level

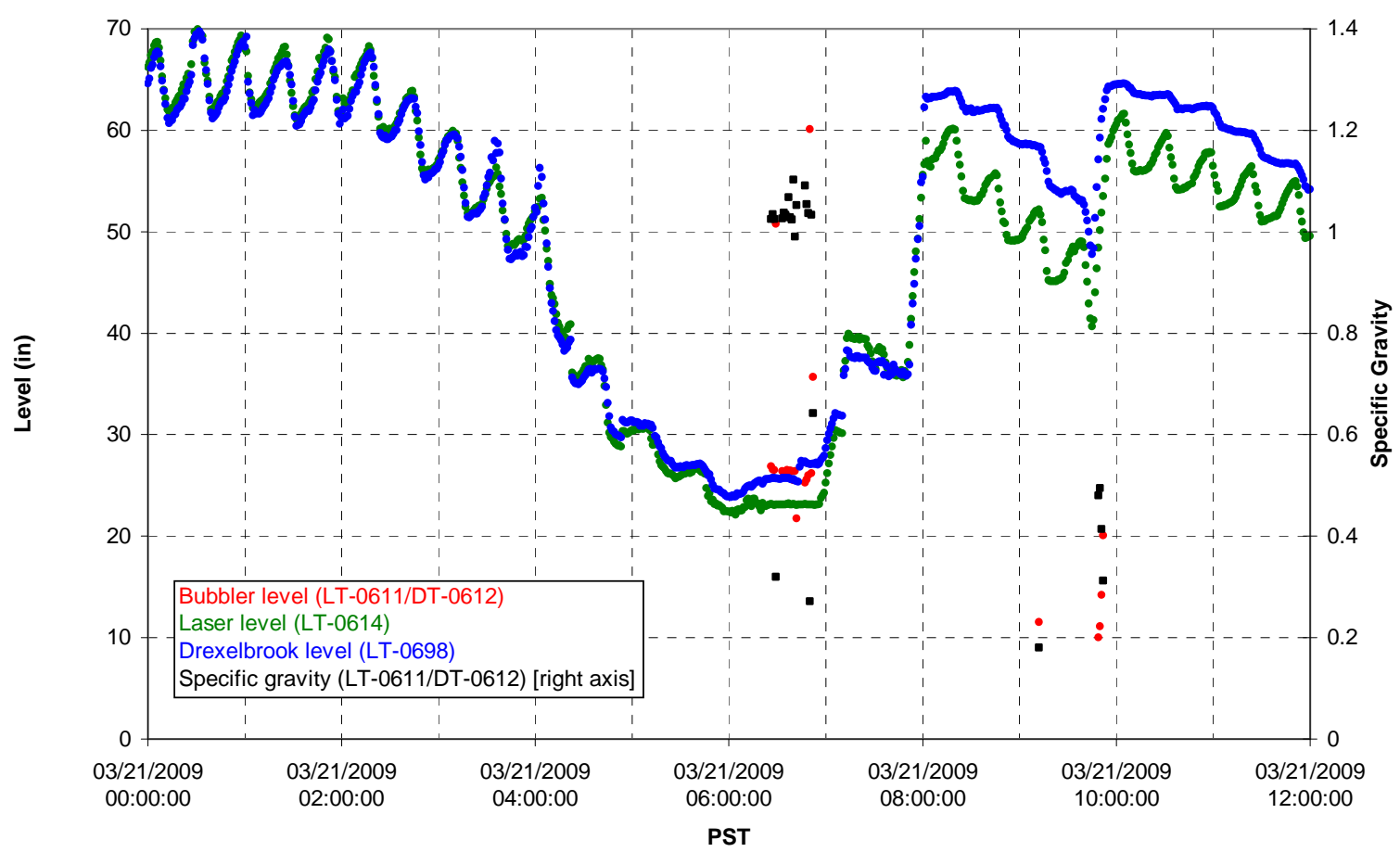

T02A temperatures

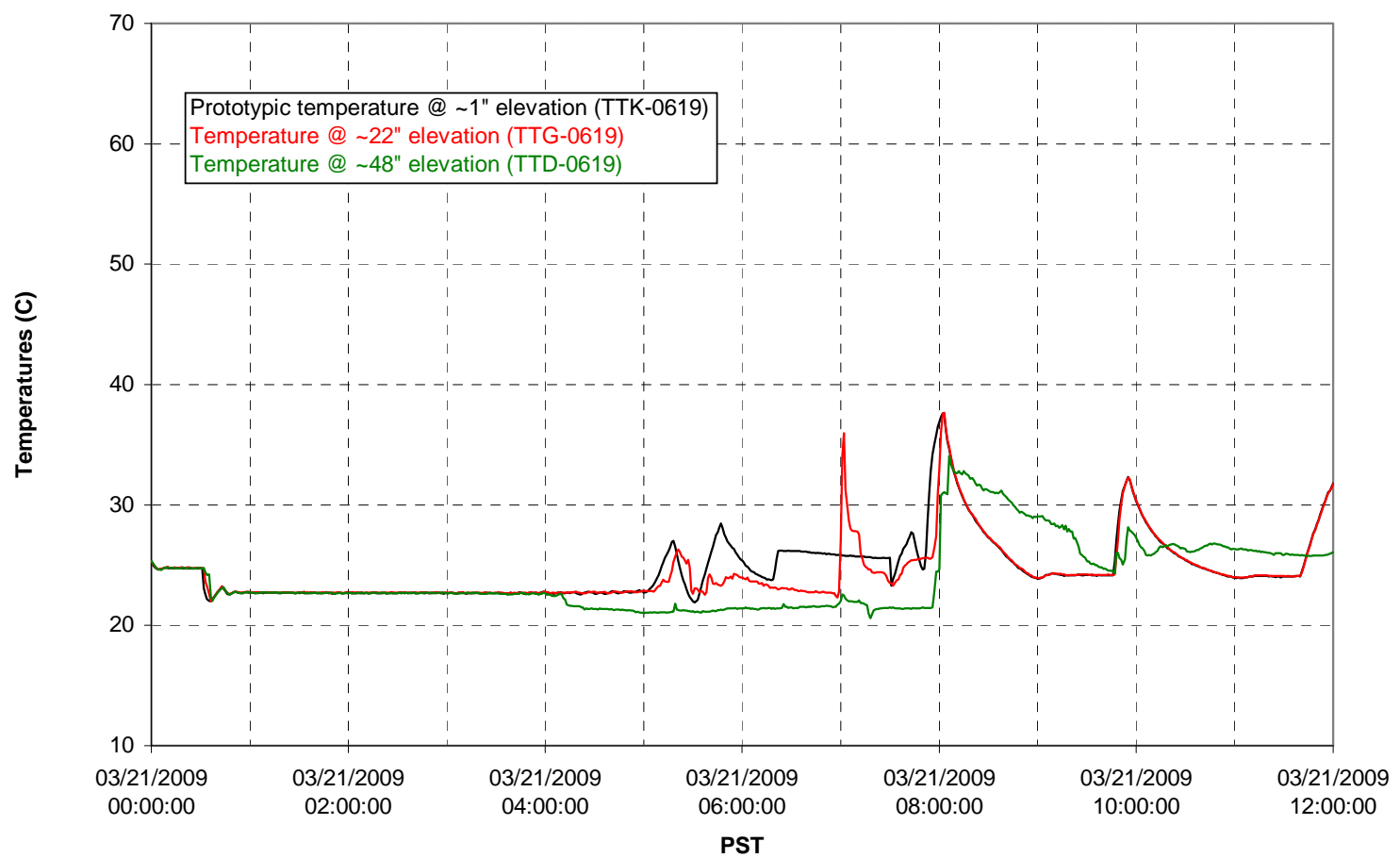


T02A and filter loop temperatures

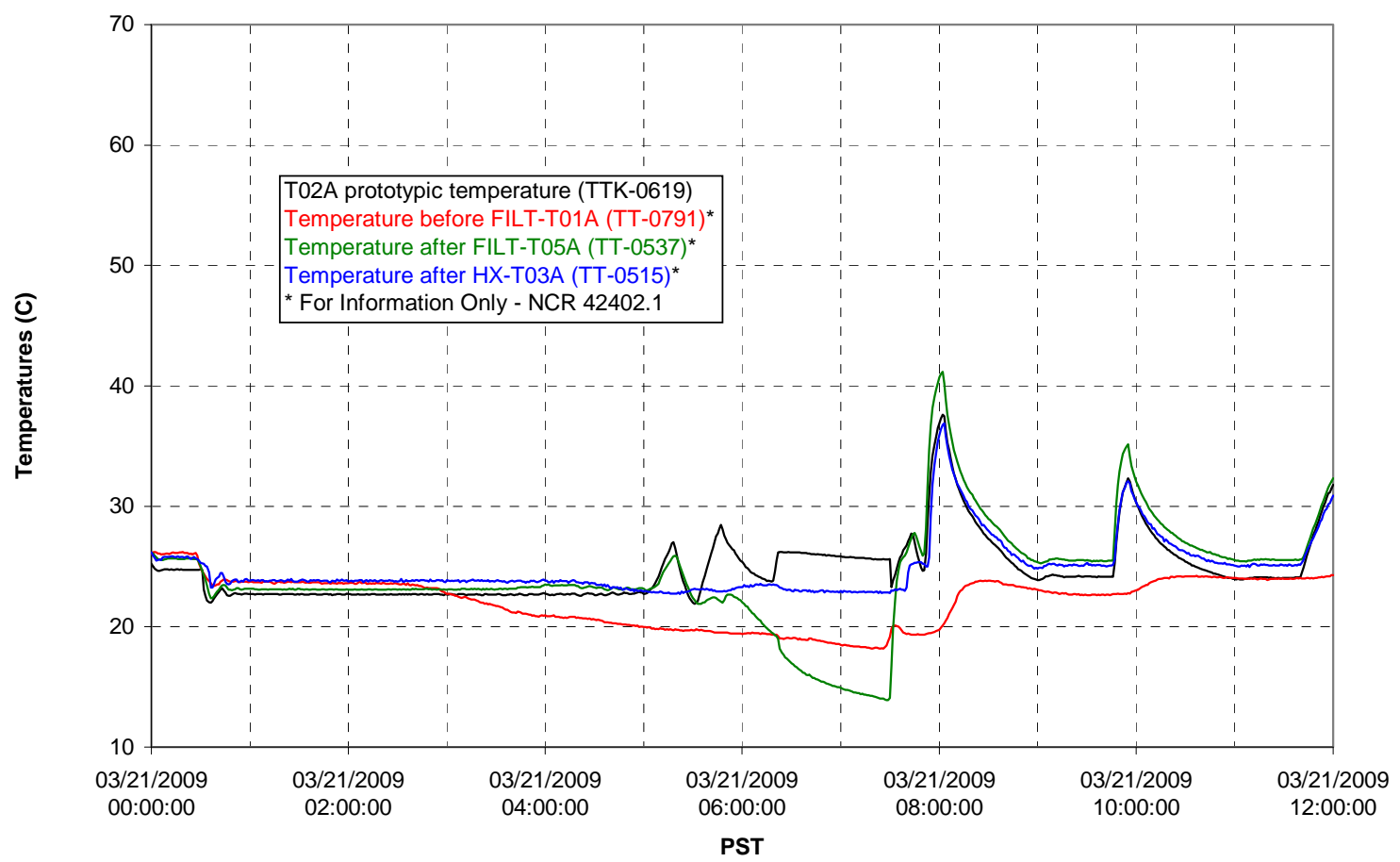

Pump Pressures and Flow

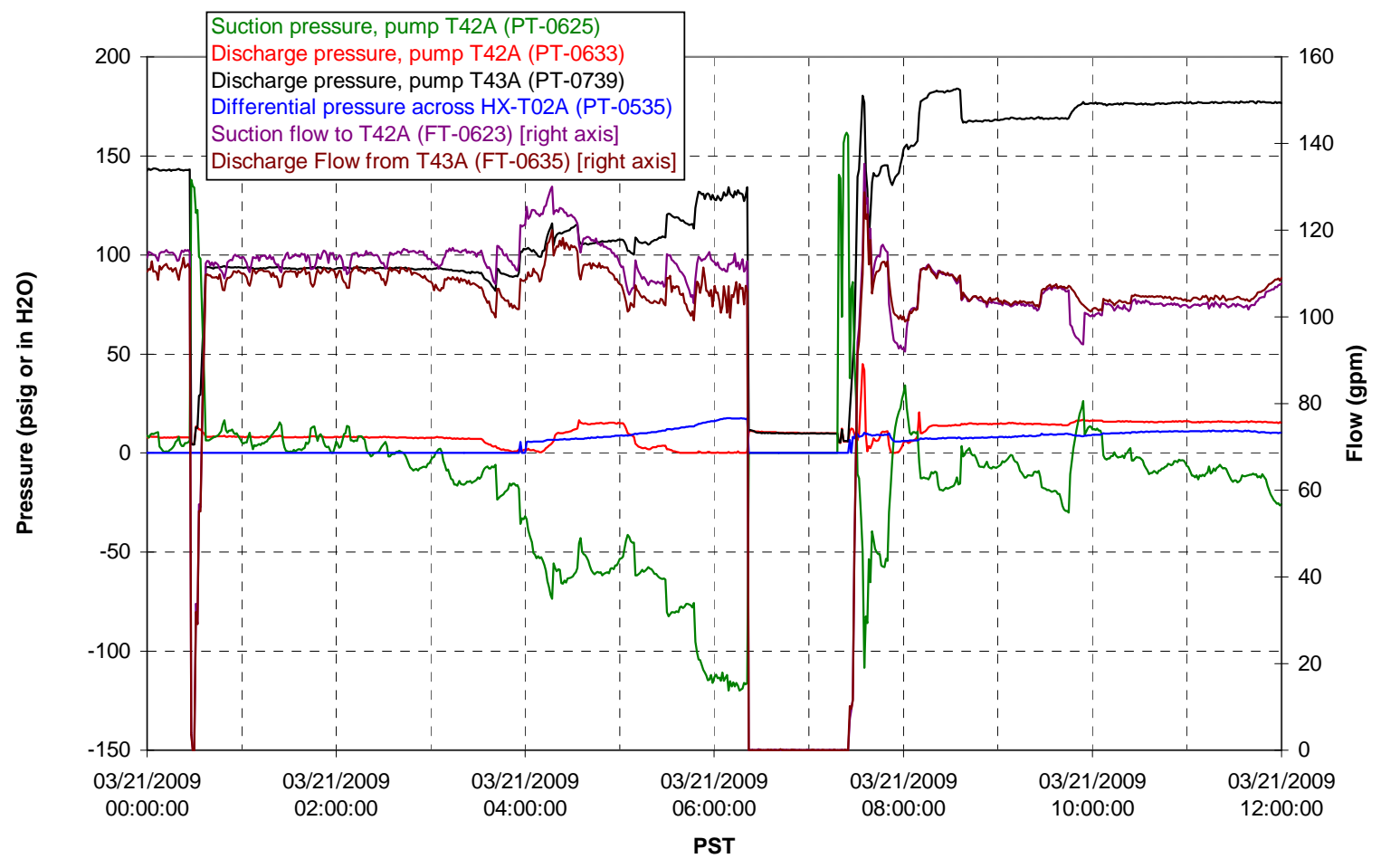




\section{Axial pressure drop}

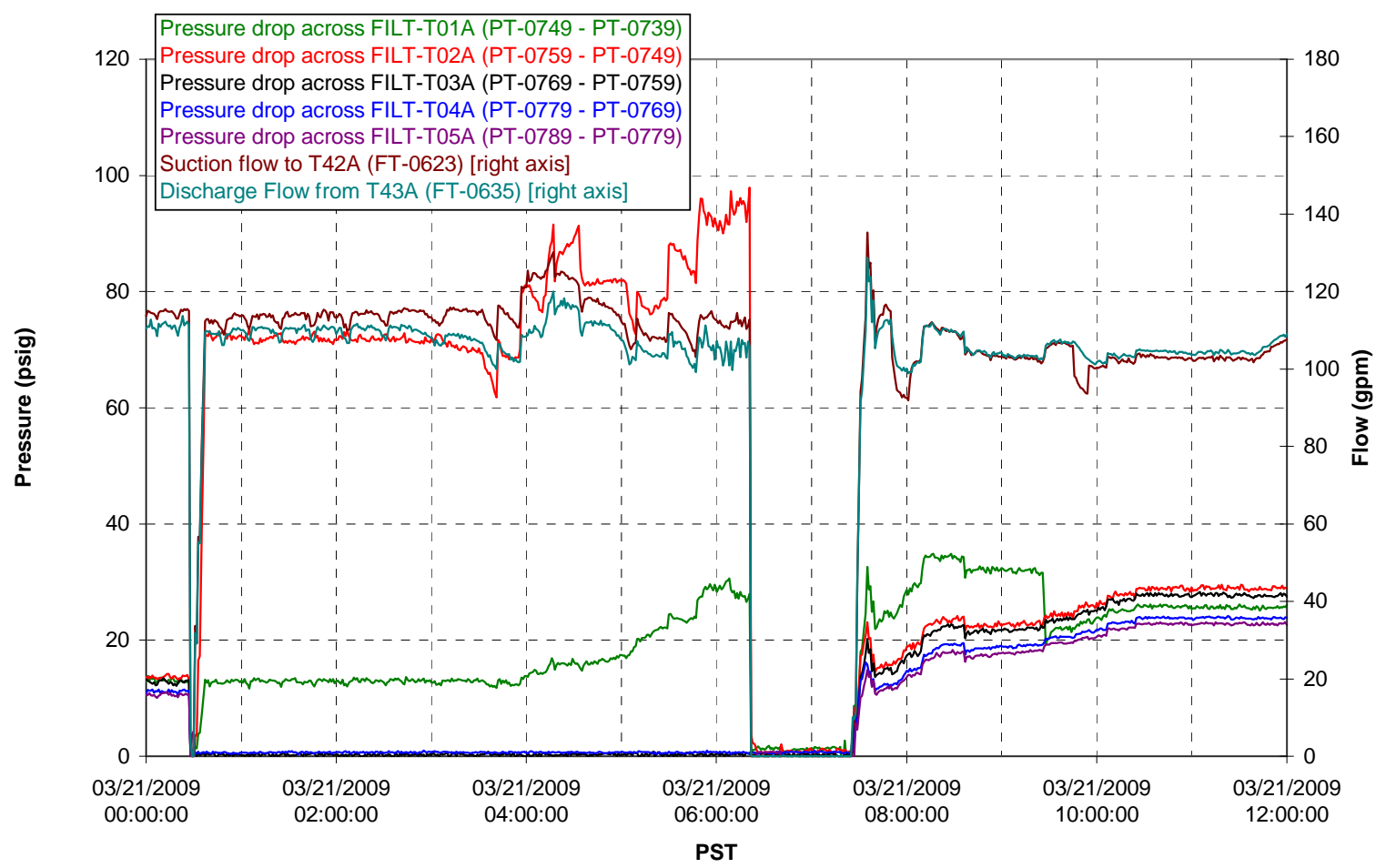

Permeate flow rates

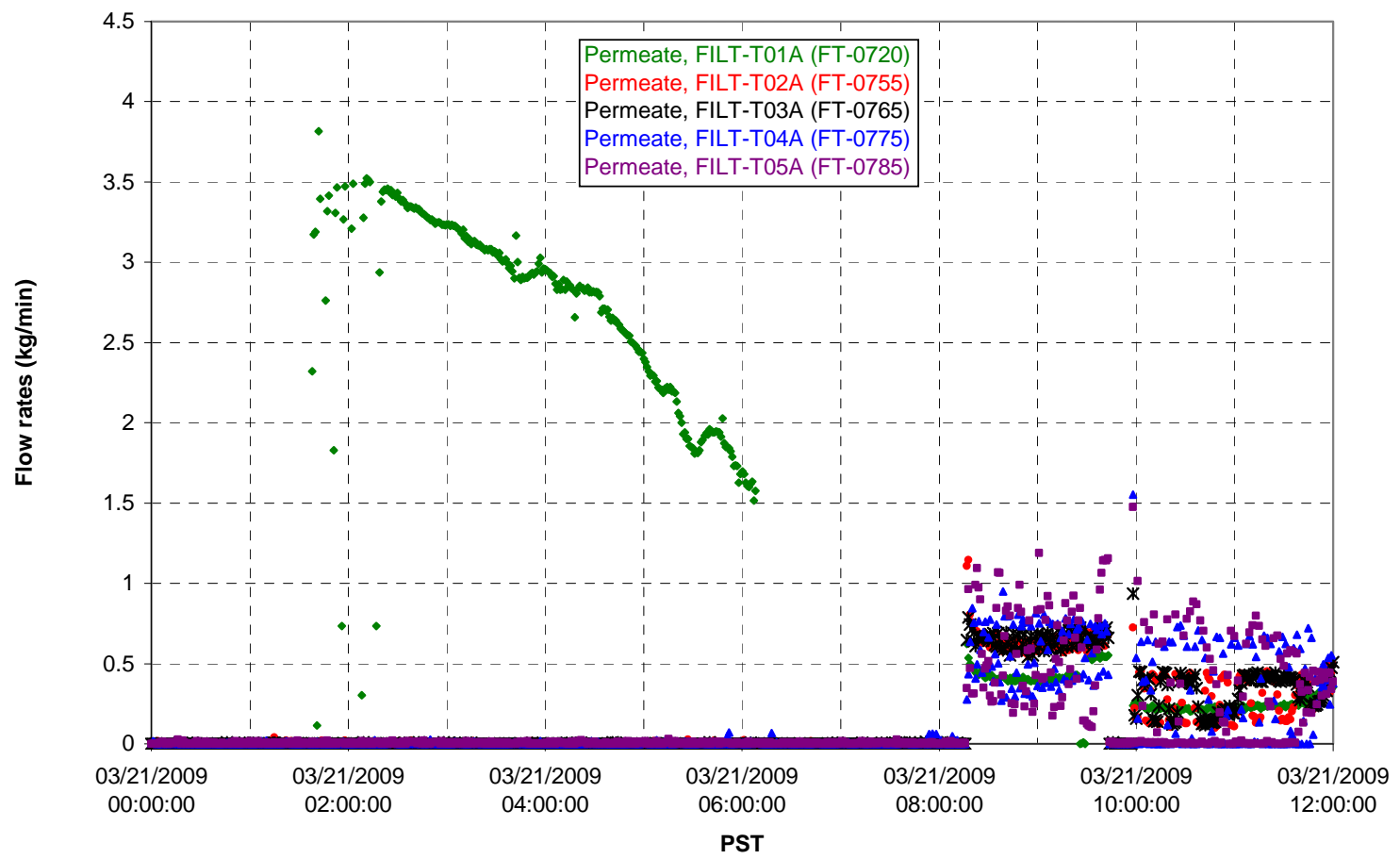


T02A Inner Temperature Tree

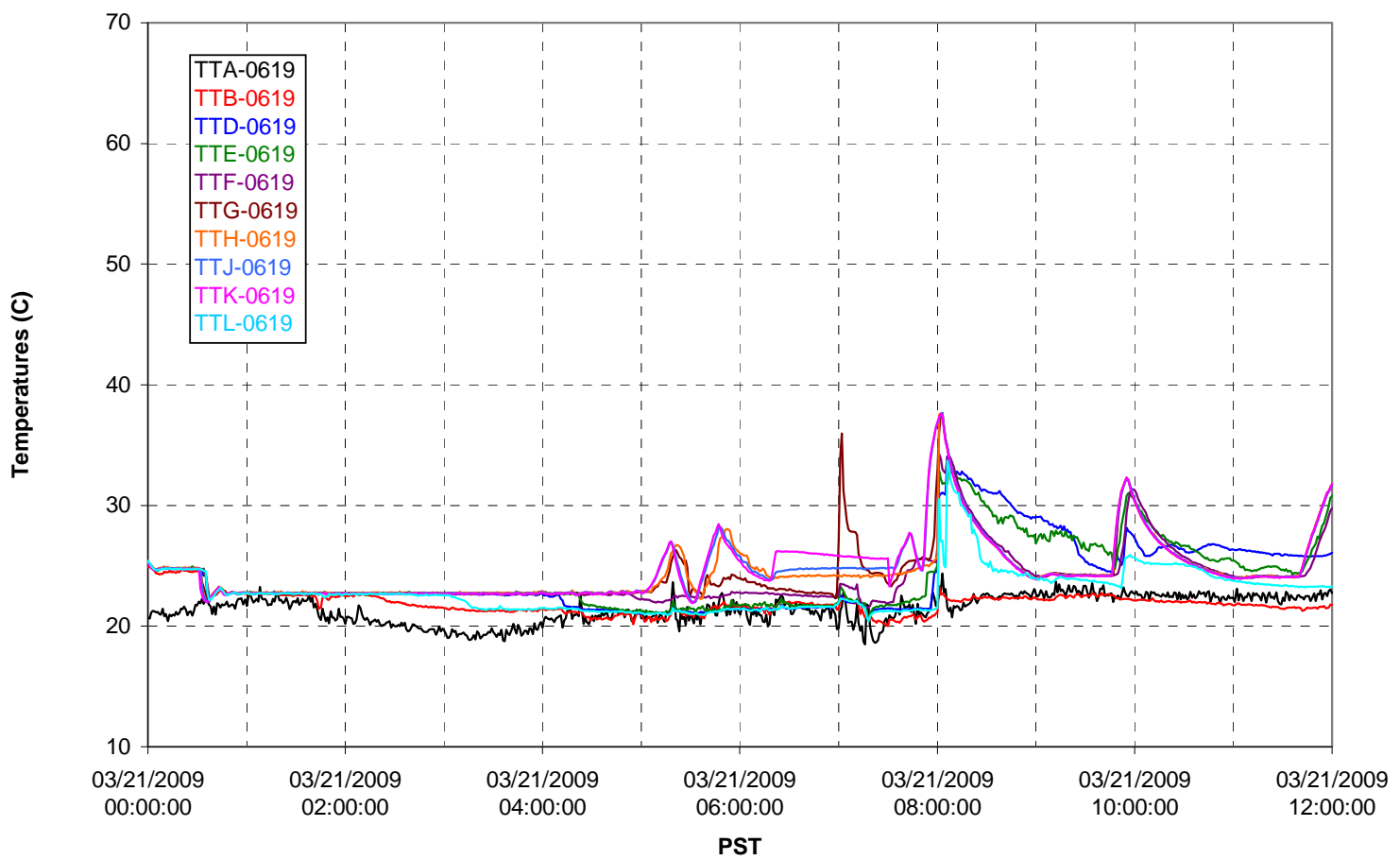

T02A Outer Temperature Tree

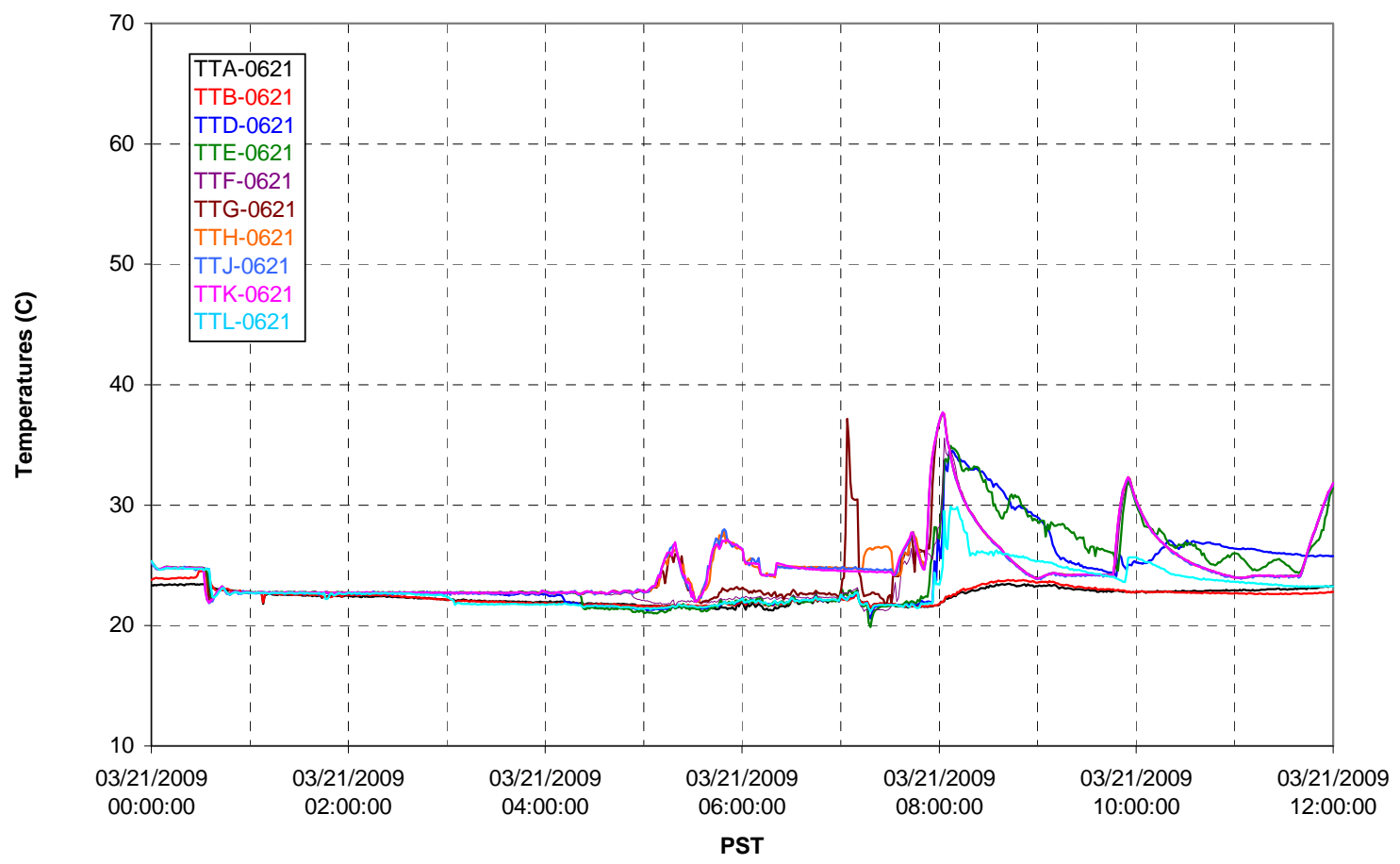


T02A temperatures

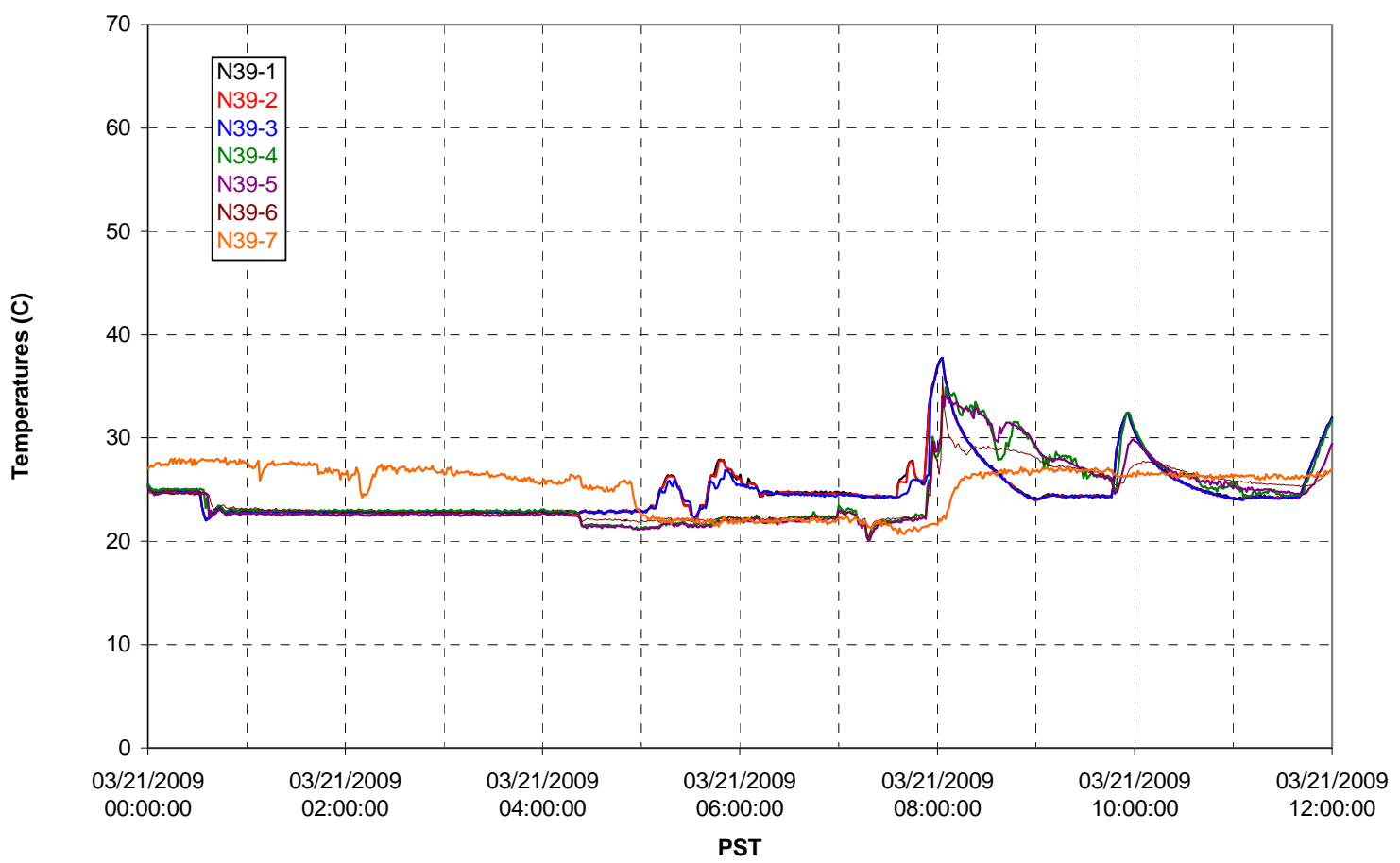

T02A temperatures

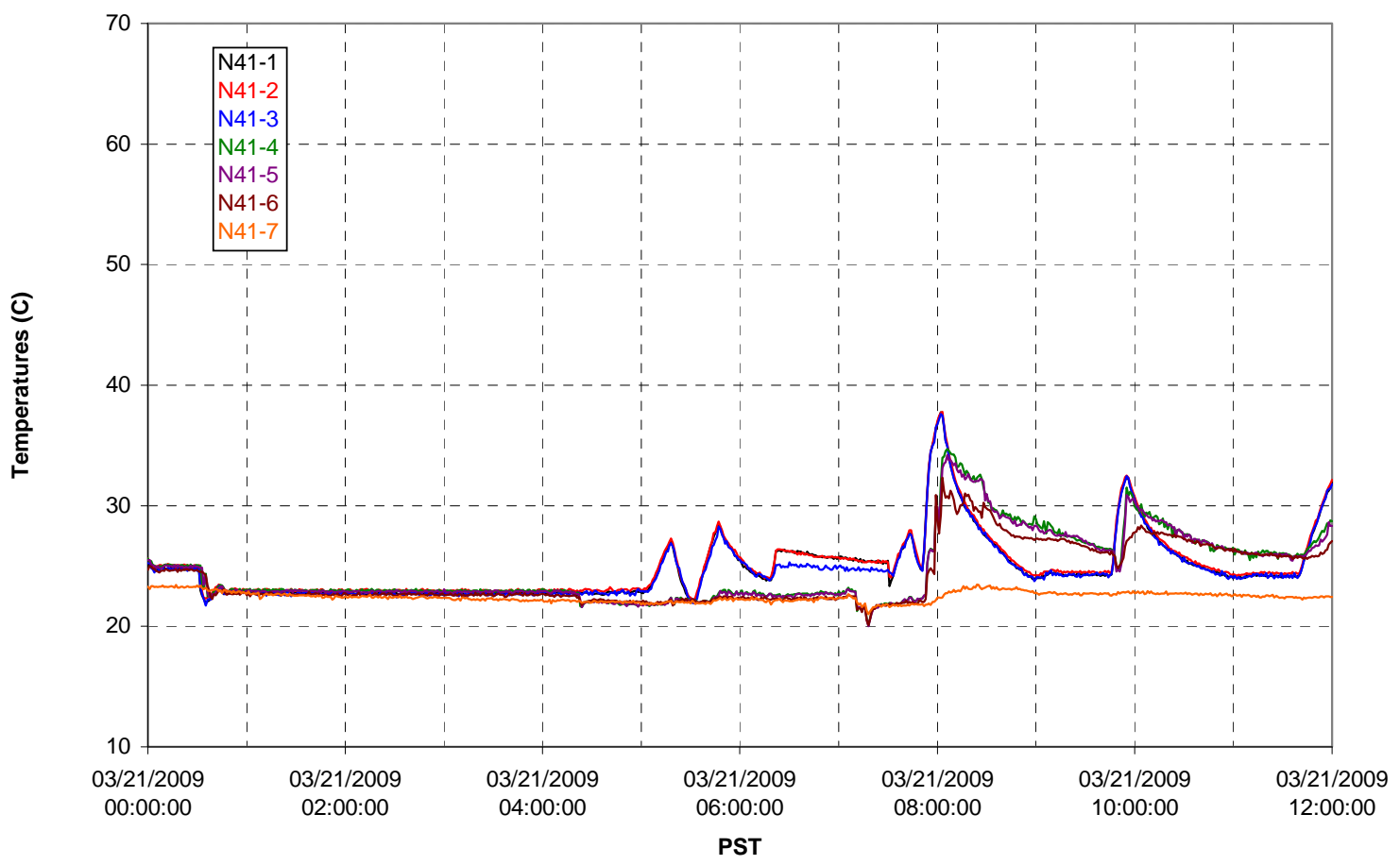


T02A temperatures

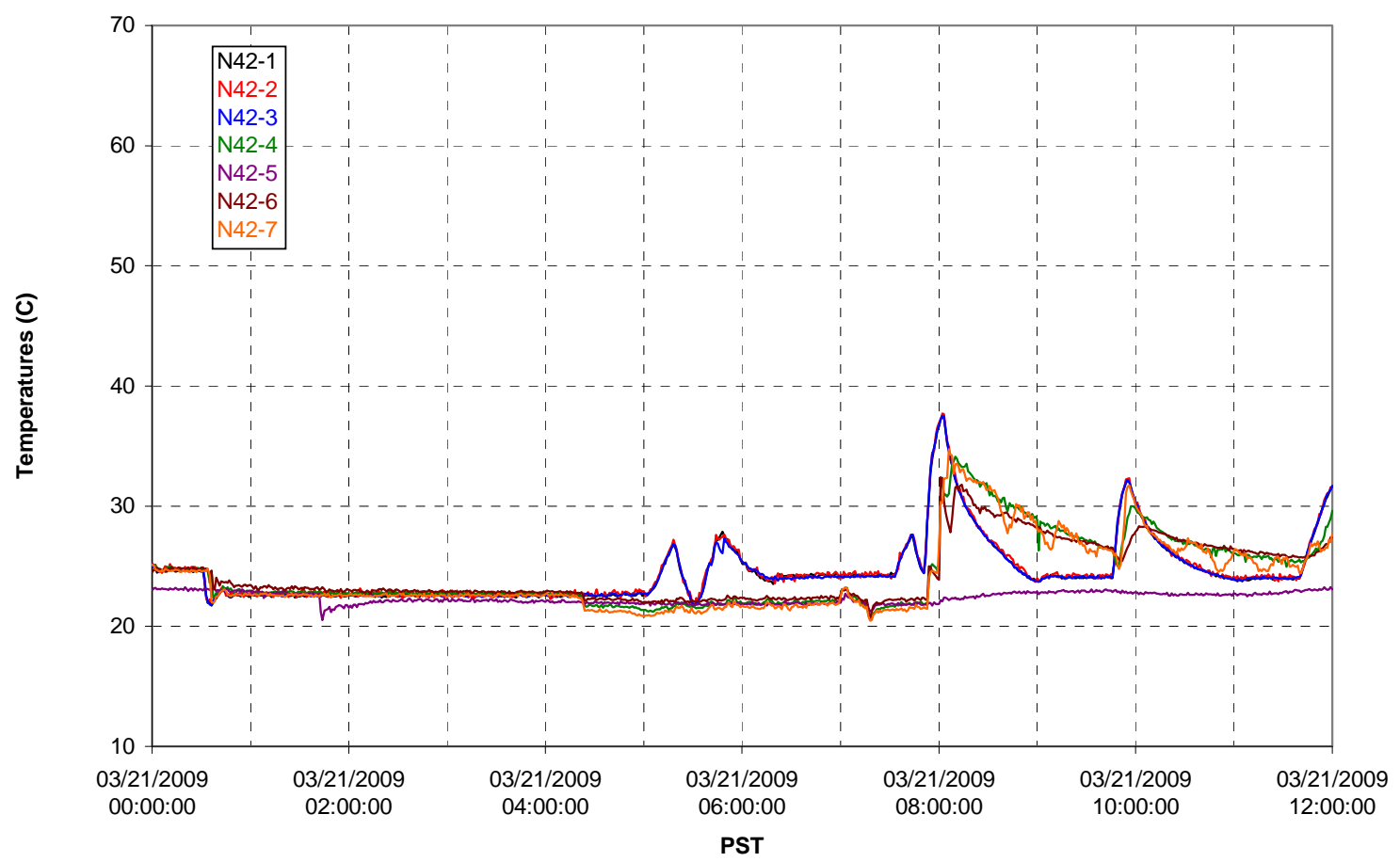

T02A temperatures

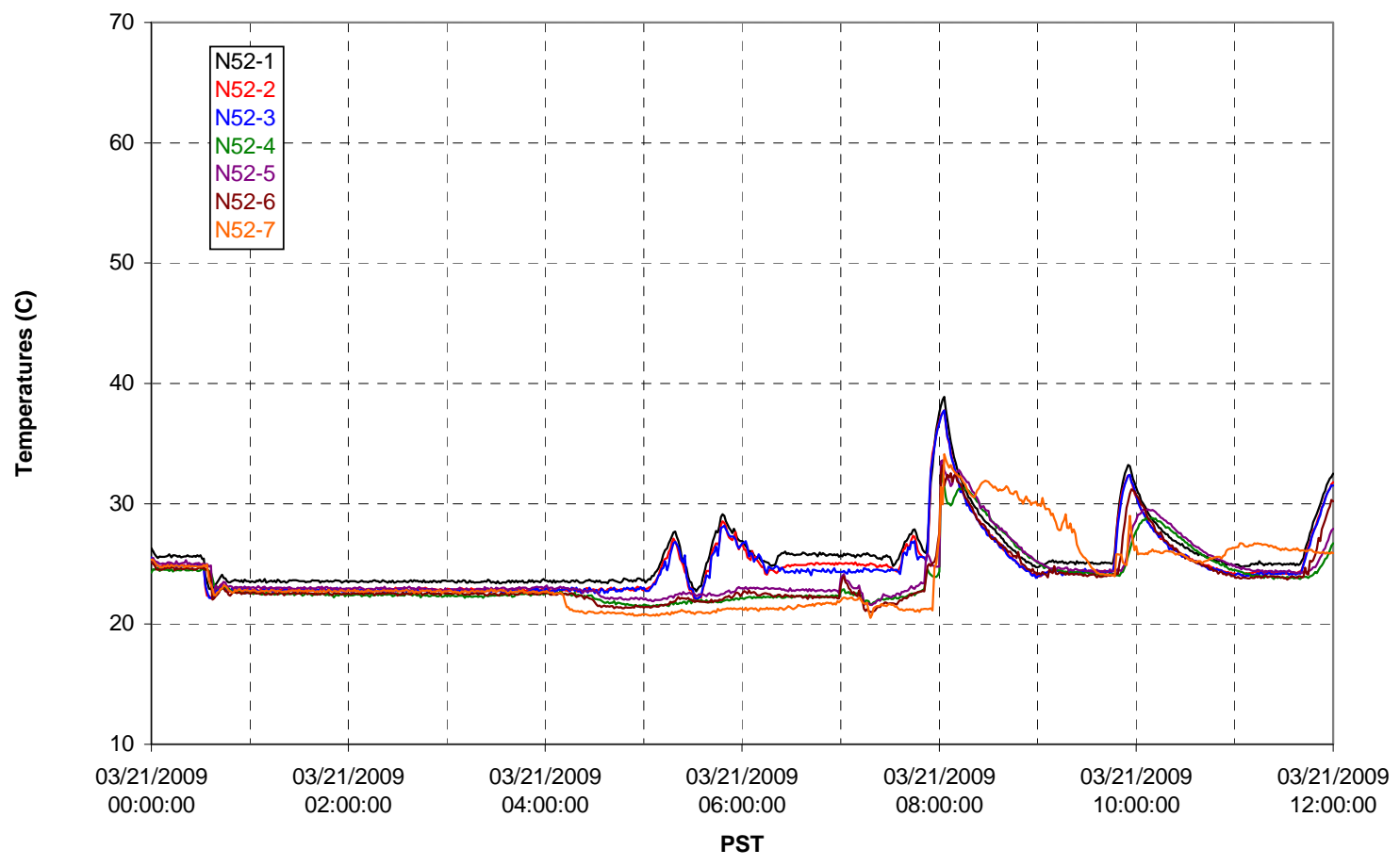


T02A Heating and Cooling

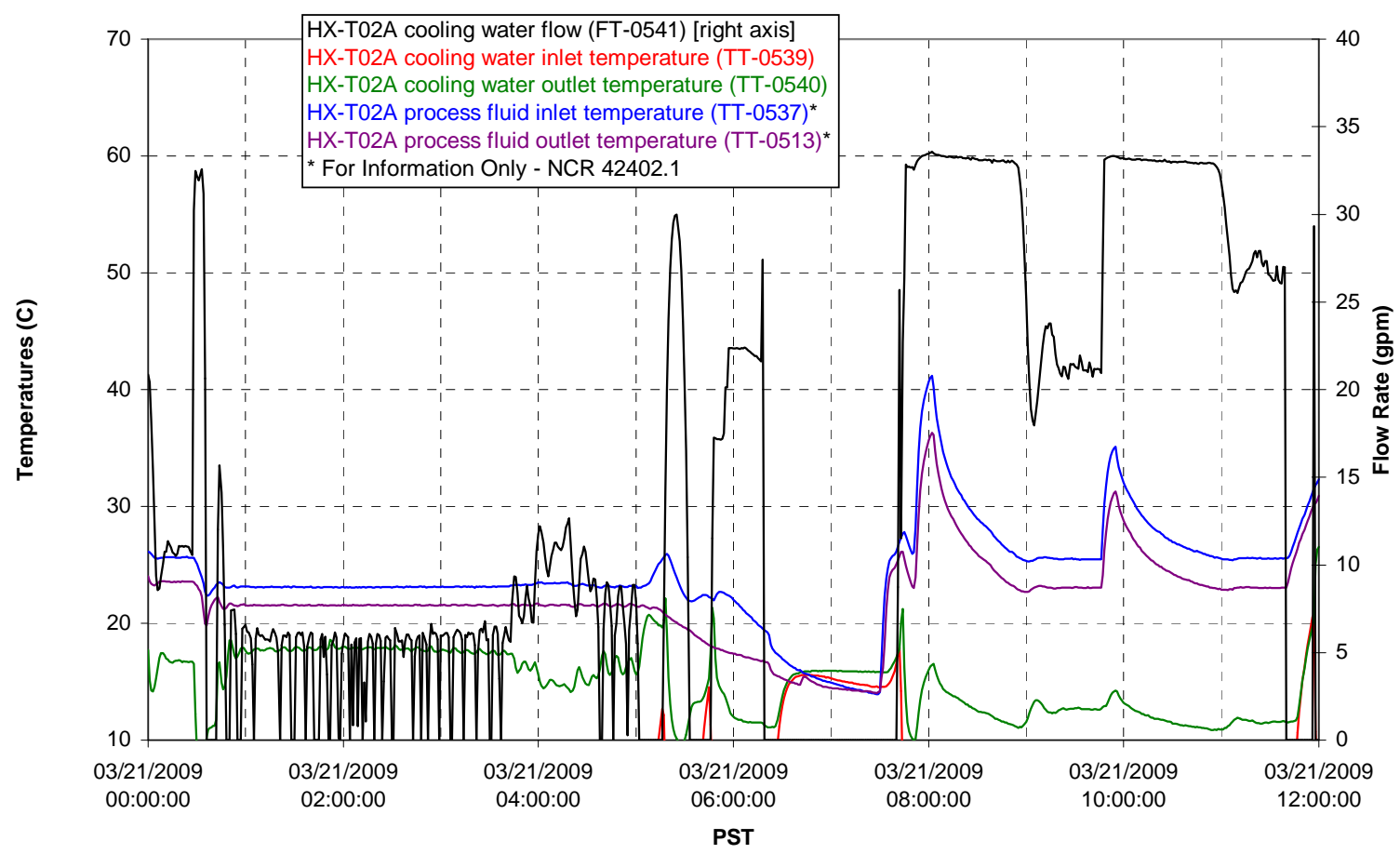

Pump Operation

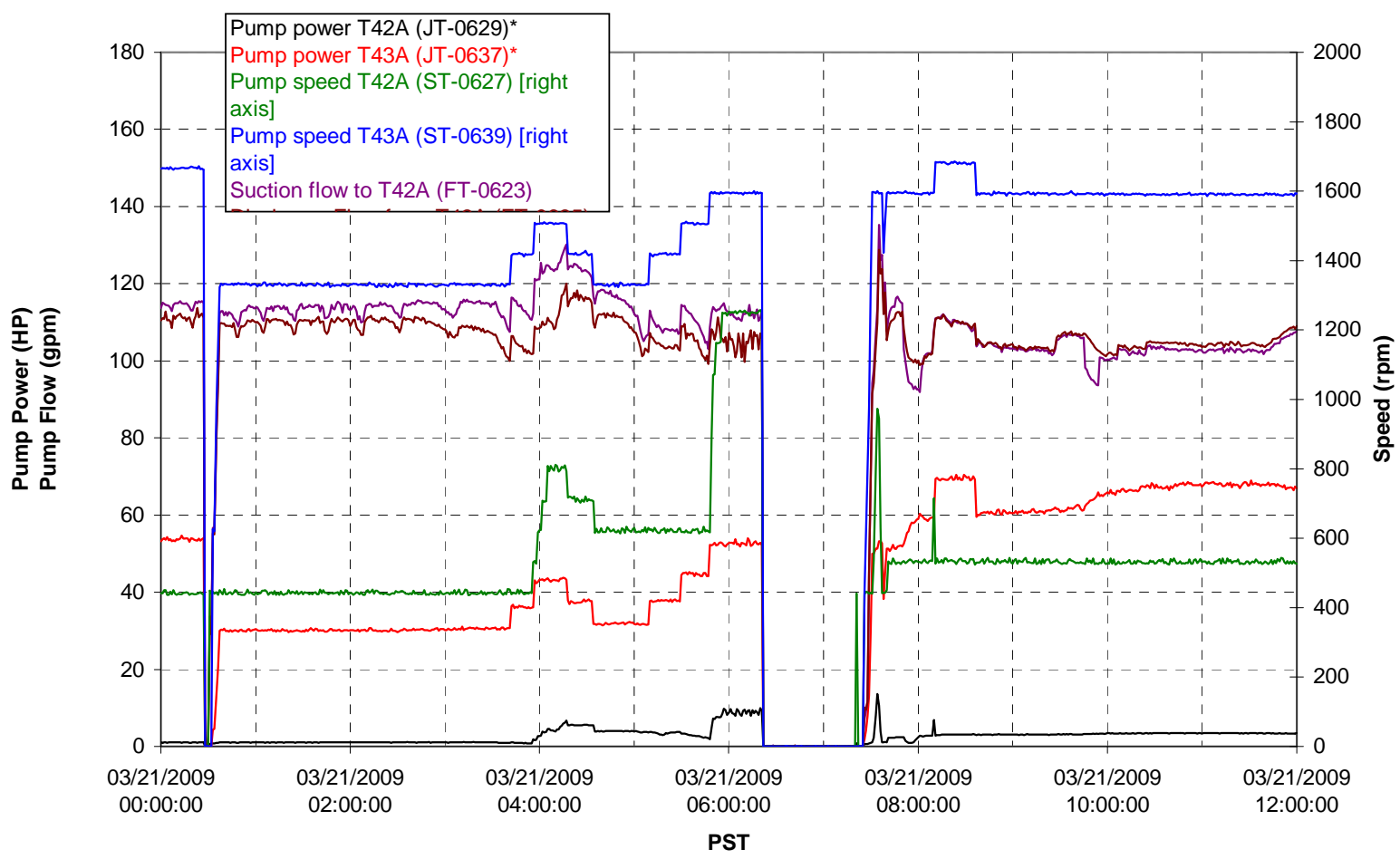


Pulsepot UFP-PP-T01A

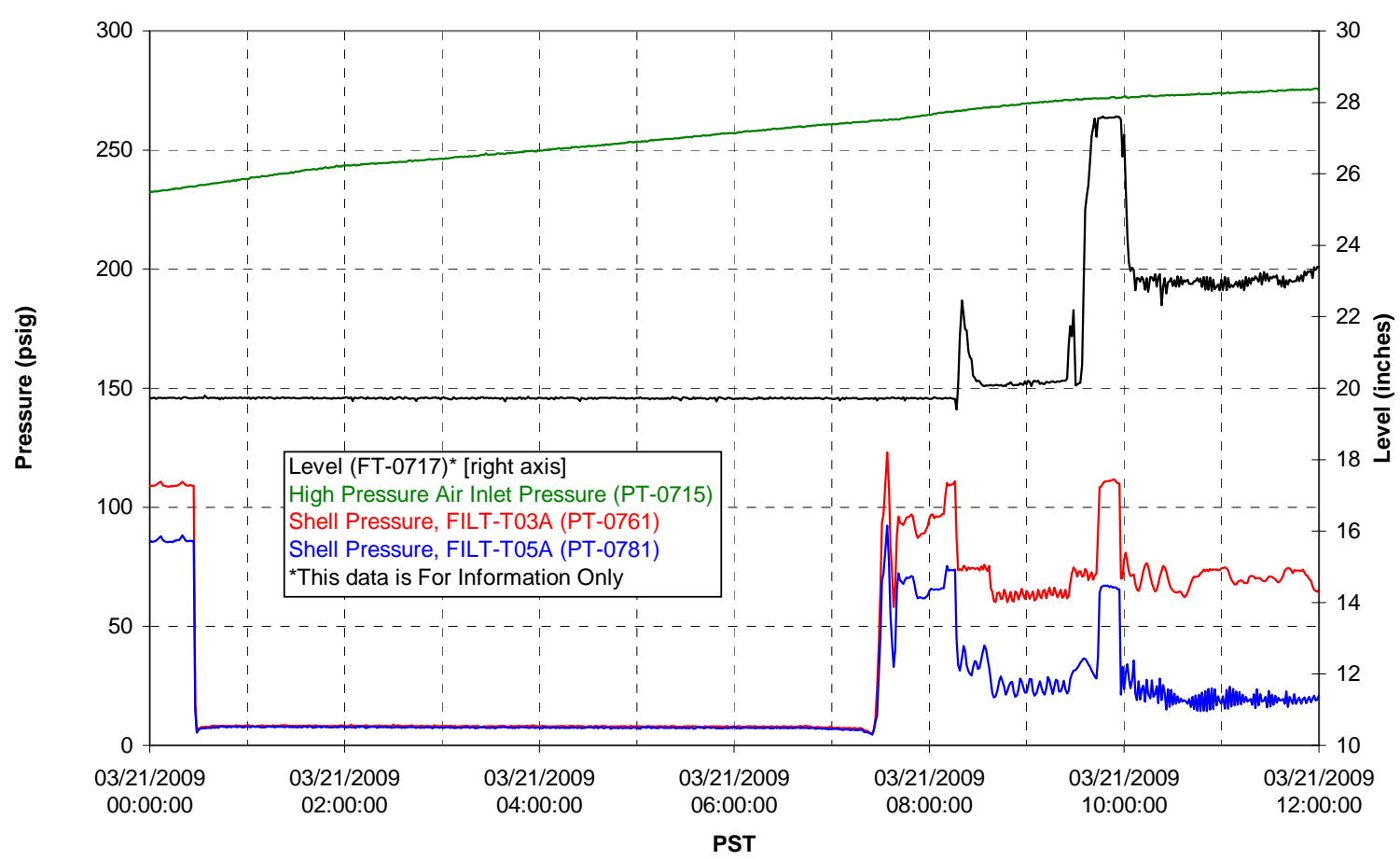

Pulsepot UFP-PP-T02A

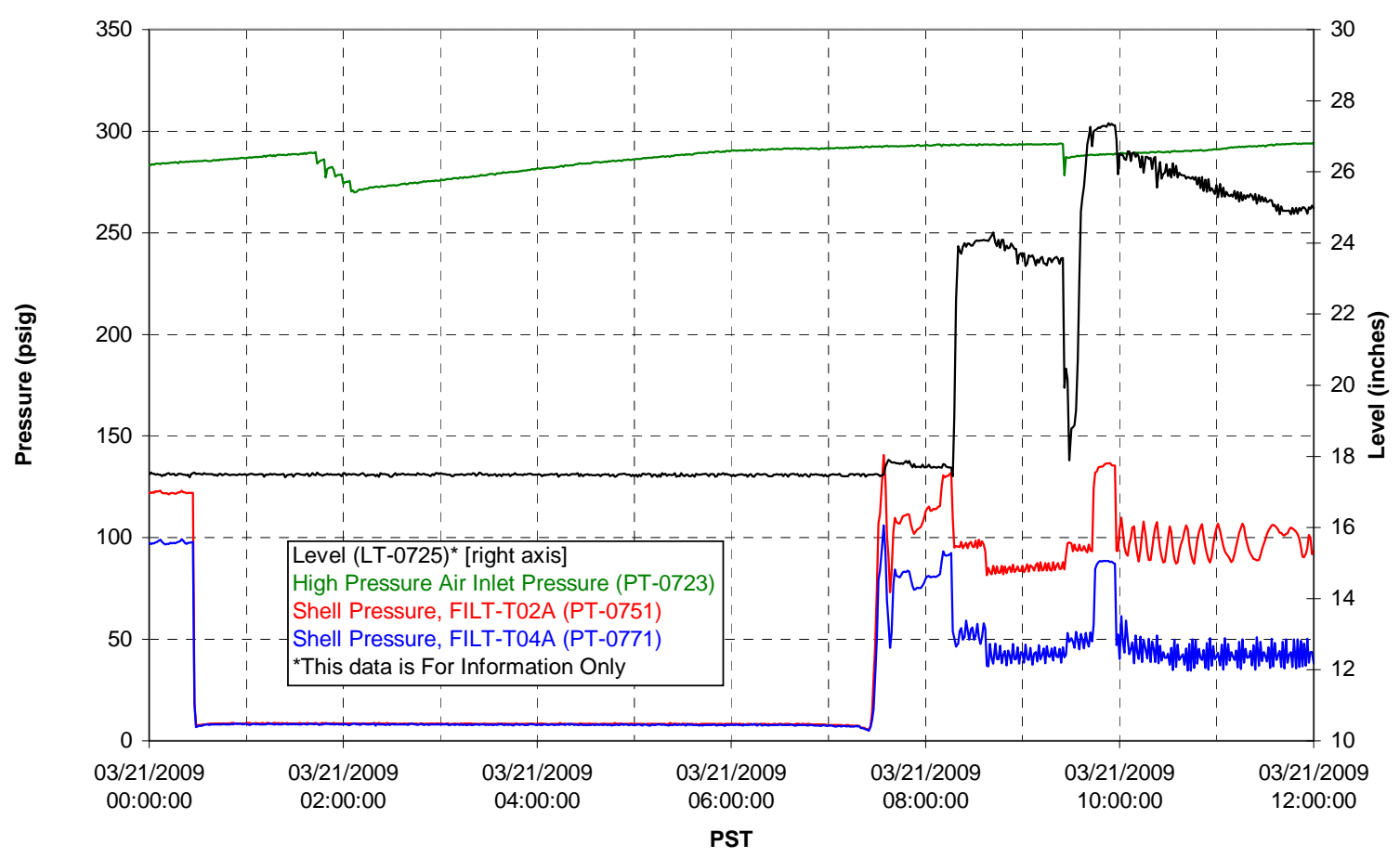


Pulsepot UFP-PP-T03A

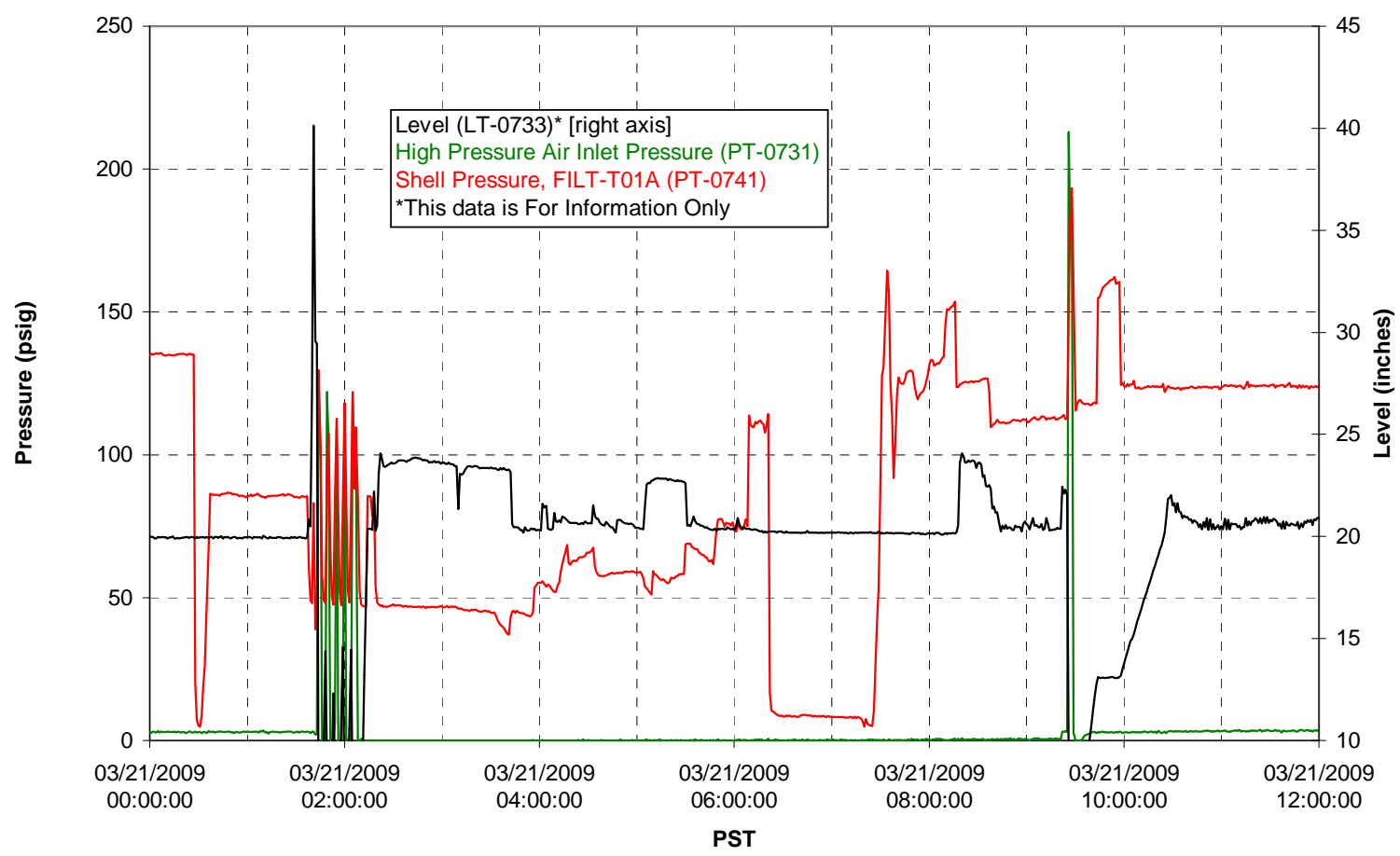

Pulsepot Levels

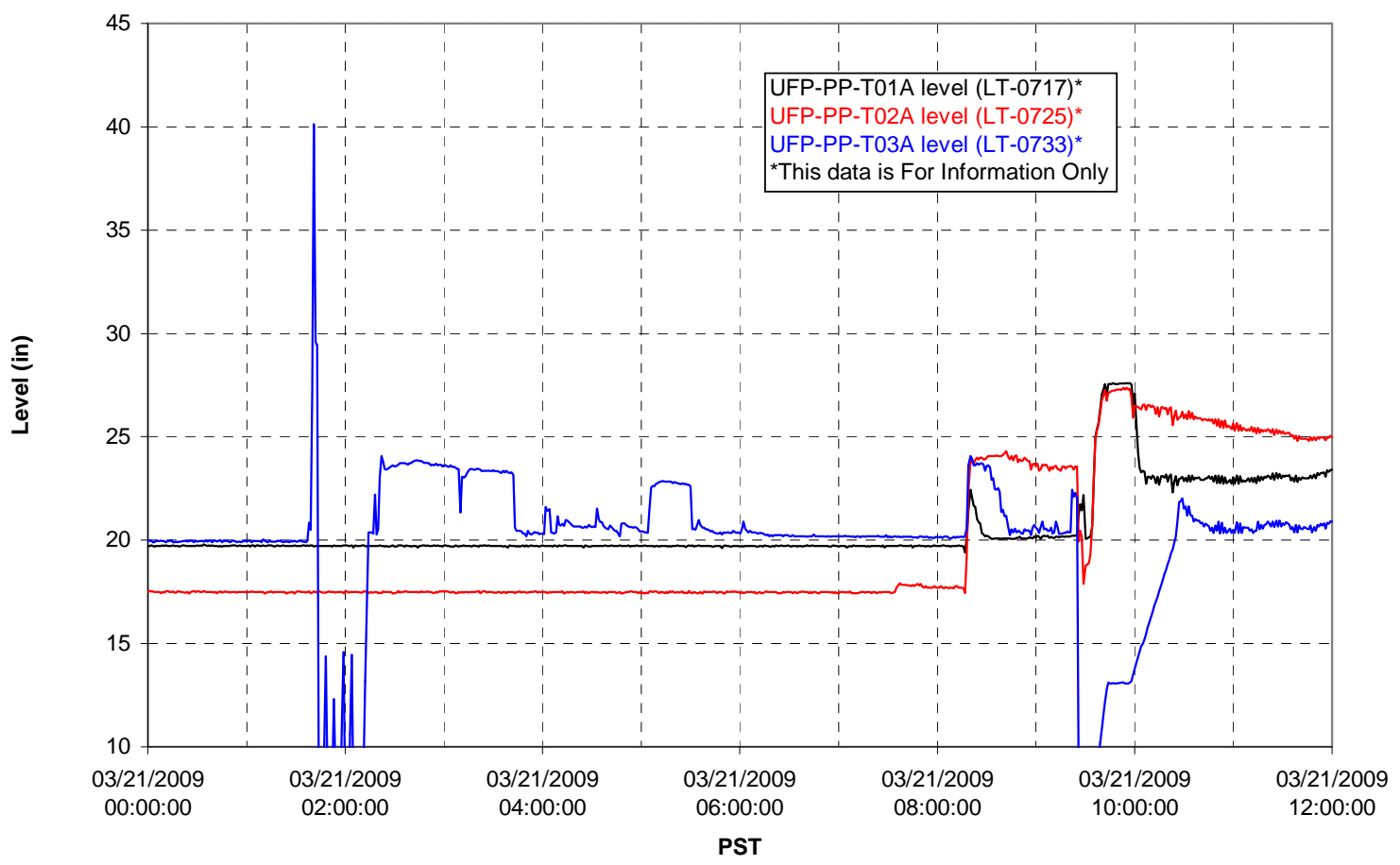


Filter UFP-FILT-T01A

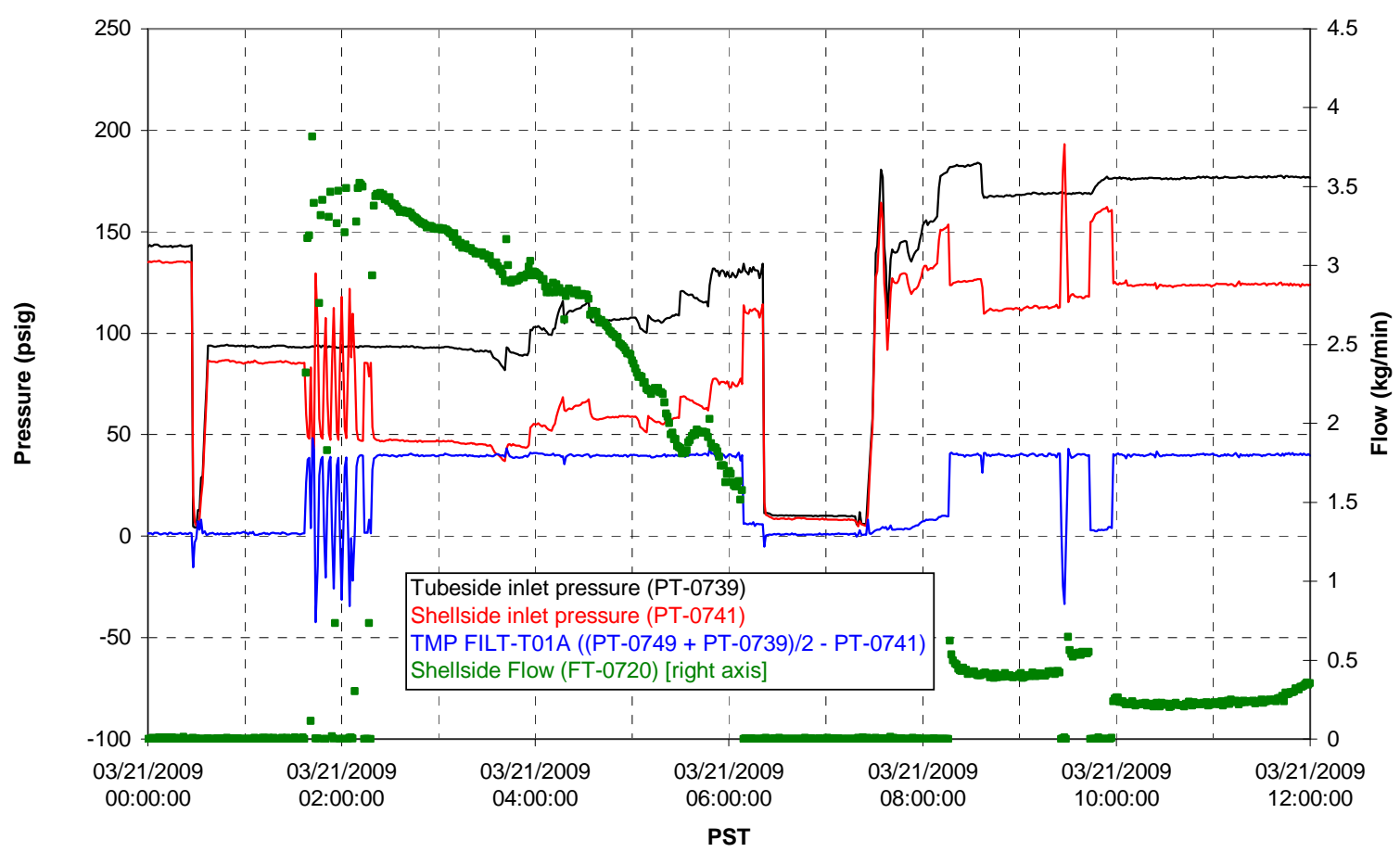

Filter UFP-FILT-T02A

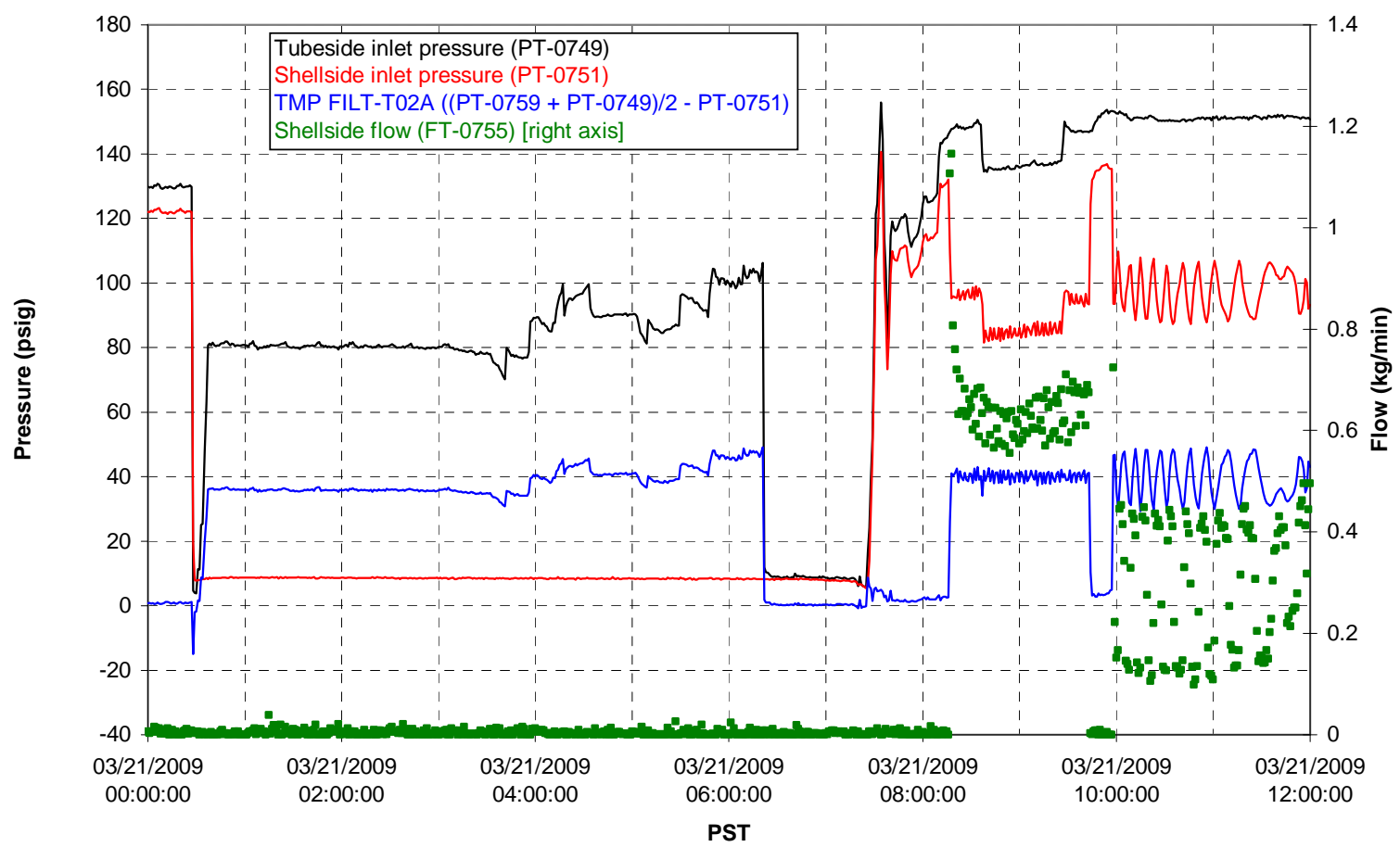


Filter UFP-FILT-T03A

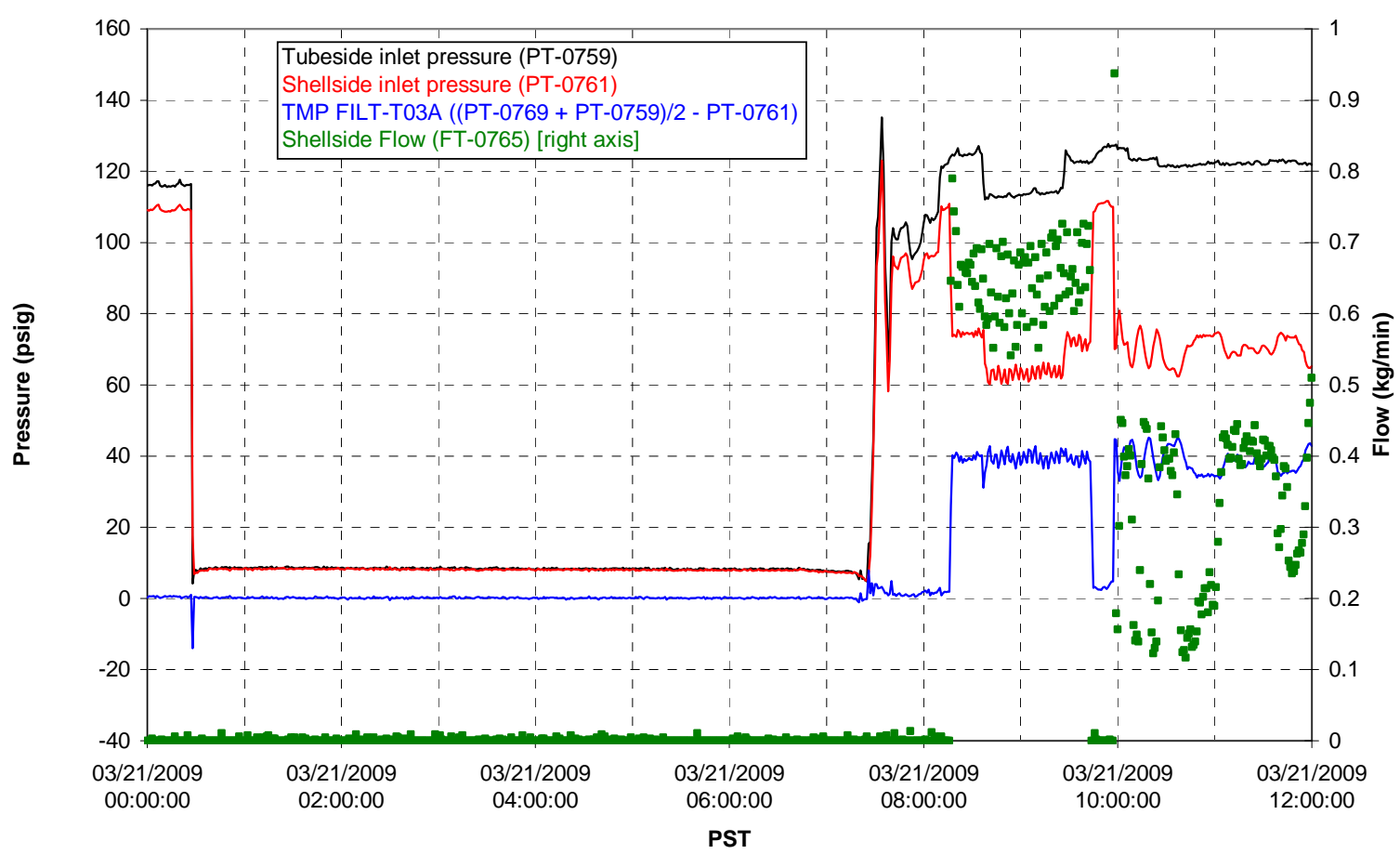

Filter UFP-FILT-T04A

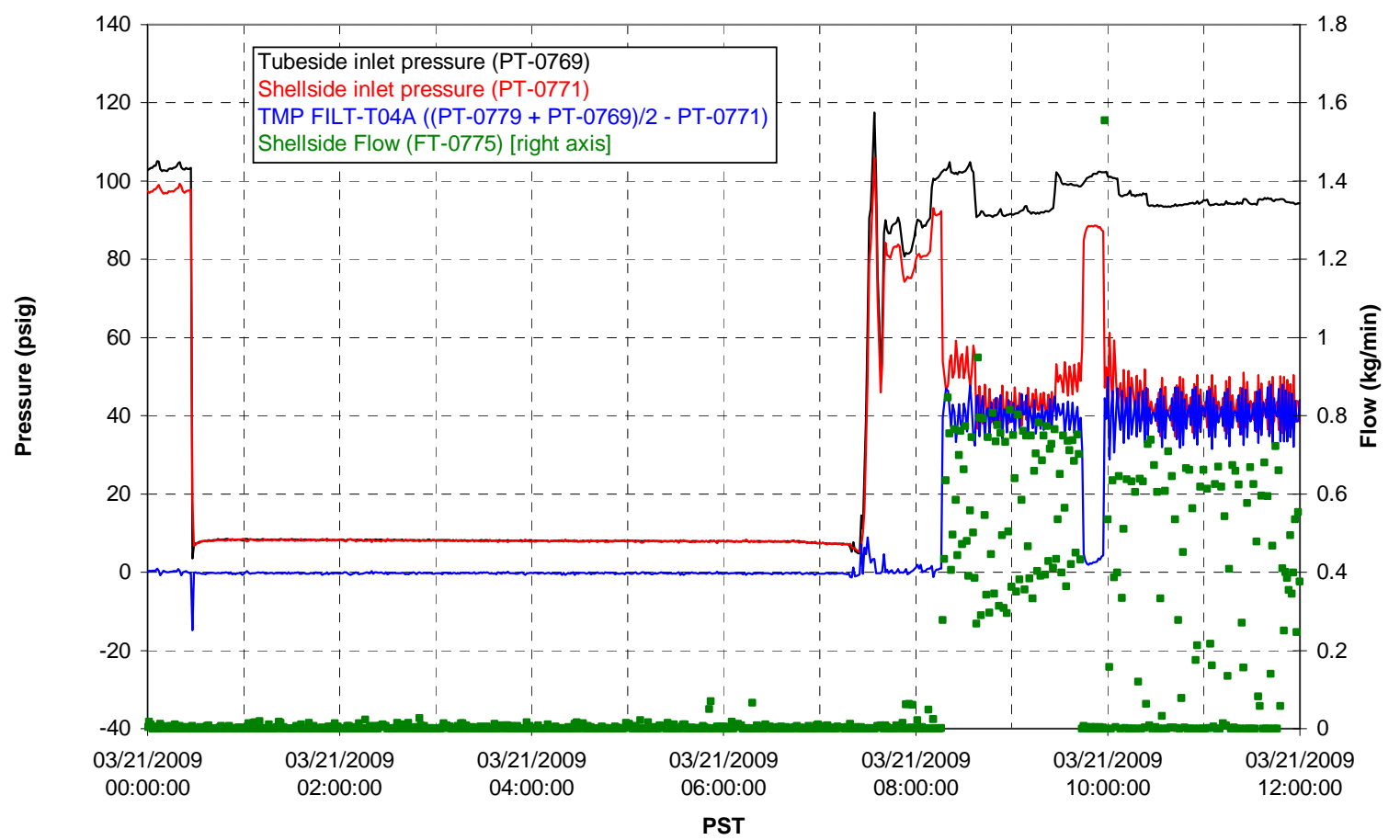


Filter UFP-FILT-T05A

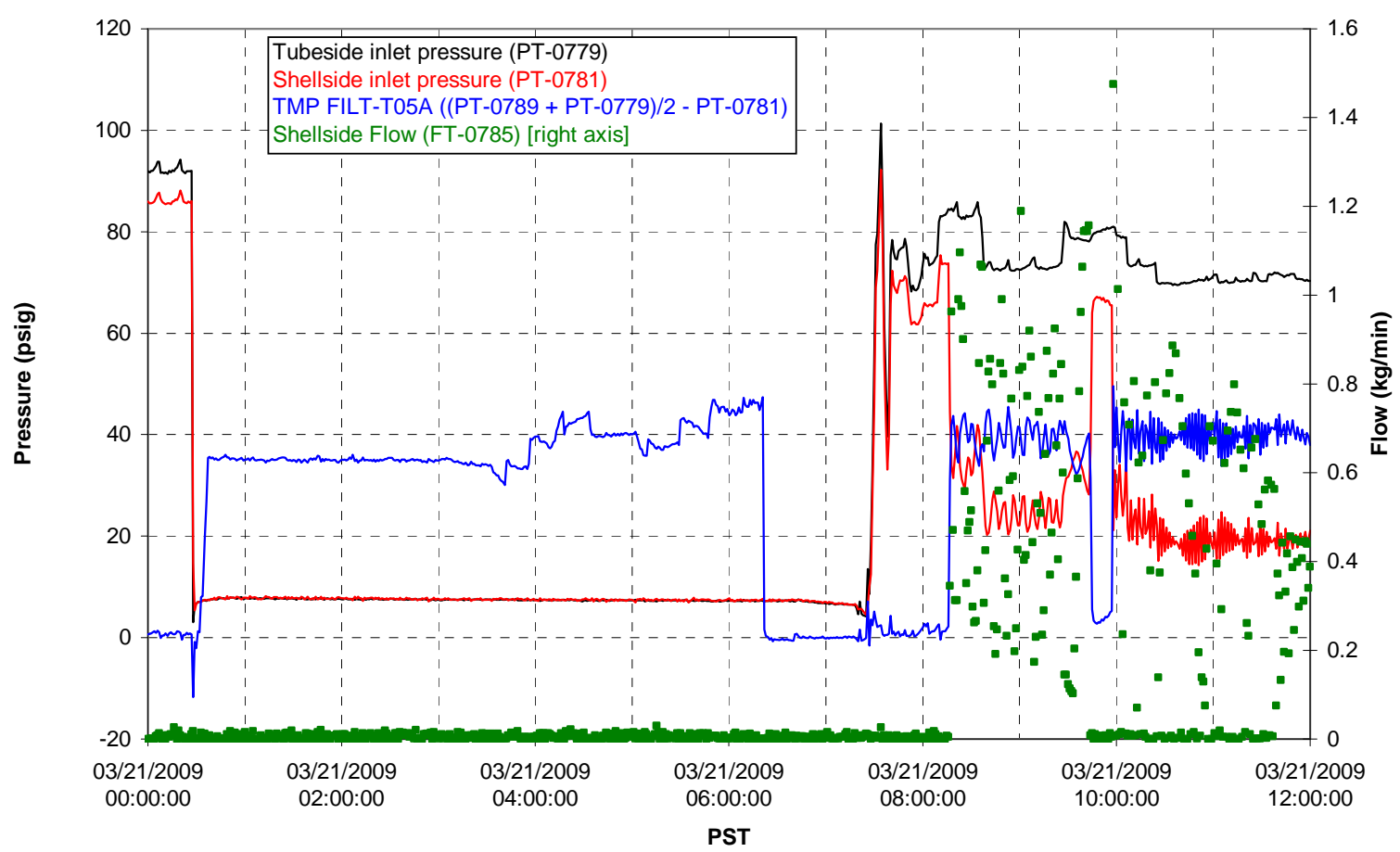

Chemical Flow

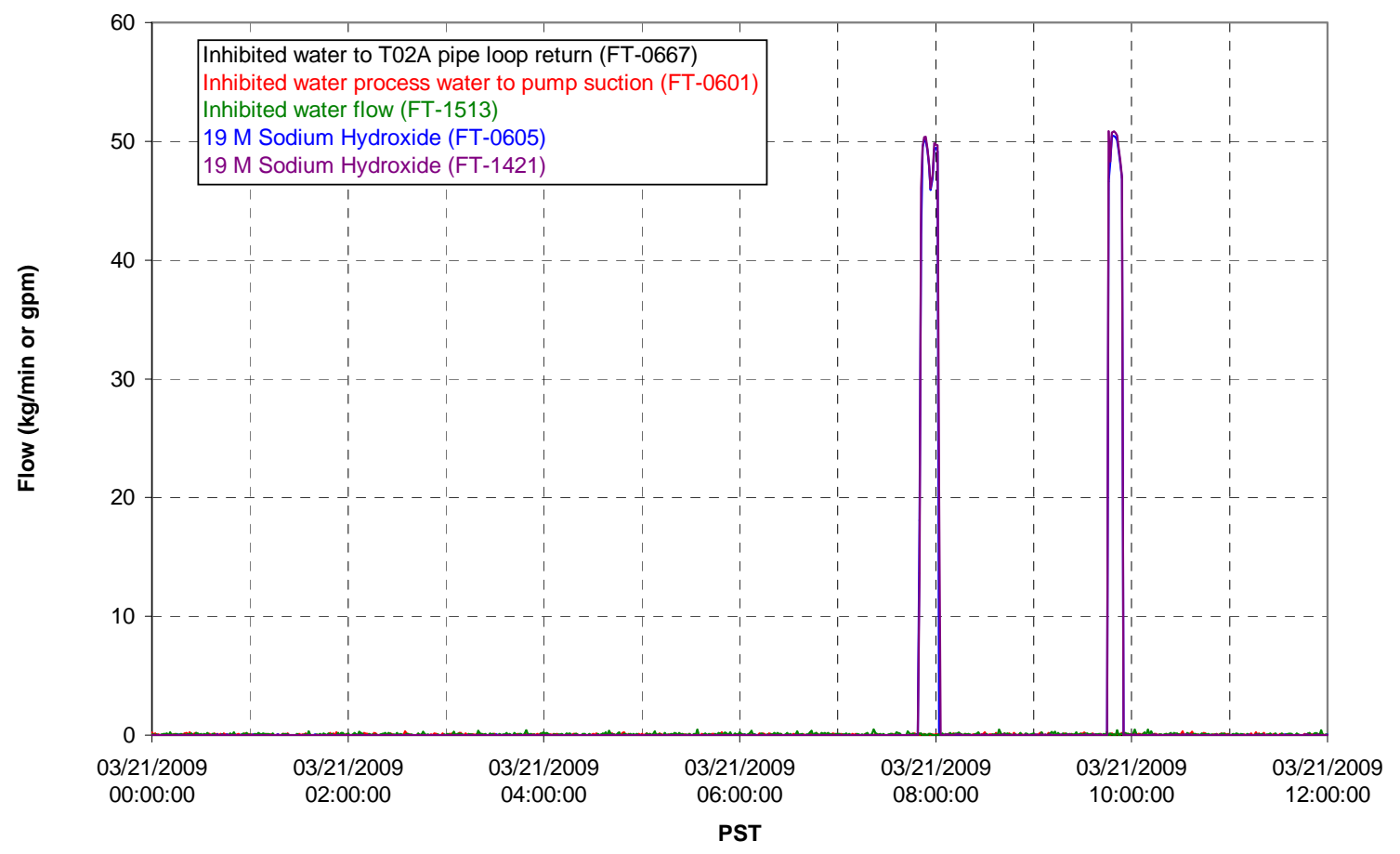




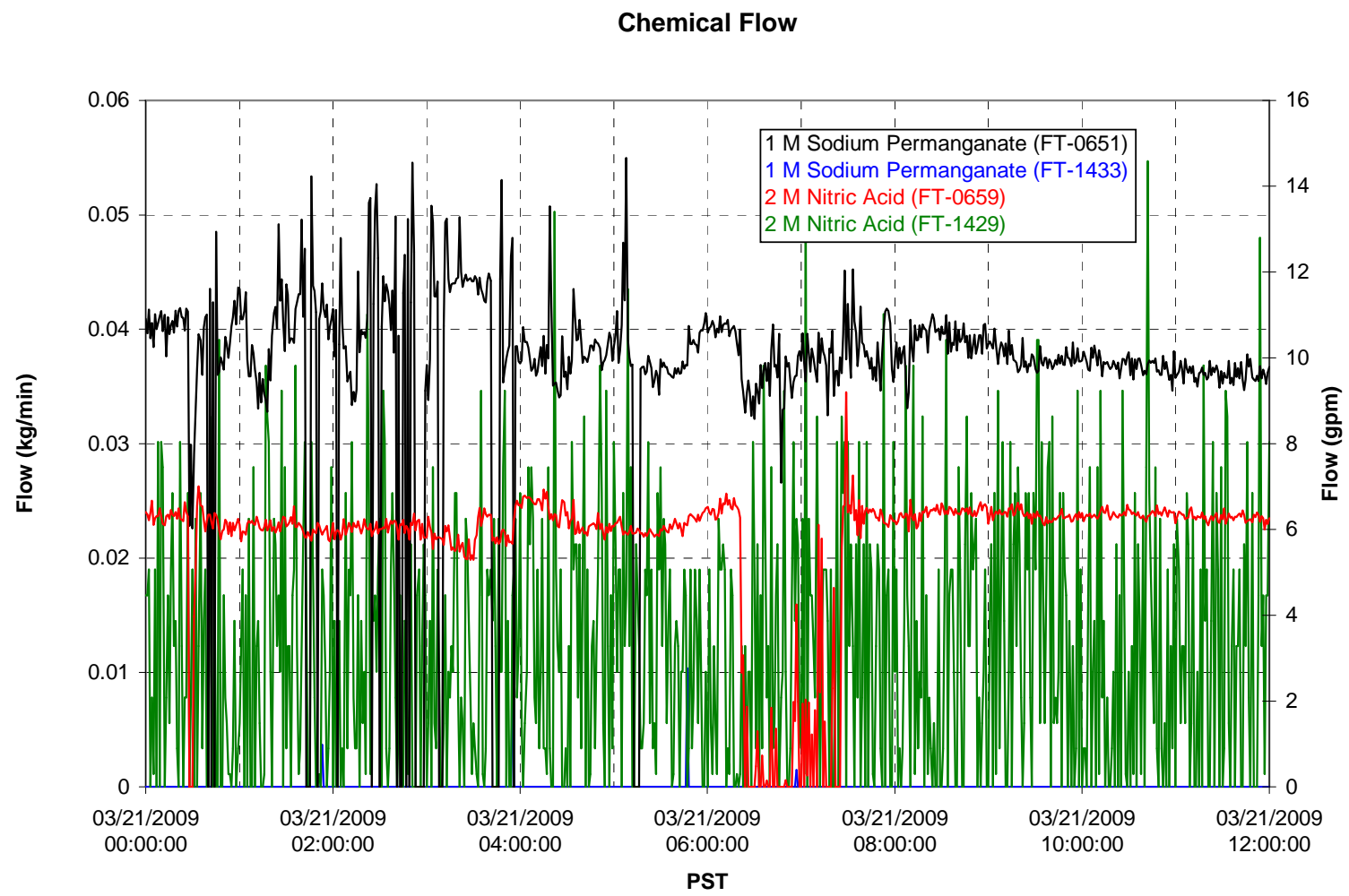

Air Flows

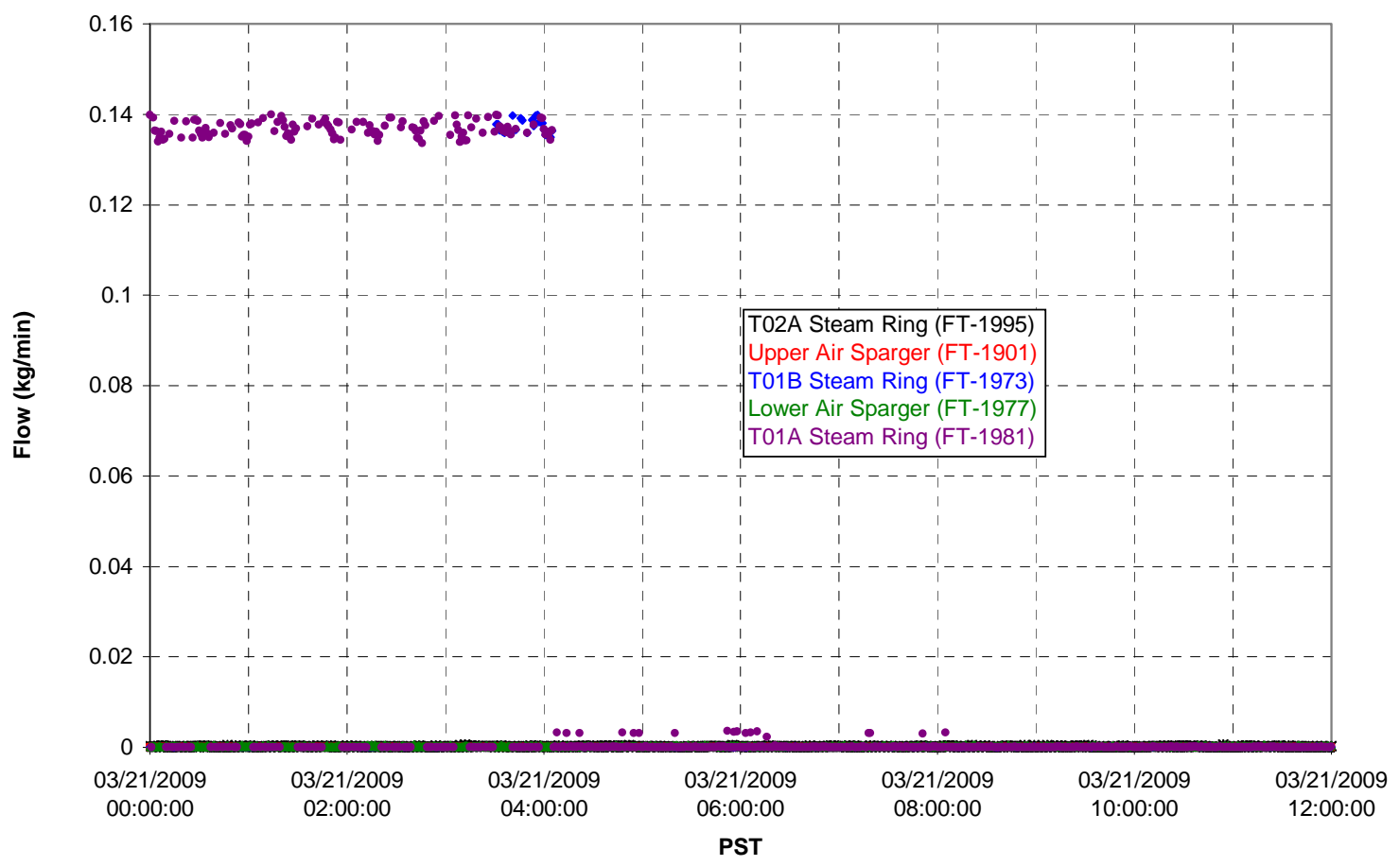


T02A Steam

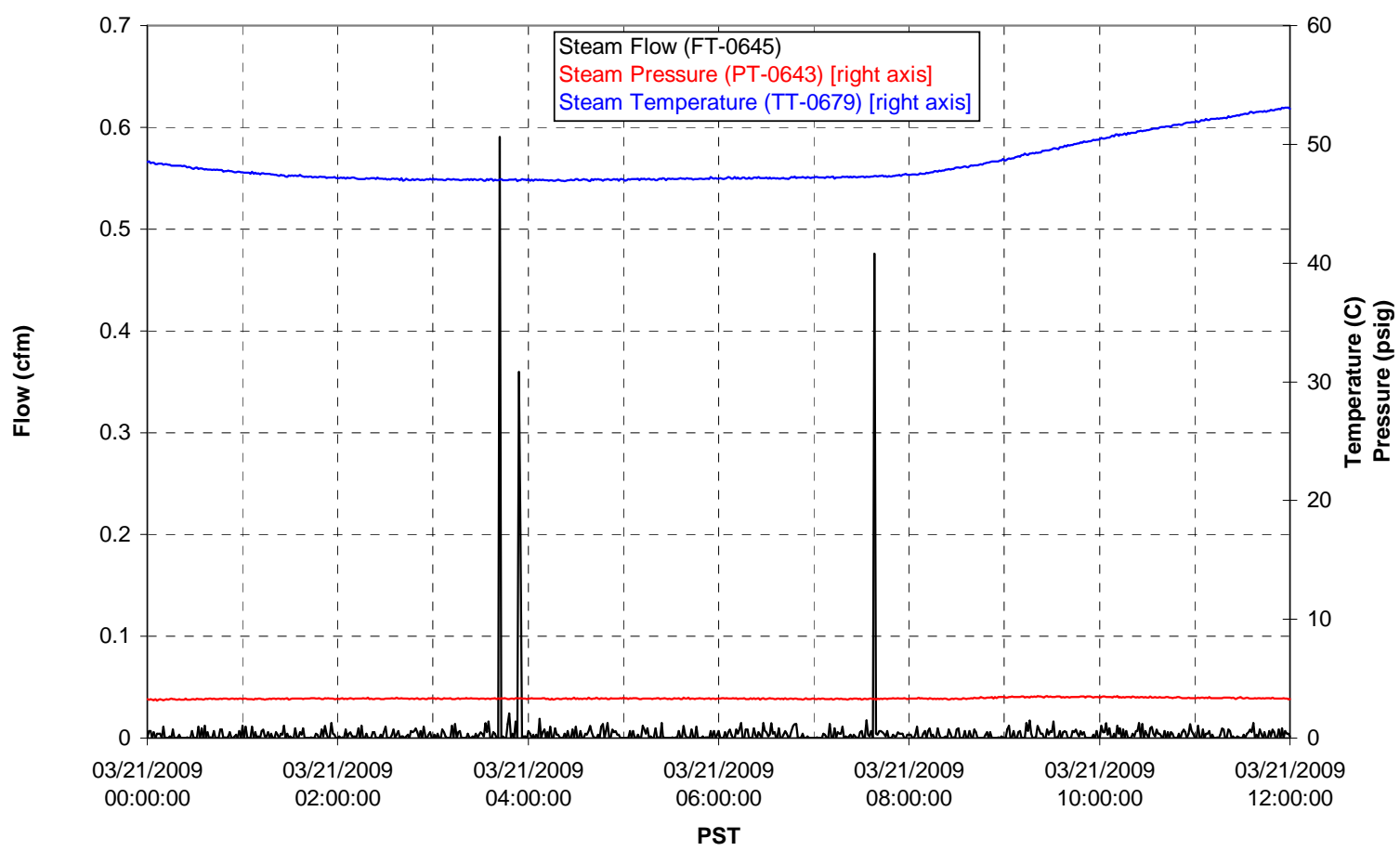

T01A Steam

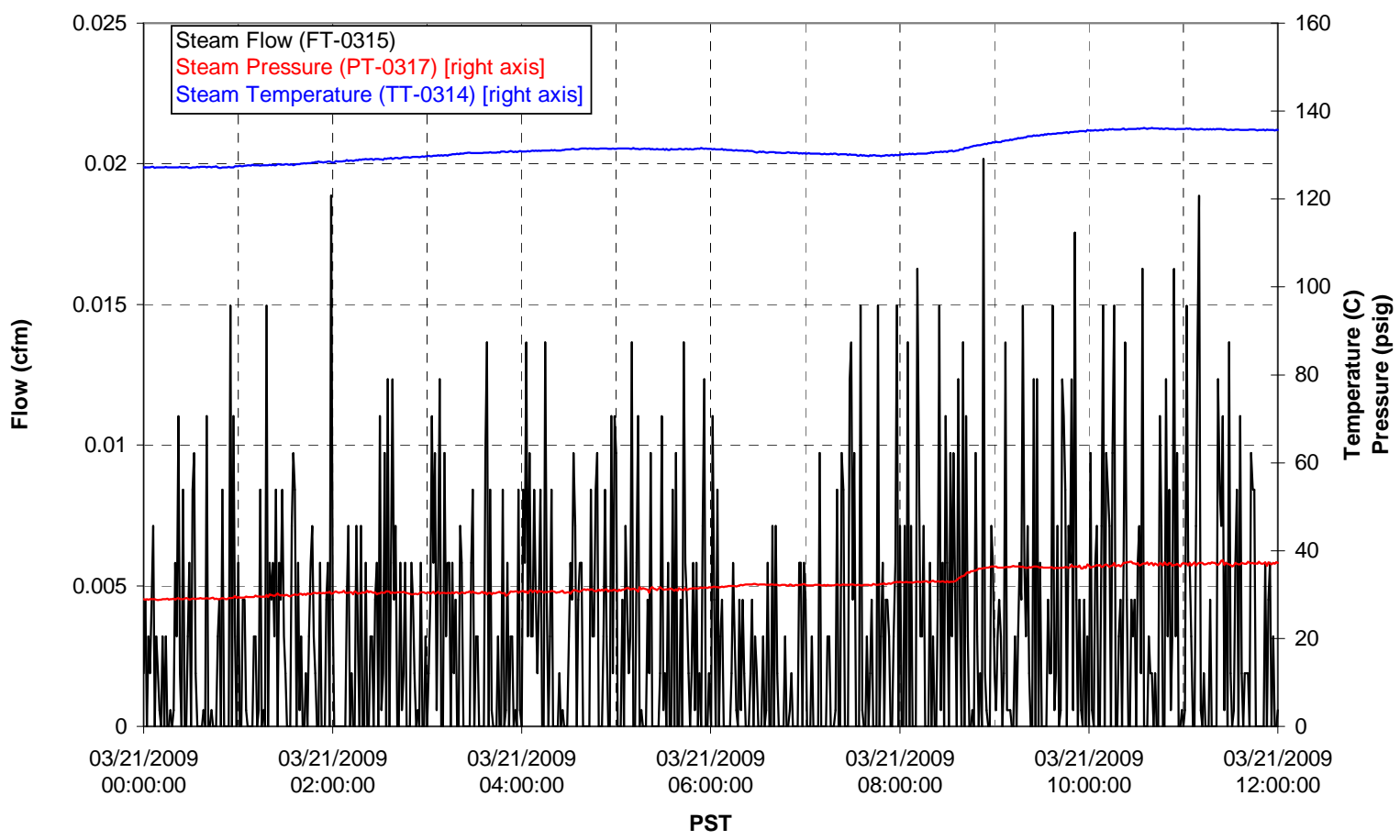


T01B Steam

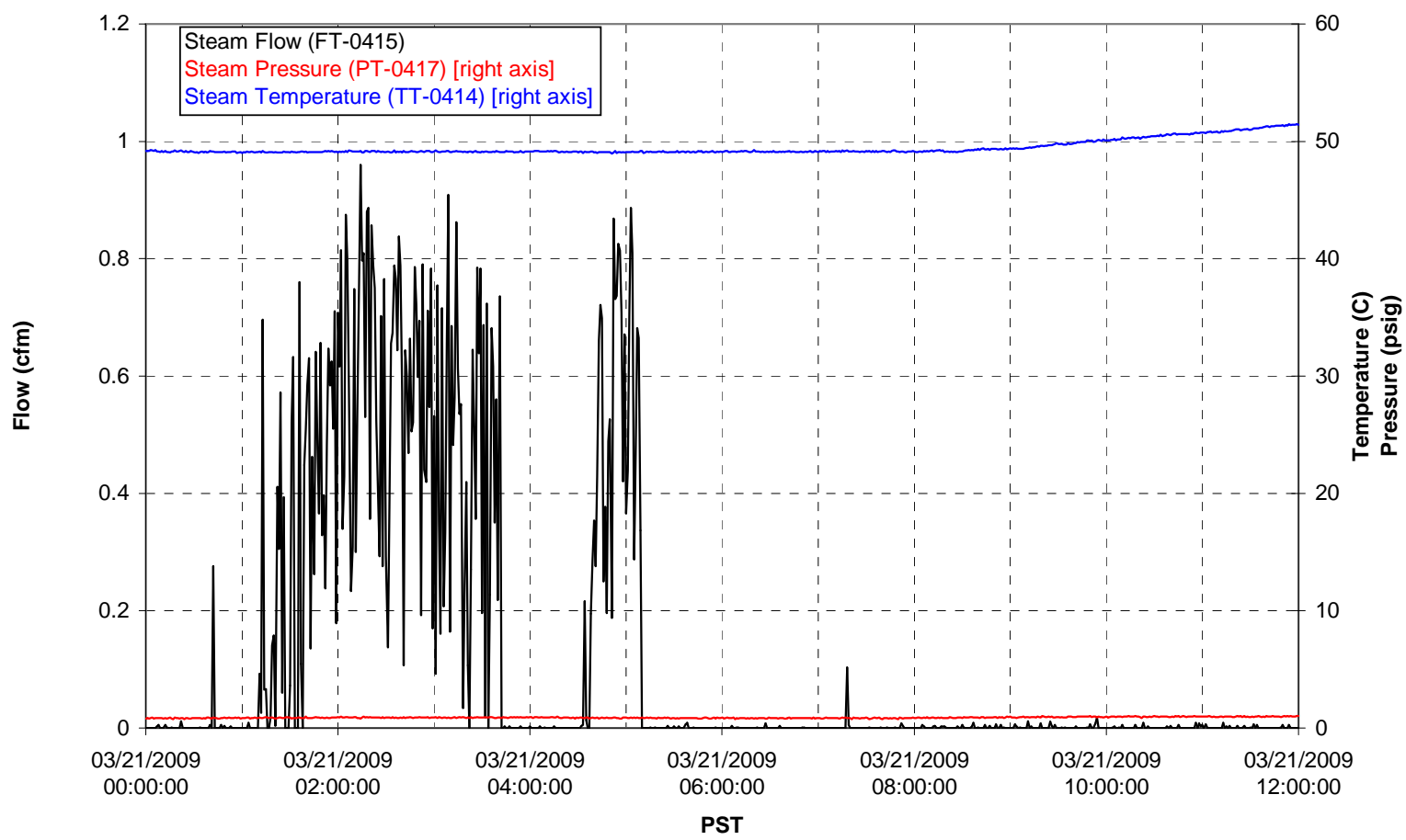




\section{Integrated Test A Data Plots 03/21/09 12:00 - 03/22/09 00:00}


T01A level

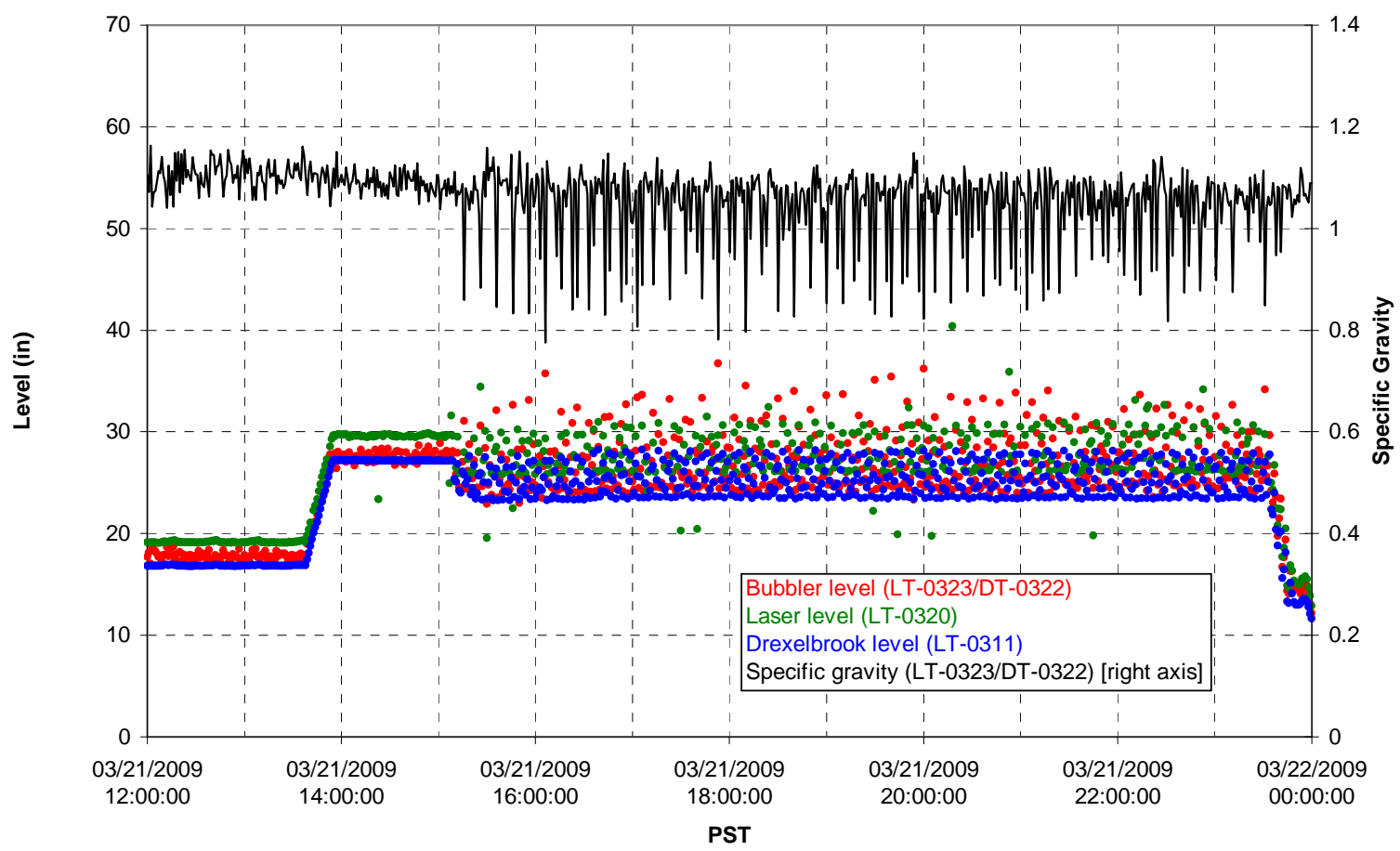

T01A temperatures

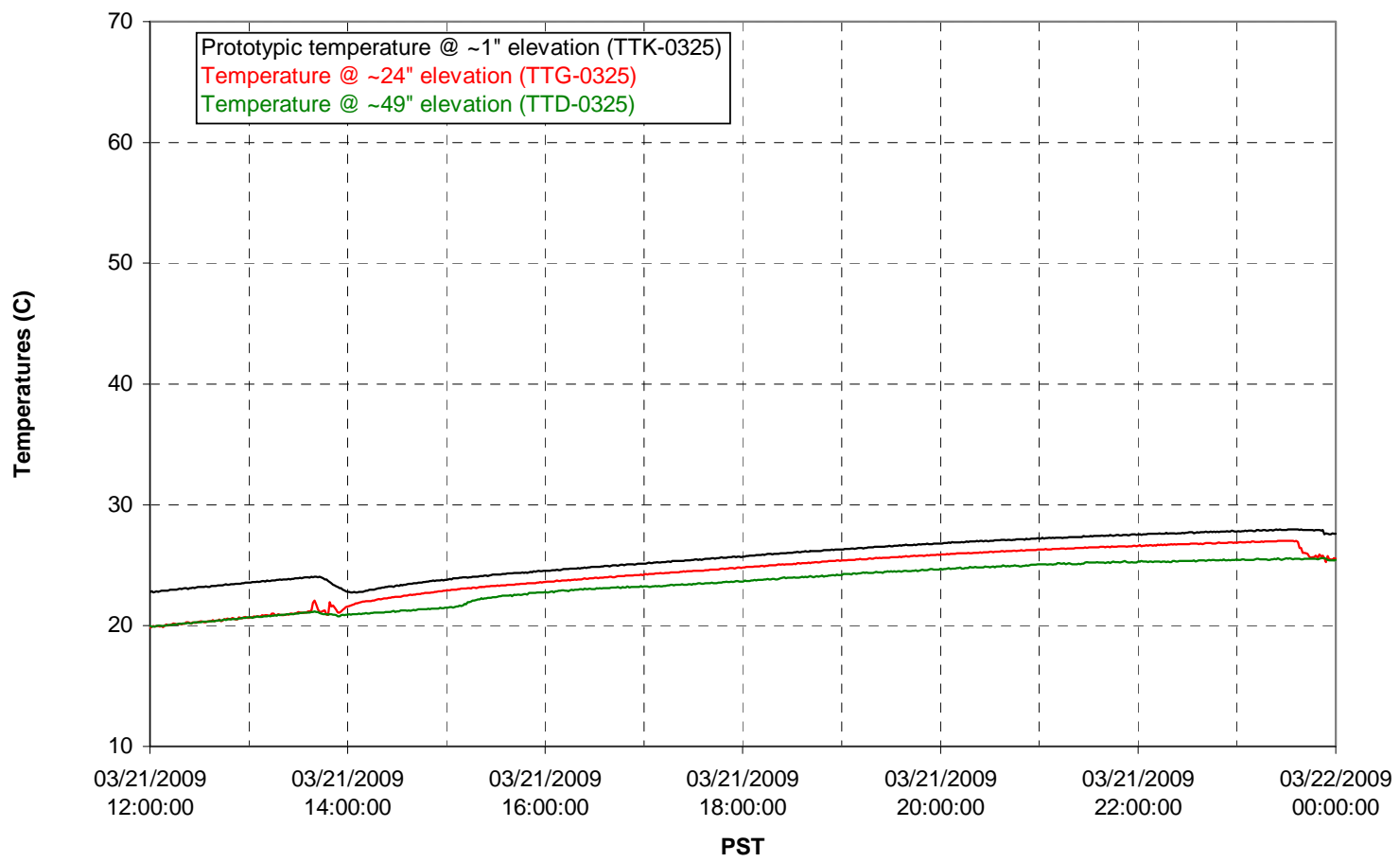


T01B level

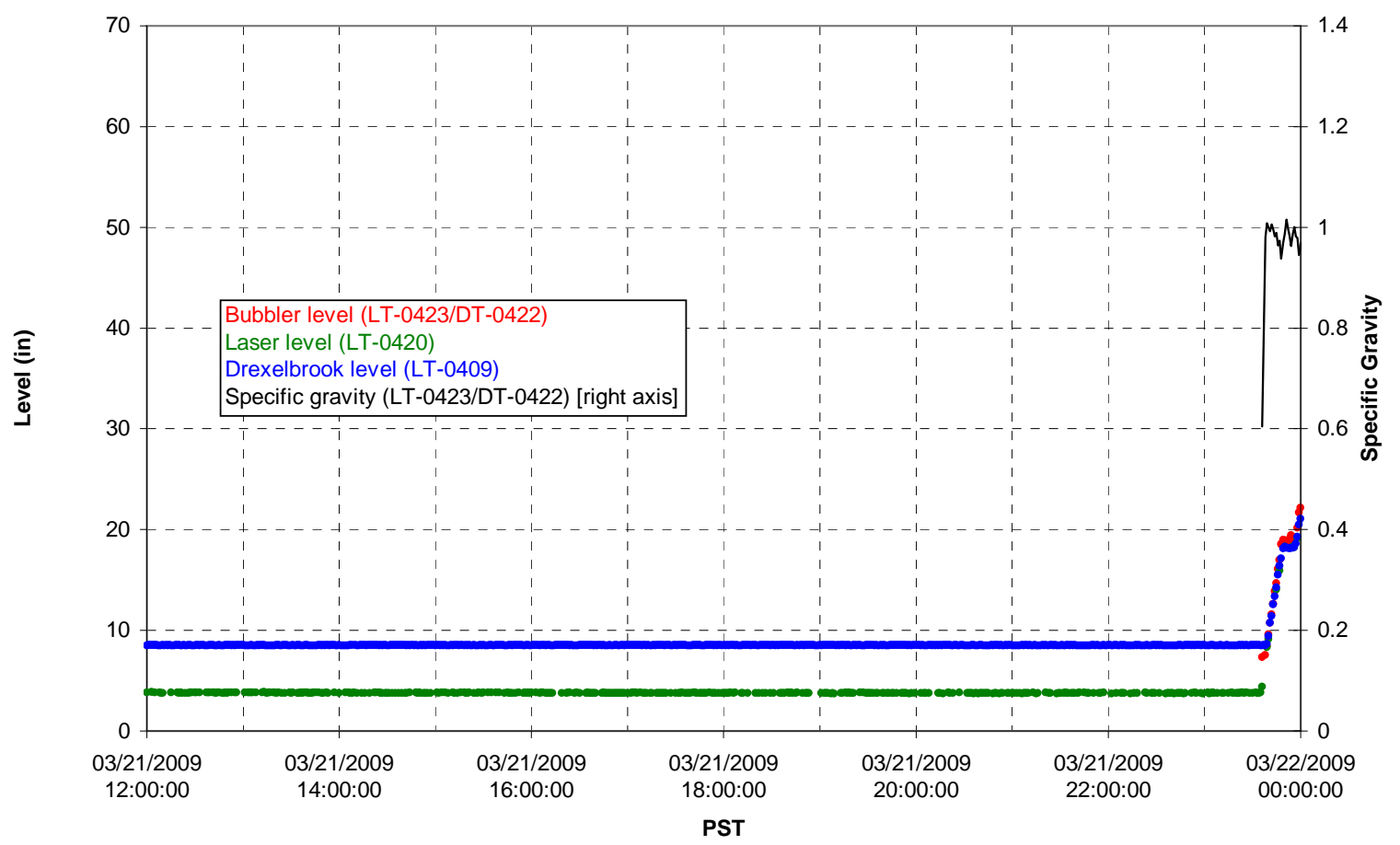

T01B temperatures

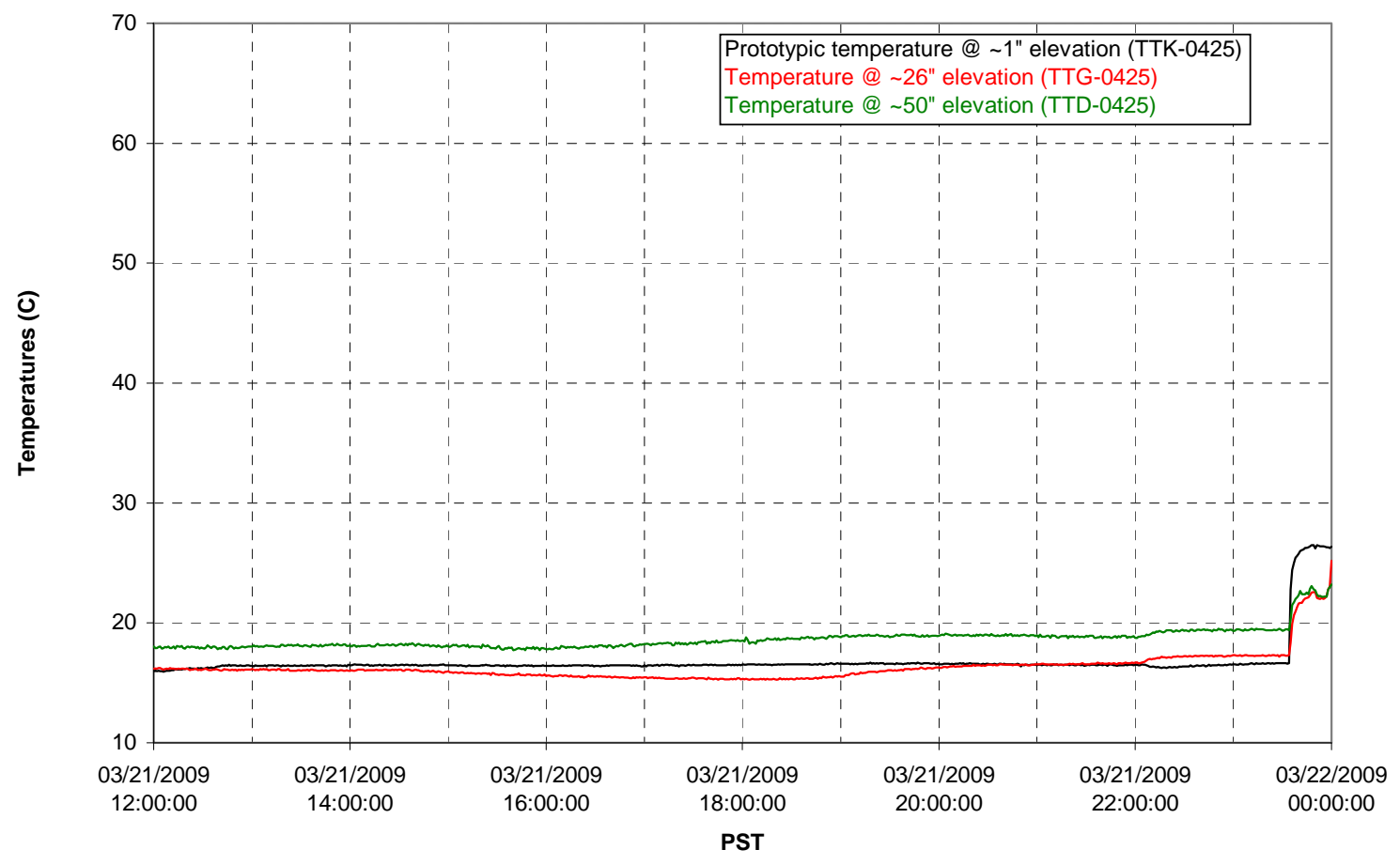


T02A level

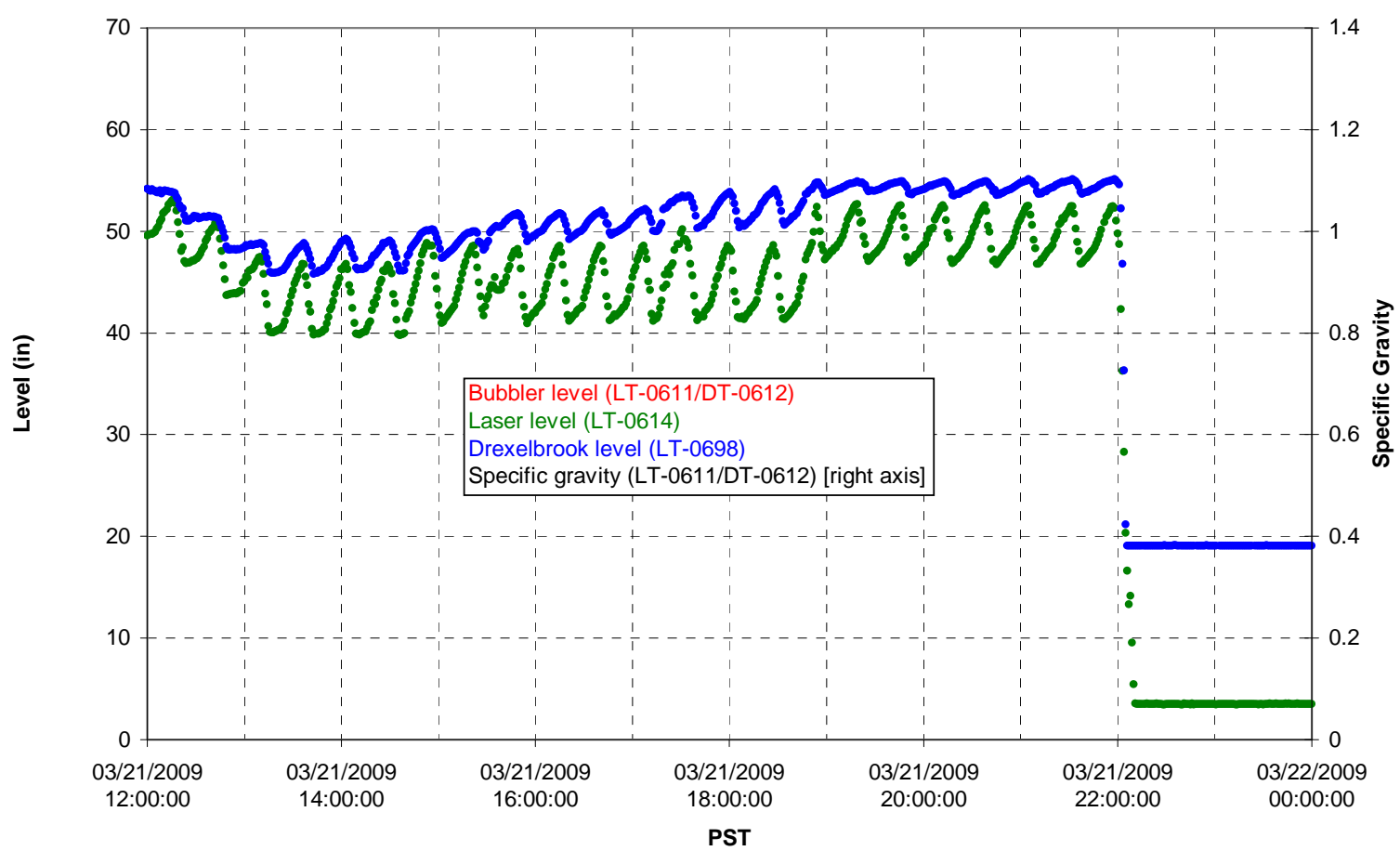

T02A temperatures

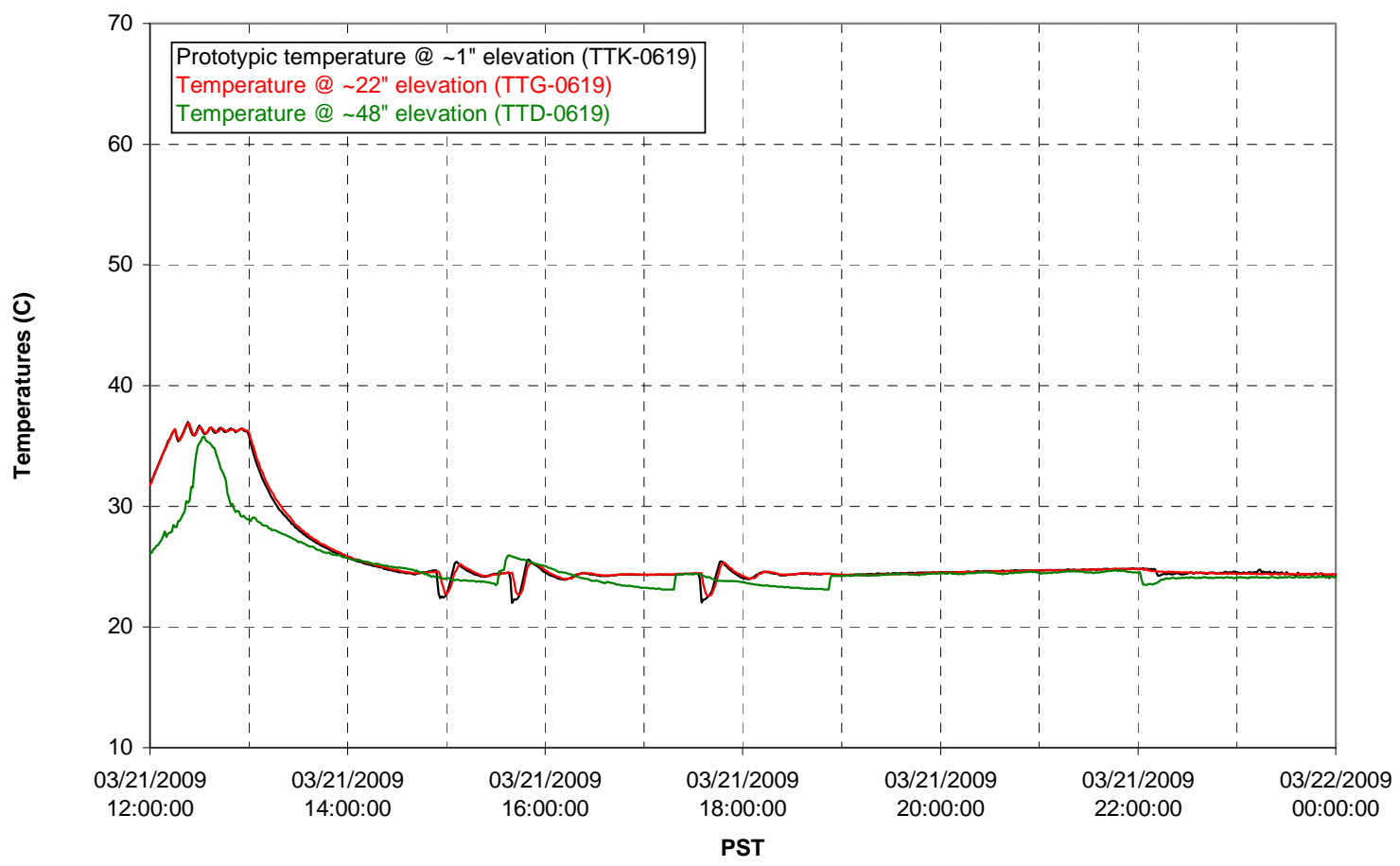


T02A and filter loop temperatures

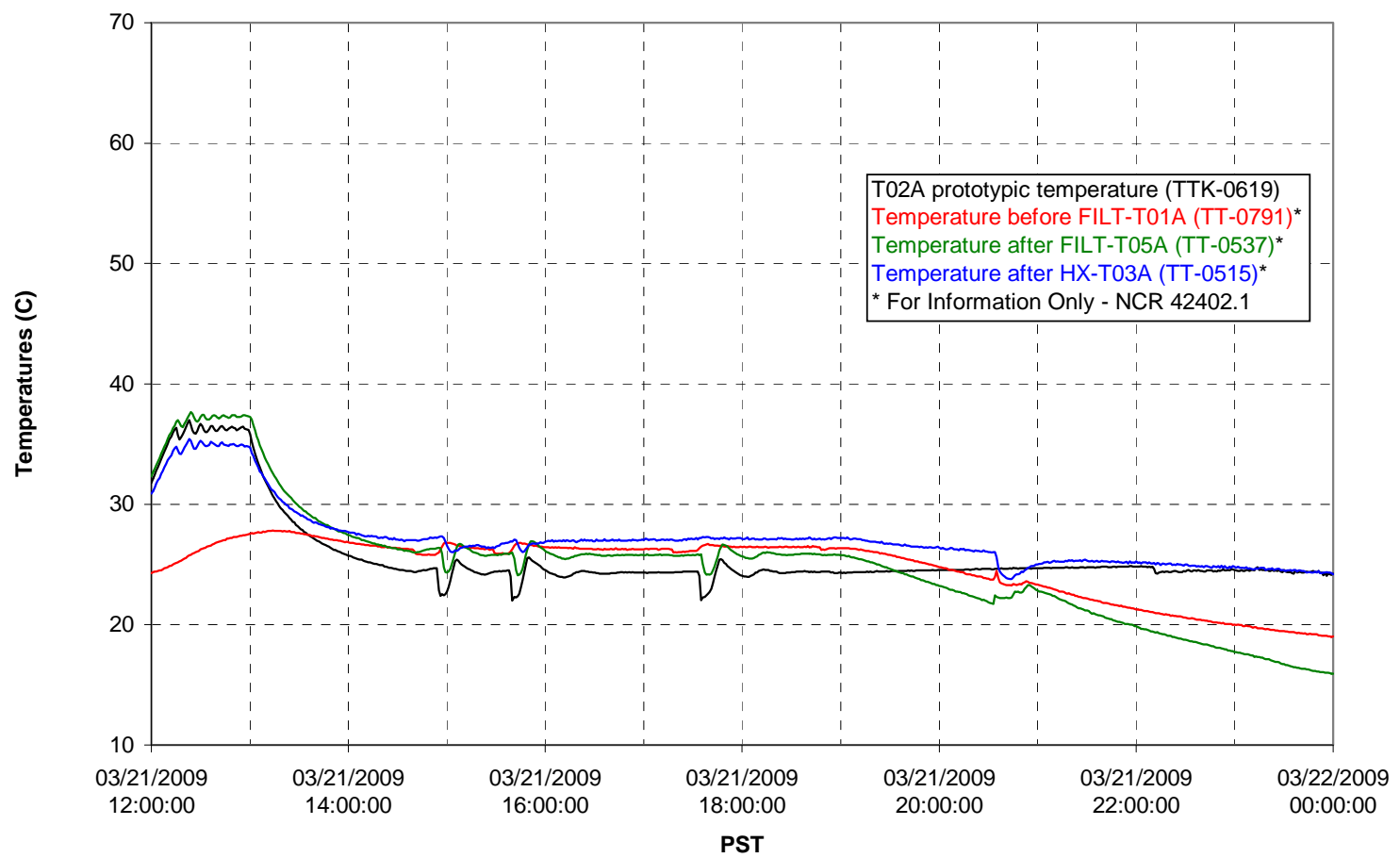

Pump Pressures and Flow

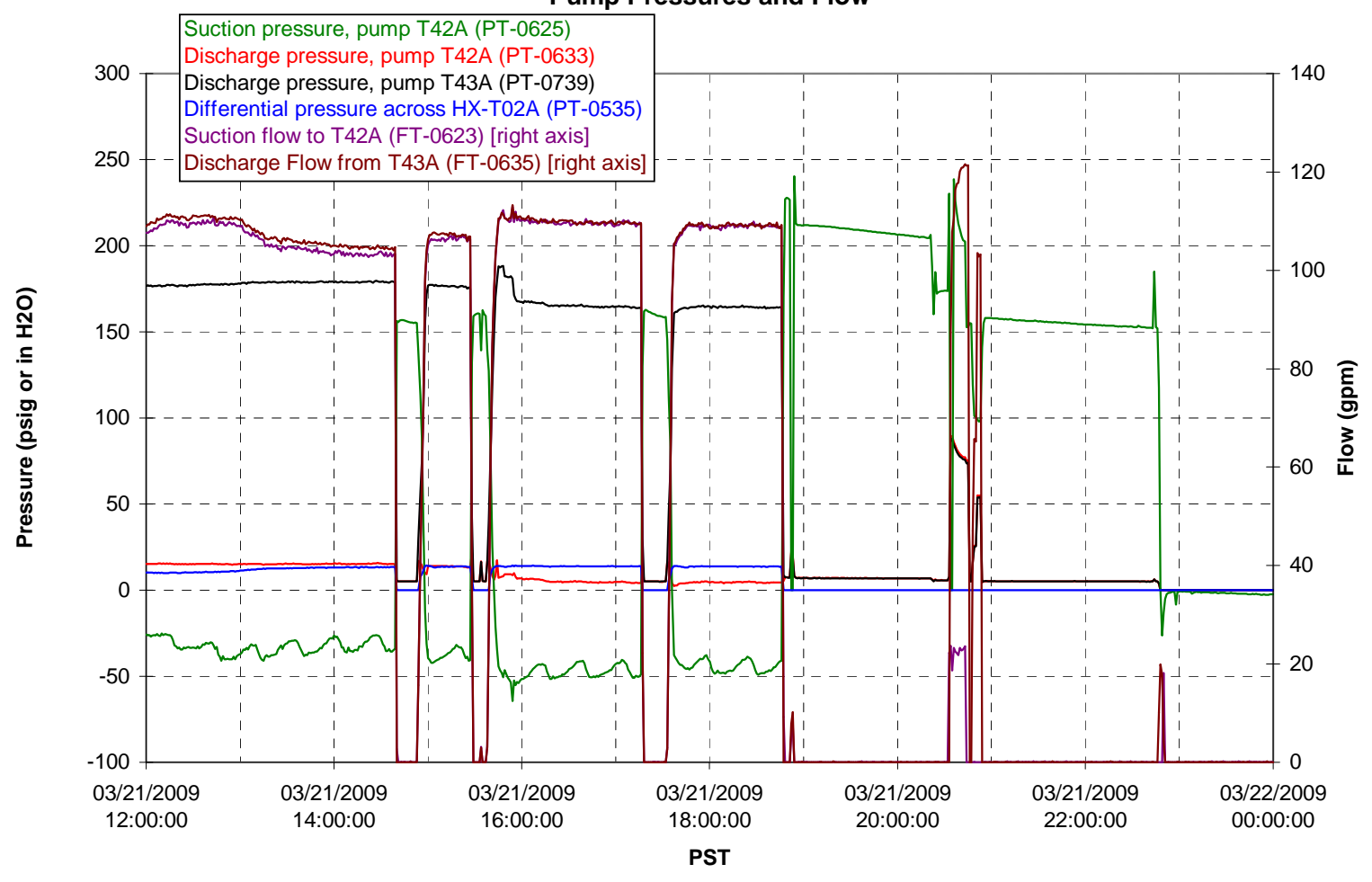


Axial pressure drop

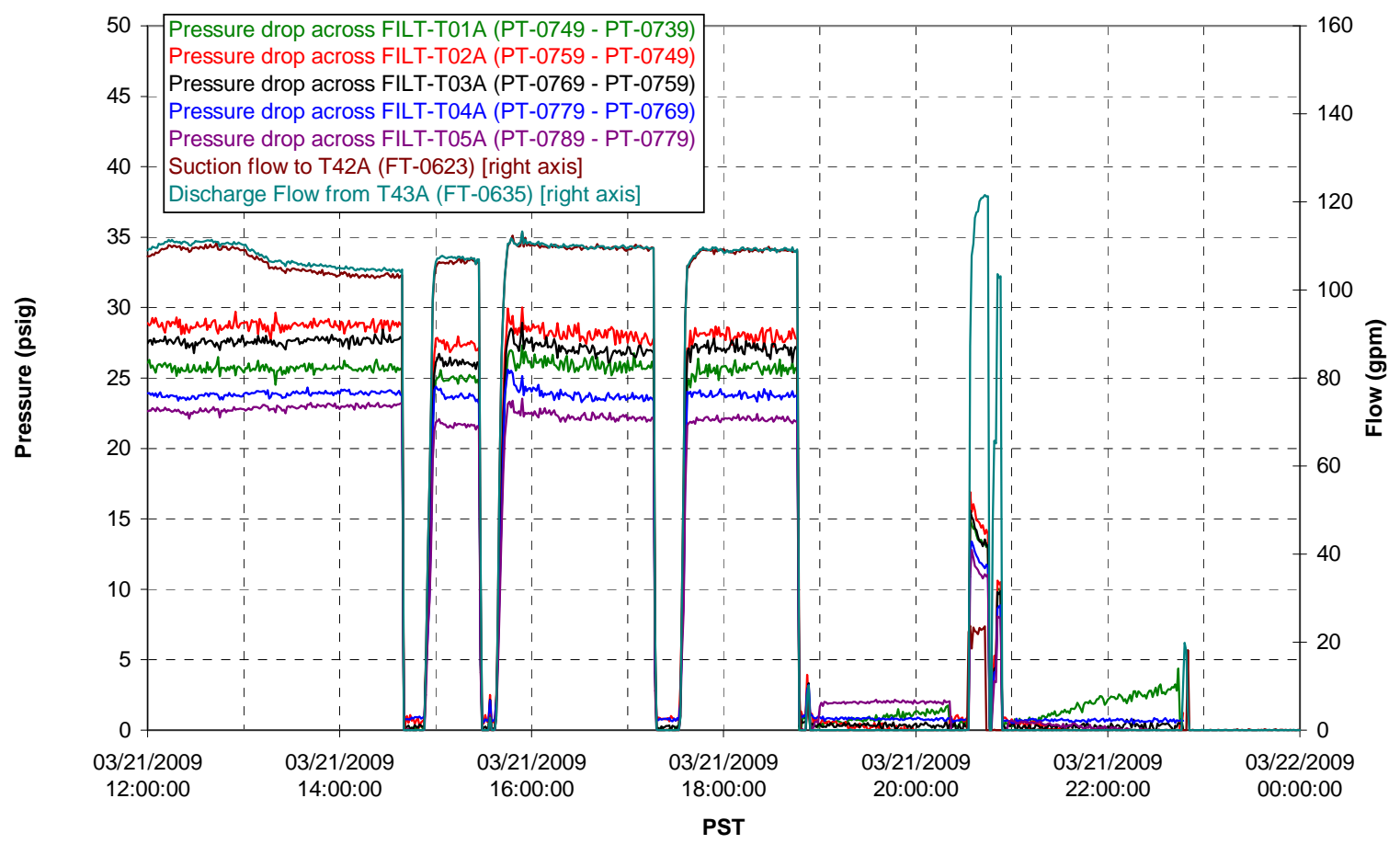

Permeate flow rates

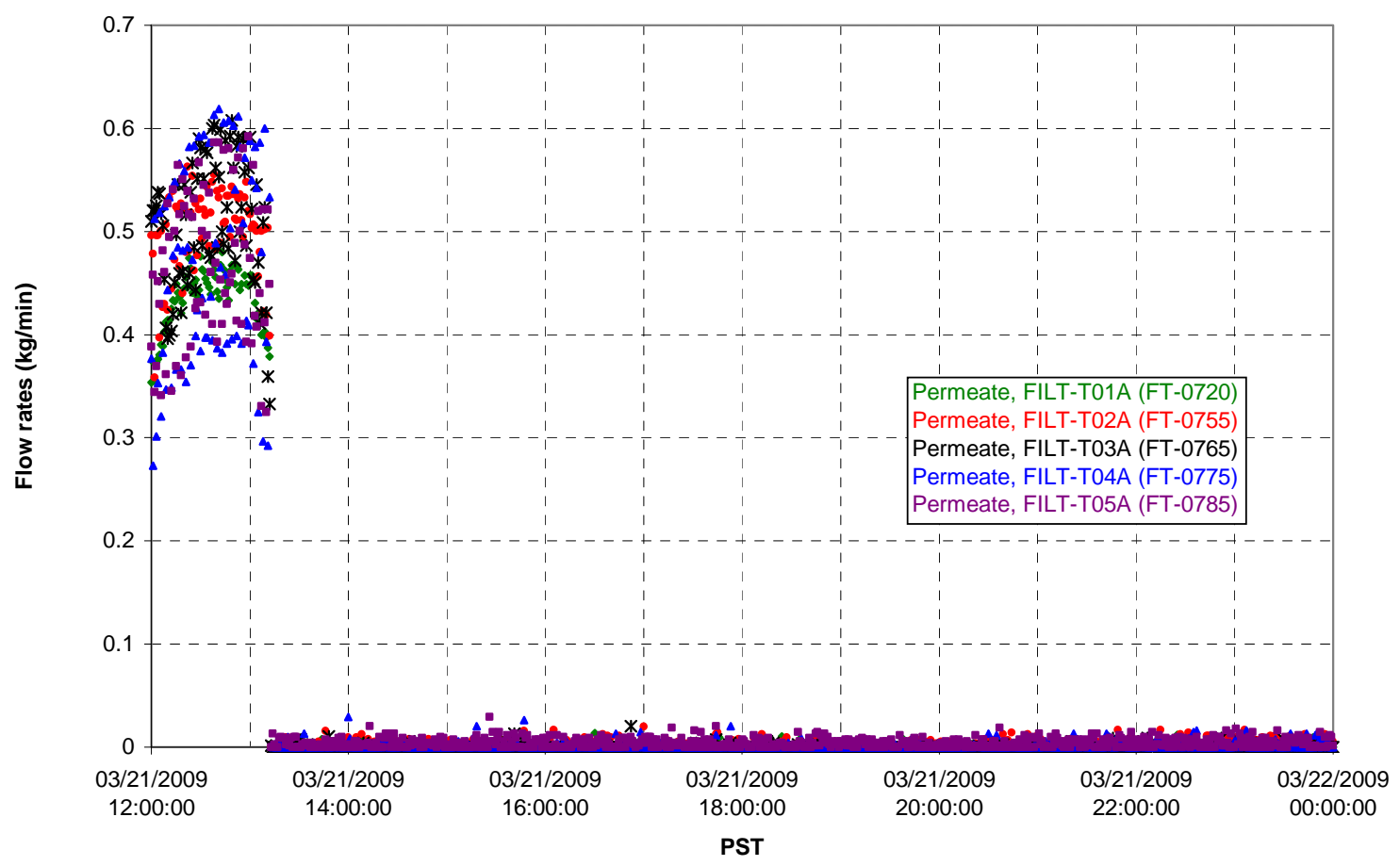


T02A Inner Temperature Tree

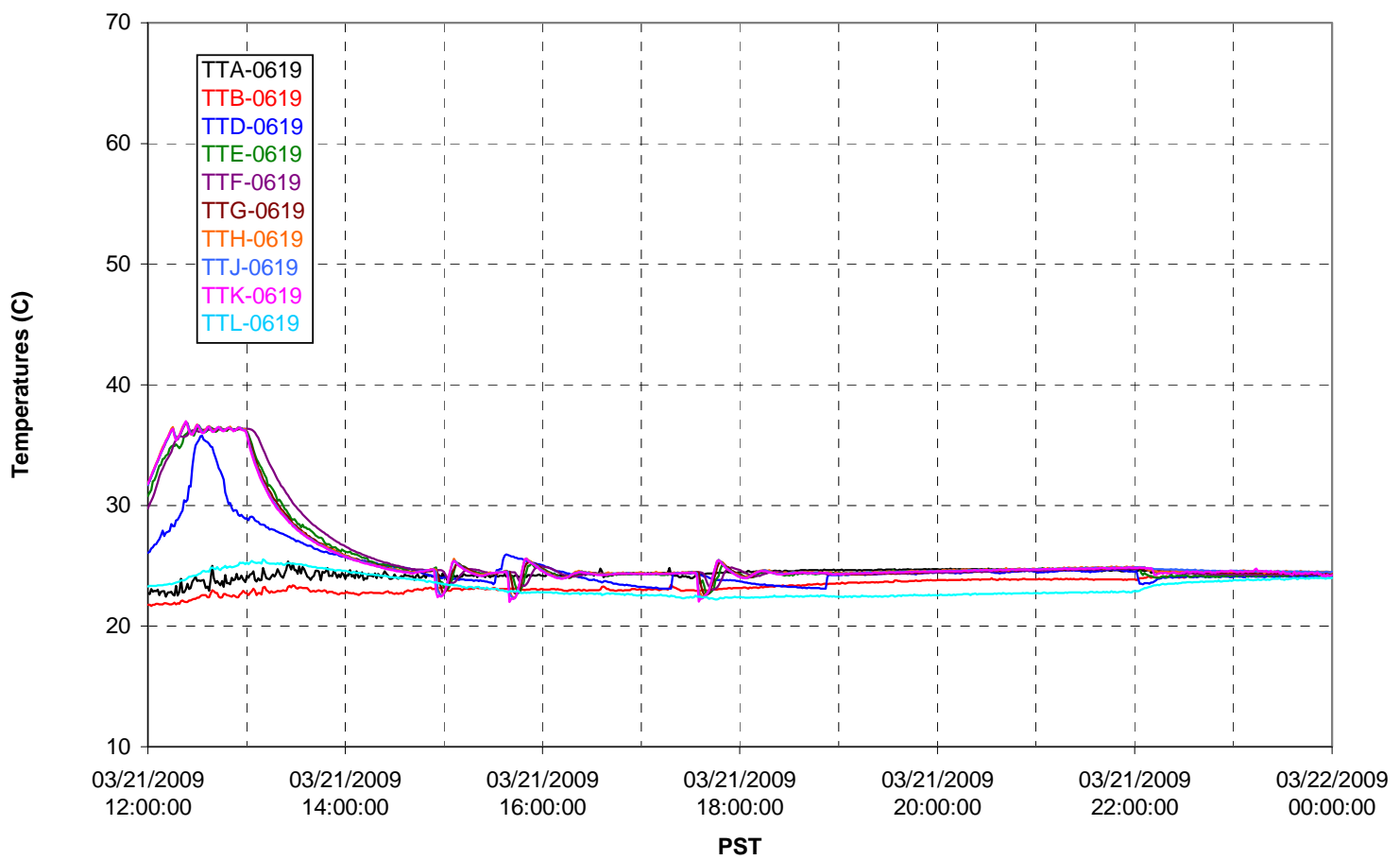

T02A Outer Temperature Tree

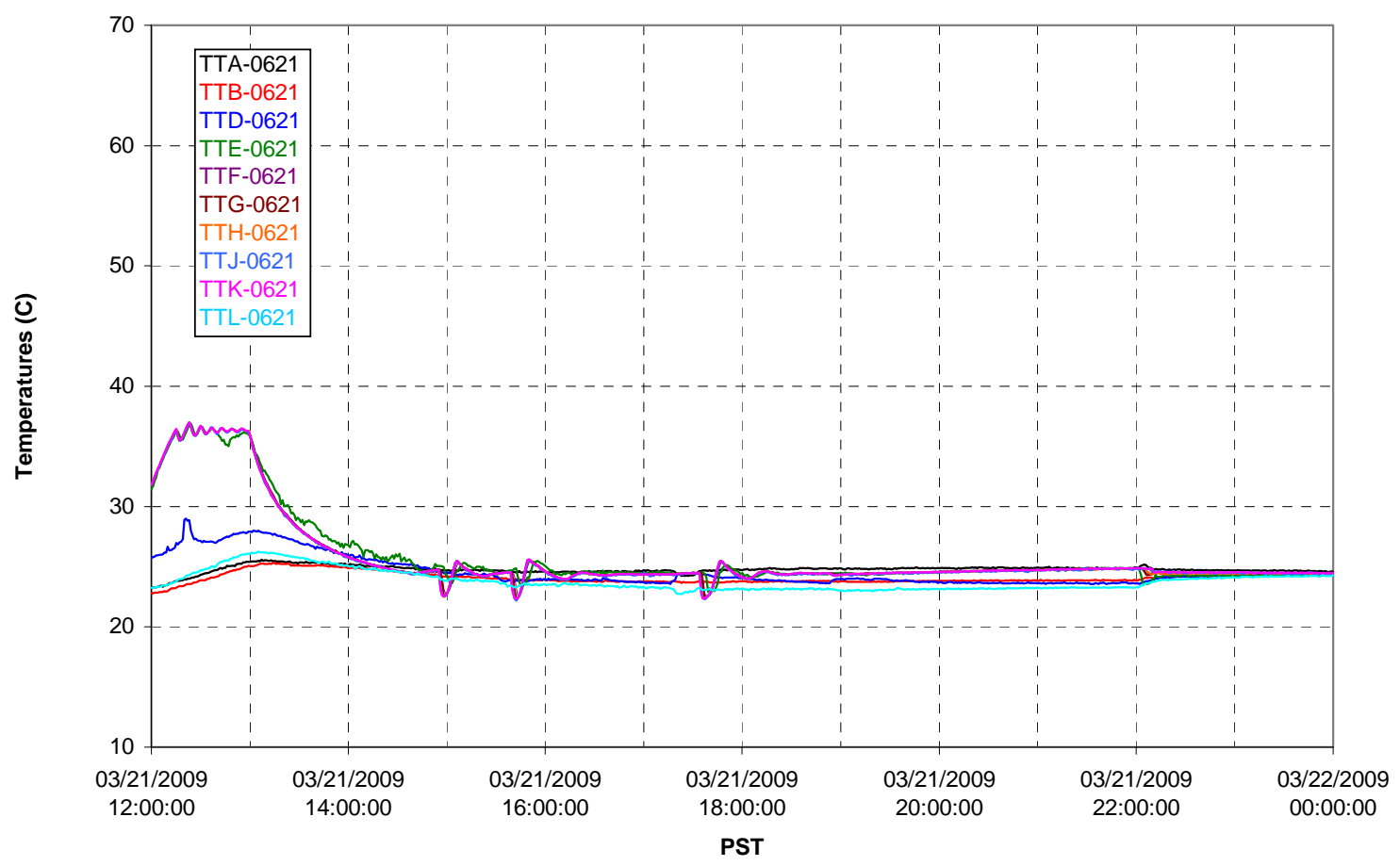


T02A temperatures

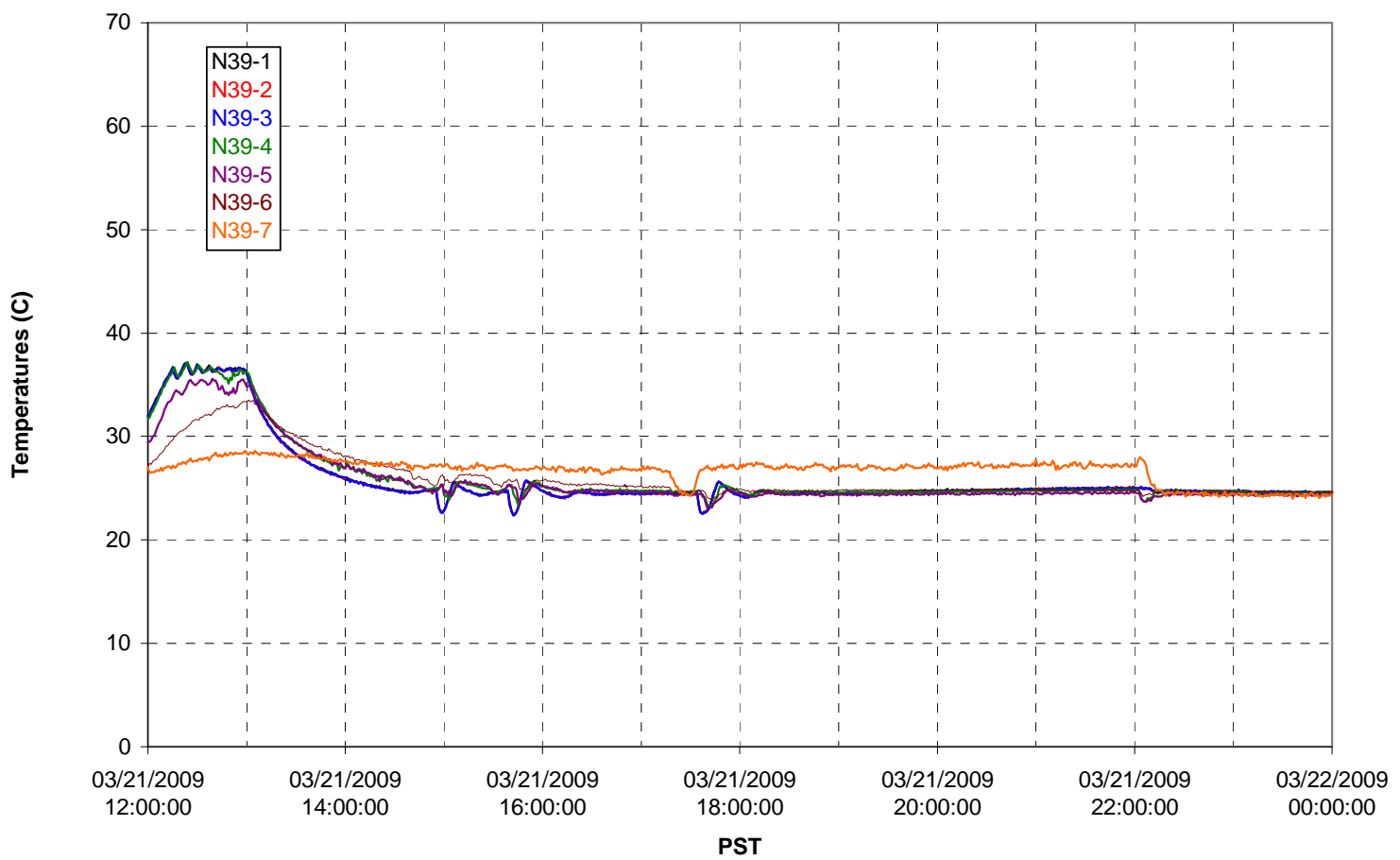

T02A temperatures

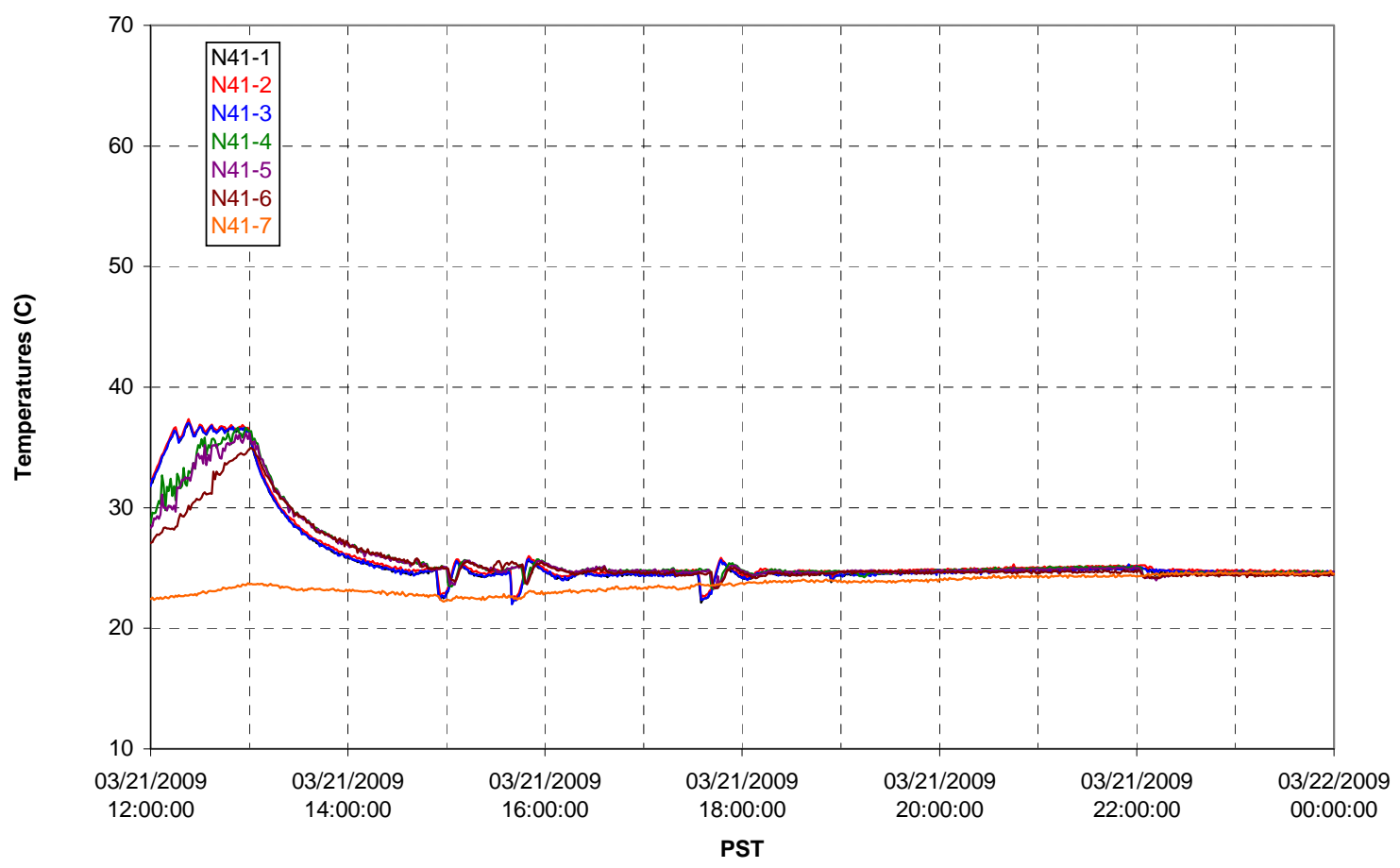


T02A temperatures

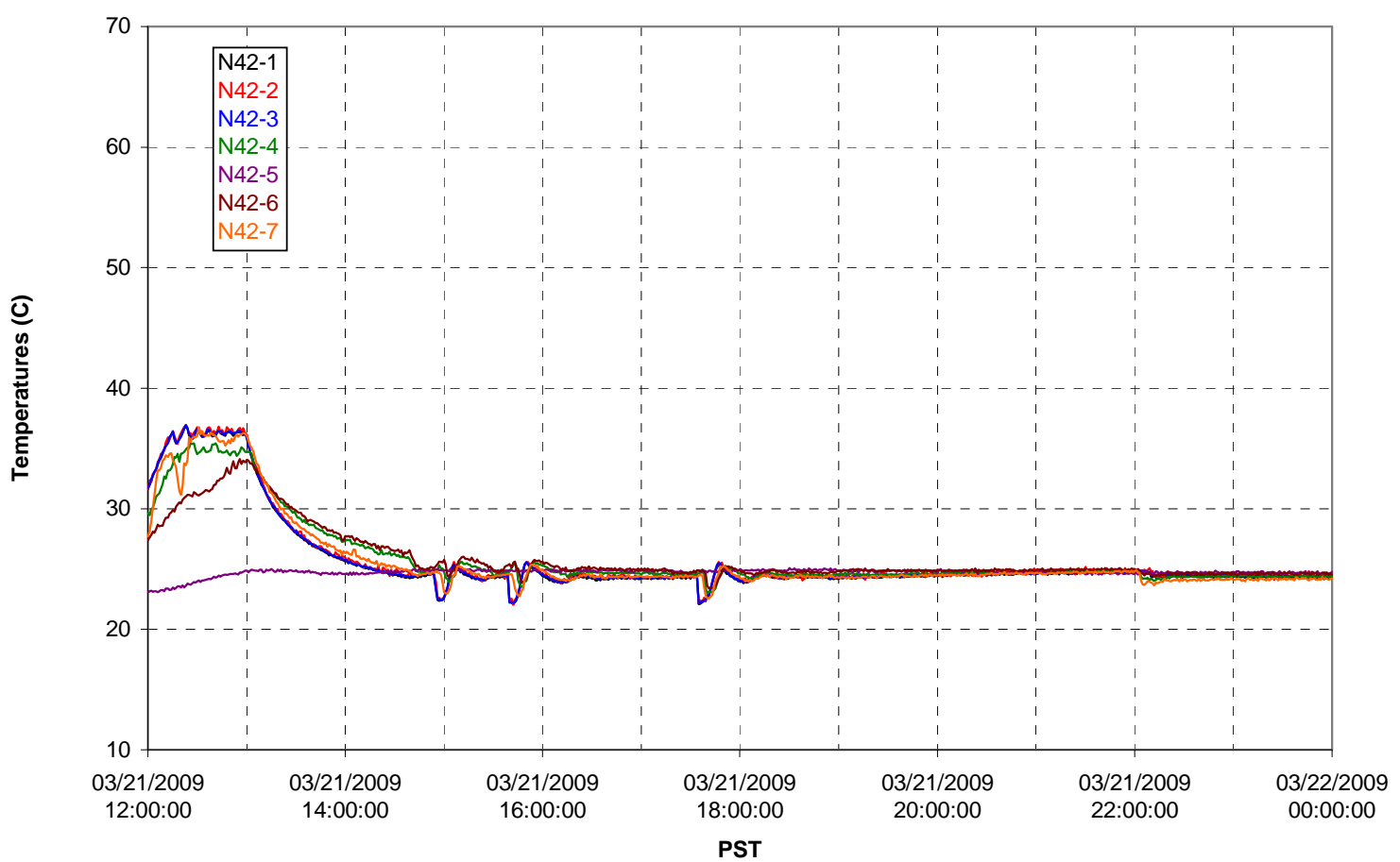

T02A temperatures

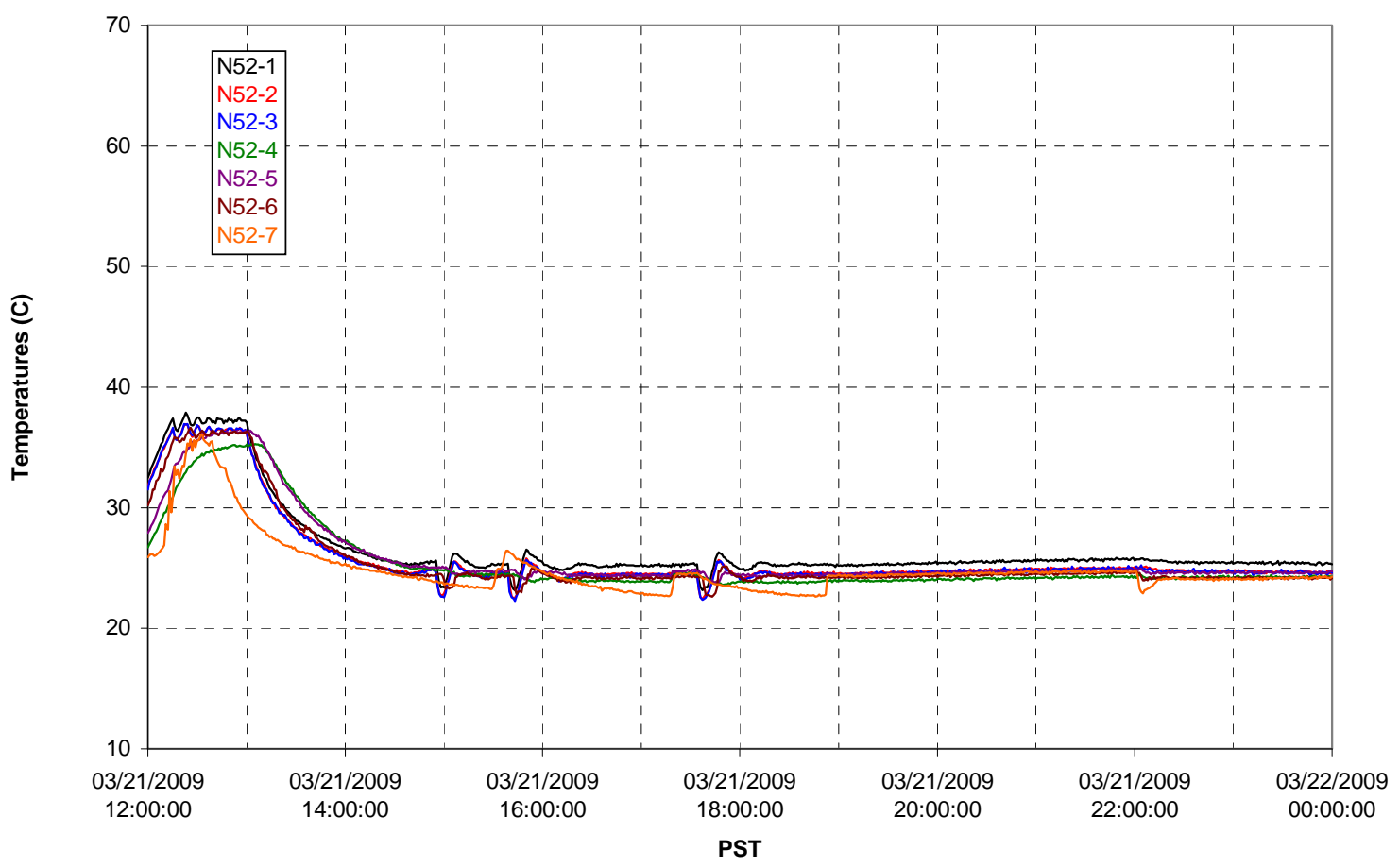


T02A Heating and Cooling

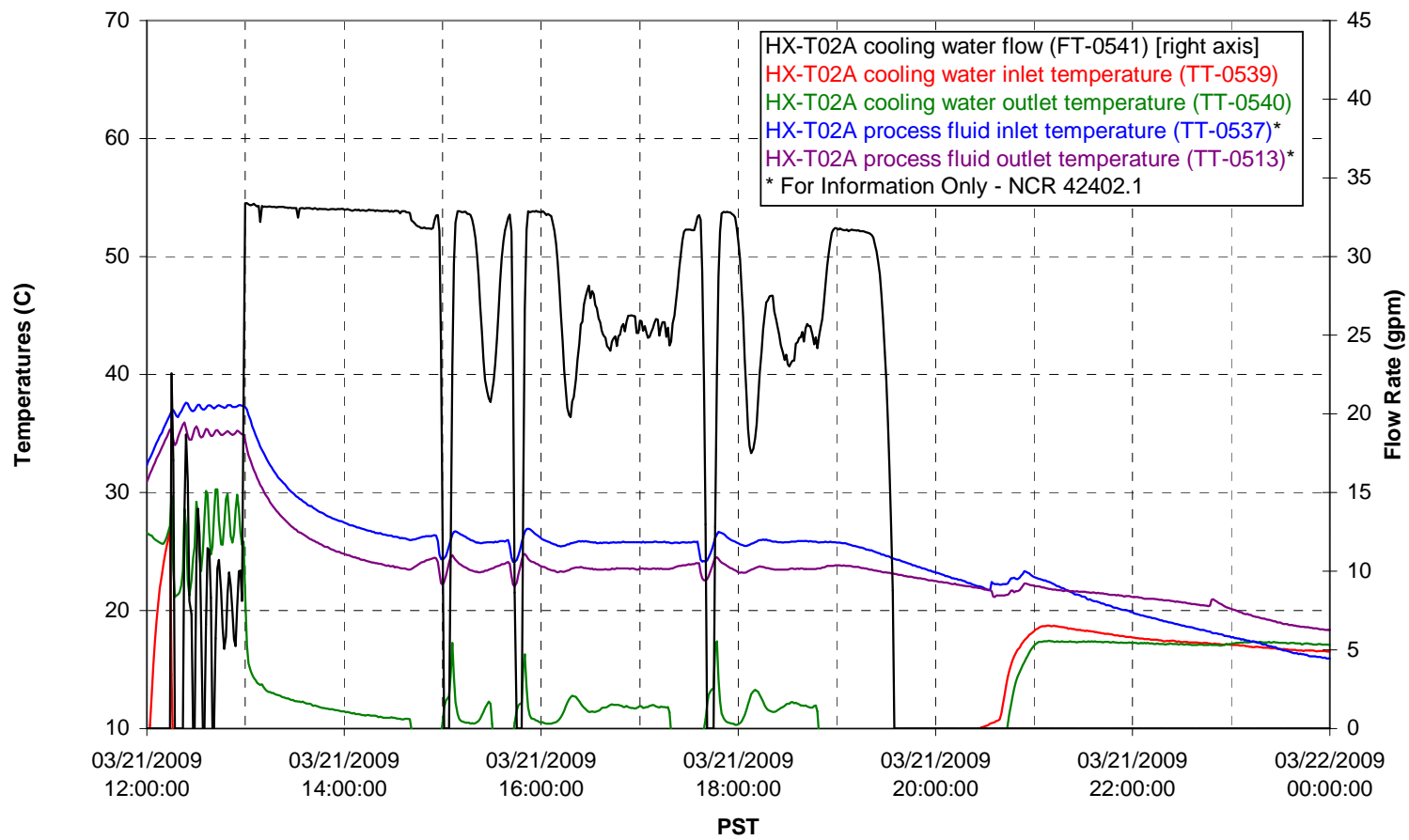

Pump Operation

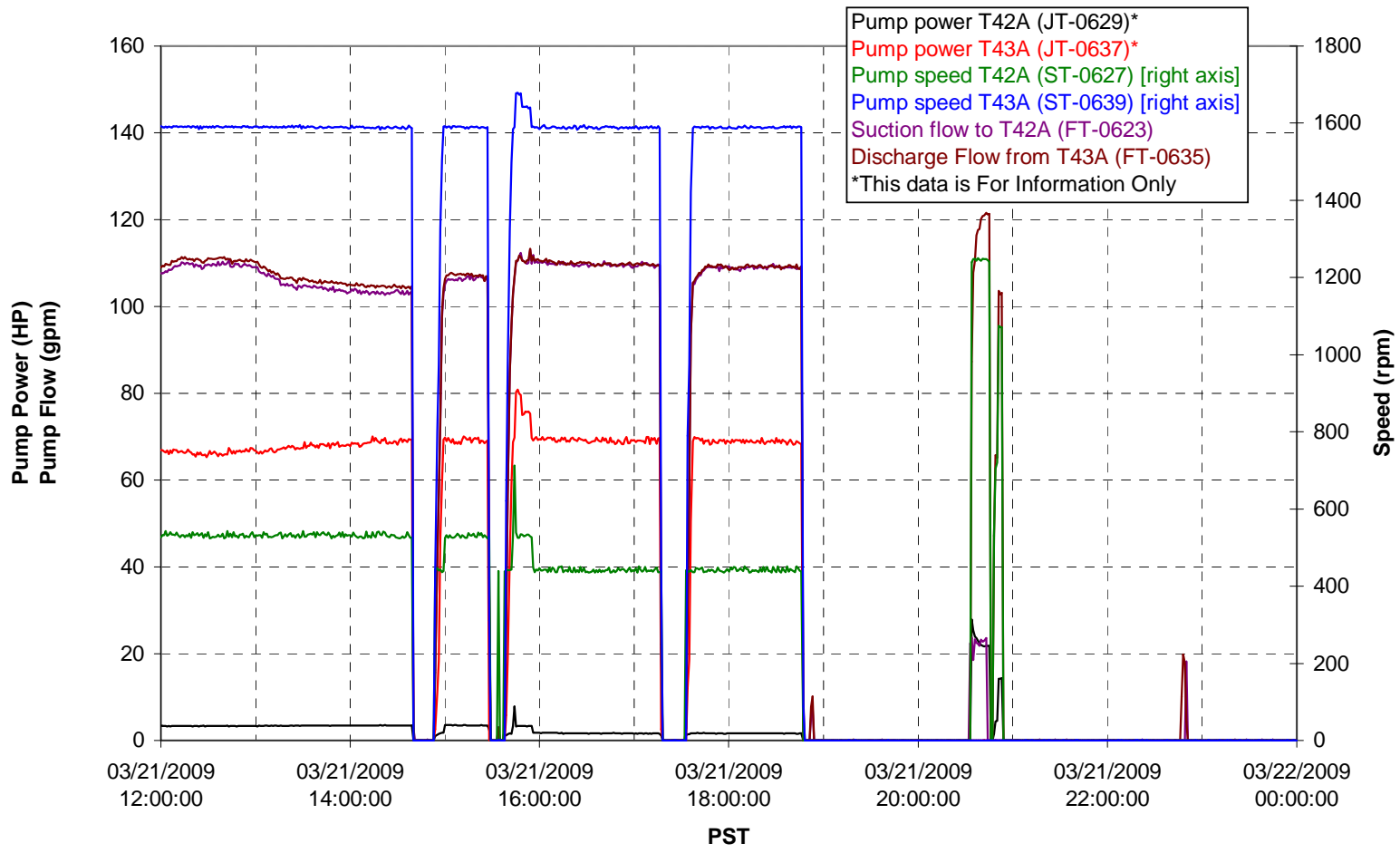


Pulsepot UFP-PP-T01A

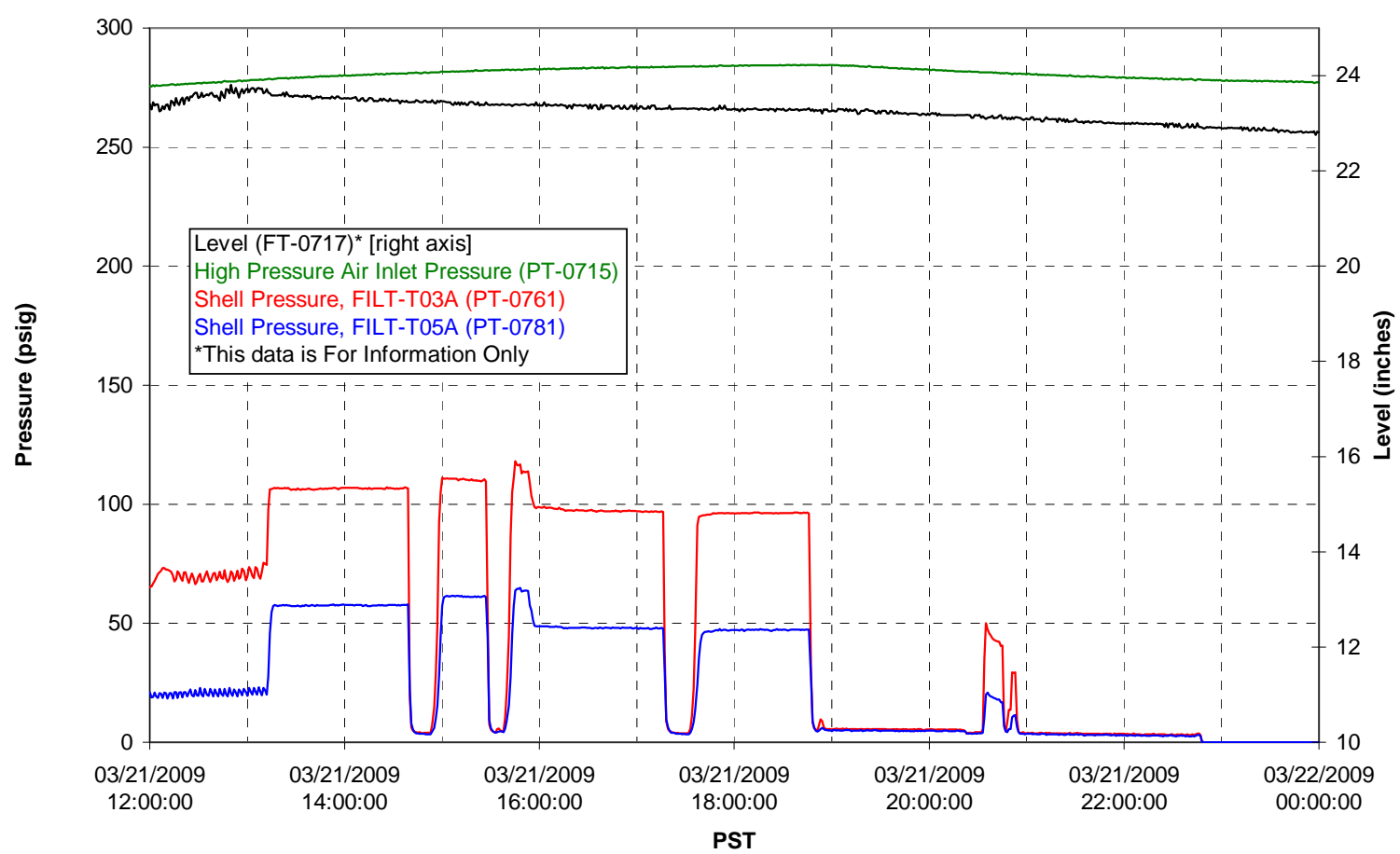

Pulsepot UFP-PP-T02A

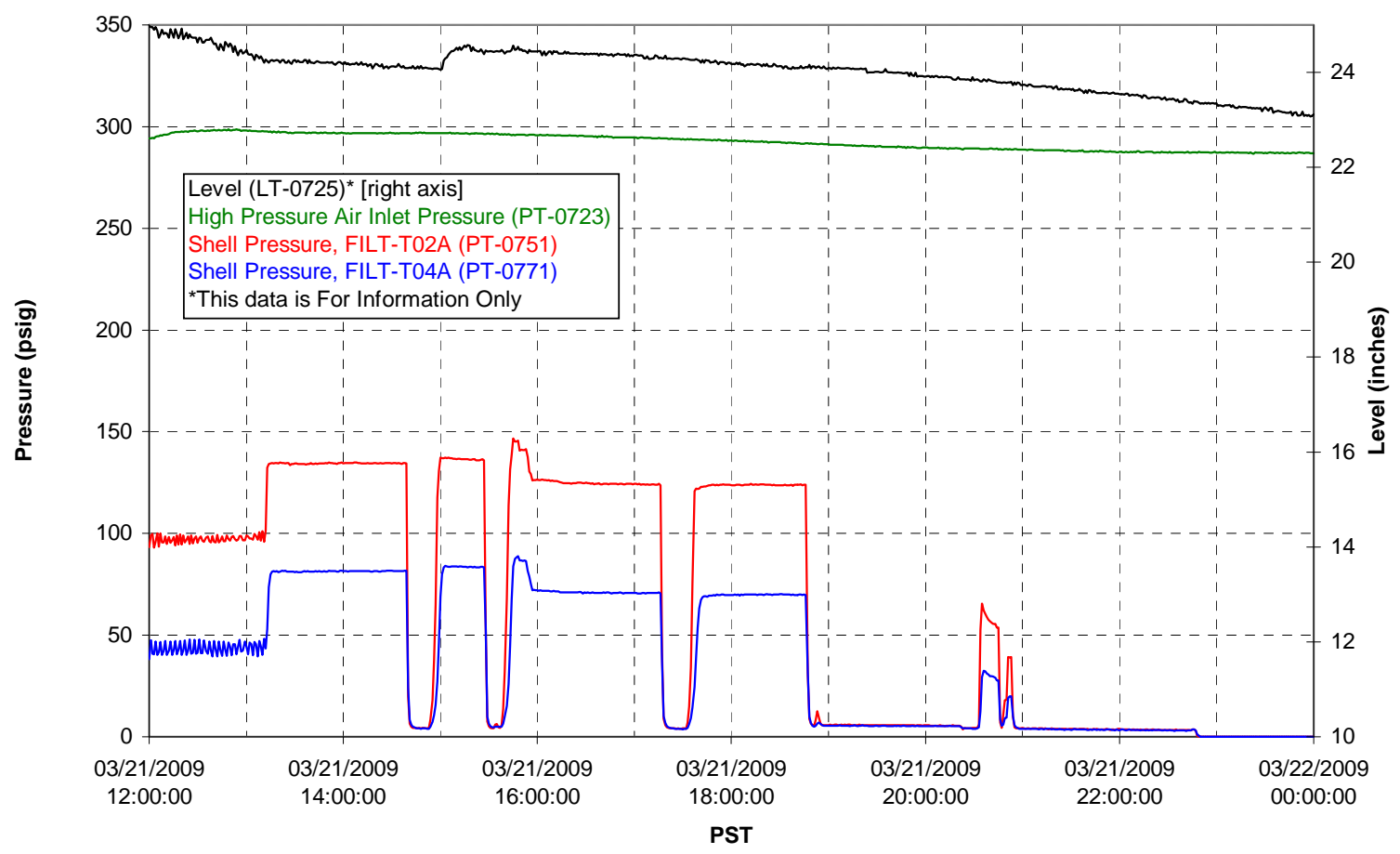


Pulsepot UFP-PP-T03A

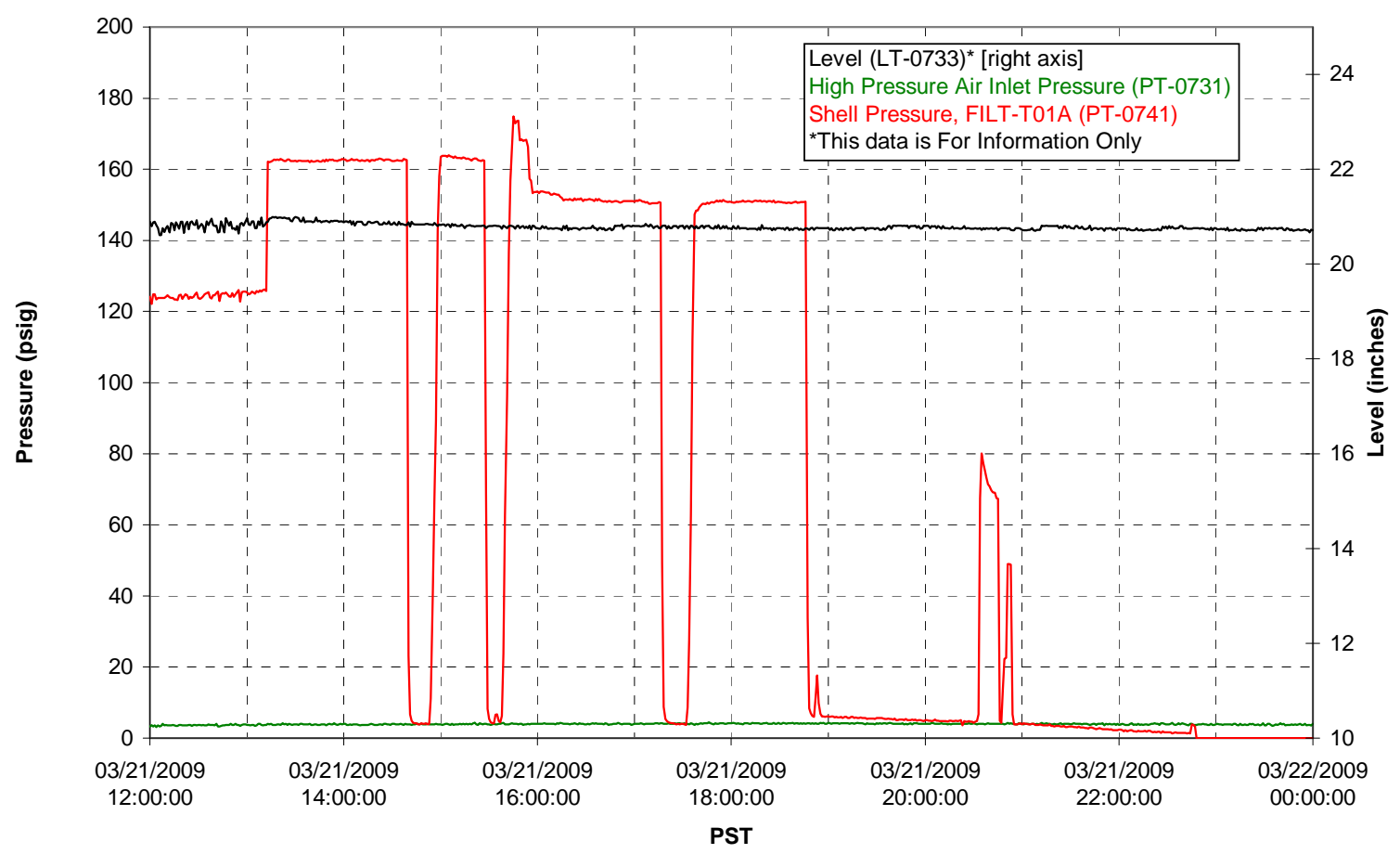

Pulsepot Levels

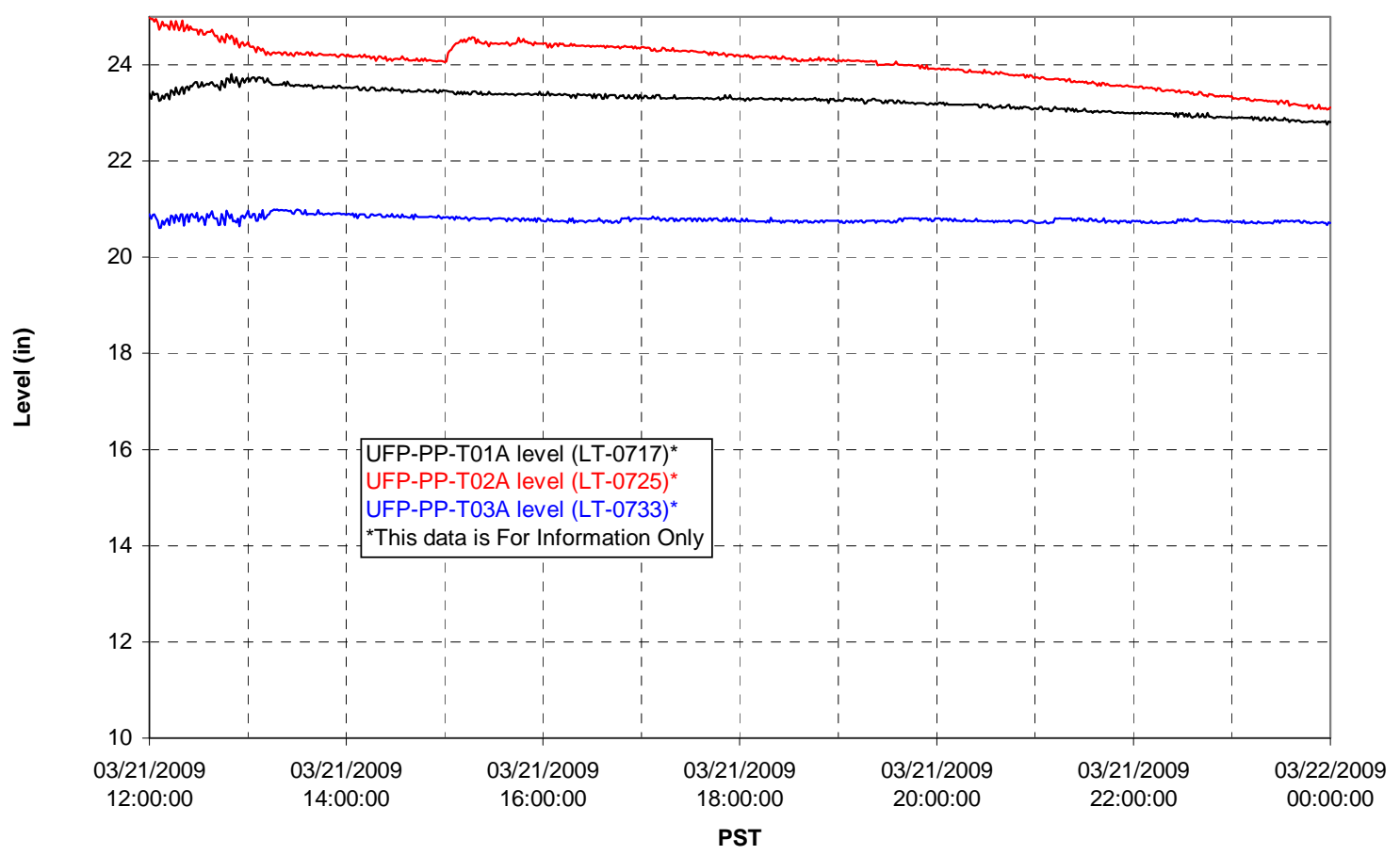


Filter UFP-FILT-T01A

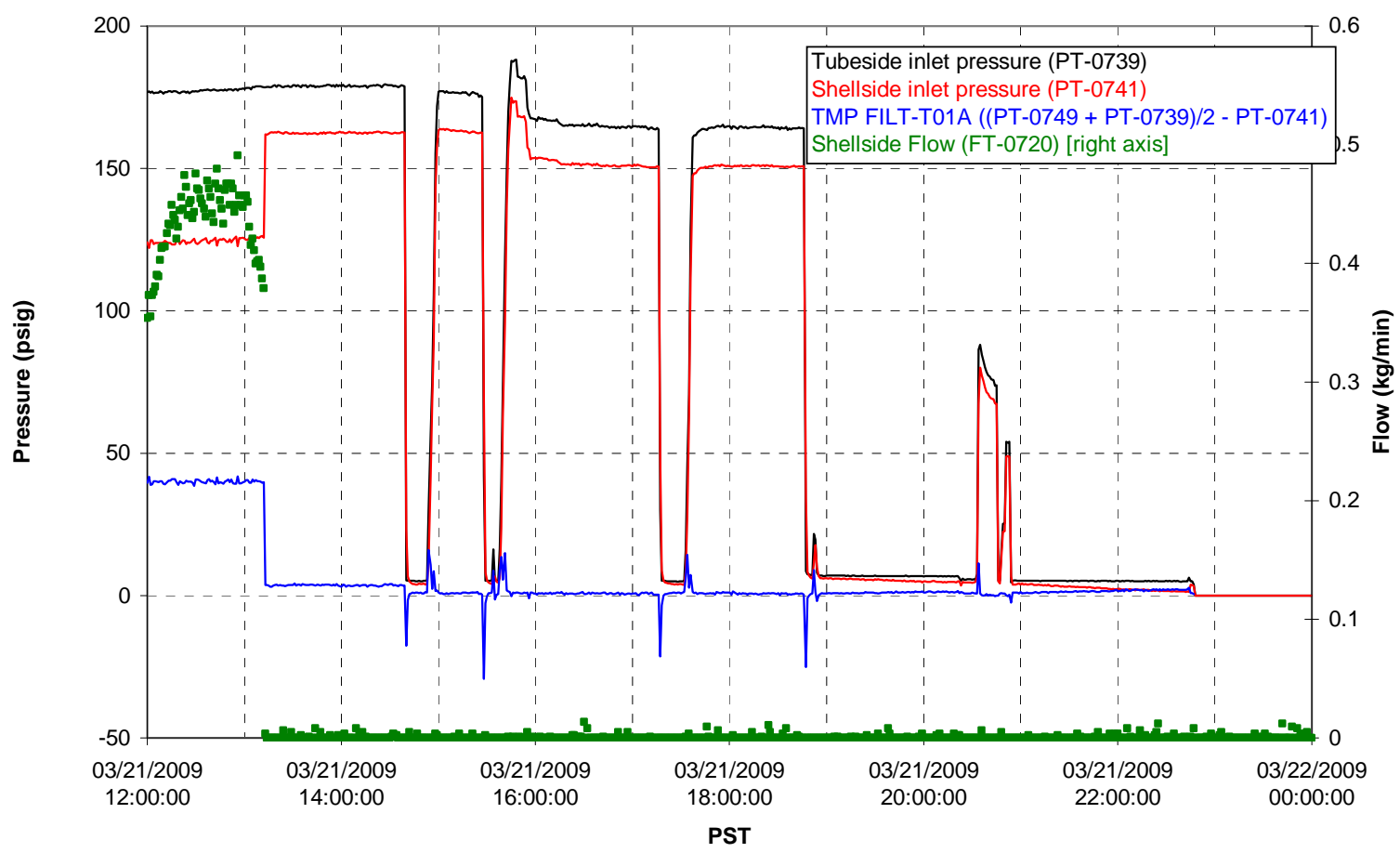

Filter UFP-FILT-T02A

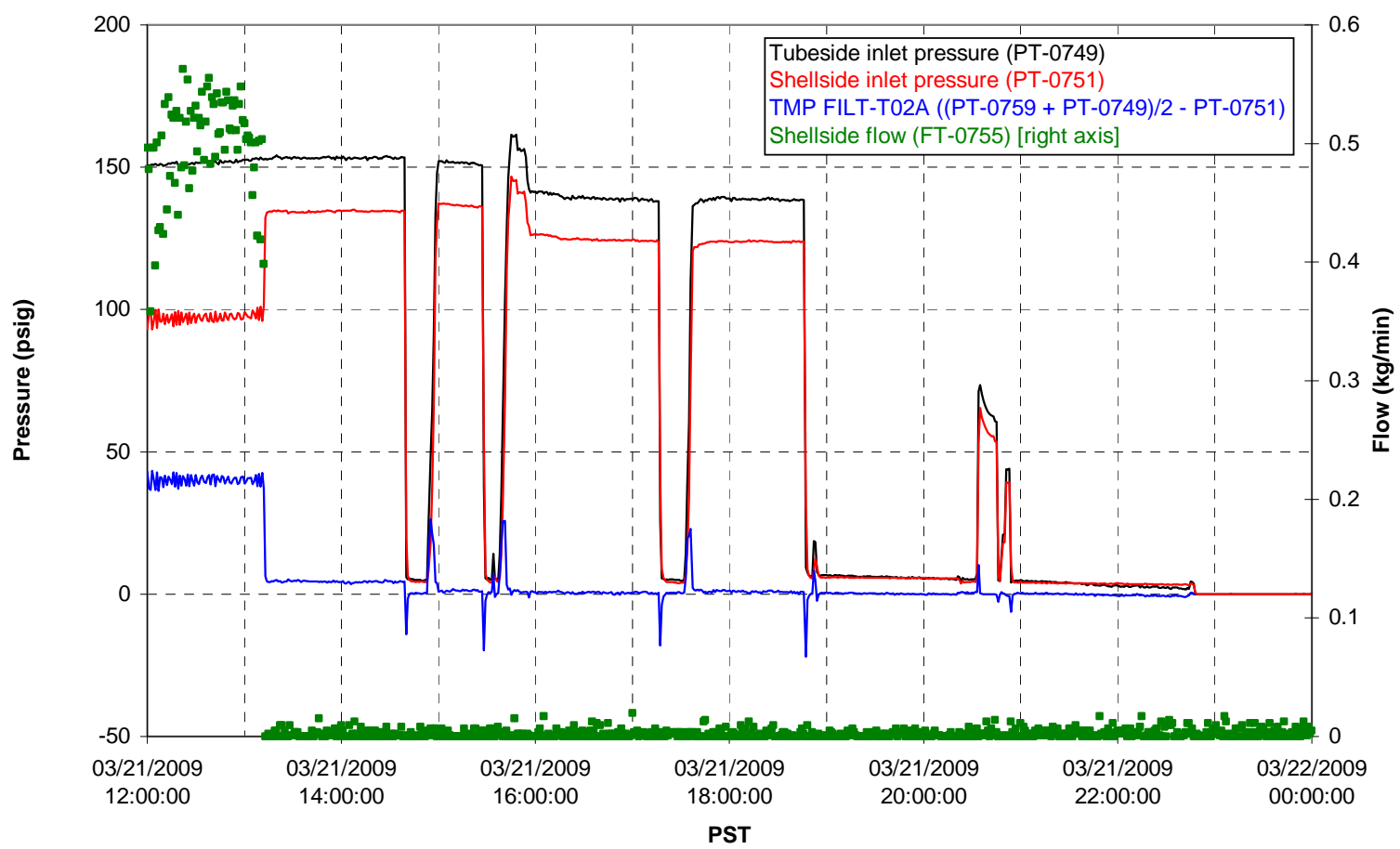


Filter UFP-FILT-T03A

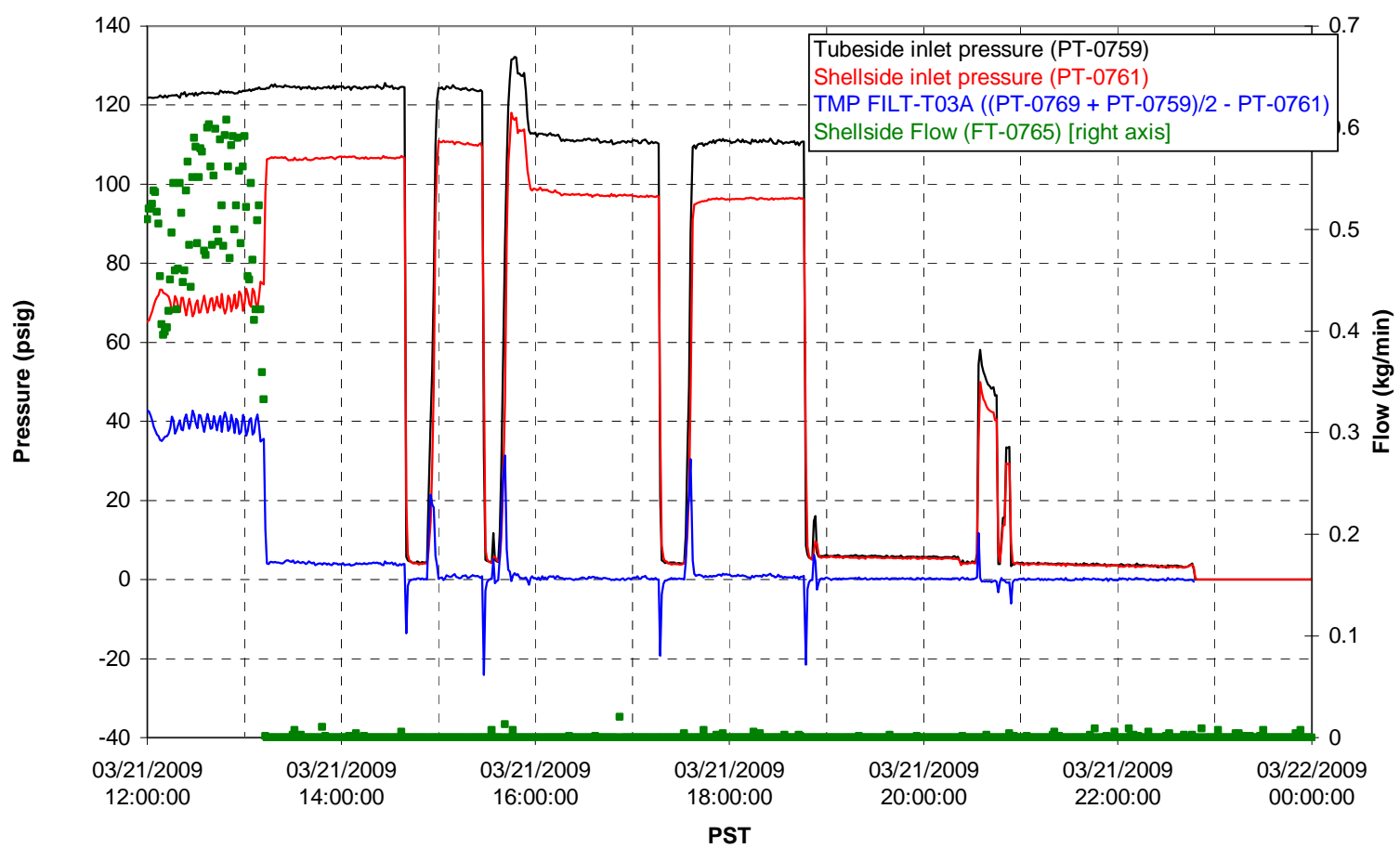

Filter UFP-FILT-T04A

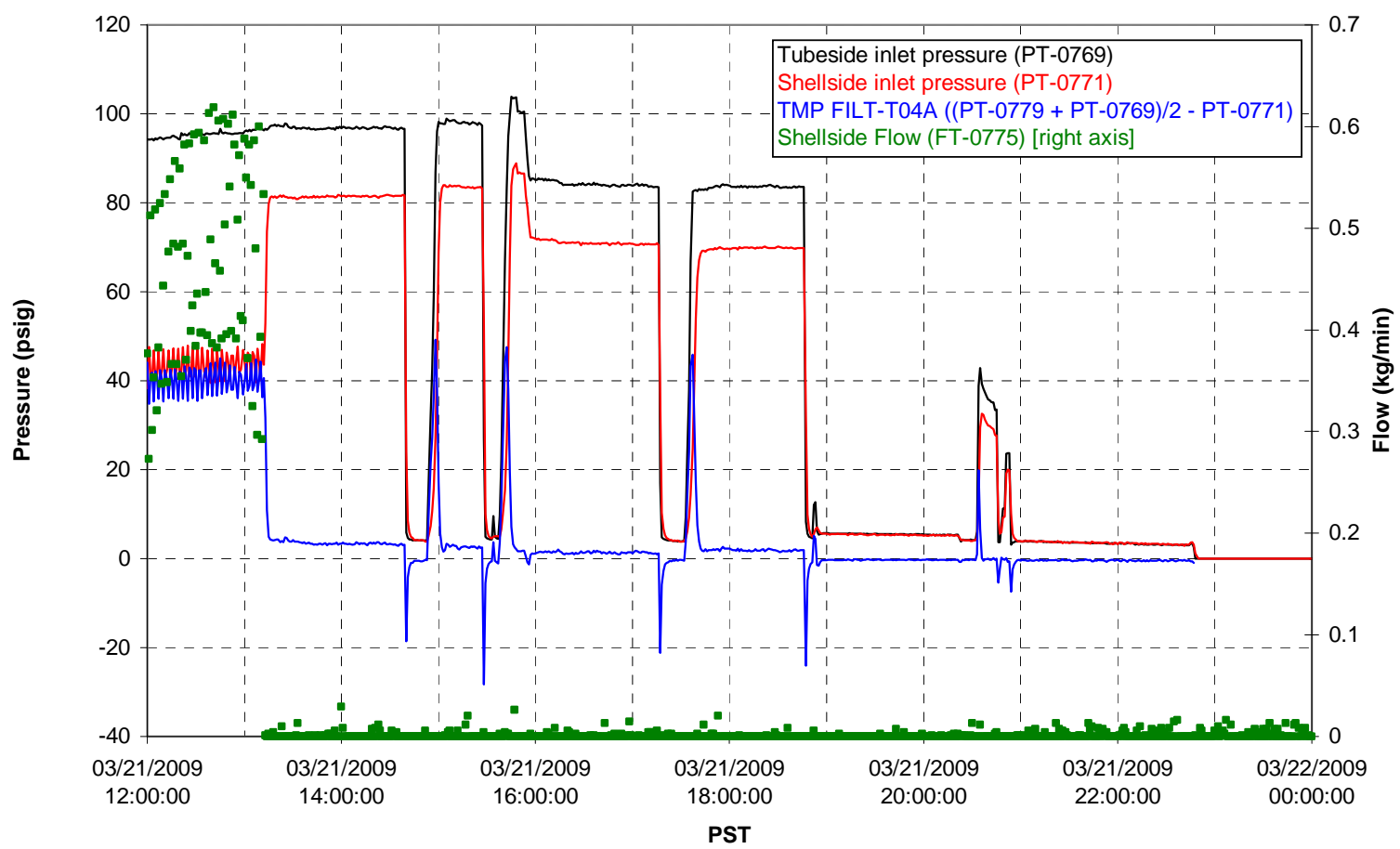


Filter UFP-FILT-T05A

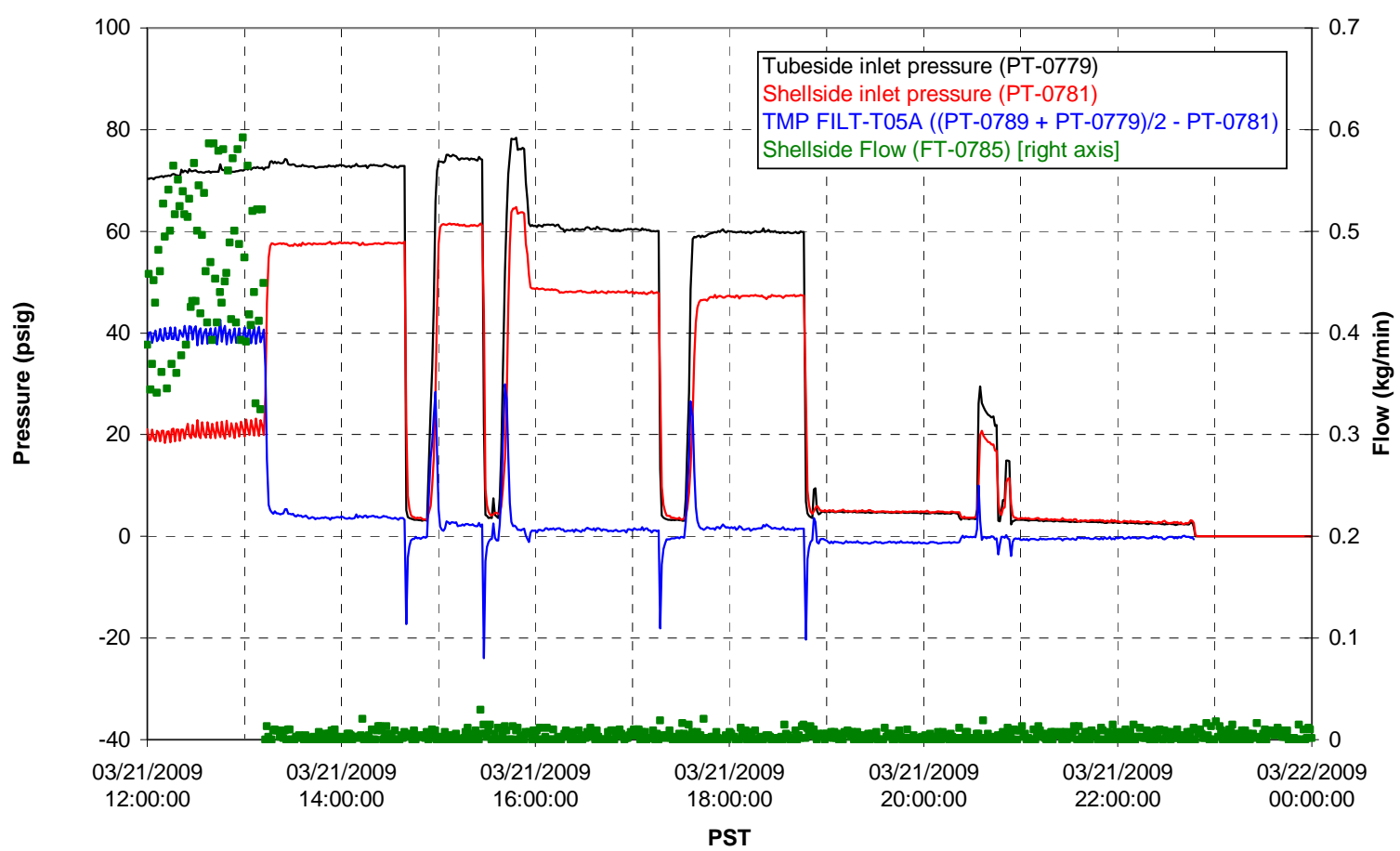

Chemical Flow

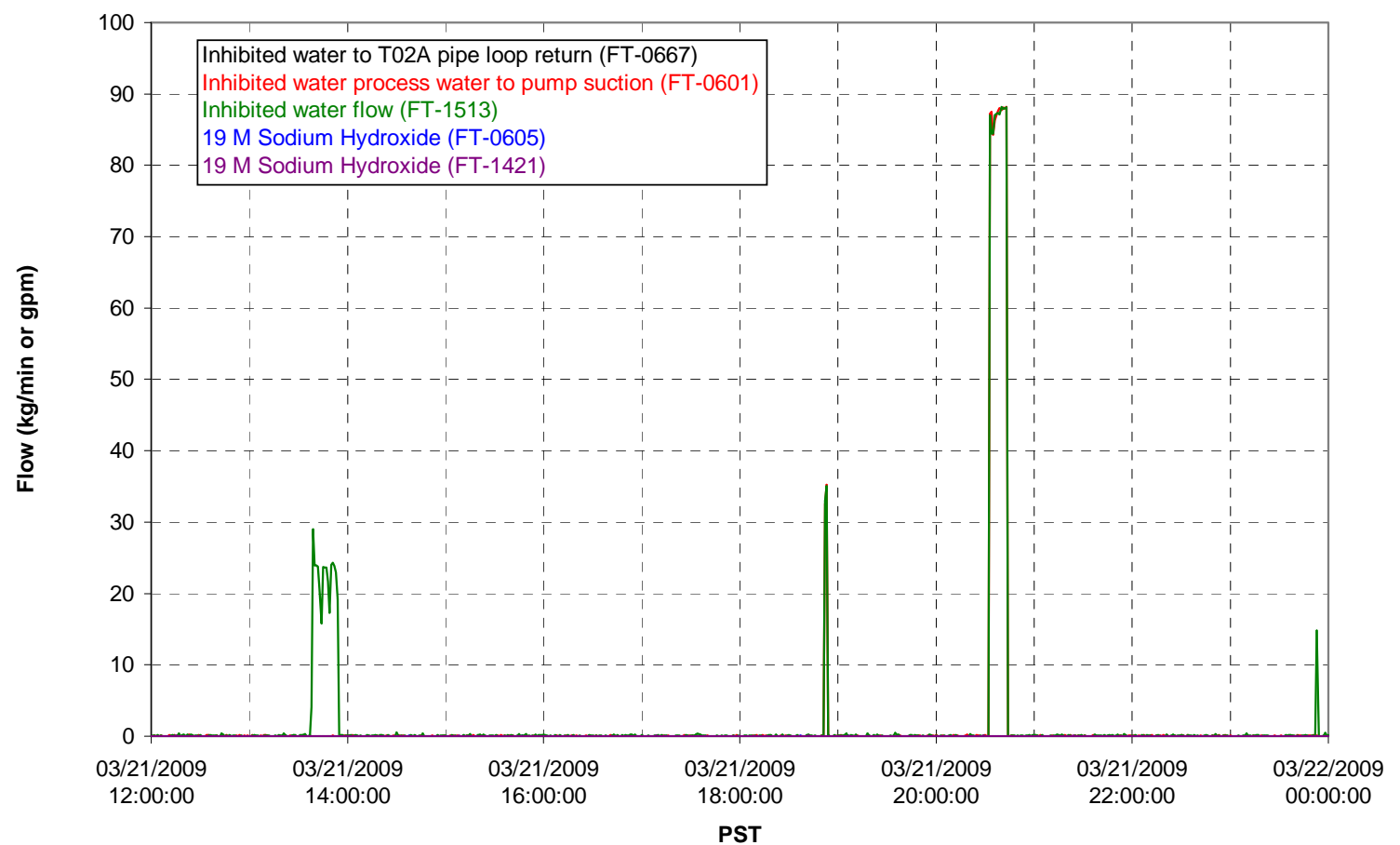




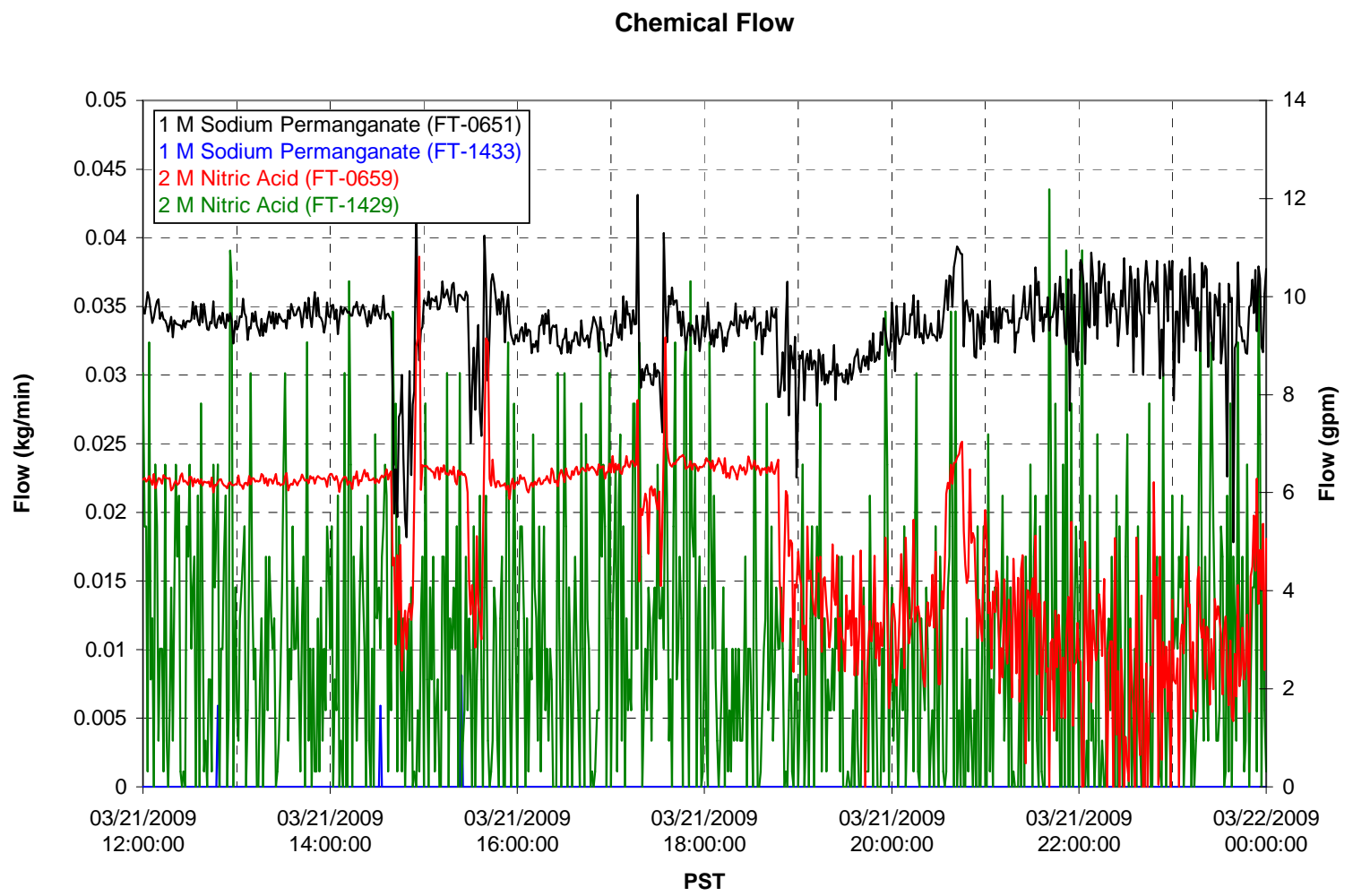

Air Flows

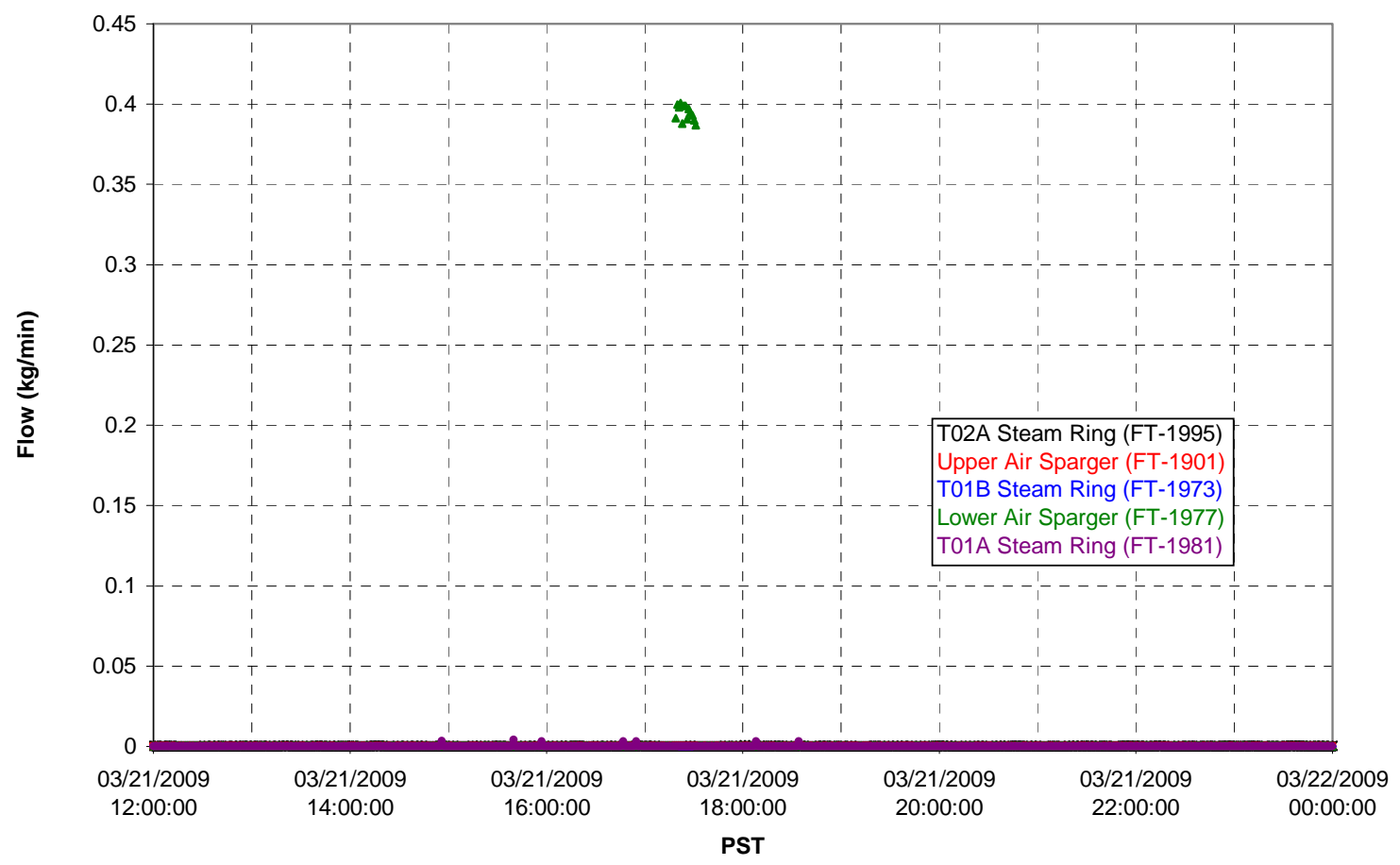


T02A Steam

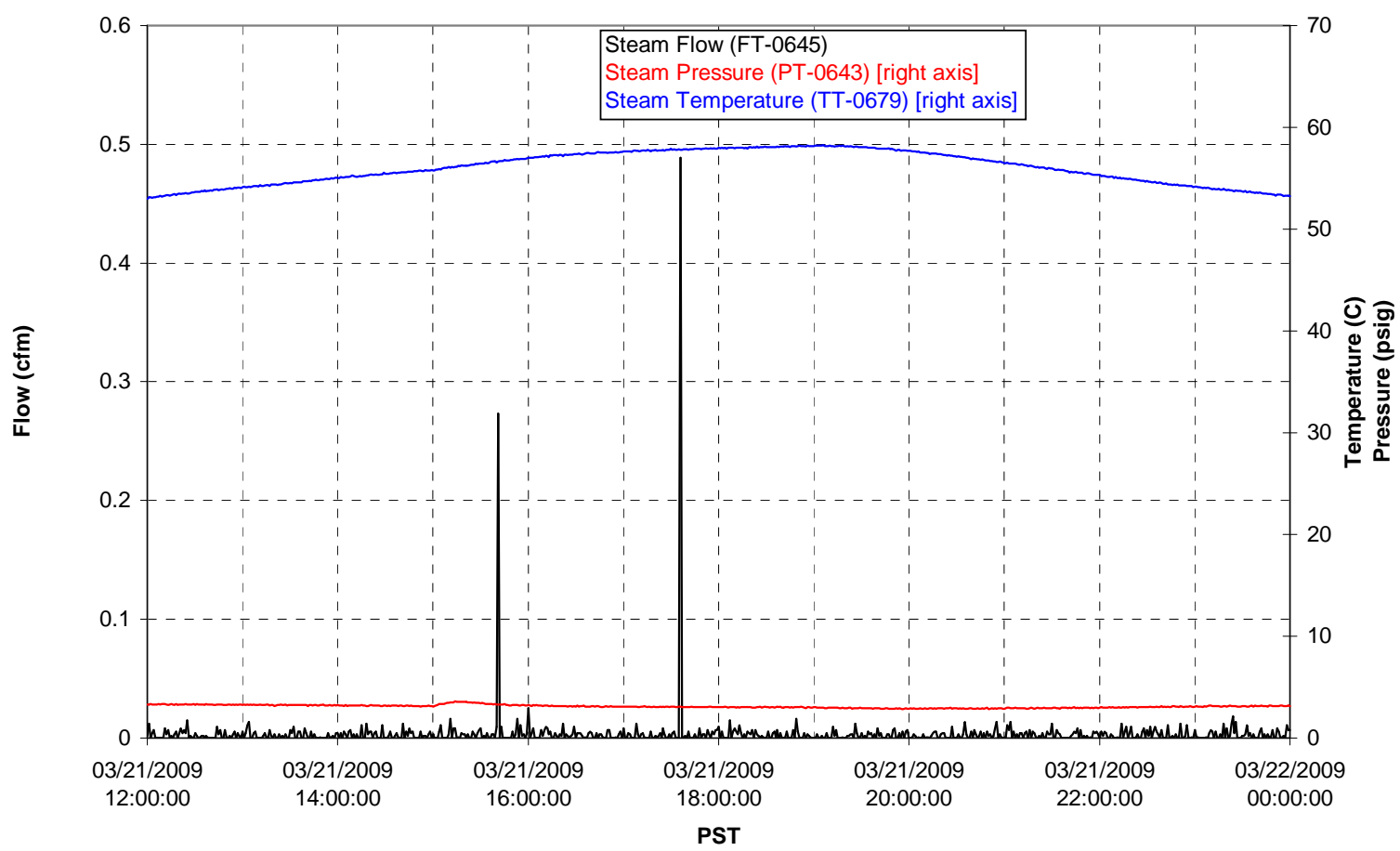

T01A Steam

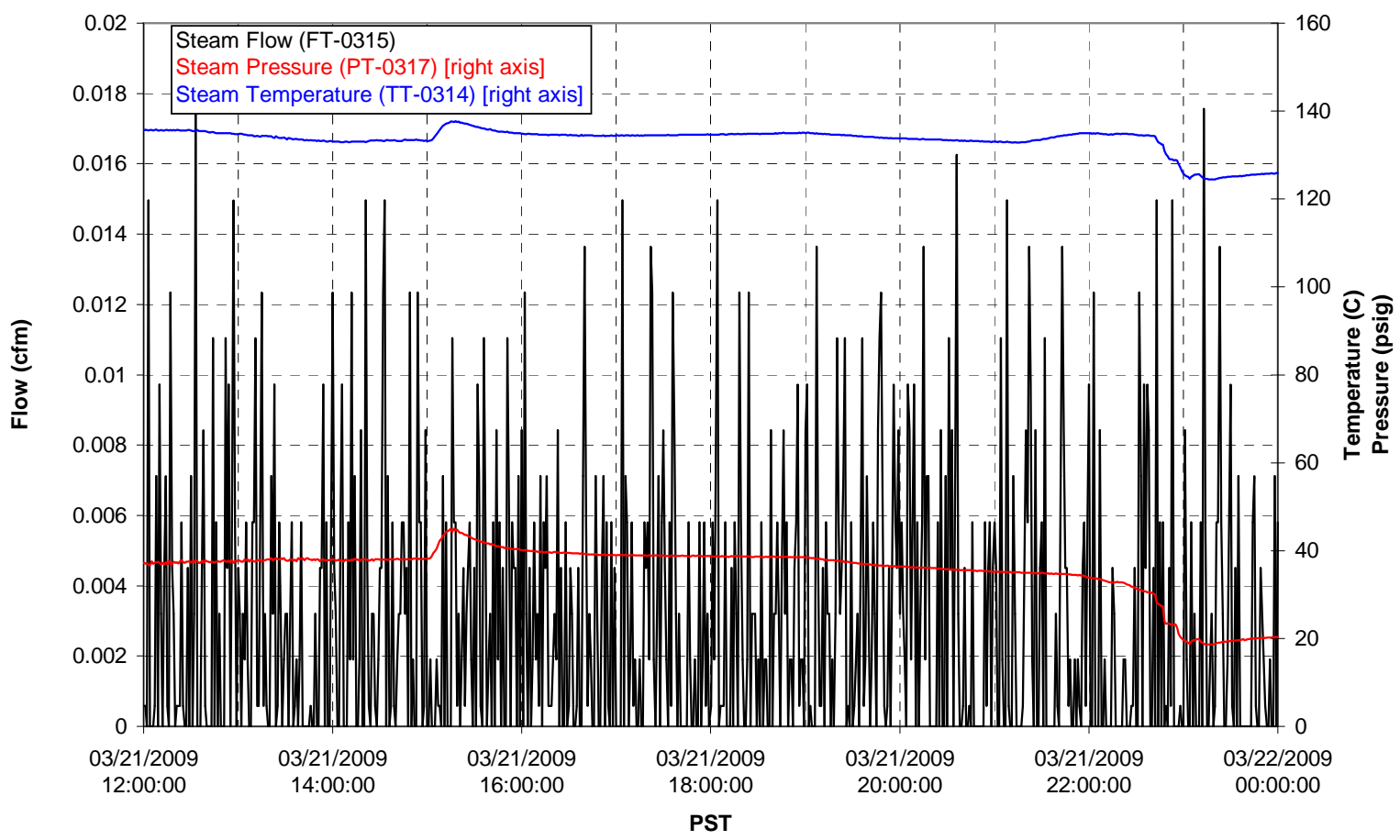


T01B Steam

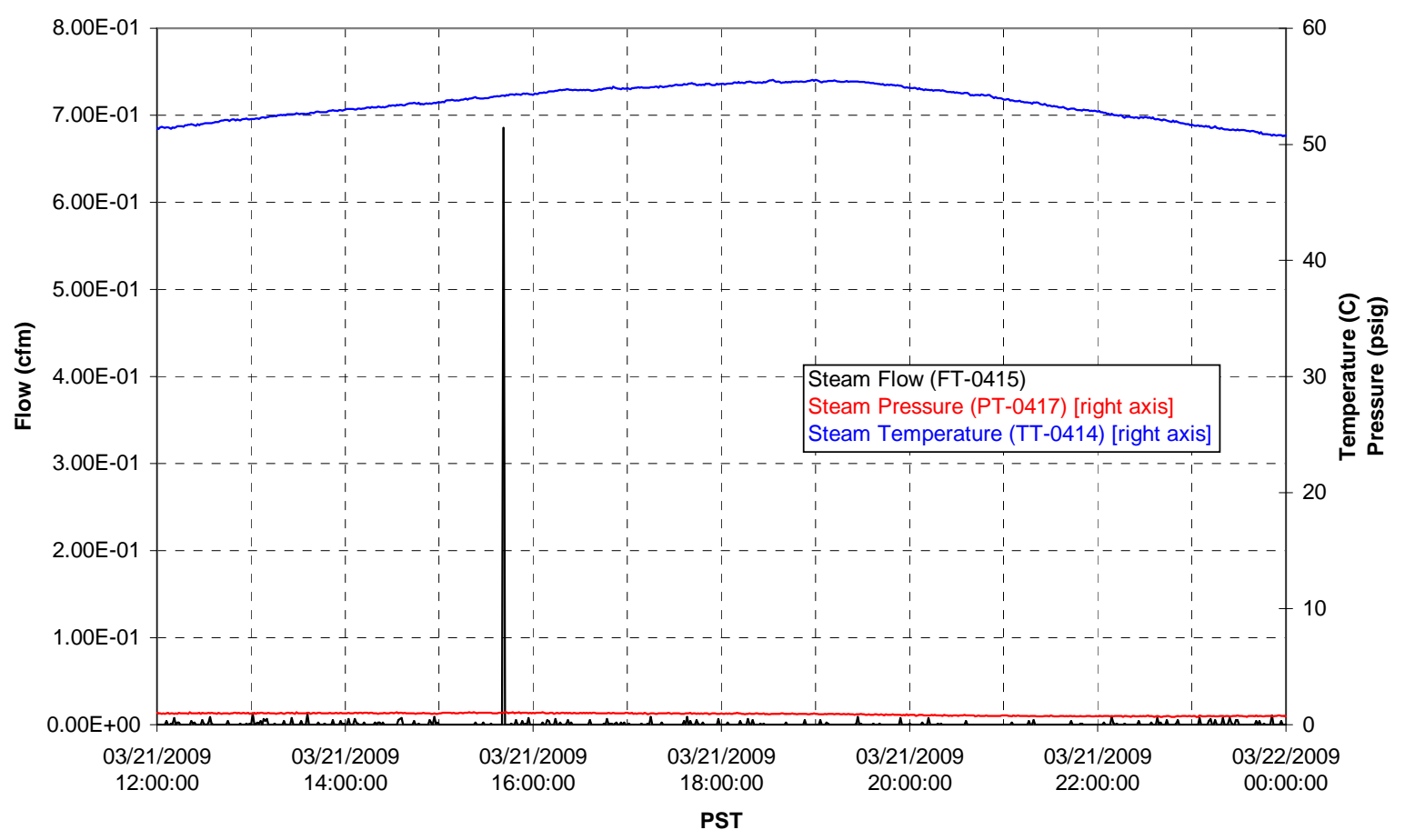


Appendix $\mathrm{H}$

\section{Physical Properties}





\section{Appendix H: Physical Properties}

\section{H.1 Rheology}

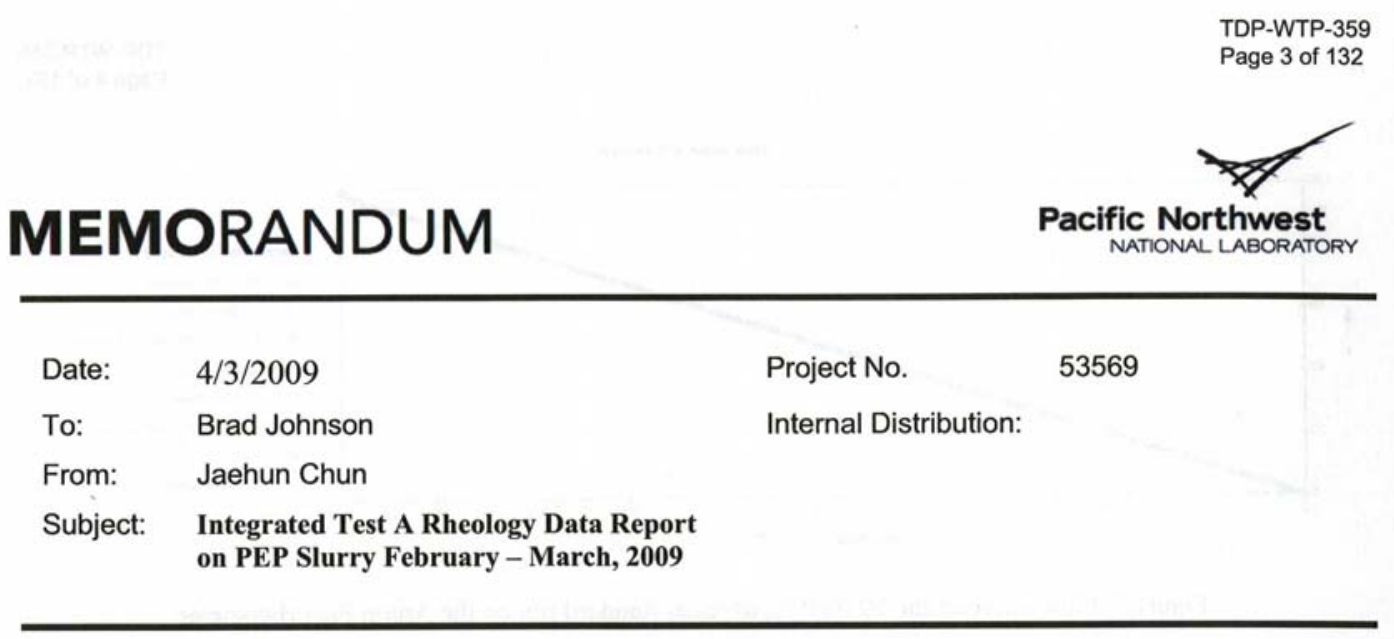

Specimens collected for the Integrated Test A were done under TI-065, Steps B.1.2, B.1.14, B.1.15, B.1.18, B.1.19, B.1.20, and B.1.21.

Multiple specimens of PEP slurry were delivered to APEL for rheological analysis between $2 / 8 / 09-2 / 23 / 09$. Specimens were analyzed to determine either their flow curves (i.e., plots of shear stress vs. shear rate) or shear strengths. The analysis was done using an Anton Parr Rheometer MCR 301 SN80371304 (for flow curve) and Haake RS-600 (for shear strength). Rheometer calibration verifications were done 3 times for Anton Parr Rheometer MCR 301 SN80371304 and 1 time for Haake RS-600 during this time period by Maria Luna in accordance with QA procedure TPR-WTP-PEP-049. Two Newtonian standards with different viscosities were used to check the calibration of these systems: a $9.4 \mathrm{cP}$ Brookfield Standard Lot \# 062408 and a $99.0 \mathrm{cP}$ Brookfield Standard Lot \#071008. All verifications were within the $10 \%$ of the reported values.

Figures 1-3 are examples of the calibration curves performed on the Anton Parr Rheometer MCR 301 SN80371304 with $9.4 \mathrm{cP}$ and $99.0 \mathrm{cP}$ Brookfield Standards and the Haake RS-600 with 99.0 cP Brookfield Standard, respectively. Note that the calibration for Haake RS-600 is only a performance check of the Haake RS-600 system, since the measurements performed on this system are shear strength measurements and Newtonian standards have a shear strength of zero.

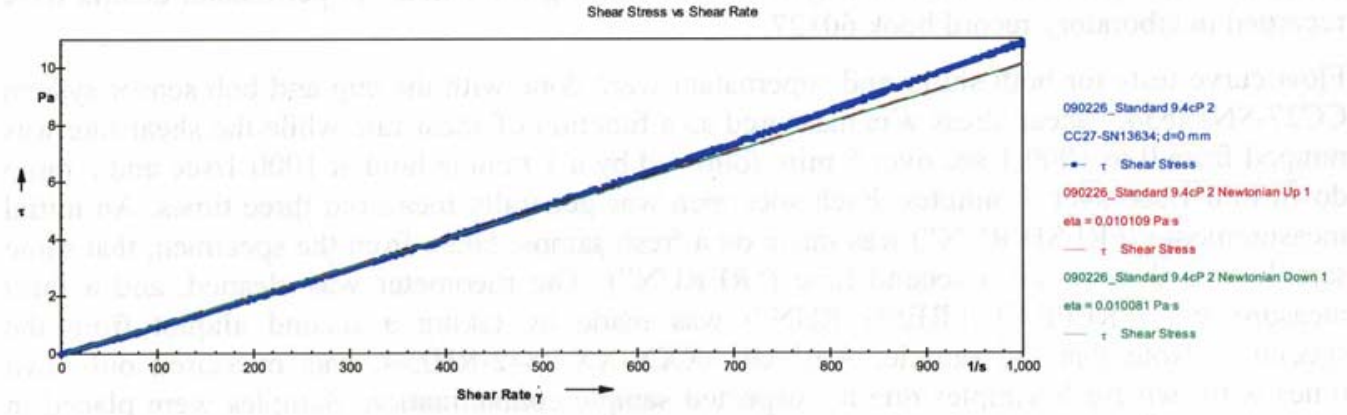

Figure 1. Flow curve of the $9.4 \mathrm{cP}$ Newtonian standard run on the Anton Paar rheometer. 


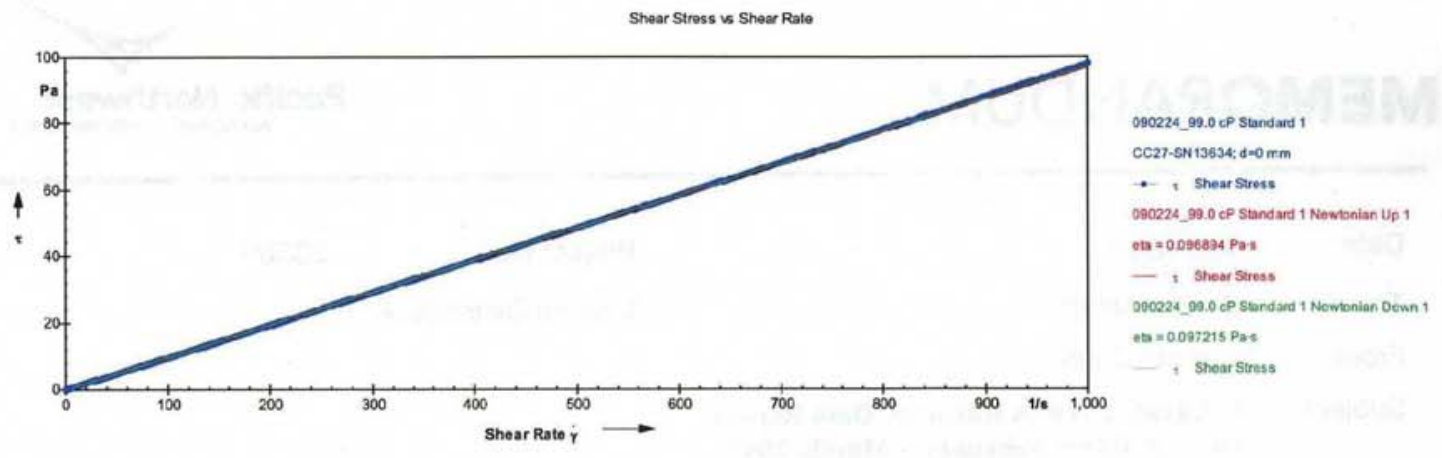

Figure 2. Flow curve of the $99.0 \mathrm{cP}$ Newtonian standard run on the Anton Paar rheometer

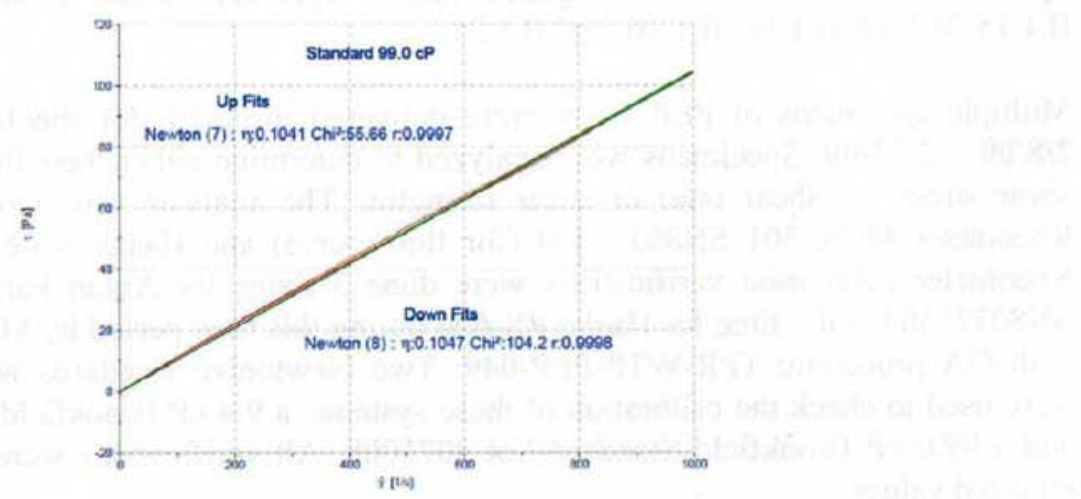

Figure 3. Flow curve of the $99.0 \mathrm{cP}$ Newtonian standard run on the Haake RS-600 rheometer.

The analysis was performed in accordance with procedure TPR-WTP-PEP-049 and the data were collected digitally using the RHEOPLUS/32 V3.21 21003751-33024 software (for flow curve) and RHEOWIN V2.96 software (for shcar strength) The same software was used to operate the corresponding rheometers. For the flow curve measurement, the specimens were prepared for analysis by shaking the bottle before extracting the sample. In order to obtain supernatant from the slurry, the specimen was centrifuged at 1000 times g for 1 hour. Experimental details were recorded in laboratory record book 60127.

Flow curve tests for both slurry and supernatant were done with the cup and bob sensor system CC27-SN13634. Shear stress was measured as a function of shear rate while the shear rate was ramped from 0 to $1000 \mathrm{1} / \mathrm{sec}$ over $5 \mathrm{~min}$, followed by a 1 minute hold at $1000 \mathrm{l} / \mathrm{sec}$ and a ramp down to $01 / \mathrm{sec}$ over 5 minutes. Each specimen was generally measured three times. An initial measurement ("FRESH RUN") was made on a fresh sample taken from the specimen, that same sample was then re-run a second time ("RERUN"). The rheometer was cleaned, and a third measurement ("REPEAT FRESH RUN") was made by taking a second aliquot from the specimen. Note that the sample, A-02ARL-XXX-XX-2842-RHE-4, was measured only two times with two fresh samples due to suspected sample contamination. Samples were placed in the cup up to the designated fill line (about 8 to $10 \mathrm{~mL}$ ). Settling cannot be prevented during the measurement if the particles are large and the viscosity low; however, no evidence of significant settling was seen in the data or the cup after testing. 


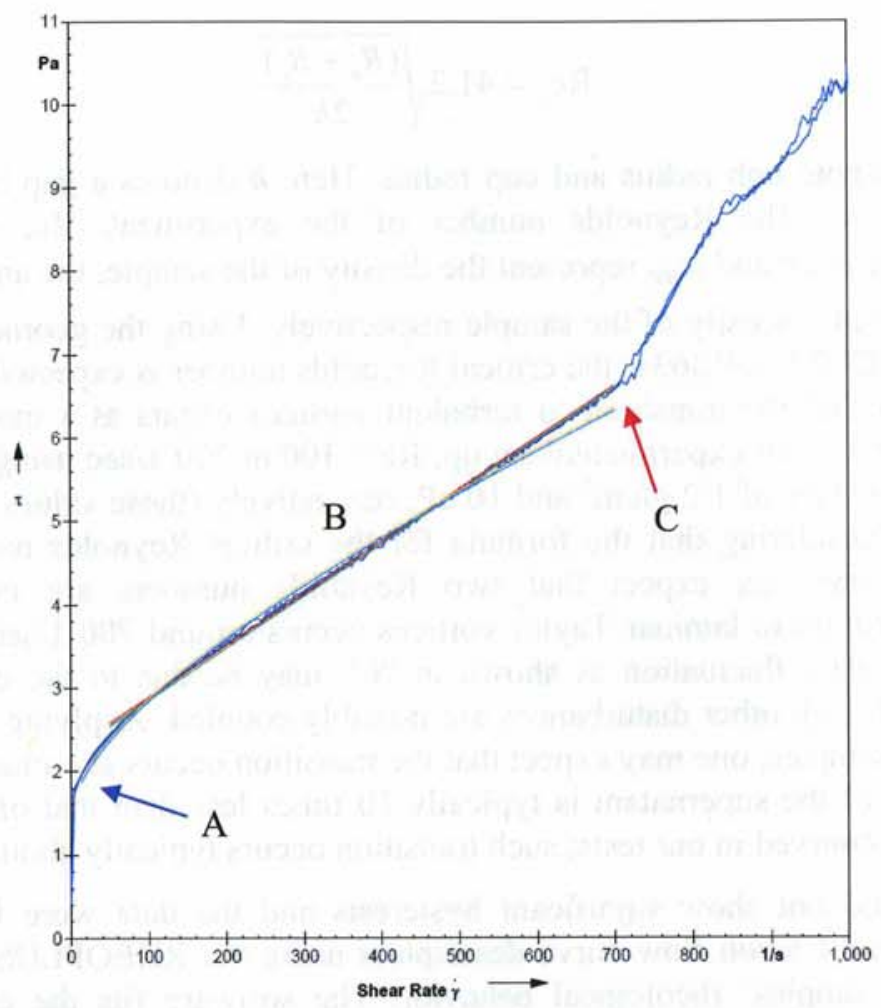

Figure 4. Typical rheogram of a slurry sample with low shear strength.

Figure 4 is a typical rheogram of a slurry sample (sample A-02AOL-015-10-2319-RHE-4). There are three features that were typically characteristic of all of the slurry specimens: i) a yield stress at the onset of flow, (indicated by the blue arrow, "A"), ii) an approximately linear region where shear stress is linearly proportional to shear rate " $\mathrm{B}$ ", and iii) a discontinuity where the slope changes and where there are more fluctuations in the data (starting about $700 \mathrm{l} / \mathrm{sec}$ in Figure 4, indicated by the red arrow, " $C$ "). The data at very low shear rates was not included in the analysis to avoid overweighting the Bingham plastic and Casson curve fit calculations performed by the RHEOPLUS/32 V3.21 21003751-33024 software. Slope changes and data fluctuation as shown in "C" above made it difficult to fit the data to a single mathematical expression because such behavior is not included in existing fitting models. Several different factors might cause such behavior: the flow instability associated with laminar Taylor vortices and other disturbances such as cavitation, minor settling, or the release of entrained gas. Previously it was presumed that these flow instabilities were associated with Taylor vortices. The data analysis team evaluated these instabilities and decided to perform curve fit analysis using only the data prior to the discontinuity (e.g., up to $7001 / \mathrm{sec}$ as shown above).

A simple hydrodynamic theory may provide a reasonable estimate and physical insights for instability behavior. The hydrodynamic transition from laminar Couette flow to laminar Taylor vortices occurs when the Reynolds number of the experiment exceeds a geometrically determined critical Reynolds number, $\mathrm{Re}_{c}$. Assuming the infinite length of the fluid column, the critical Reynolds number in our case may be described by ${ }^{1}$ 


$$
\operatorname{Re}_{c}=41.2 \sqrt{\frac{\left(R_{o}+R_{i}\right)}{2 h}}
$$

where $R_{i}$ and $R_{o}$ denote bob radius and cup radius. Here $h$ denotes a gap between the bob and cup, which is $R_{o}-R_{i}$. The Reynolds number of the experiment, Re, can be defined by $\rho h \Omega R_{i} / \mu_{a p p}$ where $\rho, \Omega$, and $\mu_{\text {app }}$ represent the density of the sample, the angular velocity of the bob, and the apparent viscosity of the sample respectively. Using the geometries of the cup and bob sensor system CC27-SN13634, the critical Reynolds number is expected to be about 145. (It is also noteworthy that the transition to turbulent vortices occurs at a much higher Reynolds number). However, for our experimental set up, $\operatorname{Re} \approx 100$ at $7001 / \mathrm{sec}$ using sample density and apparent viscosity values of $1.2 \mathrm{~g} / \mathrm{cm}^{3}$ and $10 \mathrm{cP}$, respectively (these values are typical for most slurry samples). Considering that the formula for the critical Reynolds number is based on a Newtonian fluid, one can expect that two Reynolds numbers are comparable and the hydrodynamic transition to laminar Taylor vortices occurs around $700 \mathrm{l} / \mathrm{sec}$. Consequently, the slope changes and data fluctuation as shown in " $\mathrm{C}$ " may be due to the onset of the laminar Taylor vortices although other disturbances are possibly coupled. Applying this to the rheology of the supernatant samples, one may expect that the transition occurs at a much smaller shear rate since the viscosity of the supernatant is typically 10 times less than that of the slurry samples, which was indeed observed in our tests; such transition occurs typically about $120 \mathrm{l} / \mathrm{sec}$.

The flow curves did not show significant hysteresis and the data were fit with Newtonian, Bingham Plastic, and Casson flow-curve descriptors using the RHEOPLUS curve fit software, depending on the samples' rheological behavior. The software fits the entire range of data collected. However, as explained previously, a partial range of data was used to obtain fitting parameters for most of samples in the Integrated Test A because of the slope changes and/or fluctuations. The partial ranges of data were typically $50-7001 / \mathrm{sec}$ and $0-1201 / \mathrm{sec}$ for slurry and supernatant samples respectively.

For shear strength test, a $1.6 \times 1.6 \mathrm{~cm}$ vane shear spindle was used and an enough space (at least $1.6 \mathrm{~cm}$ away from the top solid bed and the side wall and bottom of a test container) was provided for proper measurements. A and $\mathrm{M}$ factors for the tests are $116568.602 \mathrm{~Pa} / \mathrm{N} \cdot \mathrm{m}$ and 1.0 $(1 / \mathrm{sec})(\mathrm{rad} / \mathrm{sec})$ respectively indicated in RHEOWIN V2.96 software. Settling times for the test with the same sample are $1,2,4,8,16,32$, and $72 \mathrm{hrs}$. For each settling time, shear stresses were collected as a function of duration time under a constant shear rate $(0.031 / \mathrm{sec})$ during 2 minutes. Then, a maximum shear stress was obtained for each settling time.

The following table is a brief summary of samples and their specifications (chronological order). Note that all measurements were done at $25^{\circ} \mathrm{C}$.

\begin{tabular}{|l|l|l|l|}
\hline Sample Name & $\begin{array}{l}\text { Date } \\
\text { received }\end{array}$ & $\begin{array}{l}\text { Date } \\
\text { Tested }\end{array}$ & Comments \\
\hline A-02AML-007-XX-2684-UDS-2 & $02 / 08 / 09$ & $02 / 08 / 09$ & Slight Bingham \\
\hline A-000FL-015-XX-2812-RHE-4 & $02 / 15 / 09$ & $02 / 15 / 09$ & Slight Bingham \\
\hline A-000FL-007-XX-2813-RHE-4 & $02 / 15 / 09$ & $02 / 18 / 09$ & Slight Bingham \\
\hline A-000FL-014-XX-2814-RHE-4 & $02 / 15 / 09$ & $02 / 18 / 09$ & Slight Bingham \\
\hline $\begin{array}{l}\text { A-02AOL-015-XX-2294-RHE-4 } \\
\text { (supernatant) }\end{array}$ & $02 / 17 / 09$ & $02 / 20 / 09$ & Newtonian \\
\hline A-02AOL-015-XX-2293-RHE-4 & $02 / 17 / 09$ & $02 / 18 / 09$ & Slight Bingham \\
\hline
\end{tabular}




\begin{tabular}{|l|l|l|l|}
\hline Sample Name & $\begin{array}{l}\text { Date } \\
\text { received }\end{array}$ & $\begin{array}{l}\text { Date } \\
\text { Tested }\end{array}$ & Comments \\
\hline A-02AOL-014-01-2281-RHE-4 & $02 / 17 / 09$ & $02 / 19 / 09$ & Slight Bingham \\
\hline $\begin{array}{l}\text { A-02AOL-015-10-2320-RHE-4 } \\
\text { (supernatant) }\end{array}$ & $02 / 17 / 09$ & $02 / 20 / 09$ & Newtonian \\
\hline A-02AOL-015-10-2319-RHE-4 & $02 / 17 / 09$ & $02 / 19 / 09$ & Slight Bingham \\
\hline $\begin{array}{l}\text { A-02AOL-015-XX-2543-RHE-4 } \\
\text { (supernatant) }\end{array}$ & $02 / 17 / 09$ & $02 / 20 / 09$ & Newtonian \\
\hline $\begin{array}{l}\text { A-02AOL-020-XX-2451-RHE-4 } \\
\text { (supernatant) }\end{array}$ & $02 / 17 / 09$ & $02 / 20 / 09$ & Newtonian \\
\hline A-02AOL-020-XX-2450-RHE-4 & $02 / 17 / 09$ & $02 / 19 / 09$ & Slight Bingham \\
\hline A-02AOL-020-08-2436-RHE-4 & $02 / 17 / 09$ & $02 / 19 / 09$ & Slight Bingham \\
\hline $\begin{array}{l}\text { A-02AOL-020-08-2437-RHE-4 } \\
\text { (supernatant) }\end{array}$ & $02 / 17 / 09$ & $02 / 20 / 09$ & Newtonian \\
\hline A-02AOL-015-XX-2542-RHE-4 & $02 / 17 / 09$ & $02 / 20 / 09$ & Slight Bingham \\
\hline $\begin{array}{l}\text { A-02AOL-019-06-2417-RHE-4 } \\
\text { (supernatant) }\end{array}$ & $02 / 17 / 09$ & $02 / 20 / 09$ & Newtonian \\
\hline A-T22GM-002-XX-1506-RHE-4 & $02 / 17 / 09$ & $02 / 20 / 09$ & Newtonian \\
\hline A-T22GM-13C-XX-2021-RHE-4 & $02 / 17 / 09$ & $02 / 23 / 09$ & Newtonian \\
\hline A-02AOL-018-XX-2383-RHE-4 & $02 / 17 / 09$ & $02 / 26 / 09$ & Shear Strength \\
\hline A-02AOL-018-XX-2384-RHE-4 & $02 / 17 / 09$ & $02 / 26 / 09$ & Shear Strength \\
\hline A-02AOL-018-XX-2385-RHE-4 & $02 / 17 / 09$ & $02 / 23 / 09$ & Bingham \\
\hline A-T22GM-13A-XX-1772-RHE-4 & $02 / 17 / 09$ & $02 / 23 / 09$ & Newtonian \\
\hline A-02ARL-XXX-XX-2842-RHE-4 & $02 / 18 / 09$ & $02 / 18 / 09$ & Slight Bingham \\
\hline A-02AML-021-XX-2455-RHE-4 & $02 / 23 / 09$ & $03 / 03 / 09$ & Shear Strength \\
\hline A-02AML-021-XX-2456-RHE-4 & $02 / 23 / 09$ & $03 / 03 / 09$ & Shear Strength \\
\hline
\end{tabular}




\begin{tabular}{|l|l|l|l|}
\hline Sample Name & $\begin{array}{l}\text { Date } \\
\text { received }\end{array}$ & $\begin{array}{l}\text { Date } \\
\text { Tested }\end{array}$ & Comments \\
\hline A-02AML-021-XX-2457-RHE-4 & $02 / 23 / 09$ & $02 / 26 / 09$ & Bingham \\
\hline $\begin{array}{l}\text { A-02AML-021-XX-2458-RHE-4 } \\
\text { (supernatant) }\end{array}$ & $02 / 23 / 09$ & $02 / 26 / 09$ & Newtonian \\
\hline
\end{tabular}

\section{Summary of Results}

Five samples, A-02AOL-018-XX-2383-RHE-4, A-02AOL-018-XX-2384-RHE-4, B-FRPGM001-XX-2718-RHE-4 (identical to B-FRPGM-001-XX-2707-RHE-4), A-02AML-021-XX-2455RHE-4, and A-02AML-021-XX-2456-RHE-4, were tested with a $1.6 \times 1.6 \mathrm{~cm}$ vane shear spindle (Haake RS-600). Note that B-FRPGM-001-XX-2718-RHE-4 and B-FRPGM-001-XX-2707RHE-4 were mixed to obtain sufficient precipitates to measure the shear strength after removing identical amounts of supernatants from each sample bottle, since the amount of precipitates from either sample was not enough for the measurement. All samples showed continuous increases in the maximum shear strength (within the experimental uncertainty) as the settling time increases and the complete asymptotes were not observed due to insufficient settling time. Considering both the limited settling time and an asymptotic behavior as settling time approaches $72 \mathrm{hrs,} \mathrm{all}$ samples except A-02AOL-018-XX-2384-RHE4 appear to be adequate for handling in the WTP (i.e. less than $30 \mathrm{~Pa}$ of the maximum shear strength). The shear strength measurement with a longer settling time might be needed to ensure an asymptotic behavior for A-02AOL-018-XX2384-RHE4.

Some samples were run only as slurries and some only as supernatants as was needed for the matrix support for that part of the test plan. Most slurry samples were reasonably well fitted with the Bingham plastic model. All supernatants did not exhibit a yield stress and Newtonian fits appear to be the best fit.

The average rheological parameters for flow curve measurements are presented in Appendix A. More detailed data for flow curve and shear strength measurements are presented in Appendix B and the actual rheograms for each test and the curve fits are presented in Appendix C. 


\section{Appendix A}

\section{Average Rheological Parameters for Shear Stress vs. Shear Rate} Measurements 


\begin{tabular}{|c|c|c|c|c|c|c|c|c|c|c|c|}
\hline & & & & & & & & & & & \\
\hline & & & & & & & & & & & \\
\hline & & & & & & & & & & . & \\
\hline & Rheology fits & Bingham & Bingham & $\begin{array}{l}\text { Bingham } \\
\text { Down }\end{array}$ & Bingham & Casson & Casson & Casson & Casson & Newtonian & Newtonian \\
\hline & & Up & Up & $\begin{array}{l}\text { Down } \\
\text { Yield Stress }\end{array}$ & $\begin{array}{l}\text { Down } \\
\text { Consistency }\end{array}$ & Up & Up & Down & Down & Up & Down \\
\hline & & Yield Stress & Consistency & Yield Stress & Consistency & Yield Stress & Consistency & Yield Stress & Consistency & & \\
\hline & & $\tan (\mathrm{Pa})$ & eta (Pa.sec) & $\tan (\mathrm{Pa})$ & eta (Pa-sec) & $\tan (\mathrm{Pa})$ & eta ( $\mathrm{Pa} \cdot \mathrm{sec})$ & $\operatorname{tau}(\mathrm{Pa})$ & eta (Pa-Sec) & eta (Pa.sec) & eta $(\mathrm{Pa} \cdot \mathrm{sec})$ \\
\hline \# & Sample names & & & & & & & & & & \\
\hline 1 & A 02 AML 007 XX 2684 UDS 2 & & & & & & & & & & \\
\hline & Average & 1.786 & $1.410 \mathrm{E}-02$ & 1.466 & $1.402 \mathrm{E}-02$ & 0.545 & $1.017 \mathrm{E}-02$ & 0.429 & $1.034 \mathrm{E}-02$ & N/A & $\mathrm{N} / \mathrm{A}$ \\
\hline 2 & A 000FL 015 XX 2812 RHE 4 & & & & & & & & & & \\
\hline & Average & 2.771 & $1.047 \mathrm{E}-02$ & 2.307 & $1.099 \mathrm{E}-02$ & 1.380 & $5.615 \mathrm{E}-03$ & 1.068 & $6.319 \mathrm{E}-03$ & N/A & $\mathrm{N} / \mathrm{A}$ \\
\hline 3 & & & & & & & & & & +6 & \\
\hline & $\begin{array}{l}\text { A coovFL } 007 \text { XX 2813 RHE } 4 \\
\text { Average }\end{array}$ & 3.311 & $1.868 \mathrm{E}-02$ & 2.680 & $1.894 \mathrm{E}-02$ & 1.203 & $1.238 \mathrm{E}-02$ & 0.962 & & & \\
\hline & & & & & & & & 0.962 & $1.280 \mathrm{E}-02$ & N/A & N/A \\
\hline 4 & A 000FL 014 XX 2814 RHE 4 & & & & & & & & & & \\
\hline & Average & 3.309 & $1.476 \mathrm{E}-02$ & 2.756 & $1.531 \mathrm{E}-02$ & 1.417 & $8.892 \mathrm{E}-03$ & 1.138 & $9.568 \mathrm{E}-03$ & N/A & $\mathrm{N} / \mathrm{A}$ \\
\hline 5 & A $02 \mathrm{AOL} 015$ XX 2294 RHE 4 & & & & & & & & & & \\
\hline & (supernatant) & & & & & & & & & & \\
\hline & Average & N/A & N/A & N/A & N/A & N/A & N/A & N/A & N/A & $4.200 \mathrm{E}-03$ & 4.144E-03 \\
\hline 6 & A $02 A O L$ 015 XX 2293 RHE 4 & & & & & & & & & & \\
\hline & Average & 3.347 & $1.426 \mathrm{E}-02$ & 2.769 & $1.484 \mathrm{E}-02$ & 1.464 & $8.465 \mathrm{E}-03$ & 1.161 & $9.177 \mathrm{E}-03$ & N/A & $N / A$ \\
\hline & & & & & & & & 1.01 & & $\mathrm{~N} / \mathrm{A}$ & - \\
\hline 7 & A 02AOL 014012281 RHE 4 & & & & & & & & & & \\
\hline & Average & 2.988 & $1.500 \mathrm{E}-02$ & 2.685 & $1.533 \mathrm{E}-02$ & 1.341 & $8.912 E-03$ & 1.159 & $9.404 \mathrm{E}-03$ & N/A & $\mathrm{N} / \mathrm{A}$ \\
\hline 8 & A 02AOL 015102320 RHE 4 & & & & & & & & & & \\
\hline & (supernatant) & & & & & & & & & & \\
\hline & Average & $\mathrm{N} / \mathrm{A}$ & N/A & N/A & N/A & N/A & N/A & N/A & N/A & $1.222 \mathrm{E}-03$ & $1.244 \mathrm{E}-03$ \\
\hline 9 & A 02AOL 015102319 RHE 4 & & & & & & & & & & \\
\hline & Average & 2.254 & $6.188 \mathrm{E}-03$ & 2.227 & $6.204 \mathrm{E}-03$ & 1.423 & $2.485 \mathrm{E}-03$ & 1.399 & $2.511 \mathrm{E}-03$ & N/A & $\mathrm{N} / \mathrm{A}$ \\
\hline 10 & A 02AOL $015 X X 2543$ RHE 4 & & & & & & & & & & \\
\hline & (supernatant) & & & & & & & & & & \\
\hline & Average & N/A & N/A & N/A & N/A & N/A & N/A & N/A & N/A & $9.789 \mathrm{E}-04$ & $9.893 E-04$ \\
\hline 11 & A 02AOL $020 \times X 2451$ RHE 4 & 等 & & & & & & & & & \\
\hline & (supernatant) & & & & & & & & & & \\
\hline & Average & N/A & N/A & N/A & N/A & N/A & N/A & N/A & N/A & $9.466 \mathrm{E}-04$ & $9.639 \mathrm{E}-04$ \\
\hline 12 & A 02AOL $020 \times X 2450$ RHE 4 & & & & & & & & & & \\
\hline & Average & 1.687 & 4.183E-03 & 1.219 & $4.838 \mathrm{E}-03$ & 1.187 & $1.406 \mathrm{E}-03$ & 0.736 & $2.085 \mathrm{E}-03$ & $N / A$ & $N / A$ \\
\hline & & & & & & & & & & N/A & N/A \\
\hline
\end{tabular}




\begin{tabular}{|c|c|c|c|c|c|c|c|c|c|c|c|}
\hline & Rheology fits & Bingham & Bingham & Bingham & Bingham & Casson & Casson & Casson & Casson & Newtonian & Newtonian \\
\hline & & Up & Up & Down & Down & Up & Up & Down & Down & Up & Down \\
\hline & & Yield Stress & Consistency & Yield Stress & Consistency & Yield Stress & Consistency & Yield Stress & Consistency & & \\
\hline & & $\operatorname{tau}(\mathrm{Pa})$ & eta (Pa-sec) & $\operatorname{tau}(\mathrm{Pa})$ & eta ( $\mathrm{Pa} \cdot \mathrm{sec})$ & $\operatorname{tau}(\mathrm{Pa})$ & eta ( $\mathrm{Pa} \cdot \mathrm{sec})$ & $\operatorname{tau}(\mathrm{Pa})$ & eta (Pa:sec) & eta $(\mathrm{Pa} \cdot \mathrm{sec})$ & eta (Pa.sec) \\
\hline$\#$ & & & & & & & & & & & \\
\hline$\frac{\#}{13}$ & $\begin{array}{c}\text { Sample names } \\
\text { A 02AOL } 020082436 \text { RHE } 4\end{array}$ & & & & & & & & & & \\
\hline & Average & 2.640 & 4.583E-03 & 2.232 & $5.135 \mathrm{E}-03$ & 1.941 & $1.386 \mathrm{E}-03$ & \begin{tabular}{|c|}
1.517 \\
\end{tabular} & 1.807E-03 & N/A & N/A \\
\hline & & & & & & & & & & & \\
\hline 14 & A 02AOL 020082437 RHE 4 & & & & & & & & & & \\
\hline & (supernatant) & & & & & & & & & & \\
\hline & Average & N/A & N/A & N/A & $\mathrm{N} / \mathrm{A}$ & N/A & $\mathrm{N} / \mathrm{A}$ & N/A & $\mathrm{N} / \mathrm{A}$ & $9.638 \mathrm{E}-04$ & $9.838 \mathrm{E}-04$ \\
\hline & & & & & & & & & & & \\
\hline 15 & $\begin{array}{l}\text { A 02AOL } 015 \text { XX } 2542 \text { RHE } 4 \\
\text { Average }\end{array}$ & & & & & & & & & & N/A \\
\hline & Average & 1.985 & $5.151 \mathrm{E}-03$ & 2.115 & $5.037 \mathrm{E}-03$ & 1.337 & $1.859 \mathrm{E}-03$ & 1.422 & $1.798 \mathrm{E}-03$ & N/A & N/A \\
\hline 16 & A $02 \mathrm{AOL} 019062417$ RHE 4 & & & & & & & & & & \\
\hline & (supernatant) & & & & & & & & & & \\
\hline & Average & N/A & N/A & N/A & N/A & $\mathrm{N} / \mathrm{A}$ & $\mathrm{N} / \mathrm{A}$ & N/A & N/A & $1.017 \mathrm{E}-03$ & $1.021 \mathrm{E}-03$ \\
\hline & & & & & & & & & & & \\
\hline 17 & A T22GM 002 XX 1506 RHE 4 & & & & & & & & & & \\
\hline & Average & $\mathrm{N} / \mathrm{A}$ & N/A & N/A & N/A & N/A & N/A & N/A & N/A & 4.373E-03 & $4.244 \mathrm{E}-03$ \\
\hline & & & & & & & & & & & \\
\hline 18 & $\begin{array}{l}\text { A T22GM 13C XX } 2021 \text { RHE } 4 \\
\text { Average }\end{array}$ & N/A & N/A & N/A & N/A & N/A & N/A & $N / A$ & N/A & $4.340 \mathrm{E}-03$ & $4.241 \mathrm{E}-03$ \\
\hline & & & & & & & & & & & \\
\hline 19 & A $02 A O L ~ 018 X X 2385$ RHE 4 & & & & & & & & & & \\
\hline & Average & 5.733 & $6.526 \mathrm{E}-03$ & 5.751 & $6.576 \mathrm{E}-03$ & 4.382 & $1.734 \mathrm{E}-03$ & 4.339 & $1.800 \mathrm{E}-03$ & N/A & $\mathrm{N} / \mathrm{A}$ \\
\hline & & & & & & & & & & & \\
\hline 20 & A T22GM 13A XX 1772 RHE 4 & & & & & & & & & & $4.199 \mathrm{E}-03$ \\
\hline & Average & N/A & N/A & N/A & $\mathrm{N} / \mathrm{A}$ & N/A & $\mathrm{N} / \mathrm{A}$ & N/A & N/A & $4.356 \mathrm{E}-03$ & 4.199E-03 \\
\hline & & & & & & & & & & & \\
\hline 23 & A 02ARL XXX XX 2842 RHE 4 & & & & & & & & & & \\
\hline & Average & 2.456 & 4.291E-03 & 1.800 & $5.094 \mathrm{E}-03$ & 1.835 & 1.197E-03 & 1.185 & $1.902 \mathrm{E}-03$ & N/A & $\mathrm{N} / \mathrm{A}$ \\
\hline 24 & A 02AML 021 XX 2457 RHE 4 & & & & & & & & & & \\
\hline & Average & 5.175 & $5.239 \mathrm{E}-03$ & 4.515 & $6.121 \mathrm{E}-03$ & 4.051 & $1.284 \mathrm{E}-03$ & 3.333 & $1.784 \mathrm{E}-03$ & N/A & $\mathrm{N} / \mathrm{A}$ \\
\hline & & & & & & & & & & & \\
\hline 25 & A 02 AML 021 XX 2458 RHE 4 & & & & & & & & & & \\
\hline & (supernatant) & & & & & & & & & & \\
\hline & Average & $\mathrm{N} / \mathrm{A}$ & N/A & N/A & N/A & N/A & $\mathrm{N} / \mathrm{A}$ & N/A & N/A & $9.411 \mathrm{E}-04$ & 9.442E-04 \\
\hline
\end{tabular}




\section{H.2 Heat Capacity}

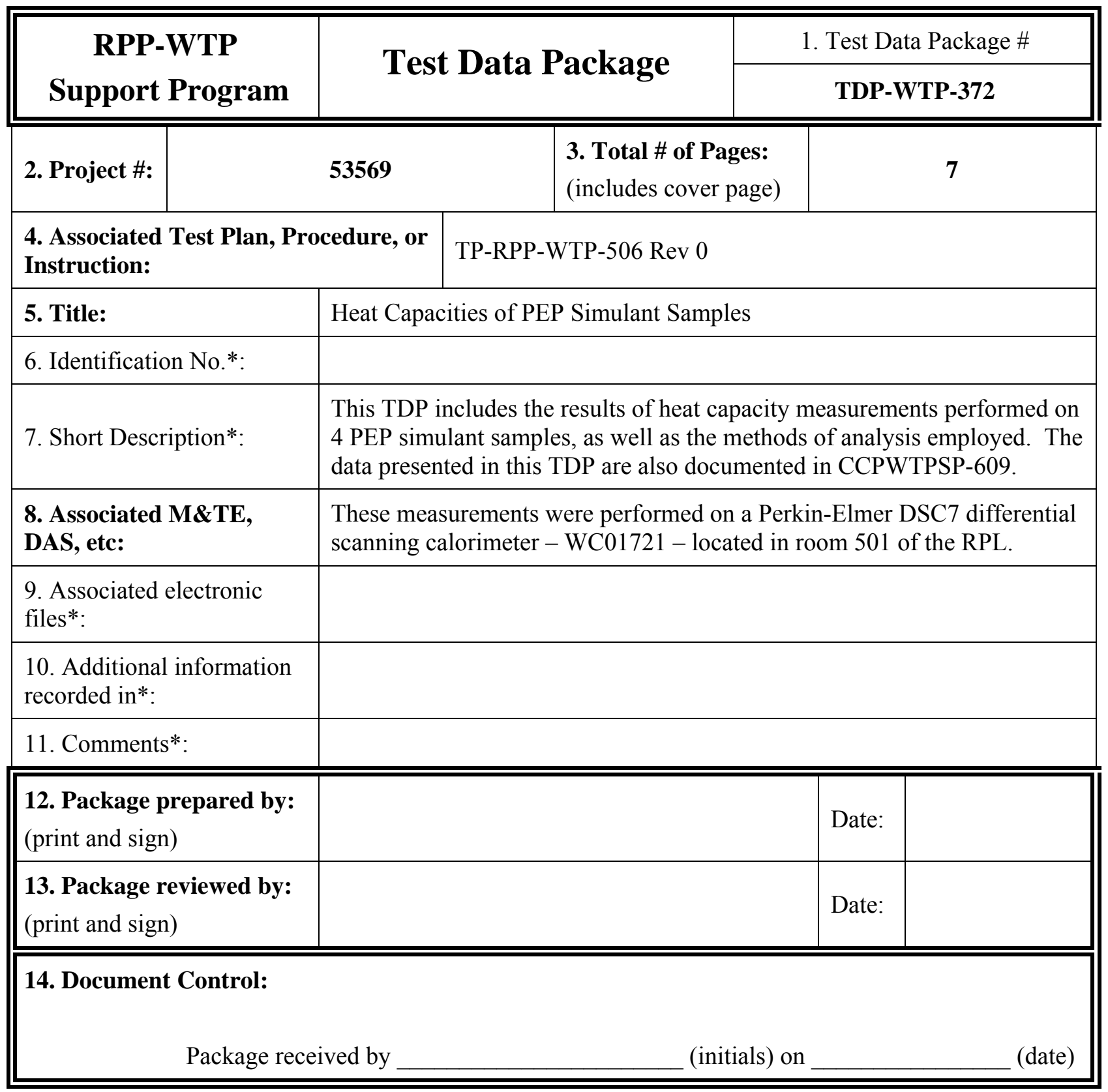




\section{PEP Slurry Sample Heat Capacity Report \\ Analysis}

This report presents results of heat capacity measurements for four PEP simulant slurry samples delivered via Chain of Custody form numbers WTP-COC-063-048 on 03/13/2009 and WTP-COC-063-051 on 03/16/2009. The samples provided were:
- B 02AML 005 XX 2949 HTC 3
- B 02AML 007 XX 2965 HTC 3
- B 02AML 008 BT 2972 HTC 3
- B 02AML 008002988 HTC 3

Three nominal $30 \mathrm{mg}$ subsamples were taken from each of these samples as they were vigorously stirred using a pipette with an enlarged tip to assure that the slurry samples were representative. We analyzed each slurry sample in triplicate, recognizing that obtaining a $30 \mathrm{mg}$ representative sample is challenging.

A Perkin-Elmer DSC7 differential scanning calorimeter (DSC) was used to determine the heat capacity of each subsample at temperatures between $20^{\circ} \mathrm{C}$ and $95^{\circ} \mathrm{C}$. The DSC7 was temperature- and enthalpy-calibrated using a gallium standard (NIST SRM 2234) (m.p. $29.8^{\circ} \mathrm{C}$ ) and/or an indium standard (NIST SRM 2232) (m.p. $156.6^{\circ} \mathrm{C}$ ).

The method used for heat capacity measurement was as provided in the DSC7 operating manual. In this method, the heat capacity of the empty sample pan is measured to provide the "baseline"; the sample pan was a gold "volatile" sample pan designed to contain volatile samples such as the water in these PEP slurries. The heat capacity of a reference or standard material such as sapphire or high purity water is then determined; the purity of these water standards was determined by measuring their electrical conductivity. Finally the heat capacity of the sample is determined. The measured heat capacity of the sample is adjusted to remove the contribution of the empty pan. We further adjusted the reported heat capacity by applying a measured response factor for the high purity water.

Optimally the heat capacity measurement approach would include analyzing the standard or reference material in the same container as the sample would be analyzed; however, because analysis of these slurry samples require a hermetic seal which cannot be broken and resealed, a different but nearly equivalent gold volatile sample pan is used for the water. In addition, we characterized a sapphire standard to demonstrate instrument performance but unfortunately, the heat capacity of sapphire $\left(0.8 \mathrm{~J} / \mathrm{g}{ }^{\circ} \mathrm{C}\right)$ is significantly lower than that of the PEP slurries $(\sim 3.5 \mathrm{~J} / \mathrm{g})$ and does not provide an adequate calibration adjustment.

\section{Results}

The expected results for the measurements can be generated by considering what the theoretical heat capacity would be for slurries with solid fraction in the range that are expected in the samples. The slurry is mostly water, which at $20^{\circ} \mathrm{C}$ is known to have a specific heat of $4.1818 \mathrm{~J} / \mathrm{g}^{\circ} \mathrm{C}$ [1]. It is also known that at $20^{\circ} \mathrm{C}$, the heat capacity of boehmite is roughly $0.9 \mathrm{~J} / \mathrm{g}^{\circ} \mathrm{C}[2]$. So for slurries with mass fraction of $10 \%$, $20 \%$, and $30 \%$ boehmite in water, the theoretical heat capacities would be, respectively: 
- $\quad 4.1818 \mathrm{~J} / \mathrm{g}^{\circ} \mathrm{C} * .9+0.9 \mathrm{~J} / \mathrm{g}^{\circ} \mathrm{C} * .1=3.85 \mathrm{~J} / \mathrm{g}^{\circ} \mathbf{C}$,

- $\quad 4.1818 \mathrm{~J} / \mathrm{g}^{\circ} \mathrm{C} * .80+0.9 \mathrm{~J} / \mathrm{g}^{\circ} \mathrm{C} * .2=3.52 \mathrm{~J} / \mathrm{g}^{\circ} \mathrm{C}$, and

- $4.1818 \mathrm{~J} / \mathrm{g}^{\circ} \mathrm{C} * .7+0.9 \mathrm{~J} / \mathrm{g}^{\circ} \mathrm{C} * .3=3.20 \mathrm{~J} / \mathrm{g}^{\circ} \mathrm{C}$.

Table 1 summarizes the results from these analyses by providing the mean measured heat capacities with their standard deviations. In general, the measured heat capacities are consistent with our expectations of hear capacities in the 3.2 to $3.8 \mathrm{~J} / \mathrm{g}^{\circ} \mathrm{C}$ assuming solids contents ranging from 10 to $30 \mathrm{wt} \%$. We used only those analyses found to be internally self-consistent, not erratic and as-such, we discarded one measurement for sample B 02AML 005 XX 2949 HTC 3, one measurement for sample B 02AML 007 XX 2965 HTC 3, and one from B 02AML 008 BT 2972 HTC 3, but replaced it with a re-analysis.

Table 1: Results of Heat Capacity Measurements.

\begin{tabular}{|l|r|r|r|r|}
\hline Temperature & $\begin{array}{l}\text { B 02AML 005 XX } \\
\text { 2949 HTC 3 } \\
\mathrm{C}_{\mathrm{p}}\left(\mathrm{J} / \mathrm{g}^{\circ} \mathrm{C}\right)\end{array}$ & $\begin{array}{l}\text { B 02AML 007 XX } \\
\text { 2965 HTC 3 } \\
\mathrm{C}_{\mathrm{p}}\left(\mathrm{J} / \mathrm{g}^{\circ} \mathrm{C}\right)\end{array}$ & $\begin{array}{l}\text { B 02AML 008 BT } \\
\text { 2972 HTC 3 } \\
\mathrm{C}_{\mathrm{p}}\left(\mathrm{J} / \mathrm{g}^{\circ} \mathrm{C}\right)\end{array}$ & $\begin{array}{l}\text { B 02AML 008 00 } \\
\text { 2988 HTC 3 } \\
\mathrm{C}_{\mathrm{p}}\left(\mathrm{J} / \mathrm{g}^{\circ} \mathrm{C}\right)\end{array}$ \\
\hline $35^{\circ} \mathrm{C}$ & $2.98 \pm 0.00$ & $3.21 \pm 0.01$ & $3.23 \pm 0.03$ & $3.22 \pm 0.09$ \\
\hline $40^{\circ} \mathrm{C}$ & $2.97 \pm 0.00$ & $3.22 \pm 0.01$ & $3.25 \pm 0.02$ & $3.23 \pm 0.09$ \\
\hline $45^{\circ} \mathrm{C}$ & $2.97 \pm 0.00$ & $3.24 \pm 0.01$ & $3.26 \pm 0.03$ & $3.23 \pm 0.09$ \\
\hline $50^{\circ} \mathrm{C}$ & $2.96 \pm 0.02$ & $3.25 \pm 0.00$ & $3.26 \pm 0.03$ & $3.23 \pm 0.09$ \\
\hline $55^{\circ} \mathrm{C}$ & $2.96 \pm 0.02$ & $3.25 \pm 0.00$ & $3.27 \pm 0.02$ & $3.24 \pm 0.08$ \\
\hline $60^{\circ} \mathrm{C}$ & $2.96 \pm 0.02$ & $3.26 \pm 0.00$ & $3.28 \pm 0.02$ & $3.23 \pm 0.09$ \\
\hline $65^{\circ} \mathrm{C}$ & $2.95 \pm 0.03$ & $3.27 \pm 0.00$ & $3.28 \pm 0.02$ & $3.23 \pm 0.09$ \\
\hline $70^{\circ} \mathrm{C}$ & $2.95 \pm 0.04$ & $3.28 \pm 0.00$ & $3.29 \pm 0.02$ & $3.23 \pm 0.08$ \\
\hline $75^{\circ} \mathrm{C}$ & $2.95 \pm 0.04$ & $3.28 \pm 0.01$ & $3.30 \pm 0.02$ & $3.23 \pm 0.08$ \\
\hline $80^{\circ} \mathrm{C}$ & $2.96 \pm 0.05$ & $3.29 \pm 0.01$ & $3.29 \pm 0.02$ & $3.23 \pm 0.08$ \\
\hline $85^{\circ} \mathrm{C}$ & $2.97 \pm 0.05$ & $3.29 \pm 0.02$ & $3.30 \pm 0.02$ & $3.22 \pm 0.08$ \\
\hline $90^{\circ} \mathrm{C}$ & $2.98 \pm 0.05$ & $3.30 \pm 0.02$ & $3.30 \pm 0.02$ & $3.22 \pm 0.08$ \\
\hline
\end{tabular}

The results from each sample measurement are presented in graphical form in Figures 1-4. In general, the heat capacity will increase nominally linearly as a function of temperature. In general, the average measured heat capacities do increase linearly with temperature, although some deviations do exist. 


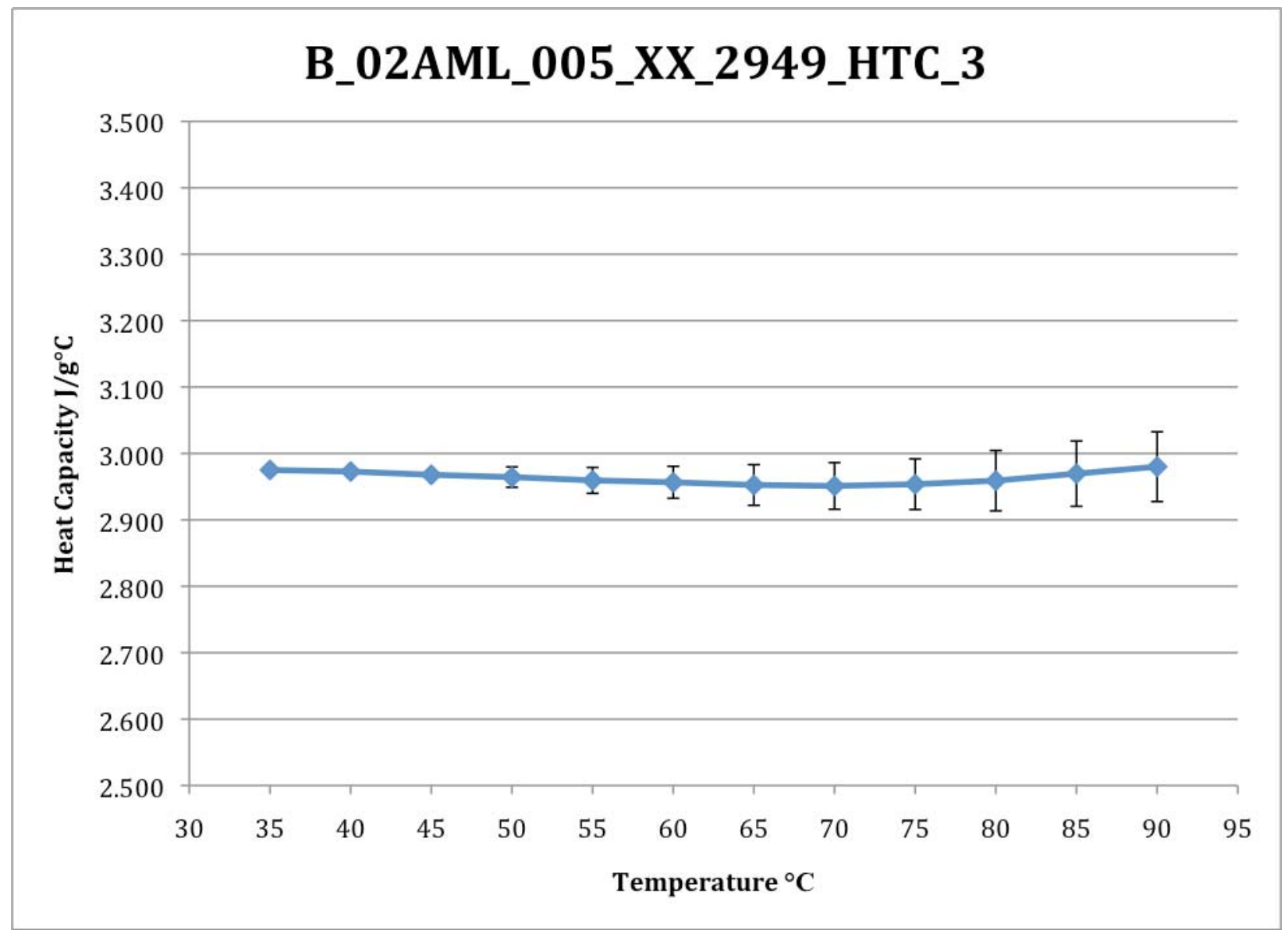

Figure 1: Heat Capacity Curve for Sample B_02AML_005_XX_2949_HTC_3 


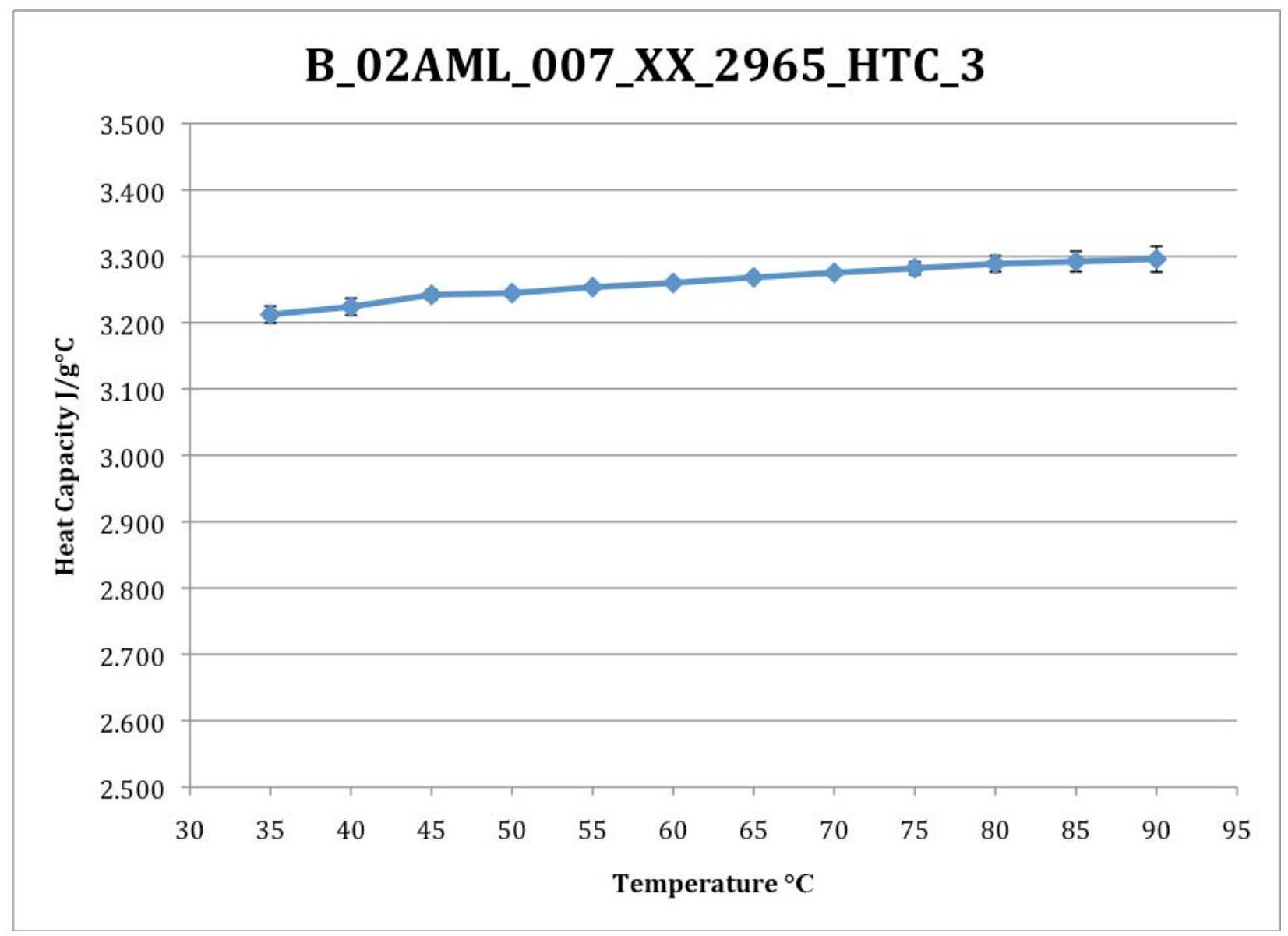

Figure 2: Heat Capacity Curve for Sample B_02AML_007_XX_2695_HTC_3 


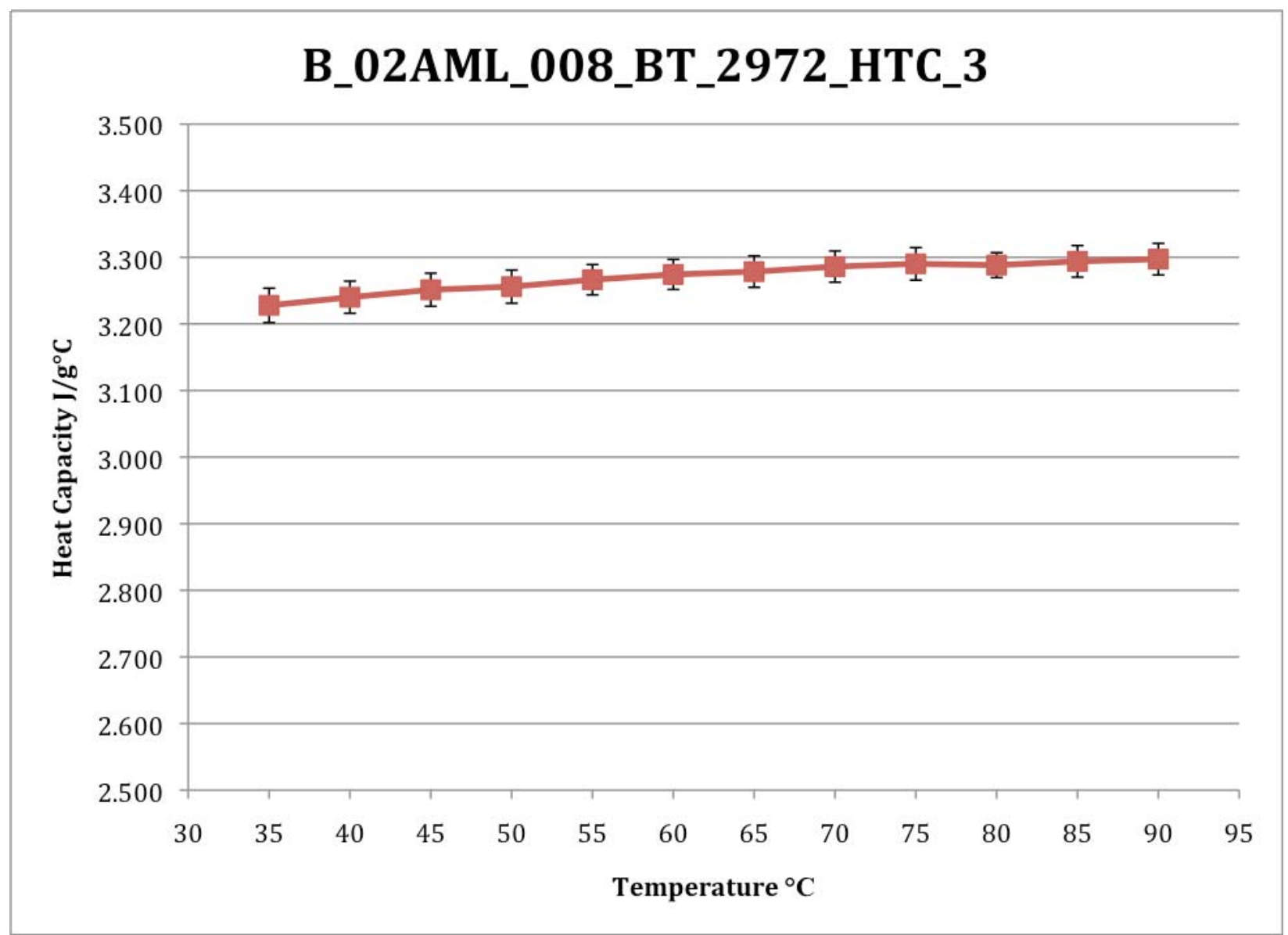

Figure 3: Heat Capacity Curve for Sample B_02AML_008_BT_2972_HTC_3 


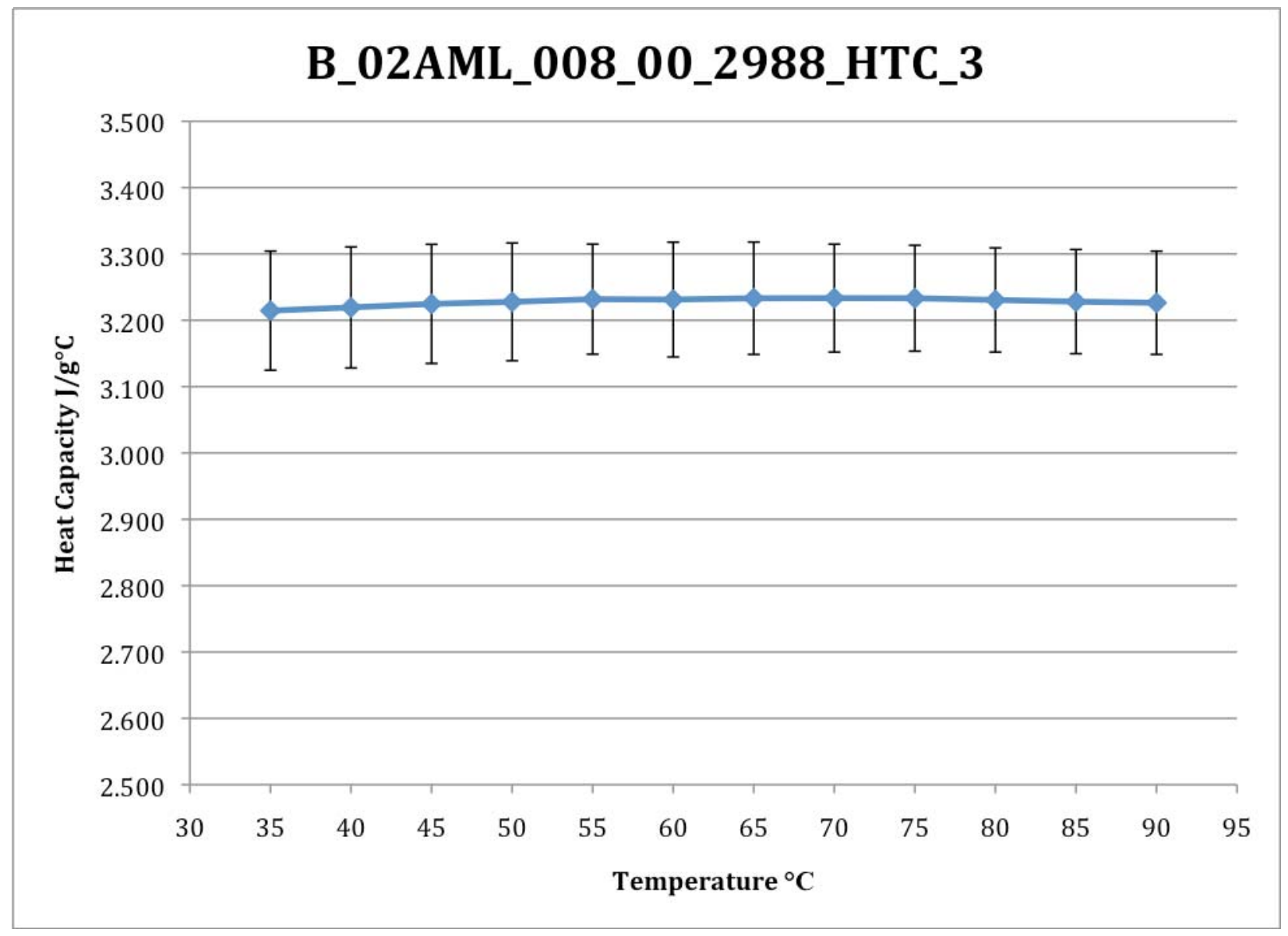

Figure 4: Heat Capacity Curve for Sample B_02AML_008_00_2988_HTC_3

\section{Conclusions}

The measured heat capacity values for the PEP slurry samples discussed in this report are well within the expected range.

\section{References}

[1] Lide, D. "CRC Handbook of Chemistry and Physics," 89 $9^{\text {th }}$ Edition, 2008-2009, 6-2.

[2] Hemingway, B.S., Robie, R.A., Apps, J.A., "Revised values for the thermodynamic properties of boehmite, $\mathrm{AlO}(\mathrm{OH})$, and related species and phases in the system Al-H-O," American Mineralogist, Volume 76, pages 445-457, 1991. 


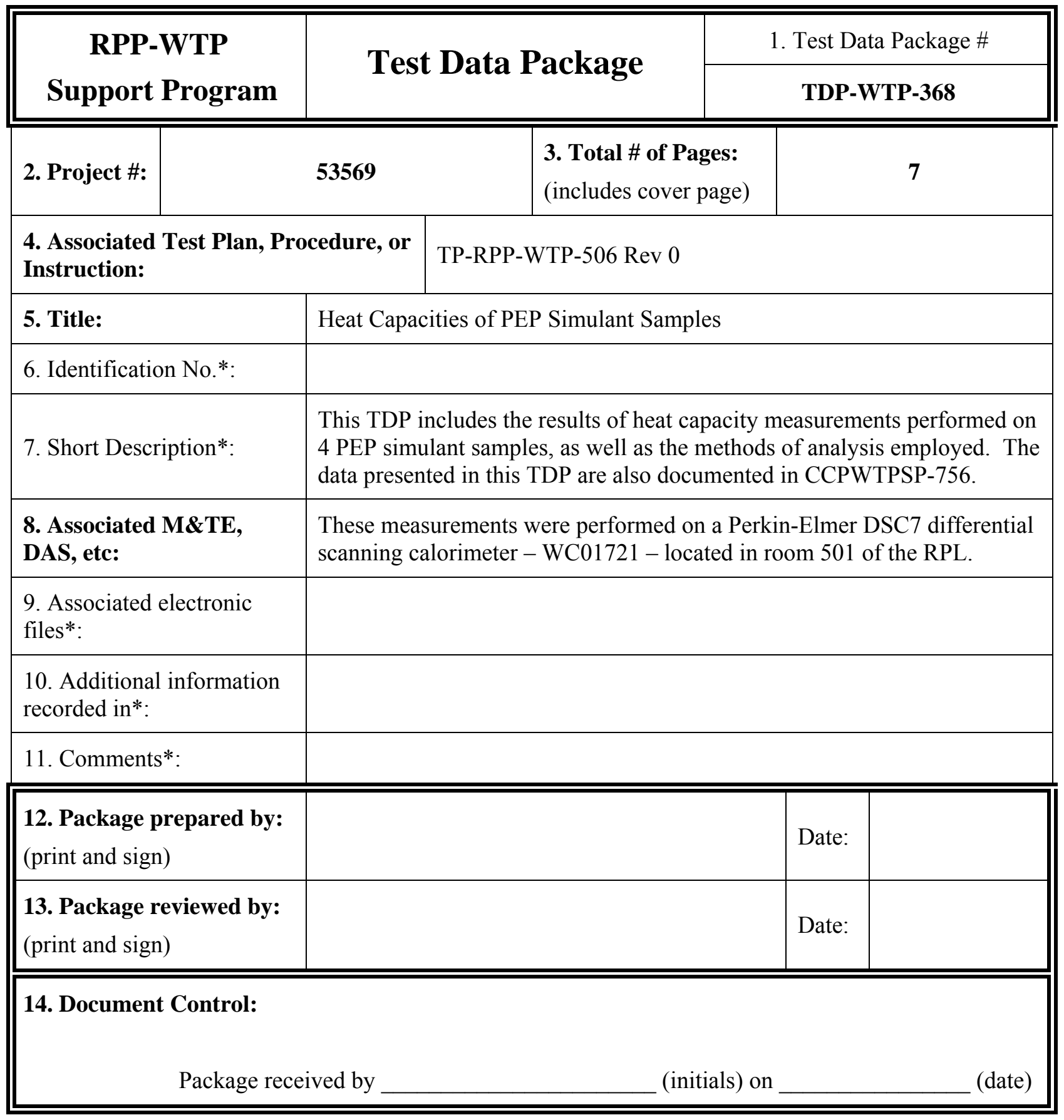




\section{PEP Slurry Sample Heat Capacity Report \\ Analysis}

This report presents results of heat capacity measurements for four PEP simulant slurry samples delivered via Chain of Custody form numbers WTP-COC-063-020 on 02/02/2009 and WTP-COC-063-024 on 02/03/2009. The samples provided were:

- A 01AIM 002 XX 1519 HTC 3

- A 01AIM 003001538 HTC 3

- A 01AIM 003 BT 1526 HTC 3

- B FRPGM 001 XX 2706 HTC 3

Three nominal $30 \mathrm{mg}$ subsamples were taken from each of these samples as they were vigorously stirred using a pipette with an enlarged tip to assure that the slurry samples were representative. We analyzed each slurry sample in triplicate, recognizing that obtaining a $30 \mathrm{mg}$ representative sample is challenging.

A Perkin-Elmer DSC7 differential scanning calorimeter (DSC) was used to determine the heat capacity of each subsample at temperatures between $20^{\circ} \mathrm{C}$ and $95^{\circ} \mathrm{C}$. The DSC7 was temperature- and enthalpy-calibrated using a gallium standard (NIST SRM 2234) (m.p. 29.8 ${ }^{\circ} \mathrm{C}$ ) and/or an indium standard (NIST SRM 2232) (m.p. $156.6^{\circ} \mathrm{C}$ ).

The method used for heat capacity measurement was as provided in the DSC7 operating manual. In this method, the heat capacity of the empty sample pan is measured to provide the "baseline"; the sample pan was a gold "volatile" sample pan designed to contain volatile samples such as the water in these PEP slurries. The heat capacity of a reference or standard material such as sapphire or high purity water is then determined; the purity of these water standards was determined by measuring their electrical conductivity. Finally the heat capacity of the sample is determined. The measured heat capacity of the sample is adjusted to remove the contribution of the empty pan. We further adjusted the reported heat capacity by applying a measured response factor for the high purity water.

Optimally the heat capacity measurement approach would include analyzing the standard or reference material in the same container as the sample would be analyzed; however, because analysis of these slurry samples require a hermetic seal which cannot be broken and resealed, a different but nearly equivalent gold volatile sample pan is used for the water. In addition, we characterized a sapphire standard to demonstrate instrument performance but unfortunately, the heat capacity of sapphire $\left(0.8 \mathrm{~J} / \mathrm{g}{ }^{\circ} \mathrm{C}\right)$ is significantly lower than that of the PEP slurries $(\sim 3.5 \mathrm{~J} / \mathrm{g})$ and does not provide an adequate calibration adjustment.

\section{Results}

The expected results for the measurements can be generated by considering what the theoretical heat capacity would be for slurries with solid fraction in the range that are expected in the samples. The slurry is mostly water, which at $20^{\circ} \mathrm{C}$ is known to have a specific heat of $4.1818 \mathrm{~J} / \mathrm{g}^{\circ} \mathrm{C}$ [1]. It is also known that at $20^{\circ} \mathrm{C}$, the heat capacity of boehmite is roughly $0.9 \mathrm{~J} / \mathrm{g}^{\circ} \mathrm{C}$ [2]. So for slurries with mass fraction of $10 \%$, $20 \%$, and $30 \%$ boehmite in water, the theoretical heat capacities would be, respectively:

- $\quad 4.1818 \mathrm{~J} / \mathrm{g}^{\circ} \mathrm{C} * .9+0.9 \mathrm{~J} / \mathrm{g}^{\circ} \mathrm{C} * .1=3.85 \mathbf{~ J} / \mathbf{g}^{\circ} \mathbf{C}$, 
- $\quad 4.1818 \mathrm{~J} / \mathrm{g}^{\circ} \mathrm{C} * .80+0.9 \mathrm{~J} / \mathrm{g}^{\circ} \mathrm{C} * .2=3.52 \mathrm{~J} / \mathrm{g}^{\circ} \mathrm{C}$, and

- $4.1818 \mathrm{~J} / \mathrm{g}^{\circ} \mathrm{C} * .7+0.9 \mathrm{~J} / \mathrm{g}^{\circ} \mathrm{C} * .3=3.20 \mathrm{~J} / \mathbf{g}^{\circ} \mathbf{C}$.

Table 1 summarizes the results from these analyses by providing the mean measured heat capacities with their standard deviations. In general, the measured heat capacities are consistent with our expectations of hear capacities in the 3.2 to $3.8 \mathrm{~J} / \mathrm{g}^{\circ} \mathrm{C}$ assuming solids contents ranging from 10 to $30 \mathrm{wt} \%$. We used only those analyses found to be internally self-consistent, not erratic and as-such, we discarded one measurement for sample A_01AIM_002_XX_1519_HTC_3, one measurement for sample A_01AIM_003_BT_1526_HTC_3 but replaced it with a reanalysis, and one measurement for Sample A_01AIM_003_00_1538_HTC_3.

Table 1: Results of Heat Capacity Measurements

\begin{tabular}{|l|r|r|r|r|}
\hline Temperature & $\begin{array}{l}\text { A 01AIM 002 XX } \\
-1519 \mathrm{HTC} \mathrm{3} \\
\mathrm{C}_{\mathrm{p}}\left(\mathrm{J} / \mathrm{g}^{\circ} \mathrm{C}\right)\end{array}$ & $\begin{array}{l}\text { A 01AIM 003 00- } \\
\text { 1538 HTC 3 } \\
\mathrm{C}_{\mathrm{p}}\left(\mathrm{J} / \mathrm{g}^{\circ} \mathrm{C}\right)\end{array}$ & $\begin{array}{l}\text { A 01AIM 003 BT- } \\
\text { 1526 HTC 3 } \\
\mathrm{C}_{\mathrm{p}}\left(\mathrm{J} / \mathrm{g}^{\circ} \mathrm{C}\right)\end{array}$ & $\begin{array}{l}\text { B FRPM 001 } \\
\text { XX- 2706 HTC 3 } \\
\mathrm{C}_{\mathrm{p}}\left(\mathrm{J} / \mathrm{g}^{\circ} \mathrm{C}\right)\end{array}$ \\
\hline $35^{\circ} \mathrm{C}$ & $3.32 \pm 0.01$ & $3.46 \pm 0.00$ & $3.33 \pm 0.09$ & $3.33 \pm 0.02$ \\
\hline $40^{\circ} \mathrm{C}$ & $3.32 \pm 0.02$ & $3.48 \pm 0.01$ & $3.35 \pm 0.09$ & $3.33 \pm 0.02$ \\
\hline $45^{\circ} \mathrm{C}$ & $3.34 \pm 0.01$ & $3.49 \pm 0.01$ & $3.36 \pm 0.09$ & $3.33 \pm 0.02$ \\
\hline $50^{\circ} \mathrm{C}$ & $3.33 \pm 0.01$ & $3.51 \pm 0.02$ & $3.36 \pm 0.09$ & $3.34 \pm 0.02$ \\
\hline $55^{\circ} \mathrm{C}$ & $3.34 \pm 0.00$ & $3.51 \pm 0.03$ & $3.37 \pm 0.09$ & $3.34 \pm 0.02$ \\
\hline $60^{\circ} \mathrm{C}$ & $3.35 \pm 0.01$ & $3.53 \pm 0.04$ & $3.39 \pm 0.09$ & $3.34 \pm 0.02$ \\
\hline $65^{\circ} \mathrm{C}$ & $3.36 \pm 0.02$ & $3.54 \pm 0.05$ & $3.40 \pm 0.09$ & $3.34 \pm 0.02$ \\
\hline $70^{\circ} \mathrm{C}$ & $3.37 \pm 0.03$ & $3.54 \pm 0.05$ & $3.41 \pm 0.09$ & $3.35 \pm 0.02$ \\
\hline $75^{\circ} \mathrm{C}$ & $3.38 \pm 0.04$ & $3.55 \pm 0.06$ & $3.43 \pm 0.10$ & $3.35 \pm 0.02$ \\
\hline $80^{\circ} \mathrm{C}$ & $3.39 \pm 0.06$ & $3.56 \pm 0.07$ & $3.43 \pm 0.10$ & $3.35 \pm 0.02$ \\
\hline $85^{\circ} \mathrm{C}$ & $3.40 \pm 0.07$ & $3.58 \pm 0.09$ & $3.44 \pm 0.10$ & $3.35 \pm 0.03$ \\
\hline $90^{\circ} \mathrm{C}$ & $3.40 \pm 0.08$ & $3.59 \pm 0.10$ & $3.45 \pm 0.10$ & $3.36 \pm 0.03$ \\
\hline
\end{tabular}

The results from each sample measurement are presented in graphical form in Figures 1-4. In general, the heat capacity will increase nominally linearly as a function of temperature. In general, the average measured heat capacities do increase linearly with temperature, although some deviations do exist. 


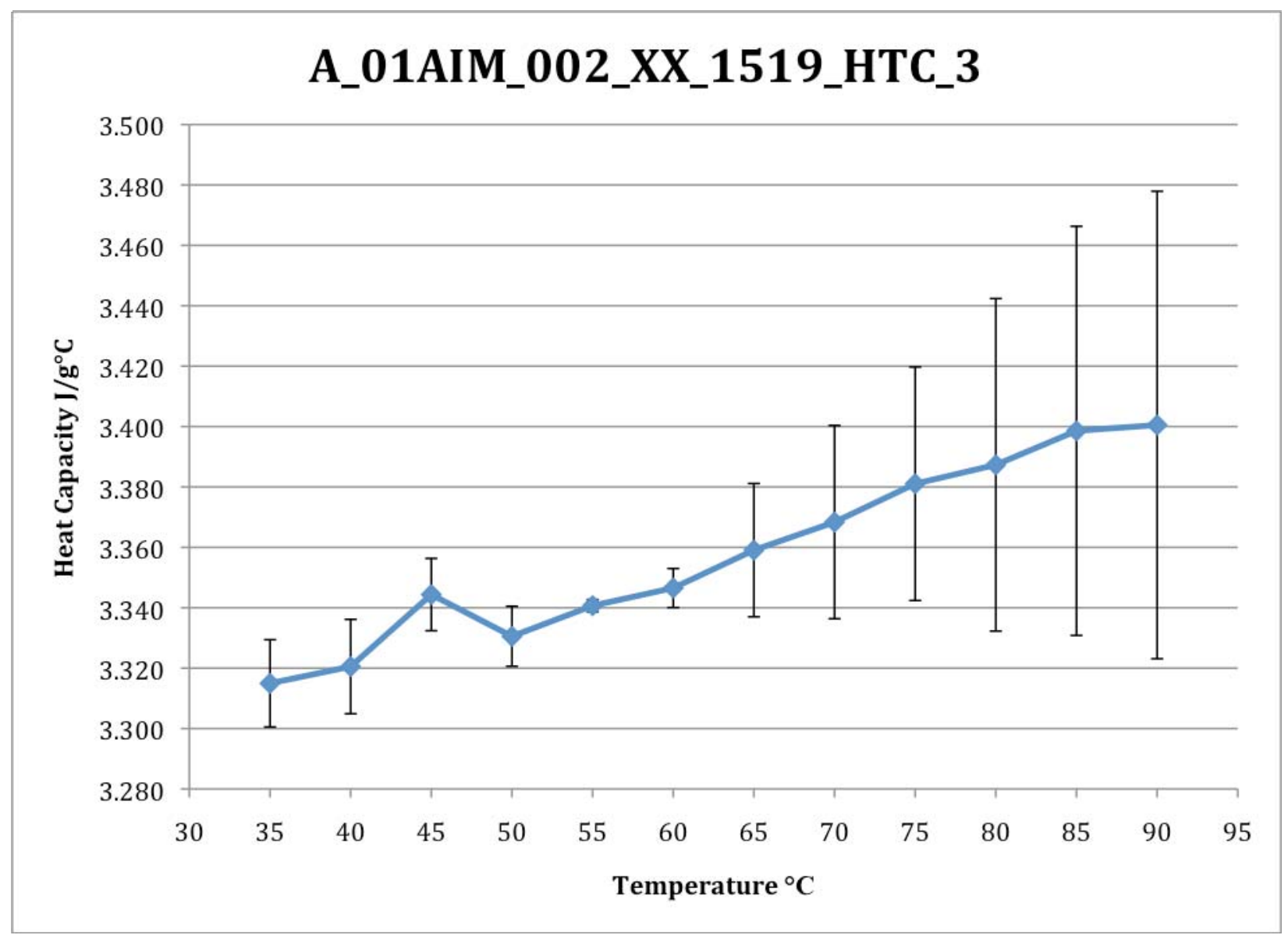

Figure 1: Heat Capacity Curve for Sample A_01AIM_002_XX_1519_HTC_3 


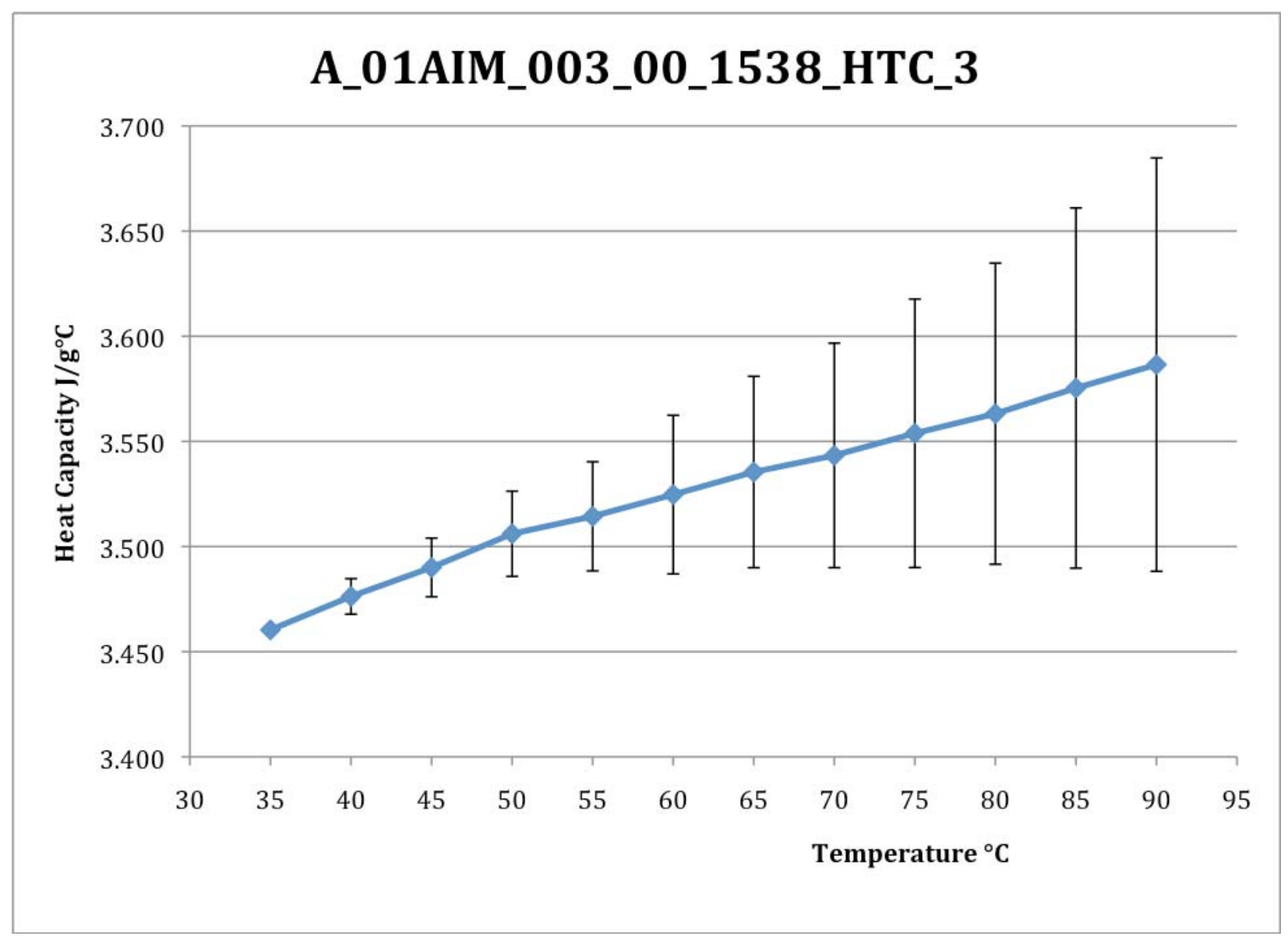

Figure 2: Heat Capacity Curve for Sample A_01AIM_003_00_1538_HTC_3 


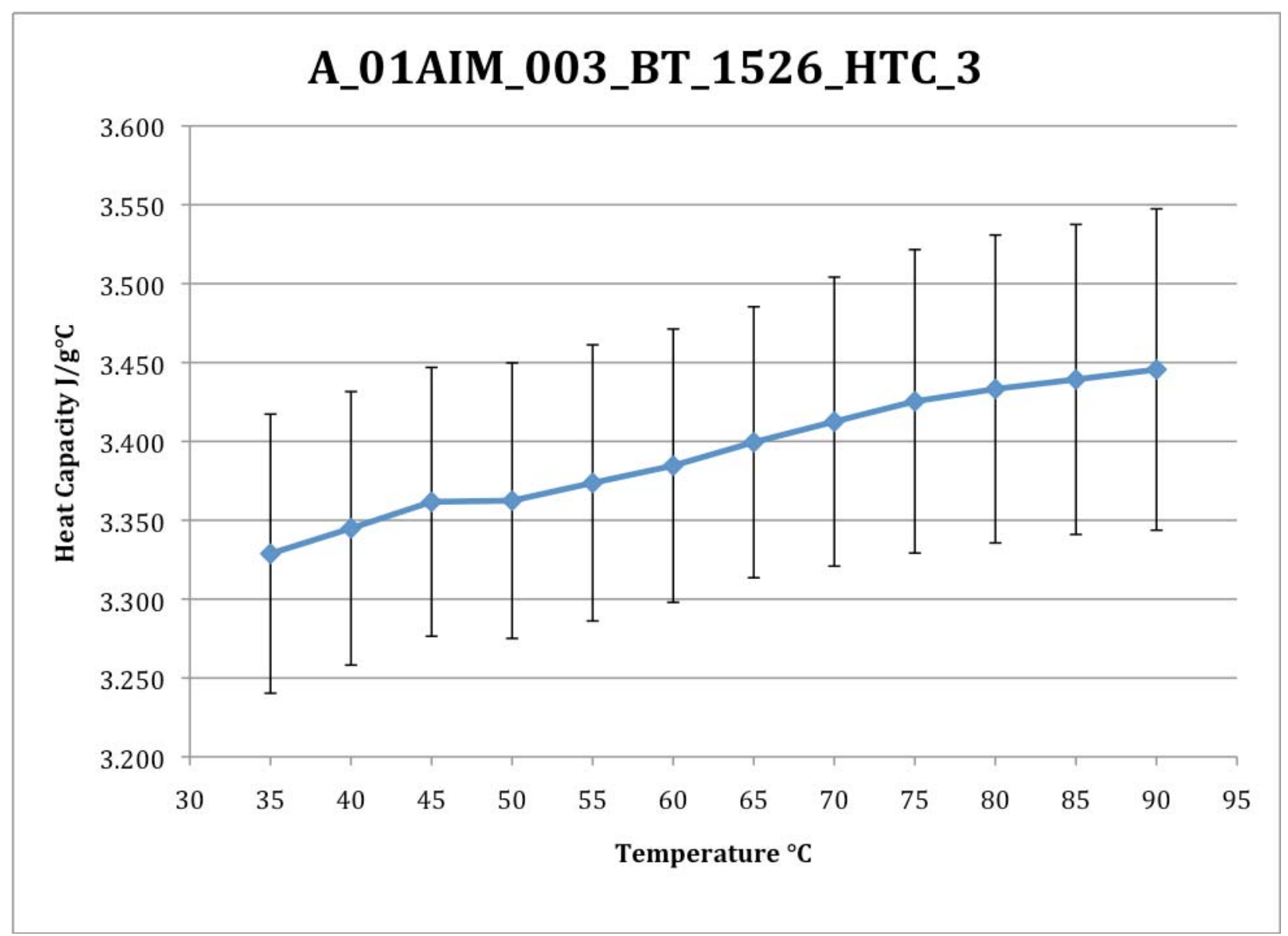

Figure 3: Heat Capacity Curve for Sample A_01AIM_003_BT_1526_HTC_3 


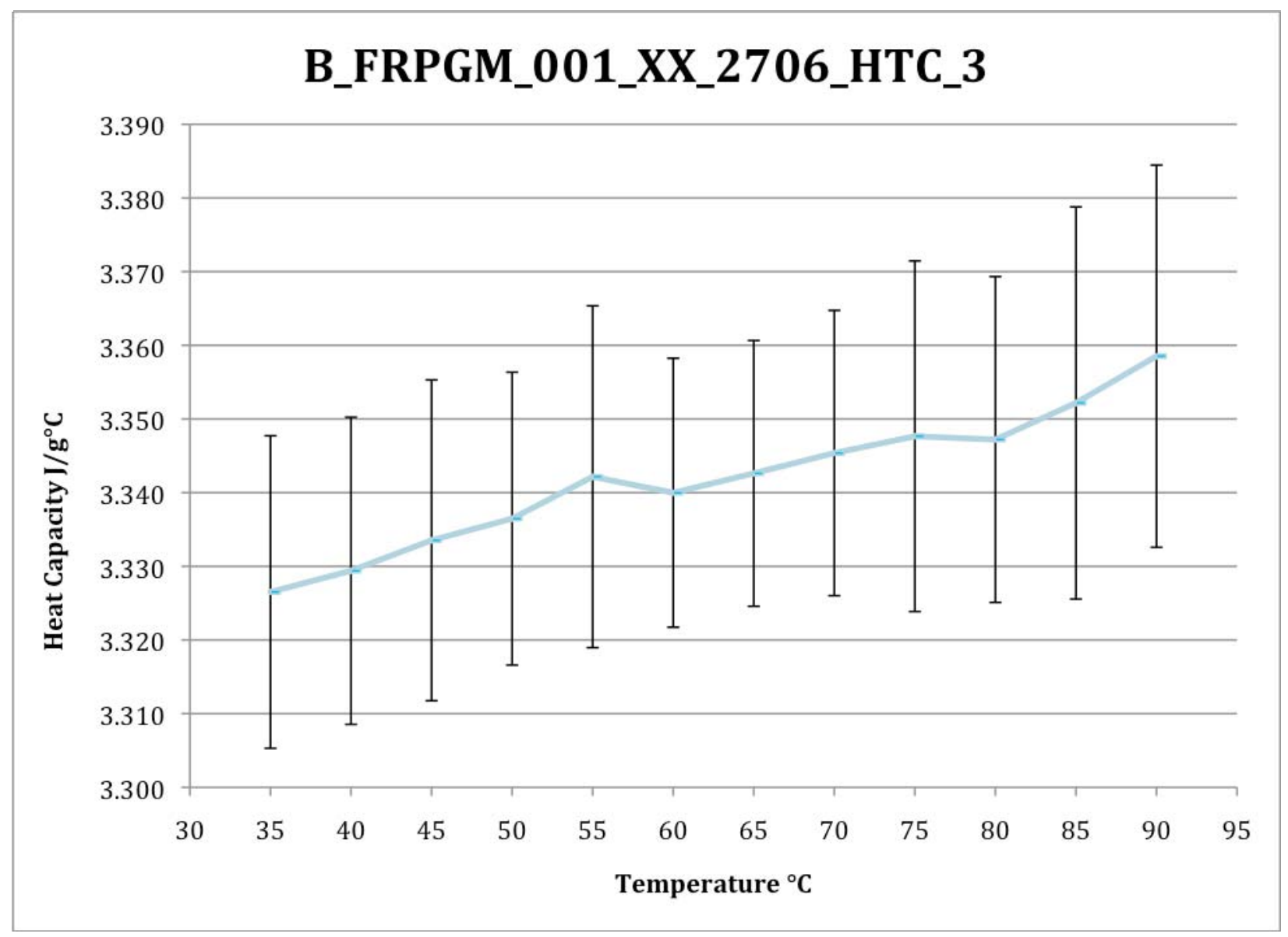

Figure 4: Heat Capacity Curve for Sample B_FRPGM_001_XX_2706_HTC_3

\section{Conclusions}

The measured heat capacity values for the PEP slurry samples discussed in this report are well within the expected range.

\section{References}

[1] Lide, D. "CRC Handbook of Chemistry and Physics," 89 $9^{\text {th }}$ Edition, 2008-2009, 6-2.

[2] Hemingway, B.S., Robie, R.A., Apps, J.A., "Revised values for the thermodynamic properties of boehmite, $\mathrm{AlO}(\mathrm{OH})$, and related species and phases in the system Al-H-O," American Mineralogist, Volume 76, pages 445-457, 1991. 


\section{H.3 Scanning Electron Microscopy}

$\begin{array}{llll}\text { Date: } & \text { March 19, } 2009 & \text { Project No.: } & 53596 \\ \text { To: } & \text { Ofelia Bredt } & \text { Internal } & \\ \text { From: } & \text { Brian Riley } & \text { Distribution: } & \\ \text { Subject: } & \text { PEP SEM Analyses for Integrated Test A, } & \\ & \text { TI-065 } & \end{array}$

Two specimens were received for scanning electron microscopy (SEM) examinations in support of work being performed at the Pretreatment Engineering Platform (PEP) during the course of Integrated Test A. The morphologies of these specimens were analyzed using a JEOL 5900 SEM in accordance with procedure APEL-102-SEM, Rev. 1.

\section{Sample Preparation}

Once received from PEP, the specimens were cleaned of nitrates. This cleaning process included 3 cycles of adding $10 \mathrm{~mL}$ of fresh $0.01 \mathrm{M} \mathrm{NaOH}$ to the centrifuge tube, vortexing for 3 sets of 15 seconds, centrifuging at $4500 \mathrm{rpm}$ for 10 minutes, decanting and then adding $10 \mathrm{~mL}$ of fresh $0.01 \mathrm{M} \mathrm{NaOH}$. These washing steps were recorded in laboratory record book BNW-60176.

After the last rinse, the $\mathrm{NaOH}$ and the pellet were vortexed, a small volume of the slurry was drawn up using a pipette, and a drop was placed on the top of an aluminum SEM sample stub. It was then placed in an oven at approximately $100^{\circ} \mathrm{C}$ to dry overnight. Once it was dry, it was coated with a thin layer of $\mathrm{Au} / \mathrm{Pd}$ using the Polaron Range plasma sputter coater in APEL/102. The Au/Pd layer was deposited using $800 \mathrm{~V}$ and $10 \mathrm{~mA}$ for $180 \mathrm{~s}$. The coated specimen was placed in an SEM sample holder and was placed in the SEM for analysis.

\section{Analysis}

Scanning electron micrographs were collected for informational purposes only using the Scan 4 option with 160 s collection times using the settings as seen below in Table 1. See Table 2 for information regarding the person that performed the analysis as well as the date of analysis. Images at the four magnifications listed in Table 1 were compiled in a $2 \times 2$ arrangement to show a macroscopic to microscopic view of the particles. See APPENDIX A: Micrograph Collages.

\section{Results}

The micrographs collected for this report compare the morphology of undissolved solids in the PEP slurry at the beginning of the PEP treatment process (B_FRPGM_001_XX_2713_XSP_4_B; "B_FRPGM" for short) and after having completed the oxidative leaching step (A_02AML_021_XX_2460_XSP_4; "A_02AML" for short). The differences between these specimens were readily apparent. Specimen B_FRPGM appeared to be single phase, and consisted of particles that were somewhat tabular and faceted, with sizes ranging from 1 to $10 \mu \mathrm{m}$ (due to similar, average atomic numbers for boehmite and gibbsite, there is not a readily apparent difference in contrast or brightness between them when imaged in an SEM). Specimen A_02AML appeared to consist of two kinds of particles that were in somewhat of a bimodal size distribution. The smaller particles were approximately $180 \mathrm{~nm}$ in apparent diameter, and were equiaxed. The larger particles had distinct facets, and were approximately $1 \mu \mathrm{m}$ in apparent 
diameter. Morphologically, the larger particles appeared similar to the particles in B_FRPGM. Based on XRD results from a separate report, specimen B_FRPGM consisted of $51.6 \mathrm{wt} \%$ boehmite and $32.1 \mathrm{wt} \%$ gibbsite (balance was amorphous), and specimen A_02AML consisted of $38.4 \mathrm{wt} \%$ boehmite, and $1.1 \mathrm{wt} \%$ hematite (balance was amorphous). Thus, the micron-scale, faceted particles in A_02AML might be residual, crystalline boehmite (orthorhombic crystal structure) that remained after the treatment process, whereas the sub-micron, equiaxed particles could be the large fraction of amorphous material ( $\sim 80 \%$, as indicated by XRD analysis) which was the product from the PEP process. More definitive chemical information about these different particles is possible using energy dispersive spectroscopy, which is available, if desired. One can conclude that the PEP treatment process had a dramatic impact on the size, shape, composition, and the atomic structure (crystalline vs amorphous) of the undissolved solids in the slurry that was tested.

Table 1. Parameters used for capturing images.

\begin{tabular}{|l|l|l|l|l|l|}
\hline Sample ID & $\begin{array}{l}\text { Voltage } \\
(\mathrm{KV})\end{array}$ & $\begin{array}{l}\text { Spot } \\
\text { Size }\end{array}$ & Detector & $\begin{array}{l}\text { Working } \\
\text { Distance } \\
(\mathrm{mm})\end{array}$ & Magnification \\
\hline B_FRPGM_001_XX_2713_XSP_4_B & 20 & 30 & SEI & 12 & $1,000 \times$ \\
\hline B_FRPGM_001_XX_2713_XSP_4_B & 20 & 30 & SEI & 12 & $2,500 \times$ \\
\hline B_FRPGM_001_XX_2713_XSP_4_B & 20 & 25 & SEI & 12 & $5,000 \times$ \\
\hline B_FRPGM_001_XX_2713_XSP_4_B & 20 & 25 & SEI & 12 & $10,000 \times$ \\
\hline A_02AML_021_XX_2460_XSP_4 & 15 & 24 & BSE & 10 & $1,000 \times$ \\
\hline A_02AML_021_XX_2460_XSP_4 & 15 & 24 & BSE & 10 & $2,500 \times$ \\
\hline A_02AML_021_XX_2460_XSP_4 & 15 & 24 & BSE & 10 & $5,000 \times$ \\
\hline A_02AML_021_XX_2460_XSP_4 & 15 & 24 & BSE & 10 & $10,000 \times$ \\
\hline
\end{tabular}

Table 2. Analysis information.

\begin{tabular}{|l|l|l|l|}
\hline Sample ID & $\begin{array}{l}\text { Date of } \\
\text { Analysis }\end{array}$ & $\begin{array}{l}\text { Analysis } \\
\text { Performed By }\end{array}$ & Signature \\
\hline B_FRPGM_001_XX_2713_XSP_4_B & $03 / 05 / 2009$ & Brian Riley & \\
\hline A_02AML_021_XX_2460_XSP_4 & $03 / 18 / 2009$ & Brad Johnson & \\
\hline
\end{tabular}




\section{APPENDIX A: Micrograph Collages}

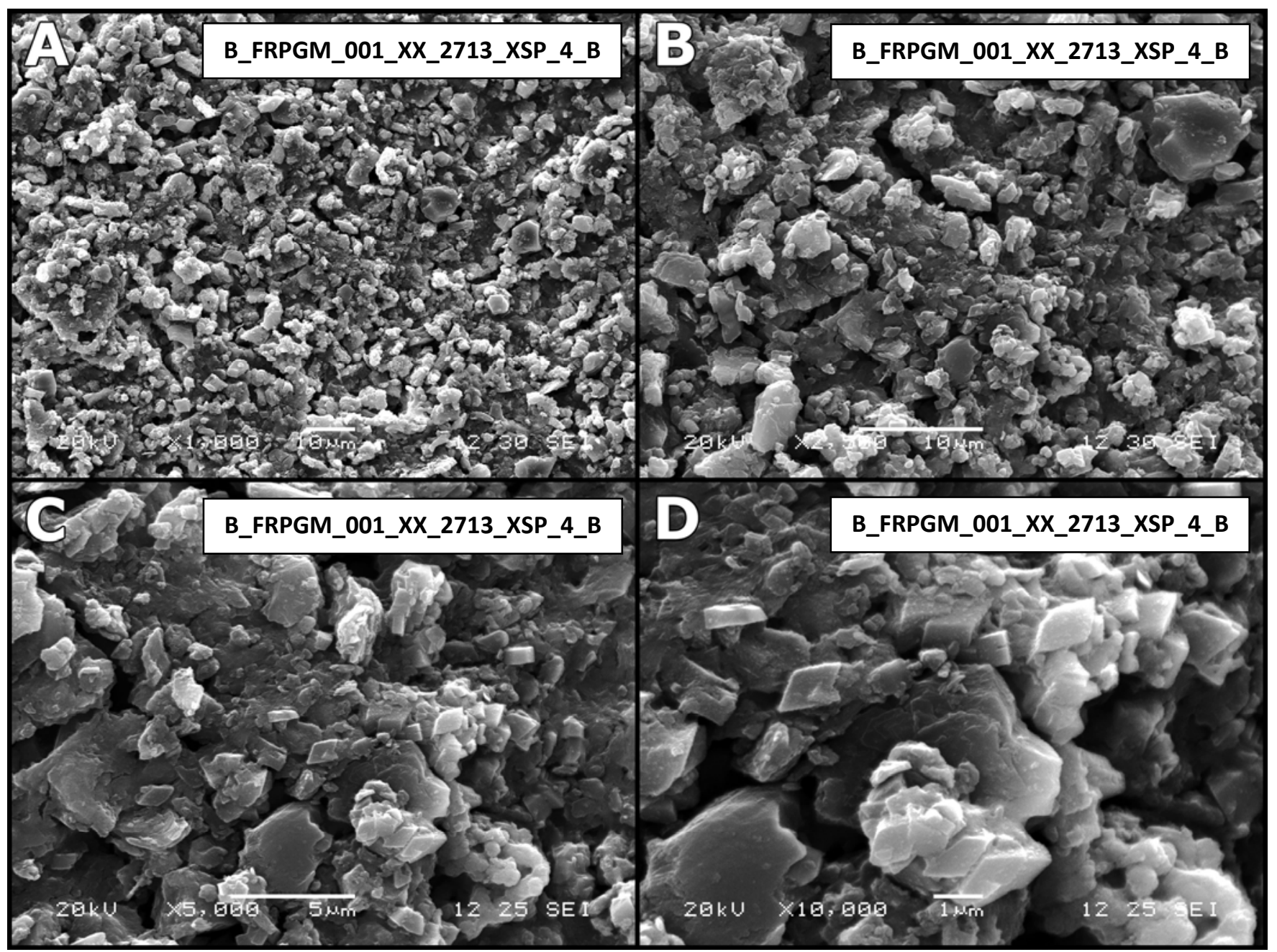

SEM micrographs from Pretreatment Engineering Platform (PEP) specimen

B FRPGM 001 XX 2713 XSP 4 B. The specimen, while in the original $50 \mathrm{~mL}$ centrifuge tube, was rinsed with $0.01 \mathrm{M} \mathrm{NaOH}$, vortexed, centrifuged ( $4500 \mathrm{rpm}$ for 10 minutes), and decanted $3 \times$ to clean off the nitrates. After the final rinse, the contents of the tube were vortexed in order to resuspend the pellet, and a drop of the suspended particles was placed on an Al SEM stub and placed in an oven at approximately $105^{\circ} \mathrm{C}$ to dry overnight. Once dry, the specimen was coated with $\mathrm{Au} / \mathrm{Pd}$ and analyzed using the APEL/102 SEM (JEOL 5900).

Figure 1. Scanning electron micrographs collected for informational purposes only on B_FRPGM_001_XX_2713_XSP_4_B at different magnifications 


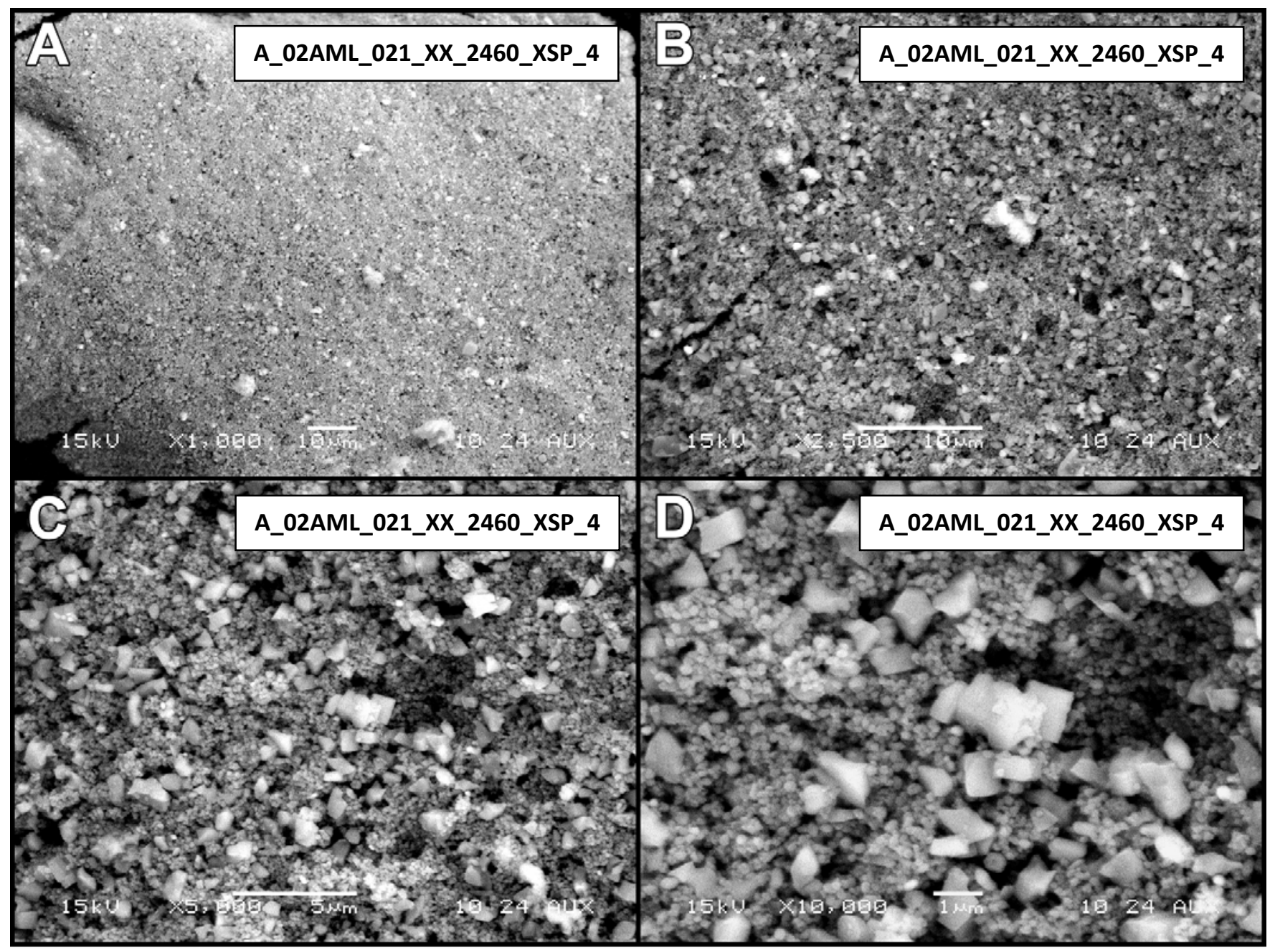

SEM micrographs from Pretreatment Engineering Platform (PEP) specimen

A_02AML_021_XX_2460_XSP_4. The specimen, while in the original $50 \mathrm{~mL}$ centrifuge tube, was rinsed with $0.01 \mathrm{M} \mathrm{NaOH}$, vortexed, centrifuged (4500 rpm for 10 minutes), and decanted $3 \times$ to clean off the nitrates. After the final rinse, the contents of the tube were vortexed in order to resuspend the pellet, and a drop of the suspended particles was placed on an Al SEM stub and placed in an oven at approximately $105^{\circ} \mathrm{C}$ to dry overnight. Once dry, the specimen was coated with $\mathrm{Au} / \mathrm{Pd}$ and analyzed using the APEL/102 SEM (JEOL 5900).

Figure 2. Scanning electron micrographs collected for informational purposes only on A_02AML_021_XX_2460_XSP_4 at different magnifications 


\section{H.4 X-Ray Diffraction}

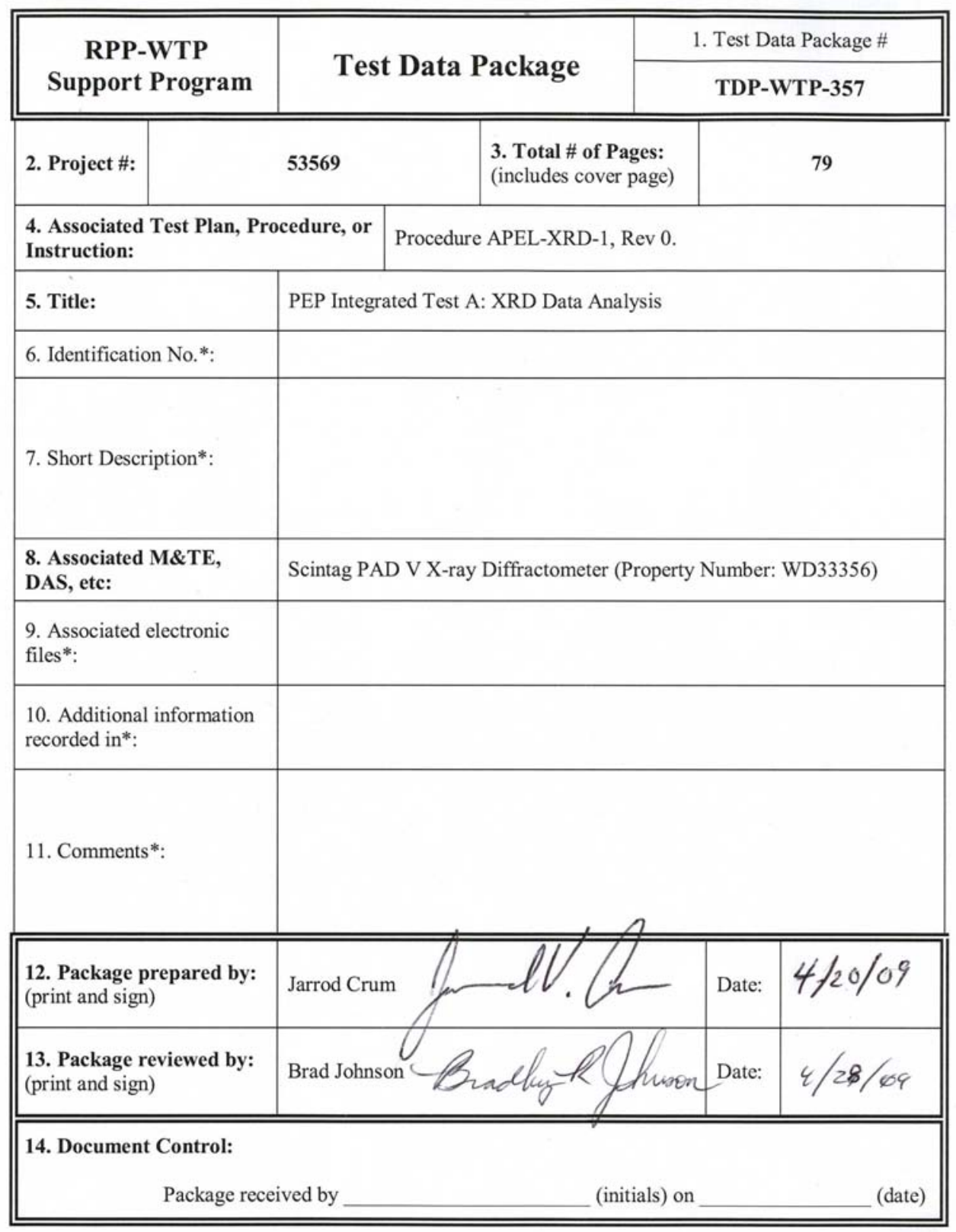

* Optional Information

QA-F1705-01, Rev. 0 
DOCUMENT REVIEW RECORD

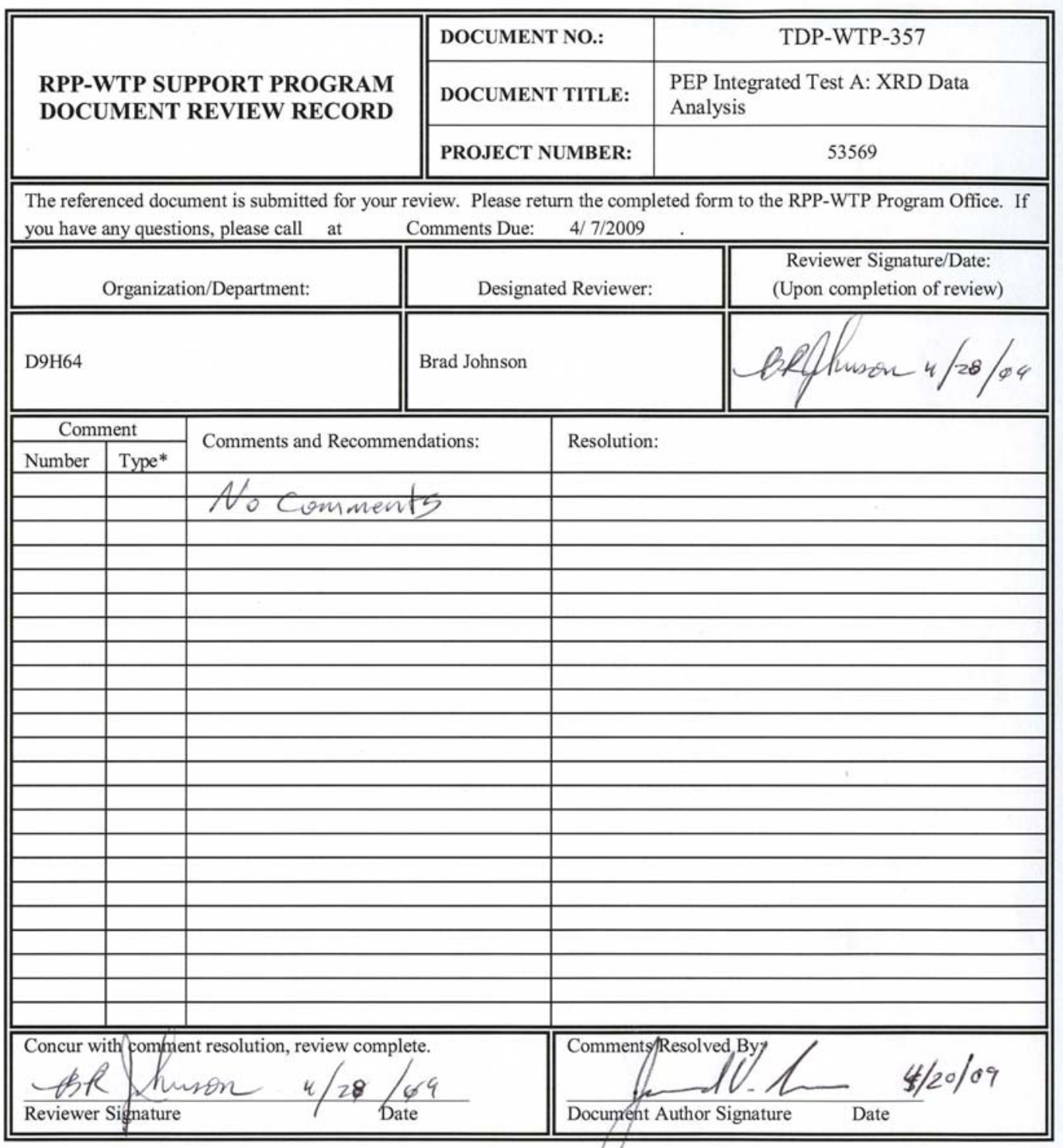

*Type: E - Editorial, addresses word processing errors that do not adversely impact the integrity of the document.

O- Optional, comment resolution would provide clarification, but does not impact the integrity of the document QA-F0604-01, Rev. 0 
Date:

March 13, 2009

Project No.:

53569

To: Ofelia Bredt

Internal

From: Jarrod Crum

Distribution:

Subject: PEP XRD Analyses

X-ray diffraction analysis was performed on a series of specimens in support of experimental work for the Pretreatment Engineering Platform tests. Sample analysis was carried out by the Pacific Northwest National Laboratory Advanced Processing and Application Group. The XRD instrument used was the Scintag PAD V X-ray Diffractometer, Property Number WD33356, located in lab 102 in the APEL building. The data range for the sample was $5^{\circ}$ to $80^{\circ}$ 2-theta, with a step size of $0.04^{\circ} 2$-theta and count time $2.0-4.0$ seconds per step. Copper $\mathrm{K} \alpha \mathrm{X}$-rays were used. The X-ray tube operating conditions were $45 \mathrm{kV}$ and $40 \mathrm{~mA}$.

\section{Sample Prep:}

The as-received samples were rinsed 3 times with a $0.01 \mathrm{M} \mathrm{NaOH}$ solution. Rinsing consisted of four steps: adding rinsate, votexing, centrifuging, and decanting of the rinsate. The process was repeated three times. The samples were then dried at $105^{\circ} \mathrm{C}$ overnight to drive off all liquid. The dried powder was placed into a tungsten carbide milling chamber and milled for $1 \mathrm{~min}$ in the angstrom shaker mill. A portion of the powder was weighted out to the nearest hundredth of a milligram. Then SRM 674b, $\mathrm{TiO}_{2}$ internal standard, was spiked in at a known mass, given in Table 1, with the dried powder and milled again for 2 min to insure a homogenous mixture of the two powders. The sample was then mounted into a sample holder. Replications were made and then summed for better counting statistics; the number of replications of each sample are shown in Table I.

Table I: Samples Examined in this Series

\begin{tabular}{|l|c|c|c|c|}
\hline Identification & XRD File ID & $\begin{array}{c}\text { Count Time per Step, } \\
\text { Seconds }\end{array}$ & $\begin{array}{c}\text { Number of } \\
\text { Replications }\end{array}$ & $\begin{array}{c}\text { Internal Standard, } \\
\text { wt }^{\text {TiO }} \text { T }^{2}\end{array}$ \\
\hline A 01AIM 003 16 1607 XSP 4 B & 020509B12 & 4 & 1 & 9.95 \\
\hline A 01AIM 13A 1880 XX XSP 4 B & 020509B10 & 4 & 1 & 8.85 \\
\hline A 01AIM 13A 16 1871 XSP 4 B & 020909b & 4 & 1 & 10.02 \\
\hline A 01AIM 13C 16 2124 XSP 4 B & 021309B & 4 & 1 & 8.92 \\
\hline A 01AIM 006 XX 1631 XSP 4 B & 021609A6 & 4 & 1 & 9.17 \\
\hline A 01AIM 13C XX 2134 XSP 4 B & 021609A7 & 4 & 1 & 8.94 \\
\hline B FRPGM 001 XX 2713 XSP 4 B & 031309A11 & 4 & 1 & 4.93 \\
\hline A 02AML 021 XX 2460 XSP 4 & 031709A11 & 4 & & 5.00 \\
\hline
\end{tabular}

Phase identification was done by use of the JADE search match routines (version 6.0, Materials Data Inc.) with comparison to the ICDD (International Centre for Diffraction Data) data base PDF-2 release 1999, which includes the ICSD (Inorganic Crystal Structure Database maintained by Fachinformationszentrum (FIZ), Karlsruhe, Germany). Searches were restricted to the PDF and ICSD Inorganic sections.

Table 2 summarizes the results of the XRD analysis to include; identified phases, mass \%, and crystallite size of each phase. Balance of material in the sample is assumed to be amorphous. Boehmite is the major phase 
Page 4 of 79

except in the first and last samples or the series. Gibbsite was only observed in the second to last sample analyzed.

Table 2. Measured Concentration and Estimated Crystallite Sizes of Identified Phases

\begin{tabular}{|c|c|c|c|c|c|c|c|}
\hline \multirow[t]{2}{*}{ Sample ID } & \multicolumn{2}{|c|}{ Boehmite } & \multicolumn{2}{|c|}{ Gibbsite } & \multicolumn{2}{|c|}{ Hematite } & \multirow[t]{2}{*}{ Other } \\
\hline & $\begin{array}{c}\text { Amount } \\
\text { (wt } \%)\end{array}$ & $\begin{array}{l}\text { Size } \\
(\AA)\end{array}$ & $\begin{array}{c}\text { Amount } \\
(w t \%)\end{array}$ & $\begin{array}{l}\text { Size } \\
(\AA)\end{array}$ & $\begin{array}{c}\text { Amount } \\
\text { (wt\%) }\end{array}$ & Size $(\AA)$ & \\
\hline A 01AIM 003161607 XSP 4 B & 48.5 & 336 & & & 2.9 & 609 & $\mathrm{FeOCl}$, trace \\
\hline A 01AIM 13A XX 1880 XSP 4 B & 47.4 & 336 & & & 3.9 & 609 & \\
\hline A 01AIM 13A 161871 XSP 4 B & 58.1 & 380 & & & 3.7 & 525 & \\
\hline A 01AIM 13C 162124 XSP 4 B & 49.3 & 335 & & & 1.6 & 555 & \\
\hline A 01AIM 006 XX 1631 XSP 4 B & 78.2 & 390 & & & 1.2 & 699 & \\
\hline A 01AIM 13C XX 2134 XSP 4 B & 51.2 & 336 & & & 2.2 & 751 & \\
\hline B FRPGM 001 XX 2713 XSP 4 B & 51.6 & 414 & 32.1 & 160 & & & \\
\hline A 02AML 021 XX 2460 XSP 4 & 38.4 & 554 & & & 1.1 & 750 & \\
\hline
\end{tabular}

Appendix A contains all of the individual reports for each sample analyzed. Individual reports contain information related to sample prep, scanning parameters, data plots, and PDF card information for phases identified.

Sincerely,

Jarrod Crum 
Page 5 of 79

Appendix A

Individual Reports of Each Sample

H.32 
Date: $\quad$ February 06, 2009

To: $\quad$ O. Bredt

From J. V. Crum

Subject: XRD Examination of: A_01AIM_003_16_1607_XSP_4_B

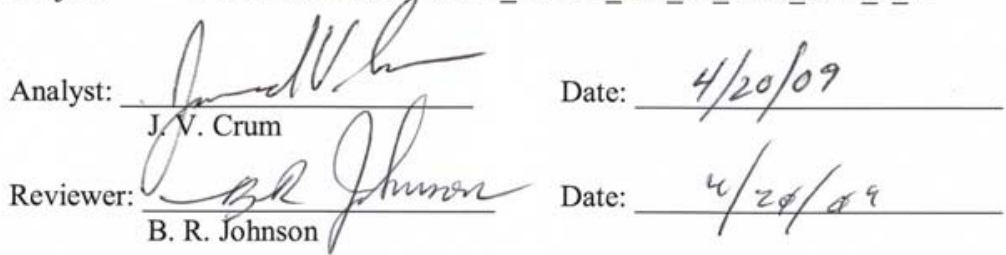

XRD data from the samples identified in Table I have been examined for identification of crystalline phases present.

Table I: Samples Examined in this Series

\begin{tabular}{|c|c|c|c|c|}
\hline Identification & XRD File ID $^{(a)}$ & $\begin{array}{c}\text { Count Time per } \\
\text { Step (Seconds) }\end{array}$ & $\begin{array}{c}\text { Number of } \\
\text { Replications }\end{array}$ & Internal Standard \\
\hline $\begin{array}{c}\text { A_01AIM_003_16_1607_- } \\
\text { XSP_4_B }\end{array}$ & 020509B12 & 4 & 1 & $9.95 \mathrm{wt} \% \mathrm{TiO}_{2}$ \\
\hline
\end{tabular}

(a) The letter appended to the XRD File ID identifies the first sample in the replication series. The letter has been dropped in subsequent text to indicate that the referenced file is the summation of data from replicate runs.

Sample analysis was carried out by the Pacific Northwest National Laboratory Advanced Processing and Application Group. The XRD instrument used was the Scintag PAD V X-ray Diffractometer, Property Number WD33356, located in lab 102 in the APEL building. The data range for the sample was $5^{\circ}$ to $80^{\circ}$ 2-theta, with a step size of $0.04^{\circ} 2$-theta and count time 4.0 seconds per step. Copper $\mathrm{K} \alpha \mathrm{X}$-rays were used. The X-ray tube operating conditions were $45 \mathrm{kV}$ and $40 \mathrm{~mA}$.

Sample 020509B12 was prepared with SRM 674b, $\mathrm{TiO}_{2}$ internal standard. The dried powder was placed into a tungsten carbide milling chamber and milled for $2 \mathrm{~min}$ in the angstrom shaker mill. The $\mathrm{TiO}_{2}$ standard was then spiked in at $9.95 \mathrm{wt} \%$ with the dried powder and milled again for $2 \mathrm{~min}$ to insure a homogenous mixture of the two powders. The sample was then mounted into standard sample holder.

Phase identification was done by use of the JADE search match routines (version 6.0, Materials Data Inc.) with comparison to the ICDD (International Centre for Diffraction Data) data base PDF-2 release 1999, which includes the ICSD (Inorganic Crystal Structure Database maintained by Fachinformationszentrum (FIZ), Karlsruhe, Germany).

The phase identification plots are shown using "stick figures" to indicate the phases present; peak positions and line intensities. The phase showing the greatest peak intensity is shown at the top of the stick figures display (except the internal standard, if used, is always the topmost). Phases present at lower peak area/height are shown in decreasing order down the display. 
The identified phases, ranked by relative peak intensities, are given below for each sample. Figure 1 shows the raw summed pattern. The background fitted pattern, along with the identified phases, are shown in figures 2,3 , and 4 .

A_01AIM_003_16_1607_XSP_4_B, File: 020509B12

- Rutile, $\overline{\mathrm{TiO}}_{2}$, card\# 21-1276

- Boehmite, syn, $\mathrm{AlO}(\mathrm{OH})$, card\# 21-1307, Excellent overall fit. Peak locations and intensities are a good match to the pattern.

- Hematite, syn, $\mathrm{Fe}_{2} \mathrm{O}_{3}$, card\# 64-6101, good fit as a minor phase.

- Iron(III) oxide chloride, $\mathrm{FeOCl}$, card\# 61-8704, possible fit to trace phase.

- Amorphous material, $36^{\circ} 2 \theta$.

The pattern was also examined using RIQAS (release 4.0.0.26, 6/10/2002, Materials Data Inc.) rietveld analysis software. The phases identified above were input into the analysis along with a cubic spline function for the background and an amorphous hump at $\sim 36^{\circ} 2 \theta$, with fitted pattern shown in figure 5 .

The results of the analysis for the crystalline phases are:

- Boehmite, $\mathrm{AlO}(\mathrm{OH}), 48.5 \mathrm{wt} \%$, with a crystallite size of $336 \AA$

- Hematite, $\mathrm{Fe}_{2} \mathrm{O}_{3}, 2.9$ wt \%, with a crystallite size of $609 \AA$

- The balance of the material is amorphous<smiles>[AlH2]=C1CCCCC1</smiles> 
8
$\vdots 0$
$\infty$
8
8
8
0

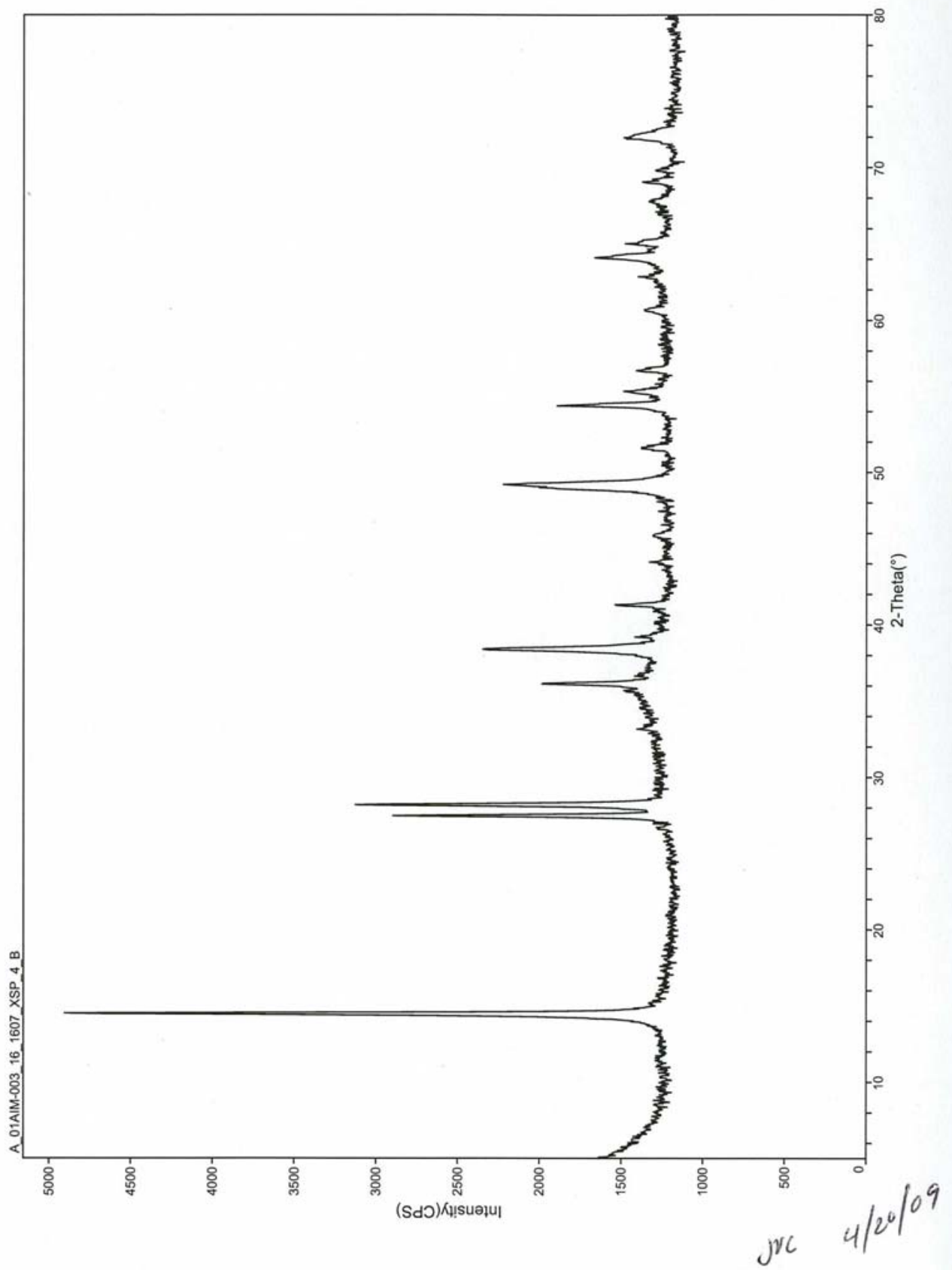

H.35 


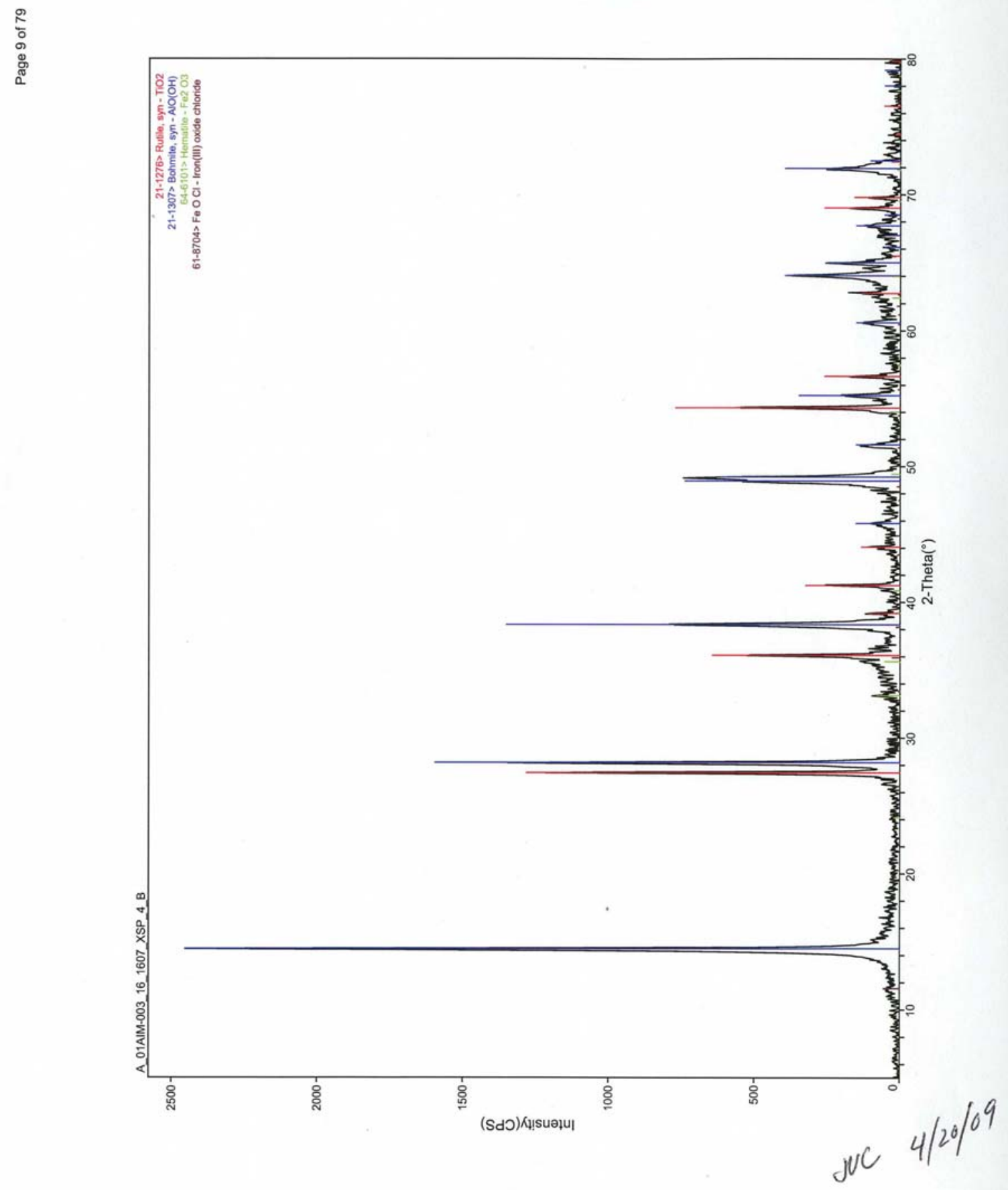

H.36 
$\frac{2}{5}$
$\frac{1}{0}$
$\frac{0}{8}$
$\frac{8}{0}$

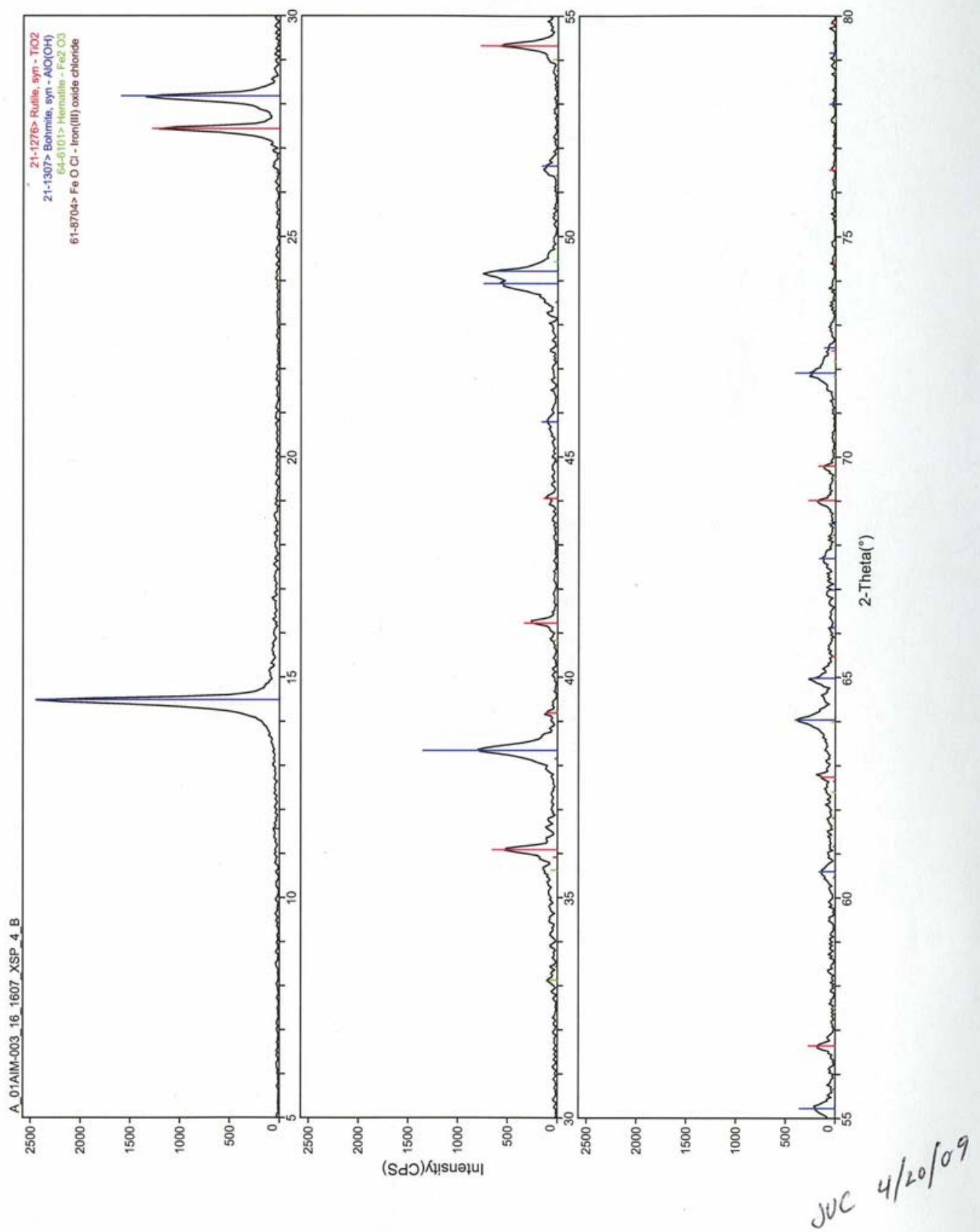


$\frac{8}{2}$
$\frac{8}{0}$
$\frac{1}{8}$
8
0

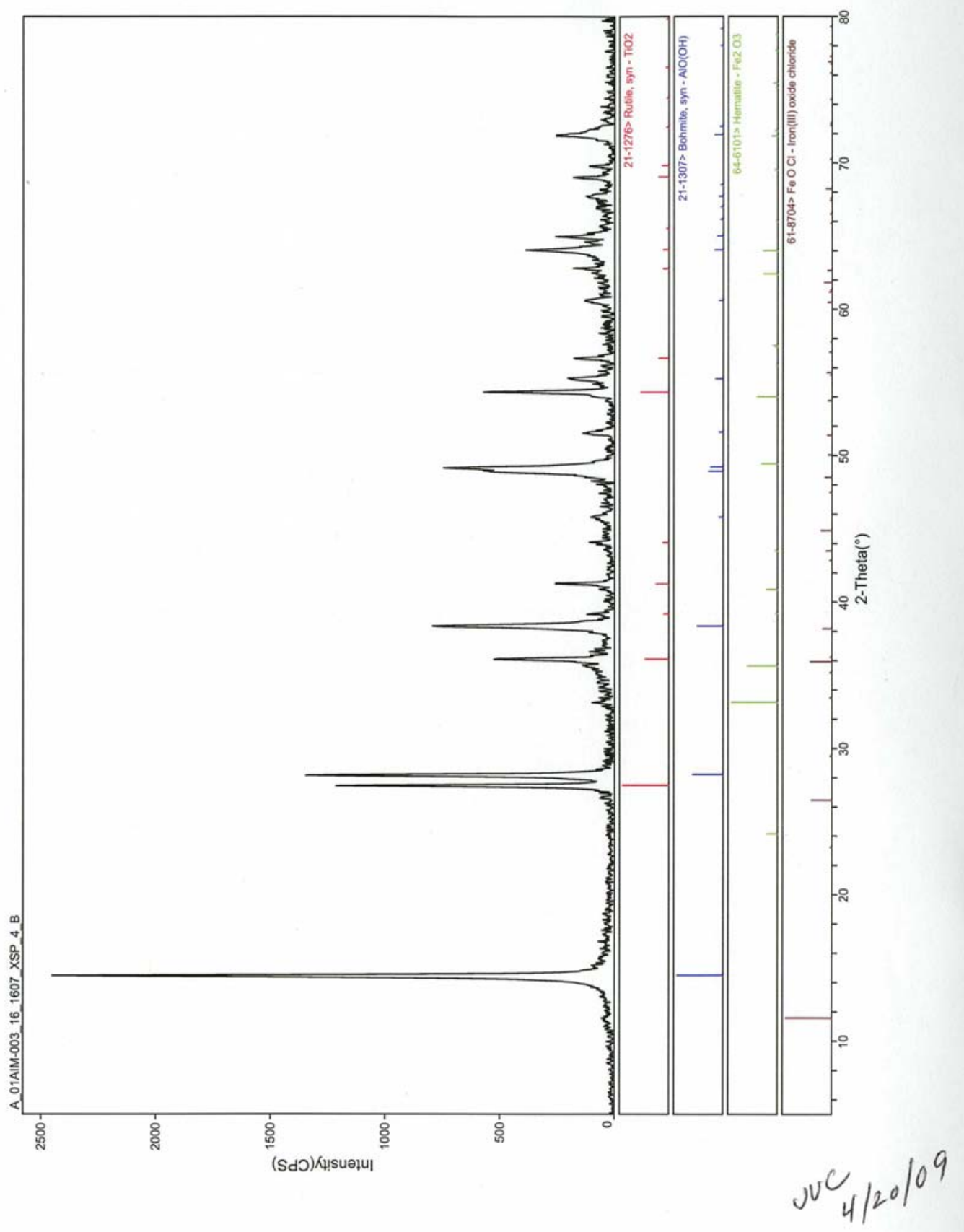




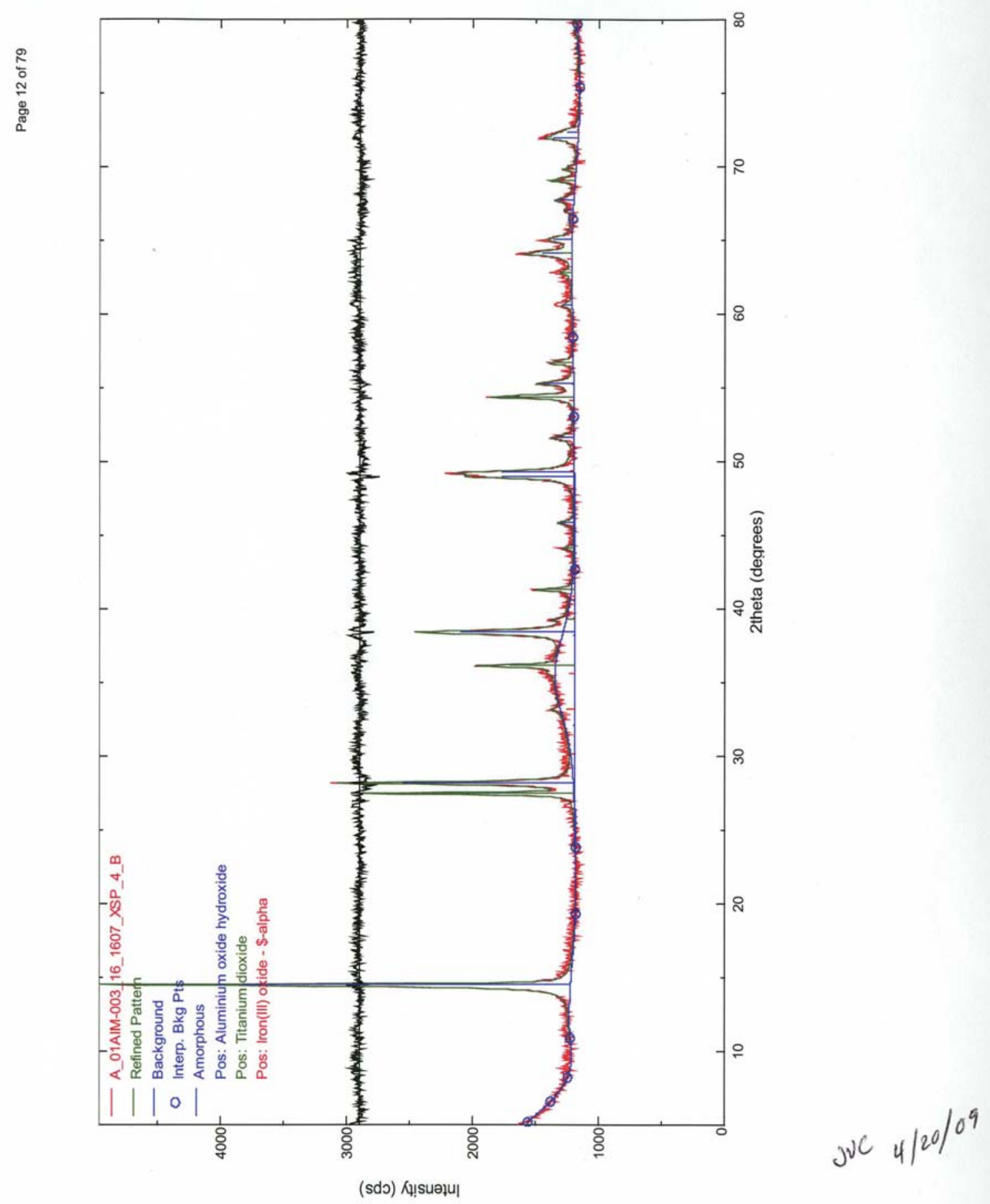


Page 13 of 79

Appendix A2

ICDD cards of interest for this sample

H.40 
- $\mathrm{PDF} \# 21-1276: \mathrm{QM}=$ Common $(+)$; $\mathrm{d}=$ Other/Unknown; $\mathrm{I}=($ Unknown $)$

Rutile, syn

$\mathrm{TiO} 2$

Radiation $=$ CuKal Lambda $=1.5406$ Filter $=$

Calibration $=\quad \mathrm{d}-$ Cutoff $=\quad \mathrm{I} / \mathrm{Ic}(\mathrm{RIR})=3.4$

Ref: Level-1 PDF

Tetragonal, $\mathrm{P} 42 / \mathrm{mnm}(136) \quad \mathrm{Z}=2 \quad \mathrm{mp}=$

CELL: $4.5933 \times 4.5933 \times 2.9592<90.0 \times 90.0 \times 90.0>\quad$ P.S $=$

Density $(\mathrm{c})=4.23$ Density $(\mathrm{m})=\quad \mathrm{Mwt}=\quad \mathrm{Vol}=62.4$

Ref: Ibid.

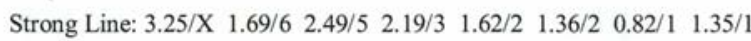

- $\mathrm{PDF} \# 21-1307$ : $\mathrm{QM}=$ Common $(+)$; $\mathrm{d}=$ Other/Unknown; $\mathrm{I}=($ Unknown)

Boehmite, syn

$\mathrm{AlO}(\mathrm{OH})$

Radiation $=$ CuKal $\quad$ Lambda $=1.5406$ Filter $=$

Calibration $=\quad \mathrm{d}-$ Cutoff $=\quad \mathrm{I} / \mathrm{Ic}(\mathrm{RIR})=$

Ref: Level-1 PDF

Orthorhombic, Amam(63) $\quad \mathrm{Z}=4 \quad \mathrm{mp}=$

CELL: $3.7 \times 12.227 \times 2.868<90.0 \times 90.0 \times 90.0>\quad$ P.S $=$

Density $(\mathrm{c})=3.07$ Density $(\mathrm{m})=\quad \mathrm{Mwt}=\quad \mathrm{Vol}=129.8$

Ref: Ibid.

Strong Line: $6.11 / \mathrm{X} \quad 3.16 / 7 \quad 2.35 / 6 \quad 1.86 / 3 \quad 1.85 / 3 \quad 1.45 / 2 \quad 1.31 / 2 \quad 1.66 / 1$

- $\mathrm{PDF} \# 64-6101: \mathrm{QM}=$ Calculated; $\mathrm{d}=$ Other/Unknown; $\mathrm{I}=($ Unknown)

Hematite - synthetic, Hematite group

$\mathrm{Fe} 2 \mathrm{O} 3$

Radiation $=\mathrm{CuKa} 1 \quad$ Lambda $=1.5406$ Filter $=$

Calibration $=\quad \mathrm{d}-$ Cutoff $=\quad \mathrm{I} / \mathrm{Ic}(\mathrm{RIR})=3.04$

Ref: Calculated from FIZ\#82134 (ICSD @08/01/01) by Jade 6.x

Hexagonal, R-3c(167) $\quad \mathrm{Z}=6 \quad \mathrm{mp}=$

CELL: $5.037 \times 5.037 \times 13.771<90.0 \times 90.0 \times 120.0>$ P.S $=$

Density $(\mathrm{c})=5.258$ Density $(\mathrm{m})=\quad \mathrm{Mwt}=\quad \mathrm{Vol}=302.6$

Ref: Ibid.

Strong Line: $2.70 / \mathrm{X} \quad 2.52 / 6 \quad 1.70 / 4 \quad 1.84 / 3 \quad 1.45 / 3 \quad 1.49 / 3 \quad 2.21 / 2 \quad 3.68 / 2$

NOTE: Effect of mechanical activation on the real structure and reactivity of iron(III) oxide with corundum-type structure, Journal of Solid State Chemistry [JSSCB] 123 (1996) 191-202, Sadykov V A, Isupova L A, Tsybulya S V, Cherepanova S V, Litvak G S, Burgina E B, Kustova G N, Kolomiichuk V N, Ivanov V P, Paukshtis E A, Golovin A V, Avvakumov E G, X-ray diffraction (powder).

$\mathrm{R}=0.053$

- PDF\#61-8704: QM=Calculated; $\mathrm{d}=\mathrm{O}$ ther/Unknown; $\mathrm{I}=($ Unknown $)$

Iron(III) oxide chloride

$\mathrm{Fe} \mathrm{OCl}$

Radiation $=$ CuKal $\quad$ Lambda $=1.5406$ Filter $=$

Calibration $=\quad$ d-Cutoff $=\quad$ I/Ic(RIR $)=5.34$

Ref: Calculated from FIZ\#37158 (ICSD @08/01/01) by Jade 6.x

Orthorhombic, Pmnm(59)Z=2

$\mathrm{mp}=$ 
CELL: $3.75 \times 7.65 \times 3.3<90.0 \times 90.0 \times 90.0>$

$\operatorname{Density}(\mathrm{c})=3.764$ Density $(\mathrm{m})=\quad \mathrm{Mwt}=\quad \mathrm{Vol}=94.7$

Ref: Ibid.

$\begin{array}{llllllll}\text { Strong Line: } 7.65 / X & 2.50 / 4 & 3.37 / 4 & 2.02 / 2 & 2.36 / 2 & 1.50 / 1 & 1.88 / 1 & 1.37 / 1\end{array}$

NOTE: Etude de quelques derives de l'oxyde ferrique ( $\mathrm{Fe} \mathrm{O} . \mathrm{OH}, \mathrm{FeO} 2 \mathrm{Na}, \mathrm{FeOCl}$ ) determination de leurs structures.,

Bulletin de la Societe Francaise de Mineralogie (-71,1948) [BSFMA] 58 (1935) 6-6, Goldsztaub M S, Dx=3.55.

At least one temperature factor missing is in the paper.

Calculated density is unusual, but tolerable.

No $\mathrm{R}$ value given in the paper. 
Date: $\quad$ February 06, 2009

To: $\quad$ O. Bredt

From J. V. Crum

Subject: XRD Examination of: A_01AIM_13a_XX_1880_XSP_4_B

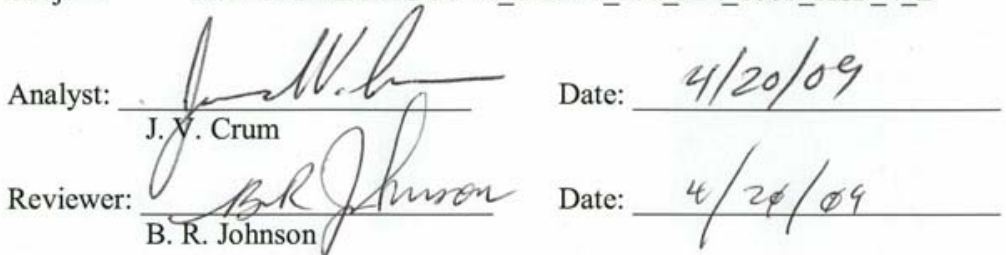

XRD data from the samples identified in Table I have been examined for identification of crystalline phases present.

Table I: Samples Examined in this Series

\begin{tabular}{|c|c|c|c|c|}
\hline Identification & XRD File ID $^{(\mathrm{a})}$ & $\begin{array}{c}\text { Count Time per } \\
\text { Step (Seconds) }\end{array}$ & $\begin{array}{c}\text { Number of } \\
\text { Replications }\end{array}$ & Internal Standard \\
\hline $\begin{array}{c}\text { A_01AIM_13a_1880_XX_- } \\
\text { XSP_4_B }\end{array}$ & 020509B10 & 4 & 1 & $8.85 \mathrm{wt} \% \mathrm{TiO}_{2}$ \\
\hline
\end{tabular}

(a) The letter appended to the XRD File ID identifies the first sample in the replication series. The letter has been dropped in subsequent text to indicate that the referenced file is the summation of data from replicate runs.

Sample analysis was carried out by the Pacific Northwest National Laboratory Advanced Processing and Application Group. The XRD instrument used was the Scintag PAD V X-ray Diffractometer, Property Number WD33356, located in lab 102 in the APEL building. The data range for the sample was $5^{\circ}$ to $80^{\circ}$ 2 -theta, with a step size of $0.04^{\circ} 2$-theta and count time 4.0 seconds per step. Copper $\mathrm{K} \alpha \mathrm{X}$-rays were used. The X-ray tube operating conditions were $45 \mathrm{kV}$ and $40 \mathrm{~mA}$.

Sample 020509B10 was prepared with $\mathrm{SRM} 674 \mathrm{~b}, \mathrm{TiO}_{2}$ internal standard. The dried powder was placed into a tungsten carbide milling chamber and milled for $2 \mathrm{~min}$ in the angstrom shaker mill. $\mathrm{The}^{\mathrm{TiO}} \mathrm{S}_{2}$ standard was then spiked in at $8.85 \mathrm{wt} \%$ with the dried powder and milled again for $2 \mathrm{~min}$ to insure a homogenous mixture of the two powders. The sample was then mounted into standard sample holder.

Phase identification was done by use of the JADE search match routines (version 6.0, Materials Data Inc.) with comparison to the ICDD (International Centre for Diffraction Data) data base PDF-2 release 1999, which includes the ICSD (Inorganic Crystal Structure Database maintained by Fachinformationszentrum (FIZ), Karlsruhe, Germany). Searches were restricted to the PDF and ICSD Inorganic sections.

The phase identification plots are shown using "stick figures" to indicate the phases present; peak positions and line intensities. The phase showing the greatest peak intensity is shown at the top of the stick figures display (except the internal standard, if used, is always the topmost). Phases present at lower peak area/height are shown in decreasing order down the display. 
The identified phases, ranked by relative peak intensities, are given below for each sample. Figure 1 shows the raw summed pattern. The background fitted pattern, along with the identified phases, are shown in figures 2,3 , and 4 .

A_01AIM_13a_XX_1880_XSP_4_B, File: 020509B10

- Rutile, $\mathrm{TiO}_{2}$, card\# 21-1276

- Boehmite, syn, $\mathrm{AlO}(\mathrm{OH})$, card\# 21-1307, Excellent overall fit. Peak locations and intensities are a good match to the pattern.

- Hematite, syn, $\mathrm{Fe}_{2} \mathrm{O}_{3}$, card\# 64-6101, good fit as a minor phase.

- Iron(III) oxide chloride, $\mathrm{FeOCl}$, card\# 61-8704, possible fit to trace phase.

- Amorphous material, $\sim 36^{\circ} 2 \theta$.

The pattern was also examined using RIQAS (release 4.0.0.26, 6/10/2002, Materials Data Inc.) rietveld analysis software. The phases identified above were input into the analysis along with a cubic spline function for the background and an amorphous hump at $\sim 36^{\circ} 2 \theta$, with fitted pattern shown in figure 5 .

The results of the analysis for the crystalline phases are:

- Boehmite, $\mathrm{AlO}(\mathrm{OH}), 47.4 \mathrm{wt} \%$, with a crystallite size of $336 \AA$

- Hematite, $\mathrm{Fe}_{2} \mathrm{O}_{3}, 3.9 \mathrm{wt} \%$, with a crystallite size of $609 \AA$

- The balance of the material is amorphous

jue $4 / 20109$ 
9
0
40
$\infty$
$\$ 0$
8
8
0

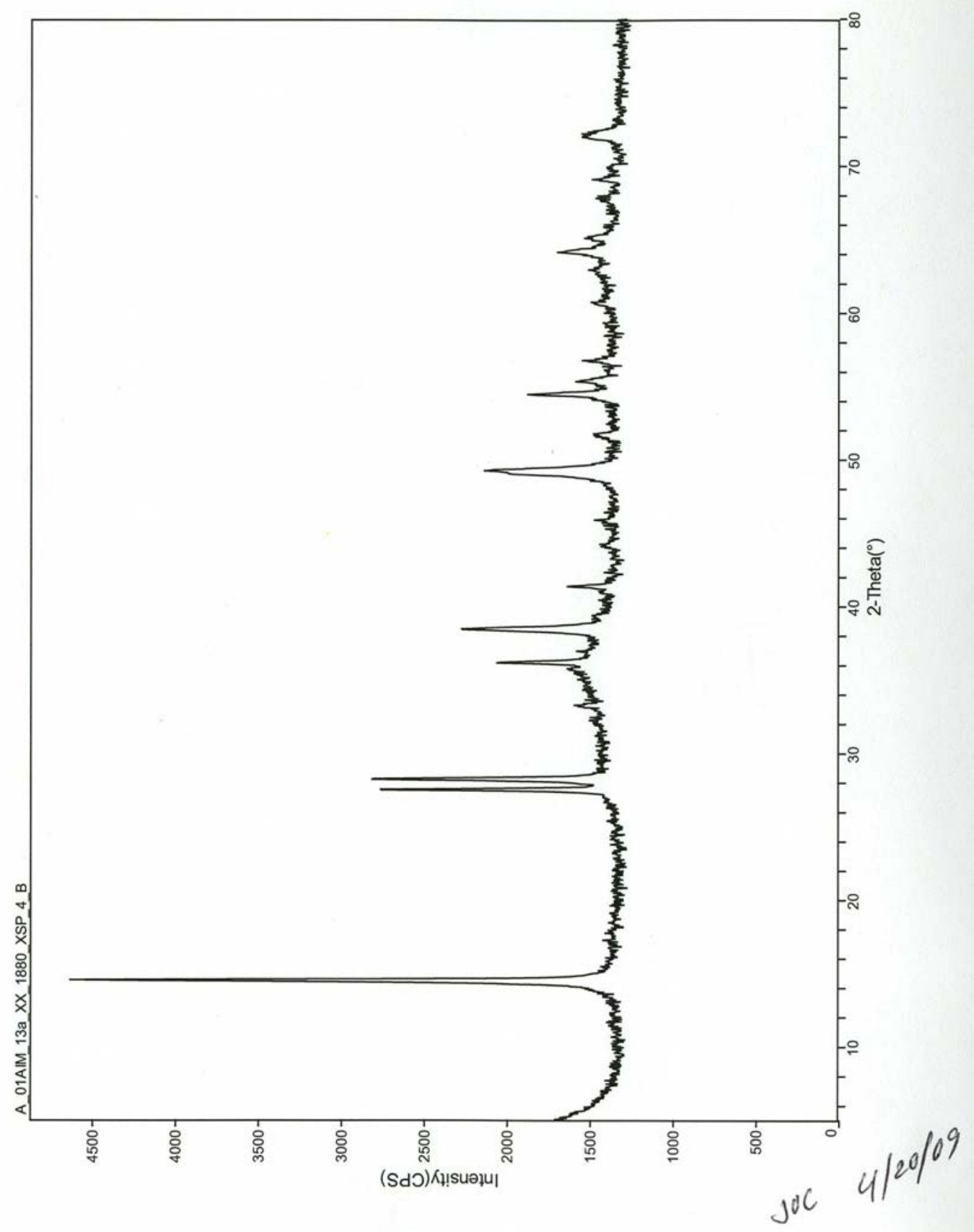

H.45 


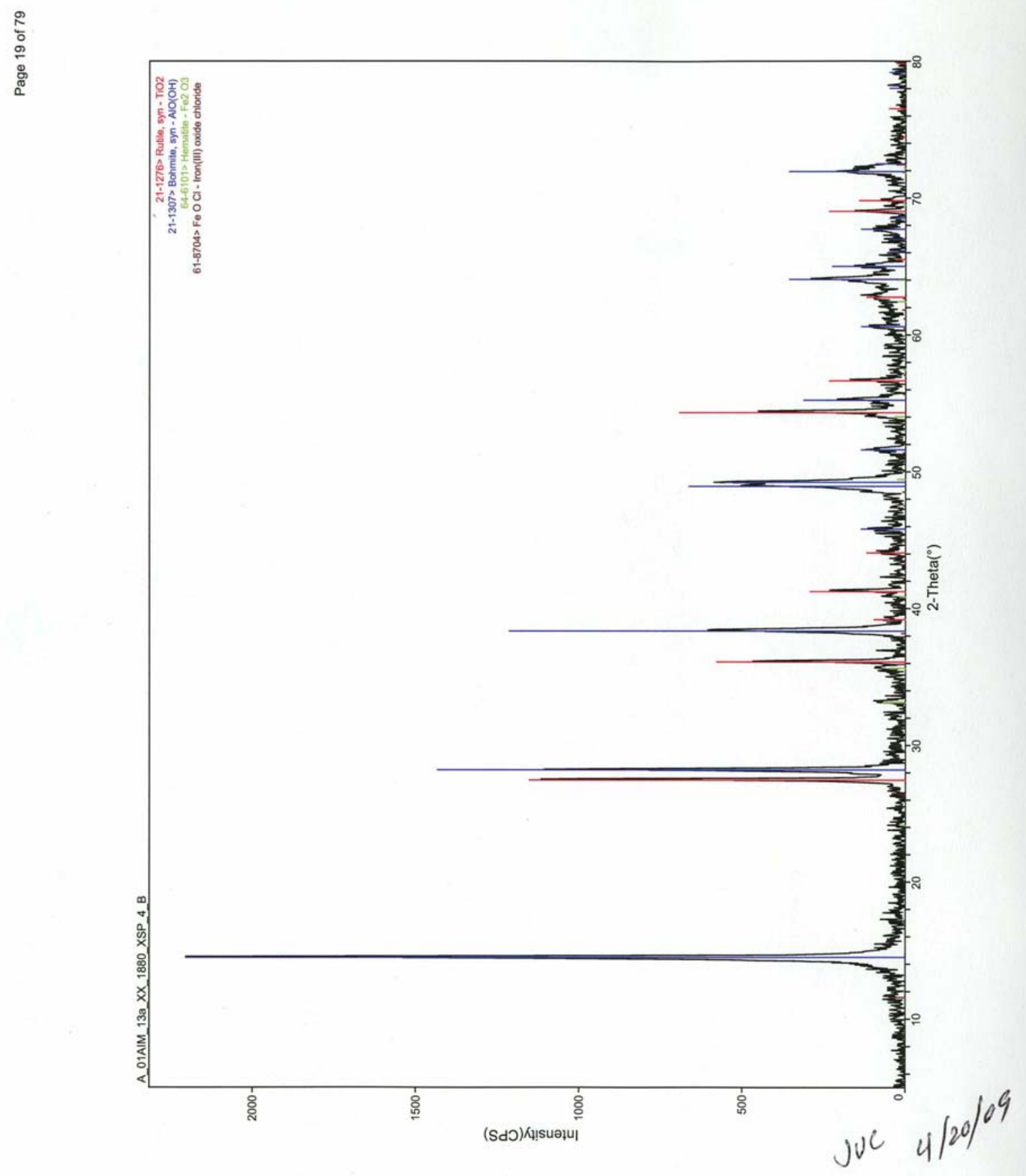

H.46 


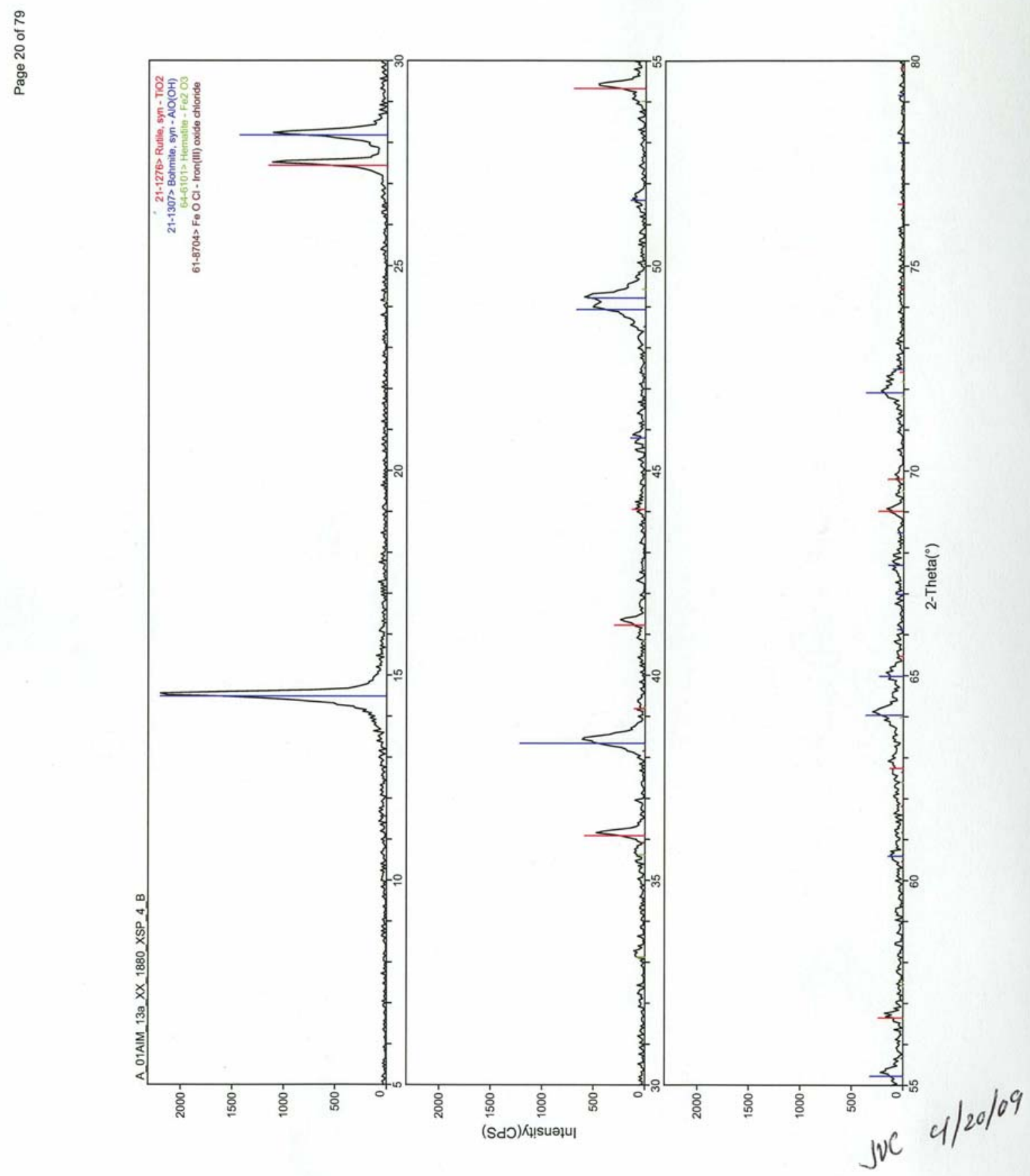


2
$\frac{2}{2}$
$\frac{0}{0}$
0
0
0

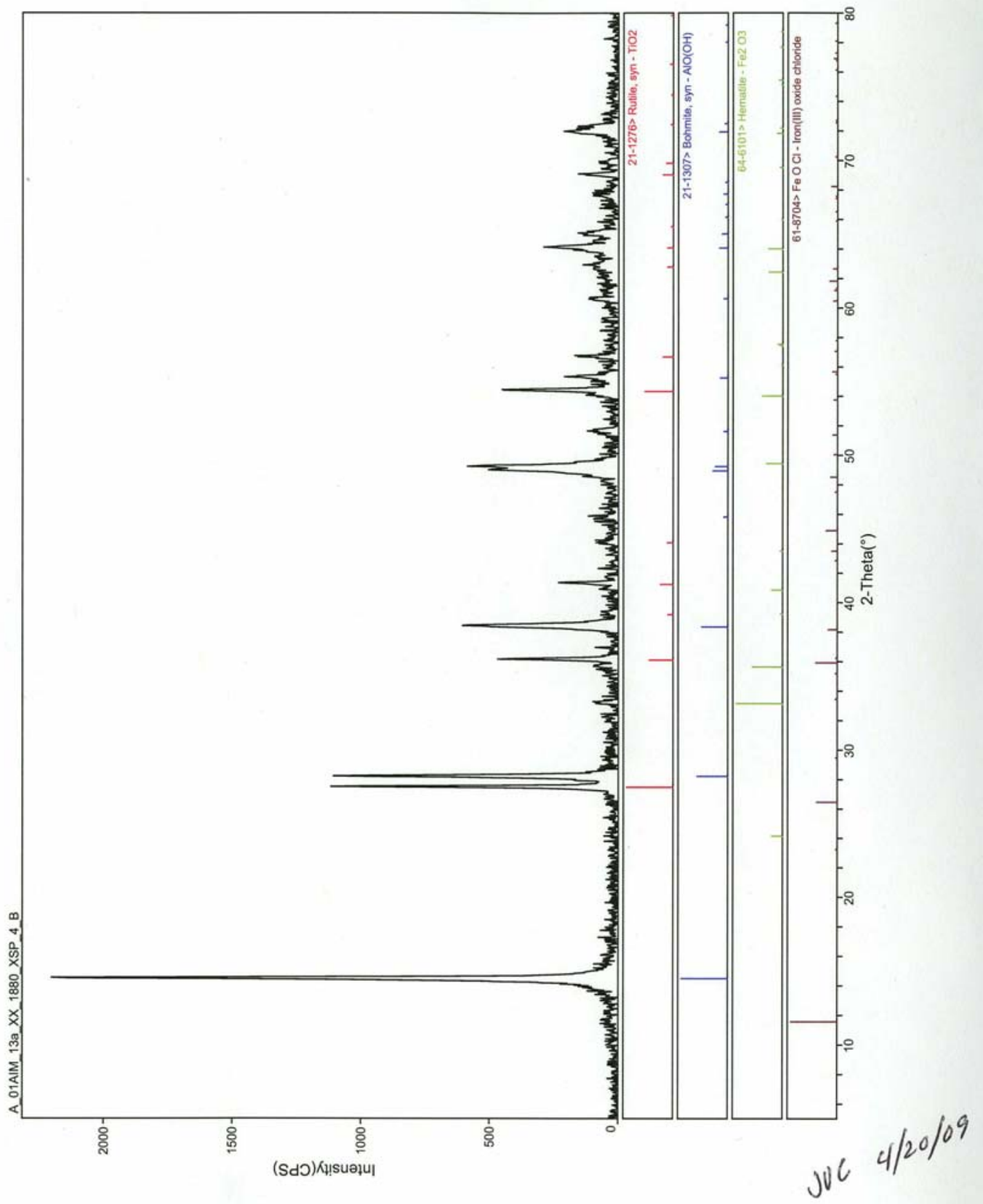




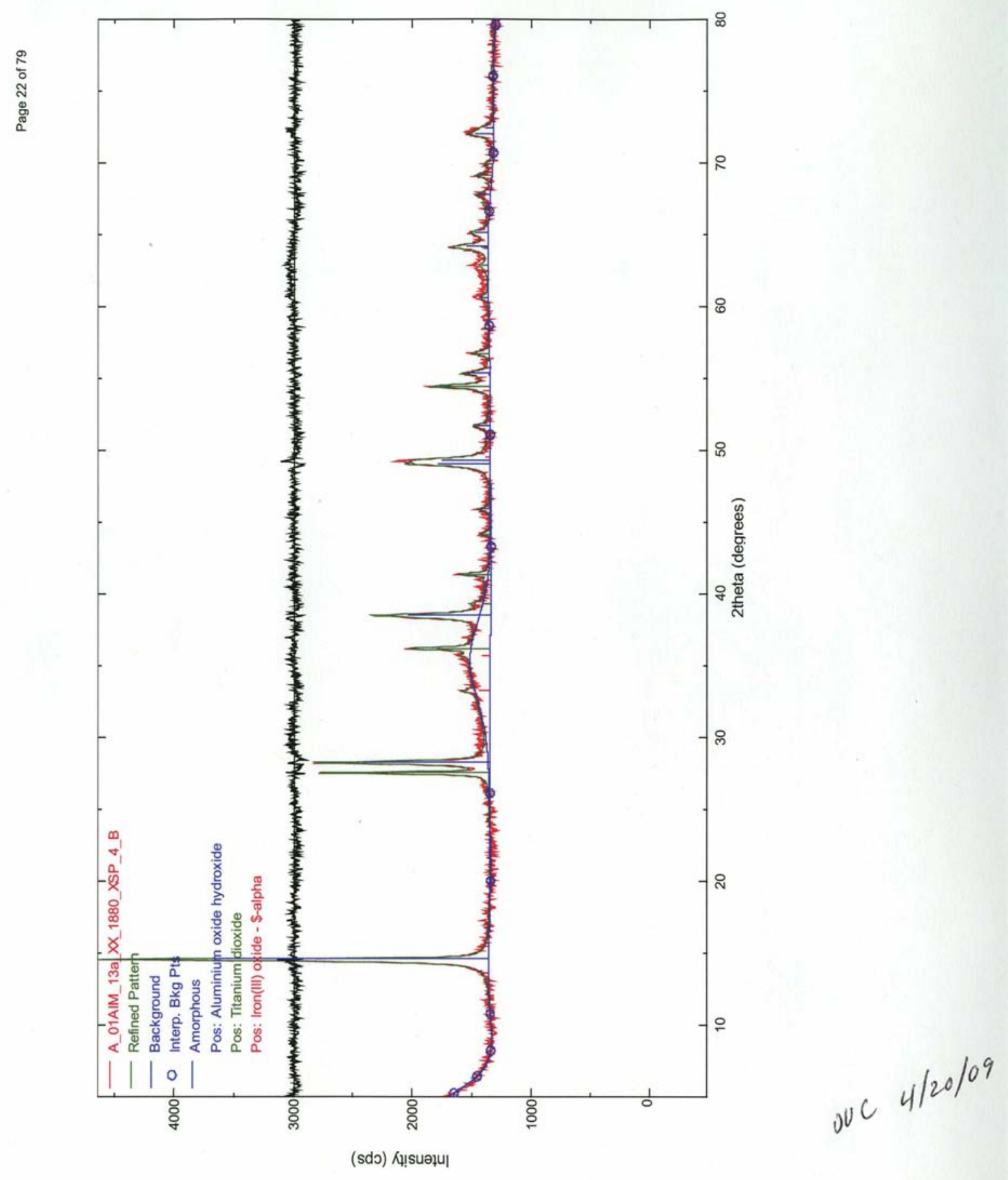


Page 23 of 79

\section{Appendix A3}

ICDD cards of interest for this sample 
- $\quad$ PDF\#21-1276: $\mathrm{QM}=$ Common(+); $\mathrm{d}=$ Other/Unknown; $\mathrm{I}=($ Unknown)

Rutile, syn

$\mathrm{TiO} 2$

Radiation $=\mathrm{CuKa} \quad$ Lambda $=1.5406$ Filter $=$

Calibration $=\quad \mathrm{d}-$ Cutoff $=\quad \mathrm{I} / \mathrm{Ic}(\mathrm{RIR})=3.4$

Ref: Level-1 PDF

Tetragonal, $\mathrm{P} 42 / \mathrm{mnm}(136) \quad \mathrm{Z}=2 \quad \mathrm{mp}=$

CELL: $4.5933 \times 4.5933 \times 2.9592<90.0 \times 90.0 \times 90.0>$

$\operatorname{Density}(\mathrm{c})=4.23$ Density $(\mathrm{m})=\quad \mathrm{Mwt}=\mathrm{Vol}=62.4$

Ref: Ibid.

Strong Line: $3.25 / \mathrm{X} \quad 1.69 / 6 \quad 2.49 / 5 \quad 2.19 / 3 \quad 1.62 / 2 \quad 1.36 / 2 \quad 0.82 / 1 \quad 1.35 / 1$

- $\quad$ PDF\#21-1307: $\mathrm{QM}=$ Common(+); $\mathrm{d}=$ Other/Unknown; $\mathrm{I}=($ Unknown)

Boehmite, syn

$\mathrm{AlO}(\mathrm{OH})$

Radiation $=$ CuKal Lambda $=1.5406$ Filter $=$

Calibration $=\quad \mathrm{d}-\mathrm{Cutoff}=\mathrm{I} / \mathrm{Ic}(\mathrm{RIR})=$

Ref: Level-1 PDF

Orthorhombic, $\operatorname{Amam}(63) \quad \mathrm{Z}=4 \quad \mathrm{mp}=$

CELL: $3.7 \times 12.227 \times 2.868<90.0 \times 90.0 \times 90.0>\quad$ P.S $=$

$\operatorname{Density}(\mathrm{c})=3.07$ Density $(\mathrm{m})=\quad \mathrm{Mwt}=\quad \mathrm{Vol}=129.8$

Ref: lbid.

Strong Line: $6.11 / \mathrm{X} \quad 3.16 / 7 \quad 2.35 / 6 \quad 1.86 / 3 \quad 1.85 / 3 \quad 1.45 / 2 \quad 1.31 / 2 \quad 1.66 / 1$

- PDF\#64-6101: QM=Calculated; $\mathrm{d}=$ Other/Unknown; $\mathrm{I}=($ Unknown)

Hematite - synthetic, Hematite group

$\mathrm{Fe} 2 \mathrm{O} 3$

Radiation $=$ CuKal Lambda $=1.5406$ Filter $=$

Calibration $=\quad \mathrm{d}-$ Cutoff $=\quad \mathrm{I} / \mathrm{Ic}(\mathrm{RIR})=3.04$

Ref: Calculated from FIZ\#82134 (ICSD@08/01/01) by Jade 6.x

Hexagonal, R-3c(167) $\mathrm{Z}=6 \quad \mathrm{mp}=$

CELL: $5.037 \times 5.037 \times 13.771<90.0 \times 90.0 \times 120.0>$ P.S $=$

$\operatorname{Density}(\mathrm{c})=5.258$ Density $(\mathrm{m})=\quad \mathrm{Mwt}=\quad \mathrm{Vol}=302.6$

Ref: Ibid.

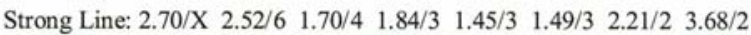

NOTE: Effect of mechanical activation on the real structure and reactivity of iron(III) oxide with corundum-type structure, Journal of Solid State Chemistry [JSSCB] 123 (1996) 191-202, Sadykov V A, Isupova L A, Tsybulya S V, Cherepanova S V, Litvak G S, Burgina E B, Kustova G N, Kolomiichuk V N, Ivanov V P, Paukshtis E A, Golovin A V, Avvakumov E G, X-ray diffraction (powder).

$\mathrm{R}=0.053$

- $\quad$ PDF\#61-8704: QM=Calculated; $\mathrm{d}=$ Other/Unknown; $\mathrm{I}=$ (Unknown)

Iron(III) oxide chloride

$\mathrm{Fe} \mathrm{OCl}$

Radiation $=$ CuKal Lambda $=1.5406$ Filter $=$

Calibration $=\quad \mathrm{d}-\mathrm{Cutoff}=\quad \mathrm{I} / \mathrm{Ic}(\mathrm{RIR})=5.34$

Ref: Calculated from FIZ\#37158 (ICSD @08/01/01) by Jade 6.x

Orthorhombic, $\operatorname{Pmnm}(59) \mathrm{Z}=2 \quad \mathrm{mp}=$ 
CELL: $3.75 \times 7.65 \times 3.3<90.0 \times 90.0 \times 90.0>$

P.S $=$

Density $(\mathrm{c})=3.764$ Density $(\mathrm{m})=\quad \mathrm{Mwt}=\quad \mathrm{Vol}=94.7$

Ref: Ibid.

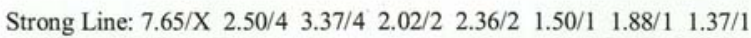

NOTE: Etude de quelques derives de l'oxyde ferrique ( $\mathrm{Fe} \mathrm{O} . \mathrm{OH}, \mathrm{FeO} 2 \mathrm{Na}, \mathrm{FeOCl})$ determination de leurs structures.,

Bulletin de la Societe Francaise de Mineralogie (-71,1948) [BSFMA] 58 (1935) 6-6, Goldsztaub M S, Dx=3.55.

At least one temperature factor missing is in the paper.

Calculated density is unusual, but tolerable.

No $R$ value given in the paper. 
Date:

February 09, 2009

To:

O. Bredt

From

J. V. Crum

Subject: XRD Examination of: A_01AIM_13a_16_1871_XSP_4_B

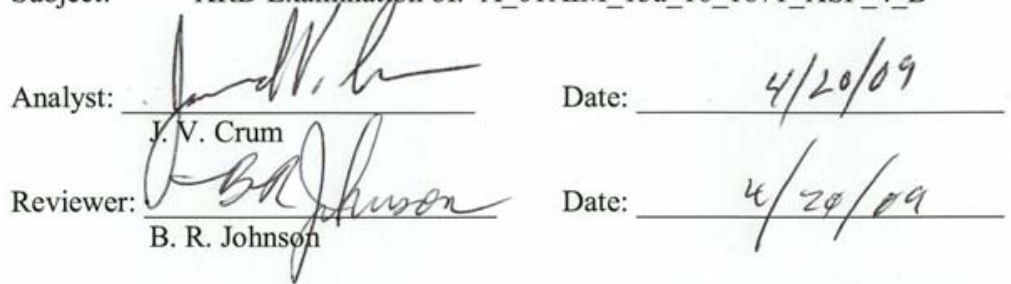

XRD data from the samples identified in Table I have been examined for identification of crystalline phases present.

Table I: Samples Examined in this Series

\begin{tabular}{|c|c|c|c|c|}
\hline Identification & XRD File ID $^{(a)}$ & $\begin{array}{c}\text { Count Time per } \\
\text { Step (Seconds) }\end{array}$ & $\begin{array}{c}\text { Number of } \\
\text { Replications }\end{array}$ & Internal Standard \\
\hline A_01AIM_13a_16_1871_XSP_4 & 020909B & 4 & 1 & $10.02 \mathrm{wt}^{\circ} \mathrm{TiO}_{2}$ \\
\hline
\end{tabular}

(a) The letter appended to the XRD File ID identifies the first sample in the replication series. The letter has been dropped in subsequent text to indicate that the referenced file is the summation of data from replicate runs.

Sample analysis was carried out by the Pacific Northwest National Laboratory Advanced Processing and Application Group. The XRD instrument used was the Scintag PAD V X-ray Diffractometer, Property Number WD33356, located in lab 102 in the APEL building. The data range for the sample was $5^{\circ}$ to $80^{\circ}$ 2-theta, with a step size of $0.04^{\circ} 2$-theta and count time 4.0 seconds per step. Copper K $\alpha$ X-rays were used. The X-ray tube operating conditions were $45 \mathrm{kV}$ and $40 \mathrm{~mA}$.

Sample 020909B was prepared with SRM 674b, $\mathrm{TiO}_{2}$ internal standard. The dried powder was placed into a tungsten carbide milling chamber and milled for $2 \mathrm{~min}$ in the angstrom shaker mill. The $\mathrm{TiO}_{2}$ standard was then spiked in at $10.02 \mathrm{wt} \%$ with the dried powder and milled again for $2 \mathrm{~min}$ to insure a homogenous mixture of the two powders. The sample was then mounted into standard sample holder.

Phase identification was done by use of the JADE search match routines (version 6.0, Materials Data Inc.) with comparison to the ICDD (International Centre for Diffraction Data) data base PDF-2 release 1999, which includes the ICSD (Inorganic Crystal Structure Database maintained by Fachinformationszentrum (FIZ), Karlsruhe, Germany). Searches were restricted to the PDF and ICSD Inorganic sections.

The phase identification plots are shown using "stick figures" to indicate the phases present; peak positions and line intensities. The phase showing the greatest peak intensity is shown at the top of the stick figures display (except the internal standard, if used, is always the topmost). Phases present at lower peak area/height are shown in decreasing order down the display. 
The identified phases, ranked by relative peak intensities, are given below for each sample. Figure 1 shows the raw summed pattern. The background fitted pattern, along with the identified phases, are shown in figures 2,3 , and 4 .

A_01AIM_13a_16_1871_XSP_4_B, File: 020909B

- Rutile, $\mathrm{TiO}_{2}$, card\# 21-1276

- Boehmite, syn, $\mathrm{AlO}(\mathrm{OH})$, card\# 21-1307, Excellent overall fit. Peak locations and intensities are a good match to the pattern.

- Hematite, syn, $\mathrm{Fe}_{2} \mathrm{O}_{3}$, card\# 64-6101, good fit as a minor phase.

- Iron(III) oxide chloride, $\mathrm{FeOCl}$, card\# 61-8704, possible fit to trace phase.

- Amorphous material, $\sim 36^{\circ} 2 \theta$.

The pattern was also examined using RIQAS (release 4.0.0.26, 6/10/2002, Materials Data Inc.) rietveld analysis software. The phases identified above were input into the analysis along with a cubic spline function for the background and an amorphous hump at $\sim 36^{\circ} 2 \theta$, with fitted pattern shown in figure 5 .

The results of the analysis for the crystalline phases are:

- Boehmite, $\mathrm{AlO}(\mathrm{OH}), 58.1 \mathrm{wt} \%$, with a crystallite size of $380 \AA$

- Hematite, $\mathrm{Fe}_{2} \mathrm{O}_{3}, 3.7 \mathrm{wt} \%$, with a crystallite size of $525 \AA$

- The balance of the material is amorphous 


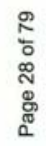

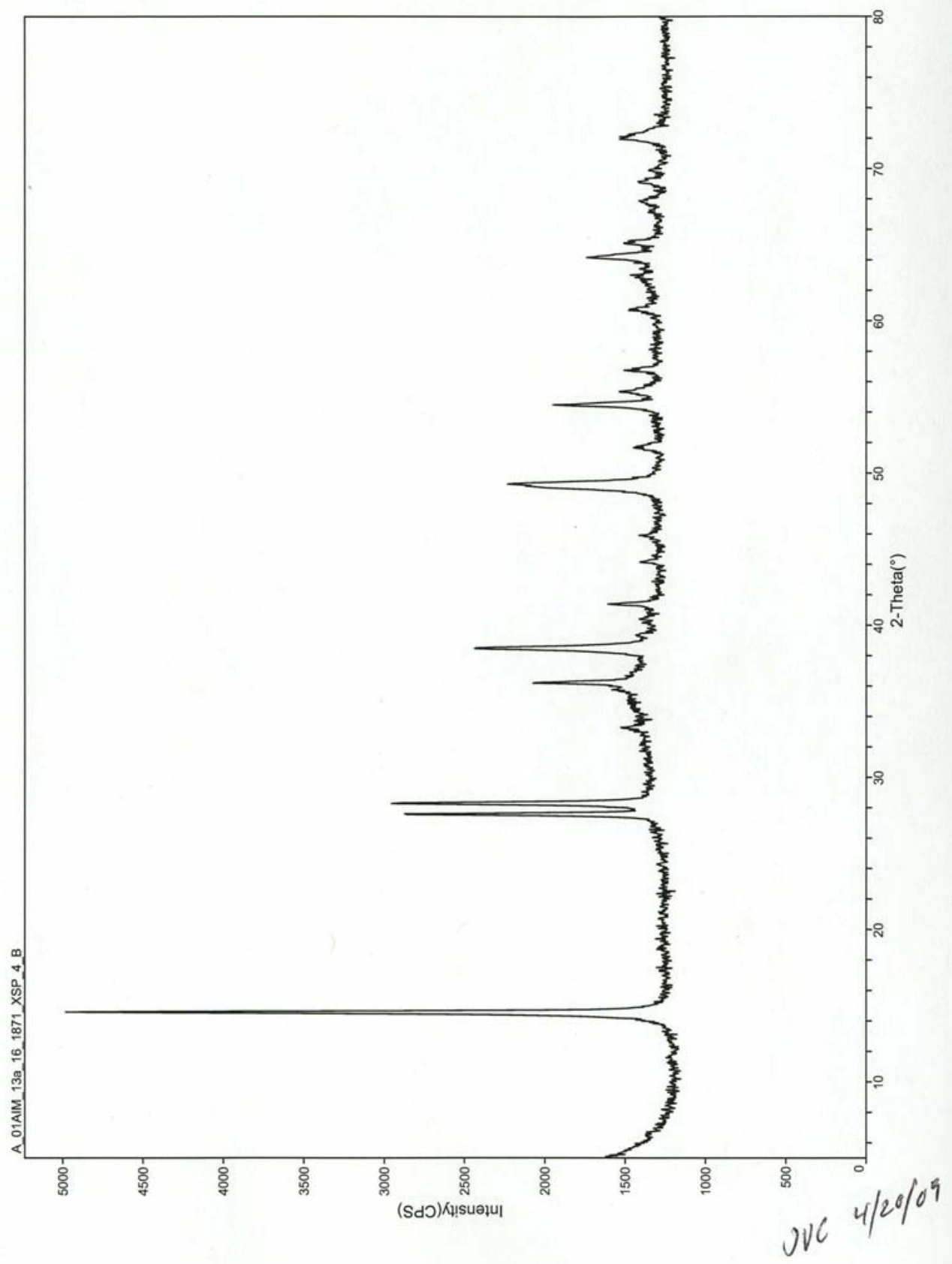

H.55 


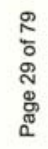

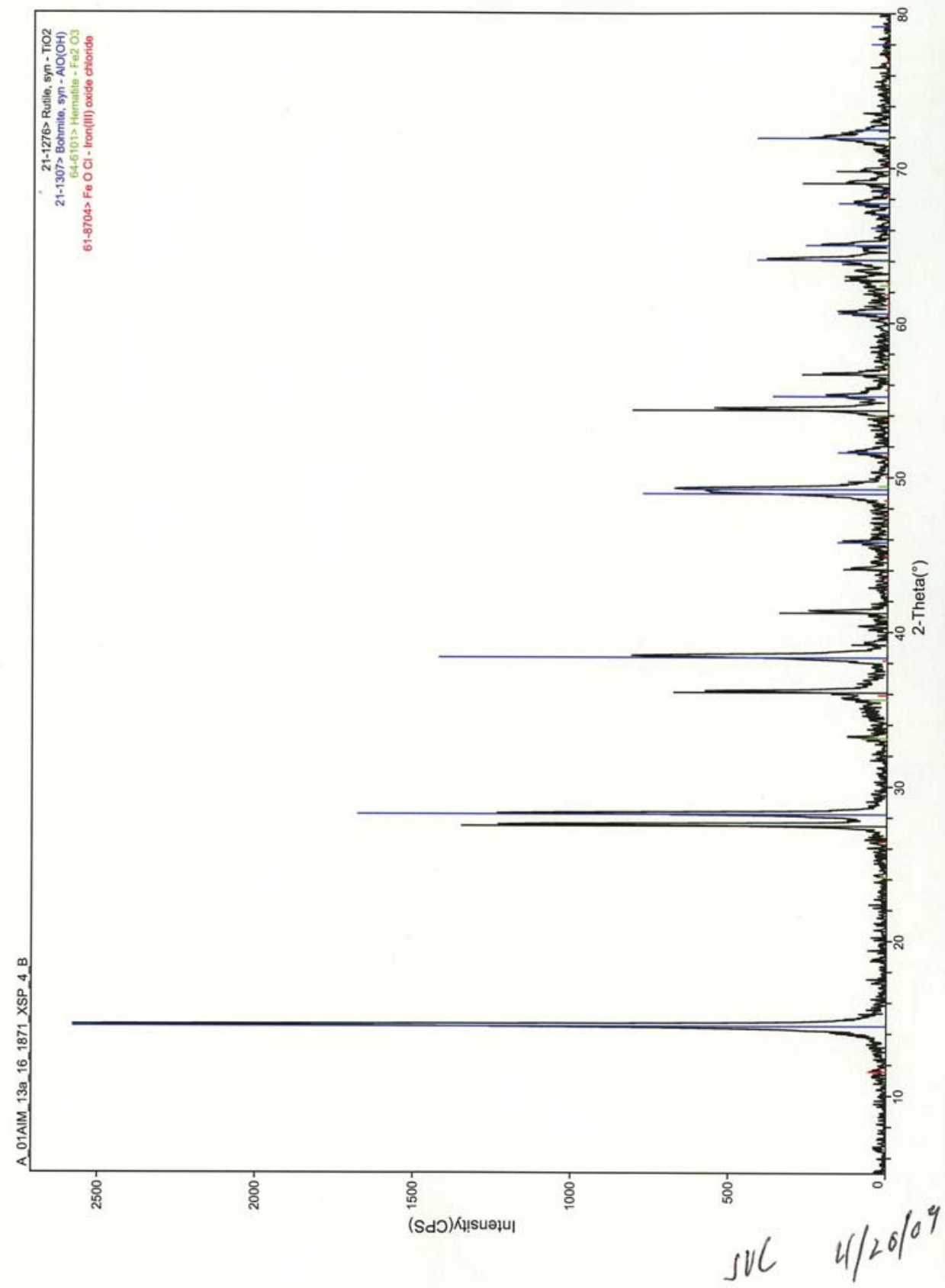

H.56 


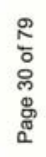

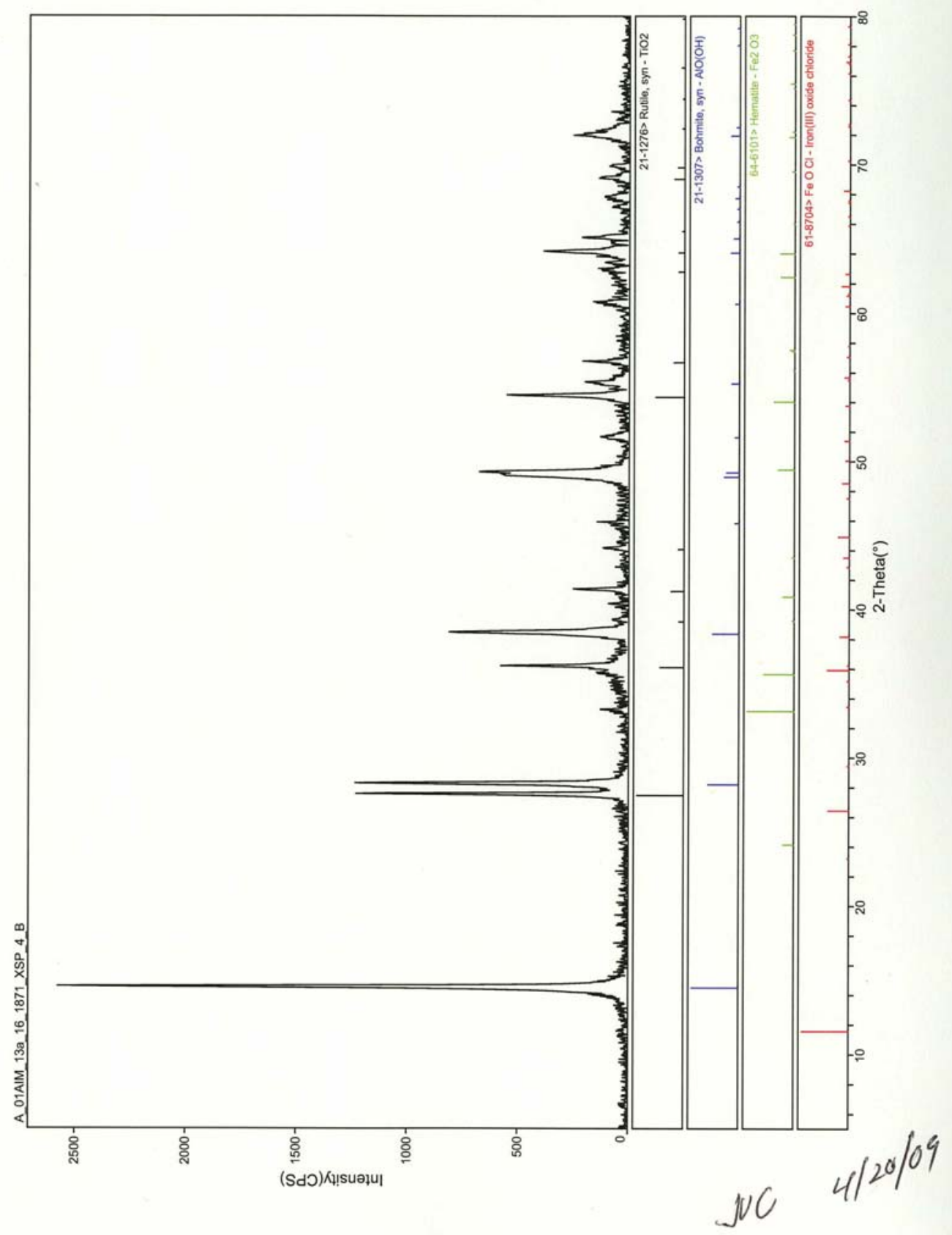




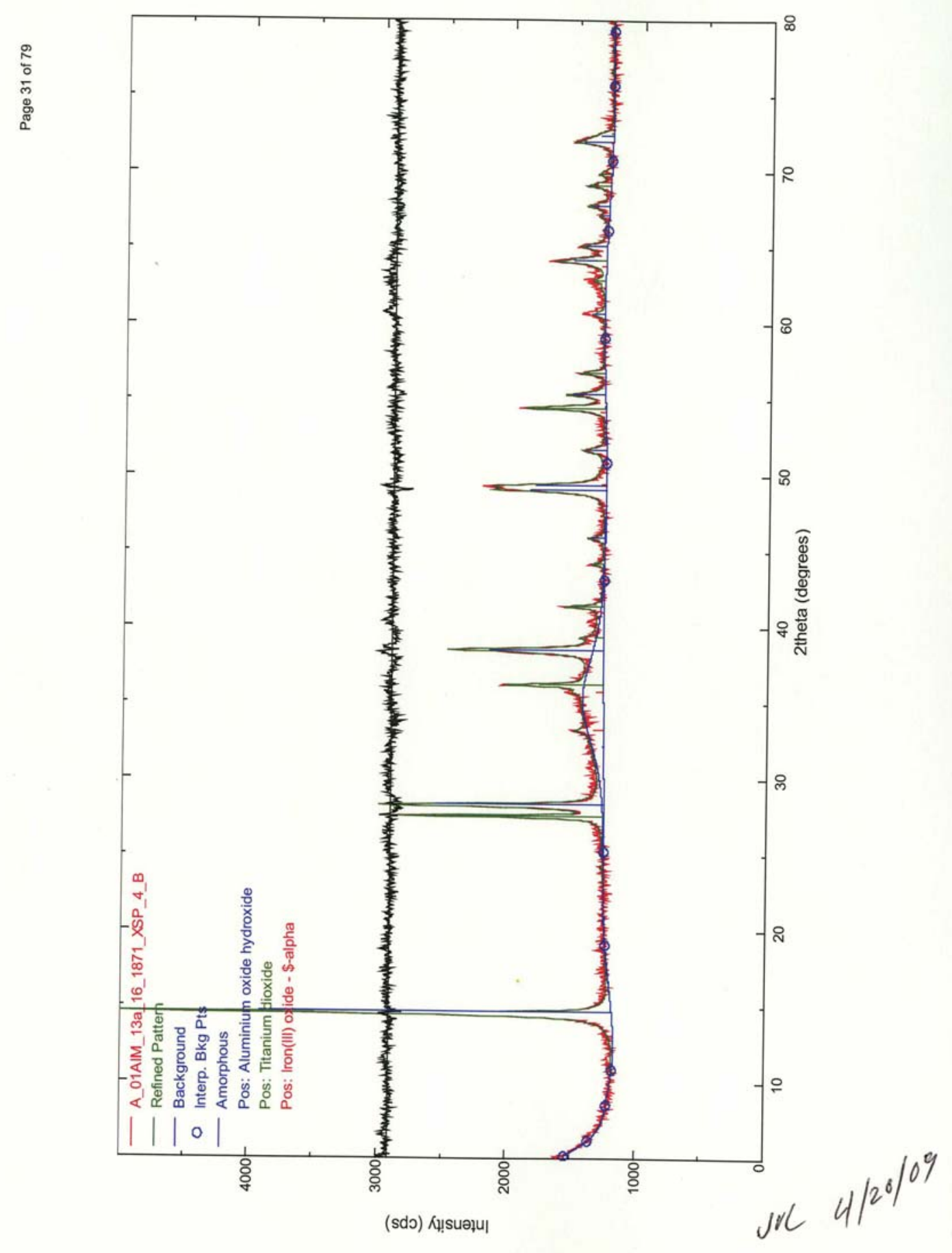


- $\mathrm{PDF} \# 21-1276$ : $\mathrm{QM}=$ Common $(+)$; $\mathrm{d}=$ Other/Unknown; $\mathrm{I}=($ Unknown $)$

Rutile, syn

$\mathrm{TiO} 2$

Radiation $=$ CuKal $\quad$ Lambda $=1.5406$ Filter $=$

Calibration $=\quad \mathrm{d}$-Cutoff $=\quad \mathrm{I} / \mathrm{Ic}(\mathrm{RIR})=3.4$

Ref: Level-1 PDF

Tetragonal, $\mathrm{P} 42 / \mathrm{mnm}(136) \quad \mathrm{Z}=2 \quad \mathrm{mp}=$

CELL: $4.5933 \times 4.5933 \times 2.9592<90.0 \times 90.0 \times 90.0>\quad$ P.S $=$

Density $(\mathrm{c})=4.23$ Density $(\mathrm{m})=\quad \mathrm{Mwt}=\quad \mathrm{Vol}=62.4$

Ref: Ibid.

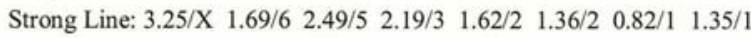

- PDF\#21-1307: QM=Common(+); $\mathrm{d}=$ Other/Unknown; $\mathrm{I}=($ Unknown)

Boehmite, syn

$\mathrm{AlO}(\mathrm{OH})$

Radiation $=$ CuKal $\quad$ Lambda $=1.5406$ Filter $=$

Calibration $=\quad \mathrm{d}-$ Cutoff $=\quad \mathrm{I} / \mathrm{Ic}(\mathrm{RIR})=$

Ref: Level-1 PDF

Orthorhombic, Amam(63) $\quad \mathrm{Z}=4 \quad \mathrm{mp}=$

CELL: $3.7 \times 12.227 \times 2.868<90.0 \times 90.0 \times 90.0>\quad$ P. $S=$

Density $(\mathrm{c})=3.07$ Density $(\mathrm{m})=\quad \mathrm{Mwt}=\quad \mathrm{Vol}=129.8$

Ref: Ibid.

Strong Line: $6.11 / \mathrm{X} \quad 3.16 / 7 \quad 2.35 / 6 \quad 1.86 / 3 \quad 1.85 / 3 \quad 1.45 / 2 \quad 1.31 / 2 \quad 1.66 / 1$

- PDF\#64-6101: QM=Calculated; d=Other/Unknown; $\mathrm{I}=($ Unknown)

Hematite - synthetic, Hematite group

$\mathrm{Fe} 2 \mathrm{O} 3$

Radiation $=$ CuKal $\quad$ Lambda $=1.5406$ Filter $=$

Calibration $=\quad \mathrm{d}-$ Cutoff $=\quad \mathrm{I} / \mathrm{Ic}(\mathrm{RIR})=3.04$

Ref: Calculated from FIZ\#82134 (ICSD @08/01/01) by Jade 6.x

Hexagonal, R-3c(167) $\quad \mathrm{Z}=6 \quad \mathrm{mp}=$

CELL: $5.037 \times 5.037 \times 13.771<90.0 \times 90.0 \times 120.0>$ P.S $=$

Density $(\mathrm{c})=5.258$ Density $(\mathrm{m})=\quad \mathrm{Mwt}=\quad \mathrm{Vol}=302.6$

Ref: Ibid.

Strong Line: $2.70 / X \quad 2.52 / 6 \quad 1.70 / 4 \quad 1.84 / 3 \quad 1.45 / 3 \quad 1.49 / 3 \quad 2.21 / 2 \quad 3.68 / 2$

NOTE: Effect of mechanical activation on the real structure and reactivity of iron(III) oxide with corundum-type structure, Journal of Solid State Chemistry [JSSCB] 123 (1996) 191-202, Sadykov V A, Isupova L A, Tsybulya S V, Cherepanova S V, Litvak G S, Burgina E B, Kustova G N, Kolomiichuk V N, Ivanov V P, Paukshtis E A, Golovin A V, Avvakumov E G, X-ray diffraction (powder).

$\mathrm{R}=0.053$

- PDF\#61-8704: QM=Calculated; $\mathrm{d}=$ Other/Unknown; $\mathrm{I}=($ Unknown)

Iron(III) oxide chloride

$\mathrm{Fe} \mathrm{OCl}$

Radiation $=$ CuKal $\quad$ Lambda $=1.5406$ Filter $=$

Calibration $=\quad \mathrm{d}$-Cutoff $=\quad \mathrm{I} / \mathrm{Ic}(\mathrm{RIR})=5.34$

Ref: Calculated from FIZ\#37158 (ICSD @08/01/01) by Jade 6.x

Orthorhombic, $\operatorname{Pmnm}(59) \mathrm{Z}=2 \quad \mathrm{mp}=$ 
CELL: $3.75 \times 7.65 \times 3.3<90.0 \times 90.0 \times 90.0>$

Density $(c)=3.764$ Density $(m)=\quad M w t=\quad \mathrm{Vol}=94.7$

Ref: Ibid.

Strong Line: $7.65 / X \quad 2.50 / 4 \quad 3.37 / 4 \quad 2.02 / 2 \quad 2.36 / 2 \quad 1.50 / 1 \quad 1.88 / 1 \quad 1.37 / 1$

NOTE: Etude de quelques derives de l'oxyde ferrique ( $\mathrm{Fe} \mathrm{O} . \mathrm{OH}, \mathrm{FeO} 2 \mathrm{Na}, \mathrm{FeOCl})$ determination de leurs structures., Bulletin de la Societe Francaise de Mineralogie (-71,1948) [BSFMA] 58 (1935) 6-6, Goldsztaub M S, Dx=3.55.

At least one temperature factor missing is in the paper.

Calculated density is unusual, but tolerable.

No $R$ value given in the paper. 
Date: $\quad$ February 16, 2009

To: $\quad$ O. Bredt

From J. V. Crum

Subject: XRD Examination of: A_01AIM_13c_16_2124_XSP_4_B

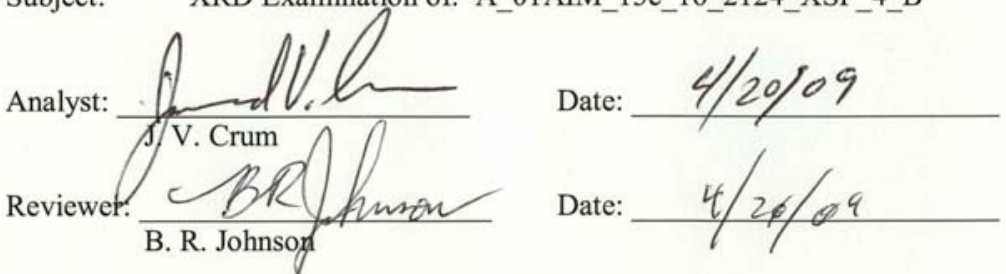

XRD data from the samples identified in Table I have been examined for identification of crystalline phases present.

Table I: Samples Examined in this Series

\begin{tabular}{|c|c|c|c|c|}
\hline Identification & XRD File ID $^{(\mathrm{a})}$ & $\begin{array}{c}\text { Count Time per } \\
\text { Step (Seconds) }\end{array}$ & $\begin{array}{c}\text { Number of } \\
\text { Replications }\end{array}$ & Internal Standard \\
\hline A_01AIM_13c_16_2124_XSP_4 & 021309B & 4 & 1 & $8.92 \mathrm{wt} \% \mathrm{TiO}_{2}$ \\
\hline
\end{tabular}

(a) The letter appended to the XRD File ID identifies the first sample in the replication series. The letter has been dropped in subsequent text to indicate that the referenced file is the summation of data from replicate runs.

Sample analysis was carried out by the Pacific Northwest National Laboratory Advanced Processing and Application Group. The XRD instrument used was the Scintag PAD V X-ray Diffractometer, Property Number WD33356, located in lab 102 in the APEL building. The data range for the sample was $5^{\circ}$ to $80^{\circ}$ 2 -theta, with a step size of $0.04^{\circ}$ 2-theta and count time 4.0 seconds per step. Copper $\mathrm{K} \alpha \mathrm{X}$-rays were used. The X-ray tube operating conditions were $45 \mathrm{kV}$ and $40 \mathrm{~mA}$.

Sample 021309B was prepared with $\mathrm{SRM} 674 \mathrm{~b}, \mathrm{TiO}_{2}$ internal standard. The dried powder was placed into a tungsten carbide milling chamber and milled for $2 \mathrm{~min}$ in the angstrom shaker mill. The $\mathrm{TiO}_{2}$ standard was then spiked in at $8.92 \mathrm{wt} \%$ with the dried powder and milled again for $2 \mathrm{~min}$ to insure a homogenous mixture of the two powders. The sample was then mounted into standard sample holder.

Phase identification was done by use of the JADE search match routines (version 6.0, Materials Data Inc.) with comparison to the ICDD (International Centre for Diffraction Data) data base PDF-2 release 1999, which includes the ICSD (Inorganic Crystal Structure Database maintained by Fachinformationszentrum (FIZ), Karlsruhe, Germany). Searches were restricted to the PDF and ICSD Inorganic sections.

The phase identification plots are shown using "stick figures" to indicate the phases present; peak positions and line intensities. The phase showing the greatest peak intensity is shown at the top of the stick figures display (except the internal standard, if used, is always the topmost). Phases present at lower peak area/height are shown in decreasing order down the display.

The identified phases, ranked by relative peak intensities, are given below for each sample. Figure 1 shows the raw summed pattern. The background fitted pattern, along with the identified phases, are shown in figures 2,3 , and 4 . 
A_01AIM_13c_16_2124_XSP_4_B, File: 021309B

- Rutile, $\overline{\mathrm{TiO}}{ }_{2}$, card\# $21-\overline{12} \overline{76}$

- Boehmite, syn, $\mathrm{AlO}(\mathrm{OH})$, card\#21-1307, Excellent overall fit. Peak locations and intensities are a good match to the pattern.

- Hematite, syn, $\mathrm{Fe}_{2} \mathrm{O}_{3}$, card\# 64-6101, good fit as a minor phase.

- Amorphous material, $\sim 36^{\circ} 2 \theta$.

The pattern was also examined using RIQAS (release 4.0.0.26, 6/10/2002, Materials Data Inc.) rietveld analysis software. The phases identified above were input into the analysis along with a cubic spline function for the background and an amorphous hump at $\sim 36^{\circ} 2 \theta$, with fitted pattern shown in figure 5 .

The results of the analysis for the crystalline phases are:

- Boehmite, $\mathrm{AlO}(\mathrm{OH}), 49.3 \mathrm{wt} \%$, with a crystallite size of $335 \AA$

- Hematite, $\mathrm{Fe}_{2} \mathrm{O}_{3}, 1.6 \mathrm{wt} \%$, with a crystallite size of $555 \AA$

- The balance of the material is amorphous

$$
\text { Jie } 4 / 20 \% 9
$$




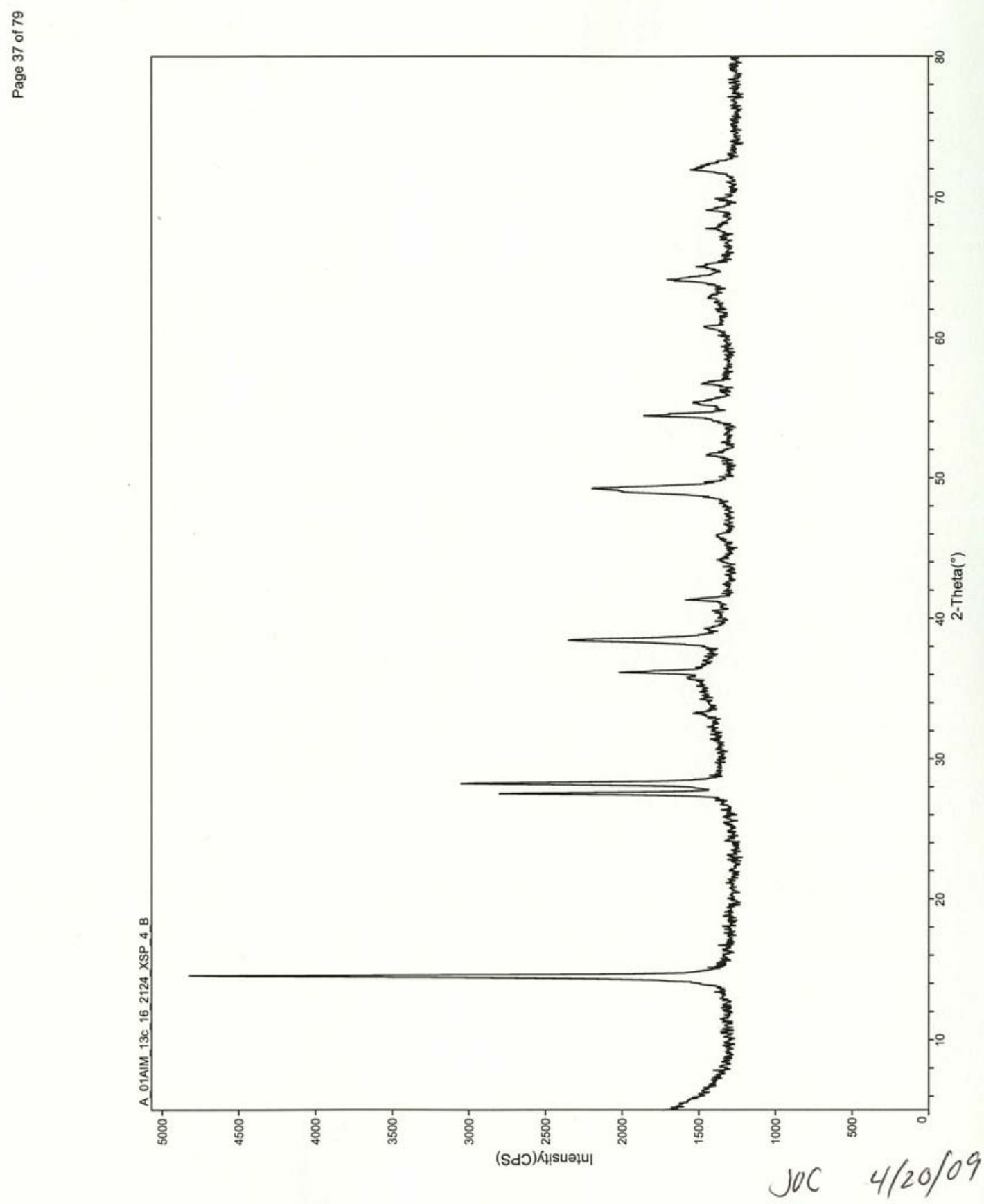

H.63 


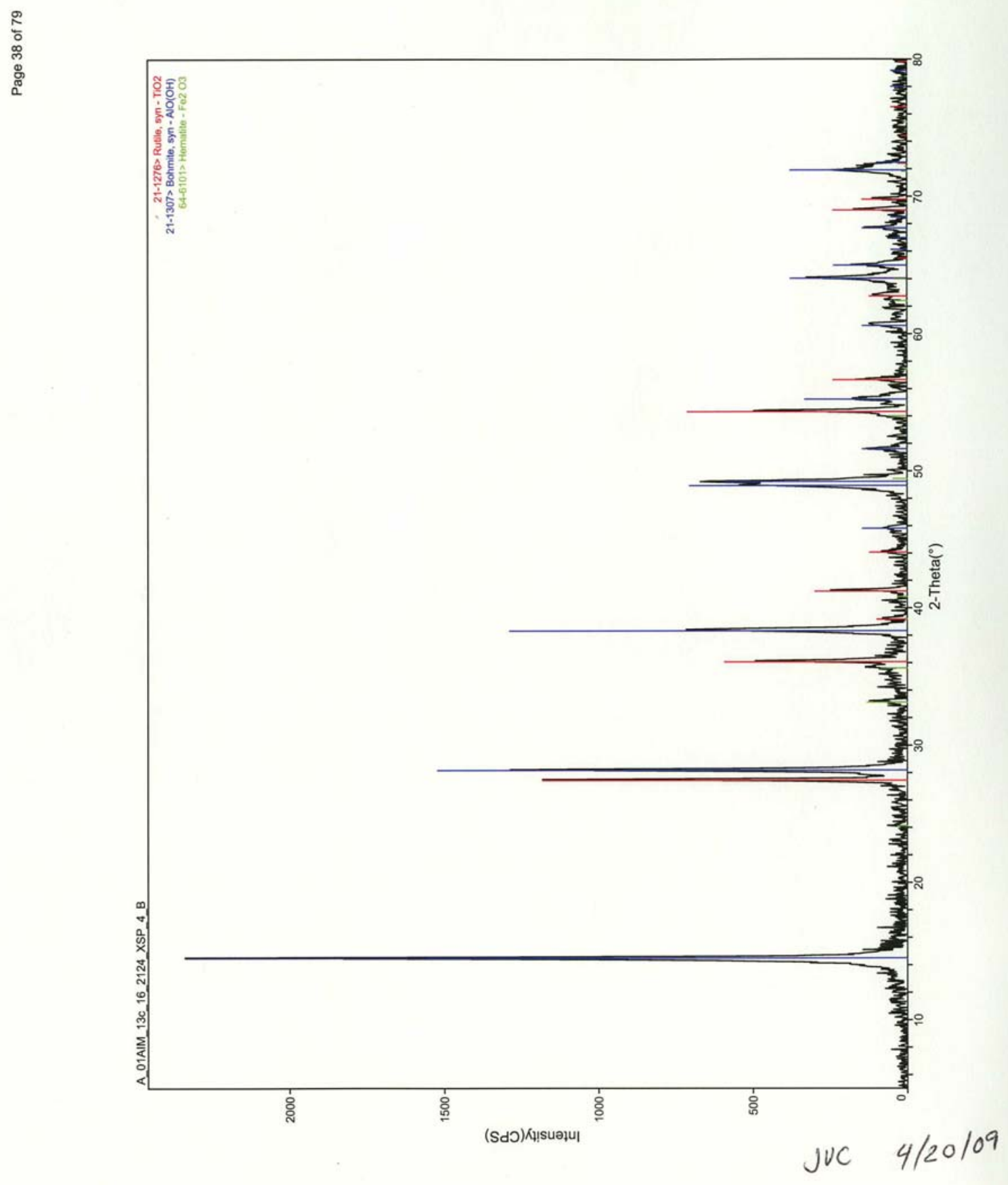

H.64 


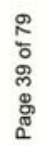

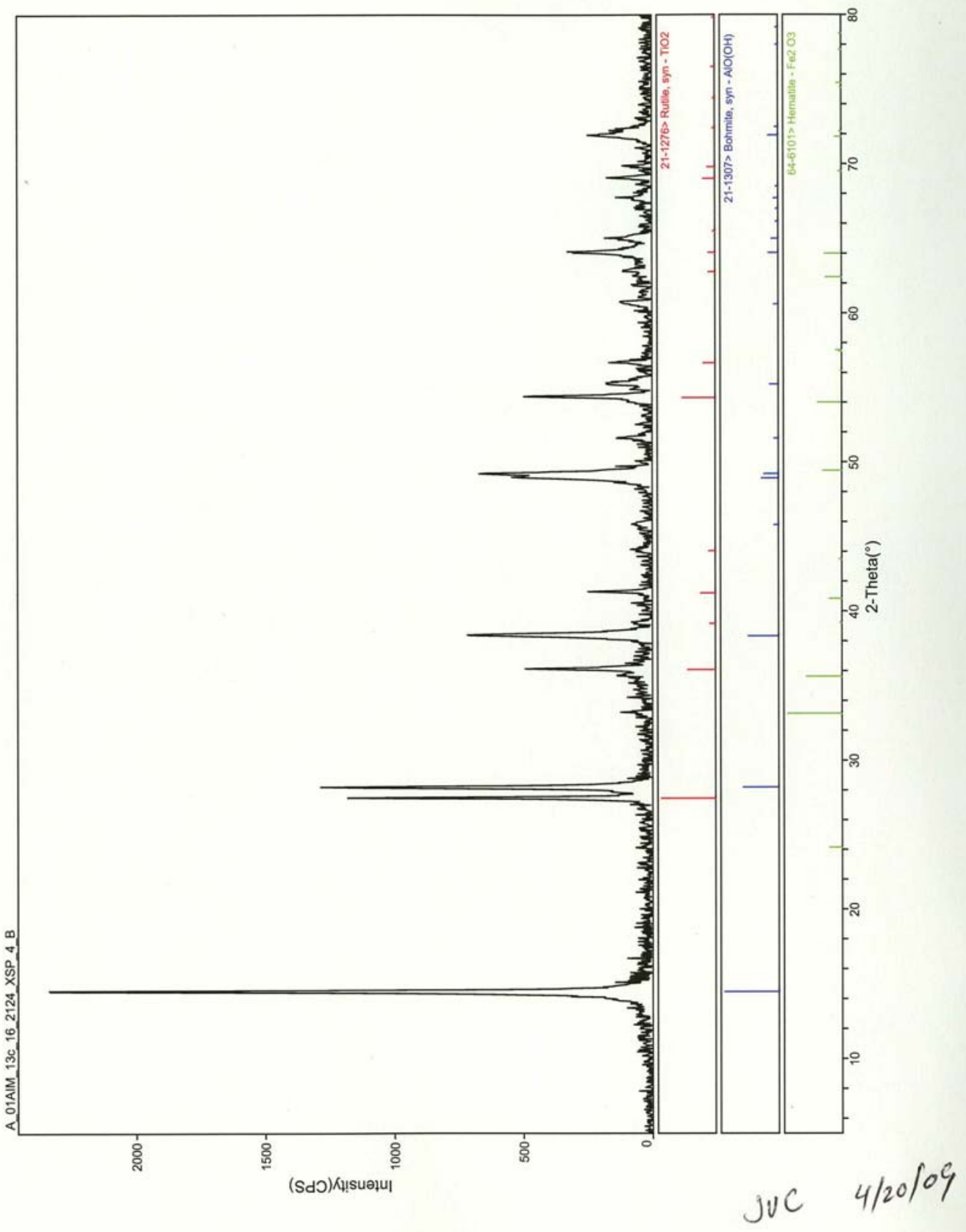




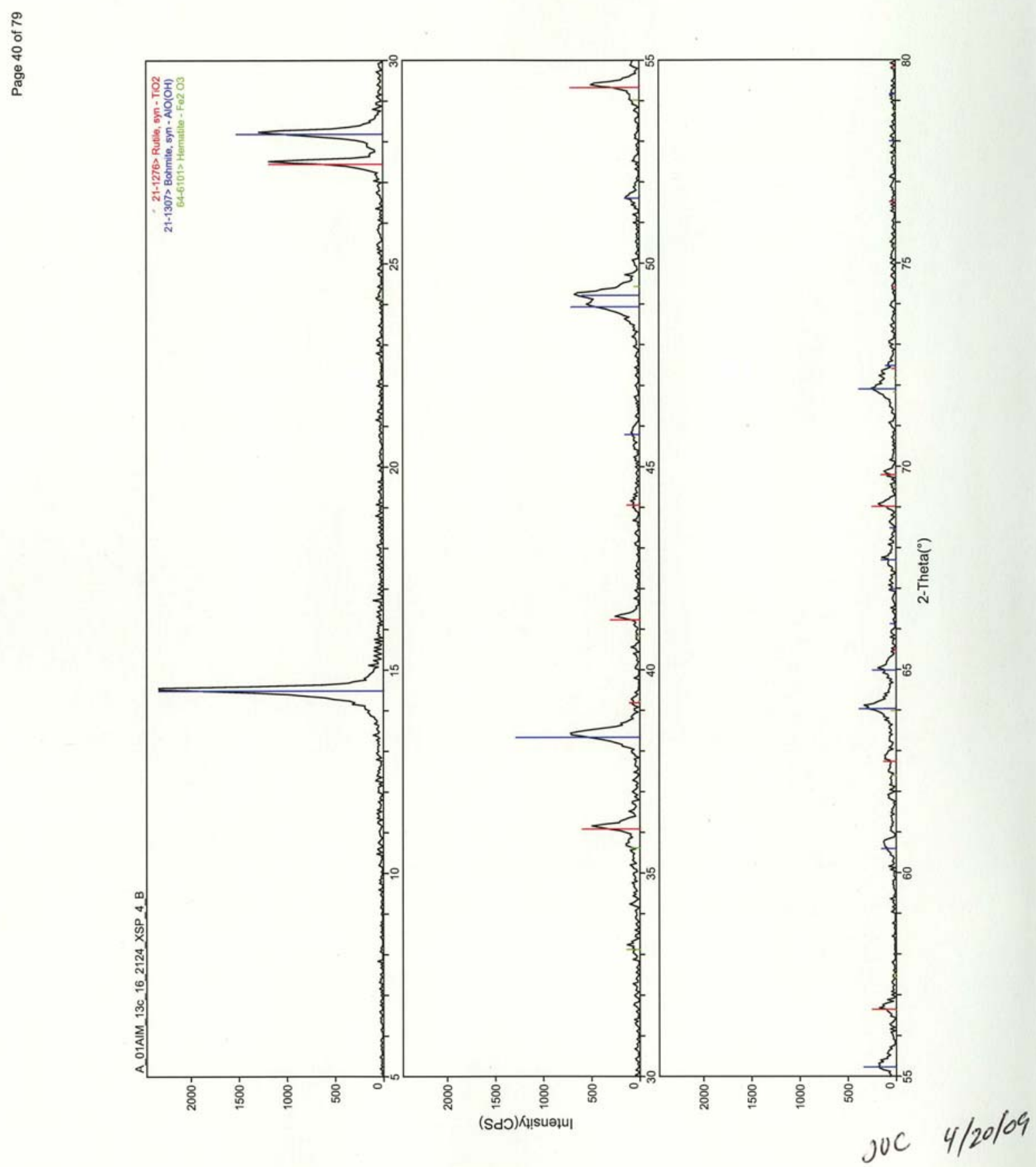

H.66 


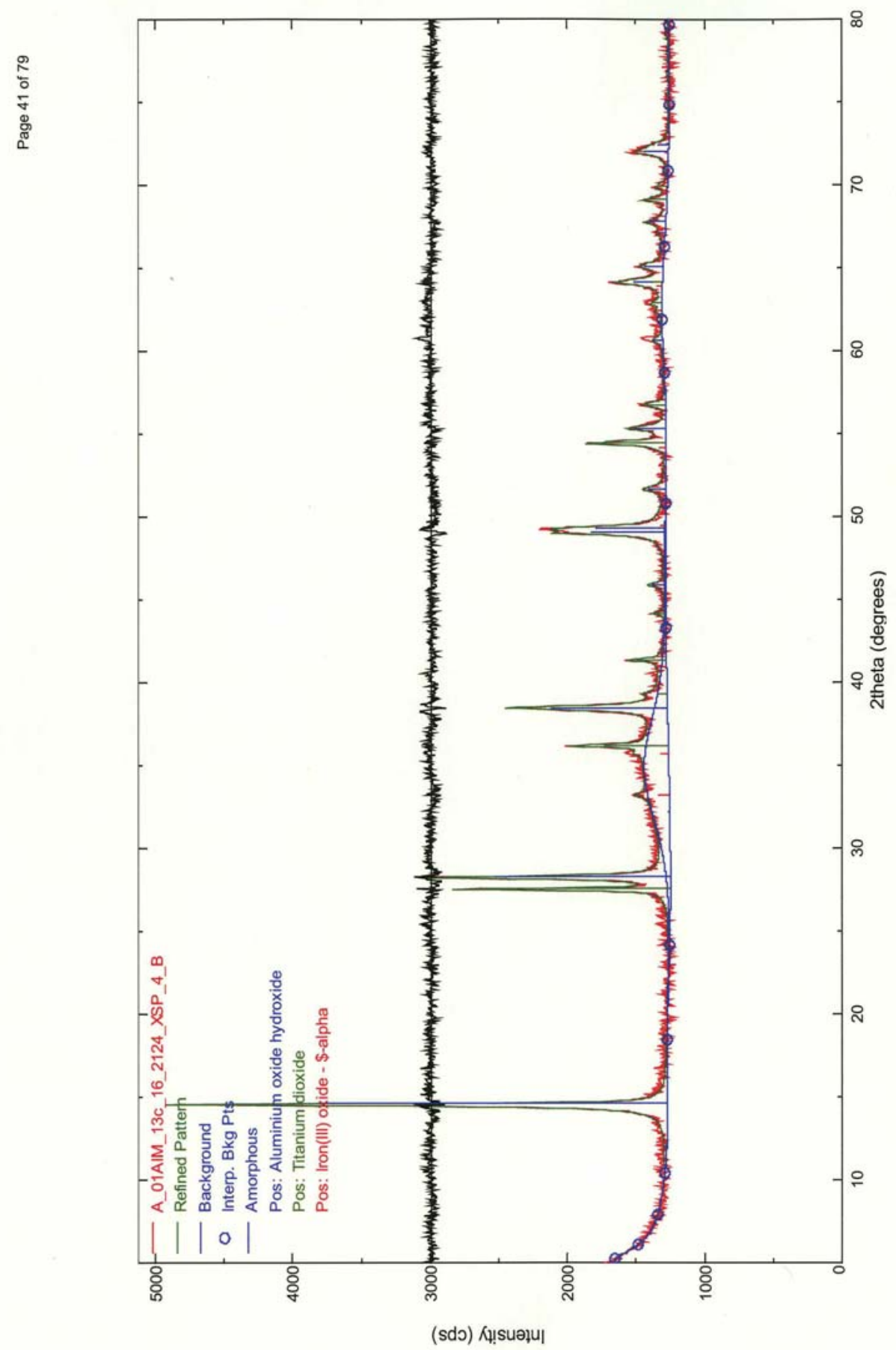

JuC 4/20109 
Page 42 of 79

Appendix A5

ICDD cards of interest for this sample

H.68 
- PDF\#21-1276: $\mathrm{QM}=$ Common(+); $\mathrm{d}=$ Other/Unknown; $\mathrm{I}=($ Unknown)

Rutile, syn

$\mathrm{TiO} 2$

Radiation $=$ CuKal Lambda $=1.5406$ Filter $=$

Calibration $=\quad \mathrm{d}-$ Cutoff $=\quad \mathrm{I} / \mathrm{Ic}(\mathrm{RIR})=3.4$

Ref: Level-1 PDF

Tetragonal, $\mathrm{P} 42 / \mathrm{mnm}(136) \quad \mathrm{Z}=2 \quad \mathrm{mp}=$

CELL: $4.5933 \times 4.5933 \times 2.9592<90.0 \times 90.0 \times 90.0>$

$\operatorname{Density}(\mathrm{c})=4.23$ Density $(\mathrm{m})=\quad \mathrm{Mwt}=\mathrm{Vol}=62.4$

P.S $=$

Ref: Ibid.

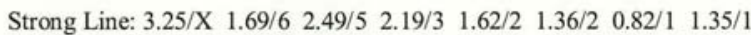

- PDF\#21-1307: $\mathrm{QM}=$ Common(+); $\mathrm{d}=$ Other/Unknown; $\mathrm{I}=($ Unknown)

Boehmite, syn

$\mathrm{AlO}(\mathrm{OH})$

Radiation $=\mathrm{CuKa} 1 \quad$ Lambda $=1.5406$ Filter $=$

Calibration $=\quad$ d-Cutoff $=\quad$ I/Ic(RIR) $=$

Ref: Level-1 PDF

Orthorhombic, Amam(63) $\quad \mathrm{Z}=4 \quad \mathrm{mp}=$

CELL: $3.7 \times 12.227 \times 2.868<90.0 \times 90.0 \times 90.0>\quad$ P.S $=$

$\operatorname{Density}(\mathrm{c})=3.07$ Density $(\mathrm{m})=\quad \mathrm{Mwt}=\quad \mathrm{Vol}=129.8$

Ref: Ibid.

Strong Line: $6.11 / \mathrm{X} \quad 3.16 / 7 \quad 2.35 / 6 \quad 1.86 / 3 \quad 1.85 / 3 \quad 1.45 / 2 \quad 1.31 / 2 \quad 1.66 / 1$

- PDF\#64-6101: QM=Calculated; $\mathrm{d}=$ Other/Unknown; $\mathrm{I}=($ Unknown $)$

Hematite - synthetic, Hematite group

$\mathrm{Fe} 2 \mathrm{O} 3$

Radiation $=$ CuKal Lambda $=1.5406$ Filter $=$

Calibration $=\quad \mathrm{d}-\mathrm{Cutoff}=\quad \mathrm{I} / \mathrm{Ic}(\mathrm{RIR})=3.04$

Ref: Calculated from FIZ\#82134 (ICSD@08/01/01) by Jade 6.x

Hexagonal, $\mathrm{R}-3 \mathrm{c}(167) \quad \mathrm{Z}=6 \quad \mathrm{mp}=$

CELL: $5.037 \times 5.037 \times 13.771<90.0 \times 90.0 \times 120.0>$ P.S $=$

Density $(\mathrm{c})=5.258$ Density $(\mathrm{m})=\quad \mathrm{Mwt}=\quad \mathrm{Vol}=302.6$

Ref: Ibid.

Strong Line: $2.70 / X \quad 2.52 / 6 \quad 1.70 / 4 \quad 1.84 / 3 \quad 1.45 / 3 \quad 1.49 / 3 \quad 2.21 / 2 \quad 3.68 / 2$

NOTE: Effect of mechanical activation on the real structure and reactivity of iron(III) oxide with corundum-type

structure, Journal of Solid State Chemistry [JSSCB] 123 (1996) 191-202, Sadykov V A, Isupova L A, Tsybulya S V,

Cherepanova S V, Litvak G S, Burgina E B, Kustova G N, Kolomiichuk V N, Ivanov V P, Paukshtis E A, Golovin A V,

Avvakumov E G, X-ray diffraction (powder).

$\mathrm{R}=0.053$ 
Date:

To:

O. Bredt

From

J. V. Crum Subject: XRD Examination gf: A_01AIM_006_XX_1631_XSP_4_B

Analyst:

Reviewer:

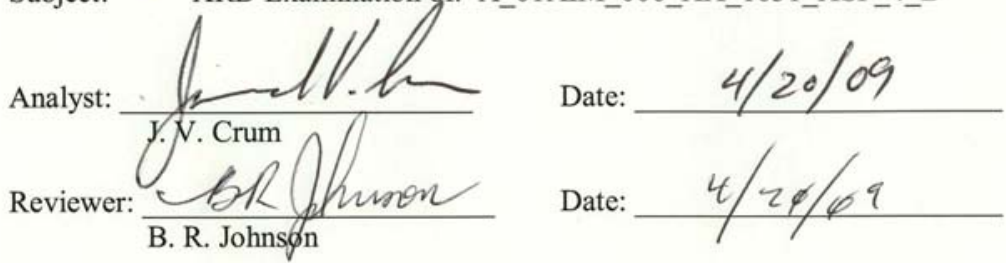

XRD data from the samples identified in Table I have been examined for identification of crystalline phases present.

Table I: Samples Examined in this Series

\begin{tabular}{|c|c|c|c|c|}
\hline Identification & XRD File ID $^{(a)}$ & $\begin{array}{c}\text { Count Time per } \\
\text { Step (Seconds) }\end{array}$ & $\begin{array}{c}\text { Number of } \\
\text { Replications }\end{array}$ & Internal Standard \\
\hline A_01AIM_006_XX_1631_XSP_4_ & 021609A6 & 4 & 1 & $9.17 \mathrm{wt}^{2} \mathrm{TiO}_{2}$ \\
\hline
\end{tabular}

(a) The letter appended to the XRD File ID identifies the first sample in the replication series. The letter has been dropped in subsequent text to indicate that the referenced file is the summation of data from replicate runs.

Sample analysis was carried out by the Pacific Northwest National Laboratory Advanced Processing and Application Group. The XRD instrument used was the Scintag PAD V X-ray Diffractometer, Property Number WD33356, located in lab 102 in the APEL building. The data range for the sample was $5^{\circ}$ to $80^{\circ}$ 2-theta, with a step size of $0.04^{\circ} 2$-theta and count time 4.0 seconds per step. Copper $\mathrm{K} \alpha \mathrm{X}$-rays were used. The X-ray tube operating conditions were $45 \mathrm{kV}$ and $40 \mathrm{~mA}$.

Sample 021609A6 was prepared with $\mathrm{SRM} 674 \mathrm{~b}, \mathrm{TiO}_{2}$ internal standard. The dried powder was placed into a tungsten carbide milling chamber and milled for $2 \mathrm{~min}$ in the angstrom shaker mill. The $\mathrm{TiO}_{2}$ standard was then spiked in at $9.17 \mathrm{wt} \%$ with the dried powder and milled again for $2 \mathrm{~min}$ to insure a homogenous mixture of the two powders. The sample was then mounted into standard sample holder.

Phase identification was done by use of the JADE search match routines (version 6.0, Materials Data Inc.) with comparison to the ICDD (International Centre for Diffraction Data) data base PDF-2 release 1999, which includes the ICSD (Inorganic Crystal Structure Database maintained by Fachinformationszentrum (FIZ), Karlsruhe, Germany). Searches were restricted to the PDF and ICSD Inorganic sections.

The phase identification plots are shown using "stick figures" to indicate the phases present; peak positions and line intensities. The phase showing the greatest peak intensity is shown at the top of the stick figures display (except the internal standard, if used, is always the topmost). Phases present at lower peak area/height are shown in decreasing order down the display. 
The identified phases, ranked by relative peak intensities, are given below for each sample. Figure 1 shows the raw summed pattern. The background fitted pattern, along with the identified phases, are shown in figures 2,3 , and 4 .

\section{A_01AIM_006_XX_1631_XSP_4_B File: 021609A6}

- Rutile, $\mathrm{TiO}_{2}$, card\# 21-1276

- Boehmite, syn, $\mathrm{AlO}(\mathrm{OH})$, card\# 21-1307, Excellent overall fit. Peak locations and intensities are a good match to the pattern.

- Hematite, syn, $\mathrm{Fe}_{2} \mathrm{O}_{3}$, card\# 64-6101, good fit as a minor phase.

- Amorphous material, $\sim 36^{\circ} 2 \theta$.

The pattern was also examined using RIQAS (release 4.0.0.26, 6/10/2002, Materials Data Inc.) rietveld analysis software. The phases identified above were input into the analysis along with a cubic spline function for the background and an amorphous hump at $\sim 36^{\circ} 2 \theta$, with fitted pattern shown in figure 5 .

The results of the analysis for the crystalline phases are:

- Boehmite, $\mathrm{AlO}(\mathrm{OH}), 78.2 \mathrm{wt} \%$, with a crystallite size of $390 \AA$

- Hematite, $\mathrm{Fe}_{2} \mathrm{O}_{3}, 1.2 \mathrm{wt} \%$, with a crystallite size of $699 \AA$

- The balance of the material is amorphous 
0
0
0
0
0
0
0
0
8

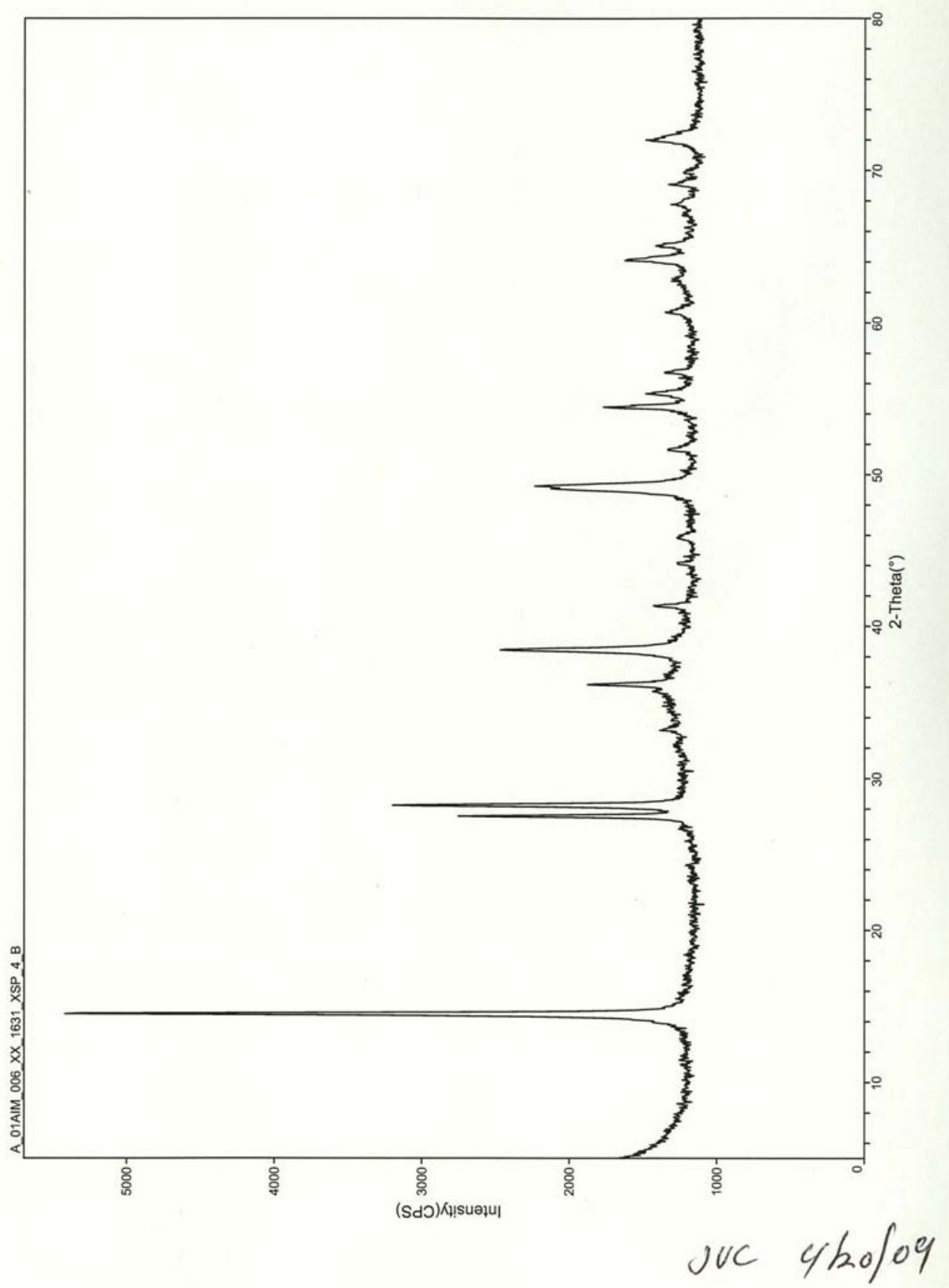

H.72 


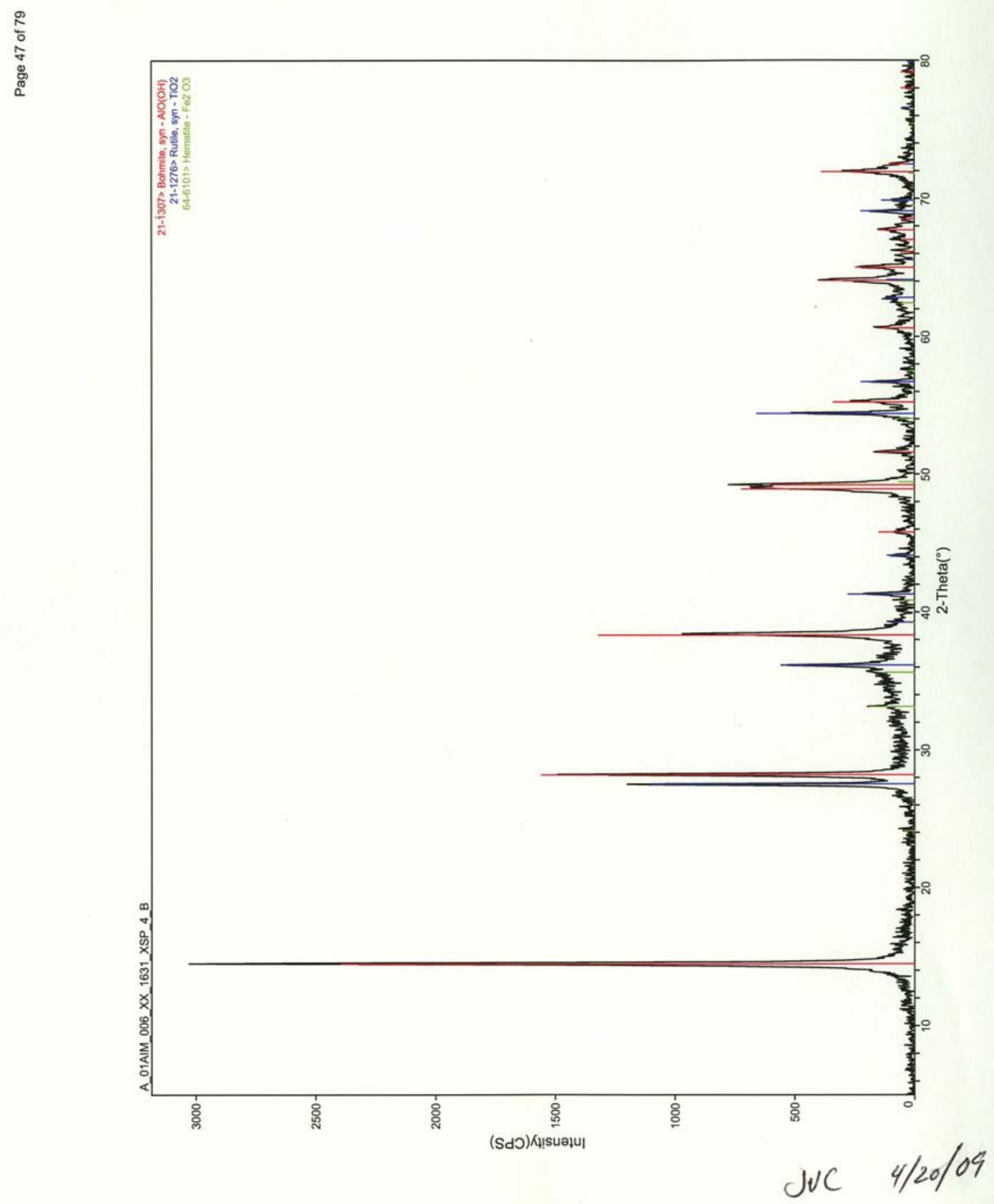

H.73 


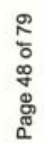

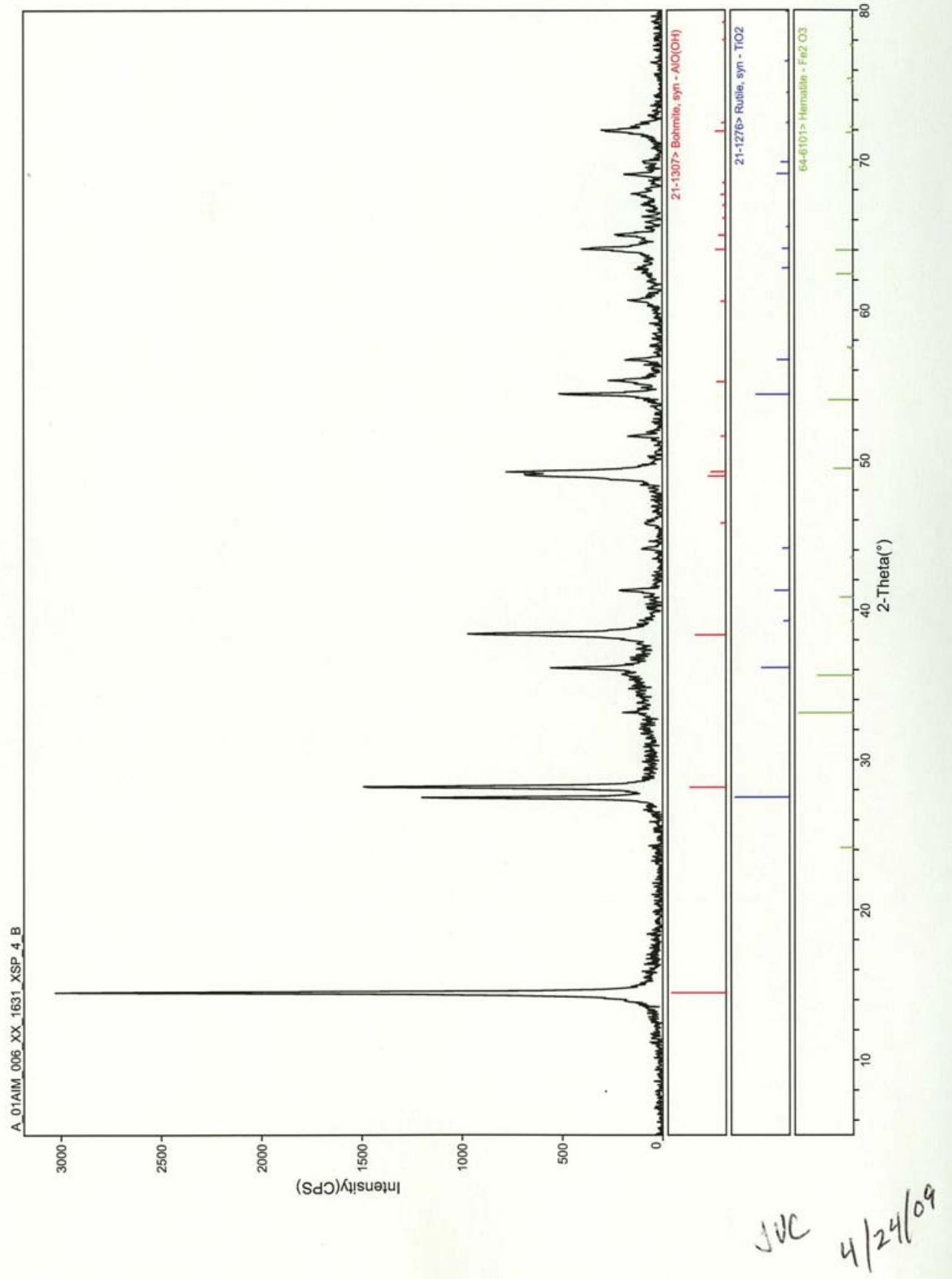


2
$\frac{8}{5}$
$\frac{8}{9}$
8
8
8

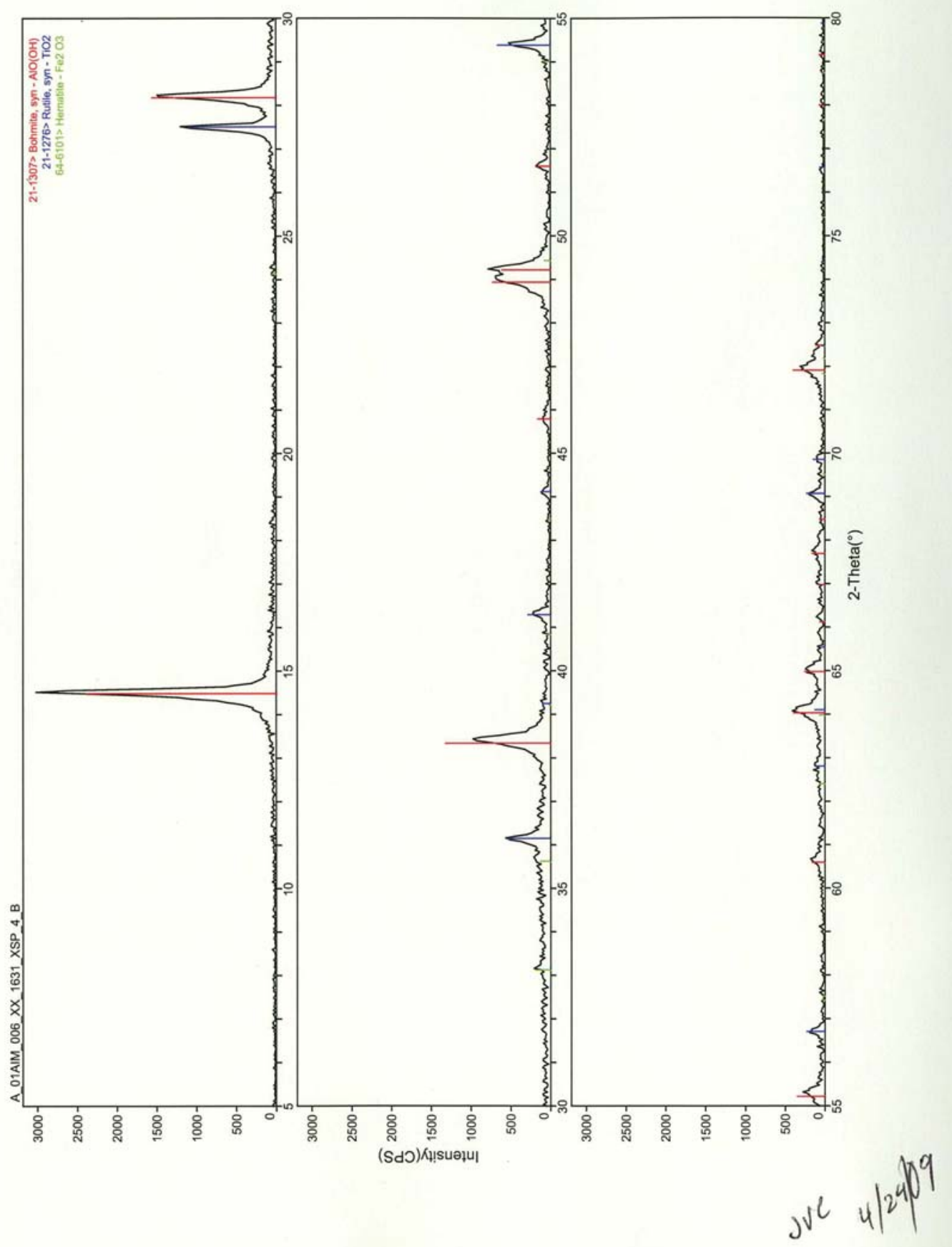

H.75 


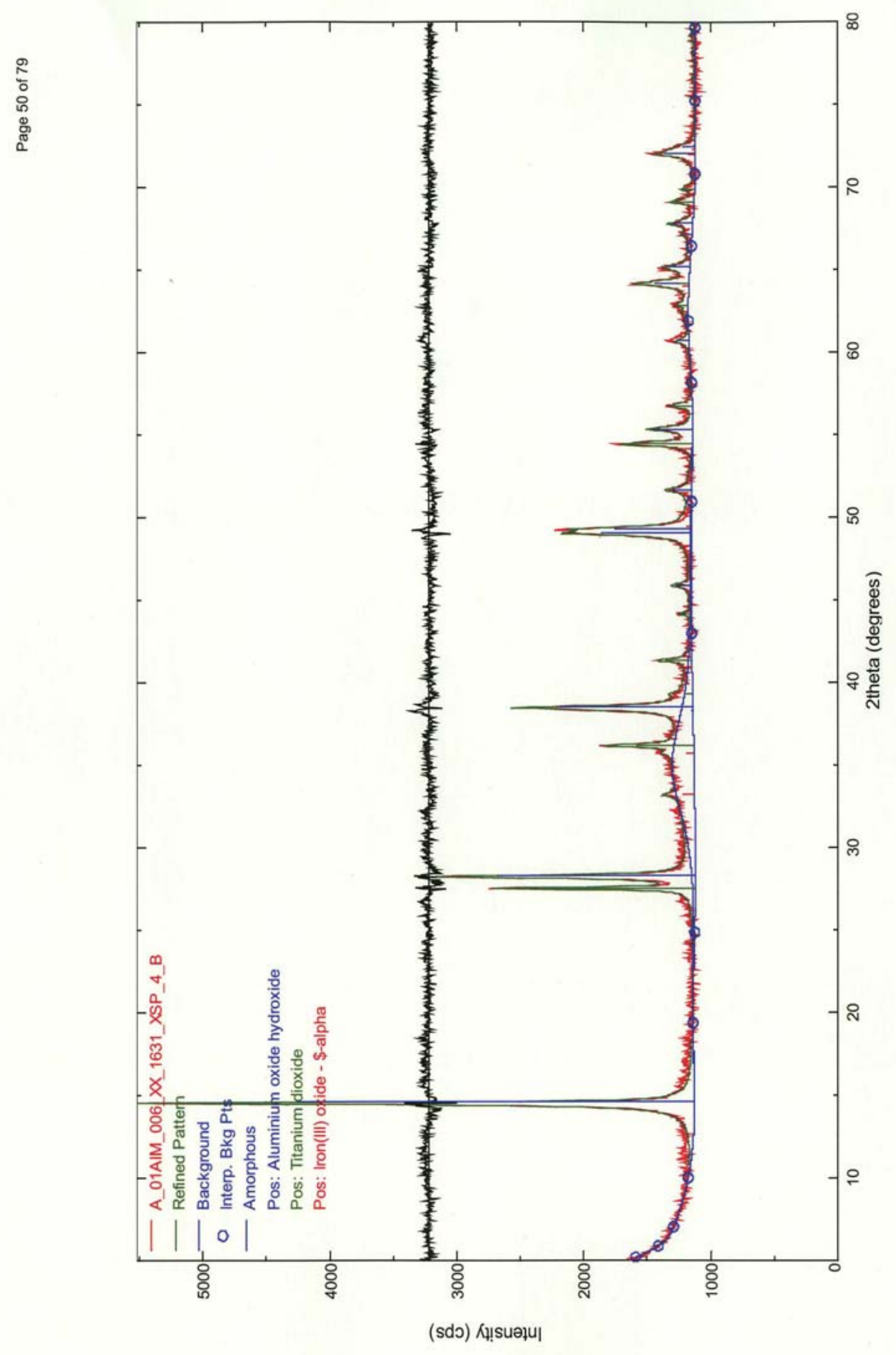

Jue $4|24|^{10^{9}}$ 
Page 51 of 79

Appendix A6

ICDD cards of interest for this sample

H.77 
- $\quad \mathrm{PDF} \# 21-1276: \mathrm{QM}=\mathrm{Common}(+)$; $\mathrm{d}=$ Other/Unknown; $\mathrm{I}=($ Unknown $)$

Rutile, syn

$\mathrm{TiO} 2$

Radiation $=\mathrm{CuKal} \quad$ Lambda $=1.5406$ Filter $=$

Calibration $=\quad \mathrm{d}-$ Cutoff $=\quad$ I/Ic $($ RIR $)=3.4$

Ref: Level-1 PDF

Tetragonal, $\mathrm{P} 42 / \mathrm{mnm}(136) \quad \mathrm{Z}=2 \quad \mathrm{mp}=$

CELL: $4.5933 \times 4.5933 \times 2.9592<90.0 \times 90.0 \times 90.0>$

$\operatorname{Density}(\mathrm{c})=4.23$ Density $(\mathrm{m})=\quad \mathrm{Mwt}=\mathrm{Vol}=62.4$

Ref: Ibid.

$\begin{array}{llllllll}\text { Strong Line: } 3.25 / \mathrm{X} & 1.69 / 6 & 2.49 / 5 & 2.19 / 3 & 1.62 / 2 & 1.36 / 2 & 0.82 / 1 & 1.35 / 1\end{array}$

- $\quad \mathrm{PDF}$ 21-1307: $\mathrm{QM}=$ Common( + ); $\mathrm{d}=$ Other/Unknown; $\mathrm{I}=($ Unknown)

Boehmite, syn

$\mathrm{AlO}(\mathrm{OH})$

Radiation $=\mathrm{CuKal} \quad$ Lambda $=1.5406$ Filter $=$

Calibration $=\quad \mathrm{d}$-Cutoff $=\quad$ I/Ic(RIR) $=$

Ref: Level-1 PDF

Orthorhombic, Amam(63)

$\mathrm{Z}=4 \mathrm{mp}=$

CELL: $3.7 \times 12.227 \times 2.868<90.0 \times 90.0 \times 90.0>\quad$ P.S $=$

$\operatorname{Density}(\mathrm{c})=3.07$ Density $(\mathrm{m})=\quad \mathrm{Mwt}=\quad \mathrm{Vol}=129.8$

Ref: Ibid.

Strong Line: $6.11 / \mathrm{X} \quad 3.16 / 7 \quad 2.35 / 6 \quad 1.86 / 3 \quad 1.85 / 3 \quad 1.45 / 2 \quad 1.31 / 2 \quad 1.66 / 1$

- PDF\#64-6101: QM=Calculated; $\mathrm{d}=$ Other/Unknown; $\mathrm{I}=($ Unknown)

Hematite - synthetic, Hematite group

$\mathrm{Fe} 2 \mathrm{O} 3$

Radiation $=$ CuKal Lambda $=1.5406$ Filter $=$

Calibration $=\quad \mathrm{d}-$ Cutoff $=\quad$ I/Ic(RIR $)=3.04$

Ref: Calculated from FIZ\#82134 (ICSD @08/01/01) by Jade 6.x

Hexagonal, R-3c(167) $\mathrm{Z}=6 \quad \mathrm{mp}=$

CELL: $5.037 \times 5.037 \times 13.771<90.0 \times 90.0 \times 120.0>$ P.S $=$

Density $(\mathrm{c})=5.258$ Density $(\mathrm{m})=\quad \mathrm{Mwt}=\quad \mathrm{Vol}=302.6$

Ref: Ibid.

Strong Line: $2.70 / X \quad 2.52 / 6 \quad 1.70 / 4 \quad 1.84 / 3 \quad 1.45 / 3 \quad 1.49 / 3 \quad 2.21 / 2 \quad 3.68 / 2$

NOTE: Effect of mechanical activation on the real structure and reactivity of iron(III) oxide with corundum-type structure, Journal of Solid State Chemistry [JSSCB] 123 (1996) 191-202, Sadykov V A, Isupova L A, Tsybulya S V, Cherepanova S V, Litvak G S, Burgina E B, Kustova G N, Kolomiichuk V N, Ivanov V P, Paukshtis E A, Golovin A V, Avvakumov E G, X-ray diffraction (powder).

$\mathrm{R}=0.053$ 
Date:

February 17, 2009

To:

O. Bredt

From

J. V. Crum

Analyst:

Reviewer:

$$
\text { B. R. Johns } \phi \text { n }
$$

Subject: XRD Examination of: A_01AIM_13c_XX_2134_XSP_4_B

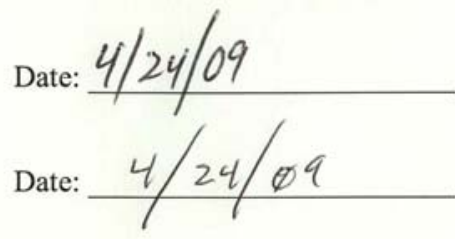

XRD data from the samples identified in Table I have been examined for identification of crystalline phases present.

Table I: Samples Examined in this Series

\begin{tabular}{|c|c|c|c|c|}
\hline Identification & XRD File ID & $\begin{array}{c}\text { Count Time per } \\
\text { Step (Seconds) }\end{array}$ & $\begin{array}{c}\text { Number of } \\
\text { Replications }\end{array}$ & Internal Standard \\
\hline A_01AIM_13c_XX_2134_XSP_4_ \\
B
\end{tabular}

Sample analysis was carried out by the Pacific Northwest National Laboratory Advanced Processing and Application Group. The XRD instrument used was the Scintag PAD V X-ray Diffractometer, Property Number WD33356, located in lab 102 in the APEL building. The data range for the sample was $5^{\circ}$ to $80^{\circ}$ 2-theta, with a step size of $0.04^{\circ} 2$-theta and count time 4.0 seconds per step. Copper $\mathrm{K} \alpha \mathrm{X}$-rays were used. The X-ray tube operating conditions were $45 \mathrm{kV}$ and $40 \mathrm{~mA}$.

Sample 021609A7 was prepared with $\mathrm{SRM} 674 \mathrm{~b}, \mathrm{TiO}_{2}$ internal standard. The dried powder was placed into a tungsten carbide milling chamber and milled for $2 \mathrm{~min}$ in the angstrom shaker mill. The $\mathrm{TiO}_{2}$ standard was then spiked in at $8.94 \mathrm{wt} \%$ with the dried powder and milled again for $2 \mathrm{~min}$ to insure a homogenous mixture of the two powders. Note: During transfer of the sample + TiO2 standard to the milling chamber, static charge caused a tiny amount of the sample and standard to leave the wax paper, used for weighing and transferring. This caused the weights to be suspect to a minor extent. Estimates of the material lost are $0.01 \mathrm{~g}$ of the total that is equal to $0.7469 \mathrm{~g}$. This does not impact the ability to identify phases in the sample only the quantification of the phases to a minor extent. The sample was then mounted into standard sample holder.

Phase identification was done by use of the JADE search match routines (version 6.0, Materials Data Inc.) with comparison to the ICDD (International Centre for Diffraction Data) data base PDF-2 release 1999, which includes the ICSD (Inorganic Crystal Structure Database maintained by Fachinformationszentrum (FIZ), Karlsruhe, Germany). Searches were restricted to the PDF and ICSD Inorganic sections.

The phase identification plots are shown using "stick figures" to indicate the phases present; peak positions and line intensities. The phase showing the greatest peak intensity is shown at the top of the stick figures display (except the internal standard, if used, is always the topmost). Phases present at lower peak area/height are shown in decreasing order down the display. 
The identified phases, ranked by relative peak intensities, are given below for each sample. Figure 1 shows the raw summed pattern. The background fitted pattern, along with the identified phases, are shown in figures 2,3 , and 4 .

A_01AIM_13c_XX_2134_XSP_4_B File: 021609A7

- Rutile, $\mathrm{TiO}_{2}$, card\# 21-1276

- Boehmite, syn, $\mathrm{AlO}(\mathrm{OH})$, card\# 21-1307, Excellent overall fit. Peak locations and intensities are a good match to the pattern.

- Hematite, syn, $\mathrm{Fe}_{2} \mathrm{O}_{3}$, card\# 64-6101, good fit as a minor phase.

- Amorphous material, $\sim 36^{\circ} 2 \theta$.

The pattern was also examined using RIQAS (release 4.0.0.26, 6/10/2002, Materials Data Inc.) rietveld analysis software. The phases identified above were input into the analysis along with a cubic spline function for the background and an amorphous hump at $\sim 36^{\circ} 2 \theta$, with fitted pattern shown in figure 5 .

The results of the analysis for the crystalline phases are:

- Boehmite, $\mathrm{AlO}(\mathrm{OH}), 51.2 \mathrm{wt} \%$, with a crystallite size of $336 \AA$

- Hematite, $\mathrm{Fe}_{2} \mathrm{O}_{3}, 2.2 \mathrm{wt} \%$, with a crystallite size of $751 \AA$

- The balance of the material is amorphous

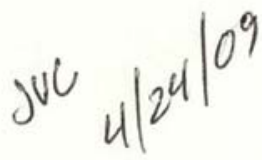


2
2
50
6
6
8
8
8
0

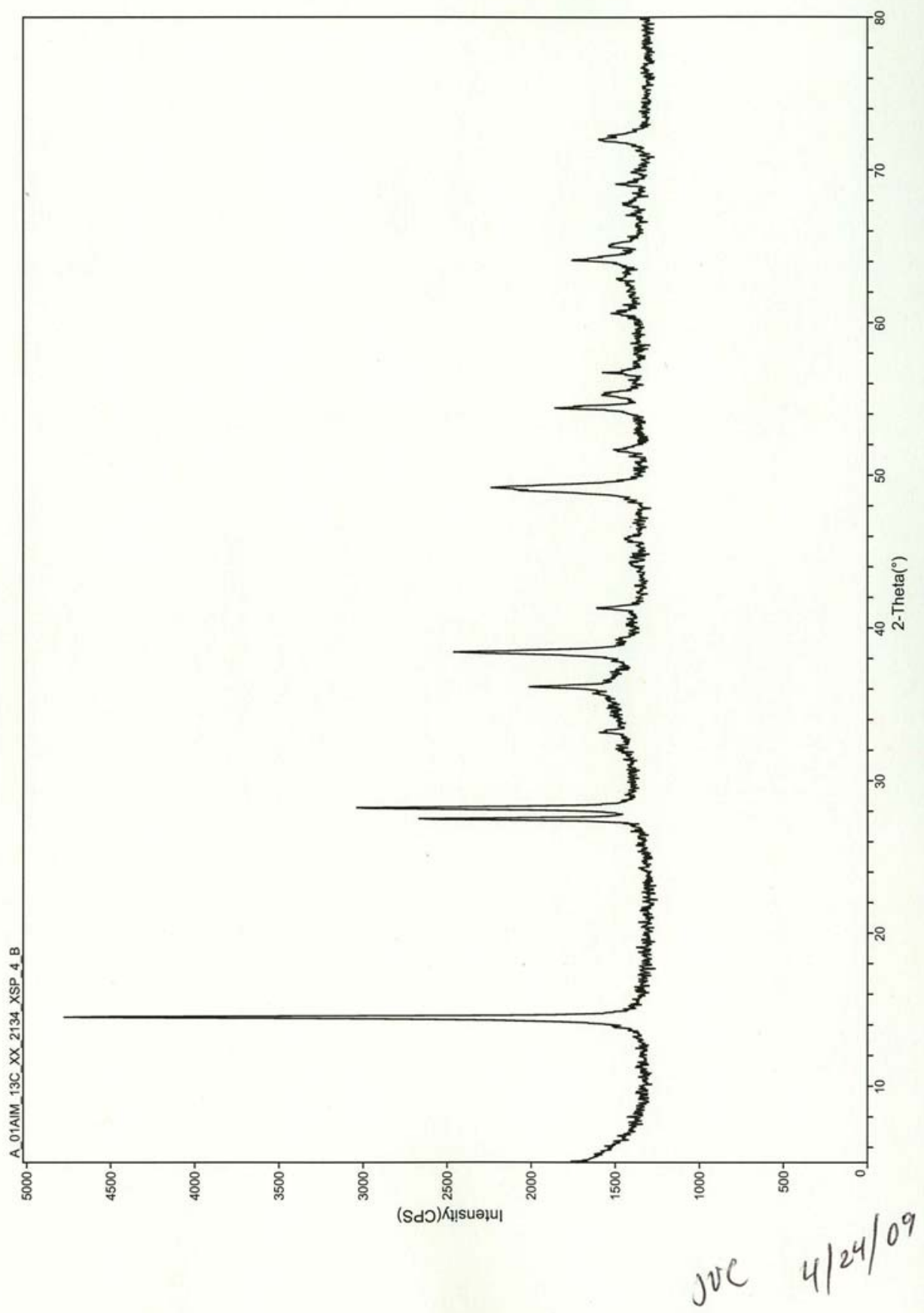

H. 81 


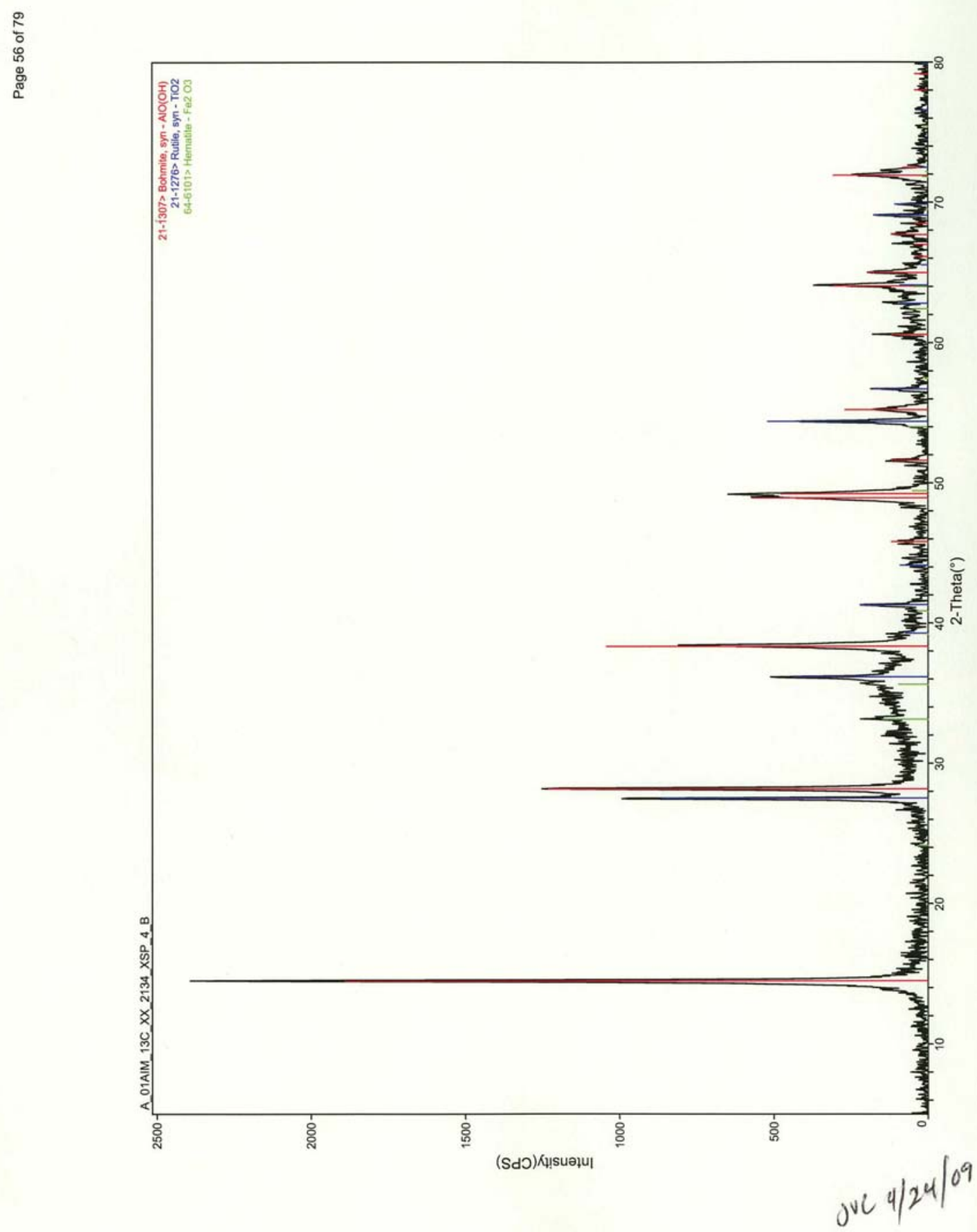

H.82 


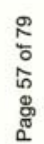

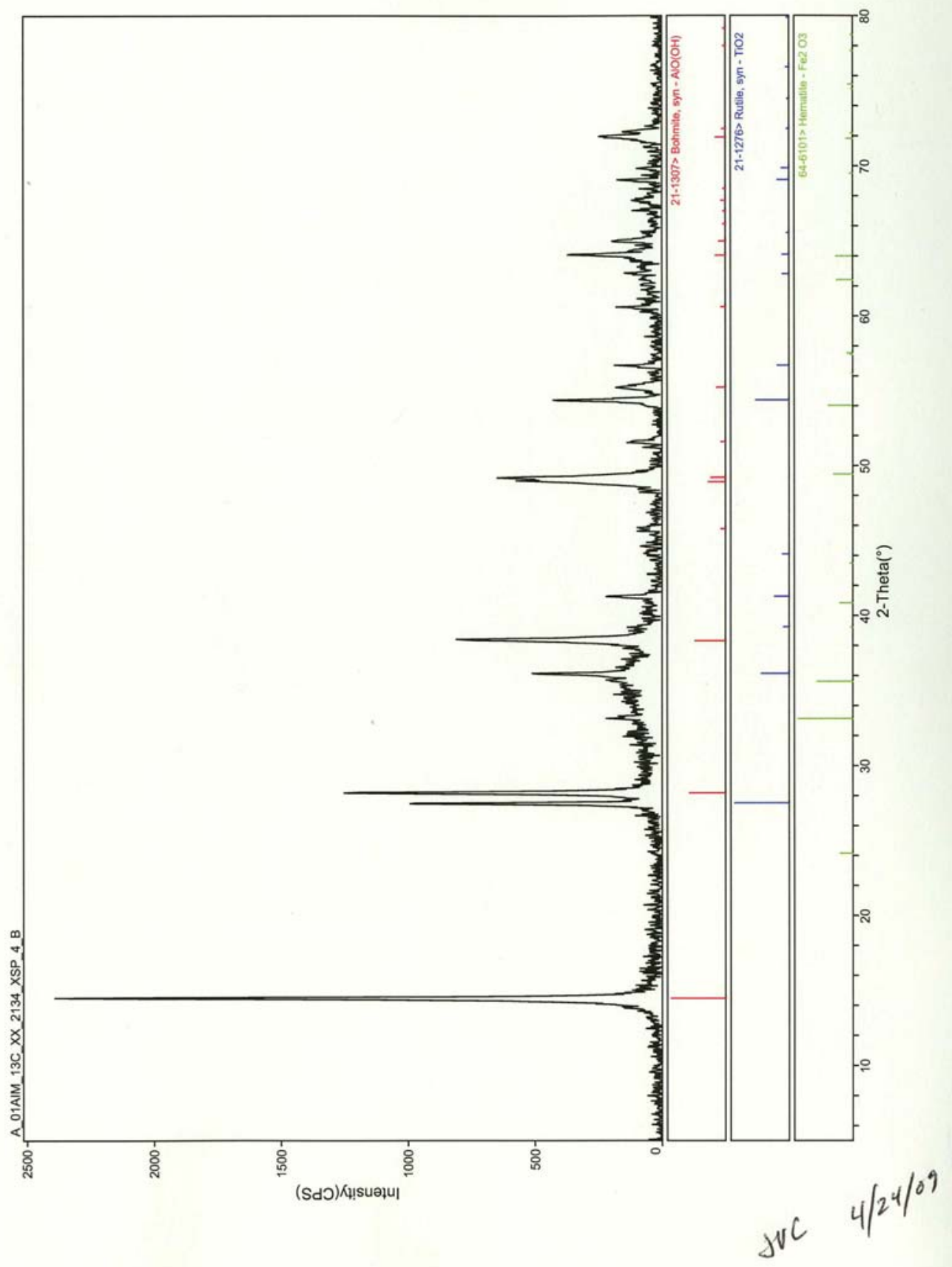




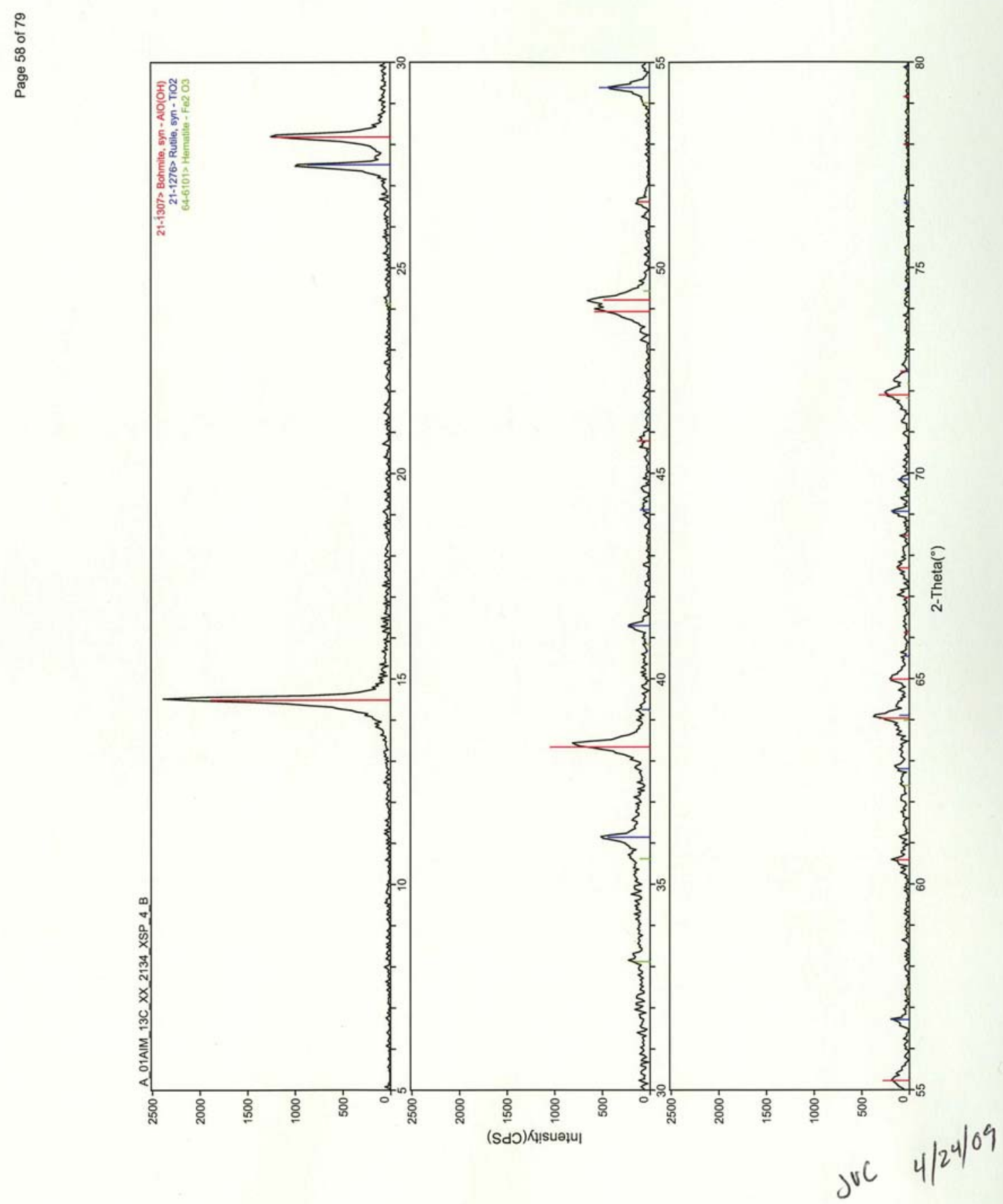




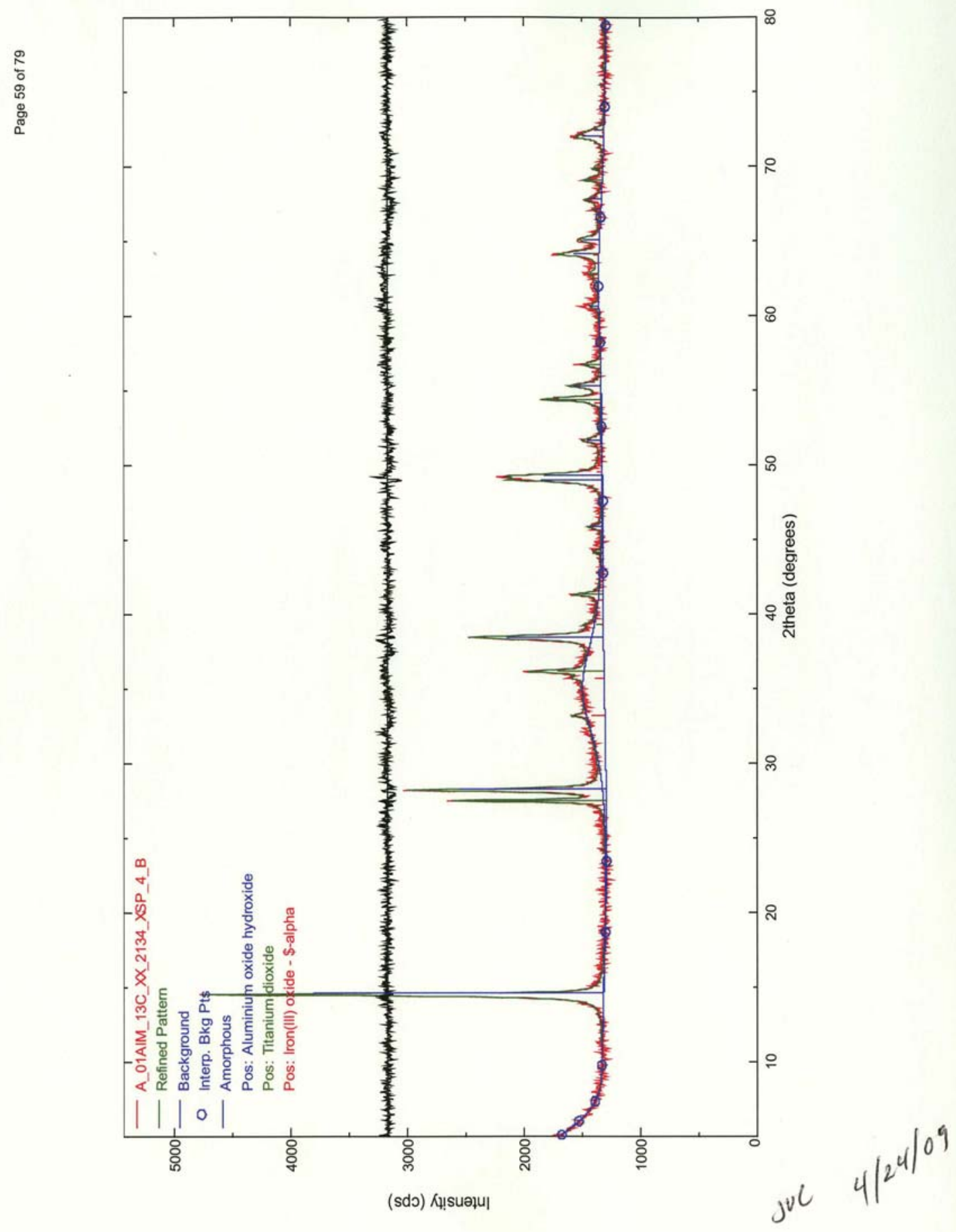


Page 60 of 79

Appendix A7

ICDD cards of interest for this sample

H.86 
- $\quad \mathrm{PDF} \# 21-1276$ : $\mathrm{QM}=$ Common( + ); $\mathrm{d}=$ Other/Unknown; $\mathrm{I}=($ Unknown)

Rutile, syn

$\mathrm{TiO} 2$

Radiation $=$ CuKal Lambda $=1.5406$ Filter $=$

Calibration $=\quad \mathrm{d}-$ Cutoff $=\quad \mathrm{I} / \mathrm{Ic}(\mathrm{RIR})=3.4$

Ref: Level-1 PDF

Tetragonal, $\mathrm{P} 42 / \mathrm{mnm}(136) \quad \mathrm{Z}=2 \quad \mathrm{mp}=$

CELL: $4.5933 \times 4.5933 \times 2.9592<90.0 \times 90.0 \times 90.0>$

$\operatorname{Density}(\mathrm{c})=4.23$ Density $(\mathrm{m})=\quad \mathrm{Mwt}=\mathrm{Vol}=62.4$

Ref: Ibid.

P.S $=$

Strong Line: $3.25 / \mathrm{X} \quad 1.69 / 6 \quad 2.49 / 5 \quad 2.19 / 3 \quad 1.62 / 2 \quad 1.36 / 2 \quad 0.82 / 1 \quad 1.35 / 1$

- $\quad$ PDF\#21-1307: $\mathrm{QM}=$ Common $(+) ; \mathrm{d}=$ Other/Unknown; $\mathrm{I}=($ Unknown $)$

Boehmite, syn

$\mathrm{AlO}(\mathrm{OH})$

Radiation $=$ CuKal Lambda $=1.5406$ Filter $=$

Calibration $=\quad \mathrm{d}$-Cutoff $=\quad$ I/Ic(RIR) $=$

Ref: Level-1 PDF

Orthorhombic, Amam(63)

$\mathrm{Z}=4 \mathrm{mp}=$

CELL: $3.7 \times 12.227 \times 2.868<90.0 \times 90.0 \times 90.0>\quad$ P.S $=$

$\operatorname{Density}(\mathrm{c})=3.07$ Density $(\mathrm{m})=\quad \mathrm{Mwt}=\quad \mathrm{Vol}=129.8$

Ref: Ibid.

Strong Line: $6.11 / \mathrm{X} \quad 3.16 / 7 \quad 2.35 / 6 \quad 1.86 / 3 \quad 1.85 / 3 \quad 1.45 / 2 \quad 1.31 / 2 \quad 1.66 / 1$

- PDF\#64-6101: QM=Calculated; $\mathrm{d}=\mathrm{Other} /$ Unknown; $\mathrm{I}=($ Unknown)

Hematite - synthetic, Hematite group

$\mathrm{Fe} 2 \mathrm{O} 3$

Radiation $=\mathrm{CuKal} \quad$ Lambda $=1.5406$ Filter $=$

Calibration $=\quad \mathrm{d}-$ Cutoff $=\quad$ I $/ \mathrm{Ic}(\mathrm{RIR})=3.04$

Ref: Calculated from FIZ\#82134 (ICSD @08/01/01) by Jade 6.x

Hexagonal, $\mathrm{R}-3 \mathrm{c}(167) \quad \mathrm{Z}=6 \quad \mathrm{mp}=$

CELL: $5.037 \times 5.037 \times 13.771<90.0 \times 90.0 \times 120.0>$ P.S $=$

$\operatorname{Density}(\mathrm{c})=5.258$ Density $(\mathrm{m})=\quad \mathrm{Mwt}=\quad \mathrm{Vol}=302.6$

Ref: Ibid.

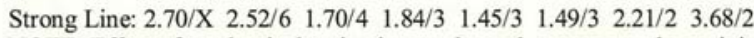

NOTE: Effect of mechanical activation on the real structure and reactivity of iron(III) oxide with corundum-type structure, Journal of Solid State Chemistry [JSSCB] 123 (1996) 191-202, Sadykov V A, Isupova L A, Tsybulya S V,

Cherepanova S V, Litvak G S, Burgina E B, Kustova G N, Kolomiichuk V N, Ivanov V P, Paukshtis E A, Golovin A V, Avvakumov E G, X-ray diffraction (powder).

$\mathrm{R}=0.053$ 
Date:

February 06, 2009

To:

O. Bredt

From

\section{J. V. Crum}

Subject: XRD Examination of: B_FRPGM_001_XX_2713_XSP_4_B
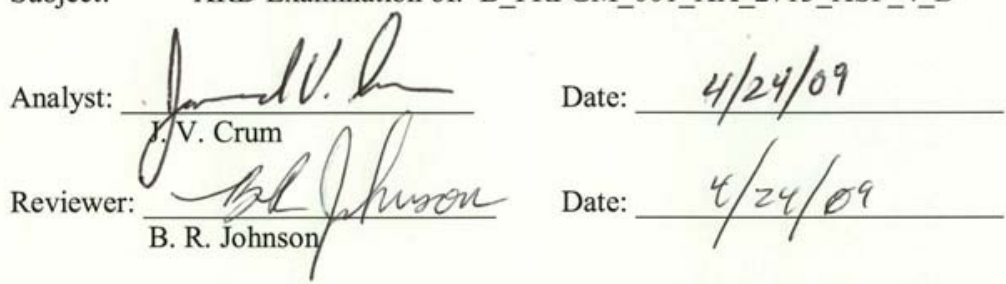

XRD data from the samples identified in Table I have been examined for identification of crystalline phases present.

Table I: Samples Examined in this Series

\begin{tabular}{|c|c|c|c|c|}
\hline Identification & XRD File ID $^{(a)}$ & $\begin{array}{c}\text { Count Time per } \\
\text { Step (Seconds) }\end{array}$ & $\begin{array}{c}\text { Number of } \\
\text { Replications }\end{array}$ & Internal Standard \\
\hline $\begin{array}{c}\text { B_FRPGM_001_XX_2713 } \\
\text { XSP_4_B }\end{array}$ & 031309A11 & 4 & 1 & $4.93 \mathrm{wt} \% \mathrm{TiO}_{2}$ \\
\hline
\end{tabular}

(a) The letter appended to the XRD File ID identifies the first sample in the replication series. The letter has been dropped in subsequent text to indicate that the referenced file is the summation of data from replicate runs.

Sample analysis was carried out by the Pacific Northwest National Laboratory Advanced Processing and Application Group. The XRD instrument used was the Scintag PAD V X-ray Diffractometer, Property Number WD33356, located in lab 102 in the APEL building. The data range for the sample was $5^{\circ}$ to $80^{\circ}$ 2-theta, with a step size of $0.04^{\circ} 2$-theta and count time 4.0 seconds per step. Copper K $\alpha$ X-rays were used. The X-ray tube operating conditions were $45 \mathrm{kV}$ and $40 \mathrm{~mA}$.

Sample 031309A11 was prepared with $\mathrm{SRM} 674 \mathrm{~b}, \mathrm{TiO}_{2}$ internal standard. The dried powder was placed into a tungsten carbide milling chamber and milled for $2 \mathrm{~min}$ in the angstrom shaker mill. The $\mathrm{TiO}_{2}$ standard was then spiked in at $4.93 \mathrm{wt} \%$ with the dried powder and milled again for $2 \mathrm{~min}$ to insure a homogenous mixture of the two powders. The sample was then mounted into standard sample holder.

Phase identification was done by use of the JADE search match routines (version 6.0, Materials Data Inc.) with comparison to the ICDD (International Centre for Diffraction Data) data base PDF-2 release 1999, which includes the ICSD (Inorganic Crystal Structure Database maintained by Fachinformationszentrum (FIZ), Karlsruhe, Germany). Searches were restricted to the PDF and ICSD Inorganic sections.

The phase identification plots are shown using "stick figures" to indicate the phases present; peak positions and line intensities. The phase showing the greatest peak intensity is shown at the top of the stick figures display (except the internal standard, if used, is always the topmost). Phases present at lower peak area/height are shown in decreasing order down the display. 
The identified phases, ranked by relative peak intensities, are given below for each sample. Figure 1 shows the raw summed pattern. The background fitted pattern, along with the identified phases, are shown in figures 2,3 , and 4 .

B_FRPGM_001_XX_2713_XSP_4_B, File: 031309A11

- Rutile, $\mathrm{TiO}_{2}$, card\# 21-1276

- Boehmite, syn, $\mathrm{AlO}(\mathrm{OH})$, card\# 21-1307, Excellent overall fit. Peak locations and intensities are a good match to the pattern.

- Gibbsite, $\mathrm{Al}(\mathrm{OH})_{3}$, card\# 60-2702, Good fit.

- Amorphous material, $\sim 36^{\circ} 2 \theta$.

The pattern was also examined using RIQAS (release 4.0.0.26, 6/10/2002, Materials Data Inc.) rietveld analysis software. The phases identified above were input into the analysis along with a cubic spline function for the background and an amorphous hump at $\sim 36^{\circ} 2 \theta$, with fitted pattern shown in figure 5 .

The results of the analysis for the crystalline phases are:

- Boehmite, $\mathrm{AlO}(\mathrm{OH}), 51.6 \mathrm{wt} \%$, with a crystallite size of $414 \AA$

- Gibbsite, $\mathrm{Al}(\mathrm{OH})_{3}, 32.1 \mathrm{wt} \%$ with a crystallite size of $160 \AA$

- The balance of the material is amorphous

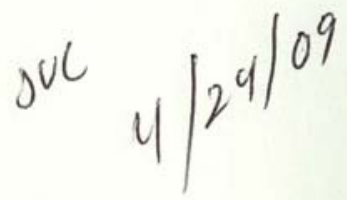




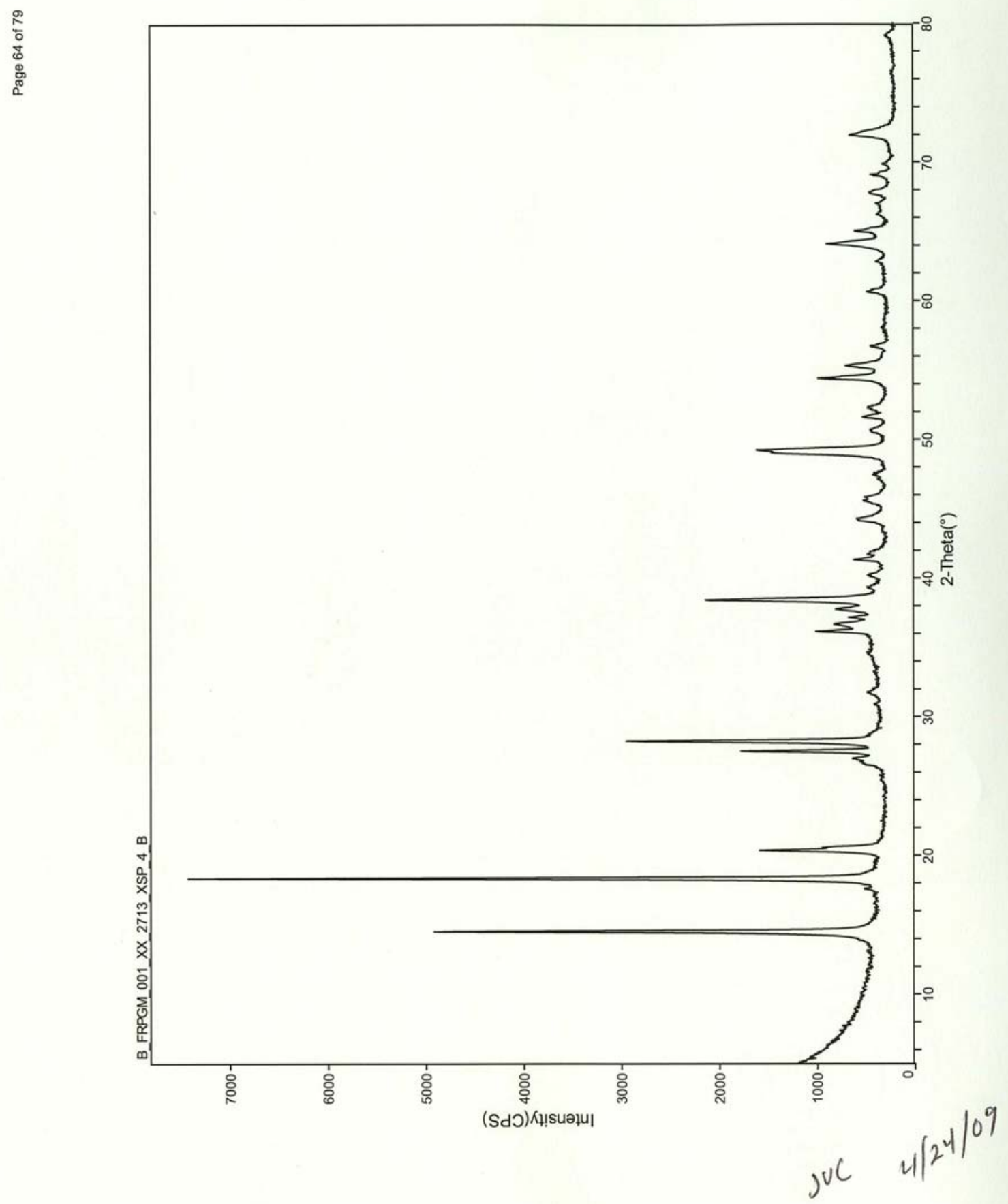

H.90 


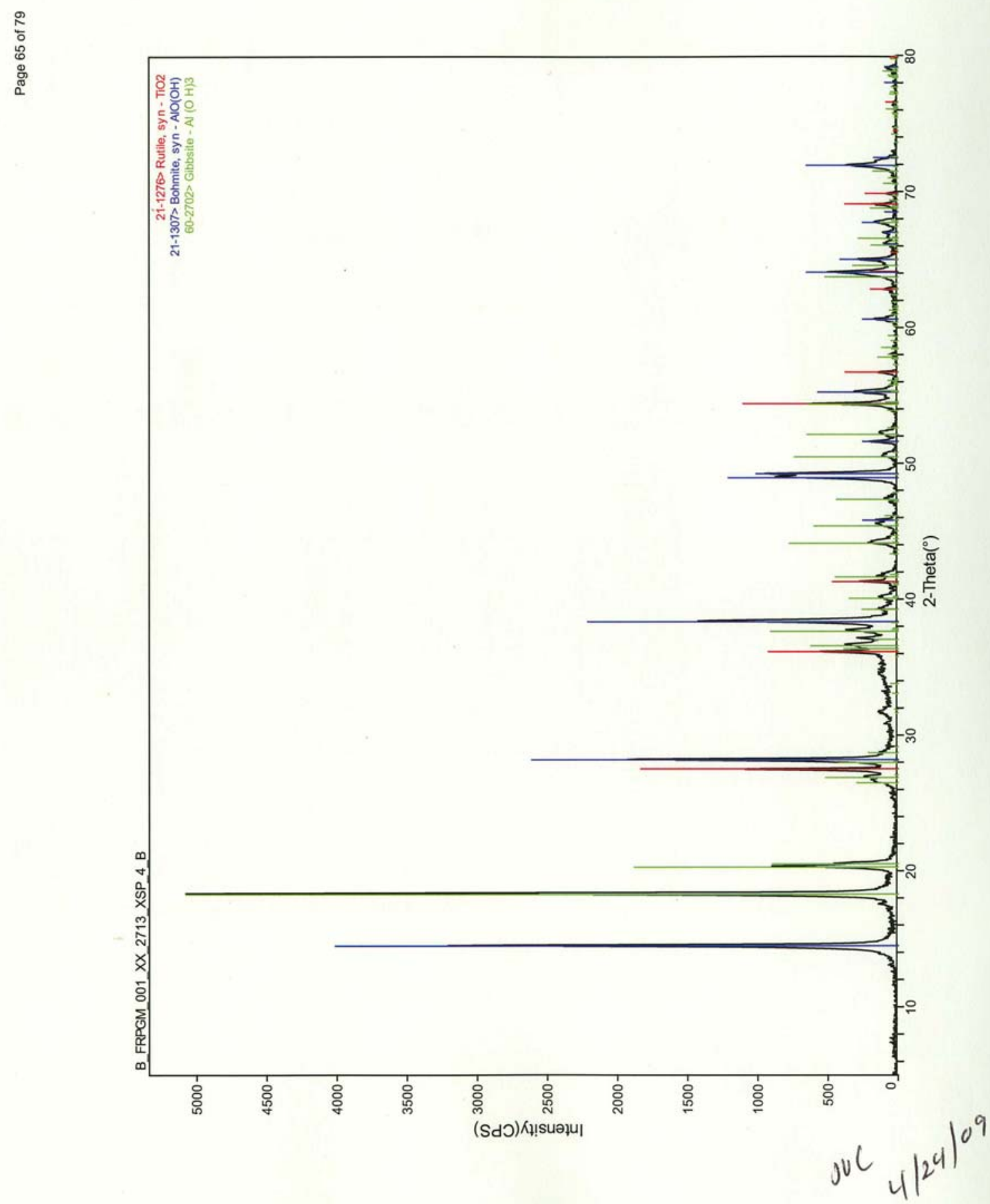

H.91 
8
2
0
8
8
8
8
0

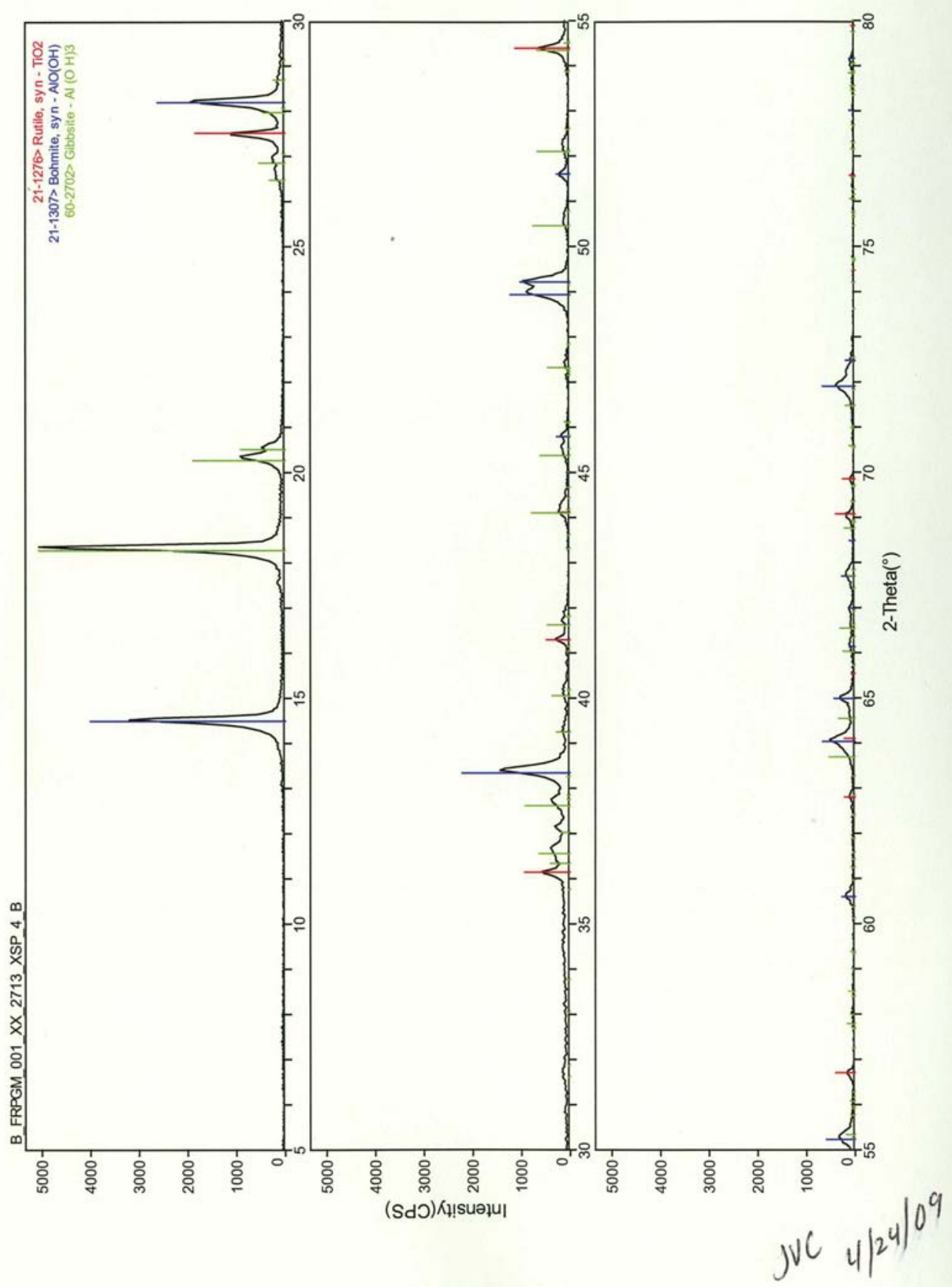


8
$\frac{8}{0}$
6
0
9
0
0

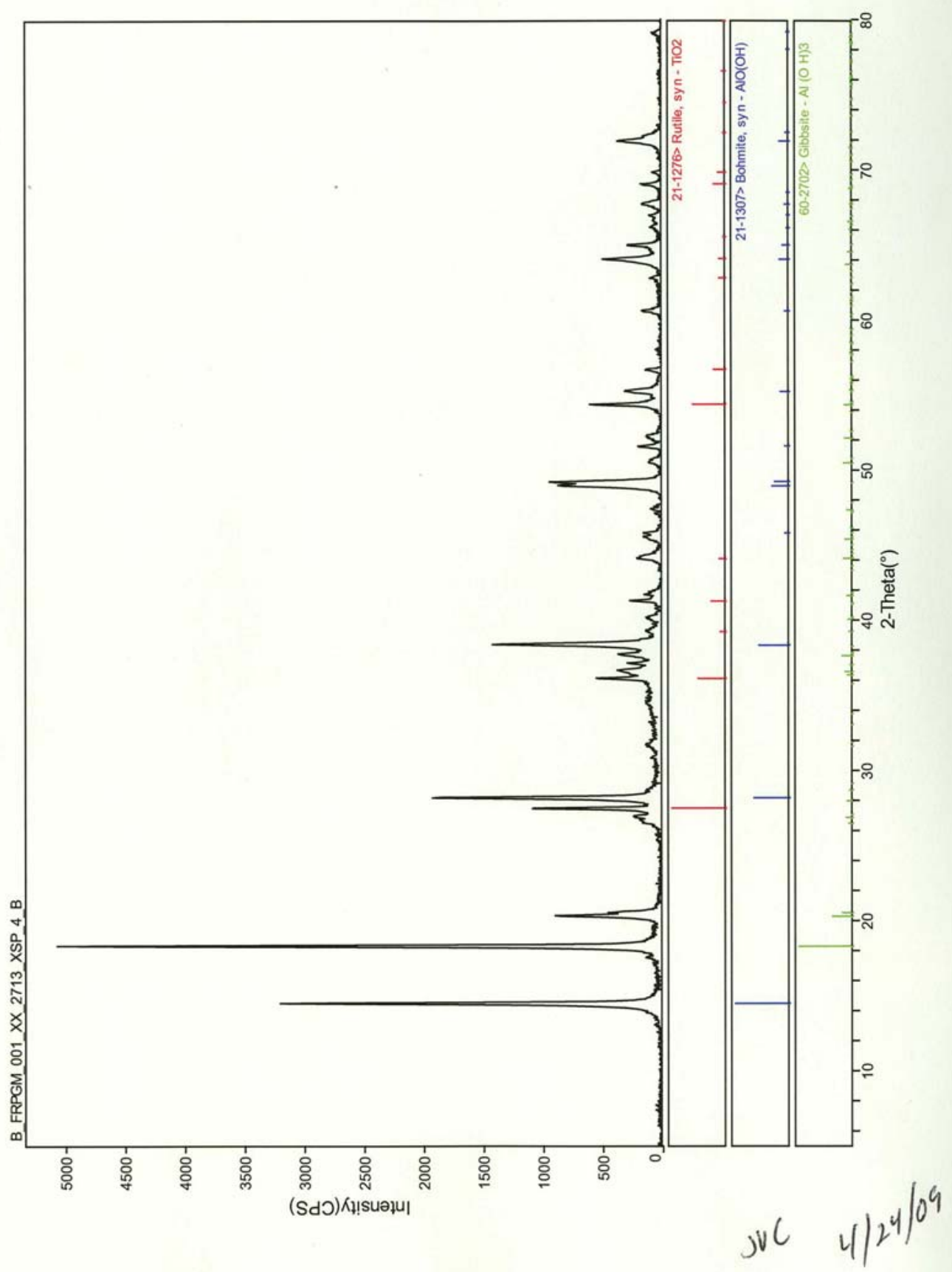


8
$\stackrel{2}{0}$
0
0
$\$$
8
0

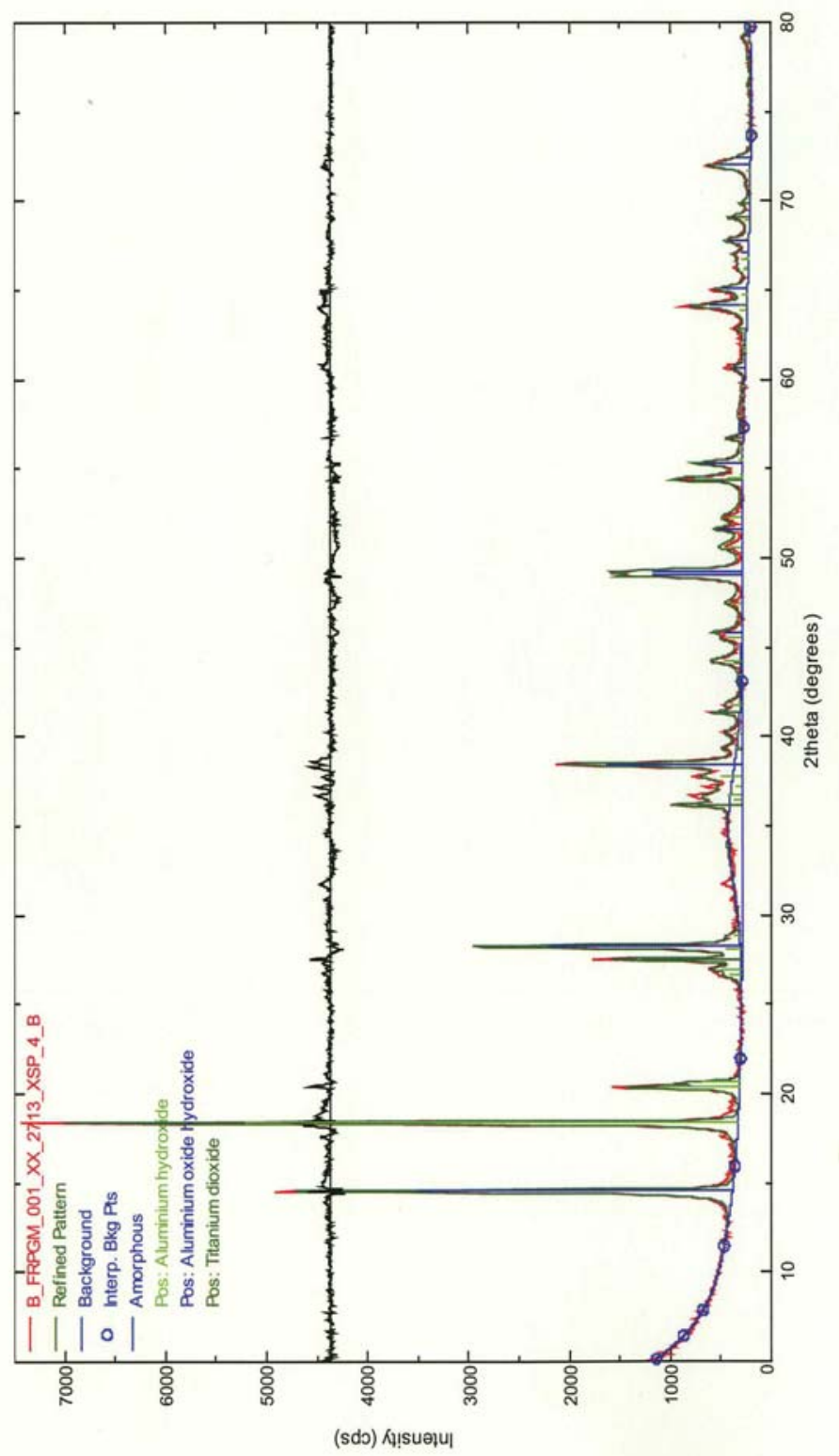

Juc 4124109 
Page 69 of 79

Appendix A8

ICDD cards of interest for this sample

H.95 
- $\quad$ PDF\#21-1276: $\mathrm{QM}=$ Common( + ); $\mathrm{d}=$ Other/Unknown; $\mathrm{I}=($ Unknown $)$

Rutile, syn

$\mathrm{TiO}_{2}$

Radiation $=\mathrm{CuKal} \quad$ Lambda $=1.5406$ Filter $=$

Calibration $=\quad \mathrm{d}$-Cutoff $=\quad \mathrm{I} / \mathrm{Ic}(\mathrm{RIR})=3.4$

Ref: Level-1 PDF

Tetragonal, $\mathrm{P} 42 / \mathrm{mnm}(136) \quad \mathrm{Z}=2 \quad \mathrm{mp}=$

CELL: $4.5933 \times 4.5933 \times 2.9592<90.0 \times 90.0 \times 90.0>$

$\operatorname{Density}(\mathrm{c})=4.23$ Density $(\mathrm{m})=\quad \mathrm{Mwt}=\quad \mathrm{Vol}=62.4$

$\mathrm{P} . \mathrm{S}=$

Ref: Ibid.

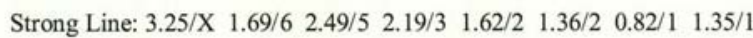

- $\quad$ PDF\#21-1307: $\mathrm{QM}=$ Common $(+)$; $\mathrm{d}=$ Other/Unknown; $\mathrm{I}=($ Unknown)

Boehmite, syn

$\mathrm{AlO}(\mathrm{OH})$

Radiation $=\mathrm{CuKa} \quad$ Lambda $=1.5406$ Filter $=$

Calibration $=\quad \mathrm{d}-$ Cutoff $=\quad \mathrm{I} / \mathrm{Ic}(\mathrm{RIR})=$

Ref: Level-1 PDF

Orthorhombic, Amam(63) $\quad \mathrm{Z}=4 \quad \mathrm{mp}=$

CELL: $3.7 \times 12.227 \times 2.868<90.0 \times 90.0 \times 90.0>\quad$ P.S $=$

$\operatorname{Density}(\mathrm{c})=3.07$ Density $(\mathrm{m})=\quad \mathrm{Mwt}=\quad \mathrm{Vol}=129.8$

Ref: Ibid.

Strong Line: $6.11 / \mathrm{X}^{3} \quad 3.16 / 7 \quad 2.35 / 6 \quad 1.86 / 3 \quad 1.85 / 3 \quad 1.45 / 2 \quad 1.31 / 2 \quad 1.66 / 1$

- PDF\#60-2702: $\mathrm{QM}=$ Calculated; $\mathrm{d}=$ Other/Unknown; $\mathrm{I}=($ Unknown $)$

Gibbsite

$\mathrm{Al}(\mathrm{O} \mathrm{H})_{3}$

Radiation $=\mathrm{CuKal} \quad$ Lambda $=1.5406$ Filter $=$

Calibration $=\quad \mathrm{d}$-Cutoff $=\quad \mathrm{I} / \mathrm{Ic}(\mathrm{RIR})=1.67$

Ref: Calculated from FIZ\#6162 (ICSD@08/01/01) by Jade 6.x

Monoclinic, $\mathrm{P} 21 / \mathrm{n}(14) \quad \mathrm{Z}=8 \quad \mathrm{mp}=$

CELL: $8.684 \times 5.078 \times 9.736<90.0 \times 94.54 \times 90.0>$ P.S $=$

$\operatorname{Density}(\mathrm{c})=2.421$ Density $(\mathrm{m})=\quad \mathrm{Mwt}=\mathrm{Vol}=428.0$

Ref: Ibid.

Strong Line: $4.85 / \mathrm{X} \quad 4.38 / 4 \quad 2.39 / 2 \quad 4.33 / 2 \quad 2.05 / 2 \quad 1.81 / 1 \quad 1.69 / 1 \quad 1.75 / 1$

NOTE: Refinement of the crystal structure of gibbsite, $\mathrm{Al}(\mathrm{O} \mathrm{H})_{3}$, Zeitschrift fuer Kristallographie, Kristallgeometrie, Kristallphysik, Kristallchemie (-144,1977) [ZEKGA] 139 (1974) 129-135, Saalfeld H, Wedde M, Dx=2.4.

M PDF 33-18.

$\mathrm{R}=0.032$. 
Date:

To:

O. Bredt

From

Subject: XRD Examination of: A_02AML_021_XX_2460_XSP_4

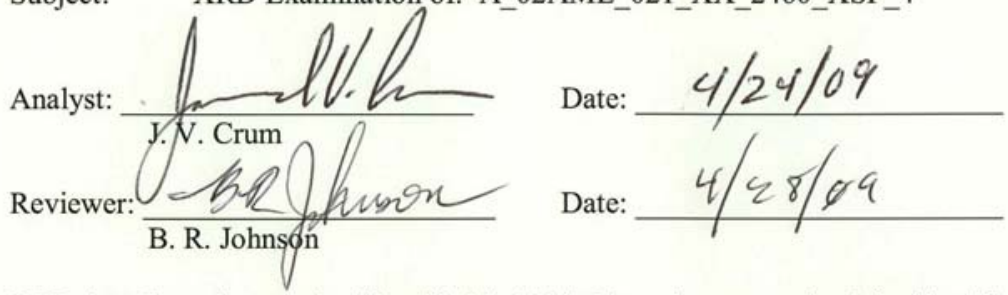

XRD data from the samples identified in Table I have been examined for identification of crystalline phases present.

Table I: Samples Examined in this Series

\begin{tabular}{|c|c|c|c|c|}
\hline Identification & XRD File ID $^{(a)}$ & $\begin{array}{c}\text { Count Time per } \\
\text { Step (Seconds) }\end{array}$ & $\begin{array}{c}\text { Number of } \\
\text { Replications }\end{array}$ & Internal Standard \\
\hline A_02AML_021_XX_2460 & 031709A11 & 4 & 1 & $5.0 \mathrm{TiO}_{2}$ \\
\hline
\end{tabular}

(a) The letter appended to the XRD File ID identifies the first sample in the replication series. The letter has been dropped in subsequent text to indicate that the referenced file is the summation of data from replicate runs.

Sample analysis was carried out by the Pacific Northwest National Laboratory Advanced Processing and Application Group. The XRD instrument used was the Scintag PAD V X-ray Diffractometer, Property Number WD33356, located in lab 102 in the APEL building. The data range for the sample was $5^{\circ}$ to $80^{\circ}$ 2-theta, with a step size of $0.04^{\circ} 2$-theta and count time 4.0 seconds per step. Copper K $\alpha \mathrm{X}$-rays were used. The X-ray tube operating conditions were $45 \mathrm{kV}$ and $40 \mathrm{~mA}$.

Sample 031709A11 was prepared with SRM 674b, $\mathrm{TiO}_{2}$ internal standard. The dried powder was placed into a tungsten carbide milling chamber and milled for $1 \mathrm{~min}$ in the angstrom shaker mill. The $\mathrm{TiO}_{2}$ standard was then spiked in at $5.0 \mathrm{wt} \%$ with the dried powder and milled again for $2 \mathrm{~min}$ to insure a homogenous mixture of the two powders. The sample was then mounted into an off-axis quartz sample holder (zero background holder).

Phase identification was done by use of the JADE search match routines (version 6.0, Materials Data Inc.) with comparison to the ICDD (International Centre for Diffraction Data) data base PDF-2 release 1999, which includes the ICSD (Inorganic Crystal Structure Database maintained by Fachinformationszentrum (FIZ), Karlsruhe, Germany). The chemistry provided for Group 7, in order of decreasing concentration, was $\mathrm{Fe}, \mathrm{Na}, \mathrm{U}, \mathrm{P}, \mathrm{Ca}, \mathrm{Al}, \mathrm{Si}, \mathrm{Bi}, \mathrm{Sr}$ and $\mathrm{Mg}$. Phase identifications first done without chemistry restrictions. Searches were restricted to the PDF and ICSD Inorganic sections.

The phase identification plots are shown using "stick figures" to indicate the phases present; peak positions and line intensities. The phase showing the greatest peak intensity is shown at the top of the stick figures display (except the internal standard, if used, is always the topmost). Phases present at lower peak area/height are shown in decreasing order down the display. Sample identification is below the scan or 
shown in the top most left of the plot figures. Scan running conditions are shown to the right on the identification.

The identified phases, ranked by relative peak intensities, are given below for each sample. Figure 1 shows the raw summed pattern. The background fitted pattern along with the identified phases are shown in figures 2,3 , and 4 .

A_02AML_021_XX_2460_XSP_4, File: 031709A11

- Rutile, $\overline{\mathrm{TiO}}_{2}$, card\# $\overline{2} 1-12 \overline{7} \overline{6}$

- Boehmite, syn, $\mathrm{AlO}(\mathrm{OH})$, card\# 21-1307, Excellent overall fit. Peak locations and intensities are a good match to the pattern.

- Hematite, $\mathrm{Fe}_{2} \mathrm{O}_{3}$, card\# 64-6101, Good fit.

The pattern was also examined using RIQAS (release 4.0.0.26, 6/10/2002, Materials Data Inc.) rietveld analysis software. The phases identified above were input into the analysis along with a polynomial background and an amorphous hump at $\sim 35^{\circ} 2 \theta$, with fitted pattern shown in figure 5 .

The results of the analysis are:

- Boehmite, $\mathrm{AlO}(\mathrm{OH}), 38.4 \mathrm{wt} \%$, with a crystallite size of $554 \AA$

- Hematite, $\mathrm{Fe}_{2} \mathrm{O}_{3}, 1.1 \mathrm{wt} \%$, with a crystallite size of $750 \AA$

$$
\text { juc } 4 / 24)^{109}
$$




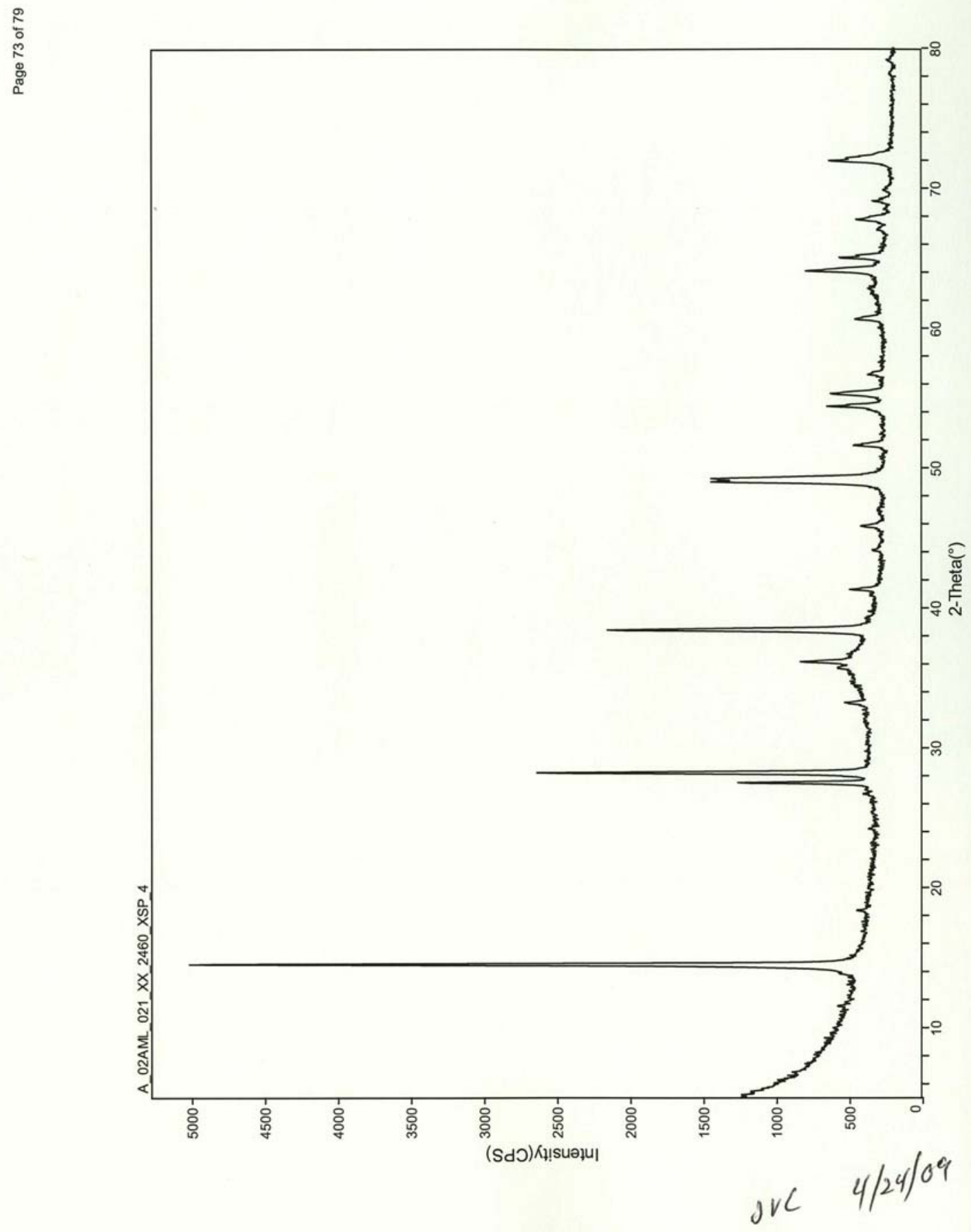

H.99 


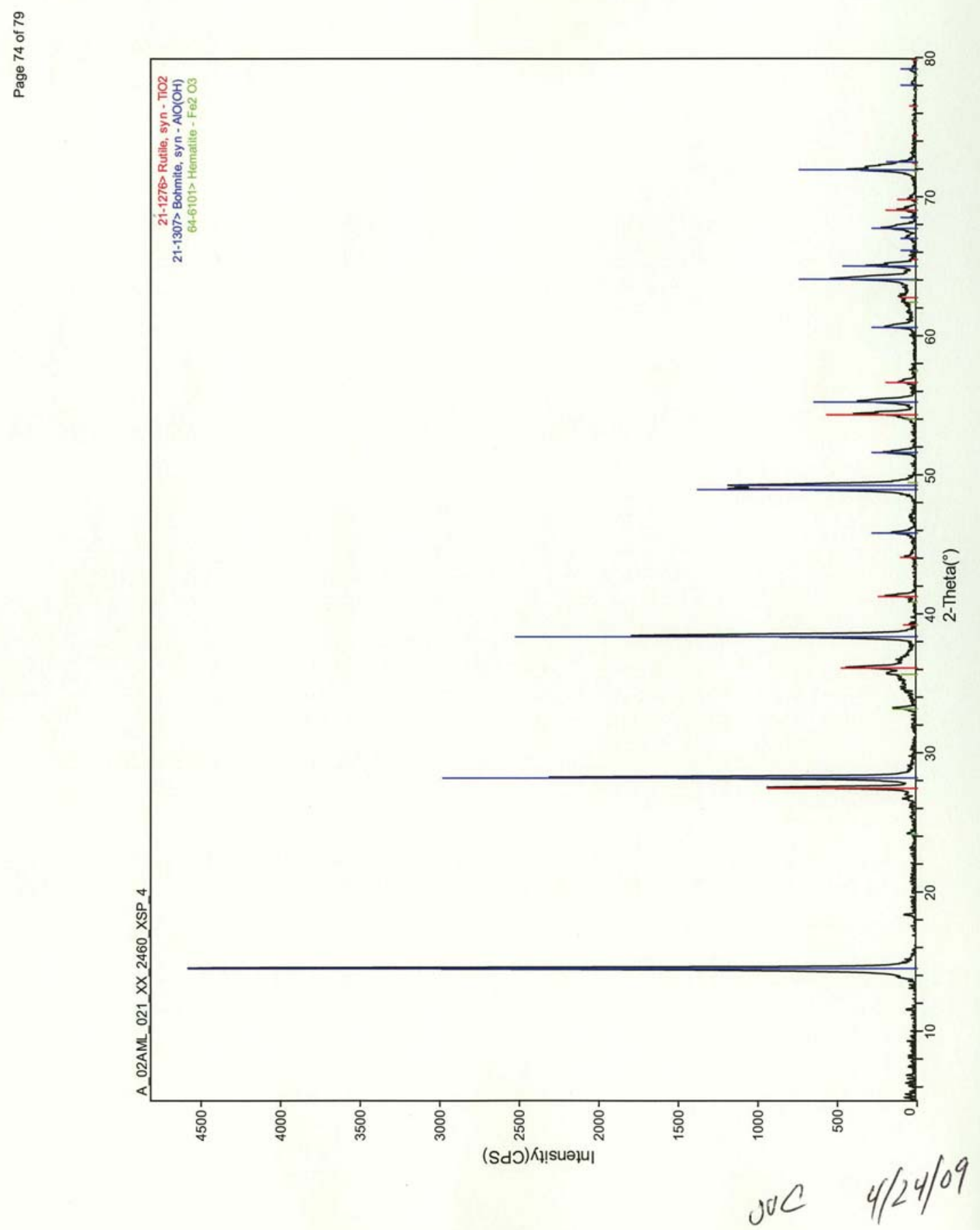

H. 100 


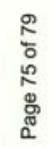

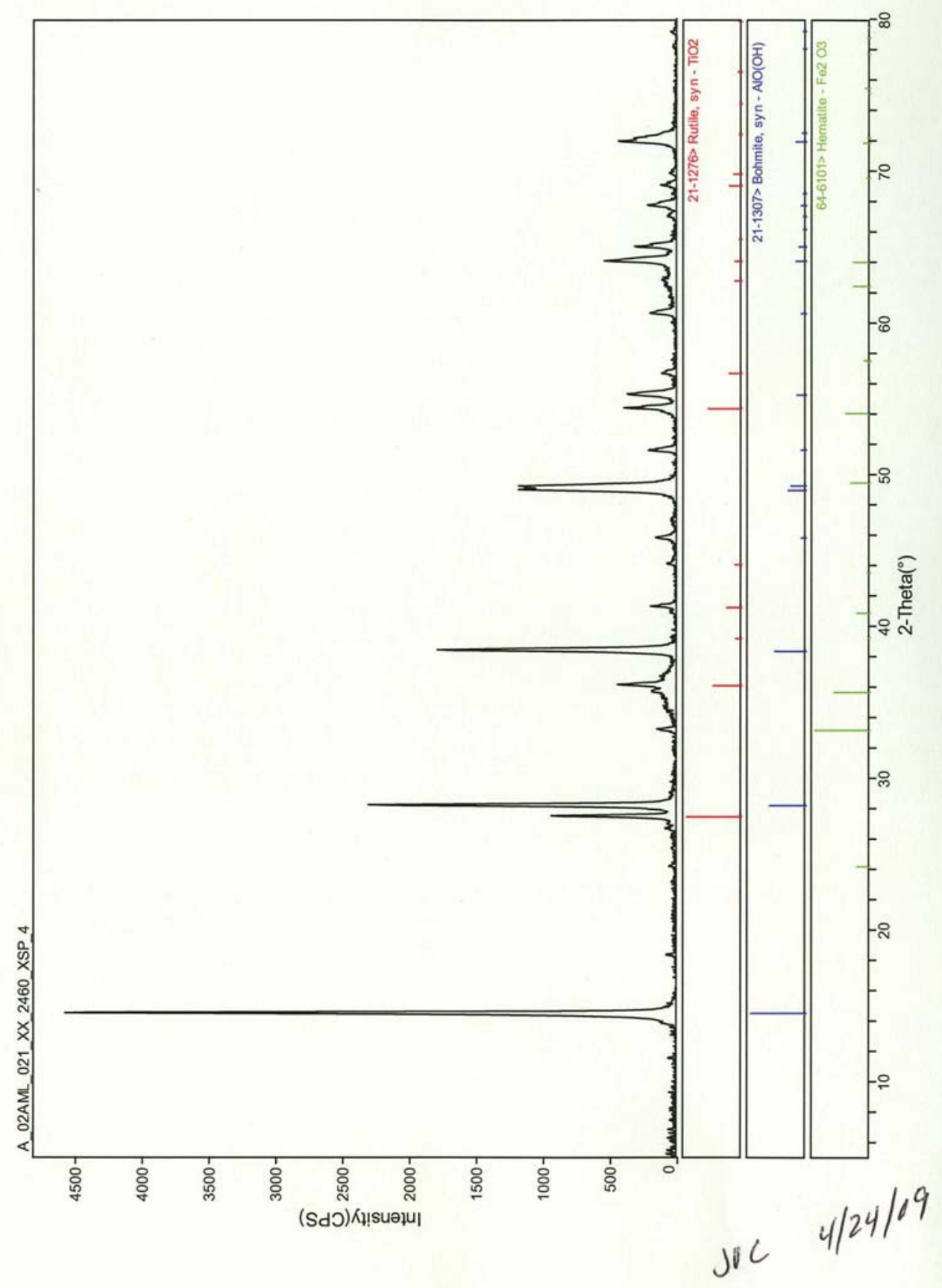

H. 101 
8
8
$\vdots$
0
0
8
0
0

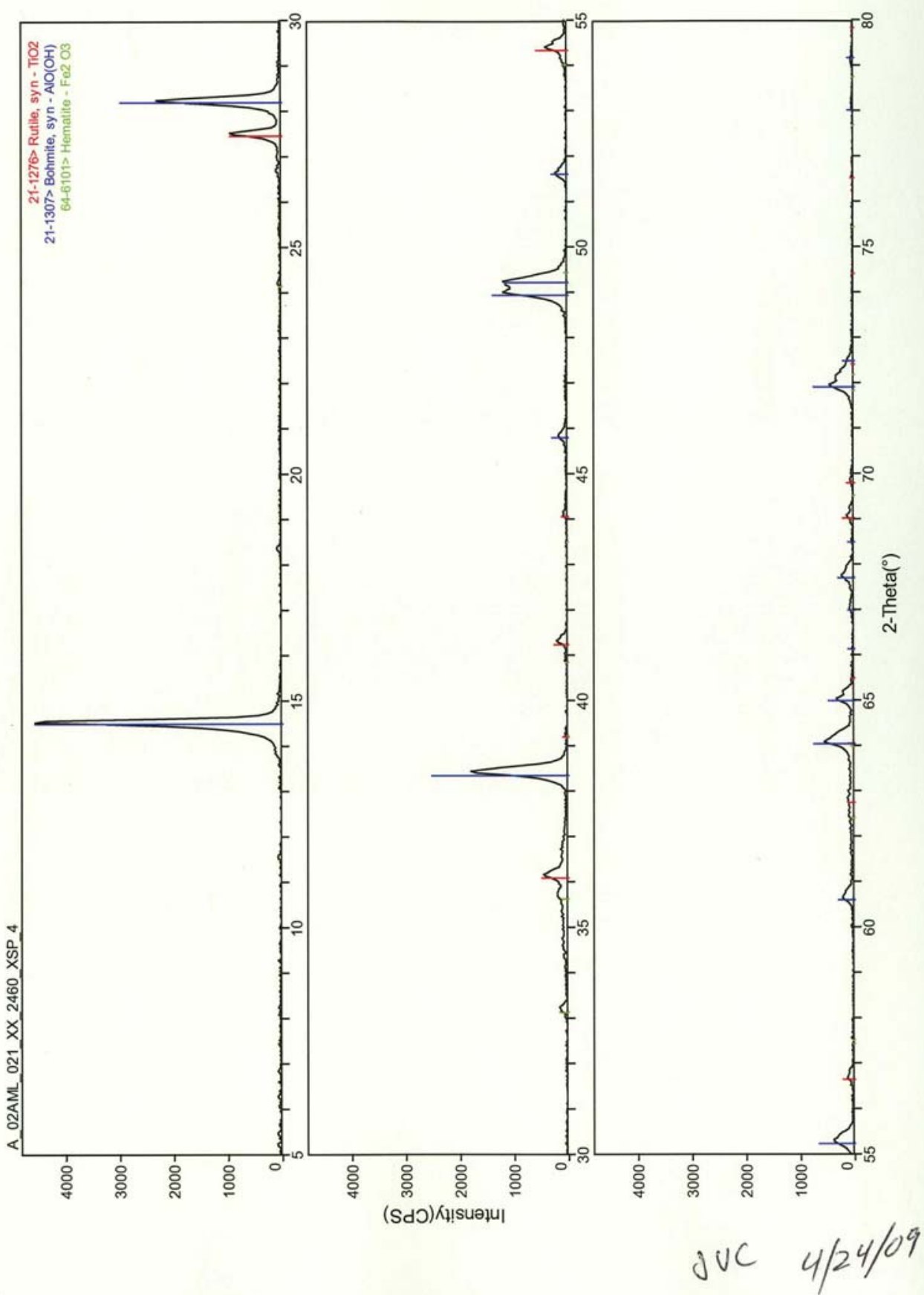

H.102 


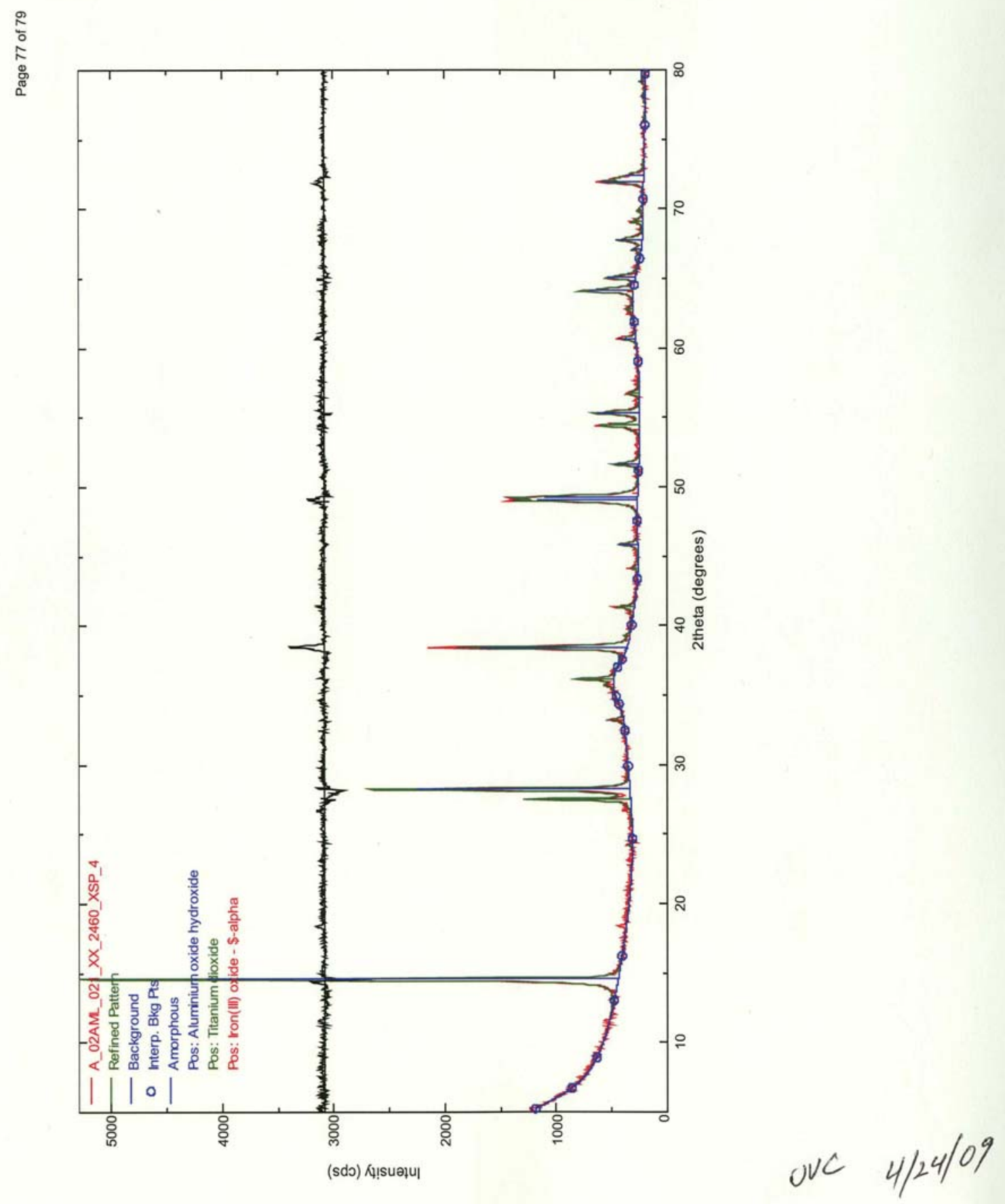

H.103 
Page 78 of 79

Appendix A

ICDD cards of interest for this sample

H.104 
- $\mathrm{PDF} \# 21-1276: \mathrm{QM}=$ Common $(+) ; \mathrm{d}=$ Other/Unknown; $\mathrm{I}=($ Unknown $)$ Rutile, syn

$\mathrm{TiO} 2$

Radiation $=$ CuKal Lambda $=1.5406$ Filter $=$

Calibration $=\quad \mathrm{d}-$ Cutoff $=\quad \mathrm{I} / \mathrm{Ic}(\mathrm{RIR})=3.4$

Ref: Level-1 PDF

Tetragonal, $\mathrm{P} 42 / \mathrm{mnm}(136) \quad \mathrm{Z}=2 \quad \mathrm{mp}=$

CELL: $4.5933 \times 4.5933 \times 2.9592<90.0 \times 90.0 \times 90.0>$

$\operatorname{Density}(\mathrm{c})=4.23$ Density $(\mathrm{m})=\quad \mathrm{Mwt}=\quad \mathrm{Vol}=62.4$

P.S $=$

Ref: Ibid.

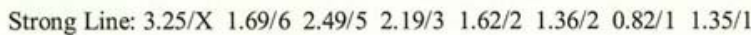

- $\quad \mathrm{PDF} \# 21-1307: \mathrm{QM}=$ Common(+); $\mathrm{d}=$ Other/Unknown; $\mathrm{I}=($ Unknown)

Boehmite, syn

$\mathrm{AlO}(\mathrm{OH})$

Radiation $=$ CuKal Lambda $=1.5406$ Filter $=$

Calibration $=\quad \mathrm{d}-$ Cutoff $=\quad \mathrm{I} / \mathrm{Ic}(\mathrm{RIR})=$

Ref: Level-1 PDF

Orthorhombic, Amam(63) Z $\quad$ =4 mp $=$

CELL: $3.7 \times 12.227 \times 2.868<90.0 \times 90.0 \times 90.0>\quad$ P.S $=$

$\operatorname{Density}(\mathrm{c})=3.07$ Density $(\mathrm{m})=\quad \mathrm{Mwt}=\quad \mathrm{Vol}=129.8$

Ref: Ibid.

Strong Line: $6.11 / \mathrm{X} \quad 3.16 / 7 \quad 2.35 / 6 \quad 1.86 / 3 \quad 1.85 / 3 \quad 1.45 / 2 \quad 1.31 / 2 \quad 1.66 / 1$

- PDF\#64-6101: $\mathrm{QM}=$ Calculated; $\mathrm{d}=\mathrm{O}$ ther/Unknown; $\mathrm{I}=($ Unknown)

Hematite - synthetic, Hematite group

$\mathrm{Fe} 2 \mathrm{O} 3$

Radiation $=\mathrm{CuKal} \quad$ Lambda $=1.5406$ Filter $=$

Calibration $=\quad$ d-Cutoff $=\quad$ I/Ic $($ RIR $)=3.04$

Ref: Calculated from FIZ $\$ 82134$ (ICSD @08/01/01) by Jade 6.x

Hexagonal, R-3c(167) $\mathrm{Z}=6 \quad \mathrm{mp}=$

CELL: $5.037 \times 5.037 \times 13.771<90.0 \times 90.0 \times 120.0>$ P.S $=$

$\operatorname{Density}(\mathrm{c})=5.258$ Density $(\mathrm{m})=\quad \mathrm{Mwt}=\quad \mathrm{Vol}=302.6$

Ref: Ibid.

Strong Line: $2.70 / X \quad 2.52 / 6 \quad 1.70 / 4 \quad 1.84 / 3 \quad 1.45 / 3 \quad 1.49 / 3 \quad 2.21 / 2 \quad 3.68 / 2$

NOTE: Effect of mechanical activation on the real structure and reactivity of iron(III) oxide with corundum-type structure, Journal of Solid State Chemistry [JSSCB] 123 (1996) 191-202, Sadykov V A, Isupova L A, Tsybulya S V, Cherepanova S V, Litvak G S, Burgina E B, Kustova G N, Kolomiichuk V N, Ivanov V P, Paukshtis E A, Golovin A V, Avvakumov E G, X-ray diffraction (powder). 


\section{H.3 Particle-Size Distribution}

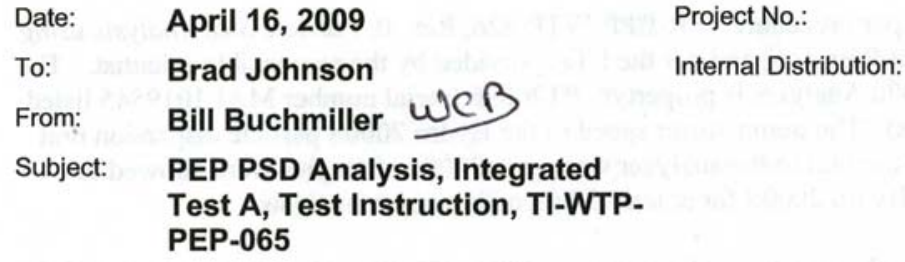

Six samples were delivered for analysis under WP\# H14568. These samples were to be analyzed per instructions provided in a 02/17/2009 Letter to Analyst (LTA). Copies of the particle distribution plots for the samples are attached in addition to a copy of the LTA. The particle size distribution plots for the performance standard calibration checks are attached as well. They were collected on 11/25/2008 and 03/09/2009 using the manufacturer specified material, QAS3001B, part No. CRM00016.

\section{SAMPLE PREP}

All 6 samples were analyzed as provided using a Malvern MS2000 Analyzer with Hydro 2000S particle dispersion unit. Settled samples delivered in conical bottom centrifuge tubes, were initially agitated with a Scientific Industries, Vortex-T, Genie 2 vibrator to the discretion of the analyst. After the initial agitation, the samples were shaken by hand and the slurry transferred to the Malvern MS2000 using a poly transfer pipette. The transfer pipette was also used to keep the sample material suspended in the centrifuge tubes during transfer.

The requester of service indicated concern over potential agglomeration issues and requested that each sample, in addition to the as-received form, be analyzed after sonication. A transfer pipette was used to mix the slurry and transfer the required amount to the analyzer. Nominally 1 to 5 drops of each sample/sub-sample were placed in the Hydro 2000S particle dispersion unit using $0.01 \mathrm{M} \mathrm{NaOH}$ as the dispersion fluid. Sample A_02AOL_015_2809_XSP_0 was analyzed using A_PF1 permeate as the dispersion fluid. Sample A_02AML_007_XX__2694_UDS_2 utilized both $0.01 \mathrm{M} \mathrm{NaOH}$ and $\mathrm{A} \_\mathrm{PF} 1$ permeate as dispersion fluids. The dispersion unit has an internal sonication device with a variable speed pump/agitator. Unless noted differently, the pump/agitator was operated at a setpoint of 3000 and the sonicator operated at $0 \%$ (as-received) or $50 \%$ maximum setpoint. Sonicator setpoints of up to $100 \%$ maximum were an option at the analyst's discretion.

The amount of sub-sample required for analysis varied with the actual concentration of the sample. Neither the amount of sample nor the amount of $0.01 \mathrm{M} \mathrm{NaOH}$ dispersion solution was critical to the analytical process since the analytical software indicates graphically the amount of sample needed for analysis. The actual amounts of sample and water did not need to be, nor were they measured or recorded. Any additional sonication required to de-agglomerate sample(s) was noted on the respective particle size distribution data plot. 
Brad Johnson

April 16, 2009

\section{ANALYSIS}

Analysis was performed per procedure TPR-RPP-WTP-626, Rev. 0, Particle Size Analysis using Malvern MS2000 (current Revision) and per the LTA provided by the responsible scientist. The ID of the Malvern MS2000 Analyzer is property\# PT20096 (serial number MAL1019545 listed on the attached data plots). The pump/stirrer speed of the Hydro 2000S particle dispersion unit (ID property\# PT20083) integral to the analyzer was set at 3000. Samples were allowed to circulate/sonicate in the Hydro $2000 \mathrm{~S}$ for at least 30 seconds prior to analysis.

The Malvern MS2000 Analyzer does not have the capability of calibration adjustment, and other than cleaning the lens or the sample chamber, or returning the equipment to the vendor, there are no adjustments, other than pump/stirrer speed and \% sonication, that will affect the operation of the equipment. Per the LTA, a QAS3001B, part No. CRM00016 standard was used to verify the analyzer performance on $11 / 25 / 2008$ and $03 / 09 / 2009$. Procedure TPR-RPP-WTP-626 requires that the performance analysis be repeated every 90 days. The following parameters were included in the standard analysis setup as per Malvern Instruction Sheet MRK704-0407-2007:

\section{Setup name: Glass Beads (typical) \\ Dispersant Name: DI Water \\ Sensitivity: Enhanced \\ Analysis model: Single mode spherical \\ Particle refractive index: 1.520 \\ Dispersant refractive index: 1.33}

Successful performance of the Malvern MS2000 as defined by the LTA, requires that standard $\mathrm{D}_{50}$ analysis results fall within $+/-10 \%$ of the manufacture's $\mathrm{D}_{50}$ specifications. The glass standard microspheres were analyzed on $11 / 25 / 2008$ and 03/09/2009 as spherical particles and the $\mathrm{D}_{50}$ values were 62.395 and 62.206 microns respectively, which fell within $+/-10 \%$ of the manufacture specified volume weighted $\mathrm{D}_{50}$ value of 60.59 microns. However, it was noted that the $03 / 09 / 2009$ standard was inadvertently analyzed using incorrect settings (Senstivity = Normal, and Analysis model $=$ General Purpose). The raw data was re-analyzed with the Mastersizer 2000 analytical software on $04 / 13 / 2009$ using the correct settings. The average $D_{50}$ results ( see LRB BNW 60175, page 51) from the repeat analysis of the raw data was 63.167 microns. This $D_{50}$ value still fell within the $+/-10 \%$ bracket on the vendor specified $D_{50}$ value. The re-analyzed results are included in Table 1 and a single typical data plot, record 2950, is included in the Performance Evaluation section of this report. 
Brad Johnson

April 16, 2009

Table 1. Standard Analysis Results from 11/25/2008 \& 03/09/2009

$M V$ and $D_{50}$ results in microns

\begin{tabular}{|c|c|c|c|c|c|c|}
\hline $\begin{array}{c}\text { Record } \\
\text { number }\end{array}$ & Sample Identification & $\begin{array}{c}\text { Measured } \\
\mathrm{D}_{50}\end{array}$ & $\begin{array}{c}\text { Vendor } \\
\text { specified } \\
\mathrm{D}_{50}\end{array}$ & $\begin{array}{c}\text { Measured } \\
\mathrm{D}_{50} \\
+10 \%\end{array}$ & $\begin{array}{c}\text { Measured } \\
\mathrm{D}_{50} \\
-10 \%\end{array}$ & Comments \\
\hline 10 & QASstd - Average & 62.395 & 60.590 & 68.6345 & 56.156 & Passed criteria \\
\hline 2946 & $\begin{array}{c}\text { QASstd, re-analyzed raw } \\
\text { data }\end{array}$ & 63.026 & 60.59 & 69.329 & 56.723 & Passed criteria \\
\hline 2947 & $\begin{array}{c}\text { QASstd, re-analyzed raw } \\
\text { data }\end{array}$ & 63.042 & 60.59 & 69.346 & 56.738 & Passed criteria \\
\hline 2948 & $\begin{array}{c}\text { QASstd, re-analyzed raw } \\
\text { data }\end{array}$ & 63.144 & 60.59 & 69.458 & 56.830 & Passed criteria \\
\hline 2949 & $\begin{array}{c}\text { QASstd, re-analyzed raw } \\
\text { data }\end{array}$ & 63.264 & 60.59 & 69.590 & 56.938 & Passed criteria \\
\hline 2950 & $\begin{array}{c}\text { QASstd re-analyzed raw } \\
\text { data }\end{array}$ & 63.360 & 60.59 & 69.696 & 57.024 & Passed criteria \\
\hline & $\begin{array}{c}\text { QASstd, re-analyzed raw } \\
\text { data - Average }\end{array}$ & 63.167 & 60.59 & 69.484 & 56.850 & \\
\hline
\end{tabular}

PEP specimens were sub-sampled a minimum of 2 times (noted as aliquots) to allow duplicate analysis of each sample. Per instructions on the LTA, 3 additional subsamples were to be analyzed if the initial duplicate MV (volume weighted mean diameter) values of the as-received or the sonicated sub-samples differed by more than $15 \%$. All duplicate analysis results varying by more than $100 \%$ were reviewed by the cognizant scientist. Any additional analysis requirements could be waived if the cognizant scientist agrees that agglomeration issues may cause results to be suspect.

The following parameters were included in the sample analysis setup:

Setup name: Ferric Oxide Hydroxide

Dispersant Name: $0.01 \mathrm{M} \mathrm{NaOH}$

Sensitivity: Normal

Analysis model: General purpose

Particle refractive index: 2.94

Dispersant refractive index: 1.33

For samples using A_PF1 permeate as the dispersion fluid:

Setup name: Ferric Oxide Hydroxide Dispersant Name: A_PF1 Permeate Sensitivity: Normal

Analysis model: General purpose

Particle refractive index: 2.94

Dispersant refractive index: 1.33 
Brad Johnson

April 16, 2009

\section{COMMENTS AND OBSERVATIONS}

Prior to completion of the Test A PSD sample analysis, discussions were initiated by the customer with regards to the effect of performing the analysis using A_PF1 Permeate as the dispersion fluid versus using $0.01 \mathrm{M} \mathrm{NaOH}$. The analysis of slurried samples versus the analysis of centrifuged solids was also discussed. Typically samples were received after the slurry form had been rinsed in $0.01 \mathrm{M} \mathrm{NaOH}$, centrifuged, and the supernatant liquid decanted. The undissolved solids in the bottom of the conical centrifuge tube (referred to as centrifuged solids) were re-suspended in $0.01 \mathrm{M} \mathrm{NaOH}$ and pipetted into the Malvern Hydro 2000S particle dispersion unit. Prior to these discussions, samples B_FRPGM_001_XX_2713_XSP_B and A T22GM_002_XX_1507_XSP_4_B had already been delivered for analysis in the centrifuged solids form. To help understand the effects of analysis with the A_PF1 Permeate and the analysis of the slurried form, multiple tests were performed on sample

A 02AML_007 XX 2694_UDS_2: 1) slurried with filtered A_PF1 permeate dispersion fluid, 2) slurried with un-filtered $\bar{A}$ PF1 permeate dispersion fluid, 3) slurried in $0.01 \mathrm{M} \mathrm{NaOH}$ dispersion fluid, and 4) washed (centrifuged 4 times replacing $0.01 \mathrm{M} \mathrm{NaOH}$ wash solution for each wash), using $0.01 \mathrm{M} \mathrm{NaOH}$ to re-suspend the centrifuged solids, and using $0.01 \mathrm{M} \mathrm{NaOH}$ as the PSD dispersion fluid. The results are shown in Table 2. NOTE that only 1 analysis, at the direction of the customer, was performed on each of the 4 pre-described conditions instead of the typical duplicate analysis.

\section{Table 2. Dispersion Fluid Evaluation Results}

\begin{tabular}{|c|c|c|c|c|c|}
\hline \multicolumn{6}{|c|}{ Diameter and D50 results in microns } \\
\hline $\begin{array}{l}\text { Record } \\
\text { number }\end{array}$ & Sample Name & $\mathrm{D}_{50}$ & $\begin{array}{c}\text { Volume } \\
\text { weighted } \\
\text { mean } \\
\text { diameter }\end{array}$ & $\begin{array}{c}\text { Ultrasonic } \\
\text { level }\end{array}$ & Analysis Condition \\
\hline 1177 & $\begin{array}{c}\text { A_02AML_007_XX_2694_UDS_2 - } \\
\text { Average }\end{array}$ & 1.882 & 11.259 & 0 & Un-washed, FilteredA PF1 \\
\hline 1201 & $\begin{array}{c}\text { A_02AML_007_XX_2694_UDS_2 - } \\
\text { Average }\end{array}$ & 2.26 & 15.994 & 0 & Un-washed, A_PF1 \\
\hline 1231 & $\begin{array}{c}\text { A_02AML_007_XX_2694_UDS_2 - } \\
\text { Average }\end{array}$ & 1.298 & 8.57 & 0 & Un-washed, $0.01 \mathrm{M} \mathrm{NaOH}$ \\
\hline 1255 & $\begin{array}{c}\text { A_02AML_007_XX_2694_UDS_2 - } \\
\text { Average }\end{array}$ & 1.25 & 1.892 & 0 & Washed, $0.01 \mathrm{M} \mathrm{NaOH}$ \\
\hline 1183 & $\begin{array}{c}\text { A_02AML_007_XX_2694_UDS_2 - } \\
\text { Average }\end{array}$ & 1.346 & 5.411 & 50 & Un-washed, FilteredA_PF1 \\
\hline 1207 & $\begin{array}{c}\text { A_02AML_007_XX_2694_UDS_2 - } \\
\text { Average }\end{array}$ & 1.413 & 4.968 & 50 & Un-washed, A_PF1 \\
\hline 1237 & $\begin{array}{c}\text { A_02AML_007_XX_2694_UDS_2 - } \\
\text { Average }\end{array}$ & 1.113 & 6.052 & 50 & Un-washed, $0.01 \mathrm{M} \mathrm{NaOH}$ \\
\hline 1261 & $\begin{array}{c}\text { A_02AML_007_XX_2694_UDS_2 - } \\
\text { Average }\end{array}$ & 1.031 & 1.485 & 50 & Washed, $0.01 \mathrm{M} \mathrm{NaOH}$ \\
\hline
\end{tabular}


Brad Johnson

April 16, 2009

It was observed that the particle size distribution (PSD) plots for the samples dispersed in permeate showed a distinct bi-modal size distribution, whereas the PSD plots for samples dispersed in $0.01 \mathrm{M} \mathrm{NaOH}$ typically showed only a single prominent peak.

As a result of the A_02AML_007_XX_2694_UDS_2 analysis, the customer decided to analyze the pre-wash slurried sample A_02AOL_015_XX_28009_XSP_0 in A_PF1 permeate, and the post-wash slurried sample A_02AOL_015_XX_28011_XSP_0 in $0.01 \overline{\mathrm{M}} \mathrm{NaOH}$. All other samples were analyzed in $0.0 \overline{1} \mathrm{M} \mathrm{NaOH}$.

Both slurried samples analyzed with the $0.01 \mathrm{M} \mathrm{NaOH}$ dispersion fluid produced results that were outside of the $+/-15 \%$ duplicate "acceptance" requirement and therefore 3 additional aliquots for each sample were analyzed. The slurried sample results analyzed with the A_PF1 permeate dispersion fluid fell within the $+/-15 \%$ duplicate "acceptance" requirement as did the centrifuged solids samples. Results are shown in Table 3.

In addition to $0 \%$ sonication and $50 \%$ sonication, most samples were analyzed at $100 \%$ sonication to observe the effects of sonication on the breakdown of potentially agglomerated particles. With the technical input of the equipment custodian, it was decided to report only the $0 \%$ and $50 \%$ sonicated results.

Table 3. Sample Analysis Results

\begin{tabular}{|c|c|c|c|c|c|c|}
\hline \multirow[b]{2}{*}{$\begin{array}{l}\text { Record } \\
\text { number }\end{array}$} & \multicolumn{6}{|c|}{ Diameter and D50 results in microns } \\
\hline & Sample Name & $\mathrm{D}_{50}$ & $\begin{array}{c}\text { Volume } \\
\text { weighted } \\
\text { mean } \\
\text { diameter }\end{array}$ & $\begin{array}{l}\text { Diameter } \\
+15 \%\end{array}$ & $\begin{array}{c}\text { Diameter } \\
-15 \%\end{array}$ & $\begin{array}{c}\text { Ultrasonic } \\
\text { level }\end{array}$ \\
\hline 1291 & B_FRPGM_001_xx_2713_XSP_4_B & 7.589 & 11.776 & 13.542 & 10.010 & 0 \\
\hline 1315 & $\begin{array}{c}\text { B_FRPGM_001_xx_2713_XSP_4_B } \\
- \text { Average }\end{array}$ & 7.21 & 11.184 & 12.862 & 9.506 & 0 \\
\hline 1297 & $\begin{array}{c}\text { B_FRPGM_001_xx_2713_XSP_4_B } \\
\text {-Average }\end{array}$ & 7.061 & 9.535 & 10.965 & 8.105 & 50 \\
\hline 1321 & $\begin{array}{c}\text { B_FRPGM_001_xx_2713_XSP_4_B } \\
- \text { Average }\end{array}$ & 6.77 & 9.005 & 10.356 & 7.654 & 50 \\
\hline 1339 & A_T22GM_002_XX_1507_XSP_4_B & 6.43 & 9.426 & 10.840 & 8.012 & 0 \\
\hline 1363 & $\begin{array}{c}\text { A_T22GM_002_XX_1507_XSP_4_B } \\
- \text { Average }\end{array}$ & 6.507 & 9.492 & 10.916 & 8.068 & 0 \\
\hline 1345 & $\begin{array}{c}\text { A_T22GM_002_XX_1507_XSP_4_B } \\
- \text { Average }\end{array}$ & 6.332 & 8.45 & 9.718 & 7.183 & 50 \\
\hline 1369 & $\begin{array}{c}\text { A_T22GM_002_XX_1507_XSP_4_B } \\
- \text { Average }\end{array}$ & 6.381 & 8.547 & 9.829 & 7.265 & 50 \\
\hline & & & & & & \\
\hline
\end{tabular}


Brad Johnson

April 16, 2009

Table 3. Sample Analysis Results (continued)

\begin{tabular}{|c|c|c|c|c|c|c|}
\hline \multicolumn{7}{|c|}{ Diameter and D50 results in microns } \\
\hline $\begin{array}{l}\text { Record } \\
\text { number }\end{array}$ & Sample Name & $\mathrm{D}_{50}$ & $\begin{array}{c}\text { Volume } \\
\text { weighted } \\
\text { mean } \\
\text { diameter }\end{array}$ & $\begin{array}{l}\text { Diameter } \\
\quad+15 \%\end{array}$ & $\begin{array}{l}\text { Diameter } \\
-15 \%\end{array}$ & $\begin{array}{c}\text { Ultrasonic } \\
\text { level }\end{array}$ \\
\hline 1639 & $\begin{array}{c}\text { A_02AOL_015_XX_2809_XSP_0 } \\
\text {-Average }\end{array}$ & 1.099 & 1.212 & 1.394 & 1.030 & 0 \\
\hline 1663 & $\begin{array}{c}\text { A_02AOL_015_XX_2809_XSP_0 } \\
\text { - Average }\end{array}$ & 1.08 & 1.203 & 1.383 & 1.023 & 0 \\
\hline 1645 & $\begin{array}{c}\text { A_02AOL_015_XX_2809_XSP_0 } \\
\text {-Average }\end{array}$ & 0.989 & 1.09 & 1.254 & 0.927 & 50 \\
\hline 1669 & $\begin{array}{c}\text { A_02AOL_015_XX_2809_XSP_0 } \\
\text {-Average }\end{array}$ & 0.97 & 1.07 & 1.231 & 0.910 & 50 \\
\hline 1519 & $\begin{array}{c}\text { A_02AOL_015_XX_2811_XSP_0 } \\
\text { - Average }\end{array}$ & 1.203 & 1.672 & 1.923 & 1.421 & 0 \\
\hline 1543 & $\begin{array}{c}\text { A_02AOL_015_XX_2811_XSP_0 } \\
\text {-Average }\end{array}$ & 1.117 & 1.463 & 1.682 & 1.244 & 0 \\
\hline 1567 & $\begin{array}{c}\text { A_02AOL_015_XX_2811_XSP_0 } \\
\text {-Average }\end{array}$ & 1.127 & 1.307 & 1.503 & 1.111 & 0 \\
\hline 1591 & $\begin{array}{c}\text { A_02AOL_015_XX_2811_XSP_0 } \\
\text {-Average }\end{array}$ & 1.103 & 2.044 & 2.351 & 1.737 & 0 \\
\hline 1615 & $\begin{array}{c}\text { A_02AOL_015_XX_2811_XSP_0 } \\
\text {-Average }\end{array}$ & 1.082 & 1.45 & 1.668 & 1.233 & 0 \\
\hline 1525 & $\begin{array}{c}\text { A_02AOL_015_XX_2811_XSP_0 } \\
\text {-Average }\end{array}$ & 0.928 & 1.04 & 1.196 & 0.884 & 50 \\
\hline 1549 & $\begin{array}{c}\text { A_02AOL_015_XX_2811_XSP_0 } \\
\text {-Average }\end{array}$ & 0.935 & 1.261 & 1.450 & 1.072 & 50 \\
\hline 1573 & $\begin{array}{c}\text { A_02AOL_015_XX_2811_XSP_0 } \\
\text {-Average }\end{array}$ & 0.932 & 1.271 & 1.462 & 1.080 & 50 \\
\hline 1597 & $\begin{array}{c}\text { A_02AOL_015_XX_2811_XSP_0 } \\
\text {-Average }\end{array}$ & 0.938 & 2.479 & 2.851 & 2.107 & 50 \\
\hline 1621 & $\begin{array}{c}\text { A_02AOL_015_XX_2811_XSP_0 } \\
\text { - Average }\end{array}$ & 0.928 & 1.278 & 1.470 & 1.086 & 50 \\
\hline & & & & & & \\
\hline 1399 & $\begin{array}{c}\text { A_02AML_021_XX_2460_XSP_4 } \\
\text {-Average }\end{array}$ & 1.409 & 1.907 & 2.193 & 1.621 & 0 \\
\hline 1423 & $\begin{array}{c}\text { A_02AML_021_XX_2460_XSP_4 } \\
\text { - Average }\end{array}$ & 1.241 & 1.671 & 1.922 & 1.420 & 0 \\
\hline 1447 & $\begin{array}{c}\text { A_02AML_021_XX_2460_XSP_4 } \\
\text { - Average }\end{array}$ & 1.215 & 1.585 & 1.823 & 1.347 & 0 \\
\hline 1471 & $\begin{array}{c}\text { A_02AML_021_XX_2460_XSP_4 } \\
\text {-Average }\end{array}$ & 1.189 & 1.375 & 1.581 & 1.169 & 0 \\
\hline 1495 & $\begin{array}{c}\text { A_02AML_021_XX_2460_XSP_4 } \\
\text {-Average }\end{array}$ & 1.179 & 1.43 & 1.645 & 1.216 & 0 \\
\hline
\end{tabular}


Brad Johnson

April 16, 2009

Table 3. Sample Analysis Results (continued)

\begin{tabular}{|c|c|c|c|c|c|c|}
\hline \multicolumn{7}{|c|}{ Diameter and D50 results in microns } \\
\hline $\begin{array}{l}\text { Record } \\
\text { number }\end{array}$ & Sample Name & $\mathrm{D}_{50}$ & $\begin{array}{c}\text { Volume } \\
\text { weighted } \\
\text { mean } \\
\text { diameter }\end{array}$ & $\begin{array}{l}\text { Diameter } \\
\quad+15 \%\end{array}$ & $\begin{array}{l}\text { Diameter } \\
-15 \%\end{array}$ & $\begin{array}{c}\text { Ultrasonic } \\
\text { level }\end{array}$ \\
\hline 1405 & $\begin{array}{c}\text { A_02AML_021_XX_2460_XSP_4 } \\
- \text { Average }\end{array}$ & 1.138 & 2.029 & 2.333 & 1.725 & 50 \\
\hline 1429 & $\begin{array}{c}\text { A_02AML_021_XX_2460_XSP_4 } \\
\text { - Average }\end{array}$ & 0.928 & 1.143 & 1.314 & 0.972 & 50 \\
\hline 1453 & $\begin{array}{c}\text { A_02AML_021_XX_2460_XSP_4 } \\
\text {-Average }\end{array}$ & 0.954 & 1.56 & 1.794 & 1.326 & 50 \\
\hline 1477 & $\begin{array}{c}\text { A_02AML_021_XX_2460_XSP_4 } \\
\text { - Average }\end{array}$ & 0.942 & 1.279 & 1.471 & 1.087 & 50 \\
\hline 1501 & A_02AML_021_XX_2460_XSP_4 & 0.954 & 1.31 & 1.507 & 1.114 & 50 \\
\hline
\end{tabular}




\section{Appendix I}

\section{PEP Operational Control Systems}





\section{Appendix I: Operational Control Systems for PEP}

The operational control systems for the Pretreatment Engineering Platform are extensive and include instrumentation, utility interfaces, chemical mixing and addition, process operations, valve alignment, and maintenance. The system is documented in the Tessenderlo Kerley Services Mechanical Data Book(s) Volumes I through XVIII as supplied by Bechtel National Inc. ${ }^{\text {(a) }}$ Testing processes were controlled by the Test Plan, ${ }^{(b)}$ the specific Test Instruction for the test being conducted, and the operating procedures for the PEP. The summary of the main operational procedures is listed below.

\section{Administrative Procedures}

OP-601 Surveillance Report Procedure

OP-602 Turnover Checklist Procedure

\section{System Startup and Shutdown Procedures}

OP-101 System Startup Procedure

OP-102 System Standby Shutdown Procedure

OP-103 System Cold Shutdown Procedure

\section{Utilities Procedures}

OP-201 Reverse Osmosis System Procedure

OP-202 Demineralized Water Procedure

SOP-203 Chilled Water System Operating Procedure (F\&O)

SOP-204 Compressed Air Operating Procedure (F\&O)

OP-205 Air Dryer Operating Procedure cancelled

SOP-206 Vacuum System Operating Procedure (F\&O)

OP-207 Vessel Vent System Procedure

SOP-208 Boiler Operating Procedure (F\&O)

OP-209 Data Acquisition System (DAS) Operating Procedure

\section{Chemical Reagents Procedures}

OP-301 Simulant Addition Procedure

OP-302 19M NaOH Operating Procedure

OP-303 Inhibited Water Procedure

OP-304 2M Caustic Operating Procedure

OP-305 Acid Procedure

OP-306 Sodium Permanganate (NaMnO4) Operating

Procedure

OP-307 Miscellaneous Chemical Addition Procedure

\section{Processing Procedures}

OP-401 Sampling Instructions Procedure

OP-402 Tank Transfer Procedure

OP-403 Tank Heating and Cooling Procedure

OP-404 Pulse Jet (PJM) Operating Procedure

OP-405 T02A Sparging Procedure

OP-406 Leaching Procedure

OP-407 T02A Leaching Procedure

OP-408 Tank T02A Recirculation Procedure

OP-409 Ultrafiltration (Dewatering) Procedure

OP-410 Solids Washing Procedure

OP-411 Tank Drain Operating Procedure

OP-412 Waste Transfer Procedure

OP-413 Flushing and Transfer of Inhibited Water Procedure

OP-414 UFP-VSL T01A \& B Recirculation Procedure

OP-415 Stable Level Measurements Procedure

\section{Process Maintenance Procedures}

OP-501 Filter Chemical Cleaning Procedure cancelled requires revision

OP-502 Pump Seal Pot Operating Procedure

OP-503 Spill Response Procedure

OP-504 Inspection of Vessel Interiors Using Video Camera or Borescope

(a) H Hazen, Letter April 15, 2009, “Contract No. DE-AC27-01RV14136 - Hanford Tank Waste Treatment and Immobilization Plant, Memorandum of Agreement (MOA), 24590-QL-HC9-WA49-00001, PNNL R\&T Support, Approval to use Subcontractor Supplied Documents for the Pretreatment Engineering Platform(PEP)", CCN 196243, Bechtel National Inc., Richland, Washington.

(b) GB Josephson, OP Bredt, JK Young, and DE Kurath. 2009. Test Plan for Pretreatment Engineering Platform (PEP) Testing (Phase I). TP-RPP-WTP-506, Rev. 0.4, Pacific Northwest National Laboratory, Richland, Washington. 
System Valve Alignment Procedures

Z001 HLP-VSL-T22 System Alignment Checklist

Z002 FRP-VSL-T01 System Alignment Checklist

Z003 UFP-VSL-T01A System Alignment Checklist

Z004 UFP-VSL-T01B System Alignment Checklist

Z005 UFP-HX System Alignment Checklist

Z006 UFP-VSL-T02A System Alignment Checklist

Z007 Ultrafilters System Alignment Checklist

Z008 UFP-VSL-T62A/B System Alignment Checklist

Z009 FEP-VSL-T01 System Alignment Checklist

Z010 HLP-VSL-T27 System Alignment Checklist

Z011 Vessel Vent System Alignment Checklist

Z012 Vacuum and Compressed Air System Alignment Checklist F\&O

Z013 Boiler System Alignment Checklist F\&O

Z014 Chemical System Alignment Checklist

Z015 Chemical System Alignment Checklist

Z016 UFP-VSL-T01A PJM System Alignment Checklist

Z017 UFP-VSL-T01B PJM System Alignment Checklist

Z018 UFP-VSL-T02A PJM System Alignment Checklist

Z019 Sparger System Alignment Checklist

Z020 UFP-VSL-T01A Coriolis Densitometer System

Alignment Checklist

Z021 UFP-VSL-T01B Coriolis Densitometer System

Alignment Checklist

Z022 UFP-VSL-T02A Coriolis Densitometer System

Alignment Checklist

Z023 Pump Seal Pot System Alignment Checklist

Z024 Pump Seal Pot System Alignment Checklist

Z025 Chilled Water System Alignment Checklist F\&O

Z026 Reverse Osmosis Unit System Alignment Checklist 


\section{Appendix $\mathbf{J}$}

Operational Process Sheets, Integrated Test A Parameters 



\section{Appendix J: Operational Process Sheet, Integrated Test A Parameters}

The Integrated Test A Test Instruction, TI-WTP-PEP-065, included a Run Sheet that provided the LTE target or setpoint values for key process operations. The LTE recorded the actual values obtained for the target setpoint values during testing from available data on the Run Sheet. Table J.1 is comprised of operational process sheets summarizing the actual target values obtained during Integrated Test A. The "actual values" column of data comes from various data sources. Majority of the times, these data were taken from the HMI, which is not NQA-1 qualified and were "real-time" values, rather than an average. Consequently, Table J.1 is for information only.

The "comments" column indicates whether the value is "for information only" or NQA-1. Values are "for information only" if taken from the HMI, valve lineup verifications, variable frequency drive (VFD) and antifoam agent or inhibited water additions. Where NQA-1values resulting from previously published reports and data analysis efforts are available, these values are provided instead. In some instances, target parameters were changed by the Test Directors during the test; these changes are shown as text strike-outs. Cross-hatched patterned cells on the operational process sheets indicate the test instruction steps associated with the target parameters were not performed per Test Director guidance.

\section{NOTE:}

HMI values are for information only.

VFD - this value was taken from the VFD readout, which was not calibrated.

The steam ring air purge and the air sparge values were read locally from the instrument (Coriolis gas flowmeter). These local readouts were calibrated and are NQA-1.

WTP-RPT-XXX - If a report is cited as the data source, the value was evaluated and checked via a computational computer program (CCP) traceable through the report referenced. 
Table J.1. Integrated Test A Operational Process Sheet

\begin{tabular}{|c|c|c|c|c|c|c|c|}
\hline \multicolumn{8}{|c|}{ Integrated Test A Run sheet (TI-065 Rev. 0) } \\
\hline & & Operation & $\begin{array}{l}\text { Target or } \\
\text { Setpoint } \\
\text { Value }\end{array}$ & $\begin{array}{c}\text { Range } \\
(-/+)\end{array}$ & Units & Actual Value & Comments \\
\hline \multirow{13}{*}{ 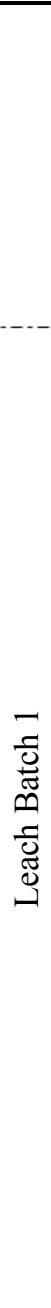 } & \multirow{3}{*}{$\begin{array}{c}\text { Simulant storage } \\
\text { in } \\
\text { HLP-VSL-T22 } \\
\text { (Test Instructions } \\
\text { step 7.1.1) }\end{array}$} & $\begin{array}{l}\text { HLP-VSL-T22 } \\
\text { Operation }\end{array}$ & - & - & - & - & ( \\
\hline & & $\begin{array}{l}\text { Minimum volume of } \\
\text { simulant in } \\
\text { HLP-VSL- } 22 \text { to } \\
\text { complete Test A. }\end{array}$ & 2,600 & - & gal & 3040 & $\begin{array}{l}\text { Value given includes allowance for } 500 \text {-gal } \\
\text { heel. } \\
\text { Value obtained from the HMI }\end{array}$ \\
\hline & & Agitator speed & 68 & $-10 /+10$ & $\mathrm{rpm}$ & 60 & $\begin{array}{l}\text { Variable Frequency Drive, } \\
\text { Information Only }\end{array}$ \\
\hline & \multirow{10}{*}{$\begin{array}{c}\text { Transfer simulant } \\
\text { from } \\
\text { HLP-VSL-T22 to } \\
\text { UFP-VSL-T01A } \\
\text { and add } 17.9 \mathrm{M} \\
\text { caustic in-line } \\
\text { (Test Instructions } \\
\text { steps 7.1.2.1 to } \\
7.1 .2 .7 \text { ) }\end{array}$} & $\begin{array}{l}\text { Total slurry transfer } \\
\text { volume (endpoint) }\end{array}$ & $388^{\mathrm{a}} 501$ & $-5 /+5$ & gal & 491 & $\begin{array}{l}\text { Assumes no previous heel in } \\
\text { UFP-VSL-T01A. } \\
\text { WTP-RPT-186 }\end{array}$ \\
\hline & & Slurry transfer rate & $7.4-9.6$ & $-1 /+1$ & gpm & 10.1 & WTP-RPT-186 \\
\hline & & $\begin{array}{l}\text { Total } 17.9 \mathrm{M} \text { caustic } \\
\text { addition (in-line after } \\
\text { HLP-PMP-T21) }\end{array}$ & 639 & $-10 /+10$ & $\mathrm{~kg}$ & 637 & WTP-RPT-186 \\
\hline & & $\begin{array}{l}17.9 \mathrm{M} \text { caustic addition } \\
\text { rate }\end{array}$ & 12.7 & $-1 /+3$ & $\mathrm{~kg} / \mathrm{min}$ & 14 & $\begin{array}{l}\text { Caustic addition should finish } 1 \text { to } 2 \text { min } \\
\text { before slurry transfer finishes to flush caustic } \\
\text { from transfer line. } \\
\text { WTP-RPT-186 }\end{array}$ \\
\hline & & $\begin{array}{l}\text { UFP-VSL-T01A } \\
\text { Operation }\end{array}$ & - & - & - & - & 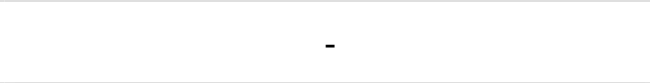 \\
\hline & & $\begin{array}{l}\text { Volume of antifoam to } \\
\text { add to } \\
\text { UFP-VSL-T01A }\end{array}$ & 1,000 & $-20 /+20$ & $\mathrm{~mL}$ & 1000 & $\begin{array}{l}\text { This value is concentrate. Dilute with } 3 \\
\text { parts IW to } 1 \text { part concentrate. Information } \\
\text { Only }\end{array}$ \\
\hline & & $\begin{array}{l}\text { Level to initiate PJM } \\
\text { operation }\end{array}$ & 15 & $-0 /+10$ & in & $40+$ & Value obtained from the HMI \\
\hline & & PJM jet velocity & 4.8 & $-0.3 /+0.3$ & $\mathrm{~m} / \mathrm{s}$ & 5.3 & NQA-1 \\
\hline & & PJM stroke $(80 \%)$ & 29.0 & $-2 /+2$ & in & $29.8(85 \%)$ & NQA-1 \\
\hline & & PJM cycle time & 35 & $-1 /+1$ & $\mathrm{~s}$ & & NQA-1 \\
\hline
\end{tabular}

\footnotetext{
${ }^{\text {a }}$ Target parameters were changed by the Test Directors during the test; these changes are shown as text strike-outs, typical for Table J.1.
} 
Table J.1. Integrated Test A Operational Process Sheet

\begin{tabular}{|c|c|c|c|c|c|c|c|}
\hline \multicolumn{8}{|c|}{ Integrated Test A Run sheet (TI-065 Rev. 0) } \\
\hline & Operation & $\begin{array}{l}\text { Target or } \\
\text { Setpoint } \\
\text { Value }\end{array}$ & $\begin{array}{c}\text { Range } \\
(-/+)\end{array}$ & Units & Actual Value & \multicolumn{2}{|l|}{ Comments } \\
\hline & \multicolumn{7}{|c|}{35.3} \\
\hline & $\begin{array}{l}\text { Steam ring air purge } \\
\text { flowrate }\end{array}$ & 0.20 & $0.02 /+0.02$ & $\mathrm{~kg} / \mathrm{min}$ & $>0.14$ & $\begin{array}{l}6.1 \mathrm{scfm} \text { below } 90^{\circ} \mathrm{C} \text { in UFP-VSL-T01A } \\
\text { WTP-RPT- } 186\end{array}$ & \\
\hline \multirow{2}{*}{$\begin{array}{l}\text { Initial heat-up of } \\
\text { UFP-VSL-T01A } \\
\text { (Non-prototypic } \\
\text { using external } \\
\text { exchanger) } \\
\text { (Test Instructions } \\
\text { steps } 7.1 .3 .1 \text { to } \\
7.1 .3 .9 \text { ) }\end{array}$} & $\begin{array}{l}\text { Initial heat-up } \\
\text { temperature (endpoint) }\end{array}$ & 57 & $-1 /+3$ & ${ }^{\circ} \mathrm{C}$ & $\sim 57.5$ & $\begin{array}{l}\text { Y (init.) } \\
\text { WTP-RPT-186 }\end{array}$ & $\begin{array}{l}\mathrm{N} \\
\mathrm{STOP}\end{array}$ \\
\hline & $\begin{array}{l}\text { Time to heat to initial } \\
\text { heat-up temperature }\end{array}$ & $<3$ & NA & $\mathrm{hr}$ & 1.6 & $\begin{array}{l}\text { Heating loop is } \sim 9 \text { gallons and will be lost } \\
\text { (unprototypic). Target and range is not } \\
\text { important. Should be complete in under } 3 \\
\text { hours. } \\
\text { WTP-RPT-186 }\end{array}$ & \\
\hline \multirow{7}{*}{$\begin{array}{l}\text { Final heat-up } \\
\text { UFP-VSL-T01A } \\
\text { (Test Instructions } \\
\text { steps } 7.1 .3 .10 \text { to } \\
7.1 .3 .13 \text { ) }\end{array}$} & $\begin{array}{l}\text { Target final } \\
\text { temperature (endpoint) }\end{array}$ & 98 & $-2 /+2$ & ${ }^{\circ} \mathrm{C}$ & 98.0 & $\begin{array}{l}\text { Y (init.) } \\
\text { WTP-RPT-186 }\end{array}$ & $\begin{array}{l}\mathrm{N} \\
\text { STOP }\end{array}$ \\
\hline & Heat-up time & 3.8 & $-0.5 /+0.5$ & $\mathrm{hr}$ & 4.4 & WTP-RPT-186 & \\
\hline & $\begin{array}{l}\text { UFP-VSL-T01A } \\
\text { Operation }\end{array}$ & - & - & - & - & - & \\
\hline & $\begin{array}{l}\text { Temperature to turn off } \\
\text { PJM suction refill }\end{array}$ & 60 & $-2 /+2$ & ${ }^{\circ} \mathrm{C}$ & 60 & Value obtained from the HMI & \\
\hline & $\begin{array}{l}\text { Temperature to reduce } \\
\text { steam ring air purge } \\
\text { rate }\end{array}$ & 90 & $-2 /+8$ & ${ }^{\circ} \mathrm{C}$ & 98 & Value obtained from the HMI & \\
\hline & $\begin{array}{l}\text { High-temperature } \\
\text { steam ring air purge } \\
\text { flowrate }\end{array}$ & 0.13 & $0.02 /+0.02$ & $\mathrm{~kg} / \mathrm{min}$ & 0.13 & $\begin{array}{l}4.0 \mathrm{scfm} \text { above } 90^{\circ} \mathrm{C} \text { in UFP-VSL-T01A } \\
\text { Local Calibrated Instrument, NQA-1 }\end{array}$ & \\
\hline & Level at end of heat-up & 61 & $-2 /+2$ & in & 62.5 & $\begin{array}{l}\text { Y (init.) } \\
\text { Anticipate accumulation of } \sim 62 \text { gal of } \\
\text { condensate during heat-up. } \\
\text { WTP-RPT-186 }\end{array}$ & $\begin{array}{l}\mathrm{N} \\
\text { STOP }\end{array}$ \\
\hline \multirow{3}{*}{$\begin{array}{l}\text { Caustic leach in } \\
\text { UFP-VSL-T01A } \\
\text { (Test Instructions } \\
\text { steps 7.1.3.14 to } \\
7.1 .3 .19 \text { ) }\end{array}$} & $\begin{array}{l}\text { Caustic leach duration } \\
\text { (endpoint) }\end{array}$ & 16 & $-0.1 /+1$ & $\mathrm{hr}$ & 16.0 & WTP-RPT-186 & \\
\hline & Temperature & 98 & $-2 /+2$ & ${ }^{\circ} \mathrm{C}$ & 97.9 & WTP-RPT-186 & \\
\hline & Volume of water to & 2.9 & $-0.1 /+0.1$ & $\mathrm{~L}$ & 2.9 & This quantity added after samples are & \\
\hline
\end{tabular}


Table J.1. Integrated Test A Operational Process Sheet

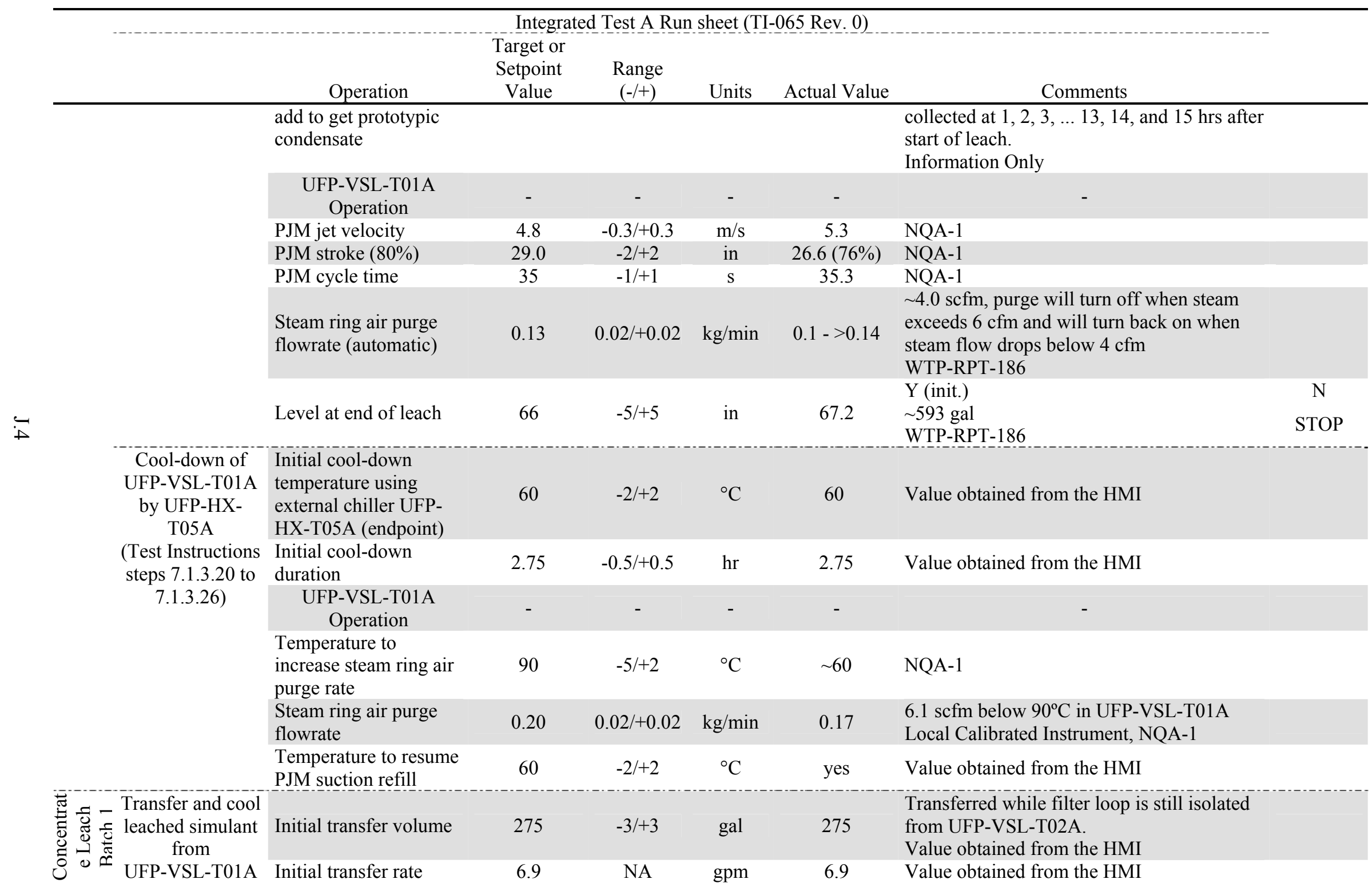


Table J.1. Integrated Test A Operational Process Sheet

\begin{tabular}{|c|c|c|c|c|c|c|c|}
\hline \multicolumn{8}{|c|}{ Integrated Test A Run sheet (TI-065 Rev. 0) } \\
\hline \multicolumn{2}{|r|}{ Operation } & $\begin{array}{l}\text { Target or } \\
\text { Setpoint } \\
\text { Value }\end{array}$ & $\begin{array}{c}\text { Range } \\
(-/+)\end{array}$ & Units & Actual Value & \multicolumn{2}{|l|}{ Comments } \\
\hline \multirow{17}{*}{$\begin{array}{c}\text { to } \\
\text { UFP-VSL-T02A } \\
\text { and concentrate } \\
\text { solids } \\
\text { (Test Instructions } \\
\text { steps } 7.1 .4 .1 \text { to } \\
7.1 .4 .8 \text { and steps } \\
7.1 .11 .1 \text { to } \\
7.1 .11 .8 \text { ) }\end{array}$} & $\begin{array}{l}\text { Make-up batch transfer } \\
\text { volume }\end{array}$ & 11 & $-0.2 /+0.2$ & gal & 11 & Value obtained from the HMI & \\
\hline & $\begin{array}{l}\text { Make-up batch } \\
\text { flowrate }\end{array}$ & 6.9 & NA & gpm & 6.4 & Value obtained from the HMI & \\
\hline & $\begin{array}{l}\text { UFP-VSL-T01A level } \\
\text { (endpoint to stop batch } \\
\text { transfers) }\end{array}$ & 10 & $-2 /+2$ & in & $\sim 10$ & $\begin{array}{l}\text { Y (init.) } \\
\text { Estimate } 23 \text { make-up batches from } \\
\text { UFP-VSL-T01A. } \\
\text { NQA-1 }\end{array}$ & $\begin{array}{c}\mathrm{N} \\
\text { STOP }\end{array}$ \\
\hline & $\begin{array}{l}\text { UFP-VSL-T02A level } \\
\text { to initiate each make- } \\
\text { up batch }\end{array}$ & 44.7 & $-1 /+1$ & in & 44.7 & Value obtained from the HMI & \\
\hline & $\begin{array}{l}\text { UFP-VSL-T02A } \\
\text { Operation }\end{array}$ & - & - & - & - & - & \\
\hline & $\begin{array}{l}\text { Level to initiate PJM } \\
\text { operation }\end{array}$ & 20 & $-2 /+10$ & in & $\sim 65$ & NQA-1 & \\
\hline & PJM jet velocity & 7.3 & $-0.4 /+0.4$ & $\mathrm{~m} / \mathrm{s}$ & 7.6 & NQA-1 & \\
\hline & PJM stroke $(80 \%)$ & 31.5 & $-2 /+2$ & in & $28.8(76 \%)$ & NQA-1 & \\
\hline & PJM cycle time & 33 & $-1 /+1$ & $\mathrm{~s}$ & 33.2 & NQA-1 & \\
\hline & $\begin{array}{l}\text { Steam ring air purge } \\
\text { flowrate }\end{array}$ & 0.14 & $0.02 /+0.02$ & $\mathrm{~kg} / \mathrm{min}$ & 0.14 & $\begin{array}{l}4.1 \mathrm{scfm} \text { below } 90^{\circ} \mathrm{C} \text { in UFP-VSL-T02A } \\
\text { Local Calibrated Instrument, NQA-1 }\end{array}$ & \\
\hline & $\begin{array}{l}\text { Total flowrate of } 5 \\
\text { lower air spargers } \\
\text { (idle) }\end{array}$ & 0.04 & $\begin{array}{l}0.005 / \\
+0.005\end{array}$ & $\mathrm{~kg} / \mathrm{min}$ & 0.04 & $\begin{array}{l}\sim 13 \text { scfh for each sparge tube } \\
\text { Local Calibrated Instrument, NQA-1 }\end{array}$ & \\
\hline & $\begin{array}{l}\text { Flowrate of upper air } \\
\text { sparger (idle) }\end{array}$ & 0.01 & $\begin{array}{l}0.005 / \\
+0.005\end{array}$ & $\mathrm{~kg} / \mathrm{min}$ & 0.01 & $\begin{array}{l}\sim 16 \text { scfh (record field transmitter value) } \\
\text { Local Calibrated Instrument, NQA-1 }\end{array}$ & \\
\hline & $\begin{array}{c}\text { Ultrafiltration Loop } \\
\text { Operation }\end{array}$ & - & - & - & - & 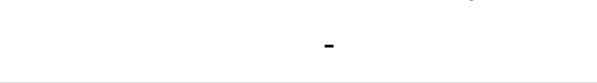 & \\
\hline & Number of filter & & & & & Y (init.) & $\mathrm{N}$ \\
\hline & $\begin{array}{l}\text { bundles } \\
\text { (UFP-FLT-T01A } \\
\text { should be aligned) }\end{array}$ & 1 & $-0 /+0$ & - & 1 & Information Only & STOP \\
\hline & Filter loop flowrate & 109 & $-10 /+10$ & gpm & 109 & Value obtained from the HMI & \\
\hline & $\begin{array}{l}\text { Trans-membrane } \\
\text { pressure }\end{array}$ & 40 & $-4 /+4$ & psi & 40 & Value obtained from the HMI & \\
\hline
\end{tabular}


Table J.1. Integrated Test A Operational Process Sheet

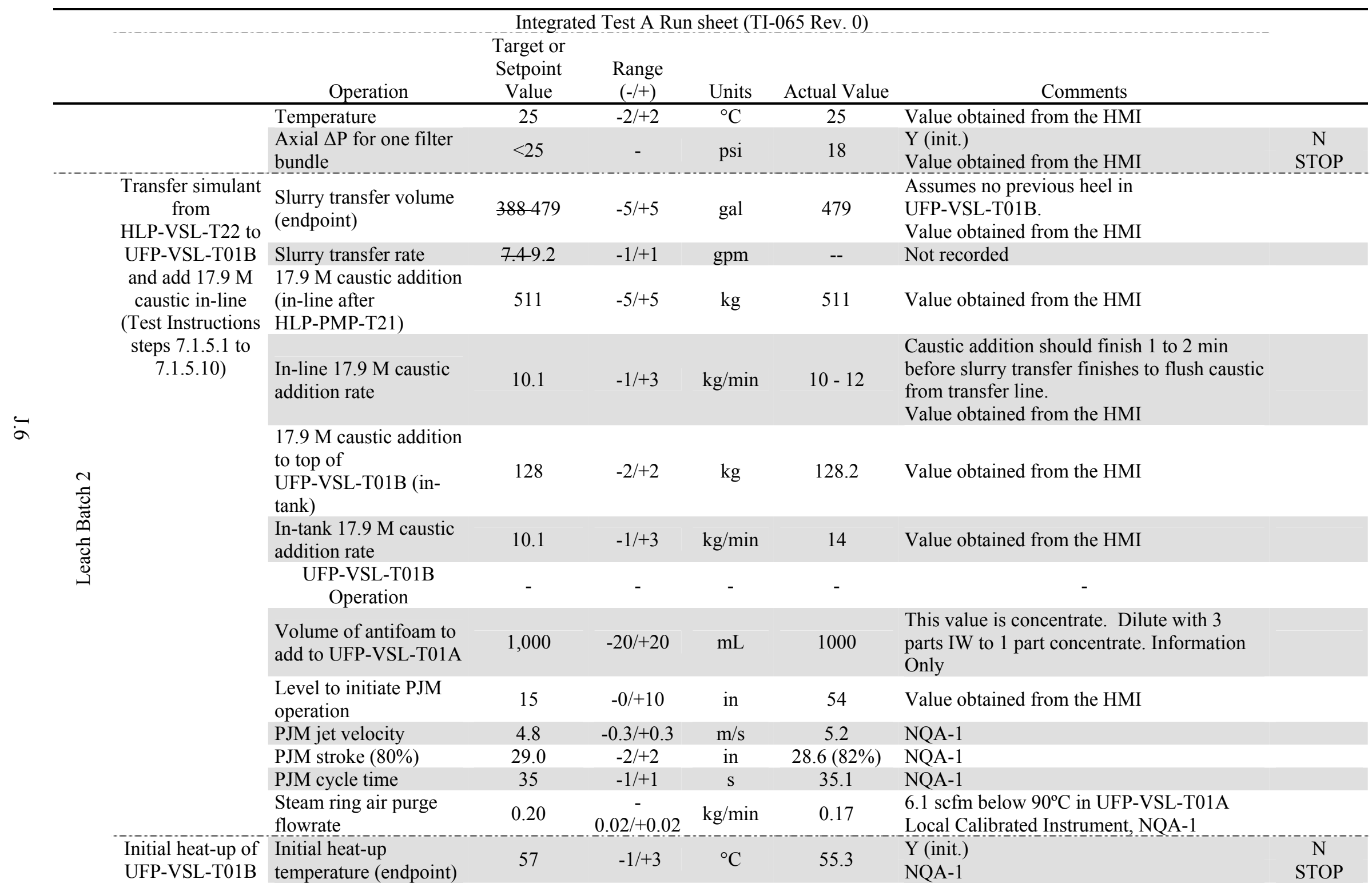


Table J.1. Integrated Test A Operational Process Sheet

\begin{tabular}{|c|c|c|c|c|c|c|c|}
\hline \multicolumn{7}{|c|}{ Integrated Test A Run sheet (TI-065 Rev. 0) } & \\
\hline & Operation & $\begin{array}{l}\text { Target or } \\
\text { Setpoint } \\
\text { Value }\end{array}$ & $\begin{array}{c}\text { Range } \\
(-/+)\end{array}$ & Units & Actual Value & \multicolumn{2}{|l|}{ Comments } \\
\hline $\begin{array}{c}\text { (Non-prototypic } \\
\text { using external } \\
\text { exchanger) } \\
\text { (Test Instructions } \\
\text { steps } 7.1 .6 .1 \text { to } \\
7.1 .6 .9)\end{array}$ & $\begin{array}{l}\text { Time to heat to initial } \\
\text { heat-up temperature }\end{array}$ & $<3$ & NA & $\mathrm{hr}$ & 1.5 & $\begin{array}{l}\text { Heating loop is } \sim 9 \text { gallons and will be lost } \\
\text { (unprototypic). Target and range is not } \\
\text { important. Should be complete in under } 3 \\
\text { hours. } \\
\text { NQA-1 }\end{array}$ & \\
\hline \multirow{7}{*}{$\begin{array}{l}\text { Final heat-up } \\
\text { UFP-VSL-T01B } \\
\text { (Test Instructions } \\
\text { steps 7.1.6.10 to } \\
\text { 7.1.6.15) }\end{array}$} & $\begin{array}{l}\text { Target final } \\
\text { temperature (endpoint) }\end{array}$ & 98 & $-2 /+2$ & ${ }^{\circ} \mathrm{C}$ & $\sim 98$ & $\begin{array}{l}\text { Y (init.) } \\
\text { NQA-1 }\end{array}$ & $\begin{array}{l}\mathrm{N} \\
\text { STOP }\end{array}$ \\
\hline & Heat-up time & 3.8 & $-0.5 /+0.5$ & $\mathrm{hr}$ & 3.8 & NQA-1 & \\
\hline & $\begin{array}{l}\text { UFP-VSL-T01B } \\
\text { Operation }\end{array}$ & - & - & - & - & - & \\
\hline & $\begin{array}{l}\text { Temperature to turn off } \\
\text { PJM suction refill }\end{array}$ & 60 & $-2 /+2$ & ${ }^{\circ} \mathrm{C}$ & 61 & Value obtained from the HMI & \\
\hline & $\begin{array}{l}\text { Temperature to reduce } \\
\text { steam ring air purge } \\
\text { rate }\end{array}$ & 90 & $-2 /+8$ & ${ }^{\circ} \mathrm{C}$ & 92 & Value obtained from the HMI & \\
\hline & $\begin{array}{l}\text { High-temperature } \\
\text { steam ring air purge } \\
\text { flowrate }\end{array}$ & 0.13 & $0.02 /+0.02$ & $\mathrm{~kg} / \mathrm{min}$ & 0.13 & $\begin{array}{l}4.0 \mathrm{scfm} \text { above } 90^{\circ} \mathrm{C} \text { in UFP-VSL-T01A } \\
\text { Local Calibrated Instrument }\end{array}$ & \\
\hline & Level at end of heat-up & 62 & $-2 /+2$ & in & 64.2 & $\begin{array}{l}\text { Y (init.) } \\
\text { Anticipate accumulation of } \sim 62 \text { gal of } \\
\text { condensate during heat-up. } \\
\text { NQA-1 }\end{array}$ & $\begin{array}{l}\mathrm{N} \\
\text { STOP }\end{array}$ \\
\hline \multirow{6}{*}{$\begin{array}{l}\text { Caustic leach in } \\
\text { UFP-VSL-T01B } \\
\text { (Test Instructions } \\
\text { steps 7.1.6.16 to } \\
7.1 .6 .19 \text { ) }\end{array}$} & $\begin{array}{l}\text { Caustic leach duration } \\
\text { (endpoint) }\end{array}$ & 16 & $-0.1 /+1$ & $\mathrm{hr}$ & 16.2 & NQA-1 & \\
\hline & Temperature & 98 & $-2 /+2$ & ${ }^{\circ} \mathrm{C}$ & $\sim 98$ & NQA-1 & \\
\hline & $\begin{array}{l}\text { Volume of water to } \\
\text { add to get prototypic } \\
\text { condensate }\end{array}$ & 2.9 & $-0.1 /+0.1$ & $\mathrm{~L}$ & 2.9 & $\begin{array}{l}\text { This quantity added after samples are } \\
\text { collected at } 1,2,3, \ldots 13,14 \text {, and } 15 \text { hrs after } \\
\text { start of leach. } \\
\text { Information Only }\end{array}$ & \\
\hline & $\begin{array}{l}\text { UFP-VSL-T01B } \\
\text { Operation }\end{array}$ & - & - & - & - & - & \\
\hline & PJM jet velocity & 4.8 & $-0.3 /+0.3$ & $\mathrm{~m} / \mathrm{s}$ & 5.4 & NQA-1 & \\
\hline & PJM stroke $(80 \%)$ & 29.0 & $-2 /+2$ & in & $21.2(60 \%)$ & NQA-1 & \\
\hline
\end{tabular}


Table J.1. Integrated Test A Operational Process Sheet

\begin{tabular}{|c|c|c|c|c|c|c|c|}
\hline \multicolumn{8}{|c|}{ Integrated Test A Run sheet (TI-065 Rev. 0) } \\
\hline & Operation & $\begin{array}{l}\text { Target or } \\
\text { Setpoint } \\
\text { Value }\end{array}$ & $\begin{array}{c}\text { Range } \\
(-/+)\end{array}$ & Units & Actual Value & \multicolumn{2}{|l|}{ Comments } \\
\hline & PJM cycle time & 35 & $-1 /+1$ & $\mathrm{~s}$ & 35.1 & NQA-1 & \\
\hline & $\begin{array}{l}\text { Steam ring air purge } \\
\text { flowrate (automatic) }\end{array}$ & 0.13 & $0.02 /+0.02$ & $\mathrm{~kg} / \mathrm{min}$ & 0.13 & $\begin{array}{l}\text { 4.0 scfm, purge will turn off when steam } \\
\text { exceeds } 6 \mathrm{cfm} \text { and will turn back on when } \\
\text { steam flow drops below } 4 \mathrm{cfm} \text { Local } \\
\text { Calibrated Instrument, NQA-1 }\end{array}$ & \\
\hline & Level at end of leach & 66 & $-5 /+5$ & in & 64.1 & $\begin{array}{l}\text { Y (init.) } \\
\sim 593 \text { gal } \\
\text { NQA-1 }\end{array}$ & $\begin{array}{c}\mathrm{N} \\
\text { STOP }\end{array}$ \\
\hline \multirow{6}{*}{$\begin{array}{l}\text { Cool-down of } \\
\text { UFP-VSL-T01B } \\
\text { by UFP-HX- } \\
\text { T05AB } \\
\text { (Test Instructions } \\
\text { steps } 7.1 .6 .20 \text { to } \\
7.1 .6 .26 \text { ) }\end{array}$} & $\begin{array}{l}\text { Initial cool-down } \\
\text { temperature using } \\
\text { external chiller UFP- } \\
\text { HX-T05B (endpoint) }\end{array}$ & 60 & $-2 /+2$ & ${ }^{\circ} \mathrm{C}$ & 60 & Value obtained from the HMI & \\
\hline & $\begin{array}{l}\text { Initial cool-down } \\
\text { duration }\end{array}$ & 2.75 & $-0.5 /+0.5$ & $\mathrm{hr}$ & 2.75 & Value obtained from the HMI & \\
\hline & $\begin{array}{l}\text { UFP-VSL-T01B } \\
\text { Operation }\end{array}$ & - & - & - & - & - & \\
\hline & $\begin{array}{l}\text { Temperature to } \\
\text { increase steam ring air } \\
\text { purge rate }\end{array}$ & 90 & $-5 /+2$ & ${ }^{\circ} \mathrm{C}$ & 90 & Value obtained from the HMI & \\
\hline & $\begin{array}{l}\text { Steam ring air purge } \\
\text { flowrate }\end{array}$ & 0.20 & $0.02 /+0.02$ & $\mathrm{~kg} / \mathrm{min}$ & 0.2 & $\begin{array}{l}6.1 \mathrm{scfm} \text { below } 90^{\circ} \mathrm{C} \text { in UFP-VSL-T01A } \\
\text { Local Calibrated Instrument, NQA-1 }\end{array}$ & \\
\hline & $\begin{array}{l}\text { Temperature to resume } \\
\text { PJM suction refill }\end{array}$ & 60 & $-2 /+2$ & ${ }^{\circ} \mathrm{C}$ & 60 & Value obtained from the HMI & \\
\hline
\end{tabular}

\begin{tabular}{|c|c|c|c|c|c|c|c|}
\hline 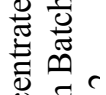 & $\begin{array}{l}\text { Transfer and cool } \\
\text { leached simulant }\end{array}$ & $\begin{array}{l}\text { Make-up batch transfer } \\
\text { volume }\end{array}$ & 11 & $-0.2 /+0.2$ & gal & -- & Not recorded \\
\hline లే & $\begin{array}{c}\text { from } \\
\text { UFP-VSL-T01B }\end{array}$ & $\begin{array}{l}\text { Make-up batch } \\
\text { flowrate }\end{array}$ & 6.9 & NA & gpm & 5.5 & Value obtained from the HMI \\
\hline
\end{tabular}


Table J.1. Integrated Test A Operational Process Sheet

\begin{tabular}{|c|c|c|c|c|c|c|c|c|}
\hline \multicolumn{9}{|c|}{ Integrated Test A Run sheet (TI-065 Rev. 0) } \\
\hline & & Operation & $\begin{array}{l}\text { Target or } \\
\text { Setpoint } \\
\text { Value }\end{array}$ & $\begin{array}{c}\text { Range } \\
(-/+)\end{array}$ & Units & Actual Value & \multicolumn{2}{|l|}{ Comments } \\
\hline & \multirow{14}{*}{$\begin{array}{c}\text { to } \\
\text { UFP-VSL-T02A } \\
\text { and concentrate } \\
\text { solids } \\
\text { (Test Instructions } \\
\text { steps } 7.1 .11 .6 \text { to } \\
7.1 .11 .8 \text { ) }\end{array}$} & $\begin{array}{l}\text { UFP-VSL-T01B level } \\
\text { (endpoint to stop batch } \\
\text { transfers) }\end{array}$ & 10 & $-2 /+2$ & in & $9.4-9.8$ & $\begin{array}{l}\text { Y (init.) } \\
\text { Estimate } 47 \text { make-up batches from } \\
\text { UFP-VSL-T01B. } \\
\text { Value obtained from the HMI }\end{array}$ & $\begin{array}{c}\mathrm{N} \\
\text { STOP }\end{array}$ \\
\hline & & $\begin{array}{l}\text { UFP-VSL-T02A level } \\
\text { to initiate each } \\
\text { make-up batch }\end{array}$ & 44.7 & $-1 /+1$ & in & 44.7 & Value obtained from the HMI & \\
\hline & & $\begin{array}{l}\text { UFP-VSL-T02A } \\
\text { Operation }\end{array}$ & - & - & - & - & - & \\
\hline & & PJM jet velocity & 7.3 & $-0.4 /+0.4$ & $\mathrm{~m} / \mathrm{s}$ & -- & Not recorded & \\
\hline & & PJM stroke $(80 \%)$ & 31.5 & $-2 /+2$ & in & -- & Not recorded & \\
\hline & & PJM cycle time & 33 & $-1 /+1$ & $\mathrm{~s}$ & 33 & Value obtained from the HMI & \\
\hline & & $\begin{array}{l}\text { Steam ring air purge } \\
\text { flowrate }\end{array}$ & 0.14 & $0.02 /+0.02$ & $\mathrm{~kg} / \mathrm{min}$ & 0.14 & $\begin{array}{l}4.1 \mathrm{scfm} \text { below } 90^{\circ} \mathrm{C} \text { in UFP-VSL-T02A } \\
\text { Local Calibrated Instrument, NQA-1 }\end{array}$ & \\
\hline & & $\begin{array}{l}\text { Total flowrate of } 5 \\
\text { lower air spargers } \\
\text { (idle) }\end{array}$ & 0.04 & $\begin{array}{c}0.005 /+ \\
0.005\end{array}$ & $\mathrm{~kg} / \mathrm{min}$ & 0.04 & $\begin{array}{l}\sim 13 \text { scfh for each sparge tube } \\
\text { Local Calibrated Instrument, NQA-1 }\end{array}$ & \\
\hline & & $\begin{array}{l}\text { Flowrate of upper air } \\
\text { sparger (idle) }\end{array}$ & 0.01 & $\begin{array}{c}-0.005 /+ \\
0.005\end{array}$ & $\mathrm{~kg} / \mathrm{min}$ & 0.01 & $\begin{array}{l}\sim 16 \text { scfh (record field transmitter value) } \\
\text { Local Calibrated Instrument, NQA-1 }\end{array}$ & \\
\hline & & $\begin{array}{l}\text { Ultrafiltration Loop } \\
\text { Operation }\end{array}$ & - & 然 & - & - & 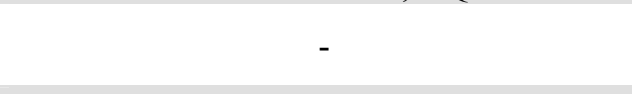 & \\
\hline & & $\begin{array}{l}\text { Number of filter } \\
\text { bundles } \\
\text { (UFP-FLT-T01A } \\
\text { should be aligned) }\end{array}$ & 1 & $-0 /+0$ & - & -- & Information Only & \\
\hline & & Filter loop flowrate & 109 & $-10 /+10$ & gpm & 106 & Value obtained from the HMI & \\
\hline & & Temperature & 25 & $-2 /+2$ & ${ }^{\circ} \mathrm{C}$ & 25 & Value obtained from the HMI & \\
\hline & & $\begin{array}{l}\text { Axial } \Delta \mathrm{P} \text { for one filter } \\
\text { bundle }\end{array}$ & $<25$ & - & psi & 18 & $\begin{array}{l}\text { Y (init.) } \\
\text { Value obtained from the HMI }\end{array}$ & $\begin{array}{c}\mathrm{N} \\
\text { STOP }\end{array}$ \\
\hline \multirow{3}{*}{ 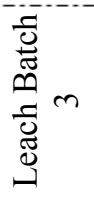 } & \multirow{3}{*}{$\begin{array}{l}\text { Transfer simulant } \\
\text { from } \\
\text { HLP-VSL-T22 to } \\
\text { UFP-VSL-T01A }\end{array}$} & $\begin{array}{l}\text { Total slurry transfer } \\
\text { volume (endpoint) }\end{array}$ & 315426 & $-5 /+5$ & gal & 426 & $\begin{array}{l}\text { Assumes previous heel in UFP-VSL-T01A. } \\
\text { Value obtained from the HMI }\end{array}$ & \\
\hline & & Slurry transfer rate & 7.4 & $-2 /+2$ & gpm & -- & Not recorded & \\
\hline & & Total $17.9 \mathrm{M}$ caustic & 629 & $-5 /+5$ & $\mathrm{~kg}$ & 629 & Value obtained from the HMI & \\
\hline
\end{tabular}


Table J.1. Integrated Test A Operational Process Sheet

\begin{tabular}{|c|c|c|c|c|c|c|c|}
\hline \multicolumn{7}{|c|}{ Integrated Test A Run sheet (TI-065 Rev. 0) } & \\
\hline & Operation & $\begin{array}{l}\text { Target or } \\
\text { Setpoint } \\
\text { Value }\end{array}$ & $\begin{array}{c}\text { Range } \\
(-/+)\end{array}$ & Units & Actual Value & \multicolumn{2}{|l|}{ Comments } \\
\hline \multirow{9}{*}{$\begin{array}{c}\text { and add } 17.9 \mathrm{M} \\
\text { caustic in-line } \\
\text { (Test Instructions } \\
\text { steps } 7.1 .7 .1 \text { to } \\
7.1 .7 .9 \text { ) }\end{array}$} & $\begin{array}{l}\text { addition (in-line after } \\
\text { HLP-PMP-T21) }\end{array}$ & & & & & & \\
\hline & $\begin{array}{l}17.9 \mathrm{M} \text { caustic addition } \\
\text { rate }\end{array}$ & 15.516 .7 & $-1 /+310$ & $\mathrm{~kg} / \mathrm{min}$ & $\sim 22$ & $\begin{array}{l}\text { Caustic addition should finish } 1 \text { to } 2 \text { min } \\
\text { before slurry transfer finishes to flush caustic } \\
\text { from transfer line. } \\
\text { Value obtained from the HMI }\end{array}$ & \\
\hline & $\begin{array}{l}\text { UFP-VSL-T01A } \\
\text { Operation }\end{array}$ & - & - & - & - & 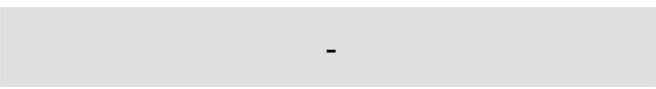 & \\
\hline & $\begin{array}{l}\text { Volume of antifoam to } \\
\text { add to } \\
\text { UFP-VSL-T01A }\end{array}$ & 890 & $-20 /+20$ & $\mathrm{~mL}$ & 890 & $\begin{array}{l}\text { This value is concentrate. Dilute with } 3 \\
\text { parts IW to } 1 \text { part concentrate. Information } \\
\text { Only }\end{array}$ & \\
\hline & $\begin{array}{l}\text { Level to initiate PJM } \\
\text { operation }\end{array}$ & 15 & $-0 /+10$ & in & 30 & Value obtained from the HMI & \\
\hline & PJM jet velocity & 4.8 & $-0.3 /+0.3$ & $\mathrm{~m} / \mathrm{s}$ & 4.6 & NQA-1 & \\
\hline & PJM stroke (80\%) & 29.0 & $-2 /+2$ & in & $25.4(72 \%)$ & NQA-1 & \\
\hline & PJM cycle time & 35 & $-1 /+1$ & $\mathrm{~s}$ & 35.3 & NQA-1 & \\
\hline & $\begin{array}{l}\text { Steam ring air purge } \\
\text { flowrate }\end{array}$ & 0.20 & $0.02 /+0.02$ & $\mathrm{~kg} / \mathrm{min}$ & 0.168 & $\begin{array}{l}6.1 \mathrm{scfm} \text { below } 90^{\circ} \mathrm{C} \text { in UFP-VSL-T01A } \\
\text { Local Calibrated Instrument, NQA-1 }\end{array}$ & \\
\hline \multirow{6}{*}{$\begin{array}{l}\text { Initial heat-up of } \\
\text { UFP-VSL-T01A } \\
\text { (Non-prototypic } \\
\text { using external } \\
\text { exchanger) } \\
\text { (Test Instructions } \\
\text { steps 7.1.7.10 to } \\
7.1 .7 .18 \text { ) } \\
\text { Final heat-up } \\
\text { UFP-VSL-T01A } \\
\text { (Test Instructions } \\
\text { steps 7.1.7.19 to } \\
7.1 .7 .22 \text { ) }\end{array}$} & $\begin{array}{l}\text { Initial heat-up } \\
\text { temperature (endpoint) }\end{array}$ & 57 & $-1 /+3$ & ${ }^{\circ} \mathrm{C}$ & $\sim 57$ & $\begin{array}{l}\text { Y (init.) } \\
\text { NQA-1 }\end{array}$ & $\begin{array}{l}\mathrm{N} \\
\text { STOP }\end{array}$ \\
\hline & $\begin{array}{l}\text { Time to heat to initial } \\
\text { heat-up temperature }\end{array}$ & $<3$ & NA & $\mathrm{hr}$ & 1.5 & $\begin{array}{l}\text { Heating loop is } \sim 9 \text { gallons and will be lost } \\
\text { (unprototypic). Target and range is not } \\
\text { important. Should be complete in under } 3 \\
\text { hours. } \\
\text { NQA-1 }\end{array}$ & \\
\hline & $\begin{array}{l}\text { Target final } \\
\text { temperature (endpoint) }\end{array}$ & 98 & $-2 /+2$ & ${ }^{\circ} \mathrm{C}$ & $\sim 98$ & $\begin{array}{l}\text { Y (init.) } \\
\text { NQA-1 }\end{array}$ & $\begin{array}{l}\mathrm{N} \\
\text { STOP }\end{array}$ \\
\hline & Heat-up time & 3.8 & $-0.5 /+0.5$ & $\mathrm{hr}$ & 3.7 & NQA-1 & \\
\hline & $\begin{array}{l}\text { UFP-VSL-T01A } \\
\text { Operation }\end{array}$ & - & - & - & - & - & \\
\hline & $\begin{array}{l}\text { Temperature to turn off } \\
\text { PJM suction refill }\end{array}$ & 60 & $-2 /+2$ & ${ }^{\circ} \mathrm{C}$ & 60 & Value obtained from the HMI & \\
\hline
\end{tabular}


Table J.1. Integrated Test A Operational Process Sheet

\begin{tabular}{|c|c|c|c|c|c|c|c|}
\hline \multicolumn{8}{|c|}{ Integrated Test A Run sheet (TI-065 Rev. 0) } \\
\hline & Operation & $\begin{array}{l}\text { Target or } \\
\text { Setpoint } \\
\text { Value }\end{array}$ & $\begin{array}{c}\text { Range } \\
(-/+)\end{array}$ & Units & Actual Value & \multicolumn{2}{|l|}{ Comments } \\
\hline & $\begin{array}{l}\text { Temperature to reduce } \\
\text { steam ring air purge } \\
\text { rate }\end{array}$ & 90 & $-2 /+8$ & ${ }^{\circ} \mathrm{C}$ & 96 & Value obtained from the HMI & \\
\hline & $\begin{array}{l}\text { High-temperature } \\
\text { steam ring air purge } \\
\text { flowrate }\end{array}$ & 0.13 & $0.02 /+0.02$ & $\mathrm{~kg} / \mathrm{min}$ & 0.13 & $\begin{array}{l}4.0 \mathrm{scfm} \text { above } 90^{\circ} \mathrm{C} \text { in UFP-VSL-T01A } \\
\text { Local Calibrated Instrument, NQA-1 }\end{array}$ & \\
\hline & Level at end of heat-up & 61 & $-2 /+2$ & in & 63.7 & $\begin{array}{l}\text { Y (init.) } \\
\text { Anticipate accumulation of } \sim 62 \text { gal of } \\
\text { condensate during heat-up. } \\
\text { NQA-1 }\end{array}$ & $\begin{array}{l}\mathrm{N} \\
\text { STOP }\end{array}$ \\
\hline \multirow{9}{*}{$\begin{array}{l}\text { Caustic leach in } \\
\text { UFP-VSL-T01A } \\
\text { (Test Instructions } \\
\text { steps 7.1.7.23 to } \\
7.1 .7 .28 \text { ) }\end{array}$} & $\begin{array}{l}\text { Caustic leach duration } \\
\text { (endpoint) }\end{array}$ & 16 & $-0.1 /+1$ & $\mathrm{hr}$ & 15.9 & NQA-1 & \\
\hline & Temperature & 98 & $-2 /+2$ & ${ }^{\circ} \mathrm{C}$ & $\sim 98$ & NQA-1 & \\
\hline & $\begin{array}{l}\text { Volume of water to } \\
\text { add to get prototypic } \\
\text { condensate }\end{array}$ & 2.9 & $-0.1 /+0.1$ & $\mathrm{~L}$ & 2.9 & $\begin{array}{l}\text { This quantity added after samples are } \\
\text { collected at } 1,2,3, \ldots 13,14 \text {, and } 15 \text { hrs after } \\
\text { start of leach. } \\
\text { Information Only }\end{array}$ & \\
\hline & $\begin{array}{l}\text { UFP-VSL-T01A } \\
\text { Operation }\end{array}$ & - & - & - & - & (1) & \\
\hline & PJM jet velocity & 4.8 & $-0.3 /+0.3$ & $\mathrm{~m} / \mathrm{s}$ & 4.5 & NQA-1 & \\
\hline & PJM stroke $(80 \%)$ & 29.0 & $-2 /+2$ & in & $26.7(76 \%)$ & NQA-1 & \\
\hline & PJM cycle time & 35 & $-1 /+1$ & $\mathrm{~s}$ & 35.3 & NQA-1 & \\
\hline & $\begin{array}{l}\text { Steam ring air purge } \\
\text { flowrate (automatic) }\end{array}$ & 0.13 & $0.02 /+0.02$ & $\mathrm{~kg} / \mathrm{min}$ & 0.13 & $\begin{array}{l}\sim 4.0 \text { scfm, purge will turn off when steam } \\
\text { exceeds } 6 \mathrm{cfm} \text { and will turn back on when } \\
\text { steam flow drops below } 4 \mathrm{cfm} \text { Local } \\
\text { Calibrated Instrument, NQA-1 }\end{array}$ & \\
\hline & Level at end of leach & 66 & $-5 /+5$ & in & 68.6 & $\begin{array}{l}\text { Y (init.) } \\
\sim 593 \text { gal } \\
\text { NQA-1 }\end{array}$ & $\begin{array}{l}\mathrm{N} \\
\text { STOP }\end{array}$ \\
\hline $\begin{array}{l}\text { Cool-down of } \\
\text { UFP-VSL-T01A } \\
\text { by UFP-HX- } \\
\text { T05A }\end{array}$ & $\begin{array}{l}\text { Initial cool-down } \\
\text { temperature using } \\
\text { external chiller UFP- } \\
\text { HX-T05A (endpoint) }\end{array}$ & 60 & $-2 /+2$ & ${ }^{\circ} \mathrm{C}$ & 60 & Value obtained from the HMI & \\
\hline
\end{tabular}


Table J.1. Integrated Test A Operational Process Sheet

\begin{tabular}{|c|c|c|c|c|c|c|c|c|}
\hline & & & Integra & ed Test A Ru & isheet $(\mathrm{T}$ & 065 Rev. 0$)$ & & \\
\hline & & Operation & $\begin{array}{l}\text { Target or } \\
\text { Setpoint } \\
\text { Value }\end{array}$ & $\begin{array}{c}\text { Range } \\
(-/+)\end{array}$ & Units & Actual Value & Comments & \\
\hline & $\begin{array}{l}\text { (Test Instructions } \\
\text { steps 7.1.7.29 to }\end{array}$ & $\begin{array}{l}\text { Initial cool-down } \\
\text { duration }\end{array}$ & 2.75 & $-0.5 /+0.5$ & $\mathrm{hr}$ & 2.75 & Value obtained from the HMI & \\
\hline & 7.1.7.35) & $\begin{array}{l}\text { UFP-VSL-T01A } \\
\text { Operation }\end{array}$ & - & - & - & - & - & \\
\hline & & $\begin{array}{l}\text { Temperature to } \\
\text { increase steam ring air } \\
\text { purge rate }\end{array}$ & 90 & $-5 /+2$ & ${ }^{\circ} \mathrm{C}$ & 84 & Value obtained from the HMI & \\
\hline & & $\begin{array}{l}\text { Steam ring air purge } \\
\text { flowrate }\end{array}$ & 0.20 & $0.02 /+0.02$ & $\mathrm{~kg} / \mathrm{min}$ & 0.169 & $\begin{array}{l}6.1 \mathrm{scfm} \text { below } 90^{\circ} \mathrm{C} \text { in UFP-VSL-T01A } \\
\text { Local Calibrated Instrument, NQA-1 }\end{array}$ & \\
\hline & & $\begin{array}{l}\text { Temperature to resume } \\
\text { PJM suction refill }\end{array}$ & 60 & $-2 /+2$ & ${ }^{\circ} \mathrm{C}$ & 60 & Value obtained from the HMI & \\
\hline$i$ & & & -- & & & & & \\
\hline & $\begin{array}{l}\text { Transfer and cool } \\
\text { leached simulant }\end{array}$ & $\begin{array}{l}\text { Make-up batch transfer } \\
\text { volume }\end{array}$ & 11 & $-0.2 /+0.2$ & gal & 11 & Value obtained from the HMI & \\
\hline & $\begin{array}{c}\text { from } \\
\text { UFP-VSL-T01A }\end{array}$ & $\begin{array}{l}\text { Make-up batch } \\
\text { flowrate }\end{array}$ & 6.9 & NA & gpm & 6.9 & Value obtained from the HMI & \\
\hline n & $\begin{array}{l}\text { to } \\
\text { UFP-VSL-T02A } \\
\text { and concentrate } \\
\text { solids }\end{array}$ & $\begin{array}{l}\text { UFP-VSL-T01A level } \\
\text { (endpoint to stop batch } \\
\text { transfers) }\end{array}$ & 10 & $-2 /+2$ & in & 9.7 & $\begin{array}{l}\text { Y (init.) } \\
\text { Estimate } 47 \text { make-up batches from } \\
\text { UFP-VSL-T01A. } \\
\text { NQA-1 }\end{array}$ & $\begin{array}{l}\mathrm{N} \\
\text { STOP }\end{array}$ \\
\hline 胥 & $\begin{array}{c}\text { (Test Instructions } \\
\text { steps } 7.1 .11 .6 \text { to } \\
7.1 .11 .8)\end{array}$ & $\begin{array}{l}\text { UFP-VSL-T02A level } \\
\text { to initiate each make- } \\
\text { up batch }\end{array}$ & 44.7 & $-1 /+1$ & in & 44.7 & Value obtained from the HMI & \\
\hline 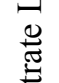 & & $\begin{array}{l}\text { UFP-VSL-T02A } \\
\text { Operation }\end{array}$ & - & - & - & - & - & \\
\hline$\overline{0}$ & & PJM jet velocity & 7.3 & $-0.4 /+0.4$ & $\mathrm{~m} / \mathrm{s}$ & 7.6 & Value obtained from the HMI & \\
\hline ש̃ & & PJM stroke $(80 \%)$ & 31.5 & $-2 /+2$ & in & 32.5 & Value obtained from the HMI & \\
\hline U & & PJM cycle time & 33 & $-1 /+1$ & $\mathrm{~s}$ & 33 & Value obtained from the HMI & \\
\hline & & $\begin{array}{l}\text { Steam ring air purge } \\
\text { flowrate }\end{array}$ & 0.14 & $0.02 /+0.02$ & $\mathrm{~kg} / \mathrm{min}$ & 0.14 & $\begin{array}{l}4.1 \mathrm{scfm} \text { below } 90^{\circ} \mathrm{C} \text { in UFP-VSL-T02A } \\
\text { Local Calibrated Instrument, NQA-1 }\end{array}$ & \\
\hline & & $\begin{array}{l}\text { Total flowrate of } 5 \\
\text { lower air spargers } \\
\text { (idle) }\end{array}$ & 0.04 & $\begin{array}{c}-0.005 /+ \\
0.005\end{array}$ & $\mathrm{~kg} / \mathrm{min}$ & 0.04 & $\begin{array}{l}\sim 13 \text { scfh for each sparge tube } \\
\text { Local Calibrated Instrument, NQA-1 }\end{array}$ & \\
\hline & & Flowrate of upper air & 0.01 & $-0.005 /+$ & $\mathrm{kg} / \mathrm{min}$ & 0.01 & $\sim 16 \mathrm{scfh}$ (record field transmitter value) & \\
\hline
\end{tabular}


Table J.1. Integrated Test A Operational Process Sheet

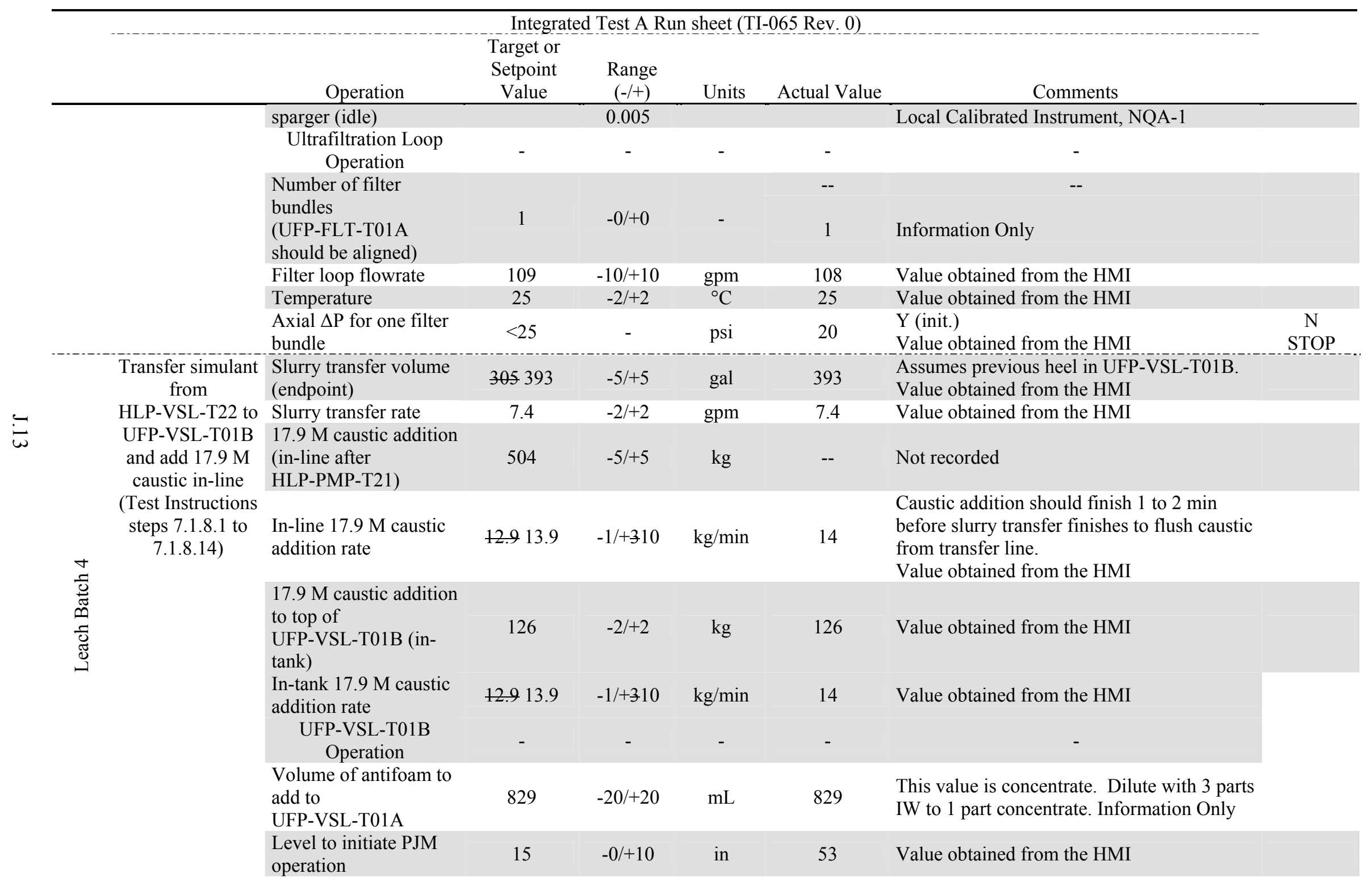


Table J.1. Integrated Test A Operational Process Sheet

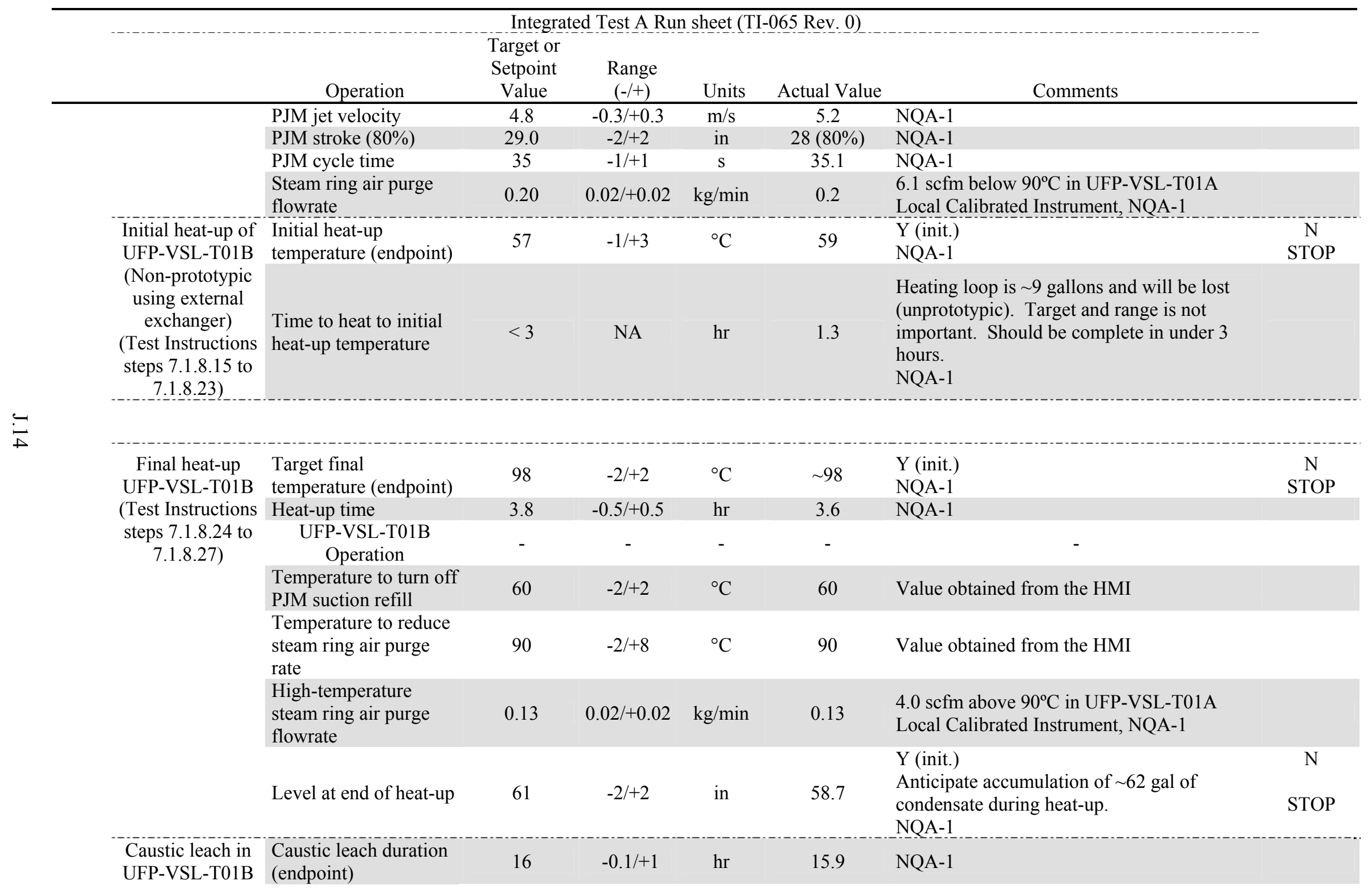


Table J.1. Integrated Test A Operational Process Sheet

\begin{tabular}{|c|c|c|c|c|c|c|c|}
\hline \multicolumn{8}{|c|}{ Integrated Test A Run sheet (TI-065 Rev. 0) } \\
\hline & Operation & $\begin{array}{l}\text { Target or } \\
\text { Setpoint } \\
\text { Value }\end{array}$ & $\begin{array}{c}\text { Range } \\
(-/+)\end{array}$ & Units & Actual Value & Comments & \\
\hline \multirow{8}{*}{$\begin{array}{c}\text { (Test Instructions } \\
\text { steps } 7.1 .8 .28 \text { to } \\
7.1 .8 .32)\end{array}$} & Temperature & 98 & $-2 /+2$ & ${ }^{\circ} \mathrm{C}$ & $\sim 98$ & NQA-1 & \\
\hline & $\begin{array}{l}\text { Volume of water to } \\
\text { add to get prototypic } \\
\text { condensate }\end{array}$ & 2.9 & $-0.1 /+0.1$ & $\mathrm{~L}$ & 2.9 & $\begin{array}{l}\text { This quantity added after samples are } \\
\text { collected at } 1,2,3, \ldots 13,14 \text {, and } 15 \text { hrs after } \\
\text { start of leach. } \\
\text { Information Only }\end{array}$ & \\
\hline & $\begin{array}{l}\text { UFP-VSL-T01B } \\
\text { Operation }\end{array}$ & - & - & - & - & 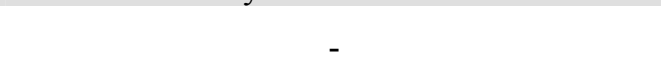 & \\
\hline & PJM jet velocity & 4.8 & $-0.3 /+0.3$ & $\mathrm{~m} / \mathrm{s}$ & 4.6 & NQA-1 & \\
\hline & PJM stroke $(80 \%)$ & 29.0 & $-2 /+2$ & in & $28.2(80 \%)$ & NQA-1 & \\
\hline & PJM cycle time & 35 & $-1 /+1$ & $\mathrm{~s}$ & 35.1 & NQA-1 & \\
\hline & $\begin{array}{l}\text { Steam ring air purge } \\
\text { flowrate (automatic) }\end{array}$ & 0.13 & $0.02 /+0.02$ & $\mathrm{~kg} / \mathrm{min}$ & 0.13 & $\begin{array}{l}\sim 4.0 \mathrm{scfm} \text {, purge will turn off when steam } \\
\text { exceeds } 6 \mathrm{cfm} \text { and will turn back on when } \\
\text { steam flow drops below } 4 \mathrm{cfm} \text { Local } \\
\text { Calibrated Instrument, NQA-1 }\end{array}$ & \\
\hline & Level at end of leach & 66 & $-5 /+5$ & in & 62.8 & $\begin{array}{l}\text { Y (init.) } \\
\sim 593 \text { gal } \\
\text { NQA-1 }\end{array}$ & STOP \\
\hline \multirow{6}{*}{$\begin{array}{c}\text { Cool-down of } \\
\text { UFP-VSL-T01B } \\
\text { by } \\
\text { UFP-HX-T05A } \\
\text { (Test Instructions } \\
\text { steps } 7.1 .8 .32 \text { to } \\
7.1 .8 .36 \text { ) }\end{array}$} & $\begin{array}{l}\text { Initial cool-down } \\
\text { temperature using } \\
\text { external chiller } \\
\text { UFP-HX-T05B } \\
\text { (endpoint) }\end{array}$ & 60 & $-2 /+2$ & ${ }^{\circ} \mathrm{C}$ & $\sim 60$ & NQA-1 & \\
\hline & $\begin{array}{l}\text { Initial cool-down } \\
\text { duration }\end{array}$ & 2.75 & $-0.5 /+0.5$ & $\mathrm{hr}$ & 2.8 & $\begin{array}{l}\text { Table } 4.4 \text { of the Run Report } \\
\text { Value obtained from the HMI }\end{array}$ & \\
\hline & $\begin{array}{l}\text { UFP-VSL-T01B } \\
\text { Operation }\end{array}$ & - & - & - & - & จ & \\
\hline & $\begin{array}{l}\text { Temperature to } \\
\text { increase steam ring air } \\
\text { purge rate }\end{array}$ & 90 & $-5 /+2$ & ${ }^{\circ} \mathrm{C}$ & $\sim 80$ & NQA-1 & \\
\hline & $\begin{array}{l}\text { Steam ring air purge } \\
\text { flowrate }\end{array}$ & 0.20 & $\begin{array}{l}-0.02 / \\
+0.02\end{array}$ & $\mathrm{~kg} / \mathrm{min}$ & 0.16 & $\begin{array}{l}6.1 \mathrm{scfm} \text { below } 90^{\circ} \mathrm{C} \text { in UFP-VSL-T01A } \\
\text { Local Calibrated Instrument, NQA-1 }\end{array}$ & \\
\hline & $\begin{array}{l}\text { Temperature to resume } \\
\text { PJM suction refill }\end{array}$ & 60 & $-2 /+2$ & ${ }^{\circ} \mathrm{C}$ & 60 & 20 & \\
\hline
\end{tabular}


Table J.1. Integrated Test A Operational Process Sheet

\begin{tabular}{|c|c|c|c|c|c|c|c|c|}
\hline & & & Integra & d Test A Ru & sheet $(\mathrm{T})$ & 065 Rev. 0) & & \\
\hline & & Operation & $\begin{array}{l}\text { Target or } \\
\text { Setpoint } \\
\text { Value }\end{array}$ & $\begin{array}{c}\text { Range } \\
(-/+)\end{array}$ & Units & Actual Value & Comments & \\
\hline & $\begin{array}{l}\text { Transfer and cool } \\
\text { leached simulant }\end{array}$ & $\begin{array}{l}\text { Make-up batch transfer } \\
\text { volume }\end{array}$ & 11 & $-0.2 /+0.2$ & gal & 11 & Value obtained from the HMI & \\
\hline & $\begin{array}{c}\text { from } \\
\text { UFP-VSL-T01B }\end{array}$ & $\begin{array}{l}\text { Make-up batch } \\
\text { flowrate }\end{array}$ & 6.9 & NA & gpm & 6.9 & Value obtained from the HMI & \\
\hline & $\begin{array}{l}\text { to } \\
\text { UFP-VSL-T02A } \\
\text { and concentrate } \\
\text { solids }\end{array}$ & $\begin{array}{l}\text { UFP-VSL-T01B level } \\
\text { (endpoint to stop batch } \\
\text { transfers) }\end{array}$ & 10 & $-2 /+2$ & in & $\sim 10$ & $\begin{array}{l}\text { Y (init.) } \\
\text { Estimate } 47 \text { make-up batches from } \\
\text { UFP-VSL-T01B. } \\
\text { NQA-1 }\end{array}$ & $\begin{array}{l}\mathrm{N} \\
\text { STOP }\end{array}$ \\
\hline & $\begin{array}{c}\text { (Test Instructions } \\
\text { steps 7.1.11.6 to } \\
7.1 .11 .8)\end{array}$ & $\begin{array}{l}\text { UFP-VSL-T02A level } \\
\text { to initiate each make- } \\
\text { up batch }\end{array}$ & 44.7 & $-1 /+1$ & in & 44.7 & Value obtained from the HMI & \\
\hline 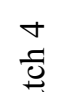 & & $\begin{array}{l}\text { UFP-VSL-T02A } \\
\text { Operation }\end{array}$ & - & - & - & - & - & \\
\hline$\stackrel{\vec{m}}{n}$ & & PJM jet velocity & 7.3 & $-0.4 /+0.4$ & $\mathrm{~m} / \mathrm{s}$ & -- & Not recorded & \\
\hline$\frac{7}{0}$ & & PJM stroke $(80 \%)$ & 31.5 & $-2 /+2$ & in & -- & Not recorded & \\
\hline 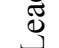 & & PJM cycle time & 33 & $-1 /+1$ & $\mathrm{~s}$ & 33 & Value obtained from the HMI & \\
\hline 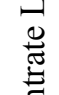 & & $\begin{array}{l}\text { Steam ring air purge } \\
\text { flowrate }\end{array}$ & 0.14 & $0.02 /+0.02$ & $\mathrm{~kg} / \mathrm{min}$ & 0.14 & $\begin{array}{l}4.1 \mathrm{scfm} \text { below } 90^{\circ} \mathrm{C} \text { in UFP-VSL-T02A } \\
\text { NQA-1 }\end{array}$ & \\
\hline ల் & & $\begin{array}{l}\text { Total flowrate of } 5 \\
\text { lower air spargers } \\
\text { (idle) }\end{array}$ & 0.04 & $\begin{array}{c}-0.005 /+ \\
0.005\end{array}$ & $\mathrm{~kg} / \mathrm{min}$ & 0.04 & $\begin{array}{l}\text { 13 scfh for each sparge tube } \\
\text { NQA-1 }\end{array}$ & \\
\hline & & $\begin{array}{l}\text { Flowrate of upper air } \\
\text { sparger (idle) }\end{array}$ & 0.01 & $\begin{array}{c}-0.005 /+ \\
0.005\end{array}$ & $\mathrm{~kg} / \mathrm{min}$ & 0.01 & $\begin{array}{l}\sim 16 \text { scfh (record field transmitter value) } \\
\text { NQA-1 }\end{array}$ & \\
\hline & & $\begin{array}{l}\text { Ultrafiltration Loop } \\
\text { Operation }\end{array}$ & - & - & - & - & 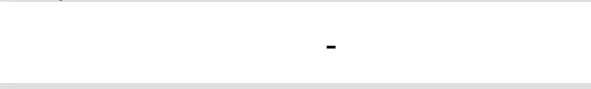 & \\
\hline & & $\begin{array}{l}\text { Number of filter } \\
\text { bundles } \\
\text { (UFP-FLT-T01A } \\
\text { should be aligned) }\end{array}$ & 1 & $-0 /+0$ & - & -- & Information Only & \\
\hline & & Filter loop flowrate & 109 & $-10 /+10$ & gpm & 110 & Value obtained from the HMI & \\
\hline & & Temperature & 25 & $-2 /+2$ & ${ }^{\circ} \mathrm{C}$ & 25.5 & Value obtained from the HMI & \\
\hline & & Axial $\Delta \mathrm{P}$ for one filter & $<25$ & - & psi & 20.5 & Y (init.) & $\mathrm{N}$ \\
\hline
\end{tabular}


Table J.1. Integrated Test A Operational Process Sheet

\begin{tabular}{|c|c|c|c|c|c|c|c|c|}
\hline \multicolumn{9}{|c|}{ Integrated Test A Run sheet (TI-065 Rev. 0) } \\
\hline & & Operation & $\begin{array}{l}\text { Target or } \\
\text { Setpoint } \\
\text { Value }\end{array}$ & $\begin{array}{c}\text { Range } \\
(-/+)\end{array}$ & Units & Actual Value & Comments & \\
\hline \multirow{15}{*}{ 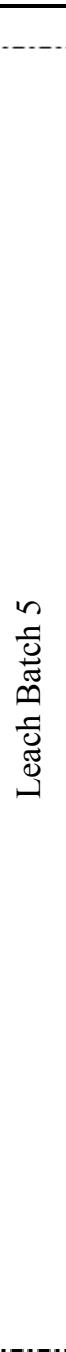 } & \multirow{12}{*}{$\begin{array}{c}\text { Transfer simulant } \\
\text { from } \\
\text { HLP-VSL-T22 to } \\
\text { UFP-VSL-T01A } \\
\text { and add } 17.9 \mathrm{M} \\
\text { caustic in-line } \\
\text { (Test Instructions } \\
\text { steps 7.1.9.1 to } \\
7.1 .9 .9 \text { ) }\end{array}$} & bundle & & & & & Value obtained from the HMI & STOP \\
\hline & & $\begin{array}{l}\text { Total slurry transfer } \\
\text { volume (endpoint) }\end{array}$ & 305415 & $-5 /+5$ & gal & 415 & $\begin{array}{l}\text { Assumes previous heel in UFP-VSL-T01A. } \\
\text { Value obtained from the HMI }\end{array}$ & \\
\hline & & Slurry transfer rate & 7.4 & $-2 /+2$ & gpm & 7.4 & Value obtained from the HMI & \\
\hline & & $\begin{array}{l}\text { Total } 17.9 \mathrm{M} \text { caustic } \\
\text { addition (in-line after } \\
\text { HLP-PMP-T21) }\end{array}$ & 625 & $-5 /+5$ & $\mathrm{~kg}$ & 625 & Value obtained from the HMI & \\
\hline & & $\begin{array}{l}17.9 \mathrm{M} \text { caustic addition } \\
\text { rate }\end{array}$ & 15.917 .2 & $-1 /+310$ & $\mathrm{~kg} / \mathrm{min}$ & 17.2 & $\begin{array}{l}\text { Caustic addition should finish } 1 \text { to } 2 \text { min } \\
\text { before slurry transfer finishes to flush caustic } \\
\text { from transfer line. } \\
\text { Value obtained from the HMI }\end{array}$ & \\
\hline & & $\begin{array}{l}\text { UFP-VSL-T01A } \\
\text { Operation }\end{array}$ & - & - & - & - & 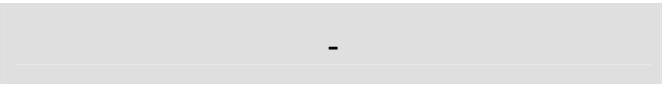 & \\
\hline & & $\begin{array}{l}\text { Volume of antifoam to } \\
\text { add to } \\
\text { UFP-VSL-T01A }\end{array}$ & 871 & $-20 /+20$ & $\mathrm{~mL}$ & 871 & $\begin{array}{l}\text { This value is concentrate. Dilute with } 3 \text { parts } \\
\text { IW to } 1 \text { part concentrate. Information Only }\end{array}$ & \\
\hline & & $\begin{array}{l}\text { Level to initiate PJM } \\
\text { operation }\end{array}$ & 15 & $-0 /+10$ & in & 42 & Value obtained from the HMI & \\
\hline & & PJM jet velocity & 4.8 & $-0.3 /+0.3$ & $\mathrm{~m} / \mathrm{s}$ & 5.1 & NQA-1 & \\
\hline & & PJM stroke $(80 \%)$ & 29.0 & $-2 /+2$ & in & $30.7(87 \%)$ & NQA-1 & \\
\hline & & PJM cycle time & 35 & $-1 /+1$ & $\mathrm{~s}$ & 35.3 & NQA-1 & \\
\hline & & $\begin{array}{l}\text { Steam ring air purge } \\
\text { flowrate }\end{array}$ & 0.20 & $0.02 /+0.02$ & $\mathrm{~kg} / \mathrm{min}$ & 0.17 & $\begin{array}{l}6.1 \mathrm{scfm} \text { below } 90^{\circ} \mathrm{C} \text { in UFP-VSL-T01A } \\
\text { Local Calibrated Instrument, NQA-1 }\end{array}$ & \\
\hline & \multirow{2}{*}{$\begin{array}{l}\text { Initial heat-up of } \\
\text { UFP-VSL-T01A } \\
\text { (Non-prototypic } \\
\text { using external } \\
\text { exchanger) } \\
\text { (Test Instructions } \\
\text { steps } 7.1 .9 .10 \text { to } \\
7.1 .9 .18 \text { ) }\end{array}$} & $\begin{array}{l}\text { Initial heat-up } \\
\text { temperature (endpoint) }\end{array}$ & 57 & $-1 /+3$ & ${ }^{\circ} \mathrm{C}$ & 57.3 & $\begin{array}{l}\text { Y (init.) } \\
\text { NQA-1 }\end{array}$ & $\begin{array}{l}\text { N } \\
\text { STOP }\end{array}$ \\
\hline & & $\begin{array}{l}\text { Time to heat to initial } \\
\text { heat-up temperature }\end{array}$ & $<3$ & NA & $\mathrm{hr}$ & 1.1 & $\begin{array}{l}\text { Heating loop is } \sim 9 \text { gallons and will be lost } \\
\text { (unprototypic). Target and range is not } \\
\text { important. Should be complete in under } 3 \\
\text { hours. } \\
\text { NQA-1 }\end{array}$ & \\
\hline & $\begin{array}{l}\text { Final heat-up } \\
\text { UFP-VSL-T01A }\end{array}$ & $\begin{array}{l}\text { Target final } \\
\text { temperature (endpoint) }\end{array}$ & 98 & $-2 /+2$ & ${ }^{\circ} \mathrm{C}$ & $\sim 98$ & $\begin{array}{l}\text { Y (init.) } \\
\text { NQA-1 }\end{array}$ & $\begin{array}{l}\mathrm{N} \\
\text { STOP }\end{array}$ \\
\hline
\end{tabular}


Table J.1. Integrated Test A Operational Process Sheet

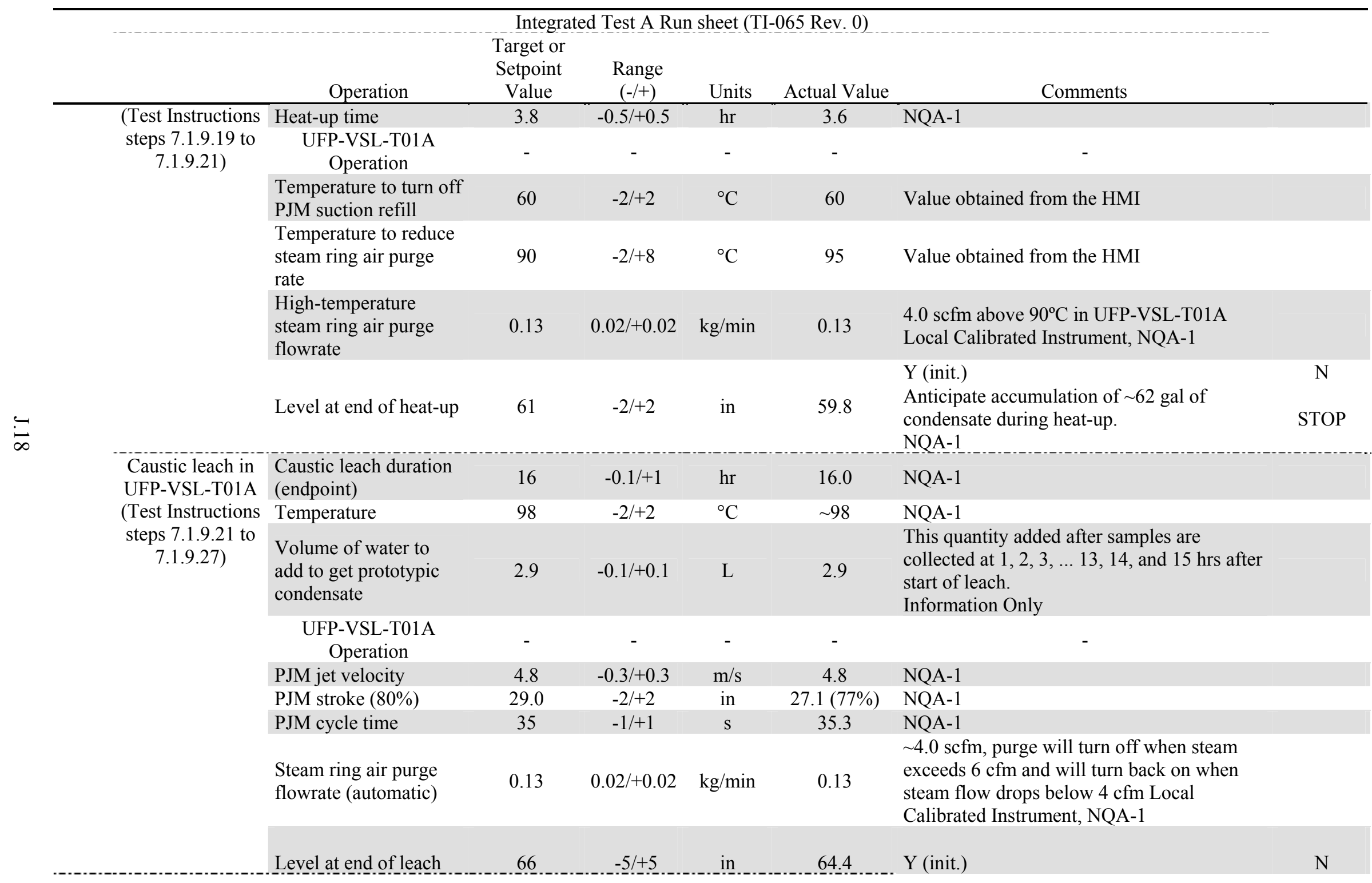


Table J.1. Integrated Test A Operational Process Sheet

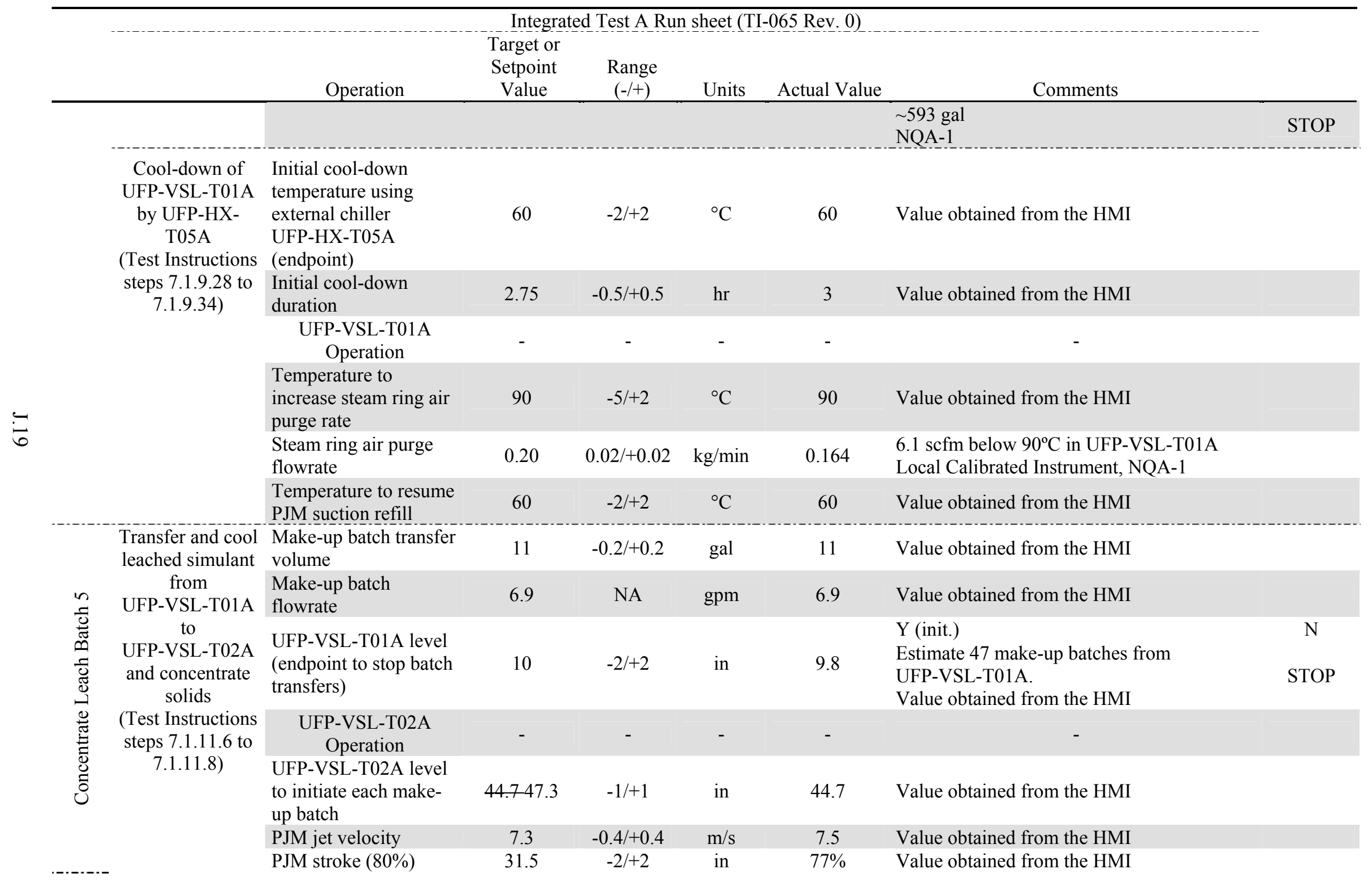


Table J.1. Integrated Test A Operational Process Sheet

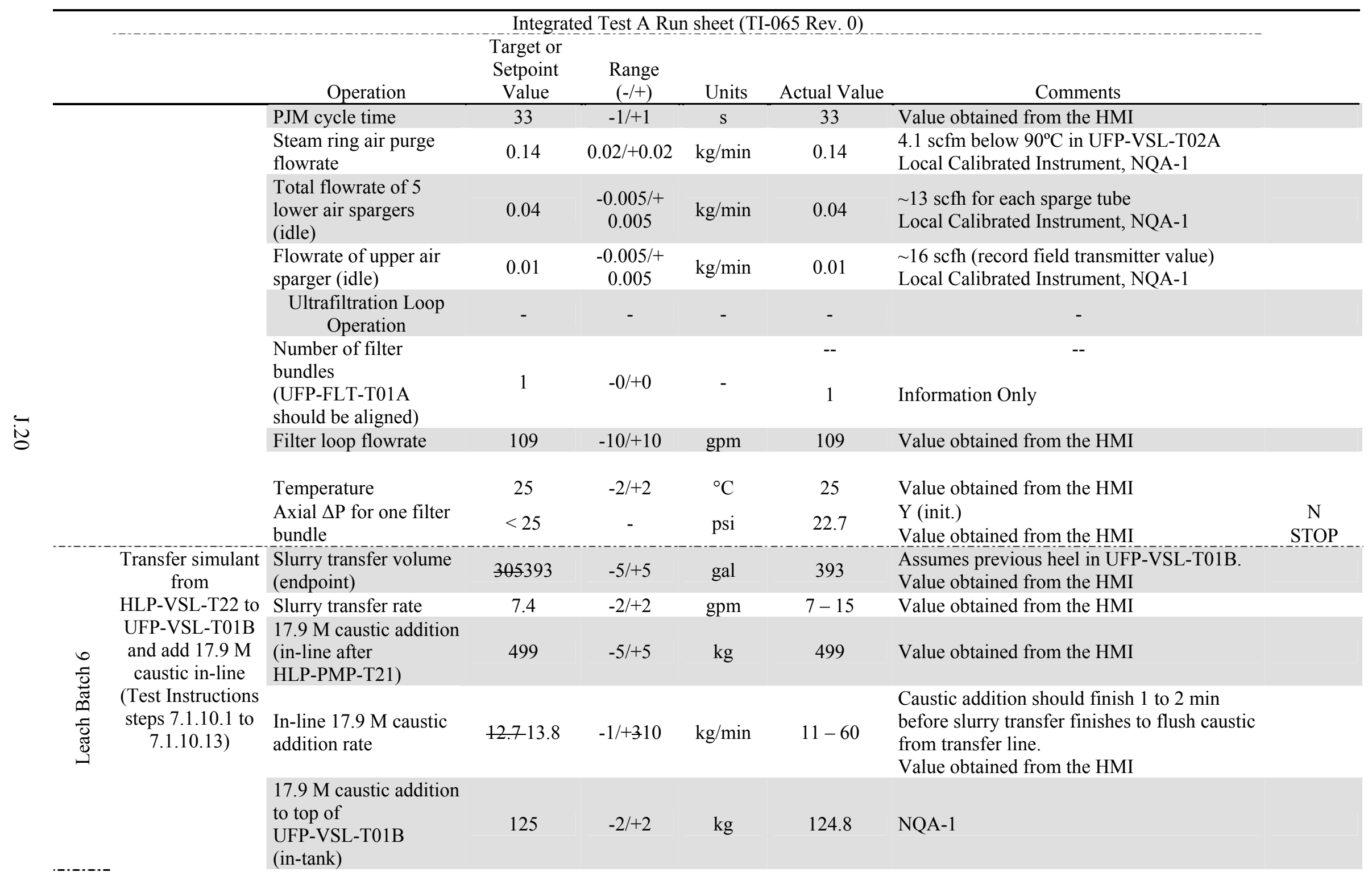


Table J.1. Integrated Test A Operational Process Sheet

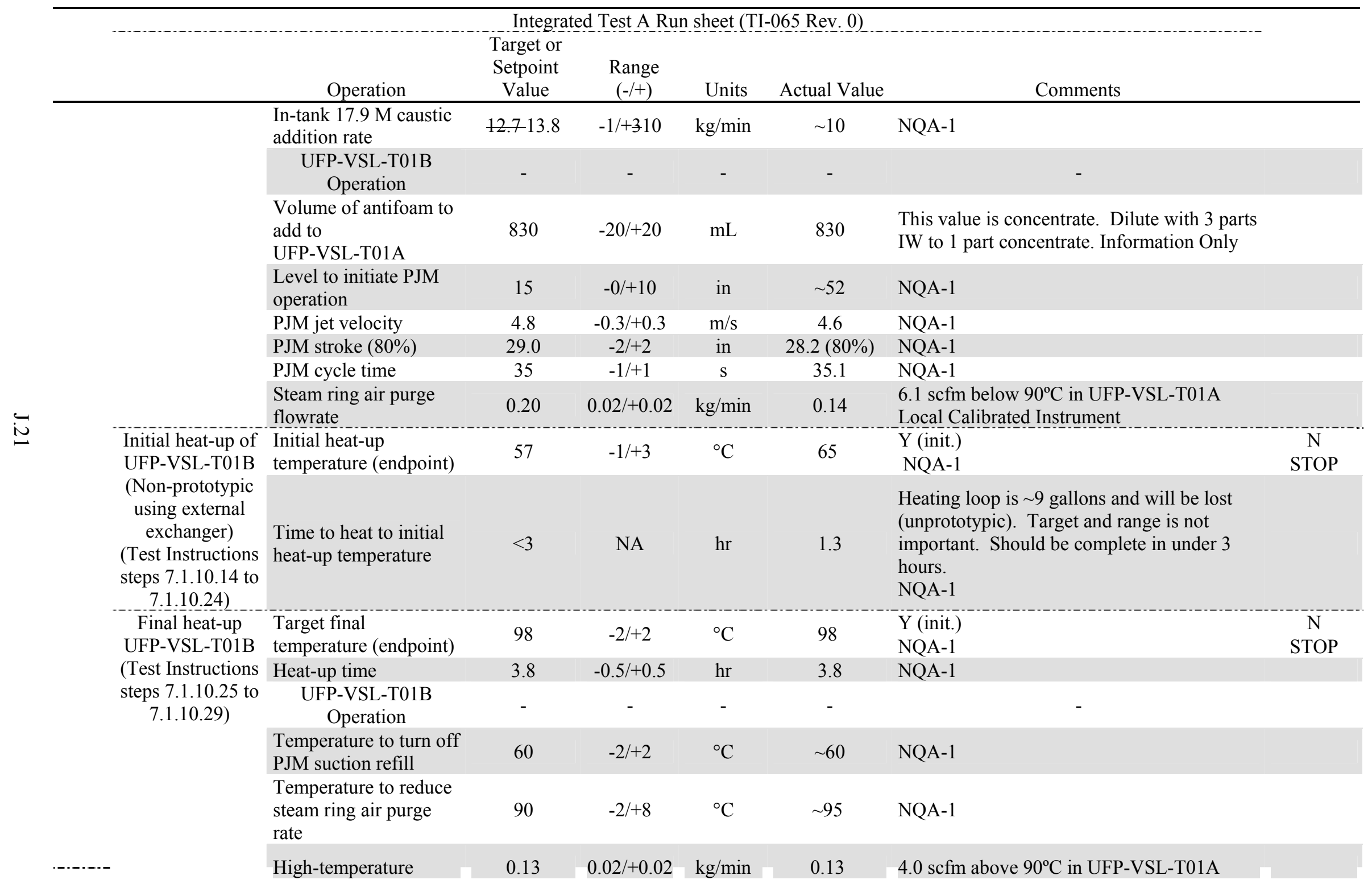


Table J.1. Integrated Test A Operational Process Sheet

\begin{tabular}{|c|c|c|c|c|c|c|c|}
\hline & & Integra & ed Test A Ru & isheet $(\mathrm{T}$ & 065 Rev. 0) & & \\
\hline & Operation & $\begin{array}{l}\text { Target or } \\
\text { Setpoint } \\
\text { Value }\end{array}$ & $\begin{array}{c}\text { Range } \\
(-/+) \\
\end{array}$ & Units & Actual Value & Comments & \\
\hline & $\begin{array}{l}\text { steam ring air purge } \\
\text { flowrate }\end{array}$ & & & & & Local Calibrated Instrument, NQA-1 & \\
\hline & Level at end of heat-up & 6162 & $-2 /+2$ & in & 59.4 & $\begin{array}{l}\text { Y (init.) } \\
\text { Anticipate accumulation of } \sim 62 \text { gal of } \\
\text { condensate during heat-up. NQA-1. }\end{array}$ & $\begin{array}{c}\mathrm{N} \\
\text { STOP }\end{array}$ \\
\hline $\begin{array}{l}\text { Caustic leach in } \\
\text { UFP-VSL-T01B }\end{array}$ & $\begin{array}{l}\text { Caustic leach duration } \\
\text { (endpoint) }\end{array}$ & 16 & $-0.1 /+1$ & $\mathrm{hr}$ & $\sim 16$ & NQA-1 & \\
\hline (Test Instructions & Temperature & 98 & $-2 /+2$ & ${ }^{\circ} \mathrm{C}$ & $\sim 98$ & NQA-1 & \\
\hline $\begin{array}{l}\text { steps } 7.1 .10 .30 \text { to } \\
\quad 7.1 .10 .33) \\
\text { Steps } 7.1 .10 .30 \text { to }\end{array}$ & $\begin{array}{l}\text { Volume of water to } \\
\text { add to get prototypic } \\
\text { condensate }\end{array}$ & 2.9 & $-0.1 /+0.1$ & $\mathrm{~L}$ & 2.9 & $\begin{array}{l}\text { This quantity added after samples are } \\
\text { collected at } 1,2,3, \ldots 13,14 \text {, and } 15 \mathrm{hrs} \text { after } \\
\text { start of leach. } \\
\text { Information Only }\end{array}$ & \\
\hline $\begin{array}{c}33 \text { are not related } \\
\text { to PJM }\end{array}$ & $\begin{array}{l}\text { UFP-VSL-T01B } \\
\text { Operation }\end{array}$ & - & - & - & - & (1) & \\
\hline operations. & PJM jet velocity & 4.8 & $-0.3 /+0.3$ & $\mathrm{~m} / \mathrm{s}$ & 4.0 & $\begin{array}{l}\text { It's cited as 7.1.10.29.2. } \\
\text { NQA-1 }\end{array}$ & \\
\hline & PJM stroke $(80 \%)$ & 29.0 & $-2 /+2$ & in & $21.7(62 \%)$ & NQA-1 & \\
\hline & PJM cycle time & 35 & $-1 /+1$ & $\mathrm{~s}$ & 35.1 & NQA-1 & \\
\hline & $\begin{array}{l}\text { Steam ring air purge } \\
\text { flowrate (automatic) }\end{array}$ & 0.13 & $0.02 /+0.02$ & $\mathrm{~kg} / \mathrm{min}$ & 0.13 & $\begin{array}{l}\sim 4.0 \mathrm{scfm} \text {, purge will turn off when steam } \\
\text { exceeds } 6 \mathrm{cfm} \text { and will turn back on when } \\
\text { steam flow drops below } 4 \mathrm{cfm} \text { Local } \\
\text { Calibrated Instrument, NQA-1 }\end{array}$ & \\
\hline & Level at end of leach & $66-66.5$ & $-5 /+5$ & in & 62.9 & $\begin{array}{l}\text { Y (init.) } \\
\sim 593 \text { gal } \\
\text { NQA-1 }\end{array}$ & $\begin{array}{c}\mathrm{N} \\
\text { STOP }\end{array}$ \\
\hline $\begin{array}{l}\text { Cool-down of } \\
\text { UFP-VSL-T01B } \\
\text { by UFP-HX- } \\
\text { T05A }\end{array}$ & $\begin{array}{l}\text { Initial cool-down } \\
\text { temperature using } \\
\text { external chiller UFP- } \\
\text { HX-T05B (endpoint) }\end{array}$ & 60 & $-2 /+2$ & ${ }^{\circ} \mathrm{C}$ & 60 & Value obtained from the HMI & \\
\hline $\begin{array}{l}\text { (Test Instructions } \\
\text { steps } 7.1 .10 .34 \text { to }\end{array}$ & $\begin{array}{l}\text { Initial cool-down } \\
\text { duration }\end{array}$ & 2.75 & $-0.5 /+0.5$ & $\mathrm{hr}$ & 2.75 & Value obtained from the HMI & \\
\hline 7.1.10.40) & $\begin{array}{l}\text { UFP-VSL-T01B } \\
\text { Operation }\end{array}$ & - & - & - & - & - & \\
\hline
\end{tabular}


Table J.1. Integrated Test A Operational Process Sheet

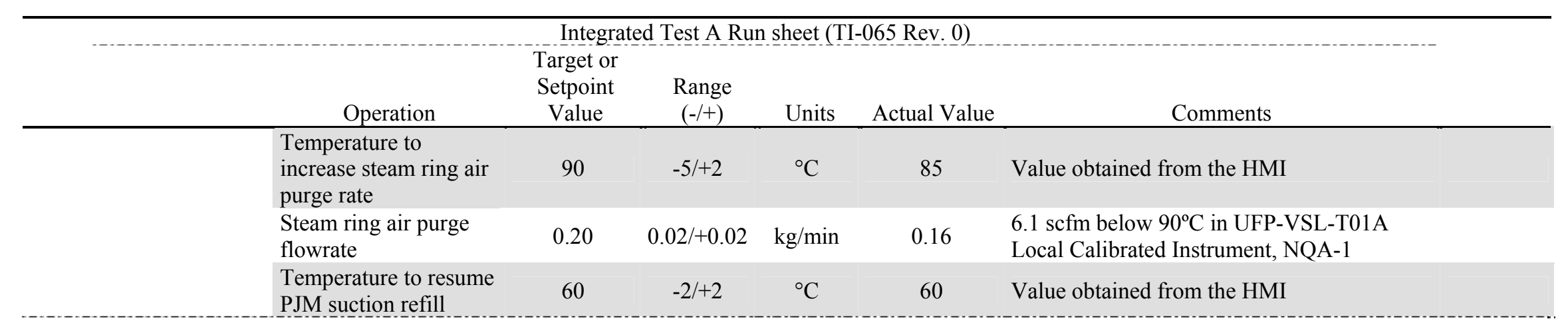

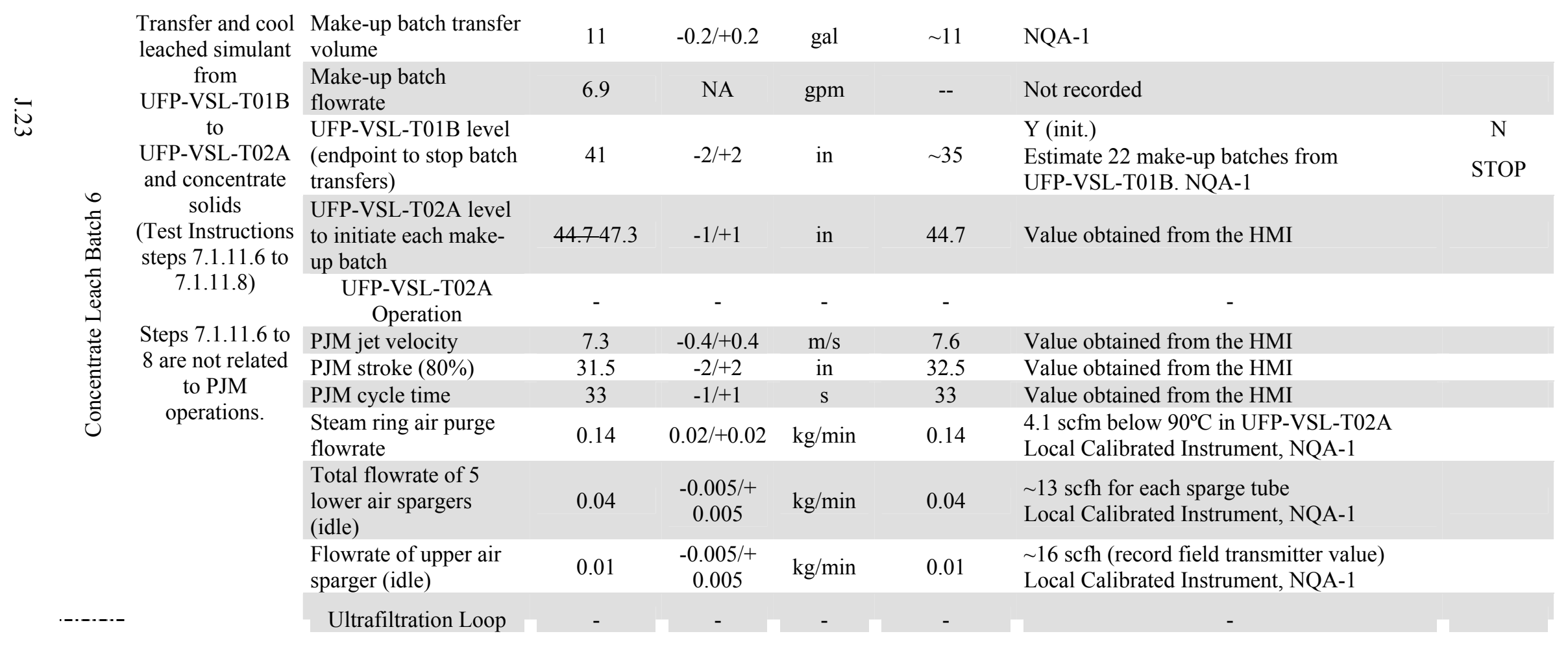


Table J.1. Integrated Test A Operational Process Sheet

\begin{tabular}{|c|c|c|c|c|c|c|c|c|}
\hline \multicolumn{9}{|c|}{ Integrated Test A Run sheet (TI-065 Rev. 0) } \\
\hline & & & $\begin{array}{c}\text { Target or } \\
\text { Setpoint } \\
\text { Value }\end{array}$ & & & & & \\
\hline \multicolumn{9}{|c|}{$\begin{array}{l}\text { Operation } \\
\text { Operation }\end{array}$} \\
\hline & & $\begin{array}{l}\text { Number of filter } \\
\text { bundles } \\
\text { (UFP-FLT-T01A } \\
\text { should be aligned) }\end{array}$ & 1 & $-0 /+0$ & - & -- & Information Only & \\
\hline & & Filter loop flowrate & 109 & $-10 /+10$ & gpm & 106 & Value obtained from the HMI & \\
\hline & & Temperature & 25 & $-2 /+2$ & ${ }^{\circ} \mathrm{C}$ & 25 & Value obtained from the HMI & \\
\hline & & $\begin{array}{l}\text { Axial } \Delta \mathrm{P} \text { for one filter } \\
\text { bundle }\end{array}$ & $<25$ & - & psi & $<25$ & Y (init.) NQA-1 & N \\
\hline \multirow{3}{*}{ 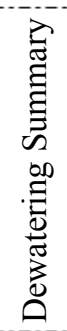 } & \multirow{3}{*}{$\begin{array}{l}\text { Summary of } \\
\text { leached solids } \\
\text { concentration } \\
\text { steps } \\
\text { (Test Instructions } \\
\text { step 7.1.11) }\end{array}$} & $\begin{array}{l}\text { Estimated total volume } \\
\text { of leached slurry } \\
\text { transferred as make-up } \\
\text { batches }\end{array}$ & 2,563 & $-100 /+100$ & gal & 2553 & Value obtained from the HMI & \\
\hline & & $\begin{array}{l}\text { Estimated total volume } \\
\text { of permeate produced }\end{array}$ & 2,563 & $-100 /+100$ & gal & $\sim 2601$ & Value obtained from the HMI & \\
\hline & & $\begin{array}{l}\text { Estimated total mass of } \\
\text { permeate }\end{array}$ & 12,244 & $-500 /+500$ & $\mathrm{~kg}$ & 12259 & NQA-1 & \\
\hline \multirow{7}{*}{ 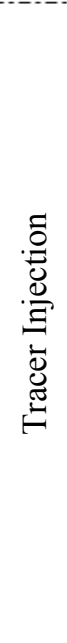 } & \multirow[t]{7}{*}{$\begin{array}{l}\text { Inject tracer into } \\
\text { filter loop } \\
\text { (Test Instructions } \\
\text { step 7.1.12) }\end{array}$} & $\begin{array}{l}\text { Mass of } \mathrm{CsBr} \text { (solid) } \\
\text { tracer to inject }\end{array}$ & 200 & $-10 /+10$ & $\mathrm{~g}$ & -- & $\begin{array}{l}\text { Tracer should be prepared as an aqueous } \\
\text { solution. Target is } 100 \text { ppm Cs when well- } \\
\text { mixed in the PEP. } \\
\text { Not recorded }\end{array}$ & \\
\hline & & $\begin{array}{l}\text { UFP-VSL-T02A } \\
\text { Operation }\end{array}$ & - & - & - & - & - & \\
\hline & & PJM jet velocity & 12 & $-0.6 /+0.6$ & $\mathrm{~m} / \mathrm{s}$ & 12 & $\begin{array}{l}\text { NOTE: Higher Jet Velocity than during } \\
\text { dewatering! } \\
\text { NQA-1 }\end{array}$ & \\
\hline & & PJM stroke (80\%) & 31.5 & $-2 /+2$ & in & $32.1(85 \%)$ & NQA-1 & \\
\hline & & PJM cycle time & 20 & $-1 /+1$ & $\mathrm{~s}$ & 20.7 & NQA-1 & \\
\hline & & $\begin{array}{l}\text { Steam ring air purge } \\
\text { flowrate }\end{array}$ & 0.100 .14 & & $\mathrm{~kg} / \mathrm{min}$ & 0.148 & $\begin{array}{l}\sim 3.1 \mathrm{scfm} \\
\text { Local Calibrated Instrument, NQA-1 }\end{array}$ & \\
\hline & & $\begin{array}{l}\text { Total lower air sparger } \\
\text { flow rate with } \\
\text { recirculation (idle) }\end{array}$ & 0.04 & $\begin{array}{c}-0.005 /+ \\
0.005\end{array}$ & $\mathrm{~kg} / \mathrm{min}$ & 0.012 & $\begin{array}{l}\sim 12.6 \text { scfh for each sparge tube } \\
\text { Local Calibrated Instrument, NQA-1 }\end{array}$ & \\
\hline
\end{tabular}


Table J.1. Integrated Test A Operational Process Sheet

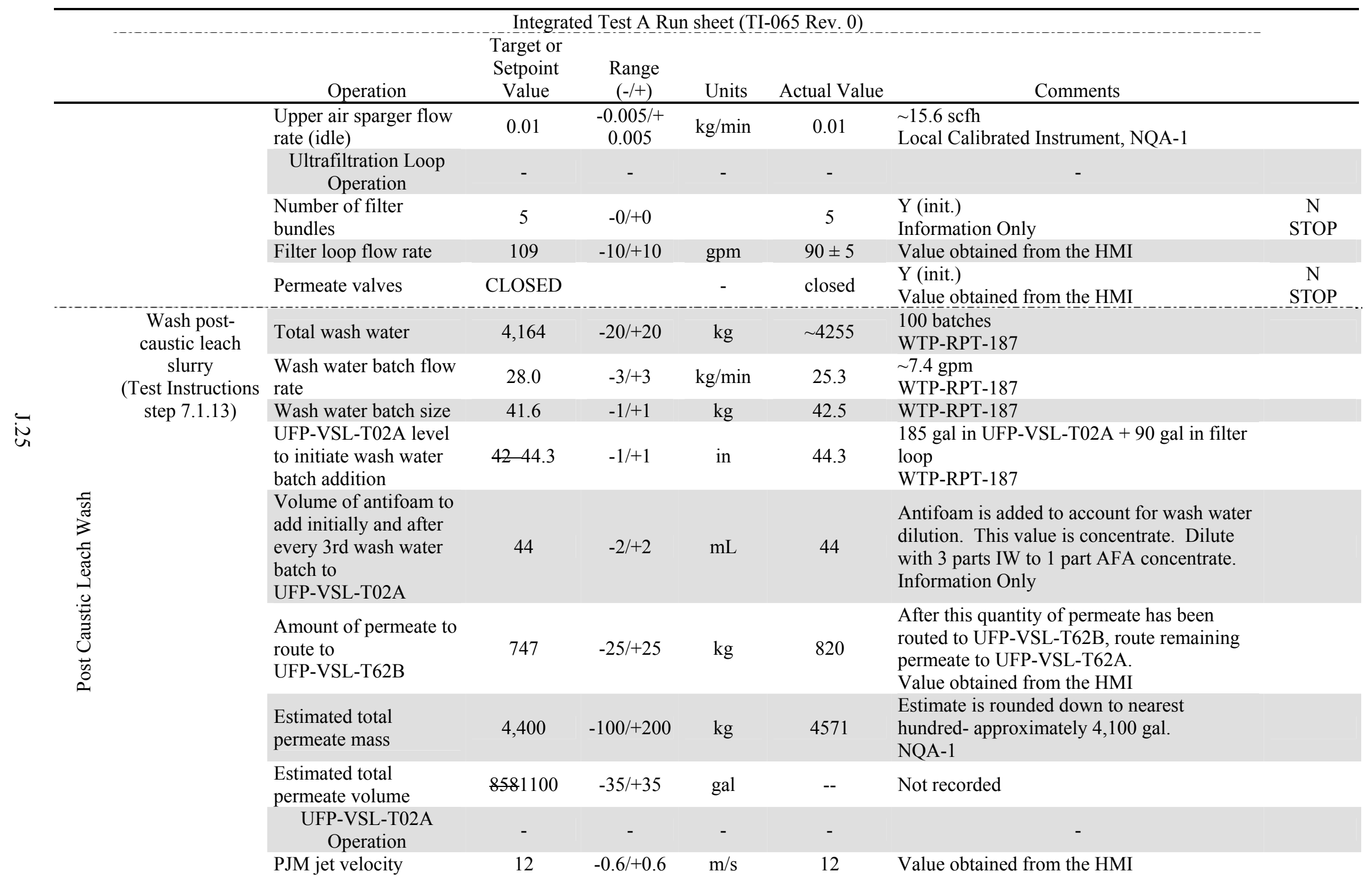


Table J.1. Integrated Test A Operational Process Sheet

\begin{tabular}{|c|c|c|c|c|c|c|c|c|}
\hline \multicolumn{9}{|c|}{ Integrated Test A Run sheet (TI-065 Rev. 0) } \\
\hline & & Operation & $\begin{array}{l}\text { Target or } \\
\text { Setpoint } \\
\text { Value }\end{array}$ & $\begin{array}{c}\text { Range } \\
(-/+)\end{array}$ & Units & Actual Value & \multicolumn{2}{|l|}{ Comments } \\
\hline & & PJM stroke $(80 \%)$ & 31.5 & $-2 /+2$ & in & -- & Not recorded & \\
\hline & & PJM cycle time & 20 & $-1 /+1$ & $\mathrm{~s}$ & 20 & Value obtained from the HMI & \\
\hline & & $\begin{array}{l}\text { Steam ring air purge } \\
\text { flowrate }\end{array}$ & 0.14 & $0.02 /+0.02$ & $\mathrm{~kg} / \mathrm{min}$ & 0.137 & $\begin{array}{l}4.1 \mathrm{scfm} \text { below } 90^{\circ} \mathrm{C} \text { in UFP-VSL-T02A } \\
\text { WTP-RPT- } 187\end{array}$ & \\
\hline & & $\begin{array}{l}\text { Total lower air sparger } \\
\text { flow rate with } \\
\text { recirculation (idle) }\end{array}$ & 0.04 & $\begin{array}{c}-0.005 /+ \\
0.005\end{array}$ & $\mathrm{~kg} / \mathrm{min}$ & 0.008 & $\begin{array}{l}\sim 12.6 \text { scfh for each sparger tube } \\
\text { WTP-RPT-187 }\end{array}$ & \\
\hline & & $\begin{array}{l}\text { Upper air sparger flow } \\
\text { rate (idle) }\end{array}$ & 0.01 & $\begin{array}{c}-0.005 /+ \\
0.005\end{array}$ & $\mathrm{~kg} / \mathrm{min}$ & 0.01 & $\begin{array}{l}\sim 15.6 \text { scfh } \\
\text { Local Calibrated Instrument, NQA-1 }\end{array}$ & \\
\hline & & $\begin{array}{l}\text { Ultrafiltration Loop } \\
\text { Operation }\end{array}$ & - & 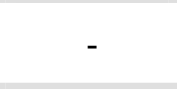 & - & - & 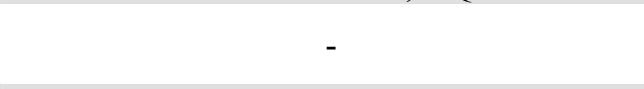 & \\
\hline & & $\begin{array}{l}\text { Number of filter } \\
\text { bundles }\end{array}$ & 5 & $-0 /+0$ & - & 5 & $\begin{array}{l}\text { Y (init.) } \\
\text { Information Only }\end{array}$ & $\begin{array}{c}\mathrm{N} \\
\text { STOP }\end{array}$ \\
\hline & & Filter loop flowrate & 109 & $-10 /+10$ & gpm & $-88.5-133.9$ & WTP-RPT-187 & \\
\hline & & $\begin{array}{l}\text { Trans-membrane } \\
\text { pressure }\end{array}$ & 40 & $-4 /+4$ & psi & 39.4 & $\begin{array}{l}\text { Lower range not applicable if back pressure } \\
\text { valve is } 100 \% \text { open. } \\
\text { WTP-RPT- } 187\end{array}$ & \\
\hline & & Temperature & 25 & $-2 /+2$ & ${ }^{\circ} \mathrm{C}$ & 24.3 & WTP-RPT-187 & \\
\hline & & $\begin{array}{l}\text { Axial } \Delta \mathrm{P} \text { for one filter } \\
\text { bundle }\end{array}$ & $<25$ & - & psi & 24.2 & $\begin{array}{l}\text { Y (init.) } \\
\text { WTP-RPT-187 }\end{array}$ & NTOP \\
\hline \multirow{6}{*}{ 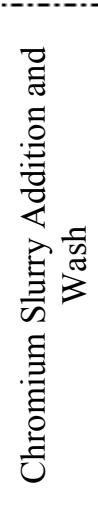 } & \multirow{6}{*}{$\begin{array}{l}\text { Add chromium } \\
\text { slurry } \\
\text { (Test Instructions } \\
\text { step 7.1.14.1) }\end{array}$} & $\begin{array}{l}\text { Volume of chromium } \\
\text { solids slurry to add } \\
\text { (endpoint) }\end{array}$ & 144 & $-1 /+1$ & gal & 145 & $\begin{array}{l}\text { with as much as } 20 \text { gal of inhibited water. } \\
\text { WTP-RPT-188 }\end{array}$ & \\
\hline & & $\begin{array}{l}\text { Chromium slurry } \\
\text { addition flowrate }\end{array}$ & $\begin{array}{l}\text { No targeted } \\
\text { rate }\end{array}$ & - & - & - & (1) & \\
\hline & & $\begin{array}{l}\text { Volume of antifoam to } \\
\text { add to } \\
\text { UFP-VSL-T02A } \\
\text { UFP-VSL-T02A } \\
\text { Operation }\end{array}$ & 230 & $-5 /+5$ & $\mathrm{~g} \mathrm{~mL}$ & 230 & $\begin{array}{l}\text { Antifoam is added to account for dilution by } \\
\text { the Cr slurry addition. Information Only }\end{array}$ & \\
\hline & & PJM jet velocity & 12 & $-0.6 /+0.6$ & $\mathrm{~m} / \mathrm{s}$ & 13.6 & Value obtained from the HMI & \\
\hline & & PJM stroke $(80 \%)$ & 31.5 & $-2 /+2$ & in & 29.5 & Value obtained from the HMI & \\
\hline & & PJM cycle time & 20 & $-1 /+1$ & $\mathrm{~s}$ & 20 & Value obtained from the HMI & \\
\hline
\end{tabular}


Table J.1. Integrated Test A Operational Process Sheet

\begin{tabular}{|c|c|c|c|c|c|c|c|}
\hline \multicolumn{8}{|c|}{ Integrated Test A Run sheet (TI-065 Rev. 0) } \\
\hline & Operation & $\begin{array}{l}\text { Target or } \\
\text { Setpoint } \\
\text { Value }\end{array}$ & $\begin{array}{c}\text { Range } \\
(-/+)\end{array}$ & Units & Actual Value & \multicolumn{2}{|l|}{ Comments } \\
\hline & $\begin{array}{l}\text { Steam ring air purge } \\
\text { flowrate }\end{array}$ & 0.14 & $0.02 /+0.02$ & $\mathrm{~kg} / \mathrm{min}$ & 0 & $\begin{array}{l}4.1 \mathrm{scfm} \text { below } 90^{\circ} \mathrm{C} \text { in UFP-VSL-T02A } \\
\text { Local Calibrated Instrument, NQA-1 }\end{array}$ & \\
\hline & $\begin{array}{l}\text { Total lower air sparger } \\
\text { flow rate with } \\
\text { recirculation (idle) }\end{array}$ & 0.04 & $\begin{array}{c}-0.005 /+ \\
0.005\end{array}$ & $\mathrm{~kg} / \mathrm{min}$ & 0.04 & $\begin{array}{l}\sim 12.6 \text { scfh for each sparge tube } \\
\text { Local Calibrated Instrument, NQA-1 }\end{array}$ & \\
\hline & $\begin{array}{l}\text { Upper air sparger flow } \\
\text { rate (idle) }\end{array}$ & 0.01 & $\begin{array}{c}-0.005 /+ \\
0.005\end{array}$ & $\mathrm{~kg} / \mathrm{min}$ & 0.01 & $\begin{array}{l}\sim 15.6 \text { scfh } \\
\text { Local Calibrated Instrument, NQA-1 }\end{array}$ & \\
\hline & $\begin{array}{l}\text { Ultrafiltration Loop } \\
\text { Operation }\end{array}$ & - & 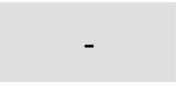 & - & - & 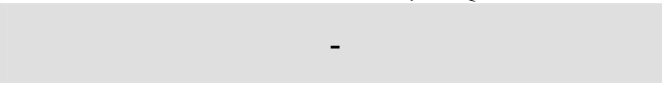 & \\
\hline & $\begin{array}{l}\text { Number of filter } \\
\text { bundles }\end{array}$ & 5 & $-0 /+0$ & - & 5 & $\begin{array}{l}\text { Y (init.) } \\
\text { Information Only }\end{array}$ & $\begin{array}{c}\mathrm{N} \\
\text { STOP }\end{array}$ \\
\hline & Filter loop flowrate & 109 & $-10 /+10$ & gpm & 103 & Value obtained from the HMI & \\
\hline & Permeate valves & CLOSED & - & - & Closed & $\begin{array}{l}\text { Y (init.) } \\
\text { Information Only }\end{array}$ & \\
\hline & Temperature & 25 & $-2 /+2$ & ${ }^{\circ} \mathrm{C}$ & 24 & Value obtained from the HMI & \\
\hline & $\begin{array}{l}\text { Axial } \Delta \mathrm{P} \text { for one filter } \\
\text { bundle }\end{array}$ & $<25$ & - & psi & $<25$ & $\begin{array}{l}\text { Y (init.) } \\
\text { Value obtained from the HMI }\end{array}$ & $\begin{array}{c}\mathrm{N} \\
\text { STOP }\end{array}$ \\
\hline \multirow{7}{*}{$\begin{array}{l}\text { Wash post- } \\
\text { chromium slurry } \\
\text { (Test Instructions } \\
\text { step 7.1.14.2 to } \\
7.1 .14 .9 \text { ) }\end{array}$} & $\begin{array}{l}\text { UFP-VSL-T02A level } \\
\text { to initiate wash water } \\
\text { batch addition }\end{array}$ & $42-44.3$ & $-1 /+1$ & in & 44.3 & Value obtained from the HMI & \\
\hline & Total wash water & $1,749-1540$ & $-10 /+10$ & $\mathrm{~kg}$ & 1593 & $\begin{array}{l}462 \text { gal of wash water in } 42 \text { wash water } \\
\text { batches } \\
\text { NQA-1 }\end{array}$ & \\
\hline & $\begin{array}{l}\text { Wash water batch flow } \\
\text { rate }\end{array}$ & 28.0 & $-3 /+3$ & $\mathrm{~kg} / \mathrm{min}$ & 28 & $\begin{array}{l}\sim 7.4 \mathrm{gpm} \\
\text { Value obtained from the HMI }\end{array}$ & \\
\hline & Wash water batch size & 41.6 & $-1 /+1$ & $\mathrm{~kg}$ & 41.6 & Value obtained from the HMI & \\
\hline & $\begin{array}{l}\text { Volume of antifoam to } \\
\text { add initially and after } \\
\text { every 3rd } 11 \text {-gal batch } \\
\text { to UFP-VSL-T02A }\end{array}$ & 44 & $-2 /+2$ & $\mathrm{~mL}$ & 44 & $\begin{array}{l}\text { Antifoam ( } 14 \mathrm{ml} \text { per } 11 \text { gallon wash batch) } \\
\text { is added to account for wash water dilution. } \\
\text { This value is concentrate. Dilute with } 3 \text { parts } \\
\text { IW to } 1 \text { part concentrate. } \\
\text { Information Only }\end{array}$ & \\
\hline & $\begin{array}{l}\text { UFP-VSL-T02A } \\
\text { Operation }\end{array}$ & - & - & - & - & 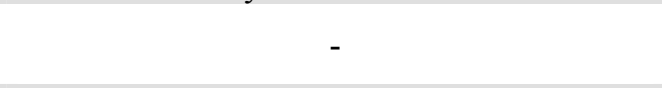 & \\
\hline & PJM jet velocity & 12 & $-0.6 /+0.6$ & $\mathrm{~m} / \mathrm{s}$ & -- & Not recorded & \\
\hline
\end{tabular}


Table J.1. Integrated Test A Operational Process Sheet

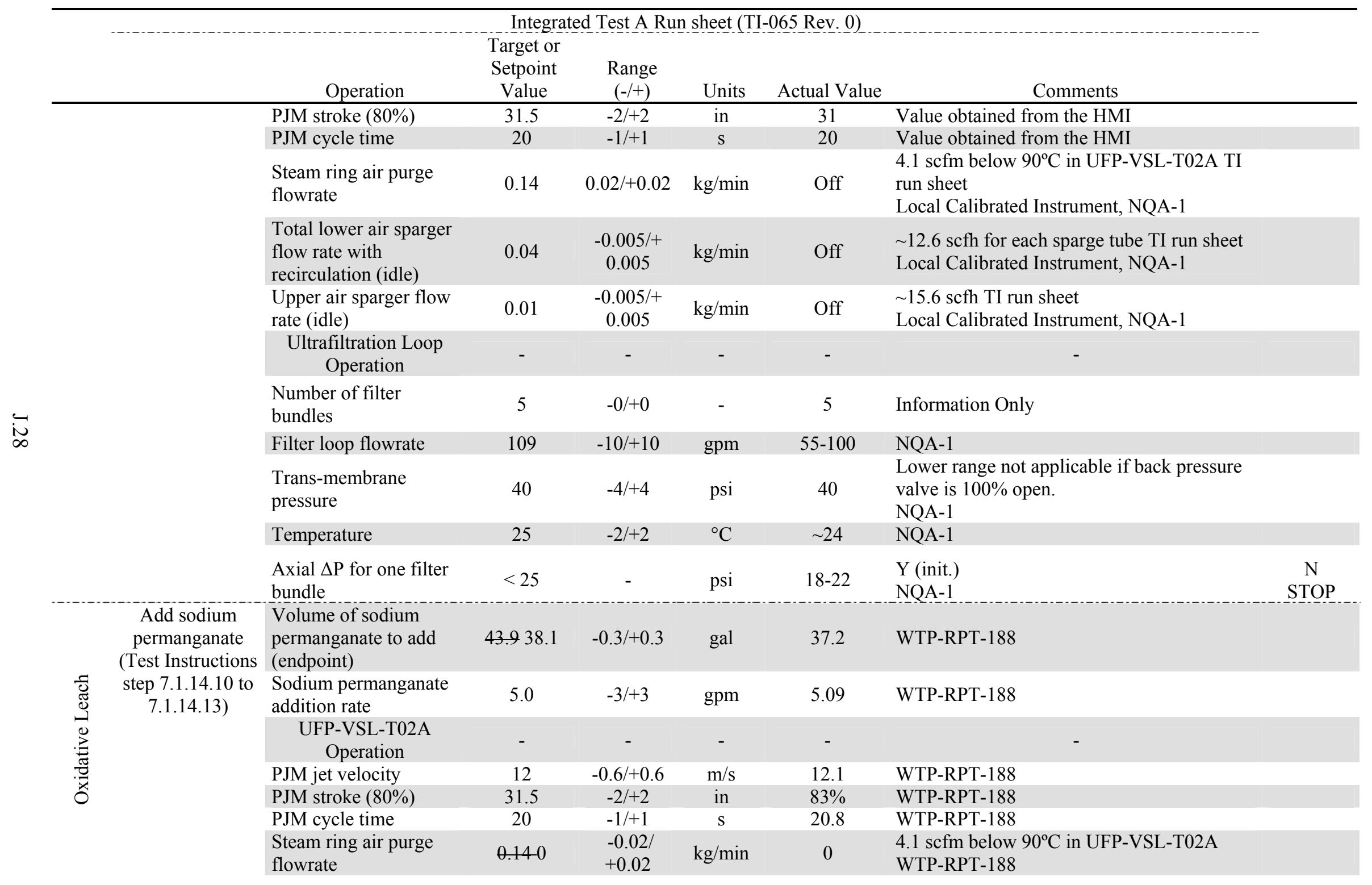


Table J.1. Integrated Test A Operational Process Sheet

\begin{tabular}{|c|c|c|c|c|c|c|c|}
\hline \multicolumn{7}{|c|}{ Integrated Test A Run sheet (TI-065 Rev. 0) } & \\
\hline & Operation & $\begin{array}{l}\text { Target or } \\
\text { Setpoint } \\
\text { Value }\end{array}$ & $\begin{array}{l}\text { Range } \\
(-/+)\end{array}$ & Units & Actual Value & \multicolumn{2}{|l|}{ Comments } \\
\hline & $\begin{array}{l}\text { Total lower air sparger } \\
\text { flow rate with } \\
\text { recirculation (idle) }\end{array}$ & 0.040 & $\begin{array}{c}-0.005 /+ \\
0.005\end{array}$ & $\mathrm{~kg} / \mathrm{min}$ & $\begin{array}{l}1.13-1.25 \\
\text { scfm }\end{array}$ & $\begin{array}{l}\sim 12.6 \text { scfh for each sparge tube } \\
\text { WTP-RPT-188 }\end{array}$ & \\
\hline & $\begin{array}{l}\text { Upper air sparger flow } \\
\text { rate (idle) }\end{array}$ & $\theta .01-0$ & $\begin{array}{c}-0.005 /+ \\
0.005\end{array}$ & $\mathrm{~kg} / \mathrm{min}$ & 0 & $\begin{array}{l}\sim 15.6 \mathrm{scfh} \\
\text { WTP-RPT-188 }\end{array}$ & \\
\hline & $\begin{array}{l}\text { Ultrafiltration Loop } \\
\text { Operation }\end{array}$ & - & - & - & - & 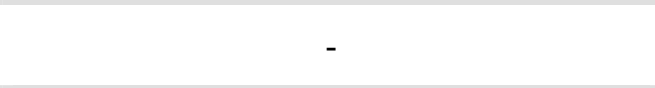 & \\
\hline & $\begin{array}{l}\text { Number of filter } \\
\text { bundles }\end{array}$ & 5 & $-0 /+0$ & - & 5 & $\begin{array}{l}\text { Y (init.) } \\
\text { Information Only }\end{array}$ & $\begin{array}{c}\mathrm{N} \\
\text { STOP }\end{array}$ \\
\hline & Filter loop flowrate & 109 & $-10 /+10$ & gpm & 114 & WTP-RPT-188 & \\
\hline & Permeate valves & CLOSED & - & - & Closed & Value obtained from the HMI & \\
\hline & Temperature & 25 & $-2 /+2$ & ${ }^{\circ} \mathrm{C}$ & 25 & Value obtained from the HMI & \\
\hline & $\begin{array}{l}\text { Axial } \Delta \mathrm{P} \text { for one filter } \\
\text { bundle }\end{array}$ & $<25$ & - & psi & $16-20$ & $\begin{array}{l}\text { Y (init.) } \\
\text { Value obtained from the HMI }\end{array}$ & $\begin{array}{l}\mathrm{N} \\
\text { STOP }\end{array}$ \\
\hline \multirow{11}{*}{$\begin{array}{c}\text { Conduct } \\
\text { oxidative leach } \\
\text { (Test Instructions } \\
\text { step 7.1.14.14) }\end{array}$} & $\begin{array}{l}\text { Oxidative leach } \\
\text { duration (endpoint) }\end{array}$ & 6 & $-0.1 /+0.5$ & $\mathrm{hr}$ & 6 & $\begin{array}{l}\text { May be extended to accommodate sampling. } \\
\text { WTP-RPT-188 }\end{array}$ & \\
\hline & Temperature & 25 & $-2 /+2$ & ${ }^{\circ} \mathrm{C}$ & $24.4-27.8$ & WTP-RPT-188 & \\
\hline & $\begin{array}{l}\text { UFP-VSL-T02A } \\
\text { Operation }\end{array}$ & - & - & - & 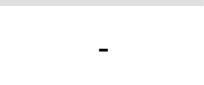 & - & \\
\hline & PJM jet velocity & 12 & $-0.6 /+0.6$ & $\mathrm{~m} / \mathrm{s}$ & 12.1 & WTP-RPT-188 & \\
\hline & PJM stroke (80\%) & 31.5 & $-2 /+2$ & in & $82 \%$ & WTP-RPT-188 & \\
\hline & PJM cycle time & 20 & $-1 /+1$ & $\mathrm{~s}$ & 20.8 & WTP-RPT-188 & \\
\hline & $\begin{array}{l}\text { Steam ring air purge } \\
\text { flowrate }\end{array}$ & $0.140-0$ & $0.02 /+0.02$ & $\mathrm{~kg} / \mathrm{min}$ & 0 & $\begin{array}{l}4.1 \mathrm{scfm} \text { below } 90^{\circ} \mathrm{C} \text { in UFP-VSL-T02A } \\
\text { WTP-RPT- } 188\end{array}$ & \\
\hline & $\begin{array}{l}\text { Total lower air sparger } \\
\text { flow rate with } \\
\text { recirculation (idle) }\end{array}$ & $\begin{array}{l}0.040-1.19 \\
\text { scfm }\end{array}$ & $\begin{array}{l}-0.005 /+ \\
0.005\end{array}$ & $\mathrm{~kg} / \mathrm{min}$ & $1.18 \mathrm{scfm}$ & $\begin{array}{l}\sim 12.6 \text { scfh for each sparge tube } \\
\text { WTP-RPT-188 }\end{array}$ & \\
\hline & $\begin{array}{l}\text { Upper air sparger flow } \\
\text { rate (idle) }\end{array}$ & 0.0100 & $\begin{array}{c}-0.005 /+ \\
0.005\end{array}$ & $\mathrm{~kg} / \mathrm{min}$ & 0 & $\begin{array}{l}\sim 15.6 \mathrm{scfh} \\
\text { WTP-RPT-188 }\end{array}$ & \\
\hline & $\begin{array}{l}\text { Ultrafiltration Loop } \\
\text { Operation }\end{array}$ & - & 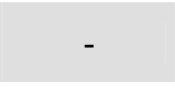 & - & - & 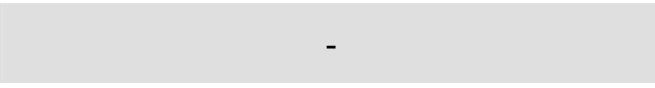 & \\
\hline & $\begin{array}{l}\text { Number of filter } \\
\text { bundles }\end{array}$ & 5 & $-0 /+0$ & - & 5 & $\begin{array}{l}\text { Y (init.) } \\
\text { Information Only }\end{array}$ & $\begin{array}{l}\mathrm{N} \\
\text { STOP }\end{array}$ \\
\hline
\end{tabular}


Table J.1. Integrated Test A Operational Process Sheet

\begin{tabular}{|c|c|c|c|c|c|c|c|c|}
\hline \multicolumn{9}{|c|}{ Integrated Test A Run sheet (TI-065 Rev. 0) } \\
\hline & & Operation & $\begin{array}{l}\text { Target or } \\
\text { Setpoint } \\
\text { Value }\end{array}$ & $\begin{array}{c}\text { Range } \\
(-/+)\end{array}$ & Units & Actual Value & Comments & \\
\hline & & Filter loop flowrate & 109 & $-10 /+10$ & gpm & 89 & WTP-RPT-188 & \\
\hline & & Permeate valves & CLOSED & - & - & -- & ${ }^{2}$ & \\
\hline & & Temperature & 25 & $-2 /+2$ & ${ }^{\circ} \mathrm{C}$ & $24.9-27.4$ & WTP-RPT-188 & \\
\hline & & $\begin{array}{l}\text { Axial } \Delta \mathrm{P} \text { for one filter } \\
\text { bundle }\end{array}$ & $<25$ & - & psi & $18-21$ & $\begin{array}{l}\text { Y (init.) } \\
\text { NQA-1 }\end{array}$ & $\begin{array}{l}\mathrm{N} \\
\text { STOP }\end{array}$ \\
\hline \multirow{14}{*}{ 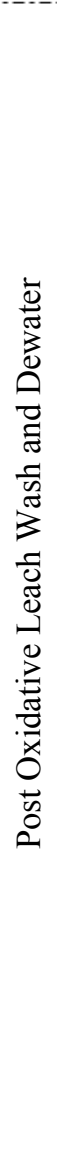 } & \multirow{14}{*}{$\begin{array}{c}\text { Wash post- } \\
\text { oxidative leach } \\
\text { slurry } \\
\text { (Test Instructions } \\
\text { step } 7.1 .15 .1 \text { to } \\
7.1 .15 .3 .6 .3 \text { ) }\end{array}$} & $\begin{array}{l}\text { UFP-VSL-T02A level } \\
\text { to initiate wash water } \\
\text { batch addition }\end{array}$ & $50.9-52.5$ & $-1 /+1$ & in & $\sim 52$ & $\begin{array}{l}231 \text { gal in UFP-VSL-T02A }+86 \text { gal in filter } \\
\text { loop } \\
\text { NQA-1 }\end{array}$ & \\
\hline & & Total wash water & 3,6223581 & $-20 /+20$ & $\mathrm{~kg}$ & 3657 & $\begin{array}{l}87 \text { batches } \\
\text { Value obtained from the HMI }\end{array}$ & \\
\hline & & $\begin{array}{l}\text { Wash water batch flow } \\
\text { rate }\end{array}$ & 28.0 & $-3 /+3$ & $\mathrm{~kg} / \mathrm{min}$ & $\sim 25$ & $\begin{array}{l}\sim 7.4 \mathrm{gpm} \\
\text { NQA-1 }\end{array}$ & \\
\hline & & Wash water batch size & 41.6 & $-1 /+1$ & $\mathrm{~kg}$ & 42.5 & WTP-RPT-187 & \\
\hline & & $\begin{array}{l}\text { Volume of antifoam to } \\
\text { add initially and after } \\
\text { every 3rd 11-gal batch } \\
\text { to UFP-VSL-T02A }\end{array}$ & 44 & $-2 /+2$ & $\mathrm{~mL}$ & 44 & $\begin{array}{l}\text { Antifoam ( } \sim 14 \mathrm{ml} \text { per } 11 \text { gallon wash batch) } \\
\text { is added to account for wash water dilution. } \\
\text { This value is concentrate. Dilute with } 3 \text { parts } \\
\text { IW to } 1 \text { part concentrate. Information Only }\end{array}$ & \\
\hline & & $\begin{array}{l}\text { Estimated total } \\
\text { permeate mass }\end{array}$ & $3,600-3500$ & $-100 /+200$ & $\mathrm{~kg}$ & 3792 & $\begin{array}{l}\text { Estimate is rounded down to nearest hundred. } \\
\text { Value obtained from the HMI }\end{array}$ & \\
\hline & & $\begin{array}{c}\text { UFP-VSL-T02A } \\
\text { Operation }\end{array}$ & - & - & - & - & 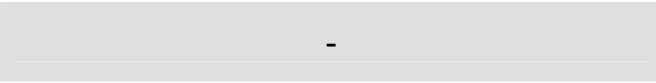 & \\
\hline & & PJM jet velocity & 12 & $-0.6 /+0.6$ & $\mathrm{~m} / \mathrm{s}$ & 12 & Value obtained from the HMI & \\
\hline & & PJM stroke $(80 \%)$ & 31.5 & $-2 /+2$ & in & -- & Not recorded & \\
\hline & & PJM cycle time & 20 & $-1 /+1$ & $\mathrm{~s}$ & -- & Not recorded & \\
\hline & & $\begin{array}{l}\text { Steam ring air purge } \\
\text { flowrate }\end{array}$ & 0.14 & $0.02 /+0.02$ & $\mathrm{~kg} / \mathrm{min}$ & Off & $\begin{array}{l}4.1 \mathrm{scfm} \text { below } 90^{\circ} \mathrm{C} \text { in UFP-VSL-T02A } \\
\text { Local Calibrated Instrument, NQA-1 }\end{array}$ & \\
\hline & & $\begin{array}{l}\text { Total lower air sparger } \\
\text { flow rate with } \\
\text { recirculation (idle) }\end{array}$ & 0.04 & $\begin{array}{c}-0.005 /+ \\
0.005\end{array}$ & $\mathrm{~kg} / \mathrm{min}$ & Off & $\begin{array}{l}\text { 12.6 scfh for each sparge tube } \\
\text { Local Calibrated Instrument, NQA-1 }\end{array}$ & \\
\hline & & $\begin{array}{l}\text { Upper air sparger flow } \\
\text { rate (idle) }\end{array}$ & 0.01 & $\begin{array}{c}-0.005 /+ \\
0.005\end{array}$ & $\mathrm{~kg} / \mathrm{min}$ & Off & $\begin{array}{l}\sim 15.6 \mathrm{scfh} \\
\text { Local Calibrated Instrument, NQA-1 }\end{array}$ & \\
\hline & & Ultrafiltration Loop & - & - & - & - & - & \\
\hline
\end{tabular}


Table J.1. Integrated Test A Operational Process Sheet

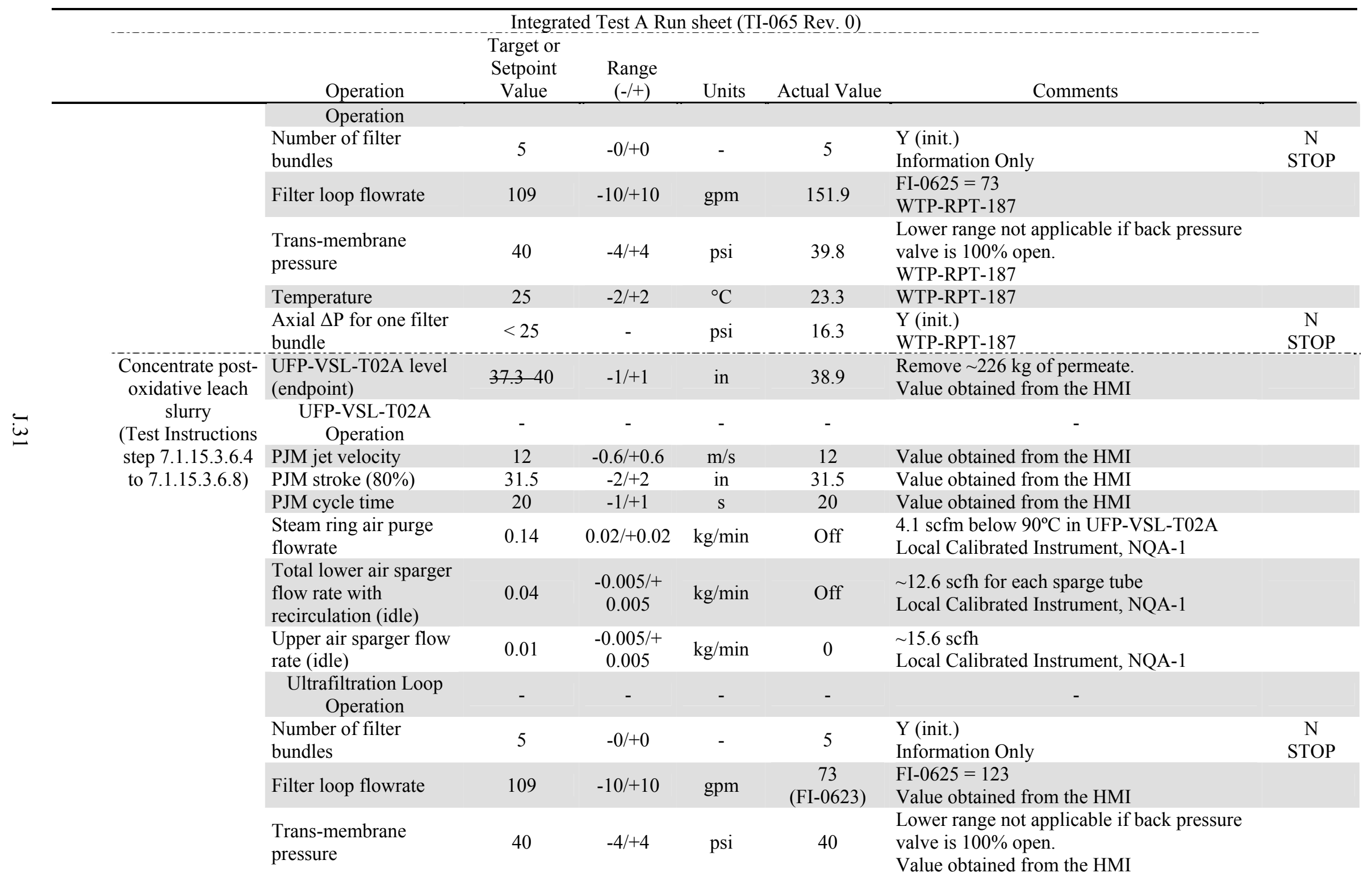


Table J.1. Integrated Test A Operational Process Sheet

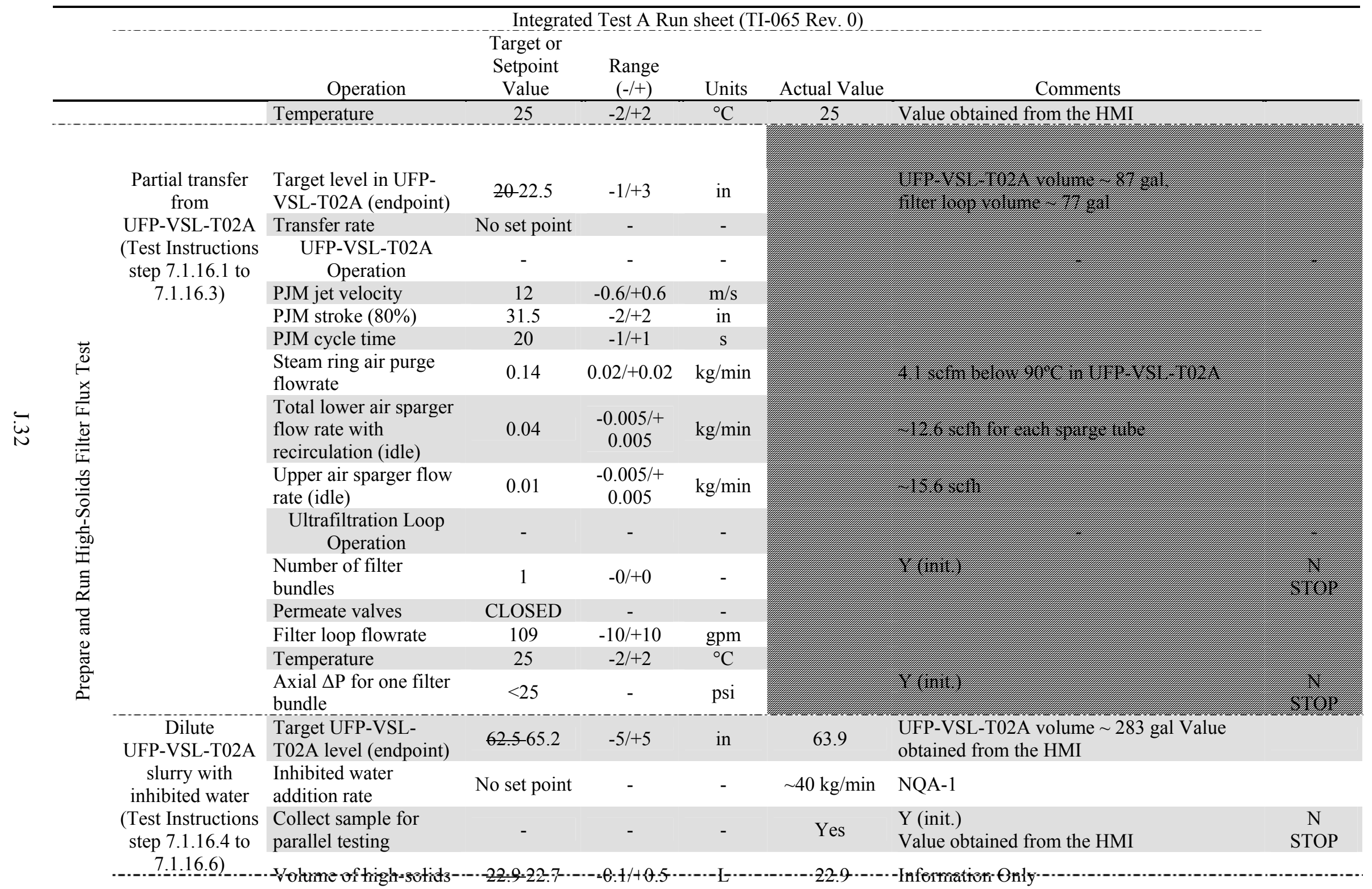


Table J.1. Integrated Test A Operational Process Sheet

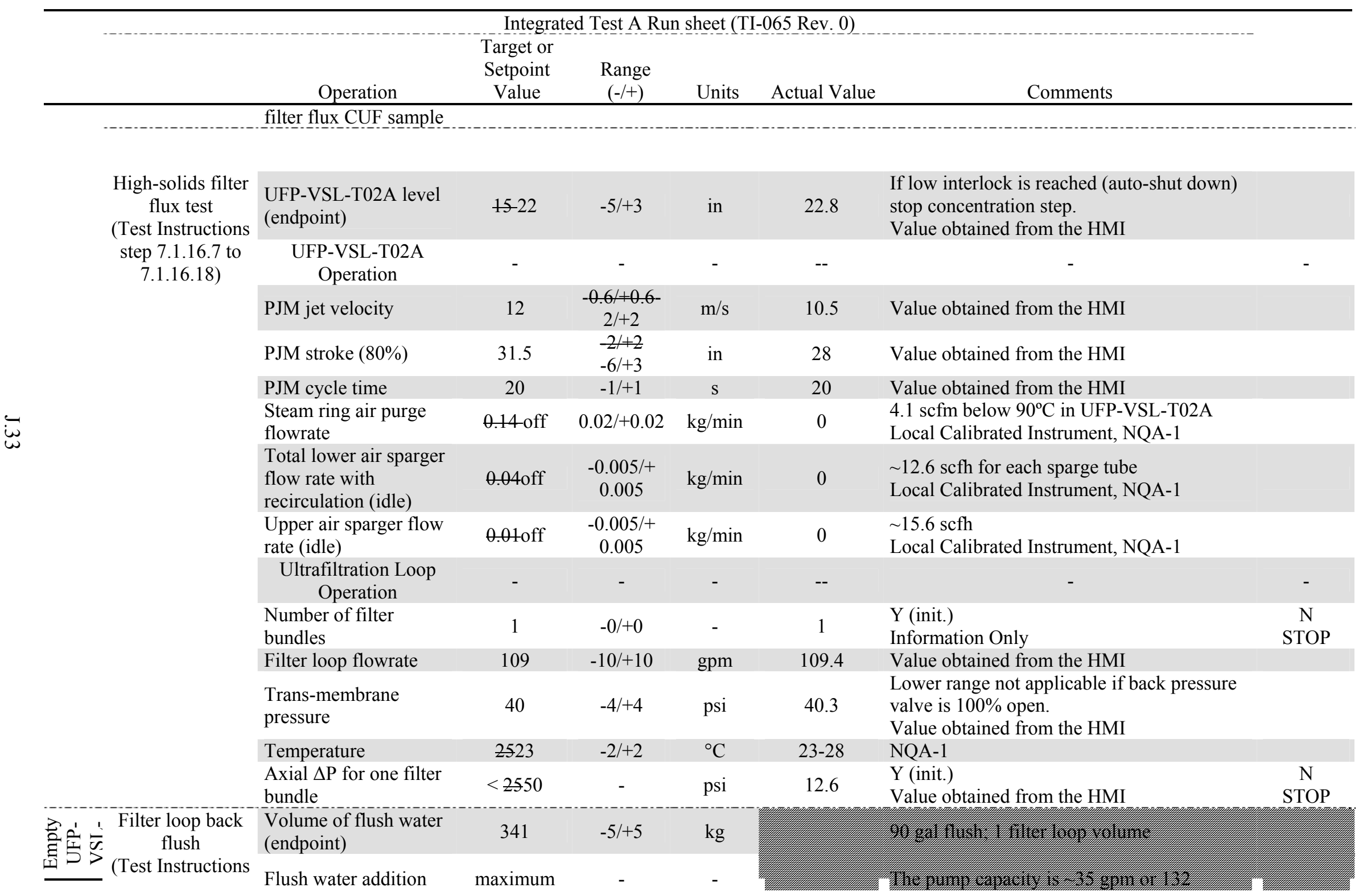


Table J.1. Integrated Test A Operational Process Sheet

\begin{tabular}{|c|c|c|c|c|c|c|c|}
\hline & & Integrat & ed Test A Ru & i sheet (T) & 065 Rev. 0) & & \\
\hline & Operation & $\begin{array}{l}\text { Target or } \\
\text { Setpoint } \\
\text { Value }\end{array}$ & $\begin{array}{c}\text { Range } \\
(-/+)\end{array}$ & Units & Actual Value & Comments & \\
\hline step 7.1.17) & rate & $\begin{array}{l}\text { achievable } \\
\text { flow }\end{array}$ & & & & $\begin{array}{l}\mathrm{kg} / \mathrm{min} \text {. In practice only } 22 \mathrm{gpm} \text { was } \\
\text { achieved. }\end{array}$ & \\
\hline & $\begin{array}{l}\text { UFP-VSL-T02A } \\
\text { Operation }\end{array}$ & (a) & - & - & - & Jum & \\
\hline & PJM jet velocity & 12 & $-0.6 /+0.6$ & $\mathrm{~m} / \mathrm{s}$ & & & \\
\hline & PJM stroke ( $80 \%)$ & 31.5 & $-2 /+2$ & in & & & \\
\hline & PJM cycle time & 20 & $-1 /+1$ & $\mathrm{~s}$ & & & \\
\hline & $\begin{array}{l}\text { Steam ring air purge } \\
\text { flowrate }\end{array}$ & 0.14 & $0.02 /+0.02$ & $\mathrm{~kg} / \mathrm{min}$ & & A. XClmbelow $90^{\circ} \mathrm{C}$ in UFP-VSU-TO2A & \\
\hline & $\begin{array}{l}\text { Total lower air sparger } \\
\text { flow rate with } \\
\text { recirculation (idle) }\end{array}$ & 0.04 & $\begin{array}{c}-0.005 /+ \\
0.005\end{array}$ & $\mathrm{~kg} / \mathrm{min}$ & & - 12.6.5efh for each sparge tube & \\
\hline & $\begin{array}{l}\text { Upper air sparger flow } \\
\text { rate (idle) }\end{array}$ & 0.01 & $\begin{array}{c}-0.005 /+ \\
0.005\end{array}$ & $\mathrm{~kg} / \mathrm{min}$ & & - - S. 6 - & \\
\hline & $\begin{array}{l}\text { Ultrafiltration Loop } \\
\text { Operation }\end{array}$ & - & - & - & - & -2 & \\
\hline & $\begin{array}{l}\text { Number of filter } \\
\text { bundles }\end{array}$ & 5 & $-0 /+0$ & - & & X(init. & STOP \\
\hline & Filter loop flowrate & OFF & - & & & & \\
\hline & Permeate valves & CLOSED & & & & & \\
\hline Transfer from & Transfer rate & No set point & N.A. & gpm & & Transfer with diaphragm pump. & \\
\hline $\begin{array}{l}\text { UFP-VSL-T02A } \\
\text { (Test Instructions }\end{array}$ & $\begin{array}{l}\text { UFP-VSL-T02A } \\
\text { Operation }\end{array}$ & - & - & - & $=$ & 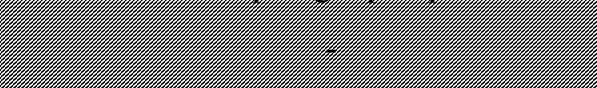 & \\
\hline step 7.1.17) & $\begin{array}{l}\text { Level to stop PJM } \\
\text { operation }\end{array}$ & 15 & $-5 /+5$ & in & & & \\
\hline & $\begin{array}{l}\text { Level to stop steam } \\
\text { ring air purge }\end{array}$ & 10 & $-5 /+0$ & in & & & \\
\hline & $\begin{array}{l}\text { Level to turn off air } \\
\text { sparge }\end{array}$ & 13 & $-5 /+5$ & in & & & \\
\hline & $\begin{array}{l}\text { Ultrafiltration Loop } \\
\text { Operation }\end{array}$ & - & - & - & $=$ & -2 & \\
\hline (2) & Number of filter & 5 & $-0 /+0$ & - & & & \\
\hline
\end{tabular}


Table J.1. Integrated Test A Operational Process Sheet

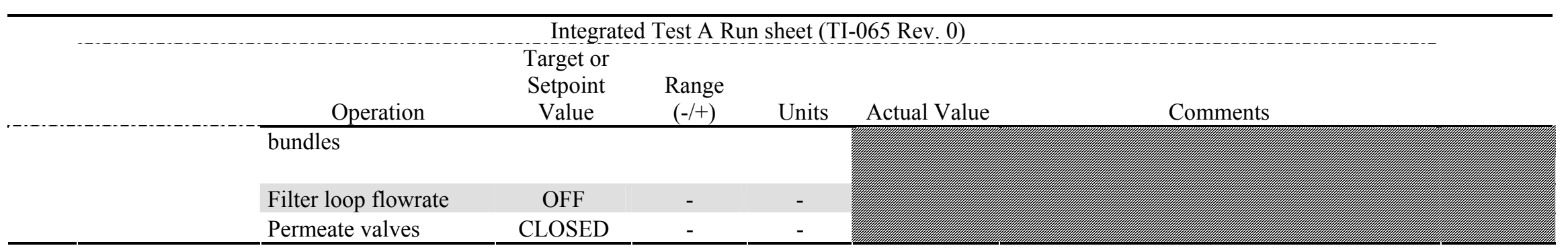



PNNL-18755

WTP-RPT-191, Rev 0

\section{Distribution}

No. of

Copies

OFFSITE
No. of

Copies

ONSITE

1 Pacific Northwest National Laboratory (staff will be notified electronically)

O.P. Bredt

K6-50

C.A. Burns

$\mathrm{P} 7-25$

R.C. Daniel

$\mathrm{P} 7-22$

J.G.H. Geeting

P7-28

E.C. Golovich

K5-25

C.E. Guzman-Leong

K5-22

G.B. Josephson

K9-69

D.E. Kurath

K3-52

G.J. Sevigny

P7-27

D.M. Smith

K6-81

Y.F Su

K2-21

P.L.J. Valdez

K5-22

S.T. Yokuda

$\mathrm{K} 7-15$

J.K. Young

K6-50

Information Release (pdf)

Project File (1)

K3-52

2 Bechtel National, Inc.

WTP R\&T Docs (1)

H4-02

S. Barnes

H4-02

Distr. 1 


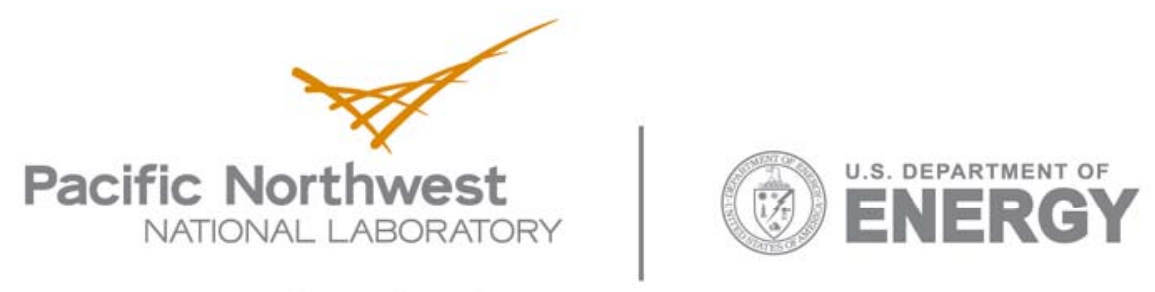

902 Battelle Boulevard

P.O. Box 999

Richland, WA 99352

1-888-375-PNNL (7665)

www.pnl.gov 